This document was prepared in conjunction with work accomplished under Contract No. DE-AC09-96SR18500 with the U. S. Department of Energy.

\title{
DISCLAIMER
}

This report was prepared as an account of work sponsored by an agency of the United States Government. Neither the United States Government nor any agency thereof, nor any of their employees, nor any of their contractors, subcontractors or their employees, makes any warranty, express or implied, or assumes any legal liability or responsibility for the accuracy, completeness, or any third party's use or the results of such use of any information, apparatus, product, or process disclosed, or represents that its use would not infringe privately owned rights. Reference herein to any specific commercial product, process, or service by trade name, trademark, manufacturer, or otherwise, does not necessarily constitute or imply its endorsement, recommendation, or favoring by the United States Government or any agency thereof or its contractors or subcontractors. The views and opinions of authors expressed herein do not necessarily state or reflect those of the United States Government or any agency thereof. 
WSRC-TR-2005-00105, REVISION 0

SRNL-RPP-2005-00012, REVISION 0

\section{FINAL REPORT: \\ RPP-WTP SEMI-INTEGRATED PILOT PLANT}

JUNE 2005

SAVANNAH RIVER NATIONAL LABORATORY

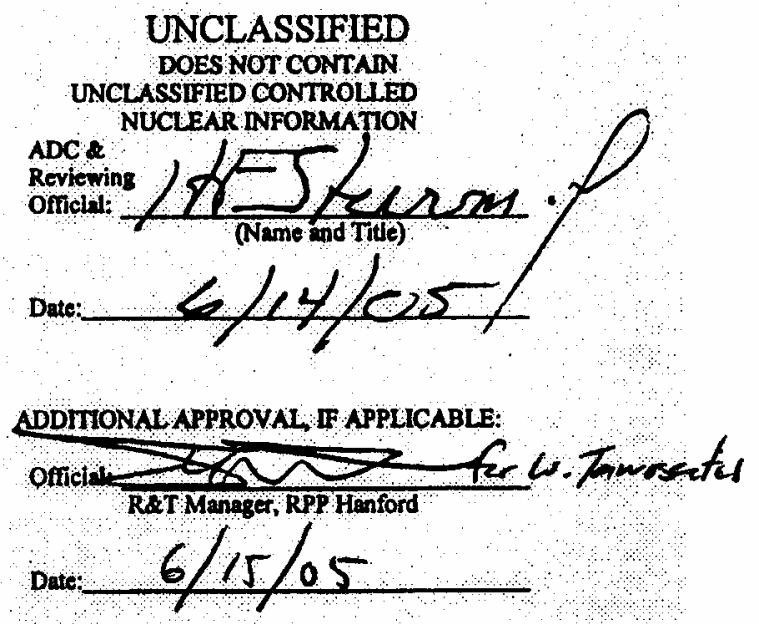

Westinghouse Savannah River Company

Savannah River Site

Aiken, SC 29808

Prepared for the U.S. Department of Energy Under Contract Number DEAC09-96SR18500

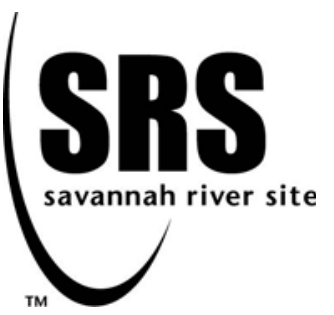


WSRC-TR-2005-00105, REVISION 0

SRNL-RPP-2005-00012, REVISION 0

\section{DISCLAIMER}

This report was prepared for the United States Department of Energy under Contract No. DE-AC09-96SR18500 and is an account of work performed under that contract. Neither the United States Department of Energy, nor WSRC, nor any of their employees makes any warranty, expressed or implied, or assumes any legal liability or responsibility for accuracy, completeness, or usefulness, of any information, apparatus, or product or process disclosed herein or represents that its use will not infringe privately owned rights. Reference herein to any specific commercial product, process, or service by trade name, trademark, name, manufacturer or otherwise does not necessarily constitute or imply endorsement, recommendation, or favoring of same by Westinghouse Savannah River Company or by the United States Government or any agency thereof. The views and opinions of the authors expressed herein do not necessarily state or reflect those of the United States Government or any agency thereof.

Printed in the United States of America

Prepared For

U.S. Department of Energy 
WSRC-TR-2005-00105, REVISION 0

SRNL-RPP-2005-00012, REVISION 0

WSRC Job Number: EES-23041

Key Words:

Ultrafiltration

Hanford Tank 241 AY-102/C-106

\section{Retention: Permanent}

Key WTP R\&T References:

Test Specifications:

24590-PTF-TSP-RT-02-015, Rev. 0, 15, Nov. 2002

24590-PTF-TSP-RT-03-002, Rev. 0, 05, Jun. 2003

24590-PTF-TSP-RT-01-003, Rev. 0, 20,Nov. 2001

(CIX chemical testing only)

Test Plans: WSRC-TR-2003-00338, Rev. 0, 04 Aug. 2003

(or SRT-RPP-2003-000165) and

WSRC-TR-2001-00340, Rev. 0, 06 Feb. 2002 (or SRT-RPP-2001-

000116) (CIX chemical testing only)

Test Exceptions: All of the following test exceptions only affect the CIX chemical testing sub-task: WSRC-TR-2001-00340

24590-WTP-TEF-RT-02-025, Rev. 0, 20 May 2002

24590-WTP-TEF-RT-02-046, Rev. 0, 01 Aug. 2002

24590-WTP-TEF-RT-03-004, Rev. 0, 07 Feb. 2003

24590-WTP-TEF-RT-03-037, Rev. 0, 30 Jul. 2003

24590-WTP-TEF-RT-03-037, Rev. 1, 17 Sept. 2003

24590-WTP-TEF-RT-03-054, Rev. 0, 17 Sept. 2003

24590-WTP-TEF-RT-03-069, Rev. 0, 14 Nov. 2003

R\&T Focus Area: RPP-WTP Pretreatment

Test Scoping Statements: S-48 \& S-134

\section{FINAL REPORT: \\ RPP-WTP SEMI-INTEGRATED PILOT PLANT}

\section{M.R. Duignan*, D.J. Adamson, T.B. Calloway, M.D. Fowley, Z.H Qureshi, J.L. Steimke, M.R. Williams, and J.R. Zamecnik, SRNL}

*Principal Author

\section{JUNE 2005}

Westinghouse Savannah River Company

Savannah River Site

Aiken, SC 29808

Prepared for the U.S. Department of Energy Under Contract Number DE-AC09-96SR18500

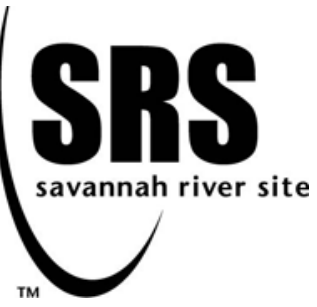


WSRC-TR-2005-00105, REVISION 0

SRNL-RPP-2005-00012, REVISION 0

\section{REVIEWS AND APPROVALS}

\section{Q) $\frac{1}{\text { D.J. Adamson, Engineering Development Laboratory RPP-WTP/SRNL }} 8 \mathrm{JuNG} 200 \mathrm{~S}$}

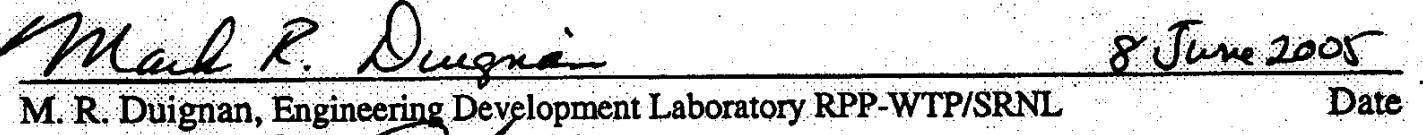

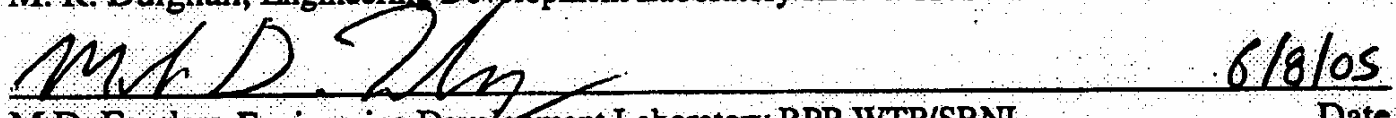

M.D. Fowley, Engineering Development Laboratory RPP-WTP/SRNL

Date

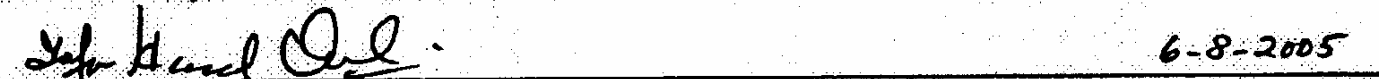

Z.H. Qureshi, Engineering Development Laboratory RPP-WTP/SRNL Date

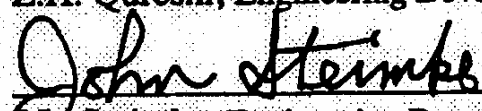

$6 / 8 / 2005$

61. Steimke, Engineering Development Laboratory RPP-WTP/SRNL

Date

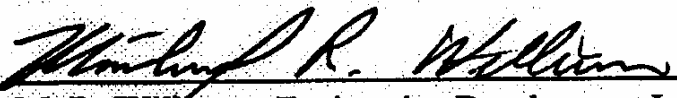

M. R. Wihiams, Engineering Development Laboratory RPP-WTP/SRNL

Date

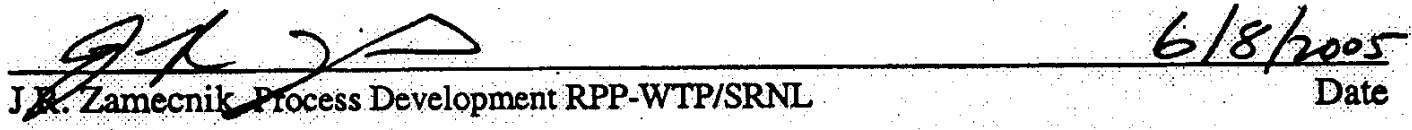

$\frac{\text { H. U. Wewer }}{\text { H.N. Guerrero, Technical Reviewer, Engineering Development Laboratory/SRNL }} 6 / 8 / 2005$

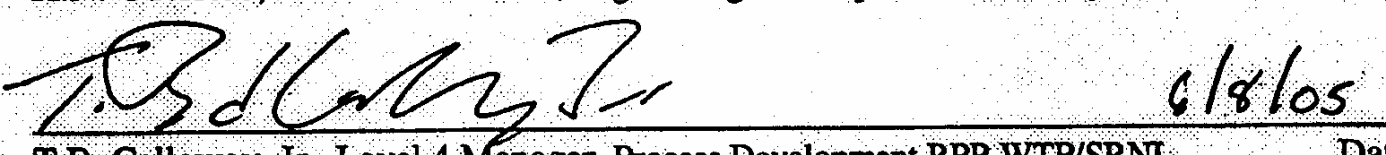

T.B. Calloway, Jr, Level 4 Manager, Process Development RPP-WTP/SRNL

Date

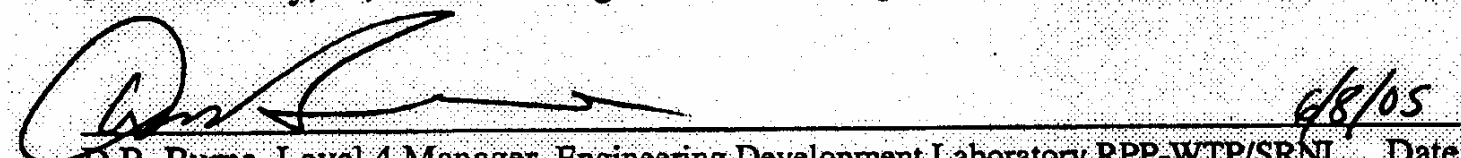

D.B. Burns, Level 4 Manager, Engineering Development Laboratory RPP-WTP/SRAL Date

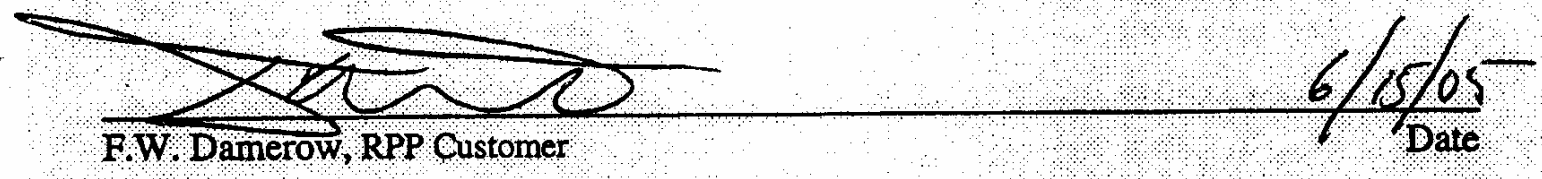




\section{ACKNOWLEDGMENTS}

The authors would like to thank all that were involved in this task. The entire crew in the Engineering Development Laboratory, including: Mike Armstrong, Vern Bush, Jerry Corbett, Susan Hatcher, Jimmy Mills, Mike Restivo, and Tim Steeper, as well as the assistance of Thaddeus Reown, Kurt Eberl, and Jeff Siler. The assistance of the many members in Aiken County Technology Laboratory was important, including: Debbie Marsh, Erich Hansen, Tim Jones, Joe Wheeler, Frances Williams, and Vickie Williams. The SRNL management of Dan Burns and Rich Edwards and the WGI management of Steve Piccolo, Walt Tamosaitis, and Fred Damerow, made this task's successful completion a sure thing. Thanks is extended to Hector Guerrero, and Dan Burns who had the unenviable task of reviewing this report and to Mimi Jones whose technical editing gave the many reports a professional sheen.

Besides direct participants in the experimental, work there were many other individuals whose participation was instrumental in completing the task. The authors thank all the SRNL personnel who gave both technical and administrative insight during the project, including: Kelly Chivers, Bill King, Charles Nash, Mike Poirier, Harold Sturm, Bill Wilmarth, and Allan Young and those who job it was to assess the project's preparedness including: Caroline Atseff, Mark Barnes, Jim Marra, and John Miller, and those whose job was to make sure it operated personnel-wise and environmentally safe including: Ray Battles, Amy Blunt, and Geoffrey Smoland. Finally, this task could not have been completed without significant input and guidance from the past and present personnel that made up the Hanford RPP-WTP Team, Don Alexander, Hani Abodishish, Dave Harty, Bill Johnson, Chi Leung, Ron Longwell, Dale Obenauer, Alan Olander, Ivan Papp, Reid Peterson, Eric Slaathaug, Murray Thorson, Paul Townson, and Todd Wright. Special thanks goes to Parameshwaran Sundar for coming on board in midstream as the immediate customer contact and lending his assistance, report reviews, and understanding to enable the final SIPP product be more useful to the overall RPP-WTP project.

The principal author would very much like to thank all the hard work put in by the many principal investigators: Dan Barnes for the upfront modeling work, Bond Calloway for his insights and keeping our feet to the fire, Zafar Qureshi for the FEP, Mark Fowley for the UFP, John Steimke for the CIX, Duane Adamson for the TLP, Mike Williams for the sampling and simulant handling, and especially Jack Zamecnik who not only had the task of developing the complex recipe for the AY102/C106 simulant, but also in developing the sample matrix, the analyzation of all those samples, and make sense of all that information in the context of each unit operation and the overall task. 


\section{TABLE OF CONTENTS}

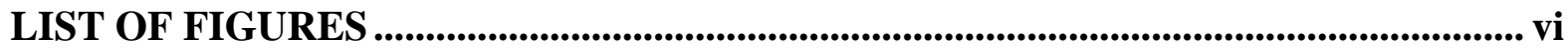

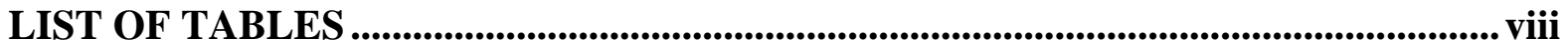

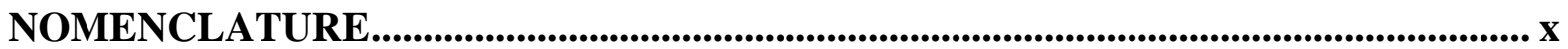

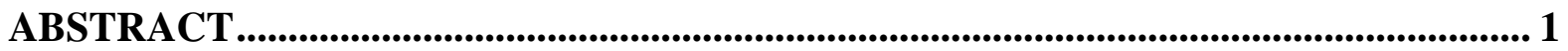

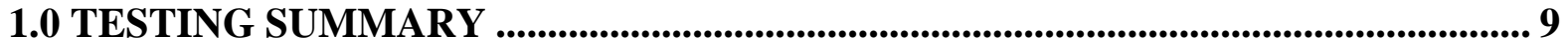

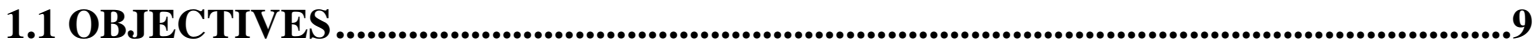

1.2 TEST EXCEPTIONS ........................................................................................14

1.3 RESULTS AND PERFORMANCE AGAINST SUCCESS CRITERIA ................15

1.4 QUALITY REQUIREMENTS......................................................................15

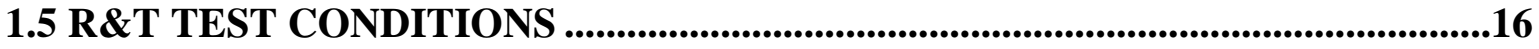

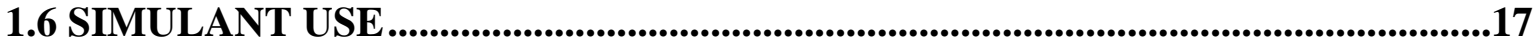

1.7 DISCREPANCIES AND FOLLOW-ON TESTS ................................................17

2.0 DISCUSSION ........................................................................................................ 19

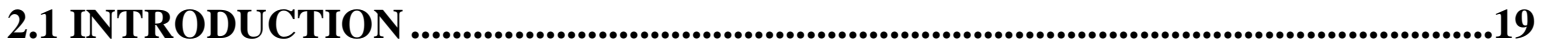

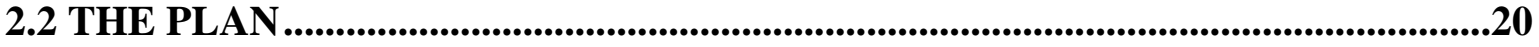

2.3 THE COMPUTATIONAL MODEL .......................................................................26

2.4 THE OVERALL SIPP TEST MATRIX ........................................................31

2.5 THE PRINCIPAL WASTE SIMULANTS: AY102 and AP101 (Author -

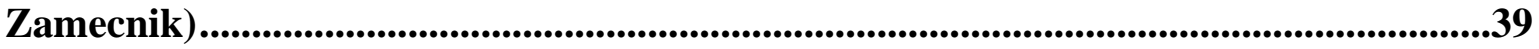

2.6 PILOT WASTE FEED EVAPORATOR (Authors - Qureshi, Williams \&

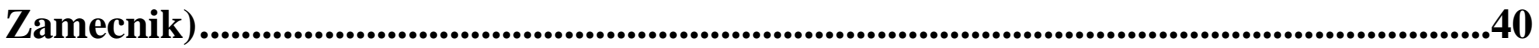

2.6.1 Chemical Description of the Pilot FEP Feed Stream (Author - Zamecnik).....41

2.6.2 Simulant Handling (Author - Williams) .........................................................47

2.6.3 Pilot FEP Equipment (Author - Qureshi) .................................................................48

2.6.4 Pilot FEP Operation (Author - Qureshi) ...................................................54

2.6.5 Pilot FEP Discussion of Results.................................................................57

2.6.6 Pilot FEP Conclusions......................................................................................63

2.7 Pilot Ultrafiltration (Authors - Fowley, Williams \& Zamecnik) ............................66

2.7.1 Chemical Description of the Pilot-Scale UFP Feed Stream (Author -

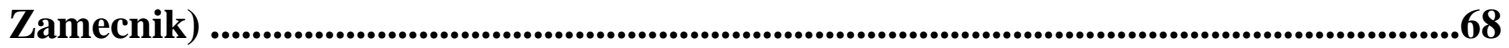

2.7.2 Simulant Handling (Author - Williams) ......................................................68

2.7.3 Pilot UFP Operation and Equipment (Author - Fowley).....................................75

2.7.4 Pilot UFP Discussion of Results ...................................................................................90

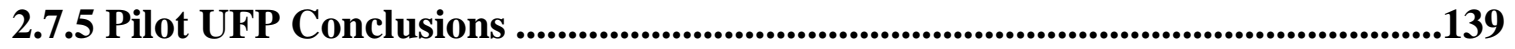

2.8 PILOT CESIUM ION EXCHANGE (Authors - Steimke \&Williams) ...............143

2.8.1 Chemical Description of the Pilot-Scale CIX Feed Stream .............................144

2.8.2 Simulant Handling (Author - Williams)...........................................................144

2.8.3 Pilot CIX Equipment (Author - Steimke).........................................................145

2.8.4 Pilot CIX Operation (Author - Steimke) ..................................................151

2.8.5 Pilot CIX Discussion of Results (Author - Steimke) ..........................................152

2.8.6 Pilot CIX Conclusions (Author - Steimke)......................................................168

2.9 PILOT TREATED LAW EVAPORATOR (Authors - Adamson, Williams \&

Zamecnik)..........................................................................................................................170

2.9.1 Chemical Description of the Pilot TLP Feed Stream (Author - Zamecnik)...170

2.9.2 Simulant Handling (Author - Williams)...............................................................172 
2.9.3 Pilot TLP Equipment (Author - Adamson)..............................................................172

2.9.4 Pilot TLP Operation (Author - Adamson) ..................................................................172

2.9.5 Pilot TLP Discussion of Results (Author - Adamson) ...........................................177

2.9.6 Pilot TLP Conclusions ...............................................................................................183

2.10 GLASS PRODUCTION BASED ON SIPP RESULTS (Author - Zamecnik)....184

2.10.1 Extrapolated Glass Production Rates based on Campaign II Data ...............184

2.10.2 Increasing Glass Production Rates.......................................................................186

2.11 SMALL-SCALE TO PILOT-SCALE COMPARISONS (Author - Calloway) .190

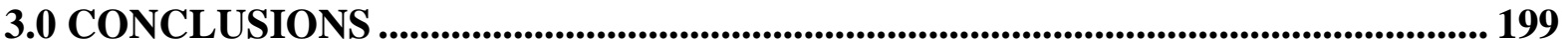

3.1 PILOT-SCALE FEP UNIT OPERATION - CAMPAIGN IV ..................................199

3.2 PILOT-SCALE UFP UNIT OPERATION - CAMPAIGN IV ..................................199

3.3 PILOT-SCALE CIX UNIT OPERATION - POST CAMPAIGN III ......................201

3.4 PILOT-SCALE TLP UNIT OPERATION - POST CAMPAIGN III ....................201

4.0 FUTURE WORK.................................................................................................................. 205

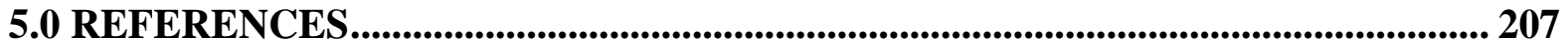

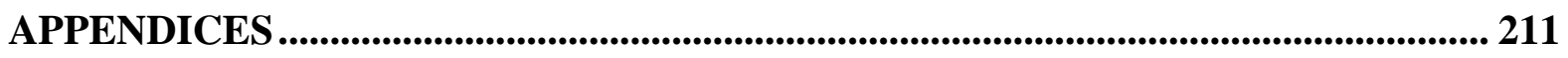

A. Semi-Integrated Pilot Plant (SIPP) Workshop - Meeting Minutes

B. Computational Model

C. WTP Discussion on Pretreatment Facility Waste Processing Residence Times

D. Sampling Matrices - Campaigns II-IV

E. Rheological and Particle Size Data

F. Dimensional Measurements of Crossflow Ultrafiltration Tubes from Mott and GKN

G. Instrument Lists and Calibration Data

H. Tabulated Raw Data from Each of the Pilot Operations

I. SIPP Interim Reports

J. Overall Chemical compositions of SIPP Campaign II Process Streams 


\section{LIST OF FIGURES}

Figure 1. Schematic of the overall task operation taken from the task plan..................... 24

Figure 2. Generation of Caustic Rinse 1 Recycle ............................................................. 42

Figure 3. FEP Feed Blending of Recycle Streams........................................................... 44

Figure 4. Pilot-scale FEP Unit Operation Feed Tank ..................................................... 48

Figure 5. SIPP Pilot-Scale FEP/TLP Unit .................................................................... 50

Figure 6. SIPP Pilot-Scale FEP/TLP Unit: The bottom half of the 15-foot tall Acrylic

Section with Ongoing Evaporation ............................................................................ 51

Figure 7. SIPP Pilot-Scale FEP/TLP Unit: Evaporating................................................. 52

Figure 8. Schematic of Ultrafiltration Process - Page 1.................................................. 69

Figure 9. Schematic of Ultrafiltration Process - Page 2.................................................. 70

Figure 10. UFP Primary Mixing Tank (Volume 1000 liters) which represents the RPP-

WTP UFP Preparation Feed Tank.

Figure 11. UFP Slurry Reservoir (Volume $~ 100$ liters) which represents the RPP-WTP

UFP Feed Tank ……………………........................................................... 73

Figure 12. GKN Filter Unit................................................................................ 75

Figure 13. Filter-Tube Bundle …………………………………………………..... 75

Figure 14. Filter Tubes and Bulkhead Stabilizing Plates................................................. 76

Figure 15. Exit (Left) and Entrance (Right) Tube Sheets of the Filter Tube Bundle ...... 76

Figure 16. GKN Sintered Metal Filter Tube for the SIPP UFP ...................................... 78

Figure 17. Exit and Entrance Tube Sheets showing Tube Ends Connections .................. 78

Figure 18. Schematic of the Campaign IV Pilot-Scale Test Rig ...................................... 79

Figure 19. Picture of the Pilot-Scale Test Rig ............................................................... 82

Figure 20. Baseline of Mott Filter prior to Removal ....................................................... 91

Figure 21. Initial Baselines of GKN Filter after Installation ............................................ 93

Figure 22. Baselines of GKN Filter after Cleaning with Acid ........................................ 94

Figure 23. Preconditioning of the GKN Filter with $20 \mathrm{wt} \%$ AN-102 ............................. 95

Figure 24. Pre-Test Acid Cleaning of Filter Unit, Permeate Flux vs. Run Time ............ 96

Figure 25. Dewatering of UFP Feed, Permeate Flux vs. Run Time with GKN filter...... 98

Figure 26. Permeate Flow during Dewatering Backpulse ................................................ 99

Figure 27. Test Rig Pressures during Dewatering Backpulse........................................ 100

Figure 28. Dewatering of UF Feed - Campaigns I , II, III (Mott Filter) and IV (GKN

Filter), Permeate Flux vs. Concentration ............................................................... 102

Figure 29. Dewatering of UF Feed - Campaigns II, III (Mott Filter) and IV (GKN

Filter), Cumulative Mean Permeate Flux vs. Concentration...................................... 103

Figure 30. Campaign I and IV Steady State Test Matrix.................................................. 104

Figure 31. Campaign IV Steady State Tests at 24 wt\% UDS ......................................... 105

Figure 32. Campaign IV High Temperature Tests (data are listed in Table 32) ........... 106

Figure 33. By using the data from Fig. 31 (Run 7@ 24 wt\% UDS) and Fig. 32 (Runs 6

and 7@19 wt\% UDS) the effect of temperature is seen at the flow conditions of V =

$15 \mathrm{ft} / \mathrm{s}$ and TMP = 50 psid.Figure 31a................................................................... 107

Figure 34. 1st Washing of Concentrated Slurry with 22 Wash Mini-batches ................ 108

Figure 35. 2nd Washing of Concentrated Slurry with 22 Wash Mini-batches............... 109

Figure 36. 1st and 2nd Washing of Concentrated Slurry, Campaigns* I, II, III, and IV111

Figure 37. 1st Caustic Rinse of the SIPP UFP ......................................................... 114

Figure 38. Acid Cleaning of the SIPP UFP …………............................................... 115 
Figure 39. 2nd and 3rd Caustic Rinses of the SIPP UFP........................................... 116

Figure 40. Campaign IV Caustic Rinse and Acid Cleaning Samples............................ 116

Figure 41. Filter Rinse and Clean, Campaigns I, II, III, and IV ................................. 118

Figure 42. Comparison of Permeability at $25^{\circ} \mathrm{C}$ during Acid Cleaning of Various

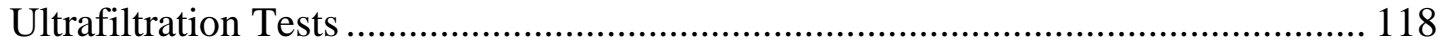

Figure 43. Baselines, Campaign III Pre- \& Post-test and Campaign IV Pre-test .......... 119

Figure 44. Pre- and Post-Test Baselines for Campaign IV .......................................... 120

Figure 45. Post-Test Baseline Solution Comparison, Campaigns II - IV ..................... 121

Figure 46. Comparison of Pre- and Post-Test Baselines, Campaign I - IV ................. 122

Figure 47. Slurry Solids and Density versus Normalized Permeate Volume ................. 125

Figure 48. Supernatant Solids vs. Sample for Temperature Tests ............................... 128

Figure 49. Total Solids vs. Sample for Temperature Tests........................................ 128

Figure 50. Undissolved Solids vs. Sample for Temperature Tests .............................. 129

Figure 51. Volume Particle Size Distribution During Temperature Tests.................... 130

Figure 52. Yield Stress and Consistency of Slurry Samples ...................................... 132

Figure 53. Viscosity of Permeate Samples ................................................................. 132

Figure 54. Pilot-scale CIX columns: The left column (COLUMN 1) is undergoing resin

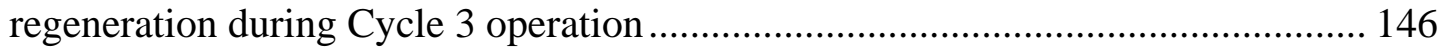

Figure 55. Schematic of Ion Exchange Facility for Integrated Testing ........................ 147

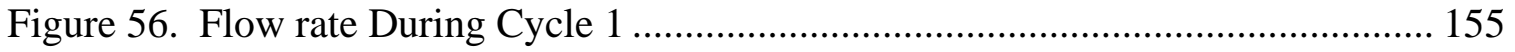

Figure 57. Pressure Drop Across Resin Bed for Lead Column and Cycle 1................. 155

Figure 58. Pressure Drop Across Resin Bed for Lag Column and Cycle 1................... 156

Figure 59. Resin Bed Height During Cycle 1 .......................................................... 156

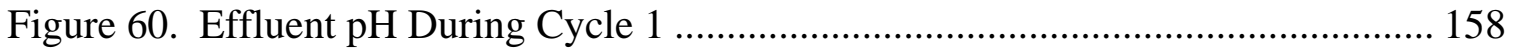

Figure 61. Effluent Conductivity During Cycle 1 ........................................................ 159

Figure 62. Test of Ability of ICP-MS to Measure Cesium........................................ 160

Figure 63. Cesium Concentration in Simulant Exiting Lead Column .......................... 162

Figure 64. Cesium Concentration Exiting Lag Column ............................................. 162

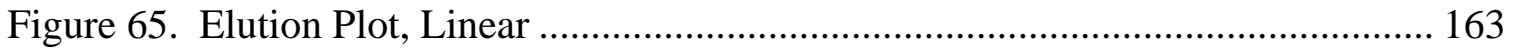

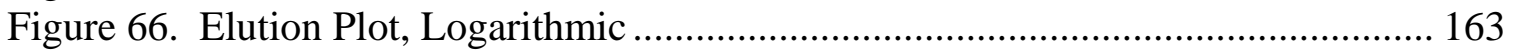

Figure 67. Cumulative Fraction of Cesium Removed during Elution .......................... 164

Figure 68. TLP Evaporator Pot Temperature during Campaigns II and III................... 173

Figure 69. TLP Evaporator Pot Pressure during Campaigns II and III ........................ 174

Figure 70. TLP Simulant Recirculation during Campaigns II and III ......................... 175

Figure 71. Pilot TLP antifoam concentration throughout Campaigns II and III testing 176

Figure 72. TLP Simulant Boiling in Clear Acrylic Section of Evaporator.................... 177

Figure 73. Comparison of Average Fluxes for Campaigns II-IV (repeat of Fig. 29) .... 187

Figure 74. Average Flux to Achieve Rates versus Undissolved Solids (at $25^{\circ} \mathrm{C}$ )......... 188

Figure 75. Average Flux to Achieve Rates versus Temperature ................................. 189

Figure 76. Predicted Combination of Filtration Temperature and Undissolved Solids

Endpoint to Meet Glass Production Targets .......................................................... 190

Figure 77. The effect of acid volume $(\mathrm{pH})$ on Al precipitation in Small Scale evaporation

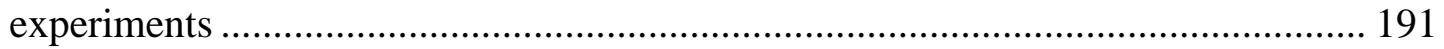

Figure 78. Solubility of sodium oxalate in $4.0 \mathrm{M} \mathrm{NaNO} 3-0.97 \mathrm{M} \mathrm{NaNO} 2$ at $25-50{ }^{\circ} \mathrm{C}$

for various sodium hydroxide concentrations (Barney, 1994)................................ 192

Figure 79. Filter Flux Comparison - Small Scale CUF vs. SIPP Cross Flow Filter...... 193 


\section{LIST OF TABLES}

Table 1. Feeds and products for planning SIPP developed for OLI/ESP computer model*

Table 2. Percent contribution for each of the recycle streams to the total FEP feed ....... 29

Table 3. Percent contribution for each of the UFP feed streams ...................................... 30

Table 4. Percent contribution for each of the TLP feed streams* .................................. 31

Table 5. Hold period before pilot-scale FEP begins .......................................................... 32

Table 6. Pilot-scale FEP test matrix with the colored columns representing Campaign IV

Table 7. Hold period before pilot-scale UFP begins........................................................ 33

Table 8. Pilot-scale UFP test matrix with the colored columns representing Campaign IV

Table 9. Hold period before pilot-scale CIX begins*

Table 10. Pilot-scale CIX (1st half) test matrix with the colored columns representing Campaign IV* ......................................................................................... 35

Table 11. Pilot-scale CIX (2nd half) test matrix with the colored columns representing Campaign IV* ................................................................................................ 36

Table 12. Hold period before pilot-scale TLP begins*...................................................... 36

Table 13. Pilot-scale TLP test matrix with the colored columns representing Campaign IV* 37

Table 14. Pilot-scale schedule for Campaign IV ............................................................. 38

Table 15. Measured Simulant Compositions ……………............................................... 39

Table 16. Recycle Streams*........................................................................................ 45

Table 17. Composition of Wash Permeate Recycles to FEP Evaporator .......................... 46

Table 18. Operating Parameters for FEP Campaigns ..................................................... 58

Table 19. Composition of Campaign IV FEP Evaporator Streams ................................... 60

Table 20. Comparison of Selected Species in Campaigns II-IV FEP............................... 62

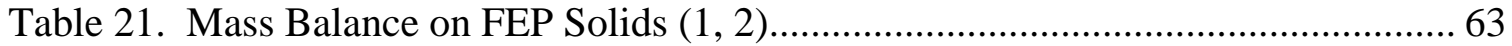

Table 22. Campaign IV UFP Test Matrix......................................................................67

Table 23. UFP Feed Constituent: AY102 Simulant...................................................... 72

Table 24. Dimensional Comparison of Mott and GKN Filter Units............................... 77

Table 25. Procedure to Operate the SIPP Backpulse System ............................................ 85

Table 26. Pilot.Scale Test Rig Backpulse Duration............................................................. 86

Table 27. SIPP UFP Measurement and Test Equipment .................................................. 87

Table 28. Uncertainties of Calculated Quantities for Campaign IV .................................. 89

Table 29. Test Matrix for Pre-Test Activities..................................................................... 92

Table 30. EDL Measured Data during Dewatering of the AY102+AP101+FEP Bottoms

Simulant ........................................................................................................... 101

Table 31. 24 wt\% UDS Steady State Test Comparison Data shown in Fig. 31 ............ 105

Table 32. 19 wt\% UDS Steady-State Test Data at Higher Temperature shown in Fig. 32

106

Table 33. Total Solids (TS) and Undissolved Solids (UDS) Concentrations of the Spent

Rinse and Cleaning Solutions from Campaign IV (also see Table 44).................... 117

Table 34. Concentrated Total Slurry and Permeate Compositions................................ 123

Table 35. Solids Content of Slurries during Ultrafiltration Dewatering.......................... 124 
Table 36. Simulant Samples Taken During Temperature Tests .................................. 126

Table 37. Solids Content of Samples from Temperature Tests ................................... 127

Table 38. Yield Stress and Consistency of Slurry From Temperature Tests................. 131

Table 39. Consistency (mP•s) of Permeate From Temperature Tests .......................... 131

Table 40. Comparison of Campaigns I to IV Washed Slurry Compositions.................. 134

Table 41. Comparison of Wash Permeate Composites from Campaigns I-IV .............. 135

Table 42. Comparison of Aluminum Concentrations in Ultrafiltration......................... 136

Table 43. Comparison of Ammonium Concentrations for Campaigns III-IV ............... 136

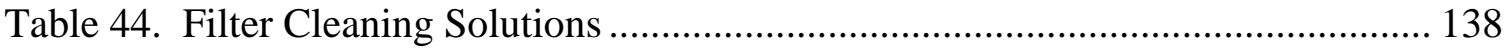

Table 45. Particle Size Distribution of the SuperLig ${ }^{\circledR 644}$ used for SIPP ..................... 150

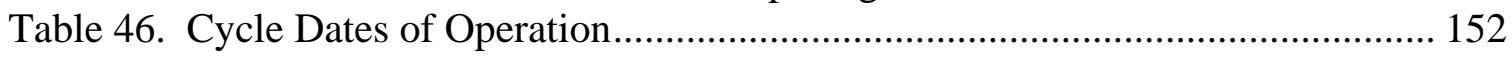

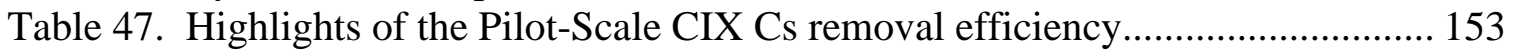

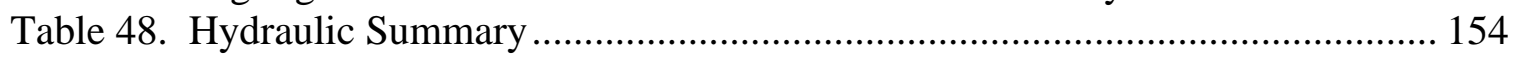

Table 49. Comparison of the Volume for Each of the CIX Regeneration Process

Solutions to the Internal Volume of Pilot-scale CIX Column ................................ 157

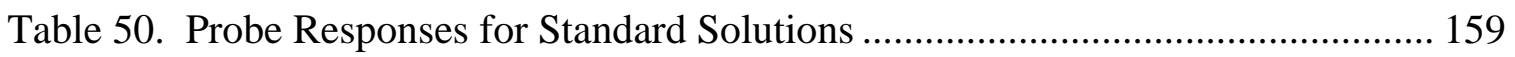

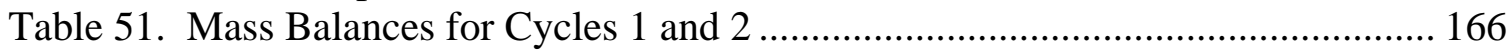

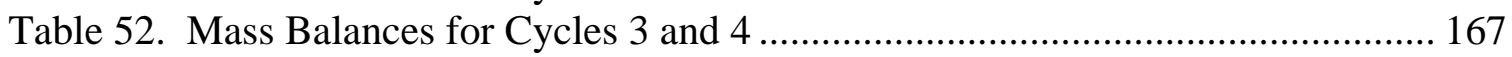

Table 53. Mass of Eluate Cesium as a function of Integration Method ........................ 168

Table 54. Measured Composition of Campaign III TLP Feeds .................................... 171

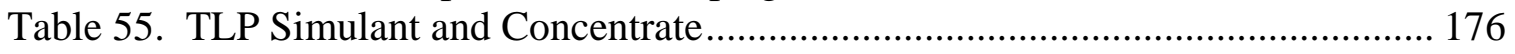

Table 56. Comparison of Campaigns II and III TLP Compositions............................. 179

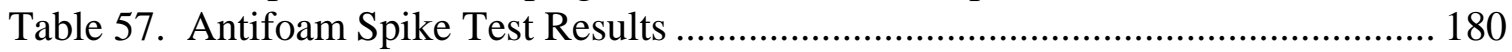

Table 58. Antifoam Concentrations in Process Samples ............................................ 182

Table 59. Glass Production Rates Based on Ultrafilter Throughput Using Campaign II

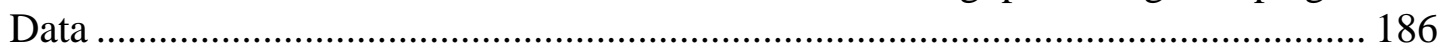

Table 60. Comparison between AP-101 and SIPP Column Tests and Expected Impacts 


\section{NOMENCLATURE}

\begin{tabular}{|c|c|}
\hline AA & Atomic absorption (spectroscopy) \\
\hline ACTL & Aiken County Technology Laboratory (part of WSRC/SRNL) \\
\hline ADS & Analytical Development Section (part of WSRC/SRNL) \\
\hline AP101 & DOE Hanford Site Tanks 241-AP-101 simulated waste \\
\hline AN102 & DOE Hanford Site Tank 241-AN-102 \\
\hline AN107 & DOE Hanford Site Tank 241-AN-107 \\
\hline AY102 & DOE Hanford Site Combined Tanks 241-AY-102 and 241-C-106 \\
\hline BNI & Bechtel National, Inc. \\
\hline CIX & Cesium Ion Exchange (Process) (part of the RPP-WTP Pretreatment Facility) \\
\hline CFD & Computational Fluid Dynamics \\
\hline CUF & $\begin{array}{l}\text { Cells Unit Filter (bench-top cross-flow filter used with both radioactively cold } \\
\text { and hot wastes) }\end{array}$ \\
\hline${ }^{\circ} \mathrm{C}$ & Degree Centigrade (or Celsius) \\
\hline $\mathrm{D}$ & Diameter \\
\hline DIF & Deionized and Filtered (0.1 micron) Water \\
\hline DOE & United States Department of Energy \\
\hline $\mathrm{dP}$ & Differential Pressure \\
\hline DS & See Solids \\
\hline EDL & Engineering Development Laboratory (part of WSRC/SRNL) \\
\hline EPDM & Ethylene Propylene Diene Monomer \\
\hline FEP & (Waste) Feed Evaporation Process (part of the RPP-WTP Pretreatment Facility) \\
\hline $\mathrm{ft}$ & Foot \\
\hline gm & Gram \\
\hline gpm & Gallons per Minute \\
\hline HLP & High Level (Activity Waste) Process \\
\hline HLW & High Level (Activity) Waste \\
\hline $\mathrm{hr}$ & Hour \\
\hline i.d. or ID & Inside Diameter \\
\hline IC & Ion Chromatography \\
\hline ICPES & Inductively coupled plasma emission spectroscopy \\
\hline ICPMS & Inductively coupled plasma mass spectrometry \\
\hline IHLW & Immobilized high level waste (glass) \\
\hline ILAW & Immobilized low activity waste (glass) \\
\hline in. & Inch \\
\hline IX & Ion Exchange \\
\hline $\mathrm{hr}$ & Hour \\
\hline l or $\mathrm{L}$ & Liter \\
\hline LAW & Low (Level) Activity Waste \\
\hline $\mathrm{m}$ & Meter \\
\hline M & Molar (mol/L) \\
\hline $\mathrm{Mg}$ & Milligram \\
\hline $\min$ & Minute \\
\hline $\mathrm{ml}$ or $\mathrm{mL}$ & Milliliter \\
\hline $\mathrm{mm}$ & Millimeter \\
\hline MTG/d & Metric tons glass/day \\
\hline MW & microwave acid dissolution \\
\hline NAS & Sodium Aluminosilicate \\
\hline
\end{tabular}


WSRC-TR-2005-00105, REVISION 0

SRNL-RPP-2005-00012, REVISION 0

nominal A "nominal" rating does not give an exact size to a filter medium, but rather an approximation to the expected performance of a filter. In the case of Mott, a nominal rated $0.1-\mu \mathrm{m}$ filter means that approximately $95 \%$ of particles greater than $0.1 \mu \mathrm{m}$ will not pass the filter.

NTU Nephelometric Turbidity Unit

o.d. or OD Outside Diameter

OLI/ESP Environmental Simulation Program software package used in the SIPP task

$\mathrm{Pa}$ Pascal

PDMS Polydimethylsiloxane - portion of Antifoam used for tracking purposes

PSD Particle Size Distribution

psi Pounds Per Square Inch

psig Pounds Force Per Square Inch Gauge

psid Pounds Force Per Square Inch Difference

PWD Plant Wash and Disposal (System)

QA Quality Assurance

RLD Radioactive Liquid (Waste) Disposal (System)

RPP River Protection Project (at the DOE Hanford Site)

s or sec Time: Second

SBS Submerged Bed Scrubber - source of LAW and HLW melter recycle streams

SIPP Semi-Integrated Pilot Plant

Solids

$\underline{\mathrm{TS}}=$ Total Solids $=$ Mass percent of all solids in a stable slurry whether dissolved or not

UDS = Undissolved Solids = Mass percent of solids in a stable slurry that are not dissolved

DS = Dissolved Solids $=$ TS - UDS $=$ Mass percent of solids in a stable slurry that are dissolved

SS = Soluble Solids = $($ TS - UDS $) /(100 \%$ - UDS $) \times 100 \%=$ Mass percent of solids in only the supernatant portion of a stable slurry

SPF sodium peroxide fusion $\mathrm{HCl}$ uptake dissolution

SRNL Savannah River National Laboratory (part of WSRC)

SS See Solids

SSM Steady-State Matrix (Applies to Campaign I only - see Fig. 33)

Std Dev Standard Deviation

Supernate Supernatant (see below for additional explanation)

TLP Treated (Feed) LAW Process (part of the RPP-WTP Pretreatment Facility)

TS See Solids

TMP Transmembrane Pressure (the average pressure drop across the thickness of the filter medium - perpendicular to the slurry flow.)

TRU Transuranic

UDS See $\underline{\text { Solids }}$

UF Ultrafiltration

UFP Ultrafiltration Process (part of the RPP-WTP Pretreatment Facility)

$\mathrm{V} \quad$ Velocity of the slurry flow along the length of the filter tubes

VSL Vitreous State Laboratory (of Catholic University of America, Wash. DC)

WGI Washington Group International

WTP Waste \& Immobilization Treatment Plant

WSRC Westinghouse Savannah River Company

XRD X-ray diffraction

Also note: Within this report, supernatant refers to the liquid (undissolved solids-free) portion of a slurry sample. When referring to the ultrafiltration process, the permeate is synonymous with the slurry supernatant; either term can thus be used, but permeate will be the preferred term herein. For the evaporation operations, the liquid fraction of the slurry feed or Bottoms product will be called the supernatant. 
WSRC-TR-2005-00105, REVISION 0 SRNL-RPP-2005-00012, REVISION 0

This page intentionally left blank.

- xii - 


\begin{abstract}
In August 2004 the last of the SIPP task testing ended - a task that formally began with the issuance of the RPP-WTP Test Specification in June 2003. The planning for the task was a major effort in itself and culminated with the input of all stakeholders, DOE, Bechtel National, Inc., Washington Group International, in October 2003 at Hanford, WA (Appendix A). This report documents the activities carried out as a result of that planning.

Campaign IV, the fourth and final step towards the Semi-Integrated Pilot Plant (SIPP) task, conducted by the Savannah River National Laboratory (SRNL) at the Savannah River Site, was to take the several recycle streams produced in Campaign III, the third step of the task, and combine them with other simulated recycle and chosen waste streams. (Campaign III was fed recycles from Campaign II, as Campaign II was fed by Campaign I.) The combined stream was processed in a fashion that mimicked the pretreatment operations of the DOE River Protection Project - Waste Treatment and Immobilization Plant (RPP-WTP) with the exception of the Ion Exchange Process. The SIPP task is considered semi-integrated because it only deals with the pretreatment operations of the RPP-WTP. That is, the pilot plant starts by receiving waste from the tank farm and ends when waste is processed to the point of being sent for vitrification. The resulting pretreated LAW and HLW simulants produced by the SIPP were shipped to VSL (Vitreous State Laboratory) ${ }^{\dagger}$ and successfully vitrified in pilot WTP melters.
\end{abstract}

Within the SIPP task these steps are referred to as Campaigns and there were four Campaigns in all. Campaign I, which is completely different than other campaigns, subjected a simulant of Hanford Tank 241-AY-102/C-106 (AY102) waste to cross-flow ultrafiltration only and in that process several important recycle streams were produced as a result of washing the simulant and cleaning the cross-flow filter. These streams were fed to subsequent campaigns and that work was the subject of the issued Campaign I interim report (Duignan et al., 2004a or Appendix I-1). The streams created in Campaign I were used for Campaign II, and during Campaign II more of the same recycle streams were produced, with the addition of recycle streams created during the pilot-scale ion exchange unit operation (Duignan et al., 2004b or Appendix I-2). Campaign III used the recycles from Campaign II and was the first campaign to use all the recycle streams (Duignan et al., 2004c or Appendix I-3).

The operation of each of the subsequent campaigns, i.e., II, III, and IV, while different from Campaign I, are very similar to each other, and can be best understood as the process of operating a series of Pretreatment Unit Operations in a somewhat prototypic manner. That is, while Campaign I studied the operation of a single, albeit important, Pretreatment Unit Operation, i.e., Ultrafiltration, subsequent campaigns were to study the four major unit operations that make-up the RPP-WTP Pretreatment Facility. They are: Waste Feed Evaporation Process (FEP), Ultrafiltration Process (UFP), Cesium Ion Exchange Process (CIX), and the Treated LAW Evaporation Process (TLP). Each of the campaigns operated basically as a separate subtask, but as with Campaign I, the recycle streams produced in one campaign were fed into the subsequent campaign. Therefore, all four campaigns were

\footnotetext{
${ }^{\dagger}$ Part of the Catholic University of America
} 
chemically connected through these recycle streams, which carry over effects of the preceding campaign.

The results of Campaign IV operations are the subject of this fourth and final report. Separate reports were issued after each of the previous campaigns, but they were treated as interim because of being limited to the results obtained from a single campaign (or past campaigns) and further limited to only highlights of that single campaign. This final report not only discusses the Campaign IV results but compares those with the previous campaigns. Also included is a more comprehensive discussion of the overall task activities, as well as abridged versions of the full databases of the accumulated results and the equipment used during the year-long SIPP task.

SIPP is a limited version of the plant's pretreatment facility in that it tests pilot-scale unit operations of FEP, UFP, CIX, and TLP only. However, an integral part of SIPP is to produce the many recycle streams that must also be processed with the stored tank farm waste. That is, it is very important to know the impact that the recycle streams will have on the operation of the several unit operations so as to better model the plant's overall pretreatment throughput, leading to a production rate of stabilized waste.

Like the interim reports, this report will begin with a summary of the overall operation of the task done during Campaign IV to understand the layout of this report. This summary is then followed with highlights of the test results.

With input and review from Hanford, SRNL developed a steady-state OLI/ESP model of the RPP-WTP Pretreatment Facility operation. That model's principal role was to determine the amounts of simulated wastes needed for each of the four unit operations. In general, it did well, but was found to be off by approximately 15\% in predicted SIPP volumes due to unknowns in the undissolved solids of the several simulants. (For a detailed discussion of model predictions differences see the Campaign II Interim Report, Duignan et al., 2004b or Appendix I-2; and Appendix B of this report.)

Each of the SIPP task interim reports contains many conclusions. The main conclusions of those reports, and those of Campaign IV, are listed below for this final report. All of the conclusions as they were reported in those interim reports can be found in this document at the end of each of the appropriate unit operations.

\section{FEP Evaporation}

- In Campaign IV a total of about $1119 \mathrm{~kg}$ of FEP, feed was concentrated down to $175 \mathrm{~kg}$.

- The feed was evaporated under vacuum (1 psia $(6.9 \mathrm{kPa}))$ at moderate temperatures ( $50^{\circ} \mathrm{C}$ ) and the entire evaporation process proceeded as designed.

- The evaporator was designed to evaporate around $0.4 \mathrm{gpm}(1.5 \mathrm{lpm})$ of water and for this campaign the average evaporation rate was met or exceeded.

- No significant foaming was observed prior to the addition of antifoam agent. During the evaporation process, the froth height remained around $1 \mathrm{ft}(0.3 \mathrm{~m})$ that is significantly less than the $5 \mathrm{ft}(1.5 \mathrm{~m})$ distance to the lower demister pad. 
- Mixing of the recycles in a different order for Campaign IV had no effect on the evaporation process as compared to the previous campaigns.

- The FEP feed and Bottoms concentrations for Campaigns III and IV were very similar, indicating that steady-state had been reached with respect to the recycle compositions.

- The precipitation of sodium oxalate in the FEP evaporator occurred in all three campaigns and accounted for most of the precipitated solids found. Dissolved oxalate, present in the feed, began to precipitate when approximately 53\% of the feed had been evaporated. A total of about $90 \mathrm{wt} \%$ of the oxalate precipitated at the end of evaporation. The oxalate appears to have precipitated as the $\mathrm{Na}$ salt $\mathrm{Na}_{2} \mathrm{C}_{2} \mathrm{O}_{4}$.

- The composition of the FEP feed was predictable by mass balance of the individual feed streams.

- Dissolved metals concentrations parallel the ratios in the AY102 simulant present in the recycles.

- About $25-40 \%$ of the dissolved $\mathrm{Al}$ in the feed to the FEP evaporator precipitated during mixing of the recycles, assumed to occur because of localized regions of low $\mathrm{pH}$. Vigorous mixing when joining caustic and acidic recycle streams in the Plant Wash and Disposal System would help to minimize Al precipitation.

- About 56-61\% of the aluminum was dissolved in the Campaign IV feed and the concentrate (Bottoms), which was similar to the Campaign III percentage. This dissolved Al fraction was less than 60-79\% realized in Campaign II, resulting in more undissolved Al fed to the UFP.

UFP

- The performance of the GKN filter unit was similar to that of the Mott filter unit under steady state conditions. In addition, the permeate flux of the GKN filter unit during dewatering was the same as the Mott filter unit, within measurement uncertainty, for the same simulated waste mixture.

- In Campaign IV, using the GKN filter unit, the average, temperature-adjusted permeate flux when dewatering from $\sim 3$ to $\sim 24 \mathrm{wt} \%$ UDS was $0.011 \mathrm{gpm} / \mathrm{ft}^{2}$. The average, temperature-adjusted permeate flux when dewatering from $\sim 3$ to $20 \mathrm{wt} \%$ UDS was 0.013 $\mathrm{gpm} / \mathrm{ft}^{2}$. The average, temperature-adjusted permeate flux when dewatering from $\sim 3$ to 17 wt\% UDS was $0.016 \mathrm{gpm} / \mathrm{ft}^{2}$.

- Permeate flux improved at higher slurry temperatures. At 19 wt\% UDS, 40 psid TMP, and a slurry velocity through the filter of $12 \mathrm{ft} / \mathrm{s}$, the permeate flux improved almost 75\% at $45^{\circ} \mathrm{C}$ compared to $25^{\circ} \mathrm{C}$.

- During the elevated temperature tests at a constant solids loading of $19 \mathrm{wt} \%$ UDS no significant changes occurred with respect to solids concentrations and particles sizes. As expected, as the simulant temperature increased from $25^{\circ} \mathrm{C}$ to $45^{\circ} \mathrm{C}$ the consistency of the slurry and the viscosity of the permeate decreased from 14 to $11 \mathrm{mPa} \bullet \mathrm{s}$ and from 2.9 to $1.8 \mathrm{mPa} \bullet \mathrm{s}$, respectively, while the slurry yield stress increased approximately 10 to $12 \mathrm{~Pa}$. 


\section{All Campaigns}

- The turbidity of the permeate from dewatering and slurry washing, when measured directly after the sample was taken, was always lower than 1 NTU. The Test Specification indicated a threshold of 2 NTU, therefore, the measured values indicated that the permeate was free of significant undissolved solids.

- The SIPP and previous UFP tests indicate that the magnitude of the permeate flux during acid cleaning of a filter appears to be more of a function of the waste dewatered than of the filter unit used, i.e., Mott or GKN.

- The increase in the adjusted permeate flux between the second and third acid cleaning steps was minimal in Campaigns II, III, and IV, possibly indicating that the third acid cleaning was unnecessary. The exception was Campaign I where whitish solids in the spent solutions of the first and second acid cleanings inhibited the permeate flux, requiring a third acid cleaning (and possibly a fourth) to reach steady conditions. The Campaign I simulant did not contain AP101 and FEP Bottoms, indicating that the necessity of a third acid cleaning was dependent on the waste dewatered.

- Cleaning the filter with $2 \mathrm{M}$ nitric acid after dewatering was sufficient to increase baseline permeate flux to approximately an order of magnitude higher than that of average waste flux during dewatering. However, it was not sufficient to return the filter to pre-test flux levels. In previous campaigns, soaking the filter unit in $0.1 \mathrm{M} \mathrm{NaOH}$ for an extended period of time (2 months or more) typically regained the pre-test flux levels. The significance of " 2 months or more" is that 2 months was typically the duration between successive SIPP campaigns.

- The compositions of the undissolved solid species in the washed sludges in Campaigns IIV were similar, as expected. The soluble species concentrations in the slurry and permeate for Campaigns II-IV were similar, as expected.

- The balances on aluminum over the process were not consistent and could not be closed without additional analytical data.

- Ammonium in the first Campaign III $0.01 \mathrm{M} \mathrm{NaOH}$ wash was mostly retained in the slurry, indicating that a slurry component has an affinity for ammonium. Approximately none of the ammonium in the second wash was retained.

- The antifoam agent did not have any observed macroscopic effects on the filter operations of the SIPP, which is also consistent with similar commercial industrial experience (Watts et al., 1995). However, since the filter was cleaned between each run, the effect of possible accumulation of antifoam on the filter media with no cleaning between runs was not determined.

\section{CIX}

- Pressure drops for the four cycles were low and the resin beds permeabilities were approximately the same as were measured for the 24-Inch Column Test with fresh resin (Fowley et al, 2004).

- The pressure drop across the columns during the flow of simulant generally increased with each cycle of the four cycles of operation. 
WSRC-TR-2005-00105, REVISION 0

SRNL-RPP-2005-00012, REVISION 0

- $\quad$ SuperLig ${ }^{\circledR} 644$ effectively removed cesium from simulated waste and the analytical ability to measure cesium concentrations in five molar sodium did allow verification of the waste acceptance criterion of 50\% breakthrough from the lead column after 100 Bed Volumes of simulated waste were treated. In fact, in the worst case, Cycle 4, even after $190 \mathrm{BV}$ of treated waste the lead column exit cesium concentration was less than $7 \%$ of the feed waste.

- $\quad$ SuperLig ${ }^{\circledR} 644$ effectively removed cesium from simulated waste, however, the analytical difficulty of measuring very low cesium concentrations in five molar sodium did not allow verification of the waste acceptance criterion of $5.2 \mu \mathrm{g} / \mathrm{L}$ from the lag column after $100 \mathrm{BV}$ of simulated waste were treated. This limitation was acknowledged before the start of testing.

- The fraction of the original cesium in the simulant that was removed by the lead column generally decreased over the four cycles of testing.

- The masses of cesium in and out of the resin beds for all four cycles balanced to within measurement uncertainty.

- During cesium elution 97\% of the Cs was removed during the first $3.4 \mathrm{BV}$.

- A post-test measurement of the dry mass of the SuperLig ${ }^{\circledR} 644$ resin found a ratio of resin in sodium form to resin in hydrogen form to be 1.19 and 1.22 for Columns 1 and 2, respectively.

- A pre-test particle-size distribution of the resin was found to be $78 \pm 4 \mathrm{wt} \%$ (Mesh > 30), $20 \pm 4 \mathrm{wt} \%$ (30 $\leq$ Mesh $\leq 40)$, and $2 \pm 1 \mathrm{wt} \%$ (Mesh < 40), where 95\% confidence limits were used. A post-test PSD on the resin found no change in the Column 2 contents, indicating that no degradation of the resin occurred. The post-test PSD on the Column 1 resin was nominally larger. (84 $\pm 4 \mathrm{wt} \%, 15 \pm 4 \mathrm{wt} \%$ and $1 \pm 1 \mathrm{wt} \%)$, although the error bars of the pre-test and post-test measurements overlap. There is no evidence for resin degradation in either column.

\section{TLP Evaporation}

The TLP was not used during Campaign IV but some overall SIPP conclusions are:

- The TLP operation met expectations by concentrating feed from a density of $1.1 \mathrm{~kg} / \mathrm{L}$ to $1.4 \mathrm{~kg} / \mathrm{L}$ or increasing the sodium concentration from approximately $2 \mathrm{M}$ to $8 \mathrm{M}$.

- Foaming was not an issue, but when the required amount of antifoam was added, i.e., 1500 ppm, a region that can be best described as the disengagement of liquid and gas, decreased from approximately $2 \mathrm{ft}$ to $1 \mathrm{ft}$.

- The lower demister pad remained free of solids throughout testing.

- The composition of products from the TLP matched what was expected from the mass balance.

- Tracking the fate of antifoam in the many simulant streams was not possible because of the ineffectiveness in the methods used to measure its concentration. However, it appears that an insignificant amount of antifoam was carried over into the evaporator condensates. This result was not surprising because of the high boiling point of the polyols fractions of the antifoam. Further, it appeared that the active component of the antifoam did not partition into the permeate stream, that is, it remained in the UFP sludge, 
WSRC-TR-2005-00105, REVISION 0

SRNL-RPP-2005-00012, REVISION 0

but the poor detection capabilities do not allow accurate quantification of the antifoam concentrations.

- The composition of the products from the TLP matched what was expected from the mass balance.

- No precipitation of solids was observed. (Feed had undissolved solids from LAW SBS recycle.)

- The concentration of aluminum in the TLP product from Campaign III was less than the result of Campaign II since the UF permeate contained less dissolved aluminum.

\section{Note on Antifoam}

In the filtration system, nearly all of the antifoam component polydimethylsiloxane (PDMS) remained in the washed concentrated sludge stream which is consistent with similar studies (Watts et al., 1995). Analysis indicates that very little if any of the PDMS was found in the UFP recycles or permeate. Nearly all of the PDMS was also retained in the FEP and TLP Bottoms concentrates, with no detectable PDMS in the condensates. Since PDMS is essentially insoluble, non-homogenous sub-samples, coupled with analytical recoveries of less than $80 \%$, did not allow for accurate quantification of the PDMS for the purposes of process material balances in all the SIPP process streams. SRNL recommends that extraction of the entire samples before analysis should be investigated for any future antifoam analytical work. Furthermore, no conclusions about the fate of the polyol fraction of the antifoam could be made since this portion was not tracked. Future work on the fate of this antifoam fraction is warranted.

\section{Observations from the SIPP task to the Overall Operation of WTP}

The SIPP operations conducted at SRNL using simulated WTP startup feed performed well without any significant incidents that caused disruption of operations. All four unit operations (Evaporation FEP\& TLP, Filtration-UFP, and Ion Exchange-CIX) performed nearly flawlessly and without significant unplanned downtime. The LAW and HLW supernate and slurry products produced by the SIPP were successfully vitrified by VSL (Matlack et al., 2005a, Matlack, et al., 2005b).

The SIPP met all success criteria with the exception of demonstration of a scaled throughput of $80 \mathrm{MTG}$ /day of LAW and 5.4 MTG/day HLW. This criterion ${ }^{\dagger}$ was partially met by the SIPP in that both evaporators preformed at or above the evaporation rate necessary to meet the desired production rates. The pilot ultrafiltration system was not able to meet the production rates given a filter concentration end point of $20 \mathrm{wt} \%$ undissolved solids (UDS). However, glass production rates can be met for simulated AY102/C106 with AP101 slurries by reducing the target ultrafiltration concentration endpoint between 15.4 and $16.5 \mathrm{wt} \%$ UDS. After the wash cycle is completed, higher undissolved solids concentrations can be achieved by concentrating the UFP slurry after washing (flux rates are much higher during washing) without reducing the overall plant production rate. Therefore, concentrating less, to

\footnotetext{
${ }^{\dagger}$ The pilot ion exchange testing was by design not required to meet full scale plant production rates (See Test Exception No. 7 in Section 1.2)
} 
a lower UDS, will not necessarily have the effect of producing a more dilute feed to the HLW melter system. Concentrating to lower UDS does result in producing less LAW feed per unit of UF feed and does result in slightly more LAW (supernatant) being recycled to the Plant Wash and Disposal (PWD) System. Increasing the operating temperature of the ultrafiltration operation also proved to increase the filter production rate beyond the desired production rate goals.

The impact of recycle solutions on SIPP operation proved to have no macroscopic effects on the overall SIPP operation or the individual unit operations. While some solids (e.g. oxalate \& aluminum) were found to precipitate in the Plant Wash \& Disposal and FEP evaporator systems, none of the these solids proved to have an impact on the overall operation of the SIPP.

Given the limits (e.g. non-prototypical piping layouts, mixing systems, simulant chemical/physical properties vs. actual waste) of the testing described in this report, the fullscale WTP Pretreatment facility unit operations that were simulated by the SIPP should also perform well using AY102/C106 feeds. However, since not all start-up and operational risks can be fully anticipated or mitigated by SIPP testing, the following observations and lessons learned should be considered by the designers and operators of the WTP.

- Precipitation of aluminum, in some tank waste compositions can increase the total number of HLW canisters produced, therefore, additional sodium hydroxide maybe necessary to prevent unwanted Al precipitation.

- Ammonium, transferred from the Duratek LAW Submerged Bed Scrubber, will partition to the concentrated/washed HLW transferred to the WTP HLW melter system. The ammonium will likely recycle back to the WTP Pretreatment facility via the HLW SBS condensate system. Incorporation of ammonium chemistry into the WTP models should be implemented to assess the fate and impact of ammonium on the overall operations of the WTP. The potential to generate ammonium nitrate in the WTP vessel vent system should be evaluated. Consideration should be given to determining if the TLP evaporator condensate and LAW melter SBS condensates can be treated and disposed of separately rather than recycling these materials to the front end of the Pretreatment facility. This would also decrease the buildup of corrosive anions (e.g. Cl, F, SO 4 ) in the TLP system.

- Maintenance of the ultrafiltration system may dislodge solids and cause a loss in filter flux. WTP should consider cleaning the filter after maintenance is conducted. A detailed filter system performance monitor program maintained by the future WTP filter system engineers would reduce plant down time when filter flux performance issues occur.

- Decon solutions that are generated from hot cell equipment maintenance were not tested in the SIPP. These solutions can carry larger concentrations of glass formers, sludge, salts, and ammonium then the nominal recycles tested by the SIPP. WTP personnel should assess the impact of processing decon solution generated from maintenance activities to determine if any unwanted reactions (e.g. precipitation) will occur. 
WSRC-TR-2005-00105, REVISION 0

SRNL-RPP-2005-00012, REVISION 0

This page intentionally left blank. 
WSRC-TR-2005-00105, REVISION 0

SRNL-RPP-2005-00012, REVISION 0

\subsection{TESTING SUMMARY}

\subsection{OBJECTIVES}

This final report contains data from all four campaigns, i.e., Campaigns I, II, III, and IV, that comprised the SIPP task. Testing was done on pilot scale unit operations of the WTP Pretreatment Facility and ranged from just ultrafiltration (UFP) in Campaign I, to just feed waste evaporation (FEP) and UFP in Campaign IV, to all four operations, i.e., FEP, UFP, Ion Exchange (CIX), and treated LAW evaporation (TLP) in Campaigns II and III. As the task evolved the simulated waste streams became chemically more complex. Campaign I filtered a single Hanford waste 241-AY-102, which was followed by combining two waste streams, 241-AY-102 and 241-AP-101 with recycle waste stream in Campaigns II, III and IV. The objectives listed below are those given in the RPP-WTP Test Specification (Longwell, 2003). For completeness, however, only the body of work approved in the WSRC Technical Task and Quality Assurance Plan (Duignan, 2003c) is required to satisfy this task. Objectives not met because they were not agreed to in the Task Plan are so noted. 
WSRC-TR-2005-00105, REVISION 0

SRNL-RPP-2005-00012, REVISION 0

\begin{tabular}{|c|c|c|}
\hline Overall Test Objectives & $\begin{array}{l}\text { Objective } \\
\text { Met (Y/N) }\end{array}$ & Discussion \\
\hline $\begin{array}{l}\text { 1. Modify the design and operation of } \\
\text { the existing pretreatment pilot facilities } \\
\text { to extent necessary to mimic the WTP } \\
\text { Pretreatment Facilities. }\end{array}$ & $\mathrm{Y}$ & $\begin{array}{l}\text { Each of the four unit operations that } \\
\text { comprise the SIPP test were either modified } \\
\text { or built to meet planned operation. }\end{array}$ \\
\hline $\begin{array}{l}\text { 2. Operate the (pilot) integrated } \\
\text { facilities using simulated AY102 } \\
\text { (Envelope A/D) and AP101 (Envelope } \\
\text { A) waste feeds. }\end{array}$ & $\mathrm{Y}$ & $\begin{array}{l}\text { Campaigns II, III, and IV involved the use } \\
\text { of simulated AY102, AP101, and recycle } \\
\text { streams. }\end{array}$ \\
\hline $\begin{array}{l}\text { 3. Collect chemical and physical } \\
\text { property data to be defined in the test } \\
\text { plan then refine and validate the } \\
\text { thermodynamic model(s) developed by } \\
\text { SRNL. }\end{array}$ & $\mathrm{Y}$ & $\begin{array}{l}\text { Sufficient property data were obtained to } \\
\text { satisfy project needs as is evidenced by the } \\
\text { customer-approved sample plan matrices } \\
\text { that are included in Appendix D. }\end{array}$ \\
\hline $\begin{array}{l}\text { 4. Demonstrate pretreatment production } \\
\text { rates required to produce } 80 \text { metric tons } \\
\text { per day of LAW glass and } 5.4 \text { metric } \\
\text { tons of HLW glass. These are contract } \\
\text { defined "expected" production } \\
\text { throughputs for 5-day sprint runs listed } \\
\text { in Table C.6-5.1 of the contract (DOE } \\
\text { 2003). These required flow rates will } \\
\text { be determined before testing begins by } \\
\text { application of the appropriate scale } \\
\text { factors to the unit operations that } \\
\text { represent an equivalence of } \\
\text { pretreatment throughput to meet } \\
\text { maximum glass production. }\end{array}$ & $\mathrm{N}$ & $\begin{array}{l}\text { The unit production rates were measured } \\
\text { but the targets were not met. The SIPP task } \\
\text { was limited to four separate unit operations } \\
\text { that comprise the principal operations of the } \\
\text { RPP-WTP Pretreatment Facility. The pilot } \\
\text { FEP and TLP evaporation units exceeded } \\
\text { the plant-modeled rate of } 0.395 \text { gpm. The } \\
\text { pilot CIX was subjected to a test exception } \\
\text { (described in Section } 1.2 \text { ), which changed } \\
\text { this objective. As shown in Campaigns II, } \\
\text { III, and IV, the pilot ultrafiltration permeate } \\
\text { rate during Campaign IV dewatering from } \\
\sim 3 \text { to } 20 \text { wt\% UDS was } 0.013 \text { gpm/ft }{ }^{2} \text {. This } \\
\text { rate is below the target rate of } 0.02 \text { gpm/ft }{ }^{2} \text {, } \\
\text { but is representative of the filter process } \\
\text { since a similar flux rate was obtained for } \\
\text { Campaign I, with no recycle streams, and } \\
\text { Campaigns II, III, and IV included several } \\
\text { recycle streams. However, if some of the } \\
\text { suggestions made in Section } 2.10 \text { to modify } \\
\text { the UFP process of either concentration to } \\
\text { less than } 20 \text { wt\% UDS or operation at } \\
\text { higher temperatures, then the stated } \\
\text { productions rates may be attainable. }\end{array}$ \\
\hline
\end{tabular}


WSRC-TR-2005-00105, REVISION 0 SRNL-RPP-2005-00012, REVISION 0

\begin{tabular}{|c|c|c|}
\hline FEP/TLP Test Objectives & $\begin{array}{l}\text { Objective } \\
\text { Met (Y/N) }\end{array}$ & Discussion \\
\hline $\begin{array}{l}\text { 1. Operate the Waste Feed Evaporator } \\
\text { and the Treated LAW Evaporator using } \\
\text { simulants derived from the integrated } \\
\text { operation of the pretreatment pilot } \\
\text { plant. }\end{array}$ & $\mathrm{Y}$ & $\begin{array}{l}\text { Feeds during Campaigns II-IV for both the } \\
\text { FEP and TLP evaporators included derived } \\
\text { simulants or recycle streams produced } \\
\text { during SIPP operations. }\end{array}$ \\
\hline $\begin{array}{l}\text { 2. Recycle secondary waste generated } \\
\text { from the pilot ultrafiltration operation } \\
\text { and the pilot ion exchange unit } \\
\text { operation to the evaporators. }\end{array}$ & Y & $\begin{array}{l}\text { Recycle streams from the pilot ultrafiltration } \\
\text { operation from Campaigns I, II, and III were } \\
\text { fed to the FEP evaporator in Campaigns II, } \\
\text { III, and IV, respectively. }\end{array}$ \\
\hline $\begin{array}{l}\text { 3. Operate the pilot evaporator using } \\
\text { simulated HLW Submerged Bed } \\
\text { Scrubber solution, simulated } \\
\text { Pretreatment Caustic Scrubbers, ion } \\
\text { exchange recycle solutions, and } \\
\text { ultrafiltration recycles generated from } \\
\text { pilot operations. }\end{array}$ & $\mathrm{Y}$ & $\begin{array}{l}\text { Campaign II, III, and IV used all the streams } \\
\text { indicated. }\end{array}$ \\
\hline $\begin{array}{l}\text { 4. Operate the pilot evaporator using } \\
\text { simulated LAW Submerged Bed } \\
\text { Scrubber solution and ion exchange } \\
\text { treated feed to produce LAW feed } \\
\text { solution. }\end{array}$ & Y & See the preceding response. \\
\hline $\begin{array}{l}\text { 5. Compare the results (solubility, } \\
\text { chemical, and physical properties, } \\
\text { which will be indicated in the test plan) } \\
\text { developed on the pilot scale with } \\
\text { results developed by experiments } \\
\text { conducted at the bench scale. }\end{array}$ & $\mathrm{Y}$ & $\begin{array}{l}\text { Limited bench-scale evaporation tests are } \\
\text { compared to SIPP results in Section 2.11. }\end{array}$ \\
\hline $\begin{array}{l}\text { 6. Determine the chemical and physical } \\
\text { properties of simulants evaporated at } \\
\text { the pilot scale as a function of sodium } \\
\text { concentration and temperature. }\end{array}$ & $\mathrm{N}$ & $\begin{array}{l}\text { The approved task plan did not require these } \\
\text { property vs. temperature evaluations. } \\
\text { However, densities and sodium } \\
\text { concentrations were measured and included } \\
\text { in this report. }\end{array}$ \\
\hline $\begin{array}{l}\text { 7. Evaluate the selected antifoam } \\
\text { reagent performance in the evaporators. }\end{array}$ & $\mathrm{Y}$ & $\begin{array}{l}\text { Antifoam was added to both evaporators } \\
\text { and those results are discussed herein. }\end{array}$ \\
\hline $\begin{array}{l}\text { 8. Evaluate the effect of in-leakage on } \\
\text { air emissions. }\end{array}$ & $\mathrm{N}$ & $\begin{array}{l}\text { This work was not included in the approved } \\
\text { task plan due to cost. }\end{array}$ \\
\hline $\begin{array}{l}\text { 9. Determine the chemical and physical } \\
\text { properties of concentrated recycle and } \\
\text { waste blends, which will be indicated } \\
\text { in the test plan, to be sent for } \\
\text { precipitation/ultrafiltration. }\end{array}$ & $\mathrm{Y}$ & $\begin{array}{l}\text { The concentrated Bottoms streams were } \\
\text { analyzed and discussed herein. }\end{array}$ \\
\hline $\begin{array}{l}\text { 10. Operate the pilot evaporator unit } \\
\text { assuming a full-scale WTP } \\
\text { Pretreatment process flow rate of } 80 \\
\text { metric tons of LAW glass per day and } \\
5.4 \text { metric tons of HLW glass per day } \\
\text { (herein written as } 80 / 5.4 \text { MTG/d). }\end{array}$ & $\mathrm{Y}$ & $\begin{array}{l}\text { Both evaporators were run at a rate of better } \\
\text { than the plant rate of } 0.395 \text { gpm, which was } \\
\text { based on a production rate of } 80 / 6 \text { MTG/d } \\
\text { (Calloway, 2004). }\end{array}$ \\
\hline
\end{tabular}


WSRC-TR-2005-00105, REVISION 0

SRNL-RPP-2005-00012, REVISION 0

\begin{tabular}{|c|c|c|}
\hline UFP Test Objectives & $\begin{array}{l}\text { Objective } \\
\text { Met (Y/N) }\end{array}$ & Discussion \\
\hline $\begin{array}{l}\text { 1. Operate with prototypical } \\
\text { wash/leach/wash/steps using simulated } \\
\text { AY102 and AP101 and concentrated } \\
\text { evaporator recycles. }\end{array}$ & $\mathrm{Y}$ & $\begin{array}{l}\text { The approved task plan made the leach } \\
\text { step optional, pending RPP-WTP guidance. } \\
\text { Before testing began RPP-WTP decided } \\
\text { against doing the leach step. All other } \\
\text { objectives were met. }\end{array}$ \\
\hline $\begin{array}{l}\text { 2. Operate with } \\
\text { prototypical/recommended } \\
\text { Ultrafiltration cleaning method. }\end{array}$ & $\mathrm{Y}$ & $\begin{array}{l}\text { RPP-WTP recommended continued use of } \\
2 \text { M nitric acid for cleaning, which was } \\
\text { done. }\end{array}$ \\
\hline $\begin{array}{l}\text { 3. Determine the chemical and physical } \\
\text { properties of the concentrated solids, } \\
\text { washed solids, leached solids, and final } \\
\text { solids product derived from each } \\
\text { campaign. }\end{array}$ & $\mathrm{Y}$ & $\begin{array}{l}\text { Properties of the concentrates were } \\
\text { measured. However no leaching was } \\
\text { performed as requested by the RPP-WTP } \\
\text { customer. }\end{array}$ \\
\hline $\begin{array}{l}\text { 4. Determine the chemical and physical } \\
\text { properties of permeate, recycles, and } \\
\text { cleaning solutions, which will be } \\
\text { indicated in the test plan, derived from } \\
\text { each campaign. }\end{array}$ & $\mathrm{Y}$ & $\begin{array}{l}\text { Properties of those streams were measured } \\
\text { and discussed herein. }\end{array}$ \\
\hline $\begin{array}{l}\text { 5. Compare the results developed on } \\
\text { the pilot scale with results developed } \\
\text { by experiments conducted at the bench } \\
\text { scale to assess the effect of recycles. }\end{array}$ & $\mathrm{Y}$ & $\begin{array}{l}\text { A limited set of bench-scale filtration } \\
\text { results are compared to SIPP Campaign I } \\
\text { for AY102/C106 in Section 2.11. }\end{array}$ \\
\hline $\begin{array}{l}\text { 6. Provide pump data (pump curves) } \\
\text { for the high solid content slurries. }\end{array}$ & $\mathrm{N}$ & $\begin{array}{l}\text { This requirement was not included in the } \\
\text { approved task plan. }\end{array}$ \\
\hline $\begin{array}{l}\text { 7. Demonstrate operation of the } \\
\text { ultrafiltration unit assuming a full-scale } \\
\text { WTP Pretreatment process flow rate } \\
\text { corresponding to production of } 80 / 5.4 \\
\text { MTG/d. }\end{array}$ & $\mathrm{N}$ & $\begin{array}{l}\text { See response } 4 \text { to the Overall Test } \\
\text { Objectives, above. }\end{array}$ \\
\hline $\begin{array}{l}\text { 8. Evaluate the downstream effects of } \\
\text { antifoam reagent. Determine the fate } \\
\text { of the reagent by tracking its } \\
\text { concentration through the integrated } \\
\text { pilot. }\end{array}$ & $\mathrm{N}$ & $\begin{array}{l}\text { The effect on filter flux seemed to be } \\
\text { insignificant, but it is discussed herein } \\
\text { along with that data used to track the } \\
\text { antifoam. }\end{array}$ \\
\hline
\end{tabular}


WSRC-TR-2005-00105, REVISION 0

SRNL-RPP-2005-00012, REVISION 0

\begin{tabular}{|c|c|c|}
\hline CIX Test Objectives & $\begin{array}{l}\text { Objective } \\
\text { Met (Y/N) }\end{array}$ & Discussion \\
\hline $\begin{array}{l}\text { 1. Operate the pilot ion exchange unit } \\
\text { using ultrafiltration permeate produced } \\
\text { using simulated AY102 and AP101. }\end{array}$ & $\mathrm{Y}$ & $\begin{array}{l}\text { This was done along with the Bottoms } \\
\text { produced by evaporating the several } \\
\text { recycle streams. }\end{array}$ \\
\hline $\begin{array}{l}\text { 2. Operate the pilot ion exchange unit } \\
\text { assuming a full-scale WTP } \\
\text { Pretreatment process flow rate } \\
\text { corresponding to production of } 80 / 5.4 \\
\text { MTG/d. }\end{array}$ & $\mathrm{N}$ & $\begin{array}{l}\text { Test Exception 24590-WTP-TEF-RT-03- } \\
069 \text { required the pilot CIX show prototypic } \\
\text { cesium removal. This required matching } \\
\text { the Plant's treated waste residence time in } \\
\text { the resin and not the full-scale flow rate. } \\
\text { Therefore, this objective could not be met. }\end{array}$ \\
\hline $\begin{array}{l}\text { 3. Demonstrate ion exchange column } \\
\text { operating parameters using the } \\
\text { recommended ion exchange resin. }\end{array}$ & $\mathrm{N}$ & $\begin{array}{l}\text { See the answer to CIX Test Objective } \\
\text { No. } 2 .\end{array}$ \\
\hline $\begin{array}{l}\text { 4. Determine the chemical and physical } \\
\text { properties, which will be indicated in } \\
\text { the test plan, of all ion exchange } \\
\text { effluents. }\end{array}$ & $\mathrm{N}$ & $\begin{array}{l}\text { Only Lead and Lag column effluents were } \\
\text { measured for Cs concentrations to } \\
\text { minimize cost since Campaign II had } \\
\text { already made the complete set of } \\
\text { measurements. The WTP approved list of } \\
\text { measurements are shown in the Sample } \\
\text { Plan given as Appendix D. }\end{array}$ \\
\hline $\begin{array}{l}\text { 5. Compare the results developed on } \\
\text { the pilot scale with results developed } \\
\text { by experiments conducted at the bench } \\
\text { scale to assess the effect of recycles. }\end{array}$ & $\mathrm{Y}$ & $\begin{array}{l}\text { Limited bench-scale ion exchange tests are } \\
\text { compared to SIPP results in Section } 2.11 \text {. }\end{array}$ \\
\hline $\begin{array}{l}\text { 6. Determine the chemical and physical } \\
\text { properties, which will be indicated in } \\
\text { the test plan, of blended ion exchange } \\
\text { secondary wastes (pre-elution rinse and } \\
\text { spent regenerant) that are recycled back } \\
\text { to the waste feed evaporation system. }\end{array}$ & $\mathrm{N}$ & $\begin{array}{l}\text { See response to Item No. } 4 \text { of CIX Test } \\
\text { Objectives. }\end{array}$ \\
\hline $\begin{array}{l}\text { 7. Determine the chemical and physical } \\
\text { properties of ion exchange eluates, } \\
\text { which will be indicated in the test plan. }\end{array}$ & $\mathrm{N}$ & $\begin{array}{l}\text { See response to Item No. } 4 \text { of CIX Test } \\
\text { Objectives. }\end{array}$ \\
\hline $\begin{array}{l}\text { 8. Evaluate the potential effects of the } \\
\text { evaporator antifoam reagent on ion } \\
\text { exchange performance. }\end{array}$ & $\mathrm{Y}$ & $\begin{array}{l}\text { The effect on ion exchanger seemed to be } \\
\text { insignificant, based on good cesium } \\
\text { removal. }\end{array}$ \\
\hline
\end{tabular}


WSRC-TR-2005-00105, REVISION 0

SRNL-RPP-2005-00012, REVISION 0

\subsection{TEST EXCEPTIONS}

\begin{tabular}{|c|c|}
\hline List Test Exceptions & Describe Test Exceptions \\
\hline $\begin{array}{l}\text { 1. 24590-WTP-TEF-RT-02-025, Rev. 0, } 20 \text { May } \\
2002\end{array}$ & $\begin{array}{l}\text { See the description for Test Exception No. 24590- } \\
\text { WTP-TEF-RT-03-069, which is No. } 7 \text { in this list. }\end{array}$ \\
\hline $\begin{array}{l}\text { 2. 24590-WTP-TEF-RT-02-046, Rev. 0, } 01 \text { Aug. } \\
2002\end{array}$ & $\begin{array}{l}\text { See the description for Test Exception No. 24590- } \\
\text { WTP-TEF-RT-03-069, which is No. } 7 \text { in this list. }\end{array}$ \\
\hline $\begin{array}{l}\text { 3. 24590-WTP-TEF-RT-03-004, Rev. 0, } 07 \text { Feb. } \\
2003\end{array}$ & $\begin{array}{l}\text { See the description for Test Exception No. 24590- } \\
\text { WTP-TEF-RT-03-069, which is No. } 7 \text { in this list. }\end{array}$ \\
\hline $\begin{array}{l}\text { 4. 24590-WTP-TEF-RT-03-037, Rev. 0, } 30 \text { Jul. } \\
2003\end{array}$ & $\begin{array}{l}\text { See the description for Test Exception No. 24590- } \\
\text { WTP-TEF-RT-03-069, which is No. } 7 \text { in this list. }\end{array}$ \\
\hline $\begin{array}{l}\text { 5. 24590-WTP-TEF-RT-03-037, Rev. 1, } 17 \text { Sept. } \\
2003\end{array}$ & $\begin{array}{l}\text { See the description for Test Exception No. 24590- } \\
\text { WTP-TEF-RT-03-069, which is No. } 7 \text { in this list. }\end{array}$ \\
\hline $\begin{array}{l}\text { 6. 24590-WTP-TEF-RT-03-054, Rev. 0, } 17 \text { Sept. } \\
2003\end{array}$ & $\begin{array}{l}\text { See the description for Test Exception No. 24590- } \\
\text { WTP-TEF-RT-03-069, which is No. } 7 \text { in this list. }\end{array}$ \\
\hline $\begin{array}{l}\text { 7. 24590-WTP-TEF-RT-03-069, Rev. 0, } 14 \text { Nov. } \\
2003\end{array}$ & $\begin{array}{l}\text { As of the completion of Campaign II there was no } \\
\text { test exception to the SIPP task plan (Duignan et } \\
\text { al., 2004). All of the listed test exceptions, } 1 \\
\text { though 7, affect only a task plan dealing solely } \\
\text { with ion exchange operation (Steimke, 2002). For } \\
\text { completeness, all the test exceptions were listed } \\
\text { but only this seventh test exception impacts SIPP } \\
\text { test. As with all the other pilot unit operations, the } \\
\text { pilot-scale CIX was to operate in a fashion to } \\
\text { demonstrate the rate of glass production. } \\
\text { However, RPP-WTP thought it was more } \\
\text { important to demonstrate cesium removal } \\
\text { efficiency. Therefore, the CIX was scaled to } \\
\text { match LAW residence time only. The pilot unit } \\
\text { was not used at a LAW flow rate that would } \\
\text { demonstrate glass production rates. This seventh } \\
\text { test exception describes how the pilot scale CIX } \\
\text { columns would operate to demonstrate cesium } \\
\text { removal. That removal efficiency is what is } \\
\text { discussed herein. }\end{array}$ \\
\hline
\end{tabular}


WSRC-TR-2005-00105, REVISION 0

SRNL-RPP-2005-00012, REVISION 0

\subsection{RESULTS AND PERFORMANCE AGAINST SUCCESS CRITERIA}

\begin{tabular}{|c|c|}
\hline List Success Criteria & $\begin{array}{c}\text { Explain How the Tests Did or Did Not Meet the } \\
\text { Success Criteria }\end{array}$ \\
\hline $\begin{array}{l}\text { 1. The integrated operation of the } \\
\text { pretreatment process produces } \\
\text { vitrification feed streams similar in } \\
\text { chemical composition to those } \\
\text { produced in bench scale experiments. }\end{array}$ & $\begin{array}{l}\text { Bench scale experiments were not part of the SIPP scope. } \\
\text { However, the SIPP task did produce both LAW and HLW feed } \\
\text { streams with simulated waste and recycle streams that were } \\
\text { processed in the same manner expected for the RPP-WTP } \\
\text { Pretreatment Facility. The chemical composition obtained is } \\
\text { that which is expected for the full-scale facility and therefore } \\
\text { this criterion was met. }\end{array}$ \\
\hline $\begin{array}{l}\text { 2. The integrated pretreatment process } \\
\text { demonstrates a scaled throughput of } 80 \\
\text { MTG/d of LAW and } 5.4 \text { MTG/d of } \\
\text { HLW. }\end{array}$ & $\begin{array}{l}\text { This success criterion was partially met in that both the pilot } \\
\text { FEP and TLP operated at, or above the evaporation rate ( } 0.395 \\
\text { gpm) necessary to meet the stated glass production rates. The } \\
\text { pilot UFP realized a dewatering filter flux rate of } 0.013 \\
\text { gpm/ft }{ }^{2} \text {. This value is below the rate needed to meet the glass } \\
\text { production rates, but is representative of rate obtained from } \\
\text { bench scale test with actual waste. The pilot CIX rate } \\
\text { purposely did not meet the full-scale plant rate as explained in } \\
\text { Test Exception No } 7 \text { above. }\end{array}$ \\
\hline
\end{tabular}

\subsection{QUALITY REQUIREMENTS}

This work was conducted in accordance with the RPP-WTP QA requirements specified for work conducted by SRNL as identified in DOE IWO MOSRLE60. SRNL has provided matrices to WTP, demonstrating compliance of the SRNL QA program with the requirements specified by WTP. Specific information regarding the compliance of the SRNL QA program with RW-0333P, Revision 10, NQA-1 1989, Part 1, Basic and Supplementary Requirements and NQA-2a 1990, Subpart 2.7 is contained in these matrices. 
WSRC-TR-2005-00105, REVISION 0

SRNL-RPP-2005-00012, REVISION 0

\subsection{R\&T TEST CONDITIONS}

\begin{tabular}{|c|c|}
\hline List R\&T Test Conditions & Were Test Conditions Followed? \\
\hline $\begin{array}{l}\text { 1. SRNL shall prepare a specific test plan containing } \\
\text { detailed information needed to implement (the) test } \\
\text { specification. The test plan shall also include, as } \\
\text { appropriate, references to documents that drive test } \\
\text { documentation requirements to assure data integrity } \\
\text { and reproducibility. This would include such items as } \\
\text { detailed test procedures, instrument calibration, } \\
\text { methods of data gathering, etc. The test plan shall } \\
\text { indicate what methodologies will be employed to } \\
\text { verify or validate data produced during testing } \\
\text { activities. } \\
\text { A draft test plan shall be submitted to WTP R \& T for } \\
\text { review and comment. Comments labeled } \\
\text { "Mandatory" by the reviewer require a disposition. } \\
\text { The changes in the test plan shall be provided } \\
\text { electronically and clearly identified by } \\
\text { redline/strikeout or highlighting to facilitate the } \\
\text { review process. } \\
\text { The final test plan must be approved by a WTP R \& T } \\
\text { representative prior to performing work. }\end{array}$ & $\begin{array}{l}\text { As required, a test plan was developed (Duignan, } \\
\text { 2003c) to meet the test specification. All } \\
\text { analytes that could not meet the listed criterion } \\
\text { were indicated in the task plan and approved } \\
\text { before testing began. Documents that were } \\
\text { available before and when the test plan was } \\
\text { completed were referenced therein, but those } \\
\text { that were not, e.g. procedures, calibration, are } \\
\text { referenced in this final report. The test plan was } \\
\text { submitted to WTP R\&T for review and } \\
\text { approval. All reviewers' comments were } \\
\text { addressed and dispositioned, after which the } \\
\text { report was approved by WTP R\&T prior to } \\
\text { testing, thus it became the principal document to } \\
\text { carry out testing. This was part of the meeting } \\
\text { content held in Hanford in October } 2003 \\
\text { (Appendix A) }\end{array}$ \\
\hline $\begin{array}{l}\text { Prior to initiation of the test campaigns, the system } \\
\text { will be simulated using the OLI Environmental } \\
\text { Simulation Program (ESP) models developed in } \\
\text { previous completed evaporation tasks. The expanded } \\
\text { waste feed evaporation model, which includes } \\
\text { ultrafiltration and recycles, along with the treated feed } \\
\text { evaporation model will simulate the pilot operations } \\
\text { under minimum ( } 48 / 3.2 \text { MTG/d) and expected (80/5.4 } \\
\text { MTG/d) operating conditions. Output of the models } \\
\text { will then be compared to above referenced ACM } \\
\text { model runs and the data obtained during the pilot } \\
\text { operations. Results of this modeling effort will be } \\
\text { discussed with WTP R\&T personnel and approval of } \\
\text { the path forward obtained PRIOR TO START of } \\
\text { testing. }\end{array}$ & $\begin{array}{l}\text { As required, SRNL developed an OLI model } \\
\text { based on several sources, included ACM and } \\
\text { sources for ACM prior to any SIPP testing. The } \\
\text { modeling results were compared to ACM results } \\
\text { by both SRNL and RPP-WTP personnel. The } \\
\text { OLI results were scaled to SIPP operation to } \\
\text { assist in completing a test plan and to determine } \\
\text { waste stream and recycle stream volumetric } \\
\text { needs. The model was also used to determine } \\
\text { scaled flowrates of each of the four unit } \\
\text { operation in order to know what operation rate } \\
\text { were necessary to meet full-scale plant glass } \\
\text { production rates of } 80 \text { MTG/d from LAW and } \\
\text { 5.4 MTG/d from HLW. Those model results are } \\
\text { discussed in Duignan et al., (2004b), Barnes } \\
\text { (2004), and in Appendix B. }\end{array}$ \\
\hline
\end{tabular}


WSRC-TR-2005-00105, REVISION 0 SRNL-RPP-2005-00012, REVISION 0

\begin{tabular}{|c|c|}
\hline List R\&T Test Conditions & Were Test Conditions Followed? \\
\hline $\begin{array}{l}\text { 3. The LAW and HLW SBS recycles will be } \\
\text { simulants generated from VSL/Duratek pilot melter } \\
\text { testing. }\end{array}$ & $\begin{array}{l}\text { Both submerged bed scrubber (SBS) recycle } \\
\text { streams were obtained from VSL and used in the } \\
\text { testing of Campaigns II, III, and IV. }\end{array}$ \\
\hline $\begin{array}{l}\text { 4. The simulant waste feeds will be based on } \\
\text { approved formulations developed under the WTP's } \\
\text { waste simulant program participated in by SRNL. }\end{array}$ & $\begin{array}{l}\text { The simulated waste feed AY102 was developed } \\
\text { and validated by SRNL (Zamecnik, et al., 2004). } \\
\text { It was filter tested at the bench scale against an } \\
\text { actual waste sample. The formulation was } \\
\text { approved by WTP R\&T before its use. The } \\
\text { formulation for the simulated feed AP101 was } \\
\text { developed by Battelle (Russell et al., 2003) and } \\
\text { approved by WTP R\&T. All other waste } \\
\text { streams used for SIPP are either recycle streams } \\
\text { or derived from recycle streams. }\end{array}$ \\
\hline $\begin{array}{l}\text { 5. Generation of initial ultrafiltration recycles will be } \\
\text { accomplished by performing the work specified in } \\
\text { AY102 Simulant Pilot Scale Ultrafiltration and } \\
\text { Washing Test, 24590-PTF-TSP-RT-02-015 (Townson, } \\
\text { 2002). }\end{array}$ & $\begin{array}{l}\text { The production of these recycle streams was } \\
\text { done during Campaign I (Duignan et al., 2004a } \\
\text { or Appendix I-1). }\end{array}$ \\
\hline $\begin{array}{l}\text { 6. An exception to the test matrix defined in (the } \\
\text { Campaign I test) specification } 24590 \text {-PTF-TSP-RT- } \\
02-015 \text { will be to not perform certain tests specified in } \\
\text { Appendix A, Table } 1.1 \text { (of that specification). The } \\
\text { tests that will not be performed are (listed in the SIPP } \\
\text { Test Specification, Longwell, 2003.) }\end{array}$ & $\begin{array}{l}\text { This condition only applied to Campaign I } \\
\text { operation, which had pilot-scale UFP test runs at } \\
\text { steady-state UDS concentration. It was not } \\
\text { applicable to subsequent campaigns. }\end{array}$ \\
\hline
\end{tabular}

\subsection{SIMULANT USE}

Many simulated waste streams were either used or produced during SIPP testing. Those include Hanford tank farm stored wastes, i.e., tank 241-AY-102/C-106, herein referred to as AY102, and tank 241-AP-101, herein referred to as AP101. During the process of making glass many recycle streams will be generated and processed. Those include streams from the: HLW and LAW Submerged Bed Scrubbers, Vessel Vent System, HLW Glass Canister Decontamination process, FEP and TLP condensate solutions, UFP wash permeate, UFP rinse solutions, UFP acid cleaning solutions, UFP loop flush solutions, CIX caustic LAW displacement solutions, CIX post elution rinse solutions, and CIX caustic regeneration solutions. These streams are discussed throughout this report.

\subsection{DISCREPANCIES AND FOLLOW-ON TESTS}

No discrepancies occurred during testing and no follow-on work is currently planned. This task involved four Campaigns and is referred to as the Semi-Integrated Pilot Plant (SIPP) task (Duignan, 2003c). 
WSRC-TR-2005-00105, REVISION 0

SRNL-RPP-2005-00012, REVISION 0

This page intentionally left blank. 
WSRC-TR-2005-00105, REVISION 0

SRNL-RPP-2005-00012, REVISION 0

\subsection{DISCUSSION}

\subsection{INTRODUCTION}

Bechtel National, Inc. (BNI) has been contracted by the Department of Energy (DOE) to design a Waste Treatment and Immobilization Plant (WTP) to stabilize liquid radioactive wastes that are stored at the Hanford Site as part of the River Protection Project (RPP). Because of its experience with radioactive waste stabilization, the Savannah River National Laboratory (SRNL) of the Westinghouse Savannah River Company (WSRC) is working with BNI and Washington Group International (WGI), to help design and test certain parts of the waste treatment facility. A major part of the waste stabilization process is the pretreatment of the wastes before vitrification, which will occur in the Pretreatment Facility where both HLW and LAW product streams will be handled. SRNL has constructed a Semi-Integrated Pilot Plant (SIPP), which imitates important unit operations of the pretreatment process. However, since the actual pilot-scale vitrification is not handled by SRNL, the pilot plant is referred to as semi integrated. After SIPP pretreats the waste streams into vitrification feed streams, they will be shipped to the Vitreous State Laboratory (VSL) of Catholic University of America to complete the entire process.

The subject of this final report is the testing of the four Campaigns that comprised the overall task. That testing involved all of the four principal unit operations that comprise the RPPWTP Pretreatment Facility, i.e., Waste Feed Evaporation Process (FEP), Ultrafiltration Process (UFP), Cesium Ion Exchange Process (CIX), and Treated LAW Evaporation Process (TLP). The operation and results of each of these unit operations are explained herein.

This technical baseline research and development work was initiated by a WTP test specification (Longwell, 2003) to test simulated non-radioactive wastes in pilot scale pretreatment unit operations in the Engineering Development Laboratory (EDL) of SRNL. While this report discusses the results of all four SIPP campaigns it principally deals with the fourth of a four-campaign task, since previous campaigns are discussed at length in the interim reports (Appendix I). Campaign IV differs from the Campaigns II and III in that a new cross-flow filter manufacturer was chosen by Bechtel while the task was ongoing. Because of the need to better quantify the performance of the new filter, Campaign IV was limited to only the UFP unit operation, as well as the FEP unit operation, which was needed to create feed for the UFP. The CIX and TLP unit operations were not used for Campaign IV.

The task first phase, Campaign I, was at one time a completely separate task (Townson, 2002) to filter a specific waste. Subsequently, that first campaign was incorporated into the SIPP task to eliminate duplication of effort and to produce several important recycle streams. While, Campaign I was limited to a single unit operation, ultrafiltration, and a single waste stream, i.e., AY102, the process of operating the filter unit allowed the production of many of the important recycle streams needed to carry out the more comprehensive phases, i.e., Campaigns II, III, and IV. After dewatering the single waste stream, the slurry was washed to produce one recycle stream. Then the filter unit was flushed, cleaned, and rinsed, thus 
creating other important recycle streams. Besides producing recycles, the Campaign I singlewaste filter results were then used to compare with filter results obtained after mixing that waste stream with others, i.e., AP101 and the many recycle streams. A further benefit to limiting Campaign I to a single waste stream was that the filter results could be compared to the pilot filter tests done with other single waste streams, (see Duignan, 2000a, Duignan, 2000b, Duignan, 2003a).

In summary, this report discusses not only Campaign IV results, but compares those results to the other unit operations' results done during Campaigns I, II, and III. There were many individuals involved and the information for each section was provided primarily by each of the principal investigators. They were:

Simulant development and sample analyses John R. Zamecnik

Simulant and sample handling - Michael R. Williams

Modeling - C. Dan Barnes (done before any testing began)

Consulting and managing - T. Bond Calloway

Technical Lead and overall reporting - M. R. Duignan

FEP operation - Zafar H. Qureshi

UFP operation - Mark D. Fowley

CIX operation - John L. Steimke

TLP operation - Duane J. Adamson

A summary of EDL task activities is as follows:

WGI Test Specification for UF Test Received - December 2002

WGI Test Specification for SIPP Received - June 2003

WSRC Task Technical \& Quality Assurance Plan Approved - August 2003

Test Procedure Approved - October 2003

Received AY102/C106 Simulant - January 2004

Campaign I - 26 January to 12 February 2004

Campaign II - 12 April to 14 May 2004

Campaign III - 7 June to 9 July 2004

Campaign IV - 2 to 6 August 2004 (Pretreatment of GKN filter)

Campaign IV - 9 to 26 August 2004 (only SIPP FEP and UFP operations were tested)

\subsection{THE PLAN}

Initial planning for the Semi-Integrated Pilot Plant (SIPP) was to decide what to study. One of the first decisions was to limit the task to the RPP-WTP Pretreatment Facility since pilot melter facilities already existed at another site ${ }^{\dagger}$. This is the reason that this task is referred to as "Semi Integrated," i.e., to indicate that vitrification is not part of the pilot operations at the Savannah River National Laboratory (SRNL). The next step was to decide which simulant(s) to test. Since the waste types at Hanford were broadly classified as envelopes A, B, C, and D (Eibling and Nash, 2001), therefore three campaigns were planned, one for each envelope,

\footnotetext{
${ }^{\dagger}$ The Vitreous State Laboratory at Catholic University of America in Washington, DC.
} 
except D. Envelope D is basically a sludge, which would not be processed alone, but would be mixed with some of the other envelopes. As such, each campaign would process a chosen simulated waste through the principal unit processes of the Pretreatment Facility. Those units are the Feed (Waste) Evaporator Process (FEP), Ultrafiltration Process (UFP), Cesium Ion Exchange (Process) (CIX), the Treated LAW Evaporation Process (TLP), and for Envelope C-types wastes, the Strontium-Transuranics (Sr/TRU) Precipitation Process.

Having a task that would test a range of possible waste types was a good comprehensive approach; however, to obtain meaningful operational data for the plant it was not just a matter of processing each of the different wastes once through each of the unit process. Steady state operation of the Pretreatment Facility would also have to include all the recycle waste streams. That is, in the process of separating the wastes into necessary components for further processing, other waste streams are produced. A few of those streams are: waste washings, filter cleaning and rinsing solutions, CIX regeneration solutions, HLW canister decontamination solutions, vessel vent solutions, submerged bed scrubber solutions from both the HLW and LAW vitrification facilities, etc. In steady state these recycle streams will be recycled to different locations in the Pretreatment Facility. Those streams need to be included to simulate the actual plant operation. This means that each of the campaigns would have to run many times until the effects of the recycle streams stabilized so that steady state operation is realized. After reevaluating the task needs, available funds and time, it was clear that the scope had to be reduced. It would be limited to processing a key waste stream with all the recycle streams and then processed enough times that steady-state operation would be attained. Focus was then directed to the wastes that the plant would use for commissioning, i.e., 241-AY-102/C-106, here referred to as AY102, and 241-AP-101, herein referred to as AP101. The first waste is the result of two waste tanks that have already been mixed together, AY-102 and C-106 and is classified as an Envelope A/D mixture. The second waste, AP101, is an Envelope A waste. With this waste mixture, all of the principal WTP Pretreatment Processes would be tested except the Sr/TRU Process because it did not include an Envelope C-type waste.

The SIPP task was dedicated to perform integrated processing of one selected waste stream combination that included recycle streams produced during the processing of waste through the four pilot-scale unit operations. As such, the testing of an "integrated" process is integrated in the sense of capturing the salient chemical processes, that is, it is important to note that the SIPP is not integrated with respect to time. The operation of the Pretreatment Facility is complex in both chemistry and time, but the many interconnected systems (e.g., piping, ventilation, etc.) and supporting processes (e.g., transfer tanks, pumps, water supply, etc.) that will exist in the plant to allow the four main unit operation to function were not included in SIPP because of schedule and expense. Just the scaling issues alone, for an entire Pretreatment facility, would have been an almost impossible task to accomplish. In short, running the four principal unit operations with simulated waste and recycle streams as chemically close as to what will result in the plant was accepted as the most efficient use of the time and money available. Time of operation was limited to the operation of each individual unit operation only, and not to their temporal interdependence on the supporting Pretreatment Facility operations, e.g. vessel fillings, waste testing and adjusting, waste transfers, line flushings, etc. However, prototypical hold times (see Appendix C) between 
the four unit operations were included to provide sufficient time for precipitation of slow forming salts, e.g., Sodium Aluminosilicate and Sodium Fluorophosphates [specifically: $\left.\mathrm{NaF}_{7}\left(\mathrm{PO}_{4}\right)_{2}\right]$.

To reiterate, the SIPP task is the operation of four individual and separate pilot-scale unit operations, each successively processing a single waste stream combination, which is chemically connected through the feedback of recycle streams. The plan is described in the RPP-WTP test specification (Longwell, 2003), in the WSRC technical task plan (Duignan, 2003c), and was discussed amongst all the stakeholders before any testing began (see Appendix A). The task's original series of three campaigns was maintained, but instead of processing different waste types, only one type would be used. Each successive campaign introduced recycle streams produced from the preceding stream. To feed the initial campaign an extra campaign was added. This addition was actually an already planned ultrafiltration test (Townson, 2002). That test became known as Campaign I and it only processed a simulant of AY102. In this way the filtration results of Campaign I served three purposes: (1.) To generate a filtration data set for a single-waste type that could be compared to past single-waste filtration tests, (2.) to become a baseline for filtration of AY102 in order to determine how additions of AP101 and recycles to that waste effect filtration, and (3.) to produce a series of recycle streams to feed to Campaign II. The last reason was very important to SIPP because the UFP produces several important recycle streams, i.e., slurry washing permeate, sludge loop flushing, loop acid cleaning, and loop caustic rinsings. Campaign I results were published in Duignan et al., 2004a or Appendix I-1, Campaign II results were published in Duignan et al., 2004b or Appendix I-2, Campaign III results were published in Duignan et al., 2004c or Appendix I-3, and finally Campaign IV results are included in this report. As summary of all the campaigns is as follows:

Campaign I UFP only with simulant of (AY102)

Campaign II All four unit operations with simulant of (AY102) + (AP101) + recycle streams (normally the CIX also has recycle streams but since Campaign I did not test the CIX they were not included in Campaign II).

Campaign III All four unit operations with simulant of $\left(\right.$ AY102) $+($ AP101 $)+2^{\text {nd }}$ generation recycle streams (but $1^{\text {st }}$ generation CIX recycle streams). Campaign IV ${ }^{\dagger \ddagger}$ Two unit operations (FEP and UFP) with simulant of (AY102) + (AP101) + $3^{\text {rd }}$ generation recycle streams (but $2^{\text {nd }}$ generation CIX recycle streams).

As shown in the preceding Section 1.1, on “Test Objectives," there were many objectives; the details of which will not be repeated here, but will be discussed in the appropriate later sections. However, the major goals were to better understand how each of the four pilotscale unit operations process the waste ${ }^{*}$, to study how the processes change with successively

\footnotetext{
† This report deals specifically with Campaign IV results, but generally covers all the SIPP work.

‡ As of May 2004 RPP-WTP informed SRNL to change the scope of Campaign IV to test a new filter made by GKN, which was chosen instead of the Mott for the RPP-WTP Pretreatment Facility Ultrafiltration Process. The number of the unit operations was reduced from four to two for Campaign IV, those being the pilot-scale FEP and UFP so that GKN performance could be compared to the Mott.

* For example, the recycle streams for this campaign, Campaign IV, contained chemicals carried over from Campaigns I, II and III.
} 
WSRC-TR-2005-00105, REVISION 0

SRNL-RPP-2005-00012, REVISION 0

complex recycle streams, and to produce throughput data to benchmark computer codes being developed by Bechtel and others, and to predict and plan for, plant operation.

Figure 1 is a schematic of the overall SIPP operation. It is broken into the four unit operations and indicates both the salient chemical and time steps. The operations are listed in a chronological order, at least with respect to the SIPP test, with the top (FEP) section being first. Of course the plant will be much more complex. A very short summary of the overall operation is as follows:

1. FEP evaporates a combined mixture of recycles.

2. UFP filters a combined mixture of the FEP concentrated product (called Bottoms) and waste from the tank farm until the mixture reaches a target concentration. The UFP produces two streams: the permeate which is sent to the CIX for LAW processing, and the concentrated slurry. That slurry is then washed to remove many of the soluble solids after which it is sent to the HLW melter facility. The slurry washing and subsequent UFP filter cleaning produces a series of recycle streams that are sent back to the FEP for processing.

3. CIX takes the permeate from the UFP and removes most of the soluble, but radioactive, cesium. When the resin in the lead CIX column is regenerated, recycle streams are created which are either sent back to the FEP or forward to the TLP for processing.

4. TLP takes the CIX product, which still has very low levels of radiation, and evaporates most of the water before it is sent to the LAW melter facility.

The evaporation of the many combined recycle streams in the FEP will be operated in a continuous sense, in that after it fills the UFP preparation tank for one filter train (there will be two separate filter trains), it will feed the preparation tank for the other filter train. However, the FEP must ultimately be a batch operation because it will be receiving waste from recycle tanks (PWD = Plant Wash and Disposal System) that will be batched through the FEP only after the feed is adjusted for $\mathrm{pH}$ and tested for acceptance. 
WSRC-TR-2005-00105, REVISION 0

SRNL-RPP-2005-00012, REVISION 0

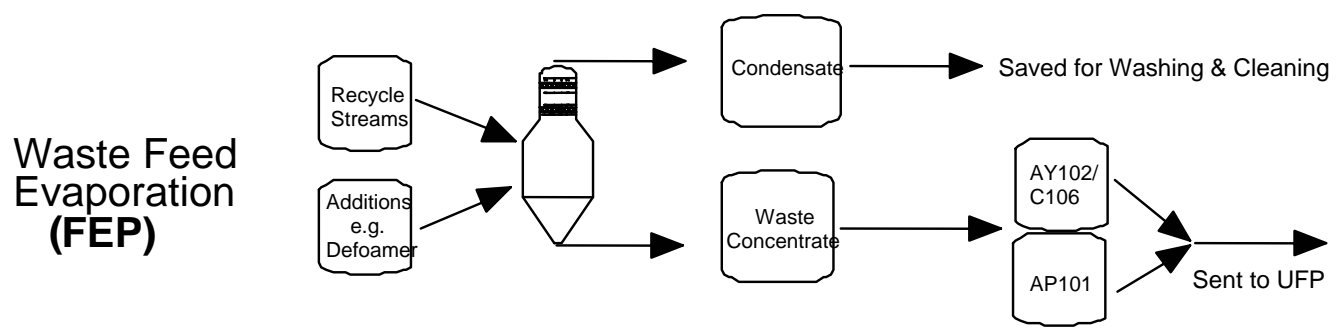

Recycle Streams: HLW SBS, UFP Wash Water, UFP Cleaning Acid, UFP Rinses \& Flushes, HLW Canister Decon, Vessel Vent, and CIX recycles

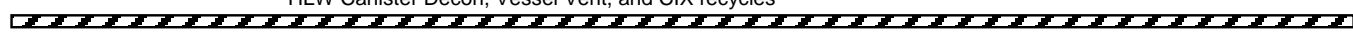

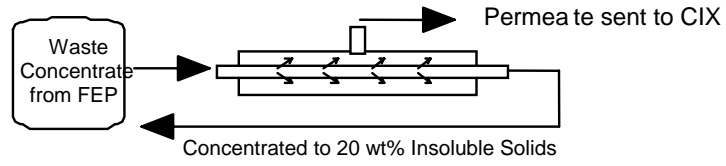

Cross-flow

Ultrafiltration waste Washing

(UFP)

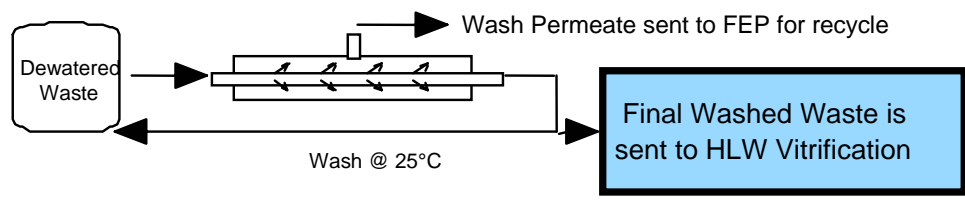

Filter Cleaning

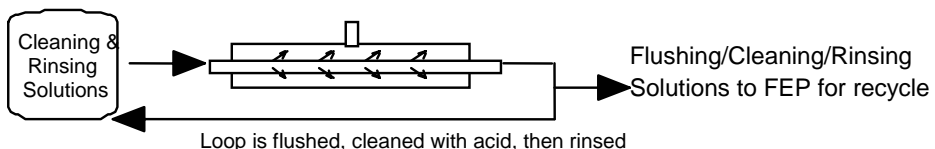

Loop is flushed, cleaned with acid, then rinsed
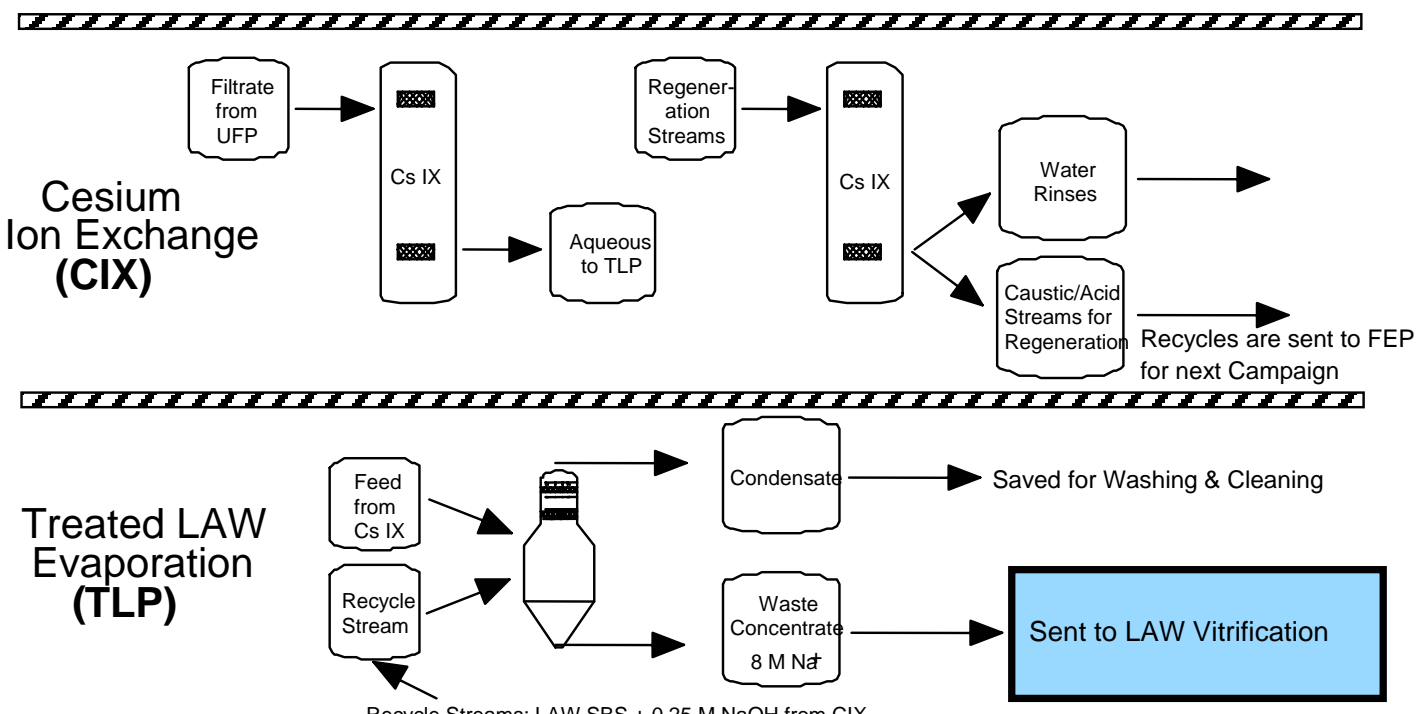

Recycle Streams: LAW SBS $+0.25 \mathrm{M} \mathrm{NaOH}$ from CIX

Figure 1. Schematic of the overall task operation taken from the task plan 
When the UFP feed and preparation tanks are full and ready for processing, the filtration can commence. This process is also batch, but the feed tank must be continuously fed with new feed as dewatering is ongoing. The feed tank will have a base volume of 21,550 gallons into which approximately 1000 gallons will be transferred from the preparation tank. Each time the feed tank level drops to 21,550 gallons another batch of 1000 gallons will be transferred until the dewatered slurry reaches its target solids concentration. For this task, the target was $20 \mathrm{wt} \%$ undissolved solids (UDS). This dewatering process is shown in the UFP section of Figure 1 as the top filtration loop. The middle loop illustrates the washing and leaching of the final concentrated slurry batch. (Note: For SIPP, the leaching process was not performed as requested by RPP-WTP (Calloway, 2004)). After the concentrated waste is washed, it is then joined with the concentrated CIX eluate before being sent to the HLW melter for further processing, but for the SIPP, the washed slurry is simply drummed to be shipped for processing in a pilot HLW melter. With the loop de-inventoried, the final loop shows that the UFP undergoes caustic rinsing and acid cleaning, in preparation for the next batch.

For the plant, the CIX does not necessarily have to wait for the entire filtration process to end. There are several filter permeate tanks to feed the CIX and they receive permeate from each of the two filter trains. However, for SIPP the CIX can begin after a fixed amount of permeate is produced from the UFP, which is approximately 200 bed volumes. A bed volume is the volume of space occupied in a CIX column by the resin, including the interstitial spaces among the resin particles. The plant will process a minimum of 100 bed volumes ( 30,000 gallons) of permeate before an CIX column is regenerated, which completes one CIX cycle; each of the SIPP campaigns contained two CIX cycles. Originally, the SIPP CIX was to demonstrate the design throughput rate of treated waste, like the other pilot unit operations, but it was thought more important ${ }^{\dagger}$ to use it for chemical testing to show cesium removal efficiency. This meant the important parameter would be a prototypic residence time for permeate in the resin, or 3 bed volumes per hour. For the 6-inch diameter pilot scale column, this resulted in a very low flow rate $(\sim 0.031 \mathrm{gpm})$, which is more than two orders of magnitude slower than the plant design flowrate of 15 gpm. SIPP used 200 bed volumes per cycle (approximately 400 bed volumes per campaign) because several past tests had indicated that cesium breakthrough will occur only at higher volumes of feed than the original plant design of 100 bed volumes. It was very important to identify the number of bed volumes of feed processed before cesium breakthrough and subsequent resin regeneration. The third section of Fig. 1 shows two columns because of the two processes that occur within CIX: the first process is the removal of cesium, the second process is a series of steps to regenerate the resin.

The final section of Fig. 1 shows the TLP operation. The CIX product is mostly 5 M solution in sodium, which is diluted to $2 \mathrm{M}$ in sodium when the product is mixed with recycle streams to make TLP feed. Then the TLP concentrates the feed sodium up to $8 \mathrm{M}$. This is the last step before the treated LAW stream is sent to the LAW melter facility. Along the way, several recycle streams are created in the UFP and the CIX, as well as from other sources, like the submerged bed scrubber quencher streams from both the HLW and LAW melter

\footnotetext{
${ }^{\dagger}$ Test Exception No. 24590-WTP-TEF-RT-03-069, Rev. 0, 14 Nov. 2003
} 
WSRC-TR-2005-00105, REVISION 0

SRNL-RPP-2005-00012, REVISION 0

facilities. These recycle streams are either fed back to the FEP evaporator or forward to the TLP evaporator in the process, which then repeat. For the SIPP, this repeating results in the subsequent campaigns.

\subsection{THE COMPUTATIONAL MODEL}

A full explanation of the computation assistance obtained at the beginning of SIPP has already been described in detail elsewhere (Duignan et al., 2004b or Appendix I-2, Barnes, 2004, and Appendix B) and will not be repeated here. However, it is important to know how the computational modeling was used for this task to understand its role for Campaign IV. The model served two purposes: 1 . To determine how much slurry to use and, 2 . To estimate the mix ratio of feeds (simulated wastes and recycle streams) for each of the unit operations. The Test Specification (Longwell, 2003) requested that the WTP Aspen Custom Modeler (ACM) Model be used to obtain the bases for the combined feed streams, i.e., the feed mix ratios. With the feed stream obtained in this manner an OLI Environmental Simulation Program (OLI/ESP) was used to determine the steady-state operation for each unit operation in each of the campaigns.

In concept, the operation of the SIPP is straightforward, but determining the exact simulant needs was quite complicated because of the differing scales of each pilot-scale unit operation and because of the chemical interaction among the recycle streams from all the campaigns. A very important part in developing the test matrix of the task plan was knowing how much simulant to use and how should all the different waste and recycle streams be mixed together. That need was satisfied by computationally predicting SIPP streams for all four campaigns. As an added benefit, once the model was made, it would be possible to evaluate changes in simulant volumes and mixing ratios to determine if the entire SIPP process comes to steady state. Steady state could be shown, at least chemically, by demonstrating that the products produced in SIPP change insignificantly from one campaign to the next.

Table 1 shows the results of the OLI/ESP model that includes WTP accepted mixture ratios for combined feed streams from the ACM model. The principal value in the entire figure is the initial "batch" size of the UFP feed tank. In Table 1 this batch size for the SIPP is shown in red as 140 liters and is listed as "UF1 SLURRY (batch at $20 \mathrm{wt} \%$ UDS)." This waste volume is very significant because for this pilot-scale task it represents the batch size of HLW sent for HLW vitrification. When this batch is dewatered to some predetermined concentration of undissolved solids (it was $20 \mathrm{wt} \%$ for SIPP), then the permeate produced during dewatering will be the batch size of LAW sent for CIX processing, which subsequently is mixed with recycles streams to be evaporated in the TLP unit operation before finally being sent to LAW vitrification. 
WSRC-TR-2005-00105, REVISION 0

SRNL-RPP-2005-00012, REVISION 0

Table 1. Feeds and products for planning SIPP developed for OLI/ESP computer model*

\begin{tabular}{|c|c|c|c|c|c|c|}
\hline \multirow{2}{*}{$\begin{array}{c}\text { WTP } \\
\text { Process }\end{array}$} & \multirow[b]{2}{*}{ Stream Type } & \multirow[b]{2}{*}{ Stream Name } & \multicolumn{4}{|c|}{ Campaign (in Liters) } \\
\hline & & & 1 & II & III & IV \\
\hline \multirow{6}{*}{ FEP } & Feed & UFP \& CIX RECYCLEs (previous campaign) & N/A & 583.1 & 602.8 & 602.8 \\
\hline & Recycle & HLW (Submerged Bed Scrubber) & $\mathrm{N} / \mathrm{A}$ & 190.6 & 190.6 & 189.9 \\
\hline & Streams & PVP (Vessel Vent System) & $\mathrm{N} / \mathrm{A}$ & 220.7 & 220.7 & 220.0 \\
\hline & & HLW DECON & N/A & 114.6 & 114.7 & 114.3 \\
\hline & Products & FEP CONDENSATE (to be process wash) & $\mathrm{N} / \mathrm{A}$ & 996.0 & 980.6 & 984.9 \\
\hline & Produced & FEP COOLED BOTTOMS (UFP feed) & $\mathrm{N} / \mathrm{A}$ & 114.4 & 150.8 & 144.9 \\
\hline \multirow{2}{*}{$\begin{array}{c}\text { UFP } \\
\text { Feeds }\end{array}$} & Feed & AY102 WASTE (at 12 wt\% UDS) & 253.5 & 252.4 & 242.2 & 241.4 \\
\hline & Feed & AP101 WASTE (at $\left.8 \mathrm{M} \mathrm{Na}^{+}\right)$ & N/A & 671.3 & 674.4 & 672.1 \\
\hline \multirow{10}{*}{ UFP } & Products & UF1 FILTRATE (routed to CIX, then TLP) & 113.5 & 922.3 & 952.2 & 943.7 \\
\hline & Produced & UF1 SLURRY (batch at 20 wt\% UDS) & 140 & 116.8 & 116.4 & 116.1 \\
\hline & $\begin{array}{l}\text { Batch } \\
\text { Waste }\end{array}$ & $\begin{array}{l}\text { FEP COND WASH (0.01 M NaOH) } \\
19 \mathrm{M} \mathrm{NaOH} \text { (to adjust FEP COND) }\end{array}$ & & $\begin{array}{c}281.1 \\
1.8\end{array}$ & $\begin{array}{c}281.5 \\
0.2\end{array}$ & $\begin{array}{c}280.7 \\
0.2\end{array}$ \\
\hline & Washing & TOTAL CAUSTIC WASH & 338.6 & 282.5 & 281.6 & 280.8 \\
\hline & \multirow{4}{*}{$\begin{array}{l}\text { Filter } \\
\text { Rinsing } \\
\quad \& \\
\text { Cleaning }\end{array}$} & $\begin{array}{l}\text { FEP COND ACID (2 M HNO3) } \\
12 \mathrm{M} \text { HNO3 (to adjust FEP COND) }\end{array}$ & & $\begin{array}{l}86.1 \\
17.0\end{array}$ & $\begin{array}{l}85.8 \\
17.0\end{array}$ & $\begin{array}{l}85.6 \\
16.9\end{array}$ \\
\hline & & TOTAL ACID CLEANING & 122.5 & 102.2 & 101.9 & 101.6 \\
\hline & & $\begin{array}{l}\text { FEP CONDENSATE TO CAUSTIC RINSE } \\
19 \mathrm{M} \mathrm{NaOH} \text { (to adjust FEP COND) } \\
\text { TOTAL CAUSTIC RINSE (0.1 M NaOH) }\end{array}$ & & $\begin{array}{c}101.8 \\
0.5 \\
102.2 \\
\end{array}$ & $\begin{array}{c}101.5 \\
0.5 \\
101.8 \\
\end{array}$ & $\begin{array}{c}101.2 \\
0.5 \\
101.5 \\
\end{array}$ \\
\hline & & TOTAL CAUSTIC RINSE (for FEP recycle)) & 117.1 & 97.6 & 97.3 & 97.0 \\
\hline & Final Filter & HLW SLURRY (to HLW melter) & 138.1 & 110.8 & 110.3 & 110.0 \\
\hline & Products & TOTAL FEP RECYCLE (for next campaign) & 583.1 & 602.8 & 604.8 & 602.3 \\
\hline \multirow{4}{*}{ CIX } & Column & CIX 0.1 M NaOH RINSE (for FEP recycle) & $\mathrm{N} / \mathrm{A}$ & 36.9 & 38.1 & 37.8 \\
\hline & Regeneration & CIX DI WATER (for FEP recycle) & & 36.9 & 38.1 & 37.8 \\
\hline & Products & $\mathrm{CIX} 0.25 \mathrm{M} \mathrm{NaOH}$ (for FEP recycle) & $\mathrm{N} / \mathrm{A}$ & 40.0 & 41.3 & 40.9 \\
\hline & Produced & $\mathrm{CIX} 0.25 \mathrm{M} \mathrm{NaOH}$ (for TLP recycle) & $\mathrm{N} / \mathrm{A}$ & 36.9 & 38.1 & 37.8 \\
\hline \multirow[b]{2}{*}{ TLP } & Feeds wFiltrate & LAW SBS (Submerged Bed Scrubber) & $\mathrm{N} / \mathrm{A}$ & 833.0 & 832.9 & 830.2 \\
\hline & $\begin{array}{l}\text { Products } \\
\text { Produced } \\
\end{array}$ & $\begin{array}{l}\text { TLP CONDENSATE (to be process wash) } \\
\text { TLP COOLED BOTTOMS (to LAW melter) }\end{array}$ & $\begin{array}{l}\mathrm{N} / \mathrm{A} \\
\mathrm{N} / \mathrm{A}\end{array}$ & $\begin{array}{c}1214.1 \\
589.3\end{array}$ & $\begin{aligned} 1231.4 \\
603.4\end{aligned}$ & $\begin{array}{c}1224.9 \\
598.1\end{array}$ \\
\hline
\end{tabular}

*Campaign IV did not used the CIX and TLP unit operations due to a scope change

This SIPP batch of 140 liters $^{\dagger}$ for Campaign I was arbitrarily chosen, but once chosen all other volumes were the result of SIPP processing. The choice was made by using existing test facilities, where the pilot UFP could contain 140 liters and still have enough room to feed in more slurry until dewatering is complete. Knowing this batch size, using the feed stream ratios from the ACM model, and the chemical makeup of the base waste slurry, i.e., AY102/C106 and AP101, the OLI/ESP model could then be iterated among all of the campaigns to result in the numbers shown in Table 1 . Note that the batch size of 140 liters, with $20 \mathrm{wt} \%$ UDS, changes from Campaign I to a value of approximately 116 liters (with 20 $\mathrm{wt} \%$ UDS) for all the other campaigns, and as such, the 116-liter volume was taken as equivalent to the full scale UFP batch ${ }^{\ddagger}$ size in those campaigns. The difference between the batch size for Campaign I (140 L) and the subsequent campaigns (116 L) was not well

\footnotetext{
${ }^{\dagger}$ For WTP this batch size was 18,000 gallons when this task began, but it currently stands at 21,550 gallons. In fact, for Campaign I operation, the 18,000-gallons size was valid and used for scaling purposes, but with Campaign II and all subsequent campaigns the latter volume was used.

₹ Same as the above footnote
} 
understood when the task was planned but because the sophisticated OLI/ESP software includes all the steady state chemistry of the processed wastes, which included dozens of compounds. It was assumed that differences in the starting waste slurries resulted in this “discrepancy." That is, Campaign I only processed a single waste, AY102, while the other campaigns included also AP101 and the concentrated recycle streams from the FEP. The inputs for the known waste chemistries, of the starting feeds, were checked many times to verify accuracy, therefore the difference was assumed to be real. In any case, the purpose of the model was to be used as a tool to plan the task and procure needed quantities of simulant, therefore further investigations on the ( 15\%) difference ${ }^{*}$ were not thought necessary. The simulated waste products were procured with enough safety margin to cover any problems if, for instance, shortages occurred during experimentation.

As shown in Figure 1, once the UFP is finished concentrating, the waste is washed with approximately $0.01 \mathrm{M} \mathrm{NaOH}$. The plant will hold the slurry volume constant and feed in two volumes of the caustic solution, each volume approximately equal to the volume of slurry, before sending the waste to the HLW Vitrification Facility. While the plant will hold the UFP volume constant, the model resulted in slightly smaller volumes, see the line in Table 1 entitled, "HLW Slurry (to HLW melter)." due to computational requirements. These are the simulated processed feeds for HLW vitrification. The other highlighted lines in Table 1 are: “AY102 WASTE (at 12 wt\% UDS),” which was the amount of feed AY102 simulant needed; “AP101 WASTE (at 8 M Na),” which was the amount of feed AP101 simulant needed; "UFP \& CIX RECYCLEs (previous campaign)," which was the portion of recycle streams carried over from the previous campaign to be mixed with other recycle streams that did not come directly from one of the four unit operations; finally, the last line contains the volumes of LAW that are treated through all four unit operations and are ready as feed for LAW vitrification.

Another feature included in Table 1, but not obvious, is the feed proportions used for each unit operation, which were supplied by the ACM model. For each of the campaigns, the proportions are slightly different, but not significantly so. The feed for the FEP was made up from only recycle streams and the volumetric proportion of each of the streams used is shown in Table 2.

While the proportions in Table 2 are close to the recycle stream values in the planning table shown as Table 1, they will not be exactly the same because of last minute changes, round off numbers, and uncertainties in some of the streams.

\footnotetext{
* In fact, the actual discrepancy was not understood until after Campaign III was complete and analysis of the SIPP data was underway. The problem turned out to be something that could not be easily resolved a priori because of the OLI/ESP. For the software package to converge on a solution the anion and cation charges of ALL simulant analytes must balance. Unfortunately, the simulated wastes streams contained many unknown complex insoluble solids compounds for which ion charge balances had to be assumed. Those assumptions caused the resulting volumetric difference once a slurry was concentrated to $20 \mathrm{wt} \%$ undissolved solids. This is a clear example of the current limitations of computational models and the need for benchmarking to experimental results of complex interactions.
} 
WSRC-TR-2005-00105, REVISION 0

SRNL-RPP-2005-00012, REVISION 0

Table 2. Percent contribution for each of the recycle streams to the total FEP feed

\begin{tabular}{|c|c|c|c|c|}
\hline \multirow{3}{*}{$\begin{array}{l}\text { List of Recycle Streams Used for } \\
\text { SIPP and Verified by WTP }\end{array}$} & \multicolumn{4}{|c|}{$\begin{array}{l}\text { Percentage of Recycle to FEP } \\
<----- \text { Campaign Number------> }\end{array}$} \\
\hline & $1^{*}$ & $\|^{\star \star}$ & III & IV \\
\hline & Vol. \% & Vol. \% & Vol. \% & Vol. \% \\
\hline (1) HLW SBS & $\mathrm{n} / \mathrm{a}$ & $17.1 \%$ & $17.5 \%$ & $17.4 \%$ \\
\hline (2) Vessel Vent & $\mathrm{n} / \mathrm{a}$ & $19.7 \%$ & $20.3 \%$ & $20.2 \%$ \\
\hline (3) HLW Can. Decon & $\mathrm{n} / \mathrm{a}$ & $10.3 \%$ & $10.5 \%$ & $10.5 \%$ \\
\hline (4) UFP Recycle (0.01 M NaOH) & $\mathrm{n} / \mathrm{a}$ & $30.3 \%$ & $21.7 \%$ & $21.6 \%$ \\
\hline (5) UFP Recycle (0.1 M NaOH) & $\mathrm{n} / \mathrm{a}$ & $10.5 \%$ & $9.0 \%$ & $8.9 \%$ \\
\hline (6) UFP Recycle (2 M HNO3) & $\mathrm{n} / \mathrm{a}$ & $11.0 \%$ & $9.4 \%$ & $9.4 \%$ \\
\hline (7) UFP Recycle (3 Loop Flushes) & $\mathrm{n} / \mathrm{a}$ & $1.2 \%$ & $1.2 \%$ & $1.2 \%$ \\
\hline (8) ClX Recycle $(0.1 \mathrm{M} \mathrm{NaOH})$ & $\mathrm{n} / \mathrm{a}$ & $0.0 \%$ & $3.4 \%$ & $3.5 \%$ \\
\hline (9) CIX Recycle (DI Water) & $\bar{n} / \mathrm{a}$ & $0.0 \%$ & $3.4 \%$ & $3.5 \%$ \\
\hline (10) CIX Recycle (0.25 M NaOH) & $\mathrm{n} / \mathrm{a}$ & $0.0 \%$ & $3.7 \%$ & $3.8 \%$ \\
\hline Total Feed to the FEP & $\mathrm{n} / \mathrm{a}$ & $100 \%$ & $100 \%$ & $100 \%$ \\
\hline $\begin{array}{l}\text { Notes: } \\
\text { Source of Streams: (1) was made fre } \\
\text { location: VSI } \\
\text { (2) and (3) were } \\
\text { (4) to (10) were } \\
\text { * Campaign I only used a single sil } \\
\text { recycles in a single unit operatior } \\
\text { ** Since Campaign I was only a filte } \\
\text { recycle streams were produced } \\
\text { by the RPP-WTP Test Specifica } \\
\text { *** VSL = Vitreous State Lab. of Ca }\end{array}$ & $\begin{array}{l}\text { nade for } \\
\text { roducts } \\
\text { ulated } \\
\text { i.e., UF } \\
\text { operati } \\
\text { on Camp } \\
\text { on (Long } \\
\text { iolic Uni }\end{array}$ & $\begin{array}{l}\text { each ca } \\
\text { of unit op } \\
\text { aste witl } \\
\text { n. there } \\
\text { ign II, a } \\
\text { well, } 200 \\
\text { ersity of }\end{array}$ & $\begin{array}{l}\text { npaign } \\
\text { erations } \\
\text { no } \\
\text { ore, no } \\
\text { indicat } \\
\text { 3). }\end{array}$ & \\
\hline
\end{tabular}

There were many routes to operating the UFP, but for SIPP, and initial plant operation, it was thought prudent to only use the FEP to process recycle streams and the UFP would be the starting point for tank farm wastes. One major benefit of this method was that it minimizes undissolved solids in the FEP and thus hopefully minimizes condenser fouling that would affect operational efficiency. This means that the feed for the UFP had three waste sources, one of which came from the FEP after the recycle streams were concentrated to a target density. The other two were mentioned above. Their individual contributions to the UFP feed are shown in Table 3. 
WSRC-TR-2005-00105, REVISION 0

SRNL-RPP-2005-00012, REVISION 0

Table 3. Percent contribution for each of the UFP feed streams

\begin{tabular}{|c|c|c|c|c|}
\hline \multirow{2}{*}{$\begin{array}{l}\text { Feed Streams Used for SIPP } \\
\text { in the UFP and Verified by WTP }\end{array}$} & \multicolumn{4}{|c|}{$\begin{array}{l}\text { Percentage of Streams to UFP } \\
<------C a m p a i g n \text { Number------> }\end{array}$} \\
\hline & \begin{tabular}{|c|} 
I $^{\star}$ \\
Vol. \%
\end{tabular} & $\begin{array}{c}\text { II }^{\star \star} \\
\text { Vol. \% }\end{array}$ & $\begin{array}{c}\text { III } \\
\text { Vol. }\end{array}$ & $\frac{\text { IV }}{\text { Vol. \% }}$ \\
\hline (1) FEP Bottoms & $0 \%$ & $11.1 \%$ & $14.3 \%$ & $13.8 \%$ \\
\hline (2) AY-102/C-106 & $100 \%$ & $24.3 \%$ & $22.6 \%$ & $22.8 \%$ \\
\hline (3) AP-101 & $0 \%$ & $64.6 \%$ & $63.1 \%$ & $63.4 \%$ \\
\hline Total Feed to the UFP & $100 \%$ & $100 \%$ & $100 \%$ & $100 \%$ \\
\hline $\begin{array}{l}\text { Notes: } \\
\text { Source of Streams: (1) was concen } \\
\text { (2) outside venc } \\
\text { (3) came from } \\
\text { * Campaign I only used a single si } \\
\text { ** EDL = Engineering Developmen } \\
\text { River National Laboratory of the } \\
\text { River Company where SIPP pilo }\end{array}$ & $\begin{array}{l}\text {, but fir } \\
\text { outside } \\
\text { ulated } \\
\text { aborato } \\
\text { estingh }\end{array}$ & $\begin{array}{l}\text { recycle } \\
\text { alized in } \\
\text { vendor } \\
\text { aste: AY } \\
\text { y of the } \\
\text { use Sav }\end{array}$ & $\begin{array}{l}102 / C 10 \\
\text { Savanna } \\
\text { annah }\end{array}$ & \\
\hline
\end{tabular}

As with the recycle percentages in Table 2, the percentages in Table 3 are slightly different than what is shown in Table 1. Those differences are mostly attributed to volumes actually created during experimentation. The computational volumes were not expected to be exact and while the values in Table 3 were used for SIPP, the actual plant mixture may still be different as its design continues to evolve.

One product of the pilot-scale UFP is the permeate produced during dewatering. This waste stream is then sent on to the CIX in its preparation for LAW vitrification. As this task evolved, the pilot-scale CIX operation was disassociated from measuring volumetric throughput to measure glass production, because WTP thought it more important to understand the cesium removal performance of the chosen resin, SuperLig ${ }^{\circledR} 644$. This requirement made the amount of permeate important, because cesium removal was to be measured. Since each SIPP campaign had two CIX cycles and each cycle required 200 bed volumes (BV) of feed, therefore it was necessary to have $400 \mathrm{BV}$ of feed for each campaign, excluding Campaign I. A bed volume is the space occupied in an ion exchange column by settled resin. For the pilot-scale CIX that volume was approximately 2.3 liters, making the produced number of bed volumes 401,414 , and $410^{\dagger}$ for Campaigns II, III, and $\mathrm{IV}^{\dagger}$, respectively. This can be seen in the Table 1 line entitled, "UF1 FILTRATE (routed to CIX, then TLP."

\footnotetext{
${ }^{\dagger}$ While this volume of CIX feed was created, it was not used since Campaign IV scope was modified to only test with the FEP and the UFP unit operations. That campaign was limited to determining the filter performance the GKN filter.
} 
Finally, the proportions of different feeds for the pilot-scale TLP were needed. Those are shown in Table 4. Note, that the scope of Campaign IV changed to only test the GKN filter performance, therefore the CIX and TLP unit operations were not used and Table 4 only represents the original plan

Table 4. Percent contribution for each of the TLP feed streams*

\begin{tabular}{|c|c|c|c|c|}
\hline \multirow{3}{*}{$\begin{array}{l}\text { Feed Streams Used for SIPP } \\
\text { in the TLP and Verified by WTP }\end{array}$} & \multicolumn{4}{|c|}{$\begin{array}{l}\text { Waste Stream Vol. \% to TLP } \\
<------C a m p a i g n ~ N u m b e r------>\end{array}$} \\
\hline & $I^{*}$ & $\| I^{\star \star}$ & III & IV \\
\hline & Vol. \% & Vol. \% & Vol. \% & Vol. \% \\
\hline (1) LAW SBS (from LAW melter) & $\mathrm{n} / \mathrm{a}$ & $47.4 \%$ & $45.7 \%$ & $45.8 \%$ \\
\hline (2) $0.25 \mathrm{M} \mathrm{NaOH}$ (recycle from CIX) & $\mathrm{n} / \mathrm{a}$ & $0.0 \%$ & $2.1 \%$ & $2.1 \%$ \\
\hline (3) Treated LAW from CIX & $\mathrm{n} / \mathrm{a}$ & $52.6 \%$ & $52.2 \%$ & $52.1 \%$ \\
\hline Total Feed to the TLP & $\mathrm{n} / \mathrm{a}$ & $100 \%$ & $100 \%$ & $100 \%$ \\
\hline $\begin{array}{l}\text { Notes: } \\
\text { Source of Streams: (1) was made fro } \\
\text { location: VSL } \\
\qquad \begin{array}{l}\text { (2) comes from a } \\
\text { (3) comes from }\end{array} \\
\text { * Campaign I did not employ the pi } \\
\text { * } \text { Since Campaign I was only a filte } \\
\text { recycle streams were produced fo } \\
\text { by the RPP-WTP Test Specificati } \\
\text { *** VSL = Vitreous State Lab. of Catl }\end{array}$ & $\begin{array}{l}\text { N melter } \\
\text { regener } \\
\text { IX after } \\
\text { t-scale } \\
\text { operatio } \\
\text { Campa } \\
\text { olic Univ }\end{array}$ & $\begin{array}{l}\text { testing } \\
\text { ated IX c } \\
\text { Cs is ren } \\
\text { TLP } \\
n \text {, there } \\
\text { ign II, as } \\
\text { vell, } 200 \\
\text { ersity of }\end{array}$ & $\begin{array}{l}\text { olumn } \\
\text { loved } \\
\text { ore, no } \\
\text { indicate } \\
\text { 3). } \\
\text { America }\end{array}$ & $\begin{array}{l}\text { CIX } \\
\text { ed }\end{array}$ \\
\hline
\end{tabular}

As with Tables 2 and 3, the percentages in Table 4 differ slightly than those from Table 1 because of limitations in the model, but all the values were sufficient to plan and begin testing.

\subsection{THE OVERALL SIPP TEST MATRIX}

To allow the reader to understand how Campaign IV was done without having to refer to any of the other SIPP reports, a portion of the overall test plan explanation is repeated here from the Campaign III report. With the plan in place and the planning results from the computational model, an overall test matrix was established. The following matrix was not the last word on how each of the unit operation was to run, but it was the starting point for each of the unit operations to develop individual test plans, as well as the simulant mixing and sample plans. As the task began with Campaign I (Duignan, et al., 2004a), and then on to other campaigns, the matrix continued to evolve as WTP needs changed, therefore, this test matrix may be slightly different than the individual test plans. However, it is instructive 
here to illustrate the overall operation of SIPP. That is, a global explanation is first given to better understand SIPP in its "integrated" form, which is then followed by discussions on each of the individual unit operations and their results.

That matrix is shown in pieces in the following series of figures:

[Note that , Table $\mathrm{X}$ >> Table Y means: table $\mathrm{x}$ indicates the wait time before using slurry in the table y unit operation.]

Table 5 (simulant aging) $\quad>>\quad$ Table 6 (pilot FEP unit operation)

Table 7 (simulant aging) $\quad>\quad$ Table 8 (pilot UFP unit operation)

[The following tables are shown for completeness but the CIX and TLP unit operations were cancelled for Campaign IV]

Table 9 (simulant aging) $\quad>\quad$ Table 10 (pilot CIX unit operation - first cycle)

$>\quad$ Table 11 (pilot CIX unit operation - second cycle)

Table 12 (simulant aging) $\quad>\quad$ Table 13 (pilot TLP unit operation)

These nine figures are in chronological order of performance.

First, Tables. 5, 7, 9, and 12 were included to show hold-up time intervals between unit operations, especially because they were not part of the computational model, which was done at steady state. In an attempt to mimic the periods that waste "waits" before being processed in each unit operation, some prototypic times were included in SIPP.

Table 5. Hold period before pilot-scale FEP begins ${ }^{\dagger}$

Waste aging may affect many processing steps, for example, the post-UFP precipitation was a key issue for the ion exchange system. Using the appropriate WTP plant hold times allowed this issue to be resolved. The many reasons for specific hold times are given in Appendix C. As an example, Table 5 allows time for the mixed multiple recycle streams to age. Recycle streams are fed from several different sources to the Plant Wash and Disposal System tanks that will feed the Waste Feed Evaporation Process. The recycle streams come in at different rates and then need to be adjusted for $\mathrm{pH}$ as the acid and alkaline streams merge in order to mitigate the precipitation of solids or gels. After the adjustment, the FEP is fed, but because of the large scale, the transfer times are not insignificant. As explained in Appendix C, the time associated in the pre-evaporation process is approximately 2 days and therefore, once the SIPP FEP feed of recycle streams is adjusted properly it is held for that number of days before evaporation begins.

\footnotetext{
${ }^{\dagger}$ Blanks in all tables in this report are intentional.
} 


\section{WSRC-TR-2005-00105, REVISION 0 SRNL-RPP-2005-00012, REVISION 0}

For each of the unit operations, a hold time is set after the appropriate feed is made ready. In summary those simulated minimum hold times are:

After joining recycle feeds and before processing in the FEP - 2 days

After making FEP Bottoms and before joining it with other UFP feeds -2 days

After joining waste feeds and before processing in the UFP - 2 days

After producing UFP permeate and before processing in the CIX - 4.5 days

After joining treated LAW with recycle streams and before processing in the TLP - 2 days

Table 6. Pilot-scale FEP test matrix with the colored columns representing Campaign IV

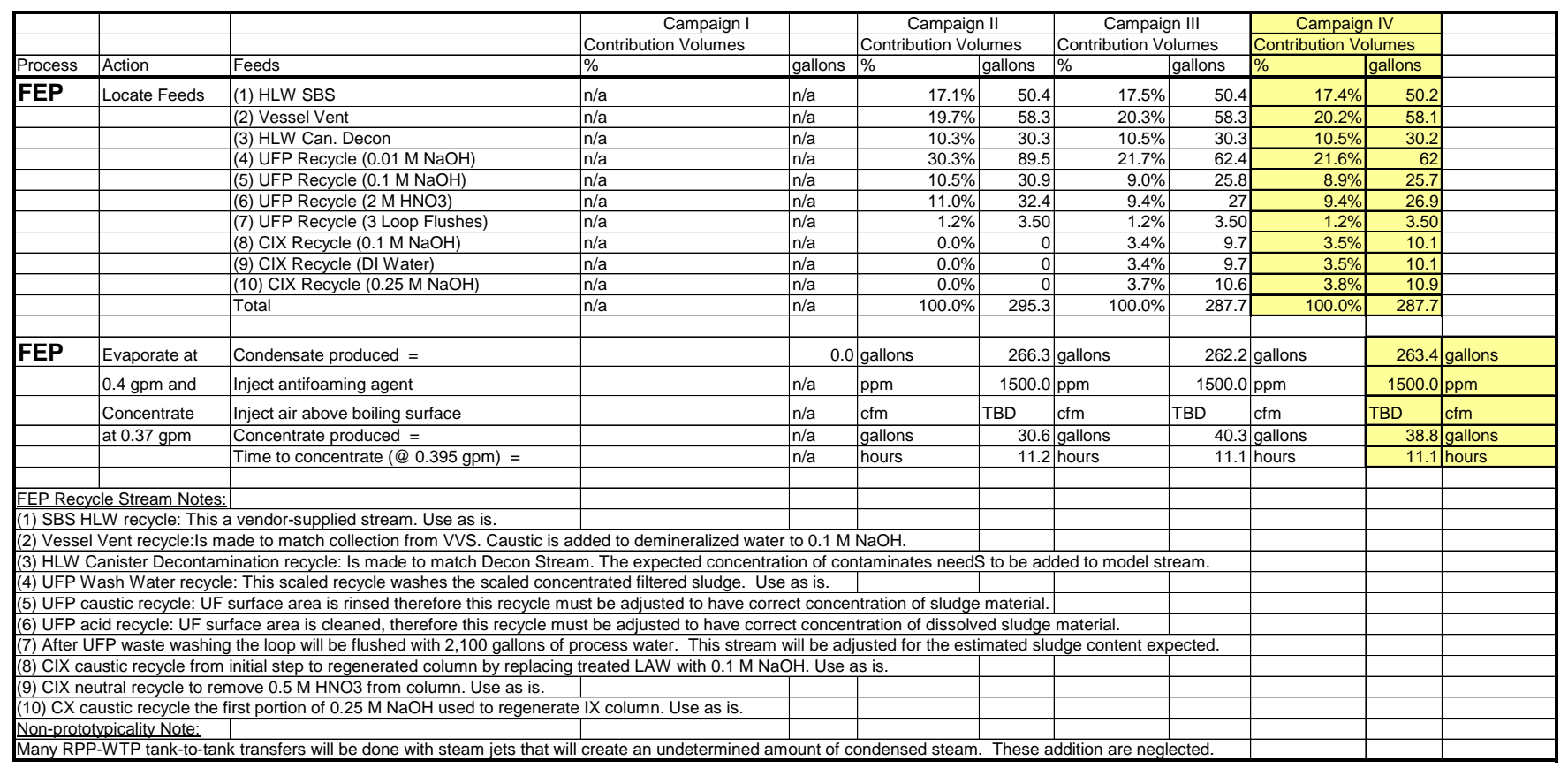

Once the recycle streams were mixed in the planned proportions, Table 2, and the appropriate hold time was realized, then the FEP test matrix, shown in Table 6, was performed. The goal was to concentrate the feed stream so that it had a sodium molarity of 5 , but the "visible" target was an evaporated slurry density of $1.266 \mathrm{~g} / \mathrm{cc}$ at $50^{\circ} \mathrm{C}$ and 1 psia (which is $1.252 \mathrm{~g} / \mathrm{cc}$ at $1 \mathrm{~atm})$. Density was used because the WTP will only be able to control concentration by measuring density.

Table 7. Hold period before pilot-scale UFP begins

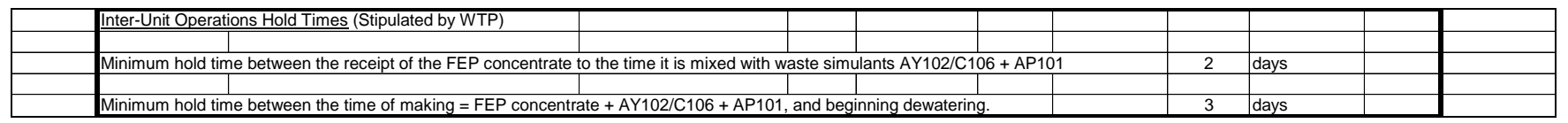


WSRC-TR-2005-00105, REVISION 0 SRNL-RPP-2005-00012, REVISION 0

When the FEP was complete its slurry product was held for 2 days, Table 7, before being mixed in the planned proportions, Table 3, with the simulated wastes of AY102 and AP101. Completing this mixture started another 3-day clock before starting the UFP.

While processing the waste through the UFP is complicated with several activities involving the washing of the concentrated slurry to the cleaning of the filter loop (see Table 8), the basic operation is to separate the waste feed stream into a HLW product, containing all of the undissolved solids, and its supernatant, containing most of the cesium. This was done by dewatering the feed from a concentration less than $5 \mathrm{wt} \%$ to $20 \mathrm{wt} \%$ undissolved solids. The details of the UFP operation will be discussed in the appropriate subsection of this report. One feature that is not immediately obvious is the process containing an internal hold time. Dewatering in WTP will be a relatively lengthy process because filtration is very slow and the volumes are very large. While the waste is waiting to be processed through the UFP, it will be sitting in the feed preparation tank, which feeds the feed tank. During this process period, chemical reactions may still be occurring within the waste and crystallization or precipitation may result. In an attempt to capture these transient phenomena, a waiting period was inserted in the dewatering process of the pilot UFP. The period is a 12-hour hold time, which is arbitrary, but is loosely connected to the planned plant operational time of the UFP, and was acceptable to the WTP project.

Table 8. Pilot-scale UFP test matrix with the colored columns representing Campaign IV

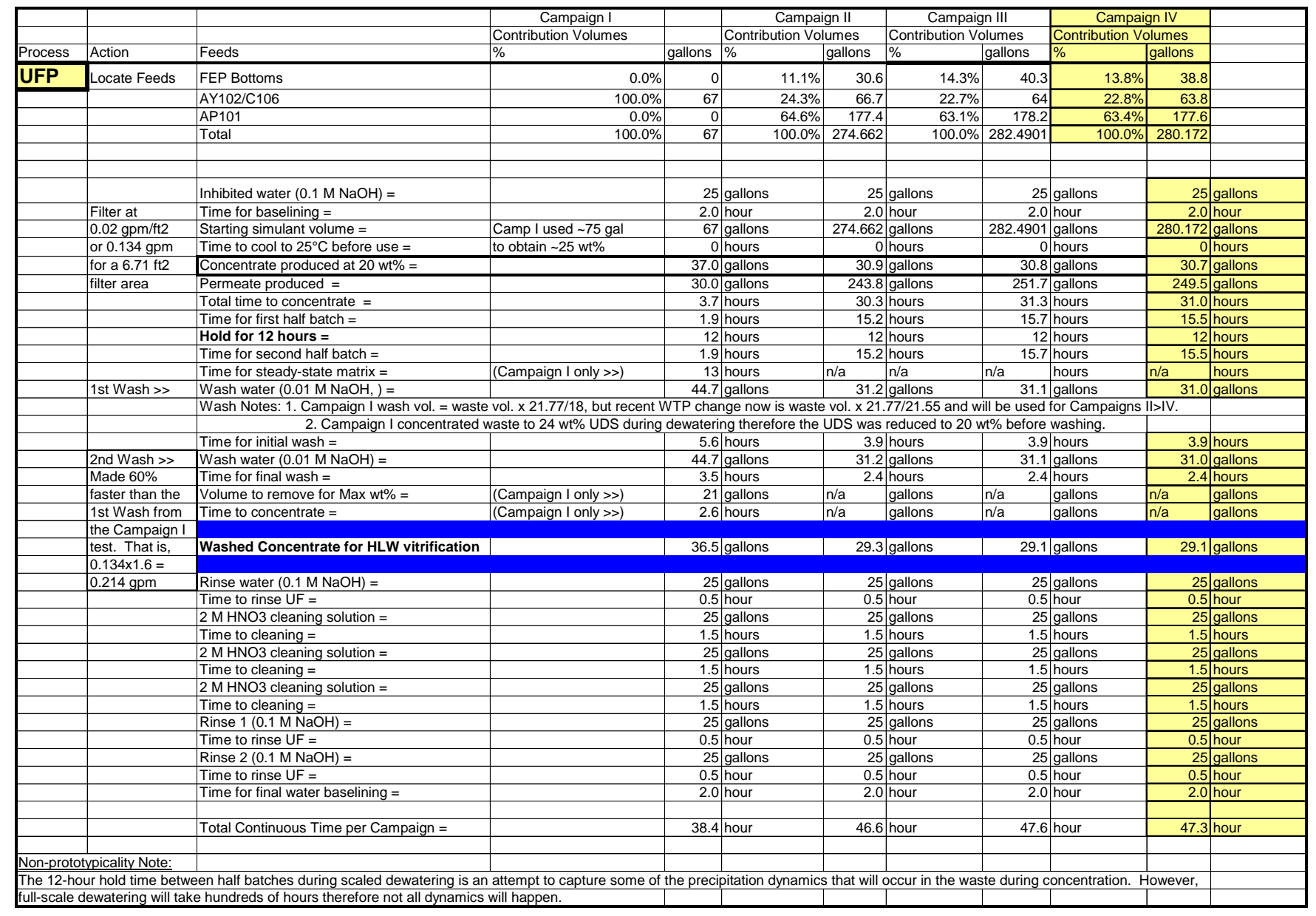


WSRC-TR-2005-00105, REVISION 0

SRNL-RPP-2005-00012, REVISION 0

The pilot-scale UFP produced permeate would have been needed for the next step in the SIPP operation, i.e., pilot CIX, if Cycle 5 and 6 were performed. After the UFP produced the permeate it would need to age at least 4.5 days, as shown in Table 9.

Table 9. Hold period before pilot-scale CIX begins*

\begin{tabular}{|l|l|l|l|l|l|l|}
\hline & \begin{tabular}{l} 
Inter-Unit Operations Hold Time (stipulated by WTP) \\
\hline $\mid$
\end{tabular} \\
\hline
\end{tabular}

*The used of CIX unit operation was for Campaign IV was removed from the task scope

As in Campaign III, if the pilot CIX operation were done then the campaign would have been broken in two parts because it was necessary to run with two complete ion exchange cycles, which allowed both CIX columns to take turns as the lead or the lag column. This is the reason there are two CIX tables, i.e., Tables 10 and 11 . The basic difference between them is that the lead column in the first cycle of operation, Table 10, became the lag column in the second cycle, Table 11. The permeate produced during a pilot UFP campaign was sufficient to satisfy the CIX need for two cycles of 400 bed volumes of feed ( 900 liters). Each cycle included the processing of 200 bed volumes of UFP permeate and then the lead column would undergo regeneration for use in the next cycle as the lag column. The colored portion of Table 10 highlights the work that would have been done for Campaign IV.

Table 10. Pilot-scale CIX (1st half) test matrix with the colored columns representing Campaign IV*

\begin{tabular}{|c|c|c|c|c|c|c|c|c|c|c|c|}
\hline & & & & & \multirow{2}{*}{\begin{tabular}{r|} 
Campaign I \\
ution Volumes
\end{tabular}} & \multicolumn{2}{|c|}{ Campaign II } & \multicolumn{2}{|c|}{ Campaign III } & \multicolumn{2}{|c|}{ Campaign IV } \\
\hline & & & & Contribution Volumes & & \multicolumn{2}{|c|}{ Contribution Volumes } & \multicolumn{2}{|c|}{ Contribution Volumes } & \multicolumn{2}{|c|}{ Contribution Volumes } \\
\hline Process & Action & Feeds & & & & & & & & & \\
\hline \multirow[t]{7}{*}{ Cs IX } & & \multicolumn{2}{|c|}{\begin{tabular}{|l|l|} 
Total UFP permeate feed & \\
\end{tabular}} & $\mathrm{n} / \mathrm{a}$ & n/a & 243.8 & gallons & \multicolumn{2}{|c|}{251.7 gallons } & \multirow[t]{2}{*}{249.5} & \multirow[t]{2}{*}{ gallons } \\
\hline & Locate Feeds & \multirow{2}{*}{\multicolumn{4}{|c|}{ 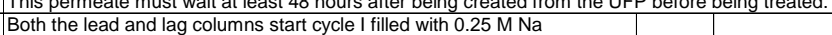 }} & & & & & & \\
\hline & & & & & & & & & & & \\
\hline & & \multicolumn{4}{|c|}{ Water used for eluate, water buffer, and $0.25 \mathrm{M} \mathrm{Na}$ must be made from fresh distilled water } & & & & & & \\
\hline & & \multicolumn{3}{|c|}{ Water used for $0.1 \mathrm{M}$ Na solution can come from evaporation condensates } & & & & & & & \\
\hline & & \multicolumn{2}{|c|}{ There will be two columns referred to as A \& B } & & & & & & & & \\
\hline & & & & & & & & & & & \\
\hline Cs IX & Treat Waste & Cs IX Test No. & & & & 1 & & 3 & & 5 & \\
\hline & Simulant and & Lead $>$ Lag Column $=$ & & $\mathrm{n} / \mathrm{a}$ & $\mathrm{n} / \mathrm{a}$ & $A>B$ & & $A>B$ & & $A>B$ & \\
\hline & regenerate as & Cycle I (treat 1/2 campaign batch) & & $\mathrm{n} / \mathrm{a}$ & $\mathrm{n} / \mathrm{a}$ & 121.9 & gallons & 125.8 & gallons & 124.7 & gallons \\
\hline & indicated & Number of BV (BV 0.617 gal. $) \gg \gg$ & Column I.D. $=5.5$ inches & $\mathrm{n} / \mathrm{a}$ & $\mathrm{n} / \mathrm{a}$ & 197.5 & BV & 204.0 & BV & 202.2 & BV \\
\hline & & Flow Rate & Bed Height $=6.0$ inches & $\mathrm{n} / \mathrm{a}$ & $\mathrm{n} / \mathrm{a}$ & 3.0 & BV/hour & 3.0 & BV/hour & 1.5 & BV/hour \\
\hline & & Total Hours to Treat & & $\mathrm{n} / \mathrm{a}$ & $\mathrm{n} / \mathrm{a}$ & 65.8 & hours & 68.0 & hours & 134.8 & hours \\
\hline & & Regeneration & & & & & & & & & \\
\hline & 4 & Column to be regenerated and eluted = & & & & A & & A & & A & \\
\hline & & $0.1 \mathrm{M} \mathrm{NaOH}$ displacement & & & & & & & & & \\
\hline & & Number of BV (BV 0.617 gal.) & & $n / a$ & $\mathrm{n} / \mathrm{a}$ & 3.0 & BV & 3.0 & BV & 3.0 & $\mathrm{BV}$ \\
\hline & & Flow Rate & & $n / a$ & $\mathrm{n} / \mathrm{a}$ & 3.0 & BV/hour & 3.0 & BV/hour & 3.0 & BV/hour \\
\hline & & Total Hours to Treat & & $\mathrm{n} / \mathrm{a}$ & $\mathrm{n} / \mathrm{a}$ & 1.0 & hours & 1.0 & hours & 1.0 & hours \\
\hline & & Distilled Water Buffer & & & & & & & & & \\
\hline & & Number of BV (BV 0.617 gal. $)$ & & $\mathrm{n} / \mathrm{a}$ & $\mathrm{n} / \mathrm{a}$ & 3.0 & BV & 3.0 & BV & 3.0 & BV \\
\hline & & Flow Rate & & $\mathrm{n} / \mathrm{a}$ & $\mathrm{n} / \mathrm{a}$ & 3.0 & BV/hour & 3.0 & BV/hour & 3.0 & BV/hour \\
\hline & & Total Hours to Treat & & $\mathrm{n} / \mathrm{a}$ & $\mathrm{n} / \mathrm{a}$ & 1.0 & hours & 1.0 & hours & 1.0 & hours \\
\hline & & $0.5 \mathrm{M} \mathrm{HNO} 3$ elution & & & & & & & & & \\
\hline & Regeneration & Number of BV (BV 0.617 gal.) & & $\mathrm{n} / \mathrm{a}$ & $\mathrm{n} / \mathrm{a}$ & 15.0 & BV & 15.0 & BV & 15.0 & $\mathrm{BV}$ \\
\hline & & Flow Rate & & $\mathrm{n} / \mathrm{a}$ & $\mathrm{n} / \mathrm{a}$ & 1.0 & BV/hour & 2.0 & BV/hour & 1.0 & BV/hour \\
\hline & & Total Hours to Treat & & $\mathrm{n} / \mathrm{a}$ & $\mathrm{n} / \mathrm{a}$ & 15.0 & hours & 7.5 & hours & 15.0 & hours \\
\hline & & Distilled Water Buffer & & & & & & & & & \\
\hline & & Number of BV (BV 0.617 gal.) & & $n / a$ & $\mathrm{n} / \mathrm{a}$ & 3.0 & BV & 3.0 & BV & 3.0 & BV \\
\hline & & Flow Rate & & $\mathrm{n} / \mathrm{a}$ & $\mathrm{n} / \mathrm{a}$ & 1.4 & BV/hour & 1.4 & BV/hour & 1.4 & BV/hour \\
\hline & & Total Hours to Treat & & $\mathrm{n} / \mathrm{a}$ & $\mathrm{n} / \mathrm{a}$ & 2.1 & hours & 2.1 & hours & 2.1 & hours \\
\hline & & $0.25 \mathrm{M} \mathrm{NaOH}$ regeneration & & & & & & & & & \\
\hline & & Number of BV (BV 0.617 gal.) & & $\mathrm{n} / \mathrm{a}$ & $\mathrm{n} / \mathrm{a}$ & 6.0 & BV & 6.0 & BV & 6.0 & BV \\
\hline & & Flow Rate & & $n / a$ & $\mathrm{n} / \mathrm{a}$ & 3.0 & BV/hour & 3.0 & BV/hour & 3.0 & BV/hour \\
\hline & $\downarrow$ & Total Hours to Treat & & $\mathrm{n} / \mathrm{a}$ & $\mathrm{n} / \mathrm{a}$ & 2.0 & hours & 2.0 & hours & 2.0 & hours \\
\hline & & & & & & & & & & & \\
\hline & & & Total CIX Cycle Flow Time & & & 87.0 & hours & 81.6 & hours & 155.9 & hours \\
\hline
\end{tabular}

*The used of CIX unit operation was for Campaign IV was removed from the task scope 
WSRC-TR-2005-00105, REVISION 0

SRNL-RPP-2005-00012, REVISION 0

Campaign IV would have included pilot CIX Cycles 5 and 6. Once again, Campaign I did not include any ion exchange work, which explains why Cycle 1 began in Campaign II, Cycle 3 began in Campaign III, and Cycle 5 would have started in Campaign IV. At the end of each campaign both columns were filled with $0.1 \mathrm{M} \mathrm{NaOH}$ to keep the resin stable for the next campaign. This inter-campaign period would be no less than 5 weeks.

Table 11. Pilot-scale CIX (2nd half) test matrix with the colored columns representing Campaign IV*

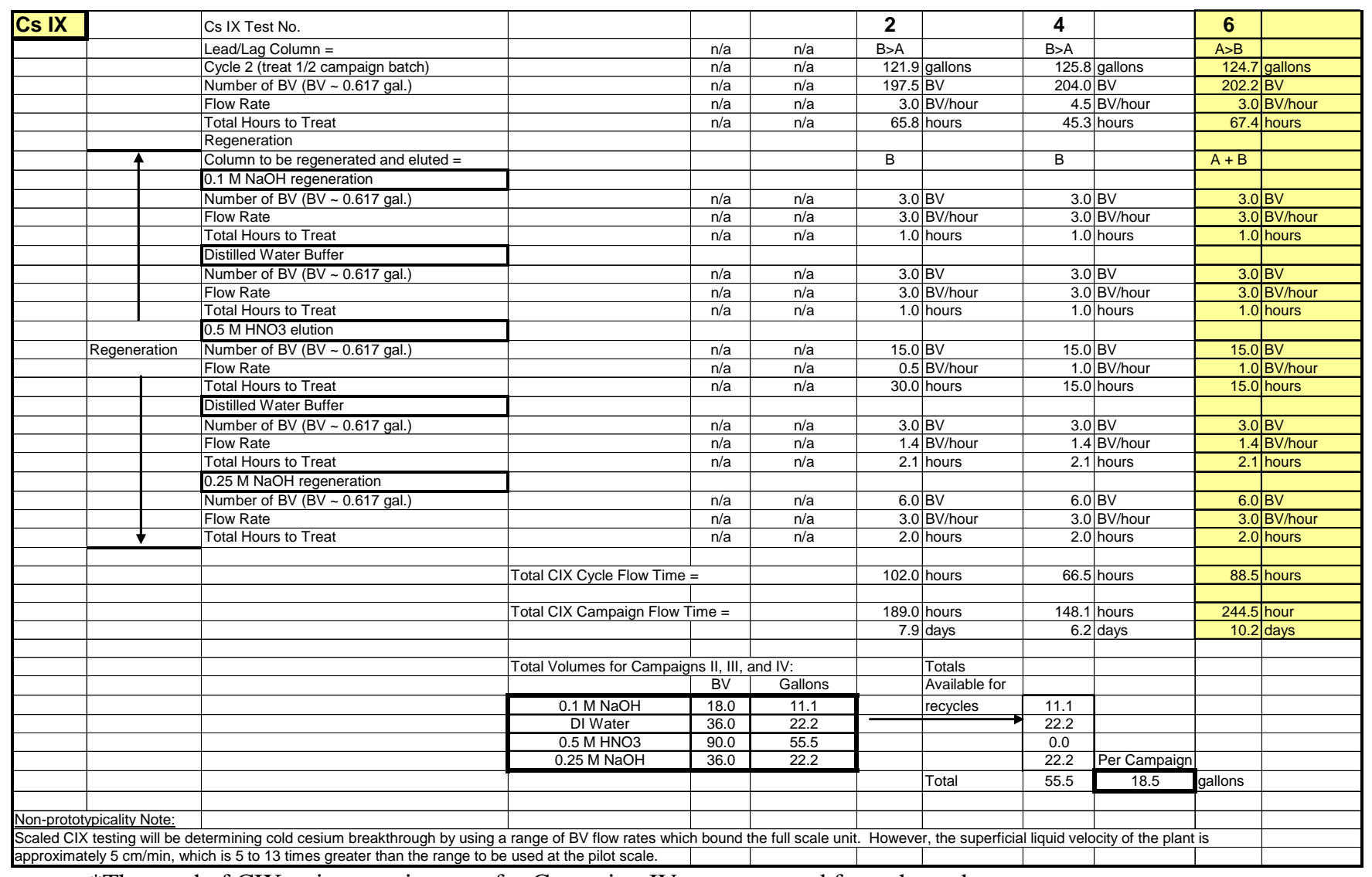

*The used of CIX unit operation was for Campaign IV was removed from the task scope

The final step in the SIPP was once again to evaporate the waste. The now treated simulated LAW product from the CIX was mixed with two recycle streams shown in Table 4. After mixing, the resulting solution was held before processing, Table 12.

Table 12. Hold period before pilot-scale TLP begins*

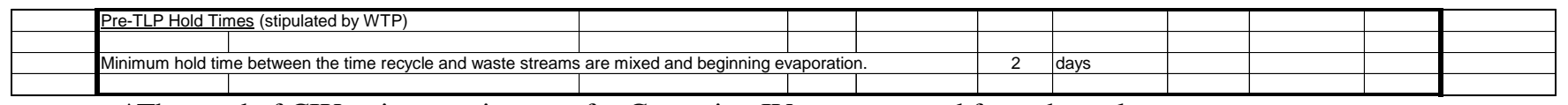

*The used of CIX unit operation was for Campaign IV was removed from the task scope

The operation of the pilot TLP, Table 13 would have been similar to the pilot FEP, Table 6 . However, in the TLP the goal was to concentrate the feed stream so that it had a sodium molarity of 8 . Once again the "visible" target was an evaporated slurry density of $1.360 \mathrm{~g} / \mathrm{cc}$ 
WSRC-TR-2005-00105, REVISION 0

SRNL-RPP-2005-00012, REVISION 0

at $50^{\circ} \mathrm{C}$ and 1 psia (which is $1.395 \mathrm{~g} / \mathrm{cc}$ at $1 \mathrm{~atm}$ ). Density was used because the WTP will only be able to control concentration by measuring density. A large difference between the pilot FEP and TLP operations was the quantity of concentrated product produced. For the FEP, the final volume of the concentrate could be completely contained within the confines of the evaporator ( $<50$ gallons), but for the TLP the concentrated volume was approximately 3 times larger. That is, the FEP was shut down just when steady-state waste concentration was reached. For the TLP, steady-state waste concentration was reached well before the waste feed was exhausted. This meant the TLP produced a continuous waste stream after the TLP contents reached the steady-state density target. The only significant impact this difference had on operation was to the defoamer chemical added to the waste. The concentration of defoamer was to be maintained at $1500 \mathrm{ppm}$, therefore, once the TLP reached steady state and concentrated waste was being removed, defoamer was periodically added.

Table 13. Pilot-scale TLP test matrix with the colored columns representing Campaign IV*

\begin{tabular}{|c|c|c|c|c|c|c|c|c|c|c|c|}
\hline & & & \begin{tabular}{|c|} 
Campaign I \\
Contribution Volumes
\end{tabular} & & $\begin{array}{r}\text { Camp } \\
\text { Contribution Vol }\end{array}$ & paign II & \begin{tabular}{|r} 
Camp \\
Contribution V
\end{tabular} & aign III & $\begin{array}{r}\text { Campa } \\
\text { Contribution Vo }\end{array}$ & aign IV & \\
\hline Process & Action & Feeds & $\begin{array}{l}\text { Contribution Volumes } \\
\%\end{array}$ & gallons & $\begin{array}{l}\text { Contribution Vol } \\
\%\end{array}$ & $\begin{array}{l}\text { lumes } \\
\text { gallons }\end{array}$ & & $\begin{array}{l}\text { /olumes } \\
\text { gallons }\end{array}$ & & 'olumes & \\
\hline TLP & Locate Feeds & Simulant & n/a & $\mathrm{n} / \mathrm{a}$ & $52.6 \%$ & 243.8 & $52.2 \%$ & 251.7 & $52.1 \%$ & 249.5 & \\
\hline & & (Recycle 1) SBS LAW & $\mathrm{n} / \mathrm{a}$ & $n / a$ & $47.4 \%$ & 220.1 & $45.7 \%$ & 220.1 & $45.8 \%$ & 219.3 & \\
\hline & & (Recycle 2) $0.25 \mathrm{M} \mathrm{NaOH}$ from CIX & $n / a$ & $n / a$ & $0.0 \%$ & 0 & $2.1 \%$ & 10 & $2.1 \%$ & 10.1 & \\
\hline & & Total & n/a & $\mathrm{n} / \mathrm{a}$ & $100.0 \%$ & 463.862 & $100.0 \%$ & \begin{tabular}{l|l}
6 & 481.7901 \\
\end{tabular} & $100.0 \%$ & 478.872 & \\
\hline TLP & Evaporate at & Condensate produced $=$ & & 0.0 & gallons & 320.8 & gallons & 325.3 & gallons & 323.6 & gallons \\
\hline & $0.4 \mathrm{gpm}$ and & Inject antifoaming agent & & n/a & ppm & 1500.0 & $\mathrm{ppm}$ & 1500.0 & $\mathrm{ppm}$ & 1500.0 & $\mathrm{ppm}$ \\
\hline & Concentrate & Inject air above boiling surface & & $\mathrm{n} / \mathrm{a}$ & $\mathrm{cfm}$ & TBD & $\mathrm{cfm}$ & TBD & $\mathrm{cfm}$ & TBD & $\mathrm{cfm}$ \\
\hline & at $0.37 \mathrm{gpm}$ & & & & & & & & & & \\
\hline & & Concentrate produced for LAW vitrification & & $\mathrm{n} / \mathrm{a}$ & gallons & 155.7 & gallons & 159.4 & gallons & 158.0 & gallons \\
\hline & & & & & & & & & & & \\
\hline & & Time to concentrate $=$ & & $\mathrm{n} / \mathrm{a}$ & hours & 13.5 & hours & 13.7 & hours & 13.7 & hours \\
\hline TLP Rec & Stream Notes & & & & & & & & & & \\
\hline (1) SBS & AW recycle: This & a vendor supplied stream. Use as is. & & & & & & & & & \\
\hline (2) Caus & CIX regeneration & recycle: This stream should have the appropriat & e concentration of caustic & 025 & $\mathrm{M} \mathrm{NaOH}$. Use as & & & & & & \\
\hline
\end{tabular}

*The used of CIX unit operation was for Campaign IV was removed from the task scope

The timetable for Campaign IV is listed in Table 14. It started on the day indicated, i.e., Monday, 9 August, 2004, but since this schedule was published before the test began minor changes occurred. However, those changes were insignificant. Campaign IV had pre-test activities which were different from the other Campaigns. A new filter from GKN was obtained due to a change in the RPP-WTP plant equipment. Before the filter could be tested it was subjected preconditioning to put it a similar used form as the Mott filter used in the previous campaigns. The preconditioning occurred from 2 to 8 August and those activities will be described in the UFP section of this report.

The only other aspect of the test not shown in all the matrix tables is the disposition of the water products. That is, the treated waste from the past campaigns using the TLP was drummed to be sent to VSL for LAW melter operations and the washed concentrated slurry from the UFP was drummed to be sent to VSL for HLW melter operation. The other product is all the boiled off and condensed water from both evaporation operations. Those waters were mixed together, as they will be in the plant and then a portion is reused to make rinse solutions for the UFP and displacement solution for the CIX. 


\section{WSRC-TR-2005-00105, REVISION 0 SRNL-RPP-2005-00012, REVISION 0}

Table 14. Pilot-scale schedule for Campaign IV

\begin{tabular}{|c|c|c|c|c|c|c|c|c|c|c|c|c|c|c|}
\hline Dates & Campaign & Start Date & & Personnel Nomeclature: & & & & & \multirow[t]{5}{*}{ Notes: } & \multirow{3}{*}{\multicolumn{5}{|c|}{$\begin{array}{l}\text { (1) Hold times are included to mimick times that } \\
\text { the plant is filling/transfer between unit operations. } \\
\text { Those times begin when a mixture is FIRST made, }\end{array}$}} \\
\hline based on & 1 & 26-Jan-04 & & \multicolumn{5}{|c|}{ da=D.Adamson, ma=M.Armstrong, $d b=D . B u r n s, v b=V . B u s h$, } & & & & & & \\
\hline a 10-week & II & 12-Apr-04 & & $\mathrm{md}=\mathrm{M}$.Duignan, $\mathrm{df}=\mathrm{D}$.Foreman, $\mathrm{n}$ & $n f=M . F$ & jwley, & & & & & & & & \\
\hline estimate & III & 7-Jun-04 & & $\mathrm{jm}=\mathrm{J}$. Mills, $\mathrm{zq}=\mathrm{Z}$. Qureshi, mw=M. & William & $\mathrm{s}, \mathrm{jz}=\mathrm{J} . \mathrm{Z}$ & amecnik & & & therefore mixing tin & nes are & also incl & uded. & \\
\hline from & IV & 2-Aug-04 & $<$ Original & & & & & & & (2) $X=$ Official sta & rt of $\mathrm{C}$ & ampaign & IV with & FEP \\
\hline 18-May-04 & & Campaign IV & & & & & & & & & & & & \\
\hline VVVVVV & Day & Date & Unit Op & Activity & Expt & Backup & Non-E. & Backup & Unit Op & Activity & Expt & Backup & Non-E. & Backup \\
\hline 2-Aug-04 & Mon & $\mathrm{X}-7$ & preUFP & New Filter Receipt/Inspection & $\mathrm{mf}$ & $\mathrm{md}$ & $\mathrm{ma}$ & $\mathrm{df}$ & & & & & & \\
\hline 3-Aug-04 & Tue & $X-6$ & preUFP & New Filter Soak & $\mathrm{mf}$ & $\mathrm{md}$ & $\mathrm{ma}$ & $\mathrm{df}$ & & & & & & \\
\hline 4-Aug-04 & Wed & $X-5$ & preUFP & New Filter Soak & $\mathrm{mf}$ & md & $\mathrm{ma}$ & df & & & & & & \\
\hline 5-Aug-04 & Thu & $\mathrm{X}-4$ & preUFP & Replacement/Checkout of Filter & $\mathrm{mf}$ & md & $\mathrm{ma}$ & df & & & & & & \\
\hline 6-Aug-04 & Fri & $x-3$ & preUFP & Baseline/Precondition Filter & $\mathrm{mf}$ & md & $\mathrm{ma}$ & df & & & & & & \\
\hline 7-Aug-04 & Sat & $X-2$ & preUFP & Precondition Filter & $\mathrm{mf}$ & $\mathrm{md}$ & $\mathrm{ma}$ & df & & & & & & \\
\hline 8-Aug-04 & Sun & $X-1$ & preUFP & Precondition Filter & $\mathrm{mf}$ & $\mathrm{md}$ & $\mathrm{ma}$ & $\mathrm{df}$ & & & & & & \\
\hline 9-Aug-04 & Mon & $\mathrm{X}$ (note 2) & UFP & Rinse/Clean (2 M HNO3) & $\mathrm{mf}$ & $\mathrm{md}$ & $\mathrm{ma}$ & df & FEP & Mix Recycles & $\mathrm{jz} / \mathrm{mw}$ & $\mathrm{mw} / \mathrm{jz}$ & df & $\mathrm{vb}$ \\
\hline 10-Aug-04 & Tue & $x+1$ & UFP & Rinse/Clean (2 M HNO3) & $\mathrm{mf}$ & md & $\mathrm{ma}$ & $\mathrm{df}$ & FEP & Admin Hold (1) & & & & \\
\hline 11-Aug-04 & Wed & $x+2$ & UFP & Baseline & $\mathrm{mf}$ & md & $\mathrm{ma}$ & df & FEP & Evaporation & $\mathrm{zq}$ & da & jm & $\mathrm{vb}$ \\
\hline 12-Aug-04 & Thu & $x+3$ & UFP & Admin Hold (1) & & & & & FEP & Product Transfers & $\mathrm{zq}$ & da & jm & $\mathrm{vb}$ \\
\hline 13-Aug-04 & Fri & $x+4$ & UFP & Mix UF feeds & $\mathrm{jz} / \mathrm{mw}$ & $\mathrm{mw} / \mathrm{jz}$ & $\mathrm{df}$ & $\mathrm{vb}$ & FEP & Clean Equipment & $\mathrm{zq}$ & da & $\mathrm{jm}$ & $\mathrm{vb}$ \\
\hline 14-Aug-04 & Sat & $x+5$ & UFP & Admin Hold (1) & & & & & & & & & & \\
\hline 15-Aug-04 & Sun & $x+6$ & UFP & Admin Hold (1) & & & & & & & & & & \\
\hline 16-Aug-04 & Mon & $x+7$ & UFP & Dewater & $\mathrm{mf}$ & $\mathrm{md}$ & $\mathrm{ma}$ & $\mathrm{df}$ & & & & & & \\
\hline 17-Aug-04 & Tue & $x+8$ & UFP & Dewater & $\mathrm{mf}$ & md & $\mathrm{ma}$ & df & & & & & & \\
\hline 18-Aug-04 & Wed & $x+9$ & UFP & Steady-state & $\mathrm{mf}$ & $\mathrm{md}$ & $\mathrm{ma}$ & $\mathrm{df}$ & & & & & & \\
\hline 19-Aug-04 & Thu & $x+10$ & UFP & Steady-state & $\mathrm{mf}$ & md & $\mathrm{ma}$ & df & & & & & & \\
\hline 20-Aug-04 & Fri & $x+11$ & & & & & & & & & & & & \\
\hline 21-Aug-04 & Sat & $x+12$ & & & & & & & & & & & & \\
\hline 22-Aug-04 & Sun & $x+13$ & & & & & & & & & & & & \\
\hline 23-Aug-04 & Mon & $x+14$ & UFP & Transfers/Wash & $\mathrm{mf}$ & md & $\mathrm{ma}$ & $\mathrm{df}$ & & & & & & \\
\hline 24-Aug-04 & Tue & $x+15$ & UFP & Wash & $\mathrm{mf}$ & md & $\mathrm{ma}$ & $\mathrm{df}$ & & & & & & \\
\hline 25-Aug-04 & Wed & $x+16$ & UFP & Rinse/Clean & $\mathrm{mf}$ & $\mathrm{md}$ & $\mathrm{ma}$ & $\mathrm{df}$ & & & & & & \\
\hline 26-Aug-04 & Thu & $x+17$ & UFP & Baseline & $\mathrm{mf}$ & $\mathrm{md}$ & $\mathrm{ma}$ & $\mathrm{df}$ & & & & & & \\
\hline
\end{tabular}




\subsection{THE PRINCIPAL WASTE SIMULANTS: AY102 AND AP101 (AUTHOR-} ZAMECNIK)

Together with the pilot-scale FEP concentrated recycle streams, the waste feed for the pilot-scale UFP contained two simulated Hanford waste streams: tank 241-AY-102/C106 referred herein as AY102, and tank 241-AP-101, referred herein as AP101.

The composition and properties, including the distribution of species between the solid and supernatant phases, of AY102 simulant has been previously described (Duignan et al., 2004a or Appendix I-1; Zamecnik, et al., 2004). This simulant was blended at SRNL from fractions manufactured by Optima Chemical (Douglas, GA) and additional reagents, as described in the references. The AP101 simulant formulation was defined by Pacific Northwest National Laboratory (Russell, et al., 2003) and the simulant was manufactured by Optima Chemical. The measured total slurry compositions of both of these simulants are shown in Table 15 (as previously shown in Duignan et al., 2004b or Appendix I-2, and 2004c).

Table 15. Measured Simulant Compositions

\begin{tabular}{|c|c|c|c|c|}
\hline All values mg/L except as noted. & \multicolumn{2}{|c|}{ AY102 Slurry } & \multicolumn{2}{|c|}{ AP101 } \\
\hline Total Solids (wt\%) & 26.80 & & 31.94 & \\
\hline Undissolved Solids (wt\%) & 11.75 & & 0.00 & \\
\hline Supernatant Solids (wt\%) & 17.05 & & 31.94 & \\
\hline Density kg/L & 1.240 & & 1.279 & \\
\hline $\mathrm{Ag}$ & 624 & & 37.3 & \\
\hline $\mathrm{Al}$ & 15996 & $17484^{*}$ & 7469 & $6932 *$ \\
\hline $\mathrm{B}$ & $<165$ & & $<142$ & \\
\hline $\mathrm{Ba}$ & 293 & 255 & $<1.53$ & \\
\hline $\mathrm{Bi}$ & 6.93 & & 0.0576 & \\
\hline $\mathrm{Ca}$ & 1131 & 1500 & 23.0 & \\
\hline $\mathrm{Cd}$ & 46.8 & & $<3.9$ & \\
\hline $\mathrm{Ce}$ & 366 & & $<127$ & \\
\hline $\mathrm{Co}$ & 151 & & $<15.6$ & \\
\hline $\mathrm{Cr}$ & 632 & 634 & 179 & 175 \\
\hline Cs & 18.7 & & 6.02 & \\
\hline Cs & $<17$ & & $<19.6$ & \\
\hline $\mathrm{Cu}$ & 77.9 & 81.8 & $<5.86$ & \\
\hline $\mathrm{Fe}$ & 35836 & 35216 & 76.4 & 68.4 \\
\hline $\mathrm{K}$ & 373 & & 30568 & \\
\hline $\mathrm{La}$ & 305 & & 13.9 & \\
\hline $\mathrm{Li}$ & $<24.8$ & & $<21.1$ & \\
\hline $\mathrm{Mg}$ & 379 & 373 & 5.51 & \\
\hline $\mathrm{Mn}$ & 7812 & 7638 & 3.00 & \\
\hline $\mathrm{Mo}$ & $<17$ & & $<10.6$ & \\
\hline $\mathrm{Na}$ (ICPES) & 84283 & & 127900 & \\
\hline $\mathrm{Na}(\mathrm{AA})$ & 91760 & & 129179 & \\
\hline Na average & 88021 & & 128540 & \\
\hline $\mathrm{Na}(\mathrm{M})$ & 3.83 & & 5.59 & \\
\hline $\mathrm{Nd}$ & 847 & & $<72.9$ & \\
\hline
\end{tabular}


WSRC-TR-2005-00105, REVISION 0

SRNL-RPP-2005-00012, REVISION 0

Table 15 continued

\begin{tabular}{|c|c|c|}
\hline All values mg/L except as noted. & AY102 Slurry & AP101 \\
\hline $\mathrm{Ni}$ & 1151 & 357 \\
\hline $\mathrm{P}$ & 1748 & 269 \\
\hline $\mathrm{Pb}$ & 1662 & $<156$ \\
\hline $\operatorname{Pr}$ & 170 & 0.0223 \\
\hline $\mathrm{Rb}$ & 4.60 & 6.06 \\
\hline $\mathrm{S}$ & 892 & 1599 \\
\hline $\mathrm{Si}$ & 11016 & 329 \\
\hline $\mathrm{Sr}$ & 279 & $<1.18$ \\
\hline $\mathrm{W}$ & $<84.7$ & $<97.7$ \\
\hline $\mathrm{Y}$ & 50.8 & 0.0728 \\
\hline $\mathrm{Zn}$ & 81.3 & 5.95 \\
\hline $\mathrm{Zr}$ & 484 & $<5.86$ \\
\hline bromide & 69.4 & $<128$ \\
\hline chloride & 165 & 1530 \\
\hline fluoride & 43.4 & 28.0 \\
\hline formate & 389 & 1380 \\
\hline nitrate & 319 & 105000 \\
\hline nitrite & 4228 & 33400 \\
\hline oxalate & 9213 & 1550 \\
\hline phosphate & 3807 & 1150 \\
\hline sulfate & 1860 & 3740 \\
\hline acetate & 415 & 1727 \\
\hline citric acid & $<81.8$ & $<104$ \\
\hline formate & 264 & 1432 \\
\hline
\end{tabular}

\subsection{PILOT WASTE FEED EVAPORATOR (AUTHORS - QURESHI, WILLIAMS \& ZAMECNIK)}

The Waste Feed Evaporator Process, or FEP, is the first unit operation in the RPP-WTP Pretreatment Facility that a waste $(<5 \mathrm{M} \mathrm{Na})$ will see on start up. However, if the incoming wastes or waste blends are greater than $5 \mathrm{M} \mathrm{Na}$, the FEP evaporators are used to concentrate WTP recycle streams for blending with the fresh tank waste. Since the AY102 and AP101 have sufficient sodium concentration, the SIPP FEP operations only included evaporation of SIPP recycles. The SIPP FEP Bottoms stream was then mixed with simulated waste streams, i.e., AY102 and AP101, which would normally come from the Hanford Tank farm to produce a feed stream for the Ultrafiltration Process (UFP) unit operation.

This section will explain the pilot-scale FEP unit operation that was done as part of Campaign IV testing. The process started with the preparation of the FEP feed product, which was a mixture of many recycle streams in a predetermined fashion, see Table 2 . A chemical explanation of the feed (recycle) stream formation will be followed by a short description on how feed formation was handled just before FEP operation and then how the waste stream was handled during and after its concentration. The following section 
WSRC-TR-2005-00105, REVISION 0

SRNL-RPP-2005-00012, REVISION 0

will discuss the physical equipment used to evaporate the waste and how the test was run. Finally, the results will be discussed and conclusions made for the FEP unit operation.

\subsubsection{Chemical Description of the Pilot FEP Feed Stream (Author - Zamecnik)}

\subsubsection{Generation of the Recycle Streams}

The feed for the FEP was a series of recycle streams to be produced from operating the RPP-WTP Pretreatment Facility. The basis for the quantities of each recycle used was described in the Campaign II report (Duignan et al., 2004b or Appendix I-2). The condensate used (that in WTP would come from the Radioactive Liquid Waste Disposal System or RLD) to generate all of the recycles from Campaign II to Campaign III employed condensate from only the Campaign II FEP evaporator, except for two synthetic streams, which used a condensate mixture that came from combining the TLP and FEP products.

The UFP pump loop recycle was approximated by mixing 4.6L of washed concentrated slurry with 8.6L of RLD condensate from the FEP evaporator, both from Campaign III. The pump loop recycle from Campaign III to Campaign IV was made using the same proportions of Campaign III material. The residual rinse in the filter loop that would be present at the start of the first $0.1 \mathrm{M} \mathrm{NaOH}$ rinse was made from approximately $0.1 \mathrm{~L}$ of washed concentrated slurry and 12.5L of FEP condensate. The UFP feed tank heel prior to each rinse and acid cleaning step was targeted to give the correct undissolved solids loading. Note, in the SIPP the amount of rinse and acid used is greater, on a proportional basis, than in the WTP. To account for using more rinse or acid, a proportionally larger heel is left.

The ejector heel of 500 gal of washed concentrated slurry left after slurry washing was simulated by the approximately 22L of washed concentrated slurry that remained in the system after draining. Note that this quantity of slurry holdup was much higher than previously assumed ( $\sim 4 \mathrm{~L})$. To this $22 \mathrm{~L}$ of slurry, 105.2L of caustic rinse $1(\sim 0.1 \mathrm{M}$ $\mathrm{NaOH}$ ), made from RLD condensate, was added. The resulting diluted slurry still had more solids than would be representative of the UFP tank after Caustic Rinse 1, so the recycle to Campaign IV needed to be diluted 1:1 with fresh caustic rinse solution. The heel from caustic rinse 1 to acid cleaning 1 was made from three quantities (1.) 10L of the diluted slurry from caustic rinse 1 , (2.) the $\sim 1.6 \mathrm{~L}$ of this slurry retained in the system, and (3.) $10 \mathrm{~L}$ of $0.1 \mathrm{M} \mathrm{NaOH}$ caustic flush. This scheme is shown in Fig. 2. The RLD condensate was essentially water, with the only significant impurity being ammonium ion at about $458 \mathrm{mg} / \mathrm{kg}$.

In the subsequent rinsing and cleaning steps, the ejector heel was approximated by putting back about $20 \mathrm{~L}$ of the drained solution plus the $\sim 1.6 \mathrm{~L}$ retained in the system. The 21.6L of heel in each step, mixed with 94.6L of caustic rinse or acid is the same ratio as the heel + filter loop $(500+700$ gal) remaining in the WTP to the rinse amount (5250 gal); both ratios are 0.228 . 
WSRC-TR-2005-00105, REVISION 0

SRNL-RPP-2005-00012, REVISION 0

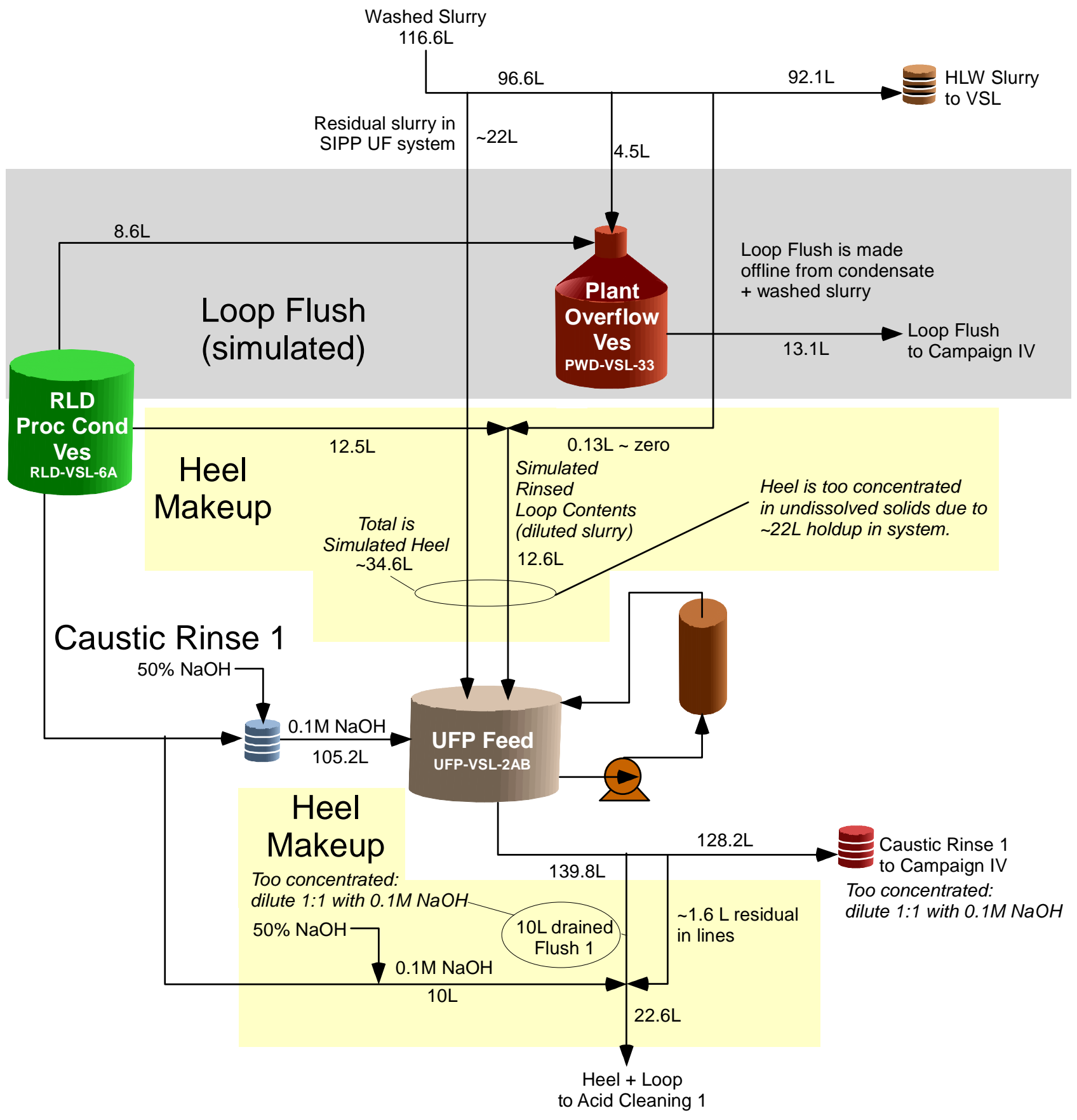

Figure 2. Generation of Caustic Rinse 1 Recycle

\subsubsection{FEP Feed Mixing}

Figure 3 shows a schematic of the FEP feed blending, and the amounts used are also shown in Table 16. The basis for the quantities of each recycle used was described in the Campaign II report (Duignan et al., 2004b or Appendix I-2). The condensate used (RLD) to generate all of the recycles from Campaign III to Campaign IV used the combined condensate from the Campaign III FEP and TLP evaporators. All of the recycles used 
were from Campaign III except for the synthetic recycles and the HLW SBS condensate from Duratek. For the CIX recycles, some synthetic recycles were used where there was insufficient material available. The approximate actual volume of the combined feeds was $1081 \mathrm{~L}$ versus the target of $1090 \mathrm{~L}$. The UFP recycles were arbitrarily split between the PWD-VSL-15 and PWD-VSL-16 tanks. Into PWD-15 were added the first 22 washes permeate, then Caustic Rinse 1, then $0.1 \mathrm{M} \mathrm{NaOH}$ prepared from condensate, and then the first and second acid cleaning solutions. The amount of Caustic Rinse 1 added was not the total amount needed, but was the amount that would give closer to the correct undissolved solids in the recycle stream when combined with the $21.3 \mathrm{~L}$ of $0.1 \mathrm{M}$ caustic prepared from condensate. This $0.1 \mathrm{M}$ caustic was added to approximate what would have been added if Rinse 1 would have had the correct undissolved solids content. (Due to the slurry holdup in the SIPP filter, the correct UDS concentration in the first caustic rinse cannot be achieved. The UDS content had to be adjusted externally to the system.) The wash permeates (117.7 L each) were to have been $140.8 \mathrm{~L}$ per the OLI model ${ }^{\dagger}$, but this much permeate was not made in Campaign III (per the model), so the total wash permeate recycled was about $46 \mathrm{~L}$ less than the model. This difference was not made up by adding any reagent solutions.

Next, the Campaign III Ion Exchange recycles were added. These consisted of the displacement solution $(\sim 0.1 \mathrm{M} \mathrm{NaOH})$ that was displaced from the column by the pre-elution rinse. The post elution rinse displaced by the regeneration solution ( $\sim$ water), and the $0.25 \mathrm{M} \mathrm{NaOH}$ regeneration solution from the column. There were insufficient amounts of all of these recycles, so synthetic recycles were generated. The amounts of recycles available were insufficient because the CIX unit operation was not scaled the same as the other unit operations in the SIPP. The amount of actual and synthetic recycles is shown in Fig. 3 and Table 16. Table 16 also shows the Campaign III quantities. The $36.7 \mathrm{~L}$ of $0.1 \mathrm{M} \mathrm{NaOH}$ synthetic recycle was made from the RLD condensate containing ammonium.

The PWD-16 tank recycles were made of the permeate from the second 22 washes, the second and third caustic rinses, and the third acid cleaning; 0.25L of additional $50 \mathrm{wt} \%$ $\mathrm{NaOH}$ was needed for $\mathrm{pH}$ adjustment. Note that the recycles were combined differently to make the PWD-15 and PWD-16 solutions in Campaign IV than in the previous campaigns. The recycles were combined differently to minimize the amount of added $\mathrm{NaOH}$ required since $\mathrm{NaOH}$ added to neutralize recycles is not counted as waste sodium. The results shown Section 2.6.5 for the FEP evaporation indicate that mixing the recycles differently had no apparent impact on the evaporation process. The elemental and anion compositions of the Campaign III recycles are not presented since they were not measured except for the solids contents, density, and acid or base content. These

\footnotetext{
${ }^{\dagger}$ As mentioned in the footnote to Table 1 , after the modeling was done, and subsequent to completing Campaign I, the WTP UFP feed-tank batch size was increased from 18,000 gallons to 21,550 gallons. However, the criterion to determine the volume of washing water per batch was not changed, which was Volume $=$ Volume of one Permeate Receipt tank $=21,770$ gallons. This means the amount of water used to wash the batch was relatively smaller, that is, $21,770 / 21,550=1.01$ instead of 21,770/18,000 $=1.209$. Since the pilot-scale UFP batch size was $\sim 117 \mathrm{~L}$, then the wash volume would be $117 \times 1.01=118 \mathrm{~L}$ instead of 117 x $1.209=1.41 \mathrm{~L}$.
} 
measured quantities for the UF recycles are shown in Table 17. The synthetic HLW recycles were made up from reagents to approximate the expected compositions. The vessel vent liquid was $0.1 \mathrm{M} \mathrm{NaOH}$, while the $\mathrm{HLW}$ canister decon liquid was $0.15 \mathrm{M}$ $\mathrm{NaOH}$ and $0.29 \mathrm{M} \mathrm{NaNO}$.

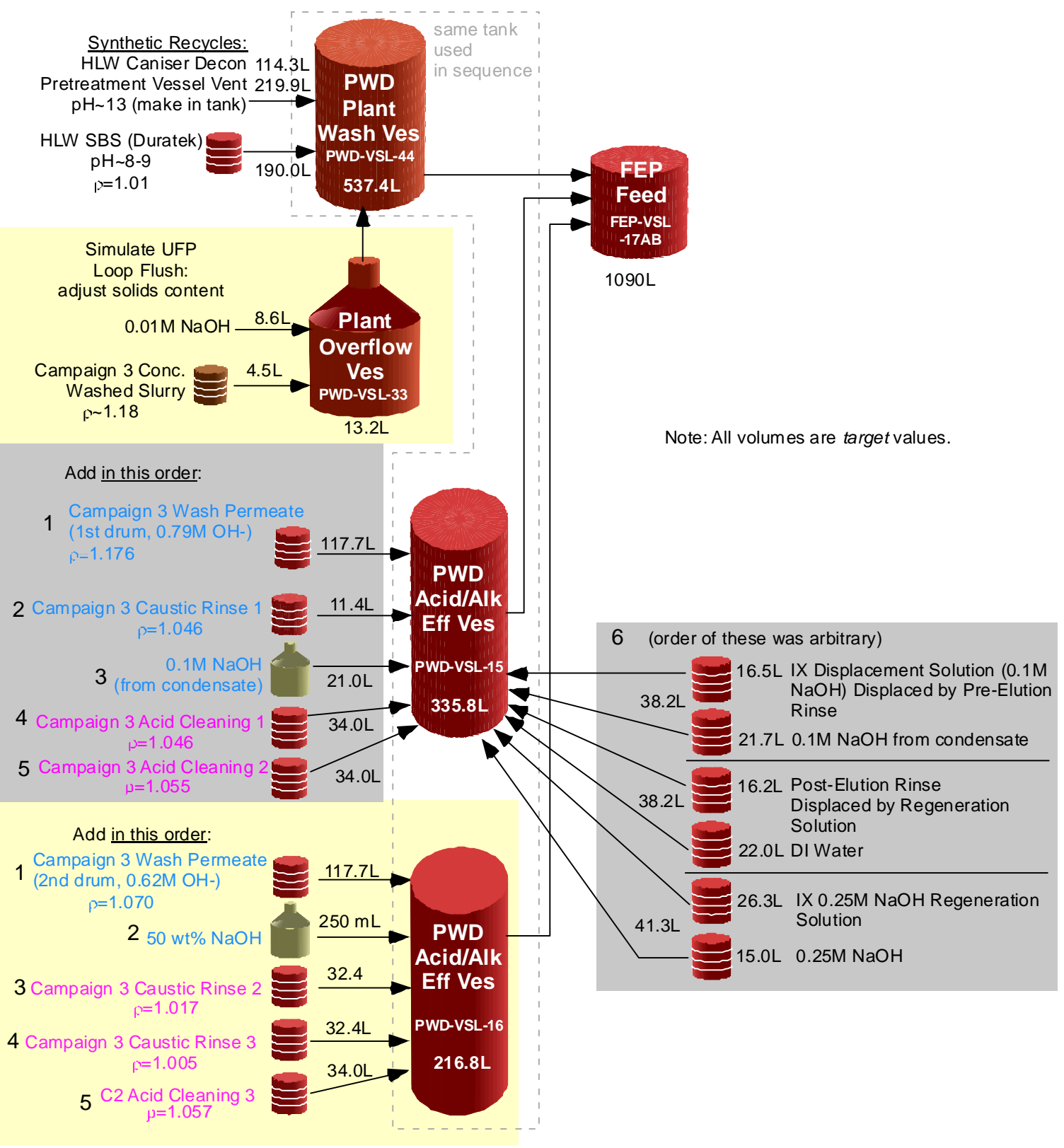

Figure 3. FEP Feed Blending of Recycle Streams 
Table 16. Recycle Streams*

\begin{tabular}{|c|c|c|c|c|}
\hline Stream & $\begin{array}{l}\text { Campaign } \\
\text { III Source }\end{array}$ & $\begin{array}{l}\text { Campaign } \\
\text { IV Volume } \\
\text { (L) }\end{array}$ & $\begin{array}{l}\text { Campaign } \\
\text { III Volume } \\
\text { (L) }\end{array}$ & $\begin{array}{c}\text { Campaign } \\
\text { II Volume } \\
\text { (L) }\end{array}$ \\
\hline HLW SBS Liquid & Duratek & 190.0 & 190.8 & 190.8 \\
\hline Vessel Vent Liquid & synthetic & 219.9 & 220.7 & 220.7 \\
\hline HLW Canister Decon Liquid & synthetic & 114.3 & 114.7 & 114.7 \\
\hline UFP Recycle - Wash Permeate & Camp. III & 235.4 & 236.2 & 338.8 \\
\hline $1^{\text {st }} 22$ Washes Permeate & & 117.7 & 118.1 & 169.3 \\
\hline $2^{\text {nd }} 22$ Washes Permeate & & 117.7 & 118.1 & 169.3 \\
\hline UFP Recycle - 0.1 M NaOH Rinse & Camp. III & 97.2 & 97.7 & 117.0 \\
\hline $1^{\text {st }}$ Rinse & & 11.4 & 11.2 & 18.2 \\
\hline $2^{\text {nd }}$ Rinse & & 32.4 & 32.6 & 49.5 \\
\hline $3^{\text {rd }}$ Rinse & & 32.4 & 32.6 & 49.5 \\
\hline Additional 0.1M Caustic & & 21.0 & 21.3 & 0 \\
\hline $50 \mathrm{wt} \%$ caustic & synthetic & 0 & 0 & 11.55 \\
\hline UFP Recycle - $2 \mathrm{M} \mathrm{HNO}_{3}$ Cleaning & Camp. III & 102.0 & 102.3 & 122.5 \\
\hline $1^{\text {st }}$ Cleaning & & 34.0 & 34.1 & 37.5 \\
\hline $2^{\text {nd }}$ Cleaning & & 34.0 & 34.1 & 37.5 \\
\hline $3^{\text {rd }}$ Cleaning & & 34.0 & 34.1 & 37.5 \\
\hline $50 \mathrm{wt} \%$ caustic & synthetic & 0.25 & 0 & 0 \\
\hline UFP Recycle - UF Loop Rinses & Camp. III & 13.1 & 13.1 & 13.2 \\
\hline RLD condensate & & 8.6 & 8.6 & 8.6 \\
\hline Washed Concentrated Slurry & & 4.5 & 4.5 & 4.6 \\
\hline CIX Recycles & Camp. III & 117.7 & 113.5 & 0 \\
\hline Displacement Solution displaced by Pre-Elution Rinse & & 16.5 & 0 & 0 \\
\hline $0.1 \mathrm{M} \mathrm{NaOH}$ (insufficient Displacement Solution) & synthetic & 21.7 & 36.7 & 0 \\
\hline Post-Elution Rinse displaced by Regeneration Solution & & 16.2 & 19.0 & 0 \\
\hline DI Water (insufficient Post-Elution Rinse) & synthetic & 22.0 & 17.7 & 0 \\
\hline 0.25M NaOH Regeneration Solution & & 26.3 & 2.0 & 0 \\
\hline $0.25 \mathrm{M} \mathrm{NaOH}$ (insufficient Regeneration Solution) & synthetic & 15.0 & 38.1 & 0 \\
\hline TOTAL & & 1090 & 1089 & 1129 \\
\hline
\end{tabular}

* This table does not show the recycle streams organized by order added. See Fig. 3 for this information. 


\section{WSRC-TR-2005-00105, REVISION 0 SRNL-RPP-2005-00012, REVISION 0}

Table 17. Composition of Wash Permeate Recycles to FEP Evaporator

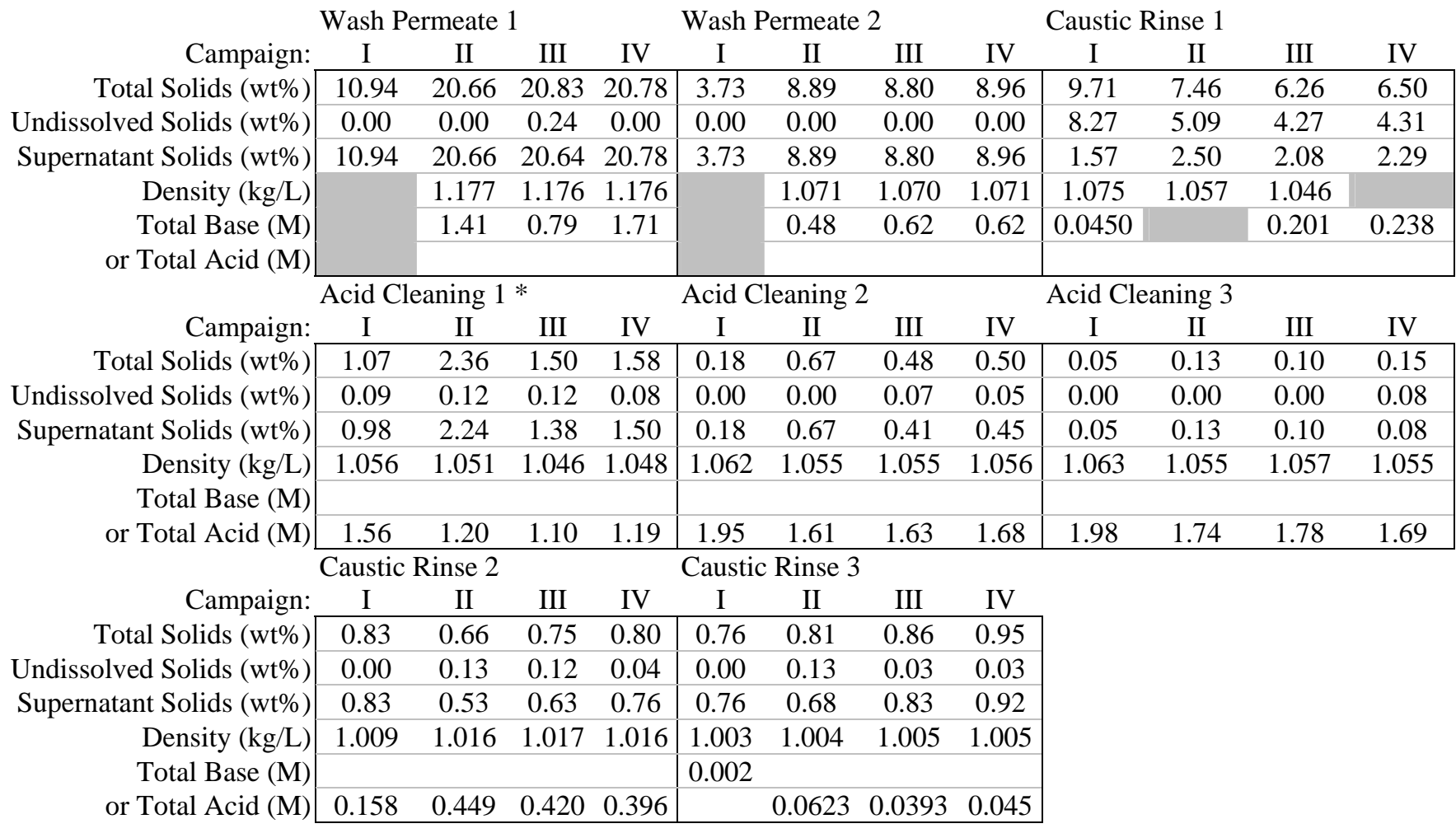




\subsubsection{Simulant Handling (Author - Williams)}

For the following description of how the recycle FEP feed stream was made at the test location, see Fig. 3. A 760-liter tank was used to mix pilot-scale quantities of simulated HLW recycles that were then transferred to the pilot-scale FEP Feed Tank, which was represented by the plant tank: FEP-VSL-17AB. On 17 Augusts 2004, pilot-scale quantities of HLW Canister Decon and HLW Vessel Vent solutions were synthesized by combining 328.8 liters of deionized and filtered (DIF) water with $2.99 \mathrm{~kg}$ of $50 \mathrm{wt} \%$ $\mathrm{NaOH}, 2.76 \mathrm{~kg}$ of sodium nitrate, and $191.9 \mathrm{~kg}$ of HLW SBS Quencher solution from VSL. At this stage, the mixing tank represented the plant tank: PWD-VSL-44. To this mixture, the contents of mock plant tank PWD-VSL-33 (Plant Overflow Vessel) were added, which contained $5.31 \mathrm{~kg}$ of Campaign III concentrated washed HLW slurry and 8.6 liters of Campaign III FEP condensate. After mixing the entire contents of the mock PWD-VSL-44, and -33, they were transferred to the FEP Feed Tank on 18 August, 2004. The Campaign IV simulant activities were documented in the laboratory notebook (Williams, 2004).

On 18 August, the then empty 760-liter mixing tank was used as a plant mock PWDVSL-15 tank and was filled with caustic and acid recycle streams from Campaign III. To that tank, the first of two drums of wash permeate was added, drum No. RM-03-269, which contained $133.0 \mathrm{~kg}$ of material. Then $21.0 \mathrm{~kg}$ of $0.1 \mathrm{M} \mathrm{NaOH}$ from condensate and the Campaign III 0.1 M caustic flush recycle stream and Campaign III Acid Cleaning 1 and 2 recycle streams were added from three drums referred to as Caustic Flush 1, and Acid Flush 1 and Acid Flush 2, respectively. The amounts added from drums Nos. RM03-281, RM-03-302, and RM-03-309, were $11.9 \mathrm{~kg}, 35.6 \mathrm{~kg}$, and $35.9 \mathrm{~kg}$, respectively. The material remaining in the drums was returned to storage. The Campaign III CIX recycles were added next including $21.7 \mathrm{~kg}$ of $0.1 \mathrm{M} \mathrm{NaOH}$ from condensate, $16.8 \mathrm{~kg}$ of Displacement Solution (0.1 M NaOH) displaced by Pre-Elution Rinse, 15.6 kg of PostElution Rinse displaced by Regeneration Solution, $22.0 \mathrm{~kg}$ of DI Water, $26.6 \mathrm{~kg}$ of $0.25 \mathrm{M} \mathrm{NaOH}$ Regeneration Solution, and $15.0 \mathrm{~kg}$ of $0.25 \mathrm{M} \mathrm{NaOH}$ from DI Water. The target $\mathrm{pH}$ for this mixture was to be greater than or equal to 12, and since the $\mathrm{pH}$ was measured at 13 no additional $\mathrm{NaOH}$ was added. The contents of this mock PWD-VSL15 mixing vessel were then added to the mixture in the FEP Feed Tank, also on 18 August.

Once again the 760-liter mixing tank was empty and this time it would represent the plant PWD-VSL-16 tank to receive the remaining caustic and acidic recycle streams. That solution has to be adjusted to be alkaline before being joined to the caustic contents of the FEP feed tank. Specifically, the empty tank was first filled with $121.4 \mathrm{~kg}$ of $0.1 \mathrm{M}$ $\mathrm{NaOH}$ wash permeate from the second of two drums of wash permeate recycle, drum No. RM-03-270. A container of $250 \mathrm{ml}$ of $50 \mathrm{wt} \% \mathrm{NaOH}$ was added to the Wash Permeate from Campaign III. While the tank agitator was running three drums of recycled Campaign III spent Caustic Flush 2, Caustic Flush 3, and 2 M nitric acid UFP cleaning solution were added with constant stirring. From the drum (referred to as Caustic Flush 
2) No. RM-03-268 an amount of $33.0 \mathrm{~kg}$ was transferred and from RM-03-312 (referred to as Caustic Flush 3) an amount of $32.6 \mathrm{~kg}$ was transferred. Finally, an amount of 35.9 kg was transferred from RM-03-310 (referred to as Acid Cleaning 3) to the PWD-VSL16 mixing tank. The acid/caustic mixture was transferred to the FEP Feed Tank on 23 August to start the clock on a 2-day holding period before evaporation began. The total material mixed in the pilot-scale FEP unit operation Feed Tank, Fig. 4, and subsequently sent to the FEP Evaporator, was 1,118.8 kg. Further, the $\mathrm{pH}$ of the feed checked with $\mathrm{pH}$ paper, indicated a $\mathrm{pH}>13$, which was the same as measured in the previous Campaigns.

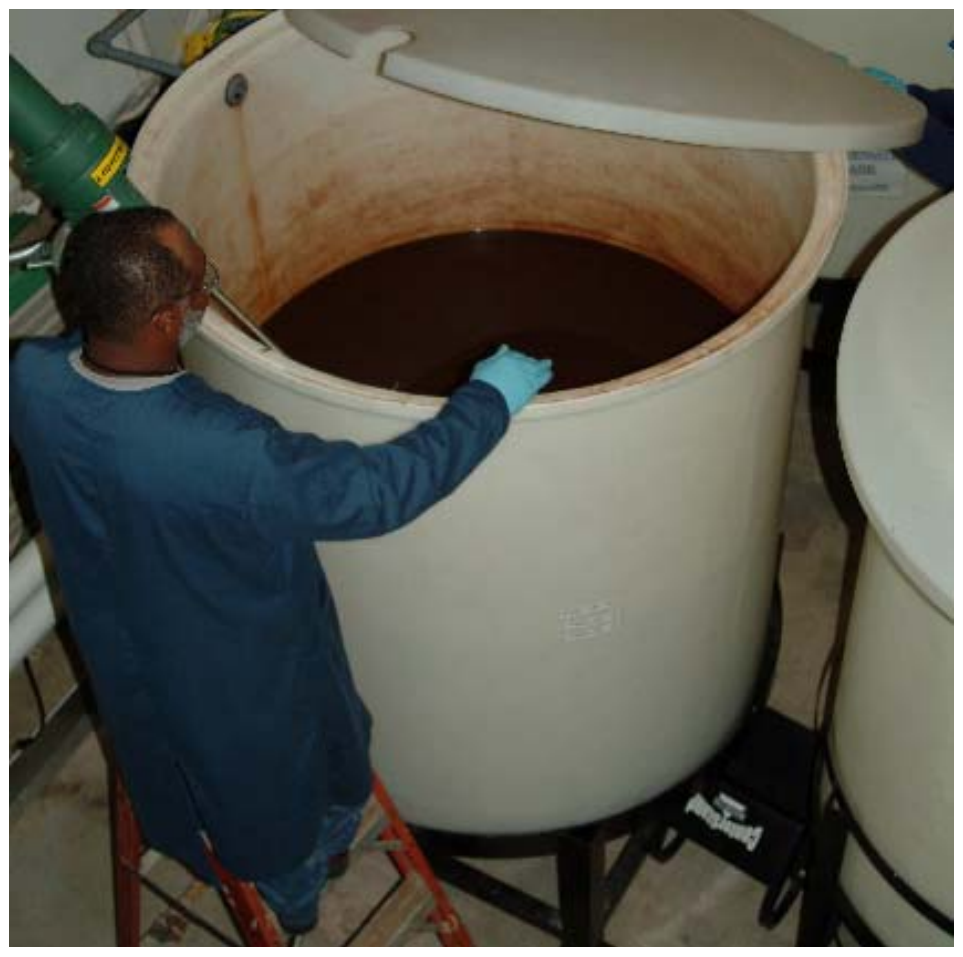

Figure 4. Pilot-scale FEP Unit Operation Feed Tank

After evaporation started on 25 August, and during the first 30 minutes of operation, 278.4 g of Dow Corning Q2-3183A antifoam were added in three batches of $92.8 \mathrm{~g}$ each. Note that, per the vendor's recommendation the antifoam was diluted to a 10:1 ratio with deionized and filtered water (DIF) before the addition was made. At the end of FEP testing, a total of $174.6 \mathrm{~kg}$ of concentrate "FEP Bottoms" were produced with a density of $1.258 \mathrm{gm} / \mathrm{ml}$, as measured by densitometer at $20^{\circ} \mathrm{C}$.

\subsubsection{Pilot FEP Equipment (Author-Qureshi)}

Three FEP evaporation campaigns (Campaigns II, III and IV) were performed in the Pilot-Scale Evaporator designed and constructed for the RPP Project. The evaporator is located in the Engineering Development Lab. of the Savannah River National Lab (SRNL). 
Figure 5 shows a schematic of the Pilot Scale Evaporator test facility. The evaporator is a forced circulation evaporator where heat is added in the reboiler. No evaporation occurs in the reboiler due to hydrostatic pressure head. The evaporator vessel is kept under vacuum (1.0 to 1.2 psia or 6.9 to $8.2 \mathrm{kPa}$ ) and the boiling temperature for water is about $105^{\circ} \mathrm{F}\left(41^{\circ} \mathrm{C}\right)$ at this pressure. The simulant has a boiling point temperature elevation of about $11^{\circ} \mathrm{F}\left(6^{\circ} \mathrm{C}\right)$. Thus the entire evaporation process occurs at moderate temperatures ( 100 to $120^{\circ} \mathrm{F}$ or 38 to $49^{\circ} \mathrm{C}$ ). The evaporator vessel is about 27 feet tall with a nominal inside diameter of 17 inches, Fig. 6. The evaporator is designed to evaporate up to $0.6 \mathrm{gpm}$ (23 lpm) of water.

The evaporator test facility consists of many sub-systems. Hardware descriptions of the facility, sub-systems, and key equipment are alphabetically described below.

\subsubsection{Antifoam System}

The evaporator recirculation loop has an injection port where antifoam agent is injected. Under normal operating conditions the pressure in the evaporator vessel vapor space is 1 psia, and despite the hydrostatic head of the concentrate, the absolute pressure at the injection port is below atmospheric pressure. The required amount of antifoam agent is diluted in a 1-liter bottle. Through a tube and a ball valve, this diluted antifoam agent is sucked in to the evaporator feed. Under certain conditions where full vacuum is not achieved yet and a positive pressure exists at the injection port, the diluted antifoam agent is injected using a large graduated syringe. See Fig. 7.

\subsubsection{Concentrate Loop}

This loop consists of a concentrate removal pump, flow meter, and a 500-gallon concentrate receipt tank. Under steady evaporator operations, the liquid level in the evaporator vessel is kept within minimum and maximum level limits. The evaporator is fed continuously and the concentrate is removed at a predetermined rate from the evaporator vessel to maintain the liquid level. However, for small batches, the evaporator is fed at a constant rate without removing any concentrate. The entire inventory of the concentrate is accumulated in the evaporator vessel until the target density is reached. At that point if the liquid level exceeds the maximum limit, concentrate is removed to bring the level to the lower limit. For the three SIPP FEP campaigns, all the concentrate was accumulated in the evaporator vessel and drained at the end of the test period. 
WSRC-TR-2005-00105, REVISION 0

SRNL-RPP-2005-00012, REVISION 0

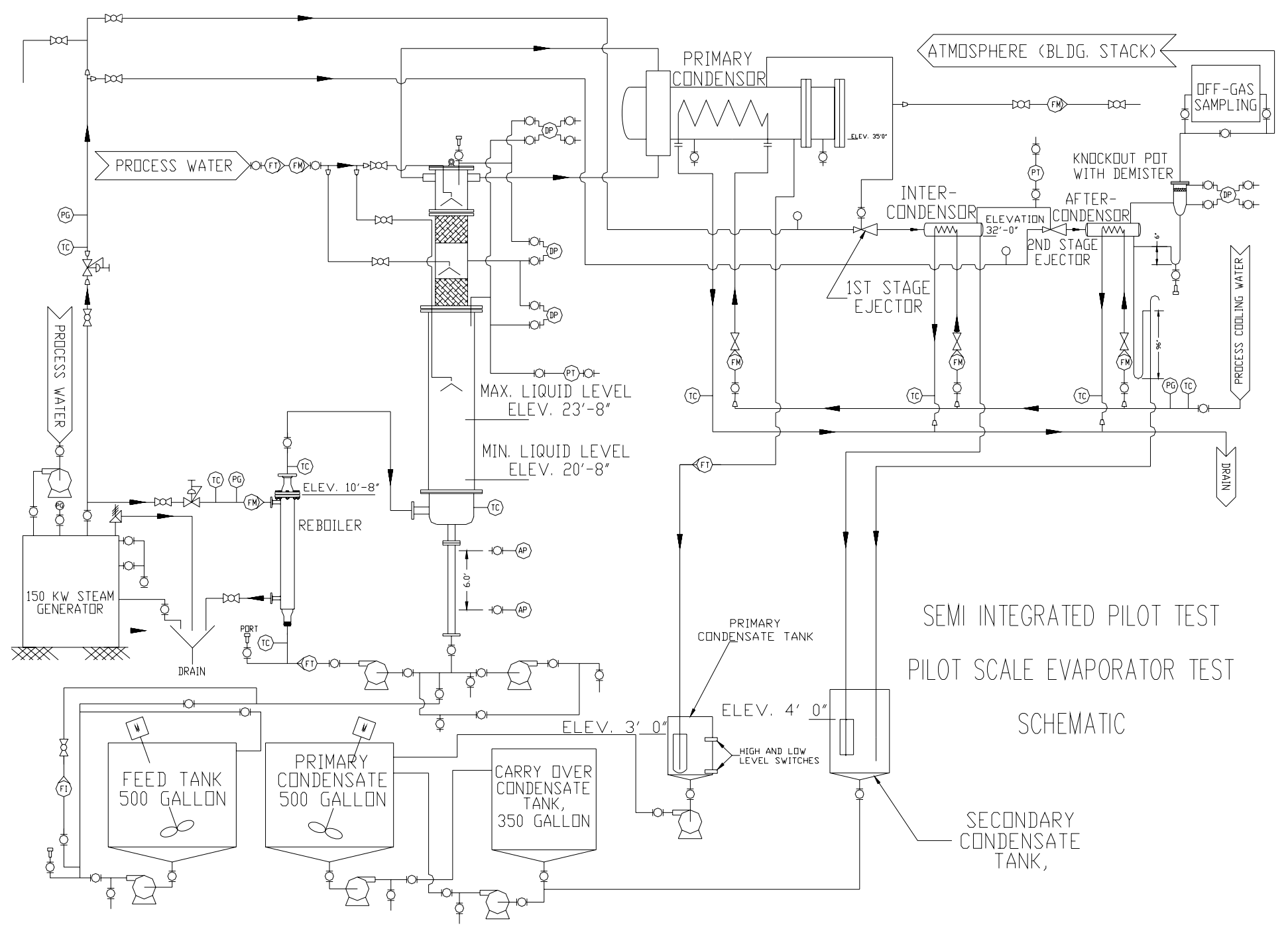

Figure 5. SIPP Pilot-Scale FEP/TLP Unit 
WSRC-TR-2005-00105, REVISION 0 SRNL-RPP-2005-00012, REVISION 0

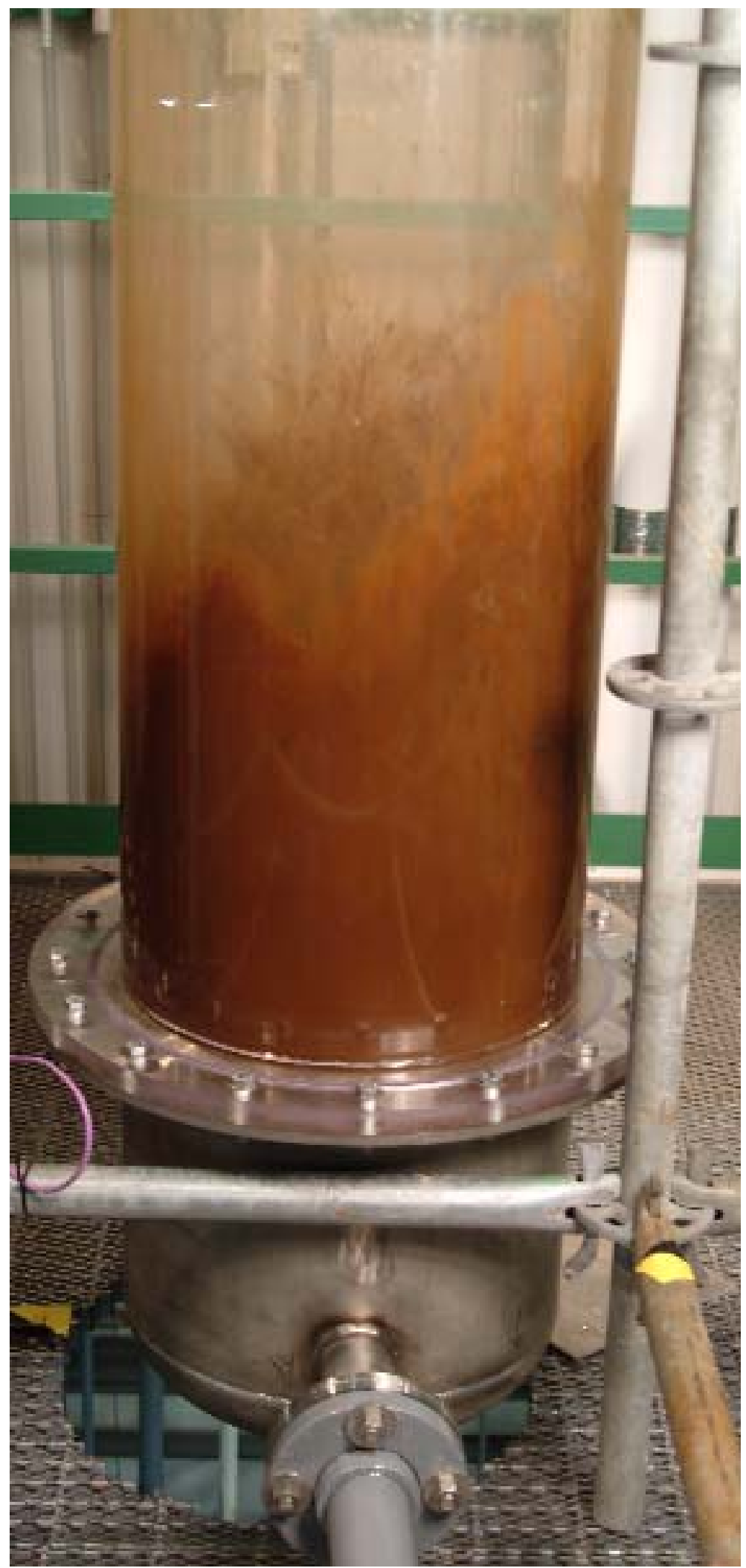

Figure 6. SIPP Pilot-Scale FEP/TLP Unit: The bottom half of the 15-foot tall Acrylic Section with Ongoing Evaporation 


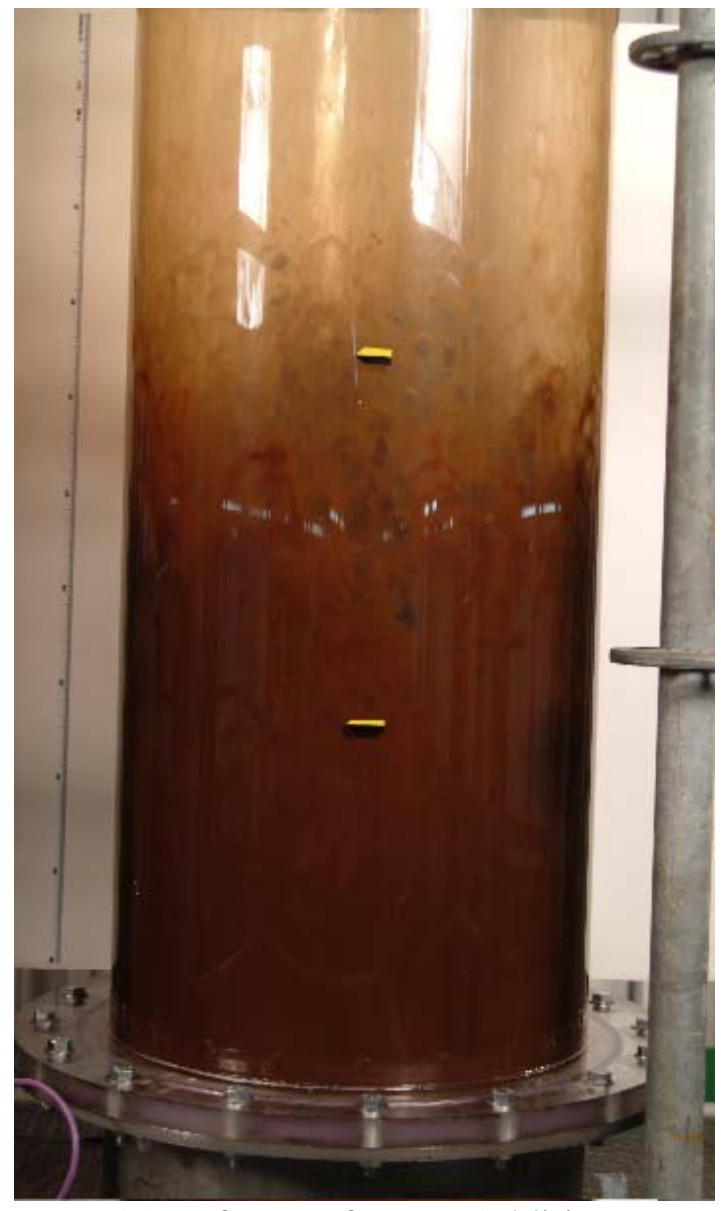

(a) Before Defoamer Addition

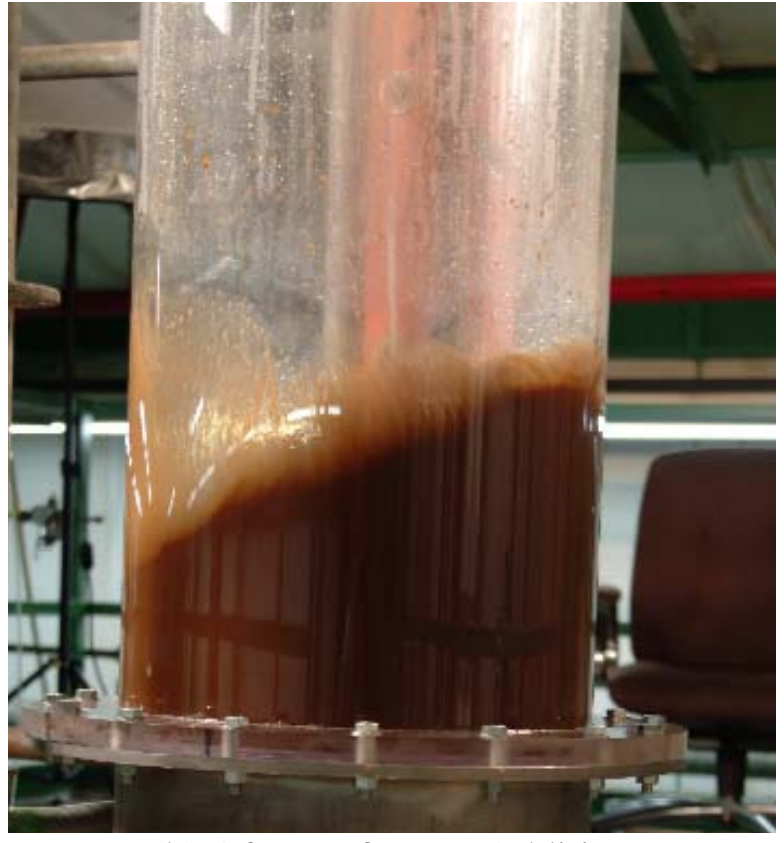

(b) After Defoamer Addition

Figure 7. SIPP Pilot-Scale FEP/TLP Unit: Evaporating

\subsubsection{Evaporator Recirculation Loop}

This loop consists of a 100 gpm variable speed drive centrifugal pump, flow meter, reboiler and the evaporator vessel. Note that the heat is not added directly to the evaporator vessel. Instead, heat is added via a vertical reboiler that is essentially a shell and tube heat exchanger. Low pressure steam condenses on the shell side and the concentrate passes through the tube side. The outlet of the tube side is about $10 \mathrm{ft}$ below the boiling surface. Due to this hydrostatic head, no boiling occurs in the reboiler. The concentrate enters the evaporator vessel through the side wall and discharges vertically through a 2-inch diameter pipe. The discharge point is about 2 feet below the boiling surface. Due to low pressure, the saturated concentrate flashes. The vapor is disengaged near the boiling surface and leaves the evaporator vessel at the top. The liquid is drawn at the bottom by the main recirculation pump. The water vapor passes through two mist eliminator pads to remove any entrained droplets. 
As stated, the evaporator vessel is about 27-foot tall with a nominal inside diameter of 17 inches. The bottom 12 feet of the vessel is stainless steel and the top 15 feet is acrylic, as seen in Fig. 6. Of the bottom 12 feet, the stainless steel vessel is only 2 feet high and the remaining height of 10 feet is taken up by a 2-inch stainless steel pipe. The boiling surface is maintained in the lower part of the acrylic section. The minimum and maximum liquid levels in the acrylic section are 5 and 40 inches, respectively. The designed capacity of evaporator vessel is about 30 gallons.

\subsubsection{Feed Loop}

This loop transfers the feed from the feed tank to the evaporator recirculation loop upstream of the main recirculation pump. It consists of the feed tank, a 10-gpm centrifugal feed pump, flow meter, and 1-inch PVC piping. Downstream of the feed pump, the feed can go either through a 1-inch PVC line or a 1/4-inch stainless steel line with 0- to 1-gpm flow meter and a throttling valve. For initial transfer of feed, the large 1-inch line is used. Once the desired liquid level is achieved in the evaporator vessel and boiling process starts, feed is transferred through the smaller line with flow meter to monitor the feed rate.

\subsubsection{Jet Pump}

The evaporator operates under vacuum (1.0 - 1.2 psia). A two-stage steam driven jet pump maintains desired vacuum in the evaporator vessel. Steam from the steam generator is supplied at 110 psig to the two stages of the jet pump. The jet pump has its own set of condensers, inter and after-condenser. A separate tank catches the condensate from the jet pump condensers. The condensate from the first stage of the jet pump is discharged to the condensate catch tank via a dip tube because the inter-condenser operates under vacuum. The condensate from the after-condenser discharges directly into the catch tank without any dip tube since this condensate is already at a few inches of water above the atmospheric pressure.

The suction side of the first stage of the jet pump is connected to the primary condenser. Any non-condensable gases and some carry over vapor from the primary condenser are sucked into the jet pump. The vacuum level is adjusted by an air bleed in to the suction line via a throttling valve. The jet pump performance degraded slightly once during an early shakedown and it was attributed to some machining debris lodged in the jet pump nozzles. However, full performance returned after a thorough cleaning of all lines.

\subsubsection{Primary Condensate Loop}

The primary condensate loop includes the primary condenser, a 1-inch clear PVC transfer line, a water-filled dip tube, primary condensate catch tank, transfer pump and a 350-gallon primary condensate storage tank. The lower end of the 1-inch condensate line is placed in the dip tube to maintain vacuum in the condenser which is placed about 35 feet above the lab floor. The primary condenser is a shell and tube type heat exchanger with water vapor condensing on the shell side and coolant water flowing through the tubes. As the vacuum is built up, water from the dip tube is sucked into the condensate line. The distance between the top of the dip tube and bottom of the primary condenser is designed to be about 32 feet. This allows sufficient water column in the 
clear PVC tube corresponding to the vacuum level. Under steady operation this water column is maintained and the condensate flows out of the dip tube into the catch tank. Once the water level is about 1 foot in the catch tank, a transfer pump automatically transfers the condensate to the 500-gallon tank. The transfer pump is controlled by two limit switches installed 1 foot apart on the catch tank.

\subsubsection{Process Water Loop}

The process-water loop includes house-water supply lines to the primary condenser, the inter-condenser, and the after-condenser. Each supply line has flow meters, ball valves and throttle valves to adjust the flow rates. Typically, the house water temperature is around 68 to $72^{\circ} \mathrm{F}\left(20\right.$ to $22^{\circ} \mathrm{C}$ ). The temperature rise through the condensers is 5 to $9^{\circ} \mathrm{F}$ $\left(3-5^{\circ} \mathrm{C}\right)$. The cooling water is discharged to the building drains after flowing through the condensers.

\subsubsection{Secondary Condensate Loop}

This loop collects condensate streams from the two jet pump condensers. All the steam supplied to the two stages of the jet pump and any carryover water vapors from the primary condenser are condensed and constitute the secondary condensate. They are collected in the secondary condensate tank. The secondary condensate is either drained through the building drains or transferred to the primary condensate tank.

\subsubsection{Steam Generator}

An electric steam generator (Electro-Steam Model LB-150) supplies steam to the reboiler and the jet pump. The supply pressure is $110-120$ psig. Steam to the reboiler is throttled down to a 3-5 psig. Steam to the jet pump is not throttled. Steam and condensate coming out the reboiler is drained through the building drains. Condensate coming out of jet pump condensers is collected in the secondary condensate tank.

\subsubsection{Spray Water}

Three water spray nozzles are provided in the evaporator vessel. Two 1-gpm conical spray nozzles are installed above each of the demister pads to wash them out as needed. The third spray nozzle is provided to clean the acrylic portion of the evaporator vessel. This nozzle is attached to a vertically traversing wand that allows washing of acrylic wall at any height.

\subsubsection{Pilot FEP Operation (Author - Qureshi)}

Typically, each campaign evaporated about 300 gallons of water in an 8- to 10-hour period from waste simulant. Feed tank supplies the simulant to the evaporator. The evaporator is designed to evaporate at a rate of up to $0.6 \mathrm{gpm}$ of water. The water vapor is condensed in the primary condenser. Under steady conditions, the concentrate is removed from the recirculation line and collected in a drum. The condensate from the primary condenser is collected in the primary condensate tank. Several sample and injection ports are provided to draw samples and inject antifoam agent as needed. 
WSRC-TR-2005-00105, REVISION 0

SRNL-RPP-2005-00012, REVISION 0

All test data were acquired by a data acquisition system (DAS) and an abridged set of those data for Campaigns II, III, and IV are listed in Appendix H1. Manual data and observations were recorded in the lab notebook issued for this task. (Qureshi, 2004)

Prior to starting the SIPP evaporation campaigns, the evaporator system was disassembled, cleaned, inspected and reassembled. The system was found to be free of any material defects, fouling and malfunction from previous evaporation runs. After assembly of the system the following tests were performed to ensure proper operation of the evaporator.

\subsubsection{Experimental Facility Leak Testing}

Objective - Determine if the system can hold vacuum, i.e., air in-leakage rate is acceptable.

\section{Test Sequence}

i) Isolated the Evaporator Recirculation (ER) Loop from rest of the system by closing appropriate valves.

ii) Disconnected the First Stage Ejector line from the primary condenser and connected a vacuum pump at the primary condenser discharge.

iii) Filled water in Evaporator vessel to the maximum operating liquid level (about 40 inches in the lower acrylic section).

iv) Filled up the primary dip tube reservoir with water.

v) Started and operated the vacuum pump until the water level in the dip tube is about 31 feet of water.

vi) Verified that there was no air bubbling into the system.

vii) Verified that the system can hold vacuum for about 30 minutes.

vii) Test complete.

\subsubsection{Evaporation Test with Water}

Objective - To shake down the system and its components using water as test fluid prior to the chemical runs.

\section{Test Sequence}

i) Isolated the Reboiler and the Jet Pump from the Steam Generator. 
ii) Started the Steam Generator.

iii) Filled the Feed Tank with Process Water to about 25\% level (about 125 gallons).

iv) Using the Feed Loop pump FL-PU1, transferred water from the Feed Tank to the evaporator vessel to a level about 40 inches in the acrylic section.

v) Filled the primary condensate and secondary condensate dip tubes with water.

vi) Started the Jet Pump Vacuum System.

vii) Started the main recirculation pump and started supplying steam to the Reboiler.

viii) Adjusted steam to the Reboiler to limit the system temperature below $60 \mathrm{C}$.

ix) Once the system pressure dropped to about 1 psia, boiled water for about 30 minutes.

x) After completing the water boiling test, turned off the steam supply to the Jet Pump and the Reboiler and shut down the Steam Generator.

xi) Test complete.

\subsubsection{SIPP-Feed Evaporation Process (FEP)Tests}

Objective - To perform pilot scale tests of the Feed Evaporation Process.

Three evaporation campaigns were performed as a part of SIPP test program. These campaigns are designated and II, III and IV. The FEP was not used for Campaign I. During the evaporation process for each campaign, several liquid samples were drawn per sample matrix provided in Appendix D. Typically, these samples included feed, concentrate, primary condensate and secondary condensate. The samples were analyzed for their chemical composition. The analytical results for all evaporation campaigns are discussed and compared in Section 2.6.5. An abridged version of the operational data are given in Appendix H1.

\section{Test Sequence}

All test campaigns were performed following EDL approved work instructions written specifically for SIPP operations. Pertinent sequence of operations is provided below.

i) Started Steam Generator and the Data Acquisition System.

ii) Ran the Feed Tank mixer for about 30 minutes. 
WSRC-TR-2005-00105, REVISION 0

SRNL-RPP-2005-00012, REVISION 0

iii) Using the Feed Loop Pump, transferred feed from the Feed Tank to the evaporator vessel to about 24" level in the clear section.

iv) Filled the primary condensate and secondary condensate dip tubes with water.

v) Supplied process water to the Jet Pump condensers to about 5 gpm each and started the Jet Pump by supplying steam to the two jets. The evaporator pressure starts dropping at this point. It took about 10 minutes to reach the target vacuum levels.

vi) Supplied process water to the Primary Condenser to about 30 gpm.

vii) Started the main recirculation pump and turned on the steam to the Reboiler.

viii) Once the feed reached the saturation temperature and boiling started, maintained the liquid level in the evaporator by feeding the appropriate amount of feed using the feed pump.

ix) Recorded all the temperature, pressure and flow date via DAS. Additionally, video taped the action near the boiling surface as needed at different times

x) Antifoam agent was added to the recirculation loop at the beginning of the evaporation campaign.

xi) Drew liquid samples according the sample matrix.

xii) Continued evaporation process until the target density for each campaign was reached.

xiii) Once the evaporation campaign was complete, the steam generator was shut down and all the concentrate was transferred into a 55 gal drum.

xiv) After removing all the concentrate, filled the system with water ( 30 gallons) and rinsed it thoroughly by running the recirculation pump. This rinse water was transferred to a drum. Subsequent water rinses were performed as needed to clear out the system.

xv) Test complete.

\subsubsection{Pilot FEP Discussion of Results}

\subsubsection{Experimental Observations (Author-Qureshi)}

For the three FEP campaigns, important test parameters are summarized in Table 18. The feed compositions and mixing process are described in section 2.6.1.2. 
WSRC-TR-2005-00105, REVISION 0

SRNL-RPP-2005-00012, REVISION 0

Table 18. Operating Parameters for FEP Campaigns

\begin{tabular}{|c|c|c|c|}
\hline Parameters & Campaign II & Campaign III & Campaign IV \\
\hline Date & $4-14-2004$ & $6-9-2004$ & $8-25-2004$ \\
\hline Total Feed Mass & $2580.1 \mathrm{lbs}$ & $2452.0 \mathrm{lbs}$ & $2466.5 \mathrm{lbs}$ \\
$(1170.3 \mathrm{~kg})$ & $(1112.2 \mathrm{~kg})$ & $(1118.8 \mathrm{~kg})$ \\
\hline $\begin{array}{c}\text { Feed Density @ 20 C, } 1 \\
\text { atm }\end{array}$ & $1.015 \mathrm{~g} / \mathrm{ml}$ & $1.0352 \mathrm{~g} / \mathrm{ml}$ & $1.0345 \mathrm{~g} / \mathrm{ml}$ \\
\hline Evaporator Pressure & $1-1.2 \mathrm{psia}$ & $1-1.2 \mathrm{psia}$ & $1-1.2 \mathrm{psia}$ \\
\hline Average Boiling Temp & $45-50 \mathrm{C}$ & $45-50 \mathrm{C}$ & $45-50 \mathrm{C}$ \\
\hline Test Duration & $8.25 \mathrm{hrs}$ & $7.5 \mathrm{hrs}$ & $7.75 \mathrm{hrs}$ \\
\hline Average Boil-off Rate & $0.54 \mathrm{gpm}$ & $0.55 \mathrm{gpm}$ & $0.54 \mathrm{gpm}$ \\
$(123 \mathrm{~kg} / \mathrm{hr})$ & $(125.6 \mathrm{~kg} / \mathrm{hr})$ & $(121.8 \mathrm{~kg} / \mathrm{hr})$ \\
\hline Total Antifoam Added $(1)$ & $1516 \mathrm{ppm}$ & $1630 \mathrm{ppm}$ & $1594 \mathrm{ppm}$ \\
\hline Final Concentrate Density & $1.25 \mathrm{~g} / \mathrm{ml}$ & $1.257 \mathrm{~g} / \mathrm{ml}$ & $1.258 \mathrm{~g} / \mathrm{ml}$ \\
@ 20 $0^{\circ}$ C, 1 atm (2) & & $375.0 \mathrm{lbs}$ & $384.9 \mathrm{lbs}$ \\
& Final Concentrate Mass & $405.2 \mathrm{lbs}$ & $(174.6 \mathrm{~kg})$ \\
\hline
\end{tabular}

(1) The listed concentrations are based on the volume (at $20^{\circ} \mathrm{C}$ and $1 \mathrm{~atm}$ ) of pilot FEP concentrate obtained at the end of each campaign. However, the target antifoam concentration was $1500 \mathrm{ppm}$ for the concentrated FEP slurry at $50^{\circ} \mathrm{C}$ and 1 psia. Based on the pilot FEP working volume of 40 gallons (151.4 L) and a target concentrate density of $1.226 \mathrm{~g} / \mathrm{ml}$ at $50^{\circ} \mathrm{C}$ and 1 psia, the mass of antifoam added in each campaign was $151,400 \mathrm{~g}$ x $1.226 \mathrm{~g} / \mathrm{mL}$ x $1500 \mathrm{ppm}=278.4$ grams

(2) The FEP final target density at the operation conditions of $50^{\circ} \mathrm{C}$ and 1 psia was $1.226 \mathrm{~g} / \mathrm{ml}$ and at ambient conditions of $20^{\circ} \mathrm{C}$ and $1 \mathrm{~atm}$ was $1.252 \mathrm{~g} / \mathrm{ml}$, as per the OLI/ESP model.

\subsubsection{FEP Waste Products (Author-Zamecnik)}

Table 19 shows the FEP feed and concentrated Bottoms total and dissolved (supernate) compositions. The slurry elemental compositions were measured on microwave acid and sodium peroxide fusion HCL uptake dissolutions. The slurry anion compositions were measured on diluted slurry that was filtered after dilution, so any water-dissolvable salts present should have been dissolved. The feed samples were taken from the feed tank prior to the evaporation, while the Bottoms samples were taken from the recirculation line sample tap at the completion of the evaporation. All samples remained at room temperature until analyzed (within approx. 30 days).

The mass ratios (g in Bottoms/g in feed as \%) of total species in the Bottoms slurry to the feed slurry are also shown. Deviation of these ratios from $100 \%$ indicates the uncertainty in the analyses since none of the species measured are lost during the evaporation (except for small amounts of entrainment). Water is the only significant species removed.

Comparison of the supernate / slurry composition ratios indicate that Ca (dissolved: 28 to $3 \%$ ) and oxalate (158 to 25\%) precipitated. Based on amount of oxalate precipitated and te Na ratio (95 to 91\%), some Na was also precipitated with the oxalate. The supernate / slurry ratio for $\mathrm{Cr}$ would also seem to indicate that some $\mathrm{Cr}$ also precipitated; this ratio in 
WSRC-TR-2005-00105, REVISION 0

SRNL-RPP-2005-00012, REVISION 0

the feed was $88 \%$ and was $81 \%$ in the Bottoms. However, the analytical uncertainty of the feed and Bottoms analyses is large enough that the difference between 88 and $81 \%$ is not significant; replicate analyses are needed to improve these values. Similarly, the apparent drop in the Al ratio from 61 to $56 \%$ is also inconclusive.

Generally, the data show that the material balance closes within about $\pm 20 \%$ for the more abundant species. The feed contains partially dissolved $\mathrm{Al}, \mathrm{Ca}, \mathrm{Li}, \mathrm{Mg}, \mathrm{Na}, \mathrm{P}, \mathrm{S}$, and Si; the remaining elements are totally undissolved. The ion analyses appear to show complete dissolution, but the analytical values are suspect. The elemental analyses show that some P (phosphate) and S (sulfate) are not dissolved. Fluoride would also be expected to be partially undissolved since some was added as $\mathrm{CaF}_{2}$. 
WSRC-TR-2005-00105, REVISION 0

SRNL-RPP-2005-00012, REVISION 0

Table 19. Composition of Campaign IV FEP Evaporator Streams

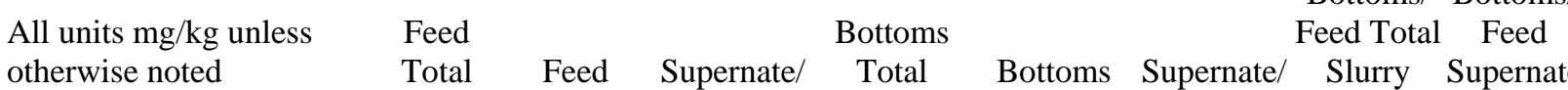

Slurry Supernate Slurry (\%) Slurry

\begin{tabular}{|c|c|c|c|c|c|c|c|c|}
\hline & Drumy & леретा1ан & simу $(10)$ & Siury & sерепाите & orumy $(70)$ & (10) & (\%) \\
\hline Total Solids (wt\%) & 4.98 & 4.78 & & 31.52 & 29.68 & & 96 & \\
\hline Undissolved Solids (wt\%) & 0.21 & 0 & & 2.61 & 0 & & 188 & \\
\hline Supernatant Solids (wt\%) & 4.78 & 4.78 & & 29.68 & 29.68 & & & \\
\hline Density $(\mathrm{kg} / \mathrm{L})$ & 1.035 & & & 1.257 & & & & \\
\hline Ag & 17.2 & $<0.58$ & & 39.0 & 1.48 & 4 & 34 & \\
\hline Al & 751 & 457 & 61 & 4865 & 2705 & 56 & 98 & 89 \\
\hline B & 74.7 & 97.7 & 131 & 334 & 628 & 188 & & 97 \\
\hline $\mathrm{Ba}$ & 3.20 & $<0.02$ & & 21.9 & $<0.02$ & & 103 & \\
\hline $\mathrm{Ca}$ & 14.0 & 3.87 & 28 & 114 & 3.47 & 3 & 123 & 14 \\
\hline $\mathrm{Cd}$ & 0.693 & $<0.03$ & & 4.63 & $<0.02$ & & 101 & \\
\hline Ce & $<8.31$ & $<1.49$ & & 25.1 & $<1.23$ & & & \\
\hline Co & $<0.20$ & $<0.09$ & & $<0.34$ & $<0.07$ & & & \\
\hline $\mathrm{Cr}$ & 22.6 & 19.8 & 88 & 155 & 126 & 81 & 103 & 97 \\
\hline $\mathrm{Cu}$ & 0.808 & $<0.10$ & & 7.98 & $<0.08$ & & 149 & \\
\hline $\mathrm{Fe}$ & 374 & $<0.09$ & & 2870 & $<0.07$ & & 116 & \\
\hline $\mathrm{K}$ & 1550 & & & 6030 & 10342 & 172 & 59 & \\
\hline $\mathrm{La}$ & 3 & $<1.35$ & & 27.3 & $<1.11$ & & 155 & \\
\hline $\mathrm{Li}$ & 20.8 & 0.478 & 2 & 128 & 0.339 & 0 & 93 & \\
\hline $\mathrm{Mg}$ & 7.90 & 0.438 & 6 & 79.8 & $<0.13$ & & 153 & \\
\hline $\mathrm{Mn}$ & 71.1 & $<0.02$ & & 537 & $<0.01$ & & 114 & \\
\hline Mo & $<2$ & 0.980 & & 2.7 & 6.58 & 244 & & 101 \\
\hline $\mathrm{Na}$ & 13641 & 12959 & 95 & 85941 & 78043 & 91 & 95 & 91 \\
\hline $\mathrm{Nd}$ & 9.02 & $<0.50$ & & 68.0 & $<0.41$ & & 114 & \\
\hline $\mathrm{Ni}$ & 11.0 & $<0.12$ & & 84.6 & 0.27 & 0 & 116 & \\
\hline $\mathrm{P}$ & 68.8 & 50.8 & 74 & 415 & 374 & 90 & 91 & 111 \\
\hline $\mathrm{Pb}$ & $<13.2$ & $<1.33$ & & 123 & 6.46 & 5 & & \\
\hline $\mathrm{S}$ & 161 & 131 & 81 & 891 & 835 & 94 & 84 & 97 \\
\hline $\mathrm{Si}$ & 124 & 7.54 & 6 & 1097 & 42.9 & 4 & 133 & 86 \\
\hline $\mathrm{Sr}$ & 8.02 & 0.08 & 1 & 59.1 & 0.547 & 1 & 111 & 98 \\
\hline $\mathrm{Ti}$ & 1 & $<0.27$ & & 8.43 & $<0.22$ & & 89 & \\
\hline $\mathrm{Zn}$ & 10.3 & $<0.72$ & & 79.8 & $<0.59$ & & 117 & \\
\hline $\mathrm{Zr}$ & 5.31 & $<0.09$ & & 36.9 & $<0.08$ & & 105 & \\
\hline bromide & $<67$ & $<96.7$ & & $<76.0$ & $<79.6$ & & & \\
\hline chloride & 131 & (371) & 283 & 934 & 1201 & 129 & 108 & 49 \\
\hline fluoride & 11 & (27.1) & 246 & 67.0 & 149 & 222 & 92 & 83 \\
\hline formate & 98 & (221) & 226 & 578 & 915 & 158 & 89 & 63 \\
\hline nitrate & 19800 & 21760 & 110 & 112000 & 125696 & 112 & 85 & 87 \\
\hline nitrite & 2270 & 2756 & 121 & 14000 & 15274 & 109 & 93 & 84 \\
\hline oxalate & 873 & (1383) & 158 & 4350 & 1074 & 25 & 75 & 12 \\
\hline phosphate & 312 & (786) & 252 & 2120 & 2291 & 108 & 103 & 44 \\
\hline sulfate & 304 & (787) & 259 & 2090 & 2235 & 107 & 104 & 43 \\
\hline ammonium & $<89$ & & & $<99$ & & & & \\
\hline
\end{tabular}

* Ratio of mass in Bottoms to mass in feed; should equal $100 \%$ for total slurry 
The results for $\mathrm{B}$ and the anions are suspect. The feed anion concentrations in the supernate (shown in parentheses) are greater than in the slurry, which is not possible. Comparison of the supernate/slurry ratios for the feed and Bottoms indicate that some Ca, $\mathrm{Cr}, \mathrm{Na}$ and oxalate precipitated. The difference in the Al ratio (61 vs. 56) is too small to conclude if any Al precipitated. As in the previous campaigns, the oxalate appears to have precipitated as sodium oxalate, lowering slightly the amount of dissolved Na. Whether or not precipitation of phosphate occurred could not be determined because the $\mathrm{P}$ and phosphate analyses data are inconclusive. The $\mathrm{S}$ and sulfate results are also inconclusive. The ammonium concentrations in the feed and concentrate were both below the detection limit. The ammonium in the condensate was $61 \mathrm{mg} / \mathrm{L}$.

The concentrations of the more prevalent species in the FEP streams for Campaigns II-IV are compared in Table 20. The Campaign III and IV undissolved solids concentrations are essentially equal, while the total and supernatant solids for Campaign IV are about $1 \mathrm{wt} \%$ higher than in Campaign III. The Campaign II UDS values are slightly higher, whereas the total and supernatant solids are lower. The elemental and anion concentrations of the Campaign III and IV feeds and concentrates are similar. The supernate/slurry wt\% values for Campaign IV are also similar to Campaign III, showing that the distribution between the undissolved and dissolved species is similar. These results indicate that the SIPP process was at steady-state with respect to the chemical composition of the recycles.

About 56-61\% of the Al was dissolved in the Campaign IV feed and concentrate compared to 53-56\% in Campaign III. This difference is small and may be totally due to analytical uncertainty. Both of these values are smaller than the Campaign II values of $60-70 \%$. Therefore, the fraction of dissolved Al in Campaign IV was definitely less than in Campaign II but similar to or slightly higher than Campaign III. The dissolved $\mathrm{Cr}$ in Campaign IV was $81-88 \%$, which is very similar to the Campaign III values of $80-81 \%$. 
WSRC-TR-2005-00105, REVISION 0

SRNL-RPP-2005-00012, REVISION 0

Table 20. Comparison of Selected Species in Campaigns II-IV FEP

\begin{tabular}{|c|c|c|c|c|c|c|c|c|c|c|c|c|c|c|c|c|c|c|}
\hline \multirow{2}{*}{$\begin{array}{c}\text { All units mg/kg } \\
\text { unless otherwise } \\
\text { noted }\end{array}$} & \multicolumn{3}{|c|}{ Campaign II } & \multicolumn{3}{|c|}{ Campaign II } & \multicolumn{3}{|c|}{ Campaign III } & \multicolumn{3}{|c|}{ Campaign III } & \multicolumn{3}{|c|}{ Campaign IV } & \multicolumn{3}{|c|}{ Campaign IV } \\
\hline & $\begin{array}{l}\text { Feed } \\
\text { Slurry }\end{array}$ & $\begin{array}{c}\text { Feed } \\
\text { Supernate }\end{array}$ & (1) & $\begin{array}{l}\text { Bottoms } \\
\text { Slurry }\end{array}$ & $\begin{array}{l}\text { Bottoms } \\
\text { Supernate }\end{array}$ & (1) & $\begin{array}{l}\text { Feed } \\
\text { Slurry }\end{array}$ & $\begin{array}{c}\text { Feed } \\
\text { Supernate }\end{array}$ & (1) & $\begin{array}{c}\text { Bottoms } \\
\text { Slurry }\end{array}$ & $\begin{array}{l}\text { Bottoms } \\
\text { Supernate }\end{array}$ & (1) & $\begin{array}{l}\text { Feed } \\
\text { Slurry }\end{array}$ & $\begin{array}{c}\text { Feed } \\
\text { Supernate }\end{array}$ & (1) & $\begin{array}{c}\text { Bottoms } \\
\text { Slurry }\end{array}$ & $\begin{array}{l}\text { Bottoms } \\
\text { Supernate }\end{array}$ & (1) \\
\hline $\begin{array}{r}\text { Total Solids } \\
\text { (wt\%) }\end{array}$ & 4.72 & 4.44 & & 28.95 & 26.73 & & 4.93 & 4.71 & & 30.75 & 28.82 & & 4.98 & 4.78 & & 31.52 & 29.68 & \\
\hline $\begin{array}{r}\text { Undissolved } \\
\text { Solids (wt\%) }\end{array}$ & 0.29 & 0 & & 3.03 & 0 & & 0.23 & 0 & & 2.70 & 0 & & 0.21 & 0 & & 2.61 & 0 & \\
\hline $\begin{array}{r}\text { Supernatant } \\
\text { Solids (wt\%) }\end{array}$ & 4.44 & 4.44 & & 26.73 & 26.73 & & 4.71 & 4.71 & & 28.82 & 28.82 & & 4.78 & 4.78 & & 29.68 & 29.68 & \\
\hline Density $(\mathrm{kg} / \mathrm{L})$ & 1.037 & 1.036 & & 1.261 & 1.214 & & 1.035 & 1.038 & & 1.255 & 1.231 & & 1.034 & & & 1.257 & 1.237 & \\
\hline $\mathrm{Al}$ & 920 & 548 & 60 & 5470 & 3823 & 70 & 811 & 452 & 56 & 5255 & 2781 & 53 & 751 & 457 & 61 & 4865 & 2705 & 56 \\
\hline $\mathrm{Ca}$ & 206 & 1.13 & 1 & 355 & $<0.05$ & 0 & 24.5 & 2.84 & 12 & 111 & 6.66 & 6 & 14.0 & 3.87 & 28 & 114 & 3.5 & 3 \\
\hline $\mathrm{Cr}$ & 51.0 & 19.6 & 38 & 188 & 124 & 66 & 25.4 & 20.4 & 80 & 156 & 126 & 81 & 22.6 & 19.8 & 88 & 155 & 126 & 81 \\
\hline $\mathrm{Fe}$ & 835 & $<0.08$ & 0 & 4600 & $<0.07$ & 0 & 635 & $<0.09$ & 0 & 3910 & $<0.07$ & 0 & 374 & $<0.09$ & 0 & 2870 & $<0.07$ & 0 \\
\hline $\mathrm{Mn}$ & 156 & $<0.02$ & 0 & 942 & $<0.02$ & 0 & 134 & $<0.02$ & 0 & 828 & $<0.01$ & 0 & 71.1 & $<0.02$ & 0 & 537 & $<0.01$ & 0 \\
\hline $\mathrm{Na}$ & 15800 & 13402 & 85 & 94200 & 88151 & 94 & 13300 & 13623 & 102 & 79400 & 80159 & 101 & 13641 & 12959 & 95 & 85941 & 78043 & 91 \\
\hline $\mathrm{Ni}$ & 208 & 0.38 & 0 & 909 & 0.29 & 0 & 15.1 & $<0.12$ & 0 & 128 & 0.72 & 1 & 11.0 & $<0.12$ & 0 & 85.0 & 0.27 & 0 \\
\hline $\mathrm{P}$ & $<160$ & 123 & & 889 & 906 & 102 & 65.3 & 54.8 & 84 & 444 & 396 & 89 & 68.8 & 50.8 & 74 & 415 & 374 & 90 \\
\hline $\mathrm{S}$ & 219 & 91.9 & 42 & 720 & 651 & 90 & 133 & 135 & 102 & 813 & 829 & 102 & 161 & 131 & 81 & 891 & 835 & 94 \\
\hline $\mathrm{Si}$ & 371 & 5.29 & 1 & 1580 & 31.3 & 2 & 147 & 9.06 & 6 & 1560 & 65.9 & 4 & 124 & 7.5 & 6 & 1097 & 42.9 & 4 \\
\hline $\mathrm{Sr}$ & 13.6 & $<0.01$ & 0 & 68.9 & 0.332 & 0 & 9.25 & 0.04 & 0 & 54.1 & 0.20 & 0 & 8.02 & 0.08 & 1 & 59.1 & 0.055 & 1 \\
\hline chloride & 83 & & & & & & 147 & 132 & 90 & 1150 & 1100 & 96 & 131 & (371) & 283 & 934 & 1201 & 129 \\
\hline fluoride & 11 & & & & & & $<17$ & 11.6 & $>68$ & 93 & 36 & 39 & 11.0 & (27.1) & 246 & 67.0 & (148.8) & 222 \\
\hline formate & 39 & & & & & & 86 & 78.3 & 91 & 732 & 667 & 91 & 98.0 & (221) & 225 & 578 & (915) & 158 \\
\hline nitrate & 14465 & & & & & & 19700 & 17101 & 87 & 120000 & 121912 & 102 & 19800 & $(21760)$ & 110 & 112000 & (125696) & 112 \\
\hline nitrite & 487 & & & & & & 2240 & 2058 & 92 & 14400 & 14422 & 100 & 2270 & $(2756)$ & 121 & 14000 & (15274) & 109 \\
\hline oxalate & 1520 & & & & 972 & & 1070 & 976 & 91 & 3860 & 924 & 24 & 873 & (1383) & 158 & 4350 & 1074 & 25 \\
\hline phosphate & 563 & & & & & & 363 & 303 & 83 & 2270 & 2279 & 100 & 312 & $(786)$ & 252 & 2120 & 2291 & 108 \\
\hline sulfate & 257 & & & & & & 350 & 318 & 91 & 2260 & 2191 & 97 & 304 & (787) & 259 & 2090 & 2235 & 107 \\
\hline
\end{tabular}

(1) All these columns are the ratio of the two preceding columns in percentage, i.e., (Supernate / Slurry) $\mathrm{x} 100 \%$ 
Table 21 presents the material balance on undissolved solids and oxalate for Campaigns IIIV. For these campaigns, the increase in undissolved solids can be accounted for by the decrease in dissolved oxalate if the oxalate precipitates as sodium oxalate. Some of the oxalate was probably also precipitated as calcium oxalate, but the amount of calcium present was very small, so most of the oxalate would have precipitated as the Na salt. For each campaign, there was a decrease in the dissolved $\mathrm{Na}$, but the decrease was on the order of the analytical uncertainty.

Table 21. Mass Balance on FEP Solids $(1,2)$

\begin{tabular}{|c|c|c|c|c|c|c|c|c|c|c|}
\hline & & Feed & ampaign & $\begin{array}{l}\text { IV } \\
\text { Change } \\
\text { (Bottoms } \\
\text { - Feed) }\end{array}$ & Feed & ampaign & $\begin{array}{l}\text { [II } \\
\text { Change } \\
\text { (Bottoms } \\
\text { - Feed) }\end{array}$ & Feed & mpaign & $\begin{array}{l}\text { II } \\
\text { Change } \\
\text { (Bottoms } \\
\text { - Feed) }\end{array}$ \\
\hline 1 & Undissolved Solids (wt\%) & 0.21 & 2.61 & & 0.23 & 2.70 & & 0.29 & 3.03 & \\
\hline 2 & Undissolved Solids Mass (g) & 2368 & 4446 & +2078 & 2567 & 4600 & +2033 & 3345 & 5626 & +2281 \\
\hline 3 & $\begin{array}{r}\text { Dissolved Oxalate as } \\
\mathrm{Na}_{2} \mathrm{C}_{2} \mathrm{O}_{4} \text { Mass (g) }\end{array}$ & 2375 & 279 & -2096 & 1653 & 239 & -1413 & 2603 & 265 & -2338 \\
\hline 4 & Dissolved Oxalate Mass (g) & 1559 & 183 & -1376 & 1085 & 157 & -928 & 1710 & 174 & -1536 \\
\hline 5 & Total Solids Mass (g) & 56.1 & 53.7 & -2.4 & 54.8 & 52.3 & -2.5 & 54.1 & 53.8 & -0.3 \\
\hline 6 & Total Solids (wt\%) & 4.98 & 31.52 & & 4.65 & 28.70 & & 4.63 & 29.80 & \\
\hline
\end{tabular}

\subsubsection{Pilot FEP Conclusions}

\subsubsection{Experimental Conclusions (Author - Qureshi)}

\section{Campaign IV}

The SIPP FEP Campaign IV was run on 25 Augusta, 2004. A total of $1118.8 \mathrm{~kg}$ of feed was concentrated down to about $174.6 \mathrm{~kg}$. The entire evaporation process proceeded without any equipment failure or operational difficulty. The average evaporation rate during this campaign was around $0.54 \mathrm{gpm}$.

Similar to the earlier campaigns, no significant foaming was observed prior to the addition of antifoam agent. During the evaporation process, the froth height remained around 1 foot. This height is significantly less than the 5-foot distance to the lower demister pad. However, during start-up and unsteady conditions, significant flashing resulted in feed splashing on the lower demister pad. Any air injection into the recirculation loop would generate instantaneous froth of 3 to 4 feet that would subside as soon as air is cut off. 
WSRC-TR-2005-00105, REVISION 0

SRNL-RPP-2005-00012, REVISION 0

Several liquid samples of feed, concentrate, primary condensate and secondary condensate were pulled during the campaign, see Appendix D.

The following conclusions were extracted from the Interim Reports of Campaign III (Duignan et al., 2004c or Appendix I-3) and Campaign II (Duignan et al., 2004b or Appendix $\mathrm{I}-2)$

\section{Campaign III}

The SIPP FEP Campaign III was run on 7 June, 2004. A total of $1112.2 \mathrm{~kg}$ of feed was concentrated down to about $170.1 \mathrm{~kg}$. The entire evaporation process proceeded without any glitch or equipment failure. The average evaporation rate during this campaign was around 0.55 gpm.

No significant foaming was observed prior to the addition of antifoam agent. During the evaporation process, the froth height remained around 1 foot that is significantly less than the 5 -foot distance to the lower demister pad. However, during start-up and unsteady conditions, significant flashing resulted in feed splashing on the lower demister pad. These conditions are associated with much larger vapor flux and vapor velocities at the interface.

The concentrate density was measured by three methods as described above. Density measurements by the density meter were used to reach the target density goal of $1.257 \mathrm{~g} / \mathrm{ml}$ at $20 \mathrm{C}$.

\section{Campaign II}

During SIPP FEP Campaign II run on 14 April, 2004, a total of about $1169.7 \mathrm{~kg}$ of feed was concentrated down to about $185.4 \mathrm{~kg}$. The feed was evaporated under vacuum (1 psia) at moderate temperatures ( $50 \mathrm{C})$. The entire evaporation process proceeded as designed. The evaporator was designed to evaporate up to $0.6 \mathrm{gpm}$ of water. The average evaporation rate during this campaign was around $0.54 \mathrm{gpm}$.

No significant foaming was observed prior to the addition of antifoam agent. During the evaporation process, the froth height remained around 1 foot that is significantly less than the 5-foot distance to the lower demister pad. However, during start-up and unsteady conditions, significant flashing resulted in feed splashing on the lower demister pad. These conditions are associated with much larger vapor flux and vapor velocities at the interface. 
WSRC-TR-2005-00105, REVISION 0

SRNL-RPP-2005-00012, REVISION 0

\subsubsection{Waste Products Conclusions (Author-Zamecnik)}

\section{Campaign IV}

- Mixing of the recycles in a different order for Campaign IV had no effect on the evaporation process as compared to the previous campaigns.

- The FEP feed and Bottoms concentrations for Campaigns III and IV were very similar, indicating that steady-state had been reached with respect to the recycle compositions, as indicated by the model in Appendix B.

- The precipitation of sodium oxalate in the FEP evaporator occurred in all three campaigns and accounted for most of the precipitated solids found. That is, while more that $90 \%$ of the oxalate was dissolved in the FEP feed less than $25 \%$ of the oxalate was still dissolved after reaching the target Bottoms concentration.

- About $56-61 \%$ of the aluminum was dissolved in the Campaign IV feed and the concentrate (Bottoms), which was similar to the Campaign III percentage. This dissolved $\mathrm{Al}$ fraction was less than 60-79\% realized in Campaign II, resulting in more undissolved Al fed to the UFP.

The following conclusions were extracted from the Interim Reports of Campaign III (Duignan et al., 2004c or Appendix I-3) and Campaign II (Duignan et al., 2004b or Appendix $\mathrm{I}-2)$

\section{Campaign III}

- As seen in Campaign II, a large portion of the dissolved oxalate in the feed was precipitated, probably as sodium oxalate.

- The oxalate concentration in the FEP feed in Campaign III was less than that in Campaign II, which is consistent with the higher oxalate concentration in the Campaign I slurry.

- About $25-40 \%$ of the dissolved $\mathrm{Al}$ in the feed to the FEP evaporator precipitated during mixing of the recycles, assumed to occur because of localized regions of low $\mathrm{pH}$. Vigorous mixing when joining caustic and acidic recycle streams in the Plant Wash and Disposal System would help to minimize Al precipitation.

- The fraction of dissolved aluminum in Campaign III FEP concentrate (Bottoms) was less than in Campaign II, resulting in more undissolved Al fed to the UFP.

\section{Campaign II}

- The composition of the FEP feed was predictable by mass balance of the individual feed streams.

- Dissolved metals concentrations parallel the ratios in the AY102 simulant present in the recycles. 
WSRC-TR-2005-00105, REVISION 0

SRNL-RPP-2005-00012, REVISION 0

- Dissolved oxalate, present in the feed, began to precipitate when approximately 53\% of the feed had been evaporated. A total of about $90 \mathrm{wt} \%$ of that oxalate precipitated at the end of evaporation. The oxalate appears to have precipitated as the $\mathrm{Na}$ salt $\mathrm{Na}_{2} \mathrm{C}_{2} \mathrm{O}_{4}$.

\subsection{PILOT ULTRAFILTRATION (Authors - Fowley, Williams \& Zamecnik)}

The RPP-WTP Ultrafiltration Process (UFP) will remove entrained solids from a combined HLW and LAW feed. The solids are removed to protect downstream cesium ion exchange (CIX) beds and to meet the specifications for LAW product. In addition, the RPP-WTP UFP will concentrate the removed solids as a slurry to minimize storage requirements for HLW and to enhance HLW vitrification efficiency.

The SIPP UFP concentrated and removed solids from combined simulated waste feed to produce two products; concentrated slurry for vitrification, and filtered permeate for the ion exchange process. The SIPP UFP waste feed consisted of a mixture of FEP Bottoms (made up of concentrated recycle streams) and simulated wastes of AY102 and AP101. The recycle streams were included in the SIPP UFP (to a limited extent) to mimic actual WTP operation.

The Campaign IV UFP tests were different from previous campaigns in that the filter unit chosen by RPP-WTP was from a different manufacturer than the one use in all previous testing. D C Fabricators, Inc. provided a new filter unit consisting of GKN sintered metal filter tubes. The filter media used in the previous campaigns (I, II, and III) was manufactured by the Mott Corporation. Both filter media were sintered 316L stainless steel with a nominal 0.1 micron porosity and made from the same size tubes. Further information about the new GKN filter unit is provided in Section 2.7.3.1 and Appendix F.

Table 22 shows the SIPP UFP Campaign IV test matrix. The numbering of the tests has been maintained and sequentially adjusted from Campaign I (Duignan, et al., 2004a). Several tests were eliminated prior to Campaign I and between Campaign I and II, which produced numerical gaps between several of the test numbers. The steady state tests X.07, X.08, X.09, and X.10 (X representing the campaign) were added back to the matrix, along with two additional steady state tests prior to Campaign IV to provide comparison with the Mott filter. In addition, seven higher-temperature tests were added to the matrix to determine the effect of slurry viscosity on permeate flux. 
WSRC-TR-2005-00105, REVISION 0 SRNL-RPP-2005-00012, REVISION 0

Table 22. Campaign IV UFP Test Matrix

\begin{tabular}{|c|c|c|c|c|c|}
\hline Test No. & Test Activity & $\begin{array}{l}\text { Trans-Membrane } \\
\text { Pressure } \\
\text { (psid) }\end{array}$ & $\begin{array}{l}\text { Slurry } \\
\text { Velocity } \\
(\mathrm{ft} / \mathrm{s})\end{array}$ & $\begin{array}{l}\text { Slurry } \\
\text { Temp. } \\
\left({ }^{\circ} \mathrm{C}\right)\end{array}$ & $\begin{array}{l}\text { Run } \\
\text { Time } \\
\text { (min) }\end{array}$ \\
\hline $4.00 \mathrm{~A}$ & Pre-test baseline $\mathrm{A}^{1}$ & 10 & 11 & 25 & 20 \\
\hline $4.00 \mathrm{~B}$ & Pre-test baseline $\mathrm{B}^{1}$ & 20 & 11 & 25 & 20 \\
\hline $4.00 \mathrm{C}$ & Pre-test baseline $C^{1}$ & 30 & 11 & 25 & 20 \\
\hline 4.03A & Dewater first half of slurry $^{2}$ & 40 & 12 & 25 & $960^{8}$ \\
\hline $4.03 \mathrm{~B}$ & Dewater second half of slurry $^{2}$ & 40 & 12 & 25 & $2300^{8}$ \\
\hline 4.07 & Steady State @ 24wt\% & 30 & 9 & 25 & 30 \\
\hline 4.08 & Steady State@ @24wt\% & 30 & 13 & 25 & 30 \\
\hline 4.09 & Steady State@ @24wt\% & 50 & 13 & 25 & 30 \\
\hline 4.11 & Steady State@ @24wt\% & 40 & 11 & 25 & 30 \\
\hline Steady5 & Steady State@ @24wt\% & 50 & 13 & 25 & 30 \\
\hline Steady6 & Steady State@ @24wt\% & 50 & 15 & $25^{7}$ & 30 \\
\hline Temp1 & High temperature test @ 20 wt\% & 40 & 12 & 30 & 30 \\
\hline Temp2 & High temperature test @ 20 wt\% & 40 & 12 & 37.5 & 30 \\
\hline Temp3 & High temperature test @ 20wt\% & 40 & 12 & 45 & 30 \\
\hline Temp4 & High temperature test @ 20 wt\% & 40 & 12 & 35 & 30 \\
\hline Temp5 & High temperature test @ 20wt\% & 40 & 12 & 25 & 30 \\
\hline Temp6 & High temperature test @ 20 wt\% & 50 & 15 & 37.5 & 30 \\
\hline Temp7 & High temperature test @ 20 wt\% & 50 & 15 & 45 & 30 \\
\hline Wash 1 & Slurry wash $1^{3}$ & 40 & 12 & 25 & $379^{8}$ \\
\hline Wash 2 & Slurry wash $2^{3}$ & 40 & 12 & 25 & $229^{8}$ \\
\hline $4.18 \mathrm{~A}$ & Caustic rinse $1^{4}$ & 20 & 11 & 25 & 30 \\
\hline $4.18 \mathrm{~B}$ & Acid clean $1^{5}$ & 40 & 11 & 25 & 90 \\
\hline $4.18 \mathrm{C}$ & Acid clean $2^{5}$ & 40 & 11 & 25 & 90 \\
\hline $4.18 \mathrm{D}$ & Acid clean $3^{5}$ & 40 & 11 & 25 & 90 \\
\hline 4.19A & Caustic rinse $2^{4}$ & 20 & 11 & 25 & 30 \\
\hline $4.19 \mathrm{~B}$ & Caustic rinse $3^{4}$ & 20 & 11 & 25 & 30 \\
\hline $4.21 \mathrm{~A}$ & Post-test baseline $\mathrm{A}^{6}$ & 10 & 11 & 25 & 20 \\
\hline $4.21 \mathrm{~B}$ & Post-test baseline $\mathrm{B}^{6}$ & 20 & 11 & 25 & 20 \\
\hline $4.21 \mathrm{C}$ & Post-test baseline $C^{6}$ & 30 & 11 & 25 & 20 \\
\hline
\end{tabular}

1. The pre-test baseline tests were conducted with $0.1 \mathrm{M} \mathrm{NaOH}$ solution made from de-ionized, filtered $(0.1 \mu \mathrm{m})$ water.

2. The slurry was dewatered to $20 \mathrm{wt} \%$ undissolved solids or greater.

3. The slurry was washed with $0.01 \mathrm{M} \mathrm{NaOH}$ solution made from FEP condensate.

4. The caustic rinse consisted of $0.1 \mathrm{M} \mathrm{NaOH}$ solution made from FEP condensate.

5. The acid clean consisted of $2 \mathrm{M} \mathrm{HNO}_{3}$ solution.

6. The post-test baseline tests were conducted with spent caustic rinse 3 solution and repeated with $0.1 \mathrm{M} \mathrm{NaOH}$ made from de-ionized, filtered water.

7. Test temperature was maintained as close to $25 \operatorname{deg} C$ as possible within the limits of the system.

8. Run time estimated from previous campaigns. 
WSRC-TR-2005-00105, REVISION 0

SRNL-RPP-2005-00012, REVISION 0

The remainder of this section will detail the pilot-scale UFP unit operation conducted as part of Campaign IV testing and compare results from all four SIPP campaigns. The process started with the preparation of the feed product. The chemical explanation of the feed stream formation will be followed by a short description on how feed formation was handled just before UFP operation and then how the waste stream was handled during and after its separation. The following section will discuss the physical equipment used to filter the waste and how the test was run. Finally, the results will be discussed and conclusions made.

\subsubsection{Chemical Description of the Pilot-Scale UFP Feed Stream (Author - Zamecnik)}

The ultrafiltration feed slurry was made from the FEP evaporator Bottoms, the AY102 simulant, and the AP101 simulant. No extensive characterization of this feed was performed; only solids and density measurements were made. Figure 8 and Fig. 9 show schematically how feed was processed through concentration, washing, rinsing and cleaning steps were performed. These steps were essentially the same as used in Campaigns II and III, with some quantities adjusted to give more correct solids loadings in the recycles.

\subsubsection{Simulant Handling (Author - Williams)}

Before testing began the simulants were prepared, which would be needed to create recycle streams from the UFP. Preparations of the simulated waste feed were then performed so as to have the accepted proportions of the constituents as per the OLI/ESP model (see Table 3).

After the dewatering portion of pilot-scale UFP testing, the slurry was adjusted in order to have a target solids concentration of $20 \mathrm{wt} \%$ undissolved solids (UDS) before washing began, as required by the Test Specification (Longwell, 2003). The UFP produced $117 \mathrm{~L}$ of concentrated slurry at $24 \mathrm{wt} \%$ UDS and approximately four 55-gallon drums containing permeate. Therefore, some of the $24 \mathrm{wt} \%$ slurry was removed and approximately $25 \mathrm{~L}$ of permeate were added back to the slurry to maintain the slurry volume at $117 \mathrm{~L}$ while attaining $19 \mathrm{wt} \%$ UDS. This section describes how the simulants were made and adjusted at the test location. 
WSRC-TR-2005-00105, REVISION 0

SRNL-RPP-2005-00012, REVISION 0

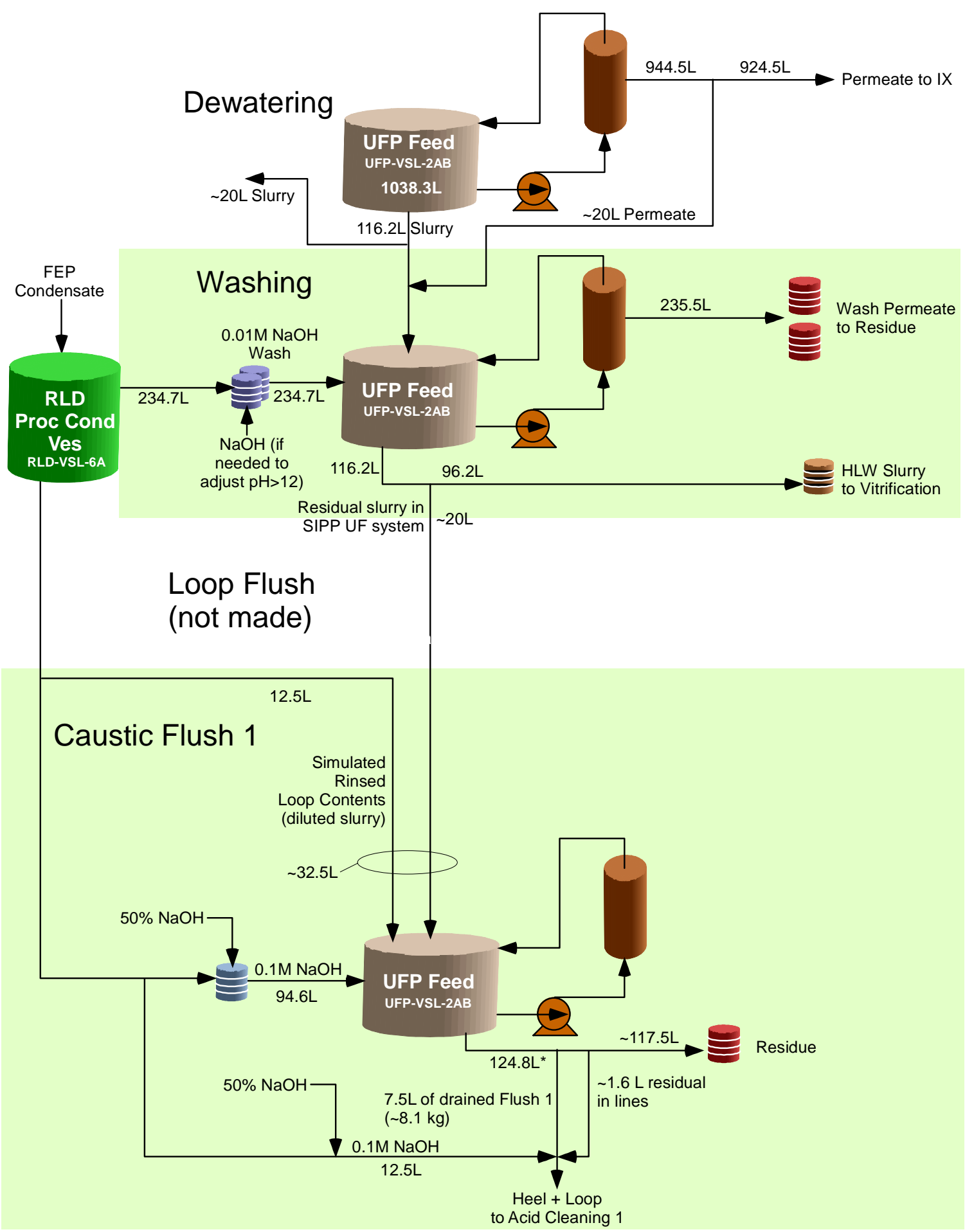

Figure 8. Schematic of Ultrafiltration Process - Page 1 
WSRC-TR-2005-00105, REVISION 0

SRNL-RPP-2005-00012, REVISION 0

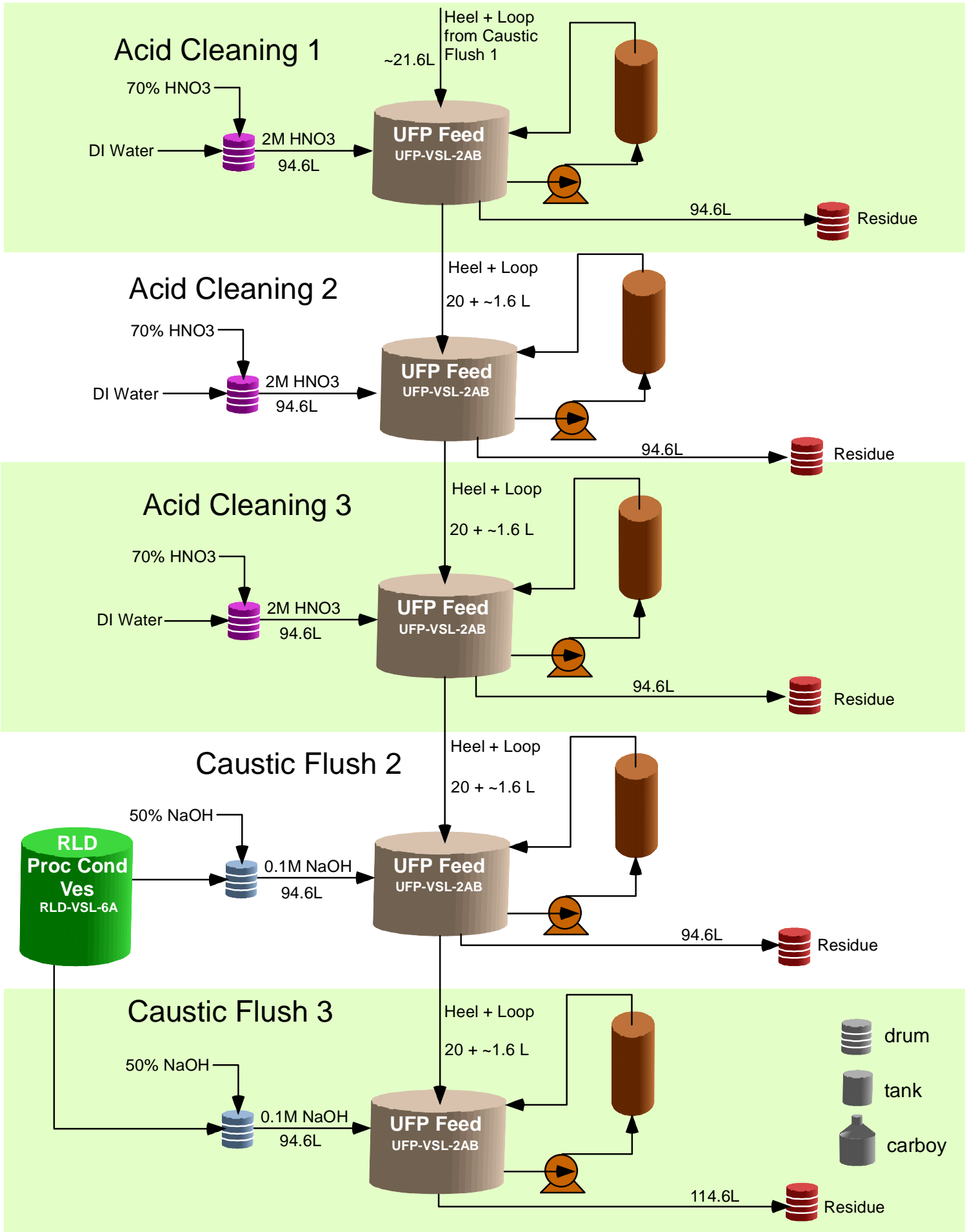

Figure 9. Schematic of Ultrafiltration Process - Page 2 


\subsubsection{Preparing for UFP Recycle Streams}

Refer to Fig. 3 (blending of recycle streams) and Figs. 8 and 9 (schematic of UFP) to better understand the following descriptions of feed preparations and use.

In preparation to create the UFP wash-water recycle stream on 17 August 2004, two 55-gallon drums, labeled “Campaign 4-UFP First or Second Wash,” were each filled with $117.4 \mathrm{~kg}$ of Campaign III RLD condensate into which were added 187.8 grams of $50 \mathrm{wt} \% \mathrm{NaOH}$ to attain a caustic concentration of $0.01 \mathrm{M} \mathrm{NaOH}^{\dagger}$. The loop rinse recycle stream from Campaign III for use in Campaign IV, was made in one carboy labeled "PWDVSL-33” and was filled with 8.6 liters of Campaign III FEP and TLP condensate. This carboy received 4.5 liters of washed Campaign III HLW slurry after washing. To create the UFP "Heel” sludge recycle stream another carboy was filled with 12.5 liters of Campaign III FEP and TLP condensate to await receipt of washed slurry. To create rinse water recycle, three 55-gallon drums, labeled "Campaign 4-0.1M NaOH for UFP Caustic Flush 1, 2 or 3," were each filled with 94.6 liters of Campaign III FEP and TLP condensate in the Caustic Flush 1 drum and 94.6 liters of FEP condensate in the Caustic Flush 2 and 3 drums. Those drums were made to have a caustic concentration of $0.1 \mathrm{M} \mathrm{NaOH}$ by adding 766.8 grams of $50 \mathrm{wt} \% \mathrm{NaOH}$ in the Caustic Flush 1 drum and 766.8 grams of $50 \mathrm{wt} \% \mathrm{NaOH}$ into each of the Caustic Flush 2 and Caustic Flush 3 drums. The final UFP recycle stream created was for acid cleaning. It was made by using three

55-gallon drums labeled "Campaign 4-UFP Acid Cleaning 1, 2 or 3,” which were each filled with 94.6 liters of DI water. To make the acid concentration $2 \mathrm{M} \mathrm{HNO}_{3}, 17 \mathrm{~kg}$ of $70 \%$ $\mathrm{HNO}_{3}$ were added into each of those drums.

\subsubsection{Preparing UFP Feed}

In preparation for pilot-scale UFP unit operation, AP101 simulated waste, which was prepared by Optima, was mixed with the FEP Evaporator Bottoms and the AY102/C106, or simply AY102, simulated waste in the UFP Primary Mixing Tank. This tank represented the plant tanks UFP-VSL-1A/B. Three major components of the AY102 simulant were also made by Optima, but those components were joined, and its final preparation was made, at the EDL test location in a separate mixing tank (UFP Secondary Mixing Tank) on 24 May in order to have just the right mix. The final adjustment is shown in Table 23:

\footnotetext{
† The concentration of $0.01 \mathrm{M} \mathrm{NaOH}$ was nominal. The real target was to have a pH of at least 12 . Due to the high acid content of the EDL water supply the initial addition of 117 grams of the $50 \mathrm{wt} \% \mathrm{NaOH}$ to the 141 liters of water still indicated a pH below 12. To be safe the amount of caustic was increased to 187.8 grams which theoretically gave a concentration of $0.02 \mathrm{M} \mathrm{NaOH}$ and a $\mathrm{pH}=12.3$. It measured as $\mathrm{pH}>12$.
} 
WSRC-TR-2005-00105, REVISION 0

SRNL-RPP-2005-00012, REVISION 0

Table 23. UFP Feed Constituent: AY102 Simulant

\begin{tabular}{|c|c|}
\hline Material & Mass Added (g) \\
\hline \multicolumn{2}{|l|}{ Wet Additions } \\
\hline AY102 Supernatant Simulant by Optima & 66,800 \\
\hline Precipitated Sludge Solids by Optima & 238,200 \\
\hline NAS, sodium aluminosilicate (wet) by Optima & 27,900 \\
\hline \multicolumn{2}{|l|}{ Dry Additions } \\
\hline $\mathrm{Al}(\mathrm{OH})_{3}$ & $5,660.0$ \\
\hline $\mathrm{Al}_{2} \mathrm{O}_{3}$ & $2,015.2$ \\
\hline Calcium Phosphate Tribasic & 135.0 \\
\hline $\mathrm{SiO}_{2}$ & $3,650.0$ \\
\hline $\mathrm{RbNO}_{3}$ & 0.46 \\
\hline Total & $344,360.66$ \\
\hline
\end{tabular}

After samples were taken, AY102 simulant in excess of the target volume of 63.8 gallons was removed. That volume was indicated by the OLI/ESP model to be used for Campaign IV and is shown at the top of Table 8. With AY102 ready, the final UFP feed preparation could be completed. The 63.8 gallons ( $304.5 \mathrm{~kg}$ ) of AY102 simulant were transferred to the UFP Primary Mixing Tank. To this slurry, $174.6 \mathrm{~kg}$ of FEP Bottoms made in the pilot-scale FEP were transferred to the UFP Primary Mixing Tank on 27 August. The last addition was $860.5 \mathrm{~kg}$ of AP101 simulant, which was also completed on 27 August. The total feed in the UFP Primary Mixing Tank was 1,339.6 kg before samples were removed for analysis.

After the UFP Primary Mixing Tank contents had settled over the weekend of 28 to 29 August, 15.8 liters of supernatant was decanted from the tank surface on 30 August, which was used to fill the permeate system of the pilot-scale UFP test facility on start up. Subsequently, the UFP Primary Mixing Tank, Fig. 10, was mixed for one hour and samples taken before initiating the transfer to the UFP Slurry Reservoir, Fig. 11, to begin dewatering operations on 30 August. 


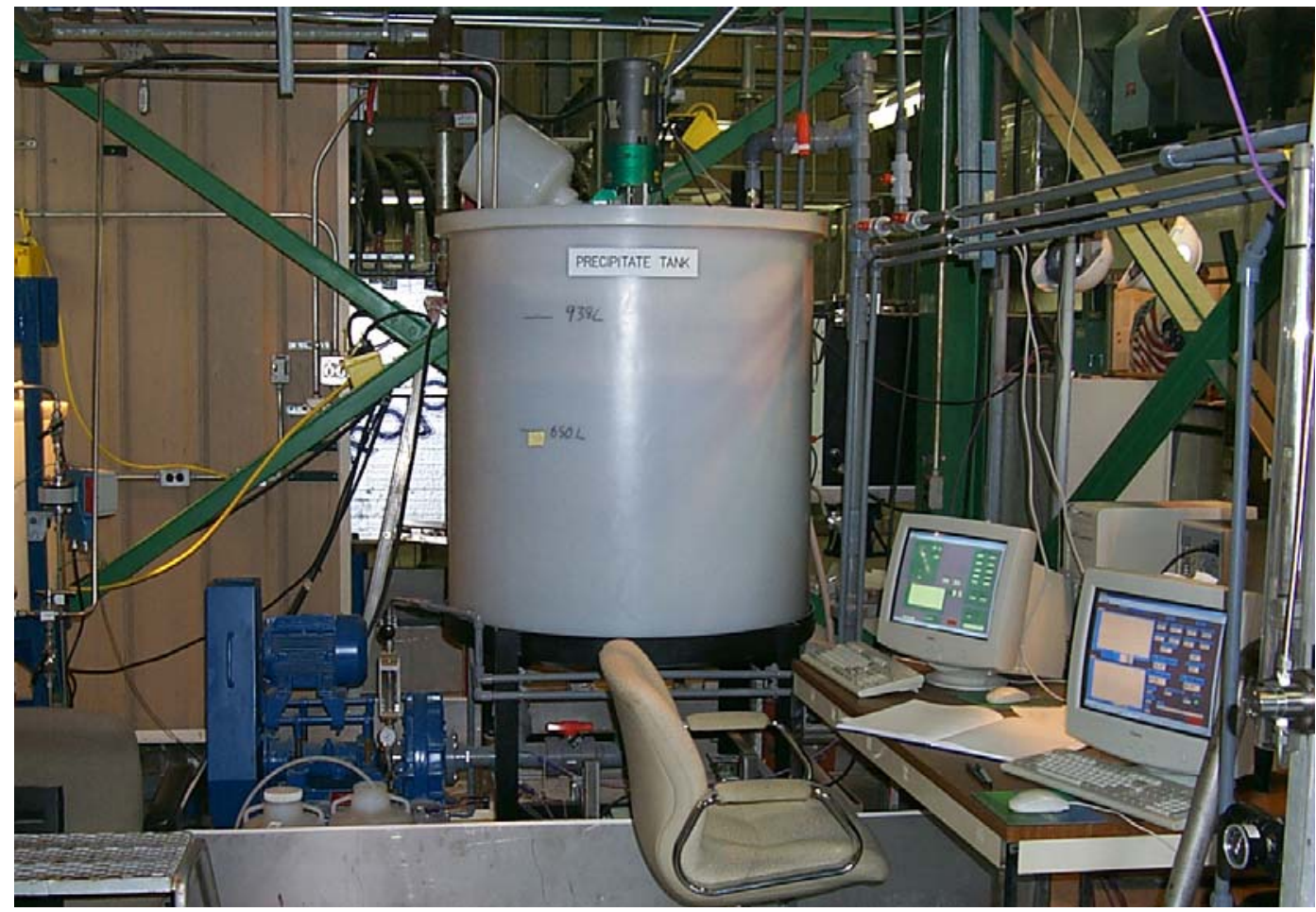

Figure 10. UFP Primary Mixing Tank (Volume 1000 liters) which represents the RPP-WTP UFP Preparation Feed Tank

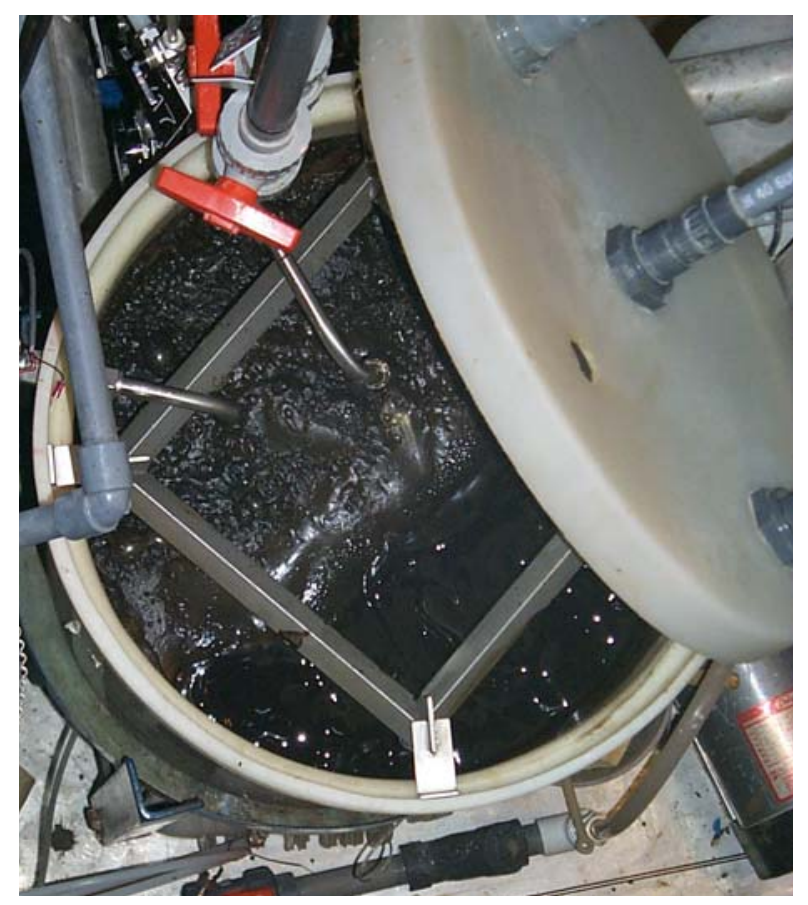

Figure 11. UFP Slurry Reservoir (Volume 100 liters) which represents the RPP-WTP UFP Feed Tank 
WSRC-TR-2005-00105, REVISION 0

SRNL-RPP-2005-00012, REVISION 0

\subsubsection{Adjusting the Final UFP Products}

The slurry produced by the UFP filter was concentrated to greater than $24 \mathrm{wt} \%$ UDS by 2 September. To bring the fixed-volume batch of slurry back to washing target of $20 \mathrm{wt} \%$ UDS, slurry was removed and permeate was added back. After the newly diluted slurry was well mixed, samples were taken and the measured solids concentration turned out to be $19 \mathrm{wt} \%$ UDS. The concentrated slurry remaining in the UFP test rig was then washed in two phases using the $0.01 \mathrm{M} \mathrm{NaOH}$ wash water from drums labeled "Campaign 4-UFP First or Second Wash" on 1 and 2 September. The water used to wash the slurry was collected in 55-gallon drums for disposal after samples were taken. The final washed HLW concentrated slurry had a density of $1.22 \mathrm{gm} / \mathrm{ml}$. After the slurry loop was drained to begin the cleaning cycle, the residual slurry was added to the carboy containing 12.5 liters of condensate to represent the simulated rinsed loop contents to the addition of the Caustic Flush 1 material.

The HLW concentrated and washed slurry was transferred to a 55-gallon drum labeled "Campaign 4 Concentrated Washed HLW Slurry" on 15 September for its shipment to VSL for eventual vitrification. The permeate produced by the UFP during dewatering was placed into six drums labeled “Campaign 4-CsIX Permeate Feed” and placed in storage.

On 7 September the filter rinse-clean-rinse sequence began. The contents of the carboy labeled "UFP Heel” were returned to the empty slurry reservoir as well as the contents from the drum marked "Caustic Flush 1" to rinse the UFP flow loop. After rinsing, all of the contents of the slurry reservoir were pumped to a drum, except 20 liters which was pumped to the carboy labeled "UFP Heel" to make the next heel. The drum was labeled "Campaign 4-Caustic Flush 1 Residue” and stored for eventual disposal. The 20 liters of "UFP Heel” were returned to the slurry reservoir along with first acid cleaning solution. This cycle repeated itself for all the Acid Cleanings 1, 2, 3 and Caustic Flush 2 and 3, that is, 20 liters of each of the preceding rinse/cleanings, simulated heel material, were fed with the next batch of cleaning or rinsing. The cleaning and rinsings were completed on 8 September. The drum contents produced were stored in drums labeled "Campaign 4-Acid Cleaning 1 or $2 \& 3$ Residual” and "Campaign 4-Caustic Flush 2 or 3 Residual” for eventual disposal. 
WSRC-TR-2005-00105, REVISION 0

SRNL-RPP-2005-00012, REVISION 0

\subsubsection{Pilot UFP Operation and Equipment (Author - Fowley)}

\subsubsection{Test Equipment}

\section{Filter Unit}

The SIPP UFP filter unit (Figs. 12, 13, 14, and 15) was manufactured by D. C. Fabricators, Inc. to meet RPP-WTP specifications (Townson, 2002, Longwell, 2003).

The filter unit had the following physical characteristics:

- 7 filter tubes with each having an inside diameter of 0.5-inch

- 92.4-inch porous length for each filter tube

- Stainless steel construction

- Nominal rated 0.1 micron filter element

The filter unit was prototypic in most of its features. The filter unit was not prototypic in three features: it only had 7 tubes instead of 241 tubes, it was mounted vertical instead of horizontal, and while the backpulse system functioned prototypically, the system design was different because of scaling. In general, filter performance data from the pilot test rig was assumed to well represent those that will be obtained in the full-scale unit.

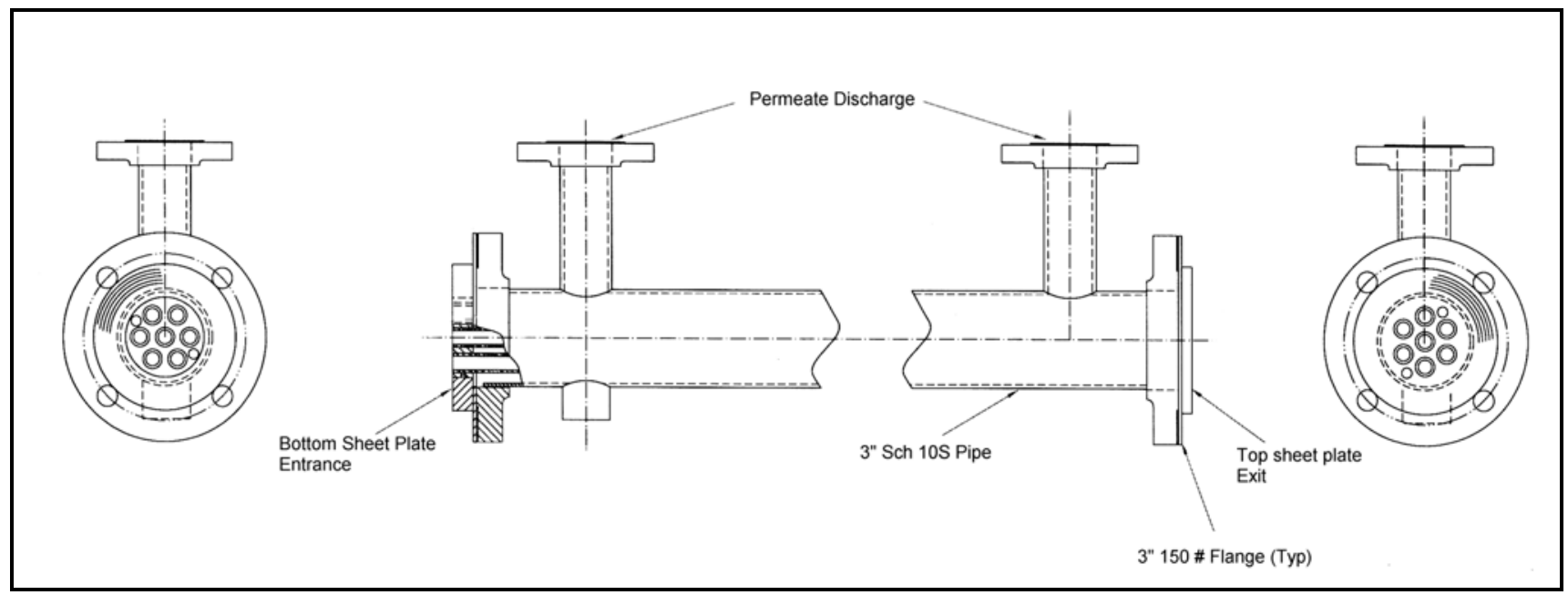

Figure 12. GKN Filter Unit

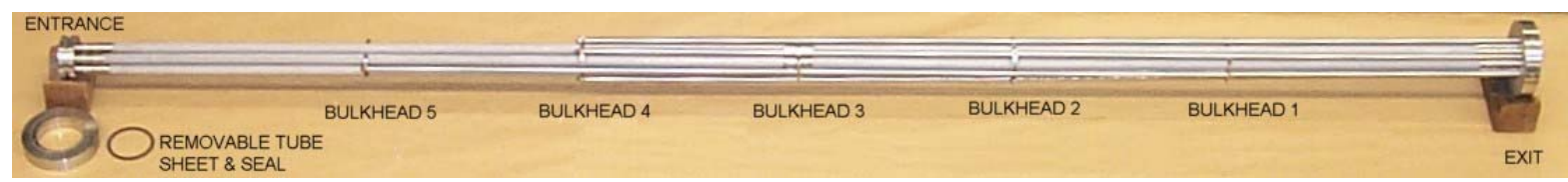

Figure 13. Filter-Tube Bundle 


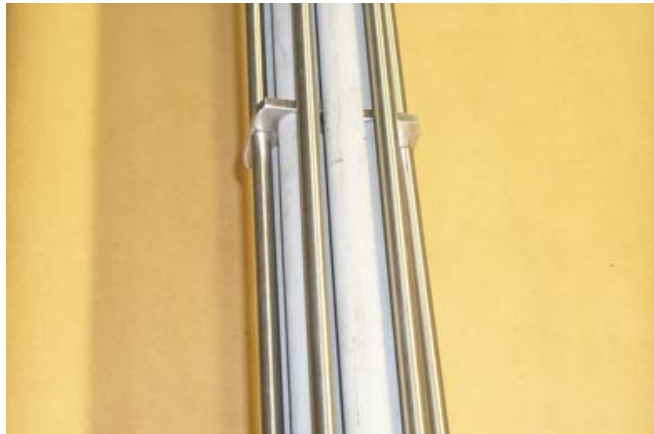

a) Bulkhead 1

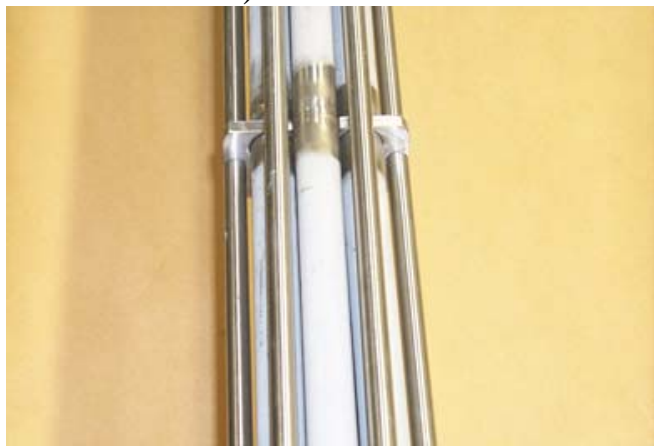

c) Bulkhead 3

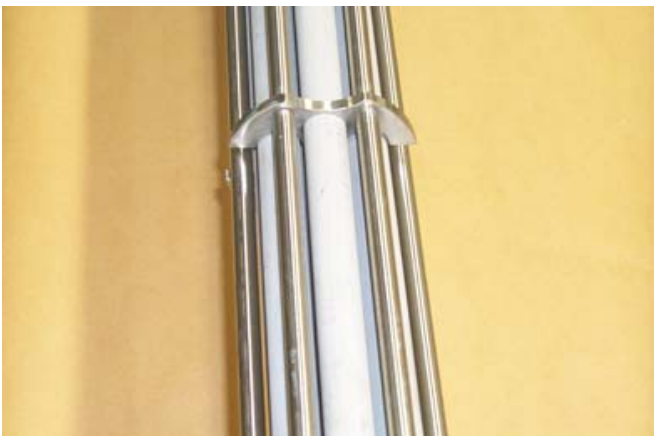

b) Bulkhead 2

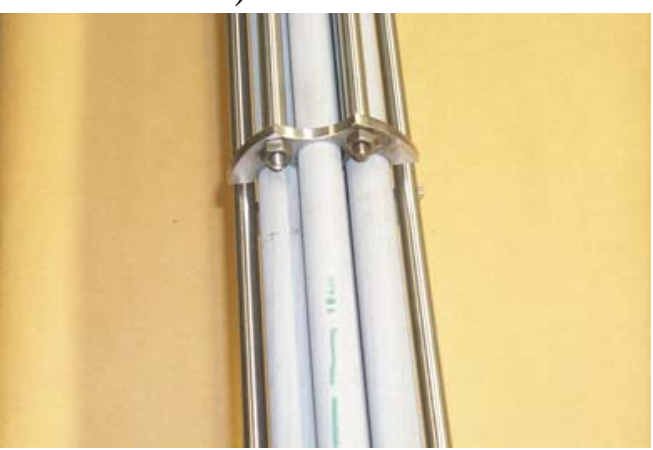

d) Bulkhead 4

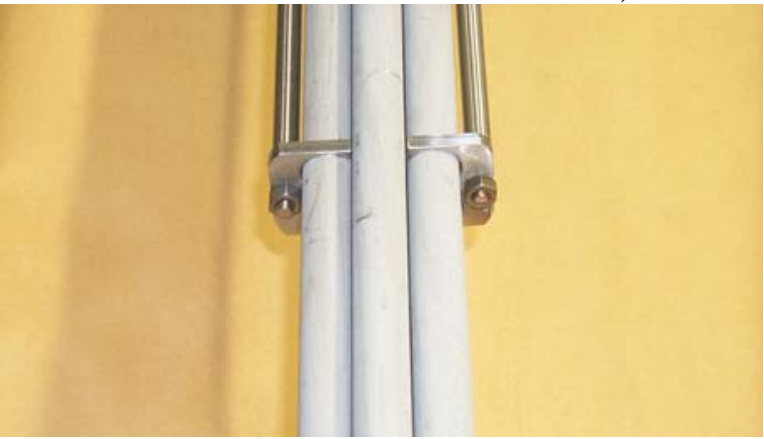

e) Bulkhead 5

Figure 14. Filter Tubes and Bulkhead Stabilizing Plates
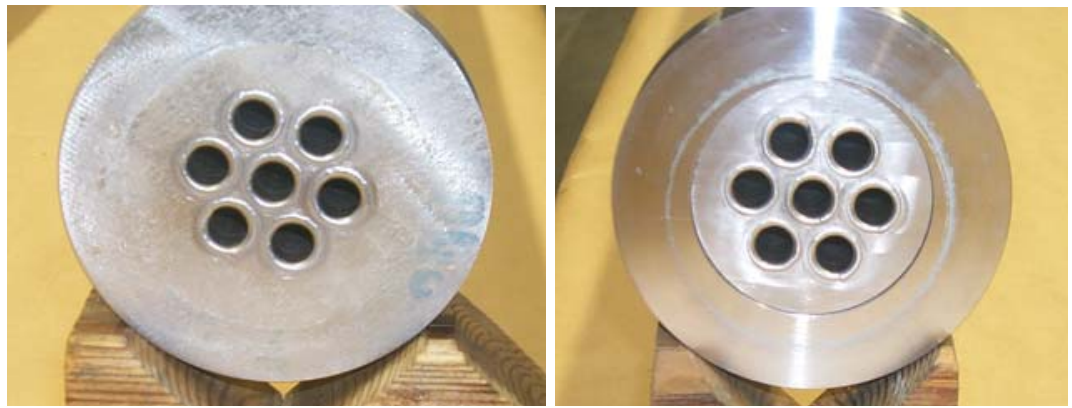

Figure 15. Exit (Left) and Entrance (Right) Tube Sheets of the Filter Tube Bundle 
Table 24 compares the physical characteristics for the filter units of the GKN, used in Campaign IV, and Mott, used in Campaigns I, II, and III. As can be seen, the filter units are similar in most of their characteristics. Further dimensional information regarding the GKN and Mott filter tubes is given in Appendix F.

Table 24. Dimensional Comparison of Mott and GKN Filter Units

\begin{tabular}{|r|c|c|l|}
\hline \multicolumn{1}{|c|}{ Parameter } & Mott & GKN & Units \\
\hline Nominal rating & 0.1 & 0.1 & micron \\
\hline Number of tubes & 7 & 7 & \\
\hline Filter construction & sintered metal - 316L & sintered metal - 316L & \\
\hline Active porous length & 90.0 & 92.4 & inches \\
\hline Tube ID - specification & 0.5 & 0.5 & inches \\
\hline -measured & 0.488 & 0.495 & inches \\
\hline Tube OD - specification & 0.625 & 0.625 & inches \\
\hline - measured & 0.654 & 0.687 & inches \\
\hline Tube Thickness & 0.083 & 0.096 & inches \\
\hline Approximate filter area & 6.8 & 7.0 & sq. ft. (based on ID) \\
\hline Overall housing length & 92.0 & 101.25 & inches \\
\hline
\end{tabular}

The GKN filter unit was constructed mainly of 316L stainless steel. Five bulkheads were equally spaced along the length of the tube bundle (Figure 14) and connected with $1 / 4$ " diameter rods to provide support and proper spacing of the tubes. The entrance tube sheet had a removable annular section to facilitate removal of the tube bundle from the housing. The annular section and the sealing o-ring are seen at the bottom left side in Figure 13. The permeate side of the filter unit had an internal volume of about $8.5 \mathrm{~L}$. Permeate exits the unit, normal to the axis, through two 1.5” schedule 40 pipes at either end of the housing. In the pilot-scale test rig only the upper pipe (nearest the unit exit) was used for dewatering, the lower pipe was isolated with a valve.

Figure 16 shows the construction of the GKN filter tube. A GKN sintered metal tube is made to a standard length. A stainless steel nipple is bonded to the ends of the tube to facilitate end connections or extra lengths. Two GKN sections were welded together to obtain the overall length required in the SIPP UFP test rig. End connections were welded to the ends of the filter tube assembly for attachment to the tube sheets. The tube-to-tube junction is shown in Figure 14c) and the end connections at the tube sheets are shown in Figure 17. The nipples of the GKN created the extra overall housing length (101.25” vs. 92.0”) compared to the Mott. The tubes of the Mott were welded together without the use of nipples. 
WSRC-TR-2005-00105, REVISION 0

SRNL-RPP-2005-00012, REVISION 0

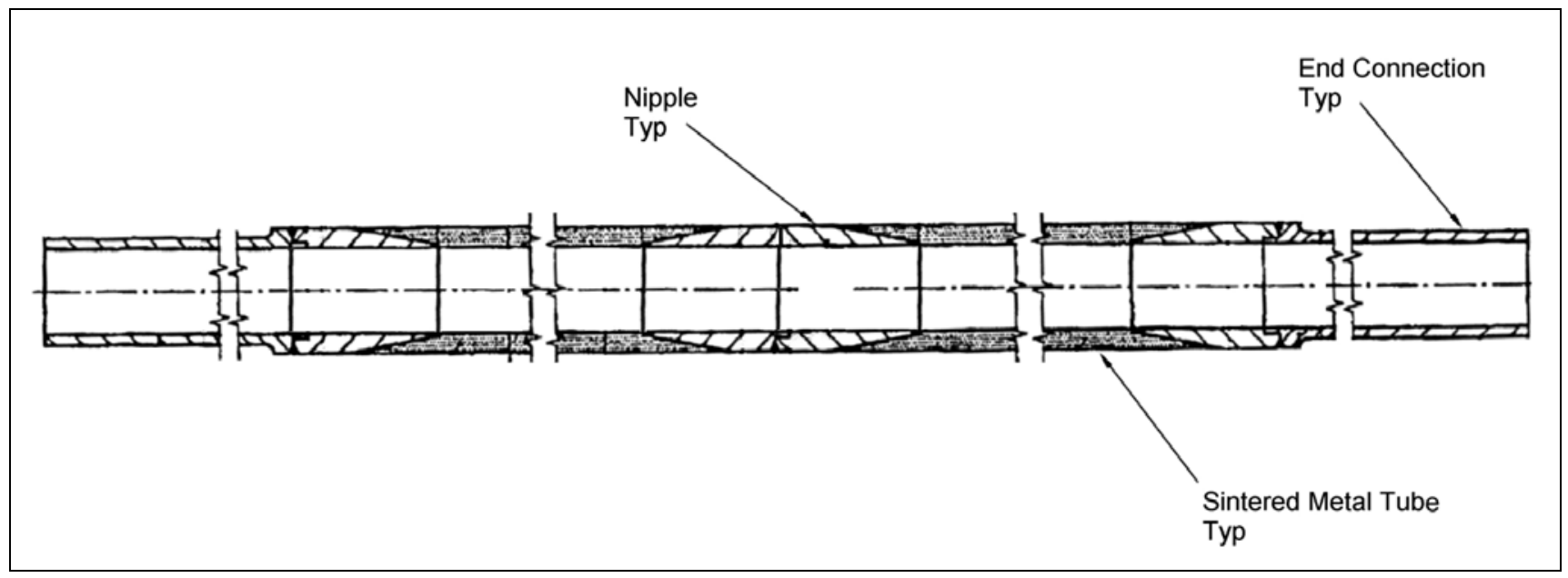

Figure 16. GKN Sintered Metal Filter Tube for the SIPP UFP
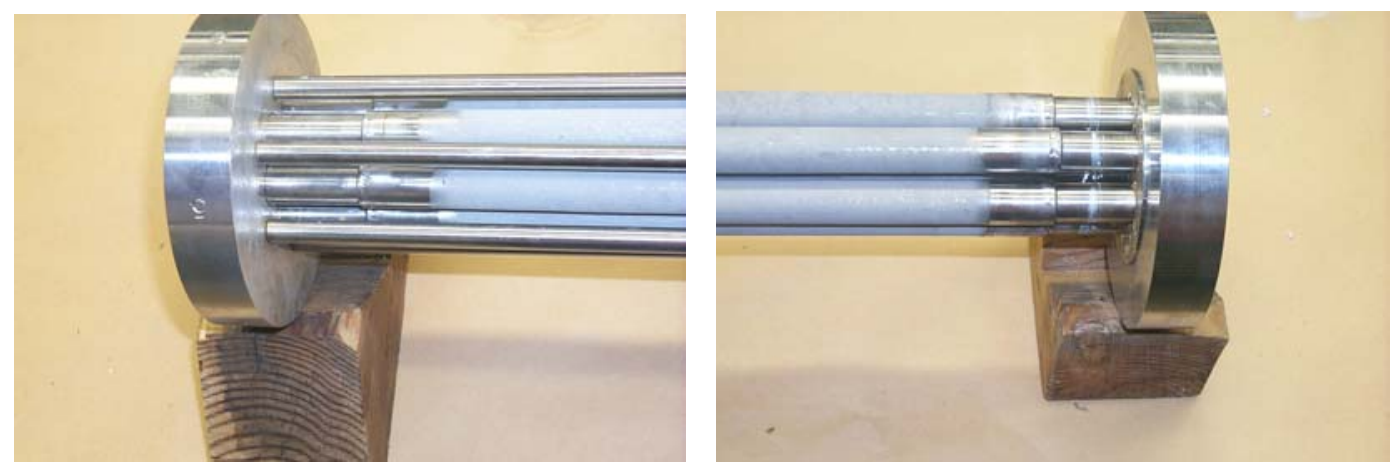

Figure 17. Exit and Entrance Tube Sheets showing Tube Ends Connections

The filter unit and associated hardware represent the pilot-scale UFP test rig. A schematic diagram of the test rig is shown in Fig. 18. The pilot-scale test rig was designed to have the following:

- Maximum axial velocity through filter tubes of $4.6 \mathrm{~m} / \mathrm{s}(15 \mathrm{ft} / \mathrm{s})$

- Maximum transmembrane pressure (TMP) of 60 psid

- Maximum axial velocity to be achievable at the maximum TMP

- Instrumentation to monitor the axial velocity, the permeate flow rate, the TMP, and the slurry temperature

- All materials compatible with the high-caustic simulants and the $2 \mathrm{M}$ nitric acid cleaning solution

All specifications were met or exceeded. 
Test Rig

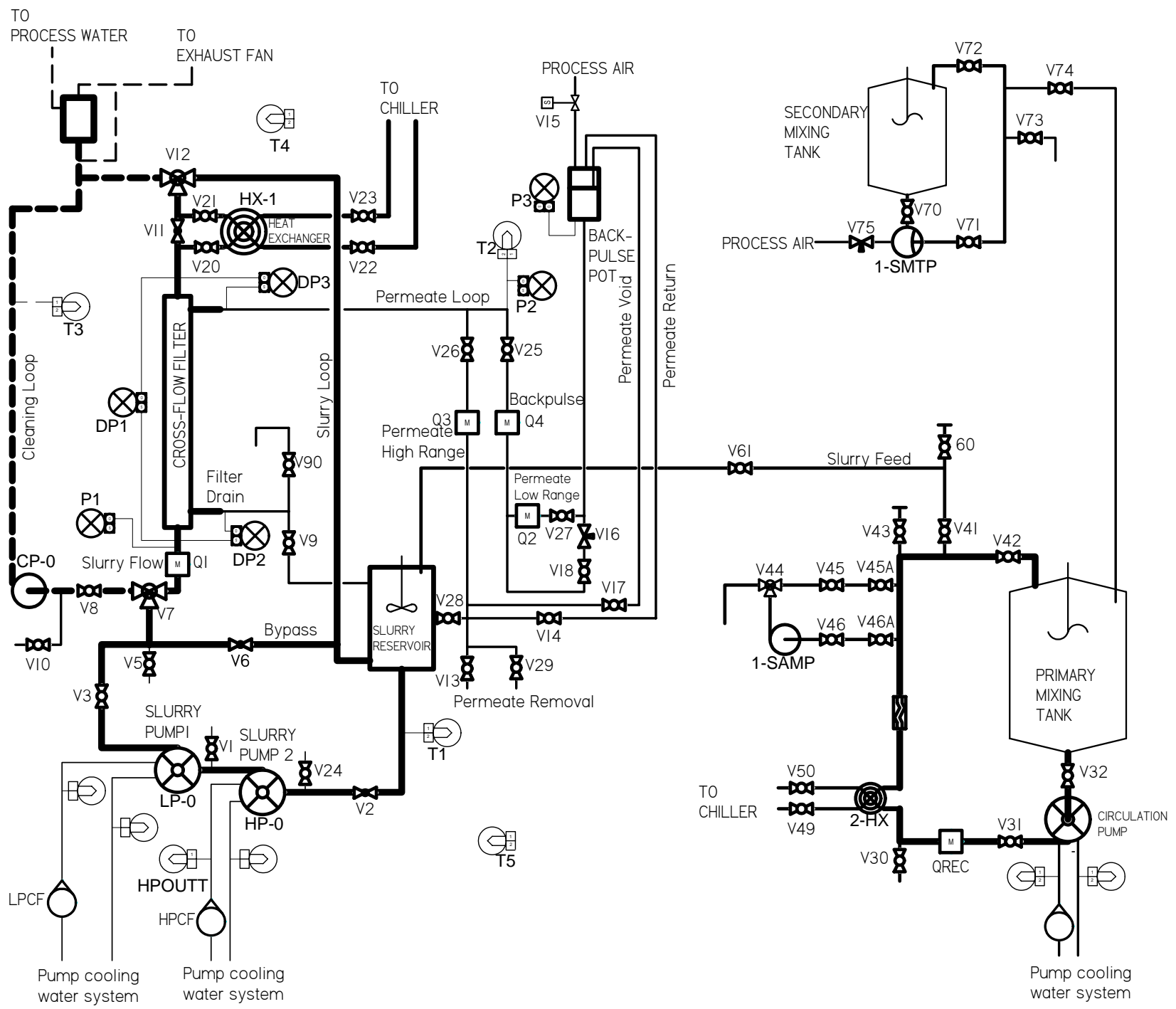

Figure 18. Schematic of the Campaign IV Pilot-Scale Test Rig

Valves in Fig. 18 are described below:

V1: $\quad$ Slurry drain valve - pump 1

V2: $\quad$ Slurry reservoir isolation valve - slurry

V3: $\quad$ Slurry pump isolation valve

V5: $\quad$ Slurry sample valve

V6: $\quad$ Slurry recirculation valve

V7: $\quad$ Slurry directional valve

V8: $\quad$ Not used - see below

V9: $\quad$ Permeate drain valve - to slurry reservoir 
V10: $\quad$ Not used - see below

V11: $\quad$ Slurry flow control valve

V12: $\quad$ Not used - see below

V13: $\quad$ Permeate drain valve - to collection drum

V14: $\quad$ Permeate return isolation valve

V15: Pulsepot pressurization valve

V16: $\quad$ Permeate flow control valve

V17: $\quad$ Pulsepot void valve

V18: $\quad$ Backpulse actuation valve

V19: $\quad$ Not used - see below

V20: Heat exchanger inlet valve

V21: Heat exchanger discharge valve

V22: Heat exchanger coolant inlet valve

V23: Heat exchanger coolant discharge valve

V24: $\quad$ Slurry drain valve - pump 2

V25: Permeate isolation valve - low flow

V26: Permeate isolation valve - high flow

V27: $\quad$ Permeate low flow meter isolation valve

V28: $\quad$ Slurry reservoir isolation valve - permeate

V29: $\quad$ Permeate sample valve

V30: $\quad$ Primary mixing tank drain valve

V31: $\quad$ Primary mixing tank pump isolation valve

V32: $\quad$ Primary mixing tank isolation valve

V41: $\quad$ Slurry feed control valve - primary mixing tank

V42: $\quad$ Slurry circulation control valve - primary mixing tank

V43: $\quad$ Slurry transfer valve - alternate

V44: $\quad$ Slurry feed sample valve

V45: $\quad$ Slurry feed sample control valve - discharge

V45A: $\quad$ Slurry feed sample isolation valve - discharge

V46: $\quad$ Slurry feed sample control valve - inlet

V46A: Slurry feed sample isolation valve - inlet

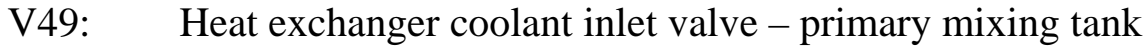

V50: Heat exchanger coolant discharge valve - primary mixing tank

V60: $\quad$ Feed line drain valve

V61: $\quad$ Slurry feed isolation valve

V70: $\quad$ Secondary mixing tank isolation valve

V71: $\quad$ Secondary mixing tank pump isolation valve

V72: $\quad$ Slurry circulation control valve - secondary mixing tank

V73: $\quad$ Slurry sample valve - secondary mixing tank

V74: $\quad$ Slurry feed control valve - secondary mixing tank

V75: $\quad$ Air control valve - secondary mixing tank positive displacement pump

V90: $\quad$ Permeate drain line - spare

Note: Valves V8, V10, V12, and V19 are for the cleaning loop. The cleaning loop was not used for SIPP, but was used for tests previous to SIPP, and is not described in this report. 
WSRC-TR-2005-00105, REVISION 0

SRNL-RPP-2005-00012, REVISION 0

The instrumentation on the pilot-scale test rig is described in Section 2.7.3.3 and in Appendix G2.

The pilot-scale test rig stood 25 feet tall and was serviced by a two level mezzanine (Figure 19). The test rig was mainly constructed of 300 series stainless steel pipe, mostly schedule 40, but some schedule 10. The pilot-scale test rig was composed of three basic flow loops; the slurry loop, the permeate loop, and the cleaning loop (not used). The slurry loop was the primary flow path for circulating slurries and contains the filter unit and the pumping system. The pumping system consisted of two 10-hp Galigher ${ }^{\circledR}$ Series 1000 slurry pumps connected in series. The impeller and impeller housing were lined with EPDM to be compatible with both the high caustic slurry and the $2 \mathrm{M}$ nitric acid cleaning solution. A recirculation loop was part of the slurry loop and was used to bypass slurry around the filter unit. The recirculation loop was primarily used during start up.

The permeate loop started at the filter housing, contained a backpulse pot and ended at the Slurry Reservoir (see Fig. 11). It consisted mostly of 3/8” stainless steel tubing, but had some 3/8" polyethylene tubing, which permitted flow visualization. The backpulse pot and associated tubing provided a pulse of permeate flow in the reverse direction to knock off built-up slurry cake on the inside of the filter tubes. Discharge valves were located in the permeate loop near the Slurry Reservoir to facilitate the extraction of permeate from the system (either for dewatering or sampling).

The backpulse pot and the method of backpulsing were designed to mimic plant operation. Extensive testing was conducted prior to the SIPP campaigns (Duignan, 2003a) to assure that the backpulse system matched, as closely as possible, reguirements specified by the RPPWTP customer.

The SIPP UFP test rig was slightly modified to accommodate the new GKN filter unit. As listed in Table 24, the overall housing length of the GKN was $91 / 4$ inches longer than the Mott filter unit. The stainless steel spool piece below the slurry flow meter (Q1, reference Figure 18) was shortened accordingly. In addition, the permeate loop was modified to fit the slightly different locations of the permeate discharge flanges.

The Primary Mixing Tank (see Fig. 10) was used to mix the combined simulated wastes during feed preparation and to transfer the mixture to the Slurry Reservoir before and during dewatering. The tank was mechanically agitated and included a slurry recirculation loop to provide additional mixing and to facilitate the transfer to the Slurry Reservoir. Pumping for the recirculation loop was provided by a 10-hp Galigher ${ }^{\circledR}$ Series 1000 slurry pump with an EPDM liner on the impeller housing and impeller. A sampling system was included in the recirculation loop to allow acquisition of a representative sample. 
WSRC-TR-2005-00105, REVISION 0 SRNL-RPP-2005-00012, REVISION 0

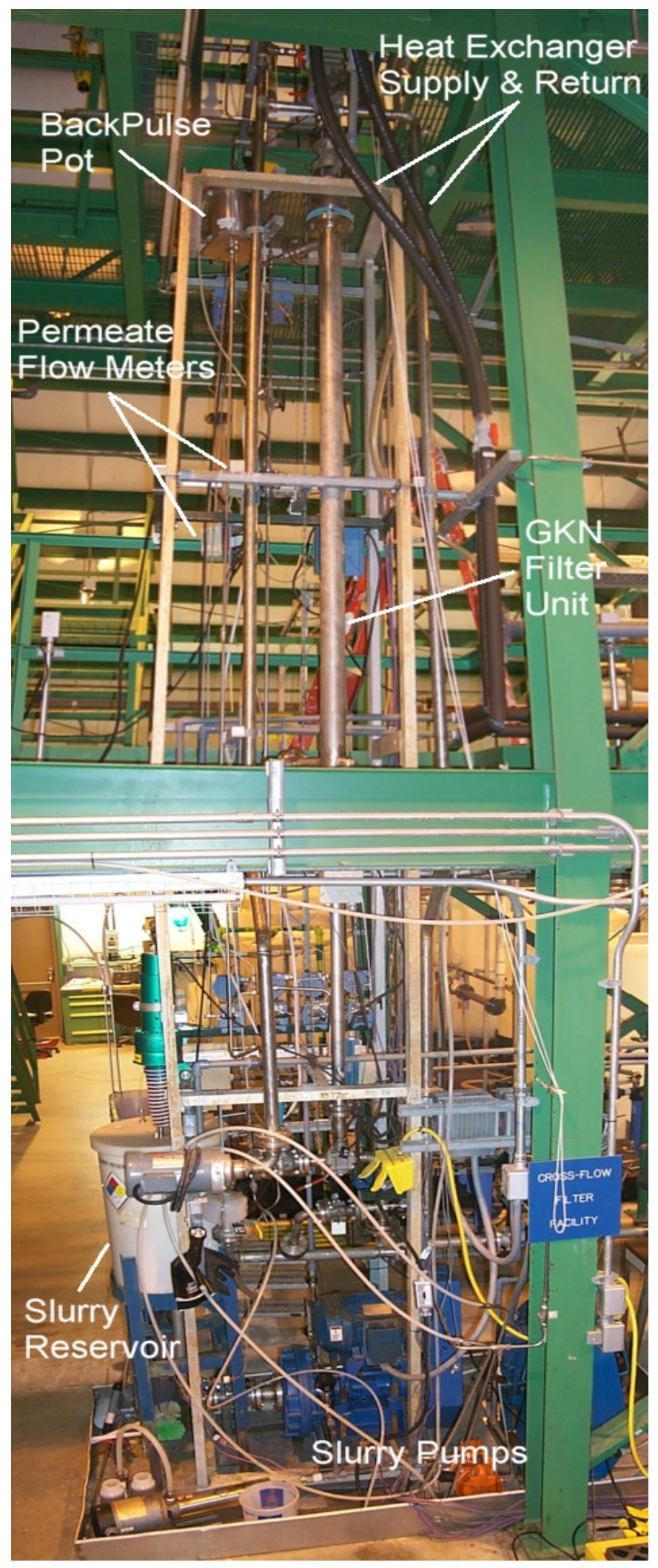

Figure 19. Picture of the Pilot-Scale Test Rig 
WSRC-TR-2005-00105, REVISION 0

SRNL-RPP-2005-00012, REVISION 0

The Secondary Mixing Tank was used to prepare and transfer the AY102 simulated waste to the Primary Mixing Tank during feed preparation. The tank was mechanically agitated and included a product recirculation loop to provide additional mixing and facilitate the transfer to the Primary Mixing Tank. Pumping for the recirculation loop was provided by a diaphragm pump with a stainless steel and Teflon wetted parts. A sample port was located in the recirculation loop to obtain representative samples.

\subsubsection{Operating Procedure}

All UFP testing activities were performed using an approved field procedure (Duignan, 2004a). The field procedure described generic activities regarding the operation of the test rig (filling, draining, dewatering, backpulsing, sampling, etc.). Work guidances were written for daily operation of the test rig. The work guidances referenced the field procedure and specified the order of the generic activities for completing a test number or series of test numbers in the test matrix. The following sections describe some of the generic activities in the SIPP UFL operating procedure. Reference Fig. 18 for the following descriptions.

\subsection{Test Preparation}

As part of the test preparation, a pre-test equipment inspection was conducted to ensure safety and proper operation at the beginning of each campaign. Specifically, a visual inspection was conducted looking for equipment leaks, damaged wires, loose nuts and bolts, and other unusual situations. M\&TE readouts on the DAS were checked for unusual readings. In addition, solution quantities were verified to ensure on-schedule and uninterrupted operation. Any work involving the chemical solution of the SIPP UFP (pretest inspection and normal operation) required the appropriate Personal Protective Equipment (PPE). This included safety glasses, a lab coat, and chemical resistant gloves. Any work involving concentrated forms of caustic or acidic solutions required the PPE listed above plus a chemical apron and a face shield. Work around the test rig that did not require interaction with any chemical solutions required safety glasses only, which was the minimum EDL requirement.

The M\&TE was checked to ensure proper operation prior to the start of each series of tests or as required. The pressure transducers were isolated and data was recorded as a zero check. The thermocouples outputs displayed on the DAS were verified prior to testing. The DASdisplayed flow rate was verified prior to start-up as a zero check.

The pressure transducer impulse lines were typically flushed with DIF (or 0.1 M NaOH depending on what was in the test rig) at the end of each campaign to clear any slurry that may have accumulated in the lines during testing. After flushing the lines were procedurally refilled with a specific quantity of DIF water using a micropump. 
WSRC-TR-2005-00105, REVISION 0

SRNL-RPP-2005-00012, REVISION 0

\subsection{Test Rig Draining}

The test rig was completely drained (slurry loop and permeate loop) or partially drained (slurry loop only) depending on the specific need of the test. The test rig was drained through valves V1 and V24 (slurry loop) using a drain line to a collection drum and through valve V9 (permeate loop), which emptied to the Slurry Reservoir. The slurry pumps were started and a low flow rate was set (10 gpm). The slurry drain valves (V1 and V24) were opened to start the draining process. If the permeate loop was to be drained then valve V9 was opened when the level in the Slurry Reservoir dropped below the reservoir discharge. Air pressure (10 to 20 psig) was applied to the backpulse pot to help clear the permeate loop. Draining was enhanced by connecting a pump to the drain line from valve V1 and V24.

\subsection{Test Rig Filling}

The test rig was filled by one of several ways via the Slurry Reservoir. 1) Slurry (simulant) was typically transferred to the Slurry Reservoir from the Primary Mixing Tank (dewatering). 2) Some solutions were pumped from storage drums to the Slurry Reservoir (slurry washing and test rig rinsing and cleaning). 3) Other solutions were poured into the Slurry Reservoir from carboys (slurry adjustment). The more involved method (1) utilized the Primary Mixing Tank slurry circulation loop. The slurry circulation control valve (V42, reference Fig. 18) was throttled to pressurize the circulation loop. Slurry was transferred by opening the slurry feed control valve (V41).

Prior to slurry pump start-up, the Slurry Reservoir was filled to at least $75 \mathrm{~L}$ and the pump cooling system was activated. The pumps were started and initially set to a low flow rate (10 - 20 gpm). The heat exchanger system was started at this point. Slurry flow bypassed the filter unit (via the recirculation loop) initially and while the Slurry Reservoir was filled to the required batch level, i.e., 117 L. Next, slurry was slowly introduced to the filter unit by closing the slurry recirculation valve (V6).

The permeate flow was started by slowly opening permeate low flow meter isolation valve (V27). At this point the permeate loop was circulating flow back to the Slurry Reservoir (permeate recirculation mode). Dewatering began when permeate was diverted from the Slurry Reservoir to a collection drum using the slurry reservoir isolation valve (V28) and the permeate drain valve (V13). A 3/8” polyethylene tube was used between the drain valve and the collection drum.

A volume of $16 \mathrm{~L}$ of supernatant from the Primary Mixing tank was added to the Slurry Reservoir to account for the permeate needed to fill the Permeate Loop prior to the start of a dewatering test. If called for, the test rig was shut down at this time by closing valve V27 to isolate the Permeate Loop, de-energizing the pumps, and closing valve V3 to isolate the Slurry Loop. The test rig was ready for testing at this point. 
WSRC-TR-2005-00105, REVISION 0

SRNL-RPP-2005-00012, REVISION 0

\subsection{Operation Mode}

The test rig could be operated in closed mode (not dewatering) or open mode (dewatering) depending on the requirements of the test. In closed mode permeate flow was directed to the Slurry Reservoir by closing the permeate isolation valve V27 and opening the permeate return valve V14 (and Slurry Reservoir isolation valve V28). The permeate mixed with the slurry in the Slurry Reservoir, which maintained the slurry at a constant concentration. In open mode permeate flow was directed to a collection drum through the permeate discharge valve V13, typically to a collection drum. As permeate flows to the collection drum the solution in the Slurry Reservoir was replenished by adding more simulant from the Primary Mixing Tank to maintain the batch volume.

\subsection{Backpulsing}

The steps and pertinant valve positions for conducting a backpulse are shown in Table 25. A backpulse was performed by first stopping dewatering (closing V13 and V14, reference Fig. 18) and closing the permeate low flow isolation valve (V27). The backpulse pot was then partially evacuated to create an air pocket (openning V17, V28 and V15); the permeate in the pulse pot was evacuated to the Slurry Reservoir. The air pocket was pressurized to 40 psig above the pressure of the slurry loop by regulating the air pressure to V15.

Table 25. Procedure to Operate the SIPP Backpulse System

\begin{tabular}{|c|c|c|c|c|c|}
\hline Mode $\backslash$ Valve & $\begin{array}{c}\text { Permeate Flow, } \\
\text { Dewatereing } \\
\text { (V27) }\end{array}$ & $\begin{array}{c}\text { Permeate Flow, } \\
\text { Backpulse } \\
\text { (V18) }\end{array}$ & $\begin{array}{c}\text { Process Air } \\
\text { (V15) }\end{array}$ & $\begin{array}{c}\text { Permeate Collection } \\
\text { (V13 / V14) }\end{array}$ & $\begin{array}{c}\text { Feed Vessel Return } \\
\text { (V17 / V28) }\end{array}$ \\
\hline Dewatering & Open & Closed & Closed & Open & Closed \\
\hline Stop Dewatering & Closed & Closed & Closed & Closed & Closed \\
\hline Void Pulse Pot & Closed & Closed & Open & Closed & Open \\
\hline Pressurise Pulse Pot & Closed & Closed & Open & Closed & Closed \\
\hline Isolate Pulse Pot & Closed & Closed & Closed & Closed & Closed \\
\hline Backpulse & Closed & Open & Closed & Closed & Closed \\
\hline Resume Dewatering & Open & Closed & Closed & Open & Closed \\
\hline
\end{tabular}

The backpulse valve (V18) was opened and closed for a short period of time (Table 26) to force permeate from the pulse pot back through the filter media to dislodge the cake from the filter walls. The pressure drop through the permeate loop (from the backpulse pot to the filter unit) was controlled by the permeate flow control valve, V16. Valve V16 was a needle valve; the valve setting was optimized during a previous filter test, before the SIPP campaigns (Duignan, 2003a). Dewatering was resumed after the backpulse was completed by openning V13, V14, and V27. 
WSRC-TR-2005-00105, REVISION 0

SRNL-RPP-2005-00012, REVISION 0

Table 26. Pilot.Scale Test Rig Backpulse Duration

\begin{tabular}{|c|c|c|c|c|c|c|c|}
\hline Pressure at the filter (P1), psig & 10 & 20 & 30 & 40 & 50 & 60 & 70 \\
\hline Duration of Pulse, seconds & 9 & 11 & 12 & 14 & 15 & 17 & 18 \\
\hline
\end{tabular}

\subsection{Sampling}

Filtrate was sampled through the permeate sample valve V29. Prior to sampling, the V29 valve and discharge tube was cleared of old permeate by momentarily opening the valve. This ensured a representative sample. The slurry was sampled through slurry sampling valve V5. In previous filtration tests the slurry was sampled from the Slurry Reservoir using a Coliwasa sampler. This method involved some risk since the Slurry Reservoir contained a mechanical agitator to provide adequate slurry mixing (possibility of breaking the glass Coliwasa by impact with the agitator blades). Through comparative sampling it was determined that a sample obtained from valve V5 was sufficiently similar to a sample obtained with a Coliwasa from the Slurry Reservoir. The sampling method was changed to the V5 method after this determination to eliminate the risk.

\subsection{Work Guidances}

Several work guidances were used per campaign to complete the testing specified in a test matrix. A work guidance was written for typical campaign activities such as; 1 ) test rig and M\&TE checkout including the pre-test baseline test, 2) the first stage of dewatering, 3) the second stage of dewatering, 4) the first slurry wash, 5) the second slurry wash, 6) rinsing and cleaning the test rig, and 7) the post-test baseline test. Other work guidances were written for campaign specific activities such as steady state testing, high temperature testing, and the installation and check out of the GKN filter unit or for off-normal activities not specified in the test matrix.

\subsubsection{Measurement Uncertainty}

The Measurement and Test Equipment (M\&TE) used on the pilot-scale test rig were calibrated using approved calibration procedures and NIST traceable standards. Calibrations were conducted before and after the SIPP campaigns to accurately determine instrument uncertainty and to qualify the SIPP campaign data. In addition, each channel of the Data Acquisition System (DAS) was calibrated before and after the SIPP campaigns and was included in the M\&TE uncertainty analysis. The M\&TE uncertainty analysis process was conducted using approved statistical methods (Coutts, 1991). 
WSRC-TR-2005-00105, REVISION 0

SRNL-RPP-2005-00012, REVISION 0

\subsection{Pressure, Flow, and Temperature Measurements}

Pressure measurements were made with Rosemount ${ }^{\circledR}$ differential pressure transmitters (model \# 1151) and gauge pressure transmitters (model \# 1144), which uses a variable capacitance principle method of pressure measurement. Flow rate measurements were made with Fisher-Porter ${ }^{\circledR}$ magnetic flow transmitters (model \# 10D1475). Temperature measurements were made with Omega ${ }^{\circledR}$ Type $E$ thermocouples with ungrounded, stainless steel sheaths. The pressure, flow rate and temperature raw data are shown in Appendix $\mathrm{H} 2$ for each test of Campaigns I to IV.

The calculated uncertainties of all EDL controlled M\&TE are shown in Table 27. Further detail of the DAS and M\&TE calibrations is provided in Appendix G. The uncertainties shown in the table represent the greatest value of before and after campaign calibrations. Typically (but not always), the after campaign calibration yields the greatest value since it includes the error associated with a zero and/or span shift of the M\&TE electronic output that may have occurred between calibrations.

Table 27. SIPP UFP Measurement and Test Equipment

\begin{tabular}{|c|c|c|c|c|}
\hline Description & Designation & Range & M\&TE \# & $\begin{array}{l}\text { M\&TE \& DAS } \\
\text { Uncertainty }\end{array}$ \\
\hline Slurry Loop Temperature & T1 & $0-100^{\circ} \mathrm{C}$ & TR-02929 & $+/-0.90^{\circ} \mathrm{C}$ \\
\hline Permeate Loop Temperature & $\mathrm{T} 2$ & $0-100{ }^{\circ} \mathrm{C}$ & TR-02927 & $+/-0.85^{\circ} \mathrm{C}$ \\
\hline Cleaning Loop Temperature & T3 & $0-100{ }^{\circ} \mathrm{C}$ & TR-02930 & $+/-0.85^{\circ} \mathrm{C}$ \\
\hline $\begin{array}{c}\text { Atmospheric Temperature } \\
\text { Upper Test Rig }\end{array}$ & $\mathrm{T} 4$ & $0-100{ }^{\circ} \mathrm{C}$ & TR-02925 & $+/-0.90^{\circ} \mathrm{C}$ \\
\hline $\begin{array}{c}\text { Atmospheric Temperature } \\
\text { Lower Test Rig } \\
\end{array}$ & T5 & $0-100^{\circ} \mathrm{C}$ & TR-02926 & $+/-0.85^{\circ} \mathrm{C}$ \\
\hline Slurry Loop Pressure & P1 & 0-100 psig & TR-02917 & +/- 0.15 psig \\
\hline Permeate Loop Pressure & $\mathrm{P} 2$ & 0-151 psig & TR-03115 & +/- 0.50 psig \\
\hline Backpulse Pot Pressure & P3 & 0-151 psig & TR-00532 & $+/-0.25$ psig \\
\hline Pressure Drop through Filter & DP1 & $0-26$ psid & TR-03495 & $+/-0.05$ psid \\
\hline $\begin{array}{c}\text { Transmembrane pressure } \\
\text { Upper Flange }\end{array}$ & DP2 & 0-101 psid & TR-03553 & +/- 0.15 psid \\
\hline $\begin{array}{c}\text { Transmembrane pressure } \\
\text { Lower Flange }\end{array}$ & DP3 & $-11-101$ psid & TR-03109 & +/- 0.20 psid \\
\hline Slurry Flow Rate & $\mathrm{Q} 1$ & 0-100 gpm & TR-20350 & $+/-0.50 \mathrm{gpm}$ \\
\hline $\begin{array}{c}\text { Permeate Flow Rate } \\
\text { Low Range }\end{array}$ & Q2 & 0-1.2 gpm & TR-20353 & +/- $0.01 \mathrm{gpm}$ \\
\hline $\begin{array}{c}\text { Permeate Flow Rate } \\
\text { High Range }\end{array}$ & Q3 & 0-15 gpm & TR-03721 & +/- $0.10 \mathrm{gpm}$ \\
\hline Backpulse Flow Rate & Q4 & $0-5 \mathrm{gpm}$ & TR-03276 & $+/-0.03 \mathrm{gpm}$ \\
\hline
\end{tabular}

It is important to note that the uncertainties listed in Table 27 represent the uncertainties of the M\&TE and DAS only. They are considered systematic uncertainties (i.e. consistent and measurable). Other factors (operator error, measurement assumptions, environmental factors, etc.) were considered incidental or insignificant and were not included in the measurement uncertainty values presented in the test results section. For example, the slurry 
temperature, measured only in the slurry loop upstream to the slurry pumps, was assumed to represent the average slurry temperature. Almost certainly, heat was added to the slurry by the pumps and removed by the heat exchanger creating isolated temperature gradients in those areas. In addition, there was an ambient temperature disparity (typically $2^{\circ} \mathrm{C}$ ) from the top of the slurry loop to the bottom due to the height of the test rig (the test rig was located in a high-roofed area of the EDL that had natural temperature gradients along it's elevation). These effects were assumed to be insignificant due to the relatively quick slurry loop velocity (slurry residence time was minimized) and the thorough mixing in the slurry loop and Slurry Reservoir (temperature variations minimized).

Measurement uncertainty was also evident as random fluctuations in the raw data. Significant fluctuations were seen in pressure and flow rate data during some tests that involved slurry (dewatering and slurry washing). The data from these tests are presented in the test results section as figures. The magnitude of the fluctuations can be seen (and quantified) directly from the figure. Any calculated value from this data, such as the mean value, will include the random fluctuation in the measurement uncertainty where appropriate. Some figures in the test results section show mean values from other tests (baselines tests, steady-state tests, higher-temperature tests). In these figures the measurement uncertainty due to random fluctuation or unsteady operation is stated or is shown as error bars in the figure (calculated as $2 \mathrm{X}$ the standard deviation of the value). Typically, the mean values and other raw data calculations will omit process interruptions such as backpulses and supply drum exchanges.

Data averages, standard deviations and other statistical information are presented in the raw data shown in Appendix H2 (bottom of the raw data file). In some cases the standard deviation of a particular parameter may seem abnormally large. This was due to the calculation being about the mean value of that parameter for the entire test. The parameter may naturally vary during the course of the test (such as permeate flux during dewatering), creating a large standard deviation about the mean value. In these cases a better representation of the standard deviation would be about a curve fit of the parameter, which is not presented in the raw data.

\subsection{Calculated Quantities}

The measurement uncertainties (95\% confidence level) of the important calculated quantities are shown below in Table 28. Two uncertainties are shown, the uncertainty based on the M\&TE and DAS, and the uncertainty based on (2X) the standard deviation about the mean value due to the data scatter. The table also shows the result of combining these two uncertainties (by the root-sum-square method), which is only relative to a mean value. The uncertainty based on the M\&TE and DAS applies to individual data points in the raw data (Appendix H2). The uncertainties based on the M\&TE and DAS were derived from the variables in the governing equations using the method of propagation of errors [described in the EDL uncertainty and calibration analysis manual (Coutts, 1991)].

The measured values in the table are taken from two different Campaign IV test points that represent opposite ends of the operational spectrum (relative to transmembrane pressure and slurry velocity). Those tests points are, 1 ) the first point of the pre-test baseline test (4.00A), 
and 2) run 7 of the high temperature test (Temp7). The uncertainty based on the M\&TE and DAS can be applied to other tests and campaigns by interpolation (or extrapolation as the case may be) between the measured values shown in the table. Note that large "\% of reading” uncertainties (double-digit) occur for some of the calculated quantities in test number Temp7. The large values are primarily due to the data scatter.

Table 28. Uncertainties of Calculated Quantities for Campaign IV

\begin{tabular}{|c|c|c|c|c|c|c|}
\hline $\begin{array}{l}\text { Calculated } \\
\text { Quantity }\end{array}$ & \multicolumn{2}{|c|}{$\begin{array}{c}\text { Filter Tube } \\
\text { Slurry Velocity } \\
\text { (V) }\end{array}$} & \multicolumn{2}{|c|}{$\begin{array}{c}\text { Transmembrane } \\
\text { Pressure } \\
\text { (TMP) }\end{array}$} & \multicolumn{2}{|c|}{$\begin{array}{c}\text { Adjusted } \\
\text { Permeate Flux } \\
\left(\mathrm{F}_{\mathrm{C}}\right)\end{array}$} \\
\hline Units & \multicolumn{2}{|c|}{$\mathrm{ft} / \mathrm{sec}$} & \multicolumn{2}{|c|}{ psid } & \multicolumn{2}{|c|}{ gpm/ft2 } \\
\hline $\begin{array}{l}\text { Governing } \\
\text { Equation }\end{array}$ & \multicolumn{2}{|c|}{ Q1/A } & \multicolumn{2}{|c|}{$(\mathrm{dP} 2+\mathrm{dP} 3) / 2$} & \multicolumn{2}{|c|}{$\begin{array}{c}(\mathrm{Q} 2 / \mathrm{A}) * \\
\mathrm{e}^{\left[2500^{*}((1 /(273+\mathrm{T}))-(1 / 298))\right]}\end{array}$} \\
\hline $\begin{array}{c}\text { Test } \\
\text { Number }\end{array}$ & $4.00 \mathrm{~A}$ & Temp7 & $4.00 \mathrm{~A}$ & Temp7 & $4.00 \mathrm{~A}$ & Temp7 \\
\hline $\begin{array}{c}\text { Measured } \\
\text { Values } \\
\end{array}$ & 10.7 & 14.8 & 10.0 & 50.1 & 0.174 & 0.016 \\
\hline $\begin{array}{c}\text { Uncertainty } \\
\text { M\&TE + DAS } \\
(+/-)\end{array}$ & 0.18 & 0.22 & 0.13 & 0.13 & $5.20 \mathrm{E}-3$ & $9.23 E-4$ \\
\hline $\begin{array}{c}\text { \% of } \\
\text { Reading }\end{array}$ & 1.7 & 1.5 & 1.3 & 0.3 & 3.0 & 5.8 \\
\hline $\begin{array}{l}2 \text { X Std. Dev. } \\
\text { Of Data } \\
(+/-)\end{array}$ & 0.06 & 2.0 & 0.26 & 0.59 & $2.44 \mathrm{E}-3$ & 2.59E-3 \\
\hline $\begin{array}{c}\% \text { of } \\
\text { Reading } \\
\end{array}$ & 0.6 & 13.5 & 2.6 & 1.2 & 1.4 & 16.2 \\
\hline $\begin{array}{c}\text { Combined } \\
\text { Uncertainty } \\
(+/-)\end{array}$ & 0.19 & 2.02 & 0.29 & 0.60 & $5.74 \mathrm{E}-3$ & $2.75 E-3$ \\
\hline $\begin{array}{c}\text { \% of } \\
\text { Reading }\end{array}$ & 1.8 & 13.6 & 2.9 & 1.2 & 3.3 & 17.5 \\
\hline
\end{tabular}

\subsection{Density, Viscosity and Turbidity Measurements by EDL}

The density of the slurry and permeate were determined by mass and volume measurements from a calibrated platform scale and volumetric flask. The viscosity of the permeate was measured by a Cannon-Fenske viscometer. The turbidity was measured by a digital turbidity meter. The uncertainty of the EDL measured quantities (95\% confidence level) is shown below. The density uncertainty was determined through calibration of the scale and flask, and by comparison to ACTL measurements. The uncertainty of the viscometer was from the manufacturer specifications. The uncertainty of the turbidity meter was from calibration with turbidity standards.

Slurry or Liquid Density $\quad=\quad \pm 0.6 \%$ of reading

Liquid Viscosity $\quad=\quad \pm 0.2 \%$ of reading

Turbidity $\quad=\quad \pm 0.2 \mathrm{NTU}$ 
WSRC-TR-2005-00105, REVISION 0

SRNL-RPP-2005-00012, REVISION 0

\subsection{Data Acquisition System}

The electronic output of the M\&TE was logged by a PC based DAS consisting of a DELL OptiPlex GX300 PC with National Instruments ${ }^{\circledR}$ LabView ${ }^{\circledR}$ for Windows software, version 6i. Data files were renamed, typically, every two hours to prevent the loss of a significant amount of data. To verify proper operation of the M\&TE a zero flow check was performed at the beginning of each test or series of tests (as directed by the Work Guidance). Other pertinent test information such as observations, non-critical calculations, chronological events, etc. was recorded in the laboratory notebook (Duignan, 2004b) and on video tape or photograph as needed. All documents, video, and electronic media for this test activity are stored and maintained by the Engineered Equipment and Systems (EES) Department Document Control System per EES procedure L9.1-2006 in Job Folder \# EES-23041.

\subsubsection{Pilot UFP Discussion of Results}

\subsubsection{Experimental Observations (Author - Fowley)}

The experimental observations of the Campaign IV SIPP UFP are presented in this section. Figures and tables are provided to aid the discussion. The SIPP UFP raw data from Campaigns I to IV are presented in Appendix H2.

\subsection{Pre-test Activities}

\subsection{Final Baseline of the Mott Filter}

Prior to the installation of the GKN filter unit a baseline test was conducted with the Mott filter unit from Campaign III, using fresh $0.1 \mathrm{M} \mathrm{NaOH}$. As seen in the test matrix (Table 22), a baseline test consists of three separate test points at the same slurry flow rate and different levels of TMP (nominally 10, 20, and 30 psid). These test parameters have been consistent throughout the SIPP campaign. The baseline test primarily provides a means of judging the effect of filter cleaning after dewatering, but can also be used to judge filter performance (with $0.1 \mathrm{M} \mathrm{NaOH}$ and DIF water) between campaigns.

The primary purpose of the Mott baseline prior to Campaign IV was to quantify the change in the baseline flux of the Mott after it had been soaking in a mild caustic solution $(0.1 \mathrm{M}$ $\mathrm{NaOH}$ ) since the end of Campaign III (22 June). As mentioned in previous Interim Reports, the flux typically improves after the filter has been idle in a mild caustic solution $(0.1 \mathrm{M}$ $\mathrm{NaOH}$ in this case) for an extended period of time (for more than a year before Campaign I, for approximately 11 weeks between Campaigns I and II, and for approximately 6 weeks between Campaigns II and III. For Campaign IV the filter was changed.). The results of the baseline are shown in Figure 20, along with Campaign III pre- and Post-test baseline results for comparison. The figure shows that the flux levels increased to Campaign III pre-test levels after the extended soaking in $0.1 \mathrm{M} \mathrm{NaOH}$. 
WSRC-TR-2005-00105, REVISION 0

SRNL-RPP-2005-00012, REVISION 0

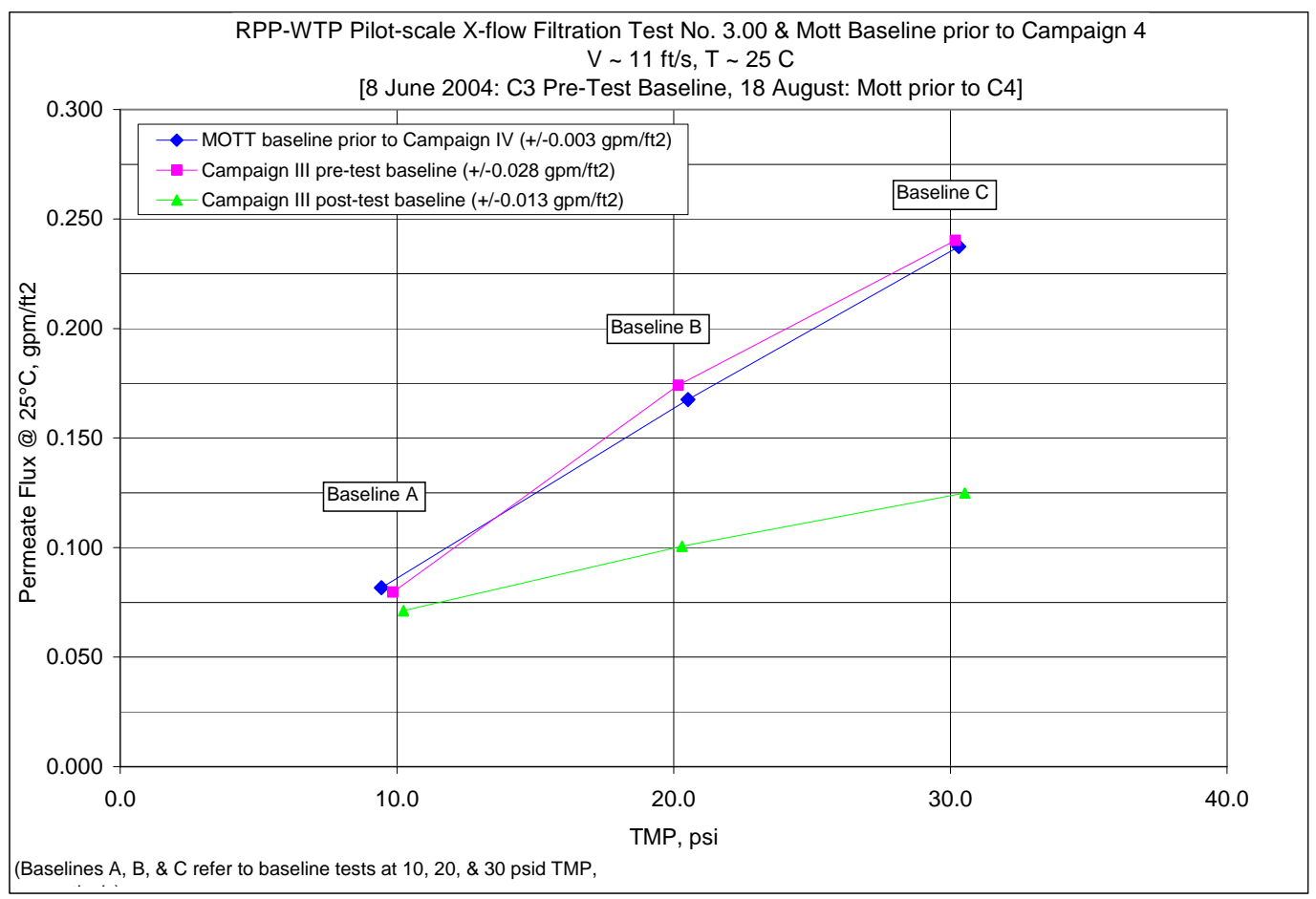

Figure 20. Baseline of Mott Filter prior to Removal

\subsection{Preconditioning the GKN Filter}

The GKN filter unit was received from the manufacturer in "pristine” condition. Before it was used for dewatering it was "preconditioned" in a manner similar to the Mott (when it was pristine). The purpose of preconditioning was to put the filter in a "used" condition and involved extended operation with a slurry with multiple backpulses. The preconditioning period was approximately 12 hours and while the exact number of backpulses was not fixed, six were preformed. Backpulsing was used to accelerate filter fouling, since after the filter cake is dislodged it is the smallest solid particles that tend to be the first to return to form the filter cake.

The series of tests that surrounded the preconditioning, and encompassed the "pre-test activities" are listed in Table 29. The original plan for the pre-test activities was to conduct a DIF water and $0.1 \mathrm{M} \mathrm{NaOH}$ baseline with the GKN filter unit prior to preconditioning, conduct the preconditioning, and clean the filter. As described later in this section, and shown in the table, additional tests were performed as warranted. 
Table 29. Test Matrix for Pre-Test Activities

\begin{tabular}{|c|c|c|c|c|c|}
\hline Activity & Test Solution & $\begin{array}{c}\text { Trans-Membrane } \\
\text { Pressure } \\
\text { (psid) } \\
\end{array}$ & $\begin{array}{c}\text { Slurry Loop } \\
\text { Velocity } \\
(\mathrm{ft} / \mathrm{s}) \\
\end{array}$ & $\begin{array}{c}\text { Slurry Loop } \\
\text { Temp. } \\
\left({ }^{\circ} \mathrm{C}\right) \\
\end{array}$ & $\begin{array}{l}\text { Run } \\
\text { Time } \\
\text { (min) }\end{array}$ \\
\hline Post-Campaign III Baseline - Mott & $0.1 \mathrm{M} \mathrm{NaOH}$ & $10,20,30$ & 11 & 25 & 20 ea \\
\hline Install GKN Filter Unit & $\mathrm{n} / \mathrm{a}$ & $\mathrm{n} / \mathrm{a}$ & $\mathrm{n} / \mathrm{a}$ & $\mathrm{n} / \mathrm{a}$ & $\mathrm{n} / \mathrm{a}$ \\
\hline Pre-preconditioning Baseline 1A & DIF Water & $10,20,30$ & 11 & 25 & 20 ea \\
\hline Pre-preconditioning Baseline 1B & $0.1 \mathrm{M} \mathrm{NaOH}$ & $10,20,30$ & 11 & 25 & 20 ea \\
\hline Clean with Acid & $0.5 \mathrm{M} \mathrm{HNO}_{3}$ & $10,20,30$ & 11 & 25 & 20 ea \\
\hline Pre-preconditioning Baseline 2A & DIF Water & $10,20,30$ & 11 & 25 & 20 ea \\
\hline Pre-preconditioning Baseline Point & DIF Water & 5 & 11 & 25 & 20 \\
\hline Pre-preconditioning Baseline 2B & $0.1 \mathrm{M} \mathrm{NaOH}$ & $10,20,30$ & 11 & 25 & 20 ea \\
\hline Preconditioning & $\begin{array}{c}\text { Old AN-102R2 } \\
\text { Simulant }\end{array}$ & 40 & 12 & 25 & Note 1 \\
\hline Caustic Rinse 1 & $0.1 \mathrm{M} \mathrm{NaOH}$ & 20 & 11 & 25 & 30 \\
\hline Acid Clean 1 & $2.0 \mathrm{M} \mathrm{HNO}_{3}$ & 40 & 11 & 25 & 90 \\
\hline Acid Clean 2 & $2.0 \mathrm{M} \mathrm{HNO}_{3}$ & 40 & 11 & 25 & 90 \\
\hline Caustic Rinse 2 & $0.1 \mathrm{M} \mathrm{NaOH}$ & 20 & 11 & 25 & 30 \\
\hline Caustic Rinse 3 & $0.1 \mathrm{M} \mathrm{NaOH}$ & 20 & 11 & 25 & 30 \\
\hline
\end{tabular}

1. The preconditioning duration was as long as possible without impacting the Campaign IV schedule.

Multiple backpulses were performed. Run time was $\sim 12$ hours and six backpulses were performed.

Prior to installation and preconditioning the GKN filter unit was disassembled for dimensional and visual inspection (Appendix F). While the tube bundle was being inspected the filter housing was installed in the pilot-scale test rig to verify the test rig modifications for the new filter unit and to flush the housing with a cleaning solution containing Alconox ${ }^{\circledR}$ (cleaning detergent). Afterwards the test rig and housing were flushed three times with deionized filtered (DIF) water.

Following the Alconox ${ }^{\circledR}$ cleaning the housing was removed, the tube bundle was inserted and the filter unit was installed in the pilot-scale test rig. The test rig was filled with $100 \mathrm{~L}$ of DIF water and left overnight to allow air to migrate out of the filter media. A baseline test was conducted the following day using the DIF water. The results of the baseline test are shown in Fig. 21. The flux levels were initially normal (compared to a Campaign III pre-test water baseline); but the rate of increase in flux with TMP was lower than that in Campaign III (the curve was flat). The rate of increase in the Campaign III baseline was $\sim 0.01 \mathrm{gpm} / \mathrm{ft}^{2}$ for every $10 \mathrm{psid}$ of TMP. The rate of increase in the Campaign IV baseline was $\sim 0.002$ $\mathrm{gpm} / \mathrm{ft}^{2}$ for every $10 \mathrm{psid}$ of TMP, lower by a factor of 5 .

$\mathrm{NaOH}$ was then added to the DIF water in the test rig to make a $0.1 \mathrm{M}$ solution and another baseline test was conducted. The results of the baseline test with $0.1 \mathrm{M} \mathrm{NaOH}$ are also shown in Figure 21. The flux levels from the $0.1 \mathrm{M} \mathrm{NaOH}$ baseline test were lower than the DIF water results and much lower than the pre-test flux levels from previous campaigns (ref. Figure 20). In addition the rate of increase in flux with TMP was low (the curve was also flat). 
It was apparent that the filter media was not sufficiently clean, or that the GKN filter unit had poor performance when compared to the Mott filter unit. Poor performance of the GKN was improbable; therefore the filter unit was cleaned by conducting a baseline test with a $0.5 \mathrm{M}$ $\mathrm{HNO}_{3}$ solution. The results of the baseline are also shown in Fig. 21. The flux levels were initially low ( $\sim 0.10 \mathrm{gpm} / \mathrm{ft}^{2}$ at 10 psid TMP) but increased considerably $\left(\sim 0.75 \mathrm{gpm} / \mathrm{ft}^{2}\right.$ at 30 psid TMP). The considerable increase in flux implied that the baseline with $0.5 \mathrm{M} \mathrm{HNO}_{3}$ successfully cleaned the filter.

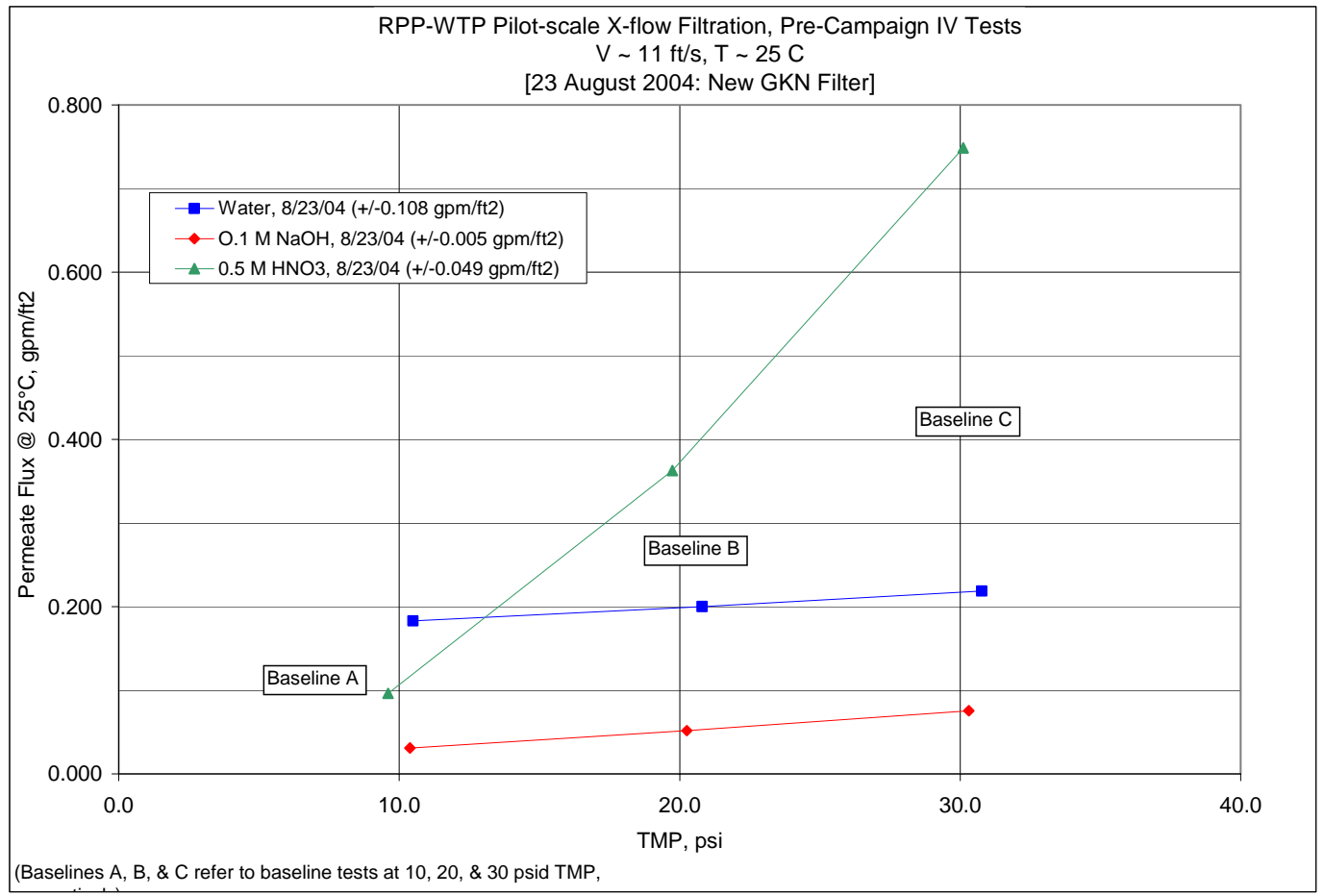

Figure 21. Initial Baselines of GKN Filter after Installation

Note that the uncertainty of the average values from the water baseline on 23 August is much higher than the other baselines. In this baseline the corrected permeate flux was not stabile; the flux was continually decreasing during the baseline test point. This was the first baseline following the cleaning with Alconox. This behavior was typically associated with solids being removed from the filter media with a backpulse and then re-depositing during continued operation. The low flux of the ensuing $0.1 \mathrm{M} \mathrm{NaOH}$ baseline and the significant increase in flux of the $0.5 \mathrm{M} \mathrm{HNO}_{3}$ implied that the filter performance was indeed hampered by solids in the system.

Following the acid baseline test the filter unit was flushed twice with DIF water. Another set of baseline tests were conducted with DIF water and $0.1 \mathrm{M} \mathrm{NaOH}$. While conducting the baseline with DIF water an addition baseline point was conducted at $11 \mathrm{ft} / \mathrm{s}$ and $5 \mathrm{psid}$ TMP. This point was comparable to a point from a pre-SIPP Mott test, when the Mott was pristine. The results of the two baseline tests, and the additional point, are shown in Fig. 22. 
WSRC-TR-2005-00105, REVISION 0

SRNL-RPP-2005-00012, REVISION 0

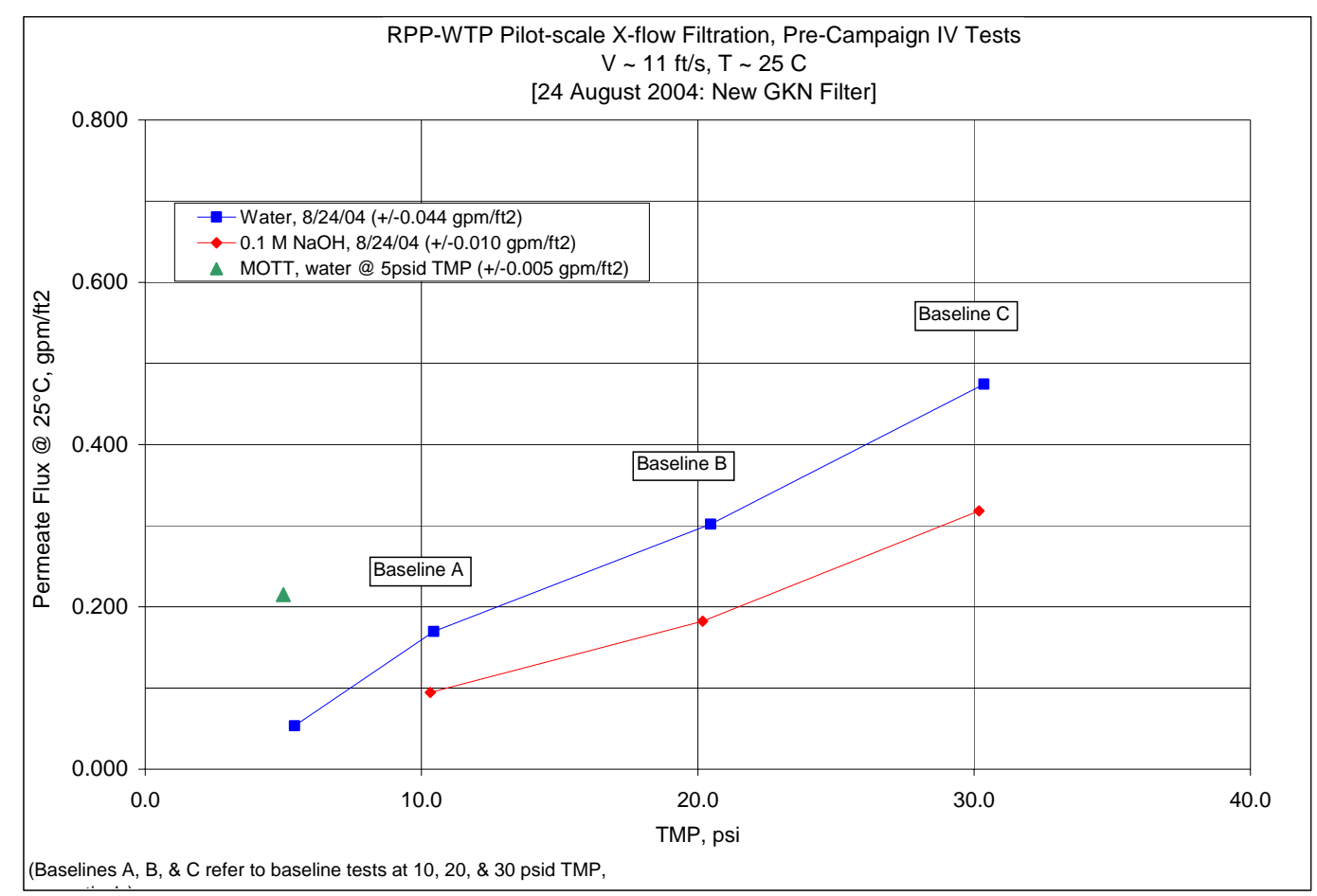

Figure 22. Baselines of GKN Filter after Cleaning with Acid

The results from the DIF water and $0.1 \mathrm{M} \mathrm{NaOH}$ baselines were much improved compared to the results of the preceding acid baseline (reference Fig. 21). The flux level of the additional DIF water baseline point ( $11 \mathrm{ft} / \mathrm{s}$ and 5 psid TMP) was lower than that of the Mott by a factor of 4. However, it has been noted in other campaigns that the results of baseline tests do not necessarily define the filter performance with slurry. A comparison to the Mott with slurry is presented later in this section.

The improved baseline results indicated that the GKN filter unit was ready for preconditioning. A volume of $75 \mathrm{~L}$ of AN-102R2 precipitated simulant was added to the pilot-scale test rig for preconditioning. This simulant was prepared in the Fall of 2002 and used in the RPP Pilot-Scale Precipitation test (Williams and Steeper, 2004). Records indicated that the solids concentration of the simulant was $20 \mathrm{wt} \%$ UDS. Given the age of the simulant some solids (carbonates) had most likely precipitated from solution, but it was suspected that not enough had precipitated to affect the wt\% UDS. AN-107 precipitated simulant was used to precondition the pristine Mott filter. Both AN102R2 and AN-107 simulants are Envelope $\mathrm{C}$ waste material and contain significant quantities of organic material which has most challenged the filter in the past.

The preconditioning process involved operating the test rig in permeate recirculation mode, that is the permeate was recirculated back to the Slurry Reservoir so that the slurry concentration did not change during the operation. Preconditioning lasted two consecutive days for a total run time of 11 hours and 48 minutes. The test rig sat idle for 15 hours and 12 minutes overnight, between days. One backpulse was conducted on the first day and five were conducted on the second day. The adjusted permeate flux during the preconditioning is 
shown in Fig. 23. The six backpulses are signified in the figure as outliers in the flux data. Included in the figure are the dewatering results from Campaign III for reference.

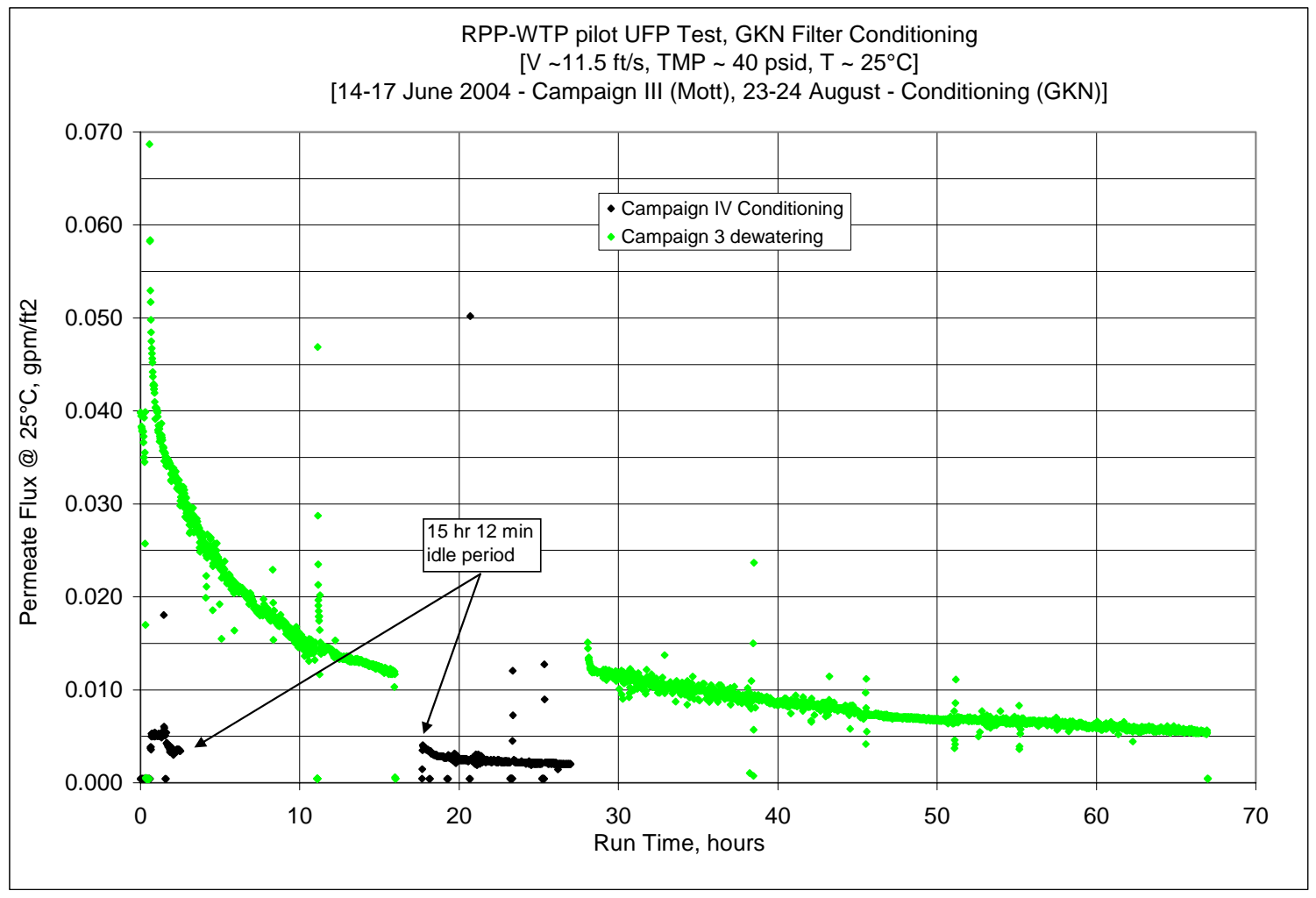

Figure 23. Preconditioning of the GKN Filter with 20 wt\% AN-102

Following the preconditioning operation the filter unit was rinsed with $0.1 \mathrm{M} \mathrm{NaOH}$ and cleaned with 2.0 M HNO3 in a fashion similar to test points 4.18 and 4.19 (however, heels were not included in each solution, reference Section 2.7.4.1.6). Only two acid cleanings were conducted. In both acid cleaning stages the test conditions of $11 \mathrm{ft} / \mathrm{s}$ and $40 \mathrm{psid}$ TMP could not be attained. At $11 \mathrm{ft} / \mathrm{s}$ and maximum pumping system output, the greatest TMP attainable was $\sim 24$ psid for both cleanings. The permeate flux was $\sim 3$ times greater than that seen during the second acid cleanings of Campaign II and III $\left(0.95 \mathrm{gpm} / \mathrm{ft}^{2} \mathrm{vs} .0 .35 \mathrm{gpm} / \mathrm{ft}^{2}\right)$. The high flux created a higher pressure in the permeate loop than that seen during the second acid cleanings of Campaign II and III (33 psig vs. -1 psig). Basically, the filter media did not provide the flow resistance necessary to attain the required TMP. The high flux of the acid cleanings possibly indicated that the filter was too clean, that is the preconditioning failed to foul the filter to a significant depth.

The filter flux results when using the rinse and cleaning solutions that followed the preconditioning are shown on Fig.24. First note the severely diminished flux of the first caustic rinse, indicating that the rinse solution and backpulse had little effect on the filter cleanliness. Then note the significant increase in flux from the first caustic rinse to the first acid cleaning, indicating that the solids obstructing the filter were dissolved and removed. There was not a notable increase of the flux from the first acid cleaning to the second, 
WSRC-TR-2005-00105, REVISION 0

SRNL-RPP-2005-00012, REVISION 0

indicating that the filter was cleaned almost to the fullest extent by the first batch of $2.0 \mathrm{M}$ $\mathrm{HNO}_{3}$. Since the second cleaning did not result in further improvement of permeate flux the planned third acid cleaning was not conducted, which also allowed the start up of Campaign IV sooner.

As seen in the figure, there was a reduction of flux from the second acid cleaning to the second caustic rinse. This was typical of all previous clean-rinse operations and was due to the heel (1.6 L, in this case) of acid solution that remained in the test rig after the second acid cleaning solution was drained. The caustic solution precipitated solids from the residual acid solution, which reduced the permeate flux.

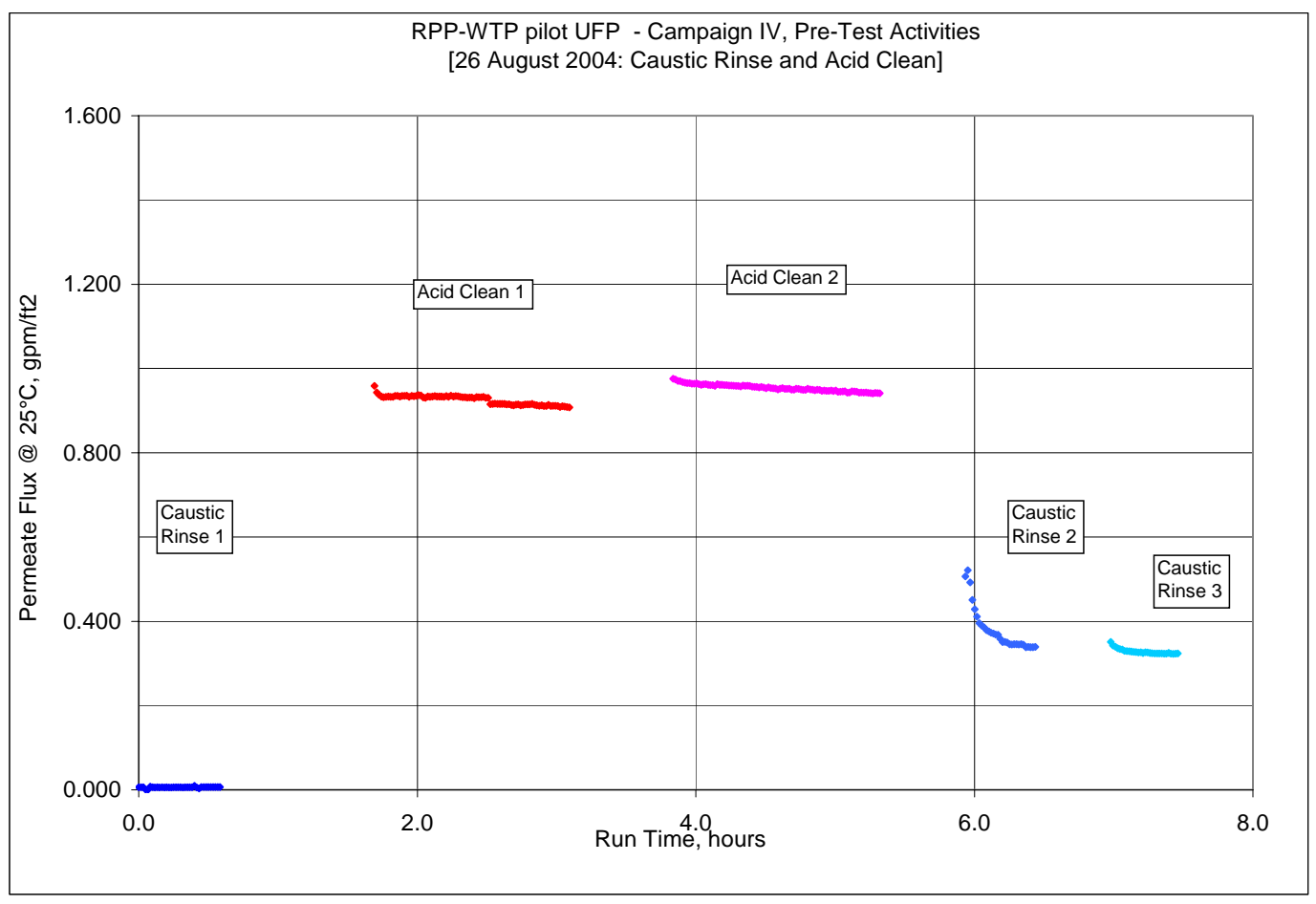

Figure 24. Pre-Test Acid Cleaning of Filter Unit, Permeate Flux vs. Run Time

Following the rinse and clean operation the test rig was flushed twice with DIF water. The GKN filter was considered ready for Campaign IV testing after the flushes. A volume of 100 $\mathrm{L}$ of $0.1 \mathrm{M} \mathrm{NaOH}$ was then added to the test rig to conduct the pre-test baseline tests (test numbers 4.00A, B, and C). The results of the pre-test baseline are shown and described in Section 2.7.4.1.5. 
WSRC-TR-2005-00105, REVISION 0

SRNL-RPP-2005-00012, REVISION 0

\subsection{Slurry Concentration (Dewatering)}

Dewatering started Monday, 30 August. The waste simulant in the Primary Mixing Tank was not agitated during the weekend before the 30th so that solids would settle out and allow supernatant to be drawn from the top of the mixture. The supernatant was added to the slurry reservoir prior to the initiation of dewatering to account for the volume of permeate necessary to fill the permeate loop ( 16 L). Doing so would assure the correct solids weight $\%$ of the waste simulant in the Slurry Reservoir after the slurry and permeate loops were completely filled and ready. After the supernatant was removed, the simulant was thoroughly agitated for one hour prior to the initial transfer of slurry to the slurry reservoir.

Per the test matrix, the dewatering was conducted in two stages. The delineation between the stages was approximately one-half of the expected permeate removed from the simulant (476 liters). A hold period, a minimum of 12 hours, was inserted between the stages to purposely extend the dewatering process. The hold period made the SIPP dewatering duration more comparable with the RPP-WTP dewatering duration, during which some waste instabilities (precipitation) could affect the UFP feed. The first stage was completed in about 15 hours and finished in the early morning of 31 August, the second stage was completed in about 38 hours and finished in the early morning of 2 September. The entire dewatering process, including the hold, and downtime for sampling, took approximately 66 hours. Excluding the hold, and sampling downtime, dewatering took a little over 53 hours (Campaign II 53 hours, Campaign III 55 hours).

A volume of about $1048 \mathrm{~L}$ of combined waste simulant was dewatered to $117 \mathrm{~L}$ to obtain a final concentration of $23.5 \mathrm{wt} \%$ UDS. The RPP-WTP UFP will concentrate waste to approximately $17 \mathrm{wt} \%$ UDS. However, all of the campaigns of SIPP have a target of at least $20 \mathrm{wt} \%$ UDS and, if possible, $25 \mathrm{wt} \%$ UDS to obtain information at an upper bound. The Campaign IV dewatering test was stopped after about 53 hours when a rough estimate of concentration indicated $25 \mathrm{wt} \%$ UDS (a real-time analysis of concentration was not available; therefore the concentration was extrapolated with previous concentration results). A volume of $914 \mathrm{~L}$ of permeate was collected in drums during dewatering. Approximately $16 \mathrm{~L}$ of permeate remained in the permeate loop and about

1 liter of samples (slurry) were taken during dewatering.

\subsection{Test Results}

Figure 25 shows the adjusted permeate flux for the entire slurry dewatering process. The flux was adjusted for temperature, as required by the RPP-WTP test specification, to account for the effect of viscosity. 
The adjustment equation is:

Adjusted Flux $=$ Flux $\left(\mathrm{gpm} / \mathrm{ft}^{2}\right) * \mathrm{e}^{(2500 \times((1 /(273+\mathrm{T}))-(1 / 298)))}$

where Flux = permeate flow rate/approximate filter area, and $\mathrm{T}=$ slurry temperature in degrees Celsius

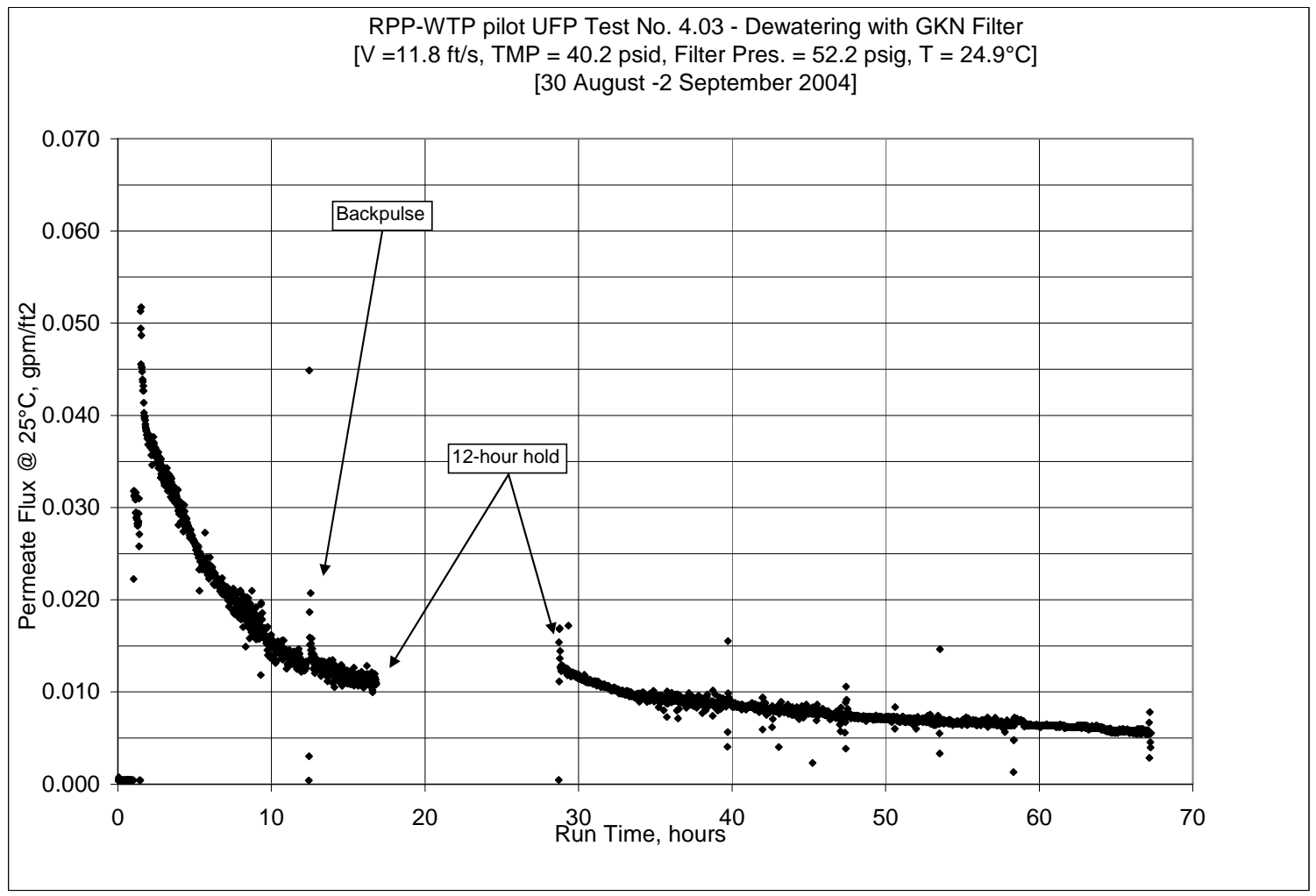

Figure 25. Dewatering of UFP Feed, Permeate Flux vs. Run Time with GKN filter

The data outliers seen in the figure were mainly from process disruptions; backpulsing, sampling, and exchanging permeate collection drums. The data scatter (or fuzziness) of the permeate flux output was from fluctuations in the permeate flow rate. Fluctuations were noticed when gas bubbles were seen in the permeate discharge line. This phenomenon was also observed (to a greater extent) during slurry washing. A detailed discussion of the bubbles is provided in the slurry washing section (Section 2.7.4.1.5).

\subsection{Backpulse}

Dewatering started with a backpulse. Another backpulse was conducted at approximately 11 hours into the first stage of dewatering. The flux recovery from the second backpulse was poor and as a result backpulsing was not attempted for the remainder of the dewatering process. Backpulses were always performed during the dewatering of the SIPP campaigns and poor recovery was evident in all. 
The permeate flow rate and some test rig pressures during the backpulse at 11 hours are shown in Figs. 26 and 27, respectivley. The time interval shown on these figures are independent of the run time shown on other figures. The backpulse started after 60 seconds, and the backpulse duration was 15 seconds in this case (i.e. the backpulse valve, V18, was opened and closed for about 15 seconds, reference Table 26). The air pocket in the pulse pot was pressurized to about 40 psig above the slurry loop pressure, prior to the backpulse (seen as P3 -P2 in Fig. 27). The asymptotic decrease of the permeate flow and pressures are indicative of the SIPP backpulse system, which was designed to be prototypic of the RPPWTP backpulse system (Duignan, 2003a). Figure 27 indicates that the filter pressure (P1) is not affected by the backpulse (since it is dominated by the pump discharge pressure) but the pressure drop through the filter is slightly affected. The permeate loop flow valve (V27) was opened to re-establish dewatering after the backpulse (effect not shown in the figures).

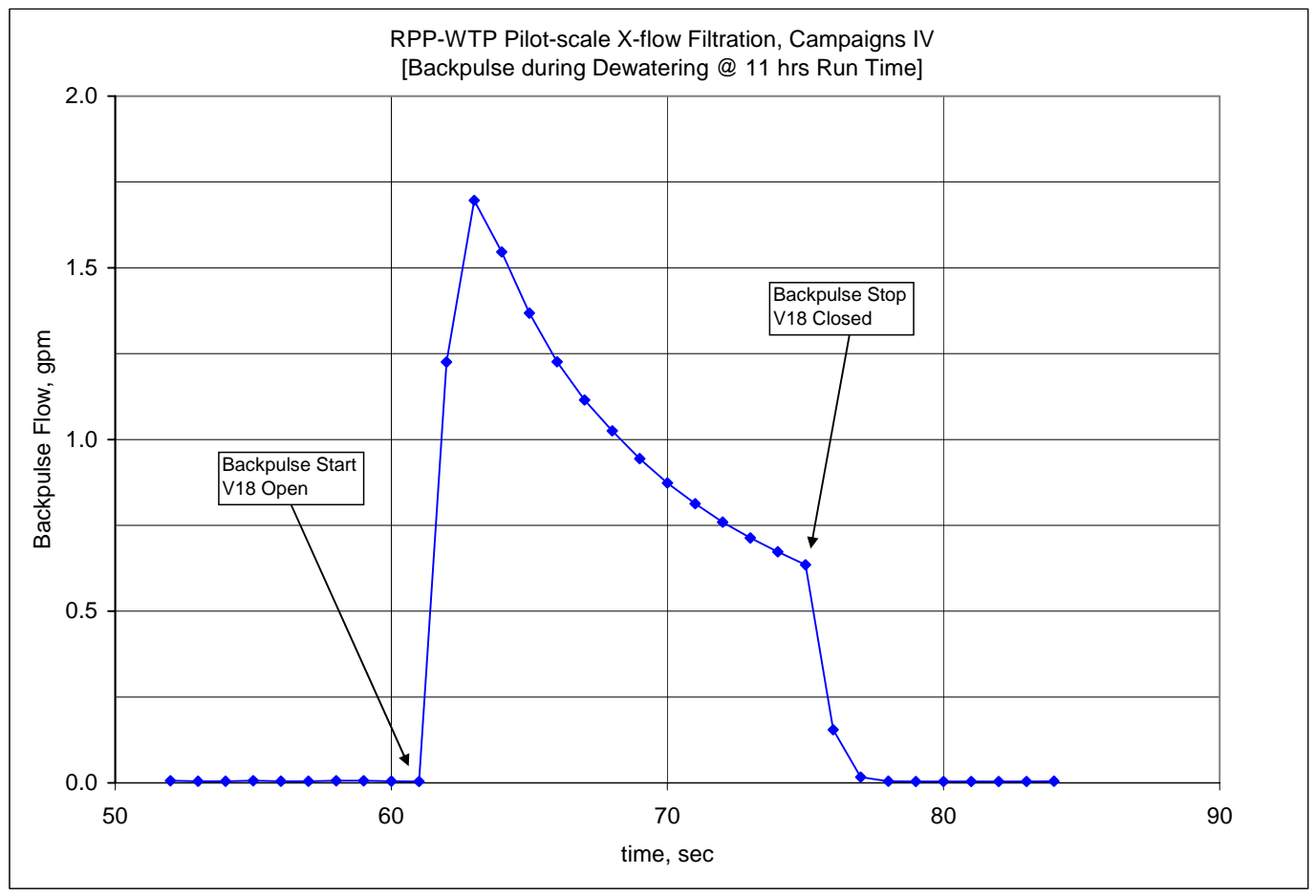

Figure 26. Permeate Flow during Dewatering Backpulse 
WSRC-TR-2005-00105, REVISION 0

SRNL-RPP-2005-00012, REVISION 0

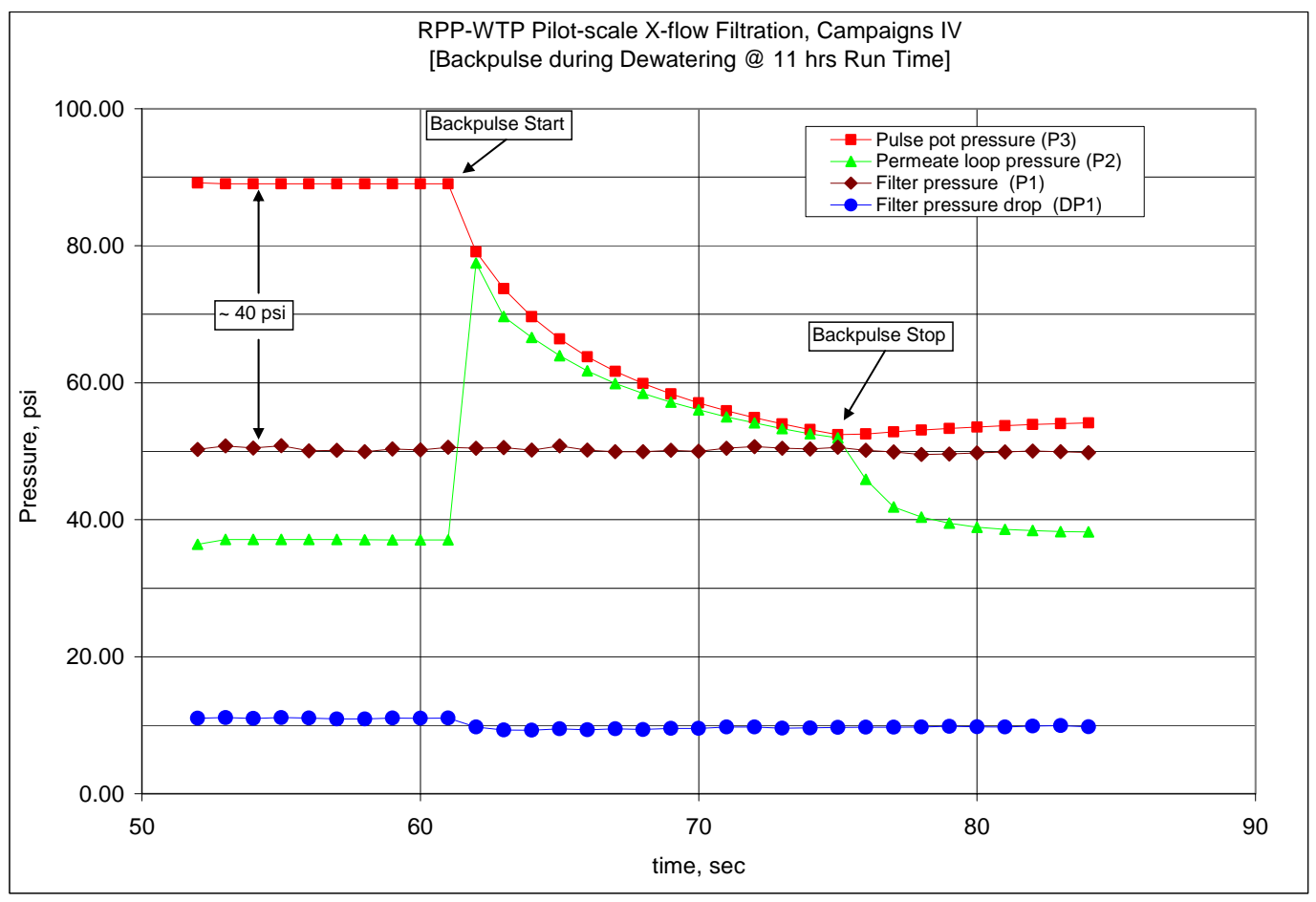

Figure 27. Test Rig Pressures during Dewatering Backpulse

\subsection{Slurry and Permeate Samples}

Samples of concentrated slurry and permeate were taken during the dewatering process in accordance to a sampling plan, Appendix D. Samples were taken at approximately 159 liters, 317 liter, and 476 liters of permeate removed for each of the two dewatering stages. Permeate density, viscosity, and turbidity, as well as slurry density, were measured in the EDL soon after the samples were taken. The results of these measurements are shown in Table 30. Additional permeate and slurry samples taken at the periods listed above were sent to Aiken County Technology Laboratory (ACTL) for measurements of density, yield stress, consistency, and weight \% solids [total solids (TS), undissolved solids (UDS), dissolved solids (DS), and supernatant solids (SS)]. The results of the separate samples are presented in Section 2.7.4.2.1. Note that the permeate and slurry densities were measured at both EDL and ACTL. 
WSRC-TR-2005-00105, REVISION 0

SRNL-RPP-2005-00012, REVISION 0

Table 30. EDL Measured Data during Dewatering of the AY102+AP101+FEP Bottoms Simulant

\begin{tabular}{|c|c|c|c|c|c|c|}
\hline $\begin{array}{c}\text { Target } \\
\text { Volume of } \\
\text { Permeate } \\
\text { Produced }\end{array}$ & $\begin{array}{c}\text { Cumulative } \\
\text { Weight of }\end{array}$ & Permeate \\
\cline { 5 - 7 } & Produced & Density & Density & Viscosity & Temp & Turbidity \\
\hline $\mathrm{L}$ & $\mathrm{kg}$ & $\mathrm{gm} / \mathrm{mL}$ & $\mathrm{gm} / \mathrm{mL}$ & $\mathrm{mPa}-\mathrm{s}$ & ${ }^{\circ} \mathrm{C}$ & NTU \\
\hline $\begin{array}{c}\text { Day1 } \\
158.7\end{array}$ & 196.65 & 1.28 & 1.25 & 2.28 & 25.0 & 0.23 \\
\hline 317.3 & 390.25 & 1.31 & 1.25 & 2.28 & 25.0 & 0.20 \\
\hline 476.0 & 586.95 & 1.34 & 1.25 & 2.29 & 26.3 & 0.37 \\
\hline Day 2 & 783.55 & 1.38 & 1.25 & 2.32 & 24.9 & 0.35 \\
\hline 158.7 & & 1.43 & 1.25 & 2.33 & 24.6 & 0.23 \\
\hline 317.3 & 980.10 & 1.46 & 1.25 & 2.36 & 25.0 & 0.38 \\
\hline 476.0 & 1142.40 & & & & & \\
\hline
\end{tabular}

The density of the slurry increased during dewatering as the lighter permeate was removed from the heavier solids. The permeate density remained constant. The turbidity measurements of the permeate were significantly lower than the test specification threshold of 2 NTU and, therefore, the permeate was considered acceptably free of undissolved solids. The permeate viscosity (and permeate temperature) fluctuated slightly throughout the process.

\subsection{Slurry Concentration}

Another way to visualize the dewatering results is to track permeate flux with the concentration of UDS. Figure 28 shows the adjusted permeate flux versus wt\% UDS concentration using the GKN filter and for comparison, the dewatering data from Campaigns I, II and III are also shown. The data trend of Campaigns II, III and IV are almost identical and the data tend of Campaign I was similar. The simulant for Campaign I contained only AY102 and was $12.5 \mathrm{wt} \%$ at the beginning of dewatering. The consistent performance of the UFP during Campaigns II, III, and IV indicates that; 1 ) the performance of the GKN filter unit was comparable to that of the Mott, 2) the rinsing and cleaning process with $0.1 \mathrm{M}$ $\mathrm{NaOH}$ and 2.0 $\mathrm{M} \mathrm{HNO}_{3}$, respectively, restored the filter unit to a repeatable performance (Mott), and 3) the addition of AP101 and FEP Bottoms to the AY102 had little effect on filter performance (Mott). 
WSRC-TR-2005-00105, REVISION 0

SRNL-RPP-2005-00012, REVISION 0

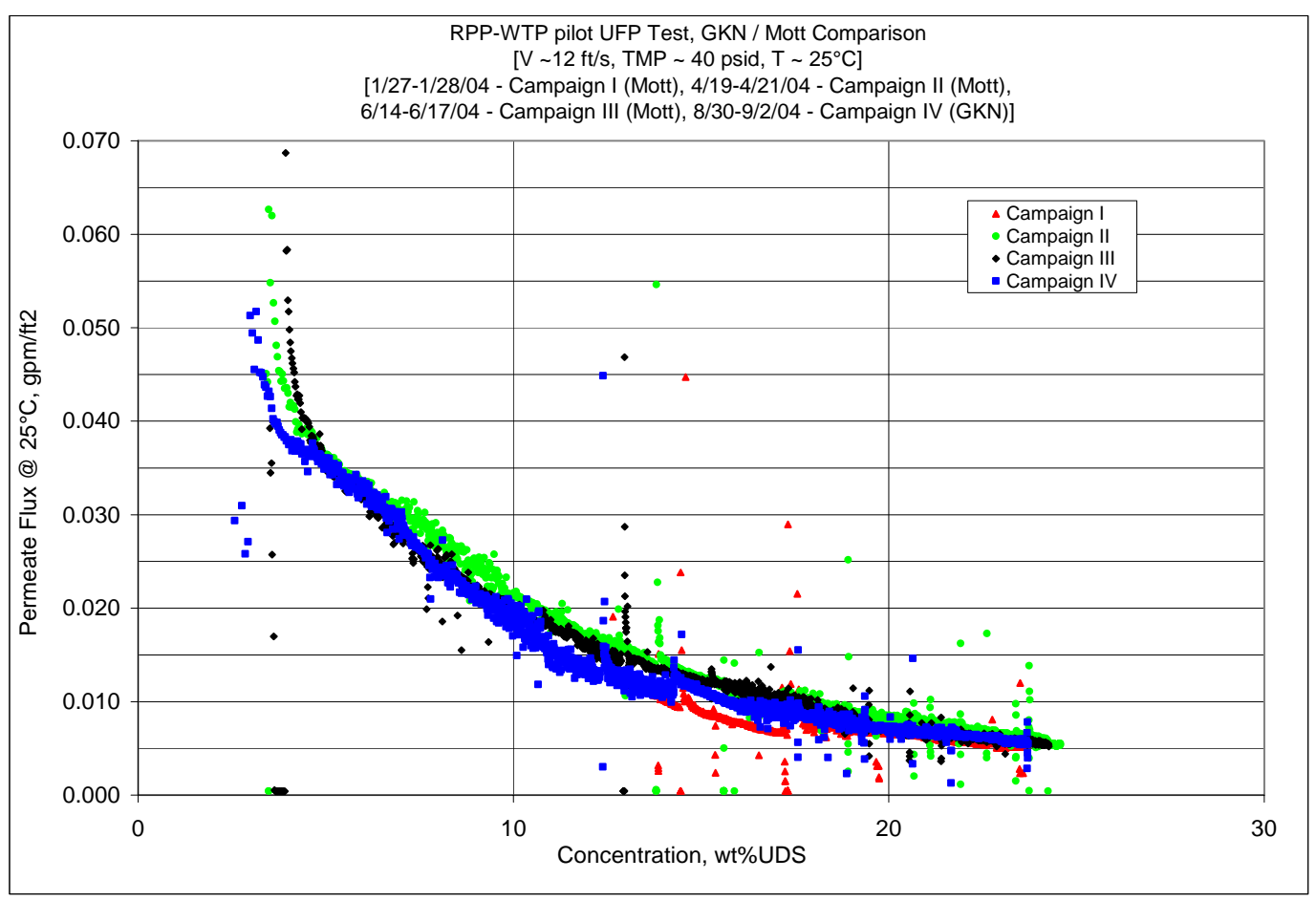

Figure 28. Dewatering of UF Feed - Campaigns I , II, III (Mott Filter) and IV (GKN Filter), Permeate Flux vs. Concentration

The average, temperature-adjusted permeate flux when dewatering from $\sim 3$ to $\sim 24 \mathrm{wt} \%$ UDS was $\sim 0.011 \mathrm{gpm} / \mathrm{ft}^{2}$ for Campaign IV. This flux value for Campaigns II and III was also $\sim 0.011 \mathrm{gpm} / \mathrm{ft}^{2}$. The average, temperature-adjusted permeate flux when dewatering from $\sim 4$ to $20 \mathrm{wt} \%$ UDS was $0.013 \mathrm{gpm} / \mathrm{ft}^{2}$ for Campaign IV. This flux value was slightly higher for Campaigns II and III, $0.015 \mathrm{gpm} / \mathrm{ft}^{2}$ and $0.014 \mathrm{gpm} / \mathrm{ft}^{2}$ respectively. The average, temperature-adjusted permeate flux when dewatering from $\sim 4$ to $17 \mathrm{wt} \%$ UDS was $0.016 \mathrm{gpm} / \mathrm{ft}^{2}$ for Campaign IV. This value was again slightly higher for Campaigns II and III, both $0.018 \mathrm{gpm} / \mathrm{ft}^{2}$. These results indicate a similarity between the GKN and Mott filter units.

Of course, the data in Fig. 28 are instantaneous results and it is useful to look at the cumulative mean fluxes. For the three SIPP campaigns that dewatered from below $5 \mathrm{wt} \%$ UDS to over $20 \mathrm{wt} \%$ (i.e., Campaigns II-IV) Fig. 29 shows mean filter fluxes at each point along the solids concentration abscissa. 
WSRC-TR-2005-00105, REVISION 0

SRNL-RPP-2005-00012, REVISION 0

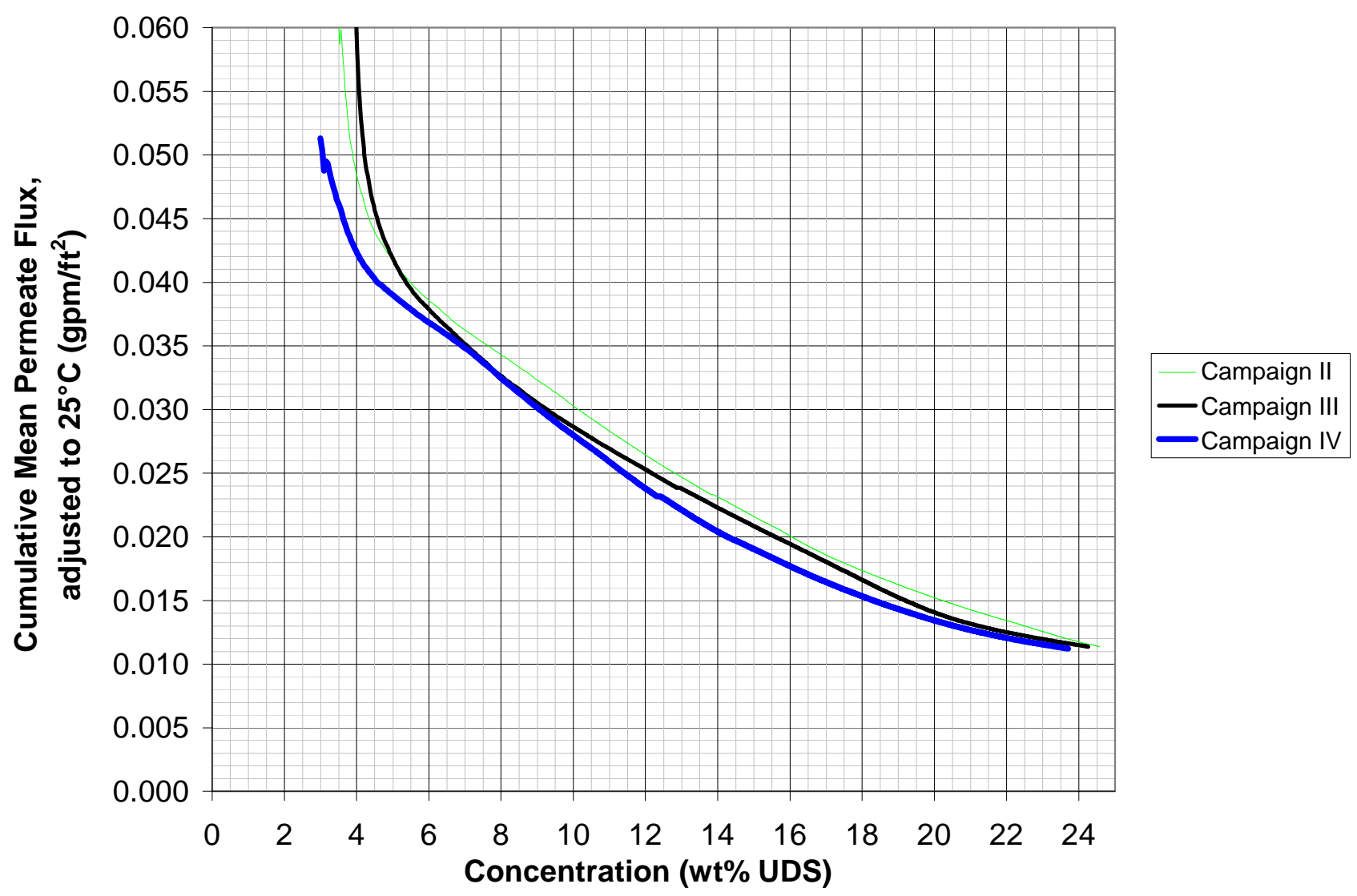

Figure 29. Dewatering of UF Feed - Campaigns II, III (Mott Filter) and IV (GKN Filter), Cumulative Mean Permeate Flux vs. Concentration

\subsection{Testing at Steady-State Solids Loading}

Six steady state tests were conducted with the GKN filter unit following dewatering to compare with steady state tests conducted with the Mott filter unit. Steady state tests with the Mott were conducted in Campaign I with AY102 slurry dewatered to approximately 24 wt\% UDS. Campaign IV steady state tests were conducted with slurry (AY102/AP101/FEP Bottoms) also dewatered to $24 \mathrm{wt} \%$ UDS.

The Campaign IV steady state tests are listed in the test matrix (Table 22) and the test plan is shown in Fig. 30, with the Campaign I steady state test points for comparison. As seen in the figure, four of the six Campaign IV tests are duplicates of Campaign I. The remaining two Campaign IV steady state tests are; a repeat of test 4.09 (Steady5), and a new test to provide useful data at a high TMP and slurry velocity (Steady6). 
WSRC-TR-2005-00105, REVISION 0

SRNL-RPP-2005-00012, REVISION 0

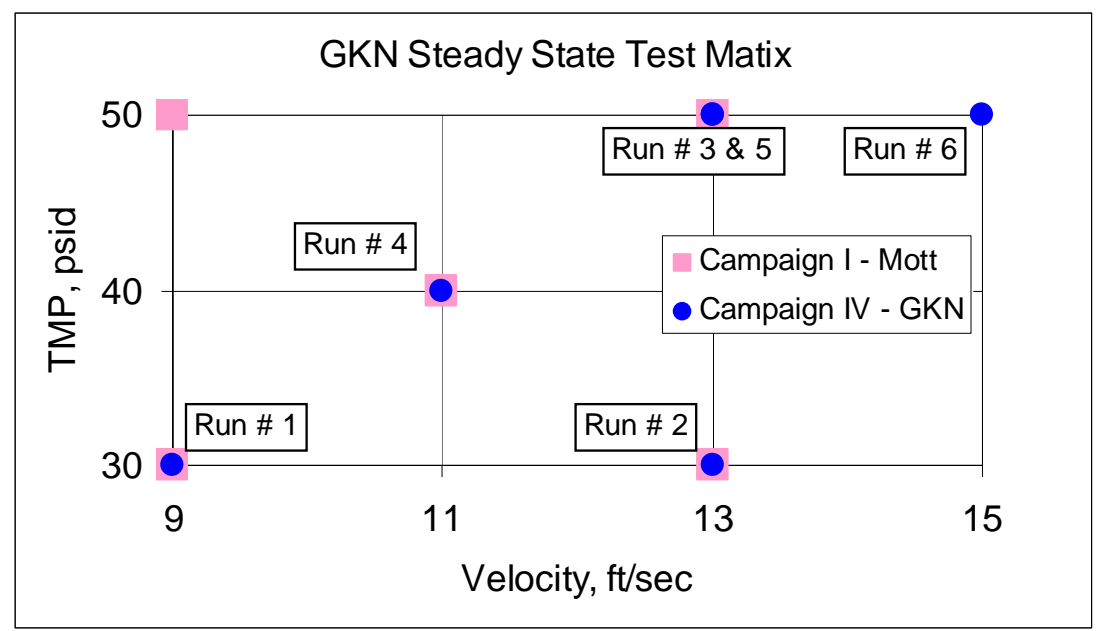

Figure 30. Campaign I and IV Steady State Test Matrix

All of the Campaign IV steady state tests were conducted at $25^{\circ} \mathrm{C}$. However, test number 4.09 and Steady6 were conducted at $31^{\circ} \mathrm{C}$ (the heat exchanger was unable to remove a sufficient amount of the system heat to maintain $25^{\circ} \mathrm{C}$ ). The mean value of the adjusted permeate flux data from the steady state tests are shown in Fig. 31. The steady state test results from the Mott filter unit are also shown in the figure. Note that the performance of the GKN filter unit was similar to the Mott at comparative data points. Also note that the Steady6 test indicated that a higher slurry velocity through the filter yields a higher permeate flux, as seen by all cross-flow filter tests done in the past.

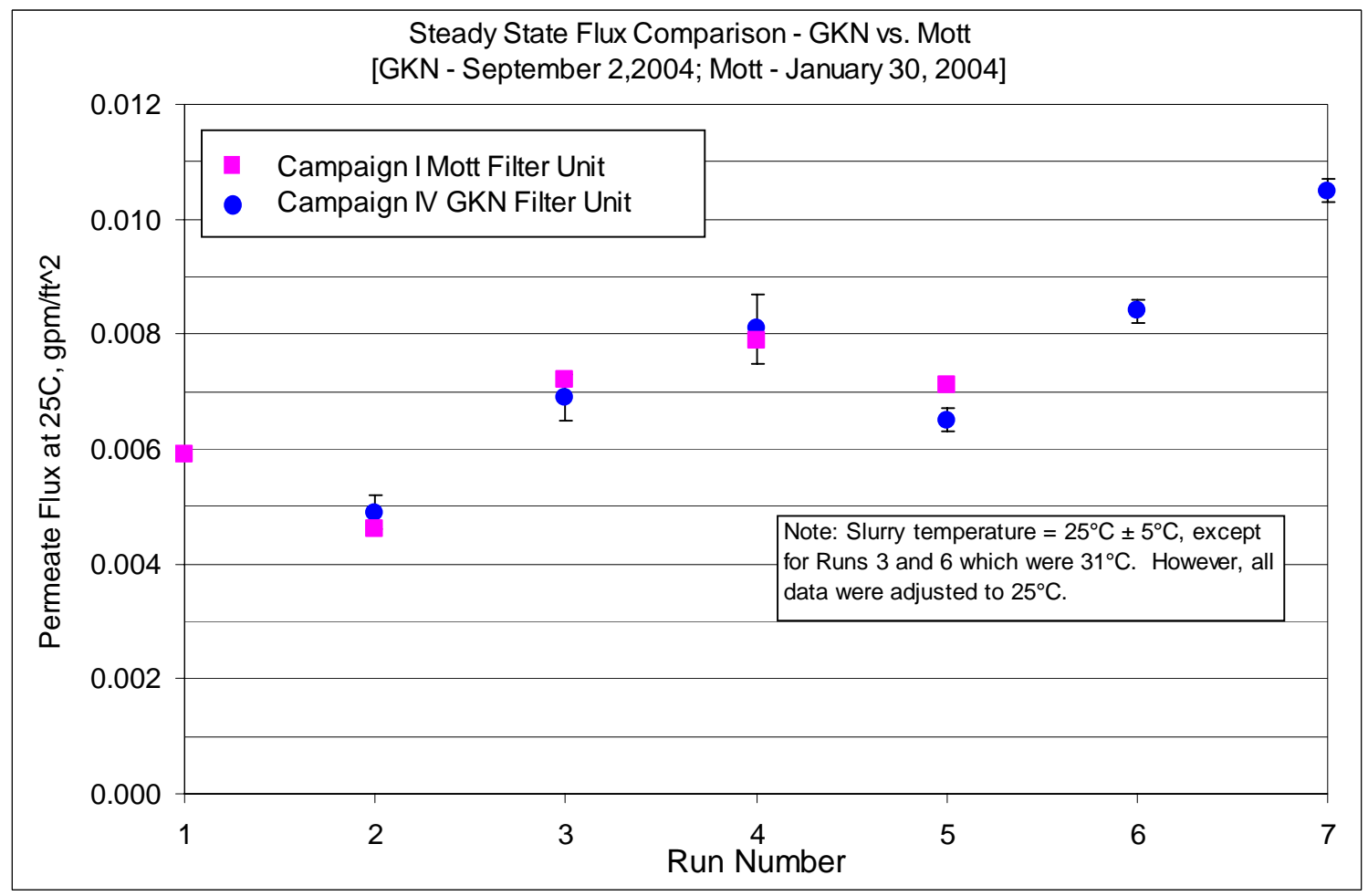


WSRC-TR-2005-00105, REVISION 0

SRNL-RPP-2005-00012, REVISION 0

Figure 31. Campaign IV Steady State Tests at $24 \mathrm{wt} \%$ UDS

(See theTable 31 for the flow conditions)

Table 31. 24 wt\% UDS Steady State Test Comparison Data shown in Fig. 31

\begin{tabular}{|c|c|c|c|c|c|c|c|c|}
\hline & \multicolumn{4}{|c|}{ Mott Filter Steady State Test } & \multicolumn{4}{|c|}{ GKN Filter Steady State Test } \\
\hline & $\begin{array}{c}\text { Campaign I } \\
\text { Test }\end{array}$ & $\begin{array}{l}\text { Slurry } \\
\text { Velocity }\end{array}$ & TMP & $\begin{array}{c}\text { Permeate } \\
\text { Flux @ } 25^{\circ} \mathrm{C}\end{array}$ & $\begin{array}{c}\text { Campaign IV } \\
\text { Test }\end{array}$ & $\begin{array}{l}\text { Slurry } \\
\text { Velocity }\end{array}$ & TMP & $\begin{array}{c}\text { Permeate } \\
\text { Flux @ } 25^{\circ} \mathrm{C}\end{array}$ \\
\hline Number & Number & $\mathrm{ft} / \mathrm{s}$ & psid & $\mathrm{gpm} / \mathrm{ft}^{\wedge} 2$ & Number & $\mathrm{ft} / \mathrm{s}$ & psid & $\mathrm{gpm} / \mathrm{ft}^{\wedge} 2$ \\
\hline 1 & 1.10 & 8.8 & 50.6 & 0.0059 & & & & \\
\hline 2 & 1.07 & 90 & 29.4 & 0.0046 & 4.07 & 89 & 29.6 & 0.0049 \\
\hline 3 & 1.08 & 13.1 & 29.0 & 0.0072 & 4.08 & 13.4 & 30.4 & 0.0069 \\
\hline 4 & 1.09 & 12.4 & 50.6 & 0.0079 & 4.09 & 12.6 & 50.0 & 0.0081 \\
\hline 5 & 1.11 & 10.9 & 40.8 & 0.0071 & 4.11 & 10.8 & 39.6 & 0.0065 \\
\hline 6 & & & & & Steady5 & 13.1 & 50.3 & 0.0084 \\
\hline 7 & & & & & Steady6 & 15.4 & 50.0 & 0.0105 \\
\hline
\end{tabular}

\subsection{Testing at Steady-State Solids Loading and at Elevated Temperatures}

Following the steady state testing, the $24 \mathrm{wt} \%$ UDS slurry was adjusted to $19 \mathrm{wt} \%$ UDS by removing slurry and adding permeate. The target wt $\%$ UDS for the adjustment was $20 \mathrm{wt} \%$, however, transfer irregularities caused the adjustment to be lower than the target concentration.

Tests were conducted with the $19 \mathrm{wt} \%$ slurry at higher slurry temperature to determine the effect on permeate flux. The high temperature tests are listed in the test matrix (Table 22). Higher slurry temperatures were attained by reducing the cooling flow to the heat exchanger. The higher temperature was expected to reduce the viscosity of the slurry, and permeate, and increase the permeate flux.

The mean value of the permeate flux data from the high temperature tests are shown in Fig. 32. The mean value of the adjusted permeate flux data of each high temperature test is also shown in the figure (adjusted to $25^{\circ} \mathrm{C}$ with the equation shown in Section 2.7.4.1.2.1). The flux level increased in all of the cases with a slurry temperature above $25^{\circ} \mathrm{C}$. At the two 45 ${ }^{\circ} \mathrm{C}$ points the flux level increased greater than $70 \%$ above the $25^{\circ} \mathrm{C}$ level. 
WSRC-TR-2005-00105, REVISION 0

SRNL-RPP-2005-00012, REVISION 0

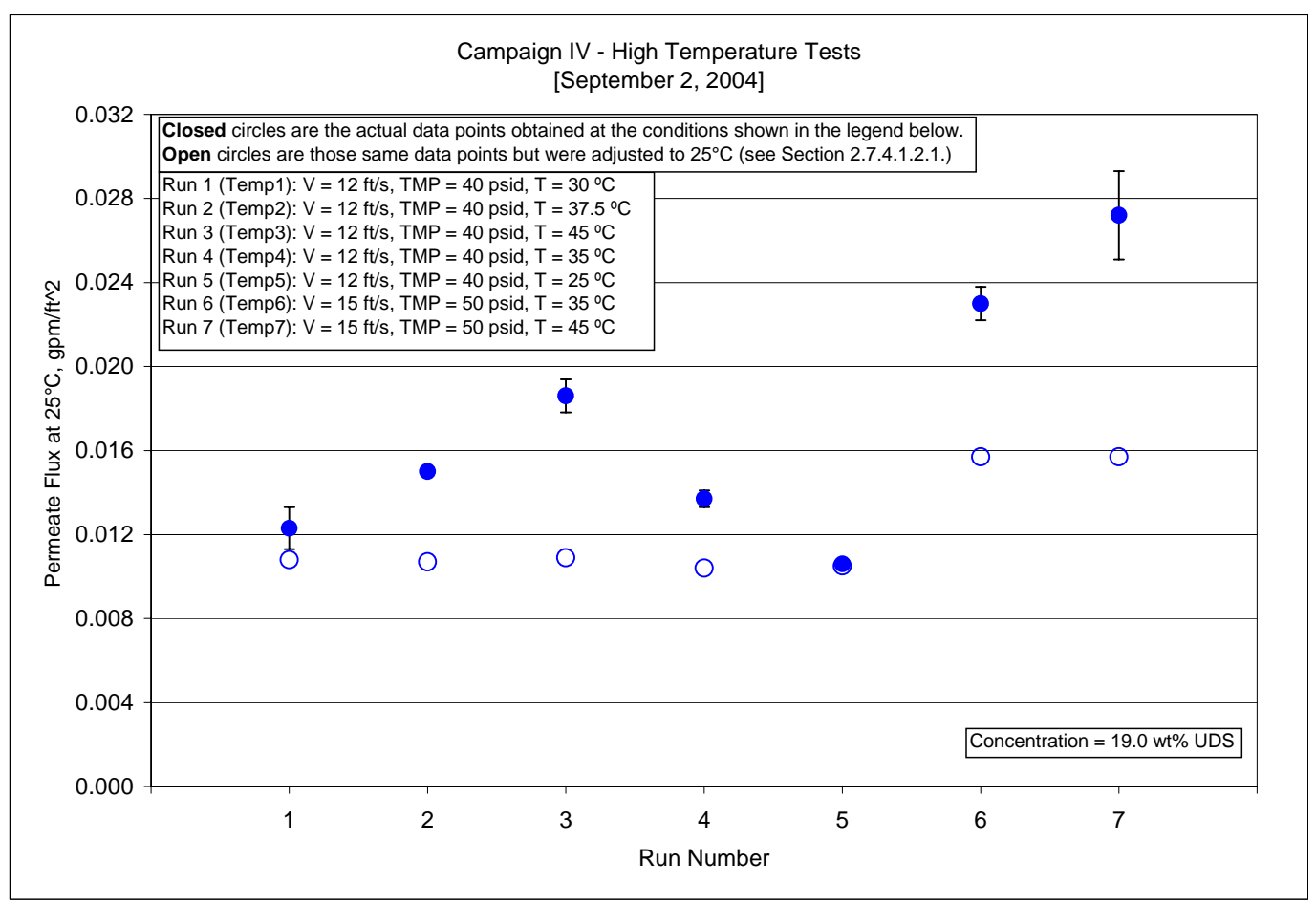

Figure 32. Campaign IV High Temperature Tests (data are listed in Table 32)

Table 32. 19 wt\% UDS Steady-State Test Data at Higher Temperature shown in Fig. 32

\begin{tabular}{|c|c|c|c|c|c|}
\hline $\begin{array}{c}\text { Run } \\
\text { Number }\end{array}$ & $\begin{array}{c}\text { Slurry } \\
\text { Velocity } \\
\mathrm{ft} / \mathrm{s}\end{array}$ & $\begin{array}{c}\text { TMP } \\
\text { psid }\end{array}$ & $\begin{array}{c}\text { Temp. } \\
\left({ }^{\circ} \mathrm{C}\right)\end{array}$ & $\begin{array}{c}\text { Actual } \\
\text { gpm/ft2 }\end{array}$ & $\begin{array}{c}\text { Temp. Adjusted } \\
\text { gpm/ft2 }\end{array}$ \\
\hline-1 & 12 & 40 & 30 & 0.0123 & 0.0108 \\
\hline 2 & 12 & 40 & 37.5 & 0.0150 & 0.0107 \\
\hline 3 & 12 & 40 & 45 & 0.0186 & 0.0109 \\
\hline 4 & 12 & 40 & 35 & 0.0137 & 0.0104 \\
\hline 5 & 12 & 40 & 25 & 0.0106 & 0.0105 \\
\hline 6 & 15 & 50 & 35 & 0.0230 & 0.0157 \\
\hline 7 & 15 & 50 & 45 & 0.0272 & 0.0157 \\
\hline
\end{tabular}

The varying conditions make it difficult to determine the effect of temperature of filter flux. However, in Fig. 33, by using data from Fig. 31 [Run $7 @ 24$ wt\% UDS at $25^{\circ} \mathrm{C}$ ] and Fig. 32

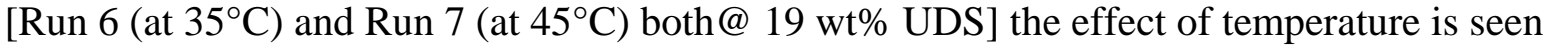
at the flow conditions of $\mathrm{V}=15 \mathrm{ft} / \mathrm{s}$ and TMP $=50$ psid. Unfortunately, both data sets were done at different concentrations of undissolved solids. If Run 7@24 wt\% UDS were done at 19 wt\% UDS then the flux in Fig. 33 for that point would be higher. 
WSRC-TR-2005-00105, REVISION 0

SRNL-RPP-2005-00012, REVISION 0

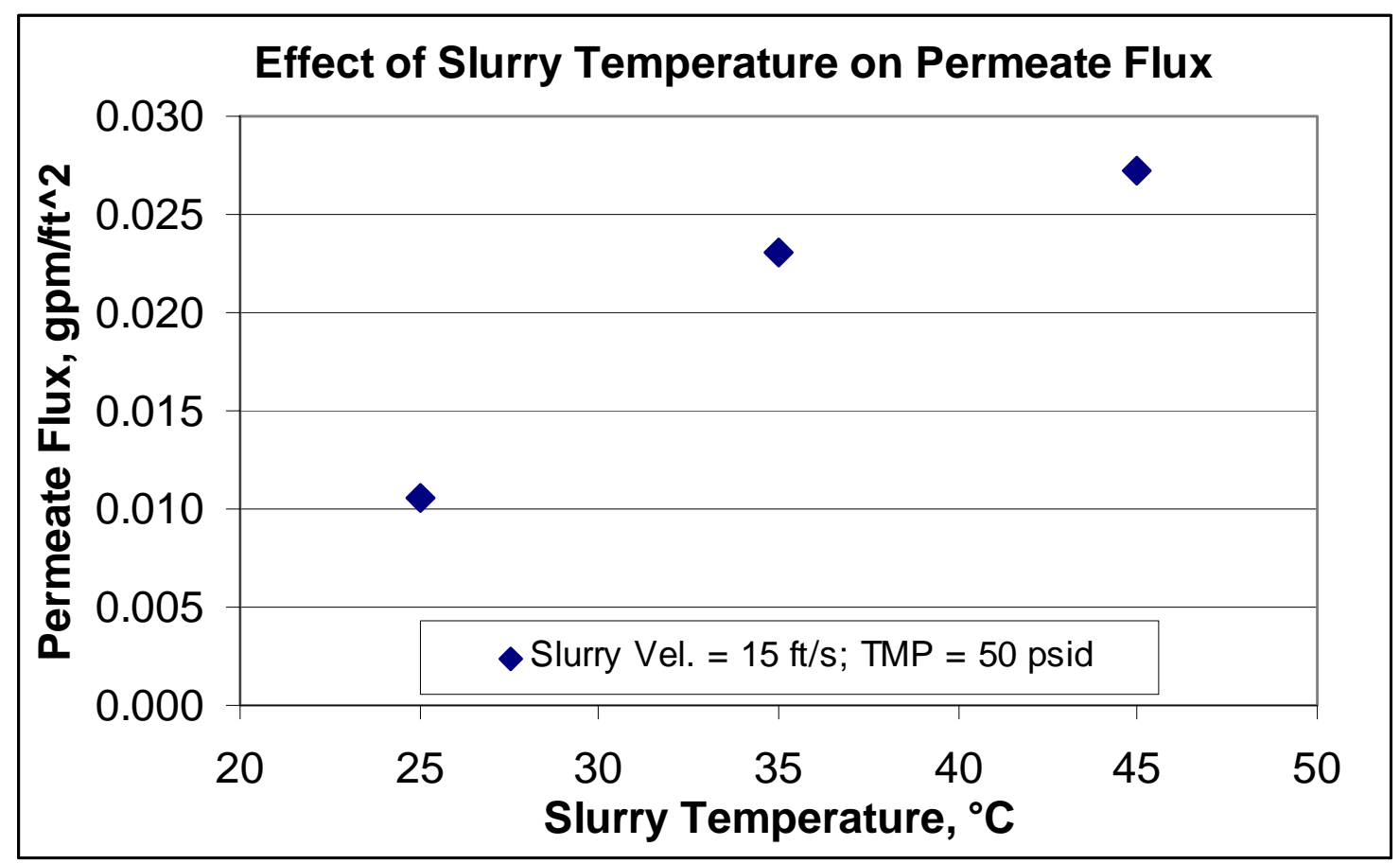

Figure 33. By using the data from Fig. 31 (Run $7 @ 24$ wt\% UDS) and Fig. 32 (Runs 6 and 7@19 wt\% UDS) the effect of temperature is seen at the flow conditions of $\mathrm{V}=15 \mathrm{ft} / \mathrm{s}$ and $\mathrm{TMP}=50$ psid. Figure 31a.

\subsection{Slurry Washing}

In the full-size design, the filtration batch size is 21,550 gallons in the RPP-WTP UFP. The concentrated slurry is washed twice with 21,770 gallons of wash solution using 22 minibatches of approximately 1,000-gallon each. The filtration batch size was $116.8 \mathrm{~L}$ in the pilot-scale SIPP UFP. To mimic the RPP-WTP process, the concentrated slurry was washed twice with $118.0 \mathrm{~L}$ using 22 mini-batches of $5.4 \mathrm{~L}$ each. In each of the two washes, a backpulse precedes the addition of the first mini-batch. The SIPP UFP wash solutions were made with condensate from the FEP evaporator.

A mini-batch of washing solution was pumped directly to the Slurry Reservoir from a drum. Adding a mini-batch raised the slurry reservoir to about $122 \mathrm{~L}$. The slurry was dewatered until the Slurry Reservoir level reached about $117 \mathrm{~L}$, and then another mini-batch was added. This basic procedure was repeated until the washing process was complete.

The wash permeate was collected in drums for storage and later use if needed. The washed slurry was removed from the test rig and stored in a drum. The washed slurry would eventually be vitrified in the Vitreous State Laboratory at Catholic University of America, located in Washington DC. The test rig was rinsed and cleaned after the slurry washing. 


\subsection{Test Results}

Figure 34 shows the adjusted permeate flux of the first washing. The flux increased from approximately 0.012 to $0.018 \mathrm{gpm} / \mathrm{ft}^{2}$ during the first wash cycle (50\% increase). The flux increased in the first wash of Campaigns I, II and III by 53\%, 64\% and 42\%, respectively. Figure 35 shows that the adjusted flux continued to increase during the second wash, from approximately 0.022 to $0.027 \mathrm{gpm} / \mathrm{ft}^{2}$ (23\% increase). The flux increased in the second wash of Campaigns I, II and III by 32\%, 25\% and 16\%, respectively.

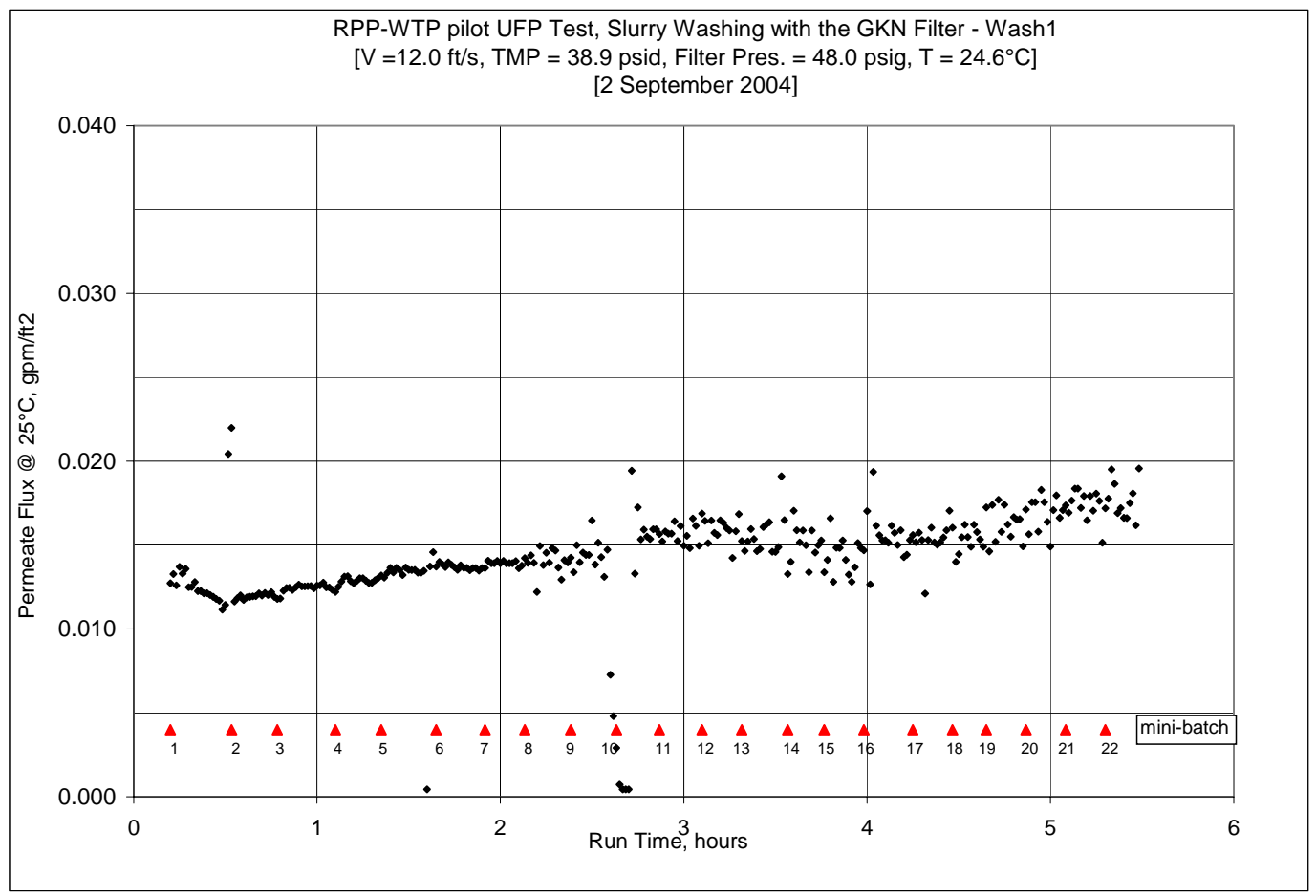

Figure 34. 1st Washing of Concentrated Slurry with 22 Wash Mini-batches 
WSRC-TR-2005-00105, REVISION 0

SRNL-RPP-2005-00012, REVISION 0

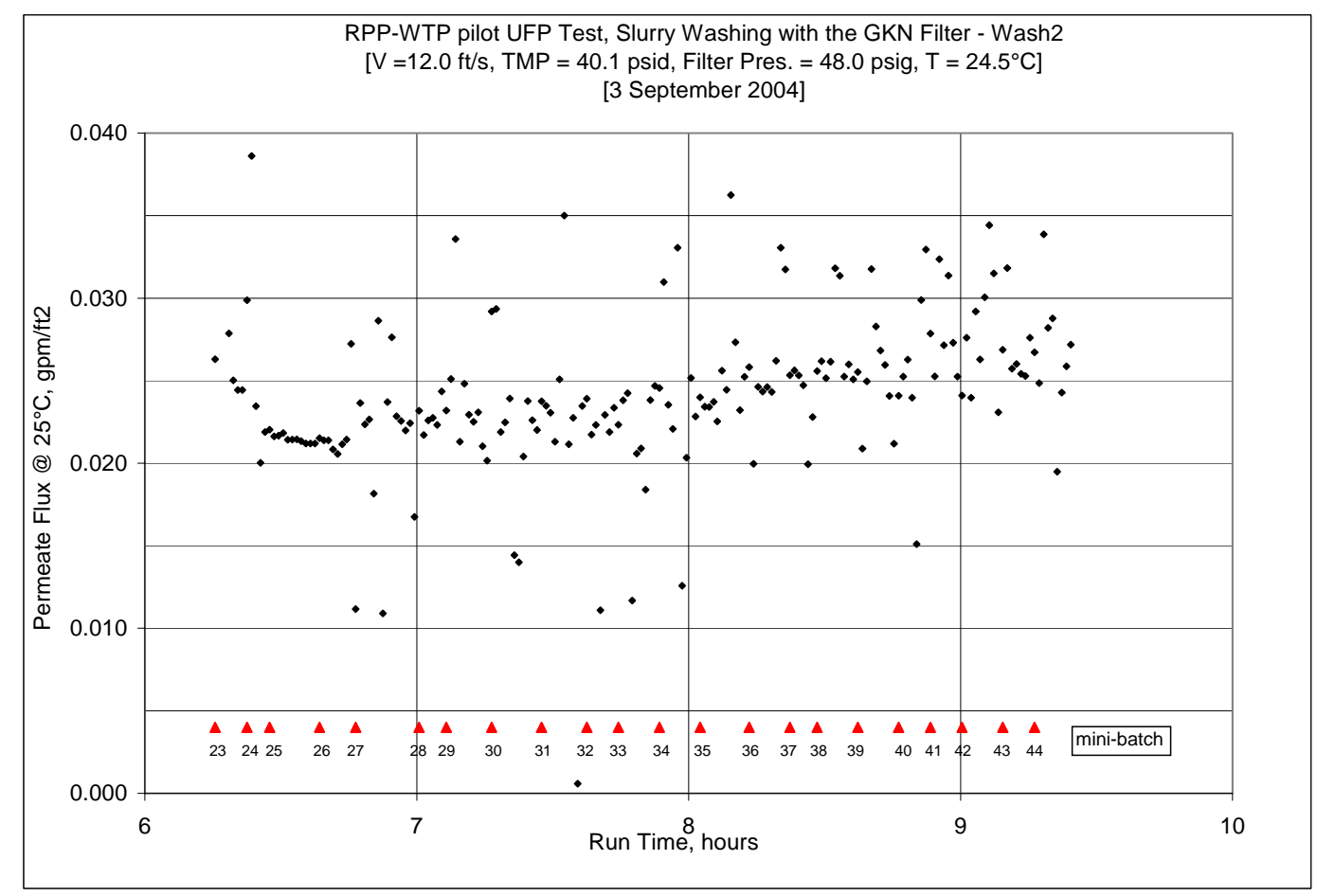

Figure 35. 2nd Washing of Concentrated Slurry with 22 Wash Mini-batches

\subsection{Data Instability}

Note in Fig. 34 that the initial flux readings are relatively stable and become progressively unstable during the first wash, and more so in the second wash (Fig. 35). This instability was systematic to the pilot-scale test rig and was attributed to gas bubbles in the small ID tubes of the permeate loop. The bubbles were small in volume at the start of the first wash but became larger and appeared more frequent as the wash progressed. Fluctuations in the permeate flow rate appeared to correspond to bubbles observed in the polyethylene section of the permeate loop, which started at the pulse pot and ending at the permeate collection drum. Similar data fluctuations were noticed in the pressure measured at the backpulse pot (P3).

During normal operation the gauge pressure at the backpulse pot was negative ( $\sim 6$ psig vacuum, P3 reference Fig. 18) due to the siphon created by the height of the backpulse pot above the permeate discharge. The vacuum at the backpulse pot would introduce air into the permeate loop if a leak existed. However, the permeate loop and filter unit were hydrostatically tested for leaks after Campaign III. The test did not reveal a leak.

A possible explanation for the gas bubbles is that the negative gauge pressure at the backpulse pot forced dissolved gasses out of the permeate. The bubbles were observed at the discharge of the backpulse pot and appeared to flow with the permeate to the collection drum. Another possible explanation is that the air is pulled into this section of the permeate loop during sampling (opening sample valve V29, reference Fig. 18). The slow moving permeate (less than $1 \mathrm{ft} / \mathrm{s}$ in the discharge line) was not able to sufficiently clear the line 
when dewatering continued after each sampling period (the discharge line would maintain oscillating air bubbles). The full-size WTP will have larger discharge pipes and higher permeate flows, which will continue to flow when a new charge of washing water is added. Because of the larger size and design of the full-size WTP UFP the instabilities attributed to the gas bubbles in the pilot-scale SIPP UFP are not expected to be a prominent in the plant unit, if at all. However, it is important to note that since the UFP tank will be partially mixed with sparging, there is the potential of entraining gas bubbles into the filter loop. The presence of gas bubbles in the loop has the potential of increase the loop pressure drop since two-phase (gas-liquid) flow is harder to push than one without gas. Also, gas may create filter problems by diverting flow from some of the many filter tubes by causing higher resistances within the filter walls.

\subsection{Permeate Flux Increase Between Washes}

In all of the campaigns to date, the adjusted flux increased from the end of the first wash to the beginning of the second wash. During the two wash cycles there was a period of time that filtering was stopped. This occurred either by shutting the permeate valve and then the pumps when washing was scheduled for different days (Campaigns I and II), or by shutting the permeate valve, but the pumps were left running when the second washing was to continue on the same day (Campaigns III and IV). For Campaigns III and IV there was still a period between the first and second washes due to the need of sampling and time to set up the next batch of caustic washing solutions. In both cases, at the start of a second wash, and initiation of filtration, a backpulse was made. The durations between the end of the first wash and the beginning of the second wash were 11 hours and 52 minutes in Campaign I, 10 hours and 42 minutes in Campaign II, 1 hour and 37 minutes in Campaign III, and 43 minutes in Campaign IV. As just stated, the first and second washes of Campaigns III and IV were conducted with very short non-filtering periods; and the washes of Campaigns I and II were conducted on consecutive days, with significant non-filtering periods. At the start of the second wash the adjusted flux increased 18\% in Campaign I, 8\% in Campaign II, 30\% in Campaign III, and 31\% in Campaign IV over the flux at the end of the first wash. It was believed that solids entrained in the filter media continued to dissolve during the non-filtering time, creating an improved flux after filtration was restarted and backpulsed to initiate the second wash. However, since the flux improvement was the same order of magnitude whether the time between the two washes were 12 hours or 43 minutes, a larger effect on flux improvement may have been the rearrangement of the filter cake from the simple action of stopping, starting, and backpulsing. During the first wash the filter cake is formed within the first 30 to 60 minutes. The solids that comprise the formed, packed filter cake are pressed against the filter substate which would minimize their movement and participation in the washing process. At the end of the first wash the filter is stopped. After the stated idle time the filter is restarted and backpulsed when, it is assumed, a considerable amount of the filter cake, if not all, is separated from the filter wall, which then mixes with the rest of the washed slurry. The new cake that forms during the second wash will contain solids that were thoroughly washed and changed through dissolution, thus changing their morphology allowing the formation of a more permeable filter cake. In light of this experience, WTP may significantly increase the permeate flux while washing if backpulsing is used throughout 
WSRC-TR-2005-00105, REVISION 0

SRNL-RPP-2005-00012, REVISION 0

the process. However, this is only recommended if washing is immediately followed by acid cleaning because increasing the backpulse frequency generally leads to faster filter fouling. When a filter is backpulsed the smallest solid particles in a slurry are the first to form the filter cake, which would increase the chance of exposing the filter to particles that could better depth foul the filter walls.

The total increase in flux from the beginning of the first wash to the end of the second wash was $125 \%$ for Campaign IV. The total increase was 107\%, 127\% and 142\% for Campaigns I, II, and III, respectively.

Figure 36 shows the linear curve fits of the adjusted permeate flux of the first and second slurry washes for Campaigns I to IV. The linear curve fits are shown to eliminate any ambiguity caused by the data scatter. To facilitate comparison, the idle time between the washes of Campaigns I, II and IV are modified to match to the start of the second wash of Campaign III. As seen by this figure and given the \% increases in flux of the washes and idle time, the GKN filter unit appeared to perform in a similar fashion to the Mott filter unit.

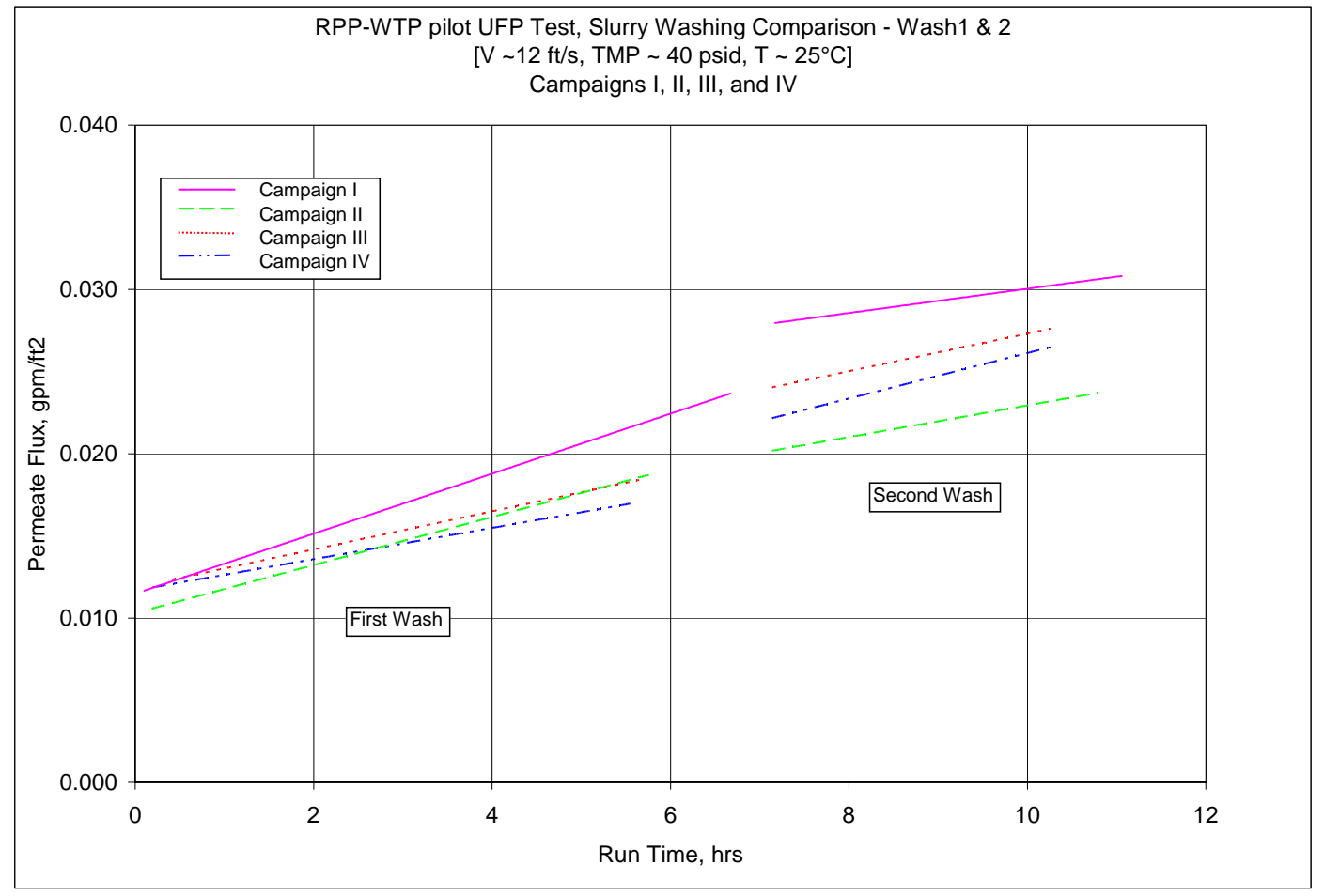

Figure 36. 1st and 2nd Washing of Concentrated Slurry, Campaigns* I, II, III, and IV

Note on Fig. 36: The measurement and curve-fit uncertainty is below.1
Campaign I $-1^{\text {st }}$ wash, +/- $0.004 \mathrm{gpm} / \mathrm{ft} 2 ; 2^{\text {nd }}$ wash, $+/-0.009 \mathrm{gpm} / \mathrm{ft} 2$
Campaign II $-1^{\text {st }}$ wash, +/- $0.003 \mathrm{gpm} / \mathrm{ft} 2 ; 2^{\text {nd }}$ wash, +/- $0.007 \mathrm{gpm} / \mathrm{ft} 2$
Campaign III $-1^{\text {st }}$ wash, +/- $0.005 \mathrm{gpm} / \mathrm{ft} 2 ; 2^{\text {nd }}$ wash, +/- $0.007 \mathrm{gpm} / \mathrm{ft} 2$
Campaign IV - $1^{\text {st }}$ wash, $+/-0.005 \mathrm{gpm} / \mathrm{ft} 2 ; 2^{\text {nd }}$ wash, +/- $0.010 \mathrm{gpm} / \mathrm{ft} 2$

Note that the duration of the first wash for Campaign I does not coincide with the durations of the other campaigns. The washes of each campaign were conducted using similar procedural steps (specified in a Work Guidance). Chronological notes in the laboratory 
WSRC-TR-2005-00105, REVISION 0

SRNL-RPP-2005-00012, REVISION 0

notebook indicated that the duration of a mini-batch in Campaign I was (on average for Wash 1) 2-3 minutes longer than in subsequent campaigns. There are several possible reasons for the extra time: 1) Taking samples and adding wash solution in Campaign I was conducted by a single technician; in Campaigns II-IV these tasks were performed by two technicians. 2) The process of taking samples and adding wash solution was more familiar by Campaign II and the subsequent campaigns, enhancing the efficiency of the sampling process. 3) The number of samples was reduced in Campaigns III and IV. The duration difference was also evident in the second wash of Campaign I and to a limited extent in the second wash of Campaign II.

\subsection{Permeate Samples}

Throughout the washing, permeate samples were taken at the end of wash numbers 1, 5, 9, 13, 17, and 22, for both washes per the sampling plan (Appendix D). These sample periods are important because while both slurry and permeate samples were taken at the same time, they are not matched samples. The permeate flux was very low and the permeate loop lengthy. It took approximately 30 minutes for permeate created at the filter to flow to the sampling point. Therefore, a permeate sample represents the permeate filtered from the slurry 30 minute previously while the slurry sample represents the slurry at the moment of the sample. However, the slurry and permeate samples can be appropriately matched by knowing the delay time.

To match the slurry sample taken at the end of each wash, the test rig was operated (in permeate recirculation mode) for an additional 30 minutes. At the end of the 30 minutes a permeate sample was taken. The analysis of the slurry and permeate samples taken during the washing are presented in Section 2.7.4.2.3.

\subsection{Filter Rinsing and Cleaning}

Following slurry washing, the slurry was drained from the test rig and the process of rinsing and cleaning the filter and test rig began. The test rig was rinsed and cleaned at $25^{\circ} \mathrm{C} \pm 5^{\circ} \mathrm{C}$ with the following sequence:

- $\quad 0.1 \mathrm{M} \mathrm{NaOH}$ rinse for 30 minutes

- $2.0 \mathrm{M} \mathrm{HNO}_{3}$ cleaning for 90 minutes

- $\quad 2.0 \mathrm{M} \mathrm{HNO}_{3}$ cleaning for 90 minutes

- $2.0 \mathrm{M} \mathrm{HNO}_{3}$ cleaning for 90 minutes

- $\quad 0.1 \mathrm{M} \mathrm{NaOH}$ rinse for 30 minutes

- $0.1 \mathrm{M} \mathrm{NaOH}$ rinse for 30 minutes

The rinse solutions were made using condensate from the FEP Evaporator. The acid solutions were made from DIF water. Each solution was pumped into the Slurry Reservoir from a drum. A separate, empty drum was positioned to receive the wash permeate during each sequence.

At the end of a particular sequence step the solution was drained from the test rig to a drum and the subsequent solution was added. For each of the rinsing and cleaning steps, it was necessary and important to have a heel, representative of what is expected in the full-scale 
plant, since it affects the chemistry of each subsequent solution. Therefore, a quantity of the previous solution was added back to each new solution to represent that heel.

The heel for the first caustic rinse consisted of a mild caustic solution (12.5 L), representing the filter-loop rinse and the residual slurry remaining in the test rig after washing (estimated to be 20-22 L and represents the concentrated slurry that will remain in the UFP filter loop and feed tank after the slurry is washed and drained). This produced a total heel of $34.5 \mathrm{~L}$ (max), and combined with the 94.6 L of $0.1 \mathrm{M} \mathrm{NaOH}$ produced a wash volume of about $129 \mathrm{~L}$ (actually about127 L, accounting for the volume change upon mixing). This was larger than the normal batch size of the SIPP UFP $(117 \mathrm{~L})$ and was unique because of the large volume of residual slurry. The 20-22 L of residual slurry produced a greater wt\% solids in the pilot-scale test rig than that expected in the RPP-WTP UFP at this stage in the process. By increasing the wash volume the excess wt\% solids were kept to a minimum (3.6 wt\% UDS vs. $1.8 \mathrm{wt} \%$ UDS in the WTP - see the footnote to Section 2.7.4.2.4.).

The wt\% UDS for the first acid cleaning was corrected back to the expected value (1.8 wt\%) with the next heel. The heel for the first acid cleaning consisted of $7.5 \mathrm{~L}$ of spent solution from the first caustic rinse, $12.5 \mathrm{~L}$ of $0.1 \mathrm{M} \mathrm{NaOH}$ (made from FEP evaporator condensate), and residual solution left in the test rig (estimated to be $1.6 \mathrm{~L}$ ). The total heel for the first acid wash was about 21.6 L. The volume of $2 \mathrm{M}$ acid cleaning solution was 94.6 L, creating a batch size of approximately $117 \mathrm{~L}$.

The heel for each subsequent solution consisted of 20 liters of the spent, previous solution and the residual solution remaining in the test rig (estimated to be $1.6 \mathrm{~L}$ ). This produced a total heel of 21.6 L. This heel added to the typical volume of subsequent cleaning or rinse solutions $(94.6 \mathrm{~L})$ produced a batch size of approximately $117 \mathrm{~L}$.

\subsection{Test Results}

The adjusted permeate flux for the first caustic rinse is shown in Fig. 37. A backpulse preceded the first mild caustic rinse. Figure 38 (a) shows a settled sample of the first rinse solution ( $\mathrm{pH} 13)$. Note the large amount of UDS. As indicated in Table 33, the concentration of the UDS was $4.3 \mathrm{wt} \%$. Table 33 gives the solids history obtained during Campaign IV testing; it is similar to the rinsing and cleaning solids results for the preceding three campaigns. It shows that the wt $\%$ TS of the $1^{\text {st }}$ acid cleaning solution was reduced from the $1^{\text {st }}$ caustic rinse, and was further reduced in the $2^{\text {nd }}$ and $3^{\text {rd }}$ cleaning. The amount of UDS remaining in the first caustic rinse of Campaign II and III was about 5\% and 4\%, respectively. The results in Table 33 indicate that the acid cleaning solution was actually about $1.70 \mathrm{M}$ and not the target $2.00 \mathrm{M}$. The total solids content of the second and third caustic rinses is higher than the third acid cleaning due to neutralization of the nitric acid. The acid cleaning solutions are $\mathrm{HNO}_{3}$, which evaporates when dried for total solids analysis. When the acid is neutralized with $\mathrm{NaOH}$, it forms $\mathrm{NaNO}_{3}$, which does not evaporate during the solids determination. 
WSRC-TR-2005-00105, REVISION 0

SRNL-RPP-2005-00012, REVISION 0

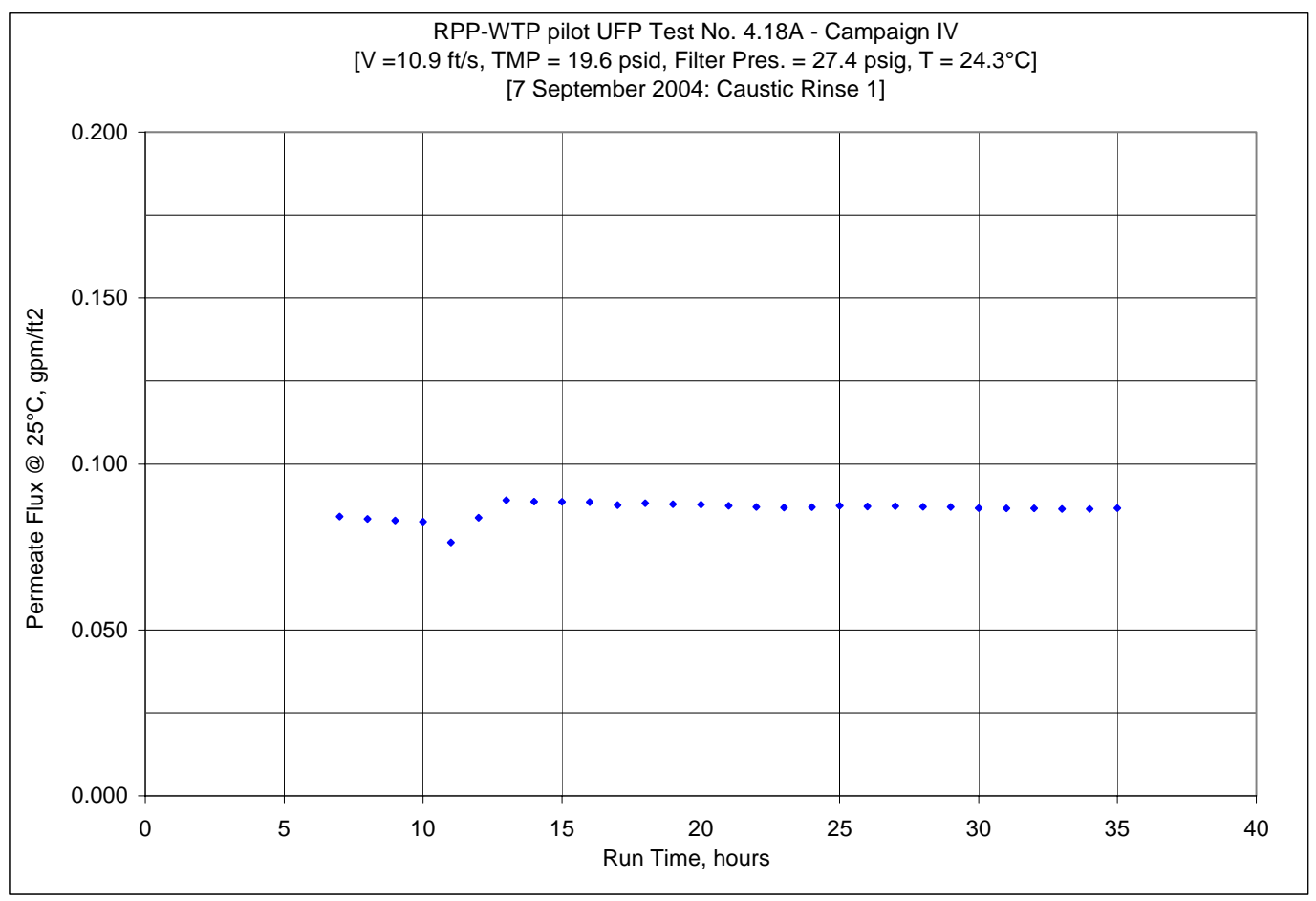

Figure 37. 1st Caustic Rinse of the SIPP UFP

Three acid cleanings followed the first caustic rinse. Figure 38 shows the adjusted permeate flux results for all three acid cleanings. A backpulse preceded each cleaning, which accounts for the higher initial flux at the beginning of each cleaning. The time gaps between the cleanings represent the times necessary to remove the old cleaning solution from the test rig and add a new cleaning solution and heel. The flux also improved between the first and second acid cleanings. This implies the residual acid solution in and on the filter media continued to dissolve solids and that backpulsing prior to cleaning removed stuck-on or lodged material. The flux did not improve between the second and third cleanings, which implies that the maximum effect of the nitric acid was realized in the second cleaning. 
WSRC-TR-2005-00105, REVISION 0

SRNL-RPP-2005-00012, REVISION 0

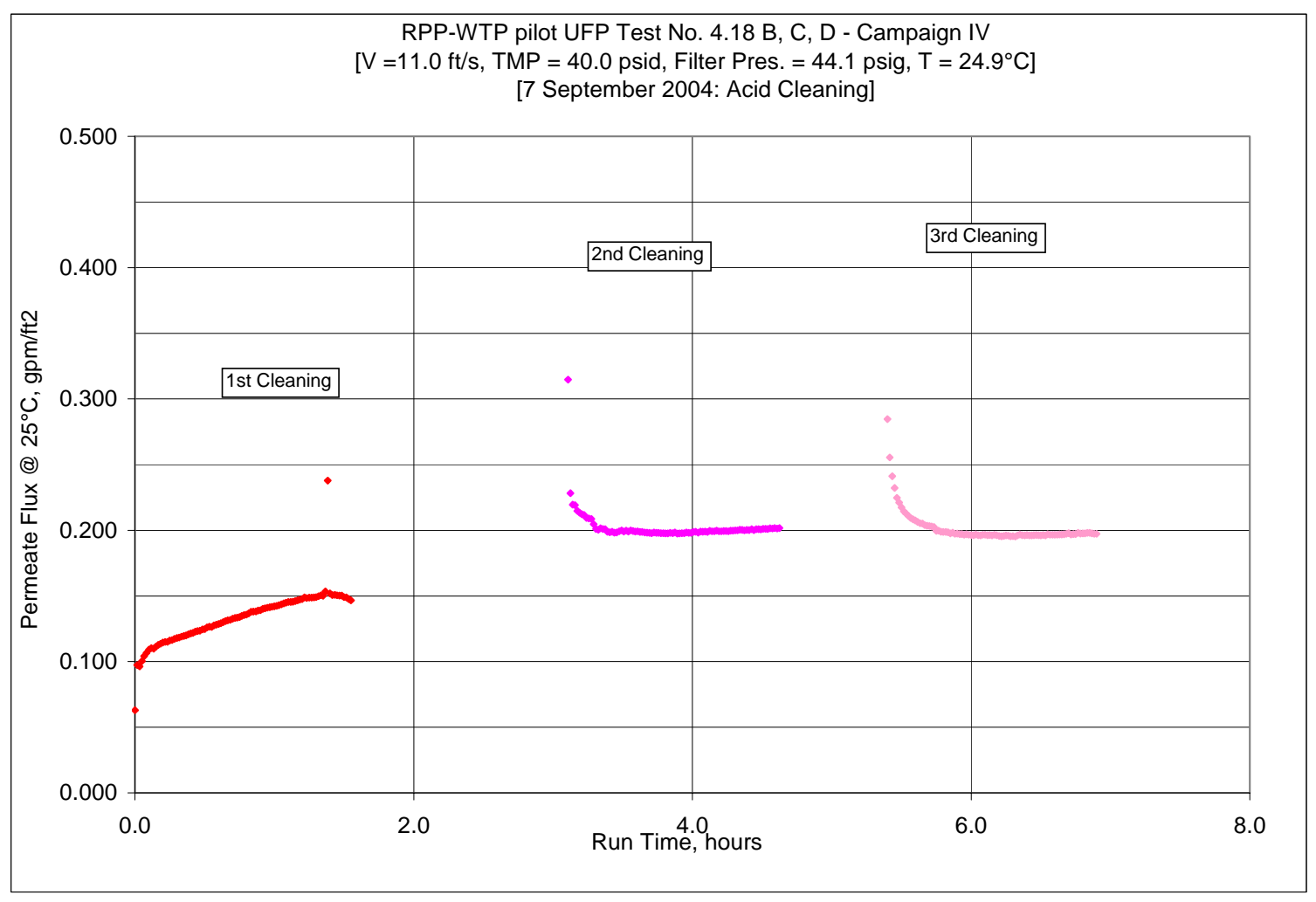

Figure 38. Acid Cleaning of the SIPP UFP

Figure 40 (b), (c), and (d) show samples of the spent acid cleaning solutions from Campaign IV. Note that the first acid cleaning solution was stained. In addition, there are small amounts of a fluffy, whitish solid on the bottom of the bottle. Spectral analysis of the whitish solid in Campaign II indicated a composition of alumina, silica and diaoyudaoite, $\mathrm{NaAl}_{11} \mathrm{O}_{17}$ (Duignan et al., 2004b or Appendix I-2). The $2^{\text {nd }}$ and $3^{\text {rd }}$ acid cleaning solutions are relatively clear. The $2^{\text {nd }}$ acid cleaning solution had a very small amount of solids collected on the bottom of the bottle. The $3^{\text {rd }}$ acid cleaning solution was visibly free of solids. The wt\% UDS results of the cleaning solutions in Table 33 are so close to zero that the results are inconclusive. The molarity of the cleaning solutions shown in the table indicates the effect of the heel.

The acid cleanings were followed by two mild caustic rinses. Figure 39 shows the adjusted flux of the last two rinses and reveals that the permeate flux of the second caustic rinse (or the first rinse after acid cleaning) was notably less than that of the third acid cleaning (ending values of $\sim 0.195 \mathrm{gpm} / \mathrm{ft}^{2}$ vs. $\sim 0.095 \mathrm{gpm} / \mathrm{ft}^{2}$ ). This trend was seen in Campaign I, II and III and was attributed to the caustic solution precipitating solids from the acid heel. The volume and concentration of the acid heel was enough to make the second caustic rinse acidic ( 0.4 molar acid). Furthermore, the volume and concentration of the second cautic rinse heel was also enough to make the third caustic rinse slightly acidic ( 0.05 molar acid). The adjusted flux during the third caustic rinse continued to decline as more solids precipitated from the more-neutral solution. At the end of the last rinse the flux had dropped to $\sim 0.08 \mathrm{gpm} / \mathrm{ft}^{2}$, which was lower than previous campaigns but still greater than the average Campaign IV 
dewatering flux of $\sim 0.011 \mathrm{gpm} / \mathrm{ft}^{2}$. Samples of the rinse solutions are shown in Figure 40 (e) and (f) and they appear to be clear. Further chemical analyses of the rinse and cleaning solutions are provided in Section 2.7.4.2.4.

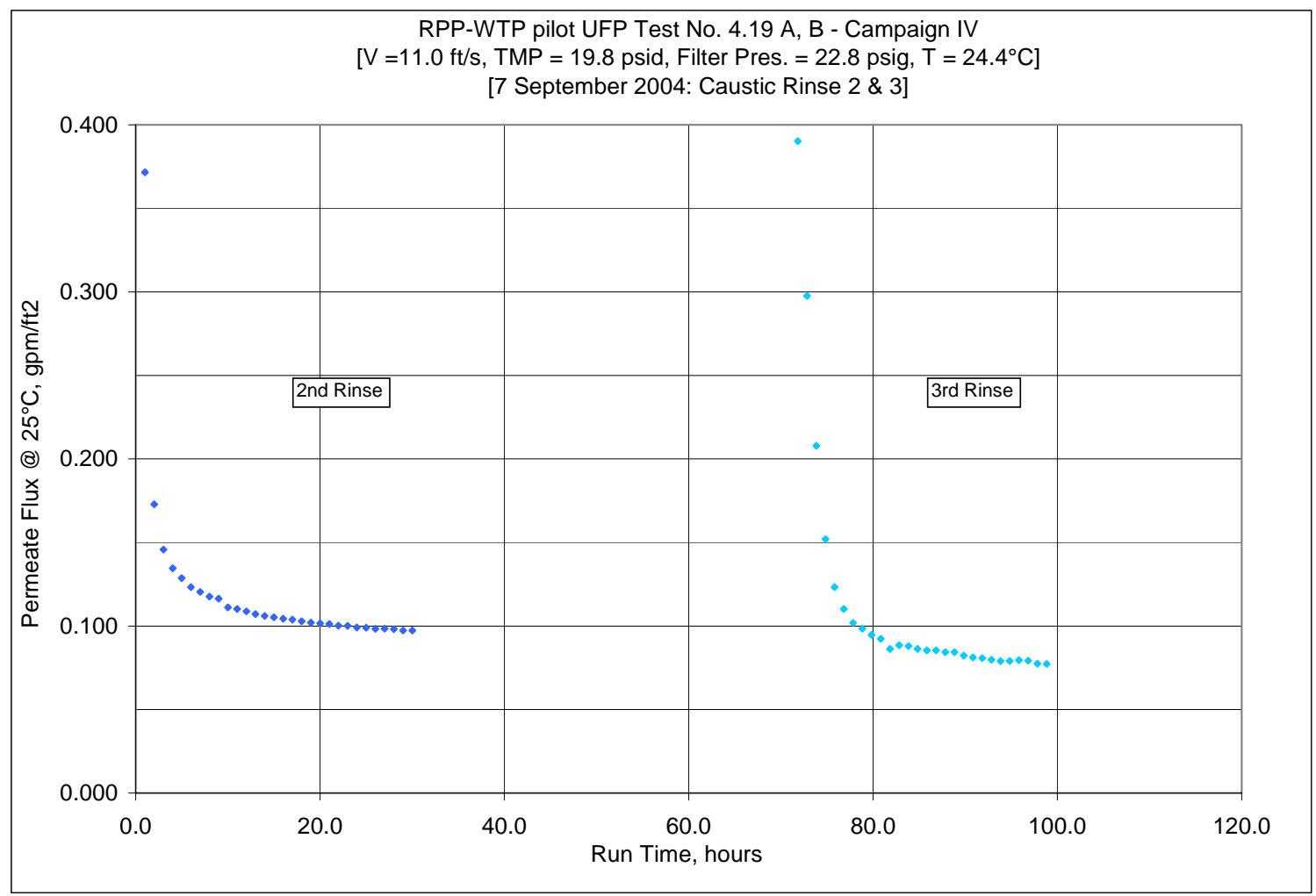

Figure 39. 2nd and 3rd Caustic Rinses of the SIPP UFP

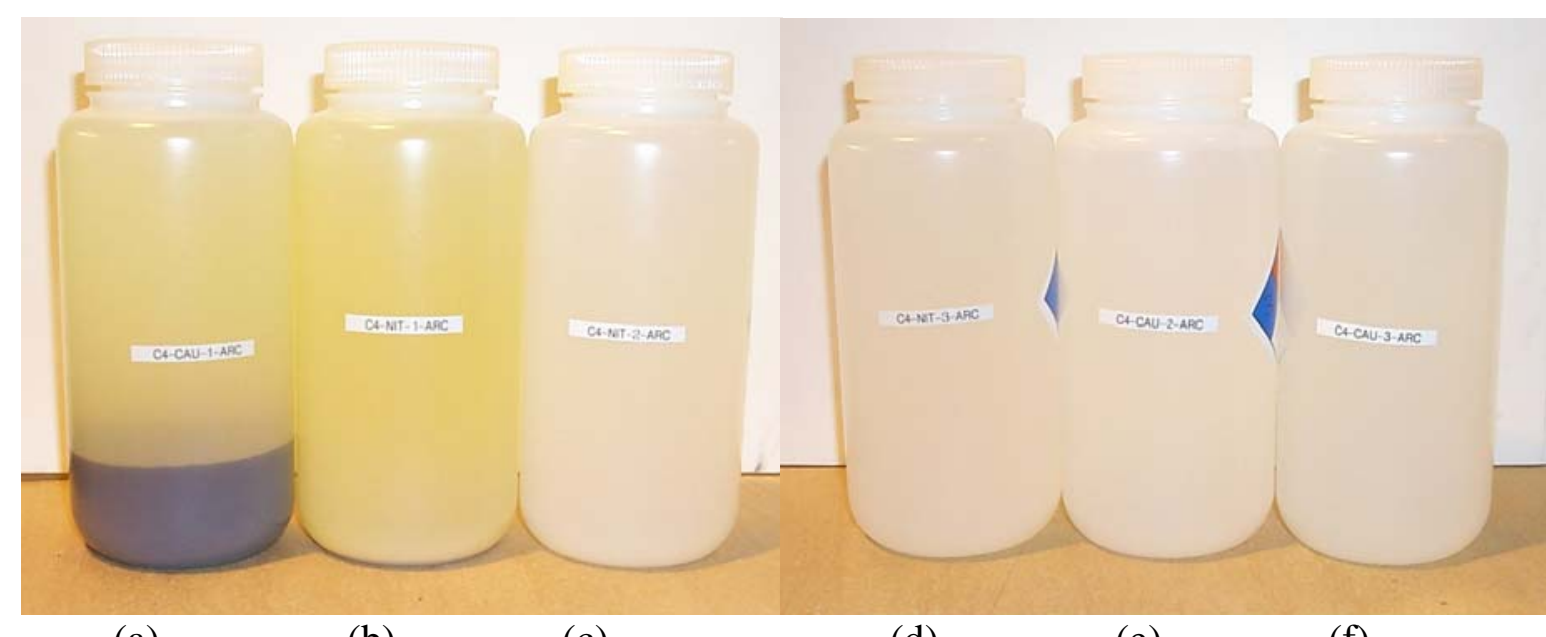

(a) (b)

(c)

(d)

(e)

(f)

Figure 40. Campaign IV Caustic Rinse and Acid Cleaning Samples

(a) first caustic rinse, (b) first $2 \mathrm{M}$ nitric cleaning, (c) second $2 \mathrm{M}$ nitric cleaning, (d) third $2 \mathrm{M}$ nitric cleaning, (e) second caustic rinse, (f) third caustic rinse 
WSRC-TR-2005-00105, REVISION 0 SRNL-RPP-2005-00012, REVISION 0

Table 33. Total Solids (TS) and Undissolved Solids (UDS) Concentrations of the Spent Rinse and Cleaning Solutions from Campaign IV (also see Table 44)

\begin{tabular}{|c|c|c|c|c|}
\hline \multirow[t]{2}{*}{ Step } & TS & UDS & $\begin{array}{c}\text { Base or Acid } \\
\text { (after } \\
\text { addition) }\end{array}$ & $\mathbf{p H}^{*}$ \\
\hline & $\mathrm{wt} \%$ & $\mathrm{wt} \%$ & $\mathrm{M}$ & --- \\
\hline First Caustic Rinse $0.1 \mathrm{M} \mathrm{NaOH}$ & 6.51 & 4.32 & 0.2 base & 13.3 \\
\hline First Acid Cleaning $2.0 \mathrm{M} \mathrm{HNO}_{3}$ & 1.57 & 0.06 & 1.19 acid & $<1$ \\
\hline Second Acid Cleaning 2.0 $\mathrm{M} \mathrm{HNO}_{3}$ & 0.47 & 0.02 & 1.68 acid & $<1$ \\
\hline Third Acid Cleaning $2.0 \mathrm{M} \mathrm{HNO}_{3}$ & 0.15 & 0.08 & 1.69 acid & $<1$ \\
\hline Second Caustic Rinse $0.1 \mathrm{M} \mathrm{NaOH}$ & 0.76 & 0.00 & 0.40 acid & $<1$ \\
\hline Third Caustic Rinse $0.1 \mathrm{M} \mathrm{NaOH}$ & 0.94 & 0.02 & 0.05 acid & 1.3 \\
\hline
\end{tabular}

The third caustic rinse solution was left in the test rig and was used for the post-test baseline test. After the baseline test the caustic solution was removed from the test rig and stored in a drum. The post-test baseline results are presented in Section 2.7.4.1.7.2.

\subsection{Acid Cleaning Comparison}

Figure 41 shows the permeate flux of the entire rinse and clean process for Campaigns I, II, III and IV. The Campaign I trend was different during the acid cleanings but similar during caustic rinses. As noted previously, the slurry of Campaign I was AY102/V106 only, where the slurry of Campaigns II and III was a mixture of AY102/C106, AP101 and FEP Bottoms. Furthermore, the Campaign I acid cleaning samples visibly contained a greater amount of the whitish solids than the Campaign II and III samples, most likely the cause of the lagging permeate flux.

The Campaign II and III data are very similar in trend and magnitude. The Campaign IV flux during the $2^{\text {nd }}$ and $3^{\text {rd }}$ acid cleanings was lower than the Campaign II and III flux levels, possibly indicating that the acid cleaning of the GKN filter was not as effective as that of the Mott. Figure 42 shows the permeability during the second acid cleaning (or for an equivalent period) of multiple tests (SIPP and before) for the GKN and the Mott (40-inch and 90-inch filter units). From this perspective the magnitude of the Campaign IV acid flux appears appropriate. The figure implies that the flux during acid cleaning was more a function of the waste simulant dewatered than the filter element used. For example, the tests using an organic-based Envelope C simulated waste, i.e., AN-102, with filter velocities from 6.5 to 12 $\mathrm{ft} / \mathrm{s}$, had permeabilities ranging between 0.02 and $0.042 \mathrm{gpm} / \mathrm{ft}^{2} / \mathrm{psi}$, while the tests using a non-organic-based Envelope D simulated waste, i.e., AY-102/C-106, with filter velocities of $11 \mathrm{ft} / \mathrm{s}$, had permeabilities below $0.01 \mathrm{gpm} / \mathrm{ft}^{2} / \mathrm{psi}$. 
WSRC-TR-2005-00105, REVISION 0

SRNL-RPP-2005-00012, REVISION 0

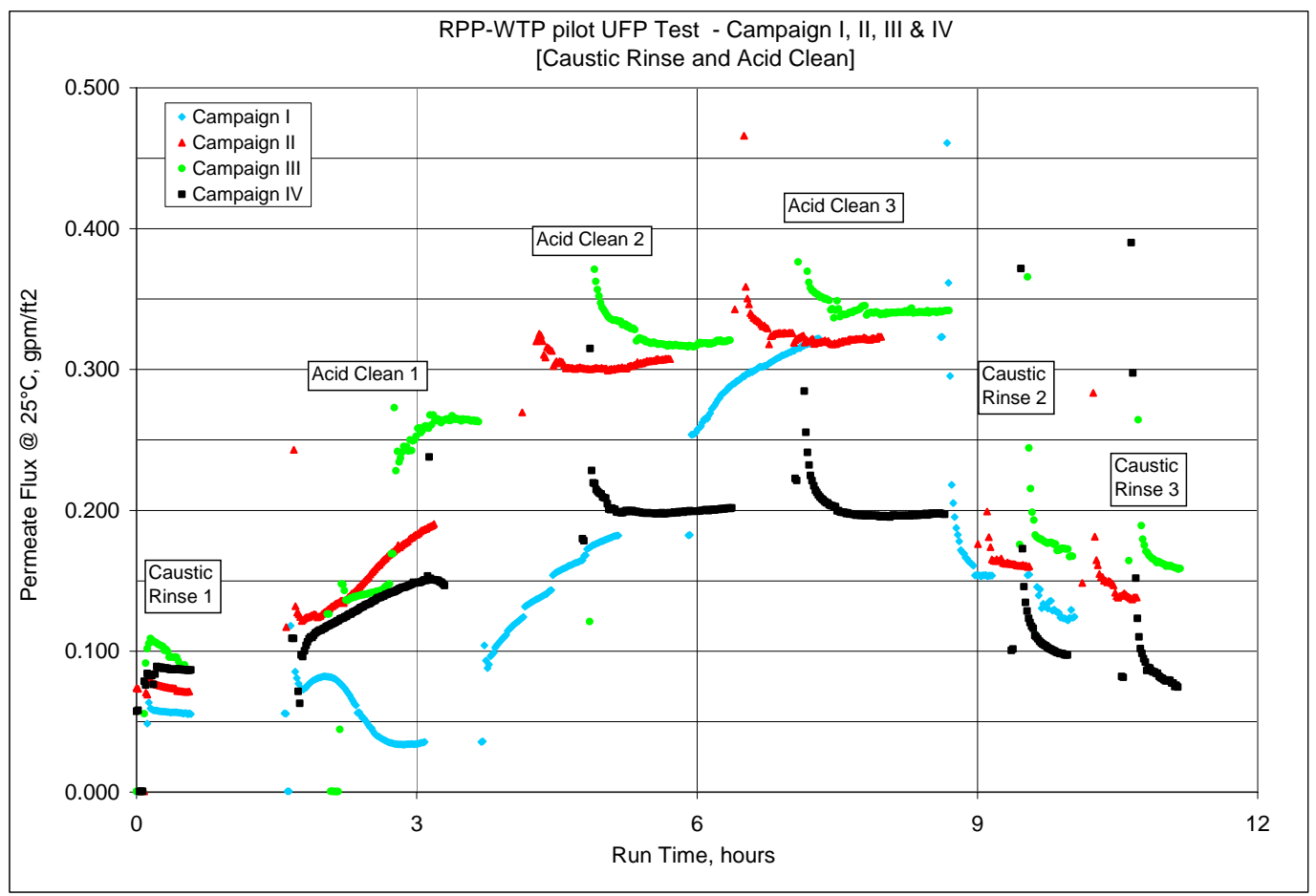

Figure 41. Filter Rinse and Clean, Campaigns I, II, III, and IV

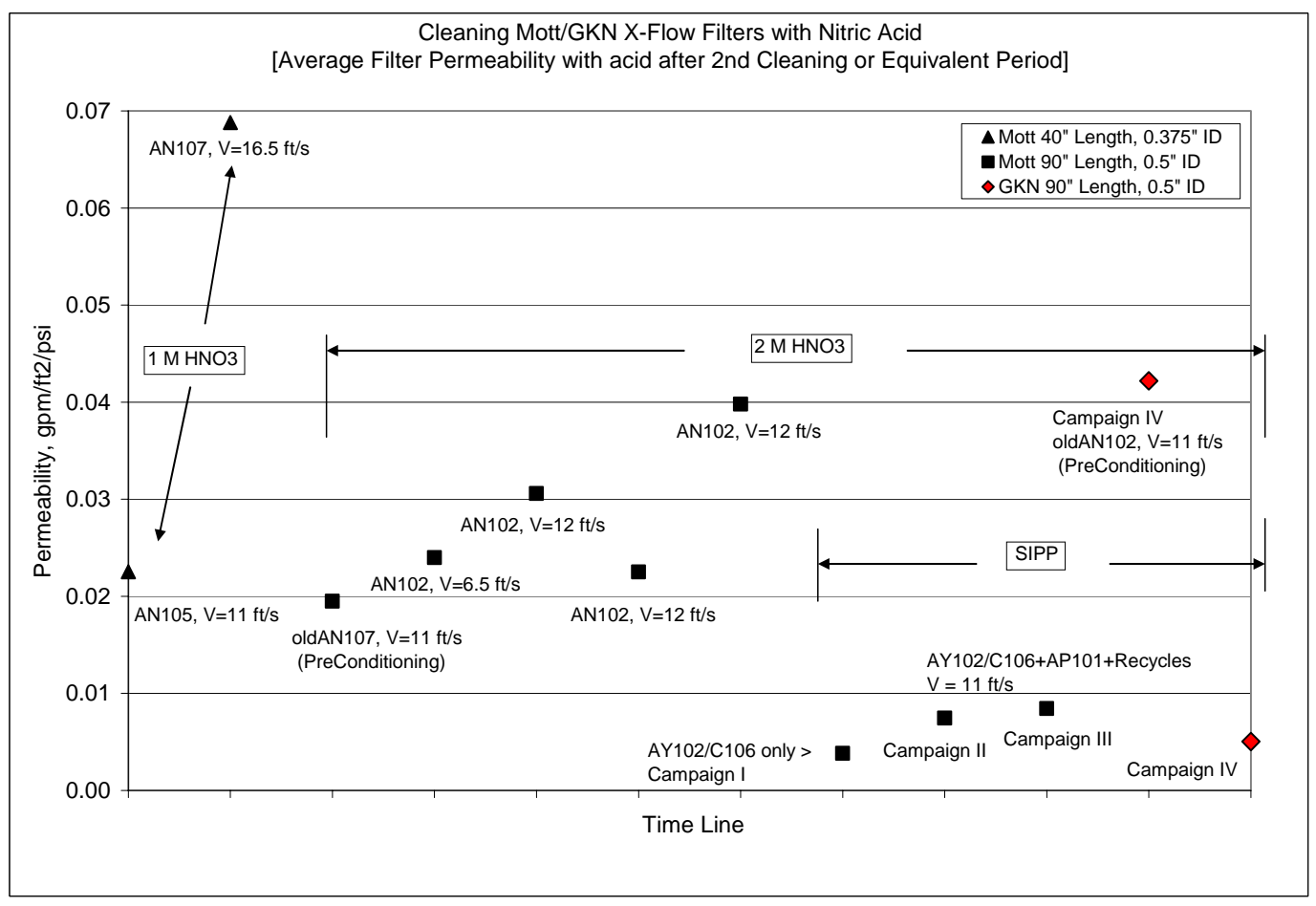

Figure 42. Comparison of Permeability at $25^{\circ} \mathrm{C}$ during Acid Cleaning of Various Ultrafiltration Tests 
WSRC-TR-2005-00105, REVISION 0

SRNL-RPP-2005-00012, REVISION 0

\subsection{Filter Baselining}

\subsection{Pre-test Filter Baseline}

A pre-test baseline test (test numbers 4.00A, B, and C) was conducted with the GKN filter unit after it was preconditioned, rinsed with $0.1 \mathrm{M} \mathrm{NaOH}$ and cleaned with $2.0 \mathrm{M} \mathrm{HNO}_{3}$. The solution for the pre-test baseline was fresh $0.1 \mathrm{M} \mathrm{NaOH}$. The adjusted flux results of this baseline are shown in Fig. 43. The pre-test baseline results are favorable and indicate an effective cleaning with the $2.0 \mathrm{M} \mathrm{HNO}_{3}$. The results of the baseline test conducted prior to the preconditioning are also shown in the figure (conducted on 8/24/04 with $0.1 \mathrm{M} \mathrm{NaOH}$ solution), as well as the pre- and post-test baseline results from Campaign III (Mott).

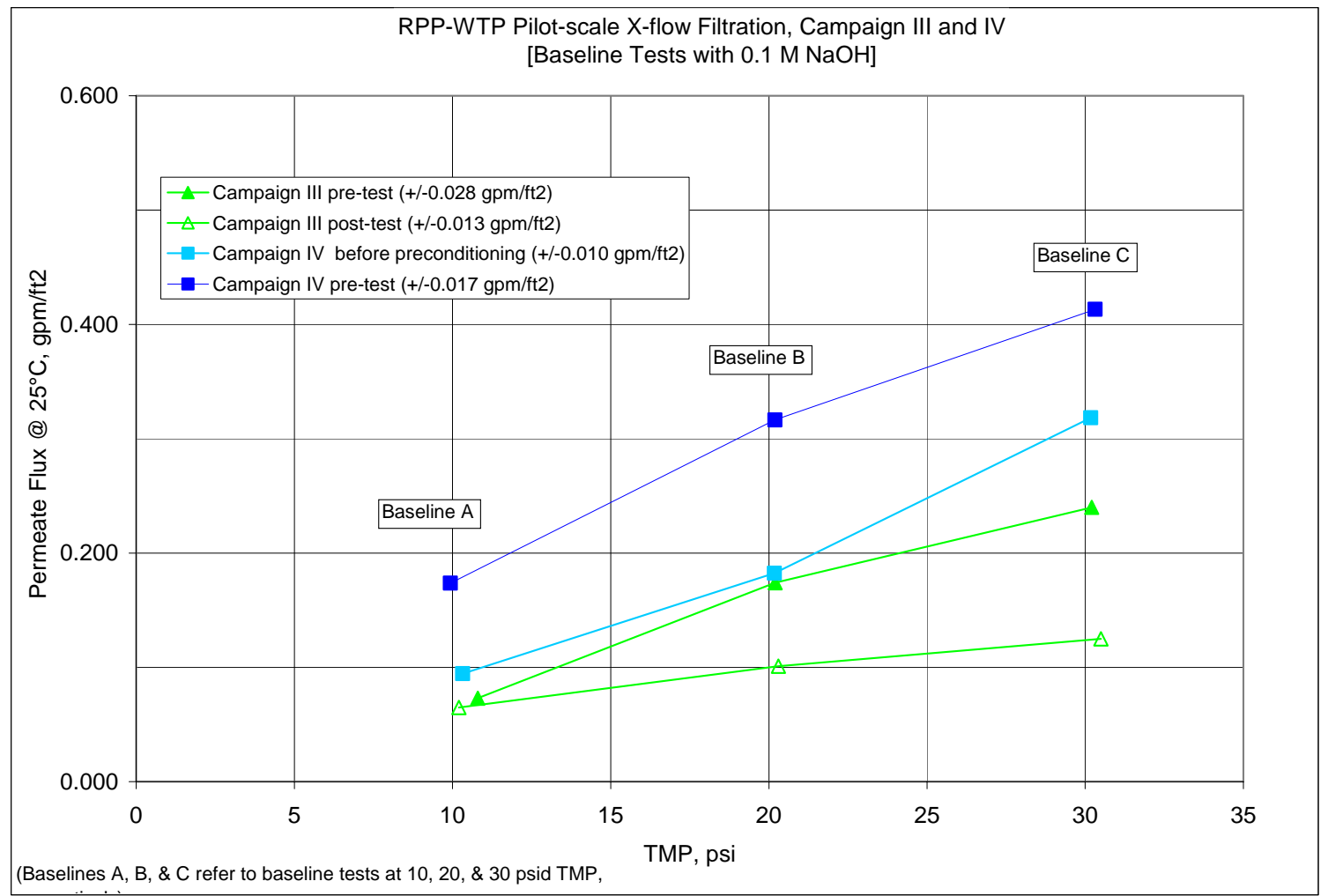

Figure 43. Baselines, Campaign III Pre- \& Post-test and Campaign IV Pre-test

\subsection{Post-Test Filter Baselines}

Two post-test baseline tests were conducted at the end of Campaign IV, after the $3^{\text {rd }}$ rinse of the rinse-clean operation (test numbers 4.18 and 4.19). The first was conducted with the $3^{\text {rd }}$ rinse solution, and the second was with fresh $0.1 \mathrm{M} \mathrm{NaOH}$. The $3^{\text {rd }}$ rinse solution included the $21.6 \mathrm{~L}$ heel from the $2^{\text {nd }}$ rinse solution. Residual nitric acid in the heel made the $3^{\text {rd }}$ rinse solution slightly acidic ( 0.05 molar acid). The results of the post-test baseline tests are shown in Fig. 44, along with the Campaign IV pre-test baseline results for comparison. 
WSRC-TR-2005-00105, REVISION 0

SRNL-RPP-2005-00012, REVISION 0

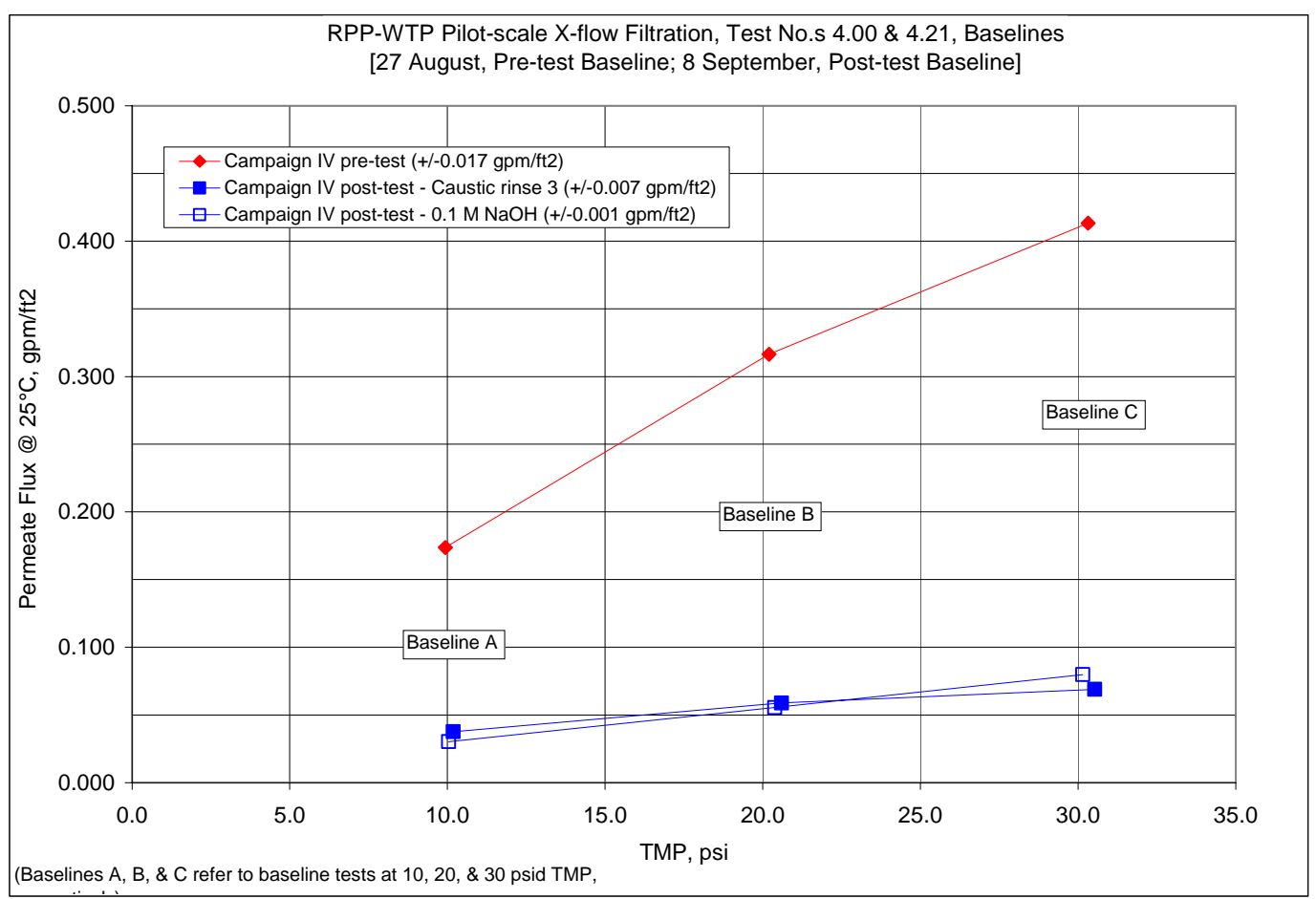

Figure 44. Pre- and Post-Test Baselines for Campaign IV

As expected the post-test baseline results show an adjusted flux lower than the pre-test baseline flux (and the curve was flatter), indicating that the filter was not completely cleaned. Note that the post-test baseline results from the test using the $3^{\text {rd }}$ rinse and the test using the fresh $0.1 \mathrm{M} \mathrm{NaOH}$ are comparable.

Dual post-test baseline test were conducted in Campaigns II, III, and IV. The results of these dual tests are shown in Fig. 45. Note that there was no specific trend in the results regarding the magnitude of the flux when comparing the baseline of the $3^{\text {rd }}$ rinse to that of the fresh $0.1 \mathrm{M} \mathrm{NaOH}$. In Campaign II the flux is higher for the $0.1 \mathrm{M} \mathrm{NaOH}$. In Campaign II the flux was higher for the $3^{\text {rd }}$ caustic rinse. In Campaign IV the fluxes were comparable. 
WSRC-TR-2005-00105, REVISION 0

SRNL-RPP-2005-00012, REVISION 0

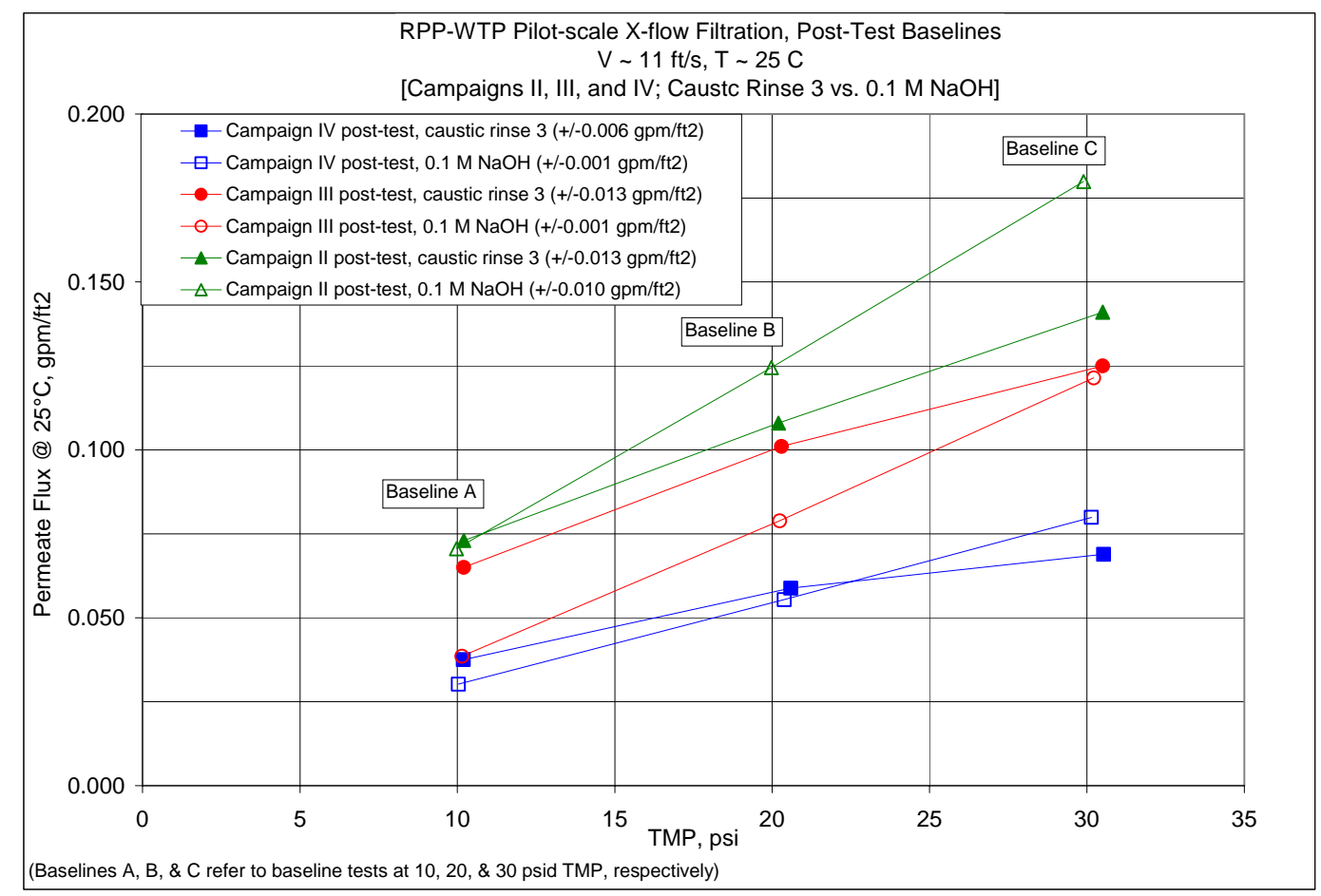

Figure 45. Post-Test Baseline Solution Comparison, Campaigns II - IV

\subsection{Baseline Comparison}

Figure 46 compares the pre- and post test baselines for Campaigns I through IV. Campaign I pre-test was omitted (it was significantly higher, most likely due to having been soaked in $0.1 \mathrm{M} \mathrm{NaOH}$ for over a year). Two pre-test baselines from Campaign III are shown; the first was prior to a pump repair, the second was after (the pump liner and impeller was replaced on each slurry pump). Note that in each campaign the post-test flux was lower than the pretest flux indicating that the filter was never cleaned to pre-test condition. 
WSRC-TR-2005-00105, REVISION 0

SRNL-RPP-2005-00012, REVISION 0

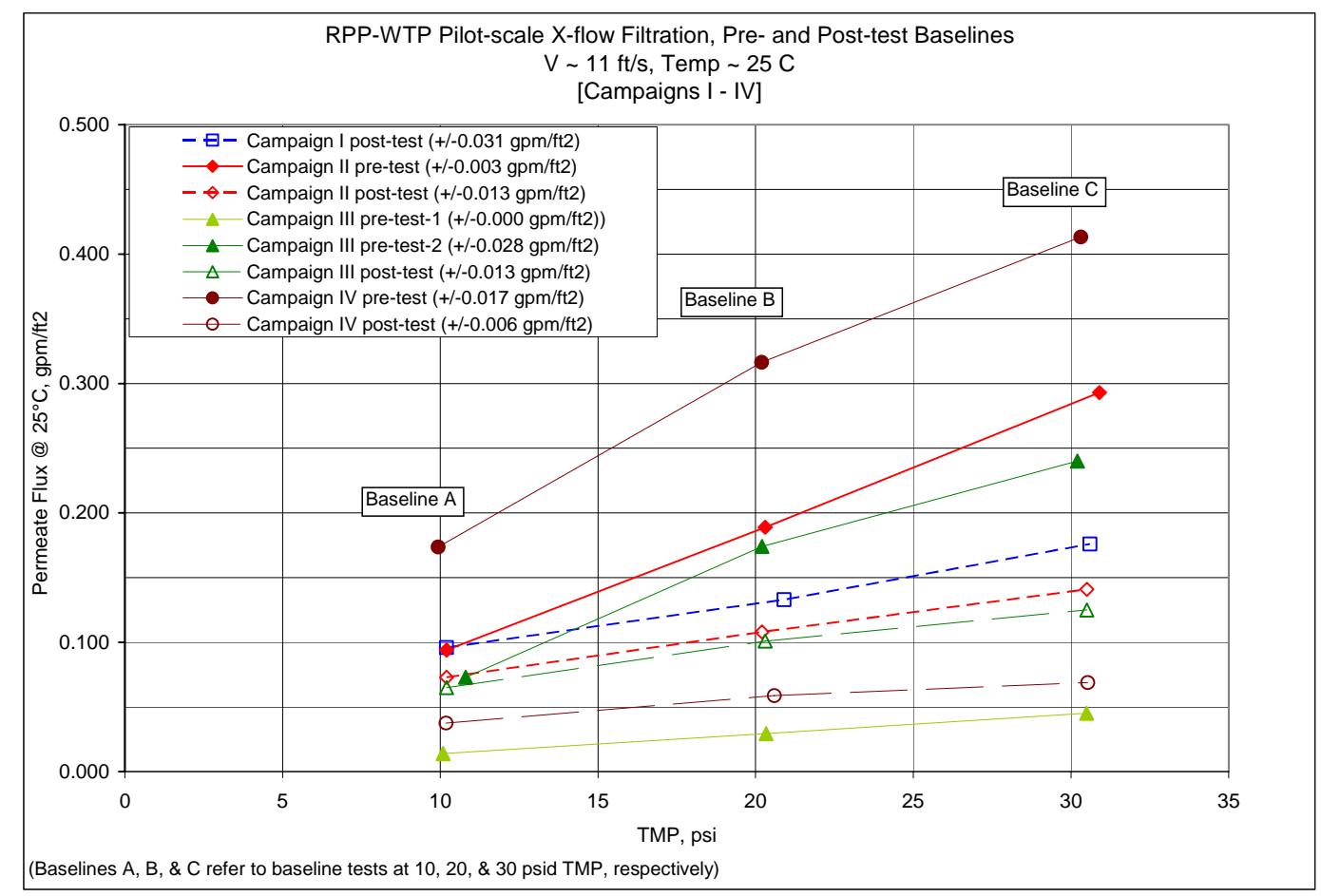

Figure 46. Comparison of Pre- and Post-Test Baselines, Campaign I - IV

\subsubsection{UFP Waste Products - Chemical Analysis (Author - Zamecnik)}

This section gives more in-depth chemical analyses for the results just presented in the preceding section, Section 2.7.4.1. In some cases the repetition is unavoidable, but it also facilitates better understanding of the many aspects of the UFP unit operation.

\subsection{Dewatering}

Table 34 shows the concentrations of the concentrated unwashed slurry for Campaigns I, II, and III (the Campaign III composition is calculated) and permeate for Campaigns I, II, III, and IV. Because of the extensive analyses done for Campaign II and that the dewater filter performance were the same in both Campaigns II and III, no slurry concentrations were done for Campaign IV to minimize cost. Note that one of the most significant differences between Campaigns II, III, and IV is the permeate Al concentrations. This also held true for the washed slurry; therefore a discussion of the aluminum concentrations will be given in that section. The Campaign I data differ significantly from the other campaigns because Campaign I did not include the AP-101 simulant, whereas the others did. The Campaign III calculated values are also likely to have more uncertainty since they were calculated from the material balance. 
WSRC-TR-2005-00105, REVISION 0 SRNL-RPP-2005-00012, REVISION 0

Table 34. Concentrated Total Slurry and Permeate Compositions

\begin{tabular}{|c|c|c|c|c|c|c|c|}
\hline \multirow[b]{2}{*}{ Campaign: } & \multicolumn{3}{|c|}{-------------Slurry------------- } & \multicolumn{4}{|c|}{----------Permeate from UF------------ } \\
\hline & I & II & III* & I & II & III & IV \\
\hline Total Solids (wt\%) & 36.91 & 45.37 & 46.27 & 17.09 & 28.15 & 28.27 & 28.24 \\
\hline Undissolved Solids (wt\%) & 23.90 & 23.51 & 24.90 & 0 & 0 & 0 & 0.00 \\
\hline Supernatant Solids (wt\%) & 17.09 & 28.58 & 28.46 & 17.09 & 28.15 & 28.27 & 28.24 \\
\hline Density $(\mathrm{kg} / \mathrm{L})$ & 1.357 & 1.470 & 1.448 & 1.168 & 1.243 & 1.248 & 1.248 \\
\hline (All values $\mathrm{mg} / \mathrm{kg} \quad \mathrm{Ag}$ & 1037 & 990 & 1070 & $<0.51$ & $<0.48$ & 0.761 & 0.686 \\
\hline unless otherwise noted) $\mathrm{Al}$ & 19117 & 17550 & 22158 & 7252 & 5670 & 5136 & 5192 \\
\hline B & 188 & $<151$ & & $<0.10$ & 70.1 & 85.7 & 89.7 \\
\hline $\mathrm{Ba}$ & 477 & 444 & 409 & $<0.03$ & $<0.03$ & $<1.3$ & $<0.018$ \\
\hline $\mathrm{Ca}$ & 2280 & 1905 & 2046 & 1.73 & $<0.05$ & $<3.6$ & 1.25 \\
\hline $\mathrm{Cd}$ & 63.5 & 70.6 & 71.6 & $<0.03$ & $<0.03$ & $<0.04$ & $<0.016$ \\
\hline $\mathrm{Ce}$ & 516 & 551 & 513 & $<1.2$ & $<1.1$ & $<1.0$ & $<0.69$ \\
\hline Co & 59.0 & $<15$ & 212 & $<0.14$ & $<0.13$ & $<0.11$ & $<0.034$ \\
\hline $\mathrm{Cr}$ & 845 & 772 & 799 & 128 & 132 & 139 & 138 \\
\hline $\mathrm{Cu}$ & 129 & 134 & 120 & $<0.05$ & 0.30 & $<0.03$ & 0.029 \\
\hline $\mathrm{Fe}$ & 58417 & 55750 & 53984 & 0.16 & 1.42 & 1.36 & 0.63 \\
\hline $\mathrm{K}$ & 330 & 13000 & 20885 & 297 & 17051 & 16346 & 15785 \\
\hline $\mathrm{La}$ & 407 & 478 & 477 & $<0.17$ & $<0.16$ & 0.849 & $<0.15$ \\
\hline $\mathrm{Li}$ & 0 & 90.9 & 91.8 & $<0.34$ & 0.93 & 1.72 & 0.79 \\
\hline $\mathrm{Mg}$ & 617 & 639 & 618 & $<0.01$ & $<0.01$ & $<0.24$ & $<0.010$ \\
\hline $\mathrm{Mn}$ & 12650 & 12300 & 11669 & $<0.02$ & 0.02 & $<0.02$ & $<0.014$ \\
\hline $\mathrm{Na}$ & 60800 & 89230 & 109462 & 60017 & 90483 & 88141 & 89744 \\
\hline $\mathrm{Nd}$ & 1288 & 1370 & 1267 & $<1.5$ & $<1.4$ & $<1.5$ & $<0.33$ \\
\hline $\mathrm{Ni}$ & 1750 & 1840 & 3135 & 0.58 & 0.303 & 0.247 & 0.25 \\
\hline $\mathrm{P}$ & 1263 & 672 & 103 & 1190 & 643 & 554 & 551 \\
\hline $\mathrm{Pb}$ & 2793 & 2625 & 2507 & $<1.4$ & 1.35 & $<0.36$ & $<1.36$ \\
\hline $\mathrm{S}$ & 680 & 862 & 1857 & 539 & 965 & 905 & 925 \\
\hline $\mathrm{Si}$ & 17983 & 15821 & 17999 & 17.0 & 43.6 & 42.1 & 48.1 \\
\hline $\mathrm{Sr}$ & 500 & 451 & 454 & 0.24 & 0.06 & 0.162 & 0.077 \\
\hline $\mathrm{Ti}$ & 22.0 & 10.9 & 66.4 & $<0.05$ & 0.07 & 0.10 & 0.065 \\
\hline $\mathrm{Zn}$ & 458 & 151 & 178 & $<0.03$ & 2.45 & 2.37 & 4.57 \\
\hline $\mathrm{Zr}$ & 797 & 745 & 722 & $<0.05$ & 0.69 & 1.43 & 0.31 \\
\hline bromide & 33.3 & $<29$ & 97.5 & 48.8 & $<8.0$ & $<80$ & $<80.1$ \\
\hline chloride & 98.0 & 738 & 45.9 & 163 & 973 & 1082 & 1178 \\
\hline fluoride & 39.0 & 52.0 & 18.4 & 38.5 & 23.3 & 44.9 & 55.3 \\
\hline formate & 168 & 700 & 467 & 299 & 981 & 1066 & 1090 \\
\hline nitrate & 224 & 49700 & 37079 & 336 & 68606 & 72917 & 108173 \\
\hline nitrite & 3107 & 14100 & 10559 & 3827 & 20268 & 23077 & 21955 \\
\hline oxalate & 14133 & 23800 & 18351 & 2748 & 600 & 658 & 897 \\
\hline phosphate & 2693 & 1070 & 846 & 2877 & 1987 & 1963 & 2027 \\
\hline sulfate & 1427 & 2080 & 209 & 2106 & 2727 & 2853 & 2732 \\
\hline total inorganic carbon (TIC) & 15133 & 3347 & 3456 & 12586 & 6233 & 6859 & \\
\hline total organic carbon (TOC) & 15300 & 5973 & 13734 & 2483 & 1609 & 801 & \\
\hline total carbon (TC) & 30433 & 9320 & 17190 & 15068 & 7842 & 7660 & \\
\hline carbonate from TIC & 75667 & 16735 & 17279 & 62928 & 31166 & 34295 & \\
\hline free hydroxide & & & & & & 19343 & \\
\hline
\end{tabular}

* Calculated by material balance; values for anions \& elements that are predominantly soluble are subject to more uncertainty than other species ; neither analyses nor material balance calculations for Campaign IV were performed. 


\subsection{Solids Concentrations during Dewatering}

The total, undissolved, and supernatant solids contents and the slurry densities during the concentration of the slurry for all four campaigns are summarized in Table 35 as a function of the amount of permeate removed. The supernatant solids content should be approximately the same for Campaigns II, III and IV. The actual mean values are the same within the analytical uncertainty of approximately $0.13 \mathrm{wt} \%$.

Table 35. Solids Content of Slurries during Ultrafiltration Dewatering

\begin{tabular}{|c|c|c|c|c|}
\hline $\begin{array}{c}\text { Permeate } \\
\text { Removed (L) }\end{array}$ & $\begin{array}{c}\text { Total Solids } \\
(\mathrm{wt} \%)\end{array}$ & $\begin{array}{l}\text { Undissolved } \\
\text { Solids (wt\%) }\end{array}$ & $\begin{array}{c}\text { Slurry Density } \\
(\mathrm{kg} / \mathrm{L})\end{array}$ & $\begin{array}{c}\text { Supernate Solids } \\
\text { (wt\%) }\end{array}$ \\
\hline \multicolumn{5}{|l|}{ Campaign IV } \\
\hline 0 & 29.87 & 2.60 & 1.268 & 28.00 \\
\hline 158 & 33.35 & 6.91 & & \multirow{5}{*}{$\begin{array}{l}\text { assumed to } \\
\text { be } 28.40\end{array}$} \\
\hline 313 & 36.16 & 10.83 & & \\
\hline 470 & 38.71 & 14.39 & & \\
\hline 628 & 40.72 & 17.20 & & \\
\hline 785 & 43.37 & 20.90 & & \\
\hline 915 & 45.42 & 23.60 & 1.448 & 28.56 \\
\hline Diluted & 41.96 & 18.96 & 1.409 & 28.39 \\
\hline \multicolumn{5}{|l|}{ Campaign III } \\
\hline 0 & 30.54 & 3.23 & 1.273 & 28.22 \\
\hline 158 & 33.54 & 7.51 & 1.270 & 28.14 \\
\hline 316 & 36.39 & 11.31 & 1.345 & 28.28 \\
\hline 474 & 38.95 & 14.79 & 1.377 & 28.35 \\
\hline 632 & 41.51 & 18.52 & 1.408 & 28.22 \\
\hline 790 & 43.44 & 21.02 & 1.366 & 28.39 \\
\hline 915 & 46.03 & 24.59 & 1.457 & 28.44 \\
\hline Diluted & 42.71 & 20.37 & 1.423 & 28.06 \\
\hline \multicolumn{5}{|l|}{ Campaign II } \\
\hline 0 & 30.59 & 3.22 & 1.276 & 28.28 \\
\hline 144 & 33.30 & 7.00 & & 28.27 \\
\hline 287 & 35.83 & 11.21 & 1.320 & 27.73 \\
\hline 463 & 38.70 & 14.89 & 1.360 & 27.98 \\
\hline 617 & 41.99 & 19.53 & 1.390 & 27.91 \\
\hline 771 & 44.00 & 22.38 & & 27.86 \\
\hline 885 & 45.80 & 23.51 & 1.470 & 29.14 \\
\hline Diluted & 42.53 & 20.80 & 1.410 & 27.44 \\
\hline \multicolumn{5}{|l|}{ Campaign I } \\
\hline 0 & 27.35 & 12.53 & 1.269 & 16.94 \\
\hline 27 & 29.21 & 14.63 & 1.279 & 17.08 \\
\hline 53 & 30.98 & 16.80 & 1.302 & 17.04 \\
\hline 80 & 32.92 & 19.06 & 1.326 & 17.12 \\
\hline 107 & 34.72 & 21.26 & 1.333 & 17.09 \\
\hline 133 & 36.30 & 23.12 & 1.367 & 17.14 \\
\hline 160 & 36.88 & 23.71 & 1.358 & 17.26 \\
\hline Diluted & 33.19 & 19.63 & 1.329 & 16.87 \\
\hline
\end{tabular}


The UDS measurements for each campaign are plotted in Fig. 47 versus permeate removed normalized on the final permeate volume. For Campaigns II, III, and IV, the TS, UDS, and densities are similar, as expected; the values for Campaign I are different because the feed slurry was limited to only AY102.

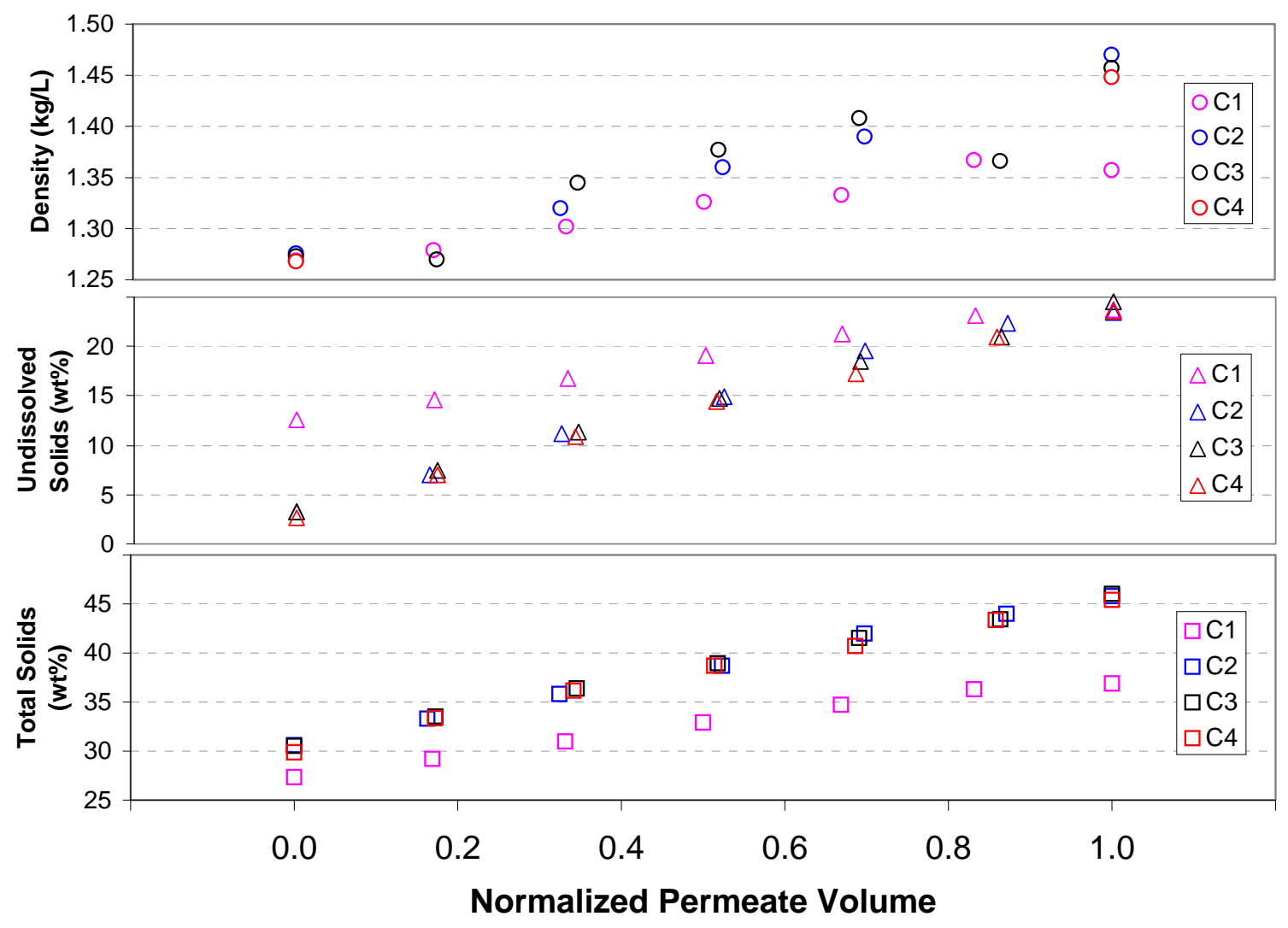

Figure 47. Slurry Solids and Density versus Normalized Permeate Volume

For Campaign IV, upon completion of concentrating the slurry, the final undissolved solids (UDS) content was 23.6 versus the target of $20 \mathrm{wt}^{\dagger}{ }^{\dagger}$. Because the final concentrated slurry was at greater than $20 \mathrm{wt} \%$ UDS, the batch of $\sim 117 \mathrm{~L}$ of slurry was diluted by first removing a calculated amount of slurry and then adding back permeate, as was done in the previous campaigns. The slurry was actually diluted back to about $19.0 \mathrm{wt} \%$ UDS.

\footnotetext{
${ }^{\dagger}$ As mentioned the Abstract, and discussed in detail in the Campaign II report (Duignan et al., 2004b), the OLI/ESP model was slightly off in predicting target values because of the difficulties in handling the complex chemical combinations of the simulated wastes. To produce the target amount of permeate during dewatering [see Table 1, Row "UF1 Filtrate (routed to CIX, than TLP)] all of the UFP feed had to be processed, which resulted in the solids content of the UFP concentrate to be $24.6 \mathrm{wt} \%$ UDS. However, the Test Specification (Longwell, 2003) required washing to be done on a UFP product at $20 \mathrm{wt} \%$ UDS and therefore, the concentrate was diluted to that value.
} 
WSRC-TR-2005-00105, REVISION 0

SRNL-RPP-2005-00012, REVISION 0

\subsection{Steady-State Tests with Elevated Temperature - Effects on Slurry}

\subsection{Solids Concentration during Elevated Temperature Tests}

The test matrix, Table 22, shows that two sets of tests were done at steady-state solids loadings: One set at $24 \mathrm{wt} \%$ UDS and $25^{\circ} \mathrm{C}$ and another set at $20 \mathrm{wt} \%$ UDS with temperatures from $25^{\circ} \mathrm{C}$ to $45^{\circ} \mathrm{C}$. The first set, done at $24 \mathrm{wt} \%$ UDS, was performed to compare to Campaign I test results, as was discussed in Section 2.7.4.1.3. The second set was done after the slurry was prepared for the washing tests by diluting the concentrated simulant from 24 to $20 \mathrm{wt} \%$ UDS (the $20 \mathrm{wt} \%$ was estimated during the tests from previous solids measurements, but the actual the measured concentration turned out to be $19 \mathrm{wt} \%$ UDS). Therefore, just before the washing tests began the second set of tests at steady-state solids loading was done at $19 \mathrm{wt} \%$ UDS. These higher-temperature filter flux results were discussed in Section 2.7.4.1.4 and shown in Fig. 32. Here, the results of the analytical measurements are discussed.

Table 36 relates the slurry sample number to each of the steady-state solids loading tests done at $19 \mathrm{wt} \%$ UDS. Along with the samples names (to relate to the overall sample test matrix in Appendix D), the table lists the temperature, linear velocity, and relation test matrix (Table 22). The measurements made include the physical properties total solids, undissolved solids, supernatant solids, particle size distribution, and rheology in order to to determine if the temperature had any effect on these properties.

Table 36. Simulant Samples Taken During Temperature Tests

\begin{tabular}{|c|c|c|c|}
\hline $\begin{array}{c}\text { Sample Name: } \\
\text { Slurry }\end{array}$ & $\begin{array}{c}\text { Temperature } \\
\left({ }^{\circ} \mathrm{C}\right)\end{array}$ & $\begin{array}{c}\text { Linear } \\
\text { Velocity (fps) }\end{array}$ & Comment \\
\hline CON2-ADJ & 25.0 & 12 & slurry properties before test \\
\hline T-30-SL & 30.0 & 12 & Temp1 from Table 22 \\
\hline T-37.5-SL & 37.5 & 12 & Temp2 from Table 22 \\
\hline $\mathrm{T}-45-\mathrm{SL}$ & 45.0 & 12 & Temp3 from Table 22 \\
\hline T-35-SL & 35.0 & 12 & Temp4 from Table 22 \\
\hline T-25-SL & 25.0 & 12 & Temp5 from Table 22 \\
\hline T-37.5(15)-SL & 37.5 & 15 & Temp6 from Table 22 \\
\hline T-45(15)-SL & 45.0 & 15 & Temp7 from Table 22 \\
\hline \multicolumn{4}{|l|}{ Permeate } \\
\hline CIX-FEED & 25.0 & 12 & permeate properties before test \\
\hline $\mathrm{T}-30-\mathrm{PE}$ & 30.0 & 12 & Temp1 from Table 22 \\
\hline $\mathrm{T}-37.5-\mathrm{PE}$ & 37.5 & 12 & Temp2 from Table 22 \\
\hline $\mathrm{T}-45-\mathrm{PE}$ & 45.0 & 12 & Temp3 from Table 22 \\
\hline T-35-PE & 35.0 & 12 & Temp4 from Table 22 \\
\hline T-25-PE & 25.0 & 12 & Temp5 from Table 22 \\
\hline T-37.5(15)-PE & 37.5 & 15 & Temp6 from Table 22 \\
\hline T-45(15)-PE & 45.0 & 15 & Temp7 from Table 22 \\
\hline
\end{tabular}


Table 37 shows the solids contents measured from samples taken during the tests. The variation in the solids contents measured is within the normal uncertainty of the measurements, so there was no apparent change in the solids contents due to the temperature tests. Note that the UDS and supernatant solids contents were measured on samples that were filtered at room temperature. Therefore, changes in the solids distribution between dissolved and undissolved that may be present at the test temperatures might not be seen when the samples are all cooled to room temperature.

Table 37. Solids Content of Samples from Temperature Tests

\begin{tabular}{|c|c|c|c|c|c|c|c|c|c|}
\hline \multirow{2}{*}{$\begin{array}{c}\text { Sample* } \\
\text { (C4-UF-...-ACTL) } \\
\text { CON2-ADJ }\end{array}$} & \multicolumn{2}{|c|}{$\begin{array}{l}\text { Supernatant } \\
\text { Solids (wt\%) }\end{array}$} & \multicolumn{2}{|c|}{$\begin{array}{c}\text { Total Solids } \\
\text { (wt\%) }\end{array}$} & \multicolumn{2}{|c|}{$\begin{array}{c}\text { Undissolved } \\
\text { Solids (wt\%) } \\
\end{array}$} & \multirow{2}{*}{$\begin{array}{c}\text { Mean } \\
\text { Supernatant } \\
\text { Solids (wt \%) }\end{array}$} & \multirow{2}{*}{$\begin{array}{c}\begin{array}{c}\text { Mean Total } \\
\text { Solids } \\
\text { (wt\%) }\end{array} \\
41.96\end{array}$} & \multirow{2}{*}{$\begin{array}{c}\begin{array}{c}\text { Mean } \\
\text { Undissolved } \\
\text { Solids (wt\%) }\end{array} \\
18.96\end{array}$} \\
\hline & 28.29 & 28.48 & 41.99 & 41.93 & 19.10 & 18.81 & & & \\
\hline T-25-SL & 28.43 & 28.07 & 42.01 & 42.08 & 18.97 & 19.48 & 28.25 & 42.05 & 19.23 \\
\hline T-30-SL & 28.52 & 28.39 & 41.88 & 41.97 & 18.69 & 18.96 & 28.46 & 41.93 & 18.83 \\
\hline T-35-SL & 28.30 & 28.22 & 41.98 & 42.15 & 19.08 & 19.41 & 28.26 & 42.07 & 19.24 \\
\hline T-37.5-SL & 28.52 & 28.33 & 41.92 & 42.01 & 18.75 & 19.09 & 28.43 & 41.97 & 18.92 \\
\hline T-37.5(15)-SL & 28.77 & 27.58 & 42.00 & 42.07 & 18.57 & 20.01 & 28.18 & 42.04 & 19.30 \\
\hline T-45-SL & 28.72 & 28.03 & 42.14 & 42.04 & 18.83 & 19.47 & 28.38 & 42.09 & 19.15 \\
\hline \multirow{2}{*}{ T-45(15)-SL } & 28.99 & 28.15 & 42.31 & 42.11 & 18.76 & 19.43 & 28.57 & 42.21 & 19.10 \\
\hline & & & & & & Mean: & 28.36 & 42.04 & 19.09 \\
\hline CIX-FEED & 28.27 & 28.24 & & & & & 28.26 & & \\
\hline T-25-PE & 28.53 & 28.55 & & & & & 28.54 & & \\
\hline T-30-PE & 28.42 & 28.54 & & & & & 28.48 & & \\
\hline T-35-PE & 28.54 & 28.55 & & & & & 28.55 & & \\
\hline T-37.5-PE & 28.38 & 28.51 & & & & & 28.45 & & \\
\hline $\mathrm{T}-37.5(15)-\mathrm{PE}$ & 28.51 & 28.42 & & & & & 28.47 & & \\
\hline T-45-PE & 28.55 & 28.42 & & & & & 28.49 & & \\
\hline \multirow[t]{2}{*}{ T-45(15)-PE } & 28.53 & 28.52 & & & & & 28.53 & & \\
\hline & & & & & & Mean: & 28.47 & & \\
\hline
\end{tabular}

These results do show that there was no permanent change in the solids ratios. Figures 48, 49 , and 50 show graphically the solids concentrations versus test number. All of the values measured are for the most part randomly distributed about the mean. The total solids do appear to increase slightly during the test, which may be due to a small amount of evaporation, but the increase is within the uncertainty of the measurements. 
WSRC-TR-2005-00105, REVISION 0

SRNL-RPP-2005-00012, REVISION 0

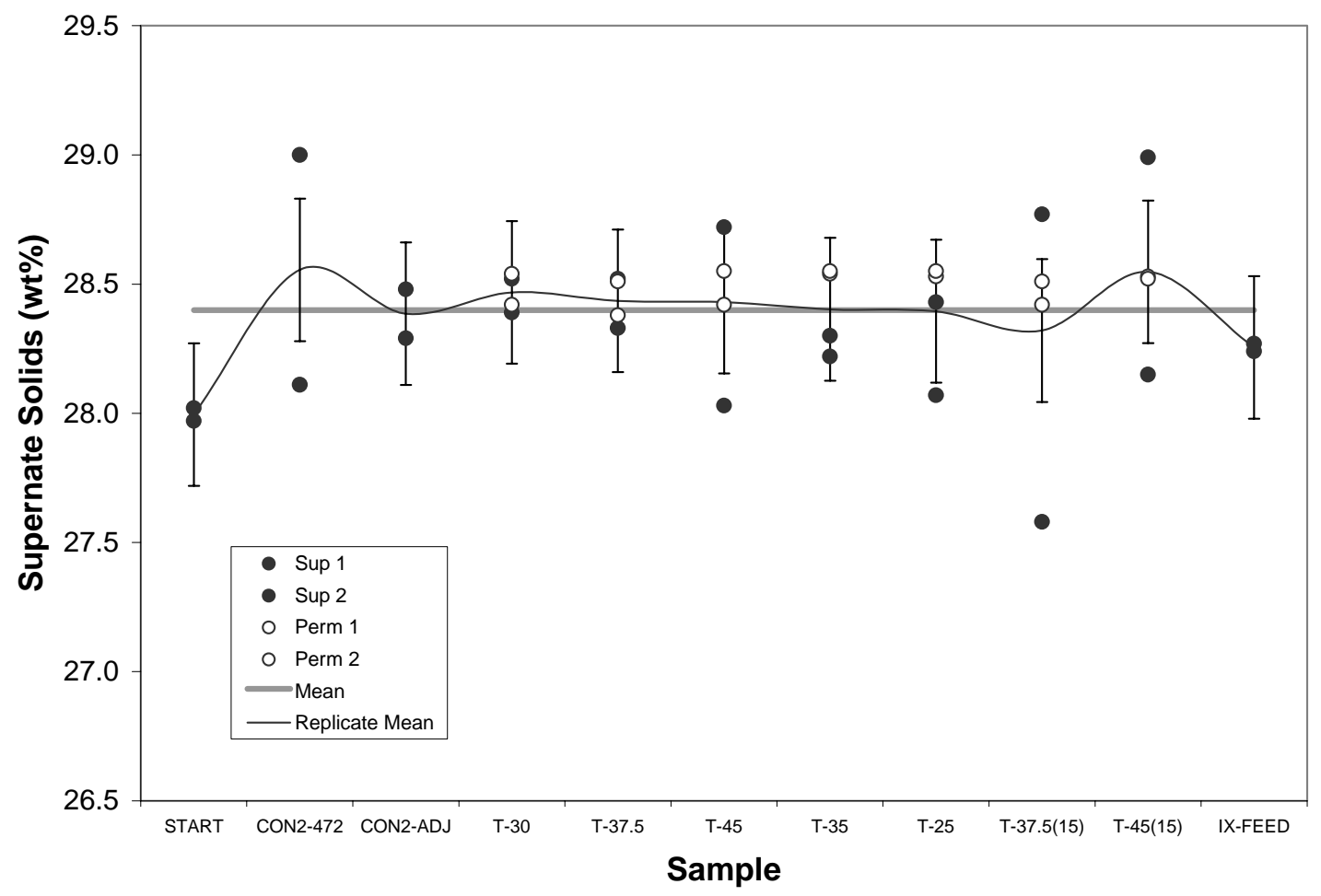

Figure 48. Supernatant Solids vs. Sample for Temperature Tests

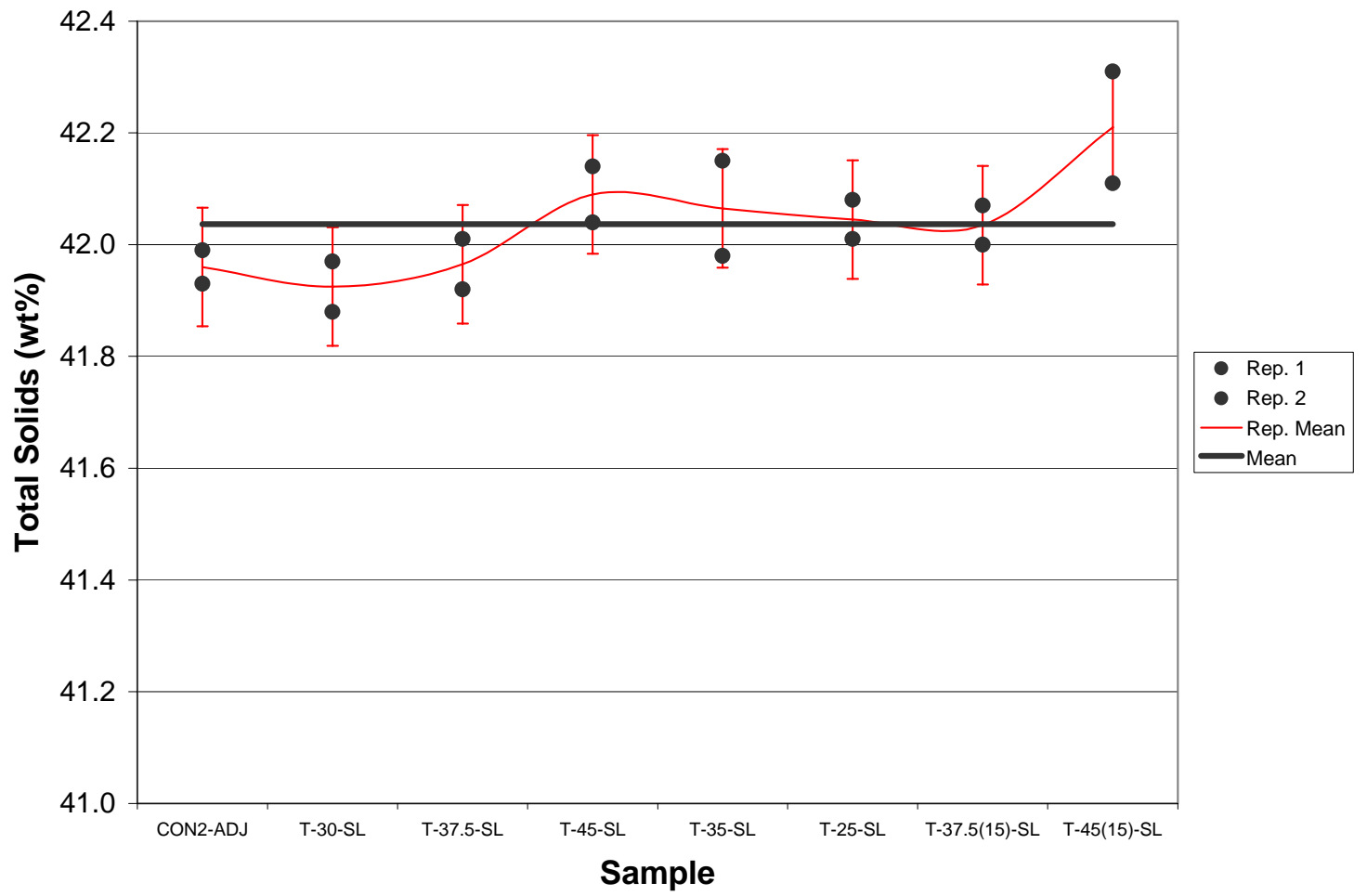

Figure 49. Total Solids vs. Sample for Temperature Tests 
WSRC-TR-2005-00105, REVISION 0

SRNL-RPP-2005-00012, REVISION 0

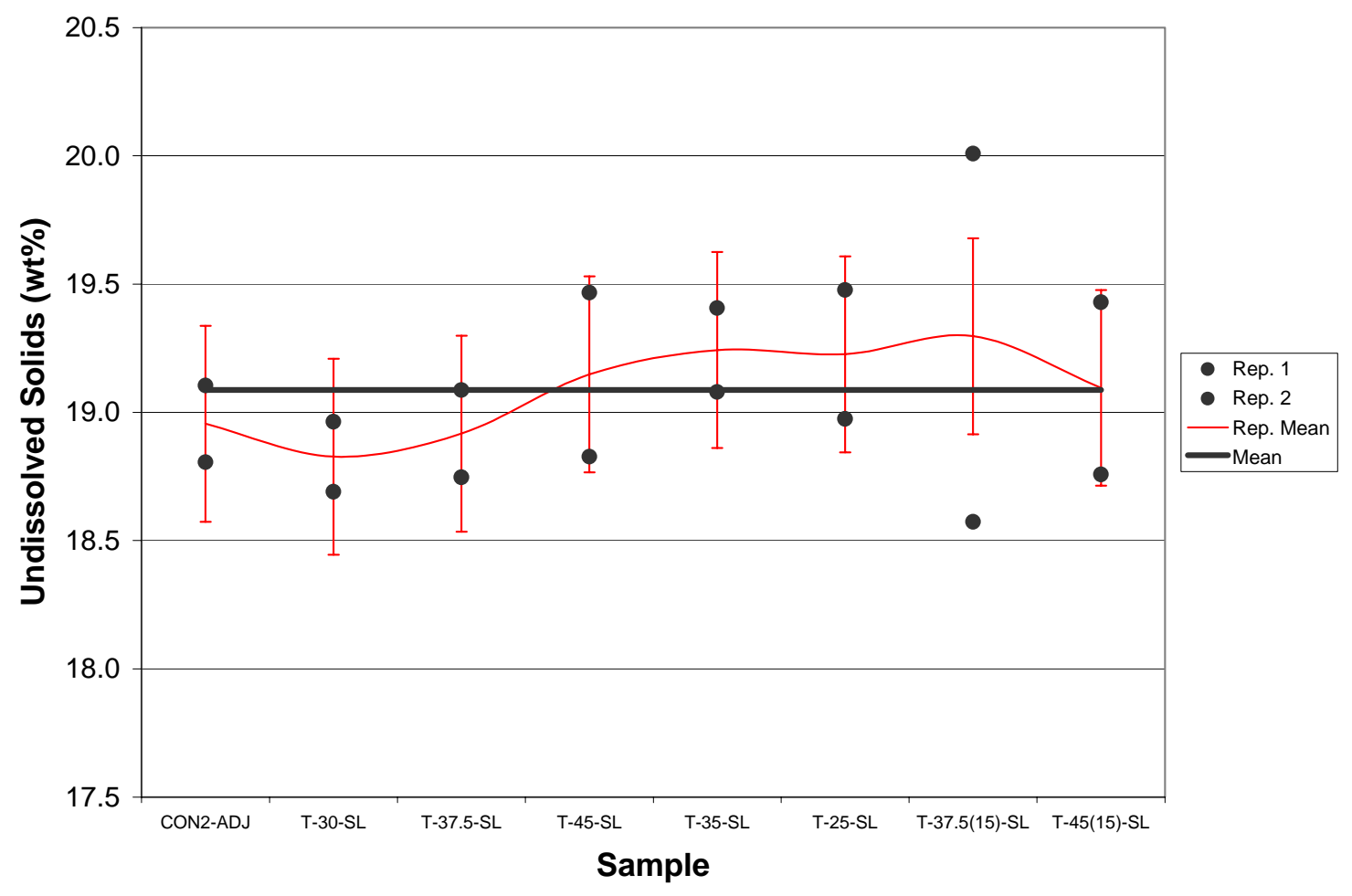

Figure 50. Undissolved Solids vs. Sample for Temperature Tests

\subsection{Particle Size during Elevated Temperature Tests}

The particle size distribution (PSD) data for each of the samples is plotted in Fig. 51. These data show that no significant change in the PSD occurred during the tests. The initial PSD showed some bimodal character that disappeared during the tests, but the differences were small. As with the solids measurements, the PSD at the actual test temperature could have been different, but was found to be the same when measured at room temperature. The raw data sheets can be found in Appendix E. 
WSRC-TR-2005-00105, REVISION 0

SRNL-RPP-2005-00012, REVISION 0

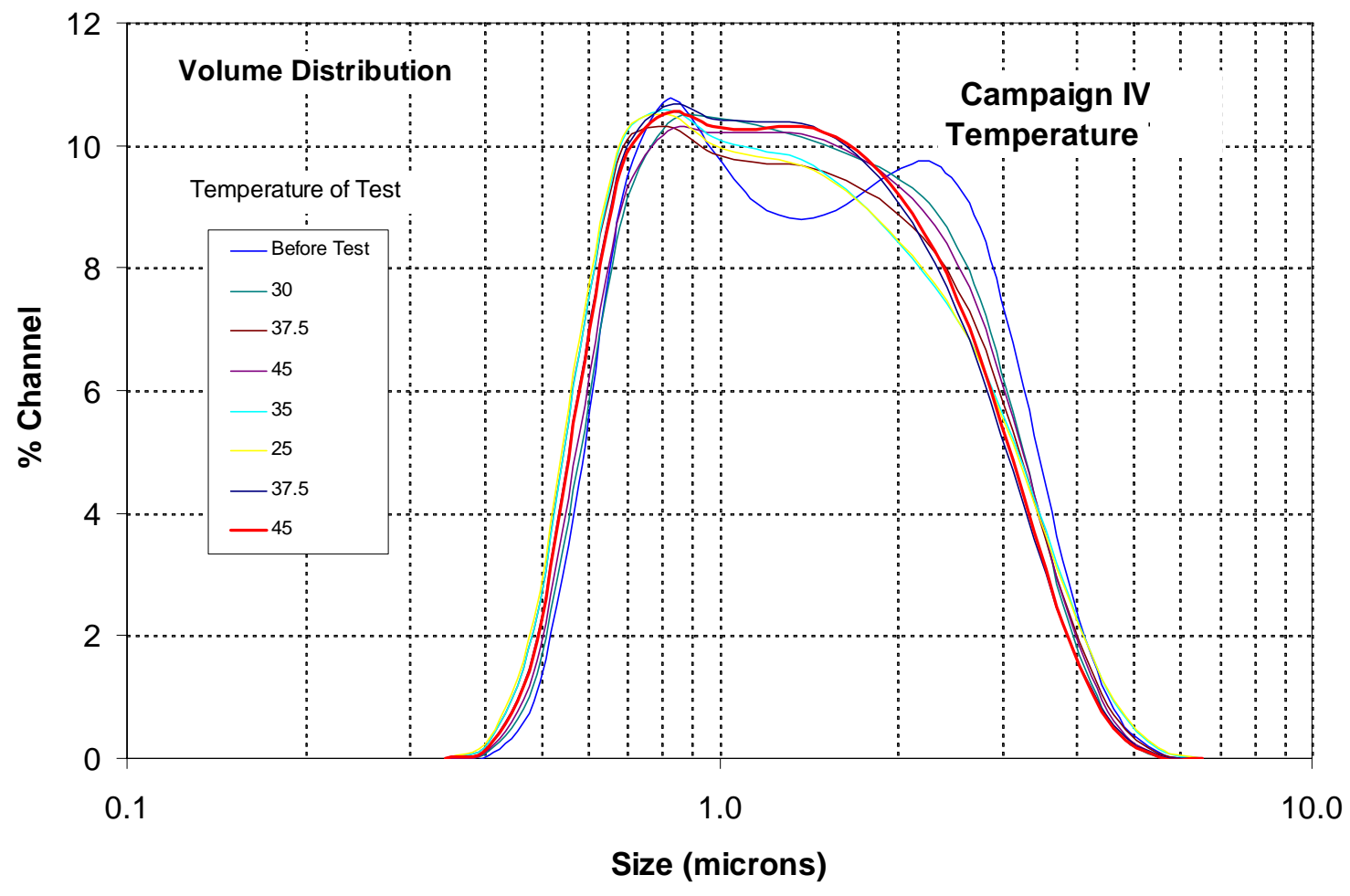

Figure 51. Volume Particle Size Distribution During Temperature Tests

\subsection{Rheology during Elevated Temperature Tests}

The rheological properties of consistency and yield stress for the slurry samples and viscosity for the permeate samples were measured at both room temperature and the test temperatures. These results are shown in Tables 38 and 39 and Figs. 52 and 53. The results show that there was no measurable change in the either the yield stress or consistency of the slurry samples at $25^{\circ} \mathrm{C}$; the same applies to the viscosity of the permeate at $25^{\circ} \mathrm{C}$. The consistency of the slurry and the viscosity of the permeate both decreased linearly with temperature. The yield stress of the slurry was found to increase linearly with temperature. 
WSRC-TR-2005-00105, REVISION 0 SRNL-RPP-2005-00012, REVISION 0

Table 38. Yield Stress and Consistency of Slurry From Temperature Tests (see Table 34 for key to Sample Names)

\begin{tabular}{|c|c|c|c|c|c|c|c|c|c|c|c|c|c|c|c|c|c|c|c|c|c|}
\hline \multirow{3}{*}{$\frac{\text { Sample Name }}{\text { (C4-UF-...-ACTL) }}$} & \multirow{2}{*}{\multicolumn{4}{|c|}{$\begin{array}{c}\text { Measured @ } 25^{\circ} \mathrm{C} \\
\text { Yield Stress }(\mathrm{Pa}) \\
\end{array}$}} & \multirow{2}{*}{\multicolumn{4}{|c|}{$\begin{array}{c}\text { Measured @ } 25^{\circ} \mathrm{C} \\
\text { Consistency (mPa•s) }\end{array}$}} & \multirow{2}{*}{\multicolumn{5}{|c|}{$\begin{array}{c}\text { Measured@ Temp } \\
\text { Yield Stress (Pa) }\end{array}$}} & \multirow{2}{*}{\multicolumn{4}{|c|}{$\begin{array}{c}\text { Measured @ Temp } \\
\text { Consistency (mPa•s) }\end{array}$}} & \multicolumn{2}{|c|}{$@ 25^{\circ} \mathrm{C}$} & \multicolumn{2}{|c|}{ @Temp } \\
\hline & & & & & & & & & & & & & & & & & & YS & Cons & YS & Cons \\
\hline & Up 1 & Dn 1 & Up 2 & Dn 2 & Up 1 & Dn 1 & Up 2 & Dn 2 & Temp & Up 1 & Dn 1 & Up 2 & Dn 2 & Up 1 & Dn 1 & Up 2 & Dn 2 & Mean & Mean & Mean & Mean \\
\hline CON2-ADJ & 8.65 & 8.18 & 8.75 & 8.50 & 14.35 & 14.50 & 14.00 & 14.01 & & & & & & & & & & 8.52 & 14.22 & & \\
\hline T-25-SL & $\mid 10.45$ & 8.90 & 10.05 & 8.91 & 13.69 & 14.81 & 13.95 & 14.68 & & & & & & & & & & 9.58 & 14.28 & & \\
\hline T-30-SL & $\mid 10.10$ & 8.84 & 9.81 & 8.81 & 14.13 & 14.87 & 14.55 & 15.06 & 30.0 & 9.84 & 9.40 & 9.84 & 9.40 & 13.95 & 14.10 & 13.55 & 13.70 & 9.39 & 14.65 & 9.62 & 13.83 \\
\hline $\mathrm{T}-35-\mathrm{SL}$ & 10.57 & 8.91 & 10.00 & 8.87 & 13.51 & 14.85 & 13.99 & 14.75 & 35.0 & 10.63 & 9.96 & 10.24 & 10.02 & $\mid 12.63$ & 13.08 & 13.22 & 13.21 & 9.59 & 14.28 & 10.21 & 13.04 \\
\hline T-37.5-SL & $\mid 10.54$ & 8.69 & 10.14 & 8.67 & 13.25 & 14.75 & 13.71 & 14.79 & 37.5 & 11.30 & 10.22 & 11.15 & 10.26 & $\mid 11.57$ & 12.25 & 11.78 & 12.21 & 9.51 & 14.13 & 10.73 & 11.95 \\
\hline T-37.5(15)-SL & 10.60 & 9.00 & 10.01 & 9.15 & 13.95 & 15.08 & 14.76 & 15.25 & 37.5 & 11.30 & 10.22 & 11.15 & 10.26 & $\mid 11.57$ & 12.25 & 11.78 & 12.21 & 9.69 & 14.76 & 10.73 & 11.95 \\
\hline T-45-SL & 9.85 & 9.14 & 9.43 & 9.04 & 14.48 & 14.79 & 14.83 & 14.87 & 45.0 & 12.04 & 11.24 & 12.03 & 11.45 & $\mid 10.60$ & 11.30 & 10.83 & 11.33 & 9.37 & 14.74 & 11.69 & 11.02 \\
\hline T-45(15)-SL & 10.19 & 9.17 & 9.85 & 9.30 & 13.99 & 14.67 & 14.78 & 14.98 & 45.0 & 12.42 & 11.50 & 12.53 & 11.71 & 10.64 & 11.35 & 10.66 & 11.38 & 9.63 & 14.61 & 12.04 & 11.01 \\
\hline
\end{tabular}

Total Solids: $42.04 \mathrm{wt} \%$; UDS: $19.09 \mathrm{wt} \%$

Table 39. Consistency (mP•s) of Permeate From Temperature Tests (see Table 34 for key to Sample Names)

\begin{tabular}{|c|c|c|c|c|c|c|c|}
\hline \multirow{2}{*}{$\begin{array}{l}\text { Sample Name } \\
\text { (C4-UF-...-ACTL) }\end{array}$} & \multicolumn{2}{|c|}{$\begin{array}{l}\text { Measured } \\
@ 25^{\circ} \mathrm{C}\end{array}$} & \multicolumn{3}{|c|}{$\begin{array}{l}\text { Measured } \\
\text { @Temp }\end{array}$} & \multirow{2}{*}{$\begin{array}{r}@ 25^{\circ} \mathrm{C} \\
\text { Mean }\end{array}$} & \multirow{2}{*}{$\frac{\text { @Temp }}{\text { Mean }}$} \\
\hline & Up 1 & Dn 1 & Temp & Up 1 & Dn 1 & & \\
\hline CON2-ADJ & 2.86 & 2.84 & & & & 2.86 & \\
\hline T-25-SL & 2.88 & 2.92 & & & & 2.90 & \\
\hline T-30-SL & 2.88 & 2.91 & 30.0 & 2.55 & 2.57 & 2.90 & 2.56 \\
\hline T-35-SL & 2.85 & 2.86 & 35.0 & 2.25 & 2.28 & 2.86 & 2.27 \\
\hline T-37.5-SL & 2.85 & 2.86 & 37.5 & 2.08 & 2.19 & 2.86 & 2.14 \\
\hline T-37.5(15)-SL & 2.85 & 2.89 & 37.5 & 2.17 & 2.11 & 2.87 & 2.14 \\
\hline T-45-SL & 2.85 & 2.83 & 45.0 & 1.82 & 1.78 & 2.84 & 1.80 \\
\hline T-45(15)-SL & 2.91 & 2.90 & 45.0 & 1.81 & 1.75 & 2.91 & 1.78 \\
\hline
\end{tabular}

Total Solids: $28.40 \mathrm{wt} \%$ 
WSRC-TR-2005-00105, REVISION 0

SRNL-RPP-2005-00012, REVISION 0

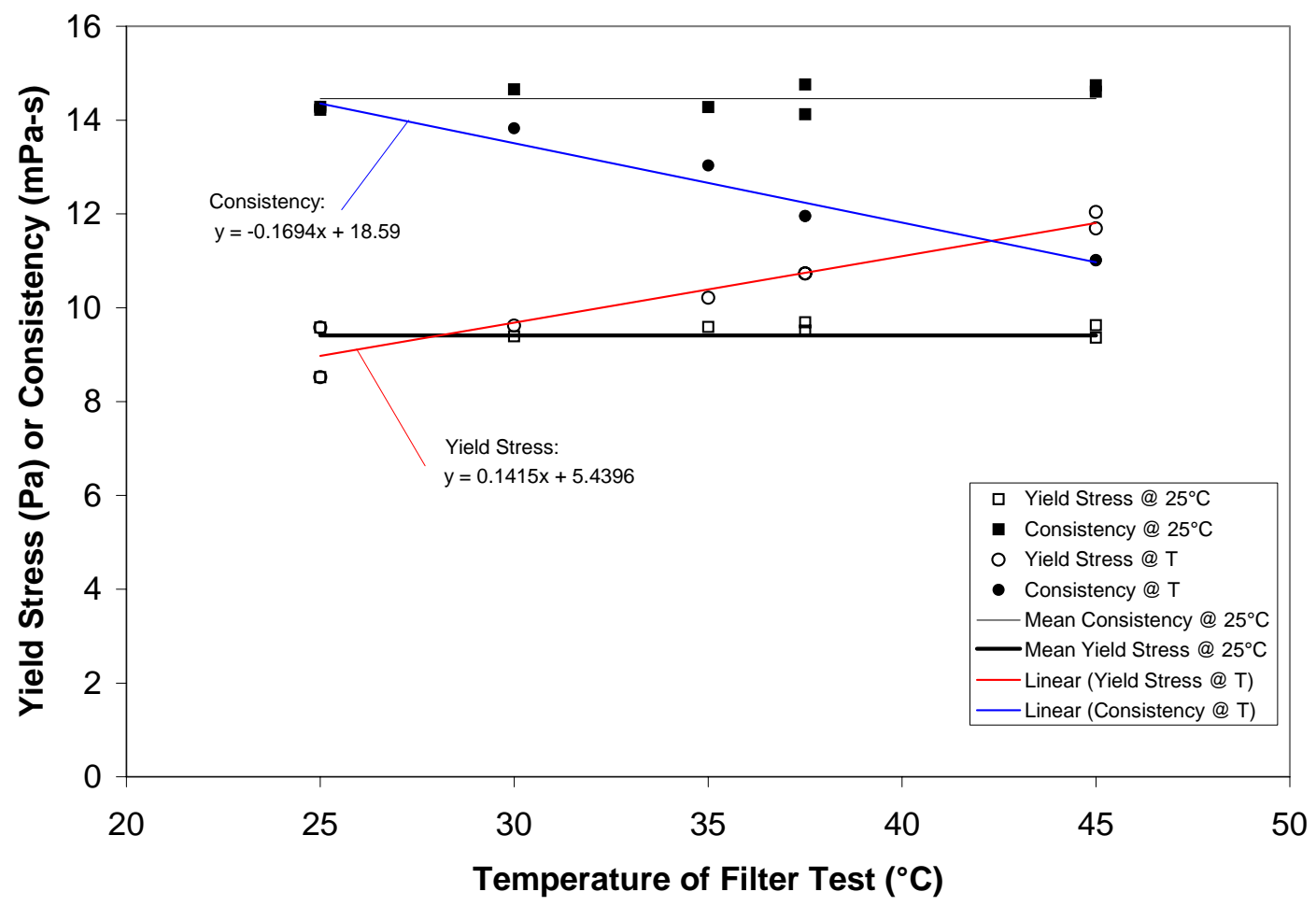

Figure 52. Yield Stress and Consistency of Slurry Samples

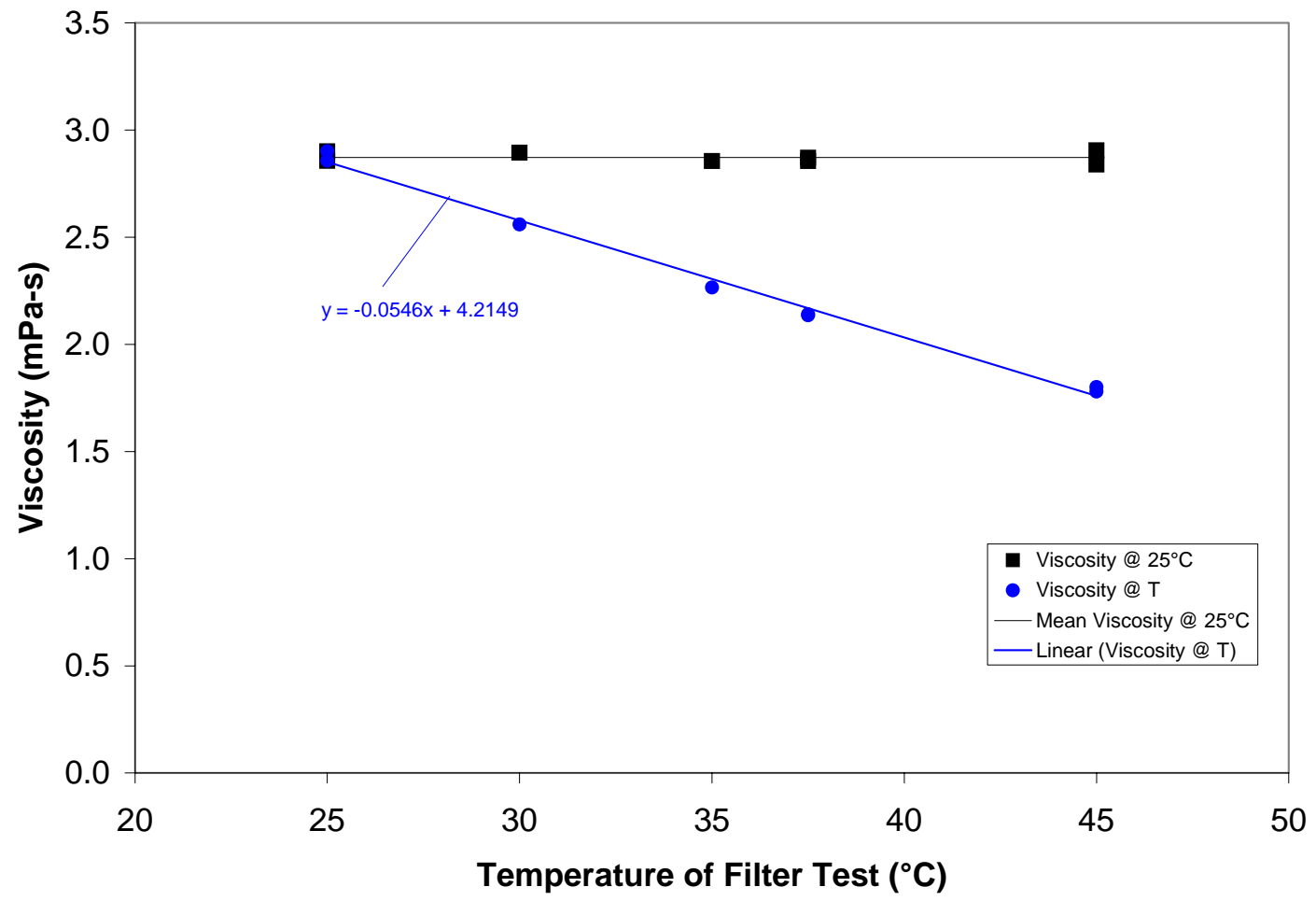

Figure 53. Viscosity of Permeate Samples 


\subsection{Washing the Concentrated Slurry}

After the dilution, the slurry was washed with 22 discrete additions of $~ 5.35 \mathrm{~L}$ of approximately $0.01 \mathrm{M} \mathrm{NaOH}$, followed by an additional 22 similar washes. The target total amount of wash water used was about 234.7L. Leaching of the solids with $\mathrm{NaOH}$ was not performed per WTP instructions. Table 39 summarizes the composition of the washed slurry after all 44 washes for all four campaigns. Table 41 shows limited characterization of the permeate composites from the first and second 22 washes and compares these same analyses to Campaigns I-III values. The Campaign II and III values for solids and density are similar, as expected. The total base values are different because the recycle compositions (FEP concentrate) to Campaign II and III were different.

The compositions of the Campaigns II-IV washed total slurries measured by the microwave acid (MW) and sodium peroxide fusion $\mathrm{HCl}$ uptake (SPF) dissolutions are shown in Table 40. These values were expected to be essentially the same since the starting slurries were expected to have similar compositions. The majority of the undissolved components for these campaigns came from the AY102 simulant, with a smaller (and comparable amount) from the FEP evaporator Bottoms. A trace amount of solids also came from the AP101 simulant for both campaigns. Examination of the data in Table 40 shows that the compositions are indeed similar, with the exception of aluminum, which was higher than expected in Campaign III. The anions in Campaign IV were comparable to Campaign II, with both being higher than Campaign III. 
WSRC-TR-2005-00105, REVISION 0

SRNL-RPP-2005-00012, REVISION 0

Table 40. Comparison of Campaigns I to IV Washed Slurry Compositions

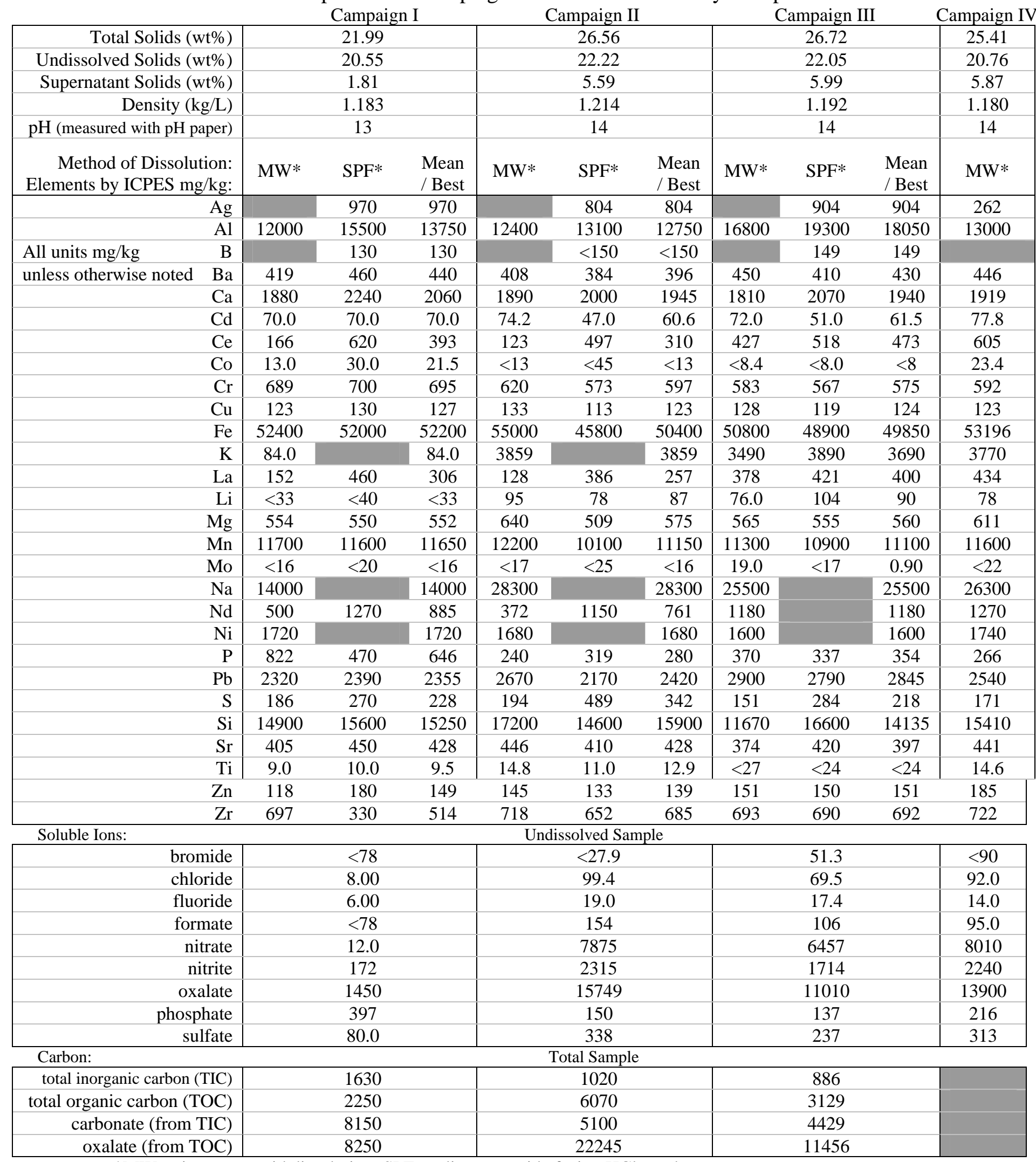

*MW: microwave acid dissolution; SPF: sodium peroxide fusion $\mathrm{HCl}$ uptake 
WSRC-TR-2005-00105, REVISION 0

SRNL-RPP-2005-00012, REVISION 0

Table 41. Comparison of Wash Permeate Composites from Campaigns I-IV

\begin{tabular}{|rccccccccc|}
\multicolumn{1}{c}{} & \multicolumn{2}{c}{ Campaign IV } & \multicolumn{2}{c}{ Campaign III } & \multicolumn{2}{c}{ Campaign II } & \multicolumn{2}{c}{ Campaign I } \\
& $1^{\text {st }} 22$ & $2^{\text {nd }} 22$ & $1^{\text {st }} 22$ & $2^{\text {nd }} 22$ & $1^{\text {st }} 22$ & $2^{\text {nd }} 22$ & $1^{\text {st } 22}$ & $2^{\text {nd }} 22$ \\
& Washes & Washes & Washes & Washes & Washes & Washes & Washes & Washes \\
\hline Total Solids wt\% & 20.78 & 8.96 & 20.83 & 8.80 & 20.66 & 8.89 & 10.94 & 3.73 \\
\hline Undissolved Solids wt\% & 0 & 0 & 0.24 & 0 & 0 & 0 & 0 & 0 \\
\hline Supernatant Solids wt\% & 20.78 & 8.96 & 20.64 & 8.80 & 20.66 & 8.89 & 10.94 & 3.73 \\
\hline Density kg/L & 1.176 & 1.071 & 1.176 & 1.070 & 1.177 & 1.071 & & \\
\hline ammonium & $<10$ & 12 & $<200$ & 1270 & & & & \\
\hline Total Base M & 1.71 & 0.62 & 0.79 & 0.62 & 1.41 & 0.48 & \\
\hline
\end{tabular}

\subsection{Aluminum and Ammonium Concentrations}

As previously mentioned, the aluminum concentration changed during the campaigns. As shown in Table 42, in Campaign III, there was more Al in the UFP slurry and less in the permeate than in Campaign II, which was attributed to precipitation of $\mathrm{Al}$ in the recycle blending. This conclusion was supported by a material balance on the mixing of the recycle stream that showed that the amount of dissolved $\mathrm{Al}$ in the FEP feed was less than expected. The results for Campaign IV are similar. Also note that as the amount of Caustic Flush 1 recycled was made more correct from Campaign II, III, and IV, there was less total $\mathrm{Al}$ in the FEP. Less dissolved $\mathrm{Al}$ in the FEP product would then result in more $\mathrm{Al}$ in the slurry and less in the permeate, as was seen in Campaign III when compared to Campaign II. However, the Campaign IV washed slurry value of $13000 \mathrm{mg} / \mathrm{kg}$ does not appear to be consistent with the permeate value of $5192 \mathrm{mg} / \mathrm{kg}$. Without analyses of the UFP feed and concentrated slurry compositions for Campaigns III and IV, it is not possible to resolve this discrepancy.

For the permeate, most of the other major species had similar concentrations in Campaigns II, III, and IV (K, Na, nitrite, phosphate, sulfate). The nitrate measured in Campaign IV was significantly higher than for Campaigns II and III; this value appears to be in error. The major undissolved species in the Campaign II and Campaign III slurries were also of similar concentration (Ca, Fe, Mn, Pb, Si, Cr, Nd). The Na and $\mathrm{K}$ are higher in Campaign II and Campaign III than in Campaign I due to the contribution of the AP101 simulant. 
Table 42. Comparison of Aluminum Concentrations in Ultrafiltration

Concentrations in $\mathrm{mg} / \mathrm{kg}$

\begin{tabular}{|c|c|c|c|}
\hline Process Stream & Campaign II & Campaign III & Campaign IV \\
\hline FEP Feed Slurry & 920 & 811 & 751 \\
\hline FEP Feed Supernate & 548 & 452 & 457 \\
\hline \% Dissolved & 60 & 56 & 61 \\
\hline FEP Concentrate Slurry & 5470 & 5255 & 4865 \\
\hline $\begin{array}{r}\text { FEP Concentrate } \\
\text { Supernate }\end{array}$ & 3823 & 2781 & 2705 \\
\hline Wt\% Dissolved & 70 & 53 & 56 \\
\hline Conc. UFP Slurry & 17550 & 22158* & \\
\hline UFP Permeate & 5670 & 5136 & 5192 \\
\hline Washed Slurry & 12750 & 18050 & 13000 \\
\hline
\end{tabular}

The ammonium concentrations in several of the process streams were measured.

Table 43 shows the streams, the concentrations, and the amount in grams in each stream. The material balance closes very well for Campaign III, but not for Campaign IV. In Campaign IV, the amount of ammonium in the wash permeates is much lower than expected based on the estimated wash water concentration ( $369 \mathrm{mg} / \mathrm{kg}$ estimated from the concentration of the Campaign III combined (RLD) condensates at the end of Campaign III.). Since this value was not measured, it appears that it must have been significantly less than expected. Although the UFP feed value is not available for Campaign IV, it should be small since it is a combination of the simulants (with no ammonium) and the FEP Bottoms.

Table 43. Comparison of Ammonium Concentrations for Campaigns III-IV

\begin{tabular}{|r|cc|} 
Process Stream & $\begin{array}{c}\text { Campaign III } \\
\text { Ammonium (mg/kg) }\end{array}$ & $\begin{array}{c}\text { Campaign IV } \\
\text { Ammonium (mg/kg) }\end{array}$ \\
FEP Feed & $<71$ & $<89$ \\
\cline { 2 - 3 } FEP Bottoms Concentrate & $<53$ & $<99$ \\
\cline { 2 - 3 } FEP Evaporator Condensate & 65 & 61 \\
UFP Feed & $<9$ & $<80$ \\
\cline { 2 - 3 } Permeate (CIX Feed) & $<160$ & 369 \\
\cline { 2 - 3 } 0.01M NaOH Wash (estimated) & 458 & $<10$ \\
Wash Permeate 1st 22 Washes & $<85$ & 12 \\
Wash Permeate 2nd 22 Washes & 342 & 70 \\
\cline { 2 - 3 } Washed Slurry & 372 & 87 \\
\hline UF Material Balance & Ammonium (g) & $\mathbf{8 7}$ \\
\hline UFP Feed & 1 & $<1$ \\
\cline { 2 - 3 } F.01M NaOH Wash (estimated) & 108 & 2 \\
Feeds & $\mathbf{1 0 9}$ & 10 \\
\cline { 2 - 3 } Wash Permeate 1st 22 Washes & $<12$ & $<\mathbf{1 3}$ \\
\cline { 2 - 3 } Wash Permeate 2nd 22 Washes & 43 & \\
Washed Slurry & 52 & \\
Products & $<\mathbf{1 0 7}$ & \\
\cline { 2 - 3 }
\end{tabular}




\subsection{Filter Rinsing and Cleaning}

Upon completion of the slurry washing, the filtration unit was drained and then cleaned. These cleaning steps are shown in Figs. 9 and 10. Limited analyses of the rinsing and cleaning solutions were performed in Campaigns III and IV. Table 44 shows the solids content and acid or base content of the rinsing and cleaning solutions for all of the campaigns. The solids contents and densities for all campaigns are similar. The Campaigns II, III, and IV acid and base concentrations are also very similar but differed for Campaign I due to use of different quantities of acid and base in the cleaning process.

The undissolved solids content of the caustic rinse 1 solution was decreased from Campaign I to Campaign IV as the remaining slurry heel amount was adjusted to be closer to the scaled target ${ }^{\dagger}$ of $3.6 \mathrm{wt} \%$. As such the Caustic Rinse 1 for Campaigns III and IV would then be diluted $1: 1$ with $0.1 \mathrm{M} \mathrm{NaOH}$ to create the recycle streams for the next campaign to get to the RPP-WTP estimated UDS concentration of $1.8 \mathrm{wt} \%$. The actual Caustic Rinse 1 values obtained in Campaign I, II, III, and IV UDS were 8.27, 5.09, 4.26 and $4.31 \mathrm{wt} \%$, respectively (see Table 44). The Caustic Rinse 1 streams from Campaigns I to III stream were then diluted appropriately to obtain $1.8 \mathrm{wt} \%$ UDS before their use as recycles. Of course, the Campaign IV Caustic Rinse 1 was discarded since SIPP was at its end. Moreover, the supernatant (dissolved) solids content in Campaigns II, III, and IV was higher than in Campaign I due to the higher content in the simulant slurry. This higher dissolved solids content also resulted in higher total solids in the Campaigns II, III, and IV cleaning solutions.

Note that the Campaign I heels were each about 10.5-12 L, whereas in the remaining campaigns, the heels were approximately 22L. Small amounts of undissolved solids in the acid cleaning solutions were indicated by the analyses, but these results were not consistent with visual examination of the solutions. Due to the approximately $\pm 0.11 \mathrm{wt} \%$ uncertainty in the total solids measurements and $\pm 0.22 \mathrm{wt} \%$ in the UDS, the measured values for low solids are inconclusive. Visual examination gives a better indication of the presence of solids; however, it does not give a good indication of the wt $\%$ of solids since the density of the settled solids can vary significantly.

\footnotetext{
${ }^{\dagger}$ The estimated UDS for the Plant Caustic Rinse 1 Heel was $1.8 \mathrm{wt} \%$, but due to differences in scale between SIPP and the Plant this concentration was not obtainable. The smaller scale indicated that the SIPP Heel concentration would be approximately double that of the Plant. Therefore, for SIPP the Caustic-Rinse-1-Heel-Solids target was set at 3.6 wt\% UDS. Once the Caustic Rinse 1 stream was produced and before its use as a recycle stream to make feed for the FEP, the stream would then be diluted with $0.1 \mathrm{M} \mathrm{NaOH}$ to obtain the needed $1.8 \mathrm{wt} \%$ UDS. (Also refer to Section 2.7.4.1.6.)
} 
WSRC-TR-2005-00105, REVISION 0

SRNL-RPP-2005-00012, REVISION 0

Table 44. Filter Cleaning Solutions

Caustic Rinse $1^{\mathrm{d}}$

\begin{tabular}{|c|c|c|c|c|c|c|c|c|c|c|c|c|}
\hline & \multicolumn{4}{|c|}{ Caustic Rinse $1^{\mathrm{a}}$} & \multicolumn{4}{|c|}{ Acid Cleaning $1^{\mathrm{d}}$} & \multicolumn{4}{|c|}{ Acid Cleaning 2} \\
\hline Campaign: & I & II & III & IV & I & II & III & IV & I & II & III & IV \\
\hline Total Solids (wt\%) & 9.27 & 7.46 & 6.26 & 6.50 & 1.07 & 2.36 & 1.50 & 1.58 & 0.18 & 0.67 & 0.48 & 0.50 \\
\hline Undissolved Solids (wt\%) & 8.27 & 5.09 & 4.26 & 4.31 & 0.09 & 0.12 & 0.12 & 0.08 & 0 & 0 & 0.08 & 0.05 \\
\hline Supernatant Solids (wt\%) & 1.57 & 2.50 & 2.08 & 2.29 & 0.98 & 2.24 & 1.38 & 1.50 & 0.18 & 0.67 & 0.41 & 0.45 \\
\hline Density $(\mathrm{kg} / \mathrm{L})$ & 1.075 & 1.057 & 1.046 & & 1.056 & 1.051 & 1.046 & 1.048 & 1.062 & 1.055 & 1.055 & 1.056 \\
\hline Total or Free ${ }^{b}$ Base (M) & $0.0450^{\mathrm{b}}$ & NM & 0.201 & 0.238 & & & & & & & & \\
\hline or $\quad$ Total Acid (M) & & & & & 1.56 & 1.20 & 1.10 & 1.19 & 1.95 & 1.61 & 1.63 & 1.68 \\
\hline $\mathrm{pH}^{\mathrm{e}}$ & 13 & 13 & 13 & 13 & 1 & $<1$ & $<1$ & $<1$ & 1 & $<1$ & $<1$ & $<1$ \\
\hline & \multicolumn{4}{|c|}{ Acid Cleaning 3} & \multicolumn{4}{|c|}{ Caustic Rinse 2} & \multicolumn{4}{|c|}{ Caustic Rinse 3} \\
\hline Campaign: & I & II & III & IV & I & II & III & IV & I & II & III & IV \\
\hline Total Solids (wt\%) & 0.05 & 0.13 & 0.10 & 0.15 & 0.83 & 0.66 & 0.75 & 0.80 & 0.76 & 0.81 & 0.86 & 0.95 \\
\hline Undissolved Solids (wt\%) & 0 & 0 & 0 & 0.08 & 0 & 0.13 & 0.12 & 0.04 & $0^{c}$ & 0.13 & 0.03 & 0.03 \\
\hline Supernatant Solids (wt\%) & 0.05 & 0.13 & 0.10 & 0.08 & 0.83 & 0.53 & 0.63 & 0.76 & 0.76 & 0.68 & 0.83 & 0.92 \\
\hline Density $(\mathrm{kg} / \mathrm{L})$ & 1.063 & 1.055 & 1.057 & 1.055 & 1.009 & 1.016 & 1.017 & 1.016 & 1.003 & 1.004 & 1.005 & 1.005 \\
\hline Total Base (M) & & & & & & & & & $0.002^{b}$ & & & \\
\hline Total Acid (M) & 1.98 & 1.74 & 1.78 & 1.69 & 0.158 & 0.449 & 0.420 & 0.396 & & 0.062 & 0.039 & 0.045 \\
\hline $\mathrm{pH}$ & 1 & $<1$ & $<1$ & $<1$ & 1 & $<1$ & $<1$ & $<1$ & 12 & 1.2 & 1.2 & 1.2 \\
\hline
\end{tabular}

${ }^{\mathrm{a}}$ acid cleaning solutions total and undissolved solids contents do not include nitrate from nitric acid (evaporates during measurement)

b estimated from $\mathrm{pH}$

c solids visible, but not quantifiable NM: not measured

d caustic rinse 1 for Campaigns III and IV diluted 1:1 with $0.1 \mathrm{M} \mathrm{NaOH}$ in recycle stream

e pH values were obtained: For Campaign I using pH paper at the test site, for Campaigns II and III pH meter from samples, and for Campaign IVby estimating from the base/acid molarities. 


\subsubsection{Pilot UFP Conclusions}

\subsubsection{ExperimentalConclusions (Author - Fowley)}

A summary of the overall conclusions for the SIPP UFP are as follows:

- The performance of the GKN filter unit was similar to that of the Mott filter unit under steady state conditions. In addition, the permeate flux of the GKN filter unit during dewatering was the same as the Mott filter unit, within measurement uncertainty, for the same simulated waste mixture.

- In Campaign IV, using the GKN filter unit, the average, temperature-adjusted permeate flux when dewatering from $\sim 3$ to $\sim 24 \mathrm{wt} \%$ UDS was $0.011 \mathrm{gpm} / \mathrm{ft}^{2}$. The average, temperature-adjusted permeate flux when dewatering from $\sim 3$ to $20 \mathrm{wt} \%$ UDS was $0.013 \mathrm{gpm} / \mathrm{ft}^{2}$. The average, temperature-adjusted permeate flux when dewatering from $\sim 3$ to $17 \mathrm{wt} \%$ UDS was $0.016 \mathrm{gpm} / \mathrm{ft}^{2}$.

- Permeate flux improved at higher slurry temperatures. At 19 wt\% UDS, 40 psid TMP, and a slurry velocity through the filter of $12 \mathrm{ft} / \mathrm{s}$, the permeate flux improved almost $75 \%$ at $45^{\circ} \mathrm{C}$ compared to $25^{\circ} \mathrm{C}$.

- The turbidity of the permeate from dewatering and slurry washing, when measured directly after the sample was taken, was always lower than 1 NTU. The Test Specification indicated a threshold of 2 NTU, therefore, the measured values indicated that the permeate was free of significant undissolved solids.

- The SIPP and previous UFP tests indicate that the magnitude of the permeate flux during acid cleaning of a filter appears to be more of a function of the waste dewatered than of the filter unit used.

- The increase in the adjusted permeate flux between the second and third acid cleaning steps was minimal in Campaigns II, III, and IV, possibly indicating that the third acid cleaning was unnecessary. The exception was Campaign I where whitish solids in the spent solutions of the first and second acid cleanings inhibited the permeate flux, requiring a third acid cleaning (and possibly a fourth) to reach steady conditions. The Campaign I simulant did not contain AP101 and FEP Bottoms, indicating that the necessity of a third acid cleaning was dependent on the waste dewatered.

- Cleaning the filter with $2 \mathrm{M}$ nitric acid after dewatering was sufficient to increase baseline permeate flux to approximately an order of magnitude higher than that of average waste flux during dewatering. However, it was not sufficient to return the filter to pre-test flux levels. In previous campaigns, soaking the filter unit in $0.1 \mathrm{M}$ $\mathrm{NaOH}$ for an extended period of time ( 2 months or more) typically regained the pretest flux levels. The significance of " 2 months or more" is that 2 months was typically the duration between successive SIPP campaigns.

The following conclusions were extracted from the Interim Reports of Campaign III (Duignan et al., 2004c or Appendix I-3), Campaign II (Duignan et at., 2004b), and Campaign I (Duignan et at., 2004a) 


\section{Campaign III [for the Mott Filter]}

- The average, temperature-adjusted permeate flux when dewatering from $\sim 3$ to $\sim 24$ wt $\%$ UDS was $0.011 \mathrm{gpm} / \mathrm{ft}^{2}$. The average, temperature-adjusted permeate flux when dewatering from $\sim 4$ to $20 \mathrm{wt} \%$ UDS was $0.014 \mathrm{gpm} / \mathrm{ft}^{2}$. The average, temperatureadjusted permeate flux when dewatering from $\sim 4$ to $17 \mathrm{wt} \%$ UDS was $0.018 \mathrm{gpm} / \mathrm{ft}^{2}$.

- Cleaning the filter with $2 \mathrm{M}$ nitric acid was sufficient to increase the $0.1 \mathrm{M} \mathrm{NaOH}$ permeate flux to an order of magnitude larger than that of waste flux. However, it was not sufficient to return the filter to pre-test flux levels.

- The turbidity of the permeate from dewatering and slurry washing, when measured directly after the sample was taken, was always lower 0.5 NTU. The Test Specification indicated a solids-free threshold of 2 NTU, therefore the measured values indicated the permeate was free of significant undissolved solids.

- Submerging the filter in a mild caustic solution over an extended period of time may improve flux. Likewise, it is probable that submerging the filter in a mild acid solution over an extended period of time will also improve flux.

\section{Campaign II [for the Mott Filter]}

- The average permeate flux when dewatering from $\sim 4$ to $\sim 24 \mathrm{wt} \%$ was $0.011 \mathrm{gpm} / \mathrm{ft}^{2}$.

- Cleaning the filter with $2 \mathrm{M}$ nitric acid was sufficient to increase the $0.1 \mathrm{M} \mathrm{NaOH}$ permeate flux to an order of magnitude larger than that of waste flux. However, it was not sufficient to return the filter to pre-test flux levels.

- The turbidity of the permeate from dewatering and slurry washing, when measured directly after the sample was taken, was always lower $0.5 \mathrm{NTU}$, indicating that the permeate was practically free of solids.

- The turbidity of the permeate from dewatering increased with time (days), indicating that solids were precipitating out of solution.

Submerging the filter in a mild caustic solution over an extended period of time may improve flux. Likewise, it is probable that submerging the filter in a mild acid solution over an extended period of time will also improve flux.

\section{Campaign I [for the Mott Filter and for the single simulant of AY102/C106]}

- Average permeate flux when dewatering from 12 to $24 \mathrm{wt} \%$ was $0.008 \mathrm{gpm} / \mathrm{ft}^{2}$.

- At no time did the permeate turbidity measure to be higher than 0.5 NTU and, therefore, the permeate was considered always free of solids.

- Steady-state solids concentration confirmed that the best permeate flux is obtained with the slurry axial velocity was at or above $11 \mathrm{ft} / \mathrm{s}$.

- When washing a batch of waste, which was concentrated to $20 \mathrm{wt} \%$ UDS, with two equal batches of $0.01 \mathrm{M} \mathrm{NaOH}$ solution, the consistency was reduced by one-half, but the yield stress remained statistically constant.

- When washing, many elements like sodium and aluminum were reduced by almost an order of magnitude. Some species in the undissolved solids actually did dissolve, like 
oxalate and fluoride, causing the concentration of undissolved solids to decrease as the dense supernatant was replaced with caustic water.

- When the filter became plugged with highly concentrated solids, recovery was possible for the vertically mounted filter by backpulsing after the downstream slurry valve was closed.

- Cleaning the filter with $2 \mathrm{M}$ nitric acid was sufficient to increase the $0.1 \mathrm{M} \mathrm{NaOH}$ permeate flux to an order of magnitude larger than that of waste flux. However, it was not sufficient to return the filter to pretest cleanliness.

\subsubsection{Waste Products Conclusions (Author - Zamecnik)}

A summary of the overall conclusions for the SIPP UFP are as follows:

- During the elevated temperature tests at a constant solids loading of $19 \mathrm{wt} \%$ UDS no significant changes occurred with respect to solids concentrations and particles sizes. As expected, as the simulant temperature increased from $25^{\circ} \mathrm{C}$ to $45^{\circ} \mathrm{C}$ the consistency of the slurry and the viscosity of the permeate decreased from 14 to 11 $\mathrm{mPa} \bullet \mathrm{s}$ and from 2.9 to $1.8 \mathrm{mPa} \bullet$, respectively, while the slurry yield stress increased approximately 10 to $12 \mathrm{~Pa}$.

- The composition of the undissolved solid species in the washed sludges in Campaigns I-IV were similar, as expected. The soluble species concentrations in the slurry and permeate for Campaigns II-IV were similar as expected.

- The balances on aluminum over the process were not consistent and could not be closed without additional analytical data.

- Ammonium in the first UFP slurry wash with $0.01 \mathrm{M} \mathrm{NaOH}$ in Campaign III was mostly retained in the slurry, indicating that a slurry component has an affinity for ammonium. Approximately none of the ammonium in the second UFP wash was retained in the slurry.

- The antifoam agent did not have any observed macroscopic effects on the filter operations of the SIPP, which is also consistent with similar commercial industrial experience (Watts et al., 1995). However, since the filter was cleaned between each run, the effect of possible accumulation of antifoam on the filter media with no cleaning between runs was not determined.

The following conclusions were extracted from the Interim Reports of Campaign III (Duignan et al., 2004c or Appendix I-3) and Campaign II (Duignan et al., 2004b or Appendix I-2)

\section{Campaign III}

- The composition of the undissolved solid species in the washed sludges in Campaigns I-III was similar, as expected. The supernatant portion species $\mathrm{Na}$ and $\mathrm{K}$ were higher in concentration in Campaigns II and III than in Campaign I due to the presence of the AP101 simulant.

- The aluminum in the Campaign III washed slurry was higher due to their being more undissolved aluminum in the FEP concentrate than in Campaign II. 
WSRC-TR-2005-00105, REVISION 0

SRNL-RPP-2005-00012, REVISION 0

- Due to higher undissolved aluminum in the FEP concentrate, the Campaign III permeate from the concentration step (CIX feed) had less aluminum than in Campaign II.

- Ammonium in the first 0.01M NaOH UFP wash of Campaign III was mostly retained in the slurry, indicating that a slurry component has an affinity for ammonium. Approximately none of the ammonium in the second wash was retained.

\section{$\underline{\text { Campaign II }}$}

- UFP produced permeate showed no tendency for post-filtration precipitation. (However, AY102-only permeate from Campaign I did post-precipitate after 2 months.)

- OLI/ESP models of the AY102 sludge need to incorporate oxides, e.g., $\mathrm{Al}_{2} \mathrm{O}_{3}$, and hydrates, e.g., FeOOH, in order to close mass and charge balances.

- Washing of concentrated slurry raised its yield stress from about 10 to $15 \mathrm{~Pa}$, but lowered the consistency from about 23 to $13 \mathrm{mPa} \bullet \mathrm{s}$.

- Campaign I data indicated that the yield stress of unwashed slurry is reduced upon extended pumping.

- Washing data matched previous SRNL and literature leaching and washing data for soluble washable species (see Table 40).

- Use of a more concentrated $\mathrm{NaOH}$ solution for the third caustic flush during filter cleaning may be warranted since the $\mathrm{pH}$ of the spent third wash was acidic.

- At least $96 \%$ of the antifoam remained in the HLW sludge slurry after filtration. 


\subsection{PILOT CESIUM ION EXCHANGE (Authors - Steimke \&Williams)}

The CIX unit operation was not used during Campaign IV activities, which were limited to FEP and UFP unit operations so that the new filter from GKN could be tested. However, for completeness the majority of Campaign III report (Duignan, et al., 2004c) is repeated here, as well as measurements made after that report was issued. New information includes measurements of the mass of dry resin on both a sodium and hydrogen basis and the resin particle size distribution.

The Cesium Ion Exchange Process, or CIX, is the third separation technology unit operation in the RPP-WTP Pretreatment Facility that the waste stream will pass through on startup. This was true for SIPP because the chosen waste type, Envelope A/D, will not be subjected to precipitation before filtration in the RPP-WTP Pretreatment process. The SIPP CIX operation removes cesium from a mixture of the liquid LAW stream coming from the UFP before it is sent to the TLP for further evaporation and eventual LAW vitrification.

The ion exchange resin used in this test was SuperLig ${ }^{\circledR} 644$, which is designed to remove cesium from caustic radioactive waste that is at a 5-molar concentration in sodium. It is manufactured by IBC Advanced Technologies. Pilot scale testing of this resin was first performed in the Engineering Development Laboratory in the year 2000 (Fowley et al., 2004). That earlier testing was intended to be focused primarily on the chemical performance of the resin on a pilot scale (more than one liter of resin); however, the pressure drop across the resin beds was unexpectedly high. Testing for the next three years was redirected to the hydraulic performance of the resin. The hydraulic issues were resolved to the satisfaction of RPP-WTP (River Protection Program Waste Treatment Plant) (Fowley et al., 2004).

In 2003, Test Exception 24590-WTP-TEF-RT-03-069 called for resumed chemical testing of SuperLig ${ }^{\circledR} 644$ resin at a pilot-scale for six chemical cycles, which would be incorporated into the four Campaigns of the SIPP task (Duignan, 2003c). A cycle is the process whereby the Cesium Ion Exchange unit operation treats enough LAW solution until the lead CIX column accumulates enough cesium to require regeneration, then it is regenerated. Later, the goals of the SIPP task were modified that reduced the number of ion exchange cycles to four; Cycles 1 and 2 would be done in Campaign II and Cycles 3 and 4 in Campaign III of the SIPP task. There were no CIX operations during Campaign I of SIPP. This report includes all four cycles of testing reported in Duignan et al.(2004b) and Duignan et al., (2004c), as well as measurements that came available only after the Campaign III report was issued.

For the actual RPP-WTP a cycle would entail the treatment of a minimum of 100 Bed Volumes (BV = a resin bed volume of 415 gallons (1571 liters) under normal operating conditions, which is subject to change) until the lead column of the three-column ion exchanger will contain enough accumulated cesium to require its regeneration. That lead column is then taken off line to be regenerated while a waiting fourth column is valved-in for the CIX to continue further LAW treatment. That fourth column, containing 
reconditioned resin, is put in the last position (called the polishing position) of the threecolumn CIX unit and the column that had been in the second position (referred to the lag position) becomes the new lead column, and so forth. The pilot scale CIX unit operation, of the SIPP task, had only two columns, the lead and lag positions. The pilot operation consisted of the treatment with approximately $190 \mathrm{BV}(\mathrm{BV}=2.34$ liters $)$ of simulated $\mathrm{LAW}$, which was then followed by the regeneration of the lead column. A new cycle begins with the newly regenerated lead column in the lag position.

This section will explain the pilot CIX unit operation that was performed as part of Campaign III testing, but Campaign II results are also included to compare the results. An abridged form of the operational raw database can be found in Appendix H3. The process started with the preparation of the feed product, which is permeate created in the UFP. An explanation of the chemical makeup of the feed stream will be followed by a short description on how feed was handled just before CIX operation and then how the waste stream was handled during and after ion exchange. That section will be followed by a discussion of the experimental hardware and how the test was run. Finally, the results will be discussed and conclusions made.

\subsubsection{Chemical Description of the Pilot-Scale CIX Feed Stream}

The feed for the pilot-scale CIX unit operation was the permeate produced by simulant waste dewatering in the pilot-scale UFP unit operation. The chemical make-up of that feed was described in detail in Section 2.7.4.2.1 and will not be repeated here. Refer to that section and specifically to Tables 34 for information on the CIX feed (simulated LAW) stream, which was the permeate produced during the UFP dewatering operation.

\subsubsection{Simulant Handling (Author - Williams)}

\subsubsection{Initial Preparations}

Before beginning the CIX Campaign III test, the solutions to be used to regenerate the lead column were made ready. Those solutions were:

1. $0.1 \mathrm{M} \mathrm{NaOH}$ Displaced the LAW in CIX column while maintaining high $\mathrm{pH}$ to prevent precipitation

2. Deionized Water Pre-elution rinse - Displaced the $0.1 \mathrm{M} \mathrm{NaOH}$ from CIX column

3. $0.5 \mathrm{M} \mathrm{HNO}_{3} \quad$ Eluent - Displaced water and stripped Cs ions from resin by replacing them with hydrogen ions. Note: eluent is pumped into the column and eluate flows out of the column.

4. Deionized Water Post-elution rinse - Displaced acid eluate from column

5. $0.25 \mathrm{M} \mathrm{NaOH}$ Displaced water and regenerated the resin by replacing hydrogen ions with sodium ions

The regeneration caustic, $0.25 \mathrm{M} \mathrm{NaOH}$, required a volume of 47.8 liters and it was made from DIF water and $50 \mathrm{wt} \% \mathrm{NaOH}$. Then it was stored in the Caustic Supply Tank 
before 25 May 2004. A large quantity of displacement caustic solution, $0.1 \mathrm{M} \mathrm{NaOH}$ was made with condensate and it was stored in a carboy labeled "Campaign 3-0.1 M $\mathrm{NaOH}$ for CsIX.” From this carboy 28.7 liters were removed and made ready for Campaign III before 2 June by adding it to the 0.1 M Caustic Supply Tank. To strip the cesium ion-exchange (CIX) resin of Cs ions, 70.1 liters of $0.5 \mathrm{M}$ HNO3 was made from DIF water and 70\% HNO3 and was placed in the Eluent Supply Tank before 2 June.

\subsubsection{Handling of Pilot-scale CIX Feed LAW Simulant}

The Simulant Supply that was produced during pilot-scale UFP operation was split into two equal parts for Cycle 3 and 4 of CIX operations. Both cycles processed 194 Bed Volumes (BV) ( 453 liters) of fresh simulant. The treated simulant was transferred to the pilot-scale TLP Evaporator Feed Tank from CIX operations.

\subsubsection{Handling CIX Regeneration and Campaign III CIX Recycle Streams}

The flow rate and duration of each of the chemical streams, was set by communication from RPP-WTP. The different chemical effluents from the column/s were collected in different containers, either jugs or tanks. There is a transit time for a fluid to pass through a column or columns, depending on flow rate. For Campaign III, the effluent hose was moved from one container to another after the transit time had elapsed, where the transit time was based on a plug flow calculation through the columns.

\subsubsection{Pilot CIX Equipment (Author-Steimke)}

\subsubsection{CIX Resin Columns}

Figure 54 is a picture of the two columns during Cycle 3 Regeneration and Fig. 55 is a schematic of the two ion exchange columns and peripheral equipment. The design is also documented in WSRC drawing EES-23041-R4-001. 
WSRC-TR-2005-00105, REVISION 0 SRNL-RPP-2005-00012, REVISION 0

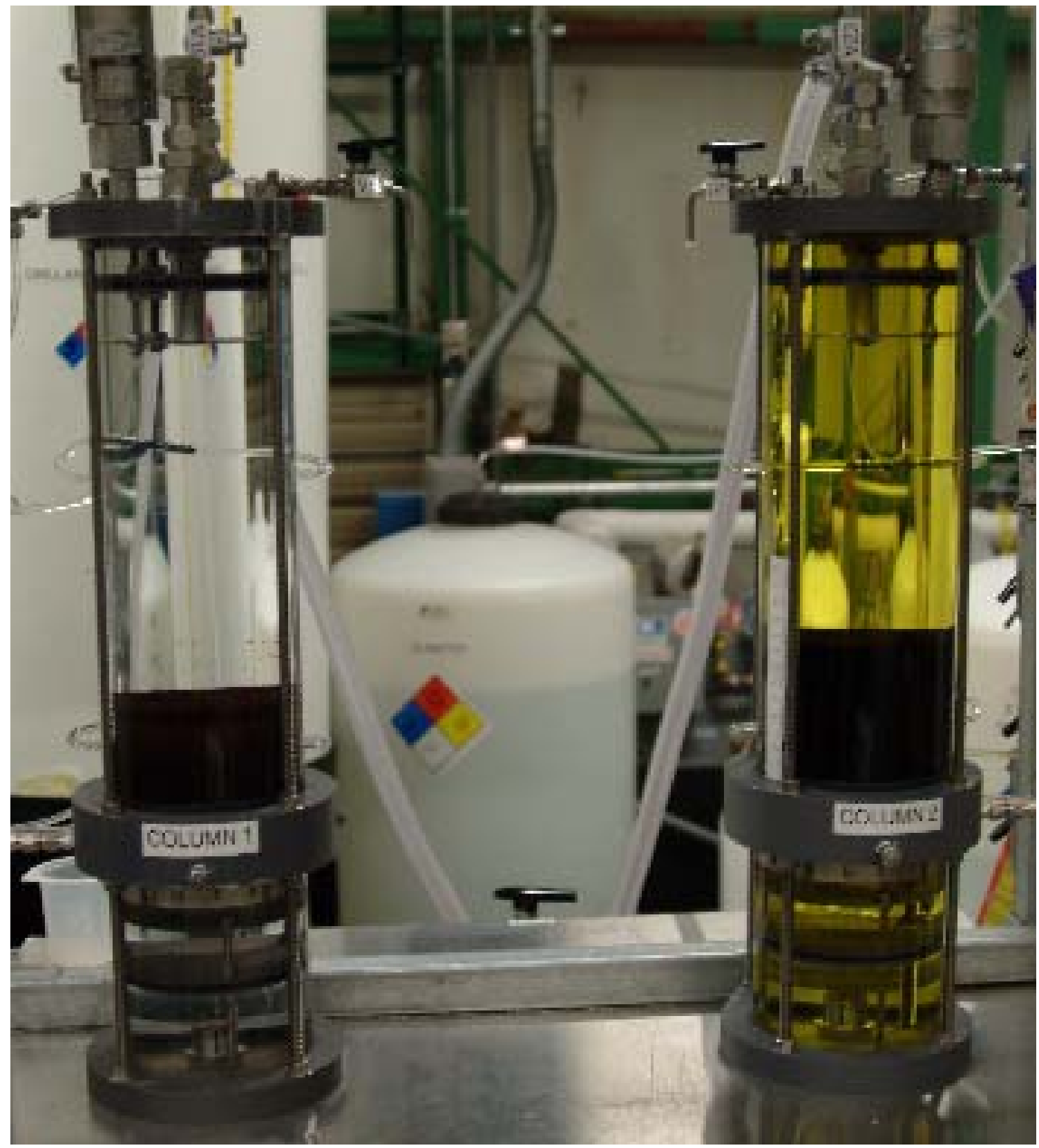

Figure 54. Pilot-scale CIX columns: The left column (COLUMN 1) is undergoing resin regeneration during Cycle 3 operation 
WSRC-TR-2005-00105, REVISION 0

SRNL-RPP-2005-00012, REVISION 0

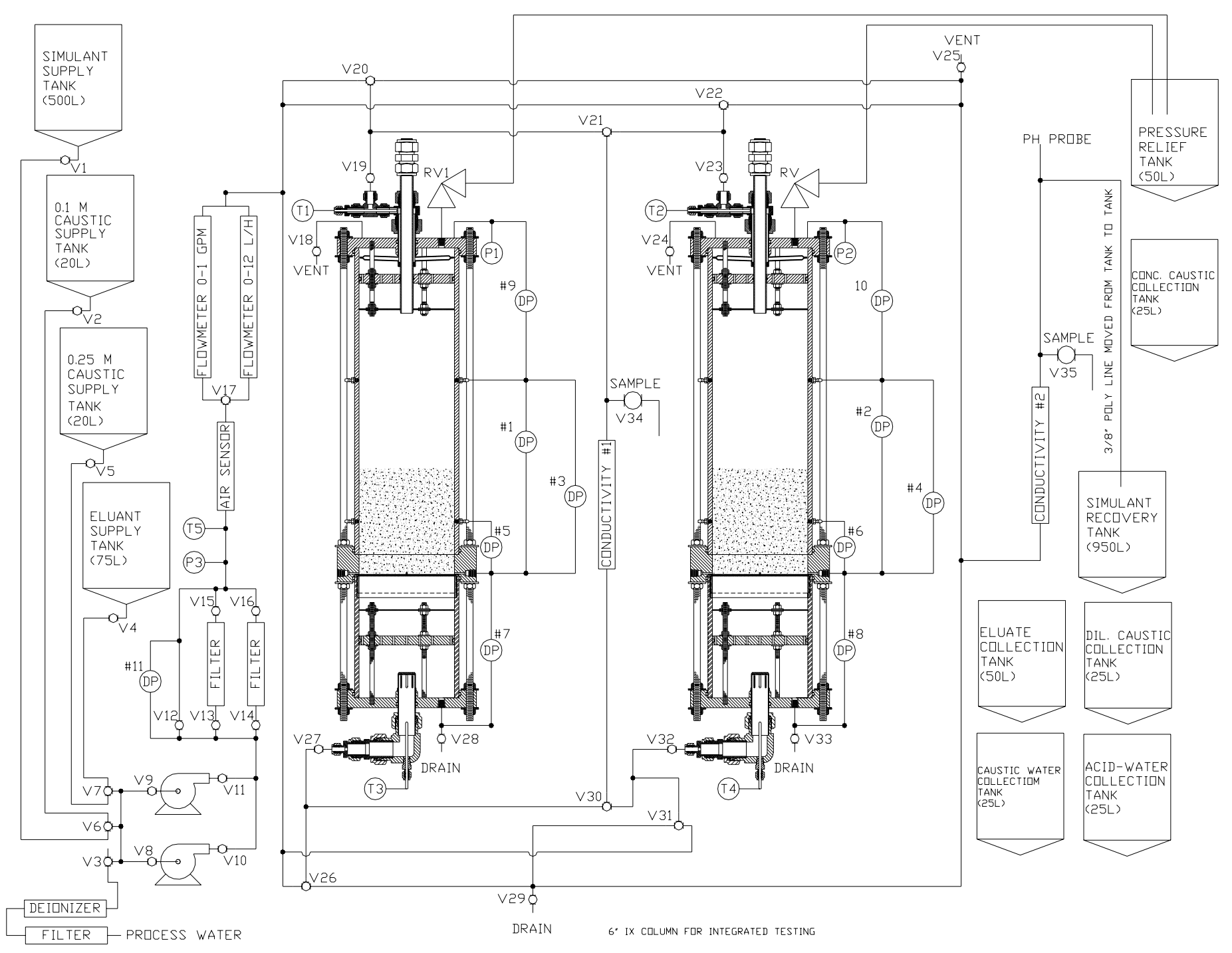

Figure 55. Schematic of Ion Exchange Facility for Integrated Testing 
Two ion exchange columns were designed and constructed for the SIPP task. The columns had an inside diameter of 5.5 inches and a target resin height in fully swollen sodium form of 6 inches ${ }^{\dagger}$, giving a height to diameter ratio of 1.1, the same ratio as for the WTP columns. Other hardware included pumps, tanks, flowmeters, pressure transducers, other instruments and a data acquisition system (DAS).

Each column had a liquid filled length of 23.75" (or an internal volume of 9.24 liters $~ 4$ $\mathrm{BV}$ ). The following hardware is positioned inside the column with the following elevations with respect to the wedge screen (also known as: V-wire screen), that supports the resin and note that the total liquid filled length of each column is 23.75”, i.e., 16.75"$(-7.00$ ').

\begin{tabular}{|c|c|}
\hline Elevation & Column Hardware Description \\
\hline$\overline{16.75 ”}$ & $\overline{\text { Top of liquid filled space }}$ \\
\hline $16.00 ”$ & Flow distributor with six perforated arms \\
\hline $14.75 ”$ & Perforated PVC plate with thirty seven 3/64” holes \\
\hline $13.25 ”$ & Perforated stainless steel plate with $3 / 32$ " holes on a $5 / 32$ " triangular pitch \\
\hline $0 ”$ & Wedge screen (Vee wire screen) with $0.010 ”$ slots to support resin \\
\hline$-2.0 ”$ & Perforated stainless steel plate with $3 / 32$ ” holes on a $5 / 32$ " triangular pitch \\
\hline$-3.50 ”$ & Perforated PVC plate with thirty seven 3/64” holes \\
\hline$-7.00 ”$ & Bottom of the liquid filled space \\
\hline
\end{tabular}

The purpose of the flow distributor and the two plates below it was to generate a more gradual transition of density when relatively dense simulant was introduced to the top of the column on top of lighter liquid. The purpose of the wedge screen was to support the resin while resisting plugging. The purpose of the bottom two plates was to generate a more uniform flow field in the event upflow operations were needed, however, the normal flow direction was down.

By opening the proper valve, feed could be drawn from one of five supply tanks, which contained either: LAW simulant, displacement $0.1 \mathrm{M} \mathrm{NaOH}$ solution, DIF water for rinsing, eluent $0.5 \mathrm{M}$ nitric acid solution, or regeneration $0.25 \mathrm{M} \mathrm{NaOH}$ solution. The solutions were pumped by one or two gear pumps connected in parallel. Most of the time only one pump was sufficient, but two-pump operation was required for upflow. The facility contained two flowmeters in parallel, a high range flowmeter for upflow with a range of 0 - $1 \mathrm{gpm}$ and a low range flowmeter for normal operation with a range of 0 - $12 \mathrm{~L} / \mathrm{hr}$. Valves allowed upflow or downflow through one column or two columns in series. The effluent line from the columns contained a $\mathrm{pH}$ probe, a conductivity probe and a thermocouple. A hose connected to the effluent line was placed in the appropriate collection jug for every step of the cycle. There were six collection jugs, one for each of the six steps of the cycle.

\footnotetext{
${ }^{\dagger}$ The actual resin height is not fixed. As explained in the following sections, and shown in Table 48, the height resin in the columns changes from 6.25 inches, when the columns are initially filled, to as short as 5.4 inches after multiple resin regenerations.
} 


\subsubsection{The Data Acquisition and Safety Circuitry}

A Dell computer with LabVIEW ${ }^{\mathrm{TM}}$ software was used to acquire data, control the flow rate, and shut down the flow if any measured pressure exceeded the setpoint of 20 psig. There was also a pressure relief valve set at 45 psig connected to the top of each of the two columns. The discharge line of the pressure relief valves was a hose leading to a jug. There was also a spill detector with a sensor placed below the columns on the floor of the containment for the facility. Any spill would disconnect power to the pumps. There was also an air detector in the line leading from the solution supply tanks to the pump. Any air in the line would disconnect power from the pumps. A manual bypass switch was used to start the pump/s when there was initially air in the line. The air detector was used because oxygen can damage the wet resin, particularly when it is in a caustic state.

\subsubsection{The Ion Exchange Resin}

To prepare the CIX resin, SuperLig ${ }^{\circledR} 644$, for use, it was taken from the 250 -Gallon Batch (C-01-11-05-02-35-60). The resin was chemically pre-treated by washing with water, treating it in $0.5 \mathrm{M}$ nitric acid, washing with water, treating it with $0.25 \mathrm{M}$ sodium hydroxide and washing it with water. The pre-treated resin was then wet sieved under a nitrogen atmosphere to remove particles finer than 40 mesh by using a 35-mesh USF screen with openings of 500 microns.

\subsection{Method of Resin Addition to the Columns}

A tank with an agitator was temporarily connected to the top of a column. The column was completely filled with $0.25 \mathrm{M} \mathrm{NaOH}$ solution, the top tank was partially filled and the valve between them was closed. Resin was added to the top tank to form a $30 \%$ by volume slurry and the agitator was energized. The bottom of the column was connected to pump suction. Pump discharge was connected to a waste container. The valve was opened and the pump was simultaneously run to create a downward velocity of $14 \mathrm{~cm} / \mathrm{min}$ (flow rate equal to $0.58 \mathrm{gpm}$ ) which deposited a resin bed with the target average resin height of 6 inches. However, the initial resin deposition resulted in a crater about 1 inch deep in both columns. An upflow for two minutes at $13 \mathrm{~cm} / \mathrm{min}$ was used to level the top of the resin beds. This process was repeated with the second resin bed. This was followed by pre-conditioning of both beds for thirty minutes with a flow rate of $15 \mathrm{~cm} / \mathrm{min}$ of $0.25 \mathrm{M} \mathrm{NaOH}$ solution. At this time, both beds had a resin height of 6.25 inches. The columns were then ready for SIPP operation.

\subsection{Dry Resin Mass on Sodium and Hydrogen Bases}

At the end of Campaign III testing $0.25 \mathrm{M} \mathrm{NaOH}$ solution was pumped through both columns followed by a rinse of deionized water. The tops of the columns were removed and full penetration "punch" samples (100 $\mathrm{mL}$ per column) were taken of the resin to determine the particle size distribution. Those results are explained the following section. Once samples were taken, the resin was removed from both columns. The resin from each column was stored in separate carboys for archiving and to determine the resin dry mass on both sodium and hydrogen basis for each column. 
The method used to determine the dry resin mass on a sodium basis was as follows: First, since months had elapsed, the resin was again treated with $0.25 \mathrm{M} \mathrm{NaOH}$ solution and then carefully rinsed with deionized water. The total contents of the wet resin from each carboy was separately filtered to a damp state and the damp resin was weighed and then mixed using a rolling drum. From the mixed damp resin four half gram samples were collected. Those samples were weighed damp, then vacuum oven dried and weighed again to determine the ratio of dry mass to damp mass. Finally the average ratio from the four samples was applied to the damp mass of the entire inventory of one column to get the sodium form dry mass in that column. The sodium form dry masses for Columns 1 and 2 were 642 grams and 713 grams, respectively, both $\pm 2 \%$. By rinsing and soaking four more half gram samples in $0.5 \mathrm{M} \mathrm{HNO}_{3}$ followed by more rinsing in deionized water, a similar procedure was followed to determine the resin dry mass on a hydrogen basis. The hydrogen form dry mass was compared to sodium form dry mass because PNL usually lists dry mass on a hydrogen basis. The sodium dry mass was $119 \% \pm 3 \%$ and $122 \% \pm 2 \%$ of the hydrogen form dry mass for the two columns, respectively.

\subsection{Resin Particle Size Distribution}

As mentioned above, before archiving the resin, a $100 \mathrm{~mL}$ sample was removed from each column using the accepted "full penetration punch"-method procedure for a particle analysis. The resin from those samples was wet sieved to compare to the wet-sieve particle size distribution (PSD) previously obtained on pre-test resin. The resulting particle size distributions by percentage mass are listed in Table 45. The uncertainties listed are 95\% confidence limits and are based on a series of replicate particle size measurements performed at SRNL.

Table 45. Particle Size Distribution of the SuperLig®644 used for SIPP

\begin{tabular}{|l|c|c|c|}
\hline \multicolumn{1}{|c|}{ Mesh size } & Pre-test & $\begin{array}{c}\text { Post-test, } \\
\text { Column 1 } \\
\text { wt\% }\end{array}$ & $\begin{array}{c}\text { Post-test, } \\
\text { Column 2 } \\
\text { wt\% } \%\end{array}$ \\
\hline Greater than 30 & $78.1 \pm 4.0$ & $84.3 \pm 4.0$ & $78.2 \pm 4.0$ \\
\hline $30-40$ & $20.0 \pm 4.0$ & $14.5 \pm 4.0$ & $19.9 \pm 4.0$ \\
\hline Less than 40 & $1.9 \pm 1.0$ & $1.2 \pm 1.0$ & $1.9 \pm 1.0$ \\
\hline
\end{tabular}

The wet-sieved PSD of the resin taken from Column 2 matched exactly to that of the pretest results, thus indicated that no degradation occurred during the four cycles of SIPP CIX unit operation; each cycle processing approximately $200 \mathrm{BV}$ of simulated LAW. The nominal results from Column 1 appeared to show a small shift to larger particle sizes from the post-test analysis. An additional sample/analysis was conducted with a similar result, an apparent small shift in the PSD to larger particles. An increase in resin PSD has not been seen in the past after other multi-cycle operations, e.g., see Fowley et al., 2004. This small shift in the resin PSD could be due to measurement uncertainty, a sampling bias, or possibly a bias in splitting the resin batch between Columns 1 and 2 during initial column loading that resulted in a slightly larger average resin particle size in Column 1, as compared to Column 2. Considering the measurement uncertainties, all 
three particle size distributions may actually be the same. In any event, it is clear there is no evidence for resin particle breakdown.

\subsubsection{Pilot CIX Operation (Author-Steimke)}

\subsubsection{Chemical Testing}

Each cycle of chemical testing had the following six chemical steps all in downflow: 1) LAW simulant, 2) 0.1 M NaOH displacement solution, 3) DIF water, 4) $0.5 \mathrm{M}$ nitric acid eluent solution, 5) DIF water, and 6) $0.25 \mathrm{M} \mathrm{NaOH}$ regeneration solution. The total volume of LAW simulant used for each cycle was about $190 \mathrm{BV}$ and simulant was always passed through both ion exchange columns connected in series. One BV was equal to 2.34 liters. All steps other than the simulant step were conducted through one column only. The $0.1 \mathrm{M} \mathrm{NaOH}$ step and the subsequent deionized water step (pre-elution rinse) were conducted at a flow rate of $3 \mathrm{BV} / \mathrm{hr}$ for one hour. The volume used for the elution step was $15 \mathrm{BV}$. The post-elution rinse was run at a flow rate of $1.4 \mathrm{BV} / \mathrm{hr}$ for 2.25 hours. The regeneration step was run at a flow rate of $3 \mathrm{BV} / \mathrm{hr}$ for 2 hours. (See Table 10 for Cycle 3 and Table 11 for Cycle 4. The information in the shaded columns of Cycle 5 and 6 of those tables would have been for Campaign IV if CIX testing continued.)

The six different chemical solutions were collected in separate jugs or tanks by moving the discharge hose of the columns. In Campaign II, for Cycles 1 and 2, the plan was to move the hose based on observing the change in the $\mathrm{pH}$ and/or conductivity probes. This proved to be difficult to implement. Therefore, in Campaign III, for Cycles 3 and 4, the timing for moving the hose to a different container was based on a plug flow calculation of when the liquid front would reach the column discharge.

There was a 39 day period between the end of Cycle 2, part of Campaign II testing, and the start of Cycle 3, part of Campaign III testing. At the end of Cycle 2, the lag column was Column 1 and it contained simulant and the lead column, Column 2, which was regenerated, contained $0.25 \mathrm{M} \mathrm{NaOH}$. At the request of RPP-WTP, both columns were displaced with $0.1 \mathrm{M} \mathrm{NaOH}$ and the resin soaked in that solution until the start of Cycle 3 for Campaign III testing. At the conclusion of all testing, the contents of both columns were displaced with $0.1 \mathrm{M} \mathrm{NaOH}$ solution. 


\subsubsection{Test Matrix Highlights}

The entire CIX test matrix is shown in Tables 10 and 11 with each cycle operation occurring on the dates listed in Table 46. For convenience some key parameters are listed below for Campaigns II and III:

- Cycles 1, 2 and 3 had a simulant flow rate of $3 \mathrm{BV} / \mathrm{hr}$. The simulant flow rate for Cycle 4 was $4.5 \mathrm{BV} / \mathrm{hr}$.

- Cycles 1 and 4 had an eluent flow rate of $1 \mathrm{BV} / \mathrm{hr}$. The eluent flow rates for Cycle 2 and 3 were $0.5 \mathrm{BV} / \mathrm{hr}$ and $2.0 \mathrm{BV} / \mathrm{hr}$, respectively.

- Column 1 was the lead column for Cycles 1 and 3. Column 2 was the lead column for Cycles 2 and 4.

Table 46. Cycle Dates of Operation

\begin{tabular}{|c|c|c|}
\hline Cycle & Start date & Finish date \\
\hline 1 & $4 / 26$ & $5 / 4$ \\
\hline 2 & $5 / 4$ & $5 / 12$ \\
\hline 3 & $6 / 21$ & $6 / 29$ \\
\hline 4 & $6 / 29$ & $7 / 7$ \\
\hline
\end{tabular}

\subsubsection{Pilot CIX Discussion of Results (Author-Steimke)}

\subsubsection{General Results}

This section presents an overview of the results. The following sections give the results and an abridged version of all the SIPP CIX operation raw data are in Appendix H3.

The CIX facility was used to run four ion exchange cycles, where a cycle consisted of six chemical steps. Table 47 lists the volumes of fresh LAW simulant that were pumped through the two beds connected in series. At 2.34 liters per bed volume, these volumes translate to about $190 \mathrm{BV}$. In addition, the final 9 liters of simulant that were pumped into the lead column, Column 1, for Cycles 1 and 3, were added to the fresh simulant in the simulant supply tank for use in Cycles 2 and 4 to mimic WTP operation. 
WSRC-TR-2005-00105, REVISION 0

SRNL-RPP-2005-00012, REVISION 0

Table 47. Highlights of the Pilot-Scale CIX Cs removal efficiency ${ }^{\dagger}$

\begin{tabular}{|l|l|l|l|l|}
\hline Cycle & $\begin{array}{l}\text { Fresh simulant } \\
\text { volume, } \mathrm{L}\end{array}$ & $\begin{array}{l}\text { Fresh simulant } \\
\text { volume, BV }\end{array}$ & $\begin{array}{l}\text { Percent cesium } \\
\text { removal by lead } \\
\text { column }\end{array}$ & $\begin{array}{l}\text { Percent cesium } \\
\text { removal by both } \\
\text { columns }\end{array}$ \\
\hline 1 & 428 & 183 & $99.45 \pm 0.25$ & $99.6 \pm 0.6$ \\
\hline 2 & 437 & 187 & $99.54 \pm 0.25$ & $99.8 \pm 0.6$ \\
\hline 3 & 453 & 194 & $98.70 \pm 0.15$ & $99.6 \pm 0.6$ \\
\hline 4 & 453 & 194 & $98.34 \pm 0.17$ & $100.0 \pm 0.6$ \\
\hline
\end{tabular}

The lead column removed more than $98 \%$ of the cesium and the lag column removed most of the remaining cesium. Note that the percentage of cesium removed by the lead column, generally decreased over the course of four cycles. Because of the measurement uncertainty no trend could be discerned for cesium removal by both columns in series.

During ion exchange operation, the axial pressure drop was measured for both resin beds. The maximum pressure drop occurred during the simulant step, because the simulant has about three times the viscosity of the other solutions. Pressure drops generally increased over the four cycles. The pressure drop across the bottom 3" of the resin column was also measured. That pressure drop was close to half of the total pressure drop for 6” of resin. This implies a resin bed with uniform permeability and no significant flow channeling in the bed.

A cesium mass balance was performed for all four cycles. The cesium masses in and out of the columns agreed within the accuracy of the ICP-MS (inductively coupled plasma mass spectroscopy) used for measuring cesium in 5 molar sodium solution. During elution of each of the lead columns fifteen bed volumes of $0.5 \mathrm{M}$ nitric acid were used. After only the first three BV, about $90 \%$ of the cesium was eluted from the bed.

\subsubsection{Hydraulic Observations}

Table 48 summarizes some important hydraulic results, including pressure drop, from Campaign III (Cycles 3 and 4) and Campaign II (Cycles 1 and 2) so that the complete set of cycles can be compared. Note, that the simulant had a density of $1.25 \mathrm{~g} / \mathrm{mL}$.

\footnotetext{
${ }^{\dagger}$ The data shown in Section 2.8.5.3.4, from Tables 51 and 52, were used to calculate the percentage removal of cesium by two columns in series listed in Table 47. The percentage was calculated as the total cesium mass entering the column minus the sum of the cesium in the simulant out and the final simulant, all divided by the total cesium entering.
} 
WSRC-TR-2005-00105, REVISION 0

SRNL-RPP-2005-00012, REVISION 0

Table 48. Hydraulic Summary

\begin{tabular}{|c|c|c|c|c|c|c|}
\hline Cycle & $\begin{array}{c}\text { Simulant } \\
\Delta \text { P Col. 1 } \\
\text { inches } \\
\text { H2O }\end{array}$ & $\begin{array}{c}\text { Simulant } \\
\Delta \text { P Col. 2 } \\
\text { inches } \\
\text { H2O }\end{array}$ & $\begin{array}{c}\text { Resin } \\
\text { height in } \\
\text { simulant, } \\
\text { Col. 1, } \\
\text { inches) }\end{array}$ & $\begin{array}{c}\text { Resin } \\
\text { height in } \\
\text { simulant, } \\
\text { Col. 2, } \\
\text { (inches) }\end{array}$ & $\begin{array}{c}\text { Simulant } \\
\text { superficial } \\
\text { velocity, } \\
\text { Col. 1, } \\
\text { (cm/hr) }\end{array}$ & $\begin{array}{c}\text { Simulant } \\
\text { superficial } \\
\text { velocity, } \\
\text { Col. 2, } \\
\text { (cm/hr) }\end{array}$ \\
\hline 1 & $0.6(\mathrm{Lead})$ & $0.5(\mathrm{Lag})$ & 5.6 & 5.8 & 46 & 46 \\
\hline 2 & $0.6(\mathrm{Lag})$ & $0.7(\mathrm{Lead})$ & 5.4 & 5.7 & 46 & 46 \\
\hline 3 & $1.0(\mathrm{Lead})$ & $0.7(\mathrm{Lag})$ & 5.6 & 5.9 & 46 & 46 \\
\hline 4 & $1.2(\mathrm{Lag})$ & $1.3(\mathrm{Lead})$ & 5.7 & 5.8 & 69 & 69 \\
\hline
\end{tabular}

Figure 56 plots the flow rates during Cycle 1, typical results, for all six solutions used. The large fluctuations in flow rate for the two water rinses occurred because the DIF water used lacked sufficient ions for the magnetic flowmeter to achieve a steady reading. However, flow checks using a volumetric cylinder and stopwatch showed that the average flow reading was correct. Figures 57 and 58 plot the pressure drops across the lead and lag column resin beds during Cycle 1. It was necessary to adjust the raw pressure drop data by the following method because the pressure drops to be measured were small. The flow was intentionally stopped about eight times per cycle and the DAS recorded the zero flow readings for the differential pressure gages. Later the raw differential pressures were corrected by the zero flow readings so that the final differential pressures were equal to zero when the flow was zero. The magnitude of the corrections was comparable to the differential pressures measured.

Figure 55 shows the locations of the pressure measurements. There were duplicate measurements of the pressure drop across the entire resin bed and one measurement of the pressure drop across the bottom 3 inches of the resin bed. The duplicate pressure drop measurements agree. The highest pressure drops were for the simulant step because of its higher viscosity. The pressure drop across the bottom 3 inches was about half of the total pressure drop, as expected. The total pressure drop across the bed for simulant was about 0.6 inches of water. By way of comparison, the 24-Inch Column Test (All-In Test) (Fowley et al., 2004) had a pressure drop for Cycle 1 of 16 inches of water for a resin height that was four times as great and a superficial velocity that was seven times as great ( $5 \mathrm{~cm} / \mathrm{min}$ vs. $0.75 \mathrm{~cm} / \mathrm{min}$ ). The 24-Inch Column Test pressure drop was 27 times as large, which agrees well with the product of the resin height ratio (4) and the velocity ratio (7). 
WSRC-TR-2005-00105, REVISION 0

SRNL-RPP-2005-00012, REVISION 0

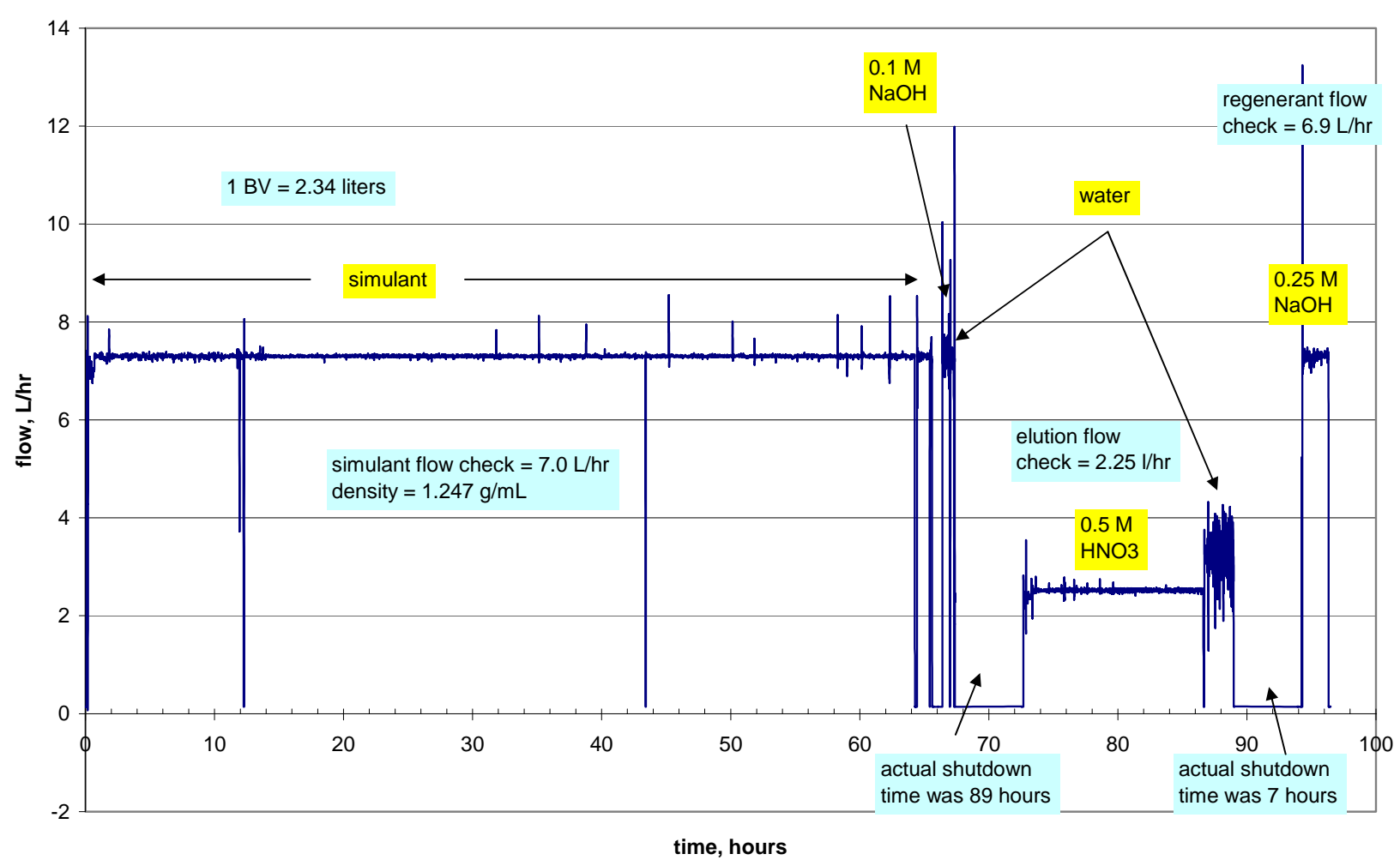

Figure 56. Flow rate During Cycle 1

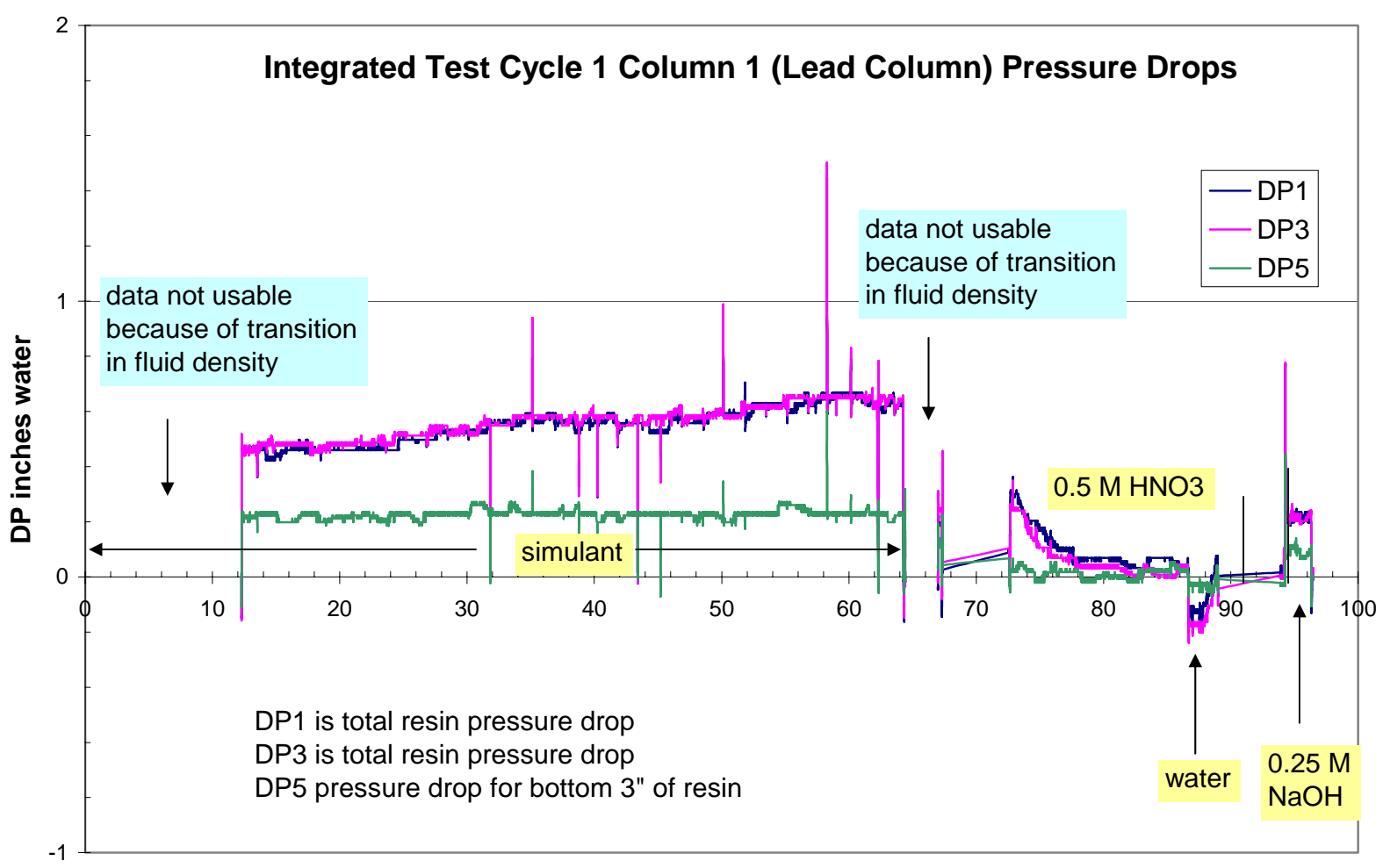

time, hours

Figure 57. Pressure Drop Across Resin Bed for Lead Column and Cycle 1 
WSRC-TR-2005-00105, REVISION 0

SRNL-RPP-2005-00012, REVISION 0

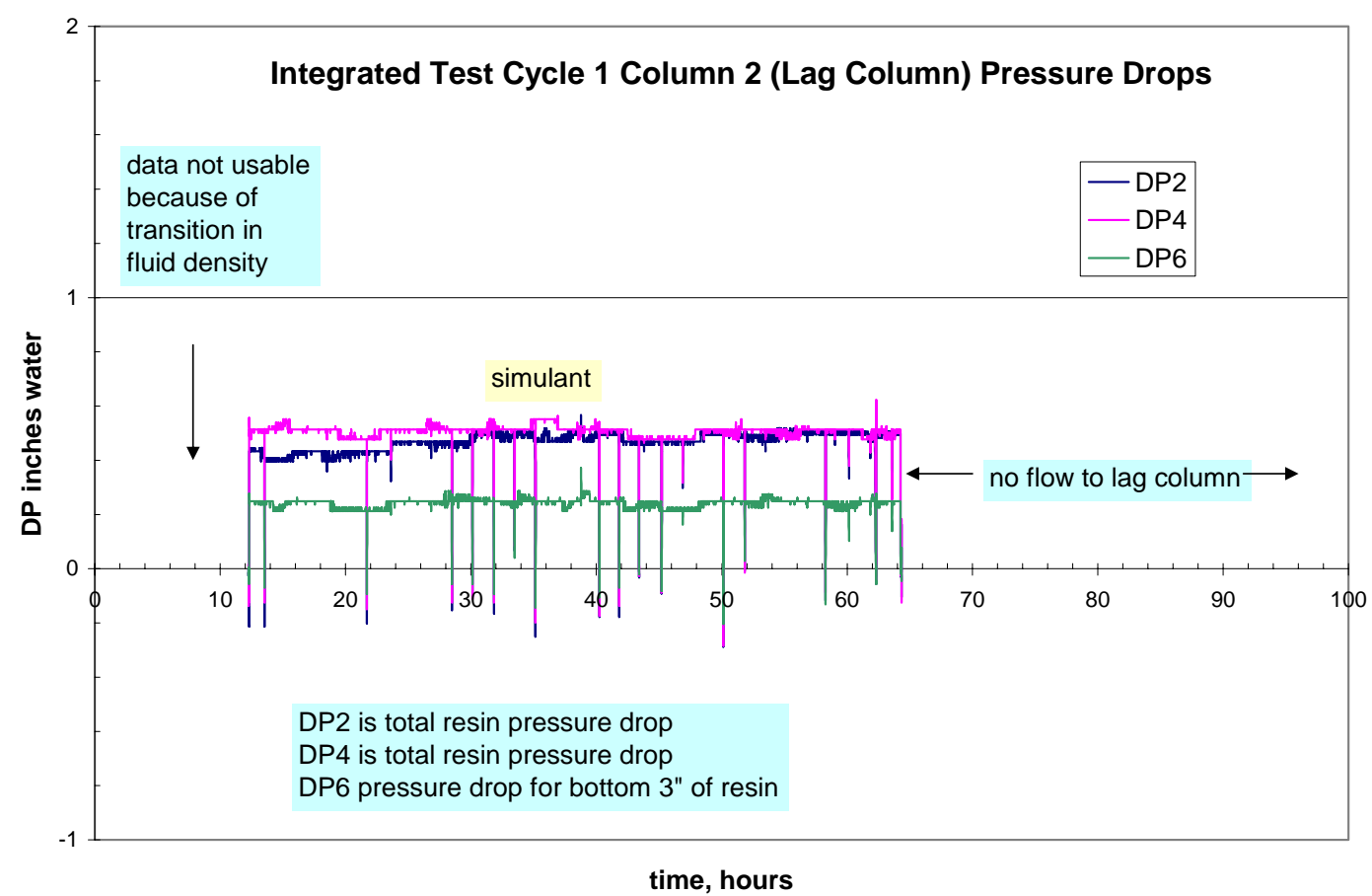

Figure 58. Pressure Drop Across Resin Bed for Lag Column and Cycle 1

Resin bed heights during Cycle 1 are plotted in Figure 59. The plot shows the expected variation with the smallest resin bed height during the elution step.

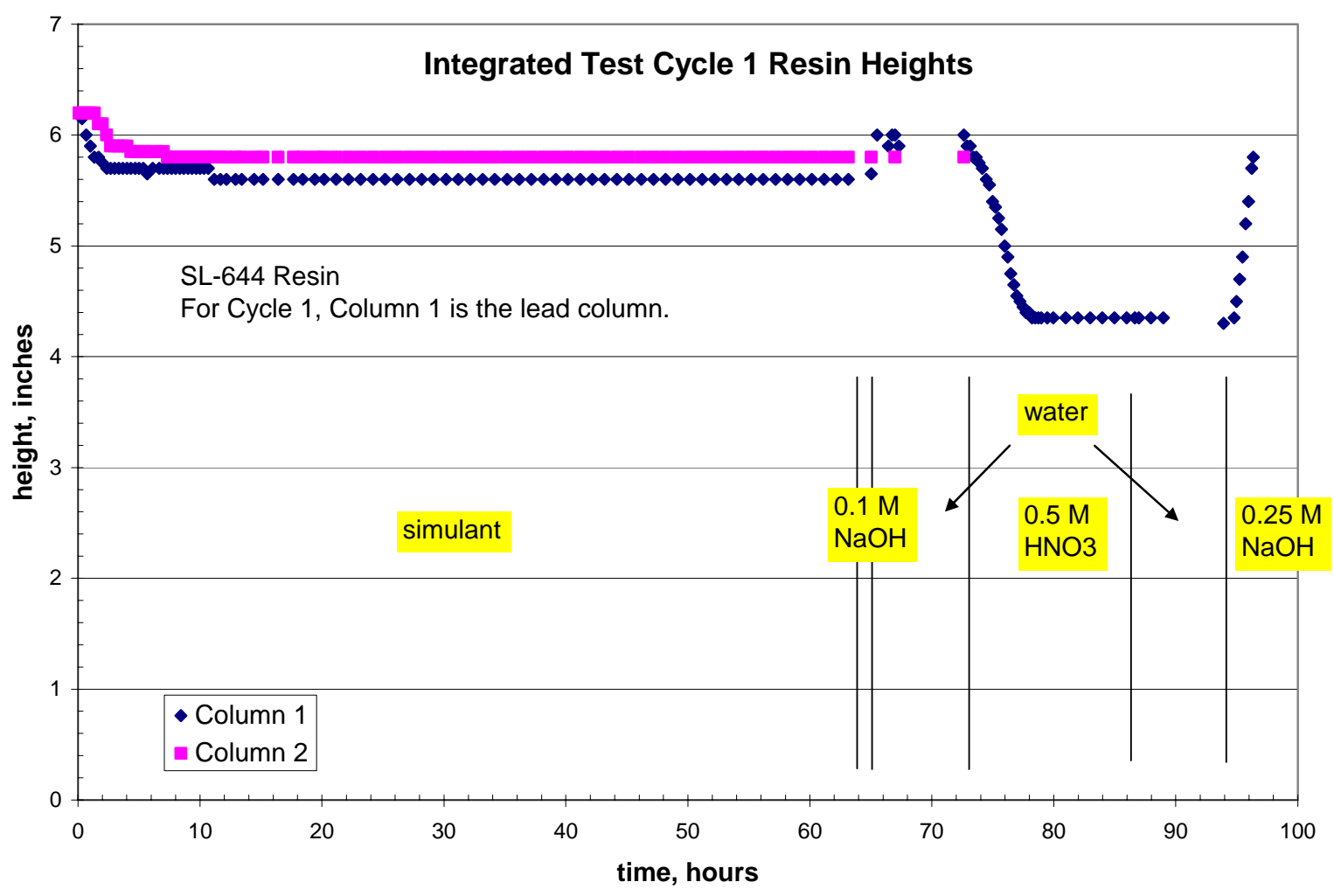

Figure 59. Resin Bed Height During Cycle 1 
Table 49 lists the volumes of the different solutions. Note that some of the "introduced" volumes were less than the internal volume of the column, which implies that some solutions could not fully displace the previous contents in the column.

Table 49. Comparison of the Volume for Each of the CIX Regeneration Process Solutions to the Internal Volume of Pilot-scale CIX Column

\begin{tabular}{|c|c|c|}
\hline $\begin{array}{c}\text { Regeneration } \\
\text { Process } \\
\text { Solution }\end{array}$ & $\begin{array}{c}\text { Volume } \\
\text { Introduced } \\
\text { into Column }\end{array}$ & $\begin{array}{c}\text { Internal } \\
\text { Internal Volume } \\
\text { of the pilot-scale } \\
\text { CIX column }\end{array}$ \\
\hline $0.1 \mathrm{M} \mathrm{NaOH}$ & 3 & BV \\
\hline Water & 3 & 4 \\
\hline $0.5 \mathrm{M} \mathrm{HNO3}$ & 15 & 4 \\
\hline Water & 3 & 4 \\
\hline $0.25 \mathrm{M} \mathrm{NaOH}$ & 6 & 4 \\
\hline
\end{tabular}

Figures 60 and 61 show the measured $\mathrm{pH}$ and conductivity of the column effluent as a function of bed volume for Cycle 1 . The $\mathrm{X}$ axis for these plots is cumulative volume reaching the exit of the column expressed in $\mathrm{BV}$. The $\mathrm{pH}$ plot has the expected shape, with one exception. The particular $\mathrm{pH}$ probe used was designed to have a range from zero to 12; we did not find a $\mathrm{pH}$ probe with a wider range that would also withstand long immersion in our solutions. Therefore, the probe read about 12 for solutions with $\mathrm{pH}$ that high and higher. The timing for changes in $\mathrm{pH}$ agrees well with the times computed using a plug flow calculation for when a new solution would reach the column exit. During the regeneration step with $0.25 \mathrm{M} \mathrm{NaOH}$, the $\mathrm{pH}$ increased to almost 12, implying that the caustic wave had broken through the column. 
WSRC-TR-2005-00105, REVISION 0

SRNL-RPP-2005-00012, REVISION 0

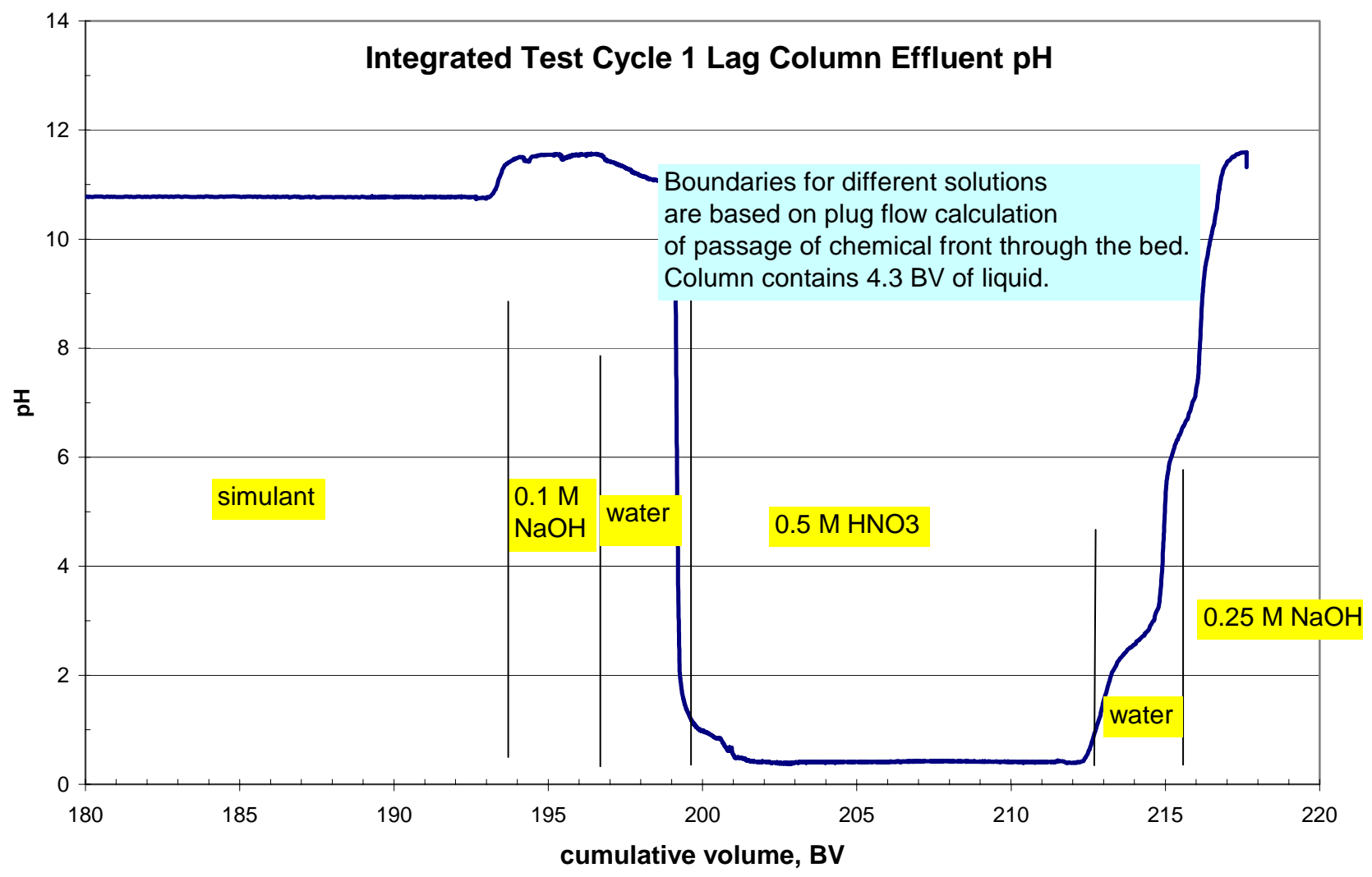

Figure 60. Effluent pH During Cycle 1

The conductivity probe reading, plotted in Fig. 61, told a somewhat different story than the $\mathrm{pH}$ probe. The conductivity plot has a noisier appearance than the $\mathrm{pH}$ plot because electrical conductivity is roughly proportional to ionic concentration and $\mathrm{pH}$ is proportional to the logarithm of hydrogen ion concentration. There are definite spikes in the measured conductivity at the simulant and $0.1 \mathrm{M} \mathrm{NaOH}$ transition and also at the acid and water transition. Conductivity of an aqueous solution increases with temperature. These spikes may have occurred as a result of heat of mixing. After acid is introduced to the column, most of the change in conductivity occurs in the first $3.5 \mathrm{BV}$, however, the conductivity appears to be changing slowly during the remaining period that acid flows through the column. The resin may continue to consume small amounts of acid after the first 3.5 BV. When $0.25 \mathrm{M} \mathrm{NaOH}$ is introduced to the column, the conductivity reading never increased very much. This implies that nearly all of the sodium ions in the $\mathrm{NaOH}$ solution are loaded on the resin and nearly all of the hydroxide ions react with residual hydrogen ions on the resin to form water. 
WSRC-TR-2005-00105, REVISION 0

SRNL-RPP-2005-00012, REVISION 0

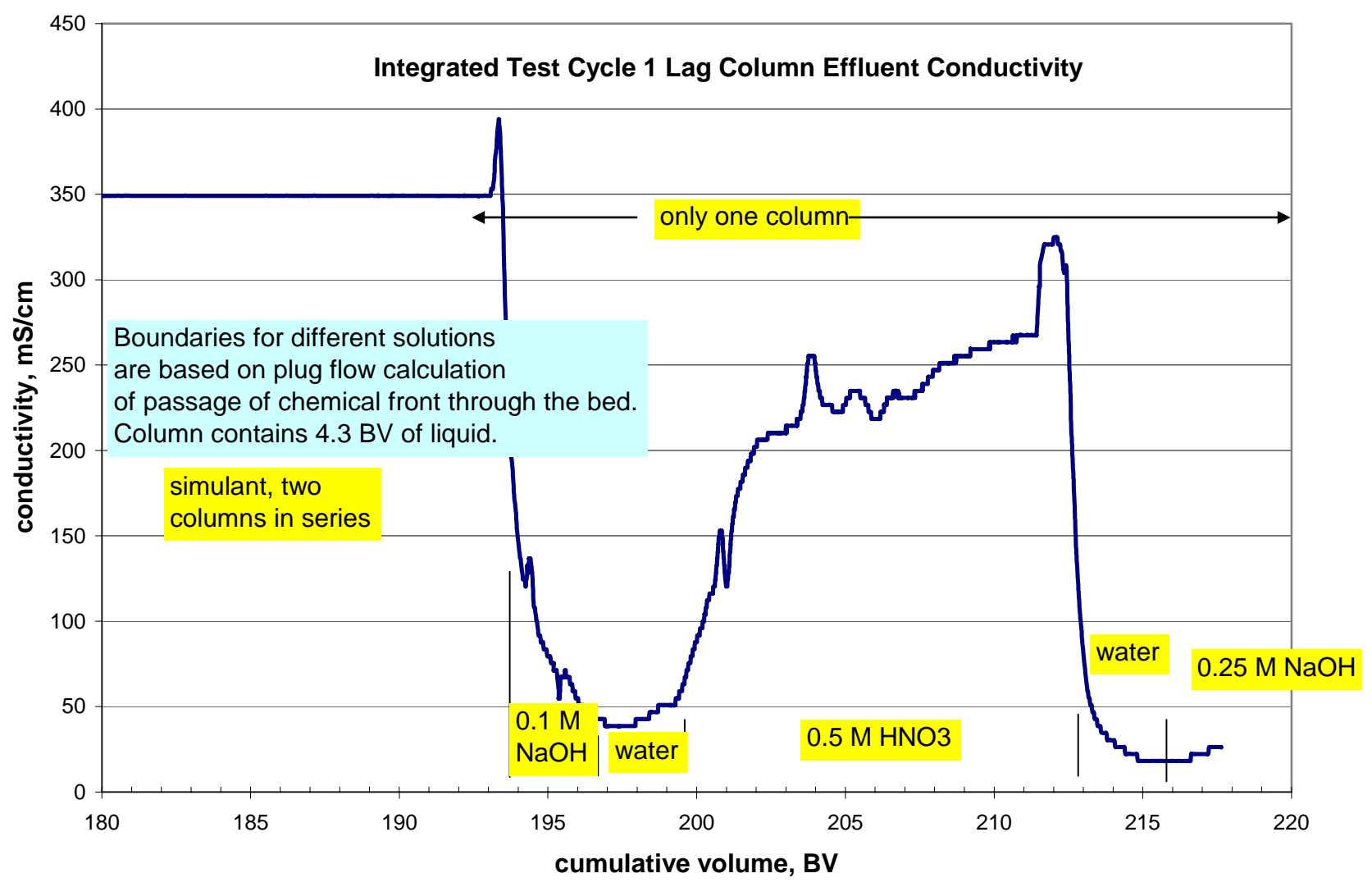

Figure 61. Effluent Conductivity During Cycle 1

Table 50 shows the response of the conductivity probe and $\mathrm{pH}$ probe when inserted into beakers of the various solutions. This table can be used to estimate how closely the transient conductivity and $\mathrm{pH}$ approached the steady state values. Note that the SI unit of the conductivity, milliSiemens/cm, was formerly referred to as mmho/cm

Table 50. Probe Responses for Standard Solutions

\begin{tabular}{|c|c|c|}
\hline solution & $\begin{array}{c}\text { Conductivity, } \\
\mathrm{mS} / \mathrm{cm}\end{array}$ & $\mathrm{pH}$ \\
\hline DI water & $<5$ & 5 to 9 \\
\hline $0.1 \mathrm{M} \mathrm{NaOH}$ & 35 & 11.1 \\
\hline $0.25 \mathrm{M} \mathrm{NaOH}$ & 76 & 10.9 \\
\hline $0.5 \mathrm{M}$ HNO3 & 250 & 0.42 \\
\hline
\end{tabular}




\subsubsection{CIX Chemical Observations}

\subsection{Measurement Uncertainty}

The purpose of the resin is to remove cesium from LAW solutions that contain a mixture of constituents and are $5 \mathrm{M}$ in sodium. Evaluation of the effectiveness of the resin requires the measurement of cesium concentration under challenging conditions, that is, with a high concentration of sodium. The samples must be heavily diluted to reduce sodium concentration to a manageable level, which also dilutes cesium. The Analytical Development Section (ADS) of SRNL claimed an accuracy of $\pm 20 \%$ of the stated value for these cesium measurements. Because of the large sodium concentration the accuracy was checked using spiked samples a well as blanks that were $5 \mathrm{M}$ in sodium. The results are shown in Fig. 62.

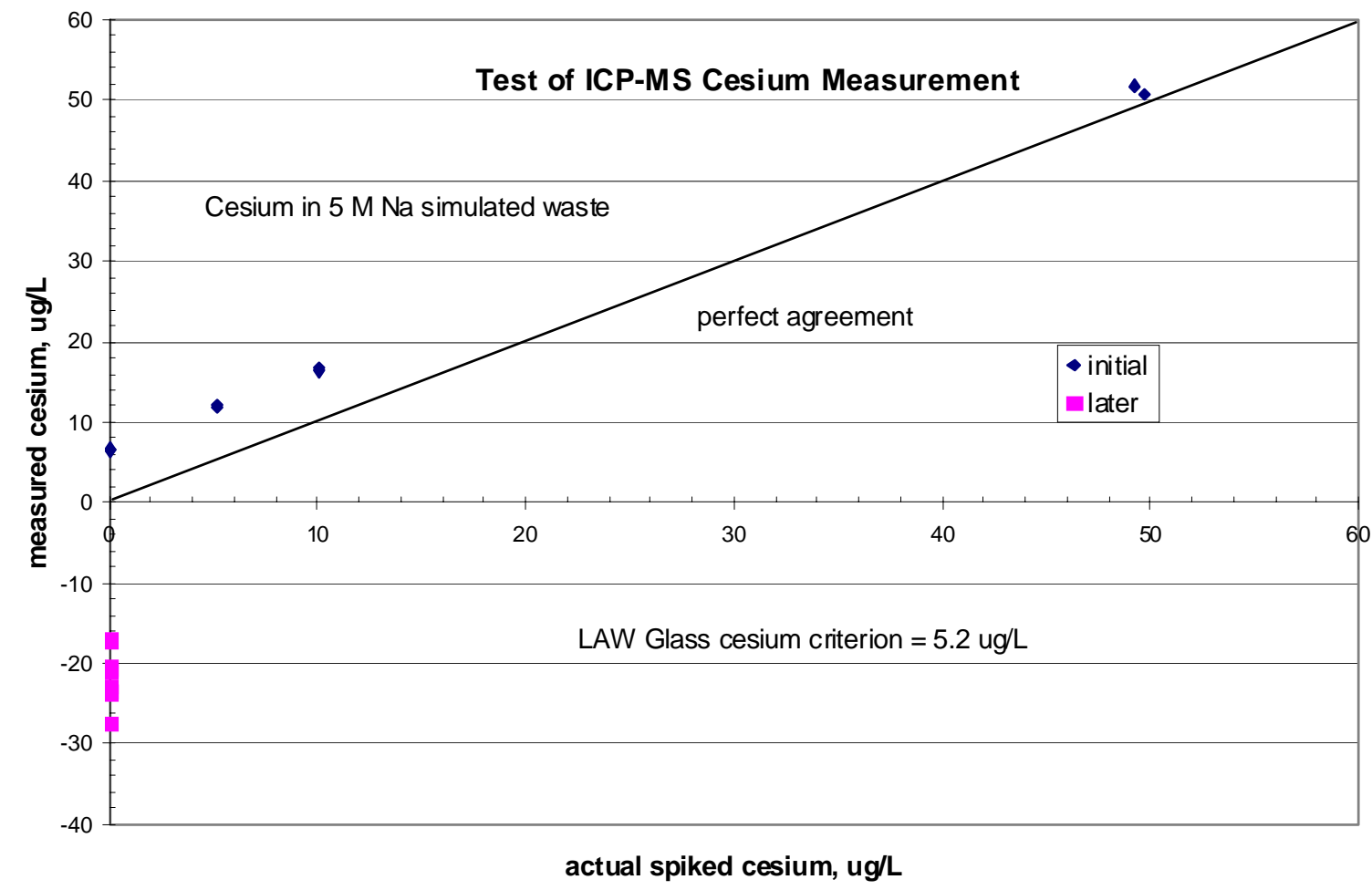

Figure 62. Test of Ability of ICP-MS to Measure Cesium

The initial results were encouraging and indicated a bias of less than $10 \mu \mathrm{g} / \mathrm{L}$ of cesium concentration for very low concentrations and less than $10 \%$ uncertainty for concentrations of $50 \mu \mathrm{g} / \mathrm{L}$. Based on the initial results it appeared possible to correct for the bias. Unfortunately, later samples were analyzed to have negative concentrations as low as $-27 \mu \mathrm{g} / \mathrm{L}$. Of course, negative concentrations are physically impossible, but provide useful information about the accuracy of the measurement. Therefore, no effort was made to correct for bias and measurement uncertainty was treated as random. Previous measurements (Steimke, 2002), not plotted, for concentrations greater than 
$7000 \mu \mathrm{g} / \mathrm{L}$ confirmed a measurement uncertainty of $\pm 10 \%$ for higher concentrations. Therefore, the estimate for the uncertainty of cesium concentration measurements in this program is: $\pm(10 \%$ reading $+30 \mu \mathrm{g} / \mathrm{L})$, which is expressed for $95 \%$ confidence limits.

It should be noted that, even the originally indicated bias of $10 \mu \mathrm{g} / \mathrm{L}$ was insufficient to meet SIPP needs. Before SIPP began, the original criterion for when a Lead column would need regeneration was when the cesium concentration of the exiting LAW stream from the lead column would exceed one-half the incoming concentration, i.e., $50 \%$ breakthrough. For Campaign III, the simulated LAW feed cesium concentration was $7760 \mu \mathrm{g} / \mathrm{L}$ [see Cs concentration column in the fresh simulant in row of Table 50], therefore, detecting a concentration $3880 \mu \mathrm{g} / \mathrm{L}$ accurately would have been no problem. However, that criterion changed to a much more stringent one. The new criterion is based on when the exiting stream of the Lag Column would contain a concentration of greater than $5.2 \mu \mathrm{g} / \mathrm{L}$ of cesium. Unfortunately, while some ADS cesium measurements appear to detect concentrations to better than $5 \mu \mathrm{g} / \mathrm{L}$, as already stated, the actual ADS uncertainty is estimated to be \pm ( $10 \%$ of a reading $+30 \mu \mathrm{g} / \mathrm{L}$ of cesium $)$. This implies that the detection limit for cesium was $30 \mathrm{ug} / \mathrm{L}$. This relatively large uncertainty must be kept in mind when evaluating such low concentrations. However, changes in cesium concentration throughout a cycle are expected to indicate when the cesium concentration is increasing in the exiting Lag Column stream.

\subsection{Cesium Removal Effectiveness}

Simulant is pumped through the lead column where most of the cesium is removed and then enters the lag column, where additional cesium is removed. Figure 63 shows the cesium concentration exiting the lead column for the four cycles, as a function of bed volumes passed.

Initially, the cesium concentration was low, or perhaps really zero when considering analytical uncertainty, and then increased. There is a general trend to more cesium escaping the lead column with additional cycles. Note that the lead column resin is fresh for Cycles 1 and 2 and has been eluted and regenerated for Cycles 3 and 4 . For all four cycles the final effluent cesium concentration was less than $7 \%$ of the inlet simulant feed concentration. Note that in Cycle 2, Column 2, the lead column, was more effective at removing cesium from the simulant, than Column 1, the lead column in Cycle 1. This is consistent with the measurement that Column 2 had more mass of resin than Column 1. The data in Fig. 63 were used to calculate the percentage of cesium removed by the lead column, as listed in Table 47. Piecewise linear fits were passed through the data for each cycle and the area under the fit was computed. Uncertainty was calculated in the following way. Each point plotted in Fig. 63 has error bars. However, the magnitude of the uncertainty associated with the fit was assumed to be less by a factor of the square root on the number of points used to make the fit.

Figure 64 shows the cesium concentration exiting the lag column. Considering the uncertainty in the analytical measurement of cesium, all of the measurements may really be zero. However, the measurement uncertainty does not allow determination of whether the RPP-WTP criterion, i.e., cesium concentration less than $5.2 \mu \mathrm{g} / \mathrm{L}$ from the lag column, is satisfied. The concentration measured for one sample, the sample collected at 
175 BV for Cycle 4 was not plotted because it was so much higher than for the other samples. This sample was analyzed twice for cesium concentration and the results were $1190 \mu \mathrm{g} / \mathrm{L}$ and $1630 \mu \mathrm{g} / \mathrm{L}$.

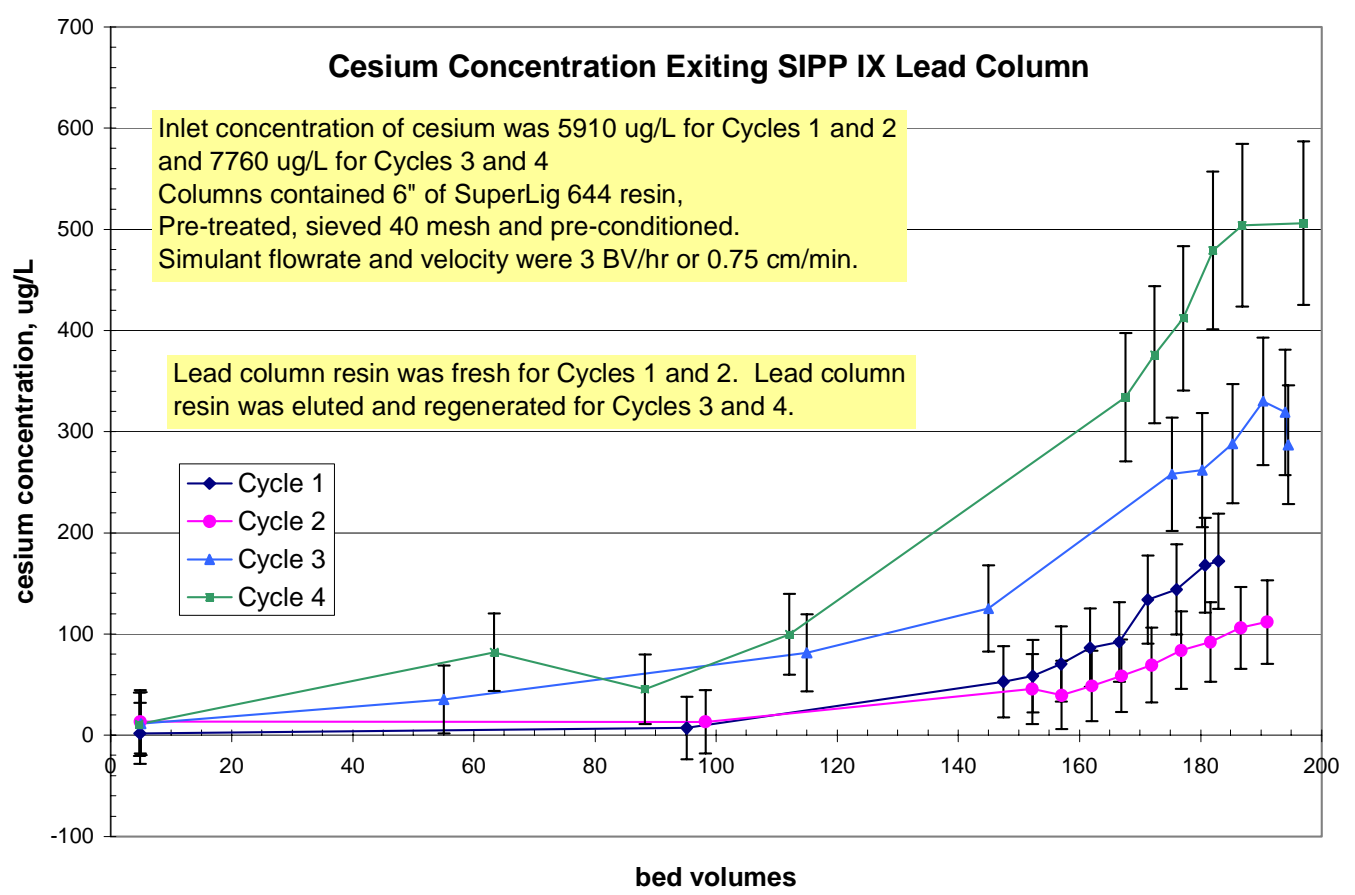

Figure 63. Cesium Concentration in Simulant Exiting Lead Column

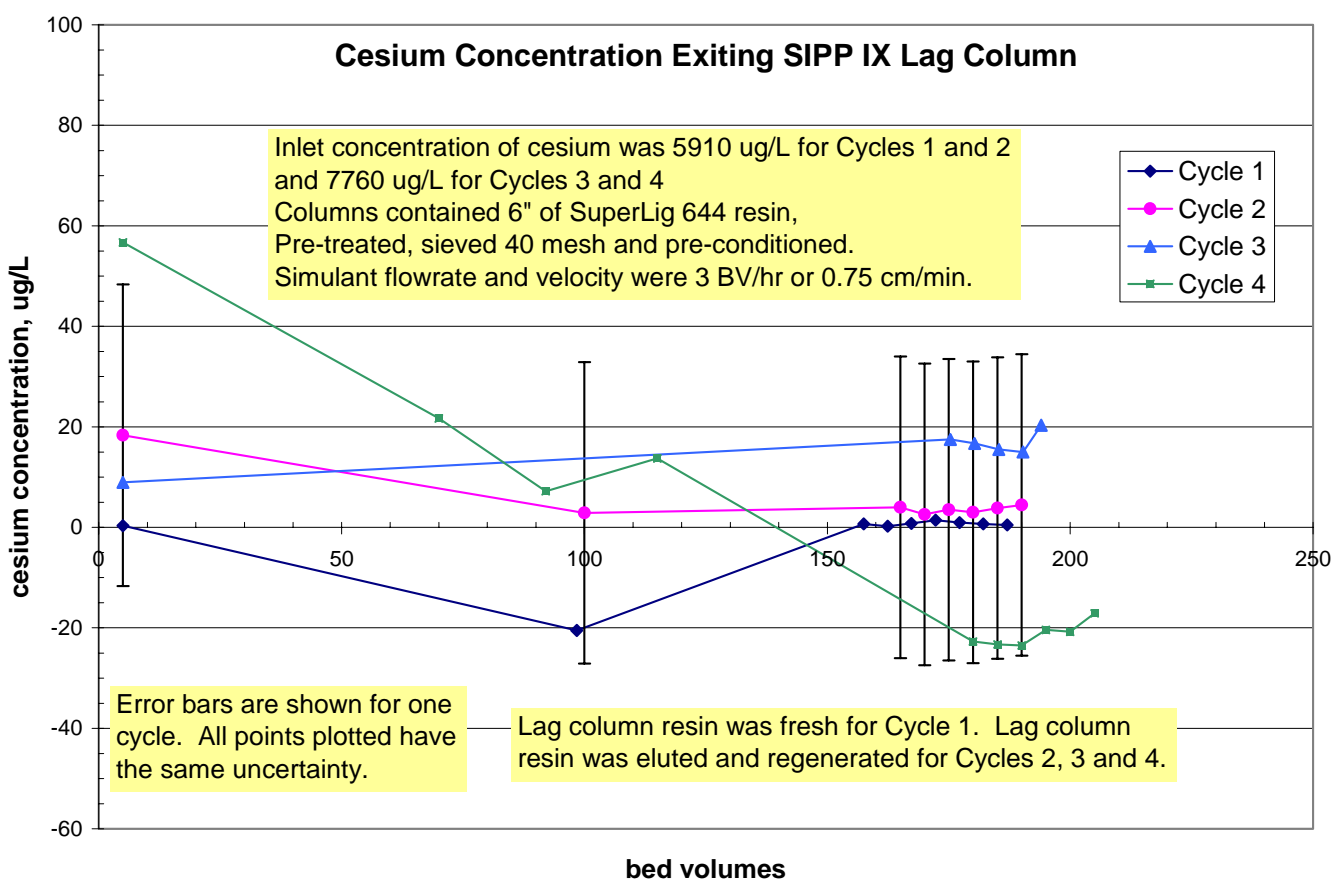

Figure 64. Cesium Concentration Exiting Lag Column 


\subsection{Elutions of Resin Beds}

In all four cycles the lead column was eluted with $0.5 \mathrm{M}$ nitric acid followed by regeneration with $0.25 \mathrm{M} \mathrm{NaOH}$. For Cycles 1 , 3 and 4 samples were collected of the eluate at regular intervals. Cesium concentrations are plotted in Figs. 65, 66, and 67 as a function of cumulative cesium removed.

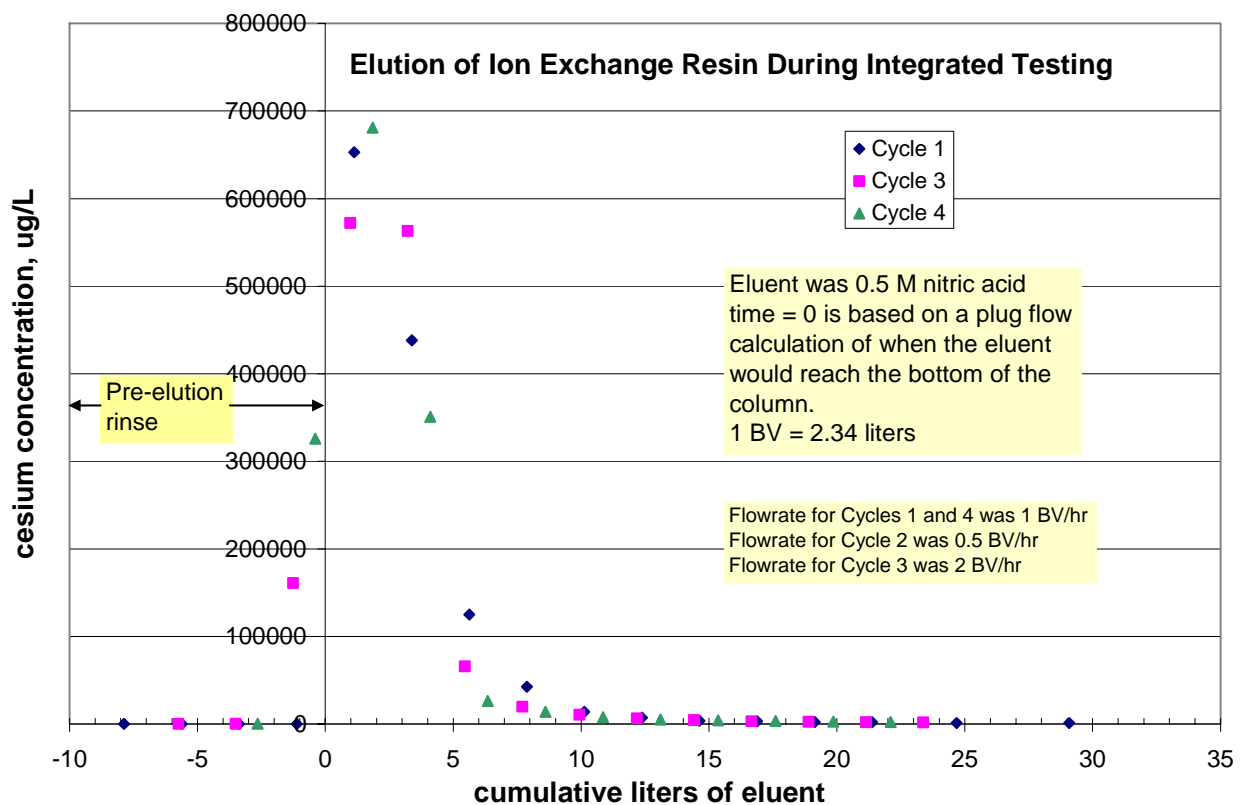

Figure 65. Elution Plot, Linear

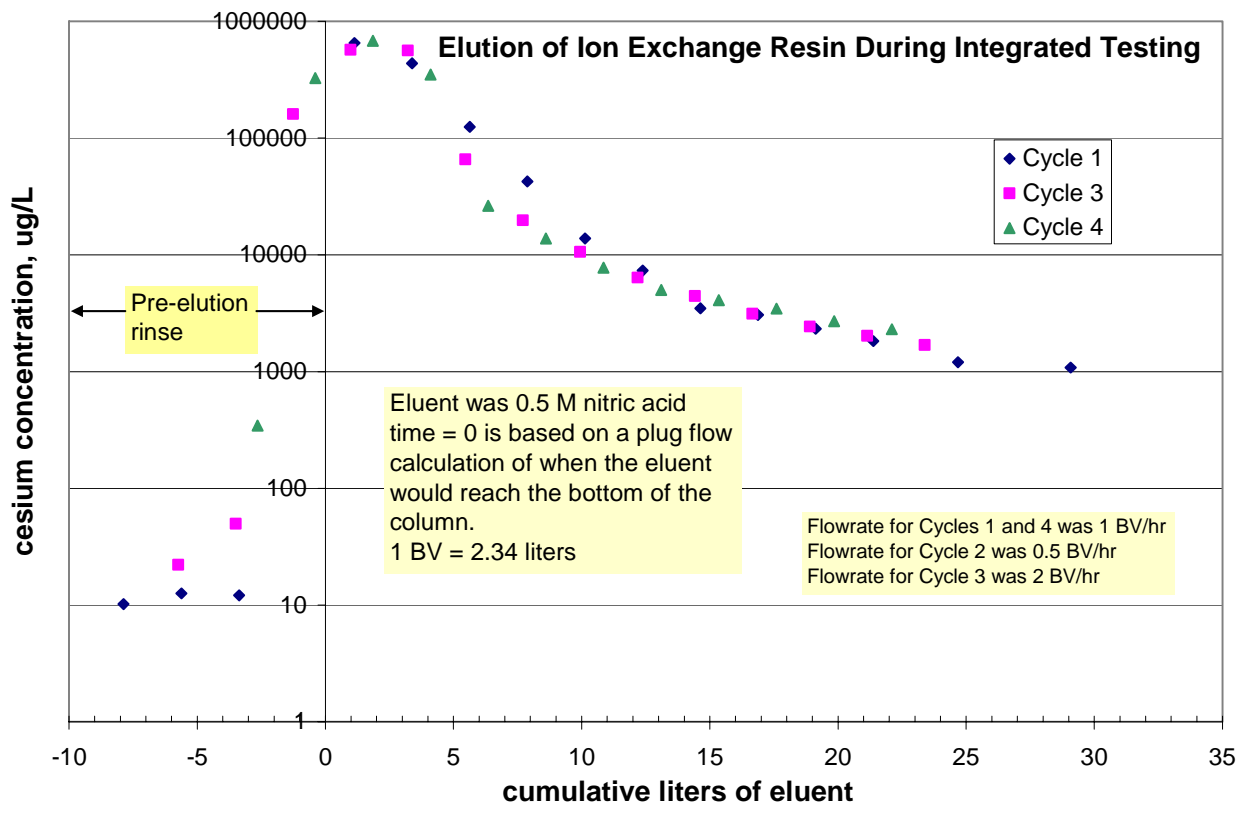

Figure 66. Elution Plot, Logarithmic 
WSRC-TR-2005-00105, REVISION 0

SRNL-RPP-2005-00012, REVISION 0

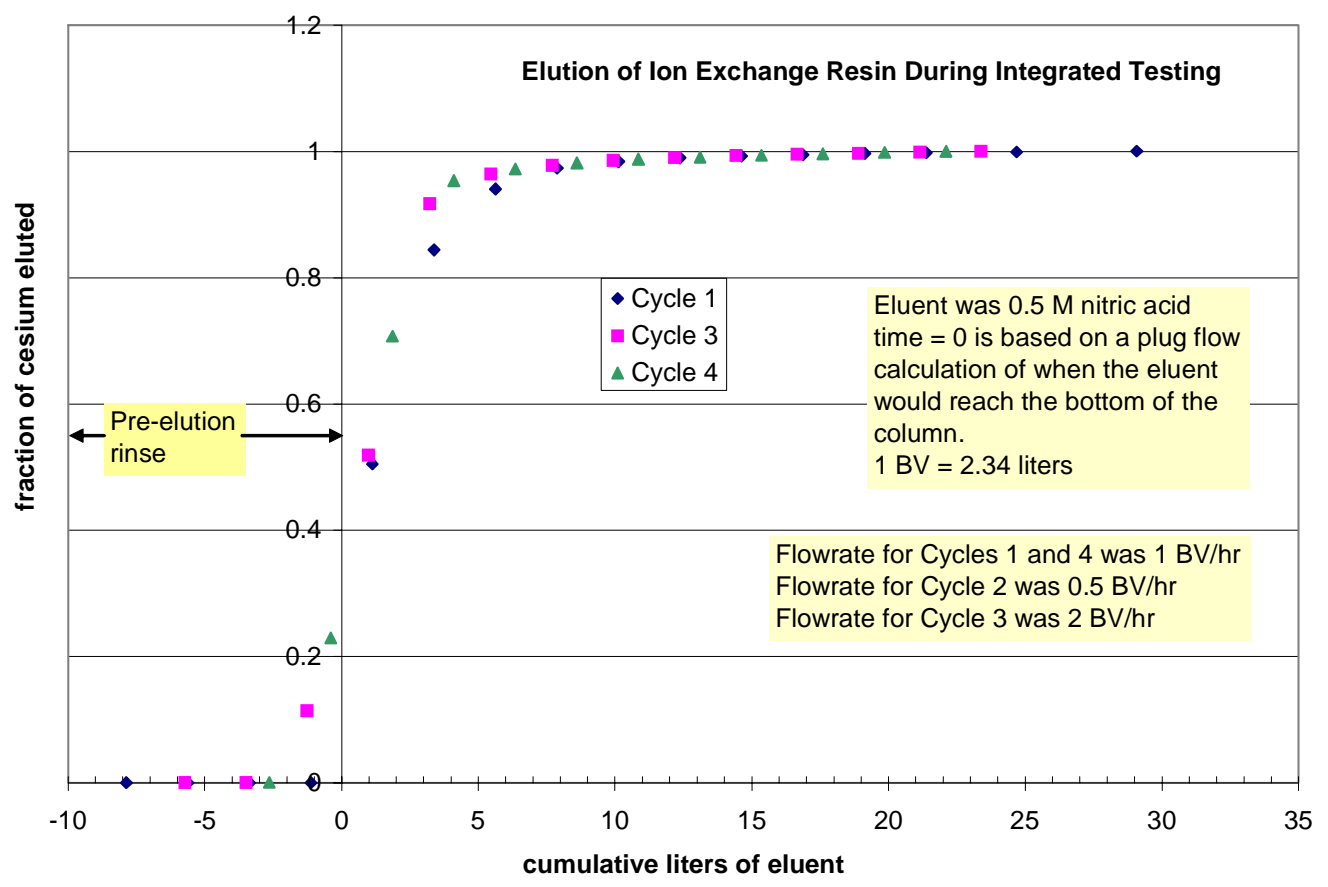

Figure 67. Cumulative Fraction of Cesium Removed during Elution

Zero volume refers to the time when a plug flow calculation indicated that acid first reached the outlet of the column. There is no obvious difference in the elution profiles for Cycles 1, 3 and 4. Note that the concentration of cesium peaks when the front of acid reaches the outlet of the column. Figure 67 shows that more than $94 \%$ of the total cesium eluted is removed by the first $6 \mathrm{BV}$ of eluent.

\subsection{Cesium Mass Balance}

In the CIX unit operation, cesium enters the two CIX columns dissolved in simulant and leaves the columns in simulant at a much lower concentration and also in eluate and other solutions. The cesium mass entering should equal the cesium mass leaving plus any accumulation, which was small. That is, the accumulation of cesium inventory change on the resin was assumed to be small. To perform this calculation, the LAW feed and receipt tanks were calibrated for volume and the results are discussed below. For some solutions the volume was calculated based on the average calibrated flow rate and the elapsed time. Mass balances are listed in Tables 51 and 52. For all four cycles the cesium mass in agrees with the cesium mass out to within measurement uncertainty, which is expressed for 95\% confidence limits. As previously mentioned in Section 2.8.5.3.1, the uncertainty of measuring cesium concentration was $\pm(10 \%$ of reading + $30 \mu \mathrm{g} / \mathrm{L}$ ). The uncertainty in measuring volume was \pm 3 liters for simulant and \pm 1 liter for the other solutions. The mass of cesium in a solution is equal to the cesium concentration multiplied by the solution volume. The cesium mass out was the sum of the masses for the various solutions. The uncertainty on the cesium mass out was computed as the root sum square of the uncertainties for the various solutions.

As a consistency check on the outlet cesium, the total cesium in the composite eluate and the composite pre-elution rinse was compared with the integral of the data plotted in 


\section{WSRC-TR-2005-00105, REVISION 0 SRNL-RPP-2005-00012, REVISION 0}

Fig. 65. In principal, the integral should give the same mass as the composite samples. The integral was calculated numerically using three methods, the Trapezoidal Rule and two variations of Simpson's Rule. The Trapezoidal Rule approximates the integral as a series of trapezoids. The area of each trapezoid is computed as the length of the base of the trapezoid multiplied by the average height. Simpson's Rule approximates segments of the data as parabolas. Simpson's Rule was applied two different ways. Recall that the equation for Simpson's Rule is:

$$
\int(x) d x=h / 3\left(f\left(x_{0}\right)+4 f\left(x_{1}\right)+2 f\left(x_{2}\right)+4 f\left(x_{3}\right)+\ldots .+4 f\left(x_{n-1}\right)+\left(f\left(x_{n}\right)\right)\right.
$$

where $\mathrm{h}$ is the difference in $\mathrm{x}$ between adjacent points. First, Simpson's Rule was applied to the data in Fig. 65. Then, two additional points with concentration equal to zero were added to the beginning and end of the data set. Then Simpson's Rule was applied again. The two applications of Simpson's Rule give different results depending on whether the data point with the largest concentration is multiplied by two or four. 
WSRC-TR-2005-00105, REVISION 0

SRNL-RPP-2005-00012, REVISION 0

Table 51. Mass Balances for Cycles 1 and 2

\begin{tabular}{|c|c|c|c|c|c|c|c|c|c|c|c|}
\hline $\begin{array}{l}\text { Cycle } 1 \\
\text { Solution }\end{array}$ & $\begin{array}{l}\text { volume } \\
\text { liters }\end{array}$ & $\begin{array}{l}\text { cesium } \\
\text { conc., } \\
\text { mg/L }\end{array}$ & $\begin{array}{c}\text { ADS } \\
\text { Sample } \\
\#\end{array}$ & $\begin{array}{l}\text { cesium } \\
\text { mass, } \\
\text { mg }\end{array}$ & $\begin{array}{c}\text { mass } \\
\text { uncertainty, } \\
\text { mg }\end{array}$ & $\begin{array}{l}\text { Cycle } 2 \\
\text { Solution }\end{array}$ & $\begin{array}{l}\text { volume } \\
\text { liters }\end{array}$ & $\begin{array}{l}\text { cesium } \\
\text { conc., } \\
\text { mg/L }\end{array}$ & $\begin{array}{c}\text { ADS } \\
\text { Sample } \\
\#\end{array}$ & $\begin{array}{c}\text { cesium } \\
\text { mass, } \\
\text { mg }\end{array}$ & $\begin{array}{c}\text { mass } \\
\text { uncertainty, } \\
\text { mg }\end{array}$ \\
\hline \multirow[t]{3}{*}{ fresh simulant in } & 428 & 5.91 & 7820 & 2529 & 284 & fresh simulant in & 437 & 5.91 & 7820 & 2583 & 289 \\
\hline & & & & & & Cycle 1 spent & 9.4 & 0.12 & 8198 & 1 & 0 \\
\hline & & & & & & total Cs mass in & & & & 2584 & 289 \\
\hline simulant out (1) & 408 & 0.0213 & 9494 & 9 & 13 & simulant out (1) & 427 & 0.00897 & 9495 & 4 & 13 \\
\hline final simulant (2) & 9.4 & 0.12 & 8198 & 1 & 1 & final simulant (2) & 20 & 0.102 & 8358 & 2 & 1 \\
\hline $0.1 \mathrm{M} \mathrm{NaOH}$ & 12.2 & 0.344 & 8509 & 4 & 1 & $0.1 \mathrm{M} \mathrm{NaOH}$ & 44.5 & 50.6 & 8359 & 2252 & 277 \\
\hline pre-elution rinse & 0.7 & 70.8 & 8497 & 50 & 76 & pre-elution rinse & 0 & 0 & 8515 & 0 & 0 \\
\hline eluate & 31.5 & 87.2 & 8200 & 2747 & 363 & eluate & 6.8 & 2.47 & 8360 & 17 & 4 \\
\hline $\begin{array}{l}\text { post-elution } \\
\text { rinse }\end{array}$ & 7.5 & 0.887 & 8201 & 7 & 2 & post-elution rinse & 5.2 & 0 & 8361 & 0 & 0 \\
\hline regenerant & 4 & 0.0336 & 8510 & 0 & 0 & regenerant & 11 & 0.0109 & 8362 & 0 & 0 \\
\hline total Cs out & & & & 2817 & 371 & total Cs out & & & & 2274 & 277 \\
\hline
\end{tabular}

(1) Simulant out refers to the amount of treated simulant during each cycle and does not include final simulant.

(2) Final simulant for Cycles 1 and 3, refers to the simulant displaced from the Lead column by caustic to begin the regeneration process. Final simulant, for Cycles 2 and 4 also includes the simulant displaced from the Lag column. 
WSRC-TR-2005-00105, REVISION 0

SRNL-RPP-2005-00012, REVISION 0

Table 52. Mass Balances for Cycles 3 and 4

\begin{tabular}{|c|c|c|c|c|c|c|c|c|c|c|c|}
\hline $\begin{array}{l}\text { Cycle } 3 \\
\text { solution }\end{array}$ & $\begin{array}{l}\text { volume } \\
\text { liters }\end{array}$ & $\begin{array}{l}\text { cesium } \\
\text { conc., } \\
\text { mg/L }\end{array}$ & $\begin{array}{l}\text { ADS } \\
\text { Sample } \\
\quad \#\end{array}$ & $\begin{array}{l}\text { cesium } \\
\text { mass, } \\
\text { mg }\end{array}$ & $\begin{array}{c}\text { mass } \\
\text { uncertainty, } \\
\text { mg }\end{array}$ & $\begin{array}{l}\text { Cycle } 4 \\
\text { solution }\end{array}$ & $\begin{array}{l}\text { volume } \\
\text { liters }\end{array}$ & $\begin{array}{l}\text { cesium } \\
\text { conc., } \\
\text { mg/L }\end{array}$ & $\begin{array}{c}\text { ADS } \\
\text { Sample } \\
\#\end{array}$ & $\begin{array}{l}\text { cesium } \\
\text { mass, } \\
\text { mg }\end{array}$ & $\begin{array}{c}\text { mass } \\
\text { uncertainty, } \\
\text { mg }\end{array}$ \\
\hline fresh simulant in & 453 & 7.76 & 9229 & 3515 & 388 & fresh simulant in & 453 & 7.76 & 9229 & 3515 & 388 \\
\hline $\begin{array}{l}\text { Cycle } 2 \text { spent } \\
\text { simulant (1) }\end{array}$ & & & & & & $\begin{array}{l}\text { Cycle } 3 \\
\text { Spent } \\
\text { simulant }\end{array}$ & 9 & 0.363 & 11124 & 3 & 0 \\
\hline & & & & & & total Cs mass in & & & & 3519 & 388 \\
\hline simulant out (2) & 434 & 0.0237 & 9496 & 10 & 14 & simulant out (2) & 442 & 0 & 9497 & 0 & 13 \\
\hline final simulant (3) & 9.4 & 0.363 & 11124 & 3 & 1 & final simulant (3) & 19.3 & 0 & 11130 & 0 & 1 \\
\hline $0.1 \mathrm{M} \mathrm{NaOH}$ & 7.7 & 41.3 & 11125 & 318 & 73 & $0.1 \mathrm{M} \mathrm{NaOH}$ & 8.8 & 0 & 11131 & 0 & 0 \\
\hline pre-elution rinse & 7.1 & 39.8 & 11126 & 283 & 68 & pre-elution rinse & 7.3 & 16.6 & 11132 & 121 & 29 \\
\hline eluate & 31.3 & 87.3 & 11127 & 2732 & 361 & eluate & 33.4 & 91.9 & 11133 & 3069 & 400 \\
\hline post-elution rinse & 7.8 & 0.694 & 11128 & 5 & 1 & post-elution rinse & 7.7 & 0.456 & 11134 & 4 & 1 \\
\hline regenerant & 13.8 & 2.23 & 11129 & 31 & 6 & regenerant & 13.2 & 0.352 & 11135 & 5 & 1 \\
\hline Total Cs out & & & & 3383 & 375 & Total Cs out & & & & 3199 & 401 \\
\hline
\end{tabular}

(1) The small volume of Cycle 2 spent simulant obtained during Cycle 2 regeneration is included in the fresh simulant. There was no separate concentration measurement made. That is, the $3515 \mathrm{mg}$ shown as the mass of Cs in the fresh simulant also includes the few milligrams of Cs from the Cycle 2 spent simulant

(2) Simulant out refers to the amount of treated simulant during each cycle and does not include final simulant.

(3) Final simulant for Cycles 1 and 3, refers to the simulant displaced from the Lead column by caustic to begin the regeneration process. Final simulant, for Cycles 2 and 4 also includes the simulant displaced from the Lag column. 
The results for cesium mass in the eluate are shown in Table 53. No results are listed for Cycle 2 because no periodic eluate samples were collected during Cycle 2. The masses of cesium calculated using numerical integration agree reasonably well with the masses determined using composite samples.

Table 53. Mass of Eluate Cesium as a function of Integration Method

\begin{tabular}{|c|c|c|c|c|}
\hline $\begin{array}{c}\text { Cycle } \\
\text { number }\end{array}$ & $\begin{array}{c}\text { Trapezoidal } \\
\text { Rule, } \\
\text { mg Cs }\end{array}$ & $\begin{array}{c}\text { Simpson's } \\
\text { Rule a, } \\
\text { mg Cs }\end{array}$ & $\begin{array}{c}\text { Simpson's } \\
\text { Rule b, } \\
\text { mg Cs }\end{array}$ & $\begin{array}{c}\text { Cs from } \\
\text { composite, } \\
\text { mg }\end{array}$ \\
\hline 1 & 2911 & 2679 & 3136 & 2797 \\
\hline 3 & 3164 & 3090 & 3236 & 3015 \\
\hline 4 & 3203 & 3188 & 3217 & 3190 \\
\hline
\end{tabular}

\subsubsection{Pilot CIX Conclusions (Author - Steimke)}

Pilot CIX operation was not part of Campaign IV testing but for completeness in reporting the following conclusions were extracted from the Interim Reports of Campaign II (Duignan et al., 2004b or Appendix I-2) and Campaign III (Duignan et at., 2004c). Also reported are measurements made after the Campaign III report was issued.

\section{$\underline{\text { Post-Campaign III Conclusions }}$}

- A post-test measurement of the dry mass of the SuperLig ${ }^{\circledR} 644$ resin found a ratio of resin in sodium form to resin in hydrogen form to be $1.19 \pm 0.03$ and $1.22 \pm 0.02$ for Columns 1 and 2, respectively.

- A pre-test particle-size distribution of the resin was found to be $78 \mathrm{wt} \%$ (Mesh > 30), $20 \mathrm{wt} \%(30 \leq$ Mesh $\leq 40)$, and $2 \mathrm{wt} \%$ (Mesh $<40$ ). A post-test PSD on the resin found no change in the Column 2 contents, indicating that no degradation of the resin occurred. The resin in Column 1 was nominally larger, but the uncertainty bars intersect, so all three samples of resin may actually have had the same size distribution. There was no evidence of particle degradation.

\section{Campaign III}

- Pressure drops for the four cycles were low and the resin beds permeabilities were approximately the same as were measured for the 24-Inch Column Test with fresh resin (Fowley et al, 2004).

- The pressure drop across the columns during the flow of simulant generally increased with each cycle of the four cycles of operation.

- SuperLig ${ }^{\circledR} 644$ effectively removed cesium from simulated waste and the analytical ability to measure cesium concentrations in five molar sodium did allow verification of the waste acceptance criterion of 50\% breakthrough from the lead column after 100 Bed Volumes of simulated waste were treated. In fact, in the worst case, Cycle 4, 
even after $190 \mathrm{BV}$ of treated waste, the lead column exit cesium concentration was less than $7 \%$ of the feed waste.

- SuperLig ${ }^{\circledR} 644$ effectively removed cesium from simulated waste, however, the analytical difficulty of measuring very low cesium concentrations in five molar sodium did not allow verification of the waste acceptance criterion of $5.2 \mu \mathrm{g} / \mathrm{L}$ from the lag column after $100 \mathrm{BV}$ of simulated waste were treated. This limitation was acknowledged before the start of testing.

- The fraction of the cesium in the simulant that was removed by the lead column generally decreased over the four cycles of testing.

- The masses of cesium in and out of the resin beds for all four cycles balanced to within measurement uncertainty.

- More than $94 \%$ of the total cesium eluted is removed by the first 6 BV of eluent.

\section{Campaign II}

- Pressure drops throughout the two cycles were low and the resin beds permeabilities were about the same as were measured for the 24-inch Test with fresh resin (Fowley et al, 2004).

- SuperLig ${ }^{\circledR} 644$ effectively removed cesium from simulated waste and the analytical ability to measure cesium concentrations in five molar sodium did allow verification of the waste acceptance criterion of $50 \%$ breakthrough from the lead column after 100 Bed Volumes of waste was treated. In fact, in the worst case, Cycle 1, even after $180 \mathrm{BV}$ of treated waste the lead column exit cesium concentration was only $3 \%$ of the feed waste.

- SuperLig ${ }^{\circledR} 644$ effectively removed cesium from simulated waste, however, the analytical ability to measure very low cesium concentrations in five molar sodium did not allow verification of the waste acceptance criterion of $5.2 \mu \mathrm{g} / \mathrm{L}$ from the lag column after $100 \mathrm{BV}$ of waste was treated.

- The masses of cesium in and out of the resin beds for Cycles 1 and 2 balanced to within measurement uncertainty.

- During cesium elution 97\% of the Cs was removed during the first 3.4 BV. 


\subsection{PILOT TREATED LAW EVAPORATOR (Authors - Adamson, Williams \& Zamecnik)}

The Treated LAW Evaporator Process, or TLP, is the last separation technology unit operation in the RPP-WTP Pretreatment Facility that a waste will see on start up. With respect to SIPP, it was be the last unit operation in line because it evaporated the treated LAW product that comes from the pilot CIX unit operation. This treated LAW product is mixed with two recycle streams to produce a feed stream for the TLP unit operation.

This section will not only highlight the pilot TLP tests carried out as part of Campaign III testing, but also includes Campaign II data for comparison. An abridged form of the raw data collected during both campaigns is given in Appendix H4. Campaign IV only dealt with FEP and UFP operations in order to test the new GKN filter, and therefore the pilot TLP was not used. However, it is useful to compare the two pilot TLP operations that were previously done (Duignan et al., 2004b or Appendix I-2 and 2004c) as part of this final report to understand the overall SIPP operation. The process started with the preparation of the TLP feed product in a predetermined fashion, see Table 4. This explanation of the feed stream preparation will be followed by a short description on how feed preparation was handled just before TLP operation and then how the waste stream was handled during and after its concentration. The following sections will discuss the equipment used to evaporate the waste and how the test was run. Finally, the results will be discussed and conclusions made.

\subsubsection{Chemical Description of the Pilot TLP Feed Stream (Author - Zamecnik)}

As shown in Table 4, the TLP evaporator feed consisted of the Cesium Ion Exchange product (Treated LAW), the LAW SBS condensate supplied by VSL, and a small amount of $0.25 \mathrm{M} \mathrm{NaOH}$ CIX regeneration solution, which is the displaced solution obtained when a newly regenerated CIX column is put into service. The compositions of the Untreated LAW CIX feed ${ }^{\dagger}$ and the LAW SBS Condensate, are given in Table 52, which were used in Campaign III. The CIX regeneration solution consisted of 20.5L of actual regeneration solution plus 17.3L of simulated regeneration solution; both were essentially $0.25 \mathrm{M} \mathrm{NaOH}$. The calculated and measured TLP Feed compositions are also shown in Table 54. The values in this table show that the measured TLP feed composition was slightly more dilute than expected from the calculation. The reason for this dilution is the small amount of $0.25 \mathrm{M} \mathrm{NaOH}$ CIX regeneration solution that was included in the treated LAW from the CIX.

\footnotetext{
${ }^{\dagger}$ Of course, the TLP feed was prepared with Treated LAW CIX feed (i.e., after being processed through the CIX), however, Table 54 shows Untreated LAW feed because only the Untreated feed samples were measured for the suite of analytes shown in the table. To minimize cost to the project, the Treated LAW feed was assumed to be chemically the same as the Untreated feed except of the concentration of cesium. Cesium was only measured in the Treated LAW CIX feed.
} 
WSRC-TR-2005-00105, REVISION 0

SRNL-RPP-2005-00012, REVISION 0

Table 54. Measured Composition of Campaign III TLP Feeds

\begin{tabular}{|c|c|c|c|c|}
\hline $\begin{array}{l}\text { All values } \mathrm{mg} / \mathrm{kg} \text { unless } \\
\text { otherwise noted }\end{array}$ & $\begin{array}{l}\text { Untreated CIX } \\
\text { LAW Feed* }\end{array}$ & $\begin{array}{l}\text { LAW SBS } \\
\text { Condensate }\end{array}$ & $\begin{array}{l}\text { TLP Feed } \\
\text { Calculated }\end{array}$ & $\begin{array}{l}\text { TLP Feed } \\
\text { Measured }\end{array}$ \\
\hline Total Solids (wt\%) & 28.29 & 0.26 & 16.32 & 16.23 to 16.24 \\
\hline Undissolved Solids (wt\%) & 0.00 & 0.17 & 0.07 & 0.07 to 0.15 \\
\hline Supernatant Solids (wt\%) & 28.29 & 0.09 & 16.26 & 16.10 to 16.18 \\
\hline Density $(\mathrm{kg} / \mathrm{L})$ & 1.248 & 1.002 & 1.130 & 1.134 \\
\hline $\mathrm{Ag}$ & 0.76 & $<3.9$ & 0.44 & $<50$ \\
\hline $\mathrm{Al}$ & 5136 & 7.53 & 2947 & 2900 \\
\hline $\mathrm{B}$ & 85.7 & 132 & 103 & \\
\hline $\mathrm{Ba}$ & $<1.3$ & $<0.26$ & 0 & $<2.0$ \\
\hline $\mathrm{Ca}$ & $<3.6$ & $<0.39$ & 0 & $<6.6$ \\
\hline $\mathrm{Cd}$ & $<0.04$ & $<0.26$ & 0 & $<2.4$ \\
\hline $\mathrm{Ce}$ & $<1.0$ & $<10$ & 0 & $<128$ \\
\hline Co & $<0.11$ & $<1.0$ & 0 & $<7.3$ \\
\hline $\mathrm{Cr}$ & 139 & 5.26 & 81.6 & 82.8 \\
\hline $\mathrm{Cu}$ & $<0.03$ & $<0.39$ & 0 & $<8.3$ \\
\hline $\mathrm{Fe}$ & 1.36 & 11.7 & 5.55 & 15.0 \\
\hline $\mathrm{K}$ & 16346 & 123 & 9420 & \\
\hline $\mathrm{La}$ & 0.85 & $<1.3$ & 0.49 & $<116$ \\
\hline $\mathrm{Li}$ & 1.72 & 11.5 & 5.68 & 1.00 \\
\hline $\mathrm{Mg}$ & $<0.24$ & 3.56 & 1.45 & $<14$ \\
\hline $\mathrm{Mn}$ & $<0.02$ & $<0.12$ & 0 & $<1.5$ \\
\hline Mo & 8.65 & $<1.3$ & 4.96 & $<17$ \\
\hline $\mathrm{Na}$ & 88141 & 587 & 50874 & 47684 \\
\hline $\mathrm{Nd}$ & $<1.5$ & $<12$ & 0 & $<43$ \\
\hline $\mathrm{Ni}$ & 0.25 & 0.34 & 0.28 & $<10$ \\
\hline $\mathrm{P}$ & 554 & $<10$ & 317 & 371 \\
\hline $\mathrm{Pb}$ & $<0.36$ & $<10$ & 0 & $<114$ \\
\hline $\operatorname{Re}$ & & 21.8 & 8.88 & \\
\hline $\mathrm{S}$ & 905 & 78.0 & 551 & 502 \\
\hline $\mathrm{Si}$ & 42.1 & 21.8 & 33.0 & 0 \\
\hline $\mathrm{Sr}$ & 0.16 & $<0.07$ & 0.09 & 8.00 \\
\hline $\mathrm{Ti}$ & 0.10 & 1.15 & 0.53 & $<23$ \\
\hline $\mathrm{Zn}$ & 2.37 & 1.29 & 1.88 & $<61$ \\
\hline $\mathrm{Zr}$ & 1.43 & 1.29 & 1.35 & $<8.0$ \\
\hline bromide & $<80$ & $<86$ & 0 & $<61$ \\
\hline chloride & 1082 & 626 & 875 & 795 \\
\hline fluoride & 44.9 & 146 & 85.1 & 56.0 \\
\hline formate & 1066 & 32.9 & 624 & 521 \\
\hline nitrate & 72917 & 135 & 41852 & 37200 \\
\hline nitrite & 23077 & 2575 & 14278 & 12300 \\
\hline oxalate & 658 & $<86$ & 377 & 374 \\
\hline phosphate & 1963 & $<86$ & 1125 & 1220 \\
\hline sulfate & 2853 & 233 & 1730 & 1570 \\
\hline ammonium & $<80$ & 990 & 827 & 785 \\
\hline
\end{tabular}

* Only the Untreated CIX LAW feed was analyzed for the tabular analytes, but it was assumed to be equivalent to the Treated CIX feed except for a higher concentration of Cs. 


\subsubsection{Simulant Handling (Author - Williams)}

To make feed for the pilot-scale TLP, in each campaign approximately $1,000 \mathrm{~kg}$ of treated simulant processed through the CIX were mixed with approximately $800 \mathrm{~kg}$ of LAW SBS Quencher from VSL, a recycle stream. Since RPP-WTP TLP feed will also recycle a portion of the $0.25 \mathrm{M} \mathrm{NaOH}$ Regeneration Solution, which is displaced from a regenerated CIX column after it is put into service, then approximately $40 \mathrm{~kg}$ of this solution was used for the pilot work. These three components make up the TLP feed.

Once the initial batch of feed was in the evaporator an amount of DOW Corning Q23183A Antifoam was added in order to have a concentration of $1500 \mathrm{ppm}$. No significant foam was observed in any of the tests but the antifoam was specifically added to mimic the intended plant operation. Despite having fewer recycle streams, the total volume of the TLP feed was larger than that for the FEP, in fact it was approximately 3.5 times larger. This meant that a steady-state evaporated concentration would be attained before exhausting the feed. Once the target slurry density was realized, then the TLP Bottoms would have to be removed while more feed was being sent into the TLP at a rate that would keep the antifoam concentration at the target level of $1500 \mathrm{ppm}$. Of course, removing concentrated slurry meant removing its antifoam too. Therefore, antifoam had to be reintroduced periodically to maintain its concentration constant. Periodically, a measured amount of antifoam was added to maintain its concentration.

Between 700 and $800 \mathrm{~kg}$ of TLP Evaporator Bottoms was produced in each campaign and shipped to VSL for LAW vitrification. After each campaign the condensate collected from both the TLP and FEP operations were combined as RLD. This condensate was used to produce wash and caustic solutions for the other unit operations, as it will be in the WTP.

\subsubsection{Pilot TLP Equipment (Author - Adamson)}

One of the requirements for SIPP as per the Test Specification (Longwell, 2003) was to utilize and modify existing equipment in the Engineering Development Laboratory of SRNL. To that end only one pilot-scale evaporator was constructed to be used for both FEP and Treated LAW Process (TLP) testing, because the design of those RPP-WTP Pretreatment Facility evaporators is the same. The task plan for those two evaporators for SIPP did not require simultaneous operation, therefore, once one unit operation test completed the test rig could be made ready for the subsequent test. As such, the description of the pilot-scale TLP is the same as for the pilot-scale FEP and therefore will not be repeated here. Please refer to equipment description for the FEP in section 2.6.3.

\subsubsection{Pilot TLP Operation (Author - Adamson)}

Prior to each of the TLP evaporation campaign, the evaporator system was cleaned, rinsed and inspected. Clean water was then added to the evaporator pot and the system was operated for approximately two hours. The spent water was visually clear with a $\mathrm{pH}$ of 7. 
WSRC-TR-2005-00105, REVISION 0

SRNL-RPP-2005-00012, REVISION 0

The TLP evaporator was operated using Work Instructions, I-EDL-132 "SIPP Treated LAW Process (TLP) Evaporation Test.” Manually recorded information was recorded in the TLP unit operation's notebook (Adamson, 2004).

Each of the pilot TLP operations took approximately 11 hours to concentrate the feed to the target density of $1.395 \mathrm{~kg} / \mathrm{L}$ at $20^{\circ} \mathrm{C}$. The total volume of the TLP concentrated product from each of the campaigns was more than 500 liters (>130 gal).

The average condensate boil off rate for the TLP campaigns was greater than $1.58 \mathrm{~L} / \mathrm{min}$ $(>0.42 \mathrm{gpm})$. The design boil off target of the RPP Pilot-scale Evaporator system was $1.5 \mathrm{~L} / \mathrm{min}(0.40 \mathrm{gpm})$.

Evaporation of the TLP simulant feed occurred at approximately $57^{\circ} \mathrm{C}$ under $-13 \mathrm{psig}$ vacuum (1.5 psia). The atmospheric pressure was approximately 14.5 psia during the campaign. However, due to pulling off the TLP concentrate and adding the cooler simulant feed, there were small fluctuations in the evaporator pot temperature and pressure as depicted in Figs. 68 and 69 for Campaigns II and III.

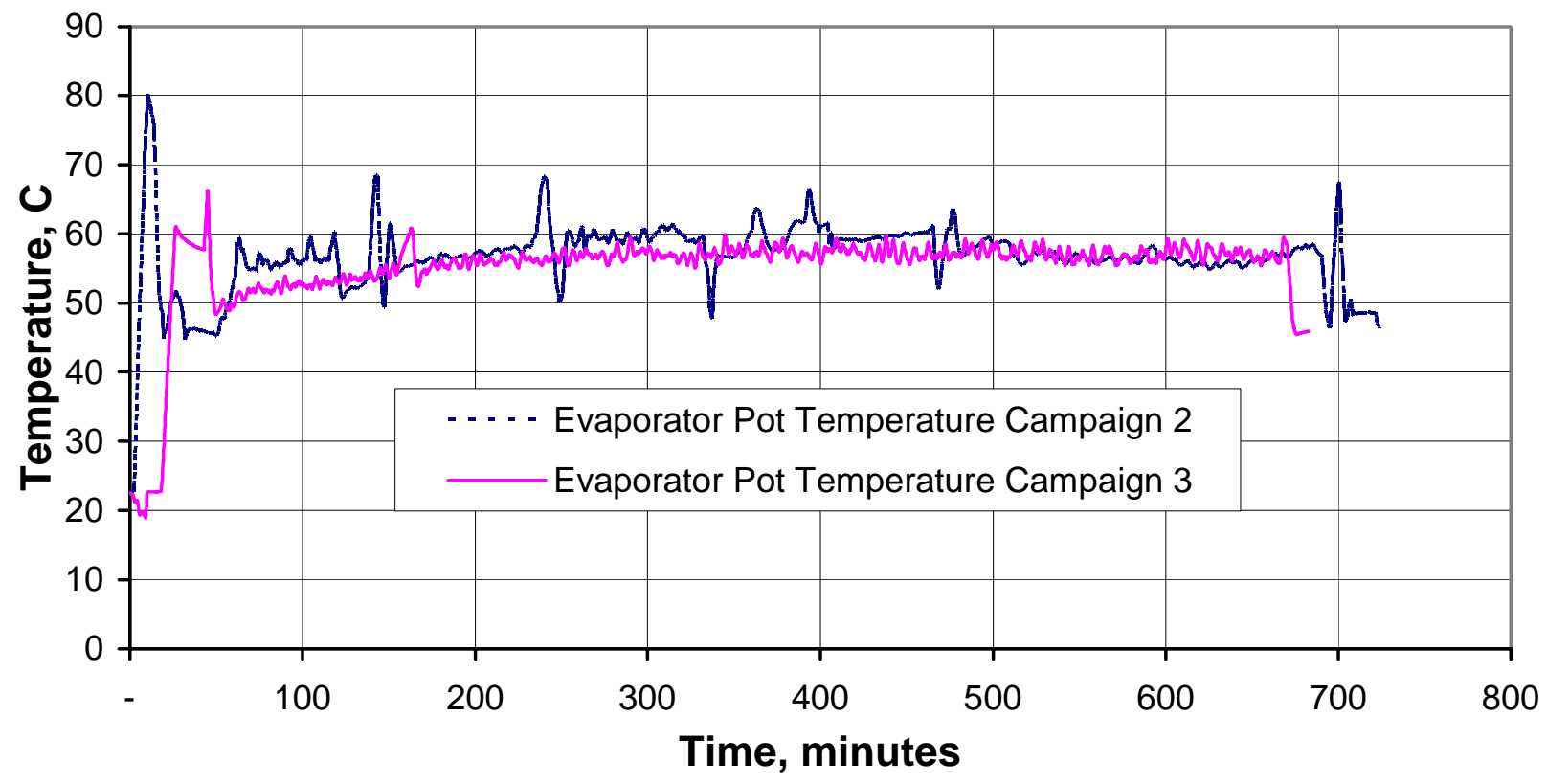

Figure 68. TLP Evaporator Pot Temperature during Campaigns II and III 
WSRC-TR-2005-00105, REVISION 0

SRNL-RPP-2005-00012, REVISION 0

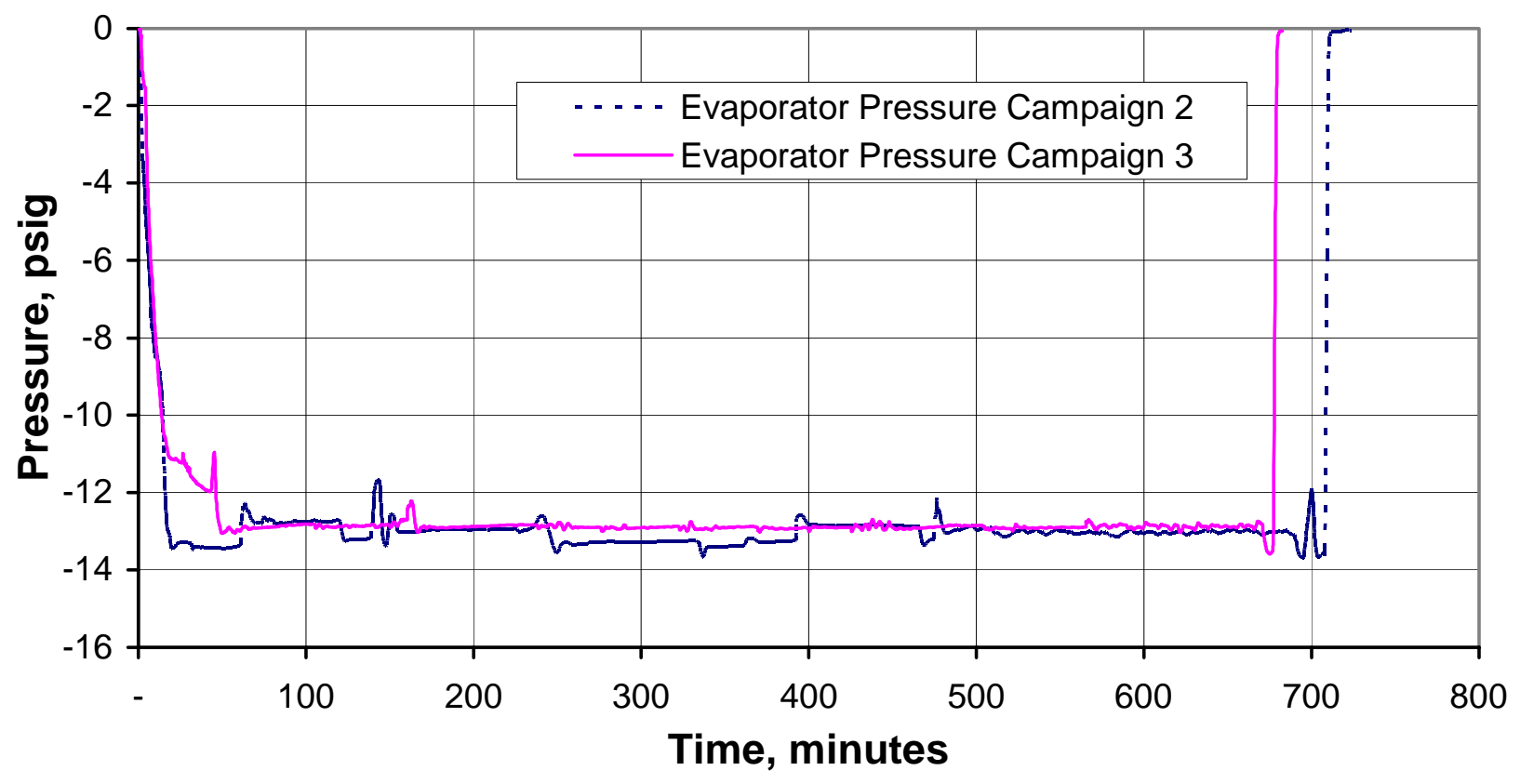

Figure 69. TLP Evaporator Pot Pressure during Campaigns II and III

For both campaigns the TLP simulant was re-circulated through the reboiler and evaporator system at approximately $90 \mathrm{gpm}$. Since the flow rate was controlled manually there were some variations during each campaign, but the fluctuations were maintained less than \pm 10 gpm. However, these fluctuations were not expected to affect the evaporation rate. As the simulant became more concentrated, the pump speed controller had to be periodically adjusted. Figure 70 depicts those flow rates. The recirculation of the simulant was continuous for the duration of the campaigns. Also recirculation continued when removing the concentrate from the evaporator system. 
WSRC-TR-2005-00105, REVISION 0

SRNL-RPP-2005-00012, REVISION 0

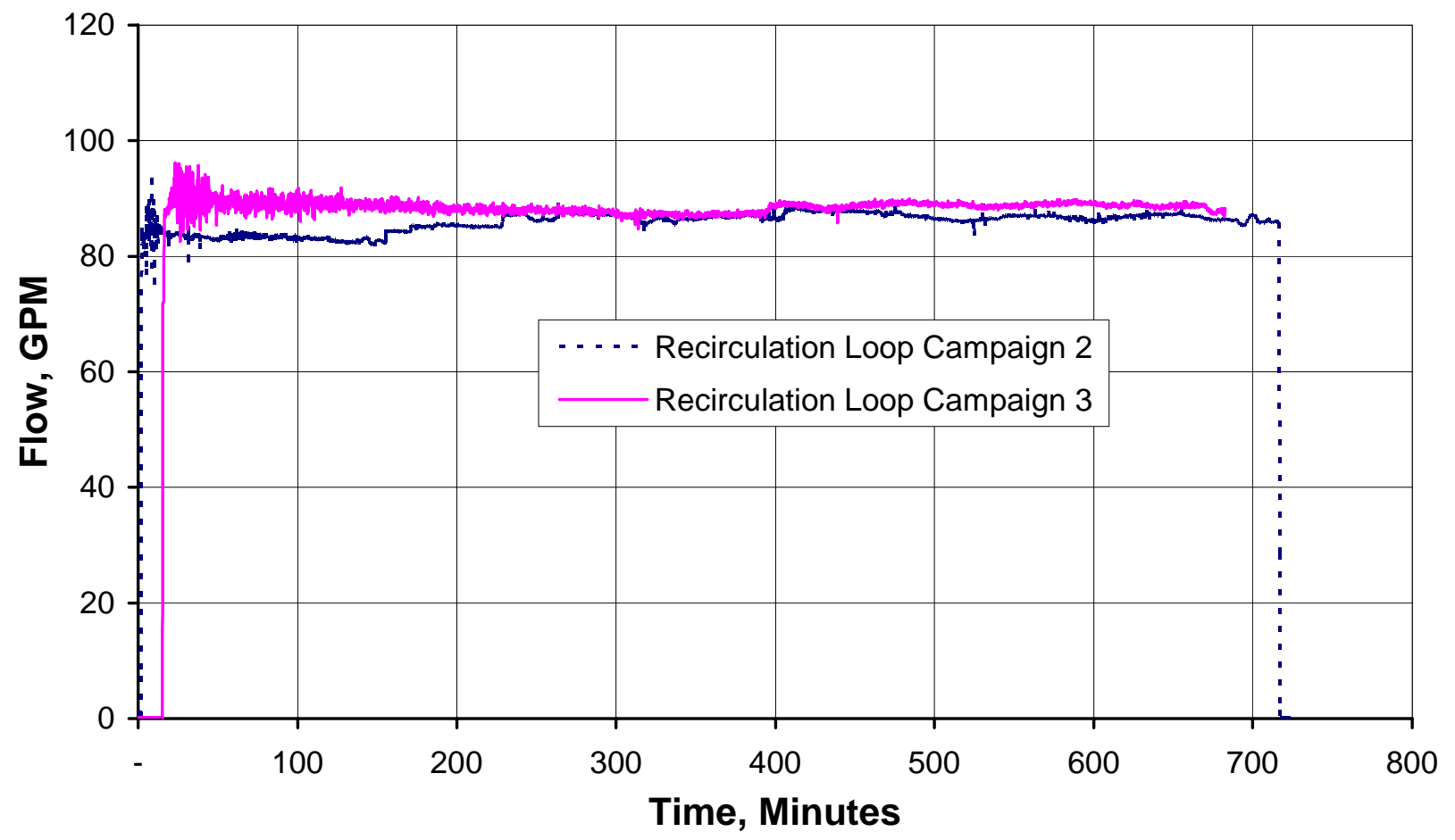

Figure 70. TLP Simulant Recirculation during Campaigns II and III

In each of the two TLP campaigns (i.e., II and III) a total of approximately 900 grams of Q2-antifoam was added to the simulant during the pilot TLP evaporation. This generally consisted of initially adding 309 grams of Q2-antifoam and then, as concentrate was removed additional Q2-antifoam was added, to maintain the concentration close to 1500 ppm. That is, the experiment starts at $1500 \mathrm{ppm}$, with a fixed evaporator volume of approximately 150 liters, and stays that way until the feed, within the evaporator, reached the target density of $1.365 \mathrm{~kg} / \mathrm{L}$ at $50^{\circ} \mathrm{C}$. In this phase, the feed rate matched the evaporation rate. Once at the target density, the batch mode operation began with slowly allowing feed to enter the evaporator slightly faster than the evaporation rate to maintain the density while allowing the evaporator volume. However, the added extra feed caused the antifoam concentration to drop to approximately $1000 \mathrm{ppm}$. This was not seen as a problem because from previous work (Qureshi et al., 2004) any reduction in foaming and/or a disengagement region occurred for concentrations of less than $300 \mathrm{ppm}$ of antifoam. Higher concentrations did not result in further reductions in foaming for the simulants under study. However, once at density some of the TLP Bottoms had to be removed to allow more feed to be processed. For continuous operation approximately 95 liters of Bottoms are removed to begin the next batch. This process repeats until all the feed is consumed. However, a further drop in antifoam concentration was not desired, therefore enough antifoam is added so that the next 95 liters of concentrated feed would also have a $1500 \mathrm{ppm}$ concentration. Figure 71 shows the fluctuations in antifoam concentrations and for both campaigns very little foaming was witnessed. 
WSRC-TR-2005-00105, REVISION 0

SRNL-RPP-2005-00012, REVISION 0

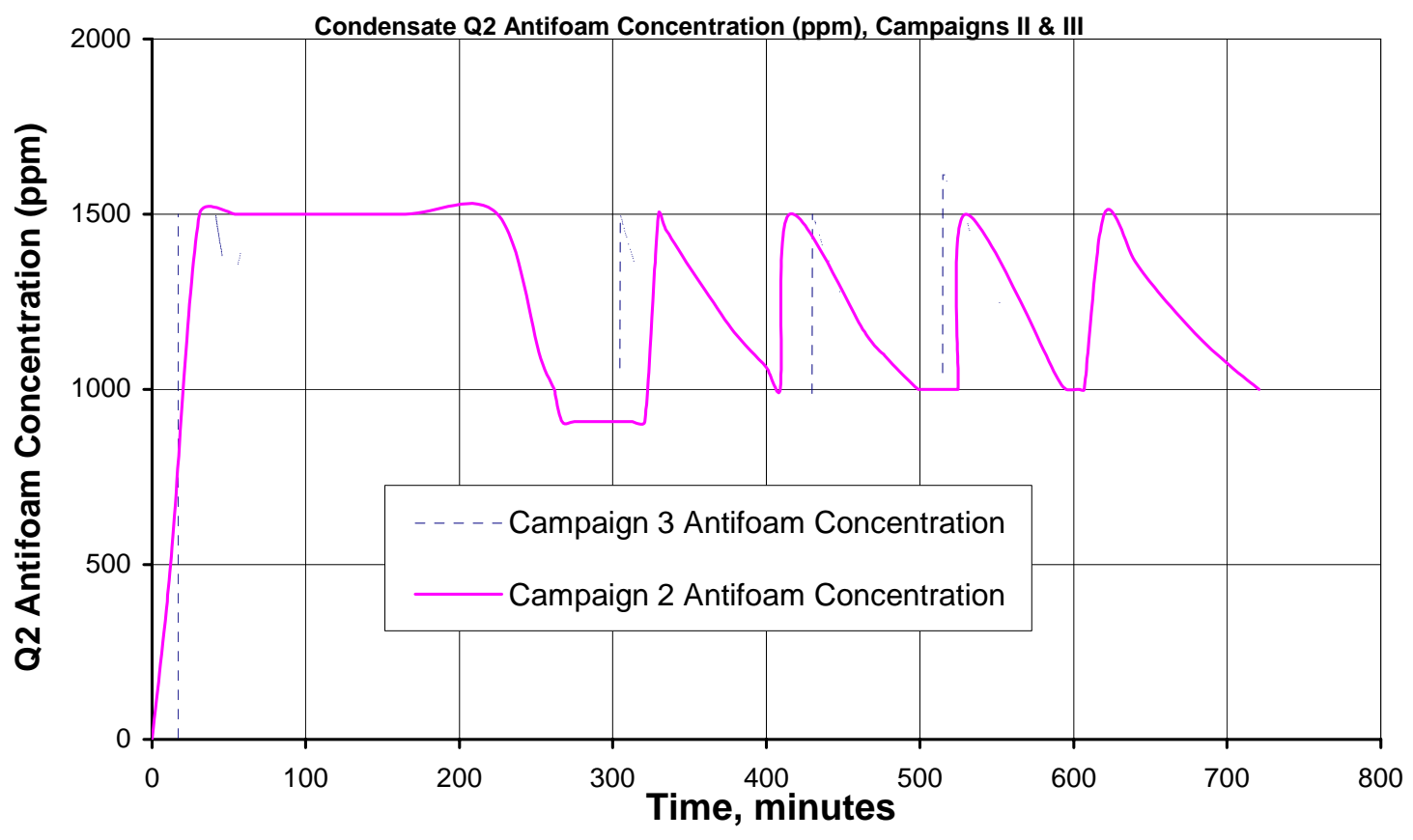

Figure 71. Pilot TLP antifoam concentration throughout Campaigns II and III testing

During testing samples from primary condensate, secondary condensate, and evaporator Bottoms were pulled every 200 liters of simulant feed pumped into the evaporator. Other samples were pulled more often to check the density of the evaporator Bottoms. The density was checked using a density meter (Anton Paar, DMA 4500) and measurements were recorded in the lab notebook (Adamson, 2004).

For both campaigns the feed simulant was concentrated down to three drums. Table 53 gives the volume, density and weight of the TLP simulant and the three drums of concentrate.

Table 55. TLP Simulant and Concentrate

\begin{tabular}{|l|l|l|l|}
\hline TLP Simulant & Volume & Density $\left(20^{\circ} \mathrm{C}\right)$ & Weight \\
\hline Campaign II TLP Feed Simulant & $1,639 \mathrm{~L} \quad(450.8 \mathrm{gal})$ & $1.14 \mathrm{~kg} / \mathrm{L}$ & $1,875 \mathrm{~kg}$ \\
\hline Drum \# 1 concentrate & $155.4 \mathrm{~L}(41.1 \mathrm{gal})$ & $1.375 \mathrm{~kg} / \mathrm{L}$ & $211.4 \mathrm{~kg}$ \\
\hline Drum \# 2 concentrate & $154.3 \mathrm{~L}(40.8 \mathrm{gal})$ & $1.385 \mathrm{~kg} / *$ & $211.4 \mathrm{~kg}$ \\
\hline Drum \# 3 concentrate & $213.4 \mathrm{~L} \quad(56.4 \mathrm{gal})$ & $1.375 \mathrm{~kg} / \mathrm{L}$ & $290.2 \mathrm{~kg}$ \\
\hline Campaign III TLP Feed Simulant & $1,707 \mathrm{~L} \quad(450.8 \mathrm{gal})$ & $1.135 \mathrm{~kg} / \mathrm{L}$ & $1,937 \mathrm{~kg}$ \\
\hline Drum \# 1 concentrate & $194.1 \mathrm{~L} \quad(51.3 \mathrm{gal})$ & $1.362 \mathrm{~kg} / \mathrm{L}$ & $264.4 \mathrm{~kg}$ \\
\hline Drum \# 2 concentrate & $193.3 \mathrm{~L} \quad(51.0 \mathrm{gal})$ & $1.366 \mathrm{~kg} / *$ & $264.0 \mathrm{~kg}$ \\
\hline Drum \# 3 concentrate & $180.1 \mathrm{~L}(47.7 \mathrm{gal})$ & $1.370 \mathrm{~kg} / \mathrm{L}$ & $247.4 \mathrm{~kg}$ \\
\hline
\end{tabular}

* Temperature at $20^{\circ} \mathrm{C}$

Both campaigns produced over 1,000 liters of condensate (water) from the simulant via evaporation to obtain more than 500 liters of concentrate, as seen in Table 53. The 
concentrate was transported to the Vitreous State Laboratory for further testing/ processing for the RPP Project.

\subsubsection{Pilot TLP Discussion of Results (Author - Adamson)}

\subsubsection{TLP Experimental Observations}

The SIPP TLP evaporation Campaign II was conducted during May 2004 and Campaign III during July 2004 in SRNL's Engineering Development Laboratory. Both evaporation tests were completed without any glitch or equipment failure. However, the Demister ${ }^{\circledR}$ instrumentation had to be purged during the campaigns due to moisture in the lines causing inaccurate readings.

As shown in Fig. 72, a region of gas/liquid disengagement of about one foot in height was witnessed throughout TLP campaigns. This disengagement region may have contained some foam or froth but most of the height was simply liquid entrainment, since upon shutdown no stable foam was evident. As previous stated antifoam was added but visual inspection would suggest that was not required to prevent foaming.

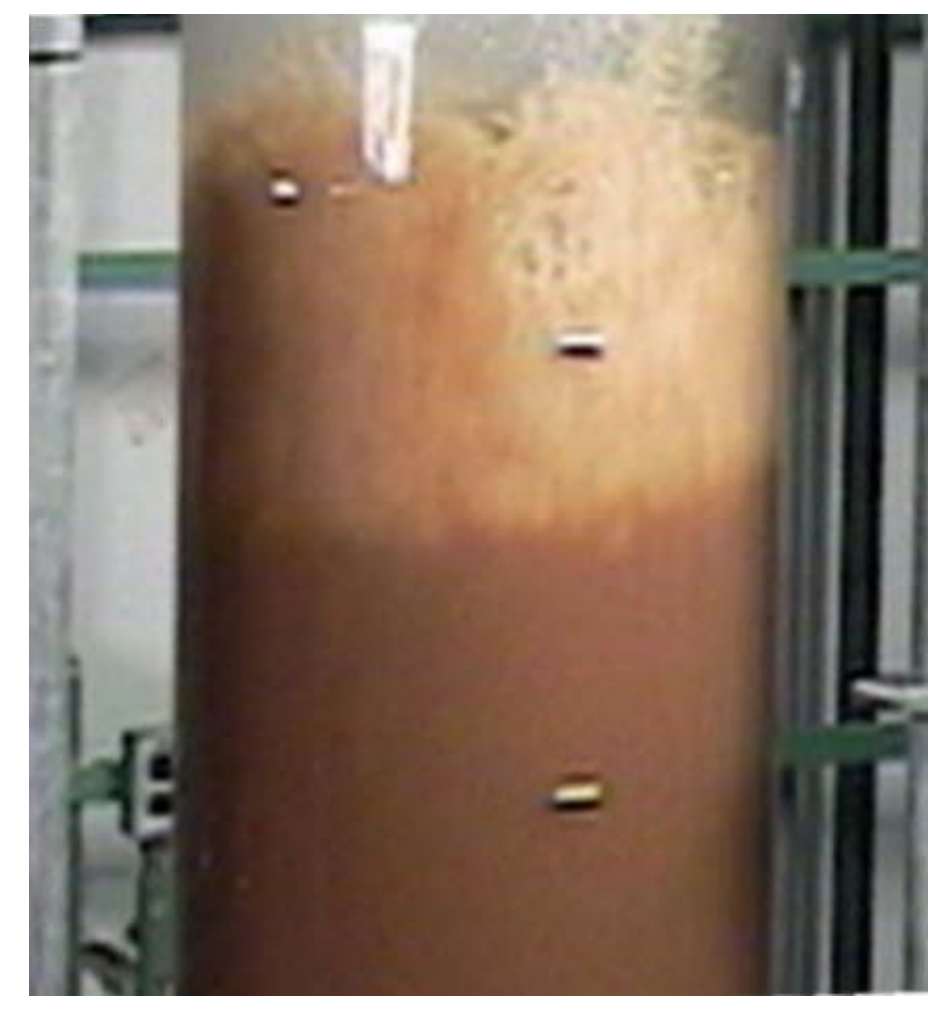

Figure 72. TLP Simulant Boiling in Clear Acrylic Section of Evaporator 


\subsubsection{TLP Waste Products (Author - Zamecnik)}

Table 56 shows the feed and Bottoms concentrate compositions for both Campaigns II and III. The Campaign III concentrate composition was calculated by material balance and is compared to the material balance from Campaign II in the adjoining columns of the table. The Campaign II material balance results in a very good agreement with the concentrate composition, so the Campaign III material balance is also expected to be accurate. (Note that the Campaign II concentrate analysis from the microwave dissolution is not shown because these values have been determined to be suspect.) The Campaign III calculated concentrate is lower in $\mathrm{Na}$ and $\mathrm{Al}$, slightly higher in $\mathrm{K}$, and about the same in most of the other significant cations and anions. Therefore, the main difference in Campaign III was a lower concentration of $\mathrm{Na}$ and $\mathrm{Al}$, which would be present as $\mathrm{Na}^{+} \mathrm{Al}(\mathrm{OH})_{4}^{-}$. The sodium concentration in Campaign III was lower than in Campaign II primarily because the final concentrate density was lower in Campaign III. However, the aluminum would have been lower in Campaign III than Campaign II, in any case, because the feed concentration for Campaign III was lower to begin with.

Two measured values for the solids content of the Campaign III feed and concentrate (Bottoms) are shown. Based on the average feed UDS value, the expected concentrate UDS is about $0.28 \mathrm{wt} \%$, which is similar to what was measured in Campaign II. The measured Campaign III values were the same as the feed values. Based on the Campaign II results, the concentrate UDS is expected to be higher. It is important to note that the analytical measurements of undissolved solids concentration are quantitatively inconclusive below $1 \mathrm{wt} \%$ UDS. Since the measurement uncertainty of the analytical method for UDS concentrations is $\pm 0.22 \mathrm{wt} \%$ UDS, then all the displayed results may be the same. Further, differences shown where there are two values in one column (which are two separate samples) may have been caused by sampling problems. Because of the uncertainties, the Bottoms UDS concentrations could be the same as the feed, or if some of the solids dissolved, but visually the concentrate appeared to have more undissolved solids. 
WSRC-TR-2005-00105, REVISION 0

SRNL-RPP-2005-00012, REVISION 0

Table 56. Comparison of Campaigns II and III TLP Compositions

\begin{tabular}{|c|c|c|c|c|c|c|}
\hline & \multicolumn{2}{|c|}{ Feed } & \multicolumn{4}{|c|}{ Bottoms } \\
\hline & \multirow{2}{*}{$\begin{array}{c}\text { Campaign III } \\
\text { Measured* }\end{array}$} & \multirow{3}{*}{\begin{tabular}{|c} 
Campaign II \\
Measured \\
16.24
\end{tabular}} & \multicolumn{2}{|c|}{ Campaign III } & \multicolumn{2}{|c|}{ Campaign II } \\
\hline & & & Measured* & Calculated & Measured & Calculated \\
\hline Total Solids (wt $\% \pm 0.11$ ) & $16.23 \quad 16.24$ & & $40.06 \quad 40.04$ & 40.42 & 41.30 & 40.54 \\
\hline Undissolved Solids (wt\% \pm 0.22 ) & $0.07 \quad 0.15$ & 0.09 & $0.15 \quad 0.07$ & 0.28 & 0.31 & 0.23 \\
\hline Supernatant Solids (wt\% \pm 0.13 ) & $16.10 \quad 16.18$ & 16.16 & $39.97 \quad 40.00$ & 40.25 & 41.12 & 40.41 \\
\hline Density $(\mathrm{kg} / \mathrm{L})$ & 1.134 & 1.136 & 1.368 & 1.368 & 1.384 & \\
\hline Volume (L) & 1706 & 1639 & & 568 & 523 & \\
\hline Sample Preparation & $\begin{array}{c}\text { Dissolved Total } \\
\text { Sample** }\end{array}$ & Supernate & & $\begin{array}{l}\text { Calculated } \\
\text { Total Sample }\end{array}$ & Supernate & $\begin{array}{c}\text { Calculated } \\
\text { Total Sample }\end{array}$ \\
\hline Concentrations in $\mathbf{m g} / \mathbf{k g}: \quad \mathrm{Ag}$ & $<50$ & $<0.5$ & & $<124$ & $<0.43$ & $<1.4$ \\
\hline $\mathrm{Al}$ & 2900 & 3319 & & 7220 & 8309 & 8537 \\
\hline $\mathrm{B}$ & & 80.7 & & & 198 & 208 \\
\hline $\mathrm{Ba}$ & $<2.0$ & $<0.04$ & & $<5.0$ & $<0.03$ & $<0.10$ \\
\hline $\mathrm{Ca}$ & $<6.6$ & 1.95 & & $<16$ & 2.57 & 5.02 \\
\hline $\mathrm{Cd}$ & $<2.4$ & $<0.04$ & & $<6.0$ & $<0.03$ & $<0.09$ \\
\hline $\mathrm{Ce}$ & $<128$ & $<15$ & & $<319$ & $<12$ & $<39$ \\
\hline Co & $<7.3$ & $<0.08$ & & $<18$ & $<0.06$ & $<0.19$ \\
\hline $\mathrm{Cr}$ & 82.8 & 79.0 & & 206 & 192 & 203 \\
\hline $\mathrm{Cu}$ & $<8.3$ & 0.143 & & $<21$ & 0.38 & 0.37 \\
\hline $\mathrm{Fe}$ & 21.0 & 0.492 & & 52.3 & 1.00 & 1.26 \\
\hline $\mathrm{K}$ & 9480 & 8389 & & 23603 & 23916 & 21579 \\
\hline $\mathrm{La}$ & $<116$ & $<0.33$ & & $<289$ & $<0.27$ & $<0.85$ \\
\hline $\mathrm{Li}$ & 1.0 & 4.09 & & 2.5 & 9.37 & 10.5 \\
\hline $\mathrm{Mg}$ & $<14$ & $<0.02$ & & $<35$ & $<0.02$ & $<0.06$ \\
\hline $\mathrm{Mn}$ & $<1.5$ & $<0.03$ & & $<3.7$ & $<0.02$ & $<0.08$ \\
\hline Mo & $<17$ & 4.79 & & $<41$ & 11.1 & 12.3 \\
\hline $\mathrm{Na}$ & 47684 & 51937 & & 118722 & 125723 & 133596 \\
\hline $\mathrm{Na}(\mathrm{AA})$ & & 54930 & & & 145954 & 141295 \\
\hline $\mathrm{Na}$ (average) & 47684 & 53433 & & 118722 & 135838 & 137445 \\
\hline $\mathrm{Na}$ (average in $\mathrm{M}$ ) & 2.4 & 2.6 & & 7.1 & 8.2 & 8.3 \\
\hline $\mathrm{Nd}$ & $<43$ & $<0.71$ & & $<107$ & $<0.59$ & $<1.8$ \\
\hline $\mathrm{Ni}$ & $<10$ & 0.24 & & $<26$ & 0.56 & 0.62 \\
\hline $\mathrm{P}$ & 371 & 355 & & 924 & 574 & 912 \\
\hline $\mathrm{Pb}$ & $<114$ & 7.13 & & $<284$ & 17.3 & 18 \\
\hline $\mathrm{S}$ & 502 & 587 & & 1250 & 1377 & 1511 \\
\hline $\mathrm{Si}$ & 0 & 36.7 & & 0 & 113 & 94 \\
\hline $\mathrm{Sr}$ & 8.0 & 0.01 & & 19.92 & 0.06 & 0.04 \\
\hline $\mathrm{Ti}$ & $<23$ & $<0.10$ & & $<58$ & 0.25 & $<0.26$ \\
\hline $\mathrm{Zn}$ & $<61$ & 11.1 & & $<152$ & 27.3 & 29 \\
\hline $\mathrm{Zr}$ & $<8.0$ & $<0.14$ & & $<20$ & 0.749 & $<0.36$ \\
\hline bromide & $<61$ & $<88$ & & $<152$ & $<72$ & $<226$ \\
\hline chloride & 795 & 663 & & 1979 & 1821 & 1705 \\
\hline fluoride & 56.0 & 61.6 & & 139 & 28.2 & 159 \\
\hline formate & 521 & 551 & & 1297 & 1171 & 1417 \\
\hline nitrate & 37200 & 37500 & & 92619 & 90318 & 96461 \\
\hline nitrite & 12300 & 11884 & & 30624 & 28974 & 30569 \\
\hline oxalate & 374 & 342 & & 931 & 831 & 881 \\
\hline phosphate & 1220 & 1136 & & 3038 & 1741 & 2921 \\
\hline sulfate & 1570 & 1514 & & 3909 & 3699 & 3895 \\
\hline ammonium & 2730 & 879 & $<40$ & & $<72$ & \\
\hline
\end{tabular}

* Solids measurements are duplicate values ** Dissolution by either microwave acid or sodium peroxide fusion HCl uptake 


\subsubsection{TLP Final Description of Dow Corning Antifoam Q2-3183 A (Author - Zamecnik)}

The behavior of the Dow Corning Q2-3183A antifoam was described in the Campaign II Interim Report (Duignan et al., 2004b or Appendix I-2), but the analytical results reported were tentative due to questionable results. Since that time, some samples were reanalyzed and several additional samples were also analyzed. All analyses were performed by Dow Corning Analytical Solutions.

In their analytical reports, Dow Corning stated that the samples were not homogeneous, so removal of representative sub-samples for analysis was questionable. This same difficulty in sampling would also apply to taking the actual samples from the SIPP process streams. Small scale evaporator samples were so inhomogeneous that most of the antifoam could be separated from the Bottoms concentrate by skimming the surface of the slurry. The SIPP samples were not nearly as inhomogeneous, but still were difficult to sample.

Dow Corning extracted the samples with n-hexane to remove the polydimethylsiloxane (PDMS) portion of the antifoam. The hexane extract was then analyzed by Fourier Transform Infrared Spectroscopy (FTIR) to measure the signal from the methyl groups at $1260 \mathrm{~cm}^{-1}$. Note that this quantitative analysis technique for PDMS has not been extensively tested since Dow Corning has not apparently had any need to perform such analyses in the past. Their analyses did not include quantification of the other components of the antifoam, such as the polyol fractions. The Dow Corning representative stated that the PDMS portion constituted about $40 \mathrm{wt} \%$ of the total antifoam mass, so the total mass of antifoam in a sample would be about 2.5 times $(1 / 0.4)$ the measured PDMS concentration. Since the non-PDMS portions of the antifoam were not actually quantified, there are no data to indicate whether these other portions remained with the PDMS.

Dow Corning performed several spike tests with supplied antifoam-free samples. These samples were spiked with known amounts of antifoam and then analyzed. The results of these spike tests are shown in Table 57. The recoveries were from $73-84 \%$, so the actual PDMS concentrations from process samples would be expected to be $15-25 \%$ higher than measured.

Table 57. Antifoam Spike Test Results

\begin{tabular}{|r|c|c|c|}
\hline Process Sample Spiked & $\begin{array}{c}\text { Spike Amount } \\
\text { (Siloxane) } \\
(\mathrm{mg} / \mathrm{kg})\end{array}$ & $\begin{array}{c}\text { Measured } \\
\text { Siloxane } \\
(\mathrm{mg} / \mathrm{kg})\end{array}$ & $\begin{array}{c}\text { Recovery } \\
(\%)\end{array}$ \\
\hline Wash Permeate & 4800 & 3900 & 81 \\
\hline IX Feed & 4800 & 3500 & 73 \\
\hline FEP Feed & 5000 & 4200 & 84 \\
\hline Washed Slurry & 5100 & 3800 & 75 \\
\hline
\end{tabular}


WSRC-TR-2005-00105, REVISION 0

SRNL-RPP-2005-00012, REVISION 0

Table 58 shows the concentrations of antifoam measured in the different process streams and the total masses actually added and calculated from the analytical data. These data show that the PDMS portion of the antifoam was retained in the FEP and TLP evaporator Bottoms and with the slurry in the filtration processes. However, quantitative measurement of the amount of antifoam was poor, with most samples showing significantly more antifoam than actually added (compare Measured Antifoam to Actual Antifoam Added). These results indicate that either the samples were either biased high in antifoam or the analytical technique on the real samples was not quantitatively reliable. The results by date for the FEP at beginning samples indicate that the ability to measure the PDMS in the samples varied over time for some reason. The replicate results for the other samples were more consistent, although incorrect. The apparent high bias may be due to non-uniform dispersal of the antifoam in the system. It appears that the samples were enriched in the antifoam. Observation of small evaporations with this antifoam show that the PDMS will float on top of the aqueous phase if not vigorously stirred. Such segregation may be the cause for the observed SIPP results.

Based on the high boiling point of polyols, we would not expect them to be found in the evaporator condensate streams. However, conclusions about the fate of these other fractions in the filtration process cannot be made with the available data, so it is not known if the non-PDMS portions of the antifoam remained with the concentrated slurry or were separated into the IX feed or wash permeate. Also, no conclusions about antifoam degradation can be made from these data. 
WSRC-TR-2005-00105, REVISION 0

SRNL-RPP-2005-00012, REVISION 0

Table 58. Antifoam Concentrations in Process Samples

\begin{tabular}{|c|c|c|c|c|c|c|c|c|c|}
\hline PROCESS STREAM & $\begin{array}{c}\text { Date } \\
\text { Analyzed } \\
(2004)\end{array}$ & $\begin{array}{c}\text { Measured } \\
\text { PDMS (1) } \\
\text { Concentration } \\
(\mathrm{mg} / \mathrm{kg})\end{array}$ & $\begin{array}{c}\text { Average } \\
\text { Measured } \\
\text { PDMS } \\
\text { Concentration } \\
\text { (mg/kg) }\end{array}$ & $\begin{array}{c}\text { Measured } \\
\text { Total } \\
\text { Antifoam (2) } \\
\text { Concentration } \\
\text { (mg/kg) }\end{array}$ & $\begin{array}{c}\text { Process } \\
\text { Amount } \\
(\mathrm{kg})\end{array}$ & $\begin{array}{l}\text { Measured } \\
\text { PDMS } \\
\text { (g) }\end{array}$ & $\begin{array}{l}\text { Measured } \\
\text { Antifoam } \\
\text { (g) }\end{array}$ & $\begin{array}{l}\text { Actual } \\
\text { Antifoam } \\
\text { Added } \\
\text { (g) }\end{array}$ & $\begin{array}{c}\text { Measured } \\
\text { / Actual } \\
(\%)\end{array}$ \\
\hline $\begin{array}{r}\text { FEP at Beginning } \\
\text { (after initial antifoam addition) }\end{array}$ & $\begin{array}{c}\text { Late July } \\
8 / 6 \\
10 / 21 \\
10 / 25\end{array}$ & $\begin{array}{l}1580 \\
1400 \\
2220 \\
2160\end{array}$ & 1840 & 4600 & 157 & 288 & 720 & 186 & 387 \\
\hline FEP Bottoms Concentrate & $\begin{array}{c}\text { Late July } \\
8 / 6 \\
10 / 21 \\
10 / 25 \\
\end{array}$ & $\begin{array}{l}2410 \\
2150 \\
2260 \\
2210 \\
\end{array}$ & 2258 & 5644 & 187 & 421 & 1053 & 278 & 379 \\
\hline FEP Condensate & Sept. & $<10$ & $<10$ & $<10$ & 1014 & & $<10$ & NA & NA \\
\hline Washed HLW Slurry & $\begin{array}{c}\text { Late July } \\
8 / 6 \\
10 / 21 \\
10 / 25 \\
\end{array}$ & $\begin{array}{c}1260 \\
1110 \\
770 \\
800 \\
\end{array}$ & 985 & 2463 & 140 & 138 & 346 & 278 & 125 \\
\hline Wash Permeate & Late July & $<10$ & $<10$ & $<10$ & 325 & & $<4$ & NA & NA \\
\hline LAW Permeate & Sept. & $<10$ & $<10$ & $<10$ & 1153 & & $<12$ & NA & NA \\
\hline $\begin{array}{r}\text { TLP Feed } \\
\text { (after initial antifoam addition) }\end{array}$ & Sept. & 650 & 650 & 1625 & 172 & 112 & 280 & 309 & 91 \\
\hline TLP Bottoms Concentrate & Sept. & 960 & 960 & 2400 & 724 & 695 & 1737 & 616 & 282 \\
\hline TLP Condensate & Sept. & $<10$ & $<10$ & $<10$ & 1214 & & $<12$ & NA & NA \\
\hline
\end{tabular}

(1) PDMS = polydimethylsiloxane

(2) Total antifoam $=$ measured siloxane fraction $\times 2.5$ 


\subsubsection{Pilot TLP Conclusions}

Pilot TLP operation was not part of Campaign IV testing but for completeness in reporting the following conclusions were extracted from the Interim Reports of Campaign II (Duignan et al., 2004b or Appendix I-2) and Campaign III (Duignan et at., 2004c), along with measurements obtained after the Campaign III report was issued.

\subsubsection{Experimental Conclusions (Author - Adamson)}

\section{Campaign III}

- $\quad$ The pilot-scale TLP evaporator operated as designed and concentrated the TLP feed simulant from a density of $1.135 \mathrm{Kg} / \mathrm{L}$ at $20^{\circ} \mathrm{C}$ and a sodium concentration of $2.4 \mathrm{M}$, to final density $1.365 \mathrm{Kg} / \mathrm{L}$ at $20^{\circ} \mathrm{C}$ and a sodium concentration of $7.1 \mathrm{M}$.

\section{Campaign II}

- The pilot-scale evaporator operated well concentrating the TLP simulant from an initial density of $1.14 \mathrm{~kg} / \mathrm{L}$ to $1.385 \mathrm{~kg} / \mathrm{L}$ at $20^{\circ} \mathrm{C}\left(1.360 \mathrm{~kg} / \mathrm{L}\right.$ at $\left.50^{\circ} \mathrm{C}\right)$.

- No significant foaming was observed prior to the addition of antifoam agent. During the evaporation process, the froth height remained around $1 \mathrm{ft}(0.3 \mathrm{~m})$, well below the $5 \mathrm{ft}(1.5 \mathrm{~m})$ distance to the lower demister pad

- The two Demisters in series operated well with no significant differential pressure build-up across the Demisters during the TLP campaign II. However, condensation had to be purged from the instrumentation lines during the campaign.

\subsubsection{Waste Products Conclusions (Author-Zamecnik)}

\section{$\underline{\text { Post-Campaign III }}$}

- Tracking the fate of antifoam in the many simulant streams was not successful because of the ineffectiveness in the methods used to measure its concentration. However, it appears that an insignificant amount of antifoam was carried over into the evaporator condensates. This result was not surprising because of the high boiling point of the polyols fractions of the antifoam. Further, it appears that the active component PDMS of the antifoam did not partition into the permeate stream, that is, it remained in the UFP sludge, but the poor detection capabilities did not allow accurate quantification of the antifoam concentrations.

\section{Campaign III}

- The concentration of aluminum in the TLP product from Campaign III was less than the result of Campaign II since the UF permeate contained less dissolved aluminum. 
WSRC-TR-2005-00105, REVISION 0

SRNL-RPP-2005-00012, REVISION 0

\section{Campaign II}

- The composition of the products from the TLP matched what was expected from the mass balance.

- No precipitation of additional solids beyond those in the feed was noted.

\subsection{GLASS PRODUCTION BASED ON SIPP RESULTS (Author - Zamecnik)}

\subsubsection{Extrapolated Glass Production Rates based on Campaign II Data}

This section repeats the data presentation made in the Campaign II Interim Report, Section 2.10 (Duignan et al., 2004b or Appendix I-2), with the addition of both Campaign III and IV data for some of the analysis and discussion. The flux-versus-slurry undissolved solids content curves for Campaigns III and IV have been shown to be very similar to the Campaign II data, but there are slight differences. The UFP flux data collected in Campaign II were used to create a model to predict the production rates of IHLW and ILAW glass assuming that the dewatering step was rate limiting. Rates were also estimated by “extrapolation” to different feed compositions. By extrapolation, we mean that different, but similar blends of the AY-102 and AP-101 simulants and the FEP Bottoms were calculated.

The rates of IHLW and ILAW glass production were estimated given the following set of assumptions:

1. The dewatering step in ultrafiltration is the rate limiting step in the production of glass.

2. ILAW glass $\mathrm{Na}_{2} \mathrm{O}$ loading of $14 \mathrm{wt} \%$.

3. IHLW glass $\mathrm{Fe}_{2} \mathrm{O}_{3}$ loading of $12.5 \mathrm{wt} \%$ (limiting condition). (Calloway, 2004)

4. WTP production rate is scaled to the SIPP production rate by the ratio of the filter surface areas $\left(710 \mathrm{ft}^{2} / 6.7 \mathrm{ft}^{2}\right)$.

5. Flux vs. time and flux vs. UDS curve-fits shown previously are used (see Figs 50 and 53).

6. Average flux is determined by integrating the area under the flux vs. time curve (see Fig 54).

7. Endpoint UDS $=20 \mathrm{wt} \%$.

8. All dissolved Na that goes to ILAW is "waste" Na.

9. WTP filter area of $710 \mathrm{ft}^{2}$ (no reduction in area assumed).

10. The HLW melter can process a lower UDS feed and maintain production rate.

Note that the entire $710 \mathrm{ft}^{2}$ surface area was assumed to be available for filtration; the $20 \%$ contingency was not included. The following analysis could also be done with the contingency in the same manner.

The "extrapolated" glass production rates were determined by calculating the blending of different quantities of the Campaign II FEP Bottoms, AY102 slurry, and AP101 together. 
Here it was assumed that the flux curves at these calculated compositions would be the same as the measured curves, i.e., the flux curves are only dependent on the undissolved solids content and not on the actual chemical composition of the feed slurry. This assumption is reasonable so long as the relative amounts of the three components are not changed significantly from the actual amounts tested. For these extrapolated situations, the composition of the FEP Bottoms was assumed to be the same even though the recycle streams would actually have slightly different compositions since the UFP feed composition would be slightly different.

For assumption No. 8, above, “waste” Na (sodium) was defined as any $\mathrm{Na}$ in the actual wastes, plus any $\mathrm{Na}$ added during washing $(0.01 \mathrm{M} \mathrm{NaOH})$ and leaching (which was not done in SIPP). Any other Na added was "non-waste" and was not counted towards the ILAW rate. However, in the calculations done for the SIPP (see Appendix B), this distinction was ignored since the only $\mathrm{Na}$ additions were small amounts of $\mathrm{NaOH}$ added to the recycle streams to maintain the $\mathrm{pH}>12$. These amounts were so small that their effect on the Na concentration in the feed was smaller than the uncertainty in the measurement of the Na.

Table 58 gives the glass production rates for several hypothetical situations. The "Actual Feed” column (1) contains the results from using the actual Campaign II measured feed composition obtained from samples after the feed mixture was made. The "Predicted Feed" column (2) gives the production rates for the feed composition predicted from the mixing of the two simulated waste feed streams and the FEP Bottoms. The compositions are essentially the same, indicating that the blending model was reasonable. Thus, the dewatering fluxes are the same $\left(0.0144 \mathrm{gpm} / \mathrm{ft}^{2}\right)$ and the glass rates for these two cases are also essentially the same. The target glass rates were $6 \mathrm{MTG} / \mathrm{d}$ of IHLW and 80 MTG/d of ILAW (Longwell, 2003), but the actual rates at WTP scale were about 6.6 MTG/d of IHLW and 59.8 MTG/d of ILAW. In the SIPP campaigns, the target rates were not reached because the ratio of undissolved solids (or Fe) to permeate (or dissolved $\mathrm{Na}$ ) was too high. The target ILAW/IHLW ratio is $13.3(80 / 6)$, but the experimentally obtained ratio turned out to be 9.1 for Campaign II. This means that there should have been more dissolved solids (permeate to LAW) relative to the undissolved solids (washed slurry to HLW).

Column 3 in Table 59 shows the predicted glass production rates with the ILAW/IHLW ratio adjusted to 13.33. To adjust this ratio, the relative amounts of the AY-102 and AP101 simulants and the FEP Bottoms had to be adjusted also. Given a fixed amount of FEP Bottoms, there will be only one blend of these three components that will result in the exact ILAW/IHLW ratio of 13.33. At this blending (rows 1-3 of column 3), the average flux is slightly higher $\left(0.0148 \mathrm{gpm} / \mathrm{ft}^{2}\right)$ because the initial UDS is higher (2.32 vs. $\sim 3.2 \mathrm{wt} \%$ ) in the actual SIPP Campaign II (higher initial UDS gives lower average flux). The values in column 3 show that the change in blending ratios gives more ILAW glass (64.2 vs. $61.3 \mathrm{mt} / \mathrm{d}$ ) and less HLW glass (4.8 vs. $6.8 \mathrm{mt} / \mathrm{d}$ ) and that the ratio of ILAW/IHLW is 13.33 . 
WSRC-TR-2005-00105, REVISION 0

SRNL-RPP-2005-00012, REVISION 0

Column 4 in Table 59 shows that an average flux of $0.0185 \mathrm{gpm} / \mathrm{ft}^{2}$ and the same blending ratio as used in column 3 will result in meeting the glass production targets.

Table 59. Glass Production Rates Based on Ultrafilter Throughput Using Campaign II Data

\begin{tabular}{|c|c|c|c|c|c|}
\hline Process Stream & Property & $\begin{array}{c}1 \\
\text { Campaign II } \\
\text { Predicted } \\
\text { Feed }^{3}\end{array}$ & $\begin{array}{l}\text { Campaign II } \\
\text { Actual Feed }\end{array}$ & $\begin{array}{c}3 \\
\text { Feed Blend to } \\
\text { meet } \\
\text { ILAW/IHLW } \\
=80 / 6(13.3)\end{array}$ & $\begin{array}{c}4 \\
\text { ILAW/IHLW = 80/6, } \\
\text { flux adjusted so ILAW }{ }^{7}= \\
80 \mathrm{mt} / \mathrm{d}\end{array}$ \\
\hline FEP Bottoms & Volume \% & 11.1 & 11.1 & 9.5 & 9.6 \\
\hline AY102 & Volume \% & 24.3 & 24.3 & 17.6 & 17.7 \\
\hline AP101 & Volume \% & 64.6 & 64.6 & 72.2 & 72.7 \\
\hline Feed & UDS (wt\%) & 3.13 & 3.22 & 2.32 & 2.32 \\
\hline & $\mathrm{Fe}(\mathrm{mg} / \mathrm{L})$ & 9315 & 9532 & 6900 & 6890 \\
\hline & $\mathrm{Na}(\mathrm{mg} / \mathrm{L})$ & 117500 & 114900 & 120300 & 120300 \\
\hline Concentrated & UDS (wt\%) & 20 & 20 & 20 & 20 \\
\hline Slurry & $\mathrm{Fe}(\mathrm{mg} / \mathrm{L})$ & 64700 & 66500 & 64600 & 64600 \\
\hline Permeate & $\begin{array}{c}\mathrm{Na}(\mathrm{mg} / \mathrm{L}) \\
(\mathrm{M})\end{array}$ & $\begin{array}{c}111900 \\
4.86\end{array}$ & $\begin{array}{c}114600 \\
4.98\end{array}$ & $\begin{array}{c}116100 \\
5.05\end{array}$ & $\begin{array}{c}116100 \\
5.05\end{array}$ \\
\hline Avg. UFP Flux & $\mathrm{gpm} / \mathrm{ft}^{2}$ & 0.0144 & 0.0144 & 0.0148 & 0.0185 \\
\hline ILAW Glass $^{1}$ & $\mathrm{mt} / \mathrm{d}^{8}$ & 60.1 & 61.3 & 64.2 & 80.0 \\
\hline IHLW Glass² & $\mathrm{mt} / \mathrm{d}$ & 6.6 & 6.8 & 4.8 & 6.0 \\
\hline ILAW/IHLW & ratio & 9.1 & 9.1 & 13.33 & 13.33 \\
\hline
\end{tabular}

1. ILAW glass rate based on $14 \mathrm{wt} \% \mathrm{Na}_{2} \mathrm{O}$ loading

2. IHLW glass rate based on $12.5 \mathrm{wt} \% \mathrm{Fe}_{2} \mathrm{O}_{3}$ loading

3. Feed composition predicted from blending of FEP Bottoms, AY102, and AP101

4. Actual UFP feed composition used

5. Feed blend adjusted to meet this ratio (feed calculated)

6. Feed blend adjusted to meet this value (feed calculated)

7. See $5+$ flux adjusted to make rates (calculated model flux not used)

8. $\mathrm{mt} / \mathrm{d}=$ metric tons / day (tonnes/day)

\subsubsection{Increasing Glass Production Rates}

In the SIPP, the feed slurry to Ultrafiltration was concentrated to greater than $20 \mathrm{wt} \%$ UDS. As the UDS concentration gets higher, the filtration flux decreases. Even at an endpoint of $20 \mathrm{wt} \%$ UDS, the average flux is not high enough to make the glass rate goals. Two potential ways to improve the rate were proposed:

1. Decrease the undissolved solids concentration endpoint at the end of the initial dewatering step (production of LAW permeate).

2. Increase the filtration temperature.

Decreasing the UDS endpoint has the effect of increasing the average flux, since the time spent operating at the lower fluxes at higher UDS values is eliminated. A lower UDS endpoint will always result in a higher average UFP flux. Starting at a lower UDS also has the effect of increasing the average flux (since the flux is higher at lower UDS), but 
this is not a variable that can be changed if the ILAW/IHLW ratio is to be maintained. Moreover, starting at a lower UDS will require more time to reach a given endpoint, even though the average flux will be higher.

Figure 73 shows the average flux versus UDS for Campaigns II-IV. These data show that the average fluxes are very similar, but that there are differences of up to about 0.0025 $\mathrm{gpm} / \mathrm{ft}^{2}$ at different UDS values. Since the model was based on the Campaign II data, the predictions will be higher than if the Campaign IV data were used. Given the data variation shown in Figure 73, along with the measurement uncertainty, then all campaigns produced approximately the same filter performance. However, a better flux to use for the modeling may be the average of the campaigns. Nonetheless, the method of this model may be applied to any data set.

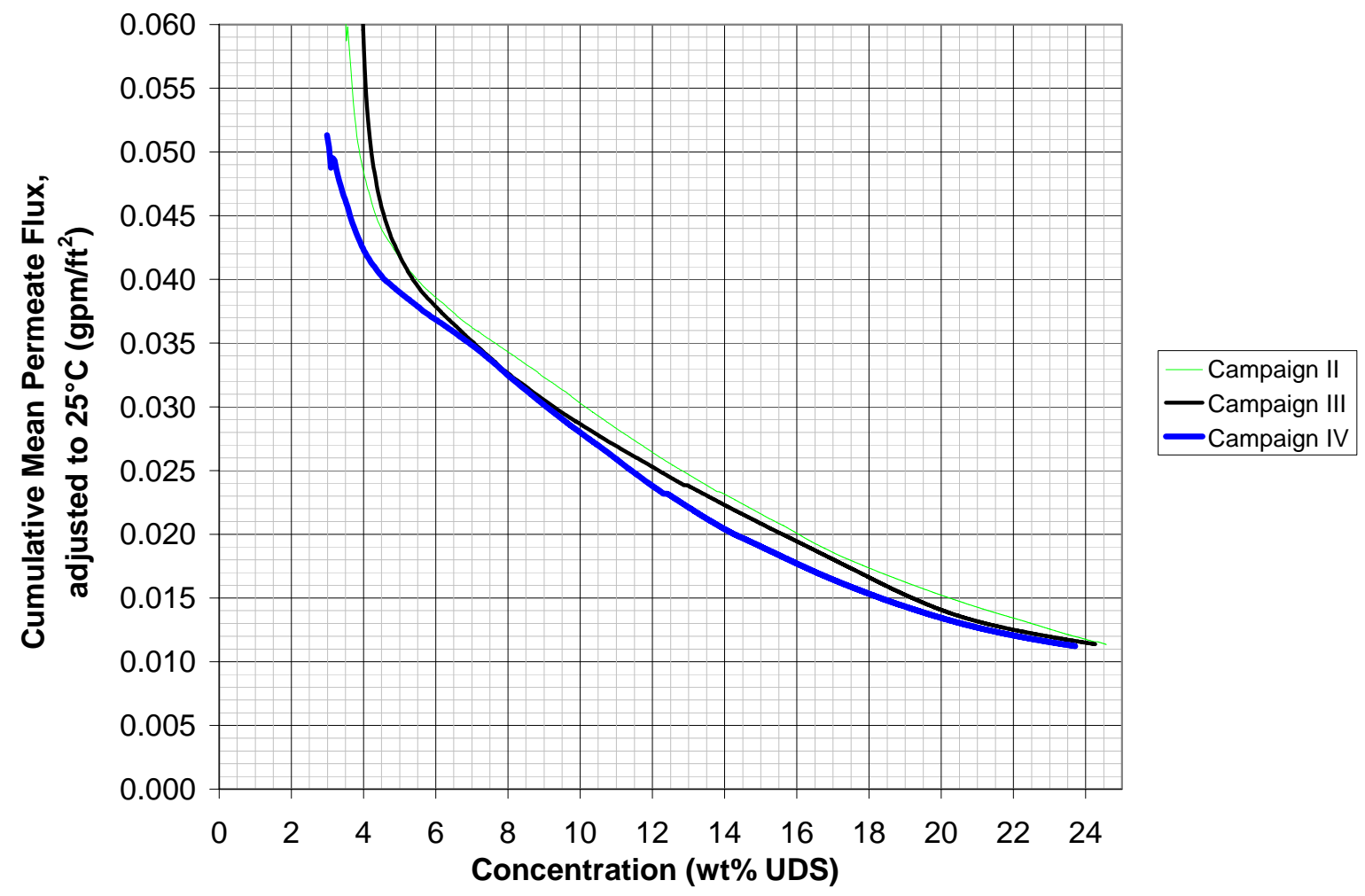

Figure 73. Comparison of Average Fluxes for Campaigns II-IV (repeat of Fig. 29)

Figure 74 shows curves describing the production rates of ILAW and IHLW as a function of the endpoint UDS at a constant processing temperature of $25^{\circ} \mathrm{C}$. The target rates of 6 MTG/d IHLW and 80 MTG/d ILAW are met for the model data when the endpoint UDS is $16.7 \mathrm{wt} \%$; the flux is $0.0185 \mathrm{gpm} / \mathrm{ft}^{2}$ (as was determined to be necessary in Table 59). The average fluxes for each of Campaigns II-IV are also shown. For the Campaign IV data, the required endpoint would be $15.4 \mathrm{wt} \%$ UDS. 
WSRC-TR-2005-00105, REVISION 0

SRNL-RPP-2005-00012, REVISION 0

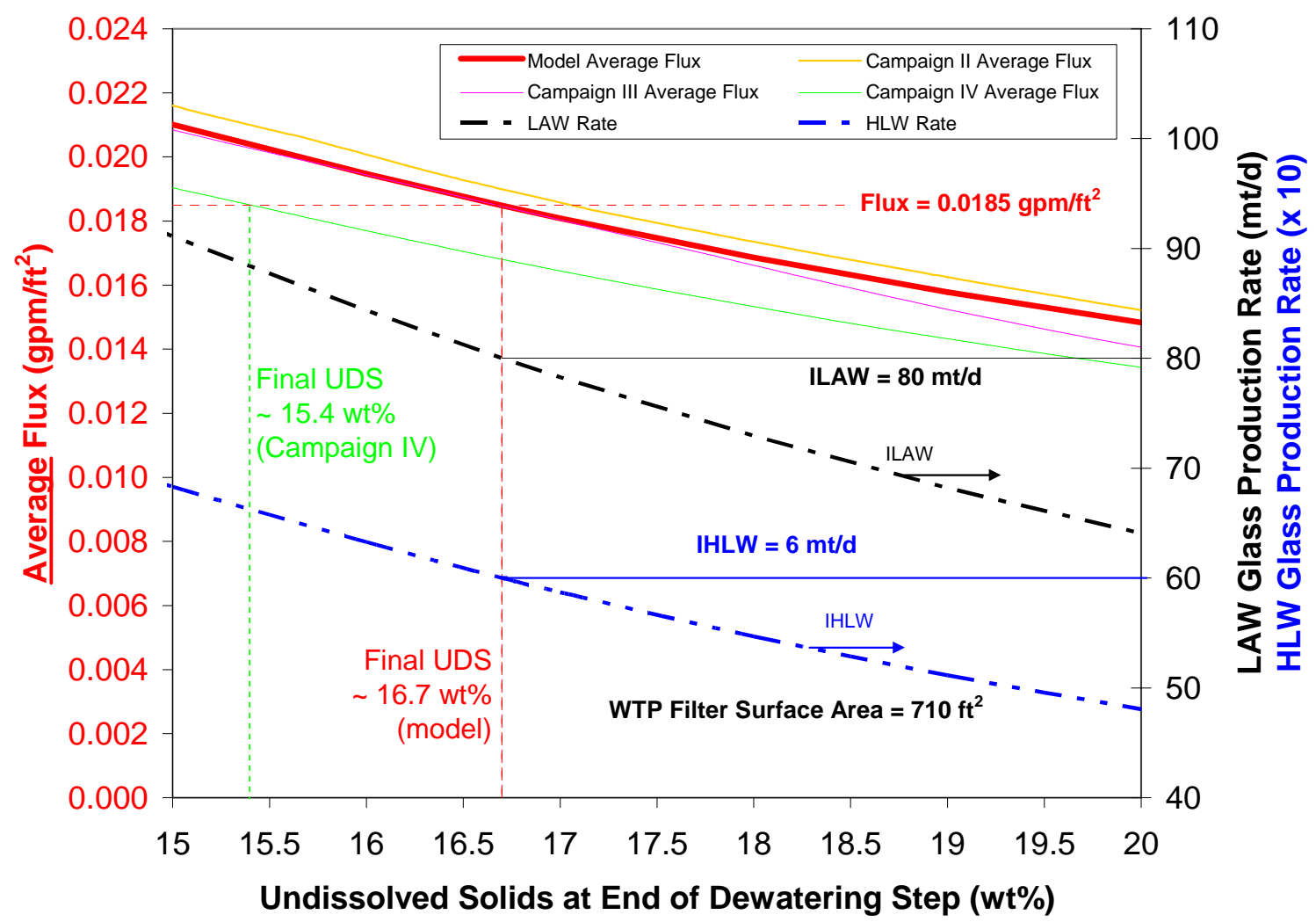

Figure 74. Average Flux to Achieve Rates versus Undissolved Solids (at $25^{\circ} \mathrm{C}$ )

Based on the operating strategy at the time of the Campaign II report, the concentration step to re-concentrate the washed slurry (dewatering of the washed slurry) will not affect the overall rate since the time to perform this step is less than that of the initial dewatering step, i.e., there is idle time at the end of the washed slurry concentration while waiting for the initial dewatering step in the other filtration train is finishing, or in other words, each train is either in initial dewatering mode or in wash/leach, but the two are never in the same mode. If the washed slurry concentration step is not idled and the next batch initial dewatering is started, the numerical results of the analysis given below do not apply, except to show that the initial dewatering step can be completed faster.

The concentration at the end of the washing step (i.e., the feed to the melter) can be higher than that at the end of the concentrating step ( $20 \%$, for example) because the rate at which the washed slurry is produced is significantly higher than the rate at which the unwashed slurry is concentrated (dewatering, LAW production). That is, the flux during washing is high enough that $20 \mathrm{wt} \%$ can be achieved even if it was not achieved during the concentrating step. Therefore, concentrating less, to lower UDS, will not necessarily have the effect of producing a more dilute feed to the HLW melter system.

Concentrating to lower UDS does result in producing less LAW feed per unit of UF feed and does result in slightly more LAW (supernatant) being recycled to the Plant Wash and Disposal (PWD) System. 


\section{WSRC-TR-2005-00105, REVISION 0 SRNL-RPP-2005-00012, REVISION 0}

Figure 75 shows, for the model data, the predicted effect of increasing the temperature during filtration while maintaining the UDS endpoint of $20 \mathrm{wt} \%$. The increase in flux due to temperature was predicted using the following WTP-supplied equation ${ }^{\dagger}$ :

$$
\text { Flux @T = Flux @25으 } \times \text { exp }\left[2500\left(\frac{1}{298}-\frac{1}{\mathrm{~T}+273}\right)\right]
$$

The minimum flux of $0.0185 \mathrm{gpm} / \mathrm{ft}^{2}$ is predicted at a temperature of about $33^{\circ} \mathrm{C}$. Figure 76 shows the combination of temperatures and ending UDS concentrations that result in predicted glass production rates meeting the target criteria. For the data from Campaign IV, the required temperature would be higher.

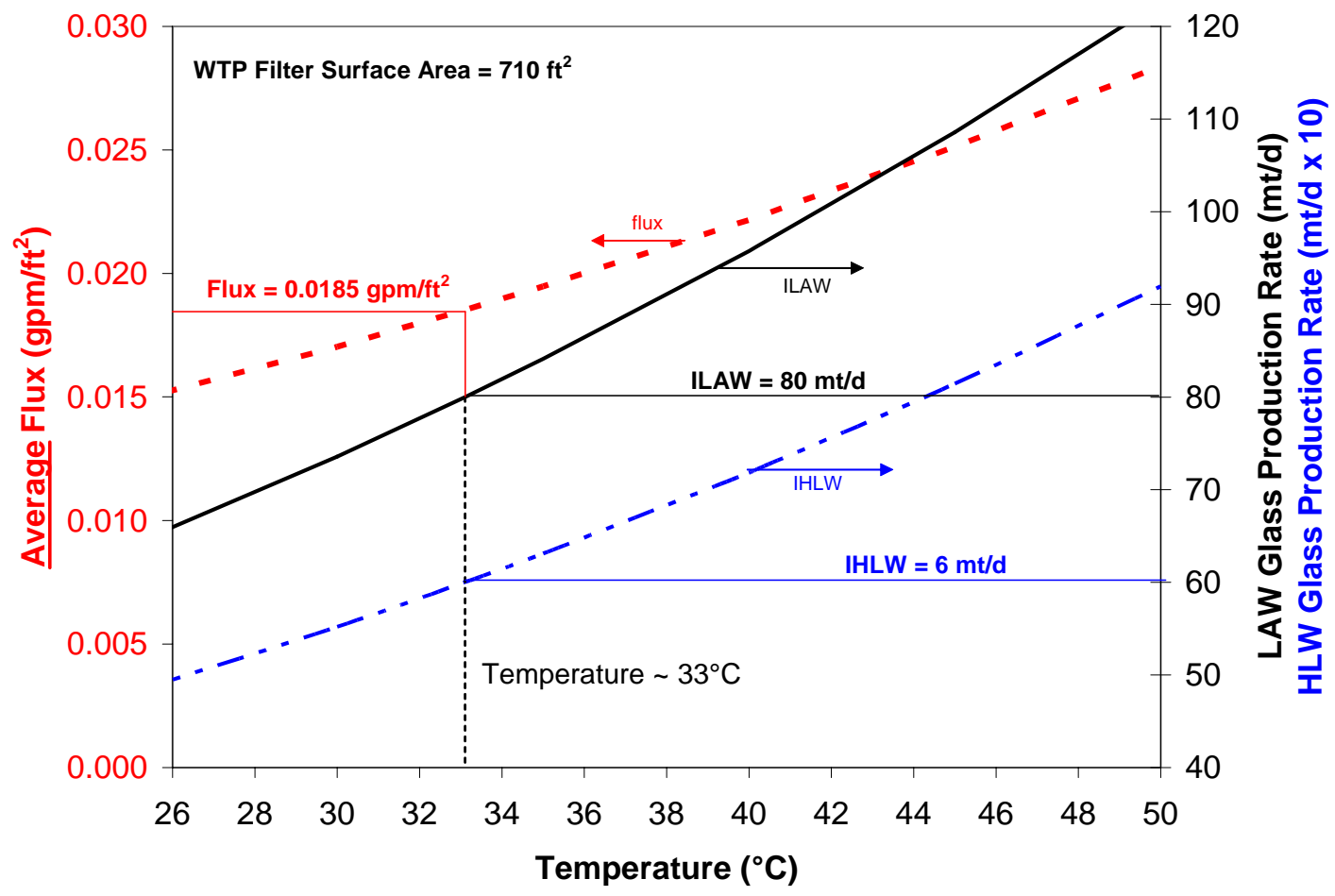

Figure 75. Average Flux to Achieve Rates versus Temperature

\footnotetext{
† A cautionary note: The "supplied" equation is required by the RPP-WTP Test Specification as a "temperature correction (that) corrects flux back to an equivalent flux at $25^{\circ} \mathrm{C}$ and accounts for changes in fluid viscosity and surface tension.” This correction has been applied to the filter data in this task, as well as all other past RPP-WTP filter tests, e.g., Duignan, 2000a, 2000b, 2003a, and Duignan et al., 2004. It is important to note that, the Test Specification also required filter testing to be carried out at $25^{\circ} \mathrm{C} \pm 5^{\circ} \mathrm{C}$, therefore the flux in that limited temperature range would only incur a correction of at most $\pm 15 \%$. In general, most data were measured within the range of $25^{\circ} \mathrm{C} \pm 2{ }^{\circ} \mathrm{C}$, which means the correction was at most $6 \%$, therefore any error in the correction itself was assumed small. This assumption may not be valid as the flux temperature differs substantially from $25^{\circ} \mathrm{C}$. How well the supplied equation actually "corrects" for viscous and surface tension is not known and may be substantially in error. Applying the equation at temperatures substantially different than $25^{\circ} \mathrm{C}$ is not recommended unless it is verified. Its use here was only to illustrate the effect of temperature changes on flux. That is, the listed flux magnitudes may contain substantial error and should not be used for actual design. However, it was shown to correct up to temperatures of $45^{\circ} \mathrm{C}$ fairly well in Section 2.7.4.1.4.
} 


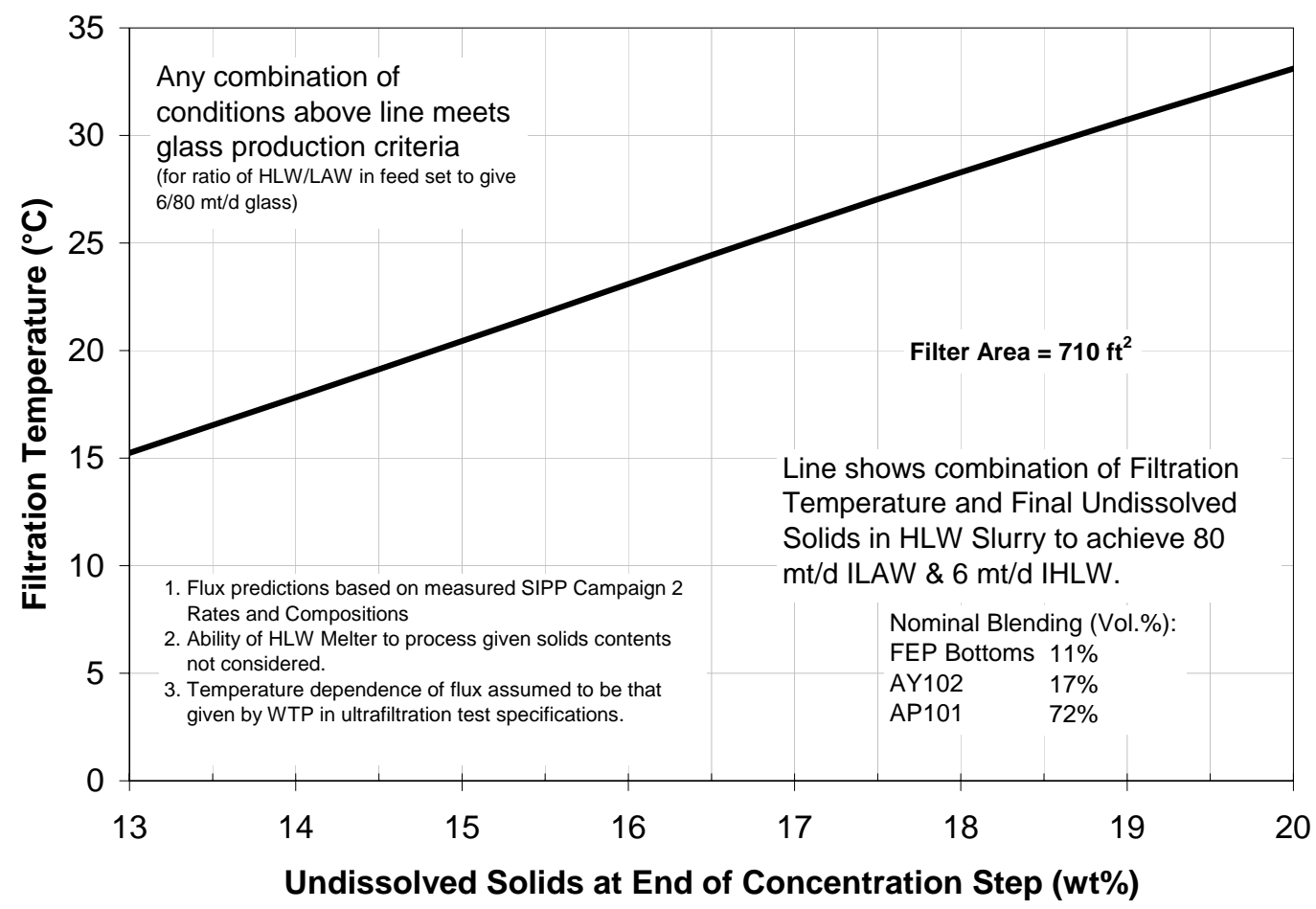

Figure 76. Predicted Combination of Filtration Temperature and Undissolved Solids Endpoint to Meet Glass Production Targets

The validity of this predictive equation was confirmed in Campaign IV as described in Section 2.7.4.1.4 and by the temperature corrected "open circle" data shown in Fig. 32 of that section. The minimum flux of $0.0185 \mathrm{gpm} / \mathrm{ft}^{2}$ needed to meet the rate targets is predicted at a temperature of about $33^{\circ} \mathrm{C}$ (with the endpoint of $20 \mathrm{wt} \%$ UDS). Figure 72 shows the combination of temperatures and ending UDS concentrations that result in predicted glass production rates meeting the target criteria.

\subsection{SMALL-SCALE TO PILOT-SCALE COMPARISONS (AUTHOR-CALLOWAY)}

Preceding the pilot-scale SIPP task a limited number of tests were done at smaller scales. Those tests are discussed and contrasted here against the SIPP experience.

\subsubsection{Evaporation of Recycle Streams}

Several observations noted during the SIPP FEP evaporation can be confirmed by previous experimental work conducted for the WTP. Aluminum (e.g. likely as amorphous aluminum hydroxide or Gibbsite) and oxalate (e.g. likely as sodium oxalate) precipitation were found to occur in the waste feed evaporator system in the SIPP and these were also found to occur in small scale evaporator studies conducted for the WTP.

$\mathrm{Al}$ was found to precipitate in the SIPP and in the small-scale experiments conducted by Stone (2004). The results from the small-scale experiments show the effect of increasing 
acid cleaning solution on aluminum precipitation in the simulated UFP recycle streams (Fig. 77). Stone found that Al precipitated as a gel when insufficient hydroxide was available to maintain the $\mathrm{Al}$ in solutions as aluminate. Additionally, recycle blends that were adjusted to maintain the $\mathrm{pH}$ greater than 13 were found to initiate precipitation of $\mathrm{Al}$ as gibbsite and bayerite after addition to simulated Envelope A mixtures. Due to Stone's experience, in blending recycles to prepare the feed for SIPP evaporation in the pilotscale FEP the feed was adjusted during preparations to maintain the $\mathrm{pH}$ greater than 13 . Even in that case, approximately $25-40 \%$ of the $\mathrm{Al}$ was found to precipitate in the SIPP. Therefore, it is likely that additional $\mathrm{NaOH}$ (UFP recycle stream $>\mathrm{pH} 14$ after blending) may be required to prevent precipitation of $\mathrm{Al}$ in the FEP evaporator system.

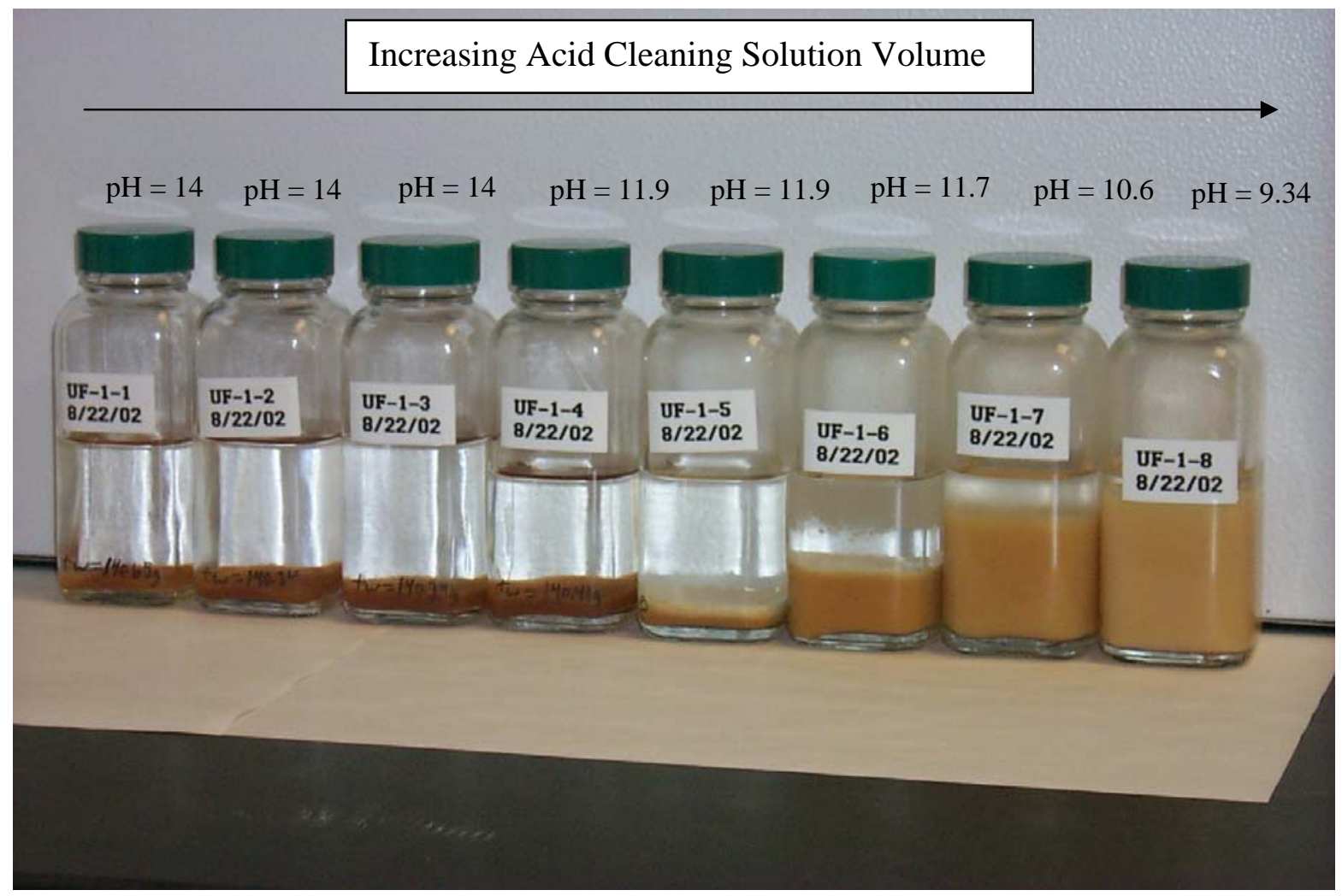

Figure 77. The effect of acid volume (pH) on Al precipitation in Small Scale evaporation experiments

Oxalate was also found to precipitate in the SIPP FEP evaporator. At the small scale Stone (2004) found that many of the recycle mixtures with Envelope A and B simulants precipitated sodium oxalate. Oxalate has been known for sometime to have limited solubility in defense HLW salt wastes. Barney (1994) conducted various organic solubility studies in the 1990's for the Hanford HLW program (see Figure 78) shows the solubility data for sodium oxalate as a function of temperature and sodium hydroxide concentration. The figure also indicates the SIPP oxalate concentrations (1.13E-02 - 1.46E-02 M) are above the solubility limit for sodium oxalate. 

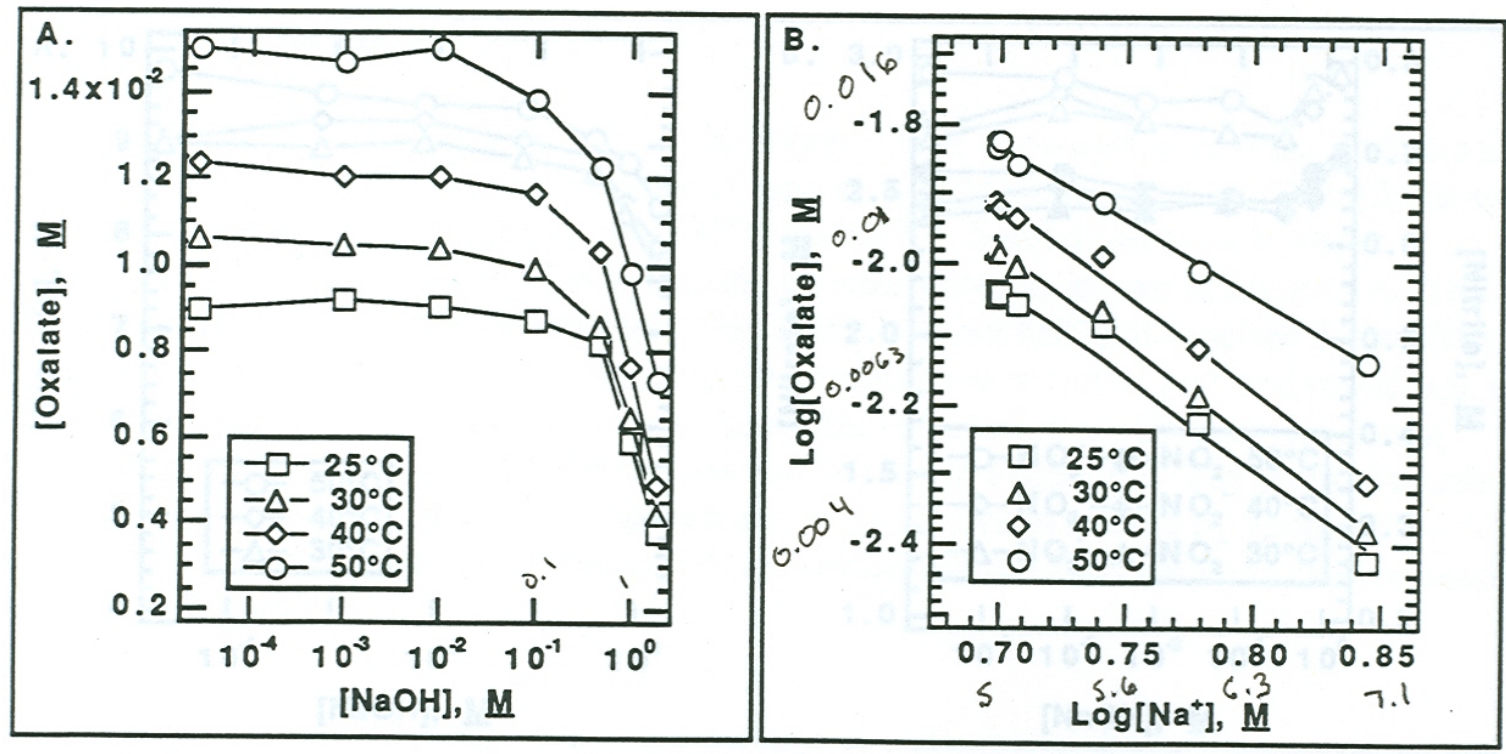

Figure 78. Solubility of sodium oxalate in $4.0 \mathrm{M} \mathrm{NaNO}-0.97 \mathrm{M} \mathrm{NaNO} 2$ at $25-50{ }^{\circ} \mathrm{C}$ for various sodium hydroxide concentrations (Barney, 1994).

\subsubsection{Filtration}

Figure 79 shows the filter flux recorded using the Cells Unit (Cross-flow) Filter (CUF) and the pilot-scale cross-flow filter located at SRNL. Actual AY102/C106 radioactive waste and simulants were used in the SRNL CUF. The pilot cross flow filter used only the AY102/C106 simulant.

Before filtration experiments began for both the radioactive CUF Actual Waste (which is the solid line in Fig. 79) and the CUF Run 1 (AY102/C106) Simulant experiment (which is the square box line in Figure 79 were both evaporated to $5 \mathrm{M} \mathrm{Na}$. As can be seen in Fig. 79, these two slurries had similar filter fluxes during dewatering. This was important to validate that the simulant represented the actual waste in its filtration performance. However, they were both difficult to filter due to precipitation of sodium carbonate that occurred during evaporation; this is the reason the data lines are very wavy.

To prevent sodium carbonate precipitation, the simulants for both the SIPP Campaign I experiment (which is the square dots in Figure 79 and CUF Run 2 (AY102/C105) Simulant experiment (which is the filled-circle line in Figure 79, were not evaporated prior to processing; that is, their sodium concentration was left at $3 \mathrm{M}$. Both data curves depict more stable flux results, however, even though the simulant was the same in both tests there was a significant difference between the small and pilot scales. The pilot-scale flux is approximately $30 \%$ lower than the small scale, but a large part of this difference can be attributed to the difference in test equipment and has been seen, and discussed, in previous work (Duignan, 2003). The CUF used of single filter tube that was shorter in length (24 inches instead of 90 inches for SIPP) and smaller in diameter (0.375 inch 
instead of 0.5 inch for SIPP). These geometric differences lead to different end effects and turbulence intensity, which result in higher fluxes for the CUF.

Since the radioactive CUF Actual Waste and the CUF Run 1 Simulant runs were evaporated, and thus changed the slurry viscosities, they can not be directly compared to the SIPP pilot data. However, in an attempt to make a useful comparison all of the filter flux results were adjusted to have the same viscosity by normalizing with respect to the Actual Waste permeate viscosity of $2.9 \mathrm{cp}$. Figure 79 implies that normalizing to viscosity does not overcome differences in scale, but that the AY102/C106 simulant is comparable to the actual waste from a filter flux perspective.

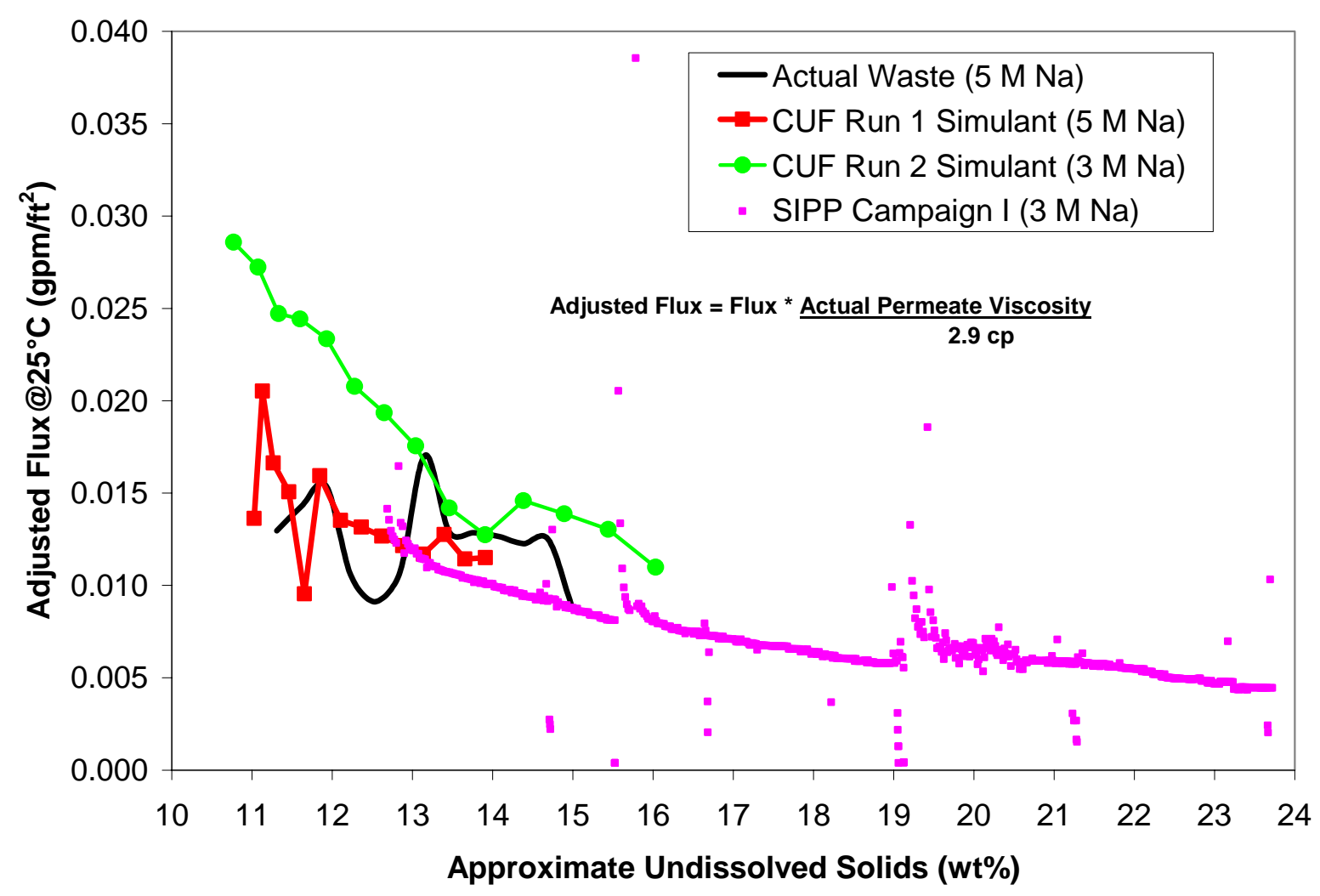

Figure 79. Filter Flux Comparison - Small Scale CUF vs. SIPP Cross Flow Filter

\subsubsection{Cesium Ion Exchange}

SIPP ion exchange column testing was conducted on two feed samples with similar compositions. Both samples were mixtures of AP-101 and AY-102/C-106 simulants and SIPP recycle streams. As previously explained in Section 2.8, Column testing was conducted only in two of the four SIPP Campaigns [i.e., II and III] and each campaign contain two cycles, i.e., Cycles 1 and 2 [Campaign II] and Cycles 3 and 4 [Campaign III]. The feed solutions for ion exchange testing were permeates generated from simulant slurry dewatering in the pilot-scale filtration process (UFP). The average molar 
concentrations for the major species were: $4.8 \mathrm{M} \mathrm{Na}^{+}, 0.53 \mathrm{M} \mathrm{K}^{+}, 1.4 \mathrm{M} \mathrm{NO}_{3}{ }^{-}, 0.59 \mathrm{M}$ $\mathrm{NO}_{2}{ }^{-}, 0.68 \mathrm{M} \mathrm{CO}_{3}{ }^{2-}$, and $1.4 \mathrm{M} \mathrm{OH}^{-}$(hydroxide only measured for Campaign III). Campaign II feed contained 4.45 E-5 M Cs, while Campaign III feed contained 5.84 E-5 M Cs. The average density of the two solutions was $1.246 \mathrm{~g} / \mathrm{mL}$. The feed for each SIPP campaign was split in half so that each cycle would process approximately the same amount of simulant. The SIPP IX tests were conducted with two 5.5" ID columns arranged in a lead/lag (sequential) configuration. The columns were eluted and regenerated between cycle and then rotated such that Column \#1 was the lead column for the first and third cycles and Column \#2 was the lead column for the second and fourth cycles. The resin used for SIPP testing was from a 250 gallon manufacturer batch of SuperLig ${ }^{\circledR} 644$ resin that had been sieved prior to use to remove particles smaller than 40 mesh. Approximately 190 resin bed volumes (BV) of solution was processed through the columns during each cycle and cesium breakthrough was monitored in the column effluent solutions.

Based on the design criterion of $>100 \mathrm{BV}$ of solution processed prior to $50 \%$ breakthrough on the Lead Column, then the cesium removal performance was acceptable for all four SIPP CIX cycles. The largest cesium breakthrough was observed for Cycle 4, where approximately $6 \%$ cesium breakthrough was measured after processing $190 \mathrm{BV}$ of solution. Less than $1 \%$ cesium breakthrough was observed in all cases after processing $100 \mathrm{BV}$ of solution. Furthermore, there was no indication of significant channeling in any test.

It is difficult to evaluate the cesium removal performance observed in the SIPP campaigns further without utilization of the existing chemical and performance models for cesium ion exchange with SuperLig ${ }^{\circledR} 644$ resin (Hamm et al., 2000). Certain qualitative evaluations can sometimes be made between column tests (by direct comparison of cesium breakthrough profiles) without the use of a chromatography model in cases where similar liquid feeds, resin samples, and processing conditions are used. Small-scale (1.5” ID) column tests were performed previously with actual Hanford AP101 waste and SuperLig ${ }^{\circledR} 644$ resin (Fiskum, et al., 2002). For comparison, the concentrations of major species in the actual AP-101 feed were: $5.0 \mathrm{M} \mathrm{Na}^{+}, 0.76 \mathrm{M} \mathrm{K}^{+}$, $1.7 \mathrm{M} \mathrm{NO}_{3}^{-}, 0.71 \mathrm{M} \mathrm{NO}_{2}^{-}, 0.45 \mathrm{M} \mathrm{CO}_{3}{ }^{2-}$, and $1.9 \mathrm{M} \mathrm{OH}^{-}$. The actual AP-101 sample contained $3.7 \mathrm{E}-5 \mathrm{M} \mathrm{Cs}^{+}$and the solution density was $1.256 \mathrm{~g} / \mathrm{mL}$. Only $110 \mathrm{BV}$ of solution was processed in the small column testing and $<1 \%$ cesium breakthrough was observed, which is comparable performance to that observed in the SIPP. However, as shown in Table 60, significant differences existed between the two data sets and any qualitative comparisons beyond that described above should be conducted utilizing the cesium performance model. The term "broader breakthrough profile" in Table 60 refers to kinetic effects upon cesium loading that impact the sharpness of the cesium breakthrough profile. A broader profile would be characterized by earlier breakthrough of relatively low cesium levels. 
WSRC-TR-2005-00105, REVISION 0

SRNL-RPP-2005-00012, REVISION 0

Table 60. Comparison between AP-101 and SIPP Column Tests and Expected Impacts

\begin{tabular}{|c|c|c|c|}
\hline $\begin{array}{l}\text { Resin Batch } \\
\text { (Feed) }\end{array}$ & $\begin{array}{l}\text { Small Scale } \\
\text { AP-101 } \\
\text { [1.5-inch } \\
\text { column] }\end{array}$ & $\begin{array}{c}\text { Pilot Scale [SIPP] } \\
\text { [AY102/C106+AP101+Recycles] } \\
\text { [6-inch column] }\end{array}$ & $\begin{array}{c}\text { Expected } \\
\text { Performance } \\
\text { Impact }^{*}\end{array}$ \\
\hline $\begin{array}{l}\mathrm{Na}^{+} / \mathrm{K}^{+} \text {mole } \\
\text { ratio }\end{array}$ & 6.5 & 9.1 & $\begin{array}{c}\text { later Cs } \\
\text { breakthrough }\end{array}$ \\
\hline $\begin{array}{l}\mathrm{Na}^{+} / \mathrm{Cs}^{+} \text {mole } \\
\text { ratio }\end{array}$ & $1.4 \mathrm{E} 5$ & $9.6 \mathrm{E} 4$ & $\begin{array}{c}\text { earlier Cs } \\
\text { breakthrough }\end{array}$ \\
\hline resin batch & $\begin{array}{c}\text { 010319SMC-IV- } \\
73\end{array}$ & C-01-11-05-02-35-60 & impact unknown \\
\hline $\begin{array}{l}\text { particle size } \\
\text { (mesh) }\end{array}$ & -40 to +70 & -20 to +40 & $\begin{array}{c}\text { broader Cs } \\
\text { breakthrough } \\
\text { profile }\end{array}$ \\
\hline $\begin{array}{l}\text { flow rate } \\
(\mathrm{BV} / \mathrm{hr})\end{array}$ & 2.8 & $\begin{array}{c}3.0 \text { (SIPP Cycles 1, 2, and 4) } \\
4.5 \text { (SIPP Cycle 3) }\end{array}$ & $\begin{array}{l}\text { minimal impact } \\
\text { broader Cs } \\
\text { breakthrough } \\
\text { profile }\end{array}$ \\
\hline
\end{tabular}

impact defined as the expected changes in SIPP cesium removal performance relative to the actual AP-101 sample

\subsection{OVERALL CHEMICAL COMPOSITIONS OF SIPP CAMPAIGN II PROCESS STREAMS (AUTHOR - ZAMECNIK)}

As explained throughout this report, the SIPP task contained four campaigns, which the first was just the operation of the ultrafiltration process and the remaining three would operate all four unit operations, i.e., FEP, UFP, CIX, and TLP. As was also discussed, the pre-task steady-state OLI/ESP computational modeling of the SIPP process showed that in preforming Campaign II, III, and IV the product streams became chemically equivalent very fast; the differences in stream composition between Campaign III and IV were less then 5\%. (Of course, this small difference cannot be fully shown experimentally because just before beginning the last campaign, the operation of Campaign IV was changed by the Hanford Project to specifically test the new GKN filter, therefore, the CIX and TLP unit operations were not used.)

The first full SIPP campaign was Campaign II and, as such, it was targeted to have its product streams chemically analyzed most extensively. Furthermore, since Campaigns III and IV were expected to be chemically similar to Campaign II, then the number samples was greatly reduced to minimize cost to the project. For those campaigns only a minimum number of samples were taken to insure proper operation the plan tests. On the other hand, the measurements made for Campaign II were extensive to capture inter-unitoperations chemically histories and are listed in Appendix J. 
WSRC-TR-2005-00105, REVISION 0

SRNL-RPP-2005-00012, REVISION 0

This section only indicates a mapping of where samples were taken in the overall process. Figure 80 shows the overall SIPP operation for the FEP and UFP unit operations of Campaign II and sample points. Figure 81 shows the overall SIPP operation for the CIX and TLP unit operations of Campaign II and sample points. These figures are repeated in Appendix $\mathrm{J}$ as well as the full table of analyzed results. The stream analyses table in that Appendix shows the measured compositions of most of the process streams, including density and solids measurements, and the approximate stream volume. 
WSRC-TR-2005-00105, REVISION 0

SRNL-RPP-2005-00012, REVISION 0

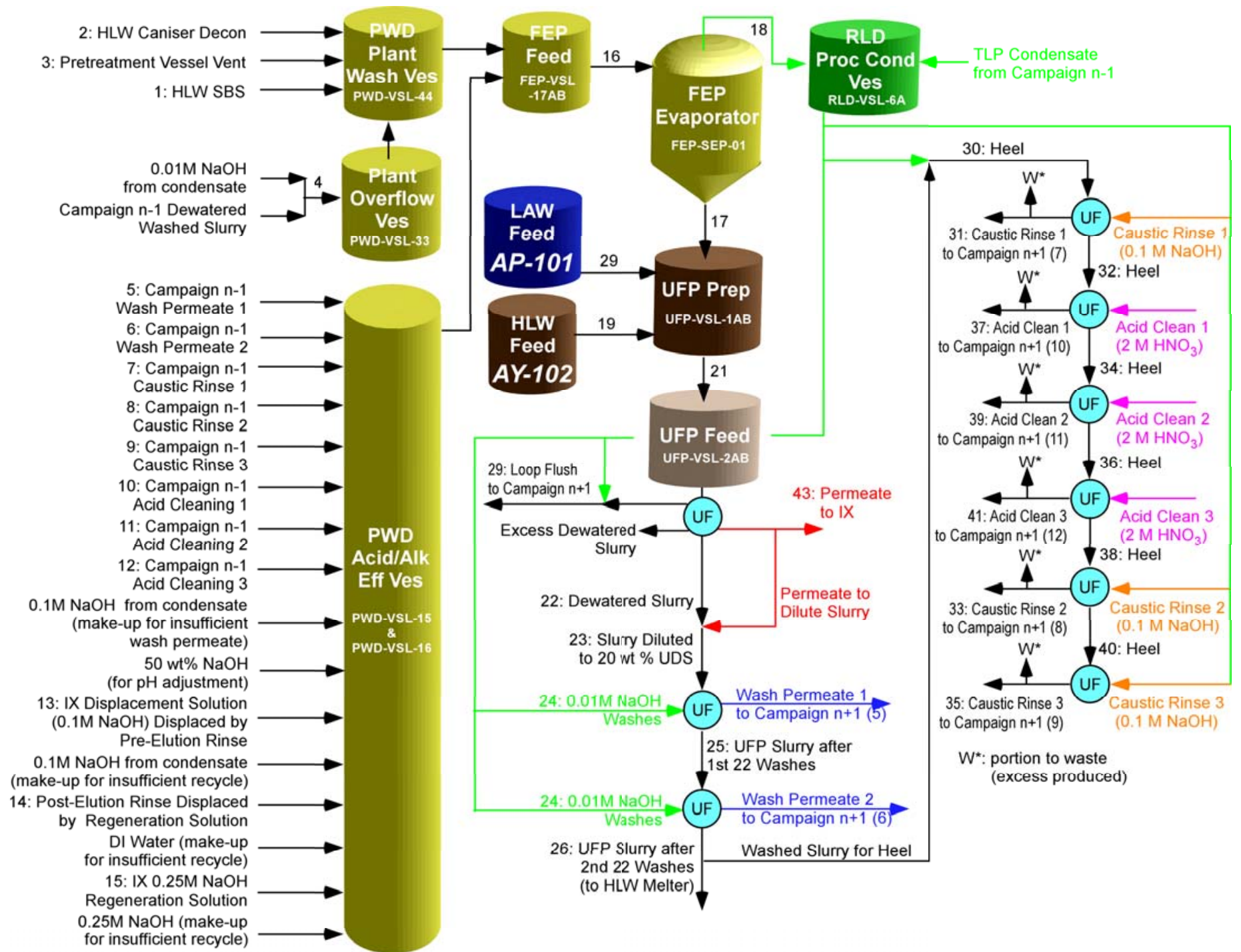

Figure 80. Product samples taken during Campaign II: FEP \& UFP. The numbers refer to column results in Appendix J 


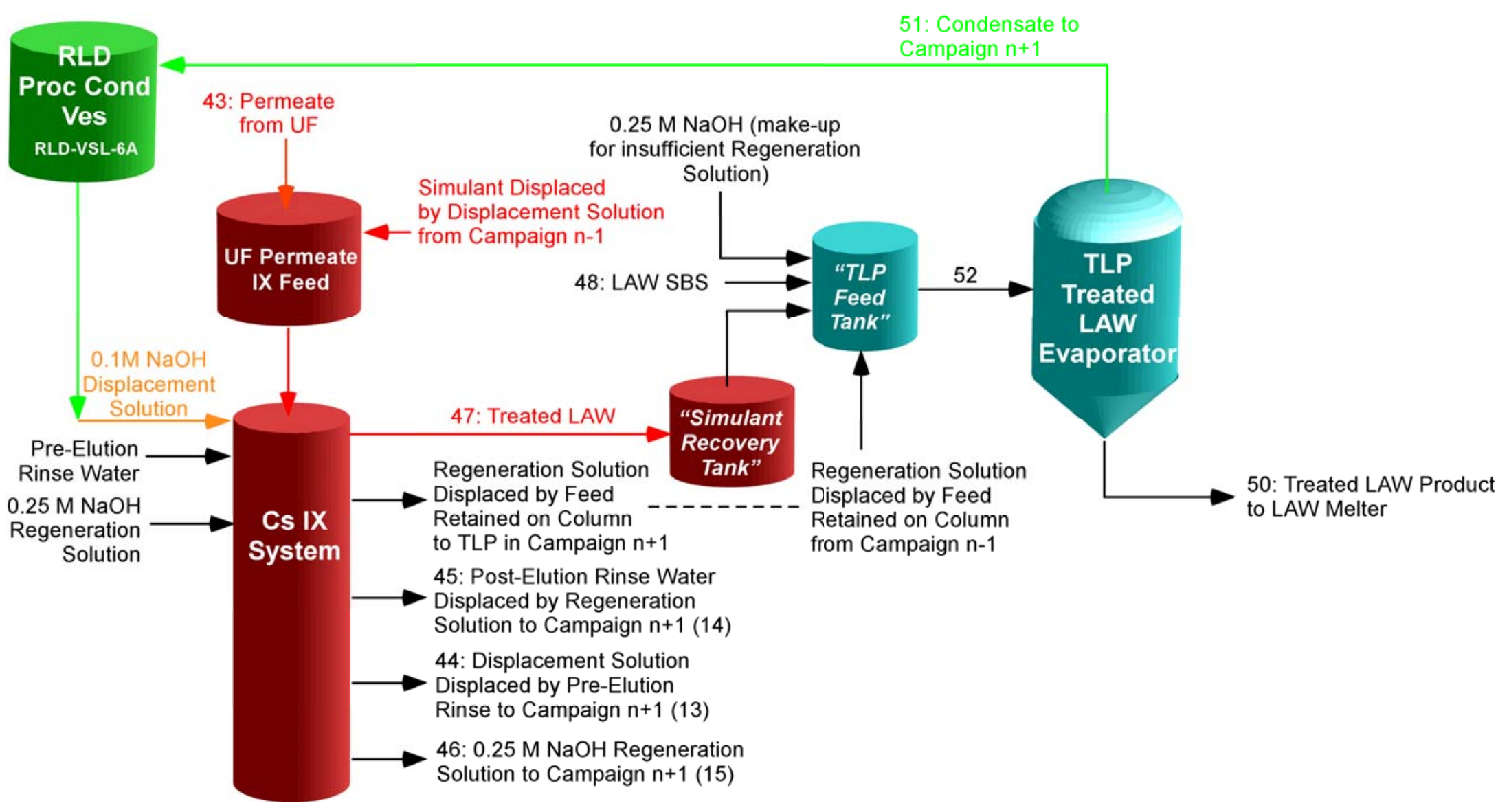

Figure 81. Product samples taken during Campaign II: CIX \& TLP. The numbers refer to column of results in Appendix J 


\subsection{CONCLUSIONS}

These are conclusion obtained from Campaign IV for the FEP and the UFP unit operations. For the CIX and TLP unit operations, which ended at Campaign III, some post task conclusions are included here. However, since the report encompasses all task activities the full list of conclusions made for Campaigns I to IV can be found at the end of each appropriate unit operation section.

\subsection{PILOT-SCALE FEP UNIT OPERATION - CAMPAIGN IV}

- A total of about $1119 \mathrm{~kg}$ of FEP feed, was concentrated down to $175 \mathrm{~kg}$.

- The feed was evaporated under vacuum (1 psia (6.9 kPa)) at moderate temperatures $\left(\sim 50^{\circ} \mathrm{C}\right)$ and the entire evaporation process proceeded as designed.

- No significant foaming was observed prior to the addition of antifoam agent. During the evaporation process, the froth height remained around $1 \mathrm{ft}(0.3 \mathrm{~m})$ that is significantly less than the $5 \mathrm{ft}(1.5 \mathrm{~m})$ distance to the lower demister pad.

- Mixing of the recycles in a different order for Campaign IV had no effect on the evaporation process as compared to the previous campaigns.

- The FEP feed and Bottoms concentrations for Campaigns III and IV were very similar, indicating that steady-state had been reached with respect to the recycle compositions.

- The precipitation of sodium oxalate in the FEP evaporator occurred in all three campaigns and accounted for most of the precipitated solids found. That is, while more that $90 \%$ of the oxalate was dissolved in the FEP feed less than $25 \%$ of the oxalate was still dissolved after reaching the target Bottoms concentration.

\subsection{PILOT-SCALE UFP UNIT OPERATION - CAMPAIGN IV}

- The performance of the GKN filter unit was similar to that of the Mott filter unit under steady state conditions. In addition, the permeate flux of the GKN filter unit during dewatering was the same as the Mott filter unit, within measurement uncertainty, for the same simulated waste mixture.

- In Campaign IV, using the GKN filter unit, the average, temperature-adjusted permeate flux when dewatering from $\sim 3$ to $\sim 24 \mathrm{wt} \%$ UDS was $0.011 \mathrm{gpm} / \mathrm{ft}^{2}$. The average, temperature-adjusted permeate flux when dewatering from $\sim 3$ to $20 \mathrm{wt} \%$ UDS was $0.013 \mathrm{gpm} / \mathrm{ft}^{2}$. The average, temperature-adjusted permeate flux when dewatering from $\sim 3$ to $17 \mathrm{wt} \%$ UDS was $0.016 \mathrm{gpm} / \mathrm{ft}^{2}$.

- Permeate flux improved at higher slurry temperatures. At 19 wt\% UDS, 40 psid TMP, and a slurry velocity through the filter of $12 \mathrm{ft} / \mathrm{s}$, the permeate flux improved almost $75 \%$ at $45^{\circ} \mathrm{C}$ compared to $25^{\circ} \mathrm{C}$.

- The turbidity of the permeate from dewatering and slurry washing, when measured directly after the sample was taken, was always lower than 1 NTU. The Test 
Specification indicated a threshold of 2 NTU, therefore, the measured values indicated that the permeate was free of significant undissolved solids.

- The SIPP and previous UFP tests indicate that the permeate flux during acid cleaning of a filter appears to be more of a function of the waste dewatered than of the filter unit used.

- The increase in the adjusted permeate flux between the second and third acid cleaning steps was minimal in Campaigns II, III, and IV, possibly indicating that the third acid cleaning was unnecessary. The exception was Campaign I where whitish solids in the spent solutions of the first and second acid cleanings inhibited the permeate flux, requiring a third acid cleaning (and possibly a fourth) to reach steady conditions. The Campaign I simulant did not contain AP101 and FEP Bottoms, indicating that the necessity of a third acid cleaning was dependent on the waste dewatered.

- Cleaning the filter with $2 \mathrm{M}$ nitric acid after dewatering was sufficient to increase baseline permeate flux to approximately an order of magnitude higher than that of average waste flux during dewatering. However, it was not sufficient to return the filter to pre-test flux levels. In previous campaigns, soaking the filter unit in $0.1 \mathrm{M}$ $\mathrm{NaOH}$ for an extended period of time (2 months or more) typically regained the pretest flux levels. The significance of " 2 months or more" is that 2 months was typically the duration between successive SIPP campaigns.

- During the elevated temperature tests at a constant solids loading of $19 \mathrm{wt} \%$ UDS no significant changes occurred with respect to solids concentrations and particles sizes. As expected, as the simulant temperature increased from $25^{\circ} \mathrm{C}$ to $45^{\circ} \mathrm{C}$ the consistency of the slurry and the viscosity of the permeate decreased from 14 to 11 $\mathrm{mPa} \bullet \mathrm{s}$ and from 2.9 to $1.8 \mathrm{mPa} \bullet$, respectively, while the slurry yield stress increased approximately 10 to $12 \mathrm{~Pa}$.

- The composition of the undissolved solid species in the washed sludges in Campaigns I-IV were similar, as expected. The soluble species concentrations in the slurry and permeate for Campaigns II-IV were similar as expected.

- The balances on aluminum over the process were not consistent and could not be closed without additional analytical data.

- Ammonium in the first Campaign III 0.01M NaOH wash was mostly retained in the slurry, indicating that a slurry component has an affinity for ammonium. Approximately none of the ammonium in the second wash was retained.

- The antifoam agent did not have any observed macroscopic effects on the filter operations of the SIPP, which is also consistent with similar commercial industrial experience (Watts et al., 1995). However, since the filter was cleaned between each run, the effect of possible accumulation of antifoam on the filter media with no cleaning between runs was not determined. 
WSRC-TR-2005-00105, REVISION 0

SRNL-RPP-2005-00012, REVISION 0

\subsection{PILOT-SCALE CIX UNIT OPERATION - POST CAMPAIGN III}

- A post-test measurement of the dry mass of the SuperLig ${ }^{\circledR} 644$ resin found a ratio of a sodium basis to hydrogen basis of 1.19 and 1.22 for Columns 1 and 2, respectively.

- A pre-test particle-size distribution of the resin was found to be $78 \mathrm{wt} \%$ (Mesh $>30$ ), $20 \mathrm{wt} \%(30 \leq$ Mesh $\leq 40)$, and $2 \mathrm{wt} \%$ (Mesh $<40)$. A post-test PSD on the resin found no change in the Column 2 contents, indicating that no degradation of the resin occurred. However, the larger distribution of Column 1 implied that some resin clumping may have occurred.

\subsection{PILOT-SCALE TLP UNIT OPERATION - POST CAMPAIGN III}

- In the filtration system, nearly all of the antifoam component polydimethylsiloxane (PDMS) remained in the washed concentrated sludge stream which is consistent with similar studies (Watts et al., 1995). Analysis indicates that very little if any of the PDMS was found in the UFP recycles or permeate. Nearly all of the PDMS was also retained in the FEP and TLP Bottoms concentrates, with no detectable PDMS in the condensates. Since PDMS is essentially insoluble, non-homogenous sub-samples, coupled with analytical recoveries of less than $80 \%$, did not allow for accurate quantification of the PDMS for the purposes of process material balances in all the SIPP process streams. SRNL recommends that extraction of the entire samples before analysis should be investigated for any future antifoam analytical work. Furthermore, no conclusions about the fate of the polyol fraction of the antifoam could be made since this portion was not tracked. Future work on the fate of this antifoam fraction is warranted.

\section{Observations from the SIPP task to the Overall Operation of WTP}

The SIPP operations conducted at SRNL using simulated WTP startup feed performed well without any significant incidents that caused disruption of operations. All four unit operations (Evaporation FEP\& TLP, Filtration-UFP and Ion Exchange, CIX) performed nearly flawlessly and without significant unplanned downtime. The LAW and HLW supernate and slurry products produced by the SIPP were successfully vitrified by VSL (Matlack 2005a, Matlack 2005b).

The SIPP met all success criteria with the exception of demonstration of a scaled throughput of $80 \mathrm{MTG} /$ day of LAW and 5.4 MTG/day HLW. This criterion ${ }^{\dagger}$ was partially met by the SIPP in that both evaporators preformed at or above the evaporation rate necessary to meet the desired production rates. The pilot ultra filter system was not able to meet the production rates given a filter concentration end point of $20 \mathrm{wt} \%$ undissolved solids. However, glass production rates can be met for simulated AY102/C106 with AP101 slurries by reducing the target ultra filter concentration

\footnotetext{
${ }^{\dagger}$ The pilot ion exchange testing was by design not required to meet full scale plant production rates (See Test Exception No. 7 in Section 1.2)
} 
endpoint to 15.4 to $16.5 \mathrm{wt} \%$ undissolved solids. After the wash cycle is completed, higher undissolved solids concentrations can be achieved by concentrating the UFP slurry after washing (flux rates are much higher during washing) without reducing the overall plant production rate. Therefore, concentrating less, to a lower UDS, will not necessarily have the effect of producing a more dilute feed to the HLW melter system.

Concentrating to lower UDS does result in producing less LAW feed per unit of UF feed and does result in slightly more LAW (supernatant) being recycled to the Plant Wash and Disposal (PWD) System. Increasing the operating temperature of the ultra filter also proved to increase the filter production rate beyond the desired production rate goals.

The impact of recycle solutions on SIPP operation proved to have no macroscopic effects on the overall SIPP operation or the individual unit operations. While some solids (e.g. oxalate \& aluminum) were found to precipitate in the Plant Wash \& Disposal and FEP evaporator systems, none of the these solids proved to have an impact on the overall operation of the SIPP.

Given the limits (e.g. non-prototypical piping layouts, mixing systems, simulant chemical/physical properties vs. actual waste) of the testing described in this report, the full-scale WTP Pretreatment facility unit operations that were simulated by the SIPP should also perform well using AY102/C106 feeds. However, since not all start-up and operational risks can be fully anticipated or mitigated by SIPP testing, the following observations and lessons learned should be considered by the designers and operators of the WTP.

- Precipitation of aluminum, in some tank waste compositions can increase the total number of HLW canister produced, therefore, additional sodium hydroxide maybe necessary to prevent unwanted $\mathrm{Al}$ precipitation.

- Ammonium, transferred from the Duratek LAW Submerged Bed Scrubber, will partition to the concentrated/washed HLW transferred to the WTP HLW melter system. The ammonium will likely recycle back to the WTP Pretreatment facility via the HLW SBS condensate system. Incorporation of ammonium chemistry into the WTP models should be implemented to assess the fate and impact of ammonium on the overall operations of the WTP. The potential to generate ammonium nitrate in the WTP vessel vent system should be evaluated. Consideration should be given to determining if the TLP evaporator condensate and LAW melter SBS condensates can be treated and disposed of separately rather than recycling these materials to the front end of the Pretreatment facility. This would also decrease the buildup of corrosive anions (e.g. $\mathrm{Cl}, \mathrm{F}, \mathrm{SO}_{4}$ ) in the TLP system.

- Maintenance of the filter system may dislodge solids and cause a loss in filter flux. WTP should consider cleaning the filter after maintenance is conducted. A detailed filter system performance monitor program maintained by the future WTP filter system engineers would reduce plant down time when filter flux performance issues occur. 
WSRC-TR-2005-00105, REVISION 0

SRNL-RPP-2005-00012, REVISION 0

- Decon solutions that are generated from hot cell equipment maintenance were not tested in the SIPP. These solutions can carry larger concentrations of glass formers, sludge, salts, and ammonium then the nominal recycles tested by the SIPP. WTP personnel should assess the impact of processing decon solution generated from maintenance activities to determine if any unwanted reactions (e.g. precipitation) will occur. 
WSRC-TR-2005-00105, REVISION 0 SRNL-RPP-2005-00012, REVISION 0

This page intentionally left blank. 
WSRC-TR-2005-00105, REVISION 0

SRNL-RPP-2005-00012, REVISION 0

\subsection{FUTURE WORK}

Given the specific scope of the SIPP (i.e., demonstrating pretreatment performance and the influences of recycle streams using AY102/C106 and AP101 simulants) several key findings of the SIPP workshop (Edwards, 2003) were deferred to future programs or tasks. These items are:

> Preliminary calculations indicate the SIPP will produce only enough HLW glass to turnover one DM-100 melter volume. Similar R\&D efforts conducted for DOE (e.g., Integrated DWPF Melter System, IDMS) typically produced sufficient glass to turnover the melter at a minimum of three volumes. This typically allows the melter system to achieve steady state operations with respect to glass composition, and production rates.

$>$ The transition of various Hanford waste feeds (e.g. mixing of Envelope D heels with Envelope C supernatant) through an integrated system is not being tested by the SIPP. Future programs or tasks should consider testing additional sludge compositions in an integrated system. Similar R\&D efforts conducted for DOE (e.g. IDMS, DWPF Cold Commissioning, and Duratek LAW Pilot Melter) tested a variety of waste chemistries and conducted testing that demonstrated these facilities could operate successfully during waste transitions.

Additionally, since only one Envelope D simulant (AY102/C106) has been tested in the SIPP, the filtration flux at a pilot scale with other wastes (e.g. AZ101, AZ102, C104, and single-shell tank sludges) is unknown and likely to be different than the filtration flux measured with in the SIPP. Because bench-top cross-flow filter fluxes, using AY102/C106 Hanford waste, are higher than pilot-scale filter fluxes using AY102/C106 simulant, it is recommended that additional pilot-scale filtration tests be conducted to determine the filter flux with other Hanford sludges. Furthermore, since it will not be practical or cost effective to conduct pilot-scale testing using all known sludge types, a correlation between bench-top radioactive filter experiments and pilot-scale data should be developed to predict the filter flux of future WTP sludge batches.

Leaching of aluminum or oxidative leaching of other metals (e.g. Cr) was not tested by the SIPP. Leaching was determined to be unnecessary for the AY102/C106 tank composition (Calloway, 2004). However, other Hanford wastes require leaching of aluminum and other metals to reduce the number of HLW canisters produced from a given waste type. While aluminum leaching and oxidative leaching of $\mathrm{Cr}$ have been demonstrated in the laboratory, pilot-scale demonstration is needed to assess the actual efficiency of these processes. Additionally, post precipitation of solids after high temperature leaching and subsequent cooling down could become a significant issue in these processes and should be assessed at a pilot scale.

An optimized filter cleaning protocol has never been developed for the WTP which balances the maintenance needs of the filter and the production goals of the plant. If the 
filter could be cleaned after every other batch, significant savings in production cycle time could be achieved. However, since no multi-batch studies have been conducted without cleaning the filter after each batch, further testing is recommended using a small scale filter (e.g., CUF) to optimize the cleaning protocols for the WTP. It has been suggested that simple flushing with dilute sodium hydroxide solutions after each batch may be sufficient for cleaning the filter, however, again there is no information to support this protocol since the SIPP testing cleaned the filter after each batch. The long term impact of the antifoam agent on the filter performance may need to be reconsidered in these future studies since it very likely that any antifoam agent left in the SIPP filter after concentration and washing was removed by the acid cleaning step. 
WSRC-TR-2005-00105, REVISION 0

SRNL-RPP-2005-00012, REVISION 0

\subsection{REFERENCES}

Adamson, D. J. (2004), “SIPP Pilot TLP,” Westinghouse Savannah River Company Notebook No. WSRC-NB-2004-00038. (began on 4 March)

Barnes, C. D. (2004) "OLI/ESP Modeling of the Semi-Integrated Pilot Plant for Estimate of Campaigns I-IV Simulant Volumes,” Westinghouse Savannah River Document No. WSRC-TR-2004-00403, Rev. 0. (August). [also SRNL-RPP-2004-00063]

Barney, G. S., (1994) "The solubilities of significant organic compounds in HLW Tank Supernate solutions,” Westinghouse Hanford Company Document No. WHC-SA-2565FP, P.O. Box 1970, Richland WA, (August)

Brooks, K. P., R. L. Myers, and K. G. Rappe (1997), "Bench-Scale Enhanced Sludge Washing and Gravity Settling of Hanford Tank C-106 Sludge,” Battelle Pacific Northwest National Laboratory Document No. PNNL-11432.

Calloway, Jr., T. B. (2004) “OLI Modeling Estimate of SIPP Run Times and Leaching Effectiveness,” Westinghouse Savannah River Company Document No. SRT-RPP-200300241, (24 March). [This is an inter-office memorandum from Calloway to Reid Peterson.]

Coleman, C. J., M. S. Hay, and K. B. Martin (2003), “Compositing and Characterization of Samples from Hanford Tank 241-AY-102/C-106," Westinghouse Savannah River Document No. WSRC-TR-2003-00205, Rev. 0. (October) [also SRT-RPP-2003-00086]

Coutts, D. A. (1991). “Uncertainty and Calibration Analysis,” Westinghouse Savannah River Company Document No. WSRC-TR-91-106, Rev. 0. (March)

Duignan, M. R. (2000a), “Final Report: Pilot-scale Cross-flow Ultrafiltration Test Using a Hanford Site Tank 241-AN-105 Waste Simulant - Envelope A + Entrained Solids,” Westinghouse Savannah River Company Document No. BNF-003-98-0221, Rev. 0, (23 February).

Duignan, M. R. (2000b), “Final Report: Pilot-scale Cross-flow Ultrafiltration Test Using a Hanford Site Tank 241-AN-107 Waste Simulant - Envelope C + Entrained Solids + Strontium-Transuranic Precipitation,” Westinghouse Savannah River Company Document No. BNF-003-0226, Rev. 0. (24 March)

Duignan, M. R. (2003a). "Final Report: Pilot-scale cross-flow ultrafiltration test using a Hanford Site tank 241-AN-102 waste simulant.” Westinghouse Savannah River Document No. WSRC-TR-2003-00204, Rev. 0. (6 May) [also SRT-RPP-2003-00087]

Duignan, M. R. (2003b), “RPP-WTP Pilot Scale Cross Flow Filtration,” Westinghouse Savannah River Company Notebook No. WSRC-NB-2003-000149. (began on 12 June) 
WSRC-TR-2005-00105, REVISION 0

SRNL-RPP-2005-00012, REVISION 0

Duignan, M. R. (2003c). “Pilot-scale Integrated Pretreatment System Test,” (Task Technical and QA Plan) Westinghouse Savannah River Document No. WSRC-TR-200300338, Rev. 0. (4 August) [also SRT-RPP-2003-00165]

Duignan, M. R. (2004a), "RPP-WTP Pilot-scale Cross-flow Ultrafiltration Test Procedure,” Westinghouse Savannah River Company Field Procedure No FP-975, (26 January)

Duignan, M. R. (2004b), “SIPP Pilot UFP,” Westinghouse Savannah River Company Notebook No. WSRC-NB-2004-00036. (began on 4 March)

Duignan, M. R., J. R. Zamecnik, and M. R. Williams (2004a). "Interim Report: RPPWTP Semi-integrated Pilot Plant - Campaign I,” Westinghouse Savannah River Document No. WSRC-TR-2004-00201, Rev. 0. (19 April) [also SRT-RPP-2004-00034]

Duignan, M. R., D. J. Adamson, C. Dan Barnes, M. D. Fowley, Z. N. Qureshi, J. L. Steimke, M. R. Williams, and J. R. Zamecnik (2004b). "Interim Report: RPP-WTP Semiintegrated Pilot Plant - Campaign II,” Westinghouse Savannah River Document No. WSRC-TR-2004-00478, Rev. 0. (10 September) [also SRT-RPP-2004-00075]

Duignan, M. R., D. J. Adamson, M. D. Fowley, Z. N. Qureshi, J. L. Steimke, M. R. Williams, and J. R. Zamecnik (2004c). "Interim Report: RPP-WTP Semi-integrated Pilot Plant - Campaign III,” Westinghouse Savannah River Document No. WSRC-TR-200400565, Rev. 0. (15 November) [also SRT-RPP-2004-00092]

Edwards, R. E., (2003) "Semi-Integrated Pilot Plant (SIPP) Workshop - Meeting Minutes,” SRT-RPP-2003-00227, Westinghouse Savannah River Company, Aiken SC 29808 (5 November)

Eibling, R. E., and C. A. Nash (2001), "Hanford Waste Simulants Created to Support the Research and Development on the River Protection Project - Waste Treatment Plant," Westinghouse Savannah River Document No. WSRC-TR-2001-00338, Rev. 0. (22 February) [also SRT-RPP-2000-00017]

Fiskum, S. K., S. T. Arm, D. L. Blanchard, Jr., B. M. Rapko (2002), "Small Column Ion Exchange Testing of SuperLig ${ }^{\circledR} 644$ for Removal of ${ }^{137}$ Cs from Hanford Waste Tank 241-AP-101 Diluted Feed,” Battelle Pacific Northwest Division Document No. PNWD3198, WPT-RPT-016 (September).

Fowley, M. D. (2004), “SIPP Pilot UFP,” Westinghouse Savannah River Company Notebook No. WSRC-NB-2004-00036. (began on 4 March) 
Fowley, M. D., D. J. Adamson, C. A. Nash, M. A. Shadday, S. E. Aleman, J. L. Steimke, L. L. Hamm, M. L. Restivo, T. J. Steeper, and W. D. King (2004), "Ion Exchange Testing With SuperLig ${ }^{\circledR} 644$ Resin (U),” Westinghouse Savannah River Document No. WSRCTR-2003-00514, Rev. 0. (July) [also SRT-RPP-2003-00233]

Hamm, L. L., F. G. Smith, D. J. McCabe, (2000) "Preliminary Ion Exchange Modeling for Removal of Cesium from Hanford Waste Using SuperLig ${ }^{\circledR} 644$ Resin,” Westinghouse Savannah River Company Document No. BNF-003-98-0220 (June)

Lumetta, G. J., M. J. Wagner, F. V. Hoopes, and R. T. Steele (1996), “Washing and Caustic Leaching of Hanford Tank C-106 Sludge,” Battelle Pacific Northwest National Laboratory Document No. PNNL-11381.

Lee, E. and M. Binsfield (2002). "Flowsheet Bases, Assumptions, and Requirements," Doc. No. 24590-WTP-RPT-PT-02-005, Rev. 1. (December)

Longwell. R. L. (2003), “Pilot Scale Integration of Pretreatment Process System,” Waste Treatment Plant - River Protection Project Document No. 24590-PTF-TSP-RT-03-002, Rev. 0. (5 June)

Matlack, K. S., W. Gong, and I. L. Pegg (2005a), “DuraMelter 100 HLW Simulant Validation Tests C-106/AY-102,” Vitreous State Laboratory Document No. VSL-05R5710-1, Catholic University of America, Wash., DC, (Draft as of 19 April).

Matlack, K. S., W. Gong, and I. L. Pegg (2005b), “DuraMelter 100 HLW Simulant Validation Tests C-106/AY-102 Blending Feed from the Semi-Integrated Pilot Plant," Vitreous State Laboratory Document No. VSL-05R-5410-1, Catholic University of America, Wash., DC, (24 February).

Poirier, M. R., P. R. Burket, J. L. Siler, and J. R. Zamecnik (2003), "Filtration, washing and leaching of a Hanford AY-102/C-106 sample,” Westinghouse Savannah River Company Document No. WSRC-TR-2003-00240, Rev. 0 (or SRT-RPP-2003-00110, Rev. 0). (June)

Qureshi, Z. H. (2004), “SIPP Pilot TLP,” Westinghouse Savannah River Company Notebook No. WSRC-NB-2004-00034. (began on 4 March)

Russell, R. L., S. K. Fiskum, L. K. Jagoda, and A. P. Polaski (2003), “AP-101 Diluted Feed (Envelope A) Simulant Development Report,” Battelle Pacific Northwest Division Document No. PNWD-3248, Rev. 0 (February) [also WTP-RPT-057]

Stallings, M. E., M. R. Poirier, and R. E. Eibling (2003). “Task Technical and Quality Assurance Plan for AY-102/C-106 Simulant Ultrafiltration and Washing (Including AY102/C-106 Simulant Development).” Westinghouse Savannah River Document No. WSRC-TR-2003-00217, Rev. 0. (31 May) [also SRT-RPP-2002-00235] 
Steimke, J. L. (2002). "Pilot Scale Ion Exchange Testing with SuperLig ${ }^{\circledR} 639$ and SuperLig ${ }^{\circledR} 644$ Resin,” (Task Technical and QA Plan) Westinghouse Savannah River Document No. WSRC-TR-2001-00340, Rev. 0. (6 February) [also SRT-RPP-200100116]

Steimke, J. L. (2004), “SIPP Pilot TLP,” Westinghouse Savannah River Company Notebook No. WSRC-NB-2004-00037. (began on 4 March)

Townson, P. S. (2002). “AY-102/C-106 Simulant Pilot Scale Ultrafiltration and Washing Test Specification,” Waste Treatment Plant - River Protection Project Document No. 24590-PTF-TSP-RT-02-015, Rev. 0. (15 November)

Watts, R.J., S. Kong, C.S. Haling, L. Gearhart, C.L. Frye \& B.W. Vigon (1995) "Fate and Effect of Polydimethylsiloxane on Pilot and Bench-Top Activated Sludge Reactors and Anaerobic/Aerobic Digestors,” Wat. Res. Vol. 29, No. 10 pp.2405-2411.

Williams, M. R. and T. J. Steeper (2004). "Pilot-Scale Precipitation Tests of Envelope C Simulants," Westinghouse Savannah River Document No. WSRC-TR-2003-00064, Rev. 0. (April) [also SRT-RPP-2003-00019]

Zamecnik, J. R., P. R. Burket, R. E. Eibling, and M. R. Poirier (2004), “Tank 241-AY102 Simulant development, ultrafiltration, and washing," Westinghouse Savannah River Company Document No. WSRC-TR-2003-00547, Rev. 0 (or SRT-RPP-2003-00240, Rev. 0). (March) 
WSRC-TR-2005-00105, REVISION 0

SRNL-RPP-2005-00012, REVISION 0

\section{APPENDICES}
A. Semi-Integrated Pilot Plant (SIPP) Workshop - Meeting Minutes
B. Computational Model
C. WTP Discussion on Pretreatment Facility Waste Processing Residence Times
D. Sampling Matrices - Campaigns II-IV
E. Rheological and Particle Size Data
F. Dimensional Measurements of Crossflow Ultrafiltration Tubes from Mott and GKN
G. Instrument Lists and Calibration Data
H. Tabulated Raw Data from Each of the Pilot Operations
I. SIPP Interim Reports 
WSRC-TR-2005-00105, REVISION 0 SRNL-RPP-2005-00012, REVISION 0

This page intentionally left blank. 


\section{APPENDIX A: SEMI-INTEGRATED PILOT PLANT (SIPP) WORKSHOP - MEETING MINUTES}

On Tuesday, 28 October, 2003, an all-day workshop was held in the WTP project offices, at Richland, WA, with representatives from WTP R\&T, Process Operations, Process Engineering, Commissioning \& Training, DOE ORP-WTP and Savannah River Technology Center (SRTC) to discuss the objectives and plans for the Semi-Integrated Pilot Plant (SIPP) ${ }^{1}$. Presentations were given concerning the test objectives, SIPP simulants (AY102/C106), pilot plant modeling and run plans. A summary of the action items developed by the workshop is listed below.

Given the specific scope of the SIPP (i.e., demonstrating pretreatment performance and the influences of recycle streams) several key findings of the workshop were deferred to future programs or tasks. These items are listed below.

> Preliminary calculations indicate the SIPP will produce only enough HLW glass to turnover one DM-100 melter volume. Similar R\&D efforts conducted for DOE (e.g. Integrated DWPF Melter System, IDMS) typically produced sufficient glass to turnover the melter at a minimum of three volumes. This typically allows the melter system to achieve steady state operations with respect to glass composition, and production rates.

$>$ The transition of various Hanford waste feeds through an integrated system is not being tested by the SIPP. Future programs or tasks should consider testing additional sludge compositions in an integrated system. Similar R\&D efforts conducted for DOE (e.g. IDMS, DWPF Cold Commissioning, and Duratek LAW Pilot Melter) tested a variety of waste chemistries and conducted testing that demonstrated these facilities could operate successfully during waste transitions.

A complete list of actions and the final resolution of these actions is listed below:

\footnotetext{
${ }^{1}$ Edwards, R. E. to W. Tamosaitis, 11/5/03, “Semi-Integrated Pilot Plant Workshop-Meeting Minutes”, SRT-RPP-2003-00277, Westinghouse Savannah River Company, Aiken SC 29808
} 
WSRC-TR-2005-00105, REVISION 0

SRNL-RPP-2005-00012, REVISION 0

SIPP Workshop Meeting Minutes - Questions/Issues/Action Items/Decisions (Revised 6/23/04-Final)

\begin{tabular}{|c|c|c|c|c|}
\hline Questions/Issues/Action Items/Decisions & Action Assigned (Status) & \multicolumn{3}{|c|}{ Discussion } \\
\hline $\begin{array}{l}\text { 1. What is the impact of other amorphous } \\
\text { components in Hanford Waste? }\end{array}$ & $\begin{array}{l}\text { SRNL, Jack Zamecnik, } \\
\text { Analysis of CUF, pilot } \\
\text { data compared to other } \\
\text { Hanford CUF data will } \\
\text { be conducted. } \\
\text { (Complete) }\end{array}$ & \multicolumn{3}{|c|}{$\begin{array}{l}\text { The direct impact of amorphous } \\
\text { components has not been addressed by the } \\
\text { current scope of the SIPP or any other } \\
\text { WTP R\&T program. Hanford waste tanks } \\
\text { contain both amorphous and crystalline } \\
\text { compounds. Amorphous compounds, such } \\
\text { as amorphous sodium aluminosilicate or } \\
\text { metal hydroxides, will tend to be more } \\
\text { gelatinous than the crystalline compounds } \\
\text { (such as boehmite, zeolite). Amorphous } \\
\text { compounds tend to be more "sticky" due } \\
\text { to the hydroxide and could make them } \\
\text { harder to pump or filter. }\end{array}$} \\
\hline $\begin{array}{l}\text { 2. Al leaching of simulant is not prototypical } \\
\text { for AY102/C106 - Resolve for pilot }\end{array}$ & $\begin{array}{l}\text { SRNL, Jack Zamecnik } \\
\text { (Complete) }\end{array}$ & $\begin{array}{l}\text { First test si } \\
\text { Final simu } \\
\text { well, as sh } \\
\text { Species } \\
\mathrm{Al} \\
\mathrm{Cr} \\
\mathrm{Fe} \\
\text { phosphate } \\
\mathrm{Na} \\
\mathrm{Ca} \\
\mathrm{Ni} \\
\mathrm{Pb} \\
\mathrm{Si} \\
\text { total mass }\end{array}$ & $\begin{array}{r}\text { rmulant dat } \\
\text { lant formul } \\
\text { own below: } \\
\% \mathrm{R} \\
\text { Radioactive } \\
53-69 \\
51-90 \\
100 \\
32-65 \\
28-48 \\
94->96 \\
99 \\
91-96 \\
91-99 \\
52\end{array}$ & $\begin{array}{l}\text { d not match. } \\
\text { on data matched } \\
\text { ined } \\
\text { Simulant } \\
53 \\
62 \\
100 \\
46 \\
9-18 \\
100 \\
100 \\
100 \\
99 \\
46\end{array}$ \\
\hline
\end{tabular}


WSRC-TR-2005-00105, REVISION 0

SRNL-RPP-2005-00012, REVISION 0

\begin{tabular}{|c|c|c|}
\hline Questions/Issues/Action Items/Decisions & Action Assigned (Status) & Discussion \\
\hline $\begin{array}{l}\text { 3. How representative was original SRNL } \\
\text { sample as compared to other AY102/C106 } \\
\text { cores taken at the same time? }\end{array}$ & $\begin{array}{l}\text { SRNL, Jack Zamecnik } \\
\text { (Complete) }\end{array}$ & See below. \\
\hline
\end{tabular}

The SRNL sample results were compared to TFCOUP, which is based on samples, and also to several core samples. The concentrations in the supernate and solids fractions compare favorably, but the undissolved solids content of the SRNL sample was about $1 / 2$ the amount given by TFCOUP.

Comparisons of the simulant and radioactive AY102/C106 are documented in SRNL technical report WSRC-TR-200300547, REV 0 (SRT-RPP-2003-00240, REV 0), which is publicly available.

Additional discussion:

1. The concentrations of most major components and many minor components agree well for both the supernate and solids.

2. The aluminate concentration in the SRNL sample was approximately 3-4X higher than shown in TFCOUP or the limited core sample analyses that were found. A possible explanation is that the Core 289 sample was taken soon after that addition of $\mathrm{NaOH}$ to the tank, so this $\mathrm{NaOH}$ in the supernate would not have equilibrated with the solids. During compositing, the solids were contacted with the supernate and a small amount of aluminum may have dissolved and resulted in the higher value. Also note that TFCOUP shows more $\mathrm{Al}$ in the solids (TFCOUP: more in solids, less in supernate; SRNL analysis: opposite).

3. It appears that the SRNL sample had more $\mathrm{Na}_{2} \mathrm{CO}_{3}$ in the supernate, but less in the solids than that predicted by TFCOUP. This difference suggests that only the distribution of $\mathrm{Na}_{2} \mathrm{CO}_{3}$ is different and that the total amount in the SRNL sample and TFCOUP are close. (Note the carbonate in the TFCOUP solids is significantly higher than in the SRNL sample. Reducing the carbonate and adding bound OH- made SRNL and TFCOUP values match better.)

4. The current TFCOUP values for Si and Zr are orders of magnitude lower that the SRNL analyses. They are also lower that the amount given by TFCOUP for tank C-106 in an earlier version of TFCOUP. It appears that the later versions of TFCOUP may have used results from less aggressive acid digestion analyses that usually underestimate Si and Zr. 
WSRC-TR-2005-00105, REVISION 0 SRNL-RPP-2005-00012, REVISION 0

5. The calculated TFCOUP undissolved solids content is $11.8 \mathrm{wt} \%$ and the total solids is $24.7 \mathrm{wt} \%$. Examination of the SRNL sample compositing data (Core 289 + Core 290 supernate) indicated that the overall composition of Core 289 was approximately $14.3 \mathrm{wt} \%$ undissolved solids and $28.3 \mathrm{wt} \%$ total solids. Note that these values are very approximate; the SRNL total solids content in the supernate also had to be assumed: $16.3 \mathrm{wt} \%$. These values are reasonably close to those calculated from TFCOUP and show that the undissolved solids in the SRNL sample (5.85 wt\%) were about $1 / 2$ of the actual amount in the tank.

6. The TFCOUP adjusted "analyses" calculated were used to calculate the expected composition of the SRNL slurry sample, which had less undissolved solids. These calculated values matched the SRNL measurements reasonably well. Without adjusting for undissolved solids, the concentrations of species mostly present in the solids were much too high in the calculated slurry composition. 
WSRC-TR-2005-00105, REVISION 0

SRNL-RPP-2005-00012, REVISION 0

\begin{tabular}{|c|c|c|}
\hline Questions/Issues/Action Items/Decisions & $\begin{array}{l}\text { Action Assigned to } \\
\text { (Status) }\end{array}$ & Discussion \\
\hline $\begin{array}{l}\text { 4. What are the commissioning simulants? } \\
\text { Has the composition been defined? }\end{array}$ & WTP R\&T (Complete) & $\begin{array}{l}\text { AY102/C106 \& AP101. No, the actual } \\
\text { simulant composition used during } \\
\text { commissioning will be defined by another } \\
\text { program. It is likely, the current simulant } \\
\text { compositions used for the pilot will be } \\
\text { modified for use by the plant. }\end{array}$ \\
\hline $\begin{array}{l}\text { 5. Check with WTP R\&T Vitrification } \\
\text { concerning the glass composition (waste } \\
\text { loading) for AY102/C106? }\end{array}$ & $\begin{array}{l}\text { SRNL, Dan Barnes, } \\
\text { Information was } \\
\text { obtained from J Perez, } \\
\text { WTP R\&T (Complete) }\end{array}$ & $\begin{array}{l}\text { Loading- Fe was limiting component @ } \\
12.5 \text { wt.\% } \mathrm{Fe}_{2} \mathrm{O}_{3}\end{array}$ \\
\hline $\begin{array}{l}\text { 6. What is the impact, if any of } \mathrm{Zr} \text { on glass } \\
\text { composition? }\end{array}$ & $\begin{array}{l}\text { SRNL, Dan Barnes, } \\
\text { (Complete), Discuss Zr } \\
\text { with J. Vienna/David } \\
\text { Peeler WTP R\&T }\end{array}$ & $\begin{array}{l}\text { The wt.\% } \mathrm{Zr} \text { is less than } 1 \% \text { and should } \\
\text { not impact glass properties. }\end{array}$ \\
\hline $\begin{array}{l}\text { 7. Comment - WTP equipment will be } \\
\text { operated on linear portion of wt.\% vs. } \\
\text { rheology curve-Curve needs to be defined } \\
\text { for each waste tank-transition between } \\
\text { linear and non-linear portion of wt.\% vs. } \\
\text { rheology curve typically defines maximum } \\
\text { operating point }\end{array}$ & WTP R\&T (Complete) & $\begin{array}{l}\text { Recommended design basis defined in } \\
\text { other project documents WPT-075 }\end{array}$ \\
\hline $\begin{array}{l}\text { 8. How will current simulant CUF data be } \\
\text { compared to pilot runs given different } \\
\text { operating conditions (no evaporation)? }\end{array}$ & $\begin{array}{l}\text { SRNL, Jack Zamecnik } \\
\text { (Complete) }\end{array}$ & $\begin{array}{l}\text { A permeate viscosity correction will be } \\
\text { used to put the data on the same basis. } \\
\text { This correction has been demonstrated for } \\
\text { the AN-102 Sr/TRU precipitate and is } \\
\text { generally accepted in filtration literature. }\end{array}$ \\
\hline 9. Adjust pilot model to $5 \mathrm{M} \mathrm{Na}$ basis & $\begin{array}{l}\text { SRNL, Dan Barnes } \\
\text { (Complete) }\end{array}$ & $\begin{array}{l}\text { A density of } 1.25 \text { yield a } \mathrm{Na} \mathrm{M} \text { of } 5.1 \mathrm{M} \\
\mathrm{Na}\end{array}$ \\
\hline
\end{tabular}


WSRC-TR-2005-00105, REVISION 0

SRNL-RPP-2005-00012, REVISION 0

\begin{tabular}{|c|l|l|}
\hline Questions/Issues/Action Items/Decisions & $\begin{array}{l}\text { Action Assigned to } \\
\text { (Status) }\end{array}$ & Discussion \\
\hline $\begin{array}{c}\text { 10. Close couple (Add a prototypical IX feed } \\
\text { tank) filtration and ion exchange in Pilot to } \\
\text { determine if filtration can exceed } \\
\text { production rate of ion exchange system }\end{array}$ & $\begin{array}{l}\text { SRNL, Mark Duignan, } \\
\text { (Complete) }\end{array}$ & $\begin{array}{l}\text { Filter flux will be measured and provided } \\
\text { as input for WTP models. }\end{array}$ \\
\hline $\begin{array}{l}\text { 11. Ratio of Waste Feed recycle streams needs } \\
\text { to be defined for pilot. }\end{array}$ & $\begin{array}{l}\text { WTP Process } \\
\text { Operations, Dave } \\
\text { Harty/Dan Barnes } \\
\text { (Complete) }\end{array}$ & Ratio has been defined. \\
\hline 12. Can simulant be simplified? & $\begin{array}{l}\text { SRNL, Jack Zamecnik, } \\
\text { (Complete) }\end{array}$ & \\
\hline
\end{tabular}

Y, Some less abundant components could be dropped to reduce price slightly. Most of the cost of producing the simulant is labor, since it involves two precipitation and washing steps. Additional R\&T efforts would be required to determine if a preparation method without precipitations could be used. Such an effort would be outside the scope of the current program and should be deferred to future tasks. Also, past experience has shown that producing hydroxide sludges with the needed properties without precipitating the hydroxides is difficult.

\begin{tabular}{|c|l|l|}
\hline Questions/Issues/Action Items/Decisions & Action Assigned (Status) & Discussion \\
\hline $\begin{array}{l}\text { 13. Do we neutralize the HLW \& LAW SBS? } \\
\text { Targeted to pH 12? }\end{array}$ & $\begin{array}{l}\text { WTP Process } \\
\text { Operations, Eric } \\
\text { Slaathaug (Complete) }\end{array}$ & $\begin{array}{l}\text { HLW SBS is } \mathrm{pH}=8 \text { and will be run as is, } \\
\text { LAW SBS is neutralized only if pH }<4 .\end{array}$ \\
\hline $\begin{array}{l}\text { 14. Should Cerium Decon solution be added to } \\
\text { pilot? }\end{array}$ & $\begin{array}{l}\text { WTP R\&T, Ron } \\
\text { Longwell (Complete) }\end{array}$ & $\begin{array}{l}\text { Ce Decon is a small contributor to the } \\
\text { total recycle flow rate and will not be used } \\
\text { in the SIPP. }\end{array}$ \\
\hline
\end{tabular}


WSRC-TR-2005-00105, REVISION 0

SRNL-RPP-2005-00012, REVISION 0

\begin{tabular}{|c|l|l|}
\hline Questions/Issues/Action Items/Decisions & Action Assigned (Status) & Discussion \\
\hline $\begin{array}{l}\text { 15. Should we increase the solids loading of } \\
\begin{array}{l}\text { SBS to simulant maintenance operations in } \\
\text { HLW/LAW vitrification? }\end{array}\end{array}$ & $\begin{array}{l}\text { WTP R\&T, Ron } \\
\text { Longwell (Complete) }\end{array}$ & $\begin{array}{l}\text {, the objective of the testing is to determine } \\
\text { if problems will occur during } \\
\text { commissioning and if the WTP } \\
\text { pretreatment facilities will meet } \\
\text { production rates. }\end{array}$ \\
\hline $\begin{array}{l}\text { 16. Flush water is added during filter } \\
\text { operations. Define filter operations/process } \\
\text { chemical/water additions. }\end{array}$ & $\begin{array}{l}\text { SRNL/WTP Process } \\
\text { Engineering, Mark } \\
\text { Duignan/Eric Slaathaug } \\
\text { (Complete) }\end{array}$ & $\begin{array}{l}\text { Information provided to SRNL to } \\
\text { calculate flush water requirements }\end{array}$ \\
\hline $\begin{array}{l}\text { 17. Define Waste Feed Evaporator Endpoint. } \\
\text { SRNL/WTP Process } \\
\text { Operations, Dan Barnes, } \\
\text { Dave Harty (Complete) }\end{array}$ & The UF feed point is defined at 5M Na \\
\hline $\begin{array}{l}\text { 18. Cr }{ }^{+3} / \mathrm{Cr}^{+6} \text { Leaching - Consider in } \\
\text { analytical plan. }\end{array}$ & $\begin{array}{l}\text { SRNL, Jack Zamecnik } \\
\text { (Complete) }\end{array}$ & See below. \\
\hline
\end{tabular}

No attempt was made to match the $\mathrm{Cr}^{+3} / \mathrm{Cr}^{+6}$ content of the simulant to specific analytical data for the waste sample since none was available. The solid phase $\mathrm{Cr}$ was precipitated from $\mathrm{Cr}^{+3}$ as $\mathrm{Cr}(\mathrm{OH})_{3}$ and the soluble $\mathrm{Cr}$ was added as $\mathrm{Cr}^{+6}\left(\mathrm{CrO}_{4}^{-2}\right)$. This is consistent with previously reported results.* Over time, $\mathrm{Cr}^{+3}$ tends to convert to $\mathrm{Cr}^{+6}$ in caustic solutions if it dissolves. We expect that any $\mathrm{Cr}$ dissolved during caustic leaching would be converted from +3 to +6 . Leaching is not being implemented in the SIPP, therefore $\mathrm{Cr}^{+3} / \mathrm{Cr}^{+6}$ is not being considered in the analytical plan. Measurements done by PNNL used X-ray Absorption, which was done at the Stanford Synchrotron Radiation Laboratory**, so SRTC cannot do this measurement in-house. These authors** note that their speciation did not attempt to determine the distribution between the solid and liquid phases. Since $\mathrm{Cr}^{+3}$ is usually insoluble and $\mathrm{Cr}^{+6}$ is usually soluble, the distributions they determined may just be the distribution between the phases. For two tanks, they report the following results:

$$
\text { Percent in Sample }
$$

$\begin{array}{lllll}\text { Tank } & \mathrm{Cr}+3 & \mathrm{Cr}+6 & \mathrm{Cr}+3 & \mathrm{Cr}+6\end{array}$

SY103 $>95<5>99<1$

T104 $84 \quad 16 \quad 96 \quad 4$

Reference: BBI. The other tanks studied have no liquid phase listed in the BBI. 
* G. J. Lumetta, B. M. Rapko, H. M. Cho, "Studies of the Fundamental Chemistry of Hanford Tank Sludges," Proceedings of ICEM '03.

** B. M. Rapko, et.al, “The Chemistry of Sludge Washing and Caustic Leaching Processes for Selected Hanford Tank Wastes”, PNNL-11089.

\begin{tabular}{|c|c|c|}
\hline Questions/Issues/Action Items/Decisions & Action Assigned (Status) & Discussion \\
\hline $\begin{array}{l}\text { 19. Compare rheology of slurry vs. wt. \% } \\
\text { solids between Campaign } 1 \text { and Campaign } \\
\text { 2. What are the effects of antifoam and } \\
\text { recycles? }\end{array}$ & $\begin{array}{l}\text { SRNL, Jack Zamecnik } \\
\text { (Complete) }\end{array}$ & This will be done as part of the SIPP task. \\
\hline $\begin{array}{l}\text { 20. Define Blending Strategy of Caustic } \\
\text { Cleaning Solutions with other UF recycles. }\end{array}$ & $\begin{array}{l}\text { SRNL/Process } \\
\text { Operations, Eric } \\
\text { Slaathaug (Complete) }\end{array}$ & $\begin{array}{l}\text { Blending scheme proposed by SRNL and } \\
\text { agreed upon by WTP (Harty and } \\
\text { Slaathaug) }\end{array}$ \\
\hline $\begin{array}{l}\text { 21. What is the hold time }(\mathrm{X}) \text { for concentrated } \\
\text { waste and recycles prior to filtration? } \\
\mathrm{X}=\text { time it takes to cool full scale } \\
\text { concentrated waste feed evaporator batch } \\
\text { to ambient }+ \text { two days of pilot filter } \\
\text { operations (concentration, total of } 48 \text { hours } \\
+\mathrm{X} \text { ) }\end{array}$ & $\begin{array}{l}\text { SRNL/Process } \\
\text { Engineering/WTP R\&T, } \\
\text { Dave Harty/Eric } \\
\text { Slaathaug, (Complete) }\end{array}$ & Hold time provided by Harty. \\
\hline $\begin{array}{l}\text { 22. Evaporator Operations will be conducted } \\
\text { on Day Shift. }\end{array}$ & $\begin{array}{l}\text { SRNL, Mark Duignan } \\
\text { (Complete) }\end{array}$ & \\
\hline $\begin{array}{l}\text { 23. What heels \& loop volumes are needed for } \\
\text { Ultrafiltration? }\end{array}$ & $\begin{array}{l}\text { WTP R\&T, Ron } \\
\text { Longwell, (Complete) }\end{array}$ & $\begin{array}{l}\text { The original response was: none - future } \\
\text { tasks will consider. Upon further } \\
\text { discussions with Harty of WTP, the } \\
\text { presence of heels in ultrafiltration were } \\
\text { included in the operational plan. }\end{array}$ \\
\hline $\begin{array}{l}\text { 24. Delete measurements required to generate } \\
\text { pump curves. }\end{array}$ & $\begin{array}{l}\text { SRNL, Mark Duignan } \\
\text { (Complete) }\end{array}$ & \\
\hline
\end{tabular}


WSRC-TR-2005-00105, REVISION 0

SRNL-RPP-2005-00012, REVISION 0

\begin{tabular}{|c|c|c|}
\hline Questions/Issues/Action Items/Decisions & Action Assigned (Status) & Discussion \\
\hline $\begin{array}{l}\text { 25. Final Cleaning Method for Filter } \\
\text { Operations - Two or Three Cleaning } \\
\text { Cycles for modeling purposes? }\end{array}$ & $\begin{array}{l}\text { WTP R\&T, Eric } \\
\text { Slaathaug (Complete) }\end{array}$ & $\begin{array}{l}\text { Cleaning methods provided by R\&T; } \\
\text { three will be used. }\end{array}$ \\
\hline 26. No pulse jets will be used. & $\begin{array}{l}\text { WTP R\&T, Ron } \\
\text { Longwell (Complete) }\end{array}$ & \\
\hline 27. Determine Scale of Melter to be used. & $\begin{array}{l}\text { WTP R\&T, Ron } \\
\text { Longwell, (Complete) }\end{array}$ & $\begin{array}{l}\text { J. Perez recommended the DM-100 at } \\
\text { VSL. The DM-100 is the smallest melter } \\
\text { that can adequately demonstrate } \\
\text { production rate. }\end{array}$ \\
\hline $\begin{array}{l}\text { 28. Define simulant hold time between } \\
\text { filtration and IX. }\end{array}$ & $\begin{array}{l}\text { WTP R\&T, Ron } \\
\text { Longwell (Complete) }\end{array}$ & A 2 day hold time will be used. \\
\hline $\begin{array}{l}\text { 29. Surge capacity between UF and IX - Item } \\
\text { associated with Ultrafiltration exceeding } \\
\text { production capacity of IX. The issue was } \\
\text { that without knowing the available surge } \\
\text { capacity between UFP and IX there is no } \\
\text { way to demonstrate with the SIPP that } \\
\text { throughput can be met. }\end{array}$ & SRNL (Complete) & $\begin{array}{l}\text { Process Engineering to provide surge } \\
\text { capacity to Process Operation- Action } \\
\text { Assigned to Eric Slaathaug - Item does } \\
\text { not impact SIPP so action is closed. } \\
\text { Filter Flux results will be provided for G2 } \\
\text { models. }\end{array}$ \\
\hline $\begin{array}{l}\text { 30. IX is operated at 3BV/hr (superficial } \\
\text { velocity will not be used)? }\end{array}$ & $\begin{array}{l}\text { WTP R\&T, Reid } \\
\text { Peterson, (Complete) }\end{array}$ & $\begin{array}{l}\text { The IX will be operated at three flowrate } \\
1.5,3.0 \text { and } 4.5 \mathrm{BV} / \mathrm{hr} \text {. }\end{array}$ \\
\hline $\begin{array}{l}\text { 31. Cs breakthrough will be determined during } \\
\text { SIPP }\end{array}$ & $\begin{array}{l}\text { SRNL/WTP R\&T, Mark } \\
\text { Duignan/Reid Peterson } \\
\text { (Complete) }\end{array}$ & \\
\hline 32. Two 6” Columns will be used. & $\begin{array}{l}\text { WTP R\&T, Reid } \\
\text { Peterson (Complete) }\end{array}$ & \\
\hline $\begin{array}{l}\text { 33. Check Cs concentration of simulant - } \\
\text { Make Cs concentration match } \\
\text { AY102/C106 within analytical error. }\end{array}$ & $\begin{array}{l}\text { SRNL, Jack Zamecnik } \\
\text { (Complete) }\end{array}$ & See below. \\
\hline
\end{tabular}


The Cs content of the AY-102 tank sample supernate was about $1.6 \mathrm{mg} / \mathrm{L}$ and the solids concentration was about $13 \mathrm{mg} / \mathrm{kg}$ of wet solids ( 42 mg/kg of dried solids without interstitial liquid). The simulant supernate Cs concentration is about $0.5-2 \mathrm{mg} / \mathrm{L}$. This concentration can be adjusted, if deemed necessary, after the portions of the slurry simulant have been combined. The Cs concentration in the solids is more difficult to achieve since most Cs compounds are soluble in water or caustic solutions. Therefore, the Cs content of the solids depends on trapping of Cs in the sodium aluminosilicate matrix. The actual concentration of Cs in the simulant batches was measured to be $<23 \mathrm{mg} / \mathrm{kg}$ and $<35 \mathrm{mg} / \mathrm{kg}$ by microwave dissolution and Atomic Absorption (AA). Note both measurements were below detection limits and both bound the target concentration (13 mg/kg). Analysis by ICP-MS could improve this detection limit.

The major source of soluble Cs in the SIPP will come from the AP-101 simulant, which will contain about $8 \mathrm{mg} / \mathrm{L}$ of Cs. Therefore, it is the AP-101 simulant Cs concentration that will have the most effect on the combined simulants.

In addition to AA for Cs, Cs by ICP-MS will be added to the analytical plan.

\begin{tabular}{|c|c|c|}
\hline Questions/Issues/Action Items/Decisions & Action Assigned (Status) & Discussion \\
\hline $\begin{array}{l}\text { 34. IX Analytical - Close material balance } \\
\text { around IX columns - analyze for post } \\
\text { precipitation. }\end{array}$ & $\begin{array}{l}\text { SRNL, Jack Zamecnik } \\
\text { (Complete) }\end{array}$ & $\begin{array}{l}\text { Analytical plan will be written to reflect } \\
\text { these needs. See analytical plan. }\end{array}$ \\
\hline $\begin{array}{l}\text { 35. Provide SBS LAW ratio for Treated Feed } \\
\text { Evaporator. }\end{array}$ & $\begin{array}{l}\text { WTP Process } \\
\text { Operations, Dave Harty } \\
\text { (Complete) }\end{array}$ & The ratio has been determined. \\
\hline $\begin{array}{l}\text { 36. Verify quantity of material sent for } \\
\text { vitrification \& associated melter turnovers, } \\
\text { forward information to J. Perez, WTP } \\
\text { R\&T. }\end{array}$ & $\begin{array}{l}\text { SRNL, Dan Barnes, } \\
\text { Bond Calloway } \\
\text { (Complete) }\end{array}$ & $\begin{array}{l}\text { Calculations complete. Bond Calloway to } \\
\text { forward information to WTP. }\end{array}$ \\
\hline 37. Determine SBS volumes needed. & $\begin{array}{l}\text { SRNL, Mark Duignan } \\
\text { (Complete) }\end{array}$ & \\
\hline $\begin{array}{l}\text { 38. Refine IX plans during SRNL quarterly- } \\
\text { Review Scope of Chemical IX testing. }\end{array}$ & $\begin{array}{l}\text { SRNL/WTP R\&T, Mark } \\
\text { Duignan/Reid Peterson } \\
\text { (Complete) }\end{array}$ & $\begin{array}{l}\text { Test exception from Reid/Sundar } \\
\text { required. }\end{array}$ \\
\hline
\end{tabular}


WSRC-TR-2005-00105, REVISION 0

SRNL-RPP-2005-00012, REVISION 0

\begin{tabular}{|c|c|c|}
\hline Questions/Issues/Action Items/Decisions & Action Assigned (Status) & Discussion \\
\hline $\begin{array}{l}\text { 39. Determine No. of Bed Volumes prior to } \\
\text { elution }\end{array}$ & $\begin{array}{l}\text { WTP R\&T, Reid } \\
\text { Peterson (Complete) }\end{array}$ & \\
\hline $\begin{array}{l}\text { 40. SRNL to document rate comparison } \\
\text { between SIPP and WTP, with and without } \\
\text { leaching, actual flux vs. design rate. }\end{array}$ & $\begin{array}{l}\text { SRNL, Dan Barnes } \\
\text { (Complete) }\end{array}$ & Letter issued. \\
\hline $\begin{array}{l}\text { 41. Document Run Plan and forward for } \\
\text { project review. }\end{array}$ & $\begin{array}{l}\text { SRNL, Mark Duignan } \\
\text { (Complete) }\end{array}$ & \\
\hline $\begin{array}{l}\text { 42. Document Sample Analytical Plan } \\
\text { (include and forward for project review). }\end{array}$ & $\begin{array}{l}\text { SRNL, Jack } \\
\text { Zamecnik/Mark Duignan } \\
\text { (Complete) }\end{array}$ & $\begin{array}{l}\text { Action item transferred to Preparedness } \\
\text { checklist. }\end{array}$ \\
\hline $\begin{array}{l}\text { 43. Decide if leaching will be performed. If } \\
\text { performed, will the prototypic heating and } \\
\text { cooling times be required (heating: } 8 \mathrm{hr} \text {, } \\
\text { leaching; } 8 \mathrm{hr} \text {, cooling: } 19 \mathrm{hr} \text { ). }\end{array}$ & $\begin{array}{l}\text { WTP R\&T, Reid } \\
\text { Peterson, Paul Townson } \\
\text { (Complete) }\end{array}$ & $\begin{array}{l}\text { Leaching will not be conducted per email } \\
\text { from WTP. }\end{array}$ \\
\hline $\begin{array}{l}\text { 44. Do IX competitors ( } \mathrm{Rb}, \mathrm{K}, \mathrm{Cr}+3 \text { ) need to } \\
\text { be at target levels for revised scope of IX } \\
\text { tests? Should Cs in AY-102 be adjusted to } \\
\text { target level? }\end{array}$ & $\begin{array}{l}\text { SRNL, Jack Zamecnik, } \\
\text { Bond Calloway, WTP } \\
\text { R\&T, Reid Peterson } \\
\text { (Complete) }\end{array}$ & $\begin{array}{l}\text { Cs will be added to adjust to target level if } \\
\text { necessary. } \\
\text { AP-101 simulant will have Rb \& } \\
\text { significant K. AY-102 supernate will have } \\
\text { Rb added (this can easily be done); K will } \\
\text { likely be low, but is so much smaller than } \\
\text { K in AP-101 effect will be small. } \\
\text { Adjustment of Cs in AY-102 can be } \\
\text { accomplished after combining simulant } \\
\text { portions at SRNL. }\end{array}$ \\
\hline
\end{tabular}




\section{Appendix B: Computational Model (AUTHOR-BARNES)}

This appendix was first included in the Campaign II report (Duignan, 2004 ${ }^{\dagger}$ ) and condensed from the modeling report (Barnes, 2004).

The volumes of AY-102 and AP-101 simulant required for the four SIPP campaigns were estimated by modeling each campaign as a steady-state process using the software package, OLI/ESP version 6.6. In addition, the minimum SIPP flow rates/fluxes necessary to prove a production rate of 80 metric tons glass per day (MTG/d) for LAW glass and 6 MTG/d HLW glass were estimated based on Campaign IV stream compositions and flow rates and the SIPP unit operation scale factors relative to the current WTP design basis. The OLI/ESP PUBLIC databook, in addition to the private databooks CARBONAT, HNO3DB, SILICA, and ZEOLITE were used to generate the chemistry model (Zamecnik, 2004) for these simulations.

The model for Campaign I simulated the concentration and wash steps of simulant AY102 (Zamecnik et al., 2004) waste feed only, while the model for Campaigns II-IV, using a blend of simulant AY-102 and AP-101 (Russell et al., 2003) waste feed. Campaigns IIIV simulated most of the pre-treatment process including the FEP evaporator, ultrafiltration, slurry wash, cesium ion-exchange column recycles, and the TLP evaporator. The estimated SIPP volumes for each campaign were based on producing 140L of washed sludge from Campaign I and the ratio of AP-101:AY-102 waste feed predicted to give a glass production ratio of 80/6 MTG/d LAW/HLW glass for each of the Campaigns II-IV.

The structures of the models for Campaigns II-IV are identical. Each of the three campaigns represent an iteration towards steady-state of the ultra-filtration and Cs-IX recycle streams, with the predicted output recycle from one campaign being used as the input recycle to the subsequent campaign. The difference in the predicted volumetric recycle flows between Campaigns III and IV was less than $0.09 \%$, indicating the Campaign IV output recycle had essentially reached steady-state.

The major assumptions used in the model are discussed in the first section below, followed by the criteria used to determine several stream flow rates. Then the model structure is described with the help of simplified flow diagrams; detailed block diagrams are used to describe certain portions of the model. Finally, the modeling results of Campaigns I and IV are given, along with estimates of the minimum SIPP flow/flux rates and process times necessary to prove rate based on the results of Campaign IV. A complete OLI/ESP listing of the properties and composition of key streams for Campaigns I and IV are given in Appendix A of Barnes (2004).

\footnotetext{
${ }^{\dagger}$ All references made in this Appendix can be found at the end of this Appendix.
} 


\section{B.1. MODEL ASSUMPTIONS}

Although the WTP pre-treatment process will have two FEP evaporator/ultra-filtration trains, only one train was included in the model. The current design basis allows only one train to operate in the concentration mode (concentrating the waste feed blend to 18,000 gals. at $20 \mathrm{wt} \%$ UDS while generating permeate for the Cs IX column) at any one time. Because this is the rate-limiting mode for this process, train "A" will complete its treat-clean mode and the stand idle while train " $\mathrm{B}$ " completes its concentration mode (the treat-clean mode consists of washing the 18,000 gal batch of 20wt\% UDS slurry, heel and treated sludge transfers, and filter cleaning). At no time will both trains perform the same function simultaneously. Therefore, a single train must be capable of sustaining a production rate of $80 \mathrm{MTG} / \mathrm{d}$ LAW glass when in the concentration mode, or $6 \mathrm{MTG} / \mathrm{d}$ HLW glass when in the treat and clean mode.

LAW glass production rate was based on $14 \mathrm{wt} \%$ loading of $\mathrm{Na}_{2} \mathrm{O}$ (Lee et al., 2003a) derived from "waste" sodium only, i.e. sodium from waste feed alone ("waste" sodium is defined to include sodium addition from the leach step, however, this step was not included in these particular models). The amount of sodium in the LAW stream considered to be "waste" sodium was calculated as the difference between the sodium in the waste feed streams and the sodium sent to HLP in the washed sludge (all sodium to HLP is assumed to be waste sodium). This is conservative, the calculated LAW glass rate would be higher for identical flow rates if some or all of the sodium going to the HLP were considered to be non-waste sodium, resulting in a corresponding increase in the amount of "waste" sodium to the LAW glass. The total amount of sodium sent to the LAW (or HLP) process is the same in either case.

HLW glass production was based on $12.5 \mathrm{wt} \%$ loading of $\mathrm{Fe}_{2} \mathrm{O}_{3}$. This was the first metal oxide to meet contract minimum, all other metal oxides and combinations thereof fell below the contract minimums (Lee et al., 2003b).

While the Cs-IX column recycle streams generated during the elution and resin regeneration sequence are modeled, the removal of Cs from the UF permeate by the column is not. This results in Cs being sent to the TLP evaporator that, in practice, would be removed from the stream and sent to the Cs eluate pot (the pot is not part of the SIPP) and eventually the HLP system. However, the concentration of Cs is small and felt to have no significant effect on the TLP bottoms with respect to the properties of interest, i.e. flow rates, density, precipitated solids, etc. No other pre-treatment process of the model is affected by this approximation.

With the exception of the filter loop, no approximations of tank heels or line volumes were included in the models. This is not likely to affect the relative feed rates of AY-102 and AP-101 a great deal; it would take a very large heel to impact the chemistry of a stream so dramatically as to shift the split of output to the LAW and HLP systems. But the lack of heels does increase the apparent efficiency of the process. Any treated sludge or Cs-IX effluent that is recycled due to heels and line volumes reduces the plant efficiency and glass production rates. Heels will also generate more recycle per unit of 
glass as compared to the model predictions. But because these recycles are largely dilute caustic, the only major impact is likely to be an increased load to the evaporators and increased condensate per unit of glass.

\section{B.2. BASES FOR MODEL INPUT/RECYCLE STREAM FLOW RATES AND COMPOSITIONS}

In general, the models of each campaign are steady-state approximations of the pretreatment process and do not introduce any dynamics. The exception is the iteration on the ultra-filtration and Cs-IX recycle stream as it changes value between Campaigns II, III, and IV (reflected in the difference between the fixed input UF and Cs-IX recycles, and the calculated output UF and Cs-IX recycles). All input streams for one particular campaign (HLW and LAW SBS, vessel vent system, recycle inputs from the previous campaign) are set to an average flow rate and do not reflect the cyclic nature of inputs that will exist under actual plant conditions. These average flow rates are based on either the glass production rate, slurry batch size, or the Cs-IX effluent batch size, and the calculations are described in the following paragraphs. The corresponding flows are listed in Tables. B1, B2, and B3.

HLW and LAW SBS recycles generated from actual Duratek melter runs are to be used in the SIPP campaigns. The analytical compositions of these streams were used as input to the model, while the flow rates were based on the most recent ACM model available at the time (dated 11/04/2003 11:42:35am, for streams RDL62 and RDL21 for HLW and LAW SBS, respectively). Because the ACM model HLW glass production rate was 5.4 MTG/d, the given HLW SBS flow rate was adjusted by a ratio of 6:5.4 to represent a flow corresponding to $6 \mathrm{MTG} / \mathrm{d}$. The vessel vent system (PVP04 and PVP06 combined) and the HLW canister decon. (HDH06) stream compositions and flow rates were also based on the ACM model, and the stream compositions given in the ACM model were approximated as a $0.15 \mathrm{M} \mathrm{NaOH}-0.3 \mathrm{M}$ NaNO3 stream for the canister decon., and a $0.1 \mathrm{M} \mathrm{NaOH}$ stream for the combined PVP04-06 vessel vent system streams. The vessel vent system flow rate is fixed as tank ventilation is not affected by the glass production rates. The flow rates for these streams are listed in Table B1.

Table B1. Campaigns II-IV Input Recycle Flow Rates as a Function of Glass Production Rate

\begin{tabular}{|c|c|c|}
\hline Stream & $\begin{array}{c}\text { Flow Rate - relative to 80/6 MTG/d } \\
\text { LAW/HLW glass production }\end{array}$ & Destination \\
\hline \hline HLW SBS (based on HLW glass) & $2.79 \mathrm{gpm}$ & FEP evaporator \\
\hline LAW SBS (based on LAW glass) & $12.2 \mathrm{gpm}$ & TLP evaporator \\
\hline PVP04 and PVP06 (vessel vent system - fixed rate) & $3.23 \mathrm{gpm}$ & FEP evaporator \\
\hline Canister decon. (based on HLW glass) & $1.68 \mathrm{gpm}$ & FEP evaporator \\
\hline
\end{tabular}




\section{WSRC-TR-2005-00105, REVISION 0 SRNL-RPP-2005-00012, REVISION 0}

The filter is cleaned once for every 18,000 gal batch of 20wt\% UDS concentrated slurry, produced using three - 5,250 gal flushes of 2M HNO3, and three - 5,250 gal flushes of $0.01 \mathrm{M} \mathrm{NaOH}$ (Lee et al., 2003c). These acid and caustic rinse streams are each modeled using an average volumetric flow ratio relative to the slurry of $3 * 5,250 / 18,000$, or 0.875 . Following the transfer of treated sludge to the HLP, 700 gals. of sludge remains in the filter loop, which gets pushed to the FEP recycle. This is modeled using an average volumetric flow ratio relative to the slurry of $700 / 18,000$. This same ratio is used for the $0.01 \mathrm{M} \mathrm{NaOH}$ caustic rinse remaining in the filter loop after cleaning, this volume gets pushed to the Cs-IX at the start of the concentration step. These streams are listed in Table B2.

Table B2. Input/Recycle Stream Flow Rates as a Function of the 20wt\% UDS Concentrated Slurry Flow Rate

(slurry batch size of 18,000 gals)

\begin{tabular}{|c|c|c|}
\hline Stream & $\begin{array}{c}\text { Volume - relative to 18,000 gals. slurry } \\
\text { (volumetric fraction relative to slurry) }\end{array}$ & Destination \\
\hline \hline Washed Sludge (in filter loop prior to cleaning) & 700 gals. $(0.03889)$ & FEP evaporator \\
\hline Acid Cleaning $\left(2 \mathrm{M} \mathrm{HNO}_{3}\right)$ & $3 * 5,250$ gals. $(0.8750)$ & FEP evaporator \\
\hline Caustic Rinse $(0.01 \mathrm{M} \mathrm{NaOH})$ & $(3 * 5,250-700)$ gals. $(0.8361)$ & FEP evaporator \\
\hline $\begin{array}{c}\text { Caustic Rinse }(0.01 \mathrm{M} \text { NaOH, remaining in filter } \\
\text { loop prior to concentration of blended waste feed. }\end{array}$ & 700 gals. $(0.03889)$ & Cs-IX column \\
\hline
\end{tabular}

The Cs-IX effluent batch size is 30,000 gals (Lee et al., 2003d). During the elution cycle, one column volume ( $\mathrm{CV}=1,200$ gals.) of $0.1 \mathrm{M} \mathrm{NaOH}$ is used to displace the feed, followed by one CV of pre-elution DI water. Both of these streams are recycled to the FEP Evaporator and modeled using a flow ratio relative to the Cs-IX effluent of $1,200 / 30,000$. The acid elution and post-elution DI rinse streams are sent to the Cs eluate pot which is not part of the SIPP, so these streams are not relevant to these models. Finally, the resin is regenerated with 2,500 gals. $0.25 \mathrm{M} \mathrm{NaOH}, 1,300$ gals of which is recycled to the FEP Evaporator, while the 1,200 gals. remaining in the column is pushed to the TLP Evaporator by the incoming feed. These streams are modeled using a flow ratio relative to the Cs-IX effluent of 1,300/30,000 and 1,200/30,000 for the FEP and TLP streams, respectively, and are listed in Table B3.

Table B3. Input/Recycle Stream Flow Rates as a Function of the Cs-IX Effluent Flow Rate

(effluent batch size of 30,000 gals, column volume of 1,200gals, liquid bed volume of 300 gals.)

\begin{tabular}{|l|c|c|}
\hline \multicolumn{1}{|c|}{ Stream } & $\begin{array}{l}\text { Volume - relative to 30,000 gals. effluent } \\
\text { (volumetric fraction relative to effluent) }\end{array}$ & Destination \\
\hline \hline $0.1 \mathrm{M} \mathrm{NaOH}$ used as feed displacement & 1,200 gals. (0.040) & FEP evaporator \\
\hline DI water - pre-elution rinse & 1,200 gals. (0.040) & FEP evaporator \\
\hline $0.25 \mathrm{M} \mathrm{NaOH}$ resin regeneration & 1,300 gals. (0.043) & FEP evaporator \\
\hline $0.25 \mathrm{M} \mathrm{NaOH}$ resin regeneration & 1,200 gals. (0.040) & TLP evaporator \\
\hline
\end{tabular}




\section{B.3. MODEL FLOW-DIAGRAMS}

A simplified flow diagram of the model for Campaign I is shown in Fig. B1, and a simplified flow diagram of the model for Campaigns II-IV is shown in Fig. B2. The model for Campaigns II-IV consisted of 294 OLI/ESP blocks (mixer, filter, etc.), of which 220 were used to represent the 44 wash steps. To simplify the block-diagram and yet convey a sense of the model's functionality, only the block diagrams detailing a single wash step (Fig. B3) and the FEP evaporator - waste feed blend concentration sequence (Fig. B4) were included here. Feed-Back and/or "Manipulate" controllers were used to set the input stream flow rates described above. Note that an OLI/ESP feed-back controller is not capable of reading the density of a stream directly, instead, it can calculate the $\mathrm{g} / \mathrm{m} 3$ of a collection of up to 100 species; 84 were used to define the permeate density and are listed (using the OLI/ESP naming convention) in Table B4.

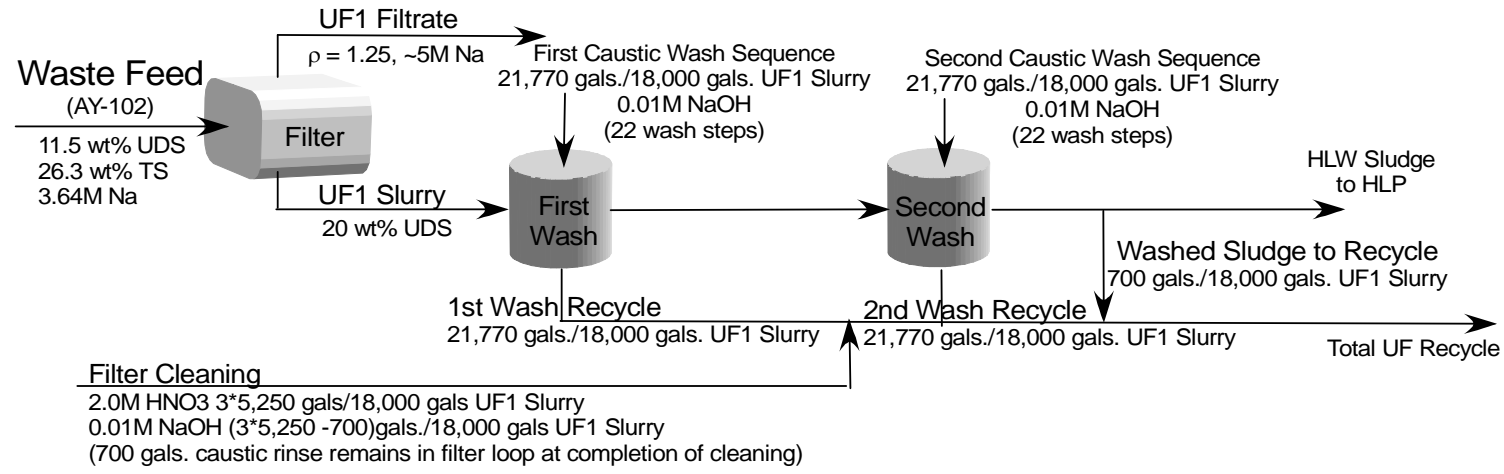

Figure B1. Simplified Flow-Diagram of Campaign I 
WSRC-TR-2005-00105, REVISION 0

SRNL-RPP-2005-00012, REVISION 0

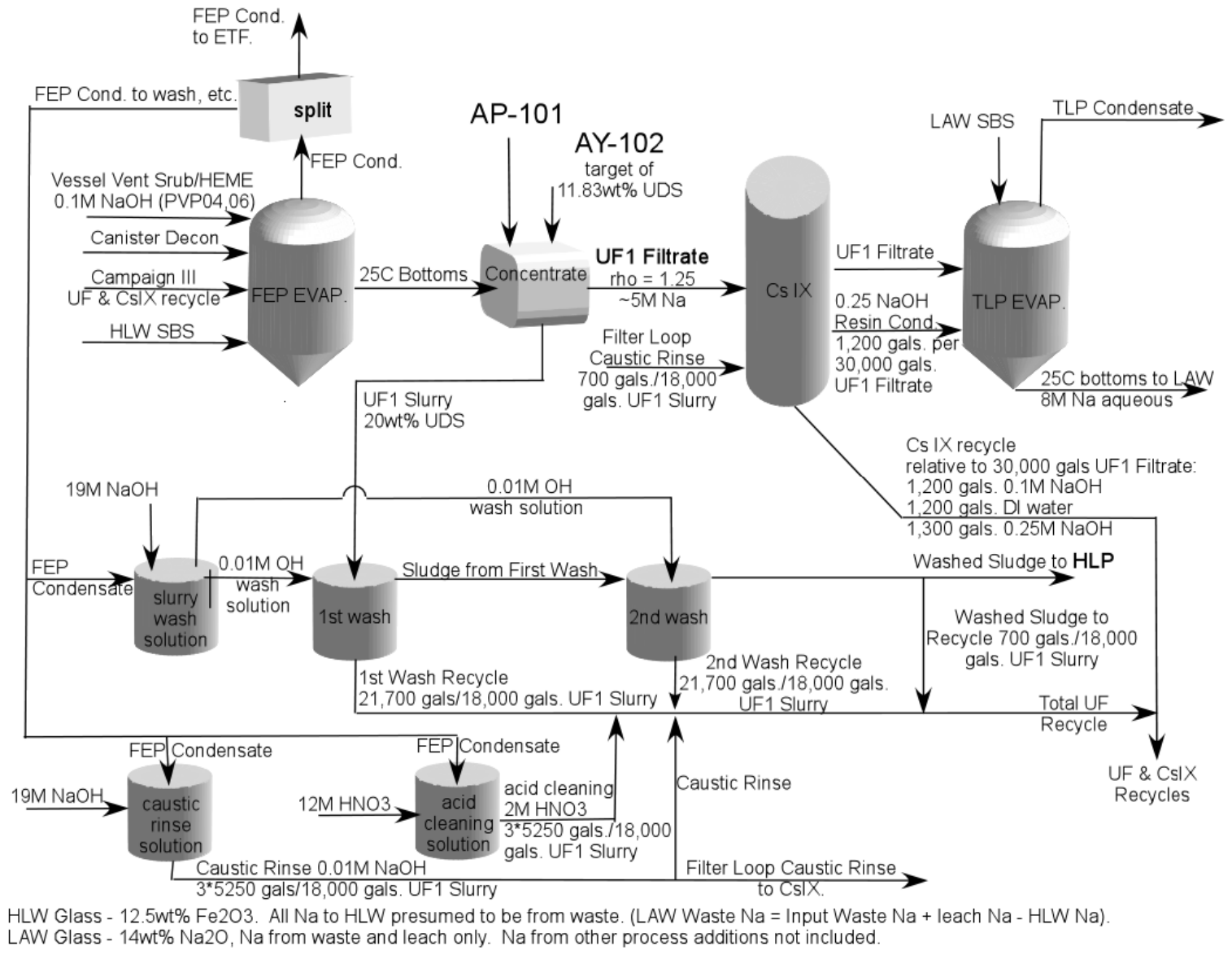

Figure B2. Simplified Flow-Diagram of Campaigns II-IV

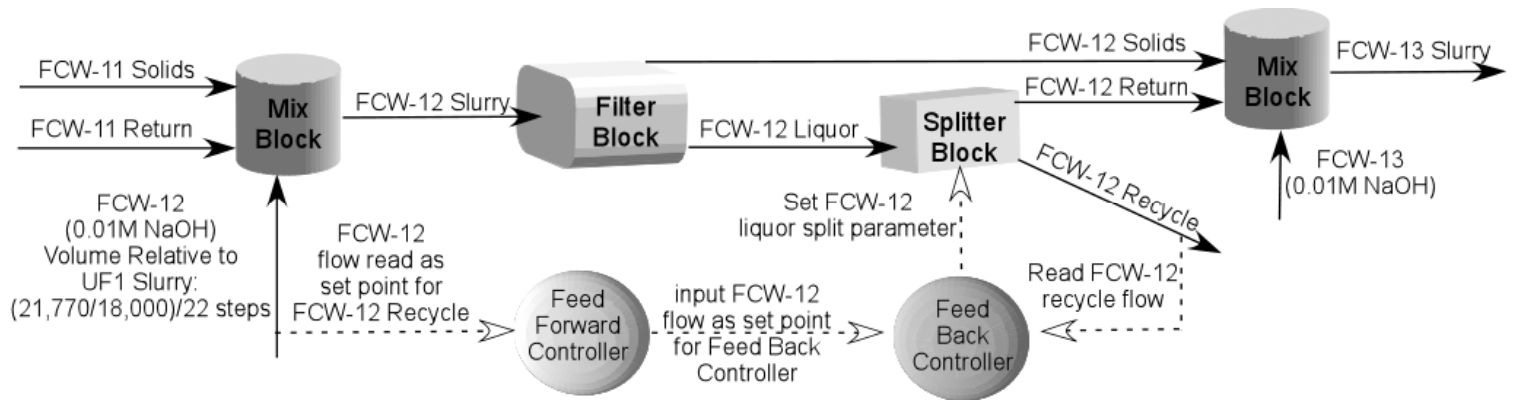

Figure B3. Example Wash Step - Step 12 of First Wash Sequence

(Sets volumetric flow of "FCW-12 Recycle" equal to "FCW-12) 
WSRC-TR-2005-00105, REVISION 0

SRNL-RPP-2005-00012, REVISION 0

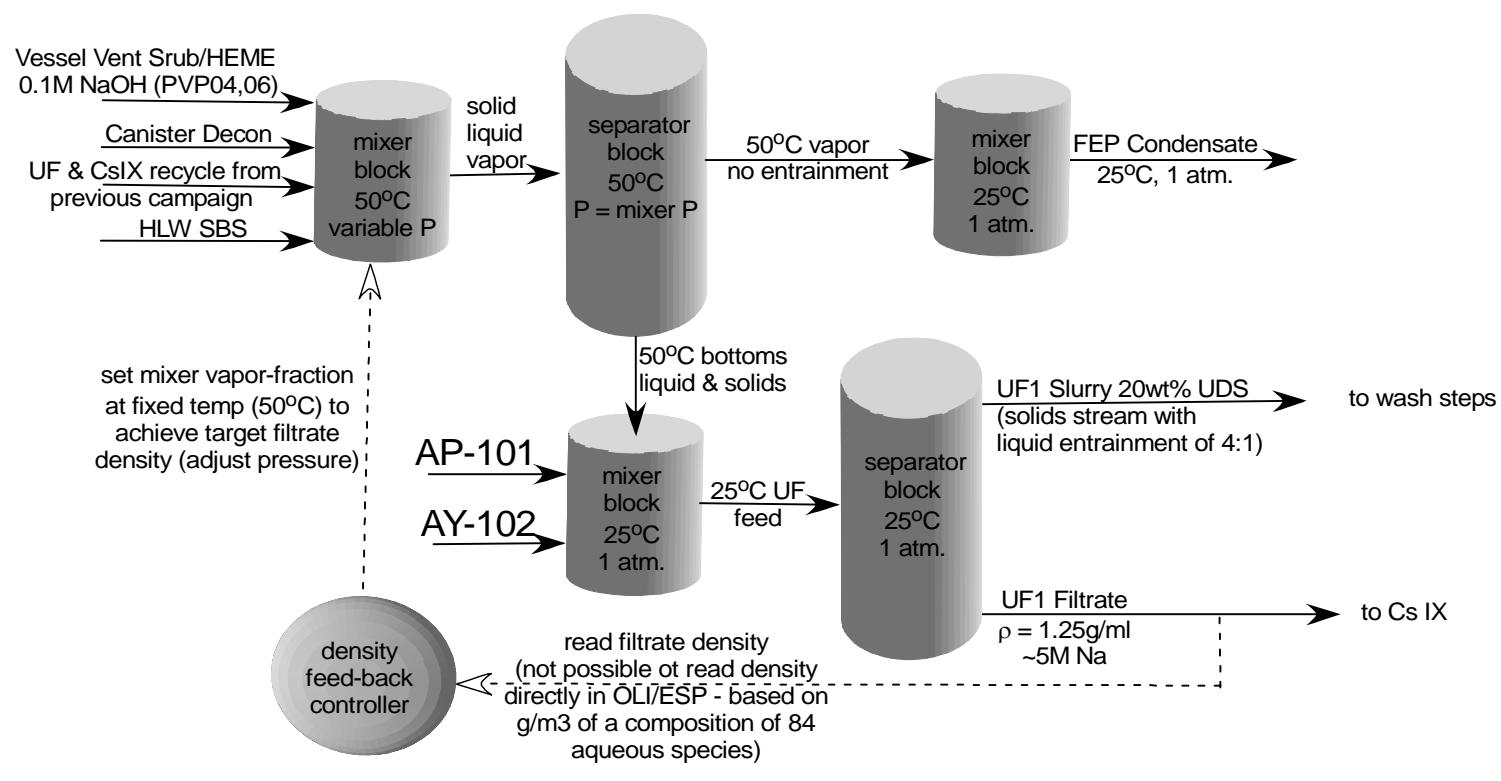

Figure B4. OLI/ESP Representation of FEP Evaporator and Slurry Concentration 
WSRC-TR-2005-00105, REVISION 0

SRNL-RPP-2005-00012, REVISION 0

Table B4. OLI/ESP Species used to Estimate Aqueous Phase Density

A suffix of AQ indicates an aqueous phase molecule; a suffix of ION indicates an aqueous phase anion/cation.

\begin{tabular}{|c|c|c|c|c|}
\hline H2O & BOH3AQ & CAC2O4AQ & CACO3AQ & CAH2SIO4AQ \\
\hline CEOH3AQ & CSCLAQ & CSNO3AQ & FEIIIH33A & KACETAQ \\
\hline KCLAQ & KCOOHAQ & KNO3AQ & LIACETAQ & LIH2BO3AQ \\
\hline LIOHAQ & NAACETAQ & NAALOH4AQ & NABOH4AQ & NABRAQ \\
\hline NACOOHAQ & NAFAQ & NAHCO3AQ & NAHSIO3AQ & NANO3AQ \\
\hline BACO3AQ & OHION & AGCL3ION & AGCL4ION & AGOH2ION \\
\hline ALACETION & ALOH4ION & ALSIO3OHION & B2OOH5ION & B4O5OH4ION \\
\hline BAION & BAOHION & BOH4ION & BRION & CAH2BO3ION \\
\hline CAION & CANO3ION & CAOHION & CAPO4ION & CEOH4ION \\
\hline CLION & CO3ION & COOHION & CRIIC2O42ION & CRIIIIN \\
\hline CRO4ION & CSION & CUOH3ION & CUOH4ION & FEIIIOH4ION \\
\hline FION & H2SIO4ION & H3SIO4ION & HCO3ION & HPBO2ION \\
\hline HPO4ION & KION & KSO4ION & LIION & LISO4ION \\
\hline MNOH3ION & MNOH4ION & NA2FION & NACO3ION & NAION \\
\hline NASO4ION & NIOH3ION & NO2ION & NO3ION & ACETATEION \\
\hline OXALATION & PO4ION & RUIIIOH2ION & SO4ION & SROHION \\
\hline WO4ION & ZNOH3ION & ZNOH4ION & ZROH5ION & \\
\hline
\end{tabular}

The structures of the models for Campaigns II-IV are identical. The campaigns are related to each other through the UF and Cs-IX recycles, with the recycle generated from one campaign being used directly, with no modifications, as input recycle to the subsequent campaign. The waste feed rates for each of these campaigns was adjusted to give the 80/6 MTG/d LAW/HLW glass ratio. The model for Campaign I was unique. The AY-102 waste feed flow rate was completely arbitrary, only the relative flow rates (acid cleaning flow, etc.) were of importance. Before using it as input to Campaign II, the (arbitrary) Campaign I UF recycle flow rate had to be adjusted to some approximation of a steady-state flow corresponding to the 80/6 MTG/d production rates of Campaigns II-IV. The was done by assuming that the amount of UF recycle generated in Campaign II would only be a function the AY-102 waste feed, and not of the AP-101 waste feed. Then the ratio of generated UF recycle to AY-102 for Campaign II could be calculated by using the same ratio of UF recycle to AY-102 from Campaign I (input UF recycle-II = AY-102-II x (output UF recycle-I)/(AY-102-I) ). This turned out to be satisfactory since the difference between the Campaign III and IV recycle volumetric flow rates was less than $0.09 \%$.

\section{B.4. MODEL RESULTS}

Campaign I was intended to serve only as a filtration baseline for the remaining campaigns and does not represent the pre-treatment process, no comparisons between the model flow rates and glass production rates were done here. The simulation results for key streams from Campaign I are summarized in Tables B5 and B6. A complete OLI/ESP listing of the properties and composition of key streams for Campaign I is given in Appendix A of Barnes (2004). 
WSRC-TR-2005-00105, REVISION 0

SRNL-RPP-2005-00012, REVISION 0

Table B5. Composition of Key Streams from the Model of Campaign I

\begin{tabular}{|c|c|c|c|c|c|c|c|}
\hline $\begin{array}{c}\mathrm{mg} / \mathrm{L} \\
\text { solution } \\
\text { @25C }\end{array}$ & $\begin{array}{c}\text { Waste Feed } \\
\text { (AY-102) }\end{array}$ & $\begin{array}{l}\text { UF1 Filtrate } \\
\text { to Cs-IX }\end{array}$ & $\begin{array}{l}\text { UF1 Slurry } \\
\text { 20wt } \% \text { UDS }\end{array}$ & $\begin{array}{l}1^{\text {st }} \text { Wash } \\
\text { Recycle }\end{array}$ & $\begin{array}{l}2^{\text {nd }} \text { Wash } \\
\text { Recycle }\end{array}$ & $\begin{array}{l}\text { Total UF } \\
\text { Recycle }\end{array}$ & $\begin{array}{c}\text { Sludge to } \\
\text { HLP }\end{array}$ \\
\hline $\mathrm{K}$ & 427.34 & 458.61 & 402.00 & 245.68 & 64.18 & 89.97 & 27.65 \\
\hline $\mathrm{Na}$ & 83620.84 & 80851.08 & 85865.68 & 45663.74 & 13618.32 & 17675.21 & 14929.21 \\
\hline $\mathrm{Ba}$ & 175.81 & 1.29 & 317.26 & 1.06 & 0.85 & 0.55 & 319.25 \\
\hline $\mathrm{Ca}$ & 965.97 & 3.44 & 1746.09 & 3.65 & 2.69 & 1.84 & 1762.15 \\
\hline $\mathrm{Zn}$ & 58.36 & 62.63 & 54.90 & 33.55 & 8.77 & 12.29 & 3.78 \\
\hline $\mathrm{Cu}$ & 67.23 & 72.15 & 63.24 & 38.65 & 6.63 & 13.15 & 8.60 \\
\hline $\mathrm{Mg}$ & 254.97 & 0.00 & 461.62 & 0.01 & 0.03 & 0.01 & 467.88 \\
\hline $\mathrm{Pb}$ & 1272.50 & 1365.61 & 1197.03 & 731.56 & 191.11 & 267.91 & 82.33 \\
\hline Al & 16182.77 & 2062.48 & 27627.02 & 862.61 & 163.30 & 297.89 & 26746.65 \\
\hline $\mathrm{Mn}$ & 37.42 & 2.54 & 65.69 & 0.55 & 0.09 & 0.18 & 65.81 \\
\hline $\mathrm{Fe}$ & 27804.11 & 45.56 & 50301.99 & 18.77 & 3.76 & 6.54 & 50961.18 \\
\hline $\mathrm{Ni}$ & 910.21 & 0.41 & 1647.59 & 0.17 & 0.03 & 0.06 & 1669.83 \\
\hline $\mathrm{F}$ & 224.30 & 240.71 & 210.99 & 128.95 & 33.69 & 47.22 & 14.51 \\
\hline $\mathrm{Cl}$ & 157.97 & 169.53 & 148.60 & 90.82 & 23.72 & 33.26 & 10.22 \\
\hline $\mathrm{Br}$ & 123.67 & 132.72 & 116.34 & 71.10 & 18.57 & 26.04 & 8.00 \\
\hline $\mathrm{Li}$ & 85.34 & 91.59 & 80.28 & 49.06 & 12.82 & 17.97 & 5.52 \\
\hline c & 0.00 & 0.00 & 0.00 & 0.00 & 0.00 & 0.00 & 0.00 \\
\hline $\mathrm{P}$ & 2108.50 & 2179.93 & 2050.60 & 1085.24 & 157.47 & 360.84 & 555.23 \\
\hline $\mathrm{s}$ & 803.08 & 818.09 & 790.90 & 438.34 & 114.64 & 160.57 & 123.83 \\
\hline $\mathrm{Cr}$ & 545.25 & 585.15 & 512.91 & 313.46 & 81.89 & 114.80 & 35.28 \\
\hline Si & 8382.35 & 28.08 & 15153.32 & 17.72 & 9.28 & 7.84 & 15327.15 \\
\hline $\mathrm{Sr}$ & 225.34 & 0.03 & 407.96 & 0.03 & 0.02 & 0.01 & 413.47 \\
\hline $\mathrm{B}$ & 8.96 & 9.62 & 8.43 & 5.15 & 1.35 & 1.89 & 0.58 \\
\hline $\mathrm{Cs}$ & 6.23 & 6.68 & 5.86 & 3.58 & 0.94 & 1.31 & 0.40 \\
\hline W & 161.10 & 172.89 & 151.55 & 92.62 & 24.19 & 33.92 & 10.42 \\
\hline $\mathrm{Ce}$ & 246.65 & 264.70 & 232.02 & 135.31 & 6.71 & 41.24 & 61.10 \\
\hline $\mathrm{Nd}$ & 359.53 & 0.00 & 650.93 & 0.00 & 0.00 & 0.00 & 659.82 \\
\hline $\mathrm{Zr}$ & 993.32 & 7.39 & 1792.40 & 3.75 & 0.98 & 1.37 & 1811.08 \\
\hline $\mathrm{Ag}$ & 488.67 & 0.02 & 884.71 & 0.00 & 0.00 & 0.00 & 896.79 \\
\hline $\mathrm{Ru}$ & 87.20 & 93.58 & 82.03 & 50.13 & 13.10 & 18.36 & 5.64 \\
\hline $\begin{array}{r}\text { density } \\
(\mathrm{g} / \mathrm{L})\end{array}$ & 1231 & 1169 & 1281 & 1098 & 1029 & 1041 & 1137 \\
\hline wt\% UDS & 11.50 & & 20.00 & 0.09 & 0.00 & 0.19 & 21.39 \\
\hline
\end{tabular}


WSRC-TR-2005-00105, REVISION 0

SRNL-RPP-2005-00012, REVISION 0

Table B6. Solids Composition of Key Streams from the Model of Campaign I

\begin{tabular}{|c|c|c|c|c|c|c|}
\hline $\begin{array}{c}\text { Solids } \\
\text { (wt } \% \text { of total UDS) }\end{array}$ & $\begin{array}{l}\text { AY-102 } \\
\text { WASTE FEED }\end{array}$ & $\begin{array}{l}\text { 20wt\% UDS } \\
\text { UF1 SLURRY }\end{array}$ & $\begin{array}{l}\text { 1st WASH } \\
\text { SEQUENCE }\end{array}$ & \begin{tabular}{|l|} 
\\
2nd WASH \\
SEQUENCE
\end{tabular} & $\begin{array}{l}\text { TOTAL UF } \\
\text { Cs-IX } \\
\text { RECYCLE }\end{array}$ & $\begin{array}{l}\text { SLUDGE } \\
\text { to HLP }\end{array}$ \\
\hline $\mathrm{Ba}(\mathrm{SO} 4)$ & 0.2097 & 0.2097 & 0.0022 & 0.0107 & 0.0134 & 0.2227 \\
\hline $\mathrm{Fe}(\mathrm{OH}) 3$ & 37.5385 & 37.5385 & 0.2297 & 0.7329 & - & 40.1081 \\
\hline $\mathrm{Al}(\mathrm{OH}) 3$ & 15.0762 & 15.0762 & 14.4437 & 65.3800 & - & 16.7198 \\
\hline $\mathrm{Mn}(\mathrm{OH}) 2$ & 0.0401 & 0.0401 & 0.0297 & 0.1011 & - & 0.0438 \\
\hline $\mathrm{Ni}(\mathrm{OH}) 2$ & 1.0149 & 1.0149 & 0.0017 & 0.0055 & - & 1.0842 \\
\hline $\mathrm{Ag} 2(\mathrm{CO} 3)$ & 0.4413 & 0.4413 & 0.0001 & 0.0002 & - & 0.4714 \\
\hline $\mathrm{Sr}(\mathrm{CO} 3)$ & 0.2683 & 0.2683 & - & - & - & 0.2865 \\
\hline $\mathrm{ZrO} 2$ & 0.9416 & 0.9416 & 0.0069 & 0.0319 & 0.0933 & 1.0060 \\
\hline $\mathrm{Mg}(\mathrm{OH}) 2$ & 0.4322 & 0.4322 & 0.0003 & 0.0273 & - & 0.4616 \\
\hline NASGEL.15.5H2O & 34.8581 & 34.8581 & 0.1099 & - & - & 37.2011 \\
\hline $\mathrm{Na} 2(\mathrm{C} 2 \mathrm{O} 4)$ & 6.9956 & 6.9956 & 85.1755 & - & - & - \\
\hline $\mathrm{Ca}(\mathrm{CO} 3)$ & 1.6988 & 1.6988 & - & - & - & 1.1021 \\
\hline $\mathrm{Nd}(\mathrm{PO} 4) \cdot 2 \mathrm{H} 2 \mathrm{O}$ & 0.4848 & 0.4848 & - & - & - & 0.5179 \\
\hline $\mathrm{Ca}(\mathrm{C} 2 \mathrm{O} 4) \cdot 1 \mathrm{H} 2 \mathrm{O}$ & - & - & - & - & 0.2793 & - \\
\hline $\mathrm{Ce}(\mathrm{PO} 4)$ & - & - & - & 24.7292 & 3.3897 & 0.0416 \\
\hline $\mathrm{Pb} 3(\mathrm{PO} 4) 2$ & - & - & - & - & 16.2916 & - \\
\hline $\mathrm{NaAl}(\mathrm{CO} 3)(\mathrm{OH}) 2$ & - & - & - & - & 79.9325 & - \\
\hline $\mathrm{Cu}(\mathrm{OH}) 2$ & - & - & - & 8.9813 & - & 0.0043 \\
\hline $\mathrm{Ca}(\mathrm{PO} 4) 2$ & - & - & - & - & - & 0.7291 \\
\hline
\end{tabular}

Campaigns II and III were intermediary steps towards the UF and Cs-IX recycle stream state-state flows of Campaign IV. As such, only the model results of Campaign IV are reported here and used to estimate the SIPP minimum flux/flow rates necessary to prove rate. These minimum rates, along with the estimated SIPP process volumes are used estimate the corresponding maximum SIPP run times as described below.

In the case of the FEP and TLP evaporators, the overhead flows predicted by the model for the $80 / 6 \mathrm{MTG} / \mathrm{d}$ glass production rates were scaled by a factor 0.13 (Table B7) to estimate the minimum SIPP overhead flows needed to prove rate.

The minimum LAW permeate flux rate necessary for the SIPP to prove rate was calculated by dividing the flow rate of the LAW permeate (going to the Cs-IX) predicted by the model for the 80/6 MTG/d glass production rates by the design basis filter area of $590 \mathrm{ft}^{2}$ (Table B7).

The flux rate for the wash permeate was calculated in a similar manner. However, as described earlier, the concentration mode is the rate-limiting step. One train will have completed the treat-clean mode and remain idle while the other finishes the concentration mode, assuming identical flux rates for the LAW and wash permeates. Instead of using identical fluxes, the calculation for the minimum wash permeate flux rate applied this idle time toward a lower wash permeate flux rate, resulting in equal process times for the 
concentration and treat-clean modes. The flux rate was calculated as volume of wash permeate produced by one treat-clean sequence (the washing of 18,000 gals. 20wt $\%$ UDS slurry) was divided by the filter area and the time required for one concentration mode, less the time needed for filter cleaning, sludge and heel transfers (i.e. the time available for washing before the train must begin sludge transfer and cleaning in preparation for the next concentration mode).

Table B7. Design Basis and SIPP Capacities

\begin{tabular}{|c|c|c|c|}
\hline Unit Operation & Design Basis & SIPP & Scale Factor \\
\hline $\begin{array}{c}\text { FEP/TLP } \\
\text { Evaporator Cond. Capacity }\end{array}$ & $30 \mathrm{gpm}$ & $0.39 \mathrm{gpm}$ & 0.13 \\
\hline Ultra-filtration Area & $590 \mathrm{ft}^{2}$ & $6.7 \mathrm{ft}^{2}$ & based only on flux rate \\
\hline
\end{tabular}

These minimum SIPP flux/flow rates where then used to calculate the time required to process the SIPP volumes estimated by the model at these rates. These volumes were based on producing 140L of 20wt\% UDS slurry in Campaign I. All flows predicted by the Campaign I model were scaled by the ratio of 140L / (model flow of the 20wt\% UDS slurry) to estimate the volumes produced by Campaign I of the SIPP. The calculated volume of the Campaign I SIPP UF recycle (which is also the input UF Recycle for the Campaign II SIPP) was then used to scale the flows predicted by the Campaign II model by the ratio of (Campaign I SIPP UF recycle) / (Campaign II model UF Recycle input). This was done for Campaigns III and IV in the same manner, and is depicted schematically in Fig. B5. 
WSRC-TR-2005-00105, REVISION 0

SRNL-RPP-2005-00012, REVISION 0

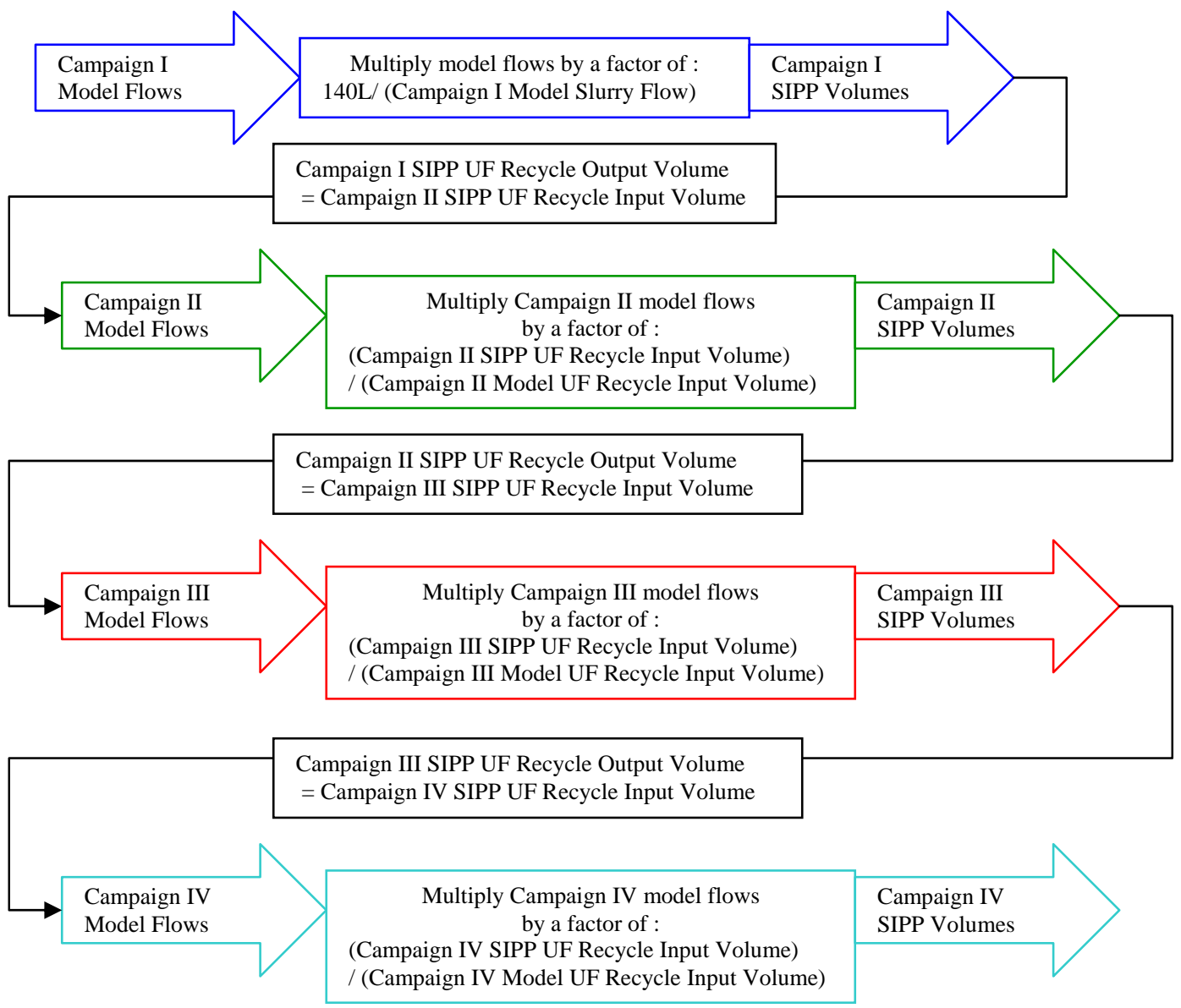

Figure B5. Algorithm for Estimation of SIPP Volumes based on Model Flow Rates

The model flow rates and corresponding SIPP volumes for Campaign IV are listed in Table B8. Table B9 shows the estimated Campaign IV SIPP run time based on values in Tables B7 and B8. The Cs column will not be operated prototypically, but in a manner to determine the break-through curve, so an estimated run-time for the Cs-IX was not included. Also, it is not possible to scale the filter cleaning or the sludge transfer times; the duration for these operations for the SIPP were set at the design basis with the understanding that they will have to be adjusted based on the actual SIPP time for these operations. Finally, these times are only estimates based on the model compositions, the actual Campaign IV SIPP stream compositions, flow, and flux rates will be used to prove or disprove the glass production rates. The model predictions of the composition for key streams of Campaign IV are given in Tables B10 and B11. A complete OLI/ESP listing of the properties and composition of key streams for Campaign IV is given in Appendix B of Barnes (2004). 
WSRC-TR-2005-00105, REVISION 0 SRNL-RPP-2005-00012, REVISION 0

Table B8. Campaign IV SIPP Volumes and Model Flow Rates

\begin{tabular}{|l|r|r|}
\hline \multicolumn{1}{|c|}{ stream name } & $\begin{array}{c}\text { SIPP Campaign IV } \\
\text { Volumes (liters) }\end{array}$ & $\begin{array}{c}\text { Model Flow Rates for } \\
\text { 80/6 MTG/d LAW/HLW } \\
\text { glass (gpm) }\end{array}$ \\
\hline AY102 & 241.39 & 3.545 \\
\hline AP101 & 672.10 & 9.870 \\
\hline RECYCLE (previous campaign) & 602.79 & 8.852 \\
\hline HLW SBS & 189.94 & 2.789 \\
\hline PVP04/06 (Vessel Vent System) & 219.98 & 3.230 \\
\hline HLW DECON & 114.26 & 1.678 \\
\hline FEP CONDENSATE & 984.86 & 14.462 \\
\hline FEP COOLED BOTTOMS & 144.93 & 2.128 \\
\hline UF1 FILTRATE to CIX & 943.65 & 13.857 \\
\hline UF1 SLURRY 20wt\% UDS & 116.09 & 1.705 \\
\hline TOTAL CAUSTIC WASH & 280.81 & 4.124 \\
\hline HLW SLURRY to HLP & 109.98 & 1.615 \\
\hline TOTAL ACID CLEANING & 101.59 & 1.492 \\
\hline TOTAL CAUSTIC RINSE & 101.54 & 1.491 \\
\hline CAUSTIC RINSE TO FEP EVAP & 97.03 & 1.425 \\
\hline CIX DI WATER & 37.75 & 0.554 \\
\hline CIX CAUSTIC RINSE & 37.75 & 0.554 \\
\hline CIX 0.25M NAOH FEP & & 0.600 \\
\hline RECYCLE & 40.89 & 0.554 \\
\hline CIX 0.25M NAOH TLP & 37.75 & 8.844 \\
\hline TOTAL UF \& CIX RECYCLE & 602.27 & 12.191 \\
\hline LAW SBS & 830.20 & 17.987 \\
\hline TLP CONDENSATE & 1224.90 & 8.783 \\
\hline TLP COOLED BOTTOMS & 598.10 & \\
\hline
\end{tabular}




\section{WSRC-TR-2005-00105, REVISION 0 SRNL-RPP-2005-00012, REVISION 0}

Table B9. Estimated Filtration Batch Cycle Times Necessary to Make WTP Glass Production Rate of 80/6 MTG/d LAW/HLW

\begin{tabular}{|c|c|c|c|c|}
\hline $\begin{array}{c}\begin{array}{c}\text { Filtration Step } \\
\text { (hrs.) }\end{array}\end{array}$ & \begin{tabular}{|} 
WTP Batch (18,000 gals.) \\
Cycle Time (hrs.) \\
Design Basis \\
(0.03 gpm/ft2)
\end{tabular} & \begin{tabular}{|c|} 
WTP Batch (18,000 gals.) \\
Cycle Time (hrs.) \\
Minimum Required Filtration Rates: \\
0.0235 and 0.00808 gpm/ft2 for the \\
concentration and wash modes \\
respectively
\end{tabular} & \begin{tabular}{|c|} 
SIPP Batch \\
Cycle Time (hrs.) \\
Design Filtration Rate \\
$\left(0.03 \mathrm{gpm} / \mathrm{ft}^{2}\right)$
\end{tabular} & $\begin{array}{c}\text { SIPP Batch } \\
\text { Cycle Time (hrs) } \\
\text { Minimum Required } \\
\text { Filtration Rates: } \\
0.0235 \text { and } 0.00808 \\
\text { gpm/ft2 for the } \\
\text { concentration and wash } \\
\text { modes respectively }\end{array}$ \\
\hline 1st concentration & 137.8 & 176.0 & 20.7 & 26.4 \\
\hline LAW subtotal & 137.8 & 176.0 & 20.7 & 26.4 \\
\hline 1st wash & 20.5 & 76.1 & 3.1 & 11.4 \\
\hline 2nd wash & 20.5 & 76.1 & 3.1 & 11.4 \\
\hline filter cleaning & 19.8 & 19.8 & 19.8 & 19.8 \\
\hline sludge heel return & 2.0 & 2.0 & 2.0 & 2.0 \\
\hline sludge trans. to HLP & 2.0 & 2.0 & 2.0 & 2.0 \\
\hline idle time & 73.0 & 0.0 & 10.9 & 0.0 \\
\hline Treat \& Clean subtotal & 137.8 & 176.0 & 40.9 & 46.6 \\
\hline Total Batch Time & 275.5 & 352.0 & 61.6 & 73.0 \\
\hline
\end{tabular}

Table B10. Composition of Key Streams from Campaign IV Model

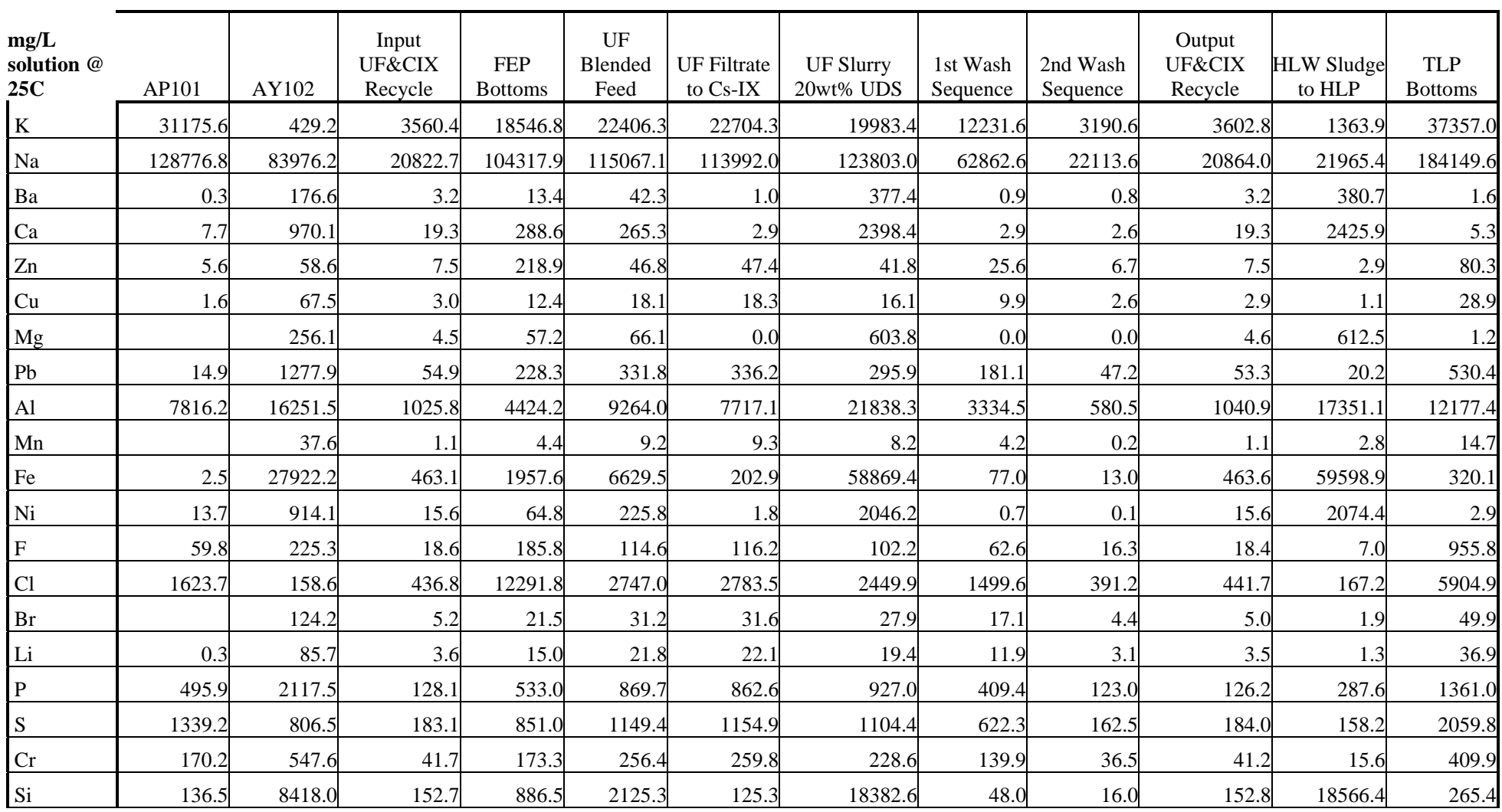


WSRC-TR-2005-00105, REVISION 0

SRNL-RPP-2005-00012, REVISION 0

\begin{tabular}{|c|c|c|c|c|c|c|c|c|c|c|c|c|}
\hline $\begin{array}{l}\text { mg/L } \\
\text { solution @ } \\
25 C\end{array}$ & AP101 & AY102 & $\begin{array}{c}\text { Input } \\
\text { UF\&CIX } \\
\text { Recycle } \\
\end{array}$ & $\begin{array}{c}\text { FEP } \\
\text { Bottoms }\end{array}$ & $\begin{array}{c}\text { UF } \\
\text { Blended } \\
\text { Feed } \\
\end{array}$ & $\begin{array}{c}\text { UF Filtrate } \\
\text { to Cs-IX }\end{array}$ & $\begin{array}{c}\text { UF Slurry } \\
\text { 20wt\% UDS }\end{array}$ & $\begin{array}{l}\text { 1st Wash } \\
\text { Sequence }\end{array}$ & $\begin{array}{l}\text { 2nd Wash } \\
\text { Sequence }\end{array}$ & $\begin{array}{c}\text { Output } \\
\text { UF\&CIX } \\
\text { Recycle } \\
\end{array}$ & $\begin{array}{c}\text { HLW Sludge } \\
\text { to HLP }\end{array}$ & $\begin{array}{c}\text { TLP } \\
\text { Bottoms } \\
\end{array}$ \\
\hline $\mathrm{Sr}$ & & 226.3 & 3.7 & 15.4 & 53.7 & 0.1 & 489.4 & 0.1 & 0.1 & 3.7 & 496.2 & 0.1 \\
\hline B & 16.0 & 9.0 & 2.1 & 8.9 & 13.4 & 13.6 & 12.0 & 7.3 & 1.9 & 2.2 & 0.8 & 1172.8 \\
\hline Cs & & 6.3 & 0.3 & 1.1 & 1.6 & 1.6 & 1.4 & 0.9 & 0.2 & 0.3 & 0.1 & 2.5 \\
\hline W & 24.6 & 161.8 & 9.5 & 39.4 & 57.9 & 58.6 & 51.6 & 31.6 & 8.2 & 9.3 & 3.5 & 92.5 \\
\hline $\mathrm{Ce}$ & & 247.7 & 10.2 & 42.6 & 62.2 & 63.1 & 55.5 & 34.0 & 8.9 & 10.0 & 3.8 & 99.5 \\
\hline Nd & & 361.1 & 5.9 & 24.5 & 85.6 & 0.0 & 781.3 & 0.0 & 0.0 & 5.9 & 792.5 & 1.0 \\
\hline $\mathrm{Zr}$ & & 997.5 & 18.3 & 76.2 & 237.6 & 24.1 & 1973.1 & 12.5 & 3.1 & 18.4 & 1982.1 & 38.1 \\
\hline $\mathrm{Ag}$ & & 490.7 & 8.0 & 33.2 & 116.3 & 0.8 & 1055.0 & 0.1 & 0.0 & 8.0 & 1069.9 & 2.7 \\
\hline $\mathrm{Ru}$ & & 87.6 & 3.6 & 15.2 & 22.0 & 22.3 & 19.6 & 12.0 & 3.1 & 3.5 & 1.3 & 35.2 \\
\hline \begin{tabular}{|l}
$\begin{array}{l}\text { density } \\
(\mathrm{g} / \mathrm{L})\end{array}$ \\
\end{tabular} & 1278 & 1236 & 1053 & 1253 & 1264 & 1250 & 1375 & 1142 & 1051 & 1053 & 1153 & 1398 \\
\hline wt\% UDS & 0.01 & 11.50 & 0.60 & 2.45 & 2.38 & 0.00 & 20.00 & 0.12 & 0.00 & 0.57 & 21.64 & 0.05 \\
\hline
\end{tabular}

Table B11. Solids Composition of Key Streams from the Model of Campaign IV

\begin{tabular}{|c|c|c|c|c|c|c|c|c|c|c|c|}
\hline wt $\%$ of total UDS & AP101 & AY102 & $\begin{array}{l}\text { Input UF \& } \\
\text { CIX Recycle }\end{array}$ & $\begin{array}{c}\text { FEP } \\
\text { Bottoms }\end{array}$ & $\begin{array}{l}\text { UF Blended } \\
\text { Feed }\end{array}$ & $\begin{array}{l}\text { 20wt\% UDS } \\
\text { UF1 Slurry }\end{array}$ & $\begin{array}{l}\text { 1st Wash } \\
\text { Sequence }\end{array}$ & $\begin{array}{l}\text { 2nd Wash } \\
\text { Sequence }\end{array}$ & $\begin{array}{c}\text { UF\&CIX } \\
\text { Recycle }\end{array}$ & $\begin{array}{l}\text { HLP } \\
\text { Sludge }\end{array}$ & $\begin{array}{c}\text { TLP } \\
\text { Bottoms }\end{array}$ \\
\hline $\mathrm{Fe}(\mathrm{OH}) 3$ & - & 37.54 & 14.10 & 12.10 & 40.83 & 40.83 & 0.97 & 1.24 & 14.65 & 45.71 & - \\
\hline $\mathrm{Al}(\mathrm{OH}) 3$ & - & 15.08 & 9.43 & 29.19 & - & - & 35.77 & 98.35 & 15.81 & 2.15 & - \\
\hline $\mathrm{Ni}(\mathrm{OH}) 2$ & 12.60 & 1.01 & 0.39 & 0.33 & 1.17 & 1.17 & 0.01 & 0.01 & 0.41 & 1.31 & - \\
\hline Ag2(CO3) & - & 0.44 & 0.16 & - & 0.49 & 0.49 & 0.01 & 0.00 & 0.17 & 0.55 & - \\
\hline $\mathrm{ZrO} 2$ & - & 0.94 & 0.39 & 0.33 & 0.96 & 0.96 & 0.01 & 0.04 & 0.41 & 1.07 & - \\
\hline $\mathrm{Mg}(\mathrm{OH}) 2$ & - & 0.43 & - & 0.45 & 0.53 & 0.53 & 0.00 & 0.01 & - & 0.59 & 0.42 \\
\hline NASGEL.15.5H2O & - & 34.86 & 14.02 & 16.74 & 39.22 & 39.22 & 2.19 & - & 14.57 & 43.91 & - \\
\hline $\mathrm{Nd}(\mathrm{PO} 4) .2 \mathrm{H} 2 \mathrm{O}$ & - & 0.48 & 0.18 & 0.15 & 0.54 & 0.54 & 0.00 & - & 0.19 & 0.61 & 0.28 \\
\hline $\mathrm{Na} 2(\mathrm{C} 2 \mathrm{O} 4)$ & 77.69 & 7.00 & - & 38.21 & 13.55 & 13.55 & 61.05 & - & - & - & 97.91 \\
\hline $\mathrm{Ba}(\mathrm{SO} 4)$ & - & 0.21 & 0.07 & 0.07 & 0.23 & 0.23 & - & 0.14 & 0.08 & 0.26 & 0.08 \\
\hline $\mathrm{Ca}(\mathrm{CO} 3)$ & 9.71 & 1.70 & 0.32 & 2.33 & 2.18 & 2.18 & - & - & 0.57 & - & - \\
\hline $\mathrm{Mn}(\mathrm{OH}) 2$ & - & 0.04 & - & 0.02 & - & - & - & 0.19 & - & 0.00 & - \\
\hline $\mathrm{Sr}(\mathrm{CO} 3)$ & - & 0.27 & 0.10 & 0.08 & 0.30 & 0.30 & - & 0.01 & 0.10 & 0.34 & 0.01 \\
\hline $\mathrm{Cu}(\mathrm{OH}) 2$ & - & - & 0.06 & - & - & - & - & - & 0.06 & - & - \\
\hline $\mathrm{Zn}(\mathrm{OH}) 2$ & - & - & 0.13 & - & - & - & - & - & 0.13 & - & - \\
\hline $\mathrm{Ca} 3(\mathrm{PO} 4) 2$ & - & - & 0.37 & - & - & - & - & 0.02 & 0.15 & 0.09 & - \\
\hline $\mathrm{Ce}(\mathrm{PO} 4)$ & - & - & 0.27 & - & - & - & - & - & 0.28 & - & - \\
\hline $\mathrm{Pb} 3(\mathrm{PO} 4) 2$ & - & - & 1.13 & - & - & - & - & - & 1.14 & - & - \\
\hline $\mathrm{NaAl}(\mathrm{CO} 3)(\mathrm{OH}) 2$ & - & - & 58.86 & - & - & - & - & - & 51.30 & - & - \\
\hline $\mathrm{CaF} 2$ & - & - & - & - & - & - & - & - & - & - & 1.30 \\
\hline $\mathrm{Ca}(\mathrm{C} 2 \mathrm{O} 4) \cdot 1 \mathrm{H} 2 \mathrm{O}$ & - & - & - & - & - & - & - & - & - & 3.41 & - \\
\hline
\end{tabular}




\section{B.5. MODEL RESULTS}

Analytical results of the stream compositions from the SIPP Campaign II run indicate a higher glass production rate by about $15 \%$ than estimated by the model. This difference is due to the adjustment made to the solids portion of the simulant recipe used in the model. While it is not necessary, even undesirable, to base the solids portion of a simulant recipe on a charge balanced analysis, the model requires all input streams to be neutral. The simulant solids recipe had an excess of cations and hydroxide was added to achieve a charge balance for the model input. This led to an inconsistency between the charge balance and the concentration of the constituent species, adding hydroxide caused the mass basis to go from $1000 \mathrm{~kg}$ to $1138 \mathrm{~kg}$ (i.e. the solids composition was given in moles/1000kg dry solids). The simulant recipe was still in development at the time of the model runs, and instead of modifying the ratio of the solids species to achieve the correct mass basis, the composition was used as given. The result was to dilute the metals concentration in the solids of the model relative to that of the final simulant composition.

\section{B.6. REFERENCES FOR APPENDIX B}

Barnes, C. D. OLI/ESP Modeling of the Semi-Integrated Pilot Plant for Estimate of Campaigns I-IV Simulant Volumes (U). WSRC-TR-2004-0403, Rev. 0, Westinghouse Savannah River Co., Aiken, 2004.

Duignan, M. R. et al., Interim Report: RPP-WTP Semi-Integrated Pilot Plant Campaign II. WSRC-TR-2004-0478, Rev. 0, Westinghouse Savannah River Co., Aiken, 2004 [also SRT-RPP-2004-00075].

Lee, E., et al. Flowsheet Bases, Assumptions, and Requirements. 24590-WTP-RPT-PT02-005, Rev. 1, pp 3.1-9, Section-3.1.3.5.1 - LAW Glass $\mathrm{Na}_{2}$ Loading, Washington Group International, Richland, WA, 2003a

Lee, E., et al. Flowsheet Bases, Assumptions, and Requirements. 24590-WTP-RPT-PT02-005, Rev. 1, pp 4.1-2, Table TS-1.1 Minimum Component Limits in High-Level Waste Glass, Washington Group International, Richland, WA, 2003b

Lee, E., et al. Flowsheet Bases, Assumptions, and Requirements. 24590-WTP-RPT-PT02-005, Rev. 1, pp 2.3-1 - 2.3.6, Ultra-Filtration Assumptions, Washington Group International, Richland, WA, 2003c

Lee, E., et al. Flowsheet Bases, Assumptions, and Requirements. 24590-WTP-RPT-PT02-005, Rev. 1, pp 2.5-33 Table 2.5-9 Cesium Removal System Process Stream Descriptions for Material Balance Calculations, Washington Group International, Richland, WA, 2003d

Russel, R.L., et al. AP-101 Diluted Feed (Envelope A) Simulant Development Report. PNWD-3248 WTP-RPT-057, Rev. 0, Battelle, Pacific Northwest Division, Richland, WA, 2003

Zamecnik, J.R., Burket, P.R., and Eibling, R.E. Tank 241-AY-102 Simulant Development, Ultrafiltration, and Washing. WSCR-TR-2003-0547, Rev. 0, Westinghouse Savannah River Co., Aiken, 2004 
WSRC-TR-2005-00105, REVISION 0

SRNL-RPP-2005-00012, REVISION 0

\title{
APPENDIX C: WTP DISCUSSION ON PRETREATMENT FACILITY WASTE PROCESSING RESIDENCE TIMES
}

----- Forwarded by Bond Calloway/WSRC/Srs on 03/16/2004 07:22 PM -----

\author{
"Harty, David"

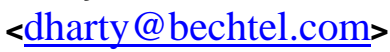 \\ 03/16/2004 06:11 PM
}

To: Bond Calloway bond.calloway@srs.gov

Cc: Harty, David dharty@bechtel.com

Olander, Alan arolande@bechtel.com

Slaathaug, Eric ejslaath@bechtel.com

Lehrman, scott slehrman@bechtel.com

Peterson, Reid rpeterso@bechtel.com

Sundar, Parameshwaran S pssundar@bechtel.com

Tadlock, Johnjatadloc@bechtel.com

\section{SUBJECT: PLANT RESIDENCE TIME DISCUSSION}

$<<$ PT Flow Diagram Rev 1b.vsd $>>\quad<$ PT Tank Vol_App B.doc $>>$

Attached (they have been removed) above are the handouts from the discussion this afternoon. Four different holdup times were discussed during the meeting.

Note: The defined production rates for SIPP are 6.0 MT IHLW/day and $80 \mathrm{MT}$ ILAW/day. Steady-state flowrates defined below are from the WTP Steady State Flowsheet.

I. Holdup Time For Permeate After Ultrafiltration.

Two permeate vessel tank volumes $(21,800$ each) plus the CXP Feed Vessel available volume (78,000 batch $-25,000$ contingency) are used for a total available holdup volume of 96,600 gallons. At a steady-state permeate flowrate of 14.8 gpm, the residence time is $96,600 /(14.8 * 60)=109$ hours $(\sim 4$ $1 / 2$ days ). 
WSRC-TR-2005-00105, REVISION 0

SRNL-RPP-2005-00012, REVISION 0

II. Holdup Time For Blended Feed With Concentrated Recycles Prior To Ultrafiltration.

Two UFP Preparation vessel tank volumes $(48,300$ each) are used for a total available holdup volume of 96,600 gallons. At a steady-state feed to UFP Feed vessels of $16.3 \mathrm{gpm}$, the residence time is $96,600 /(16.3 * 60)=99$ hours ( 4 days). This value is overly conservative and the residence time of blending the concentrated recycles with the blended feed is dependent on the dynamic nature of recycle generation, feed blending, and the processing of feed form the preparation vessel to the feed vessel. If the online UFP Feed vessel is considered full and the UF Prep vessels are both filled with blended feed and concentrated recycle, then the residence time of 4 days will result. Further consideration suggests that the residence time using one UFP Preparation vessel tank volume should be used. This would give a residence time of $48,300 /(16.3 * 60)=49$ hours ( 2 days). Use the second value.

III. Holdup Time for Blended Recycles (HLW-SBS with UFP recycles) Prior to Evaporation.

Two evaporator feed tanks with an available volume of (50,000 batch - 10,000 contingency) are used for a total available holdup volume of 80,000 gallons. At a steadystate evaporator feed flowrate of $18.5 \mathrm{gpm}$, the residence time is $80,000 /(18.5 * 60)=72$ hours ( 3 days).

IV. Holdup Time For Recycles in the Evaporator.

One evaporator with a holdup volume of 5500 gallons is used. At a steady-state concentrate flowrate of $1.8 \mathrm{gpm}$, the residence time is 5500/(1.8*60) $=51$ hours ( 2 days). It was noted that without the leaching process, there is no recycle leachate. Inclusion of the leaching recycle reduces the residence time in the evaporator because the concentrate flowrate is higher.

Comments are welcome.

Dave Harty

Pretreatment Process Operations, 371-3360 
WSRC-TR-2005-00105, REVISION 0

SRNL-RPP-2005-00012, REVISION 0

\section{APPENDIX D: SAMPLING MATRICES - Campaigns I-IV}

This appendix contains a list of all the samples taken during SIPP. Because of the large number of samples and the importance to document those that were taken, the tables have been put in a form, which is condensed. However, the tables are readable and their placement here should be useful to those wanting to better understand the data discussed in the body of this report. Immediately obvious is that the number of samples for each campaign is different and this occurred for different reasons. Campaign I has the most extensive number of samples for filtration. That campaign only involved filtration [Test Specification (Townson, 2002)] and only tested a single simulant, i.e., AY102/C106, so that it could be compared to past single-simulant filter tests and so that it would be a baseline to compare to other filtration tests in SIPP as the feed simulant included both AP101 and recycle streams.. Beginning with Campaign II a different sample matrix was devised because of the interconnected operation of four unit operations through their feed and product streams, including the recycle streams. Campaign II analyzed the largest number of sample, because it was the first campaign using all four unit operations. The number of samples analyzed in subsequent campaigns was significantly reduced to minimized cost but this was justified because of the similar filtration performance between Campaigns I and II, even with the addition of AP101 and recycle streams to the filtration feed. Finally, Campaign IV was once again just a filtration test to evaluate the new GKN filter (Feed Waste Evaporation was included in order to make feed for the filter test.) While the performance of the GKN was unknown before the test, the chemistry of the feed was not expected to be significantly different than what was measured in Campaigns II and III, therefore, only a minimum number of analytical samples were analyzed to minimize cost. This assumption was backed up be the similar filter performances of Campaigns I, II, and III, and because the model discussed in Appendix B predicted little change in chemical makeup in the streams.

Tables D2: Campaign I

Tables D3: Campaign II

Tables D4: Campaign III

Tables D5: Campaign IV

Notes on the following Sample Tables

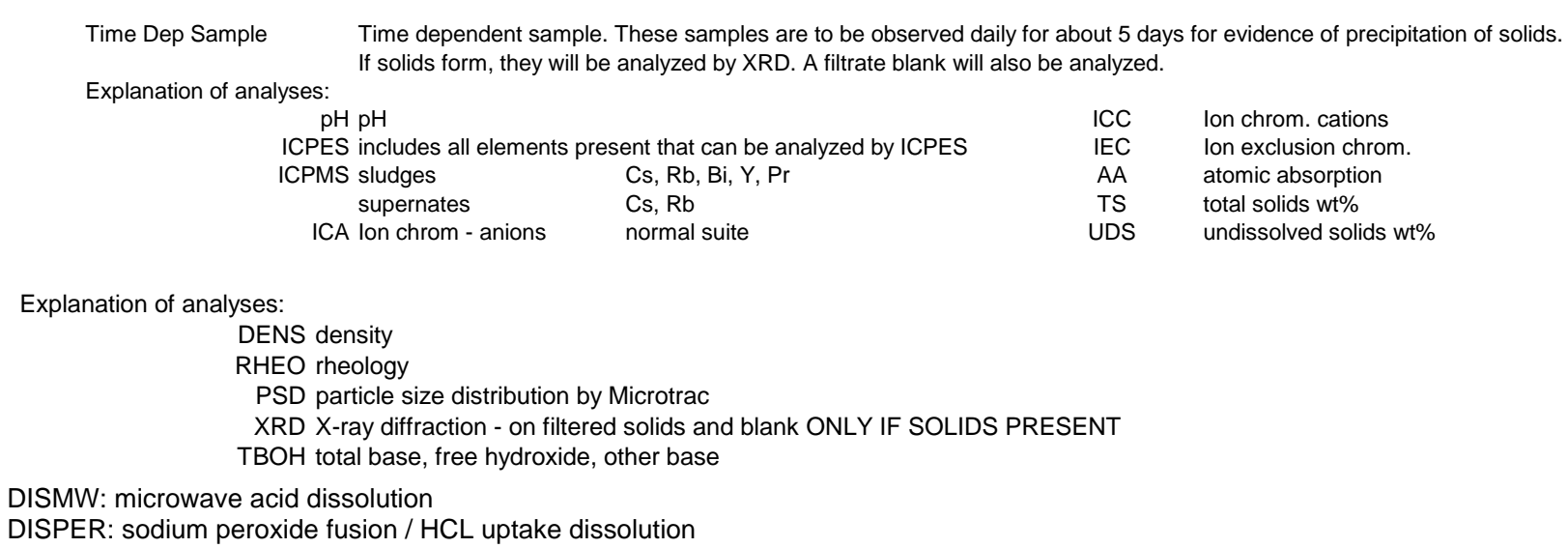


WSRC-TR-2005-00105, REVISION 0

SRNL-RPP-2005-00012, REVISION 0

\section{$\underline{\text { Note on Analysis of Sample Compositions }}$}

All analyses except total solids, undissolved solids, supernate solids, density, and rheological measurements were performed by the WSRC/SRNL Analytical Development Section per the task plan specifications. The aforementioned analyses were performed by the SRNL Immobilization Technology Section at the Aiken County Technology Laboratory. Table D1 shows the methods used for analysis of each of the species measured.

Table D1 Analysis Methods Used

\begin{tabular}{|c|c|c|}
\hline Analysis or Preparation & Analytical Method & Preparation Method \\
\hline Slurry Sample Dissolution & \multicolumn{2}{|c|}{$\begin{array}{c}\text { Microwave acid (MW) or } \\
\text { Sodium peroxide (PER) fusion/ HCl uptake }\end{array}$} \\
\hline $\begin{array}{l}\text { Ag, Al, B, Ba, Ca, Cd, Ce, Co, Cr, } \\
\text { Cu, Fe, La, Li, Mg, Mn, Mo, Nd, P, } \\
\text { Pb, Re, S, Si, Sr, Ti, W, Zn, Zr }\end{array}$ & ICPES & both \\
\hline $\mathrm{Na}$ & ICPES, also AA & MW \\
\hline $\mathrm{Ni}$ & ICPES & MW \\
\hline $\mathrm{B}$ & ICPES & PER \\
\hline $\mathrm{K}$ & AA, also ICPES & MW \\
\hline Cs & ICPMS, also AA & MW \\
\hline Bi, Pr, Rb, Y & ICPMS & MW \\
\hline $\begin{array}{r}\text { bromide, chloride, fluoride, formate, } \\
\text { nitrate, nitrite, phosphate, oxalate, } \\
\text { sulfate }\end{array}$ & Anion Chromatography (IC) & none \\
\hline acetate, citrate & Ion exclusion chromatography & none \\
\hline ammonium & Cation Chromatography & none \\
\hline $\begin{array}{r}\text { organic carbon, inorganic carbon } \\
\text { (TIC) }\end{array}$ & carbon analyzer & micronization \\
\hline "total” oxalate & $\begin{array}{l}\text { calculated from organic carbon } \\
\text { minus formate }\end{array}$ & none \\
\hline carbonate & $\begin{array}{l}\text { calculated from TIC, } \\
\text { also titration }\end{array}$ & none \\
\hline total base, free hydroxide, other base & titration & precipitation of carbonate \\
\hline total acid & titration & none \\
\hline $\begin{array}{r}\text { total solids, dissolved solids (solids } \\
\text { in supernate fraction of slurry) }\end{array}$ & halogen lamp solids analyzer & none \\
\hline undissolved solids & $\begin{array}{l}\text { calculated from total and supernate } \\
\text { solids }\end{array}$ & none \\
\hline density & $?$ & none \\
\hline rheology & Haake rheometer & none \\
\hline
\end{tabular}


WSRC-TR-2005-00105, REVISION 0

SRNL-RPP-2005-00012, REVISION 0

Table D2-1 Campaign I Sample Matrix (1 of 4 pages)

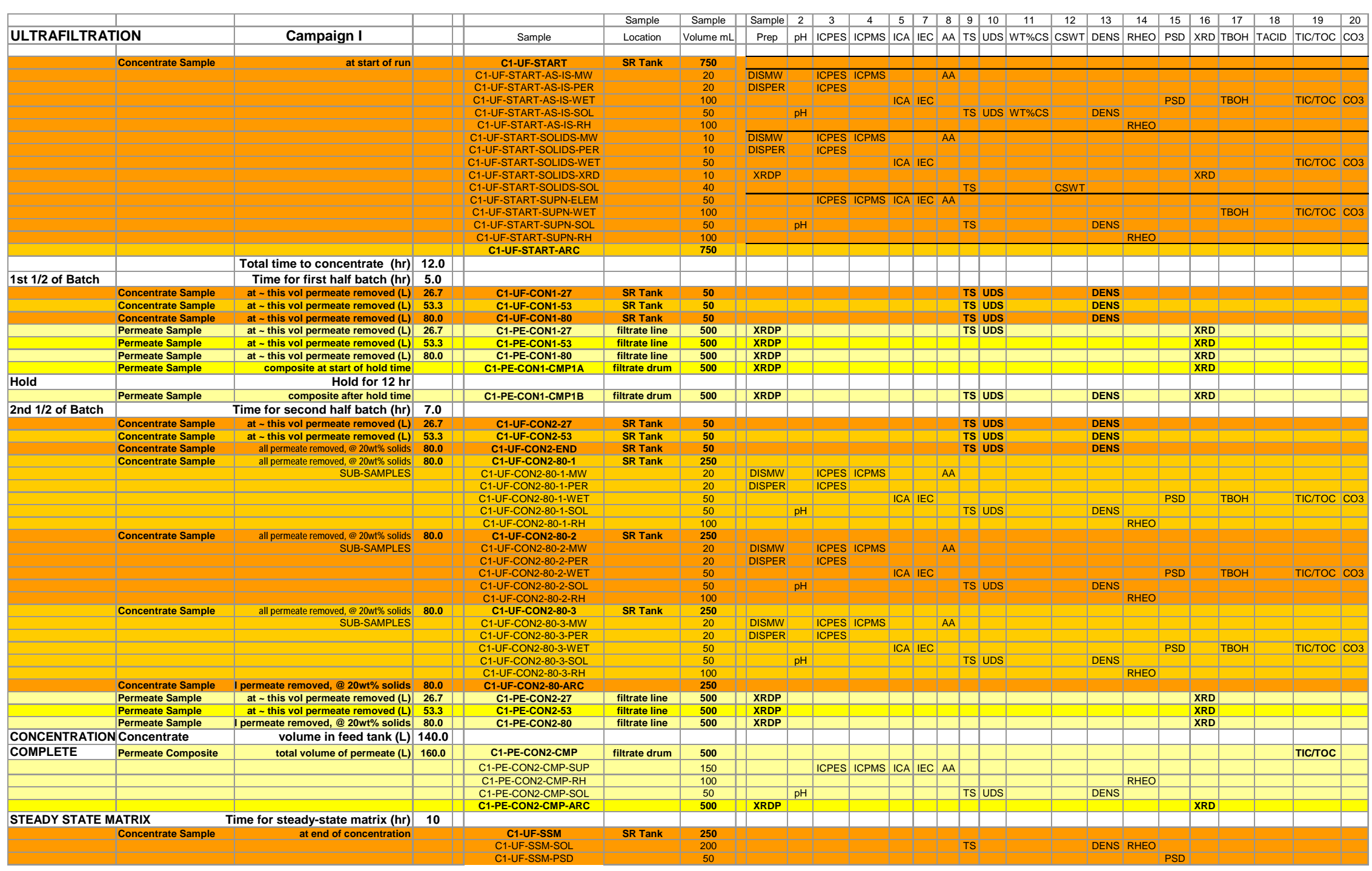


WSRC-TR-2005-00105, REVISION 0

SRNL-RPP-2005-00012, REVISION 0

Table D2-2 Campaign I Sample Matrix (2 of 4 pages)

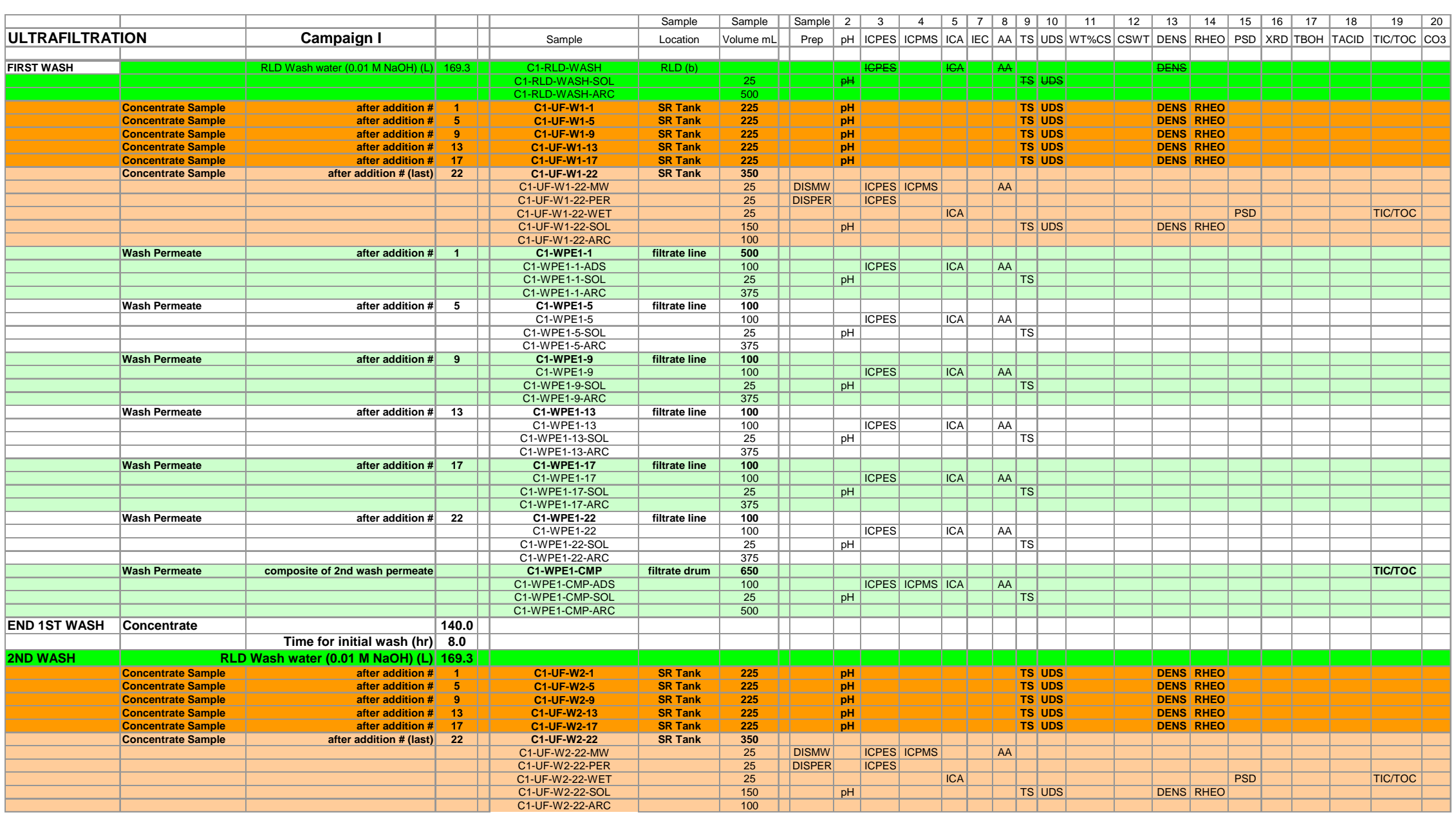


WSRC-TR-2005-00105, REVISION 0

SRNL-RPP-2005-00012, REVISION 0

\section{Table D2-3 Campaign I Sample Matrix (3 of 4 pages)}

\begin{tabular}{|c|c|c|c|c|c|c|c|c|c|c|c|c|c|c|c|c|c|c|c|c|c|c|c|c|c|}
\hline \multicolumn{2}{|c|}{ ULTRAFILTRATION } & Campaign I & & Sample & $\begin{array}{l}\text { Sample } \\
\text { Location }\end{array}$ & \begin{tabular}{|c|} 
Sample \\
Volume $\mathrm{mL}$
\end{tabular} & $\begin{array}{c}\text { Sample } \\
\text { Prep }\end{array}$ & \begin{tabular}{|c|}
2 \\
$p H$ \\
\end{tabular} & \begin{tabular}{|c|}
3 \\
ICPES
\end{tabular} & \begin{tabular}{|c|}
4 \\
ICPMS \\
\end{tabular} & \begin{tabular}{|c|}
5 \\
ICA \\
\end{tabular} & \begin{tabular}{|c|}
7 \\
IEC \\
\end{tabular} & \begin{tabular}{|l|}
8 \\
$A A$
\end{tabular} & \begin{tabular}{|c|}
9 \\
$\mathrm{TS}$ \\
\end{tabular} & \begin{tabular}{|l|}
10 \\
UDS \\
\end{tabular} & \begin{tabular}{|c|}
11 \\
WT\%CS \\
\end{tabular} & \begin{tabular}{|c|}
12 \\
CSWT
\end{tabular} & \begin{tabular}{|c|}
13 \\
DENS
\end{tabular} & \begin{tabular}{|c|}
14 \\
RHEO
\end{tabular} & $\begin{array}{l}15 \\
\text { PSD }\end{array}$ & $\begin{array}{l}16 \\
\text { XRD }\end{array}$ & $\begin{array}{c}17 \\
\text { TBOH }\end{array}$ & \begin{tabular}{|c|}
18 \\
TACID
\end{tabular} & \begin{tabular}{|c|}
19 \\
TICITOC
\end{tabular} & \begin{tabular}{|c|}
20 \\
$\mathrm{CO} 3$ \\
\end{tabular} \\
\hline & & & & & & & & & & & & & & & & & & & & & & & & & \\
\hline & Wash Permeate & after addition \# & 1 & $\begin{array}{l}\text { C1-WPE2-1 } \\
\text { C1-WPE21-ADS }\end{array}$ & filtrate line & 500 & & 一暂 & ICDEC & & ICA & & AA & $\square$ & & & & & & & & & & & \\
\hline & & & & C1-WPE2-1-SOL & & $\frac{100}{25}$ & & $\mathrm{pH}$ & ICPES & & $\mathrm{ICA}$ & & $M A$ & TS & & & & & & & & & & & \\
\hline & & & & C1-WPE2-1-ARC & & 375 & & & & & 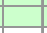 & $\square$ & & 20 & & & & & & & & & & & \\
\hline & Wash Permeate & after addition \# & 5 & $\begin{array}{l}\text { C1-WEE2-5 } \\
\text { C1-WPE2-5 }\end{array}$ & filtrate line & $\begin{array}{l}100 \\
100\end{array}$ & & & ICPES & & ICA & G & AA & 更 & - & & & & & 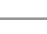 & & & & & \\
\hline & & & & $\begin{array}{l}\text { C1-WPE2-5-SOL } \\
\text { C1-WPE2-5ARC }\end{array}$ & & 25 & & $\mathrm{pH}$ & 40 & & 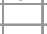 & $\square$ & 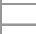 & TS & & & & & & & & & & & \\
\hline & Wash Permeate & after addition \# & 9 & $\begin{array}{l}\text { C1-WIEL-S-ARC } \\
\text { C1-WPE2-9 }\end{array}$ & filtrate line & 100 & - & 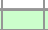 & & & & $\square$ & & $\square$ & $\square$ & & & & & & & & & & 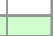 \\
\hline & & & & C1-WPE2-9 & & $\frac{100}{25}$ & & & ICPES & & ICA & & AA & & & & & & & & & & & & \\
\hline & & & & $\begin{array}{l}\text { C1-WPE2-9-SOL } \\
\text { C1-WPE-9RC }\end{array}$ & & $\begin{array}{r}25 \\
375\end{array}$ & & $\mathrm{pH}$ & & & & & & TS & & & & & & & & & & & \\
\hline & Wash Permeate & after addition \# & 13 & C1-WPE2-13 & filtrate line & 100 & & 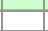 & & & & $\square$ & & $\square$ & & & & & & & & & & & \\
\hline & & & & C1-WPE2-13 & & 100 & & & ICPES & & ICA & $\square$ & AA & & & & & & & & & & & & \\
\hline & & & & $\begin{array}{l}\text { C1-WPEE-13-SOL } \\
\text { C1-WP2-13-ARC }\end{array}$ & & $\begin{array}{r}25 \\
375\end{array}$ & & $\mathrm{pH}$ & & & ( & $\square$ & & TS & & & & & & & & & & & \\
\hline & Wash Permeate & after addition \# & 17 & C1-WPE2-17 & filtrate line & 100 & & & & & & & & 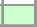 & & & & & & & & & & & \\
\hline & & & & C1-WPE2-17 & & 100 & & & ICPES & & ICA & & AA & & & & & & & & & & & & \\
\hline & & & & $\begin{array}{l}\text { C1-WPE2-17-SOL } \\
\text { C1-WP2-17-ARC }\end{array}$ & & $\frac{25}{375}$ & & $\mathrm{pH}$ & & & t & $\square$ & & TS & & & & & & & & & & & \\
\hline & Wash Permeate & after addition \# & 22 & C1-WPE2-22 & filtrate line & 100 & & 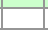 & & & & 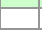 & & + & & & & & & & & & & & \\
\hline & & & & C1-WPE2-22 & & 100 & & & ICPES & & ICA & 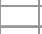 & AA & & & & & & & & & & & & \\
\hline & & & & $\begin{array}{l}\text { C1-WPE2-22-SOL } \\
\text { C1-WP2-22-ARC }\end{array}$ & & $\frac{25}{375}$ & & $\mathrm{pH}$ & & & & & & TS & & & & & & & & & & & \\
\hline & Wash Permeate & composite of 2 nd wash permeate & & $\begin{array}{l}\text { C1-WP2-22-ARC } \\
\text { C1-WPE2-CMP }\end{array}$ & filtrate drum & 375 & & 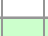 & & & & 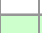 & & $\square$ & & & & & & & & & & TICTOCC & \\
\hline & & & & C1-WPE2-CMP-ADS & & 100 & & & ICPES & ICPMS & ICA & & AA & & & & & & & & & & & & \\
\hline & & & & $\begin{array}{l}\text { C1-WPE2-CMP-SOL } \\
\text { C1-WPE2-CMPARC }\end{array}$ & & $\begin{array}{r}25 \\
500\end{array}$ & & $\mathrm{pH}$ & & & t & $\square$ & & TS & & & & & & & & & & & \\
\hline \multirow{2}{*}{ END 2ND WASH } & & Time for final wash (hr) & 8.0 & & & & & & & & 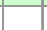 & & & 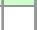 & & & & & & & & & & & \\
\hline & Concentrate & volume washed concentrate (L) & 140.0 & & & & & & & & 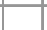 & 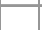 & & 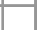 & & & & & & & & & & & \\
\hline \multirow[t]{5}{*}{ OVER-CONCENTR } & ATE & ime to remove for Max wt\% (L) & $?$ & & SR Tank & & & & & & t & L & 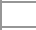 & L & & & & & & & & & & & \\
\hline & & Time to concentrate (hr) & 5.0 & & & & & & & & & & & 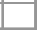 & & & & & & & & & & & \\
\hline & Concentrate & over-concentrated slurry & & C1-UF-OVER & SR Tank & 250 & & & & & 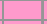 & 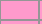 & & & & & & & & & & & & & \\
\hline & & & & & & 200 & & & & & & & & TS & & & & & RHEO & & & & & & \\
\hline & Permeate & & & $\begin{array}{l}\text { C1-UF-OVER-RARC } \\
\text { C1-PE-OVER-ARC }\end{array}$ & & $\begin{array}{r}50 \\
500\end{array}$ & & & & & 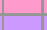 & 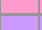 & & 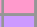 & $=$ & & & & & PSD & & & & & \\
\hline \multirow{21}{*}{ CLEANING } & $\mathrm{NaOH}$ & $50 \mathrm{wt} \% \mathrm{NaOH}, \mathrm{L}$ & 0.52 & CA-Te-UVER-ATR & & 300 & & 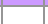 & & & & - & & & & & & & & & & & & & \\
\hline & RLD Dil $\mathrm{H} 2 \mathrm{O}$ for $\mathrm{NaOH}$ & volume needed (L) & 100 & & & & & & & & & & & 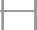 & 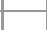 & & & & & & & & & & \\
\hline & & Time to rinse UF (hr) & 0.5 & & & & & & & & & & & & & & & & & & & & & & \\
\hline & Rinse water $(0.1 \mathrm{M} \mathrm{NaOH})$ & after rinse \& after dilution for $(\mathrm{L})$ & & C1-CAUU-1 & drum & 500 & SMW & & & & & & & & & & & & & & & & & & \\
\hline & & & 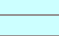 & $\begin{array}{l}\text { C1-CAUUU-1-MW } \\
\text { C1-CAU-1-PER }\end{array}$ & & $\begin{array}{l}\frac{100}{100} \\
\end{array}$ & $\begin{array}{l}\text { DISMW } \\
\text { DISPER }\end{array}$ & + & $\begin{array}{l}\text { ICPES } \\
\text { ICPES }\end{array}$ & ICPMS & & $\square$ & AA & 車 & - & & & & & & & & & & 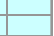 \\
\hline & & & & C1-CAUU-1-WET & & 100 & & & & & ICA & $\square$ & & 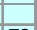 & & & & & & & & & & & \\
\hline & & & & & & 100 & & pH & & & & & & TS & UDS & & & DENS & & & & & & & \\
\hline & & & & C1-CAUU-1-ARC & & 500 & & & & & & & & -5 & & & & & & & & & & & \\
\hline & & $2 \mathrm{M} \mathrm{HNO3}$ cleaning (hr) & 1.5 & & & & & & & & & & & 工 & & & & & & & & & & & \\
\hline & 2M HNO3 clean soln & after cleaning \& after dilution for $(L)$ & 100 & C1-NITU-1 & drum & 350 & & & & & & & & & & & & & & & & & \multirow{4}{*}{ TACID } & & \\
\hline & & correction of SIPP/WTP scale & & $\begin{array}{l}\text { C1-NITU-1-SOL } \\
\text { C1NITU-1-WET }\end{array}$ & & $\begin{array}{l}50 \\
50\end{array}$ & & - & & & & 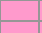 & & TS & UDS & & & DENS & & & & & & & \\
\hline & & correction or SipPIWIP scale & & $\begin{array}{l}\text { C1-NITU-1-WET } \\
\text { C1-NITU-1-ARC }\end{array}$ & & $\begin{array}{l}50 \\
150\end{array}$ & & & & & 至 & - & & 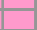 & & & & & & & & & & & \\
\hline & & & & C1-NITU-1-CMP & & 500 & & & & & & & & & & & & & & & & & & & \\
\hline & & $2 \mathrm{M} \mathrm{HNO3}$ cleaning $(\mathrm{hr})$ & 1.5 & & & & & & & & & & & & & & & & & & & & & & \\
\hline & 2M HNO3 clean soln & after cleaning \& after dilution for $(L)$ & 100 & $\begin{array}{l}\text { C1-NITU-2 } \\
\text { C1-NUT-2SO }\end{array}$ & drum & 350 & & & & & & & & TS & UDS & & & DENS & & & & & \multirow{3}{*}{ TACID } & & \\
\hline & & correction of SIPP/WTP scale & & C1-NITU-2-WET & & 50 & & & & & 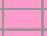 & 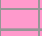 & & & 000 & & & DEIVS & & & & & & & \\
\hline & & & & $\begin{array}{l}\text { C1-NITU-2-ARC } \\
\text { C1-NITU-2-CMP }\end{array}$ & & $\begin{array}{l}150 \\
500\end{array}$ & & & & & & & & & & & & & & & & & & & \\
\hline & 2M HNO3 clean soln & $\begin{array}{l}2 \mathrm{M} \mathrm{HNO} 3 \text { cleaning (hr) } \\
\text { after cleaning \& atter dilution for ( (I) }\end{array}$ & $\begin{array}{l}1.5 \\
100\end{array}$ & C1-NITU-3 & drum & 350 & & & & & & & & & & & & & & & & & & & \\
\hline & & 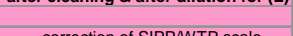 & & C1-NITU-3-SOL & - & 50 & & & & & & & & TS & UDS & & & DENS & & & & & \multirow{3}{*}{ TACID } & & \\
\hline & & correction of SIPP/WTP scale & & $\begin{array}{l}\text { C1-NIIU-3-WET } \\
\text { C1-NIU-3-ARC }\end{array}$ & & $\begin{array}{l}50 \\
150\end{array}$ & & & & & & & & & & & & & & & & & & & \\
\hline & & & & C1-NITU-3-CMP & & 500 & & & & & & & & & & & & & & & & & & & \\
\hline
\end{tabular}


WSRC-TR-2005-00105, REVISION 0

SRNL-RPP-2005-00012, REVISION 0

Table D2-4 Campaign I Sample Matrix (4 of 4 pages)

\begin{tabular}{|c|c|c|c|c|c|c|c|c|c|c|c|c|c|c|c|c|c|c|c|c|c|c|c|c|c|}
\hline \multirow{2}{*}{\multicolumn{2}{|c|}{\begin{tabular}{|l|} 
ULTRAFILTRATION \\
\end{tabular}}} & Campaign I & & Sample & $\begin{array}{l}\text { Sample } \\
\text { Location }\end{array}$ & \begin{tabular}{|l|} 
Sample \\
Volume $\mathrm{mL}$
\end{tabular} & \begin{tabular}{|l|} 
Sample \\
Prep
\end{tabular} & \begin{tabular}{|c|}
2 \\
$\mathrm{pH}$ \\
\end{tabular} & \begin{tabular}{|c|}
3 \\
ICPES
\end{tabular} & \begin{tabular}{|c|}
4 \\
ICPMS
\end{tabular} & & \begin{tabular}{|l|}
7 \\
IEC
\end{tabular} & \begin{tabular}{|c|}
8 \\
AA \\
\end{tabular} & \begin{tabular}{|l|}
9 \\
$\mathrm{TS}$ \\
\end{tabular} & \begin{tabular}{|l|}
10 \\
UDS \\
\end{tabular} & $\begin{array}{c}\frac{11}{W T \% C S} \\
W\end{array}$ & \begin{tabular}{|c|}
12 \\
$\operatorname{CSWT}$
\end{tabular} & \begin{tabular}{|c|}
13 \\
DENS
\end{tabular} & \begin{tabular}{|c|}
14 \\
RHEO
\end{tabular} & \begin{tabular}{|c|}
15 \\
PSD \\
\end{tabular} & \begin{tabular}{|c|}
16 \\
$X R D$
\end{tabular} & \begin{tabular}{|c|}
17 \\
TBOH \\
\end{tabular} & \begin{tabular}{|c|}
18 \\
TACID
\end{tabular} & \begin{tabular}{|c|}
19 \\
TIC/TOC
\end{tabular} & \begin{tabular}{|l|}
20 \\
$\mathrm{CO} 3$ \\
\end{tabular} \\
\hline & & & & & & & & & & & & & & & & & & & & & & & & & \\
\hline & $2 \mathrm{MHNO} 3$ cleaning solu & composite & & $\begin{array}{l}\text { C1-NIIU-C } \\
\text { C1-NITU-C-MW }\end{array}$ & $\begin{array}{c}\text { composite } \\
\text { bottles }\end{array}$ & $\begin{array}{l}500 \\
100\end{array}$ & DISMW & & & ICPMS & & & $A A$ & & & & & & & & & & & & \\
\hline & & & & $\begin{array}{l}\text { C1-NITU-C-PER } \\
\text { C1-NIT-C-SOL }\end{array}$ & & $\begin{array}{l}100 \\
100\end{array}$ & DISPER & & ICPES & & & & & & UDS & & & DENS & & & & & & & \\
\hline & & & & C1-NITU-C-WET & & 100 & & & & & & & & & & & & DEIVS & & & & & TACID & & \\
\hline & Add $\mathrm{NaOH}$ & $50 \mathrm{wt} \% \mathrm{NaOH}, \mathrm{L}$ & 0.52 & C1-NITU-C-ARC & & & & & & & & & & & & & & & & & & & & & \\
\hline & RLD Dil $\mathrm{H} 2 \mathrm{O}$ for $\mathrm{NaOH}$ & volume needed (L) & 100 & & & & & & & & & & & & & & & & & & & & & & \\
\hline & & Time to rinse UF (hr) & 0.5 & & & & & & & & & & & & & & & & & & & & & & \\
\hline & Rinse water (0.1M NaOH) & after rinse & & C1-CAUU-2 & drum & 500 & & & & & & & & & & & & & & & & & & & \\
\hline & & & & C1-CAUU-2-SOL & & 100 & & $\mathrm{pH}$ & & & & & & TS & UDS & & & DENS & & & & & & & - \\
\hline & & & & C1-CAUU-2-CMP & & 400 & & & & & & & & & & & & & & & & & & & \\
\hline & Add $\mathrm{NaOH}$ & 50 wt $\%$ NaOH, L & 0.52 & & & & & & & & 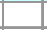 & & 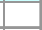 & 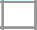 & 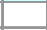 & & & & & & & & & & \\
\hline & RLD Dil $\mathrm{H} 2 \mathrm{O}$ for $\mathrm{NaOH}$ & volume needed (L) & 100 & & & & & & & & & & & & & & & & & & & & & & \\
\hline & & Time to rinse UF (hr) & 0.5 & & & & & & & & & & 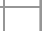 & 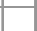 & & & & & & & & & & & \\
\hline & Rinse water (0.1M NaOH) & after rinse \& after dilution for $(L)$ & & & drum & 500 & & & & & & & & & & & & & & & & & & & \\
\hline & & & & $\begin{array}{l}\text { C1-CAUU-3-SOL } \\
\text { C1-CAUU-3MP }\end{array}$ & & 100 & & $\mathrm{pH}$ & & & & & - & TS & UDS & & & DENS & & & & & & & - \\
\hline & Rinse water composite & (post-HNO3 rinses only) & & C1-CAUU- $-2+3$ & composite & $\begin{array}{l}400 \\
500\end{array}$ & & & & & & & & & & & & & & & & & & & \\
\hline & & & & $\begin{array}{l}\text { C1-CAUU-2+3-MW } \\
\text { C1-CAUU-2+3PRR }\end{array}$ & $\begin{array}{l}\text { bottles } \\
2 \& 3\end{array}$ & $\begin{array}{l}100 \\
100\end{array}$ & $\begin{array}{l}\text { DISMW } \\
\text { DISPER }\end{array}$ & & ICPES & ICPMS & & & AA & & & & & & & & & & & & \\
\hline & & & & $\begin{array}{l}\text { C1-ACAUU-2+3-PR } \\
\text { C1-CAUU-2+3-WET }\end{array}$ & $2 \& 3$ & $\begin{array}{l}100 \\
100\end{array}$ & & & & & ICA & & & & & & & & & & & & & & \\
\hline & & & & $\begin{array}{l}\text { C1-CAUU-2+3-SOL } \\
\text { CI-CAUU-2+3-ARC }\end{array}$ & & $\begin{array}{l}100 \\
100\end{array}$ & & $\mathrm{pH}$ & & & & & & TS & UDS & & & DENS & & & & & & & \\
\hline
\end{tabular}


WSRC-TR-2005-00105, REVISION 0

SRNL-RPP-2005-00012, REVISION 0

Table D3-1 Campaign II Sample Matrix (1 of 7 pages)

\begin{tabular}{|c|c|c|c|c|c|c|c|c|c|c|c|c|c|c|c|c|c|c|c|c|c|c|}
\hline & & & Sample & $\begin{array}{l}\text { ADS } \\
\text { Travel Copy } \\
\end{array}$ & $\begin{array}{c}\text { Sample } \\
\text { Location }\end{array}$ & $\begin{array}{c}\text { Sample } \\
\text { Volume } \mathrm{mL}\end{array}$ & $\begin{array}{c}\text { Sample } \\
\text { Prep } \\
\end{array}$ & \begin{tabular}{c|c}
2 \\
$\mathrm{pH}$
\end{tabular} & \begin{tabular}{|l}
3 \\
ICPES \\
\end{tabular} & $\begin{array}{l}4 \\
\text { ICPMS } \\
\end{array}$ & \begin{tabular}{|c|}
5 \\
ICA \\
\end{tabular} & $\begin{array}{c}7 \\
\text { IEC } \\
\end{array}$ & \begin{tabular}{|l|l|}
8 & \\
$A A$
\end{tabular} & \begin{tabular}{|l|l|}
9 \\
TS \\
\end{tabular} & \begin{tabular}{|l|}
10 \\
UDS \\
\end{tabular} & \begin{tabular}{|l}
13 \\
DENS \\
\end{tabular} & $\begin{array}{c}14 \\
\text { RHEO } \\
\end{array}$ & \begin{tabular}{|l|l|}
15 \\
PSD
\end{tabular} & \begin{tabular}{|c|}
16 \\
XRD \\
\end{tabular} & \begin{tabular}{|l|l}
17 \\
TBOH
\end{tabular} & \begin{tabular}{|l}
18 \\
TACID \\
\end{tabular} & $\begin{array}{l}19 \\
\text { TICTOCC } \\
\end{array}$ \\
\hline RECYCLE BLENDING & & Campaign I UF Wash Water & $\begin{array}{l}\text { Previuusly analyeded } \\
\text { C1-WPE1 \& C1-WPE2 }\end{array}$ & & & & & & & & & & & & & & & & & & & \\
\hline & & HLW SBS & $\begin{array}{l}\text { Previously analyzed: } \\
\text { HLW-SBS-QUENCHER }\end{array}$ & & & & & & & & & & & & & & & & & & & \\
\hline & & $\begin{array}{l}\text { HLW Vessel event (PVP) } \\
\text { HLW Canister Decon }\end{array}$ & $\begin{array}{l}\text { Synthetic c no analysis } \\
\text { Synthetic - no analysis }\end{array}$ & & & & & & & & & & & & & & & & & & & \\
\hline & & $\begin{array}{l}\text { Campaing I Caustic F Fushes } \\
\text { Campaign IUF Acid Cleanings }\end{array}$ & $\begin{array}{l}\text { Previously analyzed } \\
\text { Previousy analyzed } \\
\end{array}$ & & & & \multirow{2}{*}{\multicolumn{10}{|c|}{$\begin{array}{l}\text { XRD analyses only required if solids precipitate from solutions that } \\
\text { should have no undissolved solids. } \\
\text { cost based on } 8 \text { IX samnles ner cucle: cost will increase }\end{array}$}} & & & & & & \\
\hline & Campaign Ic & 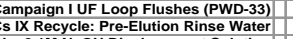 & $\begin{array}{l}\text { see below } \\
\text { NA }\end{array}$ & & & & & & & & & & & & & & & & & & & \\
\hline & Campaign I Cs IX Recyc & $\begin{array}{c}\text { E: } 0.1 \mathrm{M} \mathrm{NaOH} \text { Displacement Solution } \\
\text { COMBIEED RECYCLS }\end{array}$ & $\begin{array}{l}\text { NA } \\
\text { see below } \\
\end{array}$ & & & & & & & & & & & 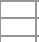 & & & & & & & & \\
\hline & PWD (doe & $\begin{array}{l}\text { es not include NaOH to neturalize HNO3) } \\
\text { Generation of Synthetic Recycles } \\
\text { HLW Canister Decon }\end{array}$ & & & & & & 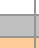 & & & & & & & & & & & & & & \\
\hline & & $\begin{array}{r}\text { DI water } \\
\mathrm{NaOH} 50 \mathrm{w} \text { t\% }(\mathrm{L})\end{array}$ & & & & & & 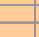 & & & & & & & & & & & & & & \\
\hline & & HLW Vessel Vent (PVP) $\operatorname{NaNO3(\mathrm {kg})}$ & & & & & & 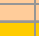 & & & & 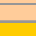 & & & & & & & & & & \\
\hline & & $\begin{array}{r}\text { D water } \\
\text { NaOH 5ow0\% (L) }\end{array}$ & & & & & & - & & & & - & Z & & & & & & & & & \\
\hline & & $\begin{array}{l}\text { Combined Canister Decon + PVP } \\
\text { UF Loop Flush }\end{array}$ & $\begin{array}{l}\text { C2-PWD-HLWRECS } \\
\text { C2-PWD-LOOPRINACTL }\end{array}$ & $\begin{array}{l}\text { ARCHIVE } \\
\text { NONE }\end{array}$ & $\begin{array}{l}\text { mixing tank } \\
\text { carboy }\end{array}$ & 50 & & - & & & & E & 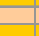 & TS & uDs & DENS & & & & & & \\
\hline & Add Campais & $\begin{array}{l}\text { Add } 0.01 \mathrm{~N} \text { NaOH to a carboy } \\
\text { gn I IF washed sludge product (20 w\%o) }\end{array}$ & & & & & & - & & & -3 & -2 & 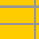 & 10 & 000 & DENS & & & & & & \\
\hline & & Combining of Recycles Sum & & & & & & & & & & & & & & & & & & & & \\
\hline & PWD-44 & W Canister Decon + PVP water to a tank & $\begin{array}{l}\text { C2-PWD-44-ACTL } \\
\text { C2-PWD-44-ARC }\end{array}$ & $\begin{array}{l}\text { NONE } \\
\text { ARCHVE }\end{array}$ & mixing tank & $\begin{array}{r}50 \\
250\end{array}$ & & $\mathrm{pH}$ & & & 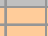 & 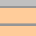 & 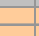 & TS & UDS & DENS & & & & & & \\
\hline & & $\begin{array}{l}\text { LW Canister Decon + PVP 50w\% NaOH } \\
\text { Add HLW Canister Decon NavO3 (kg) }\end{array}$ & & & & & & & & & & 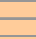 & 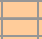 & 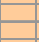 & & & & & & & & \\
\hline & & $\begin{array}{l}\text { Add HLW SSS to tank wih mixing } \\
\text { IU Loop Flushes (PWD-33) with mixing }\end{array}$ & & & & & & & & & & & 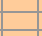 & & & & & & & & & \\
\hline & $\begin{array}{l}\text { PWD-15 } \\
\text { Add Campaign I Wash Permea }\end{array}$ & ate to a tank (use 2nd drum, $-0.05 \mathrm{M} \mathrm{OH}$ ) & $\begin{array}{l}\text { C2-PWD-15-ACTL } \\
\text { C2-PWD-15ARC }\end{array}$ & $\begin{array}{l}\text { NONE } \\
\text { ARCHIVE }\end{array}$ & mixing tank & $\begin{array}{l}50 \\
250\end{array}$ & & $\mathrm{pH}$ & & & 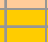 & 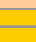 & - & TS & UDS & DENS & & & & & & \\
\hline & $\begin{array}{l}\text { Add from Camp } \\
\text { Add from Camp } \\
\text { Add from Camp }\end{array}$ & 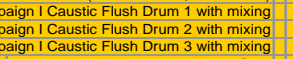 & & & & & & & & & & & E & & & & & & & & & \\
\hline & PWD-16 & Sum & C2-PWD-16-ACTL & NONE & mixing tank & 50 & & $\mathrm{pH}$ & & & - & 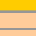 & - & TS & UDS & DENS & & 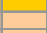 & & & & \\
\hline & Add Campaign I Wash Perm & neate to a tank (use 1st d drum, - $-1.1 \mathrm{MOH}$ ) & C2-PWD-16-ARC & ARCHIVE & & & & & & & & & & & & & & & & & & \\
\hline & $\begin{array}{l}\text { Add from Campaign I } \\
\text { Add from Campain I }\end{array}$ & I Iitric Acid Cleaning Drum 1 with mixing & & & & & & & & & & 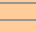 & 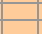 & 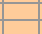 & & & & & & & & \\
\hline & Add from Campaign I & Nitric Acid Cleaning Drum 3 with mixing & & & & & & & & & & 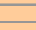 & E & - & & & & & & & & \\
\hline & Combine Recycles in (F & :EP Feed) Tank & C2-FEP-FEED-ACTL & NONE & mixing tank & 50 & & $\mathrm{pH}$ & & & & 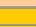 & 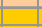 & TS & UDS & DENS & & - & & & & \\
\hline & & Add PWD-44 to tank with mixing & $\begin{array}{l}\text { C2-FEP-FEED-WET } \\
\text { C2-EP-FEF-MW }\end{array}$ & 41335 & & 100 & DISWW & & JCPES & ICPMS & ICA & & $A A$ & & & & & & & ТBOH & & TICITOC \\
\hline & & Add PWD-16 to tank with mixing & $\begin{array}{l}\text { C-2EPPFEDPER } \\
\text { C-FEP }\end{array}$ & 41337 & & 100 & DISPER & & ICPES & Arive & & -2 & 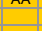 & ב & & & & & & & & \\
\hline FEP EVAPORATOR & & Starting Volume & & & & & & & & & 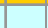 & 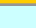 & & & & & & & & & & \\
\hline & Bottoms & After all antifoam additions & $\begin{array}{l}\text { C2-FEP-B-START-ATL } \\
\text { C2-EFP-BSTAT-ANTF }\end{array}$ & $\begin{array}{l}\text { NNNE } \\
\text { NONE } \\
\end{array}$ & $\begin{array}{l}\text { bottoms tank } \\
\text { bottoms tank }\end{array}$ & $\begin{array}{l}60 \\
100\end{array}$ & & 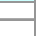 & & & 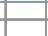 & 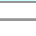 & a & TS & UDS & DENS & RHEO & & & & & \\
\hline & Bottoms & At -200 L evaporated & $\begin{array}{l}\text { C2-FEP-B-START-ARC } \\
\text { C2-EPP-B-A200-ATL }\end{array}$ & $\begin{array}{l}\text { ARCHIVE } \\
\text { NONE }\end{array}$ & $\begin{array}{l}\text { bottoms tank } \\
\text { bottoms tank }\end{array}$ & $\frac{100}{20}$ & & - & & & & - & 4 & TS & UDS & DENS & & - & & & & \\
\hline & & & C2-FEP-B-200-WET & 41338 & bottoms tank & 30 & & E & ICPES & & ICA & 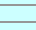 & AA & 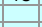 & & & & & & & & \\
\hline & Primary Condensate & & $\begin{array}{l}\text { C2-FPPB-B-200-ARC } \\
\text { C2-FEP-PC-200-ARC }\end{array}$ & $\begin{array}{l}\text { ARCHVE } \\
\text { ARCHIVE }\end{array}$ & & $\begin{array}{l}100 \\
100\end{array}$ & & 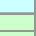 & & & 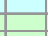 & 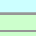 & $\theta$ & 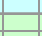 & & & & 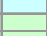 & & & & \\
\hline & $\begin{array}{l}\text { Secondary Condensate } \\
\text { Bottoms }\end{array}$ & At $\sim 400 \mathrm{~L}$ evaporated & $\begin{array}{l}\text { 22-2ERP-SC-200-AAC } \\
\text { C2-FEP-B-A00-ATLL }\end{array}$ & $\begin{array}{l}\text { ARCHVE } \\
\text { NONE }\end{array}$ & bottoms tank & $\frac{100}{20}$ & & 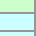 & & & 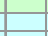 & 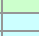 & 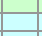 & TS & UDS & DENS & & & & & & \\
\hline & Primary Condensate & & $\begin{array}{l}\text { C2-EPPB-B-400-ARC } \\
\text { C2-FEP-PC-40-ARC }\end{array}$ & $\begin{array}{l}\text { ARCHVE } \\
\text { ARCHIVE }\end{array}$ & bottoms lank & $\begin{array}{l}100 \\
100\end{array}$ & & 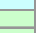 & & & $\square$ & 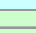 & $\theta$ & + & 至 & 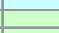 & & & & & & \\
\hline & $\begin{array}{l}\text { Secondary Condensate } \\
\text { Bottoms }\end{array}$ & At $-600 \mathrm{~L}$ evaporated & $\begin{array}{l}\text { 2C-2ERP-S-S-400-AAC } \\
\text { C2-FEP-B-60-ACTL }\end{array}$ & $\begin{array}{l}\text { ARCHVE } \\
\text { NONE } \\
\text { NONE }\end{array}$ & bottoms tank & $\begin{array}{l}100 \\
20\end{array}$ & & 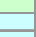 & & & & 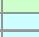 & - & TS & UDS & DENS & & & & & & \\
\hline & Primary Condensate & & 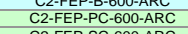 & ARCHIVE & & 100 & & 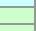 & & & 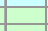 & - & 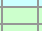 & 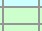 & 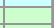 & & & 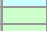 & & & & \\
\hline & Bottoms & At -800 L evaporated & $\begin{array}{l}\text { 2-2-EP-SC-600-ARC } \\
\text { C2-FPE-B-B00-ACTL }\end{array}$ & NONE & bottoms tank & $\begin{array}{l}100 \\
20 \\
20\end{array}$ & & & & & & & 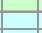 & TS & UDS & DENS & & & & & & \\
\hline & Primary Condensate & & $\begin{array}{l}\text { C2-2EPPB-8-800-ARC } \\
\text { C2-FEP-PC-800-ARC }\end{array}$ & $\begin{array}{l}\text { ARCHVE } \\
\text { ARCHIVE }\end{array}$ & bottoms tank & $\begin{array}{l}100 \\
100\end{array}$ & & & & & & 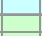 & - & & & & & & & & & \\
\hline RUSH & $\begin{array}{l}\text { Secondary Condensate } \\
\text { Bottoms }\end{array}$ & Evaporation completed & $\begin{array}{l}\text { 22-FEPPSC-800-ARC } \\
\text { C2-FEP-B-END-AOL }\end{array}$ & $\begin{array}{l}\text { ARCHIIE } \\
41339\end{array}$ & bottoms tank & $\frac{100}{60}$ & & $\mathrm{pH}$ & & & 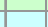 & - & 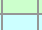 & TS & uDs & DENS & & & & & & \\
\hline & & & C2-FEP-B-END-ACTL & NONE & $\begin{array}{l}\text { bottoms tank } \\
\text { botromstank }\end{array}$ & 60 & & & & & $10 \mathrm{~A}$ & & & TS & UDS & DENS & RHEO & PSD & & $\mathrm{TBOH}$ & & 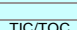 \\
\hline RUSH & & & $\begin{array}{l}\text { C2-FEP-BEND-WE } \\
\text { C2-FEP-B-END-SUP }\end{array}$ & 41341 & $\begin{array}{l}\text { bottioms tank } \\
\text { bottoms tank }\end{array}$ & 10 & FLTTER & - & ICPES & & in & - & AA & & & & & & & & & \\
\hline & & & $\begin{array}{l}\text { 2C-2EPPB-BENDD-MW } \\
\text { C2-FEP-B-END-PER }\end{array}$ & $\begin{array}{l}41342 \\
41343\end{array}$ & $\begin{array}{l}\text { bottoms tank } \\
\text { bottoms tank }\end{array}$ & $\begin{array}{l}25 \\
25 \\
25\end{array}$ & $\begin{array}{l}\text { DISSW } \\
\text { DISPER }\end{array}$ & & $\begin{array}{l}\text { ICPES } \\
\text { ICPES } \\
\end{array}$ & ICPMS & & & AA & & & & & & & & & \\
\hline
\end{tabular}


WSRC-TR-2005-00105, REVISION 0

SRNL-RPP-2005-00012, REVISION 0

Table D3-2 Campaign II Sample Matrix (2 of 7 pages)

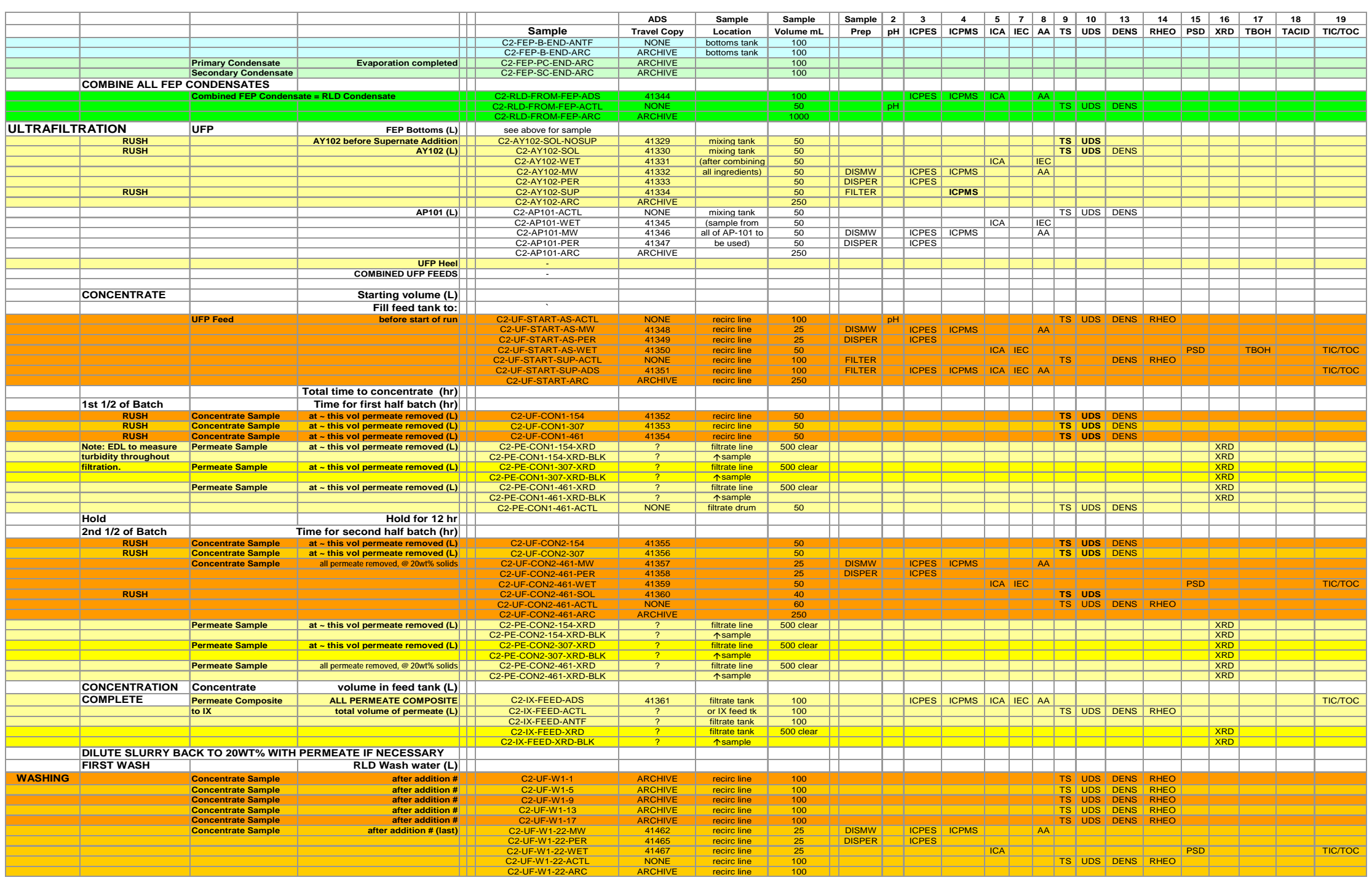


WSRC-TR-2005-00105, REVISION 0

SRNL-RPP-2005-00012, REVISION 0

Table D3-3 Campaign II Sample Matrix (3 of 7 pages)

\begin{tabular}{|c|c|c|c|c|c|c|c|c|c|c|c|c|c|c|c|c|c|c|c|c|c|c|c|}
\hline & & & & Sample & $\begin{array}{l}\text { ADS } \\
\text { Travel Copy }\end{array}$ & $\begin{array}{l}\text { Sample } \\
\text { Location }\end{array}$ & $\begin{array}{l}\text { Sample } \\
\text { Volume } \mathrm{mL}\end{array}$ & $\begin{array}{c}\text { Sample } \\
\text { Prep }\end{array}$ & $\frac{2}{\mathrm{pH}}$ & \begin{tabular}{|c|}
3 \\
ICPES \\
\end{tabular} & $\begin{array}{c}4 \\
\text { ICPMS } \\
\end{array}$ & \begin{tabular}{|c|}
5 \\
ICA \\
\end{tabular} & \begin{tabular}{|c|}
7 \\
IEC
\end{tabular} & $\begin{array}{c}8 \\
\mathrm{AA} \\
\end{array}$ & $\begin{array}{l}9 \\
\text { TS } \\
\end{array}$ & $\frac{10}{\text { UDS }}$ & \begin{tabular}{|l}
13 \\
DENS
\end{tabular} & \begin{tabular}{|c|}
14 \\
RHEO
\end{tabular} & \begin{tabular}{|l|l|}
15 \\
PSD
\end{tabular} & $\begin{array}{c}16 \\
\mathrm{XRD}\end{array}$ & $\begin{array}{l}17 \\
\mathrm{TBOH}\end{array}$ & $\begin{array}{c}18 \\
\text { TACID }\end{array}$ & \begin{tabular}{|l|l|}
19 \\
TICTIOC
\end{tabular} \\
\hline & & Wash Permeate & after addition \# & $\begin{array}{l}\text { C2-WPE1-1-ACTL } \\
\text { C2-WPE1-1-XRD }\end{array}$ & NONE & $\begin{array}{l}\text { tiltrate line } \\
\text { filtrate line }\end{array}$ & 25 & & & & & & & & & & & & & XRD & & & \\
\hline & & Wash Permeate & after addition \# & $\begin{array}{l}\text { C2-WPE1-1.XRDD-BLK } \\
\text { C-WDE1-5.ATL }\end{array}$ & NONE & $\begin{array}{l}\text { Titsample } \\
\text { fitrate line }\end{array}$ & 25 & & & & & & & & TS & & & & & XRD & & & \\
\hline & & & & $\begin{array}{l}\text { C2-WPE1-5-XRD } \\
\text { C2-WPE1-5RD-BLK }\end{array}$ & $?$ & $\begin{array}{l}\text { Muthate ine } \\
\text { filtrate line }\end{array}$ & 500 clear & & & & & & & & 15 & & & & & XRD & & & \\
\hline & & Wash Permeate & after addition \# & 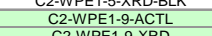 & NONE & $\begin{array}{l}\text { Tisample } \\
\text { filtrate line }\end{array}$ & 25 & & 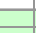 & & & & 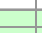 & 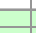 & TS & & & & & XRD & & & \\
\hline & & & ottordition & C2-WPE1-9-XRD-BLK & $?$ & $\begin{array}{l}\text { Thluale ine } \\
\text { Ts sample }\end{array}$ & & & & & & & & & & & & & & $\begin{array}{l}X R D \\
X R D\end{array}$ & & & \\
\hline & & Wash Permeate & after addition \# & C2-WPE1-13-XRD & NONE & $\begin{array}{l}\text { Intrale ine } \\
\text { filtrate line } \\
\end{array}$ & 500 clear & & & & & & - & 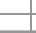 & TS & & & & & XRD & & & \\
\hline & & Wash Permeate & after addition \# & $\begin{array}{l}\text { C2-WEP1-13-XRDD-BLK } \\
\text { C2-WPE1-17-ACTL }\end{array}$ & NONE & $\begin{array}{l}\text { ssample } \\
\text { filtrate line }\end{array}$ & 25 & & & & & & & & TS & & & & & XRD & & & \\
\hline & & & & $\begin{array}{l}\text { C2-WPE1-17-XRD } \\
\text { C2-WP1-17.XR-BLK }\end{array}$ & & filtrate line & 500 clear & & & & & & & & 10 & & & & & XRD & & & \\
\hline & & Wash Permeate & after addition \# & C2-WPE1-22-ACTL & NONE & filtrate line & 25 & & & & & & & & TS & & & & & & & & \\
\hline & & & & $\begin{array}{l}\text { C2-WPE1-2-2-XRD } \\
\text { C2-WPE1-22-XRD-BLK }\end{array}$ & $?$ & $\begin{array}{l}\text { filtrate line } \\
\uparrow \text { sample }\end{array}$ & 500 clear & & & & & & & & & & & & & $\begin{array}{l}\text { XRD } \\
\text { XRD }\end{array}$ & & & \\
\hline & & Wash Permeate & $\begin{array}{l}-40 \text { minutes after last sample } \\
\text { m runnining with permeate i in recycycele }\end{array}$ & $\begin{array}{l}\text { C2-WPE1-END-ACTL } \\
\text { C2-WPE1-END-XRD }\end{array}$ & NONE & $\begin{array}{l}\text { filtrate line } \\
\text { fitrate line }\end{array}$ & $\begin{array}{ll}25 \\
500 \text { clear }\end{array}$ & & - & & & & - & Z & TS & & & & & XRD & & & \\
\hline & & Wash Permeate & composite of 1st wash permeate & $\begin{array}{l}\text { C2-WPE1-END-XRD-BLK } \\
\text { C2-WPE-CMPPADS }\end{array}$ & 41470 & filtrate drum & 100 & & 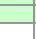 & ICPES & ICPMS & ICA & IEC & AA & & & & & & XRD & & & TICTOC \\
\hline & & & & C2-WPE1-CMP-ACTL & NONE & filtrate drum & 25 & & & & & & & & TS & & & & & & & & \\
\hline & & & & $\begin{array}{c}\text { C2-WP1-CMP-XRD } \\
\text { C2-WPE1-CMP-XRD-BLK }\end{array}$ & $?$ & $\begin{array}{l}\text { filltrate drum } \\
\text { } \text { sample }\end{array}$ & 500 clear & & & & & & & & 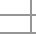 & & & & & $\begin{array}{l}\text { XRD } \\
\text { XRD }\end{array}$ & & & \\
\hline & END 1ST WASH & Concentrate & Time for initial wash (hr) & & & & & & & & & & 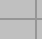 & 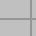 & - & & & & & 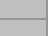 & & & \\
\hline & 2ND WASH & & RLD Wash water (L) & & & & & & & & & & & & & & & & & & & & \\
\hline & & $\begin{array}{l}\text { Concentrate Sample } \\
\text { Conste }\end{array}$ & after addition $\#$ & C2-UF-W2-1 & ARCHIVE & recirc line & 100 & & & & & & 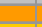 & 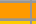 & TS & UDS & DENS & RHEO & & & & & \\
\hline & & Concentrate Sample & $\begin{array}{l}\text { atter adantion \# } \\
\text { after addition \# }\end{array}$ & $\begin{array}{l}\text { C2-UF-W2-5 } \\
\text { C2-UF-W2-9 }\end{array}$ & $\begin{array}{l}\text { ARCHVE } \\
\text { ARCHIVE }\end{array}$ & $\begin{array}{l}\text { reciric line } \\
\text { recirc ine }\end{array}$ & $\begin{array}{l}100 \\
100-l l\end{array}$ & & 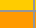 & 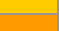 & & 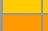 & - & 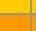 & TS & UDS & $\begin{array}{l}\text { DENS } \\
\text { DENS }\end{array}$ & $\begin{array}{l}\text { RHEO } \\
\text { RHEO }\end{array}$ & & 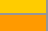 & & & \\
\hline & & $\begin{array}{l}\text { Concentrate Sample } \\
\text { Concentrate Sample }\end{array}$ & $\begin{array}{l}\text { after additition \# } \\
\text { after addition \# }\end{array}$ & $\begin{array}{l}\text { C2-UF-W2-13 } \\
\text { C2-UF-W2-17 }\end{array}$ & $\begin{array}{l}\text { ARCHVE } \\
\text { ARCHIVE }\end{array}$ & $\begin{array}{l}\text { reciric line } \\
\text { recirin ine }\end{array}$ & $\begin{array}{l}100 \\
100\end{array}$ & & $\square$ & & & & 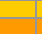 & ت & $\begin{array}{l}\text { TS } \\
\text { TS }\end{array}$ & $\begin{array}{l}\text { UDS } \\
\text { UDS }\end{array}$ & $\begin{array}{l}\text { DENS } \\
\text { DENS }\end{array}$ & $\begin{array}{l}\text { RHEO } \\
\text { RHEO }\end{array}$ & & - & & & \\
\hline & & $\begin{array}{l}\text { Concentrate Sample } \\
\text { (to HLW) }\end{array}$ & & $\begin{array}{l}\text { C2-UF-W2-22-MW } \\
\text { C2-UF-WW-PER }\end{array}$ & $\begin{array}{l}41463 \\
41466\end{array}$ & $\begin{array}{l}\text { recirc line } \\
\text { recirin line }\end{array}$ & $\begin{array}{l}25 \\
25\end{array}$ & $\begin{array}{l}\text { IISSW } \\
\text { DISPER }\end{array}$ & 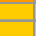 & $\begin{array}{l}\text { ICPSS } \\
\text { ICPES }\end{array}$ & ICPMS & & 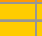 & AA & & & & & & & & & \\
\hline & RUSH & & & $\begin{array}{l}\text { C2-UF-W2-22-WET } \\
\text { C2-UE-W2-2-SOL }\end{array}$ & $\begin{array}{l}41468 \\
41471\end{array}$ & $\begin{array}{l}\text { recirc line } \\
\text { recirin line }\end{array}$ & $\begin{array}{l}25 \\
50\end{array}$ & & & & & ICA & & & TS & uDs & & & PSD & & & & TICTOC \\
\hline & & & & C2-UF-W2-22-ACTL & NONE & recirc line & 60 & & 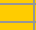 & & & 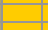 & 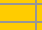 & 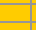 & TS & UDS & DENS & RHEO & & & & & \\
\hline & & & & $\begin{array}{l}\text { 22-2IF-W2-22-ANTF } \\
\text { C2-UF-W2-22-ARC }\end{array}$ & 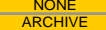 & $\begin{array}{l}\text { reciric line } \\
\text { recirc line }\end{array}$ & $\begin{array}{l}100 \\
100\end{array}$ & & & & & & & & & & & & & & & & \\
\hline & & Wash Permeate & after addition \# & $\begin{array}{l}\text { C2-WPE2-1-ACTL } \\
\text { C2-WWE2-1.XPD }\end{array}$ & NONE & $\begin{array}{l}\text { filtrate line } \\
\text { fituat line }\end{array}$ & $\frac{25}{500 \text { cear }}$ & & & & & & & & TS & & & & & & & & \\
\hline & & Wash Permeate & after addition \# & C2-WPE2-1-XRD-BLK & NONE & $\begin{array}{l}\text { Tsumple } \\
\text { Tsample }\end{array}$ & & & & & & & & & & & & & & XRD & & & \\
\hline & & 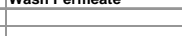 & atter addition \# & 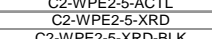 & NUNe & $\begin{array}{l}\text { mituret ine } \\
\text { filtate line }\end{array}$ & 500 clear & & & & & & 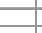 & & 15 & & & & & XRD & & & \\
\hline & & Wash Permeate & after addition \# & 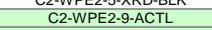 & NONE & 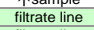 & 25 & & 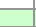 & & & & 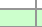 & & TS & & & & & & & & \\
\hline & & & & $\begin{array}{l}\text { C2-WPE2-9.XRD } \\
\text { C2.WPEE-9.XRD-BLK }\end{array}$ & $?$ & filtrate line & 500 clear & & & & & & & & & & & & & $\begin{array}{l}\text { XRD } \\
\text { XXP }\end{array}$ & & & \\
\hline & & Wash Permeate & after addition \# & C2-WPE2-13-ACTL & NONE & 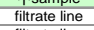 & 25 & & ${ }^{2}$ & & & & 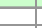 & & TS & & & & & 政 & & & \\
\hline & & & & $\begin{array}{l}\text { C2-WPE-13-XRD } \\
\text { C2-WPE2-13-XRD-BLK }\end{array}$ & $?$ & $\begin{array}{l}\text { filtrate line } \\
{ } \text {. }\end{array}$
\end{tabular}$} &{500 \text { clear }} &{ } &{\text { 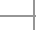 }} &{ } &{ } &{ } &{\text { - }} &{\text { - }} &{ } &{ } &{ } &{ } &{ } &{\begin{array}{l}\text { XRD } \\
\text { XRD }\end{array}} &{ } &{ } &{ } \\
{\hline} &{ } &{\text { Wash Permeate }} &{\text { after addition \# }} &{\begin{array}{l}\text { C2-WPE2-17-ACTL } \\
\text { C2-WWE2-17.XRD }\end{array}} &{\text { NONE }} &{\begin{array}{l}\text { filtrate line } \\
\text { fflthate live }\end{array}} &{25} &{ } &{\text { ב }} &{ } &{ } &{ } &{\text { Z }} &{\text { Z }} &{\text { TS }} &{ } &{ } &{ } &{ } &{\text { 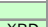 }} &{ } &{ } &{ } \\
{\hline} &{ } &{ } &{ } &{\text { C2-WPE2-17-XRD-BLK }} &{?} &{\text { 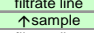 }} &{500 \text { clear }} &{ } &{\square} &{ } &{ } &{ } &{\text { 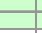 }} &{ } &{ } &{ } &{ } &{ } &{ } &{\begin{array}{l}\text { XRD } \\
\text { XRD }\end{array}} &{ } &{ } &{ } \\
{\hline} &{ } &{\text { Wash Permeate }} &{\text { atter addition \# }} &{\begin{array}{l}\text { C2-WPEE-22-ACTL } \\
\text { C2-WPE2-22-XRD }\end{array}} &{\frac{\text { NONE }}{?}} &{\text { 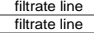 }} &{\frac{25}{500 \text { clear }}} &{ } &{ } &{ } &{ } &{ } &{ } &{\text { 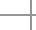 }} &{\text { TS }} &{ } &{ } &{ } &{ } &{\text { XRD }} &{ } &{ } &{ } \\
{\hline} &{ } &{ } &{ } &{\text { C2-WPE2-22-XRD-BLK }} &{\text { NONE }} &{\text { Tsample }} &{ } &{ } &{ } &{ } &{ } &{ } &{ } &{ } &{ } &{ } &{ } &{ } &{ } &{\text { XRD }} &{ } &{ } &{ } \\
{\hline} &{ } &{\text { Wash Permeate }} &{\begin{array}{l}-40 \text { minutes atter last sample } \\
\text { m running with permeatet in recycle) }\end{array}} &{\begin{array}{l}\text { C2-WPE2-END-AATL } \\
\text { C2-WPE2-END-XRD }\end{array}} &{\text { NONE }} &{\begin{array}{l}\text { 位佳ate line } \\
\text { filtrate line }\end{array}} &{\frac{25}{500 \text { clear }}} &{ } &{\text { 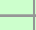 }} &{ } &{ } &{ } &{ } &{ } &{\text { TS }} &{ } &{ } &{ } &{ } &{\text { XRD }} &{ } &{ } &{ } \\
{\hline} &{ } &{\text { Wash Permeate }} &{\text { composite of } 2 \text { nd wash permeate }} &{\begin{array}{l}\text { C2-WPE2-END-XRD-BLK } \\
\text { C2-WPE2-CMPADS }\end{array}} &{41472} &{\text { filtrate drum }} &{100} &{ } &{\text { 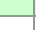 }} &{\text { ICPES }} &{\text { ICPMS }} &{\text { ICA }} &{\text { IEC }} &{\mathrm{AA}} &{ } &{ } &{ } &{ } &{ } &{\text { XRD }} &{ } &{ } &{\text { TICTOC }} \\
{\hline} &{ } &{ } &{ } &{\text { C2-WPE2-CMP-ACTL }} &{\text { NONE }} &{\text { filtrate drum }} &{25} &{ } &{ } &{ } &{ } &{ } &{ } &{-1} &{\text { TS }} &{ } &{ } &{ } &{ } &{ } &{ } &{ } &{ } \\
{\hline} &{ } &{ } &{ } &{\begin{array}{l}\text { C2-WWE2-CMPPXRD } \\
\text { C2-WPE2-CMP-XRD-BLK }\end{array}} &{?} &{\begin{array}{c}\text { filtiate durum } \\
\uparrow \text { sample } \\
\text { the }\end{array}} &{500 \text { clear }} &{ } &{ } &{ } &{ } &{ } &{ } &{ } &{ } &{ } &{ } &{ } &{ } &{\begin{array}{l}\text { XRD } \\
\text { XRD }\end{array}} &{ } &{ } &{ } \\
{\hline} &{\text { END 2ND WASH }} &{\text { Concentrate }} &{\text { Time for final wash (hr) }} &{ } &{ } &{ } &{ } &{ } &{ } &{ } &{ } &{ } &{ } &{ } &{\text { 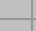 }} &{ } &{ } &{ } &{ } &{ } &{ } &{ } &{ } \\
{\hline \multirow{5}{*}{\text { CLEANING }}\text { CLEANING }} &{ } &{\text { Concentrate }} &{\text { volume washed concentrate }} &{ } &{ } &{ } &{ } &{ } &{ } &{ } &{ } &{ } &{ } &{ } &{ } &{ } &{ } &{ } &{ } &{ } &{ } &{ } &{ } \\
{\hline} &{\text { Loop Flush }} &{\text { "Flush" UF Feed Lo }} &{\text { p- offline }} &{\text { C2-LOOP-TO-C3 }} &{\text { NONE }} &{\text { carboy }} &{50} &{ } &{\text { 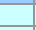 }} &{ } &{ } &{\mathrm{L}} &{\text { 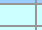 }} &{\text { 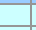 }} &{\text { TS }} &{\text { UDS }} &{\text { DENS }} &{ } &{ } &{ } &{ } &{ } &{ } \\
{\hline} &{ } &{\begin{array}{l}\text { Condensate } \\
\text { centrated Slurry }\end{array}} &{\begin{array}{l}\text { volume needed } \\
\text { volume needed }\end{array}} &{ } &{ } &{ } &{ } &{ } &{\text { 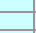 }} &{ } &{ } &{ } &{ } &{ } &{ } &{ } &{ } &{ } &{ } &{ } &{ } &{ } &{ } \\
{\hline} &{\text { UF Caustic Flush } 1} &{\begin{array}{l}\text { Save for Campaiann III } \\
\text { Flush UF System }\end{array}} &{\text { Time to Flush UF (hr) }} &{ } &{ } &{ } &{ } &{ } &{\text { 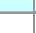 }} &{ } &{ } &{\text { 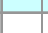 }} &{\text { 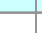 }} &{\text { 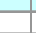 }} &{\text { 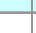 }} &{ } &{ } &{ } &{\text { 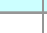 }} &{\text { 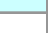 }} &{ } &{ } &{ } \\
{\hline} &{ } &{\begin{array}{l}\text { Caustic Flushing So } \\
\text { RLD Dilution Water }\end{array}} &{\text { ution volume needed for } 3 \text { flushes }} &{ } &{ } &{ } &{ } &{ } &{ } &{ } &{ } &{ } &{ } &{ } &{ } &{ } &{ } &{ } &{ } &{ } &{ } &{ } &{ } \\
$\hline$\end{array}$


WSRC-TR-2005-00105, REVISION 0

SRNL-RPP-2005-00012, REVISION 0

Table D3-4 Campaign II Sample Matrix (4 of 7 pages)

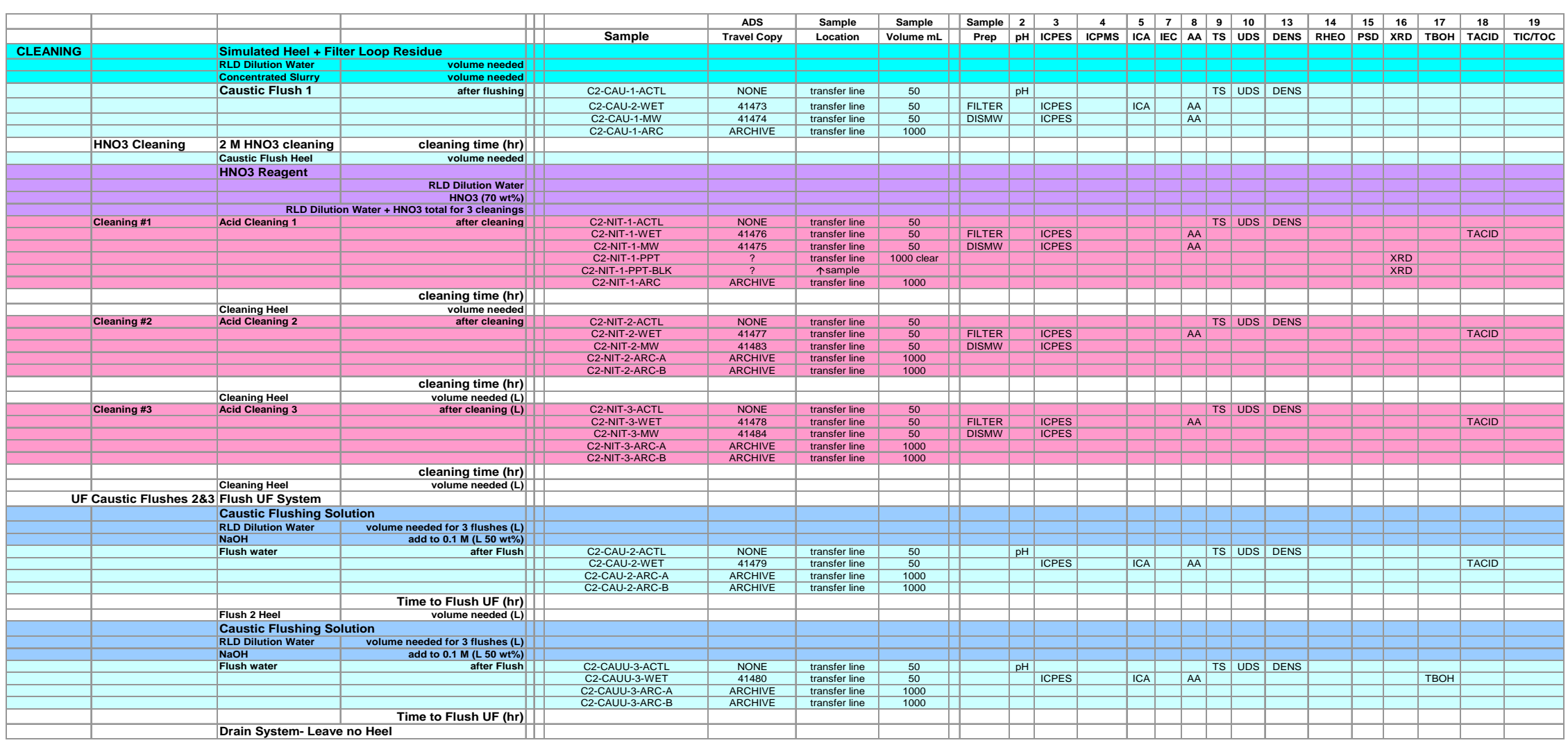


WSRC-TR-2005-00105, REVISION 0

SRNL-RPP-2005-00012, REVISION 0

Table D3-5 Campaign II Sample Matrix (5 of 7 pages)

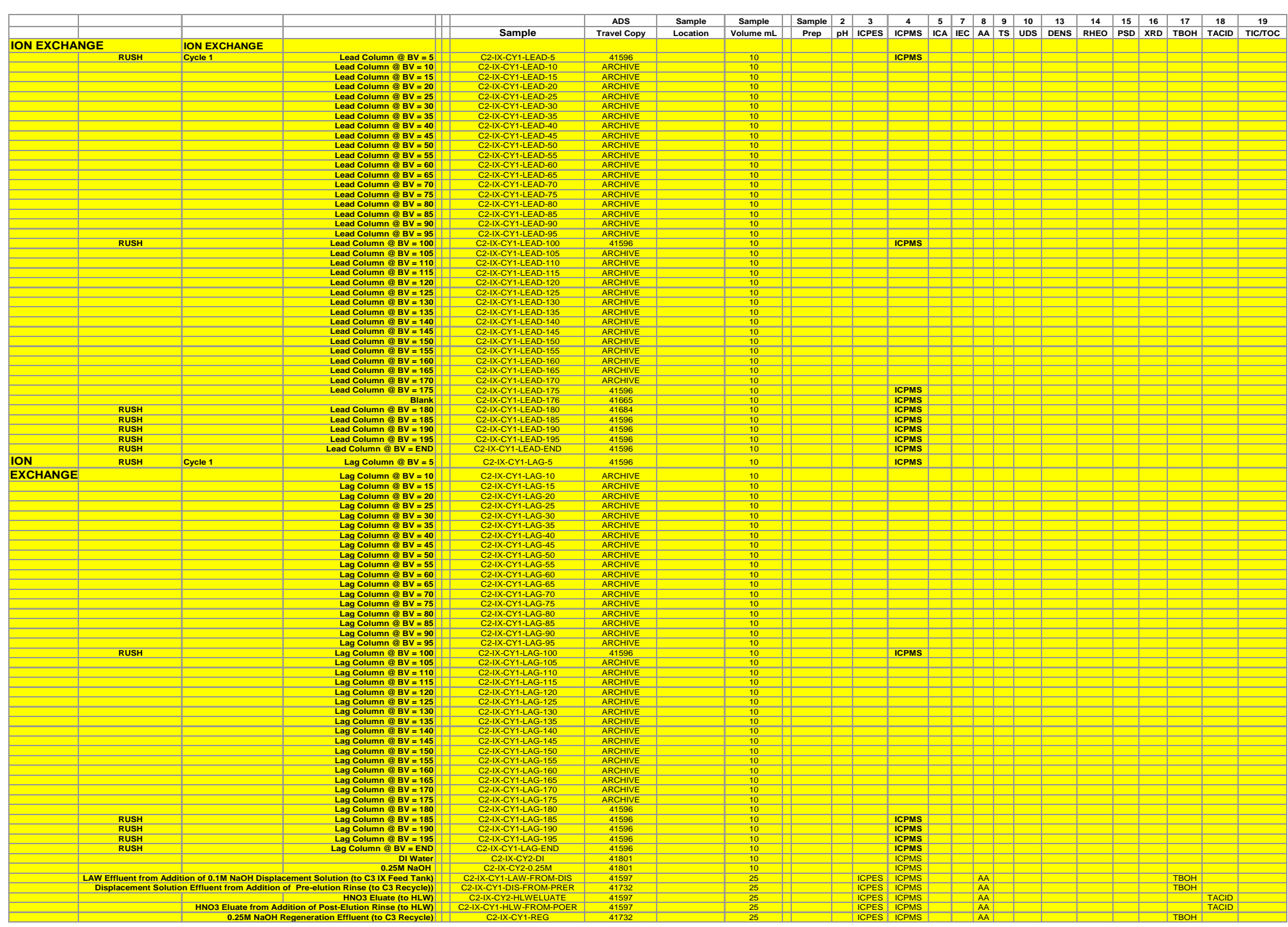


WSRC-TR-2005-00105, REVISION 0

SRNL-RPP-2005-00012, REVISION 0

Table D3-6 Campaign II Sample Matrix (6 of 7 pages)

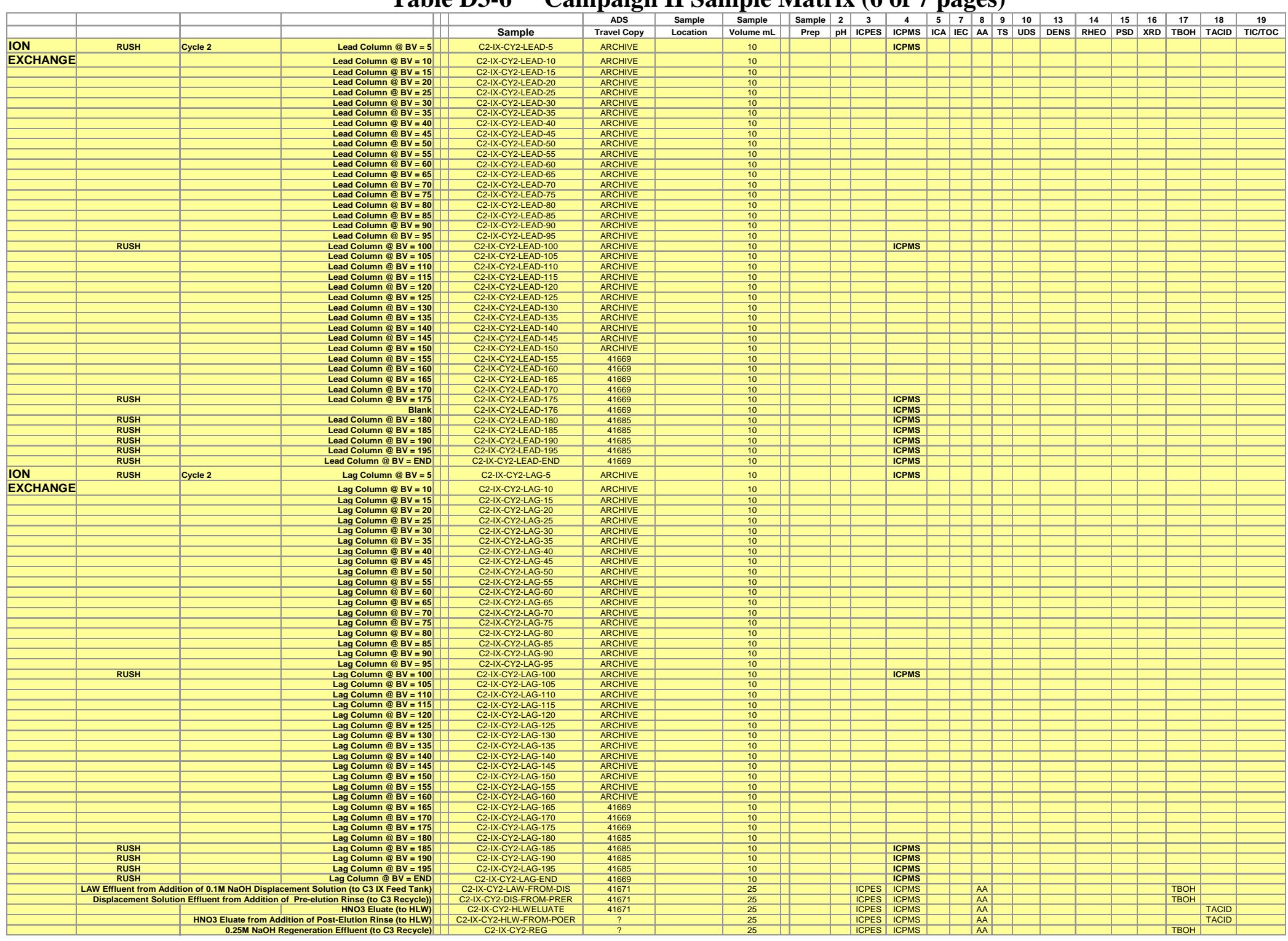


WSRC-TR-2005-00105, REVISION 0

SRNL-RPP-2005-00012, REVISION 0

Table D3-7 Campaign II Sample Matrix (7 of 7 pages)

\begin{tabular}{|c|c|c|c|c|c|c|c|c|c|c|c|c|c|c|c|c|c|c|c|c|c|c|c|}
\hline & & & & Sample & $\begin{array}{l}\text { ADS } \\
\text { Travel Copy }\end{array}$ & \begin{tabular}{|l|} 
Sample \\
Location \\
\end{tabular} & \begin{tabular}{|l} 
Sample \\
Volume $\mathrm{mL}$
\end{tabular} & $\begin{array}{l}\frac{\text { Sample }}{\text { Prep }} \\
\end{array}$ & \begin{tabular}{|c|}
$\mathrm{pH}$ \\
$\mathrm{pH}$
\end{tabular} & \begin{tabular}{|c|}
3 \\
ICPES
\end{tabular} & \begin{tabular}{|c|}
4 \\
ICPMS \\
\end{tabular} & \begin{tabular}{|c|}
5 \\
ICA \\
\end{tabular} & \begin{tabular}{|l|}
7 \\
IEC
\end{tabular} & \begin{tabular}{l|l}
8 \\
$A A$
\end{tabular} & \begin{tabular}{|l|}
9 \\
TS \\
\end{tabular} & \begin{tabular}{|l|}
10 \\
UDS \\
\end{tabular} & $\begin{array}{c}13 \\
\text { DENS }\end{array}$ & \begin{tabular}{|c|}
14 \\
RHEO
\end{tabular} & \begin{tabular}{|l|}
15 \\
PSD \\
\end{tabular} & $\begin{array}{c}16 \\
X R D\end{array}$ & $\begin{array}{c}17 \\
\text { TBOH }\end{array}$ & $\begin{array}{c}18 \\
\text { TACID }\end{array}$ & $\begin{array}{l}19 \\
\text { TICTOCC }\end{array}$ \\
\hline & IX PRODUCTS & IX Product to TLP & & C2-IX-PROD-ACTL & NONE & tank & 100 & & & & & & & & TS & UDS & DENS & RHEO & & & & & \\
\hline & & & & C2-IX-PROD-WET & 41771 & tank & 100 & & 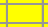 & ICPES & ICPMS & ICA & IEC & AA & & & & & & & TBOH & & TICTOC \\
\hline & & & & $\begin{array}{l}\text { C2-II-XRODDANTF } \\
\text { C2-IXPRODARC }\end{array}$ & $\begin{array}{l}\text { NONE } \\
\text { ARCHIVE }\end{array}$ & $\begin{array}{l}\operatorname{tank} \\
\operatorname{tank}\end{array}$ & $\begin{array}{l}1000 \\
500\end{array}$ & & $\square$ & & & & & & 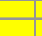 & & & & & & & & \\
\hline & & Pre-elution Rinse Water & Composite & Analyze in Campaign III & & & & & & & & & & & 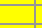 & & & & & & & & \\
\hline & & $\begin{array}{l}0.1 \mathrm{~N} \text { NaOH Displacemen } \\
0.25 \mathrm{MNaOH} \text { Regeneratic }\end{array}$ & $\begin{array}{l}\text { nt Solution Composite } \\
\text { ion Solution Composite }\end{array}$ & $\begin{array}{l}\text { Analyzzi in Campaiann III } \\
\text { Analyze in Campaign III }\end{array}$ & & & & & - & & & & 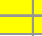 & 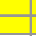 & 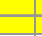 & & & & & & & & \\
\hline \multirow[t]{32}{*}{ TLP EVAPO } & PRATOR & LAW Feed (IX product) & & & & & & & + & & & & 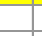 & 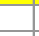 & 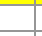 & & & & & & & & \\
\hline & & $\begin{array}{l}\text { LAW SBS } \\
\mathrm{IX.025M} \text { NaOH Regenera }\end{array}$ & ation Solution from Campaign I & & & & & & & & & & & & & & & & & & & & \\
\hline & EVAPORATE & Evaporator Feed Mixture & & C2-TLP-FEED-ACTL & NONE & feed tank & 100 & & 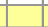 & & & & & & TS & UDS & DENS & & & & & & \\
\hline & & & & $\begin{array}{l}\text { 22-TLP-FEED-ADS } \\
\text { C2TPEDEA }\end{array}$ & $\begin{array}{l}41771 \\
A B C H Y E\end{array}$ & $\begin{array}{l}\text { teed tank } \\
\text { fedtank }\end{array}$ & 100 & & - & ICPES & ICPMS & ICA & IEC & AA & & & & & & & TBOH & & TICTIOC \\
\hline & & & & $\begin{array}{l}\text { C2-2LLPF-FEDD-ARC } \\
\text { C2-TLP-FEED-XRD }\end{array}$ & ARCHVE & $\begin{array}{l}\text { feed tank } \\
\text { feed tank }\end{array}$ & $\begin{array}{l}500 \\
1000 \text { clear }\end{array}$ & & 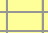 & & & & & & & & & & & \multirow{2}{*}{$\begin{array}{l}\text { XRD } \\
\text { XRD }\end{array}$} & & & \\
\hline & & Bottoms & After all antifoam additions & $\begin{array}{l}\text { C2-TLP-FED-XRD-BLK } \\
\text { C2-TLP-BSARTACACL }\end{array}$ & NONE & $\begin{array}{c}\text { Tsample } \\
\text { bottoms tank }\end{array}$ & $\frac{N A}{60}$ & & 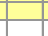 & & & & & & TS & UDS & DENS & RHEO & & & & & \\
\hline & & & & C2-TLP-B-START-ANTF & $\begin{array}{l}\text { NONE } \\
\text { NONE } \\
\end{array}$ & bottoms tank & 100 & & 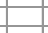 & & & & 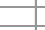 & & s & & & & & & & & \\
\hline & & Bottoms & At -200 L evaporated & $\begin{array}{l}\text { C2-TLP-B-START-ARC } \\
\text { C2-TLP-B-200-ACTL }\end{array}$ & $\begin{array}{l}\text { ARCHIVE } \\
\text { NONE }\end{array}$ & $\begin{array}{l}\text { bottoms tank } \\
\text { bottoms tank }\end{array}$ & $\begin{array}{l}100 \\
50\end{array}$ & & - & - & & $\square$ & - & $\square$ & TS & UDS & DENS & & & & & & \\
\hline & & & & $\begin{array}{l}\text { C2-TLP-B-200-XRD } \\
\text { C2TIPB }\end{array}$ & $?$ & $\begin{array}{l}\text { botoms tank } \\
\text { bottoms tank }\end{array}$ & 500 clear & & & & & & & & & & & & & XRD & & & \\
\hline & & Primary Condensate & & $\begin{array}{l}\text { C2-TLPPB-200-XRDD-BLK } \\
\text { C2-TLP-PC-200-ARC }\end{array}$ & ARCHIVE & ^sample & 100 & & - & & & & 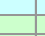 & 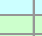 & - & & & & & XRD & & & \\
\hline & & $\begin{array}{l}\text { Secondary Condensate } \\
\text { Bottoms }\end{array}$ & At -400 L evaporated & $\begin{array}{l}\text { C2-TLPPSC-200-ARC } \\
\text { C2-TLP-B-400-ACTL }\end{array}$ & $\begin{array}{l}\text { ARCHVE } \\
\text { NONE }\end{array}$ & bottoms tank & $\begin{array}{l}100 \\
50\end{array}$ & & 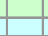 & & & & 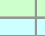 & 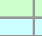 & TS & UDS & DENS & & & & & & \\
\hline & & & & $\begin{array}{l}\text { C2-TLP-B-400-XRD } \\
\text { C2TRP }\end{array}$ & $?$ & $\begin{array}{l}\text { bottoms tank } \\
\text { bosang }\end{array}$ & 500 clear & & Z & & & & & & & & & & & XRD & & & \\
\hline & & Primary Condensate & & $\begin{array}{l}\text { C2-TLP-B-400-XRDD-BKK } \\
\text { C2-TLP-PC-400-ARC }\end{array}$ & ARCHIVE & $\uparrow$ sample & 100 & & 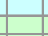 & & & & 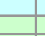 & & 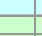 & & & & & XRD & & & \\
\hline & & $\begin{array}{l}\text { Secondary Condensate } \\
\text { Bottoms }\end{array}$ & At -600 L evaporated & $\begin{array}{l}\text { C2-TLP-SC-400-ARC } \\
\text { C2-TLP-B } 600 \text { ACTL }\end{array}$ & $\begin{array}{l}\text { ARCHIVE } \\
\text { NONE }\end{array}$ & bottoms tank & 100 & & - & & & & - & & TS & 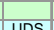 & DENS & & & & & & \\
\hline & & & At $-600 \mathrm{~L}$ evaporated & $\begin{array}{l}\text { C2-TLPPB-600-ACL } \\
\text { C2-TLP-B-60-XRD }\end{array}$ & $?$ & $\begin{array}{l}\text { boumistank } \\
\text { bottoms tank }\end{array}$ & 500 clear & & 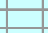 & & & & - & - & TS & UDS & DENS & & & XRD & & & \\
\hline & & Primary Condensate & & $\begin{array}{l}\text { C2-TLP-B-6-60-XRDD-BLK } \\
\text { C2-TLP-PC-600-ARC }\end{array}$ & $\begin{array}{l}? \\
\text { ARCHIVE }\end{array}$ & †sample & 100 & & - & & & & 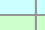 & & 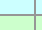 & & & & & XRD & & & \\
\hline & & Secondary Condensate & At -800 L evaporated & $\begin{array}{l}\text { C2-TLP-SC-600-ARC } \\
\text { C2-TLPC }\end{array}$ & ARCHIVE & & 100 & & - & & & & 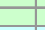 & & & & & & & & & & \\
\hline & & Bottoms & At -800 L evaporated & $\begin{array}{l}\text { C2-TLP-B-800-ACTL } \\
\text { C2-TLP-B-800-XRD }\end{array}$ & $\begin{array}{l}\text { NONE } \\
?\end{array}$ & $\begin{array}{l}\text { bottioms tank } \\
\text { bottoms tank }\end{array}$ & $\begin{array}{l}50 \\
500 \text { clear } \\
\end{array}$ & & - & & & & 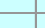 & 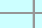 & TS & UDS & DENS & & & XRD & & & \\
\hline & & Primary Condensate & & $\begin{array}{l}\text { C2-TLP-B-800-XRD-BLK } \\
\text { C2-TID-PC-800-AC }\end{array}$ & $\frac{?}{\text { ARCHVE }}$ & ^sample & soldedit & & - & & & & 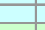 & 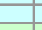 & 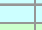 & & & & & XRD & & & \\
\hline & & Secondary Condensate & & C2-TLP-SC-800-ARC & $\begin{array}{l}\text { ARCHVE } \\
\text { ARCHIVE }\end{array}$ & & 100 & & - & & & & & - & & & & & & & & & \\
\hline & & Bottoms & At -800 L evaporated & C2-TLP-B-1000-ACTL & NONE & bottoms tank & 50 & & 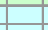 & & & & 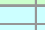 & 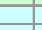 & TS & UDS & DENS & & & & & & \\
\hline & & & & $\begin{array}{l}\text { C2-TLP-B-1000-XRD } \\
\text { C2-TIPB-B }\end{array}$ & & bottoms tank & 500 clear & & & & & & & & & & & & & XRD & & & \\
\hline & & Primary Condensate & & $\begin{array}{l}\text { C2-1LPB-10-100-XRD-BLK } \\
\text { C2-TLP-PCo0-ARC }\end{array}$ & ARCHIVE & Tsample & 100 & & + & & & 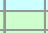 & 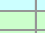 & & 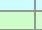 & & & & & XRD & & & \\
\hline & & $\begin{array}{l}\text { Secondary Condensate } \\
\text { Bottoms }\end{array}$ & Evaporation completed & $\begin{array}{l}\text { C2-2LPPSC-1000-ARC } \\
\text { C2-TLP-B-END-ACTL }\end{array}$ & $\begin{array}{l}\text { ARCHIVE } \\
\text { NONE }\end{array}$ & bottoms tank & $\begin{array}{l}100 \\
100\end{array}$ & & $\square$ & & & & & & & UDS & DENS & RHEO & & & & & \\
\hline & & & Evaporation completed & 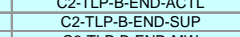 & $\begin{array}{l}\text { NONE } \\
41771 \\
4\end{array}$ & 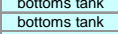 & $\begin{array}{l}100 \\
100 \\
\end{array}$ & & 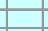 & ICPES & & ICA & IEC & AA & TS & UDS & DENS & RHEO & & & TBOH & & TICTTOC \\
\hline & & & & $\begin{array}{l}\text { C2.TLPB-BENDDMW } \\
\text { C2-TLP-BEENDPSD }\end{array}$ & $\frac{41771}{41771}$ & $\begin{array}{l}\text { bottoms tank } \\
\text { bottoms tank }\end{array}$ & $\frac{50}{25}$ & DiSMW & 1 & ICPES & ICPMS & & & $\mathrm{AA}$ & & & & & PSD & & & & \\
\hline & & & & $\begin{array}{l}\text { C2-TLP-B-END-ANTF } \\
\text { CTL-NONF }\end{array}$ & NONE & bottoms tank & 100 & & E & & & & 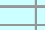 & & - & & & & 100 & & & & \\
\hline & & & & & ARCHVE & $\begin{array}{l}\text { Dobtoms tank } \\
\text { bottoms tank }\end{array}$ & 500 clear & & & & & & & & & & & & & XRD & & & \\
\hline & & & & C2-TLP-B-END-XRD-BLK & & Tsample & & & & & & & & & -5 & & & & & XRD & & & \\
\hline & & $\begin{array}{l}\text { Primary Condensate } \\
\text { Secondary Condensate }\end{array}$ & Evaporation almost completed & $\begin{array}{l}\text { C2-TLP-PC-END-ARC } \\
\text { C2-TPPSCENDARC }\end{array}$ & $\begin{array}{l}\text { ARCHIVE } \\
\text { ARCIIV }\end{array}$ & & $\begin{array}{l}100 \\
100\end{array}$ & & - & & & & & & & & & & & & & & \\
\hline & COMBINE TLP CON & $\begin{array}{l}\text { DENSATES } \\
\text { DENensate }\end{array}$ & & & & & & & & & & & & & & & & & & & & & \\
\hline & & $\begin{array}{l}\text { Combined TLP Condens } \\
\text { (Primary + Secondary) }\end{array}$ & sate = RLD Condensate & $\begin{array}{l}\text { C2-RLD-FROM-TLP-ADS } \\
\text { C2-RLD-FROM-TPA-ACTL }\end{array}$ & $\begin{array}{l}41771 \\
\text { NONE }\end{array}$ & storage tank & $\begin{array}{l}250 \\
250 \\
50\end{array}$ & & $\mathrm{pH}$ & & & & & & Ts & uDs & DENS & & & & & & ICTOC \\
\hline & COMBINE FEP \& TL & P CONDENSATES & & & & & & & & & & & & & & & & & & & & & \\
\hline & & Combined FEP + TLP Co & ondensate = RLD Condensate & $\begin{array}{l}\text { C2-RLD-TOTAL-ADS } \\
\text { C2-RD-TOTALACTL }\end{array}$ & $\begin{array}{l}41771 \\
\text { NONE } \\
\text { PON }\end{array}$ & storage tank & $\begin{array}{l}250 \\
250 \\
500\end{array}$ & & $\mathrm{pH}$ & ICPES & & ICA & & AA & TS & UDS & DENS & & & & & & TICTOC \\
\hline & & T. & ICC ammonium & $\begin{array}{l}\text { C2-RLD-TTAL_ARC } \\
\text { C2_RLD-FINAL-AGED } \\
\end{array}$ & $\begin{array}{l}\text { ARCIIVE } \\
42072 \\
\end{array}$ & storage tank & 100 & & 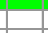 & & & & & & & & & & & & & & \\
\hline Notes: & Time Dep Sample & Time dependent sample. T & These samples are to be observed daly to & about 5 days for evidence of precil & titation of solids. & & & & & & & & & & & & & & & & & & \\
\hline & Explanation of analyses: & If solids form, they will be a & analyzed by XRD. A filtrate blank will laso & & & & & & & & & & & & & & & & & & & & \\
\hline & & pH & sent that can be analyzed by ICPES & $\begin{array}{l}\text { lon chrom } \\
\text { lon exclus }\end{array}$ & 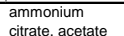 & & & & & & & - & & & & & & & & & & & \\
\hline & ICPM: & sludges & $\mathrm{Cs}, \mathrm{Rb}, \mathrm{Bi}, \mathrm{Y}, \mathrm{Pr}$ & atomic absorption & $\mathrm{Cs}, \mathrm{K}, \mathrm{Na}$ & PSD & particles s & & & & & & & & & & & & & & & & \\
\hline & ic & $\begin{array}{l}\text { lupernates } \\
\text { lon chrom - anions }\end{array}$ & $\begin{array}{l}\mathrm{Cs,} \text {, R } \\
\text { nomal suite }\end{array}$ & $\begin{array}{l}\text { total solids w w to } \\
\text { undisids wtoved solus }\end{array}$ & & $\begin{array}{c}\text { XRD } \\
\text { TBOH }\end{array}$ & $\begin{array}{l}\text { X-ray diffractior } \\
\text { totola base, free }\end{array}$ & droxide, o 0 & other $b \varepsilon_{t}$ & & & $=$ SOLI & & & & & & & & & & & \\
\hline
\end{tabular}


WSRC-TR-2005-00105, REVISION 0

SRNL-RPP-2005-00012, REVISION 0

Table D4-1 Campaign III Sample Matrix (1 of 4 pages)

\begin{tabular}{|c|c|c|c|c|c|c|c|c|c|c|c|c|c|c|c|c|c|c|c|c|c|c|c|c|}
\hline & & & & & ADS & Sample & Sample & Sample & 2 & 3 & 4 & 5 & 6 & 7 & 8 & 9 & 10 & 11 & 12 & 13 & 14 & 15 & 16 & 17 \\
\hline \multirow{38}{*}{ RECYCLE BLE } & RIFNDING & & & Sample & Travel Copy & Location & Vol mL & & $\mathrm{pH}$ & & ICPMS & & & & & TS & UDS & DENS & RHEO & PSD & XRD & TB.. & TACID & TICITOC \\
\hline & BLENDING & & Campaign II UF Wash Water & & & & & & & & & & & & & & & & & & & & & \\
\hline & & & $\begin{array}{r}\text { HLW SBS } \\
\text { HLW Vessel Vent (PVP) }\end{array}$ & & & & & & - & & & & - & & & - & & & & & & & & \\
\hline & & & & & & & & & -2 & & & & 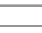 & & & - & & & & & & & & \\
\hline & & & $\begin{array}{l}\text { Campaign II Caustic Flushes } \\
\text { Campaing IUF Acid Cleaninins }\end{array}$ & & & & & & D & & & & Z & & & D & & & & & & & & \\
\hline & & & 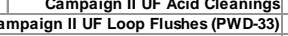 & & & & & & - & & & & - & & & - & & & & & & & & \\
\hline & Campaign II Cs IX & cle: Displacement Soluti & ion Displaced by Pre-Eution Rinse (2) & NO ANALYSIS & & & & & 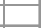 & & & & & & & 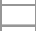 & & & & & & & & \\
\hline & Campaign II Cs IX & ycle: Post-Elution Rinse D & $\begin{array}{l}\text { Displaced + Regeneration Solutitin (5) } \\
\text { coMBBNED RECYCLES }\end{array}$ & NO ANALYSIS & & & & & - & & & & & & & 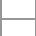 & & & & & & & & \\
\hline & & PWD & Generation of Synthetic Recyc & & & & & & 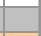 & & & & {[} & & & ב & & & & & & & & \\
\hline & & & HLW Canister Decon & NO SAMPLE & & & & & 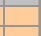 & & & & 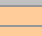 & & & - & & & & & & & & \\
\hline & & & $\begin{aligned} \text { Dl water } \\
\mathrm{NaOH} \text { 50utto }\end{aligned}$ & & & & & & 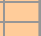 & & & & & & & - & & & & & & & & \\
\hline & & & NaNO3 (kg) & & & & & & 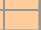 & & & & 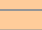 & & & 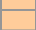 & & & & & & & & \\
\hline & & & HLW Vessel Vent (PVP) & NO SAMPLE & & & & & 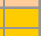 & & & & 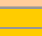 & & & 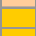 & & & & & & & & \\
\hline & & & $\begin{aligned} \text { Dl water } \\
\mathrm{NaOH} 5 \text { 5umto }\end{aligned}$ & & & & & & - & & & & & & & 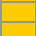 & & & & & & & & \\
\hline & & & Combined Canister Decon + PVP & & & & & & - & & & & - & & & - & & & & & & & & \\
\hline & & UF LO & oop Flush (PWD-33) from Campaign UI & NO SAMPLE & & & & & 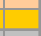 & & & & & & & 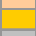 & & & & & & & & \\
\hline & & & Combining of Recycles & & & & & & & & & & & & & & & & & & & & & \\
\hline & & PWD-44 & & C3-PWD-44-ARC & NONE & mixing tank & 50 & & 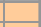 & & & & & & & 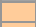 & & & & & & & & \\
\hline & & $\begin{array}{l}\text { Add HLV } \\
\text { Add HL }\end{array}$ & 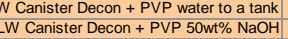 & & & & & & 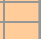 & & & & & & & 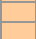 & & & & & & & & \\
\hline & & & Add HLW Canister Decon NaNO3 $(\mathrm{kg})$ & & & & & & 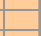 & & & & & & & 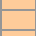 & & & & & & & & \\
\hline & & & $\begin{array}{l}\text { Add HLW SBS to tank } \\
\text { d }\end{array}$ & & & & & & 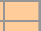 & & & & & & & 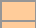 & & & & & & & & \\
\hline & & & $\begin{array}{l}\text { Add UF Loop Flush PWD-33 } \\
\text { Sum }\end{array}$ & & & & & & - & & & & & & & - & & & & & & & & \\
\hline & & PWD-15 & 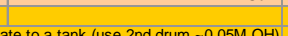 & C3-PWD-15-ARC & NONE & mixing tank & 250 & & & & & & & & & 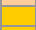 & & & & & & & & \\
\hline & & Campaign II Wash Permec & ate to a tank (use 2nd drum,, $0.05 \mathrm{O}$ OH) & & & & & & 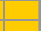 & & & & & & & E & & & & & & & & \\
\hline & & Add & 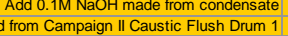 & & & & & & 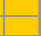 & & & & & & & - & & & & & & & & \\
\hline & & & d trom Campaign II C Cuustic Flush Drum 2 & & & & & & 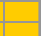 & & & & & & & 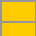 & & & & & & & & \\
\hline & & & Sum & & & & & & & & & & & & & 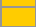 & & & & & & & & \\
\hline & & PWD-16 & & C3-PWD-16-ARC & NONE & mixing tank & 250 & & 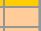 & & & & 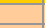 & & & 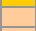 & & & & & & & & \\
\hline & & dd Campaign II Wash Perm & neate to a tank (use $1 \mathrm{st}$ drum,, $0.1 \mathrm{O} \mathrm{OH}$ ) & & & & & & & & & & & & & - & & & & & & & & \\
\hline & & Add from & 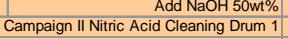 & & & & & & 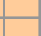 & & & & - & & & - & & & & & & & & \\
\hline & & Add from & Campaign II Nitric Acid Cleaning Drum 2 & & & & & & & & & & & & & & & & & & & & & \\
\hline & & Add from & Campaign II Nitric Acid Cleaning Drum 3 & & & & & & 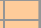 & & & & E & & & E & & & & & & & & \\
\hline & & Combine Recycles in (FE & EP Feed) Tank & C3-FEP-FEED-ACTL & NONE & mixing tank & 50 & & 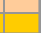 & & & & - & 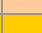 & 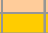 & TS & UDS & DENS & & & & & & \\
\hline & & & Add PWD-44 to tank & C3-FEP-FEED-ARC & NONE & mixing tank & 250 & & & & & & & & & & & & & & & & & \\
\hline & & & $\begin{array}{l}\text { Add PWD-15 to tank } \\
\text { Add PWD-16totank }\end{array}$ & $\begin{array}{l}\text { C3-FEP-FEED-ANTF } \\
\text { C3-FEP-FED-ADS }\end{array}$ & $\begin{array}{l}\text { NONE } \\
42007 \\
4207-3\end{array}$ & mixing tank & 100 & & - & & & & $1 \mathrm{CC}$ & & & & & & & & & & & \\
\hline & & & $\begin{array}{l}\text { Add PWDD-16 to tank } \\
\text { IX Recycles }\end{array}$ & & & & & & & & & & $\mathrm{ICC}$ & & & & & & & & & & & \\
\hline & & & $\begin{array}{r}\text { Sum } \\
\text { RID trom Campion }\end{array}$ & C2-RLD-FNAL-AGED & 42072 & & 100 & & & & & & ICC & & & & & & & & & & & \\
\hline \multirow{2}{*}{\multicolumn{2}{|c|}{ FEP EVAPORATOR }} & & RLD from Campaign II & C2-RLD-FINAL-AGED & $4<01 / 2$ & & 100 & & & & & & $\mathrm{KC}$ & & & & & & & & & & & \\
\hline & & & $\begin{array}{l}\text { Starting Volume } \\
\text { Atter all antifoam additions }\end{array}$ & C3-FEP-B-START-ARC & & bettoms tank & & & & & & & & & & & & & & & & & & \\
\hline & & Bottoms & After all antifoam additions & $\begin{array}{l}\text { C3-FEP-B-BSTART-ARC } \\
\text { C3-FEP-B-START-ANTFF }\end{array}$ & NONE & $\begin{array}{l}\text { Dottoms tank } \\
\text { bottoms tank }\end{array}$ & 100 & & - & & & & - & & & - & & & & & & & & \\
\hline & & Bottoms & At $-200 \mathrm{~L}$ fed & C3-FEP-B-200-ARC & NONE & bottoms tank & 100 & & 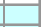 & & & 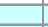 & 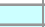 & & & 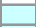 & & & & & & & & \\
\hline & & Bottoms & At $-400 \mathrm{~L}$ fed & C3-FEP-B-400-ARC & NONE & bottoms tank & 100 & & 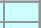 & & & & & & & 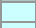 & & & & & & & & \\
\hline & & $\begin{array}{l}\text { Bottoms } \\
\text { Bottoms }\end{array}$ & At $-600 \mathrm{~L}$ fed & $\begin{array}{l}\text { C3-FEP-B-600-ARC } \\
\text { CS-BEP-B-80-ARC }\end{array}$ & NONE & $\begin{array}{l}\text { bottoms tank } \\
\text { pottoms tank }\end{array}$ & 100 & & - & & & & & & & 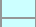 & & & & & & & & \\
\hline & & $\begin{array}{l}\text { Bottoms } \\
\text { Bottoms }\end{array}$ & $\begin{array}{l}\text { Evaporation completed } \\
\text { Eved }\end{array}$ & $\begin{array}{l}\text { C3-EPPB-8-800-ARC } \\
\text { C3-FEP-B-END-ACTL }\end{array}$ & $\begin{array}{l}\text { NONE } \\
\text { NONE }\end{array}$ & $\begin{array}{l}\text { bottoms tank } \\
\text { bottoms tank }\end{array}$ & $\begin{array}{l}100 \\
50\end{array}$ & & & & & & & & & TS & UDS & DENS & & & & & & \\
\hline & & & & $\begin{array}{l}\text { C3-FEP-B-END-WET } \\
\text { C3-FEP-B-END-MW }\end{array}$ & $\begin{array}{l}42088 \\
42089\end{array}$ & $\begin{array}{l}\text { bottoms tank } \\
\text { bottoms tank }\end{array}$ & $\begin{array}{l}50 \\
50\end{array}$ & DISMW & & & ICPMS & ICA & ICC & & AAK & & & & & & & TBOH & & TIC/TOC \\
\hline & & & & $\begin{array}{l}\text { C3-FEP-B-END-PER } \\
\text { CSFP-NOF }\end{array}$ & 42090 & bottoms tank & 50 & DISPER & & ICPES & & & & & & & & & & & & & & \\
\hline & & & & $\begin{array}{l}\text { C3-FEP-B-BND-AAC } \\
\text { C3-FEP-BENDANTF }\end{array}$ & $\begin{array}{l}\text { NNONE } \\
\text { NONE }\end{array}$ & $\begin{array}{l}\text { bottoms tank } \\
\text { bottoms tank }\end{array}$ & $\begin{array}{l}500 \\
100\end{array}$ & & & & & & & & & & & & & & & & & \\
\hline & & Primary Condensate & & C3-FEP-PC-END-ARC & NONE & & & & & & & & & & & & & & & & & & & \\
\hline & & Secondary Condensate & Composite & C3-FEP-SC-END-ARC & NONE & & 100 & & & & & & & & & & & & & & & & & \\
\hline \multirow{2}{*}{\multicolumn{4}{|c|}{$\begin{array}{l}\text { COMBINE ALL FEP CONDENSATES } \\
\text { Combined FEP Condensate }\end{array}$}} & & & & & & & & & & & & & & & & & & & & & \\
\hline & & & & $\begin{array}{l}\text { C3-RLDD-FOM-FEP-ARC } \\
\text { C3-RL-ROM-FEP-ADS }\end{array}$ & $\begin{array}{l}\text { NNNE } \\
42093\end{array}$ & & $\begin{array}{l}1000 \\
100\end{array}$ & & & & & & ICC & & & & & & & & & & & \\
\hline
\end{tabular}


WSRC-TR-2005-00105, REVISION 0

SRNL-RPP-2005-00012, REVISION 0

Table D4-2 Campaign III Sample Matrix (2 of 4 pages)

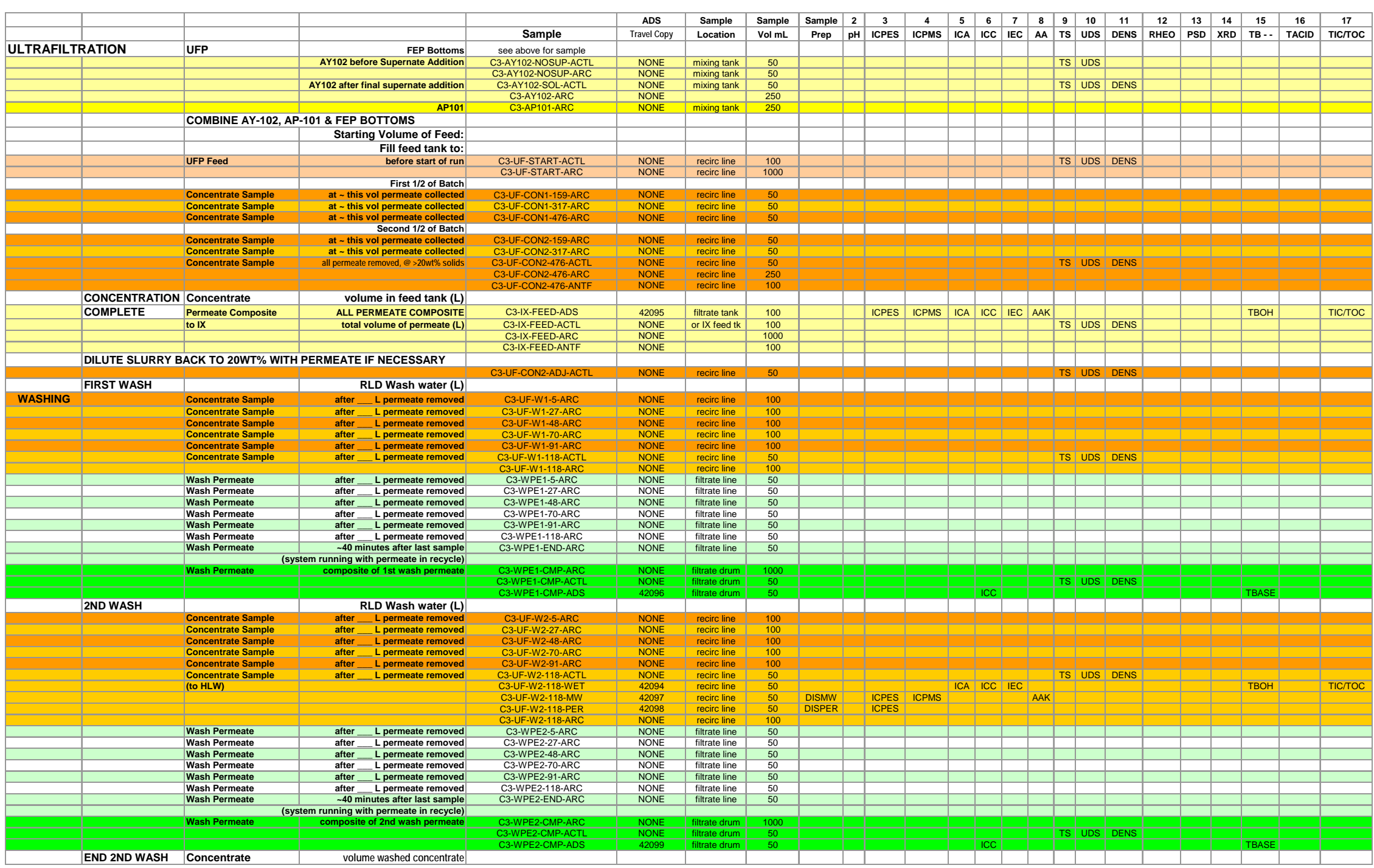


WSRC-TR-2005-00105, REVISION 0

SRNL-RPP-2005-00012, REVISION 0

Table D4-3 Campaign III Sample Matrix (3 of 4 pages)

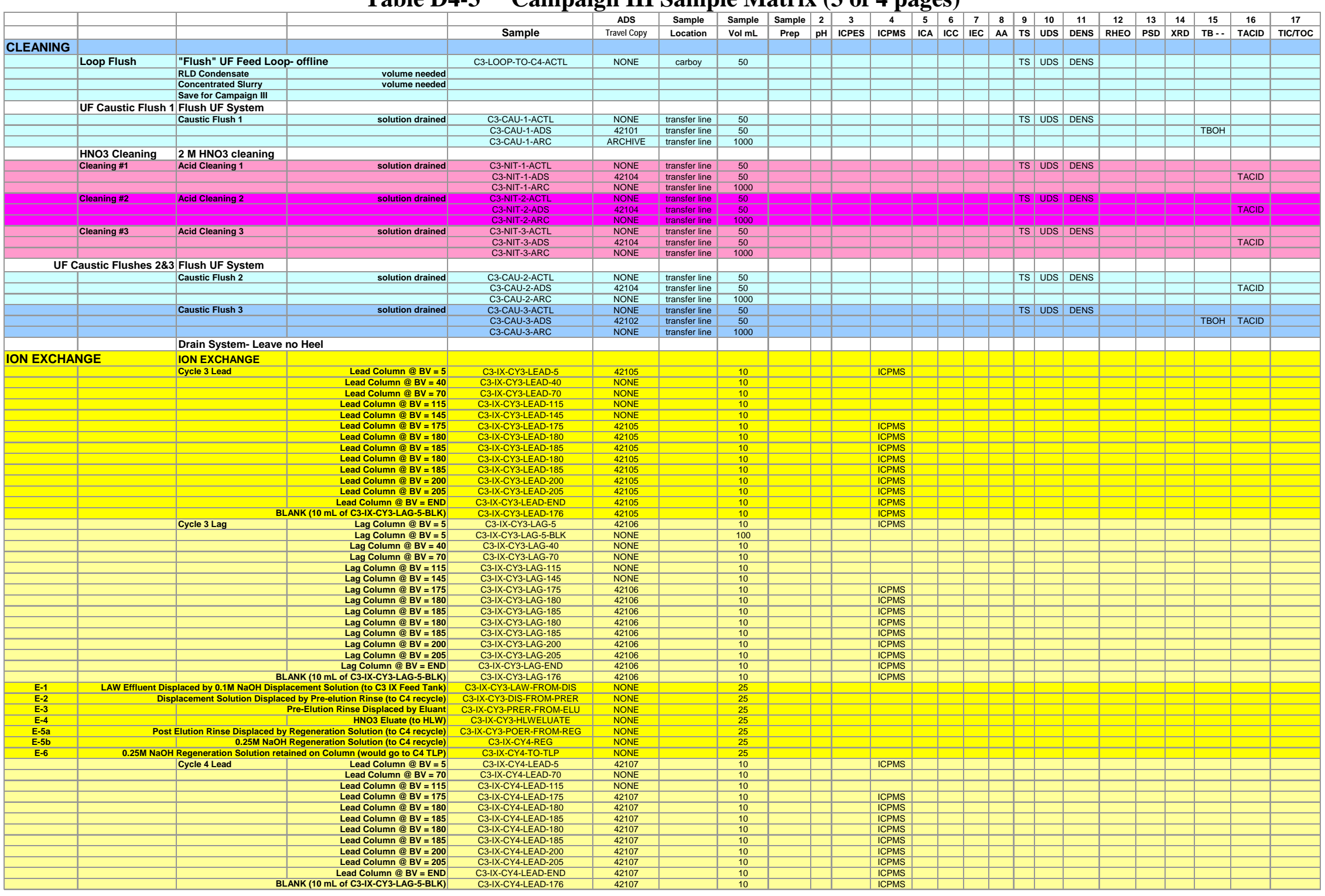


WSRC-TR-2005-00105, REVISION 0

SRNL-RPP-2005-00012, REVISION 0

\section{Table D4-4 Campaign III Sample Matrix (4 of 4 pages)}

\begin{tabular}{|c|c|c|c|c|c|c|c|c|c|c|c|c|c|c|c|c|c|c|c|c|c|c|c|c|}
\hline & & & & Sample & $\begin{array}{l}\text { ADS } \\
\text { Travel Copy }\end{array}$ & $\begin{array}{l}\text { Sample } \\
\text { Location }\end{array}$ & $\begin{array}{l}\text { Sample } \\
\text { Vol } \mathrm{mL}\end{array}$ & $\begin{array}{c}\text { Sample } \\
\text { Prep }\end{array}$ & \begin{tabular}{|c|}
2 \\
$\mathrm{pH}$
\end{tabular} & \begin{tabular}{|c|}
3 \\
ICPES \\
\end{tabular} & \begin{tabular}{|c|}
4 \\
ICPMS \\
\end{tabular} & \begin{tabular}{|c|}
5 \\
ICA
\end{tabular} & \begin{tabular}{|c}
6 \\
ICC
\end{tabular} & \begin{tabular}{|c|}
7 \\
IEC
\end{tabular} & \begin{tabular}{|c|}
8 \\
AA \\
\end{tabular} & \begin{tabular}{|l}
9 \\
TS
\end{tabular} & $\begin{array}{l}10 \\
\text { UDS }\end{array}$ & \begin{tabular}{|c|}
11 \\
DENS \\
\end{tabular} & $\begin{array}{c}12 \\
\text { RHEO } \\
\end{array}$ & \begin{tabular}{|l|}
13 \\
PSD \\
\end{tabular} & \begin{tabular}{|l|}
14 \\
XRD
\end{tabular} & \begin{tabular}{|c|}
15 \\
TB..
\end{tabular} & $\begin{array}{c}16 \\
\text { TACID }\end{array}$ & $\begin{array}{l}17 \\
\text { TIC/TOC }\end{array}$ \\
\hline & & Cycle 4 Lag & $\begin{array}{l}\text { Lag Column @ BV }=5 \\
\text { Lag Column @ BV }=70\end{array}$ & $\begin{array}{l}\text { C3-I-CY4-LAG-5 } \\
\text { C3-IX-CY4-LAG-70 }\end{array}$ & $\begin{array}{l}42108 \\
\text { NONE }\end{array}$ & & $\begin{array}{l}10 \\
10\end{array}$ & & & & & & & & & & & & & & & & & \\
\hline & & & Lag Column@BV =115 & C3-IX-CY4-LAG-115 & NONE & & 10 & & - & & & & & & & & & & & & & & & \\
\hline & & & Lag Column $@ B V=175$ & C3-I-XCYC4-LAG-175 & NONE & & 10 & & 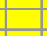 & & ICPMS & 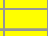 & 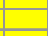 & & 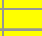 & 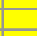 & & & & & & & & \\
\hline & & & & C3-IX-CY4-LAG-180 & 42108 & & 10 & & 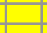 & & ICPMS & & & & 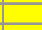 & $\square$ & & & & & & & & \\
\hline & & & $\begin{array}{l}\text { Lag Column @ BV }=185 \\
\text { Lag Column } @ \text { BV }=180\end{array}$ & $\begin{array}{l}\text { C3--1XXCY4-LAGG-185 } \\
\text { C3-IXCY4-LAG-180 }\end{array}$ & $\begin{array}{l}42108 \\
42108\end{array}$ & & 10 & & 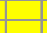 & & $\begin{array}{l}\text { ICPMS } \\
\text { ICPS }\end{array}$ & & - & & 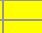 & $\square$ & & & & & & & & \\
\hline & & & Lag Column@BV = 185 & $\begin{array}{l}\text { C3-I-IXCY4-LAGG-180 } \\
\text { C3-IX-CY4-LAG-185 }\end{array}$ & 42108 & & $\begin{array}{l}10 \\
10\end{array}$ & & & & $\begin{array}{l}\text { ICPMS } \\
\text { ICPMS }\end{array}$ & & & & & & & & & & & & & \\
\hline & & & Lag Column@BV=200 & C3-IX-CY4-LAG-200 & 42108 & & 10 & & & & ICPMS & & & & 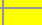 & - & & & & & & & & \\
\hline & & & Lag Column @ BV $=205$ & C3-XX-CY4-LAG-205 & 42108 & & 10 & & 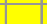 & & ICPMS & & & & 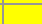 & & & & & & & & & \\
\hline & & & Lag Column @ BV = END & & 42108 & & 10 & & 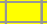 & & ICPMS & & & & 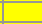 & & & & & & & & & \\
\hline$E-1$ & AW Effluent Displa & BL & LANK (10 mL of C3-IX-CY3-LAG-5-BLK & $\begin{array}{l}\text { C3-IX-CY4-LAG-176-15 } \\
\text { C3-IXCYYLAW-ROM-DIS }\end{array}$ & 42108 & & 10 & & & & ICPMS & & & & & & & & & & & & & \\
\hline$E-2$ & $\begin{array}{l}\text { LAW EEfluent Displa } \\
\text { Displa }\end{array}$ & cement Solution Displaced & cemt Solutition (to C3 IX Feed Tank & $\begin{array}{l}\text { C3-IX-CY4-LAW-FROM-DIS } \\
\text { C3-XYA-DST-FROMPPER }\end{array}$ & $\begin{array}{l}\text { NONE } \\
\text { NONE }\end{array}$ & & 255 & & & & & & & & & & & & & & & & & \\
\hline$E-3$ & & & 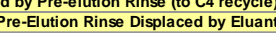 & 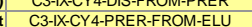 & NONE & & 25 & & & & & 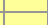 & & & 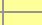 & 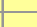 & & & & & & & & \\
\hline & & & HNO3 Eluate (to HLW) & C3-IX-CY4-HLWELUATE & NONE & & 25 & & & & & & & & & & & & & & & & & \\
\hline E-5a & Post El & ution Rinse Displaced by $\mathrm{R}$ & Regeneration Solution (to C4 recycle) & C3-IX-CY4-POER-FROM-REG & NONE & & 25 & & - & & & 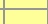 & 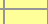 & & 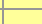 & 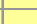 & & & & & & & & \\
\hline$E-5 b$ & & $\begin{array}{l}0.25 \mathrm{MaOHR} \\
0\end{array}$ & Regeneration Solution (to $\mathrm{C} 4$ recycle) & C3-XXCYA-REG & NONE & & 25 & & $\square$ & & & 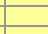 & E & & 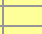 & B & & & & & & & & \\
\hline$E-6$ & $\begin{array}{l}\text { O.25M NaHR Re } \\
\text { IX PRODUCTS }\end{array}$ & $\begin{array}{l}\text { egeneration Solutiton retair } \\
\text { IX Product to TLP }\end{array}$ & $\begin{array}{l}\text { ined on Column (would go to C4 TLP) } \\
\text { IX PRODUCT }\end{array}$ & $\begin{array}{l}\text { C3-X-CY-T-TO-TLP } \\
\text { C-X-PROD-ACL }\end{array}$ & $\begin{array}{l}\text { NOOE } \\
\text { NONE }\end{array}$ & tank & 25 & & - & 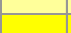 & & 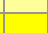 & 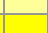 & & 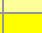 & TS & UDS & DENS & & & & & & \\
\hline & & IX Proauctro ILP & & C3-1X-PROD-ARC & NONE & tank & 500 & & - & & & & & & & & Ous & DENS & & & & & & \\
\hline & & & & C3-IX-PROD-ADS & 42110 & tank & 50 & & E & & & 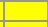 & ICC & & 2 & & & & & & & & & \\
\hline & & & on displace hy pre-elution rinse (E-2. & $\begin{array}{l}\text { C3-IX-PROD-ANTF } \\
\text { Analyze in Campaian N if needed }\end{array}$ & NONE & tank & 100 & & & & & & & & & 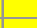 & & & & & & & & \\
\hline & & $\begin{array}{l}\text { Displacement solutio } \\
\text { Post-elution rinse displ }\end{array}$ & 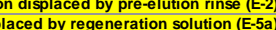 & $\begin{array}{l}\text { Anallyze in Campaign } \\
\text { Anly in needed } \\
\text { in Campaign iv if needed }\end{array}$ & & & & & & & & & & & & & & & & & & & & \\
\hline & & & Regeneration solution (E-5b) & Analyze in Campaign IV if needed & & & & & & & & & & & 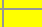 & & & & & & & & & \\
\hline TLP EVA & ORATOR & & LAW Feed (IX product) & & & & & & 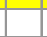 & & & & 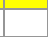 & & 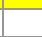 & L & & & & & & & & \\
\hline & & IX $0.25 \mathrm{M} \mathrm{NaOH}$ Reg & $\begin{array}{l}\text { LAW SBS } \\
\text { eneration Solution from Campaing }\end{array}$ & & & & & & 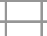 & & & & & & 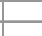 & $\square$ & & & & & & & & \\
\hline & EVAPORATE & Evaporator Feed Mixture & e & C3-TLP-FEED-ACTL & NONE & feed tank & 50 & & 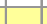 & & & & & & 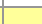 & TS & UDS & DENS & & & & & & \\
\hline & & & & C3-TLP-FEED-WET & & feed tank & 50 & & & & & ICA & ICC & & & & & & & & & TBOH & & TIC/TOC \\
\hline & & & & C3-TLP-FEED-MW & 42112 & $\begin{array}{l}\text { feed tank } \\
\text { fees }\end{array}$ & 50 & DISMW & 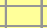 & ICPES & ICPMS & & & & AAK & & & & & & & & & \\
\hline & & & & & NONE & feed tank & 1000 & & $\mathrm{~B}_{-1}$ & & & & & & & & & & & & & & & \\
\hline & & Bottoms & After all antifoam additions & $\begin{array}{l}\text { C3-TLP-B-START-ARC } \\
\text { C3-TIPBSTAP-ANTS }\end{array}$ & NONE & bottoms tank & 100 & & $\square$ & & & & 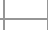 & & 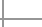 & E & & & & & & & & \\
\hline & & Bottoms & At $-200 \mathrm{~L} \mathrm{fed}$ & $\begin{array}{l}\text { C3-TLPP-BSTRRT-ANTF } \\
\text { C3-TLP-B-200-ARC }\end{array}$ & $\begin{array}{l}\text { NONE } \\
\text { NONE }\end{array}$ & $\begin{array}{l}\text { bottoms tank } \\
\text { bottoms tank }\end{array}$ & $\begin{array}{l}100 \\
50\end{array}$ & & $\square$ & & & 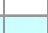 & - & & 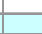 & 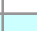 & & & & & & & & \\
\hline & & Bottoms & At $-400 \mathrm{~L}$ fed & C3-TLP-B-400-ARC & NONE & bottoms tank & 50 & & 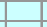 & & & & & & & - & & & & & & & & \\
\hline & & Bottoms & & $\begin{array}{l}\text { C3-TLP-B-600-ARC } \\
\text { C-3TP-B-80-ARC }\end{array}$ & NONE & bottoms tank & 50 & & & & & & & & & & & & & & & & & \\
\hline & & & $\begin{array}{l}\text { At }-800 \mathrm{~L} \text { fed } \\
\text { At } 1000 \mathrm{Lfed}\end{array}$ & $\begin{array}{l}\text { C3-TLPP-B-800-ARC } \\
\text { C3-TLP-B-100-ARC }\end{array}$ & $\begin{array}{l}\text { NONE } \\
\text { NONE }\end{array}$ & $\begin{array}{l}\text { bottoms tank } \\
\text { bottoms tank }\end{array}$ & $\begin{array}{l}50 \\
50\end{array}$ & & 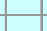 & & & & & & & & & & & & & & & \\
\hline & & $\begin{array}{l}\text { Dotoms } \\
\text { Bottoms }\end{array}$ & Evaporation completed & C3-TLP-B-END-ACTL & NONE & $\begin{array}{l}\text { bolumis dank } \\
\text { bottoms tank }\end{array}$ & 100 & & & & & & & & & TS & UDS & DENS & & & & & & \\
\hline & & & & $\begin{array}{l}\text { C3-IPP-B-END-ADS } \\
\text { C.TPPB }\end{array}$ & 42113 & bottoms tank & 50 & & & & & & ICC & & & & & & & & & & & \\
\hline & & Primary Condensate & Composite & $\begin{array}{l}\text { C3-TLPP-B-END-ARC } \\
\text { C3-TLP-PC-END-ARC }\end{array}$ & $\begin{array}{l}\text { NOOE } \\
\text { NONE }\end{array}$ & bottoms tank & $\begin{array}{l}100 \\
100\end{array}$ & & - & & & & & & & & & & & & & & & \\
\hline & & Secondary Condensate & Composite & C3-TLP-SC-END-ARC & NONE & & 100 & & & & & & & & & & & & & & & & & \\
\hline & COMBINE TLP C & ONDENSATES & & & & storane tank & & & & & & & & & & & & & & & & & & \\
\hline & & $\begin{array}{l}\text { Combined TLP Condensi } \\
\text { (Primary + Secondary) }\end{array}$ & sate $=$ RLD Condensate & 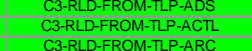 & $\begin{array}{l}42114 \\
\text { NONE } \\
\text { NOEE }\end{array}$ & storage tank & $\begin{array}{c}50 \\
50 \\
1000\end{array}$ & & & & & & & & & TS & & DENS & & & & & & \\
\hline & COMBINE FEP \& & TLP CONDENSATES & Combined condensate & C3-RLD-COMP-ARC & NONE & storage tank & 1000 & & & & & & & & & & & & & & & & & \\
\hline & & & & C3-RLD-COMP-ADS & 42115 & storage tank & 50 & & & & & & ICC & & & & & & & & & & & \\
\hline Notes: & Time Dep Sample & Time dependent sample. Th & These samples are to be observed daily f & tor about 5 days for evidence of precip & itation of solids. & & & & & & & 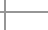 & & & 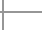 & 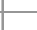 & & & & & & & & \\
\hline & & If solids form, they will be a & analyzed by XRD. A filtrate blank will als & o be analyzed. & & & & & & & & & & & & & & & & & & & & \\
\hline & Explanation of analyse & & & & & & & & & & & & & & & & & & & & & & & \\
\hline & $\begin{array}{l}\text { pp } \\
\text { ICPES }\end{array}$ & $\begin{array}{l}\text { H pH } \\
\mathrm{S} \text { includes all elements prese }\end{array}$ & ent that can be analyzed by ICPES & $\begin{array}{l}\text { lon hhrom. catitins } \\
\text { Ion exclusion chrom. }\end{array}$ & $\begin{array}{l}\text { ammonium } \\
\text { citrate, acetate } \\
\text { a }\end{array}$ & $\begin{array}{l}\text { DENS } \\
\text { RHEO }\end{array}$ & $\begin{array}{l}\text { density } \\
\text { theology }\end{array}$ & & & & & & & & & & & & & & & & & \\
\hline & ICPMS & $\mathrm{S}$ sludges & $\mathrm{Cs}, \mathrm{Rb}, \mathrm{Bi}, \mathrm{Y}, \mathrm{Pr}$ & atomic absorption & $\mathrm{CS}_{\mathrm{K}} \mathrm{K}, \mathrm{Na}$ & PSD & particle siz & e distribution & on by $\mathrm{N}$ & Microtrac & & & & & & & & & & & & & & \\
\hline & & $\begin{array}{l}\text { supernates } \\
\text { A lon chrom - anions }\end{array}$ & $\begin{array}{l}\text { Ss, Rb } \\
\text { nomal suite }\end{array}$ & $\begin{array}{l}\text { total solids w w\% } \\
\text { undissolved solids wi\% }\end{array}$ & & $\begin{array}{c}\text { XXD } \\
\text { TBOH }\end{array}$ & $\begin{array}{l}\text { llray diffica } \\
\text { total base. }\end{array}$ & $\begin{array}{l}\text { tion } \\
\text { tree hydroxic }\end{array}$ & ide, ot & ther base & & & & & & & & & & & & & & \\
\hline
\end{tabular}


WSRC-TR-2005-00105, REVISION 0

SRNL-RPP-2005-00012, REVISION 0

Table D5-1 Campaign IV Sample Matrix (1 of 3 pages)

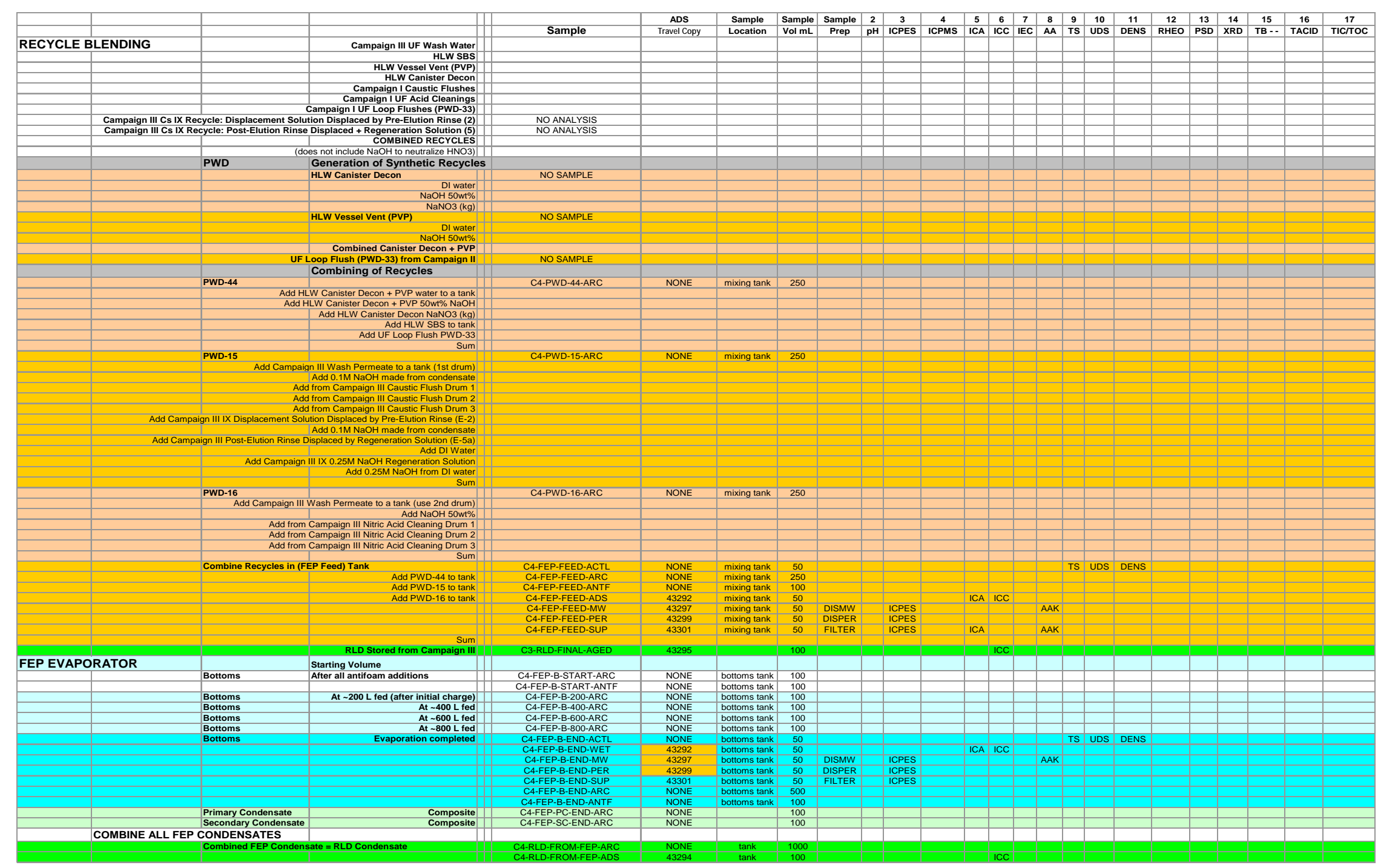


WSRC-TR-2005-00105, REVISION 0

SRNL-RPP-2005-00012, REVISION 0

Table D4-5 Campaign IV Sample Matrix (2 of 3 pages)

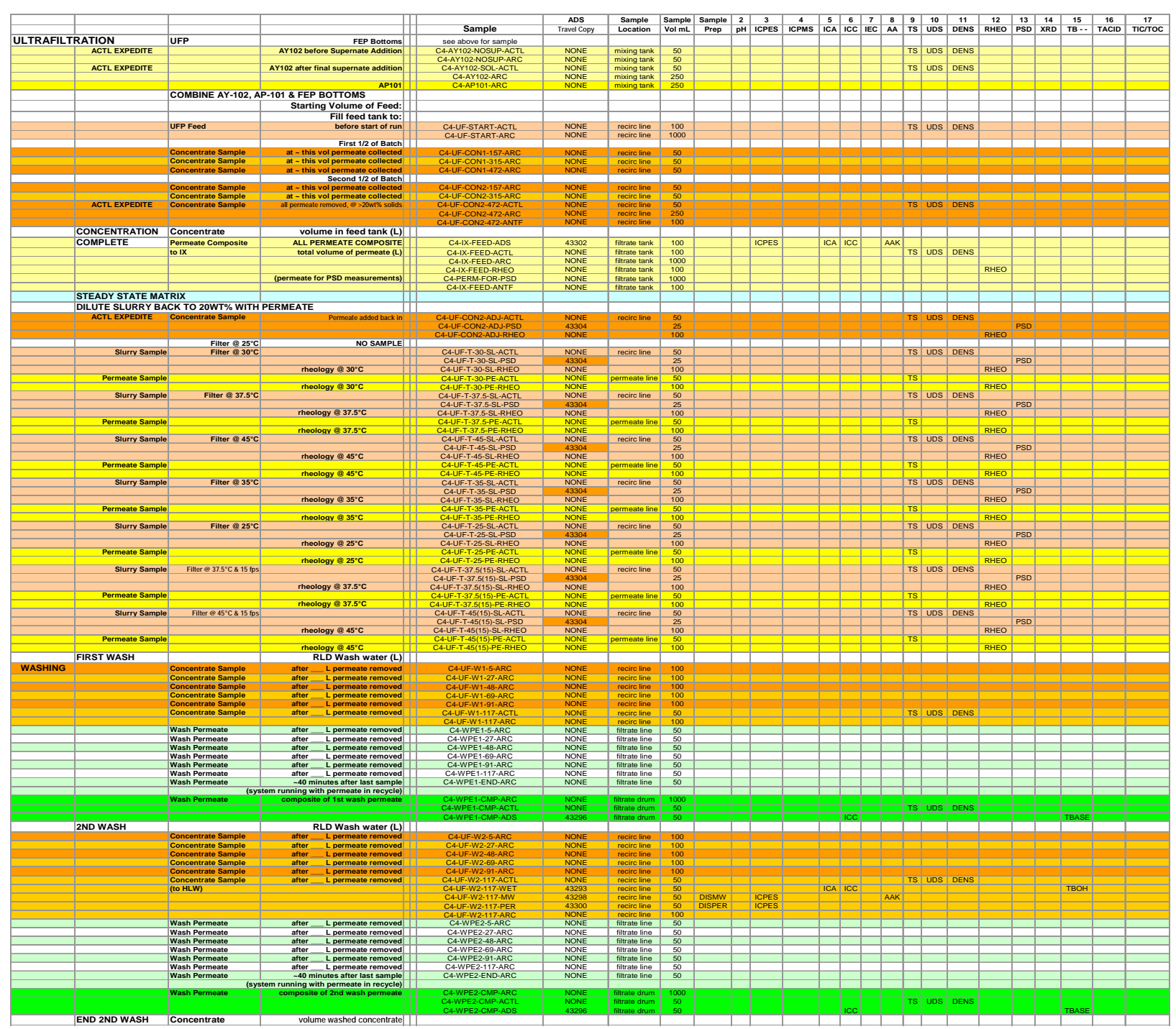


WSRC-TR-2005-00105, REVISION 0 SRNL-RPP-2005-00012, REVISION 0

Table D4-5 Campaign IV Sample Matrix (3 of 3 pages)

\begin{tabular}{|c|c|c|c|c|c|c|c|c|c|c|c|c|c|c|c|c|c|c|c|c|c|c|c|c|}
\hline & & & & Sample & $\begin{array}{c}\text { ADS } \\
\text { Travel Copy }\end{array}$ & $\begin{array}{l}\text { Sample } \\
\text { Location }\end{array}$ & $\begin{array}{l}\text { Sample } \\
\text { Vol mL }\end{array}$ & \begin{tabular}{|l|} 
Sample \\
Prep \\
\end{tabular} & \begin{tabular}{|l|}
2 \\
$\mathrm{pH}$ \\
\end{tabular} & \begin{tabular}{|c|}
3 \\
ICPES \\
\end{tabular} & \begin{tabular}{|c|}
4 \\
ICPMS \\
\end{tabular} & \begin{tabular}{|c|}
5 \\
ICA \\
\end{tabular} & \begin{tabular}{|c|}
6 \\
$1 C C$ \\
\end{tabular} & \begin{tabular}{|l|}
7 \\
IEC
\end{tabular} & \begin{tabular}{|l|}
8 \\
$A A$
\end{tabular} & \begin{tabular}{|l|l|}
9 & \\
TS
\end{tabular} & \begin{tabular}{|l|}
10 \\
UDS
\end{tabular} & $\begin{array}{c}11 \\
\text { DENS }\end{array}$ & \begin{tabular}{|c|}
12 \\
RHEO
\end{tabular} & \begin{tabular}{|l}
13 \\
PSD
\end{tabular} & \begin{tabular}{|l|}
14 \\
XRD
\end{tabular} & $\begin{array}{l}15 \\
T B . .\end{array}$ & $\begin{array}{l}16 \\
\text { TACID }\end{array}$ & $\begin{array}{l}17 \\
\text { TICITOC }\end{array}$ \\
\hline \multirow{11}{*}{ CLEANING } & & & & & & & & & & & & & & & & & & & & & & & & \\
\hline & Loop Flush & No Loop Flush Need & ds to be Made & & & & & & & & & & & & & & & & & & & & & \\
\hline & UF Caustic Flush 1 & Flush UF System & & & & & & & & & & & & & & & & & & & & & & \\
\hline & & Caustic Flush 1 & solution drained & C4-CAU-1-ACTL & NONE & transfer line & 50 & & & & & & & & & TS & UDS & DENS & & & & & & \\
\hline & & & & $\begin{array}{l}\text { C4-CAUA-1-ADS } \\
\text { C4-CAU-1-ARC }\end{array}$ & $\begin{array}{l}\text { ARCHIVE } \\
\text { ARCHE }\end{array}$ & $\begin{array}{l}\text { transfer line } \\
\text { transfer line }\end{array}$ & $\begin{array}{r}50 \\
1000 \\
\end{array}-10$ & & & & & & & & & & & & & & & TBASE & & \\
\hline & $\begin{array}{l}\text { HNO3 Cleaning } \\
\text { Cleaning } 11\end{array}$ & $\begin{array}{l}2 \mathrm{M} \mathrm{HNO3} \text { cleaning } \\
\text { Acid Cleaning } 1\end{array}$ & solution drained & C4-NIT-1-ACTL & NONE & transfer line & & & & & & & & & & TS & UDS & DENS & & & & & & \\
\hline & & & & $\begin{array}{l}\text { C4-NIT-1-ADS } \\
\text { CANIT-ARC }\end{array}$ & $\begin{array}{l}43303 \\
4303 \\
\text { NONE }\end{array}$ & $\begin{array}{l}\text { transter Inie } \\
\text { transfer line } \\
\text { transforlline }\end{array}$ & $\begin{array}{r}50 \\
50 \\
1000\end{array}$ & & & & & & & & & & & & & & & & TACID & \\
\hline & Cleaning +2 & Acid Cleaning 2 & solution drained & $\begin{array}{l}\text { C4NNIT-2-ACLL } \\
\text { C4NTL }\end{array}$ & $\begin{array}{l}\text { NUNE } \\
\text { NONE } \\
\text { NONE }\end{array}$ & 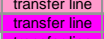 & $\begin{array}{l}1000 \\
50\end{array}$ & & & & & & & & & TS & UDS & DENS & & & & & TACID & \\
\hline & & & & $\begin{array}{l}\text { C4-NAT-2-ADS } \\
\text { C4-NIT-2ARC }\end{array}$ & $\begin{array}{l}4303 \\
\text { NONE }\end{array}$ & $\begin{array}{l}\text { transfer line } \\
\text { transfer line }\end{array}$ & $\begin{array}{c}50 \\
1000\end{array}$ & & & & & & & & & & & & & & & & TACID & \\
\hline & Cleaning \#3 & Acid Cleaning 3 & solution drained & C4-NIT-3-ACTL & NONE & $\begin{array}{l}\text { transfer line } \\
\text { lin }\end{array}$ & 50 & & & & & & & 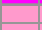 & & TS & UDS & DENS & & & & & & \\
\hline & & & & $\begin{array}{l}\text { C4-NIT-3-ADS } \\
\text { C4-NTT-ARC }\end{array}$ & $\begin{array}{l}43303 \\
\text { NONE }\end{array}$ & $\begin{array}{l}\text { transfer line } \\
\text { transfer line }\end{array}$ & $\begin{array}{r}50 \\
1000\end{array}$ & & & & & & & & & & & & & & & & TACID & \\
\hline \multirow[t]{5}{*}{ UF } & Caustic Flushes 2\&3 & Flush UF System & & & & & & & & & & & & & & & & & & & & & & \\
\hline & & Caustic Flush 2 & solution drained & C4-CAU-2-ACTL & NONE & transfer line & 50 & & & & & & & & & TS & UDS & DENS & & & & & & \\
\hline & & & & $\begin{array}{l}\text { C4-CAU-2-ADS } \\
\text { C4-CAU-2-ARC }\end{array}$ & $\begin{array}{l}43303 \\
\text { NONE }\end{array}$ & $\begin{array}{l}\text { transfer line } \\
\text { transfer line }\end{array}$ & $\begin{array}{c}50 \\
1000\end{array}$ & & & & & & & & & & & & & & & & TACID & \\
\hline & & Caustic Flush 3 & solution drained & C4-CAU-3-ACTL & NONE & transfer line & 50 & & & & & & & & & TS & UDS & DENS & & & & & & \\
\hline & & & & $\begin{array}{l}\text { C4-CAU-3-ADS } \\
\text { C4-CAU-3ARC }\end{array}$ & $\begin{array}{l}43303 \\
\text { NONE }\end{array}$ & $\begin{array}{l}\text { transfer line } \\
\text { transfer lie }\end{array}$ & $\begin{array}{c}50 \\
1000\end{array}$ & & & & & & & & & & & & & & & TBASE & TACID & \\
\hline \multirow{6}{*}{ Notes: } & Time Dep Sample & Time dependent sample. $\tau]$ & These samples are to be observed daily for & about 5 days for evidence of precipitati & tion of solids. & & & & & & & 2 & & 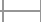 & & & & & & & & & & \\
\hline & 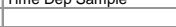 & $\begin{array}{l}\text { If solids torm, they will be } \\
\text { a }\end{array}$ & analyzed by XRD. A filtrate blank will also be & e analyzed. & . & & & & & & & & & & & & & & & & & & & \\
\hline & & & & & & & & & & & & & & & & & & & & & & & & \\
\hline & \multirow[t]{3}{*}{$\begin{array}{r}\text { ph } \\
\text { ICPES }\end{array}$} & $\begin{array}{l}\text { ph } \\
\text { includes all elements pres }\end{array}$ & sent that can be analyzed by ICPES & $\begin{array}{l}\text { lon chrom, cations } \\
\text { lon exclcusion nhrom. }\end{array}$ & $\begin{array}{l}\begin{array}{l}\text { ammonium } \\
\text { citrate, acetate }\end{array} \\
\text { a }\end{array}$ & \multirow{3}{*}{\multicolumn{6}{|c|}{$\begin{array}{l}\text { DENS density } \\
\text { RHEO rheology } \\
\text { PSD particle size distribution by Microtrac } \\
\text { XRD X-ray difraction } \\
\text { TBOH total base, free hydroxide, other base }\end{array}$}} & & & & & & & & & & & & & \\
\hline & & sludges & $\mathrm{Cs}, \mathrm{Rb}, \mathrm{Bi}, \mathrm{Y}, \mathrm{Pr}$ & atomic absorption & $\mathrm{Cs}, \mathrm{K}, \mathrm{Na}$ & & & & & & & 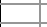 & & 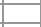 & & 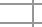 & & & & & & & & \\
\hline & & $\begin{array}{l}\text { Supernates } \\
\text { lon chrom - anions }\end{array}$ & $\begin{array}{l}\mathrm{Cs}, \mathrm{Rb} \\
\text { normal suite }\end{array}$ & $\begin{array}{l}\text { total solilds wt \% } \\
\text { undissolved solids wt\% }\end{array}$ & & & & & & & & & & & & & & & & & & & & \\
\hline
\end{tabular}


WSRC-TR-2005-00105, REVISION 0

SRNL-RPP-2005-00012, REVISION 0

\section{APPENDIX E: RHEOLOGICAL AND PARTICLE SIZE}

The following 96 pages contain the rheological and particle-size distribution analyses results taken during the several campaigns. Each analysis can be related to the point the sample was taken during a campaign by noting the sample number with the sample test matrix in the preceding appendix. The first 42 pages contain the rheological measurements made from samples taken during Campaign II. Such information was only obtained during that Campaign II because it was the first campaign to entail the use of all four unit operations and to limit cost, since rheology was not expected to significantly change. The remaining pages show the particle size distribution measured in Campaigns I, II, and IV. PSD results were not expected to change significantly from Campaigns II and III, but Campaign IV used a new filter, i.e., GKN, and therefore more samples were drawn. Note that for each PSD samples there are two pages of information, i.e., by Volume Distribution and by Number Distribution. Also note the Sample Number is listed in the row entitled "Substance" because the title information was not lined up properly. However, the sample number on those pages can be compared to the one given in the Sample Test Matrix, Appendix D. 
WSRC-TR-2005-00105, REVISION 0

SRNL-RPP-2005-00012, REVISION 0

\section{Rheological Data from SIPP Campaign II}


Company Operator

Date/Time

Substance

Sample no

Description

Comment
SRTC

ITS

15.04.2004 / 12:21:37 PM

c2-fep-b-start-act|

2
Meas ure device $\quad$ RS600

Temperature device $\mathrm{DC} 30$

Sensor $\quad \mathrm{Z41} \mathrm{Ti}$

A-factor

M-factor
6747.000 Pa/Nm

$22.430(1 / \mathrm{s}) /(\mathrm{rad} / \mathrm{s})$
1200203357007

Gap

$3.000 \mathrm{~mm}$

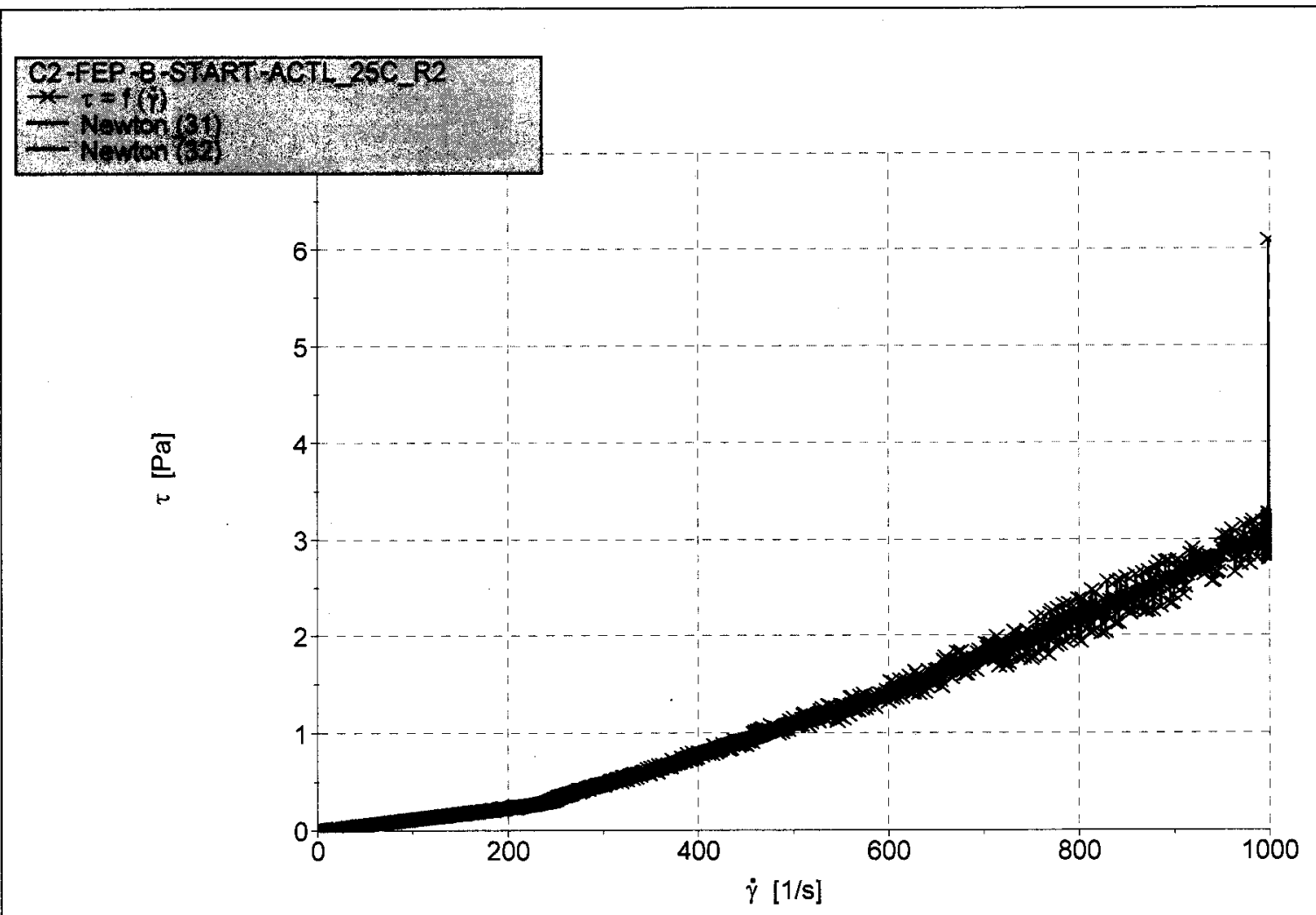

ThermoHaake RheoWin Pro 2.96

Filename: $\quad$ I:IRheologyZZaminecklapr'04/4-15-04IC2-FEP-B-START-ACTL_25C_R2.rwd (Mod)

Job:

C:IPROGRAM FILESIRHEOWINIJOBSIRPP Z41new program.rwj

I:RheologylZaminecklap r

'04/4 -15-04IC2 -FEP-B-START -ACTL_25C_R2.rwd (Mod)

Bingham (29): $\tau_{0}:-0.3522 \eta_{\mathrm{p}}: 0.003141 \mathrm{Chi}^{2}: 9.299 \mathrm{r}: 0.9888$

$$
x=\dot{\gamma}[1 / \mathrm{s}], y=\tau[\mathrm{Pa}]
$$

Bingham (30): $\tau_{0}:-0.364 \eta_{p}: 0.003147 C h i r^{2}: 13.82$ r.0.9837

$\mathrm{x}=\dot{\gamma}[1 / \mathrm{s}], \mathrm{y}=\tau[\mathrm{Pa}]$

Newton (31) : $\eta: 0.001172 \mathrm{Chi}^{2}: 0.001008$ r:0.9994

$$
x=\dot{\gamma}[1 / \mathrm{s}], y=\tau[\mathrm{Pa}]
$$

Newton (32) : $\eta: 0.001126 \mathrm{Chi}^{2}: 0.01445$ r:0.9916

$$
x=\dot{\gamma}[1 / \mathrm{s}], y=\tau[\mathrm{Pa}]
$$


Company

Operator

Date/Time

Substance

Sample no

Description

Comment
SRTC

ITS

15.04.2004/ 12:06:15 PM

c2-fep-b-start-act
Measure device RS600

Temperature device DC30

Sensor $\quad \mathrm{Z41} \mathrm{Ti}$

A-factor

M-factor
$6747.000 \mathrm{~Pa} / \mathrm{Nm}$

$22.430(1 / \mathrm{s}) /(\mathrm{rad} / \mathrm{s})$
1200203357007

Gap

$3.000 \mathrm{~mm}$

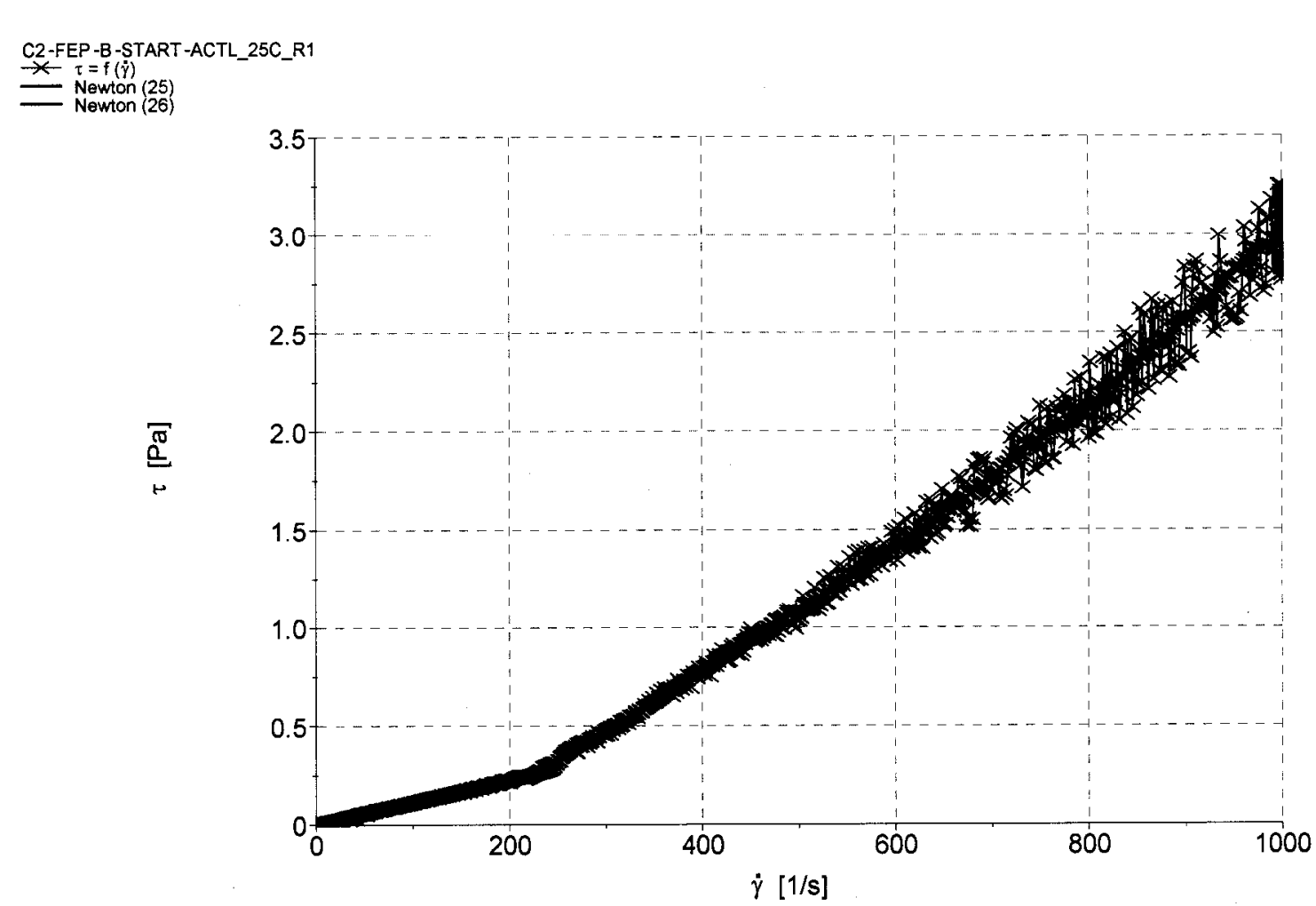

ThermoHaake RheoWin Pro 2.96

Filename: $\quad$ I:IRheologylZaminecklapr '04|4-15-04IC2-FEP-B-START-ACTL_25C_R1.rwd (Mod)

Job:

C:IPROGRAM FILESIRHEOWINIJOBSIRPP Z41new program.w

I:IRheologyZaminecklap

'04I4 -15-04IC2 -FEP-B-START -ACTL_25C_R1.rwd (Mod)

Bingham (23) : $\tau_{0}:-0.34 \eta_{p}: 0.003091 \mathrm{Ch}^{2}: 8.299$ r.0.9897

$x=\dot{\gamma}[1 / \mathrm{s}], y=\tau[\mathrm{Pa}]$

Bingham (24) : $\tau_{0}:-0.3484 \eta_{p}: 0.003097$ Chi $^{2}: 11.48$ r:0.986

$\mathrm{x}=\dot{\gamma}[1 / \mathrm{s}], \mathrm{y}=\tau[\mathrm{Pa}]$

Newton (25) : $\eta: 0.001135 \mathrm{Chi}^{2}: 0.000459$ r:0.9997

$\mathrm{x}=\dot{\gamma}[1 / \mathrm{s}], \mathrm{y}=\tau[\mathrm{Pa}]$

Newton (26) : $\eta: 0.001117 \mathrm{Chi}^{2}: 0.01577$ r:0.9906

$x=\dot{\gamma}[1 / s], y=\tau[\mathrm{Pa}]$ 
Company

Operator

Date/Time

Substance

Sample no

Description
SRTC

ITS

15.04.2004 / 12:51:08 PM c2-fep-b-end-actl

2
Meas ure device RS600

Temperature device $\mathrm{DC} 30$

Sensor $\quad \mathrm{Z41} \mathrm{Ti}$

A-factor $\quad 6747.000 \mathrm{~Pa} / \mathrm{Nm}$

M-factor
$22.430(1 / \mathrm{s}) /(\mathrm{rad} / \mathrm{s})$
1200203357007

Gap

Comment

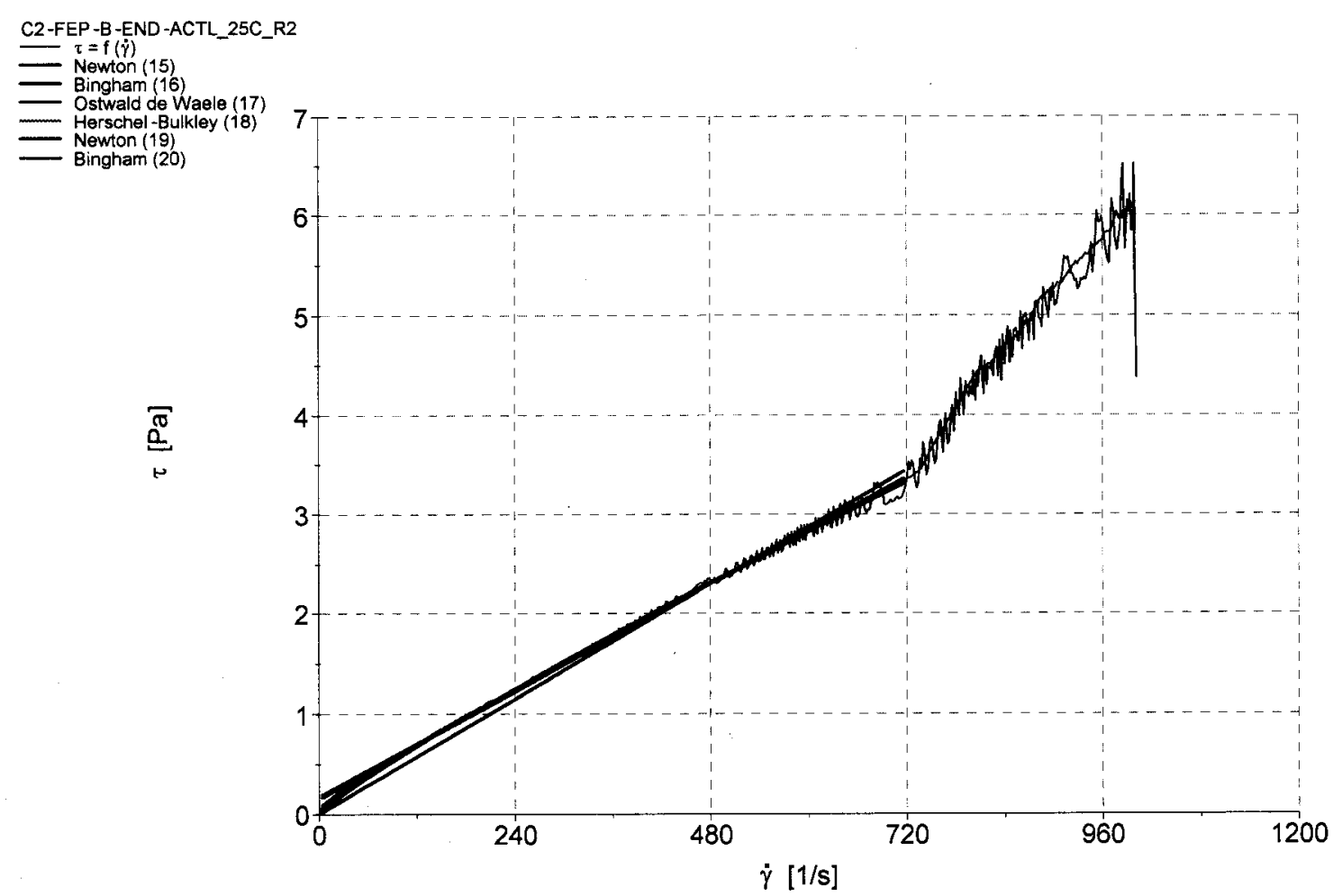

ThermoHaake RheoWin Pro 2.96

Filename:

Job:
I:IRheologyZZaminecklapr '04/4-15-04lC2-FEP-B-END-ACTL_25C_R2.rwd (Mod)

C:IPROGRAM FILESIRHEOWINIJOBSIRPP Z41new program.nj

I:IRheologyZaminecklap $r$

'0414 -15-04lC2 -FEP -B-END-ACTL_25C_R2.rwd (Mod)

Bingham (13) : $\tau_{0}:-0.1768 \eta_{p}: 0.005591 \mathrm{Chi}^{2}: 44.22 \mathrm{r}: 0.9834$

$$
x=\dot{\gamma}[1 / \mathrm{s}], y=\tau[\mathrm{Pa}]
$$

Bingham (14): $\tau_{0}:-0.1441 \eta_{\mathrm{p}}: 0.005515 \mathrm{Chi}^{2}: 47.74 \mathrm{r}: 0.9817$

$$
x=\dot{\gamma}[1 / \mathrm{s}], y=\tau[\mathrm{Pa}]
$$

Newton (15) : $\eta: 0.004787 \mathrm{Chi}^{2}: 2.23$ r.0.9964

$$
\mathrm{x}=\dot{\gamma}[1 / \mathrm{s}], \mathrm{y}=\tau[\mathrm{Pa}]
$$

Bingham (16) : $\tau_{0}: 0.148 \eta_{p}: 0.004479 \mathrm{Chi}^{2}: 0.2482 r: 0.9996$

$$
\mathrm{x}=\dot{\gamma}[1 / \mathrm{s}], \mathrm{y}=\tau[\mathrm{Pa}]
$$

Ostwald de Waele (17) : K:0.009614 n:0.8883 Chi²:0.1317 r:0.9998

$$
x=\dot{\gamma}[1 / s], y=\tau[P a]
$$

Herschel -Bulkley (18) : $\tau_{0}: 0.06383 \mathrm{~K}: 0.007338$ n:0.9271 Chi²:0.05643

r:0.9999

$$
x=\dot{\gamma}[1 / \mathrm{s}], y=\tau[\mathrm{Pa}]
$$

Newton (19) : $\eta: 0.004784 \mathrm{Chi}^{2}: 3.968$ r:0.9935

$$
x=\dot{\gamma}[1 / \mathrm{s}], y=\tau[\mathrm{Pa}]
$$

Bingham (20): $\tau_{0}: 0.1784 \eta_{\mathrm{p}}: 0.004412 \mathrm{Chi}^{2}: 1.084$ r:0.9982

$$
\mathrm{x}=\dot{\gamma}[1 / \mathrm{s}], \mathrm{y}=\tau[\mathrm{Pa}]
$$


Company

Operator

Date/Time

Substance

Sample no

Description
SRTC

ITS

15.04.2004 / 12:36:00 PM

c2-fep-bend-actl

1
Meas ure device RS600

Temperature device DC30

Sensor

A-factor

M-factor
$\mathrm{Z41} \mathrm{Ti}$

$6747.000 \mathrm{~Pa} / \mathrm{Nm}$

$22.430(1 / \mathrm{s}) /(\mathrm{rad} / \mathrm{s})$
1200203357007

Gap $\quad 3.000 \mathrm{~mm}$

\section{Comment}

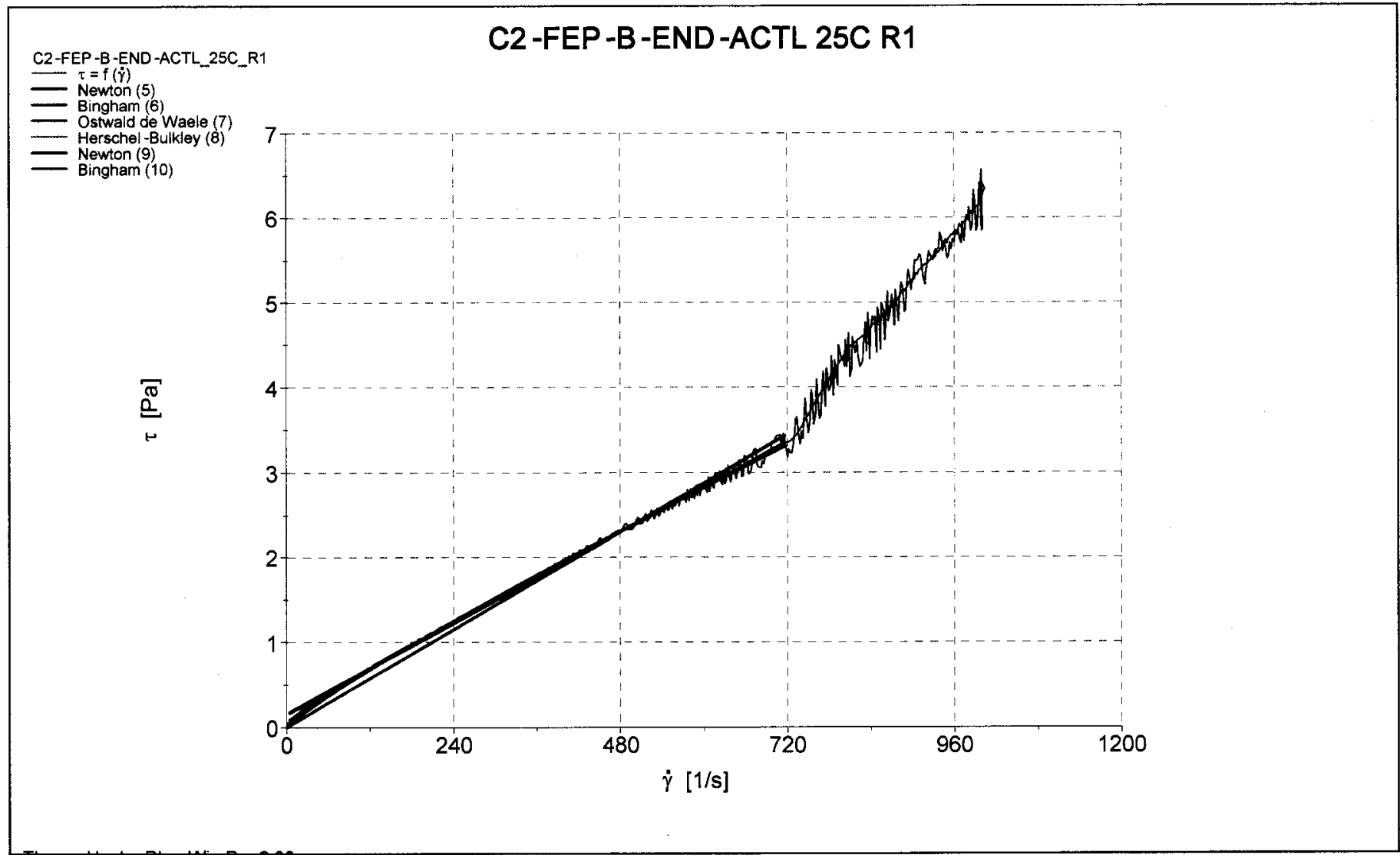

Filename:

I:IRheologylZaminecklapr '04|4-15-04|C2-FEP-B-END-ACTL_25C_R1.nwd (Mod)

Job:

C:IPROGRAM FILESIRHEOWINIJOBSIRPP Z41new program.wj

I:IRheologylZaminecklap r

'0414 -15-04IC2 -FEP-B-END-ACTL_25C_R1.rwd (Mod)

Bingham (3) : $\tau_{0}:-0.1842 \eta_{p}: 0.005617 \mathrm{Chi}^{2}: 46.08$ r.0.9828

$$
\mathrm{x}=\dot{\gamma}[1 / \mathrm{s}], \mathrm{y}=\tau[\mathrm{Pa}]
$$

Bingham (4) : $\tau_{0}:-0.1627 \eta_{\mathrm{p}}: 0.005567 \mathrm{Chi}^{2}: 49.82$ r.0.9813

$$
x=\dot{\gamma}[1 / \mathrm{s}], y=\tau[\mathrm{Pa}]
$$

Newton (5) : $\eta: 0.004785 \mathrm{Chi}^{2}: 2.261$ r.0.9964

$$
x=\dot{\gamma}[1 / \mathrm{s}], y=\tau[\mathrm{Pa}]
$$

Bingham (6) : $\tau_{0}: 0.1491 \eta_{p}: 0.004474 C h i^{2}: 0.2485$ r:0.9996

$$
x=\dot{\gamma}[1 / \mathrm{s}], y=\tau[\mathrm{Pa}]
$$

Ostwald de Waele (7) : K:0.00966 n:0.8874 Chi :0.1304 r:0.9998

$$
\mathrm{x}=\dot{\gamma}[1 / \mathrm{s}], \mathrm{y}=\tau[\mathrm{Pa}]
$$

Herschel-Bulkley (8) : $\tau_{0}: 0.06437$ K:0.007359 n:0.9265 Chi²:0.05389

r:0.9999

$$
x=\dot{\gamma}[1 / \mathrm{s}], y=\tau[\mathrm{Pa}]
$$

Newton (9) : $\eta: 0.004796 \mathrm{Chi}^{2}: 3.289$ r.0.9946

$$
x=\dot{\gamma}[1 / \mathrm{s}], y=\tau[\mathrm{Pa}]
$$

Bingham (10): $\tau_{0}: 0.1664 \eta_{\mathrm{p}}: 0.004449 \mathrm{Chi}^{2}: 0.7991$ r.0.9987

$$
\mathrm{x}=\dot{\gamma}[1 / \mathrm{s}], \mathrm{y}=\tau[\mathrm{Pa}]
$$


Company

Operator

Date/Time

Substance

Sample no

Description
SRTC

ITS

22.04.2004 / 7:40:38 AM C2-UF-START-AS-ACTL

2
Meas ure device RS600

Temperature device DC30

Sensor $\quad \mathrm{Z} 41 \mathrm{Ti}$

A-factor

$6747.000 \mathrm{~Pa} / \mathrm{Nm}$

M-factor
1200203357007

Gap

$3.000 \mathrm{~mm}$

\section{Comment}

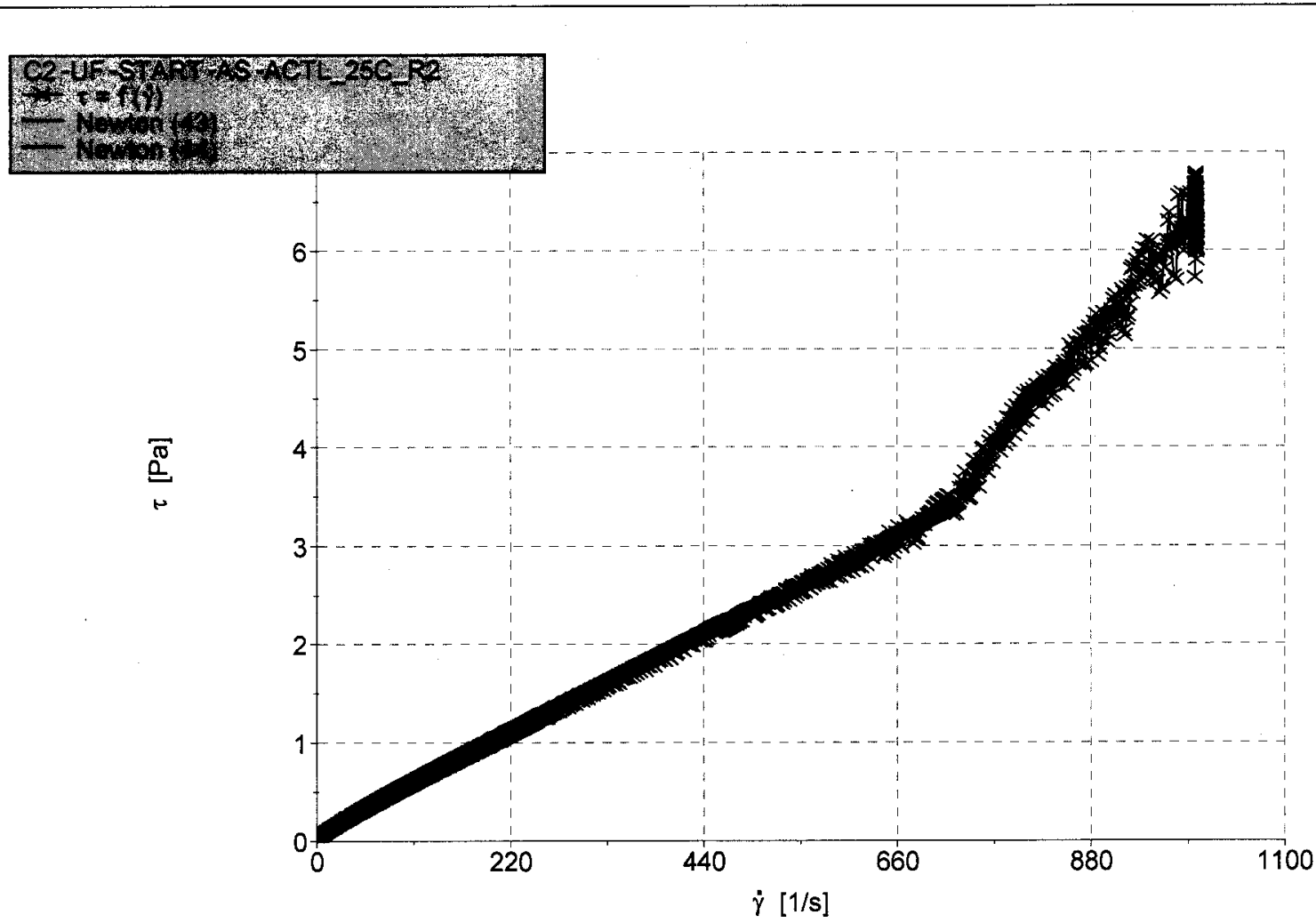

ThermoHaake RheoWin Pro 2.96

Filename:

Job:
I:IRheology'Zaminecklapr '0414-22-04IC2-UF-START-AS-ACTL_25C_R2.rwd (Mod)

C:IPROGRAM FILESIRHEOWINIJOBSIRPP Z41new program.wj

I:RheologylZaminecklapr

'04\4 -22 -04IC2 -UF -START -AS-ACTL_25C_R2.nwd (Mod)

Bingham (41): $\tau_{0}:-0.2443 \eta_{p}: 0.005768 \mathrm{Chi}^{2}: 51.68 \mathrm{r}: 0.9818$ $\mathrm{x}=\dot{\gamma}[1 / \mathrm{s}], y=\tau[\mathrm{Pa}]$

Bingham (42): $\tau_{0}:-0.2912 \eta_{p}: 0.005796 \mathrm{Chi}^{2}: 58.24 \mathrm{r}: 0.9799$ $x=\dot{\gamma}[1 / \mathrm{s}], y=\tau[\mathrm{Pa}]$

Newton (43) : $\eta: 0.004793 \mathrm{Chi}^{2}: 1.134$ r:0.9981 $\mathrm{x}=\dot{\gamma}[1 / \mathrm{s}], \mathrm{y}=\tau[\mathrm{Pa}]$

Newton (44) : ๆ:0.00469 Chi:1.067 r.0.9982 $\mathrm{x}=\dot{\gamma}[1 / \mathrm{s}], \mathrm{y}=\tau[\mathrm{Pa}]$ 
Meas ure device $\quad$ RS600

1200203357007

Operator

ITS

Date/Time

22.04.2004 / 7:22:49 AM

Temperature device $D C 30$

C2-UF-START-AS-ACTL

Sensor

$\mathrm{Z} 41 \mathrm{Ti}$

A-factor

$6747.000 \mathrm{~Pa} / \mathrm{Nm}$

Gap

$3.000 \mathrm{~mm}$ $22.430(1 / \mathrm{s}) /(\mathrm{rad} / \mathrm{s})$

Sample no 1

M-factor

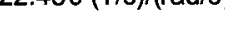

Description

Comment

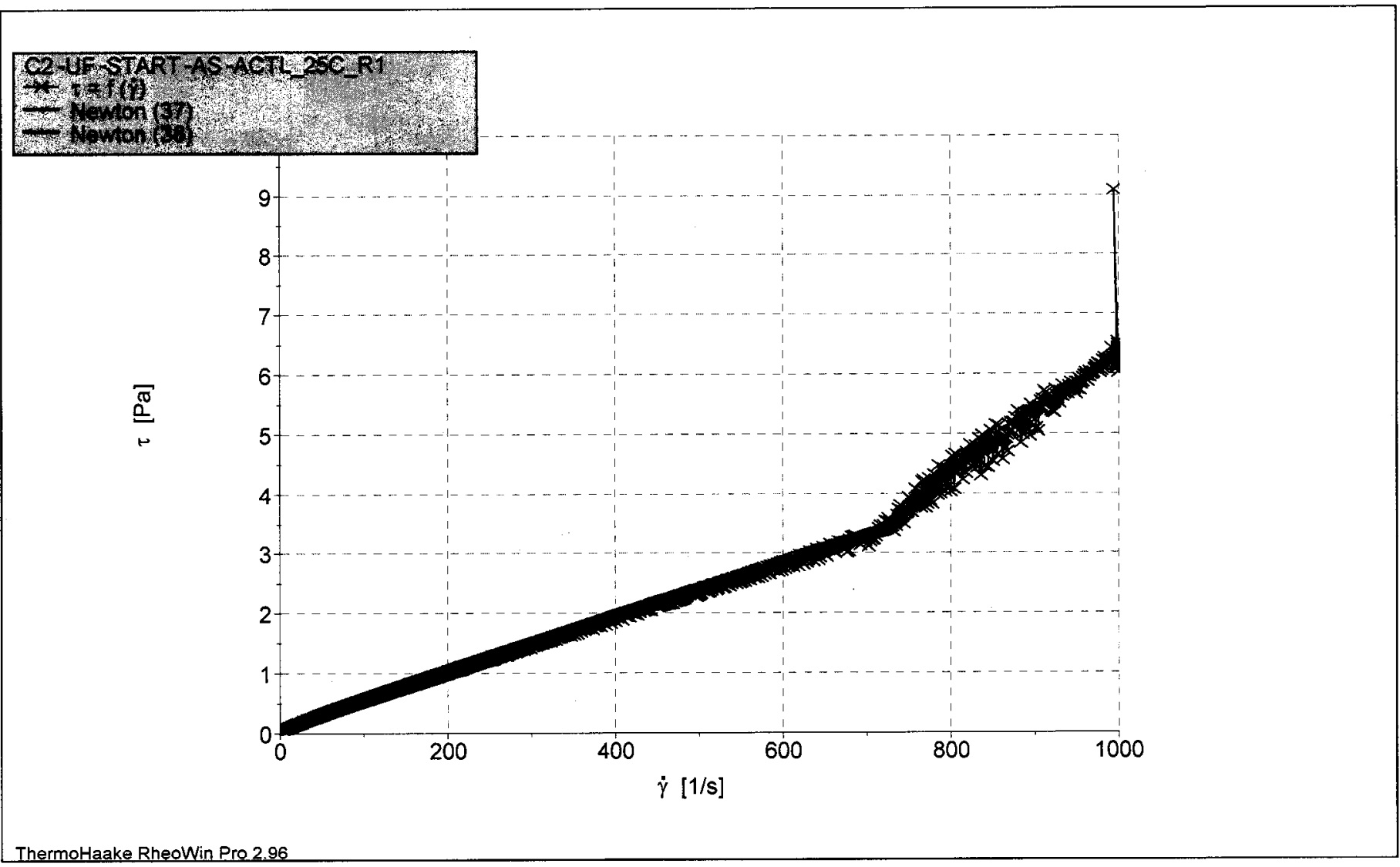

Filename:

I:IRheologyZZaminecklapr ' 0414-22-04IC2-UF-START-AS-ACTL_25C_R1.rwd (Mod)

Job:

C:IPROGRAM FILESIRHEOWINIJOBSIRPP Z41new program.rwj

I:IRheologylZaminecklapr

'0414 -22-04IC2 -UF START-AS-ACTL_25C_R1.nwd (Mod)

Bingham (35): $\tau_{0}:-0.2455 \eta_{p}: 0.005777 \mathrm{Chi}^{2}: 50.32$ r:0.9823

$x=\dot{\gamma}[1 / \mathrm{s}], y=\tau[\mathrm{Pa}]$

Bingham (36): $\tau_{0}:-0.2933 \eta_{p}: 0.005788 \mathrm{Chi}^{2}: 57.93$ r:0.9799

$\mathrm{x}=\dot{\gamma}[1 / \mathrm{s}], \mathrm{y}=\tau[\mathrm{Pa}]$

Newton (37) : $\eta: 0.0048$ Chi2:1.069 r.0.9982

$x=\dot{\gamma}[1 / \mathrm{s}], y=\tau[\mathrm{Pa}]$

Newton (38) : $\eta: 0.004681 \mathrm{Chi}^{2}: 0.9823$ r:0.9983

$x=\dot{\gamma}[1 / \mathrm{s}], y=\tau[\mathrm{Pa}]$ 


$\begin{array}{llllr}\text { Company } & \text { SRTC } & \text { Measure device } & \text { RS600 } & 1200203357007 \\ \text { Operator } & \text { ITS } & \text { Temperature device } & \text { DG4 TI } & \text { Gap } \\ \text { Date/Time } & 22.04 .2004 / 9: 15: 41 \mathrm{AM} & \text { Sensor } & \text { D.100 mm } \\ \text { Substance } & \text { C2-UF-START-SUP-ACTL } & \text { A-factor } & 3701.000 \mathrm{~Pa} / \mathrm{Nm} & \\ \text { Sample no } & 2 & \text { M-factor } & 72.670(1 / \mathrm{s}) /(\mathrm{rad} / \mathrm{s}) & \end{array}$

Description

Comment

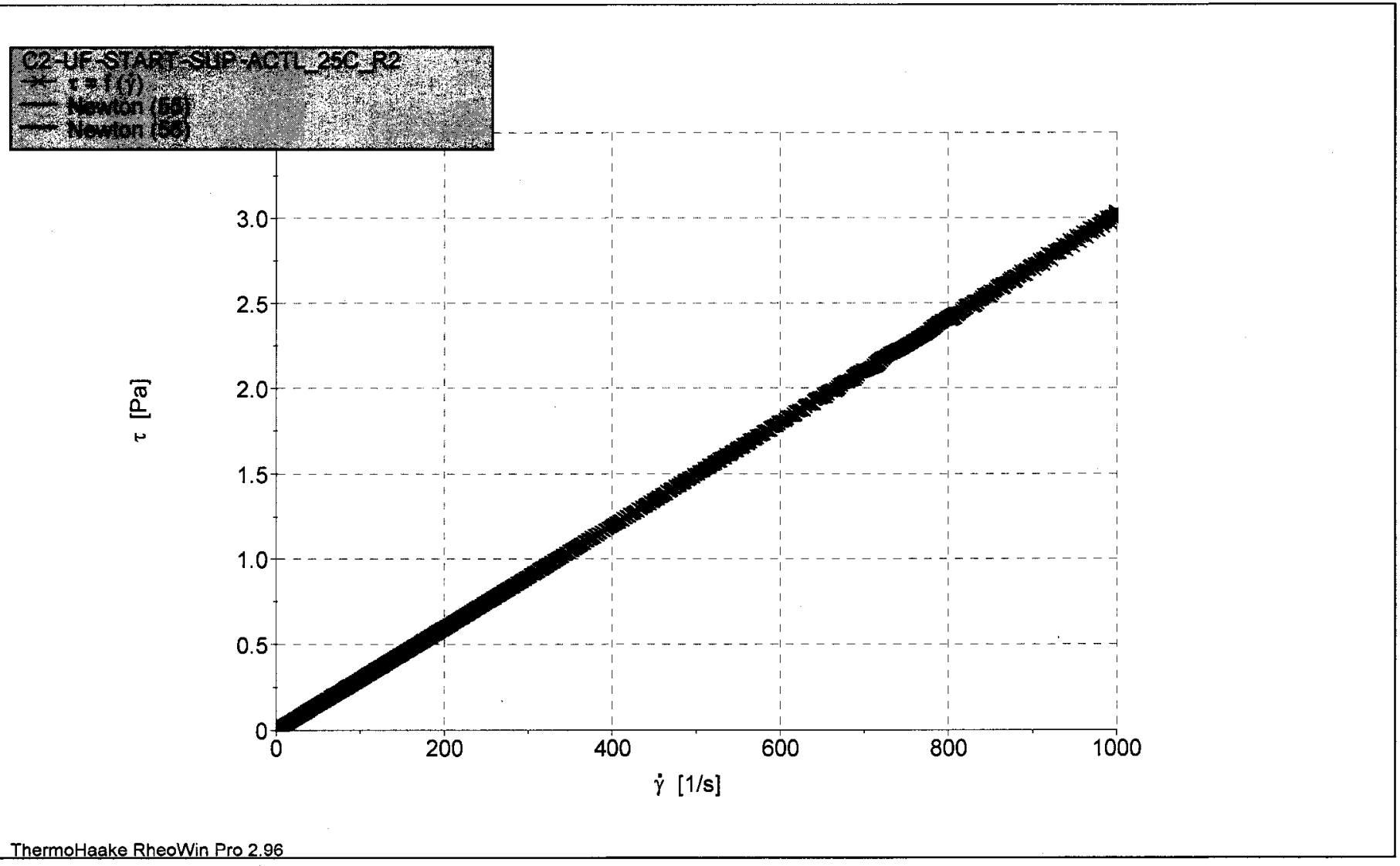

Filename: $\quad$ I:IRheologylZaminecklapr '04\4-22-04IC2-UF-START-SUP-ACTL_25C_R2.rwd (Mod)

Job:

C:IPROGRAM FILESIRHEOWINIJOBSIRPP DG41 program.rwj

I:IRheologyZaminecklap r

'0414 -22-04IC2 -UF -START -SUP-ACTL_25C_R2.nd (Mod)

Bingham (53) : $\tau_{0}:-0.001936 \eta_{p}: 0.003013 \mathrm{Chi}^{2}: 0.01675 \mathrm{r.1}$

$x=\dot{\gamma}[1 / \mathrm{s}], y=\tau[\mathrm{Pa}]$

Bingham (54): $\tau_{0}:-0.01077 \eta_{p}: 0.003018 \mathrm{Chi}^{2}: 0.0495$ r:0.9999

$\mathrm{x}=\dot{\gamma}[1 / \mathrm{s}], \mathrm{y}=\tau[\mathrm{Pa}]$

Newton (55) : $\eta: 0.00301 \mathrm{Chi}^{2}: 0.01722 \mathrm{r}: 1$

$x=\dot{\gamma}[1 / \mathrm{s}], y=\tau[\mathrm{Pa}]$

Newton (56) : $\eta: 0.003002 \mathrm{Chi}^{2}: 0.06519$ r:0.9999

$\mathrm{x}=\dot{\gamma}[1 / \mathrm{s}], \mathrm{y}=\tau[\mathrm{Pa}]$ 
Company Operator

DaterTime

Substance

Sample no

Description
SRTC

ITS

22.04.2004 / 8:51:02 AM C2-UF-START-SUP-ACTL
Meas ure device Temperature device

Sensor

A-factor

M-factor
RS600

DG41 TI

$3701.000 \mathrm{~Pa} / \mathrm{Nm}$

$72.670(1 / \mathrm{s}) /(\mathrm{rad} / \mathrm{s})$
1200203357007

Gap

$5.100 \mathrm{~mm}$

\section{Comment}

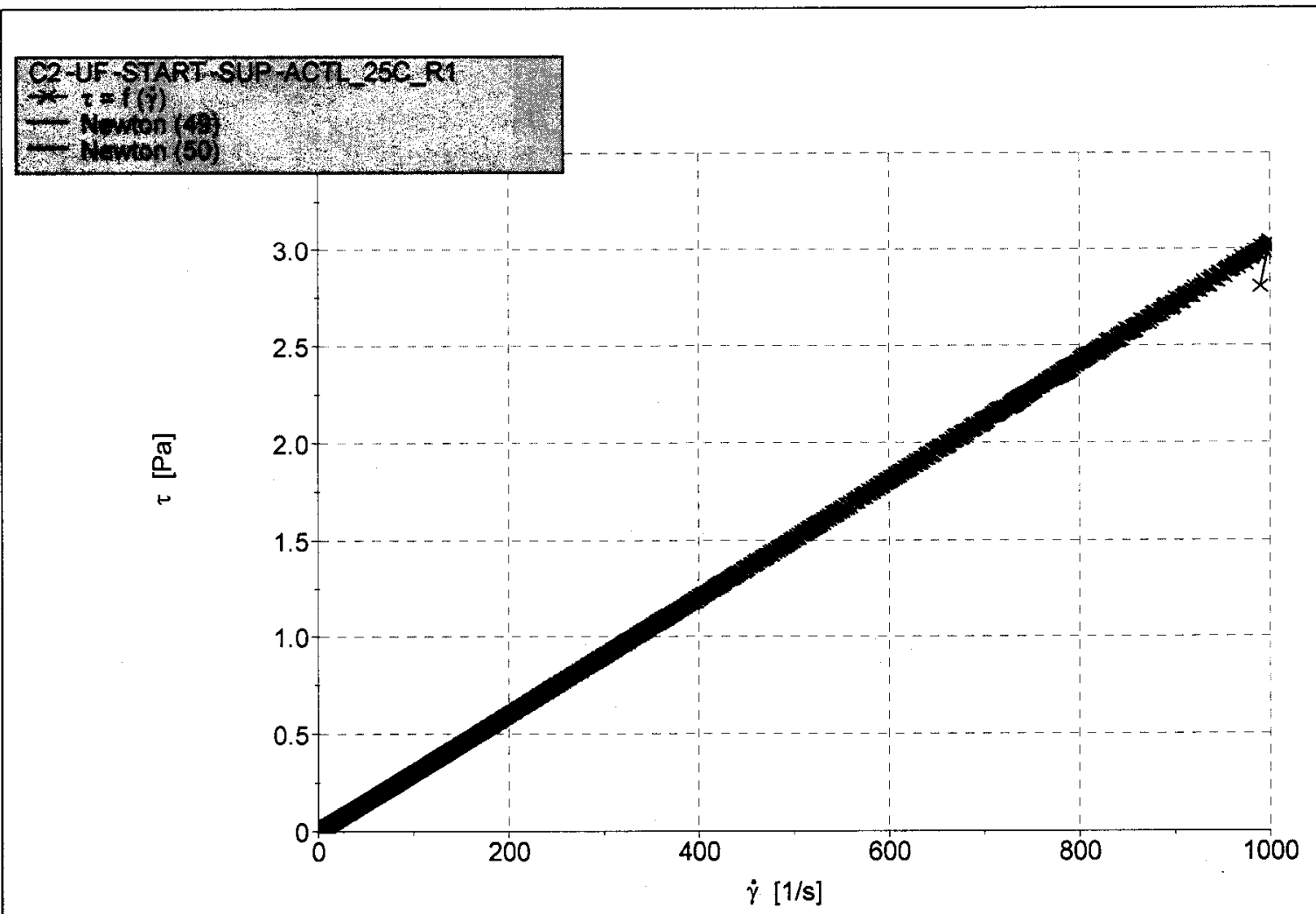

ThermoHaake RheoWin Pro 296

Filename:

Job:
I:IRheology|Zaminecklapr '04\4-22-04IC2-UF-START-SUP-ACTL_25C_R1.rwd (Mod)

C:IPROGRAM FILESIRHEOWINIJOBSIRPP DG41 program.rwj

I:IRheologylZaminecklap r

'04/4 -22-04IC2 -UF -START -SUP-ACTL_25C_R1.wd (Mod)

Bingham (47): $\tau_{0}:-0.006008 \eta_{p}: 0.003028 \mathrm{Chi}^{2}: 0.01736$ r.1

$$
x=\dot{\gamma}[1 / \mathrm{s}], y=\tau[\mathrm{Pa}]
$$

Bingham (48): $\tau_{0}:-0.007315 \eta_{p}: 0.003026 \mathrm{Chi}^{2}: 0.04681$ r.0.9999

$\mathrm{x}=\dot{\gamma}[1 / \mathrm{s}], \mathrm{y}=\tau[\mathrm{Pa}]$

Newton (49) : $\eta: 0.003019 \mathrm{Chi}^{2}: 0.02188 \mathrm{r}: 1$

$$
\mathrm{x}=\dot{\gamma}[1 / \mathrm{s}], \mathrm{y}=\tau[\mathrm{Pa}]
$$

Newton (50) : $\eta: 0.003015 \mathrm{Chi}^{2}: 0.0535$ r:0.9999

$$
\mathrm{x}=\dot{\gamma}[1 / \mathrm{s}], \mathrm{y}=\tau[\mathrm{Pa}]
$$


Company

Operator

Date/Time

Substance

Sample no

Description

Comment
Meas ure device RS600

Temperature device

Sensor

A-factor

M-factor
$238 \mathrm{Ti}$

$8007.000 \mathrm{~Pa} / \mathrm{Nm}$

$8.600(1 / \mathrm{s}) /(\mathrm{rad} / \mathrm{s})$
1200203357007

Gap

$8.000 \mathrm{~mm}$
C2-UF-CON2-461-ACTL
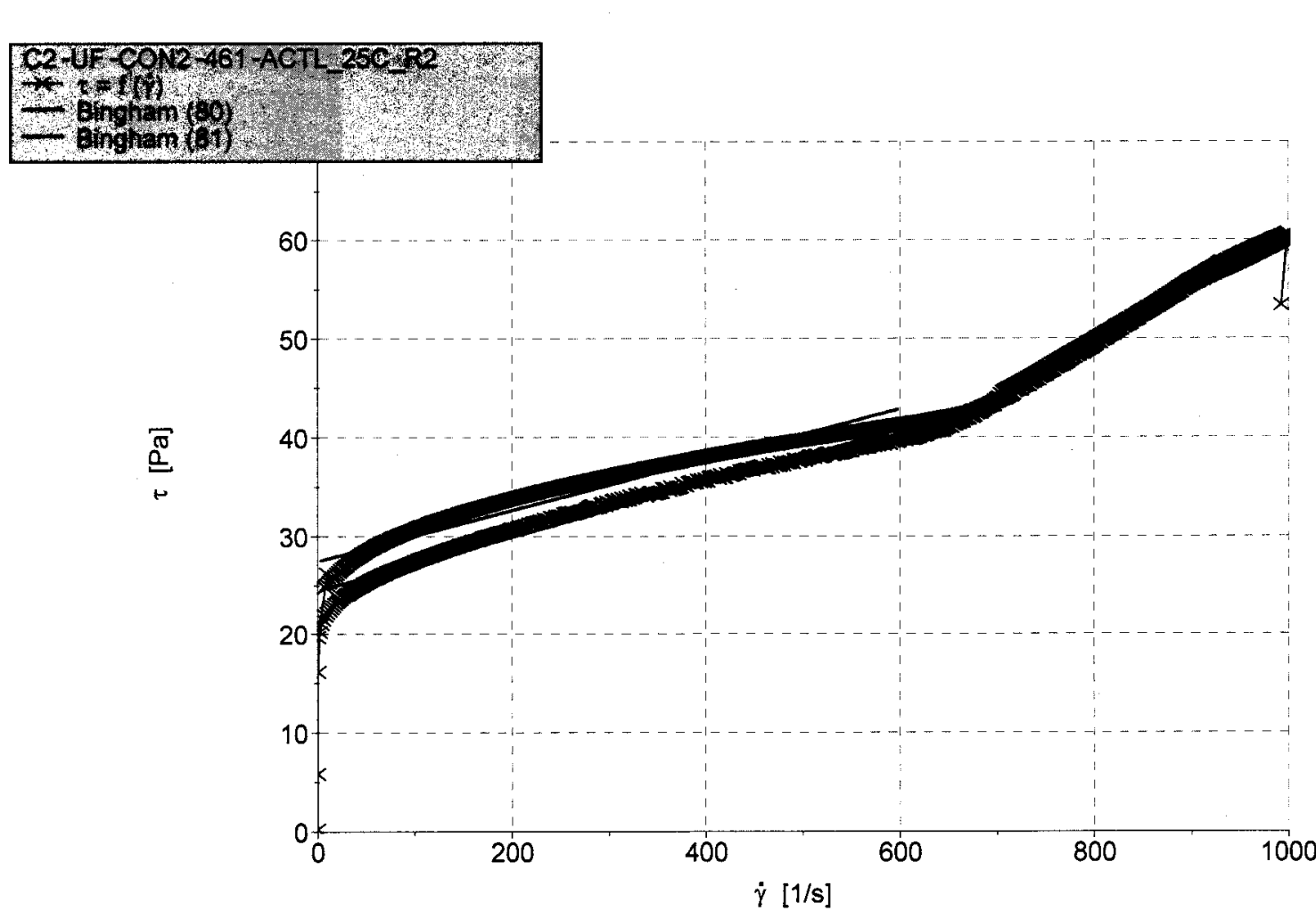

ThermoHaake RheoWin Pro 2.96

Filename:

Job:
I:IRheologyZZaminecklapr '04\4-26-04IC2-UF-CON2-461-ACTL_25C_R2.wwd (Mod)

C:IPROGRAM FILESIRHEOWINIJOBSIRPP Z38 program .rw]

I:IRheologylZaminecklap r

'04I4 -26-04IC2-UF -CON2-461-ACTL_25C_R2.rwd (Mod)

Bingham (77): $\tau_{0}: 25.91 \eta_{\mathrm{p}}: 0.03046 \mathrm{Chi}^{2}: 3270$ r:0.9601

$$
\mathrm{x}=\dot{\gamma}[1 / \mathrm{s}], \mathrm{y}=\tau[\mathrm{Pa}]
$$

Bingham (78) : $\tau_{0}: 22.6 \eta_{p}: 0.03352 \mathrm{Chi}^{2}: 1905$ r:0.9804

$$
\mathrm{x}=\dot{\gamma}[1 / \mathrm{s}], \mathrm{y}=\tau[\mathrm{Pa}]
$$

Bingham (80): $\tau_{0}: 27.47 \eta_{p}: 0.02564 \mathrm{Chi}^{2}: 1537$ r:0.8909

$$
x=\dot{\gamma}[1 / \mathrm{s}], y=\tau[\mathrm{Pa}]
$$

Bingham (81) : $\tau_{0}: 24.49 \eta_{\mathrm{p}}: 0.02722 \mathrm{Chi}^{2}: 264.8 \mathrm{r}: 0.9809$

alda

$1 \%$

i

restured
st

$$
\mathrm{x}=\dot{\bar{\gamma}}[1 / \mathrm{s}], \mathrm{y}=\tau[\mathrm{Pa}]
$$


Company

SRTC

ITS

Date/Time

Substance

Sample no

Description

\section{Comment}

1
Meas ure device $\quad$ RS600

Temperature device

26.04.2004 / 12:40:46 PM C2-UF-CON2-461-ACTL
Sensor

A-factor

M-factor
$\mathrm{Z38} \mathrm{Ti}$

$8007.000 \mathrm{~Pa} / \mathrm{Nm}$

$8.600(1 / \mathrm{s}) /(\mathrm{rad} / \mathrm{s})$
1200203357007

Gap

$8.000 \mathrm{~mm}$

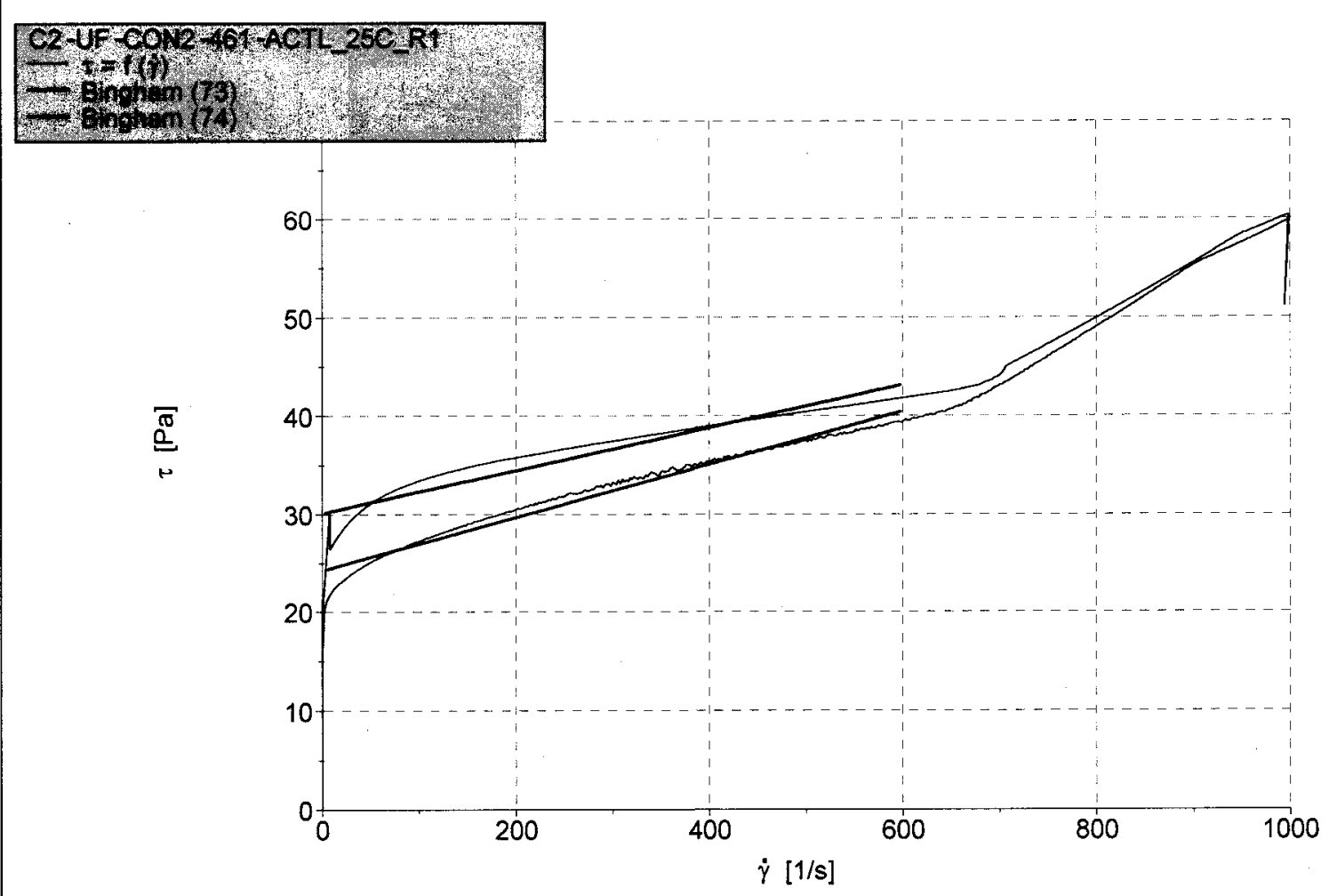

ThermoHaake RheoWin Pro 296

Filename: I:IRheologyiZaminecklapr '04I4-26-04IC2-UF-CON2-461-ACTL_25C_R1.nwd (Mod)

Job:

C:IPROGRAM FILESIRHEOWINIJOBSIRPP Z38 program.nj]

I:IRheology'Zaminecklap r

'0414 -26-04IC2 -UF -CON2 -461-ACTL_25C_R1.rwd (Mod)

Bingham (71): $\tau_{0}: 28.33 \eta_{\mathrm{p}}: 0.02743 \mathrm{Chi}^{2}: 3781$ r:0.9444

$$
\mathrm{x}=\dot{\gamma}[1 / \mathrm{s}], \mathrm{y}=\tau[\mathrm{Pa}]
$$

Bingham (72): $\tau_{0}: 22.38 \eta_{p}: 0.03343 \mathrm{Chi}^{2}: 1980$ r:0.9795

$\mathrm{x}=\dot{\gamma}[1 / \mathrm{s}], \mathrm{y}=\tau[\mathrm{Pa}]$

Bingham (73) : $\tau_{0}: 30.12 \eta_{p}: 0.02173 \mathrm{Chi}^{2}: 1901 \mathrm{r}: 0.8312$

$$
\mathrm{x}=\dot{\gamma}[1 / \mathrm{s}], \mathrm{y}=\tau[\mathrm{Pa}]
$$

Bingham (74): $\tau_{0}: 24.33 \eta_{p}: 0.02695 \mathrm{Chi}^{2}: 248.4 \mathrm{r}: 0.9817$

$x=\dot{\gamma}[1 / \mathrm{s}], y=\tau[\mathrm{Pa}]$ 


\section{Company SRTC \\ Operator ITS}

Date/Time

Substance

Sample no

Description

Comment
Meas ure device RS600

\section{Temperature device}

Sensor

A-factor

M-factor
$\mathrm{Z38} \mathrm{Ti}$

8007.000 Pa/Nm

$8.600(1 / \mathrm{s}) /(\mathrm{rad} / \mathrm{s})$
1200203357007

Gap $8.000 \mathrm{~mm}$ C2-UF-1-W1-1

1

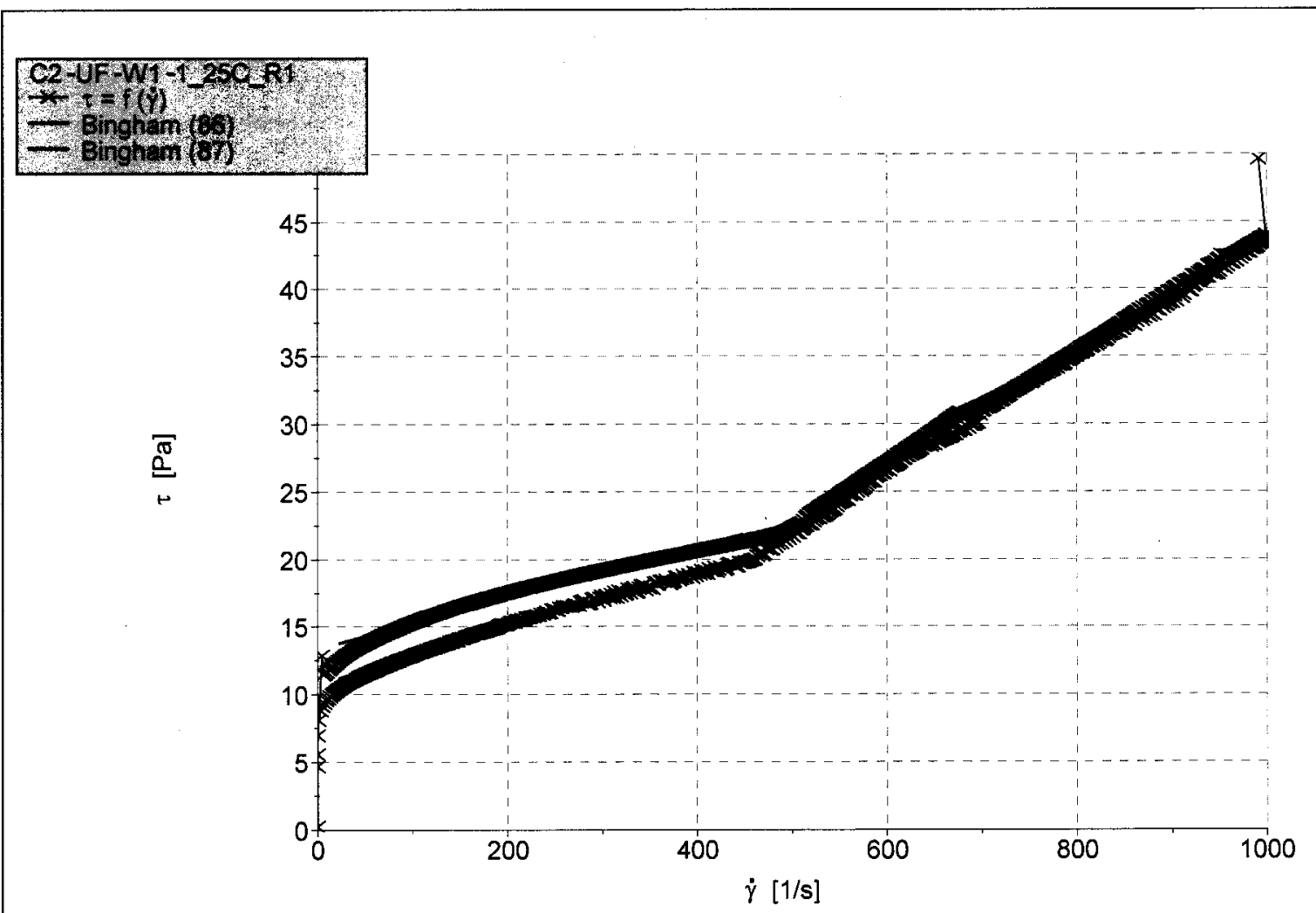

ThermoHaake RheoWin Pro 2.96

Filename: $\quad$ I:IRheologyZZaminecklapr '04I4-26-04IC2-UF-W1-1_25C_R1.rwd (Mod)

Job: $\quad$ C:IPROGRAM FILESIRHEOWINUOBSIRPP Z38 program.rwj

I:IRheologylZaminecklapr '04|4 -26 -04 IC2-UF -W1 -1_25C_R1.rwd

(Mod)

Bingham (84): $\tau_{0}: 10.14 \eta_{p}: 0.03107 \mathrm{Chi}^{2}: 1606 \mathrm{r}: 0.9805$

$$
x=\dot{\gamma}[1 / \mathrm{s}], y=\tau[\mathrm{Pa}]
$$

Bingham (85): $\tau_{0}: 7.634 \eta_{p}: 0.03347 \mathrm{Chi}^{2}: 1248 \mathrm{r}: 0.987$

$$
\mathrm{x}=\dot{\gamma}[1 / \mathrm{s}], \mathrm{y}=\tau[\mathrm{Pa}]
$$

Bingham (86): $\tau_{0}: 13.35 \eta_{p}: 0.019 \mathrm{Chi}^{2}: 31.28$ r.0.9871

$$
\mathrm{x}=\dot{\gamma}[1 / \mathrm{s}], y=\tau[\mathrm{Pa}]
$$

Bingham (87): $\tau_{0}: 10.75 \eta_{p}: 0.02096 \mathrm{Chi}^{2}: 15.79$ r:0.9946

$$
x=\dot{\gamma}[1 / \mathrm{s}], y=\tau[\mathrm{Pa}]
$$




$\begin{array}{llllr}\text { Company } & \text { SRTC } & \text { Meas ure device } & \text { RS600 } & 1200203357007 \\ \text { Operator } & \text { ITS } & \text { Temperature device } & \text { Gap } \\ \text { Date/Time } & 26.04 .2004 / 8: 28: 40 \text { AM } & \text { Sensor } & \text { Z38 Ti } & 8.000 \mathrm{~mm} \\ \text { Substance } & \text { C2-UF-1-W1-1 } & \text { A-factor } & 8007.000 \mathrm{~Pa} / \mathrm{Nm} & \\ \text { Sample no } & 2 & \text { M-factor } & 8.600(1 / \mathrm{s}) /(\mathrm{rad} / \mathrm{s}) & \end{array}$

Description

Comment

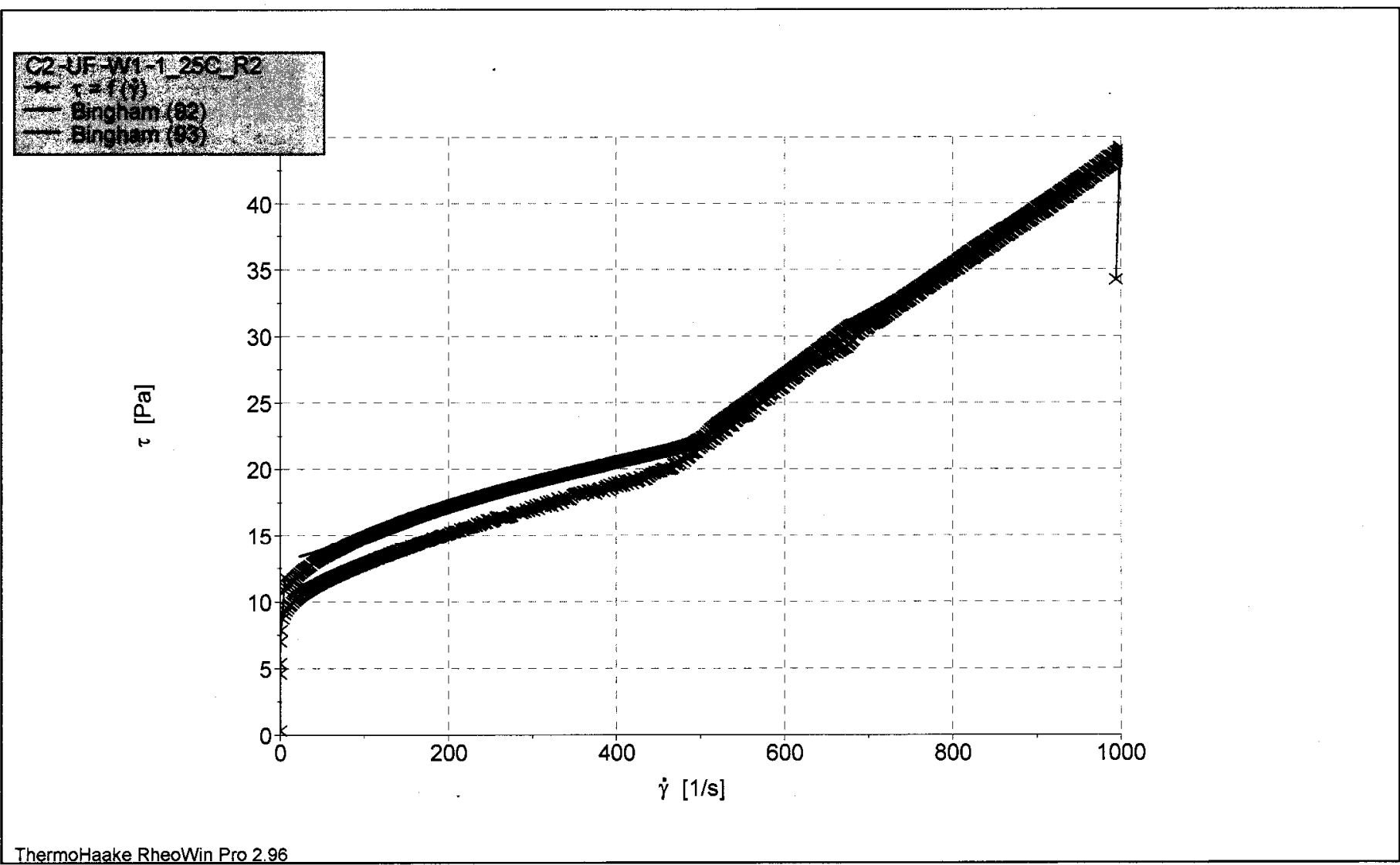

Filename:

I:IRheologylZaminecklapr '04|4-26-04IC2-UF-W1-1_25C_R2.rud (Mod)

Job:

C:IPROGRAM FILESIRHEOWINIJOBSIRPP Z38 program.rwj

I:IRheologylZaminecklap r '04/4 -26-04IC2 -UF -W1 -1_25C_R2.rwd

(Mod)

Bingham (90) : $\tau_{0}: 9.762 \eta_{p}: 0.03148 \mathrm{Chi}^{2}: 1452$ r:0.9828

$x=\dot{\gamma}[1 / \mathrm{s}], y=\tau[\mathrm{Pa}]$

Bingham (91): $\tau_{0}: 7.597 \eta_{\mathrm{p}}: 0.0333 \mathrm{Chi}^{2}: 1269 \mathrm{r}: 0.9867$

$\mathrm{x}=\dot{\gamma}[1 / \mathrm{s}], \mathrm{y}=\tau[\mathrm{Pa}]$

Bingham (92) : $\tau_{0}: 12.95 \eta_{\mathrm{p}}: 0.01923 \mathrm{Chi}^{2}: 34.32$ r:0.99

$\mathrm{x}=\dot{\gamma}[1 / \mathrm{s}], \mathrm{y}=\tau[\mathrm{Pa}]$

Bingham (93) : $\tau_{0}: 10.73 \eta_{\mathrm{p}}: 0.02074 \mathrm{Chi}^{2}: 16.3$ r:0.9959

$$
x=\dot{\gamma}[1 / \mathrm{s}], y=\tau[\mathrm{Pa}]
$$


Company

Operator

Date/Time

Substance

Sample no

Description

\section{Comment}

\section{SRTC}

ITS

26.04.2004 / 8:57:10 AM

C2-UF--W1-5

2
Meas ure device RS600

Temperature device

Sensor

A-factor

M-factor
$238 \mathrm{Ti}$

$8007.000 \mathrm{~Pa} / \mathrm{Nm}$

$8.600(1 / \mathrm{s}) /(\mathrm{rad} / \mathrm{s})$
1200203357007

Gap

$8.000 \mathrm{~mm}$

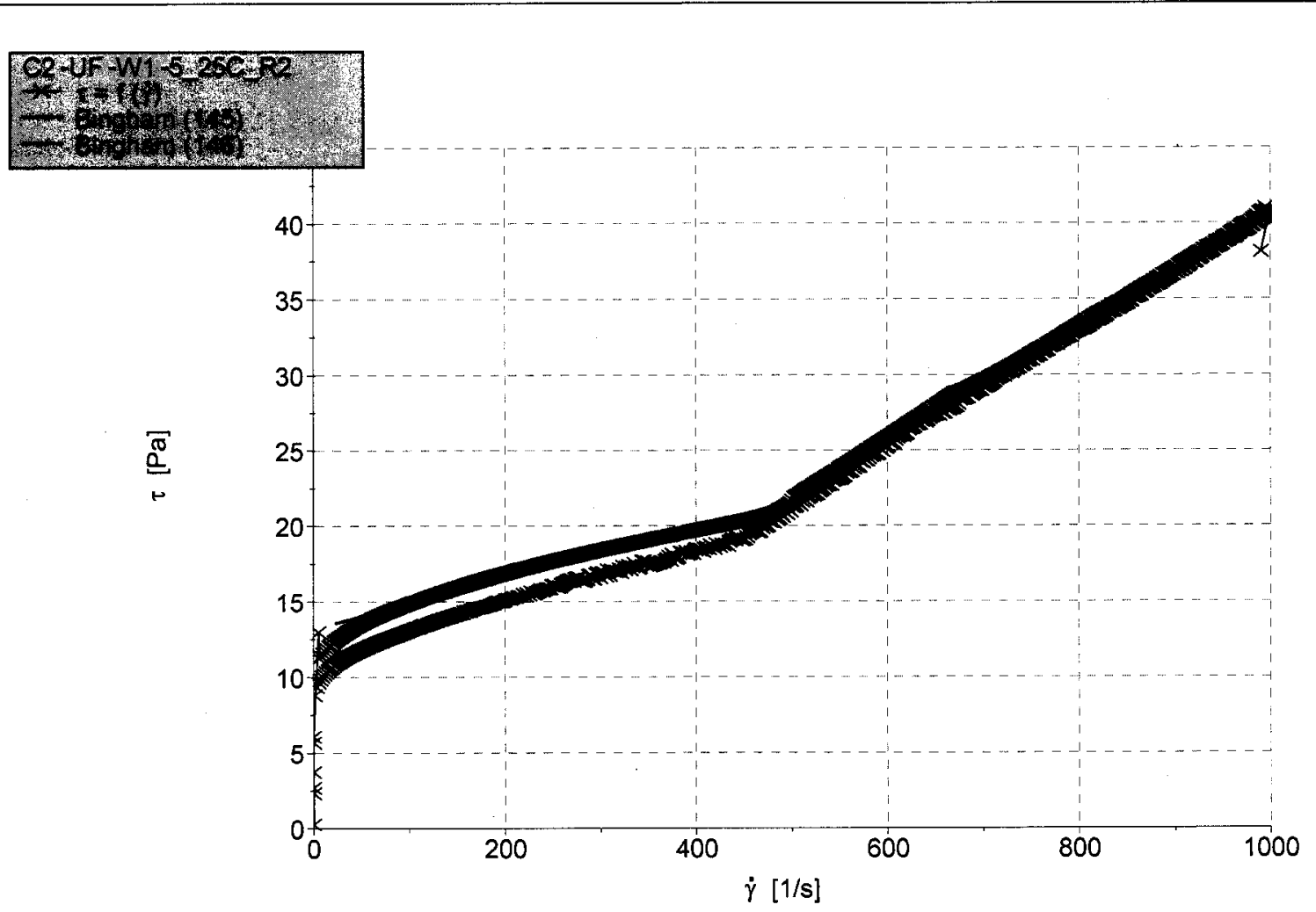

ThermoHaake RheoWin Pro 2.96

Filename: $\quad$ ::IRheologylZaminecklapr '0414-26-04IC2-UF-W1-5_25C_R2.rwd (Mod)

Job:

C:IPROGRAM FILESIRHEOWINIJOBSIRPP Z38 program.nwj

I:IRheologyIZaminecklapr '04I4-26-04IC2 -UF -W1 -5_25C_R2.rwd

(Mod)

Bingham (143): $\tau_{0}: 10.19 \eta_{\mathrm{p}}: 0.02827 \mathrm{Chi}^{2}: 1225$ r:0.982

$x=\dot{\gamma}[1 / \mathrm{s}], y=\tau[\mathrm{Pa}]$

Bingham (144): $\tau_{0}: 8.176 \eta_{\mathrm{p}}: 0.03025 \mathrm{Chj}^{2}: 1096 \mathrm{r}: 0.9862$

$\mathrm{x}=\dot{\gamma}[1 / \mathrm{s}], \mathrm{y}=\tau[\mathrm{Pa}]$

Bingham (145) : $\tau_{0}: 13.16 \eta_{\mathrm{p}}: 0.01677 \mathrm{Chi}^{2}: 24.81 \mathrm{r}: 0.9893$

$\mathrm{x}=\dot{\gamma}[1 / \mathrm{s}], \mathrm{y}=\tau[\mathrm{Pa}]$

Bingham (146) : $\tau_{0}: 11.17 \eta_{\mathrm{p}}: 0.01833 \mathrm{Chi}^{2}: 13.37 \mathrm{r}: 0.9951$

$\mathrm{x}=\dot{\gamma}[1 / \mathrm{s}], \mathrm{y}=\tau[\mathrm{Pa}]$ 
Company SRTC

Operator

Date/Time

Substance

Sample no

Description

Comment
Meas ure device RS600

\section{Temperature device}

Sensor

A-factor

M-factor
$\mathrm{Z38} \mathrm{Ti}$

$8007.000 \mathrm{~Pa} / \mathrm{Nm}$

$8.600(1 / \mathrm{s}) /(\mathrm{rad} / \mathrm{s})$
1200203357007

Gap $\quad 8.000 \mathrm{~mm}$

\section{C2-UF--W1-5}

1

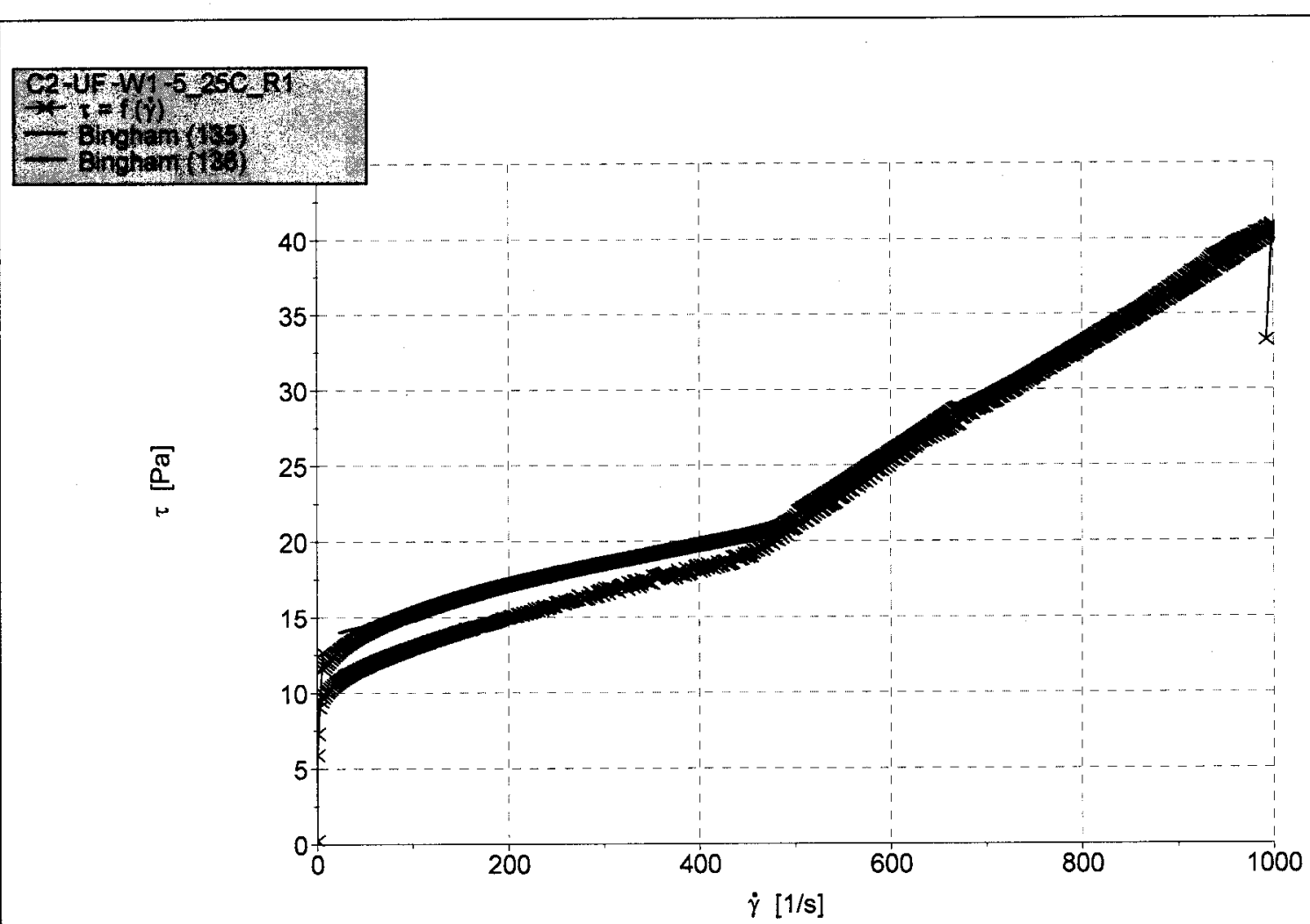

ThermoHaake RheoWin Pro 296

Filename:

Job:
I:IRheologyZaminecklapr '04\4-26-04IC2-UF-W1-5_25C_R1.rwd (Mod)

C:IPROGRAM FILESIRHEOWINIJOBSIRPP Z38 program.rwj

I:IRheologylZaminecklapr '04/4-26-04 IC2 -UF -W1 -5_25C_R1.rwd

(Mod)

Bingham (133) : $\tau_{0}: 10.51 \eta_{\mathrm{p}}: 0.0278 \mathrm{Chi}^{2}: 1355$ r:0.9795

$$
\mathrm{x}=\dot{\gamma}[1 / \mathrm{s}], \mathrm{y}=\tau[\mathrm{Pa}]
$$

Bingham (134) : $\tau_{0}: 8.246 \eta_{\mathrm{p}}: 0.02991 \mathrm{Chi}^{2}: 993.7$ r:0.987

$\mathrm{x}=\dot{\gamma}[1 / \mathrm{s}], \mathrm{y}=\tau[\mathrm{Pa}]$

Bingham (135) : $\tau_{0}: 13.62 \eta_{\mathrm{p}}: 0.01582 \mathrm{Chi}^{2}: 26.41 \mathrm{r}: 0.9872$

$$
\mathrm{x}=\dot{\gamma}[1 / \mathrm{s}], \mathrm{y}=\tau[\mathrm{Pa}]
$$

Bingham (136) : $\tau_{0}: 11.08 \eta_{\mathrm{p}}: 0.0183 \mathrm{Chi}^{2}: 13.91 \mathrm{r}: 0.995$

$$
x=\dot{\gamma}[1 / \mathrm{s}], y=\tau[\mathrm{Pa}]
$$


Company

Operator

Date/Time

Substance

Sample no

Description
SRTC

ITS

26.04.2004 / 9:39:04 AM C2-UF--W1-9

2
Meas ure device RS600

Temperature device

Sensor

A-factor

M-factor
$238 \mathrm{Ti}$

$8007.000 \mathrm{~Pa} / \mathrm{Nm}$

$8.600(1 / \mathrm{s}) /(\mathrm{rad} / \mathrm{s})$
1200203357007

Gap

$8.000 \mathrm{~mm}$

Comment

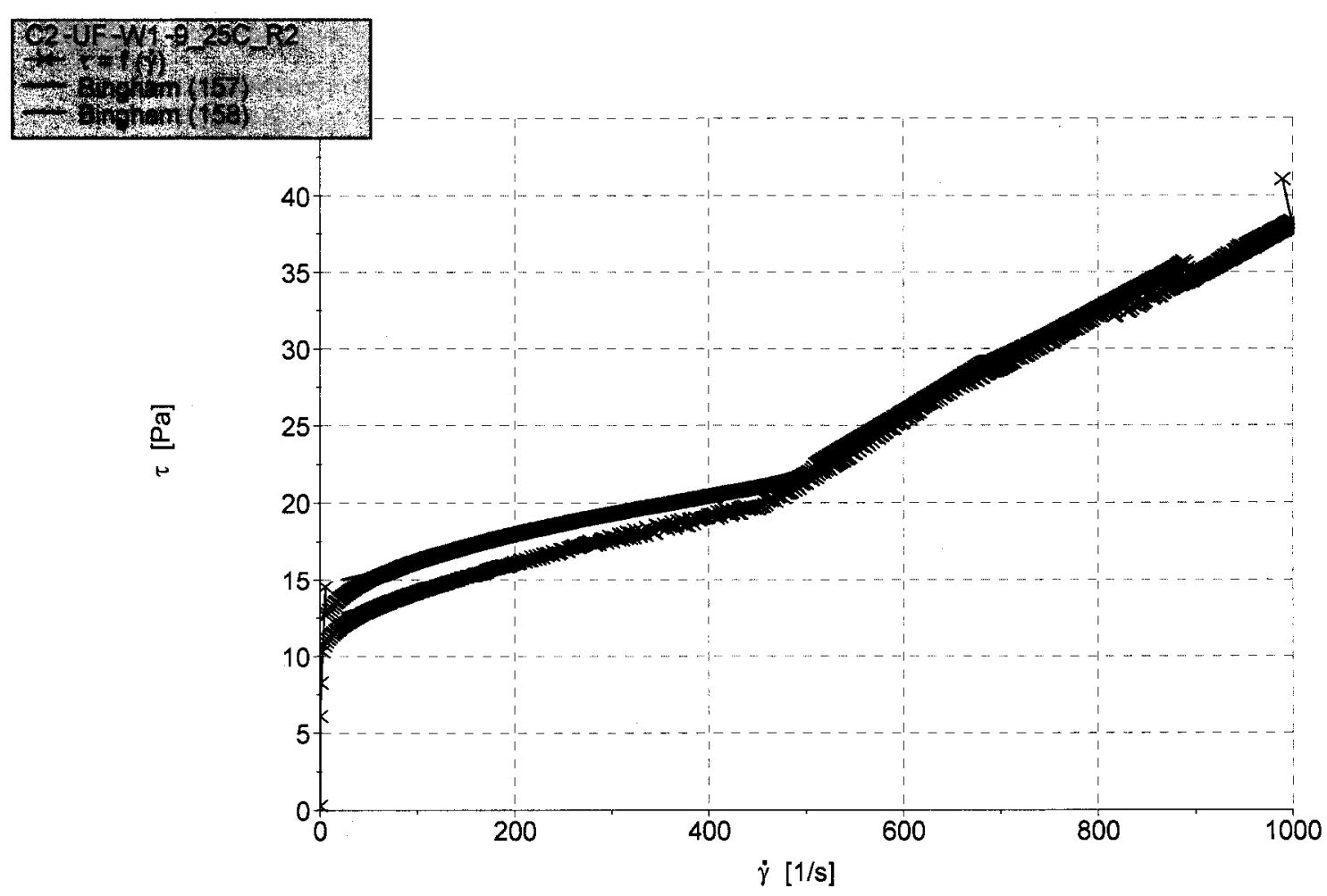

ThermoHaake RheoWin Pro 2.96

Filename:

Job:
I:IRheology Zaminecklapr '04\4-26-04|C2-UF-W1-9_25C_R2.rwd (Mod)

C:IPROGRAM FILESIRHEOWINIJOBSIRPP Z38 program.rwj

I:IRheologylZaminecklapr '04l4 -26-04 lC2 -UF -W1 -9_25C_R2.rwd

(Mod)

Bingham (155) : $\tau_{0}: 12.09 \eta_{\mathrm{p}}: 0.02475 \mathrm{Chi}^{2}: 978.1 \mathrm{r}: 0.9813$

$$
\mathrm{x}=\dot{\gamma}[1 / \mathrm{s}], \mathrm{y}=\tau[\mathrm{Pa}]
$$

Bingham (156) : $\tau_{0}: 10.13 \eta_{\mathrm{p}}: 0.02645 \mathrm{Chi}^{2}: 635.5 \mathrm{r}: 0.9893$

$$
\mathrm{x}=\dot{\gamma}[1 / \mathrm{s}], \mathrm{y}=\tau[\mathrm{Pa}]
$$

Bingham (157) : $\tau_{0}: 14.68 \eta_{p}: 0.01473 \mathrm{Chi}^{2}: 23.03$ r:0.9871

$$
x=\dot{\gamma}[1 / \mathrm{s}], y=\tau[\mathrm{Pa}]
$$

Bingham (158) : $\tau_{0}: 12.43 \eta_{p}: 0.01698 \mathrm{Chi}^{2}: 15.89$ r:0.9933

$$
x=\dot{\gamma}[1 / s], y=\tau[\mathrm{Pa}]
$$


Company

Operator

Date/Time

Substance

Sample no

Description

Comment

\section{SRTC}

ITS

26.04.2004 / 9:14:57 AM C2-UF--W1-9

1
Meas ure device RS600

Temperature device

Sensor

A-factor

M-factor
$238 \mathrm{Ti}$

$8007.000 \mathrm{~Pa} / \mathrm{Nm}$

$8.600(1 / \mathrm{s}) /(\mathrm{rad} / \mathrm{s})$
1200203357007

Gap $8.000 \mathrm{~mm}$

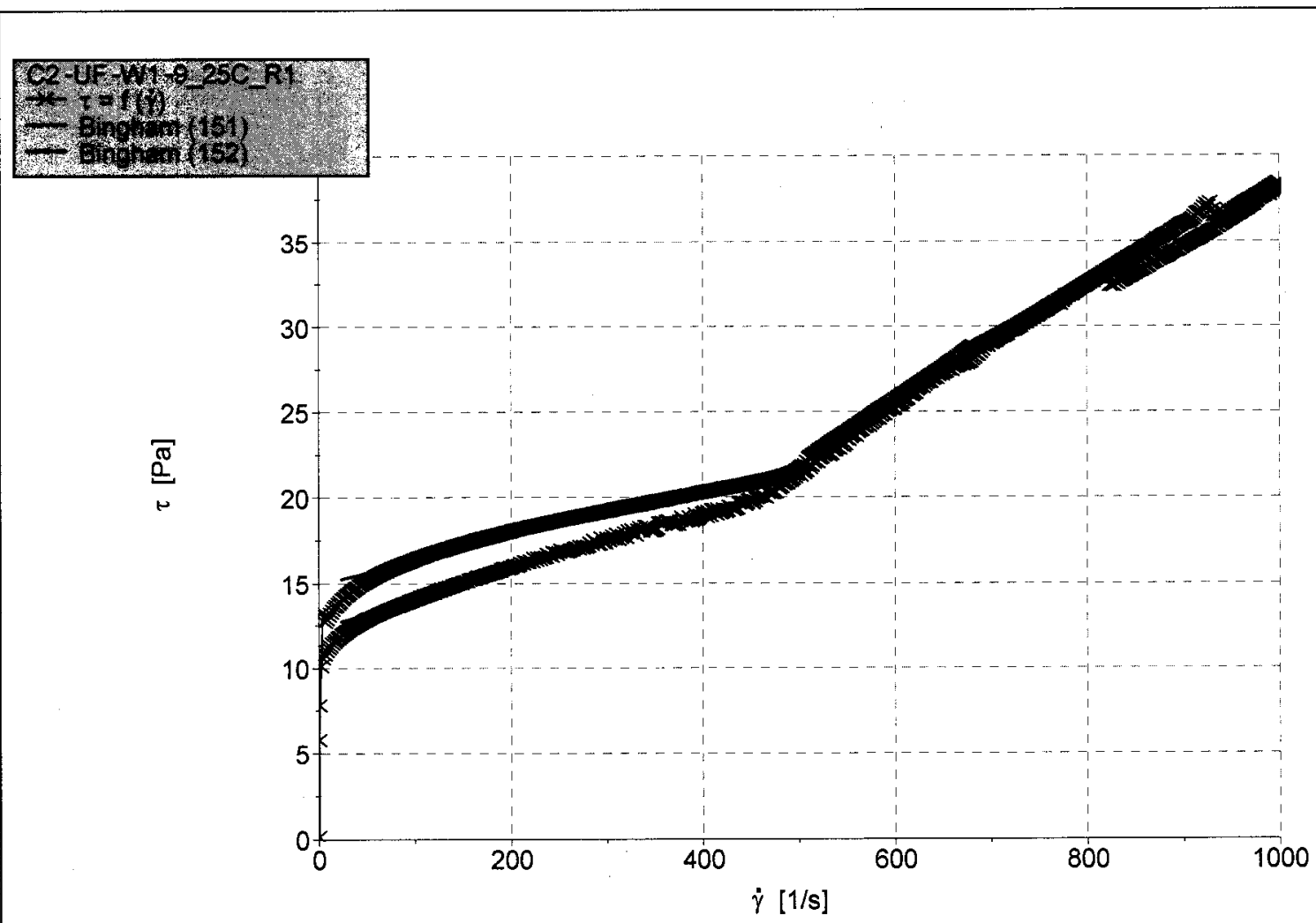

ThermoHaake RheoWin Pro 2.96

Filename:

Job:
I:IRheologylZaminecklapr '0414-26-04lC2-UF-W1-9_25C_R1.rwd (Mod)

C:IPROGRAM FILESIRHEOWINIJOBSIRPP Z38 program.rwj

I:IRheologylZaminecklapr '04l4 -26-04 IC2 -UF -W1 -9_25C_R1.rwd

(Mod)

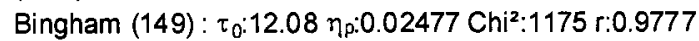
$x=\dot{\gamma}[1 / \mathrm{s}], y=\tau[\mathrm{Pa}]$

Bingham (150): $\tau_{0}: 9.975 \eta_{\mathrm{p}}: 0.02674 \mathrm{Chi}^{2}: 662.8 \mathrm{r}: 0.9891$ $\mathrm{x}=\dot{\gamma}[1 / \mathrm{s}], \mathrm{y}=\tau[\mathrm{Pa}]$

Bingham (151): $\tau_{0}: 14.93 \eta_{\mathrm{p}}: 0.01382 \mathrm{Chi}^{2}: 26.25 \mathrm{r}: 0.9836$ $\mathrm{x}=\dot{\gamma}[1 / \mathrm{s}], \mathrm{y}=\tau[\mathrm{Pa}]$

Bingham (152): $\tau_{0}: 12.34 \eta_{\mathrm{p}}: 0.01699 \mathrm{Chi}^{2}: 12.73 \mathrm{r}: 0.9946$ $\mathrm{x}=\dot{\gamma}[1 / \mathrm{s}], \mathrm{y}=\tau[\mathrm{Pa}]$ 
Company

Operator

Date/Time

Substance

Sample no

Description

Comment

\begin{tabular}{|c|c|c|c|}
\hline Meas ure device & RS600 & \multicolumn{2}{|c|}{1200203357007} \\
\hline Temperature dev & & & \\
\hline Sensor & $\mathrm{Z38} \mathrm{Ti}$ & Gap & $8.000 \mathrm{~mm}$ \\
\hline $\begin{array}{l}\text { A-factor } \\
\text { M-factor }\end{array}$ & $\begin{array}{l}8007.000 \mathrm{~Pa} / \mathrm{Nm} \\
8.600(1 / \mathrm{s}) /(\mathrm{rad} / \mathrm{s})\end{array}$ & & \\
\hline
\end{tabular}

$8007.000 \mathrm{~Pa} / \mathrm{Nm}$
$8.600(1 / \mathrm{s}) /(\mathrm{rad} / \mathrm{s})$
SRTC

ITS

26.04.2004/ 10:19:17 AM

C2-UF--W1-13

2

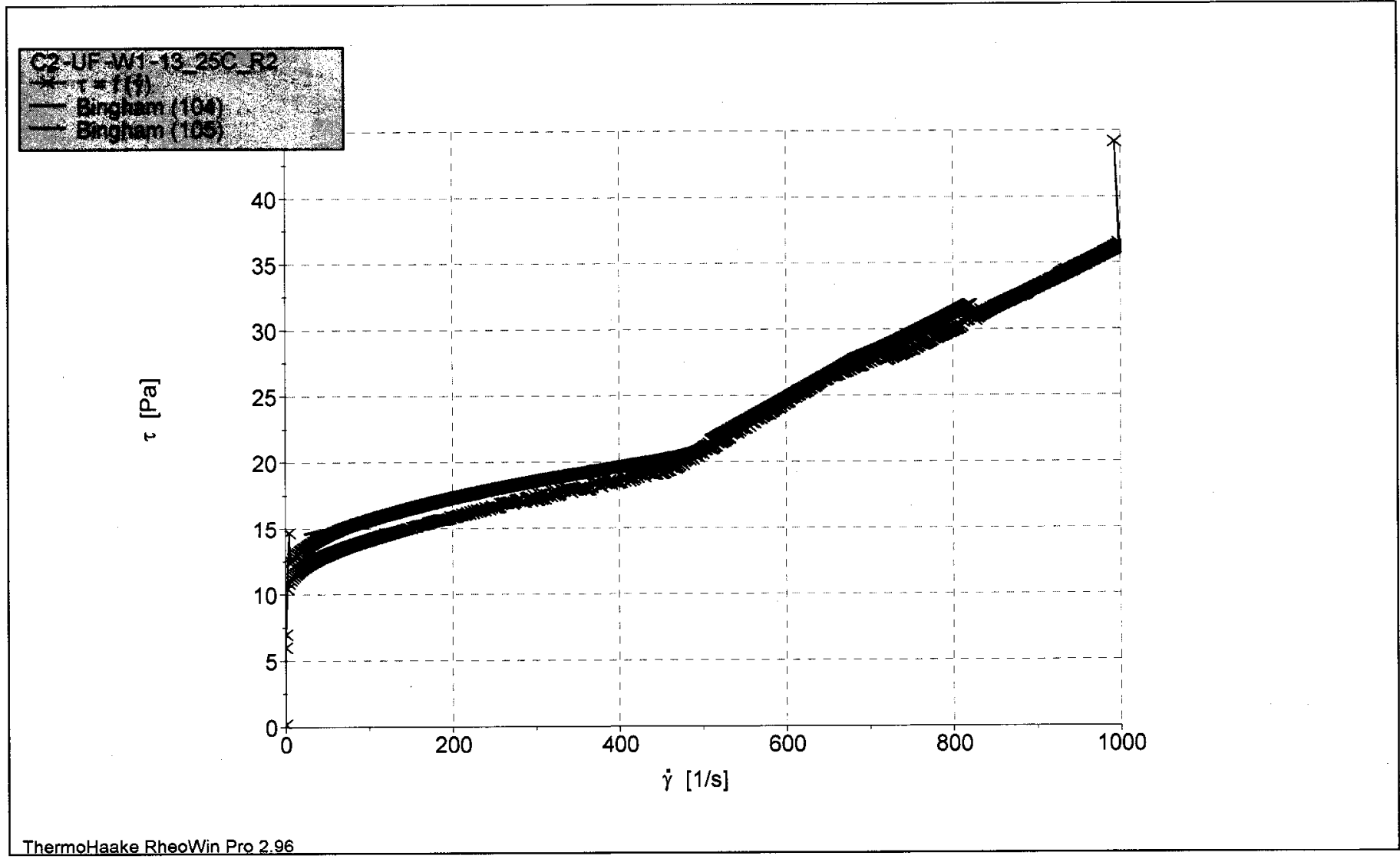

Filename:

Job:
I:IRheology Zaminecklapr '04\4-26-04\C2-UF-W1-13_25C_R2.rwd (Mod)

C:IPROGRAM FILESIRHEOWINIJOBSIRPP Z38 program.rwj

I:IRheologylZaminecklapr '04|4 -26-04IC2 -UF -W1 -13_25C_R2.rwd

(Mod)

Bingham (102) : $\tau_{0}: 11.9 \eta_{p}: 0.02293 \mathrm{Chi}^{2}: 815.7$ r:0.9818

$\mathrm{x}=\dot{\gamma}[1 / \mathrm{s}], \mathrm{y}=\tau[\mathrm{Pa}]$

Bingham (103): $\tau_{0}: 10.37 \eta_{\mathrm{p}}: 0.02433 \mathrm{Chi}^{2}: 554 \mathrm{r}: 0.989$

$\mathrm{x}=\dot{\gamma}[1 / \mathrm{s}], \mathrm{y}=\tau[\mathrm{Pa}]$

Bingham (104): $\tau_{0}: 14.24 \eta_{\mathrm{p}}: 0.01387 \mathrm{Chi}^{2}: 19.98 \mathrm{r}: 0.9874$

$\mathrm{x}=\dot{\gamma}[1 / \mathrm{s}], \mathrm{y}=\tau[\mathrm{Pa}]$

Bingham (105): $\tau_{0}: 12.52 \eta_{\mathrm{p}}: 0.01557 \mathrm{Chi}^{2}: 14.86 \mathrm{r}: 0.9926$

$\mathrm{x}=\dot{\gamma}[1 / \mathrm{s}], \mathrm{y}=\tau[\mathrm{Pa}]$ 
Company SRTC

Operator

Date/Time

Substance

Sample no

Description

Comment
Measure device RS600

Temperature device

Sensor

A-factor

M-factor
$238 \mathrm{Ti}$

$8007.000 \mathrm{~Pa} / \mathrm{Nm}$

$8.600(1 / \mathrm{s}) /(\mathrm{rad} / \mathrm{s})$
1200203357007

Gap $\quad 8.000 \mathrm{~mm}$
C2-UF--W1-13

1
26.04.2004/ 10:00:41 AM

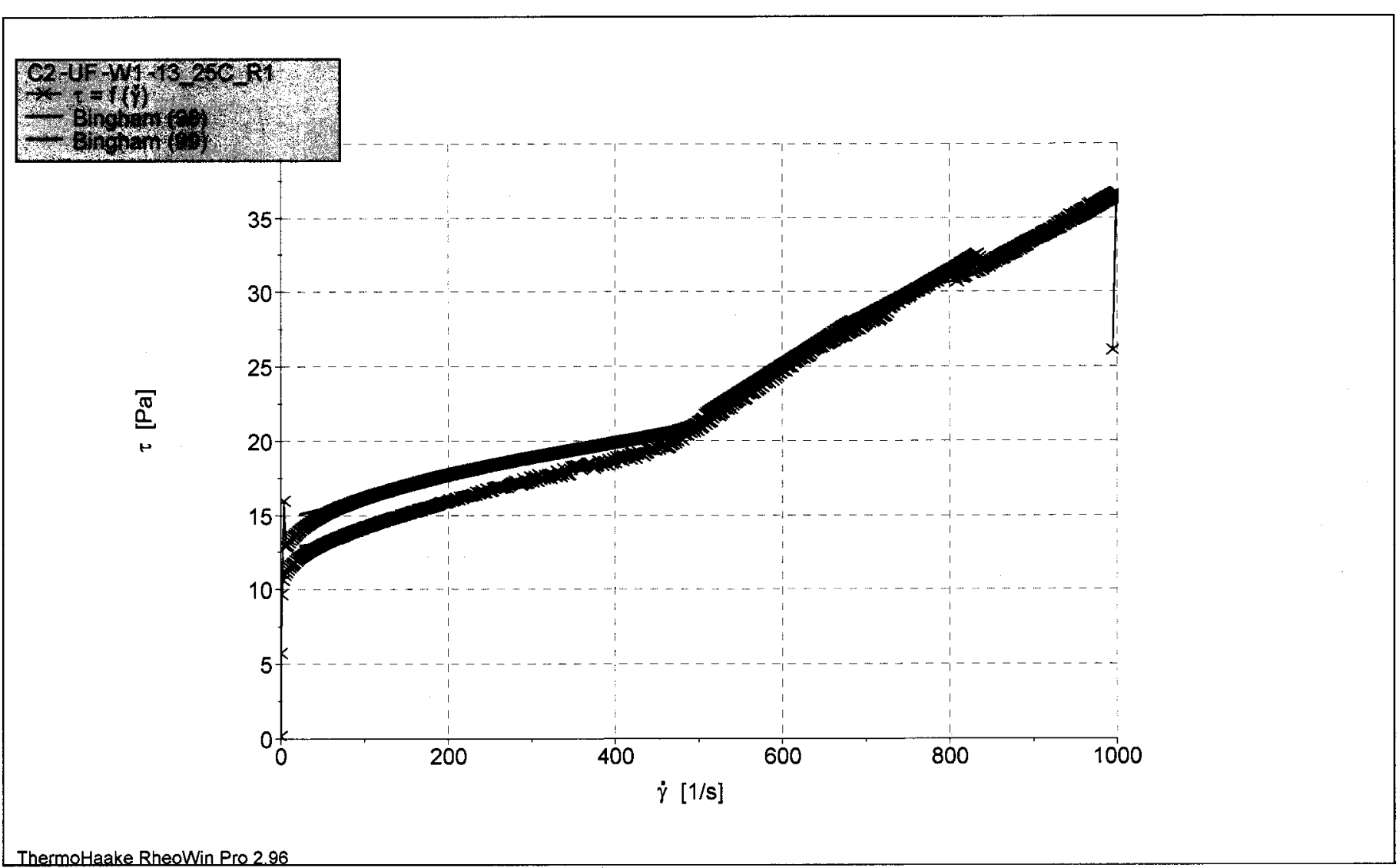

Filename: I:IRheologyZZaminecklapr '0414-26-04IC2-UF-W1-13_25C_R1.rwd (Mod)

Job: $\quad$ C:IPROGRAM FILESIRHEOWINIJOBSIRPP Z38 program.wj

I:IRheologyZZaminecklapr r $04 \backslash 4$-26-04IC2-UF -W1 -13_25C_R1.rwd

(Mod)

Bingham (96): $\tau_{0}: 12.29 \eta_{\mathrm{p}}: 0.02288 \mathrm{Chi}^{2}: 929.4$ r:0.9793

$$
x=\dot{\gamma}[1 / \mathrm{s}], y=\tau[\mathrm{Pa}]
$$

Bingham (97): $\tau_{0}: 10.37 \eta_{p}: 0.02484 \mathrm{Chi}^{2}: 565.1$ r:0.9892

$\mathrm{x}=\dot{\gamma}[1 / \mathrm{s}], \mathrm{y}=\tau[\mathrm{Pa}]$

Bingham (98) : $\tau_{0}: 14.79 \eta_{\mathrm{p}}: 0.01313 \mathrm{Chi}^{2}: 20.17 \mathrm{r}: 0.9858$

$\mathrm{x}=\dot{\gamma}[1 / \mathrm{s}], \mathrm{y}=\tau[\mathrm{Pa}]$

Bingham (99): $\tau_{0}: 12.55 \eta_{\mathrm{p}}: 0.01583 \mathrm{Chi}^{2}: 12.63 \mathrm{r}: 0.9938$

$$
x=\dot{\gamma}[1 / \mathrm{s}], y=\tau[\mathrm{Pa}]
$$




$\begin{array}{llllr}\text { Company } & \text { SRTC } & \text { Measure device } & \text { RS600 } & 1200203357007 \\ \text { Operator } & \text { ITS } & \text { Temperature device } & \\ \text { Date/Time } & 26.04 .2004 / 10: 54: 57 \text { AM } & \text { Sensor } & \text { Z38 Ti } & \text { Gap } \\ \text { Substance } & \text { C2-UF--W1-17 } & \text { A-factor } & 8007.000 \mathrm{~Pa} / \mathrm{Nm} & \\ \text { Sample no } & 2 & \text { M-factor } & 8.600(1 / \mathrm{s}) /(\mathrm{rad} / \mathrm{s}) & \end{array}$

Description

Comment

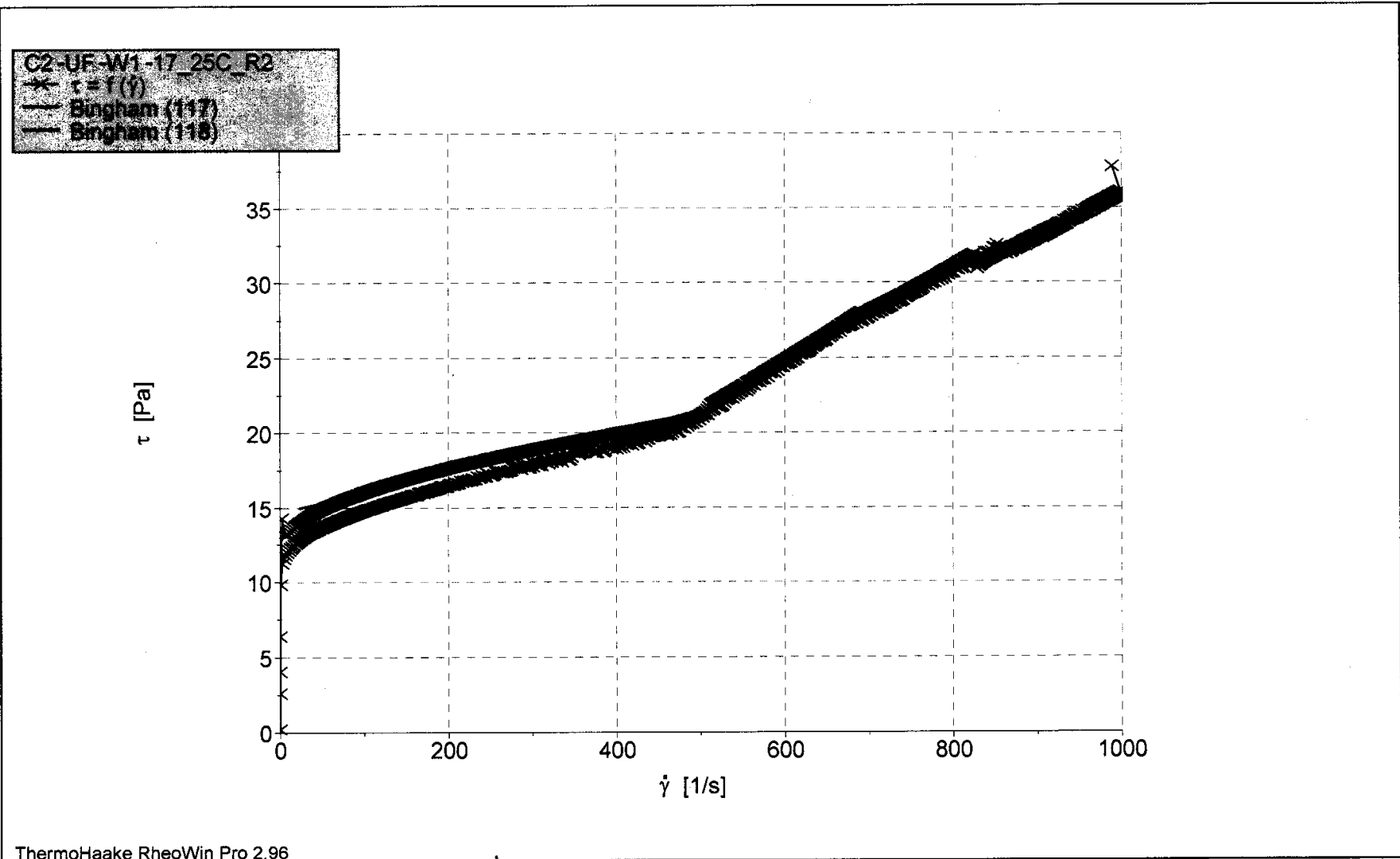

Filename: I:IRheologyZaminecklapr '04I4-26-04IC2-UF-W1-17_25C_R2.rwd (Mod)

Job:

C:IPROGRAM FILESIRHEOWINUJOBSIRPP Z38 program.nw

I:IRheologylZaminecklapr '04I4 -26-04 IC2 -UF -W1 -17_25C_R2.rwd

(Mod)

Bingham (115): $\tau_{0}: 12.39 \eta_{\mathrm{p}}: 0.02229 \mathrm{Chi}^{2}: 799.5 \mathrm{r}: 0.9812$

$$
\mathrm{x}=\dot{\gamma}[1 / \mathrm{s}], \mathrm{y}=\tau[\mathrm{Pa}]
$$

Bingham (116): $\tau_{0}: 11.06 \eta_{\mathrm{p}}: 0.02366 \mathrm{Chi}^{2}: 651.6 \mathrm{r}: 0.9865$

$\mathrm{x}=\dot{\gamma}[1 / \mathrm{s}], \mathrm{y}=\tau[\mathrm{Pa}]$

Bingham (117): $\tau_{0}: 14.67 \eta_{\mathrm{p}}: 0.01351 \mathrm{Chi}^{2}: 17.04 \mathrm{r}: 0.9886$

$x=\dot{\gamma}[1 / \mathrm{s}], y=\tau[\mathrm{Pa}]$

Bingham (118): $\tau_{0}: 13.25 \eta_{p}: 0.01506 \mathrm{Chi}^{2}: 13.76 \mathrm{r}: 0.9926$

$x=\dot{\gamma}[1 / \mathrm{s}], y=\tau[\mathrm{Pa}]$ 
Company

Operator

Date/Time

Substance

Sample no

Description
SRTC

ITS

26.04 .2004 / 10:36:22 AM

C2-UF--W1-17

1
Meas ure device RS600

Temperature device

Sensor

A-factor

M-factor
$238 \mathrm{Ti}$

$8007.000 \mathrm{~Pa} / \mathrm{Nm}$

$8.600(1 / \mathrm{s}) /(\mathrm{rad} / \mathrm{s})$
1200203357007

Gap $\quad 8.000 \mathrm{~mm}$

\section{Comment}

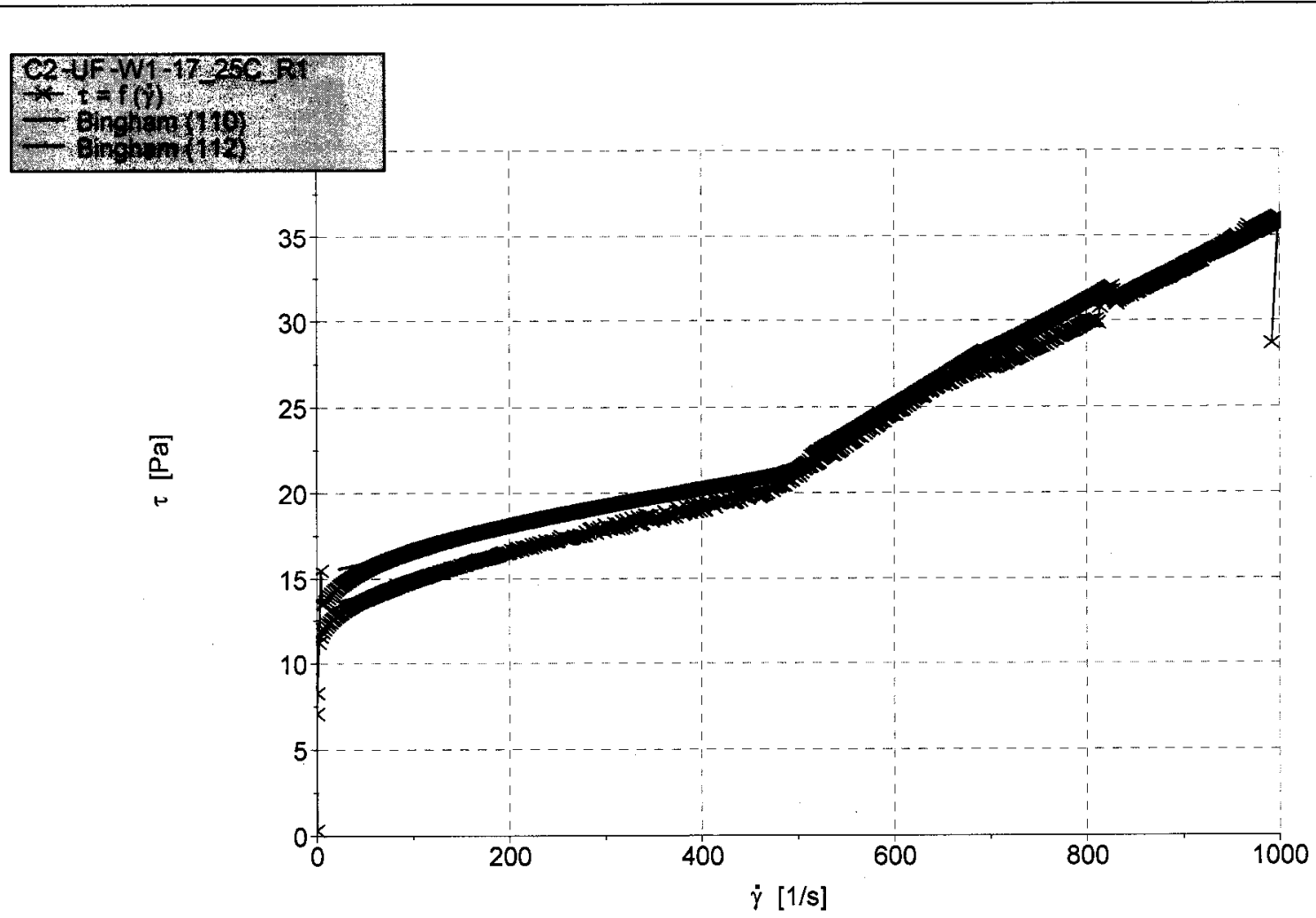

ThermoHaake RheoWin Pro 2.96

Filename: I:IRheologylZaminecklapr '04I4-26-04IC2-UF-W1-17_25C_R1.rwd (Mod)

Job:

C:IPROGRAM FILESIRHEOWINIJOBSIRPP Z38 program.IWj

I:IRheologyZZaminecklapr '04I4-26-04IC2 -UF -W1 -17_25C_R1.rwd

(Mod)

Bingham (108): $\tau_{0}: 12.95 \eta_{\mathrm{p}}: 0.02172 \mathrm{Chi}^{2}: 845.4$ r:0.9791

$\mathrm{x}=\dot{\gamma}[1 / \mathrm{s}], \mathrm{y}=\tau[\mathrm{Pa}]$

Bingham (109): $\tau_{0}: 11.3 \eta_{\mathrm{p}}: 0.02314 \mathrm{Chi}^{2}: 522.4$ r:0.9886

$x=\dot{\gamma}[1 / \mathrm{s}], y=\tau[\mathrm{Pa}]$

Bingham (110): $\tau_{0}: 15.3 \eta_{p}: 0.01271 \mathrm{Chi}^{2}: 18.09$ r:0.9864

$x=\dot{\gamma}[1 / \mathrm{s}], y=\tau[\mathrm{Pa}]$

Bingham (112): $\tau_{0}: 13.35 \eta_{p}: 0.0149 \mathrm{Chi}^{2}: 14.78 \mathrm{r}: 0.992$

$x=\dot{\gamma}[1 / \mathrm{s}], y=\tau[\mathrm{Pa}]$ 


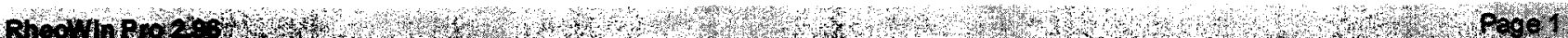

Company

Operator

Date/Time

Substance

Sample no

Description

Comment
SRTC

ITS

26.04 .2004 / 11:35:38 AM

C2-UF--W1-22

2
Meas ure device RS600

Temperature device

Sensor

A-factor

M-factor
$238 \mathrm{Ti}$

$8007.000 \mathrm{~Pa} / \mathrm{Nm}$

$8.600(1 / \mathrm{s}) /(\mathrm{rad} / \mathrm{s})$
1200203357007

Gap

$8.000 \mathrm{~mm}$

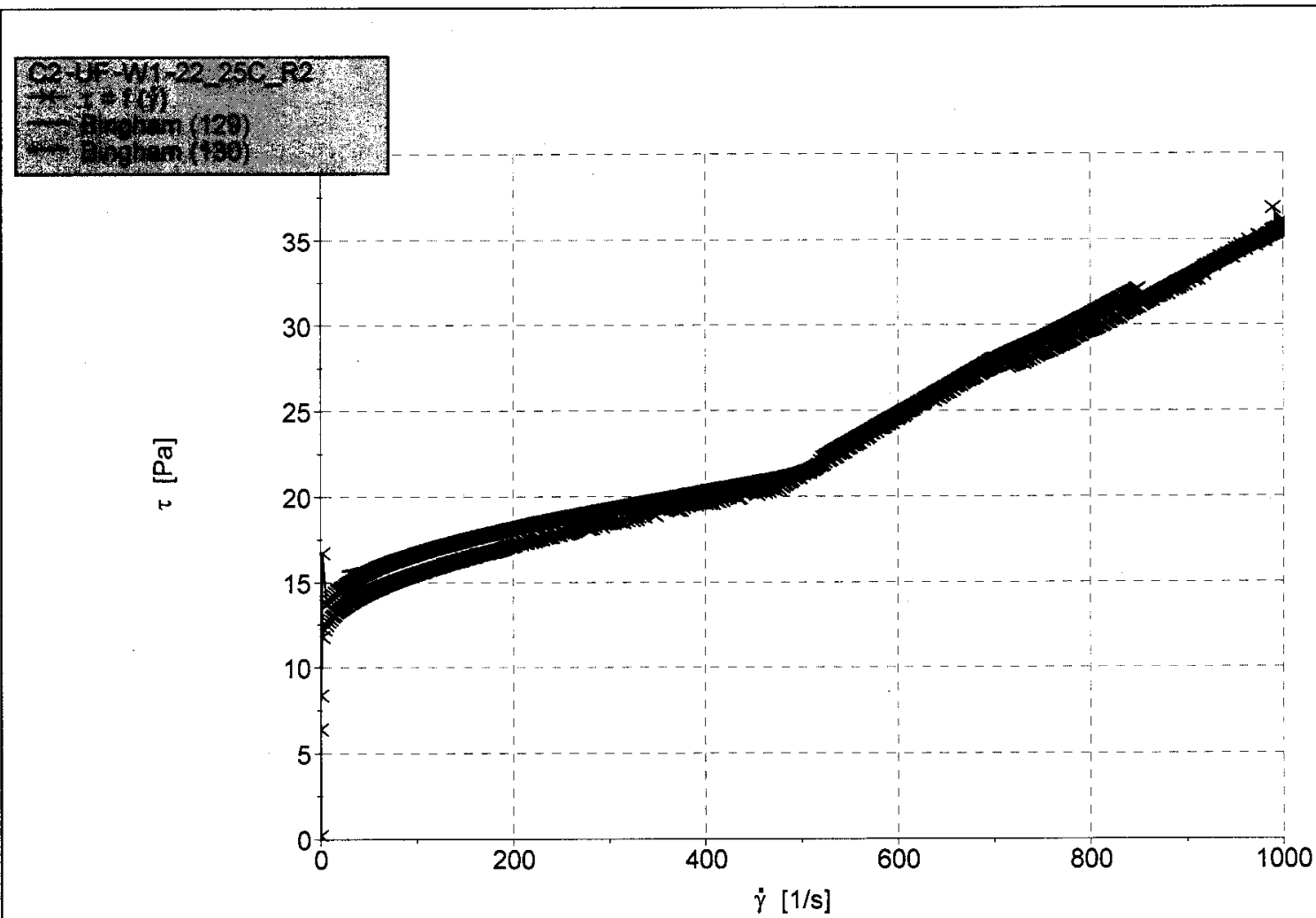

ThermoHaake RheoWin Pro 296

Filename:

Job:
I:IRheologyZZaminecklapr '0414-26-04IC2-UF-W1-22_25C_R2.rwd (Mod)

C:IPROGRAM FILESIRHEOWINIJOBSIRPP Z38 program.rwj

I:IRheologyZaminecklapr '04l4 -26-04IC2 UF -W1 -22_25C_R2.rwd

(Mod)

Bingham (127) : $\tau_{0}: 13.15 \eta_{\mathrm{p}}: 0.021 \mathrm{Chi}^{2}: 826$ r.0.9782

$$
\mathrm{x}=\dot{\gamma}[1 / \mathrm{s}], \mathrm{y}=\tau[\mathrm{Pa}]
$$

Bingham (128) : $\tau_{0}: 12.12 \eta_{\mathrm{p}}: 0.02186 \mathrm{Chi}^{2}: 487.1 \mathrm{r}: 0.988$

$\mathrm{x}=\dot{\gamma}[1 / \mathrm{s}], \mathrm{y}=\tau[\mathrm{Pa}]$

Bingham (129) : $\tau_{0}: 15.37 \eta_{\mathrm{p}}: 0.01255 \mathrm{Chi}^{2}: 14.24$ r:0.989

$\mathrm{x}=\dot{\gamma}[1 / \mathrm{s}], \mathrm{y}=\tau[\mathrm{Pa}]$

Bingham (130): $\tau_{0}: 14.02 \eta_{p}: 0.0144 \mathrm{Chi}^{2}: 14.67$ r:0.9915

$$
x=\dot{\gamma}[1 / \mathrm{s}], y=\tau[\mathrm{Pa}]
$$


Company

Operator

Date/Time

Substance

Sample no

Description

Comment
SRTC

ITS

26.04.2004/11:15:49 AM

C2-UF--W1-22

1
Measure device RS600

Temperature device

Sensor

A-factor

M-factor
$238 \mathrm{Ti}$

$8007.000 \mathrm{~Pa} / \mathrm{Nm}$

$8.600(1 / \mathrm{s}) /(\mathrm{rad} / \mathrm{s})$
1200203357007

Gap $\quad 8.000 \mathrm{~mm}$

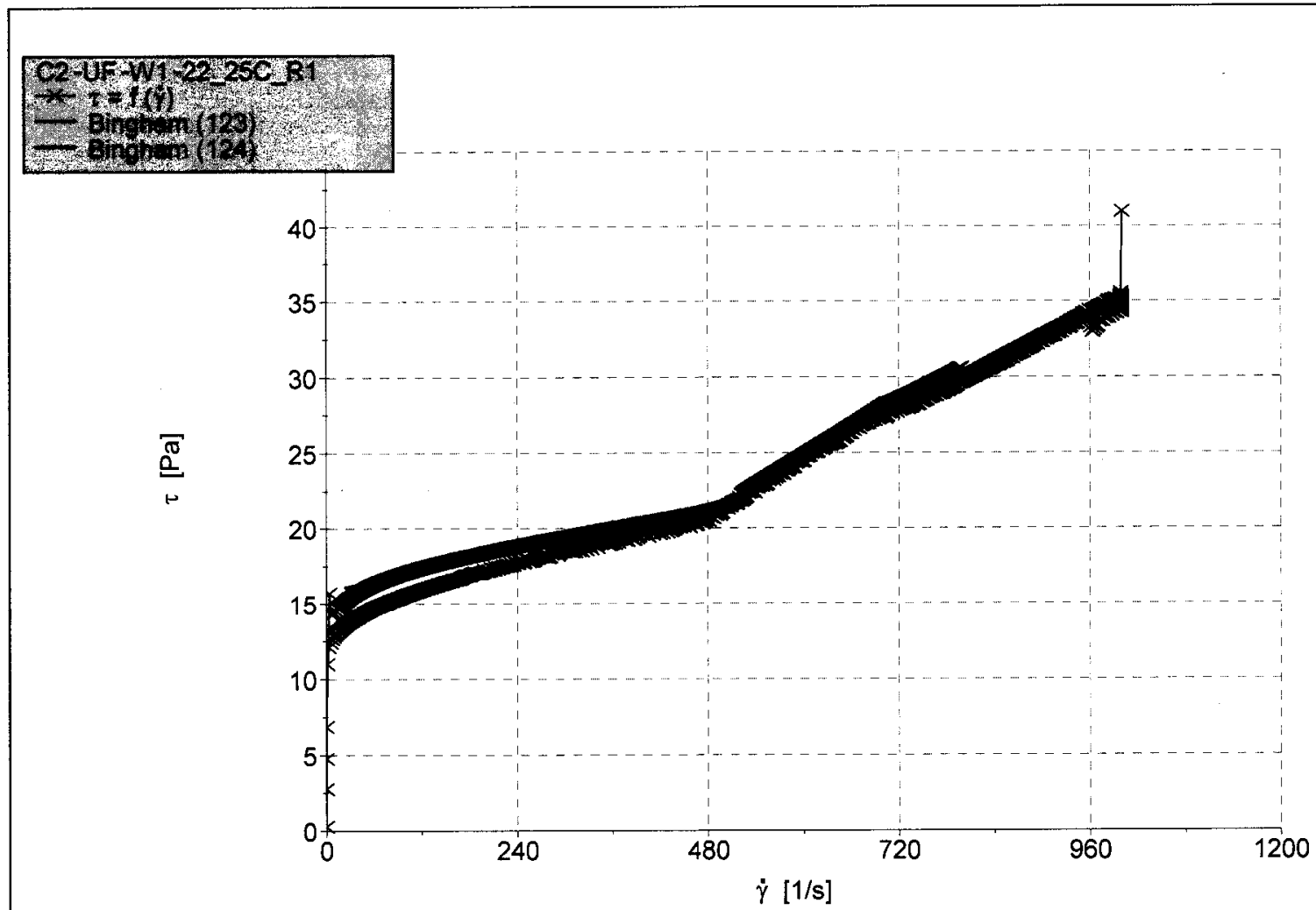

ThermoHaake RheoWin Pro 2.96

Filename: $\quad$ I:IRheologylZaminecklapr '04l4-26-04IC2-UF-W1-22_25C_R1.rwd (Mod)

Job: $\quad$ C:IPROGRAM FILESIRHEOWINIJOBSIRPP Z38 program.nj

I:IRheologylZaminecklapr '04I4-26-04 IC2 -UF -W1 -22_25C_R1.rwd

(Mod)

Bingham (121): $\tau_{0}: 13.56 \eta_{p}: 0.02029 \mathrm{Chi}^{2}: 830$ r:0.9766

$\mathrm{x}=\dot{\gamma}[1 / \mathrm{s}], \mathrm{y}=\tau[\mathrm{Pa}]$

Bingham (122): $\tau_{0}: 12.17 \eta_{\mathrm{p}}: 0.02153 \mathrm{Chi}^{2}: 550.6 \mathrm{r}: 0.9862$

$x=\dot{\gamma}[1 / \mathrm{s}], y=\tau[\mathrm{Pa}]$

Bingham (123) : $\tau_{0}: 15.84 \eta_{p}: 0.0116 \mathrm{Chi}^{2}: 13.33$ r:0.988

$x=\dot{\gamma}[1 / \mathrm{s}], y=\tau[\mathrm{Pa}]$

Bingham (124): $\tau_{0}: 14.11 \eta_{\mathrm{p}}: 0.0141 \mathrm{Chi}^{2}: 16.51$ r:0.9899

$\mathrm{x}=\dot{\gamma}[1 / \mathrm{s}], \mathrm{y}=\tau[\mathrm{Pa}]$ 
Company

Operator

Date/Time

Substance

Sample no

Description

\section{SRTC}

ITS

27.04 .2004 / 9:4 1:23 AM

C2-UF-W2-1

2
Meas ure device

Temperature device

Sensor

A-factor

M-factor
RS600

$\mathrm{Z38} \mathrm{Ti}$

$8007.000 \mathrm{~Pa} / \mathrm{Nm}$

$8.600(1 / \mathrm{s}) /(\mathrm{rad} / \mathrm{s})$
1200203357007

Gap

$8.000 \mathrm{~mm}$

\section{Comment}

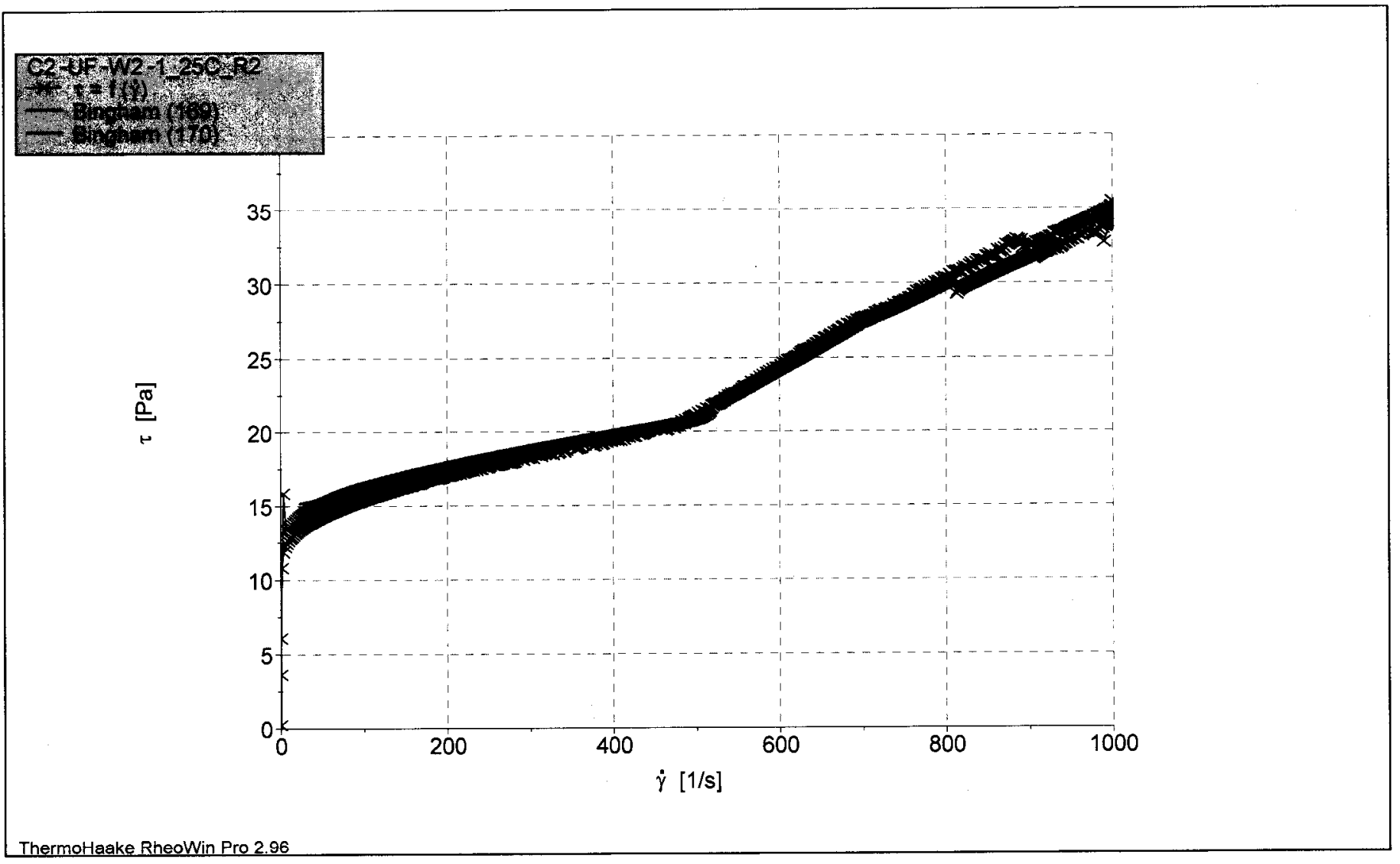

Filename:

Job:
I:IRheologylZaminecklapr '04l4-27-04IC2-UF-W2-1_25C_R2.rud (Mod)

C:IPROGRAM FILESIRHEOWINIJOBSIRPP Z38 program .nw

I:IRheologylZaminecklapr '04/4 -27 -04 IC2 -UF -W2 -1_25C_R2.rwd

(Mod)

Bingham (167) : $\tau_{0}: 12.87 \eta_{\mathrm{p}}: 0.0202 \mathrm{Chi}^{2}: 690.3 \mathrm{r}: 0.9802$

$x=\dot{\gamma}[1 / \mathrm{s}], y=\tau[\mathrm{Pa}]$

Bingham (168): $\tau_{0}: 11.89 \eta_{\mathrm{p}}: 0.02224 \mathrm{Chi}^{2}: 571.4 \mathrm{r}: 0.9865$

$x=\dot{\gamma}[1 / s], y=\tau[P a]$

Bingham (169): $\tau_{0}: 14.9 \eta_{p}: 0.01241 \mathrm{Chi}^{2}: 12.71$ r:0.9899

$x=\dot{\gamma}[1 / s], y=\tau[P a]$

Bingham (170): $\tau_{0}: 13.93 \eta_{\mathrm{p}}: 0.01424 \mathrm{Chi}^{2}: 14.82 \mathrm{r}: 0.9911$

$x=\dot{\gamma}[1 / \mathrm{s}], y=\tau[\mathrm{Pa}]$ 
Company

Operator

Date/Time

Substance

Sample no

Description

\section{SRTC}

ITS

27.04.2004 / 9:25:33 AM

C2-UF-W2-1

1
Meas ure device RS600

Temperature device

Sensor

A-factor

M-factor
$\mathrm{Z38} \mathrm{Ti}$

$8007.000 \mathrm{~Pa} / \mathrm{Nm}$

$8.600(1 / \mathrm{s}) /(\mathrm{rad} / \mathrm{s})$
1200203357007

Gap $\quad 8.000 \mathrm{~mm}$

\section{Comment}

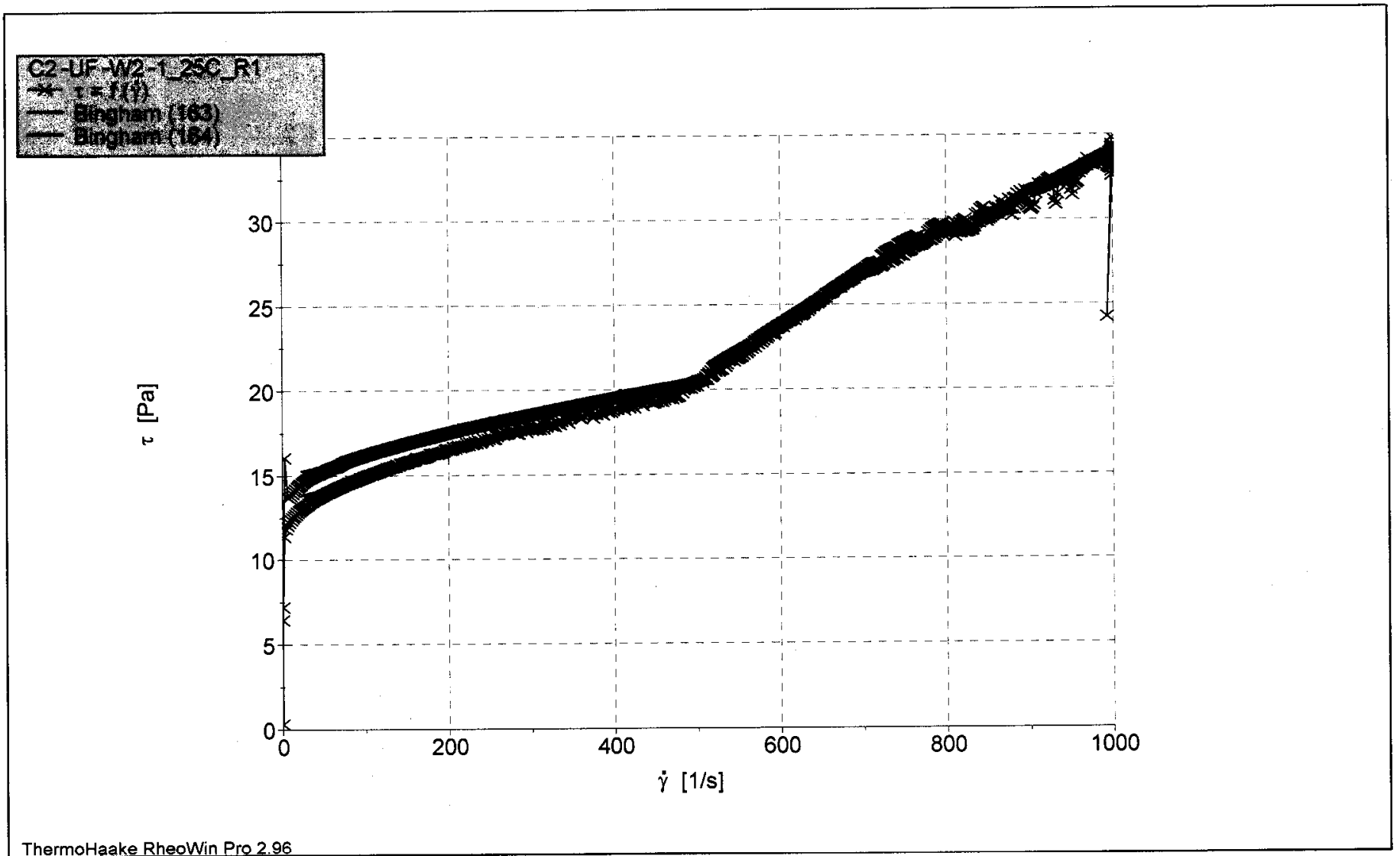

ThermoHaake RheoWin Pro 2.96

Filename:

Job:
I:IRheologyZZaminecklapr '04|4-27-04IC2-UF-W2-1_25C_R1.rwd (Mod)

C:IPROGRAM FILESIRHEOWINIJOBSIRPP Z38 program.wj

I:IRheologyZZaminecklap r '04/4 -27-04IC2 -UF -W2 -1_25C_R1.rwd

(Mod)

Bingham (161): $\tau_{0}: 12.87 \eta_{\mathrm{p}}: 0.01977 \mathrm{Chi}^{2}: 784.1 \mathrm{r}: 0.9767$

$$
\mathrm{x}=\dot{\gamma}[1 / \mathrm{s}], \mathrm{y}=\tau[\mathrm{Pa}]
$$

Bingham (162): $\tau_{0}: 11.59 \eta_{\mathrm{p}}: 0.02138 \mathrm{Chi}^{2}: 508.7$ r:0.987

$$
x=\dot{\gamma}[1 / \mathrm{s}], y=\tau[\mathrm{Pa}]
$$

Bingham (163) : $\tau_{0}: 15.01 \eta_{p}: 0.01147 \mathrm{Chi}^{2}: 9.103$ r:0.9915

$$
x=\dot{\gamma}[1 / \mathrm{s}], y=\tau[\mathrm{Pa}]
$$

Bingham (164): $\tau_{0}: 13.55 \eta_{\mathrm{p}}: 0.01353 \mathrm{Chi}^{2}: 15.46$ r:0.9899

$$
x=\dot{\gamma}[1 / \mathrm{s}], y=\tau[\mathrm{Pa}]
$$


Company

Operator

Date/Time

Substance

Sample no

Description

\section{SRTC}

ITS

27.04.2004 / 10:16:21 AM

C2-UF-W2-5

2

$\begin{array}{ll}\text { Measure device } & \text { RS600 } \\ \text { Temperature device } & \\ \text { Sensor } & \text { Z38 Ti } \\ \text { A-factor } & 8007.000 \mathrm{~Pa} / \mathrm{Nm} \\ \text { M-factor } & 8.600(1 / \mathrm{s}) /(\mathrm{rad} / \mathrm{s})\end{array}$

1200203357007

Gap

$8.000 \mathrm{~mm}$

\section{Comment}

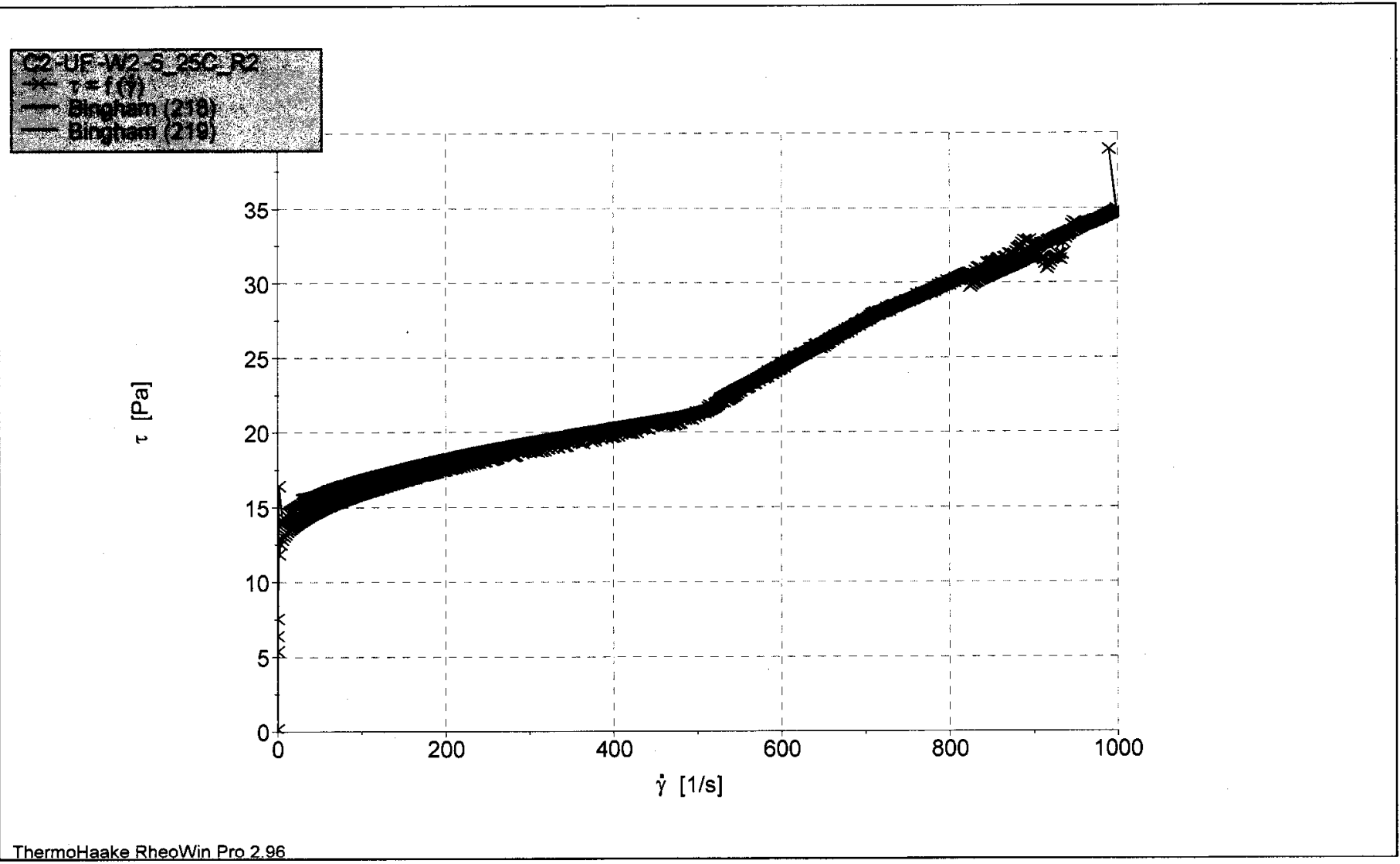

ThermoHaake Rheawin Pro 20

Filename: $\quad$ I:IRheology ZZaminecklapr '04I4-27-04IC2-UF-W2-5_25C_R2.rwd (Mod)

Job:

C:IPROGRAM FILESIRHEOWINIJOBSIRPP Z38 program.nwj

I:IRheologylZaminecklapr '04I4-27-04 IC2 -UF -W2 -5_25C_R2.rwd

(Mod)

Bingham (216) : $\tau_{0}: 13.52 \eta_{p}: 0.01968 \mathrm{Chi}^{2}: 778.3 \mathrm{r}: 0.9766$

$$
x=\dot{\gamma}[1 / \mathrm{s}], y=\tau[\mathrm{Pa}]
$$

Bingham (217): $\tau_{0}: 12.55 \eta_{\mathrm{p}}: 0.02121 \mathrm{Chi}^{2}: 546.2$ r:0.9859

$$
x=\dot{\gamma}[1 / s], y=\tau[\mathrm{Pa}]
$$

Bingham (218): $\tau_{0}: 15.58 \eta_{\mathrm{p}}: 0.01195 \mathrm{Chi}^{2}: 11.33 \mathrm{r}: 0.9903$

$$
x=\dot{\gamma}[1 / s], y=\tau[P a]
$$

Bingham (219) : $\tau_{0}: 14.51 \eta_{p}: 0.01376 \mathrm{Chi}^{2}: 18.02$ r:0.9885

$$
x=\dot{\gamma}[1 / \mathrm{s}], y=\tau[\mathrm{Pa}]
$$




$\begin{array}{llllr}\text { Company } & \text { SRTC } & \text { Measure device } & \text { RS600 } & 1200203357007 \\ \text { Operator } & \text { ITS } & \text { Temperature device } & \text { Gap } & 8.000 \mathrm{~mm} \\ \text { Date/Time } & 27.04 .2004 / 10: 01: 57 \mathrm{AM} & \text { Sensor } & \text { Z38 Ti } & 8007.000 \mathrm{~Pa} / \mathrm{Nm} \\ \text { Substance } & \text { C2-UF-W2-5 } & \text { A-factor } & 8.600(1 / \mathrm{s}) /(\mathrm{rad} / \mathrm{s}) & \end{array}$

Description

Comment

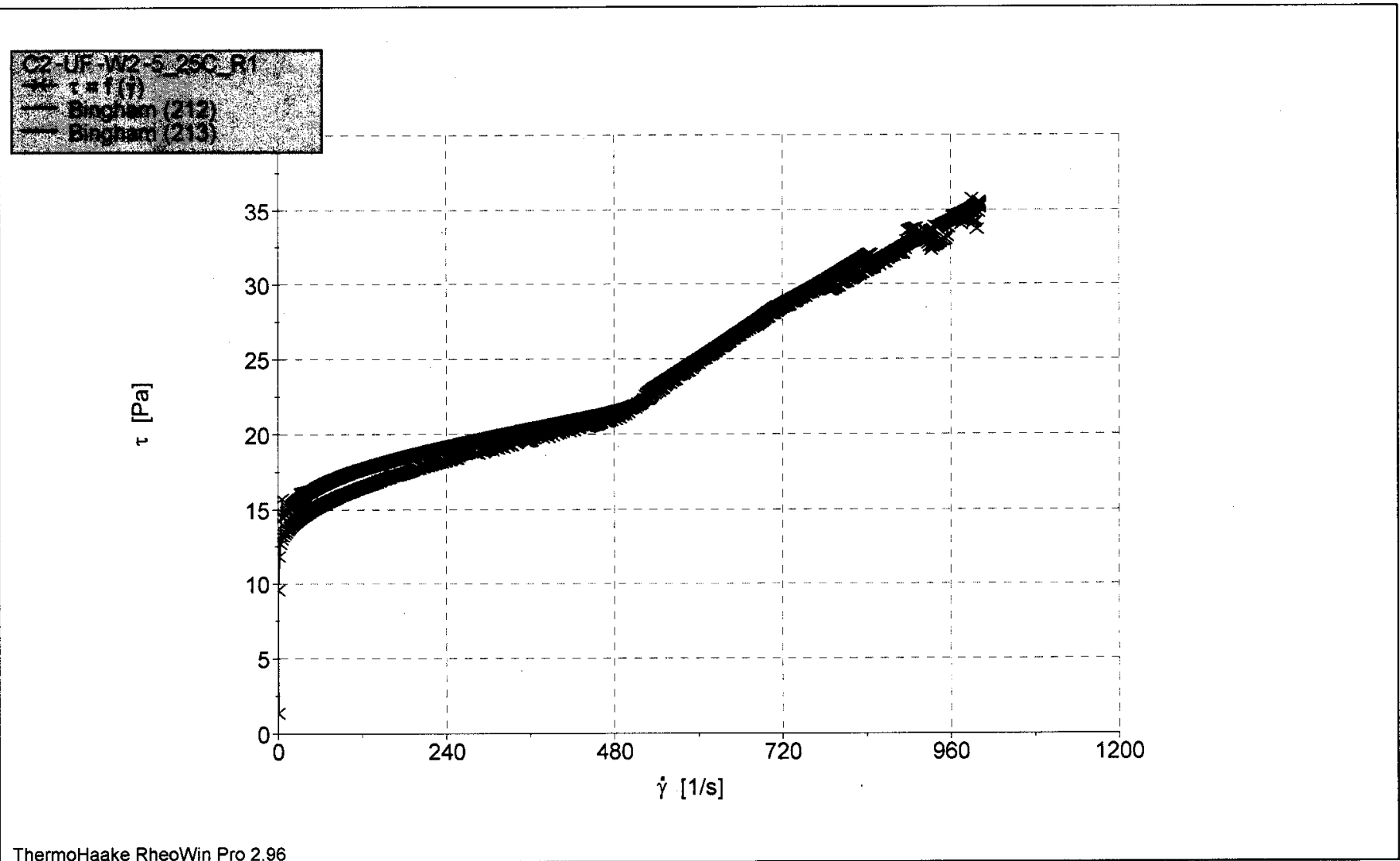

Filename: I:IRheology Zaminecklapr '0414-27-04IC2-UF-W2-5_25C_R1.rwd (Mod)

Job:

C:IPROGRAM FILESIRHEOWINIJOBSIRPP Z38 program.rwj

I:IRheologylZaminecklapr '04I4-27-04 IC2 -UF -W2 -5_25C_R1.rwd

(Mod)

Bingham (210): $\tau_{0}: 13.92 \eta_{\mathrm{p}}: 0.02009 \mathrm{Chi}^{2}: 774.4 \mathrm{r}: 0.9798$

$$
\mathrm{x}=\dot{\gamma}[1 / \mathrm{s}], \mathrm{y}=\tau[\mathrm{Pa}]
$$

Bingham (211): $\tau_{0}: 12.84 \eta_{p}: 0.02121 \mathrm{Chi}^{2}: 468.1 \mathrm{r}: 0.9877$

$$
x=\dot{\gamma}[1 / \mathrm{s}], y=\tau[\mathrm{Pa}]
$$

Bingham (212): $\tau_{0}: 16.11 \eta_{\mathrm{p}}: 0.01185 \mathrm{Chi}^{2}: 14.42 \mathrm{r}: 0.9875$

$$
x=\dot{\gamma}[1 / \mathrm{s}], y=\tau[\mathrm{Pa}]
$$

Bingham (213) : $\tau_{0}: 14.69 \eta_{\mathrm{p}}: 0.01397 \mathrm{Chi}^{2}: 17.3$ r:0.9893

$$
\mathrm{x}=\dot{\gamma}[\mathrm{1} / \mathrm{s}], \mathrm{y}=\tau[\mathrm{Pa}]
$$


Company

Operator

Date/Time

Substance

Sample no

Description

Comment
SRTC

ITS

27.04.2004/ 10:53:30 AM

C2-UF-W2-9

2
Meas ure device RS600

Temperature device

Sensor

A-factor

M-factor
$\mathrm{Z38} \mathrm{Ti}$

$8007.000 \mathrm{~Pa} / \mathrm{Nm}$

$8.600(1 / \mathrm{s}) /(\mathrm{rad} / \mathrm{s})$
1200203357007

Gap $\quad 8.000 \mathrm{~mm}$

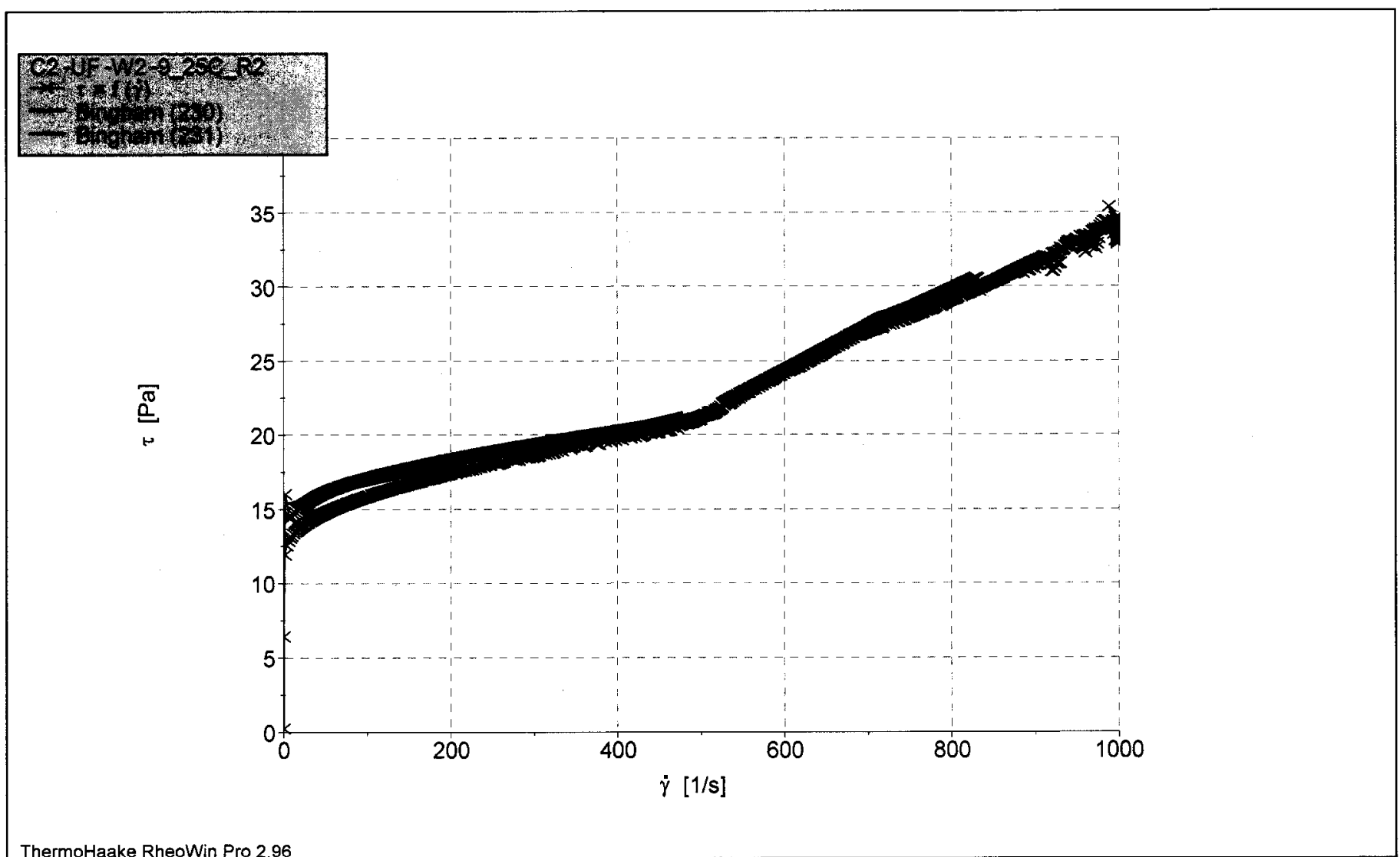

Filename: I:IRheologyZZaminecklapr '0414-27-04IC2-UF-W2-9_25C_R2.rwd (Mod)

Job:

C:IPROGRAM FILESIRHEOWINIJOBSIRPP Z38 program.rwj

I:IRheologyZZaminecklapr '04I4 -27 -04 IC2 -UF -W2 -9_25C_R2.rwd

(Mod)

Bingham (228) : $\tau_{0}: 13.84 \eta_{\mathrm{p}}: 0.01896 \mathrm{Chi}^{2}: 764.3 \mathrm{r}: 0.9753$

$\mathrm{x}=\dot{\gamma}[1 / \mathrm{s}], \mathrm{y}=\tau[\mathrm{Pa}]$

Bingham (229) : $\tau_{0}: 12.77 \eta_{\mathrm{p}}: 0.02013 \mathrm{Chi}^{2}: 379.8$ r:0.9889

$\mathrm{x}=\dot{\gamma}[1 / \mathrm{s}], \mathrm{y}=\tau[\mathrm{Pa}]$

Bingham (230): $\tau_{0}: 15.34 \eta_{p}: 0.01291 \mathrm{Chi}^{2}: 340.7$ r:0.8334

$\mathrm{x}=\dot{\gamma}[1 / \mathrm{s}], y=\tau[\mathrm{Pa}]$

Bingham (231): $\tau_{0}: 14.47 \eta_{\mathrm{p}}: 0.0135 \mathrm{Chi}^{2}: 14.66 \mathrm{r}: 0.9902$

$x=\dot{\gamma}[1 / s], y=\tau[P a]$ 
Company

Operator

Date/Time

Substance

Sample no

Description

Comment
SRTC

ITS

27.04.2004/10:30:22 AM

C2-UF-W2-9

1
Meas ure device RS600

Temperature device

Sensor

A-factor

M-factor
$238 \mathrm{Ti}$

$8007.000 \mathrm{~Pa} / \mathrm{Nm}$

$8.600(1 / \mathrm{s}) /(\mathrm{rad} / \mathrm{s})$
1200203357007

Gap

$8.000 \mathrm{~mm}$

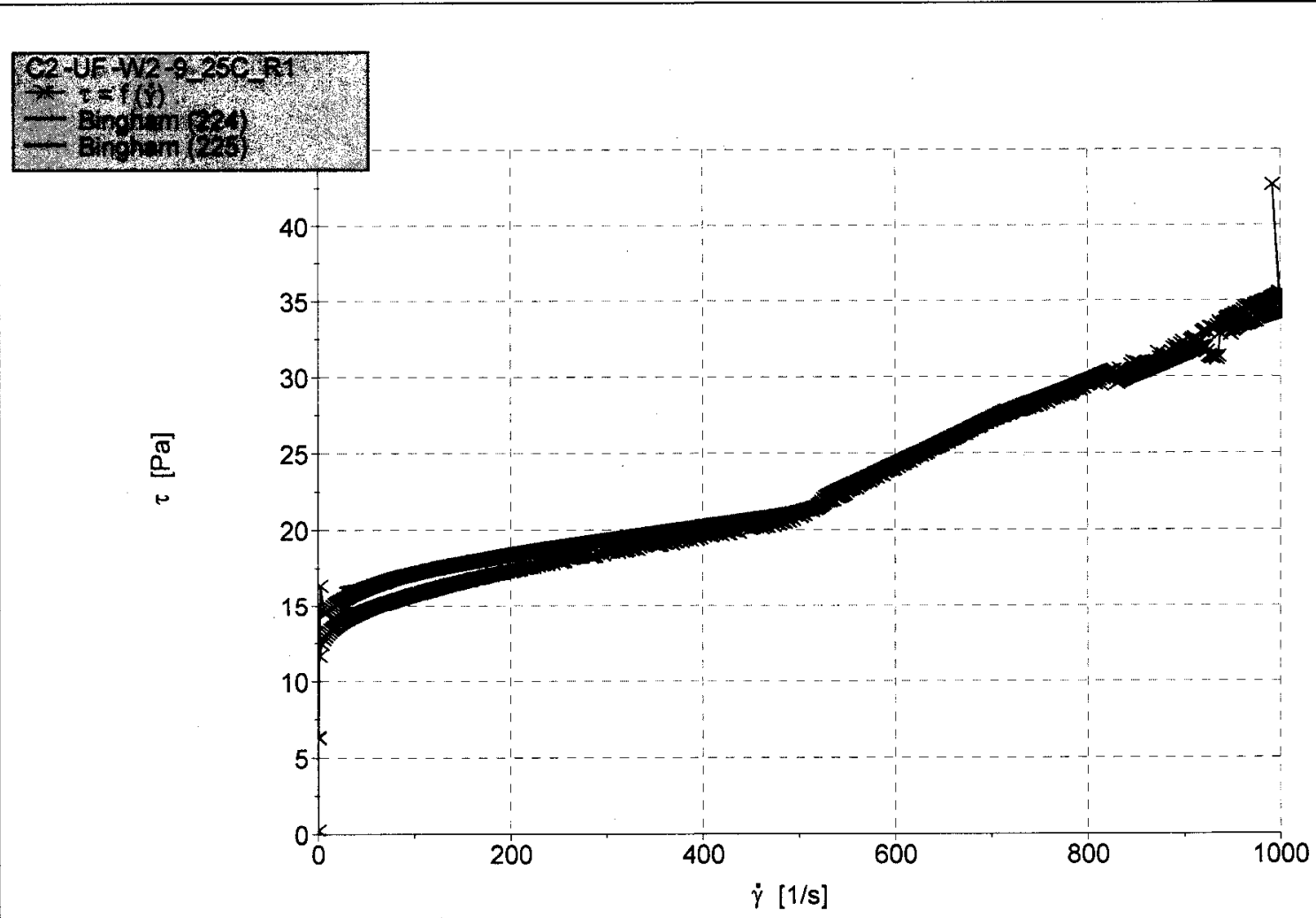

ThermoHaake RheoWin Pro 2.96

Filename:

Job:
I:IRheologylZaminecklapr '04|4-27-04IC2-UF-W2-9_25C_R1.rwd (Mod)

C:IPROGRAM FILESIRHEOWINIJOBSIRPP Z38 program .rwj

I:IRheologylZaminecklapr '04I4-27-04 IC2 -UF -W2 -9_25C_R1.rwd

(Mod)

Bingham (222): $\tau_{0}: 13.76 \eta_{\mathrm{p}}: 0.01921 \mathrm{Chi}^{2}: 893.8 \mathrm{r}: 0.972$

$$
\mathrm{x}=\dot{\gamma}[1 / \mathrm{s}], \mathrm{y}=\tau[\mathrm{Pa}]
$$

Bingham (223): $\tau_{0}: 12.48 \eta_{\mathrm{p}}: 0.02076 \mathrm{Chi}^{2}: 515.3 \mathrm{r}: 0.986$

$$
x=\dot{\gamma}[1 / \mathrm{s}], y=\tau[\mathrm{Pa}]
$$

Bingham (224): $\tau_{0}: 15.98 \eta_{\mathrm{p}}: 0.01091 \mathrm{Chi}^{2}: 11.91 \mathrm{r}: 0.9878$

$$
\mathrm{x}=\dot{\gamma}[1 / \mathrm{s}], \mathrm{y}=\tau[\mathrm{Pa}]
$$

Bingham (225) : $\tau_{0}: 14.41 \eta_{\mathrm{p}}: 0.01329 \mathrm{Chi}^{2}: 15.64$ r:0.9892

$$
x=\dot{\gamma}[1 / s], y=\tau[\mathrm{Pa}]
$$


Company

Operator

Date/Time

Substance

Sample no

Description
SRTC

ITS

27.04.2004 / 12:26:21 PM

C2-UF-W2-13

2
Meas ure device RS600

Temperature device

Sensor

A-factor

M-factor
$\mathrm{Z38} \mathrm{Ti}$

$8007.000 \mathrm{~Pa} / \mathrm{Nm}$

$8.600(1 / \mathrm{s}) /(\mathrm{rad} / \mathrm{s})$
1200203357007

Gap $\quad 8.000 \mathrm{~mm}$

\section{Comment}

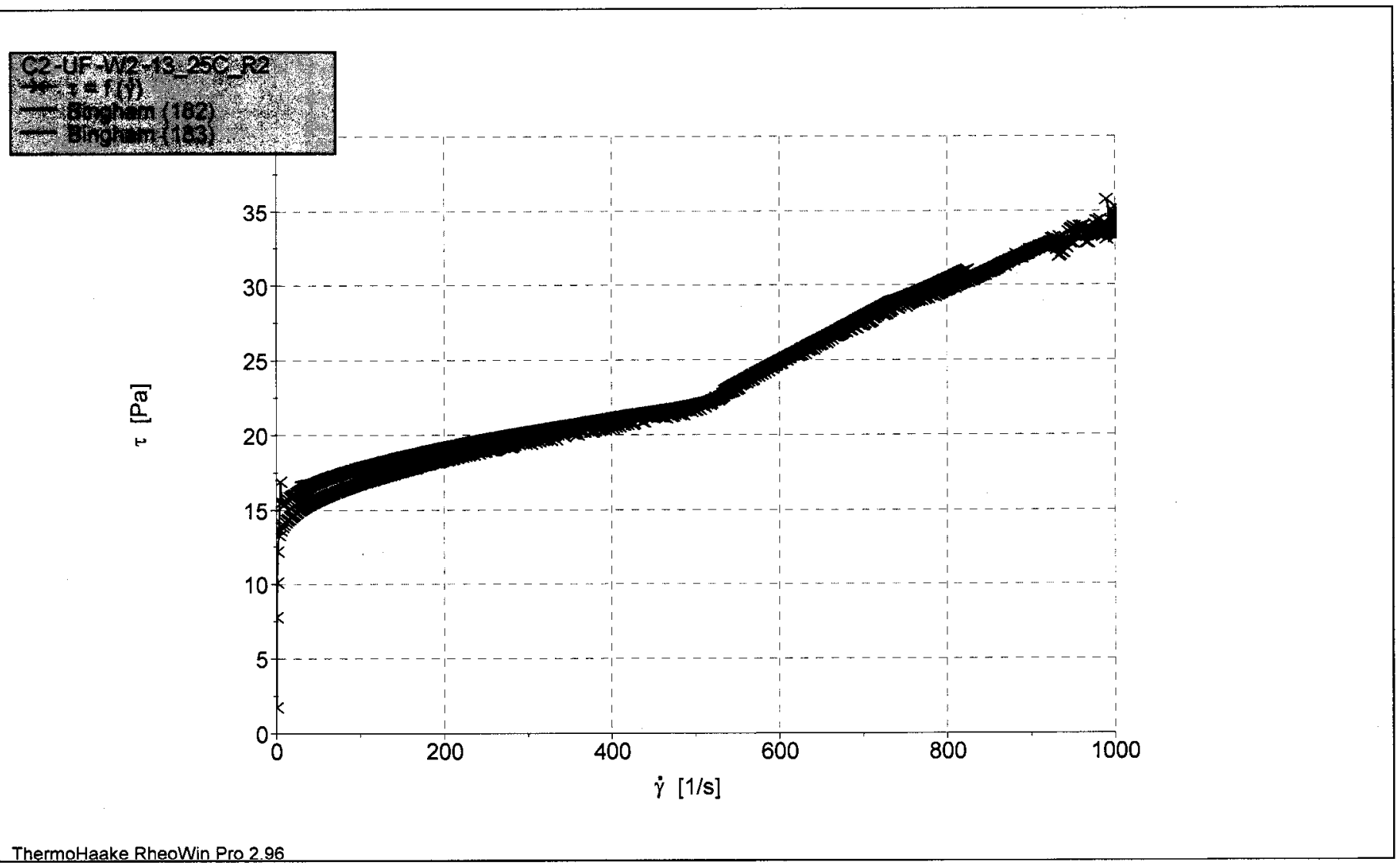

ThermaHaake RheoWin Pro 2 gs

Filename: I:IRheologyZaminecklapr '0414-27-04IC2-UF-W2-13_25C_R2.rwd (Mod)

Job:

C:IPROGRAM FILESIRHEOWINIJOBSIRPP Z38 program.nwj

I:IRheologyZaminecklapr '04I4-27-04IC2-UF -W2 -13_25C_R2.rwd

(Mod)

Bingham (180) : $\tau_{0}: 14.74 \eta_{\mathrm{p}}: 0.01853 \mathrm{Chi}^{2}: 667.4 \mathrm{r}: 0.9774$

$$
\mathrm{x}=\dot{\gamma}[1 / \mathrm{s}], \mathrm{y}=\tau[\mathrm{Pa}]
$$

Bingham (181): $\tau_{0}: 13.74 \eta_{\mathrm{p}}: 0.01958 \mathrm{Chi}^{2}: 410.9$ r:0.9874 $\mathrm{x}=\dot{\gamma}[1 / \mathrm{s}], \mathrm{y}=\tau[\mathrm{Pa}]$

Bingham (182): $\tau_{0}: 16.63 \eta_{\mathrm{p}} \cdot 0.01145 \mathrm{Chi}^{2}: 11.69 \mathrm{r}: 0.9891$

$$
\mathrm{x}=\dot{\gamma}[1 / \mathrm{s}], \mathrm{y}=\tau[\mathrm{Pa}] \text {. }
$$

Bingham (183) : $\tau_{0}: 15.38 \eta_{\mathrm{p}}: 0.01341 \mathrm{Chi}^{2}: 16.2$ r:0.9891

$$
\mathrm{x}=\dot{\gamma}[1 / \mathrm{s}], \mathrm{y}=\tau[\mathrm{Pa}]
$$


Company

Operator

Date/Time

Substance

Sample no

Description

Comment

$\begin{array}{ll}\text { Measure device } & \text { RS600 } \\ \text { Temperature device } & \\ \text { Sensor } & \text { Z38 Ti } \\ \text { A-factor } & 8007.000 \mathrm{~Pa} / \mathrm{Nm} \\ \text { M-factor } & 8.600(1 / \mathrm{s}) /(\mathrm{rad} / \mathrm{s})\end{array}$

A-factor

$8.600(1 / \mathrm{s}) /(\mathrm{rad} / \mathrm{s})$
1200203357007

Gap

$8.000 \mathrm{~mm}$
27.04.2004 / 12:09:02 PM

C2-UF-W2-13

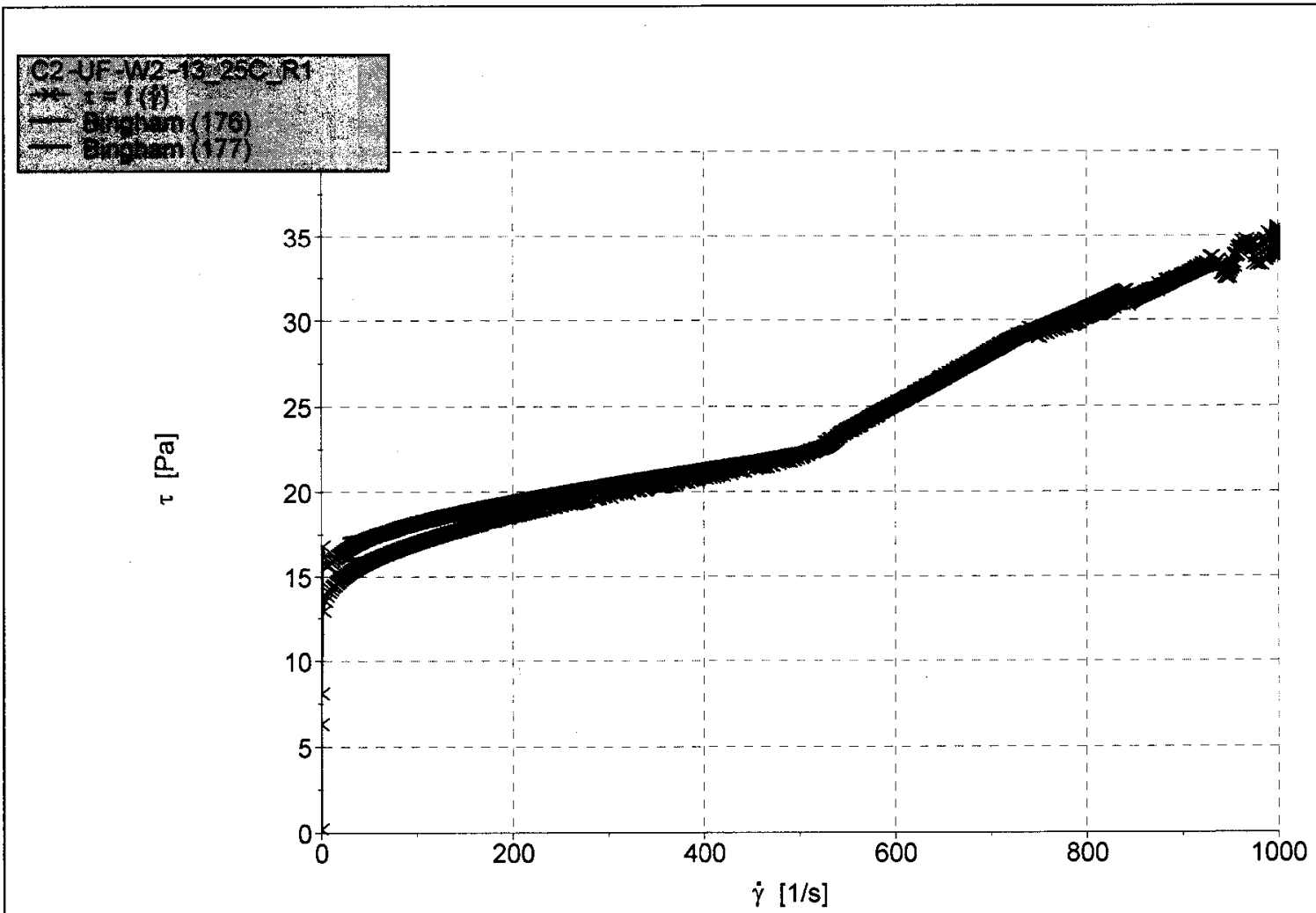

ThermoHaake Rheowin Pro 2.96

Filename: I:IRheologyZZaminecklapr '0414-27-04IC2-UF-W2-13_25C_R1.rwd (Mod)

Job:

C:IPROGRAM FILESIRHEOWINIJOBSIRPP Z38 program.wj

I:IRheologylZaminecklapr '0414 -27 -04IC2 -UF -W2 -13_25C_R1.rwd

(Mod)

Bingham (173) : $\tau_{0}: 14.93 \eta_{\mathrm{p}}: 0.01873 \mathrm{Chi}^{2}: 855.4 \mathrm{r}: 0.9718$

$$
\mathrm{x}=\dot{\gamma}[1 / \mathrm{s}], \mathrm{y}=\tau[\mathrm{Pa}]
$$

Bingham (174) : $\tau_{0}: 13.95 \eta_{\mathrm{p}}: 0.01985 \mathrm{Chi}^{2}: 421.8 \mathrm{r}: 0.9875$

$x=\dot{\gamma}[1 / \mathrm{s}], y=\tau[\mathrm{Pa}]$

Bingham (176) : $\tau_{0}: 17.04 \eta_{\mathrm{p}}: 0.01088 \mathrm{Chi}^{2}: 9.814 \mathrm{r}: 0.9899$

$x=\dot{\gamma}[1 / \mathrm{s}], y=\tau[\mathrm{Pa}]$

Bingham (177): $\tau_{0}: 15.61 \eta_{\mathrm{p}}: 0.01352 \mathrm{Chi}^{2}: 19.13 \mathrm{r}: 0.9875$

$$
x=\dot{\gamma}[1 / \mathrm{s}], y=\tau[\mathrm{Pa}]
$$


Company

Operator

Date/Time

Substance

Sample no

Description
SRTC

ITS

27.04.2004 / 13:00:55 PM

C2-UF-W2-17

2
Measure device $\quad$ RS600

Temperature device

Sensor

A-factor

M-factor
$238 \mathrm{Ti}$

8007.000 Pa/Nm

$8.600(1 / \mathrm{s}) /(\mathrm{rad} / \mathrm{s})$
1200203357007

Gap $\quad 8.000 \mathrm{~mm}$

\section{Comment}

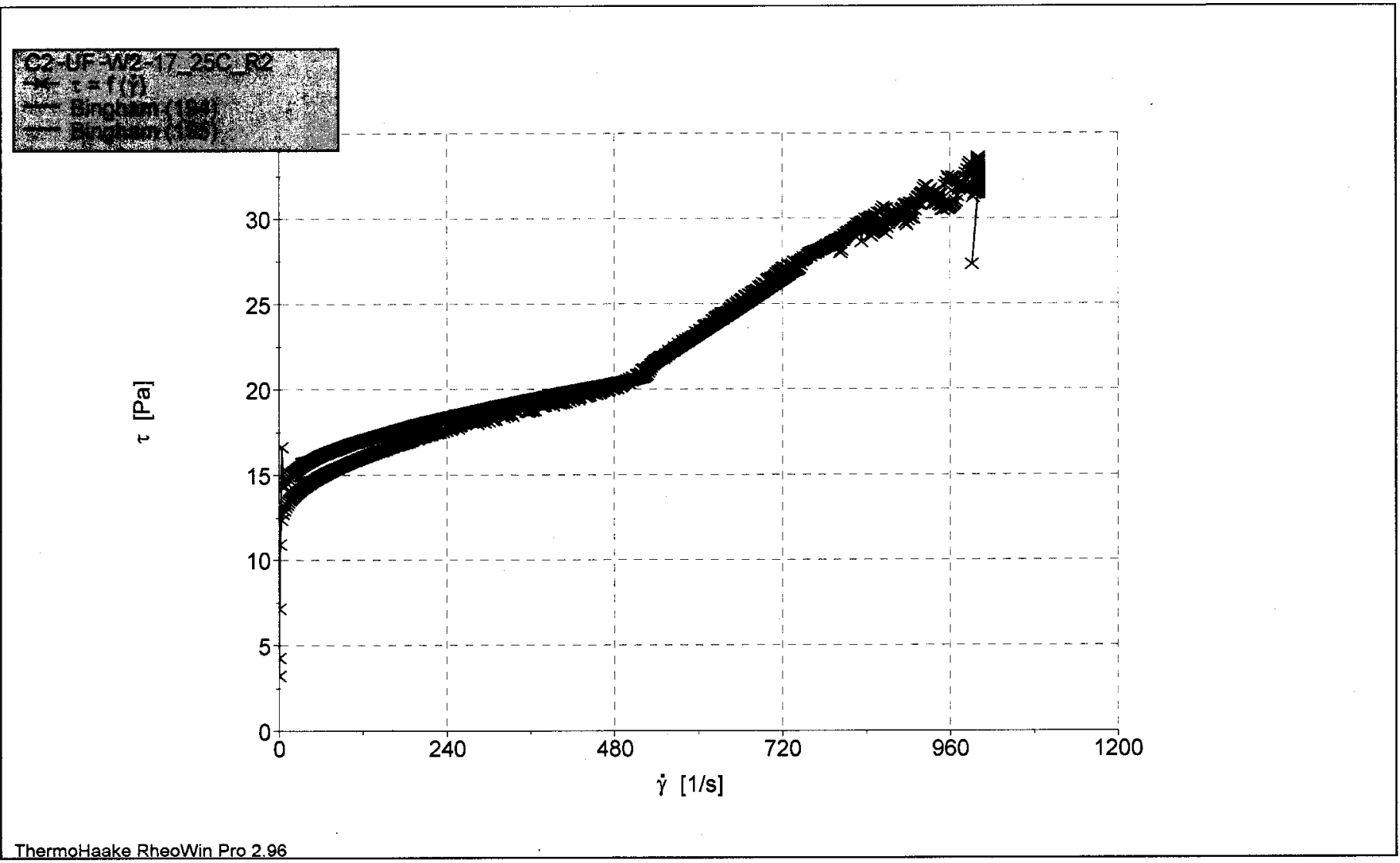

Filename: I:IRheologyZaminecklapr '0414-27-04IC2-UF-W2-17_25C_R2.rwd (Mod)

Job:

C:IPROGRAM FILESIRHEOWINIJOBSIRPP Z38 program.rwj

I:IRheologyZaminecklapr '04I4 -27-04IC2 -UF -W2 -17_25C_R2.rwd

(Mod)

Bingham (192): $\tau_{0}: 13.87 \eta_{\mathrm{p}}: 0.01735 \mathrm{Chi}^{2}: 568.9$ r:0.9781

$x=\dot{\gamma}[1 / \mathrm{s}], y=\tau[\mathrm{Pa}]$

Bingham (193): $\tau_{0}: 12.52 \eta_{\mathrm{p}}: 0.01959 \mathrm{Chi}^{2}: 600 \mathrm{r}: 0.9819$

$\mathrm{x}=\dot{\gamma}[1 / \mathrm{s}], \mathrm{y}=\tau[\mathrm{Pa}]$

Bingham (194): $\tau_{0}: 15.72 \eta_{\mathrm{p}}: 0.01026 \mathrm{Chi}^{2}: 8.261 \mathrm{r}: 0.9903$

$\mathrm{x}=\dot{\gamma}[1 / \mathrm{s}], \mathrm{y}=\tau[\mathrm{Pa}]$

Bingham (195) : $\tau_{0}: 14.42 \eta_{\mathrm{p}}: 0.01246 \mathrm{Chi}^{2}: 15.35 \mathrm{r}: 0.9881$

$\mathrm{x}=\dot{\gamma}[1 / \mathrm{s}], \mathrm{y}=\tau[\mathrm{Pa}]$ 
Company

Operator

Date/Time

Substance

Sample no

SRTC

ITS

27.04.2004 / 12:43:01 PM

C2-UF-W2-17

1
Meas ure device RS600

Temperature device

Sensor

A-factor

M-factor
1200203357007

Gap $\quad 8.000 \mathrm{~mm}$

Description

\section{Comment}

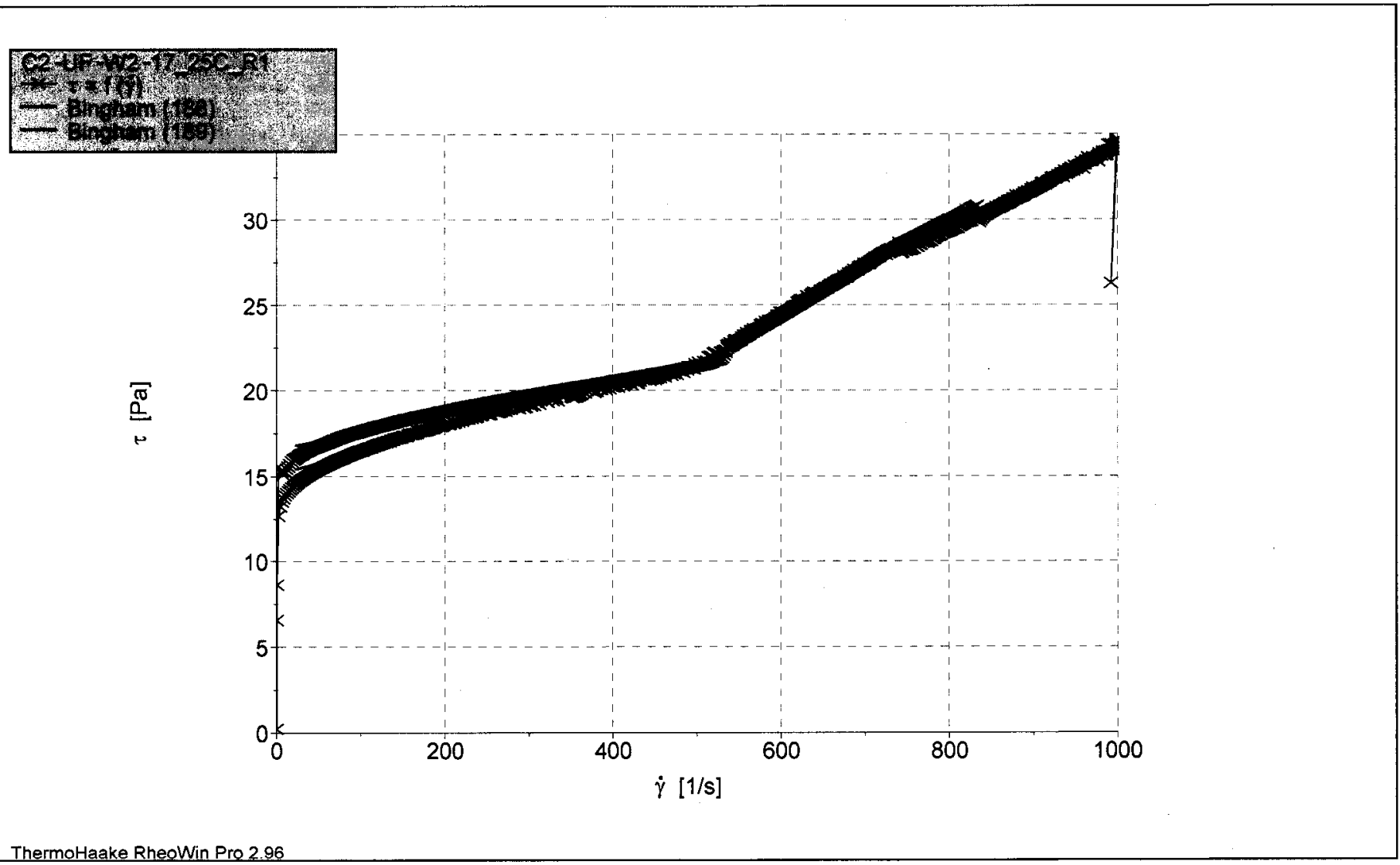

Filename: $\quad$ I:IRheologylZaminecklapr '04I4-27-04IC2-UF-W2-17_25C_R1.rwd (Mod)

Job:

C:IPROGRAM FILESIRHEOWINIJOBSIRPP Z38 program.rwj

I:VReologylZaminecklapr '04/4 -27-04 IC2 -UF -W2 -17_25C_R1.rwd

(Mod)

Bingham (186) : $\tau_{0}: 14.34 \eta_{\mathrm{p}}: 0.01851 \mathrm{Chi}^{2}: 873.7 \mathrm{r:0.9706}$

$\mathrm{x}=\dot{\gamma}[1 / \mathrm{s}], \mathrm{y}=\tau[\mathrm{Pa}]$

Bingham (187) : $\tau_{0}: 13.45 \eta_{\mathrm{p}}: 0.0196 \mathrm{Chi}^{2}: 400.4$ r:0.9878

$x=\dot{\gamma}[1 / \mathrm{s}], y=\tau[\mathrm{Pa}]$

Bingham (188) : $\tau_{0}: 16.57 \eta_{\mathrm{p}}: 0.01016 \mathrm{Chi}^{2}: 8.885 \mathrm{r}: 0.9895$

$x=\dot{\gamma}[1 / \mathrm{s}], y=\tau[\mathrm{Pa}]$

Bingham (189) : $\tau_{0}: 15.13 \eta_{\mathrm{p}}: 0.01314 \mathrm{Chi}^{2}: 17.43 \mathrm{r}: 0.9879$

$x=\dot{\gamma}[1 / s], y=\tau[P a]$ 
Company

Operator

Date/Time

Substance

Sample no

Description
SRTC

ITS

27.04.2004 / 13:34:01 PM

C2-UF-W2-22-ACTL

2
Meas ure device RS600

Temperature device

Sensor

A-factor

M-factor
$238 \mathrm{Ti}$

$8007.000 \mathrm{~Pa} / \mathrm{Nm}$

$8.600(1 / \mathrm{s}) /(\mathrm{rad} / \mathrm{s})$
1200203357007

Gap

$8.000 \mathrm{~mm}$

\section{Comment}

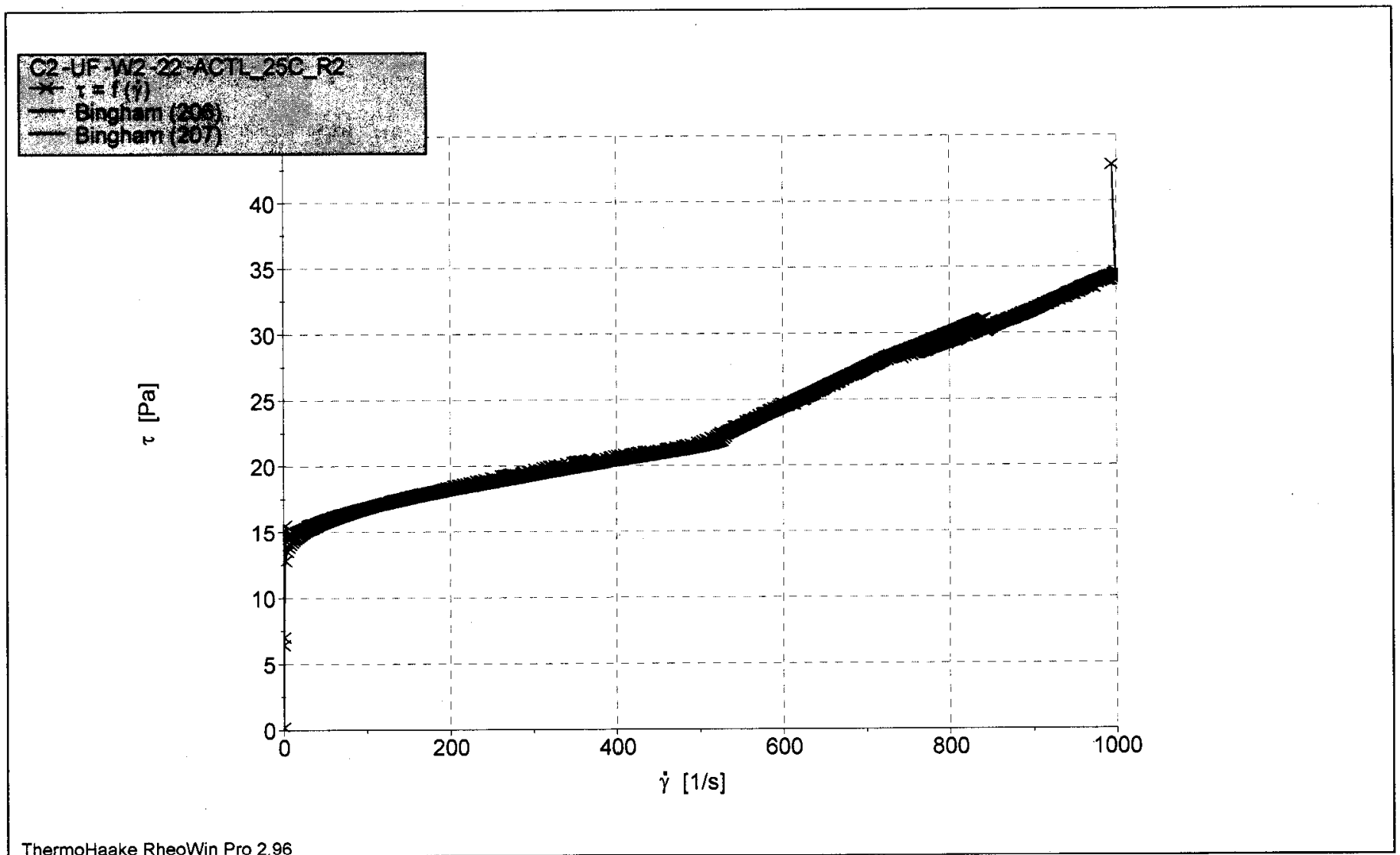

ThermoHaake RheoWin Pro 29

Filename: I:IRheologylZaminecklapr '04I4-27-04IC2-UF-W2-22-ACTL_25C_R2.rwd (Mod)

Job:

C:IPROGRAM FILESIRHEOWINIJOBSIRPP Z38 program.wj

I:IRheologyZaminecklapr

'04\4 -27-04IC2 -UF -W2-22-ACTL_25C_R2.nwd (Mod)

Bingham (204): $\tau_{0}: 13.62 \eta_{\mathrm{p}}: 0.01958 \mathrm{Chi}^{2}: 712.4 \mathrm{r}: 0.9783$

$$
\mathrm{x}=\dot{\gamma}[1 / \mathrm{s}], \mathrm{y}=\tau[\mathrm{Pa}]
$$

Bingham (205) : $\tau_{0}: 13.86 \eta_{\mathrm{p}}: 0.01913 \mathrm{Chi}^{2}: 414 \mathrm{r}: 0.9868$

$$
x=\dot{\gamma}[1 / \mathrm{s}], y=\tau[\mathrm{Pa}]
$$

Bingham (206) : $\tau_{0}: 15.58 \eta_{p} \cdot 0.01222 \mathrm{Chi}^{2}: 6.648 \mathrm{r}: 0.9945$

$$
x=\dot{\gamma}[1 / \mathrm{s}], y=\tau[\mathrm{Pa}]
$$

Bingham (207): $\tau_{0}: 15.49 \eta_{\mathrm{p}}: 0.01308 \mathrm{Chi}^{2}: 17.55 \mathrm{r}: 0.9876$

$$
x=\dot{\gamma}[1 / \mathrm{s}], y=\tau[\mathrm{Pa}]
$$


Company

Operator

Date/Time

Substance

Sample no

Description
SRTC

ITS

27.04.2004/ 13:17:39 PM

C2-UF-W2-22-ACTL

1
Meas ure device RS600

Temperature device

Sensor

A-factor

M-factor
$\mathrm{Z38} \mathrm{Ti}$

$8007.000 \mathrm{~Pa} / \mathrm{Nm}$

$8.600(1 / \mathrm{s}) /(\mathrm{rad} / \mathrm{s})$
1200203357007

Gap $7.999 \mathrm{~mm}$

Comment

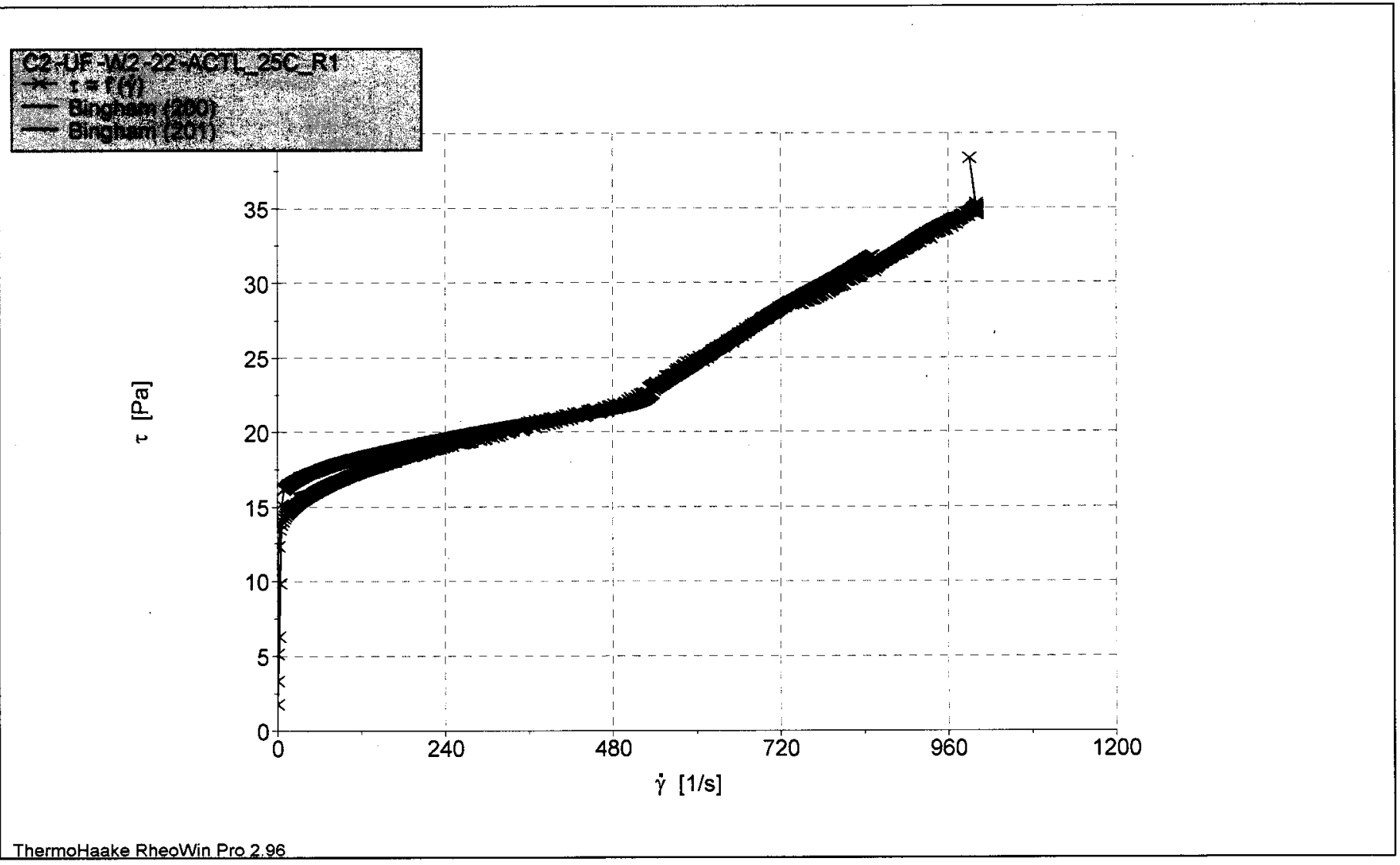

Filename: I:IRheologylZaminecklapr'0414-27-04IC2-UF-W2-22-ACTL_25C_R1.rwd (Mod)

Job:

C:IPROGRAM FILESIRHEOWINIJOBSIRPP Z38 program.wj

I:RheologylZaminecklap r

'0414 -27-04IC2 -UF -W2 -22 -ACTL_25C_R1.wwd (Mod)

Bingham (198): $\tau_{0}: 14.6 \eta_{p}: 0.01884 \mathrm{Chi}^{2}: 984.4$ r:0.9685 $x=\dot{\gamma}[1 / s], y=\tau[P a]$

Bingham (199): $\tau_{0}: 13.83 \eta_{\mathrm{p}}: 0.01969 \mathrm{Chi}^{2}: 566.6 \mathrm{r}: 0.9831$ $x=\dot{\gamma}[1 / \mathrm{s}], y=\tau[\mathrm{Pa}]$

Bingham (200) : $\tau_{0}: 17.04 \eta_{p}: 0.009802$ Chi $: 6.061$ r:0.9923 $x=\dot{\gamma}[1 / s], y=\tau[P a]$

Bingham (201): $\tau_{0}: 15.58 \eta_{\mathrm{p}}: 0.01345 \mathrm{Chi}^{2}: 17.6$ r:0.9881 $x=\dot{\gamma}[1 / s], y=\tau[\mathrm{Pa}]$ 


$\begin{array}{lllll}\text { Company } & \text { SRTC } & \text { Measure device } & \text { RS600 } & \\ \text { Operator } & \text { ITS } & \text { Temperature device } & 1200203357007 \\ \text { Date/Time } & 26.04 .2004 / 13: 34: 10 \mathrm{PM} & \text { Sensor } & \text { DG4 } 1 \mathrm{Ti} & \text { Gap } \\ \text { Substance } & \text { C2-1X-FEED-ACTL } & \text { A-factor } & 3701.000 \mathrm{~Pa} / \mathrm{Nm} & 5.100 \mathrm{~mm} \\ \text { Sample no } & & \text { M-factor } & 72.670(1 / \mathrm{s}) /(\mathrm{rad} / \mathrm{s}) & \end{array}$

Description

Comment

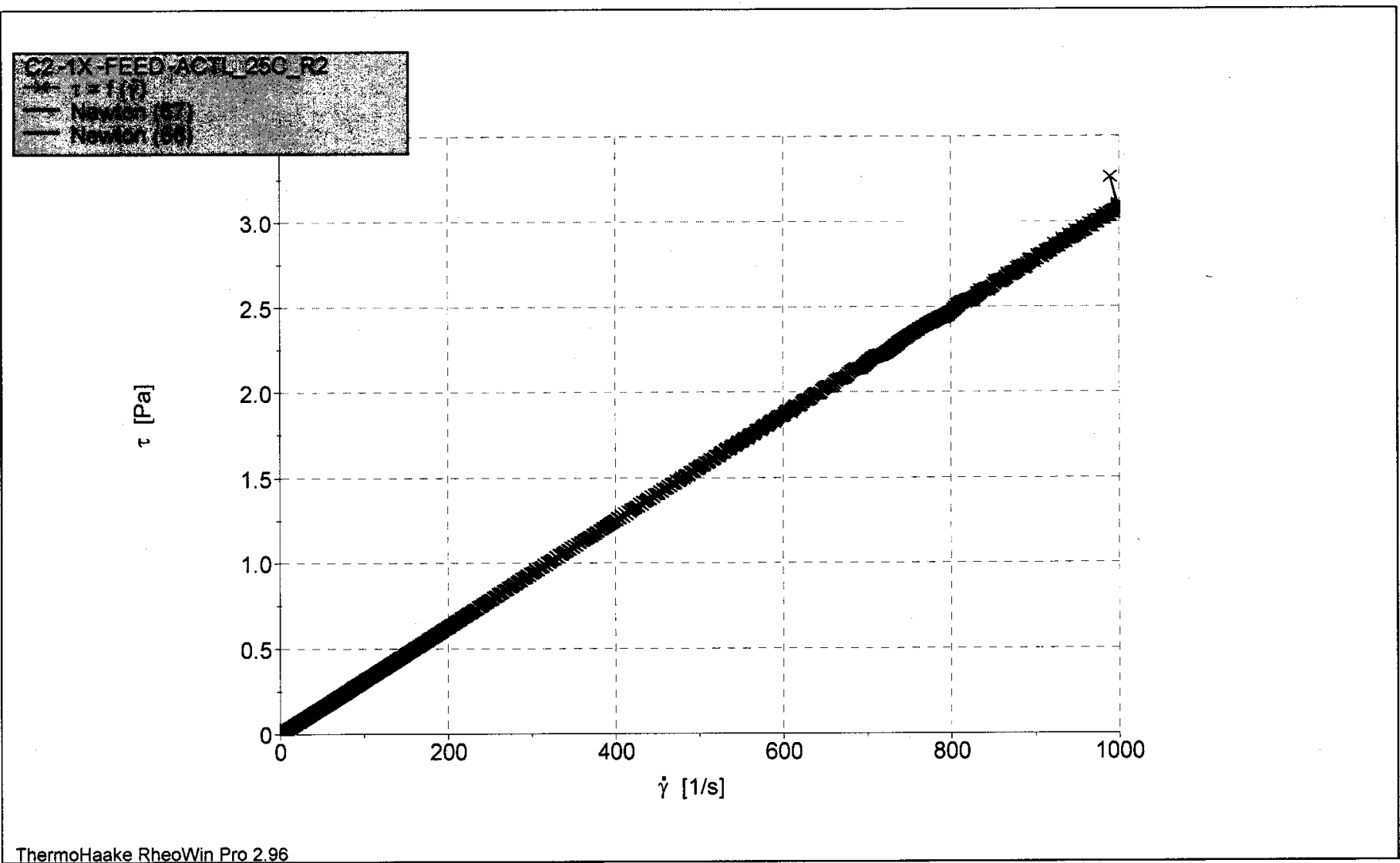

Filename: I:IRheologylZaminecklapr '04/4-26-04IC2-1X-FEED-ACTL_25C_R2.nwd (Mod)

Job: $\quad$ C:IPROGRAM FILESIRHEOWINIJOBSIRPP DG41 program.rwj

I:IRheologylZaminecklapr

'04\4 -26-04IC2 -1X-FEED-ACTL_25C_R2.nwd (Mod)

Bingham (65): $\tau_{0}: 0.01143 \eta_{\mathrm{p}}: 0.003083 \mathrm{Chi}^{2}: 0.03498 \mathrm{r}: 1$

$\mathrm{x}=\dot{\gamma}[1 / \mathrm{s}], \mathrm{y}=\tau[\mathrm{Pa}]$

Bingham (66): $\tau_{0}: 0.01163 \eta_{p}: 0.003081 \mathrm{Chi}^{2}: 0.05063$ r:0.9999

$\mathrm{x}=\dot{\gamma}[1 / \mathrm{s}], \mathrm{y}=\tau[\mathrm{Pa}]$

Newton (67) : $\eta: 0.0031 \mathrm{Chi}^{2}: 0.05135$ r:0.9999

$x=\dot{\gamma}[1 / \mathrm{s}], y=\tau[\mathrm{Pa}]$

Newton (68) : $\eta: 0.003099 \mathrm{Chi}^{2}: 0.06754$ r:0.9999

$\mathrm{x}=\dot{\gamma}[1 / \mathrm{s}], \mathrm{y}=\tau[\mathrm{Pa}]$ 
Company

Operator

Date/Time

Substance

Sample no

Description

\section{SRTC}

ITS

26.04 .2004 / 15:10:05 PM

C2-1X-FEED-ACTL

4
Meas ure device RS600

Temperature device

Sensor

A-factor

M-factor
DG41 $T$

$3701.000 \mathrm{~Pa} / \mathrm{Nm}$

$72.670(1 / \mathrm{s}) /(\mathrm{rad} / \mathrm{s})$
1200203357007

Gap

$5.100 \mathrm{~mm}$

\section{Comment}

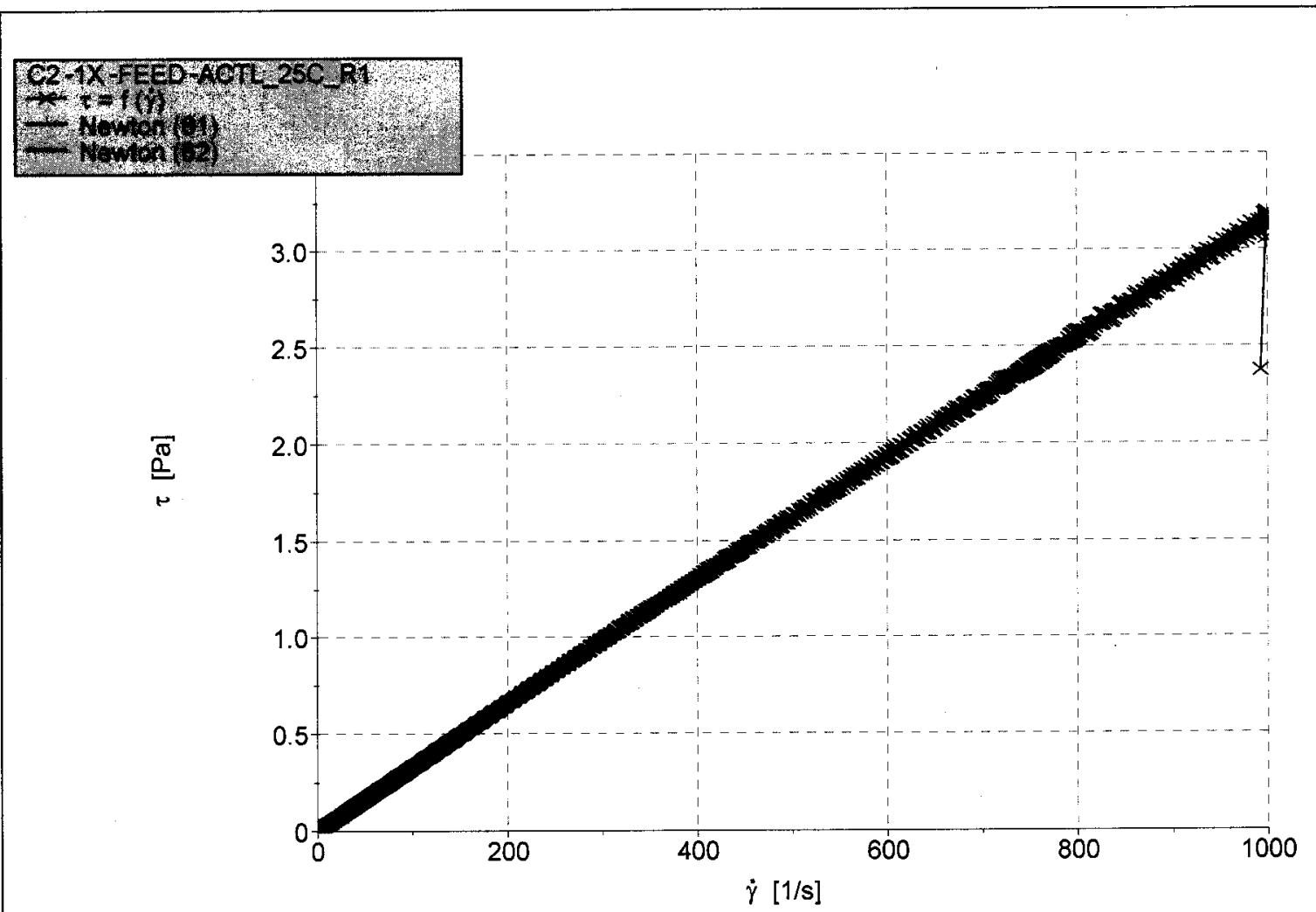

ThermoHaake RheoWin Pro 2.96

Filename:

Job:
I:IRheologylZaminecklapr '04/4-26-04IC2-1X-FEED-ACTL_25C_R1.nwd (Mod)

C:IPROGRAM FILESIRHEOWINIJOBSIRPP DG41 program.rwj

I:IRheologylZaminecklap $r$

'04/4 -26-04lC2 - 1X-FEED-ACTL_25C_R1.rwd (Mod)

Bingham (59): $\tau_{0}: 0.02752 \eta_{\mathrm{p}}: 0.003154$ Chi : 0.134 r.0.9998

$$
\mathrm{x}=\dot{\gamma}[1 / \mathrm{s}], \mathrm{y}=\tau[\mathrm{Pa}]
$$

Bingham (60): $\tau_{0}: 0.02801 \eta_{p}: 0.00315 \mathrm{Chi}^{2}: 0.22$ r:0.9997

$$
x=\dot{\gamma}[1 / \mathrm{s}], y=\tau[\mathrm{Pa}]
$$

Newton (61) : $\eta: 0.003195 \mathrm{Chi}^{2}: 0.2289$ r:0.9997

$$
\mathrm{x}=\dot{\gamma}[1 / \mathrm{s}], \mathrm{y}=\tau[\mathrm{Pa}]
$$

Newton (62) : $\eta: 0.003192 \mathrm{Chi}^{2}: 0.3181$ r:0.9996

$$
\mathrm{x}=\dot{\gamma}[1 / \mathrm{s}], \mathrm{y}=\tau[\mathrm{Pa}]
$$


Company

Operator

Date/Time

Substance

Sample no

Description

\section{Comment}

\begin{tabular}{|c|c|c|c|}
\hline \multirow{2}{*}{\multicolumn{2}{|c|}{ Measure device RS600 }} & \multicolumn{2}{|c|}{1200203357007} \\
\hline & & & \\
\hline Sensor & DG41 Ti & Gap & $5.100 \mathrm{~mm}$ \\
\hline A-factor & $3701.000 \mathrm{~Pa} / \mathrm{Nm}$ & & \\
\hline
\end{tabular}

Measure device RS600

ITS

11.05.2004 / 7:48:47 AM

c2-1x-prod-actl

2

M-factor

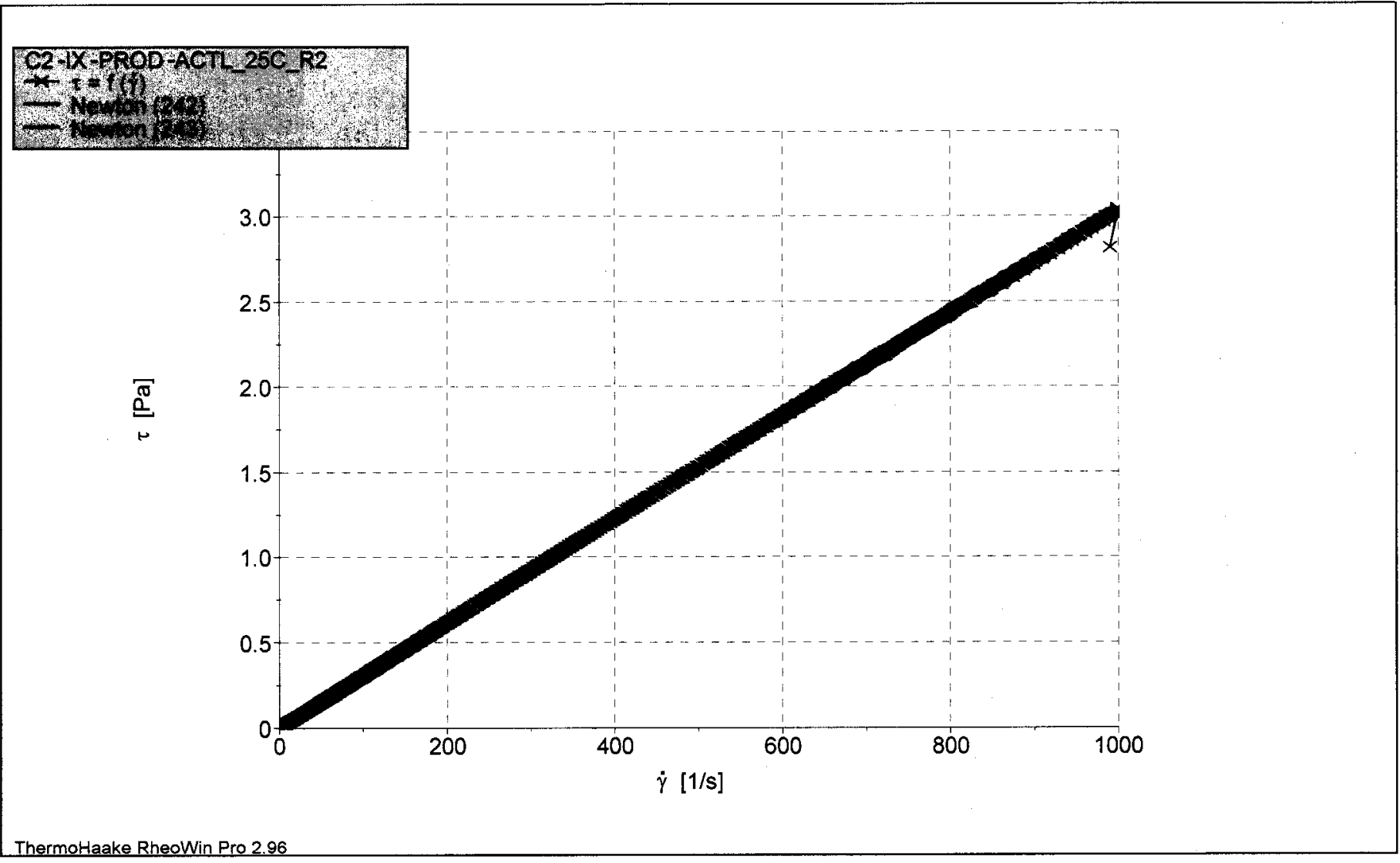

Filename: $\quad$ I:IRheologylZaminecklmay'0415-11-04IC2-IX-PROD-ACTL_25C_R2.wwd (Mod)

Job:

C:IPROGRAM FILESIRHEOWINIJOBSIRPP DG41 program.rwj

I:IRheology Zaminecklmay'0415-11-04IC2 -IX-PROD-ACTL_25C_R2.n

(Mod)

Bingham (240) : $\tau_{0}: 0.01381 \eta_{\mathrm{p}}: 0.00303 \mathrm{Chi}^{2}: 0.02406 \mathrm{r}: 1$

$x=\dot{\gamma}[1 / \mathrm{s}], y=\tau[\mathrm{Pa}]$

Bingham (241): $\tau_{0}: 0.007283 \eta_{p}: 0.003032 \mathrm{Chi}^{2}: 0.02052 \mathrm{r}: 1$

$\mathrm{x}=\dot{\gamma}[1 / \mathrm{s}], \mathrm{y}=\tau[\mathrm{Pa}]$

Newton (242) : $\eta: 0.00305 \mathrm{Chi}^{2}: 0.04794$ r:0.9999

$x=\dot{\gamma}[1 / \mathrm{s}], y=\tau[\mathrm{Pa}]$

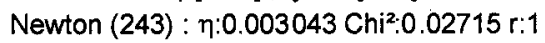

$\mathrm{x}=\dot{\gamma}[1 / \mathrm{s}], \mathrm{y}=\tau[\mathrm{Pa}]$ 
Company

Operator

Date/Time

Substance

Sample no

Description

\section{SRTC}

ITS

11.05.2004 / 7:20:46 AM

c2-1x-prod-act

1
Measure device RS600

Temperature device

Sensor

A-factor

M-factor
DG4 $1 \mathrm{TI}$

$3701.000 \mathrm{~Pa} / \mathrm{Nm}$

$72.670(1 / \mathrm{s}) /(\mathrm{rad} / \mathrm{s})$
1200203357007

Gap

$5.100 \mathrm{~mm}$

Comment

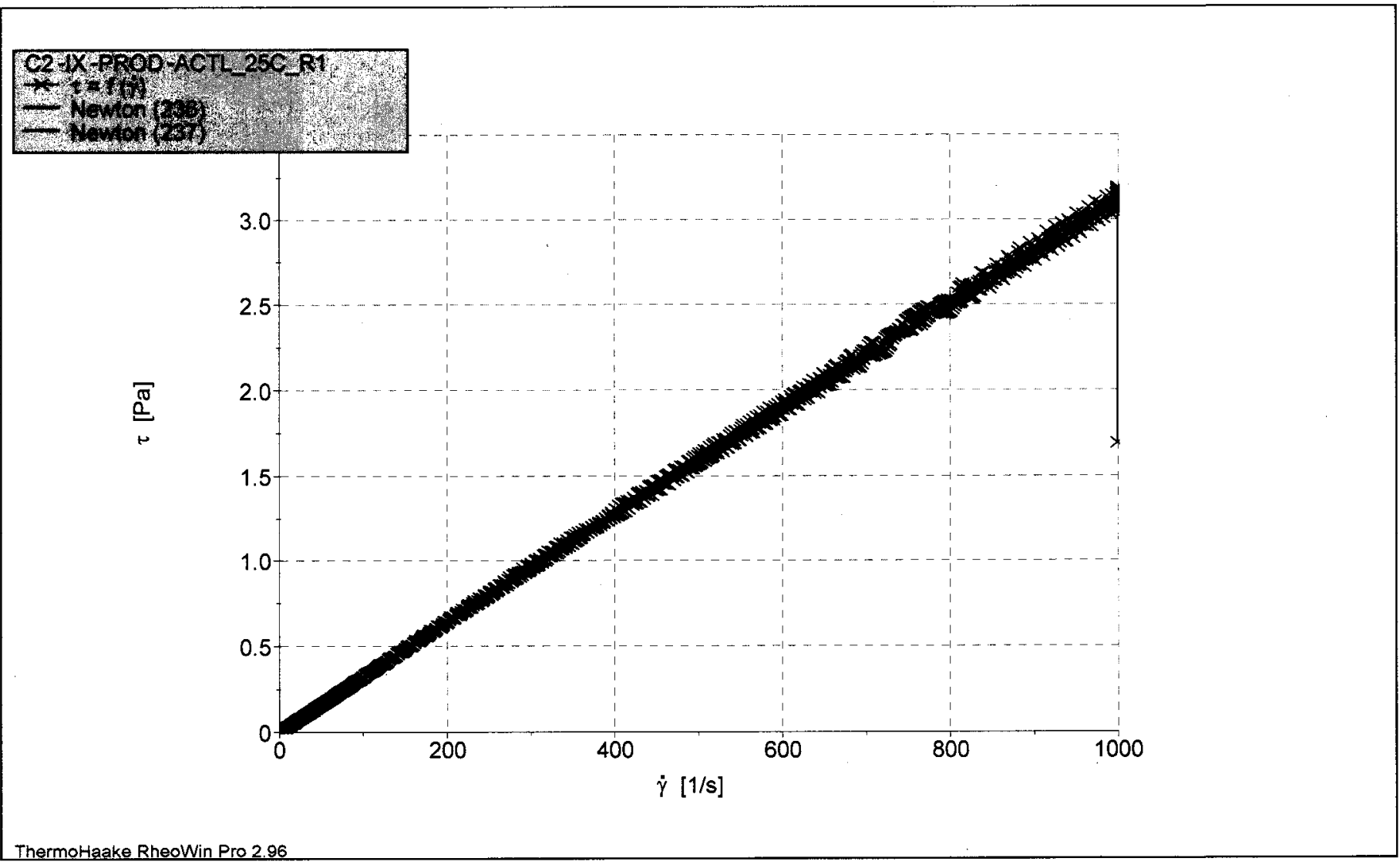

Filename:

Job:
I:IRheologylZaminecklmay'04I5-11-04IC2-IX-PROD-ACTL_25C_R1.nwd (Mod)

C:IPROGRAM FILESIRHEOWINIJOBSIRPP DG41 program.rwj

I:IRheologylZaminecklmay'0415-11-04IC2 -IX-PROD-ACTL_25C_R1.n

(Mod)

Bingham (234) : $\tau_{0}: 0.02436 \eta_{\mathrm{p}}: 0.00311$ Chi $: 0.2401$ r.0.9997

$\mathrm{x}=\dot{\gamma}[1 / \mathrm{s}], \mathrm{y}=\tau[\mathrm{Pa}]$

Bingham (235) : $\tau_{0}: 0.02087 \eta_{\mathrm{p}}: 0.003115 \mathrm{Chi}: 0.513$ r.0.9994

$x=\dot{\gamma}[1 / \mathrm{s}], y=\tau[\mathrm{Pa}]$

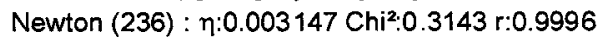

$\mathrm{x}=\dot{\gamma}[1 / \mathrm{s}], \mathrm{y}=\tau[\mathrm{Pa}]$

Newton (237) : $\eta: 0.003146 \mathrm{Chi}^{2}: 0.5674$ r:0.9993

$x=\dot{\gamma}[1 / \mathrm{s}], y=\tau[\mathrm{Pa}]$ 
Company

Operator

Date/Time

Substance

Sample no

SRTC

ITS

17.05.2004 / 11:38:09 AM

C2-TLP-B-start-act|

1
Meas ure device RS600

Temperature device

Sensor

A-factor

M-factor
DG4 $1 \mathrm{TI}$

$3701.000 \mathrm{~Pa} / \mathrm{Nm}$

$72.670(1 / \mathrm{s}) /(\mathrm{rad} / \mathrm{s})$
1200203357007

Gap $\quad 5.100 \mathrm{~mm}$

Description

Comment

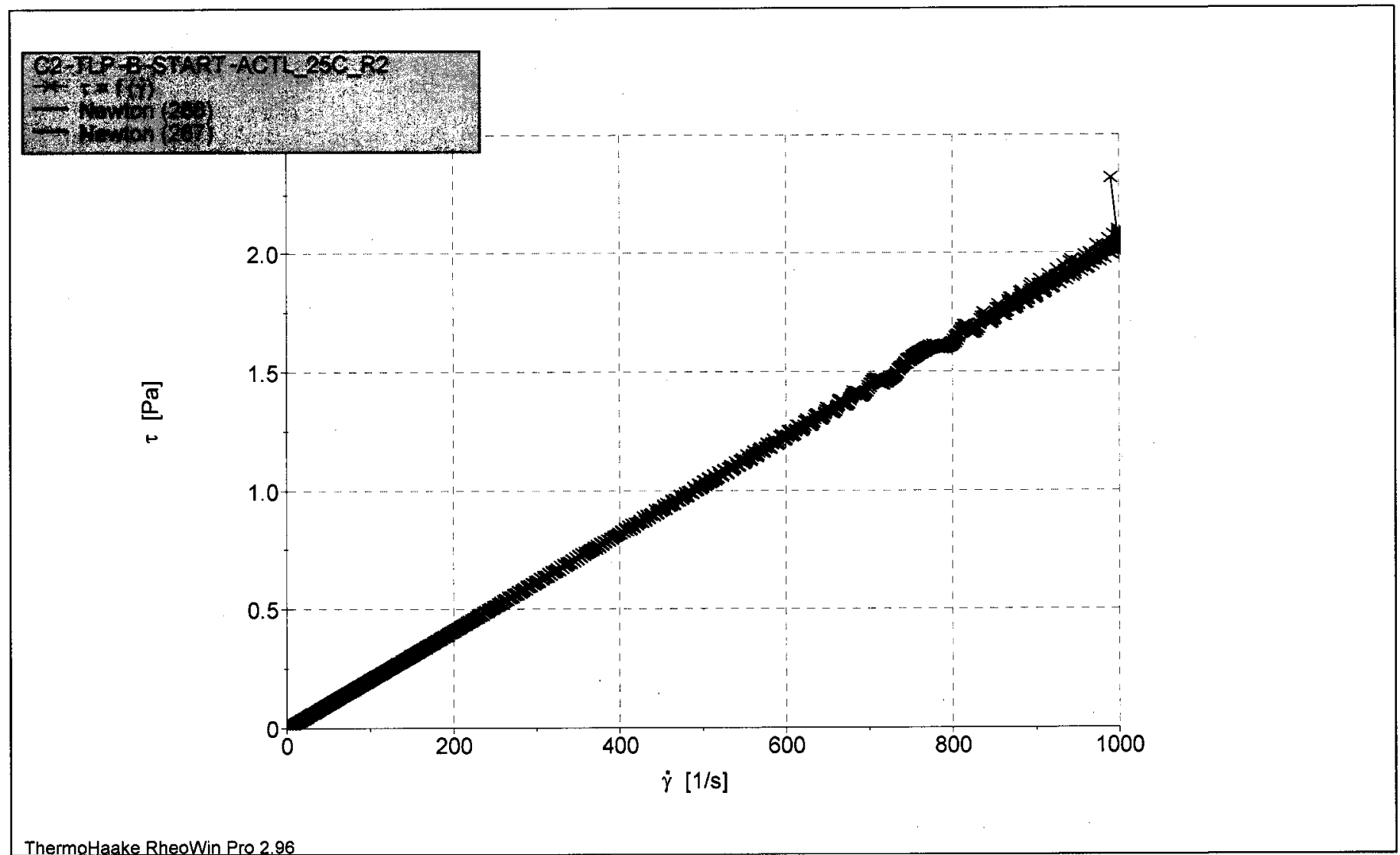

hermoHaake Rheowin Pro 2

Filename:

I:IRheologylZaminecklmay'04|5-17-04IC2-TLP-B-START-ACTL_25C_R2.nwd (Mod)

Job:

C:IPROGRAM FILESIRHEOWINIJOBSIRPP DG41 program.rwj

I:RheologylZaminecklmay'04l5 -17 -04lC2-TLP-B -START-ACTL_25C.

(Mod)

Bingham (264): $\tau_{0}:-0.0002228 \eta_{p}: 0.002049 \mathrm{Chi}^{2}: 0.033$ r.0.9999

$$
x=\dot{\gamma}[1 / \mathrm{s}], y=\tau[\mathrm{Pa}]
$$

Bingham (265) : $\tau_{0}:-0.001663 \eta_{p}: 0.002048$ Chi $^{2}: 0.1082$ r.0.9997

$$
\mathrm{x}=\dot{\gamma}[1 / \mathrm{s}], \mathrm{y}=\tau[\mathrm{Pa}]
$$

Newton (266) : ๆ:0.002049 Chi2:0.03301 r:0.9999

$$
x=\dot{\gamma}[1 / \mathrm{s}], y=\tau[\mathrm{Pa}]
$$

Newton (267) : $\eta: 0.002046 \mathrm{Chi}^{2}: 0.1085$ r:0.9997

$$
\mathrm{x}=\dot{\gamma}[1 / \mathrm{s}], \mathrm{y}=\tau[\mathrm{Pa}]
$$


Company

Operator

Date/Time

Substance

Sample no

Description
SRTC

ITS

17.05.2004 / 11:17:27 AM

C2-TLP-B-start-actl

2
Measure device RS600

Temperature device

Sensor

A-factor

M-factor
DG41 Ti

$3701.000 \mathrm{~Pa} / \mathrm{Nm}$

$72.670(1 / \mathrm{s}) /(\mathrm{rad} / \mathrm{s})$
1200203357007

Gap $\quad 5.100 \mathrm{~mm}$

Comment

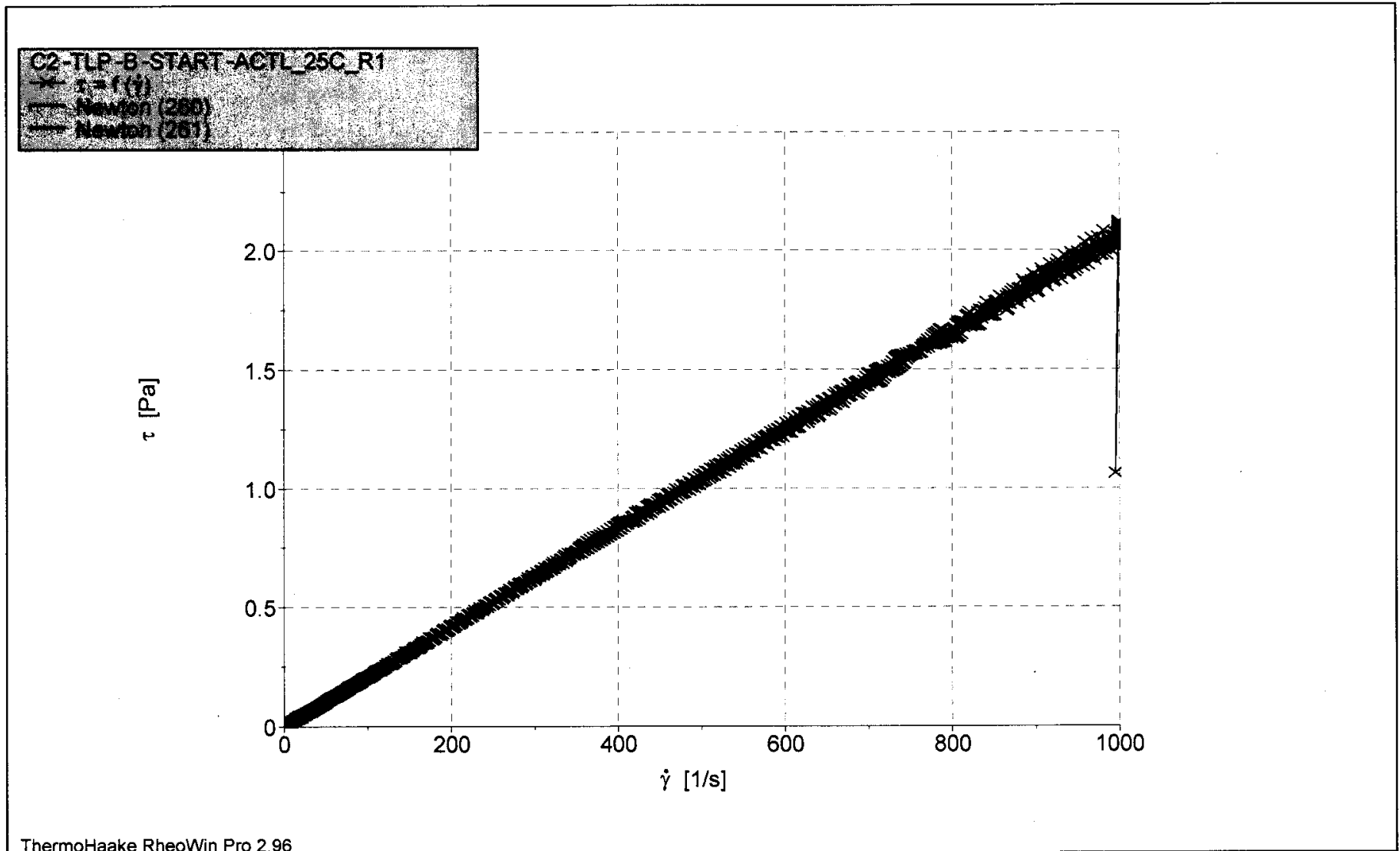

Thermo Haake RheoWin Pro 29

Filename:

Job:
I:IRheology'Zaminecklmay'0415-17-04IC2-TLP-B-START-ACTL_25C_R1.rwd (Mod)

C:IPROGRAM FILESIRHEOWINUJOBSIRPP DG41 program.rwj

I:IRheologylZaminecklmay'0415 -17 -04IC2 -TLP-B -START-ACTL_25C.

(Mod)

Bingham (258) : $\tau_{0}: 0.007913 \eta_{\mathrm{p}}: 0.002063 \mathrm{Chi}^{2}: 0.07411 \mathrm{r}: 0.9998$

$$
x=\dot{\gamma}[1 / s], y=\tau[P a]
$$

Bingham (259): $\tau_{0}: 0.003859 \eta_{\mathrm{p}}: 0.002065 \mathrm{Chi}^{2}: 0.1864$ r:0.9995

$$
x=\dot{\gamma}[1 / \mathrm{s}], y=\tau[\mathrm{Pa}]
$$

Newton (260) : $\eta: 0.002074 \mathrm{Chi}^{2}: 0.08195$ r:0.9998

$$
x=\dot{\gamma}[1 / \mathrm{s}], y=\tau[\mathrm{Pa}]
$$

Newton (261) : ๆ:0.00207 Chi:0.1882 r:0.9995

$$
x=\dot{\gamma}[1 / \mathrm{s}], y=\tau[\mathrm{Pa}]
$$


Company SRTC

Operator

Date/Time

Substance

Sample no

Description
Meas ure device RS600

Temperature device

Sensor

A-factor

M-factor
DG4 $1 \mathrm{TI}$

$3701.000 \mathrm{~Pa} / \mathrm{Nm}$

$72.670(1 / \mathrm{s}) /(\mathrm{rad} / \mathrm{s})$
1200203357007

Gap

$5.100 \mathrm{~mm}$

\section{Comment}

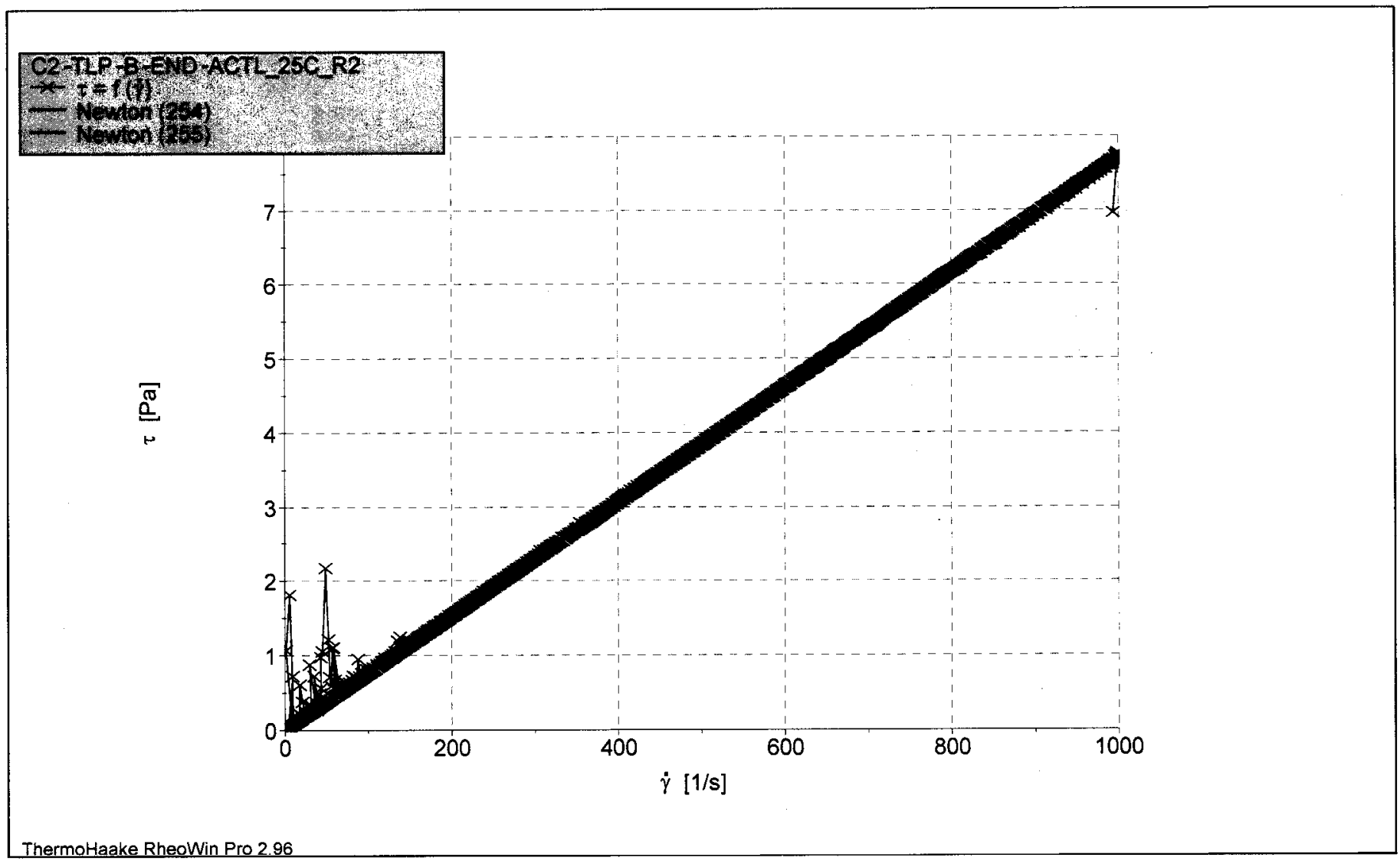

Filename:

Job:
I:IRheology|Zaminecklmay'04|5-17-04IC2-TLP-B-END-ACTL_25C_R2.rwd (Mod)

C:IPROGRAM FILESIRHEOWINIJOBSIRPP DG41 program.rwj

I:IRheologyZZaminecklmay'0415-17-04IC2 -TLP -B END -ACTL_25C_R

(Mod)

Bingham (252): $\tau_{0}: 0.1033 \eta_{\mathrm{p}}: 0.007574 \mathrm{Chi}^{2}: 10.38$ r.0.9978

$$
x=\dot{\gamma}[1 / \mathrm{s}], y=\tau[\mathrm{Pa}]
$$

Bingham (253): $\tau_{0}:-0.02319 \eta_{\mathrm{p}}: 0.007719 \mathrm{Chi}^{2}: 0.07332$ r.

$$
x=\dot{\gamma}[1 / \mathrm{s}], y=\tau[\mathrm{Pa}]
$$

Newton (254) : $\eta: 0.007729 \mathrm{Chi}^{2}: 11.71$ r:0.9976

$$
x=\dot{\gamma}[1 / \mathrm{s}], y=\tau[\mathrm{Pa}]
$$

Newton (255) : $\eta: 0.007685 \mathrm{Chi}^{2}: 0.1405 \mathrm{r}: 1$

$$
x=\dot{\gamma}[1 / \mathrm{s}], y=\tau[\mathrm{Pa}]
$$


Company SRTC

Operator

DaterTime

Substance

Sample no

Description

Comment
Meas ure device RS600

Temperature device

Sensor

A-factor

M-factor
DG4 $1 \pi$

$3701.000 \mathrm{~Pa} / \mathrm{Nm}$

$72.670(1 / \mathrm{s}) /(\mathrm{rad} / \mathrm{s})$
1200203357007

Gap

$5.100 \mathrm{~mm}$ C2-TLP-B-END-ACTL

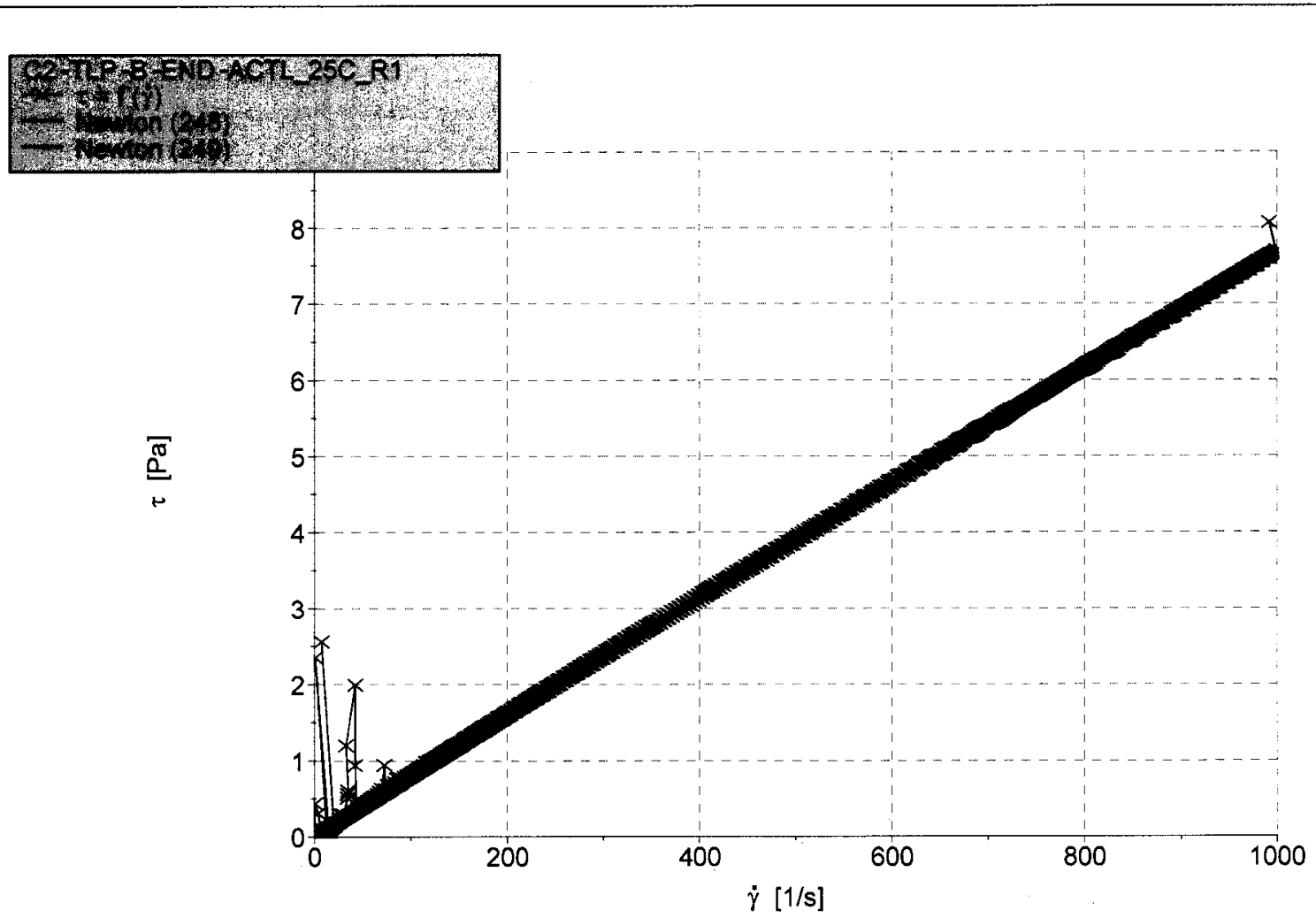

ThermoHaake RheoWin Pro 2.96

Filename: I:IRheology'Zaminecklmay'04I5-17-04IC2-TLP-B-END-ACTL_25C_R1.rwd (Mod)

Job:

C:IPROGRAM FILESIRHEOWINIJOBSIRPP DG41 program.rwj

I:IRheologylZaminecklmay'0415 - 17-04IC2 -TLP -B -END -ACTL_25C_R

(Mod)

Bingham (246) : $\tau_{0}: 0.1733 \eta_{\mathrm{p}}: 0.007506 \mathrm{Chi}^{2}: 14.3 \mathrm{r}: 0.997$

$$
\mathrm{x}=\dot{\gamma}[1 / \mathrm{s}], \mathrm{y}=\tau[\mathrm{Pa}]
$$

Bingham (247): $\tau_{0}: 0.05025 \eta_{p}: 0.007637 \mathrm{Chi}^{2}: 0.1689 \mathrm{r:1}$

$\mathrm{x}=\dot{\gamma}[1 / \mathrm{s}], \mathrm{y}=\tau[\mathrm{Pa}]$

Newton (248) : $\eta: 0.007766 \mathrm{Chi}^{2}: 18.07$ r:0.9962

$x=\dot{\gamma}[1 / \mathrm{s}], y=\tau[\mathrm{Pa}]$

Newton (249) : $\eta: 0.007713 \mathrm{Chi}^{2}: 0.4846$ r:0.9999

$$
x=\dot{\gamma}[1 / \mathrm{s}], y=\tau[\mathrm{Pa}]
$$


WSRC-TR-2005-00105, REVISION 0

SRNL-RPP-2005-00012, REVISION 0

\section{Particle-Size Distribution Data from SIPP Campaign I}


Serial Number: \$3261

MICROTRAC - 53000

Ver:9.0K

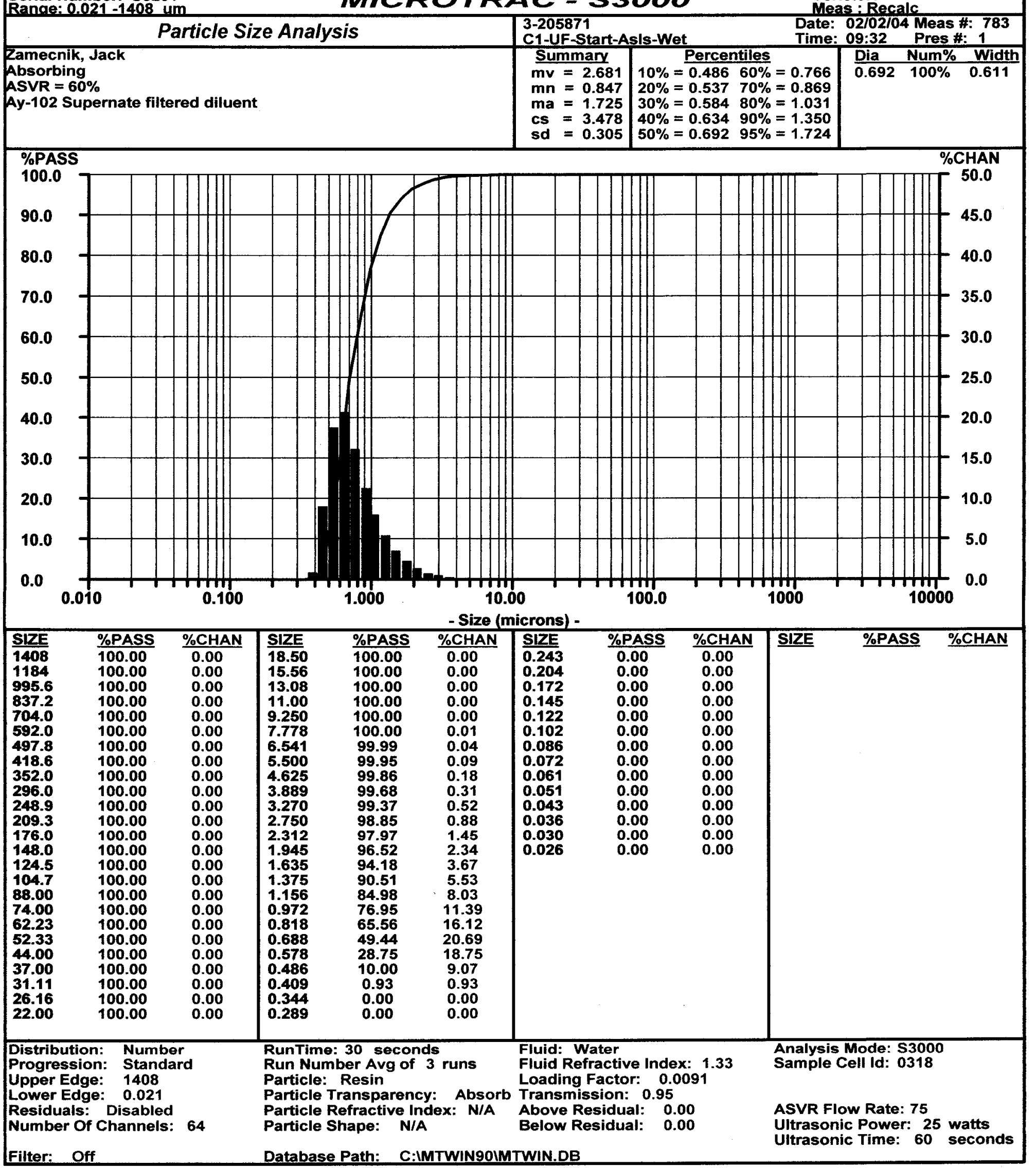




\begin{tabular}{|c|c|c|c|c|c|}
\hline Particle Size Analysis & $\begin{array}{l}\text { 3-205872 } \\
\text { C1-UF-Con2-8 }\end{array}$ & o-Wet $\quad$ Date: & $\begin{array}{l}02 / 02 / 0 \\
10: 02\end{array}$ & $\begin{array}{l}4 \text { Meas } \\
\text { Pres }\end{array}$ & $\begin{array}{l}\text { \#: } 787 \\
7: 1\end{array}$ \\
\hline $\begin{array}{l}\text { Zamecnik, Jack } \\
\text { Absorbing } \\
\text { ASVR }=60 \% \\
\text { Ay-102 Supernate filtered diluent }\end{array}$ & $\begin{array}{l}\text { Summary } \\
\text { mv }=1.761 \\
m n=0.707 \\
\text { ma }=1.207 \\
c s=4.971 \\
\text { sd }=1.074\end{array}$ & \begin{tabular}{l}
\multicolumn{3}{c}{ Percentiles } \\
$10 \%=0.61960 \%=1.823$ \\
$20 \%=0.77270 \%=2.188$ \\
$30 \%=0.96880 \%=2.630$ \\
$40 \%=1.21290 \%=3.302$ \\
$50 \%=1.49795 \%=3.910$
\end{tabular} & $\begin{array}{l}\text { Dia } \\
1.734 \\
0.581\end{array}$ & $\begin{array}{r}\text { Vol\% } \\
85 \% \\
15 \%\end{array}$ & $\begin{array}{r}\text { Width } \\
2.077 \\
0.159\end{array}$ \\
\hline
\end{tabular}

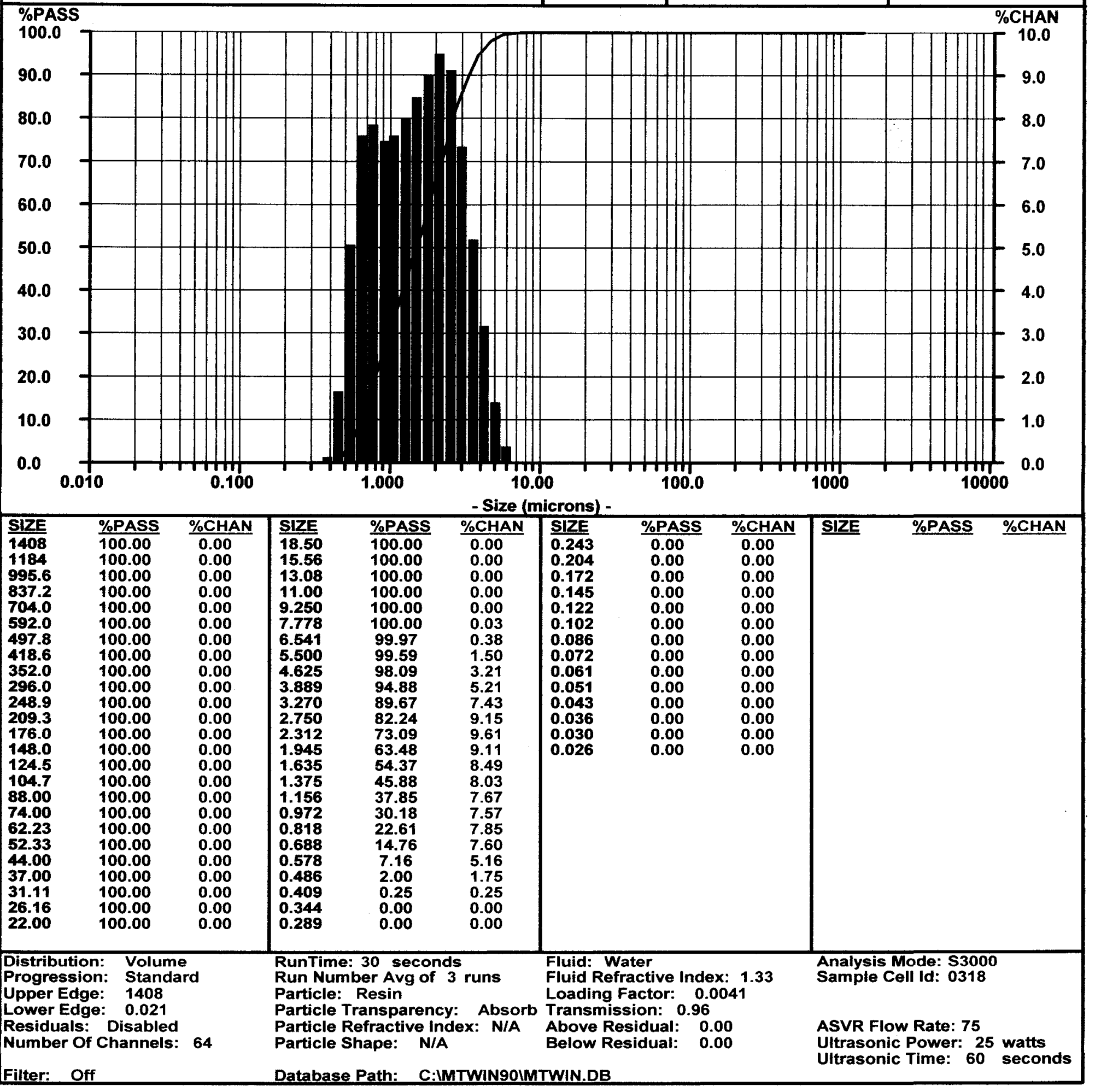




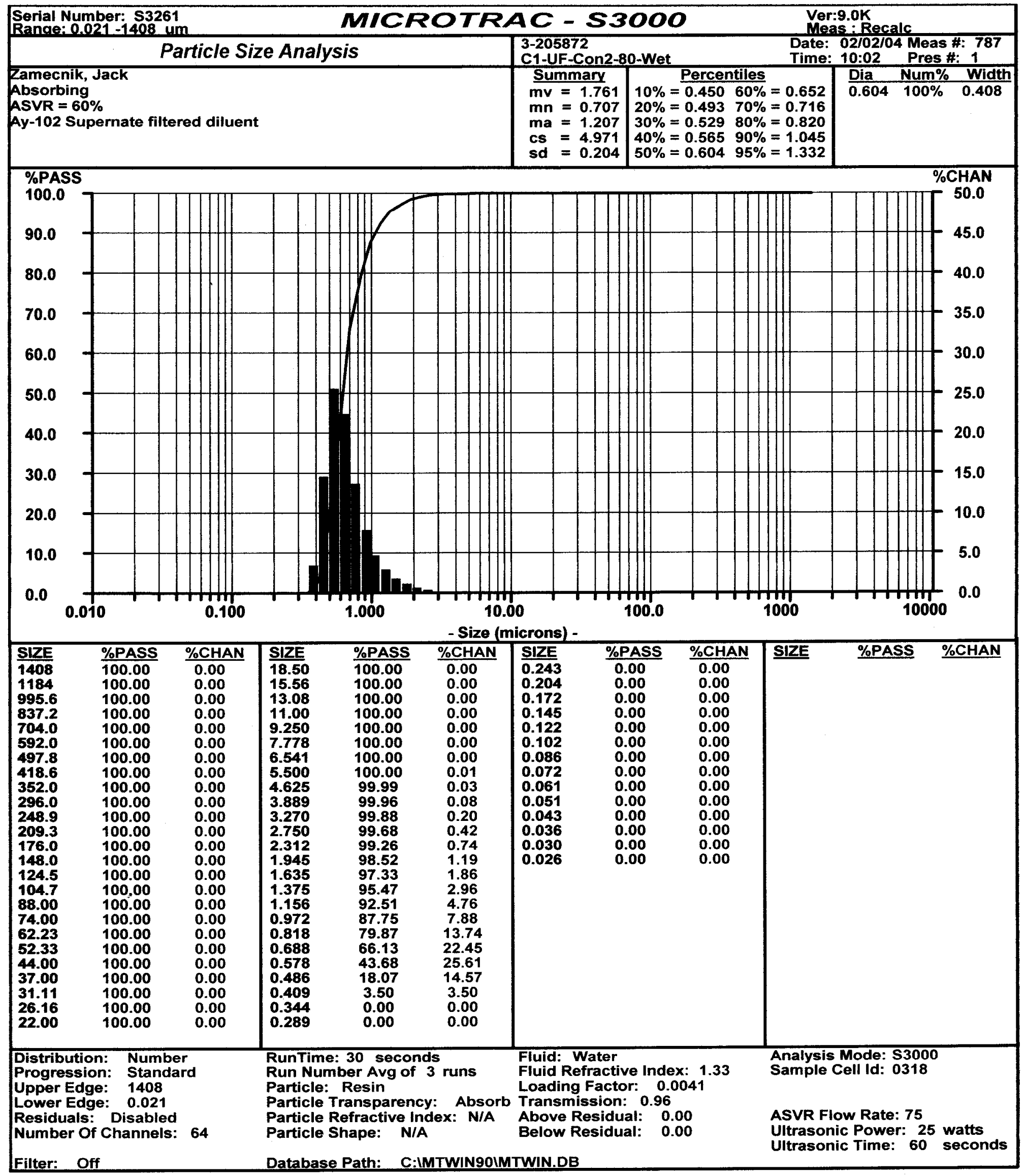




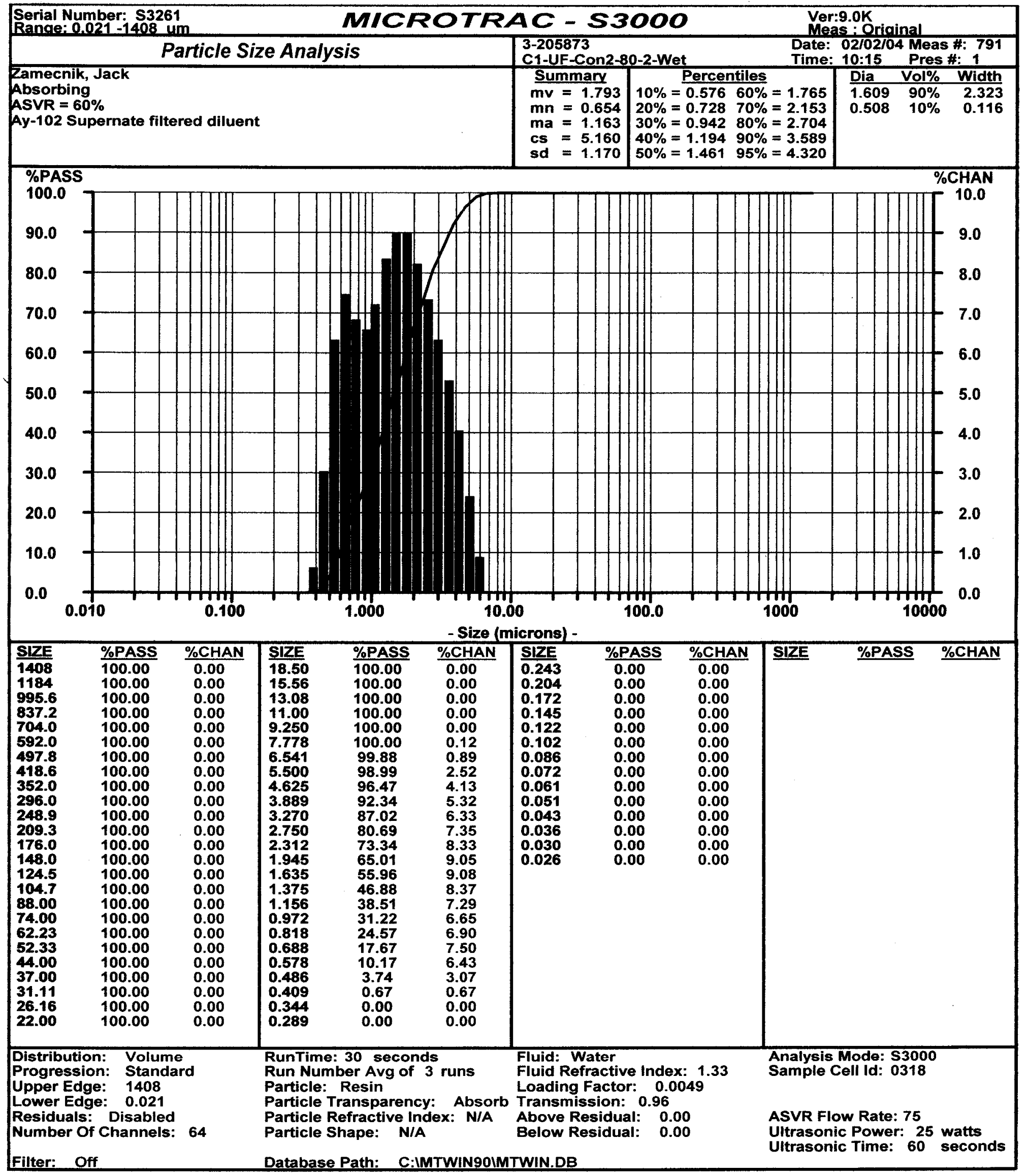


Serial Number: \$3261

MIOROTRRAC - S300O

Ver:9.0K

\begin{tabular}{|c|c|c|c|c|}
\hline Particle Size Analysis & $\begin{array}{l}\text { 3-205873 } \\
\text { C1-UF-Con2-8 }\end{array}$ & $\begin{array}{ll} & \text { Date: } \\
0-2-\text { Wet } & \text { Time: }\end{array}$ & 02/02/ & $\begin{array}{l}4 \text { Meas \#: } 791 \\
\text { Pres \#: } 1\end{array}$ \\
\hline $\begin{array}{l}\text { Zamecnik, Jack } \\
\text { Absorbing } \\
\text { ASVR }=60 \% \\
\text { Ay-102 Supernate filtered diluent }\end{array}$ & $\begin{array}{l}\text { Summary } \\
m v=1.793 \\
m n=0.654 \\
m a=1.163 \\
c s=5.160 \\
\text { sd }=0.186\end{array}$ & $\begin{array}{l}\text { Percentiles } \\
10 \%=0.418 \quad 60 \%=0.598 \\
20 \%=0.456 \quad 70 \%=0.656 \\
30 \%=0.489 \quad 80 \%=0.751 \\
40 \%=0.522 \quad 90 \%=0.974 \\
50 \%=0.556 \quad 95 \%=1.251\end{array}$ & $\frac{\text { Dia }}{0.556}$ & $\begin{array}{cc}\text { Num } \% & \text { Width } \\
100 \% & 0.373\end{array}$ \\
\hline
\end{tabular}

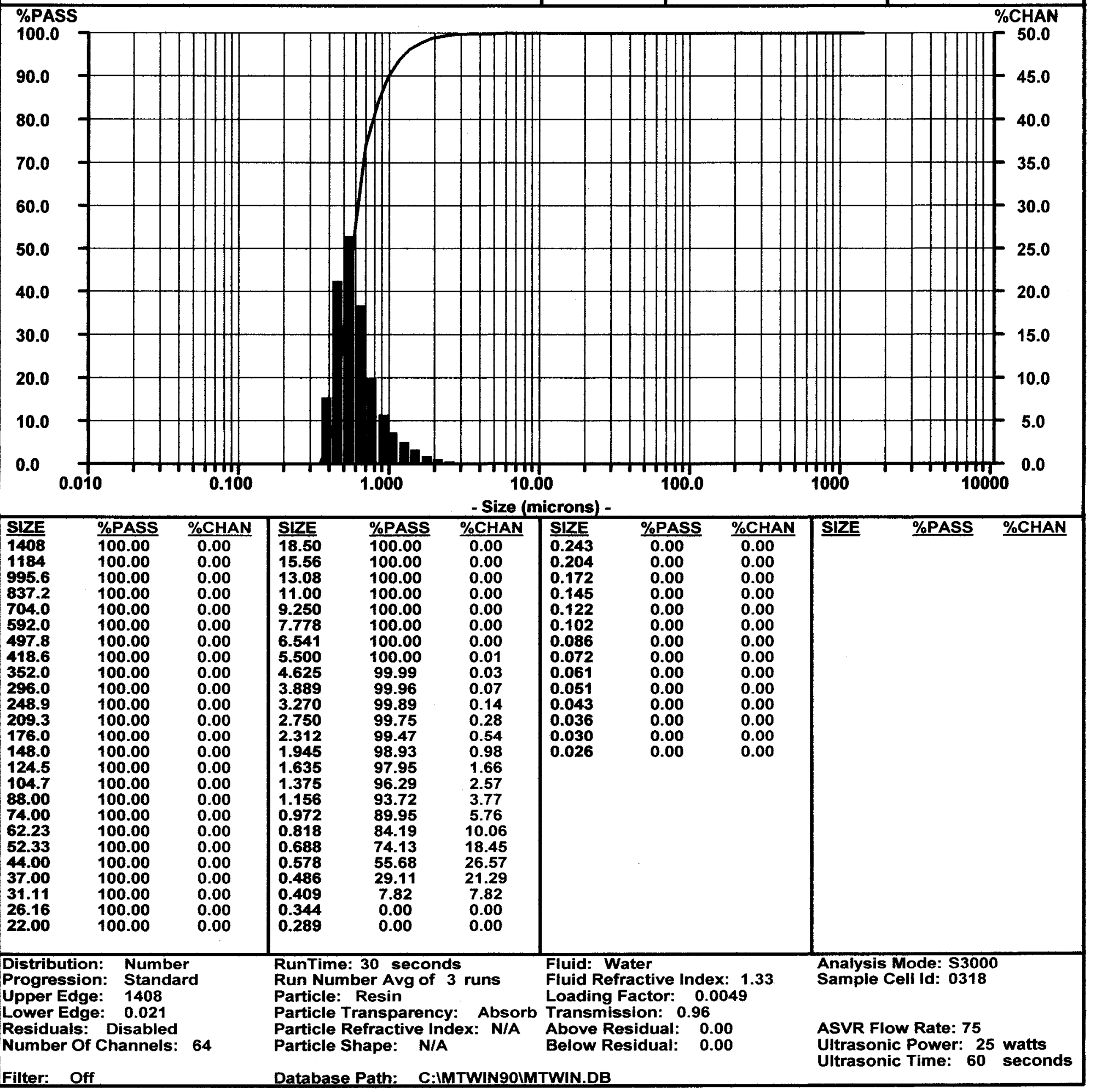




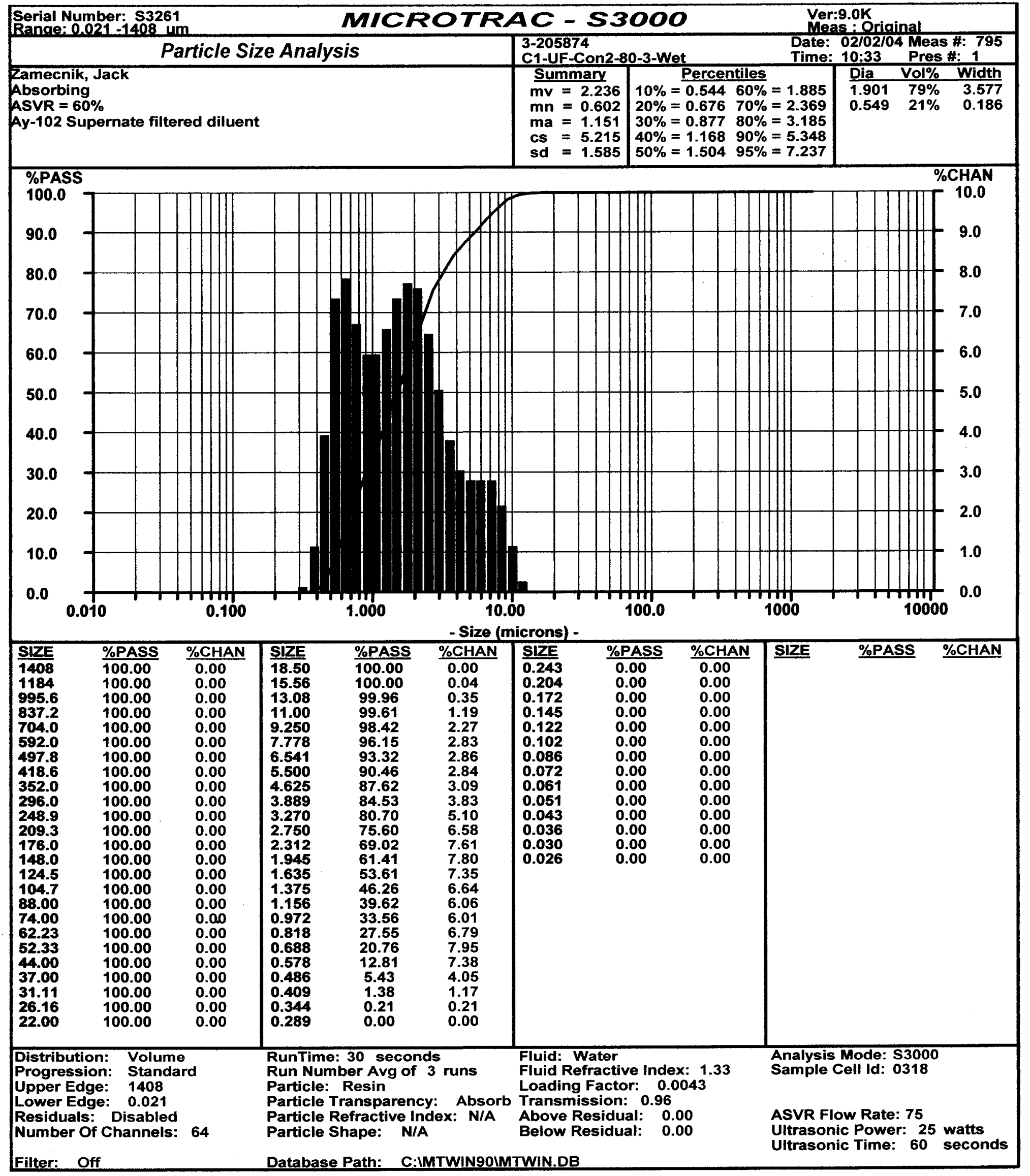


Serial Number: $\$ 3261$

MICROTRAC - 53000

Ver:9.0K

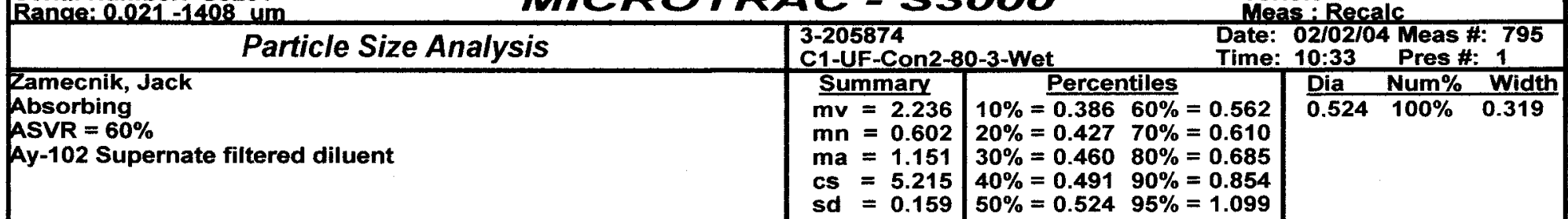

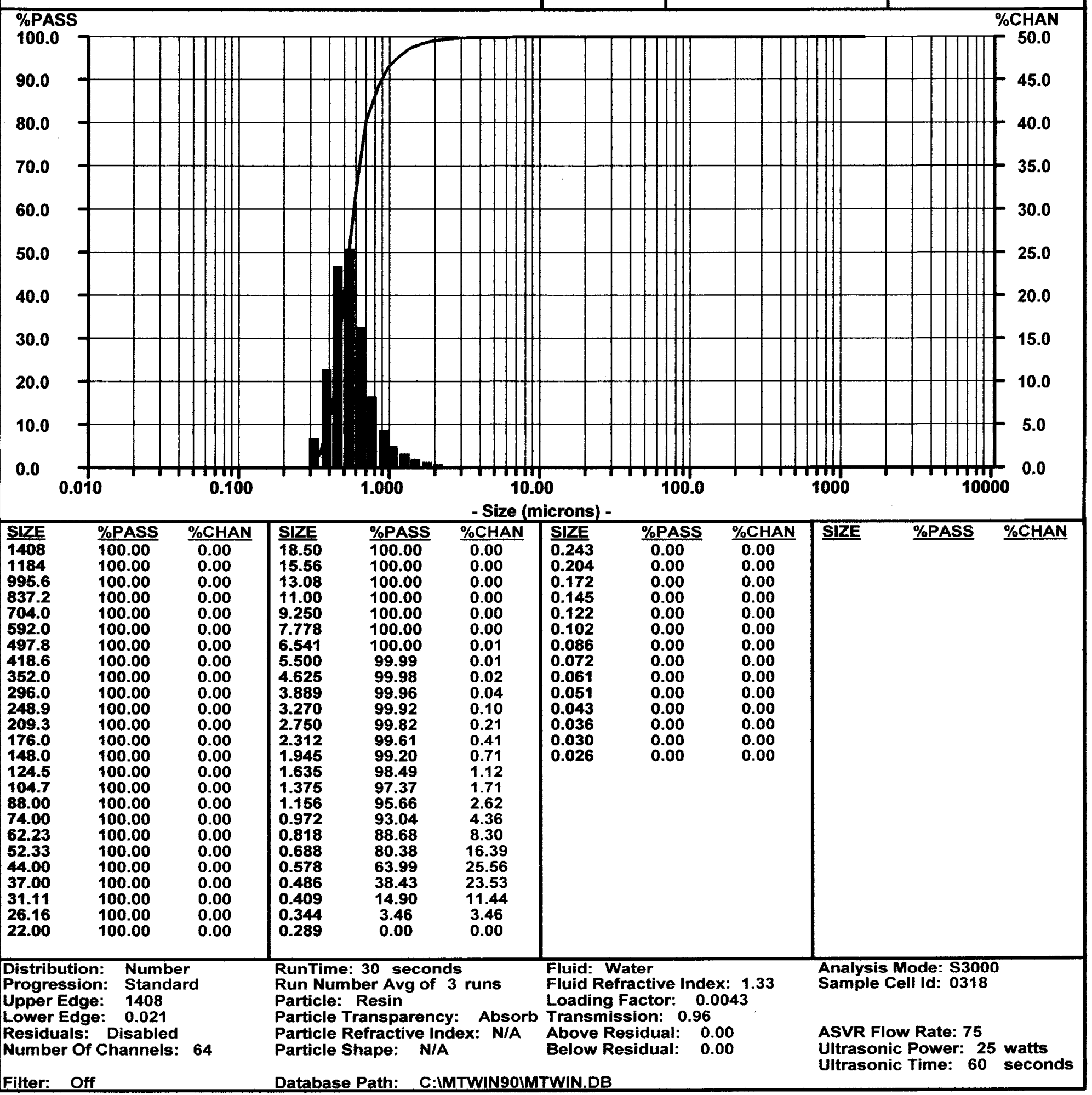


Serial Number: \$3261

MICROTRAC - S3OOO

Ver:9.0K

\begin{tabular}{|c|c|c|c|c|c|}
\hline Particle Size Analysis & $\begin{array}{l}3-205876 \\
\text { C1-UF-SSM-PS }\end{array}$ & $\begin{array}{l}\text { Date: } \\
\text { Time: }\end{array}$ & $\begin{array}{l}02 / 04 / C \\
14: 36\end{array}$ & $\begin{array}{l}4 \text { Meas } \\
\text { Pres }\end{array}$ & $\begin{array}{l}\text { \#: } 807 \\
\#: 1\end{array}$ \\
\hline $\begin{array}{l}\text { Zamecnik, Jack } \\
\text { Absorbing } \\
\text { ASVR }=60 \% \\
\text { Ay-102 Supernate Filtered Diluent }\end{array}$ & $\begin{array}{l}\text { Summary } \\
\mathrm{mv}=1.565 \\
\mathrm{mn}=0.590 \\
\mathrm{ma}=0.980 \\
\mathrm{cs}=6.120 \\
\mathrm{sd}=0.945\end{array}$ & $\begin{array}{l}10 \%=0.516 \quad 60 \%=1.418 \\
20 \%=0.61970 \%=1.740 \\
30 \%=0.75180 \%=2.198 \\
40 \%=0.936 \quad 90 \%=3.103 \\
50 \%=1.160 \quad 95 \%=4.289\end{array}$ & $\begin{array}{l}\text { Dia } \\
1.500 \\
0.544\end{array}$ & $\begin{array}{r}\text { Vol\% } \\
74 \% \\
26 \%\end{array}$ & $\begin{array}{r}\text { Width } \\
1.966 \\
0.189\end{array}$ \\
\hline
\end{tabular}

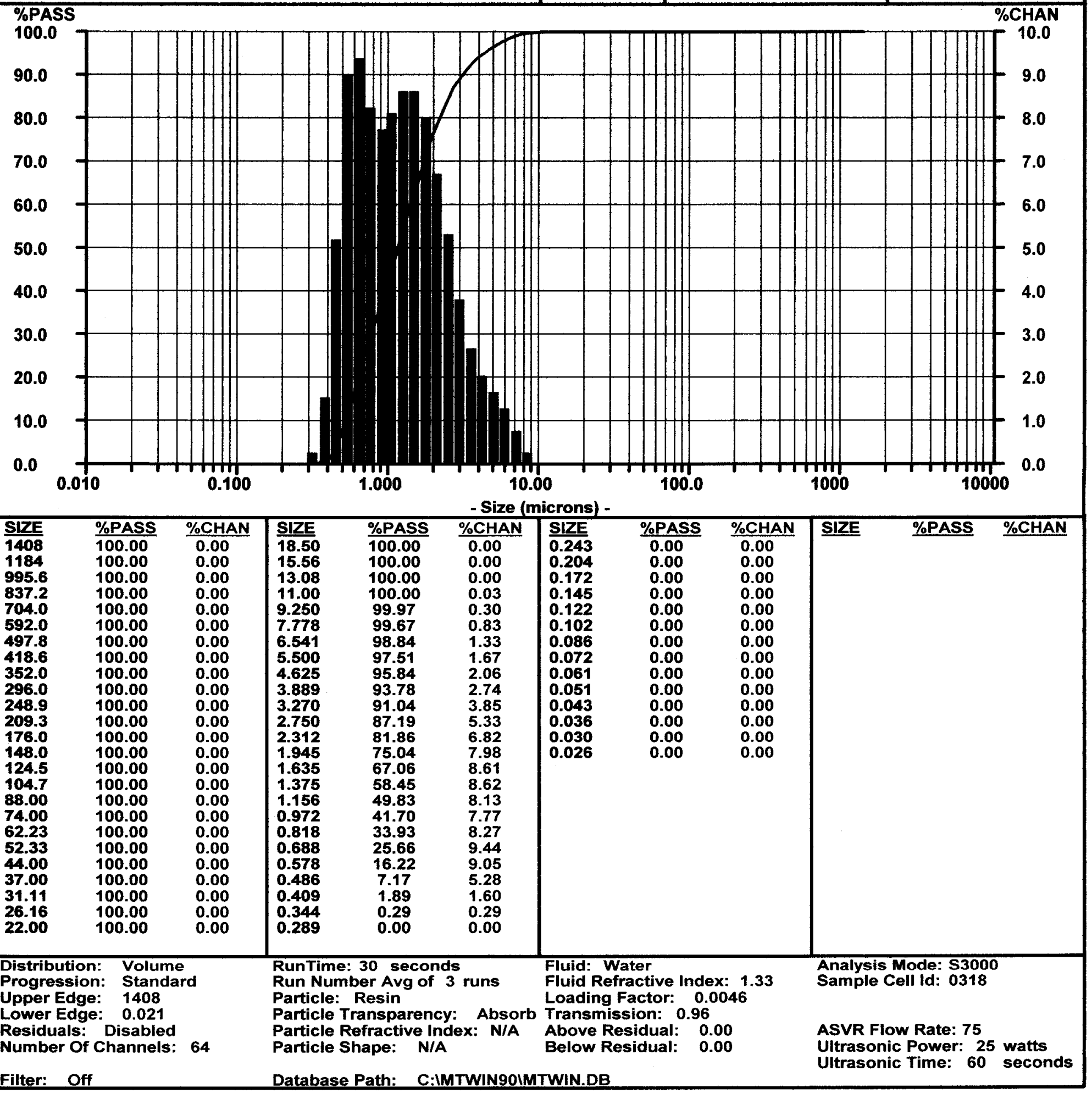


Serial Number: \$3261

MICROTRAC - S300O

Ver:9.0K

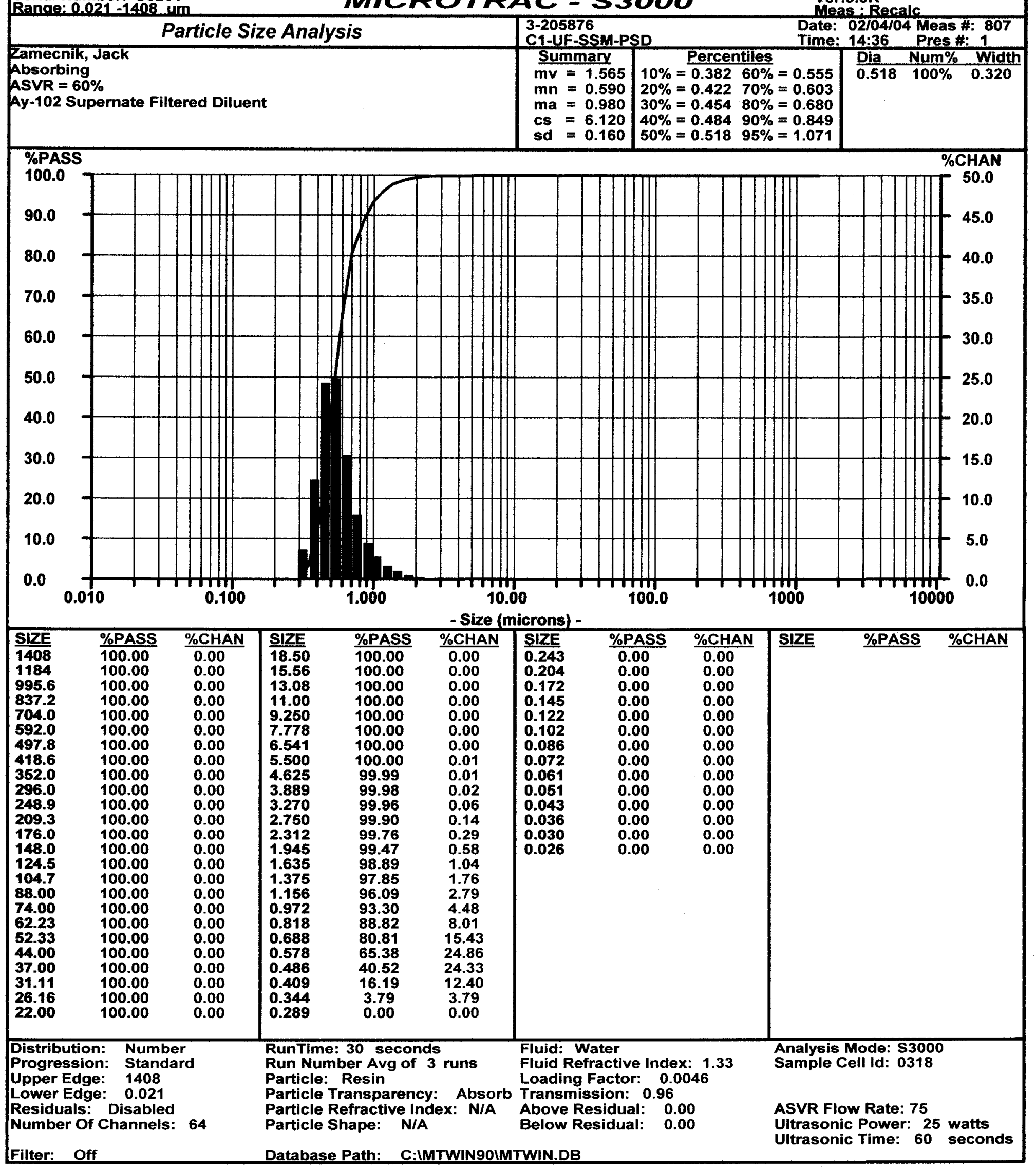




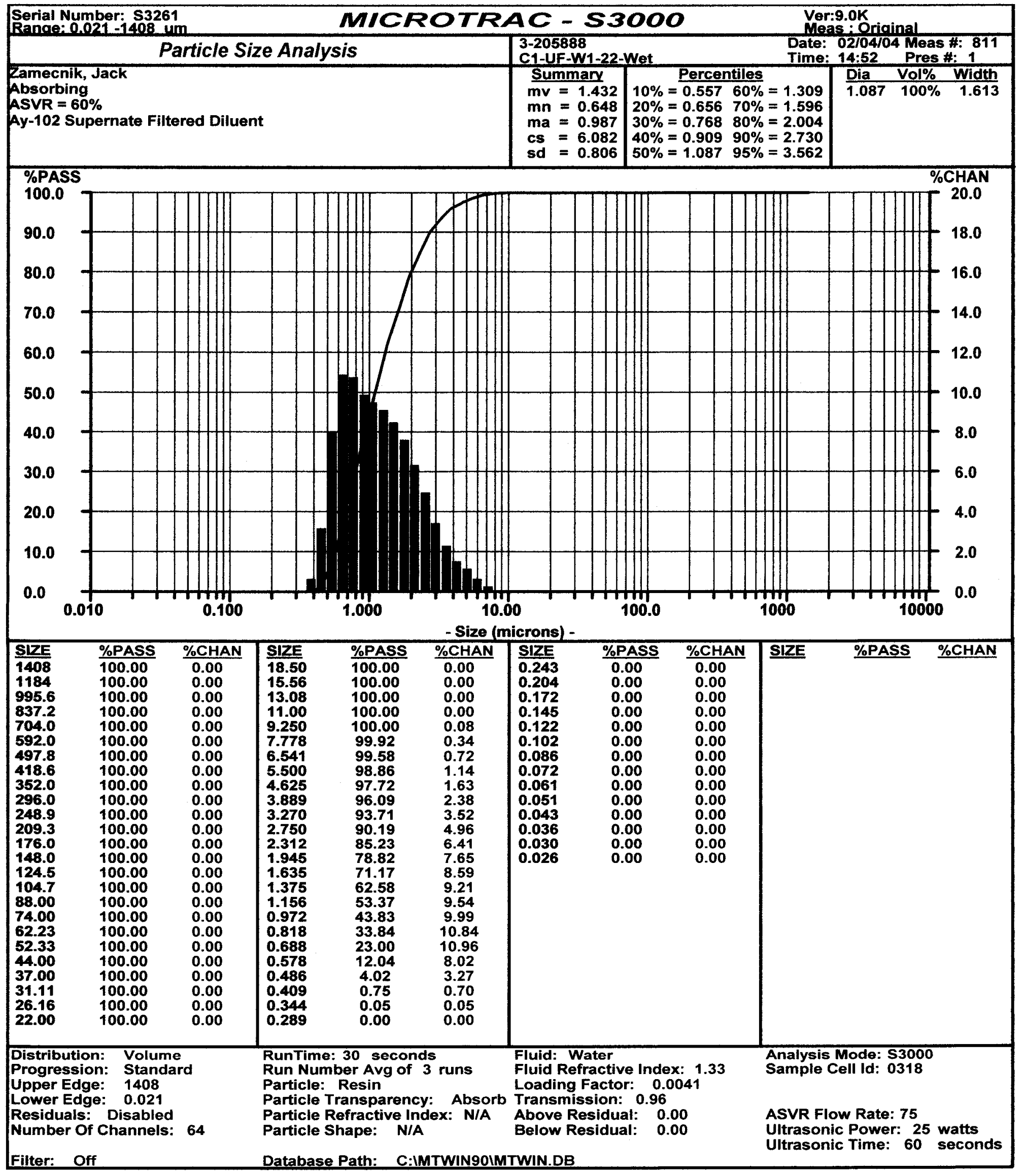




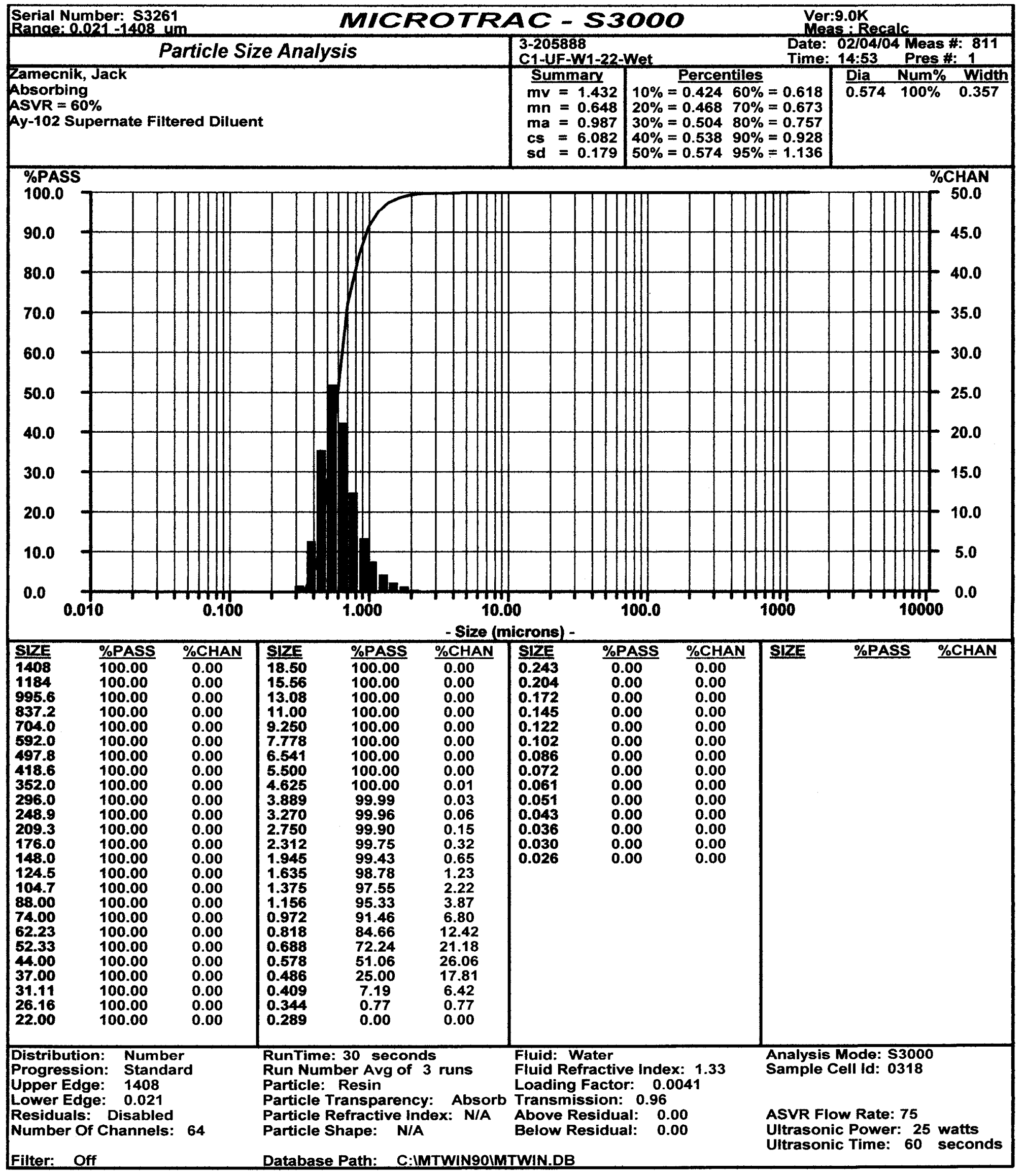


Serial Number: $\mathbf{3 2 6 1}$

MICROTRRAC - $\$ 3000$

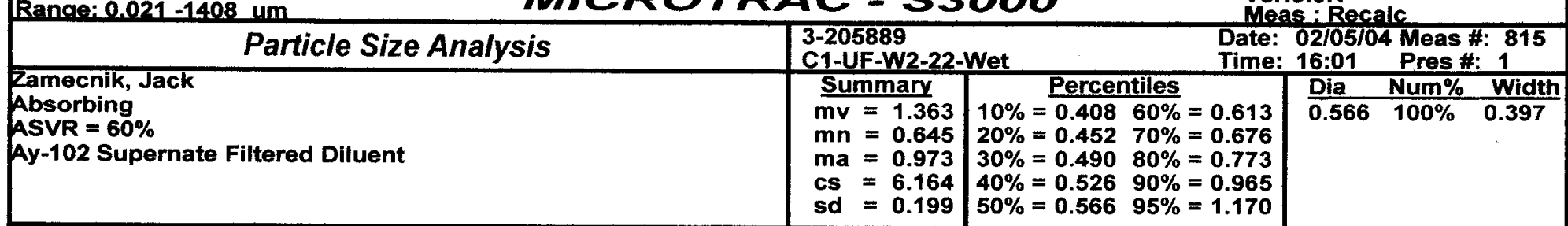

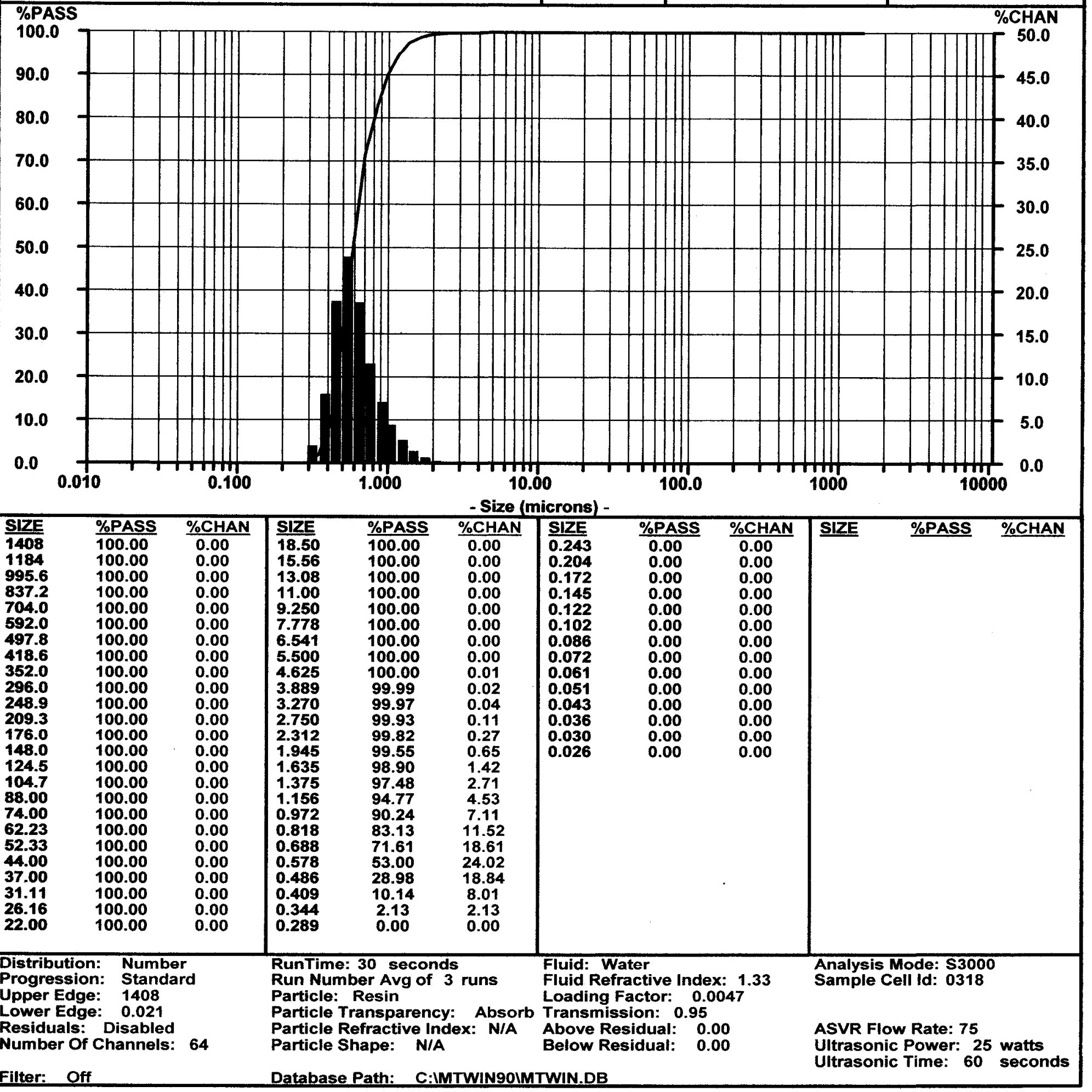




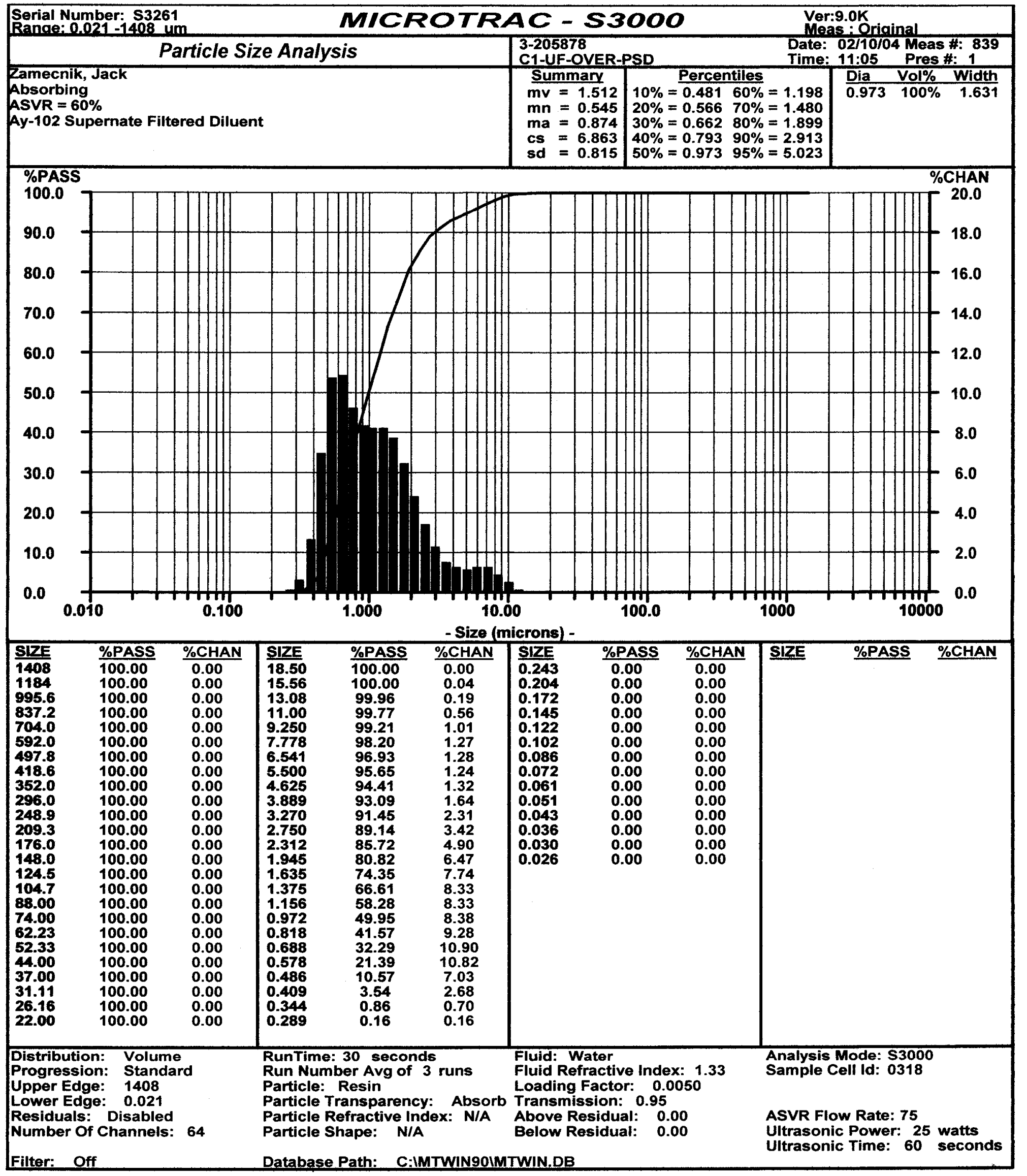




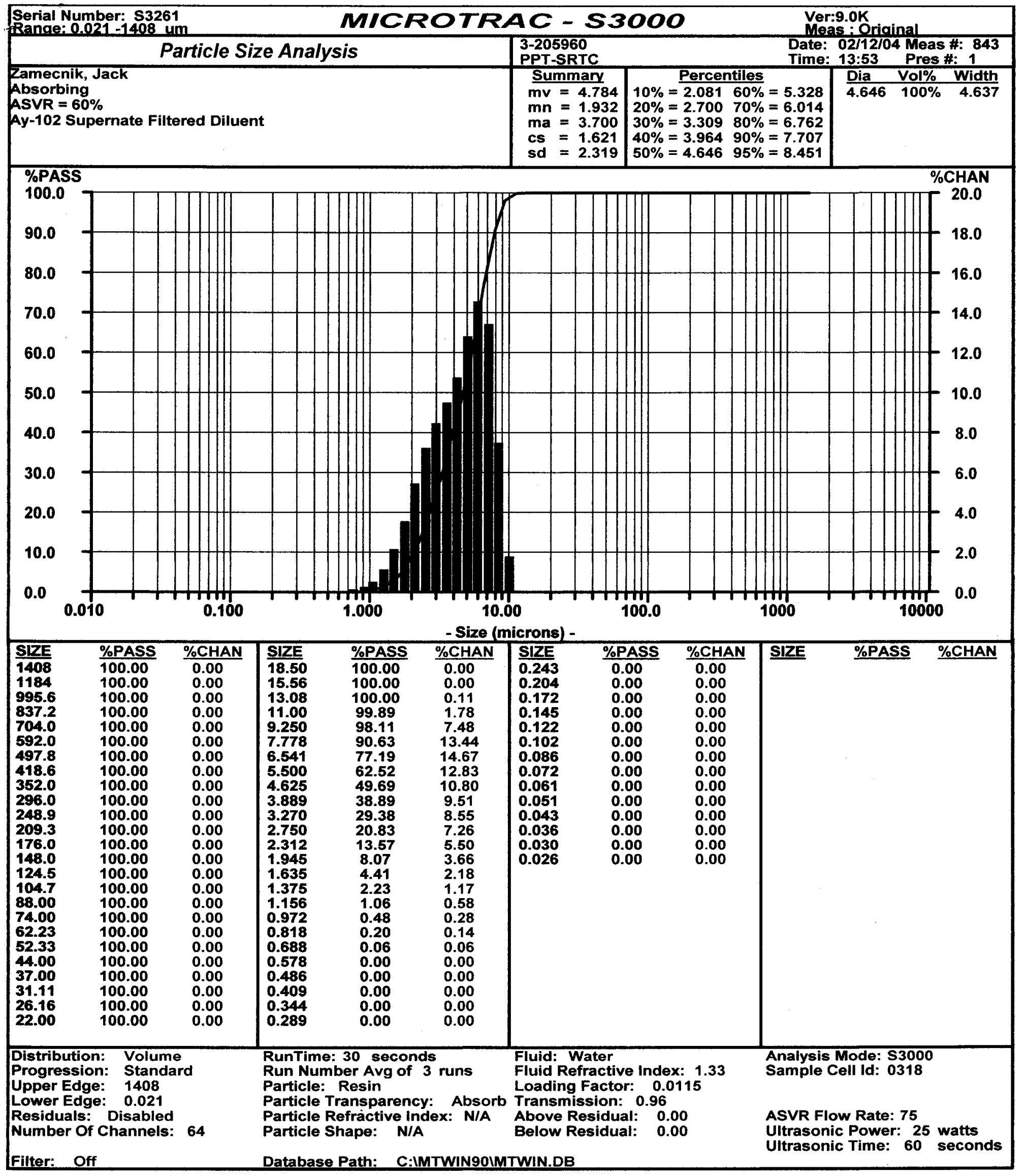


Serial Number: \$3261

MUCROTRAC - S300O

Ver:9.0K

\begin{tabular}{|c|c|c|c|}
\hline Particle Size Analysis & $\begin{array}{l}\text { 3-205960 } \\
\text { PPT-SRTC }\end{array}$ & $\begin{array}{l}\text { Date: } \\
\text { Time: }\end{array}$ & $\begin{array}{l}02 / 12 / 04 \text { Meas \#: } 843 \\
13: 57 \quad \text { Pres \#: } 1\end{array}$ \\
\hline $\begin{array}{l}\text { Zamecnik, Jack } \\
\text { Absorbing } \\
\text { ASVR }=60 \% \\
\text { Ay-102 Supernate Filtered Diluent }\end{array}$ & $\begin{array}{l}\text { Summary } \\
\text { mv }=4.784 \\
m n=1.932 \\
m a=3.700 \\
c s=1.621 \\
\text { sd }=0.946\end{array}$ & \begin{tabular}{l}
\multicolumn{3}{c}{ Percentiles } \\
$10 \%=0.799 \quad 60 \%=1.850$ \\
$20 \%=1.00470 \%=2.148$ \\
$30 \%=1.20180 \%=2.570$ \\
$40 \%=1.399 \quad 90 \%=3.379$ \\
$50 \%=1.609 \quad 95 \%=4.359$
\end{tabular} & $\begin{array}{lll}\text { Dia } & \text { Num\% } & \text { Width } \\
1.609 & 100 \% & 1.891\end{array}$ \\
\hline
\end{tabular}

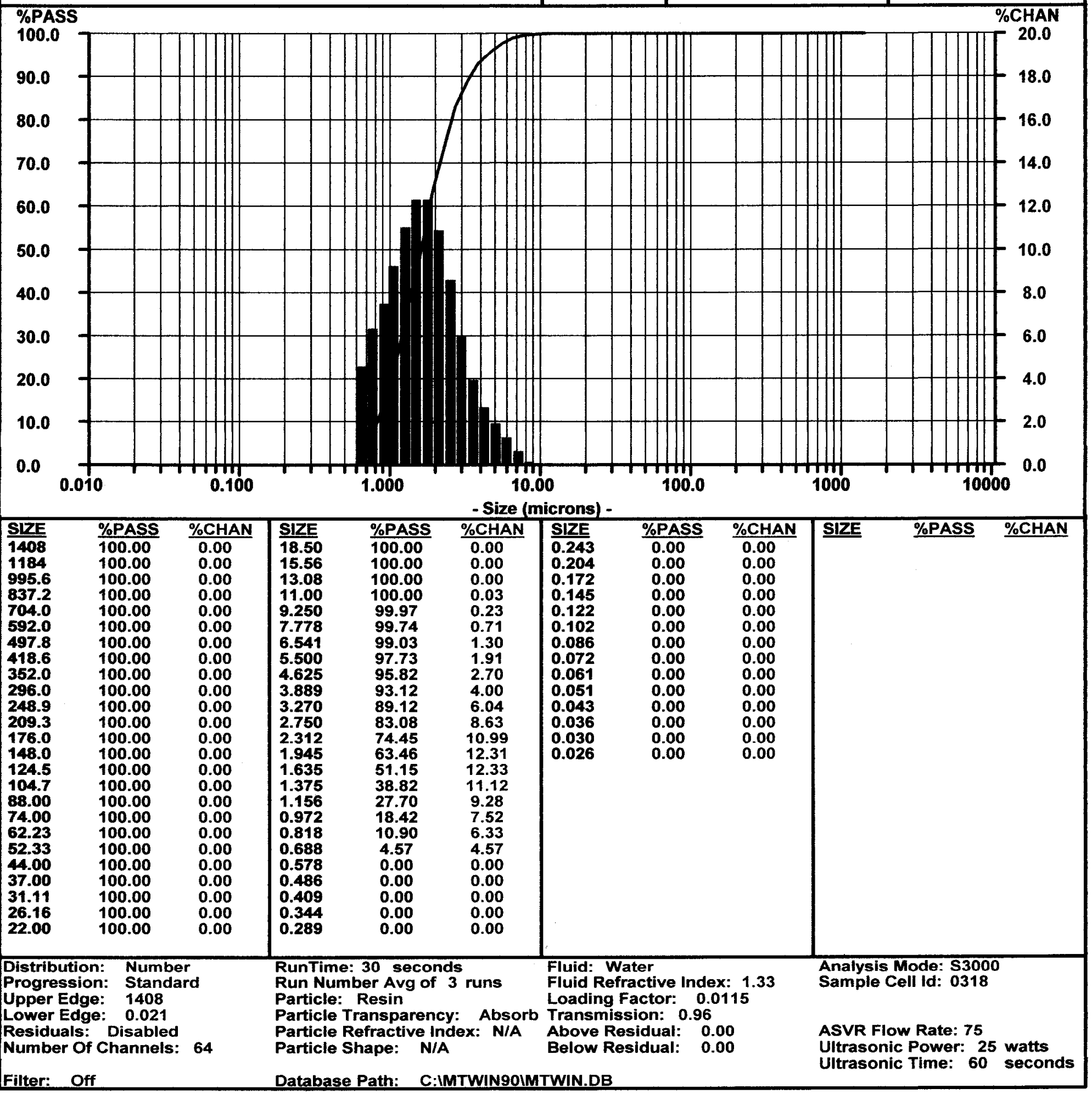




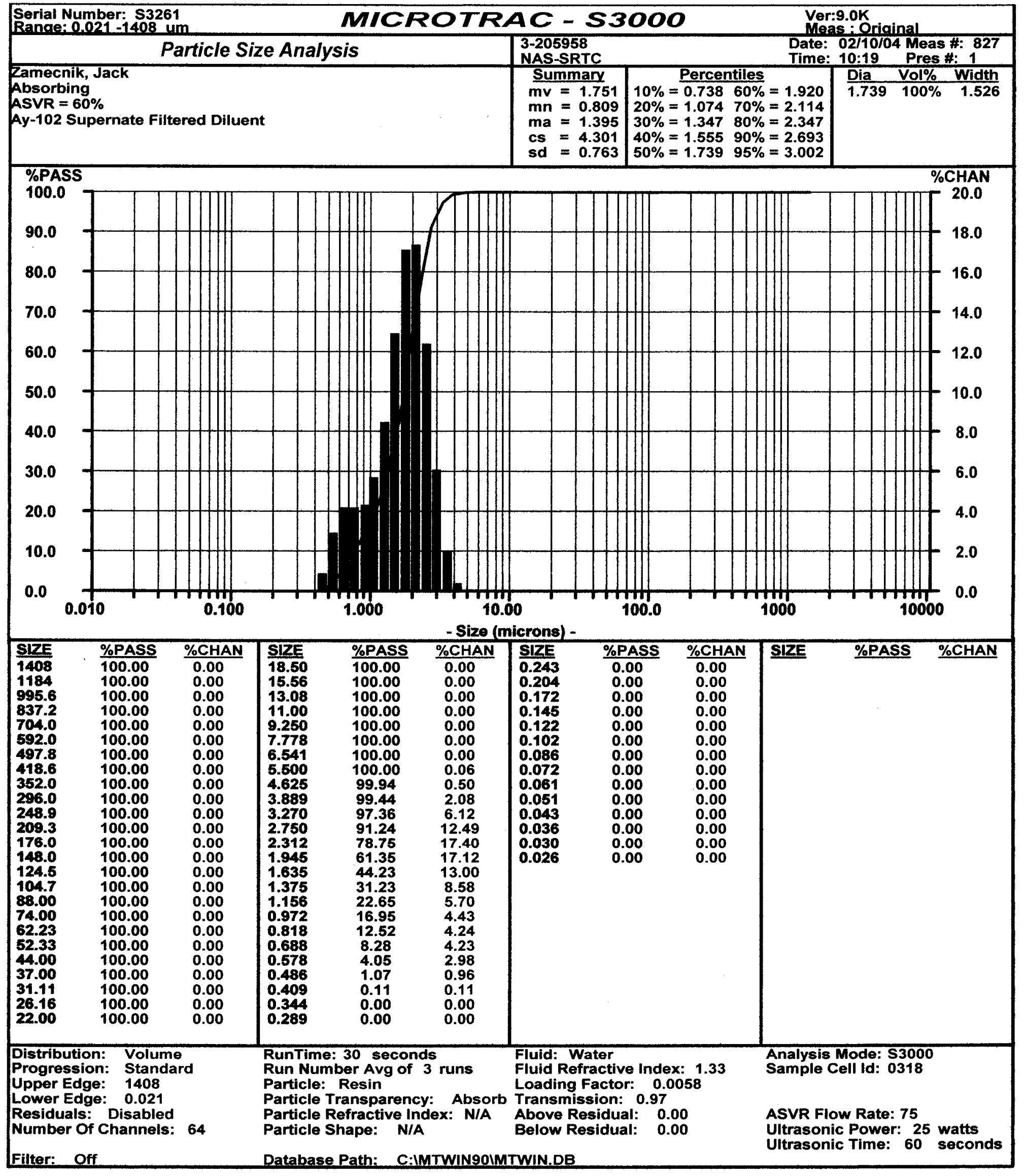


Serial Number: \$3261

MIOROTRRAC - S30OO

Ver:9.0K

\begin{tabular}{|c|c|c|c|}
\hline Particle Size Analysis & $\begin{array}{l}\text { 3-205959 } \\
\text { NAS-OPTIMA }\end{array}$ & $\begin{array}{l}\text { Date: } \\
\text { Time: }\end{array}$ & $\begin{array}{l}\text { 02/10/04 Meas \#: } 831 \\
10: 30 \quad \text { Pres \#: } 1\end{array}$ \\
\hline $\begin{array}{l}\text { Zamecnik, Jack } \\
\text { Absorbing } \\
\text { ASVR }=60 \% \\
\text { Ay-102 Supernate Filtered Diluent }\end{array}$ & $\begin{array}{l}\text { Summary } \\
\text { mv }=3.365 \\
m n=1.421 \\
m a=2.612 \\
\text { cs }=2.297 \\
\text { sd }=0.707\end{array}$ & 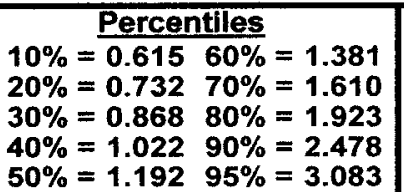 & $\begin{array}{lll}\text { Dia } & \text { Num\% } & \text { Width } \\
1.192 & 100 \% & 1.414\end{array}$ \\
\hline
\end{tabular}

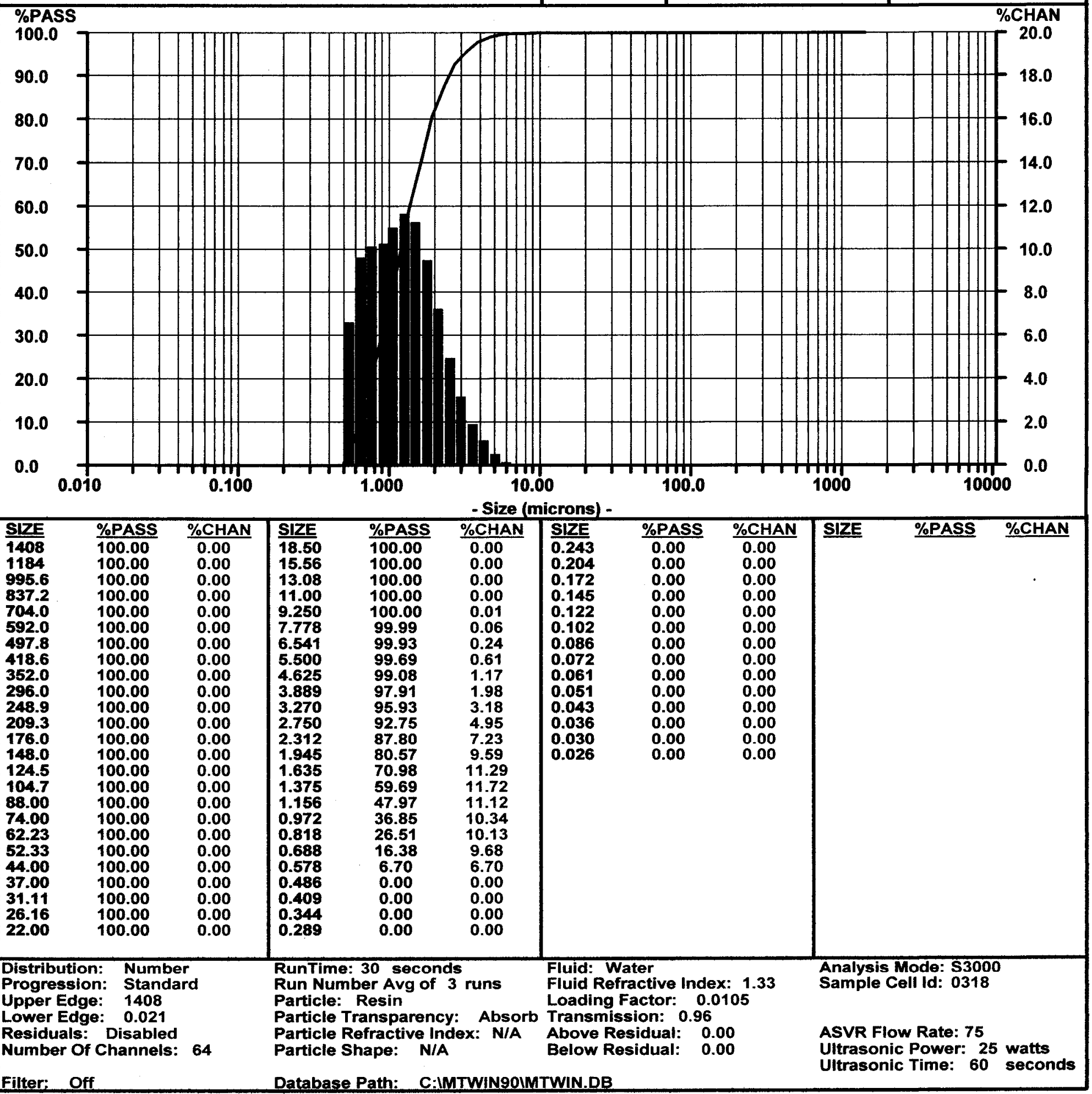


Serial Number: \$3261

\begin{tabular}{|c|c|c|c|}
\hline Particle Size Analysis & $\begin{array}{l}\text { 3-205961 } \\
\text { PPT-OPTIMA }\end{array}$ & $\begin{array}{l}\text { Date: } \\
\text { Time: }\end{array}$ & $\begin{array}{l}02 / 10 / 04 \text { Meas \#: } 835 \\
10: 42 \quad \text { Pres \#: } 1\end{array}$ \\
\hline $\begin{array}{l}\text { Zamecnik, Jack } \\
\text { Absorbing } \\
\text { ASVR }=60 \% \\
\text { Ay-102 Supernate Filtered Diluent }\end{array}$ & $\begin{array}{l}\text { Summary } \\
\text { mv }=3.708 \\
m n=0.835 \\
m a=2.177 \\
c s=2.756 \\
\text { sd }=2.511\end{array}$ & 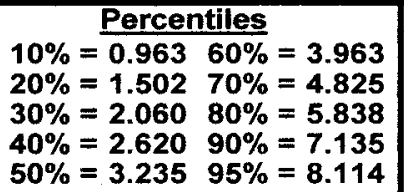 & $\begin{array}{lll}\text { Dia } & \text { Vol\% } & \text { Width } \\
3.235 & 100 \% & 5.021\end{array}$ \\
\hline
\end{tabular}

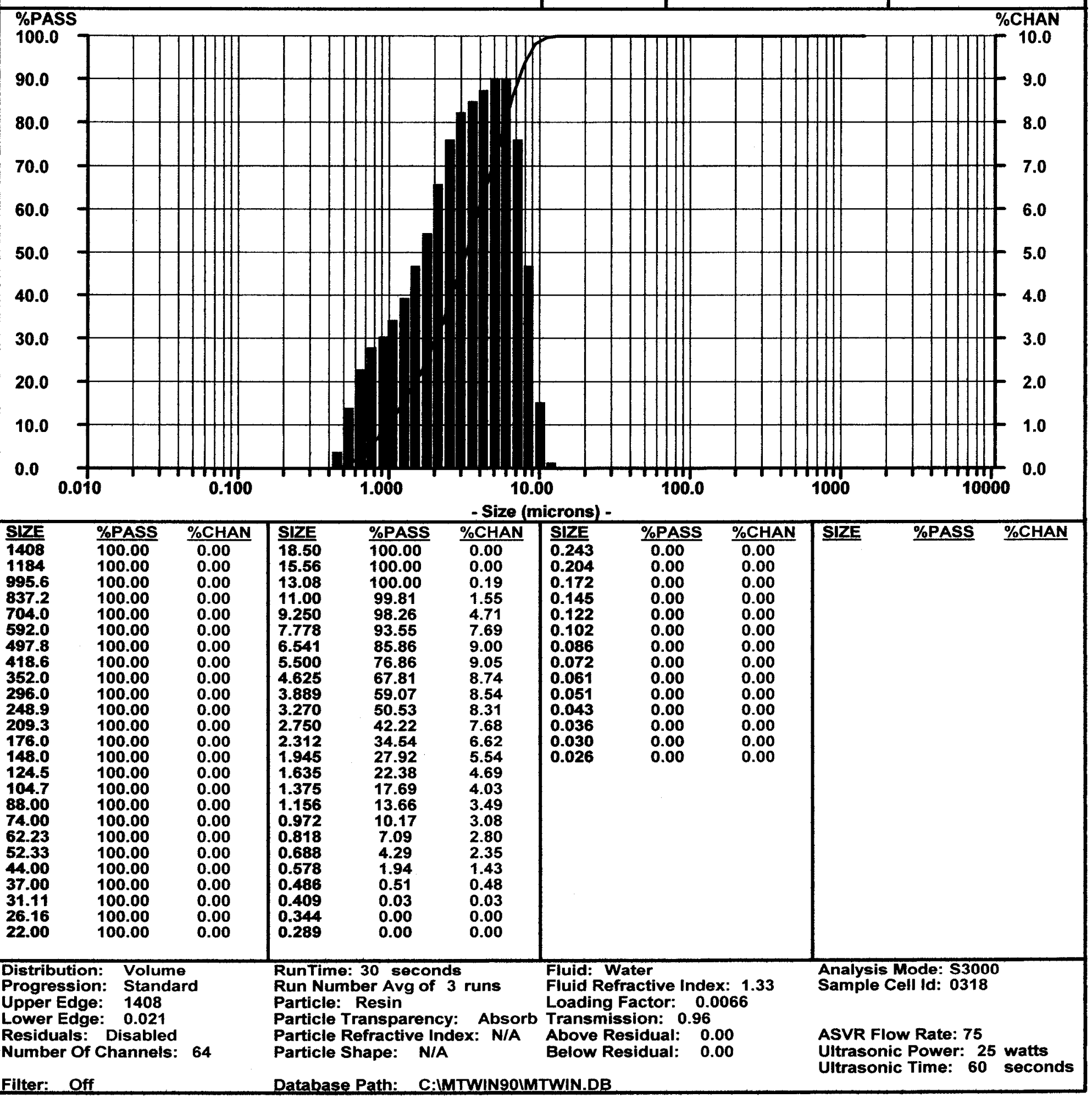


WSRC-TR-2005-00105, REVISION 0

SRNL-RPP-2005-00012, REVISION 0

\section{Particle-Size Distribution Data from SIPP Campaign II}




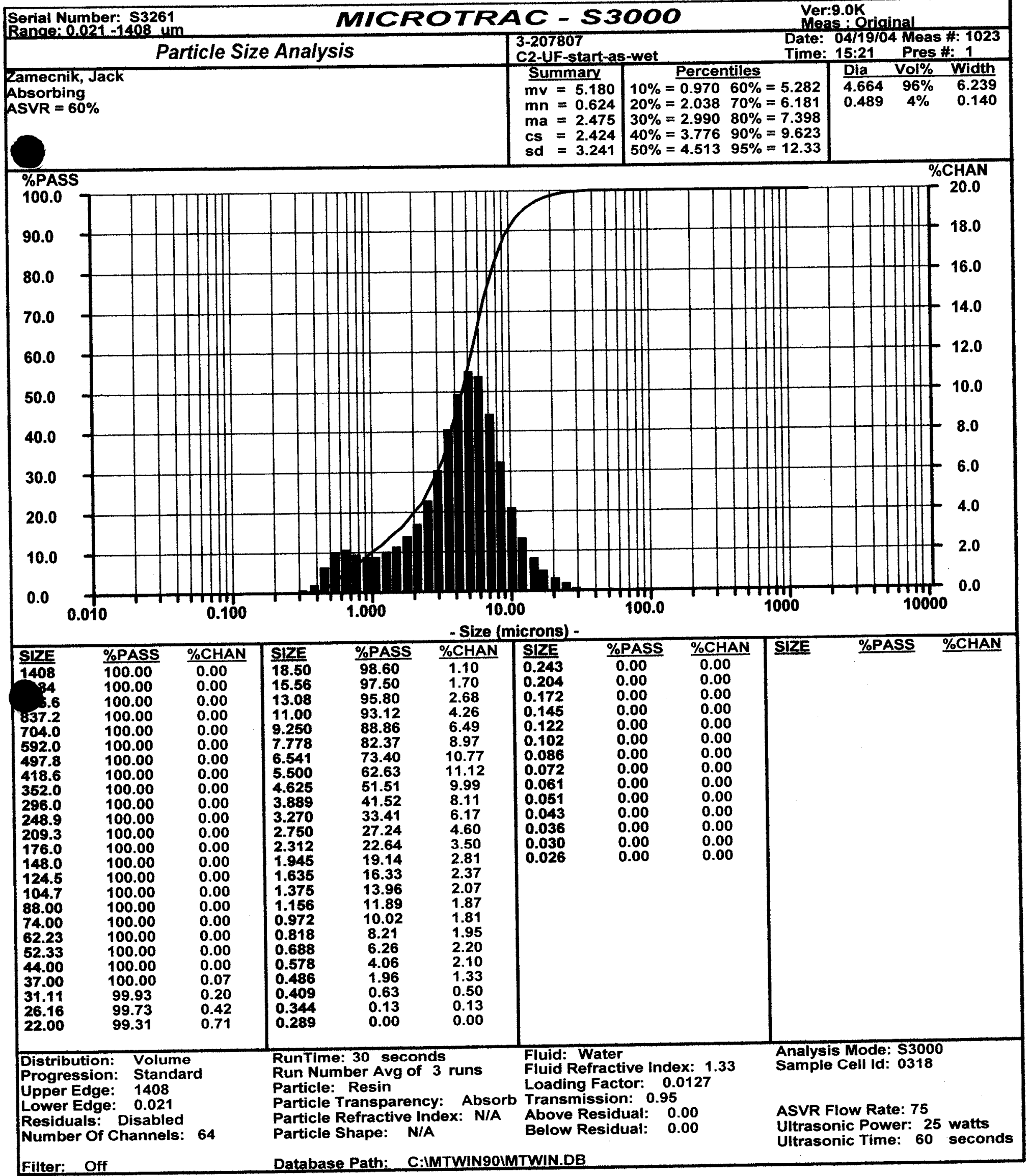




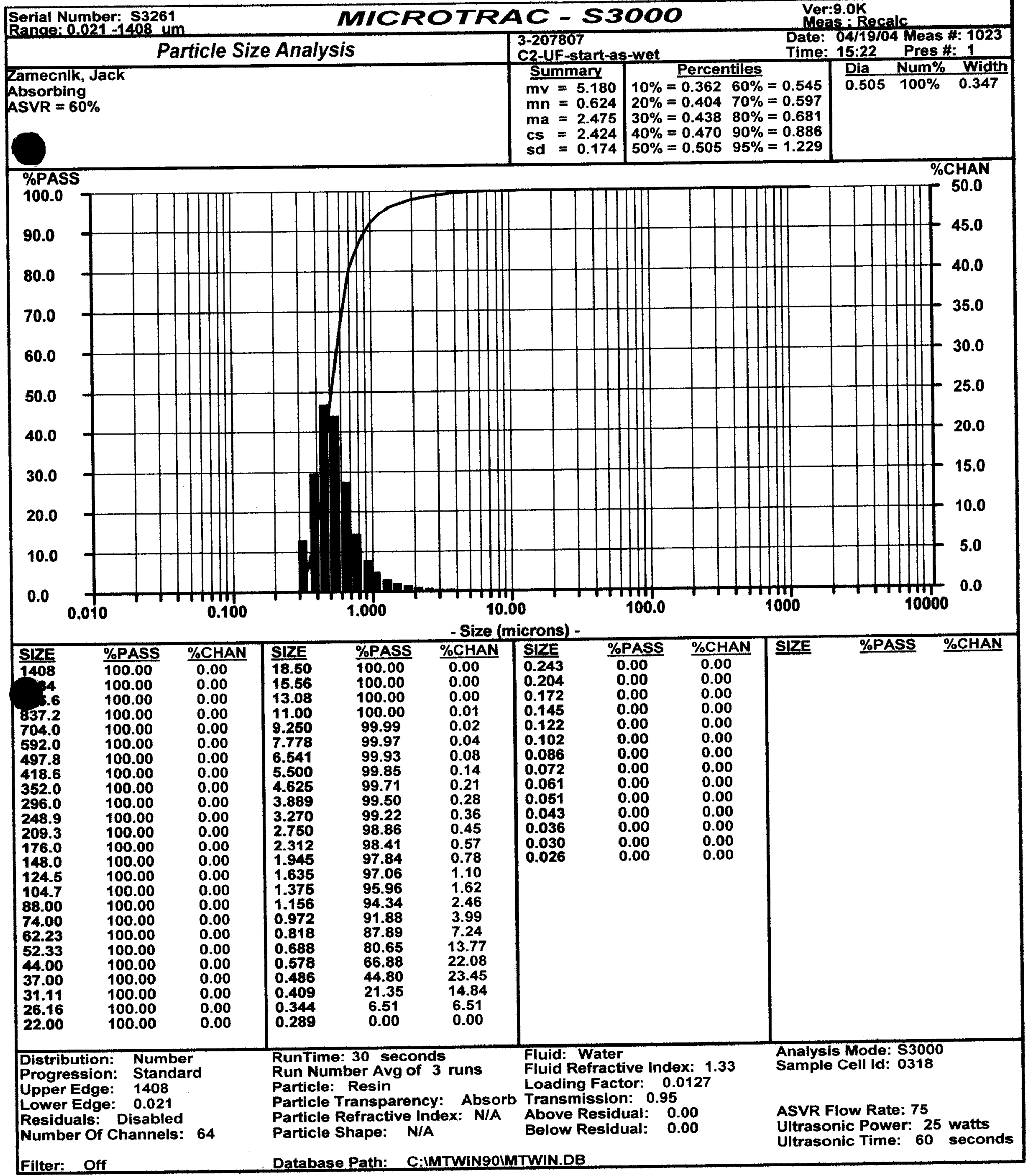


Serial Number: \$3261

WICROTRAC - S300O

Ver:9.0K

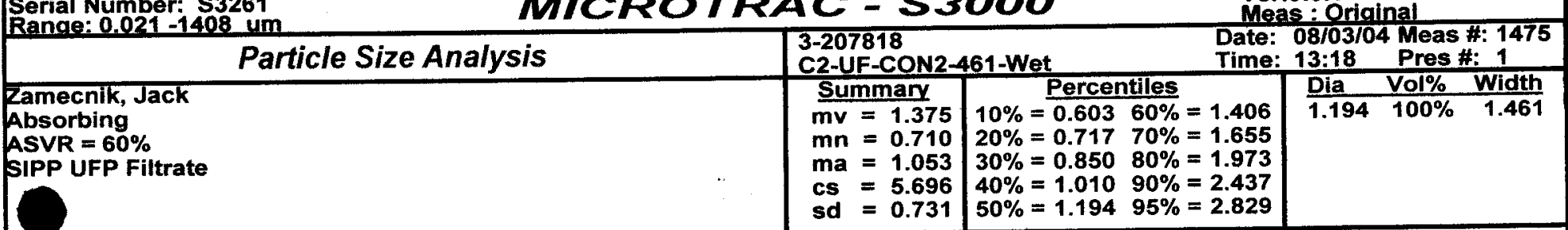

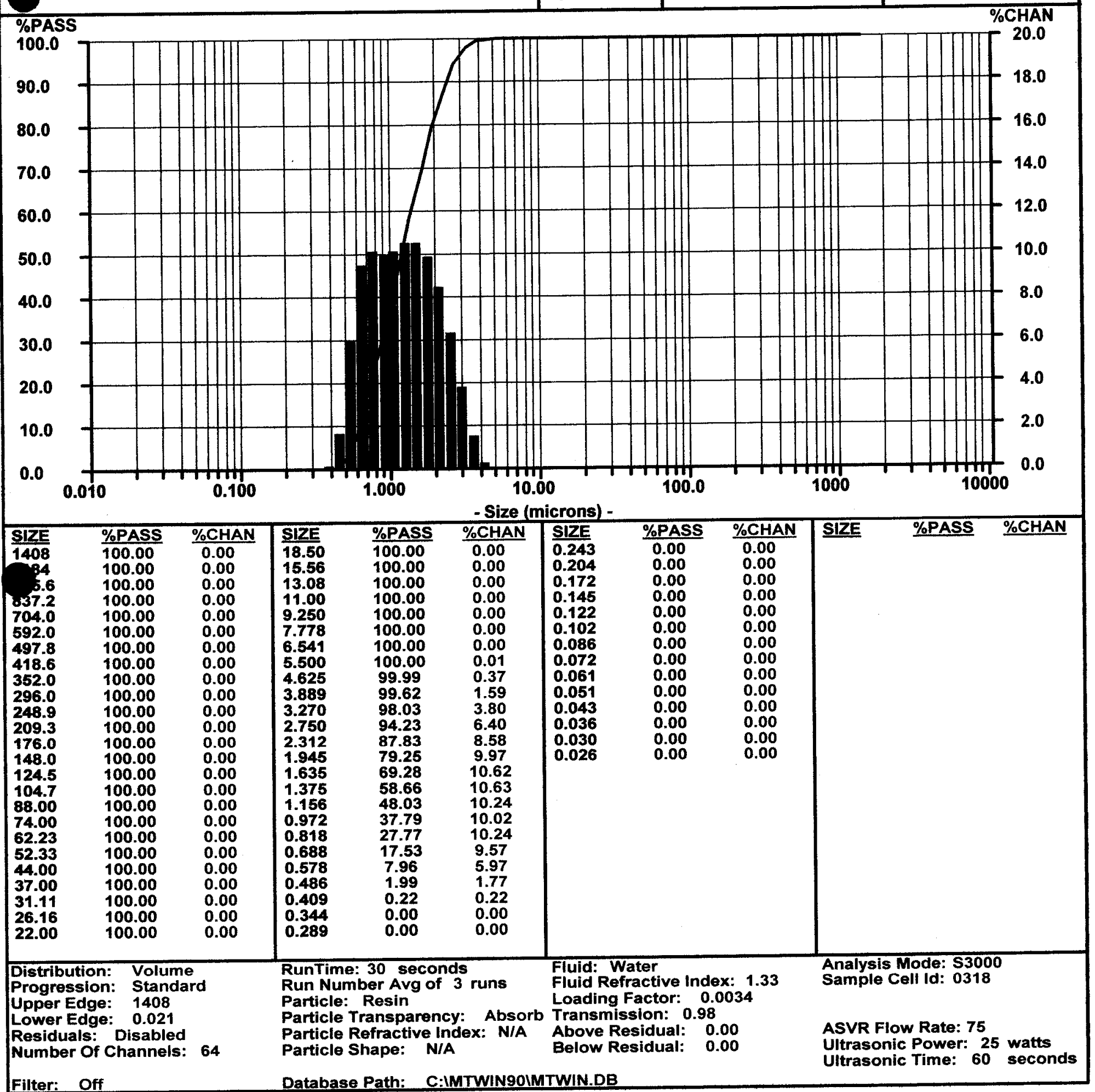


Serial Number: S3261

MICROTRAC - S3OOO

Ver:9.0K

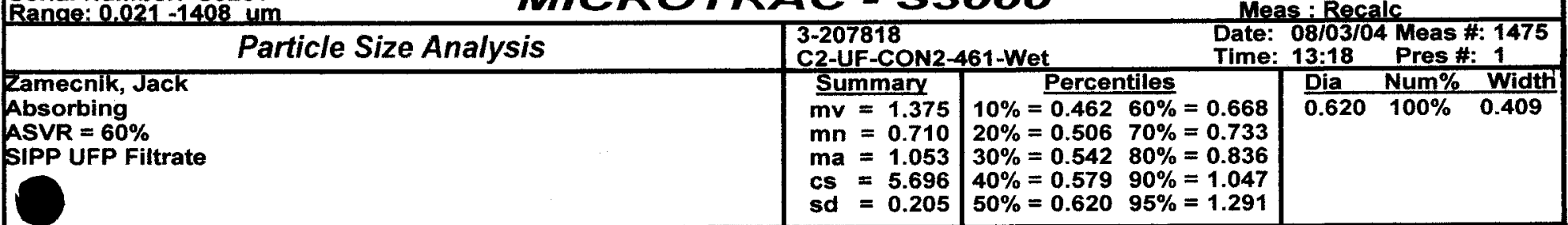

100.0

90.0
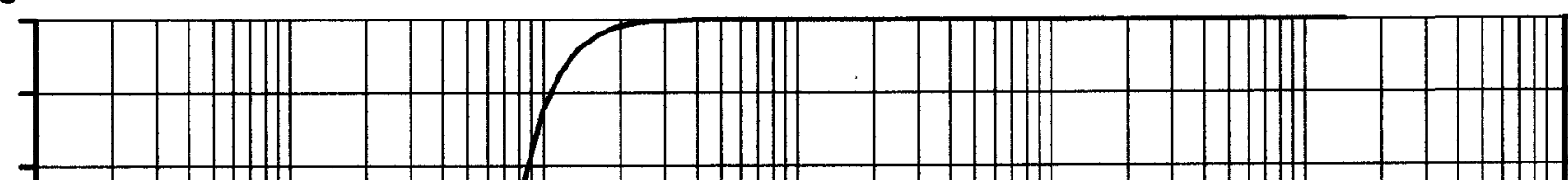

50.0

80.0

70.0

60.0

50.0

40.0

30.0

20.0

10.0

0.0
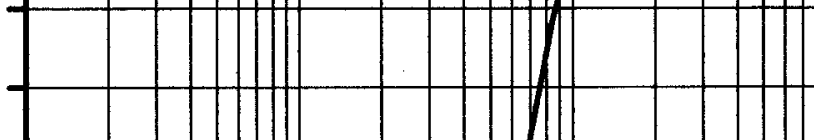

$+\quad-10$
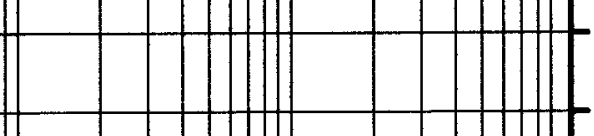

45.0

40.0
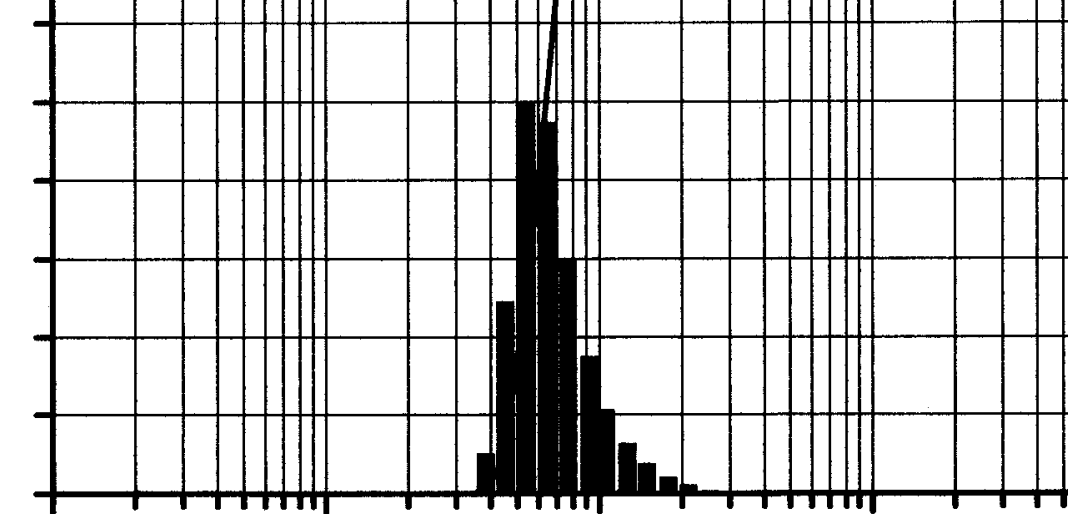

010

0.100

10.00

100.0

- Sizo (microns) -

\section{S1ZE}

1408

1408
34

3.6
37.2

704.0

592.0

497.8

418.6

352.0

296.0

248.9

209.3

176.0

148.0

124.5

104.7

88.00

74.00

62.23

52.33

44.00

37.00

31.11

26.16

22.00

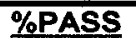

100.00

\section{\%CHAN SIZE}

100.00

100.00

100.00

100.00

100.00

100.00

100.00

100.00

100.00

100.00

100.00

100.00

100.00

100.00

100.00

100.00

100.00

100.00

100.00

100.00

100.00

100.00

100.00

100.00

\section{$0.00 \quad 15.56$}

0.00

0.00

0.00

0.00

0.00

0.00

0.00

0.00

0.00

0.00

0.00

0.00

0.00

0.00

0.00

0.00

0.00

0.00

0.00

0.00

0.00

0.00

0.00

13.08

11.00

$\mathbf{9 . 2 5 0}$

7.778

6.541

5.500

4.625

3.889

3.270

2.750

2.312

1.945

1.635

1.375

1.156

0.972

0.818

0.688

0.578

0.486

0.409

0.344

0.289

1.000

\%CHAN SIZE

\%PASS

100.00

100.00

100.00

0.00

0.00

100.00

0.00

0.00

100.00

100.00

0.00
0.00

100.00

0.00

100.00

100.00

100.00

0.00

0.00

0.02

99.98

0.09

99.89

99.64

99.08

97.99

96.03

92.74

87.41

78.65

63.59

39.84

0.25

0.56

1.09

1.96

3.29

5.33

8.76

15.06

23.75

14.97

12.38

$2.59 \quad 2.59$

0.00

0.00

0.00

Distribution: Number

Progression: Standard

Upper Edge: 1408

Lower Edge: $\quad 0.021$

Residuals: Disabled

Number Of Channels: 64

Filter: Off
RunTime: 30 seconds

Run Number Avg of 3 runs

Particle: Resin

Particle Transparency: Absorb

Particle Refractive Index: N/A

Particle Shape: N/A

Database Path: C:IMTWIN90IMTWIN.DB

\%PASS \%CHAN SIZE \%PASS \%CHAN

0.243

0.204

0.172

0.122

0.102

0.086

0.072

0.061

0.051

0.043

0.036

0.030

0.026

0.00

0.00

0.00

0.00

0.00

0.00

0.00

0.00

0.00

0.00

0.00

0.00

0.00

0.00

0.00

0.00

0.00

0.00

0.00

0.00

0.00

0.00

0.00

0.00

0.00

0.00

0.00

0.00

Fluid: Water

Fluid Refractive Index: 1.33

Loading Factor: 0.0034

Transmission: 0.98

Above Residual: 0.00

Below Residual: $\quad 0.00$
Analysis Mode: $\mathbf{5 3 0 0 0}$

Sample Cell Id: 0318

ASVR Flow Rate: 75

Uitrasonic Power: 25 watts Ultrasonic Time: 60 seconds 


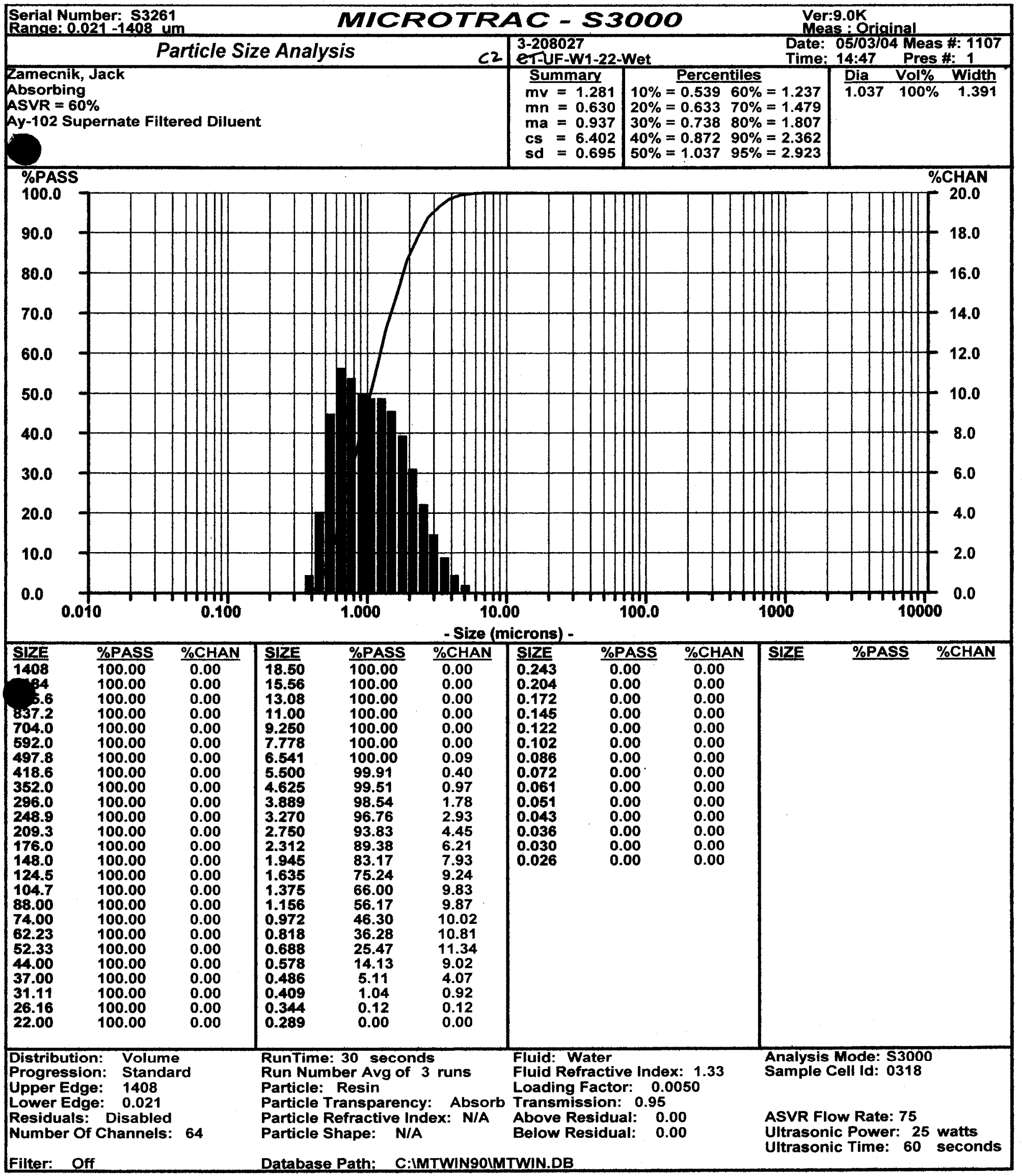




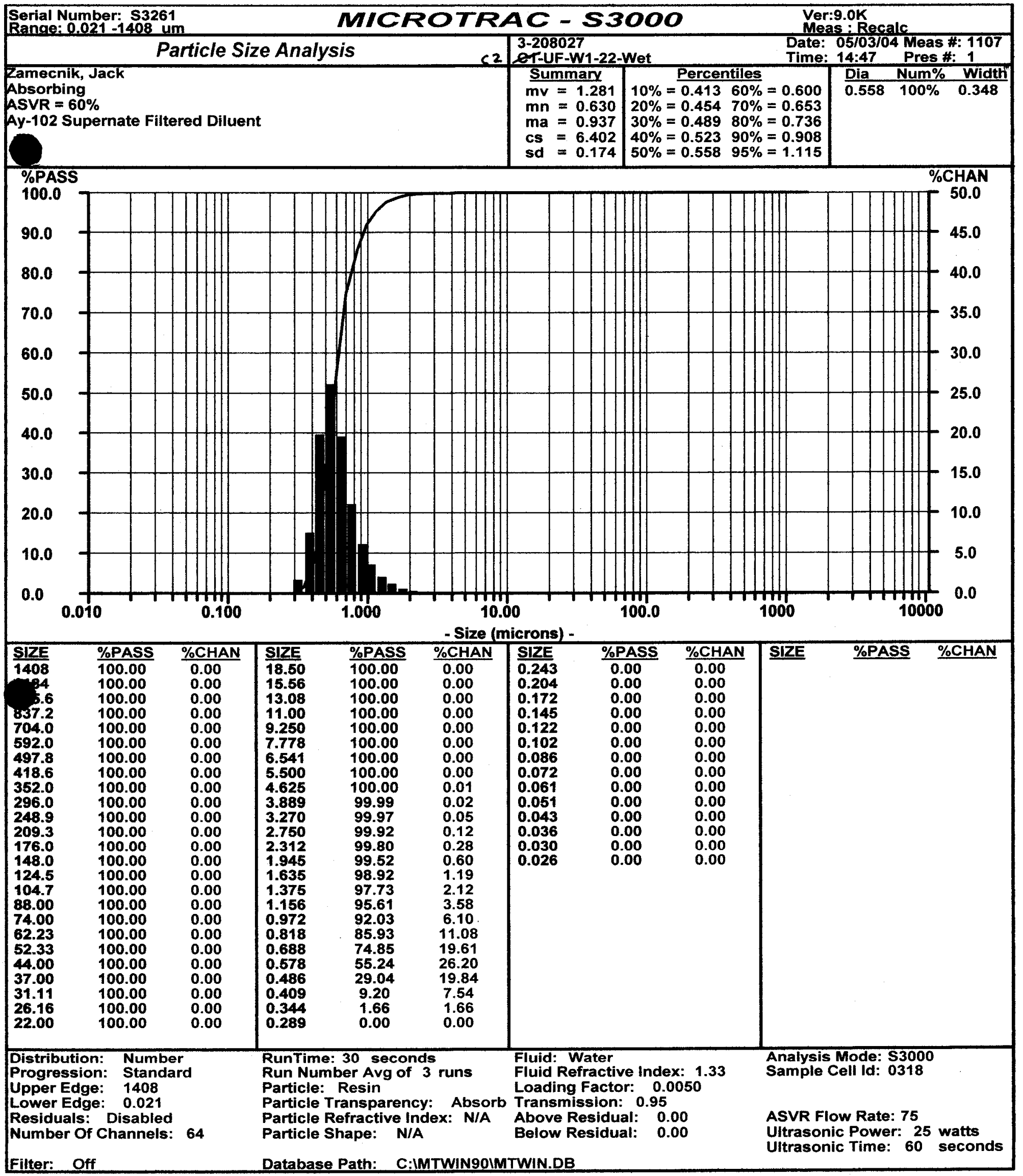




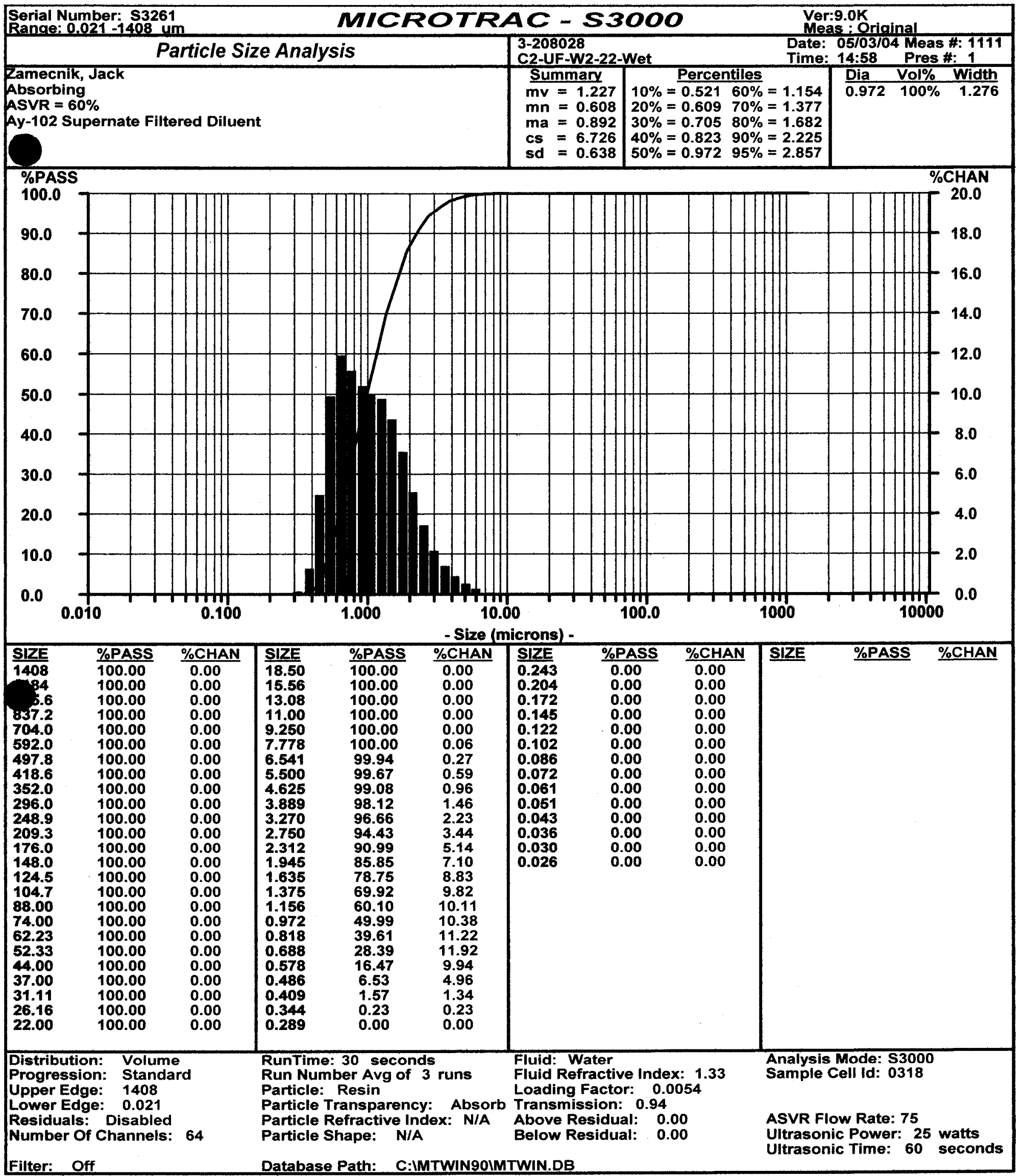




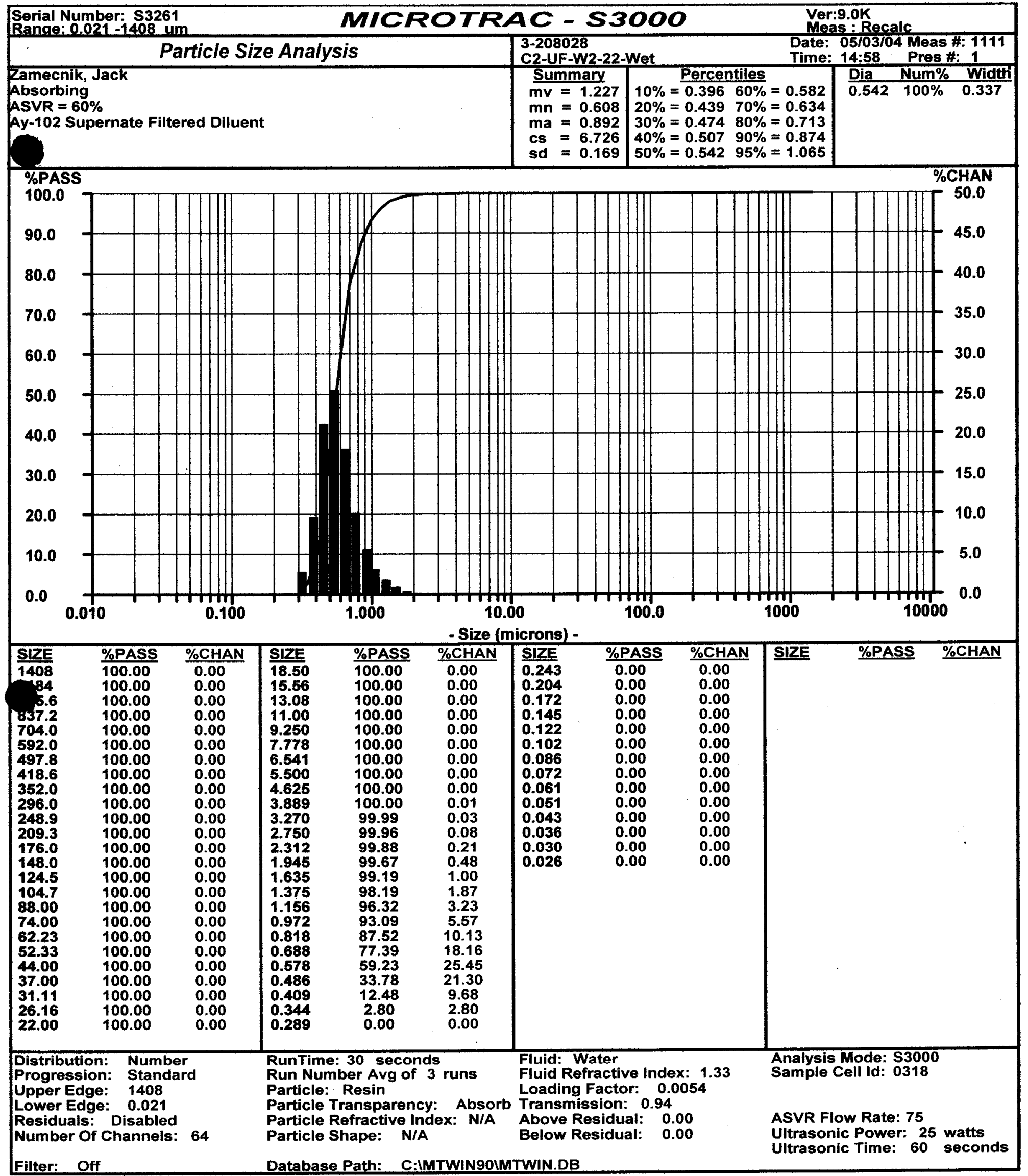




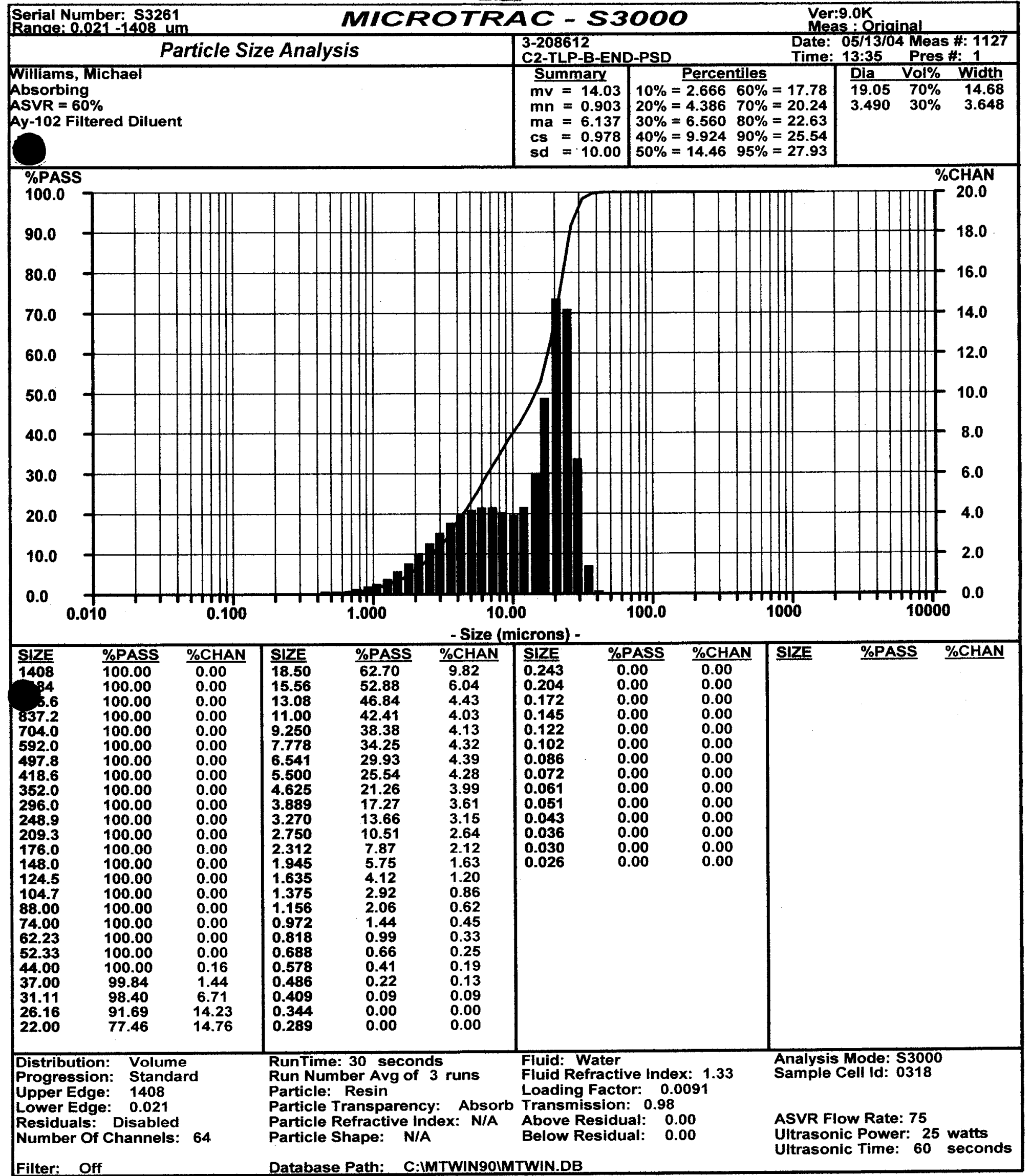


WSRC-TR-2005-00105, REVISION 0

SRNL-RPP-2005-00012, REVISION 0

\section{Particle-Size Distribution Data from SIPP Campaign IV}




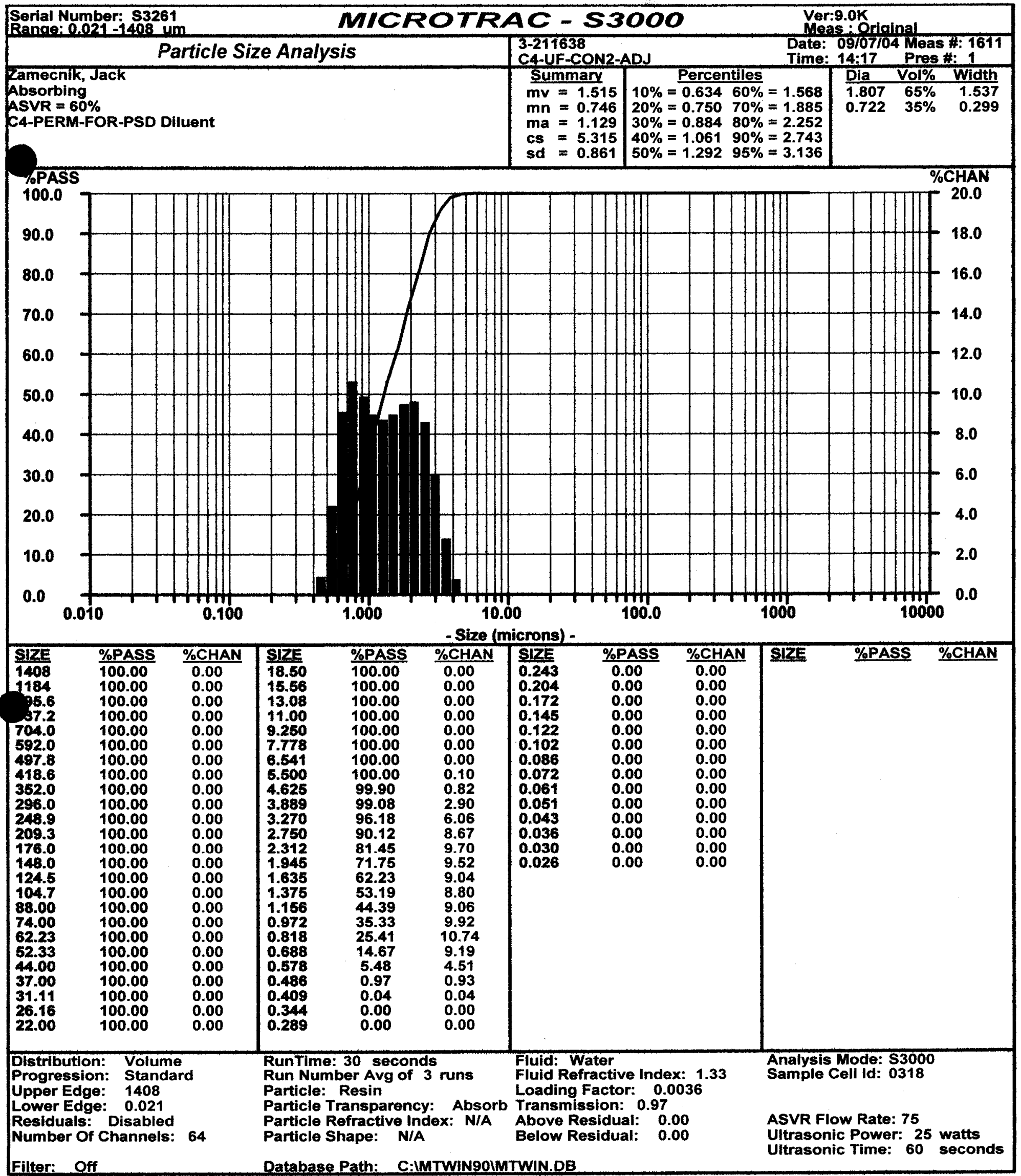


Serial Number: \$3261

Range:0.021-1408 um

MICROTRAC - $5300 O$

Ver:9.0K

\begin{tabular}{|c|c|c|c|}
\hline Particle Size Analysis & $\begin{array}{l}\text { 3-211638 } \\
\text { C4-UF-CON2- }\end{array}$ & $\begin{array}{r}\text { Date: } \\
\text { Time }\end{array}$ & $\begin{array}{l}09 / 07104 \text { Meas \#: } 1611 \\
14: 18 \quad \text { Pres \#: } 1\end{array}$ \\
\hline $\begin{array}{l}\text { Zamecnik, Jack } \\
\text { Absorbing } \\
\text { ASVR }=60 \% \\
\text { C4-PERM-FOR-PSD Diluent }\end{array}$ & $\begin{array}{l}\text { Summary } \\
\text { mv }=1.515 \\
m n=0.746 \\
m a=1.129 \\
c s=5.315 \\
\text { sd }=0.199\end{array}$ & \begin{tabular}{l}
\multicolumn{3}{c}{ Percentiles } \\
$10 \%=0.49560 \%=0.701$ \\
$20 \%=0.538 \quad 70 \%=0.764$ \\
$30 \%=0.57680 \%=0.860$ \\
$40 \%=0.61490 \%=1.067$ \\
$50 \%=0.653 \quad 95 \%=1.338$
\end{tabular} & $\begin{array}{lll}\text { Dia } & \text { Num\% } & \text { Width } \\
0.653 & 100 \% & 0.399\end{array}$ \\
\hline
\end{tabular}

100.0

90.0

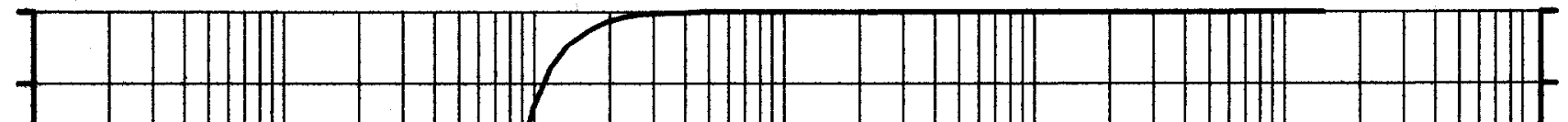

50.0

80.0

70.0

60.0
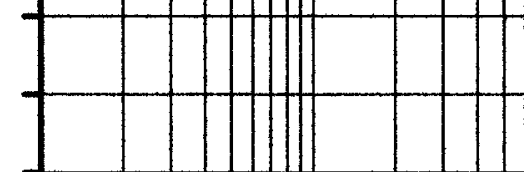

$\mathbf{5 0 . 0}$

40.0

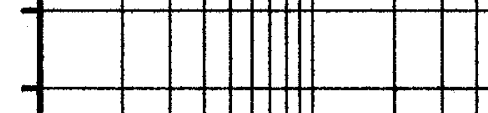

30.0

20.0

10.0

0.0
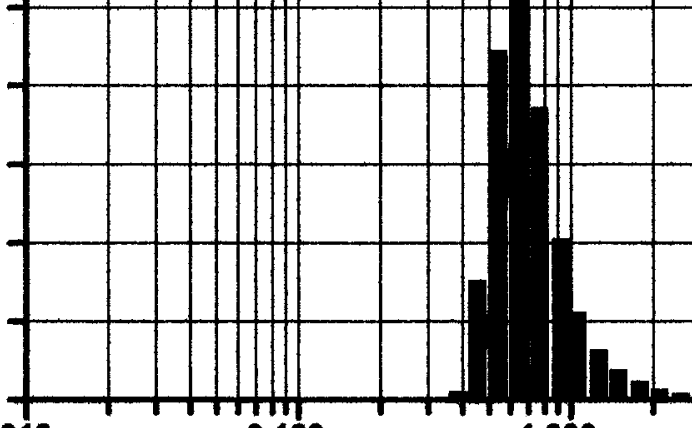

0.100

1.000

10.00

- Sizo (microns) -

\section{SIZE}

1408

1184

5.6

07.2

704.0

497.8

418.6

352.0

296.0

248.9

209.3

176.0

148.0

124.5

104.7

88.00

74.00

62.23

52.33

44.00

37.00

31.11

26.16

22.00

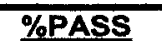

100.00

\%CHAN SIZE

100.00
100.00

100.00

100.00

100.00

100.00

100.00

100.00

100.00

100.00

100.00

100.00

100.00

100.00

100.00

100.00

100.00

100.00

100.00

100.00

100.00

100.00

100.00

100.00

100.00

\section{\%PASS}

100.00

100.00

100.00

100.00

$\begin{array}{lll}0.00 & 13.08\end{array}$

\begin{tabular}{l|l}
0.00 & 9.260
\end{tabular}

$\begin{array}{lll}0.00 & 7.778\end{array}$

0.00

0.00

0.00

0.00

0.00

0.00

0.00

0.00

0.00

0.00

0.00

0.00

0.00

0.00

0.00

0.00

0.00

0.00

100.00

100.00

100.00

6.541

5.500

4.625

3.889

3.270

2.750

2.312

1.945

1.635

1.375

1.166

0.972

0.818

0.688

0.578

0.486

0.409

0.344

0.289

95.41
$\%$ CHAN

0.00

0.00

0.00

0.00

$92.18 \quad 5.60$

$\begin{array}{ll}9.58 & 10.28\end{array}$

18.73

$\begin{array}{ll}30.55 & 27.02 \\ 8.27 & 7.28\end{array}$

$8.27 \quad 7.71$

$0.56 \quad 0.56$

$0.00 \quad 0.00$

0.00

Distribution: Number

Progression: Standard

Upper Edge: 1408

Lower Edge: $\quad 0.021$

Residuals: Disabled

Number Of Channels: 64

Filter: Off

RunTime: 30 seconds

Run Number Avg of 3 runs

Particle: Resin

Particle Transparency: Absorb Transmission: 0.97
Particle Refractive Index: N/A Above Residual: 0.00

Particle Shape: N/A

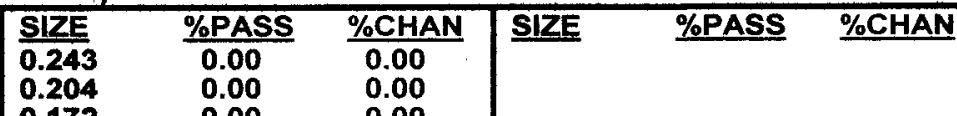

0.172

0.145

0.122

0.102

0.086

0.072

0.061

0.051

0.043

0.036

0.030

0.026

0.00

0.00

0.00

0.00

0.00
0.00

0.00

0.00

0.00

0.00

0.00

0.00

0.00

0.00

0.00

0.00

0.00

0.00

0.00

0.00

0.00

0.00

0.00

Database Path: C:IMTWIN90IMTWIN.DB

Analysis Mode: $\mathbf{S 3 0 0 0}$

Sample Cell Id: 0318

ASVR Flow Rate: 75

Ultrasonic Power: 25 watts Ultrasonic Time: 60 seconds 


\begin{tabular}{|c|c|c|c|}
\hline $\begin{array}{l}\text { Ranqe: 0.021-1408 um } \\
\text { Particle Size Analysis }\end{array}$ & $\begin{array}{l}3-211639 \\
\text { C4-UF-T-30-SL }\end{array}$ & $\begin{array}{l}\text { Date: } \\
\text { Time: }\end{array}$ & $\begin{array}{l}09 / 07 / 04 \text { Meas \#: } 1615 \\
14: 31 \quad \text { Pres \#: } 1 \\
\end{array}$ \\
\hline $\begin{array}{l}\text { Zamecnik, Jack } \\
\text { Absorbing } \\
\text { ASVR }=60 \% \\
\text { C4-PERM-FOR-PSD Diluent }\end{array}$ & $\begin{array}{l}\text { summary } \\
m v=1.444 \\
m n=0.736 \\
m a=1.098 \\
c s=5.466 \\
\text { sd }=0.790\end{array}$ & \begin{tabular}{l}
\multicolumn{3}{c|}{ Percentiles } \\
$10 \%=0.626 \quad 60 \%=1.465$ \\
$20 \%=0.748 \quad 70 \%=1.750$ \\
$30 \%=0.88280 \%=2.106$ \\
$40 \%=1.041 \quad 90 \%=2.599$ \\
$50 \%=1.233 \quad 95 \%=2.991$
\end{tabular} & $\begin{array}{lll}\text { Dia } & \text { Vol\% } & \text { Width } \\
1.233 & 100 \% & 1.580\end{array}$ \\
\hline
\end{tabular}

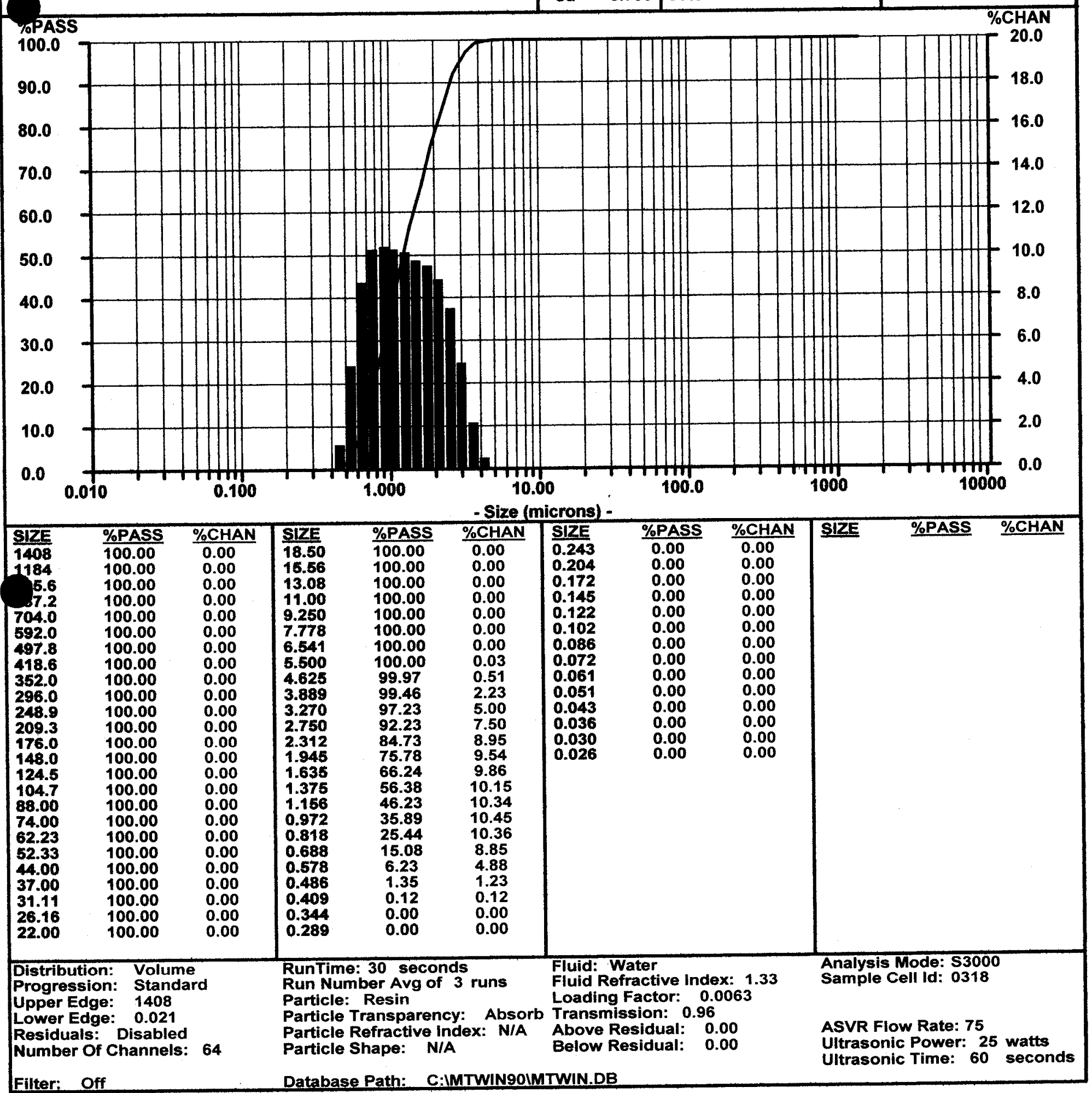


Serial Number: \$3261

Serial Number: $\$ 3261$
Range: $0.021-1408 \mathrm{um}$

MICROTRAC - S3OOO

Ver:9.0K

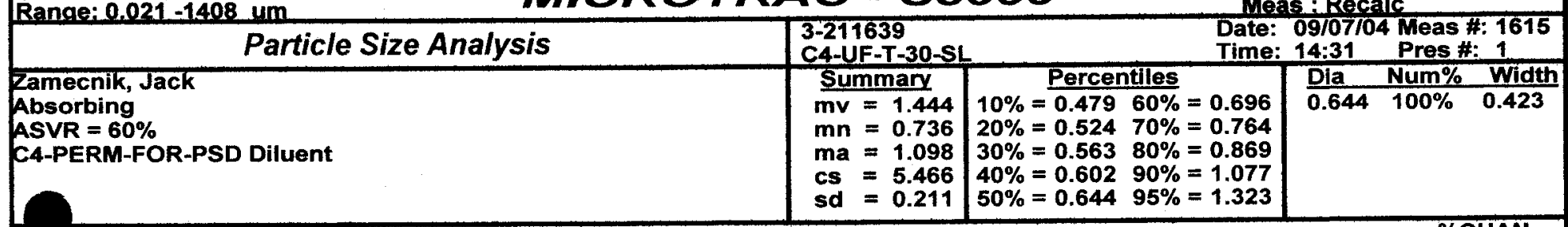

ropASS

$\%$ CHAN

100.0

90.0
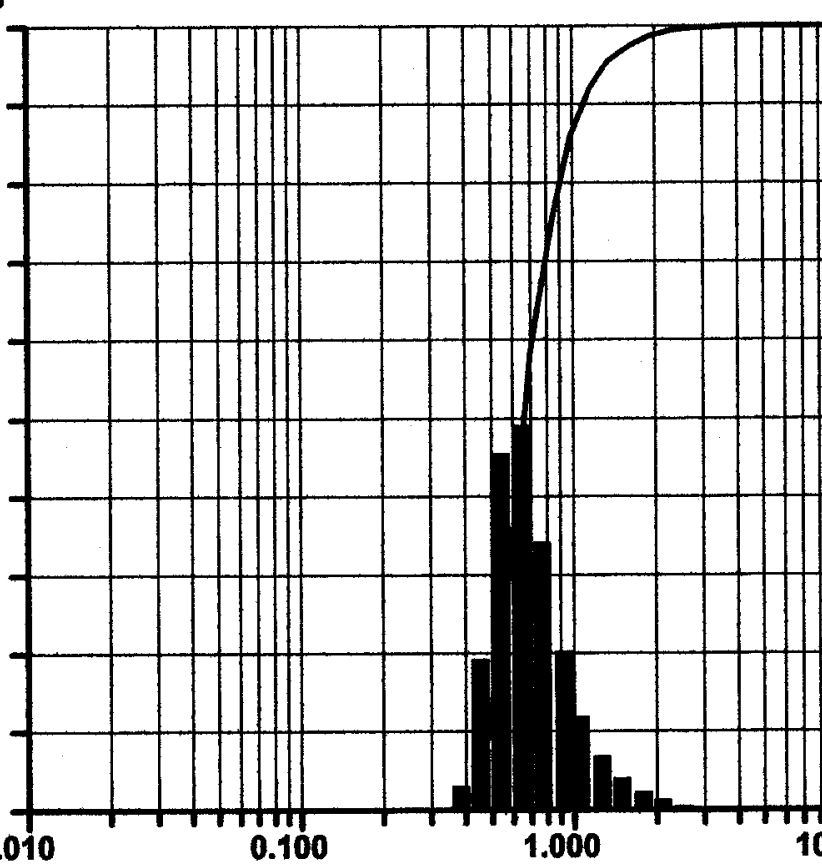

80.0

70.0

60.0

50.0

40.0

30.0

20.0

10.0

0.0

0.100

1.000

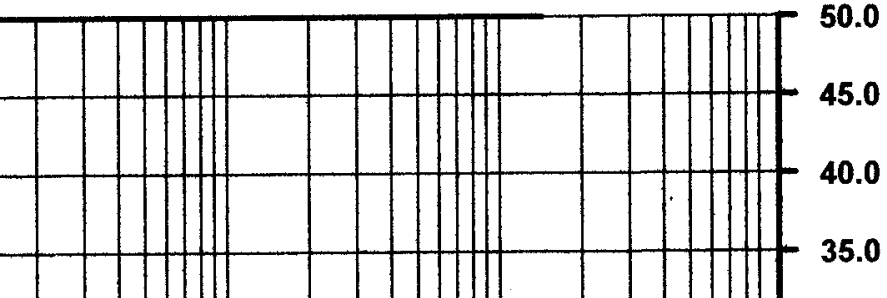

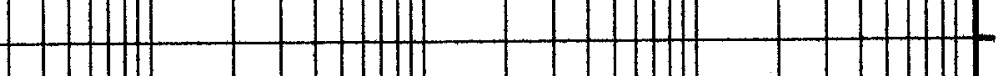

30.0

\section{SIZE}

1408
1184

1184

704.0

592.0

497.8

418.6

352.0

296.0

248.9

209.3

176.0

148.0

124.5

104.7

88.00

74.00

62.23

52.33

44.00

37.00

31.11

26.16

22.00

\begin{tabular}{l}
\hline \%PASS \\
\hline 100.00 \\
100.00 \\
100.00 \\
100.00 \\
100.00 \\
100.00 \\
100.00 \\
100.00 \\
100.00 \\
100.00 \\
100.00 \\
100.00 \\
100.00 \\
100.00 \\
100.00 \\
100.00 \\
100.00 \\
100.00 \\
100.00 \\
100.00 \\
100.00 \\
100.00 \\
100.00 \\
100.00 \\
100.00
\end{tabular}

\section{\%CHAN SIZE}

0.00

0.00

0.00

0.00

0.00

0.00

0.00

0.00

0.00

0.00

0.00

0.00

0.00

0.00

0.00

0.00

0.00

0.00

0.00

0.00

0.00

0.00

- Size (microns) -

Distribution: Number

Progression: Standard

Upper Edge: 1408

Lower Edge: 0.021

Residuals: Disabled

Number Of Channels: 64

Filter: Off
$\frac{S I Z E}{18.50}$

15.56

13.08

9.250

7.778

6.541

5.500

4.625

3.889

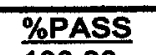

100.00

100.00

100.00

100.00

100.00

100.00

100.00

100.00

100.00

100.00

3.270

2.750

2.312

1.945

1.636

1.375

1.156

0.972

0.818

0.688

0.578

0.486

0.409

0.344

99.97

99.84

99.51

98.86

$\mathbf{9 7 . 6 9}$

95.65

92.12

86.07

75.82

58.72

34.07

11.24

1.58

0.00

0.00
RunTime: $\mathbf{3 0}$ seconds

Run Number Avg of 3 runs

Particle: Resin

Particle Transparency: Absorb

Particle Refractive Index: N/A

Particle Shape: N/A

\begin{tabular}{c} 
\%CHAN \\
\hline 0.00 \\
0.00 \\
0.00 \\
0.00 \\
0.00 \\
0.00 \\
0.00 \\
0.00 \\
0.00 \\
0.03 \\
0.13 \\
0.33 \\
0.65 \\
1.17 \\
2.04 \\
3.53 \\
6.05 \\
10.25 \\
17.10 \\
24.65 \\
22.83 \\
9.66 \\
1.58 \\
0.00 \\
0.00
\end{tabular}

Database Path:

\begin{tabular}{|l}
\hline SIZE \\
\hline 0.243 \\
0.204 \\
0.172 \\
0.145 \\
0.122 \\
0.102 \\
0.086 \\
0.072 \\
0.061 \\
0.051 \\
0.043 \\
0.036 \\
0.030 \\
0.026 \\
\end{tabular}

Fluid: Water

Fluid Refractive Index: 1.33

Loading Factor: 0.0063

Transmission: 0.96

Below Residual: 0.00

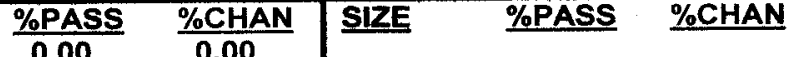

$0.00 \quad 0.00$

0.00

$0.00 \quad 0.00$

$0.00 \quad 0.00$

$0.00 \quad 0.00$

$0.00 \quad 0.00$

$0.00 \quad 0.00$

0.00

$\begin{array}{ll}0.00 & 0.00 \\ 0.00 & 0.00\end{array}$

$0.00 \quad 0.00$

Analysis Mode: $\mathbf{5 3 0 0 0}$

Sample Cell Id: 0318

ASVR Flow Rate: 75

UItrasonic Power: 25 watts Ultrasonic Time: 60 seconds 
Serial Number: S3261

MIOROTRAC - S300O

Ver:9.0K
Meas : Recalc

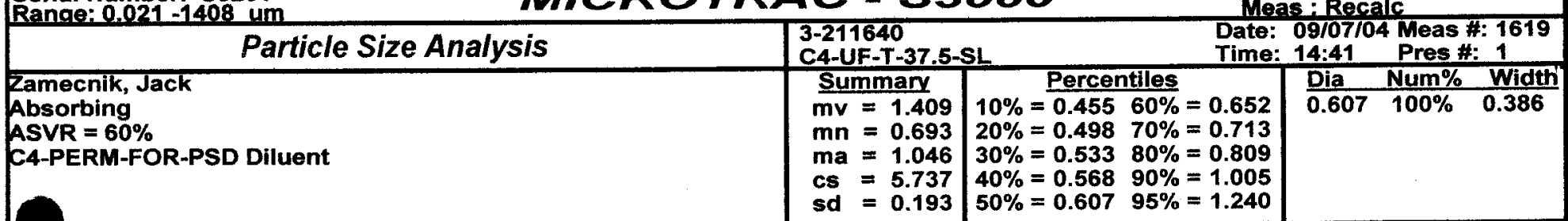

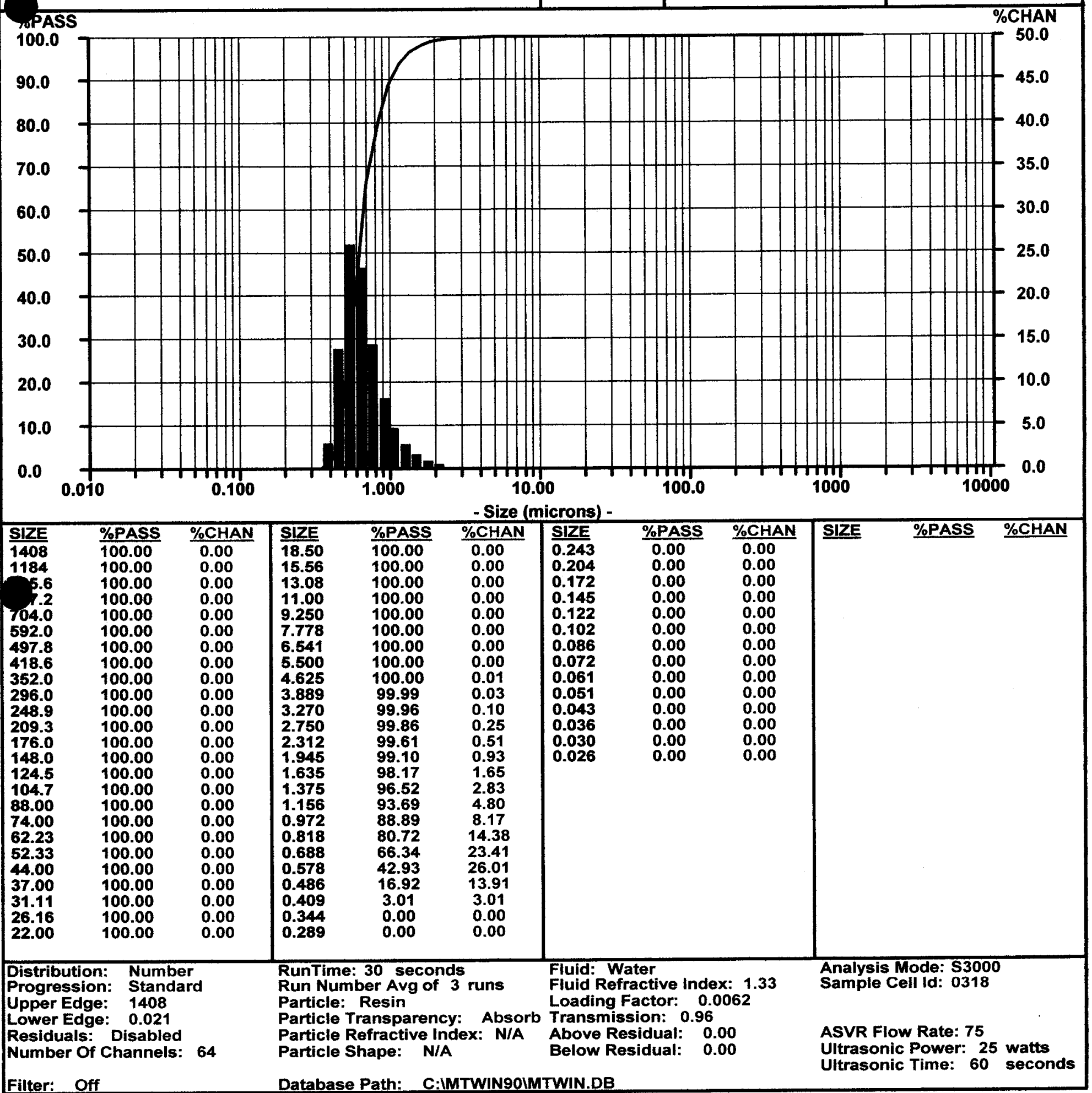


Serial Number: $\mathbf{3 2 6 1}$

MICROTRAC - S300O

Ver:9.0K

Range:0.021-1408 um

Particle Size Analysis

3-211642

Meas: Orioinal

Zamecnik, Jack

Absorbing

ASVR $=60 \%$

C4-PERM-FOR-PSD Diluent C4-UF-T-35-SL

Mate: 09/07/04 Meas \#: 1627

Summary $\quad$ Percentiles $/$ Dia Vol\% Width

mv $=1.409$

$m n=0.692$

$\mathrm{ma}=1.040$

$\mathrm{cs}=5.769$

sd $=0.802$

$10 \%=0.590 \quad 60 \%=1.389$
$20 \%=0.699 \quad 70 \%=1.677$
$30 \%=0.823 \quad 80 \%=2.062$
$40 \%=0.976 \quad 90 \%=2.632$
$50 \%=1.162 \quad 95 \%=3.103$

$1.162 \quad 100 \% \quad 1.604$

1

\%PASS

$\%$ CHAN

100.0

90.0

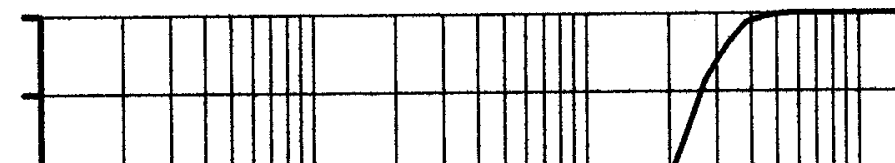

80.0

70.0

60.0

50.0

40.0

30.0

20.0

10.0

0.0
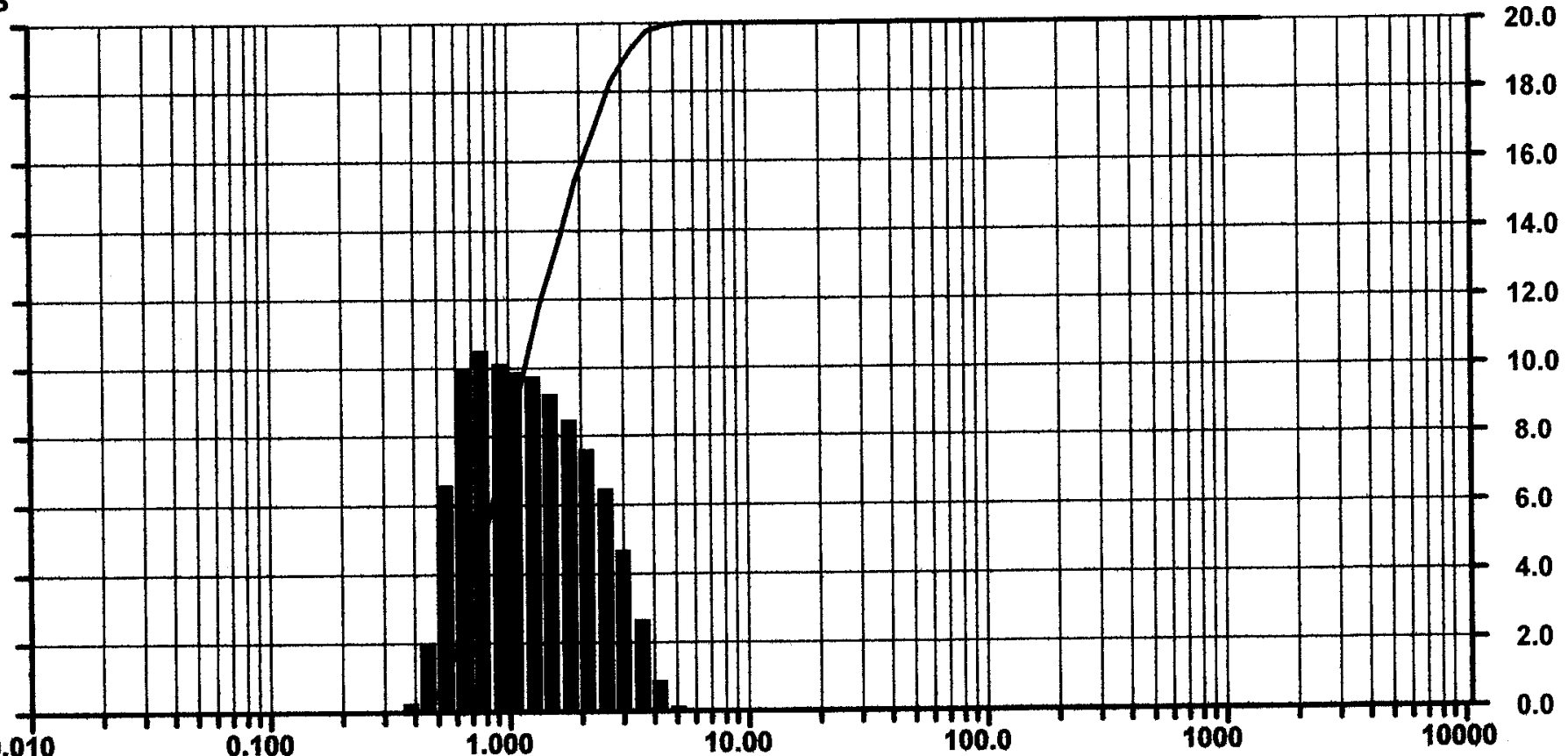

10.00

- Size (microns) -

SIZE \%PASS

$\frac{S 125}{1408}$

1184

100.00

\%CHAN SIZE

\%PASS

$\%$ CHAN

SIZE

100.00

0.00

100.00

\begin{tabular}{l|l}
0.00 & 18.50 \\
0.00 & 15.56
\end{tabular}

100.00

0.00

100.00

0.00

13.08

100.00

0.00

337.2

100.00
100.00

0.00

100.00

0.00

100.00

0.00

100.00

0.00

9.250

100.00

0.00

592.0

100.00

0.00

100.00

7.778

100.00

0.00
0.00

6.541

100.00

0.00

100.00

0.14

4.625

100.00

0.00

100.00

0.00

100.00

$\mathbf{9 9 . 8 6}$

0.14
0.93

98.93

2.68

4.76

91.49

2.750

100.00

0.00

178.0

124.5

104.7

100.00

0.00

100.00

0.00

2.312

1.945

100.00

1.635

85.00

6.49

7.70

8.56

77.30
68.74

1.376

59.47

49.71

74.00

62.23

100.00

0.00

100.00

0.00

1.156

0.972

0.818

39.77

29.64

19.06

100.00

0.00

44.00

37.00

31.11

26.16

100.00

0.00

0.688

19.06
8.98

2.39

0.486

0.409

0.344

0.28

0.00

SlZE
0.243
0.204

0.204
0.172

0.145

0.122

0.102

0.086

0.072

0.061

0.051

0.043

0.036

0.030

0.026

\begin{tabular}{ll|l} 
\%PASS & $\%$ CHAN & SIZE \\
\hline 0.00 & 0.00 & \\
0.00 & 0.00 & \\
0.00 & 0.00 &
\end{tabular}

9.27

9.76

9.94

10.13

10.58

10.08

6.59

2.11

0.28

0.00

0.00

0.289

0.00

0.00

Distribution: Volume

Progression: Standard

Upper Edge: 1408

Lower Edge: 0.021

Residuals: Disabled

Number Of Channels: 64

Filter: Off

RunTime: 30 seconds

Run Number Avg of 3 runs

Particle: Resin

Particle Transparency: Absorb

Particle Refractive Index: N/A

Particle Shape: N/A

Database Path:

\section{Fluid: Water}

Fluid Refractive Index: 1.33

Loading Factor: 0.0074

Transmission: 0.96

Above Residual: 0.00

Below Residual: $\mathbf{0 . 0 0}$
Analysis Mode: $\mathbf{3 3 0 0 0}$

Sample Cell Id: 0318

ASVR Flow Rate: 75

Ultrasonic Power: 25 watts

Ultrasonic Time: 60 seconds 


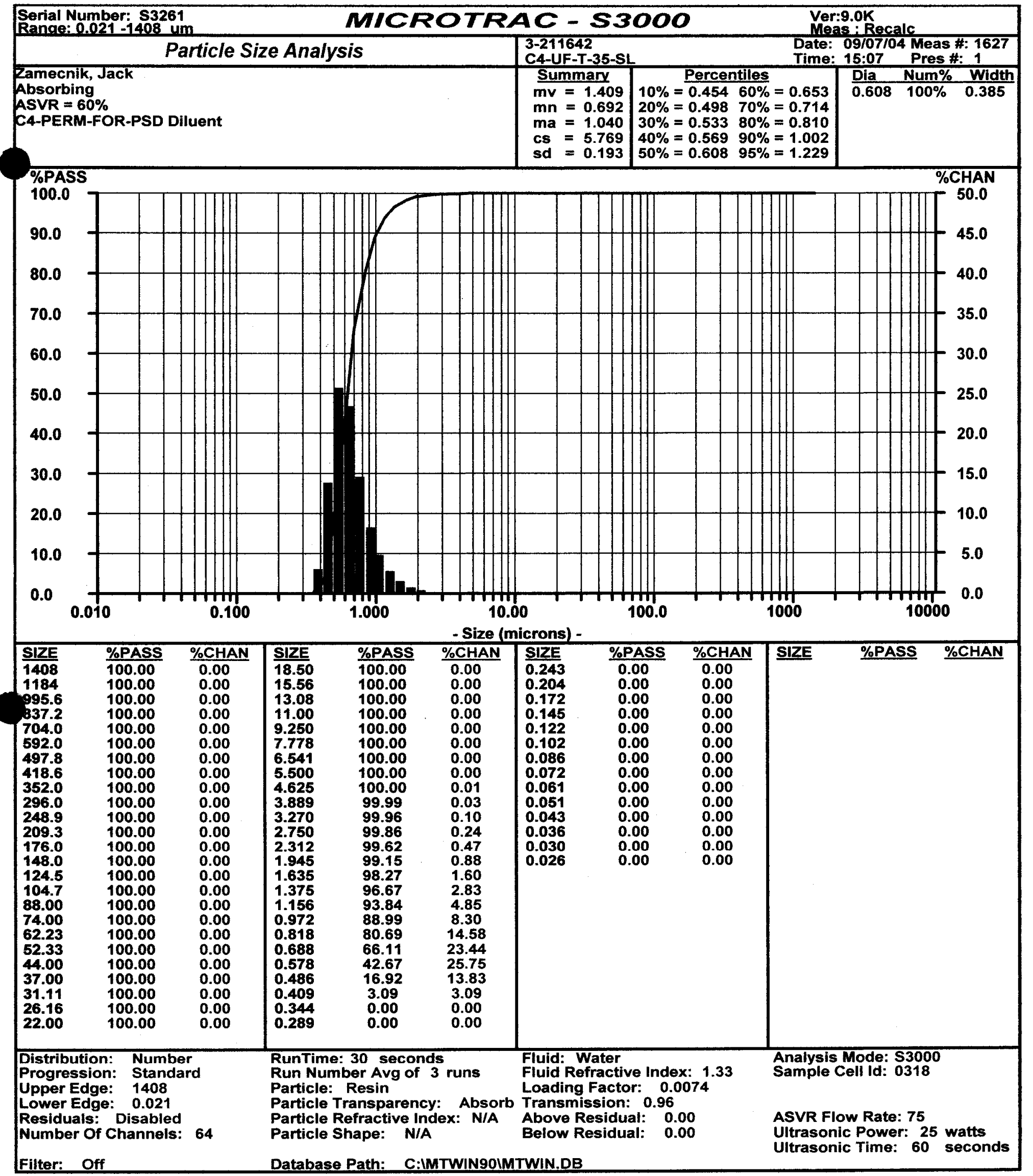




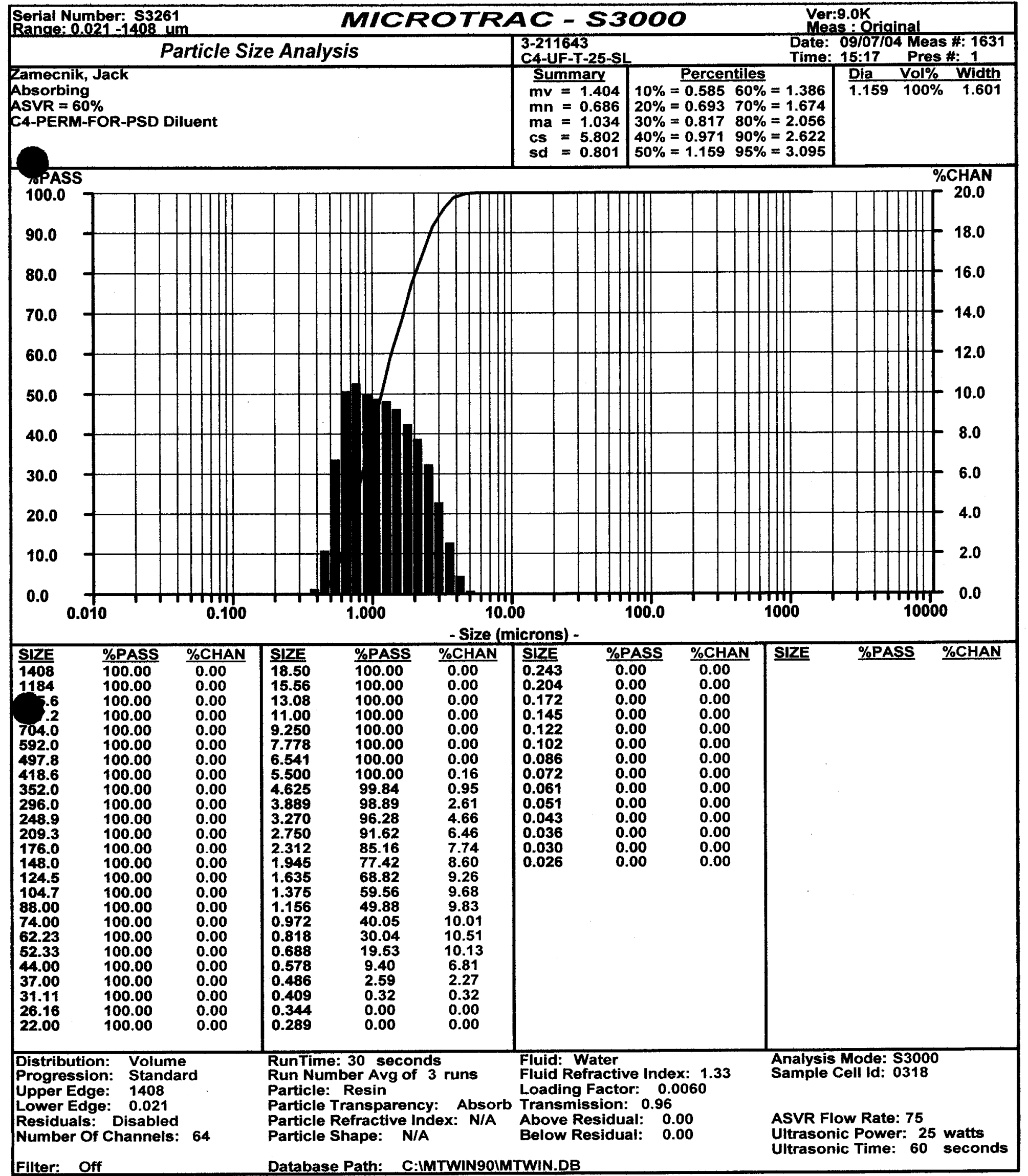


Serial Number: \$3261

Range: 0.021-1408 um

MICROTRAAC - 53000

Ver:9.0K

\begin{tabular}{|c|c|c|c|}
\hline Particle Size Analysis & $\begin{array}{l}3-211643 \\
\text { C4-UF-T-25-SL }\end{array}$ & $\begin{array}{l}\text { Date: } \\
\text { Time: }\end{array}$ & $\begin{array}{l}\text { 09/07/04 Meas \#: } 1631 \\
15: 17 \quad \text { Pres \#: } 1\end{array}$ \\
\hline $\begin{array}{l}\text { Zamecnik, Jack } \\
\text { Absorbing } \\
\text { ASVR }=60 \% \\
\text { C4-PERM-FOR-PSD Diluent }\end{array}$ & $\begin{array}{l}\text { Summary } \\
\mathrm{mv}=1.404 \\
\mathrm{mn}=0.686 \\
\mathrm{ma}=1.034 \\
\mathrm{cs}=5.802 \\
\mathrm{sd}=0.191\end{array}$ & \begin{tabular}{l}
\multicolumn{3}{c}{ Percentiles } \\
$10 \%=0.45060 \%=0.648$ \\
$20 \%=0.49370 \%=0.708$ \\
$30 \%=0.52980 \%=0.802$ \\
$40 \%=0.56490 \%=0.993$ \\
$50 \%=0.60295 \%=1.220$
\end{tabular} & $\begin{array}{lll}\text { Dia } & \text { Num\% } & \text { Width } \\
0.602 & 100 \% & 0.381\end{array}$ \\
\hline
\end{tabular}

yoPASS

$\%$ CHAN

100.0

90.0

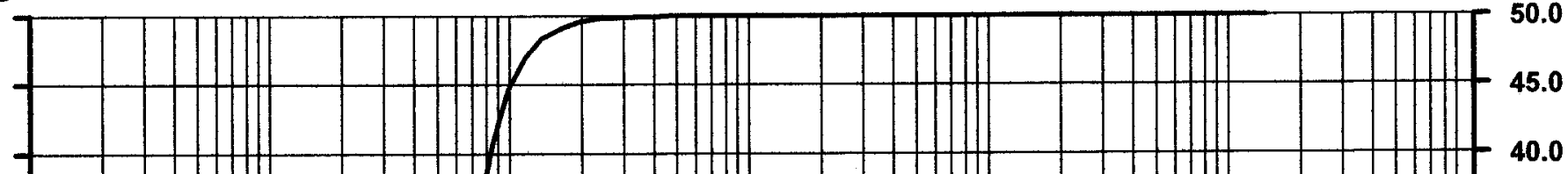

70.0

60.0
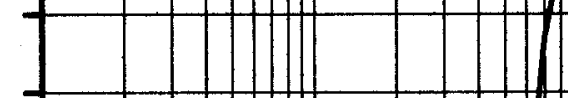

50.0
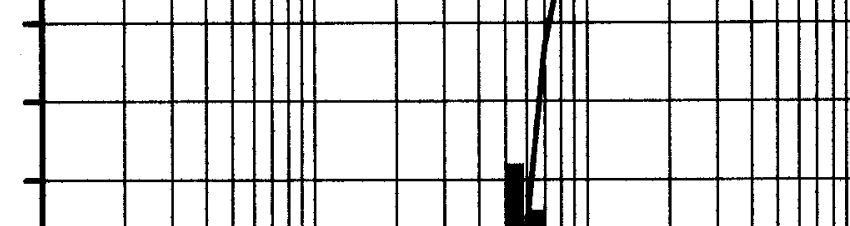

40.0

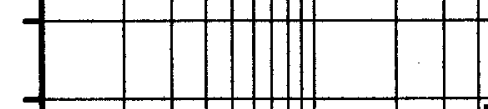

$\mathbf{3 0 . 0}$

20.0

10.0

0.0
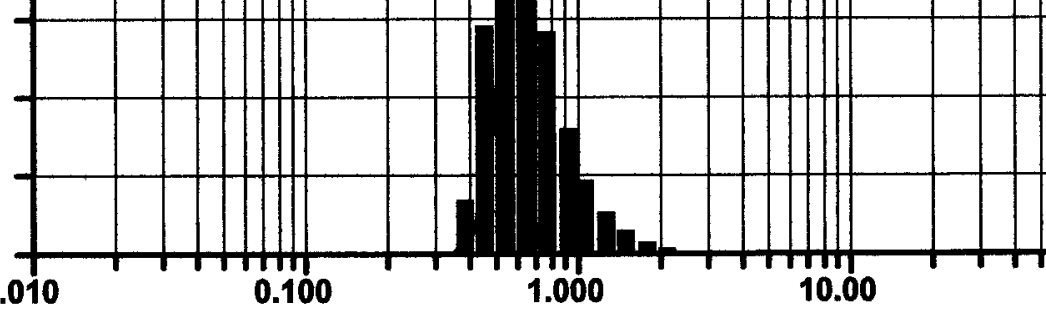

10.00

- Size (microns) -

\begin{tabular}{lll|l}
\hline SIZE & \%PASS & \%CHAN & $\frac{\text { SIZE }}{18.50}$ \\
\hline 1408 & $\frac{100.00}{14.00}$ &
\end{tabular}

1408
1184

1184
3.6

$100.00 \quad 0.00 \quad \frac{\text { SIE }}{18.50}$

\%PASS

\%CHAN

SIZE

100.00

0.00

100.00

0.00

15.56
13.08

100.00

11.00

100.00

0.00
0.00

100.00

0.00

100.00

0.00

592.0

100.00

9.250

100.00

0.00

100.00

0.00

6.541

100.00

0.00

418.6

100.00

0.00

100.00

0.00

100.00

0.00

4.625

296.0

100.00

0.00

100.00

0.00

209.3

176.0

148.0

124.5

104.7

100.00

100.00

0.00

100.00

0.01

99.99

3.270

2.750

2.312

100.00

0.00

1.945

1.635

1.375

1.156

0.972

0.818

74.00

100.00

100.00

0.00

0.00

100.00

0.00

100.00

0.00

0.00

0.00

37.00

31.11

100.00

22.00

100.00

0.00

0.688

0.578

0.486

0.409

0.344

99.96

99.86

99.63

99.17

98.31

96.75

94.00

89.30

81.27

67.09

44.04

18.01

3.45

0.10
0.23

0.46

0.86

1.56

2.75

4.70

8.03

14.18

23.05

26.03

14.56

0.00

3.45

0.00

0.00

Distribution: Number

Progression: Standard

Upper Edge: 1408

Lower Edge: 0.021

Residuals: Disabled

Number Of Channels: 64

Filter: Off
RunTime: 30 seconds

Run Number Avg of 3 runs

Particle: Resin

Particle Transparency: Absorb

Particle Refractive Index: N/A

Particle Shape: N/A

Database Path: C:IMTWIN90IMTWIN.DB

\section{\%PASS \\ \%CHAN SIZE}

0.00

0.204

0.00

0.00

0.145

0.122

0.00

0.00

0.102

0.00

0.00

0.086

0.072

0.061

0.051

0.043

0.036

0.030

0.026

0.00

$0.00 \quad 0.00$

$0.00 \quad 0.00$

$0.00 \quad 0.00$

$0.00 \quad 0.00$

$0.00 \quad 0.00$

$0.00 \quad 0.00$

$\begin{array}{ll}0.00 & 0.00\end{array}$

Fluid: Water

Fluid Refractive Index: 1.33

Loading Factor: $\mathbf{0 . 0 0 6 0}$

ransmission: 0.96

$\begin{array}{ll}\text { Above Residual: } & 0.00 \\ \text { Below Residual: } & 0.00\end{array}$
Analysis Mode: $\mathbf{\$ 3 0 0 0}$

Sample Cell Id: 0318

ASVR Flow Rate: 75

Ultrasonic Power: 25 watts

Ultrasonic Time: 60 seconds 
Serial Number: \$3261

MUCROTRAC - S300O

Ver:9.0K

Rango: 0.021 : 1408 um

Particle Size Analysis

3-211644

C4-UF-T-37.5(15)-SL

Date: 09/07/04 Meas \#: 1635

Zamecnik, Jack

Absorbing

ASVR $=60 \%$

C4-PERM-FOR-PSD Diluent

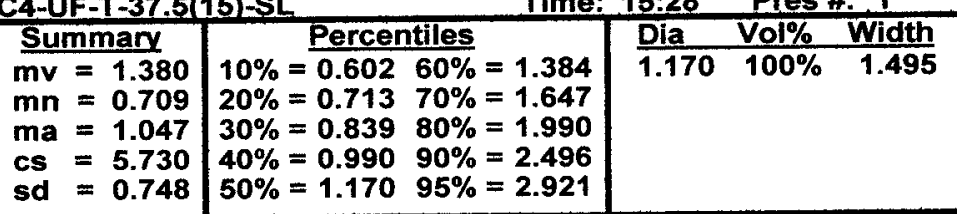

\%PASS

$\%$ CHAN

100.0

90.0
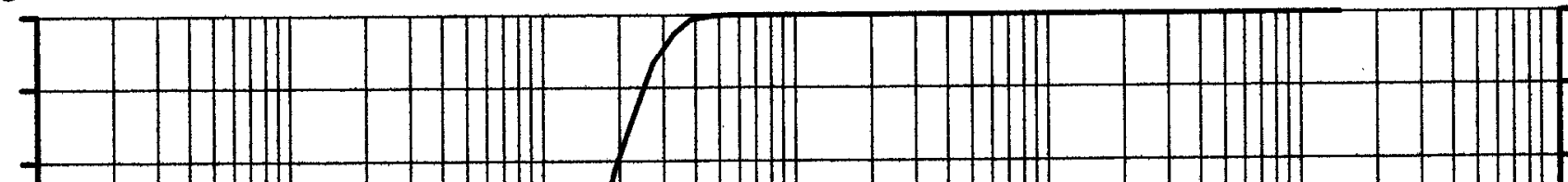

80.0

70.0

60.0
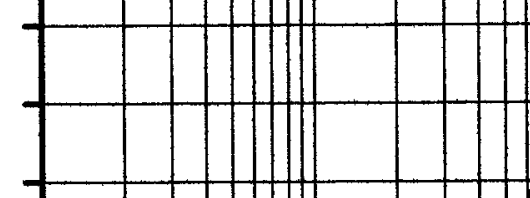

50.0

40.0

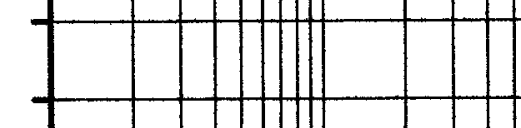

30.0

20.0

10.0

0.0

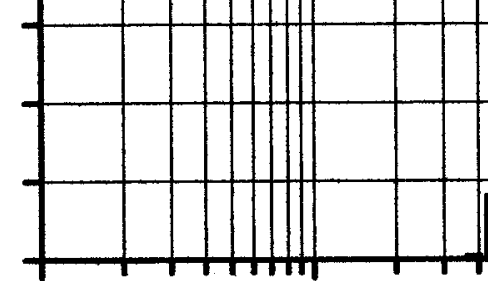

0.010

0.100

1.000
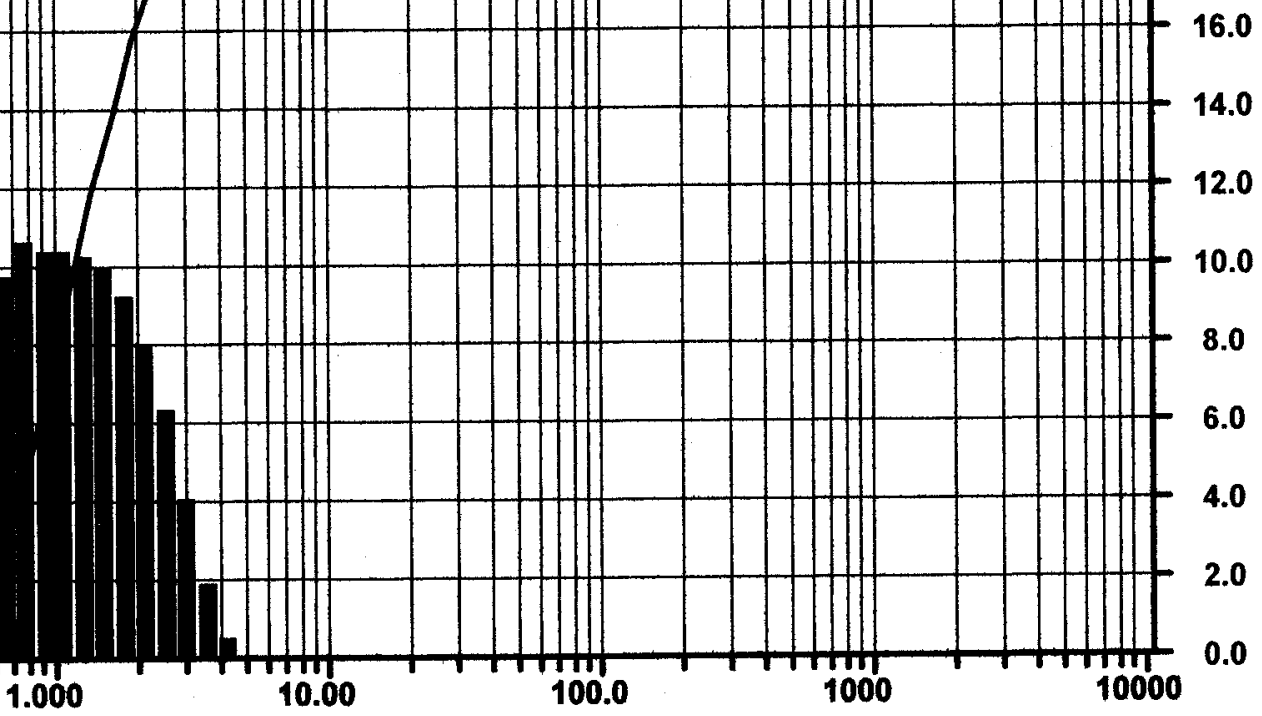

\begin{tabular}{|lll}
\hline SIZE & \%PASS & $\%$ \%CH \\
\hline 1408 & 100.00 & 0.00 \\
1184 & 100.00 & 0.00 \\
995.6 & 100.00 & 0.00 \\
837.2 & 100.00 & 0.00 \\
704.0 & 100.00 & 0.00 \\
592.0 & 100.00 & 0.00 \\
497.8 & 100.00 & 0.00 \\
418.6 & 100.00 & 0.00 \\
352.0 & 100.00 & 0.00 \\
296.0 & 100.00 & 0.00 \\
248.9 & 100.00 & 0.00 \\
209.3 & 100.00 & 0.00 \\
176.0 & 100.00 & 0.00 \\
148.0 & 100.00 & 0.00 \\
124.5 & 100.00 & 0.00 \\
104.7 & 100.00 & 0.00 \\
88.00 & 100.00 & 0.00 \\
74.00 & 100.00 & 0.00 \\
62.23 & 100.00 & 0.00 \\
52.33 & 100.00 & 0.00 \\
44.00 & 100.00 & 0.00 \\
37.00 & 100.00 & 0.00 \\
31.11 & 100.00 & 0.00 \\
26.16 & 100.00 & 0.00 \\
22.00 & 100.00 & 0.00 \\
\end{tabular}

\begin{tabular}{cc}
\hline \%PASS & \%CHAN \\
\cline { 2 - 2 } 100.00 & 0.00 \\
100.00 & 0.00 \\
100.00 & 0.00 \\
100.00 & 0.00 \\
100.00 & 0.00 \\
100.00 & 0.00 \\
100.00 & 0.00 \\
100.00 & 0.04 \\
99.96 & 0.53 \\
99.43 & 1.96 \\
97.47 & 4.15 \\
93.32 & 6.35 \\
86.97 & 8.09 \\
78.88 & 9.26 \\
69.62 & 10.00 \\
59.62 & 10.35 \\
49.27 & 10.38 \\
38.89 & 10.44 \\
28.45 & 10.65 \\
17.80 & 9.79 \\
8.01 & 6.04 \\
1.97 & 1.77 \\
0.20 & 0.20 \\
0.00 & 0.00 \\
0.00 & 0.00
\end{tabular}

SIZE

$\frac{\mathrm{SIZE}}{18.50}$

13.08

11.00

9.250

7.778

6.541

5.500

4.625

3.889

3.270

2.750

2.312

1.945

1.635

1.375

1.156

0.972

0.818

0.688

0.578

0.486

0.409

0.344

0.289

0.00

SIZE

0.243

0.204

0.172

0.145

0.122

0.102

0.086

0.072

0.061

0.051

0.043

0.036

0.030

\%PAS

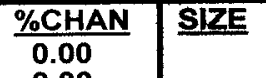

\%PASS \%CHAN

Distribution: Volume

RunTime: 30 seconds

Run Number Avg of 3 runs

Particle: Resin

Fluid: Water

Fluid Refractive Index: 1.33

Loading Factor: 0.0065

Particle Shape: N/A

Above Residual: 0.00

Below Residual: $\quad 0.00$

Database Path: C:IMTWIN90IMTWIN.DB
Analysis Mode: $\mathbf{S 3 0 0 0}$

Sample Cell Id: 0318

ASVR Flow Rate: 75

Ultrasonic Power: 25 watts

Uitrasonic Time: 60 seconds

Filter: Off 
Serial Number: \$3261

MICROTRAC - S300O

Ver:9.0K

Range: $0.021-1408$

Particle Size Analysis

3-211644

Meas : Recalc

\begin{tabular}{|c|c|c|c|}
\hline Particle Size Analysis & $\begin{array}{l}3-211644 \\
\text { C4-UF-T-37.5 }\end{array}$ & 15)-SL & $\begin{array}{l}\text { 09/07704 Meas \#: } 1635 \\
15: 29 \text { Pres \#: } 1 \\
\end{array}$ \\
\hline $\begin{array}{l}\text { Zamecnik, Jack } \\
\text { Absorbing } \\
\text { ASVR }=60 \% \\
\text { C4-PERM-FOR-PSD Diluent }\end{array}$ & $\begin{array}{l}\text { Summary } \\
\mathrm{mv}=1.380 \\
\mathrm{mn}=0.709 \\
\mathrm{ma}=1.047 \\
\mathrm{cs}=5.730 \\
\mathrm{sd}=0.201\end{array}$ & $\begin{array}{l}\text { Percentiles } \\
10 \%=0.46460 \%=0.669 \\
20 \%=0.508 \quad 70 \%=0.734 \\
30 \%=0.54480 \%=0.834 \\
40 \%=0.58190 \%=1.034 \\
50 \%=0.621 \quad 95 \%=1.267\end{array}$ & $\begin{array}{lll}\text { Dia } & \text { Num\% } \% & \text { Width } \\
0.621 & 100 \% & 0.402\end{array}$ \\
\hline
\end{tabular}

\%PASS

100.0

90.0

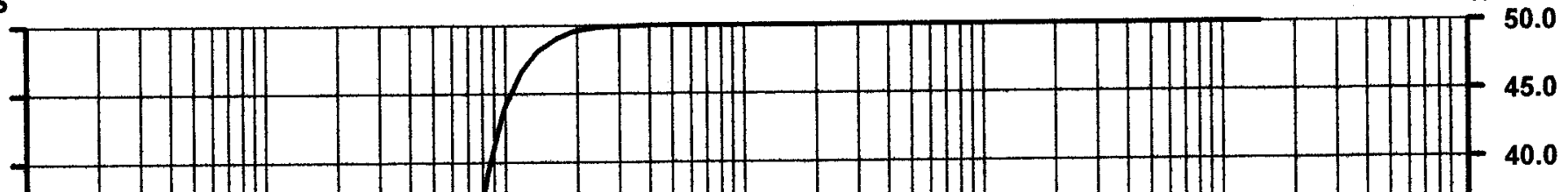

70.0

60.0

50.0

40.0

30.0

20.0

10.0

0.0

010

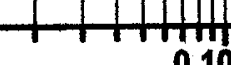

0.100

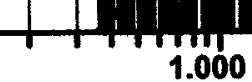

1.000

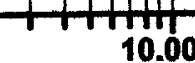

10.00

- Size (microns) -

SIZE \%PASS

1408

1184

995.6

100.00

\%CHAN SIZE

100.00

100.00

$0.00 \quad 18.50$

\%PASS

$\%$ CHAN

SIZE

100.00

0.00

100.00

0.00

15.56

100.00

0.00

837.2

100.00

0.00

11.00

100.00

0.00

100.00

0.00

692.0

497.8

100.00

0.00

100.00

0.00

100.00

0.00

352.0

296.0

100.00

0.00

100.00

0.00

100.00

0.00

209.3

176.0

148.0

124.5

104.7

100.00

0.00

0.00

0.00

100.00

0.00

100.00

0.00

88.00

74.00

62.23

$\mathbf{5 2 . 3 3}$

44.00

37.00

31.11

26.16

100.00

0.00

100.00

0.00

0.00

0.00

100.00

0.00

0.00

0.00

0.00

0.00

9.250

0.00

100.00

0.00

4.625

3.889

3.270

2.750

2312

1.945

1.635

1.375

1.166

0.872

0.818

0.688

0.578

0.486

0.409

0.344

100.00

0.00

100.00

0.00

100.00

99.97

0.03

99.64

99.12

98.12

96.30

93.14

87.80

78.79

63.34

39.37

14.53

2.32

0.243

0.204

0.172

0.145

0.122

0.102

0.086

0.072

0.061

0.051

0.043

0.036

0.030

1.00

0.026

\begin{tabular}{ll|l} 
\%PASS & $\frac{\% \text { CHAN }}{0.00}$ & SIZE \\
\hline .00 &
\end{tabular}

1000

$\begin{array}{ll}0.00 & 0.00\end{array}$

$\begin{array}{ll}0.00 & 0.00\end{array}$

$0.00 \quad 0.00$

$0.00 \quad 0.00$

$\begin{array}{ll}0.00 & 0.00\end{array}$

$\begin{array}{ll}0.00 & 0.00\end{array}$

$0.00 \quad 0.00$

$\begin{array}{ll}0.00 & 0.00\end{array}$

$0.00 \quad 0.00$

$0.00 \quad 0.00$

$0.00 \quad 0.00$

$0.00 \quad 0.00$

1.82

3.16

5.34

9.01

15.45

23.97

24.84

12.21

0.00

0.00

0.289

0.00

Distribution: Number RunTime: 30 seconds

Progression: Standard

Upper Edge: 1408

Lower Edge: $\quad 0.021$

Residuals: Disabled

Number Of Channels: 64

Filter: Off
Run Number Avg of 3 runs

Particle: Resin

Particle Transparency:

Particle Refractive Index: N/A

Particle Shape: N/A

Database Path:

C:IMTWIN90IMTWIN.DB

Fluid: Water

Fluid Refractive Index: 1.33

Loading Factor: 0.0065

Transmission: 0.95

Above Residual: $\quad 0.00$

Below Residual: $\quad 0.00$
Analysis Mode: $\mathbf{S 3 0 0 0}$

Sample Cell Id: 0318

ASVR Flow Rate: 75

Ultrasonic Power: 25 watts

Uitrasonic Time: 60 seconds 


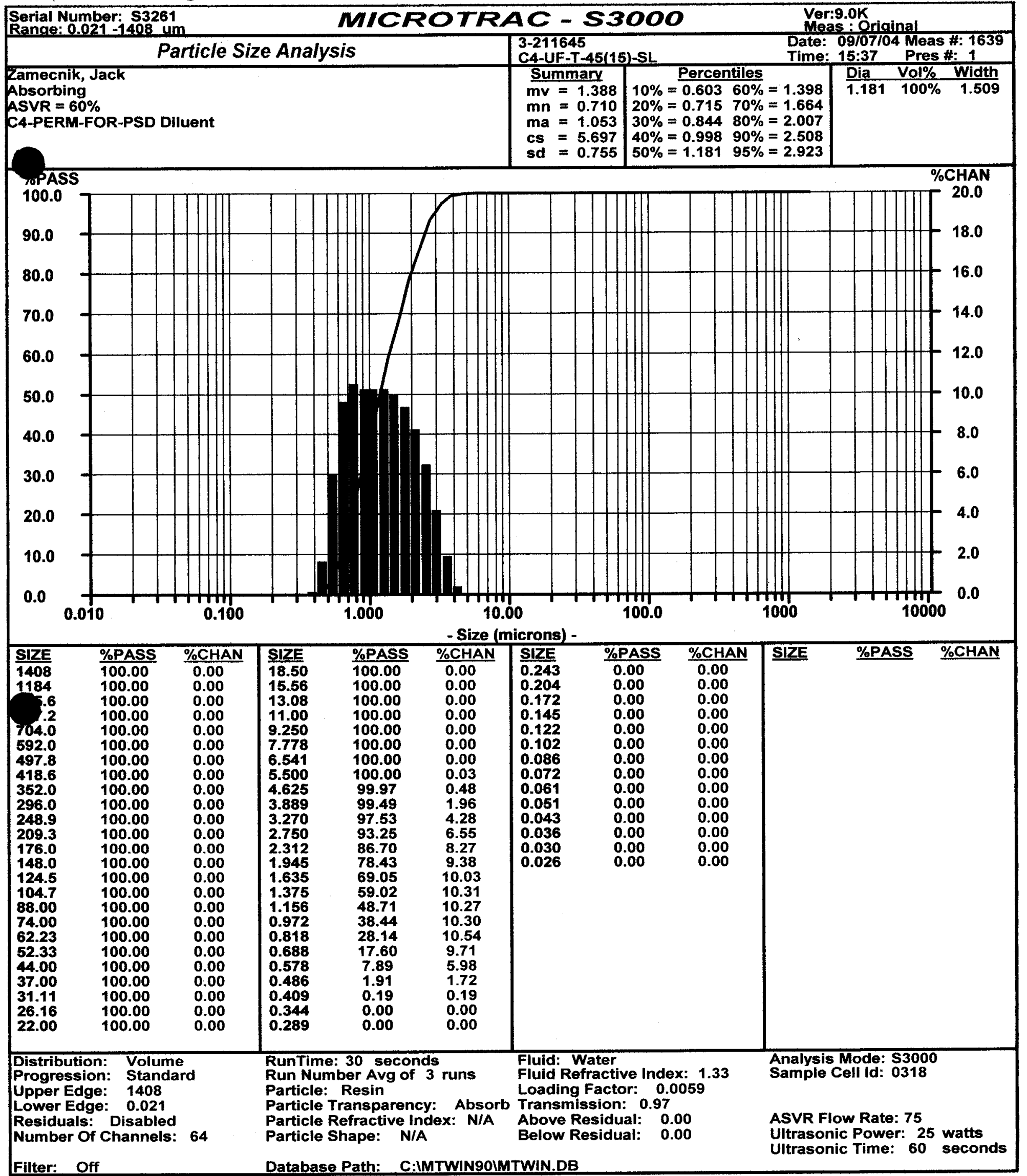


WSRC-TR-2005-00105, REVISION 0

SRNL-RPP-2005-00012, REVISION 0

\section{APPENDIX F: DIMENSIONAL MEASUREMENTS OF CROSS-FLOW ULTRAFILTRATION TUBES FROM MOTT AND GKN}

When the filter units first arrived at SRNL the filter elements were removed from the filter housing to inspect for damage. This was followed by a check on the tubes' dimensions to verify the porous lengths, tube inside diameter, and outside diameter. This was especially important ID and OD were given as nominal dimension of 0.5 inch and 0.625 inch, respectively. Further, there have been filter tests in the past which were done that the internal tube diameter was different than what was expected, e.g., receiving a 3/8-inch ID tube, when a $1 / 2$-inch tube was specified. The following two table list the measurements that were done in the Engineering Development Laboratory of the Savannah River National Laboratory before the filter units were put into service.

\section{Figure F1: MOTT FILTER USED FOR SIPP \\ Figure F2: GKN FILTER USED FOR SIPP}

Summary Measures*

\section{$\underline{\text { Filter Specified Dimensions }}$}

$$
\begin{gathered}
\text { ID }=0.5 \text { inch } \\
\mathrm{OD}=0.625 \text { inch }
\end{gathered}
$$

\section{$\underline{\text { Received Dimensions }}$}

$$
\begin{aligned}
& \text { Mott ID }=0.488 \text { inch } \pm 0.001 \text { inch (one standard deviation) } \\
& \text { Mott OD }=0.654 \text { inch } \pm 0.007 \text { inch (one standard deviation } \\
& \text { GKN ID }=0.495 \text { inch } \pm 0.002 \text { inch (one standard deviation) } \\
& \text { GKN }=0.687 \text { inch } \pm 0.007 \text { inch (one standard deviation) }
\end{aligned}
$$

*Notes:

1. The measured ID only represents the diameters at the ends of the tubes since the internal dimensions more that a couple of inches within the tubes could not be measured.

2. A much tighter tolerance on the ID was obtained and this was expected since the manufacturing process presses the 316L powder onto an accurately machined mandrel. The OD is not as good on its tolerance and has more fluctuation because the outside pressurized surface is flexible, as it has to be to compress the powder while the sintering process proceeds. 
WSRC-TR-2005-00105, REVISION 0

SRNL-RPP-2005-00012, REVISION 0

Table F1. Dimension of the new Mott Filter Elements measured on 9/10/2001

\section{Dimensions* of the SRTC 90 -inch Cross-flow Filter of 0.1-micron media [The Mott filter was designed to have a 0.5-inch I.D. and a 0.625-inch O.D.] \\ (measurements were made on 09/10/2001)}

The overall tube sheet-to-tube sheet length is 92 inches and the overall porous length is 90 inches (or approximately $895 / 8$ inches if the internal weldments are excluded).

The 14 tube-sheet bushings have inside diameters between 0.505 to 0.515 inch.

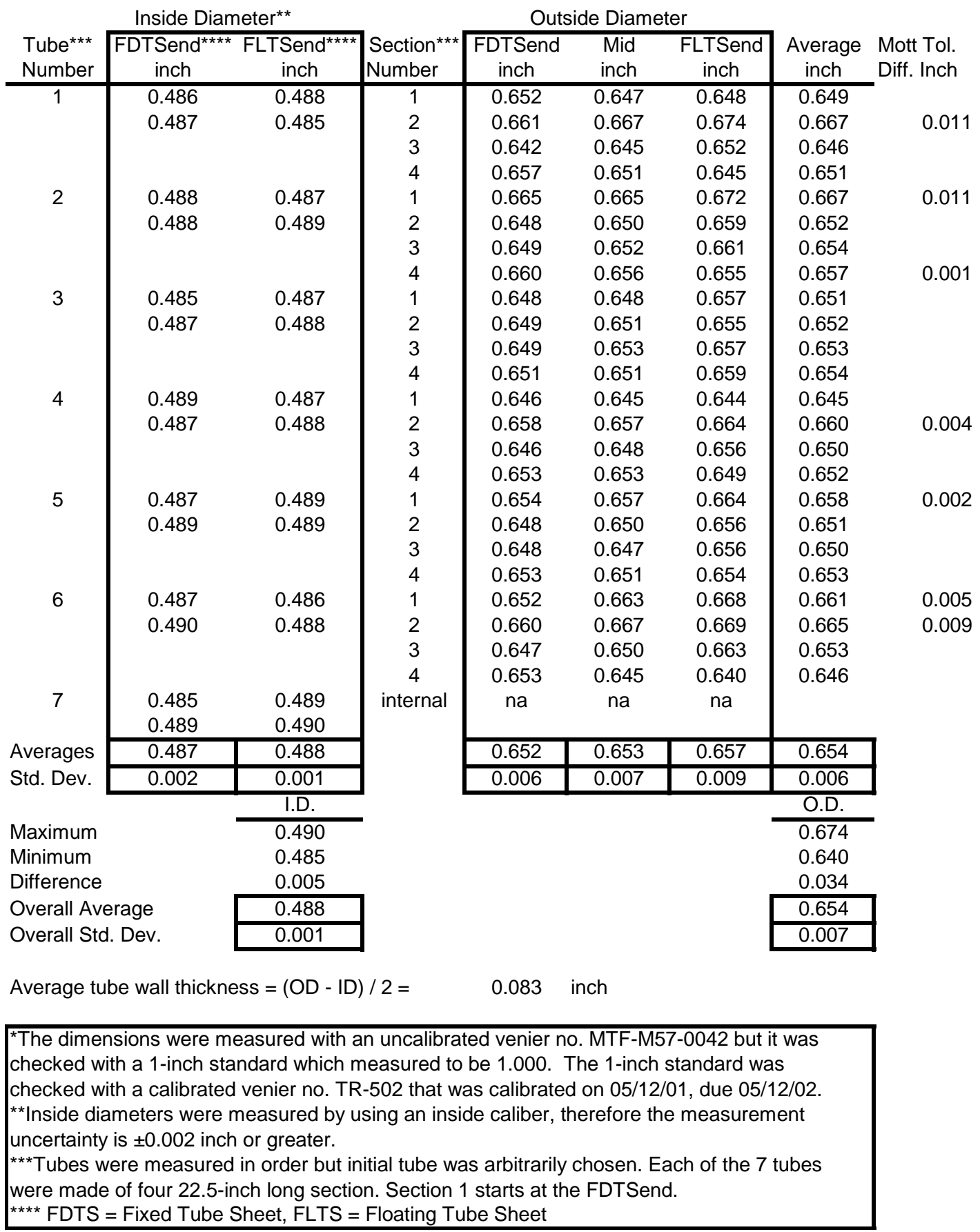


WSRC-TR-2005-00105, REVISION 0

SRNL-RPP-2005-00012, REVISION 0

Table F2. Dimension of the new GKN Filter Elements measured on 8/17-18/2004

Dimensions* of the SRTC Cross-flow Filter of 0.1-micron media

[The GKN filter was designed to have a 0.5 -inch I.D. and a 0.625-inch O.D.]

(measurements were made on 08/17/2004 and08/18/2004)

The overall tube sheet-to-tube sheet length is 101.25 inches and the overall porous length is 92.4 inches

\begin{tabular}{|c|c|c|c|c|c|c|c|}
\hline \multirow[b]{2}{*}{$\begin{array}{l}\text { Tube } \\
\text { Number }\end{array}$} & \multicolumn{2}{|c|}{ Inside Diameter } & \multicolumn{5}{|c|}{ Outside Diameter } \\
\hline & $\begin{array}{c}\text { FDTSend }^{\star \star \star} \\
\text { inch }\end{array}$ & $\begin{array}{l}\text { FLTSend }^{\star \star \star \star} \\
\text { inch }\end{array}$ & $\begin{array}{l}\text { Section }{ }^{\star *} \\
\text { Number }\end{array}$ & \begin{tabular}{|c|}
$\begin{array}{c}\text { FDTSend } \\
\text { inch }\end{array}$ \\
\end{tabular} & $\begin{array}{c}\text { FLTSend } \\
\text { inch }\end{array}$ & $\begin{array}{c}\text { Average } \\
\text { inch }\end{array}$ & $\begin{array}{l}\text { GKN Tol. } \\
\text { Diff. Inch }\end{array}$ \\
\hline \multirow[t]{6}{*}{1} & 0.497 & 0.495 & 1 & 0.698 & 0.691 & 0.695 & \\
\hline & 0.493 & 0.495 & 2 & 0.687 & 0.689 & 0.688 & \\
\hline & & & 3 & 0.677 & 0.680 & 0.679 & \\
\hline & & & 4 & 0.689 & 0.683 & 0.686 & \\
\hline & & & 5 & 0.682 & 0.685 & 0.684 & \\
\hline & & & 6 & 0.689 & 0.682 & 0.686 & \\
\hline \multirow[t]{6}{*}{2} & 0.495 & 0.497 & 1 & 0.701 & 0.692 & 0.697 & \\
\hline & 0.496 & 0.492 & 2 & 0.692 & 0.689 & 0.691 & \\
\hline & & & 3 & 0.696 & 0.689 & 0.693 & \\
\hline & & & 4 & 0.700 & 0.692 & 0.696 & \\
\hline & & & 5 & 0.691 & 0.686 & 0.689 & \\
\hline & & & 6 & 0.696 & 0.696 & 0.696 & \\
\hline \multirow[t]{6}{*}{3} & 0.495 & 0.493 & 1 & 0.679 & 0.679 & 0.679 & \\
\hline & 0.496 & 0.493 & 2 & 0.684 & 0.665 & 0.675 & \\
\hline & & & 3 & 0.678 & 0.682 & 0.680 & \\
\hline & & & 4 & 0.694 & 0.688 & 0.691 & \\
\hline & & & 5 & 0.693 & 0.689 & 0.691 & \\
\hline & & & 6 & 0.684 & 0.688 & 0.686 & \\
\hline \multirow[t]{6}{*}{4} & 0.498 & 0.491 & 1 & 0.693 & 0.686 & 0.690 & \\
\hline & 0.496 & 0.498 & 2 & 0.677 & 0.676 & 0.677 & \\
\hline & & & 3 & 0.686 & 0.665 & 0.676 & \\
\hline & & & 4 & 0.699 & 0.693 & 0.696 & \\
\hline & & & 5 & 0.684 & 0.680 & 0.682 & \\
\hline & & & 6 & 0.689 & 0.694 & 0.692 & \\
\hline \multirow[t]{6}{*}{5} & 0.498 & 0.500 & 1 & 0.695 & 0.687 & 0.691 & \\
\hline & 0.498 & 0.491 & 2 & 0.677 & 0.674 & 0.676 & \\
\hline & & & 3 & 0.676 & 0.674 & 0.675 & \\
\hline & & & 4 & 0.683 & 0.685 & 0.684 & \\
\hline & & & 5 & 0.690 & 0.689 & 0.690 & \\
\hline & & & 6 & 0.687 & 0.700 & 0.694 & \\
\hline \multirow[t]{6}{*}{6} & 0.494 & 0.495 & 1 & 0.683 & 0.684 & 0.684 & \\
\hline & 0.497 & 0.498 & 2 & 0.689 & 0.685 & 0.687 & \\
\hline & & & 3 & 0.690 & 0.693 & 0.692 & \\
\hline & & & 4 & 0.686 & 0.680 & 0.683 & \\
\hline & & & & 0.679 & 0.686 & 0.683 & \\
\hline & & & 6 & 0.690 & 0.693 & 0.692 & \\
\hline \multirow[t]{2}{*}{7} & 0.490 & 0.497 & internal & na & na & & \\
\hline & 0.494 & 0.495 & & & & & \\
\hline \multirow{2}{*}{$\begin{array}{l}\text { Averages } \\
\text { Std. Dev. }\end{array}$} & 0.496 & 0.495 & & 0.688 & 0.685 & 0.687 & \\
\hline & 0.002 & 0.003 & & 0.007 & 0.008 & 0.007 & \\
\hline \multirow{2}{*}{\multicolumn{2}{|c|}{ Maximum }} & I.D. & & & & O.D. & \\
\hline & & 0.500 & & & & 0.701 & \\
\hline \multicolumn{2}{|l|}{ Minimum } & 0.490 & & & & 0.665 & \\
\hline \multicolumn{2}{|l|}{ Difference } & 0.010 & & & & 0.036 & \\
\hline \multicolumn{2}{|c|}{ Overall Average } & 0.495 & & & & 0.687 & \\
\hline \multicolumn{2}{|c|}{ Overall Std. Dev. } & 0.002 & & & & 0.007 & \\
\hline \multicolumn{2}{|c|}{ Meas. Method Uncert. } & 0.001 & & & & 0.005 & \\
\hline \multicolumn{2}{|c|}{ Sum Square of Errors } & 0.003 & & & & 0.009 & \\
\hline \multicolumn{4}{|c|}{ Average tube wall thickness $=(O D-I D) / 2=$} & 0.096 & & 0.009 & \\
\hline $\begin{array}{l}{ }^{\star} \text { The dimel } \\
\text { uncalibrate } \\
\text { was check } \\
\text { gauges. O } \\
\text { due to inte } \\
\text { captured in } \\
\star \star \text { Tubes we } \\
\star \star \star \\
\star \star\end{array}$ & $\begin{array}{l}\text { ssions were m } \\
\text { d micrometer } \\
\text { ed with the cal } \\
\text { utside diamet } \\
\text { ference with } t \\
\text { the measure } \\
\text { ere measured } \\
\text { Fixed Tube } S\end{array}$ & $\begin{array}{l}\text { easured with c } \\
\text { no. TR-00464. } \\
\text { ibrated vernier } \\
\text { ers were made } \\
\text { he tube buldle } \\
\text { ment method u } \\
\text { in order but ini } \\
\text { heet, FLTS = }\end{array}$ & $\begin{array}{l}\text { alibrated ve } \\
\text { The accur } \\
\text { Inside dia } \\
\text { using a cu } \\
\text { tie rods. Th } \\
\text { Incertainty. } \\
\text { tial tube wa } \\
\text { Floating Tu }\end{array}$ & $\begin{array}{l}\text { nier no. TR- } \\
\text { acy of the ur } \\
\text { meters were } \\
\text { stom-made, } \\
\text { te uncertaint } \\
\text { s arbitrarily c } \\
\text { se Sheet }\end{array}$ & $\begin{array}{l}-00502 \text { and } \\
\text { ncalibrated } \\
\text { e made usir } \\
\text { adjustable } \\
\text { ty of these } \\
\text { chosen. }\end{array}$ & $\begin{array}{l}\text { with } \\
\text { nicroimeter } \\
\text { g telescopi } \\
\text { yauge block } \\
\text { nethods are }\end{array}$ & \\
\hline
\end{tabular}


WSRC-TR-2005-00105, REVISION 0

SRNL-RPP-2005-00012, REVISION 0

\section{APPENDIX G: INSTRUMENT LISTS AND CALIBRATION DATA}

This appendix contains a list of all the instruments used to carry out SIPP. The second page of each section contains a condensed figure(s) that list the instruments involved with that unit's operation, along with the pre-test calibration. The principal instruments considered critical to SIPP operation are shown on a following schematic, which is then followed by the post calibration sheets for the same.

The sections are as follows:

Section G1: Pilot Feed-Waste Evaporation Process (FEP) and Treated-LAW Evaporation Process (TLP)

(Both the FEP and TLP used the same equipment)

Section G2: Pilot Ultrafiltration Process (UFP)

Section G3: Pilot Cesium Ion Exchange Process (CIX) 
WSRC-TR-2005-00105, REVISION 0

SRNL-RPP-2005-00012, REVISION 0

\section{G1: FEP \& TLP}


WSRC-TR-2005-00105, REVISION 0 SRNL-RPP-2005-00012, REVISION 0

\begin{tabular}{|c|c|c|c|c|c|c|c|c|c|}
\hline \multicolumn{10}{|c|}{$\begin{array}{l}\text { INSTRUMENTS USED ON } \\
\text { (Printed: January 28, 2005) } \\
\end{array}$} \\
\hline \multirow{3}{*}{\begin{tabular}{|l} 
DAS Chan. \\
\end{tabular}} & \multirow{3}{*}{$\begin{array}{l}\text { Test Rig No. } \\
\end{array}$} & \multirow{3}{*}{ Instrument } & \multirow{3}{*}{\begin{tabular}{|l} 
\\
M\&TE \\
\end{tabular}} & \multirow{3}{*}{ Make } & \multirow[b]{2}{*}{ Model/Serial } & \multirow{3}{*}{ Calibrated Range } & \multirow{3}{*}{$\begin{array}{c}\text { Uncertainty } \\
\text { (pre-test) } \\
\end{array}$} & \multirow[b]{2}{*}{ Uncertainty } & \multirow{3}{*}{$\begin{array}{l}\text { Uncertainty } \\
\text { Average } \\
\end{array}$} \\
\hline & & & & & & & & & \\
\hline & & & & & & & & (post-test) & \\
\hline 0 & ER-TC2 & Thermocouple & TR-01523 & Omega & TJ36-CSXX-18U-6-SB-OST-N/None & $0-100 \mathrm{C}$ & $+/-0.9 \mathrm{C}$ & & \\
\hline 1 & ER-TC4 & Thermocouple & TR-01505 & Omega & TJ36-CSXX-18U-6-SB-OST-N/None & $0-100 \mathrm{C}$ & $+/-0.8 \mathrm{C}$ & & \\
\hline 2 & JP-TC1 & Thermocouple & TR-01509 & Omega & TJ36-CSXX-18U-6-SB-OST-N/None & $0-100 \mathrm{C}$ & $+/-0.9 \mathrm{C}$ & & \\
\hline 3 & PW-TC4 & Thermocouple & TR-01544 & Omega & TJ36-CSXX-18U-6-SB-OST-N/None & $0-100 \mathrm{C}$ & $+/-0.9 \mathrm{C}$ & & \\
\hline 4 & ER-TC3 & Thermocouple & TR-01525 & Omega & TJ36-CSXX-18U-6-SB-OST-N/None & $0-100 \mathrm{C}$ & $+/-1.0 \mathrm{C}$ & & \\
\hline 5 & ER-TC1 & Thermocouple & TR-01520 & Omega & TJ36-CSXX-18U-6-SB-OST-N/None & $0-100 \mathrm{C}$ & $+/-1.0 \mathrm{C}$ & $+/-0.77 \mathrm{C}$ & 0.885 \\
\hline 6 & PW-TC1 & Thermocouple & TR-01514 & Omega & TJ36-CSXX-18U-6-SB-OST-N/None & $0-100 \mathrm{C}$ & $+/-0.9 \mathrm{C}$ & & \\
\hline 7 & PW-TC2 & Thermocouple & TR-01515 & Omega & TJ36-CSXX-18U-6-SB-OST-N/None & $0-100 \mathrm{C}$ & $+/-1.0 \mathrm{C}$ & & \\
\hline 8 & PW-TC3 & Thermocouple & TR-01519 & Omega & TJ36-CSXX-18U-6-SB-OST-N/None & $0-100 \mathrm{C}$ & $+/-0.9 \mathrm{C}$ & & \\
\hline 9 & Spare & Thermocouple & TR-01510 & Omega & TJ36-CSXX-18U-6-SB-OST-N/None & $0-100 \mathrm{C}$ & $+/-0.8 \mathrm{C}$ & & \\
\hline 10 & ER-FT1 & Mag Flowmeter & TR-03278 & Fischer-Porter & 10D1475DK11PL29KD11CAC / 90W003447 & $0-100 \mathrm{GPM}$ & $+/-0.37 \mathrm{GPM}$ & $+/-0.43 \mathrm{GPM}$ & 0.4 \\
\hline 11 & CL-FT1 & Mag Flowmeter & TR-03703 & Fischer-Porter & 10D1475DK11PL29KD11CAC / 95W0006755 & $0-1 \mathrm{GPM}$ & $+/-0.01 \mathrm{GPM}$ & & \\
\hline 12 & FL-FT1 & Mag Flowmeter & TR-03702 & Fischer-Porter & 10D1475DK11PL29KD11CAC / 95W0006754 & $0-1 \mathrm{GPM}$ & $+/-0.06 \mathrm{GPM}$ & & \\
\hline 13 & PC-FT1 & Mag Flowmeter & TR-03705 & Fischer-Porter & 10D1475DK11PL29KD11CAC / 92W130154 & $0-1.2 \mathrm{GPM}$ & $+/-0.01 \mathrm{GPM}$ & & \\
\hline 14 & SW-FT1 & Mag Flowmeter & TR-03704 & Fischer-Porter & 10D1475DK11PL29KD11CAC / 94W0Z4465 & $0-5.3 \mathrm{GPM}$ & $+/-0.01 \mathrm{GPM}$ & & \\
\hline 15 & JP-DP1 & Pressure Transducer & TR-02151 & Rosemount & 1151DP3522M1B2/1230856 & $0-10 " \mathrm{H} 2 \mathrm{O}$ & $+/-0.02 " \mathrm{H} 2 \mathrm{O}$ & & \\
\hline 16 & JP-PT1 & Pressure Transducer & TR-01381 & Rosemount & 1151DP5E22M3B2/11210985 & $-14.5-0$ - PSIG & $+/-0.01 \mathrm{PSI}$ & & \\
\hline 17 & ER-PT1 & Pressure Transducer & TR-03554 & Rosemount & 1151DP6E22/1368954 & $-14.5-0$ PSIG & $+/-0.01$ PSI & $+/-0.009$ PSI & 0.0095 \\
\hline 18 & ER-DP1 & Pressure Transducer & TR-02150 & Rosemount & 1151DP3533M1B2/1276367 & $0-8 " \mathrm{H} 2 \mathrm{O}$ & $+/-0.01 " \mathrm{H} 2 \mathrm{O}$ & & \\
\hline 19 & ER-DP3 & Pressure Transducer & TR-03719 & Rosemount & 3051 CA1A22A1AB4/1338878 & -12 " to $12 " \mathrm{H} 2 \mathrm{O}$ & $+/-0.02 " \mathrm{H} 2 \mathrm{O}$ & & \\
\hline 20 & ER-DP2 & Pressure Transducer & TR-03720 & Rosemount & 3051 CA1A22A1AB4/1338879 & $-12 "$ to $12 " \mathrm{H} 2 \mathrm{O}$ & $+/-0.02 " \mathrm{H} 2 \mathrm{O}$ & & \\
\hline 21 & ER-AP2 & Pressure Transducer & TR-03717 & Rosemount & 3051 CA1A22A1AB4/1339363 & 0-30 PSIA & $+/-0.008$ PSI & & \\
\hline 22 & ER-AP1 & Pressure Transducer & TR-03718 & Rosemount & 3051 CA1A22A1AB4/1339364 & $0-30$ PSIA & $+/-0.009$ PSI & & \\
\hline 23 & PC-FT2 & Mag Flowmeter & TR-03562 & Fischer-Porter & 10D1475DK11PL29KD11CAC / 95W0006754 & $0-1 \mathrm{GPM}$ & $+/-0.01 \mathrm{GPM}$ & $+/-0.0083 \mathrm{GPM}$ & 0.00915 \\
\hline Spare & & Pressure Transducer & TR-03529 & Rosemount & 1151DP5S22B1/4043432 & $0-180 " \mathrm{H} 2 \mathrm{O}$ & $+/-0.14 " \mathrm{H} 2 \mathrm{O}$ & & \\
\hline & & Local Instruments & & & & & & & \\
\hline & & & & & & & & & \\
\hline & PW-FM1 & Rotameter & TR-03684 & Brooks & 1024CNE1F91AA/0102110221812/001 & 5-40 GPM & $+/-0.48 \mathrm{GPM}$ & & \\
\hline & PW-FM2 & Rotameter & TR-03682 & Brooks & 1305FL1ACJ2BA/0102110221811/002 & $2.5-25 \mathrm{GPM}$ & $+/-0.78 \mathrm{GPM}$ & & \\
\hline & PW-FM3 & Rotameter & TR-03683 & Brooks & 1305FL1ACJ2BA/0102110221811/001 & 2.5-25 GPM & \begin{tabular}{|l|l}
$+/ 1.09 \mathrm{GPM}$ \\
\end{tabular} & & \\
\hline & SW-FI1 & Rotameter & TR-20270 & Fischer-Porter & 10A4555P/91W503196 & $0-2.76 \mathrm{GPM}$ & Information Only & & \\
\hline & N/A & Weight Scale & DWB-514 & Explorer & ELM20/1118453062 & $0-32,000 \mathrm{~g}$ & $+/-0.0818 \mathrm{~g}$ & & $+/-0.0818$ \\
\hline & N/A & Density Meter & $\mathrm{N} / \mathrm{A}$ & Anton Parr & DMA4500/528030 & $0-3 \mathrm{~g} / \mathrm{mL}$ & $+/-0.00005 \mathrm{~g} / \mathrm{mL}$ & & $+/-0.00005$ \\
\hline & & & & & & & & & \\
\hline & & & & DAS Calibration & & Transfer Functions $\mathrm{f}$ & r DAS (Conversio & $\mathrm{n}$ from $\mathrm{mA}$ to Volt & \\
\hline & DAS Channel & Instrument & & Slope $(\mathrm{V} / \mathrm{mA})$ & Intercept(V) & Output & \begin{tabular}{|l|} 
Slope (unit/V) \\
\end{tabular} & & Intercept (Unit) \\
\hline & 10 & ER-FT1 & & 2.015 & 0.006 & Flow (gpm) & 12.381 & $\mathrm{xV}+$ & -24.543 \\
\hline & 11 & CL-FT1 & & 2.013 & 0.010 & Flow (gpm) & 0.133 & $\mathrm{xV+}$ & -0.263 \\
\hline & 12 & FL-FT1 & & 2.012 & 0.004 & Flow (gpm) & 0.136 & $\mathrm{xV}+$ & -0.279 \\
\hline & 13 & PC-FT1 & & 2.006 & 0.004 & Flow (gpm) & 0.150 & $\mathrm{xV}+$ & -0.302 \\
\hline & 14 & SW-FT1 & & 2.005 & -0.006 & Flow (gpm) & 0.670 & $\mathrm{xV+}$ & -1.336 \\
\hline & 15 & \begin{tabular}{|l|} 
JP-DP1 \\
\end{tabular} & & 2.010 & 0.000 & Pressure (psid) & 1.256 & $\mathrm{xV}+$ & -2.509 \\
\hline & 16 & JP-PT1 & & 2.017 & 0.010 & Pressure (psig) & -1.828 & $\mathrm{xV}+$ & 3.613 \\
\hline & 17 & ER-PT1 & & 2.008 & 0.008 & Pressure (psig) & 1.820 & $\mathrm{xV}+$ & -18.119 \\
\hline & 18 & ER-DP1 & & 2.006 & 0.004 & $\begin{array}{l}\text { Pressure (psid) } \\
\text { (a) }\end{array}$ & 1.003 & $\mathrm{xV}+$ & -2.001 \\
\hline & 19 & ER-DP3 & & 2.007 & -0.010 & Pressure (psid) & 22.579 & $\mathrm{xV}+$ & -45.076 \\
\hline & 20 & ER-DP2 & & 2.003 & 0.002 & Pressure (psid) & 3.002 & $\mathrm{xV+}$ & -17.983 \\
\hline & 21 & ER-AP2 & & 2.007 & -0.010 & Pressure (psia) & 3.766 & $\mathrm{xV}+$ & -7.538 \\
\hline & 22 & ER-AP1 & & 2.003 & -0.010 & Pressure (psia) & 3.756 & $\mathrm{xV}+$ & -7.520 \\
\hline & 23 & PC-FT2 & & 2.003 & -0.010 & Flow (gpm) & 0.127 & $\mathrm{xV+}$ & -0.256 \\
\hline
\end{tabular}

Figure G1-1. Entire FEP and TLP Instrumentation List 
WSRC-TR-2005-00105, REVISION 0

SRNL-RPP-2005-00012, REVISION 0

\begin{tabular}{|c|c|c|c|c|c|c|c|c|c|c|c|c|c|c|c|}
\hline \multirow{3}{*}{$\begin{array}{l}\text { TR-01523 } \\
\text { Temperature } \\
{ }^{\circ} \mathrm{C}\end{array}$} & \multicolumn{2}{|c|}{ Thermocouples } & \multicolumn{2}{|l|}{ TR-01505 } & \multicolumn{3}{|c|}{ TR-01509 } & \multicolumn{4}{|c|}{ TR-01544 } & \multicolumn{3}{|c|}{ TR-01525 } & \multirow{3}{*}{$\begin{array}{l}\text { Channel } 4 \\
\mathrm{~T} \text {, reading }\end{array}$} \\
\hline & \multirow{2}{*}{$\begin{array}{l}\text { Channel } 0 \\
\mathrm{~T} \text {, applied }\end{array}$} & \multirow{2}{*}{$\begin{array}{l}\text { Channel } 0 \\
\mathrm{~T} \text {, reading }\end{array}$} & \multirow{2}{*}{$\begin{array}{l}\text { Temperature } \\
{ }^{\circ} \mathrm{C}\end{array}$} & \multirow{2}{*}{$\begin{array}{l}\text { Channel } 1 \\
\mathrm{~T} \text {, applied }\end{array}$} & \multirow{2}{*}{$\begin{array}{l}\text { Channel } 1 \\
\mathrm{~T} \text {, reading }\end{array}$} & \multirow{2}{*}{$\begin{array}{l}\text { Temperature } \\
{ }^{\circ} \mathrm{C}\end{array}$} & \multirow{2}{*}{$\begin{array}{l}\text { Channel } 2 \\
\text { T, applied }\end{array}$} & \multirow{2}{*}{$\begin{array}{l}\text { Channel } 2 \\
\mathrm{~T} \text {, reading }\end{array}$} & \multirow{2}{*}{$\begin{array}{l}\text { Temperature } \\
{ }^{\circ} \mathrm{C}\end{array}$} & \multirow{2}{*}{\multicolumn{2}{|c|}{$\begin{array}{l}\text { Channel } 3 \\
\mathrm{~T} \text {, applied }\end{array}$}} & \multirow{2}{*}{$\begin{array}{l}\text { Channel } 3 \\
\mathrm{~T} \text {, reading }\end{array}$} & \multirow{2}{*}{$\begin{array}{c}\text { Temperature } \\
{ }^{\circ} \mathrm{C}\end{array}$} & \multirow{2}{*}{$\begin{array}{l}\text { Channel } 4 \\
\mathrm{~T} \text {, applied }\end{array}$} & \\
\hline & & & & & & & & & & & & & & & \\
\hline 0 & 0 & -0.40 & 0 & 0 & -0.25 & 0 & 0 & -0.42 & & 0 & 0 & -0.48 & 0 & 0 & $\quad-0.18$ \\
\hline 25 & 25 & 24.80 & 25 & 25 & 24.89 & 25 & 25 & 24.76 & & 25 & 25 & 24.73 & 25 & 25 & 24.88 \\
\hline 50 & 50 & 49.99 & 50 & 50 & 50.04 & 50 & 50 & 49.94 & & 50 & 50 & 49.94 & 50 & 50 & 49.94 \\
\hline 75 & 75 & 75.18 & 75 & 75 & 75.19 & 75 & 75 & 75.12 & & 75 & 75 & 75.15 & 75 & 75 & 75.00 \\
\hline 100 & 100 & 100.38 & 100 & 100 & 100.34 & 100 & 100 & 100.29 & & 100 & 100 & 100.36 & 100 & 100 & 100.06 \\
\hline TR-01520 & & & TR-01514 & & & TR-01515 & & & TR-01519 & & & & & & \\
\hline Temperature & Channel 5 & Channel 5 & Temperature & Channel 6 & Channel 6 & Temperature & Channel 7 & Channel 7 & Temperature & & Channel 8 & Channel 8 & & & \\
\hline${ }^{\circ} \mathrm{C}$ & $\mathrm{T}$, applied & $\mathrm{T}$, reading & ${ }^{\circ} \mathrm{C}$ & $\mathrm{T}$, applied & $\mathrm{T}$, reading & ${ }^{\circ} \mathrm{C}$ & $\mathrm{T}$, applied & $\mathrm{T}$, reading & ${ }^{\circ} \mathrm{C}$ & & $\mathrm{T}$, applied & $\mathrm{T}$, reading & & & \\
\hline 0 & 0 & -0.20 & 0 & 0 & -0.43 & 0 & 0 & -0.58 & & 0 & 0 & -0.69 & & & \\
\hline 25 & 25 & 24.88 & 25 & 25 & 24.74 & 25 & 25 & 24.64 & & 25 & 25 & 24.54 & & & \\
\hline 50 & 50 & 49.96 & 50 & 50 & 49.91 & 50 & 50 & 49.87 & & 50 & 50 & 49.78 & & & \\
\hline 75 & 75 & 75.05 & 75 & 75 & 75.07 & 75 & 75 & 75.09 & & 75 & 75 & 75.01 & & & \\
\hline 100 & 100 & 100.13 & 100 & 100 & 100.24 & 100 & 100 & 100.32 & & 100 & 100 & 100.24 & & & \\
\hline $\begin{array}{l}\text { Current } \\
\mathrm{mA}\end{array}$ & $\begin{array}{l}\text { Channel } 10 \\
V \text {, meas. }\end{array}$ & $\begin{array}{l}\text { Channel } 10 \\
\text { V, calc. }\end{array}$ & $\begin{array}{l}\text { Current } \\
\mathrm{mA}\end{array}$ & $\begin{array}{l}\text { Channel } 11 \\
\text { V, meas. }\end{array}$ & $\begin{array}{l}\text { Channel } 11 \\
\text { V, calc. }\end{array}$ & $\begin{array}{l}\text { Current } \\
\mathrm{mA}\end{array}$ & $\begin{array}{l}\text { Channel } 12 \\
\mathrm{~V}, \text { meas. }\end{array}$ & $\begin{array}{l}\text { Channel } 12 \\
\text { V, calc. }\end{array}$ & $\begin{array}{l}\text { Current } \\
\mathrm{mA}\end{array}$ & & $\begin{array}{l}\text { Channel } 13 \\
\mathrm{~V}, \text { meas. }\end{array}$ & $\begin{array}{l}\text { Channel } 13 \\
\text { V, calc. }\end{array}$ & $\begin{array}{l}\text { Current } \\
\mathrm{mA}\end{array}$ & $\begin{array}{l}\text { Channel } 14 \\
\text { V, meas. }\end{array}$ & $\begin{array}{l}\text { Channel } 14 \\
\text { V, calc. }\end{array}$ \\
\hline 4 & 1.98 & 1.998 & 4 & 1.98 & 1.998 & 4 & 1.99 & 2.004 & & 4 & 1.99 & $\quad 1.998$ & 4 & 2.00 & $\quad 2.002$ \\
\hline 8 & 3.97 & 4.003 & 8 & 3.97 & 4.001 & 8 & 3.97 & 3.996 & & 8 & 3.99 & 4.004 & 8 & 3.99 & 3.997 \\
\hline 12 & 5.95 & 5.998 & 12 & 5.96 & 6.004 & 12 & 5.96 & 5.998 & & 12 & 5.98 & 6.000 & 12 & 5.99 & 6.002 \\
\hline 16 & 7.94 & 8.003 & 16 & 7.94 & 7.997 & 16 & 7.95 & 8.000 & & 16 & 7.97 & 7.996 & 16 & 7.98 & 7.997 \\
\hline 20 & 9.92 & 9.998 & 20 & 9.93 & 10.000 & 20 & 9.94 & 10.002 & & 20 & 9.97 & 10.002 & 20 & 9.98 & 10.002 \\
\hline $\begin{array}{l}\text { Current } \\
\mathrm{mA}\end{array}$ & $\begin{array}{l}\text { Channel } 15 \\
\vee \text {, meas. }\end{array}$ & $\begin{array}{l}\text { Channel } 15 \\
\text { V, calc. }\end{array}$ & $\begin{array}{l}\text { Current } \\
\mathrm{mA}\end{array}$ & $\begin{array}{l}\text { Channel } 16 \\
\mathrm{~V} \text {, meas. }\end{array}$ & $\begin{array}{l}\text { Channel } 16 \\
\text { V, calc. }\end{array}$ & $\begin{array}{l}\text { Current } \\
\mathrm{mA}\end{array}$ & $\begin{array}{l}\text { Channel } 17 \\
\mathrm{~V} \text {, meas. }\end{array}$ & $\begin{array}{l}\text { Channel } 17 \\
\text { V, calc. }\end{array}$ & $\begin{array}{l}\text { Current } \\
\mathrm{mA}\end{array}$ & & $\begin{array}{l}\text { Channel } 18 \\
\mathrm{~V}, \text { meas. }\end{array}$ & $\begin{array}{l}\text { Channel } 18 \\
\text { V, calc. }\end{array}$ & $\begin{array}{l}\text { Current } \\
\mathrm{mA}\end{array}$ & $\begin{array}{l}\text { Channel } 19 \\
\text { V, meas. }\end{array}$ & $\begin{array}{l}\text { Channel } 19 \\
\text { V, calc. }\end{array}$ \\
\hline 4 & 1.99 & $\quad 2.000$ & 4 & 1.98 & 2.002 & (1) & 1.99 & 2.002 & & 4 & 1.99 & 1.998 & 3 & 2.00 & $\quad 2.002$ \\
\hline 8 & 3.98 & 4.000 & 8 & 3.96 & 3.999 & 8 & 3.98 & 4.000 & & 8 & 3.99 & 4.004 & 8 & 3.99 & 3.999 \\
\hline 12 & 5.97 & 6.000 & 12 & 5.94 & 5.996 & 12 & 5.97 & 5.998 & & 12 & 5.98 & 6.000 & 12 & 5.98 & 5.996 \\
\hline 16 & 7.96 & 8.000 & 16 & 7.93 & 8.003 & 16 & 7.96 & 7.996 & & 16 & 7.97 & 7.996 & 16 & 7.98 & 8.003 \\
\hline 20 & 9.95 & 10.000 & 20 & 9.91 & 10.000 & 20 & 9.96 & 10.004 & & 20 & 9.97 & 10.002 & 20 & 9.97 & 10.000 \\
\hline $\begin{array}{l}\text { Current } \\
\mathrm{mA}\end{array}$ & $\begin{array}{l}\text { Channel } 20 \\
\mathrm{~V} \text {, meas. }\end{array}$ & $\begin{array}{l}\text { Channel } 20 \\
\text { V, calc. }\end{array}$ & $\begin{array}{l}\text { Current } \\
\mathrm{mA}\end{array}$ & $\begin{array}{l}\text { Channel } 21 \\
\mathrm{~V}, \text { meas. }\end{array}$ & $\begin{array}{l}\text { Channel } 21 \\
\text { V, calc. }\end{array}$ & $\begin{array}{l}\text { Current } \\
\mathrm{mA}\end{array}$ & $\begin{array}{l}\text { Channel } 22 \\
\text { V, meas. }\end{array}$ & $\begin{array}{l}\text { Channel } 22 \\
\text { V, calc. }\end{array}$ & $\begin{array}{l}\text { Current } \\
\mathrm{mA}\end{array}$ & & $\begin{array}{l}\text { Channel } 23 \\
\mathrm{~V} \text {, meas. }\end{array}$ & $\begin{array}{l}\text { Channel } 23 \\
\text { V, calc. }\end{array}$ & & & \\
\hline 4 & 1.99 & 1.994 & 4 & 2.00 & 2.002 & 4 & 2.00 & $\quad 1.998$ & & 4 & 2.00 & 1.998 & & & \\
\hline 8 & 4.00 & 4.007 & 8 & 3.99 & 3.999 & 8 & 4.00 & 4.001 & & 8 & 4.00 & 4.001 & & & \\
\hline 12 & 5.99 & 6.000 & 12 & 5.98 & 5.996 & 12 & 6.00 & 6.004 & & 12 & 6.00 & 6.004 & & & \\
\hline 16 & 7.99 & 8.003 & 16 & 7.98 & 8.003 & 16 & 7.99 & 7.997 & & 16 & 7.99 & 7.997 & & & \\
\hline 20 & 9.98 & 9.996 & 20 & 9.97 & 10.000 & 20 & 9.99 & 10.000 & & 20 & 9.99 & 10.000 & & & \\
\hline
\end{tabular}

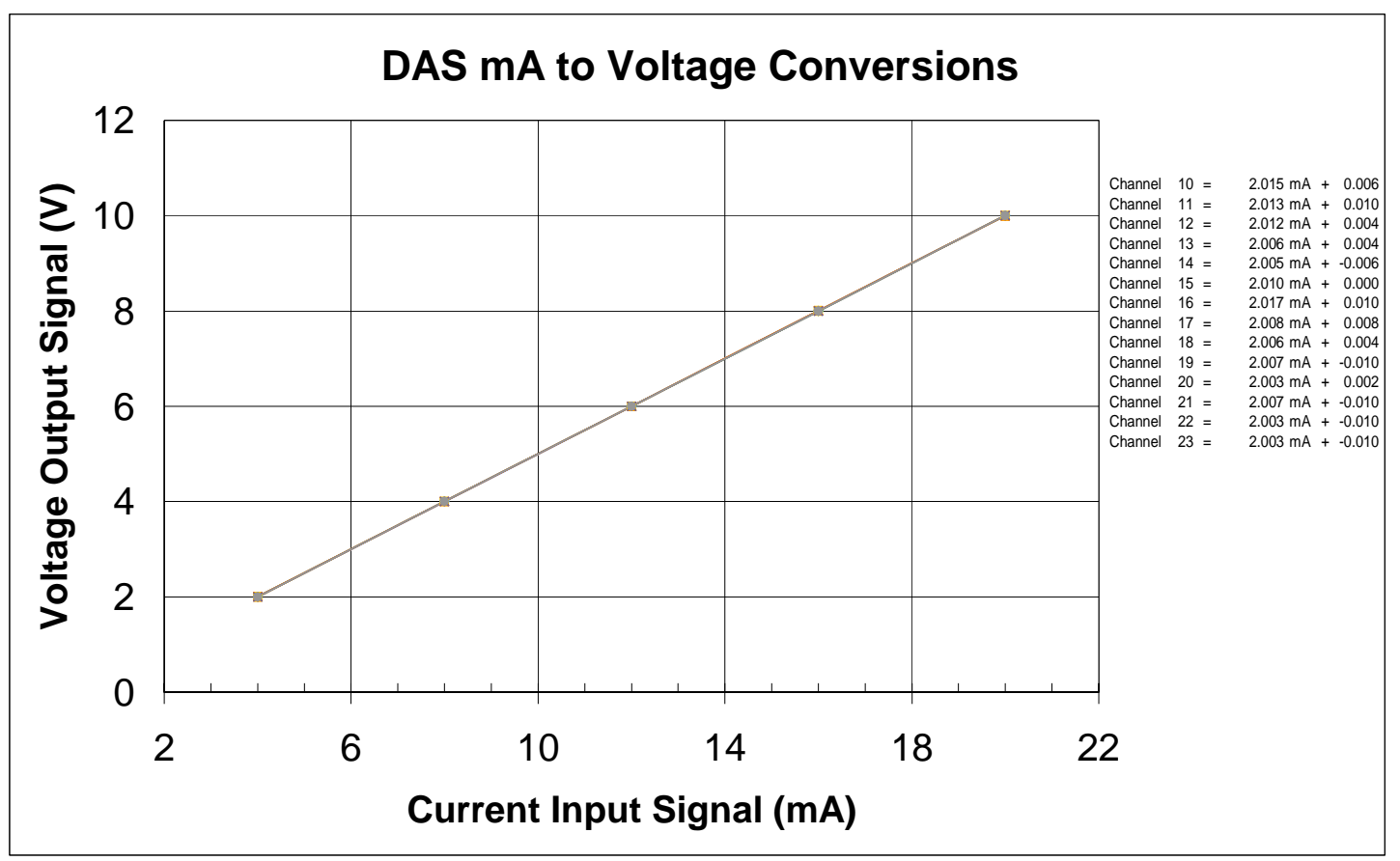

Figure G1-2. Transfer Functions for FEP and TLP Instrumentation 
WSRC-TR-2005-00105, REVISION 0

SRNL-RPP-2005-00012, REVISION 0

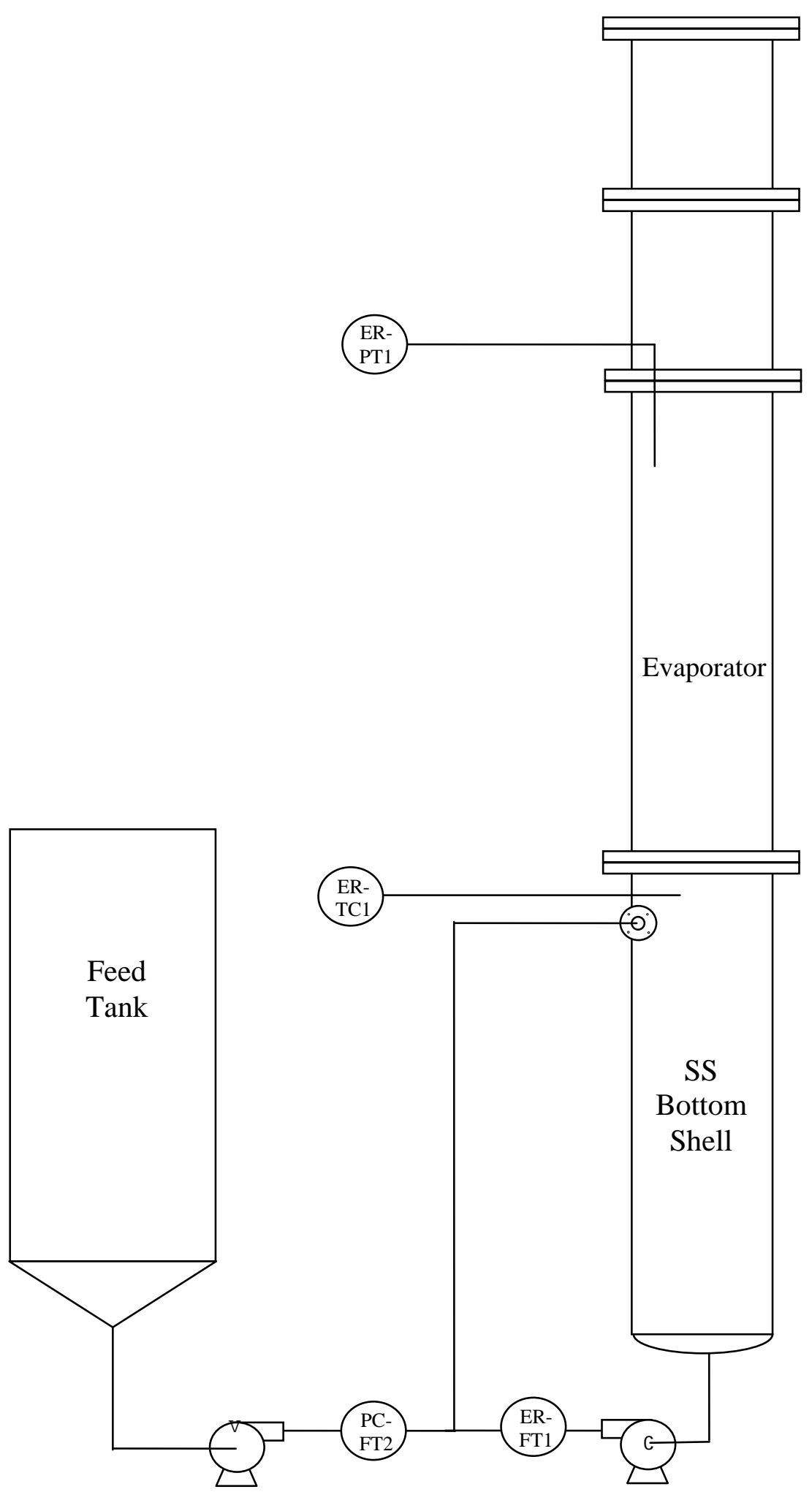

Figure G1-3. Critical Instruments Location Schematic 
WSRC-TR-2005-00105, REVISION 0

SRNL-RPP-2005-00012, REVISION 0

UNCERTAINTY ANALYSIS REF. L9.5-9114
TR-01520

Calibration Data

\begin{tabular}{|c|c|c|c|c|c|c|}
\hline Temperature & Standard & Voltage & $\begin{array}{l}\text { Calculated } \\
\text { Temperature }\end{array}$ & & Curve & Curve Fit \\
\hline Medium & $\begin{array}{c}\text { Temperature } \\
\text { (C) }\end{array}$ & $\begin{array}{l}\text { Output } \\
\text { (mV) }\end{array}$ & $\begin{array}{l}\text { (eq. 1) } \\
\text { (C) }\end{array}$ & $\begin{array}{l}\text { Error } \\
\text { (C) }\end{array}$ & $\begin{array}{l}\text { Fit } \\
\text { (C) }\end{array}$ & $\begin{array}{c}\text { Error } \\
\text { (C) }\end{array}$ \\
\hline Ice Point & 0.00 & -0.012 & -0.20 & -0.2 & -0.30 & -0.10 \\
\hline Room Temp & 20.92 & 1.230 & 20.64 & -0.3 & 20.72 & 0.08 \\
\hline Boiling Water* & 100.40 & 6.357 & 100.56 & 0.2 & 100.56 & 0.00 \\
\hline Boiling Water* & 100.40 & 6.356 & 100.55 & 0.1 & 100.56 & 0.01 \\
\hline Room Temp & 21.21 & 1.243 & 20.85 & -0.4 & 21.01 & 0.16 \\
\hline Ice Point & 0.00 & -0.013 & -0.22 & -0.2 & -0.30 & -0.08 \\
\hline Ice Point & 0.00 & -0.013 & -0.22 & -0.2 & -0.30 & -0.08 \\
\hline Room Temp & 21.21 & 1.242 & 20.83 & -0.4 & 21.01 & 0.18 \\
\hline Boiling Water* & 100.40 & 6.360 & 100.61 & 0.2 & 100.56 & -0.05 \\
\hline Boiling Water* & 100.40 & 6.360 & 100.61 & 0.2 & 100.56 & -0.05 \\
\hline Room Temp & 21.36 & 1.262 & 21.16 & -0.2 & 21.16 & 0.00 \\
\hline Ice Point & 0.00 & -0.013 & -0.22 & -0.2 & -0.30 & -0.08 \\
\hline
\end{tabular}

page calibration date $^{\text {of }}$

\begin{tabular}{|c|c|c|c|c|c|c|}
\hline \multicolumn{7}{|c|}{ Calculated } \\
\hline Temperature & Standard & Voltage & Temperature & & Curve & Curve Fit \\
\hline Medium & $\begin{array}{c}\text { Temperature } \\
\text { (C) }\end{array}$ & $\begin{array}{l}\text { Output } \\
\text { (mV) }\end{array}$ & $\begin{array}{l}\text { (eq. 1) } \\
\text { (C) }\end{array}$ & $\begin{array}{c}\text { Error } \\
\text { (C) }\end{array}$ & $\begin{array}{l}\text { Fit } \\
\text { (C) }\end{array}$ & $\begin{array}{l}\text { Error } \\
\text { (C) }\end{array}$ \\
\hline Ice Point & 0.00 & -0.012 & -0.20 & -0.2 & -0.30 & -0.10 \\
\hline Room Temp & 20.92 & 1.230 & 20.64 & -0.3 & 20.72 & 0.08 \\
\hline Boiling Water* & 100.40 & 6.357 & 100.56 & 0.2 & 100.56 & 0.00 \\
\hline Boiling Water* & 100.40 & 6.356 & 100.55 & 0.1 & 100.56 & 0.01 \\
\hline Room Temp & 21.21 & 1.243 & 20.85 & -0.4 & 21.01 & 0.16 \\
\hline Ice Point & 0.00 & -0.013 & -0.22 & -0.2 & -0.30 & -0.08 \\
\hline Ice Point & 0.00 & -0.013 & -0.22 & -0.2 & -0.30 & -0.08 \\
\hline Room Temp & 21.21 & 1.242 & 20.83 & -0.4 & 21.01 & 0.18 \\
\hline Boiling Water* & 100.40 & 6.360 & 100.61 & 0.2 & 100.56 & -0.05 \\
\hline Boiling Water* & 100.40 & 6.360 & 100.61 & 0.2 & 100.56 & -0.05 \\
\hline Room Temp & 21.36 & 1.262 & 21.16 & -0.2 & 21.16 & 0.00 \\
\hline Ice Point & 0.00 & -0.013 & -0.22 & -0.2 & -0.30 & -0.08 \\
\hline
\end{tabular}

* Temperature derived from 1967 ASME Steam Tables

$\mathrm{T}(\mathrm{C})=0.00483+17.040918^{\star} \mathrm{mV}-0.224284^{\star} \mathrm{mV}^{\wedge} 2+0.005038^{\star} \mathrm{mV} \mathrm{V}^{\wedge} 3$ (eq. 1$)$

(Limited Curve Fit, 0-100 C. From N.I.S.T. Reference Tables)

Uncertainty of the Standards:

Temperature Curve Fit: $+/-0.010 \quad \mathrm{C}$

Thermometer: $+/-\quad 0.40 \quad \mathrm{C}$

Ice Bath: $+/-\quad 0.10 \quad C$

Multimeter: $+/-(0.0045 \%$ RDG $+0.0400 \mathrm{mV})$ $=+/-\quad 0.063 \quad \mathrm{C} @ \quad 6.36 \mathrm{mV}$

Accepted Tolerance: +/- $\quad 1.7 \quad \mathrm{C}$

Statistical Info.

\begin{tabular}{|c|c|c|c|c|c|c|c|}
\hline$a$ & $b$ & $\mathrm{n}$ & $T$ & $\begin{array}{l}\text { Xbar } \\
\text { (C) }\end{array}$ & $\begin{array}{c}\text { Sxx } \\
\left(C^{\wedge} 2\right)\end{array}$ & $\begin{array}{l}\text { SEE } \\
\left(C^{\wedge} 2\right)\end{array}$ & $\begin{array}{l}\text { MSE } \\
\left(C^{\wedge} 2\right)\end{array}$ \\
\hline-0.30 & 1.00 & 12.00 & 2.179 & 33.02 & 12776.5 & 0.086 & 0.0086 \\
\hline
\end{tabular}

Calculated Uncertainties:

$\begin{array}{llll}\sigma c & \sigma E & \sigma F & \sigma T \\ (\mathrm{C}) & (\mathrm{C}) & (\mathrm{C}) & (\mathrm{C}) \\ 0.41 & 0.24 & 0.29 & 0.77\end{array}$

PASS CALIBRATION? YES

Figure G1-4. Evaporator Temperature Thermocouple Post-test Calibration (ER-TC1) 
WSRC-TR-2005-00105, REVISION 0

SRNL-RPP-2005-00012, REVISION 0

UNCERTAINTY ANALYSIS

REF. L9.5-9114, REV. 1

Calibration Data

$\begin{array}{llll}\begin{array}{l}\text { Meter } \\ \text { Output } \\ \text { (mA) }\end{array} & \begin{array}{l}\text { Water } \\ \text { Temp } \\ \text { (C) }\end{array} & \begin{array}{l}\text { Water } \\ \text { Weight } \\ \text { (lbs) }\end{array} & \begin{array}{l}\text { Time } \\ \text { Duration } \\ \text { (min) }\end{array} \\ 1.20 & 20.11 & 824.0 & 5.00 \\ 10.40 & 20.75 & 988.0 & 3.00 \\ 13.60 & 20.52 & 1479.0 & 3.00 \\ 16.80 & 21.19 & 1977.0 & 3.00 \\ 20.00 & 20.45 & 2461.0 & 3.00 \\ 7.20 & 20.08 & 825.0 & 5.04 \\ 10.40 & 20.16 & 983.0 & 3.00 \\ 13.60 & 20.21 & 1480.0 & 3.00 \\ 16.80 & 20.24 & 1970.0 & 3.00 \\ 20.00 & 20.40 & 2474.0 & 3.00 \\ 7.24 & 20.12 & 829.0 & 5.00 \\ 10.45 & 20.19 & 990.0 & 3.00 \\ 13.62 & 20.22 & 1478.0 & 3.00 \\ 16.85 & 20.32 & 1976.0 & 3.00 \\ 20.04 & 20.66 & 2472.0 & 3.00\end{array}$

TR-03278

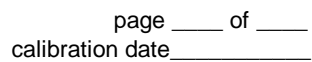

Calculated Data

$\begin{array}{llllc}\begin{array}{l}\text { Mass } \\ \text { Flow } \\ (\mathrm{lbs} / \mathrm{hr})\end{array} & \begin{array}{l}\text { Water } \\ \text { Density } \\ \left(\mathrm{lb} / \mathrm{ft}^{\wedge} 3\right)\end{array} & \begin{array}{l}\text { Volume } \\ \text { Flow } \\ \left(\mathrm{ft}^{\wedge} 3 / \mathrm{m}\right)\end{array} & \begin{array}{l}\text { Volume } \\ \text { Flow } \\ (\mathrm{GPM})\end{array} & \begin{array}{c}\text { Curve Fit } \\ (\mathrm{mA})\end{array} \\ 164.7 & 62.31 & 2.64 & 19.77 & 7.22 \\ 328.9 & 62.30 & 5.28 & 39.49 & 10.42 \\ 492.6 & 62.30 & 7.91 & 59.14 & 13.60 \\ 658.5 & 62.29 & 10.57 & 79.08 & 16.83 \\ 819.8 & 62.30 & 13.16 & 98.43 & 19.97 \\ 163.6 & 62.31 & 2.63 & 19.64 & 7.20 \\ 327.6 & 62.30 & 5.26 & 39.33 & 10.39 \\ 492.9 & 62.30 & 7.91 & 59.18 & 13.61 \\ 656.6 & 62.30 & 10.54 & 78.84 & 16.79 \\ 823.7 & 62.30 & 13.22 & 98.90 & 20.05 \\ 165.7 & 62.30 & 2.66 & 19.89 & 7.24 \\ 329.5 & 62.30 & 5.29 & 39.57 & 10.43 \\ 492.3 & 62.30 & 7.90 & 59.10 & 13.60 \\ 658.0 & 62.30 & 10.56 & 79.01 & 16.82 \\ 823.0 & 62.30 & 13.21 & 98.82 & 20.03\end{array}$

Density: $\rho=62.441-1.374 \mathrm{E}-3^{\star} \mathrm{T}-271.818 \mathrm{E}-6^{*} \mathrm{~T}^{\wedge} 2+194.093 \mathrm{E}-9^{*} \mathrm{~T}^{\wedge} 3$

7.4805 gallons $=1 \mathrm{ft}^{\wedge} 3$
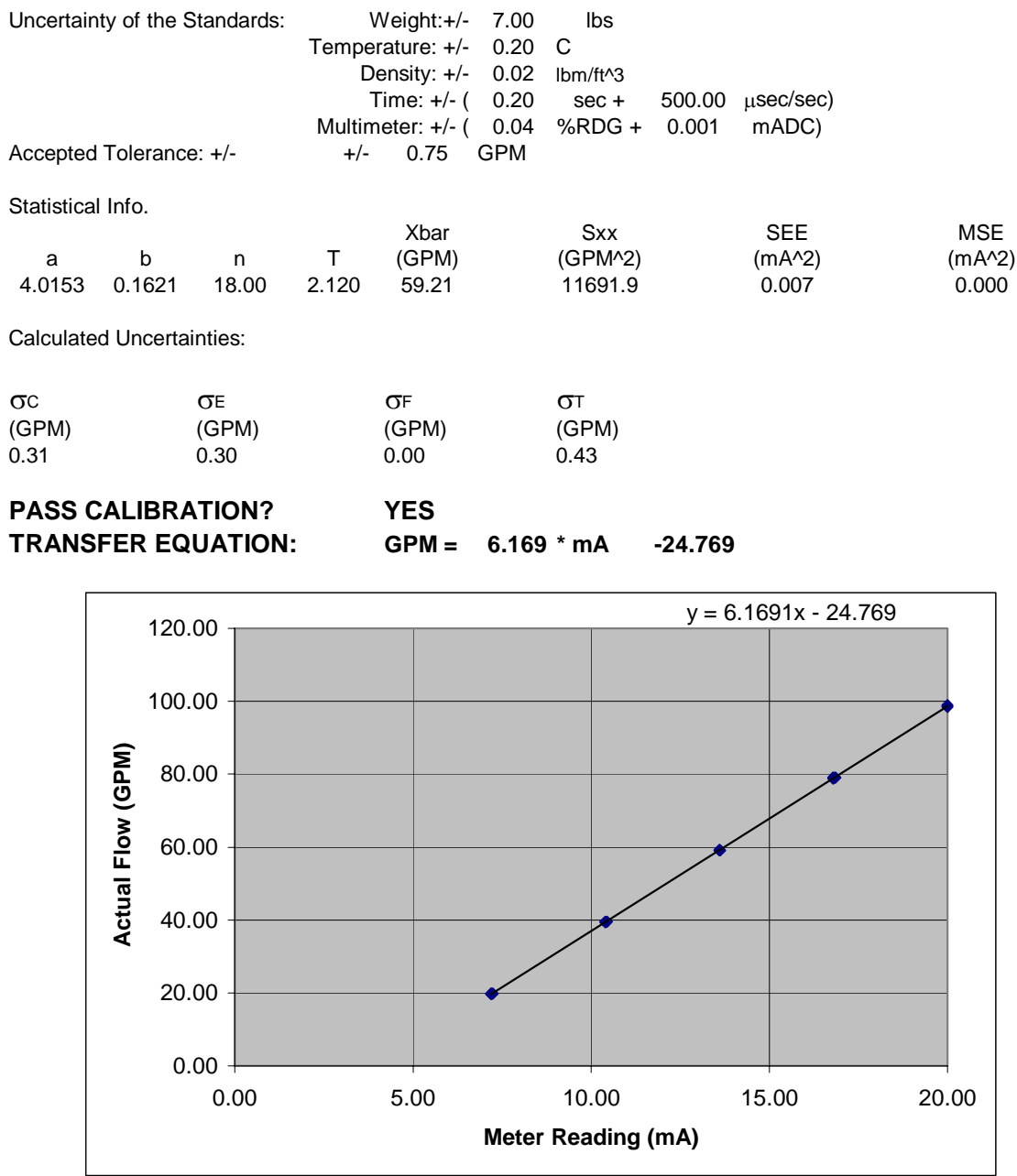

Figure G1-5. Evaporator Recirculation Pump Post-test Calibration (ER-FT1) 
WSRC-TR-2005-00105, REVISION 0 SRNL-RPP-2005-00012, REVISION 0

\begin{tabular}{|c|c|c|c|c|c|c|c|c|c|c|}
\hline $\begin{array}{l}\text { Output } \\
\text { (mA) }\end{array}$ & $\begin{array}{l}\text { Temp } \\
\text { (C) }\end{array}$ & $\begin{array}{l}\text { Weight } \\
\text { (lbs) }\end{array}$ & $\begin{array}{l}\text { Duration } \\
\text { (min) }\end{array}$ & $\begin{array}{l}\text { Flow } \\
\text { (lbs/hr) }\end{array}$ & $\begin{array}{l}\text { Density } \\
\left(\mathrm{lb} / \mathrm{ft}^{\wedge} 3\right)\end{array}$ & $\begin{array}{l}\text { Flow } \\
\left(\mathrm{ft}^{\wedge} 3 / \mathrm{m}\right)\end{array}$ & $\begin{array}{l}\text { Flow } \\
\text { (GPM) }\end{array}$ & $\begin{array}{l}\text { Curve Fit } \\
(\mathrm{mA})\end{array}$ & $\begin{array}{l}\text { Error } \\
(\mathrm{mA})\end{array}$ & $\begin{array}{l}\text { Error } \\
\text { (GPM) }\end{array}$ \\
\hline 7.24 & 19.37 & 20.7 & 12.00 & 1.7 & 62.31 & 0.03 & 0.21 & 7.26 & 0.02 & 0.001 \\
\hline 10.44 & 20.15 & 20.5 & 6.00 & 3.4 & 62.30 & 0.05 & 0.41 & 10.46 & 0.02 & 0.001 \\
\hline 13.64 & 20.88 & 25.5 & 5.00 & 5.1 & 62.30 & 0.08 & 0.61 & 13.64 & 0.00 & 0.000 \\
\hline 16.84 & 21.13 & 27.1 & 4.00 & 6.8 & 62.29 & 0.11 & 0.81 & 16.83 & -0.01 & -0.001 \\
\hline 20.03 & 21.16 & 25.5 & 3.00 & 8.5 & 62.29 & 0.14 & 1.02 & 20.06 & 0.03 & 0.002 \\
\hline 7.24 & 21.40 & 20.6 & 12.00 & 1.7 & 62.29 & 0.03 & 0.21 & 7.24 & 0.00 & 0.000 \\
\hline 10.46 & 21.32 & 20.5 & 6.00 & 3.4 & 62.29 & 0.05 & 0.41 & 10.46 & 0.00 & 0.000 \\
\hline 13.62 & 21.09 & 25.4 & 5.00 & 5.1 & 62.29 & 0.08 & 0.61 & 13.61 & -0.01 & 0.000 \\
\hline 16.84 & 20.38 & 27.2 & 4.01 & 6.8 & 62.30 & 0.11 & 0.81 & 16.84 & 0.00 & 0.000 \\
\hline 20.03 & 21.75 & 25.4 & 3.00 & 8.5 & 62.28 & 0.14 & 1.02 & 20.04 & 0.01 & 0.001 \\
\hline 7.24 & 21.35 & 20.6 & 12.00 & 1.7 & 62.29 & 0.03 & 0.21 & 7.24 & 0.00 & 0.000 \\
\hline 10.46 & 21.20 & 20.5 & 6.00 & 3.4 & 62.29 & 0.05 & 0.41 & 10.45 & -0.01 & -0.001 \\
\hline 13.62 & 20.78 & 25.4 & 5.00 & 5.1 & 62.30 & 0.08 & 0.61 & 13.60 & -0.02 & -0.001 \\
\hline 16.83 & 20.02 & 27.1 & 4.00 & 6.8 & 62.31 & 0.11 & 0.81 & 16.81 & -0.02 & -0.001 \\
\hline 20.03 & 21.48 & 25.4 & 3.00 & 8.5 & 62.29 & 0.14 & 1.02 & 20.03 & 0.00 & 0.000 \\
\hline
\end{tabular}

Density: $\rho=62.441-1.374 \mathrm{E}-3^{\star} \mathrm{T}-271.818 \mathrm{E}-6^{\star} \mathrm{T}^{\wedge} 2+194.093 \mathrm{E}-9^{*} \mathrm{~T}^{\wedge} 3$ 7.4805 gallons $=1 \mathrm{ft}^{\wedge} 3$

Uncertainty of the Standards:

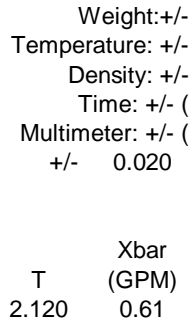

$\begin{array}{lllll}3.9868 & 15.7810 & 18.00 & 2.120 & 0.61\end{array}$

Calculated Uncertainties:

Accepted Tolerance: +/-

Statistical Info

\section{Ibs}

C

$\mathrm{lbm} / \mathrm{ft}^{\wedge} 3$

$\mathrm{sec}+500.00 \mu \mathrm{sec} / \mathrm{sec})$

$\% \mathrm{RDG}+0.001 \quad \mathrm{mADC})$

\begin{tabular}{|c|c|c|c|c|c|}
\hline $\mathrm{a}$ & b & $\mathrm{n}$ & $\mathrm{T}$ & $\begin{array}{l}\text { Xbar } \\
\text { (GPM) }\end{array}$ & $\begin{array}{r}\text { Sxx } \\
\left(\mathrm{GPM}^{\wedge}\right.\end{array}$ \\
\hline 3.9868 & 15.7810 & 18.00 & 2.120 & 0.61 & 1.2 \\
\hline \multicolumn{6}{|c|}{ Calculated Uncertainties: } \\
\hline$\sigma c$ & & $\sigma E$ & & $\sigma F$ & $\sigma \mathrm{T}$ \\
\hline (GPM) & & (GPM) & & (GPM) & (GPM) \\
\hline 0.01 & & 0.00 & & 0.00 & 0.0083 \\
\hline
\end{tabular}

\section{PASS CALIBRATION? YES \\ TRANSFER EQUATION: $\quad$ GPM $=\quad 0.063367 * \mathrm{~mA} \quad-0.25263$}

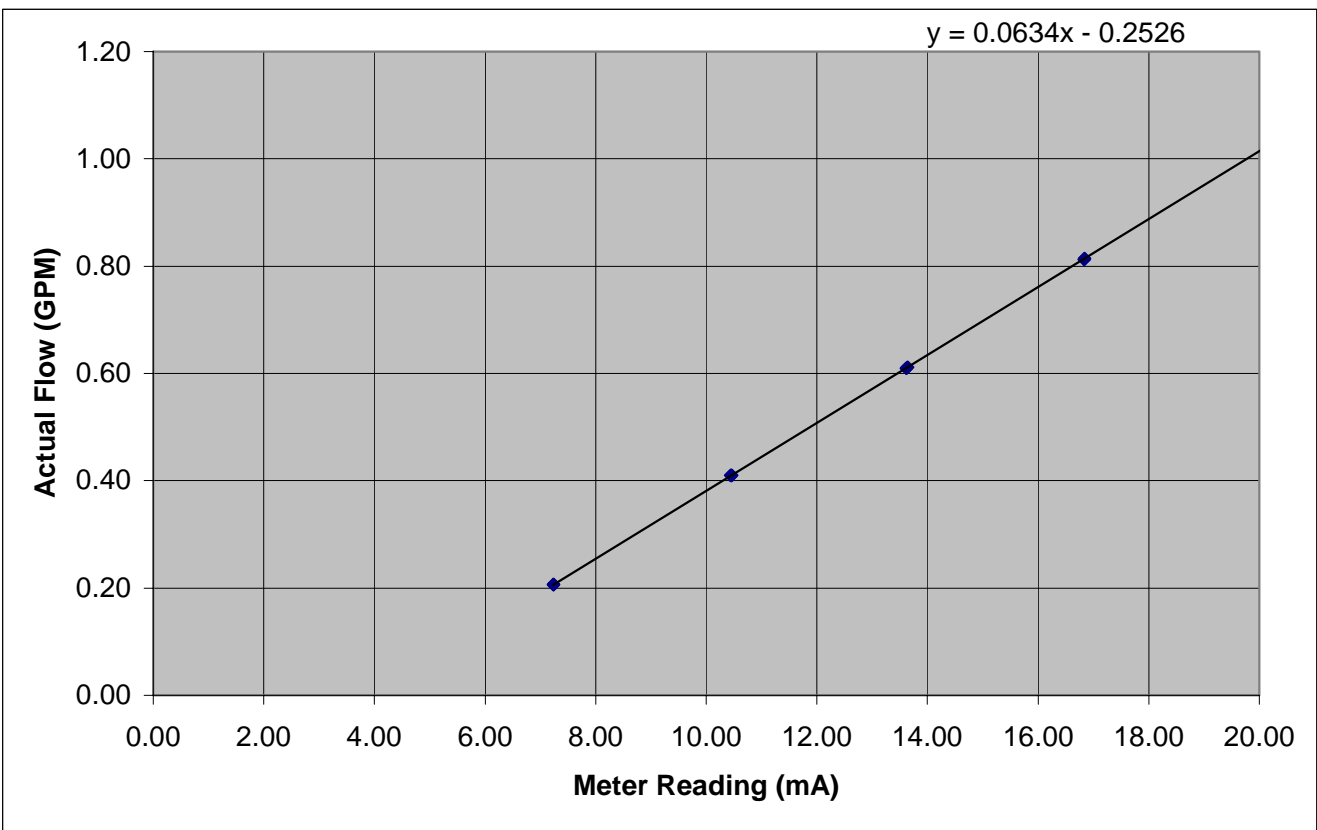

Figure G1-6. Evaporator Feed Pump Post-test Calibration (PC-FT2) 
WSRC-TR-2005-00105, REVISION 0

SRNL-RPP-2005-00012, REVISION 0

UNCERTAINTY ANALYSIS REF. L9.5-9114, REV. 1

$\begin{array}{ccc}\begin{array}{c}\text { Nominal } \\ \text { Pressure } \\ \text { (psia) }\end{array} & \begin{array}{c}\text { Applied } \\ \text { Pressure } \\ \text { (psia) }\end{array} & \begin{array}{c}\text { Gage } \\ \text { Reading } \\ \text { (mADC) }\end{array} \\ -14.5 & -14.5 & 20.03 \\ -9.5 & -9.5 & 14.51 \\ -4.5 & -4.5 & 9.00 \\ 0.0 & 0.0 & 4.04 \\ -14.5 & -14.5 & 20.03 \\ -9.5 & -9.5 & 14.51 \\ -4.5 & -4.5 & 9.00 \\ 0.0 & 0.0 & 4.04\end{array}$

TR-03554

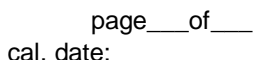

$\begin{array}{ccc}\begin{array}{c}\text { Curve } \\ \text { Fit } \\ (\mathrm{mADC})\end{array} & \begin{array}{c}\text { Error } \\ (\mathrm{mADC})\end{array} & \begin{array}{c}\text { Error } \\ (\text { psia })\end{array} \\ 20.03 & -0.003 & 0.00 \\ 14.51 & 0.004 & 0.00 \\ 9.00 & 0.000 & 0.00 \\ 4.04 & -0.002 & 0.00 \\ 20.03 & -0.003 & 0.00 \\ 14.51 & 0.004 & 0.00 \\ 9.00 & 0.000 & 0.00 \\ 4.04 & -0.002 & 0.00\end{array}$

cal. date:

\begin{tabular}{|c|c|c|c|c|c|c|}
\hline Standard Uncertainties: & & neter: +/- ( & 0.04 & $\%$ RDG + & 0.001 & mADC) \\
\hline & ad Wei & Tester: +/- & 0.04 & $\%$ RDG = & 0.000 & psi \\
\hline Statistical Info: & & & Xbar & Sxx & SEE & MSE \\
\hline $\mathrm{a}$ & $\mathrm{n}$ & $\mathrm{T}$ & psia & $\mathrm{psia}^{2}$ & $\mathrm{mADC} \mathrm{C}^{2}$ & $\mathrm{mADC}^{2}$ \\
\hline-1.1027 & 16.00 & 2.07 & -7.13 & 235.38 & 0.0001 & 0.0000 \\
\hline Calculated Uncertaintie & & & & & l Uncerta & \\
\hline$\sigma_{\mathrm{C}}$ & $\sigma_{\mathrm{E}}$ & & $\sigma_{\mathrm{F}}$ & & $\sigma_{\mathrm{T}}$ & \\
\hline psia & psia & & psia & & psia & \\
\hline 0.01 & 0.00 & & 0.00 & & 0.009 & \\
\hline Accepted Tolerance: + & & & & 0.1 & psia & \\
\hline PASS CALIBRATIC & & YES & & & & \\
\hline TRANSFER EQUA & ON: & PSIG= & -0.90688 & ${ }^{*} \mathrm{mADC}$ & 3.662 & \\
\hline
\end{tabular}

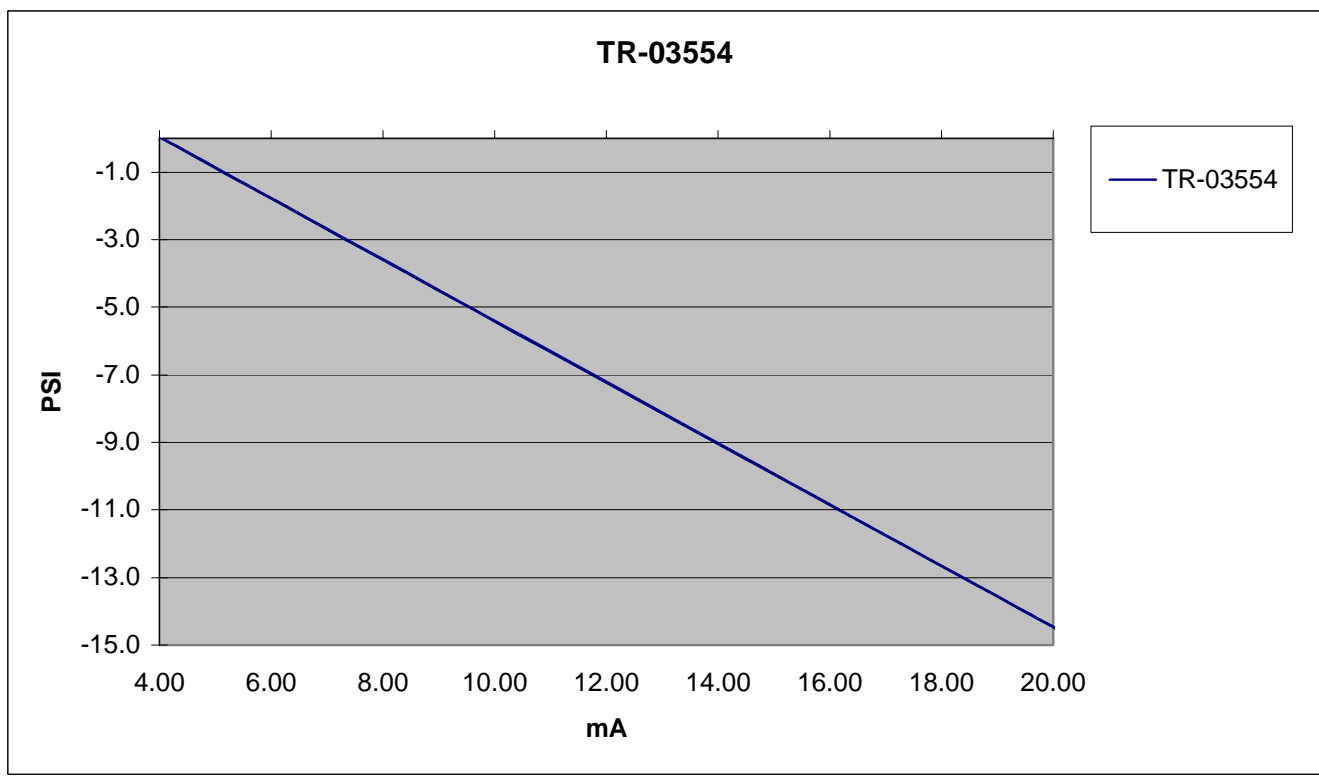

Figure G1-7. Evaporator Pot Gauge Pressure Transducer Post-test Calibration (ER-PT1) 
WSRC-TR-2005-00105, REVISION 0

SRNL-RPP-2005-00012, REVISION 0

\section{G2: UFP Instrumentation}


WSRC-TR-2005-00105, REVISION 0

SRNL-RPP-2005-00012, REVISION 0

\begin{tabular}{|c|c|c|c|c|c|c|}
\hline Channel & M\&TE \# & M\&TE Description & Instrumentation Type & Name & Range & $\begin{array}{c}\text { Tolerance } \\
( \pm)\end{array}$ \\
\hline 0 & TR-02927 & Permeate Loop Temperature & Type E Thermocouple & $\mathrm{T} 2$ & $0-100 \mathrm{C}$ & $1.7^{\circ} \mathrm{C}$ \\
\hline 1 & TR-02930 & Cleaning Loop Temperature & Type E Thermocouple & T3 & $0-100 \mathrm{C}$ & $1.7^{\circ} \mathrm{C}$ \\
\hline 2 & TR-02929 & Slurry Loop Temperature & Type E Thermocouple & $\mathrm{T} 1$ & $0-100 \mathrm{C}$ & $1.7^{\circ} \mathrm{C}$ \\
\hline 3 & TR-02925 & Atmospheric Temperature - upper & Type E Thermocouple & $\mathrm{T} 4$ & $0-100 \mathrm{C}$ & $1.7^{\circ} \mathrm{C}$ \\
\hline 4 & TR-02926 & Atmospheric Temperature - lower & Type E Thermocouple & T5 & $0-100 \mathrm{C}$ & $1.7^{\circ} \mathrm{C}$ \\
\hline 5 & Spare & & & & & \\
\hline 6 & TR-03553 & Transmembrane Pressure - upper flange & Rosemount Pressure Transmitter & $\mathrm{dP} 2$ & 0-101 psid & $0.5 \% \mathrm{FS}$ \\
\hline 7 & TR-02917 & Slurry Loop Pressure & Rosemount Pressure Transmitter & P1 & $0-100$ psig & $0.5 \% \mathrm{FS}$ \\
\hline 8 & TR-03495 & Filter Pressure Loss & Rosemount Pressure Transmitter & $\mathrm{dP} 1$ & $0-26$ psid & $0.5 \% \mathrm{FS}$ \\
\hline 9 & TR-03109 & Transmembrane Pressure - lower flange & Rosemount Pressure Transmitter & dP3 & $-11-101$ psid & $0.5 \% \mathrm{FS}$ \\
\hline 10 & TR-03115 & Permeate Loop Pressure & Rosemount Pressure Transmitter & $\mathrm{P} 2$ & 0-151 psig & $0.5 \%$ FS \\
\hline 11 & TR-00532 & Backpulse Pot Pressure & Rosemount Pr Transmitter & P3 & $0-151$ psig & $0.5 \%$ FS \\
\hline 12 & TR-20353 & Permeate Flow Rate - low range & Fischer-Porter Flow Transmitter & Q2 & $0-1.2 \mathrm{gpm}$ & $0.01 \mathrm{gpm}$ \\
\hline 13 & TR-20350 & Slurry Flow Rate & Fischer-Porter Flow Transmitter & Q1 & 0-100 gpm & $0.5 \mathrm{gpm}$ \\
\hline 14 & TR-03721 & Permeate Flow Rate - high range & Fischer-Porter Flow Transmitter & Q3 & $0-15$ gpm & $0.1 \mathrm{gpm}$ \\
\hline 15 & TR-03276 & Backpulse Flow Rate & Fischer-Porter Flow Transmitter & Q4 & $0-5$ gpm & $0.05 \mathrm{gpm}$ \\
\hline
\end{tabular}

\begin{tabular}{|c|c|c|c|c|c|c|c|c|c|}
\hline \multirow{2}{*}{ Channel } & \multirow{2}{*}{$\begin{array}{l}\text { DAS Transfer } \\
\text { Equation }^{1}\end{array}$} & \multicolumn{2}{|c|}{ DAS Uncertainty $( \pm)$} & \multirow{2}{*}{$\begin{array}{l}\text { M\&TE Transfer } \\
\text { Equation }\end{array}$} & \multicolumn{2}{|c|}{ M\&TE Uncertainty ( \pm ) } & \multirow{2}{*}{$\begin{array}{l}\text { Combined Transfer } \\
\text { Equation }\end{array}$} & \multicolumn{2}{|c|}{ Combined Uncertainty $( \pm)$} \\
\hline & & Pre-test & Post-test & & Pre-test & Post-test & & Pre-test & Post-test \\
\hline 0 & $\mathrm{~T}_{\text {in }}=0.998 * \mathrm{~T}_{\text {out }}+0.519$ & $0.14^{\circ} \mathrm{C}$ & $0.32{ }^{\circ} \mathrm{C}$ & Note 2 & $0.80^{\circ} \mathrm{C}$ & $0.63{ }^{\circ} \mathrm{C}$ & $\mathrm{T}_{\text {in }}=0.998 * \mathrm{~T}_{\text {out }}+0.519$ & $0.81^{\circ} \mathrm{C}$ & $0.71{ }^{\circ} \mathrm{C}$ \\
\hline 1 & $\mathrm{~T}_{\text {in }}=1.000 * \mathrm{~T}_{\text {out }}+0.480$ & $0.18^{\circ} \mathrm{C}$ & $0.32{ }^{\circ} \mathrm{C}$ & Note 2 & $0.80^{\circ} \mathrm{C}$ & $\mathrm{n} / \mathrm{a}$ & $\mathrm{T}_{\text {in }}=1.000 * \mathrm{~T}_{\text {out }}+0.408$ & $0.82{ }^{\circ} \mathrm{C}$ & $\mathrm{n} / \mathrm{a}$ \\
\hline 2 & $\mathrm{~T}_{\text {in }}=0.999 * \mathrm{~T}_{\text {out }}+0.520$ & $0.05^{\circ} \mathrm{C}$ & $0.14{ }^{\circ} \mathrm{C}$ & Note 2 & $0.90^{\circ} \mathrm{C}$ & $0.76{ }^{\circ} \mathrm{C}$ & $\mathrm{T}_{\text {in }}=0.999 * \mathrm{~T}_{\text {out }}+0.0520$ & $0.90^{\circ} \mathrm{C}$ & $0.77^{\circ} \mathrm{C}$ \\
\hline 3 & $\mathrm{~T}_{\text {in }}=0.999 * \mathrm{~T}_{\text {out }}+0.879$ & $0.06^{\circ} \mathrm{C}$ & $0.40^{\circ} \mathrm{C}$ & Note 2 & $0.90^{\circ} \mathrm{C}$ & $0.66^{\circ} \mathrm{C}$ & $\mathrm{T}_{\text {in }}=0.999 * \mathrm{~T}_{\text {out }}+0.879$ & $0.90^{\circ} \mathrm{C}$ & $0.77^{\circ} \mathrm{C}$ \\
\hline 4 & $\mathrm{~T}_{\text {in }}=0.997 * \mathrm{~T}_{\text {out }}+0.578$ & $0.09^{\circ} \mathrm{C}$ & $0.40^{\circ} \mathrm{C}$ & Note 2 & $0.70^{\circ} \mathrm{C}$ & $0.74{ }^{\circ} \mathrm{C}$ & $\mathrm{T}_{\text {in }}=0.997 * \mathrm{~T}_{\text {out }}+0.578$ & $0.71{ }^{\circ} \mathrm{C}$ & $0.84{ }^{\circ} \mathrm{C}$ \\
\hline 5 & Spare & & & & & & & & \\
\hline 6 & $\mathrm{~mA}=2.010 * \mathrm{~V}+0.000$ & $0.00 \mathrm{~mA}$ & $0.00 \mathrm{~mA}$ & psid=6.294*mA-24.988 & 0.10 psid & 0.12 psid & psid=12.652*V-24.988 & 0.10 psid & 0.12 psid \\
\hline 7 & $\mathrm{~mA}=2.007 * \mathrm{~V}-0.014$ & $0.03 \mathrm{~mA}$ & $0.02 \mathrm{~mA}$ & psig $=6.308 * \mathrm{~mA}-25.222$ & 0.13 psig & 0.12 psig & psig $=12.659 * V-25.145$ & 0.13 psig & 0.12 psig \\
\hline 8 & $\mathrm{~mA}=2.008 * \mathrm{~V}-0.004$ & $0.01 \mathrm{~mA}$ & $0.04 \mathrm{~mA}$ & psid=1.621*mA-6.462 & 0.05 psid & 0.04 psid & psid=3.255*V-6.469 & 0.05 psid & 0.05 psid \\
\hline 9 & $\mathrm{~mA}=2.004 * \mathrm{~V}-0.004$ & $0.01 \mathrm{~mA}$ & $0.06 \mathrm{~mA}$ & psid $=6.376 * \mathrm{~mA}-36.516$ & 0.12 psid & 0.17 psid & psid $=12.777 * V-36.547$ & $0.12 \mathrm{psid}$ & 0.18 psid \\
\hline 10 & $\mathrm{~mA}=2.010 * \mathrm{~V}-0.004$ & $0.02 \mathrm{~mA}$ & $0.04 \mathrm{~mA}$ & psig $=9.434 * m A-37.953$ & 0.50 psig & 0.40 psig & psig $=18.963 * V-37.991$ & $0.50 \mathrm{psig}$ & 0.40 psig \\
\hline 11 & $\mathrm{~mA}=2.007 * \mathrm{~V}-0.010$ & $0.01 \mathrm{~mA}$ & $0.02 \mathrm{~mA}$ & psig $=9.437 * m A-37.751$ & 0.20 psig & 0.21 psig & psig $=18.938 * V-37.846$ & 0.20 psig & 0.21 psig \\
\hline 12 & $\mathrm{~mA}=2.005 * \mathrm{~V}-0.014$ & $0.01 \mathrm{~mA}$ & $0.01 \mathrm{~mA}$ & gpm $=0.075 * \mathrm{~mA}-0.296$ & 0.005 gpm & $0.010 \mathrm{gpm}$ & gpm $=0.150 * V-0.297$ & $0.01 \mathrm{gpm}$ & $0.01 \mathrm{gpm}$ \\
\hline 13 & $\mathrm{~mA}=2.008 * \mathrm{~V}-0.004$ & $0.01 \mathrm{~mA}$ & $0.01 \mathrm{~mA}$ & gpm=6.267*mA-25.000 & $0.41 \mathrm{gpm}$ & $0.48 \mathrm{gpm}$ & gpm=12.584*V-25.025 & $0.41 \mathrm{gpm}$ & $0.48 \mathrm{gpm}$ \\
\hline 14 & $\mathrm{~mA}=2.005 * \mathrm{~V}-0.006$ & $0.01 \mathrm{~mA}$ & $0.04 \mathrm{~mA}$ & gpm=0.913*mA-3.659 & $0.05 \mathrm{gpm}$ & $0.08 \mathrm{gpm}$ & gpm=1.830*V-3.662 & $0.05 \mathrm{gpm}$ & $0.09 \mathrm{gpm}$ \\
\hline 15 & $\mathrm{~mA}=2.003 * \mathrm{~V}-0.006$ & $0.01 \mathrm{~mA}$ & $0.00 \mathrm{~mA}$ & gpm $=0.313 * \mathrm{~mA}-1.246$ & $0.014 \mathrm{gpm}$ & $0.013 \mathrm{gpm}$ & gpm $=0.627 * V 1.249$ & $0.02 \mathrm{gpm}$ & $0.01 \mathrm{gpm}$ \\
\hline
\end{tabular}

Figure G2-1. Entire UFP Instrumentation List 
WSRC-TR-2005-00105, REVISION 0

SRNL-RPP-2005-00012, REVISION 0

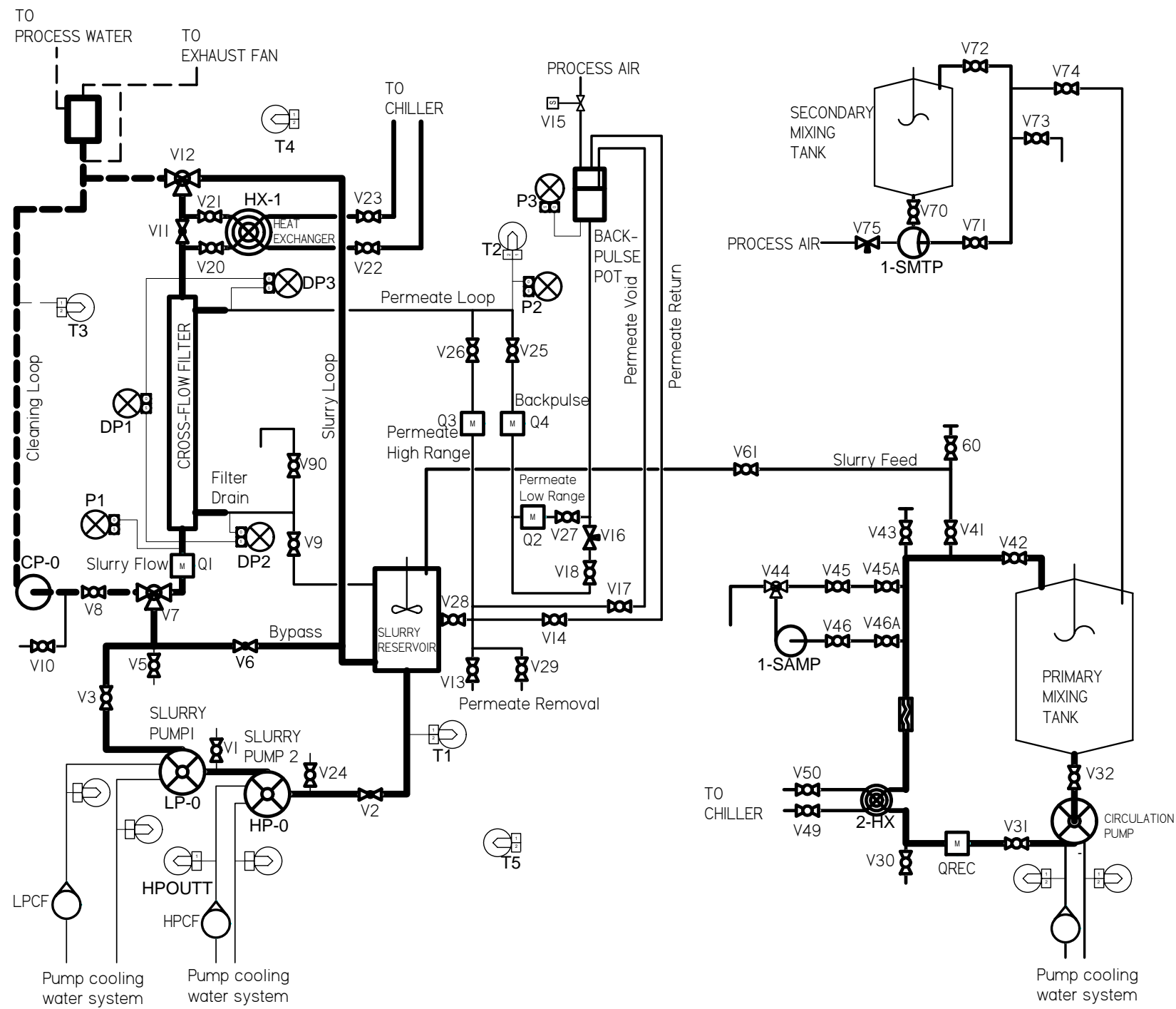

Figure G2-2. Instrument Location Schematic (shown from Section 2.7.1 for convenience) 
WSRC-TR-2005-00105, REVISION 0

SRNL-RPP-2005-00012, REVISION 0

UNCERTAINTY ANALYSIS

REF. L9.5-9114, REV. 1

TR-2929

\section{Calibration Data}

$\begin{array}{lcccc}\begin{array}{l}\text { Temperature } \\ \text { Medium }\end{array} & \begin{array}{c}\text { Standard } \\ \text { Temperature }\end{array} & \begin{array}{c}\text { Voltage } \\ \text { Output } \\ (\mathrm{mV})\end{array} & \begin{array}{c}\text { Calculated } \\ \text { Temperature } \\ (\text { eq. 1) }\end{array} & \begin{array}{c}\text { Error } \\ (\mathrm{C})\end{array} \\ \text { Ice Point } & \begin{array}{c}(\mathrm{C}) \\ \text { Room Temp }\end{array} & -0.035 & -0.59 & -0.59 \\ \text { Boiling Water } & 20.78 & 1.209 & 20.29 & -0.49 \\ \text { Boiling Water } & 100.30 & 6.360 & 100.61 & 0.31 \\ \text { Room Temp } & 100.30 & 6.359 & 100.59 & 0.29 \\ \text { Ice Point } & 20.91 & 1.212 & 20.34 & -0.57 \\ \text { Ice Point } & 0.00 & -0.036 & -0.61 & -0.61 \\ \text { Room Temp } & 0.00 & -0.036 & -0.61 & -0.61 \\ \text { Boiling Water } & 20.97 & 1.210 & 20.30 & -0.67 \\ \text { Boiling Water } & 100.30 & 6.368 & 100.73 & 0.43 \\ \text { Room Temp } & 100.30 & 6.365 & 100.68 & 0.38 \\ \text { Ice Point } & 21.06 & 1.221 & 20.49 & -0.57 \\ & 0.00 & -0.035 & -0.59 & -0.59\end{array}$

$\mathrm{T}(\mathrm{C})=0.00483+17.040918^{\star} \mathrm{mV}-0.224284^{*} \mathrm{mV}^{\wedge} 2+0.005038^{\star} \mathrm{mV} \mathrm{N}^{\wedge}$ (eq. 1 ) (Limited Curve Fit, 0-100 C. From N.I.S.T. Reference Tables)

Uncertainty of the Standards: Temperature Curve Fit: $+/-\quad 0.010$ C

Thermister: $+/-\quad 0.20 \quad \mathrm{C}$ Ice Bath: + $0.10 \quad \mathrm{C}$

Multimeter: $+/-(0.0045 \% \mathrm{RDG}+0.0040 \mathrm{mV})$

$=+/-0.063 \quad \mathrm{C} @ \quad 6.37 \mathrm{mV}$

Accepted Tolerance: +/- $\quad 1.70 \quad$ C

Statistical Info.

\begin{tabular}{|c|c|c|c|c|c|c|c|}
\hline$a$ & $\mathrm{~b}$ & $\mathrm{n}$ & $\mathrm{T}$ & $\begin{array}{l}\text { Xbar } \\
\text { (C) }\end{array}$ & $\begin{array}{c}\text { Sxx } \\
\left(C^{\wedge} 2\right)\end{array}$ & $\begin{array}{c}\text { SEE } \\
\left(C^{\wedge} 2\right)\end{array}$ & $\begin{array}{l}\text { MSE } \\
\left(C^{\wedge} 2\right)\end{array}$ \\
\hline-0.68 & 1.01 & 12.00 & 2.228 & 40.41 & 22397.0 & 0.100 & 0.0100 \\
\hline
\end{tabular}

Calculated Uncertainties:

$\begin{array}{llll}\sigma C & \sigma_{E} & \sigma_{F} & \sigma \mathrm{T} \\ (\mathrm{C}) & (\mathrm{C}) & (\mathrm{C}) & (\mathrm{C}) \\ 0.23 & 0.25 & 0.68 & 0.76\end{array}$

PASS CALIBRATION? YES

Figure G2-3. Slurry Loop Temperature [T1, Channel 2] - Post Calibration 
WSRC-TR-2005-00105, REVISION 0

SRNL-RPP-2005-00012, REVISION 0

UNCERTAINTY ANALYSIS

REF. L9.5-9114, REV. 1
TR-2927

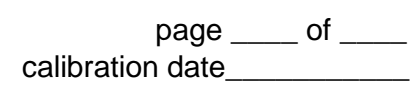

\section{Calibration Data}

$\begin{array}{lcccc}\begin{array}{l}\text { Temperature } \\ \text { Medium }\end{array} & \begin{array}{c}\text { Standard } \\ \text { Temperature }\end{array} & \begin{array}{c}\text { Voltage } \\ \text { Output } \\ (\mathrm{mV})\end{array} & \begin{array}{c}\text { Calculated } \\ \text { Temperature } \\ \text { (eq. 1) }\end{array} & \begin{array}{c}\text { Error } \\ (\mathrm{C})\end{array} \\ \text { Ice Point } & 0.00 & -0.028 & -0.47 & -0.47 \\ \text { Room Temp } & 20.78 & 1.215 & 20.39 & -0.39 \\ \text { Boiling Water } & 100.30 & 6.362 & 100.64 & 0.34 \\ \text { Boiling Water } & 100.30 & 6.360 & 100.61 & 0.31 \\ \text { Room Temp } & 20.91 & 1.222 & 20.50 & -0.41 \\ \text { Ice Point } & 0.00 & -0.028 & -0.47 & -0.47 \\ \text { Ice Point } & 0.00 & -0.028 & -0.47 & -0.47 \\ \text { Room Temp } & 20.97 & 1.217 & 20.42 & -0.55 \\ \text { Boiling Water } & 100.30 & 6.362 & 100.64 & 0.34 \\ \text { Boiling Water } & 100.30 & 6.362 & 100.64 & 0.34 \\ \text { Room Temp } & 21.06 & 1.226 & 20.57 & -0.49 \\ \text { Ice Point } & 0.00 & -0.027 & -0.46 & -0.46\end{array}$

$\mathrm{T}(\mathrm{C})=0.00483+17.040918^{\star} \mathrm{mV}-0.224284^{\star} \mathrm{mV}^{\wedge} 2+0.005038^{\star} \mathrm{mV} \mathrm{V}^{\wedge} 3 \quad$ (eq. 1$)$

(Limited Curve Fit, 0-100 C. From N.I.S.T. Reference Tables)

Uncertainty of the Standards: $\quad$ Temperature Curve Fit: $+/-\quad 0.010$ C

Thermister: $+/-\quad 0.20 \quad \mathrm{C}$

Ice Bath: + + - $0.10 \quad \mathrm{C}$

Multimeter: $+/-(0.0045 \%$ RDG $+0.0040 \mathrm{mV})$

$$
=+/-\quad 0.063 \quad \mathrm{C} @ \quad 6.36 \mathrm{mV}
$$

Accepted Tolerance: +/- $\quad 1.70 \quad$ C

Statistical Info.

\begin{tabular}{|c|c|c|c|c|c|c|c|}
\hline$a$ & b & $\mathrm{n}$ & $\mathrm{T}$ & $\begin{array}{l}\text { Xbar } \\
\text { (C) }\end{array}$ & $\begin{array}{c}S x x \\
\left(C^{\wedge} 2\right)\end{array}$ & $\begin{array}{c}\text { SEE } \\
\left(C^{\wedge} 2\right)\end{array}$ & $\begin{array}{l}\text { MSE } \\
\left(C^{\wedge} 2\right)\end{array}$ \\
\hline-0.54 & 1.01 & 12.00 & 2.228 & 40.41 & 22397.0 & 0.078 & 0.0078 \\
\hline
\end{tabular}

Calculated Uncertainties:

$\begin{array}{llll}\sigma_{C} & \sigma_{E} & \sigma_{F} & \sigma_{T} \\ (\mathrm{C}) & (\mathrm{C}) & (\mathrm{C}) & (\mathrm{C}) \\ 0.23 & 0.22 & 0.54 & 0.63\end{array}$

PASS CALIBRATION?

YES

Figure G2-4. Permeate Loop Temperature [T2, Channel 0] - Post Calibration 
WSRC-TR-2005-00105, REVISION 0

SRNL-RPP-2005-00012, REVISION 0

UNCERTAINTY ANALYSIS

REF. L9.5-9114, REV. 1

Calibration Data

$\begin{array}{llll}\begin{array}{l}\text { Meter } \\ \text { Output } \\ (\mathrm{mA})\end{array} & \begin{array}{l}\text { Water } \\ \text { Temp }\end{array} & \begin{array}{l}\text { Water } \\ \text { Weight }\end{array} & \begin{array}{l}\text { Time } \\ \text { (lbs) }\end{array} \\ \begin{array}{l}\text { Duration } \\ \text { (min) }\end{array} \\ 7.98 & 23.62 & 0 & 0.00 \\ 10.40 & 23.62 & 845 & 5.00 \\ 13.65 & 23.76 & 1001 & 3.01 \\ 16.82 & 23.58 & 1510 & 3.01 \\ 20.01 & 23.27 & 2510 & 3.00 \\ 3.98 & 23.20 & 0 & 0.00 \\ 7.22 & 23.20 & 842 & 5.01 \\ 10.46 & 23.10 & 1010 & 3.01 \\ 13.64 & 23.23 & 1508 & 3.01 \\ 16.82 & 23.67 & 2006 & 3.00 \\ 19.98 & 23.67 & 2501 & 3.00 \\ 4.00 & 23.26 & 0 & 0.00 \\ 7.22 & 23.26 & 842 & 5.01 \\ 10.45 & 23.19 & 1008 & 3.01 \\ 13.60 & 23.04 & 1501 & 3.00 \\ 16.80 & 23.77 & 2000 & 3.00 \\ 20.00 & 23.93 & 2505 & 3.00\end{array}$

TR-20350

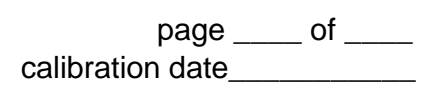

Calculated Data

\begin{tabular}{|c|c|c|c|c|}
\hline Mass & Water & Volume & Volume & \\
\hline $\begin{array}{l}\text { Flow } \\
\text { (lbs/hr) }\end{array}$ & $\begin{array}{l}\text { Density } \\
\left(\mathrm{lb} / \mathrm{ft}^{\wedge} 3\right)\end{array}$ & $\begin{array}{l}\text { Flow } \\
\left(\mathrm{ft}^{\wedge} 3 / \mathrm{m}\right)\end{array}$ & $\begin{array}{l}\text { Flow } \\
\text { (GPM) }\end{array}$ & $\begin{array}{l}\text { Curve Fit } \\
(\mathrm{mA})\end{array}$ \\
\hline 0.0 & 62.26 & 0.00 & 0.00 & 4.00 \\
\hline 168.9 & 62.26 & 2.71 & 20.29 & 7.24 \\
\hline 332.9 & 62.26 & 5.35 & 40.00 & 10.39 \\
\hline 502.2 & 62.25 & 8.07 & 60.35 & 13.64 \\
\hline 668.1 & 62.26 & 10.73 & 80.27 & 16.82 \\
\hline 835.4 & 62.26 & 13.42 & 100.37 & 20.03 \\
\hline 0.0 & 62.27 & 0.00 & 0.00 & 4.00 \\
\hline 168.1 & 62.27 & 2.70 & 20.20 & 7.23 \\
\hline 335.8 & 62.27 & 5.39 & 40.35 & 10.44 \\
\hline 501.2 & 62.26 & 8.05 & 60.22 & 13.62 \\
\hline 668.1 & 62.26 & 10.73 & 80.27 & 16.82 \\
\hline 832.6 & 62.26 & 13.37 & 100.04 & 19.98 \\
\hline 0.0 & 62.26 & 0.00 & 0.00 & 4.00 \\
\hline 168.2 & 62.26 & 2.70 & 20.20 & 7.23 \\
\hline 334.6 & 62.27 & 5.37 & 40.20 & 10.42 \\
\hline 500.1 & 62.27 & 8.03 & 60.07 & 13.60 \\
\hline 666.1 & 62.26 & 10.70 & 80.04 & 16.79 \\
\hline 834.4 & 62.26 & 13.40 & 100.27 & 20.02 \\
\hline
\end{tabular}

Density: $\rho=62.441-1.374 \mathrm{E}-3^{\star} \mathrm{T}-271.818 \mathrm{E}-6^{\star} \mathrm{T}^{\wedge} 2+194.093 \mathrm{E}-9^{\star} \mathrm{T}^{\wedge} 3$

7.4805 gallons $=1 \mathrm{ft}^{\wedge} 3$

Uncertainty of the Standards:

Weight:+/- 7.00

emperature: $+/-\quad 0.20$

Density: $+/-\quad 0.02$

Time: $+/-(0.20$

Multimeter: $+/-(0.04$

Accepted Tolerance: +/-

Statistical Info.

\begin{tabular}{|c|c|c|c|c|c|c|c|}
\hline $\mathrm{a}$ & b & $\mathrm{n}$ & $\mathrm{T}$ & $\begin{array}{l}\text { Xbar } \\
\text { (GPM) }\end{array}$ & $\begin{array}{c}\mathrm{Sxx} \\
\left(\mathrm{GPM}^{\wedge} 2\right)\end{array}$ & $\begin{array}{c}\text { SEE } \\
\left(m A^{\wedge} 2\right)\end{array}$ & $\begin{array}{c}\text { MSE } \\
\left(m A^{\wedge} 2\right)\end{array}$ \\
\hline 3.9975 & 0.1598 & 18.00 & 2.120 & 50.17 & 21062.8 & 0.004 & 0.000 \\
\hline
\end{tabular}

Calculated Uncertainties:

$\begin{array}{llll}\sigma c & \sigma_{E} & \sigma_{F} & \sigma T \\ (G P M) & (G P M) & (G P M) & (G P M) \\ 0.31 & 0.22 & 0.30 & 0.48\end{array}$

\section{PASS CALIBRATION? TRANSFER EQUATION: \\ YES}

$$
\text { GPM }=6.259 * \mathrm{~mA} \quad-25.018
$$

Figure G2-5. Slurry Flow Rate [Q1, Channel 13] - Post Calibration 
WSRC-TR-2005-00105, REVISION 0

SRNL-RPP-2005-00012, REVISION 0

Permeate Flow Rate - Low Range

UNCERTAINTY ANALYSIS

REF. L9.5-9114, REV. 1

Calibration Data

$\begin{array}{llll}\begin{array}{l}\text { Meter } \\ \text { Output } \\ (\mathrm{mA})\end{array} & \begin{array}{l}\text { Water } \\ \text { Temp }\end{array} & \begin{array}{l}\text { Water } \\ \text { Weight }\end{array} & \begin{array}{l}\text { Time } \\ \text { (lbs) }\end{array} \\ \begin{array}{l}\text { Duration } \\ \text { (min) }\end{array} \\ 7.00 & 22.25 & 0.00 & 0.00 \\ 10.41 & 22.25 & 25.05 & 12.01 \\ 13.65 & 22.95 & 24.26 & 6.00 \\ 16.84 & 23.32 & 24.16 & 4.00 \\ 20.10 & 22.09 & 30.13 & 3.00 \\ 4.00 & 21.98 & 0.00 & 0.00 \\ 7.21 & 21.98 & 24.66 & 12.01 \\ 10.42 & 22.59 & 24.32 & 6.01 \\ 13.63 & 24.14 & 24.11 & 4.00 \\ 16.86 & 23.01 & 24.12 & 3.00 \\ 19.96 & 22.20 & 29.90 & 3.00 \\ 4.00 & 22.12 & 0.00 & 0.00 \\ 7.20 & 22.12 & 24.54 & 12.00 \\ 10.47 & 22.47 & 24.48 & 6.00 \\ 13.65 & 23.91 & 24.19 & 4.01 \\ 16.86 & 22.82 & 24.15 & 3.01 \\ 20.00 & 22.44 & 30.00 & 3.00\end{array}$

TR-20353

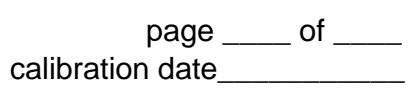

\section{Calculated Data}

\begin{tabular}{|c|c|c|c|c|}
\hline Mass & Water & Volume & Volume & \\
\hline $\begin{array}{l}\text { Flow } \\
\text { (lbs/hr) }\end{array}$ & $\begin{array}{l}\text { Density } \\
\left(\mathrm{lb} / \mathrm{ft}^{\wedge} 3\right)\end{array}$ & $\begin{array}{l}\text { Flow } \\
\left(\mathrm{ft}^{\wedge} 3 / \mathrm{m}\right)\end{array}$ & $\begin{array}{l}\text { Flow } \\
\text { (GPM) }\end{array}$ & $\begin{array}{l}\text { Curve Fit } \\
\qquad(\mathrm{mA})\end{array}$ \\
\hline 0.0 & 62.28 & 0.00 & 0.00 & 3.95 \\
\hline 2.1 & 62.28 & 0.03 & 0.25 & 7.30 \\
\hline 4.0 & 62.27 & 0.06 & 0.49 & 10.44 \\
\hline 6.0 & 62.25 & 0.10 & 0.73 & 13.65 \\
\hline 8.0 & 62.26 & 0.13 & 0.96 & 16.84 \\
\hline 10.0 & 62.28 & 0.16 & 1.21 & 20.07 \\
\hline 0.0 & 62.28 & 0.00 & 0.00 & 3.95 \\
\hline 2.1 & 62.28 & 0.03 & 0.25 & 7.25 \\
\hline 4.0 & 62.27 & 0.07 & 0.49 & 10.45 \\
\hline 6.0 & 62.25 & 0.10 & 0.72 & 13.62 \\
\hline 8.0 & 62.27 & 0.13 & 0.97 & 16.86 \\
\hline 10.0 & 62.28 & 0.16 & 1.20 & 19.94 \\
\hline 0.0 & 62.28 & 0.00 & 0.00 & 3.95 \\
\hline 2.0 & 62.28 & 0.03 & 0.25 & 7.24 \\
\hline 4.1 & 62.28 & 0.07 & 0.49 & 10.50 \\
\hline 6.0 & 62.26 & 0.10 & 0.73 & 13.65 \\
\hline 8.0 & 62.27 & 0.13 & 0.96 & 16.85 \\
\hline 10.0 & 62.28 & 0.16 & 1.20 & 20.00 \\
\hline
\end{tabular}

Density: $\rho=62.441-1.374 \mathrm{E}-3^{\star} \mathrm{T}-271.818 \mathrm{E}-6^{\star} \mathrm{T}^{\wedge} 2+194.093 \mathrm{E}-9^{\star} \mathrm{T}^{\wedge} 3$

7.4805 gallons $=1 \mathrm{ft}^{\wedge} 3$

Uncertainty of the Standards:

Weight: + /- 0.20

Temperature: $+/-\quad 0.20$

Density: $+/-\quad 0.02$

Time: $+/-(0.20$

Multimeter: $+/-(\quad 0.04$

Accepted Tolerance: +/-

Statistical Info.

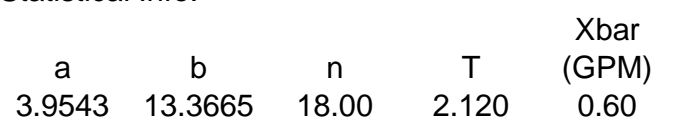

Calculated Uncertainties:

$\begin{array}{llll}\sigma c & \sigma_{E} & \sigma_{F} & \sigma T \\ (G P M) & (G P M) & (G P M) & (G P M) \\ 0.008 & 0.005 & 0.002 & 0.010\end{array}$

\section{PASS CALIBRATION? TRANSFER EQUATION:}

\section{YES}

$$
\text { GPM }=0.075 \text { * } \mathrm{mA}
$$

$-0.296$

Figure G2-6. Permeate Flow Rate - low range [Q2, Channel 11] - Post Calibration 
WSRC-TR-2005-00105, REVISION 0

SRNL-RPP-2005-00012, REVISION 0

UNCERTAINTY ANALYSIS

REF. L9.5-9114, REV. 1

Calibration Data

\begin{tabular}{|c|c|c|c|}
\hline Meter & Water & Water & Time \\
\hline $\begin{array}{l}\text { Output } \\
\text { (mA) }\end{array}$ & $\begin{array}{l}\text { Temp } \\
\text { (C) }\end{array}$ & $\begin{array}{l}\text { Weight } \\
\text { (lbs) }\end{array}$ & $\begin{array}{l}\text { Duration } \\
\text { (min) }\end{array}$ \\
\hline 4.00 & 24.55 & 0.0 & 0.00 \\
\hline 7.24 & 24.55 & 246.8 & 10.00 \\
\hline 10.45 & 24.88 & 245.8 & 5.00 \\
\hline 13.65 & 25.15 & 294.0 & 4.00 \\
\hline 16.83 & 25.25 & 292.8 & 3.00 \\
\hline 20.00 & 25.44 & 365.4 & 3.00 \\
\hline 4.00 & 24.74 & 0.0 & 0.00 \\
\hline 7.24 & 24.74 & 247.0 & 10.00 \\
\hline 10.44 & 25.05 & 245.4 & 5.00 \\
\hline 13.65 & 25.10 & 294.2 & 4.00 \\
\hline 16.84 & 25.41 & 293.4 & 3.00 \\
\hline 20.00 & 25.45 & 365.8 & 3.00 \\
\hline 4.00 & 24.27 & 0.0 & 0.00 \\
\hline 7.25 & 24.27 & 248.2 & 10.00 \\
\hline 10.43 & 25.56 & 245.0 & 5.00 \\
\hline 13.62 & 25.48 & 293.4 & 4.00 \\
\hline 16.84 & 25.18 & 293.6 & 3.00 \\
\hline 20.06 & 24.54 & 367.2 & 3.00 \\
\hline
\end{tabular}

TR-03721

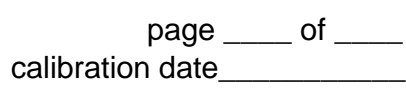

\section{Calculated Data}

\begin{tabular}{|c|c|c|c|c|}
\hline Mass & Water & Volume & Volume & \\
\hline $\begin{array}{l}\text { Flow } \\
\text { (lbs/hr) }\end{array}$ & $\begin{array}{l}\text { Density } \\
\left(\mathrm{lb} / \mathrm{ft}^{\wedge} 3\right)\end{array}$ & $\begin{array}{l}\text { Flow } \\
\left(\mathrm{ft}^{\wedge} 3 / \mathrm{m}\right)\end{array}$ & $\begin{array}{l}\text { Flow } \\
\text { (GPM) }\end{array}$ & $\begin{array}{l}\text { Curve Fit } \\
(\mathrm{mA})\end{array}$ \\
\hline 0.0 & 62.25 & 0.00 & 0.00 & 4.00 \\
\hline 24.7 & 62.25 & 0.40 & 2.97 & 7.24 \\
\hline 49.1 & 62.24 & 0.79 & 5.91 & 10.45 \\
\hline 73.4 & 62.24 & 1.18 & 8.82 & 13.64 \\
\hline 97.5 & 62.24 & 1.57 & 11.72 & 16.81 \\
\hline 121.7 & 62.23 & 1.96 & 14.63 & 19.99 \\
\hline 0.0 & 62.24 & 0.00 & 0.00 & 4.00 \\
\hline 24.7 & 62.24 & 0.40 & 2.97 & 7.24 \\
\hline 49.0 & 62.24 & 0.79 & 5.89 & 10.44 \\
\hline 73.5 & 62.24 & 1.18 & 8.84 & 13.66 \\
\hline 97.8 & 62.23 & 1.57 & 11.75 & 16.84 \\
\hline 121.9 & 62.23 & 1.96 & 14.65 & 20.01 \\
\hline 0.0 & 62.25 & 0.00 & 0.00 & 4.00 \\
\hline 24.8 & 62.25 & 0.40 & 2.98 & 7.26 \\
\hline 49.0 & 62.23 & 0.79 & 5.89 & 10.43 \\
\hline 73.3 & 62.23 & 1.18 & 8.82 & 13.63 \\
\hline 97.8 & 62.24 & 1.57 & 11.76 & 16.85 \\
\hline 122.3 & 62.25 & 1.97 & 14.70 & 20.06 \\
\hline
\end{tabular}

Density: $\rho=62.441-1.374 \mathrm{E}-3^{\star} \mathrm{T}-271.818 \mathrm{E}-6^{\star} \mathrm{T}^{\wedge} 2+194.093 \mathrm{E}-9^{\star} \mathrm{T}^{\wedge} 3$

7.4805 gallons $=1 \mathrm{ft}^{\wedge} 3$

Uncertainty of the Standards:

$$
\begin{aligned}
& \text { Density: }+/ \\
& \text { Time: }+/- \\
& \text { Multimeter: }+/-
\end{aligned}
$$$$
\text { Temperature: }+
$$$$
+/-\quad 0.30 \text { GPM }
$$

Accepted Tolerance: +/-

Statistical Info.
$\%$ RDG + 0.275

$\mathrm{C}$

$\mathrm{lbm} / \mathrm{ft}^{\wedge} 3$

\begin{tabular}{|c|c|c|c|c|c|c|c|}
\hline a & $b$ & $\mathrm{n}$ & $\mathrm{T}$ & $\begin{array}{l}\text { Xbar } \\
\text { (GPM) }\end{array}$ & $\begin{array}{c}\operatorname{Sxx} \\
\left(\mathrm{GPM}^{\wedge} 2\right)\end{array}$ & $\begin{array}{c}\text { SEE } \\
\left(\mathrm{mA}^{\wedge} 2\right)\end{array}$ & $\begin{array}{c}\text { MSE } \\
\left(m A^{\wedge} 2\right)\end{array}$ \\
\hline 3.9978 & 1.0928 & 18.00 & 2.120 & 7.35 & 450.9 & 0.001 & 0.000 \\
\hline
\end{tabular}

$\mathrm{sec}+500.00 \mu \mathrm{sec} / \mathrm{sec})$

$\% R D G+0.001$ mADC)

Calculated Uncertainties:

$\begin{array}{llll}\sigma c & \sigma_{E} & \sigma_{F} & \sigma T \\ (G P M) & (G P M) & (G P M) & (G P M) \\ 0.02 & 0.02 & 0.05 & 0.08\end{array}$

\section{PASS CALIBRATION? YES TRANSFER EQUATION:}

$-3.658$

Figure G2-7. Permeate Flow Rate - high range [Q3, Channel 14] - Post Calibration 
WSRC-TR-2005-00105, REVISION 0

SRNL-RPP-2005-00012, REVISION 0

UNCERTAINTY ANALYSIS

REF. L9.5-9114, REV. 1

Calibration Data

$\begin{array}{ccc}\begin{array}{c}\text { Nominal } \\ \text { Pressure } \\ \text { (psig) }\end{array} & \begin{array}{c}\text { Applied } \\ \text { Pressure } \\ \text { (psig) }\end{array} & \begin{array}{c}\text { Gage } \\ \text { Reading } \\ \text { (mADC) }\end{array} \\ 0.00 & 0.00 & 3.96 \\ 20.00 & 20.00 & 7.14 \\ 40.00 & 40.00 & 10.32 \\ 60.00 & 60.00 & 13.50 \\ 80.00 & 80.00 & 16.67 \\ 100.00 & 100.00 & 19.86 \\ 0.00 & 0.00 & 3.96 \\ 20.00 & 20.00 & 7.14 \\ 40.00 & 40.00 & 10.32 \\ 60.00 & 60.00 & 13.50 \\ 80.00 & 80.00 & 16.67 \\ 100.00 & 100.00 & 19.86 \\ 0.00 & 0.00 & 3.96 \\ 20.00 & 20.00 & 7.14 \\ 40.00 & 40.00 & 10.32 \\ 60.00 & 60.00 & 13.50 \\ 80.00 & 80.00 & 16.67 \\ 100.00 & 100.00 & 19.86 \\ 0.00 & 0.00 & 3.96 \\ 20.00 & 20.00 & 7.14 \\ 40.00 & 40.00 & 10.32 \\ 60.00 & 60.00 & 13.50 \\ 80.00 & 80.00 & 16.67 \\ 100.00 & 100.00 & 19.86\end{array}$

TR-3553

$\begin{array}{ccc}\begin{array}{c}\text { Curve } \\ \text { Fit } \\ \text { (mADC) }\end{array} & \begin{array}{c}\text { Error } \\ (\mathrm{mADC})\end{array} & \begin{array}{c}\text { Error } \\ \text { (psig) }\end{array} \\ 3.96 & 0.000 & 0.00 \\ 7.14 & 0.000 & 0.00 \\ 10.32 & -0.001 & -0.01 \\ 13.50 & -0.002 & -0.01 \\ 16.68 & 0.007 & 0.04 \\ 19.86 & -0.004 & -0.02 \\ 3.96 & 0.000 & 0.00 \\ 7.14 & 0.000 & 0.00 \\ 10.32 & -0.001 & -0.01 \\ 13.50 & -0.002 & -0.01 \\ 16.68 & 0.007 & 0.04 \\ 19.86 & -0.004 & -0.02 \\ 3.96 & 0.000 & 0.00 \\ 7.14 & 0.000 & 0.00 \\ 10.32 & -0.001 & -0.01 \\ 13.50 & -0.002 & -0.01 \\ 16.68 & 0.007 & 0.04 \\ 19.86 & -0.004 & -0.02 \\ 3.96 & 0.000 & 0.00 \\ 7.14 & 0.000 & 0.00 \\ 10.32 & -0.001 & -0.01 \\ 13.50 & -0.002 & -0.01 \\ 16.68 & 0.007 & 0.04 \\ 19.86 & -0.004 & -0.02\end{array}$

cal. date: page___of __

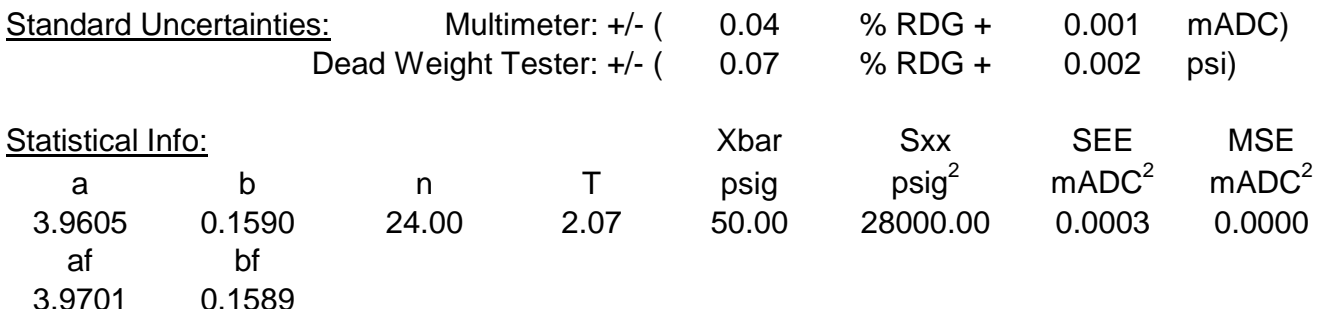

Calculated Uncertainties:

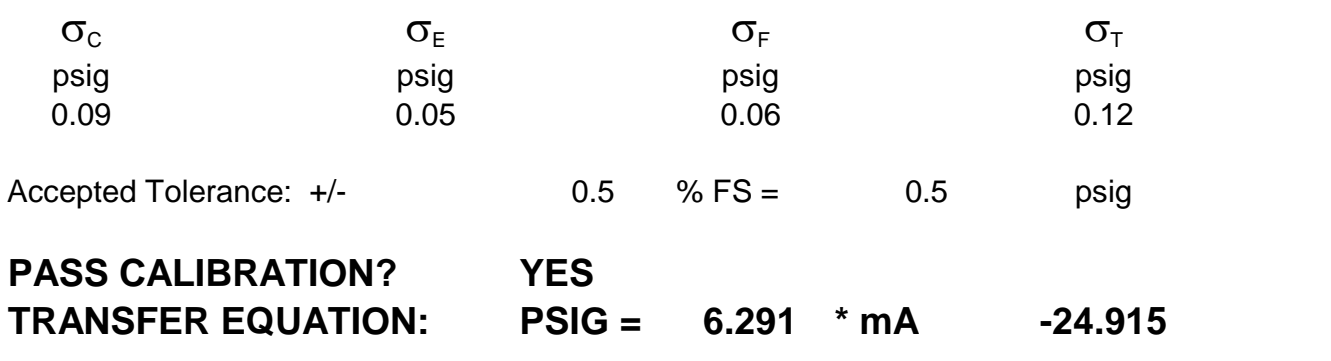

Figure G2-8. Transmembrane Pressure - upper flange [dP2, Channel 5] - Post Calibration 
WSRC-TR-2005-00105, REVISION 0

SRNL-RPP-2005-00012, REVISION 0

UNCERTAINTY ANALYSIS

REF. L9.5-9114, REV. 1

Calibration Data

$\begin{array}{ccc}\begin{array}{c}\text { Nominal } \\ \text { Pressure } \\ \text { (psid) }\end{array} & \begin{array}{c}\text { Applied } \\ \text { Pressure } \\ \text { (psid) }\end{array} & \begin{array}{c}\text { Gage } \\ \text { Reading } \\ \text { (mADC) }\end{array} \\ -11.00 & -11.00 & 4.02 \\ 0.00 & 0.00 & 5.73 \\ 11.00 & 11.00 & 7.45 \\ 31.00 & 31.00 & 10.58 \\ 61.00 & 61.00 & 15.29 \\ 91.00 & 91.00 & 20.01 \\ -11.00 & -11.00 & 4.02 \\ 0.00 & 0.00 & 5.73 \\ 11.00 & 11.00 & 7.45 \\ 31.00 & 31.00 & 10.58 \\ 61.00 & 61.00 & 15.29 \\ 91.00 & 91.00 & 20.01 \\ -11.00 & -11.00 & 4.02 \\ 0.00 & 0.00 & 5.73 \\ 11.00 & 11.00 & 7.45 \\ 31.00 & 31.00 & 10.58 \\ 61.00 & 61.00 & 15.29 \\ 91.00 & 91.00 & 20.01 \\ -11.00 & -11.00 & 4.02 \\ 0.00 & 0.00 & 5.73 \\ 11.00 & 11.00 & 7.45 \\ 31.00 & 31.00 & 10.58 \\ 61.00 & 61.00 & 15.29 \\ 91.00 & 91.00 & 20.01\end{array}$

TR-3109

$\begin{array}{ccc}\begin{array}{c}\text { Curve } \\ \text { Fit }\end{array} & \begin{array}{c}\text { Error } \\ (\mathrm{mADC})\end{array} & \begin{array}{c}\text { Error } \\ (\mathrm{psid})\end{array} \\ 4.01 & -0.014 & -0.09 \\ 5.73 & 0.001 & 0.01 \\ 7.46 & 0.006 & 0.04 \\ 10.59 & 0.012 & 0.07 \\ 15.30 & 0.006 & 0.04 \\ 20.00 & -0.010 & -0.07 \\ 4.01 & -0.014 & -0.09 \\ 5.73 & 0.001 & 0.01 \\ 7.46 & 0.006 & 0.04 \\ 10.59 & 0.012 & 0.07 \\ 15.30 & 0.006 & 0.04 \\ 20.00 & -0.010 & -0.07 \\ 4.01 & -0.014 & -0.09 \\ 5.73 & 0.001 & 0.01 \\ 7.46 & 0.006 & 0.04 \\ 10.59 & 0.012 & 0.07 \\ 15.30 & 0.006 & 0.04 \\ 20.00 & -0.010 & -0.07 \\ 4.01 & -0.014 & -0.09 \\ 5.73 & 0.001 & 0.01 \\ 7.46 & 0.006 & 0.04 \\ 10.59 & 0.012 & 0.07 \\ 15.30 & 0.006 & 0.04 \\ 20.00 & -0.010 & -0.07\end{array}$

Standard Uncertainties:

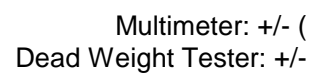

0.04

$\%$ RDG +

0.001

$\%$ RDG +

0.002

mADC)

psi)

$\begin{array}{cc}\frac{\text { Statistical Info: }}{\mathrm{a}} & \\ 5.7309 & 0.1568 \\ & \mathrm{~b} \\ \text { af } & \mathrm{bf} \\ 5.728 & 0.1568\end{array}$

Calculated Uncertainties:

$\begin{array}{cc}\sigma_{C} & \sigma_{E} \\ \text { psid } & \text { psid } \\ 0.09 & 0.14\end{array}$

Accepted Tolerance: +/-

Xbar

psid

Sxx
psid

30494.00

SEE

mADC

MSE

$\begin{array}{lll} & & \\ 24.00 & 2.15 & 30.50\end{array}$

0.0020

0.0001

PASS CALIBRATION? YES

$\begin{array}{lllll}\text { TRANSFER EQUATION: } & \text { PSIG }= & 6.3775 \quad * m A & -36.5488\end{array}$

Figure G2-9. Transmembrane Pressure - lower flange [dP3, Channel 9] - Post Calibration 
WSRC-TR-2005-00105, REVISION 0

SRNL-RPP-2005-00012, REVISION 0

UNCERTAINTY ANALYSIS

REF. L9.5-9114, REV. 1

Calibration Data

$\begin{array}{ccc}\begin{array}{c}\text { Nominal } \\ \text { Pressure } \\ \text { (psig) }\end{array} & \begin{array}{c}\text { Applied } \\ \text { Pressure } \\ \text { (psig) }\end{array} & \begin{array}{c}\text { Gage } \\ \text { Reading } \\ \text { (mADC) }\end{array} \\ 0.00 & 0.00 & 4.00 \\ 20.00 & 20.00 & 7.16 \\ 40.00 & 40.00 & 10.33 \\ 60.00 & 60.00 & 13.50 \\ 80.00 & 80.00 & 16.67 \\ 100.00 & 100.00 & 19.85 \\ 0.00 & 0.00 & 4.00 \\ 20.00 & 20.00 & 7.16 \\ 40.00 & 40.00 & 10.33 \\ 60.00 & 60.00 & 13.50 \\ 80.00 & 80.00 & 16.67 \\ 100.00 & 100.00 & 19.85 \\ 0.00 & 0.00 & 4.00 \\ 20.00 & 20.00 & 7.16 \\ 40.00 & 40.00 & 10.33 \\ 60.00 & 60.00 & 13.50 \\ 80.00 & 80.00 & 16.67 \\ 100.00 & 100.00 & 19.85 \\ 0.00 & 0.00 & 4.00 \\ 20.00 & 20.00 & 7.16 \\ 40.00 & 40.00 & 10.33 \\ 60.00 & 60.00 & 13.50 \\ 80.00 & 80.00 & 16.67 \\ 100.00 & 100.00 & 19.85\end{array}$

TR-2917

$\begin{array}{ccc}\begin{array}{c}\text { Curve } \\ \text { Fit } \\ \text { (mADC) }\end{array} & \begin{array}{c}\text { Error } \\ (\mathrm{mADC})\end{array} & \begin{array}{c}\text { Error } \\ \text { (psig) }\end{array} \\ 3.99 & -0.007 & -0.04 \\ 7.16 & 0.003 & 0.02 \\ 10.33 & 0.003 & 0.02 \\ 13.50 & 0.003 & 0.02 \\ 16.67 & 0.003 & 0.02 \\ 19.84 & -0.007 & -0.04 \\ 3.99 & -0.007 & -0.04 \\ 7.16 & 0.003 & 0.02 \\ 10.33 & 0.003 & 0.02 \\ 13.50 & 0.003 & 0.02 \\ 16.67 & 0.003 & 0.02 \\ 19.84 & -0.007 & -0.04 \\ 3.99 & -0.007 & -0.04 \\ 7.16 & 0.003 & 0.02 \\ 10.33 & 0.003 & 0.02 \\ 13.50 & 0.003 & 0.02 \\ 16.67 & 0.003 & 0.02 \\ 19.84 & -0.007 & -0.04 \\ 3.99 & -0.007 & -0.04 \\ 7.16 & 0.003 & 0.02 \\ 10.33 & 0.003 & 0.02 \\ 13.50 & 0.003 & 0.02 \\ 16.67 & 0.003 & 0.02 \\ 19.84 & -0.007 & -0.04\end{array}$

cal. date: page___ of

Standard Uncertainties: Multimeter: +/- (

0.04

$\% \mathrm{RDG}+\quad 0.001 \quad \mathrm{mADC})$ Dead Weight Tester: +/- (

0.07

$\%$ RDG + 0.002 psi)

Statistical Info:

$\begin{array}{ccccc}\mathrm{a} & \mathrm{b} & \mathrm{n} & \mathrm{T} & \mathrm{P} \\ 3.9933 & 0.1585 & 24.00 & 2.07 & 50.00 \\ \text { af } & \text { bf } & & & \\ 3.9983 & 0.1585 & & & \end{array}$

Calculated Uncertainties:

$\begin{array}{cc}\sigma_{\mathrm{C}} & \sigma_{\mathrm{E}} \\ \text { psig } & \text { psig } \\ 0.09 & 0.07\end{array}$

$\sigma_{E}$
$p s i g$
0.07

$\sigma_{\mathrm{F}}$

psig

Sxx SEE MSE

psig $^{2} \quad \mathrm{mADC}^{2} \quad \mathrm{mADC}^{2}$ 28000.00 0.0005

0.0000

Accepted Tolerance: +/-

$0.5 \% \mathrm{FS}=$

0.50

Total Uncertainty

PASS CALIBRATION? YES

$\begin{array}{lllll}\text { TRANSFER EQUATION } & \text { PSIG }= & 6.309 & *\end{array}$

Figure G2-10. Slurry Loop Pressure [P1, Channel 7] - Post Calibration 
WSRC-TR-2005-00105, REVISION 0

SRNL-RPP-2005-00012, REVISION 0

UNCERTAINTY ANALYSIS

REF. L9.5-9114, REV. 1

Calibration Data

$\begin{array}{ccc}\begin{array}{c}\text { Nominal } \\ \text { Pressure } \\ \text { (psid) }\end{array} & \begin{array}{c}\text { Applied } \\ \text { Pressure } \\ \text { (psid) }\end{array} & \begin{array}{c}\text { Gage } \\ \text { Reading } \\ \text { (mADC) }\end{array} \\ 31.00 & 0.00 & 4.00 \\ 31.00 & 31.00 & 7.31 \\ 61.00 & 61.00 & 10.51 \\ 91.00 & 91.00 & 13.69 \\ 121.00 & 121.00 & 16.86 \\ 151.00 & 151.00 & 20.01 \\ 0.00 & 0.00 & 4.00 \\ 31.00 & 31.00 & 7.31 \\ 61.00 & 61.00 & 10.51 \\ 91.00 & 91.00 & 13.69 \\ 121.00 & 121.00 & 16.86 \\ 151.00 & 151.00 & 20.01 \\ 0.00 & 0.00 & 4.00 \\ 31.00 & 31.00 & 7.31 \\ 61.00 & 61.00 & 10.51 \\ 91.00 & 91.00 & 13.69 \\ 121.00 & 121.00 & 16.86 \\ 151.00 & 151.00 & 20.01 \\ 0.00 & 0.00 & 4.00 \\ 31.00 & 31.00 & 7.31 \\ 61.00 & 61.00 & 10.51 \\ 91.00 & 91.00 & 13.70 \\ 121.00 & 121.00 & 16.86 \\ 151.00 & 151.00 & 20.01\end{array}$

TR-3115

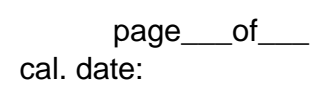

$\begin{array}{ccc}\begin{array}{c}\text { Curve } \\ \text { Fit }\end{array} & \begin{array}{c}\text { Error } \\ \text { (mADC) }\end{array} & \begin{array}{c}\text { Error } \\ \text { (mADC) }\end{array} \\ 4.02 & 0.022 & 0.20 \\ 7.31 & -0.001 & -0.01 \\ 10.49 & -0.019 & -0.18 \\ 13.67 & -0.018 & -0.17 \\ 16.85 & -0.006 & -0.06 \\ 20.04 & 0.025 & 0.24 \\ 4.02 & 0.022 & 0.20 \\ 7.31 & -0.001 & -0.01 \\ 10.49 & -0.019 & -0.18 \\ 13.67 & -0.018 & -0.17 \\ 16.85 & -0.006 & -0.06 \\ 20.04 & 0.025 & 0.24 \\ 4.02 & 0.022 & 0.20 \\ 7.31 & -0.001 & -0.01 \\ 10.49 & -0.019 & -0.18 \\ 13.67 & -0.018 & -0.17 \\ 16.85 & -0.006 & -0.06 \\ 20.04 & 0.025 & 0.24 \\ 4.02 & 0.022 & 0.20 \\ 7.31 & -0.001 & -0.01 \\ 10.49 & -0.019 & -0.18 \\ 13.67 & -0.028 & -0.26 \\ 16.85 & -0.006 & -0.06 \\ 20.04 & 0.025 & 0.24\end{array}$

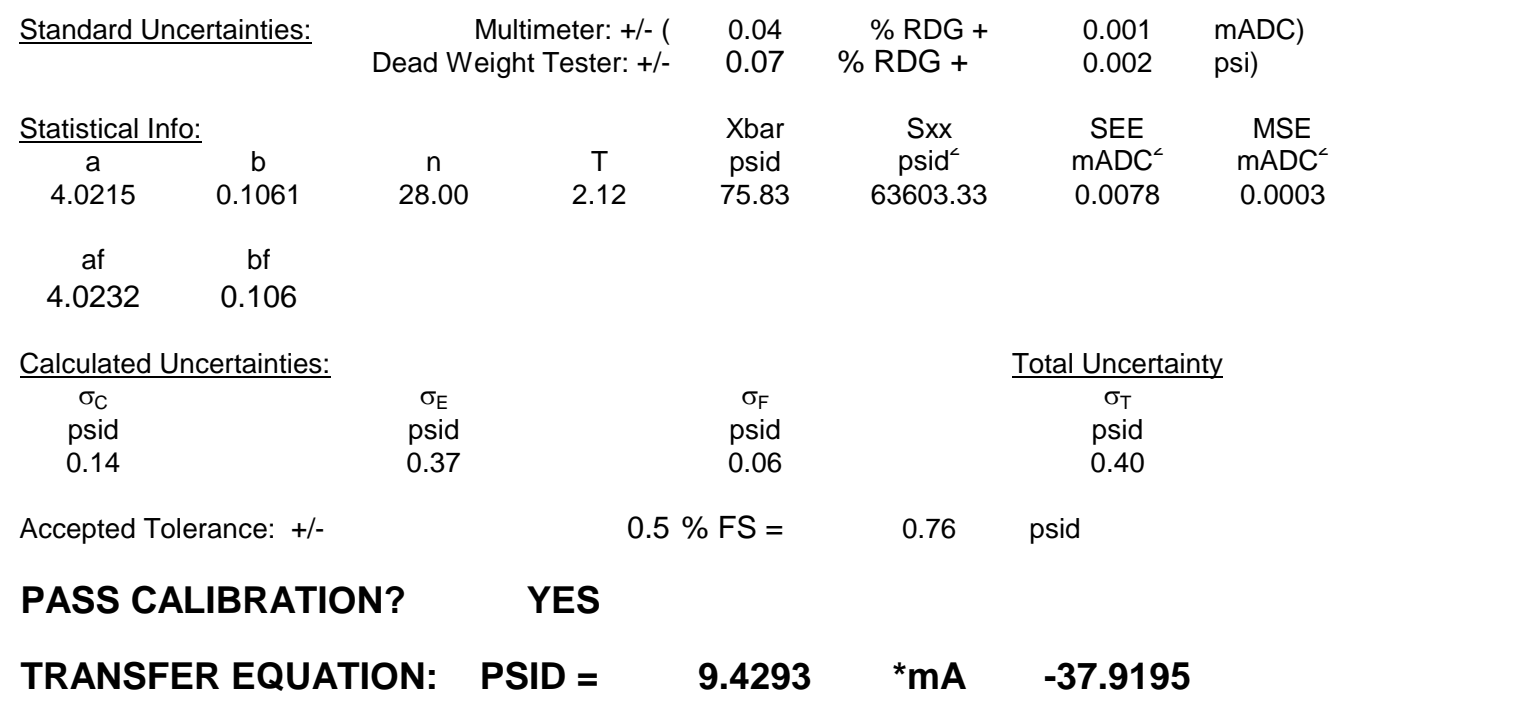

Figure G2-11. Permeate Loop Pressure [P2, Channel 10] - Post Calibration 
WSRC-TR-2005-00105, REVISION 0

SRNL-RPP-2005-00012, REVISION 0

UNCERTAINTY ANALYSIS

REF. L9.5-9114, REV. 1

Calibration Data

$\begin{array}{ccc}\begin{array}{c}\text { Nominal } \\ \text { Pressure } \\ \text { (psid) }\end{array} & \begin{array}{c}\text { Applied } \\ \text { Pressure } \\ \text { (psid) }\end{array} & \begin{array}{c}\text { Gage } \\ \text { Reading } \\ \text { (mADC) }\end{array} \\ 0.00 & 0.00 & 4.01 \\ 31.00 & 31.00 & 7.29 \\ 61.00 & 61.00 & 10.46 \\ 91.00 & 91.00 & 13.64 \\ 121.00 & 121.00 & 16.83 \\ 151.00 & 151.00 & 20.02 \\ 0.00 & 0.00 & 4.01 \\ 31.00 & 31.00 & 7.29 \\ 61.00 & 61.00 & 10.46 \\ 91.00 & 91.00 & 13.64 \\ 121.00 & 121.00 & 16.83 \\ 151.00 & 151.00 & 20.02 \\ 0.00 & 0.00 & 4.01 \\ 31.00 & 31.00 & 7.29 \\ 61.00 & 61.00 & 10.46 \\ 91.00 & 91.00 & 13.64 \\ 121.00 & 121.00 & 16.83 \\ 151.00 & 151.00 & 20.02 \\ 0.00 & 0.00 & 4.01 \\ 31.00 & 31.00 & 7.29 \\ 61.00 & 61.00 & 10.46 \\ 91.00 & 91.00 & 13.64 \\ 121.00 & 121.00 & 16.83 \\ 151.00 & 151.00 & 20.02\end{array}$

Standard Uncertainties: $\quad$ Multimeter: +/- ( Dead Weight Tester: +/- (

Statistical Info:

$\begin{array}{ccccc}\mathrm{a} & \mathrm{b} & \mathrm{n} & \mathrm{T} & \mathrm{psid} \\ 4.0019 & 0.1060 & 24.00 & 2.07 & 75.83 \\ \text { af } & \mathrm{bf} & & & \\ 4.0005 & 0.106 & & & \end{array}$

Calculated Uncertainties:

$\begin{array}{cc}\sigma_{\mathrm{C}} & \sigma_{\mathrm{E}} \\ \text { psid } & \text { psid } \\ 0.14 & 0.16\end{array}$

Accepted Tolerance: +/-
TR-00532

$\begin{array}{ccc}\begin{array}{c}\text { Curve } \\ \text { Fit } \\ \text { (mADC) }\end{array} & \begin{array}{c}\text { Error } \\ (\mathrm{mADC})\end{array} & \begin{array}{c}\text { Error } \\ \text { (psid) }\end{array} \\ 4.00 & -0.008 & -0.08 \\ 7.29 & -0.001 & -0.01 \\ 10.47 & 0.009 & 0.09 \\ 13.65 & 0.010 & 0.09 \\ 16.83 & 0.000 & 0.00 \\ 20.01 & -0.009 & -0.09 \\ 4.00 & -0.008 & -0.08 \\ 7.29 & -0.001 & -0.01 \\ 10.47 & 0.009 & 0.09 \\ 13.65 & 0.010 & 0.09 \\ 16.83 & 0.000 & 0.00 \\ 20.01 & -0.009 & -0.09 \\ 4.00 & -0.008 & -0.08 \\ 7.29 & -0.001 & -0.01 \\ 10.47 & 0.009 & 0.09 \\ 13.65 & 0.010 & 0.09 \\ 16.83 & 0.000 & 0.00 \\ 20.01 & -0.009 & -0.09 \\ 4.00 & -0.008 & -0.08 \\ 7.29 & -0.001 & -0.01 \\ 10.47 & 0.009 & 0.09 \\ 13.65 & 0.010 & 0.09 \\ 16.83 & 0.000 & 0.00 \\ 20.01 & -0.009 & -0.09\end{array}$

$\% \mathrm{RDG}+\quad 0.001 \quad \mathrm{mADC})$

$\%$ RDG +0.002 psi)

$\begin{array}{ccc}\text { Sxx } & \text { SEE } & \text { MSE } \\ \text { psid }^{2} & \text { mADC }^{2} & \text { mADC }^{2} \\ 63603.33 & 0.0013 & 0.0001\end{array}$

$\underline{\text { Total Uncertainty }}$

$\sigma_{\mathrm{F}}$

psid

0.04 cal. date:

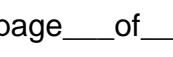


WSRC-TR-2005-00105, REVISION 0

SRNL-RPP-2005-00012, REVISION 0

\section{G3: CIX Instrumentation}


WSRC-TR-2005-00105, REVISION 0
SRNL-RPP-2005-00012, REVISION 0

INSTRUMENTS USED ON THE RPP-WTP SIPP ION EXCHANGE

(Printed: February 3, 2005)

\begin{tabular}{|c|c|c|c|c|c|c|c|c|c|}
\hline DAS Chan. & Test Rig No. & Instrument & M\&TE & Make & Model/Serial & Calibrated Range & $\begin{array}{l}\text { Uncertainty } \\
\text { (pre-test) }\end{array}$ & $\begin{array}{l}\text { Uncertainty } \\
\text { (post-test) }\end{array}$ & $\begin{array}{l}\text { Uncertainty } \\
\text { (maximum) }\end{array}$ \\
\hline 0 & P1 & Pressure Transducer & TR-3539 & Rosemount & 1144G0120A22M1/445095 & -5 to $55 \mathrm{psig}$ & $\pm .08 \mathrm{psi}$ & & \\
\hline 1 & P2 & Pressure Transducer & TR-2145 & Rosemount & 1144G-0200A22/1259930 & -5 to $55 \mathrm{psig}$ & $\pm .05 \mathrm{psi}$ & & \\
\hline 2 & P3 & Pressure Transducer & TR-2919 & Rosemount & 1151GP6E2AT0066B2/409544 & -5 to $55 \mathrm{psig}$ & $\pm .06 \mathrm{psi}$ & & \\
\hline 3 & DP1 & DP transducer & TR-3714 & Rosemount & $3051 / 1332240$ & -20 to 20 inch $\mathrm{H} 2 \mathrm{O}$ & \pm .08 in $\mathrm{H} 2 \mathrm{O}$ & \pm .13 in $\mathrm{H} 2 \mathrm{O}$ & \pm .13 in $\mathrm{H} 2 \mathrm{O}$ \\
\hline 4 & DP2 & DP transducer & TR-3713 & Rosemount & $3051 / 1332239$ & -20 to 20 inch $\mathrm{H} 2 \mathrm{O}$ & \pm .07 in $\mathrm{H} 2 \mathrm{O}$ & \pm .13 in $\mathrm{H} 2 \mathrm{O}$ & \pm .13 in $\mathrm{H} 2 \mathrm{O}$ \\
\hline 5 & DP3 & DP transducer & TR-3756 & Rosemount & 3051CD03A22A1AB4/1383987 & -20 to 20 inch $\mathrm{H} 2 \mathrm{O}$ & \pm .08 in $\mathrm{H} 2 \mathrm{O}$ & \pm .14 in $\mathrm{H} 2 \mathrm{O}$ & \pm .14 in $\mathrm{H} 2 \mathrm{O}$ \\
\hline 6 & DP4 & DP transducer & TR-3751 & Rosemount & 3051CD03A22A1AB4/1383982 & -20 to 20 inch $\mathrm{H} 2 \mathrm{O}$ & \pm .08 in $\mathrm{H} 2 \mathrm{O}$ & \pm .13 in $\mathrm{H} 2 \mathrm{O}$ & \pm .13 in $\mathrm{H} 2 \mathrm{O}$ \\
\hline 7 & DP5 & DP transducer & TR-3754 & Rosemount & 3051CD03A22A1AB4/1383985 & -20 to 20 inch $\mathrm{H} 2 \mathrm{O}$ & \pm .08 in $\mathrm{H} 2 \mathrm{O}$ & \pm .17 in $\mathrm{H} 2 \mathrm{O}$ & \pm .17 in $\mathrm{H} 2 \mathrm{O}$ \\
\hline 8 & DP6 & DP transducer & TR-3749 & Rosemount & 3051CD03A22A1AB4/1383980 & -20 to 20 inch $\mathrm{H} 2 \mathrm{O}$ & \pm .08 in $\mathrm{H} 2 \mathrm{O}$ & $\pm .09 \mathrm{in} \mathrm{H} 2 \mathrm{O}$ & \pm .09 in $\mathrm{H} 2 \mathrm{O}$ \\
\hline 9 & DP7 & DP transducer & TR-3716 & Rosemount & $3051 / 1332242$ & -20 to 20 inch $\mathrm{H} 2 \mathrm{O}$ & \pm .08 in $\mathrm{H} 2 \mathrm{O}$ & & \\
\hline 10 & DP8 & DP transducer & TR-3763 & Rosemount & $3051 / 1383984$ & -20 to 20 inch $\mathrm{H} 2 \mathrm{O}$ & \pm .08 in $\mathrm{H} 2 \mathrm{O}$ & & \\
\hline 11 & DP9 & DP transducer & TR-3752 & Rosemount & 3051CD03A22A1AB4/1383983 & -20 to 20 inch $\mathrm{H} 2 \mathrm{O}$ & \pm .08 in $\mathrm{H} 2 \mathrm{O}$ & & \\
\hline 12 & DP10 & DP transducer & TR-3755 & Rosemount & 3051CD03A22A1AB4/1383986 & -20 to 20 inch $\mathrm{H} 2 \mathrm{O}$ & \pm .08 in $\mathrm{H} 2 \mathrm{O}$ & & \\
\hline 13 & DP11 & DP transducer & TR-3750 & Rosemount & 3051CD03A22A1AB4/1383981 & -20 to 20 inch $\mathrm{H} 2 \mathrm{O}$ & \pm .08 in $\mathrm{H} 2 \mathrm{O}$ & & \\
\hline 14 & Flow 1 & Mag. Flowmeter & TR-3703 & Fischer-Porter & 1475EN01PF29KW12CAC1/95W0006755 & $0-12 \mathrm{~L} / \mathrm{hr}$ & $\pm 3.38 \mathrm{~L} / \mathrm{hr}$ & $\pm 2.72 \mathrm{~L} / \mathrm{hr}$ & $\pm 3.38 \mathrm{~L} / \mathrm{hr}$ \\
\hline 15 & Flow 2 & Mag. Flowmeter & TR-3563 & Fischer-Porter & 10D1475CDBC11BBL1/8710A0242A2 & $0-1 \mathrm{gpm}$ & $\pm .009 \mathrm{gpm}$ & & \\
\hline 16 & Cond 1 & Conductivity Probe & & Omega & CDCN-90A/60010121353 & $0-500 \mathrm{mS} / \mathrm{cm}$ & $\pm 5 \mathrm{mS} / \mathrm{cm}$ & & \\
\hline 17 & Cond 2 & Conductivity Probe & & Omega & CDCN-90A/60010121353 & $0-500 \mathrm{mS} / \mathrm{cm}$ & $\pm 5 \mathrm{mS} / \mathrm{cm}$ & & \\
\hline 18 & $\mathrm{pH}$ probe & pH Probe & & Eutech & pH200/141106 & $0-12$ & $\pm .01 \mathrm{pH}$ & & \\
\hline 20 & T1 & Thermocouple, E & TR-2814 & Omega & TJ36-CSXX-18U-6-SB-OST-N/None & $0^{\circ} \mathrm{C}-100^{\circ} \mathrm{C}$ & $\pm .83^{\circ} \mathrm{C}$ & & \\
\hline 21 & $\mathrm{~T} 2$ & Thermocouple, E & TR-2816 & Omega & TJ36-CSXX-18U-6-SB-OST-N/None & $0^{\circ} \mathrm{C}-100^{\circ} \mathrm{C}$ & $\pm .92^{\circ} \mathrm{C}$ & & \\
\hline 22 & $\mathrm{~T} 3$ & Thermocouple, E & TR-3724 & Omega & TJ36-CSXX-18U-6-SB-OST-N/None & $0^{\circ} \mathrm{C}-100^{\circ} \mathrm{C}$ & $\pm 1.02^{\circ} \mathrm{C}$ & & \\
\hline 23 & $\mathrm{~T} 4$ & Thermocouple, E & TR-1504 & Omega & TJ36-CSXX-18U-6-SB-OST-N/None & $0^{\circ} \mathrm{C}-100^{\circ} \mathrm{C}$ & $\pm .87^{\circ} \mathrm{C}$ & & \\
\hline \multirow[t]{21}{*}{24} & T5 & Thermocouple, E & TR-2933 & Omega & TJ36-CSXX-18U-6-SB-OST-N/None & $0^{\circ} \mathrm{C}-100^{\circ} \mathrm{C}$ & $\pm .99^{\circ} \mathrm{C}$ & & \\
\hline & & & & DAS Calibration & & \multicolumn{3}{|c|}{ Transfer Functions for DAS (Conversion from mA to Volts) } & \\
\hline & DAS Channel & Instrument & & Slope (V/mA) & Intercept(V) & Output & Slope (unit/V) & & Intercept (Unit) \\
\hline & 0 & P1 & & 2.005 & -0.006 & Pressure (psig) & 7.517 & $\mathrm{x} \mathrm{V}+$ & -20.029 \\
\hline & 1 & P2 & & 2.001 & -0.030 & Pressure (psig) & 7.512 & $x \mathrm{~V}+$ & -20.198 \\
\hline & 2 & P3 & & 2.007 & -0.010 & Pressure (psig) & 7.526 & $x V+$ & -20.222 \\
\hline & 3 & DP1 & & 2.002 & -0.028 & Pressure (psid) & 5.005 & $x \mathrm{~V}+$ & -30.059 \\
\hline & 4 & DP2 & & 2.499 & -0.008 & Pressure (psid) & 5.003 & $\mathrm{x} \mathrm{V}+$ & -29.999 \\
\hline & 5 & DP3 & & 2.000 & -0.012 & Pressure (psid) & 5.001 & $x \mathrm{~V}+$ & -30.026 \\
\hline & 6 & DP4 & & 2.010 & 0.000 & Pressure (psid) & 5.023 & $\mathrm{xV}+$ & -29.961 \\
\hline & 7 & DP5 & & 2.008 & -0.004 & Pressure (psid) & 5.014 & $\mathrm{x} \mathrm{V}+$ & -29.940 \\
\hline & 8 & DP6 & & 2.008 & -0.012 & Pressure (psid) & 5.014 & $x V+$ & -29.991 \\
\hline & 9 & DP7 & & 2.003 & -0.010 & Pressure (psid) & 5.007 & $x V+$ & -30.014 \\
\hline & 10 & DP8 & & 2.004 & -0.004 & Pressure (psid) & 5.009 & $x \mathrm{~V}+$ & -29.971 \\
\hline & 11 & DP9 & & 2.007 & -0.010 & Pressure (psid) & 5.017 & $x \mathrm{~V}+$ & -29.993 \\
\hline & 12 & DP10 & & 2.007 & -0.010 & Pressure (psid) & 5.017 & $x \mathrm{~V}+$ & -29.989 \\
\hline & 13 & DP11 & & 2.000 & 0.000 & Pressure (psid) & 4.996 & $x \mathrm{~V}+$ & -29.968 \\
\hline & 14 & Flow1 & & 2.000 & 0.000 & Flow (gpm) & 2.831 & $x \mathrm{~V}+$ & 3.590 \\
\hline & 15 & Flow2 & & 2.000 & 0.000 & Flow (gpm) & 31.773 & $\mathrm{xV}+$ & 3.985 \\
\hline & 16 & Cond 1 & & 2.003 & -0.010 & Conductivity $(\mathrm{mS} / \mathrm{cm})$ & 201.37 & $x \mathrm{~V}+$ & -3.82 \\
\hline & 17 & Cond 2 & & 2.001 & -0.026 & Conductivity $(\mathrm{mS} / \mathrm{cm})$ & 212.85 & $x V+$ & -8.62 \\
\hline
\end{tabular}

Figure G3-1. Instrumentation Used for the Pilot CIX 
WSRC-TR-2005-00105, REVISION 0

SRNL-RPP-2005-00012, REVISION 0

(Printed: February, 2005) (Calibration check of all DAS channels was done on 22 April 2004)

\begin{tabular}{|c|c|c|c|c|c|c|c|c|c|c|c|c|c|c|c|c|}
\hline $\begin{array}{l}\text { Current } \\
\mathrm{mA}\end{array}$ & $\begin{array}{l}\text { Channel } 0 \\
\text { V, meas. }\end{array}$ & $\begin{array}{l}\text { Channel } 0 \\
\text { V, calc. }\end{array}$ & $\begin{array}{l}\text { Current } \\
\mathrm{mA}\end{array}$ & & $\begin{array}{l}\text { Channel } 1 \\
V \text {, meas. }\end{array}$ & $\begin{array}{l}\text { Channel } 1 \\
\text { V, calc. }\end{array}$ & $\begin{array}{l}\text { Current } \\
\mathrm{mA}\end{array}$ & $\begin{array}{l}\text { Channel } 2 \\
\text { V, meas. }\end{array}$ & $\begin{array}{l}\text { Channel } 2 \\
\text { V, calc. }\end{array}$ & $\begin{array}{l}\text { Current } \\
\mathrm{mA}\end{array}$ & & $\begin{array}{l}\text { Channel } 3 \\
\text { V, meas. }\end{array}$ & $\begin{array}{l}\text { Channel } 3 \\
\text { V, calc. }\end{array}$ & $\begin{array}{l}\text { Current } \\
\mathrm{mA}\end{array}$ & $\begin{array}{l}\text { Channel } 4 \\
V \text {, meas. }\end{array}$ & $\begin{array}{l}\text { Channel } 4 \\
\text { V, calc. }\end{array}$ \\
\hline . & $\begin{array}{ll}4 & 2.00\end{array}$ & 2.00 & & 4 & 2.01 & 2.01 & & $\begin{array}{ll}4 & 2.00\end{array}$ & 2.00 & & 4 & 2.01 & $\quad 2.01$ & 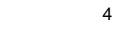 & 2.00 & 2.00 \\
\hline 8 & 3.99 & 3.99 & & 8 & 4.01 & 4.01 & & 3.99 & 3.99 & & 8 & 4.01 & 4.01 & 8 & 4.00 & 4.00 \\
\hline 12 & 5.99 & 5.99 & & 12 & 6.02 & 6.01 & & 5.98 & 5.98 & & 12 & 6.01 & 6.01 & 12 & 6.00 & 6.00 \\
\hline 16 & 7.98 & 7.98 & & 16 & 8.02 & 8.01 & & 7.98 & 7.98 & & 16 & 8.01 & 8.01 & 16 & 8.00 & 8.00 \\
\hline 20 & 9.98 & 9.98 & & 20 & 10.00 & 10.01 & & 9.97 & 9.97 & & 20 & 10.00 & 10.00 & 20 & 9.99 & 9.99 \\
\hline \multirow[t]{6}{*}{$\begin{array}{l}\text { Current } \\
\mathrm{mA}\end{array}$} & $\begin{array}{l}\text { Channel } 5 \\
V, \text { meas. }\end{array}$ & $\begin{array}{l}\text { Channel } 5 \\
\text { V, calc. }\end{array}$ & $\begin{array}{l}\text { Current } \\
\mathrm{mA}\end{array}$ & & $\begin{array}{l}\text { Channel } 6 \\
\text { V, meas. }\end{array}$ & $\begin{array}{l}\text { Channel } 6 \\
\text { V, calc. }\end{array}$ & $\begin{array}{l}\text { Current } \\
\mathrm{mA}\end{array}$ & $\begin{array}{l}\text { Channel } 7 \\
\text { V, meas. }\end{array}$ & $\begin{array}{l}\text { Channel } 7 \\
\text { V, calc. }\end{array}$ & $\begin{array}{l}\text { Current } \\
\mathrm{mA}\end{array}$ & & $\begin{array}{l}\text { Channel } 8 \\
\text { V, meas. }\end{array}$ & $\begin{array}{l}\text { Channel } 8 \\
\text { V, calc. }\end{array}$ & $\begin{array}{l}\text { Current } \\
\mathrm{mA}\end{array}$ & $\begin{array}{l}\text { Channel } 9 \\
\text { V, meas. }\end{array}$ & $\begin{array}{l}\text { Channel } 9 \\
\text { V, calc. }\end{array}$ \\
\hline & $\begin{array}{ll}4 & 2.00\end{array}$ & 2.01 & & 4 & 1.99 & 1.99 & & $\begin{array}{ll}4 & 1.99\end{array}$ & $\quad 1.99$ & & 4 & 2.00 & $\quad 2.00$ & 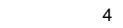 & 2.00 & 2.00 \\
\hline & 4.01 & 4.01 & & 8 & 3.98 & 3.98 & & 3.99 & 3.99 & & 8 & 3.99 & 3.99 & 8 & 4.00 & 4.00 \\
\hline & 6.01 & 6.01 & & 12 & 5.97 & 5.97 & & 5.98 & 5.98 & & 12 & 5.98 & 5.98 & 12 & 6.00 & 5.99 \\
\hline & 8.01 & 8.01 & & 16 & 7.96 & 7.96 & & 7.97 & 7.97 & & 16 & 7.97 & 7.97 & 16 & 7.99 & 7.99 \\
\hline & 10.00 & 10.01 & & 20 & 9.95 & 9.95 & & 9.96 & 9.96 & & 20 & 9.97 & 9.97 & 20 & 9.99 & 9.99 \\
\hline \multirow[t]{6}{*}{$\begin{array}{l}\text { Current } \\
\mathrm{mA}\end{array}$} & $\begin{array}{l}\text { Channel } 10 \\
\mathrm{~V}, \text { meas. }\end{array}$ & $\begin{array}{l}\text { Channel } 10 \\
\text { V, calc. }\end{array}$ & $\begin{array}{l}\text { Current } \\
\mathrm{mA}\end{array}$ & & $\begin{array}{l}\text { Channel } 11 \\
\mathrm{~V} \text {, meas. }\end{array}$ & $\begin{array}{l}\text { Channel } 11 \\
\text { V, calc. }\end{array}$ & $\begin{array}{l}\text { Current } \\
\mathrm{mA}\end{array}$ & $\begin{array}{l}\text { Channel } 12 \\
\text { V, meas. }\end{array}$ & $\begin{array}{l}\text { Channel } 12 \\
\text { V, calc. }\end{array}$ & $\begin{array}{l}\text { Current } \\
\mathrm{mA}\end{array}$ & & $\begin{array}{l}\text { Channel } 13 \\
\mathrm{~V} \text {, meas. }\end{array}$ & $\begin{array}{l}\text { Channel } 13 \\
\mathrm{~V}, \text { calc. }\end{array}$ & $\begin{array}{l}\text { Current } \\
\mathrm{mA}\end{array}$ & $\begin{array}{l}\text { Channel } 14 \\
V \text {, meas. }\end{array}$ & $\begin{array}{l}\text { Channel } 14 \\
\text { V, calc. }\end{array}$ \\
\hline & $4 \quad 2.00$ & $\quad 2.00$ & & 4 & 2.00 & 2.00 & & $\begin{array}{ll}4 & 2.00\end{array}$ & $\quad 2.00$ & & 4 & 2.00 & $\quad 2.00$ & 4 & 2.00 & 2.00 \\
\hline & 3.99 & 3.99 & & 8 & 3.99 & 3.99 & & 3.99 & 3.99 & & 8 & 4.00 & 4.00 & 8 & 4.00 & 4.00 \\
\hline & 5.99 & 5.99 & & 12 & 5.98 & 5.98 & & 5.98 & 5.98 & & 12 & 6.00 & 6.00 & 12 & 6.00 & 6.00 \\
\hline & 7.99 & 7.99 & & 16 & 7.98 & 7.98 & & 7.98 & 7.98 & & 16 & 8.00 & 8.00 & 16 & 8.00 & 8.00 \\
\hline & 9.98 & 9.98 & & 20 & 9.97 & 9.97 & & 9.97 & 9.97 & & 20 & 10.00 & 10.00 & 20 & 10.00 & 10.00 \\
\hline \multirow[t]{6}{*}{$\begin{array}{l}\text { Current } \\
\mathrm{mA}\end{array}$} & $\begin{array}{l}\text { Channel } 15 \\
\text { V, meas. }\end{array}$ & $\begin{array}{l}\text { Channel } 15 \\
\text { V, calc. }\end{array}$ & $\begin{array}{l}\text { Current } \\
\mathrm{mA}\end{array}$ & & $\begin{array}{l}\text { Channel } 16 \\
V \text {, meas. }\end{array}$ & $\begin{array}{l}\text { Channel } 16 \\
\text { V, calc. }\end{array}$ & $\begin{array}{l}\text { Current } \\
\mathrm{mA}\end{array}$ & $\begin{array}{l}\text { Channel } 17 \\
\text { V, meas. }\end{array}$ & $\begin{array}{l}\text { Channel } 17 \\
\text { V, calc. }\end{array}$ & $\begin{array}{l}\text { Current } \\
\mathrm{mA}\end{array}$ & & $\begin{array}{l}\text { Channel } 18 \\
\vee \text {, meas. }\end{array}$ & $\begin{array}{l}\text { Channel } 18 \\
\text { V, calc. }\end{array}$ & & & \\
\hline & $4 \quad 2.00$ & 2.00 & & 4 & 2.00 & 2.00 & & $4 \quad 2.01$ & 2.01 & & 4 & 1.99 & $\quad 1.99$ & & & \\
\hline & 4.00 & 4.00 & & 8 & 4.00 & 4.00 & & 4.01 & 4.01 & & 8 & 3.99 & 3.99 & & & \\
\hline & 6.00 & 6.00 & & 12 & 6.00 & 6.00 & & 6.01 & 6.01 & & 12 & 5.98 & 5.98 & & & \\
\hline & 8.00 & 8.00 & & 16 & 7.99 & 7.99 & & 8.02 & 8.01 & & 16 & 7.97 & 7.97 & & & \\
\hline & 10.00 & 10.00 & & 20 & 9.99 & 9.99 & & 10.00 & 10.01 & & 20 & 9.96 & 9.96 & & & \\
\hline \multirow{2}{*}{$\begin{array}{c}\text { TR-02814 } \\
\text { Temperature }\end{array}$} & Thermocou & iples & TR-02816 & & & & TR-03724 & & & TR-1504 & & & & TR-02933 & & \\
\hline & Channel 20 & Channel 20 & Temperatur & & Channel 21 & Channel 21 & Temperature & Channel 22 & Channel 22 & Temperature & & Channel 23 & Channel 23 & Temperature & Channel 24 & Channel 24 \\
\hline${ }^{\circ} \mathrm{C}$ & $\mathrm{T}$, applied & $\mathrm{T}$, reading & ${ }^{\circ} \mathrm{C}$ & & $\mathrm{T}$, applied & $T$, reading & ${ }^{\circ} \mathrm{C}$ & $\mathrm{T}$, applied & $\mathrm{T}$, reading & ${ }^{\circ} \mathrm{C}$ & & T, applied & $\mathrm{T}$, reading & ${ }^{\circ} \mathrm{C}$ & $\mathrm{T}$, applied & $\mathrm{T}$, reading \\
\hline 0 & 0 & 0.30 & & 0 & 0 & 0.00 & & $\begin{array}{lll}0 & 0\end{array}$ & 0.00 & & 0 & 0 & -0.10 & 0 & 0 & -0.40 \\
\hline 25 & 25 & 25.30 & & 25 & 25 & 25.00 & & 25 & 25.00 & & 25 & 25 & 24.80 & 25 & 25 & 24.60 \\
\hline 50 & 50 & 50.30 & & 50 & 50 & 50.00 & & 50 & 50.00 & & 50 & 50 & 49.80 & 50 & 50 & 49.60 \\
\hline 75 & 75 & 75.30 & & 75 & 75 & 75.00 & & 75 & 75.00 & & 75 & 75 & 74.80 & 75 & 75 & 74.60 \\
\hline 100 & 100 & 100.30 & & 100 & 100 & 100.00 & & 100 & 100.00 & & 100 & 100 & 99.90 & 100 & 100 & 99.60 \\
\hline
\end{tabular}

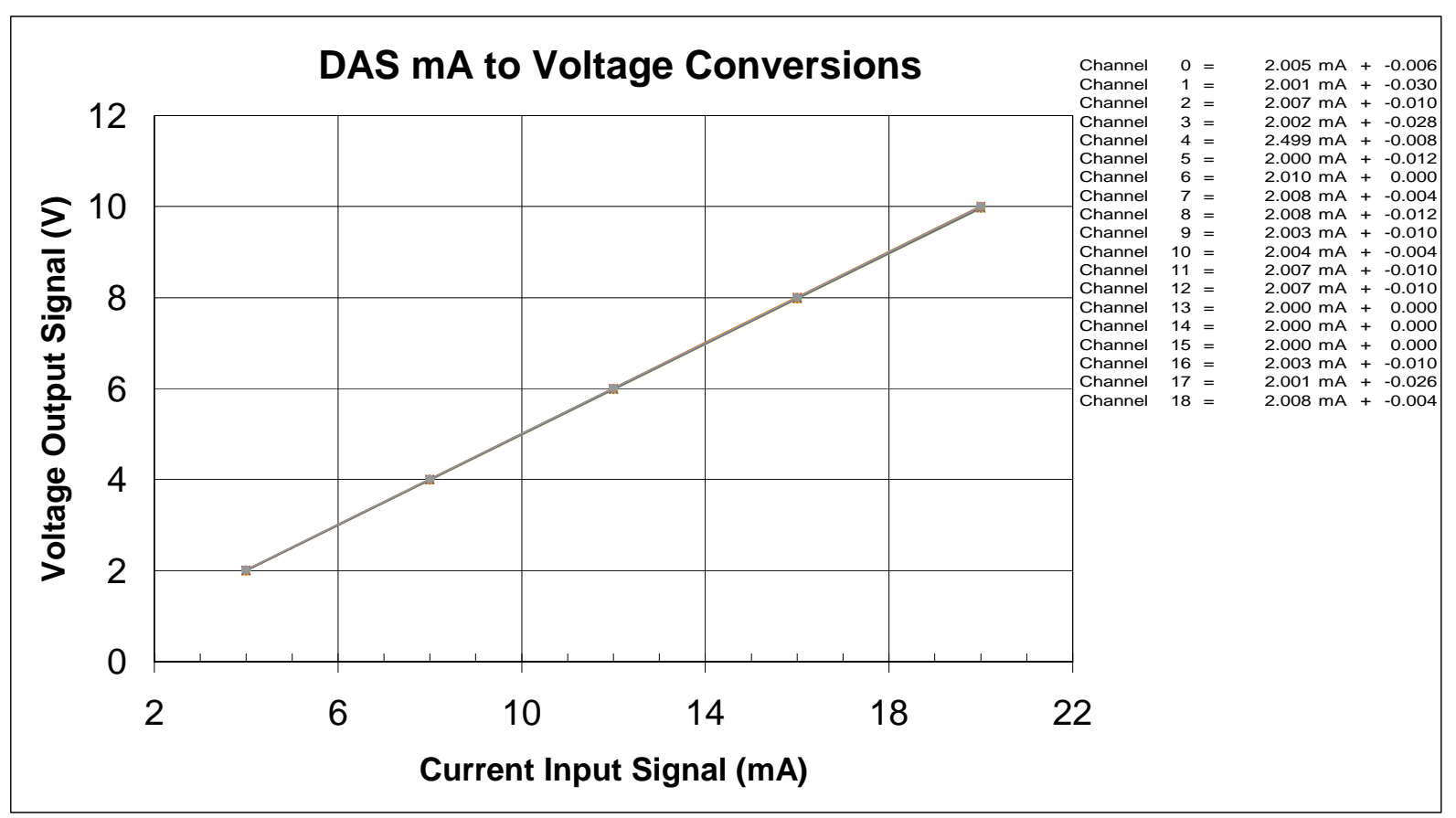

Figure G3-2. Transfer Functions for Instrumentation Used 
WSRC-TR-2005-00105, REVISION 0

SRNL-RPP-2005-00012, REVISION 0

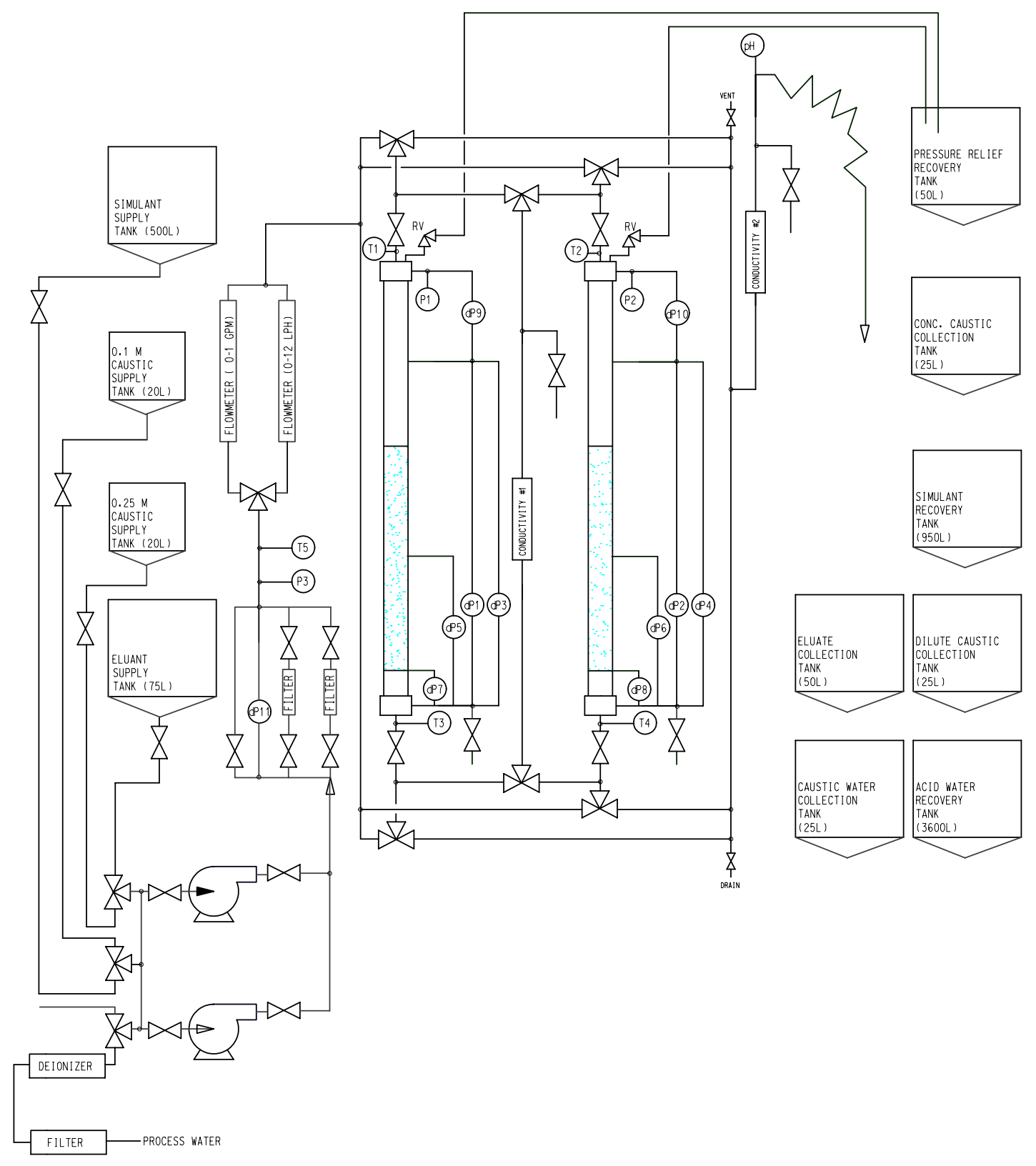

Figure G3-3. Critical Instrument Location Schematic 
WSRC-TR-2005-00105, REVISION 0

SRNL-RPP-2005-00012, REVISION 0

Calibration Data

Nominal Applied

Pressure Pressure

(in $\mathrm{H} 2 \mathrm{O}$ ) (in $\mathrm{H} 2 \mathrm{O}$ ) (mADC)

$\begin{array}{lll}-20 & -20 & 4.08\end{array}$

$\begin{array}{lll}-10 & -10 & 8.03\end{array}$

$\begin{array}{lll}0 & 0 & 11.99\end{array}$

$\begin{array}{lll}10 & 10 & 16.02\end{array}$

$20-20-20.03$

$\begin{array}{lll}-20 & -20 & 4.08\end{array}$

$\begin{array}{lll}-10 & -10 & 8.03\end{array}$

$0 \quad 0 \quad 11.99$

$10 \quad 10 \quad 16.02$

$20-20-20.03$

$-20 \quad-20 \quad 4.08$

$\begin{array}{lll}-10 & -10 & 8.03\end{array}$

11.99

16.02

20.03

4.08

8.03

11.99

16.02

20.03
Curve

Fit

(mADC)

4.05

8.04

12.03

16.02

20.01

4.05

8.04

12.03

16.02

20.01

4.05

8.04

12.03

16.02

20.01

4.05

8.04

12.03

16.02

20.01

Multimeter: +/- ( $\quad 0.04$

Standard Uncertainties:

$$
\text { Deac. }
$$

\subsection{4}

Xbar

in $\mathrm{H} 2 \mathrm{O}$

0.00

$\begin{array}{cc}a & b\end{array}$
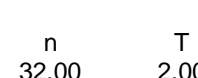

$\mathrm{T}$
2.00

$\frac{\text { Calculated Uncertainties: }}{\sigma_{c}}$ in $\mathrm{H} 2 \mathrm{O}$

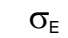

in $\mathrm{H} 2 \mathrm{O}$

0.11

$\sigma_{\mathrm{F}}$

in $\mathrm{H} 2 \mathrm{O}$

0.00

Error

(mADC)

$-0.028$

0.011

0.040

$-0.001$

$-0.022$

$-0.028$

0.011

0.040

$-0.001$

$-0.022$

$-0.028$

0.011

0.040

$-0.001$

$-0.022$

$-0.028$

0.011

0.040

$-0.001$

$-0.022$

$\%$ RDG + 0.001

$\%$ RDG

Sxx

in $\mathrm{H}_{2} \mathrm{O}^{<}$

4000.00

YES

PASS CALIBRATION?

TRANSFER EQUATION:

in $\mathrm{H} 20=2.507$

*mA

SEE

mADC ${ }^{<}$

0.0120

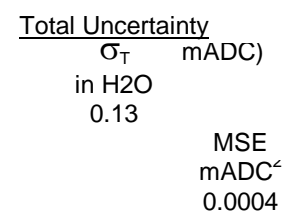

$-30.157$

cal. date:

page_of_

Error

$-0.07$

0.03

0.00

$-0.06$

0.10

$-0.07$

0.00

$-0.07$

0.03

0.00

0.0004

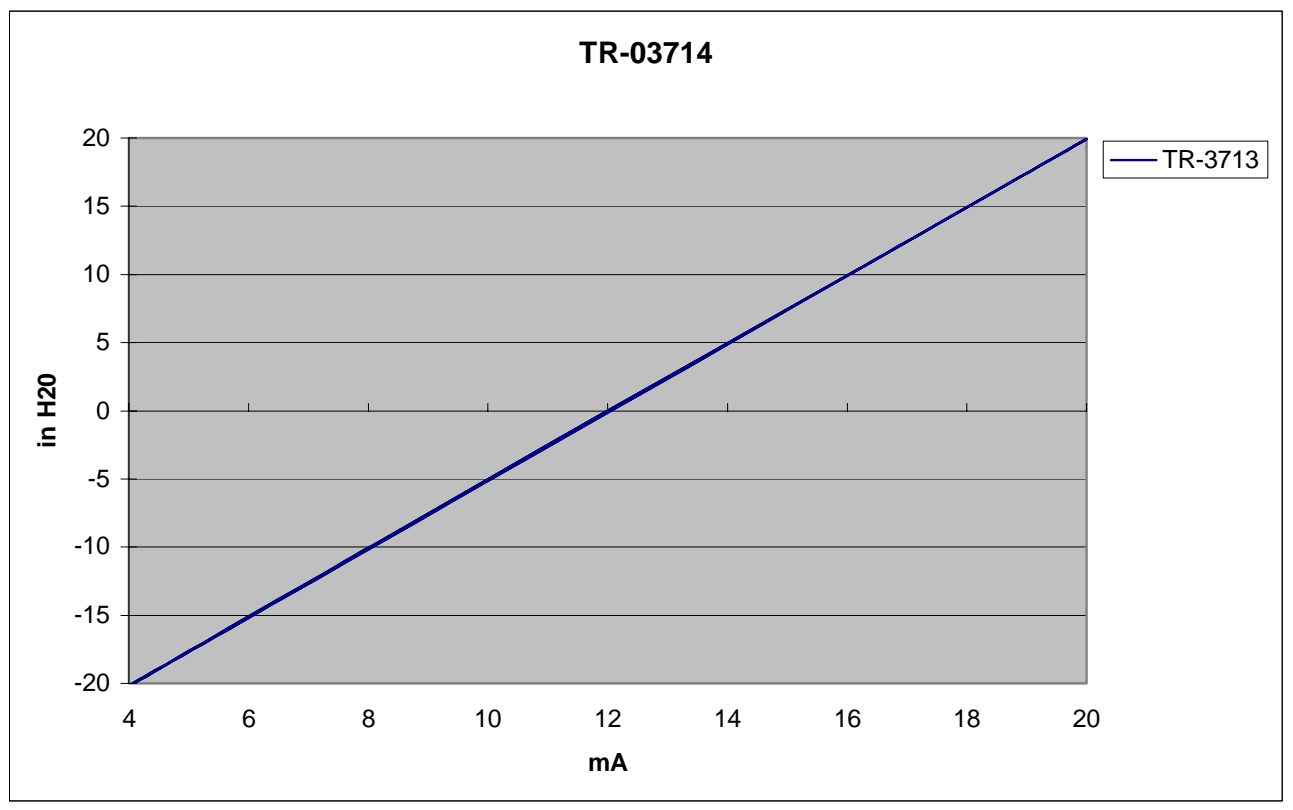

Figure G3-4. Ion Exchange Differential Pressure Post Test Calibration (DP1) 
WSRC-TR-2005-00105, REVISION 0

SRNL-RPP-2005-00012, REVISION 0

UNCERTAINTY ANALYSIS

REF. L9.5-9114, REV. 1

Calibration Data

Nominal Applied Gage

Pressure Pressure Reading

(in $\mathrm{H} 2 \mathrm{O}) \quad$ (in $\mathrm{H} 2 \mathrm{O}$ ) (mADC)

$\begin{array}{ccc}-10 & -10 & 8.03 \\ 0 & 0 & 11.99\end{array}$

$\begin{array}{ccc}0 & 0 & 11.99 \\ 10 & 10 & 16.02\end{array}$

$20 \quad 20 \quad 20.03$

$\begin{array}{lll}-20 & -20 & 4.08\end{array}$

$\begin{array}{lll}-10 & -10 & 8.03\end{array}$

$\begin{array}{lll}0 & 0 & 11.99\end{array}$

$10 \quad 10 \quad 16.02$

$\begin{array}{ccc}20 & 20 & 20.03 \\ -20 & -20 & 4.08\end{array}$

$\begin{array}{lll}-10 & -10 & 8.03\end{array}$

11.99

16.02

20.03

4.08

8.03

11.99

16.02

20.03 $\begin{array}{lll}-20 & -20 & 4.08\end{array}$

TR-3713

page__of
cal. date:

Curve

Standard Uncertainties:

Multimeter: $+/-(\quad 0.04$ Dead Weight Tester: + -

Statistical Info:

$\begin{array}{cccc}\mathrm{a} & \mathrm{b} & \mathrm{n} & \mathrm{T} \\ 12.0300 & 0.3989 & 32.00 & 2.00\end{array}$

Calculated Uncertainties:

in $\mathrm{H} 2 \mathrm{O}$

0.07

$\sigma_{\mathrm{E}}$

in $\mathrm{H} 2 \mathrm{O}$

0.11

Accepted Tolerance: +/-

PASS CALIBRATION?

TRANSFER EQUATION:
Fit

(mADC)

4.05

8.04

12.03

16.02

20.01

4.05

8.04

12.03

16.02

20.01

4.05

8.04

12.03

16.02

20.01

4.05

8.04

12.03

16.02

20.01

Error Error

(mADC) (in $\mathrm{H} 2 \mathrm{O}$ )

$\begin{array}{ll}-0.028 & -0.07\end{array}$

$0.011 \quad 0.03$

$0.040 \quad 0.10$

$\begin{array}{ll}-0.001 & 0.00\end{array}$

$\begin{array}{ll}-0.022 & -0.06\end{array}$

$-0.028 \quad-0.07$

$0.011 \quad 0.03$

$0.040 \quad 0.10$

$\begin{array}{ll}-0.001 & 0.00\end{array}$

$\begin{array}{ll}-0.022 & -0.06\end{array}$

$\begin{array}{ll}-0.028 & -0.07\end{array}$

$0.011 \quad 0.03$

$0.040 \quad 0.10$

$\begin{array}{ll}-0.001 & 0.00\end{array}$

$-0.022 \quad-0.06$

$-0.028 \quad-0.07$

$0.011 \quad 0.03$

$0.040 \quad 0.10$

$\begin{array}{ll}-0.001 & 0.00\end{array}$

$\begin{array}{ll}-0.022 & -0.06\end{array}$

$\%$ RDG + 0.001

$\%$ RDG

\section{Sxx}

in $\mathrm{H}_{2} \mathrm{O}^{<}$

SEE

mADC

0.0120

Total Uncertainty

in $\mathrm{H} 2 \mathrm{O}$

0.13

MSE

$\mathrm{mADC}^{<}$
0.0004

YES

in $\mathrm{H} 20=2.507 \quad$ *mA $\quad-30.157$

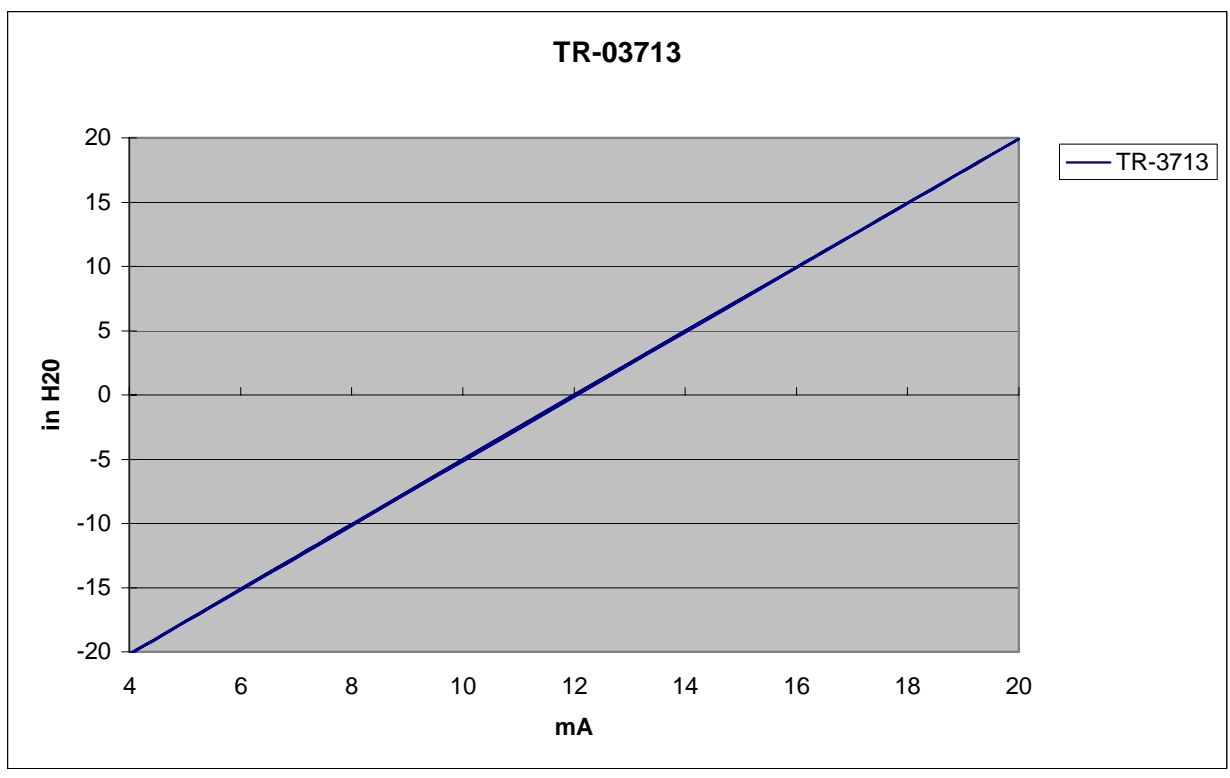

Figure G3-5. Ion Exchange Differential Pressure Post Test Calibration (DP2) 
WSRC-TR-2005-00105, REVISION 0

SRNL-RPP-2005-00012, REVISION 0

UNCERTAINTY ANALYSIS

REF. L9.5-9114, REV. 1

Nominal Applied

Pressure Pressure

$\begin{array}{lll}-10 & -10 & 7.95\end{array}$

$\begin{array}{lll}0 & 0 & 11.88\end{array}$

$\begin{array}{lll}10 & 10 & 15.9\end{array}$

$\begin{array}{lll}20 & -20 & 4.01\end{array}$

$\begin{array}{lll}-10 & -10 & 7.95\end{array}$

$\begin{array}{lll}0 & 0 & 11.88\end{array}$

$10 \quad 10 \quad 15.9$

$\begin{array}{ccc}20 & 20 & 19.9 \\ -20 & -20 & 4.01\end{array}$

$\begin{array}{lll}-10 & -10 & 7.95\end{array}$

11.88

11.88
15.9

19.9

4.01

7.95

11.88

15.9

19.9
Calibration Data

$\begin{array}{ccc}-20 & -20 & \text { (mADC) } \\ -10 & -10 & 4.01\end{array}$

TR-3756

Standard Uncertainties: Dead Weight Tester: +/-

Statistical Info:

$$
\begin{array}{ccc}
\mathrm{a} & \mathrm{b} & \mathrm{n} \\
11.9280 & 0.3973 & 32.00 \\
& \\
\text { Calculated } & \text { Uncertainties: } & \\
\hline \sigma_{\mathrm{C}} & \sigma_{\mathrm{E}} \\
\text { in } \mathrm{H} 2 \mathrm{O} & \text { in } \mathrm{H} 2 \mathrm{O} \\
0.07 & 0.12
\end{array}
$$

Accepted Tolerance: +/-

PASS CALIBRATION?

TRANSFER EQUATION:

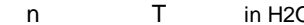

Curve

Fit

(mADC)

3.98

7.96
11.93

15.90

19.87

3.98

7.96

11.93

15.90

19.87

3.98

7.96

11.93

15.90

19.87

3.98

7.96

11.93

15.90

9.87 page_of

cal. date:
Error

(mADC)

$-0.028$

0.005

0.048

0.001

$-0.026$

$-0.028$

0.005

0.048

0.001

$-0.026$

$-0.028$

0.005

0.048

0.001

$-0.026$

$-0.028$

0.005

0.048

0.001

$-0.026$

$\%$ RDG +

$\%$ RDG

\section{Sxx}

in $\mathrm{H}_{2} \mathrm{O}^{<}$

4000.00
Error

(in $\mathrm{H} 2 \mathrm{O}$ )

$-0.07$

0.01

0.12

0.00

$-0.07$

$-0.07$

0.01
0.12

0.00

$-0.07$

$-0.07$

0.01

0.12

0.00

$-0.07$

$-0.07$

0.01

0.12

0.00

$-0.07$

0.001

SEE

mADC

0.0152

Total Uncertainty

in $\mathrm{H} 2 \mathrm{O}$

0.14

MSE

mADC
0.0005

YES

in $\mathrm{H} 20=2.517$

*mA

$-30.022$

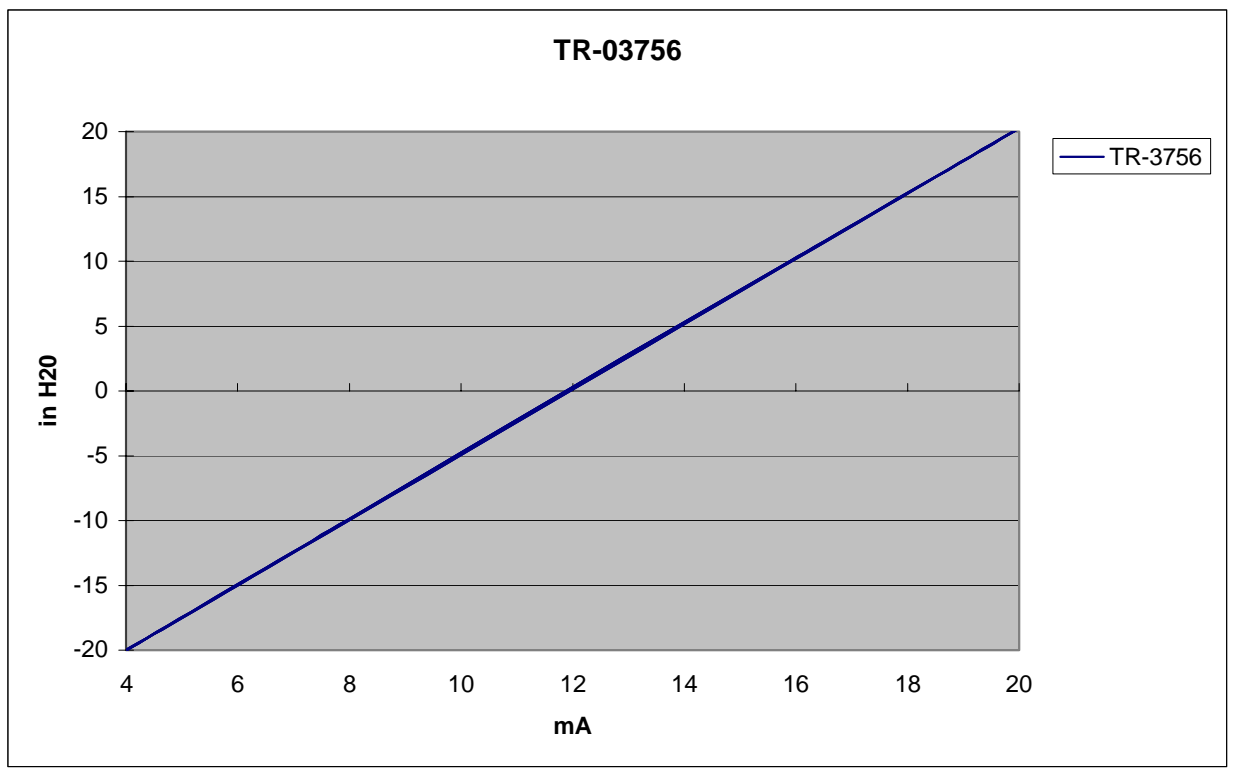

Figure G3-6. Evaporator Ion Exchange Differential Pressure Post Test Calibration (DP3) 
WSRC-TR-2005-00105, REVISION 0

SRNL-RPP-2005-00012, REVISION 0

UNCERTAINTY ANALYSIS

REF. L9.5-9114, REV. 1

Calibration Data

Nominal Applied Gage

Pressure Pressure Reading

(in $\mathrm{H} 2 \mathrm{O})$ (in $\mathrm{H} 2 \mathrm{O}$ ) (mADC)

$\begin{array}{lll}-20 & -20 & 4.08\end{array}$

$\begin{array}{ccc}-10 & -10 & 8.03 \\ 0 & 0 & 12\end{array}$

$\begin{array}{lll}10 & 10 & 15.97\end{array}$

$\begin{array}{lll}20 & 20 & 20.02\end{array}$

$\begin{array}{lll}-20 & -20 & 4.08\end{array}$

$\begin{array}{lll}-10 & -10 & 8.02\end{array}$

$\begin{array}{ccc}0 & 0 & 12 \\ 10 & 10 & 15.97 \\ 20 & 20 & 20.02\end{array}$

$\begin{array}{lll}20 & 20 & 20.02 \\ -20 & -20 & 4.08\end{array}$

$\begin{array}{lll}-10 & -10 & 8.03\end{array}$

12.01

16.03

20.02

4.08

8.03

12

16.03

20.02
TR-3751

page__of
cal. date:

Curve

Fit Error Error

(mADC) (mADC) (in $\mathrm{H} 2 \mathrm{O})$

$\begin{array}{lll}4.06 & -0.025 & -0.06\end{array}$

$\begin{array}{lll}8.04 & 0.011 & 0.03\end{array}$

$\begin{array}{lll}12.03 & 0.026 & 0.07\end{array}$

$\begin{array}{lll}16.01 & 0.041 & 0.10\end{array}$

$20.00 \quad-0.023 \quad-0.06$

$\begin{array}{lll}4.06 & -0.025 & -0.06\end{array}$

$\begin{array}{lll}8.04 & 0.021 & 0.05\end{array}$

$\begin{array}{lll}12.03 & 0.026 & 0.07\end{array}$

$\begin{array}{lll}16.01 & 0.041 & 0.10\end{array}$

$20.00 \quad-0.023 \quad-0.06$

$\begin{array}{lll}4.06 & -0.025 & -0.06\end{array}$

$\begin{array}{lll}8.04 & 0.011 & 0.03\end{array}$

$\begin{array}{lll}12.03 & 0.016 & 0.04\end{array}$

$\begin{array}{lll}16.01 & -0.019 & -0.05\end{array}$

$20.00 \quad-0.023 \quad-0.06$

$\begin{array}{lll}4.06 & -0.025 & -0.06\end{array}$

$\begin{array}{lll}8.04 & 0.011 & 0.03\end{array}$

$\begin{array}{lll}12.03 & 0.026 & 0.07\end{array}$

$16.01 \quad-0.019 \quad-0.05$

$\begin{array}{lll}20.00 & -0.023 & -0.06\end{array}$

$$
\begin{aligned}
& \begin{array}{rccc}
\text { Standard Uncertainties. } & \\
\text { Dead Weight Tester: }+/- & 0.07 & \% \text { RDG }+ & 0.001
\end{array}
\end{aligned}
$$

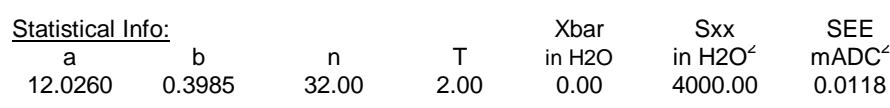

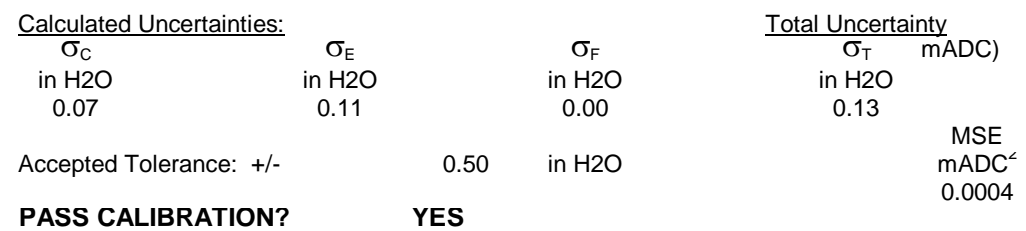

TRANSFER EQUATION: $\quad$ in $\mathrm{H} 20=2.509 \quad{ }^{*} \mathrm{~mA} \quad-30.176$

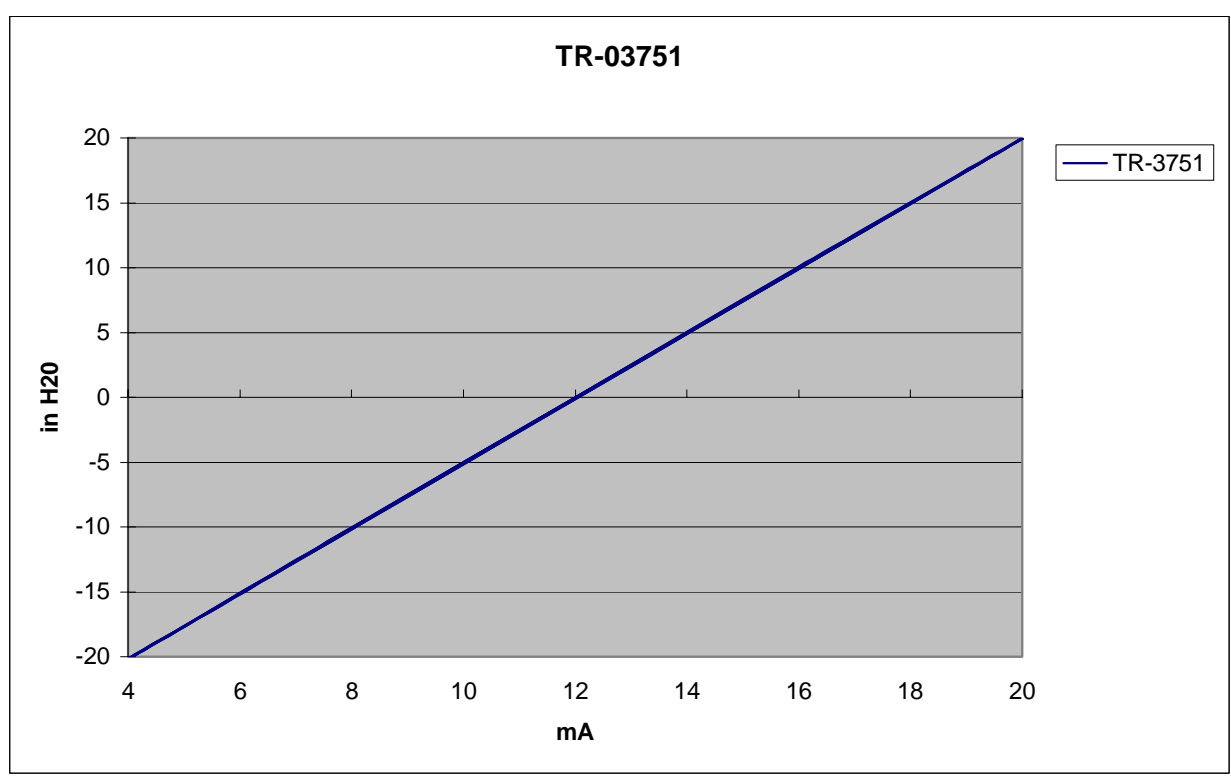

Figure G3-7. Ion Exchange Differential Pressure Post Test Calibration (DP4) 
WSRC-TR-2005-00105, REVISION 0 SRNL-RPP-2005-00012, REVISION 0

UNCERTAINTY ANALYSIS

REF. L9.5-9114, REV. 1

Calibration Data

$\begin{array}{cc}\begin{array}{c}\text { Nominal } \\ \text { Pressure }\end{array} & \begin{array}{c}\text { Applied } \\ \text { Pressure }\end{array} \\ \text { (in } \mathrm{H} 2 \mathrm{O} \text { ) } & \text { (in } \mathrm{H} 2 \mathrm{O} \text { ) } \\ -20 & -20 \\ -10 & -10 \\ 0 & 0 \\ 10 & 10 \\ 20 & 20 \\ -20 & -20 \\ -10 & -10 \\ 0 & 0 \\ 10 & 10 \\ 20 & 20 \\ -20 & -20 \\ -10 & -10 \\ 0 & 0 \\ 10 & 10 \\ 20 & 20 \\ -20 & -20 \\ -10 & -10 \\ 0 & 0 \\ 10 & 10 \\ 20 & 20\end{array}$

Gage

Reading

(mADC)

4.16

8.08

12.03

16.07

20.09

4.16

8.08

12.03

16.07

20.09

4.16

8.08

12.03

16.07

20.09

4.16

8.08

12.03

16.07

20.09

Standard Uncertainties:

Multimeter: +/- ( Dead Weight Tester: +/

Statistical Info:

$\begin{array}{cc}a & b \\ 12.0860 & 0.3985\end{array}$

Calculated Uncertainties:

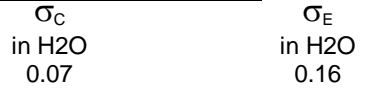

0.16

Accepted Tolerance: +/-

PASS CALIBRATION?

0.50

TR-3754

page _ of
cal date:

Curve

Fit

(mADC)

4.12

8.10

12.09

16.07

20.06

4.12

8.10

12.09

16.07

20.06

4.12

8.10

12.09

16.07

20.06

4.12

8.10

12.09

16.07

20.06

0.04

0.07

Xbar

in $\mathrm{H} 2 \mathrm{O}$

0.00

$\sigma_{\mathrm{F}}$

in $\mathrm{H} 2 \mathrm{O}$

0.00

Error Error

(mADC) (in $\mathrm{H} 2 \mathrm{O}$ )

$-0.044 \quad-0.11$

$0.021 \quad 0.05$

$0.056 \quad 0.14$

$0.001 \quad 0.00$

$-0.034 \quad-0.09$

$-0.044 \quad-0.11$

$0.021 \quad 0.05$

$0.056 \quad 0.14$

$0.001 \quad 0.00$

$-0.034 \quad-0.09$

$-0.044 \quad-0.11$

$0.021 \quad 0.05$

$0.056 \quad 0.14$

$0.001 \quad 0.00$

$-0.034 \quad-0.09$

$-0.044 \quad-0.11$

$0.021 \quad 0.05$

$0.056 \quad 0.14$

$0.001 \quad 0.00$

$\begin{array}{ll}-0.034 & -0.09\end{array}$

$\%$ RDG + 0.001

$\%$ RDG

Sxx

in $\mathrm{H}_{2} \mathrm{O}^{<}$

4000.00

SEE

$\mathrm{mADC}^{\perp}$

0.0267

Total Uncertainty

$\left.\sigma_{\mathrm{T}} \mathrm{mADC}\right)$

in $\mathrm{H} 2 \mathrm{O}$

0.17

MSE

mADC $\angle$

0.0009

TRANSFER EQUATION: $\quad$ in $\mathrm{H} 20=2.509 \quad{ }^{*} \mathrm{~mA} \quad-30.327$

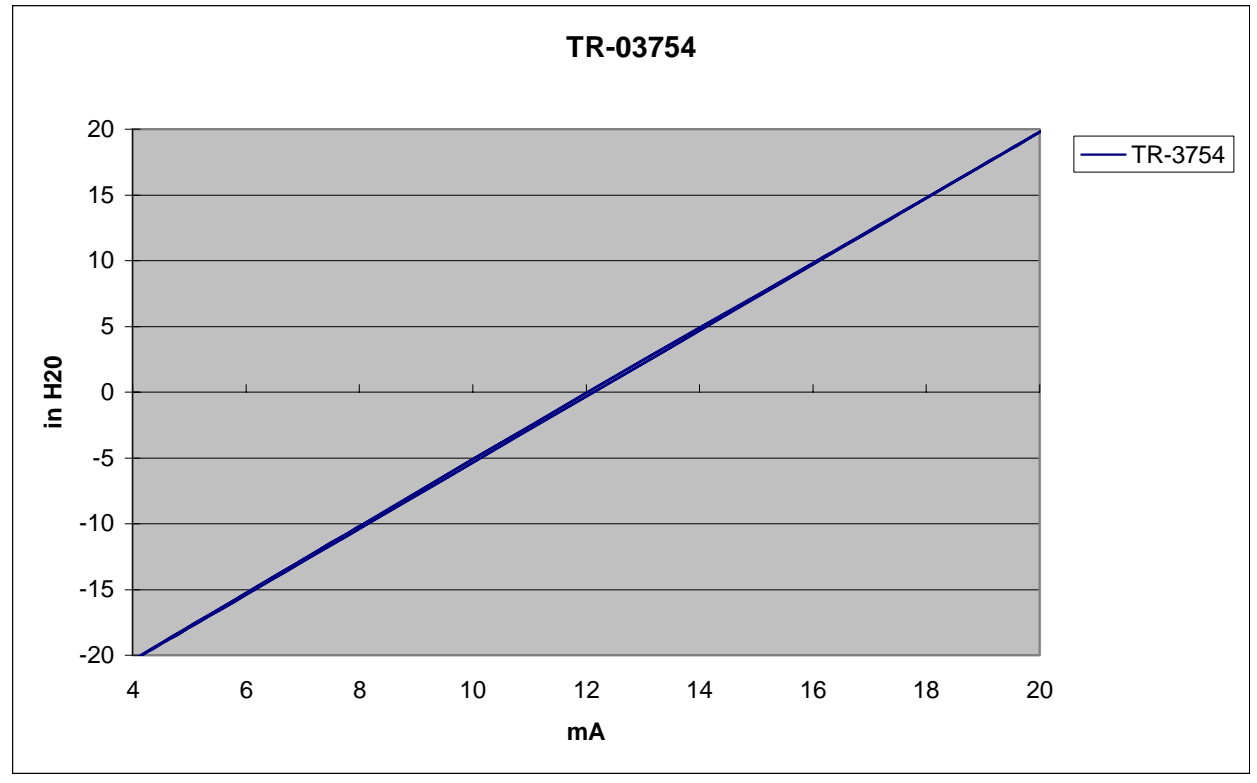

Figure G3-8. Ion Exchange Differential Pressure Post Test Calibration (DP5) 
WSRC-TR-2005-00105, REVISION 0

SRNL-RPP-2005-00012, REVISION 0

UNCERTAINTY ANALYSIS REF. L9.5-9114, REV. 1

Calibration Data

Applied Gage

(in $\mathrm{H} 2 \mathrm{O}$ ) (in $\mathrm{H} 2 \mathrm{O}$ ) (mADC)

$\begin{array}{lll}-20 & -20 & 3.97\end{array}$

$\begin{array}{lll}-10 & -10 & 7.94\end{array}$

$\begin{array}{lll}0 & 0 & 11.94\end{array}$

$\begin{array}{lll}10 & 10 & 15.97 \\ 20 & 20 & 19.97\end{array}$

$\begin{array}{lll}-20 & -20 & 3.97\end{array}$

$\begin{array}{lll}-10 & -10 & 7.94\end{array}$

$\begin{array}{lll}0 & 0 & 11.94\end{array}$

$\begin{array}{lll}10 & 10 & 15.97\end{array}$

$\begin{array}{ll}20 & 20 \\ -20 & -20\end{array}$

$\begin{array}{ll}-20 & -20 \\ -10 & -10\end{array}$

$\begin{array}{ccc}0 & 0 & 7.94 \\ 10 & 11.94\end{array}$

$\begin{array}{lll}10 & 10 & 15.97\end{array}$

$\begin{array}{lll}20 & 20 & 19.97\end{array}$

$\begin{array}{lll}-20 & -20 & 3.97 \\ -10 & -10 & 7.94\end{array}$

$\begin{array}{ccc}-10 & -10 & 7.94 \\ 0 & 0 & 11.94\end{array}$

$\begin{array}{lll}10 & 10 & 15.97\end{array}$

$\begin{array}{lll}20 & 20 & 19.97\end{array}$

Standard Uncertainties: Multimeter: $+/-($ Dead Weight Tester: $+/-$

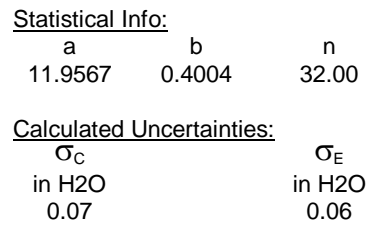

Accepted Tolerance: +/-

PASS CALIBRATION?

TRANSFER EQUATION:
TR-3749

\section{Curve}

Fit
(mADC)

3.95

7.95

11.96

15.96

19.96

3.95

7.95

11.96

15.96

19.96

3.95

7.95

11.96

15.96

19.96

3.95

7.95

11.96

15.96

0.04

0.07

Xbar
in $\mathrm{H} 2 \mathrm{O}$

$-1.05$

2.00

$\sigma_{\mathrm{F}}$

in $\mathrm{H} 2 \mathrm{O}$

0.00

0.50 in $\mathrm{H} 2 \mathrm{O}$

YES

in $\mathrm{H} 20=2.498$ page__of

cal. date:

$\%$ RDG $+\quad 0.001$ $\%$ RDG

$\begin{array}{cc}\text { Sxx } & \text { SEE } \\ \text { in } \mathrm{H}^{\circ} \mathrm{O}^{\circ} & \mathrm{mADC}^{<}\end{array}$

$3578.95 \quad 0.0036$

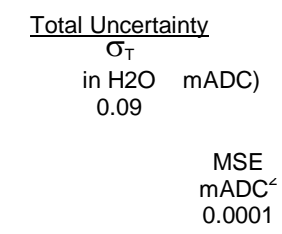

${ }^{*} \mathrm{~mA} \quad-29.863$

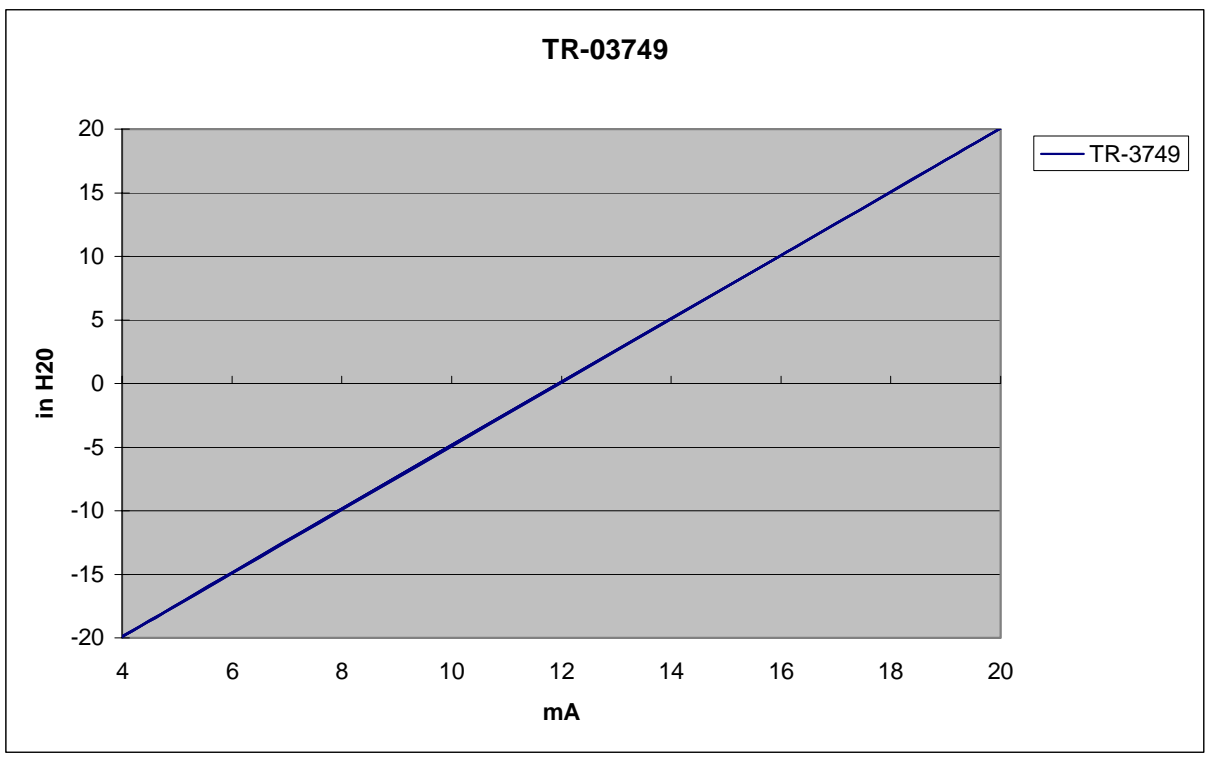

Figure G3-9. Ion Exchange Differential Pressure Post Test Calibration (DP6) 
WSRC-TR-2005-00105, REVISION 0

SRNL-RPP-2005-00012, REVISION 0

UNCERTAINTY ANALYSIS REF. L9.5-9114, REV. 1

Calibration Data

$\begin{array}{cccc}\begin{array}{c}\text { Meter } \\ \text { Output } \\ (\mathrm{mA})\end{array} & \begin{array}{c}\text { Water } \\ \text { Temp } \\ (\mathrm{C})\end{array} & \begin{array}{c}\text { Water } \\ \text { Weight } \\ \text { (lbs) }\end{array} & \begin{array}{c}\text { Time } \\ \text { Duration } \\ (\mathrm{min})\end{array} \\ 4.01 & \mathrm{~N} / \mathrm{A} & \mathrm{N} / \mathrm{A} & \mathrm{N} / \mathrm{A} \\ 7.25 & 19.87 & 0.679 & 8.00 \\ 10.40 & 19.61 & 0.669 & 4.00 \\ 13.68 & 19.62 & 0.757 & 3.00 \\ 16.88 & 19.45 & 0.836 & 2.50 \\ 20.00 & 19.68 & 0.832 & 2.00 \\ 4.01 & \mathrm{~N} / \mathrm{A} & \mathrm{N} / \mathrm{A} & \mathrm{N} / \mathrm{A} \\ 7.26 & 19.90 & 0.681 & 8.00 \\ 10.42 & 19.66 & 0.670 & 4.00 \\ 13.63 & 19.63 & 0.751 & 3.00 \\ 16.83 & 19.57 & 0.832 & 2.50 \\ 20.05 & 19.44 & 0.834 & 2.00 \\ 4.01 & \mathrm{~N} / \mathrm{A} & \mathrm{N} / \mathrm{A} & \mathrm{N} / \mathrm{A} \\ 7.28 & 19.82 & 0.685 & 8.00 \\ 10.43 & 19.70 & 0.672 & 4.01 \\ 13.63 & 19.60 & 0.752 & 3.00 \\ 16.80 & 19.61 & 0.828 & 2.51 \\ 20.00 & 19.53 & 0.831 & 2.00\end{array}$

TR-03703

$$
\begin{gathered}
\text { page } \\
\text { calibration date_08-Feb-05 }
\end{gathered}
$$

\section{Calculated Data}

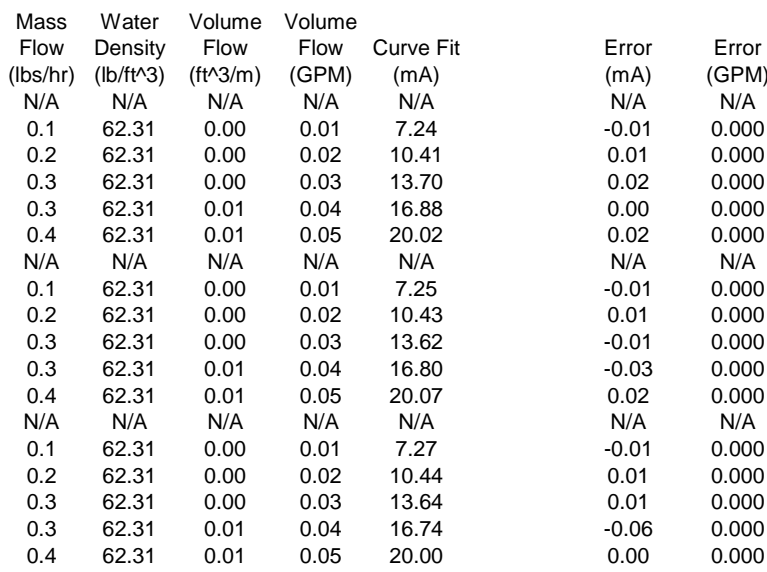

Density: $\rho=62.441-1.374 \mathrm{E}-3^{*} \mathrm{~T}-271.818 \mathrm{E}-6^{\star} \mathrm{T}^{\wedge} 2+194.093 \mathrm{E}-9^{*} \mathrm{~T}^{\wedge} 3$

7.4805 gallons $=1 \mathrm{ft}^{\wedge} 3$

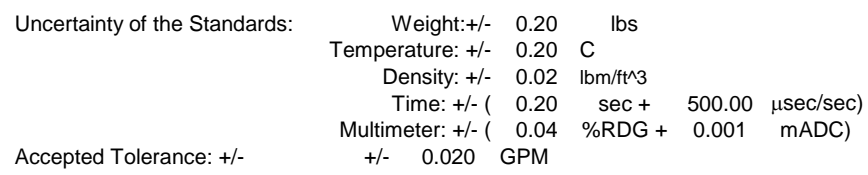

Statistical Info.

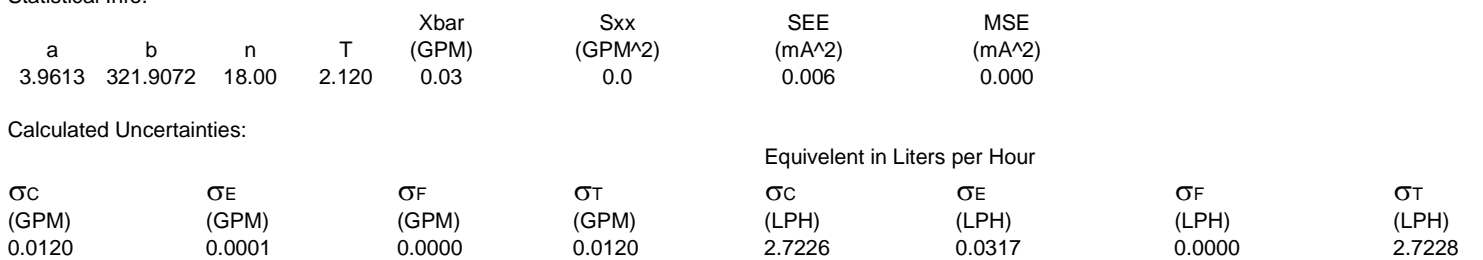

\section{$\begin{array}{ll}\text { PASS CALIBRATION? } & \text { YES } \\ \text { TRANSFER EQUATION: } & \text { GPM }=0.003 * \mathrm{~mA}-0.012\end{array}$}

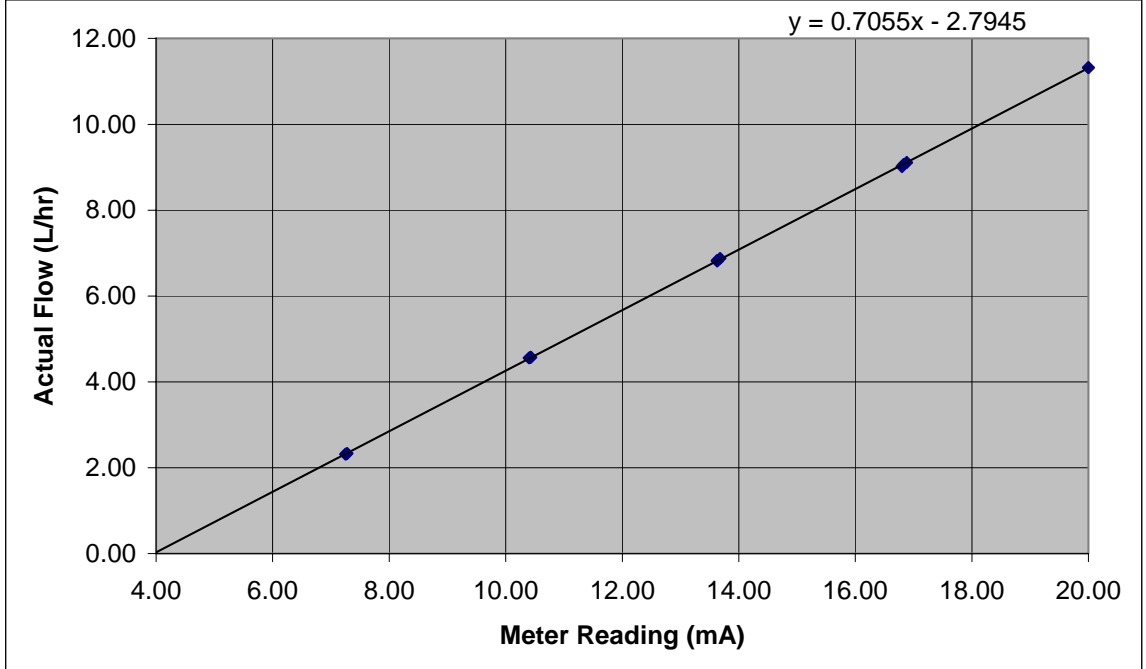

Figure G3-10. IX 0-12 L/hr (0-0.06 GPM) Flowmeter Post Test Calibration (Flowmeter) 
WSRC-TR-2005-00105, REVISION 0

SRNL-RPP-2005-00012, REVISION 0

\section{APPENDIX H: TABULATED RAW DATA FROM EACH OF THE PILOT OPERATIONS}

With time the accessibility of the raw data taken during the SIPP task will become more difficult. An attempt was made here to include the computer acquired data obtained during each of the unit operation for the four campaigns. However, because of the very large size of the files some of the data streams have been abridged. Such abridgment will be explained on the cover sheet to each of the unit operation data section. The order is as follows:

Section H1: FEP - Campaign II, III, and IV

Section H2: UFP - Campaigns I, II, III, and IV

Section H3: CIX - Campaigns II and III

Section H4: TLP - Campaigns II and III

Note that only the UFP was operated for all four campaigns, the CIX and TLP were only operated for Campaigns II and III, and the FEP was not used during Campaign I. 
WSRC-TR-2005-00105, REVISION 0

SRNL-RPP-2005-00012, REVISION 0

\section{Section H1: Pilot Feed Waste Evaporator Process (FEP) Raw Data}

FEP data were obtained ever 60 seconds, however the following tables have been reduced in size to show only the data ever 300 seconds. The full data stream is available upon request.

The short headings for used in FEP tables are described below

\section{Legend for FEP Data}

\begin{tabular}{|ll|}
\hline Conc Temp. Reboiler In & Concentrate Temperature at Reboiler Inlet \\
\hline Conc Temp. Reboiler out & Concentrate Temperature at Reboiler Outlet \\
\hline Reboiler Steam Supply Temp. & Reboiler Steam Supply Temperature \\
\hline Ejector Steam Supply Temp. & Ejector (Vacuum Pump) Steam Supply Temperature \\
\hline Cooling Water Supply Temp. & Cooling Water Supply (to all condensers) Temperature \\
\hline Evap. Vessel Temp. & Evaporator Vessel Concentrate Temperature \\
\hline Primary Condensate Temp. & Primary Condensate Temperature \\
\hline Inter. Cond. Outlet Temp. & Inter-condenser Outlet Temperature \\
\hline After Cond. Outlet Temp. & After-condenser Outlet Temperature \\
\hline Recirc. Flow Rate & Recirculation Flow Rate of Concentrate \\
\hline Feed Rate & Feed Rate to the Evaporator Recirculation Loop \\
\hline Prim. Condensate Flow & Primary Condensate Flow Rate \\
\hline Intercondenser Press. & Intercondenser Pressure \\
\hline Evaporator Press. & Evaporator Pressure \\
\hline
\end{tabular}


WSRC-TR-2005-00105, REVISION 0

SRNL-RPP-2005-00012, REVISION 0

Table H1-1a: Campaign II

\begin{tabular}{|c|c|c|c|c|c|c|c|c|c|c|c|c|c|c|c|c|}
\hline & DATE & TIME & $\begin{array}{c}\text { Conc } \\
\text { Temp. } \\
\text { Reboiler } \\
\text { In }\end{array}$ & $\begin{array}{c}\text { Conc } \\
\text { Temp. } \\
\text { Reboiler } \\
\text { out }\end{array}$ & $\begin{array}{l}\text { Reboiler } \\
\text { Steam } \\
\text { Supply } \\
\text { Temp. }\end{array}$ & $\begin{array}{l}\text { Ejector } \\
\text { Steam } \\
\text { Supply } \\
\text { Temp. }\end{array}$ & $\begin{array}{l}\text { Cooling } \\
\text { Water } \\
\text { Supply } \\
\text { Temp. }\end{array}$ & $\begin{array}{l}\text { Evap. } \\
\text { Vessel } \\
\text { Temp. }\end{array}$ & $\begin{array}{c}\text { Primary } \\
\text { Conden- } \\
\text { sate } \\
\text { Temp. }\end{array}$ & $\begin{array}{l}\text { Inter. } \\
\text { Cond. } \\
\text { Outlet } \\
\text { Temp. }\end{array}$ & $\begin{array}{l}\text { After } \\
\text { Cond. } \\
\text { Outlet } \\
\text { Temp. }\end{array}$ & $\begin{array}{c}\text { Recirc. } \\
\text { Flow } \\
\text { Rate }\end{array}$ & $\begin{array}{l}\text { Feed } \\
\text { Rate }\end{array}$ & $\begin{array}{l}\text { Prim. } \\
\text { Conden- } \\
\text { sate } \\
\text { Flow }\end{array}$ & $\begin{array}{c}\text { Intercondenser } \\
\text { Press. }\end{array}$ & $\begin{array}{l}\text { Evapo } \\
\text { rator. } \\
\text { Press. }\end{array}$ \\
\hline & & & ${ }^{\circ} \mathrm{C}$ & ${ }^{\circ} \mathrm{C}$ & ${ }^{\circ} \mathrm{C}$ & ${ }^{\circ} \mathrm{C}$ & ${ }^{\circ} \mathrm{C}$ & ${ }^{\circ} \mathrm{C}$ & ${ }^{\circ} \mathrm{C}$ & ${ }^{\circ} \mathrm{C}$ & ${ }^{\circ} \mathrm{C}$ & GPM & GPM & GPM & PSIG & PSIG \\
\hline 1 & $4 / 14 / 2004$ & $6: 47: 32$ AM & 18.80 & 19.70 & 34.50 & 169.95 & 20.27 & 18.40 & 20.12 & 21.08 & 23.14 & 32.89 & -0.01 & 0.00 & -2.78 & -4.78 \\
\hline 2 & $4 / 14 / 2004$ & $6: 52: 34 \mathrm{AM}$ & 40.97 & 45.28 & 139.10 & 169.98 & 19.79 & 42.42 & 19.79 & 20.65 & 22.60 & 83.32 & -0.01 & 0.00 & -7.52 & -8.75 \\
\hline 3 & $4 / 14 / 2004$ & $6: 57: 37 \mathrm{AM}$ & 65.94 & 67.27 & 143.32 & 169.96 & 19.55 & 66.04 & 19.48 & 20.41 & 22.40 & 83.70 & -0.01 & 0.02 & -8.43 & -10.17 \\
\hline 4 & $4 / 14 / 2004$ & 7:02:39 AM & 52.92 & 53.41 & 137.90 & 170.08 & 19.48 & 52.07 & 22.34 & 20.42 & 22.46 & 83.00 & -0.01 & 0.16 & -10.54 & -12.26 \\
\hline 5 & $4 / 14 / 2004$ & 7:07:41 AM & 36.02 & 40.09 & 142.91 & 170.14 & 19.44 & 36.02 & 22.50 & 20.34 & 22.25 & 82.45 & -0.01 & 0.23 & -11.53 & -13.63 \\
\hline 6 & $4 / 14 / 2004$ & 7:15:19 AM & 41.97 & 46.44 & 146.35 & 169.48 & 19.41 & 41.89 & 23.18 & 20.22 & 22.02 & 82.51 & -0.01 & 0.18 & -11.47 & -13.58 \\
\hline 7 & $4 / 14 / 2004$ & 7:20:21 AM & 43.71 & 48.09 & 146.64 & 168.94 & 19.40 & 43.63 & 23.17 & 20.19 & 22.00 & 82.49 & -0.01 & 0.50 & -11.26 & -13.57 \\
\hline 8 & $4 / 14 / 2004$ & $7: 25: 24 \mathrm{AM}$ & 43.77 & 48.24 & 147.20 & 169.32 & 19.40 & 43.79 & 23.35 & 20.20 & 22.02 & 82.01 & 0.09 & 0.57 & -11.35 & -13.56 \\
\hline 9 & $4 / 14 / 2004$ & 7:30:26 AM & 43.15 & 47.57 & 147.66 & 168.90 & 19.38 & 43.53 & 22.74 & 20.17 & 21.99 & 82.39 & 0.44 & 0.63 & -11.32 & -13.57 \\
\hline 10 & $4 / 14 / 2004$ & $7: 35: 28 \mathrm{AM}$ & 44.65 & 49.01 & 147.32 & 168.74 & 19.37 & 44.64 & 23.25 & 20.16 & 21.98 & 82.30 & 0.46 & 0.76 & -11.28 & -13.56 \\
\hline 11 & $4 / 14 / 2004$ & 7:40:30 AM & 44.84 & 49.21 & 147.73 & 169.52 & 19.34 & 45.04 & 22.57 & 20.17 & 22.01 & 82.15 & 0.54 & 0.83 & -11.24 & -13.54 \\
\hline 12 & $4 / 14 / 2004$ & 7:45:32 AM & 46.56 & 50.94 & 147.34 & 170.10 & 19.35 & 46.54 & 23.24 & 20.18 & 22.03 & 82.31 & 0.50 & 0.83 & -11.20 & -13.48 \\
\hline 13 & $4 / 14 / 2004$ & 7:50:35 AM & 44.50 & 48.95 & 147.40 & 169.99 & 19.33 & 44.55 & 23.15 & 20.17 & 22.01 & 82.22 & 0.55 & 0.55 & -11.34 & -13.52 \\
\hline 14 & $4 / 14 / 2004$ & 7:55:37 AM & 45.65 & 49.97 & 148.15 & 168.86 & 19.30 & 45.75 & 22.73 & 20.10 & 21.89 & 82.34 & 0.50 & 0.50 & -11.21 & -12.70 \\
\hline 15 & $4 / 14 / 2004$ & 8:00:39 AM & 45.37 & 50.31 & 147.48 & 169.91 & 19.29 & 46.62 & 22.02 & 20.06 & 21.64 & 57.12 & 0.41 & 0.45 & -10.22 & -12.21 \\
\hline 16 & $4 / 14 / 2004$ & 8:05:41 AM & 57.85 & 61.46 & 142.68 & 170.72 & 19.28 & 58.84 & 20.00 & 20.17 & 22.10 & 74.18 & 0.39 & 0.09 & -9.23 & -11.00 \\
\hline 17 & $4 / 14 / 2004$ & 8:10:43 AM & 47.44 & 51.72 & 148.73 & 169.47 & 19.28 & 47.03 & 25.23 & 20.12 & 21.95 & 82.31 & 0.46 & 0.39 & -11.36 & -12.94 \\
\hline 18 & $4 / 14 / 2004$ & 8:15:46 AM & 44.38 & 48.23 & 147.54 & 170.01 & 19.26 & 44.41 & 22.70 & 20.14 & 22.04 & 82.29 & 0.69 & 0.73 & -11.31 & -13.20 \\
\hline 19 & $4 / 14 / 2004$ & 8:20:48 AM & 41.09 & 45.13 & 148.17 & 170.84 & 19.24 & 41.16 & 22.84 & 20.17 & 22.14 & 82.15 & 0.64 & 0.18 & -11.55 & -13.36 \\
\hline 20 & $4 / 14 / 2004$ & $8: 25: 50$ AM & 42.04 & 46.03 & 148.14 & 170.88 & 19.23 & 42.20 & 22.41 & 20.14 & 22.10 & 82.19 & 0.60 & 0.35 & -11.43 & -13.37 \\
\hline 21 & $4 / 14 / 2004$ & 8:30:52 AM & 44.31 & 48.33 & 148.05 & 170.93 & 19.24 & 44.49 & 22.53 & 20.07 & 21.73 & 82.42 & 0.57 & 0.43 & -10.59 & -12.14 \\
\hline 22 & $4 / 14 / 2004$ & $8: 35: 55$ AM & 58.05 & 60.25 & 140.53 & 170.96 & 19.22 & 58.65 & 19.23 & 19.68 & 19.61 & 83.79 & 0.30 & 0.46 & -3.02 & -3.60 \\
\hline 23 & $4 / 14 / 2004$ & 8:40:57 AM & 62.86 & 63.09 & 136.94 & 171.01 & 19.21 & 62.68 & 19.16 & 19.31 & 20.17 & 83.98 & -0.01 & 0.04 & -1.13 & -1.12 \\
\hline 24 & $4 / 14 / 2004$ & 8:45:59 AM & 62.12 & 62.36 & 132.53 & 159.34 & 19.23 & 61.96 & 19.18 & 19.26 & 20.17 & 83.92 & -0.01 & 0.01 & -0.29 & -0.02 \\
\hline 25 & $4 / 14 / 2004$ & 8:51:01 AM & 61.44 & 61.66 & 128.27 & 164.95 & 19.21 & 61.27 & 19.16 & 19.80 & 21.41 & 83.71 & -0.01 & 0.02 & -1.56 & -2.13 \\
\hline 26 & 4/14/2004 & 8:56:03 AM & 60.79 & 61.02 & 124.17 & 158.07 & 19.20 & 60.63 & 19.16 & 19.59 & 20.60 & 83.69 & -0.01 & 0.01 & -0.46 & -1.55 \\
\hline 27 & $4 / 14 / 2004$ & 9:01:06 AM & 60.31 & 60.87 & 124.78 & 171.26 & 19.18 & 60.24 & 19.14 & 20.13 & 22.18 & 83.49 & -0.01 & 0.00 & -6.52 & -8.63 \\
\hline 28 & $4 / 14 / 2004$ & 9:06:08 AM & 63.63 & 64.87 & 143.73 & 171.26 & 19.18 & 63.30 & 20.70 & 20.13 & 22.16 & 83.13 & -0.01 & 0.10 & -9.30 & -11.27 \\
\hline 29 & $4 / 14 / 2004$ & 9:11:10 AM & 41.47 & 44.35 & 138.64 & 171.26 & 19.18 & 41.42 & 22.30 & 20.12 & 22.06 & 82.27 & 0.21 & 0.53 & -11.40 & -13.63 \\
\hline 30 & $4 / 14 / 2004$ & $9: 16: 12$ AM & 43.47 & 47.78 & 148.16 & 171.10 & 19.18 & 43.49 & 23.11 & 20.06 & 21.97 & 82.20 & 0.50 & 0.54 & -11.47 & -13.62 \\
\hline
\end{tabular}


WSRC-TR-2005-00105, REVISION 0

SRNL-RPP-2005-00012, REVISION 0

Table H1-1b: Campaign II

\begin{tabular}{|c|c|c|c|c|c|c|c|c|c|c|c|c|c|c|c|c|}
\hline & DATE & TIME & $\begin{array}{c}\text { Conc } \\
\text { Temp. } \\
\text { Reboiler } \\
\text { In }\end{array}$ & $\begin{array}{l}\text { Conc } \\
\text { Temp. } \\
\text { Reboiler } \\
\text { out }\end{array}$ & $\begin{array}{c}\text { Reboiler } \\
\text { Steam } \\
\text { Supply } \\
\text { Temp. }\end{array}$ & $\begin{array}{l}\text { Ejector } \\
\text { Steam } \\
\text { Supply } \\
\text { Temp. }\end{array}$ & $\begin{array}{l}\text { Cooling } \\
\text { Water } \\
\text { Supply } \\
\text { Temp. } \\
\end{array}$ & $\begin{array}{l}\text { Evap. } \\
\text { Vessel } \\
\text { Temp. }\end{array}$ & $\begin{array}{c}\text { Primary } \\
\text { Conden- } \\
\text { sate } \\
\text { Temp. } \\
\end{array}$ & $\begin{array}{l}\text { Inter. } \\
\text { Cond. } \\
\text { Outlet } \\
\text { Temp. }\end{array}$ & $\begin{array}{l}\text { After } \\
\text { Cond. } \\
\text { Outlet } \\
\text { Temp. }\end{array}$ & $\begin{array}{c}\text { Recirc. } \\
\text { Flow } \\
\text { Rate }\end{array}$ & $\begin{array}{l}\text { Feed } \\
\text { Rate }\end{array}$ & $\begin{array}{l}\text { Prim. } \\
\text { Conden- } \\
\text { sate } \\
\text { Flow } \\
\end{array}$ & $\begin{array}{c}\text { Intercondenser } \\
\text { Press. } \\
\end{array}$ & $\begin{array}{c}\text { Evapo- } \\
\text { rator. } \\
\text { Press. }\end{array}$ \\
\hline & & & ${ }^{\circ} \mathrm{C}$ & ${ }^{\circ} \mathrm{C}$ & ${ }^{\circ} \mathrm{C}$ & ${ }^{\circ} \mathrm{C}$ & ${ }^{\circ} \mathrm{C}$ & ${ }^{\circ} \mathrm{C}$ & ${ }^{\circ} \mathrm{C}$ & ${ }^{\circ} \mathrm{C}$ & ${ }^{\circ} \mathrm{C}$ & GPM & GPM & GPM & PSIG & PSIG \\
\hline 31 & $4 / 14 / 2004$ & 9:21:14 AM & 44.33 & 48.57 & 148.02 & 170.82 & 19.17 & 44.42 & 22.62 & 20.04 & 21.91 & 82.19 & 0.41 & 0.57 & -11.32 & -13.55 \\
\hline 32 & $4 / 14 / 2004$ & 9:26:17 AM & 45.04 & 49.27 & 148.25 & 170.86 & 19.17 & 45.08 & 22.83 & 20.04 & 21.92 & 82.17 & 0.43 & 0.51 & -11.30 & -13.62 \\
\hline 33 & $4 / 14 / 2004$ & 9:31:19 AM & 45.17 & 49.38 & 148.14 & 170.78 & 19.18 & 45.29 & 22.59 & 20.05 & 21.91 & 82.20 & 0.46 & 0.49 & -11.26 & -13.60 \\
\hline 34 & $4 / 14 / 2004$ & 9:36:21 AM & 45.43 & 49.67 & 148.21 & 171.08 & 19.17 & 45.45 & 22.86 & 20.04 & 21.92 & 82.06 & 0.41 & 0.52 & -11.28 & -13.57 \\
\hline 35 & $4 / 14 / 2004$ & 9:41:23 AM & 45.42 & 48.79 & 147.89 & 170.09 & 19.18 & 45.50 & 21.97 & 20.04 & 21.92 & 82.10 & 0.57 & 0.30 & -11.22 & -13.55 \\
\hline 36 & $4 / 14 / 2004$ & 9:46:26 AM & 42.72 & 46.53 & 148.37 & 171.72 & 19.19 & 42.81 & 22.46 & 20.11 & 22.08 & 81.91 & 0.54 & 0.19 & -11.41 & -13.54 \\
\hline 37 & $4 / 14 / 2004$ & 9:51:28 AM & 44.38 & 48.13 & 148.33 & 171.78 & 19.18 & 44.47 & 22.25 & 20.09 & 22.07 & 81.99 & 0.48 & 0.58 & -11.28 & -13.49 \\
\hline 38 & $4 / 14 / 2004$ & 9:56:30 AM & 44.65 & 48.39 & 148.32 & 171.87 & 19.19 & 44.75 & 22.25 & 20.09 & 22.06 & 81.96 & 0.48 & 0.66 & -11.27 & -13.48 \\
\hline 39 & $4 / 14 / 2004$ & 10:01:32 AM & 44.32 & 48.07 & 148.16 & 171.96 & 19.17 & 44.39 & 22.29 & 20.08 & 22.04 & 81.95 & 0.47 & 0.52 & -11.30 & -13.47 \\
\hline 40 & $4 / 14 / 2004$ & 10:06:34 AM & 44.56 & 48.29 & 148.33 & 172.06 & 19.17 & 44.63 & 22.23 & 20.09 & 22.05 & 81.95 & 0.43 & 0.43 & -11.29 & -13.46 \\
\hline 41 & $4 / 14 / 2004$ & $10: 11: 36$ AM & 44.56 & 48.29 & 148.34 & 172.16 & 19.17 & 44.61 & 22.32 & 20.09 & 22.05 & 81.95 & 0.36 & 0.43 & -11.29 & -13.47 \\
\hline 42 & $4 / 14 / 2004$ & 10:16:39 AM & 44.50 & 48.28 & 148.24 & 172.25 & 19.16 & 44.52 & 22.34 & 20.08 & 22.05 & 81.90 & 0.29 & 0.33 & -11.31 & -13.49 \\
\hline 43 & $4 / 14 / 2004$ & 10:21:41 AM & 44.34 & 48.20 & 148.36 & 172.30 & 19.16 & 44.36 & 22.51 & 20.07 & 22.02 & 81.95 & 0.24 & 0.19 & -11.34 & -13.53 \\
\hline 44 & $4 / 14 / 2004$ & $10: 26: 43$ AM & 45.36 & 49.18 & 148.41 & 172.41 & 19.16 & 45.36 & 22.41 & 20.06 & 22.01 & 81.90 & 0.23 & 0.18 & -11.25 & -13.52 \\
\hline 45 & $4 / 14 / 2004$ & $10: 31: 45$ AM & 48.55 & 52.20 & 148.47 & 172.39 & 19.16 & 48.67 & 21.81 & 20.05 & 22.01 & 81.87 & 0.23 & 0.66 & -10.98 & -13.45 \\
\hline 46 & $4 / 14 / 2004$ & 10:36:47 AM & 49.21 & 52.86 & 148.46 & 172.53 & 19.17 & 49.18 & 22.26 & 20.07 & 22.02 & 81.73 & 0.22 & 0.88 & -10.98 & -13.43 \\
\hline 47 & $4 / 14 / 2004$ & $10: 41: 50$ AM & 49.19 & 52.83 & 148.49 & 172.52 & 19.17 & 49.35 & 22.02 & 20.07 & 22.02 & 81.69 & 0.63 & 0.41 & -10.94 & -13.43 \\
\hline 48 & $4 / 14 / 2004$ & $10: 46: 52 \mathrm{AM}$ & 50.22 & 53.88 & 148.31 & 171.85 & 19.17 & 50.32 & 22.27 & 20.05 & 21.95 & 81.86 & 0.74 & 0.32 & -10.93 & -13.39 \\
\hline 49 & $4 / 14 / 2004$ & $10: 51: 54$ AM & 44.61 & 48.58 & 148.41 & 172.71 & 19.16 & 44.68 & 22.83 & 20.07 & 21.99 & 81.46 & 0.56 & 0.25 & -11.37 & -13.59 \\
\hline 50 & $4 / 14 / 2004$ & 10:56:56 AM & 46.10 & 50.05 & 148.40 & 172.69 & 19.15 & 46.23 & 22.27 & 20.05 & 21.97 & 81.53 & 0.47 & 0.26 & -11.20 & -13.56 \\
\hline 51 & $4 / 14 / 2004$ & $11: 01: 59$ AM & 47.31 & 51.33 & 147.79 & 172.19 & 19.17 & 47.42 & 22.56 & 20.03 & 21.93 & 84.54 & 0.38 & 0.16 & -11.13 & -13.52 \\
\hline 52 & $4 / 14 / 2004$ & 11:07:01 AM & 50.37 & 54.32 & 146.64 & 169.76 & 19.17 & 50.47 & 22.42 & 19.99 & 21.79 & 85.22 & 0.34 & 0.98 & -10.92 & -13.41 \\
\hline 53 & $4 / 14 / 2004$ & $11: 12: 03$ AM & 50.46 & 54.46 & 147.70 & 170.36 & 19.16 & 50.45 & 22.83 & 20.00 & 21.81 & 85.11 & 0.34 & 0.90 & -10.93 & -13.41 \\
\hline 54 & $4 / 14 / 2004$ & $11: 17: 05$ AM & 51.08 & 55.05 & 144.64 & 170.63 & 19.16 & 51.14 & 22.47 & 20.00 & 21.81 & 84.99 & 0.33 & 0.47 & -10.83 & -13.38 \\
\hline 55 & $4 / 14 / 2004$ & $11: 22: 07$ AM & 50.61 & 54.58 & 145.36 & 169.98 & 19.16 & 50.62 & 22.68 & 19.99 & 21.78 & 85.01 & 0.36 & 0.50 & -10.93 & -13.34 \\
\hline 56 & $4 / 14 / 2004$ & $11: 27: 10$ AM & 50.49 & 54.48 & 147.81 & 170.27 & 19.18 & 50.51 & 22.84 & 20.02 & 21.81 & 85.12 & 0.41 & 0.53 & -10.94 & -13.31 \\
\hline 57 & $4 / 14 / 2004$ & $11: 32: 12$ AM & 50.50 & 54.56 & 143.97 & 171.40 & 19.19 & 50.59 & 22.68 & 20.04 & 21.86 & 84.94 & 0.57 & 0.50 & -10.89 & -13.29 \\
\hline 58 & $4 / 14 / 2004$ & $11: 37: 14$ AM & 50.62 & 54.59 & 145.71 & 170.45 & 19.18 & 50.83 & 22.32 & 20.01 & 21.81 & 84.82 & 0.64 & 0.46 & -10.87 & -13.29 \\
\hline 59 & $4 / 14 / 2004$ & $11: 42: 16$ AM & 51.14 & 55.10 & 145.72 & 170.34 & 19.18 & 51.39 & 22.48 & 20.01 & 21.81 & 84.99 & 0.92 & 0.47 & -10.85 & -13.24 \\
\hline 60 & $4 / 14 / 2004$ & $11: 47: 18$ AM & 50.92 & 54.92 & 145.77 & 170.95 & 19.19 & 51.15 & 22.56 & 20.03 & 21.85 & 85.17 & 0.86 & 0.48 & -10.85 & -13.26 \\
\hline
\end{tabular}


WSRC-TR-2005-00105, REVISION 0

SRNL-RPP-2005-00012, REVISION 0

Table H1-1c: Campaign II

\begin{tabular}{|c|c|c|c|c|c|c|c|c|c|c|c|c|c|c|c|c|}
\hline \multicolumn{17}{|c|}{ FEP Campaign II Data (Contd...) } \\
\hline & DATE & TIME & $\begin{array}{c}\text { Conc } \\
\text { Temp. } \\
\text { Reboiler } \\
\text { In }\end{array}$ & $\begin{array}{c}\text { Conc } \\
\text { Temp. } \\
\text { Reboiler } \\
\text { out }\end{array}$ & $\begin{array}{l}\text { Reboiler } \\
\text { Steam } \\
\text { Supply } \\
\text { Temp. }\end{array}$ & $\begin{array}{l}\text { Ejector } \\
\text { Steam } \\
\text { Supply } \\
\text { Temp. }\end{array}$ & $\begin{array}{l}\text { Cooling } \\
\text { Water } \\
\text { Supply } \\
\text { Temp. }\end{array}$ & $\begin{array}{l}\text { Evap. } \\
\text { Vessel } \\
\text { Temp. }\end{array}$ & $\begin{array}{c}\text { Primary } \\
\text { Conden- } \\
\text { sate } \\
\text { Temp. }\end{array}$ & $\begin{array}{l}\text { Inter. } \\
\text { Cond. } \\
\text { Outlet } \\
\text { Temp. }\end{array}$ & $\begin{array}{l}\text { After } \\
\text { Cond. } \\
\text { Outlet } \\
\text { Temp. }\end{array}$ & $\begin{array}{c}\text { Recirc. } \\
\text { Flow } \\
\text { Rate }\end{array}$ & $\begin{array}{l}\text { Feed } \\
\text { Rate }\end{array}$ & $\begin{array}{l}\text { Prim. } \\
\text { Conden- } \\
\text { sate } \\
\text { Flow }\end{array}$ & $\begin{array}{c}\text { Intercondenser } \\
\text { Press. }\end{array}$ & $\begin{array}{c}\text { Evapo- } \\
\text { rator. } \\
\text { Press. }\end{array}$ \\
\hline & & & ${ }^{\circ} \mathbf{C}$ & ${ }^{\circ} \mathrm{C}$ & ${ }^{\circ} \mathbf{C}$ & ${ }^{\circ} \mathrm{C}$ & ${ }^{\circ} \mathrm{C}$ & ${ }^{\circ} \mathrm{C}$ & ${ }^{\circ} \mathrm{C}$ & ${ }^{\circ} \mathrm{C}$ & ${ }^{\circ} \mathrm{C}$ & GPM & GPM & GPM & PSIG & PSIG \\
\hline 61 & $4 / 14 / 2004$ & $11: 52: 21$ AM & 51.14 & 55.06 & 146.05 & 170.03 & 19.19 & 51.31 & 22.45 & 20.02 & 21.81 & 85.12 & 0.61 & 0.47 & -10.86 & -13.46 \\
\hline 62 & $4 / 14 / 2004$ & $11: 57: 23$ AM & 51.51 & 55.45 & 144.09 & 170.39 & 19.18 & 51.59 & 22.64 & 20.01 & 21.81 & 85.13 & 0.44 & 0.50 & -10.84 & -13.45 \\
\hline 63 & $4 / 14 / 2004$ & 12:02:25 PM & 51.42 & 55.35 & 143.95 & 170.52 & 19.18 & 51.51 & 22.60 & 20.02 & 21.82 & 85.40 & 0.40 & 0.50 & -10.85 & -13.48 \\
\hline 64 & $4 / 14 / 2004$ & 12:07:27 PM & 51.69 & 55.63 & 144.85 & 170.57 & 19.20 & 51.80 & 22.62 & 20.03 & 21.83 & 85.15 & 0.47 & 0.49 & -10.83 & -13.48 \\
\hline 65 & $4 / 14 / 2004$ & 12:12:29 PM & 52.01 & 55.94 & 145.08 & 170.55 & 19.18 & 52.12 & 22.56 & 20.01 & 21.81 & 84.88 & 0.49 & 0.49 & -10.79 & -13.47 \\
\hline 66 & $4 / 14 / 2004$ & 12:17:32 PM & 51.67 & 55.64 & 144.70 & 171.12 & 19.18 & 51.77 & 22.64 & 20.02 & 21.85 & 84.81 & 0.53 & 0.50 & -10.83 & -13.49 \\
\hline 67 & $4 / 14 / 2004$ & 12:22:34 PM & 51.89 & 55.79 & 141.94 & 170.22 & 19.19 & 52.02 & 22.48 & 20.02 & 21.79 & 84.83 & 0.49 & 0.48 & -10.80 & -13.49 \\
\hline 68 & $4 / 14 / 2004$ & 12:27:36 PM & 52.08 & 56.03 & 146.11 & 170.87 & 19.18 & 52.16 & 22.75 & 20.02 & 21.83 & 84.65 & 0.48 & 0.51 & -10.80 & -13.48 \\
\hline 69 & $4 / 14 / 2004$ & 12:32:38 PM & 51.71 & 55.64 & 144.26 & 170.34 & 19.18 & 51.85 & 22.45 & 20.01 & 21.79 & 84.42 & 0.48 & 0.48 & -10.81 & -13.52 \\
\hline 70 & $4 / 14 / 2004$ & $12: 37: 40 \mathrm{PM}$ & 52.64 & 56.59 & 141.45 & 171.19 & 19.20 & 52.76 & 22.51 & 20.04 & 21.85 & 84.75 & 0.47 & 0.48 & -10.73 & -13.47 \\
\hline 71 & $4 / 14 / 2004$ & 12:42:43 PM & 51.78 & 55.72 & 144.65 & 170.39 & 19.18 & 51.89 & 22.60 & 20.02 & 21.81 & 84.42 & 0.47 & 0.50 & -10.84 & -13.53 \\
\hline 72 & $4 / 14 / 2004$ & 12:47:45 PM & 52.16 & 56.10 & 141.70 & 170.79 & 19.17 & 52.24 & 22.65 & 20.01 & 21.81 & 84.34 & 0.44 & 0.49 & -10.79 & -13.51 \\
\hline 73 & $4 / 14 / 2004$ & 12:52:47 PM & 52.15 & 56.06 & 143.11 & 170.42 & 19.17 & 52.26 & 22.54 & 20.01 & 21.80 & 84.39 & 0.43 & 0.49 & -10.78 & -13.51 \\
\hline 74 & $4 / 14 / 2004$ & 12:57:49 PM & 52.68 & 56.59 & 142.41 & 170.79 & 19.18 & 52.76 & 22.55 & 20.02 & 21.83 & 84.35 & 0.42 & 0.48 & -10.75 & -13.49 \\
\hline 75 & $4 / 14 / 2004$ & 1:02:52 PM & 52.60 & 56.52 & 140.11 & 171.10 & 19.19 & 52.68 & 22.54 & 20.03 & 21.84 & 84.42 & 0.41 & 0.48 & -10.73 & -13.48 \\
\hline 76 & $4 / 14 / 2004$ & 1:07:54 PM & 52.70 & 56.61 & 142.23 & 170.74 & 19.20 & 52.74 & 22.71 & 20.04 & 21.84 & 84.18 & 0.40 & 0.50 & -10.76 & -13.48 \\
\hline 77 & $4 / 14 / 2004$ & 1:12:56 PM & 52.59 & 56.49 & 143.64 & 170.66 & 19.20 & 52.67 & 22.54 & 20.03 & 21.83 & 84.17 & 0.39 & 0.48 & -10.77 & -13.50 \\
\hline 78 & $4 / 14 / 2004$ & 1:17:58 PM & 52.39 & 56.34 & 142.69 & 171.32 & 19.19 & 52.46 & 22.60 & 20.04 & 21.87 & 84.11 & 0.39 & 0.49 & -10.78 & -13.52 \\
\hline 79 & $4 / 14 / 2004$ & 1:23:00 PM & 53.50 & 57.38 & 142.98 & 170.50 & 19.19 & 53.60 & 22.40 & 20.02 & 21.83 & 83.88 & 0.39 & 0.46 & -10.69 & -13.47 \\
\hline 80 & $4 / 14 / 2004$ & 1:28:02 PM & 53.61 & 57.49 & 141.14 & 170.56 & 19.18 & 53.69 & 22.55 & 20.02 & 21.82 & 84.08 & 0.39 & 0.48 & -10.68 & -13.44 \\
\hline 81 & $4 / 14 / 2004$ & 1:33:05 PM & 53.47 & 57.37 & 144.95 & 170.56 & 19.19 & 53.52 & 22.65 & 20.03 & 21.84 & 83.80 & 0.39 & 0.50 & -10.73 & -13.44 \\
\hline 82 & $4 / 14 / 2004$ & 1:38:07 PM & 53.52 & 57.43 & 141.65 & 171.01 & 19.20 & 53.58 & 22.49 & 20.04 & 21.85 & 83.61 & 0.37 & 0.48 & -10.71 & -13.44 \\
\hline 83 & $4 / 14 / 2004$ & 1:43:09 PM & 53.11 & 57.02 & 143.58 & 170.51 & 19.20 & 53.13 & 22.76 & 20.04 & 21.84 & 83.50 & 0.35 & 0.51 & -10.78 & -13.48 \\
\hline 84 & $4 / 14 / 2004$ & 1:48:11 PM & 54.61 & 58.47 & 138.91 & 171.24 & 19.22 & 54.68 & 22.35 & 20.06 & 21.87 & 83.99 & 0.34 & 0.45 & -10.57 & -13.41 \\
\hline 85 & $4 / 14 / 2004$ & 1:53:13 PM & 53.44 & 57.35 & 143.71 & 170.61 & 19.22 & 53.48 & 22.71 & 20.05 & 21.87 & 83.71 & 0.45 & 0.50 & -10.76 & -13.46 \\
\hline 86 & $4 / 14 / 2004$ & 1:58:16 PM & 54.01 & 57.84 & 142.79 & 170.04 & 19.23 & 54.11 & 22.46 & 20.05 & 21.84 & 84.06 & 0.47 & 0.46 & -10.68 & -13.43 \\
\hline 87 & $4 / 14 / 2004$ & 2:03:18 PM & 53.99 & 57.87 & 140.51 & 170.97 & 19.23 & 54.09 & 22.61 & 20.06 & 21.88 & 84.28 & 0.47 & 0.49 & -10.65 & -13.41 \\
\hline 88 & $4 / 14 / 2004$ & 2:08:20 PM & 54.12 & 58.00 & 141.50 & 171.14 & 19.23 & 54.20 & 22.57 & 20.07 & 21.89 & 83.75 & 0.46 & 0.48 & -10.67 & -13.40 \\
\hline 89 & $4 / 14 / 2004$ & 2:13:22 PM & 53.68 & 57.55 & 142.64 & 170.72 & 19.23 & 53.77 & 22.61 & 20.06 & 21.86 & 83.96 & 0.46 & 0.49 & -10.71 & -13.45 \\
\hline 90 & $4 / 14 / 2004$ & 2:18:25 PM & 53.78 & 57.67 & 142.48 & 171.26 & 19.23 & 53.87 & 22.58 & 20.07 & 21.89 & 84.09 & 0.46 & 0.48 & -10.70 & -13.44 \\
\hline
\end{tabular}


WSRC-TR-2005-00105, REVISION 0

SRNL-RPP-2005-00012, REVISION 0

Table H1-1d: Campaign II

\begin{tabular}{|c|c|c|c|c|c|c|c|c|c|c|c|c|c|c|c|c|}
\hline \multicolumn{17}{|c|}{ EP Campaign II Data (Contd...) } \\
\hline & DATE & TIME & $\begin{array}{c}\text { Conc } \\
\text { Temp. } \\
\text { Reboiler } \\
\text { In }\end{array}$ & $\begin{array}{c}\text { Conc } \\
\text { Temp. } \\
\text { Reboiler } \\
\text { out }\end{array}$ & $\begin{array}{l}\text { Reboiler } \\
\text { Steam } \\
\text { Supply } \\
\text { Temp. }\end{array}$ & $\begin{array}{l}\text { Ejector } \\
\text { Steam } \\
\text { Supply } \\
\text { Temp. }\end{array}$ & $\begin{array}{l}\text { Cooling } \\
\text { Water } \\
\text { Supply } \\
\text { Temp. }\end{array}$ & $\begin{array}{l}\text { Evap. } \\
\text { Vessel } \\
\text { Temp. }\end{array}$ & $\begin{array}{c}\text { Primary } \\
\text { Conden- } \\
\text { sate } \\
\text { Temp. }\end{array}$ & $\begin{array}{l}\text { Inter. } \\
\text { Cond. } \\
\text { Outlet } \\
\text { Temp. }\end{array}$ & $\begin{array}{l}\text { After } \\
\text { Cond. } \\
\text { Outlet } \\
\text { Temp. }\end{array}$ & $\begin{array}{c}\text { Recirc. } \\
\text { Flow } \\
\text { Rate }\end{array}$ & $\begin{array}{l}\text { Feed } \\
\text { Rate }\end{array}$ & $\begin{array}{l}\text { Prim. } \\
\text { Conden- } \\
\text { sate } \\
\text { Flow }\end{array}$ & $\begin{array}{c}\text { Intercondenser } \\
\text { Press. }\end{array}$ & $\begin{array}{c}\text { Evapo- } \\
\text { rator. } \\
\text { Press. }\end{array}$ \\
\hline & & & ${ }^{\circ} \mathbf{C}$ & ${ }^{\circ} \mathbf{C}$ & ${ }^{\circ} \mathbf{C}$ & ${ }^{\circ} \mathrm{C}$ & ${ }^{\circ} \mathbf{C}$ & ${ }^{\circ} \mathrm{C}$ & ${ }^{\circ} \mathrm{C}$ & ${ }^{\circ} \mathrm{C}$ & ${ }^{\circ} \mathrm{C}$ & GPM & GPM & GPM & PSIG & PSIG \\
\hline 91 & $4 / 14 / 2004$ & 2:23:27 PM & 53.73 & 57.58 & 141.68 & 170.23 & 19.24 & 53.84 & 22.55 & 20.06 & 21.85 & 84.09 & 0.47 & 0.47 & -10.72 & -13.47 \\
\hline 92 & $4 / 14 / 2004$ & 2:28:29 PM & 54.23 & 58.08 & 141.70 & 170.61 & 19.24 & 54.34 & 22.57 & 20.06 & 21.86 & 84.32 & 0.53 & 0.48 & -10.66 & -13.42 \\
\hline 93 & $4 / 14 / 2004$ & 2:33:31 PM & 53.53 & 57.40 & 144.82 & 170.43 & 19.24 & 53.64 & 22.69 & 20.05 & 21.86 & 83.99 & 0.54 & 0.50 & -10.76 & -13.48 \\
\hline 94 & $4 / 14 / 2004$ & 2:38:33 PM & 54.39 & 58.25 & 136.98 & 171.26 & 19.24 & 54.56 & 22.40 & 20.07 & 21.88 & 84.25 & 0.54 & 0.44 & -10.59 & -13.45 \\
\hline 95 & $4 / 14 / 2004$ & 2:43:36 PM & 53.87 & 57.73 & 142.15 & 170.39 & 19.23 & 54.00 & 22.60 & 20.05 & 21.84 & 84.37 & 0.55 & 0.48 & -10.69 & -13.47 \\
\hline 96 & $4 / 14 / 2004$ & 2:48:38 PM & 54.03 & 57.91 & 142.11 & 170.98 & 19.24 & 54.14 & 22.72 & 20.07 & 21.88 & 83.96 & 0.56 & 0.48 & -10.70 & -13.46 \\
\hline 97 & $4 / 14 / 2004$ & 2:53:40 PM & 53.90 & 57.75 & 142.47 & 170.72 & 19.24 & 54.04 & 22.46 & 20.06 & 21.86 & 83.72 & 0.45 & 0.46 & -10.67 & -13.47 \\
\hline 98 & $4 / 14 / 2004$ & 2:58:42 PM & 53.82 & 57.70 & 143.50 & 171.17 & 19.24 & 54.13 & 22.56 & 20.07 & 21.90 & 84.92 & -0.01 & 0.47 & -10.67 & -13.46 \\
\hline 99 & $4 / 14 / 2004$ & 3:03:44 PM & 54.96 & 58.75 & 140.09 & 170.41 & 19.25 & 54.92 & 22.48 & 20.06 & 21.85 & 84.09 & -0.01 & 0.46 & -10.61 & -13.44 \\
\hline 100 & $4 / 14 / 2004$ & 3:08:47 PM & 53.80 & 57.64 & 143.69 & 170.54 & 19.25 & 53.91 & 22.71 & 20.07 & 21.87 & 84.41 & -0.01 & 0.51 & -10.73 & -13.51 \\
\hline 101 & $4 / 14 / 2004$ & 3:13:49 PM & 53.72 & 57.58 & 141.75 & 171.02 & 19.25 & 54.04 & 22.42 & 20.08 & 21.91 & 84.32 & -0.01 & 0.45 & -10.68 & -13.49 \\
\hline 102 & $4 / 14 / 2004$ & 3:18:51 PM & 54.36 & 58.17 & 140.20 & 170.46 & 19.26 & 54.59 & 22.24 & 20.08 & 21.88 & 84.61 & -0.01 & 0.42 & -10.61 & -13.44 \\
\hline 103 & $4 / 14 / 2004$ & 3:23:53 PM & 55.15 & 58.95 & 142.58 & 170.66 & 19.25 & 55.02 & 22.87 & 20.08 & 21.89 & 83.84 & -0.01 & 0.52 & -10.63 & -13.40 \\
\hline 104 & $4 / 14 / 2004$ & 3:28:56 PM & 54.82 & 58.64 & 140.56 & 171.02 & 19.25 & 54.72 & 22.72 & 20.09 & 21.91 & 84.23 & -0.01 & 0.50 & -10.67 & -13.43 \\
\hline 105 & $4 / 14 / 2004$ & 3:33:58 PM & 53.88 & 57.72 & 143.06 & 170.43 & 19.26 & 54.10 & 22.54 & 20.08 & 21.88 & 84.14 & -0.01 & 0.47 & -10.70 & -13.48 \\
\hline 106 & $4 / 14 / 2004$ & 3:39:00 PM & 54.45 & 58.24 & 144.26 & 169.93 & 19.26 & 54.61 & 22.53 & 20.07 & 21.86 & 84.11 & -0.01 & 0.47 & -10.67 & -13.45 \\
\hline 107 & $4 / 14 / 2004$ & 3:44:02 PM & 54.50 & 58.37 & 141.22 & 171.52 & 19.26 & 54.57 & 22.62 & 20.10 & 21.93 & 84.88 & -0.01 & 0.48 & -10.66 & -13.45 \\
\hline 108 & $4 / 14 / 2004$ & 3:49:04 PM & 53.90 & 57.72 & 143.23 & 170.35 & 19.26 & 53.94 & 22.57 & 20.08 & 21.88 & 83.71 & -0.01 & 0.49 & -10.74 & -13.52 \\
\hline 109 & $4 / 14 / 2004$ & 3:54:07 PM & 53.89 & 57.77 & 142.23 & 171.26 & 19.27 & 54.01 & 22.72 & 20.10 & 21.93 & 84.76 & -0.01 & 0.49 & -10.73 & -13.50 \\
\hline 110 & $4 / 14 / 2004$ & 3:59:09 PM & 54.24 & 58.03 & 142.13 & 170.46 & 19.26 & 54.41 & 22.36 & 20.09 & 21.88 & 84.23 & -0.01 & 0.45 & -10.67 & -13.48 \\
\hline 111 & $4 / 14 / 2004$ & 4:04:11 PM & 54.81 & 58.63 & 137.98 & 171.31 & 19.26 & 54.97 & 22.45 & 20.10 & 21.91 & 84.17 & -0.01 & 0.46 & -10.59 & -13.42 \\
\hline 112 & $4 / 14 / 2004$ & 4:09:13 PM & 54.38 & 58.21 & 142.92 & 170.71 & 19.26 & 54.50 & 22.71 & 20.10 & 21.90 & 84.20 & -0.01 & 0.50 & -10.69 & -13.44 \\
\hline 113 & $4 / 14 / 2004$ & 4:14:15 PM & 54.46 & 58.24 & 140.73 & 170.57 & 19.27 & 54.65 & 22.22 & 20.09 & 21.88 & 84.60 & -0.01 & 0.43 & -10.63 & -13.45 \\
\hline 114 & $4 / 14 / 2004$ & 4:19:17 PM & 54.84 & 58.66 & 141.26 & 170.84 & 19.27 & 54.98 & 22.69 & 20.11 & 21.91 & 84.63 & -0.01 & 0.48 & -10.65 & -13.40 \\
\hline 115 & $4 / 14 / 2004$ & 4:24:20 PM & 54.47 & 58.30 & 141.20 & 171.06 & 19.29 & 54.49 & 22.58 & 20.11 & 21.94 & 84.04 & -0.01 & 0.48 & -10.66 & -13.43 \\
\hline 116 & $4 / 14 / 2004$ & 4:29:22 PM & 54.61 & 58.44 & 142.46 & 170.82 & 19.28 & 54.50 & 22.79 & 20.10 & 21.92 & 83.92 & -0.01 & 0.52 & -10.73 & -13.46 \\
\hline 117 & $4 / 14 / 2004$ & 4:34:24 PM & 55.55 & 59.31 & 139.50 & 170.77 & 19.29 & 55.48 & 22.56 & 20.11 & 21.91 & 83.42 & -0.01 & 0.48 & -10.59 & -13.40 \\
\hline 118 & $4 / 14 / 2004$ & 4:39:26 PM & 52.25 & 54.39 & 146.49 & 173.75 & 19.30 & 51.82 & 22.67 & 20.19 & 22.11 & 83.17 & -0.01 & 0.44 & -10.94 & -13.70 \\
\hline 119 & $4 / 14 / 2004$ & 4:44:29 PM & 42.48 & 42.85 & 141.86 & 175.69 & 19.30 & 42.33 & 19.78 & 20.23 & 22.25 & 82.57 & -0.01 & 0.27 & -11.34 & -13.46 \\
\hline 120 & $4 / 14 / 2004$ & 4:49:31 PM & 42.31 & 43.28 & 136.45 & 174.98 & 19.45 & 42.39 & 19.45 & 19.74 & 20.03 & 15.61 & -0.01 & 0.22 & -2.42 & -4.74 \\
\hline
\end{tabular}


WSRC-TR-2005-00105, REVISION 0

SRNL-RPP-2005-00012, REVISION 0

Table H1-2a: Campaign III

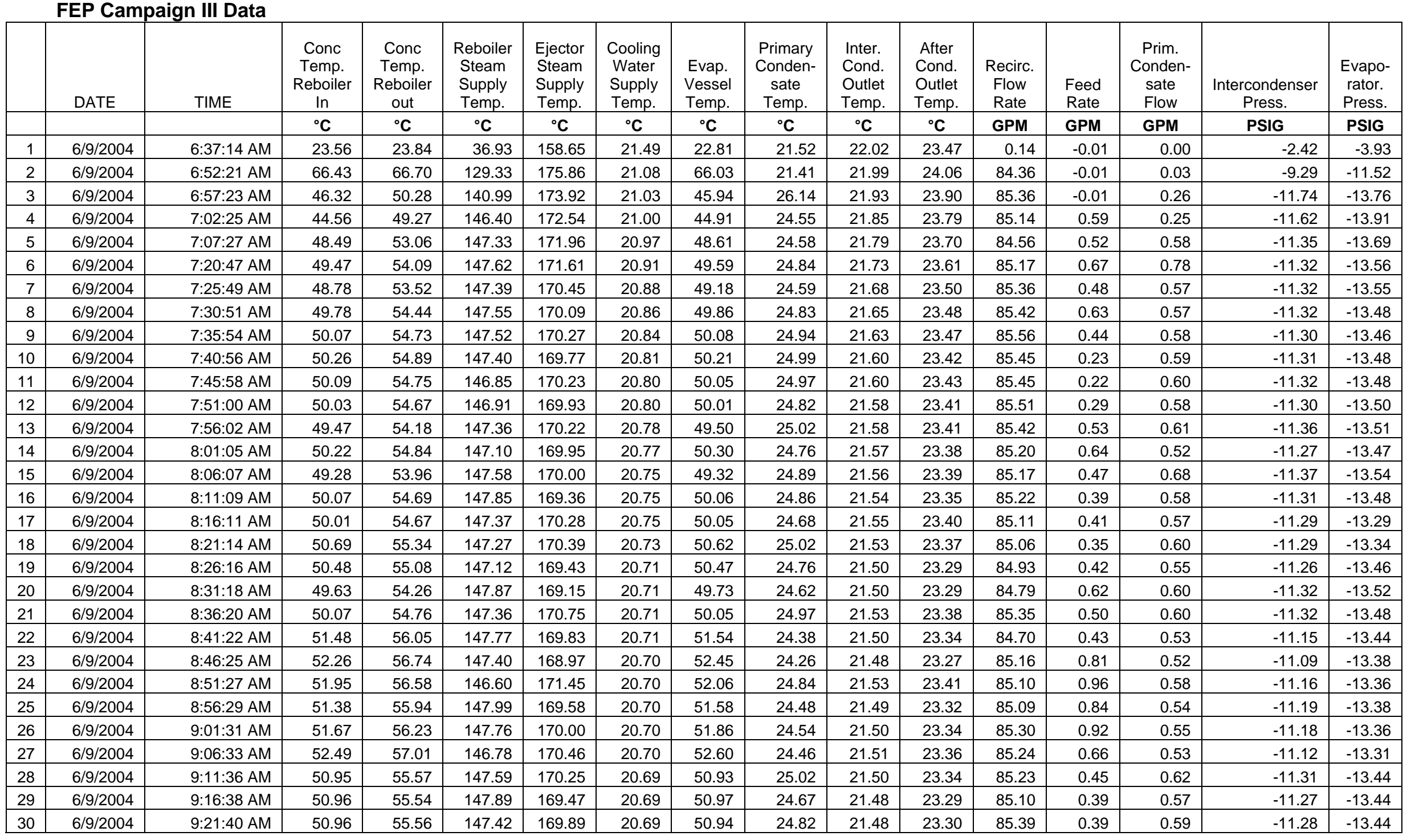


WSRC-TR-2005-00105, REVISION 0

SRNL-RPP-2005-00012, REVISION 0

Table H1-2b: Campaign III

FEP Campaign III Data (Contd..)

\begin{tabular}{|c|c|c|c|c|c|c|c|c|c|c|c|c|c|c|c|c|}
\hline & DATE & TIME & $\begin{array}{c}\text { Conc } \\
\text { Temp. } \\
\text { Reboiler } \\
\text { In }\end{array}$ & $\begin{array}{c}\text { Conc } \\
\text { Temp. } \\
\text { Reboiler } \\
\text { out }\end{array}$ & $\begin{array}{l}\text { Reboiler } \\
\text { Steam } \\
\text { Supply } \\
\text { Temp. }\end{array}$ & $\begin{array}{l}\text { Ejector } \\
\text { Steam } \\
\text { Supply } \\
\text { Temp. }\end{array}$ & $\begin{array}{l}\text { Cooling } \\
\text { Water } \\
\text { Supply } \\
\text { Temp. }\end{array}$ & $\begin{array}{l}\text { Evap. } \\
\text { Vessel } \\
\text { Temp. }\end{array}$ & $\begin{array}{c}\text { Primary } \\
\text { Conden- } \\
\text { sate } \\
\text { Temp. }\end{array}$ & $\begin{array}{l}\text { Inter. } \\
\text { Cond. } \\
\text { Outlet } \\
\text { Temp. }\end{array}$ & $\begin{array}{l}\text { After } \\
\text { Cond. } \\
\text { Outlet } \\
\text { Temp. }\end{array}$ & $\begin{array}{c}\text { Recirc. } \\
\text { Flow } \\
\text { Rate } \\
\end{array}$ & $\begin{array}{l}\text { Feed } \\
\text { Rate } \\
\end{array}$ & $\begin{array}{l}\text { Prim. } \\
\text { Conden- } \\
\text { sate } \\
\text { Flow }\end{array}$ & $\begin{array}{c}\text { Intercondenser } \\
\text { Press. }\end{array}$ & $\begin{array}{c}\text { Evapo- } \\
\text { rator. } \\
\text { Press. }\end{array}$ \\
\hline & & & ${ }^{\circ} \mathrm{C}$ & ${ }^{\circ} \mathrm{C}$ & ${ }^{\circ} \mathrm{C}$ & ${ }^{\circ} \mathrm{C}$ & ${ }^{\circ} \mathrm{C}$ & ${ }^{\circ} \mathrm{C}$ & ${ }^{\circ} \mathrm{C}$ & ${ }^{\circ} \mathrm{C}$ & ${ }^{\circ} \mathrm{C}$ & GPM & GPM & GPM & PSIG & PSIG \\
\hline 31 & $6 / 9 / 2004$ & $9: 26: 42 \mathrm{AM}$ & 51.27 & 55.89 & 146.87 & 170.41 & 20.69 & 51.30 & 24.60 & 21.48 & 23.34 & 84.68 & 0.44 & 0.55 & -11.23 & -13.42 \\
\hline 32 & $6 / 9 / 2004$ & 9:31:45 AM & 50.64 & 55.25 & 146.59 & 169.96 & 20.69 & 50.66 & 24.66 & 21.47 & 23.31 & 85.27 & 0.40 & 0.58 & -11.29 & -13.48 \\
\hline 33 & $6 / 9 / 2004$ & 9:36:47 AM & 50.79 & 55.46 & 147.06 & 170.51 & 20.67 & 50.78 & 24.84 & 21.48 & 23.33 & 84.98 & 0.43 & 0.59 & -11.29 & -13.45 \\
\hline 34 & $6 / 9 / 2004$ & 9:41:49 AM & 50.34 & 54.96 & 146.91 & 170.13 & 20.67 & 50.41 & 24.57 & 21.47 & 23.30 & 85.19 & 0.53 & 0.56 & -11.30 & -13.50 \\
\hline 35 & $6 / 9 / 2004$ & 9:46:51 AM & 50.34 & 54.98 & 147.42 & 170.37 & 20.67 & 50.36 & 24.84 & 21.48 & 23.31 & 85.04 & 0.50 & 0.59 & -11.34 & -13.49 \\
\hline 36 & $6 / 9 / 2004$ & 9:51:53 AM & 51.09 & 55.67 & 144.80 & 170.00 & 20.67 & 51.12 & 24.57 & 21.47 & 23.29 & 85.09 & 0.42 & 0.56 & -11.24 & -13.44 \\
\hline 37 & $6 / 9 / 2004$ & 9:56:56 AM & 50.53 & 55.16 & 146.32 & 170.35 & 20.67 & 50.52 & 24.71 & 21.48 & 23.30 & 84.91 & 0.37 & 0.58 & -11.30 & -13.48 \\
\hline 38 & $6 / 9 / 2004$ & 10:01:58 AM & 50.50 & 55.13 & 147.12 & 170.49 & 20.67 & 50.44 & 24.86 & 21.47 & 23.32 & 84.75 & 0.37 & 0.60 & -11.33 & -13.48 \\
\hline 39 & $6 / 9 / 2004$ & $10: 07: 00 \mathrm{AM}$ & 50.36 & 54.98 & 146.57 & 170.21 & 20.66 & 50.44 & 24.51 & 21.45 & 23.29 & 84.93 & 0.59 & 0.56 & -11.30 & -13.48 \\
\hline 40 & $6 / 9 / 2004$ & $10: 12: 02$ AM & 51.42 & 55.97 & 147.21 & 169.73 & 20.67 & 51.52 & 24.44 & 21.45 & 23.28 & 84.84 & 0.67 & 0.55 & -11.21 & -12.98 \\
\hline 41 & $6 / 9 / 2004$ & 10:17:05 AM & 51.23 & 55.84 & 145.72 & 170.40 & 20.67 & 51.32 & 24.62 & 21.47 & 23.30 & 84.87 & 0.68 & 0.57 & -11.22 & -13.42 \\
\hline 42 & $6 / 9 / 2004$ & $10: 22: 07 \mathrm{AM}$ & 50.67 & 55.33 & 145.52 & 171.30 & 20.66 & 50.73 & 24.75 & 21.48 & 23.35 & 85.07 & 0.69 & 0.58 & -11.29 & -13.47 \\
\hline 43 & $6 / 9 / 2004$ & 10:27:09 AM & 50.23 & 54.80 & 146.52 & 169.32 & 20.65 & 50.37 & 24.37 & 21.44 & 23.23 & 84.77 & 0.68 & 0.54 & -11.30 & -13.54 \\
\hline 44 & $6 / 9 / 2004$ & 10:32:11 AM & 51.56 & 56.12 & 144.08 & 170.28 & 20.65 & 51.64 & 24.58 & 21.45 & 23.28 & 84.89 & 0.66 & 0.56 & -11.20 & -13.48 \\
\hline 45 & $6 / 9 / 2004$ & $10: 37: 13$ AM & 51.24 & 55.84 & 144.74 & 170.54 & 20.66 & 51.32 & 24.66 & 21.46 & 23.29 & 85.03 & 0.65 & 0.58 & -11.25 & -13.47 \\
\hline 46 & $6 / 9 / 2004$ & $10: 42: 15$ AM & 50.69 & 55.31 & 145.70 & 170.49 & 20.65 & 50.78 & 24.57 & 21.46 & 23.30 & 85.07 & 0.64 & 0.57 & -11.28 & -13.48 \\
\hline 47 & $6 / 9 / 2004$ & $10: 47: 18$ AM & 51.01 & 55.59 & 146.10 & 170.20 & 20.65 & 51.10 & 24.53 & 21.45 & 23.28 & 84.96 & 0.64 & 0.56 & -11.26 & -13.47 \\
\hline 48 & $6 / 9 / 2004$ & 10:52:20 AM & 50.89 & 55.48 & 146.03 & 170.16 & 20.66 & 50.98 & 24.61 & 21.44 & 23.27 & 84.93 & 0.63 & 0.57 & -11.29 & -13.46 \\
\hline 49 & $6 / 9 / 2004$ & $10: 57: 22$ AM & 50.95 & 55.55 & 141.72 & 170.79 & 20.65 & 50.96 & 24.75 & 21.45 & 23.30 & 85.06 & 0.54 & 0.53 & -11.27 & -13.49 \\
\hline 50 & $6 / 9 / 2004$ & $11: 02: 24$ AM & 49.83 & 54.49 & 141.67 & 170.94 & 20.64 & 49.88 & 24.63 & 21.45 & 23.28 & 84.74 & 0.45 & 0.64 & -11.33 & -13.56 \\
\hline 51 & $6 / 9 / 2004$ & $11: 07: 27$ AM & 50.40 & 55.03 & 142.04 & 170.68 & 20.65 & 50.42 & 24.69 & 21.45 & 23.27 & 84.79 & 0.43 & 0.58 & -11.30 & -13.52 \\
\hline 52 & $6 / 9 / 2004$ & $11: 12: 29$ AM & 50.05 & 54.67 & 142.76 & 170.50 & 20.66 & 50.06 & 24.72 & 21.44 & 23.27 & 84.92 & 0.41 & 0.58 & -11.32 & -13.54 \\
\hline 53 & $6 / 9 / 2004$ & $11: 17: 31$ AM & 50.44 & 55.08 & 142.75 & 171.09 & 20.66 & 50.44 & 24.66 & 21.46 & 23.32 & 84.74 & 0.40 & 0.57 & -11.30 & -13.52 \\
\hline 54 & $6 / 9 / 2004$ & 11:22:33 AM & 49.51 & 54.14 & 145.43 & 169.80 & 20.65 & 49.51 & 24.77 & 21.43 & 23.25 & 84.75 & 0.40 & 0.60 & -11.43 & -13.59 \\
\hline 55 & $6 / 9 / 2004$ & $11: 27: 35$ AM & 50.42 & 55.04 & 144.18 & 170.55 & 20.66 & 50.43 & 24.62 & 21.46 & 23.29 & 84.52 & 0.40 & 0.57 & -11.32 & -13.53 \\
\hline 56 & $6 / 9 / 2004$ & $11: 32: 38$ AM & 50.47 & 55.06 & 142.41 & 170.35 & 20.67 & 50.52 & 24.54 & 21.45 & 23.28 & 84.53 & 0.47 & 0.56 & -11.29 & -13.51 \\
\hline 57 & $6 / 9 / 2004$ & $11: 37: 40$ AM & 51.25 & 55.82 & 143.33 & 170.56 & 20.66 & 51.28 & 24.69 & 21.45 & 23.30 & 84.49 & 0.52 & 0.57 & -11.24 & -13.46 \\
\hline 58 & $6 / 9 / 2004$ & $11: 42: 42$ AM & 50.52 & 55.11 & 143.39 & 170.49 & 20.67 & 50.61 & 24.56 & 21.45 & 23.29 & 84.49 & 0.67 & 0.56 & -11.30 & -13.51 \\
\hline 59 & $6 / 9 / 2004$ & $11: 47: 44$ AM & 50.17 & 54.76 & 145.00 & 170.16 & 20.66 & 50.29 & 24.51 & 21.44 & 23.29 & 84.31 & 0.69 & 0.56 & -11.34 & -13.54 \\
\hline 60 & $6 / 9 / 2004$ & $11: 52: 47$ AM & 51.57 & 56.10 & 144.33 & 170.82 & 20.67 & 51.68 & 24.40 & 21.46 & 23.32 & 84.67 & 0.69 & 0.53 & -11.19 & -13.46 \\
\hline
\end{tabular}


WSRC-TR-2005-00105, REVISION 0

SRNL-RPP-2005-00012, REVISION 0

Table H1-2c: Campaign III

\begin{tabular}{|c|c|c|c|c|c|c|c|c|c|c|c|c|c|c|c|c|}
\hline & DATE & TIME & $\begin{array}{c}\text { Conc } \\
\text { Temp. } \\
\text { Reboiler } \\
\text { In }\end{array}$ & $\begin{array}{c}\text { Conc } \\
\text { Temp. } \\
\text { Reboiler } \\
\text { out }\end{array}$ & $\begin{array}{c}\text { Reboiler } \\
\text { Steam } \\
\text { Supply } \\
\text { Temp. }\end{array}$ & $\begin{array}{l}\text { Ejector } \\
\text { Steam } \\
\text { Supply } \\
\text { Temp. }\end{array}$ & $\begin{array}{l}\text { Cooling } \\
\text { Water } \\
\text { Supply } \\
\text { Temp. } \\
\end{array}$ & $\begin{array}{l}\text { Evap. } \\
\text { Vessel } \\
\text { Temp. }\end{array}$ & $\begin{array}{c}\text { Primary } \\
\text { Conden- } \\
\text { sate } \\
\text { Temp. }\end{array}$ & $\begin{array}{l}\text { Inter. } \\
\text { Cond. } \\
\text { Outlet } \\
\text { Temp. }\end{array}$ & $\begin{array}{l}\text { After } \\
\text { Cond. } \\
\text { Outlet } \\
\text { Temp. }\end{array}$ & $\begin{array}{c}\text { Recirc. } \\
\text { Flow } \\
\text { Rate } \\
\end{array}$ & $\begin{array}{l}\text { Feed } \\
\text { Rate }\end{array}$ & $\begin{array}{l}\text { Prim. } \\
\text { Conden- } \\
\text { sate } \\
\text { Flow } \\
\end{array}$ & $\begin{array}{c}\text { Intercondenser } \\
\text { Press. }\end{array}$ & $\begin{array}{c}\text { Evapo- } \\
\text { rator. } \\
\text { Press. }\end{array}$ \\
\hline & & & ${ }^{\circ} \mathrm{C}$ & ${ }^{\circ} \mathrm{C}$ & ${ }^{\circ} \mathrm{C}$ & ${ }^{\circ} \mathrm{C}$ & ${ }^{\circ} \mathrm{C}$ & ${ }^{\circ} \mathrm{C}$ & ${ }^{\circ} \mathrm{C}$ & ${ }^{\circ} \mathrm{C}$ & ${ }^{\circ} \mathrm{C}$ & GPM & GPM & GPM & PSIG & PSIG \\
\hline 63 & $6 / 9 / 2004$ & 12:07:53 PM & 51.46 & 56.01 & 142.92 & 170.63 & 20.66 & 51.58 & 24.53 & 21.45 & 23.30 & 84.65 & 0.70 & 0.56 & -11.24 & -13.44 \\
\hline 64 & $6 / 9 / 2004$ & $12: 12: 55 \mathrm{PM}$ & 52.28 & 56.74 & 141.34 & 170.07 & 20.66 & 52.39 & 24.29 & 21.44 & 23.28 & 84.94 & 0.55 & 0.52 & -11.14 & -13.40 \\
\hline 65 & $6 / 9 / 2004$ & 12:17:58 PM & 51.83 & 56.35 & 145.12 & 170.74 & 20.66 & 51.81 & 24.80 & 21.46 & 23.31 & 84.52 & 0.40 & 0.59 & -11.25 & -13.43 \\
\hline 66 & $6 / 9 / 2004$ & 12:23:00 PM & 51.50 & 56.07 & 143.48 & 170.84 & 20.65 & 51.55 & 24.39 & 21.45 & 23.30 & 84.17 & 0.39 & 0.55 & -11.23 & -13.47 \\
\hline 67 & $6 / 9 / 2004$ & 12:28:02 PM & 49.33 & 53.05 & 147.86 & 172.79 & 20.64 & 49.26 & 24.45 & 21.49 & 23.43 & 84.21 & 0.49 & 0.41 & -11.44 & -13.64 \\
\hline 68 & $6 / 9 / 2004$ & 12:33:04 PM & 47.76 & 52.06 & 148.19 & 173.62 & 20.64 & 47.81 & 24.28 & 21.50 & 23.48 & 84.28 & 0.47 & 0.11 & -11.55 & -13.74 \\
\hline 69 & $6 / 9 / 2004$ & 12:38:06 PM & 48.44 & 52.90 & 145.99 & 173.14 & 20.64 & 48.55 & 24.11 & 21.49 & 23.45 & 81.97 & 0.45 & 0.32 & -11.43 & -13.72 \\
\hline 70 & $6 / 9 / 2004$ & 12:43:09 PM & 53.92 & 58.34 & 147.04 & 171.61 & 20.65 & 54.09 & 23.61 & 21.47 & 23.39 & 81.26 & 0.41 & 0.30 & -10.99 & -13.57 \\
\hline 71 & $6 / 9 / 2004$ & 12:48:11 PM & 52.63 & 57.12 & 147.30 & 170.64 & 20.65 & 52.60 & 24.99 & 21.46 & 23.32 & 81.20 & 0.41 & 0.19 & -11.21 & -13.53 \\
\hline 72 & $6 / 9 / 2004$ & 12:53:13 PM & 52.48 & 57.00 & 147.16 & 171.45 & 20.66 & 52.47 & 24.76 & 21.48 & 23.38 & 81.01 & 0.40 & 0.32 & -11.20 & -13.54 \\
\hline 73 & $6 / 9 / 2004$ & 12:58:15 PM & 53.12 & 57.54 & 147.12 & 170.21 & 20.66 & 53.15 & 24.46 & 21.45 & 23.32 & 80.64 & 0.41 & 0.97 & -11.13 & -13.54 \\
\hline 74 & $6 / 9 / 2004$ & 1:03:18 PM & 52.80 & 57.37 & 145.93 & 171.41 & 20.66 & 52.91 & 24.14 & 21.47 & 23.38 & 79.63 & 0.40 & 1.02 & -11.12 & -13.54 \\
\hline 75 & $6 / 9 / 2004$ & 1:08:20 PM & 55.63 & 60.23 & 146.73 & 171.69 & 20.66 & 55.82 & 23.93 & 21.48 & 23.39 & 77.88 & 0.66 & 0.77 & -10.88 & -13.50 \\
\hline 76 & $6 / 9 / 2004$ & 1:13:22 PM & 54.25 & 58.71 & 144.67 & 170.84 & 20.65 & 54.35 & 24.17 & 21.44 & 23.34 & 79.94 & 0.51 & 0.51 & -11.00 & -13.48 \\
\hline 77 & $6 / 9 / 2004$ & 1:18:24 PM & 53.93 & 58.41 & 146.96 & 171.10 & 20.65 & 53.94 & 24.33 & 21.46 & 23.38 & 79.96 & 0.39 & 0.53 & -11.08 & -13.49 \\
\hline 78 & $6 / 9 / 2004$ & 1:23:26 PM & 53.83 & 58.26 & 147.63 & 170.37 & 20.66 & 53.83 & 24.40 & 21.45 & 23.34 & 80.26 & 0.38 & 0.54 & -11.08 & -13.49 \\
\hline 79 & $6 / 9 / 2004$ & 1:28:29 PM & 53.75 & 58.21 & 143.36 & 171.58 & 20.67 & 53.75 & 24.36 & 21.49 & 23.38 & 80.61 & 0.37 & 0.53 & -11.06 & -13.49 \\
\hline 80 & $6 / 9 / 2004$ & 1:33:31 PM & 53.30 & 57.74 & 145.28 & 171.18 & 20.67 & 53.31 & 24.31 & 21.48 & 23.36 & 81.05 & 0.35 & 0.52 & -11.09 & -13.49 \\
\hline 81 & $6 / 9 / 2004$ & 1:38:33 PM & 52.87 & 57.33 & 146.88 & 171.04 & 20.67 & 52.84 & 24.55 & 21.48 & 23.36 & 80.67 & 0.37 & 0.56 & -11.18 & -13.49 \\
\hline 82 & $6 / 9 / 2004$ & 1:43:35 PM & 53.24 & 57.68 & 146.20 & 171.34 & 20.67 & 53.25 & 24.24 & 21.49 & 23.38 & 80.52 & 0.36 & 0.51 & -11.13 & -13.50 \\
\hline 83 & $6 / 9 / 2004$ & 1:48:37 PM & 53.23 & 57.67 & 145.95 & 170.89 & 20.67 & 53.50 & 23.98 & 21.47 & 23.35 & 80.40 & 0.18 & 0.48 & -11.07 & -13.49 \\
\hline 84 & $6 / 9 / 2004$ & 1:53:40 PM & 54.04 & 59.03 & 146.33 & 170.99 & 20.67 & 55.11 & 22.87 & 21.48 & 23.37 & 76.99 & 0.00 & 0.32 & -10.87 & -13.41 \\
\hline 85 & $6 / 9 / 2004$ & 1:58:42 PM & 55.16 & 59.66 & 145.64 & 171.50 & 20.68 & 55.02 & 25.15 & 21.50 & 23.38 & 80.55 & -0.01 & 0.65 & -11.03 & -13.28 \\
\hline 86 & $6 / 9 / 2004$ & 2:03:44 PM & 53.92 & 58.44 & 145.42 & 171.44 & 20.67 & 53.84 & 24.34 & 21.49 & 23.38 & 79.94 & -0.01 & 0.53 & -11.06 & -13.30 \\
\hline 87 & $6 / 9 / 2004$ & 2:08:46 PM & 53.32 & 57.74 & 146.22 & 170.67 & 20.66 & 53.21 & 24.51 & 21.47 & 23.35 & 80.56 & -0.01 & 0.56 & -11.16 & -13.35 \\
\hline 88 & $6 / 9 / 2004$ & 2:13:48 PM & 53.54 & 57.97 & 146.20 & 171.43 & 20.64 & 53.40 & 24.56 & 21.47 & 23.38 & 80.97 & -0.01 & 0.56 & -11.13 & -13.34 \\
\hline 89 & $6 / 9 / 2004$ & 2:18:51 PM & 54.00 & 58.37 & 143.99 & 171.02 & 20.64 & 53.88 & 24.35 & 21.46 & 23.36 & 80.81 & -0.01 & 0.53 & -11.07 & -13.31 \\
\hline 90 & $6 / 9 / 2004$ & 2:23:53 PM & 53.89 & 58.28 & 146.07 & 171.51 & 20.66 & 53.75 & 24.49 & 21.49 & 23.39 & 80.37 & -0.01 & 0.55 & -11.12 & -13.32 \\
\hline 91 & $6 / 9 / 2004$ & 2:28:55 PM & 52.58 & 54.39 & 145.09 & 153.18 & 20.68 & 52.42 & 22.55 & 21.20 & 22.13 & 80.34 & -0.01 & 0.42 & -8.00 & -9.92 \\
\hline 92 & $6 / 9 / 2004$ & 2:33:57 PM & 52.02 & 52.33 & 140.18 & 153.63 & 20.69 & 51.90 & 20.67 & 20.72 & 21.03 & 80.34 & -0.01 & 0.05 & -0.07 & -0.57 \\
\hline
\end{tabular}


WSRC-TR-2005-00105, REVISION 0

SRNL-RPP-2005-00012, REVISION 0

Table H1-3a: Campaign IV

\begin{tabular}{|c|c|c|c|c|c|c|c|c|c|c|c|c|c|c|c|c|}
\hline & DATE & TIME & $\begin{array}{c}\text { Conc } \\
\text { Temp. } \\
\text { Reboiler } \\
\text { In }\end{array}$ & $\begin{array}{c}\text { Conc } \\
\text { Temp. } \\
\text { Reboiler } \\
\text { out }\end{array}$ & $\begin{array}{l}\text { Reboiler } \\
\text { Steam } \\
\text { Supply } \\
\text { Temp. }\end{array}$ & $\begin{array}{l}\text { Ejector } \\
\text { Steam } \\
\text { Supply } \\
\text { Temp. }\end{array}$ & $\begin{array}{l}\text { Cooling } \\
\text { Water } \\
\text { Supply } \\
\text { Temp. }\end{array}$ & $\begin{array}{l}\text { Evap. } \\
\text { Vessel } \\
\text { Temp. }\end{array}$ & $\begin{array}{c}\text { Primary } \\
\text { Conden- } \\
\text { sate } \\
\text { Temp. }\end{array}$ & $\begin{array}{l}\text { Inter. } \\
\text { Cond. } \\
\text { Outlet } \\
\text { Temp. }\end{array}$ & $\begin{array}{l}\text { After } \\
\text { Cond. } \\
\text { Outlet } \\
\text { Temp. }\end{array}$ & $\begin{array}{c}\text { Recirc. } \\
\text { Flow } \\
\text { Rate }\end{array}$ & $\begin{array}{l}\text { Feed } \\
\text { Rate }\end{array}$ & $\begin{array}{l}\text { Prim. } \\
\text { Conden- } \\
\text { sate } \\
\text { Flow }\end{array}$ & $\begin{array}{c}\text { Intercondenser } \\
\text { Press. }\end{array}$ & $\begin{array}{c}\text { Evapo- } \\
\text { rator. } \\
\text { Press. }\end{array}$ \\
\hline & & & ${ }^{\circ} \mathrm{C}$ & ${ }^{\circ} \mathrm{C}$ & ${ }^{\circ} \mathrm{C}$ & ${ }^{\circ} \mathrm{C}$ & ${ }^{\circ} \mathrm{C}$ & ${ }^{\circ} \mathrm{C}$ & ${ }^{\circ} \mathrm{C}$ & ${ }^{\circ} \mathrm{C}$ & ${ }^{\circ} \mathrm{C}$ & GPM & GPM & GPM & PSIG & PSIG \\
\hline 1 & $8 / 25 / 2004$ & $7: 16: 57 \mathrm{AM}$ & 22.27 & 22.64 & 22.89 & 164.65 & 25.26 & 20.34 & 25.70 & 26.22 & 27.66 & 1.70 & -0.01 & 0.00 & -1.40 & -3.47 \\
\hline 2 & $8 / 25 / 2004$ & 7:22:00 AM & 28.06 & 31.40 & 95.82 & 173.46 & 22.25 & 28.89 & 22.33 & 23.28 & 24.79 & 71.02 & -0.01 & 0.00 & -4.82 & -7.41 \\
\hline 3 & $8 / 25 / 2004$ & 7:27:02 AM & 49.40 & 52.11 & 138.55 & 171.97 & 21.61 & 49.60 & 21.63 & 21.95 & 22.58 & 89.58 & -0.01 & 0.05 & -3.11 & -4.64 \\
\hline 4 & $8 / 25 / 2004$ & 7:32:04 AM & 66.83 & 68.74 & 113.81 & 172.37 & 21.35 & 67.00 & 21.32 & 22.33 & 23.85 & 90.23 & -0.01 & 0.00 & -3.40 & -5.87 \\
\hline 5 & $8 / 25 / 2004$ & 7:37:06 AM & 69.77 & 69.98 & 99.45 & 175.56 & 21.22 & 69.52 & 21.18 & 22.29 & 23.95 & 89.62 & -0.01 & 0.00 & -7.94 & -10.16 \\
\hline 6 & $8 / 25 / 2004$ & 7:42:09 AM & 64.49 & 64.82 & 103.26 & 175.59 & 21.13 & 63.97 & 22.43 & 22.20 & 23.86 & 89.04 & -0.01 & 0.09 & -9.63 & -11.81 \\
\hline 7 & $8 / 25 / 2004$ & 7:47:11 AM & 43.35 & 46.68 & 142.48 & 174.51 & 21.06 & 43.09 & 24.72 & 22.13 & 23.73 & 89.93 & -0.01 & 0.39 & -11.83 & -13.97 \\
\hline 8 & $8 / 25 / 2004$ & 7:52:13 AM & 45.67 & 50.19 & 145.82 & 172.67 & 21.00 & 45.63 & 24.30 & 21.99 & 23.57 & 90.28 & -0.01 & 0.59 & -11.56 & -13.95 \\
\hline 9 & $8 / 25 / 2004$ & 7:57:15 AM & 48.68 & 53.13 & 147.10 & 171.64 & 20.96 & 48.60 & 24.37 & 21.92 & 23.46 & 88.95 & -0.01 & 0.84 & -11.31 & -13.91 \\
\hline 10 & $8 / 25 / 2004$ & 8:02:17 AM & 49.35 & 53.67 & 147.56 & 170.76 & 20.91 & 49.24 & 24.40 & 21.87 & 23.37 & 90.29 & 0.10 & 0.58 & -11.28 & -13.90 \\
\hline 11 & $8 / 25 / 2004$ & 8:07:20 AM & 49.55 & 53.91 & 147.07 & 172.02 & 20.87 & 49.71 & 24.06 & 21.85 & 23.39 & 90.25 & 0.96 & 0.54 & -11.21 & -13.51 \\
\hline 12 & $8 / 25 / 2004$ & $8: 12: 22$ AM & 49.60 & 53.95 & 147.71 & 171.49 & 20.84 & 49.67 & 24.34 & 21.80 & 23.34 & 90.21 & 0.95 & 0.57 & -11.25 & -13.38 \\
\hline 13 & $8 / 25 / 2004$ & $8: 17: 24$ AM & 49.60 & 53.91 & 147.54 & 170.80 & 20.81 & 49.72 & 24.13 & 21.76 & 23.28 & 90.34 & 0.94 & 0.55 & -11.25 & -13.47 \\
\hline 14 & $8 / 25 / 2004$ & $8: 22: 26$ AM & 49.33 & 53.68 & 147.83 & 171.09 & 20.79 & 49.49 & 24.10 & 21.74 & 23.28 & 90.35 & 0.93 & 0.56 & -11.26 & -13.50 \\
\hline 15 & $8 / 25 / 2004$ & $8: 27: 28$ AM & 49.74 & 54.08 & 147.84 & 171.29 & 20.77 & 49.88 & 24.15 & 21.73 & 23.26 & 90.34 & 0.91 & 0.56 & -11.25 & -13.48 \\
\hline 16 & $8 / 25 / 2004$ & 8:32:31 AM & 50.07 & 54.36 & 147.77 & 170.74 & 20.76 & 50.21 & 23.99 & 21.71 & 23.22 & 90.33 & 0.89 & 0.54 & -11.23 & -13.46 \\
\hline 17 & $8 / 25 / 2004$ & $8: 37: 33$ AM & 50.14 & 54.45 & 147.71 & 171.21 & 20.74 & 50.27 & 24.09 & 21.70 & 23.22 & 90.37 & 0.88 & 0.56 & -11.23 & -13.46 \\
\hline 18 & $8 / 25 / 2004$ & 8:42:35 AM & 49.89 & 54.16 & 148.48 & 170.08 & 20.69 & 50.03 & 24.00 & 21.63 & 23.15 & 90.45 & 0.88 & 0.56 & -11.27 & -13.47 \\
\hline 19 & $8 / 25 / 2004$ & $8: 47: 37$ AM & 50.57 & 54.85 & 147.43 & 171.44 & 20.67 & 50.58 & 24.08 & 21.63 & 23.20 & 90.54 & 0.57 & 0.56 & -11.22 & -13.45 \\
\hline 20 & $8 / 25 / 2004$ & 8:52:40 AM & 50.39 & 54.69 & 147.82 & 171.42 & 20.69 & 50.36 & 24.20 & 21.65 & 23.21 & 90.52 & 0.36 & 0.58 & -11.26 & -13.47 \\
\hline 21 & $8 / 25 / 2004$ & $8: 57: 42$ AM & 50.13 & 54.45 & 147.93 & 171.17 & 20.70 & 50.11 & 24.21 & 21.66 & 23.19 & 90.44 & 0.34 & 0.58 & -11.29 & -13.48 \\
\hline 22 & $8 / 25 / 2004$ & 9:02:44 AM & 50.24 & 54.56 & 147.69 & 171.27 & 20.69 & 50.22 & 24.15 & 21.64 & 23.17 & 90.44 & 0.30 & 0.58 & -11.26 & -13.49 \\
\hline 23 & $8 / 25 / 2004$ & 9:07:46 AM & 50.18 & 54.48 & 148.04 & 170.76 & 20.66 & 50.17 & 24.16 & 21.61 & 23.13 & 90.32 & 0.34 & 0.58 & -11.27 & -13.49 \\
\hline 24 & $8 / 25 / 2004$ & 9:12:48 AM & 49.92 & 54.25 & 147.99 & 171.38 & 20.65 & 49.91 & 24.18 & 21.61 & 23.16 & 90.31 & 0.35 & 0.59 & -11.30 & -13.49 \\
\hline 25 & $8 / 25 / 2004$ & 9:17:50 AM & 49.90 & 54.23 & 147.98 & 170.80 & 20.66 & 49.95 & 24.07 & 21.62 & 23.14 & 90.19 & 0.55 & 0.57 & -11.29 & -13.49 \\
\hline 26 & $8 / 25 / 2004$ & 9:22:53 AM & 50.04 & 54.38 & 147.77 & 171.42 & 20.67 & 50.07 & 24.08 & 21.64 & 23.16 & 90.28 & 0.46 & 0.56 & -11.28 & -13.50 \\
\hline 27 & $8 / 25 / 2004$ & 9:27:55 AM & 50.41 & 54.65 & 147.35 & 169.94 & 20.65 & 50.46 & 24.03 & 21.59 & 23.08 & 90.25 & 0.48 & 0.56 & -11.26 & -13.48 \\
\hline 28 & $8 / 25 / 2004$ & 9:32:57 AM & 49.76 & 54.10 & 147.93 & 171.37 & 20.62 & 49.81 & 24.09 & 21.59 & 23.14 & 90.20 & 0.65 & 0.58 & -11.31 & -13.51 \\
\hline 29 & $8 / 25 / 2004$ & 9:37:59 AM & 49.78 & 54.10 & 148.06 & 170.75 & 20.61 & 49.88 & 24.00 & 21.57 & 23.12 & 90.07 & 0.67 & 0.56 & -11.29 & -13.52 \\
\hline 30 & $8 / 25 / 2004$ & 9:43:02 AM & 50.11 & 54.45 & 147.86 & 171.42 & 20.61 & 50.12 & 23.97 & 21.61 & 23.18 & 90.18 & 0.40 & 0.58 & -11.28 & -13.50 \\
\hline
\end{tabular}


WSRC-TR-2005-00105, REVISION 0

SRNL-RPP-2005-00012, REVISION 0

Table H1-3b: Campaign IV

\begin{tabular}{|c|c|c|c|c|c|c|c|c|c|c|c|c|c|c|c|c|}
\hline \multicolumn{17}{|c|}{ FEP Campaign IV Data (Contd...) } \\
\hline & DATE & TIME & $\begin{array}{c}\text { Conc } \\
\text { Temp. } \\
\text { Reboiler } \\
\text { In }\end{array}$ & $\begin{array}{c}\text { Conc } \\
\text { Temp. } \\
\text { Reboiler } \\
\text { out }\end{array}$ & $\begin{array}{l}\text { Reboiler } \\
\text { Steam } \\
\text { Supply } \\
\text { Temp. }\end{array}$ & $\begin{array}{l}\text { Ejector } \\
\text { Steam } \\
\text { Supply } \\
\text { Temp. }\end{array}$ & $\begin{array}{l}\text { Cooling } \\
\text { Water } \\
\text { Supply } \\
\text { Temp. }\end{array}$ & $\begin{array}{l}\text { Evap. } \\
\text { Vessel } \\
\text { Temp. }\end{array}$ & $\begin{array}{c}\text { Primary } \\
\text { Conden- } \\
\text { sate } \\
\text { Temp. }\end{array}$ & $\begin{array}{l}\text { Inter. } \\
\text { Cond. } \\
\text { Outlet } \\
\text { Temp. }\end{array}$ & $\begin{array}{l}\text { After } \\
\text { Cond. } \\
\text { Outlet } \\
\text { Temp. }\end{array}$ & $\begin{array}{c}\text { Recirc. } \\
\text { Flow } \\
\text { Rate }\end{array}$ & $\begin{array}{l}\text { Feed } \\
\text { Rate }\end{array}$ & $\begin{array}{l}\text { Prim. } \\
\text { Conden- } \\
\text { sate } \\
\text { Flow }\end{array}$ & $\begin{array}{c}\text { Intercondenser } \\
\text { Press. }\end{array}$ & $\begin{array}{c}\text { Evapo- } \\
\text { rator. } \\
\text { Press. }\end{array}$ \\
\hline & & & ${ }^{\circ} \mathrm{C}$ & ${ }^{\circ} \mathrm{C}$ & ${ }^{\circ} \mathrm{C}$ & ${ }^{\circ} \mathrm{C}$ & ${ }^{\circ} \mathrm{C}$ & ${ }^{\circ} \mathrm{C}$ & ${ }^{\circ} \mathrm{C}$ & ${ }^{\circ} \mathrm{C}$ & ${ }^{\circ} \mathrm{C}$ & GPM & GPM & GPM & PSIG & PSIG \\
\hline 31 & $8 / 25 / 2004$ & 9:48:04 AM & 50.28 & 54.59 & 147.81 & 171.10 & 20.60 & 50.31 & 23.67 & 21.60 & 23.21 & 90.07 & 0.44 & 0.56 & -11.27 & -13.42 \\
\hline 32 & $8 / 25 / 2004$ & 9:53:06 AM & 50.00 & 54.29 & 148.33 & 170.35 & 20.56 & 50.11 & 23.56 & 21.54 & 23.15 & 90.00 & 0.70 & 0.57 & -11.28 & -13.48 \\
\hline 33 & $8 / 25 / 2004$ & 9:58:08 AM & 50.25 & 54.56 & 147.49 & 171.32 & 20.56 & 50.32 & 23.65 & 21.57 & 23.20 & 90.04 & 0.59 & 0.56 & -11.27 & -13.49 \\
\hline 34 & $8 / 25 / 2004$ & 10:03:10 AM & 50.14 & 54.46 & 147.56 & 171.05 & 20.58 & 50.25 & 23.50 & 21.58 & 23.18 & 90.04 & 0.70 & 0.55 & -11.26 & -13.50 \\
\hline 35 & $8 / 25 / 2004$ & 10:08:13 AM & 50.40 & 54.72 & 147.90 & 171.23 & 20.59 & 50.49 & 23.65 & 21.59 & 23.18 & 89.94 & 0.70 & 0.56 & -11.26 & -13.49 \\
\hline 36 & $8 / 25 / 2004$ & $10: 13: 15$ AM & 50.32 & 54.66 & 148.03 & 171.30 & 20.58 & 50.40 & 23.67 & 21.59 & 23.19 & 90.05 & 0.62 & 0.57 & -11.28 & -13.49 \\
\hline 37 & $8 / 25 / 2004$ & 10:18:17 AM & 50.65 & 54.91 & 147.34 & 170.53 & 20.56 & 50.71 & 23.48 & 21.54 & 23.13 & 90.08 & 0.53 & 0.55 & -11.24 & -13.48 \\
\hline 38 & $8 / 25 / 2004$ & 10:23:19 AM & 50.50 & 54.79 & 147.67 & 171.03 & 20.54 & 50.58 & 23.53 & 21.54 & 23.16 & 90.02 & 0.63 & 0.56 & -11.25 & -13.48 \\
\hline 39 & $8 / 25 / 2004$ & $10: 28: 21 \mathrm{AM}$ & 50.48 & 54.78 & 147.58 & 171.04 & 20.56 & 50.56 & 23.58 & 21.56 & 23.17 & 90.00 & 0.67 & 0.56 & -11.26 & -13.48 \\
\hline 40 & $8 / 25 / 2004$ & 10:33:24 AM & 50.52 & 54.83 & 147.89 & 171.22 & 20.56 & 50.59 & 23.65 & 21.55 & 23.17 & 90.11 & 0.60 & 0.57 & -11.28 & -13.49 \\
\hline 41 & $8 / 25 / 2004$ & $10: 38: 26$ AM & 50.73 & 55.02 & 147.56 & 171.05 & 20.54 & 50.77 & 23.56 & 21.53 & 23.16 & 90.05 & 0.52 & 0.56 & -11.26 & -13.48 \\
\hline 42 & $8 / 25 / 2004$ & $10: 43: 28$ AM & 50.57 & 54.84 & 148.09 & 170.75 & 20.51 & 50.61 & 23.60 & 21.50 & 23.13 & 90.05 & 0.49 & 0.57 & -11.27 & -13.47 \\
\hline 43 & $8 / 25 / 2004$ & 10:48:30 AM & 50.96 & 55.23 & 146.80 & 171.31 & 20.52 & 51.00 & 23.53 & 21.53 & 23.18 & 90.06 & 0.49 & 0.55 & -11.23 & -13.47 \\
\hline 44 & $8 / 25 / 2004$ & 10:53:33 AM & 50.45 & 54.73 & 147.84 & 170.62 & 20.56 & 50.54 & 23.60 & 21.54 & 23.17 & 89.94 & 0.60 & 0.57 & -11.29 & -13.48 \\
\hline 45 & $8 / 25 / 2004$ & 10:58:35 AM & 50.80 & 55.09 & 147.37 & 171.30 & 20.57 & 50.84 & 23.66 & 21.56 & 23.18 & 89.96 & 0.57 & 0.57 & -11.25 & -13.47 \\
\hline 46 & $8 / 25 / 2004$ & 11:03:37 AM & 50.73 & 55.00 & 147.21 & 171.07 & 20.54 & 50.77 & 23.52 & 21.54 & 23.17 & 89.91 & 0.56 & 0.55 & -11.26 & -13.46 \\
\hline 47 & $8 / 25 / 2004$ & 11:08:39 AM & 50.96 & 55.21 & 147.63 & 170.91 & 20.53 & 51.02 & 23.55 & 21.52 & 23.17 & 89.95 & 0.55 & 0.56 & -11.23 & -13.43 \\
\hline 48 & $8 / 25 / 2004$ & 11:13:41 AM & 50.82 & 55.09 & 147.57 & 171.12 & 20.55 & 50.88 & 23.64 & 21.55 & 23.19 & 90.00 & 0.62 & 0.56 & -11.26 & -13.42 \\
\hline 49 & $8 / 25 / 2004$ & 11:18:44 AM & 50.82 & 55.10 & 147.49 & 171.25 & 20.56 & 50.87 & 23.67 & 21.57 & 23.21 & 90.02 & 0.58 & 0.57 & -11.26 & -13.44 \\
\hline 50 & $8 / 25 / 2004$ & $11: 23: 46$ AM & 51.36 & 55.57 & 144.41 & 170.78 & 20.54 & 51.43 & 23.45 & 21.53 & 23.15 & 90.01 & 0.54 & 0.54 & -11.19 & -13.44 \\
\hline 51 & $8 / 25 / 2004$ & $11: 28: 48$ AM & 51.08 & 55.32 & 146.76 & 171.13 & 20.53 & 51.13 & 23.52 & 21.53 & 23.16 & 90.00 & 0.53 & 0.55 & -11.21 & -13.44 \\
\hline 52 & $8 / 25 / 2004$ & $11: 33: 50$ AM & 51.20 & 55.45 & 145.96 & 171.19 & 20.52 & 51.22 & 23.55 & 21.52 & 23.14 & 89.99 & 0.52 & 0.56 & -11.22 & -13.43 \\
\hline 53 & $8 / 25 / 2004$ & $11: 38: 52$ AM & 51.10 & 55.33 & 144.87 & 171.02 & 20.52 & 51.18 & 23.44 & 21.51 & 23.13 & 89.94 & 0.64 & 0.54 & -11.22 & -13.46 \\
\hline 54 & $8 / 25 / 2004$ & 11:43:55 AM & 50.75 & 55.03 & 147.61 & 171.31 & 20.55 & 50.84 & 23.65 & 21.55 & 23.18 & 89.94 & 0.74 & 0.57 & -11.28 & -13.46 \\
\hline 55 & $8 / 25 / 2004$ & 11:48:57 AM & 51.33 & 55.53 & 145.21 & 170.39 & 20.56 & 51.45 & 23.46 & 21.54 & 23.12 & 90.01 & 0.74 & 0.54 & -11.19 & -13.44 \\
\hline 56 & $8 / 25 / 2004$ & 11:53:59 AM & 50.96 & 55.21 & 146.89 & 171.28 & 20.54 & 51.06 & 23.47 & 21.54 & 23.16 & 90.03 & 0.74 & 0.55 & -11.23 & -13.43 \\
\hline 57 & $8 / 25 / 2004$ & 11:59:01 AM & 51.00 & 55.26 & 147.34 & 171.36 & 20.53 & 51.10 & 23.49 & 21.53 & 23.17 & 90.06 & 0.75 & 0.55 & -11.24 & -13.43 \\
\hline 58 & $8 / 25 / 2004$ & 12:04:03 PM & 51.07 & 55.29 & 147.29 & 170.82 & 20.53 & 51.17 & 23.47 & 21.52 & 23.13 & 90.14 & 0.75 & 0.55 & -11.23 & -13.42 \\
\hline 59 & $8 / 25 / 2004$ & 12:09:06 PM & 51.08 & 55.35 & 147.03 & 171.62 & 20.56 & 51.19 & 23.60 & 21.56 & 23.19 & 90.13 & 0.74 & 0.56 & -11.24 & -13.44 \\
\hline 60 & $8 / 25 / 2004$ & 12:14:08 PM & 51.01 & 55.24 & 147.22 & 171.07 & 20.57 & 51.11 & 23.42 & 21.57 & 23.17 & 90.19 & 0.67 & 0.53 & -11.25 & -13.47 \\
\hline
\end{tabular}


WSRC-TR-2005-00105, REVISION 0

SRNL-RPP-2005-00012, REVISION 0

Table H1-3c: Campaign IV

\begin{tabular}{|c|c|c|c|c|c|c|c|c|c|c|c|c|c|c|c|c|}
\hline & DATE & TIME & $\begin{array}{c}\text { Conc } \\
\text { Temp. } \\
\text { Reboiler } \\
\text { In }\end{array}$ & $\begin{array}{c}\text { Conc } \\
\text { Temp. } \\
\text { Reboiler } \\
\text { out }\end{array}$ & $\begin{array}{c}\text { Reboiler } \\
\text { Steam } \\
\text { Supply } \\
\text { Temp. }\end{array}$ & $\begin{array}{l}\text { Ejector } \\
\text { Steam } \\
\text { Supply } \\
\text { Temp. }\end{array}$ & $\begin{array}{l}\text { Cooling } \\
\text { Water } \\
\text { Supply } \\
\text { Temp. }\end{array}$ & $\begin{array}{l}\text { Evap. } \\
\text { Vessel } \\
\text { Temp. }\end{array}$ & $\begin{array}{c}\text { Primary } \\
\text { Conden- } \\
\text { sate } \\
\text { Temp. }\end{array}$ & $\begin{array}{l}\text { Inter. } \\
\text { Cond. } \\
\text { Outlet } \\
\text { Temp. }\end{array}$ & $\begin{array}{l}\text { After } \\
\text { Cond. } \\
\text { Outlet } \\
\text { Temp. }\end{array}$ & $\begin{array}{c}\text { Recirc. } \\
\text { Flow } \\
\text { Rate }\end{array}$ & $\begin{array}{l}\text { Feed } \\
\text { Rate }\end{array}$ & $\begin{array}{l}\text { Prim. } \\
\text { Conden- } \\
\text { sate } \\
\text { Flow }\end{array}$ & $\begin{array}{c}\text { Intercondenser } \\
\text { Press. }\end{array}$ & $\begin{array}{c}\text { Evapo- } \\
\text { rator. } \\
\text { Press. }\end{array}$ \\
\hline & & & ${ }^{\circ} \mathrm{C}$ & ${ }^{\circ} \mathrm{C}$ & ${ }^{\circ} \mathrm{C}$ & ${ }^{\circ} \mathrm{C}$ & ${ }^{\circ} \mathrm{C}$ & ${ }^{\circ} \mathrm{C}$ & ${ }^{\circ} \mathrm{C}$ & ${ }^{\circ} \mathrm{C}$ & ${ }^{\circ} \mathrm{C}$ & GPM & GPM & GPM & PSIG & PSIG \\
\hline 61 & $8 / 25 / 2004$ & 12:19:10 PM & 51.57 & 55.75 & 147.65 & 170.30 & 20.56 & 51.59 & 23.54 & 21.54 & 23.12 & 90.21 & 0.47 & 0.55 & -11.23 & -13.44 \\
\hline 62 & $8 / 25 / 2004$ & 12:24:12 PM & 51.62 & 55.84 & 145.90 & 171.48 & 20.54 & 51.59 & 23.60 & 21.54 & 23.16 & 90.19 & 0.34 & 0.57 & -11.23 & -13.45 \\
\hline 63 & $8 / 25 / 2004$ & 12:29:14 PM & 51.45 & 55.67 & 146.86 & 171.37 & 20.53 & 51.40 & 23.55 & 21.53 & 23.16 & 90.04 & 0.26 & 0.57 & -11.24 & -13.46 \\
\hline 64 & $8 / 25 / 2004$ & 12:34:17 PM & 51.51 & 55.72 & 147.65 & 171.11 & 20.53 & 51.42 & 23.66 & 21.52 & 23.15 & 89.93 & 0.18 & 0.58 & -11.25 & -13.45 \\
\hline 65 & $8 / 25 / 2004$ & 12:39:19 PM & 51.62 & 55.84 & 146.23 & 171.58 & 20.53 & 51.56 & 23.59 & 21.54 & 23.19 & 89.84 & 0.22 & 0.57 & -11.22 & -13.44 \\
\hline 66 & $8 / 25 / 2004$ & 12:44:21 PM & 51.02 & 55.27 & 146.97 & 171.36 & 20.55 & 51.04 & 23.64 & 21.55 & 23.18 & 89.71 & 0.46 & 0.58 & -11.27 & -13.48 \\
\hline 67 & $8 / 25 / 2004$ & 12:49:23 PM & 51.43 & 55.56 & 145.45 & 171.02 & 20.57 & 51.41 & 23.37 & 21.56 & 23.15 & 89.70 & 0.39 & 0.53 & -11.20 & -13.48 \\
\hline 68 & $8 / 25 / 2004$ & 12:54:25 PM & 51.78 & 55.83 & 145.77 & 170.72 & 20.58 & 51.62 & 23.64 & 22.40 & 23.01 & 89.64 & 0.41 & 0.58 & -11.23 & -13.45 \\
\hline 69 & $8 / 25 / 2004$ & 12:59:28 PM & 52.02 & 56.06 & 141.10 & 171.28 & 20.57 & 51.90 & 23.35 & 22.55 & 22.98 & 89.66 & 0.44 & 0.53 & -11.15 & -13.45 \\
\hline 70 & $8 / 25 / 2004$ & 1:04:30 PM & 51.64 & 55.69 & 144.64 & 171.43 & 20.56 & 51.51 & 23.54 & 22.56 & 23.01 & 89.62 & 0.46 & 0.57 & -11.21 & -13.45 \\
\hline 71 & $8 / 25 / 2004$ & 1:09:32 PM & 51.25 & 55.32 & 146.17 & 171.57 & 20.56 & 51.15 & 23.46 & 22.57 & 23.02 & 89.59 & 0.57 & 0.56 & -11.24 & -13.48 \\
\hline 72 & $8 / 25 / 2004$ & 1:14:34 PM & 51.25 & 55.34 & 146.34 & 171.55 & 20.57 & 51.21 & 23.47 & 22.57 & 23.02 & 89.61 & 0.78 & 0.55 & -11.24 & -13.48 \\
\hline 73 & $8 / 25 / 2004$ & 1:19:36 PM & 50.92 & 54.97 & 148.01 & 170.57 & 20.57 & 50.92 & 23.39 & 22.53 & 22.99 & 89.64 & 0.78 & 0.55 & -11.29 & -13.48 \\
\hline 74 & $8 / 25 / 2004$ & 1:24:39 PM & 51.90 & 55.95 & 142.21 & 171.82 & 20.57 & 51.86 & 23.36 & 22.58 & 23.02 & 89.75 & 0.66 & 0.53 & -11.16 & -13.44 \\
\hline 75 & $8 / 25 / 2004$ & 1:29:41 PM & 51.74 & 55.75 & 146.39 & 170.70 & 20.57 & 51.55 & 23.46 & 22.53 & 22.99 & 89.69 & 0.21 & 0.56 & -11.25 & -13.47 \\
\hline 76 & $8 / 25 / 2004$ & 1:34:43 PM & 51.88 & 55.91 & 145.63 & 171.53 & 20.58 & 51.65 & 23.58 & 22.59 & 23.04 & 89.65 & 0.15 & 0.57 & -11.22 & -13.46 \\
\hline 77 & $8 / 25 / 2004$ & 1:39:45 PM & 52.01 & 56.04 & 144.34 & 171.73 & 20.59 & 51.76 & 23.57 & 22.60 & 23.03 & 89.52 & 0.09 & 0.56 & -11.22 & -13.46 \\
\hline 78 & $8 / 25 / 2004$ & 1:44:48 PM & 52.60 & 56.55 & 141.85 & 170.76 & 20.58 & 52.41 & 23.36 & 22.55 & 22.97 & 89.48 & 0.19 & 0.54 & -11.13 & -13.44 \\
\hline 79 & $8 / 25 / 2004$ & 1:49:50 PM & 51.28 & 55.31 & 145.61 & 170.92 & 20.58 & 51.27 & 23.47 & 22.56 & 23.02 & 89.27 & 0.86 & 0.57 & -11.26 & -13.46 \\
\hline 80 & $8 / 25 / 2004$ & 1:54:52 PM & 51.75 & 55.79 & 143.84 & 171.73 & 20.59 & 51.75 & 23.38 & 22.61 & 23.04 & 89.42 & 0.87 & 0.53 & -11.17 & -13.45 \\
\hline 81 & $8 / 25 / 2004$ & 1:59:54 PM & 51.56 & 55.61 & 145.93 & 171.25 & 20.63 & 51.57 & 23.38 & 22.63 & 23.05 & 89.43 & 0.88 & 0.53 & -11.21 & -13.46 \\
\hline 82 & $8 / 25 / 2004$ & 2:04:56 PM & 52.00 & 56.04 & 143.13 & 171.72 & 20.63 & 51.98 & 23.46 & 22.63 & 23.04 & 89.47 & 0.78 & 0.55 & -11.17 & -13.44 \\
\hline 83 & $8 / 25 / 2004$ & 2:09:59 PM & 51.70 & 55.72 & 146.98 & 170.89 & 20.61 & 51.67 & 23.36 & 22.59 & 23.02 & 89.49 & 0.74 & 0.53 & -11.24 & -13.46 \\
\hline 84 & $8 / 25 / 2004$ & 2:15:01 PM & 52.33 & 56.34 & 143.06 & 171.60 & 20.60 & 52.26 & 23.48 & 22.59 & 23.02 & 89.65 & 0.72 & 0.55 & -11.15 & -13.43 \\
\hline 85 & $8 / 25 / 2004$ & 2:20:03 PM & 51.94 & 55.93 & 146.26 & 171.00 & 20.59 & 51.88 & 23.32 & 22.56 & 23.01 & 89.75 & 0.57 & 0.53 & -11.20 & -13.45 \\
\hline 86 & $8 / 25 / 2004$ & 2:25:05 PM & 51.97 & 55.99 & 147.44 & 171.35 & 20.59 & 51.83 & 23.55 & 22.57 & 23.03 & 89.66 & 0.42 & 0.57 & -11.24 & -13.45 \\
\hline 87 & $8 / 25 / 2004$ & 2:30:07 PM & 52.06 & 56.07 & 146.63 & 171.45 & 20.60 & 51.91 & 23.48 & 22.58 & 23.03 & 89.50 & 0.37 & 0.56 & -11.22 & -13.44 \\
\hline 88 & $8 / 25 / 2004$ & 2:35:10 PM & 52.68 & 56.64 & 141.95 & 171.30 & 20.59 & 52.54 & 23.22 & 22.56 & 23.00 & 89.46 & 0.26 & 0.51 & -11.11 & -13.42 \\
\hline 89 & $8 / 25 / 2004$ & 2:40:12 PM & 52.32 & 56.30 & 144.56 & 170.32 & 20.59 & 52.23 & 23.56 & 22.52 & 22.97 & 89.34 & 0.58 & 0.58 & -11.20 & -13.40 \\
\hline 90 & $8 / 25 / 2004$ & 2:45:14 PM & 51.93 & 55.91 & 141.82 & 171.61 & 20.60 & 52.19 & 23.10 & 22.59 & 23.03 & 89.65 & 0.76 & 0.48 & -11.13 & -13.42 \\
\hline
\end{tabular}


WSRC-TR-2005-00105, REVISION 0

SRNL-RPP-2005-00012, REVISION 0

Table H1-3d: Campaign IV

\begin{tabular}{|c|c|c|c|c|c|c|c|c|c|c|c|c|c|c|c|c|}
\hline & DATE & TIME & $\begin{array}{c}\text { Conc } \\
\text { Temp. } \\
\text { Reboiler } \\
\text { In }\end{array}$ & $\begin{array}{l}\text { Conc } \\
\text { Temp. } \\
\text { Reboiler } \\
\text { out }\end{array}$ & $\begin{array}{l}\text { Reboiler } \\
\text { Steam } \\
\text { Supply } \\
\text { Temp. }\end{array}$ & $\begin{array}{l}\text { Ejector } \\
\text { Steam } \\
\text { Supply } \\
\text { Temp. }\end{array}$ & $\begin{array}{l}\text { Cooling } \\
\text { Water } \\
\text { Supply } \\
\text { Temp. }\end{array}$ & $\begin{array}{l}\text { Evap. } \\
\text { Vessel } \\
\text { Temp. }\end{array}$ & $\begin{array}{c}\text { Primary } \\
\text { Conden- } \\
\text { sate } \\
\text { Temp. }\end{array}$ & $\begin{array}{l}\text { Inter. } \\
\text { Cond. } \\
\text { Outlet } \\
\text { Temp. }\end{array}$ & $\begin{array}{l}\text { After } \\
\text { Cond. } \\
\text { Outlet } \\
\text { Temp. }\end{array}$ & $\begin{array}{c}\text { Recirc. } \\
\text { Flow } \\
\text { Rate }\end{array}$ & $\begin{array}{l}\text { Feed } \\
\text { Rate }\end{array}$ & $\begin{array}{l}\text { Prim. } \\
\text { Conden- } \\
\text { sate } \\
\text { Flow }\end{array}$ & $\begin{array}{c}\text { Intercondenser } \\
\text { Press. }\end{array}$ & $\begin{array}{c}\text { Evapo- } \\
\text { rator. } \\
\text { Press. }\end{array}$ \\
\hline & & & ${ }^{\circ} \mathrm{C}$ & ${ }^{\circ} \mathrm{C}$ & ${ }^{\circ} \mathrm{C}$ & ${ }^{\circ} \mathrm{C}$ & ${ }^{\circ} \mathrm{C}$ & ${ }^{\circ} \mathrm{C}$ & ${ }^{\circ} \mathrm{C}$ & ${ }^{\circ} \mathrm{C}$ & ${ }^{\circ} \mathrm{C}$ & GPM & GPM & GPM & PSIG & PSIG \\
\hline 61 & $8 / 25 / 2004$ & 12:19:10 PM & 51.57 & 55.75 & 147.65 & 170.30 & 20.56 & 51.59 & 23.54 & 21.54 & 23.12 & 90.21 & 0.47 & 0.55 & -11.23 & -13.44 \\
\hline 92 & $8 / 25 / 2004$ & 2:55:18 PM & 52.95 & 56.91 & 143.16 & 171.44 & 20.61 & 52.70 & 23.39 & 22.59 & 23.02 & 89.57 & -0.01 & 0.54 & -11.14 & -13.41 \\
\hline 93 & $8 / 25 / 2004$ & 3:00:21 PM & 52.84 & 56.80 & 145.35 & 171.26 & 20.61 & 52.56 & 23.62 & 22.58 & 23.02 & 89.48 & -0.01 & 0.59 & -11.19 & -13.41 \\
\hline 94 & $8 / 25 / 2004$ & 3:05:23 PM & 52.87 & 56.81 & 144.96 & 171.25 & 20.61 & 52.61 & 23.50 & 22.59 & 23.03 & 89.36 & -0.01 & 0.56 & -11.18 & -13.42 \\
\hline 95 & $8 / 25 / 2004$ & 3:10:25 PM & 52.79 & 56.74 & 144.94 & 171.47 & 20.62 & 52.53 & 23.54 & 22.60 & 23.04 & 89.04 & -0.01 & 0.56 & -11.17 & -13.41 \\
\hline 96 & $8 / 25 / 2004$ & 3:15:27 PM & 52.60 & 56.54 & 146.58 & 171.31 & 20.61 & 52.32 & 23.59 & 22.59 & 23.04 & 88.89 & -0.01 & 0.58 & -11.22 & -13.40 \\
\hline 97 & $8 / 25 / 2004$ & 3:20:29 PM & 52.98 & 56.89 & 143.97 & 171.31 & 20.61 & 52.69 & 23.47 & 22.58 & 23.02 & 88.66 & -0.01 & 0.55 & -11.17 & -13.40 \\
\hline 98 & $8 / 25 / 2004$ & 3:25:32 PM & 52.88 & 56.81 & 145.29 & 171.69 & 20.61 & 52.55 & 23.58 & 22.60 & 23.05 & 88.46 & -0.01 & 0.57 & -11.21 & -13.43 \\
\hline
\end{tabular}




\section{Section H2: Pilot Ultrafiltration Process (UFP) Raw Data}

This raw data section differs from the other three, i.e., H1 (FEP), H3 (CIX), and H4 (TLP) in that because of the central role and the complex set of operations of the pilot scale ultrafiltration in the SIPP task the entire UFP unit operational data base is enclosed in this section unabridged. Of course, this make for a very large appendix addition, but inquiries in the past of similar have shown the importance to make this information available. The next couple of pages attempt to orient the reader to understand the data streams and their placement in the overall SIPP task.

\section{Contents Section H2}

Appendix H2 Directory

Raw Data Notes

Raw Data Nomenclature

Instrumentation Locations

Campaign I - IV Test Matrices

Campaign I Raw Data

Campaign II Raw Data

Campaign III Raw Data

Campaign IV Raw Data

\section{Raw Data Notes}

1. The raw data appears in tabular form, in a Microsoft Excel spreadsheet. The table includes measured quantities and calculated quantities. The nomenclature in the table header is described following these notes.

2. A chart precedes each raw data set. The chart shows the permeate flux vs. time for that data set.

3. All columns of measured and calculated quantities are followed by several statistical values, calculated for each columns. The statistical values are average, maximum, medium, minimum, standard deviation and number of data points used in the statistical values. Raw data during bakpulsing were not included in the statistical values due to the significant fluctuations caused by the backpulse. 


\section{WSRC-TR-2005-00105, REVISION 0 \\ SRNL-RPP-2005-00012, REVISION 0}

Raw Data Nomenclature - reference the following figure for instrumentation locations.

\begin{tabular}{|c|c|c|}
\hline \multirow{2}{*}{\multicolumn{3}{|c|}{$\frac{\text { Column Heading }}{\text { Measured quantities }}$}} \\
\hline & & \\
\hline A & Date & Date that the data point was taken \\
\hline B & Time & Time that the data point was taken \\
\hline $\mathrm{D}$ & Filtrate - deg C - T2 & Filtrate temperature \\
\hline $\mathrm{E}$ & Cleaning - deg C - T3 & Cleaning loop temperature - not used for SIPP \\
\hline $\mathrm{F}$ & Slurry - deg C - T1 & Slurry temperature \\
\hline G & Hi Amb. - deg C - T4 & Ambient temperature measured at the top of the test rig \\
\hline $\mathrm{H}$ & Lo Amb. - deg C - T5 & Ambient temperature measure at the bottom of the test rig. \\
\hline $\mathrm{J}$ & Bot TMP - psid - dP2 & Differential pressure across the filter at the bottom permeate exit. \\
\hline K & Filter - psig - P1 & Gauge pressure in the slurry loop at the filter entrance \\
\hline $\mathrm{L}$ & Filter $\mathrm{dP}$ - psid - dP1 & Differential pressure between the filter slurry entrance and exit. \\
\hline M & Top TMP - psid - dP3 & Differential pressure across the filter at the top permeate exit. \\
\hline $\mathrm{N}$ & Filtrate - psid - P2 & Gauge pressure at the top permeate exit. \\
\hline $\mathrm{O}$ & Pulsepot - psig - P3 & Gauge pressure at the backpulse pot. \\
\hline Q & Slurry - gpm - Q1 & Slurry flow rate. \\
\hline $\mathrm{R}$ & Filtrate - gpm - Q2 & Permeate flow rate - low flow rates $(0-1.2 \mathrm{gpm})$. \\
\hline $\mathrm{S}$ & Hi Filtrate - gpm - Q3 & Permeate flow rate - high flow rates ( 0 - 5 gpm). \\
\hline $\mathrm{T}$ & Backpulse - gpm - Qbp & Permeate flow rate during backpulse - reverse direction. \\
\hline \multicolumn{3}{|c|}{ Calculated quantities } \\
\hline $\mathrm{V}$ & Time - min. & Elapsed time in minutes. \\
\hline W & Time - hour & Elapsed time in hours. \\
\hline $\mathrm{X}$ & Press. - psig & $\begin{array}{l}\text { Filter pressure corrected for head of water in the gauge tubing. } \\
\text { P1 - tubing head (psi) }\end{array}$ \\
\hline $\mathrm{Y}$ & Vel. - ft/s & $\begin{array}{l}\left.\text { Axial slurry velocity. Q1/(7.48gal/ft } \mathrm{ft}^{3}\right) /(60 \mathrm{sec} / \mathrm{min}) / \mathrm{flow} \text { area. } \\
\text { Flow area }=\pi^{*}(\text { tube } \mathrm{ID} / 12 \mathrm{in} / \mathrm{ft})^{2} / 4 \text {. }\end{array}$ \\
\hline $\mathrm{Z}$ & TMP - psi & Transmembrane pressure in psi. (dP2+dP3)/2. \\
\hline AA & TMP - bar & Transmembrant pressure in bars. TMP (psi)/(14.504bar/psi). \\
\hline $\mathrm{AB}$ & Filtrate Flux- gpm/ft ${ }^{2}$ & $\begin{array}{l}\text { Permeate flux. Q2/filter surface area } \\
\text { Filter surface area }=\pi^{*}(\text { tube ID/12in/ft)*(tube length/12in/ft)*7. }\end{array}$ \\
\hline AC & Filtrate Flux at $25 \mathrm{C}-\mathrm{gpm} / \mathrm{ft}^{2}$ & Permeate flux at $25^{\circ} \mathrm{C}$. Filtrate flux ${ }^{*} \exp (2500 *((1 / 273+\mathrm{T} 2)-(1 / 298)$. \\
\hline $\mathrm{AD}$ & Permeability - gpm- $\mathrm{ft}^{2} / \mathrm{psi}$ & Permeability. Filtrate flux @ 25ㄷTMP (psi). \\
\hline $\mathrm{AE}$ & Permeabilityx1000 & Permeability * 1000 \\
\hline $\mathrm{AF}$ & Permeability-meter-day/bar & Permeability * 851.0145 m/day/bar/gpm/ft²/bar. \\
\hline
\end{tabular}


Instrumentation locations

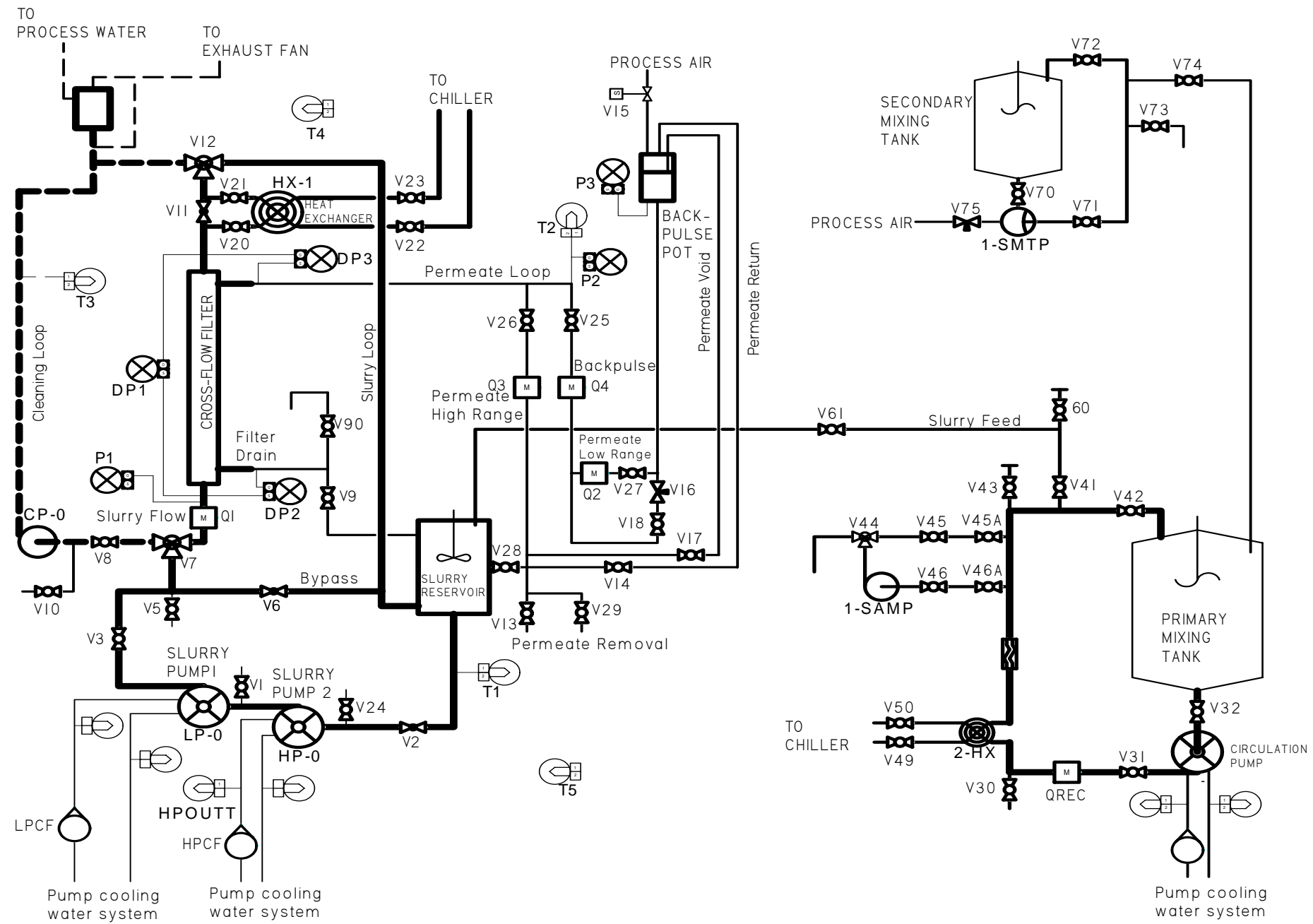


WSRC-TR-2005-00105, REVISION 0 SRNL-RPP-2005-00012, REVISION 0

Test Matrices for reference with the Raw Data Tables

Campaign I

\begin{tabular}{|c|c|c|c|c|c|}
\hline $\begin{array}{c}\text { Test } \\
\text { No. }\end{array}$ & Test Activity & $\begin{array}{c}\text { Trans-Membrane } \\
\text { Pressure } \\
(\mathrm{psid})\end{array}$ & $\begin{array}{c}\text { Slurry } \\
\text { Velocity } \\
(\mathrm{ft} / \mathrm{s})\end{array}$ & $\begin{array}{c}\text { Slurry } \\
\text { Temp. } \\
\left({ }^{\circ} \mathrm{C}\right)\end{array}$ & $\begin{array}{c}\text { Run } \\
\text { Time } \\
(\mathrm{min})\end{array}$ \\
\hline $1.00 \mathrm{~A}$ & Water & 10 & 11 & 25 & 20 \\
\hline $1.00 \mathrm{~B}$ & Water & 20 & 11 & 25 & 20 \\
\hline $1.00 \mathrm{C}$ & Water & 30 & 11 & 25 & 20 \\
\hline 1.03 & Dewater to $25 \mathrm{wt} \%$ & 40 & 12 & 25 & Note 1 \\
\hline 1.04 & $25 \mathrm{wt} \%$ & 40 & 11 & 25 & 120 \\
\hline 1.07 & $25 \mathrm{wt} \%$ & 30 & 9 & 25 & 60 \\
\hline 1.08 & $25 \mathrm{wt} \%$ & 30 & 13 & 25 & 60 \\
\hline 1.09 & $25 \mathrm{wt} \%$ & 50 & 13 & 25 & 60 \\
\hline 1.10 & $25 \mathrm{wt} \%$ & 50 & 9 & 25 & 60 \\
\hline 1.11 & $25 \mathrm{wt} \%$ & 40 & 11 & 25 & 120 \\
\hline 1.16 & $25 \mathrm{wt} \%$ & 40 & 11 & 25 & 120 \\
\hline Wash & $20 \mathrm{wt} \%$ & 40 & 12 & 25 & Note 2 \\
\hline $1.18 \mathrm{~A}$ & Water rinse & 40 & 12 & 25 & 30 \\
\hline $1.18 \mathrm{~B}$ & Acid clean & 40 & 12 & 25 & 90 \\
\hline $1.18 \mathrm{C}$ & Acid clean & 40 & 12 & 25 & 90 \\
\hline $1.18 \mathrm{D}$ & Acid clean & 40 & 12 & 25 & 90 \\
\hline $1.19 \mathrm{~A}$ & Water rinse & 20 & 12 & 25 & 30 \\
\hline $1.19 \mathrm{~B}$ & Water rinse & 20 & 12 & 25 & 30 \\
\hline $1.19 \mathrm{C}$ & Water rinse & 20 & 12 & 25 & 30 \\
\hline 1.20 & Water & $10,20,30$ & 11 & 25 & 60 \\
\hline
\end{tabular}

Note 1 Run time is the time necessary to dewater to $>20 \mathrm{wt} \%$ undissolved solids. The only test specification was to run no less than 12 hours.

Note 2 Run time was the time necessary to introduce the 22 mini-washes.

\section{Campaign II}

\begin{tabular}{|c|c|c|c|c|c|}
\hline Test No. & Test Activity & $\begin{array}{c}\text { Trans-Membrane } \\
\text { Pressure } \\
(\mathrm{psid})\end{array}$ & $\begin{array}{c}\text { Slurry } \\
\text { Velocity } \\
(\mathrm{ft} / \mathrm{s})\end{array}$ & $\begin{array}{c}\text { Slurry } \\
\text { Temp. } \\
\left({ }^{\circ} \mathrm{C}\right)\end{array}$ & $\begin{array}{c}\text { Run } \\
\text { Time } \\
(\mathrm{min})\end{array}$ \\
\hline 2.00A & Pre-test baseline A & 10 & 11 & 25 & 20 \\
\hline $2.00 \mathrm{~B}$ & Pre-test baseline B & 20 & 11 & 25 & 20 \\
\hline 2.00C & Pre-test baseline C & 30 & 11 & 25 & 20 \\
\hline 2.03A & Dewater first half of slurry & 40 & 12 & 25 & 948 \\
\hline 2.03B & Dewater second half of slurry & 40 & 12 & 25 & 2452 \\
\hline Wash 1 & Slurry wash 1 & 40 & 12 & 25 & 379 \\
\hline Wash 2 & Slurry wash 2 & 40 & 12 & 25 & 246 \\
\hline 2.18A & Caustic rinse 1 & 20 & 11 & 25 & 30 \\
\hline $2.18 \mathrm{~B}$ & Acid clean 1 & 40 & 11 & 25 & 90 \\
\hline $2.18 \mathrm{C}$ & Acid clean 2 & 40 & 11 & 25 & 90 \\
\hline $2.18 \mathrm{D}$ & Acid clean 3 & 20 & 11 & 25 & 90 \\
\hline 2.19A & Caustic rinse 2 & 20 & 11 & 25 & 30 \\
\hline $2.19 \mathrm{~B}$ & Caustic rinse 3 & 10 & 11 & 25 & 30 \\
\hline 2.21A & Post-test baseline A & 20 & 11 & 25 & 20 \\
\hline 2.21B & Post-test baseline B & & 11 & 25 & 20 \\
\hline
\end{tabular}


WSRC-TR-2005-00105, REVISION 0 SRNL-RPP-2005-00012, REVISION 0

\section{Campaign III}

\begin{tabular}{|c|c|c|c|c|c|}
\hline Test No. & Test Activity & $\begin{array}{c}\text { Trans-Membrane } \\
\text { Pressure } \\
(\mathrm{psid})\end{array}$ & $\begin{array}{c}\text { Slurry } \\
\text { Velocity } \\
(\mathrm{ft} / \mathrm{s})\end{array}$ & $\begin{array}{c}\text { Slurry } \\
\text { Temp. } \\
\left({ }^{\circ} \mathrm{C}\right)\end{array}$ & $\begin{array}{c}\text { Run } \\
\text { Time } \\
(\mathrm{min})\end{array}$ \\
\hline 3.00A & Pre-test baseline A & 10 & 11 & 25 & 20 \\
\hline 3.00B & Pre-test baseline B & 20 & 11 & 25 & 20 \\
\hline 3.00C & Pre-test baseline C & 30 & 11 & 25 & 20 \\
\hline 3.03A & Dewater first half of slurry & 40 & 12 & 25 & 960 \\
\hline 3.03B & Dewater second half of slurry & 40 & 12 & 25 & 2300 \\
\hline Wash 1 & Slurry wash 1 & 40 & 12 & 25 & 379 \\
\hline Wash 2 & Slurry wash 2 & 40 & 12 & 25 & 229 \\
\hline 3.18A & Caustic rinse 1 & 20 & 11 & 25 & 30 \\
\hline 3.18B & Acid clean 1 & 40 & 11 & 25 & 90 \\
\hline 3.18C & Acid clean 2 & 40 & 11 & 25 & 90 \\
\hline 3.18D & Acid clean 3 & 20 & 11 & 25 & 90 \\
\hline 3.19A & Caustic rinse 2 & 20 & 11 & 25 & 30 \\
\hline 3.19B & Caustic rinse 3 & 10 & 11 & 25 & 30 \\
\hline 3.21A & Post-test baseline A & 20 & 11 & 25 & 20 \\
\hline 3.21B & Post-test baseline B & 30 & 11 & 25 & 20 \\
\hline 3.21C & Post-test baseline C & & & & 25 \\
\hline
\end{tabular}

\section{Campaign IV}

\begin{tabular}{|c|c|c|c|c|c|}
\hline Test No. & Test Activity & $\begin{array}{c}\text { Trans-Membrane } \\
\text { Pressure } \\
(\mathrm{psid})\end{array}$ & $\begin{array}{c}\text { Slurry } \\
\text { Velocity } \\
(\mathrm{ft} / \mathrm{s})\end{array}$ & $\begin{array}{c}\text { Slurry } \\
\text { Temp. } \\
\left({ }^{\circ} \mathrm{C}\right)\end{array}$ & $\begin{array}{c}\text { Run } \\
\text { Time } \\
(\mathrm{min})\end{array}$ \\
\hline 4.00A & Pre-test baseline A & 10 & 11 & 25 & 20 \\
\hline 4.00B & Pre-test baseline B & 20 & 11 & 25 & 20 \\
\hline 4.00C & Pre-test baseline C & 30 & 11 & 25 & 20 \\
\hline 4.03A & Dewater first half of slurry & 40 & 12 & 25 & 960 \\
\hline 4.03B & Dewater second half of slurry & 40 & 12 & 25 & 2300 \\
\hline 4.07 & Steady State @ 24wt\% & 30 & 9 & 25 & 30 \\
\hline 4.08 & Steady State @ 24wt\% & 30 & 13 & 25 & 30 \\
\hline 4.09 & Steady State @ 24wt\% & 50 & 13 & 25 & 30 \\
\hline 4.11 & Steady State @ 24wt\% & 40 & 11 & 25 & 30 \\
\hline Steady5 & Steady State @ 24wt\% & 50 & 13 & 25 & 30 \\
\hline Staedy6 & Steady State @ 24wt\% & 50 & 15 & 257 & 30 \\
\hline Temp1 & High temperature test @ 20 wt\% & 40 & 12 & 30 & 30 \\
\hline Temp2 & High temperature test @ 20 wt\% & 40 & 12 & 37.5 & 30 \\
\hline Temp3 & High temperature test @ 20 wt\% & 40 & 12 & 45 & 30 \\
\hline Temp4 & High temperature test @ 20 wt\% & 40 & 12 & 35 & 30 \\
\hline Temp5 & High temperature test @ 20 wt\% & 40 & 12 & 25 & 30 \\
\hline Temp6 & High temperature test @ 20 wt\% & 50 & 15 & 37.5 & 30 \\
\hline Temp7 & High temperature test @ 20 wt\% & 50 & 15 & 45 & 30 \\
\hline Wash 1 & Slurry wash 1 & 40 & 12 & 25 & 379 \\
\hline Wash 2 & Slurry wash 2 & 40 & 12 & 25 & 229 \\
\hline 4.18A & Caustic rinse 1 & 20 & 11 & 25 & 30 \\
\hline 4.18B & Acid clean 1 & 40 & 11 & 25 & 90 \\
\hline 4.18C & Acid clean 2 & 40 & 11 & 25 & 90 \\
\hline 4.18D & Acid clean 3 & 40 & 11 & 25 & 90 \\
\hline 4.19A & Caustic rinse 2 & 20 & 11 & 25 & 30 \\
\hline 4.19B & Caustic rinse 3 & 20 & 11 & 25 & 30 \\
\hline 4.21A & Post-test baseline A & 20 & 11 & 25 & 20 \\
\hline 4.21B & Post-test baseline B & 30 & 11 & 25 & 20 \\
\hline 4.21C & Post-test baseline C & & 11 & 25 & 20 \\
\hline
\end{tabular}


RUN \# 1.00A, B, C; PRE-TEST BASELINE WITH DIF WATER

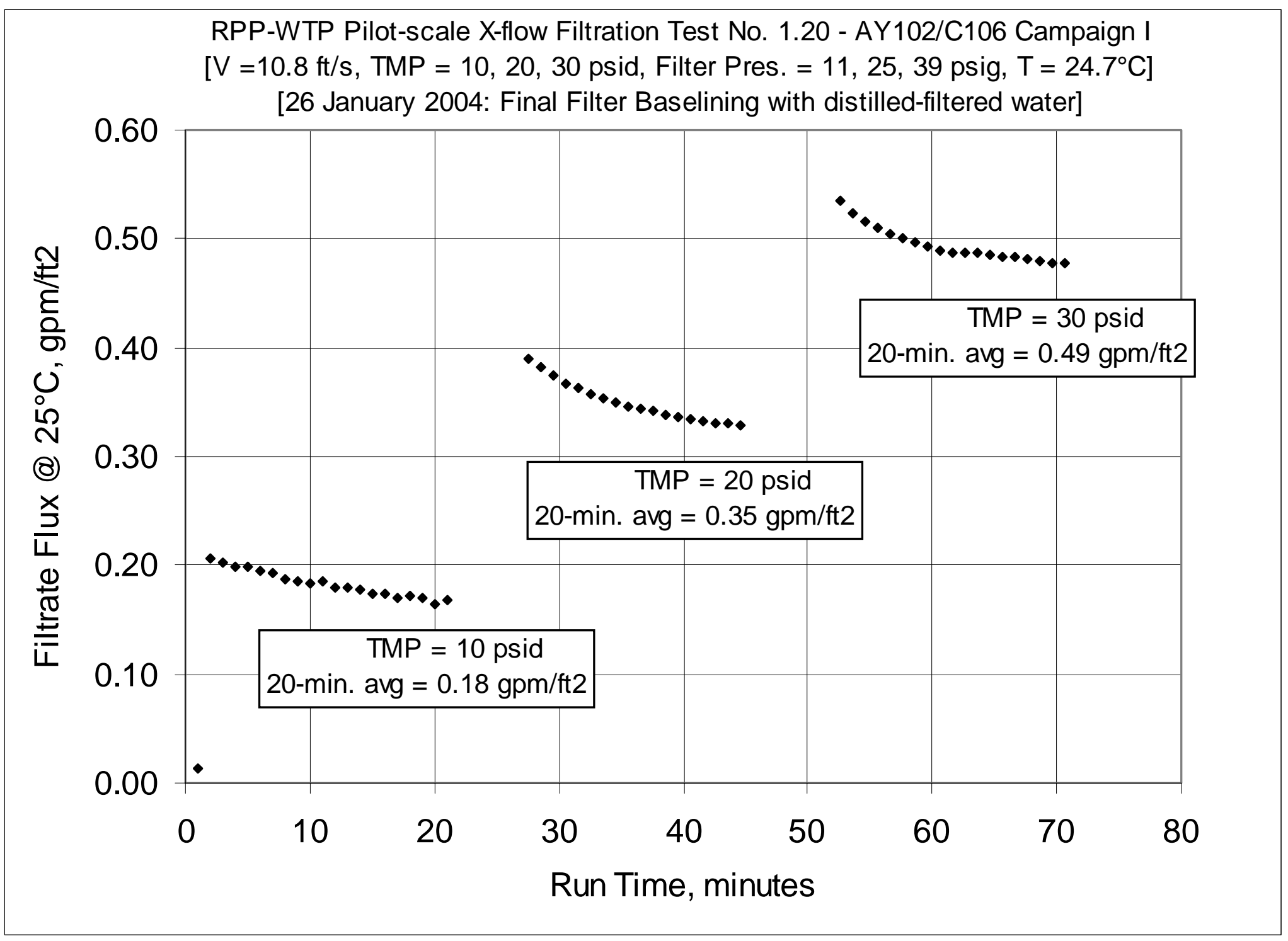


WSRC-TR-2005-00105, REVISION 0

SRNL-RPP-2005-00012, REVISION 0

RUN \# 1.00A, B, C; PRE-TEST BASELINE WITH DIF WATER - CONT.

\begin{tabular}{|c|c|c|c|c|c|c|c|c|c|c|c|c|c|c|c|c|c|c|c|c|c|c|c|c|c|c|c|c|}
\hline & A & $\mathrm{B}$ & $\mathrm{D}$ & $\begin{array}{ll}\mid & E \\
\ll<<\text { Temp }\end{array}$ & $\begin{array}{ll}\mathrm{F} \\
\text { ature }\end{array}$ & $\frac{G}{\text { asure }}$ & $\begin{array}{ll}\mid & H \\
\text { ents } \gg\end{array}$ & $\mathrm{J}$ & $\frac{K}{K \ll<\ll}$ & $\frac{L}{\text { P }} \frac{\mathrm{L}}{\text { Pressure }}$ & $\frac{M}{\text { asure }}$ & $\frac{N}{1}$ ents $\gg$ & $\frac{0}{1}$ & $\frac{Q}{2<<<<<<}$ & $\begin{array}{l}\mathrm{R} \\
<\text { Flown }\end{array}$ & $\frac{\mathrm{s}}{\text { leasureme }}$ & $T$ & $\mathrm{v}$ & $\frac{w}{s \ll \ll<s}$ & \begin{tabular}{|l|}
$x<\ll<c$ \\
$<<<$
\end{tabular} & $\frac{\mathrm{Y}}{\mathrm{C}}$ & \begin{tabular}{|l|} 
ted Fror \\
ted Fro
\end{tabular} & \begin{tabular}{|l|} 
AA \\
m Raw
\end{tabular} & $\mid \mathrm{AB}$ & $\frac{A C \mid}{A C}$ & $\frac{A D}{A}$ & $\mathrm{AE}$ & \\
\hline & DATE & TIME & Filtrate & Cleaning & Slurry & Hi Amb. & Lo Amb. & BotTMP & & Filter $\mathrm{dP}$ & \begin{tabular}{|l|l|} 
TopTMP \\
\end{tabular} & & Pulsepot & & Filtrate & & & & & & & & & & & & & \\
\hline & & & $\frac{\operatorname{deg} C}{T 2}$ & $\operatorname{deg} C$ & \begin{tabular}{|l|l|}
$\operatorname{deg} C$ \\
\end{tabular} & $\frac{\operatorname{deg} C}{T A}$ & $\operatorname{deg} C$ & psid & psig & $\begin{array}{l}\text { psid } \\
\text { sin } 1\end{array}$ & $\begin{array}{l}\text { psid } \\
\text { sp }\end{array}$ & & $\begin{array}{l}\text { psig } \\
\text { si }\end{array}$ & gpm & gpm & gpm & gpm & & & & & & & & & & & \\
\hline & & & & T3 & T1 & & & & P1 & $\mathrm{dP1}$ & $\mathrm{dP} 3$ & $\mathrm{P} 2$ & & & & Q3 & Qbp & & & & & & & & & & & \\
\hline$\frac{5}{6}$ & teros & & & & & & & & & & & & & & & & & & & & & & & & & & & \\
\hline 7 & $1 / 26 / 2004$ & 7:40:18 AM & 19.871 & 15.611 & 12.488 & 20.323 & 12.825 & $\begin{array}{l}0.056 \\
\end{array}$ & \begin{tabular}{|l|} 
\\
\end{tabular} & -0.004 & 0.024 & \begin{tabular}{|l|l|}
4 & -0.103 \\
\end{tabular} & 0.192 & -0.084 & 0.003 & $\begin{array}{ll}14.643 \\
\end{array}$ & 0.006 & & & & & & & & & & & \\
\hline & 1/26/2004 & 7:41:31 AM & 19.871 & $\begin{array}{ll}15.611 \\
19610\end{array}$ & $\begin{array}{ll}12.488 \\
12.98\end{array}$ & 20.323 & 12.825 & $\begin{array}{l}0.056 \\
\end{array}$ & \begin{tabular}{|l|}
-1.097 \\
\end{tabular} & -0.004 & 0.024 & \begin{tabular}{|c|c|c|}
4 & -0.103 \\
\end{tabular} & 0.192 & $\begin{array}{l}-0.084 \\
-0.03\end{array}$ & 0.003 & $\begin{array}{ll}14.643 \\
\end{array}$ & 0.006 & & & Convers & rsion & 851 & $\mathrm{~m} 3 / \mathrm{m} 21$ & $2 /$ day/bar & $\mathrm{g} / \mathrm{gpm} / \mathrm{tt} 2$ & ft2/barg & & \\
\hline & & $7: 41: 32 \mathrm{AM}$ & 19.867 & 15.606 & 12.488 & 20.313 & & & \begin{tabular}{|l|l|} 
& -1.101 \\
\end{tabular} & & & & & & & $\begin{array}{l}14.643 \\
\end{array}$ & & & & & & & & & & & & \\
\hline & 1/26/2004 & 7::41:33 AM & 19.872 & 15.611 & 12.488 & 20.313 & $\begin{array}{ll}12.816 \\
\end{array}$ & $\begin{array}{l}0.058 \\
\end{array}$ & \begin{tabular}{|c|} 
\\
\end{tabular} & -0.004 & 0.026 & \begin{tabular}{|l|l|}
5 & -0.144 \\
\end{tabular} & 0.192 & \begin{tabular}{|c|c|c|}
-0.084 \\
\end{tabular} & $\begin{array}{ll}0.003 \\
\end{array}$ & 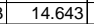 & 0.007 & & & Note & unge & & & & ately & 4 psig & & \\
\hline & $1 / 26 / 2004$ & 7:41:34 AM & 19.867 & & 12.483 & & & & \begin{tabular}{|l|l|} 
& -1.101 \\
\end{tabular} & & & \begin{tabular}{l|l}
4 & -0.1 \\
\end{tabular} & & -0.084 & 0.003 & 14.643 & 0.006 & & & & & & & & & & & \\
\hline & 1/26/2004 & 7:41:35 AM & $\frac{19.862}{10.867}$ & 15.606 & 12.483 & 20.308 & 12.81 & \begin{tabular}{|l|l|}
0.058 \\
\end{tabular} & \begin{tabular}{|l|l|}
-1.099 \\
\end{tabular} & -0.004 & 0.023 & $\begin{array}{l}3 \\
\end{array}$ & 0.195 & $\begin{array}{l}-0.084 \\
-0.091\end{array}$ & 0.003 & 14.643 & 0.005 & & & & & & & & & & & \\
\hline \begin{tabular}{|l|l|}
14 & \\
\end{tabular} & $\frac{1 / 26 / 2004}{1 / 26 / 2004}$ & 7:41:36 AM & $\frac{19.867}{19862}$ & 15.611 & 12.488 & 20.313 & $\begin{array}{l}12.81 \\
12.81 \\
\end{array}$ & 0.005 & $\begin{array}{r}-1.101 \\
1.01 \\
\end{array}$ & -0.004 & & & 0.195 & $\begin{array}{l}-0.084 \\
0.091\end{array}$ & 0.003 & & 2000 & & & 政 & & & & < Filtra & & & & \\
\hline & 112012004 & ::41:3/ AM & 19.862 & 15.606 & 12.483 & 20.318 & 12.8 & 0.056 & -1.10 & -0.004 & 0.026 & -0.1 & 0.195 & -0.084 & 0.00 & 14.643 & 0.00 & Time & & Press. & $\begin{array}{l}\text { Vel. } \\
\end{array}$ & TMP & TMP & gam & $\frac{a t z b c}{a p m}$ & & $\frac{8000}{2000}$ & \\
\hline & Data - Per N & & & & & & & & & & & & & & & & & in & Hour & psig & & psi & bar & /ft2 & 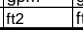 & $\mathrm{ft} 2 / \mathrm{psi}$ & $\mathrm{ft} / \mathrm{psi}$ & day/bar \\
\hline & $1 / 26 / 2004$ & 12:53:28 PM & 23.154 & 16.775 & 23.249 & 21.379 & 16.322 & 3.082 & 12.438 & & -1.461 & & 52.902 & 43.831 & 0.003 & \begin{tabular}{|c|c|c|}
-0.014 \\
\end{tabular} & 0.004 & & & 11.0 & & & & & & & & \\
\hline & 1/26/2004 & $\begin{array}{l}\text { 12:54:28 PM } \\
12.55 \cdot 28 \mathrm{PM}\end{array}$ & $\begin{array}{l}22.296 \\
23237 \\
\end{array}$ & $\begin{array}{l}16.781 \\
16.777\end{array}$ & 23.255 & $\begin{array}{r}21.4 \\
1376\end{array}$ & $\begin{array}{l}16.485 \\
16.669 \\
\end{array}$ & $\begin{array}{r}4.725 \\
11751\end{array}$ & \begin{tabular}{|l|l|}
12.415 \\
1.264
\end{tabular} & $\begin{array}{l}5.478 \\
5.465 \\
\end{array}$ & $\begin{array}{l}0.527 \\
7759 \\
\end{array}$ & 3.607 & $\begin{array}{l}0.37 \\
0.462 \\
\end{array}$ & \begin{tabular}{|l|l|}
43.845 \\
4462
\end{tabular} & 0.4 & \begin{tabular}{r|}
0.09 \\
1318
\end{tabular} & 0.004 & 1.0 & 0.01667 & $\begin{array}{ll}10.9 \\
10.8\end{array}$ & $\frac{10.7}{100}$ & 2.6 & 0.181 & \begin{tabular}{|l|l|}
0.013 \\
0197
\end{tabular} & 0.014 & 0.005 & $\frac{5.369}{2151}$ & 1.50 \\
\hline 20 & $\begin{array}{l}11 / 26 / 2004 \\
1 / 26 / 2004\end{array}$ & $\frac{12: 55: 28 \mathrm{PM}}{12: 56: 28 \mathrm{PM}}$ & & $\frac{16.1 / 7}{16.772}$ & $\begin{array}{l}23.2271 \\
23.287\end{array}$ & $\frac{21.3 / 6}{21.396}$ & $\frac{16.669}{16.932}$ & $\begin{array}{l}\mid 11.151 \\
11.826 \\
\end{array}$ & \begin{tabular}{|l|}
12.264 \\
12.297 \\
\end{tabular} & $\begin{array}{l}5.465 \\
5.473 \\
\end{array}$ & $\begin{array}{l}.1 .159 \\
7.766\end{array}$ & $\frac{-1.214}{-1.216}$ & $\begin{array}{l}0.462 \\
0.459 \\
\end{array}$ & \begin{tabular}{|l|}
44.632 \\
4.719
\end{tabular} & $\begin{array}{l}0.003 \\
0.003 \\
\end{array}$ & $\begin{array}{l}1.318 \\
1.295 \\
\end{array}$ & & 2.00 & & $\begin{array}{l}10.8 \\
10.8 \\
\end{array}$ & $\frac{10.9}{110}$ & 9.8 & & $0.197 \mid$ & $\begin{array}{l}0.206 \\
0.203 \\
\end{array}$ & $\frac{0.021}{0.021}$ & 21.151 & $\frac{18.00}{17.61} \mathrm{r}$ \\
\hline & $1 / 26 / 2004$ & 12:57:28 PM & 23.468 & 16.773 & 23.383 & 21.327 & 17.434 & $\begin{array}{l}11.020 \\
11.849\end{array}$ & \begin{tabular}{|l|}
12.249 \\
\end{tabular} & 5.444 & 7.839 & \begin{tabular}{|l|l|}
9 & -1.216 \\
\end{tabular} & 0.459 & 44.667 & 0.003 & 1.272 & 0.004 & 4.00 & $\begin{array}{l}0.05 \\
0\end{array}$ & 10.8 & \begin{tabular}{|l|l|}
3 & 10.9 \\
\end{tabular} & $\begin{array}{l}0.0 \\
9.8\end{array}$ & & 0.190 & 0.199 & & 20.167 & 17.1 \\
\hline & $1 / 26 / 2004$ & 12:58:28 PM & 23.542 & 16.772 & 23.492 & & \begin{tabular}{|l|l|}
17.327 \\
\end{tabular} & \begin{tabular}{|l|}
11.915 \\
\end{tabular} & \begin{tabular}{|l|l|}
12.289 \\
\end{tabular} & & 7.954 & -1.216 & 0.456 & 44.705 & 0.003 & 1.272 & 0.004 & 5.00 & 0.08333 & 10.8 & \begin{tabular}{|l|l}
31.1 \\
\end{tabular} & 9.9 & \begin{tabular}{|l|l|}
0.685 \\
\end{tabular} & \begin{tabular}{|l|l|} 
& 0.190 \\
\end{tabular} & 0.198 & & & \\
\hline & $1 / 26 / 2004$ & $12: 59: 28$ PM & 23.658 & 16.783 & $\begin{array}{l}3 \\
3\end{array}$ & 21.331 & 17.362 & 11.911 & & 5.401 & 7.93 & -1.214 & 0.456 & 44.619 & 0.003 & 1.258 & 0.004 & 6.00 & 0.1 & 10.7 & & & 0.684 & 0.188 & 0.195 & 0.020 & 19.643 & 16 \\
\hline & $1 / 26 / 2004$ & 1:00:28 PM & 23.785 & 16.804 & 23.75 & 21.363 & 18.056 & \begin{tabular}{|l|l|}
11.975 \\
\end{tabular} & 12.268 & 5.415 & 7.971 & -1.208 & 0.454 & \begin{tabular}{|l|l|}
44.623 \\
\end{tabular} & 0.003 & $\begin{array}{l}1.245 \\
\end{array}$ & 0.004 & m & 0.11667 & 10.8 & & & & 0.186 & 0.192 & & 19.282 & 16.41 \\
\hline & 1/26/2004 & $\begin{array}{l}\text { 1:01:28 PM } \\
10.02020\end{array}$ & $\begin{array}{r}23.856 \\
23.957\end{array}$ & $\begin{array}{r}16.79 \\
16.817\end{array}$ & 23.826 & 21.283 & $\begin{array}{r}18.32 \\
17.406\end{array}$ & $\begin{array}{r}12.089 \\
12044\end{array}$ & \begin{tabular}{|l|}
12.322 \\
12237
\end{tabular} & $\begin{array}{r}5.464 \\
5.405\end{array}$ & 8.034 & $\begin{array}{l}4 \\
\begin{array}{c}4 \\
\end{array}-1.214 \\
142\end{array}$ & 0.451 & 44.677 & 0.003 & 1.219 & 0.004 & 8.00 & 0.13333 & 10.8 & 10.9 & 0.1 & 0.694 & 0.182 & 0.188 & 0.019 & 18.673 & 15.89 \\
\hline$\frac{27}{27}$ & $\begin{array}{l}1 / 26 / 2004 \\
/ 26 / 204\end{array}$ & $1: 03: 28 \mathrm{PM}$ & $\frac{20.951}{24.023}$ & $\begin{array}{l}10.011 \\
16.802\end{array}$ & 23.960 & $\frac{21.35}{21.321}$ & $\begin{array}{l}17.400 \\
17.028\end{array}$ & 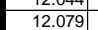 & \begin{tabular}{|l|}
12.231 \\
12.249 \\
\end{tabular} & $\begin{array}{l}5.405 \\
5.426\end{array}$ & $\begin{array}{l}.073 \\
8.071\end{array}$ & $\begin{array}{l}-1.200 \\
-1.216 \\
\end{array}$ & $\begin{array}{l}.440 \\
0.448\end{array}$ & $\begin{array}{r}44.711 \\
44.71\end{array}$ & 0.003 & $\begin{array}{l}1.210 \\
1.198 \\
\end{array}$ & 0.004 & $\frac{9.00}{10.00}$ & $\begin{array}{r}0.1567 \\
0.16667\end{array}$ & $\begin{array}{l}0.0 \\
10.8\end{array}$ & $\frac{10.9}{11.0}$ & $\frac{1.1}{10.1}$ & $\frac{0.095}{0.695}$ & $\begin{array}{l}0.101 \\
0.179 \\
\end{array}$ & $\begin{array}{l}0.100 \\
0.184 \\
\end{array}$ & $\frac{0.019}{0.018}$ & $\begin{array}{l}10.500 \\
18.252\end{array}$ & $\frac{15.75}{15.53}$ \\
\hline & $1 / 26 / 2004$ & 1:04:28 PM & 24.104 & 16.818 & & & 16.927 & & & 5.356 & 8.084 & $\frac{-1.214}{-1.214}$ & & 44.552 & 0.003 & & & & 0.18333 & & & & & 0.180 & & & & 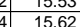 \\
\hline & $1 / 26 / 2004$ & 1:05:28 PM & 24.185 & 16.815 & 24.146 & 21.343 & 17.359 & $\begin{array}{l}12.17 \\
\end{array}$ & 12.299 & 5.433 & 8.18 & -1.211 & & 44.615 & 0.003 & 1.175 & & $\frac{12.0}{12.0}$ & 0.2 & 10. & & & & & & & & \\
\hline & $1 / 26 / 2004$ & 1:06:28 PM & 24.231 & 16.81 & 24.177 & 21.233 & 17.162 & 12.083 & 12.183 & 5.358 & 8.123 & -1.216 & & \begin{tabular}{|l|l|l|l}
44.717 \\
\end{tabular} & 0.003 & 1.172 & & 13.6 & 0.21667 & 10. & & & & 0.175 & & & & 15 \\
\hline 31 & $1 / 26 / 2004$ & $\begin{array}{ll}1: 07: 28 \\
\end{array}$ & 24.307 & 16.826 & \begin{tabular}{|l|l|}
5 & 24.298 \\
\end{tabular} & 21.259 & 17.077 & 12.313 & 12.401 & 5.42 & 8.311 & -1.216 & & 444.6 & 0.003 & $\begin{array}{l}1.166 \\
\end{array}$ & & 14.00 & 0.23333 & 10.9 & 10.6 & 10.3 & & 0.174 & & & & 14. \\
\hline & $1 / 26 / 2004$ & 1:08:28 PM & 24.375 & 16.834 & $\begin{array}{l}424.305 \\
4\end{array}$ & 21.256 & 18.02 & \begin{tabular}{|l|l|}
12.129 \\
\end{tabular} & 12.21 & 5.417 & 8.088 & -1.214 & 0.445 & 44.654 & 0.003 & 1.142 & & 15.00 & 0.25 & 10.7 & twe. & 10.1 & .697 & 0.170 & 0.174 & 0 & 17.177 & \\
\hline & $1 / 26 / 2004$ & 1:09:28 & 24.416 & 16.835 & $\begin{array}{l}54.382 \\
5\end{array}$ & 21.197 & 17.799 & 12.28 & 12.337 & 5.429 & 8.252 & -1.216 & & 44.721 & 0.003 & 1.142 & & $16.0 \mathrm{c}$ & & 100 & $\bar{F}+2$ & 10.3 & & & . & & 16.877 & \\
\hline & & 1:10:28 PM & 24.488 & & & 21.274 & & 12.245 & $\frac{12.26}{12.26}$ & 5.446 & & & & 44.775 & 0.003 & 1.127 & & 17.00 & 0.28333 & 10.8 & & $10.2 \mathrm{ra}$ & & 0.168 & & 0.017 & 16.706 & \\
\hline & 1/26/2004 & $\frac{1: 11: 28}{1.12 .28}$ & 24.539 & $\begin{array}{l}16.874 \\
16.876\end{array}$ & $\frac{24.4}{24.51}$ & $\frac{21.311}{21.313}$ & $\frac{17.69}{17.68}$ & $\frac{12.278}{12103}$ & $\frac{12.299}{12.21}$ & $\frac{5.4}{55.4}$ & $\begin{array}{r}8.209 \\
80.69\end{array}$ & -1.216 & & 44.579 & & 1.133 & & 18. & 0.3 & 10 & & & & & 0.1 & & & 14 \\
\hline 37 & 112621004 & $\frac{1.12 .201}{1.1208}$ & $\frac{24.590}{24622}$ & 10.010 & 24.51 & 21.135 & & 12016 & 12.21 & 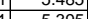 & & -1.24 & & $44.1<00$ & & & & 然 & 0 & 10 & & & & & & & & t7 \\
\hline$\frac{3}{3}$ & $1 / 26 / 2004$ & 41.1428 & 24663 & 16.878 & 24595 & 21,32 & 186 & 12181 & 12.158 & 539 & 8184 & 1.216 & & 44.575 & 0.003 & 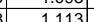 & & 210 & 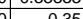 & 10 & & 10 & 704 & 0.166 & 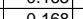 & 0.010 & & 13.74 \\
\hline & 112612004 & & & 1696 & & & & & 26497 & 5047 & & 19334 & & & & & & & & & & & & & & & 4248970 & \\
\hline$T_{1}$ & $1 / 26 / 2004$ & 1:20:0 & 22.828 & 16.978 & 25.12 & 21.354 & & 2.836 & 26.64 & 5.0 & -1755 & 19379 & & 42596 & & 146 & & & 0.44333 & 25 & & & 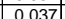 & $218 \mathrm{~K}$ & & & & \\
\hline & $1 / 26 / 2004$ & $1: 21: 0$ & 25.21 & 17.006 & 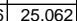 & 21.342 & & 20.511 & 25.892 & 4.95 & 17.415 & 0.587 & & 44.429 & & & & 27 & 0.46 & 24 & & 10 & & 0.391 & 0.36 & & & 17 \\
\hline & $1 / 26 / 2004$ & 1:22:04 & 25.263 & 17.019 & 24.994 & 21.299 & & 21.044 & \begin{tabular}{|l|}
26.236 \\
\end{tabular} & & 17.914 & & & 44.189 & & & & & $\overline{0.47667}$ & 24. & & & & & & & & \\
\hline 4 & $1 / 26 / 2004$ & 1:23:04 & 25.246 & 17.048 & 24.955 & 21.262 & 19.2 & 21.09 & 25.954 & 4.768 & $\begin{array}{l}8.357 \\
\end{array}$ & 0.124 & & \begin{tabular}{|l|l|l|l|}
44.031 \\
\end{tabular} & 0.003 & 2 & & 29.60 & 0.49333 & 24. & & 19.1 & .00 & 0.374 & 0.375 & 0.6 & & \\
\hline 44 & $1 / 26 / 2004$ & 1:24:04 & 25.225 & 17.072 & 24.911 & 21.26 & & 21.594 & 26.366 & & 18.519 & -0.082 & & 43.895 & & 2.458 & & & 0.51 & 24. & & & 000 & & 0.367 & & .318 & \\
\hline & 6/2004 & 1:25:04 & 25.219 & 17.102 & $\begin{array}{l}244.891 \\
24\end{array}$ & $\frac{21.27}{21.27}$ & & 21.5655 & 26.136 & 4.911 & 18.591 & -0.16 & & 43.902 & & 2.424 & & & 0.52667 & 24. & & 20. & 1.384 & 0.361 & 0.363 & & & \\
\hline & 101202004 & & 25.2 & 11.145 & 2.80 & 21.394 & & $\begin{array}{r}21.87 \\
21779\end{array}$ & & $\frac{3.09}{492}$ & 18 & -0.304 & & 43 & & 2. & & 20 & & 24 & & & & & & & & \\
\hline 4 & $\frac{1 / 2012004}{1 / 26 / 2004}$ & 1.27 .04 & $\begin{array}{l}25.175 \\
25165\end{array}$ & 11.105 & $\begin{array}{l}\quad 24.001 \\
5 \quad 2892\end{array}$ & $\frac{21.356}{21296}$ & & 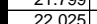 & \begin{tabular}{|l|l|l|l|}
26263 \\
\end{tabular} & & & $\begin{array}{l}-0.42 \\
-0.527\end{array}$ & & 年 & & & & 34 & 0.50 & 24.19 & & & & 0 & & & & \\
\hline & $1 / 26 / 2004$ & $\frac{1: 2904}{112: 04}$ & $\frac{2 . .125}{25.125}$ & 17.254 & 424.836 & $\frac{1.1 .205}{21.205}$ & & 22 & \begin{tabular}{|l|l|l|}
26.124 \\
\end{tabular} & $\frac{3.9}{4.9}$ & $\frac{19.059}{19.059}$ & -0.594 & & 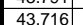 & & & & & 0.50933 & 24.0 & & & & & & & & \\
\hline & $1 / 26 / 2004$ & $1: 30: 04$ & 25.115 & 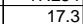 & 24.811 & 21.124 & & 22.204 & \begin{tabular}{|l|l|}
26.252 \\
\end{tabular} & & & & & 43.735 & & & & & & & & & & & & & & \\
\hline & $1 / 26 / 2004$ & $1: 31: 04 \mathrm{~F}$ & 25.083 & $\begin{array}{l}17.323 \\
\end{array}$ & 24.759 & 20.976 & & 22.061 & 26.083 & 4.8 & 19.137 & -0.724 & & 1007 & & & & & & & & & & & & & & \\
\hline & $1 / 26 / 2004$ & $1: 32: 0$ & 25.072 & 17.3 & \begin{tabular}{|l|l|}
24.748 \\
\end{tabular} & 21.066 & & 22.27 & 26.269 & & 19.094 & -0.811 & & 43.7 & & 2.2 & & 38 & 0.64333 & 24 & & & & & & & & \\
\hline & $1 / 26 / 2004$ & 1:33: & 25.05 & 17.3 & $\begin{array}{l}624 \\
6\end{array}$ & 21.13 & & 22.403 & 26.28 & & 19. & -0.8 & & & & & & & 0.66 & 24 & & & & & & & & \\
\hline & $1 / 26 / 2004$ & 1:34:04 & 25.043 & $\begin{array}{ll}17.424 \\
\end{array}$ & $\begin{array}{l}4 \mid 24.7 \\
4\end{array}$ & 21.153 & & 22.473 & 26. & 5.8 & 19.508 & -0.927 & & 43.5 & & 2.2 & & & 0.67667 & 24. & & & .44 & 32 & 0.334 & & 15.914 & \\
\hline 5 & 1/26/2004 & 1:35: $C$ & 25.019 & 17.4 & $\begin{array}{l}1 \\
11\end{array}$ & 21.224 & & 22.386 & 26.244 & & & -0.9. & & 43.6 & & 2.2 & & 41 & 0.69333 & 24 & & & & & & & & \\
\hline & $1 / 26 / 2004$ & 1:36:04 & 25.016 & $17.458 \mathrm{ra}$ & 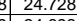 & 21.252 & & 22.48 & 26.253 & & 19.353 & -0.988 & & & & & & 42 & 0.71 & 24. & & & & & & & & \\
\hline & 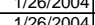 & & 25.002 & 17.469 & 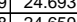 & 21.248 & & 22.454 & 26.1 & & $\frac{19.41}{19.4}$ & -0.9948 & & & & & & & & & & & & & & & & \\
\hline & $1 / 262004$ & & 24.961 & 17.46 & 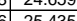 & $\frac{21.220}{21.203}$ & & $\frac{2.550}{241}$ & (20.252 & & 19.519 & 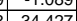 & & $\frac{43 .}{415}$ & & & & & & 24.6 & & & & & & & & \\
\hline & $\frac{1}{1 / 2612004}$ & & $\frac{24.878}{24.878}$ & $\frac{1.1 .572}{17.572}$ & 2 25.541 & $\frac{2.1 .29}{21.229}$ & & $\frac{\mid 6.45 t}{16.167}$ & & & $\frac{1.263}{12.613}$ & 8,1989 & & & & & & & & & & & & & & & & \\
\hline & $1 / 26 / 2004$ & & 25.933 & & & 21244 & & 29.682 & & & 27219 & & & & & & & & & & & & & & & & & \\
\hline & & & 26.064 & & 25.702 & 21.26 & & & & & & & & & & & & & & & & & & & & & & \\
\hline & $1 / 26 / 2004$ & & 26.141 & 17.6 & 25.724 & 21.338 & & 30.334 & & 4.5 & 27.886 & 5.008 & & 44.196 & & & & & 0.91 & & & & & & & & & \\
\hline & $1 / 26 / 200$ & 1:49:C & 26.14 & 17.6 & 25 . 25 & 21.36 & & 30.3 & & & 27.91 & & & & & & & $55>>>$ & 0.9 & & & & & & & & & \\
\hline & $1 / 26 / 2004$ & 1:50:07 P & 26.161 & 17.6 & 25.739 & 21.393 & & 30.479 & 40.169 & & 28.1 & 4.5 & & 44.0 & & 3.4 & & 56.6 & & 38. & & & & 14 & & & & \\
\hline 6 & 1/26/2004 & 1:51:07 & 26.157 & $17.667 \mathrm{r}$ & 25.74 & 21.46 & & 30.734 & 40.287 & 4.257 & 28.61 & 4.429 & & 44.046 & 0.005 & 3.425 & & 57.65 & 0.96083 & $38.8 \mathrm{r}>\mathrm{l}$ & $10.8 \mathrm{r}>\mathrm{r}$ & & & 0.511 & & & & \\
\hline & $1 / 26 / 2004$ & 1:52:07 PM & 26.144 & 17.68 & 25.722 & 21.447 & & 30.968 & 40.361 & 4.41 & 28.696 & & & & & & & 58.6 & & & & & & & & & & \\
\hline & 1120 & & 20.6167 & 11.103 & $\frac{2.5 .45}{2.571}$ & 20.1369 & & $\begin{array}{l}30.951 \\
31.67\end{array}$ & 40.289 & 4.0 & $\begin{array}{r}28.869 \\
28.967\end{array}$ & 4.188 & & $\begin{array}{r}43.96 \\
13270 \\
\end{array}$ & 0. & 3. & & 然 & 0.99417 & 0.0 .0 & 1.0. & 200 & & & & & $6=270$ & \\
\hline & $1 / 26 / 2004$ & 1:55:07 PM & 26.15 & 17.736 & \begin{tabular}{l|l}
66 & 25.703 \\
\end{tabular} & 21.372 & \begin{tabular}{l|l|l|}
2 & 18.72 \\
\end{tabular} & 31.279 & \begin{tabular}{|l|}
40.409 \\
\end{tabular} & $\begin{array}{ll}4.167 \\
\end{array}$ & 29.339 & 3.998 & & 43.795 & 0.003 & 3.332 & & 61.65 & 1.0275 & 38.9 & & 303 & & $0.497 \quad>$ & 0.487 & 0.016 - & 16070 & \\
\hline & & | 1:55:07 PM & 26.15 & 17.136 & $\begin{array}{ll}6 \mid & 25.103 \\
\end{array}$ & & & 31.279 & 40.409 & 4.167 & 29.339 & $\begin{array}{l}9 \\
9\end{array}$ & & 43.795| & & 3.332 & & & 5] 1.0275 & & & & & & & & & \\
\hline
\end{tabular}


WSRC-TR-2005-00105, REVISION 0

SRNL-RPP-2005-00012, REVISION 0

RUN \# 1.00A, B, C; PRE-TEST BASELINE WITH DIF WATER - CONT.

\begin{tabular}{|c|c|c|c|c|c|c|c|c|c|c|c|c|c|c|c|c|c|c|c|c|c|c|c|c|c|c|c|c|}
\hline & A & \begin{tabular}{l|l} 
B \\
\end{tabular} & D & $E$ & $\mathrm{~F}$ & G & \begin{tabular}{l|l}
$\mathrm{H}$ & $\mathrm{H}$ \\
$\mathrm{H}$
\end{tabular} & $\mathrm{J}$ & $\mathrm{K}$ & $\mathrm{L}$ & $\begin{array}{ll}\mathrm{M} \\
\end{array}$ & $\mathrm{N}$ & \begin{tabular}{l|l}
$\mathrm{O}$ & $\mathrm{F}$ \\
\end{tabular} & Q & $\mathrm{R}$ & $\mathrm{s}$ & \begin{tabular}{l|l}
$T$ & $\mathrm{U}$ \\
\end{tabular} & $\mathrm{v}$ & w & $x$ & $\mathrm{Y}$ & $z$ & $\mathrm{AA}$ & $A B$ & $\mathrm{AC}$ & $\mathrm{AD}$ & $\mathrm{AE}$ & AF $A$ \\
\hline & 1/26/2004 & 1:56:07 PM & 26.022 & 17.65 & 25.534 & 21.303 & 16.387 & 31.111 & 40.104 & 3.888 & \begin{tabular}{|l|}
29.357 \\
259
\end{tabular} & 3.931 & 1.445 & $\begin{array}{l}3.91 \\
3.920\end{array}$ & 0.003 & 3.322 & 0.004 & 62.65 & \begin{tabular}{|l|l|}
5 & 1.04417 \\
5
\end{tabular} & 38.6 & 10.8 & 30.2 & 2.085 & 0.495 & 0.488 & 0.016 & 16.138 & 13.73 \\
\hline & $1 / 26 / 2004$ & 1:57:07 PM & 26.007 & 17.68 & \begin{tabular}{|l|}
25.499 \\
\end{tabular} & 21.379 & 17.096 & 31.661 & 40.645 & 4.148 & 29.581 & 3.824 & 1.442 & 43.739 & 0.003 & 3.309 & 0.005 & 63.65 & \begin{tabular}{|l|l|}
5 & 1.06083 \\
\end{tabular} & 39.2 & 10.7 & 30.6 & 2.111 & 0.493 & 0.486 & 0.016 & 15.887 & 13.52 \\
\hline \begin{tabular}{|l|}
73 \\
74 \\
\end{tabular} & $\begin{array}{l}1 / 26 / 2004 \\
1 / 26 / 204\end{array}$ & \begin{tabular}{|l|}
$1: 58: 07$ PM \\
$1.59: 07$ PM
\end{tabular} & $\begin{array}{l}25.974 \\
25.938 \\
\end{array}$ & $\begin{array}{l}17.7 \\
17.73 \\
\end{array}$ & \begin{tabular}{|l|}
25.442 \\
25.425 \\
\end{tabular} & $\frac{21.381}{22.105}$ & $\begin{array}{l}17.862 \\
18.402\end{array}$ & 31.989 & $\begin{array}{l}40.838 \\
40303\end{array}$ & $\begin{array}{ll}4.043 \\
3.896\end{array}$ & \begin{tabular}{|l|l|}
30.012 \\
29.889 \\
\end{tabular} & $\begin{array}{l}3.723 \\
3.723 \\
\end{array}$ & \begin{tabular}{l|l|}
1.434 \\
1.442
\end{tabular} & $\begin{array}{ll}43.728 \\
3664\end{array}$ & $\begin{array}{l}0.003 \\
0.003\end{array}$ & 3.293 & 0.005 & 64.65 & \begin{tabular}{|l|l|}
5 & 1.0775 \\
5 & 109417
\end{tabular} & \begin{tabular}{|l|}
39.4 \\
392
\end{tabular} & \begin{tabular}{|c|}
10.7 \\
107 \\
\end{tabular} & $\begin{array}{l}731.0 \\
7308 \\
3\end{array}$ & \begin{tabular}{|l|l|}
2.137 \\
2.126
\end{tabular} & $\begin{array}{l}0.491 \\
50.489 \\
\end{array}$ & $\begin{array}{l}0.485 \\
0483\end{array}$ & $\begin{array}{l}0.016 \\
0.016\end{array}$ & \begin{tabular}{|l|}
15.642 \\
1567
\end{tabular} & $\begin{array}{l}13.31 \\
134 \\
\end{array}$ \\
\hline \begin{tabular}{|l|}
75 \\
75
\end{tabular} & $1 / 26 / 2004$ & $2: 00: 07 \mathrm{PM}$ & 25.898 & $\frac{11.15}{17.76}$ & \begin{tabular}{|l|}
25.34256 \\
25.386
\end{tabular} & $\frac{1 . .406}{21.36}$ & 10.404 & 31.512 & 40.322 & 3.898 & $\begin{array}{l}29.005 \\
29.614 \\
\end{array}$ & 3.641 & $\begin{array}{l}1.444 \\
1.434\end{array}$ & $\begin{array}{l}43.664 \\
43.559\end{array}$ & 0.003 & 3.272 & $\frac{0.005}{0.006}$ & & \begin{tabular}{|l|l|}
5 & 1.041111 \\
\end{tabular} & & & & & 0.489 & $\begin{array}{l}0.483 \\
0.483 \\
\end{array}$ & 0.016 & \begin{tabular}{|l|}
15.672 \\
15.789 \\
\end{tabular} & $\frac{13.34}{13.44}$ \\
\hline 76 & $1 / 26 / 2004$ & $2: 01: 07$ PM & 25.86 & & 25.362 & 21.256 & 18.626 & & 40.72 & 4.059 & 30.104 & 3.549 & $\begin{array}{l}1.404 \\
1.428\end{array}$ & 43.599 & 0.003 & 3.259 & 0.006 & 67.65 & \begin{tabular}{|l|l|}
5 & 1.1275 \\
\end{tabular} & 39.2 & 10.7 & & & 0.486 & $\begin{array}{l}0.400 \\
0.481\end{array}$ & & $\begin{array}{l}\frac{15.189}{15.482} \\
\end{array}$ & $\frac{13.44}{13.18}$ \\
\hline \begin{tabular}{|l|}
77 \\
\end{tabular} & $1 / 26 / 2004$ & $2: 02: 07 \mathrm{PM}$ & 25.822 & 17.79 & $\begin{array}{l}25.329 \\
\end{array}$ & 21.172 & 18.825 & 31.8 & 40.465 & 3.885 & 30.022 & 3.488 & 1.419 & 43.512 & & 3.244 & 0.006 & 68.65 & \begin{tabular}{|l|l|}
5 & 1.14417 \\
\end{tabular} & $\mid 39.0$ & \begin{tabular}{|c|c|}
10.7 \\
\end{tabular} & | & & 0.484 & 0.479 & & & $\frac{13.18}{13.19}$ \\
\hline 78 & $1 / 26 / 2004$ & 2:03:07 PM & 25.79 & 17.81 & 25.302 & 21.094 & & 31.889 & & 3.909 & 30.129 & 3.453 & 1.41 & 43.441 & 0.003 & 3.228 & & & & & 10.6 & & & & & & 年389 & $\frac{13.19}{1310}$ \\
\hline \begin{tabular}{|l|}
79 \\
\end{tabular} & $1 / 26 / 2004$ & 2:04:07 PM & 25.742 & 17.81 & 25.259 & 20.945 & 18.719 & 31.968 & 40.564 & 3.868 & 30.129 & 3.395 & 1.41 & 43.493 & 0.003 & 3.23 & & 70.65 & $\frac{1.1 .1775}{1.175}$ & 39.1 & $\frac{10.7}{10.7}$ & 31.0 & 2.141 & 0.482 & 0.478 & 0.015 & 15.398 & $\frac{13.10}{13.10}$ \\
\hline \begin{tabular}{|l|l|}
80 \\
81
\end{tabular} & & Averages & $241>>$ & & & $212>$ & $175 \mathrm{t}$ & 121 & & 5 & $210>1$ & 12 & 5054 & & $500>>>$ & 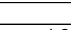 & & & & 100 & & 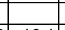 & (n) & & & 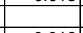 & 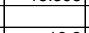 & 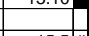 \\
\hline \begin{tabular}{|c|}
82 \\
82
\end{tabular} & & \begin{tabular}{|l|} 
Averages \\
Maximum
\end{tabular} & $\frac{24.1}{24.7}$ & 16. & 24.0 & 21.3 & 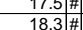 & $\frac{12.1}{12.3}$ & $\frac{12.3}{12.4}$ & 5.4 & $\frac{8.1}{8.3}$ & $\frac{-1.2}{-1.2}$ & $\frac{0.5 \#}{0.5 \#}$ & $\frac{44.7}{44.8}$ & $\frac{0.0}{0.0}$ & $\frac{1.2}{1.3}$ & & & & $\frac{10.8}{10.9}$ & & & & $\frac{0.1 / 8}{0.197}$ & $\frac{0.183}{0.206}$ & $\frac{0.018}{0.021}$ & $\frac{18.2}{212}$ & $15.5 \mathrm{HA}$ \\
\hline & & Median & & & & & & & & 5.4 & & & $0.4 \#$ & 44.6 & & & & & & & & & & & & & & 18.0 列 \\
\hline 84 & & Minimum & 23.3 & 16. & 23.3 & $\frac{21.2}{21.2}$ & 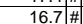 & $\frac{21.8}{11.8}$ & $\frac{12.2}{12.2}$ & 5.4 & 7.8 & $\frac{-1.2}{-1.2}>\mathrm{C}$ & $\frac{0.4 \#}{0.4 \pi}$ & 44.6 & 0.0 & 11 & $\frac{0.0}{0.0}-2$ & & & $\frac{1.0 .0}{107}>0$ & $\frac{10.3}{109}$ & & $\frac{0.053}{0.673}$ & $\frac{0.17}{0.163}$ & $\frac{0.106}{0.165}$ & $\frac{0.016}{0.016}$ & $\frac{10.02}{162}$ & $\frac{15.38}{138}$ \\
\hline & & & 0.845 & 0.07 & 0.847 & 0.104 & $0.866 \neq t$ & 0.320 & 0.115 & 0.074 & 0.304 & 0.005 & $0.011 \#$ & 0.119 & 0.000 & 0.128 & 0.000 & & & 0.115 & & & & & 0.024 & & & \\
\hline$\frac{86}{86}$ & Number c & of Points Used $\mathrm{d}^{*}$ & & & 20 & 20 & 200 & 20 & 20 & 20 & 20 & 20 & $0.01+4$ & $\frac{1.120}{20}$ & 20 & $\frac{0.120}{20}$ & 0.000 & & & $\frac{0.110}{20}$ & vas & & & 20 & $\frac{0.024}{20}$ & 20 & 20 & \\
\hline & & |* Backpulse poil & is in box & are not in & liuded & & & & & & & & & & & & & & & & & & & & & & & \\
\hline $\begin{array}{lll}88 \\
\end{array}$ & & & & & & & & & & & & & & & & & & & & & & & & & & & & \\
\hline (1) & & & & & & & & & & & & & & & & & & & & & & & $20-$ min. & 7. avg. = & 0.18 & & & \\
\hline$\frac{90}{91}$ & & An & 251 & 17 & 240 & 212 & 10 & 210 & & 50 & 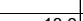 & 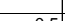 & 074 & $30^{\circ}$ & 5 & $\sqrt{2}+2>$ & 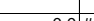 & & & $27+7>$ & & & & & & & & \\
\hline$\frac{51}{02}$ & & \begin{tabular}{|l|} 
Averages \\
Maximum
\end{tabular} & 25.1 & 17. & 22.8 .8 & $\frac{21.2}{214}$ & $\frac{19.3}{20.4 \#}$ & 21.9 & 26.2 & $\frac{5.0}{5.2}>2$ & $\begin{array}{l}18.9 \\
19.5\end{array}$ & -0.5 & $0.7 \#$ & $\begin{aligned} 43.8 \\
44 .\end{aligned}$ & 0.0 & 2.3 .3 & 0.0 & & & 24.7 & 10.7 & & & 0.348 & & & 17.2 & 14.6 丮 \\
\hline 93 & & Median & 25.1 & 17. & 24.8 & 21.4 & $\frac{20.4+1+1+1}{19.1+\#}$ & 22.0 & 26.4 & 5.0 & $\frac{19.5}{19.1}$ & $\begin{array}{l}0.6 \\
-0.6\end{array}$ & $\frac{0.1 \#}{0.7 \#}$ & $\begin{array}{c}43.4 \\
43.7 \\
\end{array}$ & 0.0 & 2.3 & 0.0 & & & $\begin{array}{l}24.9 \\
24.8\end{array}$ & \begin{tabular}{|l|}
10.9 \\
10.7 \\
\end{tabular} & 210.6 & \begin{tabular}{|l|l|l|l|} 
\\
1418
\end{tabular} & 0.391 & 0.390 & $\begin{array}{l}0.0206 \\
0.0167\end{array}$ & $\begin{array}{l}20.6 \\
16.7 \\
\end{array}$ & $\frac{17.5 \#}{14.2 \#}$ \\
\hline 94 & & Minimum & 25.0 & 17. & 24.7 & 21.0 & $18.4 \| \#$ & 20.5 & 25.9 & 4.8 & $\begin{array}{l}1.1 .4 \\
17.4\end{array}$ & -1.1 & $0.7 \#$ & 43.6 & 0.0 & 2.2 & 0.0 & & & 24.4 & 10.7 & 719.0 & 1.307 & 0.326 & 0.329 & 0.0156 & 15.6 & 13.3 \# \\
\hline 95 & & $2 \times$ Std Dev & 0.178 & 0.32 & 0.216 & 0.203 & $1.447 \#$ & 1.120 & 0.238 & 0.207 & 1.110 & 0.955 & $0.013 \#$ & 0.428 & 0.000 & 0.257 & 0.001 & & & \begin{tabular}{|l|l|}
0.238 \\
\end{tabular} & 0.105 & $\mid$ & 30.076 & \begin{tabular}{|l|l|} 
& 0.038 \\
\end{tabular} & 0.036 & 0.0028 & 2.780 & $2.366 \#$ \\
\hline \begin{tabular}{|l|}
96 \\
\end{tabular} & Number & of Points Used & 18 & 1 & 18 & 18 & 180 & 18 & 18 & 18 & 18 & 18 & 180 & 18 & 18 & 18 & 18 & & & 18 & 3 & 18 & $3 \quad 18$ & \begin{tabular}{|l|l|}
3 & 18 \\
\end{tabular} & 18 & 18 & 18 & 180 \\
\hline 97 & & * Backpulse poi & is in box & ren not ir & fluded & & & & & & & & & & & & & & & & & & & & & & & \\
\hline \begin{tabular}{|l|}
98 \\
99 \\
\end{tabular} & & & & & & & & & & & & & & & & & & & & & & & 18-min. & 1. avg. $=$ & 0.35 & & & \\
\hline & & & & & & & & & & & & & & & & & & & & & & & & & & & & \\
\hline & & Averages & 26.0 & 17. & 25.6 & 21.3 & 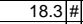 & 31.1 & 40.5 & 4.2 & 29.0 & 4.2 & $1.4 \pi$ & 43.9 & 0.0 & 3.4 & $0.0<>-0$ & & & 39.0 & 10.8 & 30.1 & 2.075 & 0.502 & 0.494 & & & 14.0 th \\
\hline & & Maximum & 26.2 & 17. & 25.8 & 21.5 & 18.8|\# & 32.0 & 40.8 & 4.5 & 30.1 & 5.6 & $1.51 \#$ & 44 & 0.6 & 3.6 & 0.6 & & & 39.4 & & & & 0.544 & 0.534 & 0.0188 & & \\
\hline $\mid \frac{103}{104}$ & & Median & 2.0 & 17. & 25.6 & 21.4 & $18.5 \mid \#$ & 31.2 & 40.4 & $\frac{4.1}{30}-3$ & $\frac{29.3}{272}$ & 4.0 & 1.4. \# & 43.9 & 0.0 & 3.3 & 0.0 & & & $38.9-9$ & 10.8 & & 2.085 & 0.497 & 0.488 & 0.0161 & 16.1 & $13.7[\#$ \\
\hline$\frac{104}{105}$ & & IMnimum & 25.1 & $\frac{113}{0.13}$ & $\begin{array}{r}2.3 \\
0.351 \\
\end{array}$ & 20.9 & $\frac{16.4[\mathrm{H}}{1.09}$ & 29.14 & 40.1 & $\begin{array}{r}3.9 \\
0.458 \\
-1\end{array}$ & $\frac{28.2}{1876}$ & $\begin{array}{r}3.4 \\
1260\end{array}$ & $\begin{array}{l}1.4^{\#} \\
0.043 \pi\end{array}$ & $\begin{array}{r}43.4 \\
0.559 \\
\end{array}$ & 0.000 & 0.238 & 000 & & & $\begin{array}{ll}38.6 \\
304\end{array}$ & 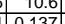 & 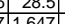 & | & 0.481 & 0.471 & 0.0154 & 15.44 & $\frac{13.11^{\#}}{1670^{*}}$ \\
\hline \begin{tabular}{|c|c|}
106 \\
\end{tabular} & Number & of Points Used & 19 & $\frac{0.10}{1}$ & & 19 & 190 & $\frac{1.425}{19}$ & $\frac{1.00}{19}$ & $\frac{4.49}{19}$ & 19 & 19 & 190 & 19 & 0.000 & $\frac{0.258}{19}$ & $\begin{array}{r}0.002 \\
19\end{array}$ & & & $\mid \begin{array}{cc}0.304 \\
19\end{array}$ & 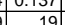 & a. 1.047 & $\begin{array}{l}9.114 \\
9.19\end{array}$ & 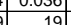 & $\mid 0.32$ & 0.0020 & $\frac{1.931}{19}$ & 1.679 \\
\hline 107 & & |* Backpulse poit & is in box & re not in & linded & & & & & & & & & & & & & & & & & & & & & & & \\
\hline & & & & & & & & & & & & & & & & & & & & & & & & & & & & \\
\hline & & & & & & & & & & & & & & & & & & & & & & & 19-min. & 1. avg. = & 0.49 & & & \\
\hline & & rages & 25.0 & 17. & 24.7 & 21.3 & 18.3/\# & 20.9 & 25.6 & 4.9 & 17.8 & 0.9 & $1.7 \#$ & 44 & 0.0 & 2.2 & $0.0 \# \#$ & & & 24.1 & 10.8 & B 19.4 & 41.337 & 0.329 & 0.328 & 0.0168 & 16.8 & 14.3 \\
\hline & & Maximum & 26.2 & 17. & 25.8 & 21.5 & $20.4 \#$ & 32.0 & 40.8 & 5.5 & 30.1 & 5.6 & $52.9 \#$ & 44.8 & 0.4 & 3.6 & $0.0 \mid \#$ & & & $\mid 39.4$ & \begin{tabular}{|l|l|}
$\mid 71$ \\
\end{tabular} & | & $\begin{array}{l}12.142 \\
12.0\end{array}$ & 0.544 & $\mid 0.534$ & \begin{tabular}{|l|l|} 
& 0.0212 \\
\end{tabular} & 21.2 & 18.4 \\
\hline 113 & & Median & 25.1 & 17. & 24.8 & 21.3 & 18.44\# & 22.0 & 26.2 & 5.0 & 18.9 & -0.6 & $0.7 \#$ & 44.0 & 0.0 & 2. & $0.0 \mid \#$ & & & 24.8 & 10.8 & B20.5 & 51.412 & 0.339 & 0.341 & 0.0169 & 16.9 & $14.3 \#$ \\
\hline 114 & & Minimum & 22.3 & 16. & 23.2 & 20.9 & $16.3 \#$ & 3.1 & 12.2 & $3.99 \mathrm{r}$ & -1.5 & $\begin{array}{r}-1.2 \\
10.5\end{array}$ & $0.4 \#$ & 43.4 & 0.0 & 0.0 & 0.0 \# & & & $\begin{array}{ll}10.7 \\
\end{array}$ & 10.6 & \begin{tabular}{|l|l|}
6 & 0.8 \\
\end{tabular} & 0.056 & -0.002 & -0.002 & -0.0027 & -2.7 & $-2.3 \#$ \\
\hline & & $2 \times \operatorname{Std} \mathrm{Dev}$ & 1.871 & 0.76 & \begin{tabular}{|l|l|}
1.474 \\
\end{tabular} & 0.212 & $1.982 \#$ & 16.797 & 23.449 & 1.107 & 18.434 & 4.959 & $13.468 \#$ & 0.867 & 0.102 & 1.958 & 0.002 \# & & & 23.450 & 0.213 & \#\#\# & 1.214 & 292 & 0.282 & 0.0066 & 6.648 & 5.657 \# \\
\hline & Number & of Points Used* & $\begin{array}{r}59 \\
5\end{array}$ & 5 & 59 & 59 & 590 & 59 & 59 & & $\begin{array}{r}59 \\
\end{array}$ & 59 & 5900 & 59 & 59 & 59 & $\begin{array}{r}59 \\
\end{array}$ & & & 59 & 59 & 59 & 59 & 59 & 59 & 59 & 59 & 5900 \\
\hline & & & & & & & & & & & & & & & & & & & & & & & & & & & & \\
\hline & & & & & & & & & & & & & & & & & & & & & & & 1-hour & avg. $=$ & 0.34 & & & \\
\hline
\end{tabular}


RUN \# 1.00A, B, C; PRE-TEST BASELINE WITH 0.1 M NAOH

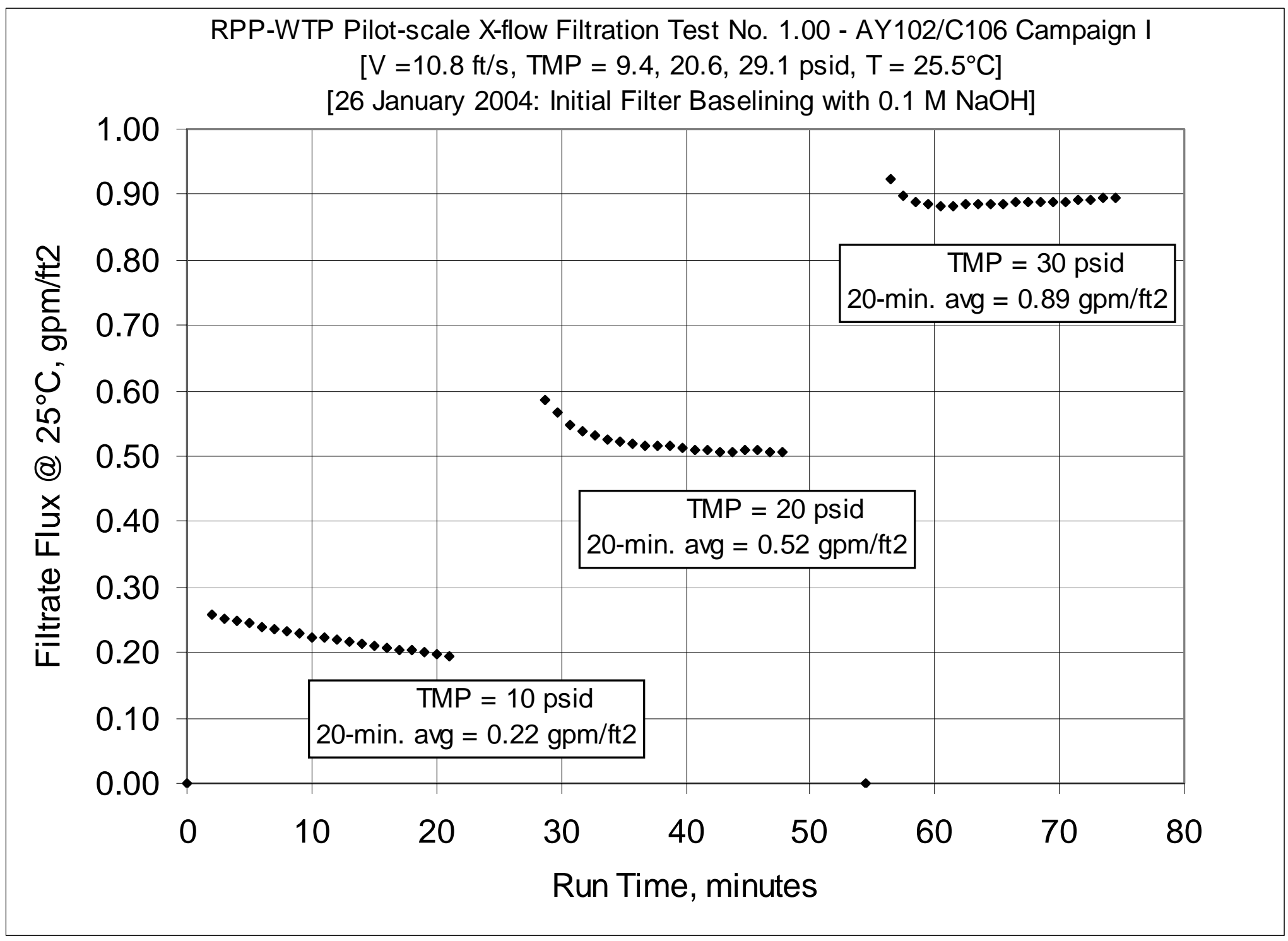


WSRC-TR-2005-00105, REVISION 0

SRNL-RPP-2005-00012, REVISION 0

RUN \# 1.00A, B, C; PRE-TEST BASELINE WITH 0.1 M NAOH - CONT.

\begin{tabular}{|c|c|c|c|c|c|c|c|c|c|c|c|c|c|c|c|c|c|c|c|c|c|c|c|c|c|c|c|c|}
\hline & & & & & & \\
\hline & $\mathrm{A}$ & B & $\mathrm{D}$ & $E$ & I $F$ & \begin{tabular}{|l|l|l|l|} 
& $G$
\end{tabular} & $\mathrm{H}$ & $\mathrm{J}$ & \begin{tabular}{l|l|}
$\mathrm{K}$ & \\
\end{tabular} & I L & \begin{tabular}{l|l} 
& \\
\end{tabular} & \begin{tabular}{|l|l|}
$\mathrm{N}$ \\
\end{tabular} & 0 & $Q$ & I $\mathrm{R}$ & $\mathrm{s}$ & $\mathrm{T}$ & $\mathrm{V}$ & 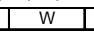 & \begin{tabular}{|l|l|}
$x$ & \\
\end{tabular} & $\begin{array}{l}I r \\
\end{array}$ & 2 & $\mid \mathrm{AA}$ & \begin{tabular}{|l|l|} 
& $A B$ \\
\end{tabular} & \begin{tabular}{|l|l|} 
& \\
\end{tabular} & AD & $\mathrm{AE}$ & $\mathrm{AF}$ \\
\hline & DATE & & 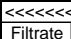 & 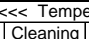 & $\begin{array}{l}\text { perature M } \\
\text { g. Slury }\end{array}$ & $\begin{array}{l}\text { Measuremer } \\
\text { H Amb }\end{array}$ & $\begin{array}{l}\text { ents } \gg \gg> \\
\text { LLAmb. }\end{array}$ & BotTMP & Filter & $\begin{array}{l}\text { Pressure } \\
\text { FititerdP }\end{array}$ & Measuremen & 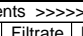 & & $\begin{array}{l}2 \ll \ll<< \\
\text { Slury }\end{array}$ & $\begin{array}{ll}\ll<<\text { Flow } \\
\text { F }\end{array}$ & 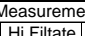 & & & & & & & Raw L & & & & & \\
\hline & & & & & & & & & & & & & & & & & & & & & & & & & & & & \\
\hline & & & $\frac{\mathrm{deg} C}{\mathrm{~T} 2}$ & $\frac{\mathrm{deg} C}{\mathrm{~T} 3}$ & $\frac{\operatorname{deg} C}{\text { T1 }}$ & $\frac{\mathrm{deg} \mathrm{C}}{\mathrm{T} 4}$ & $\frac{\operatorname{deg} C}{T 5}$ & 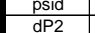 & $\frac{\mathrm{psig}}{\mathrm{P} 1}$ & $\frac{\text { psid }}{\text { dP1 }}$ & $\frac{\text { psid }}{\text { dP3 }}$ & $\frac{\text { psig }}{\text { P2 }}$ & $\begin{array}{l}\text { psig } \\
\text { P3 }\end{array}$ & gpm & $\frac{\mathrm{gpm}}{\mathrm{O} 2}$ & $\frac{\mathrm{gpm}}{\mathrm{O} 3}$ & \begin{tabular}{|l} 
gpm \\
\end{tabular} & & & & & & & & & & & \\
\hline & & & & & & & & & & & & & & & & & & & & & & & & & & & & \\
\hline & ros & & & & & & & & & & & & & & & & & & & & & & & & & & & \\
\hline 7 & $1 / 26 / 2004$ & $7: 40: 18 \mathrm{AM}$ & 19.871 & 15.611 & 12.488 & \begin{tabular}{|l|}
20.323 \\
\end{tabular} & $\begin{array}{l}12.825 \\
\end{array}$ & 0.056 & \begin{tabular}{|l|l|}
-1.097 \\
\end{tabular} & -0.004 & 0.024 & \begin{tabular}{|c|c|}
-0.103 \\
\end{tabular} & 0.192 & -0.084 & 0.003 & $\begin{array}{l}14.643 \\
\end{array}$ & 0.00 & & & Filter Su & iufface, & 6.707 & FT2 & & & & & \\
\hline 8 & 1/266/2004 & $7: 41: 31 \mathrm{AM}$ & & 15.611 & 12.488 & 20.323 & 12.825 & 0.056 & -1.097 & -0.004 & 0.024 & -0.103 & & -0.084 & 0.003 & $\begin{array}{l}14.643 \\
\end{array}$ & 0.006 & & & Convers & & & $\mathrm{m} 3 / \mathrm{m} 2 / \mathrm{c}$ & $/$ day/barg & $\mathrm{rg} / \mathrm{gpm} / \mathrm{ft}$ & tfz/larg & & \\
\hline & 1/26/2004 & 7:41:32 AM & 19.867 & & \begin{tabular}{l|l|}
6 & 12.488 \\
\end{tabular} & \begin{tabular}{|l|l|}
8 & 20.313 \\
\end{tabular} & 12.81 & 0.058 & -1.101 & -0.004 & \begin{tabular}{|l|l|}
0.024 \\
\end{tabular} & -0.106 & & & $\begin{array}{l}0.003 \\
\end{array}$ & \begin{tabular}{|l|l|}
14.643 \\
\end{tabular} & & & & & & & & & & & & \\
\hline & $1 / 26 / 2004$ & 7:41:33 AM & 19.872 & \begin{tabular}{|l|l|}
15.611 \\
\end{tabular} & \begin{tabular}{l|l|}
1 & 12.488 \\
\end{tabular} & 20.313 & $\begin{array}{l}12.816 \\
\end{array}$ & 0.058 & -1.101 & -0.004 & $\begin{array}{l}0.026 \\
\end{array}$ & -0.144 & 0.192 & $\begin{array}{l}-0.084 \\
-\end{array}$ & $\begin{array}{l}0.003 \\
\end{array}$ & \begin{tabular}{|l|l|}
14.643 \\
\end{tabular} & $\begin{array}{l}0.007 \\
\end{array}$ & & & Note: $G_{a}$ & & ressur & & approxim & imately 1.4 & 4.4 psig & & \\
\hline$\frac{11}{12}$ & $1 / 26 / 2004$ & $\begin{array}{l}7: 41: 34 \mathrm{AM} \\
7: 10: 52 \mathrm{~A}\end{array}$ & $\begin{array}{l}19.867 \\
19862\end{array}$ & & $\begin{array}{l}112.483 \\
\end{array}$ & \begin{tabular}{l|l|}
3 & 20.313 \\
\end{tabular} & 12.81 & \begin{tabular}{|c|}
0.058 \\
\end{tabular} & \begin{tabular}{|l|l|} 
& -1.101 \\
\end{tabular} & $\begin{array}{l}-0.004 \\
0.004\end{array}$ & $\begin{array}{l}0.024 \\
0033\end{array}$ & $\begin{array}{r}-0.1 \\
\end{array}$ & 0.195 & \begin{tabular}{|c|c|c|} 
\\
\end{tabular} & $\begin{array}{l}0.003 \\
\end{array}$ & $\begin{array}{l}14.643 \\
1.643\end{array}$ & 0.006 & & & & & & & & & & & \\
\hline$\frac{2}{3}$ & $\begin{array}{l}1 / 26 / 2604 \\
1 / 26 / 2004\end{array}$ & $\begin{array}{l}7: 14: 35 \mathrm{AM} \\
7: 41: 36 \mathrm{AM}\end{array}$ & $\begin{array}{l}\frac{19.862}{19.867} \\
\end{array}$ & $\frac{15.606}{15.611}$ & $\begin{array}{l}6 \\
612.483 \\
1\end{array}$ & \begin{tabular}{l|l|}
8 & 20.308 \\
8 & 20.313
\end{tabular} & $\begin{array}{r}12.81 \\
12.81 \\
\end{array}$ & $\begin{array}{l}0.058 \\
0.058\end{array}$ & \begin{tabular}{|c|}
$\mid-1.099$ \\
-1.101 \\
\end{tabular} & $\begin{array}{c}-0.004 \\
-0.004\end{array}$ & \begin{tabular}{|c|c|}
0.023 \\
0.024
\end{tabular} & \begin{tabular}{|c|c|}
-0.097 \\
-0.097
\end{tabular} & $\begin{array}{l}0.195 \\
0.195\end{array}$ & \begin{tabular}{|c|}
-0.084 \\
-0.084
\end{tabular} & \begin{tabular}{|l}
0.003 \\
0.003
\end{tabular} & $\begin{array}{r}14.643 \\
14.643 \\
\end{array}$ & $\begin{array}{l}0.005 \\
0.055\end{array}$ & & & $\begin{array}{l}\text { Pressure } \\
\text { pyyy }\end{array}$ & & & & $\frac{40.88 \mathrm{inc}}{<<\text { Fitrat }}$ & 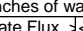 & vater tubing & 20 & \\
\hline $14 \quad$ & $1 / 26 / 2004$ & 7:41:37 AM & 19.862 & $\begin{array}{l}15.011 \\
15.606\end{array}$ & 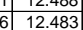 & \begin{tabular}{|c|c|}
3 & 20.313 \\
\end{tabular} & 12.81 & 0.056 & \begin{tabular}{|c|}
-1.101 \\
\end{tabular} & -0.004 & 0.026 & -0.1 & 0.195 & $\begin{array}{l}-0.064 \\
-0.084\end{array}$ & $\begin{array}{l}0.003 \\
0.003\end{array}$ & $\begin{array}{l}\frac{14.043}{14.643} \\
\end{array}$ & 0.005 & & & & & & & & at $25 \mathrm{C}$ & & 1000 & \\
\hline & & & & & & & & & & & & & & & & & & Time & Time & Press. & Vel. & TMP & TMP & gpm & \begin{tabular}{l|c}
$\mathrm{gpm}$ \\
$\mathrm{gpm}$
\end{tabular} & & $\mathrm{jpm}$ & \\
\hline & ata - Per M & & & & & & & & & & & & & & & & & Min. & Hour & sig & & psi & & & & $\mathrm{ft2/psi}$ & tit/psi & laylbar \\
\hline 17 & $1 / 26 / 2004$ & 2:29:43 PM & $\begin{array}{r}25.309 \\
23624\end{array}$ & $\begin{array}{l}18.282 \\
18.264\end{array}$ & $\begin{array}{r}24.59 \\
24.448\end{array}$ & 20.967 & $\begin{array}{l}18.206 \\
18.173\end{array}$ & \begin{tabular}{|c|}
2.827 \\
3593
\end{tabular} & $\begin{array}{l}12.523 \\
12.602\end{array}$ & $\begin{array}{l}3.747 \\
3752\end{array}$ & $\begin{array}{l}-0.267 \\
0.474\end{array}$ & \begin{tabular}{|l|l|}
5.219 \\
4947
\end{tabular} & $\begin{array}{r}52.957 \\
2064\end{array}$ & $\begin{array}{l}44.081 \\
4202\end{array}$ & \begin{tabular}{|l|l|}
0.003 \\
0088
\end{tabular} & $\begin{array}{r}-0.01 \\
14643\end{array}$ & $\begin{array}{l}0.004 \\
0.004\end{array}$ & 100 & & $\frac{11.1}{11.1}$ & 10.8 & 1.3 & 0.088 & $\frac{0.001}{2.183}$ & $\frac{-0.002}{22027}$ & $\begin{array}{l}-0.001 \\
1090\end{array}$ & $\begin{array}{r}-1.178 \\
00424\end{array}$ & -1.00 \\
\hline & $\frac{1 / 26 / 2004}{1 / 26 / 2004}$ & $2: 230: 43 \mathrm{PM}$ & & $\frac{18.264}{18283}$ & & & $\frac{18.173}{18207}$ & $\begin{array}{l}3.5933 \\
9.903\end{array}$ & & & & $\frac{4.947}{-1.214}$ & & & & & & & & & & & & & & & & 927.98 \\
\hline & $1 / 26 / 2004$ & $\begin{array}{l}2.01 .401 \\
2: 32: 43 \mathrm{PM}\end{array}$ & 24.707 & $\begin{array}{l}10.205 \\
18.255 \\
\end{array}$ & $\begin{array}{lll}5 & 24.310 \\
\end{array}$ & \begin{tabular}{c|c|} 
& 20.749 \\
\end{tabular} & $\begin{array}{l}10.2018 \\
18.482\end{array}$ & 10.129 & \begin{tabular}{|c|}
1.12 .343 \\
\end{tabular} & 3.788 & $\begin{array}{l}.552 \\
7.694 \\
\end{array}$ & $\mid$\begin{tabular}{|}
$\mid-1.214$ \\
-1.214
\end{tabular} & $\begin{array}{l}0.4562 \\
0.462\end{array}$ & 455.143 & $\begin{array}{l}.0003 \\
0.003\end{array}$ & $\begin{array}{l}1.094 \\
1.662 \\
\end{array}$ & 0.004 & 3.00 & $\begin{array}{r}0.05305 \\
0.05 \\
\end{array}$ & 10.9 & $\frac{11.1}{11.1}$ & 8.9 & $|0.614|$ & \begin{tabular}{|l|} 
\\
\end{tabular} & 0.2 & 0.028 & $\frac{29.45}{28.342}$ & $\frac{25.06}{24.12}$ \\
\hline 21 & $1 / 26 / 2004$ & 2:33:43 PM & 24.62 & 18.269 & $\begin{array}{l}924.272 \\
\end{array}$ & \begin{tabular}{l|l|}
2 & 20.707 \\
\end{tabular} & 18.152 & $\begin{array}{l}10.15 \\
\end{array}$ & \begin{tabular}{|l|l|}
12.258 \\
\end{tabular} & 3.81 & $\begin{array}{l}7.657 \\
\end{array}$ & \begin{tabular}{|l|}
-1.216 \\
\end{tabular} & 0.459 & 45.195 & 0.003 & 1.629 & 0.004 & 4.00 & \begin{tabular}{|l|l|}
0 & 0.06667 \\
\end{tabular} & 10.8 & 11.1 & 8.9 & \begin{tabular}{|l|}
0.614 \\
\end{tabular} & \begin{tabular}{|l|}
0.243 \\
\end{tabular} & 0.248 & 0.028 & 27.844 & 23.70 \\
\hline & $1 / 26 / 2004$ & 2:34:43 PM & 24.569 & $\begin{array}{l}18.277 \\
1.255\end{array}$ & $\begin{array}{l}7 \\
75 \\
5\end{array}$ & \begin{tabular}{l|l|}
5 & 20.705 \\
\end{tabular} & \begin{tabular}{|l|l|}
18.499 \\
\end{tabular} & $\begin{array}{l}10.192 \\
10.271 \\
\end{array}$ & \begin{tabular}{|r|}
12.21 \\
\end{tabular} & $\begin{array}{l}3.741 \\
3\end{array}$ & \begin{tabular}{|l|l|}
7.759 \\
\end{tabular} & \begin{tabular}{|l|l|} 
& -1.214 \\
\end{tabular} & 0.459 & 45.132 & $\begin{array}{l}0.003 \\
\end{array}$ & $\begin{array}{l}1.607 \\
\end{array}$ & 0.004 & 5.00 & \begin{tabular}{|l}
0.08333 \\
\end{tabular} & 10.7 & & & & \begin{tabular}{|l|l|} 
& 0.240 \\
\end{tabular} & & 0.027 & 27.2 & \\
\hline & $1 / 26 / 2004$ & 2:35:43 PM & 24.557 & $\begin{array}{l}18.255 \\
18.233\end{array}$ & $\begin{array}{l}5 \\
5 \\
3\end{array}$ & \begin{tabular}{c|c|}
9 & 20.598 \\
7 & 20533
\end{tabular} & $\begin{array}{l}18.346 \\
18.405\end{array}$ & \begin{tabular}{|c|}
10.347 \\
10.436
\end{tabular} & \begin{tabular}{|l|}
12.291 \\
12226
\end{tabular} & \begin{tabular}{|l|l|}
3.647 \\
3.997
\end{tabular} & \begin{tabular}{r|}
8 \\
\end{tabular} & \begin{tabular}{|r|}
$\mid-1.216$ \\
\end{tabular} & & $\begin{array}{l}44.995 \\
5.201\end{array}$ & \begin{tabular}{|l|l|l|}
0.003 \\
\end{tabular} & $\begin{array}{l}1.576 \\
1.551\end{array}$ & 0.004 & $\begin{array}{l}6.00 \\
700 \\
\end{array}$ & & 10.8 & $\frac{11.0}{1.1 .}$ & 9.2 & 0.632 & 0.235 & 0.239 & 0.026 & 26.081 & \\
\hline & $\begin{array}{l}1 / 266 / 2004 \\
1 / 26 / 2004\end{array}$ & $\begin{array}{l}2: 36: 43 \mathrm{PM} \\
2: 37: 43 \mathrm{PM}\end{array}$ & $\frac{24.5 / 5}{24.599}$ & $\begin{array}{l}18.233 \\
18227 \\
\end{array}$ & 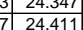 & $\frac{20.535}{20.504}$ & $\begin{array}{l}18.405 \\
18.186\end{array}$ & \begin{tabular}{|c|}
10.436 \\
10.494
\end{tabular} & \begin{tabular}{|c|}
12.266 \\
12.279 \\
\end{tabular} & $\begin{array}{l}3.797 \\
3.811 \\
\end{array}$ & $\begin{array}{r}7.92 \\
7.961 \\
\end{array}$ & $\mid$\begin{tabular}{|c|}
$\mid-1.216$ \\
-1.219 \\
\end{tabular} & & $\frac{45.201}{45.149}$ & & 1.554 & & & 0.11667 & & & & & & & & & $\frac{21.88}{2136}$ \\
\hline & $1 / 26 / 2004$ & 2:38:43 PM & 24.642 & 18.22 & \begin{tabular}{|l|l|}
2 & 24.411 \\
\end{tabular} & 20.482 & $\begin{array}{l}17.100 \\
17.912\end{array}$ & $\mid$ & \begin{tabular}{|l|}
12.318 \\
\end{tabular} & $\begin{array}{l}3.014 \\
.854\end{array}$ & 7.977 & \begin{tabular}{|c|}
-1.216 \\
\end{tabular} & 0.454 & 45.116 & $\begin{array}{l}0.003 \\
\end{array}$ & 1.51 & 0.005 & 9.00 & $\mid 0.15$ & 10.8 & $\frac{1.1}{11.1}$ & 9.3 & 0.640 & \begin{tabular}{|l|}
0.225 \\
\end{tabular} & 0.229 & 0.025 & 24.638 & $\frac{21.36}{20.97}$ \\
\hline & $1 / 26 / 2004$ & 2:39:43 PM & 24.672 & 18.22 & $\begin{array}{l}224.509 \\
\end{array}$ & 20.492 & $\begin{array}{l}17.689 \\
\end{array}$ & $\begin{array}{l}10.621 \\
\end{array}$ & \begin{tabular}{|l|l|}
12.281 \\
\end{tabular} & & 8.03 & \begin{tabular}{|l|l|} 
& -1.214 \\
\end{tabular} & & & 0.003 & $\begin{array}{l}1.483 \\
\end{array}$ & & 10.00 & 0.16667 & \begin{tabular}{l|l|l|}
10.8 \\
\end{tabular} & & 9.3 & 0.643 & 0.221 & 0.224 & 0.024 & 24.040 & \\
\hline 28 & $1 / 26 / 2004$ & 2:40:43 PM & 24.721 & $\begin{array}{l}18.229 \\
\end{array}$ & $\begin{array}{l}9 \\
9\end{array}$ & 20.546 & 17.632 & $\begin{array}{l}10.741 \\
\end{array}$ & \begin{tabular}{|l|l|}
12.353 \\
\end{tabular} & 3.931 & \begin{tabular}{|l|l|}
8.045 \\
\end{tabular} & \begin{tabular}{|l|}
-1.216 \\
\end{tabular} & 0.448 & 45.191 & $\begin{array}{l}0.003 \\
\end{array}$ & 1.474 & 0.004 & 11.00 & 0.18333 & $\begin{array}{ll}10.9 \\
\end{array}$ & 11.1 & 9.4 & \begin{tabular}{|l|}
0.648 \\
\end{tabular} & \begin{tabular}{|l|} 
\\
\end{tabular} & 0.223 & $\begin{array}{l}0.024 \\
\end{array}$ & 23.700 & 20.17 \\
\hline & $1 / 26 / 2004$ & 2:41:43 PM & $\begin{array}{r}24.72 \\
24748\end{array}$ & \begin{tabular}{|l|l|}
18.202 \\
1818
\end{tabular} & $\begin{array}{l}24.551 \\
8 \\
8\end{array}$ & 20.645 & $\begin{array}{l}17.474 \\
17194\end{array}$ & $\begin{array}{l}10.974 \\
10714\end{array}$ & \begin{tabular}{|r|}
12.55 \\
12204 \\
\end{tabular} & $\begin{array}{l}3.982 \\
38252 \\
825\end{array}$ & \begin{tabular}{|r|r|}
8.19 \\
8055
\end{tabular} & \begin{tabular}{|l|}
-1.214 \\
\end{tabular} & $\begin{array}{l}0.442 \\
0.439\end{array}$ & $\begin{array}{r}44.997 \\
.513 .13\end{array}$ & 0.003 & $\begin{array}{r}1.449 \\
1.431\end{array}$ & 0.013 & 12.00 & $\begin{array}{r}0.2 \\
\end{array}$ & $\begin{array}{l}11.1 \\
107 \\
10 .\end{array}$ & $\frac{11.0}{11.0}$ & $\begin{array}{l}9.6 \\
9.4\end{array}$ & \begin{tabular}{|l|}
0.661 \\
0647 \\
\end{tabular} & 0.216 & 0.219 & 0.023 & $\begin{array}{l}22.833 \\
2.005\end{array}$ & 19.43 \\
\hline & $\frac{1 / 26 / 2004}{1 / 262004}$ & 2:42:43 PM & $\frac{24.748}{24768}$ & $\begin{array}{r}18.18 \\
18.17 \\
\end{array}$ & $\begin{array}{r}24.58 \\
24.6 \\
\end{array}$ & $\begin{array}{r}20.745 \\
20.83\end{array}$ & $\begin{array}{r}17.194 \\
17.533\end{array}$ & $\begin{array}{l}10.714 \\
10785\end{array}$ & \begin{tabular}{|r|}
12.204 \\
1222
\end{tabular} & $\begin{array}{l}3.825 \\
3.766\end{array}$ & $\begin{array}{r}8.055 \\
8.8\end{array}$ & \begin{tabular}{|c|}
$\mid-1.214$ \\
-1216
\end{tabular} & $\begin{array}{l}0.439 \\
0.433\end{array}$ & $\begin{array}{r}45.13 \\
44828 \\
\end{array}$ & & $\begin{array}{r}1.431 \\
1.44 \\
\end{array}$ & 0.004 & $\begin{array}{l}13.00 \\
11.00\end{array}$ & 0.21667 & \begin{tabular}{l|l|l}
10.7 \\
10.7
\end{tabular} & $\frac{11.1}{110}$ & 9.4 & \begin{tabular}{|l|}
$\mid 0.647$ \\
0.654
\end{tabular} & \begin{tabular}{|l|}
$\mid 0.213$ \\
$0^{2} 111$
\end{tabular} & 0.216 & 0.023 & 23.005 & 19.58 \\
\hline & $1 / 26 / 2004$ & $\begin{array}{l}2.454 .43 \mathrm{PM} \\
2: 44: 43 \mathrm{PM}\end{array}$ & $\begin{array}{l}24.708 \\
24.797\end{array}$ & $\begin{array}{l}18.17 \\
18.153\end{array}$ & \begin{tabular}{|l|l|l|l|l} 
& 24.623 \\
\end{tabular} & $\frac{20.93}{20.915}$ & \begin{tabular}{|l|l|}
1.535 \\
\end{tabular} & $\mid$ & \begin{tabular}{|l|}
12.324 \\
\end{tabular} & $\begin{array}{l}3.100 \\
3.866\end{array}$ & \begin{tabular}{|c|c|}
8.18 \\
8.318
\end{tabular} & \begin{tabular}{|c|}
-1.210 \\
-1.216 \\
\end{tabular} & 0. & $\begin{array}{l}44.828 \\
45.068\end{array}$ & & $\begin{array}{l}.4114 \\
1.394 \\
\end{array}$ & 0.0 & $\frac{14.0}{15.0}$ & 0.25 & $\begin{array}{l}\frac{10.7}{10.9} \\
10\end{array}$ & $\frac{11.0}{11.0}$ & $\begin{array}{l}9.5 \\
9.7\end{array}$ & \begin{tabular}{|l|} 
\\
\end{tabular} & 208 & $\frac{0.213}{0.210}$ & 0.022 & $\frac{2.484}{21.749}$ & $\frac{19.13}{18.51}$ \\
\hline & 12004 & $2: 45: 43$ PM & 24.81 & 18.126 & \begin{tabular}{l|l}
624.637 \\
\end{tabular} & 21.014 & 17.606 & 10.963 & \begin{tabular}{|l|}
12.299 \\
\end{tabular} & 3.889 & 8.256 & -1.219 & & 45.022 & 0.003 & 1.381 & & 16.0 & 26667 & $0 . \varepsilon$ & & 9.6 & 0.663 & 0 & 0.208 & 022 & & \\
\hline & 1/26/2004 & 2:46:43 PM & 24.833 & & \begin{tabular}{l|l}
9 & 24.654
\end{tabular} & 21.047 & & 11.042 & \begin{tabular}{|l|}
12.357 \\
\end{tabular} & & 8.236 & -1.214 & & 44.965 & & 1.363 & & $\frac{17.00}{17.00}$ & 0.28333 & 0 & & 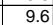 & & & $\frac{0.205}{0.205}$ & . & 21.289 & \\
\hline & & & 24.83 & 18.096 & \begin{tabular}{l|l}
6 & 24.667
\end{tabular} & 21.034 & & $|11.017|$ & \begin{tabular}{|l|}
12.252 \\
\end{tabular} & 3.816 & 8.318 & $\mid-1.214$ & & 44.972 & & 1.348 & & 18.0 & 0.3 & & 11 . & & & & & & & \\
\hline & 5/2004 & 2:48:43 PM & 24.864 & 18.084 & \begin{tabular}{l|l}
4 & 24.67 \\
\end{tabular} & 21.012 & & 11.1177 & \begin{tabular}{|l|}
12.353 \\
\end{tabular} & 3.86 & 8.414 & $|-1.216|$ & & 44.986 & & 1.333 & & 19.0 & 0.31667 & 10 & 11 & 9 & 675 & 199 & & & & 4 \\
\hline & $1 / 26 / 2004$ & 2:49: & 24.861 & 18.061 & \begin{tabular}{ll|l}
1124.703 \\
\end{tabular} & 21.045 & & 11.195 & \begin{tabular}{|l|l|}
12.316 \\
\end{tabular} & 3.883 & 8.392 & $\mid-1.214$ & & 44.945 & & 1.314 & & & & & & & & & & & & \\
\hline & $1 / 26 / 2004$ & 2:50:43 PM & 24.905 & 18.065 & $\begin{array}{l}5 \\
5\end{array}$ & 21.099 & 18.88 & 11.193 & \begin{tabular}{|l|l|}
12.293 \\
\end{tabular} & 3.873 & 8.359 & -1.214 & 0.4 & 44.88 & & & & 21.0 & 0.35 & 10.8 & 11. & 9. & 0.674 & 0.194 & $0.19 !$ & & 19.991 & \\
\hline & & 2:56:24 PM & 25.226 & 18.051 & $\begin{array}{ll}11 & 25.278\end{array}$ & 21.287 & 18.163 & 2.792 & \begin{tabular}{|r|}
32.9 \\
\end{tabular} & 3.624 & - -0.608 & 25.754 & & 41.551 & 0.003 & -0.013 & & 26.6 & 0.44 & & & & & & & -0.002 & -1.761 & \\
\hline & I2004 & & $\begin{array}{l}22^{2.3 .301} \\
2.507\end{array}$ & $\frac{18.042}{18027}$ & 25.299 & 21.278 & 18.279 & $\frac{9.634}{9.132}$ & \begin{tabular}{|}
32.481 \\
\end{tabular} & $\begin{array}{r}3.58 \\
3.268\end{array}$ & - & \begin{tabular}{|l|l|} 
\\
\end{tabular} & & 43.165 & 1.2 & -0.0 & & 27.6 & . & 2 & & 7 & & & & & & \\
\hline & & & & & & & & 10.385 & & & $\begin{array}{l}16.8944 \\
17904\end{array}$ & 1.6074 & & 44.192 & & & & 28.6 & 0.41806 & 30. & & 10.6 & & & & & & \\
\hline & & . & $\frac{23.043}{25683}$ & 18027 & \begin{tabular}{r|r|}
3 & 25.34 \\
\end{tabular} & $\frac{2.359}{21.35}$ & 10. & $\mid$ & $\mid$ & $\begin{array}{l}3.010 \\
3.422\end{array}$ & $\frac{11.904}{18782}$ & $\mid \frac{1.111}{6189}$ & & $\frac{14.49}{44.69}$ & & & & & & 305 & 111 & 18.9 & & & & & & \\
\hline & $1 / 2612004$ & 3.301.24 PM & 250.072 & 18.055 & $\begin{array}{lll}5 & 253367 \\
\end{array}$ & 21.371 & 184 & 2063 & \begin{tabular}{|l|}
31.801 \\
31
\end{tabular} & 3.487 & $\mid$ & 568 & & 445 & & & & 31.68 & 0 & 0. & & 199 & 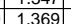 & 0.544 & & & & \\
\hline & $\frac{1 / 26 / 2004}{106}$ & 3:02:24 PM & 25.75 & 18.05 & \begin{tabular}{|l|l|}
5 & 25.403 \\
\end{tabular} & $\frac{21.412}{21.42}$ & $\frac{1.268}{18.268}$ & $\frac{21.018}{21.018}$ & \begin{tabular}{|l|l|}
32.033 \\
\end{tabular} & $\frac{3.359}{3.359}$ & 19.383 & |5.552 & & 44.204 & & $\begin{array}{l}3.603 \\
3.603\end{array}$ & & $\frac{32.6}{32.6}$ & $\frac{. .54472}{0.542}$ & 30 & $\frac{10}{10 .}$ & 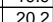 & & & & 0.026 & 3293 & \\
\hline & & & 25.77 & & & 21.437 & & 21.076 & & & 19.628 & \begin{tabular}{|l|}
5.341 \\
\end{tabular} & & $\begin{array}{l}44.154 \\
\end{array}$ & & & & & & & & & & & & & & \\
\hline & $1 / 26 / 2004$ & $3: 04 \cdot 24 \mathrm{PM}$ & 25784 & 18049 & 925462 & 21.477 & 1795 & 21671 & 32296 & 3.526 & 20.022 & 5.147 & & 4421 & & 3.557 & & 346 & 0.57806 & & 0 & & & & & & & \\
\hline & 1/26/2004 & $3: 05: 24 \mathrm{~F}$ & 25.804 & 18.054 & $\begin{array}{ll}4425.482 \\
\end{array}$ & 21.502 & $\begin{array}{ll}17 . \\
\end{array}$ & 21.584 & \begin{tabular}{|l|}
32.143 \\
\end{tabular} & 3.421 & \begin{tabular}{|c|}
19.886 \\
\end{tabular} & $\begin{array}{l}5.127 \\
\end{array}$ & & 44.264 & & & & 35.6 & 0.59472 & 30. & 10.8 & 0.7 & & & & & 25.040 & 2 \\
\hline & 1/26/2004 & 3:06 & 25.835 & 18.06 & $\begin{array}{l}625.493 \\
6\end{array}$ & 21.452 & & 21.718 & \begin{tabular}{|l|}
32.205 \\
\end{tabular} & 3.522 & 19.89 & & & 44.191 & & 3.5 & & 6. & & & & & & & & & & \\
\hline & & 3:07 & 25.85 & 18.065 & $\begin{array}{l}5 \\
5\end{array}$ & \begin{tabular}{l|l|}
2 & 21.351 \\
\end{tabular} & & 21.776 & \begin{tabular}{|l|}
32.147 \\
\end{tabular} & 3.385 & 20.167 & \begin{tabular}{|l|l|}
4.959 \\
\end{tabular} & & 44.1. & & 3.505 & & & & & & 21.0 & & & & & & \\
\hline & & 3:08:24 & 25.87 & 18.06 & \begin{tabular}{l|l|l}
6 & 25.507
\end{tabular} & 21.331 & & 21.808 & \begin{tabular}{|l|}
32.091 \\
\end{tabular} & 3.486 & 20.198 & & & 44.1 & & 3.5 & & 38. & & 30.6 & 10.8 & & & & 0.0 & & & \\
\hline & I/2004 & & 25.884 & 18.054 & $\begin{array}{ll}4 & 25.517\end{array}$ & 21.24 & & 21.916 & \begin{tabular}{|l|}
32.133 \\
\end{tabular} & 3.5 & 20.1 & & & $44 . c \mathrm{c}$ & & & & & & & & & & & & & & \\
\hline & & 3:10:24 & 25.909 & 18.059 & $\begin{array}{l}925.526 \\
\sqrt{2} 25.515\end{array}$ & 21.149 & 18. & 22.005 & $\mid$ & $\begin{array}{l}3.4 \\
3.52\end{array}$ & 20.362 & & & 44.0 & & 3.4 & & & & . & . & 21. & & & & & & \\
\hline & & & & 10.045 & & & & & & & & & & & & & & & & & & & & & & & & \\
\hline & 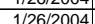 & $\begin{array}{l}3.134 \\
3.24\end{array}$ & $\begin{array}{l}25.503 \\
25.932\end{array}$ & 18057 & \begin{tabular}{|l|l|}
7 & 2559 \\
7
\end{tabular} & $\frac{20.505}{20.90}$ & 17 & $\frac{21.6}{218}$ & $\mid$ & $\begin{array}{l}0.414 \\
3.358 \\
\end{array}$ & 20.344 & $\frac{4.6}{47}$ & & 44029 & & $\frac{3.45}{3.45}$ & & & & 30.5 & 108 & & & & 0507 & & & \\
\hline & & 3:14:24 PM & 25.946 & 18.052 & $\begin{array}{ll}2 & 25.599 \\
\end{array}$ & \begin{tabular}{|l|l|}
9 & 20.834 \\
\end{tabular} & 18. & 22.388 & \begin{tabular}{|l|}
32.533 \\
\end{tabular} & \begin{tabular}{|l|l}
3 & 3.544 \\
\end{tabular} & 20.629 & \begin{tabular}{|l|} 
\\
\end{tabular} & & $\frac{44.029}{43.645}$ & & $\frac{3.4 .}{3.44}$ & & 44. & $\mid$ & 31.1 & $\frac{1.0}{10.7}$ & & & $\begin{array}{l}\frac{100}{517} \\
517\end{array}$ & $\begin{array}{l}.508 \\
0.508\end{array}$ & 0.024 & $\frac{23.950}{23.618}$ & \\
\hline & & & & 18.078 & \begin{tabular}{l|l}
8 & 25.61 \\
\end{tabular} & & & & & & & & & 43.5 & & & & & & & & & & & & & & \\
\hline & & 3:16:24 & 25.956 & 18.062 & \begin{tabular}{l|l|}
225.594 \\
\end{tabular} & 20.74 & & 22.2 & 32.2 & 3.4 & \begin{tabular}{|l|l|}
20.58 \\
\end{tabular} & & & 43.6 & & & & & & & & & & & & & & \\
\hline & & & 25.961 & 18.062 & \begin{tabular}{l|l|}
225.584 \\
\end{tabular} & 20.7 & & & 32.2 & 3.3 & 20.789 & 4.6 & & 43.6 & & & & & & & & & & & & & & \\
\hline & 1/26/2004 & $3: 24: 13$ & 26.72 & 18.129 & 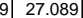 & 20.88 & 17. & 2.576 & \begin{tabular}{|l|}
62.721 \\
\end{tabular} & 3.13 & -0.511 & \begin{tabular}{|l|l|}
56.126 \\
\end{tabular} & & 38.4 & & & & 54.50 & 0.908 & & $\overline{9.4}$ & & & & & & 198 & \\
\hline & $1 / 26 / 2$ & 3:25:13 & 26.369 & 18.121 & $\begin{array}{l}11 \\
1127.032\end{array}$ & 20.968 & & 10.654 & 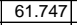 & 2.925 & 8.273 & 46.262 & & 40.4 & & -0.6 & & 55 & 0.9 & & 9.9 & & & & & & & \\
\hline & & 3:26:13 & 27.639 & 18.139 & $\begin{array}{l}9 \\
9\end{array}$ & 21.067 & 17. & 25.563 & 60. & 3. & 26.316 & \begin{tabular}{|l|}
27.514 \\
\end{tabular} & & 44. & & & & 56 & 0.94 & & 10.8 & 25.9 & & 0.979 & & & & \\
\hline & $\begin{array}{l}1.26 / 2004204 \\
11204\end{array}$ & $3: 27: 13$ & 27.676 & 18.157 & $\begin{array}{ll}7 \mid & 27.062 \\
\end{array}$ & 21.16 & & 27.0 & $\mid 60.416$ & 2.844 & 28.033 & 26 & & 43.77 & & 6. & & 0 & & & & & & & & & & \\
\hline & $\frac{11216124}{112612}$ & $\frac{3: 28.13}{3.20 .13}$ & $\frac{2 . .104}{2061}$ & $\frac{18.16}{18.172}$ & 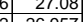 & $\frac{21.188}{206}$ & $\frac{17 .}{17 \pi}$ & $\frac{2.144}{28693}$ & \begin{tabular}{|l|}
6.008 \\
\end{tabular} & 2.135 & 28.688 & \begin{tabular}{|l|}
25.103 \\
\end{tabular} & & 43.559 & & & & & & & & 2.4 & & & & & (100 & \\
\hline & 10.212004 & $\frac{3.25 .14}{3.2012}$ & t7. & 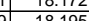 & 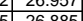 & $\frac{2.250}{21329}$ & & 20.0050 & | 0.0 .017 & 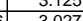 & 2.421 & \begin{tabular}{|l|l|}
2.03 \\
\end{tabular} & & 133 & & & & & & & & & & & & & & \\
\hline & 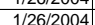 & 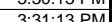 & 27552 & 18207 & 8 & 21.304 & & $\frac{20.025}{29.05}$ & 60.606 & $\begin{array}{l}3.069 \\
3.099\end{array}$ & 29.56 & 245 & & 年 & & $\frac{6.2}{6.2}$ & & 615 & 11 & & & & & & & & & \\
\hline & & $3 \cdot 32 \cdot 13$ & 27.474 & 18.235 & \begin{tabular}{l|l|}
5 & 26.75
\end{tabular} & 21.334 & & 29.118 & 60. & 3.174 & & & & & & & & & & 59.2 & 106 & 29.4 & & 929 & & & & \\
\hline 10 & 2004 & $3: 33: 13$ & 7,411 & 18.231 & 1126.711 & 2132 & & 29.431 & ||$^{61.169}$ & $\begin{array}{l}3.1 .017 \\
3\end{array}$ & 30.145 & 24.367 & & 3.169 & & 6.219 & & 5 & & & 10. & 29.8 & 2.056 & 32 & 0.884 & 0.030 & 20.671 & \\
\hline
\end{tabular}


WSRC-TR-2005-00105, REVISION 0

SRNL-RPP-2005-00012, REVISION 0

RUN \# 1.00A, B, C; PRE-TEST BASELINE WITH 0.1 M NAOH - CONT.

\begin{tabular}{|c|c|c|c|c|c|c|c|c|c|c|c|c|c|c|c|c|c|c|c|c|c|c|c|c|c|c|c|c|}
\hline 1 & A & $B$ & D & E & $F$ & $G$ & $\mathrm{H}$ & $\mathrm{j}$ & $\mathrm{k}$ & $\mathrm{L}$ & M & $\mathrm{N}$ & 0 & $Q$ & $\mathrm{R}$ & $\mathrm{s}$ & $\begin{array}{lll}T & L\end{array}$ & $\mathrm{v}$ & w & $x$ & $\mathrm{Y}$ & $z$ & $A A$ & $A B$ & $\mathrm{AC}$ & $A D$ & AE & AF $\quad A$ \\
\hline 71 & $\frac{1 / 26 / 2004}{1202001}$ & 3:34:13 PM & 27.348 & 18.253 & \begin{tabular}{|l|}
26.658 \\
\end{tabular} & \begin{tabular}{|l|}
21.297 \\
\end{tabular} & \begin{tabular}{|l|l|}
17.495 \\
\end{tabular} & 29.346 & $\begin{array}{l}60.926 \\
\end{array}$ & 3.03 & 30.038 & \begin{tabular}{|l|l|}
24.521 \\
\end{tabular} & $\begin{array}{l}-0.208 \\
-0.20\end{array}$ & \begin{tabular}{|l|l|l|l}
43.217 \\
\end{tabular} & 0.003 & 6.229 & 0.005 & 64.50 & $\begin{array}{l}1.075 \\
10057\end{array}$ & 59.5 & 10.6 & $\begin{array}{l}29.7 \\
\end{array}$ & \begin{tabular}{|l|}
2.047 \\
\end{tabular} & 0.929 & 0.887 & 0.030 & 29.859 & 25.41 \\
\hline$\frac{72}{72}$ & $\begin{array}{l}1 / 26 / 2004 \\
126004\end{array}$ & $\begin{array}{l}3: 35: 13 \mathrm{PM} \\
3: 2: 12 \mathrm{P}\end{array}$ & 27.3 & $\begin{array}{l}18.265 \\
18257\end{array}$ & \begin{tabular}{|l|}
26.605 \\
2.521 \\
\end{tabular} & \begin{tabular}{|l|l|}
21.258 \\
21.121 \\
\end{tabular} & \begin{tabular}{|l|l|}
17.871 \\
17202
\end{tabular} & 29.232 & $\begin{array}{l}60.914 \\
6989\end{array}$ & 3.164 & \begin{tabular}{|l|}
29.766 \\
3070
\end{tabular} & 24.44 & $\begin{array}{r}-0.211 \\
-0.015\end{array}$ & 43.207 & 0.003 & $\frac{6.221}{6.212}$ & 0.006 & $\begin{array}{l}65.50 \\
6650\end{array}$ & \begin{tabular}{|l|l|}
1.09167 \\
1.12823
\end{tabular} & 59.4 & \begin{tabular}{|l|l|}
10.6 \\
\end{tabular} & 29.5 & 2.034 & 0.927 & 0.887 & $\begin{array}{l}0.030 \\
.020\end{array}$ & $\frac{30.060}{32022}$ & $\begin{aligned} 25.58 \\
5.57\end{aligned}$ \\
\hline$\frac{73}{74}$ & $\frac{1 / 266 / 2004}{11 / 26 / 2004}$ & $\begin{array}{l}3: 3: 3: 13 \text { PM } \\
\text { 3:37:13 }\end{array}$ & $\begin{array}{l}27.246 \\
27.207\end{array}$ & $\begin{array}{l}\frac{18.257}{18.283} \\
\end{array}$ & \begin{tabular}{|l|}
26.531 \\
26.482 \\
\end{tabular} & \begin{tabular}{|l|}
21.174 \\
21.099 \\
\end{tabular} & \begin{tabular}{|l|}
17.923 \\
18.151 \\
\end{tabular} & \begin{tabular}{|l|}
29.127 \\
29.417
\end{tabular} & \begin{tabular}{|c|}
60.889 \\
61.038
\end{tabular} & $\begin{array}{l}2.92 \\
2.92 \\
\end{array}$ & \begin{tabular}{|r|}
29.579 \\
30.25
\end{tabular} & $\begin{array}{l}24.399 \\
24.202\end{array}$ & $\begin{array}{c}-0.015 \\
-0.021 \\
\end{array}$ & $\begin{array}{l}43.113 \\
43.096\end{array}$ & $\frac{0.003}{0.003}$ & $\frac{6.213}{6.219}$ & $\begin{array}{l}0.004 \\
0.005\end{array}$ & $\frac{66.50}{67.50}$ & $\begin{array}{r}1.10833 \\
1.125\end{array}$ & $\begin{array}{l}599.4 \\
59.6\end{array}$ & \begin{tabular}{|l|l|}
10.6 \\
10.6
\end{tabular} & $\begin{array}{r}29.4 \\
29.8 \\
\end{array}$ & $\mid$\begin{tabular}{|l|}
2.0224 \\
2.057 \\
\end{tabular} & \begin{tabular}{|l|}
0.926 \\
0.927 \\
\end{tabular} & \begin{tabular}{|l}
0.887 \\
0.889
\end{tabular} & $\frac{0.030}{0.030}$ & $\begin{array}{l}30.233 \\
29.815 \\
\end{array}$ & $\begin{array}{l}25.73 \\
25.37 \\
\end{array}$ \\
\hline 75 & $1 / 26 / 2004$ & 3:38:13 PM & 27.179 & 18.299 & \begin{tabular}{|l|}
26.459 \\
\end{tabular} & 21.04 & 18.072 & 29.313 & $\begin{array}{l}60.79 \\
\end{array}$ & 3.27 & 29.41 & 24.338 & $\begin{array}{l}-0.021 \\
-0.015\end{array}$ & \begin{tabular}{|l|}
43.090 \\
43.123 \\
\end{tabular} & 0.0003 & $\begin{array}{l}6.206 \\
6.206\end{array}$ & 0.0006 & $\frac{07.50}{68.50}$ & \begin{tabular}{|l|}
1.120 \\
1.14167 \\
\end{tabular} & 59.0 & \begin{tabular}{|l|l|}
10.6 \\
10.6
\end{tabular} & $\frac{29.0}{29.4}$ & \begin{tabular}{|l|}
2.024 \\
\end{tabular} & \begin{tabular}{|l|}
0.927 \\
0.925 \\
\end{tabular} & $\begin{array}{l}.009 \\
0.888\end{array}$ & $\begin{array}{l}0.030 \\
0.030\end{array}$ & $\frac{29.015}{30.251}$ & $\begin{array}{l}25.31 \\
25.74\end{array}$ \\
\hline 76 & 1/26/2004 & 3:39:13 PM & 27.14 & 18.31 & \begin{tabular}{|r|}
26.44 \\
\end{tabular} & \begin{tabular}{|l|}
20.955 \\
\end{tabular} & $\begin{array}{l}17.876 \\
\end{array}$ & 29.438 & 60.951 & 3.108 & 30.012 & 24.283 & $\begin{array}{l}-0.012 \\
-\end{array}$ & \begin{tabular}{|l|l|}
43.048 \\
\end{tabular} & 0.003 & 6.209 & 0.005 & 69.50 & 1.15833 & 59.5 & \begin{tabular}{|l|l|} 
\\
\end{tabular} & & \begin{tabular}{|l|l|}
2.049 \\
\end{tabular} & \begin{tabular}{|l|}
0.926 \\
\end{tabular} & $\begin{array}{l}0.889 \\
\end{array}$ & 0.030 & 29.911 & 25.45 \\
\hline 77 & $1 / 26 / 2004$ & 3:40:13 PM & 27.106 & 18.332 & 26.411 & 20.906 & 17.983 & \begin{tabular}{|l|l|}
29.178 \\
\end{tabular} & \begin{tabular}{|l|l|}
61.123 \\
\end{tabular} & 3.026 & 29.686 & \begin{tabular}{|l|l}
24.338 \\
\end{tabular} & -0.009 & \begin{tabular}{|l|l|}
43.149 \\
\end{tabular} & $\begin{array}{l}0.003 \\
\end{array}$ & 6.206 & 0.006 & 70.50 & $\begin{array}{l}1.175 \\
\end{array}$ & 59.7 & \begin{tabular}{|l|}
10.6 \\
\end{tabular} & 29.4 & & \begin{tabular}{|l|l|}
0.925 \\
\end{tabular} & 0.889 & 0.030 & 30.219 & 25.72 \\
\hline 78 & $\frac{1 / 26 / 2004}{1062001}$ & 3:41:13 PM & & 18.343 & \begin{tabular}{|l|}
26.377 \\
\end{tabular} & \begin{tabular}{|l|l|}
20.831 \\
\end{tabular} & $\begin{array}{l}17.539 \\
1770\end{array}$ & \begin{tabular}{|r|}
29.38 \\
2907
\end{tabular} & $\begin{array}{l}61.049 \\
\end{array}$ & $\begin{array}{l}3.142 \\
.2961 \\
\end{array}$ & $\begin{array}{l}29.866 \\
2070\end{array}$ & $\begin{array}{l}24.257 \\
2.2232\end{array}$ & -0.012 & \begin{tabular}{|l|l|}
43.057 \\
4
\end{tabular} & 0.003 & 6.212 & 0.004 & 71.50 & \begin{tabular}{|l|l|}
1.19167 \\
\end{tabular} & 59.6 & 10.6 & 29.6 & \begin{tabular}{|l|l|}
2.042 \\
\end{tabular} & \begin{tabular}{|l|}
0.926 \\
\end{tabular} & 0.891 & 0.030 & 30.081 & 25.60 \\
\hline $\begin{array}{ll}79 \\
80\end{array}$ & $\begin{array}{l}1 / 26 / 2004 \\
10262004\end{array}$ & 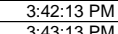 & 27.049 & $\begin{array}{l}18.355 \\
1836\end{array}$ & \begin{tabular}{|l|}
26.333 \\
26399 \\
\end{tabular} & \begin{tabular}{|l|l|}
20.828 \\
20727
\end{tabular} & \begin{tabular}{|l|l|l}
17.798 \\
17779
\end{tabular} & \begin{tabular}{|l|}
28.917 \\
2903
\end{tabular} & \begin{tabular}{|l|}
60.481 \\
6061
\end{tabular} & $\begin{array}{l}2.861 \\
3063\end{array}$ & \begin{tabular}{|l|}
29.726 \\
29255
\end{tabular} & $\begin{array}{l}24.332 \\
24393 \\
\end{array}$ & $\begin{array}{l}-0.006 \\
002\end{array}$ & \begin{tabular}{|l|l|}
42.996 \\
\end{tabular} & $\begin{array}{l}0.003 \\
\end{array}$ & 6.211 & 0.004 & 72.50 & \begin{tabular}{|l|l|}
1.20833 \\
1205
\end{tabular} & 59.0 & 10.5 & \begin{tabular}{|l|l|}
29.3 \\
\end{tabular} & \begin{tabular}{|l|l|}
2.022 \\
\end{tabular} & \begin{tabular}{|l|}
0.926 \\
\end{tabular} & $\begin{array}{ll}0.892 \\
\end{array}$ & 0.030 & & 25.89 \\
\hline $\begin{array}{ll}80 \\
1\end{array}$ & $1 / 26 / 2004$ & 3:43:13 PM & & $\begin{array}{r}18.36 \\
18.366\end{array}$ & \begin{tabular}{|l|}
26.299 \\
26.279 \\
\end{tabular} & & $\begin{array}{l}17.779 \\
17006\end{array}$ & $\begin{array}{r}29.023 \\
29046\end{array}$ & $\begin{array}{l}60.601 \\
6\end{array}$ & $\frac{3.063}{2.78}$ & $\begin{array}{r}29.255 \\
3001\end{array}$ & $\begin{aligned} 24.393 \\
20202\end{aligned}$ & & \begin{tabular}{|l|}
42.907 \\
\end{tabular} & & & 0.004 & 73.50 & \begin{tabular}{|r|}
1.225 \\
\end{tabular} & 59.1 & 10.5 & 29.1 & \begin{tabular}{|l|}
2.009 \\
\end{tabular} & \begin{tabular}{|l|}
0.927 \\
.027
\end{tabular} & 0.894 & 0.031 & 30.667 & 26.10 \\
\hline 81 & 1/26/2004 & 3:44:13 PM & 26.985 & 18.366 & 26.279 & \begin{tabular}{|l|}
20.728 \\
\end{tabular} & 17.906 & 29.046 & 60.719 & 2.78 & 30.001 & & 0.003 & 42.915 & 0.003 & 6.218 & & 74.50 & 1.24167 & 59.2 & 10.5 & & 2.036 & 0.927 & 0.894 & 0.030 & 30.295 & 25.78 \\
\hline 83 & & Averages & 24.7 & 18.2 & 24.5 & 20.8 & $18.1 \mathrm{t}$ & \begin{tabular}{|l|l|}
10.7 \\
\end{tabular} & 12.3 & 3.8 & 8.1 & -1.2 & 0.4 & 45.1 & $\begin{array}{l}0.0 \\
\end{array}$ & 1.5 & 0.0 \# & & & 10.8 & 11.0 & 9.4 & 0.646 & 0.219 & 0.223 & 0.024 & 23.8 & $20.3[\#$ \\
\hline 84 & & \begin{tabular}{|l|} 
Maximum \\
\end{tabular} & 24.9 & 18.3 & $\begin{array}{l}24.7 \\
2.5\end{array}$ & $\begin{array}{l}21.1 \\
2.7\end{array}$ & $18.9 \mathrm{~A}$ & $\begin{array}{l}11.2 \\
1107\end{array}$ & $\begin{array}{l}12.6 \\
\end{array}$ & 4.0 & $\begin{array}{l}8.4 \\
8 .\end{array}$ & $\begin{aligned}-1.2 \\
\end{aligned}$ & $\begin{array}{l}0.5 \\
\end{array}$ & 45.2 & 0.0 & 1.7 & $0.0 \#$ & & & 11.1 & 11.1 & $\begin{array}{l}9.8 \\
\end{array}$ & \begin{tabular}{|l|l|}
0.675 \\
\end{tabular} & \begin{tabular}{|l|l|}
0.253 \\
\end{tabular} & 0.257 & 0.029 & 29.5 & $25.1 \#$ \\
\hline 85 & & Median & 24.7 & 18.2 & 24.5 & 20.7 & $18.2 \#$ & 10.7 & 12.3 & 3.8 & $\begin{array}{l}8.1 \\
70\end{array}$ & -1.2 & & 45.0 & 0.0 & 1.5 & $0.0 \mid \#$ & & & 10.8 & 11.0 & 9.4 & 0.647 & \begin{tabular}{|l|l|}
0.218 \\
\end{tabular} & 0.221 & 0.023 & & $\begin{array}{ll}19.9 \\
\end{array}$ \\
\hline \begin{tabular}{|c|}
86 \\
07
\end{tabular} & & Minimum & 24.6 & 18.1 & 24.3 & 20.5 & $17.2 \#$ & $\begin{array}{r}9.9 \\
\end{array}$ & 12.2 & 3.6 & 7.6 & -1.2 & $\begin{array}{r}0.4 \\
\end{array}$ & 44.8 & 0.0 & 1.3 & $0.0 \mid \neq$ & & & 10.7 & 11.0 & 8.7 & & 0.194 & 0.195 & 0.020 & 20.0 & \\
\hline 87 & & $2 \times$ Std Dev & 0.216 & 0.142 & 0.285 & & & 0.768 & & 0.148 & 0.499 & 0.003 & & 0.213 & 0.000 & 0.233 & 0.004 & & & 0.158 & 0.052 & 0.630 & \begin{tabular}{|l|l|}
0.043 \\
\end{tabular} & \begin{tabular}{|l|l|}
0.035 \\
\end{tabular} & 0.037 & 0.006 & 5.604 & \\
\hline 88 & Number & of Points Used & 20 & 20 & 20 & & 200 & 20 & & & & & & 20 & 20 & 20 & 204 & & & 20 & 20 & 20 & 20 & 20 & 20 & 20 & 20 & \\
\hline$\frac{89}{90}$ & & Backpuise po & tis in box & re not inc & funded & & & & & & & & & & & & & & & & & & & & & & & \\
\hline 91 & & & & & & & & & & & & & & & & & & & & & & & 0-min. & avg. $=$ & 0.22 & & & \\
\hline & & & & & & & & & & & & & & & & & & & & & & & & & & & & \\
\hline$\frac{30}{94}$ & & $\begin{array}{l}\text { Averages } \\
\text { Maximum }\end{array}$ & $\frac{25.8}{26.0}$ & $\frac{18.1}{18.1}$ & $\begin{array}{l}25.5 \\
25.6 \\
\end{array}$ & 21.2 & $\frac{18.2 .7 \#}{18.9 \#}$ & 21.4 .4 & $\begin{array}{l}32.1 \\
32.5 \\
\end{array}$ & $\frac{3.4}{3.7}$ & \begin{tabular}{l|l}
$\frac{19.8}{20.8}$ &
\end{tabular} & $\begin{array}{l}5.2 \\
7.7 \\
\end{array}$ & $\begin{array}{l}1.6 \\
1.7 \\
\end{array}$ & $\frac{44.1}{44.8}$ & $\frac{0.0}{0.0}$ & $\frac{3.6}{4.0}$ & $\begin{array}{l}0.0 \\
0.0\end{array}$ & & & $\begin{array}{l}30.6 \\
31.1\end{array}$ & & & & 0.530 & 0.523 & $\frac{0.0255}{0.0332}$ & 2.5.5 & 21.7 |\#|\#| \\
\hline 95 & & Median & 25.9 & 18.1 & 25.5 & 21.3 & $18.1 \mathrm{~A}$ & 21.8 & 32.1 & 3.4 & 20.2 & 4.9 & 1.5 & 44.2 & 0.0 & 3.5 & 0.0 & & & 30.6 & \begin{tabular}{|l|}
10.8 \\
\end{tabular} & 21.0 & \begin{tabular}{|c|c|}
1.447 \\
\end{tabular} & \begin{tabular}{|l|l|}
0.522 \\
\end{tabular} & 0.515 & 0.0245 & 24.5 & $\frac{20.0}{20.9}$ \\
\hline 96 & & Minimum & 25.6 & 18.0 & 25.3 & 20.7 & $17.7 \#$ & 18.4 & 31.7 & 3.2 & 16.9 & 4.7 & 1.5 & 43.5 & 0.0 & 3.5 & 0.0 A & & & 30.2 & 10.7 & 17.6 & \begin{tabular}{|l|l|}
1.216 \\
\end{tabular} & \begin{tabular}{|l|l|}
0.515 \\
\end{tabular} & $\begin{array}{ll}0.507 \\
\end{array}$ & 0.0235 & 23.5 & 20.0 \\
\hline & & $2 \times$ Std Dev & 0.218 & 0.026 & 0.190 & 0.520 & 0.631 \# & 1.920 & 0.383 & 0.212 & 1.917 & 1.552 & 0.094 & $\begin{array}{l}7 \quad 0.680 \\
7\end{array}$ & 0.000 & 0.268 & $0.001 / \mathrm{A}$ & & & 0.383 & 0.167 & 1.915 & \begin{tabular}{|l|l|}
0.132 \\
\end{tabular} & 0.040 & 0.042 & 0.0048 & 4.806 & \\
\hline 98 & Number & 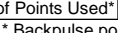 & 20 & $\frac{20}{20}$ & 20 & 20 & 200 & 20 & 20 & 20 & 20 & 20 & 20 & 20 & 20 & 20 & 20 & & & 20 & 20 & 20 & 20 & 20 & 20 & 20 & 20 & 20 \\
\hline 99 & & ${ }^{*}$ Backpulse po & ts in box & re not inc & & & & & & & & & & & & & & & & & & & & & & & & \\
\hline$\frac{1000}{101}$ & & & & & & & & & & & & & & & & & & & & & & & 18-min. & avg. $=$ & $\begin{array}{ll}0.52 \\
\end{array}$ & & & \\
\hline 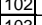 & & & & & & & & & & & & & & & & & & & & & & & & & & & & \\
\hline & & Averages & 27.3 & 18.3 & 26.6 & $\frac{2.1}{2.1}$ & $17.8: 3$ & 28.8 & 60.8 & 3.0 & 29.4 .4 & 24.7 & -0.1 & 43.3 & 0.0 & 6.3 & 0. & & & 55.3 & & 29.1 & 2.006 & & 0.890 & 0.0306 & & \\
\hline (2) & & and & 27.1 .13 & 0.4 .4 & 27.1 & $\frac{2.5}{212}$ & & 2.4 .4 & (1.2. & 3. & 30.3 & 20.8 & 0.0 & (44.1 & 0.0 & 0.0 & & & & $\frac{5.1}{5.3}$ & & 年 & 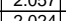 & $\mid 0.975$ & & & & 30.3 \\
\hline$\frac{106}{106}$ & & Minimum & 27.0 & $\begin{array}{l}18.0 \\
18.1\end{array}$ & $\frac{20.0}{26.3}$ & 207 & $\frac{1.05}{17.5}$ & 25.16 & 60.1 & 2.0 & $\frac{2.0 .}{26.3}$ & 224.2 & -0.0 & 44.9 & 0.0 & $\frac{6.2}{6.2}+2$ & 0. & & & $\frac{3.5}{58.6}$ & $\begin{array}{l}1.0 .5 \\
10.5\end{array}$ & 25.4 & 年 & $\mid$ & - 0.003 & - 0.0297 & 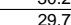 & 25.7 \\
\hline 107 & & $2 \times$ Std Dev & 0.487 & 0.144 & 0.527 & 0.408 & $0.377 \#$ & 1.917 & 0.539 & 0.280 & 1.777 & 1.553 & 0.178 & $\begin{array}{|ll|}4 & 0.587 \\
\end{array}$ & 0.000 & 0.172 & 0.001 & & & 0.539 & \begin{tabular}{|l|l|} 
& 0.144 \\
\end{tabular} & $\mid 1.834$ & 0.126 & \begin{tabular}{|l|l|}
0.026 \\
\end{tabular} & 0.018 & 0.0027 & 2.701 & $2.298|t| t$ \\
\hline 108 & Number & of Points Used ${ }^{\star}$ & 19 & 19 & & 19 & 190 & 19 & 19 & 19 & 19 & 19 & 19 & $\begin{array}{l}0 \\
\end{array}$ & 19 & 19 & 190 & & & 19 & 19 & 19 & 19 & \begin{tabular}{|l|}
19 \\
\end{tabular} & 19 & 19 & 19 & 190 \\
\hline \begin{tabular}{|l|}
109 \\
110
\end{tabular} & & ${ }^{\star}$ Backpulse po & its in box & re not inc & luded & & & & & & & & & & & & & & & & & & & & & & & \\
\hline 110 & & & & & & & & & & & & & & & & & & & & & & & & & & & & \\
\hline$\sqrt{12}$ & & & & & & & & & & & & & & & & & & & & & & & 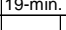 & avg. $=$ & 0.89 & & & \\
\hline \begin{tabular}{|l|l|}
113 \\
\end{tabular} & & Averages & 25.9 & 18.2 & 25.5 & 21.0 & 18.00 \# & 20.2 & 34.6 & 3.4 & 18.9 & 9.3 & 0.6 & 44.2 & 0.0 & 3.7 & 0.6 & & & 33.1 & 10.8 & 19.5 & 1.3 & 0.554 & 0.540 & 0.0266 & 26.6 & 22.6 \\
\hline 114 & & & 27.7 & 18.4 & 27.1 & 21.5 & 18.9 \# & 29.4 & 61.2 & 4.0 & 30.3 & 27.5 & 1.7 & 45.2 & 0.0 & 6.6 & 0.0 \# & & & 59.7 & 11.1 & 29.8 & 2.1 & \begin{tabular}{|l|l|l|l|} 
& 0.979 \\
\end{tabular} & & 0.0357 & 35.7 & $30.3 \#$ \\
\hline 115 & & Median & 25.9 & 18.2 & 25.5 & 21.0 & & 21.8 & 32.1 & 3.4 & 20.2 & 4.9 & 0.4 & 44.2 & 0.0 & 3.5 & & & & 30.6 & 10.8 & 21.00 & 1.4 & 0.522 & 0.514 & 0.0258 & 25.8 & \\
\hline $\mid \frac{116}{117}$ & & Minimum & $\begin{array}{r}24.6 \\
2119 \\
\end{array}$ & 18.0 & $\begin{array}{r}24.3 \\
1767\end{array}$ & 20.5 & $17.2 \#$ & $\begin{array}{r}9.9 \\
19011\end{array}$ & $\begin{array}{r}12.2 \\
30552\end{array}$ & $\frac{2.1}{0.62}$ & $\begin{array}{r}7.6 \\
17466\end{array}$ & $\mid-1.2$ & $\frac{-0.4}{1371}$ & $\begin{array}{r}42.9 \\
1552\end{array}$ & 0.0 & 1.3 & 0.023 & & & $\frac{10.7}{30.53}$ & 10.5 & 8.7 & \begin{tabular}{|r|}
0.6 \\
1110 \\
\end{tabular} & \begin{tabular}{|l|l|}
0.194 \\
0.581
\end{tabular} & 0.195 & 0.0200 & 20.0 & 1.07 \\
\hline$\frac{\mid 117}{118}$ & Number & & \begin{tabular}{|l|}
2.148 \\
59
\end{tabular} & 0.206 & $\begin{array}{r}1.767 \\
559\end{array}$ & $\begin{array}{r}0.574 \\
59\end{array}$ & 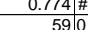 & $\begin{array}{r}14.911 \\
59\end{array}$ & $\begin{array}{r}39.552 \\
59\end{array}$ & 0.692 & $\begin{array}{r}17.466 \\
59\end{array}$ & $\begin{array}{r}21.888 \\
59\end{array}$ & 1.372 & 1.552 & 0.000 & 3.900 & 0.003 \# & & & $\frac{39.553}{59}$ & 0.380 & $\mid$ & $\begin{array}{r}1.116 \\
59\end{array}$ & \begin{tabular}{|r|r|}
0.581 \\
59
\end{tabular} & $\begin{array}{r}0.544 \\
59\end{array}$ & 0.0073 & 7.340 & 6.247 虾 \\
\hline $\mid \frac{\mid 118}{119}$ & Number & $\begin{array}{l}\text { of Points USed } \\
{ }^{\star} \text { Backpulse po }\end{array}$ & $\begin{array}{r}59 \\
t \operatorname{ts} \text { in box }\end{array}$ & $\begin{array}{r}59 \\
\text { re not inc }\end{array}$ & $\begin{array}{l}\text { luded } \\
\text { luded }\end{array}$ & 59 & 590 & 59 & 59 & 59 & 59 & 59 & 59 & 59 & 59 & 59 & 590 & & & 59 & 59 & 59 & 59 & 59 & 59 & 59 & 59 & 59 \\
\hline & & Dackpuse po & & & & & & & & & & & & & & & & & & & & & & & & & & \\
\hline 1214 & & & & & & & & & & & & & & & & & & & & & & & 1-hour a & avg. $=$ & 0.54 & & & \\
\hline$\frac{124}{123}$ & & & & & & & & & & & & & & & & & & & & & & & & Calc & 0.0313 & & & \\
\hline
\end{tabular}


RUN \# 1.03A; DEWATER SLURRY

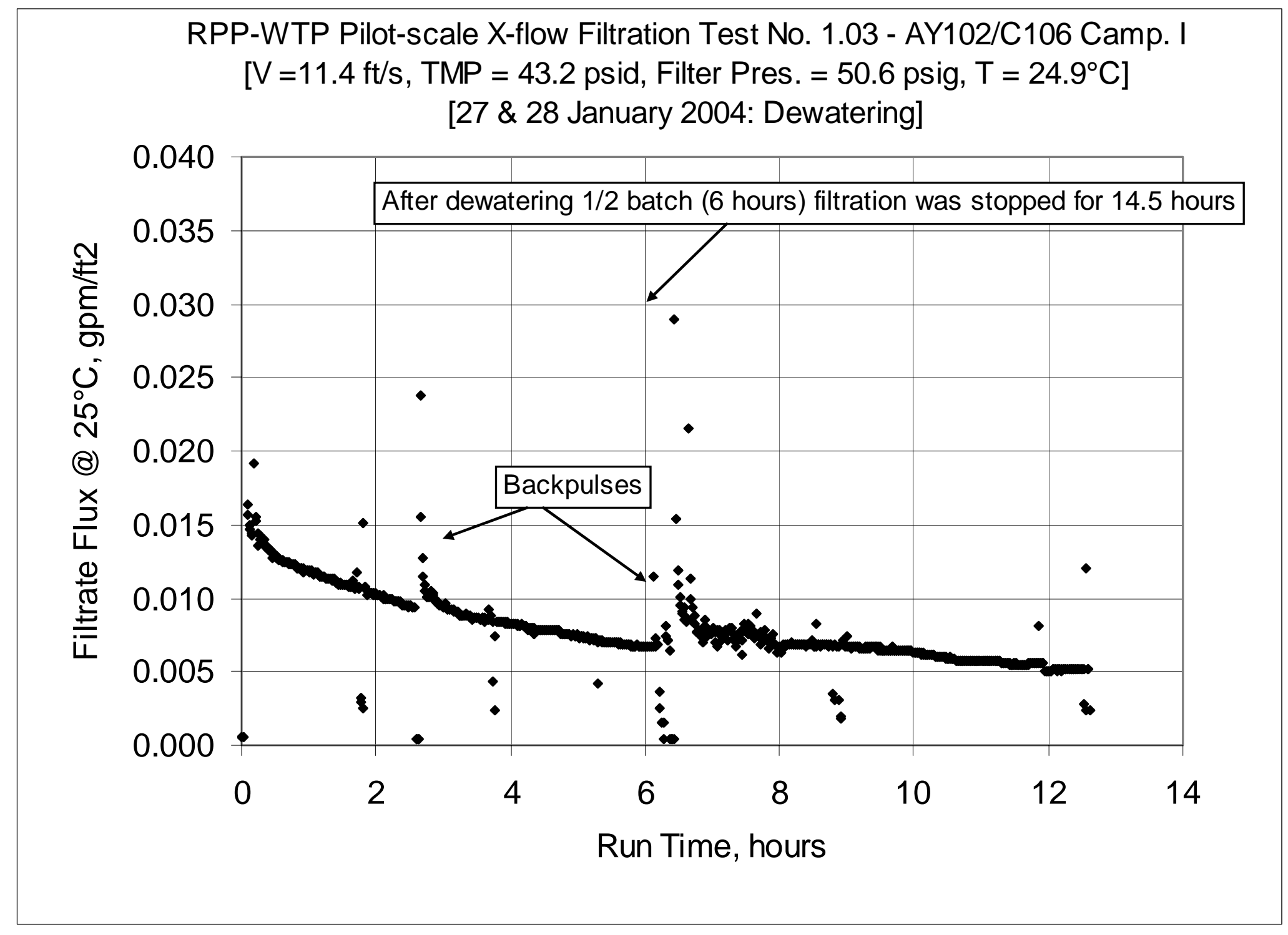


WSRC-TR-2005-00105, REVISION 0

SRNL-RPP-2005-00012, REVISION 0

RUN \# 1.03A; DEWATER FIRST HALF OF SLURRY - CONT.

\begin{tabular}{|c|c|c|c|c|c|c|c|c|c|c|c|c|c|c|c|c|c|c|c|c|c|c|c|c|c|c|c|c|}
\hline & A & B & \begin{tabular}{l|l} 
D \\
\end{tabular} & \begin{tabular}{|l|l|}
$E$ & \\
\end{tabular} & $F$ & G & $\mathrm{H}$ & \begin{tabular}{l|l} 
\\
$\leq<<<s$
\end{tabular} & \begin{tabular}{|l|}
$K$ \\
$K<<<<$
\end{tabular} & $1 \mathrm{~L}$ & \begin{tabular}{|l|}
$M$ \\
\end{tabular} & $N$ & 10 & \begin{tabular}{l|l} 
Q \\
\end{tabular} & $\begin{array}{ll}\mathrm{R} \\
\mathrm{R}\end{array}$ & $\mathrm{s}$ & \begin{tabular}{c|c}
$\mathrm{T}$ \\
\end{tabular} & $\mathrm{v}$ & $1 \mathrm{~W}$ & \begin{tabular}{l|l}
$\times$ & \\
\end{tabular} & \begin{tabular}{|l|}
$\mathrm{Y}$ \\
\end{tabular} & $\begin{array}{ll}\mathrm{z} z & \mathrm{z} \\
\end{array}$ & AA & $A B$ & $\mathrm{AC}$ & $A D$ & I $\mathrm{AE}$ & AF \\
\hline & DATE & TIME & $\begin{array}{l}\text { Filtrate } \\
\text { Fille }\end{array}$ & $\begin{array}{l}\text { k< Temper } \\
\mid \text { Cleaning| }\end{array}$ & 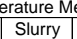 & $\begin{array}{l}\text { eleasureme } \\
\text { Hi Amb.| }\end{array}$ & 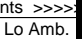 & $\begin{array}{l}k<<<<< \\
30 t T M P \mid\end{array}$ & 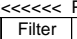 & $\begin{array}{l}\text { Pressure } 1 \\
\text { Fiter dP }\end{array}$ & \begin{tabular}{|l|} 
Measuremen \\
TopTMP
\end{tabular} & 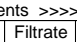 & 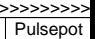 & 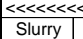 & 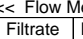 & $\begin{array}{l}\text { neasureme } \\
\text { Hi Fitate| }\end{array}$ & ntts & & & & & ed Fron & m Raw & Data >> & & & & \\
\hline & DAIL & & $\operatorname{deg} C$ & $\operatorname{deg} C$ & \begin{tabular}{|l|}
$\operatorname{deg} \mathrm{C}$ \\
\end{tabular} & $\operatorname{deg} c$ & $\operatorname{deg} C$ & psid & psig & psid & psid & psig & & gpm & $\begin{array}{l}\text { Filldale } \\
\text { gpm }\end{array}$ & mipm & & & & & & & & & & & & \\
\hline 4 & & & & & & & & $\mathrm{dP2}$ & & dP1 & & & & Q1 & & & & & & & & & & & & & & \\
\hline & $5-0126$ & & & & & & & & & & & & & & & & & & & & & & & & & & & \\
\hline 7 & $1 / 27 / 2004$ & $10: 16: 13 \mathrm{AM}$ & 21.531 & \begin{tabular}{ll|}
16.506 \\
\end{tabular} & \begin{tabular}{|l|l|}
13.839 \\
\end{tabular} & 22.26 & 14.302 & 0.052 & -0.114 & -0.001 & 0.012 & -0.171 & 0.069 & -0.02 & 0.003 & 14.643 & 0.004 & & & Filter Sur & urface, & $|6.707|$ & FT2 & & & & & \\
\hline 8 & $1 / 27 / 2004$ & 10:16:50 AM & 21.531 & 16.506 & 13.839 & 22.26 & & 0.052 & -0.114 & -0.001 & 0.012 & -0.171 & & \begin{tabular}{|c|c|c|} 
\\
\end{tabular} & 0.003 & 14.643 & 0.004 & & & Conversi & sion & & $\mathrm{m} 3 / \mathrm{m} 2 / \mathrm{c}$ & $2 /$ day/barc & $\mathrm{rg} / \mathrm{gpm} / \mathrm{tt}$ & /ft2/barg & & \\
\hline & 1/27/2004 & 10:16:51 AM & 21.531 & 16.506 & 13.824 & 22.22 & 14.31 & 0.052 & -0.112 & - -0.001 & 0.008 & -0.177 & & -0.091 & 0.003 & 14.643 & & & & & & & & & & & & \\
\hline & $1 / 27 / 2004$ & 10:16:52 AM & & 16.501 & \begin{tabular}{|l|l|l|l|l|l|} 
& 13.813 \\
\end{tabular} & 22.215 & 14.312 & 0.052 & -0.112 & - -0.001 & 0.006 & -0.177 & & $\begin{array}{l}-0.085 \\
-0.5\end{array}$ & 0.003 & 14.643 & $\tilde{0}$ & & & Vote: & uge : & esst & $P 1$ is & ipprox & atately 1.4 & 1.4 psig & & \\
\hline & 1/27/2004 & 10:16:53 AM & 21.526 & 16.495 & 13.813 & 22.21 & 14.30 & 0.054 & -0.114 & -0.002 & 0.008 & -0.189 & & -0.089 & 0.003 & 14.643 & & & & & & & & & & & & \\
\hline & $\frac{1 / 272 / 12004}{1 / 271}$ & 16:54 AM & & & & 22.1955 & & 0.054 & -0.114 & -0.002 & 0.01 & -0.18 & & -0.085 & 0.003 & 14.643 & & & & & & & & & & ir tu & & \\
\hline & $1 / 27 / 2004$ & 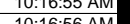 & $\frac{21.511}{2151}$ & $\frac{16.45}{1.49}$ & 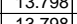 & 22.205 & & 年 & -0.114 & -0.001 & 0.01 & 0.0 .177 & & - & 0.004 & 14.643 & & & & v & & & & & & & & \\
\hline & $1 / 2 \pi / 2004$ & 10:16:56 AM & 1.516 & 16.48 & 13.798 & 22.195 & & 0.054 & -0.114 & -0.001 & 0.008 & -0.177 & & -0.083 & 0.003 & 14.643 & & & Time & Press. & $\mathrm{Vel}$ & & TMP & apm & & $\mathrm{pm}$ & 1000 & \\
\hline & ata-Per Mi & inte & & & & & & & & & & & & & & & & Min. & Hour & sig & & psi & bar & Itit2 & 12 & $\mathrm{t} 2 / \mathrm{psi}$ & $\mathrm{g} / \mathrm{p} / \mathrm{psi}$ & nay/bar \\
\hline 17 & 1/27/2004 & 1:25:00 PM & & 18.297 & 21.375 & 22.211 & 19.159 & 9.628 & 48.751 & 10.786 & -0.236 & 34.08 & & 44.894 & 0.003 & \begin{tabular}{|l|l|}
14.643 \\
\end{tabular} & & & & 47.3 & 11.0 & 4.7 & & & 0.000 & & & 0.09 \\
\hline & & :00 PM & 24.485 & 18.324 & 21.242 & 22.259 & 19.081 & 6.94 & 48.349 & 8.232 & -0.458 & 36.413 & & 54.056 & 0.003 & 14.643 & & & .0166 & 46.9 & 13.2 & 3.2 & & 0.000 & & & 0.154 & \\
\hline & 1) & 1:27:00 PM & 23.988 & 18.333 & 21.059 & 22.262 & 18.953 & 42.306 & 48.246 & 9.253 & $\begin{array}{l}33.607 \\
2.207\end{array}$ & 0.885 & & 47.107 & 0.257 & 14.643 & & 2.0 & Con & 46.8 & 11.5 & 88.0 & 2.617 & 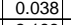 & 043 & 0.001 & 1.130 & \\
\hline & & 1.28:00 PMI & & & & & 18.986 & & & 10.1224 & 33.324] & 0.393 & & 42.869 & & & & 3.0 & & & & & & 1.83 & & & & \\
\hline & $\frac{112170004}{1027004}$ & 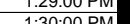 & $\frac{2.3411}{23.42}$ & & $\frac{2.1 / 11}{2019}$ & 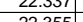 & 19.0858 & 42.98 & 48.4007 & $\frac{1.5251}{10677}$ & 每3.0924 & 0 & & 年.641 & & 14.643 & & & . & & & 年8.0 & & & & & 海.840 & \\
\hline & $\frac{1 / 27 / 2004}{1 / 27}$ & $\begin{array}{l}\text { 1.30.00 PMI } \\
\text { 1:31:00 PM }\end{array}$ & $\begin{array}{l}2.4435 \\
23.391 \\
\end{array}$ & $\begin{array}{l}18.355 \\
18.383 \\
\end{array}$ & \begin{tabular}{|l|}
20.594 \\
20.611 \\
\end{tabular} & $\begin{array}{ll}22.355 \\
22.368\end{array}$ & $\frac{19.204}{18.832}$ & $\frac{43.115}{42.98}$ & $\begin{array}{l}48.0001 \\
48.501\end{array}$ & $\frac{1.006}{10.559}$ & 32.980 & $\begin{array}{l}0.304 \\
0.367 \\
\end{array}$ & & $\begin{array}{l}40.000 \\
46.809\end{array}$ & 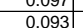 & $\begin{array}{l}14.045 \\
14.643\end{array}$ & & $\frac{3.00}{6.00}$ & $\frac{.08535}{0.1}$ & $\begin{array}{l}47.1 \\
47.0 \\
\end{array}$ & $\frac{11.4}{11.5}$ & 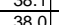 & 261 & \begin{tabular}{|l|l|}
0.014 \\
\end{tabular} & 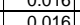 & 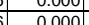 & 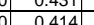 & \\
\hline & & 1:32:00 PM & 23.283 & 18.395 & \begin{tabular}{|l|}
20.643 \\
\end{tabular} & 22.319 & 19.369 & \begin{tabular}{ll|l|}
43.016 \\
\end{tabular} & \begin{tabular}{|c|}
48.552 \\
\end{tabular} & 10.76 & 32.842 & 0.373 & 0.511 & 46.302 & 0.089 & 14.643 & 0.004 & 7.00 & 0.11667 & 47.1 & 11.3 & 37.9 & $\mid 2.615$ & \begin{tabular}{|l|l|}
0.013 \\
\end{tabular} & 0.015 & 0.000 & 0.396 & \\
\hline & 1/27/2004 & 1:33:00 PM & 23.144] & 18.408 & \begin{tabular}{|l|l|}
20.665 \\
\end{tabular} & 22.256 & 19.25 & \begin{tabular}{|c|c|}
43.148 \\
\end{tabular} & \begin{tabular}{|l|l|}
48.737 \\
\end{tabular} & 10.786 & \begin{tabular}{l|l|l}
32.899 \\
\end{tabular} & 0.367 & & (43.793 & $\begin{array}{l}0.087 \\
\end{array}$ & \begin{tabular}{|l|l}
14.643 \\
\end{tabular} & & 8.00 & 0.13333 & (47.3 & 10.7 & 38.0 & 2.622 & & 0.015 & & (0.386 & \\
\hline & $\frac{1 / 127 / 20004}{1 / 2720}$ & 1:34:00 PM & 23.011 & $18.415 \mid$ & 20.753 & 22.314 & 19.545 & 43.073 & 48.679 & 10.651 & 32.912 & 0.44 & & 50.036 & 0.086 & 14.643 & & 9.0 & 0.15 & 47.2 & 12.3 & 38.0 & & & 014 & & 0.381 & \\
\hline & & & & & & & & 42.937 & 48.5444 & & $32.827]$ & 0.48 & & 4.2.205 & & & & & & & & & & & & & & \\
\hline & 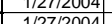 & $\begin{array}{l}1.36 .00 \mathrm{MPM} \\
1.3700 \mathrm{M}\end{array}$ & 20.2064 & 18.429 & 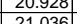 & $\frac{2.328}{2241}$ & 19.06 & 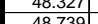 & 年8.544 & & 38.476 & & & (4.0060 & & $\frac{14.66}{14.6}>0$ & & & 18333 & & & & & & & & .0.440 & \\
\hline & $1 / 27 / 2004$ & 1:38:00 PM & $\frac{2.041}{22.602}$ & 18.463 & \begin{tabular}{|l|}
21.144 \\
\end{tabular} & $\frac{2.41}{22.412}$ & $\frac{19.550}{19.668}$ & $\frac{48.139}{48.754}$ & \begin{tabular}{|l|}
48.1587 \\
48.687 \\
\end{tabular} & $\frac{1.804}{10.846}$ & \begin{tabular}{|c|}
38.00 \\
38.707
\end{tabular} & \begin{tabular}{|l|}
-1.210 \\
-1.216 \\
\end{tabular} & $\frac{-7.0}{-7.3}$ & $\begin{array}{l}47.30<2 \\
46.409\end{array}$ & $\begin{array}{l}0.093 \\
0.092 \\
\end{array}$ & 14.643 & 0.00 & $\frac{1.2 .0}{13.0}$ & \begin{tabular}{r|}
0.21667 \\
0.25
\end{tabular} & $\frac{47.3}{47.2}$ & $\frac{11.6}{11.4}$ & $\frac{43.1}{437}$ & & & 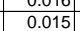 & 0.000 & 0.0 .350 & 0 \\
\hline & $1 / 27 / 2004$ & & & $\begin{array}{ll}18.459 \\
\end{array}$ & 21.215 & 22.413 & 19.8 & & \begin{tabular}{|l|l|l|l|l|} 
& 48.768 \\
\end{tabular} & 10.914 & & -1.216 & & $\begin{array}{l}45.476 \\
\end{array}$ & & & & 14.06 & & & 11.1 & 43.8 & 3.017 & & & & & \\
\hline & $1 / 27 / 2004$ & 1:40:00 PM & 22.486 & 18.487 & \begin{tabular}{|l|l|}
21.309 \\
\end{tabular} & 22.461 & 19.334 & 48.748 & 48.677 & 10.791 & $38.744 \mathrm{~S}$ & -1.216 & & 46.227 & 0.087 & 14.643 & & 15.0 & 0.25 & 47.2 & 11.3 & 43.7 & 3.016 & & . & 0.000 & & \\
\hline & 1/27/2004 & 1:41:00 PM & 22.442 & 18.494 & 21.376 & 22.493 & & 48.42 & 48.347 & 10.605 & & -1.216 & & 47.629 & & & & & 26667 & 46. & 2.1 & & & & & & & \\
\hline & 2004 & & $\frac{22.43}{2.2375}$ & 18.511 & 21.448 & 22.429 & & 48.11 & 48.656 & $10 . / 65$ & & -1.216 & & 46.054 & & & & & .28333 & 4.2 & 11.3 & 43.7 & & & & & & \\
\hline & $1 / 2120044$ & $1.43 .007 \mathrm{PM}$ & $\frac{2.3515}{2025}$ & $\mid \frac{18.528}{18.585}$ & \begin{tabular}{|r|}
21.53 \\
\end{tabular} & 2.451 & 19.4 & 48.6013 & 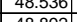 & 1.103 & & & & & & & & & 0.3 & 4.1 .1 & & 年.60 & & & & & . & \\
\hline & 1727204 & & 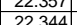 & $\mid$ & 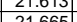 & 20.4205 & 19.5 & 48.980 & 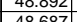 & $\mid$ & & $\mid$\begin{tabular}{|c|c|c|}
||$_{2}$ \\
\end{tabular} & & & & & & & & & & & & & & & & \\
\hline 38 & $\frac{1 / 27 / 2004}{1 / 27 / 2004}$ & $\frac{1.45 .60}{1: 46: 00}$ & $\frac{2.544}{22.33}$ & 18564 & \begin{tabular}{|l|}
21.727 \\
\end{tabular} & 22532 & & 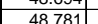 & 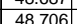 & $\frac{10.679}{1029}$ & & 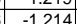 & & 4 & & $\frac{14.6}{146}$ & & & 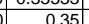 & $\frac{41}{47}$ & $\frac{1.20}{114}$ & 年 & & & & & 年 & \\
\hline 39 & $1 / 27 / 2004$ & $\begin{array}{l}1.40 .0 \\
1: 47: 0\end{array}$ & 22.333 & 18.581 & $\mid$ & 22.589 & 20.259 & 48.771 & 48.72 & 10.763 & 38.791 & $\frac{-1.214}{-1.24}$ & & 42.0 & o. 0.083 & 14.6 & & $\frac{2.0}{22.0}$ & 5.36667 & $\frac{17.2}{4.2}$ & 望. & 4 & & .012 & & 0.000 & $\frac{0.310}{0.310}$ & \\
\hline & 1/27/200 & & & & & & & & & & & & & & & & & & & & & & & & & & & \\
\hline 41 & $7 / 2004$ & 1:49:00 F & 22.331 & \begin{tabular}{l|l}
18.605 \\
\end{tabular} & 21.652 & 22.638 & 20.4 & 48.851 & 48.751 & 10.86 & & -1.216 & & 45.305 & & 14.6 & & & 0.4 & 47.3 & $\overline{l 1.1 .}$ & & & & & & & \\
\hline & & & & $\begin{array}{l}18.616 \\
\end{array}$ & 21.729 & & 20. & & 48.772 & 10.789 & & -1.216 & & & & & & & 0.41667 & 47.3 & & 43.0 & & & & & & \\
\hline & $1 / 27 / 2004$ & 1:51:00 PM & 22.324 & \begin{tabular}{|l|l|}
18.623 \\
\end{tabular} & \begin{tabular}{|l|}
21.776 \\
\end{tabular} & 22.586 & 19.92 & $\begin{array}{l}48.717 \\
\end{array}$ & \begin{tabular}{|c|}
48.65 \\
\end{tabular} & 10.718 & & -1.219 & & & & & & 26.0 & 0.43333 & 47.2 & 12.0 & 43.8 & & & & & & \\
\hline 44 & $1 / 27 / 2004$ & & 22.342 & 18.646 & \begin{tabular}{|l|}
21.849 \\
\end{tabular} & 22.689 & & 48.7 & \begin{tabular}{|l|l|}
48.741 \\
\end{tabular} & 10.84 & & $\mid-1.21$ & & & & 14.6 & & & 0.45 & & 10.6 & 43 & & & & & & \\
\hline & 1/27/2004 & & 22.353 & $\begin{array}{ll}18.67 \\
\end{array}$ & \begin{tabular}{|l|}
21.916 \\
\end{tabular} & 22.73 & 19.7 & & \begin{tabular}{|l|l|}
48.687 \\
\end{tabular} & $\begin{array}{l}10.786 \\
\end{array}$ & & & & & & & & & & 47. & 11.6 & & & & & & 0.298 & \\
\hline 40 & $1 / 27 / 2004$ & & 22.365 & 18.68 & \begin{tabular}{|l|}
21.958 \\
\end{tabular} & 22.688 & & 48.766 & 48.671 & 10.79 & 38.816 & -1.21 & & & & & & 29. & 0.48333 & 47.2 & 11.7 & 43. & & & & & & \\
\hline & $1 / 27 / 2004$ & & & 18.687 & \begin{tabular}{|l|}
21.995 \\
\end{tabular} & 22.735 & & & 48.878 & 10.864 & & -1.214 & & 46.734 & & & & & 0.5 & 47. & 11.5 & 43. & & & & & & \\
\hline & & & 22.395 & 18.109 & \begin{tabular}{|l|l|}
222.0433 \\
\end{tabular} & 22.797 & & & & 10.76 & & & & & & & & & 0.51667 & $4 r$. & & & & & & & & \\
\hline & $1 / 2,12004$ & $\frac{1.57 .00 \mathrm{PM}}{1.650}$ & $\frac{2.2,42 \pi}{2,24}$ & 18.726 | & $\frac{22.1}{2213}$ & $\frac{22.834}{22802}$ & 20. & 48.768 & $\begin{array}{ll}48.698 \\
18565\end{array}$ & $\frac{10.823}{106525}$ & $\frac{38.7}{287}$ & $-1.216 \mid$ & & & & & & & 年 & $\frac{47.2}{4.73}$ & 11.1 & 27 & & & & & $n$ & \\
\hline & & & & & & 2277 & & & & & & & & & & & & & & & & & & & & & & \\
\hline & $1 / 27 / 2004$ & $2: 00: 00 \mathrm{PM}$ & 22.477 & \begin{tabular}{|c|c|}
18.762 \\
\end{tabular} & $\mid 22.216$ & 22.815 & & 48.7 & \begin{tabular}{|l|l|}
48.716 \\
\end{tabular} & 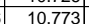 & & \begin{tabular}{|l|l|}
3 & -1.22 \\
\end{tabular} & & & & 14. & & & 0.58333 & 47.2 & \begin{tabular}{|l|}
12.3 \\
\end{tabular} & 43. & & & & & & \\
\hline & $1 / 27 / 200$ & & 22.493 & $\begin{array}{l}18.773 \\
\end{array}$ & \begin{tabular}{|l|}
22.303 \\
\end{tabular} & 22.745 & & 48.791 & \begin{tabular}{|l|l|}
48.836 \\
\end{tabular} & 10.835 & & -1.214 & & & & 14.6 & & 36.1 & 0.6 & 47.4 & \begin{tabular}{|l|l|}
10.7 \\
\end{tabular} & 43.8 & & & & & & \\
\hline 84 & $1 / 27 / 2004$ & & 22.53 & 18.785 & 22.41 & 22.808 & & & & & & -1.21 & & & & & & & & 47.1 & & 43.7 & & & & & & \\
\hline & & & 22.5/1 & 18.816 & $22.531 \mid$ & 22.828 & & 48.561 & 48.54 & 10.71 & & -1.211 & & & & & & & 63333 & 47.1 & & 43.6 & & & & & & \\
\hline & & & 22.60 & & \begin{tabular}{|l|l|}
22.603 \\
\end{tabular} & 22.8 & & & 48.809] & & & & & & & & & & & 47. & 11.2 & & & & & & & \\
\hline & & & & & & & & & & & & & & & & & & & & & & & & & & & & \\
\hline & $1 / 27 / 200$ & & & & 22.671 & & & & & 10.8 & & -1.21 & & 45.7. & & 14.6 & & & 0.68333 & 47. & 11.2 & & & & & & & \\
\hline & 1/27/20 & & 22.737 & & 22.778 & 22.959 & & 48.79 & 488.8 & 10.8 & & -1.21 & & & & $\begin{array}{lll}14.6 \\
\end{array}$ & & & $\mid 7$ & 47.3. & 10.6 & 43 & & & & & & \\
\hline & 1/27/2 & & 22.784 & 18.88 & 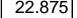 & 22.8 & & 48.6 & 48. & 10.7 & & -1.21 & & & & & & & & 47. & & & & & & & & \\
\hline & 1/27/2 & & & 18.8 & \begin{tabular}{|l|}
22.947 \\
\end{tabular} & 22.917 & & 48 & 48.8 & & & & & & & & & & & 47. & & & & & & & & \\
\hline & 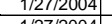 & & 2.2862 & 18.9 & 23.024 & 22.929 & & 48.586 & 48.72 & 10.66 & 38.726 & -1.214 & & & & 14.6 & & & & 477. & & & & & & & & \\
\hline & & & 2 & 18.91 & $\frac{2.101}{2.217}$ & 2.95 & & & 48.94 & 10.1. & & -1.21 & & & & & & & (1) & & 11.2 & & & & & & & \\
\hline & & & $\frac{2.2945}{20907}$ & & $\frac{2.117}{2.20}$ & 2.90 & & 年 & $\frac{48.6}{4.97}$ & & & $-\frac{-1.21}{1.21}$ & & & & & & & & & & & & & & & & \\
\hline & $1 / 27 / 20$ & & $\frac{26.90}{2304}$ & 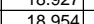 & $\mid$ & $\frac{2.540}{2293}$ & & 年6.06 & & & & $\frac{-1.2 .}{-1.2}$ & & & & 年 14.6 & & & & $47:$ & & & & & & & & \\
\hline & $1 / 1 / 27 / 2004$ & & 年 & 18965 & 23.515 & 20.951 & & 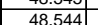 & 4869 & 1062 & 3873 & -1214 & & & & & & & & 472 & & & & & & & & \\
\hline & $\frac{1}{1 / 27 / 2004}$ & $2: 16: 00$ & 23.116 & & 23.223 & 23.018 & & 48.6 & & & & -1.219 & & & & & & & & 47.4 & & & & & & & & \\
\hline & $1 / 27 / 2004$ & & 3.143 & 18.998 & \begin{tabular}{|l|}
23.299 \\
\end{tabular} & 23.009 & & \begin{tabular}{|l|l|}
48.715 \\
\end{tabular} & 48.878 & 10.804 & 38.758 & -1.216 & & 46.45 & & & & & & 47. & & & & & & & 0.275 & \\
\hline 70 & $1 / 27 / 2004$ & 2:18:00 PM & 3.169 & \begin{tabular}{|l|}
19.014 \\
\end{tabular} & \begin{tabular}{|l|l|}
23.376 \\
\end{tabular} & 23 & & \begin{tabular}{|l|l|}
48.694 \\
\end{tabular} & \begin{tabular}{|l|l|}
48.855 \\
\end{tabular} & 10.825 & 38.691 & $\mid-1.216$ & & 45.41 & |077| & 14.643 & & & & & $11.1 .12-1$ & & & & & & 0.275 & \\
\hline
\end{tabular}


WSRC-TR-2005-00105, REVISION 0

SRNL-RPP-2005-00012, REVISION 0

RUN \# 1.03A; DEWATER FIRST HALF OF SLURRY - CONT.

\begin{tabular}{|c|c|c|c|c|c|c|c|c|c|c|c|c|c|c|c|c|c|c|c|c|c|c|c|c|c|c|c|c|}
\hline & & & & & & & & & & & & & & & & & & & & & & & & & & & & \\
\hline & & 2:19:00 PM & & & 23.443 & 23.057 & 20.692 & 48.684 & \begin{tabular}{|l|}
49.009 \\
\end{tabular} & 10.698 & 38.709 & -1.216 & -7.103 & 50.167 & 0.077 & 14.643 & 0.004 & 54.00 & 0.9 & 47.5 & $\begin{array}{l}12.3 \\
\end{array}$ & 43.7 & 3.013 & م.01 & 0.012 & 0.000 & 0.275 & \\
\hline & $1 / 27 / 2004$ & 2:20:00 PM & 23.227 & 19.037 & \begin{tabular}{|l|}
23.509 \\
\end{tabular} & 23.098 & 20.572 & & & & & & & & & 14.643 & & & & & & & & & & & & \\
\hline 73 & $1 / 27 / 2004$ & 2:21:00 PM & 23.253 & 19.059 & \begin{tabular}{|l|}
23.566 \\
\end{tabular} & 23.14 & 20.74 & 48.686 & \begin{tabular}{|l|l|}
48.899 \\
\end{tabular} & \begin{tabular}{|l|}
10.859 \\
\end{tabular} & \begin{tabular}{|c|}
38.693 \\
\end{tabular} & \begin{tabular}{|c|}
-1.216 \\
\end{tabular} & $\begin{array}{l}-7.094 \\
\end{array}$ & \begin{tabular}{|l|l}
49.06 \\
\end{tabular} & 0.076 & 14.643 & & 56.00 & 0.93333 & 47.4 & & & & & & & & \\
\hline$\frac{74}{75}$ & & $\begin{array}{l}2: 22: 00 \mathrm{PM} \\
\end{array}$ & 23.296 & 19.071 & \begin{tabular}{|l|}
23.628 \\
\end{tabular} & 23.092 & 20.656 & 48.511 & \begin{tabular}{|l|l|}
48.679 \\
\end{tabular} & \begin{tabular}{|l|l|}
10.711 \\
\end{tabular} & $\begin{array}{l}38.693 \\
3\end{array}$ & \begin{tabular}{|c|}
-1.216 \\
\end{tabular} & $\begin{array}{r}-7.086 \\
-7.086\end{array}$ & \begin{tabular}{|l|l|}
44.617 \\
\end{tabular} & 0.077 & 14.643 & 0.004 & 57.00 & 0.95 & 47.2 & 10.9 & \begin{tabular}{|l|}
43.6 \\
\end{tabular} & 3.006 & & 0.012 & 0.000 & & \\
\hline & $1 / 27 / 2004$ & $\begin{array}{l}2: 23: 00 \mathrm{PM} \\
22: 200\end{array}$ & 23.312 & 19.077 & \begin{tabular}{|l|l|}
23.644 \\
3.271
\end{tabular} & 23.123 & 20.839 & 48.684 & \begin{tabular}{|l|l|}
48.917 \\
\end{tabular} & \begin{tabular}{|l|}
10.776 \\
1092
\end{tabular} & 38.687 & \begin{tabular}{|c|}
-1.222 \\
1.21
\end{tabular} & $\begin{array}{r}-7.097 \\
7\end{array}$ & \begin{tabular}{|l|l|}
46.528 \\
\end{tabular} & 0.077 & 14.643 & 0.003 & 58.00 & \begin{tabular}{|l|}
0.966677 \\
\end{tabular} & 47.4 & 11.4 & \begin{tabular}{|l|}
43.7 \\
\end{tabular} & & 0.01 & 0.012 & 0.000 & & \\
\hline & $1 / 27 / 2004$ & 2:24:00 PM & & 19.114 & & \begin{tabular}{|l|}
23.18 \\
23.95
\end{tabular} & 21.187 & & & \begin{tabular}{|l|l|}
10.893 \\
10673
\end{tabular} & \begin{tabular}{|l|l|}
38.68 \\
38.627
\end{tabular} & \begin{tabular}{|l|l|}
-1.219 \\
1211
\end{tabular} & & & & 14.643 & & & 0.98333 & 47.6 & & & & & & & & \\
\hline & $1 / 27 / 2004$ & 2:25:00 PM & $\begin{array}{l}23.389 \\
2.2025\end{array}$ & $\begin{array}{l}19.109 \\
1012\end{array}$ & \begin{tabular}{|l|}
23.771 \\
22807
\end{tabular} & $\begin{array}{l}23.185 \\
22151\end{array}$ & 20.941 & 48.451 & \begin{tabular}{|l|l|}
48.706 \\
\end{tabular} & \begin{tabular}{|l|l|}
10.673 \\
0.09
\end{tabular} & $\begin{array}{l}38.637 \\
39.584\end{array}$ & \begin{tabular}{|c|} 
\\
\end{tabular} & $\begin{array}{r}-7.077 \\
\end{array}$ & 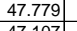 & \begin{tabular}{|c|}
0.076 \\
\end{tabular} & 14.643 & & 60.00 & & 47.2 & $\begin{array}{l}11.7 \\
1.5\end{array}$ & & 3.002 & & 0.012 & & $\begin{array}{ll}0.269 \\
\end{array}$ & \\
\hline & $\begin{array}{l}1 / 27 / 72004 \\
1 / 27 / / 2004\end{array}$ & $\begin{array}{l}2: 26: 00 \mathrm{PM} \\
2: 27: 00 \mathrm{PM}\end{array}$ & $\frac{23.425}{23.461}$ & $\begin{array}{r}19.12 \\
19147\end{array}$ & \begin{tabular}{|l|}
$\mid 23.807$ \\
23599
\end{tabular} & $\begin{array}{l}23.151 \\
23.18\end{array}$ & $\frac{21.239}{2088}$ & $\begin{array}{l}48.424 \\
48.416\end{array}$ & \begin{tabular}{|l|}
48.644 \\
48698 \\
\end{tabular} & \begin{tabular}{|r|}
10.696 \\
10.62
\end{tabular} & $\begin{array}{l}38.584 \\
38.611\end{array}$ & & $\begin{array}{r}-7.08 \\
-7007\end{array}$ & \begin{tabular}{|l}
47.107 \\
51.373
\end{tabular} & $\begin{array}{l}0.077 \\
0.077\end{array}$ & $\begin{array}{r}14.643 \\
14.643\end{array}$ & 0.004 & 61.00 & \begin{tabular}{|c|}
1.01667 \\
1.03333
\end{tabular} & 47.2 & $\frac{11.5}{126}$ & & 2.999 & & $\begin{array}{l}0.012 \\
0.121\end{array}$ & 0.000 & & 023 \\
\hline 80 & $\frac{1 / 27 / 2004}{1 / 27 / 2004}$ & $\frac{2.27 .00 \mathrm{PM}}{2: 28: 00 \mathrm{PM}}$ & $\frac{23.461}{23.503}$ & $\frac{19.14 t}{19.169}$ & \begin{tabular}{|l|}
23.389 \\
23.911 \\
\end{tabular} & $\frac{23.184}{23.189}$ & & $\frac{48.416}{48.686}$ & $\begin{array}{c}48.689 \\
48.98\end{array}$ & $\frac{10.652}{10.834}$ & $\frac{38.611}{38.67}$ & \begin{tabular}{|c|}
-1.216 \\
-1.216
\end{tabular} & $\begin{array}{l}-\frac{-1.077}{-7.074} \\
\end{array}$ & $\frac{51.3 / 3}{47.575}$ & $\frac{0.077}{0.076}$ & $\frac{14.643}{14.643}$ & .004 & $\frac{62.00}{63.00}$ & $\begin{array}{l}1.03333 \\
1.05 \\
\end{array}$ & $\begin{array}{l}4.2 \\
47.5\end{array}$ & & $\begin{array}{l}43.5 \\
43.7 \\
\end{array}$ & $\begin{array}{l}3.000 \\
3.011\end{array}$ & 01 & $\frac{0.012}{0.012}$ & $\frac{0.000}{0.000}$ & $\frac{0.272}{0.268}$ & $\frac{0.23}{0.23}$ \\
\hline 81 & $1 / 27 / 2004$ & 2:29:00 PM & 23.54 & 19.181 & \begin{tabular}{|l|}
23.953 \\
\end{tabular} & 23.221 & 20.267 & 48.872 & \begin{tabular}{|l|}
49.178 \\
\end{tabular} & & 38.689 & \begin{tabular}{|c|}
-1.219 \\
\end{tabular} & & \begin{tabular}{|l|l|}
48.676 \\
\end{tabular} & & 14.643 & 0.004 & 64.00 & \begin{tabular}{|l|}
1.06667 \\
1
\end{tabular} & 47.7 & 11.9 & & & & & & $\begin{array}{l}0.268 \\
0.267\end{array}$ & $\frac{0.23}{0.23}$ \\
\hline & $1 / 27 / 2004$ & 2:30:00 PM & 23.571 & & \begin{tabular}{|l|}
23.939 \\
\end{tabular} & 23.288 & 21.149 & 48.742 & \begin{tabular}{|l|l|}
48.99 \\
\end{tabular} & \begin{tabular}{l|l|}
10.919 \\
\end{tabular} & 38.646 & \begin{tabular}{|c|}
-1.214 \\
\end{tabular} & $\begin{array}{l}-7.057 \\
\end{array}$ & \begin{tabular}{|l|l}
47.329 \\
\end{tabular} & $\begin{array}{l}0.076 \\
\end{array}$ & 14.643 & & 65.00 & 1.08333 & 47.5 & 11.6 & 43.7 & 3.013 & $\overline{0.011}$ & 0.012 & 0.000 & 0.267 & $\begin{array}{l}0.23 \\
0.23\end{array}$ \\
\hline & $1 / 27 / 2004$ & 2:31:00 PM & & 19.209 & \begin{tabular}{|l|}
23.79 \\
\end{tabular} & 23.239 & 21.362 & 48.447 & 48.789 & $\begin{array}{l}10.695 \\
\end{array}$ & \begin{tabular}{|l|l|}
38.543 \\
\end{tabular} & \begin{tabular}{|l|l|}
-1.216 \\
\end{tabular} & & $\begin{array}{l}46.384 \\
\end{array}$ & $\begin{array}{l}0.076 \\
\end{array}$ & 14.643 & & 66.00 & & 47.3 & 11.4 & \begin{tabular}{|l|}
43.5 \\
\end{tabular} & 2.999 & & & & & $\begin{array}{l}0.23 \\
0.23\end{array}$ \\
\hline 84 & $1 / 27 / 2004$ & $2: 32: 00$ PM & 23.615 & & & 23.276 & & & & 10.756 & & & & & & & & & & & & & & & & & 0.268 & \\
\hline 86 & & & & & & & & 48.704 & \begin{tabular}{|l|l|}
49.11 \\
\end{tabular} & 10.825 & & -1.216 & & \begin{tabular}{|l|l|}
45.166 \\
\end{tabular} & & & & & & $\mid 47.6$ & & & & & & & & \\
\hline 87 & $1 / 27 / 2004$ & 2:34:00 PM & 23.622 & 19.243 & \begin{tabular}{|l|}
23.924 \\
\end{tabular} & 23.227 & 20.968 & .798 & |49.199 & 10.959 & 551 & $\mid-1.219$ & & 52.61 & & & & & 1.15 & 47.7 & 12.9 & 43. & 01. & & & & 0.264 & \\
\hline & $1 / 27 / 2004$ & 2:35:00 PM & 23.658 & 19.274 & & 23.279 & & 48.414 & 48.737 & 10.711 & 561 & -1.216 & & 46.309 & .075 & 14.6 & & & .16667 & 47.3 & & & $998 \mathrm{Cr}$ & & & 0.000 & & \\
\hline 88 & $1 / 27 / 2004$ & 2:36:00 PM & 23.668 & 19.29 & 24.016 & 23.294 & 20.58 & 48.71 & 49.154 & 10.788 & 38.625 & -1.216 & & 47.005 & 0.075 & 14.64 & & 71.00 & 1.18333 & 47.7. & 11.5 & 43. & 011 & & 0.011 & 0.000 & 0.263 & \\
\hline & $1 / 27 / 2004$ & 2:37:00 PM & \begin{tabular}{|l|l}
23.705 \\
227255
\end{tabular} & 19.317 & 24.072 & 23.296 & 21.027 & 48.615 & 48.953 & 10.853 & \begin{tabular}{|l|l|}
38.568 & \\
\end{tabular} & -1.219 & $\frac{-7.0}{7}$ & 45.779 & 0.075 & 14.643 & & .00 & $\begin{array}{r}1.2 \\
\end{array}$ & 47.5 & 11.2 & 43.6 & 005 & & & 000 & 0.263 & \\
\hline & $1 / 27 / 2004$ & 2:38:00 PM & & & & & & 48.484 & 48.87 & 10.814 & 38.541 & -1.216 & & 46.83 & 0.075 & 14.643 & & & 12667] & 47.4 & & & & & & & & \\
\hline 91 & $1 / 27 / 72004$ & 2:39:00 PM & 2. & 19.348 & 24.154 & 23.292 & 21.199 & 48.551 & 48.94 & 10.877 & $\begin{array}{l}38.492 \\
38.474\end{array}$ & -1.216 & & 43.595 & 7075 & 14.643 & & & 23333 & 47.5 & & 13.5. & 001 & & & 0.000 & 0.263 & \\
\hline & $1 / 2 / 272004$ & $2.40000 \mathrm{PM}$ & $\frac{2.3 / 12}{2320}$ & $\frac{19.354}{1026}$ & 24.18 & $\mid \frac{23.388}{2320 \mid}$ & $\frac{21.08}{2128}$ & $\frac{48.625}{4.842}$ & 4.9.0035 & $\frac{1.867}{1.891}$ & $\frac{38.411}{3891}$ & $-\frac{1.1216}{1.210}$ & & 4. & 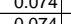 & $\frac{14.64}{1.6}$ & & & & 年, & & & & & & & & \\
\hline 94 & 1 & $2.42 .00 \mathrm{PM}$ & & & $\frac{24.211}{24247}$ & $\begin{array}{l}23.329 \\
23.31\end{array}$ & & $\frac{48.443}{48.746}$ & $\frac{48.816}{49131}$ & \begin{tabular}{r|r|}
11.07 \\
11.072
\end{tabular} & & $\frac{-1.219}{-1216}$ & & 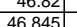 & 年 & & & & $28606 /$ & $\begin{array}{l}4.3 \\
47.7\end{array}$ & & & & & & & & \\
\hline 95 & $1 / 27 / 2004$ & 2:43:00 PM & 23.85 & 19.387 & \begin{tabular}{|l|}
24.182 \\
\end{tabular} & 23.28 & 21.353 & 48.64 & 49.112 & 10.969 & 38.449 & \begin{tabular}{|c|}
-1.216 \\
\end{tabular} & & 42.202 & 0.074 & 14.643 & & 78.00 & 1.3 & 47.6 & 10.3 & $\frac{43.5}{43.5}$ & 002 & & 0.011 & 0.000 & 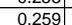 & \\
\hline & $1 / 27 / 2004$ & 2:44:00 PM & 23.875 & 19.403 & 24.093 & 23.311 & & 48.464 & 48.926 & 10.865 & 38.477 & -1.214 & & 46.84 & 0.074 & 14.643 & & & .31667 & 47.5 & 11.5 & & $\frac{997}{997}$ & .011 & 01 & & & \\
\hline & $1 / 27 / 2004$ & 2:45:00 PM & 23.882 & 19.424 & \begin{tabular}{|l|l|l|l|} 
\\
\end{tabular} & 23.337 & 21.34 & 48.48 & 48.934 & 10.897 & $\begin{array}{l}38.432 \\
\end{array}$ & \begin{tabular}{|c|}
-1.216 \\
\end{tabular} & & 44.173 & & & & & 33333 & 47.5 & & & & & & & & \\
\hline & 1/27/2004 & 2:46:00 PM & 23.893 & 19.42 & $24.17 \mid$ & 23.323 & 21.024 & 48.331 & 48.795 & 10.802 & & -1.219 & & 466.327 & & & & 81.0 & $\mid 1.35$ & 47.3 & 11.4 & 43.4 & & & & 00 & 0.260 & \\
\hline & & & & & 24.213 | & & & 48.754 & & 11.044 & & & & 48.155 & & & & & & \begin{tabular}{|l|l|}
47.8 \\
\end{tabular} & & & & & & & & \\
\hline & $1 / 27 / 2004$ & $2: 48: 00 \mathrm{~F}$ & & & & & 21.26 & 48.341 & 48.764 & & & & & 44. & & & & & | & & & & & & & & & \\
\hline & $1 / 27 / 2004$ & 2:49:00 PM & & 19.486 & 24.291 & 23.394 & 21.02 & 48.661 & 49.083 & \begin{tabular}{|l|l|}
11.023 \\
\end{tabular} & 38.465 & \begin{tabular}{|c|}
-1.214 \\
\end{tabular} & & 45.8 & & 14.6 & & & \begin{tabular}{|l|l|}
1.4 \\
\end{tabular} & \begin{tabular}{|l|l|}
47.6 \\
\end{tabular} & 1.2 & & & & & & & \\
\hline & 1/27/2004 & 2:50:00 F & 23.918 & 19.487 & 24.296 & 23.314 & 20.59 & 48.594 & 48.944 & 11.036 & 38.444 & -1.214 & & 44.959 & 0.073 & 14.643 & & & 1.41667 | & 47.5 & 11.0 & 43.5 & 000 & . 011 & 0.011 & 0.000 & 0.255 & \\
\hline & & 2:51:00 PM & 23.956] & 19.504 & \begin{tabular}{|l|}
24.344 \\
\end{tabular} & 23.382 & 21.2 & 48.71 & 49.145 & 11.085 & & -1.216 & & 46.442 & & & & & 1.43333 & & 11.4 & & & & & & 0.254 & \\
\hline & $1 / 27 / 2004$ & 2:52:00 PM & 23.962 & 19.516 & 24.365 & 23.353 & & 48.712 & 49.224 & 11.081 & 38.449 & -1.216 & & 43.43 & 0.072 & 14.643 & & 87. & 1.45 & 47.8. & & & & & & & & \\
\hline & $1 / 2 / 2004$ & $2.53: 00 \mathrm{~F}$ & 2 & 19.537 & 2.406 & 2.329 & 21.56 & 48.754 & 49.185 & 11.088 & 38.4 & -1.216 & & $\begin{array}{l}43.43 \\
43\end{array}$ & & & & & .46667 & 47.7 & 101 & 12 & & & & & .254 & \\
\hline & & & & & & & & & & & & & & & & & & & & & & & & & & & & \\
\hline & 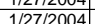 & $\frac{250.00 \mathrm{PM}}{2.56: 0}$ & 告, & 10.5051 & $\frac{2.4344}{2.240}$ & $\frac{2.5014}{23463}$ & & & 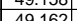 & 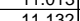 & & $\frac{-1.216}{-1216}$ & & (42.04 & & & & & $\frac{1.5}{1677}$ & $\frac{41 .}{47}$ & & 年 & & & & & 0.2010 & \\
\hline & $\frac{1}{1 / 27 / 2004}$ & $\frac{2: 57: 00}{2: 57}$ & 24.073 & 19.5977 & $\begin{array}{l}24.496 \\
2.496\end{array}$ & $\frac{23.449}{23.44}$ & & & 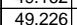 & 11.086 & $\begin{array}{r}38.403 \\
38.403 \\
\end{array}$ & $\frac{-1.216}{-1.216}$ & & $\frac{43.1}{43 .}$ & & & & & $\mid$ & 年.1. & 10.7 & 43. & & & & & 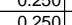 & \\
\hline & $1 / 27 / 2004$ & $2: 58: 0$ & 24.099 & 19.613 & \begin{tabular}{|l|}
24.522 \\
\end{tabular} & $\mid 23.435$ & 20.93 & & $\begin{array}{l}499.18 \\
\end{array}$ & 11.072 & & $\mid-1.219$ & & & & & & & $\mid 1.55$ & \begin{tabular}{ll|l}
47.7 \\
\end{tabular} & & $\frac{43}{43}$ & & & & & 0.251 & \\
\hline & $1 / 27 / 2004$ & 2:59:0 & 24.114 & 19.623 & 24.537 & 23.379 & 21.0 & 48.744 & 49.297 & 11.175 & 38.379 & -1.216 & & 48.6 & & & & & 1.56667 & 47.8 & & 43. & & & & & 250 & \\
\hline & & & & & & & & & & & & & & & & & & & & & & & & & & & & \\
\hline & $1 / 27 / 2004$ & $3: 0$ & 24.175 & 19.644 & \begin{tabular}{|l||}
24.578 \\
\end{tabular} & 23.4 & 21.29 & $\begin{array}{l}48.397 \\
\end{array}$ & 49.013 & \begin{tabular}{|l|l|}
10.919 \\
\end{tabular} & 38.268 & $\mid-1.219$ & & & & & & & & 47.5 & 10.9 & 43.3 & 198 & & & & 0.247 & \\
\hline & 1/27/2004 & & 24.191 & 19.65 & 24.589 & 23.436 & & & 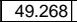 & 11. & & -1.21 & & & & & & & 61667 & 47. & & & & & & & & \\
\hline & $1 / 27 / 2004$ & :00 PM & 24.211 & 19.671 & 24.439 & 23.472 & & & 49.3 & & & & & & & & & & 63333 & & $\overline{12.0}$ & 43. & & & & & 48 & \\
\hline & 1/27/2 & & & 19.672 & 24.164 & 23.4998 & & & & 11.16 & & & & & & & & & & & & & & & & & & \\
\hline & $1 / 27 / 2004$ & & 24.198 & 19.693 & 24.236 & 23.534 & & & & 11.281 & 38.301 & & & & & & & & & & 15 & & & & & & 48 & \\
\hline & $1 / 27 / 2$ & & 24.184 & 19.699 & 24.282 & 23.53 & & 48.4 & 49.006 & 11.049 & & -1.219 & & & & & & & .68333 & 47. & 11.6 & & & & & & .246 & \\
\hline & & & 24.17 & & & & & & & 11.12 & & -1.2. & & & & & & & & & & & & & & & & \\
\hline & & & $2 \frac{2.1560}{2.369}$ & 19.137 & 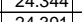 & | & & & 49.377 & & & & & & & & & & . & & 11.3 & & & & & & & \\
\hline & 1 & & 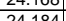 & 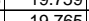 & $\mid$ & 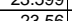 & & & & & & & & & & & & & & (1) & 110 & & & & & & 477 & \\
\hline & & & 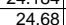 & & & 2575 & & & & & & & & & & & & & & & & & & & & & & \\
\hline & $1 / 27 / 20$ & & 24.84 & & & & & & & & & & & & & & & & & & & & & & & & & \\
\hline & $\frac{1 / 27 / 26}{1120}$ & & 24.901 & 19.796 & \begin{tabular}{|l|}
24.488 \\
\end{tabular} & 23.501 & & & & 11.191 & & & & & & & & & 18 & 47.8 & & & & & & & & \\
\hline & & & & & & & & & & 11.112 & & & & & & & & & & & & & & & & & & \\
\hline & & & 24.3 & & \begin{tabular}{|l|l|}
24.56 \\
\end{tabular} & 23.553 & & & & 11.1 & & & & & & & & & & & & & & & & & & \\
\hline & & & & & & & & & & 11.1 & & -1.2. & & & & & & & & & & & & & & & & \\
\hline & 1/27/2004 & & 24.478 & 19.865[ & 24.616 & 23.619 & $21.8 \mathrm{C}$ & & 49.409 & 11.28 & & -1.21 & & 47. & & & & & & 47. & 11.5 & & & & & & & \\
\hline & 1/27/20 & 3:18:C & 24.50 & 19.87 & $\mid 24.632$ & 23.671 & & & 49.249 & 11.14 & & -1.21 & & 45.1 & & 14.6 & & & 1.883 & 47. & & & & & & & & \\
\hline & 1/27/20 & 3:19:00 P & 24.54 & 19.88 & 24.6 & 23.681 & & 48.982 & 49.427 & 11.288 & 38.426 & -1.216 & & 48.244 & & 14.6 & & & .9 & 48. & 11.8 & & & & & & 8 & \\
\hline & 1/27/2 & & 4.561 & 19.897] & 24.674 & 23.6 & & & & 11.1 & & -1.21 & & & & & & & & 47.7 & & 43. & & & & & 8 & \\
\hline & $11 / 272$ & & 24.56 & 19.89 & 24.69 & & & & 49.294 & 11.243 & & -1.219 & & & & & & & & 4.8 & 11.7 & & & & & & 8 & \\
\hline & & & 24.58 & & 24.776 & 23.645 & & & & & & & & & & & & & & & & & & & & & & \\
\hline & 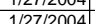 & & 2460 & 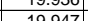 & 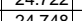 & 年 & & & | & 11.100 & | & - & & & & & & & $\mid$ & . & & & & & & & & \\
\hline & 1/172/2004 & & 24621 & 1905 & 2476 & 2370 & & 48789 & 49203 & $\frac{1.24}{1133}$ & 3829 & -1.219 & & 484 & & & & & & 477 & & 135 & & & & & & \\
\hline & $1 / 27 / 2004$ & 3.26 .00 & 24632 & 19.974 & 24.79 & 23739 & & 48.949 & 49386 & 11.459 & 3831 & -1216 & & 43568 & 0.068 & & & & & 479 & 107 & 36 & & & & & 0234 & \\
\hline & 004 & 3:27:00 PM & 4.644 & 19.996 & 4.807 & 23.71 & 2.24 & 48.822 & 49.29 & 11.311 & 38.352 & -1.219 & & 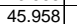 & & & & & & $47.8 \quad$ & & & & 010 & 010[ & 000 & 234 & \\
\hline & & & 4.665 & 20.002 & \begin{tabular}{|l|}
24.828 \\
\end{tabular} & 3.781 & & 48.92 & \begin{tabular}{|l|l|}
49.458 \\
\end{tabular} & \begin{tabular}{|l|l|}
11.427 \\
\end{tabular} & \begin{tabular}{|l|l|}
38.256 \\
\end{tabular} & -1.216 & & 44.029 & 0.068 & 14.643 & & & & 48.0 & 10.8 & 43.6 & & & 010 & 0.000 & 0.234 & \\
\hline
\end{tabular}


WSRC-TR-2005-00105, REVISION 0

SRNL-RPP-2005-00012, REVISION 0

RUN \# 1.03A; DEWATER FIRST HALF OF SLURRY - CONT.

\begin{tabular}{|c|c|c|c|c|c|c|c|c|c|c|c|c|c|c|c|c|c|c|c|c|c|c|c|c|c|c|c|c|}
\hline & & & & & & & & & & & & & & & & & & & & & & & & & & & & \\
\hline & \begin{tabular}{l|l} 
\\
\end{tabular} & B & $\mathrm{D}$ & $E$ & $F$ & G & $\mathrm{H}$ & $\mathrm{J}$ & $\mathrm{K}$ & $\mathrm{L}$ & $\mathrm{M}$ & $\begin{array}{ll} \\
\end{array}$ & 0 & $\mathrm{Q}$ & \begin{tabular}{l|l|l|l|}
$R$ & \\
\end{tabular} & $\mathrm{~s}$ & $\mathrm{~T}$ & $\mathrm{~V}$ & W & $x$ & $\mathrm{Y}$ & Z & $\mathrm{AA}$ & $A B$ & $\mathrm{AC}$ & $\mathrm{AD}$ & $\mathrm{AE}$ & \\
\hline 141 & $1 / 27 / 2004$ & $3: 28: 27$ PM & 24.661 & 20.013 & 24.814 & 23.797 & 22.137 & 48.567 & \begin{tabular}{|l|l|}
49.096 \\
\end{tabular} & $\begin{array}{l}11.177 \\
\end{array}$ & 38.219 & \begin{tabular}{|c|}
-1.219 \\
\end{tabular} & $\begin{array}{l}-7.386 \\
\end{array}$ & 45.468 & $\begin{array}{l}0.068 \\
\end{array}$ & 14.643 & 0.004 & 123.45 & 2.0575 & 47.6 & $\begin{array}{l}11.1 \\
\end{array}$ & & & 0.010 & 0.010 & & 0.235 & \\
\hline & & & & & 24.84 & 23.874 & 22.324 & & & & & & $\begin{array}{l}-7.389 \\
-7.399\end{array}$ & 45.766 & 0.067 & 14.643 & & & & 48.1 & & & & 0.010 & & & & \\
\hline & $1 / 27 / 2004$ & 3:30:27 PM & 24.674 & $\begin{array}{l}20.051 \\
\end{array}$ & \begin{tabular}{|l|l|}
24.521 \\
\end{tabular} & 23.911 & $\begin{array}{l}22.391 \\
\end{array}$ & 48.92 & \begin{tabular}{|l|l|}
49.425 \\
\end{tabular} & 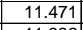 & 38.221 & \begin{tabular}{|l|l|}
-1.216 \\
\end{tabular} & $\begin{array}{l}-7.386 \\
-.7\end{array}$ & 47.936 & $\begin{array}{l}0.067 \\
\end{array}$ & \begin{tabular}{|l|l}
4.643 \\
\end{tabular} & & 125.45 & \begin{tabular}{l|l|l|}
5 & 2.09083 \\
\end{tabular} & 48.0 & \begin{tabular}{l|l|}
0 & 11.7 \\
\end{tabular} & $\begin{array}{l}43.6 \\
\end{array}$ & \begin{tabular}{|l|} 
\\
\end{tabular} & \begin{tabular}{|l|l|} 
\\
\end{tabular} & 0.010 & 0.000 & 0.232 & \\
\hline \begin{tabular}{|l|l|}
144 \\
145
\end{tabular} & $\begin{array}{l}1 / 27 / 2004 \\
1 / 27 / 2004\end{array}$ & 3:31:27 PM & $\begin{array}{r}24.65 \\
24.626\end{array}$ & $\begin{array}{r}20.063 \\
20.084 \\
\end{array}$ & \begin{tabular}{|l|}
24.462 \\
2.508
\end{tabular} & $\begin{array}{r}23.907 \\
23.073\end{array}$ & $\begin{array}{r}22.04 \\
21.986\end{array}$ & \begin{tabular}{|l|l|}
48.798 \\
48.761
\end{tabular} & \begin{tabular}{|l|}
49.295 \\
\end{tabular} & $\begin{array}{l}11.332 \\
11.327 \\
\end{array}$ & \begin{tabular}{|r|}
38.28 \\
38227 \\
\end{tabular} & \begin{tabular}{|r|}
-1.219 \\
\end{tabular} & $\begin{array}{r}-7.389 \\
73209\end{array}$ & 40.766 & \begin{tabular}{|c|c|}
0.067 \\
\end{tabular} & \begin{tabular}{|l|l|}
14.643 \\
1.643
\end{tabular} & & $\begin{array}{l}126.45 \\
127.45\end{array}$ & \begin{tabular}{r|r|r|}
5 & 2.1075 \\
\end{tabular} & \begin{tabular}{|l|l|}
47.8 \\
\end{tabular} & $\begin{array}{l}8 \quad 10.0 \\
8 \\
8\end{array}$ & $\begin{array}{l}43.5 \\
3.5\end{array}$ & $\mid$ & $\begin{array}{l} \\
\end{array}$ & 0.010 & 0.000 & $\begin{array}{l}0.233 \\
.200\end{array}$ & \\
\hline$\frac{140}{146}$ & $\begin{array}{l}1 / 272004 \\
1 / 27 / 2004\end{array}$ & $\begin{array}{l}3: 22: 27 \mathrm{PM} \\
3: 33: 27 \mathrm{PM}\end{array}$ & $\frac{24.626}{24.613}$ & & \begin{tabular}{|r|}
24.508 \\
24.57
\end{tabular} & $\begin{aligned} \frac{23.973}{23.98} \\
23\end{aligned}$ & $\begin{aligned} 21.986 \\
22.254\end{aligned}$ & \begin{tabular}{|l|}
48.762 \\
487799
\end{tabular} & \begin{tabular}{|r|}
49.224 \\
4927 \\
\end{tabular} & $\frac{11.327}{11.394} \mid$ & $38.227 \mid$ & $\begin{array}{l}-1.216 \\
-1.1214\end{array}$ & $\begin{array}{l}-7.389 \\
-7.381 \\
\end{array}$ & $\begin{array}{l}46.405 \\
45.272 \\
\end{array}$ & $\begin{array}{l}0.066 \\
0.066\end{array}$ & $\begin{array}{l}14.643 \\
14.643 \\
\end{array}$ & & $\begin{array}{l}127.45 \\
128.45\end{array}$ & \begin{tabular}{l|l|l|l}
5 & 2.12417 \\
5 & 2.14083
\end{tabular} & $\begin{array}{l}47.8 \\
47.8\end{array}$ & $\frac{11.4}{11.1}$ & $\begin{array}{l}43.5 \\
43.5\end{array}$ & \begin{tabular}{|l|}
2.999 \\
2.999 \\
\end{tabular} & \begin{tabular}{|l|l|} 
\\
0.010 \\
0.010
\end{tabular} & $\begin{array}{l}0.010 \\
0.001\end{array}$ & $\begin{array}{l}0.000 \\
0.000\end{array}$ & $\begin{array}{l}0.229 \\
0.229\end{array}$ & 0.20 \\
\hline & $1 / 27 / 2004$ & 3:34:27 PM & 24.583 & 20.112 & \begin{tabular}{|l|}
24.581 \\
\end{tabular} & $\begin{array}{r}23.98 \\
23.905 \\
\end{array}$ & $\frac{22.204}{22.285}$ & $\begin{array}{l}40.109 \\
48.978\end{array}$ & \begin{tabular}{|l|}
49.475 \\
49.45 \\
\end{tabular} & $\begin{array}{l}11.3456 \\
11.567\end{array}$ & \begin{tabular}{|l|}
38.231 \\
38.231
\end{tabular} & \begin{tabular}{|}
$\mid-1.1 .14$ \\
-1.216 \\
\end{tabular} & $\begin{array}{l}-7.301 \\
-7.386\end{array}$ & \begin{tabular}{|l}
46.272 \\
46.357
\end{tabular} & $\mid$ & $\begin{array}{l}\frac{14.643}{14.643} \\
\end{array}$ & & $\frac{128.45}{129.45}$ & $\begin{array}{rl}5 & 2.14083 \\
5 & 2.1575\end{array}$ & $\frac{4.8}{48.0}$ & $\frac{11.1}{11.4}$ & - 43.55 & \begin{tabular}{|l|l|}
3.999 \\
3.006 \\
\end{tabular} & \begin{tabular}{|l|l|} 
\\
\end{tabular} & 0 & $\begin{array}{c}0.000 \\
0.000\end{array}$ & $\begin{array}{l}0.229 \\
0.228\end{array}$ & $\frac{0.19}{0.19}$ \\
\hline 148 & $1 / 27 / 2004$ & 3:35:27 PM & 24.58 & 20.129 & \begin{tabular}{|l|}
24.618 \\
\end{tabular} & 23.933 & 22.347 & 48.891 & \begin{tabular}{|l|l|} 
\\
\end{tabular} & 11.349 & 38.315 & -1.216 & \begin{tabular}{|c|}
-7.381 \\
\end{tabular} & 45.093 & 0.066 & 14.643 & & 130.45 & \begin{tabular}{c|c|c|}
5 & 2.17417
\end{tabular} & 48.0 & 11.1 & 43.6 & 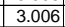 & \begin{tabular}{|l|l|} 
& 0.010 \\
\end{tabular} & 0.010 & 0.000 & 0.228 & \\
\hline & $1 / 27 / 2004$ & 3:36:27 PM & & 20.146 & \begin{tabular}{|r|}
24.66 \\
\end{tabular} & 23.944 & & & & \begin{tabular}{|l}
11.418 \\
\end{tabular} & & -1.216 & -7.384 & & 066 & & & & & & & & & & & & & \\
\hline 150 & $1 / 27 / 2004$ & 3:37:27 PM & 24.579 & & & 23.987 & & 48.571 & & \begin{tabular}{|l|l|l|l|}
11.329 \\
\end{tabular} & $\overline{143}$ & & & $\overline{029}$ & & $\overline{643}$ & & & 2.2075 & 477 & $\overline{11.3}$ & & & & $\overline{010}$ & & & \\
\hline 151 & & & & & & & & & \begin{tabular}{|l|}
49.096 \\
\end{tabular} & $\mid 11.214$ & 8.13 & & & & & & & & & 47.6 & $\frac{11.6}{11.6}$ & & & & & & & \\
\hline 152 & 1/27/2004 & 9:27 PM & 24.578 & 20.188 & 24.726 & 24.056 & & 48.878 & & 11.431 & & & & & & & & 134.45 & 2.24083 & 48.1 & 111.4 & & & 010 & & & & \\
\hline & $1 / 27 / 2004$ & 3:40:27 PM & 24.59 & 20.215 & \begin{tabular}{|l|}
24.758 \\
\end{tabular} & 24.073 & 22. & 48.866 & \begin{tabular}{|l|}
49.512 \\
\end{tabular} & 11.554 & 38.149 & -1.216 & & 433.643 & & 14.643 & & 135.45 & 2.2575 & 48.6 & & & & & & & 0.224 & \\
\hline 154 & 1/27//2004 & 3:1:1:27 PM & 24.608 & 20.227 & 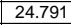 & 24.126 & 22.4 & 48.69 & \begin{tabular}{|l|l|l|l|l}
49.344 \\
\end{tabular} & 11.415 & 38.124 & $\mid-1.216$ & -7.395 & 46.513 & 0.065 & 14.643 & & 136.45 & \begin{tabular}{|l|} 
\\
\end{tabular} & 47.9 & 11.4 & 43.4 & 2.993 & . 010 & 0.010 & 0.000 & & \\
\hline 155 & $\frac{1 / 27 / 2004}{1 / 27 / 20}$ & 3:42:27 PM & 24.625 & 20.26 & 24.818 & 24.198 & 22.4 & 48.71 & 49.413 & 11.417 & 38.12 & -1.216 & & 46.624 & 0.065 & 14.643 & & 137.45 & & 47.9 & 11.4 & & 2.99 & & .010 & & & \\
\hline & $1 / 27 / 2004$ & 3:43:27 PM & & 20.282 & 24.86 & 24.16 & & 48.661 & 396 & 11.448 & 38.016 & & & 43.249 & - & 14.643 & & 138.45 & & 47.9 & 11 & & & & & & & \\
\hline & $\frac{7}{772004}$ & & & & & & & 48.48 & & 11.329 & 38.003 & & & 46.15 & & $\frac{14.643}{11.62}$ & & 139.4 & $\frac{32417}{342}$ & & 11. & & & & & & & \\
\hline & 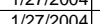 & & 24.051 & & 24.077 & 24.17 & & 48.821 & & $\begin{array}{ll}11.566 \\
11.176\end{array}$ & So.vor & -1.216 & & 44.2 & & & & 140.45 & & (48. & . & & & 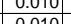 & & & 224 & \\
\hline & 1 & $\frac{3 \cdot 4.4 .27}{3 \cdot 27} \mathrm{PM}$ & 24.701 & 20.357 & & $\frac{24.253}{243}$ & $\frac{123}{223}$ & $\begin{array}{l}48.804 \\
8.804\end{array}$ & & $\frac{1.4545}{11.545}$ & $\begin{array}{l}3.0 .059 \\
38.059\end{array}$ & -1.216 & & $\begin{array}{l}43.81 \\
4463\end{array}$ & 0.005 & $\frac{14.643}{14.643}$ & & $\frac{141.45}{14245}$ & $\frac{2.3575}{23717}$ & 年 & 10.8 & & & 010 & 010 & & (. & \\
\hline 161 & $1 / 27 / 2004$ & 3:48:27 PM & 24.708 & 20.374 & \begin{tabular}{|l|}
24.931 \\
\end{tabular} & 24.277 & 22.27 & 48.536 & \begin{tabular}{|l|}
49.253 \\
\end{tabular} & 11.403 & 37.95 & -1.219 & \begin{tabular}{|c|}
-7.386 \\
\end{tabular} & 44.957 & 0.064 & 14.643 & & 143.45 & \begin{tabular}{l|l|l}
5 & 2.39083
\end{tabular} & 47.8 & 111.0 & 43.2 & 2.981 & \begin{tabular}{|l|l|}
0.010 \\
\end{tabular} & 0.010 & 0.000 & 0.221 & $\frac{4}{1}$ \\
\hline & $1 / 27 / 2004$ & 3:49:27 PM & 24.71 & 20.381 & \begin{tabular}{|l|}
24.939 \\
\end{tabular} & 24.314 & & 48.659 & \begin{tabular}{|l|l|}
49.479 \\
\end{tabular} & \begin{tabular}{|l|l|l}
11.444 \\
\end{tabular} & \begin{tabular}{|l|l|}
37.983 \\
\end{tabular} & -1.219 & & 45.748 & 0.064 & 14.643 & & 144.45 & \begin{tabular}{|l|l|l|}
5 & 2.4075 \\
\end{tabular} & 48.0 & 11.2 & & & & & & & 0 \\
\hline 163 & 1/27/2004 & $27 \mathrm{PM}$ & 24.737 & 20.403 & 24.961 & 24.371 & & 48.723 & 583 & 11.578 & 37.925 & -1.216 & & 45.881 & 0.064 & 14.643 & & 145.45 & & 48. & 11.2 & 43.3 & 2.9 & & 0.010 & & & \\
\hline 164 & $1 / 2 / 72004$ & $27 \mathrm{PM}$ & 24. 159 & 20.43 & 24.992 & 24.433 & & 48.731 & 587 & 11.546 & 37.97 & & & 45.852 & & 14.643 & & & 444083 & & & & & & 0.010 & 0.000 & 0.220 & \\
\hline & 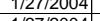 & $2: 277 \mathrm{PM}$ & & 20.432 & 24.984 & 24.424 & & 48.337 & & 11.336 & 37.816 & -1.216 & & $47.24 t$ & & & & $14 . .45$ & 2.4575 & & & & & & & & & \\
\hline & 1125170404 & 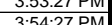 & & & & & & & & & & & & 47.116 & & & & & & & & & & & & & & \\
\hline$\frac{10 /}{168}$ & $\begin{array}{ll}1 / 27 / 2004 \\
1 / 27 / 200\end{array}$ & $3: 55: 27 \mathrm{PM}$ & 24.801 & $\frac{20.450}{20.488}$ & \begin{tabular}{|l}
24.140 \\
\end{tabular} & 24.0995 & $\frac{22.00}{22.49}$ & $\begin{aligned} \frac{40.010}{48.81} \\
\end{aligned}$ & \begin{tabular}{|r|}
49.1200 \\
49.75
\end{tabular} & $\begin{array}{l}\frac{11.004}{11.642} \\
\end{array}$ & $\begin{array}{c}31.012 \\
37.86\end{array}$ & -1.219 & $\begin{array}{l}-7.004 \\
-7.381\end{array}$ & 46.559 & $\begin{array}{l}.005 \\
0.063\end{array}$ & $\begin{array}{l}\frac{14.045}{14.643} \\
\end{array}$ & & $\begin{array}{l}1450.45 \\
150.5\end{array}$ & 25075 & $\frac{4.5}{483}$ & 10.4 & 433 & 2988 & - & . & 年 0.000 & 0218 & \\
\hline & $\frac{1 / 27 / 2004}{1}$ & 3:56:27 PM & 24.788 & 20.485 & \begin{tabular}{|l|}
24.816 \\
\end{tabular} & 24.547 & & $\begin{array}{r}48.742 \\
48.742\end{array}$ & 49.724 & 11.046 & 37.759 & $\mid-1.219$ & -7.384 & 46.962 & 0.063 & 14.643 & & 151.45 & 252417 & 483 & 115 & & & 009 & 0.009 & 0.000 & 0.218 & \\
\hline & $\frac{1 / 27 / 2004}{4}$ & 3:57:27 PM & 24.815 & 20.527 & 24.898 & $\frac{14.604}{24.604}$ & & 48.71 & 49.622 & 11.636 & 37.796 & $\mid-1.214$ & & 45.349 & & 14 & & & & 48. & & & & & & & & \\
\hline \begin{tabular}{|l|l|}
171 \\
\end{tabular} & $1 / 27 / 2004$ & & 24.802 & 20.529 & 24.89 & 24.501 & & 48.542 & & 11.571 & & -1.216 & & 48.307 & & & & 153.45 & 2.5575 & 48. & 11.8 & 43.2 & & & 0.009 & 0.000 & 0.218 & \\
\hline 172 & & & 24.819 & & & & & 48.4 & & 11.535 & 37.794 & $\frac{1.216}{-1.216}$ & & 45.197 & & & & & & 47. & & $\frac{43}{43}$ & & & & & & \\
\hline & & & & 20.558 & & 24.444 & & 46.392 & & $\begin{array}{l}11.662 \\
\end{array}$ & 35.487 & & & 43.474 & & & & 155.45 & 2.59083 & 48.2 & 10. & & & & & & & \\
\hline$[1 / 4$ & $1 / 27 / 2004$ & & & & & 24.476 & & \begin{tabular}{|l|l|}
40.641 \\
\end{tabular} & & \begin{tabular}{|l|l|l|l|l|}
11 \\
\end{tabular} & & $\begin{array}{l}3.827 \\
\end{array}$ & & 43 & & & & 156 & & & & & & & & & 0.013 & \\
\hline & 1/27/2004 & & 25.254 & 20.597 & \begin{tabular}{|l|}
24.977 \\
\end{tabular} & 24.523 & & 29.643 & & 11.58 & 18.575 & 14.741 & & 45.666 & & & & 157. & 2.62417 & 48 & 11. & 24. & & & 000 & & & \\
\hline & $1 / 27 / 2004$ & & 24.56 & & 24.994 & 24.615 & & \begin{tabular}{|l|l|l|l|}
6.766 \\
\end{tabular} & 946 & 8.197 & & 37.111 & & & & & & & & 47 & & & & & & & .162 & \\
\hline 17 & 1/27/2004 & 4:04:27 PM & 25.293 & 20.626 & 25.037 & 24.648 & & 43.364 & \begin{tabular}{|l|}
49.249 \\
\end{tabular} & 10.321 & 33.743 & 0.393 & 0.344 & 46.511 & 0.16 & & & 159 & & 47.8 & 11. & $3 \varepsilon$ & & & 0.024 & 0.001 & D.618 & \\
\hline 5 & 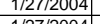 & 4: & 25.401 & 20.638 & 50.019 & 24.604 & & 43.971 & 49.804 & 11.692 & 年 & 0.324 & & 50 & 0.104 & & & 119 & 2.67. & 40. & 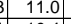 & - & & & & & $0.40 \mathrm{~s}$ & \\
\hline & & & & & & & & & & & & & & & & & & & & & & & & & & & & \\
\hline & 112170004 & & 25.499 & 20.652 & \begin{tabular}{|l|l|} 
\\
\end{tabular} & $\begin{array}{l}24.60 \\
2.59\end{array}$ & & & & 12.511 & $\frac{2.1}{310}$ & & & 46.135 & & & & & & 48.4 & & & & & & & & \\
\hline 18 & $1 / 12712004$ & & $\frac{25.355}{25547}$ & 20 & \begin{tabular}{|l|l|}
2505 \\
\end{tabular} & & & & & 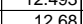 & & 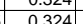 & & 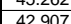 & & & & & & & .11. & 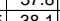 & & & & & 0.4 & \\
\hline & $1 / 27 / 2004$ & & & & 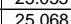 & & & & & 1288 & & & & & & & & & & & 10 & & & & & & & \\
\hline 184 & $1 / 27 / 2004$ & & 25.556 & $\begin{array}{l}20.74 \\
\end{array}$ & \begin{tabular}{|l|}
24.859 \\
\end{tabular} & 24.776 & & 44.2 & & 12.892 & 32.05 & | & & 42.4 & & & & 166 & 2.77 & 48.6 & 10. & & & & & & 0.263 & \\
\hline & & & & & & & & & & & & & & & & & & & & & & & & & & & & \\
\hline & & & 25.051 & & 24934 & & & & & $\begin{array}{l}13.198 \\
13.98\end{array}$ & & & & 424 & & & & & & & $=$ & & & & & & 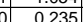 & \\
\hline & & & 25.128 & 20.807 & & & & & & $\begin{array}{l}13.078 \\
\end{array}$ & & & & 42 & & & & & & & 10. & & & & & & & \\
\hline & 1/27/2004 & & & 20.81 & & 24. & & 49 & & 12.905 & & & & & & & & 17( & & & & & & & & & & \\
\hline & $1 / 27 / 2004$ & $4: 1$ & 25.133 & 20.827 & & 24.8 & & 50.2 & & $\begin{array}{l}33.151 \\
\end{array}$ & 37.568 & -1.219 & & 46.7 & & & & 171 & & & 11. & & & & & & $\begin{array}{ll}0.234 \\
\end{array}$ & \\
\hline & $1 / 27 / 2004$ & & 25.14 & & \begin{tabular}{|l|l|}
25.048 \\
\end{tabular} & & & 49.957 & & & & & & & & & & & & & & & & & & & & \\
\hline 191 & 1/27/2004 & $4: 1$ & 25.137 & & \begin{tabular}{|l|l|} 
& 25.045 \\
\end{tabular} & 24.88 & & 50.139 & & 13.121 & 37.665 & -1.222 & & 44. & & & & 17 & 2.89 & 48 & 11.0 & 43. & & & & & 0.224 & \\
\hline & & & 25.164 & & \begin{tabular}{|l}
25.102 \\
\end{tabular} & & & & & & & & & & & & & & & & 11 & & & & & & & \\
\hline & $1 / 27 / 72004$ & $4: 2$ & 25.166 & 20.891 & \begin{tabular}{|l|} 
\\
\end{tabular} 5.109 & 24.8 & & 50.1 & 50.039 & 13.075 & 37 & -1.2 & & 42. & & & & & & & & & & & & & & \\
\hline & 112717004 & $4: 2$ & $\frac{2.1172}{25.89}$ & & 2551140 & 24.1864 & & & & $\frac{12.936}{12749}$ & & & & & & & & & & & $\frac{10.8}{10.8}$ & & & & & & 0.218 & \\
\hline 196 & $1 / 12712004$ & 告. & $\frac{2.1905}{252}$ & $\frac{20.924}{20946}$ & \begin{tabular}{|l}
2.1 .144 \\
25.59
\end{tabular} & & & (4) & & | & & $\frac{-1.29}{-1216}$ & & & & & & & & & $\frac{11 .}{11 .}$ & & & & & & & \\
\hline 197 & $1 / 27 / 2004$ & & $\frac{20.4}{25.221}$ & & 25.05 & & & 50.0221 & & 1307 & & & & & & & & & & & 11. & & & & & & & \\
\hline & $1 / 27 / 2004$ & & $\frac{25.23}{25.23}$ & 20 & \begin{tabular}{|l}
25.207 \\
\end{tabular} & 25.0 & & 50.1 & & 12.99 & & $\frac{1.216}{-1.216}$ & & $\frac{4.43}{44.3}$ & & & & 180 & & & 10. & & & & & & $\frac{.2 .213}{0.213}$ & \\
\hline & & & & & & & & & & & & & & & & & & & & & & & & & & & & \\
\hline & & & 25.24 & & 25.059 & & & 50.139 & & 13.17 & & -1.2 & & & & & & & & & & & & & & & & \\
\hline & & & & 21.019 & & & & \begin{tabular}{|l|l|}
50.32 \\
\end{tabular} & & & & & & & & & & & & & & & & & & & & \\
\hline & & & 25 & 21.021 & 25.113 & 24 & & 50.02 & 50 & 12.939 & & -1.219 & & 42 & & 14 & & & & & & & & & & & & \\
\hline 20 & $1 / 27 / 2004$ & & 25.25 & 21.02 & 25.135 & 24.956 & & $\begin{array}{l}49.7 \\
\end{array}$ & \begin{tabular}{|l|l|} 
& 49.6 \\
\end{tabular} & 12.86 & 37.745 & -1.219 & & 44. & & 14. & & 185 & & & & & & & & & 11 & \\
\hline & $1 / 27 / 2004$ & $4: 31: 27 \mathrm{~F}$ & 25.269 & 21.6 & \begin{tabular}{|l|l|}
25.173 \\
\end{tabular} & 25.074 & & \begin{tabular}{|l|l|}
49.92 \\
\end{tabular} & & \begin{tabular}{l|l|}
12.9 \\
\end{tabular} & 37.65 & -1.219 & & & & & & & & 48 & 11. & & & & & & & \\
\hline & $1 / 27 / 2004$ & & 25.256 & 21.04 & 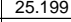 & & & 50. & \begin{tabular}{|l|l|l|} 
\\
\end{tabular} & 13.093 & & -1.219 & & 42.5 & & & & & & 48 & & & & & & & & \\
\hline & & & 25.248 & 21.04 & & 25.12 & & & & & & & & & & & & & & & 11.1 & & & & & & & \\
\hline & & & 25.26 & 21.066 & 25.239 & 25.11 & & 49.992 & 50.124 & 13.01 & 37.663 & -1.219 & & 44.2 & & & & & & 48.7 & $10.8 \mathrm{Z}$ & & & & & & & \\
\hline & & 4:35:27 PM & 25.251 & $21.0 / 3$ & \begin{tabular}{|l|}
25.245 \\
252
\end{tabular} & $24.9 / 6$ & 23. & $49.1 / 19$ & \begin{tabular}{|l|l|} 
\\
\end{tabular} & \begin{tabular}{|l|l|} 
\\
\end{tabular} & 37.685 & $\mid-1.219$ & & $43.1 / 4$ & & $\frac{14.643}{14}$ & & 190 & 3.114 & 48.4 & 10 & 4 & & & & & 0.200 & \\
\hline$\frac{209}{210}$ & $\begin{array}{l}11 / 272004 \\
1 / 27 / 2004\end{array}$ & 4:37.27 PM & \begin{tabular}{|l|}
25.254 \\
25.255
\end{tabular} & $\begin{array}{r}21.080 \\
21.087\end{array}$ & \begin{tabular}{|l|}
25.252 \\
\end{tabular} & \begin{tabular}{|r|}
25.024 \\
25.06
\end{tabular} & 23.396 & $\begin{array}{l}3.0406 \\
49.967 \\
\end{array}$ & \begin{tabular}{|l|l|}
50.211 \\
50.132 \\
\end{tabular} & \begin{tabular}{|l|}
12.0603 \\
12.963
\end{tabular} & \begin{tabular}{|r|}
37.69 \\
\end{tabular} & $\mid$\begin{tabular}{|c|}
$\mid-1.219$ \\
$\mid$
\end{tabular} & \begin{tabular}{|r|}
$\mid-7.398$ \\
\end{tabular} & \begin{tabular}{|l|l|}
4.674 \\
4.675
\end{tabular} & \begin{tabular}{|c|}
0.001 \\
0.061
\end{tabular} & \begin{tabular}{|l|l|}
14.643 \\
\end{tabular} & & 192.45 & \begin{tabular}{|l|l|}
5 & 3.10083 \\
\end{tabular} & \begin{tabular}{|l|}
48.7 \\
48.7
\end{tabular} & \begin{tabular}{|l|}
11.1 \\
10.9
\end{tabular} & \begin{tabular}{|l|l|}
43.8. \\
43
\end{tabular} & \begin{tabular}{|l|l|} 
\\
\end{tabular} .022 & \begin{tabular}{|l|}
0.0099 \\
0.009
\end{tabular} & \begin{tabular}{|c|}
.009 \\
0.009
\end{tabular} & $\begin{array}{l}0.000 \\
0.000\end{array}$ & \begin{tabular}{c|c} 
& 0.200 \\
& 0.206
\end{tabular} & $\begin{array}{l}0.1 \\
6 \\
\end{array}$ \\
\hline
\end{tabular}


WSRC-TR-2005-00105, REVISION 0

SRNL-RPP-2005-00012, REVISION 0

RUN \# 1.03A; DEWATER FIRST HALF OF SLURRY - CONT.

\begin{tabular}{|c|c|c|c|c|c|c|c|c|c|c|c|c|c|c|c|c|c|c|c|c|c|c|c|c|c|c|c|c|}
\hline \\
\hline & A & & D & $E$ & $\mathrm{~F}$ & $G$ & $\mathrm{H}$ & $\mathrm{J}$ & $\mathrm{K}$ & $\mathrm{L}$ & $\begin{array}{ll} \\
\end{array}$ & $\mathrm{N}$ & 0 & Q & \begin{tabular}{l|l|}
$\mathrm{R}$ \\
\end{tabular} & $\mathrm{s}$ & $\mathrm{T}$ & $\mathrm{v}$ & w & $x$ & $Y$ & $z$ & AA & $A B$ & AC & $\mathrm{AD}$ & AE & $\mathrm{AF} \quad \mathrm{A}$ \\
\hline & 1/27/2004 & 4:38:27 PM & $\begin{array}{l}25.277 \\
2.270\end{array}$ & $\begin{array}{l}21.114 \\
21.12\end{array}$ & 25.301 & 25.103 & 23.558 & 50.083 & 50.31 & \begin{tabular}{ll|}
13.092 \\
\end{tabular} & \begin{tabular}{|l|}
37.626 \\
3720
\end{tabular} & \begin{tabular}{|l|l|}
-1.219 \\
\end{tabular} & \begin{tabular}{l|r}
9 & -7.398 \\
\end{tabular} & $\begin{array}{l}45.754 \\
\end{array}$ & 0.061 & \begin{tabular}{|r|}
14.643 \\
\end{tabular} & 0.004 & 193.45 & 3.22417 & 48.8 & & 43.9 & 3.024 & 0.009 & 0.009 & 0.000 & 0.206 & \\
\hline & & 4:39:27 PM & $\begin{array}{r}25.278 \\
2.28\end{array}$ & & \begin{tabular}{|l|}
25.317 \\
\end{tabular} & $\begin{array}{r}25.064 \\
\end{array}$ & & & & & & \begin{tabular}{|l|}
-1.219 \\
\end{tabular} & & & 0.06 & $\begin{array}{l}14.643 \\
\end{array}$ & & $\begin{array}{l}194.45 \\
\end{array}$ & & 48.7 & & & & & & & & \\
\hline & $1 / 27 / 2004$ & 4:40:27 PM & $\begin{array}{l}25.295 \\
25207\end{array}$ & $\begin{array}{l}21.132 \\
2.121\end{array}$ & \begin{tabular}{|l|}
25.339 \\
\end{tabular} & 25.12 & 23.365 & $\begin{array}{l}49.552 \\
\end{array}$ & \begin{tabular}{|l|l|}
49.869 \\
\end{tabular} & \begin{tabular}{|l|}
12.692 \\
\end{tabular} & \begin{tabular}{|l|l|}
37.541 \\
3756
\end{tabular} & \begin{tabular}{|l|l|}
-1.219 \\
\end{tabular} & \begin{tabular}{|l|l}
-7.395 \\
7.705
\end{tabular} & $\begin{array}{l}45.587 \\
\end{array}$ & 0.06 & \begin{tabular}{|l|l|}
14.643 \\
\end{tabular} & & $\begin{array}{l}195.45 \\
\end{array}$ & 3.2575 & $\begin{array}{ll}48.4 \\
\end{array}$ & & & .002 & 0.009 & $\begin{array}{l}0.009 \\
\end{array}$ & 0.000 & 0.203 & \\
\hline & $\begin{array}{l}1 / 27 / 2004 \\
1 / 272004\end{array}$ & 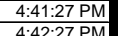 & $\begin{array}{l}25.287 \\
25.298\end{array}$ & $\begin{array}{r}21.134 \\
21.14\end{array}$ & \begin{tabular}{|r|}
25.33 \\
\end{tabular} & $\begin{array}{l}25.162 \\
25.174\end{array}$ & $\begin{array}{l}23.402 \\
23.378\end{array}$ & \begin{tabular}{|l|l|l|l|}
49.949 \\
49.959
\end{tabular} & \begin{tabular}{|l|}
50.206 \\
50.238 \\
\end{tabular} & \begin{tabular}{|r|}
13.022 \\
13
\end{tabular} & \begin{tabular}{|l|}
37.576 \\
37599
\end{tabular} & \begin{tabular}{|l|l|}
-1.222 \\
-1219 \\
\end{tabular} & $\begin{array}{r}-7.395 \\
-7935 \\
\end{array}$ & \begin{tabular}{|l|l|}
40.716 \\
45089
\end{tabular} & $\begin{array}{l}0.06 \\
\end{array}$ & \begin{tabular}{|l|l|}
14.643 \\
14632
\end{tabular} & 0.0 & \begin{tabular}{|l|l|}
196.45 \\
190.45
\end{tabular} & \begin{tabular}{|l|}
3.27417 \\
320093 \\
\end{tabular} & $\begin{array}{l}48.7 \\
8.9\end{array}$ & 10.0 & 438 & $\mid$ & \begin{tabular}{|l|l|}
0.009 \\
\end{tabular} & $\begin{array}{l}0.009 \\
\end{array}$ & 0.000 & 0.203 & 0.17 \\
\hline & $1 / 27 / 2004$ & & & & & $\begin{array}{r}25.174 \\
25.24\end{array}$ & & & \begin{tabular}{|l|}
50.238 \\
50.057 \\
\end{tabular} & & & & & $\begin{array}{l}45.989 \\
44.327\end{array}$ & 0.06 & \begin{tabular}{|c|}
14.643 \\
14.643
\end{tabular} & & $\begin{array}{r}197.45 \\
198.45\end{array}$ & \begin{tabular}{|c|}
3.29083 \\
3.3075 \\
\end{tabular} & $\begin{array}{l}48.8 \\
48.6 \\
\end{array}$ & \begin{tabular}{|l|l|}
11.3 \\
10.9
\end{tabular} & $\begin{array}{l}43.8 \\
43.7 \\
\end{array}$ & \begin{tabular}{|l|}
3.018 \\
3.014 \\
\end{tabular} & \begin{tabular}{|l|l|}
0.009 \\
0.009
\end{tabular} & $\begin{array}{l}0.009 \\
0.009\end{array}$ & 0.000 & 0.202 & 0.17 \\
\hline 217 & $1 / 27 / 2004$ & 4:44:27 PM & 25.32 & 21.163 & \begin{tabular}{|l|}
25.014 \\
\end{tabular} & 25.226 & 23.751 & 49.976 & \begin{tabular}{|l|}
50.264 \\
\end{tabular} & 13.042 & 37.566 & \begin{tabular}{|l|}
-1.222 \\
\end{tabular} & $\begin{array}{r}-7.503 \\
-7.395\end{array}$ & $\begin{array}{l}43.806 \\
\end{array}$ & $\begin{array}{l}0.06 \\
0.06\end{array}$ & $\begin{array}{l}14.643 \\
14.643 \\
\end{array}$ & & $\begin{array}{l}198.45 \\
199.45\end{array}$ & \begin{tabular}{|l|}
3.3015 \\
3.32417 \\
\end{tabular} & $\frac{48.6}{48.8}$ & \begin{tabular}{|l|l|}
10.9 \\
10.7
\end{tabular} & $\frac{43.1}{43.8}$ & $\begin{array}{l}3.014 \\
3.018 \\
\end{array}$ & \begin{tabular}{|l|l|}
0.009 \\
0.009
\end{tabular} & \begin{tabular}{|c|}
0.009 \\
0.009
\end{tabular} & $\begin{array}{l}0.000 \\
0.000\end{array}$ & & $\frac{0.17}{0.17}$ \\
\hline 218 & $1 / 27 / 2004$ & & 25.331 & 21.174 & 25.085 & 25.207 & 23.632 & 49.768 & 50.082 & $\begin{array}{l}12.898 \\
\end{array}$ & 37.533 & \begin{tabular}{|l|}
-1.222 \\
\end{tabular} & & \begin{tabular}{|l|}
43.983 \\
\end{tabular} & 0.059 & 14.643 & & 200.45 & \begin{tabular}{|l|l|}
3.34083 \\
\end{tabular} & 48.6 & \begin{tabular}{|l|l} 
\\
\end{tabular} & 43.7 & 3.010 & 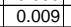 & 0.009 & 0.000 & 0.201 & \\
\hline 215 & & 4:46:27 PM & 25.343 & 21.201 & & 25.289 & 23.178 & & & & \begin{tabular}{|l|}
37.58 \\
\end{tabular} & \begin{tabular}{|l|}
-1.216 \\
\end{tabular} & & \begin{tabular}{|l|l|}
44.496 \\
\end{tabular} & & & & & $\begin{array}{l}3.3575 \\
\end{array}$ & 48.7 & & & & & & & & \\
\hline & & & 25.349 & $\begin{array}{l}21.217 \\
\end{array}$ & 25.163 & \begin{tabular}{|l|l|}
25.27 \\
\end{tabular} & 23.464 & \begin{tabular}{|l|l}
49.967 \\
\end{tabular} & 50.294 & & & & & & \begin{tabular}{|l|l|l|}
0.0599 \\
\end{tabular} & 14.643 & & & & & & & & & & & 200 & \\
\hline - & & & 25.35 & 21.223 & 25.199 & 25.241 & & & & $\begin{array}{ll}12.982 \\
\end{array}$ & 37.545 & -1.222 & & & & & & & & 48. & & & & & & & & \\
\hline & $1 / 27 / 2004$ & $27 \mathrm{PM}$ & 25.367 & 21.255 & 25.226 & 25223 & 23.593 & 866 & .267 & 13.044 & 37.404 & -1.216 & & & 0.059 & & & & 3.4075 & 48. & & & 008 & 009 & & & & \\
\hline & & 0:27 PM & 25.363 & 21.251 & 25.237 & 25.264 & & \begin{tabular}{|l|l|l|}
493 \\
\end{tabular} & 50.095 & $\mid$\begin{tabular}{|c|}
13.094 \\
\end{tabular} & 37.494 & -1.216 & & $\begin{array}{l}45.647 \\
\end{array}$ & 0 & $\begin{array}{l}14.643 \\
\end{array}$ & & & $\begin{array}{l}3.42417 \\
\end{array}$ & 48.6 & & & & 0.009 & $\mid 0.009$ & & & \\
\hline & $1 / 27 / 2004$ & 51:27 PM & 25.375 & 21.273 & 25.264 & 25.311 & 23.125 & $\begin{array}{l}49.926 \\
\end{array}$ & 50.32 & $\begin{array}{l}3.179 \\
\end{array}$ & 37.443 & 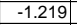 & -7.3 & 42.811 & 0.059 & 14.643 & & 206.45 & \begin{tabular}{|l|l|}
3.44083 \\
\end{tabular} & 488.8 & 10.5 & 43. & & 0.009 & \begin{tabular}{|c|c|} 
\\
\end{tabular} & & 0.200 & \\
\hline & $1 / 27 / 2004$ & 2:27 PM & $\begin{array}{l}25.387 \\
25.309\end{array}$ & $\begin{array}{l}21.285 \\
21.211\end{array}$ & 25.281 & $\begin{array}{l}25.302 \\
25202\end{array}$ & 23.066 & 49.959 & 50.3 & \begin{tabular}{|l|l|}
13.158 \\
1.90
\end{tabular} & 37.519 & & & & $\begin{array}{l}0.059 \\
\end{array}$ & $\begin{array}{l}14.643 \\
1.62\end{array}$ & & & & & & & & & & & & \\
\hline & $\begin{array}{l}1 / 2727204 \\
1 / 27 / 2004\end{array}$ & 53:27 PM & $\begin{array}{l}25.408 \\
25.398\end{array}$ & $\frac{21.511}{21.302}$ & $\frac{25.332}{25332}$ & $\frac{25.313}{25304}$ & 23.679 & 49.525 & $\begin{array}{l}49.896 \\
50.45\end{array}$ & $\begin{array}{r}12.849 \\
1309\end{array}$ & 37.443 & \begin{tabular}{|r|}
$\mid-1.216$ \\
\end{tabular} & & $\begin{array}{l}44.723 \\
6.510\end{array}$ & 0.059 & 14.643 & & & .47417 & & & & & .009 & & & & \\
\hline & 1 & $\begin{array}{l}55.27 \mathrm{PM} \\
55.27 \mathrm{PM}\end{array}$ & & & & & & & $\frac{50.453}{49711}$ & 12754 & 37408 & & & 告65.549 & & $\frac{14.643}{14643}$ & & 209.45 & 3.49083 & & & & & 0.009 & 0.009 & & & \\
\hline & (1/2004 & $27 \mathrm{PM}$ & $\frac{25.410}{25416}$ & 21319 & 25354 & 25291 & & 告5 & 5024 & 1302 & $\begin{array}{l}37.400 \\
37.349 \\
\end{array}$ & \begin{tabular}{|c|}
$\mid-1.210$ \\
-1.216 \\
\end{tabular} & & \begin{tabular}{|l|}
46.096 \\
44534 \\
\end{tabular} & $\begin{array}{l}0.059 \\
0.059\end{array}$ & $\frac{14.643}{14643}$ & & 210.45 & & & & & & & & & 201 & \\
\hline & $1 / 127 / 2004$ & $\begin{array}{l}\text { 4:56:27 PMI } \\
\text { 4:57:27 }\end{array}$ & 25.427 & 21.33 & $|25.371|$ & 25.292 & $\frac{23.51}{23.53}$ & 49.841 & \begin{tabular}{|l|}
50.42 \\
50.418 \\
\end{tabular} & \begin{tabular}{|c|}
13.02064 \\
13.06
\end{tabular} & 37.373 & $|-1.219|$ & & $\begin{array}{r}44.034 \\
48.334 \\
\end{array}$ & 0.058 & $\begin{array}{l}\frac{14.643}{14.643} \\
\end{array}$ & & $\begin{array}{l}211.45 \\
212.45\end{array}$ & \begin{tabular}{|l|}
3.52417 \\
3.54083 \\
\end{tabular} & $\begin{array}{l}48.8 \\
48.9 \\
\end{array}$ & & & $\begin{array}{l}.002 \\
007\end{array}$ & $\frac{0.009}{0.009}$ & $\begin{array}{l}0.009 \\
0.009\end{array}$ & $\frac{0.000}{0.000}$ & 200 & \\
\hline 231 & 1/27/2004 & 8:27 PM & 25.438 & 21.352 & \begin{tabular}{|l|}
25.387 \\
\end{tabular} & 25.294 & 23.559 & 49.845 & \begin{tabular}{|l|}
50.347 \\
\end{tabular} & \begin{tabular}{|l|}
13.117 \\
\end{tabular} & 37.396 & $|-1.219|$ & -7.35 & $\begin{array}{l}46.957 \\
\end{array}$ & $\mid$\begin{tabular}{|c|}
0.058 \\
\end{tabular} & \begin{tabular}{|l|l|}
14.643 \\
\end{tabular} & & $\mid 213.45$ & \begin{tabular}{|l|}
3.5575 \\
\end{tabular} & $\mid 48.9$ & 11.5 & 43.6 & $\frac{0.007}{3.007}$ & 0.009 & | 0.009 & 0.000 & 0.196 & \\
\hline & $1 / 27 / 2$ & 4:59:27 PM & 25.439 & 21.353 & $\begin{array}{l}25.393 \\
\end{array}$ & 25.224 & 23.4 & 499.525 & 50.455 & $\begin{array}{l}13.002 \\
\end{array}$ & 37.08 & -1.219 & & $\begin{array}{l}46.309 \\
\end{array}$ & 0.059 & $\begin{array}{ll}14.643 \\
\end{array}$ & & 214.45 & \begin{tabular}{|l|l|l|l}
3.7747 \\
\end{tabular} & 49.0 & 11.3 & 43.3 & 2.986 & 0.009 & $\mid 0.009$ & & 0.201 & \\
\hline & $1 / 27 / 2 / 2$ & $00: 27$ PM & 25.445 & 21.369 & 25.424 & 25.26 & 23. & 49.899 & 50.42 & $\begin{array}{l}3.122 \\
\end{array}$ & 37.381 & -1.214 & & 44.694 & 0.059 & 14.643 & & 215.45 & \begin{tabular}{|l|l}
3.59083 \\
\end{tabular} & 48.9 & 11. & 43. & & & & & & \\
\hline & $1 / 27 / 2004$ & & 25.45 & 21.374 & 25.424 & 25.306 & 23.8 & 49.793 & 50.306 & & & & & 44.483 & & 14.643 & & & 3.6075 & & & & & . 0009 & 0.009 & 0.000 & 0.199 & \\
\hline & & & & & & & & & & & & & & & & 14.643 & & & & 48 & & & & & & & 194 & \\
\hline & $1 / 27 / 2004$ & $17 \mathrm{PM}$ & 25.461 & & 24.99 & 25.342 & & 49.588 & 50.037 & 13.064 & 37.244 & & & 44.4 & 0.057 & 14.643 & & 218.45 & 3.64083 & 48.6 & & & & & & & 196 & \\
\hline & & & & & & & & & & & & -1.219 & & & $0.05 t$ & 14. & & & & 48. & & & & & & & & \\
\hline & 1/27/2004 & 5:27 PM & 25.463 & 21.417 & 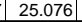 & 25.464 & 23.528 & 49.994 & 50.59 & 13.26 & 37.34 & $\begin{array}{l}-1.222 \\
\end{array}$ & & 46.632 & 0.057 & $\begin{array}{l}14.643 \\
\end{array}$ & & 220.45 & \begin{tabular}{|l|}
3.67417 \\
\end{tabular} & 49.1 & 11.4 & 43.7 & 3.011 & 0.008 & $\mid 0.008$ & 0.000 & 0.194 & \\
\hline & $1 / 27 / 2$ & 6:27 PM & 25.464 & 21.438 & 25.118 & 25.45 & 23.502 & 49.362 & 50.376 & 13.148 & 36.8911 & \begin{tabular}{|l|l|} 
& -1.219 \\
\end{tabular} & & 43.1 & 0.062 & 14.643 & & 221.45 & 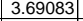 & 48.9 & & & .973 & & 0.009 & 0.000 & 0.214 & \\
\hline 24 & $1 / 27 / 2004$ & 77:27 PM & $\frac{25.45}{25}$ & 21.449 & 25.134 & 25.351 & & 49.521 & 50.182 & 12.924 & 37.262 & -1.22 & & 44.2. & & 14.643 & & 222.45 & \begin{tabular}{|l|}
3.7075 \\
\end{tabular} & 48.7 & 10. & & & & & 0.000 & 0.202 & \\
\hline & $1 / 27 / 2004$ & & & & & & & & 50.592 & & & & & & & & & & & 49.1. & & & & & & & & \\
\hline & 121727004 & (7) & 25.440 & 2.401 & 25.170 & 25.204 & 年 & - & 50.202 & 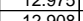 & 年 30.5300 & -1.2 & & 43.30 & 0.029 & $\begin{array}{l}14.045 \\
1.60\end{array}$ & & 224 & $\frac{3.1 / 4083}{27575}$ & 48.1. & 10.6 & 4. & & & & 00 & 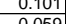 & \\
\hline & $1 / 272004$ & $7 \mathrm{PM}$ & 25.424 & $\frac{2.458}{21.488}$ & & & & & $\frac{50.03}{50.03}$ & & $\frac{34.138}{37.18}$ & $\frac{1.1 .}{-12}$ & & & & 146 & & $\frac{20.445}{2245}$ & $\mid 3.10$ & $\frac{45}{48}$ & & & & & & & & \\
\hline & $1 / 27 / 2004$ & 5:12:27 PM & 25.524 & 21.519 & \begin{tabular}{|l|l|} 
& 25.273 \\
\end{tabular} & 25.345 & & & 50.279 & $\mid 13.064$ & & \begin{tabular}{|l|l|}
-1219 \\
\end{tabular} & & 46.306 & & 14643 & & & 379 & 488 & & 43. & & & & 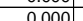 & & \\
\hline & & 3:27 PM & 25.545 & 21.51 & 25.274 & 25 & & & 50.403 & 13.17 & & \begin{tabular}{|c|}
-1.216 \\
\end{tabular} & & & & 14.6 & & & & & 11. & & & & & & 194 & \\
\hline & $1 / 27 / 2004$ & :14:27 PM & 25.56 & $21.52 !$ & 25.289 & 25.276 & 23.406 & \begin{tabular}{|l|l|l|}
49.648 \\
\end{tabular} & 50.354 & 13.158 & 37.178 & \begin{tabular}{|l|l|} 
& -1.216 \\
\end{tabular} & & 45.166 & 057 & 14.643 & & 229.44 & \begin{tabular}{|l|}
3.82417 \\
\end{tabular} & 48 & & & & & & & & \\
\hline & 1/27/2004 & 5:15:27 PM & 25.57 & 21.53 & & 25.346 & & $\begin{array}{ll}49.662 \\
\end{array}$ & \begin{tabular}{|l|l|}
50.544 \\
\end{tabular} & 13.055 & 37.16 & & & 43.605 & & & & & \begin{tabular}{|l|l|}
3.84083 \\
\end{tabular} & & \begin{tabular}{|l|}
10. \\
\end{tabular} & & & & & & & \\
\hline & & & & & & & & & & & & & & & & & & & \begin{tabular}{|l|l|}
3.8575 \\
\end{tabular} & \begin{tabular}{|l|l|}
49.0 \\
\end{tabular} & 10.7 & 43.5 & & & & & & \\
\hline & $\frac{1 / 2772004}{1 / 27004}$ & $7 \mathrm{PM}$ & 25.582 & $\frac{21.541}{21548}$ & $\frac{25.346}{25367}$ & 25.368 & & & 50.675 & $\begin{array}{r}13.135 \\
13020\end{array}$ & & -1.2 & & & & 14.6 & & 232.45 & & $\begin{array}{ll}49.2 \\
48.9\end{array}$ & & & & & & & & \\
\hline & $1 / 27 / 2004$ & & 25.3004 & $\frac{21.540}{21559}$ & \begin{tabular}{|l|l|} 
& 25393 \\
\end{tabular} & 25.3204 & & & \begin{tabular}{|l|l|} 
& 5.40267 \\
\end{tabular} & $\mid$ & & $\mid-1.2$ & & & & & & & & & & & & & & & & \\
\hline & $\frac{1 / 27 / 2004}{1 / 27}$ & & $\frac{2.004}{25.599}$ & 2157 & 25.503 & & & & 50238 & & & \begin{tabular}{|l|l|} 
& -1.2 \\
\end{tabular} & & & & & & & & & & & & & & & & \\
\hline 254 & $1 / 27 / 2004$ & $7 \mathrm{PM}$ & 25.6 & 21.57 & 25.419 & 25.325 & 23. & 49.229 & 50.153 & \begin{tabular}{|c|}
12.844 \\
\end{tabular} & 37.073 & \begin{tabular}{|l|l|} 
& -1.2 \\
\end{tabular} & & & 0.056 & & & & \begin{tabular}{|c|c|c|}
3.94083 \\
\end{tabular} & & & & & & & 000 & & \\
\hline & & & & & & & & & & & & & & & & & & & & & & & & & & & & \\
\hline & & & 25.60 & 21.587 & & & & & & & & & & & & & & & & & & & & & & & & \\
\hline & 1/27/2004 & & 25.58 & 21.566 & \begin{tabular}{|l|l|}
25.43 \\
\end{tabular} & & 23.2 & & 50. & & & & & & & & & & & 49 & & & & & & & & \\
\hline & $1 / 27 / !$ & $\mathrm{M}$ & 25.6 & & 25 & 25.36 & & & 50.2 & 13. & & \begin{tabular}{|l|} 
\\
\end{tabular} & & & & & & & & & & & & & & & & \\
\hline & $1 / 27 / 2004$ & 5:26: & 25.6 & & 25. & 25.33 & & & 50.43 & 13.1 & & \begin{tabular}{|l|} 
\\
\end{tabular} & & 43.0 & & 14.6 & & & & $4 !$ & & & & & & & 90 & \\
\hline & $1 / 27 / 2004$ & & & & 25.491 & & & 49. & 50.356 & \begin{tabular}{|l|l|l|}
13.177 \\
\end{tabular} & & $\mid-1.2$ & & & & & & & & & & & & & & & 191 & \\
\hline & 1/27/2004 & & 25.627 & 21.613 & 25.507 & 25.328 & 23.0 & 49. & 50.327 & 13.079 & & -1.21 & & & & & & & & 48 & & & & & & & 191 & \\
\hline & & & 25.63 & & & & & & & & & & & & & & & & & & & & & & & & & \\
\hline & & 5: & 25.64 & 21.624 & 25.538 & $25.4 C$ & & & 50.2 & 13.0 & 36.9 & & & & & & & & & 48 & & & & & & & & \\
\hline & & $7 \mathrm{PM}$ & 年5.6544 & $\frac{2.619}{210}$ & $\frac{25.543}{25542}$ & 25.43 & 23. & 年9.364 & 50.397 & $\begin{array}{l}13.125 \\
13252 \\
\end{array}$ & $\begin{array}{l}36.932 \\
36.858\end{array}$ & -1.2 & & & & $\frac{14.6}{14.6}$ & & & & 48. & & 4. & & & & & 1877 & \\
\hline 6 & $1 / 27 / 2004$ & $4 \mathrm{PM}$ & 25.675 & 21.636 & $\mid \begin{array}{ll}\mid 25.569 \\
\end{array}$ & 25 & 22.909 & 499 & $\mid$\begin{tabular}{|l|l|l|}
$\mid 50.49$ \\
\end{tabular} & & & \begin{tabular}{|c|c|} 
& $-1<2<5$ \\
\end{tabular} & & $\begin{array}{l}44.3 \\
458\end{array}$ & & $\frac{14 .}{14 .}$ & & & & 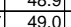 & 11.2 & & 2.981 & & 0.0 & 0.000 & 0.187 & \\
\hline & $1 / 27 / 2$ & & 25.665 & 21.626 & & & & & 50.4 & 13.1 & & -1.2 & & & & & & & & & & & & & & & & \\
\hline & $1 / 27 / 2$ & & 25.67 & 21.616 & 25.564 & & & 49. & 50.5 & 13.245 & & -1.22 & & & & & & & & 49 & & & & & & & 0.190 & \\
\hline & & & 25.68 & 21.641 & 25.589 & & & & 50.7 & & & & & & & & & & & & & & & & & & & \\
\hline & & & 25.65 & & 25.58 & & & & 50.2066 & & & -1.22 & & & & & & & & 48 & & & & & & & & \\
\hline & $\frac{11272}{112712}$ & & & 21.646 & & & 23. & 49.4 & \begin{tabular}{|}
$\mid 50.549$ \\
\end{tabular} & $\frac{13.3}{2.3}$ & & -1.21 & & & & & & & & 49 & 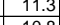 & & & & & & 37 & \\
\hline 27 & 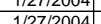 & & $\frac{2.17}{2.25}$ & 21 & $\frac{2.5344}{25209}$ & & & 49 & o.l.180 & & $\frac{30.04}{366}$ & $\frac{-1.24}{-121}+2$ & & & & & & & & & & & & & & & & \\
\hline & 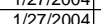 & & $\frac{25}{257}>0$ & & $\frac{2.4}{24}$ & & & 49.017 & 50.254 & 132 & & $\frac{1.21}{-121}>0$ & & & & & & & & & & & & & & & & \\
\hline 27 & & & $\frac{2.5 .681}{25.681}$ & $\frac{2.562}{21.662}$ & & $\frac{25.31}{25.31}$ & & & 50.304 & $\frac{13.4}{13.4}$ & & $\frac{-1.21}{-1.21}>$ & & & & & & & & & & & & & & & & \\
\hline & & & & & & & & 49. & & & & & & & & & & & & & & & & & & & & \\
\hline & & & 25.611 & 21.662 & & 25.36 & & & & 13.3 & & & & & & & & & & & & & & & & & & \\
\hline & & & 25.587 & & & $25.30>->$ & & 48.9 & 50.066 & 132 & 36.5 & -1219 & & & & 14 & & & & 48. & & & & & & & & \\
\hline & & & & & & & & & & & & & & & & & & & & & & & & & & & & \\
\hline 280 & $1 / 27 / 2004$ & 5:46:54 PM & 25.532 & 21.663 & 25.186 & 25.333 & 23.192 & 49.237 & 50.424 & 13.323 & 36.675 & \begin{tabular}{|c|c|} 
& -1.219 \\
\end{tabular} & & 46.428 & 0.051 & 14.643 & & 261.90 & 4.365 & 49.0 & 11.4 & & 2.962 & 0.008 & 0.008 & 0.000 & 0.176 & \\
\hline
\end{tabular}


WSRC-TR-2005-00105, REVISION 0

SRNL-RPP-2005-00012, REVISION 0

RUN \# 1.03A; DEWATER FIRST HALF OF SLURRY - CONT.

\begin{tabular}{|c|c|c|c|c|c|c|c|c|c|c|c|c|c|c|c|c|c|c|c|c|c|c|c|c|c|c|c|c|}
\hline \\
\hline & \begin{tabular}{l|l} 
\\
\end{tabular} & B & $\mathrm{D}$ & $E$ & $\mathrm{~F}$ & G & $\mathrm{H}$ & $\mathrm{J}$ & $\mathrm{K}$ & $\mathrm{L}$ & $\mathrm{M}$ & $\mathrm{N}$ & 0 & $\mathrm{Q}$ & \begin{tabular}{l|l|l|l|}
$R$ & \\
\end{tabular} & $\mathrm{~s}$ & $\begin{array}{ll}T \\
\end{array}$ & $\mathrm{v}$ & $w$ & $x$ & Y & $z$ & AA & $A B$ & $\mathrm{AC}$ & $\mathrm{AD}$ & $\mathrm{AE}$ & $\mathrm{AF}$ \\
\hline 28 & $1 / 27 / 2004$ & 5:47:54 PM & 25.511 & 21.667 & 25.22 & 25.296 & 22.85 & 49.393 & \begin{tabular}{|l|}
50.592 \\
\end{tabular} & $\begin{array}{l}13.338 \\
\end{array}$ & 36.714 & -1.219 & $\begin{array}{l}-7.441 \\
\end{array}$ & 42.375 & \begin{tabular}{|c|}
0.053 \\
\end{tabular} & $\begin{array}{r}14.643 \\
\end{array}$ & 0.004 & 262.90 & 4.38167 & 49.1 & 10.4 & & 2.968 & 0.008 & 0.008 & 0.000 & 0.182 & \\
\hline & & & & & \begin{tabular}{|r|}
25.26 \\
\end{tabular} & 25.292 & $\begin{array}{r}23.126 \\
\end{array}$ & & & & & \begin{tabular}{|r|}
-1.216 \\
\end{tabular} & $\begin{array}{r}-7.438 \\
-7.38 \\
\end{array}$ & & & \begin{tabular}{|l|}
14.643 \\
\end{tabular} & & & & 48.8 & & & & & & & 0.183 & \\
\hline & $1 / 27 / 2004$ & $5: 49: 54 \mathrm{PM}$ & 25.516 & 21.678 & 25.285 & 25.372 & 23.006 & 49.084 & 50.271 & $\begin{array}{l}13.126 \\
\end{array}$ & 36.677 & \begin{tabular}{|l|l|}
-1.216 \\
\end{tabular} & $\begin{array}{r}-7.421 \\
\end{array}$ & 45.391 & $\begin{array}{l}0.053 \\
\end{array}$ & \begin{tabular}{|l|l|}
14.643 \\
\end{tabular} & & 264.9 & 4.415 & 48.8 & \begin{tabular}{|l|}
11.1 \\
\end{tabular} & & 2.050 & \begin{tabular}{|l|}
0.008 \\
\end{tabular} & 0.008 & 0.000 & $\begin{array}{l}0.183 \\
\end{array}$ & \\
\hline $284 \mid$ & $\begin{array}{l}1 / 27 / 2004 \\
1 / 27204\end{array}$ & $\begin{array}{l}\text { 5:50:54 PM } \\
\text { 5.51.54 }\end{array}$ & $\begin{array}{r}25.521 \\
25.532\end{array}$ & $\begin{array}{r}21.678 \\
21.693\end{array}$ & $\begin{array}{r}25.32 \\
\end{array}$ & $\begin{array}{l}25.397 \\
25.363\end{array}$ & $\begin{array}{r}22.805 \\
22.801 \\
\end{array}$ & $\begin{array}{l}48.926 \\
40.78\end{array}$ & \begin{tabular}{|l|}
50.132 \\
50.31
\end{tabular} & $\begin{array}{l}13.089 \\
13.248\end{array}$ & $\begin{array}{r}36.665 \\
366^{2}\end{array}$ & \begin{tabular}{|l|} 
\\
\end{tabular} & $\begin{array}{r}-7.424 \\
-7.24\end{array}$ & $\begin{array}{r}45.81 \\
4005\end{array}$ & $\begin{array}{l}0.053 \\
\end{array}$ & $\begin{array}{r}14.643 \\
14643\end{array}$ & & $\begin{array}{r}265.90 \\
266.90\end{array}$ & 4.43167 & 48.7 & 11.2 & & \begin{tabular}{|l|l|l|} 
\\
\end{tabular} & \begin{tabular}{|l|}
0.008 \\
0008
\end{tabular} & $\begin{array}{l}0.008 \\
\end{array}$ & 0.000 & \begin{tabular}{|l|l|}
0.183 \\
\end{tabular} & \\
\hline & & 5:5:5::54 PM & $\begin{array}{r}25.532 \\
25.537 \\
\end{array}$ & $\begin{aligned} 21.683 \\
21.668\end{aligned}$ & \begin{tabular}{|l|}
25.351 \\
25.316
\end{tabular} & $\begin{array}{l}25.363 \\
25.368 \\
\end{array}$ & $\frac{22.801}{22.861}$ & $\begin{array}{r}49.078 \\
49.075 \\
\end{array}$ & \begin{tabular}{|r|}
50.31 \\
50.308 \\
\end{tabular} & $\begin{array}{l}13.248 \\
13.166\end{array}$ & $\begin{array}{r}36.63 \\
36.632 \\
\end{array}$ & $\mid$\begin{tabular}{|l|}
$\mid-1.219$ \\
-1.216
\end{tabular} & $\begin{array}{l}-7.424 \\
-7.421 \\
\end{array}$ & $\begin{array}{r}44.905 \\
45.203\end{array}$ & $\mid \begin{array}{c}0.053 \\
0.053\end{array}$ & \begin{tabular}{|l|}
14.643 \\
14.643
\end{tabular} & & 266.90 & 4.44833 & $\begin{array}{l}48.8 \\
488\end{array}$ & $\begin{array}{l}11.0 \\
111 .\end{array}$ & & \begin{tabular}{|l|}
$\mid 2.955$ \\
2.555
\end{tabular} & \begin{tabular}{|l|}
0.008 \\
0008
\end{tabular} & 0.008 & 0.000 & $\begin{array}{l}0.183 \\
0.182\end{array}$ & \\
\hline \begin{tabular}{|l|}
287 \\
\end{tabular} & $1 / 27 / 2004$ & 5:53:54 PM & 25.536 & & \begin{tabular}{|l|}
25.385 \\
\end{tabular} & 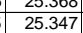 & $\frac{22.001}{22.187}$ & 499.086 & \begin{tabular}{|l|}
50.000 \\
\end{tabular} & $\frac{10.100}{13.24}$ & $\frac{36.632}{36.669}$ & \begin{tabular}{|l|l|}
-1.216 \\
-1.219 \\
\end{tabular} & $\begin{array}{l}\frac{-7.421}{-7.424} \\
\end{array}$ & $\begin{array}{l}44.203 \\
44.842\end{array}$ & $\begin{array}{l}0.0533 \\
053\end{array}$ & \begin{tabular}{|c|}
14.644 \\
14.643 \\
\end{tabular} & & $\frac{267.90}{268.90}$ & 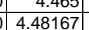 & $\begin{array}{l}48.8 \\
48.8\end{array}$ & $\frac{11.1}{11.0}$ & $\frac{42.9}{42.9}$ & $\frac{2.955}{2.956}$ & \begin{tabular}{|l|}
0.008 \\
0.008 \\
\end{tabular} & $\begin{array}{c}0.008 \\
0.008\end{array}$ & $\begin{array}{c}0.000 \\
0.000\end{array}$ & $\begin{array}{l}0.182 \\
0.182\end{array}$ & \\
\hline 288 & $1 / 27 / 2004$ & 5:54:54 PM & 25.551 & 21.662 & \begin{tabular}{|l|} 
\\
\end{tabular} & 25.357 & 22.242 & 49.105 & \begin{tabular}{|l|l|}
50.376 \\
\end{tabular} & 13.22 & 36.69 & -1.219 & & 43.099 & 0.053 & $\begin{array}{l}14.643 \\
\end{array}$ & & 269.90 & 4.49833 & 48.9 & & & 2.958 & \begin{tabular}{|l|}
0.008 \\
\end{tabular} & 0.008 & 0.000 & 0.182 & \\
\hline & & 5:55:54 PM & & & \begin{tabular}{|l|}
25.444 \\
\end{tabular} & & & & & & & & & & & & & & & & & & & & & & & \\
\hline 290 & $1 / 27 / 2004$ & 5:56:54 PM & 25.569 & 21.64 & \begin{tabular}{|l|}
25.463 \\
\end{tabular} & 25.27 & & 48.8 & \begin{tabular}{|l|l|} 
& 50.101 \\
\end{tabular} & \begin{tabular}{|l|l|}
13.068 \\
\end{tabular} & .558 & -1.219 & & & $\overline{053}$ & 643 & & & 4.53167 & 48.6 & & & $\frac{944}{944}$ & 0 & 008 & (100 & 183 & \\
\hline 291 & & 7:54 PM & & & & & & & & $\begin{array}{l}13.437 \\
\end{array}$ & & & & & & & & & & 49. & & & & & & & & \\
\hline & 1/27/2004 & 58:54 PM & 25.593 & 21.639 & \begin{tabular}{|l|l|} 
& 25.492 \\
\end{tabular} & 25.374 & & & 186 & 13.239 & & $\frac{-1.219}{-1.29}$ & & 47.206 & & & & & 4.565 & 48.7 & 11 & & & 008 & 0.008 & & 0.182 & \\
\hline & $1 / 27 / 2004$ & 5:59:54 PM & 25.598 & 21.619 & 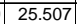 & 25.424 & 22.8 & 49.102 & \begin{tabular}{|l|}
50.389 \\
\end{tabular} & 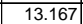 & 36.593 & -1.219 & & \begin{tabular}{|l|l|l}
44.127 \\
\end{tabular} & 0.053 & $\begin{array}{l}14.643 \\
\end{array}$ & & & \begin{tabular}{|l|l|}
4.58167 \\
\end{tabular} & 48.9 & & & 2.954 & & & & & \\
\hline 294 & $1 / 27 / 2004$ & $6: 00: 54 \mathrm{PM}$ & 25.612 & 21.633 & \begin{tabular}{|l|l|} 
& 25.532 \\
\end{tabular} & 25.353 & 22.85 & 49.158 & 50.445 & \begin{tabular}{l|l|l|l|}
13.244 \\
\end{tabular} & 36.573 & -1.216 & -7.418 & 44.21 & 0.053 & $\begin{array}{l}14.643 \\
\end{array}$ & & 275.90 & \begin{tabular}{|l|l|}
4.59833 \\
\end{tabular} & 49.0 & 10.8 & & 2.955 & D.008 & 0.008 & 0.000 & 0.182 & \\
\hline & $1 / 27 / 2004$ & $6: 01: 54 \mathrm{PM}$ & 25.606 & 21.622 & 25.536 & 25.257 & & 49.109 & \begin{tabular}{|l|l|}
50.492 \\
\end{tabular} & 13.242 & 36.521 & -1.219 & -7.41 & & 0. & 14.643 & & & 4.615 & & & & & & 0.008 & & & \\
\hline$\frac{296}{297}$ & $1 / 27 / 2004$ & 6:02:54 PM & & & & 25.288 & & 49.144 & 50.48 & 13.303 & 36.562 & -1.219 & & 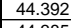 & & 14.643 & & & 63167 & & & & & & & & & \\
\hline & $\frac{17004}{12004}$ & $6: 03554 \mathrm{PM}$ & 25.641 & 21.632 & & 25.292 & & 49.405 & 50.745 & 13.412 & 36.616 & -1.216 & & 44.385 & 0.053 & 14.643 & & & 4.64833 & 49.3 & & & 2.96 & & 008 & & .181 & \\
\hline & $\frac{712004}{72004}$ & 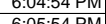 & & & \begin{tabular}{|l|l|l|} 
\\
\end{tabular} & $\frac{25.197}{25252}$ & & 49.262 & .604 & 13.381 & (5697 & \begin{tabular}{|c|c|c|} 
\\
\end{tabular} & & 44. & & & & & 4.665 & 499 & & & & & & & & \\
\hline & $1 / 27 / 2004$ & $6.00654 \mathrm{PM}$ & 25.655 & $\frac{21.045}{21.616}$ & $\mid$\begin{tabular}{|l|l|}
$\mid 25.584$ \\
25.58
\end{tabular} & & & & \begin{tabular}{|l|l|l|l|l|} 
\\
50.575
\end{tabular} & 告13.065 & $\begin{array}{l}36.597 \\
36.581\end{array}$ & $\frac{-1.219}{-1220}$ & & $\frac{4.154}{44.794}$ & & $\frac{14.645}{14.643}$ & & 200. & $\begin{array}{l}\frac{4.68101}{4.6932} \\
\end{array}$ & 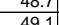 & 11 & & & : & 0.008 & & 0.182 & \\
\hline 301 & 1/27/2004 & 6:07:54 PM & 25.67 & 21.621 & 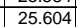 & 25.276 & 22.433 & 49.071 & \begin{tabular}{|l|}
50.403 \\
\end{tabular} & 13.223 & 36.585 & $\mid-1.219$ & -7.418 & 42.625 & 0.053 & 14.643 & & 282.90 & 4.715 & 48.9 & 10.4 & 42.8 & 2.953 & \begin{tabular}{|l|}
0.008 \\
\end{tabular} & 0.008 & 0.000 & 0.181 & 0.15 \\
\hline & $1 / 27 / 2004$ & 6:08:54 PM & 25.685 & 21.636 & \begin{tabular}{|l|} 
\\
\end{tabular} 5.6299 & 25.251 & & 49.202 & 50.621 & 13.35 & 36.517 & \begin{tabular}{|l|l|l|}
-1.219 \\
\end{tabular} & -7.415 & \begin{tabular}{|l|l|l}
42.408 \\
\end{tabular} & 0.053 & \begin{tabular}{|c|}
14.643 \\
\end{tabular} & & & & 49.1 & 10.4 & & & & & & 0.181 & \\
\hline (303 & 1/27/2004 & 6:09:54 PM & 25.684 & 21.625 & 25.638 & 25.2 & & 48.831 & \begin{tabular}{|l|}
50.151 \\
\end{tabular} & 13.241 & 36.476 & -1.219 & -7.4. & 44.736 & 0.052 & 14.643 & & & 4.74833 & 48.7 & & & 2.94 & & 0.008 & & & \\
\hline$\frac{304}{305}$ & $1 / 27 / 72004$ & 6:10:54 PM & 25.689 & & 25.648 & & & 49.405 & \begin{tabular}{|l|l|} 
\\
\end{tabular} & 13.573 & 36.573 & & & $46 . .113$ & 0.052 & 14.643 & & & 4.765 & 49.3 & & & 2.966 & 0008 & 0.008 & 0.000 & & \\
\hline & 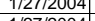 & $4 \mathrm{PM}$ & 25.693 & & 25.662 & 25.199 & & & & 13.147 & 36.435 & & & 43.62 & & 44.643 & & & 4. 18161 & & & & & & & & & \\
\hline & 112170044 & $6.12 .54 \mathrm{PM}$ & & & & & & 48.943 & $\frac{50.378}{50.464}$ & $\frac{13.229}{13137}$ & $\begin{array}{l}36.433 \\
36.48 \\
36.45\end{array}$ & & & 44.319 & & & & & 4.79833 & & & & & 008 & 008 & & & \\
\hline$\frac{301}{308}$ & $\begin{array}{ll}1 / 27 / 2004 \\
1 / 27 / 200\end{array}$ & $\begin{array}{l}0.135 .54 \mathrm{PM} \\
6: 14: 54 \mathrm{P}\end{array}$ & 25.717 & $\frac{2.003}{21.613}$ & \begin{tabular}{|l|l|}
25.060 \\
\end{tabular} & 25238 & 2252 & $\begin{array}{l}40.900 \\
49.022\end{array}$ & \begin{tabular}{|l|}
50.404 \\
50.439 \\
\end{tabular} & $\begin{array}{l}13.151 \\
13.351\end{array}$ & $\begin{array}{l}50.400 \\
36.487\end{array}$ & $\begin{array}{l}-1.219 \\
-1.219\end{array}$ & $\frac{-1.440}{-7.41}$ & $\begin{array}{l}45.041 \\
42.711\end{array}$ & $\begin{array}{l}0.052 \\
0.052\end{array}$ & $\begin{array}{l}14.045 \\
14.643\end{array}$ & & 289.90 & \begin{tabular}{|l|l}
48.015 \\
83167
\end{tabular} & 490 & $\frac{11}{10}$ & 428 & 2948 & 0.008 & 年 & 年 0.000 & 0.178 & \\
\hline & $1 / 127 / 2004$ & 6:15:54 PM & 25.727 & 21.608 & \begin{tabular}{|l|l|}
25.696 \\
\end{tabular} & 25.228 & 22.39 & 49.061 & 50.575 & 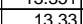 & 36.392 & $\frac{-1.222}{-1.22}$ & -7.41 & $\frac{42.11}{411}$ & 0.052 & 14.643 & & & 484833 & 49.1 & & & 294 & 008 & 0.008 & 0.00 & 0.178 & \\
\hline 310 & $1 / 27 / 2004$ & 6:6:16:54 PM & $\frac{25.742}{25.742}$ & $\frac{1.613}{21.613}$ & \begin{tabular}{|l|l|}
25.716 \\
\end{tabular} & $\frac{25.258}{25.258}$ & & 49.279 & 50.714 & $\frac{13.49}{13.49}$ & 36.511 & $\frac{-1.222}{-1.22}$ & & 44.346 & 0.052 & 14.643 & & Fi1.9 & 4.865 & 49.8 & & & & & & & & \\
\hline 311 & $1 / 127 / 2004$ & 6:17:54 PM & 25.735 & 21.596 & \begin{tabular}{|l|l|}
25.715 \\
\end{tabular} & 25.281 & & 49.127 & \begin{tabular}{|l|l|l|}
5033 \\
\end{tabular} & 13.411 & & $\frac{1.219}{-1.219}$ & & 45.264 & & & & ת & 4.88167 & 49.2 & 11 & 42. & & 008 & 0.008 & 0.000 & 0.178 & \\
\hline & & & 25.749 & 21.61 & \begin{tabular}{|l|}
25.743 \\
\end{tabular} & & & & & & 36.37 & $\frac{-1.219}{-1.219}$ & & & & & & & . & 49 & & & & & & & & \\
\hline & 1/27/2004 & & & & & 25.199 & & & \begin{tabular}{|l|l|}
50.464 \\
\end{tabular} & 13.352 & & & & 44.344 & & & & 294.90 & 4.915 & 490 & & & & 008 & & & & \\
\hline 314 & $1 / 27 / 2004$ & & 25.747 & & & 25.188 & & & \begin{tabular}{|l|}
50.8499 \\
\end{tabular} & $\begin{array}{l}13.691 \\
\end{array}$ & 36.456 & $\mid-1.219$ & & 43.134 & & & & & & & & & & & & & & \\
\hline & $1 / 27 / 2004$ & 6:21:54 PM & 25.769 & 21.609 & \begin{tabular}{|l|l|} 
& 25.763 \\
\end{tabular} & 25.234 & & & 1551 & \begin{tabular}{|l|l|l}
13.453 \\
\end{tabular} & 36.331 & $\mid-1.219$ & & $\begin{array}{lll}44.4 \\
\end{array}$ & & & & & \begin{tabular}{|l|l|l|l|}
4.94833 \\
\end{tabular} & 49.1 & 10 & & & 008 & 007 & & & \\
\hline & 1/27/2004 & $4 \mathrm{PM}$ & 25.763 & 21.604 & $25.767 \mid$ & 25.314 & & & 4.472 & 13.441 & 36.3 & & & $\begin{array}{l}44.648 \\
\end{array}$ & & & & & & & & & & & & & 0.175 & \\
\hline 31 & 1/27/12004 & 6:23:54 PM & 25.783 & 21.614 & \begin{tabular}{|l|l|} 
& 25.787 \\
\end{tabular} & 25.324 & & 49.262 & 50.826 & 13.563 & 36.273 & -1.216 & -7.398 & 43.351 & 0.052 & & & 298.9 & \begin{tabular}{|l|l|l|l|} 
\\
\end{tabular} & $\begin{array}{ll}49.4 \\
\end{array}$ & & & 29 & & 008 & & & \\
\hline$\frac{5 x}{31}$ & $27 / 2004$ & $6: 24: 54 \mathrm{PM}$ & 25.773 & 21.594 & $\frac{2.104}{25702}$ & 25.314 & & & 50.772 & 13.667 & 20250 & -1.219 & & $x+4$ & & & & (10. & . 99050 & 49 & 10 & & & & & & & \\
\hline & & & & & & & & & & & & & & & & & & & & & & & & & & & & \\
\hline & 112120004 & & 25.1899 & $\begin{array}{l}2.1 .61 \\
21.619\end{array}$ & 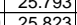 & $\frac{25.25}{25.280}$ & & & \begin{tabular}{|l|}
5.9806 \\
5.817
\end{tabular} & 13.746 & & -1.219 & & 44.1. & & & & & 5.03167 & 49.5 & 11 & & & .007 & & & & \\
\hline$\frac{25}{32}$ & 1127120 & & & $\frac{21.015}{21.605}$ & \begin{tabular}{|l|l|}
$\mid 25.025$ \\
\end{tabular} & $\frac{20.203}{2523}$ & & & (1) & 135009 & & -1216 & & 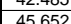 & & & & & 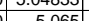 & & & & & & & & 104 & \\
\hline & 112772004 & & 25799 & 21.61 & \begin{tabular}{|l|l|}
25.033 \\
\end{tabular} & & & & 资 & 13625 & & -1214 & & $\frac{45.6}{43.1}$ & & & & & 508167 & & & & & & & & & \\
\hline 324 & $1 / 27 / 2004$ & $6: 3$ & 25.81 & 21.641 & \begin{tabular}{|l|}
$\mid 25.849$ \\
\end{tabular} & 25.255 & & & $\mid$\begin{tabular}{|r|}
$\mid 50.72$ \\
5
\end{tabular} & 13.664 & 36.261 & $\frac{-1.214}{-1.214}$ & & 43 & & & & 305.90 & 5 & & & & & & & & & \\
\hline & & & & & & & & & & & & & & 42.9 & & & & & & & & & & & & & & \\
\hline$\sqrt{32}$ & & & 25.805 & 21.615 & \begin{tabular}{|l|}
25.829 \\
\end{tabular} & & & & 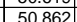 & & & & & 43. & & & & & & & & & & & & & & \\
\hline & & & 25.805 & 21.626 & \begin{tabular}{|l|l}
25.83 \\
\end{tabular} & 25.266 & & & 988 & 13.684 & & $\frac{-1.216}{-1216}$ & & 44.8 & & & & & 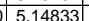 & & 11 & & & & & & & \\
\hline & 1/27/2004 & $6: 34: 5$ & & 21.616 & 25.845 & 25.22 & & & $50.5 \varepsilon$ & 13.6 & 36.1 & -1.2 & & 43 & & & & & 5.1 & & & & & & & & & \\
\hline & $1 / 27 / 2004$ & $6: 35: 54 \mathrm{~F}$ & 25. & 21.622 & 25.84 & & & & 50.81 & $\begin{array}{ll}13.609 \\
\end{array}$ & 36.2 & -1.2 & & 44.4 & 0.0 & 14. & & & \begin{tabular}{|l|l|l|}
5.181 \\
\end{tabular} & & 10 & & & & & & 0.167 & \\
\hline & $1 / 27 / 2004$ & 6:36:5 & & & 25.845 & & & & \begin{tabular}{|l|}
50.723 \\
\end{tabular} & & & & & 42.1 & & & & & & & & & & & & & & \\
\hline 331 & 1/27/2004 & $6: 37: 5$ & 25.821 & 21.627 & 25.861 & 25.282 & & & \begin{tabular}{|l|}
50.567 \\
\end{tabular} & 13.576 & & -1.219 & & 43. & & & & 312 & 5. & & 10 & & & & & & & \\
\hline & & & & & & & & & & & & & & 43. & & & & & & & & & & & & & & \\
\hline 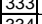 & $1 / 27 / 72004$ & & 25.816 & 21.632 & 25.41 & 25.226 & & & & 13.72 & & & & $45.6 \mathrm{r}$ & & & & & 5.24 & & & & & & & & & \\
\hline & 112717004 & 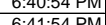 & & 21.647 & \begin{tabular}{|l|l|}
25.460 \\
\end{tabular} & 25. & & & 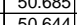 & $\frac{13.8}{136}$ & & $\begin{array}{l}-1.214 \\
1216\end{array}$ & & 42,4 & & & & & 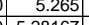 & 4 & $\frac{1}{16}$ & & & & & & (169) & \\
\hline $\mid \frac{3}{336}$ & 1 & $\frac{0.41 .54 \mathrm{P}}{6.62 .54 \mathrm{P}}$ & 25.001 & $\frac{21.044}{21637}$ & 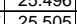 & $\frac{25.251}{25.176}$ & & & \begin{tabular}{|l|l|} 
\\
\end{tabular} & $\frac{13.06}{1367}$ & 35. & $\frac{1.210}{-1219}$ & & 437 & & & & & 5 & & 10 & & & & & & 0.10 & \\
\hline & 1 & & & & & & & & & & & & & & & & & & & & & & & & & & & \\
\hline 338 & $\frac{1}{1127 / 72004}$ & & $\frac{25.75}{25.75}$ & 21.662 & \begin{tabular}{|l|}
25.57 \\
25.57
\end{tabular} & $\frac{2.2 .201}{25.201}$ & & & $\mid$\begin{tabular}{|l|l|} 
& 50.909 \\
\end{tabular} & 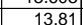 & & $\frac{-1.219}{-1.21}$ & & 43. & & & & & & & & & & & & & & \\
\hline & & & & & & & & & & & & & & & & & & & & & & & & & & & & \\
\hline 34 & & & 25.746 & 21.6 & 25.62 & 25.226 & & & & 13.8 & & -1.2 & & & & & & & & & & & & & & & & \\
\hline & & & & & 25.625 & & & & & & & \begin{tabular}{|c|c|}
-1.219 \\
\end{tabular} & & & & & & & & & & & & & & & & \\
\hline & & & & 21.668 & 25.656 & 25.157 & & & \begin{tabular}{|l|l|}
50 \\
\end{tabular} & 13.7 & & $\mid-1.219$ & & 420 & & 14 & & & 5.39833 & 44 & & & & & & & & \\
\hline \begin{tabular}{|l|l|}
343 \\
\end{tabular} & $1 / 27 / 2004$ & $6: 49: 5$ & 25.75 & 21.682 & 25.68 & 25. & & & 50.69 & 13.5 & & -1.219 & & 44.3 & & 14. & & & 5.4 & & 10 & & & & & & 0. & \\
\hline 344 & $1 / 27 / 2004$ & 6:50:5 & 25.73 & 21.67 & 25.69 & 25.14 & & & & 13.7 & & -1.219 & & & & & & & & 4 & 10 & & & & & & 66 & \\
\hline 34 & $1 / 27 / 2004$ & 6:51:5 & 25.746 & 21.687 & 25.69 & & & 48.947 & \begin{tabular}{|l|}
50.973 \\
\end{tabular} & 13.888 & & -1.219 & & 44.4 & & & & & & & 1 & & & & & & 66 & \\
\hline & & & 25.74 & 21.671 & 25 & & & & & & & & & & & & & & & & & & & & & & & \\
\hline$\frac{34}{23}$ & $1 / 2 / 72004$ & & 25.15 & 21.686 & 25.724 & 25. & & & 50.739 & 13.563 & & -1.219 & & 45.043 & & & & & 88167 & & 11.2 & & & & & & & \\
\hline $\begin{array}{ll}3448 \\
3240\end{array}$ & & $6.54: 5$ & 25.15 & $\frac{21.691}{20.71}$ & \begin{tabular}{|l|l|}
25.739 \\
\end{tabular} & 25.225 & 23.2 & $48 ., \gamma_{1}$ & 50.851 & 13.12 & 35.137 & -1.219 & & 43.322 & & 14.643 & & $\frac{29}{20}$ & ; 5.49833 & 49.4 & 10.6 & & & & & & 0.166 & 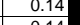 \\
\hline $\mid \frac{\mid 349}{350}$ & $1 / 27272004$ & $\begin{array}{l}6.55 .545 \mathrm{PM} \\
6: 54: 54\end{array}$ & \begin{tabular}{|l|}
25.150 \\
25.759
\end{tabular} & 21.706 & \begin{tabular}{|l|}
25.154 \\
25.768
\end{tabular} & \begin{tabular}{|l|}
25.19 \\
25.21
\end{tabular} & 23.194 & $\begin{array}{r}48.486 \\
48.474\end{array}$ & \begin{tabular}{|l|}
50.054 \\
50.716 \\
\end{tabular} & \begin{tabular}{|l|}
13.592 \\
13.562
\end{tabular} & $\begin{array}{r}35.627 \\
35.582 \\
\end{array}$ & $\mid \begin{array}{r}\mid-1.219 \\
\end{array}$ & 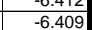 & 45.42 & \begin{tabular}{|}
0.048 \\
0.048
\end{tabular} & \begin{tabular}{|c|}
14.6433 \\
14.643 \\
\end{tabular} & & 331.90 & \begin{tabular}{|r|}
5.53167 \\
\end{tabular} & \begin{tabular}{|l|}
49.2 \\
49.2
\end{tabular} & \begin{tabular}{|l|}
$\mid 10.4$ \\
11.1 \\
\end{tabular} & \begin{tabular}{|l|} 
\\
42.0 .0 \\
\end{tabular} & \begin{tabular}{|l|}
2.8908 \\
\end{tabular} & \begin{tabular}{|l|}
0.007 \\
\end{tabular} & $\mid$\begin{tabular}{|c|}
0.007 \\
\end{tabular} & $\begin{array}{l}0.000 \\
0.000\end{array}$ & \begin{tabular}{|l|}
0.167 \\
\end{tabular} & 0.12 \\
\hline
\end{tabular}


WSRC-TR-2005-00105, REVISION 0

SRNL-RPP-2005-00012, REVISION 0

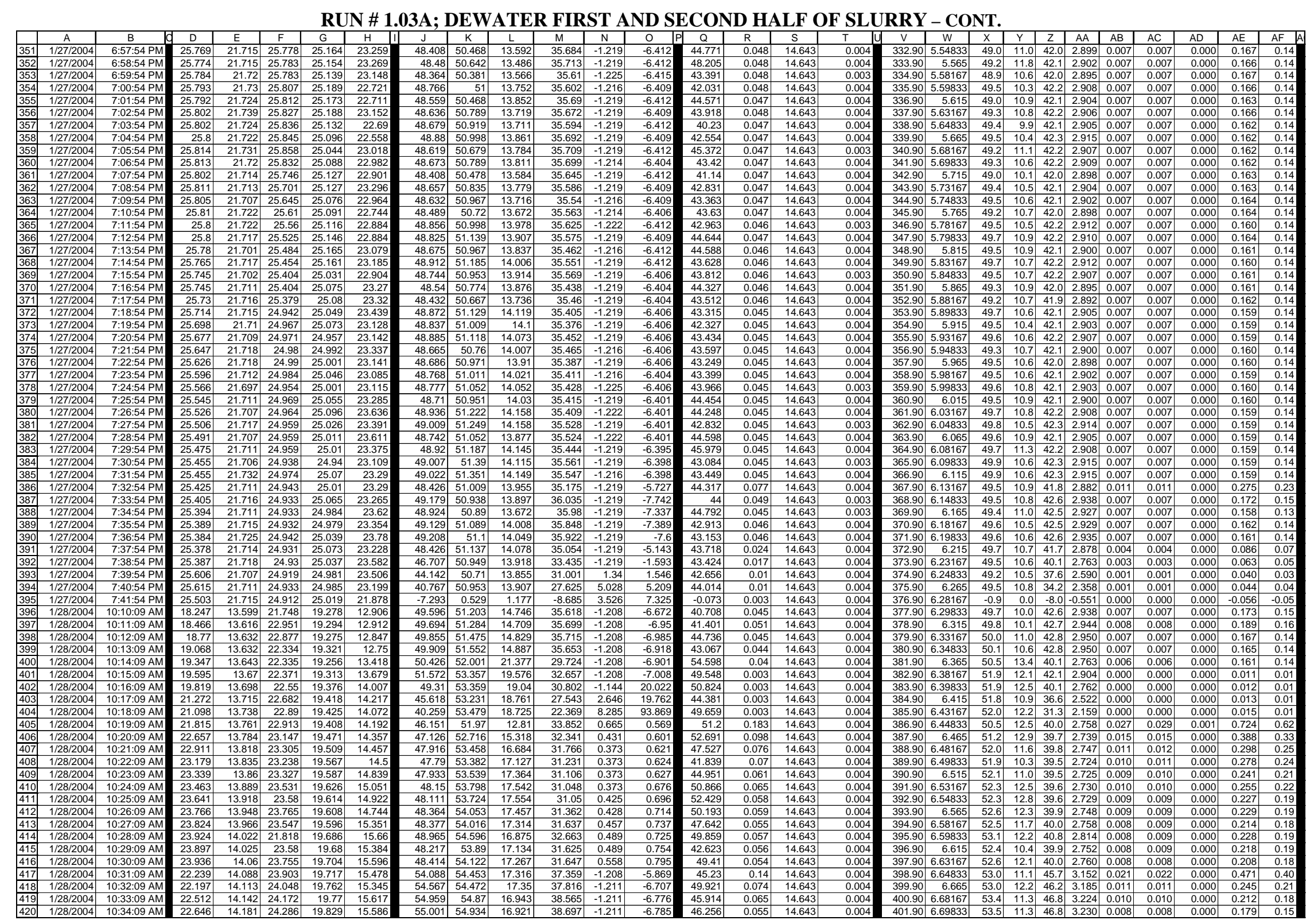


WSRC-TR-2005-00105, REVISION 0

SRNL-RPP-2005-00012, REVISION 0

RUN \# 1.03A; DEWATER SECOND HALF OF SLURRY - CONT.

\begin{tabular}{|c|c|c|c|c|c|c|c|c|c|c|c|c|c|c|c|c|c|c|c|c|c|c|c|c|c|c|c|c|}
\hline & A & $\mathrm{B}$ & D & $E$ & $\mathrm{~F}$ & \begin{tabular}{l|l}
$G$ & \\
\end{tabular} & $\mathrm{H}$ & $\mathrm{J}$ & $\mathrm{K}$ & 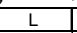 & \begin{tabular}{l|l}
$M$ \\
\end{tabular} & $\mathrm{~N}$ & $\mathrm{O}$ & \begin{tabular}{l|l} 
\\
\end{tabular} & \begin{tabular}{l|l}
$R$ & \\
\end{tabular} & \begin{tabular}{l|l}
$\mathrm{s}$ & $\mathrm{s}$ \\
$\mathrm{s}$
\end{tabular} & \begin{tabular}{l|l}
$T$ & $\mathrm{~T}$ \\
\end{tabular} & $\mathrm{v}$ & \begin{tabular}{l|l} 
w & \\
\end{tabular} & $\begin{array}{lll}x & \end{array}$ & $\mathrm{Y}$ & $z$ & $\mathrm{AA}$ & $\mathrm{AB}$ & \begin{tabular}{l|l}
$\mathrm{AC}$ & \\
\end{tabular} & $A D$ & $\mathrm{AE}$ & \\
\hline & $1 / 28 / 2004$ & 10:35:09 AM & 22.73 & 14.205 & \begin{tabular}{|l|l|}
5 & 24.409 \\
\end{tabular} & 19.899 & 15.665 & 55.036 & 54.829 & 16.909 & 38.75 & -1.211 & -6.967 & 4 & 0.062 & \begin{tabular}{|l|l|}
14.643 \\
\end{tabular} & 0.004 & 402.90 & 6.715 & 53.4 & 11.1 & & & 0.009 & 0.009 & 0.000 & & \\
\hline & & 10:36:09 AM & 22.799 & 14.224 & \begin{tabular}{|l|l|}
4 & 24.478 \\
\end{tabular} & 19.948 & 15.669 & 55.084 & & 16.955 & & -1.214 & & 45.606 & & 14.643 & & 403.90 & & 53.4 & $\frac{11.2}{11.2}$ & 46.9 & & & & & & \\
\hline \begin{tabular}{|l|l|l|l|l|l|}
424 \\
\end{tabular} & $1 / 28 / 2004$ & 10:37:09 AM & \begin{tabular}{|l|l|}
22.867 \\
202020
\end{tabular} & $\begin{array}{ll}14.252 \\
14303\end{array}$ & \begin{tabular}{|l|l|}
2 & 24.561 \\
& 24716
\end{tabular} & $\begin{array}{l}19.89 \\
1093\end{array}$ & 15.545 & \begin{tabular}{|l|l|}
55.503 \\
5200
\end{tabular} & \begin{tabular}{|l|l}
55.312 \\
5.182
\end{tabular} & 17.295 & $\begin{array}{l}38.678 \\
2037\end{array}$ & $\begin{array}{l}-1.211 \\
\end{array}$ & -6.953 & $\begin{array}{l}42.813 \\
5.830\end{array}$ & 0.055 & 14.643 & 0.004 & $\begin{array}{l}404.90 \\
0.90\end{array}$ & $\begin{array}{l}5.74833 \\
6.765\end{array}$ & 53.8 & to. & 1.1 & & .008 & 0.008 & 0.000 & 0.176 & \\
\hline$\left|\begin{array}{l}\mid 424 \\
25\end{array}\right|$ & $\begin{array}{l}1 / 28 / 2004 \\
1 / 28 / 2004\end{array}$ & $\begin{array}{l}10: 38: 09 \text { AM } \\
10.39 .09 \mathrm{AM}\end{array}$ & \begin{tabular}{l|l}
22.922 \\
2302 &
\end{tabular} & $\begin{array}{l}14.303 \\
14.326\end{array}$ & \begin{tabular}{|l|l|}
3 & 24.716 \\
6 & 24809 \\
\end{tabular} & \begin{tabular}{|c|}
19.93 \\
19938
\end{tabular} & 15.788 & \begin{tabular}{|r|r|}
52.69 \\
53.354
\end{tabular} & $\begin{array}{l}52.482 \\
53206\end{array}$ & $\begin{array}{l}20.354 \\
1945 \\
\end{array}$ & $\begin{array}{l}32.932 \\
33884\end{array}$ & $\begin{array}{l}-1.208 \\
-1.216 \\
\end{array}$ & $\begin{array}{l}-6.944 \\
-6.924\end{array}$ & \begin{tabular}{|l|l|l|}
54.838 \\
51886
\end{tabular} & 0.051 & $\begin{array}{l}14.643 \\
1.643 \\
\end{array}$ & 0.004 & \begin{tabular}{|l|l|}
405.90 & \\
\end{tabular} & \begin{tabular}{|l|l}
6.765 \\
678167
\end{tabular} & \begin{tabular}{|c|c|}
51.0 \\
51.7
\end{tabular} & 13.4 & 136 & \begin{tabular}{l|l}
2.952 \\
30207 &
\end{tabular} & $\begin{array}{l}0.008 \\
000 \\
\end{array}$ & 0.008 & 0.000 & 0.179 & \\
\hline 4 & & & & & & $\begin{array}{l}199.983 \\
19.906\end{array}$ & & & $\begin{array}{l}53.206 \\
53.065\end{array}$ & $\begin{array}{l}\frac{19.945}{19584} \\
\end{array}$ & $\begin{array}{l}33.884 \\
34.319\end{array}$ & & & 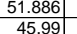 & $\begin{array}{l}0.051 \\
0.052 \\
\end{array}$ & $\begin{array}{l}14.643 \\
14.643\end{array}$ & & \begin{tabular}{|l|l|}
406.90 \\
407.90
\end{tabular} & & & & & & & \begin{tabular}{|l|l|}
0.008 & 0.008 \\
\end{tabular} & 0.000 & & 0.15 \\
\hline \begin{tabular}{|l|}
427 \\
\end{tabular} & $1 / 28 / 2004$ & 10:41:09 AM & 23.177 & 14.389 & \begin{tabular}{|l|l|}
9 & 25.046 \\
\end{tabular} & 19.909 & 15.793 & 53.385 & 53.22 & 19.155 & 34.771 & -1.211 & -6.878 & 48.42 & 0.052 & $\begin{array}{ll}14.045 \\
14.643\end{array}$ & 0.004 & 408.90 & $\frac{0.1500}{6.815}$ & $\begin{array}{l}51.6 \\
51.7 \\
\end{array}$ & $\frac{11.3}{11.9}$ & $\frac{43.8}{44.1}$ & 3.019 & 0.0008 & $\begin{array}{l}0.008 \\
0.008 \\
\end{array}$ & $\begin{array}{l}0.000 \\
0.000 \\
\end{array}$ & $\frac{0.177}{0.176}$ & $\frac{0.15}{0.15}$ \\
\hline 428 & $1 / 28 / 2004$ & & 23.251 & 14.423 & 25.095 & 19.913 & 16.111 & 53.302 & 53.181 & 19.001 & 34.859 & -1.214 & -6.828 & \begin{tabular}{|l|l|}
49.723 \\
\end{tabular} & 0.05 & 14.643 & 0.004 & 409.90 & \begin{tabular}{|c|}
6.83167 \\
\end{tabular} & 51.7 & 12.2 & 44.1 & & 0.007 & 0.007 & 0.000 & 0.169 & 0.15 \\
\hline & & 10:43:09 AM & & & & & & & & & & -1.214 & & 52.095 & 0.054 & & & 410.90 & & 51.6 & & 44.0 & & & & & & \\
\hline 430 & $1 / 28 / 2004$ & 10:44:09 AM & 23.328 & $\begin{array}{ll}14.476 \\
\end{array}$ & 25.162 & 19.975 & & 53.354 & 53.208 & 19.044 & & & & & & & & & 6.865 & & & & & & & & 0.158 & \\
\hline 431 & & & & & & & & & 53.17 & 18.923 & & -1.211 & & & & & & & (2107) & & & & & & & & & \\
\hline 432 & $1 / 28 / 2004$ & 10:46:09 AM & 23.391 & 14.55 & 25.249 & 20.053 & & 53.468 & 53.371 & 19.024 & 362 & 1211 & & & & & & & 39833 & & & & & 08 & & & 184 & \\
\hline 每334 & & 10:47:09 AM & 23.469 & 14.583 & 25.282 & 20.112 & & 53.526 & 53.401 & 18.785 & 35.331 & -1.211 & & & 0.05 & 14.6 & & & 6.915 & & & & & & & & & \\
\hline (434) & $1 / 28 / 2004$ & 10:48:09 AM & 23.488 & 14.607 & 25.336 & 20.1 & 16.552 & 53.636 & 53.56 & 18.583 & 35.577 & -1.211 & & 49.206 & 0.051 & 14.643 & & 415.90 & $6.93167 \mid$ & 52.1. & 12.1 & 44.6. & 3.07: & .008 & 0.008 & 0.000 & 0.169 & \\
\hline & $\frac{1 / 28 / 200}{1 / 28200}$ & 10:49:09 AM & $\begin{array}{ll}23.501 \\
2250\end{array}$ & 14.636 & 25.379 & 20.185 & $\frac{16.748}{1.600}$ & 53.624 & 53.587 & 18.519 & 35.575 & -1.211 & & 49.258 & 052 & 14.643 & & & & & & $x_{1}+2$ & & & & & & \\
\hline & $1 / 28 / 2004 \mid$ & 10:50:09 AM & 23.529 & $14.665 \mid$ & 25.427 & 20.203 & 16.609 & 53.213 & 53.185 & 18.195 & $\begin{array}{r}35.59 \\
25.575 \\
25\end{array}$ & -1.211 & & 50.049 & 0.052 & 14.643 & & & $\begin{array}{r}6.965 \\
60017 \\
\end{array}$ & & & 44.4 & & & & & & \\
\hline & & & & & $\frac{25.401}{25.494}$ & & $\frac{16.39}{1672}$ & $\frac{53.426}{5.26 !}$ & $\frac{53.316}{557}$ & & 35.5/57 & & & . & & 14.643 & & & 6.98167 & & & & & & 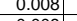 & & & \\
\hline & $\frac{1}{1 / 28 / 20004}$ & 10:53:09 AM & 23618 & & $\frac{2.5 .444}{2554}$ & 20.124 & & $\begin{array}{ll}53.106 \\
53767\end{array}$ & 53.562 & $\frac{18.305}{1.902}$ & 35.9924 & -1.214 & & 年.938 & 势 & & & & 7015 & & & & & & & & & \\
\hline & $1 / 28 / 2004$ & 10:54:09 AM & & 14.79 & 25.575 & 20.016 & $\frac{1.8 .}{16.6}$ & $\begin{array}{l}53.1698 \\
53.698\end{array}$ & $\begin{array}{l}53.689 \\
53.647\end{array}$ & $\begin{aligned} 18.026 \\
17.93\end{aligned}$ & $\begin{array}{l}36.34 t \\
36.353 \\
\end{array}$ & $\frac{-1.21}{-1.21}$ & & & 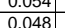 & $\frac{14.64}{14.64}$ & & & $\begin{array}{r}1.015 \\
7.03167\end{array}$ & 2 & . & & & & & & & \\
\hline 441 & $1 / 28 / 2004$ & $10: 55: 09 \mathrm{AM}$ & 23.664 & 14.797 & 25.587 & 20.157 & 16.852 & 53.65 & 53.629 & 17.892 & 36.363 & -1.211 & -6.745 & 49.172 & 0.052 & 14.643 & 0.004 & 422.90 & 7.04833 & 52.2 & 12.1 & 45.0 & 3.103 & 0.008 & 0.008 & 0.000 & 0.169 & \\
\hline & & 10:56:09 AM & 23.691 & 14.829 & 25.614 & 20.2 & 16.798 & 53.673 & 53.629 & 17.916 & 36.376 & -1.211 & & 50.021 & 0.053 & 14.643 & & & 7.065 & 52.2 & 12.3 & 45. & 3.104 & & & & & \\
\hline & 1/28/2004 & 10:57:09 AM & $\begin{array}{l}23.728 \\
20750\end{array}$ & 14.846 & 24.279 & 20.231 & $\frac{17.04}{1.0}$ & 53.781 & 53.726 & 17.946 & 36.359 & -1.214 & & 51.283 & 0.044 & 14.643 & & & 88167 & 52.3 & 12.6 & & & & & & & \\
\hline & $\frac{1 / 128 / 2004}{1 / 202}$ & 10:58: & 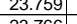 & $14.8 / 8$ & 24.18 & 20.293 & 17.83 & 53.8667 & 53.8211 & 17.263 & $37.1 / 2$ & -1.211 & & 35.41 & 0.051 & 14.643 & & & .09833 & 52.3 & & 45. 4.4 & 138 & & 0.008 & .000 & 0.171 & \\
\hline & 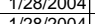 & 10:59:09 AM & 23.660 & & 20.994 & 20.285 & & $53.947 \pi$ & 53.911 & 18.143 & 36.345 & & & & & & & & & 52.4 & & & & & & & & \\
\hline & & 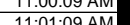 & 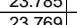 & $\frac{11.95}{14.074}$ & & & & & & & & & & & & & & & & & & & & & & & & \\
\hline 448 & $\frac{1 / 20 / 2004}{1 / 28 / 2004}$ & $11: 02: 09 \mathrm{AM}$ & | 23.747 & 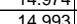 & 25.11 & 20.418 & $\frac{17.002}{16.906}$ & 53.1624 & 53.55 & $\begin{array}{l}\frac{10.004}{17.942} \\
\end{array}$ & 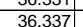 & $\begin{array}{l}-\frac{-1.211}{-1.211} \\
\end{array}$ & & 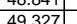 & 然0 & 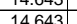 & & & 7165 & 521 & 121 & 年. & 101 & 007 & 0007 & 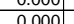 & 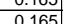 & \\
\hline 449 & $1 / 28 / 2004$ & 11:03:09 AM & 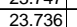 & 15.037 & 25.138 & 20 & 17.496 & 53.736 & 53.709 & $\begin{array}{ll}17.948 \\
17.948\end{array}$ & 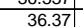 & $\begin{array}{ll}-1.214 \\
-1.214 \\
\end{array}$ & & 年. & 0.052 & 14.643 & & & \begin{tabular}{|c|c|}
71.16767 \\
\end{tabular} & 52.2 & & & 10 & & 8 & 000 & 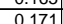 & \\
\hline & $1 / 28 / 2$ & 11:04:09 AM & 23.713 & & 25.161 & & 17.55 & & 53.952 & 18.076 & 36.382 & -1.211 & & $\begin{array}{l}49.437 \\
\end{array}$ & 0.053 & 14.6 & & & 7.19833 & & 12.1 & & & & & & & \\
\hline 451 & $1 / 28 / 2004$ & & 23.722 & 15.104 & 25.209 & 20.553 & 17.4 & 53.883 & 53.9 & 17.992 & & -1.208 & & & & & & & 7.215 & 52.4 & $\frac{12.5}{12.5}>0$ & & & & 88 & 0.000 & 0.171 & \\
\hline & & & & & 25.213 & & $17.98 \quad$ > & & 53.85 & 17.938 & & -1.211 & & & & & & & & 52.4 & & & & & & & & \\
\hline & $1 / 28 / 2004$ & 11:07:09 AM & 23.718 | & 15.162 & 25.236 & 20.656 & 17.9. & & 53.788 & & & & & & & & & & . 24833 & & & & & & & & & \\
\hline & $1 / 28 / 2004$ & 11:08 & & & & 20.67 & 17.7 & & 54.06 & 17.94 & & $\mid-1.216$ & & & & & & & & & $\overline{11.7}$ & & & & & & .176 & \\
\hline & $1 / 28 / 2004$ & 11:09 & 23.736 & & 25.274 & & & & 53.973 & $17.92 \mathrm{r}$ & & -1.214 & & & & & & & 7.28167 & 52.5 & 11.6 & & & & & & 0.176 & \\
\hline & & 11:10:09 AM & 23.75 & 15.261 & & 20.784 & & & 53.906 & 17.974 & 36. & -1.214 & & & & & & & & 52.4 & & & & & & & & \\
\hline & 1/28/2004 & 11:11:09 AM & 23.753 & 15.289 & 25.296 & 20.832 & 18.0 & 53.88 & 53.873 & 17.833 & 36.581 & -1.214 & & 49.725 & 0.051 & 14.643 & & $438.9 \mathrm{c}$ & 7.315 & 52.4 & 12.2 & & & & 08 & & & \\
\hline & 1) $128 / 20004$ & & 然. & $15.317 \mathrm{rat}$ & 20.314 & 20.88 & & 53.6 & $\begin{array}{l}53.645 \\
52720\end{array}$ & $\frac{17.6 \mathrm{C}}{17.7} \mathrm{C}$ & & -1.214 & & & & & & & (2) & 2 & 100 & & & & & & & \\
\hline & $\frac{1 / 182120044}{112020}$ & & & 15.356 & & & & & & & & & & & & & & & & & & & & & & & & \\
\hline 461 & $\frac{1 / 28 / 2004}{1 / 28 / 2004} \mid$ & 111.14 & $\frac{23.181}{23805}$ & 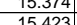 & 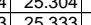 & 20.815 & & & $\begin{array}{ll}54.01 \\
5.77\end{array}$ & 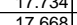 & & - & & & & & & & 7.365 & 20.6 & & 缶. & & & & & & \\
\hline & 年/28/2004 & & 资.3.827 & & 2535 & 20846 & & & 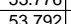 & & & $\mid-\frac{1.14+1}{-1201}$ & & & & & & & 7398 & 523 & 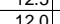 & 列 & & & & & 0 & \\
\hline & $1 / 28 / 2004$ & & $\frac{23.84}{23.84}$ & 15.488 & 25.377 & & & & 53.94 & & & $\mid \begin{array}{c}-1.208 \\
-1.208\end{array}$ & & & & & & & & & 124 & & & & & & & \\
\hline 464 & $1 / 28 / 2004$ & $11: 18: 09 \mathrm{AM}$ & 23.846 & 15.505 & \begin{tabular}{c|c|c|}
5 & 25.354
\end{tabular} & 20.875 & $18.70 \subseteq$ & 53.875 & 54.039 & 17.488 & 36.835 & -1.216 & & 49.058 & 0.042 & 14.643 & & & & 52.6 & 12.0 & & & & & & $0.1:$ & \\
\hline & $1 / 2$ & 11:19: & $23.8 / 3$ & 15.548 & 25.376 & 20.933 & & & & & & -1.211 & & & & & & & & 0.5 & & & & & & & & \\
\hline & $1128 / 2004$ & & 23.886 & & & & & & & & & & & & & & & & & 52.5 & 11.8 & & & & & & & \\
\hline & & & 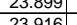 & 15.6 & $\frac{25.40 t}{2514}$ & & & & & & & & & & & & & & & 52.6. & & 45.5 .5 & & .008 & & & & \\
\hline 469 & 1128120 & & | & & $\frac{25.437}{25.437}$ & 21.064 & & & $\frac{5.46}{5.88}$ & & & $\frac{-1.41}{-121}$ & & & & & & & & 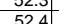 & $\frac{12.0}{122}$ & & & & & & & \\
\hline & & & 0.0050 & & 年 & 21.056 & & & 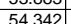 & 1766 & & & & & & & & & & 02.4 & 势, & & & & & & & \\
\hline & $1 / 28 / 2004$ & & 23.968 & 15.736 & 25.436 & 21.023 & 18.8 & 54.115 & 54.277 & 17.3 & & -1.2 & & & 0.0 & & & & 7.548 & 52.8 & 11.7 & & & & & & & \\
\hline & & & & & & & & & & & & & & & & & & & & & & & & & & & & \\
\hline & & & 23.993 & 15.812 & & 21.104 & & & $\overline{54.4}$ & 17.8 & & -1.2 & & & & & & & & 53.0 & & & & & & & & \\
\hline & & & 24.006 & 15.846 & 25.464 & 21.107 & $19.3 \mathrm{C}$ & 54.298 & 54.323 & 17.625 & 37.2. & -1.214 & & & & & & & & 02.9 & & & & & & & & \\
\hline & & 11:29:09 & & 15.879 & 25.477] & 21.196 & & & 54.147 & 17.542 & & -1.211 & & & & 14.6 & & & & 52.7 & 2.1 & & & & & & & \\
\hline & 1/28/2004 & 11:30:09 & 24.042 & 15.923 & 25.49 & 21.219 & $19.7 \mathrm{C}$ & & 54.134 & 17.63 & 37.096 & -1.21 & & 50.2 & & 14.6 & & & $\begin{array}{ll}7.6311 \\
\end{array}$ & 52.7 & 12.3 & 45.6 & 14 & & & & 0.158 & \\
\hline & 1/28/2004 & 11:31:05 & 24.056 & 15.978 & 25.494 & & & & 54.3 & & & -1.214 & & & & & & & & 52.9 & 12.0 & & & & & & & \\
\hline & 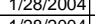 & & 24.064 & 16.001 & 25.502 & 21.241 & $19.5 \mathrm{r}$ & & & & & -1.214 & & & & & & & & 52.6 & $11.6 \mathrm{C}$ & & & & & & & \\
\hline & $1 / 2$ & & 24.002 & 16.034 & 23.807] & 21.279 & & & & & & -1.21 & & & & & & & & 03.0 & 11.. & & & & & & & \\
\hline & & & & & $\frac{23.97}{2075}$ & & & & & & & $\frac{-1.21}{1.21}$ & & & & & & & & $\begin{array}{ll}3.2 .1 \\
5\end{array}$ & & & & & & & & \\
\hline & 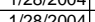 & $\begin{array}{l}11.53 . \\
1.28\end{array}$ & 4.056 & 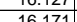 & | & 2.001 & 19.0 & & 告4.1 & & & - & & & & & & & & $\frac{52.1}{5.5}$ & $\frac{11.8}{101}$ & & & & & & & \\
\hline & $1 / 28 / 2004$ & 11:37 & 24 & & \begin{tabular}{|l|l|}
5 & 24.927 \\
\end{tabular} & 21. & & & & & & \begin{tabular}{|c|}
-1.214 \\
\end{tabular} & & & & & & & 7.748 & 526 & 11.6 & & & & & & & \\
\hline & 1/28/2004 & 11:38:05 & $\mid 3.997$ & 16.253 & 25 & 21.49 & & 54.0 & 54.2 & & & -1.20 & & & & & & & & 52.8 & 11.6 & & & & & & 0.157 & \\
\hline & & & 23.97 & $16.2 \mathrm{rt}$ & 5.013 & $21.4 \varepsilon^{2}$ & & & 54. & & & -1.214 & & & & & & & & 528 & & & & & & & & \\
\hline & & & & & & & & & & & & & & & & & & & & & & & & & & & & \\
\hline & & & 23.95 & 16.317 & 25.063 & 21.585 & & 54.0 & 54.2 & 17.5 & 37.0 & -1.214 & & & & & & & & 52.8 & & & & & & & & \\
\hline & & $11: 42: 09$ & 20.998 & $16.3 / 1$ & $1 \quad 25.136$ & 21.593 & 19.2 & 54.221 & $54.3 / 5$ & 17.03 & 37.086 & -1.214 & & 4.7 .6 & 0.044 & & & 46 & 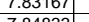 & 52.9 & 11.14 & 45 & 3.12 & & & & 0.143 & \\
\hline 490 & $\begin{array}{l}\mid 1 / 2882004 \\
1 / 28 / 2004\end{array}$ & $\begin{array}{l}\text { 11:43:09 AM } \\
11: 4: 09 \text { AM }\end{array}$ & \begin{tabular}{|l|l|}
23.979 \\
23.997
\end{tabular} & \begin{tabular}{|l|}
16.387 \\
16.431 \\
\end{tabular} & \begin{tabular}{l|l|}
1 & $25.14 t$ \\
11 & 25.165 \\
\end{tabular} & \begin{tabular}{|l|}
21.637 \\
21.63
\end{tabular} & $\frac{19.575}{20.041}$ & 54.115 5 & 54.246 & \begin{tabular}{|l|}
17.552 \\
\end{tabular} & 37.326 & $|-1.214|$ & $\begin{array}{l}-6.701 \\
-6.713\end{array}$ & 46.795 & $\mid$ & 14.643 & $\begin{array}{l}0.00 \\
0.00\end{array}$ & 471.90 & $\begin{array}{r}7.84833 \\
7.865\end{array}$ & \begin{tabular}{|c|}
52.9 \\
52.8
\end{tabular} & 11.5 & \begin{tabular}{|l|}
45.7 \\
45.7
\end{tabular} & 3.154 & $\mid$ & $\mid \begin{array}{l}0.007 \\
0.007\end{array}$ & 0.000 & $\begin{array}{l}0.159 \\
0.159\end{array}$ & \\
\hline
\end{tabular}


WSRC-TR-2005-00105, REVISION 0

SRNL-RPP-2005-00012, REVISION 0

RUN \# 1.03A; DEWATER SECOND HALF OF SLURRY - CONT.

\begin{tabular}{|c|c|c|c|c|c|c|c|c|c|c|c|c|c|c|c|c|c|c|c|c|c|c|c|c|c|c|c|c|}
\hline & A & B & $D$ & $E$ & $F$ & $G$ & $\mathrm{H}$ & $\mathrm{J}$ & $\mathrm{K}$ & $\mathrm{L}$ & $M$ & $\mathrm{~N}$ & 0 & Q & $\begin{array}{ll}R \\
\end{array}$ & $\mathrm{~s}$ & $T$ & $\mathrm{v}$ & w & $x$ & Y & z & AA & $\mathrm{AB}$ & $\mathrm{AC}$ & AD & $\mathrm{AE}$ & \\
\hline 49 & $1 / 28 / 2004$ & 11:45:09 AM & 24.004 & 16.464 & 25.207 & 21.654 & 19.87 & 54.21 & 54.468 & $\begin{array}{l}17.441 \\
\end{array}$ & 37.31 & & -6.6 & 48.459 & 0.049 & 14.643 & 0.004 & 472.90 & 7.88167 & 53.0 & 11.9 & 45.8 & & 0.007 & 0.007 & 0.000 & 0.159 & \\
\hline & & $11: 46: 09 \mathrm{AM}$ & & $\begin{array}{l}16.497 \\
16525\end{array}$ & 23.225 & 21.677 & $\begin{array}{l}19.714 \\
1906\end{array}$ & & \begin{tabular}{|l}
5.10 .10 \\
\end{tabular} & \begin{tabular}{|l|l|}
18.327 \\
1.322
\end{tabular} & & \begin{tabular}{|l|l|} 
\\
\end{tabular} & -6.756 & 52.414 & & & & & & & & & & & & & & \\
\hline & $1 / 28 / 2004$ & 11:47:09 AM & 24.02 & 16.525 & \begin{tabular}{|l|l|}
25.273 \\
\end{tabular} & 21.771 & $\begin{array}{l}19.086 \\
19.50\end{array}$ & $\begin{array}{l}53.006 \\
5.2\end{array}$ & \begin{tabular}{|l|l|}
53.187 \\
5232
\end{tabular} & 19.432 & 34.116 & \begin{tabular}{|c|} 
\\
\end{tabular} & $\begin{array}{l}-6.768 \\
-6.627\end{array}$ & $\begin{array}{l}52.207 \\
5.207\end{array}$ & 0.047 & \begin{tabular}{|l|}
14.643 \\
\end{tabular} & & $\begin{array}{l}474.90 \\
\end{array}$ & $\begin{array}{l}7.915 \\
\end{array}$ & \begin{tabular}{|l|}
51.7 \\
\end{tabular} & \begin{tabular}{|l|l}
12.8 \\
12.
\end{tabular} & & & 8.007 & 0.007 & 0.000 & 0.160 & \\
\hline \begin{tabular}{|l|}
$\mid 494$ \\
495
\end{tabular} & $\begin{array}{l}1 / 28 / 2004 \\
1 / 28 / 2004\end{array}$ & $\frac{11: 48: 09 \mathrm{AM}}{11.49}$ & $\begin{array}{r}24.023 \\
24.051\end{array}$ & $\begin{array}{l}16.548 \\
16.587\end{array}$ & 25.311 & $\begin{array}{l}21.754 \\
21.853\end{array}$ & $\begin{array}{l}19.599 \\
1.506\end{array}$ & $\begin{array}{r}53.045 \\
53045\end{array}$ & \begin{tabular}{|r|}
53.32 \\
53.326
\end{tabular} & $\begin{array}{r}19.485 \\
19389\end{array}$ & $\begin{array}{r}34.141 \\
23.109\end{array}$ & -1.214 & $\begin{array}{l}-6.632 \\
-5557\end{array}$ & $\frac{51.062}{5251}$ & 0.046 & $\begin{array}{c}4.643 \\
14643\end{array}$ & & $\begin{array}{r}475.90 \\
\end{array}$ & |7.93167| & $\begin{array}{r}51.8 \\
519\end{array}$ & $\frac{12.5}{129}$ & 136 & 3000 & \begin{tabular}{|l|}
0.007 \\
0007
\end{tabular} & 0.007 & 0.000 & $\begin{array}{l}0.156 \\
\end{array}$ & \\
\hline 4 & & & & $\begin{array}{l}10.507 \\
16.615\end{array}$ & $\begin{array}{l}25.344 \\
525.392\end{array}$ & $\begin{aligned} 21.853 \\
21.85\end{aligned}$ & $\begin{array}{l}19.50 \\
19.57\end{array}$ & $\begin{array}{l}53.045 \\
53.115\end{array}$ & \begin{tabular}{|l|}
53.326 \\
53.517 \\
\end{tabular} & $\begin{array}{l}\frac{19.389}{19397} \\
\end{array}$ & $\begin{array}{l}34.198 \\
34.172 \\
\end{array}$ & $\frac{-1.211}{-1.211}$ & & $\begin{array}{r}52.51 \\
49.877\end{array}$ & $\begin{array}{l}0.046 \\
0.043\end{array}$ & $\frac{14.643}{14.643}$ & & $\begin{array}{l}476.90 \\
477.90 \\
\end{array}$ & $\begin{array}{r}7.94833 \\
5\end{array}$ & & $\frac{12.9}{12.2}$ & $\frac{43.6}{436}$ & $\begin{array}{l}3.008 \\
3300\end{array}$ & & 0.007 & 0.000 & $\begin{array}{l}0.156 \\
0.15\end{array}$ & 0.13 \\
\hline \begin{tabular}{|l|l|}
497 \\
\end{tabular} & $1 / 28 / 2004$ & 11:51:09 AM & 24.092 & 16.643 & \begin{tabular}{|l|}
3 \\
35.42
\end{tabular} & 21.873 & 19.89 & 52.896 & 53.241 & 19.321 & $\begin{array}{l}34.1 / 2 \\
34.161 \\
\end{array}$ & $\frac{-1.211}{-1.214}$ & $\frac{-6.51}{-6.577}$ & \begin{tabular}{|l|}
4.87221 \\
52.22
\end{tabular} & $\begin{array}{l}0.043 \\
0.046\end{array}$ & $\frac{14.045}{14.643}$ & & $\frac{471.90}{478.90}$ & \begin{tabular}{|r|} 
\\
\end{tabular} & $\frac{52.0}{51.8}$ & $\frac{11.2}{12.8}$ & $\frac{43.6}{43.5}$ & $\begin{array}{l}\frac{3.000}{3.001} \\
3\end{array}$ & $\frac{0.006}{0.007}$ & $\begin{array}{l}0.006 \\
0.007\end{array}$ & $\begin{array}{c}0.000 \\
0.000\end{array}$ & 0.1456 & $\frac{0.12}{0.13}$ \\
\hline \begin{tabular}{|l|}
498 \\
\end{tabular} & & & 24.114 & 16.666 & \begin{tabular}{|l|l|}
525.452 \\
\end{tabular} & 21.845 & 19.564 & 53.263 & 53.585 & 19.435 & 34.311 & -1.214 & -6.617 & 50.328 & 0.045 & 14.643 & & 479.90 & 7.99833 & 52.1 & 12.3 & 43.8 & \begin{tabular}{|l|}
3.019 \\
\end{tabular} & \begin{tabular}{|l|l|}
0.007 \\
\end{tabular} & 0.007 & 0.000 & 0.151 & 0 \\
\hline & & & & 16.695 & 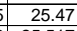 & & & & & \begin{tabular}{|l|l|}
19.304 \\
\end{tabular} & & & & & & & & & 8.015 & & & & & 0.000 & & & 0.145 & \\
\hline 500 & $1 / 28 / 2004$ & $11: 54: 09 \mathrm{AM}$ & $\begin{array}{l}24.174 \\
2.192\end{array}$ & $\begin{array}{l}16.732 \\
1675\end{array}$ & 25.517 & 21.961 & 20.15 & \begin{tabular}{|l|l|}
53.022 \\
5.020
\end{tabular} & $\begin{array}{l}53.355 \\
52.66\end{array}$ & \begin{tabular}{|l|l|}
19.23 \\
\end{tabular} & $\begin{array}{r}34.36 \\
\end{array}$ & \begin{tabular}{|c|}
-1.211 \\
\end{tabular} & & \begin{tabular}{|l|l|}
49.692 \\
5.22 \\
\end{tabular} & 0.046 & $\begin{array}{l}14.643 \\
19\end{array}$ & & 481.90 & \begin{tabular}{|l|l|} 
\\
\end{tabular} & 51.9 & 12.2 & & & 0.007 & 0.007 & 0.000 & 0.155 & \\
\hline \begin{tabular}{|l|}
501 \\
502
\end{tabular} & $1 / 28 / 2004$ & $11: 55: 09 \mathrm{AM}$ & $\begin{array}{r}24.182 \\
.201\end{array}$ & 16.75 & 25.515 & $\begin{array}{l}21.984 \\
21.072\end{array}$ & & \begin{tabular}{|l|l|}
53.062 \\
52.777
\end{tabular} & 53.446 & \begin{tabular}{|l|l|}
19.237 \\
10271
\end{tabular} & & -1.214 & & \begin{tabular}{|l|}
50.122 \\
\end{tabular} & $\begin{array}{l}0.046 \\
0.017\end{array}$ & & & 482.90 & & 52.0 & 12.3 & 43.7 & & \begin{tabular}{|l|}
0.007 \\
\end{tabular} & & & $\begin{array}{l}0.155 \\
\end{array}$ & \\
\hline$\left|\frac{502}{503}\right|$ & $\begin{array}{l}1 / 288 / 2004 \\
122004\end{array}$ & $\begin{array}{l}11: 56: 09 \mathrm{AM} \\
11157700 \mathrm{AM}\end{array}$ & $\begin{array}{r}24.21 \\
24.217\end{array}$ & $\begin{array}{l}16.788 \\
16.821 \\
\end{array}$ & $\begin{array}{l}325.548 \\
1 \\
1\end{array}$ & $\begin{aligned} 21.972 \\
22.044\end{aligned}$ & $\begin{array}{r}20.2 \\
20.83 \\
\end{array}$ & \begin{tabular}{|l}
52.777 \\
53.107
\end{tabular} & \begin{tabular}{|l|}
53.197 \\
53.554
\end{tabular} & $\begin{array}{l}\frac{19.071}{19.032} \\
\end{array}$ & $\begin{array}{r}34.36 \\
34.677 \\
\end{array}$ & $\begin{array}{l}-1.214 \\
-1.214\end{array}$ & & \begin{tabular}{|l|}
43.073 \\
99.6 \\
\end{tabular} & \begin{tabular}{|l}
0.047 \\
0.046
\end{tabular} & $\begin{array}{l}14.643 \\
14.643 \\
\end{array}$ & & $\begin{array}{r}483.90 \\
484.90\end{array}$ & \begin{tabular}{|r|}
8.065 \\
8.88167 \\
\end{tabular} & \begin{tabular}{|l|}
51.7 \\
52.1
\end{tabular} & $\frac{10.6}{12.2}$ & $\begin{array}{l}43.6 \\
43.9\end{array}$ & \begin{tabular}{|l|}
3.004 \\
3.026 \\
\end{tabular} & \begin{tabular}{|l|}
0.007 \\
0.007
\end{tabular} & $\begin{array}{l}0.007 \\
0.07\end{array}$ & $\begin{array}{l}0.000 \\
0.000\end{array}$ & $\begin{array}{l}0.158 \\
0.154\end{array}$ & 0.13 \\
\hline 504 & $1 / 28 / 2004$ & 11:58:09 AM & 24.25 & $\begin{array}{l}10.021 \\
16.865 \\
\end{array}$ & \begin{tabular}{|l|l|}
25.590 \\
\end{tabular} & 22.108 & 20.42 & 53.258 & \begin{tabular}{|l|}
53.612 \\
\end{tabular} & 19.214 & 34.676 & \begin{tabular}{|l|}
-1.214 \\
\end{tabular} & $\begin{array}{l}-0.360 \\
-6.369 \\
\end{array}$ & \begin{tabular}{|l|}
49.054 \\
\end{tabular} & 0.047 & 14.643 & 0.004 & $\begin{array}{l}485.90 \\
485.90\end{array}$ & 8.09833 & \begin{tabular}{|l|}
52.1 \\
\end{tabular} & 12.0 & $\frac{45.0}{44.0}$ & \begin{tabular}{|l|l|}
3.031 \\
3.03
\end{tabular} & & 0.007 & $\begin{array}{l}0.000 \\
0.000\end{array}$ & $\begin{array}{l}.154 \\
0.157 \\
\end{array}$ & $\begin{array}{l}0.13 \\
0.13\end{array}$ \\
\hline & $1 / 28 / 2004$ & 11:59:09 AM & 24.273 & 16.908 & \begin{tabular}{|l|l|}
25.596 \\
\end{tabular} & 22.151 & 20.36 & 52.821 & 53.255 & \begin{tabular}{|l|l|l|}
18.873 \\
\end{tabular} & 34.627 & \begin{tabular}{|l|l|} 
& -1.214 \\
\end{tabular} & & 50.726 & 0.047 & $\begin{array}{l}14.643 \\
\end{array}$ & & 486.90 & \begin{tabular}{|l|l|} 
\\
\end{tabular} & 51.8 & 12.4 & & & & 0.007 & & & \\
\hline & $1 / 28 / 2004$ & 12:00:09 PM & & & \begin{tabular}{|l|l|}
25.598 \\
\end{tabular} & 22.138 & & & 53.372 & \begin{tabular}{|l|l|l|}
18.834 \\
\end{tabular} & 34.664 & -1.219 & -6.34 & & 0.047 & $\begin{array}{l}14.643 \\
\end{array}$ & & & 8.13167 & 51.9 & 12.0 & & & & & & 0.157 & \\
\hline & $1 / 28 / 2004$ & 12:01:09 PM & 24.309 & 16.97 & 25.637 & 22.238 & & 53.001 & 53.471 & 18.871 & & & & \begin{tabular}{|l|l}
51.83 \\
\end{tabular} & 0.047 & 14.643 & & & 8.14833 & & & & & 0.007 & & 0.000 & 0.157 & \\
\hline & $1 / 28 / 2004$ & 12:02:09 PM & & $\begin{array}{l}17.008 \\
17057\end{array}$ & \begin{tabular}{|r|}
25.66 \\
\end{tabular} & $\begin{array}{l}22.286 \\
2.210\end{array}$ & & & \begin{tabular}{|l|}
53.591 \\
55720
\end{tabular} & $\begin{array}{l}18.859 \\
18.790\end{array}$ & 34.716 & $\begin{array}{r}-1.214 \\
\end{array}$ & & \begin{tabular}{|l|}
48.031 \\
\end{tabular} & 0. & $\begin{array}{l}4.643 \\
1.63\end{array}$ & & & \begin{tabular}{|r|}
8.165 \\
\end{tabular} & \begin{tabular}{|l|l|}
52.1 \\
5.2
\end{tabular} & 11.8 & & & & & & 0.160 & \\
\hline $5 \frac{509}{510}$ & $\frac{1 / 28 / 2004}{1 / 182 / 2004}$ & $\begin{array}{l}\frac{12: 03: 09 \text { PM }}{1200409} \\
\end{array}$ & $\begin{array}{r}24.36 \\
24.383 \\
\end{array}$ & $\begin{array}{l}17.057 \\
17.101\end{array}$ & \begin{tabular}{|l|}
$\mid 25.678$ \\
25.686
\end{tabular} & 22.319 & $\frac{20.34}{20.20}$ & $\begin{array}{r}53.163 \\
53.246\end{array}$ & \begin{tabular}{|l|}
53.749 \\
53.771
\end{tabular} & $\begin{array}{l}18.788 \\
18.937\end{array}$ & $\begin{array}{r}34.814 \\
34.828 \\
\end{array}$ & $\begin{array}{l}-1.211 \\
-1.216\end{array}$ & $\begin{array}{l}-6.308 \\
-6.311\end{array}$ & \begin{tabular}{|l|}
49.402 \\
50.195 \\
\end{tabular} & \begin{tabular}{|c|}
0.047 \\
0.047
\end{tabular} & & & $\begin{array}{l}490.90 \\
491.90 \\
\end{array}$ & $\mid$\begin{tabular}{|c|}
8.18167 \\
8.19833
\end{tabular} & \begin{tabular}{|l|}
52.3 \\
52.3
\end{tabular} & $\frac{12.1}{12 .}$ & $\begin{array}{l}44.0 \\
44.0\end{array}$ & \begin{tabular}{|l|} 
\\
\end{tabular} & \begin{tabular}{|l|}
0.007 \\
0.007
\end{tabular} & $\begin{array}{l}0.007 \\
0.07\end{array}$ & $\begin{array}{l}0.000 \\
0.000\end{array}$ & $\begin{array}{l}0.156 \\
0.156\end{array}$ & 0.13 \\
\hline 511 & $1 / 28 / 2004$ & 12:05:09 PM & 24.4 & 17.129 & \begin{tabular}{|l|l|}
25.7003 \\
\end{tabular} & 22.335 & 20.301 & $\begin{array}{l}53.246 \\
53.068\end{array}$ & \begin{tabular}{|l|}
53.111 \\
53.608
\end{tabular} & 18.742 & $\begin{array}{l}54.020 \\
34.863\end{array}$ & 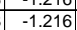 & $\begin{array}{l}-6.311 \\
-6.308\end{array}$ & \begin{tabular}{|l|}
50.1580 \\
\end{tabular} & $\begin{array}{l}0.047 \\
0.047\end{array}$ & $\begin{array}{l}14.645 \\
14.643\end{array}$ & & $\begin{array}{l}491.90 \\
492.90\end{array}$ & \begin{tabular}{|r|} 
\\
\end{tabular} & \begin{tabular}{|l|}
52.1 \\
52.1
\end{tabular} & 12.4 & $\begin{array}{l}44.0 \\
44.0 \\
\end{array}$ & \begin{tabular}{|l|}
3.030 \\
3.031 \\
\end{tabular} & \begin{tabular}{|l|}
0.007 \\
0.007 \\
\end{tabular} & 0.007 & $\begin{array}{l}0.000 \\
0.000\end{array}$ & $\begin{array}{l}0.156 \\
0.156\end{array}$ & $\begin{array}{l}0.13 \\
0.13 \\
\end{array}$ \\
\hline & $1 / 28 / 2004$ & 12:06:09 PM & 24.418 & 17.162 & 25.731 & 22.352 & 20.38 & 53.035 & \begin{tabular}{|l|}
53.591 \\
\end{tabular} & 18.757 & 34.855 & \begin{tabular}{|c|} 
\\
\end{tabular} & & 48.298 & 0.047 & 14.643 & & 493.90 & 8.23167 & \begin{tabular}{|l|}
52.1 \\
\end{tabular} & 11.8 & 43.9 & \begin{tabular}{|l|}
3.030 \\
\end{tabular} & \begin{tabular}{|l|}
0.007 \\
\end{tabular} & 0.007 & 0.000 & 0.156 & $\frac{0.13}{0.13}$ \\
\hline \begin{tabular}{|l|}
513 \\
\end{tabular} & $1 / 28 / 2004$ & 12:07:09 PM & 24.43 & 17.179 & \begin{tabular}{|l|l|}
25.733 \\
\end{tabular} & 22.429 & 19.5 & 53.149 & 53.78 & 18.83 & 34.857 & -1.214 & -6.308 & 49.996 & 0.047 & 14.643 & & 494.90 & 8.24833 & 52.3 & 12.3 & 44.0 & 3.034 & & 0.007 & & & \\
\hline 514 & $1 / 28 / 2004$ & 12:08:09 PM & 24.452 & 17.212 & 25.75 & 22.437 & 19.8 & 53.091 & & $\begin{array}{ll}18.802 \\
\end{array}$ & 34.835 & -1.214 & & \begin{tabular}{|l|l|}
48.749 \\
\end{tabular} & 0.047 & & & 495.90 & 8.265 & & & & & & & 0.000 & 0.156 & \\
\hline 515 & & & & & & & & & 53.61 & & & & & & & & & & & & & & & & & & & \\
\hline & $1 / 28 / 2004$ & 12:10:09 PM & 24.492 & & \begin{tabular}{|l|l|}
25.78 \\
\end{tabular} & 22.442 & & 52.958 & 53.599 & $\begin{array}{l}18.69 \\
\end{array}$ & 34.873 & & & \begin{tabular}{|l|l|}
50.624 \\
\end{tabular} & & & & 497.90 & 8.29833 & & 12. & & & & & & & \\
\hline & & 12:11:09 PM & & & & 22.414 & & & 53.618 & 18.701 & 34.902 & -1.214 & & 59 & & & & & & & 12. & & & & & & & \\
\hline 518 & $1 / 28 / 2004$ & 12:11:30 PM & 24.515 & 17.311 & \begin{tabular}{|l|l|} 
& 25.798 \\
\end{tabular} & 22.44 & 19. & 53.08 & 53.703 & \begin{tabular}{ll|l}
18.763 \\
\end{tabular} & 34.896 & -1.214 & & $\begin{array}{l}47.243 \\
\end{array}$ & 0.047 & & & 499.25 & 8.32083 & 52.2 & 11.6 & 44.0 & & 007 & 0.007 & 0.000 & 0.156 & \\
\hline & 1/28/2004 & $12: 12: 8$ & 24.511 & 17.317 & 25.744 & 22.411 & 20. & 52.838 & 53.488 & 18.545 & 34.896 & -1.214 & & & 0.047 & & & 500 & 8.3375 & 52.0 & 12. & & & & & & & 0.1 \\
\hline & $1 / 28 / 2004$ & $12: 13: 30$ PM & 24.534 & 17.35 & 25.731 & 22.383 & & 53.061 & 53.707 & 18.682 & 34.912 & -1.214 & & 49.94 & 0.6 & 14. & & & & 52.2 & 12. & & & & & & & \\
\hline 52 & & & 24.556 & & 25.134 & & & 52.981 & \begin{tabular}{|l}
53.593 \\
\end{tabular} & 18.793 & 34.882 & -1.214 & & 3 & & & & & 8.37083 & & & & & & & 0.000 & & \\
\hline & 8 & & 24.548 & 17.405 & & 22.448 & & 53.024 & & 18.811 & 4.853 & -1.219 & & .9 & & & & & (1) & & & . & & & & & & \\
\hline & $\frac{112810044}{102004}$ & 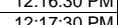 & & & & 2.2.52 & & $\frac{53.142}{55918}$ & & & $\frac{34.8}{32.1}$ & & & $\frac{2}{1.1}$ & & & & & & & & & & & & & & \\
\hline \begin{tabular}{|l|}
525 \\
\end{tabular} & $1 / 128 / 2004$ & $\frac{12.16}{12 \cdot 18}$ & 24.554 & $\begin{array}{l}17.450 \\
17.484\end{array}$ & \begin{tabular}{|l|l|}
25619 \\
\end{tabular} & $\frac{26.364}{22537}$ & & 53.022 & \begin{tabular}{|l|l|}
53723 \\
\end{tabular} & (554 & $\begin{array}{r}30.144 \\
35.19\end{array}$ & & & 76 & & & & & 8.4375 & & 11 & & & & & & & \\
\hline & $1 / 28 / 2004$ & $\frac{12.10}{12.19}$ & 24.550 & & & 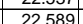 & & $\frac{0.0<4}{53.2}$ & $55^{5.914}$ & $\begin{array}{l}10.454 \\
18.619\end{array}$ & $\begin{array}{l}35.138 \\
35.15\end{array}$ & & & & & & & & & & $\frac{1}{11}$ & & & & & & & \\
\hline 52 & $1 / 28 / 2004$ & 12:20:30 PM & 24.561 & 17.545 & 24.212 & 22.642 & & 53.534 & 54.296 & 18 & 36.088 & -1.214 & & 49.757 & & & & 508 & & 52. & 12 & & & & & & 160 & \\
\hline & 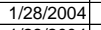 & & 24.538 & $\begin{array}{l}17.557 \\
\end{array}$ & \begin{tabular}{|l|l|}
24.835 \\
\end{tabular} & 22.653 & 21.1 & 52.937 & 53.672 & 18.698 & $\begin{array}{l}34.796 \\
\end{array}$ & & & & & & & & & & & & & & & & & \\
\hline & & 22:30 PM & & & & & & & & & & & & 49.28 & & & & & & 52.2 & 12. & & & & & & & \\
\hline & $1 / 28 / 2004$ & 12:23: & $24.44 t$ & 17.590 & \begin{tabular}{|c|}
24.545 \\
\end{tabular} & 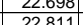 & & 53.1 & 786 & & & -1.219 & & $\begin{array}{r}49.99 \\
51.25 \\
\end{array}$ & 0.045 & & & 511.25 & \begin{tabular}{|l|l|}
8.52083 \\
\end{tabular} & & & 43.9 & \begin{tabular}{|l|l|}
3.026 \\
\end{tabular} & 0.007 & & & & 0 \\
\hline $\begin{array}{l}532 \\
532 \\
\end{array}$ & $\mid 1 / 28 / 2004$ & $\begin{array}{l}\frac{1.24}{12.25} \\
\end{array}$ & $\frac{24.424}{24392}$ & & \begin{tabular}{|l|l|l|l|l|l|l|} 
\\
\end{tabular} & & & 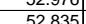 & & & & -1.214 & & 51.25 & & & & & & & $\frac{12 .}{12 .}$ & & & & & & & \\
\hline 533 & $1 / 28 / 2004$ & & 24.389 & & 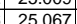 & & & & \begin{tabular}{|l|l|l|l|}
53494 \\
\end{tabular} & & & -1.211 & & 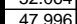 & & & & & & & & & & & & & & \\
\hline 534 & $88 / 2004$ & $12: 27: 3$ & 24.381 & 17.753 & \begin{tabular}{|l|l|}
25.064 \\
\end{tabular} & 22.848 & & 52.974 & 53 & 18.856 & & $\frac{-1.216}{-1.216}$ & & 49.4 & & & & & & 52.2 & $\frac{11}{12}$ & & & & & & 0.156 & \\
\hline & & & & & & & & & & & & & & & & & & & & & & & & & & & & \\
\hline & & & 24.374 & & & 22.8 & & 52.8 & & & & -1.216 & & 5.24 & & & & & & & 12 & & & & & & & \\
\hline & & & 24.39 & 17.843 & 25.128 & & & & & & & & & 49.1 & & & & & & & 12. & & & & & & & \\
\hline & & $12: 31$ & 24.38 & & \begin{tabular}{|l|}
25.13 \\
\end{tabular} & 22. & & 52.5 & & & & -1.214 & & 49 & & & & & & & & & & & & & & \\
\hline & $1 / 28 / 2 / 2$ & $12: 32$ & 24.379 & 17.8 & & 22. & & 52.8 & 53.56 & 18.675 & 34.9 & -1.216 & & 50 & & & & & & 52 & 12. & & & & & & 156 & \\
\hline & $1 / 28 / 2004$ & & 24.37 & & & & & 52 & & & & & & & & & & & & & 12. & & & & & & & \\
\hline 54 & $1 / 28 / 2004$ & 12:34: & 24.387 & 17.936 & 25.155 & 22.935 & & 53 & \begin{tabular}{|l|l|} 
\\
\end{tabular} 3.8833 & 18.834 & & -1.214 & & 48. & & & & & & 52.4 & 11.9 & & & & & & & \\
\hline & & & & 17.95 & & & & & & & & -1.2 & & & & & & & & & 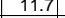 & & & & & & & \\
\hline & $1 / 28 / 2004$ & $12: 36: 30 \mathrm{P}$ & 24.395 & 18 & 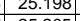 & 22.9 & & 52.9 & 53.666 & & & & & & & & & & & 52.2 & 12. & & & & & & & \\
\hline & 112820004 & & & $\frac{18.02 t}{18065}$ & & $\frac{2.2964}{2301}$ & & & \begin{tabular}{|l}
53.12 \\
53.14
\end{tabular} & $\begin{array}{l}18.125 \\
18567\end{array}$ & & - $\frac{-1.244}{-1241}$ & & & & & & & & 524 & 11. & & & & & & & \\
\hline 546 & $\mid 1 / 28 / 2004$ & $\frac{1<.30 .4}{12 \cdot 39 \cdot 3}$ & 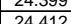 & 10.005 & & & & 52.089 & \begin{tabular}{|l|l|}
53.0414 \\
\end{tabular} & & & & & & & & & & & 52 & 111 & & & & & & & \\
\hline \begin{tabular}{|l|l|}
547 \\
\end{tabular} & $1 / 28 / 20$ & $\frac{12.05}{12: 40}$ & $\frac{24.41}{24.41}$ & & & & & & & & & & & & & & & & & & & & & & & & & \\
\hline 54 & $11 / 28 / 20$ & & 24.431 & $\begin{array}{l}18.147 \\
147\end{array}$ & $\mid$\begin{tabular}{|l|l|}
$\mid 25.244$ \\
\end{tabular} & 22.9 & & 52.5 & \begin{tabular}{|l}
53.506 \\
\end{tabular} & & & $\frac{-1.216}{-1.216}$ & & 51.4 & & & & & & & 12. & & & & & & & \\
\hline & & & & & & & & & & & & & & & & & & & & & & & & & & & & \\
\hline & & & 24.42 & & 25.242 & 23.063 & & & \begin{tabular}{|l|l|}
53.942 \\
\end{tabular} & 18.86 & & $\mid-1.214$ & & & & & & & & & & & & & & & & \\
\hline & & & & & & & & & & & & & & & & & & & & & & & & & & & & \\
\hline & & & & & & 23.12 & & & \begin{tabular}{|l|}
53.7 \\
\end{tabular} & & & -1.214 & & & & & & & & & & & & & & & & \\
\hline 55 & $1 / 28 / 2004$ & & 25.05 & 18.263 & & & & 51.36 & 53.8 & & & -1.214 & & 48.3 & & & & & & & & 42 & & & & & & \\
\hline & $1 / 28 / 2004$ & $12: 47$ & 25.62 & & 25.28 & 23.166 & & 48.8 & \begin{tabular}{|l|l|}
53.99 \\
\end{tabular} & & & -0.374 & & & & & & & & 52.4 & 11.8 & & & & & & & \\
\hline & $1 / 28 / 2$ & & 24.625 & 18.313 & 25.292 & & & 53.342 & 54.155 & 18.255 & & -1.214 & & $46.5 \mathrm{r}$ & & & & & & & 11 & & & & & & & \\
\hline & & & & & & & & & & & & & & & & & & & & & 11. & & & & & & & \\
\hline & & & 24.9 & & 24.526 & 23.299 & & 53.225 & 53.991 & & & -1.214 & & & & & & & & 52. & & & & & & & & \\
\hline & & & & & \begin{tabular}{|l}
24.7155 \\
\end{tabular} & 23. & & 53.0 & & 18.9 & & & & & & & & & & & & & & & & & 54 & 0. \\
\hline 5 & $1 / 28 / 2004$ & $12.53 \cdot 30 \mathrm{PM}$ & 24.912 & 18.427 & 24804 & \begin{tabular}{|l|}
23.3069 \\
23382
\end{tabular} & $\frac{2.1351}{20.74}$ & $\begin{array}{l}53.1113 \\
5338\end{array}$ & 54.035 & $\begin{array}{r}1.0804 \\
18.893 \\
\end{array}$ & 34802 & $\mid-1214$ & -701 & 49204 & & 14,643 & & 54125 & \begin{tabular}{|l|}
9.00417 \\
\end{tabular} & 526 & 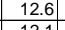 & 43.9 & & & 0007 & 5000 & 0167 & \\
\hline & $1 / 28 / 2004 \mid$ & 12:53:30 PM & & & & & & & & & & & & & & 14.643 & & 541.25 & 9.02083 & & & & & 0.007 & 0.007 & 0.000 & 0.167 & 0.1 \\
\hline
\end{tabular}


WSRC-TR-2005-00105, REVISION 0

SRNL-RPP-2005-00012, REVISION 0

RUN \# 1.03A; DEWATER SECOND HALF OF SLURRY - CONT.

\begin{tabular}{|c|c|c|c|c|c|c|c|c|c|c|c|c|c|c|c|c|c|c|c|c|c|c|c|c|c|c|c|c|}
\hline & A & B & \begin{tabular}{|l|l|} 
D \\
\end{tabular} & $E$ & $F$ & $G$ & $\mathrm{H}$ & $\mathrm{J}$ & $\mathrm{K}$ & $\mathrm{L}$ & M & $N$ & 0 & Q & $\mathrm{R}$ & \begin{tabular}{l|l|} 
&
\end{tabular} & $T$ & $\mathrm{v}$ & w & $x$ & Y & $z$ & AA & $A B$ & $\mathrm{AC}$ & $\mathrm{AD}$ & AE & \\
\hline 56. & $1 / 28 / 2004$ & 12:54:30 PM & 24.859 & 18.434 & 24.836 & 23.414 & 21.064 & 52.873 & 53.877 & 18.722 & 34.693 & -1.216 & -6.58 & 49.709 & 0.045 & \begin{tabular}{|l|l|}
14.643 \\
\end{tabular} & 0.004 & 542.25 & 9.0375 & 52.4 & 12.2 & & 3.019 & 0.007 & 0.007 & 0.000 & 0.154 & 0.13 \\
\hline & & & & 18.451 & & & 21.559 & 52.794 & \begin{tabular}{|l|l|}
53.684 \\
5872
\end{tabular} & & & $\begin{array}{r}-1.214 \\
\end{array}$ & -6.586 & & 0.044 & 14.643 & & & & & $\frac{12.1}{12.1}$ & & & & & & & \\
\hline & $\begin{array}{ll}1 / 28 / 2004 \\
1 / 282004\end{array}$ & $\begin{array}{l}12: 56: 30 \mathrm{PM} \\
12.57 \cdot 30 \mathrm{PM}\end{array}$ & $\begin{array}{l}24.757 \\
2475 \\
\end{array}$ & $\begin{array}{l}18.467 \\
18515\end{array}$ & \begin{tabular}{|l|}
24.894 \\
24942
\end{tabular} & $\begin{array}{l}23.391 \\
23.429 \\
\end{array}$ & 21.359 & 52.925 & \begin{tabular}{|l|l|}
53.879 \\
5.752
\end{tabular} & $\begin{array}{l}18.841 \\
1.712\end{array}$ & 34.625 & \begin{tabular}{|c|c|}
-1.216 \\
1.21
\end{tabular} & -6.583 & 47.838 & 0.045 & 14.643 & & & \begin{tabular}{|l|l|}
5.07083 \\
\end{tabular} & $\begin{array}{l}52.4 \\
5.2\end{array}$ & \begin{tabular}{|l|l|}
11.7 \\
\end{tabular} & 43.8 & 3.018 & 0.007 & 0.007 & 0.000 & 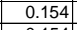 & \\
\hline$\frac{564}{565}$ & $\begin{array}{r}1 / 28 / 2004 \\
1282(204\end{array}$ & $\begin{array}{l}12: 57: 30 \mathrm{PM} \\
12.58 \cdot 30 \mathrm{PM}\end{array}$ & $\begin{array}{r}24.75 \\
24.731 \\
\end{array}$ & $\begin{array}{l}18.515 \\
18.538\end{array}$ & \begin{tabular}{|l|}
24.942 \\
24.974
\end{tabular} & $\begin{array}{l}23.429 \\
23.466 \\
\end{array}$ & $\frac{21.301}{21.64}$ & \begin{tabular}{|c|}
52.806 \\
52.979
\end{tabular} & \begin{tabular}{|l|}
53.753 \\
53.973
\end{tabular} & $\frac{18.712}{18.914}$ & $\begin{array}{l}34.654 \\
33619 \\
\end{array}$ & $\begin{array}{r}-1.216 \\
-1.16\end{array}$ & $\begin{array}{r}-6.58 \\
-6.58 \\
\end{array}$ & $\begin{array}{r}49.058 \\
50.044\end{array}$ & $\begin{array}{l}0.045 \\
0.045 \\
\end{array}$ & $\begin{array}{l}14.643 \\
14633 \\
\end{array}$ & 0.0 & $\begin{array}{r}545.25 \\
546.25\end{array}$ & \begin{tabular}{|l|l|} 
& 9.0875 \\
& 91017
\end{tabular} & & 12.0 & 43.7 & | & \begin{tabular}{|l|}
0.007 \\
0.007
\end{tabular} & 0.007 & 0.000 & $\begin{array}{l}0.154 \\
0152\end{array}$ & \\
\hline 566 & & & & & & 23.4684 & & $\begin{array}{l}53.999 \\
53.041 \\
\end{array}$ & \begin{tabular}{|l|}
$53.9 / 3$ \\
54.062 \\
\end{tabular} & & $\begin{array}{l}34.619 \\
34.705 \\
\end{array}$ & $\frac{-1.216}{-1.216}$ & $\begin{array}{l}-6.58 \\
-6.58 \\
\end{array}$ & $\begin{array}{l}50.044 \\
49.709\end{array}$ & 0.045 & \begin{tabular}{|l|}
14.643 \\
14.643
\end{tabular} & & 546.25 & \begin{tabular}{|l}
$\mid 9.10417$ \\
5912083 \\
5
\end{tabular} & $\begin{array}{l}52.5 \\
52.6\end{array}$ & $\begin{array}{l}12.3 \\
12.2 \\
\end{array}$ & $\frac{43.8}{43.9}$ & $\begin{array}{l}3.020 \\
3025 \\
3\end{array}$ & & $\begin{array}{l}0.007 \\
0.007\end{array}$ & 0.000 & 0.153 & 0.13 \\
\hline 567 & $1 / 28 / 2004$ & 1:00:30 PM & 24.706 & 18.578 & \begin{tabular}{|l|}
24.999 \\
\end{tabular} & 23.507 & 21.12 & 52.52 & \begin{tabular}{|l|l|}
53.473 \\
\end{tabular} & 18.525 & 34.635 & $\mid-1.219$ & -6.586 & 50.533 & 0.045 & \begin{tabular}{l|l}
14.643 \\
14.643
\end{tabular} & & $\begin{array}{l}544.25 \\
548.25 \\
\end{array}$ & 9.1375 & $\frac{52.6}{52.0}$ & $\frac{12.2}{12.4}$ & $\frac{43.9}{43.6}$ & $\begin{array}{l}3.025 \\
3.005 \\
\end{array}$ & \begin{tabular}{|l|}
0.007 \\
0.007 \\
\end{tabular} & $\frac{0.007}{0.007}$ & $\begin{array}{l}0.000 \\
0.000\end{array}$ & 0.153 & $\frac{0.13}{0.13}$ \\
\hline 568 & & & 24.709 & 18.596 & 25.052 & 23.565 & & 52.669 & \begin{tabular}{|l|l|}
53.664 \\
\end{tabular} & 18.723 & 34.619 & -1.214 & -6.58 & 48.332 & 0.045 & 14.643 & & 549.25 & 9.15417 & 52.2 & 11.8 & 43.6 & \begin{tabular}{|l|}
3.009 \\
\end{tabular} & \begin{tabular}{|l|}
0.007 \\
\end{tabular} & 0.007 & 0.000 & 0.153 & 0.13 \\
\hline & & & & & & & & & & & & & & & & 14.643 & & & & & 12.1 & & & & & & & \\
\hline 57. & $1 / 28 / 2004$ & 1:03:30 PM & $\begin{array}{ll}24.703 \\
2.005\end{array}$ & 18.651 & \begin{tabular}{|l|l|}
25.081 \\
25089
\end{tabular} & 23.589 & 21.8 & 52.85 & 53.912 & 18.759 & 34.656 & -1.216 & $\begin{aligned}-6.58 \\
6.571\end{aligned}$ & \begin{tabular}{|l|l|}
50.57 \\
\end{tabular} & 0.045 & $\begin{array}{ll}14.643 \\
\end{array}$ & & \begin{tabular}{|l|l|}
551.25 \\
\end{tabular} & \begin{tabular}{|c|}
9.1875 \\
\end{tabular} & 52.4 & 12.4 & 43.8 & 3.01 & 0.007 & 0.007 & 0.000 & 0.153 & \\
\hline 571 & $1 / 28 / 2004$ & 1:04:30 PM & 24.695 & 18.668 & $25.088 \mid$ & $\begin{aligned} 23.585 \\
22593 \\
\end{aligned}$ & 22.196 & \begin{tabular}{|l|l|}
52.844 \\
52672
\end{tabular} & \begin{tabular}{|l|}
53.912 \\
5070
\end{tabular} & $\begin{array}{l}18.845 \\
10709\end{array}$ & 34.609 & \begin{tabular}{|l|l|} 
& -1.216 \\
\end{tabular} & $\begin{array}{l}-6.574 \\
6571\end{array}$ & 49.31 & 0.044 & 14.643 & & & \begin{tabular}{|l|l|}
9.20417 \\
\end{tabular} & & 12.1 & & & \begin{tabular}{|l|l|} 
& 0.007 \\
\end{tabular} & 0.007 & & 0.150 & \\
\hline$\frac{572}{573}$ & 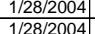 & $\begin{array}{l}\text { 1:05:30 PM } \\
1.06630 \mathrm{PM}\end{array}$ & $\begin{aligned} 24.707 \\
24.71\end{aligned}$ & $\frac{18.711}{18.743}$ & \begin{tabular}{|r|}
25.12 \\
25.122
\end{tabular} & $\begin{aligned} 23.583 \\
23.65 \\
\end{aligned}$ & $\frac{22.685}{22.391}$ & \begin{tabular}{|c|}
52.672 \\
52774 \\
\end{tabular} & \begin{tabular}{|l|}
53.678 \\
53.823 \\
\end{tabular} & $\begin{array}{l}18.708 \\
18.709\end{array}$ & ..599 625 & $\begin{array}{l}9-1.214 \\
5 \\
-1.216\end{array}$ & $\begin{array}{l}-6.571 \\
-6.568 \\
-6.5\end{array}$ & \begin{tabular}{|l|}
$\mid 48.912$ \\
45.468 \\
\end{tabular} & $\begin{array}{l}0.044 \\
0.044\end{array}$ & $\begin{array}{l}4.643 \\
.4633 \\
\end{array}$ & & $\begin{array}{l}553.25 \\
554.25 \\
\end{array}$ & \begin{tabular}{|l|l|} 
& 9.22083 \\
5.2375
\end{tabular} & \begin{tabular}{|l|}
52.2 \\
52.4 \\
\end{tabular} & \begin{tabular}{|l|}
12.0 \\
11.
\end{tabular} & $\begin{array}{ll}43.6 \\
43.7\end{array}$ & \begin{tabular}{|l|}
3.009 \\
3013
\end{tabular} & \begin{tabular}{|l|}
0.007 \\
0.007
\end{tabular} & 0.007 & 0.000 & 0.150 & 0.13 \\
\hline 574 & $1 / 28 / 2004$ & $1: 07: 30$ PM & $\frac{24.71}{24.726}$ & 18.78 & \begin{tabular}{|l|l|l|l|l|} 
& 25.169 \\
\end{tabular} & \begin{tabular}{r|r|}
23.687 \\
23.687
\end{tabular} & 22.066 & $\begin{array}{l}52.84 \\
52.782 \\
\end{array}$ & \begin{tabular}{|l|}
53.8256 \\
53.856 \\
\end{tabular} & 18.781 & $\frac{34.020}{34.66}$ & \begin{tabular}{|c|c|} 
& -1.214 \\
\end{tabular} & $\begin{array}{l}-0.000 \\
-6.568\end{array}$ & \begin{tabular}{|l|}
49.490 \\
49.794
\end{tabular} & 0.044 & $\begin{array}{l}14.643 \\
14.643 \\
\end{array}$ & $\begin{array}{l}0.004 \\
0.004\end{array}$ & 5555.25 & \begin{tabular}{|l|}
\multicolumn{1}{|c|}{$\mid 23 / 5$} \\
9.25417
\end{tabular} & $\begin{array}{l}52.4 \\
52.4\end{array}$ & $\frac{1.1}{12.2}$ & $\begin{array}{l}43.7 \\
43.7 \\
\end{array}$ & \begin{tabular}{|l|}
3.013 \\
3.014 \\
\end{tabular} & $\begin{array}{l}0.007 \\
0.007 \\
\end{array}$ & $\begin{array}{l}0.007 \\
0.007\end{array}$ & $\begin{array}{l}0.000 \\
0.000\end{array}$ & $\begin{array}{l}0.150 \\
0.149 \\
\end{array}$ & 0.13 \\
\hline & $1 / 28 / 2004$ & 1:08:30 PM & 24.729 & 18.809 & & 23.62 & & 52.819 & \begin{tabular}{|l|}
53.931 \\
\end{tabular} & 18.813 & 34.572 & -1.216 & & 47.548 & & & & & & & 11.7 & & & & 0.007 & & & $\frac{0.13}{0.13}$ \\
\hline & $1 / 28 / 2004$ & 1:09:30 PM & & 18.831 & 25.159 & & 21.946 & 52.827 & & 18.757 & 34.666 & -1.219 & -6.574 & 49.316 & 0.044 & 14.643 & & 557.25 & 9.2875 & 52.5 & 12.1 & 43.7 & & & 0.007 & & 149 & $\frac{0.13}{0.13}$ \\
\hline 577 & $1 / 28 / 2004$ & 1:10:30 PM & 24.759 & & 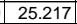 & 23.735 & 22.049 & 52.759 & 53.96 & 18.635 & & & & 48.419 & 0.045 & & & & & & & & & & & & & \\
\hline 5 & B/2004 & & & & & & & & & & 34.679 & & & $\begin{array}{l}50.814 \\
\end{array}$ & 0.044 & & & 559.2 & & 52.4 & 4. & & & & & & & \\
\hline 579 & 1/28/2004 & 1:12:30 PM & 24.788 & $\begin{array}{l}18.914 \\
\end{array}$ & & 23.775 & & 52.659 & \begin{tabular}{|l|l|} 
& 53.78 \\
\end{tabular} & \begin{tabular}{|l|l|l|l|l}
18.669 \\
\end{tabular} & & & & 49.383 & 0.044 & & & & .3375 & & 12. & & 3.0 & & & & & \\
\hline & 1/28/2004 & 1:13:30 PM & & & & & & 52.894 & \begin{tabular}{|l|l|}
54.049 \\
\end{tabular} & \begin{tabular}{|l|l|}
18.79 \\
\end{tabular} & 34.674 & -1.216 & & 110028 & 0.045 & & & & 2517 & 52.6 & & & & & & & & \\
\hline 581 & 1/28/2004 & 1:14:30 PM & 24.807 & 18.953 & 25.255 & 23.854 & 21.69 & 52.823 & \begin{tabular}{|l|l|} 
& 53.898 \\
\end{tabular} & 18.715 & 34.709 & $\mid-1.214$ & & $\begin{array}{l}48.059 \\
\end{array}$ & 0.045 & $\begin{array}{l}14.643 \\
\end{array}$ & & 562.25 & 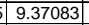 & 52.4 & 111.8 & 43.8 & 3.018 & 0.007 & 0.007 & 0.000 & 0.152 & \\
\hline & $1 / 28 / 2004$ & 1:15:30 PN & 24.815 & 18.971 & 25.253 & 23.891 & & 52.651 & 53.774 & 18.639 & 34.687 & -1.219 & & 48.707 & 0.045 & $\begin{array}{l}14.643 \\
\end{array}$ & & & 9.3875 & & 11.0 & & 3.01 & & & & & \\
\hline & $1 / 28 / 2004$ & 1:16:30 PM & 24.826 & 18.993 & 25.265 & 23.918 & & 52.665 & 53.888 & 18.611 & 34.672 & -1.216 & & 48.53 & 0.045 & 14.643 & & & 40417 & 52.4 & $11 . .5$ & & & & 0.007 & & 0.152 & \\
\hline 584 & 112282004 & $1: 17$ & 24.837 & 19.009 & 25.216 & 23.919 & & 52.865 & 54.037 & 18.779 & 34.73 & & & & 0.045 & & & & 42083 & & & 43.8 & & & 0.007 & 0.000 & 0.152 & \\
\hline & 80212004 & $\frac{1.18: 30 \mathrm{PM}}{1.1020}$ & & & 20.201 & & & 52.684 & 53.846 & 18.48 & 34.863 & & & 10 & & & & & .84375 & & & & & & & & & \\
\hline & $\frac{112810004}{102004}$ & 1.930 & & & & & & $\begin{array}{l}52.1906 \\
55806\end{array}$ & \begin{tabular}{|l|l|l} 
\\
\end{tabular} & & 34.929 & & & & & & & & & & & & & & & & & \\
\hline$\frac{501}{588}$ & $\mid$ & $\begin{array}{l}1.20 .50 \mathrm{PM} \\
1: 21: 30 \mathrm{PM}\end{array}$ & 24.889 & $\frac{19.00}{19.106}$ & \begin{tabular}{|l|l|l|}
25335 \\
2532
\end{tabular} & 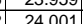 & & $\begin{array}{l}52.0068 \\
52838\end{array}$ & \begin{tabular}{|l|}
54.041 \\
54.043
\end{tabular} & $\begin{array}{l}10.050 \\
18.608\end{array}$ & 34.059 & $\begin{array}{l}-1.211 \\
-1.216 \\
\end{array}$ & & 48.785 & $=0.044$ & $\frac{14.0}{14.6}$ & & 56925 & 94875 & 526 & 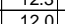 & 438 & 302 & 007 & 0006 & 年 0.000 & $\begin{array}{l}0.148 \\
0148\end{array}$ & \\
\hline & $1 / 128 / 2004$ & $1: 22: 30$ PM & 24.911 & 19.139 & 25.349 & 24018 & 22.2 & 52.819 & \begin{tabular}{|l|}
54.028 \\
5
\end{tabular} & $\begin{array}{l}18.064 \\
18.424 \\
\end{array}$ & 35.066 & -1.216 & & 48849 & 0.045 & & & & 9.50417 & 526 & 120 & & & & & & & \\
\hline & $1 / 28 / 2004$ & 1:23:30 PM & 24.919 & & 25.362 & $\frac{2.056}{24.056}$ & & $\frac{23.103}{53.103}$ & 54.366 & 18.562 & 35.142 & -1.216 & & 49.973 & 0.044 & & & 571.2 & & $\frac{32.9}{52.9}$ & $\frac{1.6}{12.2}$ & & & & & & 0.147 & \\
\hline 59 & $8 / 2004$ & & 24.926 & 19.185 & 25.345 & 24.034 & & 53.003 & \begin{tabular}{|l|l|}
54.203 \\
\end{tabular} & 10.541 & & & & 47.55 & 0.044 & & & & 9.5375 & 52.7 & 117 & 44.6 & 3.03 & & 0.006 & 0.000 & 0.148 & \\
\hline & & & & 19.207 & 25.347 & 24.086 & & & & 18.449 & & & & & & & & & & $\frac{12.8}{52.8}$ & & & & & & & & \\
\hline & $1 / 28 / 2004$ & & 24.971 & & \begin{tabular}{|l|}
25.399 \\
\end{tabular} & 24.148 & & 52.765 & \begin{tabular}{|l|}
54.014 \\
\end{tabular} & 18.416 & .058 & & & 49.815 & & & & & 57083 & & & & & & & & & \\
\hline & $1 / 28 / 2004$ & & & $\begin{array}{l}9.257 \\
\end{array}$ & & 24.14 & & & \begin{tabular}{|l|l|}
54.466 \\
\end{tabular} & 18.711 & & $\mid-1.214$ & & & & & & & & & & & & & & & 0.147 & \\
\hline & $1 / 28 / 2004$ & & 24.973 & 19.273 & 25.381 & 24.126 & & 52.781 & .093 & & .048 & & & .369 & & & & & 604 & & & & & & 006 & & 0.148 & \\
\hline & & & & & & 24.142 & & 52.769 & \begin{tabular}{|l|}
54.024 \\
\end{tabular} & & & -1.214 & & 3.954 & & & & & & & & & & & & & & \\
\hline 59 & 1/288/2004 & 1:30:30 PM & 25.001 & 19.316 & 25.414 & 24.179 & & 52.893 & \begin{tabular}{|l|}
54.254 \\
\end{tabular} & 18.52 & & & & .067 & & & & 578 & & 52 & 11. & & & & & & 148 & \\
\hline 50 & 28/2004 & 20200 & 25.022 & 19.337 & 25072 & 24.18 & & 52.806 & 51.20 & 18.409 & & -1.214 & & & & & & & & 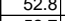 & 11.8 & & & & & & 0.140 & \\
\hline & 112882004 & & & & & & & & & & & & & & & & & & & & & & & & & & & \\
\hline$\frac{00}{60}$ & $\begin{aligned} 112802004 \\
1128004\end{aligned}$ & & 25.025 & $\begin{array}{l}1.39 \\
19.386\end{array}$ & \begin{tabular}{|l|l|}
23.333 \\
24728
\end{tabular} & 24.268 & & & & 18.529 & & $\frac{-1.210}{-1216}$ & & 48.1 & & & & & & 53.5 & $\frac{11.9}{114}$ & & & & & & & \\
\hline & $\begin{array}{l}\mid / 2 / 28 / 2004 \\
1 / 28 / 2004\end{array}$ & & $\frac{25.010}{24.991}$ & 19.300 & $\frac{24}{24}$ & 24.250 & & 5 & & & & $\frac{-1.210}{-1216}$ & & & & & & & & 529 & $\frac{1.44}{116}$ & 440 & & & & & 0.140 & \\
\hline & $1 / 28 / 2004$ & & & & 875 & & & & & & & & & & & & & & & & 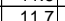 & & & & & & 0.147 & \\
\hline 60 & $1 / 28 / 2004$ & & 24.939 & 19.451 & \begin{tabular}{|l|}
24.912 \\
\end{tabular} & 24.298 & & 53.024 & & 18.249 & & $\mid-1.216$ & & 48.5 & & & & & & 53. & 11. & 44.2 & & & & & & \\
\hline & & & & & & & & & & & & & & & & & & & & & & & & & & & & \\
\hline & & & 24.9 & & & 24.40 & & & & & & & & & & & & & & 52 & 11 & & & & & & & \\
\hline & & & \begin{tabular}{|l|l|l|}
24.913 \\
\end{tabular} & & & & & & & 18.208 & & & & & & & & & & 52.8 & 11. & 44 & & & & & & \\
\hline & & & & & & 24. & & & \begin{tabular}{|l|}
54.267 \\
\end{tabular} & 1 & & -1.2 & & & & & & & & & 11 & & & & & & & \\
\hline & $1 / 28 / 2004$ & 1:42: & 24.909 & & & 24.482 & & 53. & 54.762 & 18.45 & 35.4 & -1.2 & & & & & & & & 53.3 & 11.6 & 44 & & & & & 0.144 & \\
\hline & $1 / 28 / 2004$ & & 24.91 & & & 24.545 & & & & & & & & & & & & & & & 11. & & & & & & & \\
\hline & 1/288/2004 & & 24.929 & 19.627 & 25.137 & 24.553 & & 53.062 & \begin{tabular}{|l|l} 
& 54.495 \\
\end{tabular} & & 35. & -1.216 & & & & & & & & 53 & 11. & 44 & & & & & & \\
\hline & $1 / 28 / 2004$ & & 24.93 & & & & & & & & & -1.2 & & & & & & & & & 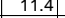 & & & & & & & \\
\hline & & & 24.949 & & & 24.5 & & 53. & & & & & & & & & & & & & & & & & & & & \\
\hline & 112820004 & & $\begin{array}{l}24.956 \\
2.969\end{array}$ & $\frac{19 .}{197}$ & & . & & 52.98 & $\begin{aligned} 54.458 \\
55617\end{aligned}$ & 18.4 & & -1.2 & & & & & & & & , & 11. & 44. & & & & & & \\
\hline & $\mid$ & 1.46. & 24.966 & & & $\frac{24.01}{2462}$ & & 5315 & 5 & 18.450 & & -1.2 & & & & & & & & 532 & & (4t. & & & & & 0.144 & \\
\hline & $\frac{1 / 20 / 2004}{1 / 28 / 2004}$ & & & & $\mid$\begin{tabular}{|l|l|l|l|}
25246 \\
\end{tabular} & & & & $\frac{54}{546}$ & & & & & & & & & & & & & & & & & & & \\
\hline & $1 / 28 / 2004$ & 1:51: & $\frac{25.01}{25.01}$ & 19.825 & \begin{tabular}{|l|l|l|}
25.278 \\
25
\end{tabular} & 24. & & 55.78 & & 18.236 & & $\frac{-1.216}{-1.216}$ & & & & & & & & 52.8 & 11. & & & & & & & \\
\hline & & & & & & & & & & & & & & & & & & & & & & & & & & & & \\
\hline & & & 25.0 & & \begin{tabular}{|l|}
25.313 \\
\end{tabular} & 24.69 & & & & 18.2 & & & & & & & & & & & & & & & & & & \\
\hline & & & & & & 24.743 & & 53.2 & 54. & & & & & & & & & & & & 11. & & & & & & & \\
\hline & 8/2004 & & & & 25.31 & 24.77 & & 53.06 & & & & -12 & & & & & & & & & & & & & & & & \\
\hline & 1/28/2004 & 1:56: & 25.074 & 20 & \begin{tabular}{|l|}
25.337 \\
\end{tabular} & 24.783 & & 53.151 & \begin{tabular}{|l|}
54.7 \\
\end{tabular} & & & -1.219 & & & & 14. & & & & 537 & 11.8 & 4 & & & & & & \\
\hline & $1 / 28 / 2004$ & & 25.092 & 20.049 & 25.36 & 24. & & 53.049 & \begin{tabular}{|l|l|}
54.648 \\
\end{tabular} & & & -1.21 & & & & & & & & 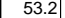 & 12. & & & & & & 144 & \\
\hline & $1 / 28 / 2004$ & & 25.1 & 20.082 & 25.363 & 24 & & 53.283 & & & & -1.219 & & & & & & & & & & & & & & & & \\
\hline & & & & & & & & & & & & & & & & & & & & & 11.9 & & & & & & & \\
\hline & & & & 20.14 & 25.385 & 24.9 & & $53.1 / 8$ & 54.7 & $18.5 \mathrm{~s}$ & 35.2 & -1.219 & & & & & & & & 53.3 & 11.8 & 44.2 & & & & & & \\
\hline & & $2001: 0$ & 25.138 & 20.167 & \begin{tabular}{|l|}
25.391 \\
\end{tabular} & 24.958 & 23.1. & 53.1655 & 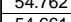 & $\begin{array}{ll}18.565 \\
18507\end{array}$ & 35.226 & \begin{tabular}{|c|c|}
5 & -1.216 \\
\end{tabular} & & $49.6 / 1$ & 0.0 & 14. & & & $\frac{87}{81}$ & \begin{tabular}{|l|l|}
3.3 .3 \\
\end{tabular} & 12.2 & 44.2 & 3.0 & & & & 0.143 & 0.1 \\
\hline 630 & $\begin{array}{l}\mid / 2 / 28 / 2004 \\
1 / 2004\end{array}$ & 2:03:07 PM & $|25.141|$ & 20.228 & \begin{tabular}{|l|}
25.39 \\
25.418 \\
\end{tabular} & \begin{tabular}{|l|}
24.926 \\
25.009 \\
\end{tabular} & 23.133 & $\begin{array}{l}53.041 \\
53.236\end{array}$ & \begin{tabular}{|r|}
54.661 \\
54.88
\end{tabular} & \begin{tabular}{|l|}
18.507 \\
18.597 \\
\end{tabular} & \begin{tabular}{|l}
335.169 \\
35.161 \\
\end{tabular} & $\mid-1.216$ & \begin{tabular}{|l|}
-6.516 \\
-6.516 \\
\end{tabular} & $\begin{array}{l}49.651 \\
48.232 \\
\end{array}$ & 0.042 & 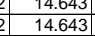 & & $\begin{array}{l}60.87 \\
610.87 \\
\end{array}$ & \begin{tabular}{|l|}
0.1811 \\
\end{tabular} & 53.4 & \begin{tabular}{|l|l|}
11.8 \\
\end{tabular} & \begin{tabular}{|l|}
44.2 \\
4.2 \\
\end{tabular} & \begin{tabular}{|l|}
3.047 \\
\end{tabular} & \begin{tabular}{|l|}
0.006 \\
\end{tabular} & \begin{tabular}{|l}
0.000 \\
0.006 \\
\end{tabular} & 0.000 & \begin{tabular}{|l|}
0.1440 \\
0.140 \\
\end{tabular} & \begin{tabular}{|l|} 
\\
\end{tabular} \\
\hline
\end{tabular}


WSRC-TR-2005-00105, REVISION 0

SRNL-RPP-2005-00012, REVISION 0

RUN \# 1.03A; DEWATER SECOND HALF OF SLURRY - CONT.

\begin{tabular}{|c|c|c|c|c|c|c|c|c|c|c|c|c|c|c|c|c|c|c|c|c|c|c|c|c|c|c|c|c|}
\hline & A & B & D & \begin{tabular}{|l|l|}
$E$ \\
\end{tabular} & $F$ & G & $\mathrm{H}$ & $\mathrm{J}$ & $\mathrm{k}$ & $\mathrm{L}$ & $\mathrm{M}$ & $\mathrm{N}$ & 0 & $Q$ & $\mathrm{R}$ & $\mathrm{s}$ & $T$ & $\mathrm{v}$ & w & $x$ & $\mathrm{Y}$ & z & AA & $\mathrm{AB}$ & $\mathrm{AC}$ & $\mathrm{AD}$ & $\mathrm{AE}$ & \\
\hline & 1/28/2004 & 2:04:07 PM & 25.192 & 20.246 & \begin{tabular}{|l|l|}
5 & 25.43 \\
\end{tabular} & 25.072 & 22.89 & 53.099 & 54.76 & $\begin{array}{l}18.378 \\
\end{array}$ & 35.19 & -1.216 & -6.513 & 47.775 & 0.042 & \begin{tabular}{|l|l|}
14.643 \\
\end{tabular} & 0.003 & 611.87 & 10.1978 & 53.3 & $\begin{array}{l}11.7 \\
\end{array}$ & 44.1 & 3.044 & 0.006 & 0.006 & 0.000 & 0.140 & \\
\hline & & & & & & 25.028 & 23.258 & & & 18.263 & & & & & & & & & 10.2144 & & 11.6 & $44 . \mathrm{C}^{2}$ & & & & & $\begin{array}{l}0.139 \\
\end{array}$ & \\
\hline & $1 / 28 / 2004$ & $2: 06: 07$ PM & $\frac{25.231}{25322}$ & \begin{tabular}{|l|}
20.306 \\
2027
\end{tabular} & $\begin{array}{l}525.459 \\
\end{array}$ & 25.046 & 23.115 & \begin{tabular}{|l|l|}
53.153 \\
5.277
\end{tabular} & \begin{tabular}{|l|}
54.818 \\
\end{tabular} & 18.245 & $\begin{array}{l}35.522 \\
.258 \\
\end{array}$ & -1.219 & $\begin{array}{l}-6.493 \\
-.949\end{array}$ & 46.682 & 0.042 & 14.643 & & 613.87 & $\begin{array}{l}0.2311 \\
1230\end{array}$ & \begin{tabular}{|l|}
53.3 \\
\end{tabular} & 11.4 & 44.3 & \begin{tabular}{|l|}
3.057 \\
\end{tabular} & 0.006 & 0.006 & 0.000 & $\begin{array}{l}0.139 \\
\end{array}$ & \\
\hline $\mid \frac{634}{635}$ & $\frac{1 / 28 / 2004}{1 / 28 / 2004}$ & 2:07:07 PM & $\begin{array}{r}25.232 \\
25254\end{array}$ & \begin{tabular}{r|}
20.297 \\
20.34
\end{tabular} & $\begin{array}{l}25.451 \\
125.463\end{array}$ & $\begin{array}{r}\frac{25.012}{25.06} \\
\end{array}$ & $\begin{array}{r}23.162 \\
23.224\end{array}$ & \begin{tabular}{|l|}
53.275 \\
53.155
\end{tabular} & \begin{tabular}{|l|}
54.916 \\
548.86
\end{tabular} & $\frac{18.215}{18.98}$ & $\begin{array}{r}35.67 \\
35.608\end{array}$ & $\begin{array}{l}-1.216 \\
-1.219\end{array}$ & $\begin{array}{l}-6.484 \\
-6.487\end{array}$ & 47.278 & $\begin{array}{l}0.042 \\
0.042\end{array}$ & $\begin{array}{l}14.643 \\
14.643\end{array}$ & & $\begin{array}{l}614.87 \\
615.87\end{array}$ & \begin{tabular}{|l|l|}
10.2478 \\
102644
\end{tabular} & $\begin{array}{l}53.4 \\
53.4\end{array}$ & 11.6 & 144 & \begin{tabular}{|l|l|}
3.066 \\
300
\end{tabular} & \begin{tabular}{|l|}
0.006 \\
0006
\end{tabular} & 0.006 & 0.000 & \begin{tabular}{|l|l|l|} 
\\
\end{tabular} & \\
\hline \begin{tabular}{|l|l|}
636 \\
\end{tabular} & & & & $\begin{array}{r}20.34 \\
20.357\end{array}$ & \begin{tabular}{|}
425.463 \\
7 \\
7
\end{tabular} & $\begin{array}{r}25.06 \\
25.087\end{array}$ & $\frac{23.224}{23.316}$ & \begin{tabular}{|l|}
5.155 \\
53.539
\end{tabular} & & $\begin{array}{l}\frac{18.198}{18.411} \\
\end{array}$ & $35.608 \mid$ & $\begin{array}{l}-1.219 \\
-1.219\end{array}$ & $\begin{array}{l}-6.487 \\
-6.551\end{array}$ & $\begin{array}{r}44.534 \\
46.428 \\
\end{array}$ & $\frac{0.042}{0.042}$ & $\begin{array}{l}14.643 \\
14.643 \\
\end{array}$ & & $\frac{615.87}{616.87}$ & 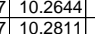 & $\begin{array}{l}53.4 \\
53.7\end{array}$ & $\begin{array}{l}10.9 \\
11.4\end{array}$ & 44.4 & \begin{tabular}{|l|}
3.060 \\
3075
\end{tabular} & \begin{tabular}{|l|}
0.006 \\
0.006
\end{tabular} & $\begin{array}{l}0.006 \\
0.066\end{array}$ & $\begin{array}{l}0.000 \\
0.000\end{array}$ & 0.139 & 0.12 \\
\hline & $1 / 28 / 2004$ & 2:10:07 PM & 25.279 & 20.384 & 25.462 & 25.139 & 23.328 & 53.356 & \begin{tabular}{|l|}
55.067 \\
\end{tabular} & 18.394 & $\begin{array}{l}3.04565 \\
35.565\end{array}$ & $\begin{array}{l}-1.219 \\
-1.219\end{array}$ & & $\begin{array}{l}0.440 \\
47.189 \\
\end{array}$ & 0.042 & $\frac{14.643}{14.643}$ & & $\frac{1010.87}{617.87}$ & \begin{tabular}{c|c|}
7 & 10.28811 \\
7 & 10.2978
\end{tabular} & $\begin{array}{l}53.1 \\
53.6\end{array}$ & $\frac{1.4}{11.6}$ & $\frac{44.6}{44.5}$ & $\begin{array}{l}3.075 \\
3.065 \\
\end{array}$ & \begin{tabular}{|l|}
0.006 \\
0.006 \\
\end{tabular} & $\begin{array}{l}0.000 \\
0.006\end{array}$ & $\begin{array}{c}0.000 \\
0.000\end{array}$ & $\begin{array}{l}0.139 \\
0.139\end{array}$ & $\frac{0.12}{0.12}$ \\
\hline \begin{tabular}{|l|}
638 \\
\end{tabular} & & & 25.291 & 20.401 & \begin{tabular}{|l|l|} 
& 25.474 \\
\end{tabular} & 25.186 & 23.22 & 53.186 & \begin{tabular}{|l|} 
\\
\end{tabular} 4.914 & $\begin{array}{l}18.267 \\
\end{array}$ & $\begin{array}{l}35.547 \\
\end{array}$ & -1.216 & -6.551 & 47.251 & 0.041 & 14.643 & & 618.87 & \begin{tabular}{|l|l|}
7 & 10.3144 \\
\end{tabular} & 53.4 & 11.6 & 44.4 & \begin{tabular}{|l|}
3.059 \\
\end{tabular} & \begin{tabular}{|l|}
0.006 \\
\end{tabular} & 0.006 & 0.000 & 0.136 & \\
\hline & & & & & & & & & & & & & $\begin{array}{l}-6.557 \\
-6.5\end{array}$ & & & & & & & & & & & & & & & \\
\hline $64 \mathrm{C}$ & $1 / 28 / 2004$ & $2: 13: 07$ PM & $\begin{array}{l}25.318 \\
\end{array}$ & \begin{tabular}{|l|l|}
20.449 \\
2070
\end{tabular} & 25.502 & $\begin{array}{l}25.194 \\
25.211\end{array}$ & 22.967 & \begin{tabular}{|l|l|l|l}
53.147 \\
5200
\end{tabular} & \begin{tabular}{|l|l|}
54.868 \\
\end{tabular} & \begin{tabular}{|l|l|}
18.394 \\
\end{tabular} & $\begin{array}{l}35.426 \\
5.20\end{array}$ & \begin{tabular}{|l|l|} 
\\
\end{tabular} & $\begin{array}{l}-6.534 \\
6.521\end{array}$ & $\begin{array}{l}46.822 \\
19.727\end{array}$ & 0.041 & 14.643 & & 620.87 & 10.3478 & 53.4 & 11.5 & & 3.053 & 0.006 & 0.006 & 0.000 & $\begin{array}{l}0.136 \\
\end{array}$ & \\
\hline 641 & $1 / 28 / 2004$ & $\begin{array}{l}2: 14: 07 \text { PM } \\
2 \cdot 015: 07\end{array}$ & $\begin{array}{r}25.33 \\
\\
\end{array}$ & 20.472 & 25.504 & $\begin{array}{r}25.211 \\
25.07\end{array}$ & $\begin{array}{r}23.27 \\
20207\end{array}$ & 53.09 & \begin{tabular}{|r|}
54.87 \\
\end{tabular} & $\begin{array}{l}18.212 \\
1.225\end{array}$ & 35.442 & & & 46.707 & 0 & 14.643 & & 621.87 & $\begin{array}{l}7 \\
7\end{array}$ & 53.4 & 11.4 & 44.3 & & \begin{tabular}{|l|}
0.006 \\
\end{tabular} & & & $\begin{array}{l}0.136 \\
\end{array}$ & \\
\hline$\left|\frac{642}{643}\right|$ & $\frac{1 / 288 / 2004}{1 / 2004}$ & 2:15:07 PM 2 & $\begin{array}{r}25.352 \\
25.358 \\
\end{array}$ & $\begin{array}{r}\mid 20.498 \\
20.51\end{array}$ & \begin{tabular}{|l|l|}
25.496 \\
1 & 25.497
\end{tabular} & $\begin{array}{l}25.197 \\
25.214\end{array}$ & $\begin{array}{l}23.347 \\
23.173 \\
\end{array}$ & $\begin{array}{r}53.271 \\
53.451 \\
\end{array}$ & \begin{tabular}{|l|}
55.017 \\
55.226
\end{tabular} & $\begin{array}{l}18.435 \\
18.485\end{array}$ & $\begin{array}{l}35.405 \\
35.419\end{array}$ & $\begin{array}{l}-1.216 \\
-1.219\end{array}$ & 536 & $\begin{array}{r}47.468 \\
48.13 \\
\end{array}$ & $\begin{array}{l}0.041 \\
0.041\end{array}$ & $\begin{array}{l}14.643 \\
14.643 \\
\end{array}$ & & $\frac{622.87}{623.87}$ & \begin{tabular}{|c|c|}
10.3811 \\
10.3978
\end{tabular} & \begin{tabular}{|l|}
53.5 \\
53.8 \\
\end{tabular} & \begin{tabular}{|l|}
11.6 \\
1.8
\end{tabular} & $\begin{array}{l}44.3 \\
44.4 \\
\end{array}$ & $\mid$\begin{tabular}{|c|c|}
3.064 \\
\end{tabular} & \begin{tabular}{|l|}
0.006 \\
0.006
\end{tabular} & $\begin{array}{l}0.006 \\
0.066\end{array}$ & $\begin{array}{l}0.000 \\
0.000\end{array}$ & $\begin{array}{l}0.136 \\
0.16\end{array}$ & 012 \\
\hline \begin{tabular}{|l|l|}
644 \\
\end{tabular} & $1 / 28 / 2004$ & $2: 17: 07 \mathrm{PM}$ & 25.371 & \begin{tabular}{|c|c|}
20.537 \\
\end{tabular} & 25.514 & $\mid 25.246$ & 23.526 & 53.346 & \begin{tabular}{|l|}
55.071 \\
\end{tabular} & 18.635 & 35..354 & -1.216 & -6.525 & \begin{tabular}{|l|}
40.10 \\
4.122
\end{tabular} & 0.041 & $\begin{array}{l}14.045 \\
14.643\end{array}$ & 0.004 & 624.87 & \begin{tabular}{|l|l|l|l|l|}
7 & 10.4144
\end{tabular} & \begin{tabular}{|l|}
53.6 \\
53.6
\end{tabular} & $\begin{array}{l}11.8 \\
11.5\end{array}$ & $\frac{44.4}{44.4}$ & \begin{tabular}{|l|}
3.0405 \\
3.058 \\
\end{tabular} & $\frac{0.006}{0.006}$ & $\begin{array}{l}0.000 \\
0.006\end{array}$ & $\begin{array}{l}0.000 \\
0.000\end{array}$ & \begin{tabular}{|l}
0.136 \\
0.136
\end{tabular} & 0.12 \\
\hline & $1 / 28 / 2004$ & 2:18:07 PM & 25.383 & 20.56 & 25.526 & 25.238 & 23.548 & 53.155 & \begin{tabular}{|l|l|}
54.882 \\
\end{tabular} & 18.486 & 35.384 & -1.219 & -6.525 & 47.341 & 0.041 & 14.643 & & 625.87 & 10.4311 & $\begin{array}{r}53.4 \\
\end{array}$ & 11.6 & 44.3 & & & 0.006 & & & 0.12 \\
\hline & $1 / 28 / 2004$ & $2: 19: 07$ PM & & 20.582 & & & 23.354 & & \begin{tabular}{|l|l|} 
& 55.336 \\
\end{tabular} & $\begin{array}{l}18.773 \\
\end{array}$ & 35.405 & \begin{tabular}{|c|}
-1.216 \\
\end{tabular} & -6.522 & 50.793 & 0.041 & 14.643 & & & $\begin{array}{ll}7 & 10.4478\end{array}$ & & 12.4 & 44.5 & 3.068 & & 0.006 & & & 0 \\
\hline \begin{tabular}{|l|l|}
647 \\
\end{tabular} & $1 / 28 / 2004$ & $2: 20: 07 \mathrm{PM}$ & 25.407 & 20.625 & \begin{tabular}{|l|l|}
25.561 \\
\end{tabular} & 25.343 & 23.21 & 53.505 & \begin{tabular}{|l|}
55.249 \\
\end{tabular} & & & & & 47.108 & & & & & & & & & & & & 0.000 & & \\
\hline & & & & & & & & & \begin{tabular}{|l|l|} 
& 55.311 \\
\end{tabular} & $\begin{array}{ll}18.663 \\
\end{array}$ & 35.38 & & & & 0.04 & & & 628.87 & 0.4811 & & 12.0 & & & & & & $\frac{132}{132}$ & \\
\hline 649 & $1 / 28 / 2004$ & 2:22:07 PM & 25.431 & \begin{tabular}{|l|l|}
20.669 \\
\end{tabular} & $25.584 \mid$ & 25.366 & & 53.397 & 55.2 & 18.722 & 35.304 & \begin{tabular}{|c|}
-1.216 \\
\end{tabular} & & 46.144 & 0.04 & & & & 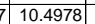 & & 11.3 & 44.4 & & & & & & \\
\hline & $1 / 28 / 2004$ & 2:23:07 PM & & & & & & 53.713 & 55.471 & 18.916 & 35.399 & -1.219 & & & 0.04 & 14. & & & 0.5144 & & 11.6 & & & & & & & \\
\hline 651 & $1 / 28 / 2004$ & 2:24:07 PM & 25.455 & 20.714 & \begin{tabular}{|l|}
25.139 \\
\end{tabular} & 25.426 & 23.991 & 53.667 & \begin{tabular}{|l|}
55.469 \\
\end{tabular} & \begin{tabular}{|l|l|l|}
18.84 \\
\end{tabular} & 35.483 & -1.214 & -6.528 & 48.58 & 0.04 & 14.643 & & 631.87 & \begin{tabular}{|l|l|}
10.5311 \\
\end{tabular} & 54.0 & 11.9 & 44.6 & 3.073 & 0.006 & 0.006 & 0.000 & \begin{tabular}{|l|l|l}
0.133 \\
\end{tabular} & \\
\hline & $1 / 28 / 2004$ & 2:25:07 PM & 25.442 & 20.72 & 24.65 & 25.373 & 24.1 & 53.46 & 55.202 & 18.967 & 35.194 & -1.216 & -6 & 46.519 & 0.04 & 14.643 & & 632.87 & 10.5478 & 53.7 & 11.4 & 44. & 3.0 & 006 & 0.006 & & 0.136 & \\
\hline $\mid \frac{653}{5}$ & $1 / 28 / 2004$ & 2:26:07 PM & 25.454 & 20.768 & 24.797 & 25.44 & & 53.01 & 54.876 & 19.334 & 34.272 & -1.219 & & 7.652 & 0.039 & 14.643 & & 633.87 & 0.5644 & & 11.7 & & & & 0.006 & & 0.134 & \\
\hline$\frac{564}{55}$ & $1 / 28 / 2004$ & 2:27:07 PM & 25.416 & 20.77 & \begin{tabular}{|l|l|} 
& 24.909 \\
\end{tabular} & 25.451 & & 53.269 & $55.0 / 5$ & 19.679 & 34.202 & -1.216 & & .248 & 0.039 & & & 634.8 & .5811 & & & & & & 0.006 & 0.000 & & \\
\hline & $\frac{1 / 28212004}{11200}$ & $2: 28: 07 \mathrm{PM}$ & 25.403 & 20.802 & & 25.434 & & 53.095 & 55.009 & & 34.315 & & & 40.000 & 0.039 & & & 635.81 & 然 & & & & & & & & & \\
\hline & $1 / 1282004$ & $\begin{array}{l}2.29 .0 \\
20.020\end{array}$ & 25.374 & & & & & $\frac{53.312}{53.285}$ & & 19.519 & $\begin{array}{l}34.324 \\
34289\end{array}$ & & & 48.121 & & & & & 10.6144 & & 11.8 & & & & & & & \\
\hline \begin{tabular}{|l|}
658 \\
658
\end{tabular} & 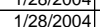 & $2.31: 07 \mathrm{PM}$ & 25.350 & $\frac{20.055}{20.857}$ & \begin{tabular}{|l|l|}
25.07 \\
25.106 \\
\end{tabular} & 25.4398 & $\frac{20.02}{23.45}$ & $\begin{array}{l}53.205 \\
53.207\end{array}$ & S5..115 & $\begin{array}{l}19.500 \\
19.391\end{array}$ & $\begin{array}{l}34.209 \\
34.338\end{array}$ & $\begin{array}{l}-1.219 \\
-1.219\end{array}$ & $\begin{array}{l}-0.054 \\
-6.534 \\
\end{array}$ & $\begin{array}{l}40.499 \\
45.625\end{array}$ & 0.039 & $\begin{array}{l}\frac{14.045}{14.643} \\
\end{array}$ & & 638.87 & 10.6478 & 53.5 & $\frac{11.9}{11.2}$ & 43.8 & 3018 & 0006 & 0.006 & 年 0.000 & o.152 & \\
\hline & $1 / 28 / 2004$ & $2: 32: 07$ PM & & 20.868 & \begin{tabular}{|l|l|}
25.147 \\
\end{tabular} & 25.404 & 23.8 & 53.271 & 55.088 & 19.482 & 34.377 & $\frac{-1.216}{-1.216}$ & & 47.4 & 0.039 & 14.6 & & 63 & 10.6644 & 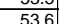 & $\frac{1.26}{1116}$ & & & 006 & 0.006 & & 0.132 & \\
\hline 660 & $1 / 28 / 2004$ & 2:33:07 PM & 25.355 & $\frac{20.89}{20.89}$ & \begin{tabular}{|l|l|}
25.188 \\
\end{tabular} & $\frac{25.421}{25.421}$ & & & 54.887 & 19.32 & 34.356 & -1.219 & & & $\frac{0.039}{0.039}$ & & & $\frac{6.0 .8}{640.87}$ & 10.6811 & & $\frac{11.2}{11.2}$ & & & & & & $\frac{0.132}{0.132}$ & \\
\hline 661 & $1 / 28 / 2004$ & 2:34:07 PM & 25.351 & 20.896 & \begin{tabular}{|l|l|}
25.205 \\
\end{tabular} & 25.477 & & 52.871 & 54.688 & 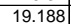 & 34.354 & & & 46.3 & 0.039 & & & & 10.6978 & 53.2 & 11.4 & 43.6 & & & 0.006 & 0.000 & 0.133 & \\
\hline & & & & 20.919 & & & & & & & & & & 47.7 & & & & & $\frac{6.7144}{.7144}$ & & & & & & & & & \\
\hline & $1 / 28 / 2004$ & & 25.366 & & \begin{tabular}{|l|}
25.294 \\
\end{tabular} & 25.547 & & 53.217 & 55.15 & 19.295 & 34.441 & & & 45.48 & & & & & & & 11.1 & 43,8 & & 006 & & & & \\
\hline 6664 _ & $1 / 28 / 2004$ & & $25.3 / 2$ & 20.96 & & 25.6 & & & 54.818 & \begin{tabular}{|l|l|}
19.15 \\
\end{tabular} & & & & $\begin{array}{lll}48.0 \\
\end{array}$ & & & & & & & 11. & & & & & & & \\
\hline & $1 / 28 / 2004$ & 2:38:C & 25.379 & 20.979 & & 25.62 & & 53.107 & \begin{tabular}{|l|}
55.017 \\
\end{tabular} & 19.272 & 455 & & & 45.737 & & & & & .7644 & & & 43.8 & & & 0.006 & & & \\
\hline & & & & 21.012 & 25.36 & & & 53.12 & \begin{tabular}{|l|l|}
55.048 \\
\end{tabular} & \begin{tabular}{|l|l|}
19.14 \\
\end{tabular} & 34.568 & & & & 0.039 & & & & & & 11. & & & & & & & \\
\hline 6677 & $1 / 28 / 2004$ & 2:40:07 PM & 25.409 & 21.05 & 25.373 & 25.745 & & & .777 & 18.991 & 34.496 & 219 & & 48.242 & 0.039 & & & & & 53 & 11. & & & & & & & \\
\hline 66 & $1 / 28 / 2004$ & & 25.417 & $\begin{array}{l}21.073 \\
20.037\end{array}$ & 20.090 & 2501 & & 53.014 & 54.947] & 19.101 & $\begin{array}{l}34.533 \\
2.165\end{array}$ & -1.216 & & & & & & & & - & $11.6 \mathrm{C}$ & & & & & & & \\
\hline & & & & & & & & 53.02 & & & & & & & & & & & & & & & & & & & & \\
\hline & $\begin{array}{ll}112820004 \\
11202004\end{array}$ & & 25.453 & $\frac{2.1 .1}{21.6}$ & $\mid$ & 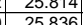 & & 53.078 & \begin{tabular}{|l}
5.036 \\
5.80
\end{tabular} & $\begin{array}{c}19.104 \\
1007\end{array}$ & - 3.4506 & -1.219 & & $41 .$. & & & & & & & 11.1 & & & & & & & \\
\hline $\mid \frac{162}{672}$ & $1 / 12812004$ & & $\begin{array}{l}25.40 \\
25.483\end{array}$ & $\frac{21.14}{212}$ & $\mid$\begin{tabular}{|c|}
25.449 \\
2548
\end{tabular} & & & 52.0601 & 资 & 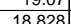 & & $\frac{-1.24}{-1210}$ & & & & & & & & & & & & & & & & \\
\hline 673 & 1 & & 25.406 & $\mid$ & 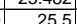 & & & & (1) & 19172 & & & & & & & & & & 0.0 .4 & 11 & & & & & & & \\
\hline 674 & $\frac{1}{1 / 28 / 2004}$ & 2:47: & 25.513 & 21.251 & 25.487 & 25.884 & $\frac{2.3}{23.3}$ & 52.939 & \begin{tabular}{|l|l|}
54.897 \\
\end{tabular} & 19.02 & 34.559 & $\mid-1.222$ & & & & & & & \begin{tabular}{|l|l|l|l|l|}
10.94 \\
\end{tabular} & 53. & 11.8 & & & & & & & \\
\hline & & & & & & & & & & & & & & & & & & & & & & & & & & & & \\
\hline & $1 / 28 / 20$ & & 25.54 & & \begin{tabular}{|l|}
25.529 \\
\end{tabular} & & & & 55.1 & 19.213 & & & & & & & & & & & 11 & & & & & & & \\
\hline & & & 25.5 & 213 & 25.546 & & & 53.242 & 55 & 193 & & & & & & & & & & 53.8 & 11. & & & & & & & \\
\hline & & & 25.574 & 21.3 & 25.543 & 25. & & & & 19.115 & & & & & & & & & & & 11. & & & & & & & \\
\hline & $1 / 28 / 2004$ & 2:52:07 & 25.601 & 21.3 & 25.575 & 25. & & & \begin{tabular}{|l|}
54.858 \\
\end{tabular} & 18.891 & & -1.2. & & 48.6 & & & & & & 53.4 & 11.9 & $\begin{array}{lll}43.6 \\
\end{array}$ & & & & & 0.131 & \\
\hline & $1 / 28 / 2004$ & $2: 53: 07$ & & & & 25.96 & & 52.997 & \begin{tabular}{|l|l|} 
& 55.054 \\
\end{tabular} & 19.179 & 34.475 & & & 47. & & & & & & 53 & 11. & & & & & & & \\
\hline 681 & $1 / 28 / 2004$ & 2:54:07 F & 25.621 & 21.421 & 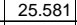 & 25.97 & & 52.869 & 54.976 & 19.026 & 34.492 & -1.219 & & 48. & & & & & 11 & 53.4 & 11. & 43. & & & & & & \\
\hline & & & 25.63 & & & & & & & & & & & & & & & & & & 11 & & & & & & & \\
\hline 然8 & $1 / 28 / 2004$ & & 25.667 & 21.44 & 25.626 & & & & & & & & & & & & & & & 53.6 & 11. & & & & & & & \\
\hline & 112820004 & & & $\frac{21}{215}$ & $|25564|$ & 26.06 & & & & & & & & & & & & & 10 & 534 & $\frac{11.7}{110}$ & & & & & & & \\
\hline$\frac{68}{68}$ & $\frac{\mid l / 20 / 2004}{1 / 28 / 2004}$ & $\frac{2.00 .007}{2.59 .07}$ & $\frac{23.001}{25704}$ & 21.527 & \begin{tabular}{|l|}
25.000 \\
$258 \mid$
\end{tabular} & & & 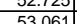 & 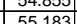 & | & & $\frac{-1.22}{-121}$ & & & & & & & & 年 & 1.5 & 128 & & & & & & \\
\hline & $\frac{1 / 20 / 2804}{1 / 28 / 2004}$ & & $\frac{2.57}{2572}$ & & $\mid$\begin{tabular}{|l|l|l|}
2566 \\
\end{tabular} & & & & & & & & & & & & & & & & & & & & & & & \\
\hline 68 & $\frac{1}{1 / 282 / 2004}$ & & $\frac{25.729}{25.729}$ & $\frac{21.614}{21.614}$ & $\mid$\begin{tabular}{|l|l|c|}
$\mid 25.638$ \\
\end{tabular} & 26. & & 52.8 & \begin{tabular}{|c|}
54.882 \\
\end{tabular} & $\begin{array}{l}19.028 \\
19.028\end{array}$ & & $\frac{-1.219}{-1.219}$ & & & & & & & & 53. & 11 & & & & & & & \\
\hline & & & & & 25.6 & & & & & & & & & & & & & & & & & & & & & & & \\
\hline & & & 25.75 & 21.65 & 25.561 & & & 53.2 & & $18.8 \subseteq$ & & & & & & & & & & & 11.6 & & & & & & & \\
\hline & & & & & & & & 52.8 & & & & & & & & & & & & & 11. & & & & & & & \\
\hline & & & & 21.708 & 25. & & & & & & & & & & & & & & & 53 & & & & & & & & \\
\hline 65 & $1 / 28 / 2004$ & 3:0 & 25.77 & 21.74 & 25.4. & 26.2 & & 53.12 & 55 & & 34.8 & -1.211 & & 47.3 & & & & & & 539 & 11.6 & & & & & & & \\
\hline & $1 / 28 / 2004$ & 3:07:07 & 25.797 & 21.769 & 25.45 & & & 53.1 & & 18.9 & & -1.216 & & 40 & & & & & & 53 & 11.7 & & & & & & & \\
\hline & $1 / 28 / 2$ & & 25.779 & 21.78 & & 26.2 & & 53.47 & & 18.81 & & & & & & & & & & 54. & 11.6 & & & & & & & \\
\hline & & & & & & & & & & & & & & & & & & & & & 11.8 & & & & & & & \\
\hline & & & & 21.816 & 25.353 & 26.3 & & 53.3. & & & & -1.219 & & 48. & & & & & & 54.1 & 11.8 & & & & & & & \\
\hline & & 3:11:0/ PM & 25.186 & 21.843 & \begin{tabular}{|r|}
25.32 \\
\end{tabular} & 26.297 & 24. & 53.462 & $55.6 / 4$ & $18.8 / 4$ & 35.1466 & -1.219 & & 46.9 & & 14.6 & & & 1.31 & 54.2 & 11.5 & 44. & & & & & 0.121 & $\pi$ \\
\hline$\frac{\mid}{7090}$ & $\begin{array}{l}1 / 281 / 2004 \\
1 / 28 / 200\end{array}$ & 3:13:07 PM & \begin{tabular}{|l|}
25.184 \\
25.784 \\
\end{tabular} & \begin{tabular}{|l|}
21.005 \\
\end{tabular} & \begin{tabular}{|l|}
25.278 \\
\end{tabular} & \begin{tabular}{|l|}
20.329 \\
26.346 \\
\end{tabular} & $\begin{array}{r}24.534 \\
24.496 \\
\end{array}$ & \begin{tabular}{|l|}
5.24060 \\
53.068 \\
\end{tabular} & \begin{tabular}{|l|l|}
54.233 \\
\end{tabular} & $\begin{array}{l}18.438 \\
18.485 \\
\end{array}$ & \begin{tabular}{|l|}
35.210 \\
\end{tabular} & \begin{tabular}{|c|}
-1.219 \\
\end{tabular} & $\begin{array}{r}-0.306 \\
-6.502 \\
\end{array}$ & $\begin{array}{l}40.980 \\
47.085 \\
\end{array}$ & 0.038 & \begin{tabular}{|l|l|}
14.645 \\
14.643 \\
\end{tabular} & & $\begin{array}{l}680.81 \\
680.87\end{array}$ & \begin{tabular}{l|l|}
7 & 11.3478 \\
\end{tabular} & 53.8 & \begin{tabular}{|l|}
11.5 \\
\end{tabular} & \begin{tabular}{|l|}
44.2 \\
44.2 \\
\end{tabular} & \begin{tabular}{|l|}
3.047 \\
\end{tabular} & \begin{tabular}{|l|}
0.006 \\
\end{tabular} & \begin{tabular}{|l}
0.006 \\
\end{tabular} & \begin{tabular}{|l} 
\\
\\
\end{tabular} & \begin{tabular}{|l|}
0.127 \\
\end{tabular} & 0.1 \\
\hline
\end{tabular}


WSRC-TR-2005-00105, REVISION 0

SRNL-RPP-2005-00012, REVISION 0

RUN \# 1.03A; DEWATER SECOND HALF OF SLURRY - CONT.

\begin{tabular}{|c|c|c|c|c|c|c|c|c|c|c|c|c|c|c|c|c|c|c|c|c|c|c|c|c|c|c|c|c|}
\hline & A & B & \begin{tabular}{l|l} 
D \\
\end{tabular} & $E$ & $F$ & $G$ & $\mathrm{H}$ & $\mathrm{J}$ & $\mathrm{K}$ & $\mathrm{L}$ & $M$ & $\mathrm{~N}$ & 0 & $Q$ & $\mathrm{R}$ & $\mathrm{s}$ & $T$ & $\mathrm{~V}$ & w & $x$ & $\mathrm{Y}$ & z & AA & $\mathrm{AB}$ & $\mathrm{AC}$ & $\mathrm{AD}$ & $\mathrm{AE}$ & \\
\hline & $1 / 28 / 2004$ & $3: 14: 07 \mathrm{PM}$ & 25.785 & 21.903 & \begin{tabular}{|l|l|}
35.254 \\
\end{tabular} & 26.387 & 24.787 & 53.113 & 55.197 & 18.616 & 35.22 & -1.219 & -6.5 & 46.911 & 0.038 & 14.643 & 0.004 & 681.87 & 1.3644 & 53.7 & 11.5 & 44.2 & 3045 & 0.006 & 0.006 & 0.000 & 0.127 & \\
\hline & & & & 21.925 & 25.231 & 26.409 & 24.469 & & & \begin{tabular}{|l|l|}
18.957 \\
\end{tabular} & 35.10 & & -6.493 & & & 14.643 & & & & 54.4 & 11.5 & 44. & & & & & 0.127 & \\
\hline & $1 / 28 / 2004$ & 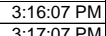 & \begin{tabular}{l|l|}
25.794 \\
257955
\end{tabular} & $\begin{array}{l}21.937 \\
21.048\end{array}$ & $\begin{array}{l}25.223 \\
25214\end{array}$ & $\begin{array}{l}26.476 \\
26517\end{array}$ & 24.27 & \begin{tabular}{|l|l|}
53.354 \\
5230
\end{tabular} & \begin{tabular}{|l|l|}
55.467 \\
5.627 \\
\end{tabular} & $\begin{array}{l}18.901 \\
18011\end{array}$ & $\begin{array}{l}35.20 \\
2.11\end{array}$ & \begin{tabular}{|l|}
-1.219 \\
\end{tabular} & $\begin{array}{l}-6.499 \\
-.40 \\
\end{array}$ & 46.811 & $\begin{array}{l}0.038 \\
0.08\end{array}$ & $\begin{array}{l}14.643 \\
1.62\end{array}$ & & $\begin{array}{l}683.87 \\
\end{array}$ & \begin{tabular}{|c|}
11.3978 \\
1.13
\end{tabular} & \begin{tabular}{|l|l|}
54.0 \\
\end{tabular} & 11.5 & & \begin{tabular}{|l|}
3.053 \\
\end{tabular} & 0.006 & 0.006 & 0.000 & 0.127 & \\
\hline & $\begin{array}{c}1 / 28 / 2004 \\
1282004\end{array}$ & $\begin{array}{l}3: 17: 07 \text { PM } \\
3.18 .07 P M\end{array}$ & $\begin{array}{r}25.795 \\
25.791\end{array}$ & $\begin{aligned} 21.948 \\
21.969\end{aligned}$ & $\begin{array}{l}35.214 \\
25.175\end{array}$ & \begin{tabular}{|l|}
26.517 \\
26.473
\end{tabular} & $\frac{24.402}{24.573}$ & $\begin{array}{r}53.395 \\
532.17\end{array}$ & \begin{tabular}{|l|}
55.627 \\
55.494
\end{tabular} & $\frac{18.811}{18.723}$ & $\begin{array}{l}35.11 \\
25.11 \\
25\end{array}$ & \begin{tabular}{|l|l|l|}
-1.219 \\
-1.225
\end{tabular} & $\begin{array}{l}-6.496 \\
-6.502\end{array}$ & $\begin{array}{l}46.699 \\
47.281\end{array}$ & $\begin{array}{l}0.038 \\
0.037\end{array}$ & $\begin{array}{l}14.643 \\
14.643\end{array}$ & & $\begin{array}{l}684.87 \\
60.97\end{array}$ & \begin{tabular}{|l|l|l}
11.4144 \\
11.1311
\end{tabular} & $\begin{array}{r}54.2 \\
540\end{array}$ & 11.4 & 44.3 & $\mid$ & \begin{tabular}{|l|l|}
0.006 \\
0000
\end{tabular} & $\begin{array}{l}0.006 \\
\end{array}$ & 0.000 & 0.127 & \\
\hline 70 & & & & & \begin{tabular}{|r|}
$23.1 / 5$ \\
25.18
\end{tabular} & 20.474 & & $\begin{array}{l}53.217 \\
53.256\end{array}$ & $\begin{array}{r}55.494 \\
55.51 \\
\end{array}$ & $\begin{array}{r}\frac{18.723}{18.71} \\
18\end{array}$ & $\begin{array}{l}35.11 \\
35.20\end{array}$ & $\mid$\begin{tabular}{|c|}
-1.225 \\
-1.219 \\
$\mid$
\end{tabular} & $\begin{array}{l}-6.502 \\
-6.496 \\
-6\end{array}$ & $\begin{array}{l}47.981 \\
7.948\end{array}$ & \begin{tabular}{|l}
0.037 \\
0.037
\end{tabular} & $\frac{14.643}{14.643}$ & & $\frac{685.87}{686.87}$ & \begin{tabular}{|l|l|}
11.4311 \\
11.4478
\end{tabular} & $\begin{array}{l}54.0 \\
54.0\end{array}$ & $\begin{array}{l}11.6 \\
11.8\end{array}$ & 44.2 & \begin{tabular}{|l|}
3.045 \\
3050
\end{tabular} & $\mid \begin{array}{l}0.006 \\
0.006\end{array}$ & $\begin{array}{l}0.005 \\
0.005\end{array}$ & $\begin{array}{l}0.000 \\
0.000\end{array}$ & $\begin{array}{l}0.124 \\
0.124\end{array}$ & \\
\hline & $1 / 28 / 2004$ & 3:20:07 PM & 25.781 & 21.984 & \begin{tabular}{|l|l}
4 & 25.15 \\
\end{tabular} & 26.443 & 24.403 & 53.414 & \begin{tabular}{|l|}
55.639 \\
\end{tabular} & 18.988 & 35.09 & \begin{tabular}{|l|}
$\mid-1.219$ \\
\end{tabular} & -6.496 & 46.861 & 0.037 & $\frac{14.045}{14.643}$ & & $\frac{6860.87}{687.87}$ & \begin{tabular}{l|l|}
11.448 \\
7 & 11.4644
\end{tabular} & $\frac{54.0}{54.2}$ & $\frac{11.8}{11.5}$ & $\frac{44.2}{44.3}$ & $\begin{array}{l}3.050 \\
3.051\end{array}$ & \begin{tabular}{|l|}
0.006 \\
0.006 \\
\end{tabular} & $\frac{0.005}{0.005}$ & $\frac{0.000}{0.000}$ & 0.124 & $\frac{0.11}{0.11}$ \\
\hline 708 & & & 25.798 & 22.011 & \begin{tabular}{|l|l|}
25.157 \\
\end{tabular} & 26.43 & 23.924 & 53.455 & \begin{tabular}{|l|}
55.618 \\
\end{tabular} & 18.765 & 35.43 & -1.216 & $\begin{array}{l}-6.499 \\
\end{array}$ & 46.647 & 0.037 & 14.643 & & 688.87 & \begin{tabular}{l|l}
7 & 11.4811 \\
\end{tabular} & 54.1 & 11.4 & 44.4 & \begin{tabular}{|l|}
3.064 \\
\end{tabular} & \begin{tabular}{|l|l|}
0.006 \\
\end{tabular} & 0.005 & 0.000 & & $\frac{0.11}{0.11}$ \\
\hline & & & & & & & & & & & & & -6.499 & & & & & & & & 11.4 & & & & & & & \\
\hline & $1 / 28 / 2004$ & 3:23:07 PM & \begin{tabular}{|l|l|}
25.783 \\
25702
\end{tabular} & $\begin{array}{l}22.012 \\
2.027\end{array}$ & $\begin{array}{l}24.366 \\
2.101\end{array}$ & $\begin{array}{l}26.475 \\
652\end{array}$ & & $\begin{array}{l}54.159 \\
55.202\end{array}$ & 56.423 & $\begin{array}{l}19.085 \\
10.071\end{array}$ & $\begin{array}{l}35.69 \\
2070\end{array}$ & \begin{tabular}{|c|}
-1.219 \\
\end{tabular} & $\begin{array}{l}-6.499 \\
6.502\end{array}$ & 45.962 & 0.037 & 14.643 & & 690.87 & 11.5144 & 55.0 & 11.3 & & 3.097 & \begin{tabular}{|l|l|}
0.006 \\
\end{tabular} & 0.006 & 0.000 & 0.125 & \\
\hline$\frac{711}{712}$ & $1 / 28 / 2004$ & $\begin{array}{l}3: 24: 07 \text { PM } \\
2: 5: 07\end{array}$ & $\begin{array}{r}25.783 \\
25750\end{array}$ & $\begin{aligned} 22.037 \\
2 ? 020\end{aligned}$ & 24.121 & 26.53 & 24.88 & $\begin{array}{l}53.232 \\
5.925\end{array}$ & .569 & 19.974 & \begin{tabular}{|l|l}
33.79 \\
3271
\end{tabular} & & & 47.762 & 0 & 14.643 & & 691.87 & $\begin{array}{l}71.5311 \\
7\end{array}$ & \begin{tabular}{|l|l|}
54.1 \\
\end{tabular} & 11.7 & & & \begin{tabular}{|l|l|} 
\\
\end{tabular} & 0.006 & & 0.126 & \\
\hline 712 & $\begin{array}{l}1 / 28 / 2004 \\
1 / 28 / 2004\end{array}$ & $\begin{array}{l}3: 25: 07 \mathrm{PM} \\
3: 26: 07 \mathrm{PM}\end{array}$ & $\begin{array}{r}25.759 \\
25.741\end{array}$ & $\begin{array}{r}22.038 \\
22.055 \\
\end{array}$ & $\begin{array}{l}324.402 \\
5 \\
54.454\end{array}$ & $\begin{array}{r}26.491 \\
26.548\end{array}$ & $\begin{array}{l}24.361 \\
2.392\end{array}$ & \begin{tabular}{|l|}
52.835 \\
52.952
\end{tabular} & \begin{tabular}{|l|}
55.125 \\
55.206
\end{tabular} & $\begin{array}{l}19.764 \\
19.733\end{array}$ & $\begin{array}{r}33.71 \\
33.91 \\
\end{array}$ & $\begin{array}{l}-1.225 \\
-1.222\end{array}$ & & \begin{tabular}{|l|}
47.16 \\
46.265 \\
\end{tabular} & 036 & $\begin{array}{l}14.643 \\
14.643 \\
\end{array}$ & & $\frac{692.87}{693.87}$ & $\begin{array}{l}7 \\
7\end{array} 11.5478$ & \begin{tabular}{|l}
53.7 \\
53.7
\end{tabular} & \begin{tabular}{|l|l|}
11.6 \\
11.3
\end{tabular} & $\frac{15.5}{43.4}$ & $\begin{array}{l}2.984 \\
2.994 \\
\end{array}$ & \begin{tabular}{|l|l|}
0.005 \\
0005
\end{tabular} & $\begin{array}{l}0.005 \\
0.005\end{array}$ & $\begin{array}{l}0.000 \\
0.009\end{array}$ & $\begin{array}{l}0.126 \\
0.125\end{array}$ & 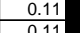 \\
\hline & $1 / 28 / 2004$ & 3:27:07 PM & 25.733 & 22.072 & 24.516 & $\frac{10.040}{26.6}$ & 24.24 & $\begin{array}{l}53.319 \\
53.519\end{array}$ & \begin{tabular}{|l|}
55.200 \\
55.55
\end{tabular} & 20.037 & 33.82 & \begin{tabular}{|l|l|}
-1.225 \\
\end{tabular} & $\begin{array}{l}-6.502 \\
-6.505\end{array}$ & \begin{tabular}{|l|}
40.200 \\
4.105
\end{tabular} & 0.036 & $\begin{array}{l}14.643 \\
14.643 \\
\end{array}$ & 0.003 & $\begin{array}{l}694.81 \\
694.87\end{array}$ & 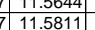 & \begin{tabular}{|l}
5.1 .1 \\
54.1
\end{tabular} & 11.3 & $\begin{array}{l}43.4 \\
3.6\end{array}$ & $\begin{array}{l}2.9944 \\
3.004\end{array}$ & \begin{tabular}{|l|l|}
0.005 \\
0.005
\end{tabular} & 0.005 & $\begin{array}{l}0.000 \\
0.000\end{array}$ & \begin{tabular}{|l}
0.125 \\
0.125
\end{tabular} & $\frac{0.11}{0.11}$ \\
\hline & $1 / 28 / 2004$ & $3: 28: 07$ PM & \begin{tabular}{|l|l|}
25.708 \\
\end{tabular} & 22.072 & 24.572 & 26.63 & & 53.105 & 55.425 & 19.781 & 33.81 & & & 46.49 & & 14.643 & & & & 54.0 & 11.4 & & 2.997 & & & & & \\
\hline & $1 / 28 / 2004$ & 3:29:07 PM & & 22.083 & & & & & 55.287 & 19.456 & & $\begin{array}{l}-1.219 \\
\end{array}$ & -6.496 & 49.36 & 0.036 & 14.643 & & 696.87 & \begin{tabular}{ll|}
7 & 11.6144 \\
\end{tabular} & 53.8 & 12.1 & 43.6 & & & 0.005 & & .124 & \\
\hline 71 & $1 / 28 / 2004$ & & $\begin{array}{l}25.67 \\
\end{array}$ & 22.089 & \begin{tabular}{|l|}
24.623 \\
\end{tabular} & 26.636 & 24.9 & 53.016 & & & 34.18 & & & & 0.036 & & & & & & & & & & & & & \\
\hline & 8/2004 & 3:31:0 & & & & & & & 55.6 & 19.626 & 34.24 & -1.219 & & $\begin{array}{l}46.918 \\
\end{array}$ & 0.036 & 14.643 & & 698.87 & 1.6478 & & 111.5 & & & & & & $\frac{\frac{124}{124}}{4}$ & \\
\hline 719 & $1 / 28 / 2004$ & 3:32:07 PM & 25.641 & 22.131 & 24.69 & 26.638 & & 52.748 & 55.14 & $\begin{array}{l}19.089 \\
\end{array}$ & 34.31 & -1.219 & & 47.2 & & & & & 1.6644 & & 11.6 & & & & & & & \\
\hline & 1/28/2004 & 3:33:07 PM & & & & 26.67 & & & 55.326 & \begin{tabular}{|l|l|}
19.33 \\
\end{tabular} & 34.32 & -1.216 & & 47280 & 036 & 14. & & & 11.6811 & & 11.6 & & & & & & 0.124 & \\
\hline & $1 / 28 / 2004$ & 3:34:07 PM & 25.631 & 22.181 & 24.715 & 26.688 & 25. & 52.935 & \begin{tabular}{|l|l|} 
& 55.216 \\
\end{tabular} & $\begin{array}{l}9.375 \\
\end{array}$ & 34.23 & $|-1.219|$ & -6.482 & 47.689 & 0.036 & 14.643 & & 701.87 & \begin{tabular}{|c|}
11.6978 \\
\end{tabular} & 53.7 & 11.7 & 43.6 & 3.005 & 0.005 & 0.005 & 0.000 & 0.124 & \\
\hline & $1 / 28 / 2004$ & 3:35:07 PM & 25.639 & 22.219 & 24.758 & 26.731 & & 53.232 & 55.546 & 18.856 & 35.08 & -1.211 & & $\begin{array}{l}47.028 \\
\end{array}$ & 0.037 & 14.643 & & 702.87 & 11.7144 & 54.1 & 11.5 & & 3.04: & 0.006 & 0.006 & & 0.126 & \\
\hline & $1 / 28 / 2004$ & 3:36:07 PM & 25.638 & 22.243 & 24.787 & 26.75 & & 53.518 & 55.84 & 19.008 & 35.02 & & & 46.83 & 0.037 & 14.643 & & 703.87 & 11.7311 & & 11.5 & & 3.05 & & 0.006 & & .125 & \\
\hline & $1 / 28 / 2004$ & 3:37:07 PM & 25.651 & 22.277 & 24.77 & 26.763 & & 53.391 & 55.796 & 18.964 & 34.98 & -1.219 & & .051 & 0.037 & & & & 1.7478 & 54.3 & 11.5 & 44. & 3.04 & & 0.006 & 0.000 & 0.126 & \\
\hline & 80212004 & 3:38:07 PM & 25.645 & & & 26.171 & & 53.373 & 55.67 & 18.848 & 35.15 & & & $46.4 / 8$ & & & & & & & 11.4 & & & & & & & \\
\hline & $\begin{array}{l}1 / 28 / 2004 \\
1 / 28 / 2004\end{array}$ & $17 \mathrm{PM}$ & & $\frac{2.353}{2232}$ & & $\begin{array}{l}2.615 \\
26776\end{array}$ & & & & & & & & 46.596 & & & & & 1.1811 & & & & & & & & & \\
\hline 72 & $1 / 28 / 2004$ & $3.40 .07 \mathrm{PM}$ & 25.671 & 22.357 & $\begin{array}{r}24.009 \\
24.8\end{array}$ & 26.763 & 25.05 & $\begin{array}{r}53.223 \\
53.23\end{array}$ & \begin{tabular}{|l|}
55.513 \\
\end{tabular} & $\frac{10.501}{18.549}$ & $\frac{35.04}{35.28}$ & \begin{tabular}{|c|}
-1.219 \\
-1.219
\end{tabular} & & $\begin{array}{l}41.051 \\
46.534\end{array}$ & 0.037 & $\begin{array}{l}14.043 \\
14.643\end{array}$ & & 708.87 & 11.8144 & 54.2 & $\begin{array}{l}11.5 \\
11.4\end{array}$ & $\frac{44.5}{44.3}$ & 3.051 & 000 & $\frac{0.000}{0.006}$ & 0.000 & 0 & \\
\hline & $1 / 28 / 2004$ & $77 \mathrm{PM}$ & 25.672 & 22.368 & 24.826 & 26.734 & 24.76 & 53.19 & 55.62 & $\begin{array}{l}18.559 \\
\end{array}$ & 35.29 & $\mid-1.219$ & & 46.719 & 0.037 & & & & 118311 & 541 & 11.4 & & & 006 & 0.006 & 0.000 & 0.125 & \\
\hline 73 & $1 / 28 / 2004$ & 3:43:07 PM & 25.684 & 22.4 & \begin{tabular}{|l|}
24.833 \\
\end{tabular} & 26.801 & & & 55.687 & \begin{tabular}{l|l}
18.243 \\
\end{tabular} & 35.50 & & & 45.964 & 0.054 & 14. & & 710.87 & & & 11.3 & & & & & & & \\
\hline 73 & 8/2004 & & 25.686 & 22.417 & $\mid 24.814$ & 26.812 & & 53.314 & \begin{tabular}{|l|l|}
55.869 \\
\end{tabular} & 18.095 & 35.8 & & & 5.821 & 0.037 & & & & 1.8644 & 54.4 & $\frac{11.4}{11.2}$ & & & & 0.006 & 0.000 & 0.124 & \\
\hline & & & & & & 26.8 & & & & \begin{tabular}{|l|l|l|l|l|}
18.084 \\
\end{tabular} & & & & & & & & & & & 11. & & & & & & & \\
\hline & $1 / 28 / 2004$ & & \begin{tabular}{|l|l|}
25.69 \\
\end{tabular} & & & 26.827 & & 53.609 & 56.186 & 17.767 & & & & 5.802 & & & & & 1.89 & & 11. & & & & & & & \\
\hline & $1 / 28 / 2004$ & & & 22.485 & & & & & \begin{tabular}{|l|l|}
56.143 \\
\end{tabular} & \begin{tabular}{|l|l|l|l|}
17.934 \\
\end{tabular} & & & & 4 & & & & & $\overline{11.9144}$ & & & & & & & & 0.123 & \\
\hline & 1/28/2004 & & 25.729 & 22.486 & 24.848 & 8.861 & & 53.841 & 56.209 & 18.043 & & -1.219 & & 45.568 & & & & & & & 11 & & & & 0.006 & & & \\
\hline & & & 25.717 & 22.478 & 24.866 & 26.884 & & & & 22.317 & 30.24 & & & 50.338 & 0.0 & & & & & & 12 & & & & & & 0.124 & \\
\hline $77^{73}$ & $1 / 28 / 2004$ & 3:50:07 PM & 25.739 & 22.51 & 24.903 & 26.9 & & 51.933 & 54.366 & 22.053 & 30.4 & -1.219 & & 50.23 & 0.0 & & & & $\begin{array}{ll}11.9644 \\
\end{array}$ & 52 & & & 28 & & & & & \\
\hline & 28/2004 & $\begin{array}{l}3: 51: 07 \mathrm{~F} \\
2: 52: 07\end{array}$ & $\begin{array}{r}25.75 \\
\end{array}$ & $\frac{22.516}{20521}$ & 24.934| & 26.92 & & $\begin{array}{r}51.94 \\
51.092\end{array}$ & 54.001 & 21.432 & 31.13 & 1.20 & & -2 & & & & & 1007 & 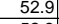 & 12. & & 20 & & & & & \\
\hline & & & & & & & & & & & & & & & & & & & & & & & & & & & & \\
\hline & $\begin{array}{ll}112820004 \\
11202004\end{array}$ & & 25.1.145 & 2.531 & \begin{tabular}{|l|l|}
24.9080 \\
\end{tabular} & & & $\begin{array}{l}51.867 \\
5.2095\end{array}$ & \begin{tabular}{|l|l|l|l|} 
\\
\end{tabular} & 21.403 & & -1.2 & & $\begin{array}{l}49.164 \\
55117\end{array}$ & & & & & 2.0144 & & 12 & & & & & & & \\
\hline 742 & $1 / 12812004$ & & 25.796 & $\frac{26.544}{22578}$ & $\mid 2.0504$ & $\frac{20.912}{26.958}$ & & & $\begin{array}{r}54.40 \\
5437\end{array}$ & 21.525 & & & & $\frac{1.1}{486}$ & & & & & & 530 & 110 & & & & & & & \\
\hline & $1 / 28 / 2004$ & & 25.798 & 2.070 & 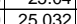 & 26.9 & & & & & & & & 4496 & & & & & & & & & & & & & & \\
\hline 74 & $1 / 28 / 2004$ & 3:57: & 25.815 & 22.612 & \begin{tabular}{|l|} 
\\
\end{tabular} & 27.012 & & 52.122 & \begin{tabular}{|l}
54.439 \\
\end{tabular} & 21.184 & & $\frac{-1.222}{-1.22}$ & & 47.873 & & & & & & 53 & 11 & & & & 0.005 & & & \\
\hline & & & & & & & & & & & & & & & & & & & & & & & & & & & & \\
\hline & $1 / 28 / 2004$ & & 25.832 & & 25.077 & & & & $\begin{array}{l}54.7 \\
\end{array}$ & & & & & & & & & & & & 12 & & & & & & & \\
\hline & & & 25.8 & 22.6 & 25.103 & & & 51. & & 20.858 & & & & 4 & & & & & & 53.0 & 12. & & & & & & & \\
\hline & & & 25.84 & & \begin{tabular}{|l|l|} 
& 25.119 \\
\end{tabular} & 27.6 & & 52.4 & \begin{tabular}{|l|l|}
54.887 \\
\end{tabular} & 21.208 & & -1.2 & & & & & & & & & & & & & & & & \\
\hline & $1 / 28 / 2004$ & & 25.865 & & 25.094 & & & 52.2 & 54.704 & 20.899 & & -1.219 & & 48.0 & & & & & 12.15 & 53.2 & 11.8 & & & & & & 0.124 & \\
\hline & $1 / 28 / 2004$ & 4:02:16 & & & & 26.988 & & 51.995 & 54.356 & & & & & 5 & & & & & & & 12 & & & & & & & \\
\hline & $1 / 28 / 2004$ & 4:03:1 & 25.893 & 22.686 & 25.162 & 27.02 & & 52.033 & 54.491 & 21.207 & & -1.219 & & 49.3 & & & & & 12.1 & 53 & 12. & & & & & & & \\
\hline & & & 20 & & & & & & & & & & & & & & & & & & $1 \pm$ & & & & & & & \\
\hline & $1 / 28 / 2004$ & & 25.916 & 22.7 & $25.1 / 6$ & 27.098 & & 52 & 54.6 & & & -1.2 & & 48. & & & & & & 53. & 12. & & & & & & & \\
\hline & 112820004 & & & $\frac{22}{22}$ & 25.214 & $\frac{27.151}{27.148}$ & & 51.1.942 & $\frac{54.292}{5448}$ & & $\frac{31.69}{31.88}$ & -1.2 & & & & & & & & & 12. & & & & & & 2124 & \\
\hline & $\mid 1 / 28 / 2004$ & $4.04 .0 .16 \mathrm{PM}$ & $\frac{2.344}{25959}$ & 22707 & $\mid$ & 27.140 & & S1.944 & $\begin{array}{l}54.40 \\
5\end{array}$ & 20.004 & $\frac{31.00}{3}$ & -1.2 & & & & & & & & 598 & 11 & & & & & & 0.124 & \\
\hline & $\frac{1 / 20 / 2804}{1 / 28 / 2004}$ & & 25.066 & 2281 & 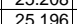 & 27.143 & & 5 & & & & & & & & & & & & & & & & & & & & \\
\hline & $\frac{1}{1 / 28 / 2004}$ & & $\frac{25.97}{25.97}$ & $\frac{2.25}{22.827}$ & $\mid$ & & & $\frac{21.9}{51.9}$ & \begin{tabular}{|l|l|}
54.464 \\
\end{tabular} & 20.4 & & -1.225 & & & & & & & & & 12. & & & & & & & \\
\hline & & & & & & & & & & & & & & & & & & & & & & & & & & & & \\
\hline & & & 26.01 & & 25.224 & 27.196 & & & & & & & & & & & & & & & 11. & & & & & & & \\
\hline & & & & & & & & 51.6 & & & & -1.2 & & & & & & & & & & & & & & & & \\
\hline & $8 / 2004$ & & & 22 & & 27.2 & & 52 & & & & 1222 & & & & 14 & & & & & & & & & & & & \\
\hline & $1 / 28 / 2004$ & & 26.06 & 22.95 & 25.26 & 27.235 & & 51.913 & \begin{tabular}{|l|l|}
54.445 \\
\end{tabular} & 20.3 & & $-1.2>$ & & 49.1 & & 14. & & & 12.3 & 5 & 12 & & & & & & & \\
\hline & & $4: 16: 16 \mathrm{P}$ & & & 25.27 & 27.258 & & 52.128 & \begin{tabular}{|l|l|}
54.723 \\
\end{tabular} & & & & & & & & & & & 53 & 12 & & & & & & 0.123 & \\
\hline & $1 / 28 / 2004$ & & 26. & 22. & 25.269 & & & 52. & 54. & & & -1.219 & & & & & & & & & $12 \quad$ & & & & & & & \\
\hline & & & & & & & & & & & & & & & & & & & & & & & & & & & & \\
\hline & & & 10 & 23.02 & 25.278 & 27.28 & & 52.5 & 55.152 & & & -1.2 & & & & & & & & 53. & 12. & & & & & & & \\
\hline & & 4:20:16 PM & 26.114 & 23.034 & $\mid 25.259$ & 27.216 & 25.6 & $52.1 / 4$ & 54.168 & 20.622 & & -1.222 & & & & 14. & & & \begin{tabular}{|l|}
12.46699 \\
\end{tabular} & 53. & 11. & & & & & & 0.123 & \\
\hline $\mid \frac{109}{770}$ & $\mid 1 / 28 / 2004$ & $\begin{array}{l}4.221 .10 \mathrm{PM} \\
4: 22: 16 \mathrm{PM}\end{array}$ & \begin{tabular}{|l|}
20.135 \\
26.145
\end{tabular} & $\begin{array}{r}23.00 \\
23.076\end{array}$ & \begin{tabular}{|l|}
25.296 \\
\end{tabular} & \begin{tabular}{|l|}
27.201 \\
27.247
\end{tabular} & $\begin{array}{l}25.524 \\
25.343\end{array}$ & \begin{tabular}{|l|}
5.265 \\
51.969
\end{tabular} & \begin{tabular}{|l|}
54.808 \\
54.553
\end{tabular} & $\begin{array}{r}20.40 \\
20.404 \\
\end{array}$ & 32.25 & $|-1.219|$ & $\begin{array}{r}-0.545 \\
-6.551 \\
\end{array}$ & \begin{tabular}{|l|}
49.07 \\
908
\end{tabular} & 0.035 & \begin{tabular}{|l|l|}
14.643 \\
\end{tabular} & & $\begin{array}{l}749.02 \\
7550.02\end{array}$ & \begin{tabular}{l|l}
2 & 12.5003
\end{tabular} & \begin{tabular}{|l|}
53.4 \\
53.1
\end{tabular} & $|12.1|$ & \begin{tabular}{|l|}
42.3 \\
22.1 \\
\end{tabular} & \begin{tabular}{|l|}
2.903 \\
\end{tabular} & \begin{tabular}{|l|l|} 
& 0.005 \\
\end{tabular} & \begin{tabular}{|}
0.005 \\
0.005
\end{tabular} & 0.000 & \begin{tabular}{|l|}
0.123 \\
\end{tabular} & 0.1 \\
\hline
\end{tabular}


WSRC-TR-2005-00105, REVISION 0 SRNL-RPP-2005-00012, REVISION 0

RUN \# 1.03A; DEWATER SECOND HALF OF SLURRY - CONT.

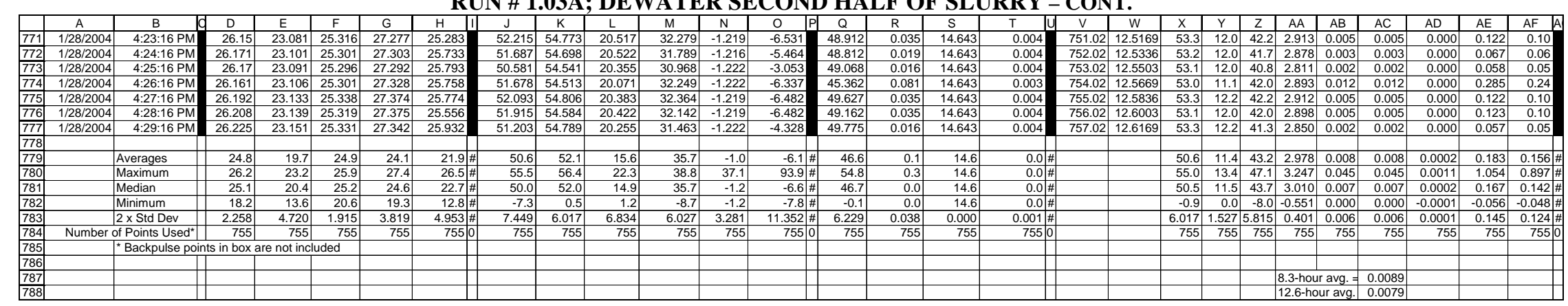


RUN \# 1.04; STEADY STATE TEST AT 25 WT\% UDS

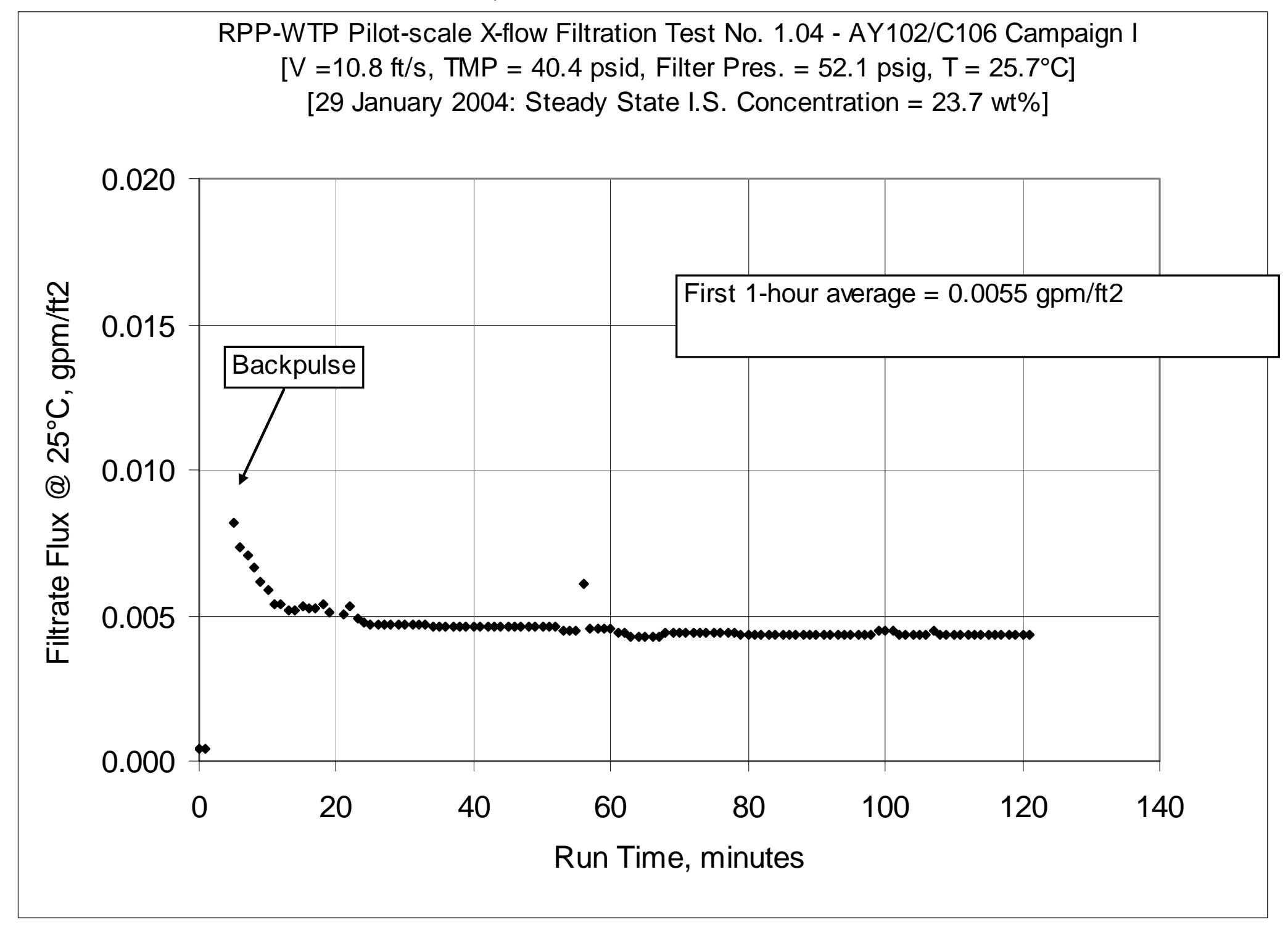


WSRC-TR-2005-00105, REVISION 0

SRNL-RPP-2005-00012, REVISION 0

RUN \# 1.04; STEADY STATE TEST AT 25 WT\% UDS - CONT.

\begin{tabular}{|c|c|c|c|c|c|c|c|c|c|c|c|c|c|c|c|c|c|c|c|c|c|c|c|c|c|c|c|c|}
\hline & A & B & \begin{tabular}{l|l} 
& \\
\end{tabular} & $\begin{array}{lll} & \\
\end{array}$ & $\mathrm{F}$ & $\mid \mathrm{G}^{\mathrm{I}}$ & $\mathrm{H}$ & \begin{tabular}{l|l}
$\mathrm{J}$ & \\
\end{tabular} & $\mathrm{K}$ & $\mathrm{L}$ & M & $\mathrm{N}$ & 0 & \begin{tabular}{l|l} 
\\
\end{tabular} & \begin{tabular}{l|l} 
\\
\end{tabular} & \begin{tabular}{|c|}
$\mathrm{s}$ \\
\end{tabular} & $\mathrm{T}$ & $\mathrm{v}$ & I w & $x$ & & & AA & $\mathrm{AB}$ & AC & $\mathrm{AD}$ & $\mathrm{AE}$ & AF \\
\hline 1 & DATE & TIME & Fitrate| & $\begin{array}{l}\text { << Tempe } \\
\text { Cleaningl }\end{array}$ & & Measurem & ents $\gg>$ en: & BotTMP & $\begin{array}{l}\ll \ll<<< \\
\text { Filter }\end{array}$ & $\begin{array}{l}\text { Pressure n } \\
\text { Fivter }\end{array}$ & Measurem & & 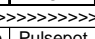 & & 《< Flow N & $\begin{array}{l}\text { Measureme } \\
\text { Hi }\end{array}$ & 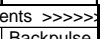 & & & & & & & & & & & \\
\hline & DAIE & IIME & - Hittate & $\frac{\text { Cleaning }}{\operatorname{deg} C}$ & \begin{tabular}{|l|} 
Segry C \\
$\operatorname{deg}$
\end{tabular} & $\mid$\begin{tabular}{|c|}
$\mid$ i Amb. \\
deg C
\end{tabular} & Lo Amb. & $\frac{\text { BotIMP }}{\text { psid }}$ & $\begin{array}{l}\text { Filter } \\
\text { psiq }\end{array}$ & $\begin{array}{l}\text { Filter r P } \\
\text { psid }\end{array}$ & \begin{tabular}{|l|} 
TopTMP \\
psid
\end{tabular} & $\begin{array}{c}\text { Filtrate } \\
\text { psig }\end{array}$ & Pulsepot & $\begin{array}{l}\text { Slurry } \\
\text { gpm }\end{array}$ & $\begin{array}{c}\text { Filtrate } \\
\text { grmm }\end{array}$ & \begin{tabular}{|c|} 
Hi Filtate \\
gam
\end{tabular} & Backpulse & & & & & & & & & & & \\
\hline & & & $\mathrm{T} 2$ & $\mathrm{~T}^{3}$ & T1 & T4 & & dP2 & $\mathrm{P} 1$ & $\mathrm{dP1}$ & $\mathrm{dP3}$ & $P^{2}$ & P3 & 01 & ${ }^{0}$ & $\mathrm{OP}^{\mathrm{O} 3 \mathrm{I}}$ & Oth & & & & & & & & & & & \\
\hline & & & & & & & & & & & & & & & & & & & & & & & & & & & & \\
\hline 7 & eros- 01/26 & & & & & & & & & & & & & & & & & & & & & & & & & & & \\
\hline & $1 / 29 / 2004$ & 7:58:21 AM & 19.97 & 14.564 & \begin{tabular}{|l|}
11.286 \\
\end{tabular} & \begin{tabular}{|l|l|}
5 & 20.431 \\
\end{tabular} & 11.485 & $\mid$\begin{tabular}{|c|c|} 
\\
\end{tabular} & -0.149 & 0.007 & $\mid 0.018$ & -0.186 & 0.098 & -0.087 & 0.003 & $\mid 14.643$ & 0.005 & & & Filter Su & Surface, & \begin{tabular}{|l|l|}
16.707 \\
\end{tabular} & FT2 & & & & & \\
\hline 8 & $1 / 29 / 2004$ & $7: 58: 50 \mathrm{AM}$ & 19.97 & 14.564 & \begin{tabular}{|l|l|}
11.286 \\
\end{tabular} & 20.431 & 11.485 & 0.059 & -0.149 & 0.007 & 0.018 & -0.186 & 0.098 & -0.087 & 0.003 & 14.643 & 0.005 & & & Convers & rsion & 851 & $\mathrm{~m} 3 / \mathrm{m} 2 /$ & 2/day/bar & $\mathrm{rg} / \mathrm{gpm} / \mathrm{ft}$ & //ft2/barg & & \\
\hline & & 7:58:52 AM & & & & 20.466 & 11.546 & 0.059 & -0.149 & & & & & & $\begin{array}{l}0.003 \\
\end{array}$ & 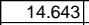 & 0.004 & & & & & & & & & & & \\
\hline & $1 / 29 / 2004$ & 7:58:53 AM & 19.981 & 14.585 & \begin{tabular}{|l|}
11.317 \\
\end{tabular} & 20.472 & 11.552 & 0.058 & -0.149 & 0.007 & 0.021 & -0.174 & 0.104 & -0.087 & \begin{tabular}{l|l}
0.003 \\
\end{tabular} & \begin{tabular}{|l|l|}
14.643 \\
\end{tabular} & 5.004 & & & Note: & unge & & $\mathrm{P} 1 \mathrm{i}$ & approx & ately 1. & 1.4 psig & & \\
\hline & $1 / 29 / 2004$ & 7:58:54 AM & 19.991 & 14.575 & \begin{tabular}{|l|l|}
11.317 \\
\end{tabular} & 20.477 & 11.55 & 0.059 & -0.149 & 0.007 & $\begin{array}{l}0.023 \\
\end{array}$ & -0.168 & 0.104 & -0.081 & $\begin{array}{l}0.003 \\
\end{array}$ & 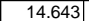 & 0.004 & & & & & & & & & & & \\
\hline & & 7:58:55 AM & 19.986 & $\begin{array}{l}14.58 \\
\end{array}$ & \begin{tabular}{|l|l|}
11.317 \\
\end{tabular} & 20.472 & 11.56 & 0.061 & -0.149 & 0.008 & 0.021 & & 0.104 & -0.087 & 0.003 & \begin{tabular}{|l|l|}
14.643 \\
\end{tabular} & & & & Press & P1is & :orrec & ted for & $0.88 \mathrm{in}$ & & er tu & & \\
\hline & & 7:58:56 AM & & & & & & & & & & & & & & & & & & VWWV & & & & < Filtra & ate Flux & $<P E$ & & $\gg>$ \\
\hline & 1/29/2004 & 7:58:57 AM & 19.986 & 14.58 & 11.312 & 20.472 & 11. & 0.059 & -0.149 & 0.008 & 0.023 & -0.1899 & 0.106 & -0.08 & 0.003 & 14.643 & & & & & & & & & & & & \\
\hline & ta - Per M & & & & & & & & & & & & & & & & & Time & ime & Press. & el. & TMP & TMP & $\mathrm{gpm}$ & om & $m$ & & eter \\
\hline$\frac{10}{17}$ & ata - Per M & ute $8.14 \cdot 42 \mathrm{AM}$ & & & & & & & & & & & & & & & & & & 53 & & 303 & 2088 & & & & & 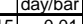 \\
\hline & $1 / 29 / 2004$ & $8: 15: 42 \mathrm{AM}$ & 19.798 & \begin{tabular}{|}
4.020 \\
14.65
\end{tabular} & 24.686 & 20.009 & $\begin{array}{l}13.007 \\
13.67\end{array}$ & $\begin{array}{r}40.101 \\
-4.01 \\
-4\end{array}$ & $\begin{array}{l}54.013 \\
52.267\end{array}$ & $\begin{array}{c}21.520 \\
9.073\end{array}$ & \begin{tabular}{|c|}
-19.801 \\
-12.718
\end{tabular} & $\begin{array}{r}8.61 \\
51.636 \\
\end{array}$ & $\begin{array}{r}9.495 \\
52.57\end{array}$ & 40.980 & $\begin{array}{l}0.003 \\
0.003\end{array}$ & $\begin{array}{l}4.643 \\
14.643 \\
\end{array}$ & 0.808 & 1.00 & 0.01667 & $\begin{array}{ll}33.1 \\
50.8\end{array}$ & \begin{tabular}{r|}
1.3 \\
9.8
\end{tabular} & $\begin{array}{l}30.5 \\
-8.4 \\
\end{array}$ & $\begin{array}{c}2.008 \\
-0.577 \\
\end{array}$ & \begin{tabular}{|l|l}
7 & 0.0000 \\
7
\end{tabular} & $\begin{array}{l}0.000 \\
0.000\end{array}$ & 0.000 & -0.054 & -0.05 \\
\hline 19 & $1 / 29 / 2004$ & $8: 16: 42 \mathrm{AM}$ & 23.187 & 14.663 & \begin{tabular}{|l|l|}
24.5844 \\
\end{tabular} & 20.731 & 13.791 & 30.709 & 51.624 & 12.027 & \begin{tabular}{|l|l|}
19.367 \\
\end{tabular} & $\begin{array}{l}6.432 \\
\end{array}$ & 0.358 & 54.431 & 0.5 & \begin{tabular}{|l|l|l|l|}
1443 \\
\end{tabular} & & 2.00 & 0.03333 & 50.2 & 13.3 & 25.0 & 1.726 & 0.075 & 0.075 & 0.003 & 3.012 & 2.56 \\
\hline & $1 / 29 / 2004$ & $8: 17: 42 \mathrm{AM}$ & 22.367 & 14.698 & \begin{tabular}{|l|}
24.548 \\
\end{tabular} & 20.791 & 13.886 & 47.844 & 53.552 & 16.609 & 31.389 & 0.263 & 0.41 & 51.427 & 0.088 & 14.643 & 0.004 & 3.0 & 0.05 & 52.1 & 12.6 & 39.6 & 2.731 & 2.183 & 2.211 & & 814 & 47.50 \\
\hline & 1/29/2004 & $8: 18: 42 \mathrm{AM}$ & 22.657| & 14.717 & \begin{tabular}{|l|}
24.5322 \\
\end{tabular} & 20.83 & 14.043 & 48.617 & 54.265 & 18.9 & 29.887 & 0.263 & & 50.658 & 0.065 & 14.643 & & 4.0 & .06667 & 52.8 & 12.4 & 39.3 & 2.706 & 2.183 & & 056 & & 47.96 \\
\hline & 1/29/2004 & $8: 19: 42$ AM & & 14.736 & 24.436 & 20.834 & 14.149 & 46.45 & 52.086 & 16.861 & 29.823 & & & & 0.054 & 14.643 & & 5.0 & & & & & & & & & & \\
\hline & $1 / 29 / 2004$ & 8:20:42 AM & 22.987 & 14. $7 / 14$ & 24.24 & 20.863 & $14.40 \pi$ & 46.881 & 52.532 & 17.218 & 29.897 & 0.246 & & 42.485 & 0.048 & 14.643 & & & & & & & & & & & & 0.16 \\
\hline & $11 / 29122004$ & $8: 21: 42$ AM & 然. & 14.181 & 24.034 & 20.863 & 14.493 & 49.206 & 54.883 & 18.583 & 30.851 & 0.231 & & 41.908 & 0.046 & 14.643 & & 1.00 & 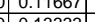 & 53.4 & $\frac{10.3}{10.3}$ & 40.0 & 2.160 & 0.001 & .001 & & & 0.15 \\
\hline & 11212004 & 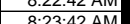 & & $\mid$ & 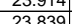 & $|0.074|$ & 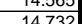 & 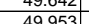 & $\begin{array}{l}53.314 \\
55607\end{array}$ & $\mid$ & $\frac{3.24}{31.030}$ & 0.20 & & 告0.940 & 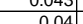 & $\begin{array}{l}14.045 \\
114643\end{array}$ & 500 & 8.00 & . & $\frac{5.9}{5.92}$ & 0.0 & 4.4 .4 & 20181 & & & & & .14 \\
\hline & $1 / 2912004$ & $8.24: 42 \mathrm{AM}$ & 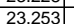 & 1486 & \begin{tabular}{|l|l|}
23.778 \\
\end{tabular} & 20.929 & $\frac{14.152}{14.666}$ & $\begin{array}{r}49.950 \\
49.98\end{array}$ & $\begin{array}{l}50.091 \\
55.718\end{array}$ & $\begin{array}{l}19.000 \\
19395\end{array}$ & 31.056 30.668 & 0.20 & & 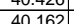 & $|0.04|$ & $\begin{array}{l}14.0545 \\
114643\end{array}$ & & 10.00 & & 5.4. & 势 & 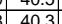 & $\frac{2.1 .24}{2780}$ & 0.000 & 然 & 然 0.0000 & & 0.13 \\
\hline 28 & $1 / 29 / 2004$ & $\begin{array}{l}\frac{6.24 .42}{8: 25: 42 \mathrm{AM}} \\
\end{array}$ & 告. 23.277 & $\mid \frac{14.000}{14.898}$ & \begin{tabular}{|l|}
23.823 \\
23.83
\end{tabular} & 20.954 & $\begin{array}{r}14.000 \\
14.99 \\
\end{array}$ & 48.648 & 54.327 & 21.265 & 27.635 & 0.26 & & 4..102 & 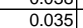 & $\begin{array}{l}14.045 \\
14.643\end{array}$ & 0.004 & 11.00 & 0.18333 & $\frac{3.2}{52.9}$ & $\frac{1.0}{105}$ & 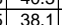 & 2630 & 0.005 & . & & $\frac{0.145}{0.141}$ & $\frac{0.12}{0.12}$ \\
\hline & $1 / 29 / 2004$ & 8:26:42 AM & 23.277 & 14.918 & \begin{tabular}{|l|l|}
24.028 \\
\end{tabular} & 21.034 & 14.878 & 49.355 & 55.048 & 21.466 & 28.052 & 0.28 & & 4216 & 0.035 & 14.643 & & 1200 & 0.2 & 53.6 & $\frac{103}{10.3}$ & $\frac{3.7}{38.7}$ & 2.668 & \begin{tabular}{|l|l|l|}
0.005 \\
\end{tabular} & 0.005 & & & $\frac{0.12}{0.12}$ \\
\hline 30 & $1 / 29 / 2004$ & $8: 27: 42 \mathrm{AM}$ & 23.276 & 14.942 & \begin{tabular}{|l|}
24.203 \\
\end{tabular} & 21.018 & 15.03 & 49.156 & 54.864 & 20.922 & 28.526 & 0.309 & & 41.419 & $\begin{array}{l}0.034 \\
\end{array}$ & 14.643 & .00 & $\begin{array}{ll}13.00 \\
1.00\end{array}$ & 1667 & 53.4 & 10.2 & 38.8 & $\begin{array}{l}2.678 \\
\end{array}$ & 0.005 & 005 & 5 & & 0.1 \\
\hline & $1 / 29 / 2004$ & 8:28:42 AM & 23.285 & 14.967 & \begin{tabular}{|l|}
24.372 \\
\end{tabular} & 21.062 & 15.075 & 49.333 & 55.094 & 21.019 & 28.505 & 0.332 & & 41.338 & 0.034 & 14.643 & & 14.00 & 23333 & 53.6 & $\frac{10.1}{10.1}$ & 38.9 & 2.683 & 0.005 & 0.005 & 0.000 & & 0.1 \\
\hline & & & & & & 21.067 & & 50.392 & 56.074 & 21.216 & 29.423 & 0.28 & & 41.315 & & 14.643 & & & 0.25 & 54.6 & 10.1 & 39.9 & & & & & & \\
\hline & $1 / 29 / 2004$ & $8: 30: 42 \mathrm{AM}$ & 24.496 & 15.026 & \begin{tabular}{|l|}
24.652 \\
\end{tabular} & 21.132 & 15.0 & 50.685 & 56.398 & 20.772 & 30.063 & 0.315 & & 40.456 & 0.035 & 14.643 & & 160 & 0.26667 & 54.9 & 9.9 & 40.4 & 2.784 & \begin{tabular}{l|l}
4 & 0.005
\end{tabular} & 0.005 & 0.000 & $\overline{0.131}$ & $\frac{0.11}{0.11}$ \\
\hline 34 & $1 / 29 / 2004$ & 8:31:42 AM & \begin{tabular}{|l|l|}
24.62 \\
\end{tabular} & \begin{tabular}{|l|l|}
15.045 \\
\end{tabular} & \begin{tabular}{|l|}
24.76 \\
\end{tabular} & 21.15 & & 50.635 & 56.362 & 20.912 & 29.889 & 0.332 & & 40.001 & & 14.643 & & 17. & 0.28333 & 54.9 & 9.9 & 40.3 & 2.776 & & 0.005 & & & 0.11 \\
\hline & $1 / 29 / 2004$ & $8: 32: 42 \mathrm{~A}$ & 24.745 & \begin{tabular}{|l|l|}
15.096 \\
\end{tabular} & \begin{tabular}{|l|}
24.865 \\
\end{tabular} & 21.2 & 15.3 & 50.656 & 56.412 & 21.103 & & 0.335 & & 40.216 & \begin{tabular}{|l|l|l|} 
\\
\end{tabular} & 14.643 & & 18.00 & \begin{tabular}{|l|l|}
0.3 \\
\end{tabular} & 54.9 & 9.9 & 40.2 & 2.773 & \begin{tabular}{l|l|l}
3 & 0.005 \\
\end{tabular} & 0.005 & 0.000 & & 0.11 \\
\hline 36 & 1/29/2004 & & 24.838 & 15.104 & \begin{tabular}{|l|}
24.934 \\
\end{tabular} & 21.188 & 15. & 50.936 & 56.673 & 21.467 & 29.657 & 0.332 & & 40.124 & 0.034 & & & 19. & 8.31667 & 55.2 & $9.8 \quad$ & 40.3 & 778 & & & & & 0.11 \\
\hline & $1 / 29 / 2004$ & 8:34:42 AM & 24.576 & 15.133 & \begin{tabular}{|l|}
25.022 \\
\end{tabular} & 21.182 & & 53.192 & 56.441 & 21.344 & 32.138 & -1.211 & & & 0.267 & 14.643 & & 20.0 & 0.33333 & 55.0 & 9.8 & 42.7 & 2.942 & \begin{tabular}{l|l|}
2 & 0.040
\end{tabular} & 0.040 & 0.001 & 932 & 0.79 \\
\hline 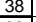 & 1/299/2004 & $8: 35: 42 \mathrm{~A}$ & & 15.167 & \begin{tabular}{|l|l|l|l|} 
\\
\end{tabular} & \begin{tabular}{l|l|} 
& 21.241 \\
\end{tabular} & & 53.265 & - & 23.957 & 29.774 & -1.211 & & 43.543 & 0.007 & 14.643 & & 21.0 & 0.00 & 52.0 & 10.1 & 41. & 2.000 & 0.00 & . & & & 0.1 \\
\hline & & & & & & & & & & & & & & & 0.036 & & & & & & $10.8 \mathrm{r}$ & & & & 0.005 & & & \\
\hline & (1)2004 & & 23.15 & 15.22 & 23.206 & 21.242 & & 52.13 & 52.133 & 23.862 & 28.604 & -1.214 & & 43.14 & & $\begin{array}{l}14.643 \\
1\end{array}$ & & & .38333 & 51.3 & 10.1 & 40.4 & 2.00 & & & & & 0.1 \\
\hline & $\begin{array}{l}112912004 \\
1291204\end{array}$ & & $\frac{2.359}{2.283}$ & & & 21.281 & & $\frac{51.1}{5.9}$ & $\begin{array}{l}52.265 \\
5251\end{array}$ & $\frac{2.30}{24.35}$ & $\frac{2.1 .11}{2012}$ & & & 44.371 & & & & & 0.4 & & $\frac{1.5}{108}$ & & 40 & & & & & 0.10 \\
\hline & $\begin{array}{ll}1 / 29 / 2004 \\
1 / 29 / 204\end{array}$ & & 势. & | & $\mid$\begin{tabular}{|l|l|l|l|}
$\mid 25.55$ \\
\end{tabular} & 21.245 & & 51.904 & $\begin{array}{l}3<.301 \\
55464\end{array}$ & 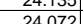 & $\frac{2.0 .14+4}{28271}$ & $\mid-1.24$ & & 44058 & 年 & & & 20 & $\begin{array}{l}0.41007 \\
0.1232\end{array}$ & | & $\begin{array}{ll}1.0 \\
108\end{array}$ & $\begin{array}{ll}4.0 .0 \\
10.1\end{array}$ & | & & 0.0005 & 0.0000 & & 0.14 \\
\hline 44 & $\frac{1 / 29 / 2004}{1 / 29}$ & $8: 41: 42 \mathrm{AN}$ & 23.572 & $\mid$ & \begin{tabular}{|l|}
25.4009 \\
25.509 \\
\end{tabular} & \begin{tabular}{|l|l|} 
& 21.3424 \\
\end{tabular} & $\frac{1.0 .5}{16.3}$ & $\frac{11.500}{51.95}$ & $\begin{array}{l}52.404 \\
52.501\end{array}$ & $\frac{24.012}{24.222}$ & $\frac{20.271}{28.148}$ & $\frac{-1.24}{-1.211}$ & & $\begin{array}{l}44.050 \\
43.918\end{array}$ & & $\frac{146}{146}$ & & $\frac{27.00}{2700}$ & $\frac{0.4505}{0.45}$ & 51.0 & $\frac{1.0}{10.8}$ & $\frac{4.1}{400}$ & 2 & $\frac{10.005}{10}$ & 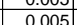 & & & $\frac{0.10}{0.10}$ \\
\hline 45 & $\begin{array}{l}1 / 29 / 2004 \\
\end{array}$ & & 24.006 & 15.368 & \begin{tabular}{|l|}
25.539 \\
\end{tabular} & 21.349 & 16.3 & 52.371 & 52.965 & 24.769 & 27.986 & -1.214 & & 45.115 & 0.0 & & & 28. & 0.46667 & 51.5 & 11.1 & 40.2 & 2.770 & & & & & 0.1 \\
\hline & 1/29/2004 & & 24.046 & 15.408 & \begin{tabular}{|l|}
25.658 \\
\end{tabular} & 21.409 & & 52.516 & 53.102 & 25.372 & 27.424 & & & 45.426 & & & & 29.0 & 0.48333 & 51.6 & $\overline{11.1}$ & 40.0 & 2.156 & & & & & \\
\hline 47 & 9/2004 & & 24.08 & & & 21.357 & & 51.985 & 52.596 & 25.256 & 27.182 & -1.216 & & & & & & & 0.5 & 51.1 & 11.2 & 39.6 & & & & & & \\
\hline & & & 24.129 & 15.462 & \begin{tabular}{|l|l|} 
\\
\end{tabular} & & & 52.288 & 52.899 & 25.043 & & & & & & & & J1.c & 0.51667 & 51.4 & 11.0 & & 2.755 & & & & & \\
\hline 49 & $1 / 29 / 2004$ & & $24.1 / 3$ & 15.492 & \begin{tabular}{|l|}
25.84 \\
\end{tabular} & 21.431 & & 52.234 & 52.81 & 24.916 & 27. & .211 & & & & & & & & 51.3 & 110 & 40.0 & $\begin{array}{l}2.757 \\
\end{array}$ & & & & & \\
\hline & & & 24.2 & 15.516 & \begin{tabular}{|l|l|}
25.874 \\
\end{tabular} & 21.41 & & 52.369 & 52.94 & & 28.1 & & & & & & & 33. & 0.55 & & 11.0 & 40.3 & 2.777 & & & & & \\
\hline & $1 / 29 / 2004$ & & 24.286 & 15.561 & \begin{tabular}{|l|l|}
25.933 \\
\end{tabular} & 21.5 & & 52.585 & 53.175 & 24.599 & 28. & -1.211 & & 44. & & 14.6 & & 34 & 0.56667 & 51.7 & 10.9 & 40 & & & & & 0.115 & 15 \\
\hline 52 & $1 / 29 / 2004$ & $8: 49: 42$ & 24.315 & 15.575 & 25.957 & 21.539 & & 52.742 & 53.311 & 24.645 & 28.399 & & & & & & & 35. & 0.58333 & 51.8 & 10.9 & & & & & & 0.114 & 0.10 \\
\hline 53 & $1 / 29 / 2004$ & $8: 50: 42 \mathrm{f}$ & 24.364 & 15.604 & 25.966 & 21.537 & & 52.682 & 53.322 & 24.617 & 28.3 & -1.211 & & 44.3 & & & & 36. & 0.6 & 51.8 & 0.9 & 40.5 & 694 & & & & 15 & 0.1 \\
\hline 54 & $1 / 29 / 20$ & & 24.417 & 15.643 & 26.0 & $21.5 \mathrm{~S}$ & & 52.39 & 52.984 & & 28.3 & -1.214 & & & & & & 37. & 0.61667 & 51.5 & $10 . \varepsilon$ & & 783 & & & & & \\
\hline & & & 24.441 & & & & & 52.4 & 53.0 & & & -1.211 & & & & & & & & 51.6 & 10.0 & & & & & & & \\
\hline 56 & & & 24.465 & 15.696] & 26.0 & 21.538 & & 52.8 & 53 & & & & & & & & & & 0.65 & 51.9 & & & & & & & & \\
\hline & & & 2 & $15 . / 31$ & 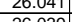 & 21.562 & & 52.319 & 52.936 & 24.371 & 28.401 & -1.214 & & & & 14. & & 40.0 & 0.66667 & 51.5 & 10.0 & 40.4 & 2.183 & & & & & 5 \\
\hline$\frac{39}{59}$ & 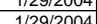 & $\frac{0.35 .44}{8.562}$ & $\frac{2.5761}{2456}$ & $\mid$ & 20.095 & 21.500 & & 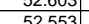 & $\begin{array}{l}53.251 \\
52121\end{array}$ & $\frac{24.534}{24665}$ & 28.405 & $\frac{-1.21}{-1214}$ & & & & $\begin{array}{l}14.643 \\
1.633\end{array}$ & & & 0.08353 & 甚1.8. & . & & $\frac{73}{701}$ & & & & & 4 \\
\hline 60 & $1 / 29 / 20$ & & 24.59 & 15.808 & \begin{tabular}{|l|l|}
26.066 \\
\end{tabular} & & & 52.641 & 53.235 & & 28.444 & & & 440 & 0.03 & & & & 0.71667 & 51.8 & 10.8 & & 95 & & & & 0.114 & 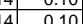 \\
\hline 6 & $1 / 29 / 20$ & 8:58: & 24.619 & 15.8 & 26.0 & \begin{tabular}{|l|l|}
5 & 21.628 \\
\end{tabular} & & 52.539 & 53.15 & 24.563 & 28.362 & $\frac{-1.214}{-1.214}$ & & & & & & & 0.73333 & 51.7 & & & & & & & & 14 \\
\hline 62 & & & 24.643 & 15.861 & 26. & 21.7 & 17. & 52.8 & 53.423 & 24.744 & 28.397 & -1.214 & & & \begin{tabular}{l|l}
0.032 \\
\end{tabular} & & & 45. & 0.75 & 52.0 & $10.7 \mathrm{Y}$ & & 2.800 & & 205 & & & $|4|$ \\
\hline 63 & & & 24.671 & & 26.1 & & & & & & & -1.211 & & & & & & & & 0 & 10.1 & & & & & & & \\
\hline 6 & & & 24.699 & 15.914] & 26.151 & 21.739 & & $52.1 / 5$ & 53.38 & & 28.861 & & & & & & & 47.00 & 78333 & 51.9 & 10.6 & & & & & & & \\
\hline & & & & & & 21.782 & & & 53.27 & 24.336 & 28.834 & -1.214 & & 43.2. & & & & & & 51.8 & & & & & & & & \\
\hline 60 & & & 24.16 & 15..965 & 26.172 & 21.810 & & 52.954 & 53.572 & 24.554 & 28.821 & & & & 0.03 & $14.6 \mathrm{C}$ & & & 0.81667 & 52 & & & 2.819 & & & & 113 & 13 \\
\hline & & & & 16.004 & 6.2 & & & 53.076 & 53.687 & 24.689 & 28.76 & & & & 0.0 & 14.6 & & & & & & & & & & & & \\
\hline & & 9:05:42 & 24.811 & 16.027 & \begin{tabular}{|l|}
26.203 \\
26202
\end{tabular} & 21.746 & $\frac{11}{170}$ & 53.008 & 53.631 & 24.66 & 28.752 & & & 43.036 & 0.03 & 14.6 & & 51.00 & 0.85 & & & & 2.819 & $\begin{array}{ll}9 & 0.005 \\
\end{array}$ & 0.005 & 0.000 & & 0.10 \\
\hline 70 & & 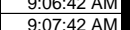 & 24.852 & 779 & 26.214 & $4[21.838$ ] & & 52.95 & 53.568 & $\frac{24.714}{24.781}$ & 28.659 & -1.216 & & 12854 & 0.031 & 14.643 & & & (8.88333 & 52.1 & & 40.8 & \begin{tabular}{|l|}
2.819 \\
2813 \\
\end{tabular} & 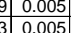 & \begin{tabular}{|c|c|c|}
0.004 \\
0.04
\end{tabular} & 0.000 & $\frac{0.113}{0.109}$ & $\frac{34}{9}$ \\
\hline
\end{tabular}


WSRC-TR-2005-00105, REVISION 0

SRNL-RPP-2005-00012, REVISION 0

RUN \# 1.04; STEADY STATE TEST AT 25 WT\% UDS - CONT.

\begin{tabular}{|c|c|c|c|c|c|c|c|c|c|c|c|c|c|c|c|c|c|c|c|c|c|c|c|c|c|c|c|c|}
\hline & $\mathrm{A}$ & $B$ & $\mathrm{D}$ & $\mathrm{E}$ & $F$ & $G$ & $\mathrm{H}$ & $\mathrm{J}$ & $\mathrm{K}$ & $\mathrm{L}$ & $\mathrm{M}$ & $\mathrm{N}$ & 0 & $\mathrm{Q}$ & $\mathrm{R}$ & $\mathrm{s}$ & $T_{T}$ & \begin{tabular}{l|l} 
& \\
\end{tabular} & w & $x$ & & $z^{\prime}$ & $\mathrm{AA}$ & $A B$ & $A C$ & $\overline{A D}$ & $\mathrm{AE}$ & AF $D A$ \\
\hline & $1 / 29 / 2004$ & 9:08:42 AM & 24.876 & 16.098 & 26.178 & 21.856 & 17.854 & 53.188 & 53.817 & 24.929 & 28.643 & & -5.424 & 42.879 & 0.031 & 14.643 & 0.004 & 54.00 & 0.9 & $\hat{52.3}$ & & & 2.82 & $\frac{0.005}{0.005}$ & 0.004 & 0.000 & 0.109 & \\
\hline & $1 / 29 / 2004$ & 9:09:42 AM & 24.903 & 16.136 & 26.11 & 21.899 & 18.215 & 52.92 & & 24.596 & 28.746 & -1.216 & $\begin{array}{l}-5.426 \\
\end{array}$ & & 0.031 & 14.643 & 0.004 & & .91667 & 52.1 & & 40. & & & 0.004 & & & \\
\hline & 1/29/2004 & 9:10:42 AM & 24.926 & 16.17 & 25.948 & 21.932 & 17.915 & 52.968 & 53.593 & 24.541 & 28.822 & $\begin{array}{l}-1.214 \\
\end{array}$ & -5.464 & \begin{tabular}{|l|l|}
42.861 \\
\end{tabular} & 0.042 & 14.643 & 0.004 & 56.00 & .93333 & 52.1 & & & & 0.006 & 0.006 & 0.000 & 0.149 & \\
\hline & 1/29/2004 & 9:11:42 AM & 24.844 & $\begin{array}{l}16.198 \\
1.207\end{array}$ & 25.861 & 21.915 & $\begin{array}{l}18.165 \\
18.07\end{array}$ & \begin{tabular}{|l|l|}
52.703 \\
\end{tabular} & \begin{tabular}{|l|l|}
53.494 \\
\end{tabular} & 24.54 & 28.608 & $\begin{array}{l}-1.214 \\
\end{array}$ & & 42.952 & 0.031 & 14.643 & 0.004 & 57.00 & 0.95 & 52.0 & & $\begin{array}{l}40.7 \\
\end{array}$ & \begin{tabular}{|l|}
2.803 \\
\end{tabular} & & & & 0.111 & \\
\hline 76 & 1/29/2004 & $\begin{array}{l}9: 12: 42 \mathrm{AM} \\
9 \cdot 13: 2 \mathrm{AM}\end{array}$ & $\begin{aligned} 24.772 \\
2471\end{aligned}$ & $\begin{array}{l}16.237 \\
16265\end{array}$ & $\begin{array}{l}25.814 \\
25757\end{array}$ & \begin{tabular}{|l|l|l|l|}
21.898 \\
21.936
\end{tabular} & $\begin{array}{l}18.067 \\
18.373\end{array}$ & $\begin{array}{r}52.92 \\
52878 \\
\end{array}$ & \begin{tabular}{|r|r|}
53.73 \\
53718 \\
\end{tabular} & $\begin{array}{l}24.627 \\
24.633\end{array}$ & $\begin{array}{l}28.639 \\
28606\end{array}$ & \begin{tabular}{|l|l|l|}
-1.214 \\
-1214
\end{tabular} & $\begin{array}{r}-5.016 \\
-5013\end{array}$ & \begin{tabular}{|l|l|l|l|l|}
43491 \\
4349
\end{tabular} & \begin{tabular}{|l}
0.031 \\
0.031
\end{tabular} & $\begin{array}{l}14.643 \\
1643\end{array}$ & \begin{tabular}{|l|l|}
0.004 \\
0.004
\end{tabular} & 58.00 & \begin{tabular}{|l|}
0.96667 \\
\end{tabular} & 52.3 & 10.6 & 40.8 & 2.812 & 0.005 & $\begin{array}{l}0.005 \\
\end{array}$ & 0.000 & $\begin{array}{l}0.111 \\
\end{array}$ & 0.09 \\
\hline$\frac{77}{77}$ & $\begin{array}{l}1 / 2992004 \\
1 / 29 / 2004\end{array}$ & $\begin{array}{l}9: 13: 42 \mathrm{AM} \\
9: 14: 42 \mathrm{AM}\end{array}$ & $\begin{aligned} 24.11 \\
24.673\end{aligned}$ & $\begin{array}{l}\frac{16.265}{16.298} \\
\end{array}$ & $\begin{array}{l}25.157 \\
25.655\end{array}$ & $\begin{array}{l}21.956 \\
21.974\end{array}$ & $\begin{array}{l}18.573 \\
18.624\end{array}$ & $\begin{array}{l}53.88 \\
53.105\end{array}$ & \begin{tabular}{|l|}
$53 . / 118$ \\
53.999
\end{tabular} & $\begin{array}{l}24.635 \\
24.716\end{array}$ & $\begin{array}{r}28.6006 \\
28.561\end{array}$ & & & \begin{tabular}{|l|}
43.249 \\
43.566 \\
\end{tabular} & $\frac{0.031}{0.031}$ & $\frac{14.643}{14.643}$ & $\begin{array}{c}0.004 \\
0.004\end{array}$ & $\begin{array}{l}59.00 \\
60.00\end{array}$ & & $\begin{array}{l}52.2 \\
5.5 \\
\end{array}$ & & & & & $\begin{array}{l}0.005 \\
0.005\end{array}$ & 0.000 & $\frac{0.111}{0.111}$ & \\
\hline & $1 / 29 / 2004$ & 9:15:42 AM & 24.65 & 16.321 & 25.547 & 22.002 & & 52.634 & \begin{tabular}{|l|}
53.427 \\
\end{tabular} & 24.429 & 28.721 & -1.214 & & \begin{tabular}{|l|}
43.706 \\
\end{tabular} & 0.03 & 14.643 & 0.004 & 61.00 & \begin{tabular}{|l|l|}
1.01667 \\
\end{tabular} & & & & \begin{tabular}{|l|}
2.815 \\
2.805 \\
\end{tabular} & & & & 0.111 & \\
\hline & $1 / 29 / 2004$ & 9:16:42 AM & 24.639 & 16.35 & 25.521 & & 18.549 & 52.979 & \begin{tabular}{|l|l}
53.794 \\
\end{tabular} & 24.491 & 28.787 & -1.214 & -5.016 & 43.851 & 0.03 & 14.643 & 0.004 & 62.00 & 1.03333 & 52.3 & 10.7 & 40.9 & 2.819 & 0.004 & 0.004 & 0.000 & 0.108 & \\
\hline & $1 / 29 / 2004$ & 9:17:42 AM & & 16.393 & 25.548 & 22.028 & 18.642 & 52.025 & \begin{tabular}{|l|l|}
52.789 \\
\end{tabular} & 25.746 & 26.734 & & & \begin{tabular}{|l|l|}
45.426 \\
\end{tabular} & 0.029 & 14.643 & 0.004 & 63.00 & 1.05 & 51.3 & & 39.4 & 2.715 & & & & & \\
\hline & $1 / 29 / 2004$ & $9: 18: 42 \mathrm{AM}$ & 24.569 & 16.411 & 25.521 & 21.96 & 18.413 & 52.367 & 53.175 & 26.014 & & & -5.0 & \begin{tabular}{|l|l|}
45.524 \\
\end{tabular} & 0.029 & 14.643 & 0.004 & 64.00 & 1.06667 & & & 39.5 & & 0.004 & 0.004 & 0.000 & & \\
\hline oc & $1 / 29 / 2004$ & 9:19:42 AM & 24.532 & $\begin{array}{l}16.44 \\
\end{array}$ & $\begin{array}{l}25.544 \\
25.567\end{array}$ & 21.973 & $\begin{array}{l}18.603 \\
18720\end{array}$ & \begin{tabular}{|l|l|}
52.101 \\
5.201
\end{tabular} & \begin{tabular}{|l|l|}
52.916 \\
5203
\end{tabular} & 25.737 & $\begin{array}{l}26.796 \\
2750\end{array}$ & & & \begin{tabular}{|l|l|}
45.389 \\
\end{tabular} & 0.029 & 14.643 & 0.004 & 65.00 & 1.08333 & & & 39.4 & \begin{tabular}{|l|l|}
2.720 \\
\end{tabular} & 0.004 & 0.004 & 0.000 & 0.108 & \\
\hline & $1 / 29 / 2004$ & $\begin{array}{l}9: 20: 42 \mathrm{AM} \\
\cdot 021: 2 \mathrm{M}\end{array}$ & $\begin{array}{l}24.524 \\
24511\end{array}$ & $\begin{array}{l}16.468 \\
16.48\end{array}$ & $\begin{array}{l}25.567 \\
25.604\end{array}$ & $\begin{array}{l}22.046 \\
22078\end{array}$ & $\begin{array}{l}18.726 \\
10.036\end{array}$ & \begin{tabular}{|l|}
52.278 \\
52184 \\
\end{tabular} & \begin{tabular}{|r|r|}
53.03 \\
53007 \\
\end{tabular} & 25.542 & & & & \begin{tabular}{|l}
45.228 \\
\end{tabular} & 0.029 & 14.643 & 0.004 & $\begin{array}{l}66.00 \\
670\end{array}$ & & 51.6 & & & \begin{tabular}{|l|}
2.738 \\
\end{tabular} & 0.004 & 0.004 & 0.000 & 0.107 & \\
\hline 85 & $\begin{array}{l}1 / 29 / 2004 \\
1 / 2912004\end{array}$ & $\begin{array}{l}9: 21: 42 \mathrm{AM} \\
9: 22: 42 \mathrm{MM}\end{array}$ & $\begin{aligned} 24.511 \\
24.509\end{aligned}$ & \begin{tabular}{r|r|r|}
16.48 \\
16.518
\end{tabular} & \begin{tabular}{|l|}
25.604 \\
25.621 \\
\end{tabular} & \begin{tabular}{|l|}
22.078 \\
22.106 \\
\end{tabular} & $\begin{array}{l}19.036 \\
19.084 \\
\end{array}$ & \begin{tabular}{|c|}
52.184 \\
52.18
\end{tabular} & \begin{tabular}{|l|}
53.007 \\
52.971 \\
\end{tabular} & $\begin{array}{l}25.145 \\
25.154\end{array}$ & $\begin{array}{l}27.518 \\
27.551 \\
\end{array}$ & $\begin{array}{l}-1.214 \\
-1.126 \\
\end{array}$ & $\begin{array}{l}-5.01 \\
-5.01\end{array}$ & $\begin{array}{l}45.324 \\
45.12\end{array}$ & \begin{tabular}{|l|l|}
0.029 \\
0.03
\end{tabular} & $\begin{array}{l}14.643 \\
14.643\end{array}$ & $\begin{array}{l}0.004 \\
0.04\end{array}$ & \begin{tabular}{|c|}
67.00 \\
6.00 \\
\end{tabular} & \begin{tabular}{|l|l|}
1.1667 \\
1.3333
\end{tabular} & $\begin{array}{l}51.5 \\
5.1 .5\end{array}$ & & & \begin{tabular}{|l|}
2.748 \\
2749
\end{tabular} & 0.004 & 0.004 & 0.000 & 0.107 & 0.09 \\
\hline & $1 / 29 / 2004$ & $9: 23: 42 \mathrm{AM}$ & 24.507 & 16.547 & \begin{tabular}{|l|}
25.021 \\
25.665 \\
\end{tabular} & 22.175 & 19.229 & 52.485 & \begin{tabular}{|l|}
53.345 \\
\end{tabular} & 25.259 & 27.611 & $\frac{1.201}{-1.208}$ & & 45.065 & 0.03 & 14.643 & $\begin{array}{l}0.004 \\
0.004\end{array}$ & 69.00 & 1.15 & 51.9 & & & $\frac{2.149}{2.761}$ & 0.004 & & & & 0.09 \\
\hline & $1 / 29 / 2004$ & 9:24:42 AM & 24.489 & 16.569 & \begin{tabular}{|l|}
25.677 \\
\end{tabular} & 22.212 & 19.18 & 52.275 & \begin{tabular}{|l|}
53.061 \\
\end{tabular} & 24.969 & 27.699 & -1.214 & & 45.289 & 0.03 & 14.643 & 0.004 & 70.00 & 1.16667 & 51.6 & & & 2.757 & & & & & 0.09 \\
\hline & $1 / 29 / 2004$ & $9: 25: 42 \mathrm{AM}$ & 24.481 & 16.587 & 25.694 & 22.21 & 18.921 & 52.412 & \begin{tabular}{|l|l|} 
& 53.228 \\
\end{tabular} & 25.013 & \begin{tabular}{l|l}
27.828 \\
\end{tabular} & & & 44.986 & 0.03 & 14.643 & & 71.00 & 1.18333 & 51.8 & & 40.1 & 2.766 & \begin{tabular}{|l|} 
\\
\end{tabular} & & & & $\frac{0.09}{0.09}$ \\
\hline & & 9:26:42 AN & 24.479 & & & & & & & & & & & 45.161 & & & & & & & & & 2.761 & & & & & \\
\hline & & & & & & 22.22 & 18.901 & 52.528 & 53.355 & 25.147 & 27.752 & -1.216 & -5.01 & & & $\frac{14.643}{14}$ & & & 21667 & & & & & & & & & $\frac{0.09}{0.09}$ \\
\hline & & & & 16.676 & & & & & & & & & & & & 14.6 & & & & & & & & & & & & \\
\hline & $1 / 29 / 2004$ & 9:29:42 AM & 24.476 & 16.708 & \begin{tabular}{|l|}
25.764 \\
\end{tabular} & 22.235 & & 52.406 & \begin{tabular}{|l|l|}
53.276 \\
\end{tabular} & 24.989 & $\begin{array}{l}27.785 \\
\end{array}$ & -1.214 & & $\overline{5.24}$ & 0.0 & 14.64 & & 75.06 & 1.25 & $\begin{array}{l}51.8 \\
5\end{array}$ & & & 2.764 & 0.004 & & & & 0.09 \\
\hline & $1 / 29 / 20$ & $9: 30: 42 \mathrm{AM}$ & & 16.741 & & & & & \begin{tabular}{|l|l|}
53.401 \\
\end{tabular} & 25.277 & & & & .2 & & 14.643 & & & & & & & & & & & & \\
\hline & $1 / 29 / 20$ & 9:31:42 AM & 24.491 & 16.764 & \begin{tabular}{|l|l|}
25.784 \\
\end{tabular} & 22.265 & $\begin{array}{l}19.435 \\
\end{array}$ & 52.361 & \begin{tabular}{|l|l|}
53.183 \\
\end{tabular} & 24.921 & 27.816 & -1.214 & & & 0.03 & $\begin{array}{l}14.643 \\
\end{array}$ & & 77.00 & \begin{tabular}{|l|l|}
1.28333 \\
\end{tabular} & 51.7 & & 40.1 & 764 & & & & 0.109 & 0.09 \\
\hline & $1 / 29 / 2$ & 9:32:42 AM & & 16.802 & \begin{tabular}{|l|}
25.811 \\
\end{tabular} & 22.307 & 19.26 & 52.352 & \begin{tabular}{|l|l|} 
& 53.16 \\
\end{tabular} & 24.96 & & 214 & & 45.56 & 0.03 & 14.643 & & 78.00 & 1.3 & & & 40. & & 0.004 & 0.004 & & & \\
\hline & $1 / 29 / 2$ & & 24.53 & 16.819 & 25.823 & 22.304 & 19.58 & 52.585 & 53.504 & 25.067 & 27.773 & & & .733 & 0.03 & 14.643 & & 79.00 & . 31667 & & & & & & & & 109 & \\
\hline & $1 / 29 / 2004$ & 34:42 AM & 24.542 & 16.846 & 25.84 & 22.356 & 19. & 52.557 & 53.438 & & 7.826 & -1.214 & & 5.243 & 0.05 & 14.643 & & .00 & & & & & & & & & & \\
\hline & 004 & & 24.559 & 16.869 & 25.852 & 22.379 & & 52.414 & 53.247 & 24.971 & 7.832 & & & & 0.03 & 14. & & 81.00 & 1.35 & 51.8 & & & & & & & & \\
\hline & 1/29/2004 & 9:36:42 AM & 24.572 & 16.891 & 25.859 & 22.381 & & 52.331 & \begin{tabular}{|l|} 
\\
\end{tabular} & 24.75 & 27.8 & -1.214 & & .424 & 0.0 & 14.6 & & 82.00 & 36667 & & & & & & & & 09 & 09 \\
\hline & & & & $\frac{16.923}{1.935}$ & & $\frac{22.352}{20302}$ & & $\begin{array}{l}52.383 \\
52.172\end{array}$ & \begin{tabular}{|l}
5.201 \\
5.201
\end{tabular} & 25.246 & 2.1 & $\frac{214}{214}$ & & .1 & & & & & 88333 & & & & & & & & & \\
\hline & $\frac{1 / 29 / 2004}{1 / 29 / 2004}$ & $\begin{array}{l}9.30 .4 \\
0.30 .1\end{array}$ & $\begin{array}{l}24.585 \\
24602\end{array}$ & $\begin{array}{l}\frac{16.945}{16073} \\
1607\end{array}$ & \begin{tabular}{|l}
25.858 \\
258
\end{tabular} & $\begin{array}{l}22.389 \\
20371\end{array}$ & & $\frac{52.172}{5217}$ & \begin{tabular}{|l|}
53.01 \\
5.054
\end{tabular} & 24.746 & 2.784 & 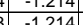 & & .5 & 0.0 & 14.6 & & & 1.4 & 516 & & & & & & & & \\
\hline & $1 / 29 / 2004$ & $9: 40 \cdot 4$ & 24.614 & 17011 & 25.902 & 22.394 & & 52.346 & \begin{tabular}{|l|l|}
53179 \\
\end{tabular} & 24.85 & & & & 1067 & 00 & & & & 4333 & & & 402 & & & & & & \\
\hline & $1 / 29 / 2004$ & 9:41:42 AM & 24.606 & 17.017 & \begin{tabular}{|l|l|}
25.893 \\
\end{tabular} & 22.38 & 19.81 & 52.381 & \begin{tabular}{|l|l}
3.301 \\
\end{tabular} & 24.596 & 28.105 & 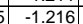 & & 45.205 & 0.03 & 14.6 & & 87.00 & 1.45 & 51.8 & 11.1 & 40.2 & 2.775 & \begin{tabular}{|l|}
0.004 \\
\end{tabular} & 0.004 & 0.000 & 0.108 & \\
\hline & & & & & 25.871 & 22.382 & & & & 24.805 & & & & & & & & & & & & & & & & & & \\
\hline & 1/29/2004 & 9:43:42 AM & 24.63 & 17.062 & \begin{tabular}{|l|l|}
25.908 \\
\end{tabular} & 22.409 & & 52.263 & 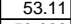 & 24.771 & & -1.216 & & 45.061 & 0.03 & & & & 1.48333 & 51.6 & 11.0 & 40.1 & & & & & 0.109 & \\
\hline & $\frac{1 / 29122004}{112004}$ & & 24.637 & 17.084 & 25.914 & 22.421 & & 52.28 & 53.092 & 24.695 & & 214 & & & & & & 90 & 1.5 & 0 & . & 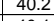 & & & & 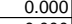 & 108 & \\
\hline & & & & 1.1.112 & & 22.4 & & & & $\frac{24.644}{20.12}$ & & & & & & & & & & & & & & & & & & \\
\hline & $\frac{1 / 29 / 2004}{1 / 29 / 2004}$ & & & & \begin{tabular}{|l|l|}
2.535 \\
502
\end{tabular} & $\frac{22.4 / 1}{22507}$ & & & 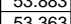 & & & & & & 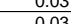 & & & & 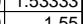 & $\begin{array}{ll}5.24 \\
5.19\end{array}$ & & & & & & & & \\
\hline & $1 / 29 /$ & & & & 25.912 & & & & & & & & & & & & & & & & & & & & & & & \\
\hline & $1 / 29 /$ & & 24.682 & & 25.905 & $\frac{22.507}{22.507}$ & & 52.5 & 53.614 & & & & & & & & & & 15833 & & & & & & & & & \\
\hline & & & $\begin{array}{l}24.694 \\
\end{array}$ & & & & & & & & & & & & & & & & & & & & & & & & & \\
\hline & & & & 17.266 & Drand & 22.576 & & & \begin{tabular}{|l|l|}
53664 \\
\end{tabular} & & & & & & & & & & 81667 & & & & & & & & & \\
\hline & & $9: 52$ & & & 25.931 & 22.588 & & 52.3 & 53.247 & & & & & & & & & & & & & & & & & & & \\
\hline & & 9:53: & & 17.311 & & & & & 53. & & & & & & & & & & & & & & & & & & & \\
\hline & $1 / 29$ & 9:54 & 24.74 & 17.343 & \begin{tabular}{|l|}
25.915 \\
\end{tabular} & 22.65 & & 52833 & 53.716 & 23.9 & & & & & & 14. & & & 66667 & & & & & & & & 10 & \\
\hline & $1 / 29 / 20$ & & 24.75 & 17 & 25.897 & & & 52.7 & \begin{tabular}{|l|l|}
53.637 \\
\end{tabular} & & & & & & & & & & 68333 & & & & & & & & & \\
\hline & 1/29/20 & 9:56:42 & 24.766 & 17.377 & 25.898 & 22.7 & & 52.431 & \begin{tabular}{|l|}
53.382 \\
\end{tabular} & 23.7 & 29.1 & & & & & & & & 1.7 & & & & & & & & 07 & \\
\hline & 1/29// & 9:57:42 & 24.78 & 17.4 & & 22.764 & & 52.487 & 53.43 & & 29.1 & & & & 0 & & & & 71667 & & 1.0 & $40 \quad-3$ & & & & & 107 & \\
\hline & 1/29// & & 24.795 & 17.447 & 25.907 & 22.7 & & 52.6 & 53.5 & & & & & & & & & 104. & 1.733 & & & & & & & & & \\
\hline & $1 / 29 / 2$ & 9:59: & & & 25.9 & & & & & & & -1.214 & & & & & & & & & & & & & & & & \\
\hline & & & & 17.492 & 25.8 & 22.7 & & 52.8 & & & & & & & & & & & & & & & & & & & & \\
\hline & & 1:01:42: & 24.821 & 1.514 & 25.914 & 22.8 & & 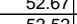 & 33.535 & & & 1.214 & & & 0.0 & & & & 年 & & .9 & & & & & & & \\
\hline & 20 & & $\begin{array}{ll}2.8 .848 \\
20.941\end{array}$ & $\begin{array}{l}11.54 t \\
1757 \\
\end{array}$ & 25.926 & $\frac{22 .}{228}$ & 20 & $\begin{aligned} 52.52 \\
5.237\end{aligned}$ & $\frac{53.595}{5230}$ & 237 & 2.2 & 121 & & & & 14.6 & & 1009 & $\frac{1.8}{36.67}$ & & & & & & & & & \\
\hline & $29 / 2$ & & 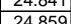 & $\frac{1.31}{17592}$ & $\frac{2.3944}{25026}$ & & 20 & $\begin{array}{ll}5.4351 \\
5.794\end{array}$ & 53. & & & 121 & & & & 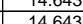 & & & & & & & & & & & & \\
\hline & $1 / 29 / 2$ & $10: 05 \cdot 42$ & & & 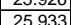 & $\frac{2.39}{229}$ & & 52547 & \begin{tabular}{|l|l|l|}
53461 \\
5
\end{tabular} & & 29. & & & & & & & & & & & & & & & & & \\
\hline & $1 / 29 / 2004$ & $\begin{array}{ll}10: 06: 42 \mathrm{AM} \\
\end{array}$ & 24.878 & 17.647 & 25.92 & 22.95 & 20 & 52.456 & 53.419 & 23.849 & 29.6 & -1.216 & & & 0. & $\frac{14.6}{14.6}$ & & 112. & 86667 & 51.9 & & & & & & & & 09 \\
\hline & 1/29/2004 & 10:07:42 AM & 24.88 & 17.665 & \begin{tabular}{|l|}
25.913 \\
\end{tabular} & \begin{tabular}{|l|}
22.968 \\
\end{tabular} & 20. & 52.564 & \begin{tabular}{|l}
53.575 \\
\end{tabular} & 23.852 & 29.086 & \begin{tabular}{|l|l|}
5 & -1.222 \\
\end{tabular} & & $\begin{array}{ll}4.0045 \\
4.844\end{array}$ & & $\frac{14.045}{14.643}$ & & 3.00 & 8333 & & & & & 0.004 & 0.004 & .000 & 107 & 0.09 \\
\hline
\end{tabular}


WSRC-TR-2005-00105, REVISION 0 SRNL-RPP-2005-00012, REVISION 0

RUN \# 1.04; STEADY STATE TEST AT 25 WT\% UDS - CONT.

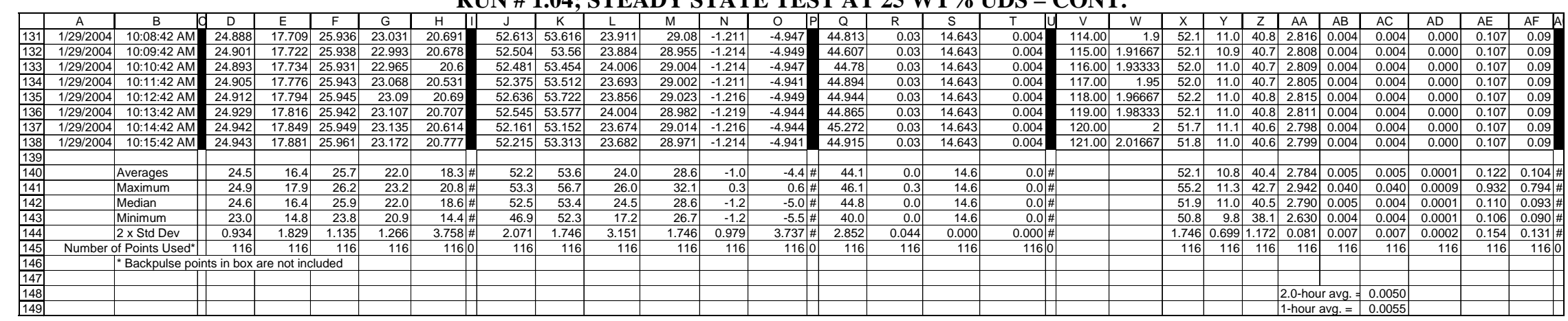


RUN \# 1.07; STEADY STATE TEST AT 25 WT\% UDS

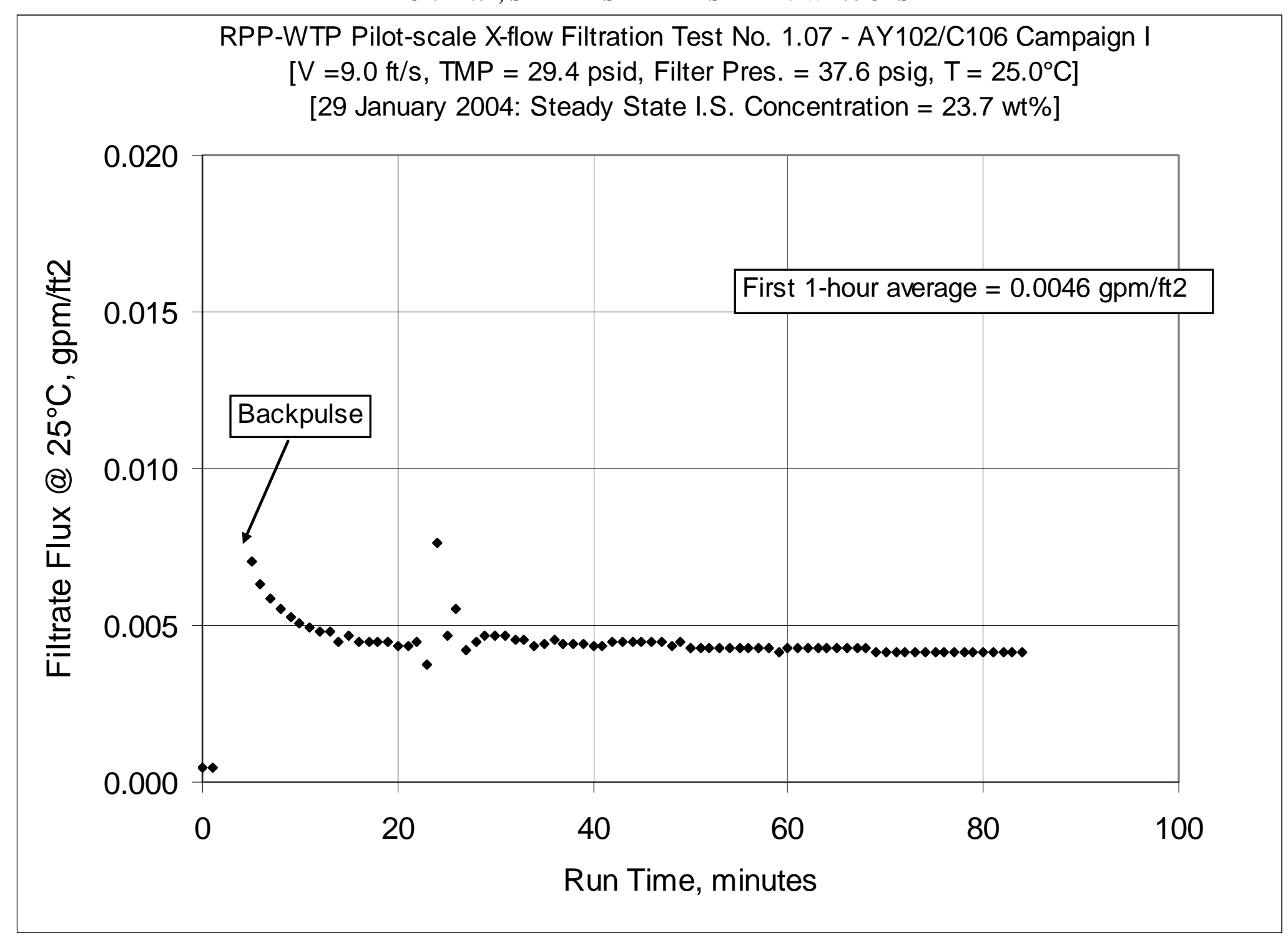


WSRC-TR-2005-00105, REVISION 0

SRNL-RPP-2005-00012, REVISION 0

RUN \# 1.07; STEADY STATE TEST AT 25 WT\% UDS - CONT.

\begin{tabular}{|c|c|c|c|c|c|c|c|c|c|c|c|c|c|c|c|c|c|c|c|c|c|c|c|c|c|c|c|c|}
\hline & A & B & $\mathrm{D}$ & E & $\mathrm{F}$ & \begin{tabular}{|l|l} 
\\
\end{tabular} & $\mathrm{H}$ & $\mathrm{J}$ & $\mathrm{K}$ & $1 \mathrm{~L}$ & I M & IN & 0 & Q & $\mathrm{R}$ & \begin{tabular}{l|l}
$\mathrm{s}$ & \\
\end{tabular} & $\mathrm{T}$ & $\mathrm{v}$ & $\mathrm{w}$ & $x$ & $Y$ & $z$ & $\mathrm{AA}$ & $A B$ & $\mathrm{AC}$ & $A D$ & $\mathrm{AE}$ & $\mathrm{AF}$ \\
\hline & DATE & & & $\begin{array}{l}\ll<\text { Temp } \\
\text { Cleaning }\end{array}$ & & Measureme & ents \>>> & BotTMP & Filter & $\begin{array}{l}\text { Pressure e } \\
\text { Finter dP. }\end{array}$ & $\begin{array}{l}\text { Measureme } \\
\text { TonTMP }\end{array}$ & 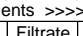 & & $\ll \ll<\ll<4$ & $\begin{array}{l}<<\text { Flow } \\
\text { Fittrate }\end{array}$ & $\begin{array}{l}\text { Measureme } \\
\text { Hititate }\end{array}$ & & & & & & & & & & & & \\
\hline & DAIL & & & 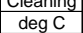 & \begin{tabular}{|l|} 
Sulug C \\
$\operatorname{deg}$
\end{tabular} & Heg $C$ & & psid & & 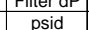 & \begin{tabular}{|l|l|} 
psid \\
\end{tabular} & \begin{tabular}{|l|l|l|l|l|l|} 
psig \\
\end{tabular} & psisgo & $\frac{\text { Sury }}{\text { gpm }}$ & Filuate & Hillilate & & & & & & & & & & & & \\
\hline & & & $\mathrm{T} 2$ & T3 & T1 & T4 & T5 & $\mathrm{dP2}$ & $\mathrm{P} 1$ & $\mathrm{dP1}$ & $\mathrm{dP3}$ & $\frac{p s y}{P 2}$ & $\frac{P_{0} 19}{\mathrm{P} 3}$ & $\frac{\mathrm{g} P \mathrm{m1}}{\mathrm{Q} 1}$ & $\mathrm{QP}^{\mathrm{Q} 2}$ & $\mathrm{Q}^{\mathrm{g} 3 \mathrm{I}}$ & gpp & & & & & & & & & & & \\
\hline & & & & & & & & & & & & & & & & & & & & & & & & & & & & \\
\hline & Seros- 01/26 & & & & & & & & & & & & & & & & & & & & & & & & & & & \\
\hline 7 & $1 / 29 / 2004$ & 7:58:21 AM & 19.97 & 14.564 & 11.286 & 20.431 & 11.485 & 0.059 & -0.149 & 0.007 & 0.018 & -0.186 & 0.098 & -0.087 & 0.003 & 14.643 & 0.005 & & & Filter Su & Surface & 告.707 & FT2 & & & & & \\
\hline 8 & $1 / 29 / 2004$ & 7:58:50 AM & 19.97 & 14.564 & 11.286 & 20.431 & 11.485 & 0.059 & -0.149 & 0.007 & 0.018 & -0.186 & 0.098 & -0.087 & 0.003 & 14.643 & 0.005 & & & Convers & rsion & 851 & $|\mathrm{~m} 3 / \mathrm{m} 2|$ & I/day/barc & $\mathrm{rg} / \mathrm{gpm} / \mathrm{t}$ & ftit/barg & & \\
\hline 9 & & & & & & & & & -0.149 & & & & & & & 14.643 & & & & & & & & & & & & \\
\hline 10 & $1 / 29 / 2004$ & 7:58:53 AM & 19.981 & 14.585 & 11.317 & 20.472 & 11.552 & 0.058 & -0.149 & 0.007 & 0.021 & $\mid-0.174$ & 0.104 & -0.087 & 0.003 & 14.643 & 0.004 & & & Note: G & Gauge & essur & re $P 1$ is & approxin & imately 1 . & .4 psig & & \\
\hline & 1/29/2004 & 7:58:54 AM & 19.991 & 14.575 & 11.317 & 20.477 . & 11.552 & 0.059 & -0.149 & 0.007 & & -0.168 & 0.104 & -0.081 & 0.003 & 14.643 & 0.004 & & & & & & & & & & & \\
\hline & 1/29/2004 & 7:58:55 AM & & 14.58 & 11.317 & 20.472 & 11.562 & 0.061 & -0.149 & 0.008 & 0.021 & -0.177 & 0.104 & -0.087 & 0.003 & 14.643 & 0.005 & & & Pressur & ure Plis & scorrec & ted fo & & & ter tub & & \\
\hline & 112912004 & 7:58:56 AM & & & & & $11.5 / 2$ & & -0.149 & 0.008 & & & & & 0.003 & 14.643 & 0.005 & & & WWV & & & & < Filtrat & ate flux & $<P E R \mid$ & & \\
\hline & 1/29/2004 & 7:58:57 AM & 19.986 & 14.58 & 3) 11.312 & 20.472 & 11.572 & 0.059 & $\begin{array}{ll}-0.149 \\
\end{array}$ & 0.008 & 0.023 & -0.189 & 0.104 & -0.087 & 0.003 & 14.643 & 0.005 & & & & & & & & & & & \\
\hline$\frac{15}{16}$ & Data - PerM & & & & & & & & & & & & & & & & & IIme & IIme & Press. & vel. & IMP & IMP & gpm & gpm & om & & eter \\
\hline & $1 / 29 / 2004$ & & & & & & & & & & & & & & & & & & & & & & & & & & & \\
\hline 18 & $1 / 29 / 2004$ & $10: 22: 21 \mathrm{AM}$ & 23.588 & 18.045 & 24.582 & 23.279 & 21.824 & $\frac{30.039}{6.481}$ & \begin{tabular}{|l|}
39.999 \\
39.862 \\
\end{tabular} & $\frac{1.905}{8.321}$ & $\begin{array}{l}-17.4902 \\
-1.502\end{array}$ & \begin{tabular}{|l|} 
\\
28.4273 \\
\end{tabular} & 46.126 & $\begin{array}{l}3.011 \\
36.92\end{array}$ & 0.003 & \begin{tabular}{l|l}
14.044 \\
14.643 \\
\end{tabular} & 0.004 & $1.0 \mathrm{P}-\mathrm{s}$ & 0.01667 & $\begin{array}{l}30.5 \\
38.4 \\
\end{array}$ & 9.4 & $\frac{2.03}{2.5}$ & $\frac{1.011}{0.172}$ & 0.000 & 0.000 & 0.000 & 0.0182 & \\
\hline 19 & $1 / 29 / 2004$ & 10:23:21 AM & 25.262 & 18.067 & 24.603 & 23.286 & 21.695 & 29.803 & 39.352 & 9.343 & 21.072 & 4.374 & 0.329 & 39.225 & 0.493 & 14.643 & & 2.0 & 0.03333 & 37.9 & 9.6 & 25.4 & 1.754 & 0.074 & 0.074 & & & \\
\hline 20 & 1/29//2004 & 10:24:21 AM & 25.718 & 18.089 & 24.585 & 23.282 & 21.636 & 33.735 & 39.441 & 11.349 & 22.8 & 0.208 & 0.387 & 39.285 & 0.074 & 14.643 & 0.004 & 3.0 & & 38.0 & 9.6 & 28.3 & 1.949 & 2.183 & & & 88.140 & \\
\hline & 29/2004 & 10:25:21 AM & 25.665 & 18.121 & 24.597 & 23.359 & 21.739 & 33.662 & 39.337 & 12.702 & 21.396 & 0.202 & 0.399 & 39.505 & 0.056 & 14.643 & 0.004 & 4.0 & 0.06667 & 37.9 & 9] & 27.5 & 1.898 & 2.183 & 2.208 & 0.080 & 30.209 & 68.26 \\
\hline 22 & $1 / 29 / 2004$ & 10:26:21 AM & & 18.132 & 24.693 & 23.33 & 22.081 & 34.089 & 39.766 & 13.621 & 20.881 & 0.202 & 0.402 & 38.883 & 0.047 & 14.643 & 0.00 & 5.0 & 833 & 38.3 & & 27.5 & 1.895 & & & 0.000 & 0.257 & \\
\hline & $\mid 122172004$ & 10:27:21 AM & & & $24 . / 399$ & 23.351 & 22.138 & 34.141 & 39.826 & & 20.407 & 0.199 & & 38.349 & 0.042 & 14.643 & & & & & & & & 0.006 & .006 & & & \\
\hline 24 & 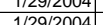 & $10.28 .21 \mathrm{AM}$ & $\frac{25.574}{2551}$ & 18.185 & 24.91 & & 22.204 & 34.284 & 39.963 & 14.608 & 20.063 & 0.02 & 0.4353 & 3.978 & 0 & 14.643 & & & . 11060 & 年.5 20.5 & & 17.4. & 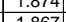 & 0.006 & & & 0.215 & \\
\hline 26 & (1) & $10.30 .21 \mathrm{AM}$ & & 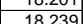 & & 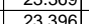 & & 年44.401 & $\frac{40.189}{40.05}$ & 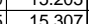 & $\frac{19.685}{19424}$ & 0.205 & & 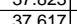 & 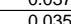 & 10.045 & & & 年 & 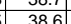 & & & & 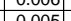 & & & 0.205 & \\
\hline 27 & 1/29/2004 & $10: 31: 21 \mathrm{AM}$ & 25.483 & $\begin{array}{c}10.205 \\
18.26 \\
\end{array}$ & 24.805 & 23.462 & $\frac{22.240}{22.213}$ & $\frac{34.044}{34.51}$ & 40.249 & $\frac{1.007}{15.652}$ & $\begin{array}{ll}19.4243 \\
19.223\end{array}$ & o.214 & & 37.487 & 0.034 & $\begin{array}{l}\frac{14.045}{14.643} \\
\end{array}$ & 0.00 & 100 & 0.1667 & 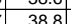 & & & & 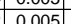 & & & 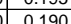 & \\
\hline 28 & $1 / 29 / 2004$ & $10: 32: 21 \mathrm{AM}$ & 25.459 & 18.271 & \begin{tabular}{|l|}
24.076 \\
\end{tabular} & 23.498 & 22.465 & 34.486 & \begin{tabular}{|l|l|}
40.181 \\
\end{tabular} & 15.009 & 18.993 & 0.237 & $\begin{array}{l}0.41 \\
0.48 \\
\end{array}$ & 37.412 & 0.033 & $\begin{array}{ll}14.643 \\
14.643\end{array}$ & 0.003 & 11.0 & 0.18333 & 38.7 & 9.2. & 26.7 & \begin{tabular}{|l|l|}
1.846 \\
1.844
\end{tabular} & 0.005 & 0.005 & 0.000 & 0.185 & \\
\hline & 9/2004 & & & 18.313 & 24.757 & 23.455 & 22.181 & 34.656 & 40.372 & & $\begin{array}{ll}18.977 \\
\end{array}$ & 0.257 & 0.491 & 37.25 & 0.032 & 14.643 & 0.00 & & 0.2 & $\begin{array}{lll}38.9 \\
\end{array}$ & $\overline{91}$ & $26.8 \mathrm{r} \quad \mathrm{l}$ & 1.849 & & & & 0.179 & 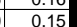 \\
\hline 30 & $1 / 29 / 2004$ & 10:34:21 AM & 25.442 & 18.346 & 24.759 & 23.512 & 21.931 & 34.469 & 40.173 & 15.993 & 18.928 & 0.257 & 0.5 & 37.049 & 0.032 & 14.643 & 0.00 & 13.0 & $0.21667 \mathrm{C}$ & |38.7 & 7t & $\frac{26.7}{26.7}-2$ & 1.84 & 0.005 & 0.005 & 0.000 & 0.180 & \\
\hline & 9/2004 & & & & & & 22.134 & & \begin{tabular}{|l|l|}
40.007 \\
\end{tabular} & & & & & & & & & & & & & & & & & & & \\
\hline 32 & 9/2004 & 10:36:21 AM & 25.391 & 18.385 & 24.748 & 23.541 & & & 39.901 & 16.58 & 18.008 & 0.257 & 0.52 & 37.853 & 0.031 & 14.643 & & 15.0 & 0.25 & 38.4 & $\overline{9.3} \mathrm{~S}>\mathrm{s}$ & 26.1 & 79 & 0.005 & 005 & & $\begin{array}{ll}0.178 \\
\end{array}$ & \\
\hline 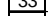 & $1 / 29 / 2004$ & & & & & & & & 39.945 & 16.604 & 18.06 & 0.254 & & 37.805 & 0.03 & 14.64 & & & .26667 & 38.5 & & & & 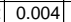 & & & $\overline{0.172}$ & \\
\hline 34 & $\mid 1 / 29 / 2004$ & 10:38:21 AM & 25.369 & 18.439 & 24.766 & & 22.175 & 34.234 & 39.972 & 16.51 & 18.214 & 0.257 & & 37.458 & 0.03 & 14.64 & & 17.00 & 0.28333 & 38.5 & 9.2 & & $1.808 \quad$ & & & & 0.172 & \\
\hline 35 & $1 / 29 / 2004$ & 10:39:21 AM & 25.361 & 18.481 & & 23.566 & & 34.374 & 40.175 & 16.592 & 18.222 & 0.286 & & 37.415 & 0.03 & 14.64 & & 18.0 & & 38.7 & $9.2 \mathrm{P}>\mathrm{C}$ & & & & 0.004 & & 0.171 & \\
\hline 36 & 1/29//2004 & 10:40:21 AM & 25.348 & 18.493 & 24.785 & 23.608 & 22.354 & 34.371 & 40.154 & 16.651 & 18.14 & 0.327 & 0.561 & 37.377 & 0.03 & 14.643 & & 19.0 & 0.31667 & 38.7 & 9.2 & & 1.810 & 0.004 & 0.004 & 0.000 & 0.171 & \\
\hline 37 & 1/29//2004 & 10:41:21 AM & 25.33 & 18.521 & 24.767 & 23.59 & 22.176 & 34.305 & 40.081 & 16.657 & 18.136 & \begin{tabular}{|l|l|} 
& 0.327 \\
\end{tabular} & & 37.31 & 0.029 & 14.643 & 0.00 & 20.0 & 0.3333 & 38.6 & 9.1 & 26.2 & 1.808 & 0.004 & .004 & & 0.166 & 0.1 \\
\hline 38 & 1/29/2004 & 10:42:21 AM & 25.332 & 18.548 & 24.764 & 23.557 & 21.7. & 34.429 & 40.241 & 16.813 & 18.037 & $\begin{array}{l}3 \\
\end{array}$ & 0. & 37.352 & 0.029 & 14. & & 21.0 & 0.35 & 38.8 & 9.2 & 26.2 & 1.809 & | 0.004 & 004 & & 0.166 & 0.1 \\
\hline & 1/299/2004 & $10: 43$ & 25.308 & 18.559 & 24.745 & 23.613 & & 34. & 40.111 & 16.757 & 17.992 & $2 \quad 0.327$ & & 37.31 & 0.03 & & & & 0.36667 & 38.6 & & & 303 & & & & & \\
\hline 400 & 1/29/2004 & 10:44:21 AM & 25.311 & 18.597 & 24.663 & 23.611 & & 34.257 & 40.26 & 16.848 & 17.84 & 0.547 & & 37.2 & 0.0 & & & 23. & & 38.8 & - & & 1.796 & 0.004 & & & 0.144 & \\
\hline$\frac{7 t}{42}$ & & & 25.322 & 18.619 & & 23.618 & 22.9 & 36.01 & 40.231 & 16.907 & 19.465 & -1.214 & & 37.225 & & & & & 0.4 & 38.8 & 9.1. & & & & & & & \\
\hline & $\mid$ & 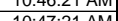 & 20.34 & 18.054 & 24.181 & 23.00 & & 37.395 & 41.00 & 1.33 & 20.344 & - & & 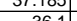 & 0.031 & & & 260 & 0.41001 & 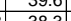 & 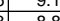 & 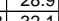 & 10 & & & & 0.161 & \\
\hline \begin{tabular}{|l|l|l|l|l|}
44 &
\end{tabular} & 年 & 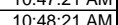 & $\frac{25.35}{25.8}$ & (18.035 & 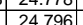 & 年 & & & 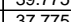 & 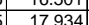 & & $\frac{-1.29}{-126}$ & & $\frac{50.1}{3734}$ & & & & $\frac{2.6}{270} \mathrm{ra}$ & & & & & & & & & & \\
\hline & 1/29/2004 & $10.49 .21 \mathrm{AM}$ & 25349 & 18728 & & 23.886 & & 37.957 & 37.915 & 17.654 & 20986 & $\mid-1214$ & & (37.016 & $\frac{0.020}{0.03}$ & 14 & & $\frac{21}{28}$ & & 364 & 9.1 & 2.5 & 032 & 04 & 05 & & 0153 & 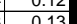 \\
\hline 46 & $1 / 29 / 2004$ & $10.50 .21 \mathrm{AM}$ & 25.37 & $18.7 \mathrm{r}-\mathrm{s}$ & 124.682 & 23.807 & & & \begin{tabular}{|l|l|}
38.179 \\
\end{tabular} & 17.028 & 21.91 & $\mid-1.211$ & & 36.086 & & & & 20 & 0.4833 & 36.7 & S. & 30.1 & .073 3 & & & & 0.155 & \\
\hline 47 & $1 / 29 / 2004$ & 10:51:21 AM & 25.362 & 18.756 & $\mid 24.619$ & 23.748 & & 38.528 & \begin{tabular}{|l|l|}
38.498 \\
\end{tabular} & 16.956 & 22.277 & $\frac{-1.216}{-1.216}$ & & 35.838 & 0.031 & & & 30.0 & & 37.0 & 8.8 & $\frac{30.4}{30.4}-2$ & .096 & $\mid$\begin{tabular}{|c|}
0.005 \\
\end{tabular} & 005 & 0.000 & 0.154 & \\
\hline 488 & & 20 & 25.344 & & & & & & & & 21.9 & $\mid-1.216$ & & & & & & & 0.51667 & 36.8 & 3.0 & So. & & & & & & \\
\hline 49 & $1 / 29 / 2004$ & 10:53:21A & 25.321 & 18.81 & 24.598 & 23.767 & & 38.136 & 38.083 & 17.246 & 21.6 & -1.219 & & 36.901 & 0.03 & & & 32. & & 36.6 & 9.0 & & $2050^{\circ}-2$ & 204 & 05 & & .151 & \\
\hline & & $10: 54$ & 25.263 & & & 23.794 & & & 38.241 & 17.272 & 21.7 & $\mid-1.216$ & & & & & & & 0.55 & 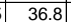 & & & & & & & & \\
\hline & $1 / 29 / 200$ & 10:55 & 25.22 & 18.845 & 24.582 & 23.832 & & 38.227 & 38.17 & $\begin{array}{ll}17.54 \\
\end{array}$ & 21.382 & -1.216 & & 37.05 & 0.029 & 14.6 & & 34. & 0.56667 & 36.7 & 9.1 & $29.8 \mathrm{P} \quad \mathrm{C}$ & & & & & .147 & \\
\hline & $1 / 29 /$ & & 25.203 & 18.873 & 24.564 & 23.824 & & & 38.166 & 17.6. & 21.3 & $\mid-1.216$ & & & 0.029 & 14.6 & & 35.0 . & 0.58333 & 36.7 & 9.1 & $29.8>-2$ & & & 004 & & 0.147 & \\
\hline & 1/29/200 & $10: 57$ & 25.19 & 18. & 24.536 & 23.856 & & $38.3 \mathrm{r}>\mathrm{r}$ & 38.284 & 17.534 & 21.5 & -1.21 & & 36.87 & 0.03 & & & 36. & & 36. & & 9.9 & & & & & & \\
\hline 54 & 1/29/2004 & 10:58:21 & 25.181 & 18.916 & 24.503 & 23.938 & & 38.2 & 38.228 & 17 & 21.569 & -1.219 & & 36.6 & 0.029 & 14. & & 37. & 0.61667 & 36.8 & 9.0 & 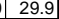 & 062 & & & & 0.147 & \\
\hline 55 & 1/29/2004 & $10: 59$ & 25.178 & 18.9 & 24.49 & 23.98 & & 38.366 & 38.361 & 17.344 & 21.7 & -1.219 & & 36.4 & 0.029 & & & 38 & 0.63333 & 36.9 & 8.9 & 0.6 & & & & & & \\
\hline 56 & 1/29/2004 & & 25.165 & $18.986 \mathrm{rat}$ & 24.5 & 24.022 & & 38.05 & 38 & 17.505 & 21.226 & | -1.216 & & 37.463 & 0.029 & & & 39 . & 0.65 & 36.5 & 9.2 & 29.6 & & & & & 148 & \\
\hline 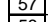 & & & 25.171 & & 24.6 & & & 38. & 0.2 & & & -1.2 & & & 0.029 & & & & 0.66667 & 30.6 & 9.0 & S & 2.006 & & & & 146 & \\
\hline 58 & & & 25.143 & 19.05 & 24.79 & 24.015 & & & 38.583 & 1.6 & 21.6. & -1.216 & & & & & & & & 37.1 & 9.1 & 30.1 & & & & & & \\
\hline & & & 2.174 & & & 24.06 & & & 30.05 & 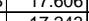 & 2.1. & -1.210 & & & & & & & 0.1 & 31.2 & & & & & & & 0.149 & \\
\hline$\frac{50}{601}$ & 年 & 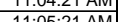 & $\frac{2.20}{2520}$ & 19.125 & 24.033 & 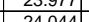 & & & 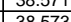 & & 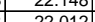 & -1.210 & & So.4 3 & & & & & 年 0.11001 & & 8.9 & 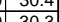 & & & & & 0.148 & \\
\hline & 年 & 11.066.21 A & 25289 & 1920 & 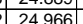 & 23.064 & & & 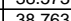 & 势 & & $\mid-1214$ & & $6.68-25$ & & & & & & & & 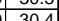 & & & & & & \\
\hline 63 & | & $11.0721 \mathrm{~A}$ & 2534 & 19219 & 25023 & 24.032 & & & 387 & 1738 & 219 & -1244 & & & $\frac{0.03}{0.03}$ & & & 460 & 0.7667 & $37 .>2>$ & (a) & 303. & & & & & 0.147 & \\
\hline \begin{tabular}{|l|l|l|l|}
64 \\
\end{tabular} & $\frac{1 / 2 / 2004}{1 / 2 / 2004}$ & $11: 08: 21$ & $\frac{25.392}{25.392}$ & 19.246 & 25.065 & 24.0499 & & & & & & $\frac{-1.216}{-1.216}$ & & 36.664 & 0.03 & 14.6 & & $4700 \quad-2$ & 07833 & 3723 & $900-0$ & & & & & & 0.147 & \\
\hline & $1 / 29 / 2004$ & & 25.444 & 19.2 & & & & & & & & & & & 0.029 & & & 48. $(>->)$ & & $37.2 \quad 25$ & & 30 & & & & & 0142 & \\
\hline 66 & $1 / 29 / 2004$ & & 25.477 & & 25.134 & 24.044 & & & & & & & & & & & & 49.6 - $>$ & 0.81667 & & & $30.6-2>3$ & & & & & 5146 & \\
\hline & & 11:11:21 AM & 25.508 & 19.328 & & & & & & & & & & & & & & & & 374 & & & & & & & 0.141 & \\
\hline $\begin{array}{ll}68 \\
\end{array}$ & $1 / 29 / 2004$ & 11:12:21 AM & $\begin{array}{l}25.53 \\
\end{array}$ & 19.361 & 25.198 & 24.208 & & 38.698 & 38.668 & 17.302 & 22.105 & -1.214 & & & 0.029 & $14.6>>>$ & & 51.0 & 0.85 & 37.2 & 8.9 & 30.4 & & & & & 0.141 & \\
\hline & & & 5547 & 19.372 & 2518 & 242 & & & 39002 & & & & & 250 & & 14643 & & 520 & 86667 & & 80 & 306 & & 004 & 0004 & & 0.141 & 0 \\
\hline (70 & 1/29/2004 & 11:14:21 AM & 25.569 & 19.4 & 25.202 & 24.267 & 22.556 & 38.965 & 38.956 & 17.512 & 22.125 & $\mid-1.216$ & -5.149 & 36.401 & 0.029 & 14.643 & 00 & 530 & 0.88333 & 37.5 & & 30.5 & 2.10 & $\mid \begin{array}{ll}0.004 \\
\end{array}$ & 0.004 & 0.000 & 0.141 & 0 \\
\hline
\end{tabular}


WSRC-TR-2005-00105, REVISION 0

SRNL-RPP-2005-00012, REVISION 0

RUN \# 1.07; STEADY STATE TEST AT 25 WT\% UDS - CONT.

\begin{tabular}{|c|c|c|c|c|c|c|c|c|c|c|c|c|c|c|c|c|c|c|c|c|c|c|c|c|c|c|c|c|}
\hline & $\mathrm{A}$ & $\mathrm{B}$ & $D^{-}$ & $E$ & $t+$ & & & $\mathrm{J}$ & $\mathrm{K}$ & $L$ & M & IV & 0 & $\mathrm{Q}$ & $\begin{array}{l}R \\
\end{array}$ & $\mathrm{~s}$ & $\mathrm{~T}$ & $\mathrm{v}$ & $w$ & $x$ & $\mathrm{Y}$ & $\mathrm{Z}$ & $\mathrm{AA}$ & $\mathrm{AB}$ & $\begin{array}{ll}\mathrm{AC} \\
\end{array}$ & $\mathrm{AD}$ & $\mathrm{AE}$ & \begin{tabular}{ll|} 
AF & $A$ \\
\end{tabular} \\
\hline & $\frac{1 / 29 / 2004}{1 / 29 / 2004}$ & $\begin{array}{ll}\frac{11: 15: 21 \mathrm{AM}}{11 \cdot 16 \cdot 21 \mathrm{AM}} \\
\end{array}$ & $\frac{25.5}{25.22}$ & $\begin{array}{l}\frac{19.411}{19.423} \\
19\end{array}$ & $\frac{25.203}{25.23}$ & $\frac{24.293}{2420}$ & $\frac{22.623}{22.529}$ & $\frac{38.895}{38.88}$ & $\frac{38.871}{38.84}$ & $\begin{array}{r}17.47 \\
17.433\end{array}$ & & $\begin{array}{l}-1.219 \\
-1.216\end{array}$ & $\begin{array}{l}-5.149 \\
5.149\end{array}$ & $\begin{array}{l}36.457 \\
637\end{array}$ & 0.029 & $\begin{array}{r}14.643 \\
14642\end{array}$ & \begin{tabular}{|c|}
0.004 \\
004
\end{tabular} & $\begin{array}{l}54.00 \\
55.00\end{array}$ & & \begin{tabular}{|l|}
37.4 \\
37.4
\end{tabular} & \begin{tabular}{|l|}
8.9 \\
8.9
\end{tabular} & 30.5 & & \begin{tabular}{|c|}
0.004 \\
0.04
\end{tabular} & & & & \\
\hline & & & 25.63 & & \begin{tabular}{|l|}
25.230 \\
25.237 \\
\end{tabular} & $\begin{array}{r}24.29 \\
24.327\end{array}$ & $\frac{22.529}{22.717}$ & $\begin{array}{l}0.00 \\
38.93\end{array}$ & 38.91 & & & $\frac{-1.216}{-1.222}$ & $\begin{array}{l}-5.149 \\
-5.152\end{array}$ & $\frac{36.267}{36.267}$ & $\begin{array}{l}0.029 \\
0.029\end{array}$ & $\begin{array}{l}\frac{14.643}{14.643} \\
\end{array}$ & \begin{tabular}{|c|}
0.004 \\
0.003
\end{tabular} & 53.00 & & 37.4 & & & 2.107 & & 0.004 & & $\begin{array}{l}0.141 \\
0.141\end{array}$ & $\frac{0.12}{0.12}$ \\
\hline 74 & $1 / 29 / 2004$ & & 25.67 & 19.473 & 25.259 & 24.369 & 22.438 & 38.899 & 38.898 & 17.291 & 22.207 & -1.216 & -5.146 & 36.199 & 0.029 & 14.643 & 0.004 & 57.00 & & 37.4 & 8.9 & & 2.107 & 0.004 & & & 0.140 & 0.12 \\
\hline & $1 / 29 / 2004$ & & 25.69 & 19.495 & 25.276 & 24.376 & 23.082 & 38.971 & 38.981 & 17.235 & 22.396 & -1.219 & -5.143 & 35.986 & 0.029 & $\begin{array}{l}14.643 \\
\end{array}$ & 0.004 & 58.00 & \begin{tabular}{|l|}
0.96667 \\
\end{tabular} & 37.5 & 8.8 & 30.7 & 2.116 & \begin{tabular}{|l|l|}
0.004 \\
\end{tabular} & 0.004 & & 0.140 & \\
\hline 76 & $1 / 29 / 2004$ & 11:20:21 AM & 25.72 & 19.522 & \begin{tabular}{|l|}
25.313 \\
\end{tabular} & 24.338 & 23.295 & 38.631 & 38.637 & $\begin{array}{l}17.768 \\
17.70\end{array}$ & $\begin{array}{l}21.53 \\
\end{array}$ & -1.219 & -5.152 & 37.586 & 0.028 & 14.643 & & & \begin{tabular}{|l|l|}
0.98333 \\
\end{tabular} & 37.2 & 9.2 & 30.1 & 2.074 & $\begin{array}{ll} & 0.004 \\
\end{array}$ & 0.004 & & 0.138 & \\
\hline & $1 / 29 / 2004$ & 11:21:21 AM & & & 25.329 & 24.399 & 23.366 & 38.571 & 38.587 & 17.666 & 21.552 & -1.216 & -5.146 & & & $\begin{array}{l}14.643 \\
\end{array}$ & 0.004 & & & 37.1 & 9.1 & & & & & & & \\
\hline 78 & $1 / 29 / 2004$ & $\begin{array}{l}11: 22: 21 \mathrm{AM} \\
11: 2021\end{array}$ & $\begin{array}{l}25.74 \\
25.77 \\
\end{array}$ & $\begin{array}{l}19.561 \\
19.602\end{array}$ & $\begin{array}{l}25.317 \\
25220\end{array}$ & 24.286 & $\begin{array}{l}23.944 \\
23.190\end{array}$ & 38.824 & $\begin{array}{l}38.842 \\
38.723\end{array}$ & $\begin{array}{l}17.617 \\
1575\end{array}$ & $\begin{array}{l}21.844 \\
2109\end{array}$ & \begin{tabular}{|l|l|} 
\\
\end{tabular} & & \begin{tabular}{|l|}
36.762 \\
\end{tabular} & & & & 61. & \begin{tabular}{|l|l|} 
\\
\end{tabular} & 37.4 & 9.0 & 30.3 & 2.091 & 0.004 & 0.004 & & 0.141 & \\
\hline & & $\begin{array}{l}11: 23: 21 \mathrm{AM} \\
111: 21: 21\end{array}$ & & & $\begin{array}{r}25.349 \\
25.361\end{array}$ & 24.404 & & & $\begin{array}{l}38.763 \\
28.72\end{array}$ & & \begin{tabular}{|l|l|}
21.949 \\
21.897 \\
\end{tabular} & & & & & 14.643 & 0.00 & & & 37.3 & 9.0 & 30.4 & 2.094 & & & & 0.141 & 0.12 \\
\hline$\frac{8}{8}$ & $1 / 29 / 2004$ & $\begin{array}{l}11: 24: 21 \mathrm{AM} \\
111.25\end{array}$ & $\begin{array}{l}25.78 \\
2579\end{array}$ & $\begin{array}{l}19.625 \\
19647\end{array}$ & $\begin{array}{r}25.361 \\
25.347\end{array}$ & $\begin{array}{l}24.471 \\
24.432\end{array}$ & $\begin{array}{l}23.437 \\
23.644\end{array}$ & $\begin{array}{l}38.708 \\
38739 \\
3\end{array}$ & $\begin{array}{r}38.73 \\
38713\end{array}$ & $\begin{array}{r}17.474 \\
17.51\end{array}$ & \begin{tabular}{|l|}
21.897 \\
21.065 \\
\end{tabular} & $\begin{array}{r}-1.219 \\
-1216\end{array}$ & $\begin{array}{l}-5.143 \\
5.143\end{array}$ & \begin{tabular}{|l}
36.666 \\
36518
\end{tabular} & 0.029 & $\begin{array}{r}14.643 \\
14643\end{array}$ & 0.004 & 63.00 & $\begin{array}{r}1.05 \\
10667\end{array}$ & $\begin{array}{l}37.3 \\
37.2 \\
\end{array}$ & $\begin{array}{l}9.0 \\
8.9 \\
\end{array}$ & $\frac{30.3}{30.4}$ & $\begin{array}{l}2.089 \\
2003\end{array}$ & \begin{tabular}{|l|l|}
0.004 \\
0004
\end{tabular} & 0.004 & 0.000 & 0.141 & 0.12 \\
\hline & $\begin{array}{l}1 / 2 / 29 / 2004 \\
1 / 29 / 2004\end{array}$ & $\frac{11.25 .21 \mathrm{AM} M}{11.26 \cdot 21 \mathrm{AM}}$ & $\frac{25.19}{25.82}$ & $\begin{aligned} 19.647 \\
19.68\end{aligned}$ & \begin{tabular}{|l|}
25.341 \\
25339 \\
\end{tabular} & $\frac{24.432}{24.435}$ & $\frac{23.644}{23.912}$ & $\begin{array}{l}38.139 \\
38.859 \\
\end{array}$ & $\begin{array}{l}38.113 \\
38.877 \\
\end{array}$ & $\begin{array}{r}17.51 \\
17.454\end{array}$ & $\frac{21.965}{22.064}$ & $\frac{-1.216}{-1.222}$ & $\begin{array}{l}-5.143 \\
-5.146\end{array}$ & $\frac{36.518}{36.297}$ & & $\frac{14 .}{14.6}$ & & $\frac{64 .}{65}$ & \begin{tabular}{|l|l|}
1.068613 \\
1.0333
\end{tabular} & $\begin{array}{l}37.2 \\
37.4\end{array}$ & 8. & & $\frac{2.093}{2100}$ & 0.004 & $\mid \begin{array}{l}0.004 \\
0.004\end{array}$ & & 0.141 & 0.12 \\
\hline & $1 / 29 / 2004$ & $\begin{array}{l}11: 27: 21 \mathrm{AM} \\
\end{array}$ & 25.85 & $\begin{array}{l}19.00 \\
19.713\end{array}$ & 25.342 & 24.538 & 23.564 & 38.762 & 38.79 & 17.442 & 21.986 & -1.219 & -5.143 & \begin{tabular}{|l|}
36.251 \\
6.532
\end{tabular} & 029 & $\begin{array}{l}14.045 \\
14.643\end{array}$ & & 66.00 & \begin{tabular}{|l|l|}
1.0000 \\
\end{tabular} & $\begin{array}{l}37.4 \\
37.3\end{array}$ & 9.0 & 30.4 & 2.094 & \begin{tabular}{|l|}
0.004 \\
0.004
\end{tabular} & & & 0.141 & $\begin{array}{l}0.12 \\
0.12 \\
\end{array}$ \\
\hline 8 & $1 / 29 / 2004$ & $11: 28: 21 \mathrm{AM}$ & 25.86. & 19.74 & 25.344 & 24.57 & 23.551 & 38.708 & 38.705 & $\begin{array}{l}17.407 \\
\end{array}$ & 21.981 & \begin{tabular}{|l|l|} 
& -1.219 \\
\end{tabular} & -5.14 & \begin{tabular}{|l|}
36.497 \\
\end{tabular} & 029 & 14.643 & & 67.00 & \begin{tabular}{|l|l|}
1.11667 \\
\end{tabular} & 37.2 & 8.9 & 30. & 2.092 & 0.004 & 0.004 & & 0.141 & 0.1 \\
\hline & $1 / 29 / 2004$ & 11:29:21 AM & 25.86 & 19.763 & 25.341 & 24.572 & 24.099 & & 39.022 & $\begin{array}{l}17.373 \\
\end{array}$ & 22.23 & -1.219 & & 36.119 & & & & 68.00 & 1.13333 & 37.5 & 8.9 & 30.6 & 2.110 & & $\begin{array}{l}0.004 \\
\end{array}$ & & 0.140 & \\
\hline & $1 / 29 / 2004$ & 11:30:21 AM & 25.87. & 19.8 & 25.363 & 24.609 & 23.685 & 38.44 & 38.465 & $\begin{array}{l}17.643 \\
\end{array}$ & 21.481 & \begin{tabular}{|l|l|}
-1.214 \\
\end{tabular} & -5.143 & 37.325 & & 14.643 & & 69.00 & 1.15 & 37.0 & 9.1 & & 2.066 & \begin{tabular}{|c|c|} 
\\
5
\end{tabular} & 0.004 & & 0.138 & \\
\hline 87 & $1 / 29 / 2004$ & $\begin{array}{l}11: 31: 21 \mathrm{AM} \\
11: 2020\end{array}$ & $\begin{array}{l}25.87 \\
25\end{array}$ & 19.827 & 25.38 & 24.631 & 23.522 & 38.353 & 38.365 & 17.623 & & $\begin{array}{l}-1.219 \\
.210\end{array}$ & -5.143 & 37.235 & & & & 70.00 & 1.16667 & 36.9 & 9.1 & 29.9 & 2.060 & & 0.004 & & 0.138 & 0.12 \\
\hline & $1 / 29 / 2004$ & $\begin{array}{l}11: 32: 21 \text { AM } \\
11: 23: 21: M\end{array}$ & 25. & 19.856 & $\begin{array}{r}25.398 \\
25.416\end{array}$ & 24.634 & 22.613 & 38.613 & 38.583 & $\begin{array}{r}17.761 \\
1766\end{array}$ & $\begin{array}{r}21.54 \\
21.09\end{array}$ & $\begin{array}{r}-1.219 \\
\end{array}$ & $\begin{array}{l}-5.143 \\
5.123\end{array}$ & \begin{tabular}{|l|}
37.106 \\
6.029
\end{tabular} & 0.028 & 14.643 & 0.0 & 71.00 & 1.18333 & 37.1 & 9.1 & 30.1 & 2.074 & $\begin{array}{ll} \\
\end{array}$ & 0.004 & & 0.137 & 0.12 \\
\hline & $\begin{array}{l}\mid 1 / 29 / 2004 \\
1 / 2 / 2004\end{array}$ & 11:33:21 AM & $\frac{25.91}{25.91}$ & $\begin{array}{l}19.899 \\
19.921\end{array}$ & \begin{tabular}{|l|}
25.416 \\
25.403
\end{tabular} & $\frac{24.687}{24.684}$ & $\frac{23.312}{23.209}$ & $\begin{array}{l}38.685 \\
38.642 \\
-104\end{array}$ & $\begin{array}{r}38.689 \\
3867\end{array}$ & $\begin{array}{r}17.66 \\
17.625 \\
\end{array}$ & \begin{tabular}{r|}
21.69 \\
21.63 \\
\end{tabular} & $\begin{array}{r}-1.219 \\
-1.219 \\
\end{array}$ & $\begin{array}{r}-5.143 \\
-5.143 \\
\end{array}$ & \begin{tabular}{|l}
36.939 \\
36.912
\end{tabular} & 0.028 & $\begin{array}{l}14.643 \\
14643 \\
\end{array}$ & 0.0 & $\begin{array}{l}72.00 \\
7300\end{array}$ & \begin{tabular}{|r|}
1.2 \\
121667
\end{tabular} & $\begin{array}{l}37.2 \\
372 \\
37\end{array}$ & 9.1 & $\frac{30.2}{302}$ & 2.081 & 0.004 & & 0.0 & 0.137 & 0.12 \\
\hline & $1 / 29 / 2004$ & $11: 34: 21 \mathrm{AM}$ & $\begin{array}{l}25.91 \\
25.95 \\
\end{array}$ & $\frac{19.9<1}{19.943}$ & $\begin{array}{r}25.403 \\
25.4\end{array}$ & $\frac{24.684}{24.721}$ & $\frac{23.09}{23.422}$ & $\begin{array}{l}38.642 \\
38.791 \\
\end{array}$ & $\begin{array}{l}38.61 \\
38.836 \\
\end{array}$ & $\begin{array}{l}\frac{17.625}{17.566} \\
\end{array}$ & $\begin{array}{l}21.603 \\
21.852 \\
\end{array}$ & $\frac{-1.219}{-1.216}$ & & $\frac{3.6912}{36.647}$ & & & & 74. & \begin{tabular}{|l|l|}
1.2106 \\
1.23333 \\
\end{tabular} & 37.2 & $\frac{9.0}{9.0}$ & & & & & & $\frac{0.137}{0.136}$ & 0.12 \\
\hline & $1 / 29 / 2004$ & 11:36:21 AM & 25.95 & 19.97 & 25.401 & 24.758 & 23.263 & 38.824 & 38.871 & $\begin{array}{ll}17.511 \\
\end{array}$ & 21.983 & -1.214 & -5.1 & 36.334 & & & & 75 & 1.25 & 37.4 & & & & & & & 136 & 0.12 \\
\hline & 1/29/2004 & 11:37:21 AM & 25.96 & 19.992 & 25.368 & 24.805 & 23.45 & 38.845 & 38.85 & 17.419 & 22.09 & -1.211 & -5.1 & \begin{tabular}{|l|l|}
36.407 \\
\end{tabular} & & & & 76.0 & 1.26667 & 37.4 & & & & & & & $\frac{130}{136}$ & $\frac{0.12}{0.12}$ \\
\hline & $1 / 29 / 2004$ & 11:38:21 AM & 25.97. & 20.025 & 25.366 & 24.807 & & 38.857 & 38.865 & 17.303 & 22.209 & -1.216 & -5.1 & 36.105 & & & & & & 37.4 & & & & & & & & 0.1. \\
\hline & $1 / 29 / 2004$ & 11:39:21 AM & 25.96. & & & 24.779 & & & & 17.345 & & & & 36.088 & & & & & 1.: & (1) & & & & & & & $\left.\frac{0.13}{0.13}\right)>(1)$ & \\
\hline & $1 / 29 / 2004$ & & & & 25.325 & 24.736 & 23.69 & 38.99 & 39.026 & 17.315 & 22.31 & -1.216 & & & & & & & & & & & & & & & & \\
\hline & 1/29/2004 & 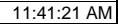 & 25.93 & 20.087 & 25.333 & 24.769 & & 38.951 & 38.983 & & & -1.216 & & & & & & & & & & & & & & & 0.135 & \\
\hline & 1/29/2004 & $11: 42: 21 \mathrm{AM}$ & 25.90 & 20.11 & 25.34 & 24.791 & $23.8 \mathrm{Y}$ & 38.938 & 38.962 & 17.307 & 22.314 & -1.219 & & 35.948 & & & & 81. & 1.35 & 37. & & & & & & & & \\
\hline & & 11:43:21A & & 20.117 & 25.317 & 24.818 & & 38.98 & & & & -1.216 & & & & & & & & & & & & & & & & \\
\hline & & 11:44:21A & & 20.139 & 25.304 & 24.875 & & 38.971 & 38.979 & 17.369 & 22.304 & -1.21 & & 35.966 & & & & 83.00 & $\mid 1.38333$ & & & & & & & & 135 & \\
\hline & $1 / 29 / 2004$ & 11:45:21 AM & .51 & 20.151 & 25.286 & 24.908 & 23.829 & 38.892 & 38.946 & 17.314 & 22.252 & -1.219 & -5.13 & 35.946 & 0.028 & 14.643 & & 84.00 & 1.4 & 37.5 & $8.8 \mathrm{r}$ & 30.6 & 2.10 & 0.004 & 0.002 & 0.00 & 0.135 & 0.1 .78 \\
\hline & & Averag & 25. & 19.2 & 25.0 & 24.1 & 22.9 & 37.6 & 39.0 & 17.1 & 21.1 & \begin{tabular}{c|c|c|}
-0.9 &
\end{tabular} & -3. & 36.8 & 0.0 & 14.6 & & & & 37.6 & & 29. & & & & & 15 & \\
\hline & & & 26. & 20.2 & 25.4 & 24.9 & & 40.0 & 41.1 & & 24.3 & 0.5 & & & & & & & & 39.6 & 9.4 & & & & & & & \\
\hline & & Med & 25. & 19.2 & 25.0 & 24.0 & & 3 & 38.9 & & 21.8 & -1.2 & -5.1. & & & & & & & 37.4 & 9.0 & & & & & & 146 & \\
\hline & & Minim & 24. & 18.2 & 24.5 & 2 & $1.8 \mathrm{~s}$ & 34.1 & 37.8 & 1 & 17.8 & -1.2 & -5.4 & 35.8 & ( & 14.6 & & & & 36.3 & 8.8 & 26.0 & 1.796 & & & & 0.135 & \\
\hline 10 & & & 0.57 & 1.193 & 0.607 & 0.915 & 1.234 & $3.6 \mathrm{~F}-2 \mathrm{c}$ & 1.45 & 1.389 & 2.944 & & & & & .000 & & & & 1.452 & .282 & & & & & & .048 & \\
\hline & Number o & & & & & & & 79 & 79 & 79 & 79 & 79 & & & 79 & & & & & 79 & 79 & 79 & 79 & 79 & & & 79 & \\
\hline & & & ts in bo & not & ided & & & & & & & & & & & & & & & & & & & & & & & \\
\hline & & & & & & & & & & & & & & & & & & & & & & & & & & & & \\
\hline & & & & & & & & & & & & & & & & & & & & & & & & g. $=$ & .0046 & & & \\
\hline
\end{tabular}


RUN \# 1.08; STEADY STATE TEST AT 25 WT\% UDS

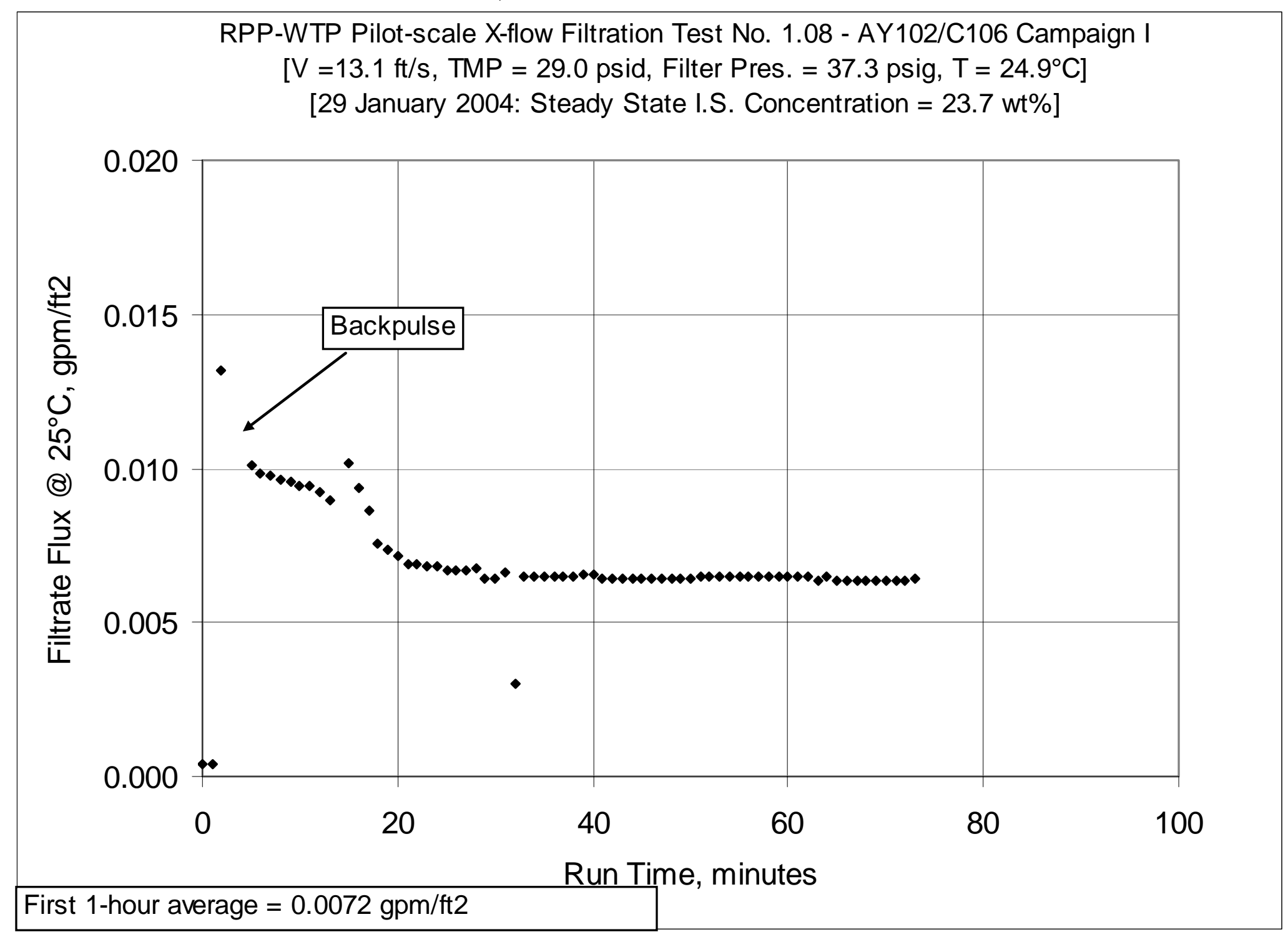


WSRC-TR-2005-00105, REVISION 0

SRNL-RPP-2005-00012, REVISION 0

RUN \# 1.08; STEADY STATE TEST AT 25 WT\% UDS - CONT.

\begin{tabular}{|c|c|c|c|c|c|c|c|c|c|c|c|c|c|c|c|c|c|c|c|c|c|c|c|c|c|c|c|c|}
\hline & A & B & D & E & $F$ & G & $\mathrm{H}$ & $\mathrm{J}$ & $\mathrm{K}$ & $\mathrm{L}$ & $\mathrm{M}$ & $\mathrm{N}$ & 0 & Q & $R$ & $\mathrm{~s}$ & $\mathrm{~T}$ & $\mathrm{v}$ & w & $x$ & & $\mathrm{z}$ & $\mathrm{AA}$ & $A B$ & $A C$ & $\mathrm{AD}$ & $\mathrm{AE}$ & AF \\
\hline & & & & & & & & & & & & & & & & & & & & & & & & & & & & \\
\hline & DATE & TIME & Filtrate & Cleaning & Slurry & Hi Amb & Lo Amb. & BotTMP & $\begin{array}{l}\text { Filter } \\
\text { ncim }\end{array}$ & Filter dP & \begin{tabular}{|l|} 
TopTMP \\
\end{tabular} & \begin{tabular}{|l|} 
Filtrate \\
\end{tabular} & \begin{tabular}{|l|l|} 
Pulsepot \\
\end{tabular} & Slurry & Filtrate & \begin{tabular}{|l|l} 
Hi Filtate \\
\end{tabular} & Backpulse & & & & & & & & & & & \\
\hline & & & $\frac{\operatorname{deg} C}{\mathrm{~T} T}$ & $\operatorname{deg} C$ & $\operatorname{deg} C$ & $\operatorname{deg} C$ & \begin{tabular}{|l|}
$\operatorname{deg} C$ \\
$T 5$
\end{tabular} & $\begin{array}{l}\text { psid } \\
\text { P2 }\end{array}$ & psig & \begin{tabular}{|l|l|} 
psid \\
$d P 1$
\end{tabular} & \begin{tabular}{|l|} 
psid \\
d?
\end{tabular} & \begin{tabular}{|l|} 
psig \\
p2
\end{tabular} & \begin{tabular}{|l|} 
psig \\
p2
\end{tabular} & $\mathrm{gpm}$ & $\mathrm{gpm}$ & \begin{tabular}{|l|l}
$\mathrm{gpm}$ \\
\end{tabular} & gpm & & & & & & & & & & & \\
\hline & & & & & & & & & & & & & & & & & & & & & & & & & & & & \\
\hline & Zeros- $01 / 26$ & 2004 & & & & & & & & & & & & & & & & & & & & & & & & & & \\
\hline 7 & $1 / 29 / 2004$ & $7: 58: 21 \mathrm{AM}$ & 19.97 & 14.564 & 11.286 & 20.43 & 11.485 & 0.059 & -0.149 & 0.007 & 0.018 & -0.186 & 0.098 & -0.087 & 0.003 & $\begin{array}{ll}14.643 \\
\end{array}$ & & & & Filter $S$ & Surface & 16.707 & FT2 & & & & & \\
\hline & 1/29/2004 & 7:58:50 AM & 19.97 & 14.564 & 11.286 & 20.43. & 11.485 & 0.059 & -0.149 & 0.007 & 0.018 & -0.186 & & -0.087 & 0.003 & 14.643 & & & & Conver & rsion & & $\mathrm{m} 3 / \mathrm{m} 2$ & l/day/bar & $\mathrm{rg} / \mathrm{gpm} / \mathrm{t}$ & ftr/barg & & \\
\hline & $1 / 29 / 2004$ & 7:58:52 AM & 19.981 & 14.574 & 11.307 & 20.466 & 11.546 & 0.059 & -0.149 & 0.007 & 0.021 & -0.168 & 0.10 & -0.083 & 0.003 & 14.643 & & & & & & & & & & & & \\
\hline & 1/29/2004 & 7:58:53 AM & 19.981 & 14.585 & 11.317 & 20.47: & 11.552 & 0.058 & -0.149 & 0.007 & 0.021 & -0.174 & 0.104 & -0.087 & 0.00 & 14.643 & & & & |Note: & & ressur & re P1 is & aporc 24 & mately 1 & 4 psig & & \\
\hline & $1 / 29 / 2004$ & 7:58:54 AM & & 14.575 & 11.317 & & 11.552 & 0.059 & -0.149 & 0.007 & 0.023 & -0.168 & & -0.081 & 0.003 & 14.643 & & & & & & & & & & & & \\
\hline & $1 / 29 / 2004$ & 7:58:55 AM & 19.986 & 14.58 & 11.317 & 20.472 & 11.562 & 0.061 & -0.149 & 0.008 & & & & & 0.003 & 14.643 & & & & & & & & & & & & \\
\hline & 1/29/2004 & 7:58:56 AM & 19.986 & 14.58 & 11.317 & & 11.572 & - 0.058 & & 0.008 & .023 023 & -0.168 & & .081 & 0.003 & 14.643 & & & & WWW & & & & < Filtra & & & & \\
\hline 14 & $1 / 29 / 2004$ & 7:58:57 AM & 19.986 & 14.58 & 11.312 & 20.472 & 11.572 & 0.059 & -0.149 & 0.008 & 0.02 & -0.189 & & & 0.00 & 14.643 & & & & & & & & & & & & \\
\hline & ata - Per M & & & & & & & & & & & & & & & & & ime & Time & $\begin{array}{l}\text { Press. } \\
\text { nsig- }\end{array}$ & $\begin{array}{l}\text { Vel. } \\
\frac{\text { tils }}{2}\end{array}$ & TMP & TMP & gpm & $\mathrm{om}$ & $\mathrm{pm}$ & & neter \\
\hline & 1/29/2004 & $12: 40: 59$ PM & 25.833 & & & & 23.433 \# & & & & & $\begin{array}{ll}0.327 \\
\end{array}$ & 86.355 & $\begin{array}{lll}62.357 \\
\end{array}$ & 0.003 & 14.643 & 0.004 & & & 44.5 & 5 & & & 0.000 & 0.000 & $\frac{0.000}{0.000}$ & & $\frac{\mathrm{a} / \mathrm{yba}}{0.01}$ \\
\hline & $1 / 29 / 2004$ & 12:41:59 PM & 24.888 & 20.813 & 27.51 & 25.925 & 23.503 \# & 14.279 & 37.619 & 16.604 & -1.925 & 18.062 & 8.337 & 70.703 & & 14.643 & & 1.0 & 0.01667 & 36.1 & 17.3 & 6.2 & 0.426 & 0.000 & 0.000 & & 0.068 & 0.06 \\
\hline & 1/29//2004 & 12::42:59 PM & 26.907 & 20.838 & 27.54 & 25.915 & 23.504 \# & 33.036 & 38.825 & 19.7 & 14.082 & 0.228 & 0.295 & 70.019 & 0.095 & $\begin{array}{ll}14.643 \\
\end{array}$ & & 2.0 & 0.03333 & 37.4 & 17.2 & 23.6 & 1.624 & 0.014 & 0.013 & 0.001 & 0.560 & 0.48 \\
\hline & $1 / 29 / 2004$ & 12:43:59 PM & 27.46 & & 27.619 & 25.919 & 23.638|\# & 33.459 & 39.257 & 20.679 & 13.495 & 0.202 & & 63.554 & 0.081 & 14.643 & & 3.0 & 0.05 & $37.8 \mathrm{r}$ & 15.6 & 235 & $1.61 \mathrm{~T}$ & 2.183 & 2.029 & 0.086 & & \\
\hline & 1/29/2004 & 12:44:59 PM & 27.703 & 20.918 & 27.689 & 25.988. & 23.658|\# & 33.498 & 39.304 & 21.001 & 13.264 & 0.234 & 0.367 & & 0.075 & 14.643 & & & 0.06667 & 37.8 & & & & 2.183 & & & 6.624 & \\
\hline & 1129720004 & 12:45:59 PM & 27.843 & 20.948 & 27.734 & 26.072 & 23.683|\# \# & 33.48 & 39.319 & 21.092 & 13.1612 & 0.254 & $0.3 / 8$ & 72.855 & 0.003 & 14.643 & & & 833 & $3 . .8 \mathrm{r}, \mathrm{l}$ & 17.9 & & & & .010 & & & \\
\hline$\frac{24}{24}$ & $\mid 112129004$ & 118.46.59 PMM & 2.948 & 20.919 & 2.164 & 26.1 & 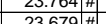 & 3.503 & 39.416 & 21.251 & 13.083 & 0.231 & & 62.76 & 0.071 & 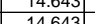 & & 6.0 & 0. & 37.9 & 15.4 & 20 23.3 & 1.608 & & . & & & \\
\hline & $\begin{array}{l}1 / 29 / 2004 \\
1 / 29 / 2004\end{array}$ & 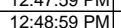 & 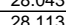 & 21.005 & $\mid \begin{array}{l}27.014 \\
27.924\end{array}$ & $\frac{26.14}{26.14}$ & 23.096 \#\# & \begin{tabular}{r|}
33.4 .0 \\
33.492
\end{tabular} & 年39.451 & $\frac{21.300}{21199}$ & 13.044 & 年 & & 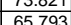 & 0.071 & $\frac{14.045}{14643}$ & & 80 & 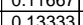 & $\begin{array}{l}3.060 \\
379\end{array}$ & $\frac{1.1}{161}>>$ & 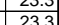 & $\frac{1.000}{1604}$ & & 些010 & 0.000 & & \\
\hline 26 & $1 / 29 / 2004$ & $12: 49: 59 \mathrm{PM}$ & 28.197 & $\frac{21.045}{21.075}$ & \begin{tabular}{|l|}
2.524 \\
28.019 \\
\end{tabular} & $\frac{2.14}{26.17}$ & $\frac{20.001+1+1}{24.085}$ & 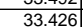 & 39.333 & $\frac{21.159}{21.218}$ & $\begin{array}{l}10.044 \\
12.989\end{array}$ & 0.324 & 0.4462 & 67.324 & 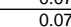 & $\begin{array}{l}\frac{14.045}{14.643} \\
\end{array}$ & & $\frac{0.0}{9.0}$ & $\frac{0.1500}{0.15}$ & $\frac{0.5}{37.9}$ & $\frac{1.1}{16.5}$ & 23.2 & $\frac{1.004}{1.600}$ & 0.010 & 0.010 & 0.000 & 0 & $0.35 \mathrm{r}-\mathrm{s}$ \\
\hline & $1 / 29 / 2004$ & 12:50:59 PM & 28.282 & 21.105 & \begin{tabular}{|l|}
2.0104 \\
\end{tabular} & 26.1 & $24.06 \#$ & 33.453 & 39.389 & 21.235 & 12.978 & 0.324 & 0.485 & 66.045 & 0.069 & 14.643 & & 10.0 & 0.16667 & 37.9 & 162 & 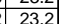 & 1601 & 0.010 & 0.009 & & 0.406 & 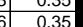 \\
\hline 28 & 1/29/2004 & 12:51:59 PM & 28.396 & 21.14 & 28.193 & 26.1 & $24.259 \#$ & 33.525 & 39.505 & 21.261 & 12.943 & 0.361 & 0.514 & 68.444 & 0.069 & 14.643 & & 11.0 & 0.18333 & 38.0 & $16.8 \mathrm{P}>\mathrm{l}$ & $3.2-2 \times 3$ & 1.60 & & 0.009 & & 0.405 & \\
\hline & $1 / 29 / 2004$ & 12:52:59 PM & 28.47 & 21.175 & 28.247 & 26.14 & $24.254 \#$ & 33.498 & $\begin{array}{l}39.499 \\
39\end{array}$ & 21.299 & 12.937 & 0.358 & & 62.436 & 0.068 & $\begin{array}{l}14.643 \\
\end{array}$ & & & 0.2 & $\frac{38.0}{38}$ & 15.3 & & $\frac{1.60}{1.60}>0$ & & 0.009 & 0.000 & 0.399 & \\
\hline 30 & 1/29/2004 & 12:53:59 PM & 28.561 & 21.241 & 28.253 & 26.289 & 24.145 \# & 32.725 & 38.707 & 20.875 & 12.613 & 0.387 & & 62.722 & 0.066 & 14.643 & & 13.00 & 0.21667 & 37.2 & 15.4 & $22.7 \mathrm{r}-\mathrm{l}$ & 1.563 & 0.010 & 0.009 & & 0.396 & \\
\hline & $1 / 29 / 2004$ & 12:54:59 PM & 28.168 & 21.267 & 28.159 & 26.286 & $24.446 \#$ & 39.637 & & 20.944 & 19.201 & & -5.473 & 61.262 & 0.105 & $\begin{array}{ll}14.643 \\
\end{array}$ & & 14.00 & 0.23333 & 38.1 & 15.0 & 29.4 & $2.028 \mathrm{~S}$ & 0.016 & 0.014 & & 0.487 & \\
\hline & 1/29/2004 & & 27.846 & 21.299 & 27.842 & 26.342 & & 39.27 & 38.952 & 19.064 & 20.656 & -1.216 & & 61.667 & & 14.643 & & & & 37.5 & 15.1 & 30.0 & & & & & & \\
\hline & 1/29/2004 & 12:56:59 PM & 27.722 & 21.34 & 27.219 & $26.38: 5 \mathrm{~s}$ & $24.678 \| \#$ & 39.162 & 38.616 & 17.821 & 21.848 & -1.219 & -5.846 & 47.297 & 0.067 & 14.643 & & 16.0 & 0.26667 & 37.1 & 11.6 & 30.5 & 2.103 & & 0.009 & & 0.308 & 0.26 \\
\hline 34 & 1/299/2004 & 12:57:59 PM & 27.642 & 21.36 & 26.775 & $26.305 \mathrm{~s}$ & 24.633|\#| & 39.187 & 38.597 & 17.647 & 22.035 & -1.222 & & 51.515 & 0.061 & 14.643 & & 17.00 & 0.28333 & 37.1 & 12.6 & 30.6 & & 0.009 & 0.009 & 0.000 & & 0.2 \\
\hline & $1 / 29 / 2004$ & 12:58:59 PM & 27.548 & 21.416 & 26.436 & 26.394 & $24.809 \#$ & 39.544 & 38.898 & 18.186 & 21.852 & . 219 & -5.929 & 50.313 & & 14.643 & & 18.0 & 0.3 & 37.4 & 12.3 & 30.7 & 2.117 & & & & 0.247 & \\
\hline 36 & 1/29//2004 & 12:59:59 PM & 27.488 & 21.456 & 26.101 & $26.43 \mathrm{~s}$ & 25.034|\#1 & 39.677 & 39.02 & 18.395 & 21.784 & 216 & -5.938 & 51.84 & 0.0 & 14. & & 19.0 & 0.31667 & 37.5 & 12. & 30.7 & $2.11 \mathrm{C}$ & 0.008 & 0.007 & 0.000 & 0.240 & \\
\hline & 1/29//2004 & 1:00:59 PM & 27.428 & 21.481 & 25.846 & 26.41 & 25.163|\# & 39.144 & & & 21.501 & -1.219 & -5.869 & ( & 0.049 & $14.6 \mathrm{~s}-\mathrm{r}$ & & 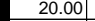 & 0.33333 & 37.1 & 12.3 & 30.3 & .091 & & - > & inc & 0.200 & \\
\hline 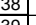 & & & 27.329 & 21.517 & 25.561 & $26.398 \mathrm{c}$ & & 39.448 & 38.778 & 18.845 & 21.085 & & & & & & & & & & & & & & & & & 0.15 \\
\hline$\frac{39}{10}>>>$ & 1121292004 & $1: 02: 59 \mathrm{PM}$ & 27.264 & 21.551 & 25.466 & 26.47 & $25.118|+|+\mid$ & 39.5 & 38.821 & 18.993 & 21.013 & -1.216 & & $51.8 / 8$ & $0.04 \pi$ & 14.6 & & 2.00 & 0.36067 & 3.7 .4 & $\frac{12.1}{12.1}>0$ & 30.3 & 2.0000 & 0.001 & 0.007 & & 0.229 & 0.15 \\
\hline 列 & 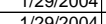 & (59PM & 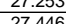 & 21.591 & $\mid 25.354$ & 20.472 & & $\frac{3.49}{30.661}$ & 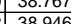 & 18.997 & & - & & (49.9009 & & & & $\frac{23.00}{20}$ & 0.38335 & $\begin{array}{l}\frac{3.3}{375} \\
375\end{array}$ & 123 & & & & & & & \\
\hline$\frac{4 x}{42}$ & $\begin{array}{ll}1 / 29 / 2004 \\
1 / 2004\end{array}$ & - & $\frac{21.440}{27316}$ & $\frac{21.025}{21665}$ & 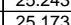 & $\frac{20.48}{265}$ & 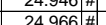 & (39.064) & 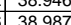 & 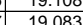 & $\frac{21.068}{2106}$ & $\frac{-1.22}{-1201}$ & & 年 & & & & 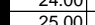 & & 年7.5 375 & $\begin{array}{ll}1.2 .3 \\
126\end{array}$ & 3. 3.4 & 2.094 & ( & 0.007 & 0.0 & 0.224 & 0.19 \\
\hline 43 & $1 / 29 / 2004$ & $1: 06: 59 \mathrm{PM}$ & 年 & 21.704 & 25086 & 26.556 & $25.029 \#$ & 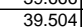 & 38.521 & 18891 & $\frac{2.006}{21.124}$ & -121 & & (51.4502 & & & & $\frac{2.00}{260}$ & 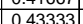 & 告, 35 & 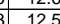 & 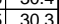 & $\frac{2.054}{2090}$ & 0.007 & & & & $\frac{0.1 .15}{0.11}$ \\
\hline 44 & $1 / 29 / 2004$ & 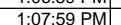 & 27.035 & 21.728 & $\begin{array}{l}25.026 \\
25.026\end{array}$ & 26.60 & 25.054 \#\# & 2039.728 & 39.055 & 18.877 & $\frac{21.329}{21.329}$ & $\mid-1.219$ & & 49 & & & & 2700 & $\frac{0.40 .45}{0.45}$ & $\frac{1.6}{37.6}$ & $\frac{121}{121}+2$ & $\frac{30.5}{30.5}-3$ & & \begin{tabular}{|l|l|l|}
0.007 \\
\end{tabular} & 0.007 & 0.000 & & 0 \\
\hline & $1 / 29 / 2004$ & 1:08:59 PM & 26.915 & 21.768 & 24.915 & 26.638 & $25.158 \| \#$ & 40.021 & 39.331 & & $\frac{21 .}{21 .}$ & $\frac{216}{216}$ & & & & & & & 0.46667 & 37.9 & 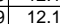 & & & & & & & \\
\hline 46 & $1 / 29 / 2004$ & 1:09:59 PM & 26.779 & 21.812 & \begin{tabular}{|l|l|}
24.884 \\
\end{tabular} & 26.65 & $25.292 \| \#$ & 39.119 & 38.417 & 19.043 & & $\frac{-1.216}{-1.216}$ & -5.98 & 52.082 & & & & & 0.48333 & 369 & 128 & 298 & & 0006 & 006 & & & \\
\hline & & $1: 10: 5$ & 26.669 & 21.832 & & & & 38.928 & & & & -1.2 .20 & & 49.5 & & & & & 0.5 & $\frac{36.8}{36.8}$ & 12.2 & 29.6 & & & & & 0.218 & $\frac{0.1}{0.1}+2$ \\
\hline 48 & $1 / 29 / 2004$ & 1:11:59 PM & 26.552 & 21.866 & 24762 & 26.445 & $25.425 \#$ & 39.15 & 38.467 & 19.259 & & -1222 & & $52.2>>$ & 0.04 & & & 31. & 051667 & 37.0 & 128 & 298 & 2053 & 0.007 & & & & \\
\hline 49 & $1 / 29 / 2004$ & 1:12:59 PM & 26.441 & 21.889 & \begin{tabular}{|l|}
24.675 \\
\end{tabular} & $26.278 \mathrm{~S}$ & & 39.299 & & 19.335 & $20.8>-2$ & -1.222 & & 51.97 & & & & 32.0 & 0.53333 & 37.1 & 12.7 & 29.9 & 2.061 & (c) & & & 0.101 & \\
\hline 50 & $1 / 29 / 2004$ & 1:13:59 PM & 26.354 & 21.928 & 24.648 & $26.177 \mathrm{r}$ & & 38.8 & 38.4 & & & & & & & & & & 0.55 & 37.1 & 12.6 & 29.5 & .032 & 56 & & & 0.220 & \\
\hline & $1 / 29 / 2004$ & 1:14:59 PM & 26.267 & 21.966 & \begin{tabular}{|l|}
24.586 \\
\end{tabular} & 26.04 & $25.27 \mid \#$ & 38.839 & 38.626 & 19.281 & 20.0 & -1.219 & -5.52 & 51.083 & & & & 34. & 0.56667 & 37.2 & 12.5 & 29.4 & 2.030 & $\begin{array}{l}0.006 \\
\end{array}$ & 0.006 & & 0.220 & 0.15 \\
\hline & $1 / 29 / 2004$ & 1:15:59 PM & 26.185 & 21.978 & 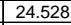 & 25.94 & 25.38. & 38.832 & 38.5 & 19.2 & & & -5.5 & 53.578 & & 14. & & 35.0 & 0.58333 & 37.1 & 13.1 & 29.4 & .05 & $\begin{array}{ll}0.006 \\
\end{array}$ & 506 & 0.0 & 0.22 & 0. \\
\hline & $1 / 29 / 2004$ & 1:16:59 PM & 26.097 & 22.001 & 24.47 & $25.79 \mathrm{~s}$ & 25.20 & 38.868 & 38.6 & 19.265 & & & & 52.456 & & & & 36.0 & 0.6 & 37.2 & 12.9 & 29.5 & 2.03 & & & & & \\
\hline 54 & 1/29//2004 & $1: 17: 5$ & 26.045 & 22.055 & 24.454 & & & & 38. & & & -1.219 & & 51.354 & & & & & 0.61667 & 37.2 & 12.6 & 29.5 & & & & & & \\
\hline$\frac{55}{50}$ & $1 / 29 / 2004$ & 1:18:59 PM & 25.968 & 22.067 & 24.396 & 25.62 & 25.5 & 38.832 & 38. & & & & & & & & & 38. & 0.63333 & 37.1 & $13.2 \mathrm{Cl}$ & 29.5 & 2.03 & & & & 0.22 & \\
\hline & $1 / 29 / 2004$ & $1: 19: 59 \mathrm{PM}$ & 25.891 & 22.096 & $\mid 24.354$ & $25.54 \mathrm{C}$ & 24.62 & 38.8 & 38.589 & 19.1 & & -1.2 & & 50. & & & & 39.04 & 0.65 & 3.7.1 & 12.5 & 29.5 & 2.030 & & & & $0.2 \angle 1$ & 0 \\
\hline & $\begin{aligned} 112912004 \\
11202004\end{aligned}$ & $\begin{array}{l}1.202 .59 \\
1.259\end{array}$ & $\begin{array}{l}\frac{25.808}{25735} \\
257\end{array}$ & $\begin{array}{l}22.101 \\
20125\end{array}$ & \begin{tabular}{|l|l|}
24.301 \\
2.272
\end{tabular} & 25.454 & 25.162 & | & & 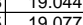 & & $-\frac{-1.2<2}{1210}$ & & 096 & & 14.6 & & & 0.06061 & $\begin{array}{ll}3.1 .1 \\
272\end{array}$ & & & 25 & & & & & \\
\hline & 1120004 & $\frac{1.25}{1.5259} \mathrm{PM}$ & 25683 & $\frac{26.155}{22132}$ & 24.475 & 25306 & $\frac{20.125}{25245}$ & 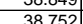 & (3). & 189 & & -1.219 & & & & $\frac{14.0}{146}$ & & 年.1.0 & 0 & S.t. & $\frac{1.5}{120}$ & 20.0 & \begin{tabular}{|l|l|l|}
2030 \\
2032
\end{tabular} & & 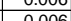 & & 0.210 & \\
\hline & 1 & $\frac{1.25}{1.23 .59} \mathrm{PM}$ & 2559 & $\frac{1254}{22144}$ & 24.287 & 25265 & 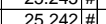 & 38737 & & 18.9 & & & & 4597 & & & & & & 27.1 & & & & & & & & \\
\hline & $\frac{1}{1.29 / 20004}$ & $\begin{array}{l}1.24 .59 \mathrm{~F} \\
\end{array}$ & 25.532 & 22.161 & 24.144 & 25.1 & $25.124 \| \#$ & 38.893 & 38. & 19.118 & & & & & & & & 44.00 & 07333 & 372 & 133 & 296 & & & & & & \\
\hline & $1 / 29 / 2$ & & 25.469 & & & & & & & & & & & & & & & & & & & & & & & & & \\
\hline 63 & & $1.26: 5$ & 25.4 & 22.164 & 24.052 & 24895 & 25.39 & 38.847 & & 19.045 & & -1225 & & & & & & & 0.766 & 37.2 & 10.5 & & & & & & & \\
\hline & & & 25.337 & & \begin{tabular}{|l|l|}
24.039 \\
\end{tabular} & & & & & & & & & & & 14. & & & & 372 & 131 & & & & & & & \\
\hline & $1 / 29 / 2004$ & $1.28 .59 \mathrm{PM}$ & 25.277 & 22.192 & \begin{tabular}{|l|l|}
239994 \\
\end{tabular} & $24.80^{7}$ & 25.234 & 38.743 & 38.5 & 18.9 & 20 & $\frac{-1.219}{-1.219}$ & & 51.2 & & 14. & & 480 & 0.8 & 371 & 126 & 29.5 & & & & & & \\
\hline & $1 / 29 / 2004$ & 1:29:59 PM & 25.219 & 22.209 & 23.966 & 24.77 & 25.271 & 38.984 & 38.7 & 19.09 & 20.364 & -1.219 & & 144 & & 14.6 & & & 0.81667 & 37.3 & & 29.7 & 046 & & & $\overline{0}$ & & \\
\hline 67 & $1 / 29 / 2004$ & 1:30:59 P| & 25.137 & 22.237 & 23.944 & $24.7 \mathrm{r}$ & 25.529 & 38.864 & 8.6 & 18.9 & 20. & & -5. & 54.5 & 0.0 & 14.6 & & 50 & 0.83333 & 37.2 & 13.4 & 29.6 & 2043 & 0.0 & 0.006 & 0000 & 0.218 & \\
\hline 68 & 1/29/2004 & 1:31:59 PM & 25.068 & 22.243 & 23.91 & 24.69 & 25.56 \# & 38.779 & 38.5 & 18.869 & 20.407 & -1.219 & & 52.65 & 0.0 & 14.6 & & 51.0 & 0.85 & \begin{tabular}{l|l}
37.1 \\
\end{tabular} & 12.9 & 29.6 & 2.040 & \begin{tabular}{|l|l|l|}
0.006 \\
\end{tabular} & 0.006 & 0.000 & 0.218 & 0.1 \\
\hline 69 & 1/29/2004 & 1:32:59 PM & 24.99 & \begin{tabular}{|l|}
22.26 \\
\end{tabular} & 23.881 & 24.65 & $25.581 \#$ & 38.857 & 38.682 & 18.885 & 20.45 & \begin{tabular}{|l|l|l|} 
\\
\end{tabular} & & 51.636 & 0.04 & \begin{tabular}{|l|l|l|l|}
14.643 \\
\end{tabular} & & 52.0 & 0.86667 & 37.2 & 12.7 & 29.7 & 2.045 & \begin{tabular}{|l|l|}
0.006 \\
\end{tabular} & 0.006 & 0.000 & \begin{tabular}{l|l} 
\\
\end{tabular} & 0.1 \\
\hline 70 & $1 / 29 / 2004$ & 1:33:59 PM & 24.941 & 22.251 & \begin{tabular}{|l|l|}
23.843 \\
\end{tabular} & 24.58 & 25.403 \# & \begin{tabular}{|l|l}
38.994 \\
\end{tabular} & 38.803 & 18.985 & 20.496 & $|-1.222|$ & -5.478 & 50.952 & 0.042 & 14.643 & & 530 & 0.88333 & 37.3 & 12.5 & 29.7 & 2.051 & \begin{tabular}{|l|l|l|l} 
\\
\end{tabular} & 0.006 & 0.000 & $\begin{array}{ll}0 & 0.218 \\
\end{array}$ & \\
\hline
\end{tabular}


WSRC-TR-2005-00105, REVISION 0

SRNL-RPP-2005-00012, REVISION 0

RUN \# 1.08; STEADY STATE TEST AT 25 WT\% UDS - CONT.

\begin{tabular}{|c|c|c|c|c|c|c|c|c|c|c|c|c|c|c|c|c|c|c|c|c|c|c|c|c|c|c|c|c|}
\hline & A & & $\mathrm{D}$ & & $\mathrm{F}$ & $f(x)$ & & $\mathrm{J}$ & K & 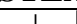 & $M$ & Nt & $R^{2}$ & $e^{-1}$ & 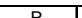 & 5 & & & We & & & & $\omega_{1}$ & $A B$ & $A C$ & & & \\
\hline 71 & $1 / 29 / 2004$ & 1:34:59 PM & 24.908 & 22.273 & 23.814 & 24.572 & 25.514 & 38.949 & 38.79 & 18.877 & 20.514 & -1.222 & -5.478 & 51.189 & 0.042 & 14.643 & 0.004 & 54.00 & 0.9 & 37.3 & 12.5 & & $\mathrm{mon}$ & 0.006 & 0.006 & & 0.218 & \\
\hline 72 & $1 / 29 / 2004$ & 1:35:59 PM & 24.864 & 22.295 & 23.801 & 24.578 & 25.381 & 38.897 & \begin{tabular}{|l|}
38.743 \\
\end{tabular} & 18.865 & 20.514 & -1.219 & -5.476 & 51.373 & 0.042 & 14.643 & 0.004 & 55.00 & 0.91667 & 37.3 & 12.6 & & .04 & 0.006 & 0.006 & 0.0 & & \\
\hline & 9/2004 & 1:36:59 PM & & 22.301 & 23.783 & 24.605 & 25.268 & 38.992 & & 18.924 & & -1.219 & \begin{tabular}{|l}
-5.473 \\
\end{tabular} & & & 14.643 & 0.004 & 56.00 & 0.93333 & 37.4 & & & & & & & & \\
\hline & 9:/2004 & 1:37:59 PM & 24.792 & 22.313 & 23.739 & 24.541 & 25.454 & 38.92 & \begin{tabular}{|l|}
38.753 \\
\end{tabular} & 18.875 & 20.508 & -1.222 & & 52.214 & & 14.6 & & 57.00 & 0.95 & \begin{tabular}{|l|} 
\\
\end{tabular} & \begin{tabular}{|l|l|} 
& 12.8 \\
\end{tabular} & & 2.049 & & & & 218 & \\
\hline 75 & $1 / 29 / 2004$ & 1:38:59 PM & $\begin{array}{l}24.734 \\
2.705\end{array}$ & 22.325 & $\begin{array}{l}23.715 \\
23701\end{array}$ & 24.513 & 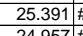 & $\begin{array}{l}027 \\
7955\end{array}$ & 38 & $\begin{array}{r}18.945 \\
1.901\end{array}$ & \begin{tabular}{|l|}
20.506 \\
2000
\end{tabular} & $\begin{array}{l}1.222 \\
1216\end{array}$ & \begin{tabular}{|l|l|}
-5.467 \\
5.61
\end{tabular} & \begin{tabular}{|l|}
52.589 \\
5.26 \\
\end{tabular} & & \begin{tabular}{|l|l|}
14.643 \\
\end{tabular} & & 58.00 & \begin{tabular}{|l|}
0.96667 \\
\end{tabular} & \begin{tabular}{|l|}
37.4 \\
\end{tabular} & \begin{tabular}{|l|}
12.9 \\
\end{tabular} & 29.8 & & & & & 0.218 & \\
\hline $\begin{array}{ll}76 \\
77 \\
\end{array}$ & $1 / 29 / 2004$ & $\begin{array}{ll}1: 39: 59 \mathrm{PM} \\
1.40 .59\end{array}$ & $\begin{array}{l}24.705 \\
24664\end{array}$ & $\begin{array}{l}22.341 \\
22.325 \\
\end{array}$ & \begin{tabular}{|l|}
23.701 \\
23651
\end{tabular} & $\begin{array}{r}24.479 \\
2.388\end{array}$ & $\begin{array}{l}24.957 \\
23.901 t\end{array}$ & $\begin{array}{l}785 \\
034 \\
0.5\end{array}$ & \begin{tabular}{|l|}
38.612 \\
38859 \\
\end{tabular} & $\begin{array}{r}18.81 \\
19.034\end{array}$ & $\begin{array}{r}20.492 \\
20.51\end{array}$ & \begin{tabular}{|c|}
-1.216 \\
-1.216
\end{tabular} & $\begin{array}{l}-5.46 \\
-5.46\end{array}$ & \begin{tabular}{|l|}
52.266 \\
51.965 \\
\end{tabular} & $\begin{array}{l}0.042 \\
0.042\end{array}$ & \begin{tabular}{|l|l|}
14.643 \\
14643
\end{tabular} & & $\begin{array}{l}59.00 \\
6000\end{array}$ & 0.98333 & $\begin{array}{r}37.1 \\
37.4\end{array}$ & \begin{tabular}{|l|}
12.8 \\
12.7 \\
\end{tabular} & $\begin{array}{l}329.6 \\
7298\end{array}$ & $\frac{2.043}{2.053}$ & 006 & $\begin{array}{l}0.006 \\
0.007\end{array}$ & 500 & & $\frac{0.19}{0.19}$ \\
\hline 78 & $1 / 29 / 2004$ & 1:41:59 PM & $\begin{array}{r}24.064 \\
24.64\end{array}$ & 22.3231 & \begin{tabular}{|l|l|} 
& 23.63 \\
\end{tabular} & \begin{tabular}{|l|}
24.300 \\
24.238
\end{tabular} & 24.287 & & \begin{tabular}{|l|l|}
38.659 \\
38.583
\end{tabular} & $\begin{array}{l}\frac{19.034}{18.772} \\
\end{array}$ & . 0.51 & $\begin{array}{l}-1.216 \\
-1.219 \\
\end{array}$ & $\begin{array}{l}-5.461 \\
-5.458\end{array}$ & \begin{tabular}{|l|}
51.965 \\
52718
\end{tabular} & $\begin{array}{l}0.042 \\
0.042\end{array}$ & $\begin{array}{c}14.663 \\
14643\end{array}$ & & & \begin{tabular}{|r|}
1 \\
101667
\end{tabular} & $\frac{37.4}{37.1}$ & $\frac{12.1}{129}$ & $\frac{29.8}{29.6}$ & & & & & & $\begin{array}{l}0.19 \\
0.19\end{array}$ \\
\hline $\begin{array}{l}79 \\
\end{array}$ & $1 / 29 / 2004$ & 1:42:59 PM & 24.584 & 22.325 & 23.606 & \begin{tabular}{|l|l|} 
& 24.177 \\
\end{tabular} & 24.532 & & & & & -1.216 & & \begin{tabular}{|l|}
51.719 \\
\end{tabular} & & 14.643 & .004 & & \begin{tabular}{|l|l|}
.01606 \\
\end{tabular} & | & \begin{tabular}{|l|l|}
12.7 \\
12.7
\end{tabular} & \begin{tabular}{|l|}
29.6 \\
29.8
\end{tabular} & 2.05 & & & & & \\
\hline 80 & $1 / 29 / 2004$ & 1:43:59 PM & 24.549 & 22.33 & \begin{tabular}{|l|l|}
3 & 23.59 \\
\end{tabular} & 24.111 & $24.491 \neq$ & 015 & $3 \varepsilon$ & 18.891 & 20.584 & -1.219 & & 50.343 & & 14.643 & & 63.00 & \begin{tabular}{|l|} 
\\
\end{tabular} & 37.4 & 12.3 & 29.8 & $\frac{2.055}{2.055}$ & & 0.006 & & .213 & $\frac{0.19}{0.18}$ \\
\hline & $1 / 29 / 2004$ & 1:44:59 PM & 24.509 & 22.335 & & 24.061 & 24.871 & & 8.78 & 18.793 & & -1.219 & & 55.411 & & $\begin{array}{l}14.643 \\
\end{array}$ & & 64.00 & \begin{tabular}{|c|}
1.06667 \\
\end{tabular} & 37.3 & 13.6 & 29.8 & & & & & & \\
\hline & $1 / 29 / 2004$ & 1:45:59 PM & 24.477 & 22.338 & \begin{tabular}{|l|l|}
23.564 \\
\end{tabular} & 24.09 & 24.905 \# & & & & & -1.216 & & & & & & & $\mid 1.08333$ & 37.2 & 12.6 & & & & & & 214 & \\
\hline & & & 24.422 & & & & $24.265:$ & & & & & -1.21 & & & & & & & & & & & & & & & & \\
\hline & $1 / 29 / 2004$ & 1:47:59 PM & 24.371 & 22.317 & 23.477 & 24.049 & 23.753 & & & 18.796 & & -1.22 & \begin{tabular}{|c|}
-5.45 \\
\end{tabular} & 51.1. & & 14. & & & .11667 & & & & 2056 - & & & & 0.215 & \\
\hline & $1 / 29 / 2004$ & 1:48:59 PM & 24.367 & & \begin{tabular}{|l}
3 \\
23.493
\end{tabular} & 24.105 & $24.84 A$ & & & 18.84 & & -1.22 & & & & & & & \begin{tabular}{|l|}
1.100 \\
1.13333
\end{tabular} & 37.3 & $\frac{12.9}{12.9}$ & & & & & & $\begin{array}{l}0.214 \\
0.214\end{array}$ & \\
\hline & 1/29/2004 & 1:49:59 PM & 24.347 & 22.349 & 23.483 & 24.206 & 25.035 & & & 18.826 & & -1.216 & & 52.6 & & & & & 1.15 & 37.3 & 12.9 & & & & & & & \\
\hline & 1/29/2004 & $1: 50: 59$ PM & 24.324 & 22.351 & \begin{tabular}{|l|l|} 
& 23.466 \\
\end{tabular} & 24.303 & 25.447 & & & 18.746 & & -1.219 & & 50.0 & & & & & \begin{tabular}{|l|l|}
1.16667 \\
\end{tabular} & & 12.3 & 25 & & & & & 0.215 & \\
\hline & $1 / 29 / 2004$ & 1:51:59 PM & 24.286 & 22.362 & 23.452 & \begin{tabular}{|l|l|}
24.38 \\
\end{tabular} & 25.513 & & \begin{tabular}{|l|}
38.714 \\
\end{tabular} & 18.778 & & -1.222 & & 53.515 & & 14.6 & & 71.00 & .18333 & 37.2 & 13.1 & 9.7 & 2.0 & & & & 0.215 & \\
\hline 89 & $1 / 29 / 2004$ & 1:52:59 PM & 24.283 & 22.38 & \begin{tabular}{|l|l|}
33.444 \\
\end{tabular} & 24.417 & 25.171 & & & 18.903 & & -1.219 & -5.4 & 52.049 & & & & & & 37.4 & & & 2.0 & & & & 0.214 & \\
\hline 90 & $1 / 29 / 2004$ & 1:53:59 PM & 24.265 & 22.386 & \begin{tabular}{|l|l|}
23.431 \\
\end{tabular} & 24.489 & 25.008 & 38.677 & 38.535 & 18.666 & 20.557 & -1.222 & -5.429 & 50.643 & 0.041 & 14.643 & & & \begin{tabular}{|l|l|}
1.21667 \\
\end{tabular} & 37.1 & 12.4 & 49.6 & 2.042 & 0.006 & 0.006 & & 0.216 & \\
\hline $9 ?$ & & Averaces & 261 & 219 & & & $24.96>0$ & & & & & & $-4.99-4$ & & & & & & & & & & & & & & 0.248 & \\
\hline & & Maximum & 28.6 & 22.4 & 28.3 & & 25.6 & 40.0 & $\begin{array}{l}30.8 \\
39.5\end{array}$ & 21.3 & 22.0 & 0.4 & & 73 & & & & & & 38.1 & $\frac{13.1}{18.1}$ & \begin{tabular}{|l|}
30.7 \\
30.7
\end{tabular} & & & & & $\begin{array}{l}0.248 \\
0.487\end{array}$ & \\
\hline & & Median & 25.8 & 22.1 & 24.3 & 25 & $25.1 \mathrm{t}$ & 38.9 & 38 & 19. & & -1.2 & -5.5 & 5 & & 14 & & & & 37.3 & 12.7 & 29.7 & 2.048 & & & & 0.219 & \\
\hline & & Minimum & 24.3 & 21.0 & 23.4 & 24.0 & $23.7 A$ & 32.7 & 38.2 & 17. & 2.6 & -1.2 & -6.0 & 4 & & 14.6 & & & & $\overline{36.8}$ & 10.5 & \begin{tabular}{|l|}
22.7 \\
\end{tabular} & 1.563 & & & & 0.101 & \\
\hline & & $2 \times \mathrm{St}$ & 2.706 & 0.868 & 3.057 & 1.806 & 1.028 & 3.684 & 0.566 & 1.651 & 5.019 & 0.999 & 3.950 & 10.838 & 0.025 & 0.000 & 0.001 & & & 0.567 & 2.656 & i. 4.337 & 0.299 & \begin{tabular}{|l|l|}
0.004 \\
\end{tabular} & 0.003 & 0.0001 & 0.143 & \\
\hline & Number 0 & is Used & 68 & 68 & 68 & 68 & 68 - & 68 & 68 & 68 & 68 & 68 & 68 & 68 & 68 & 68 & 68 & & & 68 & 68 & \begin{tabular}{|l|l}
3 & 68 \\
\end{tabular} & 68 & 68 & 68 & 68 & 68 & \\
\hline 9 & & ${ }^{*}$ Backpulse po & oints in box? & are not inc & & & & & & & & & & & & & & & & & & & & & & & & \\
\hline & & & & & & & & & & & & & & & & & & & & & & & & & & & & \\
\hline $101 \mid$ & & & & & & & & & & & & & & & & & & & & & & & 1-hour a & avg. $=$ & \begin{tabular}{|l|l|l|} 
\\
0.0072
\end{tabular} & & & \\
\hline
\end{tabular}


RUN \# 1.09; STEADY STATE TEST AT 25 WT\% UDS

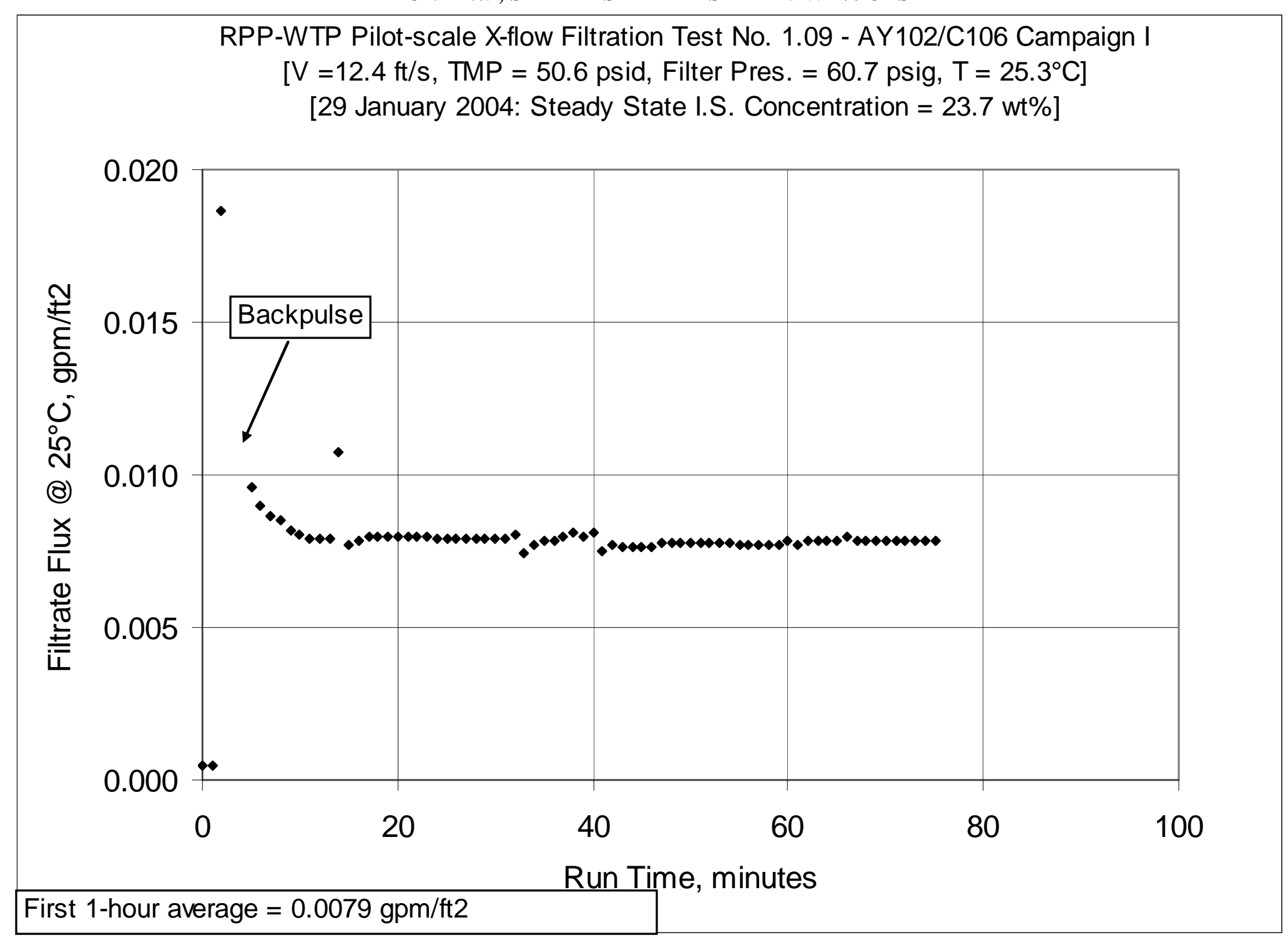


WSRC-TR-2005-00105, REVISION 0

SRNL-RPP-2005-00012, REVISION 0

RUN \# 1.09; STEADY STATE TEST AT 25 WT\% UDS - CONT.

\begin{tabular}{|c|c|c|c|c|c|c|c|c|c|c|c|c|c|c|c|c|c|c|c|c|c|c|c|c|c|c|c|c|}
\hline & $\mathrm{A}$ & B & $\mathrm{D}$ & $E$ & $F$ & $G$ & $\mathrm{H}$ & J & $\mathrm{K}$ & $\mathrm{L}$ & $\mathrm{M}$ & $\mathrm{N}$ & 0 & $\mathrm{Q}$ & $\mathrm{R}$ & $\mathrm{s}$ & $\mathrm{T}$ & $\mathrm{v}$ & w & $\mathrm{x}$ & & $z$ & $\mathrm{AA}$ & $A B$ & $\mathrm{AC}$ & $A D$ & $\mathrm{AE}$ & \\
\hline & DATE & TIME & & $\begin{array}{l}\text { << Temp } \\
\text { Cleaning }\end{array}$ & \begin{tabular}{|} 
erature I \\
Slurry
\end{tabular} & $\begin{array}{l}\text { Measurem } \\
\text { Hi Amb. }\end{array}$ & $\begin{array}{l}\text { ents >>> } \\
\text { Lo Amb. }\end{array}$ & & & $\begin{array}{l}\text { Pressure } \\
\text { Filter dPP }\end{array}$ & $\begin{array}{l}\text { Masurem } \\
\text { TopTMP }\end{array}$ & & & & & $\begin{array}{l}\text { teasureme } \\
\text { Hi Filtate }\end{array}$ & & & & & & & & & & & & \\
\hline & & & $\frac{1}{\operatorname{deg} C}$ & $\operatorname{deg} C$ & \begin{tabular}{|l|}
$\operatorname{deg} C$ \\
\end{tabular} & \begin{tabular}{|l|}
$\operatorname{deg} C$ \\
\end{tabular} & $\operatorname{deg} C$ & psid & psig & psid & & \begin{tabular}{|l|l|l|l|l|l|l|l} 
psig \\
\end{tabular} & & glury & Fing & gpm & & & & & & & & & & & & \\
\hline & & & $\mathrm{T} 2$ & T3 & & T4 & & $\mathrm{dP2}$ & P1 & $\mathrm{dP1}$ & $\mathrm{dP3}$ & P2 & & Q1 & & $\mathrm{Q3}^{3}$ & & & & & & & & & & & & \\
\hline 5 & 70 & not & & & & & & & & & & & & & & & & & & & & & & & & & & \\
\hline$\frac{0}{7}$ & $\begin{array}{c}\text { Zeros- } 01 / 26 \\
1 / 29 / 2004\end{array}$ & 7:2004 & 19.97 & 14.564 & 111.286 & 20.431 & 11.485 & 0.059 & -0.149 & 0.007 & 0.018 & -0.186 & 0.098 & \begin{tabular}{|c|c|}
-0.087 \\
\end{tabular} & 0.003 & 14.643 & 0.005 & & & Filter Su & Surface & 6.707 & FT2 & & & & & \\
\hline 8 & $1 / 29 / 2004$ & 7:58:50 AM & 19.97 & 14.564 & 11.286 & 20.431 & 11.485 & 0.059 & -0.149 & 0.007 & 0.018 & -0.186 & 0.098 & $\begin{array}{ll}-0.087 \\
\end{array}$ & 0.003 & 14.643 & 0.005 & & & Convers & rsion & 851 & $\mathrm{~m} 3 / \mathrm{m} 21$ & $2 /$ day/bar & $\mathrm{rg} / \mathrm{gpm} / \mathrm{ft}$ & fft2/barg & & \\
\hline 9 & $1 / 29 / 2004$ & 7:58:52 AM & 19.981 & 14.574 & 11.307 & 20.466 & 11.546 & 0.059 & & 0.007 & 0.021 & -0.168 & 0.101 & -0.083 & 0.003 & 14.643 & 0.004 & & & & & & & & & & & \\
\hline 10 & $1 / 29 / 2004$ & 7:58:53 AM & & 14.585 & \begin{tabular}{|l|l|l|l|}
11.317 \\
\end{tabular} & 20.472 & 11.55 & & -0.149 & 0.007 & 0.021 & -0.174 & 0.10 & -0.087 & 0.003 & 14.643 & 0.00 & & & Note: & ayde & & & & hately & 4 psig & & \\
\hline & $1 / 29 / 2004$ & 7:58:54 AM & & 14.575 & 11.317 & 20.477 & 11.552 & 0.059 & -0.149 & 0.007 & 0.023 & -0.168 & 0.10 & -0.081 & 0.003 & $\begin{array}{l}14.643 \\
\end{array}$ & 0.004 & & & & & & & & & & & \\
\hline 12 & $1 / 29 / 2004$ & 7:58:55 AM & 19.986 & 14.58 & 11.31 & 20.472 & & 0.061 & -0.149 & 0.008 & 0.021 & -0.177 & 0.104 & -0.087 & 0.003 & 14.643 & & & & & & & $d e^{2}$ & & & & & \\
\hline & & 7:58:56 AM & & 14.58 & & 20.477 & 11.572 & & & & & & & & & 14.643 & & & & VVVV & & & & < Filtr & & & & \\
\hline 14 & $1 / 29 / 2004$ & 7:58:57 AM & 19.986 & 14.58 & 11.312 & 20.472 & 11.572 & 0.059 & -0.149 & 0.008 & 0.023 & -0.189 & 0.104 & $\begin{array}{l}-0.087 \\
\end{array}$ & 0.003 & $\begin{array}{l}14.643 \\
\end{array}$ & 0.005 & & & & & & & & at 25C & & 1000 & \\
\hline$\frac{15}{15}$ & thar & & & & & & & & & & & & & & & & & Time & rime & Press & & TMP & MP & gpm & & $m$ & & ieter \\
\hline & $1 / 29 / 2004$ & 1:59:46 PM & 24349 & 22466 & 233.997 & 25.386 & 25.227 & 24.072 & 61.374 & & 3715 & 331903 & \begin{tabular}{l|l}
3 & 100,665
\end{tabular} & 50.023 & 0.003 & 14,643 & 0.003 & & & $\frac{\operatorname{sig}}{59.9}$ & 123 & $\frac{139}{139}$ & 0.958 & 30.000 & 0.000 & $2 / \mathrm{ps}$ & 0.033 & 0.03 \\
\hline 17 & $1 / 29 / 2004$ & 2:00:46 PM & 24.674 & & & 25.43 & 25.421 & & & 10.31 & -0.025 & 43.848 & 46.135 & . & 0.003 & 14.643 & 0.005 & 1.0 & 0.01667 & 57.6 & 13.0 & \begin{tabular}{|l|l|} 
& 5.0 \\
\end{tabular} & \begin{tabular}{|l|l|}
0.344 \\
\end{tabular} & 0.000 & 0.000 & 0.000 & 0.092 & 0.08 \\
\hline & $1 / 29 / 2004$ & 2:01:46 PM & 24.643 & 22.515 & 24.121 & 25.42 & 25.451 & 54.565 & \begin{tabular}{|l|l|}
60.487 \\
\end{tabular} & 15.611 & 39.588 & 0.315 & 0.323 & 51.148 & 0.122 & 14.643 & 0.004 & 2.0 & 0.03333 & 59.0 & 12.5 & 47.1 & 3.246 & \begin{tabular}{|l|}
0.018 \\
\end{tabular} & $\mid 0.019$ & & 0.396 & 0.34 \\
\hline & $1 / 29 / 2004$ & 2:02:46 PM & 24.744 & 22.536 & 24.152 & 25.41 & 25.786 & 55.608 & 61.469 & 18.562 & 37.628 & 0.251 & 0.352 & 51.757 & 0.084 & 14.643 & & 3.4 & 0.05 & 60.0 & 12. & & 3.214 & $\begin{array}{ll}42.183 \\
\end{array}$ & 2.236 & & & 40.82 \\
\hline & $1 / 29 / 2004$ & 2:03:46 PM & 24.804 & 22.577 & 24.187 & 25.451 & 25.956 & 56.006 & 61.878 & 20.01 & 36.575 & 0.214 & 0.378 & \begin{tabular}{|l|l|}
48.639 \\
\end{tabular} & 0.07 & $\begin{array}{l}14.643 \\
\end{array}$ & & & & 60.4 & 11. & 46. & 3,192 & 2.18 & & & & 41.0 \\
\hline 22 & $1 / 29 / 2004$ & 2:04:46 PM & 24.855 & 22.602 & 24.233 & 25.326 & 26.176 & 56.336 & 62.193 & 20.731 & 36.201 & 0.217 & 0.407 & 51.907 & 0.063 & 14.643 & & 3.0 & .08 & 60. & & 46. & & & & & & \\
\hline 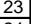 & $1 / 29 / 2004$ & 2:05:46 PM & & 22.644 & 24.264 & 25.312 & 26.082 & 56.595 & 62.489 & 21.163 & 36.013 & 0.254 & & 45.7 & 0.059 & 14.643 & & 6.00 & & 61.0 & 11. & 46. & & & & & 0.194 & 0.1 \\
\hline & $1 / 29 / 2004$ & 2:06:46 PM & & & & 25.26 & 26.35 & 56.456 & & & 35.797 & 0.257 & & 47.936 & 0.057 & 14.643 & & & 0.11667 & & & & 3.180 & 0.008 & & & 0.188 & \\
\hline & & & & & & & 25.90 & & & & & & & 46.92 & 0.056 & 14.643 & & 8.0 & & & & & & & & & & \\
\hline & $1 / 29 / 2004$ & 2:08:46 PM & 25.003 & 22.706 & 24.327 & 25.134 & 25.59 & 56.869 & 62.775 & 21.772 & 35.727 & 0.324 & & 46.428 & 0.054 & 14.643 & & 9.0 & 0.15 & 61.3 & & & 3.192 & & & & & \\
\hline & $1 / 29122004$ & 2:09:46 PM & & 22.106 & 24.331 & 25.024 & 25.35 & 56.186 & $\frac{62.111}{6.020}$ & 21.604 & 35./.13 & 0.324 & & 47.596 & & 14.643 & & & 0.16661 & & & & 3.1899 & & & & & 1.1 \\
\hline$\frac{20}{29}$ & $1 / 29 / 2004$ & 2:11:40 PM & 25.094 & 22741 & 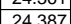 & 24.9388 & $\begin{array}{l}25.58 \\
25.58\end{array}$ & $\begin{array}{c}50.904 \\
57.23\end{array}$ & \begin{tabular}{|l|l|}
03252 \\
6325
\end{tabular} & $\begin{aligned} 21.04 \\
21.834\end{aligned}$ & 35.887 & 0.324 & 0.503 & $\begin{array}{r}45.9249 \\
48499\end{array}$ & 0.052 & $\begin{array}{r}14.643 \\
14643 \\
\end{array}$ & 0.004 & 11.00 & $\frac{18333}{0.2}$ & $\begin{array}{l}61.4 \\
61.8 \\
6.9\end{array}$ & \begin{tabular}{|l|l|l|}
11.3 \\
3 & 11.9
\end{tabular} & \begin{tabular}{|l|l|}
46.3 \\
6.6
\end{tabular} & $\frac{3.1924}{3.210}$ & $\mid 0.008$ & $\begin{array}{l}0.0008 \\
0.008\end{array}$ & 0.000 & $\frac{0.170}{0.169}$ & 0.15 \\
\hline 30 & $1 / 29 / 2004$ & $2: 12: 46 \mathrm{PM}$ & 25.123 & 22.756 & 24.406 & 24.802 & 25.565 & 57.068 & 63.041 & 21.585 & 36.072 & 0.361 & & 46.893 & 0.052 & 14.643 & & 13.00 & 0.21667 & $\frac{61.6}{61.6}$ & 11.5 & $\frac{46.6}{46.6}$ & 3.211 & \begin{tabular}{|l|l|} 
& 0.008 \\
\end{tabular} & $\mid 0.008$ & 0.000 & 0.169 & 0.14 \\
\hline & $1 / 29 / 2004$ & 2:13:46 PM & 25.119 & 22.762 & 24.427 & 24.753 & 25.63 & 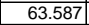 & 63.43 & 21.819 & \begin{tabular}{|l|l|l}
41.847 \\
\end{tabular} & -1.219 & & 46.939 & & 14.643 & & 14.00 & & 62.0 & 11.5 & & 3.635 & & & & 204 & 0.1 \\
\hline 32 & $1 / 29 / 2004$ & 2:14:46 PM & 25.119 & 22.772 & 24.473 & 24.698 & 25.716 & 61.658 & \begin{tabular}{|l|l|}
61.2211 \\
\end{tabular} & 25.01 & 36.723 & -1.222 & & 53.219 & 0.051 & 14.643 & & 15.00 & 0.25 & 59.7 & $\overline{130}$ & 49.2 & 3.392 & 0.008 & 0.008 & 0.000 & 0.157 & 0.1 \\
\hline 30 & $1 / 29 / 2004$ & 2:15:46 PM & 25.185 & 22.784 & 24.529 & 24.624 & 25.677 & 62.429 & 61.83 & & & -1.222 & & 54.111 & & 14.643 & & 16.00 & 0.26667 & 60.4 & 13.3 & . & 3.448 & .008 & 0.008 & 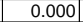 & 0.157 & 0.1 \\
\hline & $1 / 29 / 2004$ & 2:16:46 PM & & & 24.575 & & 25.489 & 62.251 & & 24.48 & 37.857 & & & 50.316 & & & & & 0.28333 & 60.2 & & & 3.451 & & & & 0.160 & 0.1 \\
\hline 35 & $1 / 29 / 2004$ & 2:17:46 PM & 25.268 & 22.811 & 24.632 & 24.547] & 25. & & \begin{tabular}{|l}
62.168 \\
\end{tabular} & 24.154 & 38.767 & -1.222 & & 52.049 & 0.053 & 14.643 & & 18.0 & 0.3 & 60.7 & $12.8 \mathrm{CP}$ & & 3.501 & 0.008 & 0.008 & & 157 & 0.1 \\
\hline & $\frac{1 / 29122004}{12004}$ & 2:18:46 PM & 25.308 & 22.812 & 24.667 & 24.527 & 25.42 & & & 23.6 & & -1.219 & & .529 & & 14.6 & & & 0.31667 & 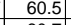 & & & 0 & & & & 0.157 & 0.1. \\
\hline & $11 / 29122004$ & $2.19: 46 \mathrm{PM}$ & 25.36 & & 24.09 & 24.488 & 25.6 & & 62.162 & $23.57 \mathrm{~g}$ & & -1.219 & & 50.6 & & & & 20.0 & 0.33333 & 60.1 & $12.4 \mathrm{Y}$ & & 3.513 & & 0.008 & & 0.156 & 0.13 \\
\hline 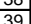 & $1 / 299 / 2004$ & $2: 20.46 \mathrm{PM}$ & $\frac{25.385}{2541}$ & 22.839 & 24.759 & 24.484 & 25.58 & & \begin{tabular}{|l|l|l|l|}
6.1240 \\
\end{tabular} & 23.4 & & -1.216 & & .292 & & & & 21.0 & $\begin{array}{r}0.35 \\
03667\end{array}$ & $\frac{60.6}{607}$ & $\frac{12.6}{120}$ & S1.0 & 3.513 & 008 & & & 0.156 & 0.13 \\
\hline 40 & $\frac{1 / 29 / 2004}{1 / 29 / 2004}$ & $\frac{2.2 .456}{2.22 .46}$ & $\begin{aligned} 25.41 \\
25.449\end{aligned}$ & 22.835 & \begin{tabular}{|l|l|}
24.109 \\
4803
\end{tabular} & $\frac{24.463}{24.407}$ & $\frac{2.29}{24.33}$ & $\begin{array}{l}62.155 \\
62935 \\
625\end{array}$ & \begin{tabular}{|l|l|l|l|}
62406 \\
\end{tabular} & $\frac{23.338}{23191}$ & $\begin{array}{l}39.563 \\
39.674 \\
\end{array}$ & \begin{tabular}{|l|l|l|l|l|} 
& -1.219 \\
1 & -1219
\end{tabular} & & $\begin{array}{l}49.964 \\
49172\end{array}$ & & $\begin{array}{l}14.045 \\
114643\end{array}$ & & & $\begin{array}{l}0.36060 \\
0.3823\end{array}$ & (60.t) & $\frac{1.2}{121}$ & & 3.557 & & & & D..155 & 0.1 \\
\hline 41 & $1 / 29 / 2004$ & $\frac{2.22 .40 \mathrm{PM}}{2.23: 46 \mathrm{PM}}$ & 25.464 & 22.005 & \begin{tabular}{|l|l|l|}
2483 \\
\end{tabular} & 24.467 & $\frac{2.35}{249}$ & $\begin{array}{c}02.950 \\
62.9 \\
\end{array}$ & \begin{tabular}{|l|l|l|l|}
62251 \\
\end{tabular} & $\frac{2.191}{23304}$ & $\begin{array}{l}39.014 \\
39.715 \\
\end{array}$ & $\mid-1219$ & & (55.172 & 0 & $\begin{array}{l}14.045 \\
14643\end{array}$ & & $\frac{25.0}{240}$ & | & 60.9 & $\frac{1<.1}{127}$ & $\frac{51.3}{513}$ & 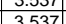 & 0.008 & | & & 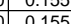 & 0.1. \\
\hline & $1 / 29 / 2004$ & $2: 24: 46$ PM & 25.493 & 22.842 & 24.868 & 24.362 & & 62.869 & \begin{tabular}{|l|l|}
62199 \\
\end{tabular} & 23.167 & 39.803 & -1.219 & & 49.809 & & & & & 041667 & 607 & thent & & 353 & 8 & & & 0.154 & 0.1 \\
\hline & $\frac{1 / 29 / 2004}{1}$ & $2: 25: 46$ PM & & 22.847 & 24888 & 24372 & 25.24 & 62.765 & & 23.065 & & -1219 & & & & & & & & 00.1 & 13.4 & & & & & & & 0.1 \\
\hline 44 & $\frac{1 / 29 / 2004}{1 / 2904}$ & $\frac{2 \cdot 26: 46 \mathrm{PM}}{2}$ & 25.57 & 22.858 & 24.919 & 24.433 & & & \begin{tabular}{|l|l|}
62108 \\
\end{tabular} & & & -1.222 & & & & & & & 0.45 & 606 & 123 & & & & & & & \\
\hline & $1 / 29 / 2$ & $2 \cdot 27 \cdot 46$ PM & 25.576 & & 24.95 & 24.555 & 2466 & 62689 & 62145 & 23014 & 39795 & -1219 & & 50.558 & & & & 2800 & 0.46667 & 607 & 124 & 512 & 353 & 30008 & & & 154 & 0.1 \\
\hline 46 & $1 / 29 / 2$ & $2.28 .46 \mathrm{PM}$ & 25.528 & 22877 & \begin{tabular}{|l|l|}
24.983 \\
\end{tabular} & 24.617 & 2528 & & & & 39.819 & -1.222 & & 55297 & & & & 2900 & 0.48333 & & 13 & & 3536 & & & & & 01 \\
\hline & $1 / 29 / 2004$ & $2 \cdot 29 \cdot 46 \mathrm{PM}$ & 25.585 & 22.883 & 25029 & 24,689 & 25382 & 62.937 & $\mid 62354$ & 23.93 & 39.858 & -1216 & & 50.939 & 0.053 & & & 3000 & 0.40 & , & 125 & 514 & 3544 & 100 & & & 0.154 & 0.1 \\
\hline 48 & $1 / 29 / 2004$ & $2: 30: 46 \mathrm{PM}$ & 25.625 & 22.889 & 2505 & 24.684 & 25. & 62.942 & \begin{tabular}{|l|}
62.419 \\
\end{tabular} & 23.0 & 39.854 & \begin{tabular}{|c|}
4.219 \\
\end{tabular} & & & & 146 & & 31. & \begin{tabular}{|l|l|}
0.51667 \\
\end{tabular} & 60.9 & 124 & \begin{tabular}{|l|}
51.4 \\
\end{tabular} & 3544 & 10.008 & 0.008 & & 0154 & $\frac{0.1}{0.11}$ \\
\hline 49 & $1 / 29 / 2004$ & 2:31:46 PM & 25.652 & 22.901 & 25.077 & 24.7 & 25. & & 62.6 & 22 & 40.462 & -1.222 & & & & 14.6 & & 32 & 0.53333 & 61.2 & 12.4 & 1.9 & 3.575 & & & 10 & 0.155 & 0.13 \\
\hline 50 & $1 / 29 / 2004$ & $2: 32: 46$ & 25.69 & 22.9 & 25.124 & 24.854 & & 61 & \begin{tabular}{|l|l|}
60.738 \\
\end{tabular} & 25.334 & 36. & \begin{tabular}{|l|l|}
3 & -1. \\
\end{tabular} & & 56.648 & 2.05 & 14.643 & & 33. & 0.55 & 59.3 & 13.9 & 4 & 363 & & & & 0.152 & 0.1 \\
\hline & $1 / 29 / 2004$ & & 25.727 & 22.931 & 25.182 & 24.862 & 26.16 & 62.541 & \begin{tabular}{|l|l|}
61.948 \\
\end{tabular} & 24. & 38.473 & \begin{tabular}{|l|l|}
3 & -1. \\
\end{tabular} & & & & & & 34. & 0.566 & 60.5 & 12.4 & & 482 & & & & 0.153 & $\overline{0.1}$ \\
\hline & $1 / 29 / 2$ & & 25.781 & 22. & & 24.9 & & & & 23. & 39 & $\mid-1.219$ & & & & & & & 0.58333 & 60.7 & & & & & & & 0.154 & \\
\hline & $1 / 29 / 200$ & & 25.819 & 22.963 & 25.234 & 24.9 & & 62.958 & 62.4 & 22.8 & & \begin{tabular}{|l|l|} 
& -1.219 \\
\end{tabular} & & & & & & 36.00 & 0.6 & 60.9 & & & & & & & & \\
\hline 54 & 1/29/2 & & 25.848 & 22.993 & 25.273 & 25.0 & & & & 22.8 & & -1.222 & & & & & & & 0.61667 & 61.1 & & 01. & 3.567 & & & & 154 & U. \\
\hline & & & 25.911 & 23.001 & 25.281 & 25.0 & & & & & & -1.219 & & & & & & & 0.633 & 60.9 & & & & & & & 0.156 & \\
\hline & & & 25..931 & 23.025 & 25.296 & 25.1 & & 63.39 & & 22. & & -1.222 & & & & & & & & 61.3 & & & & & & & & \\
\hline & $1 / 29122$ & 2:39:46 & 25.943 & 23.037 & 25.308 & 25.168 & & 63.429 & 62.858 & 21.8 & 41.6 & -1.219 & & $51.5 \mathrm{~s}$ & & 14. & & & 0.66667 & 61.4 & 12.6 & & 3.022 & & & & & \\
\hline & 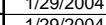 & $2: 40.46 \mathrm{~F}$ & $2 \frac{2.944}{20.501}$ & 23.049 & 25.355 & 25.12 & & 62.0 & 61.426 & 24.2 & $\frac{33.894}{3721}$ & 12 & & & & 14.64 & & & 33 & 50.0 & & & .445 & & & & & \\
\hline 60 & $\frac{112912004}{11201204}$ & $\frac{2.41 .46}{2.10 .46}$ & $\frac{25.961}{2509}$ & $\frac{23.046}{23092}$ & 25.360 & 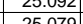 & $\frac{26.1}{35}$ & $\frac{62.0}{62.2}$ & \begin{tabular}{|l}
6.3 .35 \\
61508 \\
\end{tabular} & 24.14 & $\frac{37.921}{38245}$ & 1210 & & 503 & & 14.6 & & $\frac{42 .}{43}$ & $\begin{array}{r}0.1 \\
\end{array}$ & S9.9 & $\frac{12.5}{2.32}$ & 50.0 & & & & 0.000 & 0.154 & 0.1 \\
\hline 61 & 11292004 & $\frac{2.42 .40}{2 \cdot 2 \cdot 46}$ & $\frac{2.9500}{26025}$ & 23.083 & 20.438 & 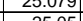 & $\frac{25}{25.5}$ & 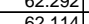 & & & 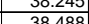 & -1.2 & & 507 & & 14.6 & & 1 & $\mid 0.110$ & - & 12 & 15 & 3.400 & & & & $0.15<2$ & 0.1 \\
\hline & 11292004 & & $\frac{26.025}{26097}$ & $\frac{2.00}{23.118}$ & $\frac{2.44}{25.202}$ & $\frac{25.035}{25.38}$ & & $\frac{62.1}{624}$ & & & & & & & & & & & 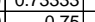 & 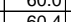 & 12 & & & & & & & \\
\hline & $1 / 292004$ & & 26098 & 23110 & $\frac{20.050}{25.24}$ & 25124 & & $\frac{0.421}{6251}$ & 61005 & & 30.021 & 4.1210 & & 51308 & & & & & & 0.0 .4 & $12 f^{2}$ & & & & & & 0.151 & \\
\hline & & & 26117 _ & 23123 & & 25143 & & & & & & & & & & & & & & 6033 & & & & & & & 0.153 & \\
\hline 65 & $1129 / 2004$ & $2 \cdot 44 \cdot 46 \mathrm{P}$ & 6.14 & 23.117 & 25.56 & 25213 & & 62494 & 61888 & 23658 & 38859 & -1222 & & 5294 & 0.053 & 14643 & & 00 & & & & 50 & $\frac{3.49}{3.99}$ & 0.008 & | & 0.000 & 0.153 & \\
\hline
\end{tabular}


WSRC-TR-2005-00105, REVISION 0

SRNL-RPP-2005-00012, REVISION 0

RUN \# 1.09; STEADY STATE TEST AT 25 WT\% UDS - CONT.

\begin{tabular}{|c|c|c|c|c|c|c|c|c|c|c|c|c|c|c|c|c|c|c|c|c|c|c|c|c|c|c|c|c|}
\hline & A & $\mathrm{B}$ & $\mathrm{D}$ & $E$ & $\mathrm{~F}$ & G & $\mathrm{H}$ & $\mathrm{J}$ & $\mathrm{K}$ & $\mathrm{L}$ & $M$ & $\mathrm{~N}$ & 0 & $\mathrm{Q}$ & $R$ & $\mathrm{~s}$ & $\mathrm{~T}$ & $\mathrm{~V}$ & W & $x$ & & $z$ & $\mathrm{AA}$ & $A B$ & $\mathrm{AC}$ & $A D$ & $\mathrm{AE}$ & \\
\hline 66 & $1 / 29 / 2004$ & \begin{tabular}{ll|l|l|}
$2: 48$ PM \\
$2: 4: 46 \mathrm{P}$
\end{tabular} & $\begin{array}{l}26.199 \\
26209\end{array}$ & $\frac{23.139}{23.15}$ & $\frac{25.619}{25.5}$ & $\frac{25.205}{252}$ & $\begin{array}{l}24.947 \\
25187\end{array}$ & 62.334 & & & $\begin{array}{l}38.816 \\
38.826\end{array}$ & $\begin{array}{l}-1.219 \\
.220\end{array}$ & \begin{tabular}{|l|}
-5.944 \\
5047
\end{tabular} & & 0.053 & $\begin{array}{l}14.643 \\
14643\end{array}$ & $\begin{array}{l}0.004 \\
0.094\end{array}$ & 49 & $\begin{array}{l}8.81667 \\
88^{23232}\end{array}$ & 60.3 & 122 & 50 & & 0008 & $\begin{array}{l}0.008 \\
000\end{array}$ & 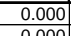 & 0.154 & \\
\hline$\frac{61}{68}$ & $\begin{array}{l}1 / 2992004 \\
129204\end{array}$ & & 26.209 & & 25.65 & 25.2 & & & & 23.577 & & & & & 0.053 & 14.643 & & & & & & & 3.488 & . 0008 & 0.008 & 0.00 & 0.153 & 0.13 \\
\hline 68 & $\begin{array}{l}1 / 291 / 204 \\
1 / 29 / 2004\end{array}$ & $\begin{array}{l}2: 50: 46 \mathrm{PM} \\
2.51 .46 \mathrm{PM}\end{array}$ & $\frac{26.229}{26.254}$ & $\begin{array}{r}23.14 \\
23.144\end{array}$ & \begin{tabular}{|l|}
25.67 \\
25.674 \\
\end{tabular} & $\begin{array}{l}25.105 \\
24.999\end{array}$ & 258 & $\frac{62.465}{62.668}$ & $\frac{61.070}{62.07}$ & $\frac{23.183}{23.234}$ & 39495 & $\begin{array}{l}-\frac{1.219}{-1.222} \\
\end{array}$ & & 1605 & 0.053 & 14643 & & & 866677 & & & & & & & & & 0.13 \\
\hline 70 & $1 / 29 / 2004$ & 2:52:46 PM & 26.279 & 23.14 & \begin{tabular}{|l|}
25.705 \\
\end{tabular} & $\frac{24.550}{24.9}$ & $\frac{25.806}{25.592}$ & 62.641 & \begin{tabular}{|l|l|}
62.031 \\
\end{tabular} & 23.227 & 30 & $\frac{-1.242}{-1.222}$ & $\begin{aligned}-5.941 \\
-5.95\end{aligned}$ & 51.409 & 0.053 & $\frac{14.643}{14.643}$ & $\frac{0.003}{0.003}$ & 53.00 & $\begin{array}{l}0.8667 \\
0\end{array}$ & $\frac{60.6}{60.6}$ & $\frac{12.0}{12.6}$ & $\frac{51.1}{51.1}$ & \begin{tabular}{|l|}
.0 .024 \\
3.520 \\
\end{tabular} & \begin{tabular}{|l|}
0.008 \\
\end{tabular} & $\frac{0.008}{0.008}$ & 0.000 & $\frac{0.152}{0.152}$ & $\frac{0.13}{0.13}$ \\
\hline 71 & $1 / 29 / 2004$ & 2:53:46 PM & 26.304 & 23.145 & 25.725 & 24.839 & 25.342 & 62.293 & \begin{tabular}{|l|l|}
61.71 \\
\end{tabular} & 23.001 & 39.39 & \begin{tabular}{|l|}
-1.225 \\
\end{tabular} & -5.953 & 49.852 & & 14.643 & & 54.00 & \begin{tabular}{|ll}
0 & 0.9
\end{tabular} & 60.2 & 12.2 & & & & & & 0.152 & \\
\hline 72 & $1 / 29 / 2004$ & 2:54:46 PM & 26.329 & 23.155 & 25.755 & 24.87 & 25.467 & 62.543 & 61.942 & 23.215 & 39.448 & \begin{tabular}{|l|l|}
-1.219 \\
\end{tabular} & -5.944 & 49.755 & 0.053 & 14.643 & 0.004 & 55.00 & \begin{tabular}{|l|l}
0 & 0.91667
\end{tabular} & 60.5 & 12.2 & 51.0 & \begin{tabular}{|l|l|} 
& 3.516 \\
\end{tabular} & 0.008 & 0.008 & 0.000 & 0.152 & $010^{2}$ \\
\hline 73 & $1 / 29 / 2004$ & 2:55:46 PM & & 23.161 & & & 25.513 & & & 23.149 & & & -5.944 & & 0.053 & 14.643 & 0.004 & & \begin{tabular}{|l|l}
0 & 0.9333 \\
\end{tabular} & 60.5 & & 51.0 & 3.515 & \begin{tabular}{|l|l|} 
\\
\end{tabular} & 0.008 & & & \\
\hline 74 & $1 / 29 / 2004$ & 2:56:46 PM & 26.364 & 23.155 & 25.785 & 24.779 & 25.712 & 62.525 & 61.907 & 23.212 & 39.438 & \begin{tabular}{|l|l|}
-1.211 \\
\end{tabular} & -5.944 & 48.344 & 0.053 & 14.643 & 0.004 & 57.00 & \begin{tabular}{|l|l}
0 & 0.95 \\
\end{tabular} & 60.4 & 11.8 & 51.0 & \begin{tabular}{|l|}
3.515 \\
\end{tabular} & \begin{tabular}{|l|l|}
0.008 \\
\end{tabular} & 0.008 & 0.000 & 0.152 & \\
\hline 75 & $1 / 29 / 2004$ & $2: 57: 46$ PM & 26.399 & 23.155 & 25.8 & 24.739 & 25.522 & 62.608 & 62.013 & 23.215 & 39.491 & \begin{tabular}{|l|}
-1.219 \\
\end{tabular} & -5.947 & 51.058 & 0.053 & 14.643 & 0.003 & 58.00 & \begin{tabular}{c|c|c|}
0 & 0.96667 \\
\end{tabular} & 60.5 & 12.5 & 51.0 & 3.520 & \begin{tabular}{|l|l|}
0.008 \\
\end{tabular} & 0.008 & 0.000 & 0.151 & \\
\hline 76 & $1 / 29 / 2004$ & 2:58:46 PM & 26.403 & 23.154 & 25.814 & 24.794 & 25.786 & 62.647 & 62.006 & 23.154 & 39.661 & \begin{tabular}{|l|l|}
-1.219 \\
\end{tabular} & -5.944 & 52.126 & 0.053 & 14.643 & 0.004 & 59.00 & 0.98333 & 60.5 & 12.8 & 51.2 & & & 0.008 & & 0.151 & \\
\hline 77 & $1 / 29 / 2004$ & 2:59:46 PM & 26.469 & 23.17 & 25.84 & 24.824 & 25.792 & 62.382 & 61.782 & 22.884 & $\begin{array}{l}39.635 \\
\end{array}$ & \begin{tabular}{|l|l|}
-1.219 \\
\end{tabular} & -5.947 & 54.3 & $\begin{array}{l}0.054 \\
\end{array}$ & 14.643 & 0.003 & 60.00 & & 60.3 & 13.3 & 51.0 & \begin{tabular}{|l|}
3.517 \\
\end{tabular} & 0.008 & 0.008 & 0.000 & 0.154 & \\
\hline 78 & $1 / 29 / 2004$ & 3:00:46 PM & $\begin{array}{r}26.51 \\
651\end{array}$ & & \begin{tabular}{|l|l|}
25.856 \\
2570
\end{tabular} & 24.851 & $\begin{array}{l}25.673 \\
25.771\end{array}$ & 62.481 & & 23.105 & $\begin{array}{r}39.52 \\
3072\end{array}$ & \begin{tabular}{|l|l|}
.222 \\
\end{tabular} & \begin{tabular}{|l|l|}
-5.944 \\
5094
\end{tabular} & 50.013 & 0.053 & 14.643 & 0 & 61.00 & $\begin{array}{ll}0 & 1.01667 \\
\end{array}$ & 60.4 & 12.3 & & & & 0.008 & & 0.151 & \\
\hline 79 & $1 / 29 / 2004$ & 3:01:46 PM & $\begin{array}{l}26.516 \\
26.538 \\
25\end{array}$ & $\begin{array}{l}23.183 \\
23.189 \\
\end{array}$ & \begin{tabular}{|l|}
25.878 \\
2589
\end{tabular} & $\begin{array}{l}24.867 \\
24.878\end{array}$ & $\begin{array}{l}25.774 \\
2576\end{array}$ & $\begin{array}{l}62.602 \\
62514\end{array}$ & 61.956 & 23.015 & \begin{tabular}{|l|}
39.729 \\
30.095
\end{tabular} & \begin{tabular}{|l|} 
\\
\end{tabular} & \begin{tabular}{|l|l|} 
& -5.944 \\
5.944
\end{tabular} & $\begin{array}{l}52.558 \\
5.5001\end{array}$ & $\begin{array}{l}0.054 \\
\end{array}$ & 14.643 & & 62.00 & \begin{tabular}{l|l}
0 & 1.03333 \\
\end{tabular} & 60.5 & 12.9 & \begin{tabular}{|l|l|}
51.2 \\
512
\end{tabular} & \begin{tabular}{|l|}
3.528 \\
\end{tabular} & 0.008 & $\begin{array}{l}0.008 \\
\end{array}$ & & 0.154 & 0.13 \\
\hline $80 \mid$ & $1 / 29 / 2004$ & $\begin{array}{l}3: 02: 46 \text { PM } \\
3 \cdot 0.0346 \mathrm{PM}\end{array}$ & 26.538 & $\frac{23.189}{23207}$ & \begin{tabular}{|l|}
25.889 \\
250.07
\end{tabular} & $\frac{24.878}{24.862}$ & $\begin{array}{r}25.76 \\
25.853\end{array}$ & $\frac{62.514}{62340}$ & \begin{tabular}{|l|l|}
61.865 \\
61.877
\end{tabular} & $\begin{array}{r}22.81 \\
22.689\end{array}$ & $\begin{array}{r}39.885 \\
39.848\end{array}$ & \begin{tabular}{|r|}
-1.219 \\
1222 \\
\end{tabular} & $\begin{array}{r}-5.944 \\
5.947\end{array}$ & $\begin{array}{r}52.091 \\
49454\end{array}$ & 0.054 & $\frac{14.643}{14643}$ & $\begin{array}{l}0.003 \\
0.003\end{array}$ & $\frac{63.00}{6400}$ & \begin{tabular}{r|r} 
& 1.05 \\
\end{tabular} & $\frac{60.4}{60.2}$ & 12.8 & \begin{tabular}{|l|}
51.2 \\
51.1
\end{tabular} & $\begin{array}{l}3.530 \\
353 \\
5\end{array}$ & \begin{tabular}{|l|l|}
0.008 \\
0.008
\end{tabular} & $\begin{array}{l}0.008 \\
0.008\end{array}$ & & $\frac{0.153}{0.154}$ & 0.13 \\
\hline$\frac{81}{82}$ & $\begin{array}{l}11 / 29 l 2004 \\
1 / 29 / 2004\end{array}$ & $\begin{array}{l}3: 03: 46 \mathrm{P} \\
3: 04: 46 \mathrm{PM}\end{array}$ & 20 & $\frac{23.201}{23.218}$ & $\mid \frac{25.901}{25.938}$ & $\begin{aligned} 24.862 \\
24.918 \\
\end{aligned}$ & $\begin{array}{l}25.853 \\
25.615\end{array}$ & $\frac{62.349}{62.546}$ & \begin{tabular}{|l|l|}
61.8876 \\
61.876
\end{tabular} & 22.689 & \begin{tabular}{|}
39.848 \\
39.891 \\
\end{tabular} & \begin{tabular}{|c|}
$\mid-1.222$ \\
-1.219 \\
\end{tabular} & $\begin{array}{l}-5.947 \\
-5.938\end{array}$ & $\begin{array}{l}\begin{array}{l}49.454 \\
50.693\end{array} \\
5\end{array}$ & $\mid$ & $\frac{14.643}{14.643}$ & $\begin{array}{l}0.003 \\
0.004\end{array}$ & $\frac{64.00}{65.00}$ & \begin{tabular}{l|l|l|}
0 & 1.06667
\end{tabular} & $\frac{60.2}{60.4}$ & \begin{tabular}{|l|l}
12.1 \\
12.4
\end{tabular} & $\frac{51.1}{51.2}$ & \begin{tabular}{|l|l|}
3.523 \\
3.531
\end{tabular} & \begin{tabular}{|l|}
0.008 \\
0.008
\end{tabular} & $\frac{0.008}{0.008}$ & 0.0000 & 0.154 & 0 \\
\hline 83 & $1 / 29 / 2004$ & 3:05:46 PM & 26.624 & 23.231 & 25.946 & 24.87 & 25.388 & 62.602 & 62.083 & 22.694 & \begin{tabular}{|l|l|}
40.064 \\
\end{tabular} & \begin{tabular}{|l|}
-1.216 \\
\end{tabular} & & 48.628 & 0.055 & 14.643 & & 66.00 & \begin{tabular}{|l|l|}
0 & 1.1
\end{tabular} & 60.6 & 11.9 & 51.3 & \begin{tabular}{|l|} 
\\
\end{tabular} & \begin{tabular}{|l|l|}
0.008 \\
\end{tabular} & 0.008 & & 0.156 & \\
\hline 84 & $1 / 29 / 2004$ & 3:06:46 PM & 26.616 & 23.217 & 25.932 & 24.796 & 25.899 & 62.755 & 62.114 & 22.741 & \begin{tabular}{|l|l|}
40.164 \\
\end{tabular} & \begin{tabular}{|l|}
-1.219 \\
\end{tabular} & -5.9 & 48.518 & 0.054 & 14.643 & & 67.00 & $\begin{array}{l}0 \\
0\end{array}$ & & 11.9 & & \begin{tabular}{|l|l|}
3.5 \\
\end{tabular} & & & & 0.152 & \\
\hline 85 & $1 / 29 / 2004$ & $3: 07: 46 \mathrm{PM}$ & 26.621 & 23.223 & 25.928 & 24.812 & 25.879 & 63.02 & 62.5 & 22.57 & \begin{tabular}{|l|l}
40.437 \\
\end{tabular} & \begin{tabular}{|l|l|}
-1.219 \\
\end{tabular} & & 51.94 & 0.054 & 14.643 & & 68.00 & $\begin{array}{lll}0 & 1.13333 \\
\end{array}$ & 61.0 & $127,+3$ & & 3.56 & \begin{tabular}{|l|l|}
0.008 \\
\end{tabular} & 0.008 & & 0.152 & \\
\hline 86 & $1 / 29 / 2004$ & 3:08:46 PM & 26.618 & 23.2 & 25.945 & & 25.796 & 63.089 & 62.62 & & & \begin{tabular}{|l|l|}
-1.219 \\
\end{tabular} & & & 0.054 & 14.6 & & 69.00 & $\begin{array}{ll}0 & 1.15 \\
\end{array}$ & 61.1 & 12.9 & & 3. & & & & 0.151 & \\
\hline 87 & 1/29/2004 & 3:09:46 PM & 26.625 & 23.221 & 25.916 & 24.735 & 25.563 & 62.732 & 62.124 & 22.478 & & -1.225 & & 51.269 & 0.054 & & & 70 & $\begin{array}{l}0 \\
0\end{array}$ & 60.7 & \begin{tabular}{|l|}
12.6 \\
\end{tabular} & 51.6 & \begin{tabular}{|l|l|} 
\\
\end{tabular} & & & & 0.152 & \\
\hline 88 & 1/29/2004 & 3:10:46 PM & 26.666 & 23.238 & 25.947 & 24.716 & 25.739 & 62.682 & 62.145 & 22.433 & 40.347 & -1.222 & -5.94 & 47.039 & 0.054 & 14.643 & & 71.00 & 0.18333 & $\begin{array}{l}60.7 \\
\end{array}$ & 11.5 & \begin{tabular}{|l|l|}
51.5 \\
\end{tabular} & \begin{tabular}{|l|}
3.552 \\
\end{tabular} & 0.008 & 0.008 & & 0.152 & 0 \\
\hline \begin{tabular}{|l|l|}
89 \\
00
\end{tabular} & $1 / 29 / 2004$ & 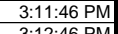 & $\begin{array}{l}26.67 \\
25.521\end{array}$ & $\frac{23.237}{23232}$ & & $\begin{array}{r}24.676 \\
22.671\end{array}$ & $\begin{aligned} 25.928 \\
25.964\end{aligned}$ & $\begin{array}{l}62.79 \\
66002\end{array}$ & & $\begin{array}{l}22.462 \\
2009\end{array}$ & 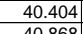 & \begin{tabular}{|c|}
-1.219 \\
.221
\end{tabular} & -5.5 & $\begin{array}{l}51.496 \\
50.472\end{array}$ & 0.054 & $\begin{array}{l}14.643 \\
11643\end{array}$ & & 72.00 & $\begin{array}{r}0.1 .2 \\
0\end{array}$ & 60.8 & 12.6 & 51.6 & \begin{tabular}{|l|}
3.557 \\
\end{tabular} & \begin{tabular}{|l|l|}
0.008 \\
\end{tabular} & 0.008 & & 0.152 & 0.13 \\
\hline \begin{tabular}{|l|}
90 \\
91
\end{tabular} & $1 / 29 / 2004$ & $\begin{array}{l}3: 12: 46 \text { PM } \\
2: 12: 40 P\end{array}$ & 26.521 & $\begin{aligned} 23.233 \\
23238\end{aligned}$ & $\begin{array}{l}25.937 \\
2.092\end{array}$ & 24.671 & $\begin{array}{l}25.864 \\
25.069\end{array}$ & 62.902 & 62.392 & $\begin{array}{r}22.089 \\
20.169\end{array}$ & \begin{tabular}{|l|}
40.868 \\
40.919
\end{tabular} & \begin{tabular}{|c|}
-1.222 \\
1.222
\end{tabular} & $\frac{941}{928}$ & $\begin{array}{l}50.472 \\
55.641\end{array}$ & 0.054 & $\begin{array}{l}14.643 \\
14643\end{array}$ & 003 & 73.00 & \begin{tabular}{l}
0 \\
\hdashline
\end{tabular} & 60.9 & 12.4 & \begin{tabular}{|l|}
51.9 \\
519
\end{tabular} & \begin{tabular}{|l|}
3.577 \\
357
\end{tabular} & 0.008 & 0.008 & 0.000 & 0.151 & 0.13 \\
\hline \begin{tabular}{|l|}
91 \\
92
\end{tabular} & $\begin{array}{l}1 / 29 / 2004 \\
1129204\end{array}$ & $\begin{array}{l}3: 13: 46 \mathrm{PM} \\
3 \cdot 14 \cdot 46 \mathrm{PM}\end{array}$ & $\frac{26.341}{26.232}$ & $\frac{23.238}{23.248}$ & $\begin{array}{l}25.942 \\
25.958 \\
\end{array}$ & $\frac{24.691}{24.697}$ & $\begin{array}{l}25.969 \\
26059\end{array}$ & $\frac{62.894}{63.267}$ & $\frac{62.321}{62715}$ & $\frac{22.136}{22243}$ & $\begin{array}{l}40.919 \\
41.098\end{array}$ & $\begin{array}{l}-1.222 \\
-1.222\end{array}$ & & $\begin{array}{l}52.641 \\
48.251\end{array}$ & 0.054 & $\frac{14.643}{14643}$ & 0.004 & $\begin{array}{l}74.00 \\
75.00\end{array}$ & $\begin{array}{l}01.23333 \\
0.125\end{array}$ & 60.8 & \begin{tabular}{|l|l|}
12.9 \\
11.8
\end{tabular} & \begin{tabular}{|l|}
51.9 \\
52.2
\end{tabular} & \begin{tabular}{|l|}
3.579 \\
3598
\end{tabular} & $\mid 0.008$ & 0.008 & 0.000 & & 0. \\
\hline 93 & & & & & & & & & & & & & & & & & & & 1.25 & & & & & 0.008 & & & 0.150 & 0.1 \\
\hline \begin{tabular}{|l|}
94 \\
\end{tabular} & & Averages & 25.9 & 23.0 & 25.3 & 24.8 & 25.6 & 62.0 & 62.1 & 23.0 & 39.1 & \begin{tabular}{|c|c|}
-1.0 \\
\end{tabular} & & 50.6 & $\begin{array}{ll} & \\
\end{array}$ & 14.6 & & & & 60.7 & 12.4 & & 3.48 & 0.008 & 0.008 & & 157 & \\
\hline 95 & & Maximum & 26.7 & 23.2 & 26.0 & 25.3 & 26 & 63.6 & 63.4 & 25.3 & 41.8 & $\begin{array}{l}0.4 \\
\end{array}$ & & 56.6 & 0.1 & 14.6 & & & & 62.0 & 13.9 & 52.7 & 3.635 & 0.011 & 0.011 & & 0.204 & \\
\hline 96 & & Median & 25.9 & 23.0 & 25.3 & 24.8 & 25.6 \# & 62.6 & 62.1 & 23.1 & 39.5 & $\begin{array}{r}-1.2 \\
\end{array}$ & & 50.7 & 0.1 & & & & & 60.6 & \begin{tabular}{|l|}
12.4 \\
\end{tabular} & & 3.520 & 0.008 & 0.008 & & 0.154 & \\
\hline 97 & & Minimum & 24.9 & 22.6 & 24.3 & 24.4 & $24.3 \mathrm{~A}$ & 56.5 & 60.7 & 21.2 & 35.7 & $\begin{array}{l}-1.2 \\
\end{array}$ & & 45.7 & 0.1 & $\begin{array}{l}14.6 \\
\end{array}$ & & & & 59.3 & 11.2 & 46.1 & 3.180 & 0.007 & 0.007 & 0.0002 & 0.150 & \\
\hline 98 & & $2 \times$ Std Dev & 1.080 & 0.367 & 1.114 & 0.482 & 0.779 & 3.771 & 0.910 & 1.710 & 3.075 & 0.975 & & 4.584 & 0.005 & 0.000 & 0.00 & & & 0.910 & 1.123 & 3.302 & 0.228 & 0.001 & 0.001 & 0.0000 & 0.021 & \\
\hline 99 & Number of & Points Used $\mathrm{d}^{\star}$ & 70 & 70 & 70 & 70 & & 70 & & 70 & & 70 & & 70 & 70 & & & & & 70 & 70 & 70 & 70 & 70 & 70 & & 70 & \\
\hline & & * Backpulse pc & nts in box & are noti & ncluded & & & & & & & & & & & & & & & & & & & & & & & \\
\hline$\frac{101}{102}$ & & & & & & & & & & & & & & & & & & & & & & & & & & & & \\
\hline$\frac{104}{103}$ & & & & & & & & & & & & & & & & & & & & & & & $\begin{array}{l}1.3 \text {-hour } \\
1 \text {-hour a }\end{array}$ & avg. = & \begin{tabular}{|l|}
0.0079 \\
0.0079 \\
\end{tabular} & & & \\
\hline
\end{tabular}


RUN \# 1.10; STEADY STATE TEST AT 25 WT\% UDS

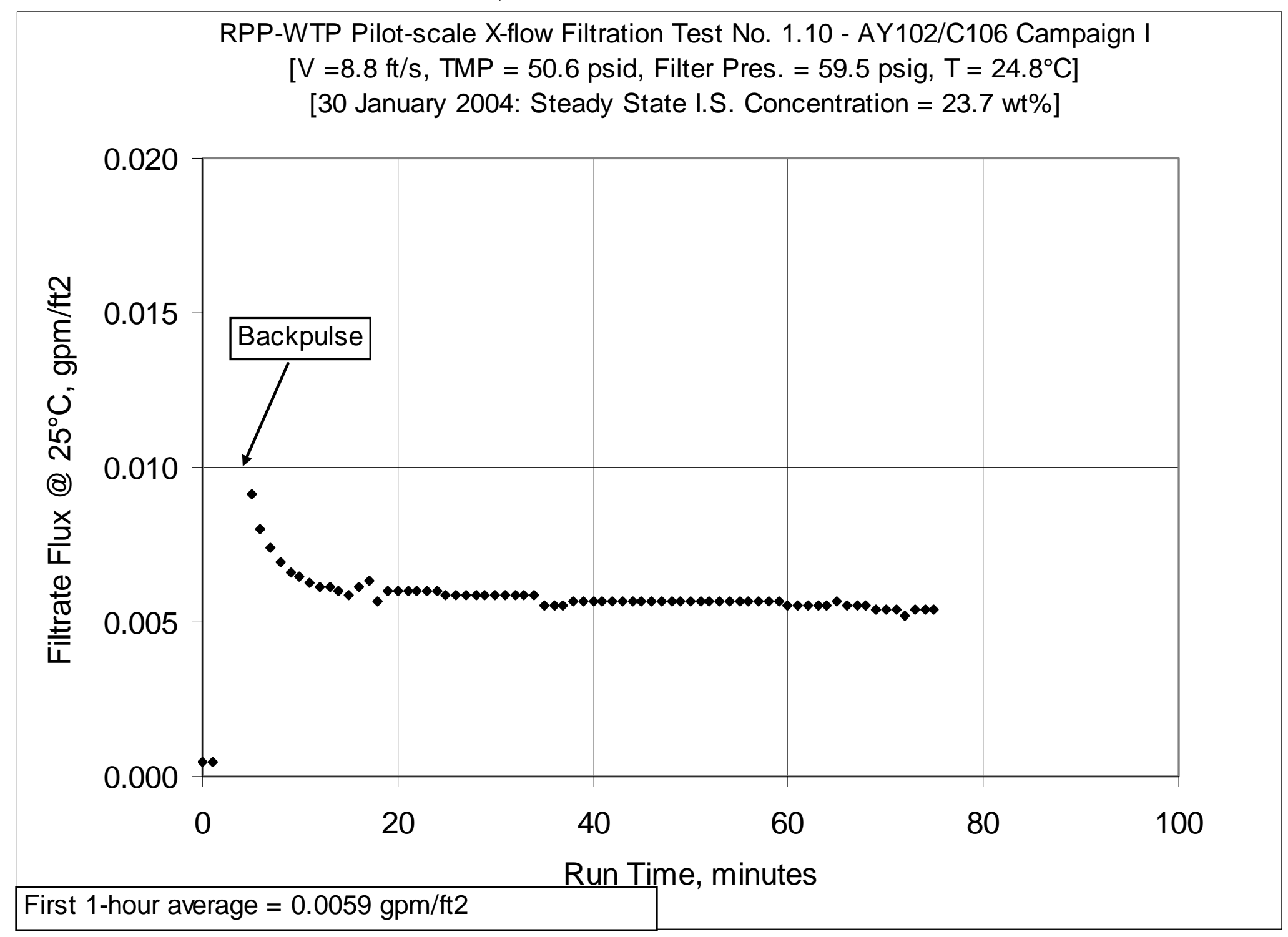


WSRC-TR-2005-00105, REVISION 0

SRNL-RPP-2005-00012, REVISION 0

RUN \# 1.10; STEADY STATE TEST AT 25 WT\% UDS - CONT.

\begin{tabular}{|c|c|c|c|c|c|c|c|c|c|c|c|c|c|c|c|c|c|c|c|c|c|c|c|c|c|c|c|c|}
\hline & A & B & D & $\frac{E}{K}$ & $F$ & G & $\mathrm{H}$ & J & $\mathrm{K}$ & $\frac{L}{\text { Presu }}$ & $\mathrm{M}$ & IN & 0 & $\mathrm{Q}$ & $\begin{array}{ll}\mathrm{R} \\
<\mathrm{El}\end{array}$ & $s$ & \begin{tabular}{|l}
1 \\
\end{tabular} & $\mathrm{v}$ & w & $x$ & & $\mathrm{Zz}$ & AA & AB & $\mathrm{AC}$ & $A D$ & $\mathrm{AE}$ & \\
\hline & DATE & TIME & Filtrate & $\begin{array}{l}\ll<\text { Temp } \\
\text { Cleaning }\end{array}$ & \begin{tabular}{|l|l|} 
erature Ilury \\
\end{tabular} & $\begin{array}{l}\text { Measurem } \\
\text { Hi Amb. }\end{array}$ & $\begin{array}{l}\text { ients } \gg \gg> \\
\text { Lo Amb }\end{array}$ & BotTMP & Filter & $\begin{array}{l}\text { Pressure } \\
\text { Filter dPP }\end{array}$ & $\begin{array}{l}\text { Measurem } \\
\text { ToptMP }\end{array}$ & 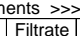 & Pulsepot & Slurry & $\begin{array}{l}\text { << Flow } \\
\text { Filtrate }\end{array}$ & $\begin{array}{l}\text { Neasurume } \\
\text { Hi Eiltate }\end{array}$ & & & & & & & & & & & & \\
\hline & & & $\operatorname{deg} C$ & $\operatorname{deg} \mathrm{C}$ & $\operatorname{deg} \mathrm{C}$ & \begin{tabular}{|l|}
$\operatorname{deg} C$ \\
\end{tabular} & & psid & psig & psid & & & & $\mathrm{gpm}$ & $\mathrm{gpm}$ & $\mathrm{gpm}$ & & & & & & & & & & & & \\
\hline & & & & & & & & $\mathrm{dP2}$ & & $\mathrm{dP1}$ & $\mathrm{dP3}$ & & & & & & & & & & & & & & & & & \\
\hline 5 & $20 \mathrm{~S} 012$ & 004 & & & & & & & & & & & & & & & & & & & & & & & & & & \\
\hline 7 & $\frac{e r s=-01 / 26 /}{1 / 30 / 2004}$ & $\begin{array}{l}004 \\
7: 55: 26 \text { AM }\end{array}$ & 23.13 & 17.795 & 14.599 & 23.739 & 14.969 & 0.05 & -0.06 & 0.008 & & -0.212 & 0.04 & -0.079 & 0.003 & \begin{tabular}{|l|l|}
14.643 \\
\end{tabular} & 0.005 & & & Filter S & Surface & 6.707 & FT2 & & & & & \\
\hline 8 & $1 / 30 / 2004$ & 7:55:43 AM & 23.13 & 17.795 & \begin{tabular}{|l|l|}
14.599 \\
\end{tabular} & 23.739 & 14.969 & 0.05 & -0.06 & 0.008 & & -0.212 & 0.04 & -0.079 & 0.003 & $\begin{array}{l}14.643 \\
\end{array}$ & 0.005 & & & Conver & rsion & 851 & $\mathrm{~m} 3 / \mathrm{m} 21$ & $/ d a y / b a r$ & $\mathrm{rg} / \mathrm{gpm} / \mathrm{ft}$ & fft2/barg & & \\
\hline 9 & $1 / 30 / 2004$ & 7:55:45 AM & 23.12 & 17.785 & \begin{tabular}{|l|l|}
14.599 \\
\end{tabular} & 23.664 & 14.989 & 0.048 & -0.06 & 0.008 & & -0.177 & 0.037 & -0.077 & $\begin{array}{ll}0.003 \\
\end{array}$ & $\begin{array}{l}14.643 \\
\end{array}$ & 0.004 & & & & & & & & & & & \\
\hline & $1 / 30 / 2004$ & 7:55:46 АM & 23.125 & 17.79 & 14.599 & 23.664 & 14.989 & 0.05 & -0.058 & & 0.002 & -0.206 & 0.04 & -0.083 & 0.003 & 14.643 & 0.00 & & & Note: & 140e & & & $\mathrm{nnne}$ & hately & 4 psig & & \\
\hline & $1 / 30 / 2004$ & 7:55:47 AM & & 17.795 & 14.599 & 23.659 & 14.989 & 0.05 & -0.06 & 0.008 & & \begin{tabular}{|c|c|}
-0.206 \\
\end{tabular} & 0.04 & \begin{tabular}{l|l|l|}
-0.077 \\
\end{tabular} & 0.003 & $\begin{array}{ll}14.643 \\
\end{array}$ & 0.005 & & & & & & & & & & & \\
\hline & $1 / 30 / 2004$ & 7:55:48 AM & 23.125 & & & 23.644 & 14.989 & 0.05 & -0.06 & 0.008 & & -0.174 & & -0.081 & 0.003 & 14.643 & & & & & & & $d e^{2}$ & 888 & & & & \\
\hline & & 7:55:49 AM & & & 14.599 & & & & -0.06 & & & -0.2 & & & 0.003 & 14.643 & & & & VWVV & & & & Filtra & & & & \\
\hline & $1 / 30 / 2004$ & 7:55:50 AM & 23.125 & 17.785 & 14.589 & 23.644 & 14.984 & 0.05 & -0.06 & 0.008 & & -0.189 & 0.04 & -0.081 & 0.003 & 14.643 & 0.005 & & & & & & & & at $25 \mathrm{C}$ & & $\times 1000$ & \\
\hline & tor Per & & & & & & & & & & & & & & & & & Time & Time & Press. & & TMP & TMP & $\mathrm{gpm}$ & & $m$ & & \\
\hline & $1 / 30 / 2004$ & une $8.15 \cdot 11 \mathrm{AM}$ & 24381 & 18168 & 23866 & 23788 & 19.606 & 44067 & 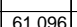 & 17.935 & 26.443 & 11.661 & 100.208 & 36136 & 0.003 & 14,643 & 0.004 & & & $\frac{39}{596}$ & 89 & (3513 & 2431 & 40.000 & 0,000 & $2 \mathrm{PSI}$ & 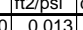 & 0 \\
\hline & $1 / 30 / 2004$ & 8:16:11 AM & 22.997 & 18.193 & & 23.893 & 19.747 \# & 4.022 & \begin{tabular}{|l|}
60.174 \\
\end{tabular} & 6.349 & -2.073 & 52.267 & $\frac{170.200}{57.328}$ & S0.150 & & $\begin{array}{l}14.045 \\
14.643\end{array}$ & 0.004 & 1.00 & 0.01667 & 58.7 & & \begin{tabular}{|l|}
53.5 \\
\end{tabular} & $\begin{array}{l}2.41 \\
0.067\end{array}$ & \begin{tabular}{|l|l|} 
\\
\end{tabular} & 0.000 & & \begin{tabular}{|ll}
0 & 0.474 \\
& 0.474
\end{tabular} & 0.40 \\
\hline & $1 / 30 / 2004$ & $8: 17: 11 \mathrm{AM}$ & \begin{tabular}{|l|l|}
24.23 \\
\end{tabular} & 18.239 & 24.051 & 23.863 & $19.954 \#$ & 49.036 & \begin{tabular}{|l|l|} 
& 59.747 \\
\end{tabular} & 8.37 & 40.864 & 5.633 & 0.451 & 39.263 & 0.655 & 14.643 & 0.004 & 2.0 & 0.03333 & 58.3 & 9. & & 3.099 & 0.098 & 0.100 & 0.002 & 2.232 & \\
\hline & $1 / 30 / 2004$ & $8: 18: 11 \mathrm{AM}$ & 24.556 & 18.28 & 24.122 & 23.863 & $19.964 \#$ & 54.617 & \begin{tabular}{|l|l|} 
& 60.41 \\
\end{tabular} & 10.893 & 43.79 & $\mid$\begin{tabular}{|l|l|} 
\\
\end{tabular} & 0.48 & 37.955 & 0.099 & 14.643 & 0.00 & 3.0 & 0.05 & 58.9 & 9.3 & 49.2 & 3.392 & .183 & 2.238 & & & \\
\hline & $1 / 30 / 2004$ & 8:19:11 AM & 24.707 & 18.331 & 24.273 & 23.929 & 20.136 \# & 54.756 & \begin{tabular}{|l|l|}
60.584 \\
\end{tabular} & 12.488 & $\begin{array}{l}42.309 \\
\end{array}$ & $\mid 0.228$ & 0.509 & 37.095 & 0.072 & \begin{tabular}{l|l}
14.643 \\
\end{tabular} & & & & 59. & & 48.5 & & & & & & \\
\hline & $1 / 30 / 2004$ & $8: 20: 11 \mathrm{AM}$ & 24.832 & 18.366 & 24.367 & 23.898 & 20.145 \# & 55.011 & \begin{tabular}{|l|l|}
60.729 \\
\end{tabular} & 14.213 & 40.852 & 0.248 & 0.529 & 36.837 & 0.06 & 14.643 & & 5.0 & 0.083 & 59.3 & & 47. & & 005 & & & & \\
\hline & $1 / 30 / 2004$ & $8: 21: 11 \mathrm{AM}$ & 24.922 & 18.422 & 24.433 & 23.934 & 20.357 \# & 55.243 & 60.928 & 15.466 & 39.846 & 0.234 & 0.046 & 36.38 & 0.053 & 14.643 & 0.004 & 6.00 & & 59.5 & & 47.5 & & & & & 0.169 & \\
\hline & $1 / 30 / 2004$ & 8:22:11 AM] & & & 24.558 & & & & & & 39.29 & 0.257 & & & 0.049 & 14.643 & & & 0.11667 & & & & 3.266 & & & & & \\
\hline & & 8:23:11 AM & & & 24.648 & 23.958 & & & & 16.987 & & & & & 0.046 & 14.643 & & 8.0 & & & & & & & & & & \\
\hline & $1 / 30 / 2004$ & 8:24:11 AM & 25.122 & 18.563 & 24.728 & 23.973 & $20.931 \mid \#$ & 55.502 & 61.308 & 17.362 & 38.198 & 0.26 & 0.58 & 35.648 & 0.044 & 14.643 & & 9.0 & & 59.8 & & & 0.200 & & & & 141 & \\
\hline & 113012004 & $8.25: 11$ AM & 25.1927 & 18.609 & 24.134 & 24.019 & 21.060 /\# & $\begin{array}{c}5.5653 \\
55.639\end{array}$ & 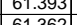 & $\frac{11.181}{11252}$ & $\frac{37.979}{32215}$ & 0.28 & & 35.55 & 0.043 & 14.643 & & & \begin{tabular}{|l}
0.16661 \\
.19232
\end{tabular} & & & & & . & & & & \\
\hline & $1 / 13 / 2004$ & 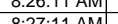 & $\frac{25.241}{2523}$ & $\begin{array}{l}18.654 \\
18695\end{array}$ & \begin{tabular}{|l|l|l|l|} 
\\
\end{tabular} & 24.029 & $2.111+4$ & 55.538 & 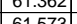 & $\begin{array}{l}17.252 \\
17007\end{array}$ & $\begin{array}{l}38.315 \\
37.827\end{array}$ & $\begin{array}{l}0.280 \\
0.020\end{array}$ & 0 & 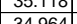 & 0.044 & $\begin{array}{l}14.045 \\
114643\end{array}$ & & 11.00 & 0.18333 & S6.9. & 8.6 & & $\frac{3.350}{3205}$ & 0.000 & 0.000 & & $\mid$ & 0.1 \\
\hline 30 & $1,30 / 2004$ & $8 \cdot 28 \cdot 11 \mathrm{AM}$ & $\begin{array}{r}25.352 \\
25388\end{array}$ & 18751 & 24.054 & 24.03 & 20.81037 & 55.1309 & \begin{tabular}{|l|l|}
61726 \\
\end{tabular} & $\frac{11.901}{17.778}$ & 31.021 & o. & 0.024 & 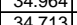 & 0.0041 & $\begin{array}{l}14.045 \\
114643\end{array}$ & & 12.00 & 10.2 & $\frac{60.1}{60.3}$ & $\frac{8.6}{8.5}$ & 460 & 3.245 & \begin{tabular}{|l|l|l|} 
\\
\end{tabular} & 0.0006 & 0 & 0.131 & 0.1 \\
\hline & $1 / 130 / 2004$ & $\frac{0.20 .11 \mathrm{AV}}{8.29 \cdot 11 \mathrm{AM}}$ & $\begin{array}{r}5.300 \\
25.442\end{array}$ & $\frac{10.151}{18.801}$ & 24.919 & 24.014 & $\frac{21.001}{21.263}$ & 5 & \begin{tabular}{|l|l|l|l|l|}
61.559 \\
\end{tabular} & 17.775 & $\begin{array}{l}30.020 \\
37.788\end{array}$ & 0.327 & & 3.1.150 & 0.044 & 14643 & & 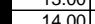 & 0.2333 & 0.0 & 0.4 & 46. & | & \begin{tabular}{|l|l|l|} 
\\
\end{tabular} & 0.006 & & & 0.1 \\
\hline & $1 / 30 / 2004$ & $8: 30: 11 \mathrm{AM}$ & 25.483 & 18.842 & 24.809 & 24.07 & $21.44 \#$ & 56.193 & \begin{tabular}{|l|l|}
62.037 \\
\end{tabular} & 18.374 & 37.753 & 0.327 & 0.653 & 34.411 & 0.039 & 14.643 & & 15.00 & 0.25 & 60.6 & 8.4 & 470 & 332 & 0.006 & 0.006 & 0.000 & 0124 & 0.1 \\
\hline & 1/30/2004 & $1: 11 \mathrm{AM}$ & & & 24.779 & 24.095 & $21.525 \mathrm{\#}$ & & 61.753 & & $43.50 \Omega$ & -1.216 & & & & & & 16.00 & 6667 & & 8. & & & & & & & \\
\hline 34 & $1 / 30 / 2004$ & $2: 11 \mathrm{AM}$ & 24.862 & & 24.764 & & & & \begin{tabular}{|l|l|}
61.559 \\
\end{tabular} & \begin{tabular}{|l|l|}
18.64 \\
\end{tabular} & & & & 34.146 & & & & $17.0 \mathrm{c}$ & & 60.1 & 8.4 & 52 & 3.608 & 006 & 0.006 & & & \\
\hline 35 & $1 / 30 / 2$ & $8: 33: 11 \mathrm{AM}$ & \begin{tabular}{l|l|}
24.886 \\
\end{tabular} & & 24.798 & \begin{tabular}{|l|l|}
24.184 \\
\end{tabular} & 21.624|\#| & 192 & & 23.508 & & -1.216 & & & 0.038 & 14.643 & & 18.00 & 0.3 & 58.6 & 9 & 48. & 3.335 & 0.006 & 0.006 & & 0.118 & \\
\hline & & $34: 11 \mathrm{AM}$ & & & 24.849 & & & & & & & & & & 0.04 & & & & & 58. & & & 3.480 & & & & 119 & \\
\hline 37 & $1 / 30 / 2004$ & $8: 35: 11 \mathrm{AM}$ & 24.932 & 19.059 & \begin{tabular}{|l|l|l|}
24.844 \\
\end{tabular} & 24.225 & $21.509 \#$ & 60.61 & \begin{tabular}{|l|l|}
60.39 \\
\end{tabular} & 20.486 & 40.162 & -1.216 & & 36.76 & 0.04 & 14.6 & & 20.00 & \begin{tabular}{|l|l|l}
0.33333 \\
\end{tabular} & $\frac{58.9}{58.9}$ & $\overline{9.6}$ & 50.4 & 3.474 & \begin{tabular}{|l|l}
0.006 \\
\end{tabular} & $\frac{0.006}{0.006}$ & & 0.119 & \\
\hline 38 & $1 / 30 / 2004$ & $8: 36: 11 \mathrm{AM}$ & & & 24.849 & 24.194 & & & & 20.1 & & & & 36.566 & 0.04 & & & 21.8 & 0.35 & 59.1 & 9. & 50.7 & 3.495 & & & & & \\
\hline & $1 / 30 / 2004$ & $8: 37: 11 \mathrm{AM}$ & 24.936 & 19.134 & 24823 & 24.108 & $21.649 \#$ & 61.45 & \begin{tabular}{|l|}
61.262 \\
\end{tabular} & & & \begin{tabular}{|c|c|}
-1.219 \\
\end{tabular} & & 36.132 & 0.04 & & & & 0.36667 & 59.8 & & $\overline{51.3}$ & 3.540 & \begin{tabular}{|l|l|}
0.006 \\
\end{tabular} & & & 0.117 & \\
\hline 40 & $1 / 30 / 2004$ & $8: 38: 11 \mathrm{AM}$ & 24.94 & $\begin{array}{l}19.174 \\
\end{array}$ & \begin{tabular}{|l|l|l|}
24.802 \\
\end{tabular} & 24.203 & $21.593 \#$ & \begin{tabular}{|l|l|}
61.05 \\
\end{tabular} & \begin{tabular}{|l|l|l|}
60.833 \\
\end{tabular} & 18.9 & & $\begin{array}{l}3 \\
3\end{array}$ & & 35.554 & 0.04 & $\begin{array}{l}14.643 \\
\end{array}$ & & 23.00 & 0.38333 & 59.4 & 8.7 & 51.5 & 3.553 & \begin{tabular}{|l|l|} 
\\
\end{tabular} & $\begin{array}{l}0.006 \\
\end{array}$ & & \begin{tabular}{|c|}
0.116 \\
\end{tabular} & \\
\hline 41 & 1/301/2004 & $8: 39: 11 \mathrm{AM}$ & 24.949 & 19.213 & 24.811 & \begin{tabular}{l|l}
24.167 \\
\end{tabular} & $21.577 \|$ & 61.492 & \begin{tabular}{|l|l|}
61.353 \\
\end{tabular} & 18.8 & & -1.216 & & 34.841 & 0.04 & & & 24. & 0.4 & 59.9 & & 52.6 & 3.583 & & & & $\mid$ & \\
\hline 42 & $1 / 30 / 2004$ & $8: 40: 11 \mathrm{AM}$ & 24.953 & 19.242 & 24.815 & 24.21 & & 60.864 & \begin{tabular}{|l|l|}
60.696 \\
\end{tabular} & 19.8 & & $\begin{array}{l}9 \\
9\end{array}$ & & & & & & & 0.41667 & 59.2 & 8.8 & 50.9 & 3.509 & D006 & | & & 0.115 & \\
\hline & $30 / 2004$ & 8:41:11 AM] & 24.952 & 19.272 & 24.815 & 24.255 & & 61.328 & \begin{tabular}{|l}
61.279 \\
\end{tabular} & 20.305 & & & & 36. & & & & 26. & & 59.8 & 0.8 & ol. & & & & & 114 & \\
\hline 44 & & 8:42:11 AM & & & 24.844 & & & & & & 41.435 & -1.216 & & & & & & & 0.45 & 59.6 & 8.8 & 51.3 & & & & & 0.114 & \\
\hline 45 & & 8:43:11 AM & 24.986 & 19.356 & 24.838 & 24.259 & 21.8 & & & & & -1.216 & & & & & & & 0.46667 & 59.9 & 8.8 & 51.5 & 3.553 & 006 & & & 113 & \\
\hline 46 & $1 / 3012004$ & 8:44:11 AM & 24.985 & 19.386 & 24.802 & 24.308 & 22.31 & & 60.976 & & & -1.216 & & 35.485 & & & & 29.6 & 0.48333 & 59.5 & 0. & 51.1 & 3.526 & & & & 0.114 & \\
\hline & $1 / 30 / 2004$ & $8: 45: 11 \mathrm{AM}$ & 24.989 & 19.415 & 24.182 & 24.302 & & & & & & & & 35.596 & & & & & & 59.5 & 8. & & 3.555 & & & & & \\
\hline & $1 / 3012004$ & $8.46 .11 \mathrm{AM}$ & 25.009 & 19.465 & 24.831 & 24.372 & 22.50 & 6..09 & & & & -1.216 & & 35 & & & & 31. & 0.510621 & 59.4 & 8. & 51.4 & 3.545 & & & & & \\
\hline 50 & $1 / 13 / 2004$ & 8.47 .11 AM & $\mid 20.028$ & $\begin{array}{r}19.5 \\
19.50\end{array}$ & 24.851 & $\begin{array}{l}24.382 \\
24365\end{array}$ & 22.00 & | & & & 41.872 & -1.2 & & $\begin{array}{l}35.445 \\
32476\end{array}$ & & & & & 0.53333 & (59.6 & & & 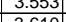 & & & & \begin{tabular}{|l|l|l|l|} 
\\
\end{tabular} & \\
\hline & $1 / 30 / 2004$ & 8.48 .1 .14 Alv & 2502071 & $\begin{array}{l}19.359 \\
1959\end{array}$ & 24.035 & $\frac{24.305}{24.12}$ & $\frac{2.34}{22.41}$ & $\mid 0.2025$ & \begin{tabular}{|c|}
61.93 \\
6123
\end{tabular} & 19.6 & $\begin{array}{l}42.08 \\
12.077\end{array}$ & $\frac{-1.2}{1.2}$ & & | & & & & . & 0.53 & 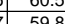 & & 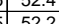 & 步 & & & & 0.1 .112 & \\
\hline & $\frac{1 / 10 / 2004}{1 / 30 / 2004}$ & $8.450 .11 \mathrm{AM}$ & $\frac{\mid 2.0504}{2504}$ & & 24.014 & 24.444 & & & & & & & & & & & & & 0.50033 & & & & & & & & & \\
\hline & $1 / 30 / 2004$ & $8.51 .11 \mathrm{AM}$ & 250.044 & 19642 & 24. & 24.4 & 2266 & & & 20 & 39.906 & $\frac{1.2}{6}-12$ & & & & & & 36 & 0.000 & 59 & & & & & & & n110 & \\
\hline 54 & & $8.52 \cdot 11 \mathrm{Al}$ & & & & & & & & & & & & & & & & & 0.61667 & & & & & & & & & \\
\hline 55 & $1 / 30 / 2$ & $8: 53: 11 \mathrm{AM}$ & 25.052 & & & 24.485 & & & & & & & & 36.5 & & & & & & 59.4 & & & & & & & & \\
\hline & & $8 \cdot 54 \cdot 11 \mathrm{~A}$ & & & 224873 & 24,489 & & & & & & & & & & & & & & & & & & & & & & \\
\hline 57 & & $8: 55 \cdot 11 \mathrm{AM}$ & 25.059 & 19753 & 24.871 & 24.5 & & & 60 & 20.2 & & -1.216 & & 36.611 & & & & & 0.66 & 59.3 & & & & & & & & \\
\hline & $1 / 30 / 2004$ & $8.56 \cdot 11 \mathrm{AM}$ & 25077 & 19.792 & 2487 & & 22.976 & 60 & 60. & & 40,6 & -12 & & 36 & & & & & 068 & & & & & & & & & \\
\hline & $1 / 30 / 2004$ & $8.57 \cdot 11 \mathrm{AM}$ & 25075 & 19.816 & 24.853 & 24.514 & 233 & 60834 & 60 & 19 & & -12 & & 36.68 & & $\begin{array}{l}14,643 \\
\end{array}$ & & & 0.7 & 59.2 & & & 3.499 & & & & 0.112 & \\
\hline 60 & $1 / 30 / 2004$ & $8: 58: 11 \mathrm{AM}$ & 25.083 & 19.844 & 24.8 & 24.507 & 23.24 & 60.702 & & 20.0 & 40.5 & -1.2 & & $\overline{36.4}$ & & 14.6 & & 43 & 0.716 & 59.0 & & & & & & & 0.112 & \\
\hline 61 & $1 / 30 / 2004$ & $8: 59: 11 \mathrm{AM}$ & 25.097 & $\begin{array}{l}9.898 \\
\end{array}$ & 24.89 & 24.566 & 23.30 & 60.681 & \begin{tabular}{|l|l|}
60.615 \\
\end{tabular} & 19.9 & 40.7 & -1.214 & & 36.4 & & 14.6 & & 44. & 0.73333 & 59.1 & 1 & 50.7 & 3.497 & & & & 0.112 & \\
\hline 62 & $1 / 30 / 2004$ & 9:00:11 AM & 25.1 & 19.917 & & & & & 60 & $19 . \varepsilon$ & 40. & -1.2 & & 36. & & & & & & & & & 3.504 & & & & 0.112 & \\
\hline & $1 / 30 / 2004$ & $9: 01: 11 \mathrm{AM}$ & 25.114 & 19.951 & 24.882 & 24.538 & 23.07 & & 60.667 & 19.782 & & -1.216 & & 36.203 & & & & & 0.76667 & 59.2 & 2 & & 3510 & & 0.006 & & 0.112 & \\
\hline 64 & $1 / 30 / 2004$ & 9:02:11 AM & 25.128 & & & 24.572 & 23.297 \# & 007 & \begin{tabular}{|l|l|}
60.841 \\
\end{tabular} & & $\begin{array}{l}41.248 \\
\end{array}$ & & & & & & & 47.00 & & 59.4 & & 51 & & & 0.006 & & 0.111 & \\
\hline 65 & $1 / 30 / 2004$ & $9: 03: 11 \mathrm{AM}$ & 25.126 & 20.028 & 24.864 & 24.61 & $23.18 \| \#$ & 60.679 & \begin{tabular}{|l|l|}
60.574 \\
\end{tabular} & 19.421 & 41.26 & $\begin{array}{l}6 \\
6\end{array}$ & -5.565 & 36.126 & 0.038 & 14.643 & & 48.00 & & 59.1 & & & 3.514 & & 0.006 & .000 & 112 & \\
\hline
\end{tabular}


WSRC-TR-2005-00105, REVISION 0

SRNL-RPP-2005-00012, REVISION 0

RUN \# 1.10; STEADY STATE TEST AT 25 WT\% UDS - CONT.

\begin{tabular}{|c|c|c|c|c|c|c|c|c|c|c|c|c|c|c|c|c|c|c|c|c|c|c|c|c|c|c|c|c|}
\hline & A & B & $\begin{array}{ll}19 \\
\end{array}$ & $E$ & $F$ & G & $\mathrm{H} \mid \mathrm{I}$ & \begin{tabular}{l|l|l|}
$\mathrm{J}$ & \\
\end{tabular} & $\mathrm{K}$ & $\begin{array}{ll}\mathrm{L} \\
\end{array}$ & \begin{tabular}{l|l|} 
M \\
\end{tabular} & $\mathrm{N}$ & \begin{tabular}{l|l}
$\mathrm{O}$ & $\mathrm{F}$ \\
\end{tabular} & Q & $R$ & s & $\begin{array}{l}T^{\prime} \\
\end{array}$ & $\mathrm{V}$ & w & $x$ & & $z$ & $\mathrm{AA}$ & $\mathrm{AB}$ & $\mathrm{AC}$ & $A D$ & $\mathrm{AE}$ & AF $A$ \\
\hline \begin{tabular}{l|l}
66 \\
67
\end{tabular} & $1 / 30 / 2004$ & 9:04:11 AM & \begin{tabular}{|l|}
25.135 \\
25143
\end{tabular} & \begin{tabular}{|l|l|}
20.057 \\
2009
\end{tabular} & $\begin{array}{l}24.872 \\
2895\end{array}$ & 24.634 & 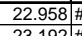 & $\begin{array}{ll}60.864 \\
60627\end{array}$ & \begin{tabular}{|l|}
60.626 \\
60523
\end{tabular} & \begin{tabular}{|l|l|}
19.573 \\
\end{tabular} & 41.248 & \begin{tabular}{|l|l|}
-1.219 \\
\end{tabular} & -5.562 & 36.153 & 0.038 & 14.643 & 0.004 & 49.00 & 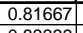 & 59.2 & $\begin{array}{l}8.9 \\
9.7\end{array}$ & 51.1 & 3.520 & \begin{tabular}{|l|l|}
0.006 \\
\end{tabular} & 0.006 & 0.000 & 0.111 & 0.09 \\
\hline$\frac{67}{68}$ & $\begin{array}{l}1 / 30 / 2004 \\
1 / 30 / 2004\end{array}$ & $\begin{array}{l}9: 05: 11 \mathrm{AM} \\
9006\end{array}$ & $\left|\begin{array}{|c|}25.143 \\
25.151\end{array}\right|$ & $\frac{20.08}{20.114}$ & $\frac{24.885}{24.894}$ & $\begin{array}{r}24.662 \\
24.69 \\
\end{array}$ & $\frac{\left.23.192\right|^{7}}{23.211}$ & $\frac{60.627}{61.479}$ & $\begin{array}{r}\mid 6.533 \\
61.37 \\
\end{array}$ & \begin{tabular}{r|r|r|}
19.2 .29 \\
19.439
\end{tabular} & $\begin{array}{l}41.502 \\
41.767\end{array}$ & \begin{tabular}{|c|}
-1.219 \\
-1211 \\
\end{tabular} & $\begin{array}{l}-5.562 \\
5.56\end{array}$ & \begin{tabular}{|r|r|r|}
35.64 \\
35.539
\end{tabular} & $\mid 0.038$ & $\begin{array}{l}14.643 \\
14643\end{array}$ & $\frac{0.004}{0.004}$ & 50.00 & \begin{tabular}{|c|c|c|}
0.83333 \\
\end{tabular} & $\begin{array}{l}59.1 \\
59.9 \\
\end{array}$ & $8.7 \mid$ & 51.1 & \begin{tabular}{|c|}
3.521 \\
3.599 \\
\end{tabular} & \begin{tabular}{|l|l|}
0.006 \\
\end{tabular} & 0.006 & & 0.111 & 0.09 \\
\hline 69 & $1 / 30 / 2004$ & $9: 07: 11 \mathrm{AM}$ & 25.159 & 20.142 & 24.907 & 24.693 & 23.484 & 61.087 & 61.043 & $\begin{array}{l}18.436 \\
\end{array}$ & 42.621 & -1.211 & -5.554 & 35.241 & 0.038 & 14.643 & 0.004 & 52.00 & 0.06667 & 59.6 & 8.6 & & & 0.000 & 0.006 & & & 0.09 \\
\hline & $1 / 30 / 2004$ & & 25.162 & 20.171 & 24.86 & 24.716 & $23.237{ }^{\#}$ & 61.395 & \begin{tabular}{|c|}
61.217 \\
\end{tabular} & 18.627 & & -1.216 & & \begin{tabular}{|l|l|}
35.043 \\
\end{tabular} & & $\begin{array}{l}14.643 \\
\end{array}$ & 0.003 & & 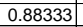 & $\begin{array}{ll}99.7 \\
\end{array}$ & 8.6 & & & & & & & \\
\hline & $1 / 30 / 2004$ & 9:09:11 AM & $\begin{array}{l}25.17 \\
\end{array}$ & 20.204 & 24.862 & 24.674 & $23.299 \pi$ & 61.444 & \begin{tabular}{|l|l|}
61.299 \\
\end{tabular} & 18.945 & 42.391 & -1.214 & -5.554 & 35.08 & & 14.643 & 0.004 & 54.00 & & 59.8 & 8.6 & & & & & & & \\
\hline 72 & & 9:10:11 AM & & & 24.861 & & & & & & & & & & & & & & & & & & & & & & & \\
\hline & $1 / 30 / 2004$ & $9: 11: 11 \mathrm{AM}$ & & 20.261 & 24895 & 24.771 & $23892+$ & 61.426 & 61.295 & 19238 & 42.175 & -1.214 & -5.554 & 24.076 & & 14.643 & & & & & & & & & & & & \\
\hline 74 & $1 / 30 / 2004$ & 9:12:11 AM & 25.189 & 20.279 & 24.862 & 24.789 & $23.87 \|$ & 61.579 & 61.515 & 19.02 & |42.344 & 1.222 & 5.56 & 35.087 & |038 & 14.643 & & 00 & 0.95 & 60.0 & 8.6 & 52.0 & 3.583 & 006 & $\mid$ & $\overline{000}$ & .109 & \\
\hline & $1 / 30 / 2004$ & 9:13:11 AM & 25.192 & 20.312 & 24.845 & 24.747 & $23.532 \mid \#$ & 519 & 61.422 & 19.172 & 229 & 1.222 & & 0.00 & & 14.6 & & & 0.96667 & & & & & & & & & \\
\hline 76 & $1 / 30 / 2004$ & 9:14:11 AM & 25.2 & 20.33 & 24.823 & 24.799 & $23.49 \mid \pi$ & 61.218 & \begin{tabular}{|c|}
61.028 \\
\end{tabular} & 19.236 & 42.067 & \begin{tabular}{|l|l|l|l|}
-1.219 \\
\end{tabular} & -5.55 & 34.928 & & 14.643 & 5.004 & 59.00 & 0.98333 & 59.6 & 8.6 & & 561 & 5.006 & $\mid .006$ & & D.110 & \\
\hline 77 & $1 / 30 / 2004$ & 9:15:11 AM & 25.218 & 20.364 & 24.841 & 24.803 & $23.984 \mid \#$ & 61.395 & 61.341 & 18.982 & 42.333 & -1.222 & -5.559 & 35.099 & 0.037 & 14.643 & 0.003 & 60.00 & & 59.9 & 8.6 & 51.9 & 3.576 & \begin{tabular}{|l|l|}
0.006 \\
\end{tabular} & 0.006 & & 0.107 & \\
\hline 78 & 1/30/2004 & $9: 16: 11 \mathrm{AM}$ & 25.22 & 20.396 & 24.848 & 24.805 & $24.287 \mid \#$ & 61.241 & 61.067 & 18.981| & 42.173 & -1.219 & -5.559 & 35.222 & 0.037 & 14.643 & 0.004 & 61.00 & 1.01667| & 59.6 & 8.6 & & 3.565 & 0.006 & & & 0.107 & \\
\hline 79 & $1 / 30 / 2004$ & 9:17:11 AM & 25.233 & 20.434 & 24.871 & 24.778 & $24.425 \mid \#$ & 61.181 & 61.015 & 19.244 & 41.937 & 1.216 & -5.556 & 35.372 & 0.037 & 14.643 & 0.004 & 62.00 & 1.03333 & 59.5 & 8.7 & 51.6 & 3.555 & 0.006 & .0006 & 0.000 & 0.107 & \\
\hline & $1 / 30 / 2004$ & 9:18:11 AM & 25.237] & 20.458 & 4.87 & 24.846 & $24.558 \mid \#$ & 61.397| & 61.329 & 19.133 & 42.03 & -1.216 & & 3.372 & & 14.643 & & 63.00 & 1.05 & 59.9 & & & & & & & & \\
\hline & $1 / 30 / 2004$ & $9: 19: 11 \mathrm{AM}$ & 25.24 & & & 24.889 & 24.566 & & 61.244 & & 2.247 & & & 34.947 & & 14.643 & & & & 59.8 & & & & & & & & \\
\hline 82 & $1 / 30 / 2004$ & 9:20:11 AM & 25.247 & 20.0359 & 24.85 & 24.907 & 24.53447 & 61.208 & 61.088 & & 42.578 & -1.219 & -5.55 & $\begin{array}{l}3.859 \\
2.87\end{array}$ & & 14.643 & & & .08333 & 59.6 & 8.5 & & $3.5 / 8$ & & .006 & & .110 & \\
\hline & $\frac{1 / 3012004}{11202}$ & $9: 21: 11 \mathrm{AM}$ & 2 & 20.53 & 24.849 & 24.911 & $24.59 / 7$ & 61.299 & $\mid 61.25$ & $\frac{18.869}{1.857}$ & $\frac{42.298}{4.225}$ & - & 5.55 & $\frac{3.010}{2.782}$ & & $\frac{1.045}{1.642}$ & & $\frac{66}{67}$ & $\frac{1.1}{107}$ & 5.8 .8 & & t5. & (35) & & & & 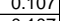 & \\
\hline & $\frac{11503004}{1312004}$ & 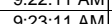 & & & & $\frac{24.510}{24961}$ & $\frac{24.001+1}{20202 t}$ & $\frac{61.365}{61.496}$ & & $\frac{18.857}{18904}$ & & & & $\frac{34.882}{34814}$ & & $\frac{14.643}{14643}$ & & & & & & & & & & & & \\
\hline 8 & $\frac{170004}{1 / 30 / 2004}$ & 9:24:11 AM & $\frac{25.240}{25.25}$ & 20.688 & $\frac{24.053}{24893}$ & 24.995 & $\frac{24.002 \pi}{24.351 \#}$ & & $\begin{array}{l}60.5744 \\
6.45\end{array}$ & $\frac{10.504}{20.602}$ & $\frac{4.475}{39.828}$ & $\begin{array}{l}-1.214 \\
-1.214\end{array}$ & -5.55 & $\frac{34.014}{36.753}$ & & $\frac{14.045}{14.643}$ & & $\frac{68.00}{69.00}$ & $\begin{array}{l}1.15005 \\
1.15\end{array}$ & $\begin{array}{l}59.8 \\
59.1\end{array}$ & & & & & & & $\begin{array}{l}01 \\
07 \\
\end{array}$ & \\
\hline & $1 / 30 / 2004$ & 9:25:11 AM & 25.242 & 20.701 & 22845 & 24.992 & $24.058 \#$ & 60.389 & 60.416 & 20.825 & 39.577 & -1.219 & -5.559 & 36.651 & & 14.643 & & 70.00 & $\mid$\begin{tabular}{|l|}
1.16667 \\
\end{tabular} & 58.9 & 9.0 & & & & & & 0.108 & \\
\hline & $1 / 30 / 2004$ & $9: 26: 11 \mathrm{AM}$ & 25.255 & 20.744 & 24.883 & 24.98 & $24.286 \#$ & & 60.916 & 20.547 & 40.242 & \begin{tabular}{|l|l|}
-1.219 \\
\end{tabular} & & 36.574 & & 14.643 & & 71.00 & 1.18333 & 59.4 & 9.0 & 50.6 & 3.487 & \begin{tabular}{|l|}
0.005 \\
\end{tabular} & 0.005 & 0.000 & 0.106 & \\
\hline & $1 / 30 / 2004$ & 9:27:11 AM & 25.262 & 20.776 & 24.906 & 25.027 & $24.429 \| \#$ & 60.567 & 60.555 & 20.669 & & \begin{tabular}{|l|}
-1.216 \\
\end{tabular} & -5.626 & 36.906 & & 14.6 & & 00 & 1.2 & 59.1 & 9.0 & 50.2 & 3.459 & \begin{tabular}{|l|l|}
0.005 \\
\end{tabular} & \begin{tabular}{l|l}
0.005 \\
\end{tabular} & & 0.104 & \\
\hline & $1 / 30 / 2004$ & 9:28:11 AM & 25.275 & 20.799 & 24.908 & 25.02 & $24.326 \#$ & 60.416 & 60.385 & 20.749 & 39.641 & \begin{tabular}{|l|l|}
-1.222 \\
\end{tabular} & & 36.941 & & & & 73.00 & \begin{tabular}{|l|l|}
1.21667 \\
\end{tabular} & 58.9 & 9.1 & & & & & & & \\
\hline & 1/30/2004 & $9: 29: 11 \mathrm{AM}$ & 25.298 & 20.827 & & 24.897 & $24.029 \mid \#$ & & 60.371 & 21.299 & 39 & \begin{tabular}{|l|l|} 
& -1.219 \\
\end{tabular} & & 36.939 & & 14.643 & 0.004 & 74.00 & & 58.9 & 9.1 & 49.9 & 3.441 & 0.005 & 0.005 & & 0.108 & \\
\hline 92 & $1 / 30 / 2004$ & 9:30:11 AM & 25.316 & 20.855 & 24.924 & 24.835 & $24.122 \#$ & 60.289 & $\begin{array}{l}60.164 \\
\end{array}$ & 21.083 & 39.327 & \begin{tabular}{|l|l|} 
\\
\end{tabular} & -5.629 & 37.035 & 0.036 & 14.643 & & 75.00 & 1.25 & 58.7 & 9.1 & 49.8 & 3.434 & 0.005 & 0.005 & & 0.108 & 0.09 \\
\hline 94 & & Averages & & 19.7 & & 245 & $22.8 \| \pi$ & & \begin{tabular}{ll|}
61.0 \\
\end{tabular} & & 40.8 & -1.0 & & & & 14.6 & & & & 59.5 & 8.8 & & 3.485 & & & & 115 & \\
\hline & & Maximum & & 20.9 & 24.9 & 25.0 & & & 62.0 & 2355 & 43.5 & 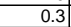 & & & & 14.6 & & & & 606 & 9.5 & 527 & 3.635 & & & 0.0002 & 0.169 & \\
\hline$\sqrt{6}$ & & Median & 25.1 & 19.8 & 24.8 & 24.5 & $23.0 \#$ & 60.9 & 61.0 & 19.2 & 41.1 & -1.2 & -5.8 & 35.6 & 0.0 & 14.6 & 0. & & & 59.5 & 8.7 & 51.0 & 3.517 & \begin{tabular}{|l|l|}
0.006 \\
\end{tabular} & 0.006 & 0.0001 & 0.112 & 0.095 \\
\hline & & Minimum & 24.8 & 18.4 & 24.4 & 23.9 & $20.4 \|$ & 55.2 & 60.1 & 15.5 & 36.5 & -1.2 & & & 0.0 & 14.6 & & & & 58.6 & 8.4 & 46.7. & 3.219 & & 0.005 & 0.0001 & 0.104 & \\
\hline & & $2 \times$ Std Dev & 0.280 & 1.383 & 0.151 & 0.650 & $2.386 \#$ & 3.890 & 0.878 & 2.467 & 3.125 & 1.052 & 4.313 & 1.746 & 0.006 & 0.06 & & & & 0.878 & 0.428 & 298 & 0.227 & 0.001 & 0.001 & . 00000 & 0.023 & \\
\hline & Number of & Points Uned & 107 & 70 & 70 & 70 & $70 \mid \#$ & 70 & 70 & & 70 & 70 & $70[\mathrm{C}$ & 70 & 70 & 70 & 70 & & & 70 & 70 & 70 & 70 & 70 & 70 & 70 & 70 & 0 \\
\hline & & se pc & in box & re not in & clluded & & & & & & & & & & & & & & & & & & & & & & & \\
\hline 102 & & & & & & & & & & & & & & & & & & & & & & & & & 658 & & & \\
\hline$\sqrt{3}$ & & & & & & & & & & & & & & & & & & & & & & & & y. $=$ & .0059 & & & \\
\hline
\end{tabular}


RUN \# 1.11; STEADY STATE TEST AT 25 WT\% UDS

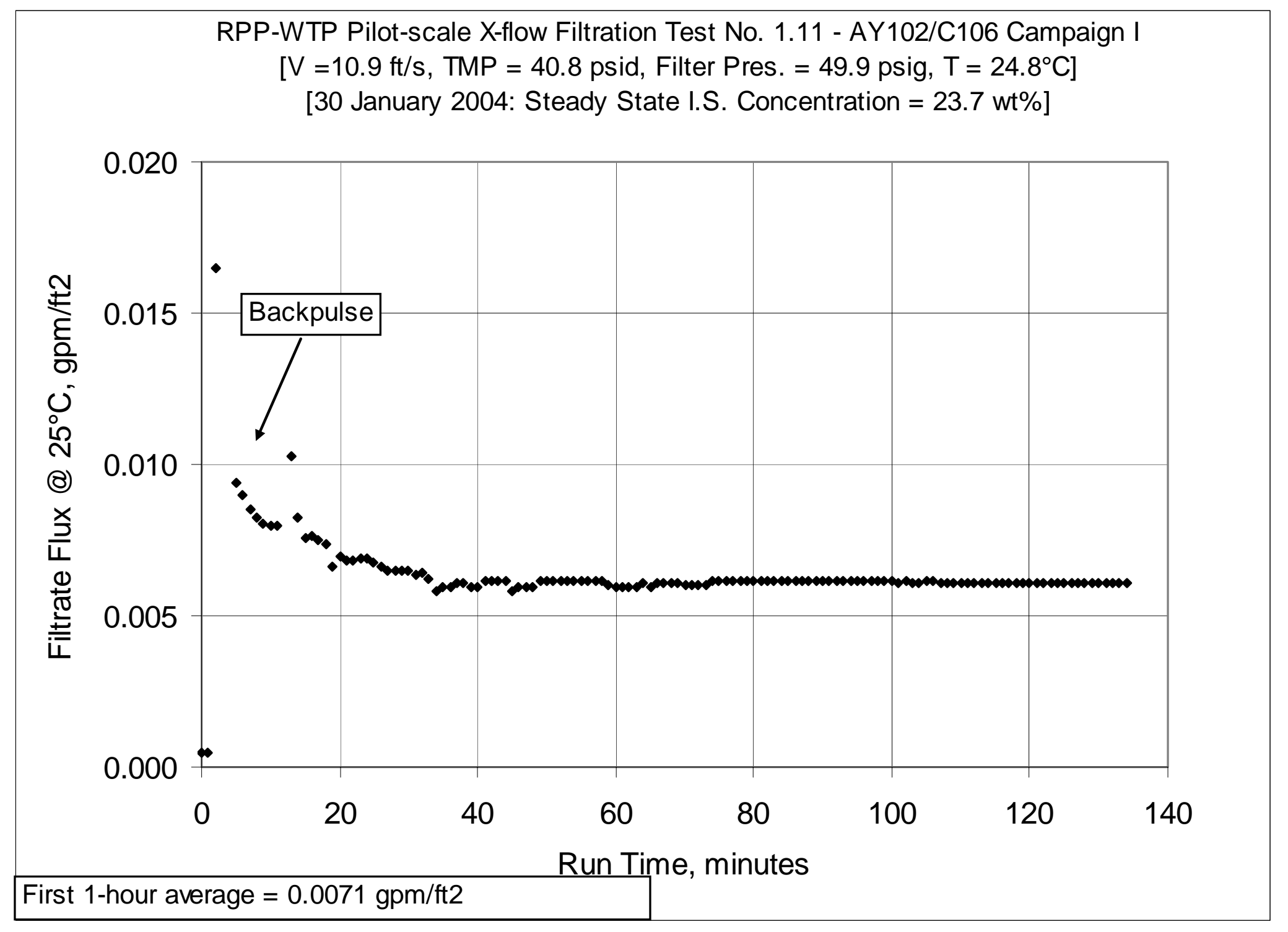


WSRC-TR-2005-00105, REVISION 0

SRNL-RPP-2005-00012, REVISION 0

RUN \# 1.11; STEADY STATE TEST AT 25 WT\% UDS - CONT.

\begin{tabular}{|c|c|c|c|c|c|c|c|c|c|c|c|c|c|c|c|c|c|c|c|c|c|c|c|c|c|c|c|c|}
\hline & A & B & D & $E$ & $F$ & $\mathrm{G}$ & $\mathrm{H}$ & $\mathrm{J}$ & $\mathrm{K}$ & $\mathrm{L}$ & $\mathrm{M}$ & $\mathrm{N}$ & $\mathrm{O}$ & Q & $R$ & $\mathrm{~s}$ & $T^{T}$ & $\mathrm{v}$ & w & $x$ & & $z$ & $\mathrm{AA}$ & $A B$ & & $A D$ & $\mathrm{AE}$ & AF \\
\hline & DATE & TIME & Filtrate & $\frac{1<<<\text { Temp }}{k}$ & $\begin{array}{l}\text { perature } \mathrm{M} \\
1 \text { Slurgy }\end{array}$ & Measurem & nents >> & & & $\begin{array}{l}\text { Pressure } \\
\text { Prostion }\end{array}$ & Measurem & & & & $<$ <lown & Measurem & & & & & & & & & & & & \\
\hline & DATE & TIME & $\begin{array}{l}\text { Fillate } \\
\operatorname{deg} C\end{array}$ & \begin{tabular}{|c|} 
Cleaning \\
$\operatorname{deg} C$
\end{tabular} & \begin{tabular}{|l|} 
Surry C \\
deg
\end{tabular} & 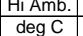 & $\begin{array}{l}\text { Lo Amb. } \\
\operatorname{deg} \text { C }\end{array}$ & $\begin{array}{c}\text { BottMP } \\
\text { psid }\end{array}$ & \begin{tabular}{|l|} 
Filter \\
psig
\end{tabular} & \begin{tabular}{|l} 
Filter dP \\
sid
\end{tabular} & TopTMP & Filtrate & Pulsepot & Slurry & Filtrate & Hi Filtate & e Backpulse & & & & & & & & & & & \\
\hline & & & $\mathrm{T} 2$ & T3 & & T4 & & $\mathrm{dP2}$ & Dig & $\mathrm{dP1}$ & $\mathrm{dP} 3$ & & & gpm & $\mathrm{gpm}$ & gpm & gpm & & & & & & & & & & & \\
\hline & & & & & & & & & & & & & & & & & & & & & & & & & & & & \\
\hline & $1 / 29 / 2004$ & $7 \cdot 58.21 \mathrm{AM}$ & 19.97 & 14.564 & 11.286 & 20,431 & 11.48 & 0.059 & -0.149 & 0.007 & 0.018 & -0.186 & & -0.087 & 0.003 & 14.643 & 0.005 & & & & & & & & & & & \\
\hline & $1 / 29 / 2004$ & 7:58:50 AM & 19.97 & 14.564 & 11.286 & 20.431 & 11.48 & 0.059 & $\begin{array}{r}-0.149 \\
\end{array}$ & 0.007 & 0.018 & -0.186 & 0.0 & -0.087 & 0.003 & 14.643 & 0.005 & & & Convers & rsion & & $\mathrm{n} 3 / \mathrm{m} 21$ & 2//day/barg & $\mathrm{rg} / \mathrm{gpm} / \mathrm{ft}$ & fft2/barg & & \\
\hline & & 7:58:5 & & 14.574 & 11.307 & 20.466 & 11.54 & & -0.149 & 0.007 & & -0.168 & & & 0.003 & 14.643 & 0.002 & & & & & & & & & & & \\
\hline & $1 / 29 / 2004$ & 7:58:53 AM & 19.981 & 14.585 & 511.317 & 20.472 & 11.55 & 0.058 & \begin{tabular}{|l|l|} 
& -0.149 \\
\end{tabular} & 0.007 & 0.021 & -0.174 & & -0.087 & 0.003 & 14.643 & 0.004 & & & & & & & & ate & & & \\
\hline & $1 / 29 / 2004$ & $\begin{array}{l}7: 58: 54 \mathrm{AM} \\
7.58 .55 \mathrm{AM}\end{array}$ & 19.991 & \begin{tabular}{r|}
14.575 \\
14.58
\end{tabular} & $\begin{array}{l}\mid 11.317 \\
11.317 \\
3\end{array}$ & \begin{tabular}{|l|}
20.477 \\
20.47 \\
\end{tabular} & & $\begin{array}{l}0.059 \\
0.061\end{array}$ & 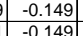 & $\begin{array}{l}0.007 \\
0.008 \\
\end{array}$ & $\begin{array}{l}0.023 \\
0.021\end{array}$ & $\begin{array}{l}-0.168 \\
-0.177 \\
\end{array}$ & 0.104 & \begin{tabular}{|c|}
-0.081 \\
\end{tabular} & 0.003 & 14.643 & 0.004 & & & & & & & & & & & \\
\hline & $\begin{array}{l}1 / 29 / 2004 \\
1 / 29 / 2004\end{array}$ & 7:58:55 AM & $\frac{19.986}{19.986}$ & $\begin{array}{r}14.58 \\
14.58 \\
\end{array}$ & \begin{tabular}{|l|l|}
11.317 \\
11317
\end{tabular} & $\begin{array}{l}20.472 \\
20.477 \\
\end{array}$ & $\frac{11.562}{11.572}$ & $\begin{array}{l}0.061 \\
0.058 \\
\end{array}$ & $\begin{array}{l}-0.149 \\
-0.149\end{array}$ & $\begin{array}{l}0.008 \\
0.008 \\
\end{array}$ & \begin{tabular}{|l|l}
0.021 \\
0.023
\end{tabular} & $\begin{array}{l}-0.177 \\
-0.168\end{array}$ & 0.104 & \begin{tabular}{|c|}
-0.087 \\
\end{tabular} & $\begin{array}{l}0.003 \\
0.003 \\
\end{array}$ & $\begin{array}{r}14.643 \\
14643\end{array}$ & $\begin{array}{l}0.005 \\
0.005 \\
\end{array}$ & & & (1) & & & ted for 4 & Filtrat & ches of $v$ & $\begin{array}{l}\text { ter tubin } \\
\text { PFPA }\end{array}$ & & \\
\hline 14 & $1 / 29 / 2004$ & 7:58:57 AM & 19.986 & $\begin{array}{l}44.50 \\
14.58 \\
\end{array}$ & 11.312 & 20.472 & 11.57 & 0.050 & \begin{tabular}{|l|}
-0.149 \\
-0.149 \\
\end{tabular} & 0.008 & 0.023 & -0.189 & 0.104 & $\begin{array}{l}-0.001 \\
-0.087 \\
-\end{array}$ & $\begin{array}{l}0.0003 \\
0.003\end{array}$ & $\begin{array}{l}14.045 \\
14.643 \\
\end{array}$ & 0.005 & & & & & & & & at $25 \mathrm{C}$ & & $\times 1000$ & \\
\hline & & & & & & & & & & & & & & & & & & Time & Time & Press. & Vel. & TMP & TMP & gpm & gpm & $\mathrm{gpm}$ & gpm & meter \\
\hline 16 & Data-Per M & linute & 25557 & & & & & 50226 & & & & 0321 & & 43690 & & & & & Hour & psig & $\mathrm{ft/s}$ & psi & $\frac{b a r}{2000}$ & & & tit/psi & & day/bar \\
\hline & $\frac{1 / 30 / 2004}{1 / 30 / 2004}$ & \begin{tabular}{|l} 
99:445:25 AM \\
9:45:25 AM
\end{tabular} & $\frac{23.531}{24.465}$ & $\frac{21.144}{21.168}$ & 25.4619 & $\begin{array}{r}24.18 \\
24.168\end{array}$ & $\frac{24.102}{24.68}$ & $\begin{aligned} \frac{50.226}{7.396} \\
\end{aligned}$ & $\begin{array}{l}56.068 \\
54.493\end{array}$ & $\frac{26.093}{8.413}$ & $\begin{array}{l}\frac{19.656}{-0.852} \\
-\end{array}$ & $\frac{0.321}{42.065}$ & $\frac{96}{87}$ & $\begin{array}{l}43.689 \\
50.605 \\
\end{array}$ & $\begin{array}{l}0.003 \\
0.003\end{array}$ & $\frac{14.643}{14.643}$ & 0.003 & 1.00 & 0.01667 & $\begin{array}{l}54.6 \\
530 \\
\end{array}$ & $\frac{10.7}{12.4}$ & $\frac{34.9}{3.3}$ & 2.409 & 0.000 & 0.000 & $\frac{0.000}{0.000}$ & $\frac{0.013}{0.135}$ & 0.01 \\
\hline & $1 / 30 / 2004$ & & & & 25.744 & & & 45.394 & 51.276 & & 29.844 & 0.327 & & 52.912 & 0.113 & 14.643 & 然 & & & & & & & & & & 0.135 & 0.37 \\
\hline & $1 / 30 / 2004$ & & 26.142 & & & 24.15 & & 46.354 & 52.231 & 17.83 & 28.598 & 257 & & 51.457 & 081 & 14.643 & & 3.0 & 0.05 & 50.8 & 126 & & 2.584 & 183 & $.127 \mathrm{~s}$ & & & \\
\hline & & & & & & 24.138 & & & 52.859 & 18.797 & & & & 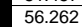 & & 14.643 & & & & & & & & & & & & \\
\hline & & & & & & 24.146 & & 47.0 & & & & 0.254 & & 18.791 & & 14.643 & & & & & & & & & & & & \\
\hline & $1 / 30 / 2004$ & & 26.48 & 21.239 & 26.086 & 24.124 & 24.38 & 47.408 & 53.268 & 19.396 & 28.235 & 0.251 & & 47.155 & 0.062 & 14.643 & & 6 & & 51 & & & 2.60 & 0.009 & 0.009 & & & \\
\hline & & 9:51:25 & & 21.267 & 26.163 & 24.172 & & 47.70 & 53.579 & 19.733 & 28.138 & & & 51.5 & & 14.64 & & & D.11667 & & & & & & & & & \\
\hline & $1 / 30 / 2004$ & 9:52:25 AM & 26.621 & 21.28 & 26.166 & 24.139 & & 47.839 & 53.805 & 20.008 & 27.931 & 0.298 & & 48.209 & 0.057 & 14.643 & & 8.4 & 0.1333 & & & & 2.612 & .000 & . & & & \\
\hline & $1 / 30 / 2004$ & 9:53:25 & 26.698 & 21.308 & $\begin{array}{l}326.199 \\
\end{array}$ & 24.157 & 24.54 & 47.933 & 53.823 & 20.23 & 27.789 & 0.324 & & 50.61 & 0.056 & 14.643 & & & 0.15 & & & & 2.610 & .008 & 0.008 & & & 1 \\
\hline & $1 / 30 / 2004$ & & 26.715 & 21.305 & 26.151 & 24.069 & & 47.839 & 53.827 & 20.057 & & & & 50.096 & & 14.643 & & 10 & tovor & & & & 2.609 & & & & & \\
\hline & $1 / 30 / 200$ & & 26.742 & 21.317 & 26.123 & 24.071 & 24.5 & 48.14 & \begin{tabular}{|l|l|l|} 
& 54.113 \\
\end{tabular} & 20.343 & 27.826 & 0.35 & & 48.782 & 0.055 & 14.643 & & $11.0 \mathrm{r}$ & 0.18333 & 52. & & & 2.619 & 0.008 & D.008 & 0.000 & 0.209 & \\
\hline & $1 / 30 / 2004$ & 9:56:25 AM & 26.669 & 21.339 & 26.065 & 24.073 & 24.4 & 52.504 & 53.821 & 20.287 & 32.208 & -1.219 & & 49.116 & & 14.643 & & 12. & & & & & 920 & & & & & \\
\hline & $1 / 30 / 2004$ & 25 AM & & 21.352 & 25.997 & 24.115 & & $53.7 / 1$ & 914 & 20.5 & 33.143 & -1.2 & & & & 14.643 & & & 21667 & & & & 996 & & & & & \\
\hline & $\frac{13 / 2012}{13212}$ & & 26.15 & 21.39 & 25.94 & 24.119 & & & & & & & & & & & & & & & & & & & & & & \\
\hline & 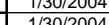 & & & $\frac{21.403}{21.42}$ & $\begin{array}{l}35.779 \\
25.759\end{array}$ & 24.192 & & 49.718 & 49.846 & $\frac{19.414}{19.710}$ & & -1.21 & & 1406 & & 16 & & & & & & & & & & & & \\
\hline & & & & & & & & & & & & & & & & & & & 200 & & & & & & & & & \\
\hline & $\frac{1 / 30 / 2004}{1 / 30 / 2004}$ & & $\frac{2.0301}{2001}$ & 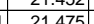 & 20.300 & $\frac{24.10}{24.188}$ & & 50.654 & 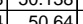 & 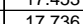 & & & & 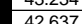 & & & & & & & & & 势. & & & & & 1 \\
\hline 36 & 1/30/2004 & $10: 03: 25 \mathrm{AM}$ & 25.938 & $\frac{21.401}{21.478}$ & \begin{tabular}{|c|}
25.298 \\
25
\end{tabular} & \begin{tabular}{|l|}
24.150 \\
24.156 \\
\end{tabular} & 25.35 & \begin{tabular}{|l}
0.0523 \\
49.523
\end{tabular} & 4 & $\frac{11.150}{19.728}$ & 29.727 & $\frac{-1.219}{-1.219}$ & & \begin{tabular}{|l|}
42.051 \\
47.483 \\
\end{tabular} & 0.045 & $\frac{14.045}{14.643}$ & & & 0.31667 & 48 & $\frac{10.6}{11.6}$ & & $\frac{2.001}{2.732}$ & & 007 & & 0.168 & th \\
\hline & $1 / 30 / 2$ & \begin{tabular}{|l|l|}
$10: 04: 25$ \\
\end{tabular} & 25.917 & 21.517 & 25.231 & 24.194 & 25.3 & 50.249 & 50.202 & 18.632 & 31 & \begin{tabular}{l|l|}
-1.222 \\
\end{tabular} & & \begin{tabular}{|l|l|}
43.645 \\
\end{tabular} & & & & 20. & \begin{tabular}{|l|l|} 
& 0.33333 \\
\end{tabular} & 48. & 10. & 40 & 2.821 & & & & 0.170 & 0.1 \\
\hline & & & 25.885 & 21.53 & 25.169 & 24.183 & & 50 & 50.379 & 18. & & -1.219 & & 42. & & & & & $\begin{array}{ll} & 0.35 \\
\end{array}$ & 48. & 10.5 & 4 & 2.830 & & & & $\begin{array}{l}0.166 \\
\end{array}$ & 0.1 \\
\hline & $1 / 30 /$ & $10: 06$ & 25.843 & 21.553 & $\begin{array}{l}325.072 \\
\end{array}$ & 24.166 & 25.5 & 50.737 & 50.762 & 18.6 & 31.978 & & & 43. & & & & & 0.36667 & 49.3 & 10.7 & & 2.851 & & & & 165 & 0.1 \\
\hline & & & & 21.576 & 24.995 & 24.1 & & 50.938 & 50.942 & 18.2 & & -1. & & & & & & & 0.38333 & 49. & 10.4 & & & & & & 64 & 0.1 \\
\hline & & & 25.763 & 21.599 & 24. & 24.151 & & 51. & 51.106 & 18. & & & & 41.9 & & & & & 0.4 & 49 & 10. & & 96 & & & & 0.164 & \\
\hline & $1 / 30 / 2004$ & 10:09:25 AM & 25.716 & 21.617 & 24.815 & 24.179 & & & & 18 & 32.934 & \begin{tabular}{|l|l|} 
\\
.1219 \\
\end{tabular} & & 42.095 & & & & 2000 & $0.41667 \mid$ & 49.9 & 20 & & 2.904 & & 007 & & 0.160 & 0.1 \\
\hline & $\frac{1 / 30 / 2004}{1 / 3 / 2004}$ & 10:10:25 AM & $\begin{array}{l}25.678 \\
25.255 \\
\end{array}$ & 21.639 & $\begin{array}{l}24.732 \\
24618 \\
\end{array}$ & 24.151 & & & \begin{tabular}{|l|l|}
51.434 \\
151.27
\end{tabular} & 18.3 & & \begin{tabular}{|c|c|}
-1.219 \\
12251
\end{tabular} & & \begin{tabular}{|l|}
41.699 \\
41.223
\end{tabular} & & & & & 0.43333 & 50.0 & 10.2 & & .915 & & & & 156 & 0.1 \\
\hline & & & & & $\begin{array}{l}74.618 \\
74.59 \\
7\end{array}$ & & & & & & & $\begin{array}{l}-1.225 \\
-1.222 \\
\end{array}$ & & & & & & & & $\frac{44.8}{49.7}$ & 10.1 & & & & & & $\frac{0.154}{0.154}$ & 0.1 \\
\hline & & & 25.548 & 21. & & 24. & & & & & & & & & & & & 25 & 0.48333 & 50.1 & & & & & & & & 0.1 . \\
\hline & & & & & & 24.0 & & & & & & \begin{tabular}{l|l|}
6 & -1.225 \\
\end{tabular} & & & & & & & & & y & & & & & & & 0.1 \\
\hline & & & & 21.724 & & 24.0 & 24. & & & 18. & & & & & & & & & 0.51 & 50.3 & & & & & & & $\begin{array}{l}0.150 \\
\end{array}$ & \\
\hline & & & 25.399 & 21. & & & & & & 17.9 & & & & & & & & 32.00 & 0.53333 & 50.5 & 9 & & 2.950 & 0 & & & 0.149 & 0.1 \\
\hline & & & & & & & & & & & & & & & & & & & & 50. & 9. & & 445 & & & & & \\
\hline & & $10: 18$ & 25.294 & 21. & 24.23 & 24.1 & & & 50.687 & & & & & & & & & & 0.56 & 49.2 & & & & & & & & 0.1 \\
\hline & $1 / 301$ & & 25.256 & 21.798 & 24.1 & 24.1 & & 51.133 & 51.2 & 21.5 & & -1.219 & & & & & & 35.00 & 0.58333 & 49.8 & 11. & 40.3 & 78 & & & & 48 & \\
\hline & & & $\begin{array}{l}25.208 \\
25.179 \\
\end{array}$ & 21.8 & & 24. & & & & & & & & & & & & & 0.6 & 0.4 & & 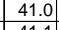 & & & & & 45 & the \\
\hline & $\frac{1 / 30 / 2}{1 / 20 / 2}$ & \begin{tabular}{|l|l|l|l|}
$10: 21: 25$ \\
$10 \cdot 22 \cdot 25$
\end{tabular} & $\begin{array}{l}25.179 \\
25.132 \\
\end{array}$ & $21.837 \mid$ & 24.182 & $\frac{24.1}{24.1}$ & 25.7 & $\begin{array}{r}51.348 \\
51.546\end{array}$ & $\begin{array}{l}31.512 \\
51.664 \\
5\end{array}$ & $\begin{array}{r}20.36 \\
20012\end{array}$ & & $\begin{array}{l}-1.219 \\
-1.219\end{array}$ & & & & & & & $0.61667 \mid$ & 50.0 & $\frac{10 .}{10}$ & & & & & & 148 & 0.1 \\
\hline & $\frac{1 / 100 / 2}{1 / 30 / 2}$ & & 25.132 & $\begin{array}{l}21.844 \\
21.861\end{array}$ & & $\frac{24.1}{24.1}$ & & $\begin{array}{l}51.566 \\
51.384 \\
\end{array}$ & & $\frac{20.012}{21.349}$ & & 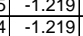 & & & & & & & $\begin{array}{rl}63333 & 0.65 \\
\end{array}$ & 50.2 & & & 804 & & & & $\mid \frac{0.147}{0.146}$ & \\
\hline & & & 25. & & & 24. & & 51. & $\begin{array}{ll}2 \\
2\end{array}$ & 20 & & & & 44. & & & & & & 50.1 & & & 20 & & & & 0.146 & 0.1 \\
\hline & & & 25.014 & & & 24. & & & & & & & & & & & & & 0.68333 & 49 & & & & & & & 0.149 & \\
\hline & & & 24. & & & & & & & & & & & & & & & & $\mid .7$ & $49 . \mathrm{s}$ & 10. & & & & & & & \\
\hline & & & & & 24. & & & 51 & & & & & & & & & & & & 49. & & & & & & & & \\
\hline & $1 / 30 / 2$ & & 24.913 & 21.931 & 24.0 & 24.0 & & & & & & & & & & & & & & 50. & 10.6 & & 57 & & & & 148 & \\
\hline & & 10:29:25 A & 24.869 & 21.942 & 24.036 & 24.051 & & & & & & -1.219 & & & & & & & & & & & & & & & & \\
\hline & $1 / 30 / 2$ & \begin{tabular}{|l}
$10: 30: 25 \mathrm{AM}$ \\
\end{tabular} & 24.85 & 21.953 & 24.038 & 24.042 & 25.277 & 51.118 & 51.384 & 21.0 & 29.909 & \begin{tabular}{|l|l|}
-1.219 \\
\end{tabular} & & 44.944 & & 14.6 & & & $0.76667 \mid$ & 49.9 & 11.0 & 40.5 & 2.793 & & & & 0.147 & 0.1 \\
\hline & $1 / 30 / 2004$ & 10:31:25 AM & 24.82 & 21.975 & 24.034 & 24.054 & 25.5 & \begin{tabular}{|l|}
51.205 \\
50.867 \\
\end{tabular} & 51.446 & 20.86 & $\begin{array}{l}30.236 \\
30531\end{array}$ & -1.219 & & 46.6 & & 14.643 & & $\begin{array}{l}47.00 \\
400\end{array}$ & 0.78333 & 50.0 & 11.4 & 40.7 & 2.808 & & 0.00 & 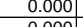 & 0.147 & 7 \\
\hline & $\begin{array}{l}1 / 30 / 2004 \\
1 / 30 / 2004\end{array}$ & & $\frac{24.809}{24.776}$ & $\begin{array}{r}r 2.998 \\
22.01\end{array}$ & \begin{tabular}{|l|}
$\mid 24.032$ \\
23.998 \\
\end{tabular} & \begin{tabular}{|l|}
$\mid 24.172$ \\
24.189 \\
\end{tabular} & $\frac{25.6}{25.2}$ & \begin{tabular}{|l}
50.867 \\
51.073
\end{tabular} & 5 & $\begin{array}{l}20.337 \\
20.187 \\
\end{array}$ & $\begin{array}{l}30.051 \\
30.952 \\
\end{array}$ & 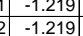 & & \begin{tabular}{|l|l|}
4.1 .5 \\
4.5
\end{tabular} & 0.0 & $\begin{array}{l}14.643 \\
\end{array}$ & & $\begin{array}{l}48.00 \\
49.00\end{array}$ & \begin{tabular}{r|r}
0.81667 \\
\end{tabular} & $\begin{array}{l}49.6 \\
49.8 \\
\end{array}$ & \begin{tabular}{l|l}
3 & 10.9
\end{tabular} & & $\frac{2.806}{2.828}$ & & $\frac{6}{6}$ & & & \\
\hline & $1 / 30 / 2004$ & $10: 34: 25$ AM & 24.758 & 22.011 & $23.96 \mid$ & \begin{tabular}{|l|l|}
24.146 \\
\end{tabular} & 25. & 51.041 & 51.334 & 20.073 & 30.931 & $1-1.222$ & -5. & \begin{tabular}{|l|l|}
43.284 \\
\end{tabular} & 0.04 & 14.643 & 0.0 & 50.00 & 0.83333 & 49.9 & \begin{tabular}{|l|}
9 \\
\end{tabular} & 41.0 & 2.826 & \begin{tabular}{|l|l|}
6 & 0.006 \\
\end{tabular} & $\mid 0.006$ & 0.000 & 0.150 & 0.1 \\
\hline & $1 / 30 / 2004$ & $10: 35: 25 \mathrm{AM}$ & 24.74 & \begin{tabular}{|l|l|}
4 & 22.039 \\
\end{tabular} & 23.972 & \begin{tabular}{|l|l|l|l|} 
\\
\end{tabular} & 25.17 & 51.035 & \begin{tabular}{|l|l|} 
\\
51.405
\end{tabular} & \begin{tabular}{|l|l|}
20.031 \\
\end{tabular} & 30.863 & \begin{tabular}{|c|c|}
3 & -1.222 \\
\end{tabular} & & 43.662 & 0.04 & 14.643 & & 51.00 & 0.85 & 49.9 & \begin{tabular}{|l|l|}
$y$ & 10.7 \\
\end{tabular} & 40.9 & 2.823 & \begin{tabular}{|c|c|}
3 & 0.006 \\
\end{tabular} & \begin{tabular}{|l|}
0.006 \\
\end{tabular} & \begin{tabular}{|l|l|} 
& 0.000 \\
\end{tabular} & 0.150 & 0. \\
\hline
\end{tabular}


WSRC-TR-2005-00105, REVISION 0

SRNL-RPP-2005-00012, REVISION 0

RUN \# 1.11; STEADY STATE TEST AT 25 WT\% UDS - CONT.

\begin{tabular}{|c|c|c|c|c|c|c|c|c|c|c|c|c|c|c|c|c|c|c|c|c|c|c|c|c|c|c|c|c|c|}
\hline & A & & $\mathrm{D}$ & E & $\mathrm{F}$ & \begin{tabular}{|l|l} 
\\
\end{tabular} & $\mathrm{H}$ & $\mathrm{J}$ & $\mathrm{K}$ & $\mathrm{L}$ & \begin{tabular}{l|l}
$\mathrm{M}$ \\
\end{tabular} & $\mathrm{N}$ & $\mathrm{O}$ & Q & $\mathrm{R}$ & & & $\mathrm{v}$ & W & $x$ & $\begin{array}{r}Y \\
Y\end{array}$ & $z$ & & $\mathrm{AA}$ & $A B$ & $\mathrm{AC}$ & & $\mathrm{AE}$ & \\
\hline$\frac{69}{70}$ & $\begin{array}{l}1 / 30 / 2004 \\
1 / 30 / 2004\end{array}$ & 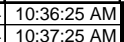 & \begin{tabular}{l|}
24.716 \\
24.699
\end{tabular} & $\frac{22.045}{22.063}$ & $\begin{array}{l}23.959 \\
23.946 \\
\end{array}$ & \begin{tabular}{|l|}
24.084 \\
24.086 \\
\end{tabular} & $\begin{array}{l}25.089 \\
25.011\end{array}$ & \begin{tabular}{|l|l|}
51.056 \\
51.073
\end{tabular} & $\begin{array}{l}51.417 \\
51.392 \\
\end{array}$ & $\begin{array}{l}19.996 \\
20.069 \\
\end{array}$ & \begin{tabular}{|l|}
30.859 \\
30.931
\end{tabular} & $\begin{array}{l}-1.219 \\
-1.222 \\
\end{array}$ & $\begin{array}{l}-5.533 \\
-5.533 \\
\end{array}$ & \begin{tabular}{|l|}
43.234 \\
44.112 \\
\end{tabular} & \begin{tabular}{|l|}
0.04 \\
0.04 \\
\end{tabular} & \begin{tabular}{l|l}
14.643 \\
14.643
\end{tabular} & $\begin{array}{l}0.004 \\
0.003 \\
\end{array}$ & \begin{tabular}{l|}
52.00 \\
53.00
\end{tabular} & \begin{tabular}{|l|}
0.86667 \\
0.88333 \\
\end{tabular} & $\begin{array}{l}49.9 \\
49.9\end{array}$ & \begin{tabular}{|l|}
10.6 \\
10.8
\end{tabular} & & & .824 & \begin{tabular}{l|l}
0.006 \\
0.006
\end{tabular} & $\begin{array}{l}0.006 \\
0.006\end{array}$ & $\begin{array}{l}0.000 \\
0.000\end{array}$ & $\begin{array}{l}0.150 \\
0.150\end{array}$ & \\
\hline & & & & $\frac{22.003}{22.049}$ & & & & & & & $\begin{array}{l}30.931 \\
30.943\end{array}$ & $\begin{array}{l}-1.224 \\
-1.222\end{array}$ & & & $\begin{array}{l}0.04 \\
0.04 \\
\end{array}$ & & & 54.00 & & & \begin{tabular}{|l|l|}
10.8 \\
10.8
\end{tabular} & & & 828 & & & & 0.150 & \\
\hline$\frac{71}{72}$ & $\frac{1 / 30 / 2004}{1 / 30 / 2004}$ & 10:39:25 AM & 24.0055 & $\frac{2.049}{22.085}$ & $\begin{array}{l}23.992 \\
23.913 \\
\end{array}$ & \begin{tabular}{|l|}
24.137 \\
24.139 \\
\end{tabular} & $\frac{25.14}{25.323}$ & $\begin{array}{l}51.095 \\
51.042\end{array}$ & \begin{tabular}{|l|}
51.45 \\
51.407
\end{tabular} & $\mid \begin{array}{l}2.025 \\
19.784\end{array}$ & $\begin{array}{l}30.945 \\
31.196\end{array}$ & $\begin{array}{l}-1.224 \\
-1.222\end{array}$ & $\begin{array}{l}-3.533 \\
-5.528 \\
\end{array}$ & $\begin{array}{l}4.253 \\
42.464 \\
\end{array}$ & $\begin{array}{l}0.04 \\
0.04 \\
\end{array}$ & $\begin{array}{l}14.043 \\
14.643 \\
\end{array}$ & 0.004 & $\begin{array}{l}55.00 \\
55.00\end{array}$ & $\begin{array}{r}0.9 \\
.91667 \\
\end{array}$ & $\begin{array}{ll}30.0 \\
49.9\end{array}$ & \begin{tabular}{|l|l|}
10.8 \\
10.4
\end{tabular} & $\begin{array}{ll}41 \\
41\end{array}$ & & 8.835 & 0.006 & 0.000 & 0.000 & $\begin{array}{l}0.150 \\
0.150\end{array}$ & \\
\hline 73 & $1 / 30 / 2004$ & $10: 40: 25 \mathrm{AM}$ & 24.642 & 22.097 & 23.905 & 24.135 & 25.59 & 51.255 & 51.68 & 19.867 & 31.264 & 1.216 & & $\begin{array}{l}4.4045 \\
44.075\end{array}$ & $\begin{array}{l}0.04 \\
0.04\end{array}$ & $\begin{array}{l}\frac{14.045}{14.643} \\
\end{array}$ & 0.003 & 56.00 & 0.91001 & 50.5 & \begin{tabular}{|l|l|}
10.4 \\
10.8
\end{tabular} & 41. & & .845 & 0.006 & 0.006 & 0.000 & 0.149 & \\
\hline 74 & $1 / 30 / 2004$ & 10:41:25 AM & & & 23.905 & 24.136 & & 51.284 & 51.697 & 19.865 & 31.305 & 1.222 & & 257 & 0.04 & 14.643 & & 57.00 & 0.95 & 50.2 & 10.6 & & & 2.847 & .006 & 0.006 & & 0.149 & \\
\hline 75 & $1 / 30 / 2004$ & 10:42:25 AM & 44.604 & 22.124 & 23.871 & 24.157 & 25.80 & 50.382 & 50.808 & 19.7 & 30.591 & 1.222 & & 44.842 & 0.04 & 14.643 & 0.003 & 58.00 & 0.96667 & & 11.0 & 40. & & 2.791 & 0.006 & 0.006 & & 0.152 & \\
\hline 76 & $1 / 30 / 2004$ & 10:43:25 AM & 4.596 & 22.135 & & 24.124 & 26.03 & 50.548 & 51.009 & 20.559 & 29.93 & -1.219 & & 44.068 & 0.039 & 14.643 & 0.004 & & 0.98333 & & & & & & & & & 0.149 & \\
\hline 77 & 1/30/2004 & 10:44:25 AM & & & 24.03 & 24.186 & 26.21 & 50.701 & 51.091 & 20.453 & 30.203 & & & 45.078 & & 14.643 & & 60.00 & & & 11.0 & & & & & & & 0.148 & \\
\hline & 1/30/26 & & 4.578 & 22.173 & 24.076 & 24.156 & 25.0 & 50.643 & 51.048 & 20.369 & 30.166 & 1.219 & & .638 & 0.039 & 14.643 & & 61.00 & 01667 & 49.6. & 10.9 & 40. & & 2.786 & .006 & 0.006 & 0.000 & 0.148 & \\
\hline 19 & & & & & & 24.145 & & 50.664 & & & 30.141 & 1.222 & & .369 & $0.03 \mathrm{~S}^{-1}$ & 14.643 & & & & 49.6 & & & & & & & & & \\
\hline 然1 & & & & & & 24.139] & & 50.538 & & & & & & & & .643 & & & & & & & & & & & & .148 & \\
\hline & & & & 22.191 & 24.254 & 24.104] & & 50.188 & 10.253 & 20.45 & .211 & 1.214 & & & 0.04 & 14.643 & & & .0606/ & 49.8 & & & & & 006 & .006 & & 0.150 & \\
\hline & $\frac{1 / 33120004}{1202004}$ & $\begin{array}{ll}1.49 .25 \mathrm{Am} \\
10.25 \mathrm{~A}\end{array}$ & 2157 & 22.216 & 24.329 & $24.129 \mid$ & & 50.853 & $\begin{array}{l}51.326 \\
50770 ?\end{array}$ & 20.489 & 30.246 & 1.2199| & & (4) & 0.039 & 14.643 & & 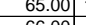 & & $49.9 ?$ & 11.2 & & & $778+2>$ & .006 & & & 0.146 & \\
\hline 84 & $\frac{113010004}{112004}$ & \begin{tabular}{|l|}
$10: 50: 25$ AM \\
$10.51 \cdot 25$ |
\end{tabular} & $\frac{24.57}{24.54}$ & $\frac{22.224}{2204}>0$ & $\frac{24.354}{2.432}$ & $\mid \frac{2.123}{2.203}$ & & 50.33 & $\begin{array}{ll}50.62 \\
51005 \\
\end{array}$ & $\frac{2.111}{2023}$ & $\frac{30.199}{30297}$ & $\frac{1.219}{1219}$ & & $\frac{4.986}{209}$ & 0.04 & $\frac{14.643}{14.633}$ & & $\frac{66.00}{6700}$ & $\begin{array}{r}1.1 \\
11667\end{array}$ & $\frac{49.3}{495}$ & 11.0 & & & 7788 & .006 & & & 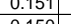 & \\
\hline 85 & $1 / 30 / 2004$ & $10: 52: 25 \mathrm{AM}$ & $\begin{array}{l}24.504 \\
24.573\end{array}$ & $\frac{2.2204}{22.214}$ & 24.453 & $\begin{array}{l}24.2026 \\
24.146\end{array}$ & $\frac{24.956}{24.786}$ & 50.791 & 51.214 & 20.364 & $\frac{30.291}{30.377}$ & |..2192 & & 44.039 & 0.04 & $\frac{1.0045}{14643}$ & 等 & 6800 & - & $\frac{49.5}{49.7}$ & 10.8 & & & 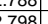 & 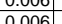 & 等 0.0006 & & 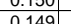 & \\
\hline 86 & $1 / 30 / 2004$ & 10:53:25 AM & 24.593 & 22.234 & 24.467 & 24.066 & 25.206 & 50.77 & 51.241 & 20.318 & 30.375 & -1.219 & -5.516 & 44.972 & 0.04 & $\begin{array}{l}14.045 \\
14.643\end{array}$ & 0.004 & 6.00 & 1.15 & 49.8 & $\frac{1.0}{11.0}$ & 40 & & .797 & 0.006 & 0.006 & 0.000 & 0.149 & \\
\hline & & 10:54:25 AM & & 22.249 & 24.512 & 24.071 & & 50.681 & 51.253 & 20.196 & 30.385 & -1.219 & & 44.214 & 0.04 & 14.643 & & & & 49.8 & & & & .795 & & & & 0.149 & \\
\hline $88 \longrightarrow$ & 1/30/2 & 0:55:25 AM & 4.608 & 22.228 & 24.517 & 24.045 & 24. & 50.863 & 51.394 & 20.357 & 30.459 & 1.222 & & 44.64 & 0.04 & 14.643 & & & 1.18333 & & & & & & .006 & 0.006 & & 0.149 & \\
\hline & 1/30/2004 & & 4.6211 & 22.236 & 24.57 & 24.074 & & 50.78 & 51.278 & 20.219 & 30.43 & 1.216 & & 383 & 0.04 & 14.643 & & 72.00 & 1.2 & 49.8 & 11.1 & & & & .006 & & & 0.149 & \\
\hline 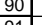 & $1 / 30 / 2004$ & $10: 57: 25$ AM & 24.647 & 22.252 & 24.616 & 24.089 | & & 50.363 & 0.934 & 19.868 & 30.398 & 1.216 & & to & 0.04 & 14.643 & & & 11667 & 49.5 & & & & 784 & .006 & 0.006 & & 0.149 & \\
\hline & $1 / 30 / 2004$ & 10:58:25 AM & 24.666 & & & & & & & & & & & & & & & & 3333 & & 0.9 & & & & .006 & & & 0.151 & \\
\hline & $1 / 3$ & & & & & & & & & & & & & & & & & & & & & & & & & & & 151 & \\
\hline 然 & . & 1:00:25 AM & 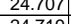 & 2.203 & 24.721 & 24.07 & & 50.345 & & $19.4877 \mathrm{r}$ & 30.911 & 1.222 & & & & 1.643 & & & (20067 & 49.4 & 0.9 & & & & & & & & \\
\hline & $\mid$ & $\mid \frac{1.01 .25 \mathrm{MM}}{11.025 \mathrm{AM}}$ & & $\frac{2.254 \mid}{2220 \mid}$ & 247454 & 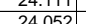 & & & | & 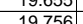 & & \begin{tabular}{rl|l}
-1.219 \\
1.25
\end{tabular} & & 势 & & $\frac{14.6}{14.6}$ & & 7800 - & & & & & & & & & & & \\
\hline 96 & $\frac{1 / 30 / 2004}{1 / 30 / 2004}$ & 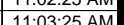 & $\begin{array}{l}24.74 \\
24.74 \\
4\end{array}$ & $\frac{2<.25}{22301}$ & 24.47 & 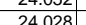 & $\frac{2.35}{247}+3$ & 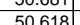 & 5 & 19730 & | & $\frac{-1.25}{-1219}$ & & (4) & & 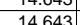 & & 7900 & 1316.5 & 年 49.6 & 然108 & & & 807 & 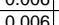 & & & 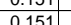 & \\
\hline 97 & $\frac{1}{1 / 30 / 2004}$ & 11:04:25 AM & 24.756 & 22.312 & $24,755 \mathrm{r}$ & 23.98 & 25.01 & 50.672 & 51.27 & 19.826 & 30.833 & -1.222 & & & 0.041 & 14643 & & 80.00 & 1.133333 & 49.8 & $\frac{10.9}{10.9}$ & & & & 0.006 & 0.006 & & 0.151 & \\
\hline & $1 / 30 / 2004$ & 11:05:25 AM & 24.75 & 22.28 & 2.459 & 23.866 & 24.74 & 50.681 & 51.334 & 19.817 & 30.744 & -1.222 & & 44.346 & $\frac{0.041}{0.041}$ & 14.6 & & 81.00 & 1.35 & 49.9 & 10.9 & & & 807 & & & & & \\
\hline & $1 / 30 / 2$ & 11:06:25 AM & 24.79 & 22.31 & 24.799 & 23.851 & 24.7 & 50.592 & 51.21 & 19.756 & 30.761 & $\frac{1.219}{1.29}$ & & 44.181 & 0.041 & 14.643 & & $\frac{82.0}{82.0}$ & 36667 & & $\frac{0.8}{10.8}-3$ & 40 & & 805 & 0.006 & 0.006 & & 0.151 & \\
\hline & $1 / 30 / 26$ & $1: 07: 25 \mathrm{AM}$ & 24.793 & 22.309 & 24.812 & 23.779 & & 50.637 & & 19.874 & 30.746 & 1.222 & & 918 & 0.041 & & & 83 & & $\frac{49.8}{49.8}>$ & & & & & & & & & \\
\hline & $1 / 30 / 2004$ & 8:25 AM & & & & 23.764 & & & 1.454 & 19.961 & & & & 088 & & 3 & & 84.00 & 1.4 & & $\frac{11.0}{11.0}-2$ & & & & & & & & \\
\hline & & $1: 09$ & & 22.286 & 24.84 & 23.686 & & & & \begin{tabular}{|c|c|}
19.76 \\
\end{tabular} & & & & 267 & & 43 & & & & 49.8 & $\overline{0.8}$ & & & .806 & & & & 0.151 & \\
\hline & & & & & & & & & & 19.541 & & & & 5.043 & & & & & & & 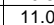 & & & & & & & & \\
\hline & $1 / 30 / 2004$ & 11:11:25 AM & 24.835 & 22.285 & 24.884 & 23.666 & & $\begin{array}{l}50.179 \\
\end{array}$ & 50.913 & 19.45 & 30.787 & -1.222 & & 46.002 & & $\overline{14.6} \times>>$ & & 87.00 & 1.45 & $\mid 49.4$ & 11.3 & & & 4.791 & 0.006 & & & $\frac{151}{151}$ & \\
\hline & & & 24.834 & 22.275 & & 23.62 & & & & & & & & & & & & & 1.46667 & & & & & & & & & .151 & \\
\hline & 1/30/2004 & 11:13:25 AM & 24.839 & 22.275 & 24.904 & 23.58 & & 50.604 & 51.288 & & & -1.222 & & 4.3 & & & & & 1.48333 & 49.8 . & 10.9 & & & .804 & & & & .151 & \\
\hline & 1/30/2004 & 11:14:25 AM & 24.855 & 22.285 & & 23.595] & & 50.2 & 50.977 & 19.496] [ & 30.763 & -1.219 & & 49.339 & & 14.6 & & 90. & 1.5 & 49.5] & 12.1 & & & .794 & 0.006 & & & 0.151 & \\
\hline & 1/30/2 & 11:15:25 AM & 24.828 & 22.253 & 24.907 & 23.553 & & 50.5 & 51.295 & 19.629 & 30.824 & -1.219 & & 44.759 & & & & 91. $\mathrm{s}$. & \begin{tabular}{|c|c|c|}
1.51667 \\
\end{tabular} & & 11.0 & & & & & & & $\begin{array}{l}0.151 \\
\end{array}$ & \\
\hline & 1/30/2004 & 11:16:25 AM & 24.828 & 22.243 & 24.912 & 23.493 & & 50. & 51.029 & 19.475 & 30.855 & -1.219 & & 46.211 & & & & & & & 11.3 & & & & 06 & & & 0.151 & \\
\hline & $1 / 3 c$ & 11:17:25 AM & 24.8266 & 22.231 & 24.895| & 23.446] & & 50.5 & 51.282 & $19.483]$ & 31.063 & -1.219 & & 4 & & & & 93 & 1.55 & $49.8 \mathrm{r}-\mathrm{x}$ & & & & & & & & 0.150 & \\
\hline & 1/30/2 & & & & & & & & & 19.234 & & 1.219 & & & & & & & & 49.7 & 10.6 & & & & & & & 1000 & \\
\hline & & & 24.8433 & & 24.932 & 23.463 & & & & & & & & & & & & & .58333 & & & & & & & & & & \\
\hline & 1130124 & 11:20:2 & 24.837 & 22.2227 & 24.932 & 23.552 & & 50. & 51.21 & 19.338 & 31.089 & 1.2199| & & 3.5 & & & & 96. & 1.6 & & $\frac{10.1}{3}$ & & & & & & & .150 & \\
\hline & & & & & & & & & & 19.395 & & & & & & & & & & & 10.1 & & & & & & & & \\
\hline & 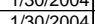 & & 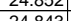 & 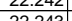 & 24.912 & th.0000 & & & & 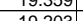 & & -1.214 & & & & & & & $\frac{1.05030}{165}$ & & 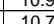 & & & & & & & 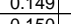 & \\
\hline & (1) & & & 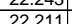 & 24.9095 & 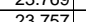 & & & & & & & & & & & & & 1.006 & & $\frac{1.1}{114}$ & & & & & & & 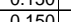 & \\
\hline & 1) & & 2484 & $\frac{22.41}{2224}$ & 24991 & 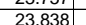 & & & & & & & & & & & & & (1.00030 & & $\frac{\frac{1.4}{110}}{110}>0$ & & & & & & & 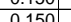 & \\
\hline & $\frac{130 / 26}{1130 / 26}$ & 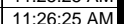 & & 22.22 & 24.97 & $\frac{23.912}{23.93}$ & & & & $\frac{29.214}{19214}$ & & $\frac{-1.216}{-1216}$ & & & & & & & $\begin{array}{ll}17 \\
17\end{array}$ & & & & & & & & & 0 & \\
\hline & $\frac{1}{1 / 30 / 26}$ & & 24.847 & $\frac{2.237}{22.237}$ & & 24.059 & & $\frac{50.0}{50}$ & & 19.151 & & $\frac{-1.219}{-1.219}$ & & & & & & & 171667 & & & & & & & & & 0151 & \\
\hline & & & & & & 24.169 & & & & & & & & & & & & & & & $=$ & & & & & & & & \\
\hline & & & 24.8 & & & & & & & & & & & & & & & & 175 & 50. & & & & & & & & & \\
\hline & & $11: 30$ & 24.872 & & 24.987 & 24.366 & & & 51.585 & & & -1.222 & & & & & & & 176667 & 50.1 & 0.7 & & & & & & & 149 & \\
\hline & & $11 \cdot 3 \cdot 3$ & & 22.289 & & 24.402 & & 50.6 & & $19.4 \times>$ & & & & & & & & & & & 10.0 & & & & & & & & \\
\hline & & $11: 32: 2$ & & 22.296 & 25.015 & 24.379 & & 50. & 51.261 & 19.3 & 31.0 & 1.222 & & & & & & & 1.8 & 49.8 & 10.9 & & & & & & & & \\
\hline & 1/30/2 & 11:33:25 & 24.89 & 22.276 & & $24.344 \mid$ & & 50.6 & 51.61 & 19.454] & 31.0 & -1.21 & & 44.1. & & 14.6 & & 109 & 81667 & & $10.8 \mathrm{r}-1$ & & & & & & & 0.150 & \\
\hline & 1/30/2004 & $11: 34: 25 \mathrm{~A}$ & 24.907 & 22.287 & 25.011 & 24.35 & $24.4 .4>0$ & 50.531 & 51.3 & 19.37 & 31.085 & -1.219 & & 45.1. & & 14.6. & & 110 & 1.83333 & 49. & $1.1 .12>$ & 40 & & $.81 \mathrm{P}>\mathrm{C}$ & 0.0 & & & 0.150 & \\
\hline & 1/30/2004 & 11:35: & 24. & 22.325 & & 24.333 & & 50.5 & 51.405 & 19.508 & 31.0 & $1.2 .2-3$ & & & & & & 111 & 1.85 & 498 & $0.8 \mathrm{C}-\mathrm{x}$ & & & & & & & 0.150 & \\
\hline & & 11:36 & & 22.32 & & 24.288 & & 50.5 & 51.45 & 19.387 & & -1.2 & & & & & & & & & & & & & & & & 0.150 & \\
\hline & & & & 321 & & 24.2 & & 50. & & & & & & & & & & & 1.88333 & & .3 & & & & & & & 150 & \\
\hline & & & 24. & & 25.046 & 24.234 & & 50.4 & 51.363 & & & 1.22 & & & & & & & 1.9 & & $11.2+24$ & & & & & & & 150 & \\
\hline & & & & & & 24. & & & & & & & & & & & & & & & & & & & & & & & \\
\hline & & & 24. & 358 & 25.067 & 24.2 & 20 & & $51.4 / 1$ & 19.3 & & & & & & & & & & 50.0 & $10.8 \mathrm{~s}-\mathrm{p}$ & & & & & & & & \\
\hline & & 11.41.25 AM & 1000 & 20.343 & (5).042 & 2 & & & & 19.421 & & & & & & & & & $\frac{1.95}{1097}$ & $108-2$ & & & & & & & & .150 & \\
\hline 136 & $1 / 30 / 2004$ & \begin{tabular}{|l}
$1.42 .25 \mathrm{HIM}$ \\
$11.425 \mathrm{MM}$
\end{tabular} & 24078 & 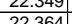 & 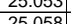 & 20.1000 & & $\begin{array}{l}50.394 \\
50.533 \\
\end{array}$ & 51469 & 19418 & 30.972 & 1210 & & 44803 & & 1.4065 & & & & & & & & & & & & 0.150 & \\
\hline & $1 / 30 / 2004$ & 11:43:25 AM & 24.978 & $22.364 \mid$ & $25.058]$ & $24.08 \mid$ & & $50.533 \mid$ & 51.469] & 19.418] & 30.972 & $-1.219 \mid$ & & 44.803 & & $14.643 \mid$ & & & 1.98333 & & & & & & 0.006 & 0.006 & 0.000 & 0.150 & \\
\hline
\end{tabular}


WSRC-TR-2005-00105, REVISION 0

SRNL-RPP-2005-00012, REVISION 0

RUN \# 1.11; STEADY STATE TEST AT 25 WT\% UDS - CONT.

\begin{tabular}{|c|c|c|c|c|c|c|c|c|c|c|c|c|c|c|c|c|c|c|c|c|c|c|c|c|c|c|c|c|}
\hline & A & B & $\mathrm{D}$ & $E$ & $F$ & $G$ & $\mathrm{H}$ & $\mathrm{J}$ & $\mathrm{K}$ & $\mathrm{L}$ & $\mathrm{M}$ & $\mathrm{N}$ & 0 & $\mathrm{Q}$ & $\mathrm{R}$ & \begin{tabular}{l|l} 
\\
\end{tabular} & $T$ & $\mathrm{~V}$ & w & $x$ & $\mathrm{Y}$ & $z$ & AA & $\mathrm{AB}$ & $\mathrm{AC}$ & $\mathrm{AD}$ & $\mathrm{AE}$ & AF $|A|$ \\
\hline $131 \mid$ & $1 / 30 / 200$ & 11:38:25 AM & 24.941 & 22.337 & 25.046 & 24.234 & 24.383 & 50.459 & 51.363 & 19.318 & 31.069 & -1.219 & -5.473 & 45.898 & 0.041 & 14.643 & 0.00 & 114.0 & 1.9 & 49.9 & 11.2 & 40.8 & 2.811 & & 0.006 & & & 0.13 \\
\hline & $1 / 30 / 20$ & \begin{tabular}{|l|l}
$11: 39: 25 \mathrm{AM}$ \\
\end{tabular} & 24.962 & 22.338 & 25.056 & 24.25 & 24.619 & 50.349 & 51.338 & 19.344 & 30.968 & -1.219 & -5.47 & 44.25 & 0.041 & 14.643 & 0.00 & & \begin{tabular}{|l|l|}
1.91667 \\
\end{tabular} & 49.9 & 10.8 & 40.7 & 2.803 & 0.006 & 0.006 & 000 & 0.150 & 0.13 \\
\hline 133 & $1 / 30 / 2004$ & \begin{tabular}{|l|}
$11: 40: 25 \mathrm{AM}$ \\
\end{tabular} & 24.973 & 22.358 & \begin{tabular}{|l|}
25.067 \\
\end{tabular} & 24.2 & & 50.517 & 51.471 & 19.365 & 30.997 & -1.219 & -5.467 & & 0.041 & 14.643 & 0.00 & & 1.93333 & 50.0 & & 40.8 & & 0.006 & 0.006 & & 0.150 & \\
\hline 134 & $1 / 30 / 2004$ & \begin{tabular}{|l|}
$11: 41: 25 \mathrm{AM}$ \\
\end{tabular} & 24.958 & 22.343 & \begin{tabular}{|l|}
25.042 \\
\end{tabular} & 24.195 & 25.055 & 50.575 & 51.533 & & 30.999 & -1.219 & -5.467 & 44.961 & 0.041 & 14.643 & & & 1.95 & 50.1 & 11.0 & 40.8 & & 0.006 & 0.006 & & 0.150 & \\
\hline 135 & $1 / 30 / 2004$ & \begin{tabular}{|l|}
$11: 42: 25 \mathrm{AM}$ \\
$11: 4325 \mathrm{AM}$
\end{tabular} & \begin{tabular}{l|l}
24.968 \\
24078
\end{tabular} & $\begin{array}{l}22.349 \\
22364 \\
\end{array}$ & \begin{tabular}{|l|l|}
25.053 \\
25058
\end{tabular} & $\frac{24.186}{2408}$ & 25.4 & 50.392 & 51.315 & $\begin{array}{l}19.369 \\
19.19\end{array}$ & 31.009 & \begin{tabular}{|l|l|}
-1.219 \\
\end{tabular} & -5.464 & $\begin{array}{ll}44.696 \\
4.907\end{array}$ & 0.041 & 14.643 & 0.004 & 118.00 & \begin{tabular}{|l|l|}
1.96667 \\
\end{tabular} & 49.8 & 11.0 & & 2.806 & $\begin{array}{ll}0.0066 \\
\end{array}$ & 0.006 & 0.000 & 0.150 & 0.13 \\
\hline $\begin{array}{ll}\frac{136}{137} \\
137\end{array}$ & $1 / 30 / 2004$ & \begin{tabular}{|l|}
$11: 43: 25 \mathrm{AM}$ \\
$11.44 \cdot 25 \mathrm{AM}$
\end{tabular} & 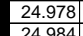 & $\begin{array}{ll}22.364 \\
22.37 \\
\end{array}$ & 25.058 & $\begin{array}{r}24.08 \\
24.006\end{array}$ & $\begin{array}{r}25.19 \\
25.656\end{array}$ & \begin{tabular}{|l|l|}
50.533 \\
50.4
\end{tabular} & 51.469 & $\begin{array}{l}19.418 \\
1935\end{array}$ & 30.972 & \begin{tabular}{|l|l|}
-1.219 \\
\end{tabular} & $\begin{array}{l}-5.464 \\
.450\end{array}$ & \begin{tabular}{ll|l|}
44.803 \\
4.507
\end{tabular} & 0.041 & 14.643 & & 119.00 & 1.98333 & 50.0 & 11.0 & & 2.810 & & 0.006 & & 0.150 & 0.13 \\
\hline \begin{tabular}{|c|}
138 \\
138 \\
\end{tabular} & 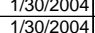 & $\begin{array}{l}\text { 11:44:25 AM } \\
11.45 .25 \mathrm{AM}\end{array}$ & $24.984 \mid$ & \begin{tabular}{r|r|}
22.37 \\
2373
\end{tabular} & 25.068 & 24.006 & 25.656 & $\begin{array}{r}50.44 \\
50.172\end{array}$ & $\frac{51.413}{51.195}$ & $\begin{array}{l}19.356 \\
19157 \\
\end{array}$ & $\begin{array}{ll}30.956 \\
30.915\end{array}$ & \begin{tabular}{|c|}
$\mid-1.216$ \\
-1222
\end{tabular} & & $\frac{45.072}{45015}$ & 0.041 & $\begin{array}{l}14.643 \\
14634 \\
\end{array}$ & 0.004 & $\frac{120.00}{1210}$ & 2 & & $\frac{11.0}{110} r y-3$ & & 2.806 & 0.006 & & & 0.150 & 0.13 \\
\hline & $1 / 30 / 2004$ & \begin{tabular}{|l}
$1.47 .26: 25 \mathrm{AM}$ \\
\end{tabular} & 25.004 & 22.375 & \begin{tabular}{|l|}
25.079 \\
\end{tabular} & $\frac{20.501}{23.851}$ & $\frac{25.526}{25.062}$ & So.135 & 51.1.176 & $\frac{19.107}{19.159}$ & $\frac{30.915}{30.878}$ & \begin{tabular}{|l|}
-1.2219 \\
-1.219 \\
\end{tabular} & 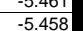 & $\frac{45.015}{43.486}$ & $\begin{array}{l}0.041 \\
0.041 \\
\end{array}$ & $\frac{14.643}{14.643}$ & & $\frac{122.0}{122.0}$ & 2.001061 & $\frac{49.1}{49.7}$ & 11.0 & & & & & & $\frac{0.150}{0.151}$ & $\frac{0.13}{0.13}$ \\
\hline 40 & $1 / 30 / 2004$ & 11:47:25 AM & 25.016 & 22.387 & 25.101 & 23.807 & 25.188 & & 51.27 & 19.28 & 31.011 & \begin{tabular}{|l|}
-1.219 \\
\end{tabular} & & 46.98 & 0.041 & 14.643 & & & 2.05 & 49.8 & 11.5 & & & & 0.006 & & 0.150 & - \\
\hline 41 & $1 / 30 / 2004$ & 11:48:25 AM & 25.011 & 22.371 & 25.08 & 23.762 & 25.842 & 50.471 & 51.502 & 19.414 & 30.9 & \begin{tabular}{|l|l|}
-1.219 \\
\end{tabular} & & 42.176 & 0.041 & 14.643 & & 124.00 & \begin{tabular}{|l|l|}
2.06667 \\
\end{tabular} & 50.0 & $\frac{\frac{1.5}{10.3}}{10.3}$ & 40 & 2.807 & & 0.006 & & 0.150 & \\
\hline 42 & $1 / 30 / 2004$ & 11:49:25 AM & 25.006 & 22.367 & \begin{tabular}{|l|}
25.086 \\
\end{tabular} & & 25.078 & 50.488 & 51.541 & 19.435 & 30.939 & \begin{tabular}{|l|l|}
-1.219 \\
\end{tabular} & -5.452 & 44.241 & 0.041 & 14.643 & & 125.00 & 2.08333 & 50.1 & 10.8 & 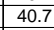 & 2.807 & & 0.006 & & 0.150 & \\
\hline 143 & $1 / 30 / 2004$ & 11:50:25 AM & 25.018 & 22.379 & 25.092 & 23.699 & 25.37 & 49.971 & 51.112 & 18.972 & 30.874 & \begin{tabular}{|l|l|}
-1.219 \\
\end{tabular} & -5.452 & & 0.041 & 14.643 & & 126.00 & 2.1 & 49.6 & 10.8 & 40.4 & 2.787 & 0.006 & 0.006 & 0.000 & 0.151 & 0.13 \\
\hline 144 & $1 / 30 / 2004$ & 11:51:25 AM & 25.014 & 22.38 & 25.084 & 23.69 & 24.886 & 50.1 & 51.222 & 19.128 & & \begin{tabular}{|l|l|}
-1.222 \\
\end{tabular} & -5.45. & 44.442 & 0.041 & & & 127.00 & \begin{tabular}{|l|}
2.11667 \\
\end{tabular} & 49.7 & 10.9 & 40.5 & 2.790 & & 0.006 & & 0.151 & \\
\hline & $1 / 30 / 2004$ & 11:52:25 AM & 25.015 & 22.381 & \begin{tabular}{|l|l|}
25.079 \\
\end{tabular} & 23.701 & 25.102 & 50.405 & 51.5 & 19.344 & & \begin{tabular}{|l|l|}
-1.219 \\
\end{tabular} & & 45.783 & 0.041 & 14.6 & & 128.00 & 2.13333 & 50.0 & 11.2 & 40.7 & 2.803 & & 0.006 & & 0.150 & \\
\hline 146 & $1 / 30 / 2004$ & 11:53:25 AM & 25 & 22.371 & \begin{tabular}{|l|}
25.064 \\
250
\end{tabular} & $\begin{array}{l}23.67 \\
23701 \\
\end{array}$ & 25.142 & 50.027 & 51.162 & 19.025 & 30.894 & \begin{tabular}{|l|l|}
-1.219 \\
\end{tabular} & -5.455 & $\begin{array}{l}44.876 \\
\end{array}$ & 0.041 & $\begin{array}{ll}14.643 \\
\end{array}$ & 0.003 & 129.00 & 2.15 & 49.7 & 11.0 & 40.5 & 2.790 & \begin{tabular}{|l|l|l|l|l}
0.006 \\
\end{tabular} & 0.006 & 0.000 & 0.151 & 0.13 \\
\hline $\mid \frac{147}{148}$ & $1 / 30 / 2004$ & 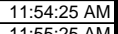 & \begin{tabular}{r|r|}
25.01 \\
25012
\end{tabular} & $\begin{array}{l}22.381 \\
22307 \\
\end{array}$ & \begin{tabular}{|r|}
25.07 \\
25.071
\end{tabular} & 23.701 & 25.512 & $\begin{array}{l}50.314 \\
50.114\end{array}$ & $\begin{array}{l}51.375 \\
5\end{array}$ & $\begin{array}{l}19.295 \\
10210\end{array}$ & $\begin{array}{l}30.941 \\
30.95 \\
\end{array}$ & \begin{tabular}{|c|}
-1.219 \\
\end{tabular} & & $\begin{array}{r}44.35 \\
5\end{array}$ & 0.041 & $\begin{array}{l}14.643 \\
14643\end{array}$ & & $\begin{array}{l}130.00 \\
131.00\end{array}$ & \begin{tabular}{|l|}
2.16667 \\
213223
\end{tabular} & 49.9 & 10.9 & \begin{tabular}{|l|l|}
40.6 \\
\end{tabular} & $\begin{array}{l}2.801 \\
2793 \\
\end{array}$ & & 0.006 & & 0.150 & 0.13 \\
\hline \begin{tabular}{|c|}
149 \\
\end{tabular} & $\begin{array}{l}\mid 1 / 30 / 2004 \\
1 / 30 / 2004\end{array}$ & \begin{tabular}{|l|}
$11: 55: 25 \mathrm{AM}$ \\
$115625 \mathrm{AM}$
\end{tabular} & $\frac{25.012}{25.007}$ & $\frac{22.387}{22.388}$ & $\left|\begin{array}{|l}25.011 \\
25.072\end{array}\right|$ & $\frac{23.692}{23.653}$ & 25.528 & & \begin{tabular}{|l|}
51.222 \\
51.218
\end{tabular} & $\begin{array}{l}19.219 \\
19.159\end{array}$ & $\begin{array}{l}30.905 \\
30.917 \\
\end{array}$ & $\mid$\begin{tabular}{|c|}
$\mid-1.221$ \\
-1.219 \\
\end{tabular} & & $\frac{45.084}{44.241}$ & & $\frac{14.6}{14.6}$ & & $\begin{array}{l}131.00 \\
132.00\end{array}$ & \begin{tabular}{|l|} 
\\
\end{tabular} & $\begin{array}{l}49.1 \\
49.7 \\
\end{array}$ & & 40.5 & $\begin{array}{l}2.93 \\
2.795 \\
\end{array}$ & & & & $\begin{array}{l}0.151 \\
0.150\end{array}$ & \\
\hline 150 & $1 / 30 / 2004$ & 11:57:25 AM & 25.008 & 22.384 & \begin{tabular}{|l|}
25.067 \\
\end{tabular} & 23.618 & 25.759 & 50.087 & 51.174 & 19.246 & & \begin{tabular}{|l|}
-1.222 \\
\end{tabular} & -5. & 45.41 & 0.041 & 14.643 & & 133.0 & \begin{tabular}{|l|}
2.21667 \\
\end{tabular} & 49.7 & 11.1 & & & & 0.006 & & 0.151 & \\
\hline & $1 / 30 / 2004$ & 11:58:25 AM & 25.019 & 22.405 & \begin{tabular}{|l|}
25.103 \\
\end{tabular} & 23.604 & 25.826 & 50.226 & 51.384 & 19.284 & 30.827 & -1.219 & -5.441 & 44.264 & 0.041 & 14.643 & 0.004 & 134.00 & 2.23333 & 49.9 & 10.8 & \begin{tabular}{|l|l|}
40.5 \\
\end{tabular} & 2.794 & 0.006 & 0.006 & 0.000 & 0.150 & 0.13 \\
\hline & & Ayoracs & 251 & 2217 & & 210 & & & $51+2$ & 106 & 300 & 11 & & 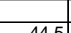 & 00 & & & & & 100 & 100 & 108 & 2911 & 0007 & 000 & & 0161 & \\
\hline & & $\begin{array}{l}\text { Mverages } \\
\text { Maximum }\end{array}$ & $\frac{25.1}{26.7}$ & $\frac{22.1}{22.4}$ & $\begin{array}{l}24.8 \\
26.2 \\
\end{array}$ & $\frac{24.0}{24.4}$ & $\frac{25.1 \#}{26.4 \#}$ & $\begin{array}{l}50.6 \\
53.8\end{array}$ & $\begin{array}{l}54.4 \\
54.1 \\
\end{array}$ & $\begin{array}{l}\frac{19.6}{22.0} \\
\end{array}$ & $\begin{array}{l}30.9 \\
33.8 \\
\end{array}$ & $\begin{aligned}-1.1 \\
0.4 \\
\end{aligned}$ & & $\begin{array}{l}44.5 \\
51.6\end{array}$ & 0.0 & $\begin{array}{l}\frac{14.6}{14.6} \\
\end{array}$ & 0.0 & & & $\frac{49.9}{52.6}$ & 10.9 & $\frac{4.8}{43.5}$ & $\begin{array}{l}2.811 \\
2.996\end{array}$ & \begin{tabular}{|l|}
0.0037 \\
0.037
\end{tabular} & 0.001 & & $\begin{array}{l}0.181 \\
0.837 \\
\end{array}$ & 0.13 \\
\hline 55 & & Median & 24.9 & 22.2 & 24.9 & 24.1 & & 50.6 & 51.3 & 19.5 & 30.9 & $\begin{array}{c}-1.2 \\
\end{array}$ & & 44.3 & 0.0 & 14.6 & & & & 49.8 & 10.9 & 40.7 & & & 0.006 & & & \\
\hline 156 & & Minimum & 24.6 & 21.2 & 23.9 & 23.4 & $23.9 \#$ & 47.4 & 49.2 & 17.2 & 27.8 & -1.2 & -5.9 & 40.2 & 0.0 & 14.6 & 0.0 & & & 47.7 & 9.9 & \begin{tabular}{|l|}
37.8 \\
\end{tabular} & 2.608 & & 0.006 & 0.0001 & & \\
\hline 157 & & $2 \times \operatorname{Std} \mathrm{Dev}$ & \begin{tabular}{|l|}
1.073 \\
\end{tabular} & 0.663 & 1.123 & 0.457 & 1.057 & 1.662 & 1.473 & 1.611 & 2.190 & 0.641 & 2.610 & 3.893 & 0.037 & 0.000 & 0.001 & & & 1.473 & 0.954 & 1.769 & 0.122 & & 0.005 & 0.0001 & & \\
\hline & Number of & f Points Used* $*$ & & 129 & & & & 129 & & 129 & & & & 129 & 129 & 129 & & & & 129 & 129 & 129 & 129 & & 129 & 129 & 129 & \\
\hline & & Backpulse po & bints in box & $x$ are not in & ncluded & & & & & & & & & & & & & & & & & & & & & & & \\
\hline & & & & & & & & & & & & & & & & & & & & & & & & & & & & \\
\hline 162 & & & & & & & & & & & & & & & & & & & & & & & 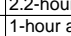 & $\begin{array}{l}\text { ir avg. = } \\
\text { avg. }\end{array}$ & \begin{tabular}{|l|} 
\\
0.006661 \\
0.0071
\end{tabular} & & & \\
\hline
\end{tabular}


RUN \# 1.16; STEADY STATE TEST AT 25 WT\% UDS

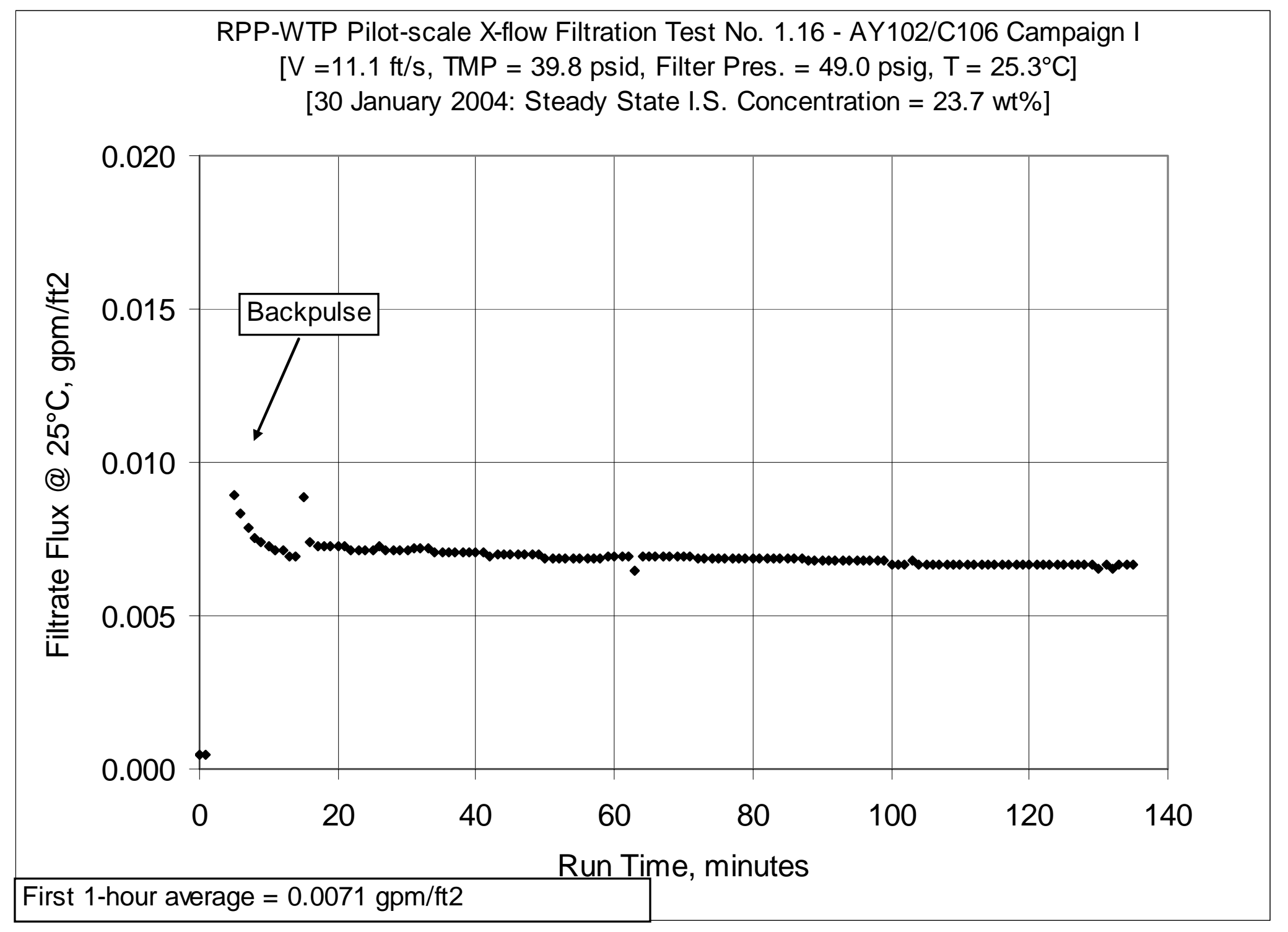


WSRC-TR-2005-00105, REVISION 0

SRNL-RPP-2005-00012, REVISION 0

RUN \# 1.16; STEADY STATE TEST AT 25 WT\% UDS - CONT.

\begin{tabular}{|c|c|c|c|c|c|c|c|c|c|c|c|c|c|c|c|c|c|c|c|c|c|c|c|c|c|c|c|c|}
\hline & A & B & $D$ & $\frac{E}{K S T e m}$ & $\mathrm{~F}$ & $\frac{G}{d e a s m}$ & $\frac{\mathrm{H}}{\text { ents }>}$ & $\mathrm{J}$ & $\frac{K}{K \ll \ll<}$ & \begin{tabular}{|c|}
$\mathrm{L}$ \\
Pressure
\end{tabular} & \begin{tabular}{|c|}
$M$ \\
Measure
\end{tabular} & \begin{tabular}{|l|}
$\mathrm{N}$ \\
ents $\gg \gg>$
\end{tabular} & 0 & $\mathrm{Q}$ & $\begin{array}{ll} & R \\
\ll<\text { flowe }\end{array}$ & $\frac{S}{\text { easure }}$ & $\frac{T}{2}$ & $\mathrm{~V}$ & $w$ & $\frac{1 x}{<\ll<<}$ & 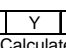 & $\mathrm{z}$ & AA & $A B$ & \begin{tabular}{|l|l|}
$A C \mid$ \\
$\gg \gg \gg \gg \gg$
\end{tabular} & $A D$ & $\mathrm{AE}$ & $\mathrm{AF}$ \\
\hline & DATE & TIME & Filtrate | & Cleaning & 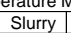 & Hi Amb. & Lo Amb. & BotTMP & Filter & \begin{tabular}{|l|l|l} 
Filter $\mathrm{dP}$ \\
\end{tabular} & TopTMP & Filtrate & \begin{tabular}{|l} 
Pulsepot \\
\end{tabular} & Slurry & Filtrate & $\begin{array}{l}\text { Nieasureme } \\
\text { Hi Filtate }\end{array}$ & & & & & & & & & & & & \\
\hline & & & & & $\operatorname{deg} C$ & & & psid & psig & \begin{tabular}{|l|} 
psid \\
\end{tabular} & \begin{tabular}{|l|} 
psid \\
\end{tabular} & \begin{tabular}{|l|} 
psig \\
\end{tabular} & psig & $\mathrm{gpm}$ & & & & & & & & & & & & & & \\
\hline & & & $\mathrm{T} 2$ & T3 & T1 & $\frac{T 4}{T 4}$ & & $\mathrm{dP2}$ & $\mathrm{P1}$ & $\mathrm{dP1}$ & $\mathrm{dP3}$ & P2 & & & $\mathrm{Q}^{2}$ & $\mathrm{Q}_{3}$ & Qbp & & & & & & & & & & & \\
\hline & & & & & & & & & & & & & & & & & & & & & & & & & & & & \\
\hline \begin{tabular}{|l|l}
7 \\
\end{tabular} & $\mid=\frac{\mid c r s-01 / 26}{1 / 29 / 2004}$ & 7:58:21 AM & \begin{tabular}{|l|}
19.97 \\
\end{tabular} & 14.564 & 11286 & 20.431 & 1.485 & 0.059 & -0.149 & 0.007 & 0.018 & -0186 & 0.098 & -0.087 & 0003 & \begin{tabular}{|l|l}
14.643 \\
\end{tabular} & & & & ter S & & 6707 & $\mathrm{~T}_{2}$ & & & & & \\
\hline 8 & $1 / 29 / 2004$ & 7:58:50 AM & 19.97 & 14.564 & 11.286 & 20.431 & 11.485 & 0.059 & -0.149 & 0.007 & 0.018 & -0.186 & 0.098 & -0.087 & 0.003 & 14.643 & 0.6 & & & Convers & rsion & & $\mathrm{m} 3 / \mathrm{m} 2$ & e/day/barc & $\arg / \mathrm{gpm} / \mathrm{f}$ & //tt/barg & & \\
\hline 9 & 1/29/2004 & 7:58:52 AM & 19.981 & 14.574 & 11.307 & 20.466 & 11.546 & & -0.149 & 0.007 & 0.021 & -0.168 & 0.101 & -0.083 & 0.003 & \begin{tabular}{|l|l|l|l|}
1443 \\
\end{tabular} & & & & & & & & & & & & \\
\hline 10 & $1 / 29 / 2004$ & 7:58:53 AM & 19.981 & 14.585 & 11.317 & 20.472 & 11.552 & 0.058 & -0.149 & 0.007 & & -0.174 & 0.104 & -0.087 & 003 & $\begin{array}{l}14.643 \\
\end{array}$ & & & & & & & & & & 1.4 psig & & \\
\hline & $1 / 29 / 2004$ & 7:58:54 AM & 19.991 & 14.575 & 11.317 & 20.477 & 11.552 & 0.059 & -0.149 & 0.007 & 1.023 & -0.168 & 0.104 & -0.081 & 0.003 & & 0.004 & & & 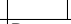 & & & & & & & & \\
\hline & 1/29/2004 & 7:58:55 AM & 19.986 & $\begin{array}{l}14.58 \\
1.58 \\
\end{array}$ & $\frac{11.317}{11317}$ & 20.472 & $\frac{11.562}{11.572}$ & 0.061 & -0.149 & 0.008 & .021 & -0.177 & 0.104 & -0.087 & 0.003 & 14.643 & 0.005 & & & Pressu & e P1is & & & 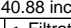 & inches of $w$ & water tubin & & F \\
\hline 14 & $1 / 292004$ & $7.50 .57 \mathrm{Al}$ & | & 4.450 & 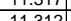 & 20.411 & 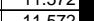 & 0 & - & $\begin{array}{l}0.008 \\
0.08\end{array}$ & 0.025 & 年 & ( & - & $\begin{array}{l}0.0035 \\
0.002\end{array}$ & $\begin{array}{l}14.643 \\
1.643\end{array}$ & & & & & & & & Filliat & aree flux & & & \\
\hline & & & & & & & & & & & & & & & & & & ime & & Press & Vel. & TMP & TMP & $\mathrm{gnm}$ & & pm & & \\
\hline 16 & Data - Per Mi & & & & & & & & & & & & & & & & & & Hour & psig. & $\begin{array}{lll}\mathrm{N} f l . s \\
\mathrm{fts}\end{array}$ & psi & bar & |lit2 & & $\mathrm{ftz} / \mathrm{psi}$ & $2 / \mathrm{psi}$ & lay/b \\
\hline & $1 / 30 / 2004$ & $\begin{array}{l}02: 54 \text { PM } \\
2: 54 \mathrm{PM}\end{array}$ & $\begin{array}{l}25.387 \\
24762\end{array}$ & 22.387 & \begin{tabular}{|l|l|}
25.071 \\
25.067
\end{tabular} & $\begin{array}{l}23.552 \\
23557\end{array}$ & 24.604 & $\begin{array}{l}39.152 \\
7304 \\
\end{array}$ & \begin{tabular}{|l|}
50.725 \\
5634
\end{tabular} & $\begin{array}{l}19.529 \\
\end{array}$ & \begin{tabular}{r|r|}
19.8 \\
1850 \\
\end{tabular} & \begin{tabular}{rl|l}
6.134 \\
7030
\end{tabular} & 91.466 & $\begin{array}{l}44.711 \\
3505\end{array}$ & 0.003 & \begin{tabular}{|l|l|}
14.643 \\
14633
\end{tabular} & $\begin{array}{ll}0.004 \\
164\end{array}$ & & & 49.3 & $\begin{array}{ll}11.0 \\
10.7\end{array}$ & 29.5 & 2.032 & 0.000 & 0.000 & 0.000 & & 0. \\
\hline & 1/1/30/2004 & $03: 54 \mathrm{PMM}$ & & & & & & & & & & 70.294 & & $\frac{43.505}{48.632}$ & & & & & & & & & & & & & & -0.0 \\
\hline$\frac{15}{20}$ & $1 / 30 / 2004$ & $2: 05: 54 \mathrm{PM}$ & $\begin{array}{l}25.677 \\
25.677\end{array}$ & $\frac{22.398}{22.418}$ & \begin{tabular}{|l|}
25.88 \\
25.442
\end{tabular} & $\frac{0.045}{23.713}$ & 24.934 & 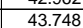 & $\mid$\begin{tabular}{|l|}
48.942 \\
49603
\end{tabular} & $\begin{array}{l}11.999 \\
14.419\end{array}$ & $\frac{30.915}{29.312}$ & 0.35 & 8 & $\frac{48.632}{44.6}$ & $\begin{array}{l}0.153 \\
0.088\end{array}$ & $\frac{14.645}{14.643}$ & & $\frac{2.00}{3.06}$ & 0.05 & 48. & $\frac{11.9}{10.9}$ & 36 & & 183 & & & & 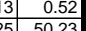 \\
\hline & $1 / 30 / 2004$ & 12:06:54 PM & 25.84 & 22.396 & \begin{tabular}{l|}
25.5745 \\
25.57
\end{tabular} & 23.721 & 23.671 & $\begin{array}{l}44.37 \\
\end{array}$ & 50.215 & \begin{tabular}{|l|}
14.419 \\
16.028 \\
\end{tabular} & 28.255 & 0.208 & 416 & 45.151 & 0.07 & $\begin{array}{l}14.045 \\
14.643\end{array}$ & & 4.06 & 0.06667 & 48.7 & $\frac{11.3}{11.1}$ & & & $\mid$\begin{tabular}{|l}
2.105 \\
2183
\end{tabular} & $\frac{1.148}{148}$ & .059 & 5915 & \\
\hline & & & 25.96 & & & & & & & & & & & & & $\begin{array}{l}14.045 \\
14.643 \\
\end{array}$ & & & & & & & & & & & & \\
\hline & 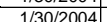 & 12:08:54 PM & & 22.406 & \begin{tabular}{|l|}
25.745 \\
\end{tabular} & 23.836 & & 44.903 & \begin{tabular}{|l|l|}
350.76 \\
\end{tabular} & $\mid \begin{array}{l}\mid 17.692 \\
19\end{array}$ & 27.159 & $\frac{0.254}{0.254}$ & 468 & 45.589 & 0.057 & $\begin{array}{l}14.045 \\
14.643 \\
\end{array}$ & & 600 & 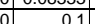 & $\frac{45.3}{49.3}$ & $\frac{\frac{1.1}{112}}{112}$ & 360 & 年 2484 & 0.008 & 0.008 & 000 & $\frac{0.241}{0.231}$ & \\
\hline & & & 26.169 & & & & & 905 & & $18.019 \mid$ & & 0.254 & & 45.783 & 0.054 & 6013 & & & & & & & & & & & & \\
\hline & $\frac{\pi}{1 / 2}$ & :54 PM & 26.249 & 22.416 & & 23.906 & 2 & & \begin{tabular}{|l|}
50.975 \\
\end{tabular} & $\mid 18.237$ & 26.6 & 0.251 & & 45.579 & 0.052 & & & .06 & 0.1333 & & $\frac{1.2}{11.2}$ & & & & & & & \\
\hline & 30/2004 & & & 22.442 & 25.94 & & & & \begin{tabular}{|l|l|} 
& 51.133 \\
\end{tabular} & \begin{tabular}{|c|}
18.515 \\
\end{tabular} & & & & .026 & \begin{tabular}{l|l}
0.051 \\
\end{tabular} & & & & 0.15 & & $10.3 \mathrm{r}$ & & & & & & & \\
\hline & $1 / 30 / 2$ & 12:12:54 PM & 26.358 & 22.41 & 25.939 & 24.011 & 2 & 353 & 51.278 & 18.608 & & 0.318 & & 45.145 & 0.05 & & & & 0.16667 & 49.8 & 11.1 & 36.0 & 2.483 & & & & & \\
\hline & $1 / 5$ & 2:13:54 PM & 26.415 & 22.427 & 25.986 & 24.023 & 25.123 & .253 & 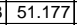 & \begin{tabular}{|l|l|l|l|}
1886 \\
\end{tabular} & 26.603 & 0.324 & 53 & 44.233 & 0.049 & \begin{tabular}{l|l}
14.643 \\
\end{tabular} & & 11.00 & 0.1833 & 49.7 & 10.8 & 35.9 & 92.477 & 0.007 & 0.007 & 0.000 & 0.198 & \\
\hline & & & 26.451 & 22.433 & . & 24.014 & & & 353 & & & 324 & & 45.612 & 049 & & & 12.00 & & & 11.2 & (0.0.0 & 2.48 & & & & 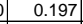 & \\
\hline 30 & & & 26.497] & & & & & & & & & 0.338 & & & & & & & 0.21667 & & & & & & & & & \\
\hline & & 12:16:5 & & 22.436 & 26.004 & 24.207 & & 45. & \begin{tabular}{|l|} 
\\
\end{tabular} 1.083 \mid & $\begin{array}{r}18.74 \\
10.020\end{array}$ & 26.494 & 0.338 & & 4.5 & & & & 14.6 & 2.23335 & 49 & 10.9 & 35. & 2.471 & & & & & \\
\hline , & & & 25.5627 & 22.4 & . & & & & $\mid \begin{array}{l}\mid 51.23 \\
507\end{array}$ & & & $\frac{222}{210}$ & & & & & & & & & & & & & & & & \\
\hline 34 & & $\begin{array}{l}12.10 .54 \mathrm{PM} \\
12 \cdot 19: 54 \mathrm{PM}\end{array}$ & $\begin{array}{l}25.094 \\
25768\end{array}$ & $\begin{array}{l}22.453 \\
22.47\end{array}$ & \begin{tabular}{|l|}
25.944 \\
25.963
\end{tabular} & $\begin{array}{l}24.445 \\
24.462\end{array}$ & & & & \begin{tabular}{|l|}
19.34 \\
20.312
\end{tabular} & & $\begin{array}{l}-1.219 \\
-1.219 \\
\end{array}$ & & & $\frac{0.051}{0.05}$ & & & & $\frac{0.20001}{0.28333}$ & & $\frac{11.2}{11.5}$ & 404 & 12.020 & & & & . & \\
\hline & 10 & & 25.814 & $\frac{2.2 .4 !}{22.486}$ & $\mid$ & 24.458 & & & $\mid$\begin{tabular}{|l|l|}
$\mid$ \\
$\mid 50.47$
\end{tabular} & $\mid$ & & $\begin{array}{l}-1.219 \\
-1.219 \\
\end{array}$ & & 08 & 0.05 & & & 180 & 00 & & 1116 & 40.4 & 2786 & & 0.07 & 000 & & 30 \\
\hline & & & & & & & & & & & & & & & & & & & & & 112 & & & & & & & \\
\hline & & & 25842 & 22509 & $|25.947|$ & & & & & & & 219 & & 17 & 0.05 & & & 20. & 333 & . & 116 & 405 & 793 & & & & (179 & \\
\hline & & & & & & & & & & & & & & & & & & 21. & & & $\frac{11.4}{11.4}$ & & & & & & & \\
\hline & & & \begin{tabular}{|l|}
25.839 \\
\end{tabular} & & 5.939 & & & & & & & & & & & & & & & & 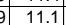 & & 788 & & & & & \\
\hline & $1 / 30 / 20$ & & 25.845 & 22.532 & 25.935 & & & & & & & & & & & & & 23. & & & 1.9 & & 2.789 & & & & & \\
\hline & & 12:26:54 & 25.857 & 22.544 & & & & & & & & & & & & & & 24. & 0.4 & & 105 & & 2797 & & & & & \\
\hline $\begin{array}{lll}4 \\
\end{array}$ & & & 25.852 & 22.5 & \begin{tabular}{|l|}
25.892 \\
\end{tabular} & 24.3 & & & & & & & & & 0.049 & & & & \begin{tabular}{|l|}
0.41667 \\
\end{tabular} & 49 & $\overline{11.5}$ & & 790 & & 0.007 & & & \\
\hline 45 & & 12.20.0 & 25.853 & & & & & & & & & & & & & & & & & 49 & 11.0 & & 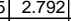 & & & & & \\
\hline & & & & & & & & & & & & & & & & & & & & & 11.4 & & & & & & & \\
\hline & & & & & & & & & & 19. & & & & & & & & & & & & & & & & & & \\
\hline$\frac{4}{4}$ & & & & & & & & & & & & & & & & & & & & & $\begin{array}{ll}11.5 \\
112\end{array}$ & & & & & & & \\
\hline$\frac{4}{4}$ & & & $\begin{array}{l}20.021 \\
25811\end{array}$ & & & & & & & & & & & & & & & & & & & & & & & & & \\
\hline 4 & & & & $\frac{22.4}{22.5}$ & & & & & & & & & & & & & & & & & & & & & & & & \\
\hline & & & & & & & & & & & & & & & & & & & & & & & & & & & & \\
\hline & & & 25.72 & 22.5 & & & & & & & & & & & & & & & & & & & & & & & & \\
\hline & & & & & & & & & & & & & & & & & & & & & 11.4 & & & & & & & \\
\hline & & & 25.6 & & & & & & & & & & & & & & & & & & $\frac{11.1}{11.1}$ & & & & & & & \\
\hline 5 & $1 / 30 / 2$ & & 25.626 & 22.5 & & & & & & & & & & & & & & & & 486 & 11.0 & & & & & & 0.176 & \\
\hline & & & 25.597 & 22.5 & & & & & & & & & & & & & & & 0.63 & & 11.6 & & & & & & & \\
\hline & & & & & & & & & & & & & & & & & & & & & & & & & & & & \\
\hline & & & 25.544 & & & & & & & & & & & & & & & & & & & & & & & & & \\
\hline & & & 25.5 & 22.5 & & 23.6 & & & & & & & & & & & & & & & & & & & & & & \\
\hline & & & 25.4 & 22. & & $\frac{2.6}{3.6}$ & & & & & & & & & & & & & & & 11.3 & & & & & & & \\
\hline & & & 25.4 & $\frac{24 .}{20}$ & & & & & & & & & & & & & & & & & 0.4 & & & & & & & \\
\hline & & & 25.44 & $\frac{22}{225}$ & & 23. & & & & & & & & & & & & & & & & & & & & & & \\
\hline & & & $\frac{20.404}{25405}$ & 226 & & & & & & & & & & & & & & & & & & & & & & & & \\
\hline & & & & & & & & & & & & & & & & & & & & & & & & & & & & \\
\hline & & & 25342 & & & 23.582 & & & & & & & & & & & & & & & & & & & & & & \\
\hline & & & & & & 23.622 & & & & 19. & 36 & & & & & & & & & 8.7 & & & & & & & & \\
\hline & & & 25.297 & 22614 & 24 & 23.76 & & & & 19. & 30.6 & -1.219 & & & & & & & & . & & & & & & & & \\
\hline 68 & $1 / 30 / 2004$ & & 25.262 & 22.614 & 24.931 & 23.793 & & & $7 \quad 50.2$ & \begin{tabular}{|l|l|}
19.7 \\
\end{tabular} & 30.0 & -1.219 & & 47.9 & 0.0 & & & 51 & 0.85 & 189 & & & & & & & $\begin{array}{l}0.172 \\
\end{array}$ & \\
\hline & $1 / 30 / 2$ & $2: 54: 4$ & 25.253 & $\overline{22.631}$ & & 23.83 & & & & & 30.07 & -1.222 & -5.9 & 44.227 & & & & 52. & & 48.8 & 10.8 & & & & 0.0 & & 0.172 & \\
\hline & & & 25.224 & 22.636 & 24.898 & 24.011 & & & \begin{tabular}{|l|l|l|}
549.939 \\
\end{tabular} & \begin{tabular}{|r|}
19.59 \\
\end{tabular} & 30.057 & \begin{tabular}{|l|}
-1.219 \\
\end{tabular} & & 44.425 & 0.046 & \begin{tabular}{|c|c|}
5 & 14.643
\end{tabular} & & 53.00 & $\begin{array}{ll}0 & 0.8833 \\
\end{array}$ & 48.5 & \begin{tabular}{l|l}
5 & 10.9 \\
\end{tabular} & \begin{tabular}{|l|l|}
99.8 \\
\end{tabular} & \begin{tabular}{|l|l|}
3 & 2.74 \\
\end{tabular} & & \begin{tabular}{|l|}
0.007 \\
\end{tabular} & 0.000 & $\begin{array}{l}0.173 \\
\end{array}$ & \\
\hline
\end{tabular}


WSRC-TR-2005-00105, REVISION 0

SRNL-RPP-2005-00012, REVISION 0

RUN \# 1.16; STEADY STATE TEST AT 25 WT\% UDS - CONT.

\begin{tabular}{|c|c|c|c|c|c|c|c|c|c|c|c|c|c|c|c|c|c|c|c|c|c|c|c|c|c|c|c|c|}
\hline & & & & & & & & & & & & & & & & & & & & & & & & & & & & \\
\hline & & & & & & & & & & & & & & & & & & & & & & & & & 0.007 & & 0.17 & \\
\hline & & $54 \mathrm{PM}$ & 25.187 & & & 23.979 & & & & 19.60 & & & 9 & & & 14.643 & & & .91667 & & & & 2.753 & 0.007 & 0.007 & 0.000 & 0.173 & \\
\hline & & & 25.174 & & & & 25.461 & & & & & & -5.941 & & & 14.643 & & & 3333 & & & & 2.751 & & & & & \\
\hline & $1 / 30 / 2004$ & 12:59:54 PM & 25.155 & 22.668 & \begin{tabular}{|l|}
24.809 \\
\end{tabular} & 24.077 & 26.056 & 49.751 & 50.192 & 19.59 & 30.133 & \begin{tabular}{|l|}
-1.219 \\
\end{tabular} & -5.944 & 41.49 & 0.046 & 14.643 & & 57.00 & 0.95 & 48.7 & & 39. & 2.154 & & 0.007 & 0.000 & 0.173 & \\
\hline & & & 25.143 & 22.675 & \begin{tabular}{|l|}
24.787 \\
271 \\
\end{tabular} & 24.085 & & 835 & \begin{tabular}{|r}
50.31 \\
5
\end{tabular} & 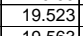 & & \begin{tabular}{|r|}
-1.219 \\
\end{tabular} & $\begin{array}{l}-5.944 \\
\end{array}$ & 45.804 & 0.046 & 14.643 & & & \begin{tabular}{l|l}
0 & 0.96667 \\
\end{tabular} & 48.8 & & & & & & & & \\
\hline & 1/30/2004 & 1:01:54 PM & & 22.675 & \begin{tabular}{|l|}
24.742 \\
\end{tabular} & 24.145 & 25.135 & & & & & & $\begin{array}{l}-5.944 \\
\end{array}$ & & & & & & \begin{tabular}{l|l}
0 & 0.98333 \\
\end{tabular} & 49.0 & & & & & 0.007 & 0.000 & & \\
\hline & $1 / 30 / 2 \mid$ & 1:02:54 PM & 25.116 & 22.709 & \begin{tabular}{|r|}
24.72 \\
\end{tabular} & 24.254 & 25.008 & & 50.202 & \begin{tabular}{|l|l|}
19.233 \\
\end{tabular} & & -1.219 & \begin{tabular}{|c|}
-5.944 \\
\end{tabular} & 45.583 & & 4.643 & & 60.00 & & 48.7 & & & & .007 & 0.007 & 0.000 & .172 & \\
\hline & 30/2004 & 1:03:54 PM & \begin{tabular}{|l|}
25.086 \\
2000
\end{tabular} & $\begin{array}{l}22.704 \\
\end{array}$ & \begin{tabular}{|l|l|}
24.7 \\
2.0
\end{tabular} & 24.224 & $\begin{array}{r}25.663 \\
\end{array}$ & 49.704 & \begin{tabular}{|l}
50.248 \\
\end{tabular} & \begin{tabular}{|l|l|}
19.133 \\
\end{tabular} & & & $\begin{array}{r}-5.944 \\
\end{array}$ & & & 1.643 & & 61.00 & $\begin{array}{l}01667 \\
0207\end{array}$ & & & & & & & 0.000 & & \\
\hline & $\begin{array}{l}1 / 30 / 2004 \\
13 / 2004\end{array}$ & $\begin{array}{l}1: 04: 54 \mathrm{PM} \\
1 \cdot 105.54 \mathrm{PM}\end{array}$ & 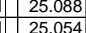 & \begin{tabular}{l|}
22.726 \\
2717
\end{tabular} & \begin{tabular}{|l|}
24.692 \\
24.658 \\
\end{tabular} & $\begin{array}{l}24.246 \\
24.26\end{array}$ & $\begin{array}{r}25.59 \\
26.095 \\
\end{array}$ & & \begin{tabular}{|r|}
50.233 \\
5024 \\
5
\end{tabular} & \begin{tabular}{|l|l|}
19.364 \\
19295
\end{tabular} & & & .944 & \begin{tabular}{|l|l|}
43.716 \\
45116
\end{tabular} & & & & 62.00 & .03333 & & & 40. & 2.767 & .007 & & 0.000 & & D.15 \\
\hline & $1 / 30 / 2004$ & $\begin{array}{l}1.05 .54 \\
1: 06: 54 \mathrm{PM}\end{array}$ & \begin{tabular}{|l}
25.054 \\
25.062
\end{tabular} & 22.75 & \begin{tabular}{|l|}
24.658 \\
24.666 \\
\end{tabular} & $\begin{array}{r}24.262 \\
24.35\end{array}$ & $\frac{26.095}{26.293}$ & $\begin{array}{l}\frac{49.669}{49.694} \\
\end{array}$ & \begin{tabular}{|l|}
50.22 \\
50.275 \\
\end{tabular} & \begin{tabular}{|l|l|}
9.295 \\
19.105 \\
\end{tabular} & $\begin{array}{l}30.332 \\
30.445 \\
\end{array}$ & \begin{tabular}{|l|}
-1.225 \\
-1.222 \\
\end{tabular} & $\begin{array}{l}-5.8 / 4 \\
-5.895 \\
\end{array}$ & \begin{tabular}{|l|}
44.287 \\
44.287
\end{tabular} & $\begin{array}{l}0.043 \\
0.046\end{array}$ & $\begin{array}{l}\frac{14.643}{14.643} \\
\end{array}$ & 0.003 & $\frac{63.00}{64.00}$ & \begin{tabular}{l|l} 
& 1.05 \\
0 & 1.06667
\end{tabular} & $\begin{array}{l}48.8 \\
48.8\end{array}$ & & & $\frac{2.758}{2.763}$ & & $\frac{0.006}{0.007}$ & $\frac{0.000}{0.000}$ & $\begin{array}{l}0.162 \\
0.173\end{array}$ & 0.14 \\
\hline & $1 / 30 / 2004$ & 1:07:54 PM & 25.049 & 22.767 & \begin{tabular}{|l|}
24.638 \\
\end{tabular} & 24.392 & 26.31 & & 50.443 & 19.325 & & & -5.938 & \begin{tabular}{|l|l|}
46.914 \\
\end{tabular} & 046 & 14.643 & & & $\begin{array}{ll}0 & 1.08333 \\
\end{array}$ & & & & & & & 0.000 & & \\
\hline & & 1:08:54 PM & & & & \begin{tabular}{|l|l|}
24.43 \\
\end{tabular} & 26.582 & & & & & & & & & & & 66.00 & & & & 40. & & & & & & \\
\hline & $1 / 30 / 2$ & 1:09:54 PM & \begin{tabular}{|l|}
25.024 \\
\end{tabular} & 22.802 & \begin{tabular}{|l|}
24.648 \\
\end{tabular} & 24.392 & 26.764 & 50.135 & 50.783 & 9.535 & & .222 & \begin{tabular}{|c|}
-5.938 \\
\end{tabular} & 317 & & 643 & & 67.00 & 11667 & & & & & .007 & 0.007 & 0.000 & 0.172 & \\
\hline & $1 / 30 / 2004$ & 1:10:54 PM & \begin{tabular}{l|l|} 
& 25.011 \\
\end{tabular} & \begin{tabular}{|l|l|}
22.8099 \\
22.92
\end{tabular} & \begin{tabular}{|l|l|}
24.675 \\
2.70
\end{tabular} & \begin{tabular}{|l|}
24.449 \\
24.919
\end{tabular} & 26.002 & $\begin{array}{l}49.754 \\
4932\end{array}$ & \begin{tabular}{|l|l|}
50.296 \\
50382
\end{tabular} & $\begin{array}{l}19.388 \\
19.461\end{array}$ & & \begin{tabular}{|l|l|}
-1.219 \\
\end{tabular} & \begin{tabular}{|l|}
-5.938 \\
5.941 \\
\end{tabular} & \begin{tabular}{|l|l|}
42.108 \\
4519. \\
\end{tabular} & & 4.643 & & & $\frac{13333}{1.15}$ & & & & & & & 0.000 & $\begin{array}{l}0.173 \\
0.723\end{array}$ & 1.15 \\
\hline & $\begin{array}{l}\frac{1 / 301 / 2004}{1 / 30 / 2004} \\
\end{array}$ & $\begin{array}{l}1: 11: 54 \mathrm{PM} \\
11 \cdot 254 \mathrm{PM}\end{array}$ & \begin{tabular}{l|l} 
& 25.00 \\
\end{tabular} & $\begin{array}{r}22.82 \\
22885\end{array}$ & \begin{tabular}{|l|}
24.706 \\
24.726
\end{tabular} & \begin{tabular}{|r|}
24.491 \\
24.42
\end{tabular} & $\frac{24.849}{24.148}$ & & \begin{tabular}{|l|}
50.383 \\
50.509 \\
\end{tabular} & $\begin{array}{l}19.461 \\
19.497\end{array}$ & & $\begin{array}{l}-1.222 \\
-1.222 \\
\end{array}$ & $\begin{array}{l}-5.941 \\
-5.938\end{array}$ & \begin{tabular}{|l|l|l|}
.418 \\
023
\end{tabular} & & & & & $\begin{array}{r}1.15 \\
1.6667\end{array}$ & & & & 2.763 & & $\frac{0.007}{0.007}$ & & & D.15 \\
\hline & $1 / 30 / 2004$ & 1:13:54 PM & 24.994 & 22.832 & \begin{tabular}{|l|}
24.773 \\
\end{tabular} & $\begin{array}{ll}24.427 \\
2.427\end{array}$ & $\begin{array}{l}24.140 \\
24.901\end{array}$ & & & $\begin{array}{l}19.457 \\
19.293 \\
\end{array}$ & & \begin{tabular}{|l|l|} 
& -1.222 \\
\end{tabular} & -5.941 & 44.26 & & & & 71.00 & 1.18333 & \begin{tabular}{|l|l|}
48.7 \\
\end{tabular} & & & & & & & $\frac{0.172}{0.173}$ & $\frac{0.15}{0.15}$ \\
\hline & & 1:14:54 PM & 24.996 & 22.859 & \begin{tabular}{|l|l|}
24.815 \\
\end{tabular} & 24.474 & 25.967 & 49.542 & 50.14 & 19.191 & 30.412 & \begin{tabular}{|l|l|l|}
-1.222 \\
\end{tabular} & -5.941 & 46.772 & 0.046 & 14.643 & & 72.00 & \begin{tabular}{|lll}
0 & 1.2 \\
\end{tabular} & & & 40.0 & 2.756 & & & & & \\
\hline & $1 / 30 / 2004$ & 1:15:54 PM & 24.992 & 22.866 & & & $26.39 \varepsilon$ & & 50.466 & 19.42 & & & & 47.326 & & & & & 1.21667 & & & & & & & & & \\
\hline & & 1:16:54 PM & 24.994 & 22.883 & \begin{tabular}{|l|l|} 
& \\
\end{tabular} & 24.422 & 26.80 & & & 9.429 & & & 94 & (48.244 & & & & & & & & & & & 007 & & & \\
\hline & & & & & & & & & & & & & & 846 & & & & & & & & & & & & & & \\
\hline & & 1:18:5 & & & & & & & & & & & & & & & & & & & & & & & & & & \\
\hline & & & & & & 577 & & & 50.441 & & & & & & & & & & & & & & & & & & & \\
\hline & 1 & 1:20:54 PM & 25.031 & 22.945 & \begin{tabular}{|l|}
25.006 \\
\end{tabular} & \begin{tabular}{|l|}
24.495 \\
\end{tabular} & 5.73 & & 50.312 & \begin{tabular}{|l|}
19.347 \\
\end{tabular} & & \begin{tabular}{|l|}
-1.219 \\
\end{tabular} & -5 & 44.552 & & & & 78.00 & & & & 39.9 & 2.75 & & 0.007 & & 0.172 & \\
\hline & & 1:21:54 PM & 25.049 & 22.973 & \begin{tabular}{|l|}
25.039 \\
\end{tabular} & \begin{tabular}{|l|l|}
24.458 \\
\end{tabular} & 26.564 & & 50.387 & & & & 338 & & & & & & .31667 & & & & & & & & & \\
\hline & & 1:22:54 PM & 25.061 & 22.985 & & & & & & & & & & & & & & & & & & & & & & & 0.171 & \\
\hline & & 1:23:54 PM & \begin{tabular}{|l|l|l}
1 & 25.077 \\
20.09
\end{tabular} & 23.007 & \begin{tabular}{|l|}
25.092 \\
2.05 \\
\end{tabular} & \begin{tabular}{|l|}
24.436 \\
2.317 \\
\end{tabular} & & & 50.337 & & & \begin{tabular}{|l|}
-1.219 \\
\end{tabular} & & .339 & & & & & 1.35 & & & & & & 0.007 & 0.000 & 0.171 & \\
\hline & & $\begin{array}{l}1: 24: 54 \mathrm{PM} \\
1 \cdot 25.54 \mathrm{PM}\end{array}$ & & & \begin{tabular}{|r|}
25.054 \\
251 \\
\end{tabular} & & $\frac{26.35}{26.44}$ & & $\begin{array}{r}50.42 \\
50.271 \\
\end{array}$ & \begin{tabular}{r|}
19.36 \\
19157 \\
\end{tabular} & & & & $\begin{array}{l}5.217 \\
1719 \\
170\end{array}$ & & & & & .36667 & & & & & & & & 0.171 & .15 \\
\hline & $\frac{1 / 30 /}{1 / 30 /}$ & $\begin{array}{l}1: 25: 544 \mathrm{M} \\
1.26654 \mathrm{PM}\end{array}$ & \begin{tabular}{l|l} 
& 25.096 \\
1 & 25.097
\end{tabular} & $\frac{23.035}{23.041}$ & \begin{tabular}{|r|}
25.1 \\
25.102
\end{tabular} & $\begin{array}{r}24.264 \\
24.23 \\
\end{array}$ & & & \begin{tabular}{|l|l|}
50.271 \\
50.345
\end{tabular} & $\begin{array}{r}19.157 \\
19.2\end{array}$ & & \begin{tabular}{|c|}
-1.219 \\
-1.222 \\
\end{tabular} & $\begin{array}{l}-5.94 \\
-5.94 \\
\end{array}$ & \begin{tabular}{|l|}
44.749 \\
4553 \\
\end{tabular} & & & & & .38333 & & & 39.9 & & & & & 0.171 & $\frac{15}{15}$ \\
\hline & $1 / 30 / 26$ & $1: 27: 54 \mathrm{PM}$ & \begin{tabular}{|l|l|} 
& 25.108 \\
\end{tabular} & 23.058 & \begin{tabular}{|l|}
25.102 \\
25.118 \\
\end{tabular} & \begin{tabular}{|r|}
24.23 \\
24.241 \\
\end{tabular} & $\frac{26.3}{26.4}$ & & \begin{tabular}{|l|l|}
50.345 \\
50.244
\end{tabular} & $\begin{array}{r}19.2 \\
18.91 \\
\end{array}$ & & \begin{tabular}{|l|l|}
-1.2 \\
\end{tabular} & -5.94 & \begin{tabular}{|l|}
43.497 \\
43.457
\end{tabular} & & & & & \begin{tabular}{|r|}
1.4 \\
1.41667
\end{tabular} & & & & & & & & 171 & $\frac{1.15}{15}$ \\
\hline & & $1: 28: 5$ & 25.114 & 23.064 & & 24.207 & & & & 19.152 & & & & & & & & & & & & & & & & & & \\
\hline & & & 25.126 & & 25. & & & & & & & & & & & & & & 1.45 & & & & & & & & & \\
\hline & & & 25.142 & & \begin{tabular}{|l|}
25.137 \\
\end{tabular} & & & & 50. & & & & & & & & & & & & & & & & & & & \\
\hline & $\frac{1 / 30}{1 / 20}$ & & \begin{tabular}{|l|l|}
25.148 \\
25.139
\end{tabular} & \begin{tabular}{|l|l|}
23.103 \\
23093
\end{tabular} & \begin{tabular}{|l|l|}
25.143 \\
25.132
\end{tabular} & $\begin{array}{l}24.156 \\
2410\end{array}$ & 26.2 & & \begin{tabular}{|l|l|}
50.638 \\
50759
\end{tabular} & 1 & & & -5 & \begin{tabular}{|l|}
45.27 \\
43209
\end{tabular} & & & & 9.00 & 88333 & & & 40.3 & 878 & & & 000 & 170 & 14 \\
\hline & & $\begin{array}{l}1: 32: 54 \mathrm{PM} \\
1.3355 \mathrm{PM}\end{array}$ & $\begin{array}{l}25.138 \\
25.159\end{array}$ & $\begin{array}{l}23.093 \\
23.099\end{array}$ & \begin{tabular}{|l|}
25.133 \\
25.159
\end{tabular} & \begin{tabular}{|l|}
24.106 \\
24.071 \\
\end{tabular} & & & \begin{tabular}{|l|l|}
50.758 \\
50.269
\end{tabular} & & & \begin{tabular}{|c|}
-1.219 \\
-1219
\end{tabular} & & & & & & & & & & & & & & & 169 & $1 \frac{14}{14}$ \\
\hline & & & $\begin{array}{l}25.159 \\
25.163\end{array}$ & (3) & \begin{tabular}{|l|}
25.1 .15 \\
25.15
\end{tabular} & & & & \begin{tabular}{|l|}
50.269 \\
50.634 \\
\end{tabular} & & & & & & & & & & & & & & & & & & & $\frac{14}{14}$ \\
\hline & & $1: 35: 5$ & 25.167 & & 25.1 & 24.049 & & & & 18.7 & & & & & & & & & & & & & & & & & & \\
\hline & & & 25.16 & & & & & & & & & & & & & & & & & & & & & & & & & \\
\hline & & & 25.165 & & 25.1 & & & & 50.5 & & & & & & & & & & 1.58333 & & & & & & & & & \\
\hline & $\frac{1 / 30}{1 / 30}$ & $\begin{array}{l}1: 38: 5 \\
1.39\end{array}$ & $\begin{array}{l}25.163 \\
25.168\end{array}$ & $\begin{array}{l}23.113 \\
23113\end{array}$ & \begin{tabular}{|l|}
25.188 \\
25118 \\
\end{tabular} & \begin{tabular}{|l|}
24.055 \\
24.055 \\
\end{tabular} & $\begin{array}{l}25 . \\
25\end{array}$ & 49. & \begin{tabular}{|l|}
50.034 \\
150.478 \\
\end{tabular} & \begin{tabular}{|l|l|}
18.6 \\
18.9
\end{tabular} & & \begin{tabular}{|l|l|}
-1.22 \\
-1.2
\end{tabular} & $\begin{array}{r}-5.94 \\
-5.9 \\
\end{array}$ & & & & & 96 & $\begin{array}{r}1.6 \\
61667\end{array}$ & & & & & & & & 0.171 & \\
\hline & & & $\begin{array}{l}25.168 \\
25.193\end{array}$ & & & & & & & & & & & & & & & & & & & & & & & & & \\
\hline & & & 25.191 & & & & & & & & & & & & & & & & & & & & & & & & & \\
\hline & & & 25.182 & & & & & & & & & & & & & & & & & & & & & & & & & \\
\hline & & & & & & & & & & & & & & & & & & & & & & & & & & & & \\
\hline & & & 25.175 & 23. & 25.2 & 23.952 & & & & & & & & & & & & & & & & & & & & & & \\
\hline & & & $\begin{array}{l}25.183 \\
25.161\end{array}$ & $\frac{23.16}{230}$ & $\begin{array}{l}25 . \\
25.5\end{array}$ & \begin{tabular}{|l|l}
23.945 \\
23.912
\end{tabular} & & $\frac{49}{49}$ & $\begin{array}{r}50.515 \\
55049 \\
5\end{array}$ & \begin{tabular}{|l|l|}
18.8 \\
18.9
\end{tabular} & $\frac{3}{30}$ & \begin{tabular}{|c|}
-1.219 \\
-1216 \\
\end{tabular} & $-\frac{-5.9}{-5.9}$ & & & & & $\begin{array}{l}103.00 \\
10400\end{array}$ & $\begin{array}{l}1.71667 \\
173233\end{array}$ & & & & & & & & 170 & 1 \\
\hline & & \begin{tabular}{|l}
$:: 4:$ \\
$1: 47:$
\end{tabular} & & & & 23.912 & & & $\begin{array}{r}50.49 \\
50615 \\
\end{array}$ & & & -1.2 & & & & & & & & & & & & & & & & \\
\hline & & & & & & & & & & & & & & & & & & & & & & & & & & & & \\
\hline & & & & & & & & & & & & & & & & & & & & & & & & & & & & \\
\hline & & & & & & & & & & & & & & & & & & & & & & & & & & & & \\
\hline & & & \begin{tabular}{|l|l|}
25.199 \\
\end{tabular} & & & & & & 50.6 & & & & & & & & & & 667 & & & & & & & & & \\
\hline & & & $\begin{array}{r}25.204 \\
25.2\end{array}$ & & 25.2 & \begin{tabular}{|l|}
24.026 \\
24042
\end{tabular} & & & 50.5 & 18. & & -1.22 & $\frac{-5}{50}$ & & & & & & & & & & & & & & & \\
\hline & & & $\frac{25}{25.20}$ & & & & & & & & & & & & & & & & & & & & & & & & & \\
\hline & & & & & & & & & & & & & & & & & & & & & & & & & & & & \\
\hline & & & & & & & & & & & & & & & & & & & & & & & & & & & & \\
\hline & & & & & & & & & & & & & & & & & & & & & & & & & & & & \\
\hline & & & & & & & & & & & & & & & & & & & & & & & & & & & & \\
\hline & & & & & & & & & & & & & & & & & & & & & & & & & & & & \\
\hline & & & & & & & & & & & & & & & & & & & & & & & & & & & & \\
\hline & & & & & & & & & & & & & & & & & & & & & & & & & & & & \\
\hline & & & & & & & & & & & & & & & & & & & & & & & & & & & & \\
\hline & & & 25.216 & & & & & & & & & & & & & & & & & & & & & & & & 67 & \\
\hline & & & & & & & & & & & & & & & & & & & & & & & & & & & & \\
\hline
\end{tabular}


WSRC-TR-2005-00105, REVISION 0

SRNL-RPP-2005-00012, REVISION 0

RUN \# 1.16; STEADY STATE TEST AT 25 WT\% UDS - CONT.

\begin{tabular}{|c|c|c|c|c|c|c|c|c|c|c|c|c|c|c|c|c|c|c|c|c|c|c|c|c|c|c|c|c|}
\hline \multirow{2}{*}{\multicolumn{29}{|c|}{ 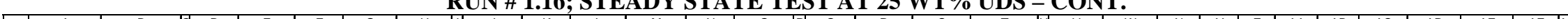 }} \\
\hline & A & B & Id $\mathrm{D}$ & & & $G$ & \begin{tabular}{l|l}
$\mathrm{H}$ & $\mathrm{I}$ \\
\end{tabular} & $\mathrm{J}$ & $\mathrm{K}$ & $L$ & $\mathrm{M}$ & & & Q & \begin{tabular}{l|l|}
$\mathrm{R}$ \\
\end{tabular} & $\mathrm{s}$ & $T_{1}$ & $\mathrm{~V}$ & w & $x$ & $\mathrm{Y}$ & z & \begin{tabular}{|l|} 
AA \\
\end{tabular} & $\mathrm{AB}$ & $\mathrm{AC}$ & $\mathrm{AD}$ & AE & $\begin{array}{ll}\mathrm{AF} & \mathrm{A} \\
\end{array}$ \\
\hline \begin{tabular}{|l|}
141 \\
12
\end{tabular} & $1 / 30 / 2004$ & $2: 06: 54 \mathrm{PM}$ & 25.228 & \begin{tabular}{|l|l|}
23.098 \\
22101
\end{tabular} & \begin{tabular}{|l|}
25.263 \\
35277 \\
\end{tabular} & 24.05 & 25.985 \# & 49.326 & \begin{tabular}{|r|}
50.47 \\
50.21
\end{tabular} & $\begin{array}{l}19.016 \\
1976\end{array}$ & $\begin{array}{l}30.318 \\
30217\end{array}$ & \begin{tabular}{|l|}
-1.222 \\
\end{tabular} & \begin{tabular}{|l|}
-5.941 \\
.591
\end{tabular} & \begin{tabular}{|l|l|}
45.176 \\
\end{tabular} & 0.045 & $\begin{array}{l}14.643 \\
\end{array}$ & $\begin{array}{l}0.003 \\
0.04\end{array}$ & 124.00 & \begin{tabular}{|l|}
2.06667 \\
\end{tabular} & 49.0 & 11.1 & \begin{tabular}{|l|l|}
1139.8 \\
30
\end{tabular} & \begin{tabular}{|l|}
2.746 \\
\end{tabular} & 0.007 & $\begin{array}{l}0.007 \\
\end{array}$ & 0.000 & 0.167 & 0.14 \\
\hline$\frac{142}{143}$ & $1 / 30 / 2004$ & 2:07:54 PM & 25.229 & & 25.274 & & $26.044 \#$ & & & 18.746 & 30.217 & -1.219 & & \begin{tabular}{|l|l|} 
& 44.356 \\
\end{tabular} & & 14.643 & & 125.00 & & & & & & & & & 0.168 & \\
\hline$\frac{143}{144}$ & /2004 & 2:08:54 PM & 25.234 & 23.104 & 25.274 & \begin{tabular}{|l|l|}
24.021 \\
\end{tabular} & 26.09 \# & 49.275 & \begin{tabular}{|l|l|}
50.434 \\
\end{tabular} & 18.938 & 30.287 & -1.219 & -5.94 & & & 14.643 & 0.004 & 126.00 & 2.1 & 49.0 & & & 2.74 & & & & .167 & \\
\hline$\frac{\mid 444}{145}$ & & 2:09:54 PM & 25.234 & 23.104 & 5.294 & .076 & & 9.245 & 0.551 & 18.837 & & 216 & & 43.315 & & & & & 11667 & 49. & & & & & & & & \\
\hline$\frac{445}{146}$ & $\frac{1 / 30 / 2004}{1 / 30 / 2004}$ & 2:10:54 PM & 25.234 & 23.104 & 25.279 & 24.016 & 26.345 \# & 49.368 & \begin{tabular}{|l|}
5.602 \\
5
\end{tabular} & 18.982 & 30.297 & -1.219 & & 44.388 & & 14.643 & & $\frac{128.00}{180}$ & & & & & & & & & & \\
\hline & $\frac{1 / 30 / 2004}{1 / 30 / 2004}$ & $2.11545 \mathrm{PM}$ & $\frac{25.234}{25324}$ & $\frac{23.104}{2.1002}$ & $\frac{25.284}{25279}$ & $\frac{23.961}{2300}$ & $\frac{26.1260}{26051}$ & 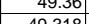 & & 19.016 & & $\frac{-1.219}{1.210}$ & & & & $\frac{14.643}{1.612}$ & & $\frac{122.00}{1200}$ & & & 11. & & & & & & & \\
\hline & $\frac{1 / 30 / 2004}{1 / 30 / 2004}$ & $\frac{2.12 .54 \mathrm{MM}}{2.13 .54 \mathrm{PM}}$ & $\frac{25.224}{25218}$ & $\frac{23.09}{23.10}$ & $\frac{25.279}{25288}$ & $\frac{23.945}{23.995}$ & $\frac{2.0 .059 \text { \# }}{26069 \mathrm{~A}}$ & $\frac{49.518}{49.32}$ & \begin{tabular}{|l|}
50.549 \\
50596 \\
\end{tabular} & $\frac{19.059}{18.091}$ & & $\frac{-1.219}{-1.219}$ & & $\frac{44.559}{44.3}$ & & $\frac{14.643}{14.643}$ & & & $\frac{2.1066}{2.1833}$ & $\frac{49.1}{40.1}$ & & & & & & & & \\
\hline 149 & $1 / 30 / 2004$ & $2.153 .54 \mathrm{PM}$ & $\frac{25.210}{25.222}$ & $\frac{23.103}{23.097}$ & $\begin{array}{l}25.280 \\
25.292\end{array}$ & $\begin{array}{r}2.995 \\
24.03\end{array}$ & $26.044 \#$ & $\begin{array}{l}49.394 \\
49.494\end{array}$ & \begin{tabular}{|l|}
50.756 \\
50.762
\end{tabular} & $\begin{array}{l}18.991 \\
19.157\end{array}$ & $\begin{array}{l}30.217 \\
30.192\end{array}$ & $\frac{1.219}{-1.219}$ & $\frac{-5.94}{-5.94}$ & $\begin{array}{r}44.3 \\
45.841\end{array}$ & $\begin{array}{l}0.045 \\
0.044 \\
\end{array}$ & $\begin{array}{l}\frac{14.045}{14.643} \\
\end{array}$ & & $\begin{array}{l}131.100 \\
132.00\end{array}$ & 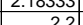 & 449.1 & 112 & & & 0.007 & 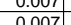 & 年 & 0 & \\
\hline & $1 / 30 / 2004$ & $2.145 .54 \mathrm{PM}$ & 25.2222 & 23.102 & 25 & 24.05 & 25.939 \# & $\frac{45.454}{49.223}$ & \begin{tabular}{|l|}
50.567 \\
\end{tabular} & 18.918 & 年 & $\frac{-1.213}{-1.222}$ & & 4 & & $\begin{array}{l}14.045 \\
14.643\end{array}$ & & 1330 & 20.4 & 491 & 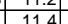 & 年, & $\mid$ & & & & 0.168 & \\
\hline & $1 / 30 / 2004$ & $2: 16: 54 \mathrm{PM}$ & $\frac{25.22}{25.22}$ & 23.08 & 25.28 & 24.012 & 25.427 \# & 4 & \begin{tabular}{|l|l|}
50.472 \\
\end{tabular} & $\begin{array}{l}10.510 \\
18.845\end{array}$ & $\begin{array}{l}30.199 \\
30.199\end{array}$ & -1.219 & & 43.021 & & $\begin{array}{l}14.045 \\
14.643\end{array}$ & & $\begin{array}{l}134.00 \\
134.00\end{array}$ & 2.23333 & 4490 & $\frac{1.4}{1.5}$ & $\frac{5.14}{39.7}$ & 2735 & & & & 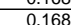 & \\
\hline & $1 / 30 / 2004$ & $2: 17: 54$ PM & 25.236 & 23.095 & 25.295 & 24.028 & 25.143 \# & 49.43 & \begin{tabular}{|l|l|} 
\\
\end{tabular} & 19.12 & 30.213 & -1.214 & -5.9 & 43.877 & 0.045 & 14.643 & & 1355.00 & 2.25 & & & & 2.746 & $\frac{0.007}{0.007}$ & 0.007 & 0.000 & $\frac{0.100}{0.167}$ & \\
\hline & & & & & & & & & & & & & & & & & & & & & & & & & & & & \\
\hline 154 & & Averages & 25.4 & 22.8 & 25.3 & 24.1 & $25.6 \#$ & 49.5 & 50.5 & 19.2 & 30.2 & -1.1 & -5.5 & 45.2 & 0.0 & & & & 1.2 & 49.0 & 11.1 & $\overline{39.8}$ & 4.745 & .007 & .007 & & .174 & \\
\hline & & Maximum & 26.5 & 23.1 & 26.0 & 24.6 & & 51.1 & 51.4 & 20.3 & 32.1 & 0.3 & 0.6 & 48.6 & 0.1 & 14.6 & & 135.0 & & & & & & & & & & \\
\hline & & Median & 25.2 & 22.8 & 25.2 & 24.1 & $25.7[\#$ & 49.7 & 50.5 & 19.2 & 30.4 & -1.2 & -5.9 & 45 & 0 & 14.6 & & 70.5 & & & 11.0 & 40. & 2.762 & 0.007 & & & 172 & \\
\hline & & Minimum & $\begin{array}{l}25.0 \\
0737\end{array}$ & 22.4 & & & 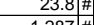 & 44.9 & & $\begin{array}{l}17.7 \\
0.79\end{array}$ & & $=1.2$ & $\begin{array}{r}-6.0 \\
270\end{array}$ & 41.5 & & 14.6 & $0.0 \|$ & 6.0 & 0.1 & 48.5 & & & 2.471 & & & & 0.162 & \\
\hline & Numbor & $2 \times$ Sta Dev & 0 & 0.315 & | & - 0.493 & 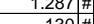 & 2.464 & 0.553 & 0.814 & $\frac{1.960}{100}$ & 0.170 & 3.216 & 2.506 & 0.005 & 0.000 & 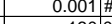 & 75.053 & 1.251 & 0.553 & $\left.0.614\right|^{2}$ & $\begin{array}{l}42.185 \\
40.03\end{array}$ & 0.151 & 0.001 & 0.001 & 0.0000 & 0.021 & \\
\hline & & P & 130 & & 130 & & & & & & & & & & & & & 130 & 130 & 130 & & & & & & 130 & 130 & \\
\hline & & & & & & & & & & & & & & & & & & & & & & & & & & & & \\
\hline & & & & & & & & & & & & & & & & & & & & & & & & & & & & \\
\hline & & & & & & & & & & & & & & & & & & & & & & & & & & & & \\
\hline
\end{tabular}


RUN \# 1.17; STEADY STATE TEST AT <20 WT \% UDS

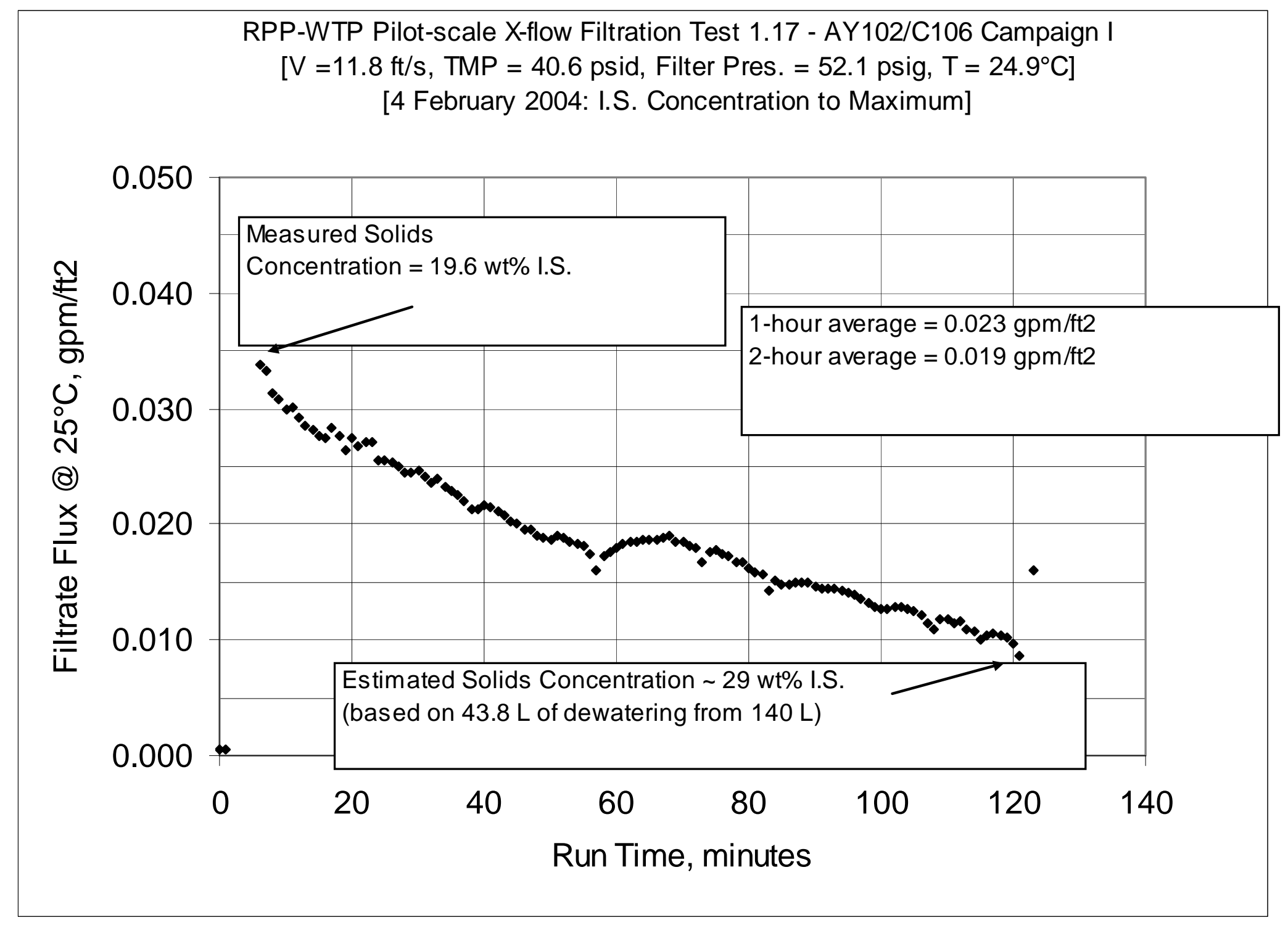


WSRC-TR-2005-00105, REVISION 0

SRNL-RPP-2005-00012, REVISION 0

RUN \# 1.17; STEADY STATE TEST AT <20 WT \% UDS - CONT.

\begin{tabular}{|c|c|c|c|c|c|c|c|c|c|c|c|c|c|c|c|c|c|c|c|c|c|c|c|c|c|c|c|c|}
\hline & $\mathrm{A}$ & $\mathrm{B}$ & $\mathrm{D}$ & $\frac{E}{K}$ & $F$ & $\frac{G}{G}$ & $\frac{\mathrm{H}}{\mathrm{t}-\mathrm{s}}$ & $\mathrm{J}$ & $K$ & $\frac{\mathrm{L}}{\mathrm{s}}$ & $M$ & $\mathrm{~N}$ & 0 & $\frac{Q}{Q}$ & $\begin{array}{ll} & R \\
<<\text { Flow }\end{array}$ & $\frac{\mathrm{S}}{\text { Measure }}$ & $\mathrm{T}$ & $\mathrm{v}$ & w & $\frac{1 x}{x<\ll<}$ & & $\frac{\mathrm{z}}{\text { ated Fro }}$ & \begin{tabular}{|c|} 
AA \\
$m$ Raw
\end{tabular} & \begin{tabular}{|l|}
$A B$ \\
Data
\end{tabular} & $A C$ & $A D$ & $\mathrm{AE}$ & AF \\
\hline & DATE & TIME & Filtrate & $\begin{array}{l}\text { Cleaning } \\
\text { Cleaning }\end{array}$ & 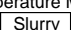 & $\begin{array}{l}\text { Neasurem } \\
\text { Hi Amb. }\end{array}$ & $\begin{array}{l}\text { Lo Amb. } \\
\text { Lo Amb. }\end{array}$ & BotTMP & Filter & \begin{tabular}{|l} 
Pressure \\
Filter dP
\end{tabular} & 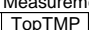 & $\begin{array}{l}\text { Diltrate } \\
\text { | }\end{array}$ & Pulsepot & Slurry & $\begin{array}{l}\text { \& Fllow } \\
\text { Filtrate }\end{array}$ & $\begin{array}{l}\text { eleasurem } \\
\text { Hi Filtate }\end{array}$ & Backpulse & & & & & & & & & & & \\
\hline & & & $\operatorname{deg} C$ & $\operatorname{deg} \mathrm{C}$ & $\operatorname{deg} \mathrm{C}$ & $\operatorname{deg} C$ & $\operatorname{deg} C$ & psid & psig & psid & psid & & psig & $\mathrm{gpm}$ & gpm & gpm & & & & & & & & & & & & \\
\hline$\frac{4}{5}$ & & & & & & & & & & & $\mathrm{dP3}$ & & & & & & & & & & & & & & & & & \\
\hline & Zeros & & & & & & & & & & & & & & & & & & & & & & & & & & & \\
\hline 7 & $2 / 4 / 2004$ & 8:01:53 AM & 22.179 & 17.007 & \begin{tabular}{|l|l|}
13.889 \\
\end{tabular} & 22.838 & 14.707 & 0.054 & -0.052 & 0.009 & 0.006 & -0.174 & 0.075 & -0.081 & 0.003 & 14.643 & 0.005 & & & Filter Si & Surface & 6.707 & FT2 & & & & & \\
\hline 8 & 2/4/2004 & 8:02:24 AM & 22.179 & 17.007 & \begin{tabular}{|l|l|}
13.889 \\
\end{tabular} & 22.838 & 14.707 & 0.054 & -0.052 & 0.009 & 0.006 & -0.174 & 0.075 & -0.081 & 0.003 & 14.643 & 0.005 & & & Convers & rision & 851 & $\mathrm{~m} 3 / \mathrm{m} 2 /$ & $2 / \mathrm{day} / \mathrm{ba}$ & $\mathrm{rg} / \mathrm{gpm} / \mathrm{tt}$ & fft2/barg & & \\
\hline 9 & $2 / 4 / 2004$ & 8:02:25 AM & 22.168 & 16.992 & 13.853 & 22.757 & 14.733 & 0.052 & -0.052 & 0.008 & 0.002 & -0.183 & & $\begin{array}{l}-0.083 \\
\end{array}$ & 0.003 & 14.643 & 0.004 & & & & & & & & & & & \\
\hline & $2 / 4 / 2004$ & 8:02:26 AM & & 17.003 & 13.859 & 22.763 & 14.743 & & -0.05 & 0.008 & & -0.174 & 0.06 & -0.083 & 0.003 & 14.643 & 000 & & & Note: & 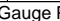 & & & & nately & 4 psig & & \\
\hline & $2 / 4 / 2004$ & $8: 02: 27 \mathrm{AM}$ & & 16.992 & 133.853 & 22.757 & 14.738 & 0.052 & -0.05 & 0.008 & 0.002 & -0.174 & 0.06 & \begin{tabular}{l|l|}
-0.083 \\
\end{tabular} & 0.003 & $\begin{array}{l}14.643 \\
\end{array}$ & 0.004 & & & & & & & & & & & \\
\hline & $2 / 4 / 2004$ & 8:02:28 AM & 22.168 & 16.992 & 13.858 & 22.757 & & 0.052 & -0.05 & 0.008 & 0.002 & $\begin{array}{r}-0.18 \\
\end{array}$ & & -0.083 & 0.003 & 14.643 & & & & & & & $d e^{2}$ & & & & & \\
\hline 13 & $2 / 4 / 2004$ & 8:02:29 AM & & 16.997 & 13.864 & & 14.743 & & & & 0.002 & -0.215 & & & & 14.643 & & & & WVWV & & & & Filt & & & & \\
\hline & $2 / 4 / 2004$ & 8:02:30 AM & 22.168 & 16.997 & 13.853 & 22.757 & 14.728 & 0.054 & -0.052 & 0.008 & & -0.197 & 0.069 & -0.083 & 0.003 & 14.643 & 0.006 & & & & & & & & at $25 \mathrm{C}$ & & : & \\
\hline$\frac{15}{16}$ & $D$ & & & & & & & & & & & & & & & & & Time & rime & Press & & TMP & IMP & gpm & & $\mathrm{m}$ & & eter \\
\hline 17 & $\frac{10 a t-P e r}{2 / 4 / 2004}$ & ute $8 \cdot 11: 49 \mathrm{AM}$ & 22263 & 17062 & 222403 & 22887 & 16.238 & 9.476 & \begin{tabular}{|l|l|}
48841 \\
\end{tabular} & 10.662 & -1.287 & 34.694 & 88.495 & 49.621 & 0.003 & 14,643 & 0.004 & & & $\frac{\operatorname{sig}}{47.4}$ & 122 & $\frac{10}{41}$ & 0282 & 0.000 & 0.000 & IPSI & 0118 & 010 \\
\hline 18 & $2 / 1 / 2004$ & 8:12:49 AM & & & \begin{tabular}{|l|}
22.313 \\
\end{tabular} & 22.943 & 16.422 & -5.892 & \begin{tabular}{|l|}
48.405 \\
\end{tabular} & 7.12 & -13.062 & 49.93 & & 50.249 & 0.003 & 14.643 & 0.966 & 1.0 & \begin{tabular}{|l|}
0.01667 \\
\end{tabular} & 46.9 & 12.3 & -9.5 & -0.653 & $\begin{array}{l}30.000 \\
\end{array}$ & 0.000 & & $\begin{array}{lll}0 & -0.051\end{array}$ & $\begin{array}{l}-0.04 \\
-0.04\end{array}$ \\
\hline & $2 / 1 / 2004$ & 8:13:49 AM & 22.457 & 17.121 & 22.1 & 22.895 & 16.592 & 41.999 & 48.405 & 8.692 & 32.891 & 1.548 & & 51.411 & 0.537 & 14.643 & 0.004 & 2.0 & \begin{tabular}{|l|l|}
0.03333 \\
\end{tabular} & 46.9 & 12.6 & 37.4 & 2.582 & \begin{tabular}{|l|l|} 
& 0.080 \\
\end{tabular} & 0.087 & & 2.322 & 1.98 \\
\hline & $2 / 4 / 2004$ & $8: 14: 49 \mathrm{AM}$ & 22.526 & 17.16 & 21.962 & 22.939 & 16.858 & 43.559 & \begin{tabular}{|l|l|}
49.253 \\
\end{tabular} & 11.501 & 31.539 & 0.665 & 0.40 & 48.015 & 0.238 & 14.643 & & 3.6 & & 47.8 & 11.8 & 37.5 & 2.589 & .183 & 2.380 & & 63.388 & 3.94 \\
\hline & $2 / 4 / 2004$ & 8:15:49 AM & 22.534 & 17.198 & 21.789 & 22.982 & 16.891 & 43.837 & \begin{tabular}{|l|l|} 
& 49.357 \\
\end{tabular} & 12.257 & 31.153 & 0.668 & 0.47 & 50.387 & 0.206 & 14.643 & & & & & & & & & & 0.0 & & \\
\hline 22 & $2 / 4 / 2004$ & 8:16:49 AM & 22.416 & 17.237 & 21.601 & 22.95 & 16.895 & 46.209 & \begin{tabular}{|l|l|}
49.278 \\
\end{tabular} & 12.633 & 33.154 & -1.214 & & \begin{tabular}{|l|l|} 
\\
\end{tabular} & 0.442 & 14.643 & & 5.0 & .083 & 47. & & 39.7 & & . 066 & & & & \\
\hline 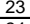 & $2 / 4 / 2004$ & 8:17:49 AM & 22.354 & 17.271 & 21.388 & 22.968 & 17.095 & 48.54 & \begin{tabular}{|l|}
49.707 \\
\end{tabular} & 13.103 & 35.048 & -1.214 & & 46.64 & 0.205 & 14.643 & & 6.0 & & 48.2 & 11. & 41.8 & & & & & & \\
\hline & $2 / 4 / 20004$ & $8: 18: 49 \mathrm{AM}$ & 22.358 & & 21.215 & 23.037] & 17.175 & 48.294 & \begin{tabular}{|l} 
\\
\end{tabular} & 13.254 & 34.599 & & & & & 14.643 & & & 0.11667 & & & & 2.858 & 0.030 & & & & \\
\hline & & 8:19:49 AM & & & & 23.016 & 17.234 & 48.862 & & & & & & 49.187 & 0.188 & 14.643 & & 8.0 & & & & & & & & & & \\
\hline & $2 / 4 / 2004$ & 8:20:49 AM & 22.22 & 17.414 & 20.946 & 23.065 & 17.395 & 48.93 & 49.462 & 13.599 & 34.894 & -1.214 & & 53.332 & 0.184 & 14.643 & & $9.6 \mathrm{C}$ & & 48. & 13. & 41.9 & 89 & 027 & & & & \\
\hline & $2 / 2 / 42004$ & 8:21:49 AM & & 17.449 & & 23.054 & & 49.19 & $49.6 / 8$ & $\begin{array}{l}13.196 \\
132027\end{array}$ & & -1.214 & & 41.669 & 0.188 & 14.643 & & & $\frac{0.16661}{16232}$ & & & & & & & & & 6 \\
\hline$\frac{20}{29}$ & $2 / 4 / 2004$ & $\begin{array}{l}0.22 .45 \mathrm{AM} \\
8: 23: 49 \mathrm{~A}\end{array}$ & 22.035 & $\frac{17.487}{17.531}$ & \begin{tabular}{|l|}
20.727 \\
20644
\end{tabular} & $\begin{array}{r}23.095 \\
23.086\end{array}$ & $\begin{array}{l}17.453 \\
17.568\end{array}$ & $\begin{array}{r}\frac{49.409}{49.27} \\
\end{array}$ & \begin{tabular}{|r|}
49.703 \\
493 \\
\end{tabular} & $\begin{array}{l}13.937 \\
13.992\end{array}$ & $\begin{array}{l}3.071 \\
34.835\end{array}$ & $\frac{-1.214}{-1.211}$ & $\begin{array}{l}-6.068 \\
-6.019\end{array}$ & $\begin{array}{r}40.59 \\
49.2\end{array}$ & $\begin{array}{l}.179 \\
0.173\end{array}$ & $\begin{array}{l}\frac{14.643}{14.643} \\
\end{array}$ & 0.004 & $\begin{array}{l}11.00 \\
12.00\end{array}$ & $\frac{18333}{0.2}$ & $\begin{array}{l}48.3 \\
48.2 \\
\end{array}$ & $\frac{11.4}{12.1}$ & $\frac{42.2}{42.1}$ & $\frac{2.912}{2.899}$ & 0.027 & $\begin{array}{l}0.030 \\
0.029\end{array}$ & $\frac{0.001}{0.001}$ & 0.114 & 0.61 \\
\hline 30 & $2 / 4 / 2004$ & $8: 24: 49 \mathrm{AM}$ & 21.983 & 17.566 & 20.558 & 23.105 & 17.648 & 49.669 & 50.08 & 14.246 & 34.951 & \begin{tabular}{|l|} 
\\
\end{tabular} & & 48.507 & 0.168 & 14.643 & & 13.00 & 0.21667 & 48.6 & 11.9 & 42.3 & 2.917 & 0.025 & 0.028 & 0.001 & 0.672 & 0.5 \\
\hline & $2 / 4 / 2004$ & 8:25:49 AM & 21.937 & 17.615 & 20.521 & 23.134 & 17.808 & 48.148 & 48.61 & 15.783 & 31.904 & -1.216 & & 51.826 & 166 & 14.643 & & 14.00 & & 47.1 & 12.7 & 40.0 & & & & & & , \\
\hline 32 & $2 / 4 / 2004$ & 8:26:49 AM & 21.911 & 17.685 & 20.5 & 23.178 & 18.039 & 48.789 & \begin{tabular}{|l|l|}
49.154 \\
\end{tabular} & $\begin{array}{l}15.127 \\
\end{array}$ & 33.174 & \begin{tabular}{|c|c|}
-1.214 \\
\end{tabular} & -6.1 & 50.628 & 0.163 & $\begin{array}{l}14.643 \\
\end{array}$ & & 15.00 & 0.25 & 447. & $\overline{12.4}$ & $\overline{41.0}$ & 2.826 & 0.024 & 0.028 & 0.001 & 0.674 & \\
\hline 30 & $2 / 4 / 2004$ & $\mathrm{AM}$ & 21.819 & 17.709 & 20.438 & 23.142 & 10.24 & 48.605 & \begin{tabular}{|l|l|} 
& 49.011 \\
\end{tabular} & & 33.421 & $\begin{array}{l}-1.219 \\
\end{array}$ & & 51.55 & 0.102 & 14.643 & & 16.0 & 0.26667 & 47.5 & 12.0 & 41.0 & 2.020 & .024 & . & - & 0.011 & 0.0 \\
\hline & $2 / 4 / 2004$ & 8:28:49 & 21.773 & 17.759 & 20.448 & 23.075 & & 48.885 & & 14.8 & 33.55 & & & 49.946 & & & & & 0.28333 & & $12.2 \mathrm{rl}$ & $41.2 \mathrm{r}$ & 2.842 & & & & 0.688 & 0.54 \\
\hline 35 & $2 / 4 / 2004$ & 8:29:49 AM & 21.711 & 17.793 & 20.472 & 23.175 & $18.2 \mathrm{rt}$ & 48.843 & \begin{tabular}{|l|l|l|} 
\\
\end{tabular} & 14.782 & 33.671 & -1.214 & & 47.485 & 0.163 & 14.643 & & 18.0 & 0.3 & 47. & 11.6 & & 2.845 & 5 & 0.028 & & 0.670 & \\
\hline & $2 / 2 / 20004$ & 8:30:49 AM & $\frac{21.671}{21.672}$ & 17.853 & 20.501 & $\frac{23.1944}{2020}$ & 18.31 & 49.057 & \begin{tabular}{|l}
49.338 \\
\end{tabular} & 15.01 & 33.609 & -1.214 & & 45.201 & & 14.66 & & & 0.31667 & 47. & 11.1 & 41.3 & 2.850 & & & & 0.640 & \\
\hline & $2 / 1412004$ & $8.31: 49 \mathrm{~F}$ & 21.639 & & 20.525 & 23.228 & & 49.389 & \begin{tabular}{|l}
44.68 \\
\end{tabular} & 15.106 & 33.702 & -1.216 & & 51.615 & & 14.643 & & & 0.33333 & 48. & 12.6 & & 2.864 & & .027] & & 0.661 & .56 \\
\hline$\frac{30}{39}$ & $\frac{2 \mid 1 / 2 / 2004}{2 / 1 / 2004}$ & 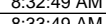 & $\frac{21.608}{21.592}$ & $\frac{17.942}{1.902}$ & 20.554 & $\frac{23.323}{23201}$ & 18. & 49.32 & \begin{tabular}{|l|l}
4.5081 \\
\end{tabular} & $\frac{15.218}{15.191}$ & $\begin{array}{l}33.597 \\
23201\end{array}$ & -1.214 & & $\begin{array}{l}499.974 \\
50202\end{array}$ & 0.158 & $\frac{14.643}{1.612}$ & & 21.0 & $\begin{array}{r}0.35 \\
026567\end{array}$ & $\frac{48}{10}$ & $\frac{12 .}{12}$ & 告1.5 & 2.858 & & 年 & & 0.645 & $0.5 \mathrm{~s}$ \\
\hline 40 & $2 / 1 / 42004$ & 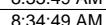 & $\frac{21.592}{21.545}$ & 18016 & $\mid$\begin{tabular}{|l|l|}
$\mid 2.599$ \\
2077
\end{tabular} & $\frac{23.301}{23.234}$ & & $\frac{49.476}{49218}$ & 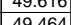 & 年 & $\begin{array}{l}33.891 \\
32.441\end{array}$ & $\frac{-1.214}{1216}$ & & S0.393 & $\begin{array}{l}0.16 \\
0.16\end{array}$ & $\begin{array}{l}14.645 \\
11643\end{array}$ & & 22.0 & 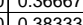 & 48.1 & 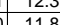 & 41.13 & $\frac{2.884}{2050}$ & $\begin{array}{l}0.024 \\
0.022\end{array}$ & & & 0 & \\
\hline 41 & $2 / 4 / 2004$ & 8:35:49 AM & 21.529 & 18.066 & 20.596 & 23.239 & 18.656 & 49.814 & \begin{tabular}{|l|}
50.146 \\
\end{tabular} & 15.816 & 33.431 & $\mid$\begin{tabular}{|c|c|}
-1.214 \\
\end{tabular} & -6. & 48.036 & 0.151 & 14.643 & & 24.00 & 0.00 .4 & 48.7 & 118 & 41.6 & $\mid 2870$ & 0.023 & 0.026 & & 0.613 & $\frac{0.5}{0.5}$ \\
\hline & $2 / 4 / 2004$ & 8:36:49 & 21.523 & 18.105 & 20.626 & 23.288 & & 49.733 & 49.956 & 15.872 & 33.386 & -1.214 & & 48.388 & & & & & 0.41667 & 48.5 & 11. & 41.6 & 2.865 & & 226 & & 0.614 & 0.5 \\
\hline & $2 / 4 / 2004$ & $8: 37: 49 \mathrm{~A}$ & 21.527 & 18.154 & 20.66 & 23.322 & & 49.532 & \begin{tabular}{|l|l}
49.707 \\
\end{tabular} & 15. & 33.223 & -1.216 & & 45.239 & 0 & & & 26.0 & & 48. & I. & & 2.030 & & & & & \\
\hline 44 & & & & 18.193 & 20.694 & & & & & & & -1.214 & & & & & & & 0.45 & 48. & 12.5 & 41.4 & & & & & & \\
\hline & & & 21.519 & & 20.693 & 23.34 & & 49.801 & 50.064 & & & & & 47.75 & & & & & & 48. & $11.7 \mathrm{rt}$ & & 2.865 & & & & 0.588 & \\
\hline 46 & 2/4/20004 & 8:40:4 & 21.519 & 18.263 & 20.707 & 23.324 & & 49.623 & 49.885 & 16. & 33.121 & -1.216 & & 47.8 & & & & 29.0 & 0.48333 & 48. & 11.7 & 41.4 & 2.852 & & & & 0.591 & \\
\hline & & & $\frac{21.518}{21512}$ & & & & & & & & & & & & & & & & 0.5 & & T. & & & & & & & \\
\hline$\frac{40}{49}$ & $\frac{21 / 4 / 2004}{2 / 1 / 2004}$ & $\begin{array}{l}8: 42.49 \mathrm{AM} \\
883449\end{array}$ & $\frac{21.543}{21.551}$ & & $\frac{20.131}{2073}$ & & & $\begin{array}{r}49 \\
49.7\end{array}$ & \begin{tabular}{|l|}
49.134 \\
50.039
\end{tabular} & & & & & & & & & & & $\frac{48.3}{48.6}$ & $\frac{11.5}{11.9}$ & $\begin{array}{ll}5 & 41.3 \\
9 & 41.4\end{array}$ & $\frac{2.846}{2.856}$ & & $\mid \begin{array}{ll}0.024 \\
0.024\end{array}$ & & 0.583 & 0.50 \\
\hline 50 & $2 / 4 / 2004$ & $8: 44: 4$ & 21.576 & 18. & 20.754 & 23.3 & 20 & 49.955 & \begin{tabular}{|l|}
50.302 \\
\end{tabular} & 16. & 32. & -1.214 & & & & & & 33 & 0.55 & 48.8 & 11.7 & \begin{tabular}{l|l}
74.1 .5 \\
\end{tabular} & 2.859 & & $\mid 0.024$ & & 0.576 & $0.44^{0.4}$ \\
\hline & $2 / 4 / 20$ & $8: 45: 49$ & $\begin{array}{l}21.6 \\
\end{array}$ & 18.496 & 20.773 & 23.3 & 20 & 50.154 & \begin{tabular}{|l|l|}
50.449 \\
\end{tabular} & 16.7 & 32.965 & $5-1.216$ & & 46. & & & & 34 & 0.56667 & 49.0 & & \begin{tabular}{l|l}
4 & 41.6
\end{tabular} & & & & & 0.559 & 0.48 \\
\hline & $2 / 4 / 2$ & & 21.624 & $\begin{array}{l}18.546 \\
\end{array}$ & 20.803 & 23.4 & 19 & 50.235 & 5 & 16. & 32. & \begin{tabular}{|c|c|}
3 & -1.216 \\
\end{tabular} & & & & & & & 0.58333 & & & & & & & & & \\
\hline & $2 / 4 / 2004$ & & 21.699 & 18.596 & 20.827 & 23.403 & 19 & 50.1 & 50.347 & & & -1.214 & & & & & & 36. & 0.6 & 48.9 & & & & & & & 0.543 & \\
\hline 54 & & & 21.75 & & & & & 50.071 & 50.347 & & & -1.216 & & 46.945 & & & & & 0.61667 & 48.9 & & 40.1. & & & & & 0.540 & \\
\hline & $2 / 4 / 20$ & & 21.772 & 18.6 & 20.916 & 23.401 & & 49.592 & & & & & & & & & & & & & & & & & & & & \\
\hline & & & 1.785 & & 20.959 & 23.43 & & 49.801 & 50.151 & $17.8 \mathrm{C}$ & & & & & & & & & & & & & & & & & & \\
\hline & $2 / 4 / 2$ & 8:51:49 & 21.789 & 18.783 & 20.983 & 23.453 & & 49.49 & 49.871 & $17.7 \mathrm{rt}$ & 31.202 & -1.216 & & & & 14 & & & 0.66667 & 48.4 & 11.9 & 40.5 & .782 & & & & & \\
\hline & $2 / 1 / 42004$ & $\frac{8.52 .49 k}{8.62 .49}$ & $\frac{21.93}{21912}$ & $\frac{18.8}{1.98}$ & $\mid 2.1 .1212$ & $\frac{23.432}{23207}$ & 20. & $\frac{49.89 ?}{50.95}$ & 50.2 & $\frac{1.93}{10.910}$ & 31 & 121 & & & & 14.643 & & & 333 & & & & & & & & & \\
\hline 60 & $\frac{21 / 42 \angle 004}{2 / 1 / 2004}$ & $\frac{8.53: 497}{8.54: 49}$ & $\frac{21.842}{21.841}$ & & \begin{tabular}{|l|}
21.092 \\
21.106
\end{tabular} & 23.507 & $\frac{20 .}{21}$ & $\begin{array}{l}5.195 \\
49855\end{array}$ & & $\frac{18.514}{18.521}$ & $\frac{31.467}{30.87}$ & \begin{tabular}{|l|l|} 
& -1.211 \\
-1216
\end{tabular} & & $\frac{4.1 .1}{431}$ & & $\frac{14.6}{14.6}$ & & $\frac{42}{43}$ & 071 & 488 & $\overline{0.6}$ & & $\frac{815}{783}$ & & & & $\frac{0.518}{0.516}$ & 4 \\
\hline 61 & $2 / 14 / 20$ & 8:55:49 & 21.864 & 18.975 & & 23.448 & & 50. & \begin{tabular}{|l|}
50.37 \\
\end{tabular} & & & \begin{tabular}{|c|}
-1.216 \\
\end{tabular} & & & & $\frac{14.045}{14.643}$ & & 44. & 07333 & 489 & 123 & \begin{tabular}{|l|l|}
3 & 404
\end{tabular} & \begin{tabular}{|l|}
2787 \\
\end{tabular} & 80.018 & | & $i t$ & $\begin{array}{l}0.510 \\
0.502\end{array}$ & 04 \\
\hline & $2 / 4 / 20$ & $8: 56: 49$ & & & & & & 50.284 & & & & $\mid$\begin{tabular}{|c|}
$\mid-1.214$ \\
$\mid$
\end{tabular} & & & & & & & & 49.3 & 11.2 & $\begin{array}{l}24.04 \\
\text { 2. } 40.4\end{array}$ & & & & & & \\
\hline 63 & $2 / 4 / 2$ & $8: 57: 49$ & 21.933 & & 21.178 & 23.622 & & 50.475 & & 19.468 & & & & 48. & & & & & 0.76 & 49.4 & \begin{tabular}{|l|l|}
4 & 11 \\
\end{tabular} & \begin{tabular}{|l|l|}
8 & 40.5 \\
\end{tabular} & \begin{tabular}{|l|l|}
2.79 \\
\end{tabular} & 30 & 0.020 & & (0.484 & \\
\hline 54 & $2 / 4 / 2004$ & 8:58:49 AM & 21.941 & 19.118 & 21.091 & 23.611 & 21. & 50.517 & \begin{tabular}{|l|l|} 
& 50.864 \\
\end{tabular} & 19. & 30 & & & & & & & & 3333 & 49.4 & 412 & 40.4 & 2.784 & & & & & \\
\hline & & $8: 59: 49 \mathrm{~A}$ & & & 21014 & & & & & & 30.135 & 1.219 & & 46.767 & 0.114 & 14.643 & & & & & & & 2.78 & & $\mid 0.019$ & 0.000 & \begin{tabular}{|l|l|}
0 & 0.405 \\
\end{tabular} & \\
\hline
\end{tabular}


WSRC-TR-2005-00105, REVISION 0

SRNL-RPP-2005-00012, REVISION 0

RUN \# 1.17; STEADY STATE TEST AT <20 WT \% UDS - CONT.

\begin{tabular}{|c|c|c|c|c|c|c|c|c|c|c|c|c|c|c|c|c|c|c|c|c|c|c|c|c|c|c|c|c|}
\hline & A & B & $\mathrm{D}$ & $E$ & $\mathrm{~F}$ & G & $\mathrm{H}$ & $\mathrm{J}$ & $\mathrm{K}$ & $\mathrm{L}$ & $M$ & $\mathrm{~N}$ & O & Q & $\mathrm{R}$ & $\mathrm{s}$ & \begin{tabular}{l|l|l|}
$T$ \\
\end{tabular} & $\mathrm{~V}$ & W & $x$ & Y & $z$ & AA & $A B$ & $A C$ & $A D$ & $\mathrm{AE}$ & \\
\hline 66 & $2 / 4 / 2004$ & 9:00:49 AM & 21.954 & $\begin{array}{l}19.207 \\
19266\end{array}$ & & $\begin{array}{l}23.558 \\
23.592\end{array}$ & $\begin{array}{l}21.144 \\
20.876\end{array}$ & \begin{tabular}{|l}
50.73 \\
5121
\end{tabular} & & $\begin{array}{l}20.255 \\
21960\end{array}$ & $\begin{array}{l}29.926 \\
.901\end{array}$ & 1.216 & -6.3 & 45.904 & 0.1 & 14.643 & 0.004 & & & 49.8 & 11.2 & & & 017 & & & & \\
\hline & & & 21.968 & & & $\frac{23.592}{23601}$ & & & 1.527 & 21.868 & & & & & & & & & & 50.1 & & & 2.762 & & & 000 & 0.467 & 0.40 \\
\hline$\frac{69}{69}$ & $2 / 1 / 4 / 2004$ & $\begin{array}{l}9: 02: 49 \text { AM } \\
9.03: 49\end{array}$ & $\begin{array}{l}21.907 \\
21.981\end{array}$ & $\begin{array}{r}19.3 \\
19.355\end{array}$ & \begin{tabular}{|l|}
21.08 \\
21.11
\end{tabular} & $\frac{23.601}{23.615}$ & & $\frac{51.3 / 5}{51.46}$ & \begin{tabular}{|r|}
51.78 \\
51788
\end{tabular} & $21.389 \mid$ & $\frac{29.447}{29.891}$ & $\frac{1.216}{1.216}$ & & & & & & & & $50^{3}$ & 11 & & 2804 & & & & 0.470 & \\
\hline 70 & $2 / 4 / 2004$ & 9:04:49 AM & 21.989 & 19.399 & 21.12 & 23.683 & $\frac{21.015}{21.48}$ & 51.456 & \begin{tabular}{|l|l|l|l|}
51.933 \\
\end{tabular} & $\frac{21.194}{21.331}$ & $\begin{array}{r}29.891 \\
29.7\end{array}$ & $\frac{-1.216}{-1.216}$ & $\frac{-0.308}{-6.302}$ & $\frac{4.896}{49.31}$ & 0.111 & $\frac{14.643}{14.643}$ & 0.004 & 53.00 & \begin{tabular}{|l|}
0.8806331 \\
0.88333
\end{tabular} & $\frac{50.3}{50.5}$ & $\frac{11.7}{12.1}$ & $\frac{40.1}{40.6}$ & $\frac{2.804}{2.798}$ & \begin{tabular}{|l|l|}
0.017 \\
\end{tabular} & $\mid \frac{0.019}{0.018}$ & $\frac{0.000}{0.000}$ & 0.463 & $\frac{0.39}{0.39}$ \\
\hline & $2 / 4 / 2004$ & 9:05:49 AM & & 19.443 & \begin{tabular}{|l|}
21.17 \\
\end{tabular} & 23.737 & 21.554 & 51.85 & \begin{tabular}{|l|}
52.246 \\
\end{tabular} & & 29.726 & & & & 0.11 & 14.643 & 0.004 & 54.00 & & 50.8 & & & 2.812 & 0.016 & 0.018 & & 0.448 & \\
\hline 72 & $2 / 4 / 2004$ & 9:06:49 AM & 2.037 & 19.487 & 21.23 & 23.741 & 22.12 & 51.597 & 52.022 & 21.926 & 29.269 & -1.214 & -6.317 & 46.599 & 0.109 & 14.643 & 0.004 & 55.00 & \begin{tabular}{|l|l|}
0.91667 \\
\end{tabular} & 50.5 & 11.4 & 40.4 & 2.788 & 0.016 & 0.018 & 0.000 & 0.448 & \\
\hline 73 & $2 / 4 / 2004$ & 9:07:49 AM & 22.055 & & 21.27 & & & 50.351 & 50.837 & 21.394 & & -1.216 & & 45.733 & 0.105 & 14.643 & & 56.00 & \begin{tabular}{|l|l|}
0.93333 \\
\end{tabular} & 49.4 & 11.2 & 39.4 & 2.716 & 0.016 & & & 0.442 & \\
\hline 74 & & & & & & & 21.83 & & & 21.853 & & -1.214 & -6.268 & 44.529 & 0.097 & 14.643 & & & & 50.2 & 10.9 & & & 0.014 & & & & \\
\hline 75 & $2 / 4 / 2004$ & $9: 09: 49 \mathrm{AM}$ & 22.107 & 19.608 & \begin{tabular}{|l|}
21.87 \\
\end{tabular} & 23.846 & 21.246 & 52.456 & \begin{tabular}{|l|l|}
52.961 \\
\end{tabular} & 23.636 & 28.354 & -1.216 & -6.305 & 51.198 & 0.106 & 14.643 & 0.004 & 58.00 & \begin{tabular}{|l|}
0.96667 \\
\end{tabular} & 51.5 & 12.5 & 40.4 & 2.786 & 0.016 & 0.017 & 0.000 & 0.427 & \\
\hline 76 & $2 / 1 / 2004$ & 9:10:49 AM & & 19.651 & \begin{tabular}{|l|}
22.21 \\
\end{tabular} & & 21.239 & 51.788 & & & 28.542 & -1.216 & & & 0.109 & 14.643 & & & \begin{tabular}{|l|l|} 
\\
\end{tabular} & 50.7 & & & 2.769 & 0.016 & & & 0.438 & \\
\hline & $2 / 4 / 2004$ & 9:11:49 AM & 22.274 & 19.696 & \begin{tabular}{|l|l|}
22.53 \\
\end{tabular} & 23.878 & $21.6 \mathrm{~L}$ & 51.848 & 52.428 & \begin{tabular}{|l|l|}
22.313 \\
2.73
\end{tabular} & & -1.214 & & 50.839 & 0.112 & 14.643 & $\begin{array}{l}0.004 \\
\end{array}$ & 60.00 & & 51.0 & 12.5 & & 2.787 & & 0.018 & & 0.443 & \\
\hline 78 & 2/4/2004 & $9: 12: 49$ AM & 22.408 & 19.734 & 22.78 & 23.906 & 21.487 & 51.535 & 52.128 & 21.794 & 29.261 & -1.216 & & 48.13 & 0.115 & 14.643 & & & 1.01667 & 50.7 & 11.8 & & 2.785 & 0.017 & 0.018 & & 0.452 & \\
\hline 80 & $2 / 4 / 2004$ & $\begin{array}{l}9: 13: 49 \text { AM } \\
9 \cdot 14: 49 A\end{array}$ & $\frac{22.558}{2.676}$ & $\begin{array}{l}19.773 \\
19791\end{array}$ & \begin{tabular}{|l|}
23.01 \\
23.18 \\
\end{tabular} & $\begin{array}{l}23.955 \\
23.913\end{array}$ & $\begin{array}{l}21.607 \\
22.138\end{array}$ & $\begin{array}{l}51.705 \\
51.473\end{array}$ & \begin{tabular}{|l|}
52.246 \\
52074 \\
\end{tabular} & $\begin{array}{l}21.421 \\
21.195\end{array}$ & $\begin{array}{l}29.852 \\
22846 \\
\end{array}$ & -1.214 & & $\begin{array}{l}51.861 \\
5.051\end{array}$ & 0.117 & $\begin{array}{l}14.643 \\
1.612\end{array}$ & 0.004 & 62.00 & & 50.8 & $\begin{array}{l}12.7 \\
12.5\end{array}$ & & \begin{tabular}{|l|l|}
2.812 \\
2803
\end{tabular} & \begin{tabular}{|l|l|l|} 
\\
\end{tabular} & 0.018 & & 0.453 & \\
\hline & $\frac{2 / 4 / 2004}{2 / 1 / 2004}$ & $\begin{array}{l}9: 14: 49 \mathrm{AM} \\
0 \cdot 15: 49\end{array}$ & $\frac{22.676}{22824}$ & $\begin{array}{l}19.791 \\
19835\end{array}$ & \begin{tabular}{|l|}
23.18 \\
23.35 \\
\end{tabular} & $\begin{aligned} 23.913 \\
23.991\end{aligned}$ & $\frac{21.378}{21.391}$ & $\begin{array}{l}51.473 \\
51218\end{array}$ & \begin{tabular}{|l|}
52.074 \\
51885
\end{tabular} & $\frac{21.195}{20.785}$ & $\begin{array}{r}29.846 \\
29.944\end{array}$ & $\begin{array}{r}-1.216 \\
-1216\end{array}$ & & $\begin{array}{r}51.054 \\
50.445\end{array}$ & 0.118 & $\frac{14.643}{11643}$ & 0.004 & 63.00 & \begin{tabular}{|r|}
1.05 \\
10667
\end{tabular} & $\begin{array}{l}50.6 \\
50.4\end{array}$ & $\frac{12.5}{12.4}$ & & $\begin{array}{l}2.803 \\
27908\end{array}$ & \begin{tabular}{|l|l|}
0.018 \\
0018
\end{tabular} & 0.019 & 0.000 & $\begin{array}{l}0.455 \\
0.458\end{array}$ & 0.39 \\
\hline 82 & $2 / 4 / 2004$ & $\begin{array}{l}9: 15: 49 \text { AM } \\
9: 16: 49\end{array}$ & $\frac{22.824}{22.958}$ & $\frac{19.835}{19.873}$ & \begin{tabular}{|l|}
23.35 \\
23.49 \\
\end{tabular} & $\begin{array}{r}23.991 \\
24.02\end{array}$ & $\frac{21.391}{21.375}$ & $\begin{array}{l}51.218 \\
51.365\end{array}$ & \begin{tabular}{|l|}
51.885 \\
52.076
\end{tabular} & $\begin{array}{c}20.185 \\
20.67\end{array}$ & $\begin{array}{r}29.944 \\
30.25\end{array}$ & $\frac{-1.216}{-1.214}$ & $\frac{-6 .}{-6 .}$ & $\begin{array}{r}50.445 \\
50.56 \\
\end{array}$ & 0.119 & $\begin{array}{l}14.643 \\
14.643 \\
\end{array}$ & $\frac{0.004}{0.004}$ & $\begin{array}{l}64.00 \\
65.00\end{array}$ & \begin{tabular}{|l|}
1.06667 \\
1.108333
\end{tabular} & $\begin{array}{l}50.4 \\
50.6 \\
\end{array}$ & $\begin{array}{l}12.4 \\
12.4\end{array}$ & & $\frac{2.798}{2.814}$ & \begin{tabular}{|l|}
0.018 \\
0.018
\end{tabular} & & & $\frac{0.458}{0.458}$ & 0.39 \\
\hline 83 & $2 / 4 / 2004$ & 9:17:49 AM & 23.108 & 19.918 & \begin{tabular}{|l|l|}
23.63 \\
\end{tabular} & 24.028 & 21.419 & 51.504 & \begin{tabular}{|l|}
52.209 \\
\end{tabular} & 20.497 & 30.523 & -1.214 & & 51.185 & 0.121 & 14.643 & 0.004 & 66.00 & \begin{tabular}{|l|} 
\\
\end{tabular} & 50.7 & 12.5 & 41.0 & 2.828 & \begin{tabular}{|l|l|}
0.018 \\
\end{tabular} & 0.019 & & & 0.39 \\
\hline & $2 / 4 / 2004$ & 9:18:49 AM & & 19.945 & 23.76 & 24.086 & & 51.496 & 52.256 & $\begin{array}{l}20.388 \\
\end{array}$ & 30.615 & -1.211 & & 54.237 & 0.122 & 14.643 & & 67.00 & & 50.8 & 13.3 & & 2.831 & \begin{tabular}{|l|l|}
0.018 \\
\end{tabular} & 0.019 & & & $\begin{array}{l}0.39 \\
0.39\end{array}$ \\
\hline 85 & $2 / 4 / 2004$ & 9:19:49 AM & 23.349 & 19.969 & 23.85 & 24.069 & 21.54 & 51.496 & 52.331 & 20.376 & 30.554 & -1.216 & -6.192 & 48.876 & 0.123 & 14.643 & & 68.00 & 1.13333 & 50.9 & 12.0 & 41. & 2.829 & \begin{tabular}{|l|l|}
0.018 \\
\end{tabular} & 0.019 & & $\frac{0.459}{0.462}$ & $\begin{array}{l}0.39 \\
0.39\end{array}$ \\
\hline & 2/4/2004 & 9:20:49 AM & 23.468 & 20.008 & 23.97 & 24.078 & 21.523 & 51.601 & 52.402 & 20.645 & 30.5 & -1.211 & & 51.2 & 0.121 & 14.643 & & & $\begin{array}{l}1.15 \\
\end{array}$ & & 12.5 & 41. & & 0.018 & 0.019 & & & 0.39 \\
\hline & 2/4/2004 & 9:21:49 AM & & & 24.06 & 24.03 & 21.65 & 51.952 & & & 30.551 & -1.219 & & & 0.121 & 14.643 & & 70.00 & \begin{tabular}{|l|l|}
1.16667 \\
\end{tabular} & 51.4 & 12.6 & 41. & 2.844 & & 0.019 & & 0.449 & \\
\hline 88 & 2/4/2004 & 9:22:49 AM & 23.669 & 20.058 & 24.18 & & & & & 21.188 & & & & & 0.119 & & & & $\begin{array}{l}1.18333 \\
\end{array}$ & & & & & & & 8.000 & 0.440 & \\
\hline & & 9:23:49 AM & & & & 24.142 & & 51.977 & & \begin{tabular}{|l|l}
21.427 \\
\end{tabular} & 30.061 & -1.214 & & & 0.118 & $\begin{array}{l}14.643 \\
\end{array}$ & & & & & 120 & & & & 18 & & 437 & \\
\hline 90 & $2 / 4 / 2$ & 9:24:49 AM & & & 24.42 & 24.09 & 22.3 & & & 21.749 & 29.973 & 1.216 & & & 0 & 14.643 & & & & 51. & & & & & & & 406 & \\
\hline & $2 / 4 / 2$ & $9: 25: 49 \mathrm{AM}$ & & 20.174 & & & & 52.512 & & 22.133 & 29.874 & & & & & 14.643 & & & & & & & & & & & & \\
\hline & $2 / 4 / 2004$ & 9:26:49 AM & 24.098 & 20.213 & \begin{tabular}{|l|l|}
24.63 \\
\end{tabular} & 24.156 & 22.8 & 52.427 & \begin{tabular}{|l|l|}
53.342 \\
\end{tabular} & 22.338 & 29.612 & -1.219 & & & 0.1 & 14.643 & & 75.4 & 1.25 & 51.9 & 11.9 & & & & & & & \\
\hline 93 & $2 / 4 / 2004$ & 9:27:49 AM & 24.196 & 20.257 & \begin{tabular}{|l|l|} 
& 24.7 \\
\end{tabular} & 24.195 & 22.47. & 52.495 & \begin{tabular}{|l|l|} 
& 53.313 \\
\end{tabular} & 22.694 & 29.341 & -1.219 & & .156 & 0.116 & 14.643 & & 76 & 1.26667 & 51.8 & 12.0 & & 2.821 & & & & 0.425 & \\
\hline 94 & $2 / 4 / 2004$ & 9:28:49 AM & 24.285 & 20.286 & 24.87 & 24.154 & 22.63 & 52.887 & 53.753 & 23.06 & 29.335 & -1.219 & & 49.17 & 0.115 & 14.643 & & 77.00 & 1.28333 & 52.3 & 12.0 & $\begin{array}{lll}41.1 \\
\end{array}$ & 2.834 & & 17 & & 0.419 & \\
\hline & $2 / 4 / 2004$ & 9:29:49 AM & 24.389 & 20.33 & 24.98 & 24.177 & & 53.047 & \begin{tabular}{|l}
53.886 \\
\end{tabular} & 23.352 & 29.246 & -1.216 & & & & 14.643 & & 78. & 1.3 & 52.4 & 11.7 & 41 & 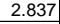 & & & & & \\
\hline 9 & $2 / 1 / 1 / 2$ & 9:30:49 AM & 24.492 & 20.368 & 25.10 & 24.246 & 23.418 & 52.956 & \begin{tabular}{|l|}
53.807 \\
\end{tabular} & 23.597 & 28.906 & 1.219 & & & 0.112 & 14.643 & & & 1667 & 52.3 & 1.4 & 40 & 82 & & 117 & & 0.407 & \\
\hline & 2/4/2004 & 9:31:49 AM & & 20.397 & 25.21 & 24.289 & 2. & 53.176 & 54.047 & 23.962 & 28.77 & 1.216 & & .634 & & 14.6 & & & & & 1.4 & 41 & & 016 & & & 394 & \\
\hline & 2/4/2004 & & 24.68 & 20.452 & & 24.218 & & 52.925 & 53.832 & 24.139 & 28.473 & -1.216 & & & & $14.6 \mathrm{C}$ & & 81.0 & 1.35 & 52.4 & 1.2 & & 806 & & & & & \\
\hline 99 & $2 / 4 / 2 / 2$ & & 24.768 & & 25.46 & 24.06 & 22.6 & & & 24.662 & 28.233 & -1.216 & & 6.063 & 0.106 & 14.643 & & & & 53.1 & 1.3 & & & & & & & \\
\hline & $2 / 1420004$ & 9:34:49 AM & & 20.518 & $\mid$\begin{tabular}{|l|l|}
$\mid 25.58$ \\
27
\end{tabular} & 23.898 & 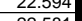 & 53.561 & \begin{tabular}{|l|l}
54.654 \\
\end{tabular} & 25.046 & 27.853 & $\frac{-1.216}{-120}$ & & 6006 & $n^{2}{ }^{2}$ & & & & 333 & 51 & $\frac{1.1}{112}$ & & 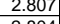 & & & & & \\
\hline & & 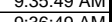 & $\begin{array}{lll}44.945 \\
\end{array}$ & & & & & $\begin{array}{l}53.030 \\
5.2777\end{array}$ & & 23.454 & & $=\frac{-1.219}{1.202}$ & & 40.040 & & & & & 1.4 & 53.1 & 11.3 & & 2.004 & & & & 年 & \\
\hline & $2 / 4 / 42004$ & 3.50 .45 & 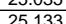 & 20.501 & $\mid$\begin{tabular}{|l|l|}
26.08 \\
\end{tabular} & 年. & 237 & 54505 & \begin{tabular}{|l|l|}
5.0479 \\
5569
\end{tabular} & 26093 & $\frac{20.241}{25448}$ & -1.216 & & $\begin{array}{l}4.0604 \\
49047\end{array}$ & & 146 & & 告 & \begin{tabular}{|l|}
1.41667 \\
133333
\end{tabular} & 5.4. & $\frac{11.4}{120}$ & & 2750 & & & & 0.000 & \\
\hline & $2 / 4 / 2004$ & $3.58 .494 \mathrm{AM}$ & 年 & & & 23.04 & & $\frac{54.034}{5424}$ & \begin{tabular}{|l|l}
55392 \\
5539
\end{tabular} & 26093 & 25.4361 & -1.219 & & 50.679 & 0.104 & 146 & & 87 & 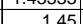 & $\frac{54.2}{539}$ & $\frac{1.50}{124}$ & & $\frac{700}{744}$ & & & & 135 & \\
\hline & $2 / 4 / 2$ & 9:39:49 AM & 0.200 & 20.646 & 26.57 & 23.287 & 23.1 & 54.524 & \begin{tabular}{|l}
55.507 \\
557
\end{tabular} & 26.093 & 25.521 & -1.216 & & 49.5 & & 14.643 & & & 1.46667 & $\frac{3.2}{54.2}$ & 21 & & & & & & 0.374 & \\
\hline & $2 / 4 / 2$ & & 5.526 & 20.684 & \begin{tabular}{|l|l|}
26.78 \\
\end{tabular} & 23.275 & & 54.598 & 55.817 & 26.093 & 25.677 & -1.219 & & 9.7 & & & & & & 54.3 & 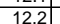 & & 767 & & & & & \\
\hline & $2 / 4 / 2$ & & & & 27.00 & 23.237 & & & \begin{tabular}{|l|l|}
55.683 \\
\end{tabular} & 26.093 & 25.955 & -1.216 & & & & & & & 1.5 & $F$ & & & & & & & & \\
\hline & $2 / 4 / 2$ & & & & & 23.179 & & 54.611 & & & & & & & & 14.6 & & & & $\begin{array}{ll}54.4 \\
\end{array}$ & & & & & & & & \\
\hline & $2 / 4 / 2$ & & 25.964 & 20.748 & 27.39 & 23.172 & 23. & 54.75 & \begin{tabular}{|l|l|}
55.985 \\
\end{tabular} & 26.093 & 26.092 & -1.219 & & 8.4 & 0.1 & 14.6 & & 92.00 & 1.53333 & 54.5 & 11.9 & & 2.787 & 16 & & & 0.3 & \\
\hline & & & & 20.787 & & 22.935 & & 55.042 & 56371 & 26.093 & 26.232 & -1.219 & & & & & & & & 54.9 & & & & & & & & \\
\hline & $2 / 4 / 2$ & & 26.256 & 20.811 & \begin{tabular}{|l|l|}
27.75 \\
\end{tabular} & 22.909 & & 54.737 & \begin{tabular}{|l|}
55.989 \\
\end{tabular} & 26.093 & 26.045 & -1.219 & & & & & & & 56667 & 54.5 & $\frac{12.0}{12.0}$ & 40.4 & 2785 & & & & & \\
\hline & $2 / 4 / 2$ & & 26.379 & 20.829 & 27.91 & 22.902 & & 54.835 & \begin{tabular}{|l|l|}
55.875 \\
\end{tabular} & 26.093 & 25.774 & $\begin{array}{l}-1.219 \\
\end{array}$ & & 48.08 & 0.1 & 14.6 & & & \begin{tabular}{|l|l|l|l}
1.583333 \\
\end{tabular} & 54.4 & \begin{tabular}{l|l|}
11.8 \\
\end{tabular} & 40 & 2.779 & \begin{tabular}{|l|l|}
0.015 \\
\end{tabular} & & & 0.351 & \\
\hline & $2 / 4 / 2$ & & & & 28.09 & 22.884 & & & 57. & 26. & 25.5 & -1.2 & & & 0.1 & 14.6 & & & 1.6 & & 1.7 & 40 & 2.796 & & & & 0.344 & \\
\hline & $2 / 4 / 2004$ & & & 20.886 & & 22.847 & & 54.7 & 56.039 & 26. & 25.1 & -1.2 & & & & 14.6 & & & 1.61667 & 54.6 & 11.6 & & 755 & & & & 0.341 & \\
\hline & $2 / 4 / 2$ & 9:49:49 & 26.802 & 20.904 & 28.43 & 22.82 & & 55.125 & 56.543 & 26.093 & 25.283 & -1.2 & & & 0.0 & 14.6 & & 98 & 1.63333 & b. & 1.5 & & 12 & & & & & \\
\hline & $2 / 4 / 2$ & 9:50:49 & 26.94 & 20.937 & 28.61 & 22.767 & & 55.49 & \begin{tabular}{|l|}
56.88 \\
\end{tabular} & 26.093 & 25.2 & -1.216 & & & & & & & 1.65 & & .4 & 0.4 & & & & & & \\
\hline & $2 / 4 / 2$ & 9:51:4 & 2.0 .6 & 20.96 & 28.76 & 22.725 & & 55.561 & 57.048 & 26.093 & 25.146 & -1.2 & & & & $14.6 \mathrm{C}$ & & 100 & 1.66667 & 55.6 & 1.3 & 40.4 & .882 & & & & 0.313 & \\
\hline & $2 / 4 / 2$ & $9: 52: 49$ & & 20.988 & 29.06 & 22.728 & & 56.631 & \begin{tabular}{|l}
58.066 \\
\end{tabular} & 26.0 & 24.077 & -1.214 & & & & & & 0.1 .00 & 8333 & 50. & & & & & & & & \\
\hline & & & & 21.001 & 29.3 & 22.106 & & & & 26. & & & & & & & & & $1.7 \mathrm{t}$ & & & & & & & & & \\
\hline & & & 27.51 & & 29.54 & 22.649 & & 56.149 & & 26.0 & & -1.2 & & & & & & & & 56.8 & & & & & & & & \\
\hline & $2 / 142004$ & & 4.000 & 21.043 & 29.106 & 2.65 & & 56.8362 & \begin{tabular}{|l|l|}
58.5344 \\
\end{tabular} & 26.093 & 24.5 & $=-1.216$ & & 172 & & 14. & & & 333 & 58.1 & 1.88 & 4 & 2.005 & & & & & \\
\hline & $\frac{2442}{21 / 2}$ & $\frac{9.30 .496}{9.57 \cdot 19}$ & .820 & $\frac{2.051}{21062}$ & \begin{tabular}{|l|l|}
2.917 \\
3017
\end{tabular} & $20.64 \mid$ & & $\frac{30.935}{57.89}$ & 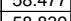 & 20.093 & 24.551 & $\frac{-1.219}{-1210}$ & & $68-2$ & & 14.0 & & $\frac{105}{106}$ & \begin{tabular}{|l|l|l|} 
\\
7
\end{tabular} & 57 & 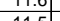 & 4c & 2.809 & & & & & \\
\hline & $2 / 41 / 2004$ & S.5.45A & 80.96 & $\frac{21.003}{21091}$ & 30.175 & $\frac{2.026}{226}$ & & 567672 & 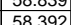 & 20.099 & 21.456 & -1.216 & & & & 1464 & & 年 & 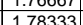 & 56. & 17 & & 2711 & & & & & \\
\hline & $2 / 21 / 2004$ & 9.59 .49 & 2826 & 2112 & 30.48 & 22593 & & 58308 & 60095 & 26099 & 23065 & 12 & & & & 年 & & 108 & | & 586 & & & & & & & & \\
\hline & $2 / 2 / 42$ & $10 \cdot 00.49$ & & 21.138 & $\mid 3063$ & 22576 & & 57.919 & \begin{tabular}{|l|}
59592 \\
\end{tabular} & & 2233 & -1.219 & & & & & & & & & & & & & & & & \\
\hline & & $\frac{10: 01: 4}{10: 01}$ & 28.558 & $\frac{21.153}{21.153}$ & & $\frac{25.661}{22.661}$ & & 58.201 & 59.5 & $\frac{26.093}{26.093}$ & 23.2 & $\frac{-1.216}{-1.216}$ & & & & & & & & 855 & & & & & & & & \\
\hline & & & & 21.177 & & 22685 & & 58.53 & & 26.093 & 23.266 & -1.219 & & & & & & & 1.85 & & & & & & & & & \\
\hline & $2 / 4 / 2$ & & & & & & & & & & & & & & & & & & & & & & & & & & & \\
\hline 130 & $2 / 4 / 2$ & $10: 04: 49$ & 8.934 & 21.211 & 31.44 & 22.694 & & 59.476 & \begin{tabular}{|l|l|}
61.196 \\
\end{tabular} & 26.093 & 22.905 & -1.216 & & & 0.088 & 14.643 & & & & & $1.8 \mathrm{r}$ & & 2.840 & 13 & 011 & 0.000 & 0.267 & \\
\hline
\end{tabular}


WSRC-TR-2005-00105, REVISION 0 SRNL-RPP-2005-00012, REVISION 0

RUN \# 1.17; STEADY STATE TEST AT <20 WT \% UDS - CONT.

\begin{tabular}{|c|c|c|c|c|c|c|c|c|c|c|c|c|c|c|c|c|c|c|c|c|c|c|c|c|c|c|c|c|}
\hline & & & & & & & & & & & & & & & & & & & W & $x$ & & & AA & & $\mathrm{AC}$ & $A D$ & AE & AF \\
\hline & $2 / 4 / 2004$ & $0: 05: 49 \mathrm{AM}$ & & 21.241 & & & & & & & & & & & & 14.643 & & & & & $\overline{11.2}$ & 40.5 & & 0.013 & 0.011 & 000 & 0.261 & $\frac{\pi 12}{0.2}$ \\
\hline 32 & 2/4/2004 & 10:06:49 AM & 29.111 & 21.245 & 32.10 & 22.773 & 26.013 & 60.27 & \begin{tabular}{|l|l|}
61.928 \\
\end{tabular} & 26.093 & 20.773 & -1.219 & -6.689 & 47.75 & 0.082 & 14.643 & 0.004 & 115.00 & 1.91667 & 60.5 & $\begin{array}{ll}11.7 \\
\end{array}$ & 40.5 & $\mid 2.794$ & $\mid 0.012$ & 0.010 & 0.000 & \begin{tabular}{|l|l|}
0.248 \\
\end{tabular} & 0.21 \\
\hline 33 & $2 / 4 / 2004$ & $10: 07: 49$ AM & 29.305 & 21.275 & 32.85 & 22.742 & 25.852 & 59.9 & 61.917 & 26.093 & 20.37 & -1.219 & -6.351 & 49.663 & 0.086 & 14.643 & & 116.00 & 1.93333 & 60.4 & 12.2 & 40.2 & 2.770 & 0.013 & $\begin{array}{ll}0.010 \\
\end{array}$ & 0.000 & 0.257 & \\
\hline 134 & 2/4/2004 & 10:08:49 AM & 29.601 & 21.298 & 33.52 & 22.756 & 25.881 & 59.84 & 61.756 & 26.093 & 20.699 & -1.214 & -6.346 & 49.585 & 0.089 & 14.643 & 0.004 & 117.00 & 1.95 & 60.3 & 12.2 & 40.3 & 2.776 & & 0.011 & & \begin{tabular}{|l|l|}
0.261 \\
\end{tabular} & 0.22 \\
\hline 135 & 2/4//2004 & $10: 09: 49$ AM & 29.907 & 21.301 & 34.16 & 22.698 & 26.024 & 59.83 & 61.952 & 26.093 & 20.617 & -1.219 & -6.351 & 49.062 & 0.089 & 14.643 & 0.004 & $\begin{array}{ll}118.00 \\
\end{array}$ & 1.96667 & 60.5 & 12.0 & 40.2 & 2.774 & 0.013 & 0.010 & 0.000 & 0.257 & 0.22 \\
\hline 136 & 2/4/2004 & 10:10:49 AM & 30.252 & 21.309 & 34.86 & 22.681 & 26.151 & 60.25 & 62.425 & 26.093 & 20.61 & -1.219 & -6.363 & 48.305 & 0.09 & 14.643 & & 119.00 & 1.98333 & 61.0 & 11.8 & 40.4 & \begin{tabular}{|l|l|}
2.788 \\
\end{tabular} & 0.013 & 0.010 & 000 & 0.254 & 0.22 \\
\hline 137 & 2/4/2004 & 10:11:49 AM & 30.568 & 21.347 & 36.08 & 22.746 & 26.348 & 58.27 & 60.431 & 26.093 & 19.872 & -1.216 & -6.38 & 44.071 & 0.087 & 14.643 & 0.004 & 120.00 & & 59.0 & 10.8 & 39.1 & 2.694 & 0.013 & 0.010 & .000 & 0.246 & 0.21 \\
\hline 138 & 2/4/2004 & 10:12:49 AM & 30.963 & 21.371 & 37.92 & 22.763 & 26.257 & 58.48 & 60.709 & 26.093 & 19.209 & -1.216 & -6.317 & 42.2311 & 0.082 & 14.643 & & 121.00 & & 59.2 & 10.3 & $38.8 \mathrm{r}$ & 2.678 & 0.012 & 0.009 & .000 & & \\
\hline & 2/4/20004 & 10:13:49 AM & 31.401 & 21.393 & 39.87 & 22.77 & 26.169 & 54.20 & 58.215 & 26. & 16.233 & -1.156 & -6.6 & 39.064 & & 14.643 & & 2.00 & & 56.7 & 9.6 & 33. 3. & 2.4 & & & 000 & 0.009 & 0.01 \\
\hline $1 \frac{140}{140}+3$ & $2 / 1420004$ & 10:14:49 AM & 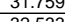 & 21.399 & 41.85 & 22.706 & 20.315 & 52.77 & 58.105 & 26.093 & $\frac{14.6}{10.67}$ & 0.312 & -1.096 & 38.046 & 68 & 14.643 & & & 2.05 & 56.6 & 9. & 33.7 & & 0.025 & 0.016 & 0.000 & 0.474 & 0.40 \\
\hline$\frac{141}{142}$ & 2/4/2004 & 10:15:49 AM & 32.533 & 21.416 & 43.8 & 22.647 & 26.247 & 55.50 & 58.52 & 26.093 & 16.557 & -1.219 & & 37.108 & & 14.643 & & 124.00 & & 57.1 & 9. & $36 . c$ & 2.484 & 0.005 & 0.003 & 0.000 & 0.090 & 0.08 \\
\hline 1143 & & Averages & 24.3 & 19.7 & 24. & $23.3 \mathrm{r}-\mathrm{l}$ & 21.9 & 52. & 53.6 & 21.3 & 28.6 & $\begin{array}{c}-1.2 \\
\end{array}$ & -6.2 & 48.1 & 0.1 & 14.6 & & & & 52.1 & 11.8 & 40.6 & 2.802 & 0.018 & 0.019 & 0.0005 & 0.453 & $0.386 \#$ \# \\
\hline 144 & & Maximum & 32.5 & 21.4 & 43. & 24.3 & 26.3 & 60. & 62.4 & 26.1 & 35.1 & 0.3 & & 54.2 & & 14.6 & & & & 61.0 & 13.3 & 42.3 & & & 0.034 & & & \\
\hline 145 & & Median & 23.0 & 19.9 & 23. & $23.3 \times 3$ & $21.8 \mid$ & 51. & & 21.7 & 29.7 & -1.2 & -6. & \begin{tabular}{ll|l|}
48.4 \\
\end{tabular} & & 14.6 & & & & 50.8 & 11.9 & 40.7 & 2.807 & 0.017 & 0.018 & 0.0004 & 0.448 & \\
\hline 146 & & Minimum & 21.5 & 17.3 & 20. & $22.6 \mathrm{x}-\mathrm{s}$ & $17.1 \#$ & 48. & 48.6 & 13.1 & 14.6 & $\begin{array}{lll}-1.2 & \end{array}$ & -6.6 & 37.1 & 0.0 & 14.6 & & & & 47.1 & 9.1 & 33.7 & 2.323 & 0.000 & 0.000 & 0.0000 & 0.009 & \\
\hline 147 & & $2 \times \mathrm{Std}$ Dev & 5.865 & 2.524 & 10.01 & 0.98 & $5.060 \#$ & 6.54 & 7.713 & 8.761 & 9.004 & 0.279 & 1.062 & 5.160 & 0.061 & 0.000 & 0.001 & & & 7.716 & (1.265 & 2.215 & & 0.009 & 0.012 & & 0.295 & 0.25 \\
\hline 148 & Number o & Jf Points Used ${ }^{\star}$ & 119 & 119 & 11 & 11 & $119]$ & 11 & 119 & 119 & 119 & 119 & 119 & 119 & 119 & 119 & 11 & & & 119 & 119 & 119 & 119 & 119 & 119 & 119 & 119 & 119 \\
\hline$\frac{149}{150}$ & & & & & & & & & & & & & & & & & & & & & & & & & 185 & & & \\
\hline \begin{tabular}{|l|}
151 \\
\end{tabular} & & & & & & & & & & & & & & & & & & & & & & & 1-hour a & & 0.0231 & & & \\
\hline
\end{tabular}


RUN \# WASH1; CAMPAIGN I SLURRY WASH 1

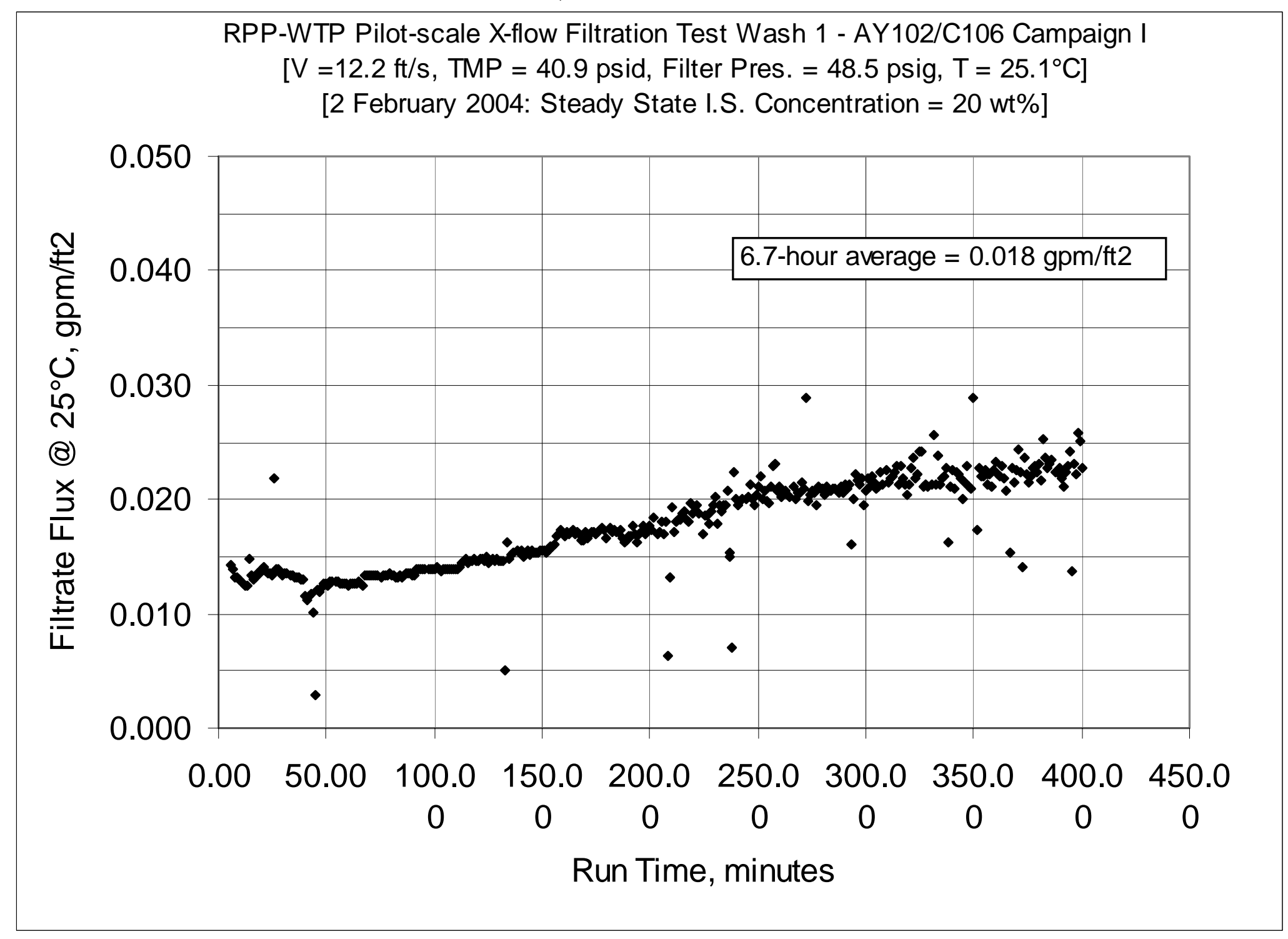


WSRC-TR-2005-00105, REVISION 0

SRNL-RPP-2005-00012, REVISION 0

RUN \# WASH1; CAMPAIGN I SLURRY WASH 1 - CONT.

\begin{tabular}{|c|c|c|c|c|c|c|c|c|c|c|c|c|c|c|c|c|c|c|c|c|c|c|c|c|c|c|c|c|}
\hline & A & B & D & $\mathrm{E}$ & $\mid \mathrm{F}$ & G & $\mathrm{H}$ & \begin{tabular}{l|l}
$\mathrm{J}$ & 1 \\
\end{tabular} & $\mathrm{~K}$ & $\begin{array}{ll} \\
\end{array}$ & $\mathrm{M}$ & $\mathrm{N}$ & 0 & $\mathrm{Q}^{\mathrm{Q}}$ & $\mathrm{R}$ & $\mathrm{s}$ & $\mathrm{T}$ & $\mathrm{v}$ & W & $\mathrm{x} \mid \mathrm{x}$ & $\mid Y$ & $\mid z$ & $\mathrm{AA}$ & $A B$ & $\mathrm{AC}$ & $\mathrm{AD}$ & $\mathrm{AE}$ & $\mathrm{AF}$ \\
\hline & & & 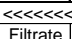 & $\begin{array}{l}\text { «e Temp } \\
\text { CCleaning }\end{array}$ & 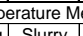 & easurem & ents >>> & 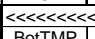 & $\begin{array}{l}2 \ll<\ll< \\
\text { Eilter } \\
\end{array}$ & Pressure & Measuremer & & Pulser & 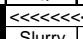 & 《< Flow M & Measureme & & & & & & & & & & & & \\
\hline & DAIE & & & & & HIA Amb. & Lo Amb. & & & & & & & & & & Backpuls & & & & & & & & & & & \\
\hline 4 & & & $\mathrm{~T}_{2}$ & $\frac{\text { aegC }}{\mathrm{T} 3}$ & $\frac{\mathrm{deg} C}{\mathrm{~T} 1}$ & $\frac{\mathrm{deg}}{\mathrm{d} 4}$ & $\frac{\mathrm{deg} C}{\mathrm{~T} 5}$ & $\begin{array}{l}\text { psid } \\
d \mathrm{P} 2\end{array}$ & $\begin{array}{l}\frac{p s i g}{P 1} \\
1\end{array}$ & $\begin{array}{l}\text { psid } \\
\text { dP1 }\end{array}$ & $\begin{array}{l}\text { psid } \\
d P 3\end{array}$ & $\frac{p s i g}{P 2}$ & $\frac{p s i g}{\text { p3 }}$ & $\frac{\mathrm{gpm}}{\mathrm{O} 1}$ & $\frac{\mathrm{gpm}}{\mathrm{O} 2}$ & gpm & gpm & & & & & & & & & & & \\
\hline & & & & & & & & & & & & & & & & & & & & & & & & & & & & \\
\hline & & & & & & & & & & & & & & & & & & & & & & & & & & & & \\
\hline 7 & $2 / 2 / 2004$ & 10:20:32 AM & 22.477 & 17.831 & 14.99 & 22.905 & 15.993 & 0.048 & -0.056 & 0.016 & 0 & -0.177 & 0.055 & $\begin{array}{ll}-0.083 \\
\end{array}$ & 0.003 & 14.643 & 0.004 & & & Filter Su & Surface, & 6.707 & FT2 & & & & & \\
\hline 8 & 2/2/2004 & 10:20:56 AM & 22.477 & 17.831 & 14.99 & 22.905 & 15.99 & 0.048 & -0.056 & 0.016 & & -0.177 & & -0.083 & 0.003 & 14.643 & 0.00 & & & Convers & & & $\mathrm{m} 3 / \mathrm{m} 2$ & 2/day/bar & $\mathrm{rg} / \mathrm{gpm} / \mathrm{ft}$ & ftrl/barg & & \\
\hline & $2 / 2 / 2004$ & 10:20:57 & 22.473 & 17.831 & 14.971 & 22.901 & 16.004 & 0.048 & -0.054 & 0.014 & -0.004 & -0.212 & 0. & -0.085 & 0.003 & 14.643 & 0.0 & & & & & & & & & & & \\
\hline & 2/2/2004 & 10:20:58 AM & & 17.826 & 14.966 & 22.896 & 15.994 & 0.05 & -0.056 & 0.014 & -0.004 & \begin{tabular}{|l|l|}
-0.174 \\
\end{tabular} & & -0.077 & 0.003 & 14.643 & & & & Note: & auge & ress & eP1i & appro & nately 1.2 & $4 \mathrm{psig}$ & & \\
\hline & 2/2/2004 & 10:20:59 AM & & 17.826 & 14.971 & 22.886 & 15.994 & 0.046 & -0.056 & 0.014 & -0.004 & \begin{tabular}{|l|l|} 
& -0.183 \\
\end{tabular} & & -0.081 & 0.003 & 14.643 & & & & & & & & & & & & \\
\hline & & & 22.468 & & 14.966 & 22.901 & 15.994 & 0.046 & -0.056 & 0.014 & $\begin{array}{l}-0.004 \\
-0.021\end{array}$ & -0.192 & & -0.081 & 0.003 & 14.643 & & & & & & & & & & r tub & & \\
\hline & $\frac{2 / 21 / 2004}{2(212004}$ & 10.01010 & $\frac{2.4863}{2.2073}>$ & & $14.9 / 1$ & 22.906 & & 0.048 & -0.056 & 0.014 & -0.004 & $|-0.1 / 4|$ & & & 0.003 & 14.643 & & & & VWV & & & & $F=1$ & & & & \\
\hline & 2/2/2004 & 10:21: & 22.473 & 17.837 & 14.971 & 22.906 & & 0.048 & -0.054 & 0.015 & -0.004 & -0.218 & & -0.079 & 0.003 & 14.643 & & Time & Time & Press. & Vel. & TMP & TMP & gapm & & $\mathrm{gpm}$ & $\frac{1000}{n m}$ & \\
\hline 16 & ata-Per Mi & & & & & & & & & & & & & & & & & Min. & Hour & psig & ftts & psi & bar & 年12 & 12 & $\mathrm{ft2} / \mathrm{psi}$ & tz/psi & $\begin{array}{l}\text { meter } \\
\text { day/bar }\end{array}$ \\
\hline 17 & 2/2/2004 & & & & 25.549 & & 20.105 & 31.876 & 52.721 & 19.285 & 12.939 & \begin{tabular}{|l|l|}
15.497 \\
\end{tabular} & & 49.498 & 0.003 & 14.643 & & & & 51.2 & & 22.4 & & & 0.000 & & & \\
\hline & 2/2/2004 & 12:04 & 23.589 & 18.973 & 25.559 & 24.044 & 20.126 & $\begin{array}{ll}9.248 \\
9\end{array}$ & 50.316 & $\begin{array}{ll}10.747 \\
\end{array}$ & & 35.953 & 92.27 & 59.414 & 0.003 & 14.643 & & 1 & D.016 & 48.8 & 14.6 & 4.1 & 0.282 & 0.000 & 0.000 & & 0.108 & \\
\hline & $\frac{2 / 2 / 2004}{2 / 2 / 2004}$ & $\begin{array}{l}12: 05: 31 \mathrm{PM} \\
12: 06.31 \mathrm{PM}\end{array}$ & $\begin{array}{l}23.769 \\
25.711 \\
\end{array}$ & $\begin{array}{l}19.008 \\
19058 \\
\end{array}$ & $\begin{array}{l}325.564 \\
3\end{array}$ & $\begin{array}{l}24.048 \\
2403\end{array}$ & $\begin{array}{l}20.483 \\
20.765 \\
\end{array}$ & \begin{tabular}{rl|l}
9.192 \\
45.423 \\
\end{tabular} & $\begin{array}{l}50.575 \\
51.274\end{array}$ & $\begin{array}{l}\frac{10.686}{1273} \\
\end{array}$ & \begin{tabular}{|c|}
-1.172 \\
32699 \\
\end{tabular} & \begin{tabular}{|l|}
36.202 \\
0.327
\end{tabular} & \begin{tabular}{|l|}
5.394 \\
0.251 \\
\end{tabular} & \begin{tabular}{|l|l|}
52.931 \\
52.927
\end{tabular} & \begin{tabular}{|c|}
0.003 \\
0.168
\end{tabular} & $\begin{array}{l}14.643 \\
14.643 \\
\end{array}$ & 0.006 & $\frac{2.00}{300}$ & $\frac{03333}{0.05}$ & 49.1 & $\frac{13.0}{130}$ & \begin{tabular}{r|r|}
3.0 \\
39.0
\end{tabular} & 0.276 & $\frac{0.000}{2183}$ & $\begin{array}{l}0.000 \\
2149\end{array}$ & $\frac{0.000}{0.055}$ & 0.110 & $\frac{0.0}{46.8}-8$ \\
\hline & 2/2/2004 & 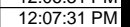 & 25.881 & 19.114 & 4.574 & 23.998 & 20.583 & 45.709 & 51.512 & 13.959 & 31.643 & \begin{tabular}{|l|}
0.26 \\
\end{tabular} & & 52.987 & \begin{tabular}{|c|}
0.100 \\
0.12
\end{tabular} & $\begin{array}{l}14.045 \\
14.643 \\
\end{array}$ & & & 0.06667 & 49.0 & & (39.0 & 2.091 & & & & 55.544 & \\
\hline & $2 / 2 / 2004$ & 12:08:31 PM & 25.976 & & | 25.549 & 24.069 & $3208>$ & $\mid 45.78$ & \begin{tabular}{|l|l|}
51.574 \\
\end{tabular} & 14.504 & 31.208 & 0.251 & & 49.517 & 0.104 & 14.6 & & 5 & 0.08333 & & & 38.5 & & & & & & \\
\hline 23 & $2 / 2 / 2004$ & 12:09:31 PM & 26.035 & 19.194 & 425.509 & 24.088 & 20.849 & 46.074 & 51.892 & $\begin{array}{ll}14.778 \\
\end{array}$ & 31.206 & 0.254 & & 56.369 & | 0.097 & 14. & & 6.00 & 0.1 & 50.4 & 13.8 & 38.6 & & 0.014 & \begin{tabular}{|c|c|c|}
0.014 \\
\end{tabular} & 0.000 & $\begin{array}{ll}0.369 \\
\end{array}$ & \\
\hline & $2 / 2 / 2004$ & 12:10:31 PM & 26.04 & 19.234 & & 24.147 & 20.36 & 46.077 & 51.885 & 14.666 & 31.385 & \begin{tabular}{|l|l|} 
& 0.257 \\
\end{tabular} & & 53.129 & 0.094 & 14.643 & & 7.6 & 0.11667 & 50.4 & 13.0 & 38.7 & 2.670 & 0.014 & 0.014 & 0.000 & 0.357 & 0. \\
\hline & 2/2/2004 & 12:11:31 PM & 26.052 & 19.272 & 25.485 & 24.14 & 20.745 & 46.17 & 51.995 & 14.971 & 31.104 & 0.286 & & 50.816 & 0.09 & 14.643 & & 8.0 & 0.1333 & 50. & 12.5 & 38.6 & 2.664 & & & 0.000 & 0.343 & \\
\hline & 2/2/20004 & 12:12:31 PM & 26.056 & & 25.474 & 24.153 & 21.403 & 46.431 & 52.292 & 15.05 & 31.264 & 0.327 & & 53.025 & 0.089 & 14.643 & & 9.0 & 0.15 & 50. & 13. & 38.8 & 2.678 & & 0.013 & 0.000 & 337 & \\
\hline 27 & 2.12/20004 & 12:113:31PMM & 26.055 & $19.3 / 1$ & 25.458 & 24.067 & & 46.4199 & 52.314 & $14.938 \mathrm{r}$ & 31.413 & 0.338 & & 49.167 & 0.088 & 14.6 & & & 0.16667 & & & 38.99 & & & & & 0.333 & \\
\hline & 21212004 & 年.14. & 20.044 & $\frac{19.41}{10961}$ & 25.4424 & 24.096 & 21.86 & | 46.189 & \begin{tabular}{|l|l|}
52.082 \\
52301
\end{tabular} & (14.9935 & & 0.358 & & 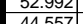 & 0 & 14.6 & & & . 1833 & . & . & 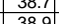 & & - & & & & \\
\hline & $\frac{21 / 1 / 20044}{2 / 21 / 2004}$ & 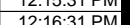 & $\frac{2.0493}{26053}$ & $\frac{19.401}{19516}$ & $\frac{2.4242}{25477}>0$ & $\frac{24.121}{24.16}$ & 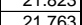 & & \begin{tabular}{|l|l|l|l|l|}
52416 \\
5
\end{tabular} & 15.104 & 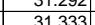 & $\frac{0.304}{0408}$ & & (54.5304 & 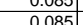 & $\frac{14.6}{146}>3$ & & & $\frac{1.4}{02166}$ & & & 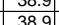 & & & & & & \\
\hline$\overline{31}$ & 2/2/2004 & 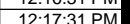 & 25.056 & 19.545 & 年. & 24.471 & $\frac{2.18}{218}$ & $\begin{array}{ll}50.4563 \\
51.562\end{array}$ & \begin{tabular}{|l|l|l|l|l|}
52596 \\
\end{tabular} & 15119 & (3.504 & $\frac{-.461}{-1.21}$ & & 498 & 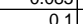 & 146 & & $\frac{140}{140}$ & 0.2333 & 511 & 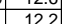 & 440 & 303 & 0.010 & & & 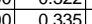 & \\
\hline 32 & $2 / 2 / 2004$ & 12:18:31 PM & 25.448 & 19.611 & 25.471 & 24.241 & 22.124 & 51.726 & \begin{tabular}{|l|}
52.681 \\
\end{tabular} & 15.296 & 36.304 & \begin{tabular}{|l|l|}
-1.219 \\
\end{tabular} & & 50.359 & 0.091 & 14.643 & & 15.06 & 0.25 & $\frac{11.2}{51.2}$ & 12.3 & 44.0 & 3.035 & .014 & 0.0 & 0.000 & 0.304 & \\
\hline & & & 25.463 & & & & & \begin{tabular}{|c|}
51.485 \\
\end{tabular} & & 15.218 & 36.212 & \begin{tabular}{|l|l|}
-1.219 \\
\end{tabular} & & & & & & 16.6 & & & 12.6 & & & & & & & \\
\hline & $2 / 2 / 200$ & 12 & 25.479 & 19.744 & 425.217 & 24.343 & & \begin{tabular}{|c|}
51.16 \\
\end{tabular} & \begin{tabular}{|l|}
52.147 \\
\end{tabular} & 14.86 & & & & 49.9 & & & & 7 & 0.28333 & 50.7 & 12.2 & 43.7 & & & 001 & 0.000 & 302 & \\
\hline & $2 / 2 / 2004$ & & 25.441 & 19.80 & 125.244 & & & & 52.171 & 14.696 & 36.534 & & & & & & & 18 & & 50 & & 43.9 & & & & & & \\
\hline 36 & $2 / 2 / 2004$ & 12:22: & 25.407 & 19.873 & 25.26 & 24.415 & 23.1 & \begin{tabular}{|c|}
51.28 \\
\end{tabular} & \begin{tabular}{|l|}
52.227 \\
\end{tabular} & 14.601 & & \begin{tabular}{|l|l|} 
& -1.216 \\
\end{tabular} & & & & & & & 0.31667 & 50. & 14.1 & 44.0 & & 0.014 & & & 310 & \\
\hline & $2 / 2 / 2004$ & & 25.384 & 19.95 & & 24.502 & & 51.386 & \begin{tabular}{|l|l|}
52.288 \\
\end{tabular} & 14.51 & & -1.2 & & & & 14.6 & & & 0.33333 & 50.8 & & $\begin{array}{l}44.2 \\
\end{array}$ & & 0.014 & 0.014 & 0.000 & & \\
\hline 38 & $2 / 2 / 2004$ & & 25.355 & & 25.253 & 24.494 & & \begin{tabular}{|l|}
52.089 \\
\end{tabular} & 53.104 & 13.755 & & -1.2 & & & & & & & 0.35 & 51.6 & 12.4 & $\begin{array}{l}45.2 \\
\end{array}$ & & & & & 0.311 & \\
\hline 39 & 2/2/2004 & 12:25:31 & 25.336 & 20.084 & $\begin{array}{l}425.264 \\
\end{array}$ & 24.51 & 23.917 & 52.4 & 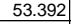 & 13.633 & 38.689 & \begin{tabular}{|l|l|} 
& -1.222 \\
\end{tabular} & & 48.734 & 0.093 & 14.6 & & & 0.36667 & 51.9] & 11.9 & 45.5 & 3.140 & 0.014 & 0.014 & 0.000 & 0.302 & \\
\hline 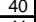 & & & & & & & & 51.747 & \begin{tabular}{|l|l|}
52.0717 \\
\end{tabular} & & & & & & & & & & & 01.2 & 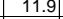 & 44.0 & & & & & & \\
\hline & & & 25.326 & 20.24 & 25.309 & & & & 52.302 & 14.3 & 30.088 & & & & & & & & & 50.8 & & & & & & & & \\
\hline$\frac{42}{43}$ & $2 / 212004$ & 10.20 .31 & $\frac{25.323}{2532}$ & $\frac{20.308}{3020}$ & $\frac{25.306}{25212}$ & 24.481 & 24.0 & 51.715 & 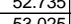 & $\frac{14.519}{14266}$ & $\begin{array}{l}37.051 \\
3.005\end{array}$ & $\frac{-1.219}{-1219}$ & & & & 14. & & 260 & 0.41067 & 51. & $\frac{12.4}{1.4}$ & 44.4 & 111 & & & 0000 & 0.005 & 0 \\
\hline$\frac{74}{44}$ & $\begin{array}{ll}2 / 1 / 2004 \\
2 / 2004\end{array}$ & & $\begin{array}{l}25.35 \\
25251\end{array}$ & 20.59 & & & & & & & & & & & & & & & & & & 45 & & & & & & \\
\hline 45 & 2.1/2/2004 & $\frac{12.00 .}{12: 31: 3}$ & 25.288 & $\frac{0.444}{20.514}$ & 4 & 24.567 & 23. & 52. & \begin{tabular}{|l|l|}
52712 \\
\end{tabular} & 14056 & & -1.216 & & 49.7 & 年. & 144 & & & 0.46667 & 512 & 412.0 & 45.5 & & & 0.01 & & 0.306 & \\
\hline 46 & 2/2/2004 & $12: 32: 3$ & 25.294 & 20.581 & \begin{tabular}{|l|l|}
125.237 \\
252.237
\end{tabular} & & & & \begin{tabular}{|l|l|}
52.928 \\
\end{tabular} & & 37.958 & & & & & & & & 0 & 51.5 & & 45.3 & & & & & & \\
\hline & & & 25.31 & 20.643 & 25.268 & 24.634 & & 52.464 & \begin{tabular}{|l|l|}
52.646 \\
\end{tabular} & & & 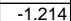 & & & & & & & 0.5 & 512 & & 45.3 & & & & & 294 & \\
\hline 48 & & & 25.317 & 20.695 & 525.245 & & & & 52.847 & 14.6 & 38.504 & & & 46.9 & 91 & & & & 0.51667 & 51.4 & & 45,6 & & & & & 296 & \\
\hline & 2/2/2004 & & $\begin{array}{l}25.323 \\
\end{array}$ & 20.762 & 25.261 & 24.642 & 24.8 & & \begin{tabular}{|l|}
53.019 \\
\end{tabular} & 14.1 & & \begin{tabular}{|l|l|}
-1.2 \\
\end{tabular} & & & 0.091 & & & & 0.53333 & 51.5 & $\overline{12.4}$ & 4כ. & & & & & & \\
\hline & & & 25.339 & 20.824 & 425.288 & 24.6 & & & 53.092 & & & \begin{tabular}{|l|l|} 
\\
\end{tabular} & & & (1) & & & & 0.55 & 516 & 138 & 45.7 & & & & & & \\
\hline & $2 / 2 / 200$ & $12: 37$ & 25.336 & 20.881 & $\begin{array}{l}125.289 \\
\end{array}$ & 24.71 & & & \begin{tabular}{|l|l|}
53.061 \\
\end{tabular} & 14. & $38.6 !$ & $\mid-1.2$ & & & & 14. & & 34 & 0.56667 & 51.6 & 12.8 & 45.8 & 3.154 & & & & & \\
\hline & $2 / 2 / 2004$ & 12:38: & 25.356 & 20.942 & 25.315 & 24.846 & 23.7 & 52. & \begin{tabular}{|l|l|}
53.038 \\
\end{tabular} & $\begin{array}{l}14.113 \\
\end{array}$ & 38.6 & -1.2 & & 47.5 & 0.0 & 14. & & 35. & 0.58333 & $\begin{array}{l}51.6 \\
\end{array}$ & & 45 & & & & & .288 & \\
\hline & 2/2/2004 & & 25.366 & 20.986 & \begin{tabular}{|l|l|l|} 
& 25.339 \\
\end{tabular} & 24.87 & 23. & \begin{tabular}{|l|}
52.63 \\
\end{tabular} & \begin{tabular}{|l|l|}
52.988 \\
\end{tabular} & 14.3 & 38.1 & -1.21 & & 48 & & 14. & & & 0.6 & 51.5 & $11 . .0$ & 45.4. & 3.1 & & & & & \\
\hline 54 & & & 25.376 & & 25.329 & & & & 52.907 & & & & & & & & & & & 51.4 & & 45.6 & & & & & 288 & \\
\hline & & & 25.385 & 21.012 & \begin{tabular}{|l|l|}
25.349 \\
\end{tabular} & 24.85 & & & 52.839 & 14. & 38.3 & & & & & & & & 0.63333 & 51.4 & $11.6 \mathrm{r}$ & 45.5 & & 0.013 & & & & \\
\hline & & & 25.3899 & 21.09 & 5. & 24.7 & & & & 14. & & -1.2 & & & & & & & 0.65 & 51. & $\frac{12.2}{212}$ & 45.8 & & & & & 884 & \\
\hline & & & 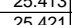 & $\begin{array}{l}21.15 \\
201158\end{array}$ & $\begin{array}{l}25.435 \\
325415\end{array}$ & 24.932 & & & & 17.1 & & & & & & & & & 0 & 48. & & & & & & & & \\
\hline 59 & 2.1/21/2) & & $\mid \frac{2.412}{2546}$ & 21.1213 & 325.444 & $\frac{2.0}{24}$ & & & & 157 & & & & & & & & & 0 & . & & & & & & & & \\
\hline & 2 & & 25.448 & 21231 & 125427 & 249 & & & & & & -1219 & & & & & & & 071667 & 478 & & & & & & & & \\
\hline & & & 25.457 & & & & & & & & & & & & & & & & & 484 & & & & & & & & \\
\hline & & & 25.935 & 21308 & $\mid 25,434$ & 24.995 & & 46.301 & 49.605 & 15.155 & & 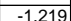 & & & & & & & & & & & & & & & & \\
\hline & & & 25.444 & 21.327 & 25.438 & 24.979 & & & & 15228 & & -1.216 & & 52 & & 146 & & & & 48 & & 412 & & & & & & \\
\hline & & & 25.469 & 21.383 & 25.357 & 24.893 & & & 49,396 & 14.957 & $\begin{array}{l}34.094 \\
\end{array}$ & \begin{tabular}{|l|l|} 
& -1.21 \\
\end{tabular} & & 55.125 & & 146 & & & 0.78333 & 47 & & 41.6 & & & & & 888 & \\
\hline & & & & $\begin{array}{l}21.434 \\
\end{array}$ & 25. & 24.8 & & & & 14. & & $\mid-1.21$ & & & & 14. & & & & $\begin{array}{lll}47.4 \\
\end{array}$ & & & & & & & & \\
\hline 66 & $2 / 2 / 2$ & & 25.5 & 21.475 & 25.229 & 24.8 & & & & 14.7 & & -1.2 & & & & 14.6 & & & & 47.6 & 1 & & & & & & & \\
\hline 67 & 2/2/2004 & & 25.521 | & 21.531 & 25.225 & 24.8 & & 48. & & 14.7 & 34.038 & \begin{tabular}{|l|l|} 
& -1.219 \\
\end{tabular} & & & & & & & 0.83333 & $47.7 \mid$ & 13. & 41.4 & & & 0.013 & 0.000 & 0.304 & \\
\hline & $2 / 2 / 2$ & & & 21.582 & & 24.7 & & 48. & & 14.446 & & & & & & & & & & 47.2 & 12.6 & & & & & & 0.303 & \\
\hline 70 & & & 25.487 & 21.1613 & \begin{tabular}{|c|c|}
3 & 25.1711 \\
\end{tabular} & 24.6 & & 48.383 & \begin{tabular}{|l|l|} 
\\
\end{tabular} & 14.025 & & -1.219 & & & & & & & & & 11. & 41.4 & & & & & 308 & \\
\hline 70 & & \begin{tabular}{|l|l|}
$12: 56: 3$ \\
\end{tabular} & 25.478 & 21 & \begin{tabular}{l|l}
4 & 25.161 \\
\end{tabular} & \begin{tabular}{|l|}
24.637 \\
\end{tabular} & & 48.383 & \begin{tabular}{|l|l|}
48.826 \\
\end{tabular} & 14.006 & .385 & -1.219 & & 51.79 & 0.086 & 14.643 & & & .883 & & 12.7 & & & & & 1.000 & 0.308 & \\
\hline
\end{tabular}


WSRC-TR-2005-00105, REVISION 0

SRNL-RPP-2005-00012, REVISION 0

RUN \# WASH1; CAMPAIGN I SLURRY WASH 1 - CONT.

\begin{tabular}{|c|c|c|c|c|c|c|c|c|c|c|c|c|c|c|c|c|c|c|c|c|c|c|c|c|c|c|c|c|}
\hline & A & $\mathrm{B}$ & D & $E$ & $\mathrm{~F}$ & $G$ & $\mathrm{H}$ & $\mathrm{J}$ & $\mathrm{K}$ & $\mathrm{L}$ & \begin{tabular}{l|l}
$M$ \\
\end{tabular} & $\mathrm{~N}$ & 0 & \begin{tabular}{l|l} 
\\
\end{tabular} & $R$ & s & $T$ & $\mathrm{v}$ & W & \begin{tabular}{c|c|}
$x$ &
\end{tabular} & $\begin{array}{l}\mathrm{Y} \\
\end{array}$ & & $\mathrm{AA}$ & $\overline{A B}$ & \begin{tabular}{ll|}
$A C$ & \\
\end{tabular} & & & $\overline{A F} \mid A$ \\
\hline & 2/2/2/2004 & 12:57:31 PM & 25.474 & 21.715 & 25.153 & 24.658 & 25.375 & 48.617 & \begin{tabular}{|l|l|}
49.125 \\
\end{tabular} & \begin{tabular}{ll|}
14.197 \\
\end{tabular} & 34.334 & $\mid-1.219$ & -7.644 & 53.267 & 0.086 & 14.643 & 0.004 & 54.00 & 0.9 & 47.7 & 13.1 & 41.5 & 2.860 & 0.013 & 0.013 & 0.00 & 0.308 & 0.26 \\
\hline & $2 / 2 / 2004$ & \begin{tabular}{|l|}
$12: 58: 31 \mathrm{PM}$ \\
$12.59 \cdot 31 \mathrm{PM}$
\end{tabular} & $\begin{array}{l}25.448 \\
25.432 \\
\end{array}$ & \begin{tabular}{|l|l|}
21.74 \\
21.785
\end{tabular} & \begin{tabular}{|l|}
25.117 \\
25107
\end{tabular} & \begin{tabular}{|l|}
24.607 \\
24589 \\
\end{tabular} & $\begin{array}{l}24.865 \\
25115 \\
\end{array}$ & 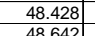 & \begin{tabular}{|l|}
48.969 \\
4094 \\
\end{tabular} & $\begin{array}{l}14.078 \\
14071\end{array}$ & \begin{tabular}{|l|}
34.291 \\
34506
\end{tabular} & $\mid-1.219$ & $\begin{array}{l}-7.589 \\
-7632 \\
\end{array}$ & 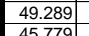 & 0.086 & \begin{tabular}{|l|l|}
14.643 \\
11643
\end{tabular} & & \begin{tabular}{|l|l|l|}
55.00 & \\
5600 &
\end{tabular} & 0.91667 & 47.5 & $\frac{12.1}{112}$ & 41.4 & & & 0.013 & & & \\
\hline & $\begin{array}{l}2 / 2 / 2004 \\
2 / 2 / 2004\end{array}$ & \begin{tabular}{|c|}
$12: 59: 31 \mathrm{PM}$ \\
$1 \cdot 0031 \mathrm{PM}$
\end{tabular} & $\begin{array}{l}25.433 \\
25.413 \\
\end{array}$ & 21.785 & \begin{tabular}{|l|l|}
25.107 \\
2507
\end{tabular} & \begin{tabular}{|l|}
24.582 \\
24557
\end{tabular} & & & & & $\begin{array}{l}34.596 \\
32.311\end{array}$ & \begin{tabular}{|c|c|c|}
-1.222 \\
130
\end{tabular} & & & & 14.643 & & 56.00 & & 17 & & & & & & & & \\
\hline & $\frac{2 / 2 / 2004]}{2 / 2 / 2004}$ & $\begin{array}{l}1: 00: 31 \mathrm{PM} \\
1: 01: 31 \mathrm{PM}\end{array}$ & 25.398 & 21.815 & $\mid$\begin{tabular}{|l}
25.097 \\
25.087
\end{tabular} & \begin{tabular}{|l|}
24.527 \\
24.472 \\
\end{tabular} & $\frac{25.065}{25.235}$ & $\begin{array}{l}48.416 \\
48.265\end{array}$ & 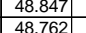 & $\begin{array}{l}14.106 \\
14.196 \\
\end{array}$ & $\frac{34.511}{34.038}$ & $\mid \begin{array}{l}\mid-1.219 \\
-1.222 \\
\end{array}$ & & $\begin{array}{l}49.967 \mid \\
50.681\end{array}$ & $\begin{array}{l}0.085 \\
0.085 \\
\end{array}$ & $\frac{14.6}{14.6}$ & & $\frac{57.00}{58.00}$ & \begin{tabular}{r|r|r|}
96967 & \\
96667
\end{tabular} & $\begin{array}{l}47.4 \\
47.3\end{array}$ & $\frac{12.2}{12.4}$ & $\frac{41 .}{41}$ & & & \begin{tabular}{|l|l|}
0.013 & 0.013 \\
\end{tabular} & & $\begin{array}{l}0.306 \\
0.307\end{array}$ & \\
\hline & 2/2/2/2004 & $1: 02: 31$ PM & 25.372 & 21.875 & \begin{tabular}{|l|}
25.076 \\
2507
\end{tabular} & \begin{tabular}{|l|}
24.416 \\
246
\end{tabular} & 25.923 & .312 & $\frac{.802}{822}$ & $\begin{array}{l}14.1909 \\
14.199\end{array}$ & 34.060 & $|-1.222|$ & & 年. & . & $\begin{array}{l}4.640 \\
14.643\end{array}$ & & 59.00 & 0.98333 & 47.3 & \begin{tabular}{|l|}
12.6 \\
11.6 \\
\end{tabular} & 41.2. & 2.840 & 013 & 0 & 0.000 & 0.307 & 26 \\
\hline & & & & & & 24.4 & & & & & & & & 48.346 & 0.084 & 14.643 & & & & & & & & & & & & \\
\hline 78 & $2 / 2 / 2004$ & 1:04:31 PM & 25.356 & 21.949 & 25.075 & 24.354 & 25.133 & 48.439 & \begin{tabular}{|l|}
48.973 \\
\end{tabular} & 14.267 & 34.061 & -1.219 & -7.606 & \begin{tabular}{|l|l|}
51.675 \\
\end{tabular} & 0.085 & 14.643 & & 61.00 & 1.01667 & 47.5 & 12.7 & 41.3 & 2.844 & 0.013 & 0.013 & 0.000 & 0.307 & 0.26 \\
\hline & $2 / 212004$ & & & 21.973 & 25.064 & & & 48.246 & \begin{tabular}{|l|l|}
48.863 \\
\end{tabular} & 14.165 & 34.001 & & & \begin{tabular}{|l|l}
50.883 \\
\end{tabular} & 0.085 & 14.643 & & & & & & & & & & & & \\
\hline (0) & 2/2/2004 & 1:06:31 PM & 25.329 & 21.992 & \begin{tabular}{|l|l|}
25.048 \\
\end{tabular} & & 25.411 & $\begin{array}{l}48.468 \\
\end{array}$ & \begin{tabular}{|l|l|}
49.004 \\
\end{tabular} & 14.305 & \begin{tabular}{l|l}
34.135 \\
\end{tabular} & \begin{tabular}{|c|} 
\\
\end{tabular} & & \begin{tabular}{l|l|}
50.341 \\
\end{tabular} & 0.085 & $\begin{array}{l}14.643 \\
\end{array}$ & & 63. & 1.05 & 47.5 & 12.3 & & 2.848 & 0.013 & 0.013 & & 0.306 & \\
\hline & $2 / 2 / 2004$ & 1:07:31 PM & & & \begin{tabular}{|l|l|}
25.026 \\
2505
\end{tabular} & \begin{tabular}{ll|l}
24.26 \\
24.20
\end{tabular} & 25.789 & 48.601 & 49.201 & 14.241 & $\begin{array}{l}34.336 \\
2.25\end{array}$ & $\begin{array}{l}-1.219 \\
\end{array}$ & & 52.134 & $\begin{array}{l}0.085 \\
\end{array}$ & \begin{tabular}{ll|l}
14.643 \\
\end{tabular} & & 64. & 1.06667 & 47.7 & 12.8 & & & & 0.013 & 0.000 & 0.305 & \\
\hline 83 & $2 \frac{2 / 2 / 2004}{2 / 2 / 2004}$ & $\begin{array}{l}1: 08: 31 \text { PM } \\
1.09 .31 \mathrm{PM}\end{array}$ & $\begin{array}{l}25.306 \\
25.309\end{array}$ & \begin{tabular}{r|r|}
22.05 & \\
22.063 &
\end{tabular} & $25.025 \mid$ & $\frac{24.249}{24.227}$ & 25.693 & $\begin{array}{ll}48.244 \\
48213\end{array}$ & & $\begin{array}{l}14.001 \\
1394 \\
\end{array}$ & & 222 & $\begin{array}{r}-7.592 \\
7.759 \\
\end{array}$ & \begin{tabular}{|l|l|l|l|l}
49.986 & \\
55063
\end{tabular} & $\begin{array}{l}0.086 \\
0.085\end{array}$ & $\frac{14.6}{146}$ & 0.06 & 65.00 & 1.08333 & $\begin{array}{l}47.3 \\
47.4\end{array}$ & $\frac{12.2}{12 .}$ & (4.1.4 & 2.844 & 0.013 & 0.013 & 0.000 & 0.311 & 26 \\
\hline 84 & $2|2 / 2 / 2004|$ & $\begin{array}{l}\text { 1:09:31 PM } \\
1: 10: 31 \mathrm{PM}\end{array}$ & 25.309 & $\begin{array}{l}22.063 \\
22.076\end{array}$ & \begin{tabular}{|c|c|}
24.948 & 2.806 \\
2
\end{tabular} & 24.272 & 25.216 & $\begin{array}{l}48.213 \\
47.586\end{array}$ & $\begin{array}{l}.832 \\
.192\end{array}$ & \begin{tabular}{|l|l|}
13.944 \\
13.657
\end{tabular} & $\begin{array}{l}34.235 \\
33.929\end{array}$ & \begin{tabular}{|c|}
-1.219 \\
-1.219
\end{tabular} & $\begin{array}{r}-7.589 \\
-7.554 \\
\end{array}$ & \begin{tabular}{|l|l|l}
52.063 & \\
51.392 &
\end{tabular} & $\begin{array}{l}085 \\
083\end{array}$ & & & 66.00 & & \begin{tabular}{ll|l}
47.4 \\
467
\end{tabular} & $\frac{12.8}{126} \mathrm{r} \mathrm{l}$ & \begin{tabular}{ll|}
41.2 \\
40.8
\end{tabular} & 2.842 & 0.013 & \begin{tabular}{l|l|l|}
0.013 \\
0.012
\end{tabular} & 0.000 & $\begin{array}{l}0.308 \\
0.305\end{array}$ & 0.26 \\
\hline 85 & $2 \mid 2 / 21 / 2004$ & $\begin{array}{l}1: 10.31 \mathrm{PM} \\
1: 1131 \mathrm{PM}\end{array}$ & $\frac{25.307}{25.289}$ & $\begin{array}{l}22.0708 \\
22.108\end{array}$ & $\mid$\begin{tabular}{|l|}
$\mid 24.8080$ \\
\end{tabular} & \begin{tabular}{|l|}
24.245 \\
24.337 \\
\end{tabular} & $\frac{25.244}{25.381}$ & \begin{tabular}{|r|}
47.586 \\
47.7
\end{tabular} & $\frac{48.1924}{48.277}$ & $\begin{array}{l}13.65 / 4 \\
13.795 \\
\end{array}$ & $\begin{array}{l}33.929 \\
33.886\end{array}$ & \begin{tabular}{|l|l|}
-1.21 .219 \\
-1.21
\end{tabular} & $\begin{array}{l}-\frac{-1.554}{-7.56} \\
\end{array}$ & $\frac{51.392}{50.22}$ & $\begin{array}{l}0.083 \\
0.089 \\
\end{array}$ & \begin{tabular}{|l|l|}
14.6433 \\
14.643
\end{tabular} & .003 & $\frac{67.00}{68.00}$ & \begin{tabular}{|l|}
1.11606 \\
1.13333 \\
\end{tabular} & $\frac{46.1}{46.8}$ & $\frac{12.6}{12.3}$ & $\frac{40.8}{40.8}$ & 2.8813 & $\frac{0.012}{0.013}$ & \begin{tabular}{|c|c|}
0.012 & 013 \\
0.013
\end{tabular} & $\begin{array}{l}0.000 \\
0.000\end{array}$ & $\begin{array}{l}\frac{0.305}{0.327} \\
\end{array}$ & $\frac{0.26}{0.28}$ \\
\hline & $2 / 2 / 2004$ & 1:12:31 PM & & & 24.811 & 24.461 & 25.749 & \begin{tabular}{|l|l|}
47.817 \\
\end{tabular} & 48.484 & 13.65 & 34.122 & -1.216 & & 53.511 & \begin{tabular}{|c|}
0.089 \\
\end{tabular} & 14.643 & & 69.00 & \begin{tabular}{|l|}
1.15 \\
\end{tabular} & 47.0 & 13.1 & 41.0 & & 0.013 & 0.013 & 0.000 & & 0.28 \\
\hline & 2/2/2004 & 1:13:31 PM & 25.25 & 22.149 & 24.809 & & 25.762 & & 48.267 & 13.76 & 33.893 & & -7.548 & 48.311 & 0.089 & 14.643 & & & 1.16667 & 46.8 & & 40.8 & 2.811 & & & & & 0.28 \\
\hline & 2/2/2004 & 1:14:31 PM & & 22.162 & 24.831 & 24.606 & 26.198 & $\begin{array}{l}47.958 \\
\end{array}$ & & & & & & 52.155 & & & & & 1.18333 & $\begin{array}{l}47.2 \\
\end{array}$ & & & & & & & & \\
\hline o & & 1:15:31 PM & & & 24.889 & & & 47.676 & & 13.724 & & -1.219 & & 49.423 & 089 & & & & & & & & 816 & & & & & \\
\hline & 2/2/2004 & 1:16:31 PM & 25.192 & & 24.951 & 24.706 & 26.138 & 47.806 & & 13.797 & 33.86 & -1.219 & & 48.835 & 0.09 & & & 73.6 & 1.21667 & & & 40.8 & 815 & .013 & & 500 & 0.329 & $\frac{20}{28}$ \\
\hline & 2/2/20004 & 1:17:31 PM & 25.199 & 22.254 & 25.003 & $24.784 \mid$ & 25.9 & & 304 & 13.576 & 33.813 & -1.216 & & 47.302 & 0.09 & 14.6. & & & .23333 & $46.8 \mathrm{r}>\mathrm{s}$ & & & & & & & 0.330 & \\
\hline & 2/2/2004 & 1:18:31 PM & 25.211 & 22.276 & 25.07 & 24.891 & 26.307 & 47.644 & 3.374 & 13.731 & 33.862 & -1.219 & & 51.208 & 0.089 & 14.643 & & 75.0 & 1.25 & 46.9 & & 40.8 & 8.810 & & 0.013 & 0.000 & & \\
\hline$\overline{94}>4$ & & 1 PM & & 22.293 & 25.122 & 24.878 & 26.613 & & .366 & 13.664 & 33.87 & & & 46.152 & 0.09 & & & & & 46.9 & & & & & & & & \\
\hline & | & 1:20:31PM PM & $\begin{array}{l}25.22 \\
25.22\end{array}$ & $22.321 \mid$ & 25.175 & 24.8355] & 26.426 & 47.717 & 48.449 & 13.76 & 33.966 & -1.222 & & 51.277 & 0.09 & 14.643 & & & 1.28333 & 47.0 & 12.6 & $40 . \varepsilon$ & 81 & & . & 000 & 327 & \\
\hline$\overline{96}$ & $2 / 212$ & $1: 21: 3$ & & & 25.236 & & 25.883 & 47.692 & 48.443 & 13.666 & 34.032 & -1.21 & & 638 & .09 & 14.643 & & & & & & & & & & & S & \\
\hline & . & $\frac{1: 2.31 \mathrm{PM}}{1.231}$ & & 2.2306 & $\frac{25.214}{2532]}$ & 2.4.955 & $\begin{array}{r}26.43 \\
25.704\end{array}$ & & 48.48 & $\frac{13.581}{13.55}$ & 34.112 & -1.222 & & 0.37 & 091 & 14.6 & & & & & & & & & & & & \\
\hline 98 & $\frac{24 / 212004}{2 / 2 / 2004}$ & $\frac{1.25 .3170}{1 \cdot 24 \cdot 31}$ & & $\frac{2.240}{2243}$ & 25.324 & 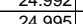 & $\frac{2.17}{263^{2}}>0$ & & $\begin{array}{l}48.501 \\
48.478\end{array}$ & $\begin{array}{l}13.055 \\
13.596\end{array}$ & $\begin{array}{l}34.015 \\
33.96 \\
\end{array}$ & -1.219 & & 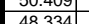 & & $\frac{14.6}{14.6}$ & & & & 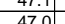 & & & & & & & & \\
\hline & 2/2/2/2004 & $\begin{array}{l}1.24 .01 \mathrm{PM} \\
1: 25: 31 \mathrm{PM}\end{array}$ & 25.344 & $\begin{array}{ll}22.451 \\
22.486\end{array}$ & $\frac{25.01}{25.429}$ & 24.964 & 26.07 & 47.0259 & 年. & 13.650 & 33.858 & $\begin{array}{l}-1.219 \\
-1.219 \\
\end{array}$ & $\frac{-7.488}{-1.48}$ & 年. & o.051 & $\begin{array}{l}14.0443 \\
14.643\end{array}$ & & & 13667 & 470 & & $40.7-7-3$ & & & 0.013 & 0,000 & | & \\
\hline & & 1:26:31 PM & 25.367 & 22.514 & $\mid$ & 24.967 & $\frac{20.1}{26.1}$ & & & 13.613 & & $\mid-1.219$ & & & & & & & & & & & & & & & & \\
\hline & $2 / 2 / 2004$ & 1:27:31 PM & $25.39 \mid$ & 22.532 & 25.505 & 24.925 & 25.99 & 47.522 & 48.474 & $13.627 \mid$ & 33.761 & $\mid-1.219$ & & 52.61 & 0.091 & 14.6 & & $84.6 \quad-3$ & 1.4 & $\frac{47.0}{47.0}-2$ & & 40. & 30 & & 013 & 000 & 0.329 & \\
\hline & 2/2/2004 & 1:28:31 PM & & 22.565 & 25.548 & 24.878 & 25.8 & 47.742 & & 13.729 & & -1.2 & & & & & & & .41667 & & & & & & & & 324 & \\
\hline & & $1: 2$ & 25.446] & 22.583 | & 25.586 & & & & & 13.71 & & & & 7.639| & & & & & 43333 & 47.2 & & & & & & & . 327 & \\
\hline & $2 / 2 / 2 C$ & $1: 30$ & & & & & $\overline{26.4} \mathrm{r}>\mathrm{s}$ & & $48.714 \mid$ & 13.649 & 34.079 & & & 13 & & 14.6 & & & 1.45 & $47.2=8$ & & & & & & & & \\
\hline & 2/2/2004 & & & & 25.642 & 24.847 & & & & & 34.342 & & & & & & & & 466 & 47.6 & & & & & & & & \\
\hline & 2/2/2004 & 1:32:31 PM & $25.525 \mid$ & 22.663 & 25.68 & 24.83 & 26 & & & 13.581 & 34.211 & -1.219 & & 53.628 & & 14.6. & & & .48333 & 47.3 & 3.1 & 41. & 828 & 0.014 & & 0.000 & 0.332 & 28 \\
\hline & $2 / 2 / 2 / 2)$ & 1:33:31 PM & 25.563 & 22.691 & 25.728 - & 24.868 & 26.2 & & 48.495 & 13.79 & 33.728 | & -1.21 & & 47.813 & & & & & 1.5 & & & & & & & & & \\
\hline & 2/2/20 & 1:34:3 & 25.587 & 22.7 & 25.652 & 24.807 & 26.5 & 47. & 48.208 & 13.5644 & 33.611 & & & 56.6 & & & & & .51667 & & & & & & & & 0.330 & \\
\hline & 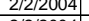 & & 20.001 & $22 . / 24$ & 25.526 & 24.79 & & & & & & & & & & & & & & & 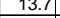 & & & & & & 344 & \\
\hline & 2/2/2/2004 & 1.36:31 PM & 20.625 & 20.53 & 2.5.585 & & & & & & 33.451 & & & 48.8/ & & & & & & 46.5 & & & & & & & & \\
\hline & 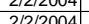 & 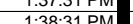 & 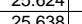 & $\frac{2.0212}{2086}$ & $\frac{2.0009}{2562}$ & 2.140 & & & & $\frac{1.034}{1.251}$ & 33.51 325 & & & & & & & & (50001 & 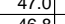 & & & & & & & & \\
\hline 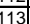 & $\frac{2121240}{2 / 20}$ & & 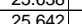 & 资, & | & $\frac{24}{24}, 4>$ & & & & $\frac{15.51}{133}$ & $\frac{5.40}{333}$ & & & & & & & & & & 18 & & & & & & 然 & \\
\hline & 212120 & $1 \mathrm{PM}$ & 25.6 & 22.83 & 25.571 & 24.736 & 26.8 & & & 13.396 & 33.615 & & & & & & & & $\frac{61.67}{6167}$ & & 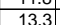 & & & & & & 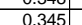 & \\
\hline & $2 / 2 / 2004$ & 1:41 & $\frac{25.645}{25}$ & 22.859 & 25.57 & 24.755 & $\frac{27.0}{27.0}-1$ & 46.854 & 47.995 & 13.325 & 33.48 & $\frac{-1.2}{-1.2}<\mathrm{c}$ & & 51.548 & 0.095 & & & & 1.63333 & & 12.6 & & & & & & 0.347 & \\
\hline & & & & & & & & & & & & & & & & & & & & & & & & & & & & \\
\hline & 2/2/2004 & $1: 43$ & 25.653 & $22.907 \mid$ & 25.558 & 24.683 | & 26.1 & & & 13.513 & 33.48 & -1.22 & & & & & & & 66667 & 46.9 & 12.4 & & & & & & 0.346 & \\
\hline & $2 / 2 / 20$ & & 25.64 & 22.925 & 25.5 & & & & & 13.3 & & & & & & & & & 6835 & & & & & & & & & \\
\hline & 2 & & & 22.943 & & & & & & 13.7 & 32.6 & & & & & & & & & & & & & & & & & \\
\hline & 2/2/2004 & & & 976 | & 25.5 & & & & & 13.63 & & & & & & & & & & & & & & & & & $|8|$ & \\
\hline & & & 25.6 & 22.983 & & & & & & & & & & & & & & & & & & & & & & & & \\
\hline & & & 25.6 & 25 & 25.516 & & & & 47.8 & 13.67 & & & & & & & & & & & & & & & & & . & \\
\hline & & & & & & & & & & 13.6 & & & & & & & & & & & & & & & & & & \\
\hline & $2 / 2 / 2 / 2$ & & 25.645 & $\frac{23.039}{23}$ & 25.53 & 24.789 & 26. & & & 13.6 & 32.9 & & & 48 & & & & & & 46.6 & 1.8 & & & & & & & \\
\hline & & & 势. & $\frac{2.0442}{2304}$ & & 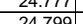 & & & & $\frac{13.6}{1271}$ & 32.09 & & & & & & & & & 4. & & & & & & & 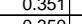 & \\
\hline & & & $\frac{2.03}{2564}$ & $\frac{2.0044}{2307}$ & & & & & & $\frac{13.14}{1348}$ & & & & & & & & & & & & & & & & & & \\
\hline & & & & & & & & & & & & & & & & & & & & & & & & & & & & \\
\hline & & & 25.646 & $\frac{2.3 .116}{23.16}$ & 25.321 & 24.806 & & & & $\mid 13.32$ & 32.84 & -1.219 & & & & & & & 1866 & 464 & & & & & & & & \\
\hline & & & & & & & & & & & & & & & & & & & & & & & & & & & & \\
\hline & $\sqrt{12}$ & & 25.60 & 23.139 & 25.289 & & & & & $\overline{13.3}$ & 32.84 & & & & & & & & & & & & & & & & & \\
\hline & & & & & & & & & & & & & & & & & & & & & & & & & & & & \\
\hline & 2/2/2004 & 1:59:3 & 25.594 & 23.179 & 25.284 & 24.763 & 26.4 & 46.388 & 48.0 & 13.37 & 32.811 & -1.2 .2 & & 3.3 & 0.0. & 14.6 & & & 1.93333 & 46.6 & 3.1 & & & & & & 6 & \\
\hline & 2/2/200 & 2:00:31 F & 25.5 & 23.2 & 25.295 & 24.70 & & 46.42 & 48.0 & $13.3 \mathrm{~K}$ & & -1.22 & & 51.47 & & 14.6 & & & 1.95 & & & & & & & & $\begin{array}{lll}9 & \end{array}$ & \\
\hline & & & & 23.1 & & & & 46. & & & & & & & 0.09 & & & & & & & & & & & & 2 & \\
\hline & 2/2/20 & & 25.57 & 23.212] & 25.292 & & & & & 13.25 & 32.733 & & & 52.896 ] & 1 & & & & & $46.2 \mathrm{C}$ & 130 & & & & & & 0.375 & \\
\hline & & & 25.584 & & & & & & & & & & & & & & & & & & & & & & & & & \\
\hline & 2/2/2/2004 & 2:03:35 PM & 23.566 & 23.242 & 2 & $24.686]$ & 25.7 & 46.195 & \begin{tabular}{|l|l|l|l|} 
\\
\end{tabular} & 13.118 & 32.895 & -1.219 & -1.3 & 4.12 & 0.099 & 14.6 & & & 2.00111 & 46.4 & 1.2 .0 & 3.5 & 2.12 & 0.010 & 0.015 & .000 & 0.370 & 0.3 \\
\hline & & $\begin{array}{l}\text { 2:04:35 PM } \\
\text { 2:05:35 PM }\end{array}$ & $\begin{array}{rl}25.568 & 25.56 \\
\end{array}$ & \begin{tabular}{|c|}
23.269 \\
2.261
\end{tabular} & \begin{tabular}{|r|}
25.293 \\
25.29
\end{tabular} & \begin{tabular}{|l|}
$\mid 24.687$ \\
24.709 \\
\end{tabular} & \begin{tabular}{|l}
26.189 \\
26.156
\end{tabular} & $\begin{array}{l}46.149 \\
46.172\end{array}$ & $\left|\begin{array}{l}47.937 \\
44.881\end{array}\right|$ & \begin{tabular}{|l|}
13.169 \\
13.144
\end{tabular} & \begin{tabular}{|l|}
32.801 \\
32.908
\end{tabular} & $\mid$\begin{tabular}{|l}
$\mid-1.222$ \\
-1.2219 \\
$\mid$
\end{tabular} & 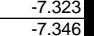 & $\begin{array}{l}44.857 \\
51.467\end{array}$ & & \begin{tabular}{|l|}
14.643 \\
14.643
\end{tabular} & & & \begin{tabular}{|l|}
2.01748 \\
2.03444 \\
\end{tabular} & $\begin{array}{l}46.5 \\
46.4 \\
\end{array}$ & & \begin{tabular}{|l|}
39.5 \\
39.5
\end{tabular} & \begin{tabular}{|l|l|}
2.722 \\
2.726
\end{tabular} & $\mid$\begin{tabular}{|l}
$\mid 0.015$ \\
0.015 \\
\end{tabular} & $\begin{array}{l}0.015 \\
0.015 \\
\end{array}$ & $\begin{array}{l}0.000 \\
0.000\end{array}$ & \begin{tabular}{|l|}
0.375 \\
0.374 \\
\end{tabular} & 0.32 \\
\hline
\end{tabular}


WSRC-TR-2005-00105, REVISION 0

SRNL-RPP-2005-00012, REVISION 0

RUN \# WASH1; CAMPAIGN I SLURRY WASH 1 - CONT.

\begin{tabular}{|c|c|c|c|c|c|c|c|c|c|c|c|c|c|c|c|c|c|c|c|c|c|c|c|c|c|c|c|c|}
\hline & & & & & & & & & & & & & & & & & & & & & & & & & & & & \\
\hline & A & B & D & $E$ & $\mathrm{~F}$ & G & $\mathrm{H}$ & $\mathrm{J}$ & $\mathrm{K}$ & \begin{tabular}{l|l|l|l|} 
L & \\
\end{tabular} & $\mathrm{M}$ & $\mathrm{N}$ & 0 & Q & \begin{tabular}{l|l|l|}
$R$ & \\
\end{tabular} & \begin{tabular}{l|l|} 
\\
\end{tabular} & $\mathrm{T}$ & v & w & $x$ & $\mathrm{Y}$ & $z$ & AA & $A B$ & $\mathrm{AC}$ & $A D$ & $\mathrm{AE}$ & \\
\hline 14 & $2 / 2 / 2004$ & 2:06:35 PM & 25.557 & 23.278 & \begin{tabular}{|l|l|}
35.292 \\
\end{tabular} & 24.696 & 26.118 & 46.2 & \begin{tabular}{|l|l}
48.043 \\
\end{tabular} & 13.173 & 32.949 & & $\begin{array}{l}-7.346 \\
\end{array}$ & 52.372 & 0.099 & 14.643 & & 123.0 & .05111 & 46.6 & 12.8 & & 2.730 & & 0.015 & 0.000 & 0.370 & \\
\hline & $2 / 212004$ & & & & 25.294 & 24.728 & 26.115 & 40.04 & \begin{tabular}{|l|l|}
48.126 \\
\end{tabular} & $\begin{array}{l}13.244 \\
324\end{array}$ & 32.942 & \begin{tabular}{|l|l|}
-1.219 \\
\end{tabular} & $\begin{array}{r}-7.337 \\
-737\end{array}$ & & 0.101 & \begin{tabular}{|l|}
14.643 \\
\end{tabular} & & & & 46.7 & 111.6 & & & & & & 0.377 & \\
\hline & 2/2/2004 & $2: 08: 35$ PM & 25.545 & 23.311 & 25.305 & 24.71 & 26.466 & 46.394 & \begin{tabular}{|l|l|}
48.246 \\
\end{tabular} & $\begin{array}{l}13.254 \\
\end{array}$ & 32.973 & \begin{tabular}{|c|}
-1.222 \\
\end{tabular} & $\begin{array}{r}-7.346 \\
\end{array}$ & 57.627 & $\begin{array}{l}0.098 \\
\end{array}$ & \begin{tabular}{ll|}
14.643 \\
\end{tabular} & & 125. & 2.08444 & 46.8 & $\begin{array}{l}14.1 \\
\end{array}$ & & 2.736 & & 0.014 & 0.000 & 0.365 & \\
\hline$\frac{144}{145}$ & $\begin{array}{l}2 / 2 / 2004 \\
2 / 212004\end{array}$ & 2:09:35 PM & $\begin{array}{l}25.548 \\
25.549\end{array}$ & \begin{tabular}{|l|l|}
23.324 \\
22.34
\end{tabular} & 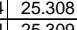 & $\begin{array}{l}824.762 \\
\end{array}$ & $\begin{array}{l}26.513 \\
25.906\end{array}$ & 46.101 & \begin{tabular}{|l|}
48.016 \\
\end{tabular} & $\begin{array}{l}13.103 \\
1260\end{array}$ & $\begin{array}{l}32.829 \\
32709\end{array}$ & \begin{tabular}{|c|} 
\\
\end{tabular} & $\begin{array}{r}-7.34 \\
-7.324\end{array}$ & \begin{tabular}{|l|l|}
46.409 \\
5.516
\end{tabular} & $\begin{array}{l}0.099 \\
\end{array}$ & \begin{tabular}{l|l|}
14.643 \\
14.64
\end{tabular} & & $\begin{array}{l}126.07 \\
12707\end{array}$ & $2.10111 \mid$ & 46.5 & 11.4 & $\begin{array}{r}39.5 \\
39.5 \\
3\end{array}$ & 52771 & 0.015 & \begin{tabular}{|l|l|}
0.015 \\
015
\end{tabular} & 0.000 & 0.371 & \\
\hline$\frac{145}{146}$ & $\begin{array}{l}2 / 2 / 2004 \\
2 / 2 / 2004\end{array}$ & $\begin{array}{l}2: 10: 35 \mathrm{PM} \\
2: 11: 35 \mathrm{PM}\end{array}$ & 25.549 & $\begin{array}{r}23.34 \\
23.346\end{array}$ & $\begin{array}{l}4 \quad 25.309 \\
5 \\
5\end{array}$ & \begin{tabular}{rl|} 
& 24.774 \\
& 24.74
\end{tabular} & $\frac{25.906}{26.007}$ & $\begin{array}{l}46.195 \\
46.342 \\
\end{array}$ & \begin{tabular}{|l|}
48.055 \\
48.244 \\
\end{tabular} & \begin{tabular}{|l|}
13.268 \\
13.317
\end{tabular} & $\begin{array}{r}32.729 \\
32.782 \\
\end{array}$ & \begin{tabular}{|c|}
$\mid-1.212$ \\
\\
\end{tabular} & $\begin{array}{r}-7.334 \\
-7.337 \\
\end{array}$ & $\begin{array}{l}50.516 \\
53.943 \\
\end{array}$ & $\begin{array}{r}0.1 \\
0.099\end{array}$ & \begin{tabular}{|c|}
14.643 \\
14.643
\end{tabular} & & $\begin{array}{l}127.07 \\
11807\end{array}$ & 2.11778 & $\begin{array}{l}46.6 \\
46.8\end{array}$ & $\frac{12.4}{13.2}$ & $\begin{array}{l}39.5 \\
39.6\end{array}$ & \begin{tabular}{|c|}
2.721 \\
2778 \\
\end{tabular} & \begin{tabular}{|l|l|}
0.015 \\
0.015 \\
\end{tabular} & $\begin{array}{l}0.015 \\
0.155\end{array}$ & 0.000 & $\begin{array}{l}0.375 \\
0.370\end{array}$ & \\
\hline & $2 / 2 / 2004$ & $2: 12: 35$ PM & 25.557 & 23.364 & 25.317 & \begin{tabular}{|l|l|}
77 & 24.752 \\
\end{tabular} & 25.894 & $\begin{array}{l}40.042 \\
46.216\end{array}$ & \begin{tabular}{|l|}
40.2403 \\
48.103 \\
\end{tabular} & 13.233 & $\begin{array}{l}32.862 \\
32.869\end{array}$ & \begin{tabular}{|l|}
-1.219 \\
-1.222 \\
\end{tabular} & $\begin{array}{l}-7.331 \\
-7.337 \\
\end{array}$ & \begin{tabular}{|l}
35.943 \\
56.077
\end{tabular} & $\frac{0.099}{0.1}$ & \begin{tabular}{|l|l|}
14.643 \\
14.643
\end{tabular} & & $\frac{128.07}{129.07}$ & 2.1 .154411 & $\begin{array}{l}46.8 \\
46.6\end{array}$ & $\frac{13.4}{13.7}$ & $\frac{39.6}{39.5}$ & $\mid \frac{2.778}{2.726}$ & \begin{tabular}{|l|}
0.01 \\
0.01 \\
\end{tabular} & $\frac{0.015}{0.015}$ & $\frac{0.000}{0.000}$ & 0.370 & \\
\hline \begin{tabular}{|l|}
148 \\
\end{tabular} & $2 / 21 / 2004$ & & 25.558 & 23.369 & 25.323 & 24.793 & 25.78 & 45.927 & \begin{tabular}{|l|}
47.898 \\
\end{tabular} & 13.161 & 32.61 & -1.219 & $\begin{array}{l}-7.308 \\
\end{array}$ & 50.047 & 0.099 & 14.643 & & 130.07 & 2.16778 & 46.4 & 12.3 & 39.3 & $\mid 2.707$ & \begin{tabular}{|l|l|}
0.015 \\
\end{tabular} & 0.015 & 0.000 & & 0.32 \\
\hline & & & & & \begin{tabular}{|l|l|}
25.314 \\
\end{tabular} & & & 46.209 & \begin{tabular}{|r|}
48.19 \\
\end{tabular} & & & -1.219 & & & 0.099 & & & & 2.18444 & 46.7 & 12.3 & & & & & & & \\
\hline \begin{tabular}{|l|}
150 \\
151
\end{tabular} & $2 / 2 / 2004$ & $2: 15: 35 \mathrm{PM}$ & $\begin{array}{l}25.581 \\
25617\end{array}$ & $\begin{array}{l}23.412 \\
2.200\end{array}$ & \begin{tabular}{|l|l|}
25.341 \\
\end{tabular} & 24.745 & 26.1 & 46.263 & \begin{tabular}{|l|l|l|}
48.246 \\
\end{tabular} & $\begin{array}{r}13.37 \\
\end{array}$ & $\begin{array}{l}32.686 \\
20011\end{array}$ & & $\begin{array}{l}-7.326 \\
\end{array}$ & 49.175 & 0.099 & \begin{tabular}{ll|}
14.643 \\
\end{tabular} & & 132 & \begin{tabular}{|l|l|} 
\\
\end{tabular} & 46.8 & 12.1 & & & 01 & 0.015 & 0.000 & 0.370 & \\
\hline $\mid \frac{151}{152}$ & $2 / 2 / 2004$ & $2: 16: 35 \mathrm{PM}$ & $\begin{array}{r}25.617 \\
25.523\end{array}$ & $\begin{array}{r}23.398 \\
23.301\end{array}$ & $\begin{array}{l}35.322 \\
\end{array}$ & $\begin{aligned} 24.726 \\
2.662\end{aligned}$ & 26.313 & 42.603 & \begin{tabular}{|l|}
47.792 \\
\end{tabular} & \begin{tabular}{|l|l|}
13.082 \\
\end{tabular} & $\begin{array}{l}29.314 \\
2207\end{array}$ & & $\begin{array}{l}-0.532 \\
7.69\end{array}$ & 46.574 & 034 & & & & 2.21778 & 46.3 & 11.4 & 36. & \begin{tabular}{|l|l|}
2.479 \\
\end{tabular} & & 0.005 & & 0.140 & \\
\hline$\left|\frac{152}{153}\right|$ & $\begin{array}{l}2 / 2 / 2004 \\
2 / 21 / 2004\end{array}$ & $\frac{2: 17: 35 \mathrm{PM}}{2 \cdot 18 \cdot 35 \mathrm{PM}}$ & $\begin{array}{l}25.623 \\
25.648\end{array}$ & $\begin{array}{l}23.404 \\
23.419 \\
\end{array}$ & 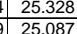 & $\begin{array}{l}24.662 \\
24.677\end{array}$ & $\frac{26.787}{26.264}$ & $\begin{array}{l}45.996 \\
45.373 \\
\end{array}$ & \begin{tabular}{|l|}
48.204 \\
47.553 \\
\end{tabular} & \begin{tabular}{|l|}
13.302 \\
12.921
\end{tabular} & $\begin{array}{l}32.497 \\
32265\end{array}$ & \begin{tabular}{|c|}
$\mid-1.216$ \\
-1.219
\end{tabular} & $\begin{array}{r}-7.268 \\
-7.32 \\
\end{array}$ & $\begin{array}{r}50.9 \\
47.938\end{array}$ & 0.11 & \begin{tabular}{|c|}
14.643 \\
14.633
\end{tabular} & & $\begin{array}{l}134.07 \\
13507\end{array}$ & 2.23444 & $\begin{array}{l}46.7 \\
46.1\end{array}$ & 12.5 & $\begin{array}{l}39.2 \\
38.8\end{array}$ & $\begin{array}{l}2.706 \\
2676\end{array}$ & 0.016 & 0.016 & 0.000 & $\begin{array}{l}0.414 \\
0.383\end{array}$ & 0.35 \\
\hline 154 & $2 / 2 / 2004$ & $\begin{array}{l}2.18 .35 \mathrm{PM} \\
2: 19: 35 \mathrm{PM}\end{array}$ & 25.629 & $\frac{23.419}{23.435}$ & 25.139 & 24.623 & 20.204 & 45.807 & \begin{tabular}{|l|l|}
41.050 \\
4808 \\
\end{tabular} & 12.041 & 32.442 & $\mid-1.219$ & $\begin{array}{c}-1.32 \\
-7.305 \\
\end{array}$ & 52.856 & $\begin{array}{r}0.1 \\
0.102 \\
\end{array}$ & $\begin{array}{l}14.643 \\
14.643\end{array}$ & 0.00 & $\begin{array}{l}1356.07 \\
136.07\end{array}$ & $\frac{2.25111}{2.26778}$ & \begin{tabular}{|}
46.1 \\
46.6 \\
\end{tabular} & \begin{tabular}{|l|l|l|l}
13.0 \\
\end{tabular} & $\frac{38.8}{39.1}$ & $\left|\begin{array}{|l|}2.667 \\
2.697\end{array}\right|$ & 0.01 & 0.015 & 0.000 & $\begin{array}{l}.383 \\
0.387 \\
\end{array}$ & $\begin{array}{l}0.33 \\
0.33 \\
\end{array}$ \\
\hline & $\begin{array}{l}2 / 212004 \\
212004\end{array}$ & $\begin{array}{ll}2: 20: 35 \mathrm{PM} \\
2: 2035 \mathrm{P}\end{array}$ & $\begin{array}{r}25.6 \\
55.6\end{array}$ & 23.442 & 25.135 & 24.664 & & 45.405 & \begin{tabular}{|r|}
47.63 \\
\end{tabular} & \begin{tabular}{|l|}
12.972 \\
1.972
\end{tabular} & 32.267 & & & 52.456 & & 14.643 & & & 2.28444 & 46.2 & & & 2.678 & & & & 0.394 & \\
\hline & 2/2/2004 & $2: 21: 35$ PM & & & 25.136 & & & 45.405 & \begin{tabular}{|l|l|}
47.668 \\
\end{tabular} & 12.797 & & & -7.291 & & 104 & 14.643 & & & \begin{tabular}{|l|l|} 
& 2.30111 \\
\end{tabular} & 46.2 & 12.9 & & 2.682 & & & & 0.397 & \\
\hline 157 & 2/2/2004 & $2: 22: 35 \mathrm{PM}$ & 25.577 & 23.464 & & 24.486 & & 45.413 & \begin{tabular}{|l|l|} 
\\
\end{tabular} & 12.854 & & & & 52.076 & & & & & 2.31778 & & 12.8 & & 2.683 & & & 0.000 & 0.393 & \\
\hline & $2 / 2 / 2004$ & $2: 23: 35 \mathrm{PM}$ & $\begin{array}{l}25.569 \\
25.557\end{array}$ & \begin{tabular}{|l|l|}
23.471 \\
23023
\end{tabular} & \begin{tabular}{|l|}
25.139 \\
25.10 \\
\end{tabular} & $\begin{array}{l}24.533 \\
2.556\end{array}$ & $\frac{26.61}{25.01}$ & & \begin{tabular}{|l|l}
47.585 \\
17529
\end{tabular} & \begin{tabular}{|l|l|}
12.851 \\
1.766
\end{tabular} & $\begin{array}{l}32.224 \\
2021\end{array}$ & \begin{tabular}{|c|}
-1.219 \\
.2202
\end{tabular} & & 51.648 & & & & & \begin{tabular}{|l|l|}
2.33444 \\
225111
\end{tabular} & & 12.7 & 38.8 & & & 0.016 & & $\begin{array}{ll}0.402 \\
\end{array}$ & \\
\hline$\frac{150}{160}$ & $\begin{array}{l}2 / 2 / 2004 \\
2 / 21 / 2004\end{array}$ & $\frac{2: 24: 35 \mathrm{PM}}{2 \cdot 25 \cdot 35 \mathrm{PM}}$ & $\begin{array}{l}25.557 \\
25.543\end{array}$ & $\begin{aligned} 23.493 \\
23.495\end{aligned}$ & \begin{tabular}{|l|}
25.142 \\
25.133
\end{tabular} & $\frac{24.556}{24.622}$ & $\begin{array}{l}25.918 \\
25.755 \\
\end{array}$ & $\begin{array}{r}45.235 \\
45.261\end{array}$ & \begin{tabular}{|r|}
47.529 \\
47.58 \\
\end{tabular} & \begin{tabular}{|l|}
12.762 \\
12.814 \\
\end{tabular} & $\begin{array}{l}32.331 \\
32.318 \\
\end{array}$ & $\frac{-1.222}{-1.222}$ & $\begin{array}{l}-7.265 \\
-7.285 \\
\end{array}$ & \begin{tabular}{|l|l}
47.775 \\
52.139 \\
\end{tabular} & $\frac{101}{103}$ & 14 & & $\begin{array}{l}141.07 \\
142.07\end{array}$ & 2.35111 & $\frac{46.1}{46.1}$ & $\begin{array}{l}11.7 \\
12.8\end{array}$ & $\begin{array}{l}38.8 \\
38.8 \\
3\end{array}$ & \begin{tabular}{|l|l|}
$\mid 3.674$ \\
$|3|$
\end{tabular} & 0.015 & 0.015 & 0.000 & $\begin{array}{l}0.387 \\
0.394\end{array}$ & 0.33 \\
\hline 161 & $2 / 2 / 2004$ & $\begin{array}{l}2.255 .35 \mathrm{PM} \\
2: 26: 35 \mathrm{PM}\end{array}$ & 25.539 & 23.521 & \begin{tabular}{|l|l|l|l|l|l|} 
\\
\end{tabular} & 24.0294 & 26.315 & $\begin{array}{r}45.261 \\
45.16\end{array}$ & \begin{tabular}{|l|l|}
47.464 \\
\end{tabular} & \begin{tabular}{|l|l|}
12.014 \\
12.767
\end{tabular} & 32.257 & $\mid$\begin{tabular}{|l|}
$\mid-1.222$ \\
\end{tabular} & $\begin{array}{l}-1.200 \\
-7.277\end{array}$ & \begin{tabular}{|l|}
51.062 \\
\end{tabular} & 0.104 & \begin{tabular}{|l|}
14.6453 \\
14.643
\end{tabular} & & $\begin{array}{l}\frac{142.07}{143.07} \\
\end{array}$ & $\mid$\begin{tabular}{|l|l|}
2.358748 \\
2.3844
\end{tabular} & $\frac{46.1}{46.0}$ & $\begin{array}{l}11.8 \\
12.5 \\
\end{array}$ & $\frac{38.8}{38.7}$ & $\left|\begin{array}{l}2.604 \\
2.669\end{array}\right|$ & \begin{tabular}{|l|l|}
0.016 \\
0.016
\end{tabular} & O. & $\begin{array}{l}0.000 \\
0.000\end{array}$ & $\begin{array}{l}0.394 \\
0.399\end{array}$ & $\begin{array}{l}0.34 \\
0.34\end{array}$ \\
\hline & $2 / 2 / 2004$ & 2:27:35 PM & 25.525 & 23.522 & 25.135 & 24.644 & 26.18 & 45.251 & \begin{tabular}{|l|l|}
47.539 \\
\end{tabular} & 12.928 & 32.187 & $\mid-1.219$ & & 53.111 & \begin{tabular}{|l|}
0.102 \\
\end{tabular} & \begin{tabular}{|l|}
14.643 \\
\end{tabular} & & 144.07 & 2.40111 & 46.1 & \begin{tabular}{|l|}
13.0 \\
\end{tabular} & 38.7 & 2.670 & & & & & $\frac{0.34}{0.33}$ \\
\hline \begin{tabular}{|l|l|}
163 \\
\end{tabular} & $2 / 2 / 2004$ & $2: 28: 35 \mathrm{PM}$ & & 23.533 & \begin{tabular}{|l|}
25.136 \\
\end{tabular} & 24.685 & 25.98 & 45.073 & \begin{tabular}{|l|l|}
47.473 \\
\end{tabular} & 12.916 & 32.062 & -1.222 & -7.268 & & 0.104 & 14.643 & & 145.07 & 2.41778 & 46.0 & 12.4 & 38.6 & 2.659 & & 0.015 & & & \\
\hline 164 & 2/2/2004 & 2:29:35 PM & 25.516 & 23.548 & 25.136 & 24.681 & & & 47.668 & $\begin{array}{l}12.86 \\
\end{array}$ & 32.329 & & & 49.696 & & & & & 2.43444 & & & & & & & 0.000 & 0.394 & \\
\hline 165 & & & & & & & & & \begin{tabular}{|l|l|} 
& 47.752 \\
\end{tabular} & & & & & & & & & & & & 11.5 & & & & & & & \\
\hline & $2 / 2 / 2004$ & $2: 31: 3$ & $\begin{array}{l}25.513 \\
\end{array}$ & 23.57 & 25.148 & 24.63 & & 45.154 & \begin{tabular}{|l|}
47.686 \\
\end{tabular} & $\begin{array}{ll}12.878 \\
\end{array}$ & 32.06 & -1.219 & & 0.678 & 0.103 & & & & 2.46778 & & 12. & & & & & & & \\
\hline & 2/2/2004 & & & & & 24.633 & & & \begin{tabular}{|l|l|} 
& 47.742 \\
\end{tabular} & & 32.319 & & & & & & & & 2.48444 & & & & & & & & & \\
\hline 168 & $2 / 2 / 2004$ & 2:33:35 PM & 25.501 & 23.593 & 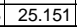 & 24.695 & 27.0 & 45.214 & \begin{tabular}{|l|}
47.79 \\
\end{tabular} & 12.903 & 32.082 & -1.219 & & 54.929 & 0.104 & $\begin{array}{l}14.643 \\
\end{array}$ & & 150.0 & 2.50111 & 46.3 & 13.5 & 38.6 & \begin{tabular}{|l|l|}
52.66 \\
\end{tabular} & 0.016 & 0.015 & 0.000 & 0.399 & \\
\hline 169 & $2 / 2 / 2004$ & $2: 34: 3$ & 25.502 & 23.604 & \begin{tabular}{|l|}
25.142 \\
\end{tabular} & 24.646 & 27.16 & 45.058 & \begin{tabular}{|l|}
47.587 \\
\end{tabular} & 12.868 & 32.082 & -1.222 & -7.242 & 50.209 & 0.104 & 14. & & 151. & \begin{tabular}{|l|l|}
2.51778 \\
\end{tabular} & 46.1 & 12.3 & 38.6 & & 0.016 & 0.015 & 0.000 & 0.400 & 0 \\
\hline 170 & $2 / 2 / 2004$ & $2: 35: 35 \mathrm{PM}$ & 25.499 & 23.616 & \begin{tabular}{|l|l|}
25.149 \\
\end{tabular} & 24.658 & & 45.195 & \begin{tabular}{|l|}
47.806 \\
\end{tabular} & 12.887 & 32.093 & -1.219 & & 51.267 & & & & & \begin{tabular}{|l|l|}
2.53444 \\
\end{tabular} & 46.3 & 12.6 & 38.6 & 2.664 & & 0.015 & & 0.396 & \\
\hline 171 & 2/2/2004 & $2: 36: 35$ PM & 25.504 & 23.632 & \begin{tabular}{|l|}
24.974 \\
\end{tabular} & 24.648 & 26.8 & 44.737 & \begin{tabular}{|l|}
47.346 \\
\end{tabular} & $\begin{array}{l}12.748 \\
\end{array}$ & 31.785 & $\begin{array}{l}-1.219 \\
\end{array}$ & & 48.905 & & & & 153.07 & 2.55111 & 45.9 & 12.0 & 38.3 & \begin{tabular}{|l|l|}
2.638 \\
\end{tabular} & & 0.016 & 0.000 & 0.406 & \\
\hline \begin{tabular}{|l|l|}
172 \\
172
\end{tabular} & $2 / 2 / 2004$ & $2: 37: 35 \mathrm{PM}$ & $\begin{array}{l}25.486 \\
25.161\end{array}$ & & \begin{tabular}{|r|}
24.98 \\
\end{tabular} & 24.735 & 26.50 & $\begin{array}{l}44.785 \\
4.562\end{array}$ & \begin{tabular}{|l|}
47.46 \\
\end{tabular} & \begin{tabular}{|l|l|}
12.779 \\
1.993
\end{tabular} & 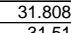 & $\begin{array}{l}-1.222 \\
.210\end{array}$ & & \begin{tabular}{|l|l}
55.983 \\
\end{tabular} & 0 & & & $\begin{array}{l}154.07 \\
15.07\end{array}$ & 2.56778 & 46.0 & \begin{tabular}{|l|}
13.7 \\
\end{tabular} & 38.3 & 2.640 & 0.016 & 0.016 & & 0.413 & 5 \\
\hline$\left|\frac{173}{174}\right|$ & $\begin{array}{r}2 / 2 / 2004 \\
2 / 204\end{array}$ & $\frac{2: 38: 35 \mathrm{PM}}{2 \cdot 39 \cdot 35 \mathrm{PM}}$ & $\frac{25.461}{25.437}$ & $\begin{aligned} 23.639 \\
23.654\end{aligned}$ & \begin{tabular}{|l|}
24.981 \\
24067
\end{tabular} & 24.726 & $\frac{26.34}{26.36}$ & $\begin{array}{r}44.563 \\
44583\end{array}$ & \begin{tabular}{|r|}
47.24 \\
47.209
\end{tabular} & $\begin{array}{r}12.883 \\
1266 \\
\end{array}$ & $\begin{array}{r}31.51 \\
331729\end{array}$ & \begin{tabular}{|c|}
-1.219 \\
\end{tabular} & & \begin{tabular}{|l|l|l}
46.367 \\
4052
\end{tabular} & & & & 155.0 & 2.58444 & $\begin{array}{r}45.8 \\
45.8\end{array}$ & $\frac{11.4}{10.8}$ & & 2.622 & 0.016 & 0.016 & 0.000 & $\begin{array}{l}0.420 \\
0.422\end{array}$ & 36 \\
\hline 175 & $\begin{array}{l}2 / 21 / 2004 \\
2 / 2004\end{array}$ & $2 \frac{2.395 .3}{2: 40: 3}$ & $\begin{array}{l}25.437 \\
25.448\end{array}$ & $\frac{23.654}{23.68}$ & \begin{tabular}{|l|}
24.967 \\
24.987 \\
\end{tabular} & $\frac{24.116}{24.772}$ & & $\frac{44.583}{46.243}$ & \begin{tabular}{|l|l|}
47.296 \\
9116
\end{tabular} & $\begin{array}{c}\frac{12.66}{11.344} \\
\end{array}$ & & $\frac{-1.222}{-1.219}$ & & $\begin{array}{l}\frac{44.052}{45.727} \\
\end{array}$ & & & & & \begin{tabular}{|l|l|l|l|l|l|}
261778 \\
\end{tabular} & & $\frac{10.8}{112}$ & & & & & & & 0.36 \\
\hline & $2 / 2 / 2004$ & 2:41:35 PM & 25.444 & 23.691 & \begin{tabular}{|l|}
24.974 \\
\end{tabular} & $\frac{24.171}{24.819}$ & & & & 11..944 & & & & 50.472 & & & & & 63444 & & $\frac{11}{12}$ & & & & & & & $\frac{0.35}{0.36}$ \\
\hline \begin{tabular}{|l|}
177 \\
\end{tabular} & $2 / 2 / 2004$ & 2:42:35 PM & 25.451 & 23.714 & \begin{tabular}{|l|}
24.991 \\
\end{tabular} & 24.805 & 26.98 & 46.939 & \begin{tabular}{|l|}
49.809 \\
\end{tabular} & $\begin{array}{l}11.837 \\
\end{array}$ & 34.851 & -1.216 & & 48.953 & & & & & & 48 & . & & & & & 00 & & \\
\hline \begin{tabular}{|l|}
178 \\
\end{tabular} & $2 / 2 / 2004$ & & 25.438 & 23.726 & & & & & & 12.191 & & & & & & & & & & & & & & & & & & \\
\hline & & & & & \begin{tabular}{|l|l|}
24.993 \\
\end{tabular} & 24.75 & & 46.51 & & 11.841 & 34.453 & & & & 0.115 & & & & 268 & $\frac{4}{4 \varepsilon}$ & 12. & & & & & & & \\
\hline & $2 / 2 / 2004$ & & 25.431 & 23.748 & 25 & 24.78 & & & & 12.00 & 34.426 & & & & & & & & & & 12.1 & & & & & & & \\
\hline & 304 & & 25.426 & 23.754 & 24.986 & 24.78 & & & & & & & & & & & & & & & 11. & & & & & & & \\
\hline 182 & $2 / 2 / 2004$ & & 25.423 & 23.76 & 24.987 & 24.85 & & & & $\mid 11.908$ & & & & 47.8 & & & & & & & 11.7 & & & & & & & \\
\hline & $2 / 2 / 2004$ & & & & & 24.82 & & & & & & & & & & & & & & & & & & & & & & \\
\hline 184 & |l2004 & 2:49:3 & 25.43 & 23.793 & 24.99 & 24.8 & & 47.2 & \begin{tabular}{|l|}
50.306 \\
\end{tabular} & 12.052 & 34.853 & -1.225 & & 3.0 & & & & & 2.76 & & 13. & & & & & & 0.418 & \\
\hline & & & & & 25.006 & & & & & & & & & & & & & & & & & & & & & & & \\
\hline & & & & & \begin{tabular}{|l|l|} 
& 25.001 \\
\end{tabular} & & & & & & & & & & & & & & & & 12. & & & & & & & \\
\hline & 4 & & & & & & & & & & & & & & & & & & & & & & & & & & & \\
\hline $\mid$\begin{tabular}{|l|l|}
189 \\
\end{tabular} & $\frac{21 / 120044}{21 / 2004}$ & & 25.4039 & $\frac{2.034}{23847}$ & 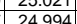 & $\frac{24}{24}$ & & & & 12.005 & & & & 45. & & & & & & & $\frac{1.1}{118}$ & & & & & & & \\
\hline & $2 / 2 / 2004$ & & 20.4050 & & & & & & & & & & & & & & & & & & 11. & & & & & & & \\
\hline 191 & $2 / 2 / 2004$ & $2: 56: 3$ & 25.425 & 23.883 & $\mid \begin{array}{r}24.05 \\
\mid\end{array}$ & 24.96 & & 46.599 & \begin{tabular}{|l|l|}
49 \\
\end{tabular} & 11.76 & 34.506 & $\frac{-1.2}{-1.2}$ & & $\frac{4.3}{49.3}$ & & & & & 2.88444 & & 12.1 & & & & & & & \\
\hline & & & & & & & & & & & & & & & & & & & & & & & & & & & & \\
\hline & 2004 & & 25.404 & 23.91 & & 25.6 & & & & $\frac{1.1 .6}{11.6}$ & & & & & & & & & & & $\frac{21}{11}$ & & & & & & & \\
\hline & & & & 23 & & & & & & & & & & & & & & & & & 11 & & & & & & & \\
\hline & $2 / 2 / 2004$ & & & & \begin{tabular}{|l|}
24.855 \\
\end{tabular} & 24.94 & & 46.7 & & 11.5 & 34. & & & 48 & & & & & & & 11.8 & & & & & & & \\
\hline 1 & $2 / 2 / 2004$ & & 25.38 & 23.952 & 24.84 & 24.92 & & 46 & & 11.4 & 35.1 & & & 46.0 & & & & & & & 11. & & & & & & & \\
\hline & $2 / 2 / 2004$ & & & & & 25. & & 46. & & & & & & & & & & & & & 11 & & & & & & & \\
\hline 198 & 2/2/2004 & & 25.38 & 23.968 & & 25 & & 46.8 & & 11.4 & & & & 48 & & & & & & & 11. & & & & & & & \\
\hline & $2 / 2$ & & 25.361 & & & & & & & & & & & & & & & & & & 11 & & & & & & & \\
\hline & $2 / 2$ & & 25.368 & & & 24 & & & & & & & & & & & & & & & & & & & & & & \\
\hline & 212120 & & & 24 & 24.8 & & & 47.184 & & 11.396 & & & & & & & & & & & 11 & & & & & & & \\
\hline$\frac{20}{20}$ & $\frac{21212004}{212104}$ & & 25 & $\frac{24.019}{24026}$ & 24.64 & & & 470 & & $\sqrt{116}$ & & & & & & & & & & & & & & & & & & \\
\hline & 2 & & & & & & & 47.13 & & & & & & & & & & & & & & & & & & & & \\
\hline & $\frac{21 / 2}{212}$ & & $\frac{25.369}{25.369}$ & $\frac{24.043}{24.043}$ & $\mid$\begin{tabular}{|l|l|}
$\mid 24.824$ \\
\end{tabular} & & & 47.5 & & 11.859 & & & & & & & & & & & & & & & & & & \\
\hline & & & & & & & & & & & & & & & & & & & & & & & & & & & & \\
\hline & $2 / 2 / 2004$ & & & & & & & 47.595 & & & & & & & & & & & & & & & & & & & & \\
\hline & & & & 2406 & 246 & 25131 & & 46.896 & & $\frac{114}{114}$ & 352 & & & 2 & & & & & & & & & & & & & & \\
\hline & & & & & & & & & & & & & & & & & & & & & & & & & & & & \\
\hline 210 & 2/2/2004 & 3:15:35 PM & 323 & 24.077 & $|24.663|$ & \begin{tabular}{|l|l|}
3 & 25.128
\end{tabular} & 27.496 & 46.719 & 50.333 & 11.34 & 35.157 & $|-1.222|$ & & 46.928 & \begin{tabular}{|c|c|c|}
0.118 \\
\end{tabular} & 14.643 & & & 0111 & 48.9 & 11.5[ & & & . & 0.018 & 0.000 & 0.434 & \\
\hline
\end{tabular}


WSRC-TR-2005-00105, REVISION 0

SRNL-RPP-2005-00012, REVISION 0

RUN \# WASH1; CAMPAIGN I SLURRY WASH 1 - CONT.

\begin{tabular}{|c|c|c|c|c|c|c|c|c|c|c|c|c|c|c|c|c|c|c|c|c|c|c|c|c|c|c|c|c|}
\hline & & & & & & & & & & & & & & & & & & & & & & & & & & & & \\
\hline & $\mathrm{A}$ & B & $\mathrm{D}$ & $E$ & $\mathrm{~F}$ & G & $\mathrm{H}$ & $\mathrm{J}$ & $\mathrm{K}$ & \begin{tabular}{l|l|l|l|} 
L & \\
\end{tabular} & $\mathrm{M}$ & $\mathrm{N}$ & 0 & Q & $\begin{array}{ll}R \\
\end{array}$ & \begin{tabular}{l|l|} 
\\
\end{tabular} & $\mathrm{T}$ & $\mathrm{v}$ & w & $x$ & Y & $z$ & AA & $A B$ & $\mathrm{AC}$ & $\mathrm{AD}$ & $\mathrm{AE}$ & \\
\hline 21 & $2 / 2 / 2004$ & 3:16:35 PM & 25.31 & 24.084 & 24.67 & 25.166 & 27.353 & 46.305 & \begin{tabular}{|l|l|}
4995 \\
\end{tabular} & 11.982 & 33.997 & & -7.07. & $\begin{array}{r}45.547 \\
\end{array}$ & 0.113 & 14.643 & & 193.07 & 3.21778 & 48.5 & 11.2 & & 2.768 & & 0.017 & 0.000 & 0.424 & \\
\hline & $2 / 2 / 2004$ & & & 24.095 & \begin{tabular}{|l|l|}
24.686 \\
\end{tabular} & 25.166 & $\begin{array}{l}27.493 \\
27101\end{array}$ & \begin{tabular}{|l|l|}
45.469 \\
\end{tabular} & \begin{tabular}{|l|}
49.108 \\
\end{tabular} & & 32.345 & $\begin{array}{l}-1.219 \\
-1.237\end{array}$ & $\begin{array}{r}-7.112 \\
-7.112\end{array}$ & 47.11 & & \begin{tabular}{|l|}
14.643 \\
\end{tabular} & & & 3.23444 & 47.6 & 11.5 & & & & & & 0.418 & \\
\hline \begin{tabular}{|l|}
213 \\
12
\end{tabular} & $2 / 2 / 2004$ & $3: 18: 35 \mathrm{PM}$ & 25.282 & $\begin{array}{l}24.101 \\
\end{array}$ & \begin{tabular}{|l|l}
24.732 \\
\end{tabular} & 25.168 & $\begin{array}{l}27.171 \\
\end{array}$ & $\begin{array}{l}47.564 \\
\end{array}$ & \begin{tabular}{|l|}
51.228 \\
\end{tabular} & \begin{tabular}{ll|}
14.028 \\
\end{tabular} & 33.285 & 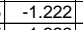 & $\begin{array}{r}-7.008 \\
-1.0\end{array}$ & 52.821 & 0.113 & \begin{tabular}{ll|}
14.643 \\
\end{tabular} & & 195.0 & 3.25111 & $\begin{array}{l}49.8 \\
\end{array}$ & 12.9 & & \begin{tabular}{|l|l|}
2.787 \\
\end{tabular} & & 0.017 & 0.000 & $\begin{array}{l}0.420 \\
\end{array}$ & \\
\hline \begin{tabular}{|l|l|}
214 \\
215
\end{tabular} & $\begin{array}{l}2 / 2 / 2004 \\
2 / 212004\end{array}$ & $\begin{array}{l}3: 19: 35 \text { PM } \\
3 \cdot 20: 35 \text { PM }\end{array}$ & $\begin{array}{r}25.278 \\
25289\end{array}$ & \begin{tabular}{|l|}
24.082 \\
24833
\end{tabular} & 24.788 & $\begin{array}{r}25.128 \\
251 .\end{array}$ & $\begin{array}{l}27.271 \\
27.362\end{array}$ & $\begin{array}{r}47.597 \\
4769\end{array}$ & \begin{tabular}{|r|}
51.32 \\
51.477 \\
\end{tabular} & $\begin{array}{r}13.59 \\
1355\end{array}$ & $\begin{array}{l}33.757 \\
32012\end{array}$ & \begin{tabular}{|c|}
$\mid-1.222$ \\
\end{tabular} & \begin{tabular}{|l|l|}
-7.011 \\
6047
\end{tabular} & \begin{tabular}{|l|l|}
48.449 \\
51.25
\end{tabular} & $\begin{array}{l}0.114 \\
0.118\end{array}$ & \begin{tabular}{l|l|}
14.643 \\
14.643
\end{tabular} & & $\begin{array}{l}196.07 \\
10707\end{array}$ & $\begin{array}{ll}3.26778 \\
32844\end{array}$ & \begin{tabular}{|l|l}
49.8 \\
50.
\end{tabular} & \begin{tabular}{|l|l|}
11.9 \\
\end{tabular} & \begin{tabular}{|l|l|}
40.7 \\
0.9
\end{tabular} & 2810 & 0019 & \begin{tabular}{|l|l|l|}
0.017 \\
0019
\end{tabular} & 0.000 & 0.420 & \\
\hline$\frac{\mid 210}{216}$ & $\frac{2 / 2 / 2004}{2 / 2 / 2004}$ & & & $\begin{array}{r}24.083 \\
24.09\end{array}$ & $\begin{array}{l}34.869 \\
24.966\end{array}$ & $\begin{array}{r}25.1 \\
25.107\end{array}$ & $\begin{array}{l}27.362 \\
27.369 \\
\end{array}$ & $\begin{array}{r}47.69 \\
46.788\end{array}$ & \begin{tabular}{|l|}
51.477 \\
50.501
\end{tabular} & $\frac{13.505}{12.982}$ & $\begin{array}{r}33.913 \\
33.62 \\
\end{array}$ & \begin{tabular}{r|}
-1.219 \\
-1.225
\end{tabular} & $\begin{array}{l}-6.947 \\
-6.956\end{array}$ & $\begin{array}{r}51.25 \\
46.797\end{array}$ & 0.118 & \begin{tabular}{|c|}
14.643 \\
14.643
\end{tabular} & & $\begin{array}{l}197.07 \\
19807\end{array}$ & 3.28444 & 50.0 & $\begin{array}{l}12.6 \\
11.5\end{array}$ & $\frac{40.8}{402}$ & & & 0.018 & 0.000 & 0.433 & \\
\hline \begin{tabular}{|l|}
217 \\
\end{tabular} & $2 / 2 / 2004$ & $3: 22: 35$ PM & 25.332 & 24.106 & \begin{tabular}{|l|l|l|l|l}
24.900 \\
\end{tabular} & \begin{tabular}{|l|l|l|l|l|}
25.112 \\
\end{tabular} & 27.151 & \begin{tabular}{|l|}
46.8689 \\
46.869
\end{tabular} & \begin{tabular}{|l|}
50.501 \\
50.72 \\
\end{tabular} & $\begin{array}{ll}\frac{12.982}{12.827} \\
\end{array}$ & $\begin{array}{l}33.62 \\
33.759 \\
\end{array}$ & $\begin{array}{l}-1.225 \\
-1.216\end{array}$ & $\begin{array}{l}-6.956 \\
-6.976\end{array}$ & $\begin{array}{r}46.191 \\
49.41 \\
\end{array}$ & $\begin{array}{l}0.113 \\
0.115\end{array}$ & \begin{tabular}{|l|}
14.6434 \\
14.643
\end{tabular} & & $\frac{198.07}{199.07}$ & $\begin{array}{l}3.30111 \\
3.31778\end{array}$ & $\frac{4990}{49.2}$ & $\frac{11.5}{12.1}$ & $\frac{40.2}{40.3}$ & $\frac{2.772}{2.780}$ & & $\frac{0.017}{0.017}$ & $\begin{array}{c}0.000 \\
0.000\end{array}$ & 0.420 & $\frac{0.36}{0.36}$ \\
\hline \begin{tabular}{|l|}
218 \\
\end{tabular} & $2 / 21 / 2004$ & & 25.339 & 24.103 & \begin{tabular}{|l|l|}
3 & 24.979 \\
\end{tabular} & 25.064 & 27.332 & 47.002 & 50.743 & 12.953 & 33.78 & -1.222 & -6.97 & 50.499 & 0.119 & 14.643 & & 200.07 & 3.33444 & 49.3 & 12.4 & 40.4 & \begin{tabular}{|l|}
4.785 \\
\end{tabular} & 0.018 & 0.018 & 0.000 & 0.440 & \\
\hline \begin{tabular}{|l|}
219 \\
\end{tabular} & & & & & & 25.049 & & 46.908 & \begin{tabular}{|l|}
50.679 \\
\end{tabular} & & & & & & & \begin{tabular}{|l|}
14.643 \\
\end{tabular} & & & & 49.2 & 12.4 & & & & & & & \\
\hline 220 & $2 / 2 / 2004$ & $3: 25: 35 \mathrm{PM}$ & 25.369 & 24.098 & \begin{tabular}{|l|l|}
25.024 \\
\end{tabular} & 25.044 & 26.68 & 47.097 & \begin{tabular}{|l|}
51.104 \\
\end{tabular} & $\begin{array}{l}12.812 \\
\end{array}$ & 33.919 & -1.222 & -6.753 & $\begin{array}{l}48.805 \\
\end{array}$ & 0.124 & \begin{tabular}{ll|}
14.643 \\
\end{tabular} & & & 3.36778 & 49.6 & 12.0 & & 2.79 & & $\begin{array}{l}0.018 \\
\end{array}$ & 0.000 & 0.456 & \\
\hline $\mid \frac{221}{322}$ & $2 / 2 / 2004$ & 3:26:35 PM & $\begin{array}{r}25.379 \\
25.301\end{array}$ & $\begin{array}{l}24.088 \\
\end{array}$ & 25.049 & 25.004 & & 47.25 & \begin{tabular}{|l|}
50.975 \\
\end{tabular} & 12.576 & & \begin{tabular}{|l|l|} 
& -1.219 \\
\end{tabular} & $\begin{array}{r}-6.99 \\
\end{array}$ & \begin{tabular}{|l|l|}
45.793 \\
\end{tabular} & 0.114 & & & 203.07 & 3.38444 & 49.5 & 11.2 & & 2.81 & 0.01 & \begin{tabular}{|l|l|}
0.017 \\
\end{tabular} & & $\begin{array}{l}0.415 \\
\end{array}$ & \\
\hline $\mid \frac{222}{223}$ & $\begin{array}{l}2 / 2 / 2004 \\
2 / 21 / 2004\end{array}$ & $\begin{array}{l}\text { 3:27:25 PM } \\
\text { 3:28:35 }\end{array}$ & $\begin{array}{r}25.394 \\
25.408\end{array}$ & \begin{tabular}{|l|l|}
24.088 \\
24.097
\end{tabular} & \begin{tabular}{|l|l|l|l|}
25.064 \\
725.093
\end{tabular} & 24.963 & $\frac{26.434}{26.857}$ & \begin{tabular}{|l|}
47.281 \\
47.165
\end{tabular} & \begin{tabular}{|l|}
51.158 \\
51.042 \\
\end{tabular} & \begin{tabular}{|c|}
12.653 \\
12.503
\end{tabular} & $\begin{array}{r}34.43 \\
34.391 \\
\end{array}$ & $\begin{array}{l}-1.219 \\
-1222\end{array}$ & \begin{tabular}{|c|c|}
-6.597 \\
-6.713
\end{tabular} & \begin{tabular}{|l|}
50.474 \\
53837
\end{tabular} & $\begin{array}{l}0.115 \\
0.121\end{array}$ & $\frac{14.643}{14643}$ & & $\begin{array}{l}204.07 \\
20507\end{array}$ & \begin{tabular}{|c|}
3.40111 \\
3
\end{tabular} & $\begin{array}{l}49.7 \\
496\end{array}$ & $\begin{array}{l}12.4 \\
132\end{array}$ & 408 & $\mid \frac{2.011}{2812}$ & 0.01 & \begin{tabular}{|c|c|c|} 
\\
\end{tabular} & 0.000 & $\begin{array}{l}0.419 \\
0.441\end{array}$ & 0.36 \\
\hline 224 & $2 / 2 / 2004$ & $\begin{array}{l}3: 28.35 \mathrm{PM} \\
3: 29: 35 \mathrm{PM}\end{array}$ & 25.421 & $\begin{array}{l}24.085 \\
24\end{array}$ & 25.107 & \begin{tabular}{|l|}
24.905 \\
\end{tabular} & 27.105 & 47.572 & \begin{tabular}{|l|l|}
51.396 \\
\end{tabular} & 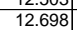 & 34.518 & \begin{tabular}{|c|}
-1.221 \\
-1.219 \\
\end{tabular} & $\begin{array}{l}-6.713 \\
-6.935\end{array}$ & \begin{tabular}{|l|}
53.8311 \\
49.882 \\
\end{tabular} & $\begin{array}{l}.121 \\
0.114\end{array}$ & $\begin{array}{l}14.643 \\
14.643\end{array}$ & 0.0 & $\begin{array}{l}205.07 \\
206.07\end{array}$ & \begin{tabular}{|l|}
$3.411 / 8$ \\
3.43444
\end{tabular} & $\begin{array}{l}49.6 \\
49.9\end{array}$ & 12.2 & & \begin{tabular}{|l|}
2.812 \\
2.830 \\
\end{tabular} & & $\begin{array}{l}.018 \\
0.017\end{array}$ & 0.000 & $\begin{array}{l}0.441 \\
0.413\end{array}$ & $\frac{0.38}{0.35}$ \\
\hline & $\frac{1 / 2 / 2004}{212200}$ & $\begin{array}{ll}3: 30: 35 \mathrm{PM} \\
3: 350\end{array}$ & 25.426 & & 25.117 & 24.976 & & & & & 34.588 & & & \begin{tabular}{|l|l|}
50.777 \\
\end{tabular} & 0.121 & & & & 3.45111 & 49.9 & 12.4 & & & & 0.018 & & & \\
\hline & $2 / 2 / 2004$ & & & & & & 27.41 & 43.92 & \begin{tabular}{|l|l|}
51.135 \\
\end{tabular} & 12.444 & 31.286 & & & 47.085 & 0.043 & 14.643 & & & \begin{tabular}{|l|l|}
3.46778 \\
\end{tabular} & 49.7 & 11. & & & & 0.006 & & 0.170 & \\
\hline \begin{tabular}{|l|}
227 \\
\end{tabular} & $2 / 2 / 2004$ & $3: 32: 35$ PM & 25.556 & 24.08 & 25.117 & 24.976 & 27.1 & $\begin{array}{l}46.749 \\
\end{array}$ & \begin{tabular}{|l|}
51.077 \\
\end{tabular} & $\begin{array}{l}12.453 \\
\end{array}$ & 34.028 & -1.106 & & 53.599 & 0.089 & \begin{tabular}{|l|l|}
14.643 \\
\end{tabular} & & & \begin{tabular}{|l|l|}
3.48444 \\
\end{tabular} & 49.6 & 13.1 & & 2.785 & & 0.013 & 0.000 & 0.327 & \\
\hline $2 \frac{228}{220}$ & $2 / 2 / 2004$ & 3:33:35 PM & $\begin{array}{l}25.517 \\
25.520\end{array}$ & \begin{tabular}{|l|l|}
24.071 \\
2.092
\end{tabular} & \begin{tabular}{|l|}
25.012 \\
25052
\end{tabular} & $\begin{array}{l}24.962 \\
21.070\end{array}$ & $\begin{array}{l}27.191 \\
2701\end{array}$ & & & \begin{tabular}{|l|l|}
12.353 \\
1.107
\end{tabular} & & $\frac{222}{221}$ & & \begin{tabular}{|l|l|}
48.584 \\
\end{tabular} & 129 & & & & 3.50111 & 49.4 & 11.9 & & & $\overline{0.01}$ & 0.019 & & \begin{tabular}{|l|l|l|}
0.469 \\
\end{tabular} & \\
\hline$\frac{229}{230}$ & $\begin{array}{l}2 / 2 / 2004 \\
2 / 21 / 2004\end{array}$ & 3:3:3::35 PM & $\begin{array}{r}25.528 \\
25.527 \\
\end{array}$ & 24.092 & \begin{tabular}{|l|}
25.053 \\
25.057 \\
\end{tabular} & $\frac{24.978}{24.942}$ & $\frac{27.017}{26.573}$ & \begin{tabular}{|l|}
46.873 \\
47.402 \\
\end{tabular} & \begin{tabular}{|l|l|}
50.642 \\
50.992
\end{tabular} & \begin{tabular}{|c|}
12.197 \\
12.23 \\
\end{tabular} & $\begin{array}{r}34.385 \\
34.951 \\
\end{array}$ & $\begin{array}{l}-1.222 \\
-1.222 \\
\end{array}$ & & \begin{tabular}{|l|}
51.809 \\
50.036
\end{tabular} & $\begin{array}{l}0.115 \\
0.121\end{array}$ & 6433 & & $\frac{211.07}{2107}$ & \begin{tabular}{|l|}
3.51778 \\
35444
\end{tabular} & \begin{tabular}{|l|}
49.2 \\
495
\end{tabular} & $\frac{12.7}{12 .}$ & 40.6 & 2.801 & 0.017 & \begin{tabular}{|c|c|c|} 
\\
\end{tabular} & 0.000 & $\begin{array}{l}0.421 \\
0.437\end{array}$ & 0.36 \\
\hline 231 & $2 / 2 / 2004$ & $\begin{array}{l}0.50 .55 \mathrm{FM} \\
3: 36\end{array}$ & 25.537 & 24.081 & \begin{tabular}{|l|l|}
25.085 \\
\end{tabular} & 24.9432 & 26.832 & \begin{tabular}{|l|l|}
4.46 .995 \\
\end{tabular} & \begin{tabular}{|l|l|}
50.654 \\
\end{tabular} & 12.101 & 34.611 & \begin{tabular}{|l|l|} 
& -1.2222 \\
\end{tabular} & $\begin{array}{l}-6.889 \\
-6.907\end{array}$ & \begin{tabular}{|l|}
55.035 \\
\end{tabular} & 0.121 & $\begin{array}{l}14.643 \\
14.643 \\
\end{array}$ & & 213.07 & \begin{tabular}{|c|}
3.535444 \\
3.55111
\end{tabular} & $\begin{array}{l}49.2 \\
49.2\end{array}$ & 11.3 & $\begin{array}{ll}41.2 \\
40.8\end{array}$ & 2.813 & 0.018 & $\frac{0.018}{0.018}$ & $\begin{array}{l}0.000 \\
0.000\end{array}$ & $\begin{array}{l}0.437 \\
0.445 \\
\end{array}$ & $\frac{0.37}{0.38}$ \\
\hline & 2/2/2004 & $3: 37: 35 \mathrm{PM}$ & 25.536 & 24.065 & \begin{tabular}{|l|}
25.092 \\
\end{tabular} & 24.946 & 27.021 & & \begin{tabular}{|l|l|}
50.627 \\
\end{tabular} & 12.025 & 34.859 & $|-1.219|$ & & \begin{tabular}{|l|l|}
48.737 \\
\end{tabular} & 0.122 & 14.643 & & 214. & 3.56778 & 49.2 & 11.9 & & & & & & $\begin{array}{l}.445 \\
0.443 \\
\end{array}$ & $\frac{0.38}{0.38}$ \\
\hline \begin{tabular}{|l|l|}
233 \\
\end{tabular} & $2 / 2 / 2004$ & 3:38:35 PM & 25.542 & 24.071 & \begin{tabular}{|l|}
25.107 \\
\end{tabular} & 24.922 & 27.10 & 47.155 & 50.884 & 12.169 & 34.722 & -1.219 & & 47.473 & 0.126 & 14.643 & & & 3.58444 & 49.4 & 11.6 & & & & & & & \\
\hline 234 & 2/2/2004 & $3: 39: 35 \mathrm{PM}$ & 25.548 & 24.067 & 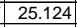 & 24.928 & 27.232 & 47.043 & \begin{tabular}{|l|}
50.776 \\
\end{tabular} & \begin{tabular}{|l|l|l|}
12.133 \\
\end{tabular} & 34.633 & -1.222 & & 49.765 & 0.128 & \begin{tabular}{|l|l|}
14.643 \\
\end{tabular} & & & 3.60111 & 49.3 & 12. & & & & 0.019 & 0.000 & 0.466 & \\
\hline & & & & & & & & & & & & & & & & & & & & & & & & & & & 0.443 & \\
\hline 236 & $2 / 2 / 2004$ & & 25.543 & $\begin{array}{l}24.067 \\
\end{array}$ & 25.153 & 24.898 & & \begin{tabular}{|l|l|l|}
47.284 \\
\end{tabular} & 1116 & $\begin{array}{ll}12.229 \\
\end{array}$ & 34.775 & & & 43.747 & 0.122 & & & & $\begin{array}{l}3.63444 \\
\end{array}$ & & & & & & 018 & & & \\
\hline & 2/2/2004 & & & & & 24.947 & & & 766 & & 34.8 & & & & & & & & & 49.3 & 1L. & & & & & & 0.479 & \\
\hline \begin{tabular}{|l|l|}
238 \\
\end{tabular} & $2 / 2 / 2004$ & 3:43:35 PM & 25.542 & 24.056 & 25.162 & 24.877 & 27.70 & $\begin{array}{l}47.383 \\
\end{array}$ & \begin{tabular}{|l|l|} 
\\
\end{tabular} & \begin{tabular}{ll|l|}
12.296 \\
\end{tabular} & 34.767 & -1.222 & & 50.412 & 0.126 & $\begin{array}{l}14.643 \\
\end{array}$ & & & 3.66778 & $\begin{array}{ll}49.8 \\
\end{array}$ & 12.4 & 41.1 & 2.832 & D19 & 0.019 & 0.000 & $\begin{array}{l}0.455 \\
\end{array}$ & \\
\hline & 2/2/2004 & 3:44:35 PM & 25.548 & 24.062 & 25.168 & 24.92 & 27.7 & 47.288 & 51.208 & 12.101 & 34.834 & -1.228 & & 50.808 & 0.13 & & & 221.0 & 3.68444 & 49.7 & & & & & 0.019 & & & \\
\hline |240 & $2 / 2 / 2004$ & & 25.568 & 24.082 & 25.214 & 24.973 & & 47.375 & \begin{tabular}{|l|l|} 
& 51.179 \\
\end{tabular} & 12.032 & 35.122 & & & 49.805 & 0.132 & 14. & & & 3.70111 & 49.7 & 12. & & & & & & 0.474 & \\
\hline |241 & 2/2/2004 & & 25.559 & 24.063 & 25.19 & 25.094 & & 47.338 & 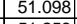 & 12.336 & 34.74 & 225 & & & 0.127 & & & & & 49.6 & & & & & & 0.000 & 0.459 & \\
\hline & & & $25.5 / 6$ & 24.085 & 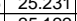 & 25.051 & 27.11 & 4r.352 & 2 & 12.341 & 34.648 & & & 51.135 & 0.1 & & & & 3. 3.34444 & 499 & & & & & & & & \\
\hline & 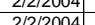 & & & 24.035 & 25.122 & $\frac{25.060}{2009}$ & & & & 12.091 & $\frac{34.397}{32404}$ & & & 52.4 & 0.1 & & & & & & & & & & & & & \\
\hline 245 & $2 / 2 / 2 / 2004$ & & 25.356 & 24089 & \begin{tabular}{|l|l|l|l|l|} 
\\
\end{tabular} & 24.5503 & & $\frac{40.017}{46.45}$ & 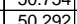 & 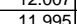 & $\begin{array}{l}34.404 \\
33209\end{array}$ & -1. & & $\begin{array}{l}32.025 \\
49327\end{array}$ & $\frac{0.125}{012}$ & & & & 378444 & 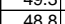 & 12 & & 278 & & & & 0.450 & \\
\hline & 2/1/2/2004 & & 25.557 & & 25.132 & $\frac{25.00}{2512}$ & & & . & 年 & $\begin{array}{l}33.259 \\
33999\end{array}$ & & & 27 & 0.128 & & & & & 年 48.6 & 12 & & & & & & & \\
\hline 2477 & $2 / 2 / 2004$ & 3:52:35 PM & 25.563 & 24.107 & $\frac{25.133}{25.13}$ & 25.103 & & 46.773 & | 50.667 & 12.189 & 34.295 & -1.228 & & 51.358 & 0.131 & 14 & & 22 & & 49. & & & & & & 500 & 0.480 & \\
\hline 248 & & 3:53:35 PM & & & & & & & & & & & & & & & & & & & 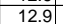 & & & & & & & \\
\hline & $2 / 2 / 2004$ & 3:54:35 PM & 25.571 & 24.13 & 25.166 & 25.181 & & 46.863 & 50.681 & 12.109 & 34.441 & -1.225 & & 48.858 & 0.12 & & & & & 49 & 12 & & & & & & 0.438 & \\
\hline & $2 / 2 / 2004$ & & 25.572 & 24.146 & 25.177 & 25.292 & & & & 11.924 & 34.1 & & & 44.8 & 0.1 & & & & & & 11. & & & & & & & \\
\hline & $2 / 2 / 2004$ & & & 24.142 & 25.183 & 25.188 & & & & & & & & 48.5 & & & & & & & 11 & & & & & & & \\
\hline 25 & 2/2/2004 & & 25.589 & 24.154 & 25.2 & 25.16 & & & & \begin{tabular}{|l|l|}
12.057 \\
\end{tabular} & & & & 5 & & & & & & & 12.6 & & & & & & & \\
\hline & $2 / 2 / 2004$ & & & 24.1 & & & & & & & & & & 6 & & & & & & & & & & & & & & \\
\hline 254 & 2/2/2004 & 3:59:35 PM & 25.608 & 24.182 & 25.228 & 25.128 & & 46.958 & \begin{tabular}{|l|l|} 
\\
\end{tabular} & 12.134 & 34.508 & -1.225 & & 53.903 & 0.14 & & & & 3.93444 & & 13. & & $2.8 \mathrm{C}$ & & & & 0.509 & \\
\hline & $2 / 2$ & & 25.61 & 24.19 & & 25. & & & & 12 & & & & & 0. & & & & & & & & & & & & & \\
\hline$\frac{25}{55}$ & & & & & & & & & & & & & & & & & & & & & 12 & & & & & & & \\
\hline & | & & & & \begin{tabular}{|l|l|l|l} 
\\
\end{tabular} & & & & & $\frac{12.0}{110}$ & & & & 50.5 & & & & & & & & & & & & & & \\
\hline \begin{tabular}{|l|}
259 \\
\end{tabular} & $\frac{21 / 120044}{21 / 2004}$ & & 25.000 & $\frac{24.219}{24.219}$ & \begin{tabular}{|l|}
2.250 \\
\end{tabular} & $\frac{25.250}{2529}$ & & & & $\mid$ & $\frac{3.4}{352}$ & & & 年 & & & & & & & 告.4. & & & & & & & \\
\hline & $2 / 2 / 2004$ & & & & 25.208 & 25.359 & & & & & & & & 481 & & & & & & & & & & & & & & \\
\hline 261 & $2 / 2 / 2004$ & & 25.62 & 24.231 & \begin{tabular}{|l|l|} 
& 25.13 \\
\end{tabular} & 25.407 & & & & 11.873 & 35.268 & $\frac{1.222}{-1.222}$ & & $\frac{5.9}{5.9}$ & 0.134 & & & & 8 & & 13. & & & & & & & \\
\hline & & & & & & & & & & & & & & & & & & & & & & & & & & & & \\
\hline 263 & 2004 & & 25.61 & 24.24 & \begin{tabular}{|l|}
25.127 \\
\end{tabular} & 25.36 & & & & $\frac{11.64}{11.64}$ & & $\frac{-1.2}{-1.2}$ & & $\overline{51.1}$ & 0.1 & & & & & & $\frac{12}{12}$ & & & & & & & \\
\hline & & & & & & & & & & & & & & $\overline{511}$ & & & & & & & 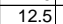 & & & & & & & \\
\hline & $2 / 2 / 2004$ & & & $24.2 \varepsilon$ & \begin{tabular}{|l|l|}
25.167 \\
\end{tabular} & 25.442 & & & & 11.5 & & & & & & 14 & & & 4.10 & & 12 & & & & & & & \\
\hline 2 & $2 / 2 / 2004$ & & 25.6 & 24.313 & \begin{tabular}{|l|l|}
25.204 \\
\end{tabular} & 25.449 & & 47.3 & 50 & 11.6 & & -1.21 & & 49.8 & 0.1 & & & & 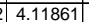 & & 12 & & & & & & 0.4 & \\
\hline 267 & 2/2/2004 & $4: 11: 3$ & 25.6 & 24 & $\begin{array}{l}25.185 \\
\end{array}$ & 25.396 & & & & 11.4 & & -1. & & & & & & & & & 12 & & & & & & & \\
\hline 26 & 2/2/2004 & & 25.64 & & 25.197 & & & & \begin{tabular}{|l|}
50.7141 \\
\end{tabular} & 11. & & & & 48.6 & & & & & & 4 & 11 & & & & & & & \\
\hline & 2/21/2004 & & & & 25.192 & 25.468 & & & & & & & & & & & & & & & 12 & & & & & & & \\
\hline & $2 / 2$ & & 25.65 & 24.327 & \begin{tabular}{|l|}
25.188 \\
\end{tabular} & 25.5 & & & & & & & & & & & & & & & 11 & & & & & & & \\
\hline & 212120044 & & & 24.344 & 25.2. & & & & & 11. & & & & & 0.1 & & & & & & 16 & & & & & & & \\
\hline 势 & $\frac{21212004}{212104}$ & & 2565 & $\frac{24.535}{24348}$ & $\frac{23.200}{25194}$ & & & $\begin{array}{l}4.087 \\
47.85\end{array}$ & 451.10 & $\frac{11.095}{11.743}$ & 年3.. & & & 487 & 0.1 & & & & & & & & & & & & & \\
\hline 年 & 2 & & & 2438 & $|2526|$ & & & 47.835 & & 1178 & $\frac{3.15}{35}$ & $\mid$ & & & & & & & & & 12 & & & & & & & \\
\hline 275 & $2 / 2$ & & & 24.382 & & & & & & 11.406 & 35. & -1.219 & & & & & & & & & & & & & & & & \\
\hline & & & & & & & & & & & & & & & & & & & & & & & & & & & & \\
\hline \begin{tabular}{|l|}
277 \\
\end{tabular} & 2/2/2004 & & & 24.38 & 25.0 & 25. & & & & 11.405 & & -1.2 & & & & & & & & & 12.9 & & & & & & & \\
\hline & & & & 24.44 & 25.06 & 25. & & & 50 & 11.399 & 35.446 & -1.2 & & & 0.14 & & & & & & & & & & & & & \\
\hline & A & & & & & & & & & & & & & & & & & & & & & & & & & & & \\
\hline \begin{tabular}{|l|l|}
280 \\
\end{tabular} & 2/2/2004 & 4:24:38 PM & 5.633 & \begin{tabular}{|l|}
24.403 \\
\end{tabular} & \begin{tabular}{|l|}
25.069 \\
\end{tabular} & 25.154 & & 47.437 & \begin{tabular}{|l|l|} 
& 50.683 \\
\end{tabular} & 11.458 & 35.633 & $\mid-1.219$ & & 49.481 & 0.136 & \begin{tabular}{|l|l|}
6 & 14.643 \\
\end{tabular} & & & 35194 & 49.2 & 12. & & 2.864 & .020 & 0.020 & 0.000 & 0.487 & 0.4 \\
\hline
\end{tabular}


WSRC-TR-2005-00105, REVISION 0

SRNL-RPP-2005-00012, REVISION 0

RUN \# WASH1; CAMPAIGN I SLURRY WASH 1 - CONT.

\begin{tabular}{|c|c|c|c|c|c|c|c|c|c|c|c|c|c|c|c|c|c|c|c|c|c|c|c|c|c|c|c|c|}
\hline & \\
\hline & \begin{tabular}{ll|} 
\\
\end{tabular} & $\begin{array}{lll}\mathrm{B} & \mathrm{C}\end{array}$ & \begin{tabular}{l|l} 
\\
\end{tabular} & $E$ & $\mathrm{~F}$ & G & $\mathrm{H}$ & $\mathrm{J}$ & K & \begin{tabular}{l|l|l|l|} 
&
\end{tabular} & \begin{tabular}{l|l}
$M$ \\
\end{tabular} & $\mathrm{~N}$ & 0 & \begin{tabular}{l|l|l|}
$Q^{2}$ & \\
\end{tabular} & \begin{tabular}{l|l}
$R$ & \\
\end{tabular} & $\mathrm{~s}$ & \begin{tabular}{l|l|l|} 
\\
\end{tabular} & $\mathrm{V}$ & $w$ & 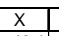 & $\mathrm{Y}$ & $z$ & AA & $A B$ & $A C$ & \begin{tabular}{ll|}
$\mathrm{AD}$ \\
\end{tabular} & $\mathrm{AE}$ & AF $A$ \\
\hline 8 & 2/2/2/2004 & 4:25:38 PM & \begin{tabular}{|l|}
25.623 \\
5
\end{tabular} & 24.413 & $\frac{25.074}{25.74}$ & 25.013 & 27.526 & 47.263 & 50.532 & $\begin{array}{l}11.366 \\
\end{array}$ & 35.575 & \begin{tabular}{|c|}
-1.222 \\
\end{tabular} & -6.583 & \begin{tabular}{|c|}
57.775 \\
\end{tabular} & 0.138 & 14.643 & 0.003 & 262.1 & 4.36861 & 49.1 & 14.2 & & 2.856 & & 0.021 & 0.000 & 0.496 & 0.42 \\
\hline & & & \begin{tabular}{|l|}
25.619 \\
5619
\end{tabular} & 24.409 & & & 27.452 & 47.532 & & \begin{tabular}{|l|}
11.48 \\
\end{tabular} & & & & & & 14.643 & & & & 49.4 & 1200 & & & & & & & \\
\hline & $2 / 2 / 2004$ & $4: 27: 38 \mathrm{PM}$ & 25.619 & 24.414 & \begin{tabular}{|r|}
25.08 \\
\end{tabular} & $\begin{array}{l}25.029 \\
2 .\end{array}$ & 27.492 & $\begin{array}{l}47.4 \\
\end{array}$ & \begin{tabular}{|l|}
50.619 \\
\end{tabular} & $\begin{array}{l}11.605 \\
\end{array}$ & 35.52 & \begin{tabular}{|l|l|}
-1.219 \\
\end{tabular} & $\begin{array}{l}-6.493 \\
\end{array}$ & $\begin{array}{l}50.134 \\
\end{array}$ & $\begin{array}{l}0.137 \\
\end{array}$ & 14.643 & 0.004 & 264.12 & \begin{tabular}{|l|l|l}
4.40194 \\
\end{tabular} & 49.1 & $\begin{array}{ll}12.3 \\
\end{array}$ & & & 0.020 & 0.020 & 0.000 & \begin{tabular}{l|l|l}
0.492 \\
\end{tabular} & 0.42 \\
\hline$\frac{284}{285}$ & $\begin{array}{l}2 / 2 / 20004 \\
2 / 2 / 2004\end{array}$ & $\begin{array}{l}4: 28: 38 \mathrm{PM} \\
4: 29: 38 \mathrm{PM}\end{array}$ & $25.619 \mid$ & $\begin{array}{l}24.409 \\
24.403\end{array}$ & \begin{tabular}{|c|}
25.075 \\
25.804 \\
\end{tabular} & $\begin{array}{l}25.034 \\
25.049\end{array}$ & 27.4426 & 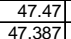 & \begin{tabular}{|l|}
50.747 \\
50778
\end{tabular} & \begin{tabular}{|c|}
11.478 \\
11.599
\end{tabular} & \begin{tabular}{|l|}
35.688 \\
35.454
\end{tabular} & \begin{tabular}{|l|}
-1.222 \\
-1219
\end{tabular} & \begin{tabular}{|l|l|}
-6.612 \\
-6.51
\end{tabular} & \begin{tabular}{r|r|}
50.8 \\
53661
\end{tabular} & $\begin{array}{l}0.136 \\
0.142\end{array}$ & $\begin{array}{l}14.643 \\
14643\end{array}$ & 0.00 & \begin{tabular}{|l|l}
265.12 \\
26612
\end{tabular} & \begin{tabular}{|l|}
4.41861 \\
4.43528 \\
\end{tabular} & $\begin{array}{l}49.3 \\
493 \\
49\end{array}$ & \begin{tabular}{l|}
12.4 \\
132
\end{tabular} & (1) & \begin{tabular}{|l|l|}
2.867 \\
2.067
\end{tabular} & 年 & 0.020 & $\begin{array}{l}0.000 \\
\end{array}$ & $\begin{array}{l}0.487 \\
\end{array}$ & \\
\hline & 2/2/2/2004 & & & & \begin{tabular}{|r|}
25.084 \\
25.578 \\
\end{tabular} & $\begin{array}{l}25.049 \\
25.003\end{array}$ & 27.5966 & 47.3877 & \begin{tabular}{|c|}
50.748 \\
50.001 \\
\end{tabular} & \begin{tabular}{|l|}
11.579 \\
11.595
\end{tabular} & & \begin{tabular}{r|}
-1.219 \\
-1.225
\end{tabular} & & \begin{tabular}{|l|}
53.661 \\
52.353
\end{tabular} & $\begin{array}{l}0.142 \\
0.135\end{array}$ & $\frac{14.643}{14.643}$ & & \begin{tabular}{l|}
266.12 \\
267.12
\end{tabular} & \begin{tabular}{|l|}
4.43528 \\
4.45194
\end{tabular} & $\begin{array}{l}49.3 \\
49.4\end{array}$ & & & \begin{tabular}{|l|l|}
2.856 \\
2865
\end{tabular} & 0.021 & $\frac{0.021}{0.020}$ & \begin{tabular}{|l|l|}
0.001 \\
0.000
\end{tabular} & \begin{tabular}{|l|l|l|}
0.510 & \\
\end{tabular} & \begin{tabular}{|l|} 
\\
\end{tabular} \\
\hline 287 & $2 / 2 / 2004$ & 4:31:38 PM & 25.618 & 24.398 & \begin{tabular}{|l|}
25.088 \\
\end{tabular} & 25.048 & 27.465 & 47.474 & & 11.465 & 35.619 & -1.222 & -6.684 & $\mid \frac{\mid}{51.64}$ & $\mid$ & $\frac{14.643}{14.643}$ & 0.00 & $\frac{267.12}{268.12}$ & $\frac{4.45194}{4.46861}$ & 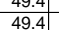 & $\frac{12.8}{12.7}$ & $\begin{array}{l}41.6 \\
41.5\end{array}$ & $\frac{2.865}{2.864}$ & $\frac{0.020}{0.021}$ & $\begin{array}{l}0.020 \\
0.021\end{array}$ & $\mid \frac{0.000}{0.000}$ & $\frac{0.483}{0.494}$ & $\frac{0.41}{0.42}$ \\
\hline 288 & 2/2/2/2004 & & 25.607 & 24.387 & 25.082 & 25.002 & 27.455 & 47.649 & \begin{tabular}{|l|}
50.828 \\
\end{tabular} & 11.505 & 35.828 & -1.222 & -6.536 & \begin{tabular}{|l|l|}
49.905 \\
\end{tabular} & 0.138 & 14.643 & & 269.1 & \begin{tabular}{|l|l|}
4.48528 \\
\end{tabular} & 49.4 & 12.2 & & 2.878 & 0.021 & 0.021 & 0.000 & 0.492 & $\frac{0.42}{0.42}$ \\
\hline & 2/2/2004 & $33: 38$ PM & & & \begin{tabular}{|l|}
25.117 \\
\end{tabular} & 24.967 & & & & & 35.126 & -1.219 & & & & & & & & & 12.1 & & & & & & & \\
\hline 290 & & & 25.582 & & 25.097 & 24.937 & & & & 111.695 & & & & & 141 & 14.6 & & & 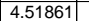 & \begin{tabular}{l|l}
49.5 \\
\end{tabular} & & & & & & D01 & 500 & $\frac{0.40}{0.43}$ \\
\hline 291 & & & & 24.366 & 25.107 & 24.941 & & 47.267 & & 11.634 & 35.344 & -1.222 & & 49.354 & 0.194 & & & & & 49.4 & & & & & & & & \\
\hline 292 & 2/2/2004 & $38 \mathrm{PM}$ & 25.55 & 24.36 & \begin{tabular}{|l|}
25.055 \\
\end{tabular} & 24.809 & 27.537 & 47.732 & 50.913 & \begin{tabular}{|l|l|}
11.72 \\
\end{tabular} & \begin{tabular}{l|l}
35.653 \\
\end{tabular} & -1.222 & & & 133 & & & 270 & .55194 & $\mid 49.4$ & & & & 020 & & 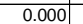 & 0.475 & $\frac{59}{40}$ \\
\hline 20 & & $38 \mathrm{PM}$ & 25.55 & 24.365 & 24.965 & 24.859 & $27.4 !$ & 47.441 & 50.609 & \begin{tabular}{|l|l|l|}
11.434 \\
\end{tabular} & 35.635 & -1.222 & & 49.998 & 0.137 & 14.64 & & 274.1 & 4.56861 & 49.1 & & & 2.864 & & & & 0.492 & 0.42 \\
\hline 294 & $2 / 2 / 2004$ & 8:38 PM & 25.533 & 24.353 & \begin{tabular}{|l|l|}
24.969 \\
\end{tabular} & 24.798 & 27.486 & 47.478 & \begin{tabular}{|l|l|l|} 
\\
5755
\end{tabular} & 11.536 & \begin{tabular}{|l|}
35.61 \\
\end{tabular} & -1.225 & & \begin{tabular}{|l|l|}
44.99 \\
\end{tabular} & $\begin{array}{l}0.139 \\
\end{array}$ & 14.643 & & 275.1. & $\mid$ & 49.1 & 11.0 & 41.5 & 2.864 & & 0.021 & 0.000 & 0.499 & 0.42 \\
\hline & | & $38 \mathrm{PM}$ & 25.543 & 24.363 & 25.008 & 24.792 & & 47.441 & 50.667 & 11.474 & 35.604 & -1.219 & & 53.052 & 0.138 & 14.643 & & 276.1 & 4.60194 & 49.2 & & & & & & & & 0.42 \\
\hline & $2 \frac{2 / 2 / 2004}{2 / 2 / 2004}$ & $\frac{38 \mathrm{PM}}{38}$ & & 24.342 & 24.977 & & & 47.2077 & $\begin{array}{l}50.312 \\
5\end{array}$ & $\mid 11.389$ & $\begin{array}{l}35.602 \\
307\end{array}$ & & & 48.922 & 0.131 & 14.643 & & & 4.61861 & 48.8 & & & & & & & .472 & \\
\hline & 2121212004 & 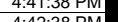 & & & $\frac{2.4911}{2.975}$ & & & 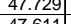 & ST1.044 & $\frac{11.506}{11.06}$ & $\frac{35.7 / 4}{32.74}$ & & & - 47.562 & (1) & 14.643 & & 288. & 4.63528 & 49.6 & & & & & & 001 & & \\
\hline & 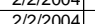 & 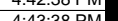 & 20.3400 & $\frac{24.525}{2.210}$ & 25.4750 & 24.197 & $\frac{2.368}{27217}$ & | & $50.010 \mathrm{O}$ & 1.1.455 & $\frac{35.1044}{25651}$ & -1.225 & & $\frac{5.002}{5.51}$ & 0.14 & & & (1) & 4.65194 & 49.4 & & & & & & & & \\
\hline & $2 / 2 / 20004$ & $8 \mathrm{PM}$ & 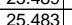 & 24,323 & 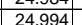 & 24,708 & 27.282 & 年 47.509 & $\frac{1.6044}{511}$ & $\frac{11.54}{11.548}$ & S & -1.252 & & 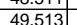 & & $\begin{array}{ll}1.46 \\
1146\end{array}$ & & 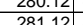 & 4.66801 & $\begin{array}{lll}4.2 \\
496\end{array}$ & & & 861 & & & & 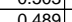 & \\
\hline 301 & $2 / 2 / 2004$ & $5: 38 \mathrm{PM}$ & 25.483 & 24.317 & 25.008 & 24.762 & 27.331 & 47.267 & 50.615 & 11.394 & 35.584 & $\frac{-1.222}{-1.22}$ & -6.487 & 43.912 & 0.142 & 14.643 & & 282.12 & \begin{tabular}{|c|}
4.70194 \\
\end{tabular} & 49.1 & $\frac{10.8}{10.8}$ & $\frac{01.4}{41.4}$ & 2.856 & 0.021 & 0.021 & 0.001 & 0.511 & 43 \\
\hline & $2 / 2 / 20$ & 4:46:38 PM & 25.461 & 24.291 & 24.981 & 24.761 & 27.349 & 47.308 & 50.783 & \begin{tabular}{|c|}
11.357 \\
\end{tabular} & 35.534 & $\begin{array}{l}-1.222 \\
\end{array}$ & & 50.234 & \begin{tabular}{|c|c|}
0.139 \\
\end{tabular} & 14.643 & & 283.12 & 4.71861 & 49.3 & 12.3 & & 2856 & 02 & 0.021 & 0.001 & & $\frac{0.45}{0.43}$ \\
\hline 30 & & B PM & 25.454 & 24.289 & 24.989 & 24.743 & 27.631 & 47.501 & 50.793 & $11.497 \mid$ & 35.715 & & & 48.697| & $\mid$ & 14.643 & & 284. & 4.73528 & 49.3 & 11.9 & & & & & & |.498 & \\
\hline 30 & 2/2/2004 & & & 24.282 & 25.012 & 24.706 & 27.694 & $47.398 \mid$ & 50.928| & 11.431 & 35.528| & -1.216 & & 47.662 & \begin{tabular}{|c|}
0.14 \\
\end{tabular} & 14.643 & & 285.1 & $\begin{array}{l}4.75194 \\
\end{array}$ & 49.5 & $\begin{array}{lll}11.7 \\
\end{array}$ & $\begin{array}{lll}41.5 \\
\end{array}$ & 8.859 & & 0.021 & 0.001 & \begin{tabular}{|c|}
0.503 \\
\end{tabular} & \\
\hline & & & 25.46 & & & & & & & & & & & 47.06 & 0.141 & 14.64 & & & & 49.1 & 11.5 & & & & & & & \\
\hline & $\frac{2 / 2 / 2004}{4}$ & BPM & $\begin{array}{l}25.473 \\
2\end{array}$ & 24.282 & \begin{tabular}{|c|}
24.878 \\
\end{tabular} & 24.812 & 27.699 & 47.053 & 50.592 & $\begin{array}{ll}11.392 \\
\end{array}$ & 35.309 & $\frac{-1.219}{-1.21}$ & & \begin{tabular}{|l|l|l}
48.115 \\
\end{tabular} & 0.137 & 14.6 & & 287.1 & \begin{tabular}{|l|}
4.78528 \\
\end{tabular} & 49.1 & $\begin{array}{ll}11.8 \\
\end{array}$ & & & & & & | & \\
\hline & & & & 24.27 & & & & & J0.5T & & 35.243 & -1.219 & & & 0.141 & 14.6 & & & & 40. & 11.7 & & & & & & & \\
\hline 30 & $2 / 2 / 2004$ & $8 \mathrm{PM}$ & 25.459 & 24.269 & 24.869 & 24.894 & 27.79 & 47.221 & 50.621 & 11.397 & 35.502 & -1.222 & & \begin{tabular}{|l|l|}
51.54 \\
\end{tabular} & 0.137 & 14.643 & & 289.12 & 4.81861 & 49.1 & 12.6 & $11.4 \quad-1$ & 2.852 & 020 & 0.021 & 0.000 & 0.496 & \\
\hline & 2/2/2/2004 & & 25.463 & 24.268 & \begin{tabular}{|l|l|l|}
2473 \\
\end{tabular} & 24.963 & 27.864 & 47.097 & 50.548 & 11.254 & 35.541 & -1.22 & & 51.772 & 0.142 & 14.6 & & 290.1 & & 49.1 & 12.7 & & 2.849 & & 0.02 & 0.001 & & 0.44 \\
\hline & 004 & & 25.458 & 24.267 & \begin{tabular}{|l|}
24.878 \\
\end{tabular} & 25.018 & 27.859 & 46.587 & .099 & 11.161 & 35.107 & -1.219 & & 51.517 & 0.14 & 14.6 & & 291.1 & & 48.6 & 12.6 & & & & & 501 & 0.513 & \\
\hline 311 & 2/2/2/2004 & & 25..452 & 24.267 & 24.882 & 24.982 & & 47.004 & 50.428 & 11.226 & & & & 53.816 & 0.142 & & & & 86861 & 49.0 & & & .846 & & & 001 & 1.515 & \\
\hline & $|2| 2121204$ & & 20.468 & 24.283 & 20.414 & 25.159| & 28.0 & 46.521 & 50.325 & 11.25 & 34.962 & -1.219 & & 4.516 & & 14.6 & & 293 & 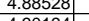 & 48.9 & 11.6 & & 000 & & & & 0.396 & \\
\hline & $\frac{21212044}{212004 \mid}$ & & $\frac{2.5434 \mid}{25239}$ & $\frac{24.289}{2.2079}$ & 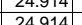 & & & & 50.283 & & & & & & & 14.6 & & & & 4.8 .8 & & & & & & & & \\
\hline & 2 & & $\frac{2.3459}{2545}$ & 24.275 & $\mid$\begin{tabular}{|c|}
24.314 \\
2493
\end{tabular} & 25216 & $\frac{2.1}{281}$ & & . & $\mid 11.521$. & & $\mid-1.219$ & & 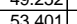 & | & $\frac{14.0}{116}$ & & $\frac{250}{296}$ & & (45.4 & $\frac{1.4 .1}{131}$ & & & & & & (0.54) & \\
\hline & 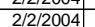 & & 25.467 & $\frac{24.250}{24.312}$ & \begin{tabular}{|}
$\mid 24.951$ \\
24.947 \\
\end{tabular} & 25.192 & & & .482 & $\mid \frac{1.1 .24}{11.31}$ & | & -1.222 & & $\frac{1.45}{50.5}$ & & $\frac{14.6}{146}$ & & & & $\begin{array}{l}4.5 .6 \\
490\end{array}$ & 势. & & & & & & & \\
\hline & $2 / 2 / 2004$ & 5:01:38 PM & 25.457 & 24.302 & 24.942 & 25.273 & $\frac{17.873}{27.873}$ & $\frac{17.234}{47}$ & $|50.725|$ & $\frac{11.407}{11.407}$ & 35.491 & -1.1222 & & $\frac{51.31}{5571}$ & 0 & 14.64 & & 298. & & $\frac{49.3}{49.3}$ & 12.6 & & & & & 01 & & \\
\hline & & & & & & & & & & & & & & & & & & & & & & & & & & & & \\
\hline & $1 / 2004$ & & 25.473 & 24.322 & 24.888 & 25.333 & & 46.778 & 211 & 11.297 & $35.14 !$ & -1.22 & & 47.748 & 0.139 & & & & & 48.7 & 11.7 & & & & & & |.508 & \\
\hline & $2 / 2 / 2004$ & & 25.463 & 24.323 & 24.833 & 25.339 & & 46 & \begin{tabular}{|l|l|}
50.339 \\
\end{tabular} & 11.251 & 35.1 & & & 48.2 & & & & & & 48.9 & 11.8 & & & & & & & \\
\hline & & & 25.428 & 24.308 & & & & 46.7 & & \begin{tabular}{|l|l|}
11.236 \\
\end{tabular} & & & & & & & & & & 48.9 & 3.1 & & & & & & & \\
\hline & 2/2/20004 & & 25.439 & 24.339 & \begin{tabular}{|l|}
24.839 \\
\end{tabular} & 25.154 & & & 163 & \begin{tabular}{|l|l|}
11.17 \\
\end{tabular} & & -1.219 & & & & & & & & 48.7 & 12.5 & & & & & & 0.539 & \\
\hline & 21212004 & & 25.435 & 24.34 & 24.84 & & & & & \begin{tabular}{|c|}
11.13 \\
\end{tabular} & & & & & & & & & & & & & & & & & & \\
\hline 324 & $2 / 2 / 2004$ & & 25.436 & 24.341 & 24.846 & 25.292 & 28.0 & 46.954 & \begin{tabular}{|l|}
50.519 \\
\end{tabular} & 11.111 & 35.5 & -1.219 & & 50.0 & 14 & & & & | & 49.0 & 12.3 & & & & & & 0.508 & \\
\hline & 21212004 & & 25.4443 & 24.358 & & & & & & 11.00 & & & & & & & & & & & . & & & & & & , & \\
\hline & $\frac{14212044}{21212004}$ & & $\frac{25.438}{22.38}$ & 24.363 & 24.858 & 25.3 & & & 110 & $10.98 \mathrm{r}$ & & & & & & & & & & 48.7 & 12.8 & & & & & & & \\
\hline & ZIZZZZOU4 & & 20.5435 & 24.508 21282 & & & & & & & & & & & & & & & & & 11.80 & & & & & & & \\
\hline & $2 / 212 / 20$ & 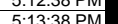 & (2.3.400 & $\frac{24.505}{24385}$ & & $\frac{25.2}{25}$ & & & & (10.900 & & $\frac{1.42}{-12}+2$ & & $\frac{4.1}{55^{2}}$ & & & & & & $\frac{4.0}{490}$ & 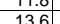 & & & & & & & \\
\hline & & & 25.45 & 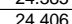 & 2487 & & & & $\frac{1}{4}$ & 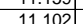 & & & & & & & & & & 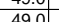 & & & & & & & & \\
\hline 31 & 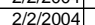 & & $\frac{25.442}{25.42}$ & 24.407 & 24.877 & 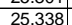 & 28.2 & 46.5 & $\frac{5.007}{50.207}$ & $\frac{1.1 .058}{11.058}$ & & $\frac{-1.222}{-1.22}$ & & & & $14.6 \mathrm{~T}-\mathrm{C}$ & & & | & 48.7 & 年. & & & & & & 0.538 & \\
\hline & & & & & & & & & & & & & & & & & & & & & & & & & & & & \\
\hline & $\frac{2 / 2 / 2 / 1}{20}$ & & 25.449 & 24.40 & $24.8 \mathrm{r}-\mathrm{l}$ & 25.365 & & & 50 & $\frac{11.095}{11.095}$ & & & & & & & & & & $48.8 \mathrm{~s} \quad \mathrm{~s}$ & 0.6 & & & & & & & \\
\hline & $\sqrt{2}$ & & 25.461 & 24.431 & 24.901 & & & 47.0 & & 11.136 & $\begin{array}{l}35.608 \\
35.608\end{array}$ & & & & & & & & & $\frac{49.1}{49.1}-2$ & 3.4 & & & & & & & \\
\hline & & & 25.466 & 24.426 & \begin{tabular}{|l|l|}
24.756 \\
\end{tabular} & 25.412 & 28.1 & 46.73 & & 10.976 & & -1.219 & & & & & & & & 48.9 & 11.9 & & & & & & 0.559 & \\
\hline 336 & $2 / 2 / 2004$ & $5: 20: 3$ & 25.45 & 24.449 & \begin{tabular}{|l|l|}
24.784 \\
\end{tabular} & 25.365 & 28.1 & 46.5 & 50.1 & 10.80 & & -1.225 & & 48.0 & & 14.6 & & & & 48. & 11.8 & 41 & & & & & 0.534 & \\
\hline & 2/2/2/2 & 5:21:3 & 25.444 & 24.459 & & & & 46.4 & & 10.7 & & & & & & & & & & 48. & $2.2 .2>$ & & & & & & & \\
\hline $338-2+2$ & 2/2/2 & & 25.436] & 24.4 & 24.791 & & & 46.7 & \begin{tabular}{|l|l|} 
& 50.611 \\
\end{tabular} & 10.909 & & & & & & & & & 5.31861 & 49. & 12.9 & & & & & & 0.496 & \\
\hline 200 & 2/2/2/2004] & & 25.411 & 24.451 & 24.77 & & & 46.7 & & 10.94 & & -1.222 & & & & & & & & 49.1 & 20.5 & & & & & & & \\
\hline 340 & & & 25.42 & 24.48 & 24.80 & 25.423 & 2.67 & & & 10.9 & & -1.222 & & & & & & & & 48.9 & & & & & & & & \\
\hline 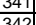 & 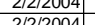 & & 25.4 & 24.488 & $\frac{24.7}{247}$ & $\frac{25.3}{252}>2>$ & $\frac{21}{37}$ & 46.90 & & 10.97 & 35.5 & -1.219 & & & & 14. & & & & 49.3 & 11.8 & 41. & 2.80 & & & & $\frac{5}{4}$ & \\
\hline 343 & 212120044 & & $\frac{2.4 .4}{25.3}-2>$ & $\frac{24.479}{24.84}$ & $\mid$ & $\frac{23.34}{2533} \mathrm{rat}$ & $\frac{2.1}{275}+2$ & (40.6465 & $\mid \begin{array}{ll}350.04 \\
504\end{array}$ & 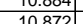 & 年3.0.6. & $\mid-\frac{1.25}{-1201}$ & & & & 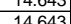 & & & & 49.2 & 泣. & & & & & & $\frac{1}{1}$ & \\
\hline 344 & 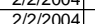 & & 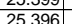 & 24.445 & & & & 46879 & 50.44 & & 35.50 & -1.21 & & & & & & & & 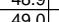 & 1. & & & & & & 5 & \\
\hline & & & $\frac{2.539}{25.39}$ & $\frac{2.4 .4}{24.4}-3$ & & & & $\frac{76.045}{47.045}$ & $\frac{5.548}{50.88}$ & | & 356 & -1.21 & & & & & & & & 494 & $\frac{1.34}{114}$ & & & & & & & \\
\hline & & & & & & & & & & 10.944 & & & & & & & & & & 490 & 119 & & & & & & & \\
\hline & $2 / 2 / 20$ & & 25401 & 24,497 & 24.816 & 25.172 & & 46.943 & & 1103 & & -1222 & & & & & & & & 492 & 112 & & & & & & & \\
\hline & & & 25391 | & 24.487 & 24811 & 25117 & & 46.57 & \begin{tabular}{|l|l|l|}
50213 \\
\end{tabular} & 10.913 & 35.38. & & & & & & & & & 48.7 & 114 & & & & & & & \\
\hline & & & & & & & & & & & & & & $10.5,5>$ & & & & & & & 2 & & & & & & & \\
\hline 350 & 2/2/2004 & 5:34:38 PM & 25.371 & 24.491 & 24.826 & 25.066 & 27.085 & 46.966 & \begin{tabular}{|l|l|}
50.872 \\
\end{tabular} & $\mid 11.348$ & 35.317 & -1.219 & & 47.9 & 0.171 & 14.643 & & 11.12 & 5.51861 & 49.4 & 111.7 & $41.1]$ & $2.837 \mid$ & 0.025 & 0.026 & 0.001 & 0.623 & \\
\hline
\end{tabular}


WSRC-TR-2005-00105, REVISION 0

SRNL-RPP-2005-00012, REVISION 0

RUN \# WASH1; CAMPAIGN I SLURRY WASH 1 - CONT.

\begin{tabular}{|c|c|c|c|c|c|c|c|c|c|c|c|c|c|c|c|c|c|c|c|c|c|c|c|c|c|c|c|c|}
\hline & & & & & & & & & & & & & & & & & & & & & & & & & & & & \\
\hline & A & $\mathrm{B}$ & $\mathrm{D}$ & $\mathrm{E}$ & $F$ & $G$ & $\mathrm{H}$ & $\mathrm{J}$ & $\mathrm{K}$ & \begin{tabular}{ll|}
$\mathrm{L}$ \\
\end{tabular} & $M$ & $\mathrm{~N}$ & 0 & $\mathrm{Q}$ & $\begin{array}{ll}R \\
\end{array}$ & $\mathrm{~s}$ & $T$ & $\mathrm{v}$ & w & $x$ & $\mathrm{Y}$ & $z$ & AA & $\mathrm{AB}$ & $\mathrm{AC}$ & $\mathrm{AD}$ & $\mathrm{AE}$ & AF $A$ \\
\hline & $2 / 212004$ & 5:35:38 PM & 25.379 & 24.469 & \begin{tabular}{|l|l|}
24.719 \\
\end{tabular} & 24.984 & 27.188 & 46.448 & 50.277 & 10.938 & 35.19 & -1.222 & $\begin{array}{l}-6.068 \\
-6.0\end{array}$ & 53.63 & 0.142 & 14.643 & 0.003 & 332.1 & .53528 & 48.8 & 13.1 & & & & 0.021 & 0.001 & 0.523 & 0.44 \\
\hline & $2 / 212004$ & 5:36:38 PM & & 24.474 & \begin{tabular}{|l|l|}
24.713 \\
\end{tabular} & \begin{tabular}{|l|l|}
24.958 \\
\end{tabular} & 27.063 & & \begin{tabular}{|r|}
50.49 \\
\end{tabular} & 10.934 & & & $\begin{array}{r}-5.724 \\
\end{array}$ & 48.499 & 0.159 & 14.643 & 0.003 & & $\begin{array}{l}5.55194 \\
\end{array}$ & 49.0 & \begin{tabular}{|l|}
11.9 \\
\end{tabular} & & & & 0.024 & & 0.584 & 0.50 \\
\hline & 2/2/2004 & $5: 37: 38 \mathrm{PM}$ & 25.368 & 24.468 & \begin{tabular}{|l|l|}
24.723 \\
\end{tabular} & \begin{tabular}{|l|}
24.923 \\
\end{tabular} & 27.201 & 46.776 & 50.503 & \begin{tabular}{ll|}
10.863 \\
\end{tabular} & 35.493 & -1.219 & -6.184 & 45.068 & 0.142 & 14.643 & 0.004 & 334.12 & \begin{tabular}{|l|}
5.56861 \\
\end{tabular} & 49.0 & \begin{tabular}{|l|l|}
11.0 \\
\end{tabular} & 41.1 & \begin{tabular}{|l|}
2.836 \\
\end{tabular} & & 0.021 & 0.001 & 0.519 & 0.44 \\
\hline$\frac{354}{355}$ & $\frac{2 / 2 / 20044}{2 / 2 / 2004}$ & $\begin{array}{l}5: 38: 38 \mathrm{PM} \\
5: 39: 38 \mathrm{PM}\end{array}$ & $\begin{array}{l}25.356 \\
25.341\end{array}$ & $\begin{array}{l}24.466 \\
24.456\end{array}$ & \begin{tabular}{|l|}
24.726 \\
24.705 \\
\end{tabular} & \begin{tabular}{|l|}
24.861 \\
24.795 \\
\end{tabular} & $\frac{27.379}{27.204}$ & $\begin{array}{l}46.854 \\
46.549\end{array}$ & \begin{tabular}{|l|}
50.463 \\
50.236 \\
\end{tabular} & $\begin{array}{l}\frac{10.961}{10.787} \\
\end{array}$ & $\begin{array}{l}35.532 \\
35.395 \\
\end{array}$ & $\begin{array}{l}-1.222 \\
-1.225 \\
\end{array}$ & $\begin{array}{l}-6.354 \\
-6.288 \\
-6.5\end{array}$ & $\begin{array}{l}50.236 \\
46513\end{array}$ & $\begin{array}{l}0.145 \\
0.147\end{array}$ & $\begin{array}{l}14.643 \\
14.643 \\
\end{array}$ & \begin{tabular}{|l}
0.003 \\
0.003
\end{tabular} & $\begin{array}{l}335.12 \\
336.12 \\
\end{array}$ & \begin{tabular}{|l|l|}
5.58528 \\
560194 \\
\end{tabular} & $\begin{array}{l}49.0 \\
448.8\end{array}$ & \begin{tabular}{|l|l|}
12.3 \\
114
\end{tabular} & $\begin{array}{ll}41.2 \\
41.2\end{array}$ & \begin{tabular}{|l|l|}
2.840 \\
2825
\end{tabular} & \begin{tabular}{|l|}
0.02 \\
0.02
\end{tabular} & \begin{tabular}{|l|l|}
0.022 \\
0022
\end{tabular} & 0.001 & $\begin{array}{l}0.529 \\
0.539 \\
\end{array}$ & $\begin{array}{l}0.45 \\
0.46\end{array}$ \\
\hline 356 & $2 / 2 / 212004$ & $5: 40: 38$ PM & 25.329 & 24.430 & & & & & & & & & & & & & & & & & & & & & & & $\begin{array}{l}0.539 \\
0.553\end{array}$ & $\begin{array}{l}0.46 \\
0.47\end{array}$ \\
\hline & $2 / 2 / 21 / 2004$ & & & 24.444 & \begin{tabular}{|l|}
24.724 \\
\end{tabular} & \begin{tabular}{|l|}
24.473 \\
\end{tabular} & 27.332 & 46.985 & \begin{tabular}{|l|}
50.449 \\
\end{tabular} & 10.905 & 35.692 & -1.219 & $\begin{array}{c}-5.779 \\
\end{array}$ & $\begin{array}{l}5.1 .952 \\
4\end{array}$ & 0.108 & 14.643 & 0.00 & & & 49.0 & 11.3 & & & 0.016 & 0.016 & & & $\frac{0.47}{0.33}$ \\
\hline & $2 / 2 / 2004$ & 5:42:38 PM & 25.313 & 24.433 & 24.723 & 24.471 & 27.44 & 47.047 & \begin{tabular}{|l|}
50.671 \\
\end{tabular} & 11.006 & 35.536 & -1.222 & -6.123 & 43.791 & 0.141 & 14.643 & 0.00 & 339.12 & 5.65194 & 49.2 & 10.7 & & $\begin{array}{l}2.847 \\
\end{array}$ & 0.02 & 0.021 & 0.001 & 0.513 & $\frac{0.33}{0.44}$ \\
\hline & $2 / 2 / 2004$ & & & & & & & & & & & & & & & & & & & & & & & & & & & \\
\hline 360 & & & & 24.401 & 24.77. & 24.5 & & & 50.654 & 10.979 & 35.801 & & & & 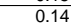 & 10 & & & & 40.0 & 118 & & & & & & & $\frac{0.47}{0.43}$ \\
\hline 361 & $2 / 2 / 2004$ & 5:45:38 PM & & 24.39 & 24.821 & 24.429 & & 46.96 & 50.495 & & & & & & 0.149 & $\frac{643}{643}$ & & & 5.7.70194 & 49.0 & 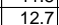 & & $\frac{2.847}{2.847}$ & & & $\frac{0.00}{0.00}$ & $\frac{.541}{0.541}$ & $\frac{0.43}{0.46}$ \\
\hline & $2 / 2 / 2004$ & 5:46:38 PM & & 24.38 & & 24.399 & & & & 11.065 & & & & 51.354 & 0.148 & & & & & 180 & & & & & & & & \\
\hline 363 & $2 / 2 / 2004$ & 5:47:38 PM & 25.325 & 24.37 & 24.695 & 24.398 & 27.139 & 46.593 & \begin{tabular}{|l|l|}
50.242 \\
\end{tabular} & 10.782 & 35.43 & -1.228 & & 49.5 & 0.145 & 14.643 & & 344 & 5.73528 & 48.8 & 12.1 & 41.0 & 2.828 & .02 & 0.022 & 0.001 & 0.532 & 0.45 \\
\hline 364 & $2 / 2 / 2004$ & 5:48:38 PM & 25.32 & 24.35 & 24.785 & 24.454 & 27.1 & 46.452 & 50.049 & 10.792 & 35.339 & -1.219 & -6 & 48.159 & 0.134 & 14.643 & & 345 & 5.75194 & 48.6 & 11.8 & & 2.82 & & & 0.00 & 0.491 & 0.42 \\
\hline 365 & $2 / 2 / 2004$ & 5:49:38 PM & 25.312 & 24.337 & 24.812 & 24.486 & 27.2 & 46.325 & $\begin{array}{l}49.997 \\
\end{array}$ & 10.715 & 35.333 & -1.219 & & 49.755 & 0.143 & $\begin{array}{l}14.643 \\
\end{array}$ & & 346. & 5.76861 & 48.5 & 12. & & & & $\overline{0.021}$ & 0.00 & 0.525 & 0.45 \\
\hline 366 & 2/2/2004 & 5:50:38 PM & 25.319 & 24.324 & 24.834 & 24.573 & & 46.34 & 50.128 & 10.624 & 35.337 & & & & 0.153 & 14.643 & & 347 & 88528 & 48.7 & 12 & & & & & & & \\
\hline 367] & 2/2/212004 & 5:51:38 PM & & & & & & & & & & & & & & & & & & & & & & & & & & \\
\hline & $\frac{2 / 212004}{2121204}$ & 5:52:38 PM & $\begin{array}{l}25.36 \\
5.200\end{array}$ & 24.335 & 24.905 & 24.779 & & 46.659 & $\begin{array}{r}50.37 \\
-5.006\end{array}$ & 10.741 & $\frac{35.528}{3.52}$ & & & 8.086 & 0.14 & 14.643 & & 349 & 5.81861 & 48.9 & 11.8 & & & & & & & 0.43 \\
\hline 年 & $\frac{2 \mid 2 / 2004}{2 / 2 / 2004}$ & $\begin{array}{l}5.53: 38 \mathrm{PM} \\
5.54 \cdot 38 \mathrm{PM}\end{array}$ & $\begin{aligned} 25.388 \\
25396\end{aligned}$ & $\frac{24.333}{24316}$ & $\begin{array}{l}24.964 \\
24036\end{array}$ & 24.848 & 217.416 & $\frac{44.251}{46.317}$ & $\begin{array}{l}50.406 \\
55126\end{array}$ & 10.1899 & $\begin{array}{l}36.158 \\
35305\end{array}$ & 年.1.219 & & 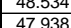 & $\begin{array}{l}0.193 \\
0.116\end{array}$ & $\frac{14.643}{14643}$ & & & 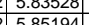 & $\begin{array}{ll}48.9 \\
1487\end{array}$ & $\frac{11.9}{117}$ & 408 & 2814 & (1) & - & 0.001 & 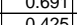 & 0 \\
\hline & 2 & $\begin{array}{l}5.54 .50 \mathrm{PM} \\
5.55: 38 \mathrm{PM}\end{array}$ & 25.43 & $\frac{24.010}{24.325}$ & $\begin{array}{l}24.450 \\
24.99\end{array}$ & $\begin{aligned} 24.020 \\
24.86\end{aligned}$ & 27.072 & $\begin{array}{l}40.011 \\
47.001\end{array}$ & 50.1529 & $\begin{array}{l}10.004 \\
10.666\end{array}$ & $\begin{array}{l}35.005 \\
36.058\end{array}$ & $\begin{array}{l}-1.220 \\
-1.222\end{array}$ & $\begin{array}{l}-3.100 \\
-6.299 \\
-6\end{array}$ & $\frac{41.950}{45.332}$ & 0.1152 & $\frac{14.045}{14.643}$ & & $\frac{351.14}{352.12}$ & $\begin{array}{l}5.05144 \\
5 \\
5.86861\end{array}$ & 48.19 & $\frac{1.1 .1}{11.1}$ & $\frac{4.0}{415}$ & $\frac{2.014}{2863}$ & & & 0.001 & 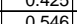 & 0.36 \\
\hline 372 & $2 / 2 / 2004$ & 5:56:38 PM & 25.468 & 24.328 & 25.034 & 24.878 & 27.795 & 47.051 & 50.439 & 10.783 & 35.924 & $\frac{-1.222}{-1.222}$ & -6.291 & 51.073 & 0.148 & 14.643 & & 3531 & 588528 & 490 & 125 & 415 & 2860 & & 0,22 & 0001 & 0.531 & 0.46 \\
\hline & $2 / 2 / 2004$ & 5:57:38 PM & 25.488 & 24.323 & 25.043 & & & & 50.339 & & & & & & 0.148 & 14.643 & & & 5.90194 & & & & & & & & & \\
\hline [374 & $2 / 2 / 2004$ & 5:58:38 PM & 25.513 & 24.323 & 25.083 & 24.973 & 27.923 & 46.945 & 50.464 & 10797 & 35.156 & - & & $\frac{1.5058}{44.528}$ & 0.152 & 14643 & & $\frac{354}{355}$ & 5.591861 & 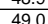 & $\frac{15}{119}$ & 41. & $\frac{2.045}{2853}$ & & 0.023 & 0.000 & 0.546 & 046 \\
\hline & & & & & & & & & & & & & & & 0.143 & 14.643 & & & & & & & & & & & & \\
\hline \begin{tabular}{|c|c|}
376 \\
\end{tabular} & $2 / 2 / 2004$ & 5:59:57 PM & 25.558 & 24.338 & \begin{tabular}{|l|}
25.128 \\
\end{tabular} & 24.998 & & 46.678 & \begin{tabular}{|r|}
50.25 \\
\end{tabular} & 10.918 & 35.378 & & & 47.681 & 0149 & 14.643 & & 356. & 5.940 & & & & & & & & & \\
\hline & $2 / 2 / 2004$ & 6:00:57 PM & & 24.348 & 25.148 & 25.088 & & 47.12 & 50.826 & 11.092 & 35.53 & & & & 0.15 & 14.643 & & 357.4 & 5.95 & 49.4 & & & 2.84 & & & & & \\
\hline 378 & $2 / 2 / 2004$ & 6:01:57 PM & 25.588 & 24.348 & 24.994 & 25.099 & & 46.299 & 49.925 & 735 & 35.185 & & & 7.877 & 0.142 & 14.643 & & 358.43 & & 48.5 & 11.7 & & & & & & & 0.44 \\
\hline$[379$ & $\mid$ & 6:02:57 PM & 25.588 & 24.348 & 25.039 & 25.089 & 27.48 & $\begin{array}{l}46.325 \\
\end{array}$ & 50.018 & 10.673 & 35.266 & -1.222 & & 44.427 & 0.152 & 14.643 & & 359.4 & 5.99056 & 48.5 & 10.9 & 40.8 & 2.81 & 0 & 0.023 & 0.001 & 0.555 & $\overline{0.47}$ \\
\hline sou & $\mid$ & $7 \mathrm{PM}$ & 25.573 & 24.348 & 25.049 & 25.069 & & 46.392 & 50.018 & 10.71 & & -1.219 & & 促 & 0.156 & 14.643 & & & 6.00 & 48. & & 40.9 & & & & & 0.568 & \\
\hline $\mid 381$ & 2/2/2/2004 & 6:04:57 PM & 25.59 & 24.365 & 25.09 & & & 46.243 & 50.01 & 10.709 & 35.165 & & & 48.88 & 0.149 & 14.643 & & & 6.02 & 48.5 & & & 2.806 & & & 0.001 & 0.544 & \\
\hline & 2 & $6: 05: 57 \mathrm{PM}$ & 25.59 & & & & & & 50.111 & 10.13 & 35.241 & & & 49.011 & 0.148 & & & & & & & & $m$ & & & & & \\
\hline $\begin{array}{ll}383 \\
384\end{array}$ & $\frac{21212004}{21204}$ & $6: 00.57 \mathrm{PM}$ & 25.606 & 24.360 & & & & & & & & $\frac{-1.222}{1.120}$ & & 49.84 & $\begin{array}{l}0.154 \\
0147\end{array}$ & & & & $6.05 / 22$ & & & & & & & & & \\
\hline 385 & $\begin{array}{l}2 / 1 / 20044 \\
2 \mid 2 / 2004\end{array}$ & 0.060 .50 & $\frac{23.010}{25643}$ & 24.301 & \begin{tabular}{|l}
2.12121 \\
25.52
\end{tabular} & $\frac{23.001}{25198}$ & 告 & $\begin{array}{l}40.304 \\
4634\end{array}$ & $\begin{array}{l}50.23 \\
50.194\end{array}$ & 10.1546 & $\frac{35.252}{25268}$ & $-\frac{1.129}{-120}$ & & 267 & $0.14 t$ & $\frac{14.045}{11643}$ & & & 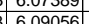 & 4.6 & $\frac{1.4}{126}$ & 408 & $\frac{2.01}{3.81}$ & & & 00 & 0 & 0.46 \\
\hline 386 & $\frac{21 / 1 / 20044}{21 / 2004}$ & $6.00 .5 .57 \mathrm{PM}$ & $\begin{array}{l}23.045 \\
25679\end{array}$ & $\begin{array}{l}24.300 \\
24379\end{array}$ & 20.135 & $\frac{2.150}{2528}$ & & $\begin{array}{l}46.354 \\
46.255\end{array}$ & \begin{tabular}{|l|l|l|}
350.451 \\
\end{tabular} & & 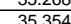 & $\mid-1.419$ & & & 0.103 & & & & 6.0.07072 & $\frac{46.1}{490}$ & $\begin{array}{ll}\frac{1.0}{117} \\
17\end{array}$ & $\begin{array}{l}4.0 .0 \\
408\end{array}$ & & 011 & . & 0.000 & $=0.35$ & \\
\hline & $2 / 2 / 21 / 2004$ & 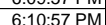 & & 24.396 & 25.196 & 25.326 & & $\frac{1.6 .55}{46.45}$ & \begin{tabular}{|l|l|}
50.134 \\
\end{tabular} & & & & & & & & & & & 40.0 & & & & & & & & \\
\hline 388 & $2 / 2 / 2004$ & 6:11:57 PM & 25.708 & 24.398 & \begin{tabular}{|l|}
25218 \\
\end{tabular} & 25.308 & & 46.7 & 50.333 & 10639 & 35766 & & & 0.497 & 0145 & 14 & & 3684 & 6614056 & 年 & & & 284 & & & non & 0.521 & 0 \\
\hline & 2004 & & & & & 25.314 & & & & & & & & & & & & & & & & & & & & & & \\
\hline 390 & $2 / 212004$ & $6 \cdot 13 \cdot 5$ & 25735 & 24,411 & 25241 & 25.336 & 27.9 & 46.9 & \begin{tabular}{|l|l|}
50.584 \\
\end{tabular} & 10783 & 35.735 & -122 & & 48.603 & 0.165 & & & 3704 & 6 6.17389 & 49.1 & 119 & 413 & 2849 & 1802 & 0.024 & 0.001 & 0.591 & 50 \\
\hline & $2 / 2 / 2004$ & 6:14:5 & 25.737 & 24.412 & \begin{tabular}{|l|}
25.247 \\
\end{tabular} & 25.373 & & & & & 35.4 & -1.219 & & & 0.151 & & & 371. & & 48 & 12. & & & & & 0.0 & 0.545 & 0.46 \\
\hline 392 & $2 / 2 / 2004$ & 6:15:57 PM & $\begin{array}{l}25.773 \\
\end{array}$ & 24.423 & \begin{tabular}{|l|}
25.169 \\
\end{tabular} & 25.389 & 27.9 & & 50. & 10.7 & 35.2 & & & 50.535 & & 14. & & 372 & & 48.7 & 12.4 & 40.8 & \begin{tabular}{|l|}
2.81 \\
\end{tabular} & & & & 0.345 & 0.29 \\
\hline [393] & 2/2/2004] & 6:16:57 PM & 25.755 & 24.431 & & 25.301 & & & & 10.524 & & -1.219 & & 47.967 & 0.159 & 14.6 & & 373. & 389 & 48.4 & 11.8 & & & & 0.024 & & 0.577 & \\
\hline 394] & $\mid 2004$ & 6:17:57 PM & 25.753 & 24.444 & 25.194 & 25.349 & & 46.576 & 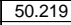 & 10.57 & 35.612 & -1.219 & & 47 & 0.15 & & & & & 48. & 11. & 41. & & & & & 0.541 & \\
\hline & 004 & & 25.736 & 24.451 & 25.197 & 25.3 & & & & & & & & & & & & $37 \mathrm{C}$ & & & & & & & & & & \\
\hline 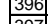 & $2 / 2$ & $6: 19: 5$ & 25.748 & 24.459 & 25.209 & 25.444 & & 46 & 49.92 & & 3 & -1.222 & & 45. & & & & & 6.27 & & & & & & & & & \\
\hline & $\frac{2 / 212004}{212004}$ & $6: 20: 57 \mathrm{PM}$ & $\frac{25.161}{25763}$ & 24.461 & 25.217 & $2 \frac{2.5382}{2524}$ & & 告6.415.41 & $\begin{array}{l}50.101 \\
50.107\end{array}$ & 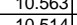 & & 12 & & & & 14.6 & & & & 48.6 & 11. & & & & & & 0.554 & 0.41 \\
\hline 390 & 4 & $\frac{0.2 .57}{6.257}$ & $\frac{2.1035}{2575}$ & 24.474 & 2.254 & $\frac{25.254}{25165}$ & & $\begin{array}{r}40.41 \\
16419\end{array}$ & \begin{tabular}{|l|l|}
50200 \\
\end{tabular} & $\mid$ & $\frac{35.526}{25402}$ & - & & $\frac{40.193}{4878}$ & $\begin{array}{l}0.135 \\
0.151\end{array}$ & $\frac{14.045}{14643}$ & & 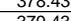 & | & 40.0 & $\frac{1.5}{120}$ & 41.0 & & & & 0.00 & 0.50 & \\
\hline & $\frac{21 / 21 / 2004}{21 / 2004}$ & 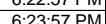 & 25.777 & $\frac{24.455}{24,777}$ & & $\frac{23.105}{25122}$ & & $\frac{40.419}{46301}$ & \begin{tabular}{|l|l|l|l|}
50.664 \\
\end{tabular} & & 35.403 & & & 40.10 & & & & & & & & & & & & & & $0.4 \mathrm{f}$ \\
\hline 401 & 12004 & 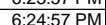 & 25.772 & 24,467 & 25.238 & 25.097 & & $\frac{76.519}{46.419}$ & \begin{tabular}{|l|l|l|}
50.173 \\
\end{tabular} & $\frac{10.654}{10.62}$ & . & & & 45.6 & & & & & & & & & & & & & $\frac{0.528}{0.528}$ & \\
\hline 402 & 12004 & & 25.788 & & & 25.068 & & & & & & & & & & & & & & & & & & & & & & \\
\hline 403 & |2004 & $6.26 .57 \mathrm{~F}$ & 25.793 & 24.469 & 25.269 & 25.019 & & & 50 & 10.642 & & & & & & & & & & & $\frac{10.4}{114}$ & & & & & & 87 & \\
\hline 404 & $\mid 2004$ & & 25.814 & 24.464 & 25.28 & 24.949 & & 46. & 50. & 10.8 & & & & 470 & 0.154 & 14 & & 384 & & 48.9 & 11.5 & & & & & & & \\
\hline (405 & $2 / 2 / 2004$ & $6: 28: 57 \mathrm{PM}$ & 25.8 & 24.474 & \begin{tabular}{|l|}
25.155 \\
\end{tabular} & 24.934 & & 46.2 & \begin{tabular}{|l|l|}
49.8 \\
\end{tabular} & & 35.2 & & & & 0.1 & 14.6 & & 385 & 6 & 48.4 & & 40.7 & & & & & & \\
\hline |406 & $2 / 2 / 2004$ & $6: 29: 57 \mathrm{~F}$ & 25.785 & 24.45 & 25.17 & 24.87 & & 46.209 & \begin{tabular}{|l|}
49.908 \\
\end{tabular} & 10.456 & 35.3 & 222 & & 505 & 0.1 & 14.6 & & 386 & 6.44 & 48.4 & 12.4 & 40.8 & & 0.024 & & 00 & $\begin{array}{l}0.575 \\
\end{array}$ & 0.49 \\
\hline 407 & $2 / 2 / 2004$ & $6: 30: 57 \mathrm{PM}$ & 25.759 & 24.454 & 25.18 & 24.874 & 27. & 46.278 & 50.1 & 10.4 & 35.415 & -1.219 & -5.5 & 46.2 & 0.151 & & & 387 & & 48. & 11.3 & & & & & 86 & \begin{tabular}{|l|l|l|}
0.548 \\
\end{tabular} & 0.47 \\
\hline 4008 & 2/2/2004 & $6: 31: 5$ & 25.763 & 24.449 & \begin{tabular}{|l|l|} 
& 25.189 \\
\end{tabular} & 24.893 & & 46.2 & & 10.48 & 35.305 & -1.216 & & 45.8 & & & & 388 & & 48.6 & 11. & 40. & & & 0.022 & 0.001 & 0.549 & 0.47 \\
\hline & $2 / 21212004$ & 6:32:57 F & 25.758 & 24.438 & 25.198 & 24. & & & 50.078 & 10 & 5.214 & 219 & & & & & & & & & & & & & & & 0.557 & \\
\hline & & & & & & & & & & & & & & & & & & & & & & & & & & & 0.536 & \\
\hline
\end{tabular}


WSRC-TR-2005-00105, REVISION 0 SRNL-RPP-2005-00012, REVISION 0

RUN \# WASH1; CAMPAIGN I SLURRY WASH 1 - CONT.

\begin{tabular}{|c|c|c|c|c|c|c|c|c|c|c|c|c|c|c|c|c|c|c|c|c|c|c|c|c|c|c|c|c|}
\hline & & & & & & & \\
\hline & $\mathrm{A}$ & B & D & $E$ & $\mathrm{~F}$ & $\mathrm{G}$ & \begin{tabular}{ll|}
$\mathrm{H}$ \\
\end{tabular} & $\mathrm{J}$ & $\mathrm{K}$ & $\mathrm{L}$ & M & $\mathrm{N}$ & 0 & \begin{tabular}{l|l} 
\\
\end{tabular} & \begin{tabular}{l|l|}
$R$ \\
\end{tabular} & $\begin{array}{ll} \\
\end{array}$ & $\mathrm{T}$ & $\mathrm{V}$ & $\mathrm{W}$ & $x$ & $\begin{array}{r}Y \\
Y\end{array}$ & $z$ & $\mathrm{AA}$ & $A B$ & $\mathrm{AC}$ & $A D$ & $\mathrm{AE}$ & $\mathrm{AF} \quad \mathrm{A}$ \\
\hline \begin{tabular}{|l|}
411 \\
\end{tabular} & $\frac{2 / 2 / 2004}{2 / 2200}$ & 6:34:57 PM & 25.76 & 24.431 & \begin{tabular}{|l|}
25.201 \\
\end{tabular} & \begin{tabular}{|l|l}
24.77 \\
\end{tabular} & $\begin{array}{l}27.194 \\
7752\end{array}$ & 45.962 & \begin{tabular}{|l|l|}
49.838 \\
\end{tabular} & 10.458 & $\begin{array}{r}35.12 \\
3.14\end{array}$ & \begin{tabular}{|l|l|}
-1.219 \\
\end{tabular} & $\begin{array}{l}-5.947 \\
-9.09\end{array}$ & \begin{tabular}{|l|l|}
48.949 \\
\end{tabular} & 0.142 & \begin{tabular}{|l|l|}
2 & 14.643 \\
\end{tabular} & 0.004 & 391.43 & 6.52389 & 48.4 & $\frac{12.0}{12.0}$ & \begin{tabular}{|l|l}
40.5 \\
\end{tabular} & 2.795 & 0.021 & 0.021 & 0.001 & 0.519 & 0.44 \\
\hline $\mid$ & $\frac{2 / 2 / 2004}{2 / 2 / 2004}$ & $6: 35: 57 \mathrm{PM}$ & $\frac{25.764}{25751}$ & $\frac{24.424}{24.401}$ & $\begin{array}{r}25.21 \\
25.182\end{array}$ & $\begin{aligned} \frac{24.713}{24.63} \\
24\end{aligned}$ & $\frac{27.153}{26861}$ & $\begin{array}{r}45.84 \\
45.936\end{array}$ & \begin{tabular}{|l|l|}
49.777 \\
4935
\end{tabular} & \begin{tabular}{|l|l|}
10.414 \\
10.338
\end{tabular} & \begin{tabular}{|c|}
35.071 \\
352277
\end{tabular} & $\frac{-1.222}{-1225}$ & $\begin{array}{l}-5.953 \\
6.034\end{array}$ & \begin{tabular}{|l|l|}
47.969 \\
52875
\end{tabular} & $\begin{array}{l}0.151 \\
0.155 \\
\end{array}$ & $\begin{array}{ll}1 & 14.643 \\
5 & 14664\end{array}$ & $\begin{array}{r}0.003 \\
\end{array}$ & $\begin{array}{r}392.43 \\
393.43\end{array}$ & \begin{tabular}{|c|}
6.54056 \\
6.5572
\end{tabular} & 48.3 & $\frac{11 .}{13}$ & 40.5 & 2.789 & & 0.022 & 0.001 & 0.553 & $\frac{0.47}{0.48}$ \\
\hline 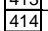 & $\frac{1 / 2 / 2004}{2 / 2004}$ & 6:37:57 PM & $\frac{25.171}{25.773}$ & $\begin{array}{l}24.401 \\
24.403\end{array}$ & 25.218 & $\frac{24.00}{24.612}$ & $\begin{array}{l}20.001 \\
26.693\end{array}$ & $\begin{array}{l}45.900 \\
46.062\end{array}$ & $\mid$ & $\begin{array}{l}10.300 \\
10.468\end{array}$ & $\mid$\begin{tabular}{|l|}
35.451 \\
35.198
\end{tabular} & $\begin{array}{l}\frac{1.220}{1.219} \\
\end{array}$ & $\begin{array}{l}-0.074 \\
-5.961\end{array}$ & $\begin{array}{l}32.075 \\
48.693\end{array}$ & $\begin{array}{l}.163 \\
0.163\end{array}$ & $\begin{array}{l}\frac{14.045}{14.643} \\
\end{array}$ & 0.003 & $\begin{array}{l}39.45 \\
394.43 \\
\end{array}$ & $\frac{0.05129}{6.57389}$ & $\frac{48.4}{48.6}$ & & & & & $\begin{array}{l}0.023 \\
0.024\end{array}$ & & $\begin{array}{l}0.500 \\
0.594 \\
0.59\end{array}$ & \\
\hline 415 & $2 / 2 / 2004$ & 6:38:57 PM & 25.773 & 24.393 & 25.208 & \begin{tabular}{|l|l|}
24.566 \\
\end{tabular} & 26.822 & 46.159 & 49.93 & 10.428 & 35.343 & \begin{tabular}{|l|}
-1.219 \\
\end{tabular} & $\begin{array}{l}-5.9 \\
-5.3 \\
-1\end{array}$ & 50.050 & $\begin{array}{l}.109 \\
0.092 \\
\end{array}$ & $\begin{array}{l}14.045 \\
14.643\end{array}$ & & $\begin{array}{l}3945.45 \\
395.43\end{array}$ & 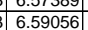 & $\begin{array}{l}40.0 \\
48.5\end{array}$ & & 40 & & & 0.014 & & 0.0 .335 & 0.5 \\
\hline 416 & $2 / 2 / 2004$ & 6:39:57 PM & 25.783 & 24.393 & 25.239 & \begin{tabular}{|l|}
24.577 \\
\end{tabular} & 26.828 & 46.354 & & & 35.454 & \begin{tabular}{|l|}
-1.216 \\
\end{tabular} & & 49.938 & 0 & & & $\frac{35.45}{396.43}$ & & 48.6 & $\frac{12 .}{12.2}$ & $\frac{4.0}{40.9}$ & & & & & & 0.48 \\
\hline \begin{tabular}{|l|}
417 \\
\end{tabular} & $2 / 2 / 2004$ & 6:40:57 PM & 25.784 & 24.369 & \begin{tabular}{|r|}
25.22 \\
\end{tabular} & \begin{tabular}{|l|}
24.568 \\
\end{tabular} & 26.988 & 46.174 & 49.976 & 10.473 & 35.337 & \begin{tabular}{|c|}
-1.219 \\
\end{tabular} & -5.86 & 49.794 & 0.15 & 14.643 & & 397.43 & 6.62389 & 48.5 & 12.2 & 40.8 & 2.810 & 0.022 & & & 0.545 & 0.46 \\
\hline 418 & $2 / 2 / 2004$ & 6:41:57 PM & 25.79 & 24.36 & \begin{tabular}{|l|}
25.236 \\
\end{tabular} & $\begin{array}{r}24.62 \\
\end{array}$ & 27.114 & 46.439 & 49.979 & 10.445 & 35.602 & 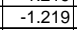 & -5.828 & 48.23 & 0.174 & \begin{tabular}{|l|l|}
4 & 14.643 \\
\end{tabular} & 0.004 & 398.43 & $\mid 6.64056$ & 48.5 & 11.8 & 41.0 & 2.828 & \begin{tabular}{|l|l|}
8 & 0.026 \\
\end{tabular} & 0.026 & 0.001 & 0.628 & 0.53 \\
\hline \begin{tabular}{|l|}
419 \\
\end{tabular} & $2 / 2 / 2004$ & & 25.793 & 24.353 & \begin{tabular}{|l|}
25.243 \\
\end{tabular} & \begin{tabular}{|l|}
24.712 \\
\end{tabular} & & & 50.18 & 10.566 & & \begin{tabular}{|l|}
-1.222 \\
\end{tabular} & & & & & & 399.43 & & 48.7 & & 40.9 & \begin{tabular}{|l|l|}
9 & 2.818 \\
\end{tabular} & & 0.025 & & 0.616 & \\
\hline \begin{tabular}{|l|}
420 \\
\end{tabular} & $2 / 2 / 2004$ & 6:43:57 PM & 25.81 & 24.35 & 25.26 & \begin{tabular}{|l|l|}
24.749 \\
\end{tabular} & 26.471 & 46.551 & 50.291 & 10.672 & 35.475 & \begin{tabular}{|c|}
-1.222 \\
\end{tabular} & -5.906 & 47.316 & 0.154 & 14.643 & 0.003 & 400.43 & 6.67389 & 48.8 & 11.6 & & 2.828 & 0.02 & 0.0 & 0.001 & 0.556 & \\
\hline \begin{tabular}{|l|}
421 \\
422 \\
\end{tabular} & & Averages & 25.5 & 23.4 & 25.1 & 24.9 & $26.7 \#$ & 47.2 & 50.0 & 123 & 34.7 & -1.2 & -6.6 & 49.7 & 0.1 & 14.6 & & & & 48.5 & 122 & 40.9 & & 0.018 & 0.018 & & & \\
\hline \begin{tabular}{|l|l|}
423 \\
\end{tabular} & & Maximum & 26.1 & $\frac{25.4}{24.5}$ & 25.7 & $\begin{array}{l}25.6 \\
25.6\end{array}$ & 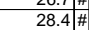 & $\frac{47.2}{52.9}$ & $\begin{array}{l}53.4 \\
53.4\end{array}$ & $\begin{array}{l}17.1 \\
17.1\end{array}$ & $\frac{34.1}{38.7}$ & $\begin{array}{l}1.2 . \\
1.8\end{array}$ & 0.6 & $\begin{array}{l}49.1 \\
57.8\end{array}$ & 0.1 & $\begin{array}{l}14.6 \\
14.6\end{array}$ & $0.0 \mid \#$ & & & $\begin{array}{l}48.5 \\
51.9 \\
\end{array}$ & $\frac{11.2}{14.2}$ & $\frac{40.9}{45.8}$ & & $\frac{0.018}{0.029}$ & & & $\begin{array}{l}0.432 \\
0.698\end{array}$ & \\
\hline 424 & & Median & 25.5 & 24.1 & 25.1 & 24.9 & & 46.9 & 50.3 & 12.0 & 35.1 & -1.2 & -6.7 & 49.8 & 0.1 & 14.6 & & & & 48.8 & & 40.9 & & 0.017 & & & 0.423 & \\
\hline \begin{tabular}{|l|}
425 \\
\end{tabular} & & Minimum & 25.2 & 19.2 & 24.7 & 24.1 & $20.4 \#$ & 42.6 & 47.2 & 10.3 & 29.3 & -1.2 & $-7.8 \#$ & 42.1 & 0.0 & 14.6 & $0.0 \#$ & & & 45.8 & \begin{tabular}{|l|l|}
8 & 10.3 \\
\end{tabular} & 36.0 & \begin{tabular}{ll|}
0 & 2.479 \\
\end{tabular} & 0.003 & 0.003 & 0.0001 & 0.072 & \\
\hline \begin{tabular}{|l|}
426 \\
\end{tabular} & & $2 \times \operatorname{Std}$ Dev & 0.316 & 2.618 & 0.448 & 0.606 & 2.816 \# & 3.060 & 2.684 & 2.754 & 2.937 & 0.535 & 2.599 \# & 5.452 & 0.053 & 0.000 & 0.001 & & & 2.684 & & & \begin{tabular}{|l|l|}
1 & 0.188 \\
\end{tabular} & 0.008 & 0.008 & 0.0002 & 0.192 & \\
\hline \begin{tabular}{|l|}
427 \\
\end{tabular} & Number & of Points Used* & 398 & 398 & 398 & \begin{tabular}{|l|l|}
398 \\
\end{tabular} & 3980 & 398 & 398 & 398 & 398 & 398 & 3980 & 398 & 398 & 398 & 3986 & & & 398 & \begin{tabular}{|l|l|}
8 & 398 \\
\end{tabular} & 398 & \begin{tabular}{|l|l|}
8 & 398 \\
\end{tabular} & 398 & 398 & 398 & 398 & \\
\hline \begin{tabular}{|l|}
428 \\
429 \\
\end{tabular} & & & & & & & & & & & & & & & & & & & & & & & 6.7-ho & ravg.: & & & & \\
\hline$\frac{\mid 49}{430} \mid$ & & & & & & & & & & & & & & & & & & & & & & & $\begin{array}{l}\text { b. } \\
1 \text {-hour }\end{array}$ & ravg. $=$ & $\begin{array}{ll}0.0129 \\
\end{array}$ & & & \\
\hline
\end{tabular}


RUN \# WASH2; CAMPAIGN II SLURRY WASH 2

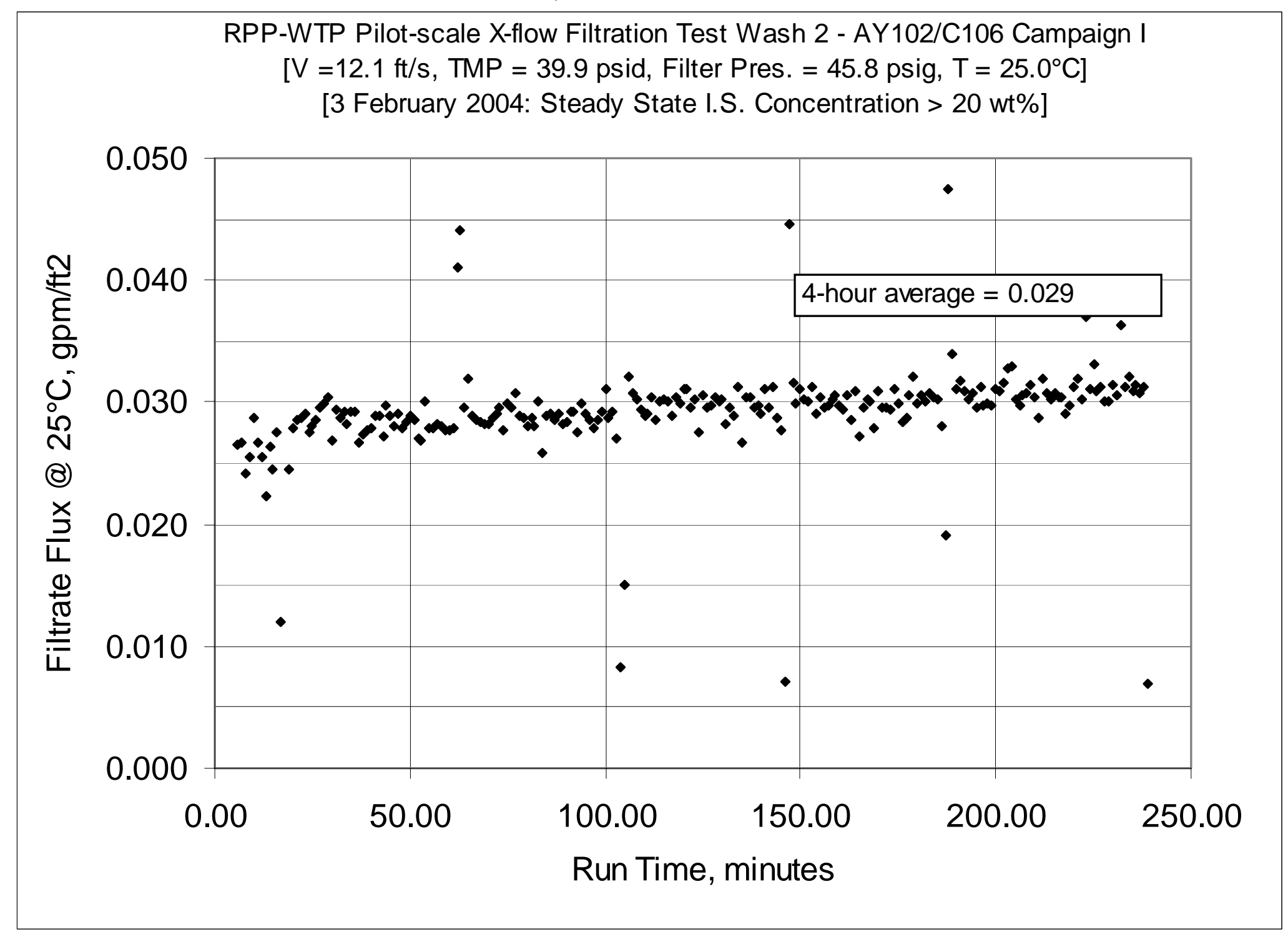


WSRC-TR-2005-00105, REVISION 0

SRNL-RPP-2005-00012, REVISION 0

RUN \# WASH2; CAMPAIGN II SLURRY WASH 2 - CONT.

\begin{tabular}{|c|c|c|c|c|c|c|c|c|c|c|c|c|c|c|c|c|c|c|c|c|c|c|c|c|c|c|c|c|}
\hline & A & B & $D$ & $E$ & $\mathrm{~F}$ & $G$ & $\mathrm{H}$ & $\mathrm{J}$ & $\mathrm{K}$ & $\mathrm{L}$ & $M$ & $\mathrm{~N}$ & 0 & Q & $\mathrm{R}$ & $\mathrm{s}$ & $T$ & $\mathrm{v}$ & w & $x$ & Y & $z$ & $\mathrm{AA}$ & $A B$ & $A C$ & $A D$ & $\mathrm{AE}$ & AF \\
\hline & & & $\mathrm{T} 2$ & T3 & $\mathrm{T} 1$ & T4 & T5 & $\mathrm{dP} 2$ & P1 & $\mathrm{dP1}$ & $\mathrm{dP3}$ & $\mathrm{P} 2$ & P3 & Q1 & Q2 & Q3 & Qbp & & & & & & & & & & & \\
\hline & & & & & & & & & & & & & & & & & & & & & & & & & & & & \\
\hline & |12004 & $8: 11: 15 \mathrm{AM}$ & 23.157 & \begin{tabular}{|l|l|l|}
1877 \\
\end{tabular} & 15.664 & 23.677 & $\begin{array}{ll}16.209 \\
\end{array}$ & 0.048 & -0.019 & 0.008 & -0.002 & -0.206 & 0.0 & -0.077 & 0.00 & $\begin{array}{l}14.643 \\
\end{array}$ & & & & Filter & face & & $=\frac{\mathrm{TT} 2}{2}$ & & & & & \\
\hline & $2 / 3 / 2004$ & $8: 11: 28 \mathrm{AM}$ & 23.157 & \begin{tabular}{|l|l|}
18.127 \\
\end{tabular} & \begin{tabular}{|l|l|}
15.6644 \\
\end{tabular} & 23.677 & 16.209 & 0.048 & -0.019 & 0.008 & $\begin{array}{l}-0.002 \\
\end{array}$ & -0.206 & 0.0 & -0.077 & 0.003 & $\begin{array}{l}14.643 \\
\end{array}$ & 0.0 & & & Convers: & & & $\mathrm{m} 3 / \mathrm{m} 2$ & 2/day/bar & $\mathrm{ag} / \mathrm{gpm} / \mathrm{ft}$ & 2/barg & & \\
\hline & & & 23.152 & \begin{tabular}{|l|l|l|l|} 
\\
10.127
\end{tabular} & \begin{tabular}{|l|l|}
15.644 \\
1.50
\end{tabular} & 23.667 & 16.245 & & & 0.008 & & & & -0.085 & & 14.643 & 0.0 & & & & & & & & & & & \\
\hline & $\frac{2 / 3 / 2004}{2 / 32004}$ & $8: 11: 30 \mathrm{AM}$ & $\begin{array}{l}23.152 \\
23.152\end{array}$ & \begin{tabular}{|l|}
18.127 \\
18.127
\end{tabular} & \begin{tabular}{|l|}
15.649 \\
15.644 \\
\end{tabular} & $\begin{array}{l}23.667 \\
23.662\end{array}$ & $\begin{array}{r}16.255 \\
16.25 \\
\end{array}$ & $\begin{array}{l}0.048 \\
0.048\end{array}$ & $\begin{array}{r}-0.019 \\
-0.019 \\
\end{array}$ & $\begin{array}{l}0.008 \\
0008\end{array}$ & $\begin{array}{l}-0.006 \\
-0.002\end{array}$ & $\begin{array}{r}-0.215 \\
-0.218\end{array}$ & $\begin{array}{l}0.046 \\
0.046\end{array}$ & $\begin{array}{r}-0.079 \\
-0.083\end{array}$ & 0.003 & $\begin{array}{r}14.643 \\
14.643\end{array}$ & $\begin{array}{l}0.004 \\
0.094\end{array}$ & & & Vote: & uge & & & & mately 1 & psig & & \\
\hline & $\frac{2 / 3 / 2 / 204}{2 / 3 / 2004}$ & $\begin{array}{l}8: 11: 31 \mathrm{AM} \\
8: 1132 \mathrm{AM}\end{array}$ & $\frac{23.152}{23.152}$ & \begin{tabular}{|l|}
18.127 \\
18.122
\end{tabular} & \begin{tabular}{|c|}
15.6444 \\
15.6399 \\
\end{tabular} & $\begin{array}{l}23.662 \\
23.657\end{array}$ & $\begin{array}{l}16.25 \\
16.25\end{array}$ & \begin{tabular}{|l}
0.048 \\
0.048
\end{tabular} & $\begin{array}{c}-0.019 \\
-0.019\end{array}$ & $\begin{array}{l}0.008 \\
0.008\end{array}$ & $\begin{array}{c}-0.002 \\
-0.004 \\
\end{array}$ & $\begin{array}{r}-0.218 \\
-0.18\end{array}$ & $\begin{array}{l}0.046 \\
0.046\end{array}$ & $\begin{array}{l}-0.083 \\
-0.079\end{array}$ & \begin{tabular}{|l}
0.003 \\
0.003
\end{tabular} & $\begin{array}{r}14.643 \\
14.643 \\
\end{array}$ & $\begin{array}{l}0.004 \\
0.004\end{array}$ & & & & & orre & ted for & & & & & \\
\hline & $2 / 3 / 2004$ & $11: 33 \mathrm{AM}$ & 23.152 & 18.122 & \begin{tabular}{|l|}
15.644 \\
\end{tabular} & 23.657 & 16.255 & 0.05 & \begin{tabular}{|l|l|} 
& -0.019 \\
\end{tabular} & 0.008 & -0.006 & $\begin{array}{r}-0.18 \\
\end{array}$ & 0.046 & -0.083 & 0.003 & \begin{tabular}{|c|}
14.643 \\
\end{tabular} & 0.00 & & & WVVV & & & & $<$ Filtra & & $1<<$ PERM & MIABILITY & $Y \gg$ \\
\hline & $2 / 3 / 2004$ & $8: 11: 34 \mathrm{AM}$ & 23.152 & \begin{tabular}{|l|l|}
18.127 \\
\end{tabular} & \begin{tabular}{|l|l|}
15.649 \\
\end{tabular} & 23.677 & 16.25 & 0.048 & -0.021 & 0.008 & -0.004 & -0.174 & 0.043 & -0.079 & 0.003 & $\begin{array}{l}14.643 \\
\end{array}$ & 0.00 & & & & & & & & at 25C & & & \\
\hline & & & & & & & & & & & & & & & & & & Time & Time & Press. & Vel. & TMP & TMP & $\mathrm{gpm}$ & gpm & gpm & gpm & \\
\hline & $\frac{2 \text { ata - Per M }}{2 / 2 / 20}$ & & & & & & & & & & & & & & & & & & & osig & & & & & & $2 \mid p s i$ & & \\
\hline & $\begin{array}{l}2 / 3 / 2004 \\
2 / 3 / 2004 \\
\end{array}$ & $\begin{array}{l}\text { 8:30:50 AM } \\
8: 31: 50 \mathrm{AM}\end{array}$ & $\begin{array}{l}24.627 \\
23.417\end{array}$ & \begin{tabular}{r|}
18.2 \\
18.202 \\
\end{tabular} & \begin{tabular}{|l|}
24.604 \\
24.736 \\
\end{tabular} & \begin{tabular}{|l|}
23.744 \\
23.671
\end{tabular} & \begin{tabular}{|l|}
18.109 \\
18.146
\end{tabular} & $\begin{array}{l}\frac{10.051}{-4.176} \\
\end{array}$ & \begin{tabular}{|l|l|}
50.151 \\
49.465 \\
\end{tabular} & \begin{tabular}{|r|}
11.697 \\
7.167 \\
\end{tabular} & $\begin{array}{r}-1.547 \\
-11.232 \\
\end{array}$ & \begin{tabular}{|r|}
35.25 \\
49.169 \\
\end{tabular} & $\begin{array}{l}89.94 \\
49.85 \\
\end{array}$ & $\begin{array}{l}\frac{49.677}{53.196} \\
5\end{array}$ & 0.003 & \begin{tabular}{|l}
14.643 \\
14.643
\end{tabular} & & & & $\begin{array}{l}48.7 \\
48.0 \\
\end{array}$ & $\frac{12.2}{13.9}$ & & & & 0.000 & & & \\
\hline & $2 / 3 / 2004$ & $\begin{array}{l}8.31 .50 \mathrm{AMI} \\
8: 32: 50 \mathrm{Am}\end{array}$ & 24.417 & $\begin{array}{r}18.204 \\
18.24 \\
\end{array}$ & \begin{tabular}{|l|}
24.130 \\
24.679 \\
\end{tabular} & $\begin{array}{l}23.011 \\
23.718 \\
\end{array}$ & $\begin{array}{l}18.3746 \\
18.36\end{array}$ & $\begin{array}{l}-4.180 \\
43.841 \\
\end{array}$ & \begin{tabular}{|l|}
49.405 \\
49.856 \\
\end{tabular} & $\begin{array}{r}1.061 \\
10.061 \\
\end{array}$ & \begin{tabular}{|c|}
-1.232 .447 \\
33.4
\end{tabular} & \begin{tabular}{|r|}
49.1909 \\
0.801 \\
\end{tabular} & $\frac{49.85}{0.33}$ & 51.936 & $\begin{array}{l}0.003 \\
0.324 \\
\end{array}$ & \begin{tabular}{|l|}
14.6434 \\
14.643
\end{tabular} & & 2.00 & \begin{tabular}{|l|l|} 
& 0.03333 \\
\end{tabular} & 48.4 & $\frac{13.6}{12.7}$ & 38.6 & $\begin{array}{l}-0.531 \\
2.664 \\
\end{array}$ & 0.048 & 0.049 & & $\begin{array}{l}-0.030 \\
1.261 \\
\end{array}$ & \\
\hline & $2 / 3 / 2004$ & & 24.954 & & \begin{tabular}{|l|}
24.671 \\
\end{tabular} & 23.785 & & $\begin{array}{l}44.643 \\
\end{array}$ & 50.412 & 12.429 & & & & & 0.203 & $\begin{array}{l}14.643 \\
\end{array}$ & & 3.00 & \begin{tabular}{|l|l|}
0.05 \\
\end{tabular} & 48.9 & 12.5 & & 2.637 & & & & 57.606 & \\
\hline & $2 / 3 / 2004$ & 4:50 AM & 25.057 & 18.296 & 24.699 & 23.819 & 18.331 & $\begin{array}{l}44.948 \\
\end{array}$ & 50.747 & \begin{tabular}{|l|l|}
13.174 \\
\end{tabular} & 31.381 & 0.573 & 0.46 & 47.844 & 0.178 & 14.643 & & 4.0 & \begin{tabular}{|l|}
0.06667 \\
\end{tabular} & 49.3 & 11.7 & 38.2 & 2.631 & 2.183 & & 0.058 & 57.691 & \\
\hline & $2 / 3 / 2004$ & $8: 35: 50 \mathrm{AM}$ & 25.125 & 18.308 & 24.726 & 23.786 & $\begin{array}{l}18.247 \\
\end{array}$ & 44.66 & 50.526 & $\begin{array}{l}13.366 \\
\end{array}$ & 30.816 & 0.596 & 0.52 & 45.026 & 0.168 & $\begin{array}{l}14.643 \\
\end{array}$ & & 5.00 & 0.08333 & 49.1 & 11.6 & 37.7 & 2.602 & & & & 0.669 & \\
\hline & $2 / 3 / 2004$ & $8: 36: 50 \mathrm{AM}$ & 24.757 & $\begin{array}{l}18.326 \\
19320\end{array}$ & \begin{tabular}{|l|l|}
24.749 \\
\end{tabular} & 23.733 & 18.245 & 48.75 & 50.843 & 13.852 & 34.455 & -1.216 & & 45.032 & 0.177 & 14.643 & & 6.00 & \begin{tabular}{c|l}
0.1 \\
\end{tabular} & 49.4 & 11. & 41.6 & 2.868 & $\begin{array}{l}0.026 \\
\end{array}$ & 0.027 & & 0.639 & \\
\hline & $2 / 3 / 2004$ & & $\frac{24.854}{2.897}$ & \begin{tabular}{|l|l|l|l|l|}
18.301 \\
\end{tabular} & \begin{tabular}{|l|}
24.461 \\
2.501
\end{tabular} & 23.746 & $\begin{array}{l}18.489 \\
1.69\end{array}$ & 48.121 & 50.128 & $\begin{array}{l}13.219 \\
1.20\end{array}$ & $\begin{array}{l}34.449 \\
2.015\end{array}$ & -1.214 & $\begin{array}{l}-5.08 \\
\end{array}$ & 51.171 & 0.176 & 14.643 & & 7.00 & \begin{tabular}{|c|c|c|}
0.11667 \\
\end{tabular} & 48.7 & 12. & 41.3 & 2.846 & 0.026 & 0.027 & & 0.645 & \\
\hline & $2 / 3 / 2004$ & & 24.887 & \begin{tabular}{|c|}
18.391 \\
\end{tabular} & \begin{tabular}{|l|}
24.503 \\
2.144
\end{tabular} & $\begin{array}{r}23.808 \\
2372\end{array}$ & $\begin{array}{l}18.618 \\
18711\end{array}$ & $\begin{array}{r}48.207 \\
\end{array}$ & 50.097 & $\begin{array}{l}12.948 \\
12833\end{array}$ & & & & 55.071 & 0.16 & 14.643 & & 8.00 & 0.13333 & 48.6 & 13.5 & & 2.865 & 0.024 & 0.024 & & 0.582 & \\
\hline & $\frac{2 / 3 / 2004}{2 / 32004}$ & $\begin{array}{l}8: 39: 50 \\
8.0 .50\end{array}$ & $\begin{aligned} 24.834 \\
24.822\end{aligned}$ & \begin{tabular}{|r|}
18.388 \\
18.427
\end{tabular} & \begin{tabular}{|l|}
24.144 \\
24.222 \\
\end{tabular} & $\begin{array}{r}23.72 \\
23.753 \\
\end{array}$ & \begin{tabular}{|l|}
18.741 \\
18941
\end{tabular} & $\begin{array}{r}48.48 \\
49.142 \\
\end{array}$ & \begin{tabular}{|l|l}
49.856 \\
50.267
\end{tabular} & \begin{tabular}{|l|}
12.833 \\
12.771
\end{tabular} & \begin{tabular}{|l|}
35.302 \\
36.099
\end{tabular} & \begin{tabular}{|r|}
-1.219 \\
\end{tabular} & $\begin{array}{r}-5.866 \\
\end{array}$ & $\begin{array}{l}47.808 \\
46.311\end{array}$ & 0.167 & \begin{tabular}{|l|l|}
14.643 \\
14643
\end{tabular} & 0.00 & $\begin{array}{r}9.00 \\
1000\end{array}$ & \begin{tabular}{|r|r|}
5 & 0.15 \\
\end{tabular} & $\begin{array}{ll}48.4 \\
8.8\end{array}$ & $\frac{11.7}{11.7}$ & 41.9 & 2.888 & $\frac{0.025}{1028}$ & 0.026 & 0.001 & 0.609 & \\
\hline & $\frac{2 / 3 / 2004}{2 / 32004}$ & & $\frac{24.822}{24.799}$ & \begin{tabular}{|l|}
18.427 \\
18.439
\end{tabular} & \begin{tabular}{|l|}
24.222 \\
24.29 \\
\end{tabular} & $\begin{array}{l}23.753 \\
23.695 \\
\end{array}$ & $\begin{array}{l}18.941 \\
18999\end{array}$ & $\begin{array}{l}49.142 \\
49.061 \\
\end{array}$ & $\begin{array}{l}50.267 \\
50.032\end{array}$ & $\begin{array}{l}12.7711 \\
12.649\end{array}$ & \begin{tabular}{|}
$\mid 36.049$ \\
36.132 \\
\end{tabular} & \begin{tabular}{|l|}
-1.216 \\
-1.219
\end{tabular} & $\begin{array}{r}-6.49 \\
-6.502\end{array}$ & $\begin{array}{r}46.311 \\
49.89\end{array}$ & $\frac{0.188}{0.176}$ & $\begin{array}{r}14.643 \\
14.643\end{array}$ & 0.00 & $\begin{array}{l}\frac{10.00}{11.00} \\
\end{array}$ & \begin{tabular}{l|l|} 
& 0.16667 \\
\end{tabular} & \begin{tabular}{ll|l}
48.8 \\
48.6
\end{tabular} & $\frac{11.2}{12.2}$ & $\frac{42.6}{42.6}$ & $\frac{2.937}{2.937}$ & 0.028 & 0.029 & 0.001 & $\begin{array}{l}0.673 \\
0.628 \\
\end{array}$ & \\
\hline & $2 / 3 / 2004$ & $8: 42: 5$ & 24.797 & \begin{tabular}{|l|l|l|l}
18.473 \\
\end{tabular} & \begin{tabular}{|l|}
24.404 \\
\end{tabular} & $\begin{array}{l}23.095 \\
23.794 \\
\end{array}$ & $\begin{array}{r}19.1349 \\
19.133\end{array}$ & $\begin{array}{l}49.001 \\
47.846 \\
\end{array}$ & 48.032 & \begin{tabular}{|l|}
14.2494 \\
14.243
\end{tabular} & 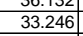 & \begin{tabular}{|l|}
-1.219 \\
-1.214 \\
\end{tabular} & $\begin{array}{l}-0.064 \\
-6.643\end{array}$ & $\begin{array}{r}52.879 \\
52.879\end{array}$ & $\begin{array}{l}0.110 \\
0.168 \\
\end{array}$ & $\begin{array}{l}\frac{14.045}{14.643} \\
\end{array}$ & & 12.00 & \begin{tabular}{|l|l|}
0.10333 \\
\end{tabular} & $\begin{array}{l}48.6 \\
47.3 \\
\end{array}$ & $\frac{12 .}{13 .}$ & $\frac{42.6}{40.5}$ & $\begin{array}{l}2.931 \\
2.796\end{array}$ & $\begin{array}{l}.026 \\
025\end{array}$ & $\begin{array}{l}0.021 \\
0.025\end{array}$ & $\begin{array}{l}0.001 \\
0.001\end{array}$ & \begin{tabular}{|c|}
0.628 \\
0.628 \\
\end{tabular} & \\
\hline & $2 / 3 / 2004$ & & 24.775 & 18.496 & \begin{tabular}{|l|}
24.472 \\
\end{tabular} & 23.776 & & 46.628 & 47.469 & 13.108 & & \begin{tabular}{|c|}
-1.214 \\
\end{tabular} & & 54.377 & 0.147 & 14.643 & & 13.00 & 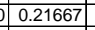 & 46.0 & 13.3 & 200 & 2.752 & & & & & \\
\hline & $2 / 3 / 2004$ & & 24.823 & \begin{tabular}{|l|}
18.534 \\
\end{tabular} & \begin{tabular}{|l|l|}
24.5544 \\
\end{tabular} & 23.769 & 18.9 & 46.599 & 47.535 & 13.014 & 33.263 & -1.216 & & 50.38 & 0.174 & $\begin{array}{l}14.643 \\
\end{array}$ & & \begin{tabular}{ll|}
14.00 \\
\end{tabular} & 0.23333 & 46.1 & 12. & 39.9 & 2.753 & .026 & 0.026 & 0.001 & 0.658 & \\
\hline & $2 / 3 / 2004$ & & 24.85 & \begin{tabular}{|l|l|}
18.531 \\
18550
\end{tabular} & \begin{tabular}{|l|}
24.611 \\
2.5
\end{tabular} & 23.731 & 19.4 & $\begin{array}{l}47.155 \\
\end{array}$ & \begin{tabular}{|l|l}
48.13 \\
\end{tabular} & $\begin{array}{l}12.787 \\
\end{array}$ & $\begin{array}{l}33.966 \\
2015\end{array}$ & -1.214 & & 46.417 & & 14.6 & & 15.00 & \begin{tabular}{c|l}
0 & 0.25 \\
\end{tabular} & 46.7 & 11.4 & 40.6 & 2.797 & .024 & 0.025 & & 0.606 & \\
\hline & $2 / 3 / 26$ & & 24.903 & & $24.665 \mid$ & 23.749 & & 47.25 & 48.032 & 12.597 & & -1.214 & & 46.709 & 0.183 & & & 16.00 & \begin{tabular}{|l|l|l}
0.26667 \\
\end{tabular} & 46.6 & 11.4 & 40.8 & 2.812 & .027 & 0.028 & & 0.675 & \\
\hline & $\frac{2 / 3 / 2004}{2 / 32004}$ & $\begin{array}{l}8: 47: 4 \\
8: 8: 5\end{array}$ & $\begin{array}{r}24.971 \\
24.838\end{array}$ & \begin{tabular}{|r|}
18.587 \\
18.62
\end{tabular} & \begin{tabular}{|l|}
24.718 \\
24805 \\
\end{tabular} & $\begin{array}{l}23.787 \\
23.789 \\
\end{array}$ & $\begin{array}{l}19.601 \\
19.537\end{array}$ & $\begin{array}{l}46.182 \\
45.187\end{array}$ & $\begin{array}{l}48.125 \\
48.026\end{array}$ & $\begin{array}{l}12.385 \\
12221 \\
\end{array}$ & \begin{tabular}{|l|}
33.355 \\
32.67
\end{tabular} & \begin{tabular}{|c|}
-1.216 \\
-1211
\end{tabular} & \begin{tabular}{|c|}
-3.767 \\
3.25
\end{tabular} & $\begin{array}{l}48.945 \\
50.115\end{array}$ & $\begin{array}{r}0.08 \\
0.41 \\
\end{array}$ & 14.6 & 0.0 & $\begin{array}{l}17.00 \\
1800\end{array}$ & \begin{tabular}{r|r}
0.28333 \\
\end{tabular} & $\begin{array}{l}46.7 \\
466\end{array}$ & $\frac{12.6}{12 .}$ & 39.8 & \begin{tabular}{|l|l|}
2.742 \\
2684
\end{tabular} & .012 & 0.012 & & 0.302 & 0.2 \\
\hline & $\frac{2 / 3 / 2 / 204}{2 / 3 / 2004}$ & & $\begin{aligned} 24.838 \\
24.955\end{aligned}$ & $\begin{array}{r}18.62 \\
18.632\end{array}$ & \begin{tabular}{|l|}
24.805 \\
24.672 \\
\end{tabular} & $\begin{aligned} 23.789 \\
23.776\end{aligned}$ & $\begin{array}{l}19.537 \\
19.938\end{array}$ & $\begin{array}{l}45.187 \\
47.126\end{array}$ & \begin{tabular}{|l|l|l}
48.026 \\
47.896
\end{tabular} & $\frac{12.211}{12.121}$ & $\begin{array}{l}32.657 \\
34.676\end{array}$ & \begin{tabular}{|l|}
-1.211 \\
-1.216 \\
\end{tabular} & $\frac{-2.62}{-6.34}$ & $\begin{array}{l}50.115 \\
49.047\end{array}$ & 0.4111 & $\frac{14 .}{14 .}$ & 0.0 & $\begin{array}{l}18.00 \\
19.00\end{array}$ & $\begin{array}{r}0.3 \\
0.31667\end{array}$ & $\begin{array}{l}46.6 \\
46.4 \\
4\end{array}$ & $\frac{12.3}{120}$ & $\frac{38.9}{40.9}$ & $\begin{array}{l}2.684 \\
2.820\end{array}$ & & 0.062 & 0.002 & $\frac{1.583}{0.600}$ & \\
\hline & $2 / 3 / 2004$ & $8: 50$ & 25.023 & 18.661 & \begin{tabular}{|r|}
24.73 \\
\end{tabular} & 23.819 & 20.051 & 47.049 & \begin{tabular}{|l|l|}
47.839 \\
\end{tabular} & \begin{tabular}{|c|}
11.95 \\
1.12
\end{tabular} & 34.6 & -1.214 & & 51.346 & 0.185 & $\frac{14.6}{14.6}$ & & 20. & \begin{tabular}{|l|}
$\mid .31006$ \\
\end{tabular} & $\begin{array}{l}40.4 \\
46.4 \\
\end{array}$ & $\frac{11.0}{12.6}$ & & \begin{tabular}{|l|}
2.8218 \\
\end{tabular} & D28 & & & $\begin{array}{l}0.680 \\
0.680\end{array}$ & \\
\hline & $2 / 3 / 2004$ & & 25.035 & 18.667 & \begin{tabular}{|l|}
24.797 \\
\end{tabular} & 23.81 & & 47.354 & 48.101 & 11.996 & & -1.214 & & 48.369 & 0. & 14. & & 21. & \begin{tabular}{|l|l}
5 & 0.35 \\
\end{tabular} & 46.6 & 11.0 & & & & & & & \\
\hline & $2 / 3 / 2004$ & 8:52: & 25.063 & \begin{tabular}{|l|l|}
18.716 \\
\end{tabular} & 24.865 & 23.834 & & 47.246 & 47.968 & 12.086 & & & & 48.169 & & 14. & & 22. & 0.36667 & 46.5 & 11.8 & & 2.830 & \begin{tabular}{|l|l|} 
& 0.029 \\
\end{tabular} & 0.029 & & 00 & \\
\hline & & & 25.09 & & \begin{tabular}{|l|}
24.932 \\
\end{tabular} & 23.841 & 20.1 & $\begin{array}{l}47.01 \\
\end{array}$ & & $\begin{array}{l}11.963 \\
\end{array}$ & & -1.214 & & 49.7 & & 14. & & 23.00 & 0.38333 & 46.3 & 12.2 & 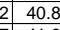 & 2.814 & & & & 0.710 & \\
\hline & & & 25.133 & & \begin{tabular}{|l|l|}
24.995 \\
\end{tabular} & & & & 47.842 & & & & & & & & & & $\begin{array}{r}0.4 \\
\end{array}$ & & & & & & & & & \\
\hline & $2 / 3 / 2004$ & & $\frac{25.171}{25.182}$ & 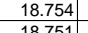 & \begin{tabular}{|l|}
25.018 \\
25024
\end{tabular} & $\frac{23.812}{23778}$ & $\begin{array}{r}20.11 \\
19.914\end{array}$ & & & $\begin{array}{l}11.972 \\
12037\end{array}$ & & \begin{tabular}{|r|}
-1.214 \\
\end{tabular} & & 49.162 & & 14. & & & & & & & & & & & 0.684 & \\
\hline & $\frac{2 / 3 / 2 / 204}{2 / 3 / 2004}$ & & $\begin{array}{l}25.182 \\
25.266\end{array}$ & \begin{tabular}{|r|}
18.751 \\
1879
\end{tabular} & \begin{tabular}{|l|}
25.024 \\
25088 \\
\end{tabular} & $\begin{array}{r}23.778 \\
23877\end{array}$ & $\begin{array}{l}19.914 \\
20.371\end{array}$ & & \begin{tabular}{|l|}
47.989 \\
47752 \\
\end{tabular} & & & & & $\begin{array}{l}47.973 \\
50.956\end{array}$ & & & & 26.00 & 0.43333 & & 11.8 & & 2.827 & & & & 0.698 & \\
\hline & $2 / 3 / 2004$ & & $\frac{25.266}{25.315}$ & $\frac{18}{18.8}$ & \begin{tabular}{|l|}
25.148 \\
25.147 \\
\end{tabular} & $\frac{23.871}{23.881}$ & $\frac{20.3}{20.1}$ & & $\mid$\begin{tabular}{|l|l|}
4.1 .1 \\
47.9
\end{tabular} & $\frac{11.911}{12.063}$ & & \begin{tabular}{|l|}
-1.214 \\
-1.214 \\
\end{tabular} & & $\begin{array}{l}50.856 \\
47.911\end{array}$ & & 14. & & & \begin{tabular}{|l|}
0.46667 \\
\end{tabular} & & $\frac{12.5}{11.7}$ & & & & & & & \\
\hline & & & 25.365 & & \begin{tabular}{|l|}
24.952 \\
\end{tabular} & 23.856 & 20.5 & 46.751 & 47. & 11.838 & & \begin{tabular}{|l|}
-1.216 \\
\end{tabular} & & 46.8 & & & & & \begin{tabular}{|l}
0.48333 \\
\end{tabular} & 46 & 11.5 & & & & & & & \\
\hline & $2 / 3 / 2$ & & & 18. & \begin{tabular}{|l|l|}
25.069 \\
\end{tabular} & 23.828 & & & 47. & 11.82 & & & & 49.5 & 0.181 & 14. & & 30. & 0.5 & 46.0 & 12.2 & & & & & & & \\
\hline & & & 25.367 & & 25.12 & 23.793 & & & & 11.861 & & & & 48. & & 14. & & 31. & \begin{tabular}{|l|l|}
5 & 0.51667 \\
\end{tabular} & 46.0 & 11.8 & & & & & & & \\
\hline & $2 / 3 / 2$ & & 25.389 & 18.899 & 25.166 & 23.84 & & 47.085 & 47.7 & 11.8 & & & & & & & & 32 & \begin{tabular}{ll|l} 
& 0.53333 \\
\end{tabular} & 46 & 11.6 & & & & & & & \\
\hline & $2 / 3 / 2004$ & & 25.421 & $\begin{array}{l}18.922 \\
18.028\end{array}$ & \begin{tabular}{|l|}
25.194 \\
25.225
\end{tabular} & $\begin{aligned} 23.893 \\
23.870\end{aligned}$ & 20.4 & 46.883 & 47.668 & $\begin{array}{l}11.728 \\
1165\end{array}$ & $\begin{array}{l}34.716 \\
3479 \\
\end{array}$ & \begin{tabular}{|l|}
-1.214 \\
\end{tabular} & & 46.421 & 0.197 & 14. & & 33.00 & $\begin{array}{r}0.55 \\
\end{array}$ & 46.2 & 11.4 & 40.8 & 2.010 & & & & 0.716 & \\
\hline & $\frac{2 / 3 / 2004}{2 / 32004}$ & & $\frac{25.453}{25.48}$ & $\begin{array}{r}\mid 8.928 \\
18.95\end{array}$ & \begin{tabular}{|l|}
25.235 \\
25.272
\end{tabular} & $\frac{23.879}{23.911}$ & & $\begin{array}{r}46.8 \\
4708\end{array}$ & \begin{tabular}{|l|l|} 
& 47.7 \\
\end{tabular} & $\begin{array}{l}11.658 \\
11.683 \\
\end{array}$ & & -1.216 & & 51.361 & & 14. & & & | $0.56667 \mid$ & 46.2 & $\frac{12.6}{127}$ & 40.8 & & & & & & \\
\hline & $2 / 2 / 3 / 2$ & & $\frac{25.4}{25.52}$ & & \begin{tabular}{|l|}
25.319 \\
25.219 \\
\end{tabular} & $\frac{23.9}{23.9}$ & & & & $\frac{11.66}{11.6}$ & & & & & & & & & \begin{tabular}{|l|r|}
0.58333 \\
0
\end{tabular} & & $\frac{12 .}{12 .}$ & & & & & & & \\
\hline & $2 / 3 / 2$ & & & & & 23 & & 46. & & 11. & & -1.214 & & 49.099 & & 14. & & & \begin{tabular}{|l|l|}
0.61667 \\
\end{tabular} & 46 & 12. & & & & & & & \\
\hline & $2 / 3 / 2$ & & 25.596 & & 25.384 & & 21. & 47 & & 11.95 & & -1.216 & & 53.649 & & 14.6 & & 38 & 0.63333 & 46 & 13. & 40.9 & & & & & & \\
\hline & & & & & & 23. & & 46 & 47.7 & 11.995 & & & & & & & & & 0.65 & 46 & 12. & & 07 & & & & & \\
\hline & & & & & & & & & & & & -1.214 & & 54.1 & & 14. & & & 0.66667 & 46 & 13. & & & & & & & \\
\hline & $2 / 3 / 26$ & 9:11:5 & 25.617 & \begin{tabular}{|c|}
19.07 \\
\end{tabular} & 25.295 & 23.8 & 21.2 & & 47. & & & \begin{tabular}{|l|l|}
-1.219 \\
\end{tabular} & & & & & & & 0.68333 & 46. & 11.6 & & 2.809 & & & & & \\
\hline & $2 / 3 / 20$ & & $\begin{array}{r}25.62 \\
25.642\end{array}$ & $\begin{array}{l}19.092 \\
1009\end{array}$ & $25.347 \mid$ & $\frac{23.5}{230}$ & $\frac{21}{211}$ & $\begin{array}{l}46.643 \\
46.639 \\
\end{array}$ & 47.676 & 11.5 & & -1.214 & & 48.5 & 0. & 14.6 & & & \begin{tabular}{|l|r|} 
& 0.7 \\
\end{tabular} & $\begin{array}{r}46.2 \\
4.2 \\
4.9\end{array}$ & $\frac{12.6}{12.0}$ & 4 & 2.804 & & 29 & 001 & 0.711 & tr \\
\hline & $\frac{2 / 3 / 20}{2 / 3 / 20}$ & & $\frac{25.642}{25.674}$ & & \begin{tabular}{|l|}
25.374 \\
25.39 \\
\end{tabular} & $\frac{23.5}{23.9}$ & $\frac{21 .}{21 .}$ & $\frac{46.6}{46.5}$ & \begin{tabular}{|r|r|}
47.68 \\
\end{tabular} & $\frac{11.503}{11.524}$ & & $\begin{array}{l}-1.214 \\
-1.219 \\
\end{array}$ & & & 0. & & & & $\begin{array}{l}0.71667 \\
0\end{array}$ & & $\frac{12.5}{12 .}$ & & & & & & & \\
\hline & $2 / 3 / 20$ & & $\frac{25.014}{25.696}$ & 19. & \begin{tabular}{|l|}
25.419 \\
25.319 \\
\end{tabular} & $\frac{23.916}{23.932}$ & $\frac{21}{21}$. & 46. & \begin{tabular}{|l|l|}
41.151 \\
7.672 \\
\end{tabular} & $\begin{array}{l}11.524 \\
11.522\end{array}$ & 34. & \begin{tabular}{|l|}
-1.219 \\
-1.216 \\
\end{tabular} & & 48.5 & 0.196 & $\begin{array}{l}14.6 \\
14.6\end{array}$ & & 45.1 & $\begin{array}{r}0.13353 \\
0.75\end{array}$ & $\begin{array}{l}40.3 \\
46.2 \\
\end{array}$ & $\begin{array}{l}12.1 \\
11.9\end{array}$ & $\frac{40.6}{40.4}$ & 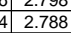 & & & & $\begin{array}{l}0.154 \\
0.714 \\
\end{array}$ & \\
\hline & $2 / 3 / 2$ & & 25.7 & 19.1 & 25.4 & 23.96 & & 46.498 & 47. & 11.502 & $\begin{array}{l}34.644 \\
\end{array}$ & \begin{tabular}{|l|l|}
-1.216 \\
\end{tabular} & & 47. & 0 & 14.6 & & 46 & \begin{tabular}{|l|l|}
0.76667 \\
\end{tabular} & 46.1 & 11.7 & & \begin{tabular}{l|l|}
6 & 2.797 \\
\end{tabular} & & & & 89 & \\
\hline & $2 / 3 / 2 / 2$ & & 25.7 & 19.174 & 25.4 & 23.943 & & 46.5 & 47 & & & -1.2 & & & & 14. & & & 0.78333 & & & & & & & & & \\
\hline & & & & & & 23.9 & & & & & & & & & & & & & & & & & & & & & & \\
\hline & & & b. & 19.6 & $\frac{2.5}{2.5}$ & 23.96 & & & & 11.8 & & - 1.216 & & & & & & & & & & & & & & & & \\
\hline & & & & & & 20.8 & & & & & & -1.219 & & & & 14. & & & & & & & & & & & & \\
\hline & $2 / 120004$ & & & 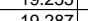 & 25.535 & 20.004 & & 4 & | & 11.516 & 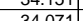 & 1216 & & 5015 & 0.190 & 146,63 & & & 86667 & & & & & & & & & \\
\hline & $2 / 3 / 2004$ & $9: 23: 50 \mathrm{AM}$ & 25.749 & $\begin{array}{l}19.299 \\
\end{array}$ & \begin{tabular}{|l|}
25.473 \\
\end{tabular} & 23.916 & 21.321 & 45.985 & 47.553 & 11.496 & |33.958 & \begin{tabular}{|l|}
-1.214 \\
\end{tabular} & -0.40 & 48.676 & 0.182 & \begin{tabular}{|l|}
14.643 \\
14.643
\end{tabular} & & 53.00 & 0.88333 & \begin{tabular}{|l|}
4.0 .1 \\
\end{tabular} & 11.9 & \begin{tabular}{l|l}
9 & 40.0 \\
\end{tabular} & \begin{tabular}{l|l|}
0 & 2.756 \\
\end{tabular} & 0.027 & 0.027 & 0.001 & 10.670 & f \\
\hline
\end{tabular}


WSRC-TR-2005-00105, REVISION 0

SRNL-RPP-2005-00012, REVISION 0

RUN \# WASH2; CAMPAIGN II SLURRY WASH 2 - CONT.

\begin{tabular}{|c|c|c|c|c|c|c|c|c|c|c|c|c|c|c|c|c|c|c|c|c|c|c|c|c|c|c|c|c|}
\hline & & & & & & & & & & & & & & & & & & & & & & & & & & & & \\
\hline & A & $B$ & D & $E$ & $\mathrm{~F}$ & G & $\mathrm{H}$ & $\mathrm{J}$ & $\mathrm{K}$ & $\mathrm{L}$ & $\mathrm{M}$ & $\mathrm{N}$ & $\mathrm{O}$ & Q & $\begin{array}{ll}R \\
\end{array}$ & $\mathrm{~s}$ & $T$ & $\mathrm{v}$ & w & $x$ & $Y$ & $z$ & AA & $A B$ & $\mathrm{AC}$ & $\mathrm{AD}$ & $\mathrm{AE}$ & AF $A$ \\
\hline 71 & $2 / 3 / 2004$ & 9:24:50 AM & 25.757 & 19.312 & 25.475 & 23.898 & 21.55 & 46.018 & 47.439 & $\begin{array}{l}11.401 \\
\end{array}$ & 34.202 & -1.216 & -6.378 & 49.825 & 0.204 & 14.643 & 0.004 & 54.00 & 0.9 & 46.0 & 12.2 & 40.1 & 2.765 & & & & 0.748 & \\
\hline 72 & & & 25.759 & 19.329 & 25.502 & 23.895 & 21.441 & 45.875 & \begin{tabular}{|l|l|}
47.468 \\
\end{tabular} & 11.446 & 33.991 & & & 50.647 & 0.19 & \begin{tabular}{|l|l|}
14.643 \\
\end{tabular} & & 55.00 & 0.91667 & 46.0 & 12.4 & 39.9 & 2.753 & 0.028 & 0.028 & 0.001 & 0.699 & \\
\hline & $2 / 3 / 2004$ & 9:26:50 AM & 25.78 & \begin{tabular}{|l|l|}
19.34 \\
\end{tabular} & & 23.916 & 21.502 & 46.054 & \begin{tabular}{|l|l|}
47.763 \\
\end{tabular} & \begin{tabular}{|l|l|}
11.527 \\
\end{tabular} & & -1.214 & $\begin{array}{l}-6.404 \\
\end{array}$ & 41.376 & 0.19 & \begin{tabular}{|l|l|}
14.643 \\
\end{tabular} & & & & 46.3 & \begin{tabular}{|l|l|}
10.1 \\
\end{tabular} & 40.0 & & & & & 0.698 & \\
\hline 74 & 2/3/2004 & $9: 27: 50 \mathrm{AM}$ & 25.803 & \begin{tabular}{|l|l|}
19.368 \\
\end{tabular} & 25.541 & \begin{tabular}{|l|}
23.9699 \\
\end{tabular} & 21.797 & 45.907 & \begin{tabular}{|l|l|}
47.591 \\
\end{tabular} & \begin{tabular}{|l|l|}
11.449 \\
\end{tabular} & 34.014 & \begin{tabular}{|l|}
-1.216 \\
\end{tabular} & & 49.725 & 0.192 & 14.643 & 0.004 & 57.00 & $\begin{array}{l}0.95 \\
\end{array}$ & 46.1 & 12.2 & 40.0 & \begin{tabular}{|l|}
2.755 \\
\end{tabular} & \begin{tabular}{|l|l|} 
\\
\end{tabular} .029 & $\begin{array}{l}0.028 \\
\end{array}$ & 0.001 & 0.706 & \\
\hline 75 & $2 / 3 / 2004$ & $9: 28: 50$ AM & 25.814 & 19.38 & & \begin{tabular}{|l|}
23.936 \\
\end{tabular} & 21.662 & & \begin{tabular}{|l|l} 
& 47.369 \\
\end{tabular} & $\begin{array}{l}1.432 \\
\end{array}$ & 33.747 & \begin{tabular}{|c|}
$\mid-1.216$ \\
\end{tabular} & & 51.4 & 0.191 & 14.643 & & 58.00 & 0.96667 & 45.9 & \begin{tabular}{|l|}
12.6 \\
\end{tabular} & 39.7 & 2.736 & 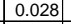 & \begin{tabular}{|c|}
0.028 \\
\end{tabular} & & & \\
\hline$\frac{76}{777}$ & $2 / 3 / 2004$ & $9: 29: 50$ AM & $\begin{array}{r}25.82 \\
25.859\end{array}$ & 19.391 & \begin{tabular}{|l|}
25.564 \\
25011
\end{tabular} & \begin{tabular}{|l|}
23.952 \\
\end{tabular} & 21.644 & $\begin{array}{l}45.797 \\
\end{array}$ & \begin{tabular}{|l|l}
47.583 \\
\end{tabular} & $\begin{array}{r}11.523 \\
1.557\end{array}$ & $\begin{array}{l}33.806 \\
23.91\end{array}$ & \begin{tabular}{|c|}
$\mid-1.216$ \\
\end{tabular} & -6.415 & 49.894 & 0.189 & \begin{tabular}{|l|l|} 
\\
\end{tabular} & 0.004 & 59.00 & 0.98333 & 46.1 & 12.2 & 39.8 & \begin{tabular}{|l|} 
\\
\end{tabular} & \begin{tabular}{|l|} 
\\
\end{tabular} & 0.028 & 0.001 & 0.697 & 0.59 \\
\hline$\frac{11}{78}$ & $2 \frac{21 / 2004}{2 / 3 / 2004}$ & $\begin{array}{l}9: 30: 50 \mathrm{AM} \\
9.31: 50 \mathrm{M}\end{array}$ & $\frac{25.858}{25.87}$ & $\frac{19.424}{19.446}$ & & $\frac{23.97}{24.042}$ & $\frac{22.1899}{21839}$ & $\begin{array}{r}45.878 \\
45.94 \\
\end{array}$ & \begin{tabular}{|l|}
47.649 \\
47.723 \\
\end{tabular} & $\frac{11.55}{11.745}$ & $\begin{array}{r}33.884 \\
33.718 \\
\end{array}$ & \begin{tabular}{|c|}
-1.2114 \\
-1.216 \\
\end{tabular} & $\begin{array}{l}-6.395 \\
-6.453 \\
\end{array}$ & $\begin{array}{l}50.359 \\
50.447\end{array}$ & $\begin{array}{c}0.189 \\
0.19\end{array}$ & $\begin{array}{l}14.643 \\
14.643 \\
\end{array}$ & $\begin{array}{l}0.004 \\
0.004\end{array}$ & $\frac{60.00}{66.00}$ & \begin{tabular}{|r|}
1 \\
\end{tabular} & $\begin{array}{l}46.2 \\
46.3 \\
\end{array}$ & $\frac{12.3}{12.4}$ & $\begin{array}{l}39.9 \\
39.8\end{array}$ & 2.750 & $\frac{0.028}{0.028}$ & $\mid \begin{array}{l}0.028 \\
0.028 \\
\end{array}$ & $\frac{0.001}{0.001}$ & $\mid \begin{array}{l}0.695 \\
0.699\end{array}$ & 0.59 \\
\hline & $2 / 3 / 2004$ & 9:32:50 AM & & 19.473 & \begin{tabular}{|l|l|}
25.615 \\
\end{tabular} & \begin{tabular}{|l|}
23.994 \\
\end{tabular} & 21.7 & $\begin{array}{l}4.54 \\
4.54\end{array}$ & \begin{tabular}{|l}
47.815 \\
\end{tabular} & 11.567 & 32.557 & & & 49.873 & 0.28 & 14.643 & & & 1.03333 & $\begin{array}{l}40.5 \\
46.3 \\
\end{array}$ & $\frac{1.4}{12.2}$ & $\frac{3.6}{38.5}$ & & & & & & $\frac{0.59}{0.91}$ \\
\hline 80 & $2 / 3 / 2004$ & $9: 33: 50 \mathrm{AM}$ & 25.639 & 19.49 & 25.392 & 24.036 & 22.305 & 45.307 & \begin{tabular}{|l|}
47.29 \\
\end{tabular} & 11.358 & 33.431 & -1.216 & -4.903 & 51.844 & 0.299 & 14.643 & & & 1.05 & 45.8 & 12.7 & 39.4 & 2.714 & .045 & $\begin{array}{l}0.041 \\
0.044\end{array}$ & 0.00 & $\begin{array}{l}\frac{1.004}{1.120} \\
\end{array}$ & $\begin{array}{l}0.91 \\
0.95 \\
\end{array}$ \\
\hline 81 & 2/3/2004 & 9:34:50 AM & & 19.508 & \begin{tabular}{|l|} 
\\
\end{tabular} & \begin{tabular}{|l|}
24.053 \\
\end{tabular} & 22.197 & 46.726 & 47.386 & \begin{tabular}{|l|l}
11.448 \\
\end{tabular} & & & -6.349 & & & 14.643 & & 64.00 & \begin{tabular}{|l|}
1.06667 \\
\end{tabular} & 45.9 & 12.6 & & & & & & & \\
\hline 82 & $2 / 3 / 2004$ & $9: 35: 50 \mathrm{AM}$ & 25.814 & 19.526 & \begin{tabular}{|l|l|}
25.387 \\
\end{tabular} & 24.036 & & $\begin{array}{l}46.294 \\
\end{array}$ & & 11.411 & 34.5 & & & $\begin{array}{ll}49.951 \\
\end{array}$ & 0.216 & 14.643 & & & \begin{tabular}{|l|l|}
$\mid$ \\
\end{tabular} & \begin{tabular}{|l|l|}
45.7 \\
\end{tabular} & & 40.4 & 785 & 0.032 & 0.032 & 0.00 & 0.789 & \\
\hline & & 9:36:50 AM & & 19.549 & & 24.034 & & & 396 & 11.333 & & & & & & 14.643 & & & & 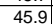 & & & .000 & & & & & \\
\hline \begin{tabular}{|l|l|}
84 \\
\end{tabular} & $2 / 3 / 2004$ & $9: 37: 50 \mathrm{AM}$ & 25.794 & 19.577 & \begin{tabular}{|l|}
25.367 \\
\end{tabular} & 24.066 & 21.758 & 46.446 & 47.286 & 11.316 & 34.779 & $\frac{1.216}{-1.216}$ & & 48.313 & 0.194 & 14.643 & & $\frac{6.6}{67 .}$ & $\begin{array}{l}1.11667 \\
\end{array}$ & 45.8 & $\frac{\frac{\pi}{11.8}}{11.8}$ & $\frac{0.6}{40.6}$ & 2.800 & $\frac{.029}{.029}$ & $\frac{\mid .029}{0.029}$ & & 0.705 & \\
\hline & $2 / 3 / 2004$ & 9:38:50 AM & & 19.594 & \begin{tabular}{|l|l|}
25.345 \\
\end{tabular} & 23.998 & 21.42 & 46.377 & \begin{tabular}{|l|l|}
47.431 \\
\end{tabular} & \begin{tabular}{|l|l|}
11.48 \\
\end{tabular} & 34.397 & \begin{tabular}{|l|}
$\mid-1.222$ \\
\end{tabular} & & & 0.192 & 14.643 & & 68.0 & 1.13333 & 46.0 & & 40.4 & 2.785 & & & & & \\
\hline 86 & $2 / 3 / 2004$ & 9:39:50 AM & 25.774 & 19.627 & \begin{tabular}{|l|}
25.377 \\
\end{tabular} & 24.031 & \begin{tabular}{l|l}
21.657 \\
\end{tabular} & 46.63 & 47.64 & 11.518 & 34.689 & \begin{tabular}{|l|l|l|}
$\mid 1216$ \\
\end{tabular} & & 50.549 & 0.191 & $\begin{array}{l}14.643 \\
\end{array}$ & & 69.00 & 1.15 & $\begin{array}{l}46.2 \\
\end{array}$ & $\overline{12.4}$ & & 2.803 & 0.028 & $\mid$ & 0.001 & \begin{tabular}{|l|l|l|}
0.693 \\
\end{tabular} & \\
\hline & $2 / 3 / 2004$ & 9:40:50 AM & 25.756 & 19.639 & \begin{tabular}{|l|}
25.349 \\
\end{tabular} & 24.083 & 21.8 & 46.51 & 47.603 & 11.532 & 34.512 & -1.219 & & 49.066 & 0.191 & 14.643 & & & 1.16667 & 46.1 & & 40.5 & 2.793 & & & & 0.696 & \\
\hline 88 & 2/3/2004 & 9:41:50 AM & 25.752 & 19.666 & 25.175 & 24.15 & 22.038 & 46.006 & 47.023 & 11.415 & 34.163 & -1.216 & & 47.946 & 0.193 & 14.643 & & & 1.18333 & 45.6. & & 40. & 1.764 & 0.029 & 0.029 & & 0.714 & \\
\hline & 2/3/2004 & 9:42:50 AN & 25.689 & 19.672 & 25.127 & 24.1111 & 22.2 & 45.857 & 46.988 & 11.385 & 34.022 & -1.2 .2 & & 49.289 & 0.196 & 14.643 & & & 1.2 & 45.5. & 12. & & 755 & & 0.049 & & & \\
\hline 90 & $2 / 3 / 2004$ & $9: 43: 50 \mathrm{AN}$ & & & & & & 46.062 & 47.184 & & 34.198 & & & 50.159 & 0.199 & 14.643 & & & & & & & & .030 & 0.030 & & 0.736 & \\
\hline 91 & $2 \mid 31 / 2004$ & 9:44:50 AM & 25.638 & 19.727 & 25.161 & 24.15 & 22.2699 & 46.346 & \begin{tabular}{|l|l} 
& 47.394 \\
\end{tabular} & 11.416 & 34.599 & & & 50.009 & 0.187 & 14.643 & & 14.0 & (1) & 45.9 & & & 700 & 0.028 & .028 & & & \\
\hline & 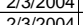 & 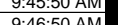 & & $\mid \frac{19.144}{10765}$ & $\frac{25.161}{25164}$ & 24.132 & $\frac{2.2406}{2.492}$ & $\frac{45.515}{45027}$ & \begin{tabular}{|l|}
46.181 \\
47108
\end{tabular} & $\frac{11.250}{11.402}$ & $\frac{33.87}{34102}$ & $\begin{aligned}-1.219 \\
12.6\end{aligned}$ & & 49.683 & 0.2011 & 14.643 & & 75.00 & & $\frac{45.3}{4.6}$ & & & .739 & 0.030 & 0.0300 & & & \\
\hline $\begin{array}{l}04 \\
94\end{array}$ & 2 & $\begin{array}{l}9.40 .50 \mathrm{AM} \\
9.47: 50 \mathrm{~A}\end{array}$ & $\frac{25.000}{25.608}$ & $\begin{array}{l}19.1003 \\
19782\end{array}$ & $\begin{array}{l}25.104 \\
25.171\end{array}$ & $\frac{24.100}{24.145}$ & $\frac{2.49}{21.973}$ & $\frac{43.927}{46.087}$ & \begin{tabular}{|l|}
47.100 \\
47.518 \\
\end{tabular} & $\begin{array}{ll}11.404 \\
11.461\end{array}$ & $\begin{array}{l}34.102 \\
34.088 \\
\end{array}$ & $\mid$ & & $\begin{array}{r}40.545 \\
45.46\end{array}$ & $\begin{array}{l}0.199 \\
0.207\end{array}$ & $\begin{array}{l}14.045 \\
14.643\end{array}$ & & & $\mid$ & 4 & & & 764 & & & & | & \\
\hline & $2 / 3 / 2004$ & 9:48:50 AM & 25.589 & 19.794 & \begin{tabular}{|l|}
25.171 \\
25.172
\end{tabular} & 24.106 & $\frac{21.5126}{22.526}$ & 446.141 & $\frac{4.010}{47.39}$ & $\begin{array}{l}11.401 \\
11.423\end{array}$ & $\begin{array}{l}34.000 \\
34.295\end{array}$ & $\mid-1.216$ & & $\begin{array}{l}5.40 \\
50.14\end{array}$ & 0.191 & $\begin{array}{l}14.045 \\
14.643\end{array}$ & & & 1.20000 & 45.9 & $\frac{1.1}{12.3}$ & $\frac{4.1}{40.2}$ & 704 & $\begin{array}{l}029 \\
029 \\
\end{array}$ & & & 0.719 & \\
\hline 96 & $2 / 3 / 2004$ & $9: 49: 50 \mathrm{AM}$ & 25.585 & 19.821 & 25.173 & 24.128 & 21.775 & 46.018 & \begin{tabular}{|l|l|} 
& 47.278 \\
\end{tabular} & 11.434 & 34.159 & \begin{tabular}{|c|c|}
-1.216 \\
\end{tabular} & & 50.128 & 0.193 & 14.643 & & 79. & 1.31667 & 45.8 & 12.3 & 40.1 & 2.764 & 0.029 & 0.029 & 0.00 & 0.714 & \\
\hline 97 & $2 / 3 / 20$ & 9:50:50 AM & 25.591 & 19.847 & 25.189 & 24.189 & 21.866 & 45.967 & 47.321 & 11.491 & 34.024 & -1.214 & & & $\begin{array}{l}0.189 \\
\end{array}$ & 14.643 & & & & 45.8 & $2.4 \quad$ & 40.0 & 758 & & & & & \\
\hline 98 & $2 / 3 / 2004 \mid$ & $51: 50 \mathrm{AM}$ & 25.587| & 19.848 & 25.165 & 24.135 & & 46.234 & 47.663 & 11.537 & 34.205 & & & 678 & 0.193 & $\begin{array}{l}14.643 \\
\end{array}$ & & & 1.35 & 46.2 & & 40.2 & & 0.029 & 0.029 & 0.00 & & \\
\hline 99 & & 9:52:50 AM & 25.579 & 19.865 & 25.167 & 24.136 & 21.9 & & 47.359 & & & & & 49.139 & 0.189 & 14.643 & & & & $45 .$. & & & 764 & & & & & \\
\hline & $2 / 3 / 2004$ & 9:53:50 AM & 25.575 & 19.891 & & & & 45.656 & \begin{tabular}{|l|l|}
46.954 \\
\end{tabular} & 11.241 & 34.024 & & & 48.649 & & 14.643 & & & 38333 & 45.5 & & & 1.747 & $\frac{0.030}{60}$ & & & 55 & \\
\hline & $2 / 3 / 2004$ & & 25.526 & & & 24.219 & 22.34 & 46.006 & \begin{tabular}{|l|l|}
47.481 \\
\end{tabular} & $\begin{array}{l}11.41 \\
\end{array}$ & 34.135 & -1.214 & & $\begin{array}{l}47.487 \\
\end{array}$ & & $\begin{array}{l}14.643 \\
\end{array}$ & & & $\mid 1.4$ & 46.6 & & 40. & & & & & & \\
\hline & $2 / 3 / 2004$ & 9:55:50 AM & 25.503 & 19.925 & \begin{tabular}{|l|}
25.021 \\
\end{tabular} & 24.276 & 22.6. & 45.73 & \begin{tabular}{|l|l|}
47.203 \\
\end{tabular} & 11.335 & 33.944 & \begin{tabular}{|l|l|} 
\\
\end{tabular} & & $\begin{array}{ll}41.966 \\
\end{array}$ & 0.194 & $\begin{array}{l}14.643 \\
\end{array}$ & & & 1.41667 & 45. & 10.3 & & 1.747 & $\overline{229}$ & & & 726 & \\
\hline & $2 / 3 / 2004$ & 9:56:50 AM & 25.505 & 19.966 & 25.053 & 24.313 & 22.25 & 45.539 & 46.936 & \begin{tabular}{|l|l|l|l|}
11.188 \\
\end{tabular} & 33.932 & -1.216 & & 46.984 & 0.195 & $\begin{array}{l}14.643 \\
\end{array}$ & & & 1.43333 & 45.5 & $\overline{11.5}$ & 39.7 & 2.740 & 0.029 & \begin{tabular}{|l|l|l|}
0.029 \\
\end{tabular} & & 0.731 & \\
\hline 10 & 2/3/2004 & 9:57:50 AM & 25.466 & 19.952 & \begin{tabular}{|l|}
25.019 \\
\end{tabular} & 24.269 & & 45.761 & \begin{tabular}{|l|l}
47.28 \\
\end{tabular} & 11.228 & 34.014 & & & 47.763 & 0.192 & 14.643 & & & 1.45 & 45.8 & & & & & & & & \\
\hline & $2 / 3 / 2 \mathrm{C}$ & 9:58:50 AM & 25.482 & 19.989 & 25.055 & 24.28 & & 45.878 & 47.34 & 11.236 & 34.213 & -1. & & 48.173 & 0.195 & 14.643 & & 88 & 1.46667 & 45.9 & & 40. & 76 & 229 & & .00 & 725 & \\
\hline & 2/3/2004 & 9:59:50 AM & 25.474 & 20.001 & 25.062 & 24.272 & & 45.564 & \begin{tabular}{|l|l} 
\\
\end{tabular} & 11.192 & 30.095 & -1.219 & & 51.550 & 0.19 & 14.643 & & 09. & & 45.7. & $12.6 \mathrm{C}$ & 09.1 & 2.109 & 0.020 & 0.020 & 0.00 & 0.712 & \\
\hline & $2 / 3 / 2004$ & & & & & & & & & & & & & 53.194 & & & & & & & & & & & & & & \\
\hline & 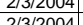 & $0.01 .50 \mathrm{AM}$ & $\frac{2.486}{2.897}$ & 20.049 & & 24.31 & $\frac{2.49}{2.29}$ & & & 11.281 & 34.069 & \begin{tabular}{|c|c|c|c|} 
\\
\end{tabular} & & $\frac{49.951}{52520}$ & & 14.6 & & & 1.151606/1 & 45.9 & & (1) & & & & & 0 & \\
\hline & 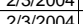 & 0.020 .50 & $\frac{2.0406}{25455}$ & 20.083 & 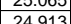 & 24.01 & & & 41 & 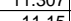 & 34.042 & & & 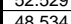 & & 14.6 & & & $\mid$ & 40.0 & & & 4.105 & & $0.0 \angle 9$ & & 0 & \\
\hline & 2 & 10.00 & $\frac{2.40406}{25.426}$ & 20.094 & \begin{tabular}{|l|l|}
24.929 \\
\end{tabular} & 24274 & $\frac{251}{2216}$ & & \begin{tabular}{|l|l|l|}
47135 \\
\end{tabular} & 11.1.158 & $\frac{3.006}{33.948}$ & $\frac{-1.219}{-1.214}$ & & 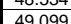 & 0.1044 & 14643 & & 94 & 15667 & 年 45. & $\frac{1.10}{120}>0$ & 年3. & 2740 & & & & & \\
\hline & & to. & $\frac{2.460}{25.42}$ & & & $\frac{2.671}{2431}$ & & & & 11.1.141 & & $\frac{-12}{-12}$ & & 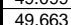 & 0.195 & $\frac{1.46}{146}$ & & & & & & & & & & & & \\
\hline & $\frac{2 / 3 / 2}{2 / 2}+2$ & 10:06:50 AM & $\frac{25.398}{25}$ & $\frac{20.116}{20.116}$ & \begin{tabular}{|l|l|}
24.946 \\
\end{tabular} & 24.336 & & & 47.236 & 11.21 & 33.812 & $\frac{-1.216}{-1.216}$ & & 48.328 & 0.191 & 144.6 & & & $\frac{1.6}{1.6}$ & $\frac{45.8}{45}$ & & & & & $\mid 0.029$ & & 0.719 & \\
\hline & & $\frac{1.50 .50 \mathrm{AM}}{10.07 .50}$ & 25.399 & & & & & & & 11266 & & & & 10 & & $14.6 \mathrm{C}>\mathrm{C}$ & & & 1.61667 & 45.7 & & & & & & & & \\
\hline 115 & $2 / 320044$ & $10.08 .50 \mathrm{AM}$ & 25395 & 20.158 & 24.978 & 24.358 & 220 & 45 & 4684 & 11197 & 33.488 & -1219 & & 48036 & & 146 & & & 163333 & 454 & 118 & 393 & & & & & 25 & \\
\hline & $2 / 3 / 2004$ & 10:09:50 AM & 25.386 & 20.165 & 24.979 & 24.369 & $\overline{22.1}$ & 45.419 & \begin{tabular}{|l|}
47.097 \\
\end{tabular} & 11.278 & 33.681 & -1.216 & & 47.247 & 0.196 & 14.6 & & & 1.65 & 45.6 & $111.6 \mathrm{C}-\mathrm{s}$ & & & & & & & \\
\hline & $2 / 3 / 2004$ & 10:10:50 AM & 25.377 & 20.176 & 24.985 & & & 448 & & 11.278 & 33.683 & & & 45.739 & & 14.6 & & & 66667 & 45.7 & 112 & 396 & 2.728 & & & & 784 & \\
\hline 11 & $2 / 3 / 2004$ & 10:11:50 AM & 25.378 & 20.181 & \begin{tabular}{|l|l|}
24.971 \\
\end{tabular} & 24.361 & & & \begin{tabular}{|l|l|l} 
& 47.093 \\
\end{tabular} & 11.253 & 33.531 & -1.2. & & 50.4. & & 14.643 & & & 1.68333 & 45.6 & 12.4 & $\overline{39.4}$ & 19 & & & & 0.727 & \\
\hline 119 & 2/3/2004| & 10:12:50 AM & 25.379 & 20.208 & \begin{tabular}{|l|l|}
24.997 \\
\end{tabular} & 24.362 & & & 47.564 & 11.275 & 34.09 & -1.216 & & 51.5. & & 14.6 & & 102. & 1.7 & 46.1 & 12.6 & 40.0 & 755 & & & & 0.731 & \\
\hline 12 & 2/3/2004 & 10:13:50 AM & 25.385 & 20.219 & 25.003 & 24.328 & & 45.488 & & 11.365 & 33.667 & -1.2 & & 48.6 - 45 & 0.1 & 14.6 & & & 1.71667 & 45.7. & 11. & & & & & & & \\
\hline 12 & 2/3/2004 & 10:14:50 AM & 25.411 & 20 & 25.004 & 24.369 & & & 47.454 & 11.274 & 29.351 & 1.1 & & 49. & & $14.6 \mathrm{C}>\mathrm{C}$ & & 104. & $\begin{array}{l}1.73333 \\
\end{array}$ & 46.0 & & 35. & & D08 & 0.008 & & 233 & \\
\hline & 2/3/2004 & 10:15:50 A & 25.298 & 20.257 & 24.87 & 24.401 & & & & 11.099 & 32.692 & -1.216 & & $47.4 .42>$ & & 14. & & & 1.75 & 45.6. & $1+$. & & & & & & & \\
\hline$\frac{123}{122}>2$ & & & 25.243 & 20.263 & 24.851 & 24.4 & & & & 11.093 & 34.49 & & & & & & & & 1.66667 & 45.4. & & & & & & & & \\
\hline & 2/3/212004] & 10:1:50 AM & (35.01 & 20.275 & \begin{tabular}{|l|l|}
24.863 \\
\end{tabular} & 24.449 & 22.8 & 46.1 & 46.994 & 11.151 & 34.635 & -1.216 & & $48 . \pi$ & 0.205 & $14.6 \mathrm{C}>\mathrm{C}$ & & 10000 & & 45.5 & 1.0 & & & & & & & \\
\hline & & $\frac{10.18 .50 \mathrm{~A}}{10.601}$ & 2.350 & 20.291 & 24.884 & 24.40 & & & 4.085 & 11.22 & 34.5 & & & $\frac{5.1}{51}>>19$ & & 14.6 & & & 1.8 & 45.60 & & & & & & & & \\
\hline & 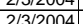 & 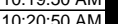 & $\frac{2.53}{2533}$ & $\frac{20.304}{20313}$ & 24.005 & $\frac{24.4606}{24406}$ & & & & $\frac{1.0011}{111}$ & $\frac{34 .}{33 .}$ & -1.2 & & & & 4.0 & & 110 & | & 45.4 & 10 & & & & & & & \\
\hline 128 & 年 & 10.5.50 AM & $\frac{25.53}{2533}>0$ & 20.338 & 24.926 & 24,417 & $\frac{2.5}{225}$ & 4 & & $\mid \frac{11.1}{11096}$ & $\frac{34.56}{346}$ & -12 & & 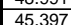 & & $\frac{140}{146}$ & & & $\mid \frac{1.0055}{1.85}$ & 年 45.5 & & & & & & & 0.700 & \\
\hline 129 & $2 / 3 / 2004$ & $10: 2250 \mathrm{AM}$ & 25.334 & 20.344 & 24.932 & 24,408 & & 46.093 & 47.158 & 11.179 & 34.447 & -12 & & $50.4>->$ & & 1146 & & 112 & 186667 & 45.7 & 12.4 & & & & & & & \\
\hline 130 & $2 / 3 / 2004$ & 10:23:50 AM & 25.335 & 20.355 & \begin{tabular}{|l|l|}
24.923 \\
\end{tabular} & 24.409 & & 46.097 & & 11.085 & & $\frac{-1.219}{-1.219}$ & & & & & & & 1.88333 & 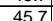 & & & & & & & & \\
\hline 131 & & $10: 24: 50$ & 25.342 & 20.372 & 24.94 & 24.395 & & 46.168 & & 11.216 & 34.5 & -1.219 & & 47.23 & & & & & D 1.9 & 45.7 & 11.6 & & & & & & & \\
\hline & & & & 20.3 & 24 & 24.436 & & 46.16 & & 11.347 & & $-1.22>0$ & & & & 14 & & & & 45.6 & & & & & & & & \\
\hline 133 & $2 / 3 / 2004$ & $10: 26: 50 \mathrm{~A}$ & 25.339 & 20.409 & \begin{tabular}{|l|l|}
24.796 \\
\end{tabular} & 24.447 & $22.8 \mathrm{~s}>\mathrm{l}$ & 45.622 & 46 & 11.044 & 34.188 & -1.2 & & 45.99 & 0.2 & 14.6 & & 116 & 1.93333 & 45.1 & 11.3 & & & & & & & \\
\hline & & 10:27:50 & 25.30 & 20.42 & 24.817 & 24.49 & & 46.02 & \begin{tabular}{|l|l|}
47.129 \\
\end{tabular} & 10.988 & 34.6 & $\begin{array}{l}-1.219 \\
\end{array}$ & & 49.5 & 0.1 & 14.6 & & & 1.95 & 45.7 & & & & & & & & \\
\hline 135 & 2/3/2004] & $\begin{array}{ll}10: 28: 50 \mathrm{~A} \\
\end{array}$ & 25.284 & 20.425 & 24.822 & 24.488 & 22. & 46.058 & & \begin{tabular}{|c|}
11.08 \\
\end{tabular} & 34.496 & \begin{tabular}{|l|l|} 
& -1.225 \\
\end{tabular} & & 53.636 & 0.203 & $\begin{array}{l}14.643 \\
\end{array}$ & & 118. & 1.96667 & 45.7 & 13.1 & $46>$ & & \begin{tabular}{|l|} 
\\
\end{tabular} & 0.030 & & 755 & \\
\hline & & 10:29:5 & 25.28 & 20.441 & \begin{tabular}{|l|}
24.853 \\
\end{tabular} & \begin{tabular}{|l|}
24.514 \\
\end{tabular} & 22.6 & 45.724 & 46. & 11.103 & 34.239 & -1.216 & & 48.9 & 0.2 & 14.6 & & 119. & 1.98333 & 45.3 & 12. & & & & & & 0.749 & \\
\hline & 004 & 10:30:50 & 25.276 & & \begin{tabular}{|l}
24.859 \\
\end{tabular} & 24.535 & & 45.871 & \begin{tabular}{|l|}
46.981 \\
\end{tabular} & 11.106 & 34.301 & -1.219 & & 1.4 & 0.2 & 14.6 & & & & 45.5 & 12.6 & & 164 & & & & & \\
\hline & & $10: 31: 50$ AM & 25.277 & 20.479 & & & & 45.965 & & 11.1117 & 34.4 & \begin{tabular}{|l|l|} 
& -1.216 \\
\end{tabular} & & 49.8 & & 14. & & & & 45.6 & 12.2 & & & & & & & \\
\hline & & 10:32: & 5.2.283 & & 24 & 24.502 & & & & 11.055 & $\frac{34.57}{24.57}$ & -1. & & & & 14.6 & & & & 45.7 & & & & & & & & \\
\hline & & & & & & & & & & & & & & & & & & & & & & & & & & & & \\
\hline
\end{tabular}


WSRC-TR-2005-00105, REVISION 0

SRNL-RPP-2005-00012, REVISION 0

RUN \# WASH2; CAMPAIGN II SLURRY WASH 2 - CONT.

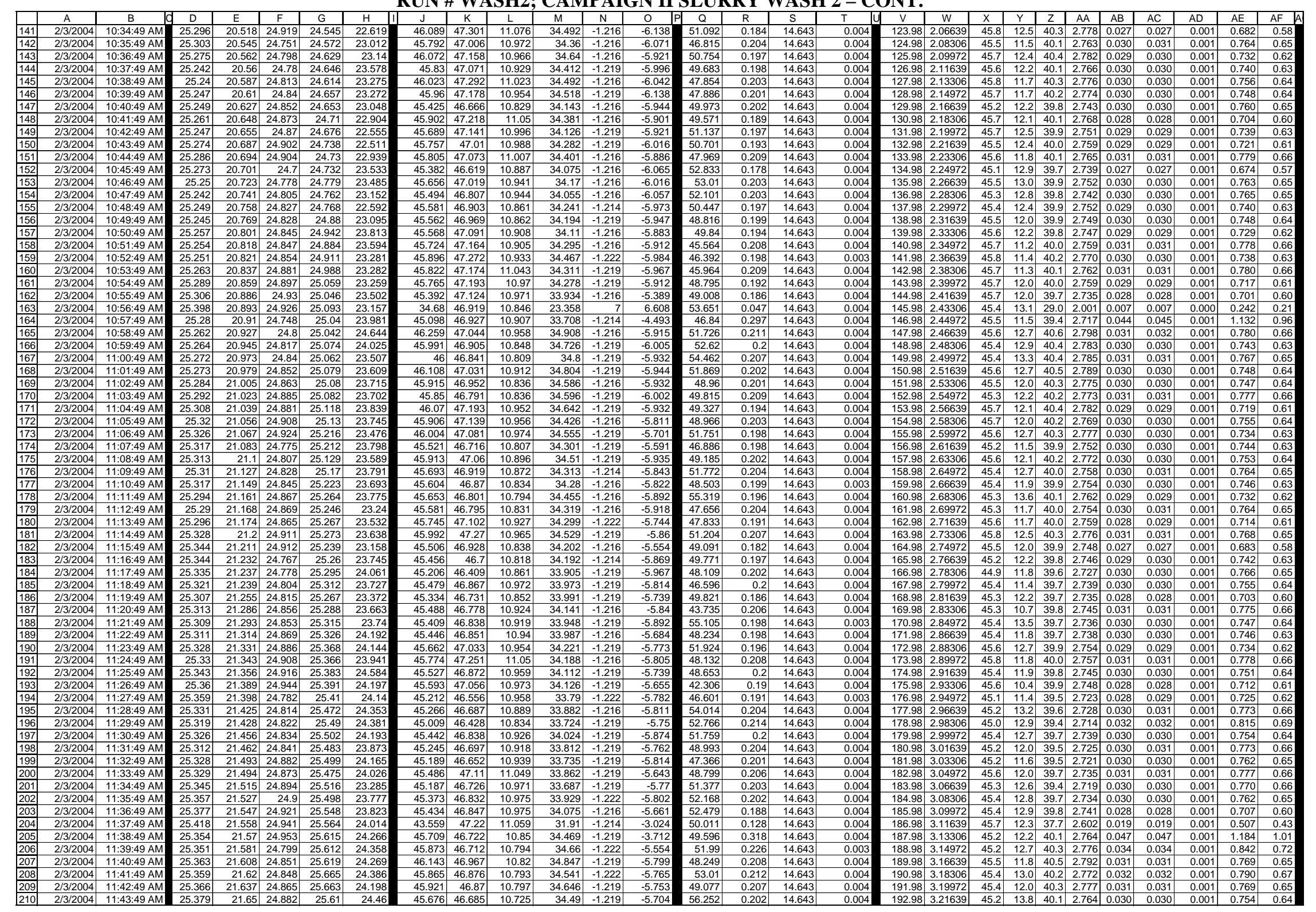


WSRC-TR-2005-00105, REVISION 0

SRNL-RPP-2005-00012, REVISION 0

RUN \# WASH2; CAMPAIGN II SLURRY WASH 2 - CONT.

\begin{tabular}{|c|c|c|c|c|c|c|c|c|c|c|c|c|c|c|c|c|c|c|c|c|c|c|c|c|c|c|c|c|}
\hline & & & & & & & \\
\hline & $\mathrm{A}$ & $\mathrm{B}$ & $\mathrm{D}$ & $E$ & $\mathrm{~F}$ & $G$ & $\mathrm{H}$ & $\mathrm{J}$ & $\mathrm{K}$ & $\mathrm{L}$ & $\mathrm{M}$ & $\mathrm{N}$ & 0 & $\mathrm{Q}$ & $\mathrm{R}$ & $\mathrm{s}$ & \begin{tabular}{l|l|l|} 
\\
\end{tabular} & $\mathrm{v}$ & W & $x$ & & $z$ & AA & $A B$ & $\mathrm{AC}$ & $\mathrm{AD}$ & $\mathrm{AE}$ & AF $A$ \\
\hline & 2/3/2004 & $11: 44: 49 \mathrm{AM}$ & 25.386 & 21.678 & 24.905 & 25.537 & \begin{tabular}{|l|l|}
24.238 \\
\end{tabular} & 46.008 & 47.166 & 10.844 & 34.621 & -1.219 & -5.759 & 47.06 & 0.206 & $\begin{array}{l}14.643 \\
\end{array}$ & 0.004 & 193.98 & 3.23306 & 45.7 & 11.5 & 40.3 & & & 0.031 & 0.00 & 0.764 & 0.65 \\
\hline & 2/3/2004 & 11:45:49 AM & 25.389 & 21.68 & 24.918 & \begin{tabular}{|l|l}
25.475 \\
\end{tabular} & 24.236 & 45.763 & 46.874 & 10.755 & 34.588 & -1.219 & $\begin{array}{r}-5.617 \\
-5.617 \\
\end{array}$ & 49.45 & 0.198 & 14.643 & 0.004 & 194.98 & $\frac{3.24972}{3.24972}$ & 45.4 & & 40.2 & & & & & 0.736 & \\
\hline & $2 / 3 / 2004$ & 11:46:49 AM & 25.407 & 21.713 & 24.966 & 25.433 & 24.459 & 45.583 & 46.828 & $\begin{array}{l}10.781 \\
\end{array}$ & 34.323 & -1.216 & -5.551 & 46.261 & 0.209 & 14.643 & $\begin{array}{l}0.004 \\
\end{array}$ & 195.98 & 3.26639 & 45.4 & & 40.0 & 2.100 & & & 0.001 & 0.781 & 0.66 \\
\hline 214 & $2 / 3 / 2004$ & 11:47:49 AM & 25.405 & 21.716 & 24.743 & 25.345 & $\begin{array}{l}24.892 \\
\end{array}$ & 45.394 & 46.712 & 10.756 & 34.104 & -1.219 & $\begin{array}{l}-5.609 \\
\end{array}$ & 54.483 & 0.198 & 14.643 & 0.004 & \begin{tabular}{|l|l|}
196.98 \\
\end{tabular} & 3.28306 & 45.2 & \begin{tabular}{|l|l|} 
& 13.4 \\
\end{tabular} & 39.7 & \begin{tabular}{|l|l|} 
& 2.741 \\
\end{tabular} & 0.030 & 0.030 & 0.001 & 0.748 & 0.64 \\
\hline$\frac{215}{216}$ & $2 / 3 / 2004$ & 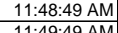 & $\begin{array}{r}25.373 \\
25.37\end{array}$ & $\begin{array}{l}21.719 \\
21.752\end{array}$ & \begin{tabular}{|l|}
24.801 \\
2.879
\end{tabular} & \begin{tabular}{|l|}
25.248 \\
25.201 \\
\end{tabular} & $\begin{array}{l}24.779 \\
\end{array}$ & 45.235 & \begin{tabular}{|l|l|}
46.4433 \\
46501
\end{tabular} & $\begin{array}{l}10.696 \\
10614\end{array}$ & $\begin{array}{l}34.069 \\
3254\end{array}$ & $\begin{array}{r}-1.219 \\
.210\end{array}$ & \begin{tabular}{|c|}
-5.684 \\
5
\end{tabular} & 45.045 & $\begin{array}{l}0.199 \\
0.190\end{array}$ & 14.643 & 0.004 & 197.98 & $\begin{array}{l}3.29972 \\
.2020\end{array}$ & 45.0 & 11.0 & 39.7 & \begin{tabular}{|l|l|} 
& 2.734 \\
\end{tabular} & & 0.030 & 0.001 & 0.752 & 0.64 \\
\hline$\frac{\mid 210}{217}$ & $\begin{array}{l}2 / 3 / 2004 \\
2 / 3 / 2004\end{array}$ & $\begin{array}{l}11: 49: 49 \mathrm{AM} \\
111.50\end{array}$ & $\begin{array}{r}25.37 \\
25.363\end{array}$ & $\frac{21.752}{21.759}$ & \begin{tabular}{|l|}
24.829 \\
24.841
\end{tabular} & \begin{tabular}{|l|}
25.201 \\
25.168
\end{tabular} & $\begin{array}{l}24.047 \\
23.769\end{array}$ & $\begin{array}{l}45.289 \\
45268\end{array}$ & \begin{tabular}{|l|}
46.501 \\
46.538 \\
\end{tabular} & $\begin{array}{l}\frac{10.614}{10.652} \\
\end{array}$ & $\begin{array}{r}34.254 \\
33.153 \\
\end{array}$ & \begin{tabular}{|l|l|}
-1.219 \\
-1.219
\end{tabular} & $\begin{array}{l}-5.704 \\
-5.5698\end{array}$ & $\begin{array}{r}51.475 \\
44.102\end{array}$ & $\begin{array}{l}0.199 \\
0.208\end{array}$ & $\begin{array}{r}14.643 \\
14.643\end{array}$ & $\begin{array}{l}0.004 \\
0.004\end{array}$ & \begin{tabular}{|l|l|}
198.98 \\
19998
\end{tabular} & 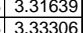 & $\begin{array}{l}45.0 \\
45.1\end{array}$ & & $\begin{array}{l}39.8 \\
3397\end{array}$ & $\frac{2.742}{2.738}$ & $\begin{array}{l}0.030 \\
\end{array}$ & $\begin{array}{l}0.030 \\
0.031\end{array}$ & $\begin{array}{l}0.001 \\
0.001\end{array}$ & $\begin{array}{l}0.750 \\
0.784\end{array}$ & 0.64 \\
\hline 218 & 2/3/2004 & $11: 51: 49 \mathrm{AM}$ & 25.364 & & \begin{tabular}{|l|}
24.862 \\
\end{tabular} & 25.129 & & 45.413 & & 10.728 & 34.243 & & -5.649 & 51.988 & $\begin{array}{l}0.200 \\
0.207\end{array}$ & $\begin{array}{l}14.045 \\
14.643\end{array}$ & $\begin{array}{l}0.004 \\
0.004\end{array}$ & & & $\begin{array}{l}5.1 \\
45.3\end{array}$ & & & $\frac{2.138}{2.746}$ & $\frac{0.031}{0.031}$ & $\frac{0.031}{0.031}$ & $\frac{0.001}{0.001}$ & 0.0 .7784 & 0.67 \\
\hline & $2 / 3 / 2004$ & & & 21.778 & 24.87 & 25.071 & & 45.529 & \begin{tabular}{|l|l|}
46.959 \\
\end{tabular} & 10.759 & 34.243 & -1.219 & & 49.093 & 0.211 & 14.643 & & 201.98 & 3.36639 & 45.5 & & & & & & & & $\frac{0.66}{0.67}$ \\
\hline 220 & $2 / 3 / 2004$ & 11:53:49 AM & 25.373 & 21.805 & 24.897 & 25.043 & 24.005 & 45.149 & 466.869 & 10.787 & 33.864 & -1.219 & -5.198 & 51.577 & 0.219 & 14.643 & 0.004 & 202.98 & 3.38306 & 45.4 & & \begin{tabular}{|l|l|}
6 & 39.5 \\
\end{tabular} & 2.724 & \begin{tabular}{|l|l|} 
\\
\end{tabular} & 0.033 & 0.001 & 0.829 & 0.67 \\
\hline 221 & $2 / 3 / 2004$ & 11:54:49 AM & 25.38 & 21.827 & 24.924 & 25.035 & & & & & & -1.216 & -5.545 & 52.691 & & & & & & & & & & & & & & $\frac{0.71}{0.71}$ \\
\hline 222 & 2/3/2004 & 11:55:49 AM & 25.397 & 21.834 & 24.921 & 25.022 & 24.459 & 45.496 & 46.961 & 10.814 & 34.188 & -1.219 & -5.4 & 52.076 & 202 & $\begin{array}{l}14.643 \\
\end{array}$ & & 204.98 & 3.41639 & 45.5 & & & 747 & 030 & 030 & .00 & 0.758 & $\frac{0.11}{0.64}$ \\
\hline$\frac{223}{224}$ & $2 / 3 / 2004$ & 11:56:49 AM & 25.399 & & & 25.019 & 24.446 & 45.425 & & & 34.174 & -1.219 & & & & & & & & & & & & & & & & \\
\hline 2224 & $2 / 3 / 2004$ & 11:57:49 AM & 25.402 & 21.854 & 24.715 & 24.997 & 24.569 & 45.382 & \begin{tabular}{|l|l|l|}
46.756 \\
\end{tabular} & 10.737 & 34.174 & $\frac{-1.219}{-1.219}$ & & 51.179 & & 14.643 & & 206.98 & .44972 & 45.3 & & 39. _ _ & 6743 & 030 & & .001 & & 0.04 \\
\hline$\frac{225}{226}$ & $2 / 3 / 2004$ & $11: 58: 49 \mathrm{AM}$ & 25.384 & 21.881 & 24.812 & 24.989 & 24.361 & 45.064 & 46.67 & 10.734 & 33.732 & -1.216 & -5 & 50.801 & & $\begin{array}{l}14.643 \\
\end{array}$ & & & $\begin{array}{l}3.46639 \\
\end{array}$ & 45.8 & & & $2.716 \mathrm{~S}$ & & & & & \\
\hline$\frac{226}{227}$ & $2 / 3 / 2004$ & 11:59:49 AM & 25.336 & 21.874 & \begin{tabular}{|l|l|}
24.825 \\
\end{tabular} & \begin{tabular}{|l|}
25.001 \\
\end{tabular} & 24.153 & 45.093 & \begin{tabular}{|l|l|l}
46.521 \\
\end{tabular} & 10.749 & 33.915 & -1.216 & -5.623 & 52.587 & 0.21 & $\begin{array}{l}14.643 \\
\end{array}$ & .00 & 208.98 & \begin{tabular}{|l|l|}
3.48306 \\
\end{tabular} & 45.0 & 12.9 & $\begin{array}{l}9 \\
9\end{array}$ & 2.724 & \begin{tabular}{|l|}
0.031 \\
\end{tabular} & 0.031 & 0.001 & 0.796 & 0.68 \\
\hline & & 12:00:49 PM & & 21.885 & \begin{tabular}{|l|}
24.831 \\
\end{tabular} & 24.997 & 23.944 & 45.166 & \begin{tabular}{|l|l|}
46.668 \\
\end{tabular} & 10.727 & 33.948 & $\begin{array}{l}-1.219 \\
\end{array}$ & -5 & 48.941 & 0.203 & 14.643 & & 209.98 & 3.49972 & 45.2 & & & 72 & & & & 1.769 & \\
\hline & $\frac{2 / 3 / 2004}{2 / 2004}$ & 12:01:49 PM & 25.33 & 21.897 & 24.843 & 24.924 & 24.146 & & 46.542 & 10.693 & 33.878 & -1.222 & -5.389 & 49.312 & 0.192 & 14.643 & & 210.9 & 3.51639 & 45. & & 39. & 1.72 & & & & ..729 & 0.62 \\
\hline & $\frac{2 / 3 / 2004}{2 / 32004}$ & 12:02:49 PM & 25.33 & $\frac{21.908}{22}$ & $\frac{24.849}{24.855}$ & 24.895 & 24.122 & 45.104 & \begin{tabular}{|l|l|}
46.546 \\
\end{tabular} & 10.186 & 100 & -1.219 & & 6002 & 0.2 & 14.643 & & (1) & 50072 & 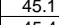 & & & & & & & & 年 \\
\hline & $\frac{2131204}{21 / 204}$ & $12: 03: 4 \mathrm{PM}$ & 20.351 & & & & & & \begin{tabular}{|l|l}
46.869 \\
79
\end{tabular} & & & $\frac{-1.222}{-1212}$ & & 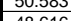 & 0.2 & 14.643 & & & & & & & & & & & & 0.66 \\
\hline & $\frac{1512004}{2 / 2004}$ & 12:04449M & 20.301 & $\frac{2.9305}{21051}$ & $\frac{2.001}{2.012}$ & 24.054 & 24.074 & 4 & 46.604 & 10.095 & $\frac{33.911}{2.120}$ & $\frac{-1.219}{-1.211}$ & & 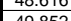 & 0.2 & 14.0 & & 11108 & 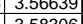 & 45.2 & & & & & & , & & \\
\hline & $2 / 3 / 2004$ & $\begin{array}{ll}120.06449 \mathrm{PM} \\
10\end{array}$ & $\frac{25.505}{25365}$ & $\frac{2.51968}{21.968}$ & 24.918 & $\frac{24.004}{24824}$ & 24.56 & & 40.3148 & & & -1219 & & 878 & & $\frac{14.6}{146}$ & & & & & & & & & & & & \\
\hline & $2 / 3 / 2004$ & 12:07:49 PM & 25.365 & 21.973 & \begin{tabular}{|l|}
24.929 \\
\end{tabular} & 24.83 & 24.072 & 45.278 & 46.514 & 10.766 & 34.02 & -1.219 & -5.626 & 50.339 & 0. & 14,643 & & & 1639 & 45.3 & $\mid 12.3$ & \begin{tabular}{l|l}
3 & 39.6 \\
\end{tabular} & 2.7 & 0.030 & 0.030 & 0.001 & 0.765 & \\
\hline & & 12:08:49 PM & & 21.99 & 24.72 & 24.776 & 24.734 & 44.847 & 46 & 10.64 & 33.687 & -1.219 & & & & 14.6 & & & & 45.0 & & & & & & 0.001 & 0.739 & 0.6 \\
\hline$\frac{23}{3}$ & $2 / 3 / 2004$ & 12:09:49 PM & 25.353 & 22.012 & 24.802 & 24.753 & $\begin{array}{r}24.43 \\
\end{array}$ & 44.952 & 46.538 & 10.663 & 33.817 & -1.216 & & 52.295 & 0.198 & 14.643 & & 218.98 & 3.64972 & 45.1 & 129 & 39.2 & & 030 & 0.030 & 0.00 & 0.754 & \\
\hline & 3/2004 & 12:10:49 PM & & 22.002 & \begin{tabular}{|l|l|} 
& \multicolumn{1}{|l}{817} \\
\end{tabular} & 24.708 & 24. & 44.897 & 46.59 & 10.687 & 33.681 & -1.219 & & .008 & & 14.6 & & .90 & 3.66639 & 45. & & & & & & & & \\
\hline$\frac{23}{3}$ & 2/3/2004 & $12: 111: 49$ PM & 25.35 & 22.044 & 24.864 & 24.755 & 24.132 & 44.977 & 46 & 10.71 & 33.794 & -1.219 & & 49.963 & 0.21 & 14.6 & & & 3.68306 & 45.2. & & & .7 & & & 0.00 & 309 & \\
\hline & $2 / 3 / 2004$ & 12:12:49 PM & $\frac{25.321}{25200}$ & 22.0366 & 24.856 & 24.711 & 24.859 & 45.347 & 47 & 10.867 & 33.909 & -1.219 & & 1.071 & & 14.6 & & 201.90 & $3.699 / 2$ & 45.6 & & & & & & & & \\
\hline & $\frac{21312004}{212004}$ & 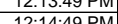 & 25.329 & $\frac{2.2048}{22050}$ & & 24.123 & 24. & $\begin{array}{l}44.6060 \\
4.510\end{array}$ & \begin{tabular}{|l|l|l|l|l|}
4681 \\
\end{tabular} & & & -1.2 & & . 3088 & & & & & & & & & & & & & & \\
\hline$\frac{241}{242}$ & $2 / 3 / 2 / 2004$ & $\begin{array}{ll}12.44 .49 \\
12.159 \mathrm{PM}\end{array}$ & $\frac{23.321}{25343}$ & $\frac{2.039}{22082}$ & 24.679 & 24.065 & $\frac{24.114}{2304}$ & $\frac{45.345}{45420}$ & \begin{tabular}{|l|l|}
46.014 \\
1954
\end{tabular} & 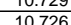 & $\frac{34.4}{34.1}$ & $\frac{-1.219}{-120}$ & & 5 & & 14. & & & & & & & & & & & & \\
\hline 243 & $2 / 3 / 2004$ & $12: 16: 49$ PM & 25.355 & $\frac{22.089}{22.089}$ & \begin{tabular}{|l|l|}
24.893 \\
\end{tabular} & 24.629 & 24.261 & 45.427 & \begin{tabular}{|l|l|}
46.903 \\
\end{tabular} & 10.806 & 34.126 & \begin{tabular}{|c|c|}
-1.219 \\
\end{tabular} & & 52.902 & & 14.4 & & & 376639 & 45.4 & $\frac{13.0}{130}$ & 39.8 & 2.7 & & & & & \\
\hline 244 & $2 / 3 / 2004$ & 12:17:49 PM & 25.361 & 22.1 & \begin{tabular}{|l|l|}
24.845 \\
\end{tabular} & 24.625 & 24.188 & 45.458 & 46.83 & 10.716 & 34.315 & \begin{tabular}{|l|l|l|} 
\\
\end{tabular} & -5.58 & 49.306 & 0.209 & 14.643 & & 226.98 & 3.78306 & 45.4 & \begin{tabular}{|l|}
12.1 \\
\end{tabular} & 39.9 & 2.750 & & & 0.001 & 0.785 & \\
\hline 245 & $2 / 3 / 2004$ & 12:18:4 & 20.373 & & & & & 44.928 & & 10. & 33.691 & -1.219 & & . & & & & & 3.7 & 45.1 & & & & & & & & \\
\hline 2 & $2 / 3 / 2004$ & & 25.329 & 22.118 & 24.778 & 24.608 & 24.68 & 45.056 & & & 33.79 & & & .299 & & 14. & & & & & & & & & & & & \\
\hline 2 & 2 & $12: 20: 4$ & 20.320 & 22.145 & 24.19 & 24.59 & 24. & 45.125 & & & 33.929 & & & 46.6 & & $14.6 \mathrm{C}$ & & & 20 & 45.22 & & & & & & & & \\
\hline & $\begin{array}{ll}213120004 \\
21 / 2004\end{array}$ & & $\frac{25.301}{25270}$ & $\begin{array}{l}2.145 \\
22167\end{array}$ & 24.79 & & & 45.448 & & & & & & 51.4 & & & & & & & & & & & & & & \\
\hline$\frac{240}{250}$ & $2 / / 3 / 2004$ & $\begin{array}{l}12.22 \\
12.23 \\
\end{array}$ & $\frac{2.2510}{25248}$ & $\frac{2.1 .18}{22182}$ & $\frac{24}{24}$ & & 24. & & & & $\begin{array}{l}33.425 \\
33999\end{array}$ & $\frac{-1.2}{-1.2}$ & & 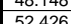 & & & & & & & 10 & & & & & & & \\
\hline 251 & 27512004 & $\frac{12.25 .45}{12.24 .49}$ & $\begin{array}{l}25.240 \\
25259\end{array}$ & 22184 & 24848 & 24 & 24. & & & & & -1.2 & & 245 & & 146 & & & & & & & & & & & & \\
\hline 252 & & $\begin{array}{ll}12.25 \\
2549 \mathrm{PM}\end{array}$ & $\frac{25.254}{25.264}$ & $\frac{25.149}{22.199}$ & 24.853 & & $\frac{25}{23}$ & 45.353 & & & 34.057 & -1.219 & & 4 & & & & & & & & & & & & & & \\
\hline & & $12: 26: 44$ & 25.264 & 22.189 & & 24. & & & & & & -1.219 & & & & & & & & & & & & & & & & \\
\hline & 82004 & $12: 27: 49$ & $\frac{25.259}{25}$ & $\frac{22.199}{22}$ & 24.868 & & 24. & 45,421 & & & & & & & & & & & & & & & & & & & & \\
\hline & & & & 209 & & & & 45.17 & & & 33.9 & -1.216 & & & & & & & & 45.3 & & & & & & & & \\
\hline & $2 / 3 / 2004$ & 12:29:49 PM & 5.431 & 22.215 & 24.91 & 24.464 & 24.46 & 31.667 & 46.863 & $\begin{array}{ll}10.757 \\
\end{array}$ & 20.467 & 9.863 & & 52.983 & 0.046 & 14.643 & & & $983 \mathrm{C}$ & & & & & & .007 & & 264 & \\
\hline & & & & & & & & & & & & & & & & & & & & & & & & & & & & \\
\hline & & Averages & 25.4 & 20.5 & $\begin{array}{r}25.0 \\
256\end{array}$ & 24.6 & 22.6 & $\frac{45.8}{49.1}$ & $\begin{array}{l}47.2 \\
50.8\end{array}$ & $\frac{11.2}{14.2}$ & $\begin{array}{l}34.1 \\
36.1\end{array}$ & $\frac{-1.1}{9.9}$ & 5.8 & $\frac{49.6}{56.3}$ & 0.2 & 14.6 & & & & $\frac{45.8}{49.4}$ & \begin{tabular}{|l|}
12.1 \\
138 \\
\end{tabular} & $\begin{array}{r}39.9 \\
426\end{array}$ & $\frac{2.754}{2.937}$ & & $\frac{0.029}{0.062}$ & $\frac{0.0007}{0.0016}$ & $\begin{array}{l}0.735 \\
1.533 \\
\end{array}$ & 0.625 \\
\hline & & Median & $\begin{array}{l}25.9 \\
25.3\end{array}$ & $\frac{22.4}{20.5}$ & $\begin{array}{l}24.0 \\
24.9\end{array}$ & 24.5 & & 45.1 & 47 & $\begin{array}{l}14.4 \\
11.1\end{array}$ & $\begin{array}{l}3.1 \\
34.2\end{array}$ & $\frac{9.9}{-1.2}$ & & 49.6 & & 12 & & & & 45.4 & $\begin{array}{l}13.6 \\
121\end{array}$ & 40.6 & & & $\frac{0.002}{0.030}$ & & 738 & \\
\hline 2 & & & 24.8 & $\begin{array}{l}18.3 \\
\end{array}$ & 24.1 & 23.7 & 18.2 & 31.7 & $\frac{46.4}{46.4}$ & 10.6 & 20.5 & -1.2 & & 41.4 & & 12 & & & & $\frac{74.9}{44.9}$ & & & & & & & & \\
\hline 26 & & $2 \times S t$ & 0.432 & 2.259 & 0.491 & 1.120 & 3.059 & 2.875 & 1.271 & 1.131 & 2.547 & 1.822 & & 4.881 & 0.060 & 0.0 & & & & 1.271 & 1.1 & & & & & & 224 & \\
\hline 26 & Number & ff Points Used* & 234 & 234 & 234 & 234 & $234 \mathrm{C}$ & 234 & 234 & 234 & & 234 & & 234 & 234 & 234 & & & & 234 & 234 & 234 & 234 & 234 & 234 & 234 & 234 & \\
\hline$\frac{2}{2}$ & & & & & & & & & & & & & & & & & & & & & & & & & & & & \\
\hline$\frac{265}{266}$ & & & & & & & & & & & & & & & & & & & & & & & 1-hour & $q=$ & 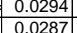 & & & \\
\hline & & & & & & & & & & & & & & & & & & & & & & & 1-hour & gg. $=$ & $\mid 0.0287$ & & & \\
\hline
\end{tabular}


RUN \# 1.18; FIRST SLURRY RINSE WITH 0.1 M NAOH

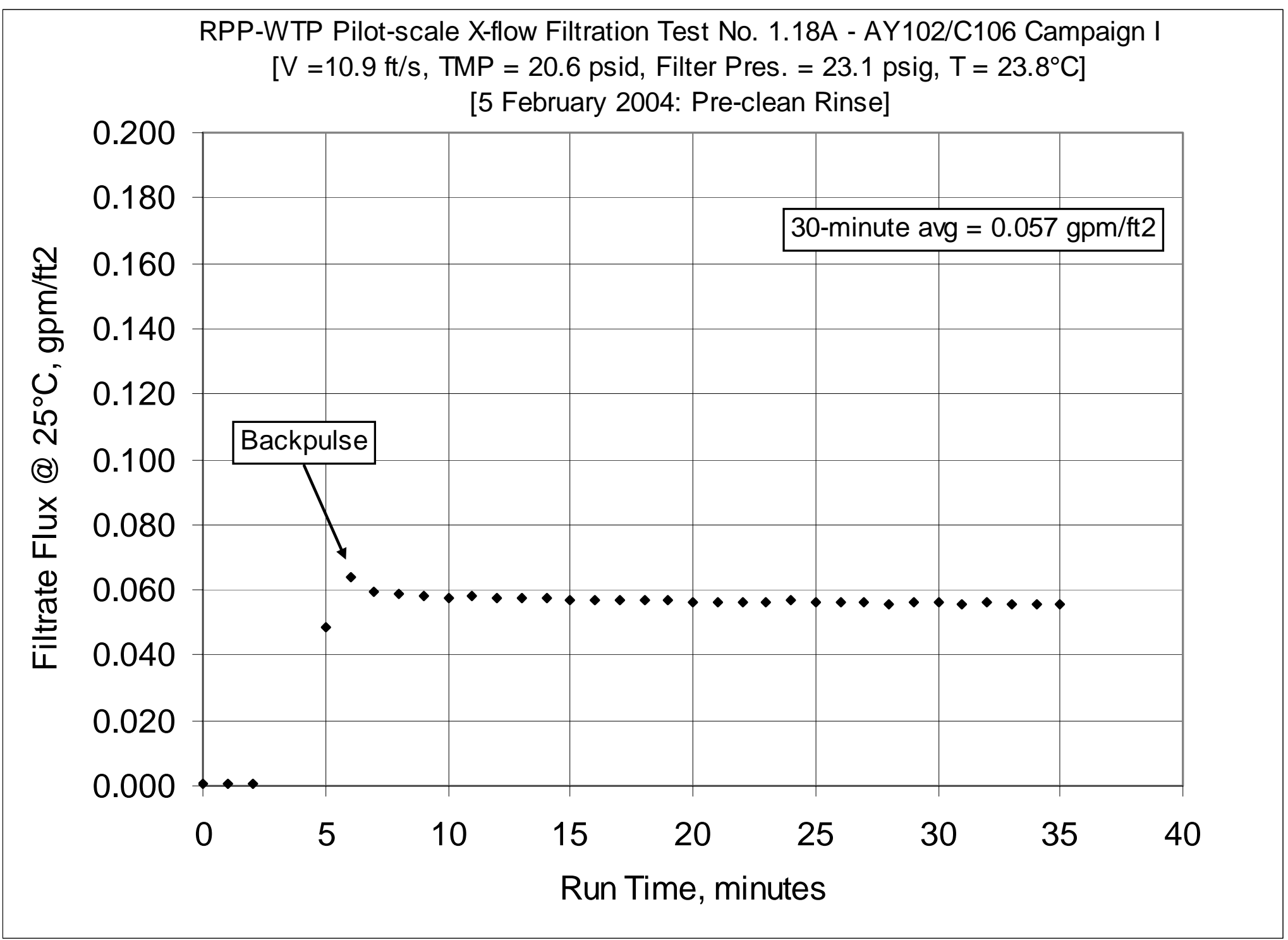


WSRC-TR-2005-00105, REVISION 0

SRNL-RPP-2005-00012, REVISION 0

RUN \# 1.18; FIRST SLURRY RINSE WITH 0.1 M NAOH - CONT.

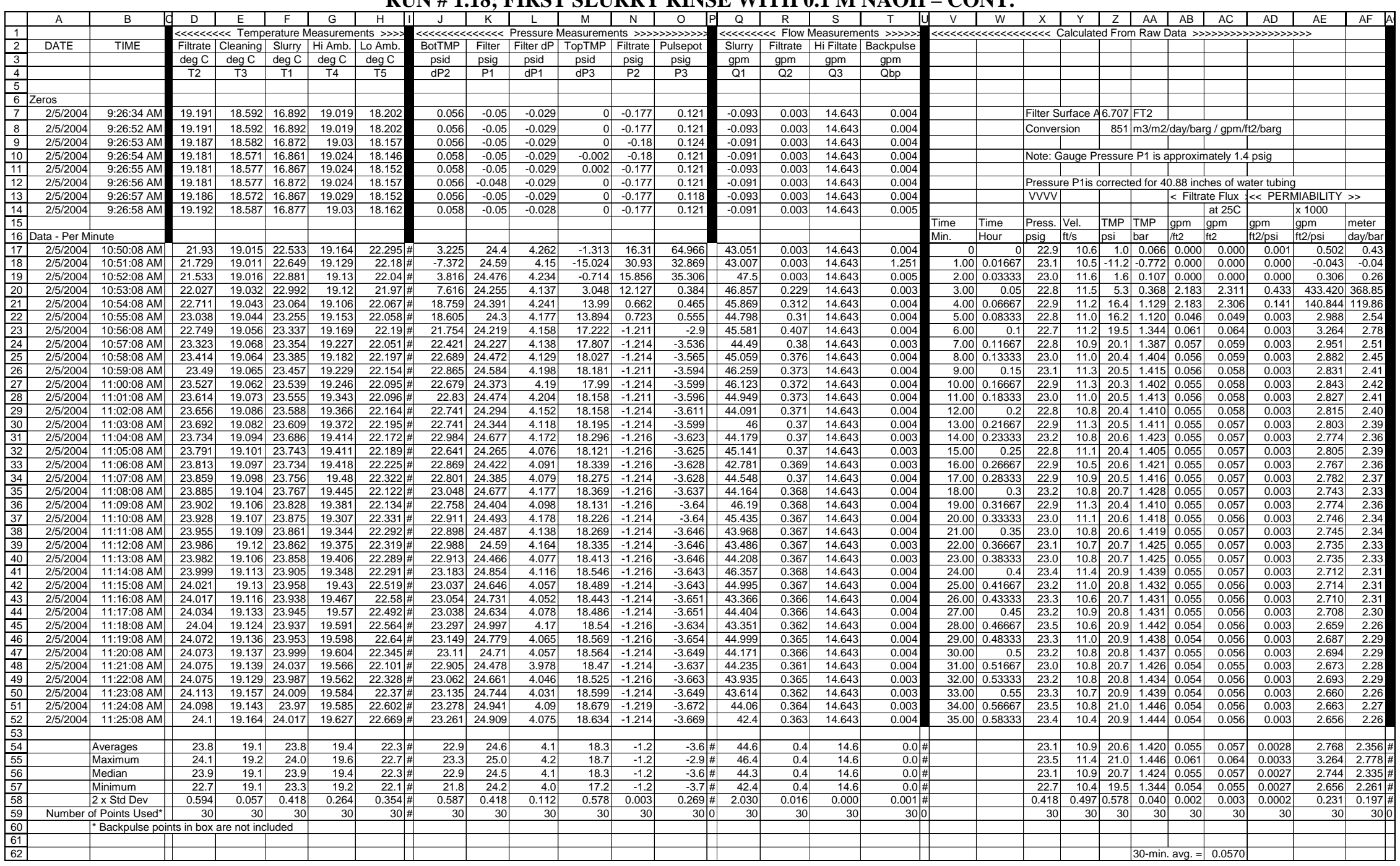


RUN \# 1.18; FIRST ACID CLEAN WITH 2.0 M HNO3

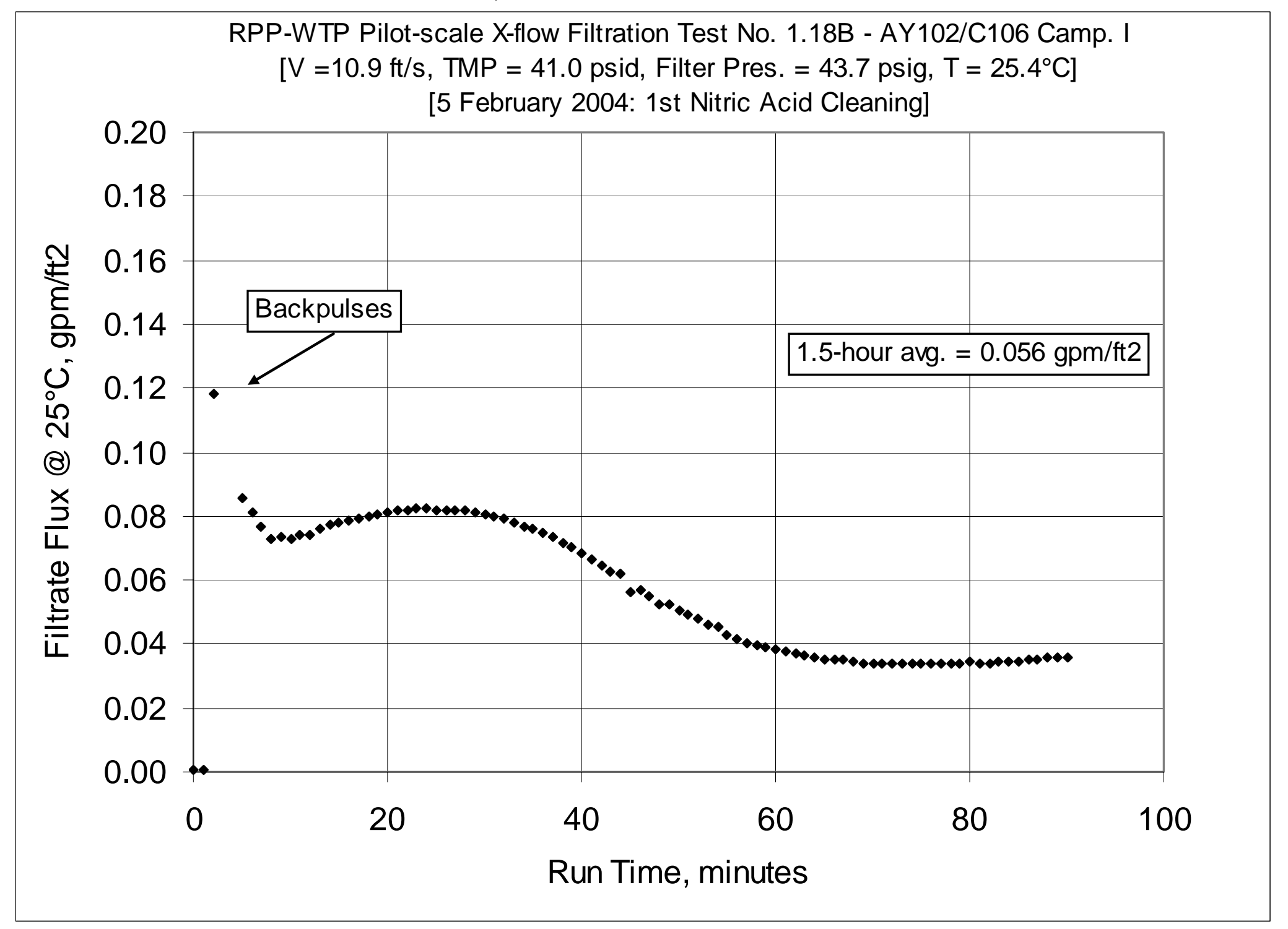


WSRC-TR-2005-00105, REVISION 0

SRNL-RPP-2005-00012, REVISION 0

RUN \# 1.18; FIRST ACID CLEAN WITH 2.0 M HNO3 - CONT.

\begin{tabular}{|c|c|c|c|c|c|c|c|c|c|c|c|c|c|c|c|c|c|c|c|c|c|c|c|c|c|c|c|c|}
\hline & $\mathrm{A}$ & B & D & 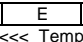 & \begin{tabular}{|l|l|} 
erature \\
\end{tabular} & $\frac{G}{\text { easurem }}$ & $\frac{H}{\text { nents } \gg \gg \geqslant}$ & 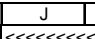 & $\frac{1 \mathrm{~K}}{3 \ll \ll \ll<}$ & $\frac{\mathrm{L}}{\mathrm{L} \text { ressure }}$ & easurem & $\frac{N \mathrm{~N}}{\mathrm{n} \text { ents } \gg>}$ & 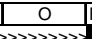 & $\frac{Q}{Q}$ & 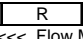 & $\frac{\mathrm{s}}{\text { Measureme }}$ & $T$ & $\frac{v}{1 \ll \ll \ll \ll}$ & 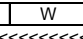 & $\frac{1 x}{1 \leq x<<}$ & Y P & $\frac{1 z}{z \text { ted }}$ & AA & $A B$ & AC & 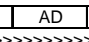 & $\frac{A E}{D \gg}$ & $\mathrm{AF}$ \\
\hline$\frac{1}{2}$ & DATE & TIME & Filtrate $]$ & & \begin{tabular}{|l|} 
Slutury \\
\end{tabular} & $\begin{array}{l}\text { neasurem } \\
\text { Hi Amb. }\end{array}$ & $\begin{array}{l}\text { nents }>>> \\
\text { Lo Amb. }\end{array}$ & $\begin{array}{l}\text { BotTMP } \\
\text { BotTe } \\
\end{array}$ & K Filter & & $\begin{array}{l}\text { asurem } \\
\text { DTTMP }\end{array}$ & $\begin{array}{l}\text { enenss }>>> \\
\mid \text { Filtrate } \\
\end{array}$ & ulsepot & & $\begin{array}{l}\ll<\text { Hllow I } \\
\text { Iiltrate }\end{array}$ & $\frac{\text { Measureme }}{\text { | Hi Filtate }}$ & & & & & & & & & & & & \\
\hline 3 & & & $\operatorname{deg} C$ & $\operatorname{deg} C$ & $\operatorname{deg} C$ & $\operatorname{deg} \mathrm{C}$ & $\operatorname{deg} \mathrm{C}$ & psid & psig & psid & psid & psig & psig & $\mathrm{gpm}$ & $\mathrm{gpm}$ & $\mathrm{gpm}$ & $\mathrm{gpm}$ & & & & & & & & & & & \\
\hline \begin{tabular}{l|}
4 \\
5
\end{tabular} & & & & & & & & & & & & & & & & Q3 & & & & & & & & & & & & \\
\hline & eros & & & & & & & & & & & & & & & & & & & & & & & & & & & \\
\hline 7 & $2 / 5 / 2004$ & :26:34 AM & 19.19 & & $16.892 \mathrm{r}$ & 19.019 & 8.202 & 0.056 & -0.05 & -0.029 & & .177 & .12 & -0.093 & 0.00 & 14.643 & & & & & & & & & & & & \\
\hline 8 & 2/5/2004 & $6: 52 \mathrm{AM}$ & 19.191 & 18.592 & 16.892 & 19.019 & 18.202 & 0.056 & -0.05 & -0.029 & & \begin{tabular}{|l|l|}
-0.177 \\
\end{tabular} & 0.121 & -0.093 & 0.003 & 14.643 & 0.004 & & & Conver: & ision & & $\mathrm{m} 3 / \mathrm{m} 2 /$ & 2/day/bart: & $\mathrm{arg} / \mathrm{gpm} / \mathrm{s}$ & l/ftz/barg & & \\
\hline & $2 / 5 / 2004$ & $6: 53 \mathrm{AM}$ & 19.187 & 18.582 & 16.872 & 19.03 & 18.157 & & -0.05 & -0.029 & & $\begin{array}{l}-0.18 \\
\end{array}$ & 0.124 & & 0.003 & 14.643 & 0.004 & & & & & & & & & & & \\
\hline 10 & 5/2004 & 8:54 AM & 19.181 & 18.571 & 16.861 & 19.024 & 18.146 & 0.058 & -0.05 & -0.029 & -0.002 & $\begin{array}{ll}-0.18 \\
\end{array}$ & & $\begin{array}{l}-0.091 \\
-1\end{array}$ & 0.003 & 14.643 & & & & & & & & & & psig & & \\
\hline 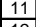 & $2 / 5 / 2004$ & 9:26:55 & 19.181 & 18.577 & 16.867 & 19.024 & 18.152 & $\begin{array}{l}0.058 \\
\end{array}$ & -0.05 & & 0.002 & -0.177 & 0.121 & $\begin{array}{r}-0.093 \\
-0.021\end{array}$ & 0.003 & 14.643 & 0.004 & & & & & & & & & & & \\
\hline$\frac{12}{13}$ & $\begin{array}{l}\mid 2 / 5 / 2004 \\
25 / 2004 \\
\end{array}$ & $\begin{array}{l}\text { 9:26:5 AM } \\
9: 26: 57 \mathrm{AM}\end{array}$ & $\begin{array}{l}19.181 \\
191166\end{array}$ & $\begin{array}{l}18.577 \\
18.572\end{array}$ & $\begin{array}{ll}16.872 \\
16.867\end{array}$ & $\begin{array}{l}19.024 \\
19.029 \\
\end{array}$ & \begin{tabular}{|l|}
18.157 \\
18.152 \\
\end{tabular} & \begin{tabular}{|l|l}
0.056 \\
0.056
\end{tabular} & \begin{tabular}{|l|}
-0.048 \\
-0.05 \\
\end{tabular} & \begin{tabular}{|l|}
$\mid-0.029$ \\
-0.029
\end{tabular} & & \begin{tabular}{|l|}
$\mid-0.177$ \\
\\
-0.1177
\end{tabular} & $\begin{array}{l}0.121 \\
0.118 \\
\end{array}$ & $\begin{array}{l}-0.091 \\
-0.093 \\
-1\end{array}$ & $\begin{array}{l}0.003 \\
0.003 \\
\end{array}$ & $\frac{14.643}{14.643}$ & 0.004 & & & $\begin{array}{l}\text { Pressut } \\
\text { Vwvy }\end{array}$ & & scorrec & cted for 4 & $\frac{40.88 \text { itc }}{4<\text { Fitra }}$ & $\begin{array}{l}\text { nches of } y \\
\text { ate Flux }\end{array}$ & «PPEPN & N & \\
\hline 14 & $2 / 5 / 2004$ & 9:26:58 AM & 19.192 & 18.587 & 16.877 & $\begin{array}{ll}19.03 \\
\end{array}$ & 18.162 & \begin{tabular}{|c|c|}
0.058 \\
\end{tabular} & $\begin{array}{ll} & -0.05 \\
\end{array}$ & $\mid-0.028$ & & \begin{tabular}{|l|l|} 
& -0.177 \\
\end{tabular} & 0.121 & -0.091 & 0.003 & $\frac{14.049}{14.643}$ & 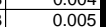 & & & & & & & & & & & \\
\hline 15 & & & & & & & & & & & & & & & & & & Time & Time & Press. & vel. & TMP & TMP & $\mathrm{gpm}$ & $\mathrm{gpm}$ & gpm & $\mathrm{pm}$ & \\
\hline & ata - Per Mi & & & & & & & & & & & & & & & & & & Hour & osig & & psi & bar & tit2 & & tipsi & $2 / 2 \mathrm{psi}$ & day/ba \\
\hline 17 & $2 / 5 / 2004$ & 1:13:09 PM & 24.162 & 19.004 & $\begin{array}{l}25.401 \\
25.527\end{array}$ & $\begin{array}{l}19.315 \\
19427 \\
\end{array}$ & $\begin{array}{l}20.826 \\
20707\end{array}$ & \begin{tabular}{|r|}
3.14 \\
2742
\end{tabular} & \begin{tabular}{|l|}
47.004 \\
46.965
\end{tabular} & 3.387 & -0.263 & 39.355 & 87.228 & 41.188 & 0.003 & $\begin{array}{l}14.643 \\
1.63\end{array}$ & & & & 45.5 & 10. & 1.4 & & 0.000 & 0.000 & 0.000 & 0.307 & 0.26 \\
\hline \begin{tabular}{|l|l|}
19 \\
\end{tabular} & $\begin{array}{r}2 / 5 / 2004 \\
2 / 5 / 2004\end{array}$ & $\begin{array}{l}\text { 1:14:00 PM } \\
1.15509 \mathrm{PM}\end{array}$ & $\begin{array}{l}23.281 \\
25356\end{array}$ & 19.016 & 25.527 & $\begin{array}{l}19.427 \\
19505\end{array}$ & 20.707 & $\begin{array}{r}2.742 \\
38.424 \\
\end{array}$ & \begin{tabular}{|l|}
46.965 \\
46.921 \\
\end{tabular} & $\begin{array}{l}3.298 \\
3.333 \\
\end{array}$ & $\begin{array}{r}-0.657 \\
3489 \\
\end{array}$ & $\frac{39.1 .024}{3.404}$ & $\frac{43.15}{0.543}$ & \begin{tabular}{ll|l}
45.862 \\
42.156
\end{tabular} & 0.003 & $\begin{array}{r}14.643 \\
14.643 \\
\end{array}$ & & & 0.0333 & $\frac{45.5}{45.4}$ & $\frac{11.2}{10 .}$ & 367 & 2527 & 0.020 & & $\frac{000}{003}$ & & 0.36 \\
\hline & $2 / 5 / 2004$ & 1:16:09 PM & 25.812 & 19.034 & 25.715 & 19.613 & 20.922 & 40.164 & \begin{tabular}{|l|l|}
47.071 \\
\end{tabular} & $\mid 3.42$ & 36.22 & $\begin{array}{l}0.448 \\
1.586\end{array}$ & $\begin{array}{c}0.545 \\
-0.272\end{array}$ & \begin{tabular}{|l|}
42.794 \\
42.19
\end{tabular} & 0.68 & 14.643 & & $\frac{2.0}{30}$ & 0.0 .05 & & $\frac{10.5}{6}$ & & 2.633 & 2.183 & & & & 2.74 \\
\hline & $2 / 5 / 2004$ & 1:17:09 PM & 26.059 & 19.056 & 25.812 & 19.766 & 20.808 & 41.284 & \begin{tabular}{|l|l|}
46.898 \\
\end{tabular} & 3.341 & 37.609 & \begin{tabular}{|l|l|} 
& 0.367 \\
\end{tabular} & -1.133 & \begin{tabular}{|l|l|}
42.911 \\
\end{tabular} & 0.622 & $\begin{array}{ll}14.643 \\
\end{array}$ & & 4.0 & 0.06667 & 45.4 & \begin{tabular}{l|l}
4 & 10.5 \\
\end{tabular} & \begin{tabular}{|l|l|}
39.4 & \\
\end{tabular} & 2.720 & 2.183 & $\frac{2.140}{2.134}$ & 0.056 & 64.097 & $\frac{47.68}{46.04}$ \\
\hline $22->>$ & $2 / 5 / 2004$ & 1:18:09 PM & 26.166 & 19.053 & 25.874 & 19.783 & $\begin{array}{ll}21.071 \\
\end{array}$ & \begin{tabular}{|l|l|l|l|l|}
41.73 \\
\end{tabular} & \begin{tabular}{|l|l|}
46.697 \\
\end{tabular} & 3.262 & 38.171 & -0.082 & -1.434 & 42.825 & 0.588 & 14.643 & & 5.0 & 0.08333 & 45.2 & $\begin{array}{l}2 \\
2\end{array}$ & \begin{tabular}{|l|l|}
5 & 39.9 \\
\end{tabular} & 2.754 & 40.088 & 5.086 & & $\frac{4.091}{2.141}$ & $\frac{46.04}{1.82}$ \\
\hline & 2/5/2004 & $9 \mathrm{PM}$ & 26.243 & 19.06 & 25.936 & 19.846 & 993 & 42.171 & 46.722 & 3.351 & 38.43 & -0.536 & -1.72 & \begin{tabular}{|l|l|l|l|}
42.706 \\
\end{tabular} & 0.557 & 14.643 & 0.0 & 6.0 & 0.1 & 45.2 & $10 !$ & 40.3 & & & & & & $\frac{1.82}{171}$ \\
\hline 24 & $2 / 5 / 2004$ & & & & & & & & & & & $\begin{array}{l}-0.991 \\
\end{array}$ & & 41.893 & & 14.643 & & & 11667 & 45.4 & 10. & & & . 079 & $\frac{0.077}{0.077}$ & 0.002 & 1.879 & \\
\hline & & & & & & & & \begin{tabular}{|c|c|c|}
42.748 \\
\end{tabular} & & 3.655 & 38.709 & & & $4.9 .9-3$ & & 14.643 & & 8.6 & & 45.0 & 11. & & & & & & & \\
\hline 26 & $2 / 5 / 2004$ & & 26.413 & 19.081 & 26.067 & 20.069 & & \begin{tabular}{|l|l|}
42.686 \\
\end{tabular} & \begin{tabular}{|l|l|}
46.053 \\
\end{tabular} & 3.395 & 39.083 & -1.216 & & 45.0 & 0.507 & 14.643 & & & 0.15 & 44.6 & & 40.9 & & & & & 1.794 & \\
\hline 27 & $2 / 5 / 2004$ & & 26.399 & & 26.023 & 20.116 & & 387 & 46.967 & 3.504 & 39.462 & -1.216 & & 44.584 & 0.502 & 14.643 & & & .16667 & 45.5 & $10.5 \mathrm{P}-\mathrm{P}$ & & & & & & 1.756 & 49 \\
\hline & $2 / 5 / 2004$ & गM & 26.421 & 19.105 & 25.98 & 20.092 & 20.161 & .177 & 46.799 & 3.456 & 39.343 & -1.216 & -2.49 & & 0.51 & 14.643 & & 11.0 & 0.1833 & 45.3 & 11.0 & 41. & 2.845 & $\begin{array}{l}50.076 \\
5\end{array}$ & 0.074 & 002 & 1.793 & \\
\hline 2 & 2/5/2004 & 5:0 & 26.428 & 19.121 & 25.932 & 20.013 & 20.943 & .034 & 46.583 & 3.683 & 39.076 & -1.216 & & 42.402 & 0.511 & 14.643 & & 12. & $0.2-2>0$ & 45.1 & & 41 & .8 & 076 & 0.074 & 002 & $.808 \mathrm{~s}$ & \\
\hline & & & 26.423 & & & & & & & 3.5 & 535 & -1.214 & & & & 14.643 & & & & 44. & & & & & & & & \\
\hline 31 & $2 / 5 / 200$ & & 26.395 & & & & & & \begin{tabular}{|l|}
46.192 \\
\end{tabular} & 3.553 & 38.789 & -1.214 & & 1.0 & & & & & 23333 & 44. 4.4 & & & & & & & & \\
\hline & $\frac{2512004}{215004}$ & & $\frac{20.412}{26368}$ & $\begin{array}{l}19.135 \\
19147\end{array}$ & & & & & \begin{tabular}{|l|l|}
46.318 \\
16379
\end{tabular} & 3.591 3604 & $\frac{38.16}{38.161}$ & & & & .535 & $\frac{14.643}{14643}$ & & & $\frac{0.25}{2667}$ & $\frac{44.8}{44.8}$ & & & & & & & & \\
\hline 34 & $2 / 5 / 5 / 2004$ & $\frac{1.29 .09}{1: 30: 09}$ & $\begin{array}{c}20.500 \\
26.34\end{array}$ & $\frac{19.147}{19.164}$ & $\frac{2.1710}{2579}$ & 20.166 & & \begin{tabular}{|c|c|c|c|c|c|}
42.663 \\
\end{tabular} & $\mid \frac{40.671}{46521}$ & $\begin{array}{l}3.044 \\
3.715 \\
\end{array}$ & $\begin{array}{l}30.401 \\
38652\end{array}$ & 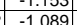 & & 448 & 0.545 & $\frac{14.045}{14.643}$ & & . & 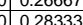 & 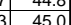 & 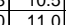 & $\frac{40}{40}$ & 8803 & 0.081 & 080 & 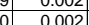 & & \\
\hline 35 & $2 / 5 / 2004$ & & 26.308 & 19.182 & & & & & 414 & 3.586 & 38.477 & & & 437 & 0.549 & $\frac{14.045}{14.643}$ & & & & 44.9 & 11. & & & & & & & \\
\hline & $2 / 5 / 2004$ & $1: 32: 0$ & 26.28 & & 25.714 & 19.444 & & 42.244 & 46.204 & 3.499 & 38.455 & -0.976 & & 46682 & & 14.643 & & 19 & $\frac{1667}{1667}>2$ & 44.7 & t & 70 & & & 00 & $0027+2$ & & \\
\hline & $2 / 5 / 2004$ & & 26.253 & & 25.682 & 19.25 & & $\mid 42.223$ & 446.19 & 3.51 & 38.529 & -0.933 & & 45.0 & 0.555 & 14.643 & & & 3333 & & & & & 0.08 & 0.08 & 0.002 & & \\
\hline 38 & & & 26.22 & & & & & & & 3.541 & & -0.875 & & & & & & & & 44 & & & & & & & & \\
\hline 39 & $215 / 2004$ & & 26.195 & & & & & & & 3 & & -0.881 & & & & & & & 0.36667 & $\frac{45}{45}-2$ & 10. & & & 083 & & 0.002 & 016 & \\
\hline 40 & 2/5/2004 & & 26.144 & $\begin{array}{l}9.148 \\
\end{array}$ & 25.573 & & & & & 3.48 & 38.492 & \begin{tabular}{|c|c|c|}
-0.878 \\
\end{tabular} & & & & & & & & 45. & & & & & & & & \\
\hline \begin{tabular}{|lll}
41 & \\
\end{tabular} & 2/5/2004 & & 26.128 & 19.137 & & 19.23 & & & & 3.512 & & $\mid-0.855$ & & & & & & 24 & 0.4 & 44.9 & $10.8 \mathrm{~g}$ & & & & & & & \\
\hline 4 & $2 / 5 / 2004$ & & 26.118 & 19.121 & \begin{tabular}{|l|}
25.546 \\
\end{tabular} & 19.27 & & & & 3.469 & 38.198 & -0.875 & & 43.8 & 0.558 & 14.643 & & 25 & 0.41667 & 44.9 & 10.8 & & & & & 0.002 & 041 & \\
\hline 43 & $2 / 5 / 200$ & & 20.090 & 19.089 & 25.504 & 19.243 & & & & & 38.416 & & & & & & & & & 44.8. & 11. & & & & & . & . & \\
\hline 44 & & & 26.0899 & & & & & & & & & & & & & & & & & & & & & & & & & \\
\hline$\frac{45}{46}$ & & & 26.06 & & $\frac{25}{25}$ & & & & & & & & & & & & & & 10007 & $44-2$ & & & & & & & & \\
\hline 47 & $\begin{array}{r}2 / 5 / 2004 \\
2 / 5 / 2004\end{array}$ & & & & & & & & & & & & & & & & & & . & 年.5.5 & & & & & & & & \\
\hline \begin{tabular}{|l|l|}
48 \\
\end{tabular} & $2 / 5 / 2004$ & & 260 & & & & & & & & & & & & & & & & 0.51667 & & & & & & & & & \\
\hline \begin{tabular}{|l|l|}
49 \\
\end{tabular} & & & 25.983 & & & 19.168 & & & & & & & & & & & & & 0.53333 & 45. & & & & & & & & \\
\hline & & & & & & & & & & & & & & & & & & & & & & & & & & & & \\
\hline & & & 25.95 & \begin{tabular}{|l|l|l|l}
18.868 \\
\end{tabular} & 25.4 & 19 & & & & & & & & & & & & & & & & & & & & & & \\
\hline & & & 25.967 & 18.862 & \begin{tabular}{|l|l|l|}
25.44 \\
\end{tabular} & \begin{tabular}{l|l|}
19.264 \\
\end{tabular} & & & & 3.29 & & & & 43. & & & & & & 44 & & & & & & & & \\
\hline & $2 / 5 / 2$ & & 5.946 & & 25.399 & & & & & 3.351 & & -1.216 & & & & 14.6 & & & 0.6 & 45. & 10 & & & & & & & \\
\hline \begin{tabular}{|ll}
5 \\
\end{tabular} & $2 / 5 / 200$ & & 25.91 & & 25. & & & & & 3.4 & & 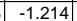 & & 44.9 & & & & 37 & 0.61667 & 45.5 & $11.0 \mathrm{C}$ & 41.7 & & & & 02 & & \\
\hline 5 & & & 25.913 & & & $\begin{array}{l}19.189 \\
\end{array}$ & & & & & & & & & & & & & & 44 & & & & & & & & \\
\hline 56 & & & 25.876 & & 25.364 & & & & & & & & & & & & & & & 45. & 10. & & & & & & & \\
\hline 51 & & & & & & & & & & & & & & & & & & & & & & & & & & & & \\
\hline$\frac{58}{50}$ & & & & & & & & & & 3.4 & & & & & & & & & & 45. & & & & & & & & \\
\hline & & & & & 20.37 & & & & & & & & & & & & & & & & & & & & & & & \\
\hline & & & & & 20.3044 & & & & & & & & & & & & & & & 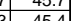 & & & & & & & & \\
\hline \begin{tabular}{|l|l|}
6 \\
\end{tabular} & 25152044 & & 25.809 & $\begin{array}{l}18.650 \\
18.657\end{array}$ & 25383 & $\frac{1.19}{19.1}$ & & & & & & -1214 & & & & & & & & 434 & & & & & & & & \\
\hline & & & & & & & & & & & & & & & & & & & & & & & & & & & & \\
\hline 64 & & & 25.7 & 18.647 & 25.393 & 19.322 & & & & & & & & & & & & & & 442 & & & & & & & & \\
\hline & & & & 18 & 25.395 & 19.203 & & & & & & & & & & & & & & & & & & & & & & \\
\hline & & & 5573 & 18.6 & 253 & & & & & 39 & & -1.2 & & & & & & & & 20 & & & & & & & & \\
\hline & 2/5/2004 & & 25.67 & 18.608 & 25.364 & 19. & & & & & & -1.219 & & & & 14.6 & & & 0.83 & 44.1 & & 442 & & & & & & \\
\hline 6 & 2/5/2004 & & 25.74 & 18.5 & 25.367 & 19.205 & & 44.239 & & $\begin{array}{l}4.033 \\
\end{array}$ & & -1.216 & & & & & & 51 & 0.85 & \begin{tabular}{l|l|}
44.4 \\
\end{tabular} & 113 & 420 & & & & 01 & 1.178 & \\
\hline & 2/5/2004 & & 25.715 & 18.597 & \begin{tabular}{|l|}
25.373 \\
\end{tabular} & 19.20 & & & & 4.023 & 40.06 & -1.211 & & & 0.32 & 14 & & 52 & & 44. & 11. & & & & & 0.001 & 1.127 & \\
\hline & $\mid$ & & 25.646 & \begin{tabular}{|l|l|} 
\\
\end{tabular} & \begin{tabular}{|l|l|} 
& 25.379 \\
\end{tabular} & 19.212 & & 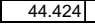 & & 3.894 & 40.289 & \begin{tabular}{|l|l|} 
& -1.214 \\
\end{tabular} & & 45.973 & & 14.643 & & & & 3.9 & & & & & & 0.001 & 1.08 & \\
\hline
\end{tabular}


WSRC-TR-2005-00105, REVISION 0

SRNL-RPP-2005-00012, REVISION 0

RUN \# 1.18; FIRST ACID CLEAN WITH 2.0 M HNO3 - CONT.

\begin{tabular}{|c|c|c|c|c|c|c|c|c|c|c|c|c|c|c|c|c|c|c|c|c|c|c|c|c|c|c|c|c|}
\hline & A & B & $\mathrm{D}$ & $E$ & $\mathrm{~F}$ & $G_{G}$ & $\mathrm{H}$ & & $\mathrm{K}$ & $\mathrm{L}$ & $\mathrm{M}$ & $\mathrm{N}$ & $\mathrm{O}$ & $s+$ & $R$ & $\mathrm{~s}$ & $\mathrm{~T}$ & $\mathrm{~V}$ & w & $x$ & & & ${ }_{A A}$ & $A B$ & $A C$ & $A D$ & $\mathrm{AE}$ & \\
\hline 71 & $\frac{215 / 2004}{2 / 5 / 2004}$ & $2: 07: 09$ PM & $\frac{25.566}{2.506}$ & $\begin{array}{l}18.577 \\
18507\end{array}$ & \begin{tabular}{|l|l|}
25.379 \\
25509
\end{tabular} & $\begin{array}{l}19.444 \\
19.626\end{array}$ & 21.478 & 44.60 & $\begin{array}{l}45.817 \\
3602\end{array}$ & 3.904 & 40.25 & -1.214 & $\begin{array}{l}-4.279 \\
-290\end{array}$ & & 0.307 & 14.643 & 0.004 & 54. & 0.9 & 44. & 11. & 42. & & 0.046 & 0.045 & & 1.067 & \\
\hline & & & 5.406 & & 25.359 & & 21.694 & & & & 38.781 & & $\begin{array}{r}-4.389 \\
-4.429\end{array}$ & & & & & & & 42. & & & & & 0.043 & & 1.057 & \\
\hline 74 & $\frac{215 / 2004}{2 / 5 / 2004}$ & $\begin{array}{l}2: 00: 09 \mathrm{PM} \\
2: 10: 09 \mathrm{P}\end{array}$ & $\frac{25.197}{25092}$ & $\begin{array}{r}\frac{18.623}{18.65} \\
\end{array}$ & 25.300 & $\begin{array}{l}\frac{19.854}{19.946} \\
\end{array}$ & 21.992 & $\begin{array}{l}42.677 \\
42968\end{array}$ & $\begin{array}{l}43.191 \\
43.978\end{array}$ & $\begin{array}{l}3.683 \\
3.777 \\
\end{array}$ & $\begin{array}{l}38.119 \\
38.736\end{array}$ & $\begin{array}{l}-1.214 \\
-1.214\end{array}$ & $\begin{array}{r}-4.429 \\
-4.501\end{array}$ & $\begin{aligned} 44.702 \\
45.33\end{aligned}$ & $\frac{0.281}{0.274}$ & 14.643 & & 5700 & 0.95 & 425 & & & 2817 & & $\begin{array}{l}0.041 \\
0.049\end{array}$ & 0.001 & \begin{tabular}{|c|c|}
1.019 \\
0.99
\end{tabular} & 0.87 \\
\hline 75 & $2 / 5 / 2004$ & 2:11:09 PM & 25.028 & 18.681 & 25.322 & 20.104 & 22.194 & 42.87 & 43.539 & 3.749 & 38.841 & -1.214 & & 44.386 & 0.268 & $\frac{14.043}{14.643}$ & $\begin{array}{l}0.004 \\
0.004\end{array}$ & 58.00 & \begin{tabular}{|l|}
0.96667 \\
\end{tabular} & $\begin{array}{l}42.5 \\
42.1\end{array}$ & $\frac{11.1}{10.9}$ & 40.9 & & & $\begin{array}{l}0.040 \\
0.040\end{array}$ & & \begin{tabular}{|c|c|}
0.990 \\
0.969
\end{tabular} & $\begin{array}{l}0.84 \\
0.82\end{array}$ \\
\hline 76 & $2 / 5 / 2004$ & 2:12:09 PM & 24.95 & 18.713 & 25.303 & 20.141 & 22.271 & 42.87 & 43.7 & 3.782 & 38.658 & -1.216 & -4.571 & $\begin{array}{l}44.200 \\
44.208\end{array}$ & 0.265 & 14.643 & 0.004 & 59.00 & \begin{tabular}{|l|}
0.98333 \\
\end{tabular} & $\begin{array}{l}42.1 \\
42.2\end{array}$ & $\frac{10.5}{10.8}$ & & 2.81 & 0 & $\begin{array}{l}.040 \\
0.039\end{array}$ & 0.001 & $\begin{array}{l}0.961 \\
0.961\end{array}$ & \\
\hline 77 & $2 / 5 / 2004$ & 2:13:09 PM & 24.896 & 18.734 & 25.259 & 20.167 & 22.342 & 43.02 & 43.615 & 3.742 & 38.949 & -1.216 & $\begin{array}{l}-4.603 \\
\end{array}$ & 44.018 & 0.258 & 14.643 & 0.004 & 60.00 & & 42.1 & 10.8 & & 2.826 & 0.038 & 0.038 & 0.001 & 0.932 & \\
\hline 78 & $2 / 5 / 2004$ & 2:14:09 PM & 24.862 & 18.791 & \begin{tabular}{|l|}
25.26 \\
\end{tabular} & 20.264 & 22.394 & 43.016 & 43.543 & 3.742 & 38.884 & -1.216 & $\begin{array}{l}-4.637 \\
\end{array}$ & 44.45 & 0.254 & 14.643 & 0.004 & 61.00 & \begin{tabular}{|l|l|}
1.01667 \\
\end{tabular} & 42.1 & 10.9 & 41.0 & 2.823 & \begin{tabular}{|l|}
0.038 \\
\end{tabular} & 0.038 & 0.001 & 0.918 & \\
\hline 79 & $2 / 5 / 2004$ & 2:15:09 PM & 24.834 & 18.819 & 25.232 & 20.296 & 22.331 & 42.8 & 43.261 & 3.635 & 38.955 & -1.214 & -4.663 & 42.821 & 0.25 & 14.643 & 0.004 & 62.00 & 1.03333 & 41.8 & 10.5 & & 2.820 & \begin{tabular}{|l|}
0.037 \\
\end{tabular} & 0.037 & 0.001 & 0.905 & \\
\hline & $2 / 5 / 2004$ & 2:16:09 PM & & 18.846 & 25.21 & 20.36 & 22.469 & 42.862 & 43.3 & & & -1.214 & & & & 14.643 & & 63.00 & 1.05 & 41.8 & & & & & & & & \\
\hline 81 & $2 / 5 / 2004$ & 2:17:09 PM & $\frac{24.824}{2.821}$ & 18.895 & \begin{tabular}{|l|}
25.208 \\
2.1019
\end{tabular} & 20.403 & 22.472 & 42.758 & 43.45 & 3.633 & $\begin{array}{l}38.672 \\
20701\end{array}$ & $\frac{-1.214}{.214}$ & \begin{tabular}{|l|l|}
-4.712 \\
\end{tabular} & \begin{tabular}{|l|l|}
44.788 \\
\end{tabular} & 0.242 & 14.643 & 0.004 & 64.00 & 1.06667 & 42.0 & 11.0 & & & 0.036 & 0.036 & 0.001 & 0.881 & \\
\hline 82 & $2 / 5 / 2004$ & $\begin{array}{ll}\text { 2:18:09 PM } \\
21: 00\end{array}$ & $\begin{array}{l}24.801 \\
247686\end{array}$ & $\begin{array}{l}18.906 \\
18.918\end{array}$ & \begin{tabular}{|l|}
25.184 \\
25171
\end{tabular} & $\begin{array}{l}20.424 \\
20.467\end{array}$ & 22.458 & 42.8 & 43.551 & $\begin{array}{l}3.664 \\
3632\end{array}$ & & \begin{tabular}{|c|}
-1.214 \\
1216
\end{tabular} & $\begin{array}{r}-4.73 \\
\end{array}$ & 44.613 & 0.238 & $\begin{array}{l}14.643 \\
1.612\end{array}$ & 0.004 & & 1.08333 & 42.1 & & & & & & & $\begin{array}{l}0.865 \\
\end{array}$ & 0.74 \\
\hline$\frac{83}{84}$ & $2 / 5 / 2004$ & $\begin{array}{ll}2: 19: 09 \text { PM } \\
2 \cdot 20.09 P M\end{array}$ & $\frac{24.768}{24.76}$ & $\begin{array}{l}18.918 \\
18.95\end{array}$ & \begin{tabular}{|l|}
25.171 \\
25162
\end{tabular} & $\begin{array}{l}20.467 \\
20.438\end{array}$ & $\begin{array}{l}22.676 \\
22.657\end{array}$ & $\begin{array}{l}43.00 \\
4324\end{array}$ & $\frac{43.632}{43833}$ & $\begin{array}{l}3.632 \\
3659\end{array}$ & $\begin{array}{l}38.904 \\
339138\end{array}$ & $\frac{-1.216}{1.214}$ & $\begin{array}{l}-4.744 \\
.4753\end{array}$ & $\begin{array}{r}45.02 \\
\end{array}$ & 0.236 & $\begin{array}{l}14.643 \\
1643\end{array}$ & 0.004 & $\begin{array}{l}66.00 \\
6700\end{array}$ & $\begin{array}{r}1.1 \\
\end{array}$ & 42.2 & $\begin{array}{ll}11.0 \\
107\end{array}$ & 41.0 & $\begin{array}{l}2.824 \\
2.84 \\
\end{array}$ & \begin{tabular}{|l|l|} 
\\
0.035
\end{tabular} & 0.035 & 0.001 & 0.855 & 0.73 \\
\hline $\begin{array}{l}84 \\
85\end{array}$ & $\frac{2 / 5 / 2004}{2 / 5 / 2004}$ & $\begin{array}{l}2: 20: 09 \mathrm{PM} \\
2 \cdot 2 \cdot 109 \mathrm{PM}\end{array}$ & $\frac{24.764}{24.756}$ & $\begin{array}{r}18.95 \\
18.967\end{array}$ & \begin{tabular}{|l}
25.162 \\
25.154
\end{tabular} & $\frac{20.438}{20.42}$ & $\frac{22.657}{22.634}$ & $\begin{array}{l}43.242 \\
43306\end{array}$ & $\frac{43.833}{43.647}$ & $\begin{array}{r}3.659 \\
3.694\end{array}$ & $\begin{array}{r}39.138 \\
39.333 \\
\end{array}$ & $\frac{-1.214}{-1.214}$ & $\begin{array}{r}-4.753 \\
-4.77\end{array}$ & \begin{tabular}{|l|}
43.612 \\
44.083 \\
\end{tabular} & 0.235 & $\frac{14.643}{14.643}$ & $\begin{array}{l}0.004 \\
0.004\end{array}$ & & 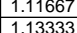 & $\begin{array}{l}42.4 \\
42.2\end{array}$ & $\frac{10.7}{10.8}$ & & $\frac{2.840}{2849}$ & \begin{tabular}{|l|}
0.035 \\
0.035
\end{tabular} & 0.035 & 0.001 & 0.847 & 0.72 \\
\hline 86 & $2 / 5 / 2004$ & $2: 22: 09 \mathrm{PM}$ & $\frac{24.150}{24.763}$ & $\frac{10.907}{18.984}$ & \begin{tabular}{|l|l|l|l|l|}
25.136 \\
\end{tabular} & $\frac{20.44}{20.436}$ & 22.721 & 43.08 & $\frac{43.04 t}{43.503}$ & $\begin{array}{l}3.044 \\
3.622\end{array}$ & $\frac{39.050}{39.101}$ & $\frac{-1.214}{-1.216}$ & $\begin{array}{r}-4.11 \\
-4.782 \\
\end{array}$ & $\begin{array}{l}44.005 \\
45.005\end{array}$ & $\frac{0.324}{0.23}$ & $\frac{14.043}{14.643}$ & & 68.00 & $\begin{array}{r}1.1 .13333 \\
1.15\end{array}$ & $\frac{42.2}{42.0}$ & $\frac{1.8}{11.0}$ & $\frac{41.3}{41.1}$ & & & $\begin{array}{l}0.034 \\
0.034\end{array}$ & & & 0.71 \\
\hline 87 & $2 / 5 / 2004$ & 2:23:09 PM & 24.765 & 19.021 & 25.143 & 20.469 & 22.678 & 43.16 & 43.611 & 3.635 & 39.173 & -1.216 & -4.793 & 45.685 & 0.229 & 14.643 & 0.0 & $\begin{array}{l}69.00 \\
70.00\end{array}$ & $\frac{1.10}{1.16667}$ & 42.1 & $\frac{11.6}{11.2}$ & & & \begin{tabular}{|l|}
0.034 \\
0.034
\end{tabular} & & & & $\frac{0.11}{0.70}$ \\
\hline 88 & $2 / 5 / 2004$ & 2:24:09 PM & 24.782 & 19.034 & 25.145 & 20.546 & 22.519 & 43.15 & 43.398 & 3.672 & 39.185 & -1.214 & -4.79 & 45.005 & 0.228 & 14.643 & & 71.00 & 1.18333 & 41.9 & 11.0 & 41 & $\begin{array}{l}2.039 \\
2.839 \\
\end{array}$ & \begin{tabular}{|l|}
0.034 \\
0.034
\end{tabular} & 0.034 & 0.001 & 0 & $\begin{array}{l}0.10 \\
0.70\end{array}$ \\
\hline 89 & 2/5/2004 & 2:25:09 PM & 24.813 & 19.045 & 25.141 & 20.558 & 22.51 & 42.825 & 43.11 & 3.642 & 38.927 & -1.216 & -4.796 & \begin{tabular}{|l|l|}
44.377 \\
\end{tabular} & 0.228 & 14.643 & 0.00 & 72.00 & 1.2 & 41.6 & 10.9 & & & \begin{tabular}{|l|}
0.034 \\
\end{tabular} & 0.034 & & & \\
\hline & $2 / 5 / 2004$ & 2:26:09 PM & 24.861 & 19.058 & 25.109 & 20.596 & 22.608 & 42.736 & 43.13 & 3.565 & 38.918 & -1.219 & -4.793 & 45.209 & 0.228 & 14.643 & & 73.00 & 21667 & $\begin{array}{ll}41.7 \\
\end{array}$ & 11.1 .1 & & & & & & & \\
\hline 91 & & 2:27:09 PM & 24.828 & 19.095 & 25.126 & 20.699 & & & & 3.597 & & & & & & & & & 1.23333 & & & & & 034 & $\frac{0.034}{0.034}$ & & & \\
\hline & 2/5/2004 & 2:28:09 PM & 24.835 & & & & & 43.188 & 43.684 & 3.605 & 39.212 & -1.216 & & 46.442 & 0.227 & 14.643 & & & & 42.2 & $\frac{1.4}{1.4}>2 \mathrm{~s}$ & & 2.841 & & & & & \\
\hline 93 & $2 / 5 / 2004$ & 2:29:09 PM & 24.821 & & 25.079 & 20.681 & 22.81 & 42.986 & 43.559 & 3.561 & 38.96 & & & & 0.228 & & & & 1.26667 & & & & & & & & & \\
\hline $\begin{array}{lll}94 & & \end{array}$ & $2 / 5 / 2004$ & 2:30:09 PM & & 19.141 & & 20.779 & 22.87 & 43.011 & 43.452 & 3.514 & & -1.214 & & 44.465 & & & & & 1.28333 & & & & & & & & & \\
\hline 95 & $2 / 5 / 2004$ & 2:31:09 PM & 24.94 & 19.158 & 25.088 & 20.807 & 22.843 & 43.488 & 44.194 & 3.747 & 39.196 & -1.219 & & 44.65 & 0.229 & 14.643 & & 78.00 & 1.3 & 422.7 & 10.9 & 41. & 2.85 & 0.034 & 0.034 & 0.001 & 0.824 & \\
\hline 96 & $2 / 5 / 2004$ & 2:32:09 PM & & 19.18 & 25.075 & 20.89 & & 43.198 & 43.763 & & 39.142 & -1.216 & & $\begin{array}{lll}43.518 \\
\end{array}$ & & & & & 1.31667 & & & & & & & & & \\
\hline 97 & 2/5/2004 & 2:33:09 PM & 25.37 & 19.188 & 25.068 & 20.938 & 22.953 & 43.06 & 43.503 & 3.524 & 39.245 & -1.216 & -4.788 & 43.979 & 0.23 & 14.643 & & 80.00 & 1.33333 & 42.0 & 10.8 & & 2837 & 0.034 & 0.034 & 0.001 & 0.832 & \\
\hline 98 & 2/5/2004 & 2:34:09 PM & 20.090 & 19.216 & 25.076 & 20.976 & 22.6 & 42.49. & 43.064 & 3.714 & 38.436 & -1.216 & & & 0. & & & & 1.35 & & 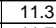 & & & & & & & \\
\hline 99 & 2/5/2004 & & 25.425 & 19.229 & & 21.019 & 22.7 & & 43.3 & & 38.442 & -1.216 & & & & & & & & & 11.7 & & & & & & & \\
\hline$\frac{100}{101}>>2$ & $2 / 5 / 2004$ & 2:36:09 PM & 25.428 & 19.252 & 25.096 & $21.07 / 7$ & 22.902 & 42.335 & 42.12 & 3.668 & 38.36 & -1.216 & & 46.2 & 0.23 & & & & 1.38333 & 41.2 & 11.3 & & & & & & & \\
\hline 101 & $25 / 2004$ & 2:37:09 PM & 20.326 & 19.27 & 25.104 & 21.07 & 22.914 & 42.995 & 43.696 & 3.901 & & -1.216 & & & & & & & 1.4 & 42. & & & & & & & & \\
\hline & $\frac{215 / 2}{215 / 3}$ & $\begin{array}{l}2.50 .099 \mathrm{PM} \\
2.3909 \mathrm{M}\end{array}$ & $20.4<4$ & $\begin{array}{l}19.250 \\
19301\end{array}$ & $\frac{23.114}{2514}$ & $\frac{2.006}{21045}$ & $\frac{23.14}{2312}$ & $\frac{4.406}{4.655}$ & 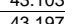 & $\frac{3.125}{387}$ & $\frac{30.30}{38309}$ & $\frac{1.210}{1216}$ & & & & & & & & 41. & 望. & & & & & & & \\
\hline 104 & $\frac{215 / 2}{2 / 5 / 2}$ & \begin{tabular}{|l|}
$2.09 .09 \mathrm{PM}$ \\
$240: 09 \mathrm{PM}$
\end{tabular} & 25.450 25.414 & $\frac{19.301}{1939}$ & $\frac{20.14}{25122}$ & $\frac{21.045}{20983}$ & $\frac{2.14}{23033}$ & . & $\frac{15.131}{43215}$ & $\frac{3.07}{3.878}$ & 年0.355 & $\frac{-1.210}{-1216}$ & & 45.014 & & 14. & & & 145 & 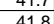 & $\frac{1.4}{115}>>3$ & & & & & 0001 & & \\
\hline & 2/5/2004 & 2:41:09 PM & 25.426 & $\frac{15.029}{19.346}$ & 20 & 21015 & 22.975 & 42.6 & 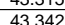 & $\begin{array}{l}0.070 \\
3.672 \\
\end{array}$ & 38.684 & 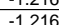 & & 444.557 & & & & & 1.4667 & $\frac{41.0}{41.9}-2$ & & & & & & & & \\
\hline 10 & $2 / 5 / 2004$ & 2:42:09 PM & 25.444 & 19.364 & 25.117 & 21.133 & 22.902 & 42. & 43619 & 3.816 & & -1.214 & & 44.742 & & & & 8900 & 1.48333 & 42 & 10 & & & & & & & \\
\hline & $2 / 5 / 2004$ & 2:43:09 PM & 25.456 & 19.362 & 25.094 & 21.156 & 22.91 & 42.65 & 43.381 & 3.738 & 38.477 & -1.216 & -4.718 & 46.2 & 0.242 & 14.643 & & 90.00 & 1.5 & 41. & 11.3 & 40. & 2.191 & $\frac{0.036}{0.036}$ & 0.036 & 0.001 & 0.087 & \\
\hline & & & & & & & & & & & & & & & & & & & & & & & & & & & & \\
\hline 109 & & Averages & 25.6 & 19.0 & 25.4 & 19.9 & $20.6 \mid t$ & 43.6 & 45.1 & 3.6 & 39.1 & -1.2 & -3.5 & 44.5 & 0.4 & 14.6 & & & & 43.7 & 10.9 & 41.0 & 2.830 & 0.057 & 0.05 & 0.0014 & $1.36 \mathrm{C}$ & \\
\hline & & & 26.4 & 19.4 & 26.1 & 21.2 & & 44.6 & & 4.6 & 41.0 & -0.5 & & 48.1 & 0.6 & & & & & $\begin{array}{l}45.7 \\
\end{array}$ & $11.6 \mathrm{C}>\mathrm{s}$ & & & & & & & \\
\hline & & Median & $25.8 \mathrm{r}-\mathrm{l}$ & .0 & 25.4 & 19.9 & & 4 & & $\begin{array}{ll}3.6 \\
3.2\end{array}$ & & -1.2 & \begin{tabular}{|c|}
-3.8 \\
\end{tabular} & 44.5 & 0.4 & & & & & 44.2 & & & $2.8 \mathrm{~S}-\mathrm{s}$ & & & 0.0013 & & \\
\hline & & Maninum & 24.0 & 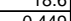 & 2.11 & 19.0 & 10. & 42. & 42.1 & & 3.1 .1 & -1.4 & & 41.2 & 0.2 & 14.6 & & & & 41.2 & 10.1. & 40.1 & 2.100 & & 0.034 & & & \\
\hline 1114 & Number of & $2 x$ Sta & 1.110 & 0 & 0.545 & 1.000 & & 1.20 & 2.919 & 0.433 & $1.1 \%$ & 97 & & 2.908 & 0.221 & 0.000 & 0.001 & & & 2.980 & 0.713 & 164 & $\begin{array}{ll}4 & 0.080 \\
4\end{array}$ & 0.040 & 0.039 & 0.0010 & 0.966 & \\
\hline & TNumber on & (a) & 85 & 0 & 63 & & & & & & & & & & & & & & & 85 & 85 & 85 & & & 85 & & 85 & \\
\hline & & & & are not in & & & & & & & & & & & & & & & & & & & & & & & & \\
\hline & & & & & & & & & & & & & & & & & & & & & & & $15 \mathrm{hc}$ & in avg. & 0.0561 & & & \\
\hline
\end{tabular}


RUN \# 1.18; SECOND ACID CLEAN WITH 2.0 M HNO3

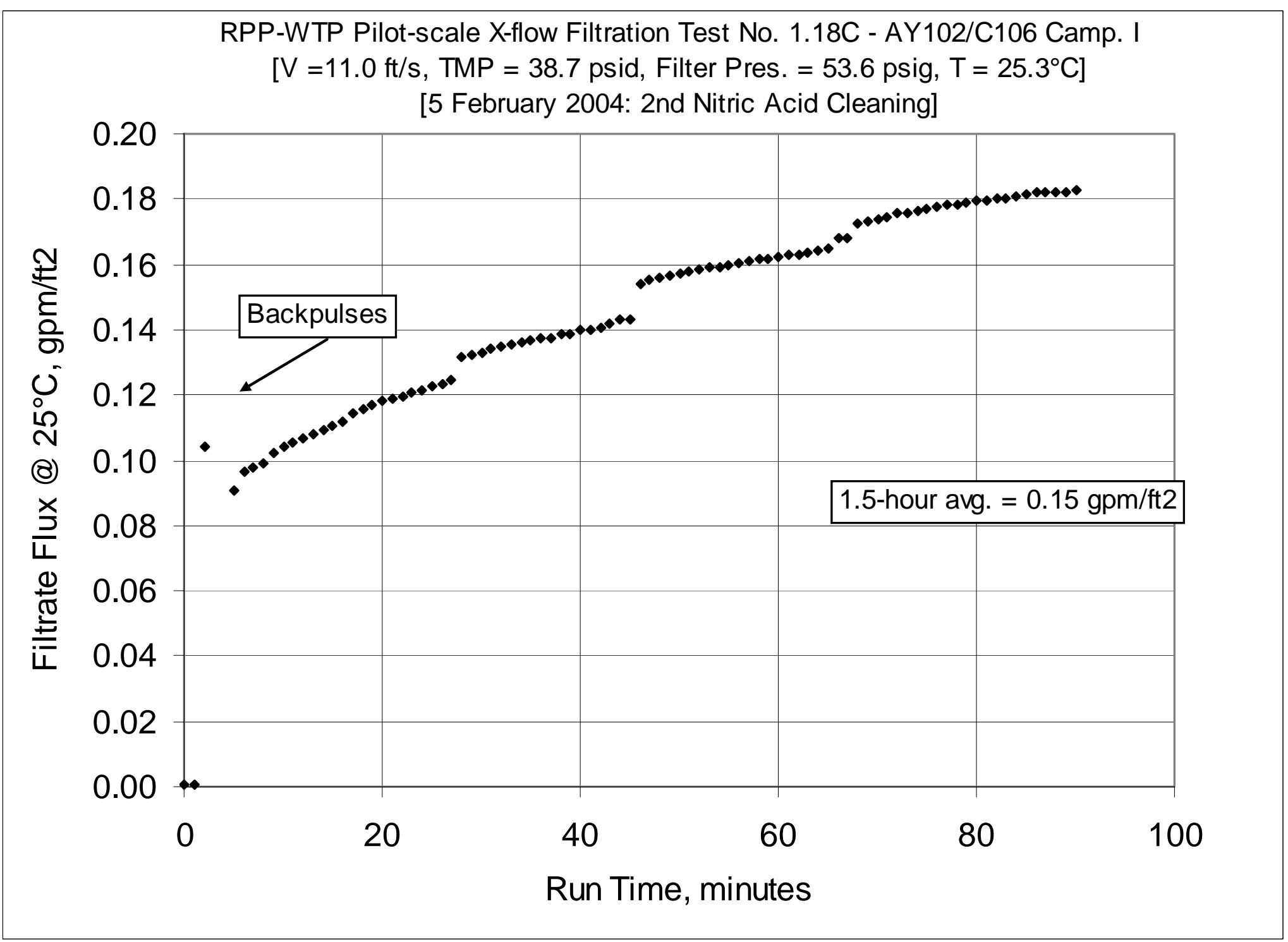


WSRC-TR-2005-00105, REVISION 0

SRNL-RPP-2005-00012, REVISION 0

RUN \# 1.18; SECOND ACID CLEAN WITH 2.0 M HNO3 - CONT.

\begin{tabular}{|c|c|c|c|c|c|c|c|c|c|c|c|c|c|c|c|c|c|c|c|c|c|c|c|c|c|c|c|c|}
\hline & $\mathrm{A}$ & B & $\mathrm{D}$ & 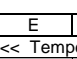 & \begin{tabular}{|l|}
$\mathrm{F}$ \\
eerature $\mathrm{N}$
\end{tabular} & \begin{tabular}{|l|} 
Geasurem \\
Mas
\end{tabular} & $\begin{array}{ll}\mathrm{H} \\
\text { nents } \gg \gg\end{array}$ & $\mathrm{J}$ & $\begin{array}{ll}K \\
<\ll<\ll<<\end{array}$ & $\frac{\mathrm{L}}{\mathrm{L}} \mid$ & \begin{tabular}{|c|}
$\mathrm{M}$ \\
Measureme
\end{tabular} & \begin{tabular}{|l|}
$\mathrm{N}$ \\
ents $\gg \gg$ \\
\end{tabular} & $\mathrm{O}$ & $\frac{Q}{<\ll<\ll<s}$ & \begin{tabular}{l|}
$\mathrm{R}$ \\
$<$ Flow N
\end{tabular} & \begin{tabular}{l|}
$\mathrm{s}$ \\
easureme
\end{tabular} & 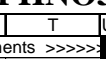 & $\frac{v}{R \ll<\ll<s}$ & $\frac{|w|}{\ll<\ll<\ll<<<~}$ & \begin{tabular}{|c|}
$x<\ll<c$ \\
$x<<$
\end{tabular} & $\begin{array}{l}\frac{1}{\mid} \mathrm{Y} \mid \\
\text { Calculate }\end{array}$ & \begin{tabular}{|l|}
$z$ \\
ted Fror \\
\end{tabular} & \begin{tabular}{l|l|} 
AA \\
I Raw
\end{tabular} & \begin{tabular}{l|}
$A B$ \\
Data $\gg>$
\end{tabular} & $\begin{array}{ll}A C \\
>2 ?\end{array}$ & \begin{tabular}{l|}
$\mathrm{AD}$ \\
$\gg \gg \gg \gg \lambda$
\end{tabular} & AE & AF \\
\hline & DATE & TIME & Filtrate | & Cleaning & Slurry & Hi Amb. & Lo Amb. & BotTMP & & \begin{tabular}{|l|} 
Filter $d \mathrm{~d}$ \\
\end{tabular} & \begin{tabular}{|l|l|} 
TopTMP \\
\end{tabular} & Filtrate & & & & Hi Filtate & & & & & & & & & & & & \\
\hline 3 & & & $\operatorname{deg} C$ & $\operatorname{deg} C$ & \begin{tabular}{|l|}
$\operatorname{deg} C$ \\
\end{tabular} & \begin{tabular}{|l|}
$\operatorname{deg} \mathrm{C}$ \\
\end{tabular} & $\operatorname{deg} C$ & psid & psig & psid & psid & \begin{tabular}{|l|} 
psig \\
\end{tabular} & psig & gpm & gpm & gpm & gpm & & & & & & & & & & & \\
\hline \begin{tabular}{|l|l}
4 \\
\end{tabular} & & & T2 & T3 & T1 & T4 & T5 & $\mathrm{dP2}$ & P1 & $\mathrm{dP1}$ & $\mathrm{dP3}$ & $P^{3}$ & P3 & $\mathrm{Q}_{1}$ & $\mathrm{Q} 2$ & $\mathrm{Q} 3$ & Qbp & & & & & & & & & & & \\
\hline & & & & & & & & & & & & & & & & & & & & & & & & & & & & \\
\hline $\begin{array}{l}0 \\
7 \\
\end{array}$ & $\frac{103 / 2004}{2 / 5 / 200}$ & 9:26:34 AM & 19.191 & 18.592 & \begin{tabular}{|l|l|}
16.892 \\
\end{tabular} & \begin{tabular}{|l|}
19.019 \\
\end{tabular} & 18.202 & $\mid 0.056$ & -0.05 & -0.029 & 0 & -0.177 & & -0.093 & 0.003 & $\begin{array}{l}14.643 \\
\end{array}$ & 0.004 & & & Filter Su & $\frac{1}{\text { Surface } 16}$ & 6.707 & $\mathrm{FT2}$ & & & & & \\
\hline 8 & $2 / 5 / 2004$ & 9:26:52 AM & 19.191 & 18.592 & \begin{tabular}{|l|l|}
16.892 \\
\end{tabular} & \begin{tabular}{|l|l|}
19.019 \\
\end{tabular} & 18.202 & 0.056 & -0.05 & -0.029 & 0 & -0.177 & 0.12 & -0.093 & 0.003 & 14.643 & 0.004 & & & Convers & rsion & & $\mathrm{m} 3 / \mathrm{m} 2 / \mathrm{l}$ & day/bar! & $\mathrm{g} / \mathrm{gpm} / \mathrm{f}$ & t2/barg & & \\
\hline 9 & $2 / 5 / 2004$ & & 19.187 & 18.582 & \begin{tabular}{|l|l|}
16.872 \\
\end{tabular} & \begin{tabular}{|l|}
19.03 \\
\end{tabular} & \begin{tabular}{|l|} 
\\
\end{tabular} & 0.056 & -0.05 & & & -0.18 & 0.12 & -0.091 & 0.000 & 14.643 & 0.004 & & & & & & & & & & & \\
\hline 10 & 2/5/2004 & 9:26:54 AM & 19.181 & 18.571 & \begin{tabular}{|l|l|}
16.861 \\
307
\end{tabular} & \begin{tabular}{|l|}
19.024 \\
\end{tabular} & 18.146 & 0.058 & -0.05 & -0.029 & -0.002 & \begin{tabular}{|l|}
-0.18 \\
\end{tabular} & & -0.091 & 0.003 & 14.643 & 0.004 & & & & & & & & ately & 4 psig & & \\
\hline & & 9:26:55 AM & & 18.577 & 16.867 & & & & & -0.029 & 0.002 & & & -0.093 & 0.003 & 14.643 & & & & & & & & & & & & \\
\hline & $2 / 5 / 2004$ & 9:26:56 AM & 19.181 & $\mid 18.577$ & \begin{tabular}{|l|}
16.872 \\
\end{tabular} & \begin{tabular}{|l|}
19.024 \\
\end{tabular} & 18.157 & 0.056 & \begin{tabular}{|l|l|} 
& -0.048 \\
\end{tabular} & $\mid-0.029$ & & $\mid-0.177$ & & -0.091 & 0.003 & 14.643 & & & & Press & $\begin{array}{l}\text { Plis } \\
\end{array}$ & orrec & ed for 2 & & 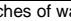 & er tubi & & \\
\hline & 2/5/2004 & $9: 26: 57 \mathrm{AM}$ & 19.186 & 18.572 & \begin{tabular}{|l|l|l|} 
\\
\end{tabular} & 19.029 & 18.152 & 0.056 & -0.05 & -0.029 & & -0.177 & & -0.093 & 0.003 & 14.643 & & & & NVV & & & & Filtra & te Flux & PEF & ABILI & \\
\hline 4 & $25 / 2004$ & 9:26:58 AM & 19.192 & 18.587 & \begin{tabular}{|l|l|}
16.877 \\
\end{tabular} & $\begin{array}{ll}19.03 \\
\end{array}$ & 18.162 & 0.058 & -0.05 & -0.028 & & -0.177 & 0.12 & -0.091 & 0.003 & 14.643 & 0.005 & & & & & & & & & & & \\
\hline & & & & & & & & & & & & & & & & & & Time & Time & Press. & vel. & TMP & TMP & $\mathrm{pm}$ & $\mathrm{m}$ & $\mathrm{pm}$ & $\mathrm{m}$ & eter \\
\hline & $\frac{\text { Data - Per Mil }}{2 / 52004}$ & $\begin{array}{l}\text { inute } \\
\text { i.1.3734 PM }\end{array}$ & & & & & & & & & & & & & & & & & Hour & osig & & 10 & & & & $2 / p s i$ & & 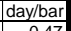 \\
\hline \begin{tabular}{|l|}
17 \\
18 \\
\end{tabular} & $\frac{25 / 20004}{2 / 5 / 2004}$ & $\begin{array}{l}\text { 3:11::34 PM } \\
\text { 3:18:34 PM }\end{array}$ & $\begin{array}{l}22.824 \\
22.265\end{array}$ & $\begin{array}{l}19.758 \\
19.762\end{array}$ & \begin{tabular}{|l|}
$\mid 23.3224$ \\
23.42 \\
\end{tabular} & \begin{tabular}{|l|}
20.3977 \\
20.532 \\
\end{tabular} & 22.019 & $\begin{array}{l}2.663 \\
2.877 \\
\end{array}$ & \begin{tabular}{|l|}
43.137 \\
43.533 \\
\end{tabular} & $\begin{array}{l}3.644 \\
3.675 \\
\end{array}$ & $\begin{array}{c}-0.977 \\
-0.903 \\
\end{array}$ & \begin{tabular}{|l|}
35.823 \\
35.774 \\
\end{tabular} & $\frac{83}{42}$ & \begin{tabular}{|l|}
43.8311 \\
43.301 \\
\end{tabular} & $\begin{array}{l}0.003 \\
0.003 \\
\end{array}$ & $\frac{14.643}{14.643}$ & 0.005 & 1.00 & 0.01667 & $\begin{array}{l}41.7 \\
42.1 \\
\end{array}$ & $\begin{array}{l}\frac{10.7}{10.6} \\
\end{array}$ & $\frac{0.8}{1.0}$ & $\frac{0.058}{0.068}$ & .000 & $\frac{0.000}{0.000}$ & 0.001 & 0.556 & 0.47 \\
\hline 19 & 2/5/2004 & 3:19:34 PM & 23.52 & 19.772 & 23.552 & & 22.409 & 35.504 & 42.992 & 3.604 & 31.881 & 2.368 & 0.569 & 45.237 & 0.671 & 14.643 & $\begin{array}{l}0.005 \\
0.004\end{array}$ & 2.00 & $\begin{array}{l}0.01001 \\
0.03333 \\
\end{array}$ & $\begin{array}{l}42.1 \\
41.5 \\
\end{array}$ & 11.1 & 33.7 & 2.323 & 0.100 & 0.004 & $\begin{array}{l}0.003 \\
0.003 \\
\end{array}$ & $\begin{array}{l}0.474 \\
3.093 \\
\end{array}$ & \\
\hline & $2 / 5 / 2004$ & 3:20:34 PM & 23.695 & 19.771 & \begin{tabular}{|l|}
23.637 \\
\end{tabular} & 20.44 & & 38.104 & 43.205 & & \begin{tabular}{|l|l|l|}
34.19 \\
\end{tabular} & & & 44.753 & 0.603 & & & 3.00 & & 41.7 & 11.0 & & 2.492 & 2.183 & 2.269 & 0.063 & 62.770 & \\
\hline 21 & 2/5/2004 & 3:21:34 PM & 23.926 & & \begin{tabular}{|l|l|}
23.723 \\
\end{tabular} & 20.467 & 22.189 & 38.807 & 43.346 & 3.725 & 34.832 & -0.646 & -1 & 44.838 & 0.569 & 14.643 & & 4.00 & 0.06667 & 41.9 & 11.0 & & 2.539 & 2.183 & 2.263 & 0.061 & 61.473 & \\
\hline 22 & $2 / 5 / 2004$ & & $\begin{array}{l}24.046 \\
2.119\end{array}$ & 19.772 & \begin{tabular}{|l|}
23.813 \\
2301 \\
\end{tabular} & \begin{tabular}{|l|}
20.411 \\
20.91
\end{tabular} & 22.459 & & 44.764 & $\begin{array}{ll}4.096 \\
350\end{array}$ & $\begin{array}{l}35.436 \\
3756\end{array}$ & \begin{tabular}{|l|} 
\\
\end{tabular} & & \begin{tabular}{|l|}
50.56 \\
\end{tabular} & $\begin{array}{ll}0.587 \\
\end{array}$ & & & 500 & 0.08333 & 43.3 & 12.4 & & 2.593 & & & 0.002 & 2.406 & \\
\hline & $\begin{array}{l}2 / 5 / 2004 \\
25 / 5004\end{array}$ & 3:34 PM & $24.141 \mid$ & 19.782 & \begin{tabular}{|l|}
23.913 \\
24018 \\
\end{tabular} & \begin{tabular}{|l|}
20.481 \\
20.577 \\
\end{tabular} & $\frac{22.309}{22.23}$ & $\begin{array}{ll}41.107 \\
41.246\end{array}$ & \begin{tabular}{|l|}
46.662 \\
46.845 \\
\end{tabular} & $\begin{array}{l}3.505 \\
3.34 \\
34\end{array}$ & $\begin{array}{l}37.506 \\
33762\end{array}$ & 0.327 & & $\begin{array}{l}44.581 \\
43607\end{array}$ & $\begin{array}{ll}0.627 \\
0.67\end{array}$ & $\frac{14.643}{14643}$ & 0.004 & 6.00 & $\begin{array}{rr}0.1 \\
1667\end{array}$ & $\frac{45.2}{454}$ & 10.9 & $\begin{array}{l}39.3 \\
39.4 \\
\end{array}$ & $\frac{2.710}{2.70}$ & 093 & $\begin{array}{ll}0.096 \\
0098\end{array}$ & 0.002 & 2.452 & \\
\hline $\begin{array}{l}24 \\
25 \\
\end{array}$ & $\frac{25 / 2004}{2 / 5 / 2004}$ & $\begin{array}{l}\text { 3:2:2:34 PM } \\
\text { 3:25:34 PM }\end{array}$ & $\begin{array}{l}24.276 \\
24.432\end{array}$ & $\begin{array}{l}19.987 \\
19.797\end{array}$ & $\mid$\begin{tabular}{|}
24.1818 \\
24.144 \\
\end{tabular} & \begin{tabular}{|l|}
$\mid 20.577$ \\
20.587 \\
\end{tabular} & $\begin{array}{l}22.123 \\
21.988\end{array}$ & $\begin{array}{l}41.246 \\
40.825\end{array}$ & \begin{tabular}{|l|}
46.845 \\
4691 \\
\end{tabular} & $\begin{array}{l}3.434 \\
3.278\end{array}$ & $\begin{array}{l}37.642 \\
37.537\end{array}$ & $\mid \begin{array}{l}0.578 \\
0.726 \\
\end{array}$ & & $\mid$\begin{tabular}{|l|}
$\mid 43.167$ \\
3.194
\end{tabular} & $\begin{array}{l}0.637 \\
0.649 \\
\end{array}$ & $\begin{array}{l}14.643 \\
14.643\end{array}$ & & $\frac{7.00}{8.00}$ & $\mid 0.116334$ & $\begin{array}{l}45.4 \\
45.2 \\
\end{array}$ & 10.6 & $\begin{array}{ll}39.4 \\
39.2\end{array}$ & $\frac{2.720}{2.701}$ & $\begin{array}{l}0.095 \\
0.097 \\
\end{array}$ & $\begin{array}{l}0.098 \\
0.099 \\
\end{array}$ & $\begin{array}{l}0.002 \\
0.003 \\
\end{array}$ & $\frac{2.475}{2.530}$ & \\
\hline 26 & & 3:26:34 PM & 24.542 & 19.803 & 24.24 & \begin{tabular}{|l|}
20.593 \\
\end{tabular} & & 41.999 & \begin{tabular}{|l|}
48.399 \\
\end{tabular} & 3.555 & 38.196 & 1.181 & & $\begin{array}{ll}44.367 \\
\end{array}$ & 0.673 & 14.643 & & 9.00 & 0.15 & 46.9 & 10.9 & 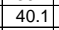 & 2.765 & 0.100 & 0.103 & 0.003 & 2.557 & \\
\hline & & & 24.704 & & & & & & 48.905 & & & 1.464 & & & & & & 10.00 & & 47.4 & 10. & 40 & 2.790 & & & 0.003 & 2.580 & \\
\hline 28 & 2/5/2004 & 3:28:34 PM & 24.819 & $\begin{array}{ll}19.809 \\
\end{array}$ & \begin{tabular}{|l|l|}
24.457 \\
\end{tabular} & \begin{tabular}{|l|l|}
20.539 \\
\end{tabular} & 21.839 & 41.798 & 48.729 & 3.439 & 38.235 & 1.716 & & $\begin{array}{l}45.176 \\
\end{array}$ & $\begin{array}{ll}0.697 \\
\end{array}$ & \begin{tabular}{|l|l|}
14.643 \\
\end{tabular} & & 11.00 & 0.18333 & 47.3 & 11.1 & & 2.759 & 0.104 & $\overline{0.1}$ & 0.003 & 2.637 & \\
\hline 29 & 2/5/2004 & $\begin{array}{l}3: 29: 34 \mathrm{PM} \\
3: 23: 34 \mathrm{PM}\end{array}$ & \begin{tabular}{|l|l|l}
24.886 \\
2.907
\end{tabular} & $\begin{array}{l}19.811 \\
19791\end{array}$ & \begin{tabular}{|l|}
24.538 \\
2461 \\
\end{tabular} & \begin{tabular}{|l|}
20.364 \\
20203
\end{tabular} & 22.338 & 41.313 & 48.339 & $\begin{array}{l}3.432 \\
342 \\
\end{array}$ & $\begin{array}{l}37.743 \\
28057\end{array}$ & $\begin{array}{l}1.873 \\
203\end{array}$ & -0.25 & 44.494 & $\begin{array}{l}0.708 \\
0717\end{array}$ & $\begin{array}{l}14.643 \\
14643 \\
\end{array}$ & 0.003 & 12.00 & $\begin{array}{r}0.2 \\
\end{array}$ & 46.9 & 10.9 & $\frac{39.5}{390}$ & 2.725 & $\begin{array}{ll}0.106 \\
\end{array}$ & 0.107 & 0.003 & 2.705 & \\
\hline & $\frac{2 / 5 / 2004}{2 / 5 / 2004}$ & $\begin{array}{l}\text { 3:30:34 PM } \\
\text { 3:31:34 PM }\end{array}$ & $24.976 \mid$ & \begin{tabular}{|c|}
19.791 \\
1977
\end{tabular} & \begin{tabular}{|l|}
$\mid 24.614$ \\
24.69 \\
\end{tabular} & \begin{tabular}{|l|}
20.203 \\
20112 \\
\end{tabular} & \begin{tabular}{|l|}
22.403 \\
21.927
\end{tabular} & $\begin{array}{r}41.68 \\
41.663 \\
\end{array}$ & \begin{tabular}{|l|}
49.044 \\
48.988 \\
\end{tabular} & $\begin{array}{ll}3.452 \\
3531 \\
\end{array}$ & 38.057| & \begin{tabular}{|l|}
2.02 \\
2233 \\
\end{tabular} & -0.15 & $\begin{aligned} 44.534 \\
4435\end{aligned}$ & $0.717 \mid$ & & & $\frac{13.00}{1400}$ & & $\begin{array}{l}47.6 \\
47.5 \\
\end{array}$ & 10.9 & $\frac{39.9}{39.8}$ & $\frac{2.749}{2.746}$ & & & $\begin{array}{ll}0.003 \\
0.003\end{array}$ & 2.711 & \\
\hline 32 & $\mid \frac{25 / 2004}{2 / 5 / 2004}$ & 3:3:32:34! & $\begin{array}{l}25.0082 \\
25.162\end{array}$ & 19.777 & \begin{tabular}{|l|}
24.799 \\
24.775 \\
\end{tabular} & $\mid$\begin{tabular}{|l|}
20.114 \\
20.284 \\
\end{tabular} & 21.927 & . 3634 & \begin{tabular}{|l|}
48.988 \\
48.932 \\
\end{tabular} & $\begin{array}{l}3.531 \\
3.443 \\
\end{array}$ & 853 & \begin{tabular}{|l|}
2.243 \\
2.414 \\
\end{tabular} & & $\frac{44.035}{44.617}$ & $\begin{array}{l}0.128 \\
0.736 \\
\end{array}$ & & & & 0.2335 & & $\begin{array}{ll}10.8 \\
10.9 \\
\end{array}$ & & & & & & & \\
\hline 33 & & 3:33:34 & 25.256 & 19.771 & $\mid$\begin{tabular}{|l|}
24.844 \\
\end{tabular} & 20.465 & 21.861 & .045 & \begin{tabular}{|l|}
48.899 \\
\end{tabular} & 3.418 & 465 & 2.64 & & & 0.746 & & & & & & 106 & & $\frac{2.70}{.70}$ & & & 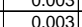 & 2.846 & \\
\hline & $2 / 5 / 2004$ & & 25.316 & 19.776 & \begin{tabular}{|l|}
24.92 \\
\end{tabular} & & 21.776 & & \begin{tabular}{|l|l|}
49.446 \\
\end{tabular} & & & 3.054 & & & & & & & 28 & & & & & & & & & \\
\hline & & & 25.406 & & 25 & & 21.67 & & & 448 & & & & & & & & & & & 08 & & & & & & & \\
\hline & 2004 & & 25.471 & & 25.07 & 20.849 & & & \begin{tabular}{|l|l|}
49 \\
\end{tabular} & & & 3.456 & & & & & & & .31667 & & & & & & & & $\frac{5}{55}$ & \\
\hline 37 & & & 25.562 & & $\mid 25.14$ & 20.91 & & & & 3.478 & & & & & 0.794 & & & & 0.33333 & & $\frac{10.7}{10.7}$ & & & & & & 3.011 & \\
\hline 38 & $2 / 5 / 2004$ & & $25.627 \mid$ & & \begin{tabular}{|l|l|}
25.186 \\
\end{tabular} & D11 & & & & 3.455 & & 3.835 & & & & & & & & & 10.9 & & & & & & & \\
\hline & & & 25.703 & & \begin{tabular}{|l|}
25.242 \\
\end{tabular} & & & & \begin{tabular}{|l|l|}
49.748 \\
\end{tabular} & 3.486 & & & & & 0.809 & & & & & & & & & & & & & \\
\hline 40 & & & 25.73 & & \begin{tabular}{|l|}
25.288 \\
\end{tabular} & & & & & & & .157 & & & & & & & 0.38333 & & & & & & & & 3.138 & \\
\hline 41 & & & 25.81 & & & & & & & & & & & 45.1 & & & & & 0.4 & & & & & & & & 3.192 & \\
\hline $44^{42}$ & & & $\frac{25.84}{25596}$ & & & 21.165 & & & 50.101 & 3.506 & & & & & & & & & & & & & & & & & & \\
\hline 海 & & & & $\frac{19.816}{1983}$ & \begin{tabular}{|r|}
25.41 \\
25.47
\end{tabular} & 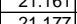 & & & & $\frac{3.5306}{3501}$ & & & & & & & & & & & & & & & & & & \\
\hline 475 & & & 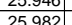 & & \begin{tabular}{|l|l|l|}
25.416 \\
\end{tabular} & 21.17 & & & \begin{tabular}{|l}
4.93 .451 \\
53007
\end{tabular} & $\mid$ & & 4.0 & & & & & & & & & & & & & & & & \\
\hline 46 & & & & & & \begin{tabular}{|l|l|}
21.213 \\
\end{tabular} & & & 0.0 & 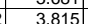 & & & & & $\frac{0.004}{0.904}$ & & & & . & & 11.1. & & & & & & & \\
\hline & $2 / 5 / 2004$ & & 资.6.149 & & $\begin{array}{l}25.703 \\
25.703\end{array}$ & 201.26 & & & 0.072 & 3.736 & & 551 & & $\frac{25.2}{45.2}$ & 0.007 & & & 年 & - & & & & & & & & & \\
\hline 48 & & & & & \begin{tabular}{|l|}
25.759 \\
\end{tabular} & 281 & & & 52.55 & 3.629 & & & & & & & & & 67 & 51. & $11:$ & 38.8 & & & & & & \\
\hline 49 & & & & & & & & & & & & & & & & & & & & & & & & & & & & \\
\hline 5 & & & 26.381 & & 25.886 & 21.308 & & & & 3.645 & & & & & & & & & & & 17 & & & & & & & \\
\hline & $2 / 51 /$ & & 26.42 & & \begin{tabular}{|l|l|}
25.942 \\
\end{tabular} & & & & & 3.7 & & & & & & & & & 667 & & & & & & & 0.004 & & \\
\hline \begin{tabular}{|ll}
5 \\
\end{tabular} & & & & & & & & & & & & & & & & & & & 0.5833 & & & & & & & & & \\
\hline 53 & & & 26.544 & & & & & & & 3.56 & & & & & & & & & 0.6 & 51. & 11 & & & & & & & \\
\hline 54 & & & & & & & & & & & & & & & & & & & & & & & & & & & & \\
\hline 55 & & & & & & & & & & & & & & & & & & & & & & & & & & & & \\
\hline$\frac{56}{57}$ & & & & & 26.217 & & & & & & & & & & & & & & & & & & & & & & & \\
\hline 51 & & & & & $\frac{20.245}{26289}$ & & & & & & & & & & & & & & 689 & & & & & & & & & \\
\hline 59 & & & & & & & & & & & & & & & & & & & & & & & & & & & & \\
\hline 60 & & & & & 26.105 & & & & & & & & & & & & & & & & & & & & & & & \\
\hline \begin{tabular}{|l|}
61 \\
\end{tabular} & $2 / 5 / 2004$ & & & & & & & & & & & & & & & & & & & & & & & & & & & \\
\hline 62 & & & & & 25.9 & & & & & 3.63 & & & & & & & & & & & & & & & & & & \\
\hline & & & & & 25.9 & & & & & & & & & & & & & & & & & & & & & & & \\
\hline \begin{tabular}{|l|l|}
64 & \\
\end{tabular} & & & 26. & & & 21.73 & & & & & & & & & & & & & 0.783 & & & & & & & & & \\
\hline 65 & $2 / 5 / 2004$ & & 26.536 & 20.054 & 25.7 & 21.746 & & $40.7 \mathrm{C}$ & 56.6 & 3.245 & & & & 44.5 & 1.0 & 14. & & & $\overline{0.8}$ & $55 ? 2$ & 109 & 911 & & & & & & \\
\hline \begin{tabular}{|l|l|}
66 \\
\end{tabular} & & & & & & & & & & & & & & & 1.073 & & & & & & & & & & & & & \\
\hline 67 & & & & & & & & & & & & & & & & & & & & & & & & & & & & \\
\hline 68 & $2 / 5)$ & & & 20 & 25. & & & 40.62 & & & & & & & & & & & & & & & & & & & & \\
\hline & & & & & & & & & & & & & & & & & & & & & & & & & & & (2) & \\
\hline & & & & & & & & & & & & & & & & & & $3.6 \mathrm{C}$ & & & & & & 162 & 0.159 & .004 & 4.092 & \\
\hline
\end{tabular}


WSRC-TR-2005-00105, REVISION 0

SRNL-RPP-2005-00012, REVISION 0

RUN \# 1.18; SECOND ACID CLEAN WITH 2.0 M HNO3 - CONT.

\begin{tabular}{|c|c|c|c|c|c|c|c|c|c|c|c|c|c|c|c|c|c|c|c|c|c|c|c|c|c|c|c|c|}
\hline & A & B & D & $E$ & $\mathrm{~F}$ & $\mathrm{G}_{\mathrm{G}}$ & $\mathrm{H}$ & $\mathrm{J}$ & $\mathrm{K}$ & $\mathrm{L}$ & $\mathrm{M}$ & $\mathrm{N}$ & $\mathrm{O}$ & Q & $\mathrm{R}$ & 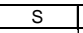 & \begin{tabular}{l|l}
$\mathrm{T}$ & $\mathrm{T}$ \\
\end{tabular} & & $\mathrm{w}$ & & & $L_{2}$ & AA & $A B$ & $A C$ & $A D$ & $\mathrm{AE}$ & AF \\
\hline 71 & $2 / 5 / 2004$ & 4:11:34 PM & $\frac{26.414}{2.44}$ & $\frac{20.128}{20125}$ & $\begin{array}{l}25.684 \\
25665\end{array}$ & $\begin{array}{l}21.77 \\
21.791\end{array}$ & $\begin{array}{l}22.302 \\
2027\end{array}$ & 40.5 & $\begin{array}{l}57.336 \\
57255\end{array}$ & 3.449 & $\begin{array}{l}37.018 \\
32715\end{array}$ & $\begin{array}{ll}1.724 \\
11849\end{array}$ & $\frac{5.58}{5.6}$ & $\begin{aligned} 43.76 \\
32.201\end{aligned}$ & 1.08 & $\begin{array}{l}14.643 \\
14643\end{array}$ & 0.003 & & $\frac{0.9}{016.97}$ & 55. & & & & & 0.159 & 0.004 & 4.105 & 3.49 \\
\hline 73 & $\frac{21512004}{215 / 2004}$ & $\begin{array}{l}4: 12: 34 \mathrm{PM} \\
1 \cdot 23: 34 \mathrm{PM}\end{array}$ & & & $\frac{25.005}{25.621}$ & & & & 7.225 & $\frac{3.356}{3.303}$ & & & & & & & & & & & & & & 1.163 & 0.160 & 0.004 & 4.110 & 3.50 \\
\hline 74 & 2/5/2004 & $\begin{array}{l}\text { 4.13:34 PMI } \\
\text { 4:14:34 PM }\end{array}$ & $\frac{20.511}{26.372}$ & 20.167 & $\frac{25.021}{25.652}$ & 21.823 & 21.747 & 40.110 & $\begin{array}{l}50.020 \\
57.193\end{array}$ & 3.005 & $\begin{array}{r}37.020 \\
36.79 \\
\end{array}$ & \begin{tabular}{|l|}
1.1 .092 \\
12.043 \\
\end{tabular} & $\begin{array}{l}5 . / 78 \\
5.787\end{array}$ & $\begin{array}{r}44.295 \\
44.6 \\
\end{array}$ & $\begin{array}{l}1.096 \\
1.099 \\
\end{array}$ & $\begin{array}{l}14.643 \\
14.643\end{array}$ & 0.00 & 57.00 & 0.95 & 55.7 & 10.9 & 38.5 & \begin{tabular}{|l|}
2.656 \\
\end{tabular} & \begin{tabular}{|l|}
0.163 \\
0.164 \\
\end{tabular} & $\begin{array}{l}0.101 \\
0.161 \\
\end{array}$ & $\begin{array}{l}0.0004 \\
0.004\end{array}$ & $\begin{array}{l}4.1 .10 \\
4.176 \\
\end{array}$ & $\begin{array}{l}3.54 \\
3.55 \\
\end{array}$ \\
\hline 75 & $2 / 5 / 2004$ & 4:15:34 PM & 26.349 & 20.173 & 25.639 & 21.805 & & 40.116 & 57.237 & 3.312 & 36.702 & & & & 1.102 & $\begin{array}{l}14.045 \\
14.643 \\
\end{array}$ & & 58.00 & 0.96667 & & 0.9 & $\begin{array}{l}38.5 \\
38.4 \\
\end{array}$ & \begin{tabular}{|l|}
2.0548 \\
2.648 \\
\end{tabular} & & $\begin{array}{l}0.101 \\
0.161\end{array}$ & $\begin{array}{l}0.004 \\
0.004 \\
\end{array}$ & $\begin{array}{l}4.1100 \\
4.202 \\
\end{array}$ & \\
\hline 76 & $2 / 5 / 2004$ & 4:16:34 PM & 26.34 & 20.184 & 25.625 & 21.796 & 22.117 & 39.88 & 57.204 & 3.381 & 36.339 & 12.188 & 5.871 & 46.392 & 1.105 & $\begin{array}{l}14.045 \\
14.643 \\
\end{array}$ & 0.004 & 59.00 & 0.98333 & 55.7 & 11.4 & $\begin{array}{l}0.4 \\
38.1 \\
\end{array}$ & 2.628 & \begin{tabular}{|l|}
0.165 \\
\end{tabular} & 0.162 & 0.004 & 4.248 & 361 \\
\hline 77 & $2 / 5 / 2004$ & 4:17:34 PM & 26.331 & 20.191 & 25.596 & & & 39.859 & 56.92 & 3.383 & 36.517 & 12.306 & 5.961 & 43.931 & 1.108 & 14.643 & 0.004 & 60.00 & & 55.4 & 10.8 & 38.2 & 2.633 & 0.165 & 0.162 & 0.004 & 4.254 & 3.6 \\
\hline 78 & $2 / 5 / 2004$ & & 26.317 & & 25.587 & 21.813 & 21.848 & 39.946 & 57.368 & 3.485 & 36.304 & 12.332 & 5.998 & 46.571 & 1.11 & 14.643 & 0.004 & 61.00 & 1.01667 & 55.9 & 11.4 & 38.1 & 2.629 & 0.165 & 0.163 & 0.004 & 4.270 & 3.6 \\
\hline 79 & & 4:19:34 PM & 26.309 & 20.213 & 25.584 & 21.799 & 21.824 & 39.898 & 57.419 & 3.513 & 36.146 & \begin{tabular}{|l|}
12.477 \\
\end{tabular} & 6.059 & 44.131 & 1.113 & 14.643 & 0.004 & 62.00 & 1.03333 & 55.9 & 10.8 & $\frac{38.0}{38.0}$ & 2.621 & 1.66 & 0.163 & 0.004 & & 3.05 \\
\hline$\frac{80}{19}$ & $2 / 5 / 2004$ & 4:20:34 PM & 26.29 & 20.214 & & $\begin{array}{l}21.79 \\
2.79\end{array}$ & 21.599 & & 57.079 & 3.434 & 35.91 & $\begin{array}{l}12.55 \\
1\end{array}$ & 6.134 & 43.912 & 1.117 & 14.643 & (2) & 63.00 & 1.05 & 55.6 & & & 2.601 & & 0.164 & 0.004 & 4.344 & 3.70 \\
\hline 81 & $2 / 5 / 2004$ & $\begin{array}{l}4: 21: 34 \mathrm{PM} \\
4: 22: 34 \mathrm{PM}\end{array}$ & $\begin{array}{ll}26.286 \\
26266\end{array}$ & 20.235 & \begin{tabular}{|l|l|}
25.58 \\
25.55
\end{tabular} & $\begin{array}{l}21.786 \\
21751\end{array}$ & $\begin{array}{ll}21.961 \\
21821\end{array}$ & $\begin{array}{l}39.575 \\
32757 \\
3\end{array}$ & 57.289 & 3.437 & 36.008 & 12.634 & 6.163 & 44.118 & 1.12 & 14.643 & & 64.00 & 1.06667 & 55.8 & & & $\begin{array}{ll}2.606 \\
2.07\end{array}$ & & 0.164 & 0.004 & 4.347 & 3.70 \\
\hline 82 & 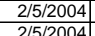 & 4:22:34 PM & 26.266 & & 25.555 & 21.751 & 21.821 & 39.757 & 57.382 & 3.381 & 36.437 & $\begin{array}{ll}12.686 \\
1321\end{array}$ & 6.235 & 43.564 & 1.122 & 14.643 & 0.00 & & 1.08333 & & & 38.1 & 2.627 & & & 0.004 & 4.323 & 3.68 \\
\hline$\frac{05}{84}$ & $\frac{2 / 5 / 2004}{2 / 5 / 2004} \mid$ & $\frac{4: 23: 34 \mathrm{PM}}{4: 24,34 \mathrm{PM}}$ & & $\frac{20.246}{20.268}$ & \begin{tabular}{|l|}
25.551 \\
2567
\end{tabular} & $\frac{21.777}{21.783}$ & & $\begin{array}{l}40.002 \\
40.429\end{array}$ & $58.464 \mid$ & & & $\begin{array}{l}13.424 \\
13.482\end{array}$ & 6.614 & $\begin{array}{l}45.387 \\
44.49 \\
\end{array}$ & & $\frac{14.643}{14.643}$ & 0.00 & 66.00 & & & & & 2.632 & & 0.168 & 0.004 & 4.396 & 3.74 \\
\hline 85 & $2 / 5 / 2004$ & $\begin{array}{l}\text { 4.24.34 PMI } \\
\text { 4:25:34 PM }\end{array}$ & 26.293 & $\frac{20.200}{20.273}$ & \begin{tabular}{|l|}
25.601 \\
25.607 \\
\end{tabular} & 21.793 & 22.094 & $4 \frac{40.429}{41.091}$ & $\begin{array}{l}50.189 \\
60.651\end{array}$ & 3.623 & $\begin{array}{l}30.083 \\
37.289\end{array}$ & $\begin{array}{l}\frac{13.482}{14.547} \\
1\end{array}$ & 7.314 & $\begin{array}{r}44.49 \\
45.155 \\
\end{array}$ & $\frac{1.140}{1.177}$ & $\begin{array}{l}\frac{14.643}{14.643} \\
\end{array}$ & & 68.00 & 1.13333 & 59.2 & $\frac{11.9}{11.1}$ & 39.2 & $\begin{array}{l}2.0005 \\
2.702 \\
\end{array}$ & 0.175 & $\frac{0.168}{0.173}$ & $\begin{array}{l}0.004 \\
0.004 \\
\end{array}$ & 4.402 & $\begin{array}{l}3.70 \\
3.75 \\
\end{array}$ \\
\hline 86 & $2 / 5 / 2004$ & 4:26:34 PM & 26.319 & 20.284 & 25.548 & 21.885 & 21.894 & 40.726 & 60.354 & 3.601 & 37.028 & 14.593 & 7.354 & 45.802 & 1.179 & 14.643 & & 69.0 & 1.15 & 58.9 & & 38.9 & 2.680 & & & & 4.452 & \\
\hline 87 & $2 / 5 / 2004$ & 4:27:34 PM & 26.274 & 20.285 & 25.414 & 21.976 & 21.649 & 40.664 & 60.294 & 3.525 & 37.123 & 14.657 & 7.383 & 45.543 & 1.18 & 14.643 & & 70.00 & $\begin{array}{ll}1.16667 \\
\end{array}$ & 58.8 & & & \begin{tabular}{|l|}
2.682 \\
\end{tabular} & \begin{tabular}{|l|l|}
0.176 \\
\end{tabular} & 0.174 & & 4.471 & \\
\hline 88 & $2 / 5 / 2004$ & 4:28:34 PM & 26.184 & 20.285 & 25.299 & 21.916 & 21.91 & 40.481 & 60.114 & 3.578 & 36.842 & 14.663 & & 45.18 & 1.182 & 14.643 & 0.004 & 71.00 & 1.18333 & 58.6 & 11.1 & 38.7 & \begin{tabular}{|l|}
2.666 \\
\end{tabular} & \begin{tabular}{|l|l|}
0.176 \\
\end{tabular} & 0.175 & 0.005 & 4.520 & 3.8 \\
\hline 89 & $2 / 5 / 2004$ & 4:29:34 PM & 26.116 & 20.311 & 25.225 & 21.942 & 21.765 & 40.912 & 60.875 & 3.711 & 36.948 & 14.715 & 7.45 & 44.824 & 1.184 & 14.643 & & 72.00 & 1.2 & 59.4 & & & \begin{tabular}{|l|}
2.684 \\
\end{tabular} & \begin{tabular}{|l|}
0.177 \\
\end{tabular} & 0.175 & & & \\
\hline 90 & $2 / 5 / 2004$ & 4:30:34 PM & 26.042 & 20.317 & 25.141 & 21.943 & $\frac{21.555}{2.772}$ & 40.388 & 60.145 & 3.514 & 36.87 & 14.744 & & 46.776 & 1.184 & & & 73.00 & 1.21667 & 58.7 & 111.5 & 38.6 & \begin{tabular}{|l|}
2.663 \\
\end{tabular} & \begin{tabular}{|l|l|}
0.177 \\
\end{tabular} & 0.176 & 0.005 & 4.552 & \\
\hline 91 & $\begin{array}{l}2 / 5 / 2004 \\
2 / 12001 \\
\end{array}$ & 4:31:34 PM & 25.963 & 20.323 & \begin{tabular}{|l|l|}
25.062 \\
2.000
\end{tabular} & 21.939 & 21.773 & 40.784 & 60.682 & 3.69 & 36.905 & \begin{tabular}{|l|}
14.88 \\
14.82
\end{tabular} & 7.501 & 45.595 & 1.185 & 14.643 & & 74.00 & 1.23333 & 59.2 & 11.2 & 38.8 & \begin{tabular}{|l|}
2.678 \\
.677 \\
\end{tabular} & \begin{tabular}{|l|}
0.177 \\
\end{tabular} & 0.176 & 0.005 & 4.540 & 3.86 \\
\hline$\frac{92}{93}$ & $\begin{array}{ll}2 / 5 / 2004 \\
25 / 2004 & \end{array}$ & $\begin{array}{l}4: 32: 34 \mathrm{PM} \\
4 \cdot 34: 34\end{array}$ & $\begin{aligned} 25.894 \\
2583 \\
\end{aligned}$ & $\frac{20.334}{20.335}$ & \begin{tabular}{|l|l|}
24.998 \\
243838
\end{tabular} & $\begin{array}{l}21.975 \\
21.991 \\
\end{array}$ & 21.723 & $\begin{array}{l}40.695 \\
40.628\end{array}$ & $\begin{array}{l}60.468 \\
66096 \\
\end{array}$ & $\begin{array}{r}3.63 \\
3.589 \\
\end{array}$ & 36.961 & \begin{tabular}{|l|l|}
14.883 \\
14.99
\end{tabular} & 7.53 & $\begin{array}{l}44.273 \\
44.972 \\
\end{array}$ & $\begin{array}{l}1.187 \\
1.188 \\
\end{array}$ & $\begin{array}{l}14.643 \\
1.643 \\
\end{array}$ & & 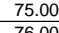 & $\begin{array}{r}1.25 \\
126667\end{array}$ & & & & & & 0.177 & 0.005 & 4.558 & $\frac{3.88}{3.80}$ \\
\hline $\begin{array}{l}93 \\
94 \\
\end{array}$ & $\frac{2 / 5 / 2004}{2 / 5 / 2004}$ & $\begin{array}{l}\text { 4:3:3:34 PM } \\
4: 34 \text { PM }\end{array}$ & $\frac{25.83}{25.761}$ & $\frac{20.335}{20.346}$ & \begin{tabular}{|l|}
24.938 \\
24.884 \\
\end{tabular} & $\frac{21.991}{21.992}$ & $\frac{21.679}{21.504}$ & $\frac{40.628}{40.236}$ & $\frac{60.296}{60.228}$ & $\frac{3.589}{3.619}$ & \begin{tabular}{|l|l|}
37.433 \\
3.487 \\
\end{tabular} & $\begin{array}{r}14.99 \\
15.005 \\
\end{array}$ & 7.614 & $\frac{44.972}{44.938}$ & $\frac{1.188}{1.19}$ & $\frac{14.643}{14.643}$ & & & $\frac{1.26667}{1.28333}$ & & & & & & & & $\frac{4.563}{4.640}$ & $\frac{3.88}{3.95}$ \\
\hline 95 & $2 / 5 / 2004$ & $4: 35: 34$ PM & 25.696 & 20.352 & \begin{tabular}{|l|l|}
24.83 \\
\end{tabular} & 22.028 & 21.645 & 40.45 & 60.45 & 3.607 & & 15.005 & 7. & 45.489 & 1.19 & 14.643 & & & 1.3 & 59.0 & 11.1 & 38.6 & 2.66 & & 0.178 & & 4.613 & \\
\hline 96 & $2 / 5 / 2004$ & 4:36:34 PM & 25.648 & 20.353 & 24.781 & 22.019 & 21.657 & 40.116 & 60.045 & & & 15.068 & 7.62 & 45.954 & 1.192 & & & & 1.31667 & 58.6 & 11.3 & & & & & & & \\
\hline 97 & $2 / 5 / 2004$ & 4:37:34 PM & 25.584 & 20.359 & 24.727 & 22.07 & 21.396 & 40.558 & 60.64 & 3.672 & 813 & 15.06 & 7.6 & 44.227 & 1.193 & 14. & & 80.00 & 1.33333 & 59.2 & 10.8 & 38.7 & \begin{tabular}{|l|}
2.667 \\
\end{tabular} & \begin{tabular}{|l|l|}
0.178 \\
\end{tabular} & 0.179 & 0.005 & 4.633 & \\
\hline 98 & $2 / 5 / 2004$ & 4:38:34 PM & 25.545 & 20.375 & 24.698 & 22.071 & & 40.467 & 60.553 & 3.632 & & 15.135 & & 45.735 & 1.195 & & & & $\begin{array}{r}1.35 \\
\end{array}$ & 59.1 & 40 & 38.1 & 2.66 & & & & 4.647 & \\
\hline 99 & $2 / 5 / 2004$ & 4:39:34 PM & 25.511 & 20.391 & 24.674 & 22.012 & 21.546 & & $\begin{array}{ll}60.852 \\
6.025\end{array}$ & 3.607 & & 15.239 & & 45.193 & 1.197 & & & & 1.36667 & 59.4 & 11.1 & 38.5 & 2.65 & & & & 4.679 & \\
\hline$\frac{100}{101}$ & $2 / 5 / 2004$ & 4:40:34 PM & 25.466 & 20.387 & 24.634 & 21.982 & & 39.867 & 59.95 & 3.492 & & \begin{tabular}{|l|l|l|}
15.207 \\
\end{tabular} & & 44.617 & 1.198 & 14. & & & 1.38333 & 58.5 & 10.9 & 38.1 & 2.627 & 0.179 & & & 4.736 & \\
\hline$\frac{101}{102}$ & $\begin{array}{ll}2 / 5 / 2004 \\
25 / 2004 & \end{array}$ & & 25.417 & 20.398 & & 22.004 & 21.837 & 40.359 & $\begin{array}{ll}60.684 \\
6500\end{array}$ & $\frac{3.588}{3.57}$ & & $\begin{array}{l}15.274 \\
15392 \\
\end{array}$ & & 44.871 & 1.2 & \begin{tabular}{|l|l|l}
14.643 \\
14.612
\end{tabular} & & & \begin{tabular}{|r|}
1.4 \\
141667
\end{tabular} & 59.2 & $\begin{array}{l}11.0 \\
112 \\
\end{array}$ & 38.6 & & $\begin{array}{ll}0.179 \\
\end{array}$ & 0. & & $\begin{array}{ll}4.693 \\
4779\end{array}$ & 然 \\
\hline$\frac{102}{103}$ & \begin{tabular}{|l|l|}
$2 / 5 / 2004$ \\
$2 / 5 / 2004$
\end{tabular} & 4:42:34 PM & $\frac{25.398}{25.353}$ & $\frac{20.409}{20.404}$ & \begin{tabular}{|l|}
24.556 \\
2.451 \\
\end{tabular} & \begin{tabular}{r|}
22 \\
2201 \\
\end{tabular} & $\frac{21.798}{21748}$ & 39.888 & $\begin{array}{l}60.508 \\
60.572 \\
\end{array}$ & $\begin{array}{r}3.57 \\
3.565 \\
\end{array}$ & $\frac{36.103}{36.485}$ & $\frac{15.392}{15.392}$ & & 45.622 & $\frac{1.203}{1.203}$ & $\frac{14.643}{14643}$ & & & 1.41667 & 59.0 & 11.2 & 38.0 & & & & & $\begin{array}{l}4.779 \\
4746\end{array}$ & 4.0 \\
\hline & $2 / 5 / 2004$ & $4: 43.3434 \mathrm{PM}$ & 25.314 & $\frac{20.404}{20.41}$ & \begin{tabular}{|l|l|}
24.492 \\
24.42
\end{tabular} & $\frac{22.01}{22.04}$ & $\frac{21.740}{21.663}$ & $\frac{40.153}{40.162}$ & $\begin{array}{l}\mid \frac{60.012}{60.883} \\
\end{array}$ & 3.005 & $\begin{array}{l}\frac{50.405}{36.32} \\
3\end{array}$ & $\begin{array}{l}15.094 \\
15.43 \\
\end{array}$ & & $\frac{45.024}{45.222}$ & $\frac{1.200}{1.204}$ & $\begin{array}{l}\frac{14.0}{14.6} \\
\end{array}$ & & & $\begin{array}{r}1.45000 \\
1.45 \\
\end{array}$ & $\begin{array}{l}59.4 \\
59.4 \\
\end{array}$ & $\begin{array}{l}11.0 \\
11.1\end{array}$ & \begin{tabular}{|l|}
38.3 \\
38.2 \\
\end{tabular} & & & & & $\begin{array}{l}4.446 \\
4.762 \\
\end{array}$ & $\frac{4.04}{4.05}$ \\
\hline & $2 / 5 / 2004$ & $4: 45: 34$ PM & 25.305 & 20.435 & 24.472 & 22.056 & 21.8 & 39.604 & 60.164 & & & 15.505 & & 46.957 & 1.204 & & & & 1.46667 & 58.7 & 11.5 & 37.8 & & & & & 4.819 & 4.1 \\
\hline 106 & $2 / 5 / 2004$ & 4:46:34 PM & 25.275 & 20.435 & 24.437 & 22.081 & & 39.517 & 59.981 & 3.575 & & 15.505 & & 45.153 & 1.204 & & & & 1.48333 & 58. & 11.1 & 37.7 & & 0.180 & & & 4.8 & 4 \\
\hline 107 & 2/5/2004 & 4:47:34 PM & 25.246 & 20.452 & 24.418 & 22.082 & & 40.035 & 60.607 & 3.651 & 36.347 & 15.627 & & 44.961 & 1.204 & 14.643 & & & 1.5 & 59. & & 38.2 & 2.63 & 0.180 & 0.182 & 0.005 & 4.778 & \\
\hline 109 & & Averages & & & & & & & & & & & & & & & & & & 536 & & & & & & 00038 & & \\
\hline$\frac{109}{110}$ & & $\begin{array}{l}\text { Averagess } \\
\text { Maximum }\end{array}$ & $\frac{2.5}{26.8}$ & 20.5 & 26.3 & 22.1. & & 42.3 & $\begin{array}{l}0.1 \\
60.9\end{array}$ & $\frac{0.0}{3.8}$ & 38.8 & $\begin{array}{l}9.5 \\
15.6 \\
\end{array}$ & & & $\frac{1.0}{1.2}$ & & & & & $\begin{array}{l}35.0 \\
59.4 \\
\end{array}$ & & \begin{tabular}{|l|}
38.1 \\
40.5 \\
\end{tabular} & & & $\begin{array}{l}0.148 \\
0.182 \\
\end{array}$ & & & \\
\hline & & Mediar & 26.1 & 20.1 & 25.5 & 21.8 & & 40.5 & 56.8 & 3.5 & 36.9 & 11.1 & & & & 14.6 & & & & 55.4 & 1.0 & \begin{tabular}{|l|}
38.7 \\
\end{tabular} & & & 0.156 & 0.0040 & 3.971 & \\
\hline$\overline{112}$ & & Minimum & 24. & 19.8 & 2208 & 20.1 & & 39.0 & 46.7 & 0.4 & 35. & 0. & & & & 12 & & & & $4 !$ & 0.4 & & & & & & 2.452 & \\
\hline & & $2 \times \mathrm{SStd} D \mathrm{D}$ & 1.268 & 0.452 & 1.183 & 1.066 & & 1.376 & 8.966 & 0.260 & 1.41 & 9.65 & & $\begin{array}{ll}1.916 \\
\end{array}$ & 0.351 & 0.000 & & & & 8.966 & \begin{tabular}{l|l|l|l|l|l}
0.470 \\
\end{tabular} & 1.383 & & .052 & 0.0 & 0.0014 & 1.398 & \\
\hline 14 & Number o & Points UsE & 0 & 85 & 85 & 85 & 85 & 85 & 85 & 85 & 85 & 85 & & 85 & 85 & 85 & & & & 85 & 85 & 85 & 85 & 85 & 85 & 85 & 85 & \\
\hline & & & ing & ire not i & uded & & & & & & & & & & & & & & & & & & & & & & & \\
\hline & & & & & & & & & & & & & & & & & & & & & & & & & & & & \\
\hline 117 & & & & & & & & & & & & & & & & & & & & & & & 1.5 -hour & ur avg. & 0.1480 & & & \\
\hline
\end{tabular}


RUN \# 1.18; THIRD ACID CLEAN WITH 2.0 M HNO3

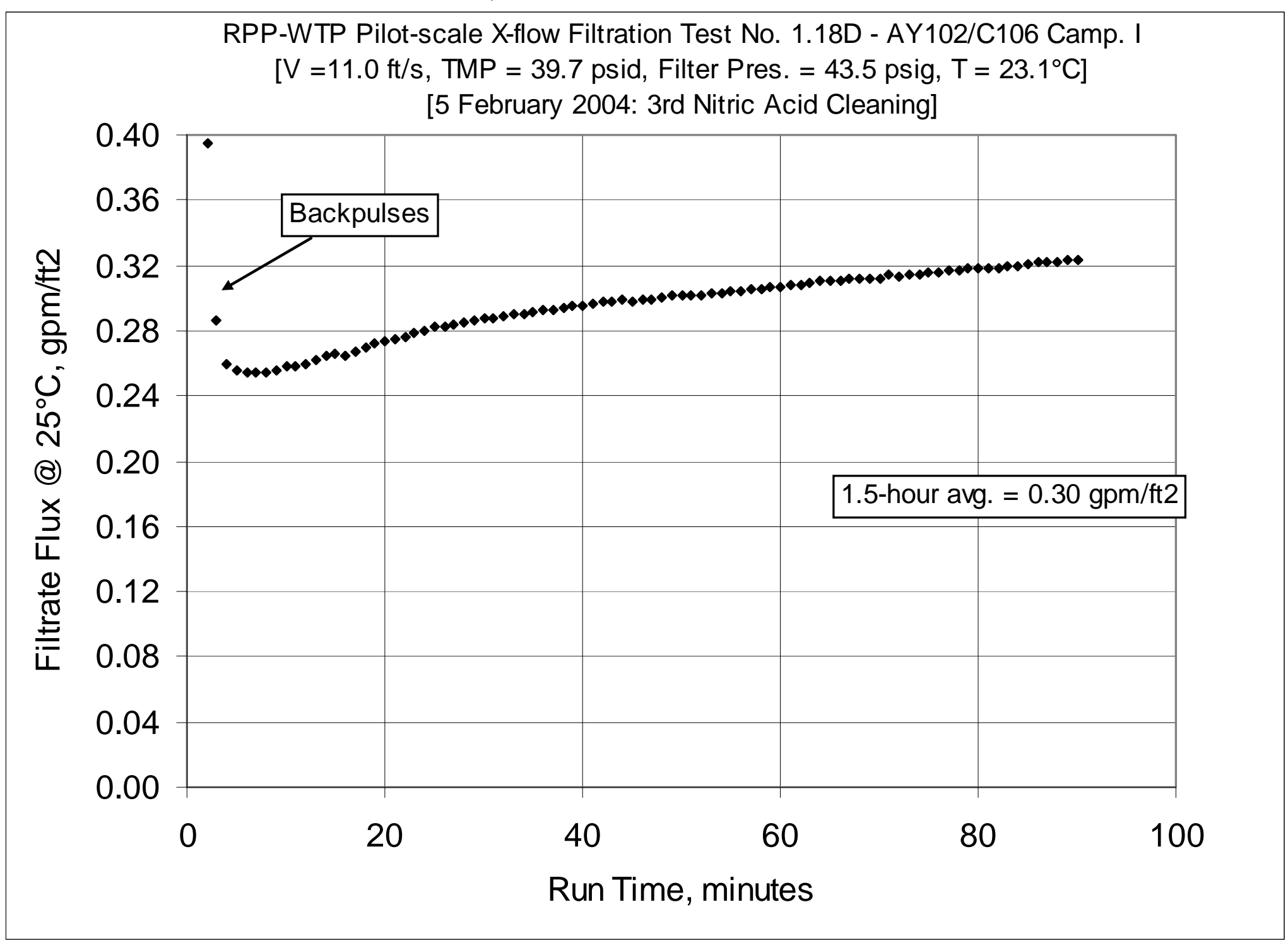


WSRC-TR-2005-00105, REVISION 0

SRNL-RPP-2005-00012, REVISION 0

RUN \# 1.18; THIRD ACID CLEAN WITH 2.0 M HNO3 - CONT.

\begin{tabular}{|c|c|c|c|c|c|c|c|c|c|c|c|c|c|c|c|c|c|c|c|c|c|c|c|c|c|c|c|c|}
\hline & A & B & $\mathrm{D}$ & E & $\mathrm{F}$ & \begin{tabular}{|l|l|} 
& \\
\end{tabular} & $\mathrm{H}$ & $\mathrm{J}$ & $\mathrm{K}$ & $\begin{array}{ll}\mathrm{L} \\
\end{array}$ & $\mathrm{M}$ & $\mathrm{N}$ & 0 & Q & $\mathrm{R}$ & $\mathrm{s}$ & $\mathrm{T}$ & $\mathrm{v}$ & $\mid$\begin{tabular}{l|l} 
\\
\end{tabular} & $x$ & $\mathrm{Y}$ & $\mathrm{z}$ & AA & $A B$ & AC & $\begin{array}{ll}A D \\
\end{array}$ & $\mathrm{AE}$ & AF \\
\hline \begin{tabular}{|l}
$\frac{1}{2}$ \\
\end{tabular} & & TIME & & k<< Temp & & Measurem & ments >>> & 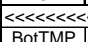 & & \begin{tabular}{|l|} 
Pressurut \\
Filter dP
\end{tabular} & Measureme & & & & 㲾< Flow & $\begin{array}{l}\text { Measurem } \\
\text { tit Fittate }\end{array}$ & 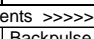 & & & & & & & & & & & \\
\hline & DATE & MIME & Filtrate & Cleaning & f) Slurry & Hi Amb. & Lo Amb. & BotTMP & Filter & Filiter dP & ToрTMP & Filtrate & Pulsepot & & Filtrate & Hi Filtate & Backpulse & & & & & & & & & & & \\
\hline \begin{tabular}{|l|}
3 \\
4
\end{tabular} & & & $\frac{\operatorname{deg} C}{T 2}$ & $\operatorname{deg}($ & $\operatorname{deg} C$ & $\operatorname{deg} C$ & $\operatorname{deg} C$ & $\begin{array}{l}\text { psid } \\
\text { pid? }\end{array}$ & psig & psid & psid & psig & psig & gpm & $\mathrm{gpm}$ & $\mathrm{gpm}$ & & & & & & & & & & & & \\
\hline & & & & & & & & & & dP1 & $\mathrm{dP3}$ & & & & Q2 & Q3 & & & & & & & & & & & & \\
\hline & eros & & & & & & & & & & & & & & & & & & & & & & & & & & & \\
\hline $\begin{array}{ll}7 \\
\end{array}$ & $2 / 5 / 2004$ & 9:26:34 AM & 19.191 & 18.592 & $\begin{array}{ll}216.892 \\
2\end{array}$ & \begin{tabular}{|c|}
19.019 \\
\end{tabular} & $\begin{array}{l}98.202 \\
\end{array}$ & 0.056 & -0.05 & -0.029 & & \begin{tabular}{|l|l|} 
& -0.177 \\
\end{tabular} & 0.121 & -0.093 & 0.003 & 14.643 & 0.004 & & & & & & & & & & & \\
\hline 8 & $2 / 5 / 2004$ & 9:26:52 AM & 19.191 & 18.592 & \begin{tabular}{|l|l|}
2 & 16.892 \\
\end{tabular} & $\begin{array}{l}19.019 \\
\end{array}$ & \begin{tabular}{|l|l|}
98.202 \\
\end{tabular} & 0.056 & -0.05 & -0.029 & & -0.177 & 0.121 & -0.093 & 0.003 & $\begin{array}{l}14.643 \\
\end{array}$ & 0.004 & & & Conver & & & $\mathrm{m} 3 / \mathrm{m} 2$ & /day/barg & $\mathrm{g} / \mathrm{gpm} / \mathrm{ft}$ & /ft2/barg & & \\
\hline & $2 / 5 / 2004$ & 9:26:53 AM & & & & & & & -0.05 & \begin{tabular}{|l|l|l|}
-0.029 \\
\end{tabular} & & & & -0.091 & 0.003 & $\begin{array}{l}14.643 \\
\end{array}$ & & & & & & & & & & & & \\
\hline & $2 / 5 / 2004$ & 9:26:54 AM & 19.181 & 18.571 & $\begin{array}{l}1 \\
1\end{array}$ & 19.024 & 18.146 & 0.058 & -0.05 & -0.029 & -0.002 & -0.18 & 0.121 & -0.091 & 0.003 & 14.643 & 0.004 & & & & & & $\mathrm{e} P 1$ is & approxin & mately 1. & 1.4 psig & & \\
\hline+4 & 2/5/2004 & 9:26:55 AM & 19.181 & 18.577 & 16.867 & 19.024 & 18.152 & 0.058 & -0.05 & -0.029 & 0.002 & -0.177 & 0.12 & -0.093 & 0.003 & 14.643 & & & & & & & & & & & & \\
\hline & $2 / 5 / 2004$ & 9:26:56 AM & 19.181 & 18.577 & 716.872 & 19.024 & 18.157 & 0.056 & -0.048 & -0.029 & & -0.177 & 0.121 & -0.091 & 0.003 & 14.643 & 0.004 & & & Press & e Plis & correct & ted for & $0.88 \mathrm{in}$ & esc & er tu & & \\
\hline 13 & & 9:26:57 AM & 19.186 & 18.572 & $\begin{array}{l}216.867 \\
\end{array}$ & & & & & -0.029 & & -0.177 & & -0.093 & 0.003 & 14.643 & 0.0 & & & VVVV & & & & $<$ Filtrat & te Flux & $<P E$ & & \\
\hline & $2 / 5 / 2004$ & 9:26:58 AM & 19.192 & 18.587 & $\begin{array}{l}7 \mid \\
76.877\end{array}$ & 19.03 & 18.162 & 0.058 & -0.05 & -0.028 & & -0.177 & 0.121 & -0.091 & 0.003 & 14.643 & 0.00 & & & & & & & & at $25 \mathrm{C}$ & & $\times 1000$ & \\
\hline$\frac{15}{16}$ & Jata - Per Mi & & & & & & & & & & & & & & & & & $\frac{\text { Time }}{\text { Min. }}$ & \begin{tabular}{|l|} 
Time \\
Hour
\end{tabular} & \begin{tabular}{|l|} 
Press. \\
psig
\end{tabular} & 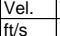 & $\frac{T \text { TMP }}{\text { psi }}$ & $\begin{array}{l}\text { TMP } \\
\text { bar }\end{array}$ & \begin{tabular}{|l|l}
$g p m$ \\
$/ 1 / 2$
\end{tabular} & gpm & $\frac{g \mathrm{gm}}{\mathrm{ft} / \mathrm{psi}}$ & $\mid \mathrm{gt} / \mathrm{fm}$ & lay/lat \\
\hline & $2 / 5 / 2004$ & $5: 26: 33 \mathrm{PM}$ & 23.116 & 20.441 & & 21.477 & $21.945 \#$ & 2.678 & 45.645 & 3.071 & -0.536 & 38.263 & 85.913 & 40.702 & 0.003 & \begin{tabular}{|c|c|c|}
-0.012 \\
\end{tabular} & 0.004 & & & 44.2 & 10.0 & 1.1 & \begin{tabular}{|l|} 
\\
\end{tabular} & & -0.002 & -0.002 & -1.784 & 34 \\
\hline 18 & $2 / 5 / 2004$ & 5:27:33 PM & 23.212 & 20.447 & 722.692 & \begin{tabular}{|l|l|}
21.473 \\
\end{tabular} & $21.921 \#$ & 2.564 & 45.912 & 3.111 & \begin{tabular}{|c|}
-0.692 \\
\end{tabular} & 38.423 & \begin{tabular}{|l|l|l|l}
42.544 \\
\end{tabular} & 40.278 & 0.003 & $\begin{array}{l}14.643 \\
\end{array}$ & & 1.0 & 0.01667 & 44.4 & & & 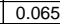 & \begin{tabular}{|l|l|}
2.183 \\
\end{tabular} & 2.331 & 2.490 & 2490.261 & \\
\hline 19 & $2 / 5 / 2004$ & 5:28:33 PM & 23.272 & 20.431 & 122.682 & 21.417 & 21.629 \# & $\begin{array}{l}38.623 \\
\end{array}$ & $\begin{array}{l}44.666 \\
\end{array}$ & 3.044 & 36.156 & 0.483 & 1.734 & 41.54 & 0.004 & $\begin{array}{l}2.478 \\
\end{array}$ & & 2.0 & 0.03333 & 43.2 & 10.2 & & & 0.369 & & 0.011 & & \\
\hline & $2 / 5 / 2004$ & 5:29:33 PM & 23.332 & 20.452 & 222.717 & 21.448 & 22.006 \# & 42.091 & 45.075 & 3.157 & 39.195 & -1.219 & 1.295 & 41.759 & 0.003 & 1.803 & 0.00 & 3.0 & 0.05 & 43.6 & & 40.6 & 2.802 & 0.269 & 0.287 & 0.007 & 7.057 & \\
\hline 21 & 15/2004 & 5:30:33 PM & 23.364 & 20.463 & & 21.524| & 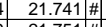 & 41.493 & 43.914 & 3.735 & 37.872 & -1.219 & .208 & 42.402 & 0.003 & 1.628 & & 4. & 0.0666 & 42.4 & & 39.7 & 2.73 & 443 & & 0.007 & & \\
\hline & $2 / 5 / 2004$ & 5:31:33 PM & 23.369 & & & 21.519 & $\left.21.751\right|_{t}$ & & 44.19 & 3.565 & 38.089 & & & 43.099 & 0.003 & 1.606 & & & 0.0833 & & & & & & & & & \\
\hline$\frac{23}{21}$ & $2 / 5 / 2004$ & 5:32:33 PM & 23.364 & 20.438 & $\begin{array}{l}822.748 \\
8\end{array}$ & 21.494 & 21.736 \# & 42.144 & 44.552 & 3.542 & 38.617 & -1.219 & 1.191 & 45.207 & 0.003 & 1.597 & & & & 43.1 & & 40. & & 0.238 & & .006 & & \\
\hline$\frac{24}{25}$ & $\frac{2512004}{2151004}$ & $\begin{array}{c}5.353 .353 \\
5.232 \mathrm{PM}\end{array}$ & 2.3599 & 20.458 & $\begin{array}{ll}5 \\
\end{array}$ & | & 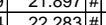 & 42.061 & $\begin{array}{ll}44.474 \\
44562\end{array}$ & $\begin{array}{l}3.5559 \\
3.470\end{array}$ & $\begin{array}{r}38.51 \\
38.72\end{array}$ & -1.216 & & $\begin{aligned} 43.62 \\
3.807\end{aligned}$ & 0.0 & 1.599 & & 1.00 & 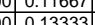 & 年 & & & $\frac{6778}{770}$ & 0 & & 0.006 & & \\
\hline 26 & 2 & $5.35 .535 \mathrm{PM}$ & $\frac{20.419}{23433}$ & 20.403 & f) 22.18 & $\frac{2.4454}{21.483}$ & 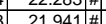 & $\begin{array}{l}42.410 \\
4203\end{array}$ & $\begin{array}{l}44.3053 \\
4446\end{array}$ & $\frac{1.471}{3.361}$ & $\mid$ & $\frac{-1.210}{-1216}$ & & $\begin{array}{l}43.001 \\
42969\end{array}$ & 然 0.003 & $\frac{1.000}{161}$ & & $\frac{0.00}{900}$ & 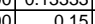 & 告5.1 & $\begin{array}{ll}1.0 .6 \\
105\end{array}$ & 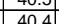 & $\frac{2.471}{2786}$ & 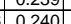 & & 0.0006 & & \\
\hline 27 & $2 / 5 / 2004$ & 5:36:33 PM & 23.455 & 20.404 & 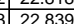 & $\frac{2.450}{21.519}$ & $\frac{21.541+1}{21.741 \#}$ & $\begin{array}{r}42.259 \\
42.259\end{array}$ & \begin{tabular}{|l|l|l|l|}
44757 \\
\end{tabular} & $\begin{array}{l}3.001 \\
.412\end{array}$ & \begin{tabular}{|l|}
38.849 \\
389
\end{tabular} & \begin{tabular}{|l|}
-1.210 \\
-1.214 \\
\end{tabular} & & 年 433905 & & 1.01 & & 1000 & & 433 & 106 & 4.4 & 27.106 & 0 & & 0.006 & & \\
\hline 28 & $2 / 5 / 2004$ & 5:37:33 PM & 23.464 & 20.453 & $\begin{array}{l}322.854 \\
25\end{array}$ & 21.504 & $21.58 \#$ & $\begin{array}{l}42.377 \\
\end{array}$ & 44.988 & 3.515 & 38.904 & -1.219 & 1.194 & $\begin{array}{l}43.199 \\
\end{array}$ & 0.003 & 1.632 & & 11.00 & 0.18333 & 43.5 & 10.6 & 40.6 & \begin{tabular}{|l|l|}
2.802 \\
\end{tabular} & $\mid 0.243$ & 0.259 & 0.006 & 6.363 & \\
\hline & $2 / 5 / 2004$ & & & 20.457 & & 21.483 & 21.775 \# & & & 3.31 & & \begin{tabular}{|l|}
-1.216 \\
\end{tabular} & & & & 1.64 & & & & 43.2 & 10.6 & & & & & & & \\
\hline 30 & $2 / 5 / 2004$ & 5:39:33 PM & 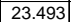 & 20.457 & $7 \quad 22.873$ & 21.493 & $322.222 \#$ & \begin{tabular}{l|l}
41.914 \\
\end{tabular} & \begin{tabular}{|l|l|l}
44.556 \\
\end{tabular} & 3.336 & 38.726 & $\mid-1.214$ & 1.205 & 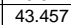 & 0.003 & $\begin{array}{l}1.657 \\
\end{array}$ & & 13.00 & 0.21667 & 43.1 & $\frac{10.6}{10.6}$ & 40.3 & 2.78 & 0.247 & 0.262 & 0.007 & 6.508 & \\
\hline & & & & & & 21.548 & & & \begin{tabular}{|l|}
44.824 \\
\end{tabular} & & & & & & & & & & & & & & & & & & & \\
\hline & $2 / 5 / 2004$ & 5:41:33 PM & 23.537 & 20.476 & & 21.532 & $21.955 \#$ & \begin{tabular}{|l|l}
41.96 \\
\end{tabular} & 444.71 & 3.395 & 38.707 & & 1.208 & 43.232 & 0.003 & 1.677 & & 15.00 & 0.25 & 43.2 & & & & 0.250 & & & & \\
\hline 33 & 2/5/2004 & 5:42:33 PM & 23.531 & 20.47 & $\begin{array}{l}7 \\
22.901\end{array}$ & \begin{tabular}{l|l}
21.502 \\
\end{tabular} & 21.829 \# & 41.744 & & 3.766 & \begin{tabular}{|l|l|}
38.106 \\
\end{tabular} & 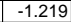 & & 44.942 & & 1.675 & & & 0.26667 & & 11.0 & & & & & & & \\
\hline 34 & $2 / 5 / 2004$ & 5:43:33 PM & 23.531 & 20.465 & $\begin{array}{l}522.916 \\
\end{array}$ & 21.512 & 21.703 \# & 41.958 & 44.888 & 3.821 & 38.243 & \begin{tabular}{|l|} 
\\
\end{tabular} & 1.20 & 46.098 & 0.003 & $\begin{array}{l}1.69 \\
\end{array}$ & & 17.00 & 0.28333 & \begin{tabular}{ll|l} 
&
\end{tabular} & 11.3 & 40.1 & 2.765 & 252 & 0.267 & & 6.666 & \\
\hline 35 & $2 / 5 / 2004$ & 5:44:33 PM & 23.562 & 20.475 & \begin{tabular}{|c|c|}
22.957 \\
\end{tabular} & 21.532 & 21.673\#\# & 41.858 & 44.737 & 3.794 & 38.276 & \begin{tabular}{|l|} 
\\
\end{tabular} & 1.214 & 45.96 & 0.6 & 1.703 & & 18.00 & & 43.3 & 11.3 & & 2.762 & 254 & & & & \\
\hline 36 & 2/5/2004 & 5:45:33 PM| & 23.582 & 20.475 & 22.992 & 21.522 & 21.728 \# & 42.057 & 45.066 & 3.755 & 38.483 & 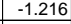 & & 45.42 & 0.003 & 1.723 & & 19.00 & 0.31667 & 43.6 & $11+5$ & 40. & . .776 & 0.257 & & & & \\
\hline 37 & 2/5/2004 & 5:46:33 PM & 23.612 & 20.481 & 23.027 & 21.512 & 21.573\#\# & 41.684 & 44.726 & 3.632 & 38.227 & -1.216 & & 45.443 & & 1.7 & & 20.00 & \begin{tabular}{l|l|l}
0 & 0.33333
\end{tabular} & 43.3 & 地. & 40.0 & 2.100 & 0.259 & 0.273 & & 42 & 12 \\
\hline & $2 / 5 / 2004$ & 7:33 PM & 23.638 & & & 21.528 & $21.5999 \#$ & & 44.542 & & 8.161 & -1.211 & & 46.069 & & 1.744 & & 21.00 & 0.35 & 43.1 & 11 & & & & 275 & & 到 & \\
\hline 39 & 2/5/2004 & 5:48:33 PM & 23.654 & 20.493 & $\begin{array}{l}33.054 \\
\end{array}$ & 21.519 & 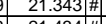 & 41.846 & 44.776 & 3.663 & 38.424 & -1.216 & 1.2. & 45.188 & 0.003 & 1.756 & & 22.0 & 0.3666 & 43.3 & & $40.1]$ & 2.76 & 0.262 & & & & \\
\hline & & 5.45 .353 & $\frac{23.059}{2369}$ & $\frac{0.4}{204}$ & 23.049 & $\frac{2.483}{2152}$ & $\frac{21.434 \pi}{21967+\pi}$ & $\frac{41.628}{4181}$ & & $\begin{array}{l}3.619 \\
3.659\end{array}$ & $\begin{array}{l}38.145 \\
38202\end{array}$ & $\frac{-1.216}{-1214}$ & 122 & $\frac{45.512}{4523}$ & & $\frac{1.168}{1770}$ & & 23.00 & 0.38333 & 43.4 & 110 & 39.9 & & 0.264 & & & 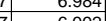 & \\
\hline 42 & $2 / 5 / 2004$ & $5.5 .51 \cdot 3 \mathrm{PM}$ & 23.096 & 20.4994 & 123.076 & $\frac{1.50}{215}$ & $\frac{2.01}{21.591}$ & $\begin{array}{c}41.01 \\
41.806\end{array}$ & 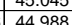 & $\begin{array}{l}.0547 \\
3.647\end{array}$ & $\begin{array}{l}0.02 \\
38.395 \\
\end{array}$ & \begin{tabular}{|l|}
-1.214 \\
-1.216 \\
\end{tabular} & & $\frac{45.25}{45.211}$ & 0.003 & 1.179 & 08 & 2500 & 0.4667 & 43.0 & $\frac{1.1}{11.1}$ & 40. & & 0.267 & 0.282 & 0.007 & 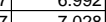 & \\
\hline 43 & $2 / 5 / 2004$ & $5 \cdot 5 \cdot 33 \mathrm{PM}$ & 23.711 & 20.495 & $\frac{15.091}{23091}$ & 2146 & $51.546 \#$ & & $\mid \begin{array}{l}49.506 \\
45166\end{array}$ & 3.695 & $\mid$ & $\mid-1214$ & & $\frac{45.524}{44824}$ & & 17.78 & & 2600 & \begin{tabular}{|l|l|l|l}
0.4333 \\
\end{tabular} & 43. & $\frac{1.1}{110}$ & 40.1 & & & & & & \\
\hline & $2 / 5 / 2004$ & 5:53:33 PM & 23.717 & 20.491 & \begin{tabular}{|l|l|}
23.108 \\
\end{tabular} & 21.456 & b. & 41.572 & \begin{tabular}{|l|l|}
44.844 \\
\end{tabular} & 3.655 & 38.114 & \begin{tabular}{|l|l|}
-1.214 \\
\end{tabular} & & 45.22 & & 1.803 & & 27.00 & 0.45 & 43.4 & & & \begin{tabular}{|l|l|}
2.74 \\
\end{tabular} & 0.269 & 0.284 & & 7.118 & 8 \\
\hline 45 & $2 / 5 / 2004$ & 5:54:33 PM & 23.738 & 20.496 & & 21.472 & & & & & & & & & & & & & & & & & & & & & & \\
\hline 46 & & 5:55:33 PM & 23.738 & 20.496 & & 21.497 & $21.794 \#$ & $\begin{array}{lll}41.5 \\
\end{array}$ & \begin{tabular}{|l|l|}
44.869 \\
\end{tabular} & 3.616 & \begin{tabular}{|l|l|l|}
38.118 \\
\end{tabular} & \begin{tabular}{|l|l|} 
& -1.219 \\
\end{tabular} & & 43.916 & & 1.817 & & 29.00 & \begin{tabular}{|l|l}
0.48333 \\
\end{tabular} & 43.4 & 10. & & & & 0.286 & & 7.173 & \\
\hline $4 r^{4}$ & & & 23.748 & & & 21.503 & & 41.587 & & & & \begin{tabular}{|l|}
-1.216 \\
\end{tabular} & & 45.2 & & 1.83 & & 30.0 & & 43.5 & 11.1 & 300 & 4.753 & & & & & \\
\hline \begin{tabular}{|l|l|}
48 \\
\end{tabular} & $2 / 5 / 2004$ & 5:57:33 PM & 23.749 & 20.482 & 23.129 & 21.478 & 21.665 \#\# & $\begin{array}{l}411.433 \\
\end{array}$ & \begin{tabular}{|l|}
44.944 \\
\end{tabular} & 3.588 & 37.958 & -1.222 & 1.231 & 44.792 & & 1832 & & 31.00 & 0.51667 & 43.5 & 11.0 & \begin{tabular}{|l|}
39.7 \\
\end{tabular} & 2.737 & 0.273 & 0.288 & & 7255 & \\
\hline \begin{tabular}{|l|l|}
49 & \\
\end{tabular} & 2/5/2004 & $5: 58: 3$ & \begin{tabular}{|c|c|}
23.754 \\
\end{tabular} & 20.477 & 23.134 & 21.473 & 21.67 & 41.4 & 44.8 & 3.619 & 38.057 & -1.219 & & 44.8 & & 1.8 & & 32. & & 43.3 & 11.0 & 39.7 & 2.739 & & & & 883 & \\
\hline \begin{tabular}{|l}
50 \\
\end{tabular} & 2/5/2004 & 5:59: & 23.785 & 20.504 & 23.16 & 21.54 & 421.71 & \begin{tabular}{ll|l}
41.678 \\
\end{tabular} & 45. & 3.693 & & -1.216 & & 44.4 & & 1.8 & & 33 & 0.55 & 43.6 & 10.9 & 40.0 & & $\mid .275$ & 0.290 & & 7.257 & $\overline{7}$ \\
\hline 51 & $2 / 5 / 2004$ & 6:00:33 PM| & 23.791 & 20.514 & & 21.53 & 21.63 & 41.877 & & 3.742 & & -1.216 & & 45.2 & & & & & & 43.9 & 11.1 & 40.11 & $2.767 \mid$ & ).276 & & & 235 & \\
\hline 52 & 2/5/2004 & 6:01:33 PM & 23.796 & 20.51 & 23.157 & 21.511 & $21.702 \#$ & 441.518 & 45.016 & 3.562 & 38.303 & \begin{tabular}{|l|l|} 
& -1.219 \\
\end{tabular} & 1.246 & 44.882 & & 1.858 & & 35.00 & 0.58333 & 43.5 & 11.0 & 39.9 & 2.752 & 0.277 & 0.292 & & 7.313 & 3 \\
\hline 50 & $2 / 5 / 2004$ & 6:02:33 PM & 23.800 & 20.515 & & 21.541 & & & & & & \begin{tabular}{|l|l|} 
\\
\end{tabular} & & & & & & 36.00 & 0.6 & 43.5 & 11. & & 2.104 & & & & 7.370 & \\
\hline 54 & & & 23.811 & & & 21.531 & & & & & & & & & & & & & & 43.6 & 11. & & & & & & & \\
\hline & & & 23.817 & 20.505 & & 21.521 & 21.9399 & 41.659 & 45.236 & 3.674 & 38.184 & \begin{tabular}{|l|l|l|l|} 
\\
\end{tabular} & & 45.2 & & & & 38.0 & & 43.0 & & & & & & & & \\
\hline$\frac{30}{57}$ & 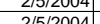 & 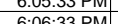 & 年 & 20.500 & & $\frac{21.531}{21577}$ & $\frac{21.093}{21023}$ & 41.742 & \begin{tabular}{|l|l}
45.54 \\
951
\end{tabular} & 3. & $\mid$ & $\mid-1.242$ & & 45.14 & & & & & & (3.5.9 & 111 & & & & & & & \\
\hline 58 & $2 / 5 / 2$ & 6.00 .53 & 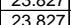 & 20.521 & $\begin{array}{l}15.25 \\
23193\end{array}$ & 2158 & $\frac{21.03}{21.77}$ & 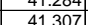 & $\mid \begin{array}{l}44.051 \\
44985\end{array}$ & $\mid$ & $\mid$ & \begin{tabular}{|l|l|} 
\\
-121219
\end{tabular} & & 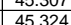 & & $\frac{1.001}{1.89}$ & & 年 40.000 & 0.0002 & 43.4 & $\frac{1.1}{111}$ & 年 & & & $\frac{250}{207}$ & & 7.454 & \\
\hline 59 & 21515200 & $6 \cdot 60.33 \mathrm{PM}$ & 23827 & 20.51 & 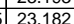 & 21526 & & 41.543 & & 3695 & & $\frac{1.250}{1219}$ & & & & 18 & & 4200 & & 147 & $1+.1$ & $398 \mathrm{l}$ & 174 & & & & & \\
\hline \begin{tabular}{|l|l|}
60 & \\
\end{tabular} & $2 / 5 / 200$ & $6 \cdot 09 \cdot 33 \mathrm{PM}$ & 23.842 & 20.52 & 23193 & 21.537 & 21.56 & 41.439 & & 3.67 & 38.0 & -1.219 & & & & 1894 & & & 667 & 438 & 1104 & 397 & & & & & 7481 & \\
\hline 61 & & & 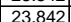 & 20.5 & & 21.497 & & & & & & -1.214 & & & & 1.903 & & & & 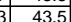 & 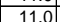 & & & & & & & \\
\hline 62 & & & 23.842 & & 23.203 & 21.517 & & 41.369 & & & & & & & & & & & & & 1110 & & & & & & & \\
\hline $\begin{array}{ll}63 \\
\end{array}$ & $2 / 5 / 2004$ & $6: 12: 33 \mathrm{PM}$ & 23.847 & 20.526 & & 21.466 & & 40.971 & 44 & 3.503 & & -1.219 & & 44.919 & & 1.907 & & & 0.76 & 430 & 11.0 & & & & & & 81 & \\
\hline 64 & $2 / 5 / 2004$ & $6: 13: 33$ & 23.847 & 20.53 & 23.232 & 21.446 & $21.552 \#$ & 41.13 & 44 & 3.562 & 37.784 & -1.216 & & 45.147 & & & & & & 43.5 & 11.1 & & & & & & & \\
\hline $\begin{array}{ll}65 \\
\end{array}$ & $2 / 5 / 2004$ & $6: 14: 33 \mathrm{PM}$ & 23.847 & 20.52 & 23.227 & 21.486 & 21.59 & \begin{tabular}{|l|l}
41.489 \\
\end{tabular} & 45.2 & 3.66 & \begin{tabular}{|l|l|}
38.108 \\
\end{tabular} & \begin{tabular}{|l|} 
\\
\end{tabular} & & 45.0 & & 1.916 & & 48.00 & $\overline{0.8}$ & \begin{tabular}{l|l|}
43.8 \\
\end{tabular} & \begin{tabular}{ll|}
11.0 \\
\end{tabular} & & 2.744 & & & & 7.547 & \\
\hline \begin{tabular}{|l}
66 \\
\end{tabular} & $2 / 5 / 200$ & $6: 15: 33 \mathrm{PI}$ & 23.851 & 20.525 & 23.212 & 21.435 & \begin{tabular}{|l|l}
521.627 \\
\end{tabular} & 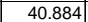 & \begin{tabular}{|l|}
44.581 \\
\end{tabular} & 3.495 & \begin{tabular}{|c|}
37.704 \\
\end{tabular} & \begin{tabular}{|l|} 
\\
\end{tabular} & 1.2 & $\begin{array}{l}44.36 \\
\end{array}$ & 0.0 & $\begin{array}{ll}1.92 \\
\end{array}$ & 0.0 & 49.00 & \begin{tabular}{ll|l}
0 & 0.81667
\end{tabular} & \begin{tabular}{|l|l}
43.1 \\
\end{tabular} & \begin{tabular}{|l|l|}
10.9 \\
\end{tabular} & \begin{tabular}{|l|}
39.3 \\
\end{tabular} & \begin{tabular}{|l|l|}
2.709 \\
\end{tabular} & 0.286 & 0.36 & 0.0 & 7.663 & 6.5 \\
\hline & $2 / 5 / 2004$ & 6:16:33 PM| & 23.857 & 20.536 & 23. & 21.466 & 21.48 & 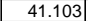 & & 3.609 & 37.806 & 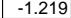 & & & & 1.9 & & & $\mid 0.83$ & \begin{tabular}{|l|l|}
43.4 \\
\end{tabular} & 11.2 & 395 & & & & & 7.654 & 4 \\
\hline 68 & 2/5/2004 & 6:17:33 PM| & 23.867 & 20.536 & 23.228 & 21.537] & 21.487|\#| & 41.427 & 45.168 & 3.73 & 37.917 & -1.214 & & 45.339 & 0.0 & 1.925 & & 51.00 & 0.85 & 43.7 & 11.1 & $39.7>$ & 2.735 & 0.287 & 0.302 & 0.008 & 7.607 & $6.4-4-2$ \\
\hline 69 & $2 / 25 / 200$ & 6:18:33 PM & 23.872 & 20.541 & & 21.537] & 21.6 & 41.2 & & 3.6 & & & & & & & & & & 43.6 & & & 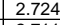 & & & & 47 & \\
\hline & & & & & & & & & & & & & & & & & & & & & & & & & & & 698 & \\
\hline
\end{tabular}


WSRC-TR-2005-00105, REVISION 0

SRNL-RPP-2005-00012, REVISION 0

RUN \# 1.18; THIRD ACID CLEAN WITH 2.0 M HNO3 - CONT.

\begin{tabular}{|c|c|c|c|c|c|c|c|c|c|c|c|c|c|c|c|c|c|c|c|c|c|c|c|c|c|c|c|c|}
\hline & A & $\mathrm{B}$ & D & $E$ & $\mathrm{~F}$ & $G$ & $\begin{array}{lll}\mathrm{H}_{1} & \\
\end{array}$ & $\mathrm{~J}$ & $\mathrm{~K}$ & $\mathrm{~L}$ & $\mathrm{M}$ & $\mathrm{N}$ & $\mathrm{O}$ & \begin{tabular}{l|l} 
\\
\end{tabular} & $R$ & $\mathrm{~s}$ & $\mathrm{~T}$ & $\mathrm{v}$ & w & $x$ & $Y$ & $z$ & $\mathrm{AA}$ & $A B$ & $\mathrm{AC}$ & $\mathrm{AD}$ & $\mathrm{AE}$ & \\
\hline$\frac{71}{72}$ & $2 / 5 / 2004$ & 6:20:33 PM & 23.858 & 20.531 & 23.238 & 21.542 & 21.694 \# & 41.025 & \begin{tabular}{|l|l|}
44.88 \\
\end{tabular} & $\begin{array}{l}3.601 \\
\end{array}$ & $\begin{array}{l}37.775 \\
37709\end{array}$ & -1.216 & 1.272 & \begin{tabular}{|l|l|}
45.059 \\
\end{tabular} & 0.003 & $\begin{array}{ll}1.932 \\
\end{array}$ & 0.004 & 54.00 & \begin{tabular}{|l|l|}
0 & 0.9 \\
\end{tabular} & $\begin{array}{l}43.4 \\
\end{array}$ & 11.0 & 39.4 & \begin{tabular}{|l|l|}
4 & 2.716 \\
\end{tabular} & 0.288 & 0.303 & $\begin{array}{l}0.008 \\
\end{array}$ & 7.685 & 6.54 \\
\hline$\frac{72}{73}$ & $\begin{array}{l}2 / 5 / 2004 \\
2 / 1 / 204\end{array}$ & 6:21:33 PM & $\begin{array}{l}23.862 \\
23877 \\
\end{array}$ & 20.531 & \begin{tabular}{|l|}
23.238 \\
23213 \\
\end{tabular} & 21.517 & 21.703 \# & $\begin{array}{ll}40.859 \\
41097\end{array}$ & \begin{tabular}{|l|}
44.623 \\
44.959 \\
\end{tabular} & $\begin{array}{l}3.501 \\
3681 \\
\end{array}$ & \begin{tabular}{|l|l|}
37.728 \\
37648
\end{tabular} & $\begin{array}{r}-1.216 \\
.219 \\
\end{array}$ & $\begin{array}{l}1.272 \\
1269 \\
\end{array}$ & \begin{tabular}{|l|}
44.823 \\
45687 \\
\end{tabular} & 0.003 & $\begin{array}{ll}1.937 \\
1942\end{array}$ & 0.004 & $\begin{array}{l}55.00 \\
5600\end{array}$ & \begin{tabular}{|c|c|} 
& 0.91667 \\
\end{tabular} & \begin{tabular}{|l|l|}
43.1 \\
43.
\end{tabular} & \begin{tabular}{|l|l|}
11.0 \\
112
\end{tabular} & $\begin{array}{ll}39.3 \\
33.4\end{array}$ & \begin{tabular}{|l|l|}
3 & 2.709 \\
1 &
\end{tabular} & \begin{tabular}{|l|l|}
0.289 \\
0209
\end{tabular} & $\begin{array}{l}0.304 \\
0305\end{array}$ & $\begin{array}{l}0.008 \\
0.008\end{array}$ & $\begin{array}{l}7.726 \\
7736 \\
\end{array}$ & 6.57 \\
\hline 74 & 2/5/2004 & 6.22.33 PM & $\frac{20.041}{23.867}$ & & \begin{tabular}{|l|l|}
23.232 \\
232
\end{tabular} & & & $\begin{array}{l}41.097 \\
40.857\end{array}$ & \begin{tabular}{|l|}
44.9559 \\
44.654 \\
\end{tabular} & $\begin{array}{l}3.681 \\
3.622 \\
\end{array}$ & $\begin{array}{l}37.648 \\
37.531 \\
\end{array}$ & $\frac{-1.219}{-1.216}$ & 1.275 & & 0.003 & $\begin{array}{l}1.944 \\
1.946\end{array}$ & 0.005 & & \begin{tabular}{|l|r|}
0 & 0.933 \\
0
\end{tabular} & \begin{tabular}{|l|}
43.5 \\
43.2
\end{tabular} & & $\begin{array}{l}39.4 \\
39.2 \\
\end{array}$ & & & $\begin{array}{l}0.305 \\
0.305\end{array}$ & $\begin{array}{l}0.008 \\
0.008\end{array}$ & $\begin{array}{l}7.736 \\
7.783 \\
\end{array}$ & $\frac{6.58}{6.62}$ \\
\hline 75 & & & 23.877 & 20.546 & 23.247 & 21.496 & 21.863 \# & 41.269 & \begin{tabular}{|l|}
45.141 \\
\end{tabular} & 3.683 & 37.857 & -1.216 & 1.28 & 45.287 & 0.003 & 1.952 & 0.005 & 58.00 & & 43.7 & & 39.6 & \begin{tabular}{|l|l|}
6 & 2.728 \\
\end{tabular} & 0.291 & 0.306 & 0.008 & 7.730 & 6.58 \\
\hline 76 & $2 / 5 / 2004$ & & 23.877 & 20.536 & & 21.497 & 21.668 \# & 41.213 & 45.141 & 3.687 & 37.724 & -1.216 & 1.277 & 45.503 & 0.003 & 1.954 & 0.005 & 59.00 & 0.98333 & 43.7 & 11.2 & 39.5 & \begin{tabular}{|l|l|}
5 & 2.721 \\
\end{tabular} & 0.291 & 0.306 & 0.008 & 7.762 & 6.61 \\
\hline 77 & $2 / 5 / 2004$ & 6:26:33 PM & & 20.551 & 23.243 & 21.542 & & 41.108 & 45.075 & 3.576 & 37.845 & -1.214 & 1.283 & 45.451 & 0.003 & 1.961 & 0.005 & 60.00 & & 43.6 & 11.1 & 39.5 & 52.722 & 0.292 & 0.307 & 0.008 & 7.784 & \\
\hline 78 & $2015 / 2004$ & 6:27:33 PM & 23.874 & 20.542 & \begin{tabular}{|l|}
23.234 \\
\end{tabular} & 21.508 & 21.684 \# & 41.338 & 45.207 & 3.632 & 38.026 & -1.216 & 1.283 & \begin{tabular}{|l|l|}
44.776 \\
\end{tabular} & 0.003 & 1.964 & 0.004 & 61.00 & \begin{tabular}{|l|l|}
0 & 1.01667 \\
\end{tabular} & 43.7 & \begin{tabular}{|l|l|}
11.0 \\
\end{tabular} & 39.7 & $\begin{array}{ll}72.736 \\
\end{array}$ & 0.293 & 0.308 & 0.008 & 7.757 & 6.60 \\
\hline 79 & $\begin{array}{l}2 / 5 / 2004 \\
2 / 1 / 204\end{array}$ & 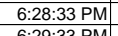 & & 20.547 & \begin{tabular}{|l|}
23.244 \\
23200
\end{tabular} & \begin{tabular}{l|l|}
21.528 \\
21.523 \\
\end{tabular} & $\begin{array}{l}21.508 \text { \# } \\
21.549 . \#\end{array}$ & $\begin{array}{ll}41.445 \\
41219\end{array}$ & \begin{tabular}{|l|l|}
45.317 \\
45239
\end{tabular} & $\begin{array}{ll}3.604 \\
3545\end{array}$ & \begin{tabular}{|l|l|}
38.19 \\
3797
\end{tabular} & $\begin{array}{l}-1.216 \\
.210\end{array}$ & 1.283 & \begin{tabular}{|l|l|}
45.191 \\
4507
\end{tabular} & 0.003 & $\begin{array}{ll}1.968 \\
1.07\end{array}$ & & 62.00 & $\begin{array}{ll}0 & 1.03333 \\
\end{array}$ & \begin{tabular}{ll|}
43.8 \\
\end{tabular} & 11.1 & 39.8 & \begin{tabular}{|l|l|}
8.745 \\
8.720
\end{tabular} & \begin{tabular}{|l|l|}
0.293 \\
\end{tabular} & & 0.008 & 7.745 & 6.59 \\
\hline 80 & $\begin{array}{l}2 / 5 / 2004 \\
2 / 5 / 2004 \\
\end{array}$ & \begin{tabular}{|l|}
$6: 29: 33$ PM \\
$6630.33 \mathrm{MM}$ \\
\end{tabular} & 23.858 & 20.537 & \begin{tabular}{|l|}
23.229 \\
2.215
\end{tabular} & $\begin{array}{ll}21.523 \\
21.519 \\
\end{array}$ & $\frac{21.548 \mid \#}{21.62}$ & \begin{tabular}{|l|l|l|l|l|}
41.219 \\
4.122
\end{tabular} & \begin{tabular}{|l|}
45.238 \\
45077 \\
\end{tabular} & $\begin{array}{l}3.545 \\
3533 \\
\end{array}$ & $\begin{array}{l}37.917 \\
37991 \\
\end{array}$ & -1.219 & $\begin{array}{ll}1.286 \\
1283\end{array}$ & \begin{tabular}{|l|l|}
45.074 \\
\end{tabular} & 0.003 & 1.97 & 0.006 & 63.00 & $\begin{array}{r}1.05 \\
10667\end{array}$ & $\begin{array}{l}43.8 \\
\end{array}$ & & $\begin{array}{l}39.6 \\
30.5 \\
\end{array}$ & & \begin{tabular}{|l|l|} 
& 0.294 \\
\end{tabular} & & 0.008 & 7.805 & 6.64 \\
\hline \begin{tabular}{|l|l|}
82 \\
\end{tabular} & $\begin{array}{l}225 / 2004 \\
2 / 5 / 2004\end{array}$ & 6:30:33 PM & $\begin{array}{r}23.88 \\
23.874 \\
\end{array}$ & 20.548 & \begin{tabular}{|r|}
23.245 \\
23.24
\end{tabular} & 21.519 & $\frac{21.62 / \#}{21.72 \mid \#}$ & $\frac{41.122}{40.811}$ & $\begin{array}{l}45.017 \\
44.786\end{array}$ & $\begin{array}{l}3.533 \\
3.493 \\
\end{array}$ & \begin{tabular}{|l}
37.729 \\
37.609
\end{tabular} & & $\begin{array}{l}1.283 \\
1.286 \\
\end{array}$ & \begin{tabular}{|l|l|}
45.007 \\
4.959
\end{tabular} & 0.003 & $\begin{array}{l}1.978 \\
1.979 \\
\end{array}$ & 0.005 & & $\begin{array}{l}0.06667 \\
0\end{array}$ & & & & $\frac{2.725}{2703}$ & 0.295 & & 0.008 & 7.841 & 6.67 \\
\hline 83 & $2 / 5 / 2004$ & 6:32:33 PM & 23.874 & 20.533 & & 21.513 & 21.845 \#\# & 40.991 & \begin{tabular}{|l|}
44.867 \\
4.807
\end{tabular} & $\begin{array}{l}3.492 \\
3.492 \\
\end{array}$ & 37.892 & -1.214 & 1.292 & $\begin{array}{ll}44.949 \\
\end{array}$ & 0.003 & 1.982 & & 66.00 & & 43.4 & \begin{tabular}{|l|}
11.0 \\
11.0
\end{tabular} & & & & & 0.008 & & $\frac{6.73}{6.70}$ \\
\hline 84 & $2 / 5 / 2004$ & 6:33:33 PM & 23.869 & 20.527 & 23.204 & 21.493 & 21.695 \#\# & 40.9 & 44.822 & 3.506 & 37.802 & -1.222 & 1.289 & 45.164 & 0.003 & 1.984 & 0.005 & 67.00 & \begin{tabular}{|l|l|}
0 & 1.11667 \\
\end{tabular} & 43.3 & \begin{tabular}{|l|}
11.1 \\
\end{tabular} & 39.4 & $\begin{array}{ll}42.713 \\
\end{array}$ & \begin{tabular}{|l|l|}
0.296 \\
\end{tabular} & 0.311 & 0.008 & 8.809 & $\frac{6.70}{6.73}$ \\
\hline 85 & $2 / 5 / 2004$ & 6:34:33 PM & 23.874 & 20.543 & \begin{tabular}{|l|}
23.224 \\
\end{tabular} & 21.503 & 21.675 \# & 41.085 & 45.048 & & 37.821 & -1.216 & 1.292 & 44.922 & & 1.988 & & 68.00 & \begin{tabular}{|l|l|}
0 & 1.13333 \\
\end{tabular} & 43.6 & 11.0 & 39.5 & 52.72 & 0.296 & 0.312 & 0.008 & & $\frac{6.73}{6.72}$ \\
\hline 86 & $2 / 5 / 2004$ & 6:35:33 PM & 23.884 & & 23.229 & 21.468 & $21.644 \#$ & & & 3.547 & & & 1.292 & 44.638 & 0.003 & 1.992 & & 69.00 & & & & & & & & 0.008 & 7.907 & $\frac{72}{73}$ \\
\hline 87 & & 6:36:33 PM & & & & & 21.283|\# & 41.107 & 45.106 & & & & & & & 1.994 & & & & & & & & 297 & & & & \\
\hline $\begin{array}{lll}88 & & \\
\end{array}$ & $2 / 5 / 2004$ & 6:37:33 PM & & 20.548 & \begin{tabular}{|l|l|}
2.3 .25 \\
2.25
\end{tabular} & & 21.66 \# & 41.008 & $\mid 45.166$ & 3.542 & 37.728 & -1.216 & 1.298 & 45.518 & 0.0 & 2.003 & & 00 & $\begin{array}{l}0.18333 \\
0.1833\end{array}$ & & & 89.4 & & $\frac{0.299}{0.299}$ & $\frac{0.314}{0.314}$ & & & \\
\hline 89 & $2 / 5 / 2004$ & 6:38:33 PM & 23.88 & 20.544 & & 21.494 & & 40.94 & \begin{tabular}{|l|l|} 
& 45.054 \\
\end{tabular} & 3.485 & 37.786 & -1.219 & & 44.415 & & & & & 0.1 .2 & & & & & & & & & \\
\hline 90 & $2 / 5 / 2004$ & 6:39:33 PM & 23.885 & 20.548 & 23.245 & $\frac{21.514}{21}$ & $21.791 \mid \#$ & 41.114 & \begin{tabular}{|l|l|}
455.054 \\
\end{tabular} & 3.501 & 38.046 & -1.216 & 1.298 & 44.738 & 0.0 & 2.008 & & 00 & \begin{tabular}{|l|l|}
0 & 1.21667 \\
\end{tabular} & 43.6 & 110 & 39.6 & & 299 & 0.315 & & & $\frac{6.76}{6.76}$ \\
\hline & $2 / 5 / 2004$ & 6:40:33 PM & 23.865 & 20.528 & 23.215 & 21.463 & 21.449 \#\# & 40.975 & 45.118 & 3.575 & 37.706 & -1.216 & 1.298 & 44.821 & & 2.008 & & 74. & 1.23333 & & 11.0 & 39.3 & & 299 & & 0.008 & 8.004 & 6.8 \\
\hline 9 & & 6:41:33 PM & 23.88 & 20.548 & 23.22 & 21.489 & 21.379 \# & 40.722 & $\begin{array}{ll}44.902 \\
\end{array}$ & 3.494 & 37.552 & -1.216 & 1.301 & $\begin{array}{l}44.721 \\
\end{array}$ & 0.003 & 2.01 & & 75.00 & 1.25 & 43.4 & & 39.1 & & & & 0.008 & 8.053 & \\
\hline & 2/5/2004 & 6:42:33 PM & & 20.559 & & 21.464 & 21.314 & 40.977 & & 3.524 & 31.124 & -1.216 & & 44.746 & & 2.012 & & & 1.26667 & & & & & & & & & \\
\hline & & 3:33 PM & 23.88 & & 23.231 & 21.434 & 21.746 & 41.02 & 45.259 & 3.597 & 37.665 & -1.216 & 1.303 & 44.775 & 0.005 & 2.02 & & & 1.28333 & & & & & 301 & 0.317 & 0.008 & 8.048 & \\
\hline & 2/5/2004 & 6:44:33 PM & 23.875 & 20.539 & 23.216 & 21.439 & 21.736|\# & 40.601 & 44.762 & 3.441 & 37.57 & -1.222 & 1.301 & 45.205 & 0.003 & 2.02 & & 78.00 & 1.0 & & & & & 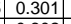 & 0.317 & 0.008 & 8.105 & 6.90 \\
\hline & 2 & 6:54:33 PM & $\frac{23.865}{2037}$ & 20.543 & & 21.474 & 22.19227 & 41.159 & & & $\begin{array}{r}37.81 \\
27.656\end{array}$ & -1.219 & 1.306 & 44.805 & & & & & & & & & & & & & & \\
\hline$\frac{91}{98}$ & $\frac{21 / 2 / 2004}{2 / 5 / 2004}$ & $\begin{array}{l}0.46 .35 \mathrm{MM} \\
6.0732 \mathrm{M}\end{array}$ & $\frac{23.87}{2387}$ & 20.549 & $\frac{23.230}{23245}$ & $\frac{2.5 .54}{21504}$ & $\frac{22.303+1}{20.197}$ & $\frac{41.101}{41.025}$ & $\frac{45}{45}>>2$ & $\frac{3.581}{361}$ & $\begin{array}{ll}37.650 \\
37602\end{array}$ & -1.210 & 1.300 & (44.819 & & 2.0 & & 80.00 & 1.33333 & & 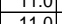 & 39 & & 2303 & 然. & & 8.068 & 60 \\
\hline 99 & \begin{tabular}{|c|}
$/ 5 / 5 / 2004$ \\
$2 / 5004$
\end{tabular} & $\begin{array}{l}0.4 .435 \mathrm{PM} \\
6 \cdot 48 \cdot 33 \mathrm{PM}\end{array}$ & $\frac{2.01}{23.875}$ & $\frac{20.548}{20.548}$ & \begin{tabular}{|r|}
23.245 \\
2324 \\
\end{tabular} & $\frac{21.504}{21.489}$ & $\frac{22.18171 \#}{21.941 \#}$ & $4 \frac{41.025}{40.693}$ & \begin{tabular}{|l|}
45 \\
44 \\
\end{tabular} & $\begin{array}{l}3.01 \\
3.458 \\
\end{array}$ & $\begin{array}{l}37.093 \\
37.601 \\
\end{array}$ & $\frac{-1.216}{-1216}$ & 1.509 & $\begin{array}{ll}44.951 \\
44.88\end{array}$ & & & & & $\begin{array}{l}1.35 \\
136667 \\
\end{array}$ & & $\frac{11.6}{110}$ & & & & & & 每. 8935 & 6.89 \\
\hline & $2 / 5 / 2004$ & 6::49:33 PM & $\frac{23.885}{23.885}$ & 20.563 & 23.245 & $\frac{21.474}{21.47}$ & 21.66 \# & 40.778 & 45. & $\frac{3.492}{3.492}$ & 37.543 & -1.219 & 1.309 & 44.738 & & & & & 13833 & & & & & & & & 8162 & \\
\hline & $2 / 5 / 2004$ & $6: 50: 33 \mathrm{PM}$ & 23.871 & 20.544 & & & & & & & & & & 45.3 & & & & & & $43.4>-3$ & & & & & & & & \\
\hline & $2 / 5 / 2004$ & 6:51:33 PM & 23.881 & 20.544 & 23.226 & 21.46 & $21.701 \neq$ & 40.915 & 45.342 & 3.599 & 37.55 & -1.219 & 1.312 & 44.532 & & 2.0 & & & 1.41667 & 43.5 & $\frac{\frac{10.9}{109}}{2}$ & & & & & & & \\
\hline & 2004 & & 23.876 & 20.555 & & 21.455 & & & & 3.4 & & -1.219 & 1315 & 45.107 & & & & & 1.43333 & & 11. & & & & & & & \\
\hline & $\frac{2 / 5}{2 / 5}$ & 6:5:3:33 PM & 23.881 & 20.555 & 23.242 & $\frac{11.49}{21.49}$ & 21.848 \# & 40.643 & \begin{tabular}{|l|l|l|}
44.983 \\
\end{tabular} & 3.49 & 37.55 & $\frac{-1.216}{-1.216}$ & 1.315 & 44.951 & & 2.054 & & 87.00 & $\frac{1.45}{1.45}$ & & & & & 56 & $\frac{0.322}{0.322}$ & & 233 & \\
\hline & $2 / 5 / 2004$ & 6:54:33 PM & & 20.555 & 247 & & & 40.96 & 45. & & 37.759 & -1.216 & & 44.871 & & & & & 1.46667 & & 1 & & & & & & 3.180 & \\
\hline & $2 / 5 / 2004$ & 6:55:33 PM & 23.867 & 20.55 & 23.237 & & $21.895 \mathrm{~S}$ & 40.492 & \begin{tabular}{|l|l|l|l|l|l|}
44.687 \\
\end{tabular} & 3.579 & 37.287 & -1.222 & & 44.62 & & & & & 1.48333 & & $0 c 0$ & & & & & & $8.302 \quad$ & \\
\hline & $2 / 5 / 2004$ & 6:56:33 PM & 23.877 & 20.55 & 23.247 & 21.466 & 21.979 & 40.628 & 45.039 & 3.475 & 37.422 & -1.216 & 32. & 44.725 & 0.003 & 2.063 & & & 1.5 & $43.6 .6-2$ & 11.0 & $39.6>>2$ & 69 & 0.308 & 0.323 & 0.008 & 8.283 & 7.0 \\
\hline & & Average & & & & & $21.7>>>$ & 41.3 & & 3.6 & 38.0 & -12 & $1.3 t \mid$ & 44.9 & 0.0 & 1.9 & & & & 43.5 & 110 & $3977>$ & & 0281 & 0296 & 0075 & 746 & \\
\hline & & & 23.9 & 20.6 & $\frac{23.1}{23.3}$ & 21.6 & & $\begin{array}{l}4.1 .3 \\
42.4\end{array}$ & $\frac{45.0}{45.5}$ & 3.0 & 390 & $\frac{-1.4}{-1.2}$ & & 466 & & & & & & & & & & & & & & \\
\hline 111 & & Median & 238 & 20.5 & 232 & 215 & 217 & 41.3 & 450 & 36 & 37.5 & -12 & 13 & 450 & 00 & 19 & & & & 435 & 110 & & & 0286 & & & & \\
\hline & & & & 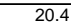 & & 21.4 & & 40.4 & & 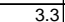 & 37.3 & & & & & & & & & 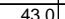 & (1) & 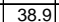 & & & & & & \\
\hline & & $2 \times$ Std Dev & & $\frac{2.06}{0.06}$ & 5 & 0.061 & $0.409 \#$ & 0.908 & 0.475 & 0.09 & 0.773 & 0.005 & $0.075 \#$ & 1395 & 0.000 & 0.264 & 000 & & & \begin{tabular}{l|l}
0.475 \\
\end{tabular} & \begin{tabular}{|l|l|}
0.342 \\
\end{tabular} & 0.828 & 0.0 & & $\frac{0.5040}{0.040}$ & $\frac{0011}{0011}$ & 资 & 96 \\
\hline & Number of & Points! & 85 & 85 & 85 & $\frac{55}{85}$ & 85 \# & 85 & 85 & 85 & 85 & 85 & 850 & 85 & 85 & 85 & & & & 85 & 85 & 85 & & 85 & 85 & 85 & 85 & \\
\hline & & sengen & in he & are not if & ncluded & & & & & & & & & & & & & & & & & & & & & & & \\
\hline & & & & & & & & & & & & & & & & & & & & & & & & & & & & \\
\hline & & & & & & & & & & & & & & & & & & & & & & & $1.5-\mathrm{ho}$ & ir avg. & 0.2961 & & & \\
\hline
\end{tabular}


RUN \# 1.19; SECOND SLURRY RINSE WITH 0.1 M NAOH

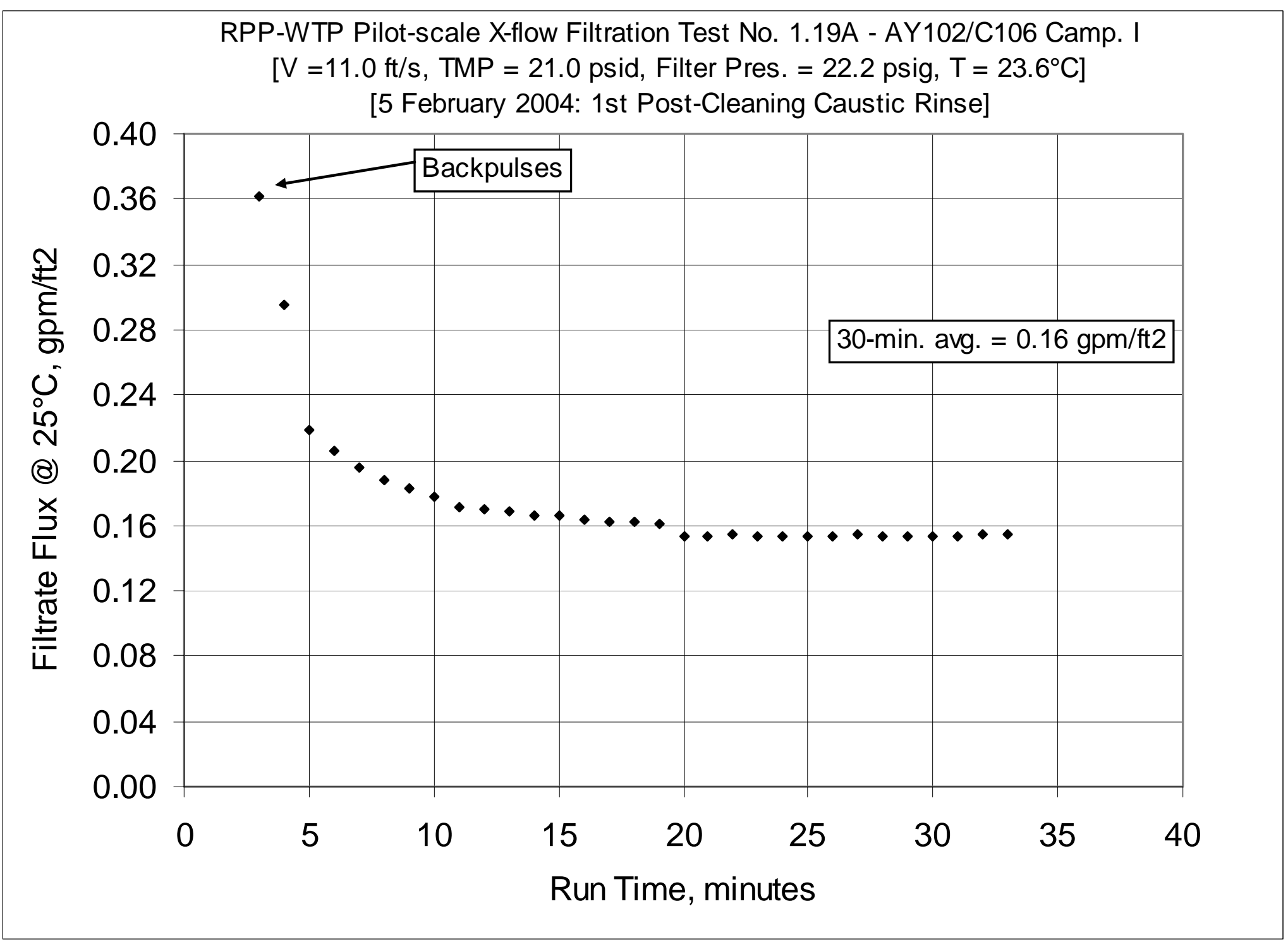


WSRC-TR-2005-00105, REVISION 0

SRNL-RPP-2005-00012, REVISION 0

RUN \# 1.19; SECOND SLURRY RINSE WITH 0.1 M NAOH - CONT.

\begin{tabular}{|c|c|c|c|c|c|c|c|c|c|c|c|c|c|c|c|c|c|c|c|c|c|c|c|c|c|c|c|c|}
\hline & $\mathrm{A}$ & $\mathrm{B}$ & $\frac{D}{1<\ll<\varepsilon}$ & $\mathrm{E}$ & $\frac{F}{1}$ & $G$ & $\mathrm{H}$ & $\mathrm{J}$ & $\mathrm{K}$ & $\mathrm{L}$ & M & $\frac{1 \mathrm{~N}}{\mathrm{n}}$ & 0 & Q & $\mathrm{R}$ & $\mathrm{s}$ & $1 \quad T$ & $\mathrm{v}$ & w w & \begin{tabular}{|c|}
$x$ \\
\end{tabular} & $\begin{array}{ll}r \\
C\end{array}$ & $z$ & AA & AB & $A C$ & $\mathrm{AD}$ & $\mathrm{AE}$ & $\mathrm{AF}$ \\
\hline 1 & DATE & TIME & Filtrate & $\begin{array}{l}\text { Cleaning } \\
\text { Cleang }\end{array}$ & & $\begin{array}{l}\text { Measurem } \\
\text { Hi Amb. }\end{array}$ & $\begin{array}{l}\text { nents > >> } \\
\text { Lo Amb. }\end{array}$ & BotTMP & $\begin{array}{l}\text { Filter } \\
\text { File }\end{array}$ & $\begin{array}{l}\text { Pressure } \\
\text { Fitter dP } \\
\end{array}$ & $\begin{array}{l}\text { Measurem } \\
\text { TopTMP }\end{array}$ & 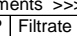 & Pulsepot & Slurry & $\begin{array}{l}\text { < Flltow I } \\
\text { Fitrate }\end{array}$ & $\begin{array}{l}\text { Measureme } \\
\text { |Hi Fittate }\end{array}$ & ents >>>>> & & & & & & & & & & & \\
\hline & DATL & & $\operatorname{deg} \mathrm{C}$ & deg C & $\operatorname{deg} C$ & $\operatorname{deg} \mathrm{C}$ & $\operatorname{deg} C$ & $\frac{p s i d}{p s i d}$ & psig & psid & psid & psig & psig & gpm & $\mathrm{gpm}$ & 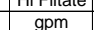 & gom & & & & & & & & & & & \\
\hline & & & & & $\mathrm{T} 1$ & & & $\mathrm{dP2}$ & P1 & $\mathrm{dP1}$ & dP3 & P2 & P3 & Q1 & $\mathrm{Q} 2$ & \begin{tabular}{|l}
$\mathrm{g} m \mathrm{~m}$ \\
$\mathrm{Q} 3$
\end{tabular} & Qbp & & & & & & & & & & & \\
\hline & & & & & & & & & & & & & & & & & & & & & & & & & & & & \\
\hline & & & & & & & & & & & & & & & & & & & & & & & & & & & & \\
\hline 7 & $2 / 5 / 2004$ & 9:26:34 AM & 19.191 & 18.592 & 16.892 & 19.019 & 18.202 & 0.056 & -0.05 & -0.029 & & -0.177 & 0.121 & -0.093 & 0.003 & 14.643 & 0.004 & & & Fititer S & urface & A6.707 & FT2 & & & & & \\
\hline 8 & 2/5/2004 & 9:26:52 AM & 19.191 & 18.592 & 16.892 & 19.019 & 18.202 & 0.056 & -0.05 & -0.029 & & -0.177 & 0.121 & -0.093 & 0.003 & 14.643 & 0.004 & & & Convers & rsion & 851 & $\mid \mathrm{m} 3 / \mathrm{m} 2$ & //day/barg & $\mathrm{rg} / \mathrm{gpm} / \mathrm{ttt}$ & ftr/barg & & \\
\hline & 2/5/2004 & 9:26:53 AM & & 18.582 & 16.872 & & & 0.056 & & -0.029 & & -0.18 & & & 0.003 & 14.643 & 0.004 & & & & & & & & & & & \\
\hline 10 & 2/5/2004 & 9:26:54 AM & 19.181 & 18.571 & 16.861 & 19.024 & 18.146 & 0.058 & -0.05 & -0.029 & -0.002 & -0.18 & 0.121 & -0.091 & 0.003 & 14.643 & 0.004 & & & Note: $G$ & Gauge P & Pressur & e $P 1$ is a & approxin & nately 1 & 4 psig & & \\
\hline & 2/5/2004 & 9:26:55 AM & 19.181 & 18.577 & 16.867 & 19.024 & 18.152 & 0.058 & -0.05 & -0.029 & 0.002 & -0.177 & 0.121 & -0.093 & 0.003 & 14.643 & 0.004 & & & & & & & & & & & \\
\hline & $2 / 5 / 2004$ & 9:26:56 AM & 19.181 & 18.577 & 16.872 & 19.024 & 18.157 & 0.056 & \begin{tabular}{|c|c|}
-0.048 \\
\end{tabular} & $\begin{array}{l}-0.029 \\
0.020\end{array}$ & & $\begin{array}{l}-0.177 \\
0.177\end{array}$ & 0.121 & -0.091 & 0.003 & 14.643 & & & & & ure Plis & orrec & ted for 4 & & & er tu & & \\
\hline & & 9:26:57 AM & 19.186 & 18.572 & $\frac{16.867}{167}$ & 19.029 & 10.154 & & & -0.029 & & & 0.118 & & 0.0000 & 14.643 & 0.004 & & & VwW & & & & Filtrate & ate Flux & 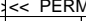 & & \\
\hline & 2/5/2004 & 9:26:58 AM & 19.192 & 18.587 & 16.877 & 19.03 & 18.162 & 0.058 & -0.05 & -0.028 & & -0.177 & 0.121 & -0.091 & 0.003 & 14.643 & 0.005 & & & & & & & & & & & \\
\hline & Data - PerM & linute & & & & & & & & & & & & & & & & Inme & Home & Press. & vel. & MPP & IMP & $\mathrm{gpm}$ & $\mathrm{pm}$ & 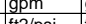 & $\mathrm{pm}$ & tele \\
\hline \begin{tabular}{|l|l|}
17 \\
17
\end{tabular} & $2 / 5 / 2004$ & $7: 23: 35 \mathrm{PM}$ & & & 21.849 & 20.603 & 23.468 & 3.317 & 23.517 & 3.284 & -0.425 & 15.627 & 61.641 & 43.682 & 0.003 & -0.01 & 0.007 & & & 22.0 & 10.7 & 1.4 & & & -0.002 & $\frac{2 \mathrm{PSS}}{-0.001}$ & $\frac{2 \mid \mathrm{PSS}}{-1.128}$ & \\
\hline 18 & $2 / 5 / 2004$ & 7:24:35 PM & 22.068 & 20.503 & 22.004 & 20.571 & 23.657 & 2.786 & 23.506 & 3.077 & -0.811 & 16.067 & 25.931 & 43.461 & 0.003 & 14.643 & 0.084 & 1.00 & 0.01667 & 22.0 & 10.7 & 1.0 & \begin{tabular}{|l|l|} 
\\
\end{tabular} & 2.183 & 2.377 & 2.407 & \begin{tabular}{|l|l|}
7 & 2407.393 \\
\end{tabular} & \begin{tabular}{|c|c|}
-0.048. \\
2
\end{tabular} \\
\hline$\frac{19}{n}>>$ & $2 / 5 / 2004$ & $\begin{array}{l}\text { 7:25:35 PMM } \\
\end{array}$ & 22.268 & 20.487 & 22.179 & 20.51 & 23.676 & 15.273 & 22.924 & 3.336 & 12.135 & 2.333 & 1.567 & 46.14 & 0.005 & 2.853 & תf & 2.00 & 0.03333 & 21.5 & 11.3 & 13.7 & 0.945 & 0.425 & 0.461 & 0.034 & \begin{tabular}{|l|l|}
4 & 33.630 \\
\end{tabular} & 28.62 \\
\hline & $2 / 5 / 2004$ & 7:26:35 PM & 22.563 & 20.506 & 22.314 & 20.559 & 23.254 & 19.344 & 24.296 & 2.672 & 16.654 & -0.449 & 1.419 & 42.519 & 0.003 & 2.247 & 0.004 & 3.00 & 0.05 & 22.8 & 10.4 & 18.0 & 1.241 & 0.335 & 0.362 & 0.020 & 20.088 & 1710 \\
\hline & $2 / 5 / 2004$ & 7:27::35 PM & 22.774 & 20.51 & 22.554 & 20.568 & 22.988 & 23.139 & 26.354 & 3.175 & 19.839 & -1.214 & 1.228 & 44.98 & 0.003 & 1.849 & 0.00 & 4.00 & 0.06667 & $24 . \mathrm{C} . \mathrm{C}$ & 11.0 & 21.5 & 1.482 & 0.276 & 0.295 & 0.014 & 13.15 & \\
\hline & $\frac{215 / 2004}{25 / 2004}$ & 7:28:35 PM & 22.878 & 20.484 & 22.654 & 20.593 & 23.308 & 21.3 & $\mid 23.044$ & 3.14 & 17.9619 & -1.216 & 1.006 & 44.433 & 0.003 & 1.369 & 0.0 & & 0.08333 & 21.6 & & & 1.35. & 0.204 & & & & \\
\hline$\frac{23}{24}$ & $\frac{2 / 1 / 2004}{2 / 5 / 2004}$ & $=122: 35 \mathrm{PM}$ & $2 \frac{23.018}{220}>$ & 20.494 & 22.169 & 20.617 & 23.132 & 22.146 & $\mid \frac{2.3111}{32917}$ & 3.1133 & 18.811 & -1.216 & 0.983 & 43.010 & 0.003 & 1.292 & & 6.00 & 0.1 & $\frac{2.2}{2.2}$ & 11.0 & $20.5 \mathrm{r}-\mathrm{r}$ & 1.412 & 0.193 & & 0.010 & 10.021 & \\
\hline & (2) & $7.31 .5 \mathrm{PM}$ & 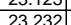 & 20.410 & t) 22.904 & 20.006 & 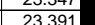 & 22.422 & $\mid \frac{20.011}{2354}$ & 3.092 & 19.1305 & $\frac{-1.216}{-1.211}$ & & $\begin{array}{l}44.138 \\
44.127\end{array}$ & 然 0003 & $\frac{1.54}{1189}$ & 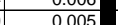 & 8.00 & - & 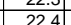 & 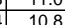 & $\frac{2.0}{20.9}-9$ & $\frac{1.433}{1.444}$ & 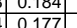 & 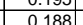 & - & & \\
\hline 26 & $215 / 2004$ & $7 \cdot 3 \cdot 3 \cdot 35 \mathrm{PM}$ & 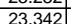 & 20.487 & 23074 & 20.727 & 23.27 & 22.787 & 24016 & 3.11 & 19385 & -1214 & & 43824 & 0.003 & 116 & 0.0 & 900 & $\frac{0.150 .15}{0.15}$ & $\frac{2.4}{22.5}$ & 107 & 211 & 1.454 & 0.173 & 0.183 & 0.009 & $\frac{562}{662}$ & \\
\hline 27 & $2 / 5 / 2004$ & 7:33:35 PM & 23.417 & 20.476 & \begin{tabular}{|l|l|}
23.203 \\
\end{tabular} & 20.68 & 23.284 & 23.118 & 244.279 & 3.241 & 19.566 & \begin{tabular}{|c|c|}
5 & -1.216 \\
\end{tabular} & & 43.82 & 0.003 & 1.135 & 0.0 & 10.00 & 0.16667 & 22.8 & 10.7 & 21.3 & 1.471 & 0.169 & 0.178 & 0.008 & 8.343 & \\
\hline & 2/5/2004 & 7:34:35 PM & 23.511 & 20.48 & 23.268 & 20.66 & 22.135 & 22.772 & 23.759 & 3.302 & 19.26 & & & $\begin{array}{l}44.567 \\
\end{array}$ & 0.003 & & & 11.00 & 0.18333 & 22.3 & 10.9 & 21.0 & 1.449 & 0.164 & & 0.008 & & \\
\hline & 2/5/2004 & 7:35:35 PM & 23.595 & 20.469 & 23.316 & & 22.249 & 22.855 & 23.923 & 3.153 & 19.439 & -1.219 & 0.90 & 44.832 & 0.003 & 1.088 & & 12.00 & 0.2 & 22.4 & 11.0 & $\frac{21.1}{21.1}$ & 1.458 & 0.162 & 0.170 & 0.008 & 8.045 & 685 \\
\hline & 2/5/2004 & 7:36:35 PM & 23.676 & 20.449 & 23.403 & 20.467 & 19.981 & 22.805 & 23.875 & 3.015 & 19.513 & -1.216 & & 44.03 & 0.003 & 1.085 & 0.0 & 13.0 & 0.21667 & 22.4 & 10.8 & 21.2 & 1.459 & & & 0.008 & 7.999 & \\
\hline & 2/5/2004 & 7:37:35 PM & 23.739 & 20.407 & 23.456 & 20.399 & 19.44 & 22.894 & 23.821 & 3.15 & 19.566 & -1.216 & & 43.845 & 0.003 & 1.07 & 0.0 & 14.00 & 0.23333 & 22.3 & 10.7 & 21.2 & 1.464 & \begin{tabular}{|c|}
0.160 \\
\end{tabular} & 0.167 & 0.008 & 7.850 & 66 \\
\hline & $2 / 5 / 2004$ & 7:38: & 23.8. & 20.375 & \begin{tabular}{|l|l|}
23.49 \\
\end{tabular} & 20.3 & 19.4 & 23.176 & & 3.16 & & .216 & & 43.8 & & & & 10.4 & & 22.6 & & 21.5 & 1.40 & & 0.166 & & & \\
\hline 33 & $2 / 5 / 2004$ & 7:39:35 PM & 23.866 & 20.358 & $\begin{array}{l}323.527 \\
\end{array}$ & 20.275 & 20.3 & 23.064 & 23..968 & 3.101 & 19. & -1.219 & & 43.781 & 0.003 & & & 16.00 & 0.26667 & 22.5 & 10.7 & 21.4 & 1.477 & & 0.164 & & & \\
\hline 34 & 2/5/2004 & $\begin{array}{r}7: 40: 35 \\
\end{array}$ & 23.909 & 20.331 & 23.611 & 20.258 & 21.6 & 23.033 & & & & -1.216 & & 43.824 & & & & & 0.28333 & 22.4 & & 21.4 & $1.47 \mathrm{~s}$ & 0.156 & 0.163 & & & \\
\hline 35 & $2 / 5 / 2004$ & 7:411:35 PM & 23.95 & 20.296 & 23.641 & 20.223 & 22.3 & & \begin{tabular}{|l|}
23.919 \\
\end{tabular} & 3.151 & 19.619 & -1.219 & 0. & 44.1 & 0.003 & & 0.0 & 18.6 & 0.3 & 22.4 & 10.8 & 21.3 & & & 0.162 & & 7.598 & 0.4 \\
\hline$\frac{36}{37}$ & 2/5/2004 & 7:42:35 PM & 24.012 & 20.298 & $\begin{array}{l}323.678 \\
3\end{array}$ & 20.265 & 22.4 & 23.22 & \begin{tabular}{|l}
24.163 \\
\end{tabular} & 3.167 & 19.79 & $\begin{array}{l}9 \\
\end{array}$ & 0.88 & 43.901 & & 1.039 & & 19.00 & & 22.1 & 10.8 & 1.5 & .483 & & 0.101 & & & \\
\hline 37 & $2 / 5 / 2004$ & 7:43:35 PM & 24.046 & 20.277 & 23.697 & 20.33 & 22.47 & 22.569 & \begin{tabular}{|l}
23.345 \\
\end{tabular} & 3.517 & 18.827 & -1.219 & & 46.267 & 0.003 & 0.995 & 0.00 & 20.0 & 0.33333 & 21.9 & 11.3 & & .427 & 48 & & & 7.436 & \\
\hline & 2/5/2004 & $7: 4$ & & & & 20.42 & & 22.556 & & & & -1.216 & & & & 0.997 & & 21.00 & 0.05 & 21.9 & 1100 & & 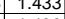 & & 0.154 & & 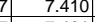 & \\
\hline 列 & & & $\frac{2.105}{2.157}$ & & 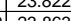 & & $22 . .05$ & 22.081 & 23.401 & 3.450 & 18.975 & & & 46.019 & 0.003 & & & & & & 11.5 & & & & & & & \\
\hline & 21512004 & $\frac{1.40 .035}{7.750}$ & 年 & 20.204 & \begin{tabular}{|l|l|l|l|l|l} 
\\
\end{tabular} & 20.492 & 202012 & $\frac{22.66}{22612}$ & $\begin{array}{r}23.5 \\
320\end{array}$ & $\begin{array}{l}3.399 \\
3.216\end{array}$ & 19 & $\frac{-1.216}{-1.26}$ & & 45.015 & .4 & - & & 20 & 0 & $\frac{2.0}{2.0}$ & $\frac{11.2}{112}$ & 20.8 & 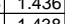 & $\mid 0.149$ & 0.154 & & . & .2 \\
\hline 42 & 215152004 & $\frac{1 \cdot 4 \cdot 6 \cdot 35}{7 \cdot 4 \cdot 35}$ & 年 & 20.249 & 23866 & 20.5 & & 22764 & & & & & & & & 1 & & & & & & & & & & & & \\
\hline & $2 / 5 / 2004$ & $7: 49:$ & 24.23 & 20.246 & 23.897 & 20.576 & 23. & 22.934 & & & & -1.219 & & & & & & 26.00 & 0 & 22.3 & 11.0 & 211. & 1.454 & & 0.154 & & & 0.2 \\
\hline 44 & $2 / 5 / 2004$ & $7: 50: 35$ & $\frac{24.261}{24}$ & & & & & 22.724 & 23.494 & & & & & & & 1.004 & & 2700 & & 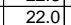 & 11.2 & 51.0 & 10 & & 0.154 & & & \\
\hline & $2 / 5 / 2004$ & 7:51: & 24.272 & 20.2 & \begin{tabular}{l|l|}
323.974 \\
\end{tabular} & 20.6 & & 22.585 & 23.405 & & & & & & & & & 28.00 & 0.46667 & 21.9 & 11.2 & & 433 & & & & & 0.4 \\
\hline 40 & & $7: 5$ & Z4.509 & & & & & 22.612 & & & & -1.214 & & & & & & & 0.48333 & 22.0 & & & .437 & & & & & \\
\hline & 2/5/2004 & & 24.315 & & & & & 22.708 & & & 195 & & & 45.7 & & & & 30.00 & & 22.0 & 11.2 & & 445 & & & & & \\
\hline 48 & & $7: 54: 35$ & 24.346 & 20.246 & & 20.738 & & 22.662 & 23.494 & 3.356 & 19.059 & -1.216 & & & & & & 31.00 & \begin{tabular}{|l|}
0.51667 \\
\end{tabular} & 22.0 & 11.2 & 20.9 & .438 & & & & & \\
\hline & $2 / 5 / 2004$ & $7: 55: 35$ & 24.352 & 20.237 & 24.044 & 20.679 & & 22.54 & 23.303 & 3.275 & & & & & 0. & 1.006 & & 32.00 & 0.53333 & 21.8 & 11.2 & 20.8 & 1.436 & & & & & \\
\hline & 2/5/2004 & 7:56:35 PM & 24.373 & 20.243 & 24.065 & 20.771 & 23.133 & 22.795 & 23.587 & 3.34 & 19.209 & -1.214 & 0.8 & 45.789 & 0.003 & 1.008 & & 33.00 & 0.55 & 22.1 & 11.2 & 21.0 & 1.448 & 0.150 & 0.154 & 0.007 & 7.347 & \\
\hline & & & 23.9 & & 23.6 & & & 22.8 & 23.7 & & & 12 & & 44.9 & 0.0 & 1.1 & & & & 222 & 110 & & .44 & 0.158 & 0.164 & & & \\
\hline & & & 24.4 & 20.5 & 24.1 & 20.8 & & 23.2 & 24.3 & 3.5 & & & & & & 1.3 & & & & 22.8 & 11.3 & 21.5 & 1.48 & & & & & \\
\hline 54 & & & 24.0 & 20.3 & 23.7 & 20.6 & & 22.7 & 23.7 & & 19.2 & -1.2 & 0 & 45 & 0.0 & 1.0 & & & & 22.3 & $\frac{11.0}{11.0}$ & $\frac{21.0}{21.0}$ & 1.446 & & & & 457 & \\
\hline 55 & & Minimum & & 20.2 & 22.8 & 20.2 & 19.4 & 22.1 & 23.3 & 3.0 & 18.8 & -1.2 & 0.9 & 43.8 & 0.0 & 1.0 & & & & 21.8 & 10.7 & 20.5 & 1.412 & 0.148 & . & & 7.294 & \\
\hline & & $2 \times$ Std Dev & 0.791 & 0.196 & 0.731 & 0.319 & 2.304 & 0.470 & 0.538 & 0.261 & 0.565 & 0.004 & 0.057 & 1.673 & 0.000 & 0.156 & & & & $\mid 0.538$ & 0.410 & & 0.035 & |23 & 028 & 014 & 1.358 & \\
\hline & Number of & f Points Used* & 28 & 28 & $\quad 28$ & & & 28 & & 28 & & 28 & $28]$ & 28 & & 28 & 28 & & & 28 & 28 & 28 & 28 & 28 & 28 & 28 & 28 & \\
\hline & & Backpuse po & nts in box & kare not I & incluaed & & & & & & & & & & & & & & & & & & & & & & & \\
\hline 60 & & & & & & & & & & & & & & & & & & & & & & & & 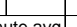 & 01641 & & & \\
\hline & & & & & & & & & & & & & & & & & & & & & & & 3 & unte avg.| & 0.1641 & & & \\
\hline
\end{tabular}


RUN \# 1.19; THIRD SLURRY RINSE WITH 0.1 M NAOH

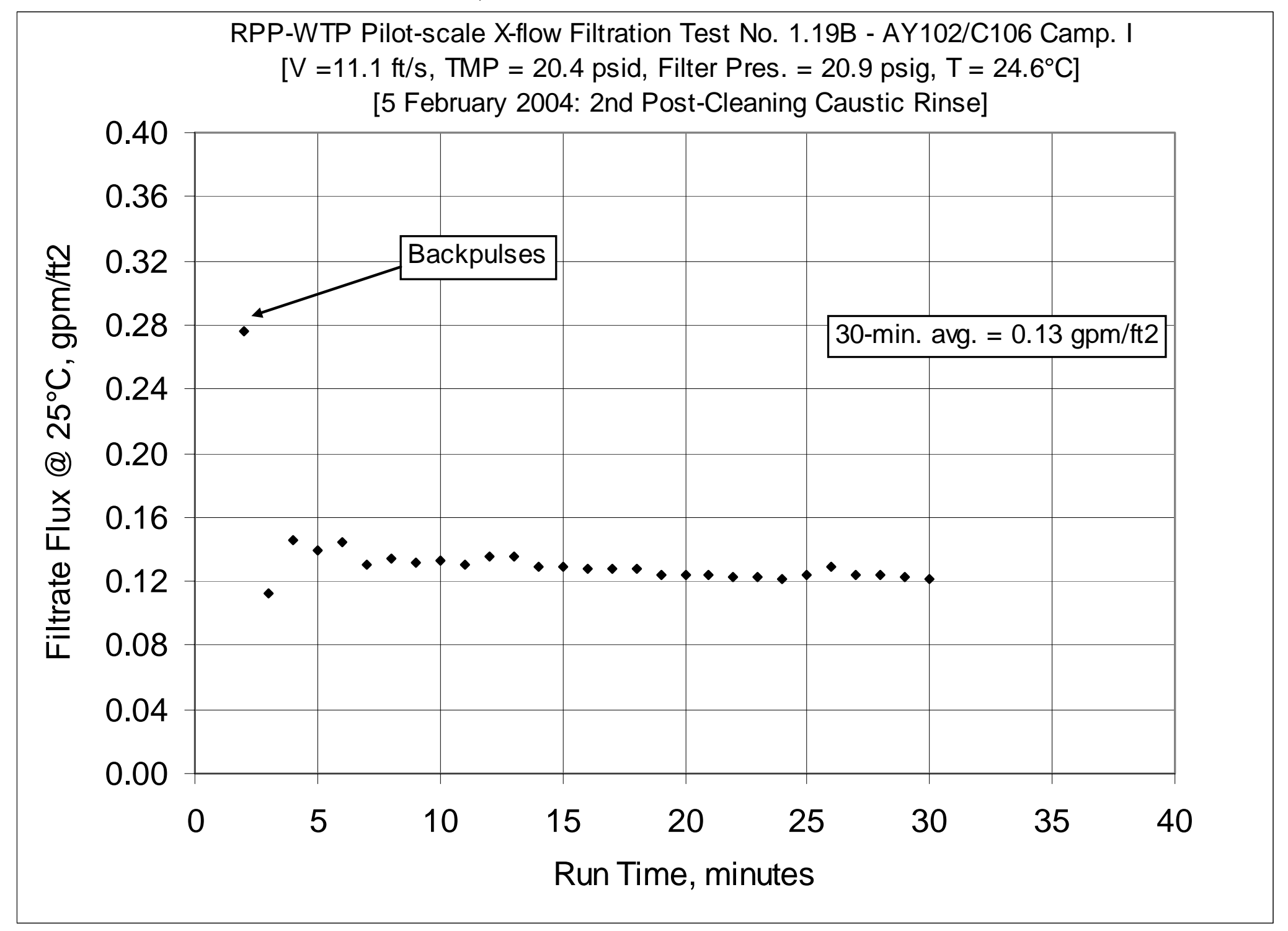


WSRC-TR-2005-00105, REVISION 0

SRNL-RPP-2005-00012, REVISION 0

RUN \# 1.19; THIRD SLURRY RINSE WITH 0.1 M NAOH - CONT.

\begin{tabular}{|c|c|c|c|c|c|c|c|c|c|c|c|c|c|c|c|c|c|c|c|c|c|c|c|c|c|c|c|c|}
\hline & A & $\mathrm{B}$ & $\mathrm{D}$ & $\begin{array}{l}\mathrm{E} \\
\mathrm{KTem}\end{array}$ & \begin{tabular}{|l|l|} 
perature \\
\end{tabular} & $\frac{1}{|c|}$ & $\frac{H}{\text { ents }>}$ & $\mathrm{J}$ & $\frac{K}{K \ll<<<}$ & \begin{tabular}{|l|l|} 
Pressure \\
\end{tabular} & \begin{tabular}{|c|} 
M \\
Measur
\end{tabular} & $\mathrm{N}$ & $\mathrm{O}$ & $\frac{Q}{Q<\ll<<}$ & $\begin{array}{l}\mathrm{R} \\
<\text { Flow! }\end{array}$ & $\frac{S}{\text { asure }}$ & $\mathrm{T}$ & $\mathrm{V}$ & $\frac{w}{m \ll \ll<<}$ & $\frac{x}{<\ll<<<~}$ & $\frac{\mathrm{Y} \mid}{\text { alculat }}$ & \begin{tabular}{|l|}
$z$ \\
ted Fron \\
\end{tabular} & \begin{tabular}{|l|} 
AA \\
m Raw \\
\end{tabular} & \begin{tabular}{|l|}
$A B$ \\
Data : \\
\end{tabular} & $A C$ & $A D$ & $\frac{A E}{D \supset}$ & AF \\
\hline 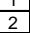 & DATE & TIME & Filtrate & Cleaning & Slurry & Hi Amb. & Lo Amb. & BotTMP & Filter & $\begin{array}{l}\text { Pressure } \\
\text { Filter dP }\end{array}$ & TopTMP & \begin{tabular}{|l|l|l|l|l|l|} 
Fitrate \\
\end{tabular} & Pulsepot & & Filtrate & Hi Filtate & $\begin{array}{l}\text { ents } \gg \gg \gg>\text { | } \\
\text { Backpulse }\end{array}$ & & & & & & & & & & & \\
\hline & & & $\frac{\operatorname{deg} C}{\mathrm{~d}}$ & $\operatorname{deg} C$ & $\operatorname{deg} C$ & \begin{tabular}{|l|}
$\operatorname{deg} \mathrm{C}$ \\
\end{tabular} & $\operatorname{deg} \mathrm{C}$ & psid & \begin{tabular}{|l} 
psig \\
Rg
\end{tabular} & psid & $\begin{array}{l}\text { psid } \\
\end{array}$ & \begin{tabular}{|l|} 
psig \\
\end{tabular} & psig & $\mathrm{gpm}$ & gpm & gpm & gpm & & & & & & & & & & & \\
\hline & & & & & & & & & & & & & & Q1 & Q2 & & & & & & & & & & & & & \\
\hline & & & & & & & & & & & & & & & & & & & & & & & & & & & & \\
\hline 7 & $2 / 5 / 2004$ & $9: 26: 34 \mathrm{AM}$ & 19.191 & 1859 & \begin{tabular}{|l|l|}
16.892 \\
\end{tabular} & 19019 & 18202 & 0056 & -0.05 & -0029 & & 0.0177 & 012 & -0093 & 8003 & 14,643 & 0004 & & & Filter & urface & & $\mathrm{T} T 2_{2}$ & & & & & \\
\hline 8 & $2 / 5 / 2004$ & 9:26:52 AM & \begin{tabular}{|l|l|}
19.191 \\
\end{tabular} & 18.592 & 16.892 & \begin{tabular}{|l|l|}
2 & 19.019 \\
\end{tabular} & 18.202 & 0.056 & -0.05 & -0.029 & & -0.177 & 0.121 & $\begin{array}{l}-0.093 \\
\end{array}$ & 0.003 & 14.643 & 0.004 & & & Convers & & & $\mathrm{m} 3 / \mathrm{m} 2 / \mathrm{c}$ & Idayybart & $\mathrm{rg} / \mathrm{gpm} / \mathrm{f}$ & ft/2/barg & & \\
\hline & $2 / 5 / 2004$ & $9: 26: 53 \mathrm{AM}$ & & & 16.872 & \begin{tabular}{|l|l|} 
& 19.03 \\
\end{tabular} & $\begin{array}{l}18.157 \\
\end{array}$ & & & -0.029 & & -0.18 & 0.124 & -0.091 & 0.003 & $\begin{array}{l}14.643 \\
\end{array}$ & 0.004 & & & & & & & & & & & \\
\hline $10 \mathrm{P}$ & $2 / 5 / 2004$ & 9:26:54 AM & 19.181 & 18.571 & \begin{tabular}{|l|}
16.861 \\
\end{tabular} & \begin{tabular}{|l|l|}
1 & 19.024 \\
\end{tabular} & 18.146 & 0.058 & -0.05 & -0.029 & -0.002 & $\begin{array}{l}-0.18 \\
\end{array}$ & 0.121 & -0.091 & 0.003 & $\begin{array}{l}14.643 \\
\end{array}$ & 0.004 & & & & auge & & & & & .4 psig & & \\
\hline 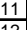 & $2 / 5 / 2004$ & 9:26:55 AM & 19.181 & $\begin{array}{l}18.577 \\
8.97\end{array}$ & \begin{tabular}{|l|l|}
16.867 \\
\end{tabular} & $\begin{array}{l}7 \\
7\end{array}$ & 18.152 & 0.058 & -0.05 & $\begin{array}{r}-0.029 \\
-020\end{array}$ & 0.002 & \begin{tabular}{|l|l|}
-0.177 \\
\end{tabular} & 0.121 & -0.093 & 0.003 & 14.643 & 0.004 & & & & & & & & & & & \\
\hline & $2 / 5 / 2004$ & $\frac{9: 26: 56 \mathrm{AM}}{9 \cdot 26.57 \mathrm{AM}}$ & \begin{tabular}{|l|l|}
19.181 \\
19186
\end{tabular} & $\frac{18.577}{18.572}$ & \begin{tabular}{|l|l|}
16.872 \\
16867
\end{tabular} & $\begin{array}{r}2 \\
7 \\
7\end{array}$ & $\begin{array}{l}18.157 \\
18.152\end{array}$ & $\begin{array}{l}0.056 \\
0.056\end{array}$ & $\begin{array}{l}-0.048 \\
-0.05\end{array}$ & $\begin{array}{l}-0.029 \\
-0.029\end{array}$ & & $\begin{array}{l}-0.177 \\
-0.177\end{array}$ & $\begin{array}{l}0.121 \\
0.118\end{array}$ & $\begin{array}{r}-0.091 \\
-0.093\end{array}$ & $\begin{array}{l}0.003 \\
0.003 \\
\end{array}$ & $\begin{array}{l}14.643 \\
14.643 \\
\end{array}$ & 0.004 & & & $\begin{array}{l}\text { Pressur } \\
\text { Vwayl }\end{array}$ & & & ted for & Filtrat & ate Flux & ter tubi & & \\
\hline$\frac{15}{14}$ & $2 / 5 / 2004$ & $\begin{array}{l}9.26 .5: 58 \mathrm{AM} \\
\end{array}$ & $\frac{19.100}{19.192}$ & $\frac{10.517}{18.587}$ & \begin{tabular}{|l|}
10.001 \\
16.877 \\
\end{tabular} & \begin{tabular}{|l|}
7 \\
7
\end{tabular} & $\begin{array}{l}10.152 \\
18.162\end{array}$ & $\begin{array}{l}0.050 \\
0.058\end{array}$ & $\begin{array}{l}-0.05 \\
-0.05\end{array}$ & $\begin{array}{l}-.029 \\
-0.028\end{array}$ & & $\begin{array}{l}-.171 \\
-0.177\end{array}$ & $\begin{array}{l}0.110 \\
0.121\end{array}$ & $\begin{array}{l}-0.095 \\
-0.091\end{array}$ & $\begin{array}{l}0.0003 \\
0.003\end{array}$ & $\frac{14.045}{14.643}$ & 0.004 & & & & & & & Filua & at $25 \mathrm{C}$ & & $\times 1000$ & \\
\hline 15 & & & & & & & & & & & & & & & & & & Time & Time & Press. & Vel. & TMP & TMP & $\mathrm{gpm}$ & gpm & gpm & $\mathrm{gpm}$ & meter \\
\hline$\frac{16}{17}$ & Data - Per Mi & inute & & & 23237 & 20199 & 23549 & & 22601 & 3407 & & 14038 & 63234 & 44094 & 8003 & 14643 & & Min. & Hour & psig & $\frac{\mathrm{ft} / \mathrm{s}}{11}$ & psi & bar & Itit2 & & $\mathrm{ft} 2 / \mathrm{psi}$ & ft2/psi & day/bar \\
\hline$\frac{11}{18}$ & $2 / 5 / 2004$ & $\begin{array}{l}\text { 8.19.20 PM } \\
8: 20: 26 \mathrm{PM}\end{array}$ & $\frac{2.027}{22.095}$ & $20.28 \varepsilon$ & \begin{tabular}{|l|l|}
23.351 \\
2357
\end{tabular} & & $\begin{array}{r}23.549 \\
23.488\end{array}$ & & $\frac{2.2 .01}{22.63}$ & $\begin{array}{l}3.4019 \\
3.419\end{array}$ & $\begin{array}{l}-0.909 \\
-0.893\end{array}$ & $\frac{14.958}{14.903}$ & $\frac{03.214}{27.026}$ & $\begin{array}{l}44.994 \\
44.834\end{array}$ & $\begin{array}{l}0.003 \\
0.003\end{array}$ & $\begin{array}{l}14.045 \\
14.643\end{array}$ & 0.006 & 1.00 & 0.01667 & $\frac{21.1}{21.2}$ & $\frac{11.0}{11.0}$ & 1.0 & 0.072 & 2.183 & 2.295 & $\frac{2.350}{2.200}$ & $\begin{array}{l}\frac{2544.900}{2200.173} \\
\end{array}$ & $\frac{004.11}{8072.38}$ \\
\hline 19 & $2 / 5 / 2004$ & $8: 21: 26 \mathrm{PM}$ & 23.576 & 20.298 & $\begin{array}{l}23.534 \\
\end{array}$ & 20.346 & 23.684 & 18.991 & 21.948 & 3.281 & 15.853 & -1.216 & & 46.037 & 0.003 & 1.779 & 0.004 & 2.00 & 0.03333 & 20.5 & 11.3 & 17.4 & 1.201 & 0.265 & 0.276 & 0.016 & 15.869 & 13.50 \\
\hline 20 & $2 / 5 / 2004$ & 8:22:26 PM & 23.896 & 20.288 & 23.643 & 20.35 & 23.363 & 21.652 & 22.364 & 3.333 & & -1.216 & & 45.326 & 0.003 & & 0.003 & 3.00 & 0.05 & 20.9 & 11. & 19.9 & 1.374 & 0.108 & 0.112 & & & \\
\hline & $2 / 5 / 2004$ & 8:23:26 PM & 23.976 & 20.287 & \begin{tabular}{|l|l|}
23.803 \\
20.802
\end{tabular} & 20.395 & $\begin{array}{r}23.427 \\
\end{array}$ & $\begin{array}{r}21.741 \\
\end{array}$ & 22.227 & $\begin{array}{l}3.357 \\
.251\end{array}$ & $\begin{array}{r}18.24 \\
\end{array}$ & -1.216 & $\begin{array}{l}0.829 \\
\end{array}$ & $\begin{array}{l}45.395 \\
5.277\end{array}$ & 0.003 & 0.945 & 0.004 & 4.00 & 0.06667 & 20.8 & 11. & 20.0 & 1.378 & 0.141 & 0.146 & 0.007 & 7.290 & \\
\hline$\frac{24}{23}$ & $\begin{array}{l}2 / 5 / 2004 \\
2 / 5 / 2004\end{array}$ & $\begin{array}{l}8: 24: 26 \mathrm{PM} \\
8.25 \cdot 26 \mathrm{PM}\end{array}$ & $\begin{array}{r}24.095 \\
24.19\end{array}$ & $\frac{20.296}{20281}$ & \begin{tabular}{|l|l|}
23.882 \\
23.972
\end{tabular} & \begin{tabular}{|l|} 
\\
2
\end{tabular} & $\begin{array}{r}23.115 \\
23.276\end{array}$ & 21.843 & 22.25 & 3.354 & $\frac{18.316}{18468}$ & $\frac{-1.216}{-1222}$ & & 45.437 & & 0.96 & & & 0.08333 & 20.8 & 11. & 2.1 & $\frac{1.384}{1.302}$ & 0.135 & 0.140 & & & \\
\hline 24 & $2 / 5 / 2004$ & 8.25 .26 & 2 & 20.25 & 24077 & $\frac{20.454}{20.404}$ & $\frac{2.51}{23 .}$ & 21.963 & 220270 & & $\begin{array}{l}10.4040 \\
11825\end{array}$ & -126 & & (45.045 & 0 & & 0.6 & 7.6 & $\frac{1}{0.167}$ & 20. & 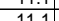 & 20.4 & t..530 & & 0.121 & & $\frac{7.116}{6.464}$ & $\frac{6.06}{5.0}$ \\
\hline 25 & $2 / 5 / 2004$ & 8:27:26 PM & 24.364 & 20.274 & \begin{tabular}{|l|l|} 
& 44.147 \\
\end{tabular} & 20.388 & 23.17 & 21.76 & 22.031 & 3.287 & 18.306 & -1.216 & & 45.107 & 0.003 & 0.875 & & 8.00 & 0.13333 & 保 & 11 & 20.6 & 1.381 & 136 & 0.134 & & & 5.50 \\
\hline & $2 / 5 / 2004$ & 8:28:26 PM & 24.423 & 20.263 & 24.215 & 20.442 & 23.048 & 22.027 & 222.221 & 3.293 & 18. & -1.219 & & 45.335 & & & 0.0 & 9.0 & & 20 & & 20. & 1.401 & & & & & \\
\hline & 2/5/2004 & & 24.503 & & 24.29 & 20.477 & & 21.857 & 22.095 & 3.265 & 18.445 & -1.219 & & 45.284 & & & & 10.0 & 0.16667 & 20, & 11. & 20.2 & 1.389 & 13 & 0.13 & & & $\frac{5.51}{5.60}$ \\
\hline 28 & $2 / 5 / 2004$ & 8:30:26 & 24.582 & 20.272 & 24.365 & 20.452 & 23.14 & 22.013 & 22.368 & 3.37 & & -1.21 & & 45. & & & & 11. & 0.18333 & & & 20. & $1.38 \mathrm{~s}$ & & 0.13 & & & \\
\hline & $2 / 5 / 2004$ & $8: 31: 26$ & \begin{tabular}{l|l|}
24.642 \\
\end{tabular} & 20.277 & 24.435 & 20.461 & & 22.003 & 222.254 & 3.22 & 18.624 & -1.219 & & 45.168 & 0.0 & & & 12. & 0.2 & 20. & 11.1 & 20.3 & 1.401 & 0.13 & & & & \\
\hline & $2 / 5 / 2004$ & $\begin{array}{l}8: 32: 26 \\
\end{array}$ & 24.702 & 20.282 & \begin{tabular}{|l|}
24.48 \\
\end{tabular} & 20.517 & & 22.1 & 22.377 & & & -1.2 & & 45.28 & & & & & 0.21667 & & & & 1.40 & & & & & \\
\hline$\frac{3}{2}$ & 2/5/2004 & $8: 3$ & 24.747 & 20.271 & 24.509 & \begin{tabular}{l|l|} 
& 20.516 \\
\end{tabular} & & 22.218 & 22.449 & & & -1.216 & & & & & & 14. & 0.23 & 21. & 11. & 20. & 1.408 & & & & & \\
\hline & $2 / 5 / 2004$ & $8: 34: 26$ & 24.816 & 20.26 & 24.518 & \begin{tabular}{l|l|}
3 & 20.515 \\
\end{tabular} & 23.05 & 22.154 & \begin{tabular}{|l|l|}
22.428 \\
\end{tabular} & 3.318 & 18.593 & -1.219 & & 45.138 & 0. & & 0.0 & 15 & 0.25 & 21. & 11. & 20.4 & 1.405 & $.128 \mathrm{~s}$ & 0. & 06 & & \\
\hline 34 & $2 / 5 / 2004$ & $8: 35: 26$ & 24.851 & 20.266 & & 20.536 & & 22.262 & 22.455 & 3.36 & 18.725 & -1.216 & & 45.214 & & & & & 0.26667 & 21 & +4 & 20. & 1.41 & & $0.12 \varepsilon$ & & & \\
\hline & $2 / 5 / 2004$ & 8:36:26 PM & 24.886 & 20.27 & 24.639 & 20.56 & 22.85 & 22.254 & 22.482 & 3.286 & $18.83 \mathrm{~S}$ & -1.219 & & 45.239 & 0.0 & & & 17.0 & 0.2833 & & & 20. & $1.4 \pm$ & & $0.12 \varepsilon$ & & & 5.2 \\
\hline$\overline{36}$ & $\begin{array}{l}2 / 5 / 2004 \\
2 / 5 / 2004\end{array}$ & 8:37:26 & & 20.27 & & 20.58 & & 22.034 & & 0.2 & & -1.21 & & & & & & 18 & 0.3 & 2.0 & 11. & 200 & 1.7 & & & & & \\
\hline & $2 / 5 / 2004$ & $8.83 .26 \mathrm{~F}$ & $\frac{24.965}{24.995}$ & 20.27 & \begin{tabular}{|l|}
24.068 \\
24.708 \\
\end{tabular} & $\begin{array}{r}20.55 \\
20.555\end{array}$ & 22.8964 & 21.832 & $\mid \frac{22.097}{2043}$ & $3.1 / 1$ & $\begin{array}{l}18.513 \\
18.686\end{array}$ & $=\frac{1.219}{1014}$ & & $\begin{array}{r}45.46 \\
45.082\end{array}$ & $\begin{array}{l}0.003 \\
0.003\end{array}$ & & & & $\frac{0.31606}{0.33333}$ & $\begin{array}{l}20.6 \\
2110\end{array}$ & $\frac{11 .}{11 .}$ & $\frac{2.4}{20.4}$ & $\mid$\begin{tabular}{|l}
1.1931 \\
1.409
\end{tabular} & $\mid 0.123$ & 0.124 & 0.006 & & \\
\hline 38 & $2 / 5 / 2004$ & 8:40: & 25.015 & 20.264 & \begin{tabular}{|l|l|}
24.768 \\
\end{tabular} & 20.585 & 22.91 & 22015 & 22211 & 3.236 & & -1.21 & & 45. & & & & 2100 & 0.35 & 207 & 11. & 20.3 & 1.402 & 0.123 & 0.123 & & & 5.16 \\
\hline$\overline{39}$ & 2/5/2004 & & 25.04 & 20.26 & \begin{tabular}{|l|l|}
24.778 \\
\end{tabular} & 20.55 & & 22.2 & & & & -1.2 & & & & & & & 0.36667 & 2. & 11. & 20.4 & & & & & & \\
\hline 40 & $2 / 5 / 2004$ & 8:42:2 & 25.07 & 20.25 & 24.763 & 20.549 & & 22.129 & 22.343 & & 18. & -1.2 & & 45.0 & & & & 23.00 & 0.38333 & 20.6 & 11.6 & 20.4 & \begin{tabular}{|l|l|}
1.408 \\
\end{tabular} & & 0.1 & & & 5.11 \\
\hline 41 & $2 / 5 / 2004$ & & \begin{tabular}{|l|l|}
25.09 \\
\end{tabular} & 20.255 & & 20.57 & & 22.013 & 22.29 & 3.2 & & & & 45.0 & & & & & 0.4 & & $11.0 \mathrm{C}$ & 20.3 & 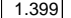 & & & & & \\
\hline 42 & $2 / 5 / 2004$ & & 25.13 & 20.265 & 24.838 & 20.6 & & 22.289 & 22.489 & & & -1.2 & & 45.0 & & & & 25.0 & 0.41667 & 21. & 11. & 20.5 & $1.4 \mathrm{Y}$ & & & & & \\
\hline 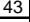 & 2/5/2004 & & 25.145 & 20.26 & & 20.62 & & 22.181 & 22.362 & & & & & & & & & & 0.43333 & & & & & & & & & \\
\hline 44 & 2/5/2004 & & 25.155 & 20.265 & & 20 & & 22.2 & & & & & & & & & & & & & & & & & & & & \\
\hline 4 & $2 / 5 / 2004$ & & $25.179 \mid$ & 20.248 & 24.8 & 20.624 & & 22.202 & 22.323 & 3.3. & & & & & & & & 28.00 & 0.46667 & 20. & 111. & 20. & & & & & & \\
\hline & 2/5/2004 & & 55.194 & & 24.887 & 20.609 & & 22.175 & 22.412 & & & & & & & & & 29.00 & 0.48333 & & 11. & 20.5 & 410 & & & & & \\
\hline & $2 / 5 / 2004$ & 8:49:26 PM & 25.208 & 20.252 & 24.926 & 20.608 & & 22.341 & 22.611 & 3.268 & 18.839 & -1.216 & & 45.02 & 0.003 & 0.815 & & 30.00 & 0.5 & 21. & & 20.6 & $\begin{array}{l}1.420 \\
\end{array}$ & 0.12 & 0.12 & 0.006 & 5.914 & \\
\hline$\frac{70}{49}$ & & & 24.8 & 203 & 24.6 & 20 & & 221 & 223 & 3.3 & & & & & & & & & & 209 & & & & & & & & \\
\hline & & & & & 2 & & & & 22.6 & 0.0 & & & & & & 0.0 & & & & 21.1 & 11.1 & 20.6 & $\frac{1.444}{1.420}$ & 0.140 & 0.144 & & & \\
\hline & & & $\begin{array}{l}24.9 \\
\end{array}$ & 20.3 & 2 & 20.6 & & 2 & 22.4 & 3.3 & & & & & & 0.8 & 0.0 & & & $\begin{array}{l}20.9 \\
\end{array}$ & 11.1 & 20.4 & \begin{tabular}{|l|l}
1.405 \\
\end{tabular} & $\begin{array}{ll}0.126 \\
\end{array}$ & 0.128 & \begin{tabular}{|l|l|}
0.0063 \\
\end{tabular} & 6.2 & \\
\hline & & & 24.2 & 20.2 & 24.0 & 20.4 & & 21.8 & & 3.2 & 18.3 & -1.2 & 0.1 & 44.7 & & 0.8 & & & & 20.6 & 11.0 & 20.0 & 1.381 & 0.121 & 0.122 & | 0.0059 & 5.914 & \\
\hline & & $2 \times \mathrm{Stc}$ & 0.607 & 0.021 & 0.541 & 0.142 & 0.346 & 0.302 & 0.268 & 0.115 & 0.271 & 0.005 & 0 & 0.314 & 0.000 & 0.059 & 0.002 & & & 0.268 & 0.077 & 0.276 & \begin{tabular}{|l|l|}
0.019 \\
\end{tabular} & 0.009 & 0.011 & 0.0006 & 0.572 & \\
\hline & Number of & Points Used ${ }^{*}$ & 25 & 25 & 25 & 25 & & & & & & 25 & & 25 & 25 & 25 & & & & 25 & 25 & 25 & 25 & 25 & 25 & 25 & 25 & \\
\hline & & * Backpulse po & nts in box & $x$ are not & ncluded & & & & & & & & & & & & & & & & & & & & & & & \\
\hline 57 & & & & & & & & & & & & & & & & & & & & & & & 30-minu & ute avg. & 0.128 & & & \\
\hline
\end{tabular}


RUN \# 1.21A, B, C; POST-TEST BASELINE WITH THE THIRD CAUSTIC RINSE SOLUTION

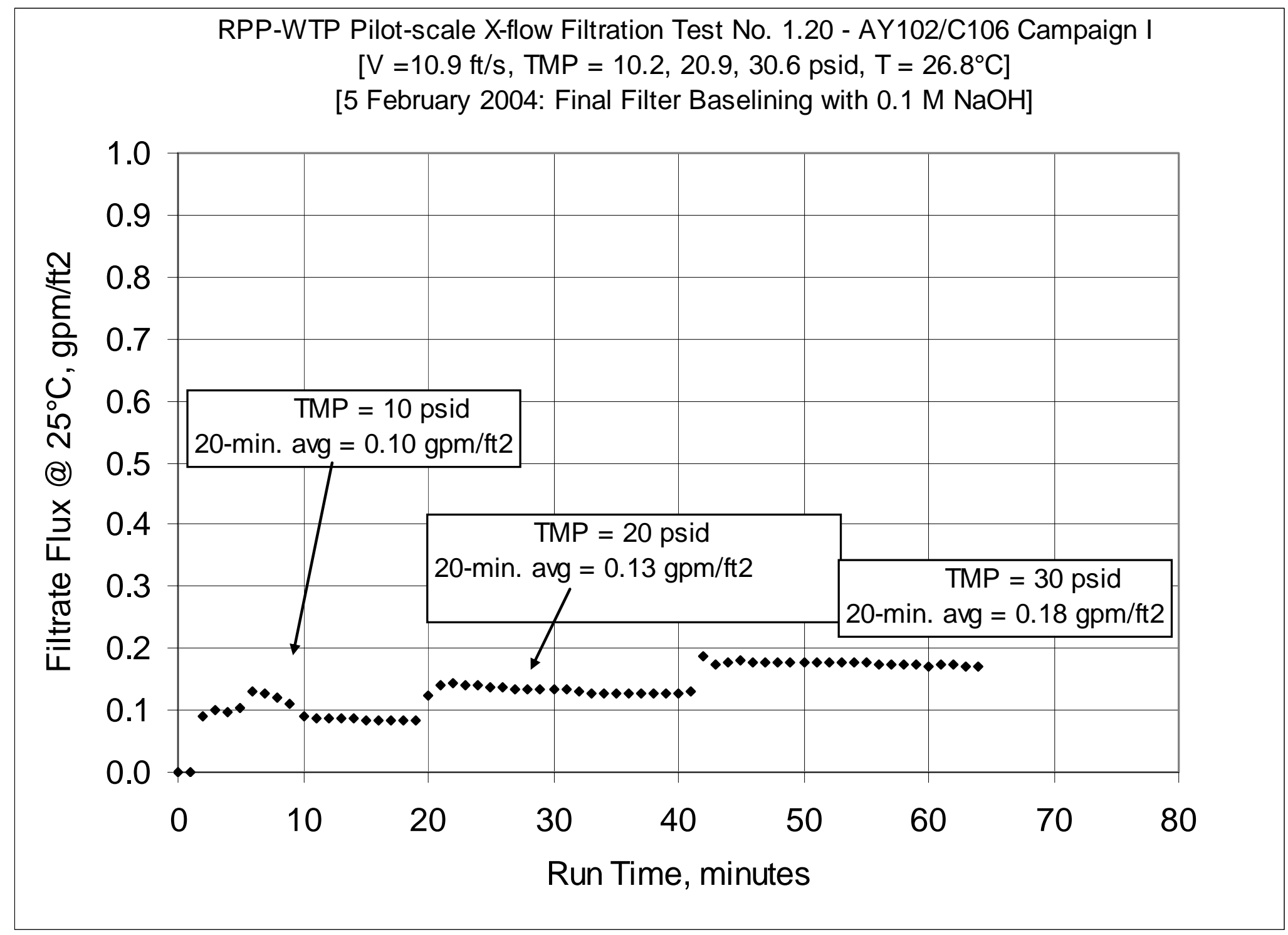


WSRC-TR-2005-00105, REVISION 0

SRNL-RPP-2005-00012, REVISION 0

RUN \# 1.21A, B, C; POST-TEST BASELINE WITH THE THIRD CAUSTIC RINSE SOLUTION - CONT.

\begin{tabular}{|c|c|c|c|c|c|c|c|c|c|c|c|c|c|c|c|c|c|c|c|c|c|c|c|c|c|c|c|c|}
\hline & A & B & D & $\mathrm{E}$ & $F$ & $\begin{array}{ll}\mathrm{G} \\
\end{array}$ & $\mathrm{H}$ & $\mathrm{J}$ & $\mathrm{K}$ & L & M & $\mid \mathrm{N}$ & 0 & Q & $\mathrm{R}$ & $\mathrm{s}$ & I & $\mathrm{v}$ & \begin{tabular}{l|l|} 
& \\
\end{tabular} & $|x|$ & \begin{tabular}{|r|} 
\\
\end{tabular} & $z$ & & $\mathrm{AB}$ & $A C$ & $A D$ & AE & AF \\
\hline & & & & & & neasurem & & & & $\begin{array}{l}\text { Pressure } \\
\end{array}$ & Measurem & & & & $<<$ Flow! & Measureme & & & & & & & & Data & & & & \\
\hline 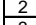 & DATE & TIME & & & Slurry & Hi Amb. & $\begin{array}{l}\text { Lo Amb. } \\
\text { al }\end{array}$ & & Filter & Filter dP & $\begin{array}{l}\text { TортMP } \\
\end{array}$ & Filtrate & Pulsepot & Slurry & \begin{tabular}{|l|} 
Filtrate \\
\end{tabular} & \begin{tabular}{|l|} 
Hi Filtate \\
\end{tabular} & Backpulse & & & & & & & & & & & \\
\hline & & & $\frac{\operatorname{deg} C}{T 2}$ & \begin{tabular}{l|}
$\operatorname{deg} C$ \\
$T 3$
\end{tabular} & $\operatorname{deg} C$ & \begin{tabular}{|l|}
$\operatorname{deg} \mathrm{C}$ \\
\end{tabular} & $\operatorname{deg} C$ & psid & $\begin{array}{l}\text { psig } \\
\text { p1 }\end{array}$ & \begin{tabular}{|l|} 
psid \\
P1P1
\end{tabular} & \begin{tabular}{|l|} 
psid \\
dP3
\end{tabular} & \begin{tabular}{|l|} 
psig \\
P2
\end{tabular} & & $\mathrm{gpm}$ & gpm & $\begin{array}{l}\mathrm{gpm} \\
\mathrm{n}\end{array}$ & & & & & & & & & & & & \\
\hline \begin{tabular}{|l|}
4 \\
5
\end{tabular} & & & & & & T4 & & $\mathrm{dP} 2$ & & & $\mathrm{dP3}$ & P2 & & & & Q3 & Qbp & & & & & & & & & & & \\
\hline & & & & & & & & & & & & & & & & & & & & & & & & & & & & \\
\hline \begin{tabular}{|l|}
7 \\
\end{tabular} & $2 / 5 / 2004$ & 9:26:34 AM & 19.191 & \begin{tabular}{|l|l|}
18.592 \\
\end{tabular} & 16.892 & $\begin{array}{ll}19.019 \\
\end{array}$ & 18.202 & 0.056 & -0.05 & -0.029 & & -0.177 & 0.121 & -0.093 & 0.003 & \begin{tabular}{|l|l|}
14.643 \\
\end{tabular} & 0.004 & & & Fitter Si & Surface & 6.707 & $17 \mathrm{FT2}$ & & & & & \\
\hline \begin{tabular}{|l}
8 \\
\end{tabular} & 215/2004 & 9:26:52 AM & 19.191 & $\begin{array}{l}18.592 \\
\end{array}$ & 16.892 & \begin{tabular}{|l|l|}
19.019 \\
\end{tabular} & 18.202 & 0.056 & -0.05 & -0.029 & 0 & -0.177 & 0.121 & -0.093 & 0.003 & $\begin{array}{ll}14.643 \\
\end{array}$ & 0.004 & & & Convers & ersion & & $1 \mathrm{~m} 3 / \mathrm{m} 2$ & //day/barg & $\mathrm{rg} / \mathrm{gpm} / \mathrm{t}$ & ftt2/barg & & \\
\hline \begin{tabular}{|l|}
9 \\
10
\end{tabular} & $2 / 5 / 2004$ & 9:26:53 АM & 19.187 & $\begin{array}{l}18.582 \\
18571\end{array}$ & 16.872 & $\begin{array}{r}19.03 \\
\end{array}$ & 18.157 & 0.056 & -0.05 & $\begin{array}{l}-0.029 \\
-020\end{array}$ & & \begin{tabular}{|c|} 
\\
\end{tabular} & 0.124 & $\begin{array}{r}-0.091 \\
\end{array}$ & 0.003 & $\begin{array}{l}14.643 \\
1.9\end{array}$ & 0.004 & & & & & & & & & & & \\
\hline \begin{tabular}{|l|l|}
11 \\
11
\end{tabular} & $\begin{array}{r}2 / 5 / 2004 \\
2 / 552004\end{array}$ & $\begin{array}{l}9: 26: 54 \mathrm{AM} \\
9 \cdot 26.55 \mathrm{~A}\end{array}$ & $\frac{19.181}{19.181}$ & $\begin{array}{l}18.571 \\
18577\end{array}$ & $\frac{16.861}{16.867}$ & $\begin{array}{l}19.024 \\
19.024\end{array}$ & $\begin{array}{l}18.146 \\
18.152\end{array}$ & \begin{tabular}{|c|c|}
0.058 \\
0.058
\end{tabular} & $\begin{array}{l}-0.05 \\
-0.05 \\
\end{array}$ & $\begin{array}{l}-0.029 \\
-0.029\end{array}$ & $\begin{array}{l}-0.002 \\
0.002\end{array}$ & \begin{tabular}{|r|}
-0.18 \\
\end{tabular} & $\begin{array}{l}0.121 \\
0.121\end{array}$ & -0.091 & $\begin{array}{l}0.003 \\
0.003\end{array}$ & $\begin{array}{r}14.643 \\
14643 \\
\end{array}$ & 0.004 & & & & & & & & & 4 psig & & \\
\hline \begin{tabular}{|l|l}
12 \\
\end{tabular} & $2 / 15 / 2004$ & $\begin{array}{l}9.20 .55 \\
9: 26: 56 \mathrm{AM}\end{array}$ & $\begin{array}{l}\frac{19.181}{19.181} \\
\end{array}$ & 18.577 & \begin{tabular}{|l|}
16.007 \\
16872
\end{tabular} & 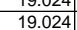 & $\begin{array}{l}18.152 \\
18.157\end{array}$ & $\begin{array}{l}0.056 \\
0.056\end{array}$ & $\begin{array}{r}-0.05 \\
-0.048\end{array}$ & $\begin{array}{l}-0.029 \\
-0.029\end{array}$ & & $\begin{array}{l}-0.1 / 7 \\
-0.177\end{array}$ & & $\frac{-0.093}{-0.091}$ & $\begin{array}{l}0.003 \\
0.003\end{array}$ & $\frac{14.045}{14643}$ & $\begin{array}{l}0.004 \\
0.004\end{array}$ & & & Pressur & & scorrec & ccted for & & & & & \\
\hline 13 & $2 / 5 / 2004$ & 9:26:57 AM & 19.186 & 18.572 & \begin{tabular}{|l|l|} 
\\
\end{tabular} & 19.029 & 18.152 & 0.056 & -0.05 & -0.029 & 0 & $\mid-0.177$ & 0.118 & -0.093 & 0.003 & 14.643 & 0.004 & & & VWVV & $v \mid$ & & & < Filtrat & te Flux & $<<$ PERM & रMIABIUT & $Y \gg$ \\
\hline 14 & $2 / 5 / 2004$ & 9:26:58 AM & 19.192 & \begin{tabular}{ll|l}
18.587 \\
\end{tabular} & 16.877 & \begin{tabular}{|l|l|}
19.03 \\
\end{tabular} & $\begin{array}{l}18.162 \\
\end{array}$ & 0.058 & -0.05 & -0.028 & & $\mid-0.177$ & 0.121 & -0.091 & 0.003 & $\begin{array}{l}14.643 \\
\end{array}$ & 0.005 & & & & & & & & at $25 \mathrm{C}$ & & $\times 1000$ & \\
\hline 15 & & & & & & & & & & & & & & & & & & Time & Time & Press. & Vel. & IMP & TMP & $\mathrm{gpm}$ & & gpm & gpm & 1eter \\
\hline & Data - Per M & nute & & & & & & & & & & & & & & & & & Hour & psig & & & & & & & & \\
\hline 17 & & 9:23:33 PM & 22.362 & 20.208 & 22.725 & 20.114 & 23.72 & 2.676 & 13.769 & 3.295 & -0.919 & 6.221 & 52.28 & 44.133 & 0.003 & \begin{tabular}{|l|}
14.643 \\
\end{tabular} & & & & 12.3 & & 0 & 0.06 & & & & 0.543 & $\frac{0.46}{0.0}$ \\
\hline & 2/5/2004 & 9:24:33 PM & & 20.188 & & & & 2.761 & 13.566 & 3.446 & -0.985 & 5.897 & & 44.623 & 0.003 & \begin{tabular}{|l|l|}
5.654 \\
\end{tabular} & & 1.0 & .01661 & 12.1 & 10.9 & & 0.061 & & & & & $\begin{array}{l}0.40 \\
0.46\end{array}$ \\
\hline & $2 / 5 / 2004$ & 9:25:33 PM & 22.961 & 20.194 & 23.008 & 20.101 & 24.1 & $\begin{array}{l}6.598 \\
\end{array}$ & 13.3 & 3.332 & 2.998 & 1.742 & & 48.086 & 0.578 & 14.643 & & & 0.03333 & 11.8 & & & & 0.086 & & & 9.004 & 16.17 \\
\hline 20 & $2 / 5 / 2004$ & 9:26:33 PM & 23.127 & 20.179 & 23.139 & \begin{tabular}{|l|l|}
20.071 \\
\end{tabular} & & 8.041 & 13.605 & 3.364 & 4.308 & $\begin{array}{l}0.639 \\
\end{array}$ & & $\begin{array}{l}44.696 \\
\end{array}$ & 0.646 & 14.643 & & 3.00 & 0.05 & 12.1 & 11.0 & 6.2 & 0.426 & 0.096 & 0.102 & 0.016 & 16.443 & 13.99 \\
\hline 21 & $2 / 5 / 2004$ & 9:27:33 PM & 23.313 & 20.185 & \begin{tabular}{|l|l} 
& 23.29 \\
\end{tabular} & \begin{tabular}{|l|}
20.076 \\
\end{tabular} & 24.148 & 8.615 & 13.707 & 3.475 & 4.671 & 0.107 & -1.24 & 45.116 & & 14.643 & & 4.00 & 0.06667 & 12.2 & 11.1 & 6.6 & $\begin{array}{ll}60 & 0.458 \\
\end{array}$ & 0.092 & 0.097 & & 14.558 & 12.39 \\
\hline & 2/5/2004 & 9:28:33 PM & 23.393 & 20.159 & 23.4 & 20.066 & 24.05 & 9.547 & 15.547 & 3.248 & 5.995 & 1.019 & -0.645 & 44.429 & $\begin{array}{l}0.668 \\
\end{array}$ & 14.643 & & 5.0 & 0.08333 & 14.1 & 10.9 & 7.8 & 0.536 & 0.100 & 0.104 & & 13.410 & 11.41 \\
\hline 23 & $2 / 5 / 2004$ & 9:29:33 PM & 23.593 & 20.169 & 23.591 & 20.04 & 22.98 & 12.268 & 21.832 & 3.38 & 8.623 & 4.574 & 1.474 & 45.307 & 0.844 & 14.643 & & 6.00 & 0. & 20.4 & 11.1 & 10.4 & $\begin{array}{ll}4 & 0.72 \mathrm{C} \\
\end{array}$ & 0.126 & 0.131 & 013 & 12.536 & 10.67 \\
\hline 24 & $2 / 3 / 2004$ & 9:30:33 PM & 23.764 & 20.159 & 23.791 | & 20.035 & 22.398 & 13.103 & 22.043 & 3.321 & & 3.98 & & 45.007 & 0.816 & 14.643 & & & 0.11667 & 20.6 & 11.0 & 11.2 & 0.775 & 0.122 & 0.126 & & 1.1.196 & \\
\hline 25 & $2 / 5 / 2004$ & 9:31:33 PM & 23.989 & 20.159 & 24.002 & 20.05 & 22.57 & 14.008 & $22.126 \mid$ & 3.28 & 10.468 & 3.21 & & 44.694 & 0.781 & 14.643 & & 8.00 & 0.1333 & 20.7 & & & 0.844 & 0.116 & 0.120 & & 9.787 & 8.33 \\
\hline & 21012004 & $9: 32: 33 \mathrm{PM}$ & 2.109 & 20.199 & $\mid \begin{array}{l}24.192 \\
252\end{array}$ & 20.053 & $\frac{22.92}{200}$ & 13.920 & 2.878 & 3.034 & $\frac{1.193}{0.91}$ & 2.1166 & & 41.005 & 0.124 & 14.643 & & 9.00 & .15 & 19.4 & 11.1 & (11.9. & & 0.108 & 0.110 & . & 9.312 & 7.92 \\
\hline & 21515004 & $\frac{3.353 .30}{0.320}$ & 24.305 & $\frac{2.140^{2}}{20129}$ & \begin{tabular}{|l|l|l|l|l|} 
\\
\end{tabular} & 10.084 & & 12749 & 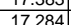 & $\frac{1.003}{3.303}$ & $\begin{array}{l}8.864 \\
9.058\end{array}$ & $\begin{array}{l}-0.351 \\
=0.778\end{array}$ & & $\begin{array}{l}45.249 \\
45.152\end{array}$ & 0.054 & $\begin{array}{l}14.045 \\
114643\end{array}$ & & 1100 & 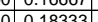 & 1.5. & & & & 0 & 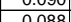 & & 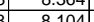 & 7.12 \\
\hline 2 & $21 / 52004$ & $9: 35.33 \mathrm{PM}$ & 24.574 & 20.148 & $\begin{array}{l}24.472 \\
24622\end{array}$ & 20.014 & 2383 & 13.143 & \begin{tabular}{|l|l|}
17.5 \\
17
\end{tabular} & 3.432 & $\begin{array}{l}0.0373 \\
9373\end{array}$ & -0.559 & -1.69 & 44807 & 0.578 & 14643 & & 1200 & 0.100 & 160 & 110 & $\frac{11.3}{11.3}$ & & 然 & 0.007 & 0 & & 0.90 \\
\hline & $2 / 15 / 2004$ & $9: 36: 33$ PM & 24.72 & 20.143 & 24.758 & 20.025 & & 12.946 & \begin{tabular}{|l|}
17.269 \\
\end{tabular} & 3.289 & 9.282 & $\frac{-0.646}{-0.646}$ & & $\begin{array}{r}44.001 \\
44.874\end{array}$ & $\begin{array}{l}0.570 \\
0.573\end{array}$ & $\begin{array}{l}\frac{14.045}{14.643} \\
\frac{1}{10}\end{array}$ & & $\frac{12.00}{13.00}$ & $\begin{array}{r}0.2 \\
0.21667\end{array}$ & $\frac{10.6}{15.8}$ & $\begin{array}{ll}11.0 \\
11.0\end{array}$ & $\begin{array}{ll}\frac{11.5}{11.1} \\
1.1\end{array}$ & 0.766 & $\begin{array}{l}0.000 \\
0.085 \\
\end{array}$ & 0.086 & 0.000 & $\begin{array}{l}7.151 \\
7.739\end{array}$ & $\frac{0.30}{659}$ \\
\hline 31 & $2 / 5 / 2004$ & 9:37:33 PM & 24.83 & 20.123 & 24.883 & 20.004 & 24.16 & 13.043 & $\mid$\begin{tabular}{|l|l|}
17.344 \\
\end{tabular} & 3.259 & 9.405 & $\mid-0.704$ & & 44.705 & 0.572 & 14.643 & & $\frac{1.00}{14.00}$ & \begin{tabular}{|l|l|l}
0.23333 \\
\end{tabular} & $\begin{array}{l}1.0 .6 \\
15.9\end{array}$ & $\frac{11.0}{11.0}$ & $\frac{1.4}{11.2}$ & 0.774 & 0.085 & 0.086 & 0.008 & 7.623 & 6.49 \\
\hline & 21512004 & $9.38 \cdot 33 \mathrm{PM}$ & 24.954 & & & & & & & & & -0765 & & 44803 & & 14643 & & & & 158 & 110 & 112 & & & & & & $\frac{0.49}{6.42}$ \\
\hline 33 & $2 / 5 / 2004$ & $9: 39: 33 \mathrm{PM}$ & 25.115 & 20.133 & \begin{tabular}{|l|}
25.163 \\
\end{tabular} & \begin{tabular}{|l|}
20.014 \\
\end{tabular} & & 13.245 & 17.446 & 3.288 & $\begin{array}{l}9.543 \\
\end{array}$ & \begin{tabular}{|c|}
-0.8 \\
\end{tabular} & & 44.842 & 0.565 & 14.643 & & 16.00 & 0.26667 & 16.0 & 11.0 & $\overline{11.4}$ & \begin{tabular}{l|l|}
4 & 0.786
\end{tabular} & 0.084 & 0.084 & 0.007 & 7.359 & 6.26 \\
\hline 34 & & & & 20.122 & & & & $\begin{array}{ll}13.052 \\
\end{array}$ & 17.185 & & & -0.814 & & $\begin{array}{l}44.876 \\
\end{array}$ & $\begin{array}{l}0.562 \\
\end{array}$ & 14.643 & & 17.0 & 0.28333 & 15.7 & & 11.3 & & & & & 7345 & 0.25 \\
\hline & $2 / 5 / 2004$ & 9:41:33 PM & 25.324 & 20.117 & 25.407 & \begin{tabular}{|l|}
19.968 \\
\end{tabular} & 24.1 & 13.421 & & 3.348 & 9.686 & -0.878 & & 44.871 & 0.561 & 14.643 & & 18.00 & 0.3 & 16.1 & $\overline{11.0}$ & 11.6 & \begin{tabular}{l|l}
6 & 0.797
\end{tabular} & 0.084 & 0.083 & 0.007 & & $\begin{array}{ll}6.09 \\
\end{array}$ \\
\hline \begin{tabular}{|l|l}
36 \\
\end{tabular} & $2 / 5 / 2004$ & 9:42:33 PM & 25.494 & 20.112 & 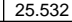 & \begin{tabular}{|c|}
19.978 \\
\end{tabular} & & 13.34 & \begin{tabular}{|l|l|}
17.535 \\
\end{tabular} & 3.227 & 9.709 & \begin{tabular}{|c|c|c|}
-0.814 \\
\end{tabular} & & & 0.561 & 14.643 & & 19.00 & 0.31667 & 16.1 & 10.9 & 11.5 & & 0.084 & & & 7.150 & 6.08 \\
\hline 37 & 2/5/2004 & 9:43:33 PM & 25.598 & 20.116 & \begin{tabular}{|l|}
25.682 \\
\end{tabular} & 19.987 & 23.8 & 17.861 & \begin{tabular}{|l|l|}
27.208 \\
\end{tabular} & 2.908 & 14.731 & 4.423 & & 43.134 & 0.838 & 14.643 & & 20.00 & 0.33333 & 25.7 & 10.6 & 16.3 & 1.124 & 0.125 & 0.123 & 0.008 & & 6.40 \\
\hline 38 & 2/5/2004 & 9:44:33 PM & 25.788 & 20.106 & 25.937 & 19.987 & 22.58 & 20.995 & \begin{tabular}{|l|}
33.517 \\
\end{tabular} & 3.364 & 17.292 & 7.55 & & 44.823 & 0.965 & 14.643 & & 21.00 & 0.35 & 32.0 & 11.0 & 19.1 & 1.320 & 0.144 & 0.140 & 0.007 & 7.320 & 6.23 \\
\hline & $2 / 5 / 2004$ & 9:45:33 PM & 26.108 & 20.127 & 26.262 & 20.059 & & 22.432 & 35.49 & 3.288 & 18.938 & 8.245 & & 44.761 & 0.992 & 14.643 & & 22.0 & 0.36667 & 34.0 & 11.0 & 20.7 & 1.426 & 0.148 & 0.143 & 0.007 & & 5.87 \\
\hline 40 & $2 / 5 / 2004$ & 9:46:33 PM & 26.392 & 20.11 & 26.551 & 19.987 & & 22.442 & 35.432 & 3.11 & 19.143 & 8.039 & & 44.352 & 0.986 & 14.643 & & 23.00 & \begin{tabular}{l|l|l}
0 & 0.38333 \\
\end{tabular} & 34.0 & 10.9 & 20.8 & 1.434 & 0.147 & 0.141 & & 6.7 & \\
\hline 41 & $2 / 3 / 2004$ & 9:47:33 PM & $26.6 / 6$ & 20.111 & 26.831 & 19.951 & 22.0 & 22.521 & 35.258 & 3.06 & 19.35 & 7.941 & & 43.95 & 0.981 & 14.643 & & 24.00 & 0.4 & 33.8 & 10.8 & 20.9 & $\begin{array}{l}91.443 \\
\end{array}$ & 0.146 & 0.139 & 0.007 & 6.631 & 5.65 \\
\hline 42 & 213502004 & $\begin{array}{l}9.48 .33 \mathrm{P} \\
-\end{array}$ & 27.01 & & 27.105 & 19.941 & & 22.826 & 35.17 & 3.113 & 19.297 & 1.889 & & 44.1 & 0.978 & 14.643 & & 25.00 & 0.41607 & 34.3 & 10.8 & 21.1 & 1.452 & 0.140 & 0.137 & & & \\
\hline 43 & $2 / 1 / 20004$ & $9: 49,33 \mathrm{PM}$ & $\begin{array}{l}27.204 \\
27527\end{array}$ & 20.104 & $\mid 27.354$ & $\begin{array}{l}11.96 \\
19.965\end{array}$ & & 22.859 & \begin{tabular}{|c|}
35.559 \\
3572
\end{tabular} & $\frac{3.066}{3.060}$ & $\frac{19.588}{1958}$ & $\frac{1.816}{7819}$ & & 43. & 0.967 & $\frac{14.643}{1.642}$ & & 26.00 & 0.43333 & $\frac{34.1}{3.2}$ & $\frac{1.8}{10.8}$ & $\frac{21.2}{20.2}$ & .1.463 & 0.146 & 0 & & 0.420 & 5.46 \\
\hline 4 & $21 / 52004$ & $9.51 .33 \mathrm{PM}$ & 27742 & 20.135 & $\left\{\begin{array}{l}27.042 \\
27.922\end{array}\right.$ & 19.95 & & 年 & $\mid$\begin{tabular}{|l|l|}
35779 \\
35
\end{tabular} & 3.019 & 19677 & 7.0409 & & $\frac{44.041}{1201}$ & 0.078 & $\begin{array}{l}14.045 \\
14643\end{array}$ & & 2800 & 0.45 & 势, & 10.0 & $\frac{2.4}{213}$ & $\frac{1.405}{1.470}$ & & & & 0 & $\begin{array}{l}5.45 \\
536\end{array}$ \\
\hline $\begin{array}{ll}46 \\
\end{array}$ & $215 / 2004$ & $9 \cdot 52 \cdot 33 \mathrm{PM}$ & 28036 & 2013 & 28.156 & 1996 & & 23.89 & \begin{tabular}{|l|l|}
36125 \\
\end{tabular} & 30.055 & 19.816 & 7883 & & & 0.978 & 14643 & & 2000 & 0.48832 & 347 & 10.6 & 215 & 1.478 & 0 & 年 & 0.006 & 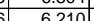 & $\begin{array}{l}3.300 \\
5.520\end{array}$ \\
\hline \begin{tabular}{|l|}
47 \\
\end{tabular} & $2 / 15 / 2004$ & 9:53:33 PM & 28.334 & 20.129 & 28.415 & 20.026 & & $\frac{23.044}{23}$ & \begin{tabular}{|c|}
35.803 \\
\end{tabular} & 3.008 & 19.835 & \begin{tabular}{|c|c|}
5 & 7.8899 \\
\end{tabular} & & & 0.98 & 14.643 & & 3000 & & 34.3 & 107 & 214 & $\begin{array}{lll}4 & 1.478 \\
\end{array}$ & 0,146 & 0.13 & & 6197 & 5 \\
\hline \begin{tabular}{|l|l|}
48 &
\end{tabular} & $2 / 5 / 2004$ & 9:54:33 PM & 28.533 & 20.134 & \begin{tabular}{|l|}
28.669 \\
\end{tabular} & \begin{tabular}{|r|r|}
19.999 \\
\end{tabular} & & 22.909 & \begin{tabular}{|l|l|}
35.783 \\
\end{tabular} & 2.96 & $\begin{array}{l}19.708 \\
\end{array}$ & 7.944 & & 45 & 0.982 & 14.643 & & 3100 & 0.51667 & 343 & 107 & nit & \begin{tabular}{l|l}
3 & 1.469 \\
\end{tabular} & 0.146 & 0.132 & 0.006 & 6204 & 5.28 \\
\hline \begin{tabular}{|l|l|}
49 \\
\end{tabular} & $2 / 5 / 2004$ & & 2007 & 20.139 & \begin{tabular}{|l|}
28.928 \\
\end{tabular} & $\begin{array}{l}19.99 \\
\end{array}$ & & 23.031 & & 2.989 & 19.81 & 8.004 & & & 0.984 & 14.643 & & & & 34.5 & 107 & 21.4 & & 147 & & & & \\
\hline 50 & $2 / 5 / 2004$ & $9: 56: 33 \mathrm{~F}$ & 29.026 & 20.139 & & \begin{tabular}{|l|}
20.021 \\
\end{tabular} & & 22.341 & 34.504 & 3.212 & \begin{tabular}{l|l}
18.88 \\
\end{tabular} & 7.252 & & & 0.956 & & & 33.00 & \begin{tabular}{l|l}
0 & 0.55
\end{tabular} & 33.0 & $\overline{11.1}$ & 200 & \begin{tabular}{l|l}
6 & 1.421
\end{tabular} & & 0.127 & & & 52 \\
\hline \begin{tabular}{|l|l|}
51 & \\
\end{tabular} & & & 29.268 & 20.128 & \begin{tabular}{|l|}
29.384 \\
\end{tabular} & & & 22.419 & \begin{tabular}{|c|}
34.709 \\
\end{tabular} & & & 7318 & & & & 14.643 & & & 0.56667 & $\overline{33.2}$ & 111 & & 1.425 & 0.143 & 0.126 & & & \\
\hline 52 & $2 / 5 / 2004$ & 9:58:33 PM & 29.48 & 20.126 & \begin{tabular}{|l|}
29.303 \\
\end{tabular} & \begin{tabular}{|l|l|}
20.179 \\
\end{tabular} & 22. & 22.227 & \begin{tabular}{|l|}
34.448 \\
\end{tabular} & 3.144 & 18.823 & 7.272 & & 44.761 & \begin{tabular}{l|l}
0.958 \\
\end{tabular} & 14.643 & & 35.00 & \begin{tabular}{|l|l|l|}
0.58333 \\
\end{tabular} & 33.0 & $\overline{11.0}$ & 20.5 & 1.415 & & 0127 & & \begin{tabular}{|c|}
6.176 \\
\end{tabular} & 5.26 \\
\hline & $2 / 5 / 2004$ & 9:59:33 F & 29.503 & 20.129 & \begin{tabular}{|l|l|} 
& 9222 \\
\end{tabular} & 20.314 & 22. & 22.5 & 34.6 & 3.189 & 19.149 & 7.249 & & & 0.9 & 14.643 & & 36. & 0.6 & 33.1 & 11.1 & $0.8 \quad-8$ & 1.437 & 0.143 & & & 6.113 & \\
\hline 54 & $2 / 5 / 20$ & 10:00: & 29.416 & 20.132 & 28 & 20.432 & & 22.48 & 34. & 3.197 & 19 & 7.202 & & & 0.956 & 14.643 & & 37. & \begin{tabular}{c|c}
0.61667 \\
\end{tabular} & 33.3 & 11.0 & 20.7 & \begin{tabular}{l|l}
7 & 1.430 \\
\end{tabular} & $\mid 0.143$ & 0.128 & 06 & 6.157 & 5.24 \\
\hline 5 & 2/5/2004 & 10:01:33 F & 29.31 & 20.135 & \begin{tabular}{|l|l|} 
& 28.794 \\
\end{tabular} & 20.527 & & 22.575 & \begin{tabular}{|l|}
34.564 \\
\end{tabular} & 3.166 & 19.274 & 7.136 & & & 0.9 & 14.643 & & 38. & \begin{tabular}{l|l}
0 & 0.63333
\end{tabular} & 33.1 & 11.0 & & \begin{tabular}{l|l}
9 & 1.443 \\
\end{tabular} & 0.142 & 0.128 & & 6.111 & 5.20 \\
\hline 56 & 2/5/2 & & 29.189 & 20.139 & 28.643 & 20.585 & & 22. & & 3.218 & 19.182 & 7.133 & & & 0.95 & 14.643 & & 39.00 & 0.65 & 33.4 & 11.0 & 20.9 & $\begin{array}{l}9 \\
9\end{array}$ & 0.142 & 0.128 & 0.006 & 6.110 & 5.2 \\
\hline 57 & $2 / 5 / 2$ & 10:03:33 & 29.089 & 20.148 & 28 & 20.625 & & 22.967 & 34. & & 19.41 & 7.055 & & & 0.949 & & & 40.00 & $\begin{array}{ll}0 & 0.66 \\
0\end{array}$ & 33.5 & 11.0 & 21.2 & $\begin{array}{l}2 \\
21.461\end{array}$ & 0.141 & 0.128 & & & \\
\hline 58 & $2 / 5 / 2004$ & & 28.979 & 20.147 & 28. & 20.654 & & $22.6 / 2$ & & 3.108 & 19.309 & 1.046 & & 44.995 & 0.95 & 14.643 & & & 0.0 .68333 & 33.2 & 11.0 & 21.0 & $1.44 I^{\prime}$ & & 0.129 & & & \\
\hline 59 & $2 / 5 / 2$ & 10:05:33 PM & 28.859 & 20.166 & 28.292 & 20.689 & & 33.129 & 34.637 & 3.194 & 29.704 & -1.216 & & & 0.8 & 1.367 & & 42.00 & 0.7 & 33.2 & 11.1 & & & & 0.186 & & & \\
\hline & & & & & & & & & & & & & & & & & & 43.00 & \begin{tabular}{l|l}
0 & 0.71667
\end{tabular} & & 11.1 & 30.1 & & & & & & \\
\hline 61 & 2/5/2004 & $0: 07: 33 \mathrm{P}$ & 28.62 & 20.161 & 28.068 & 20.734 & & 31.933 & | 33.063 & 3.153 & 28.713 & -1.216 & $-4.8 \quad 2$ & & & 1.2 & & 44 & 0.73333 & 31.6 & 11.0 & & 191 & & & & & \\
\hline \begin{tabular}{|l|l|}
62 \\
\end{tabular} & $2 / 5 / 20$ & 10:08: & 28.52 & 20.166 & \begin{tabular}{|l|}
27.973 \\
\end{tabular} & 20.759 & & 32.148 & 36 & 3.6 & 28.7 & -1.216 & & & & 1.306 & & 45. & 0.75 & 32.1 & 10.9 & 30.5 & 00 & 0.195 & 0.179 & & 5.884 & 5.0 \\
\hline 63 & 2/5/2004 & 10:09:33 P & 28.411 & 20.166 & 27.879 & 20.78 & & 32.063 & 33.35 & 3.133 & 28.659 & -1.216 & & 44. & 0.0 & 1.284 & & 46. & $\mid 0.76667$ & 31.9 & 10.9 & 30.4 & \begin{tabular}{l|l|l|l|}
4 & 2.093
\end{tabular} & 0.191 & 0.177 & & 5.819 & .95 \\
\hline 64 & $2 / 5 / 2$ & 10:10:33F & 28.3 & 20.172 & 27.809 & 20.821 & & 31.9 & 33.1 & 3.074 & 28.6 & -1.216 & & 44.4 & 0.6 & 1.285 & & 47.00 & \begin{tabular}{l|l|l}
0 & 0.78333 \\
\end{tabular} & 31.7 & 10.9 & 30.3 & $\begin{array}{ll}3 & 2.096\end{array}$ & 0.192 & 0.177 & 0.006 & 5.843 & 4.9 \\
\hline 65 & $2 / 5 / 2$ & 10:11:33F & 28.247 & 20.182 & 27.73 & & & 32.136 & \begin{tabular}{|l|}
33.374 \\
\end{tabular} & 3.073 & 28.858 & -1.214 & & & & 1.278 & & 48.00 & & 31.9 & 10.9 & & & & & & & \\
\hline | & $2 / 3 / 2004$ & & 28.158 & 20.188 & 27.645 & 20.841 & & 32.192 & & 3. & & & & $44.2 \mathrm{r}$ & & $1.2 / 4$ & & 49.0 & $0.8166 /$ & 31.9 & 10.8 & 30.6 & & 90 & $0.1 / 6$ & & $5 . / 74$ & \\
\hline \begin{tabular}{|l|l|}
67 \\
\end{tabular} & $2 / 5 / 2$ & 10:13:33 & 28.089 & 20.188 & 27.576 & 20.847 & & 32.511 & 33.847 & 3.082 & 29.152 & -1.216 & & & & 1.279 & & 50.00 & \begin{tabular}{l|l}
0 & 0.83333 \\
\end{tabular} & 32.4 & 10.8 & & 2.126 & 191 & & & 56 & \\
\hline 68 & & 10:14:33 PM & 28.025 & 20.204 & 27.522 & 20.8/8 & & 32.204 & 33.399 & 2.989 & 28.963 & -1.216 & & 44.062 & 0.003 & $1.2 / 4$ & & 51.00 & 0.85 & 31.9 & 10.8 & 30.6 & $2.10 \mathrm{~s}$ & 0.190 & 0.111 & 0.006 & 5.788 & 4.93 \\
\hline & & - & & 20195] & 27.433 & 20.884 & & 32.268 & & 3050 & 2000 & & & 43791 & & & & & 86667 & & & & & & 0177 & nnos & 5.784 & 492 \\
\hline $\begin{array}{ll}70 \\
\end{array}$ & $2 / 5 / 2004$ & 10:16:33 PM & 27.902 & 20.211 & 27.399 & 20.94 & \begin{tabular}{|l|}
22.715 \\
\end{tabular} & 32.268 & 33.488 & 2.966 & 29.086 & 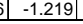 & & 43.791 & 0.003 & 1.268 & & 53.00 & \begin{tabular}{l|l|l|l|l}
0 & 0.8833
\end{tabular} & 32.0 & 10.7 & 30.7 & $\begin{array}{l}7 \mid 2.115 \\
7\end{array}$ & 0.189 & 0.177 & 0.006 & 5.763 & 4.9 \\
\hline
\end{tabular}


WSRC-TR-2005-00105, REVISION 0

SRNL-RPP-2005-00012, REVISION 0

RUN \# 1.21A, B, C; POST-TEST BASELINE WITH THE THIRD CAUSTIC RINSE SOLUTION - CONT.

\begin{tabular}{|c|c|c|c|c|c|c|c|c|c|c|c|c|c|c|c|c|c|c|c|c|c|c|c|c|c|c|c|c|}
\hline & A & $\mathrm{B}$ & D & $E$ & $\mathrm{~F}$ & G & $\mathrm{H}$ & 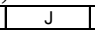 & $\mathrm{K}$ & $\mathrm{L}$ & \begin{tabular}{|l|} 
\\
\end{tabular} & $\mathrm{N}$ & \begin{tabular}{l|l}
$\mathrm{P}$ \\
\end{tabular} & Q & $\mathrm{R}$ & $\mathrm{s}$ & \begin{tabular}{l|l}
$\mathrm{T}$ & $\mid \mathrm{U}$
\end{tabular} & $\mathrm{v}$ & w & $x$ & Y & $z$ & AA & $A B$ & $A C$ & $A D$ & AE & AF $\quad A$ \\
\hline 71 & $2 / 5 / 2004$ & 10:17:33 PM & 27.843 & 20.201 & 27.335 & 20.951 & 22.756 & 32.333 & 33.449 & 2.919 & \begin{tabular}{|l|}
29.326 \\
\end{tabular} & \begin{tabular}{|l|} 
\\
\end{tabular} & -5.013 & 43.804 & 0.003 & 1.264 & 0.004 & 54.00 & 0.9 & 32.0 & 10.7 & 30.8 & 2.126 & 0.188 & 0.177 & 0.006 & 5.727 & 4.87 \\
\hline \begin{tabular}{|c|}
72 \\
2
\end{tabular} & $2 / 5 / 2004$ & 10:18:33 PM & 27.799 & \begin{tabular}{|l|}
20.213 \\
\end{tabular} & \begin{tabular}{|l|}
27.291 \\
\end{tabular} & $\begin{array}{l}20.927 \\
\end{array}$ & 22.812 & 32.188 & 33.358 & 2.922 & 29.057 & $\mid-1.219$ & -5.016 & 43.614 & 0.003 & 1.257 & 0.003 & 55.00 & $\begin{array}{ll}0 & 0.91667 \\
0\end{array}$ & 31.9 & 10.7 & 30.6 & 2.111 & $\begin{array}{l}0.187 \\
\end{array}$ & 0.176 & 0.006 & 5.741 & 4.89 \\
\hline 73 & $2 / 5 / 2004$ & \begin{tabular}{|l|}
$10: 19: 33 \mathrm{PM}$ \\
\end{tabular} & 27.749 & 20.223 & \begin{tabular}{|l|}
27.256 \\
\end{tabular} & $\begin{array}{r}20.982 \\
\end{array}$ & 22.351 & 32.43 & 33.627 & 3.021 & 29.174 & \begin{tabular}{|l|}
-1.214 \\
\end{tabular} & -5.013 & 43.564 & 0.003 & 1.254 & 0.005 & 56.00 & $\begin{array}{ll}0 & 0.93333 \\
\end{array}$ & 32.2 & 10.7 & 30.8 & 2.124 & \begin{tabular}{|l|l|} 
& 0.187 \\
\end{tabular} & 0.176 & 0.006 & 5.699 & 4.85 \\
\hline 74 & $2 / 5 / 2004$ & 10:20:33 PM & 27.711 & 20.229 & 27.213 & 20.979 & 22.508 & 32.463 & 33.722 & 3.045 & 29.172 & -1.216 & -5.016 & 43.599 & 0.003 & 1.249 & 0.005 & 57.00 & $\begin{array}{ll}0 & 0.95 \\
\end{array}$ & 32.3 & 10.7 & 30.8 & 2.125 & 0.186 & 0.175 & 0.006 & 5.680 & 4.83 \\
\hline 75 & $2 / 5 / 2004$ & 10:21:33 PM & 27.678 & 20.241 & 27.179 & 21.036 & 22.62 & 32.571 & 33.863 & 3.026 & 29.254 & -1.214 & -5.013 & 43.699 & 0.003 & 1.244 & 0.009 & 58.00 & \begin{tabular}{l|l}
0 & 0.96667 \\
\end{tabular} & 32.4 & 10.7 & 30.9 & 2.131 & 0.185 & 0.175 & 0.006 & 5.645 & 4.80 \\
\hline 76 & $\frac{2 / 5 / 2004}{2 / 52004}$ & 10:22:33 PM & 27.643 & $\begin{array}{ll}20.252 \\
20253\end{array}$ & 27.145 & $\begin{array}{l}21.051 \\
21.037 \\
\end{array}$ & $\begin{array}{r}22.56 \\
22.071 \\
\end{array}$ & $\begin{array}{r}31.993 \\
3223\end{array}$ & 33.155 & $\begin{array}{l}2.978 \\
3.137 \\
\end{array}$ & 28.768 & \begin{tabular}{|l|l|} 
& -1.216 \\
\end{tabular} & $\begin{array}{l}-5.022 \\
.020\end{array}$ & 44.175 & 0.003 & $\begin{array}{l}1.233 \\
1221 \\
\end{array}$ & 0.008 & 59.00 & \begin{tabular}{l|l}
0 & 0.98333 \\
\end{tabular} & 31.7 & $\begin{array}{l}7 \\
7 \\
\end{array}$ & 30.4 & \begin{tabular}{|l|}
2.095 \\
\end{tabular} & 0.184 & 0.173 & 0.006 & 5.699 & 4.85 \\
\hline$\frac{77}{78}$ & $\frac{2 / 5 / 2004}{21 / 52004}$ & 10:23:33 PM & $\begin{array}{r}27.6 \\
27.565 \\
\end{array}$ & \begin{tabular}{|l|}
20.253 \\
20.258 \\
\end{tabular} & \begin{tabular}{|l|}
27.121 \\
27.09 \\
\end{tabular} & \begin{tabular}{|l|}
21.037 \\
21.08 \\
\end{tabular} & $\begin{array}{r}22.471 \\
22511 \\
\end{array}$ & \begin{tabular}{|r|}
32.32 \\
32.154 \\
\end{tabular} & \begin{tabular}{|l|l|}
33.465 \\
33231
\end{tabular} & $\begin{array}{l}3.137 \\
3.131 \\
\end{array}$ & $\begin{array}{l}28.916 \\
28778 \\
\end{array}$ & -1.216 & $\begin{array}{l}-5.025 \\
5022 \\
\end{array}$ & \begin{tabular}{|l|}
44.654 \\
44.394
\end{tabular} & 0.003 & \begin{tabular}{|l|}
1.221 \\
1.226 \\
\end{tabular} & 0.004 & 60.00 & $\begin{array}{l}0 \\
0 \\
\end{array}$ & $\begin{array}{l}32.0 \\
31.8\end{array}$ & $\begin{array}{l}0 \\
0 \\
8\end{array}$ & \begin{tabular}{|l|}
30.6 \\
30.5 \\
\end{tabular} & \begin{tabular}{|l|}
2.111 \\
2101
\end{tabular} & 0.182 & 0.172 & 0.006 & $\begin{array}{l}5.603 \\
567 \\
567\end{array}$ & 4.77 \\
\hline \begin{tabular}{|l|}
79 \\
79 \\
\end{tabular} & $2 \frac{215 / 2004}{2 / 5 / 2004}$ & \begin{tabular}{|l|}
$10: 24: 33$ PM \\
102533 PM
\end{tabular} & $\frac{27.565}{27.546}$ & \begin{tabular}{|l|}
20.258 \\
20.274 \\
\end{tabular} & \begin{tabular}{|l|}
27.097 \\
27.092 \\
\end{tabular} & 21.028 & $\frac{22.511}{22.492}$ & $\begin{array}{r}32.154 \\
32.08\end{array}$ & \begin{tabular}{|l}
33.231 \\
33.21 \\
3
\end{tabular} & $\begin{array}{l}3.131 \\
.071 \\
\end{array}$ & \begin{tabular}{|l|}
28.787 \\
28.782 \\
\end{tabular} & $\frac{-1.216}{-1.214}$ & $\begin{array}{l}-5.022 \\
-5.022 \\
\end{array}$ & \begin{tabular}{|l|}
44.394 \\
44.279
\end{tabular} & $\begin{array}{l}0.003 \\
0.003 \\
\end{array}$ & $\begin{array}{l}1.226 \\
1.225 \\
\end{array}$ & 0.004 & $61.00 \mid$ & $\begin{array}{ll}0 & 1.01667 \\
0 & 10333\end{array}$ & $\begin{array}{l}31.8 \\
31.7 \\
\end{array}$ & 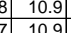 & \begin{tabular}{|l|}
30.5 \\
30.4 \\
\end{tabular} & \begin{tabular}{|l|}
$\mid 2.101$ \\
2098 \\
\end{tabular} & \begin{tabular}{|l|l|} 
& 0.183 \\
& 183
\end{tabular} & $\begin{array}{l}0.172 \\
0.172 \\
\end{array}$ & 0.006 & $5.657 \mid$ & $\begin{array}{l}4.81 \\
4.82 \\
\end{array}$ \\
\hline \begin{tabular}{|l|}
80 \\
\end{tabular} & $2 / 5 / 2004$ & \begin{tabular}{|l|}
10.25 .35 \\
$10: 26: 33 \mathrm{PM}$
\end{tabular} & 27.522 & 20.28 & \begin{tabular}{|l|}
27.078 \\
\end{tabular} & $\begin{array}{l}21.053 \\
21.009\end{array}$ & 222.473 & $\begin{array}{l}32.1962 \\
32.192 \\
\end{array}$ & 33.364 & $\begin{array}{l}3.011 \\
3.066\end{array}$ & 28.782 & $\mid$\begin{tabular}{|l|}
$\mid-1.214$ \\
-1.211 \\
\end{tabular} & $\begin{array}{l}-3.024 \\
-5.022\end{array}$ & \begin{tabular}{|l|}
44.279 \\
44.398 \\
\end{tabular} & $\begin{array}{l}0.003 \\
0.003\end{array}$ & $\begin{array}{l}1.253 \\
1.222 \\
\end{array}$ & $\begin{array}{l}0.005 \\
0.004\end{array}$ & $\begin{array}{l}62.00 \\
63.00\end{array}$ & 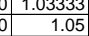 & $\begin{array}{l}31.7 \\
31.9 \\
\end{array}$ & 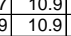 & \begin{tabular}{|l|}
30.4 \\
30.5 \\
\end{tabular} & \begin{tabular}{|l|}
2.1098 \\
2.102 \\
\end{tabular} & \begin{tabular}{|l|}
0.183 \\
0.182 \\
\end{tabular} & $\begin{array}{l}0.1 / 2 \\
0.172\end{array}$ & $\begin{array}{l}0.060 \\
0.006 \\
\end{array}$ & $\begin{array}{l}5.661 \\
5.638\end{array}$ & 4.82 \\
\hline 81 & $2 / 5 / 2004$ & 10:27:33 PM & 27.488 & 20.271 & 27.049 & 20.97 & 22.514 & 32.418 & 33.441 & 3.082 & 29.216 & -1.216 & -5.025 & 44.298 & 0.003 & 1.222 & 0.004 & 64.00 & \begin{tabular}{l|l}
0 & 1.06667 \\
\end{tabular} & 32.0 & 10.9 & 30.8 & 2.125 & 0.182 & 0.172 & 0.006 & 5.583 & \\
\hline $\begin{array}{l}82 \\
83 \\
\end{array}$ & & Averages & & & & & & & 176 & & & & -0.9 & & 0.6 & & & & & & & & & & 0.096 & & & \\
\hline $\begin{array}{l}\frac{63}{84} \\
\end{array}$ & & $\begin{array}{l}\text { Mverages } \\
\text { Maximum }\end{array}$ & $\begin{array}{l}24.5 \\
25.5 \\
\end{array}$ & 20.1 & $\begin{array}{l}24.5 \\
25.5 \\
\end{array}$ & $\begin{array}{l}20.0 \\
20.1\end{array}$ & & $\begin{array}{l}12.0 \\
14.0\end{array}$ & 22.1 & $\begin{array}{l}3.3 \\
.9\end{array}$ & $\begin{array}{r}8.5 \\
10.5\end{array}$ & $\begin{array}{l}0.0 \\
4.6\end{array}$ & $\begin{array}{r}-0.9 \\
1.5 \\
\end{array}$ & $\frac{4.2}{48.1}$ & 0.6 & $\begin{array}{l}14.6 \\
14.6 \\
\end{array}$ & 0.0 & & & $\frac{16.1}{20.7}$ & $\begin{array}{l}11.1 \\
1.8 \\
\end{array}$ & $\begin{array}{l}\frac{10.2}{12.2} \\
\text { r }\end{array}$ & \begin{tabular}{|l|l|}
0.103 \\
0.844
\end{tabular} & 0.094 & $\begin{array}{l}0.096 \\
0.131 \\
\end{array}$ & $\frac{0.0101}{0.0190}$ & $\frac{10.1}{19.0}$ & $\frac{8.6}{16.2}$ \\
\hline 85 & & Median & 24.4 & 20.1 & 24.4 & 20.0 & 24.0 & 13.0 & 17.4 & 3.3 & $\begin{array}{r}10.5 \\
9.4 \\
\end{array}$ & \begin{tabular}{r|r|}
-0.4 \\
-0.4
\end{tabular} & $\mid-1.6$ & $\frac{40.1}{44.9}$ & 0.0 & $\begin{array}{l}14.6 \\
14.6\end{array}$ & 0.0 & & & 15.9 & $\begin{array}{ll}11.0 \\
11.0\end{array}$ & $\begin{array}{l}\frac{12.2}{11.2} \\
\end{array}$ & 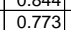 & $\begin{array}{l}0.120 \\
0.087 \\
\end{array}$ & 0.081 & 0.0082 & $\frac{19.0}{8.2}$ & $\frac{10.2}{7.0}$ \\
\hline \begin{tabular}{|l|}
86 \\
\end{tabular} & & Minimum & 23.0 & 20.1 & 23.0 & 20.0 & & 6.6 & 13.3 & 3.2 & 3.44 & $\begin{array}{l}-0.4 \\
\end{array}$ & $-1.9 \#$ & 44.4 & 0.6 & $\begin{array}{l}44.6 \\
14.6\end{array}$ & (2) & & & 11.8 & 10.9 & 4.8 & 0.331 & 0.084 & 0.082 & 0.0071 & $\frac{0.2}{7.1}$ & 6.1 \\
\hline 87 & & $2 \times$ Std Dev & 1.546 & 0.048 & 1.587 & 0.072 & 1.249 & 4.300 & 5.236 & 0.290 & 4.300 & 3.487 & $2.253 \#$ & 2.010 & 0.183 & 0.000 & 0.001 & & & 5.236 & $\begin{array}{ll}6 & 0.493 \\
\end{array}$ & 4.297 & \begin{tabular}{|l|l|}
0.296 \\
\end{tabular} & 0.027 & 0.031 & 0.0070 & 7.015 & 5.970 \\
\hline \begin{tabular}{|l|}
88 \\
\end{tabular} & Number o & Points Used* & 18 & & 18 & 18 & 18 & 18 & 18 & 18 & 18 & 18 & 180 & 18 & 18 & 18 & 18 & & & 18 & \begin{tabular}{|l|l|}
8 & 18 \\
\end{tabular} & 18 & 18 & 18 & 18 & 18 & 18 & 18 \\
\hline \begin{tabular}{|l|}
89 \\
\end{tabular} & & * Backpulse $p$ & ints in box & xare not in & ncluded & & & & & & & & & & & & & & & & & & & & & & & \\
\hline \begin{tabular}{|l|}
90 \\
\end{tabular} & & & & & & & & & & & & & & & & & & & & & & & & & & & & \\
\hline$\frac{91}{92}$ & & & & & & & & & & & & & & & & & & & & & & & 18-min. & avg. $=$ & 0.10 & & & \\
\hline$\frac{92}{93}$ & & Averages & 28.2 & 20.1 & 28.1 & 20.1 & 21.9 & 222.6 & 35.2 & 3.1 & 19.2 & 7.6 & 3.2 \# & 44.4 & 1.0 & 14,6 & 0.0 & & & 33.7 & 10.9 & 20.9 & 1.443 & 0.144 & 0.133 & 0.0063 & & 5.4 \\
\hline \begin{tabular}{|l|}
94 \\
94
\end{tabular} & & Maximum & 29.5 & 20.1 & $\frac{20.1}{29.4}$ & 20.7 & & 23.2 & $\begin{array}{l}0.4 \\
36.1\end{array}$ & $\frac{0.1}{3.4}$ & $\begin{array}{ll}19.4 \\
19.8\end{array}$ & 8.2 & $\frac{3.4 \pi}{3.6 \#}$ & $\begin{array}{l}44.4 \\
45.2 \\
\end{array}$ & 1.0 & $\begin{array}{l}14.6 \\
14.6\end{array}$ & & & & 34.7 & $\frac{1.5}{11.1}$ & & & & 0.143 & & 7.3 & 6. \\
\hline \begin{tabular}{|l|}
95 \\
\end{tabular} & & Median & 28.5 & 20.1 & 28.4 & 20.0 & 21.9 & 22.7 & 35.3 & 3.1 & 19.3 & $\begin{array}{ll}0.2 \\
7.8\end{array}$ & $3.4 \#$ & 44.7 & 1.0 & 14.6 & 0.0 & & & 33.8 & 11.0 & 20.5 & $\begin{array}{l}1.404 \\
1.444 \\
\end{array}$ & 0.146 & 0.132 & 0.0062 & 6.2 & \\
\hline \begin{tabular}{|l|}
96 \\
\end{tabular} & & Minimum & 25.8 & 20.1 & 25.9 & 19.9 & 21.3 & 21.0 & 33.5 & 3.0 & 17.3 & 7.0 & $2.9 \#$ & 43.7 & 0.9 & 14.6 & 0.0 & & & 32.0 & 10.7 & 19.1 & 1.320 & \begin{tabular}{|l|l|}
0.141 \\
\end{tabular} & 0.126 & 0.0061 & 6.1 & 5.2 \\
\hline \begin{tabular}{|l|}
97 \\
\end{tabular} & & $2 \times$ Std Dev & 2.375 & 0.024 & $\begin{array}{ll}2.047 \\
\end{array}$ & 0.499 & 0.543 & 0.894 & 1.307 & 0.208 & 1.073 & 0.765 & $0.490 \#$ & 1.085 & 0.027 & 0.000 & 0.001 & & & 1.307 & $\begin{array}{ll}7 & 0.266 \\
\end{array}$ & 0.978 & 0.067 & \begin{tabular}{|l|l|}
0.004 \\
\end{tabular} & 0.010 & 0.0006 & 0.634 & 0.539 \\
\hline \begin{tabular}{|l|}
98 \\
\end{tabular} & Number o & P Points Used ${ }^{*}$ & 21 & & & 21 & 21 & 21 & 21 & 21 & 21 & 21 & 210 & 21 & 21 & 21 & 21 & & & 21 & \begin{tabular}{ll|}
1 & 21 \\
\end{tabular} & 21 & 21 & 21 & 21 & 21 & 21 & 21 \\
\hline \begin{tabular}{|l|}
99 \\
\end{tabular} & & * Backpulse p & ints in box & xare not in & ncluded & & & & & & & & & & & & & & & & & & & & & & & \\
\hline & & & & & & & & & & & & & & & & & & & & & & & & & & & & \\
\hline$\frac{101}{102}$ & & & & & & & & & & & & & & & & & & & & & & & 21-min. & avg. $=$ & 0.13 & & & \\
\hline $\begin{array}{l}\frac{102}{103} \\
103\end{array}$ & & ages & 28.0 & 20.2 & 27.5 & 20.9 & $22.5 \#$ & 32.2 & 33.5 & 3.1 & 29.0 & \begin{tabular}{|c|}
-1.2 \\
\end{tabular} & $-5.0 \#$ & 44.3 & 0.0 & 1.3 & 0.0 & & & 32.0 & 10.8 & & 2.110 & \begin{tabular}{|l|} 
\\
\end{tabular} & 0.176 & 0.0057 & & \\
\hline \begin{tabular}{|l|l|}
104 \\
\end{tabular} & & Maximum & 28.9 & 20.3 & 28.3 & 21.1 & $22.8 \#$ & 33.1 & 34.6 & 3.2 & 29.7 & -1.2 & $-4.9 \#$ & 45.2 & 0.0 & 1.4 & & & & & 11.1 & & \begin{tabular}{|l|} 
\\
\end{tabular} & \begin{tabular}{|l|l|} 
& 0.204 \\
\end{tabular} & 0.186 & 0.0059 & 5.9 & \\
\hline 105 & & Median & 27.9 & 20.2 & 27.4 & 20.9 & $22.5 \#$ & 32.2 & 33.4 & 3.1 & 28.9 & -1.2 & $-5.0 \#$ & 44.3 & 0.0 & 1.3 & 0.0 & & & 31.9 & 10.9 & 30.6 & 2.109 & \begin{tabular}{|l|l|}
0.189 \\
\end{tabular} & 0.176 & 0.0058 & 5.8 & 4.9 \\
\hline 106 & & Minimum & 27.5 & 20.2 & 27.0 & 20.7 & 21.8 \# & 31.8 & 33.0 & 2.9 & 28.4 & -1.2 & $-5.0 \#$ & 43.6 & 0.0 & 1.2 & $0.0 \#$ & & & 31.6 & 10.7 & 30.1 & 2.074 & 0.182 & 0.172 & 0.0056 & 5.6 & 4.8 \\
\hline $107 \mid$ & & $2 \times$ Std Dev & 0.813 & 0.077 & 0.754 & 0.223 & 0.571 \# & 0.536 & 0.661 & 0.156 & 0.548 & 0.003 & $0.104 \#$ & 0.937 & 0.000 & 0.067 & $0.003 \#$ & & & 0.661 & \begin{tabular}{l|l|}
1 & 0.230 \\
\end{tabular} & 0.533 & \begin{tabular}{|l|l|}
0.037 \\
\end{tabular} & 0.010 & 0.006 & 0.0002 & 0.178 & 0.151 \\
\hline 108 & Number o & f Points Used* & 23 & & & 23 & 23 & & 23 & 23 & 23 & 23 & 230 & 23 & 23 & 23 & 230 & & & 23 & \begin{tabular}{|l|l|}
3 & 23 \\
\end{tabular} & 23 & 23 & 23 & 23 & 23 & 23 & \\
\hline 109 & & * Backpulse p & ints in box & $x$ are not in & ncluded & & & & & & & & & & & & & & & & & & & & & & & \\
\hline$\frac{110}{111}$ & & & & & & & & & & & & & & & & & & & & & & & & & 018 & & & \\
\hline$\frac{111}{112}$ & & & & & & & & & & & & & & & & & & & & & & & 2s-mint. & avg.- & & & & \\
\hline \begin{tabular}{|l|l|}
113 \\
\end{tabular} & & & 27.0 & 20.2 & 26.8 & 20.4 & $22.6 \# \#$ & 23.0 & 29.4 & 3.2 & 19.6 & 2.3 & $-1.0 \#$ & 44.6 & 0.5 & 9.8 & 0.0 & & & 27.9 & 10.9 & 21.3 & 1.470 & \begin{tabular}{|l|}
0.146 \\
\end{tabular} & 0.138 & 0.0072 & 7.2 & \\
\hline 114 & & & 29.5 & 20.3 & 29.4 & 21.1 & $24.2 \#$ & 33.1 & 36.1 & 3.9 & 29.7 & 8.2 & $3.6 \#$ & 48.1 & 1.0 & 14.6 & $0.0 \#$ & & & 34.7 & 11.8 & 31.4 & 2.166 & 0.204 & 0.186 & 0.0190 & 19.0 & 16.2 \\
\hline 115 & & Median & 27.6 & 20.1 & 27.2 & 20.1 & $22.5 \#$ & 22.8 & 33.4 & 3.1 & 19.4 & -0.5 & $-1.6 \#$ & 44.7 & 0.6 & 14.6 & $0.0 \#$ & & & 32.0 & 10.9 & 21.1 & 1.452 & 0.146 & 0.134 & 0.0062 & 6.2 & 5.2 \\
\hline 116 & & & 23.0 & 20.1 & 23.0 & 19.9 & 21.3 \# & 6.6 & 13.3 & 2.9 & 3.0 & -1.2 & $-5.0 \#$ & 43.1 & 0.0 & 1.2 & $0.0 \mid \#$ & & & 11.8 & \begin{tabular}{|l|l|}
8 & 10.6 \\
\end{tabular} & 4.8 & 0.331 & \begin{tabular}{|l|l|} 
& 0.084 \\
\end{tabular} & 0.082 & & 5.6 & 4.8 \\
\hline 117 & & $2 \times \mathrm{Std}$ Dev & 3.821 & 0.092 & \begin{tabular}{|l|}
3.511 \\
\end{tabular} & $\begin{array}{ll}0.849 \\
\end{array}$ & 1.681|\# & 16.421 & 15.373 & 0.323 & \begin{tabular}{|l|l|l|}
16.743 \\
\end{tabular} & 7.925 & $6.977 \#$ & 1.621 & 0.831 & 12.882 & $0.002 \#$ & & & 15.374 & $\begin{array}{ll}4 & 0.397 \\
\end{array}$ & \#\#\# & \begin{tabular}{|l|l|}
1.143 \\
\end{tabular} & 0.077 & $\begin{array}{l}0.067 \\
\end{array}$ & 0.0053 & 5.302 & 4.512 \\
\hline 118 & Number o & f Points Used* & 63 & 63 & 63 & 63 & 630 & 63 & 63 & 63 & 63 & 63 & $63 \mid 0$ & 63 & 63 & 63 & 630 & & & 63 & 63 & 63 & 63 & 63 & 63 & 63 & 63 & \\
\hline 119 & & * Backpulse p & ints in box & $x$ are not in & ncluded & & & & & & & & & & & & & & & & & & & & & & & \\
\hline$\frac{120}{121}$ & & & & & & & & & & & & & & & & & & & & & & & 1-hour a & avg. = & 0.14 & & & \\
\hline
\end{tabular}


WSRC-TR-2005-00105, REVISION 0

SRNL-RPP-2005-00012, REVISION 0

RUN \# 2.00A, B, AND C; PRE-TEST BASELINE WITH 0.1 M NAOH

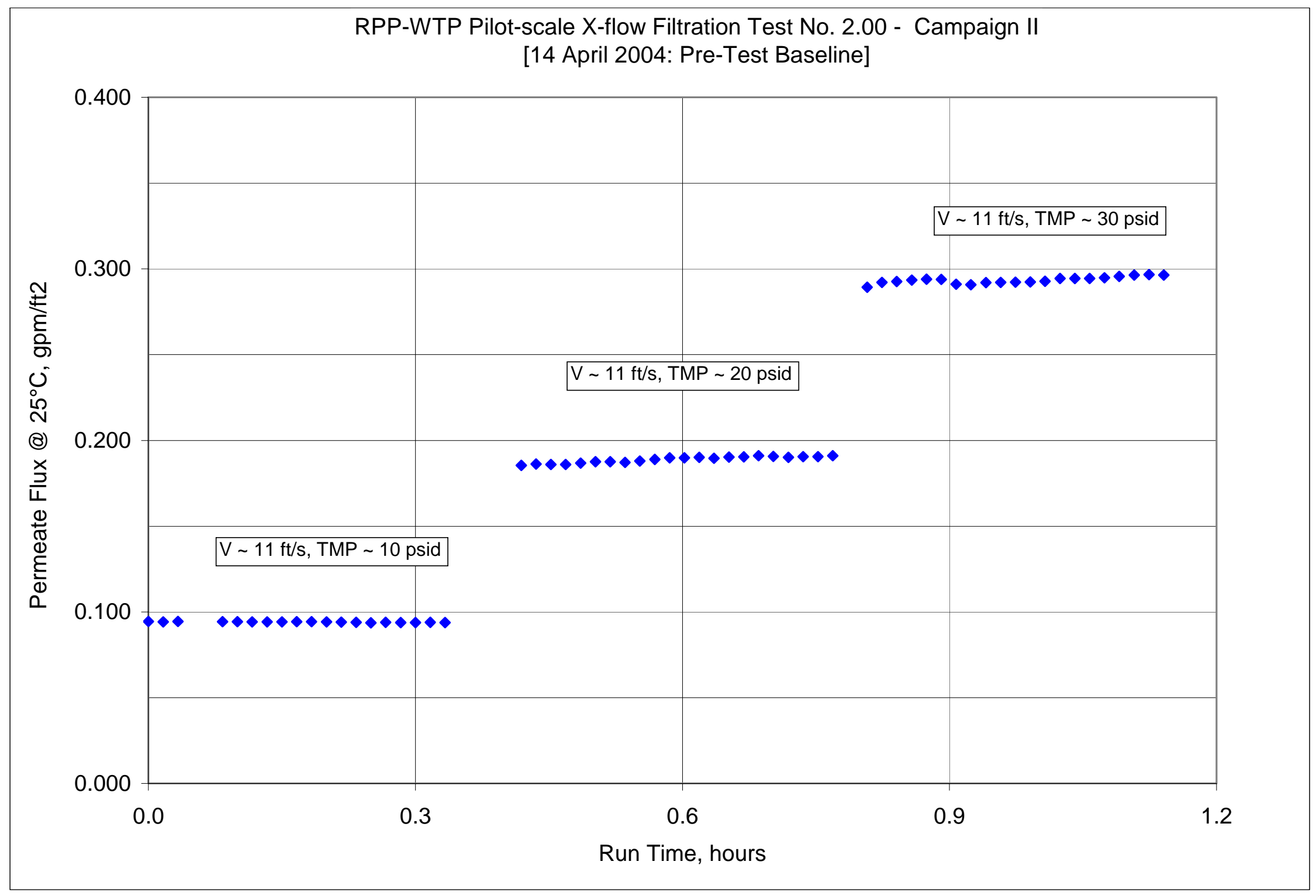


WSRC-TR-2005-00105, REVISION 0

SRNL-RPP-2005-00012, REVISION 0

RUN \# 2.00A, B, AND C; PRE-TEST BASELINE WITH 0.1 M NAOH - CONT.

\begin{tabular}{|c|c|c|c|c|c|c|c|c|c|c|c|c|c|c|c|c|c|c|c|c|c|c|c|c|c|c|c|c|}
\hline & A & B & $\frac{D}{S}$ & $\begin{array}{ll}E \\
\end{array}$ & $|F|$ & G & H & $\frac{j}{1}$ & $1 K$ & $\mid \frac{\mathrm{L}}{1}$ & M & \begin{tabular}{|l|} 
\\
\end{tabular} & \begin{tabular}{|l|}
0 \\
\end{tabular} & \begin{tabular}{|l|}
$Q$ \\
$<<<<<$
\end{tabular} & $\begin{array}{ll}R \\
\end{array}$ & $|s|$ & \begin{tabular}{|c|}
$\mathrm{T}$ \\
\end{tabular} & y $v$ & $1 \mathrm{w}$ & $1 \times$ & $\begin{array}{ll} & Y \\
\end{array}$ & $z$ & AA & $A B$ & AC & AD & $\begin{array}{ll}\mathrm{AE} \\
\end{array}$ & $\mathrm{AF}$ \\
\hline & DATE & TIME & $\begin{array}{l}\text { Filtrate } \\
\text { Fiste }\end{array}$ & $\begin{array}{l}\text { s< Temp } \\
\text { CCleaning }\end{array}$ & 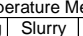 & enasureme & $\begin{array}{l}\text { nnts }>>>> \\
\text { Lo Amb. }\end{array}$ & $\begin{array}{l}\text { BotTM } \\
\text { BotTMP }\end{array}$ & $\begin{array}{l}k \ll<<< \\
\text { d Filter }\end{array}$ & $\begin{array}{l}\text { Pressure } \\
\text { Filter } \mathrm{dP}\end{array}$ & $\begin{array}{l}\text { Measureme } \\
\text { TopTMP }\end{array}$ & 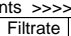 & 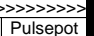 & $\begin{array}{l}k<\ll<< \\
\text { Slurry }\end{array}$ & $\begin{array}{l}\text { «< Fllow I } \\
\text { | Filtrate }\end{array}$ & $\begin{array}{c}\text { Measuremer } \\
\text { IHi Fittate }\end{array}$ & & & & & & & Raw & Data 》 & & & & \\
\hline & DAII & & & & $\operatorname{deg} C$ & $\operatorname{deg} C$ & deg $C$ & posid & & psid & & & & somy & \begin{tabular}{|l|} 
Filuate \\
gom
\end{tabular} & plom & $\begin{array}{l}\text { Dackpulse } \\
\text { gom }\end{array}$ & & & & & & & & & & & \\
\hline & & & $\mathrm{T} 2$ & & $=1$ & $\mathrm{~T}_{4}$ & & $\mathrm{dF}$ & $\frac{1}{P 1}$ & $\mathrm{dP1}$ & dP3 & P2 & & Q1 & $\mathrm{Q} 2$ & $\mathrm{Q3}$ & Qbp & & & & & & & & & & & \\
\hline & & & & & & & & & & & & & & & & & & & & & & & & & & & & \\
\hline & & & & & & & & & & & & & & & & & & & & & & & & & & & & \\
\hline & $4 / 14 / 2004$ & 1:30:58 PM & 24.687 & 21.862 & 24.835 & 24.226 & 22.583 & 0.054 & 0.02 & $\begin{array}{l}-0.021 \\
\end{array}$ & -0.005 & $\begin{array}{l}-0.179 \\
\end{array}$ & 0.099 & $\begin{array}{l}-0.08 \\
\end{array}$ & 0.003 & 0.086 & 0.005 & & & & urface & 670 & ET2 & & & & & \\
\hline & $4 / 19 / 2004$ & $7: 37: 13 \mathrm{AM}$ & 22.595 & 22.228 & 23.926 & 21.772 & 22.175 & 0.046 & 0.082 & -0.025 & -0.009 & $\begin{array}{r}-0.179 \\
-.0\end{array}$ & 0.143 & -0.086 & 0.003 & 14.643 & 0.003 & & & Convers: & ersion & 85 & $\mathrm{~m} 3 / \mathrm{m} 21$ & e/day/bart] & $\mathrm{rg} / \mathrm{gpm} / \mathrm{ft}$ & tit/2/barg & & \\
\hline & $4 / 19 / 2004$ & $8: 21: 39 \mathrm{AM}$ & 22.248 & 22.333 & 22.324 & 22.194 & 22.199 & -0.008 & 0.059 & & 0.007 & -0 & 0.131 & & 0.003 & 14.6 & & & & & & & & & & & & \\
\hline & 4/20/2004 & 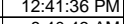 & 22.7772 & 22.767 & 22.049 & 23.603 & 24.746 & -0.004 & 0.045 & & -0.007 & -0.176 & 0.108 & -0.084 & 0.003 & $14.6 \mathrm{C}$ & 0.005 & & & & & & & & & & & \\
\hline & $41 / 21212004$ & $8.4 .434 \mathrm{AM}$ & & 21.446 & & 22.710 & & 0.01 & 0.028 & & & -0.205 & & 46.562 & 0.043 & 14.643 & & & & & & & & & & & & \\
\hline & $\begin{array}{l}4 / 2 / 26 / 2004 \\
4 / 2\end{array}$ & $\begin{array}{l}8.53 .20 \mathrm{AM} \\
900: 47 \mathrm{AM}\end{array}$ & $\frac{21.548}{22.379}$ & $\frac{21.131}{22.907}$ & $\frac{2.0 .045}{2345}$ & 21.89 & $\frac{21.369}{21.819}$ & $\begin{array}{l}-\frac{-0.004}{-0.012} \\
-\end{array}$ & 0.026 & 0.003 & $\begin{array}{l}-0.005 \\
-0.009\end{array}$ & $\begin{array}{l}-0.196 \\
-0.205\end{array}$ & & $\begin{aligned}-0.082 \\
-0.08\end{aligned}$ & 0 & $\frac{14 .}{14 .}$ & 0.005 & & & volow & & & tor & 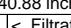 & te Flux & $\mathbb{t}_{1<<\text { PFN }}$ & $\mid \mathrm{ABI}$ & \\
\hline & $4 / 27 / 2004$ & $9: 14: 59 \mathrm{AM}$ & 20.374 & 19.305 & 19.368 & 20.841 & 19.777 & -0.002 & 0.02 & -0.001 & 0.005 & -0.193 & 0.111 & $\begin{array}{r}-0.084 \\
-0.084\end{array}$ & 0.00 & $\begin{array}{l}14.040 \\
14.643\end{array}$ & 0.006 & & & & & & & & 1 a 250 c & & 1000 & \\
\hline & & & & & & & & & & & & & & & & & & Time & Time & Press. & $\begin{array}{l}\text { Vel. } \\
\text { Vin }\end{array}$ & TMP & TMP & gpm & $\mathrm{gpm}$ & gpm & gpm & meter \\
\hline & iaseline $1 \mathrm{~A}$ & 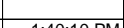 & 20201 & & 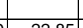 & 22521 & & & 16786 & & & & & 11891 & & & & & our & ssig & & osi & bar & & & 2 & & ay/bal \\
\hline & $4 / 14 / 2004$ & 1:40:10 PM & $\begin{array}{l}23.391 \\
20210\end{array}$ & $\frac{21.99}{21.902}$ & & 23.631 & & $\frac{12.056}{12.128}$ & 16.786 & & 1.996 & - & & 44.821 & 0 & -0.014 & 0.003 & & (20) & 15.3 & & & & & & & & \\
\hline & $\frac{4 / 1 / 24 / 2004}{4 / 1 / 204}$ & $\frac{1.4 .10 \mathrm{PM}}{1: 42 \cdot 10 \mathrm{PM}}$ & $\frac{23.19}{23.044}$ & $\frac{21.983}{22.014}$ & $\frac{22.819}{22784}$ & $\frac{23.675}{23.64}$ & $\frac{24.357}{24.997}$ & $\frac{12.138}{12.124}$ & \begin{tabular}{|l|l|}
16.828 \\
16.821
\end{tabular} & $\frac{3.142}{3.765}$ & & $\begin{array}{l}-0.274 \\
-0.0207\end{array}$ & $\frac{-1.52}{-1.51}$ & $\frac{44.906}{44.841}$ & 0.5 & & 0.0 & & 0.01667 & 15.4 & & $\frac{10.2}{10 .}$ & 0.702 & & 0.094 & 0.009 & $\frac{9.249}{9308}$ & 7.87 \\
\hline 20 & 4/1/4/2004 & 1:43:10 PM & 22.93 & $\frac{22.014}{22.015}$ & $\frac{22.144}{22.735}$ & $\begin{array}{r}23.64 \\
23.666\end{array}$ & $\begin{array}{l}24.997 \\
25.438\end{array}$ & $\frac{12.124}{12.358}$ & 17.066 & 3.798 & 8.395 & $\begin{array}{l}-0.201 \\
-0.239\end{array}$ & $\frac{-1.51}{-1.51}$ & $\begin{array}{l}44.641 \\
44.798\end{array}$ & $\begin{array}{l}.595 \\
0.595\end{array}$ & & $\frac{0.003}{0.003}$ & 300 & 0.0335 & 15.3 & $\frac{11.0}{110}$ & $\frac{1.1}{104}$ & 0.70 & $\mid$ & 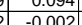 & 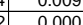 & 1969 & 7.92 \\
\hline & $4 / 14 / 2004$ & 1:44:10 PM & 22.83 & 22.031 & \begin{tabular}{|l|l|}
22.786 \\
\end{tabular} & \begin{tabular}{|l|l|} 
& 23.677 \\
\end{tabular} & 25.37 & 12.058 & 16.728 & 3.748 & 8.22 & -0.236 & -1.56 & \begin{tabular}{|l|l|l|l}
4479 \\
\end{tabular} & 0.595 & & & 4.00 & 0.06667 & 153 & $\frac{110}{110}$ & 101 & 0.699 & -0.001 & $\frac{-0.001}{-0.001}$ & 0.000 & - & $\begin{array}{l}-0.17 \\
0.12\end{array}$ \\
\hline & $4 / 14 / 2004$ & 1:45:10 PM & 22.79 & 22.025 & 22.886 & \begin{tabular}{|l|l|} 
& 23.697 \\
\end{tabular} & 24.844 & 12.163 & 16.91 & 3.756 & $\begin{array}{l}8.204 \\
\end{array}$ & $\begin{array}{c}-0.213 \\
\end{array}$ & -1.49 & 44.648 & 0.596 & $\mid-0.013$ & 0.003 & 5.0 & 0.08333 & 15.4 & 10.5 & 10.2 & 0.702 & & & 0.009 & $\begin{array}{l}-0.141 \\
9.265 \\
\end{array}$ & $\frac{0.12}{7.88}$ \\
\hline & $4 / 14 / 2004$ & & 22.758 & 22.019 & 22.97 & 23.67 & 24.458 & 12.136 & 16.896 & & 8.173 & -0.216 & -1.49 & $\begin{array}{l}44.842 \\
\end{array}$ & & & & 6.00 & & 15.4 & & & & & & & & . \\
\hline & & 1:47:10 PM & & & & & & & & 3.759 & 8.079 & & $\begin{array}{l}-1.48 \\
\end{array}$ & \begin{tabular}{|l|l|l}
44.708 \\
\end{tabular} & $\begin{array}{l}0.597 \\
\end{array}$ & -0.0 & & 70 & 0.11667 & 15.2 & 11.6 & 10.0 & 0.692 & $\mid 0.089$ & 9 & 0.009 & 9.379 & $\frac{7.98}{7.98}$ \\
\hline & $4 / 14 / 2004$ & 1:48:10 PM & 22.777 & 21.917 & 23.004 & 23.558 & $\begin{array}{l}18.924 \\
\end{array}$ & 12.188 & $\begin{array}{l}16.987 \\
\end{array}$ & 3.812 & 8.142 & $\begin{array}{l}-0.187 \\
\end{array}$ & -1.48 & 44.944 & & & & 8.00 & 0.1333 & 15.5 & 11.0 & $10.2 \mathrm{C}$ & 0.701 & \begin{tabular}{|l|l|}
10.089 \\
\end{tabular} & & 0.009 & 9.266 & 7.85 \\
\hline & $4 / 14 / 2004$ & & 22.806 & 21.845 & & 23.502 & & & & & 8.21 & & & $\begin{array}{l}44.62 \\
\end{array}$ & & & & & 0.15 & 15.5 & 10.9 & & & & & & & 7.86 \\
\hline & 4/114/2004 & 1:50:10 & 22.858 & 21.767 & 23.085 & \begin{tabular}{|l|l|l|l|l|}
23.43 \\
\end{tabular} & & 12.136 & 5.956 & 3.749 & 8.222 & -0.106 & & \begin{tabular}{l|l}
44.841 \\
\end{tabular} & 0.5 & & & 10.00 & 0.16667 & 15.5 & 11.0 & 10.2 & $\begin{array}{ll}0.702 \\
\end{array}$ & \begin{tabular}{l|l|}
2 & 0.089 \\
\end{tabular} & 0.094 & 0.009 & 9.263 & 7.88 \\
\hline & $4 / 14 / 2004$ & 1:51:10 PM & 22.899 & 21.642 & 23.076 & 23.344 & 16.5 & 12.277 & 17.004 & 3.859 & 8.3 & $\mid-0.126$ & & $\begin{array}{l}44.775 \\
\end{array}$ & 0.599 & -0.0 & & 11.00 & 0.18333 & 15.5 & 111.0 & 10.3 & & & 0.094 & 0.009 & 9.166 & 7.86 \\
\hline 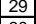 & $4 / 14 / 2004$ & & 22.939 & 21.541 & 23.181 & 23.248 & 18. & 12.143 & & 3.806 & 8 & & & 44.7 & 0.6 & & & 12.00 & & 15.5 & 11.0 & & & & & 009 & & \\
\hline & $4 / 144 / 2004$ & & 22.989 & 21.47 & 23.241 & 23.238 & & 12.16 & & & 13 & & & & 0.6 & & & & 0.21667 & & 11.0 & & & & & & & 7.9 \\
\hline & $41 / 14212$ & 1.54: & $\frac{23.015}{23019}$ & 21.405 & 23.266 & 23.304 & 22.042 & $\frac{11.983}{12066}$ & 16.7 & 3.734 & 8.052 & -0.109 & -1. & 44.731 & 0.6 & & & 14.00 & 0.23333 & 15.3 & 11.0 & & & & & & & 7.9 \\
\hline & 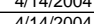 & $\begin{array}{l}1.55: 10 \\
1.56 \cdot 10\end{array}$ & $\frac{2.049}{23072}$ & $\frac{21.359}{21342}$ & $\frac{2.3014}{23249}$ & $\frac{23.419}{23692}$ & & $\frac{12.0}{120}$ & $\begin{array}{l}10.8 \\
\end{array}$ & & 8.187 & & & $\begin{array}{ll}44.1 \\
447\end{array}$ & & & & & & 15.4 & 11.6 & & & & & 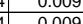 & $\frac{254}{274}$ & 7.87 \\
\hline 34 & $4 / 144 / 2004$ & $\frac{1.5 .10}{1.5710}$ & 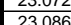 & $\frac{21.342}{21.325}$ & | & 23.0937 & $\frac{24.5}{242}$ & $\frac{12.041}{12209}$ & $\begin{array}{l}10.001 \\
17062\end{array}$ & 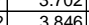 & $\frac{0.2}{81}$ & $=0$ & & (44.154 & 0.0601 & & & 1700 & $\mid \begin{array}{l}.20003 \\
02333\end{array}$ & 15.4 & 11.00 & $\begin{array}{ll}9 & 1.1 \\
\end{array}$ & & & & & & 7.8 \\
\hline & $4 / 142004$ & $1: 5 \cdot 10$. & 23095 & 21.315 & 23.427 & 24.123 & 246 & $\frac{12.13}{1213}$ & 16968 & 3.375 & 8 & $\mid-0.109$ & & 44693 & 0.002 & & & 1800 & & 15.5 & 10.5 & & & & & 0.0009 & & $7.8 \mathrm{~s}$ \\
\hline & $4 / 1 / 42004$ & $1: 59: 10 \mathrm{PM}$ & 23111 & 21.316 & 23.438 & 24.42 & $\begin{array}{l}24.090 \\
25.064\end{array}$ & 12.17 & 17.008 & 3.752 & $\begin{array}{l}0.105 \\
8.239\end{array}$ & $\begin{array}{l}-0.109 \\
-0.103 \\
-\end{array}$ & -1.4 & 44.566 & 0.603 & - & 0.0 & 1900 & 0.31 .567 & 15.5 & 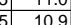 & 102 & 0.704 & $\begin{array}{l}0.090 \\
0.090\end{array}$ & 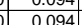 & 然 0.009 & 势 & $\frac{7.8}{78}$ \\
\hline 37 & $4 / 14 / 2004$ & $2: 00: 10 \mathrm{PM}$ & 23.127 & 21.332 & 23.434 & \begin{tabular}{|l|} 
\\
\end{tabular} 4.631 & 25.14 & 12.192 & 17.024 & 3.837 & 8.148 & -0.109 & -1.436 & 44.781 & 0.602 & -0.013 & 0.003 & 20.00 & 0.33333 & 15.6 & 11.0 & \begin{tabular}{l|l}
0 & 10.2 \\
\end{tabular} & \begin{tabular}{|l|}
2 \\
2
\end{tabular} & \begin{tabular}{|l|l|} 
& 0.090 \\
\end{tabular} & 0.094 & 0.009 & \begin{tabular}{l|l}
9 & 9.225 \\
\end{tabular} & \\
\hline & & & & & & & & & & & & & & & & & & & & & & & & & & & & \\
\hline & & & 229 & 216 & 232 & & & 121 & 169 & 38 & 82 & & & & nf & 000 & & & & 155 & 110 & & & & 80 & (2) & & \\
\hline & & & 23.1 & 22.0 & $\begin{array}{l}23.4 \\
\end{array}$ & 24.6 & & 12.3 & 17.1 & 3.9 & 8.3 & .1 & & & 0.6 & 0.0 & & & & 15.6 & 11.0 & \begin{tabular}{l|l|l|}
0 & 10.3 \\
\end{tabular} & & 0. & 0.0 & 0.009 & $\frac{9.4}{9.4}$ & \\
\hline & & Median & 23.0 & 21.5 & $\begin{array}{l}23.2 \\
\end{array}$ & 23.6 & 23 & 12.1 & $\begin{array}{l}17.0 \\
\end{array}$ & 3.8 & 8.2 & -0.1 & & 4 & 0.6 & 0.0 & & & & 15.5 & 11.0 & 10.2 & \begin{tabular}{|l|l|}
20.701 \\
\end{tabular} & 10.0 & & 0.009 & 9. & \\
\hline & & & 22.7 & & & 23.2 & & & & & & -0.2 & & & & 0.0 & & & & 15.2 & & 10.0 & $\begin{array}{l}0 \\
0\end{array}$ & & 0.094 & 0.009 & 9.2 & \\
\hline & & $2 \times \operatorname{Std} \mathrm{Dev}$ & 0.271 & 0.542 & 0.358 & 0.792 & 6.415 & 0.151 & 0.176 & 0.092 & 0.121 & 0.082 & 0.046 & 0.193 & 0.004 & 0.002 & 0.000 & & & 0.176 & 0.047 & 70.123 & $\mid$ & 01 & 0.000 & 0.000 & 0.107 & 0.091 \\
\hline & Number & $\begin{array}{l}\text { of Points Used } \\
\text { t* } \text { Backpuse }^{*}\end{array}$ & $\frac{16}{1 \text { ts in box }}$ & $\frac{16}{\text { are notinc }}$ & $\begin{array}{l}516 \\
\text { cluded }\end{array}$ & & & & & & & & & & & & & & & 16 & & $\begin{array}{l}6 \quad 16 \\
6\end{array}$ & 16 & 16 & & 16 & 16 & \\
\hline
\end{tabular}


WSRC-TR-2005-00105, REVISION 0

SRNL-RPP-2005-00012, REVISION 0

RUN \# 2.00A, B, AND C; PRE-TEST BASELINE WITH 0.1 M NAOH - CONT

\begin{tabular}{|c|c|c|c|c|c|c|c|c|c|c|c|c|c|c|c|c|c|c|c|c|c|c|c|c|c|c|c|c|}
\hline & A & B & D & $E$ & $\mathrm{~F}$ & G & $\mathrm{H}$ & \begin{tabular}{l|l}
$\mathrm{J}$ & \\
\end{tabular} & \begin{tabular}{|l|l}
$\mathrm{K}$ \\
\end{tabular} & \begin{tabular}{l|l} 
\\
\end{tabular} & $M$ & $\mathrm{~N}$ & \begin{tabular}{l|l} 
\\
\end{tabular} & $Q$ & $R$ & \begin{tabular}{l|l|} 
& \\
\end{tabular} & \begin{tabular}{c|c}
$\mathrm{T}$ & $\mathrm{U}$ \\
\end{tabular} & $\mathrm{V}$ & w & $x$ & $Y$ & 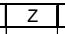 & $\mathrm{AA}$ & $A B$ & $A C$ & AD & $\mathrm{AE}$ & AF $A$ \\
\hline $\begin{array}{l}49 \\
50\end{array}$ & $\begin{array}{c}\text { Baseline } 1 B \\
4 / 14 / 2004\end{array}$ & & & $21.53 \varepsilon$ & 24.03 & 25.097 & & & 23.193 & 3.744 & & -1.218 & -4.743 & & & & & & & 21.7 & & & & & & & & \\
\hline 51 & $4 / 14 / 2004$ & 2:06:18 PM & 23.705 & 21.595 & 24.048 & 25.069 & 26.5 & 22.066 & \begin{tabular}{|l|}
23.242 \\
\end{tabular} & 3.731 & 18.29 & -1.218 & -4.74 & 44.816 & 0.003 & 1.216 & 0.003 & 26.13 & 0.43556 & 21.8 & 11.0 & & 1.391 & 0.181 & 0.186 & $\begin{array}{l}0.009 \\
0.009\end{array}$ & $\begin{array}{l}\frac{9.240}{9.230} \\
\end{array}$ & 7.85 \\
\hline 52 & $4 / 14 / 2004$ & $2: 07: 18 \mathrm{PM}$ & $23.751 \mid$ & 21.641 & \begin{tabular}{|l|}
24.059 \\
\end{tabular} & 24.999 & 26.74 & 22.243 & \begin{tabular}{|l|}
23.487 \\
\end{tabular} & 3.755 & \begin{tabular}{|c|}
18.414 \\
\end{tabular} & $\begin{array}{l}-1.218 \\
\end{array}$ & -4.737 & 44.869 & $\begin{array}{l}0.003 \\
\end{array}$ & 1.215 & 0.003 & 27.13 & 0.45222 & 22.0 & 11.0 & 20.3 & 1.402 & 0.181 & 0.186 & 0.009 & 9.151 & 7.79 \\
\hline 53 & $4 / 14 / 2004$ & $2: 08: 18$ PM & 23.788 & \begin{tabular}{|l|}
21.708 \\
1775
\end{tabular} & 24.081 & $\begin{array}{l}24.876 \\
2.771\end{array}$ & 26.846 & \begin{tabular}{l|l|}
22.056 \\
201
\end{tabular} & \begin{tabular}{|l|}
23.294 \\
3220
\end{tabular} & \begin{tabular}{|l|l|}
3.713 \\
355
\end{tabular} & 18.262 & \begin{tabular}{|c|}
-1.221 \\
\end{tabular} & \begin{tabular}{|l|l|} 
& -4.737 \\
.721
\end{tabular} & 44.737 & 0.003 & 1.215 & 0.005 & 28.13 & \begin{tabular}{|l|l|}
0.468899 \\
\end{tabular} & 21.8 & 11.0 & & 1.390 & 0.181 & 0.186 & 0.009 & 9.222 & 7.85 \\
\hline $\begin{array}{l}54 \\
55\end{array}$ & $\begin{array}{l}4 / 14 / 2004 \\
41 / 42004\end{array}$ & $\begin{array}{l}2: 09: 18 \mathrm{PM} \\
22 \cdot 10 \cdot 18 \mathrm{PM}\end{array}$ & \begin{tabular}{|l|l|}
23.834 \\
22359
\end{tabular} & \begin{tabular}{|l|l|}
21.754 \\
21.805
\end{tabular} & 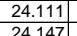 & $\begin{array}{l}24.771 \\
24681\end{array}$ & $\begin{array}{l}26.668 \\
26.43\end{array}$ & \begin{tabular}{|l|l|}
22.174 \\
22257
\end{tabular} & \begin{tabular}{|l|}
23.429 \\
2358 \\
\end{tabular} & \begin{tabular}{|l|l|}
3.655 \\
3595
\end{tabular} & $\begin{array}{r}18.45 \\
18.623\end{array}$ & \begin{tabular}{|c|}
-1.218 \\
-1.218
\end{tabular} & $\begin{array}{l}-4.731 \\
-4726\end{array}$ & \begin{tabular}{ll|l}
44.702 & 44527
\end{tabular} & 0.6 & \begin{tabular}{l|l|}
1.222 \\
1228
\end{tabular} & $\begin{array}{l}0.005 \\
0.003\end{array}$ & $\begin{array}{l}29.13 \\
30.13\end{array}$ & \begin{tabular}{|c|}
0.48556 \\
0.552222
\end{tabular} & $\begin{array}{l}22.0 \\
22.0 \\
20\end{array}$ & \begin{tabular}{l|l|}
11.0 \\
109
\end{tabular} & \begin{tabular}{|l|}
20.3 \\
20.4 \\
\end{tabular} & $\begin{array}{l}1.400 \\
1.409\end{array}$ & $0183 \quad-\quad>$ & $\begin{array}{l}0.187 \\
0.188\end{array}$ & \begin{tabular}{|l|l|}
0.009 \\
0.009
\end{tabular} & \begin{tabular}{|c|}
9.198 \\
9175 \\
\end{tabular} & $\begin{array}{l}7.83 \\
781\end{array}$ \\
\hline 56 & $\begin{array}{l}4 / 14 / 2004 \\
4 / 14 / 2004\end{array}$ & 2:10:18 PM & $23.859 \mid$ & $\frac{21.805}{21344}$ & \begin{tabular}{|c|}
24.147 \\
24.176
\end{tabular} & $\begin{aligned} 24.681 \\
24.585\end{aligned}$ & $\frac{26.433}{26138}$ & $22.257 \mid$ & \begin{tabular}{|r|}
23.458 \\
23.342 \\
\end{tabular} & $\begin{array}{l}3.595 \\
3.586\end{array}$ & $\frac{18.623}{18391}$ & $\begin{array}{l}-1.218 \\
-1.212\end{array}$ & $\begin{array}{l}-4.726 \\
-4.723\end{array}$ & $\begin{array}{l}44.527 \\
457\end{array}$ & $\begin{array}{l}0.003 \\
0.003\end{array}$ & $\begin{array}{l}1.228 \\
1229\end{array}$ & $\begin{array}{l}0.003 \\
0.004\end{array}$ & $\begin{array}{l}30.13 \\
31.13 \\
\end{array}$ & 0.50222 & $\begin{array}{l}22.0 \\
221.9 \\
\end{array}$ & $\begin{array}{l}10.9 \\
10.9\end{array}$ & \begin{tabular}{|l|}
20.4 \\
20.2
\end{tabular} & 1.4099 & $\begin{array}{ll}0.183 \\
0.183\end{array}$ & $\begin{array}{l}0.188 \\
0.188 \\
\end{array}$ & 0.009 & 9.175 & 7.81 \\
\hline 57 & $4 / 14 / 2004$ & 2:12:18 PM & 23.938 & 21.909 & 24.241 & 24.519 & $\frac{20.100}{26.203}$ & 22.015 & \begin{tabular}{|l|l|}
23.242 \\
\end{tabular} & 3.528 & 18.411 & -1.215 & $\begin{array}{l}-4.1725 \\
-4.717\end{array}$ & 44.476 & 0.003 & $\frac{1.229}{1.229}$ & $\frac{0.004}{0.004}$ & $\frac{31.13}{32.13}$ & $\begin{array}{l}0.51889 \\
0.53556\end{array}$ & $\frac{21.9}{21.8}$ & $\frac{10.9}{10.9}$ & \begin{tabular}{|l|l|}
20.2 \\
20.2
\end{tabular} & $\frac{1.394}{1.394}$ & \begin{tabular}{|l|}
0.183 \\
0.183 \\
\end{tabular} & $\begin{array}{l}0.188 \\
0.187 \\
\end{array}$ & $\mid \frac{0.009}{0.009}$ & 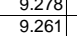 & $\frac{7.90}{7.88}$ \\
\hline 58 & $4 / 14 / 2004$ & 2:13:18 PM & 23.946 & 21.928 & \begin{tabular}{|l|l|}
24.219 \\
\end{tabular} & 24.412 & 26.326 & 22.085 & \begin{tabular}{|r|}
23.3 \\
\end{tabular} & 3.617 & 18.457 & $\mid-1.218$ & $\begin{array}{l}-4.717 \\
\end{array}$ & 44.464 & 0.003 & 1.233 & 0.003 & 33.13 & 0.55222 & 21.8 & \begin{tabular}{l|l|}
10.9 \\
\end{tabular} & 20.3 & 1.398 & \begin{tabular}{|l|l|}
0.184 \\
\end{tabular} & 0.188 & 0.009 & 9.271 & 7.89 \\
\hline 59 & $4 / 14 / 2004$ & $2: 14: 18 \mathrm{PM}$ & 23.975 & 21.977 & \begin{tabular}{|l|l|}
23.902 \\
\end{tabular} & 24.351 & 26.22 & 21.936 & \begin{tabular}{|l|l|}
23.155 \\
\end{tabular} & 3.586 & 18.323 & \begin{tabular}{|l|l|} 
& -1.221 \\
\end{tabular} & $\begin{array}{l}-4.717 \\
\end{array}$ & 44.524 & & 1.228 & 0.004 & 34.13 & 0.56889 & 21.7 & $\begin{array}{l}10.9 \\
\end{array}$ & 20.1 & 1.388 & 0.183 & 0.189 & 0.009 & 9.382 & 7.98 \\
\hline 60 & $4 / 14 / 2004$ & $2: 15: 18 \mathrm{PM}$ & 24.004 & 22.016 & 23.916 & 24.31 & 26.189 & 22.334 & \begin{tabular}{|l|}
23.692 \\
\end{tabular} & 3.675 & 18.514 & -1.218 & $\begin{array}{l}-4.711 \\
\end{array}$ & $\begin{array}{l}44.41 \\
\end{array}$ & \begin{tabular}{|l|l|}
0.003 \\
\end{tabular} & 1.235 & 0.005 & 35.13 & 0.58556 & 22.2 & 10.9 & 20.4 & 1.408 & 0.184 & 0.190 & 0.009 & 9.296 & 7.91 \\
\hline $\begin{array}{ll}61 \\
62 \\
2\end{array}$ & $4 / 14 / 2004$ & $\begin{array}{l}2: 16: 18 \mathrm{PM} \\
2.171 .18 \mathrm{PM}\end{array}$ & $\begin{array}{l}24.018 \\
24.024\end{array}$ & \begin{tabular}{|l|}
22.055 \\
22095
\end{tabular} & \begin{tabular}{|l|l|}
23.92 \\
23099
\end{tabular} & \begin{tabular}{l|l}
24.294 \\
24273
\end{tabular} & $\begin{array}{l}26.123 \\
25748\end{array}$ & \begin{tabular}{|l|}
22.185 \\
2286
\end{tabular} & \begin{tabular}{|l|}
23.431 \\
23.64 \\
\end{tabular} & \begin{tabular}{|l|l|}
3.591 \\
36757
\end{tabular} & $\begin{array}{l}18.557 \\
18537\end{array}$ & \begin{tabular}{|c|}
-1.218 \\
1.215
\end{tabular} & \begin{tabular}{|l|} 
\\
\end{tabular} & \begin{tabular}{|l|l|l}
44.406 \\
419
\end{tabular} & \begin{tabular}{|l|l|}
0.003 \\
0033
\end{tabular} & $\begin{array}{l}1.235 \\
1230\end{array}$ & 0.005 & $\begin{array}{l}36.13 \\
37.12 \\
37\end{array}$ & 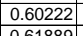 & 22.0 & 10.9 & 20.4 & $\begin{array}{l}1.405 \\
1.407 \\
\end{array}$ & 0.184 & $\begin{array}{l}0.190 \\
0.100\end{array}$ & 0.009 & 9.319 & 7.93 \\
\hline$\frac{62}{63}$ & 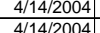 & $2: 17: 18 \mathrm{PM}$ & \begin{tabular}{ll|}
24.042 \\
24067
\end{tabular} & $\begin{array}{l}22.095 \\
22.134\end{array}$ & 23.969 & 24.273 & $\begin{array}{l}25.748 \\
25747\end{array}$ & \begin{tabular}{|l|l|}
22.286 \\
2220
\end{tabular} & \begin{tabular}{r|r}
23.64 & \\
23.444
\end{tabular} & \begin{tabular}{|c|}
3.657 \\
3638
\end{tabular} & $\begin{array}{r}18.537 \\
1858\end{array}$ & \begin{tabular}{|c|}
-1.215 \\
1201
\end{tabular} & $\begin{array}{l}-4.702 \\
-4705\end{array}$ & \begin{tabular}{|l|}
44.318 \\
4355
\end{tabular} & & \begin{tabular}{|l|l|}
1.239 \\
1.237
\end{tabular} & 0.005 & $\begin{array}{l}37.13 \\
38.13 \\
3\end{array}$ & 0.61889 & \begin{tabular}{l|l|}
22.2 \\
220
\end{tabular} & $\begin{array}{l}10.9 \\
10.9\end{array}$ & 20.4 & $\begin{array}{l}1.407 \\
1.47 \\
\end{array}$ & & 0.190 & 0.009 & \begin{tabular}{|c|c|c|} 
\\
\end{tabular} & 7.93 \\
\hline $\begin{array}{ll}64 \\
64\end{array}$ & $\begin{array}{r}\mid 4 / 1442004 \\
/ 14 / 2004\end{array}$ & $\frac{2: 18: 18 \mathrm{PM}}{2: 19: 18 \mathrm{PM}}$ & $\frac{24.067}{24.075}$ & \begin{tabular}{|l|}
22.134 \\
22.147
\end{tabular} & & $\frac{24.277}{24.25}$ & $\begin{array}{l}25.747 \\
25.075\end{array}$ & $\frac{2.222}{22.29}$ & \begin{tabular}{|l|}
23.3444 \\
23.562
\end{tabular} & \begin{tabular}{|c|c|}
3.638 \\
3.644
\end{tabular} & $\frac{18.58}{18.561}$ & & $\begin{array}{r}-4.705 \\
-4.7\end{array}$ & \begin{tabular}{|}
$\mid 44.355$ \\
44.358 \\
\end{tabular} & $\mid \begin{array}{l}0.003 \\
0.003\end{array}$ & & $\frac{0.004}{0.006}$ & & 0.63556 & & \begin{tabular}{|c|c|}
10.9 \\
10.9
\end{tabular} & & $\frac{1.407}{1.408}$ & $\mid \frac{0.184}{0.185}$ & $\frac{0.190}{0.190}$ & \begin{tabular}{|c|}
0.009 \\
0.009
\end{tabular} & & 7.91 \\
\hline 65 & $4 / 14 / 2004$ & 2:20:18 PM & 24.093 & \begin{tabular}{|c|}
22.17 \\
\end{tabular} & 24.019 & 24.188 & 25.198 & $\begin{array}{l}22.29 \\
22.079\end{array}$ & \begin{tabular}{|l|}
23.3808 \\
\end{tabular} & 3.517 & 焉. & -1.215 & $\begin{array}{l}-4.697 \\
-4.697\end{array}$ & \begin{tabular}{|l|l|}
44.138 \\
\end{tabular} & 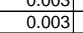 & $\begin{array}{l}\frac{1.241}{1.242} \\
\end{array}$ & $\begin{array}{l}0.0004 \\
0.004\end{array}$ & 40.13 & 0.66889 & $\begin{array}{l}22.1 \\
21.9\end{array}$ & $\begin{array}{l}\frac{10.9}{10.8} \\
\end{array}$ & \begin{tabular}{|l|}
$\mid 20.4$ \\
\end{tabular} & $\frac{1.408}{1.399}$ & $\mid$\begin{tabular}{|l|}
0.1885 \\
0.185
\end{tabular} & $\begin{array}{l}0.190 \\
0.190\end{array}$ & $\begin{array}{l}0.009 \\
0.009\end{array}$ & $\begin{array}{l}9.312 \\
9.383\end{array}$ & $\begin{array}{l}7.92 \\
7.98\end{array}$ \\
\hline 66 & $4 / 14 / 2004$ & 2:21:18 PM & $24.111 \mid$ & 22.193 & 24.017 & 24.155 & 25.736 & 22.097 & 23.358 & 3.575 & 18.459 & -1.218 & $\begin{array}{r}-4.7 \\
\end{array}$ & 44.166 & $\begin{array}{l}0.003 \\
\end{array}$ & 1.246 & 0.006 & 41.13 & 0.68556 & 21.9 & 10.8 & & 1.398 & \begin{tabular}{|l|l|}
0.186 \\
\end{tabular} & & 0.009 & 9.419 & $\frac{7.98}{8.02}$ \\
\hline 67 & $4 / 14 / 2004$ & $2: 22: 18 \mathrm{PM}$ & 24.129 & 22.227 & 24.031 & 24.164 & 25.579 & \begin{tabular}{|l|}
22.274 \\
\end{tabular} & \begin{tabular}{|l|}
23.562 \\
\end{tabular} & 3.566 & \begin{tabular}{|l|l}
18.608 \\
\end{tabular} & -1.215 & -4.694 & 444.203 & $\begin{array}{ll}0.003 \\
\end{array}$ & 1.244 & 0.004 & 42.13 & & 22.1 & $\begin{array}{ll}10.8 \\
\end{array}$ & 20.4 & $\begin{array}{lll}1.409 & \\
\end{array}$ & & 0.191 & \begin{tabular}{l|l|}
0.009 \\
\end{tabular} & & $\frac{8.02}{7.94}$ \\
\hline 68 & 4/14/2004 & 2:23:18 PM & 24.152 & 22.26 & 24.054 & & & & & & \begin{tabular}{|c|c|}
18.58 \\
\end{tabular} & & & & $\mid 0.003$ & 1.242 & & & & 22.0 & $10.8 \mathrm{C} \quad \mathrm{C}$ & & 1.406 & & & & 327 & $\begin{array}{l}7.94 \\
7.94\end{array}$ \\
\hline & $4 / 14 / 2004$ & 2:24:18 PM & 24.15 & & & 24.139 & 25.49 & 22.234 & 23.58 & 3.555 & 18.592 & -1.218 & & 15000 & & 1.244 & & 44.13 & & 22.1 & & & & & 0.191 & & & 7.95 \\
\hline & $4 / 14 / 20$ & 2:25:18 PM & 24.151 & 22.285 & 24.053 & 24.121 & 25.307 & 22.199 & 23.51 & 3.587 & 18.518 & -1.218 & -4.685 & 444.184 & & 1.244 & & & & 22.0 & & & & & & & & 7.96 \\
\hline 71. & 4/14/2004 & $2: 26: 18 \mathrm{PM}$ & 24.163 & 22.312 & 24.09 & 24.118 & 25.429 & 22.153 & 23.533 & 3.561 & 18.492 & -1.218 & -4.68 & 44.174 & 0.0 & 1.249 & & 46.1 & .76889 & 22.1 & 10. & & .40 & .186 & 0.191 & 0.009 & 9.402 & (1) \\
\hline$\frac{72}{73}$ & & Averages & 24.0 & & 24.1 & 24.4 & 26.0 & 22.2 & 23.4 & 3.6 & 18.5 & & & & & & 0.0 & & & 22.0 & $\overline{10 .} \mathrm{C}$ & & & 184 & & & 9.3 & \\
\hline 74 & & Maximum & & & 24.2 & 25.1 & 26.8 & 22.3 & & 3.8 & 18.6 & -1.2 & & 45.1 & & & & & & 22.2 & 110 & & & & & 009 & & $\begin{array}{l}7.9 \\
8.0\end{array}$ \\
\hline 75 & & Median & 24.0 & 22.0 & 24.0 & 24.3 & 26.2 & 22.2 & 23.4 & 3.6 & 18.5 & -1.2 & -4.7 & 44.4 & 0.0 & 1.2 & 0.0 & & & 22.0 & 10.9 & 20.3 & 401 & 184 & 0.190 & 009 & 9.3 & 9 \\
\hline 76 & & & & 21.5 & & & 25.1 & 21.9 & & & 18.1 & -1.2 & -4.7 & $\begin{array}{lll}44.0 \\
\end{array}$ & 0.0 & 1.2 & & & & 21.7 & & & & & & Dog & 9.2 & \\
\hline & & d Dev & 0.306 & 0.471 & 0.173 & 0.640 & & 0.218 & 0.285 & 0.137 & 0.250 & 0.003 & .03 & $\overline{0.527}$ & & 0.022 & & & & 0.285 & 0.129 & & & & 0.004 & & D.141 & \\
\hline & Number o & ff Points Used & 22 & & & & 22 & & 22 & 22 & 22 & 22 & 22 & 22 & 22 & 22 & & & & 22 & 22 & 22 & & & 22 & 22 & 22 & 22 \\
\hline 79 & & Backpulse point & ts in box at & re not incli & Iuded & & & & & & & & & & & & & & & & & & & & & & & \\
\hline 80 & & & & & & & & & & & & & & & & & & & & & & & & & & & & \\
\hline & Baseline 1C & & & & & & & & & & & & & & & & & & & & & & & & & & & \\
\hline 82 & 4/14/2004 & 2:28:37 PM & 24.373 & 22.366 & 24.36 & 24.087| & 25.977 & 32.187 & \begin{tabular}{|l|}
35.755 \\
\end{tabular} & 3.665 & $\begin{array}{l}28.482 \\
2.20\end{array}$ & -1.215 & -4.471 & 44.927| & 0.003 & 1.906 & 0.005 & 48.45 & \begin{tabular}{|l|} 
\\
\end{tabular} & 34.3 & 11.0 & 30.3 & 2.091 & 0.284 & 0.289 & 0.010 & 9.538 & 8.12 \\
\hline 84 & $4 / 14 / 2004$ & $\frac{2: 29: 37 \mathrm{PM}}{2.3037 \mathrm{P}}$ & $\frac{24.4515}{24517}$ & $22.3 / 4 \mid$ & $\frac{24.312}{24284}$ & 24.089 & $\frac{25.999}{26116}$ & \begin{tabular}{l|l}
32.602 \\
32544
\end{tabular} & $\frac{36.0 / 4}{304011}$ & $\begin{array}{l}3.453 \\
3.473 \\
\end{array}$ & $\frac{29.389}{29163}$ & \begin{tabular}{|c|c|c|}
-1.224 \\
1.21
\end{tabular} & $-\frac{-4.466}{446}$ & $\frac{44.097}{43.984}$ & 0.0 & $\frac{1.922}{1.924}$ & & $\frac{49.45}{50.45}$ & $\frac{0.824}{0.846}$ & 34.6 & & & $\frac{13}{12}$ & & 0.29 & 09 & .9.426] & 8.02 \\
\hline 85 & $4 / 114 / 2004$ & $2: 31: 37 \mathrm{P}$ & 24.515 & $\frac{22.438}{22.438}$ & 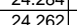 & (24.179 & $\frac{26.116}{26.567}$ & $\frac{3.544}{33.066}$ & 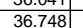 & $\frac{3.473}{3.565}$ & $\begin{array}{r}29.103 \\
29.51\end{array}$ & $\begin{array}{l}-1.221 \\
-1.221 \\
\end{array}$ & $\begin{array}{l}-4.46 \\
-4.45 \\
\end{array}$ & $\begin{array}{l}43.9844 \\
43853\end{array}$ & & & & 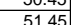 & 0.84 & $\begin{array}{l}34.6 \\
35.3 \\
\end{array}$ & $\frac{10 .}{10}$ & & 15 & & & $\begin{array}{ll}099 \\
09\end{array}$ & & 8.07 \\
\hline 86 & $4 / 14 / 2004$ & 2:32:37 PM & 24.503 & 22.482 & 24.245 & 24.293 & 26.506 & 32.728 & 36.226 & 3.461 & 29.411 & -1.221 & -4.45 & 43.69 & 0.0 & 193 & & 52.45 & 0.87 & $\frac{348}{34.8}$ & 10. & & & & 0.294 & & 9.461 & \\
\hline 87 & 4/114/2004 & 2:33:37 PM & 24.481 & & $\frac{2.212}{24.212}$ & $\frac{24.325}{24}$ & 26.499 & 32.552 & 36.101 & 3.431 & 29.175 & -1.218 & $-4.4 !$ & 43.751 & & $\frac{1.92}{1.92}$ & & $\frac{23.45}{53.45}$ & $\frac{0.89}{0.89}$ & $\frac{34.6}{34.6}$ & $\frac{\frac{10}{10}}{10}$ & 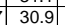 & $\frac{2.12}{2.12}+2$ & & & & & \\
\hline ( & 4/14/2004 & & & 22.563 & & 24.354 & & & & & & & & 44.718 & & & & 54.45 & & 34.6 & & & & & & & & \\
\hline & & 2:35:3 & 24.456 & $22.611 \mid$ & 24.203 & 24.366 & 26.53 & 32.452 & 35.987 & 3.552 & $28.88 \mathrm{~S} \quad \mathrm{C}$ & -1.221 & & 44.606 & 0.0 & 1.907 & & 55.45 & & 34.5 & $\overline{10.9}$ & $30.7 \mathrm{~s}-2=$ & 2.114 & 0.284 & 0.291 & 09 & 9.482 & \\
\hline 90 & 4/14/2004 & 2:36: & 24.413 & 22.628 & 24.145 & 24.3 & 26.6 & 32.216 & 35.751 & 3.48 & & -1.221 & & 44.20 & & & & 56.4 - $>2$ & & 34.3 & & & 1 & & 0.2 & & & 3.14 \\
\hline & $4 / 14 / 2004$ & & & 22.666 & 24.132 & 24.335 & & 32.625 & 36.07 & & & & & 44.257 & & & & & & 34.6 & & & & & & & .450 & . \\
\hline 92 & 4/14/2004 & & $24.393 \mid$ & $22.714 \mid$ & 24.135 & & 26.68 & 32.394 & 35.85 & 3.49 & $28.92 \mathrm{r}>\mathrm{C}$ & & & 44.2 & & & & & & 34.4 & & & & & & & 9.530 & \\
\hline 93 & $4 / 144 / 2$ & 2:39:37 & 24.386 & 22.762 & 24.133 & 24.376 & 26.56 & 32.608 & 36.049 & 3.53 & 29.167 & -1.218 & -4.4 & $44.38 \mathrm{~s}-\mathrm{s}$ & & & & 59.45 & 5.99 & 34.6 & 10 & & 10 & & & & 9.468 & 8.06 \\
\hline 94 & & & 24.353 & 22.794 & 24.115 & 24.3 & & 32.343 & 35.743 & 3.42 & 29.6 & & & 44.0 & & & & 60.45 & & 34.3 & $10.8 \mathrm{r}>\mathrm{r}$ & & & & & & 9.538 & \\
\hline & 4/14t/ & & 24.361 & 22.848 & 24.118 & 24.42 & 26.65 & 32.656 & $30.12]$ & & & & & & & & & & & & & & & & & & & \\
\hline 966 & & & 24.339| & 22.88 & 24.106 & 24.449 & 26.38 & 32.525 & 36.004 & $3.38 \mathrm{~s}-\mathrm{l}$ & 29. & -1.21 & & 43.67 & & 1.92 & & 62.45 & & 34.5 & 10. & & 2.13 & & & & 9.526] & 8.11 \\
\hline $\begin{array}{ll}97 \\
98\end{array}$ & 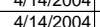 & & 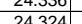 & $\frac{2.953}{22956}$ & 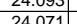 & $\frac{2.51}{245}$ & $\begin{array}{r}26.4 \\
26.328\end{array}$ & 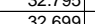 & 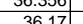 & 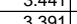 & $\frac{29.4}{29.3}$ & $\begin{array}{l}-1.21 \\
-1.21\end{array}$ & $\begin{array}{l}-4.4 \\
-44\end{array}$ & 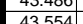 & & & & $\frac{63.45}{64.45}$ & & 34.9 & 10 & 100 & 2140 & & & & 9.468 & 8.06 \\
\hline 99 & $4 / 114 / 2004$ & & 24.312 & 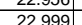 & 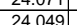 & & & 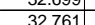 & & & & - & & 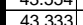 & & & & & & & & & & & & & & \\
\hline & $4 / 14 / 2004$ & & 24.299 & 23.021 & 2036 & 24605 & & 33.008 & & $\frac{3.42}{3.42}-2$ & & -1.1218 & & 43.1 & & & & & & & & & & & & & & \\
\hline & & & 24.307 & 23.065 & $\frac{24.039}{20.93}$ & $\frac{14.578}{24.5}$ & & \begin{tabular}{|c|}
32.37 \\
\end{tabular} & $\mid$ & $\frac{3.32}{3.32} \mathrm{C}$ & & $\frac{-1.221}{-1.221}$ & & & & & & & & & & & & & & & & \\
\hline & $4 / 14 / 2004$ & 2:48:37 PM & 24.289 & 23.087 & \begin{tabular}{|l|l|}
24.021 \\
\end{tabular} & 24.55 & 27.001 & $\begin{array}{l}32.996 \\
\end{array}$ & \begin{tabular}{|l|l|}
36.595 \\
\end{tabular} & 3.39 & 29.66 & -1.218 & -4.41 & 43.142 & 0.003 & 1.934 & 0.004 & 68.45 & 1.1408 & 35.1 & 10.6 & 31.3 & 2.160 & & 0.296 & 0.009 & 9.462 & 8.05 \\
\hline $1(1$ & & race & & & 242 & & & & & & & & & & & & & & & & & & & & & & & \\
\hline & & 部 & 24.4 & $\frac{2.1}{231}$ & 24.4 & $2.4 .64-3$ & 200 & 3. & $\frac{30}{36}$ & 年.5 & 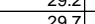 & -1. & & & & & & & & 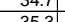 & & & & & & & & \\
\hline & & Median & 24.4 & 22.7 & 24.1 & 24.4 & & $\frac{\mid}{32.6}$ & 36.1 & 3.5 & $\frac{29.2}{29.2}$ & $\frac{-1.2}{-1.2}$ & -4.4 & 43.9 & 0.0 & 1.9 & 0 & & & 34.6 & $\frac{\mid 10.7}{10.7}$ & $\mid$\begin{tabular}{|c|}
$\mid 70.9$ \\
30
\end{tabular} & 2.1. & & & 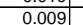 & a.5. & \\
\hline & & Minimum & 24.3 & 22.4 & 24.0 & 24.1 & & 32.2 & 35.7 & 3.3 & 28.5 & $\begin{array}{lll}-1.2 \\
\end{array}$ & & 43.1 & 0.0 & 1. & & & & 34.3 & $\begin{array}{ll}10.6 \\
\end{array}$ & & 2.05 & & & 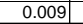 & 9.4 & \\
\hline & & $2 \times \mathrm{Stc}$ & 0.148 & 0.466 & 0.188 & 0.299 & 0.682 & 0.468 & 0.547 & 0.160 & 0.557 & $\mid 0.004$ & 0.034 & 1.006 & 0.000 & $\begin{array}{l}0.019 \\
\end{array}$ & 0.00 & & & 0.547 & 0.247 & 0.501 & \begin{tabular}{|l|l|}
0.035 \\
\end{tabular} & $\begin{array}{ll} & 0.003 \\
\end{array}$ & 0.004 & 0.000 & 0.109 & 0.093 \\
\hline & Number o & of Points & $21 \mid$ & 21 & 21 & 21 & 21 & 21 & 21 & 21 & 21 & 21 & 21 & 21 & 21 & 21 & 21 & & & 21 & 21 & 21 & 21 & 21 & 21 & 21 & 21 & 21 \\
\hline & & ${ }^{*}$ Backpulse poir & sin box a & re not inc & & & & & & & & & & & & & & & & & & & & & & & & \\
\hline & & & & & & & & & & & & & & & & & & & & & & & & & & & & \\
\hline 1 & & & 23.8 & 22.1 & 23.9 & 24 & & 23.2 & 26.2 & 3.6 & 19.5 & -0.9 & & $\begin{array}{lll}44.3 \\
\end{array}$ & 0.2 & 1.1 . & 0.0 & & & 24.7 & 10.9 & 21.3 & $1.47 \mathrm{r}$ & \begin{tabular}{|l|l|}
0.195 \\
\end{tabular} & 0.200 & $\mid 0.009$ & 9.4 & 8.0 \\
\hline & & & 24.5 & & \begin{tabular}{ll|}
24.4 \\
\end{tabular} & 25.1 & & 33.1 & 36.7 & 3.9 & 29.7 & -0.1 & $-1.44,4$ & & 0.6 & 1.9 & $\overline{0.0}$ & & & 35.3 & \begin{tabular}{ll|l}
11.0 \\
\end{tabular} & 31.3 & 2.16 & 0.28 & & & 9.6 & 8.2 \\
\hline & & & 24.1 & & 10 & 24.3 & & 22.2 & & 3.6 & 18.5 & \begin{tabular}{l|l|}
-1.2 \\
\end{tabular} & -4. & 44.4. & & 1.2 & & & & 22.0 & 10.9 & 20.4 & 1.46 & 0.185 & 0.190 & 0.009 & 9.3 & 7.9 \\
\hline & & & 22.7 & 21.3 & 22.9 & 23.2 & 16.5 & 12.0 & \begin{tabular}{|l|}
16.7 \\
\end{tabular} & 3.3 & 8.1 & -1.2 & $\begin{array}{r}-4.7 \\
\end{array}$ & 43.1 & 0.0 & 0.0 & & & & 15.2 & 10.6 & (10.0 & \begin{tabular}{|l|l|}
0.691 \\
\end{tabular} & 0.089 & 0.094 & 009 & 9.2 & 7.8 \\
\hline & & $2 \times$ Std Dev & 1.183 & 1.032 & $\begin{array}{l}0.847 \\
\end{array}$ & 0.885 & 5.368 & 16.138 & 15.654 & 0.27 & 16.587 & 0.964 & 2.786 & & & & 02 & & & 15.654 & 0.236 & 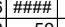 & \begin{tabular}{|r|r|}
1.128 \\
\end{tabular} & 0.156 & 0.157 & 000 & 0.238 & 0.202 \\
\hline & Numbe & $\begin{array}{l}\text { of Points Used } \\
\text { |* Backpulse poin }\end{array}$ & $\begin{array}{l}59 \\
\text { ts in box a }\end{array}$ & $\frac{59}{\text { ree not incll }}$ & luded 59 & & 59 & & 59 & & 59 & 59 & & & & 59 & 59 & & & 59 & 59 & 59 [ & 59 & 59] & 59 & 59 & 59] & 99 \\
\hline
\end{tabular}


WSRC-TR-2005-00105, REVISION 0 SRNL-RPP-2005-00012, REVISION 0

RUN \# 2.03A AND B; FIRST AND SECOND HALF OF SLURRY DEWATERING

RPP-WTP Pilot-scale X-flow Filtration Test No. 2.03 - Campaign II

$\left[\mathrm{V}=12.0 \mathrm{ft} / \mathrm{s}, \mathrm{TMP}=40.1 \mathrm{psid}\right.$, Filter Pres. $\left.=51.6 \mathrm{psig}, \mathrm{T}=25.1^{\circ} \mathrm{C}\right]$

[19-22 April 2004: Dewatering]

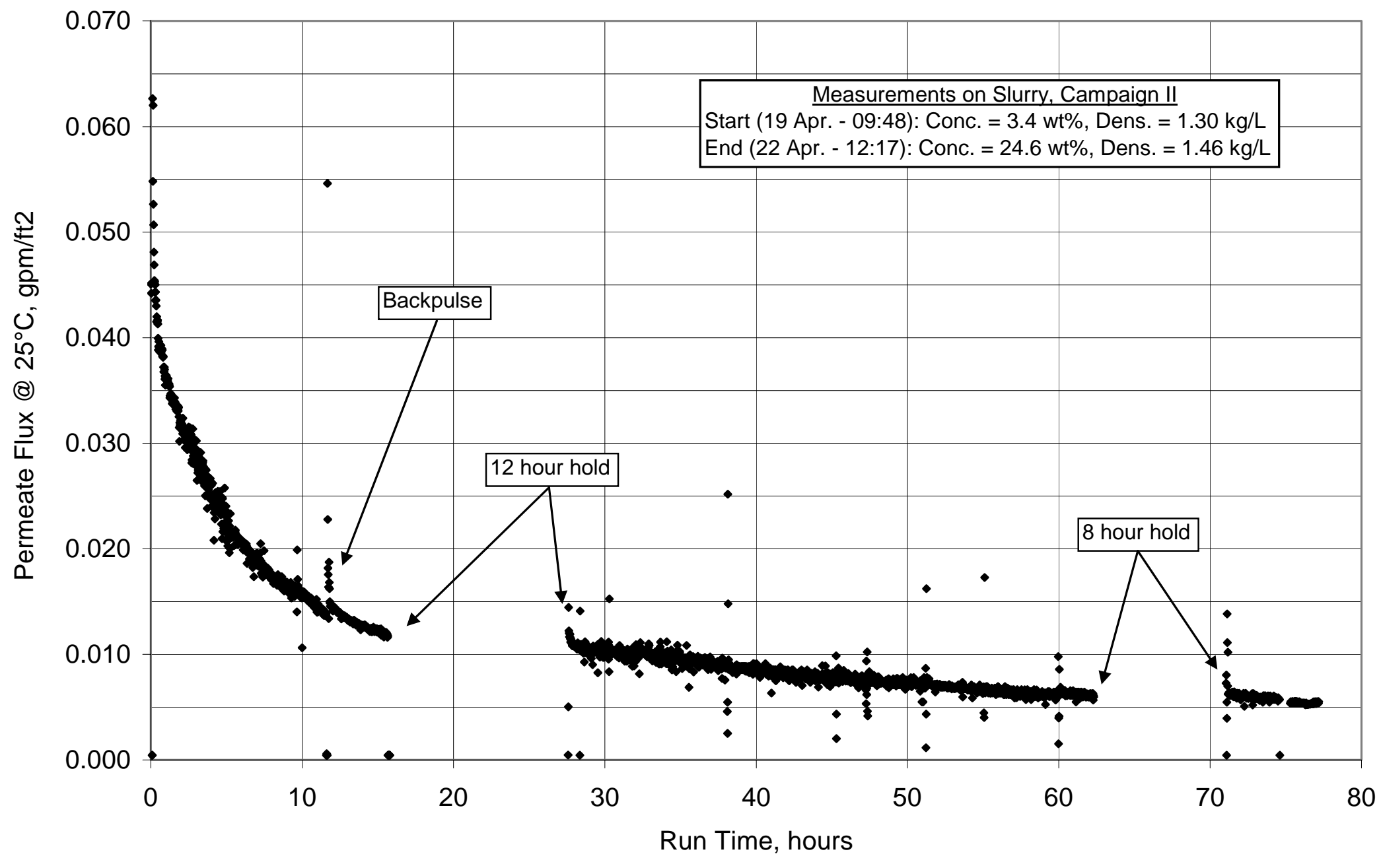


WSRC-TR-2005-00105, REVISION 0

SRNL-RPP-2005-00012, REVISION 0

RUN \# 2.03A AND B; FIRST AND SECOND HALF OF SLURRY DEWATERING - CONT.

\begin{tabular}{|c|c|c|c|c|c|c|c|c|c|c|c|c|c|c|c|c|c|c|c|c|c|c|c|c|c|c|c|c|}
\hline & A & B & $\mathrm{D}$ & $E$ & $F$ & $G$ & $\mathrm{H}$ & $\mathrm{J}$ & $\mathrm{K}$ & $\frac{\mathrm{L}}{\mathrm{gscH}}$ & $M$ & $\frac{1}{N}$ & 0 & $\mathrm{Q}$ & $R$ & $\mathrm{~s}$ & $T$ & $\mathrm{v}$ & $\mid w$ & $x$ & \begin{tabular}{|l|l|}
$Y$ \\
\end{tabular} & & AA & $A B$ & $\mathrm{AC}$ & $A D$ & $\mathrm{AE}$ & \\
\hline & DATE & TIME & Filtrate & Cleaning & Slurry & Hi Amb. & $\begin{array}{l}\text { ents }>>> \\
\text { Lo Amb. }\end{array}$ & BotTMP & Filter & Filter dP & $\begin{array}{l}\text { Measureme| } \\
\text { TopTMP }\end{array}$ & 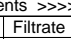 & 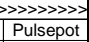 & Slurry & $\begin{array}{l}<<<\text { Flow I } \\
\text { | Filtrate }\end{array}$ & $\begin{array}{l}\text { Measureme } \\
\text { Hi Filtate }\end{array}$ & Backpulse & & & & & & & & & & & \\
\hline & & & $\frac{\operatorname{deg} C}{\operatorname{deg}}$ & deg $\mathrm{C}$ & deg $\mathrm{C}$ & $\operatorname{deg} \mathrm{C}$ & $\operatorname{deg} C$ & psid & psig & psid & psid & psig & psig & $\mathrm{gpm}$ & & $\mathrm{gpm}$ & & & & & & & & & & & & \\
\hline & & & & & $\mathrm{T} 1$ & & T5 & & $\mathrm{P1}$ & $\mathrm{dP1}$ & & $\mathrm{P2}$ & & & $\mathrm{Q}^{2}$ & 03 & Qbp & & & & & & & & & & & \\
\hline & & /2004 & & & & & & & & & & & & & & & & & & & & & & & & & & \\
\hline 77 & $4 / 14 / 2004$ & 1:30:58 PM & 24.687 & 21.862 & 24.835 & 24.226 & 22.583 & $\begin{array}{ll}0.054 \\
\end{array}$ & 0.02 & -0.021 & -0.005 & \begin{tabular}{|l|l|} 
& -0.179 \\
\end{tabular} & 0.099 & $\begin{array}{c}-0.08 \\
\end{array}$ & $\begin{array}{l}0.003 \\
\end{array}$ & $\begin{array}{l}0.086 \\
\end{array}$ & 0.00 & & & & & & & & & & & \\
\hline & 4/19/2004 & 7:37:13 AM & 22.595 & 22.228 & 23.926 & 21.772 & 22.175 & 0.046 & 0.082 & -0.025 & $\begin{array}{ll}-0.009 \\
\end{array}$ & \begin{tabular}{|l|l|} 
& -0.179 \\
\end{tabular} & 0.143 & -0.086 & 0.003 & 14.643 & 0.003 & & & Conver: & irsion & 851 & $\mathrm{~m} 3 / \mathrm{m} 2$ & $2 /$ day/barc & $\mathrm{rg} / \mathrm{gpm} / \mathrm{tt}$ & ft2/barg & & \\
\hline & & & & & & & & & & & & & & & & \begin{tabular}{l|l|l|l|l}
14.643 \\
\end{tabular} & & & & & & & & & & & & \\
\hline & $4 / 20 / 2004$ & 12:41:36 PM & 22.772 & 22.767 & 22.049 & 23.603 & 24.746 & -0.004 & 0.045 & -0.009 & -0.007 & -0.176 & 0.108 & -0.084 & 0.003 & \begin{tabular}{|l|l|l|l|l}
14.643 \\
\end{tabular} & & & & Note & auge & (ces & & ppro & ately 1 & 4 psig & & \\
\hline & $4 / 22 / 2004$ & 8:40:43 AM & 22.558 & 21.446 & & & 22.489 & 0.01 & & 0.001 & 0.001 & & 0.096 & 46.562 & 0.043 & $\begin{array}{l}14.643 \\
\end{array}$ & & & & & & & & & & & & \\
\hline & $4 / 23 / 2004$ & 8:53:20 AM & 21.548 & 21.737 & 22.041 & 21.89 & 21.369 & -0.004 & 0.026 & 0.003 & -0.005 & -0.196 & 0.08 & -0.082 & 0.003 & 14.643 & 0.00 & & & & & & & & & er th & & \\
\hline & $4 / 2012004$ & 9:00:47 AM & & & & & & & & & & & & & & & 0.000 & & & WWV & & & & & & & & \\
\hline & $4 / 27 / 2004$ & 9:14:59 AM & 20.374 & 19.305 & 19.368 & 20.841 & 19.777 & -0.002 & 0.02 & -0.001 & 0.005 & -0.193 & 0.111 & -0.084 & 0.003 & 14.643 & 0.006 & & & & & & & & at $25 \mathrm{C}$ & & $\begin{array}{l}\times 1000 \\
\end{array}$ & \\
\hline 16 & Data - Per M & inute & & & & & & & & & & & & & & & & $\begin{array}{l}\text { Mine } \\
\text { Min. }\end{array}$ & $\begin{array}{l}\text { Time } \\
\text { Hour }\end{array}$ & $\begin{array}{l}\text { Press } \\
\text { psig }\end{array}$ & 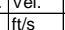 & $\frac{\mathrm{TMP}}{\mathrm{psi}}$ & $\begin{array}{l}\text { TMPP } \\
\text { bar }\end{array}$ & $\frac{\mathrm{gpm}}{1 \mathrm{tin}}$ & $\mathrm{gpm}$ & $\frac{p m}{2 / p s i}$ & $\mathrm{gpm}$ & $\begin{array}{l}\text { meter } \\
\text { dayphas }\end{array}$ \\
\hline 17 & $4 / 19 / 2004$ & 9:42:07 AM & & 22.072 & 26.109 & 23.471 & 23.694 & 43.936 & 45.793 & 9.157 & 35.531 & -1.215 & -4.679 & 49.877 & 0.312 & $\begin{array}{ll}14.643 \\
\end{array}$ & 0.00 & & & 44.3 & 12.2 & 39.7 & 2.739 & 0.047 & 0.045 & 0.001 & 1.135 & \\
\hline & $4 / 19 / 2004$ & 9:43:14 AM & & & & & 23.694 & & & & & & -4.679 & & & 14.643 & & & 0.01861 & $\begin{array}{lll}4.3 \\
\end{array}$ & & 39.7 & & 0.047 & 0.045 & 0.001 & & \\
\hline & $4 / 19 / 2004$ & 9:44:14 AM & 25.711 & 22.117 & $\begin{array}{r}25.889 \\
25.795 \\
\end{array}$ & 23.331 & 23.629 & 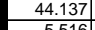 & 45.876 & 8.933 & 35.946 & \begin{tabular}{|l|l|} 
& -1.215 \\
\end{tabular} & -4.882 & 49.531 & 0.304 & 14.643 & 0.003 & & 0.03528 & 44.4 & 12.1 & 40.0 & 2.761 & 0.045 & 0.044 & 0.001 & 1.104 & \\
\hline & 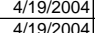 & $\begin{array}{l}9: 45: 14 \mathrm{AM} \\
\cdot 96 \cdot 14 \mathrm{AM}\end{array}$ & 26.071 & $\frac{22.123}{22.128}$ & \begin{tabular}{|r|}
25.785 \\
25.69
\end{tabular} & $\frac{23.282}{23.262}$ & $\begin{array}{r}23.625 \\
23.44 \\
\end{array}$ & \begin{tabular}{|c|}
5.516 \\
6.464 \\
\end{tabular} & \begin{tabular}{|l|}
45.957 \\
46678 \\
\end{tabular} & 8.655 & -2.532 & \begin{tabular}{|l|l|}
35.022 \\
34791
\end{tabular} & $\begin{array}{r}19.234 \\
20578\end{array}$ & $\begin{array}{r}49.82 \\
7511\end{array}$ & 0.004 & $\begin{array}{r}14.643 \\
14643\end{array}$ & 0.000 & & $\begin{array}{l}0.05194 \\
0.06861\end{array}$ & 44.5 & 12.2 & $\begin{array}{l}1.5 \\
24\end{array}$ & & $\frac{2.183}{2.183}$ & $\begin{array}{r}2.136 \\
2.41\end{array}$ & 1.431 & & \\
\hline & $\begin{array}{l}4 / 1 / 19204 \\
4 / 1 / 2004\end{array}$ & $9.40 .14 \mathrm{Am}$ & $\frac{25.897}{25.838}$ & $\begin{aligned} 22.128 \\
22.16\end{aligned}$ & $\begin{array}{r}25.69 \\
25.651 \\
\end{array}$ & $\frac{23.262}{23.228}$ & $\begin{array}{r}23.64 \\
23.651\end{array}$ & $\begin{array}{l}6.464 \\
6.126 \\
\end{array}$ & \begin{tabular}{|l|}
45.6486 \\
55.406
\end{tabular} & $\begin{array}{l}8.132 \\
8.863 \\
\end{array}$ & $\begin{array}{c}-1.596 \\
-2.049 \\
\end{array}$ & \begin{tabular}{|l|l|}
34.191 \\
\end{tabular} & $\begin{array}{l}20.5 / 8 \\
19.188\end{array}$ & $\frac{47.511}{49.952}$ & $\begin{array}{l}0.003 \\
0.003\end{array}$ & $\frac{14.643}{14.643}$ & & & . 0.06861 & $\frac{45.2}{43 .}$ & 11.6 & 2.4 & $\frac{0.168}{0.141}$ & 2.183 & & 0.880 & & \\
\hline 23 & $4 / 19 / 2004$ & 9:48:14 AM & 25.144 & 22.156 & 25.562 & 23.214 & 23.447 & 5.884 & 45.723 & 8.557 & -1.965 & 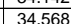 & 46.307 & 50.267 & 0.003 & 14.643 & & & 0.10194 & 44 & 123 & & 0.14 & 0.000 & 500 & $\frac{0.000}{0.000}$ & 0.225 & \\
\hline & $4 / 19 / 2004$ & 9:49:14 AM & 25.49 & 22.156 & 25.488 & 23.214 & 23.593 & & 45.671 & 8.52 & 30.425 & 1.706 & 40.001 & & 0.426 & 14.643 & & &. .10154 & 44. & 12. & & & 0.064 & & & & \\
\hline & & & & 22.173 & \begin{tabular}{|l|}
25.419 \\
\end{tabular} & & 23.69 & 38.345 & 45.675 & 8.572 & 30.436 & 1.585 & & 49.218 & 0.372 & 14.643 & & & & 44.2 & & 34. & & & & & & \\
\hline & $4 / 19 / 2004$ & 9:51:14 AM & 25.852 & \begin{tabular}{l|l}
22.184 \\
\end{tabular} & \begin{tabular}{|l|}
25.351 \\
\end{tabular} & 23.242 & 23.811 & \begin{tabular}{l|l|}
42.996 \\
\end{tabular} & 446.166 & 8.507 & 35.199 & -1.218 & -3.743 & 47.995 & 0.42 & $\begin{array}{l}14.643 \\
\end{array}$ & & 9 & $\overline{0.15194}$ & 44.7 & 11.8 & 39.1 & $2.696 \mathrm{C}$ & 0.063 & 0.062 & & & \\
\hline & $4 / 19 / 2004$ & 9:52:14 AM & & 22.174 & & & 20.011 & & & & & & & & 0.356 & 14.643 & & & .1000 & & & 40. & & & & & & \\
\hline & $4 / 19 / 2004$ & 9:53:14 AM & 25.717 & 22.179 & 25.21 & 23.222 & 23.851 & 44.234 & 46.328 & 8.488 & 36.401 & -1.218 & $-4.78 \mathrm{E}$ & 48.312 & 0.342 & 14.643 & & 11 & 0.18528 & 44. & 11.8 & 40.3 & 2.78 & 0.051 & 0.051 & 0.001 & 1.257 & \\
\hline & 4/199/2004 & 9:54:14 AM & 25.634 & 22.18 & 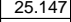 & 23.218 & 23.882 & 44.286 & 46.175 & 8.542 & 36.514 & -1.221 & -4.91 & 48.775 & 0.324 & 14.643 & & & 0.20194 & 44. & 12. & 40.4 & 2.785 & 0.048 & 0.048 & 0.001 & 1.191 & \\
\hline & $4 / 199 / 2004$ & 9:55:14 AM & 25.594 & 22.206 & 25.162 & 23.269 & 23.842 & 44.639 & & & & & & 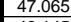 & 0.316 & 14.643 & & & 21861 & 44.8 & 11.5 & 40.8 & & .047 & 0.047 & & & \\
\hline & 4119922004 & $\begin{array}{l}9: 5: 14: 14 \mathrm{AM} \\
\end{array}$ & $\frac{2.54}{25.511}$ & $\begin{array}{ll}22.201 \\
22102\end{array}$ & 25.168 & 203.295 & 23.844 & 44.697? & \begin{tabular}{|l|l|l|} 
\\
\end{tabular} & 9.28 & $\begin{array}{l}36.054 \\
36.290\end{array}$ & -1.218 & 5 & $\begin{array}{l}49.145 \\
5.211\end{array}$ & 0.306 & 14.643 & & . & 0 & 44.8 & 12.0 & 40.4 & 2.1844 & 0.046 & 0.045 & 001 & & \\
\hline & 411912004 & & 25.511 & $\begin{array}{l}22.193 \\
22194\end{array}$ & & & 23.845 & (44.8506 & \begin{tabular}{|l|l|}
40.478 \\
\end{tabular} & $\begin{array}{l}9.244 \\
0201\end{array}$ & - & $\frac{-1.218}{-1210}$ & & 52.811 & $\begin{array}{l}0.305 \\
0308\end{array}$ & $\begin{array}{l}14.643 \\
1.643\end{array}$ & & & 0.25194 & & & 40.6 & & & 0.045 & & & \\
\hline & $\begin{array}{l}4 / 1 / 19 / 2004 \\
\end{array}$ & $9.0 .0 .4 \mathrm{Am}$ & $\begin{array}{l}25.404 \\
25483\end{array}$ & $\frac{26.194}{22195}$ & $\frac{25.12}{25.111}$ & $\frac{15.242}{23228}$ & $\frac{24.030}{2.112}$ & 年 45.2035 & $\frac{44.6}{46.778}$ & $\frac{.41}{9.12}$ & $\begin{array}{l}30.005 \\
36773\end{array}$ & - & $\frac{-301}{-505}$ & 4659 & $\begin{array}{l}0.290 \\
0.303\end{array}$ & 14.045 & & 17 & $\frac{0.2801}{02858}$ & 年 45. & $\frac{12 .}{114}$ & 年 & $\mid$ & 0.045 & 0 & 0.001 & t..010 & \\
\hline & $4 / 19 / 2004$ & $10: 00: 14 \mathrm{AM}$ & 25.435 & 22.196 & \begin{tabular}{|l|}
25.111 \\
\end{tabular} & 23.239 & 24.204 & 45.321 & 46.871 & $\begin{array}{r}9.118 \\
\end{array}$ & 37.038 & \begin{tabular}{|l|}
-1.218 \\
\end{tabular} & -5.08 & 50.223 & 0.298 & $\begin{array}{ll}14.045 \\
14.643\end{array}$ & 0.00 & 18 & 0.30194 & 45.4 & \begin{tabular}{l|l}
4.43 \\
\end{tabular} & 41.2. & 2.839 & 0.044 & 0.044 & 0.001 & 1.076 & \\
\hline & & & & & & & & & & & & & & & & 14.643 & & 10 & & 45.4 & & 110 & & .044 & & & & \\
\hline 37 & $4 / 19 / 2004$ & 10:02:14 & $\begin{array}{l}25.418 \\
25\end{array}$ & 22.204 & 25.11 & 23.338 & 24.467 & 45.419 & 47.113 & 9.107 & & -1.21 & & $\frac{48.4}{48.4}$ & 0.293 & 14.6 & & 20 & 33528 & 45. & 11.5 & 41 & & & $\overline{0 .}$ & & & \\
\hline & $4 / 19 / 2004$ & $10: 03: 14$ & & & 25.087 & 23.4 & 24.554 & 45.539 & \begin{tabular}{|l|l|l|}
46.838 \\
\end{tabular} & & & $\frac{-1.21}{-1.21}$ & & 45.9 & 0.293 & 14.6 & & 21 & .35194 & 45.4 & 11.3 & 41.4 & & 044 & 0.044 & & & \\
\hline & $4 / 19 / 2004$ & $10: 04: 14$ & 25.35 & 22.217 & 25.063 & 23.501 & & \begin{tabular}{|l|l|}
44.942 \\
\end{tabular} & \begin{tabular}{|l|l|l}
46.423 \\
\end{tabular} & 8.949 & & -1.21 & & & & 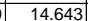 & & 22 & & 45.6 & 12.1 & 40.8 & & & 0.043 & & & \\
\hline 40 & $4 / 19 / 2004$ & 10:05:14 AM & 25.322 & 22.244 & \begin{tabular}{|l|}
25.054 \\
\end{tabular} & 23.598 & 24.816 & $\begin{array}{l}44.776 \\
\end{array}$ & \begin{tabular}{|l|l|l|}
46.085 \\
\end{tabular} & 9.528 & 35.958 & -1.215 & & 50.188 & 0.279 & $\begin{array}{l}14.643 \\
\end{array}$ & & 23 & & 44. & 12.3 & & & & 0.042 & & & \\
\hline & 4/199/2004 & 10:06:14 AM & 25.325 & 22.267 & $\begin{array}{l}25.042 \\
\end{array}$ & 23.641 & 24.864 & 44.902 & 46.156 & 9.19 & 36.621 & -1.218 & & 52.824 & 0.282 & \begin{tabular}{l|l|l|l}
14.643 \\
\end{tabular} & & 24. & 0.40194 & 44.7 & 12.9 & 40.8 & 2.810 & 0.042 & 0.042 & 0.001 & 1.030 & \\
\hline 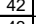 & $4 / 19 / 2004$ & 10:07:14 AM & 20.011 & 22.284 & 20.00 & 23.679 & 24.921 & 45.23 & 46.577 & 9.211 & 36.826 & -1.218 & 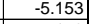 & 47.442 & 0.279 & 14.643 & & 20 & 0.41001 & 45. & 11.6 & $4 \pm$. & 2.02 & 0.042 & 0.044 & 0.00 & 1.012 & \\
\hline & & & & & & & & & & & & & & & & & & & & & 12.5 & & & & & & & \\
\hline & 411992004 & $10: 0: 0: 14 \mathrm{~A}$ & 25.312 & 22.339 & 25.01 & 23.648 & 24.960 & 45.1266 & 46.43 & 9.136 & $\frac{36.713}{37.207}$ & - -1.1218 & -5.1 & 48.342 & 0.28 & 14.643 & & 27. & 0.45194 & 45.6 & 11.8 & 40.5 & & & 0.042 & & & \\
\hline 46 & $\begin{array}{ll}4 / 1 / 19 / 2004 \\
\end{array}$ & 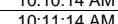 & $\frac{25.204}{25225}$ & $\frac{2.32}{22.352}$ & 25012 & $\frac{20.304}{23546}$ & $\frac{24.071}{24794}$ & 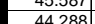 & & $\frac{3.254}{9.74}$ & & $\frac{1.244}{-1218}$ & & $\frac{46.035}{51742}$ & $\frac{0.271}{0268}$ & $\frac{14.043}{14643}$ & & $\frac{2.1}{291}$ & 0.40010 & 45. & $\frac{11.0}{127}$ & 41. & & & & & & \\
\hline & $4 / 1 / 912004$ & 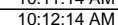 & 25.107 & 22365 & 25.075 & 23.533 & & 44.124 & 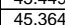 & 9.53 & & $\frac{1.2118}{-1218}$ & & & $\frac{.2 .261}{0.261}$ & & & $\frac{20}{30}$ & & 439 & 12. & 39.7 & & 039 & 0.079 & & & \\
\hline & $4 / 11 / 2004$ & $10 \cdot 13 \cdot 14$ & 25.059 & 22367 & 25057 & 23.495 & & 44139 & 45159 & 9514 & 35. & -1218 & -5 & & & & & & & 43 & 117 & & & & & & & \\
\hline 49 & & $10.14 \cdot 14$ & 25026 & 22369 & 25.039 & 23.497 & & 44.622 & 45847 & 9366 & & -121 & & & & & & & & 44.4 & 123 & & & & & & & \\
\hline 50 & 4/19/2004 & $10: 15 \cdot 14$ & 25.004 & 22.377 & 25.011 & 23.495 & 2470 & 44.49 & 455.544 & 9.138 & & -1.218 & & 48.9 & 0.263 & 14.6 & & 33. & 0.55194 & 44.1 & 120 & 40.3 & 2781 & 0.039 & 0.039 & & & \\
\hline & $4 / 19 / 2004$ & $10: 16: 14$ & 24.975 & 22.378 & 24.983 & 23.486 & & 44.514 & 455.6 & & & -1.22 & & & & & & & 0.56 & 44.2 & 12.3 & 40.3 & & & & & & \\
\hline & 4/19/2004 & 10:17:14 & 24.951 & 22.375 & \begin{tabular}{|l|}
24.964 \\
\end{tabular} & 23.478 & & $\begin{array}{l}44.301 \\
\end{array}$ & 45.489 & 9.159 & 35.993 & $-1.21 \varepsilon$ & & 49.19 & 0.259 & 14. & & 35 & 0.58 & 44. & 12.1 & & 2.768 & 339 & 039 & & & \\
\hline & $4 / 199 / 2004$ & 10:18:14 & 24.948 & 22.386 & \begin{tabular}{|l}
25.026 \\
\end{tabular} & 23.469 & & \begin{tabular}{|l|l|}
48.78 \\
\end{tabular} & 45.92 & 9.227 & 36. & -1.21 & -5. & 49.5 & 0.262 & 14.643 & & 36 & 0.60194 & 44.4 & 12.1 & & 2.14 & .039 & 0.039 & 0.001 & 1.963 & \\
\hline & & 10:19:14 & & & & 23.436 & & 44.5 & & & & -1.2 & & & & & & & & & 12.0 & & & & & & & \\
\hline 55 & $4 / 199 / 2004$ & 10:20:14 & 24.927 & 22.376 & 24.975 & 23.423 & & 44.753 & 45.772 & & & -1.215 & & $49.8 \mathrm{r}$ & 0.261 & & & & & 44. & 12.2 & 40. & 2.194 & 339 & & & & \\
\hline & & $10: 21.14$ & & 22,3 & 24.952 & 23.435 & & & 45.28 & & & & & & & & & & & & 11.8 & & & & & & & \\
\hline 8. & 4 & 10.22 .44 & $\frac{24.96}{2488}$ & $\frac{22.305}{22.35}$ & $\frac{2.493}{2491}$ & $\frac{23.422}{23403}$ & & 年45.144 & 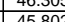 & & & & & & & & & & & 44. & $\frac{12.1}{116}$ & & & & & & & \\
\hline 59 & $4 / 19 / 2004$ & $10: 24: 14$ & 24.802 & 22.351 & \begin{tabular}{|l|}
24.911 \\
\end{tabular} & 23.440 & & 45..002 & \begin{tabular}{|l|l}
4.0 .06 \\
46.17
\end{tabular} & 9.12 & 36.574 & -1.215 & & 49.2 & $\frac{0.200}{0.26}$ & $\frac{14.0}{14.6}$ & & 42.1 & & 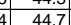 & $\frac{1.50}{121}$ & 40.8 & 3812 & 0.039 & 0.039 & & & \\
\hline 60 & $4 / 19 / 2004$ & $10: 25: 14$ & 24.859 & 22.347 & 24.972 & 23.385 & & 44.973 & 46.065 & 8.95 & & -1.218 & & & 0.26 & & & & & 44.6 & 11.8 & 40. & & & & & & \\
\hline & & $10: 26: 14$ & 24.859 & 22.332 & 24.952 & 23.4 & & 45.182 & 46.293 & 8.925 & 36 & -1.221 & & 48.5 & 0.261 & & & & & 44.8 & 11.9 & & & 39 & & & & \\
\hline & & & 25026 & 22.349 & 24.939 & 23.472 & & 45.578 & $46.52^{\circ}$ & 8.976 & 37.306 & -1.218 & & 50.703 & 0.26 & & & & & 45 & 124 & & & & & & & \\
\hline & $4 / 19 / 2004$ & 10:28:14 & 24.938 & 22.351 & 24.911 & 23.504 & & 45.134 & 46.079 & 8.86 & 37.023 & -1.224 & -5. & & 0.256 & 14.6 & & & 076 & 44. & 11.8 & & & & & & & \\
\hline 64 & $4 / 1912$ & $10: 29: 14$ & 24.92 & 22.3 & 24. & 23.651 & & 45.657 & 46.747 & & & $-1.21 \varepsilon$ & & & & & & & & & $\overline{12.1}$ & & & & & & & \\
\hline 6 & $4 / 199 / 2004$ & 10:30: & 24.87 & 22.374 & 24.879 & 23.708 & & 45.416 & 46.293 & 8.908 & 37.2 & -1.221 & & & 0.2 & & & 48 & & 44.8 & 11.4 & 41.3 & $82-3$ & 0.038 & 0.038 & & 23 & \\
\hline 66 & 4/199/2004 & $10: 31: 14 \mathrm{~A}$ & 24.847 & 22.38 & 24.93 & 23.805 & & 45.4211 & 46.236 & 8.8 & & & & $48 . \varepsilon$ & 0.256 & 14. & & & & 44.8 & 12.0 & 14.5 & & & & & & \\
\hline & $4 / 19 / 2004$ & 10:32:14A & 24.864 & 22.418 & \begin{tabular}{|l}
24.942 \\
\end{tabular} & 23.882 & & 45.1671 & 46.672 & 8.134 & 37.877 & -1.215 & & 47.365 & & & & $5 c$ & & 45.2 & $11.6 \mathrm{C}$ & 年1.8 & 2.000 & 0.038 & & & & \\
\hline & $4 / 19 / 2004$ & 10.33 .14 & 24.8057 & 22.435 & 24.925 & 23.87 & & $45.1 \pi$ & & 9.089 & & - -1.218 & & 48.646 & & & & 51 & & 44.6 & 11.9 & 41.0 & & 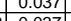 & & & 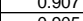 & \\
\hline & & $10 \cdot 35 \cdot 14 \mathrm{AM}$ & 2484 & 22469 & 24913 & 23888 & & 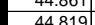 & 45523 & 9351 & $\begin{array}{l}36.311 \\
36212 \\
\end{array}$ & -1218 & & $\frac{15.25}{49.02}$ & 0246 & 14.643 & & 53.1 & 1088528 & 440 & 120 & 40.5 & 2793 & 0.037 & 0.037 & 0.001 & 0.907 & \\
\hline & & & & & & & & & & & & & & & & & & & & & & & & & & & & \\
\hline
\end{tabular}


WSRC-TR-2005-00105, REVISION 0

SRNL-RPP-2005-00012, REVISION 0

RUN \# 2.03A AND B; FIRST AND SECOND HALF OF SLURRY DEWATERING - CONT.

\begin{tabular}{|c|c|c|c|c|c|c|c|c|c|c|c|c|c|c|c|c|c|c|c|c|c|c|c|c|c|c|c|c|}
\hline & & & & & & & & & & & & & & & & & & & & & & & & & & & & \\
\hline & A & B & D & $E$ & $F$ & $G$ & $\mathrm{H}$ & $\mathrm{J}$ & $\mathrm{K}$ & $\mathrm{L}$ & $\mathrm{M}$ & $\mathrm{N}$ & $\mathrm{O}$ & $\frac{Q}{Q}$ & $\mathrm{R}$ & $\mathrm{s}$ & \begin{tabular}{ll|}
$\mathrm{T}$ \\
\end{tabular} & & w & $x$ & S & $\mathrm{z}$ & AA & $\mathrm{AB}$ & $A C$ & $A D$ & $\mathrm{AE}$ & \\
\hline & $\begin{array}{l}4 / 1 / 19 / 2004 \\
4 / 1 / 2004\end{array}$ & $\begin{array}{l}10: 36: 14 \mathrm{AM} \\
10.37 .14 \mathrm{AM}\end{array}$ & $\begin{aligned} 24.837 \\
24.82\end{aligned}$ & & \begin{tabular}{|r|}
24.91 \\
24.999 \\
\end{tabular} & \begin{tabular}{|r|}
23.87 \\
23.863 \\
\end{tabular} & $\begin{array}{l}25.257 \\
2.385 \\
\end{array}$ & $\begin{array}{l}45.062 \\
45.091\end{array}$ & \begin{tabular}{|r|}
45.99 \\
46007
\end{tabular} & $\begin{array}{l}9.245 \\
9.069 \\
\end{array}$ & $\begin{array}{ll}36.577 \\
36.77 \\
\end{array}$ & \begin{tabular}{|l|l|}
-1.218 \\
-1.218 \\
\end{tabular} & $\begin{array}{l}-5.469 \\
-5.463 \\
\end{array}$ & $\begin{array}{ll}48.033 \\
48.917\end{array}$ & $\begin{array}{l}0.249 \\
0.28\end{array}$ & $\begin{array}{ll}14.643 \\
14.643 \\
\end{array}$ & $\begin{array}{l}0.003 \\
0.003 \\
\end{array}$ & 54. & $\begin{array}{l}0.90194 \\
0.91861 \\
\end{array}$ & $\begin{array}{l}44.5 \\
4.5 \\
\end{array}$ & \begin{tabular}{|l|l|l|}
11.8 \\
120
\end{tabular} & \begin{tabular}{|l|l|}
40.8 \\
40.9
\end{tabular} & & $\frac{0.037}{0.037}$ & $\begin{array}{l}0.037 \\
0.037 \\
\end{array}$ & $\begin{array}{l}0.001 \\
0.001\end{array}$ & \begin{tabular}{|l|l|}
0.912 \\
0.903
\end{tabular} & $\begin{array}{l}0.78 \\
0.77 \\
\end{array}$ \\
\hline & & & $\frac{24.82}{24.829}$ & $\begin{array}{ll}22.499 \\
22.517\end{array}$ & \begin{tabular}{|l|}
24.999 \\
24.997 \\
\end{tabular} & \begin{tabular}{|l|}
23.8636 \\
23.856 \\
\end{tabular} & $\frac{25.385}{25.448}$ & $\begin{array}{l}454.091 \\
44.485 \\
\end{array}$ & $\begin{array}{l}45.3014 \\
45.314 \\
\end{array}$ & $\begin{array}{l}9.0695 \\
9.525\end{array}$ & $\frac{35.17}{35.722}$ & \begin{tabular}{|l|l|}
-1.218 \\
-1.218 \\
\end{tabular} & $\begin{array}{l}-5.463 \\
-5.547 \\
\end{array}$ & $\begin{array}{l}\frac{48.917}{52.797} \\
\end{array}$ & $\begin{array}{l}0.248 \\
0.242 \\
\end{array}$ & \begin{tabular}{|l|}
14.6433 \\
14.643 \\
\end{tabular} & & & & & & & & & & & & \\
\hline 7 & $4 / 19 / 2004$ & \begin{tabular}{|l|l|}
$10: 39: 14 ~ A M$ \\
\end{tabular} & 24.842 & 22.526 & & 23.849 & 25.361 & $\begin{array}{r}44.4059 \\
\end{array}$ & 45.708 & 9.449 & 36.08 & \begin{tabular}{|l|l|}
-1.218 \\
\end{tabular} & -5.532 & 49.975 & 0.244 & \begin{tabular}{|l|}
14.643 \\
\end{tabular} & & 57 & 0.95194 & 44.2 & 12.2 & 40.4 & 2.788 & 0.036 & 0.036 & 0.001 & 0.900 & 0.77 \\
\hline & $4 / 19 / 2004$ & $10: 40: 14 \mathrm{AM}$ & 24.824 & & 24.987 & \begin{tabular}{|l|l|}
23.847 \\
\end{tabular} & 25.154 & 45.197 & 45.988 & 9.18 & 36.77 & -1.215 & -5.469 & 48.006 & 0.238 & 14.643 & & 58.1 & 0.96861 & 44.5 & \begin{tabular}{|l|l|}
11.8 \\
\end{tabular} & & & & & 0.001 & 0.866 & 0.74 \\
\hline & $4 / 19 / 2004$ & & & & & & & & 46.085 & 9.281 & & & & & 0.243 & 14.643 & & 59.1 & & 44.6 & & & & & & & & \\
\hline & $4 / 19 / 2004$ & \begin{tabular}{|l|l|}
$10: 42: 14 ~ A M$ \\
\end{tabular} & 24.784 & $\begin{array}{l}22.513 \\
22525 \\
2.525\end{array}$ & \begin{tabular}{|l|}
24.937 \\
2.030
\end{tabular} & $\begin{array}{ll}23.822 \\
23780\end{array}$ & $\begin{array}{l}25.029 \\
2526\end{array}$ & 45.226 & $\begin{array}{l}46.266 \\
\end{array}$ & $\begin{array}{ll}9.194 \\
\end{array}$ & 36.82 & -1.221 & -5.526 & 49.357 & $\begin{array}{ll}0.244 \\
\end{array}$ & \begin{tabular}{|l|}
14.643 \\
\end{tabular} & & 60.1 & $\begin{array}{ll}1 & 1.00194 \\
\end{array}$ & 44.8 & 12.1 & 41.0 & \begin{tabular}{|l|l|}
2.828 \\
\end{tabular} & 0.036 & 0.036 & 0.001 & $\begin{array}{ll}0.888 \\
\end{array}$ & \\
\hline $70+2+3$ & 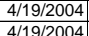 & $\begin{array}{l}\text { 10:43:14 AM } \\
\text { 10:44:14 AM }\end{array}$ & 24.831 & $\begin{array}{l}22.525 \\
22.537\end{array}$ & \begin{tabular}{|l|}
24.939 \\
25.021 \\
\end{tabular} & \begin{tabular}{|l|}
23.788 \\
23.77 \\
\end{tabular} & $\begin{array}{l}25.036 \\
25268\end{array}$ & $\begin{array}{l}45.058 \\
45313 \\
\end{array}$ & \begin{tabular}{|l|}
45.876 \\
46.26 \\
\end{tabular} & $\begin{array}{l}9.203 \\
9242 \\
\end{array}$ & $\begin{array}{l}36.588 \\
36783 \\
\end{array}$ & $\begin{array}{l}-1.215 \\
-1.218 \\
\end{array}$ & $\begin{array}{l}-5.526 \\
-5.541 \\
\end{array}$ & $\begin{array}{r}51.36 \\
49779 \\
\end{array}$ & $\begin{array}{l}0.244 \\
0.244 \\
\end{array}$ & \begin{tabular}{|l|}
14.643 \\
14.643 \\
\end{tabular} & & \begin{tabular}{|l|l|}
61.1 \\
62.1 \\
\end{tabular} & \begin{tabular}{ll|l|l|l|l|}
1 & 1.01861 \\
1 & 1.03528
\end{tabular} & $\begin{array}{l}44.4 \\
448\end{array}$ & 12.6 & \begin{tabular}{|l|l|}
40.8 \\
410
\end{tabular} & \begin{tabular}{|l|}
2.814 \\
2830
\end{tabular} & 0.036 & $\begin{array}{l}0.036 \\
0.036\end{array}$ & \begin{tabular}{|l}
0.001 \\
0.001
\end{tabular} & $\begin{array}{ll}0.893 \\
0.886\end{array}$ & $\begin{array}{l}0.76 \\
0.75 \\
\end{array}$ \\
\hline 80 & $\begin{array}{l}4 / 19 / 2004 \\
4 / 19 / 2004\end{array}$ & & 24.000 & 22.517 & \begin{tabular}{|l|}
23.021 \\
24.997 \\
\end{tabular} & \begin{tabular}{|l|}
23.776 \\
\end{tabular} & $\begin{array}{l}25.200 \\
25.188 \\
\end{array}$ & $\begin{array}{l}45.313 \\
45.215 \\
\end{array}$ & $\begin{array}{l}4.2000 \\
45.795 \\
\end{array}$ & $\begin{array}{l}9.242 \\
9.151\end{array}$ & $\begin{array}{l}36.100 \\
36.89 \\
\end{array}$ & & $\begin{array}{l}-5.541 \\
-5.547 \\
\end{array}$ & $\frac{49.7 / 9}{49.015}$ & $\begin{array}{l}0.2443 \\
0.243\end{array}$ & \begin{tabular}{|l|}
14.04643 \\
14.64
\end{tabular} & & $\frac{62.1}{6.1}$ & $\frac{1.03528}{1.05194}$ & $\begin{array}{l}44.8 \\
44.3 \\
\end{array}$ & \begin{tabular}{|l|l|}
12.2 \\
12.0
\end{tabular} & $\begin{array}{ll}41.0 \\
41.1\end{array}$ & \begin{tabular}{|l|}
2.830 \\
2.831 \\
\end{tabular} & 0.036 & $\frac{0.036}{0.036}$ & 0.001 & $\begin{array}{l}0.886 \\
0.833 \\
\end{array}$ & 0.75 \\
\hline & 4/19/2004 & & 24.875 & & & & & & 45.994 & 9.258 & 36.66 & -1.218 & & 46.979 & 0.242 & 14.643 & & $\frac{5.4}{64 .}$ & & 44.5 & & & & & & & & $\frac{0.75}{0.75}$ \\
\hline 82 & $4 / 19 / 2004$ & 10:47:14 AM & 24.881 & 22.52 & 24.979 & 23.728 & 25.226 & 45.197 & 46.21 & 9.116 & 36.72 & -1.218 & -5.538 & 47.66 & 0.242 & 14.643 & & & 1.08528 & 44.7 & 11.7 & & & & & & & 0.75 \\
\hline 83 & $4 / 19 / 2004$ & 10:48:14 AM & 24.873 & & 24.976 & & & & & 9.33 & & -1.218 & & & & & & & 1.10194 & & & & & 036 & & 0 & - 0.0877 & 0.75 \\
\hline r & $4 / 19 / 2004$ & 10:49:14 AM & & & \begin{tabular}{|l|l|}
24.967 \\
\end{tabular} & 23.711 & & 45.28 & 46.239 & 9.327. & 36.64 & -1.218 & & 48.752 & 0.24 & 14.643 & & & $\frac{1.11861}{1.1861}$ & 44.8 & & & & & & & 0.874 & \\
\hline & $4 / 19 / 2004$ & 10:50:14 AM & 24.867 & 22.516 & 24.97 & 23.724 & 22.473 & 44.631 & 45.385 & 912 & 36.276 & & & & & 14.643 & & & & & & & & & & & & \\
\hline 86 & $4 / 19 / 2004$ & $10: 51: 14 \mathrm{AM}$ & 24.854 & 22.508 & 25.042 & 23.746 & 22.334 & 45.197 & 46.145 & 9.214 & 36.666 & -1.218 & -5.558 & 53.293 & 0.239 & 14.643 & & 69.1 & 1.15194 & 44.7 & 13.1 & 40.9 & 2.822 & 0.036 & 0.036 & 0.001 & 0.870 & \\
\hline & $4 / 19 / 2004$ & 10:52:14 AM & 24.861 & 22.5 & 25.029 & 23.778 & 22.296 & 45.047 & 45.86 & 9.198 & 36.52 & -1.218 & $-5.57:$ & 48.704 & 0.238 & 14.643 & & 70. & 1.16861 & 44.4 & & 40.8 & & & & 0.001 & 0.869 & 0.74 \\
\hline & & & & & & & & & & 9.146 & 36.855 & & & & & 14.643 & & & & 44.6 & & & & & & & & \\
\hline & $4 / 19 / 2004$ & 10:54:14 AM & 24.885 & 22.499 & \begin{tabular}{|l|l|}
25.008 \\
\end{tabular} & 23.742 & 22.299 & 45.097 & 46.199 & 9.224 & 36.541 & -1.21 & & 48.736 & 0.239 & 14.643 & & & . & 44.7. & & & & 036 & 036 & 0.00 & 0.873 & 0.74 \\
\hline & $\frac{411912004}{11 / 2004}$ & $\begin{array}{l}10.55 .14 \mathrm{AM} \\
0.56 \mathrm{AMM}\end{array}$ & 2497 & $\frac{2.49}{22.476}$ & $\begin{array}{ll}24.919 \\
2.071\end{array}$ & $\frac{2.3138}{2375}$ & 22.32. & (45.305 & (46.2724 & 9.199 & $\frac{30.828}{3.6819}$ & $\frac{-1.21}{-122}$ & & & 0.250 & 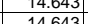 & & & 1.1861 & $44.8>3$ & & & & & & & & \\
\hline$\frac{11}{92}$ & $\begin{array}{l}4 / 19 / 2004 \\
4 / 19 / 2004 \\
\end{array}$ & $10.50 .14 \mathrm{AM}$ & 24878 & $\frac{22.410}{22482}$ & $\begin{array}{l}24.911 \\
24997\end{array}$ & $\frac{23.755}{23.771}$ & $\frac{22.282}{22343}$ & $\begin{array}{l}\frac{45.174}{44.307} \\
\end{array}$ & $\mid$ & $\begin{array}{l}9.2024 \\
9.684 \\
\end{array}$ & 36.818 & $\frac{-1.224}{-1.218}$ & & $\frac{70.54}{52.54}$ & $\frac{0.237}{0.23}$ & $\begin{array}{l}14.643 \\
14.643 \\
\end{array}$ & & & $\frac{1.23528}{1.25194}$ & $\frac{44.5}{437}$ & & & & .035 & 0.034 & 0001 & $\begin{array}{l}0.863 \\
0.861 \\
\end{array}$ & 0.73 \\
\hline & $4 / 19 / 2004$ & $10: 58: 14 \mathrm{AM}$ & 24.884 & & \begin{tabular}{|l|}
25.088 \\
\end{tabular} & 23.782 & 22.409 & 44.541 & 45.455 & 9.613 & 35.702 & -1.218 & -5.63 & 50.644 & 0.232 & $\begin{array}{ll}14.045 \\
14.643\end{array}$ & & 76.1 & 1.26861 & 44.0 & 12.4 & \begin{tabular}{|l|l}
40.1 \\
\end{tabular} & & & & & 0.860 & 0.73 \\
\hline & $4 / 19 / 2004$ & $10: 59: 14 \mathrm{AM}$ & & 22.473 & 25.083 & 23.797 & & 44.931 & 45.998 & & 36.088 & & & & 0.231 & 14.643 & & 77.1 & & 44.5 & 12.5 & 40.5 & 2.79 & 0.034 & & & 0.848 & \\
\hline & $4 / 199 / 2004$ & $11: 00: 14 \mathrm{AM}$ & 24.9 & 22.463 & 25.063 & & 22.696 & 44.514 & $\begin{array}{ll}45.426 \\
\end{array}$ & $\begin{array}{l}9.42 \\
\end{array}$ & & & -5.628 & 50.244 & 0.232 & 14.643 & & 78.1 & 1.30194 & 44.0 & 12.3 & 40.2 & & & & 0.001 & 0.859 & \\
\hline & 4/19/2004 & 11:01:14 AM & $\frac{24.915}{24.915}$ & $\begin{aligned} 22.483 \\
22.488\end{aligned}$ & \begin{tabular}{|l|}
25.068 \\
25043
\end{tabular} & $\frac{23.983}{24.053}$ & $\begin{array}{r}22.836 \\
23032\end{array}$ & $\begin{array}{l}45.251 \\
45.91\end{array}$ & $\begin{array}{r}46.517 \\
46144 \\
\end{array}$ & $\begin{array}{l}9.386 \\
9319\end{array}$ & $\begin{array}{r}36.58 \\
36565 \\
\end{array}$ & $\begin{array}{r}-1.218 \\
.218 \\
\end{array}$ & \begin{tabular}{|c|c|}
-5.62 \\
562
\end{tabular} & 50.052 & $\begin{array}{l}0.232 \\
0233 \\
\end{array}$ & $\begin{array}{l}14.643 \\
11643\end{array}$ & & 79.1 & \begin{tabular}{|l|l|}
1.31861 \\
132528
\end{tabular} & $\begin{array}{l}45.0 \\
447\end{array}$ & 12.3 & 40.9 & 2.821 & 0.035 & U35 & 0.001 & $\begin{array}{ll}0.844 \\
0848\end{array}$ & 0.12 \\
\hline 98 (9) & $\begin{array}{l}4 / 19 / 2004 \\
4 / 19 / 2004 \\
\end{array}$ & $11: 03: 14$ AM & $\frac{24.915}{24.925}$ & 22.488 & 250.035 & $\frac{24.053}{24.139}$ & $\frac{23.032}{23047}$ & $\frac{45.191}{45.244}$ & \begin{tabular}{|l|}
46.164 \\
46.336
\end{tabular} & $\begin{array}{l}9.319 \\
9.413 \\
\end{array}$ & $\frac{36.658}{36.5}$ & $\frac{-1.218}{-1.218}$ & $\begin{array}{l}-5.625 \\
-5.57 \\
\end{array}$ & $\begin{array}{l}\frac{4.952}{52.169} \\
\end{array}$ & 0.233 & $\frac{14.643}{14.643}$ & & 80.1 & $\begin{array}{ll}1.33528 \\
1.35194\end{array}$ & 44.1 & 11.8 & 40.9 & 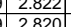 & & 的 & & $\begin{array}{l}0.848 \\
0.845 \\
\end{array}$ & 0.72 \\
\hline 99 & $4 / 19 / 2004$ & 11:04:14 AM & 24.932 & 22.531 & \begin{tabular}{|l|}
25.050 \\
25.051
\end{tabular} & 24.176 & 22.0419 & 45.085 & $\begin{array}{l}4.500 \\
46.243 \\
\end{array}$ & $\begin{array}{l}9.410 \\
9.365 \\
\end{array}$ & 36.428 & -1.218 & -5.636 & 51.159 & 0.231 & $\begin{array}{l}14.045 \\
14.643\end{array}$ & & $\frac{12}{82}$ & 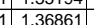 & $\frac{4.38}{44.8}-1$ & & 40.8 & 2810 & 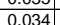 & & (1) & $\frac{0.044}{0.84}$ & 0.72 \\
\hline & $4 / 19 / 2004$ & $11: 05: 14 \mathrm{AM}$ & & 22.56 & 25.144 & 24.22 & 22.942 & & 46.344 & 9.39 & 36.63 .5 & -1.218 & & 49.22 & & & & & & 44.9 & & & & & & & & \\
\hline$\overline{10}$ & 4/19/20004 & 11:06:14 AM & 24.958 & 22.562 & 25.127 & 24.242 & 22.87 & 45.128 & 46.349 & 9.16 & 36.6 & -1.218 & 6.628 & 49.895 & $\frac{0.232}{0.232}$ & $\begin{array}{l}14.045 \\
14.643 \\
\end{array}$ & & 84 & 1.40194 & 44.9 & 12. & 40.9 & & 年 & $\frac{0.034}{0.034}$ & $\frac{0.001}{0.001}$ & 0.043 & \\
\hline & $4 / 19 / 2004$ & 11:07:14 AM & 24.954 & 22.563 & 25.103 & 24.238 & 22.776 & 45.251 & 46.405 & 9.208 & 36.717 & $-1.21 \varepsilon$ & & 49.236 & 0.227 & 14.643 & & & 1.41861 & 44.9 & $12-2>$ & 41 & & & & - & & \\
\hline & $4 / 19 / 2004$ & $11: 08: 14 \mathrm{AM}$ & 24.966 & 22.59 & & 24.195 & & 45.139 & & 9.344 & & & & & 0.229 & 14.643 & & & 143528 & 44.9 & & 40 & & & & & & \\
\hline & $4 / 19 / 2004$ & 11:09:14 AM & 24.977 & 22.606 & 25.09 & 24.171 & 22.683 & 45.199 & 46.373 & 9.419 & 36.46 & -1.221 & & 48.594 & 0.231 & 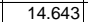 & & 87.1 & 1.45194 & 44.9 & 11.9 & 40.8 & 1815 & 0.034 & 0.034 & 0.001 & 0.841 & \\
\hline & 4/19/2004 & 11:10:14 AM & 24.977 & 22.606 & 25.065 & 24.141 & 22.110 & $\begin{array}{l}44.948 \\
\end{array}$ & 466.096 & 9.305 & 36.327 & -1.22 & & 49.83 & 0.228 & 14.643 & & & 1.46861 & $\begin{array}{lll}44.6 & -2\end{array}$ & 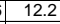 & 40 & & & & & 0.835 & \\
\hline & $4 / 19 / 2 / 2$ & 11:11:14 AM & 24.988 & 22.632 & 25.086 & 24.166 & 22.744 & 44.74 & 45.967 & 9.264 & 36.198 & -1.21 & & & 0.229 & 14.643 & & & & 44.5 & 12.4 & 40. & 2.790 & & & & 0.842 & \\
\hline & 4/11992/2004 & 11:12:14 AM & 24.998 & 22.623 & $25.177 \mathrm{Cl}$ & 24.167 & & 45.261 & 46.3711 & 9.31 & & -1.2. & & 49.61. & 0.229 & 14.643 & & & .50194 & 44.9 & 12.2 & 41. & $m_{2}$ & 334 & & 0.0 & 0.829 & \\
\hline & & & & & & 24.178 & & & & & & & & & 0.229 & & & & & & & & & & & & & \\
\hline & $\begin{array}{l}4 / 19 / 2004 \\
4 / 19 / 2004 \\
\end{array}$ & $\begin{array}{l}11.14 .4 \mathrm{AMM} \\
11.15 .4 \mathrm{MM}\end{array}$ & $\frac{23.015}{25036}$ & $\frac{2.03}{22.636}$ & 25.144 & $\frac{24.135}{24.125}$ & $\frac{22.521}{2502}$ & 年 & $\mid \begin{array}{l}4 \\
4.03562\end{array}$ & 年.250 & 36.611 & $\frac{-1.21}{-121}$ & & 48876 & $=.26$ & $\frac{14.643}{14.643}$ & & $\frac{94}{93}$ & 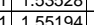 & 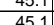 & $\frac{11.8}{120}$ & 40. & 2835 & $\frac{034}{034}$ & 0,034 & - & 0.882 & \\
\hline & $4 / 19 / 2004$ & $11: 16: 14$ AM & 25.046 & 22.651 & 25.155 & 24.15 & & & $\frac{46.05}{46455}$ & 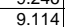 & 36.88 & -121 & & 4704 & $\frac{0.231}{0.231}$ & 14643 & & & 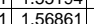 & 45 & $\frac{1.6}{115}>0$ & 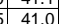 & & & & & & \\
\hline & $4 / 19 / 2004$ & $11: 17: 14 \mathrm{AM}$ & 25.037 & 22.642 & 25.105 & 24.101 & & & 46.618 & 9.139 & 37.015 & -1.21 & & 51.562 & 0.228 & 14.643 & & 95 & 1.58528 & 45. & & 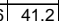 & $2.8 \mathrm{2}-\mathrm{P}$ & & & & 0.823 & \\
\hline & $4 / 19 / 2004$ & 11:18:14 AM & & 22.643 & 25.132 & 24.097 & & & & 9.296 & & -1.21 & & & & 14.6 & & & 1.60194 & & & & & & & & & \\
\hline & 4/19/2004 & 11:19:14 AM & 25.038 & 22.633 & 25.167 & 24.092 & & & 45.967 & 9.187 & & -1.22 & & 48.7 & & & & & & & & & & & & & 819 & \\
\hline & 4/19/2004 & 11:20:14 AM & 25.054 & 22.633 & 25.172 & 24.128 & 22 & & 46.145 & 9.249 & & -1.21 & & 48. 5 & & & & & 1.63 & 44. & $12.0 \mathrm{P}-\mathrm{C}$ & $407-3$ & .80 & & & 0. & 327 & \\
\hline & 4/19/2004 & 11:21:1 & 25.075 & 22.645 & 25.164 & 24.134 & 22.54 & 45.054 & 46.313 & & & -1.21 & & 49.5 & & & & $9 \varsigma$ & $\mid 94$ & 44 & 12.1 & 40 & & & & & 0.813 & \\
\hline 11 & 4/19/2004 & 11:22:14 AM & 25.06 & 22.644 & 25.148 & 24.1 & & & $\begin{array}{lll}46.336 \\
\end{array}$ & 9.148 & 36.607 & -1.22 & & $48.8 \quad-3$ & 0.226 & 14 & & 100 & 1.66 & 44.9 & $12.8 \mathrm{C}>\mathrm{l}$ & 40. & & & & & 322 & \\
\hline $1 \pm$ & 4/199/2004 & 11:23:14 AM & 25.07 & 22.645 & 25.154 & 24.119 & & 45.3 & 46.568 & 9.153 & & -1.21 & & 48.60 & 0.225 & & & 101 & 1.685 & 45. & 11.9 & 41.2 & & & & & 0.811 & \\
\hline & 4/19/2004 & $11: 24: 144$ & 25.05 & 22.62 & \begin{tabular}{|l|l|}
25.109 \\
\end{tabular} & 24.109 & & & 45.982 & 9.11 & & & & 48.8 & & & & & & & & 40. & & & & & & \\
\hline & $4-119922004$ & $11: 256$ & 25.066 & $\frac{22.63}{22.63}$ & 25.224 & 24.109 & & & 46.686 & & & -1.21 & & & 0.223 & & & 10 & $\frac{1.71}{1.72}$ & $45.2 .2>3$ & 12.4 & 41. & & & & & 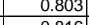 & \\
\hline & $\frac{41912004}{4192004}$ & $\frac{11.26 .14}{1127.14}$ & 年3.081 & $\frac{26.041}{22635}$ & 25 & $\frac{24.1}{24.1}$ & & & 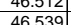 & 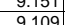 & & & & & & & & & & & & & & & & & & \\
\hline & 4 & 11.28.14 & 25096 & 2263 & 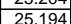 & 24.14 & & & 年 47.116 & & & & & & 0.224 & & & & & & $\frac{14.1}{117}$ & & & & & & & \\
\hline & $\frac{4 / 199 / 2004}{4}$ & 11:29:14 AM & 25.095 & $\frac{22.629}{22.629}$ & 25.178 & $\frac{24.128}{24.128}$ & $\frac{22.545}{22.545}$ & 45.742 & 477.136 & 9.183 & 37.3 & $\frac{-1.218}{-1.218}$ & & 49.499 & $\frac{0.226}{0.226}$ & & & 107 & & 45 & 121 & 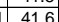 & & & & & 307 & \\
\hline & & & & & & & & & & & & & & & & & & & & & & & & & & & & \\
\hline & $4 / 19 / 2004$ & $11: 31: 14 \mathrm{AM}$ & 25.113 & 22.638 & 25.187 & 24.157 & & 45 & 47.043 & 9.166 & 37.265 & $-1.218 \quad$ & & & 0.223 & 14. & & & & 45,6 & $11.9 \mathrm{rat}$ & $110>-1$ & & & & & (98 & \\
\hline & $4 / 19 / 2$ & $11: 32: 14 \mathrm{~A}$ & 25.108 & 22.637 & 25.181 & 24.151 & & & 46.825 & & 37.25 & -1.2 & & & & & & 11 & & & 11.8 & $\frac{41}{41}>2>$ & & & & & & \\
\hline & 4/19/2004 & 11:33:14 AM & 25.12 & 22.643 & 25.307 & 24.172 & & 45.62 & 47.097 & 9.128 & 37.28 & -1.21 & & 46.7. & 0.2 & 14.643 & & 111 & 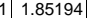 & 45.6 & 11.5 & 41.5 & 2.858 & 0.034 & & & 0.806 & \\
\hline 12 & 4/19/2004 & 11:34:14 AM & 25.138 & 22.64 & \begin{tabular}{|l|}
25.297 \\
\end{tabular} & 24.152 & & 45.244 & 46.82 & 9.3 & 36 & -1.21 & & & 0.22 & & & 112 & 1.86 & 45 & 12.1 & 40 & & & & & 0.797 & \\
\hline 13 & & 11:35:1 & 25.153 & 22.648 & 25.297 & 24.182 & & 44 & 45.69 & 9.751 & 35 & $-1.218 \mathrm{y} \quad \mathrm{x}$ & & 46. & 0.2 & & & 113 & & $44.2 \mathrm{r}-\mathrm{s}$ & 11.5 & 39 & & & & & 0.790 & \\
\hline & 4/119/20004 & 11:36:14 AM & 25.153 & 22.648 & 25.287 & 24.192 & & 44.234 & 45.515 & 9.581 & 35.426 & -1.221. & & 49.1. & 0.204 & 14.6 & & 11 & 1.90194 & 44.0 & 11. & & & & & & & \\
\hline & $\frac{411912004}{41 / 2004}$ & $\begin{array}{l}11.3: 144 \mathrm{AM} \\
11.38\end{array}$ & 25158 & 2.642 & $\mid 25.271$ & 24.202 & & 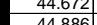 & 40.251 & $\begin{array}{l}9.609 \\
9657\end{array}$ & 36 & 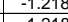 & & 40.5 & 0.216 & 14.6 & & $\frac{11}{11}$ & 61 & 44.8 & 12.0 & 10 & & & & & & \\
\hline & 4 & & 年3.150 & $\frac{2.050}{22013}$ & \begin{tabular}{|l|l|}
2.30202 \\
\end{tabular} & 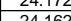 & & (44.000 & 40.0000 & & & & & & & & & & & 45 & 12.1 & & & & & & & \\
\hline 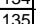 & $\frac{4151204}{41 / 92004}$ & 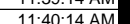 & 25.15 & 年2.0458 & $\mid$ & 24.187 & & $\frac{44.4517}{4917}$ & 年 4650 & 9.569 & & -1.21 & & & 0.0217 & & & $\frac{1}{11}$ & & (4) & $\frac{1.1}{113}>>$ & & & & & & & \\
\hline 136 & & & 25.16 & 22.638 & & & & 44 & 46208 & 9.381 & & -12118 & & & & & & 116 & & & 108 & & & & & & & \\
\hline$\overline{13}$ & $4 / 19 / 2004$ & & 25.1 & & & 24.204 & & & 46.208 & & & & & & & & & & & & & & & & & & & \\
\hline 138 & & & 25.16 & & 25.189 & 24.244 & & & & 9.447 & & & & & & & & & & 446 & & & & & & & & \\
\hline 12 & $4 / 10 / 2004$ & 11.44 .14 & 25143 & 22618 & $202+2$ & & & & 45967 & 376 & & -1.221 & & 9099 & 021 & 14.643 & & 12 & 203528 & 44.5 & 120 & & & 231 & 031 & 0001 & 776 & \\
\hline 140 & $4 / 19 / 2004$ & 11:45:14 AM & 25.14 & 22.614 & 25.229 & 24.284 & & 44.581 & 46.156 & 9.573 & 35.667 & \begin{tabular}{|l|l|} 
\\
\end{tabular} & & 49.735 & 0.215 & 14.643 & & & 2.05194 & 44.7 & & & 2.766 & 0.032 & & 0.001 & 0.794 & \\
\hline
\end{tabular}


WSRC-TR-2005-00105, REVISION 0

SRNL-RPP-2005-00012, REVISION 0

RUN \# 2.03A AND B; FIRST AND SECOND HALF OF SLURRY DEWATERING - CONT.

\begin{tabular}{|c|c|c|c|c|c|c|c|c|c|c|c|c|c|c|c|c|c|c|c|c|c|c|c|c|c|c|c|c|}
\hline & & & & & & & & & & & & & & & & & & & & & & & & & & & & \\
\hline & A & \begin{tabular}{|c|} 
B \\
\end{tabular} & D & $E$ & 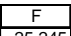 & G & $\mathrm{H}$ & $\mathrm{J}$ & $\mathrm{K}$ & $\mathrm{L}$ & $\mathrm{M}$ & $\mathrm{N}$ & 0 & Q & $\mathrm{R}$ & $\mathrm{s}$ & $\mathrm{T}$ & $\mathrm{V}$ & W & $\mathrm{X}$ & 10 & $z$ & AA & $A B$ & $A C$ & $A D$ & $\mathrm{AE}$ & \\
\hline$\frac{141}{142}$ & $\begin{array}{l}4 / 1 / 9 / 2004 \\
4 / 192004 \\
\end{array}$ & \begin{tabular}{|l|}
$11: 46: 14 \mathrm{AM}$ \\
$11.47 .14 \mathrm{AM}$
\end{tabular} & $\begin{array}{l}25.147 \\
25.149 \\
\end{array}$ & $\begin{array}{l}22.611 \\
22.603\end{array}$ & \begin{tabular}{|l|}
25.245 \\
25.232 \\
\end{tabular} & \begin{tabular}{|l|}
24.346 \\
24.363 \\
\end{tabular} & $\begin{array}{l}22.773 \\
22.765 \\
\end{array}$ & \begin{tabular}{|c|}
44.193 \\
44.574 \\
\end{tabular} & $\begin{array}{l}45.621 \\
46.071 \\
\end{array}$ & $\begin{array}{l}9.453 \\
9.569 \\
\end{array}$ & $\begin{array}{l}35.455 \\
35.833 \\
\end{array}$ & \begin{tabular}{|l|l|} 
& -1.218 \\
-1.221 & \\
\end{tabular} & $\begin{array}{l}-5.752 \\
-5.726 \\
-\end{array}$ & 50.233 & $\frac{0.213}{0.211}$ & \begin{tabular}{|l|}
14.643 \\
14.643 \\
\end{tabular} & $\begin{array}{l}0.003 \\
0.003 \\
\end{array}$ & $\frac{124.1}{125.1}$ & \begin{tabular}{ll|l|l|l|l|l|l}
1 & 2.06861 \\
& 2.08528 \\
\end{tabular} & $\begin{array}{l}44.1 \\
44.6 \\
\end{array}$ & 12.3 & \begin{tabular}{|l|}
39.8 \\
40.2
\end{tabular} & $\frac{2.746}{2.772}$ & 0.032 & 0.032 & $\begin{array}{l}0.001 \\
0.001\end{array}$ & $\begin{array}{l}0.792 \\
0.777 \\
\end{array}$ & $\begin{array}{l}0.67 \\
0.66\end{array}$ \\
\hline$\frac{\frac{142}{143}}{3} x-3$ & & & 25.1491 & & & \begin{tabular}{|l|}
24.3436 \\
24.466 \\
\end{tabular} & $\begin{array}{l}22.765 \\
22.748 \\
\end{array}$ & & \begin{tabular}{|l|l|}
46.158 \\
46.0
\end{tabular} & $\begin{array}{l}9.569 \\
9.594 \\
\end{array}$ & & & & 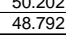 & $\begin{array}{l}0.211 \\
0.209 \\
\end{array}$ & \begin{tabular}{|l|}
14.6433 \\
14.643
\end{tabular} & & & 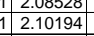 & & & & & & & & & \\
\hline 144 & $4 / 19 / 2004$ & \begin{tabular}{|l|l|}
$11: 48: 18 \mathrm{AM}$ \\
\end{tabular} & 25.171 & 22.626 & \begin{tabular}{|l|}
25.315 \\
\end{tabular} & \begin{tabular}{|l|}
24.471 \\
\end{tabular} & 22.722 & 44.699 & 46.357 & 9.538 & 35.839 & -1.215 & -5.723 & 47.13 & 0.213 & 14.643 & & 126.2 & $\begin{array}{l}2 \quad 2.10306 \\
2\end{array}$ & 44.9 & 11.5 & & 2.776 & 0.032 & 0.031 & 0.001 & 0.782 & \\
\hline 145 & $4 / 19 / 2004$ & \begin{tabular}{|l|l}
$11: 49: 18 \mathrm{AM}$ \\
\end{tabular} & 25.183 & 22.622 & \begin{tabular}{|l|l|}
25.302 \\
\end{tabular} & \begin{tabular}{|l|l|}
24.493 \\
\end{tabular} & 22.539 & 44.519 & 46.029 & 9.546 & $\begin{array}{ll}35.632 \\
\end{array}$ & $\mid-1.221$ & -5.755 & 51.456 & $\begin{array}{l}0.219 \\
0.219 \\
\end{array}$ & \begin{tabular}{|l|}
14.643 \\
\end{tabular} & & 127.2 & \begin{tabular}{|l|l|}
2 & 2.11972 \\
\end{tabular} & 44.6 & \begin{tabular}{|l|l|}
12.6 \\
\end{tabular} & \begin{tabular}{|l|}
40.1 \\
\end{tabular} & 2.763 & & & & 0.808 & 0.69 \\
\hline & & & 25.175 & & & 24.51 & & & 46.092 & 9.522 & 35.644 & & & & & \begin{tabular}{|l|}
14.643 \\
\end{tabular} & & $\begin{array}{ll}128.2 \\
\end{array}$ & 2.13639 & $\begin{array}{lll}44.6 \\
\end{array}$ & 12.5 & 40.0 & 2.760 & & & & & \\
\hline 147 & $4 / 19 / 2004$ & 11:51:18 AM & 25.202 & 22.641 & 25.315 & 24.537 & 22.929 & $\begin{array}{l}44.38 \\
\end{array}$ & & 9.446 & 35.701 & -1.215 & -5.781 & 48.865 & 0.21 & 14.643 & & & 2.15306 & $\begin{array}{ll}44.4 \\
\end{array}$ & 12.0 & 40.0 & 2.761 & 0.031 & 0.031 & 0.001 & 0.775 & \\
\hline$\frac{148}{149}$ & \begin{tabular}{|l|}
$4 / 19 / 2004$ \\
$4 / 192004$ \\
\end{tabular} & $\begin{array}{l}\text { 11:52:18 AM } \\
\text { 11.53:18 AM }\end{array}$ & $\begin{array}{l}25.204 \\
252006\end{array}$ & $\begin{array}{l}22.633 \\
22.636\end{array}$ & \begin{tabular}{|l|}
25.287 \\
25.28
\end{tabular} & \begin{tabular}{|l|}
24.549 \\
24581 \\
\end{tabular} & $\begin{array}{l}22.966 \\
22888 \\
\end{array}$ & $\begin{array}{l}44.726 \\
44548\end{array}$ & \begin{tabular}{|l|}
46.359 \\
46015 \\
\end{tabular} & $\begin{array}{l}9.482 \\
9389 \\
\end{array}$ & \begin{tabular}{|r|}
35.96 \\
36.11 \\
\end{tabular} & \begin{tabular}{|l|l|}
-1.218 \\
-1218
\end{tabular} & $\begin{array}{l}-5.656 \\
-5.804\end{array}$ & $\begin{array}{r}45.92 \\
4.502 \\
\end{array}$ & $\begin{array}{l}0.205 \\
0.21 \\
\end{array}$ & \begin{tabular}{|l|l|}
14.643 \\
14643
\end{tabular} & & $\frac{130.2}{1312}$ & \begin{tabular}{|l|l|}
2.16972 \\
218639
\end{tabular} & $\begin{array}{l}44.9 \\
44.5 \\
\end{array}$ & $\begin{array}{l}11.3 \\
1119\end{array}$ & \begin{tabular}{|l|}
40.3 \\
403 \\
\end{tabular} & $\frac{2.782}{2.777}$ & 0.031 & $\begin{array}{l}0.030 \\
0.031 \\
\end{array}$ & $\begin{array}{l}0.001 \\
0.001\end{array}$ & $\begin{array}{l}0.752 \\
0771\end{array}$ & 0.64 \\
\hline & $\begin{array}{l}4 / 19 / 2004 \\
4 / 19 / 2004\end{array}$ & & 25.214 & 222.636 & \begin{tabular}{|r|}
25.28 \\
25.282 \\
\end{tabular} & \begin{tabular}{|l|}
$\mid 24.010$ \\
24.659 \\
\end{tabular} & $\begin{array}{l}22.888 \\
23.326\end{array}$ & $\begin{array}{r}44.548 \\
44.55 \\
\end{array}$ & $\begin{array}{l}46.15 \\
46.154 \\
\end{array}$ & $\begin{array}{l}9.035 \\
9.504\end{array}$ & $\begin{array}{l}5.011 \\
35.792 \\
\end{array}$ & & $\begin{array}{l}-5.804 \\
-5.784 \\
\end{array}$ & $\begin{array}{l}40.5064 \\
46.604\end{array}$ & $\begin{array}{r}0.21 \\
0.209\end{array}$ & $\begin{array}{l}14.643 \\
14.643\end{array}$ & & $\begin{array}{l}131.2 \\
12.2 \\
\end{array}$ & \begin{tabular}{l|l|} 
& 2.18639 \\
2 & 2.20306 \\
\end{tabular} & $\begin{array}{l}44.5 \\
44.7 \\
\end{array}$ & $\begin{array}{l}11.9 \\
11.4\end{array}$ & & $\frac{2.777}{2.770}$ & $\frac{0.031}{0.031}$ & $\frac{0.031}{0.031}$ & $\frac{0.001}{0.001}$ & 0.7710 & 0.66 \\
\hline 151 & 4/19/2004 & 11:55:18 AM & & & & \begin{tabular}{|l|l|}
24.668 \\
\end{tabular} & & & 45.808 & & 35.41 & -1.218 & & 49.307 & 0.205 & 14.643 & & 133.2 & \begin{tabular}{|l|l|}
2 & 2.21972 \\
\end{tabular} & 44.3 & & 39.8 & & & & & & $\begin{array}{l}0.65 \\
0.65 \\
\end{array}$ \\
\hline 152 & $4 / 19 / 2004$ & $11: 56: 18 \mathrm{AM}$ & $\begin{array}{ll}25.227 \\
\end{array}$ & 22.666 & 25.29 & $\begin{array}{ll}24.677 \\
\end{array}$ & 22.878 & 44.543 & 46.289 & 9.5 & 35.757 & \begin{tabular}{|l|}
-1.221 \\
\end{tabular} & -5.749 & 48.73 & 0.211 & \begin{tabular}{|l|l|}
14.643 \\
\end{tabular} & & 134 & 2.23639 & 44.8 & 11. & 40.2 & 2.768 & & & & & 0.65 \\
\hline 153 & $4 / 19 / 2004$ & 11:57:18 AM & 25.226 & & & 24.697 & & & & & & & & & 0.208 & & & 135 & 2.25306 & $\begin{array}{ll}44.8 \\
\end{array}$ & & & & & & 0.00 & 0.764 & 0.66 \\
\hline & $4 / 19 / 2004$ & 11:58:18 AM & & & 25.274 & 21721 & & 44.604 & \begin{tabular}{ll|l}
46.243 \\
\end{tabular} & 9.507 & 35.804 & -1.218 & & 50.857 & 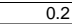 & $\begin{array}{ll}14.643 \\
\end{array}$ & & & 26972 & 44.8 & & & & & & & 0.736 & \\
\hline & $4 / 19 / 2004$ & $11: 59: 18 \mathrm{AM}$ & 25.214 & 22.664 & 25.233 & 24.705 & 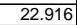 & 44.38 & 45.876 & 9.429 & 35.714 & -1.221 & & $48.7 .7-3$ & & 14.643 & & tol.c. & 2.28639 & 44.4 & & 40.0 & 4.761 & & & & & \\
\hline & $4 / 199 / 2004$ & 12:00:18 PM & 25.222 & 22.687 & 25.236 & 24.713 & 22.919 & 44.857 & 46.517 & 9.62 & 35.956 & -1.218 & -5.82. & 477.017 & 0.21 & 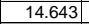 & & 138.2 & 2.30306 & 45.0 & 11.5 & 40.4 & 2.786 & 0.031 & 0.031 & 0.001 & 0.770 & \\
\hline & $4 / 19 / 2004$ & 12:01:18 PM & 25.227 & 22.691 & 25.23 & 24.742 & 22.85 & 44.541 & 46.106 & 9.527 & 35.734 & -1.218 & & 49.468 & 0.205 & 14.643 & & 139.2 & 2.31972 & 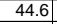 & & 40.1 & 2.767 & & & 0.00 & 0.757 & 0.64 \\
\hline & 4/199/2004 & & & & & & & 44.46 & 45.93 & 9.48 & & & & & & 14.643 & & 140.2 & & 44. & & 40. & & & & & & \\
\hline & $4 / 19 / 2004$ & 12:03:18 PM & 25.224 & 22.709 & 25.218 & 24.825 & 22.886 & 44.732 & 46.2933 & 9.433 & 5.026 & -1.218 & & 49.41 & 0.211 & 14.643 & & & 35306 & 44.8 & & 40. & & & & & 0.774 & 0.66 \\
\hline & $\frac{411912004}{11 / 2004}$ & $\frac{12.04 .18 \mathrm{PM}}{1205 \mathrm{M}}$ & & $\frac{22.108}{20723}$ & 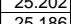 & 24.8699 & & $\frac{44.502}{4.42}$ & (45.9495 & $\frac{9.403}{902}$ & & - 1.2. & & 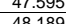 & $\frac{0.201}{0.020}$ & $\frac{14.64}{1.642}>>0$ & & & & 44. & & 40,0 & & & & & & \\
\hline 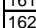 & $\begin{array}{l}4 / 19 / 2004 \\
4 / 19 / 2004 \\
\end{array}$ & $\frac{12.0561 .18 \mathrm{PM}}{12.06 \mathrm{PM}}$ & $\frac{25.228}{25.227}$ & $\frac{22.123}{22.722}$ & \begin{tabular}{|l|}
25.180 \\
25.17
\end{tabular} & 24.0359 & 22.938 & $\mid \begin{array}{ll}44.15 \\
488 .\end{array}$ & 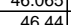 & $\begin{array}{cc}3.4060 \\
9.58\end{array}$ & $\begin{array}{l}36.155 \\
36112 \\
\end{array}$ & $\frac{-1.215}{-1.218}$ & & 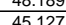 & $\begin{array}{r}0.208 \\
0.21 \\
\end{array}$ & $\begin{array}{l}14.643 \\
14.643 \\
\end{array}$ & & 1442 & $\frac{2.38639}{2.40306}$ & & & $\begin{array}{l}40.4 \\
40.5 \\
\end{array}$ & 2792 & & & 0001 & $\begin{array}{l}0.763 \\
0.769 \\
\end{array}$ & 0.65 \\
\hline 163 & $4 / 19 / 2004$ & \begin{tabular}{|l|}
$12: 07: 18$ PM \\
\end{tabular} & 25.225 & 22.741 & 25.154 & \begin{tabular}{|l|l|}
24.896 \\
\end{tabular} & 23.113 & 44.475 & 46.011 & 9.506 & 35.634 & \begin{tabular}{|l|l|}
-1.224 \\
\end{tabular} & -5.758 & 49.653 & 0.198 & $\begin{array}{ll}14.045 \\
14.643\end{array}$ & & 145.2 & \begin{tabular}{|l|l|}
2 & 2.41972 \\
\end{tabular} & 44.5 & $\frac{11.2}{12.2}$ & 40.1 & & 0.030 & & 0.001 & 0.734 & 0.65 \\
\hline & $4 / 19 / 2004$ & 12:08:18 PM & 25.219 & 22.739 & \begin{tabular}{|l|}
25.137 \\
\end{tabular} & \begin{tabular}{|l|}
24.925 \\
\end{tabular} & & 44.518 & 46.189 & 9.61 & 35.607 & $-1.21 \varepsilon$ & & 49.839 & 0.206 & 14.643 & & 146.2 & \begin{tabular}{|l|l|}
2 & 2.43639 \\
\end{tabular} & 44.7 & 12.2 & 40.1 & 2.762 & & & & 0.764 & \\
\hline & $4 / 19 / 2004$ & 12:09:18 PM & 25.212 & $\frac{22.742}{22.741}$ & 25.126 & 24.948 & 23.1 & 44.799 & 46.376 & 9.549 & 36.083 & -1.215 & -5.77 & 48.51 & 0.203 & 14.643 & & 147.2 & 2.45306 & 44.9 & 11.5 & 40.4 & 2.788 & 0.030 & & 0.001 & 0.746 & \\
\hline & 4/19/2004 & 12:10:18 PM & $\begin{array}{l}25.196 \\
25205\end{array}$ & $\frac{22.741}{22.77}$ & \begin{tabular}{|l|}
25.089 \\
25108
\end{tabular} & 24.992 & $\frac{23.534}{23242}$ & $\begin{array}{l}44.753 \\
4.964\end{array}$ & $\begin{array}{l}46.204 \\
46.463 \\
\end{array}$ & $\frac{9.473}{9.477}-\mathrm{l}$ & $\frac{36.122}{36.266}$ & -1.221 & $\begin{array}{r}-5.76 \\
5.82 \\
\end{array}$ & 48 & $\begin{array}{l}0.201 \\
0.201 \\
\end{array}$ & 14.643 & & $\frac{148.2}{1492}$ & 2.46972 & 44.7 & 11.9 & 40.4 & $\frac{2.788}{2800}$ & 0.030 & & 0.001 & 0.739 & 0.00 \\
\hline & $\begin{array}{l}4 / 19 / 2004 \\
4 / 19 / 2004 \\
\end{array}$ & $12: 12: 18$ PM & 25204 & 22.775 & 25077 & 25.071 & & $\begin{array}{ll}44.964 \mid \\
44705\end{array}$ & $\begin{array}{r}46.463 \\
46204 \\
\end{array}$ & $\frac{5.441}{9.47}$ & $\frac{30.200}{35.7}$ & $\begin{array}{l}-1.21 \mathrm{c} \\
-1.221 \\
\end{array}$ & $=-5.06$ & $\frac{14.024}{5011}$ & 0.212 & $\frac{14.045}{14.643}$ & & 149.2 & $\frac{2.48639}{250306}$ & 45.0 & $\frac{12.2}{124}$ & $\begin{array}{ll}40.6 \\
40.3\end{array}$ & 2.800 & & & & & 0.63 \\
\hline & $4 / 19 / 2004$ & 12:13:18 PM & 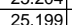 & 22.79 & $\mid \begin{array}{l}25.072 \\
25.072\end{array}$ & 25.076 & 23.117 & 44.666 & 年 46.10 & $\frac{9.41}{9.552}$ & 35.86 & -1.218 & -5.8 & 53.32 & 0.023 & 14.643 & & 1512 & $\frac{1.50502}{251972}$ & $\frac{4.16}{44.6}-1$ & 势 & 年 & . & 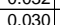 & & 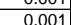 & 0 & $\frac{0.67}{0.64}$ \\
\hline & $4 / 19 / 2004$ & 12:14:18 PM & & 22.79 & 25.062 & 25.076 & & & 46.587 & 9.542 & 36.091 & $\frac{-1.21}{-1.21}>0$ & & 46.779 & 0.204 & $\frac{14.6}{14.6}$ & & & 2.53639 & 45.1 & & $40:$ & 793 & & & & & \\
\hline 171 & 4/199/2004 & 12:15:18 PM & 25.194 & 22.804 & \begin{tabular}{|l|}
25.037 \\
\end{tabular} & 25.075 & 191 & 44.769 & 46.21 & 9.428 & 36.163 & $\frac{-1.218}{-1.218}$ & & 49.518 & $\frac{.2 .205}{0.205}$ & 14.643 & & $\frac{15.2}{153.2}$ & $\frac{2.55306}{2.55306}$ & 44.7 & $\frac{1.1}{12 .}$ & 40.5 & & & & 0.001 & 0.075 & \\
\hline & $4 / 19 / 2004$ & 12:16:18 PM & 25.188 & 22.809 & \begin{tabular}{|l|l|}
25.026 \\
\end{tabular} & 25.094 & 23.16 & $\begin{array}{ll}44.923 \\
\end{array}$ & 46.307 & 9.457 & $\begin{array}{l}36.348 \\
6\end{array}$ & $\frac{-1.215}{-1.25}$ & & 49.785 & 0.202 & 14.643 & & 154 & 2.56972 & 44.8 & & 40. & & & & & & \\
\hline 17 & $4 / 19 / 2004$ & 12:17:18 PM & 25.198 & 22.829 & \begin{tabular}{|l|}
25.011 \\
\end{tabular} & 25.13 & & 44.966 & 46.492 & 9.545 & 36.241 & $-1.218 \quad \mathrm{C}$ & & & 0.204 & 14.643 & & & 2.58639 & 45.6 & & 40. & & & & & & \\
\hline & $4 / 19 / 2004$ & 12:18:18 PM & 25.188 & 22.834 & 24.986 & 25.145 & 23.286 & 44.954 & 46.413 & 9.507 & 36.229 & -1.218 & & 48.187 & 0.211 & 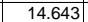 & & 156.2 & \begin{tabular}{l|l|l|}
2 & 2.60306 \\
\end{tabular} & 44.9 & 11.8 & 40.6 & 2.799 & .031 & 0.031 & 0.001 & | 0.775 & \\
\hline & 4/19/2004 & 12:19:18 PM & & & 24.965 & 25.154 & & 45.284 & 46.869 & 9.51 & 36.561 & -1.218 & & 49.211 & 0.207 & 14.643 & & 15 & 2.01912 & 45.4 & 12. & 40 & & & & & & \\
\hline 1776 & $4 / 19 / 2 / 2$ & 12:20:18 PM & 25.158 & 22.844 & 24.946 & & & 444.693 & 46.03 & 9.544 & 35.886 & -1.22 & & 47.272 & & & & 15 & & 44.6 & $11.6 \mathrm{r}-\mathrm{s}$ & & & & & & 0.752 & \\
\hline$\frac{17}{17}$ & 4/11992/2004 & $8 \mathrm{PM}$ & 25.174 & 22.869 & 24.942 & 25.18 & & 45.027 & 46.521 & 9.595 & 36.1 & -1.2. & & 45.21 & 0.199 & 14.643 & & 15 & & 45. & 11. & 40. & .79 & & & $0 . c$ & & \\
\hline & & & & & & & & & & & & & & & $\frac{0.200}{0.106}$ & & & & & & & & & & & & & \\
\hline 180 & $\begin{array}{l}4 / 19 / 2004 \\
4 / 19 / 2004 \\
\end{array}$ & $\frac{12.251 .18}{12.24 .18 \mathrm{M}}$ & 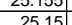 & $\frac{2.091}{22896}$ & \begin{tabular}{|l|l|l|}
24.908 \\
\end{tabular} & 25.2517 & $\frac{25}{23}$ & 年44.013 & $\mid \begin{array}{ll}46.32 \\
4653\end{array}$ & $\frac{9.45}{9542}>>$ & $\begin{array}{l}3.067 \\
36252\end{array}$ & $\frac{-1.21}{-121}$ & & 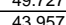 & 0.190 & $\begin{array}{l}14.643 \\
14.643 \\
\end{array}$ & & $\frac{161.2}{162.2}$ & | & 年4.6 & $\frac{12.2}{108}$ & $\frac{40.5}{40.6}$ & 2802 & $\frac{0.029}{0.028}$ & 0.028 & 0.001 & 0.724 & \\
\hline 181 & $4 / 19 / 2004$ & $12: 25: 18 \mathrm{PM}$ & 25.13 & 22.896 & \begin{tabular}{|l|}
24.006 \\
24.863 \\
\end{tabular} & 25.237 & 18 & 45.035 & $\begin{array}{l}4.517 \\
46.517\end{array}$ & 0.046 & & -122 & & 45.252 & 0.19 & $\begin{array}{ll}14.045 \\
14.643 \\
\end{array}$ & & $\frac{10.2}{163.2}$ & 2.10000 & & $\frac{10.6}{111}$ & 40.0 & & & & & & \\
\hline & $4 / 19 / 2004$ & $12: 26.18 \mathrm{PM}$ & 25.131 & 22.907 & 24.904 & 25.213 & 189 & 45.056 & 46.539 & $\frac{9.532}{9.532}$ & 36.184 & $\frac{-1.21}{-1.21}>0$ & & 47.557 & 0.198 & 14.643 & & 164 & 2.73639 & & 11 & 40 & & & & 0.00 & 0.729 & \\
\hline & $4 / 19 / 2004$ & & & & \begin{tabular}{|l|l|}
24.903 \\
\end{tabular} & & & 45.195 & 46.689 & 9.56 & & & & & & 14. & & & & & & & & & & & & \\
\hline & 4/19/2004 & 12:28:18 PM & 25.137 & & 24.945 & 25.264 & & 45.045 & 46.811 & 9.517 & 36.231 & -1.21 & & $48.8 \mathrm{r}-\mathrm{Y}$ & 0.21 & & & & & & & & & & & & 0.772 & \\
\hline & 4/19/2004 & 12:29:18 PM & 25.138 & 22.944 & \begin{tabular}{|l|l|}
24.941 \\
\end{tabular} & 25.255 & & 45.072 & 46.61 & 9.535 & 36.268 & -1.21 & & 50.2 & 0.188 & 14. & & 167 & 2.78639 & & 12.3 & 40. & 2.804 & & & 0. & $\begin{array}{ll}0.690 \\
\end{array}$ & \\
\hline & 4/19/2004 & 12:30:18 PM & 25.144 & 22.956 & \begin{tabular}{|l|l|}
24.957 \\
\end{tabular} & 25.261 & & & 46.257 & 9.521 & & -1.2. & & 51 & & & & 168.2 & & 4 & 12. & 40 . & .791 & & & & 0.712 & \\
\hline 187 & 4/19/2004 & 12:31:18 PM & 25.14 & 22.972 & 24.983 & 25.242 & & 45.3 & 46.732 & 9.565 & 36.678 & -1.21 & & 49.007 & 0.196 & 14 & & 169. & & & $12.8 \mathrm{C}>\mathrm{l}$ & 41. 41. & & & & & 713 & \\
\hline 188 & 4/19/2004 & 12:32:18 PM & 25.151 & 22.977 & 24.994 & 25.253 & & 45.33 & 46.823 & 9.595 & & -1.21 & & 48. & 0.197 & & & & & 45 & 11.. & 40 & & 029 & & & 0.718 & \\
\hline & 4/19/2004 & $P$ & 25.15 & 22.977 & 24.973 & 25.222 & & & 46.2644 & 9.486 & & & & $48.8-3>$ & 0.204 & & & & & & & & & & & & & \\
\hline$\frac{19}{10}$ & $\frac{411992004}{1119204}$ & $12: 34: 18 \mathrm{PM}$ & 25.124 & 22.998 2009 & \begin{tabular}{|l|l|}
25.03 \\
\end{tabular} & 25.299 & & 45.054 & $\begin{array}{r}46.672 \\
46619\end{array}$ & $\frac{9.508}{9528}$ & & -1.215 & & 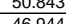 & 0.202 & & & 172 & & & . & 40 & $700+2+3$ & 30 30 & & & - 0.740 & \\
\hline & $\frac{41912004}{4192004}$ & $\frac{12.3 .10 \mathrm{P}}{12.36 \mathrm{PM}}$ & $\frac{23.16}{2518}$ & 告23.999 & 250.035 & $\frac{23.244}{25344}>0$ & & $\frac{44.950}{4511}$ & $\frac{4.0 .0}{467}$ & 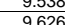 & & $\frac{-1.41}{-121}>>$ & & & & & & & & & & 40 & & & & & & \\
\hline$\sqrt{15}$ & 4 & $12.37 .18 \mathrm{PM}$ & 25.17 & 23015 & 25.042 & 252955 & & 45.132 & & $\frac{3.050}{9562}$ & & -122 & & & & & & & & 45 & 11.0 & & & & & & $\begin{array}{l}0.724 \\
0.688 \\
\end{array}$ & \\
\hline 194 & $\frac{4 / 199 / 2004}{4}$ & 12:38:18 PM & 25.179 & $\frac{23.035}{23.035}$ & \begin{tabular}{|l|l|}
25.047 \\
\end{tabular} & $\mid 25.29$ & & $\frac{4.92}{44.92}$ & $\frac{46.5}{46.5}$ & $\frac{9.637}{9.637}$ & & $\frac{-1.218}{-1.218}$ & & & 0.195 & & & 17 & & & 123 & & & & & & & \\
\hline & $4 / 19 / 2004$ & & & & & & & & & & & & & & & & & & & & & & & & & & & \\
\hline 196 & 4/19/2004 & 12:40:18 PM & 25.198 & 23.035 & 25.071 & 25,33 & & & 46.643 & 9.661 & & $-1.218 \quad$ & & & 0.195 & & & & & & 12. & & & & & & & \\
\hline & $4 / 19 / 2$ & 12:41:18 $\mathrm{F}$ & 25 & 23.05 & 25.061 & 25.3 & & & 46.7 & & & -1.21 & & 45. & & & & & & & tit & & & 0.030 & & & & \\
\hline 198 & 4/19/2004 & 12:42:18 PM & & 23.054 & 5.09 & 25.213 & & 45.17 & 46.745 & 9.6 & 36.262 & -1.21 & & 48.9 & 0.196 & \begin{tabular}{|c|c|}
14.643 \\
\end{tabular} & & 180 & 3.00 & $4 !$ & $12.8>>>$ & 40. & $2.807 \mid$ & 0.029 & & & 0.716 & \\
\hline 199 & 4/199/2004 & 12:43:18 PM & 25.225 & 23.037 & \begin{tabular}{|l|l|}
25.044 \\
\end{tabular} & 25.142 & & 44.946 & 46.487 & & & -1.21 & & 47.937 & & & & 18 & 3.01 & 45. & 11. & $40+2-3$ & & & & & 0.746 & \\
\hline & & 12:44:18 PM & 25.234 & 23.03 & 25.037 & 25.095 & & 44.749 & 46.081 & 9.852 & 35 & -1.21 & & 50.801 & 0 & & & 182.2 & $\begin{array}{c}2 \mid \\
3.03\end{array}$ & 44.6. & 12.4 & 40 & 2.7 & 0.029 & & & 0.726 & \\
\hline 20 & 4/119/20004 & 12:45:18 PM & 25.23 & 23.0244 & 25.075 & 25.004 & & 44.641 & 46.085 & 9.99 & 35.433 & -1.218 & & 51.4 & 0.178 & 14.6 & & & & 44.6 & 1E. & & & & & & & \\
\hline & $\frac{411912004}{41 / 2004}$ & $12: 46: 18 \mathrm{PM}$ & 25051 & 20.012 & \begin{tabular}{|l|l|}
25.069 \\
\end{tabular} & 24.932 & mo & (44.618 & 46.125 & 9.966 & 35.324 32. & -1.218 & & 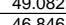 & 0.1 & $14.6 \mathrm{C}$ & & & 1208 & 44.7. & $\frac{12.0}{1.6}>\mathrm{r}$ & 20 & & 0.020 & & & $\frac{11}{12}$ & \\
\hline & 4 & 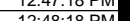 & & $\frac{2.012}{23001}$ & \begin{tabular}{|c|c|}
2.105 \\
25.102
\end{tabular} & 24.954 & & 44.110 & (40.197 & & & & & 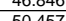 & & & & & & & & & & & & & & \\
\hline $20: 5+3$ & $\frac{4151204}{41 / 92004}$ & 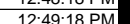 & & (2) & 20 & 24.971 & & $\begin{array}{ll}44.054 \\
468\end{array}$ & $\begin{array}{ll}40.34 \\
46.26\end{array}$ & & & -12 & & 50 & & 14 & & & & & 年 & & & & & & & \\
\hline $20 \mathrm{c}$ & & $12: 50: 18$ & 25.25 & 22.996 & 25 & & & & $\mid 46.359$ & & & $\frac{-1.21}{-1.21}>0$ & & & & & & & & & 115 & & & 2028 & & & & \\
\hline 20 & $4 / 19 / 2004$ & & & 22.964 & & 24.964 & & & & & & & & & & & & & & & 120 & & & & & & 80 & \\
\hline 20 & & & & 22979 & 25.111 & & & & & & & & & & & & & & & 45.5 & $120 \mathrm{P}=\mathrm{l}$ & & & & & & & \\
\hline 20 & $41191212>$ & $12.53 \cdot 18 \mathrm{PM}$ & 25257 & 22958 & 251 & 059 & & 45.2 & 46.827 & 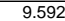 & 360 & 1218 & & 48.59 & & 14.643 & & th & 318 & 45.4 & 110 & & & 020 & & & 703 & \\
\hline 10 & & 12:54:18 PM & & & 25.084 & 25.157 & & 45,344 & 46.792 & 9.553 & 36.555 & -1.221 & & 47.175 & 0.195 & $\begin{array}{l}14.643 \\
\end{array}$ & & & \begin{tabular}{|l|l|}
2 & 3.20306 \\
\end{tabular} & & $\frac{11.6}{11.6}$ & 40.9 & $2.88 \mathrm{Y}-\mathrm{x}$ & 0.029 & 0.029 & 0.001 & 0.708 & \\
\hline
\end{tabular}


WSRC-TR-2005-00105, REVISION 0

SRNL-RPP-2005-00012, REVISION 0

RUN \# 2.03A AND B; FIRST AND SECOND HALF OF SLURRY DEWATERING - CONT.

\begin{tabular}{|c|c|c|c|c|c|c|c|c|c|c|c|c|c|c|c|c|c|c|c|c|c|c|c|c|c|c|c|c|}
\hline & & & & & & & & & & & & & & & & & & & & & & & & & & & & \\
\hline & A & \begin{tabular}{|c|} 
B \\
\end{tabular} & D & $E$ & $r$ & G & $\mathrm{H}$ & $\mathrm{J}$ & $\mathrm{K}$ & $\mathrm{L}$ & $\mathrm{M}$ & $\mathrm{N}$ & 0 & $\mathrm{Q}$ & $\mathrm{R}$ & \begin{tabular}{|c|}
$\mathrm{s}$ \\
\end{tabular} & $\mathrm{T}$ & $\mathrm{v}$ & w & $x$ & 1 & $z$ & AA & $A B$ & $A C$ & $A D$ & $\mathrm{AE}$ & \\
\hline$\frac{2}{2 .}$ & \begin{tabular}{|l|}
$4 / 19 / 2004$ \\
$4 / 19 / 2004$ \\
\end{tabular} & \begin{tabular}{|c|}
$12: 55: 18 \mathrm{PM}$ \\
$12: 56: 18 \mathrm{PM}$ \\
\end{tabular} & $\begin{array}{l}25.275 \\
25.265\end{array}$ & $\begin{array}{l}22.956 \\
22.946\end{array}$ & \begin{tabular}{|l|}
25.148 \\
25.128 \\
\end{tabular} & \begin{tabular}{|l|}
25.212 \\
25.232 \\
\end{tabular} & $\begin{array}{l}23.142 \\
23.363 \\
\end{array}$ & $\begin{array}{l}45.215 \\
45.332\end{array}$ & \begin{tabular}{|l|}
46.765 \\
46.854 \\
\end{tabular} & \begin{tabular}{|l|}
9.615 \\
9.629 \\
\end{tabular} & $\begin{array}{l}36.293 \\
36.43 \varepsilon \\
\end{array}$ & \begin{tabular}{|c|c|}
-1.218 \\
3.218 \\
\end{tabular} & $\begin{array}{l}-5.873 \\
-5.865 \\
\end{array}$ & $\begin{array}{l}49.284 \\
49.823\end{array}$ & $\begin{array}{l}0.189 \\
0.182\end{array}$ & \begin{tabular}{|l|}
14.643 \\
14.643 \\
\end{tabular} & $\begin{array}{l}0.003 \\
0.003\end{array}$ & $\begin{array}{l}193.2 \\
194.2 \\
\end{array}$ & $\begin{array}{l}2 \\
2.21972 \\
2.23639\end{array}$ & $\begin{array}{l}45.3 \\
45.4\end{array}$ & \begin{tabular}{|l|l}
12.2 \\
12.2
\end{tabular} & $\begin{array}{l}40.8 \\
40.9\end{array}$ & 2.810 & $\begin{array}{l}0.028 \\
0.027\end{array}$ & $\begin{array}{l}0.028 \\
0.027\end{array}$ & $\begin{array}{l}0.001 \\
0.001\end{array}$ & $\begin{array}{l}0.689 \\
0.661 \\
\end{array}$ & $\begin{array}{l}0.59 \\
0.56 \\
\end{array}$ \\
\hline & & & & & \begin{tabular}{|l|}
25.128 \\
25.148 \\
\end{tabular} & \begin{tabular}{|l|}
25.232 \\
25.267 \\
\end{tabular} & & $\frac{45.332}{45.132}$ & \begin{tabular}{|l|}
46.6544 \\
46.614
\end{tabular} & $\begin{array}{l}.629 \\
9.605 \\
\end{array}$ & $\frac{30.456}{36.245}$ & & & 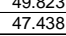 & $\begin{array}{ll}0.182 \\
0.187\end{array}$ & \begin{tabular}{|l|}
14.6433 \\
14.643
\end{tabular} & & & & & \begin{tabular}{|l|l|}
12.6 \\
11.6 \\
\end{tabular} & & & & & & & \\
\hline$\frac{14}{214}$ & $4 / 19 / 2004$ & 12:58:18 PM & 25.279 & 22.956 & \begin{tabular}{|l|}
25.158 \\
\end{tabular} & \begin{tabular}{|l|}
25.251 \\
\end{tabular} & 23.016 & 45.694 & 47.271 & 9.634 & 36.894 & \begin{tabular}{|l|l|} 
& -1.215 \\
\end{tabular} & -5.74 & 48.919 & 0.179 & \begin{tabular}{|l|}
14.643 \\
\end{tabular} & 0.004 & 196.2 & 23.26972 & 45.8 & 12.0 & 41.3 & 2.847 & & & 0.001 & 0.643 & 0.55 \\
\hline & $4 / 19 / 2004$ & \begin{tabular}{|l|}
$12: 59: 18 \mathrm{PM}$ \\
\end{tabular} & 25.279 & 22.945 & \begin{tabular}{|l|}
25.127 \\
\end{tabular} & 25.255 & 22.991 & 45.518 & 47.02 & 9.498 & 36.78 & \begin{tabular}{|c|}
$3-1.218$ \\
\end{tabular} & -5.896 & 47.1 & 0.196 & 14.643 & & 197.2 & 23.28639 & 45.5 & \begin{tabular}{|l|l}
11.5 \\
\end{tabular} & & 2.837 & & & 0.001 & 0.708 & 0.60 \\
\hline & & & & & 25.147 & & & 44.56 & 46.059 & 10.08 & & & & 47.491 & & \begin{tabular}{|l|}
14.643 \\
\end{tabular} & & 198.2 & \begin{tabular}{|l|l|}
2 & 3.30306 \\
\end{tabular} & 44.6 & 11.6 & 39.8 & & & & & & 0.60 \\
\hline 217 & $4 / 19 / 2004$ & 1:01:18 PM & 25.294 & 22.95 & 25.092 & 25.271 & 222.981 & $\begin{array}{l}44.429 \\
\end{array}$ & 45.938 & 10.194 & 35.024 & \begin{tabular}{|l|l|} 
& -1.218 \\
\end{tabular} & -5.946 & 50.25 & 0.189 & \begin{tabular}{|l|}
14.643 \\
\end{tabular} & & 199.2 & 3.31972 & 44.5 & 12.3 & 39.7 & 2.739 & 0.028 & & 0.001 & $\begin{array}{ll}0.707 \\
\end{array}$ & 0.60 \\
\hline$\frac{218}{219}$ & \begin{tabular}{|l|}
$4 / 19 / 2004$ \\
$4 / 192004$ \\
\end{tabular} & $\begin{array}{l}\text { 1:02:18 PM } \\
1.03 \cdot 18 \mathrm{PM}\end{array}$ & $\begin{array}{l}25.298 \\
25.303\end{array}$ & $\begin{array}{l}22.964 \\
22.964\end{array}$ & \begin{tabular}{|l|}
25.121 \\
25.141 \\
\end{tabular} & \begin{tabular}{|r|}
25.29 \\
25295 \\
\end{tabular} & $\begin{array}{l}23.01 \\
2306 \\
\end{array}$ & \begin{tabular}{|l|l|l|l|}
44.792 \\
\end{tabular} & \begin{tabular}{|l|}
46.108 \\
46.172 \\
\end{tabular} & $\begin{array}{ll}9.988 \\
9969\end{array}$ & $\begin{array}{l}35.494 \\
35.59\end{array}$ & \begin{tabular}{|l|l|} 
& -1.215 \\
-1218 &
\end{tabular} & $\begin{array}{l}-5.983 \\
-5.836 \\
\end{array}$ & $\begin{array}{l}50.139 \\
48487\end{array}$ & $\begin{array}{l}0.186 \\
0.178 \\
\end{array}$ & \begin{tabular}{|l|l|}
14.643 \\
14643
\end{tabular} & & 200.2 & $\begin{array}{l}23.33639 \\
2\end{array}$ & $\begin{array}{l}44.6 \\
447\end{array}$ & 12.3 & $\begin{array}{l}40.1 \\
40.2 \\
\end{array}$ & $\frac{2.765}{2.771}$ & 0.028 & 0.028 & $\begin{array}{l}0.001 \\
0.001\end{array}$ & $\begin{array}{l}0.689 \\
0658\end{array}$ & 0.59 \\
\hline$\frac{220}{220}$ & $\begin{array}{l}4 / 19 / 2004 \\
4 / 19 / 2004\end{array}$ & 1:04:18 PM & 25.298 & 22.98 & \begin{tabular}{|l|}
25.141 \\
25.141 \\
\end{tabular} & \begin{tabular}{|l|}
25.290 \\
25.305 \\
\end{tabular} & $\begin{array}{l}23.06 \\
23.075 \\
\end{array}$ & \begin{tabular}{|l|}
44.1594 \\
44.894
\end{tabular} & $\begin{array}{l}46.12 \\
46.344 \\
\end{array}$ & 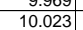 & $\begin{array}{l}35.605 \\
35.67 \\
\end{array}$ & $\begin{array}{l}-1.210 \\
-1.221 \\
\end{array}$ & $\begin{array}{l}-5.836 \\
-5.96\end{array}$ & $\begin{array}{l}58.015 \\
52.015\end{array}$ & $0.1 / 8$ & \begin{tabular}{|l|}
14.643 \\
14.643 \\
\end{tabular} & 0.0 & \begin{tabular}{r|}
201.2 \\
202.2 \\
\end{tabular} & $\begin{array}{l}23.35306 \\
23.36972 \\
2\end{array}$ & $\frac{44.7}{44.9}$ & \begin{tabular}{|l|l|}
11.9 \\
12.7
\end{tabular} & $\begin{array}{l}40.2 \\
40.3 \\
\end{array}$ & $\frac{2.771}{2.778}$ & $\frac{0.027}{0.028}$ & $\frac{0.026}{0.028}$ & 0.001 & $\begin{array}{l}0.658 \\
0.66 \\
\end{array}$ & \\
\hline 22 & $4 / 19 / 2004$ & 1:05:18 PM & & & & & & 44.896 & 46.173 & & & & & 50.782 & 0.184 & \begin{tabular}{|l|l|}
14.643 \\
\end{tabular} & & & $\begin{array}{l}23.00639 \\
2.3839\end{array}$ & 44.7 & & $\begin{array}{r}40.3 \\
40.3 \\
\end{array}$ & & & & & $\begin{array}{l}0.666 \\
0.677 \\
\end{array}$ & 0.58 \\
\hline & $4 / 19 / 2004$ & 1:06:18 PM & 25.303 & 22.98 & \begin{tabular}{|l|l|}
25.141 \\
\end{tabular} & 25.33 & 23.05 & 45.149 & & 9.815 & 36.11 & $\mid-1.221$ & -5.98 & 49.551 & 0.181 & \begin{tabular}{|l|l|}
14.643 \\
\end{tabular} & & & 3.40306 & 45.0 & 12.1 & & & & & & & 0.58 \\
\hline 22 & $4 / 19 / 2004$ & 1:07:18 PM & 25.324 & & & & & & $\begin{array}{ll}46.784 \\
\end{array}$ & & & -1.218 & & 49.822 & 0.189 & 14.643 & & & 3.41972 & 45.3 & 12.4 & 40.8 & & & & 0.00 & $\begin{array}{l}0.002 \\
0.686 \\
\end{array}$ & 0.56 \\
\hline & $4 / 19 / 2004$ & 1:08:18 PM & & 23.012 & \begin{tabular}{|l|l|}
25.194 \\
\end{tabular} & 25.342 & & $\begin{array}{l}45.257 \\
\end{array}$ & $\begin{array}{l}466.651 \\
\end{array}$ & \begin{tabular}{|l|l|}
9.537 \\
\end{tabular} & 36.53. & -1.218 & & 46.6 & 4 & 14.643 & & & & & 11 & & & & & & 0.689 & \\
\hline 225 & $4 / 19 / 2004$ & 1:09:18 PM & 25.33 & 22.991 & 25.193 & 25.267 & 22.741 & 45.2. & 46.672 & & 36.4 & & & 47.774 & & $\begin{array}{l}14.643 \\
\end{array}$ & & & & & & & & & & & 0.694 & \\
\hline$\frac{226}{227}$ & $4 / 19 / 2004$ & 1:10:18 PM & 25.335 & 22.991 & \begin{tabular}{|l|l|}
25.178 \\
\end{tabular} & 25.162 & 22.53 & 45.327 & |46.975 & 9.69 & 36.375 & $\begin{array}{l}-1.218 \\
\end{array}$ & -5 & 49.216 & 0.179 & \begin{tabular}{|l|l|l|}
14.643 \\
\end{tabular} & & 208.2 & $\begin{array}{l}2 \\
3.46972\end{array}$ & 45.5 & 12.1 & $\begin{array}{l}40.9 \\
\end{array}$ & 2.817 & j.027 & 0.027 & 0.001 & $\begin{array}{l}0.650 \\
\end{array}$ & 0.55 \\
\hline & $4 / 19 / 2004$ & 1:11:18 PM & 25.338 & 22.99 & \begin{tabular}{|l|l|}
25.106 \\
\end{tabular} & 25.125 & & 45.5 & 46.885 & 9.657 & 36.73 & -1.218 & -5.9 & 49.975 & 0.187 & \begin{tabular}{|l|l|}
14.643 \\
\end{tabular} & & 209.2 & 3.48639 & 45.4 & 12.2 & 41.1. & & & & 0.00 & 0.676 & 0.58 \\
\hline & $4 / 19 / 2004$ & 1:12:18 PM & & & & & & & & 9.741 & 36.155 & & & 48.279 & 0.186 & 14.643 & & & & 45. & & & & & & & & \\
\hline & $4 / 199 / 2004$ & 1:13:18 PM & 25.34 & 22.966 & 25.133 & $\frac{25.021}{2021}$ & 22.274 & 45.576 & 46.972 & $\frac{9.651}{9692}$ & $\begin{array}{l}33.687 \\
30.678\end{array}$ & & & 49.611 & 0.185 & 14.643 & & & & 45. & & & & 028 & & 0.00 & 0.667 & \\
\hline & $\begin{array}{l}4 / 1 / 19 / 2004 \\
/ 1204\end{array}$ & $\frac{1.14 .18 \mathrm{PM}}{1 \cdot 15 \cdot 18 \mathrm{P}}$ & 25335 & & & & $\frac{22.447}{2049}$ & $\begin{array}{r}45.5 \\
45259\end{array}$ & $\frac{46.995}{46.68}$ & $\begin{array}{l}9.583 \\
9596\end{array}$ & $\frac{36.64}{36.461}$ & $\frac{-1.21}{-121}$ & & $\frac{46 .}{512}$ & 0.177 & $\frac{14.645}{14643}$ & & & & & & & & & & & & \\
\hline & $4 / 19 / 2004$ & $1: 16.18 \mathrm{PM}$ & 25.328 & 22.919 & 25.151 & 24.869 & 22.815 & 45.591 & $\begin{array}{l}47.155 \\
47.55\end{array}$ & 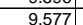 & $\begin{array}{l}3.6 .747 \\
3.677\end{array}$ & & & $\begin{array}{l}4.1 .002 \\
4.002\end{array}$ & 0.176 & 14.643 & & & 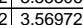 & & $\frac{1.5}{11.5}$ & $\frac{41.2}{41.2}$ & 2.839 & .026 & & 0.001 & $\mid$ & 0.54 \\
\hline & $4 / 19 / 2004$ & 1:17:18 PM & 25.338 & 22.919 & 25.216 & 24.894 & 23.045 & 44.682 & 46.002 & \begin{tabular}{|l|l|}
10.04 \\
\end{tabular} & 35.543 & \begin{tabular}{|l|l|}
3 & -1.218 \\
\end{tabular} & -5.98 & 50.822 & 0.179 & 14.643 & & 215.2 & 2) 3.58639 & 44.5 & 12.5 & 40.1 & 2.766 & & & & 0.661 & 0.56 \\
\hline & $4 / 19 / 2004$ & 1:18:18 PM & 25.345 & 22.921 & 25.253 & & 22.936 & 44.979 & 46.552 & 10.021 & 35.72 & & & 50.736 & 0.169 & 14.643 & & 216.2 & $\begin{array}{l}2 \\
3.60306\end{array}$ & 45.1 & 12.4 & \begin{tabular}{|l|l|} 
& \\
\end{tabular} & 2.78 & & & & 0.620 & \\
\hline & $4 / 19 / 2004$ & 1:19:18 PM & 25.349 & $\begin{array}{r}22.89 \\
22.870\end{array}$ & \begin{tabular}{|l|l|}
25.247 \\
\end{tabular} & $\begin{array}{r}24.99 \\
\end{array}$ & 22.76 & $\begin{array}{r}44.784 \\
\end{array}$ & 46.324 & $\begin{array}{ll}10.061 \\
\end{array}$ & 35.457 & -1.218 & -5.98 & 49.074 & 0.186 & \begin{tabular}{|l|l|}
14.643 \\
\end{tabular} & & 217.2 & 3.61972 & 44.9 & 12.0 & 40.1 & 2.766 & & & 0.001 & 0.686 & \\
\hline & $\begin{array}{l}4 / 199 / 2004 \\
4\end{array}$ & $\begin{array}{l}1: 20: 18 \text { PM } \\
1 \cdot 12 \cdot 18 \text { M }\end{array}$ & $\frac{25.343}{25.342}$ & 22.879 & \begin{tabular}{|l|}
25.221 \\
25165 \\
\end{tabular} & $\frac{25.035}{25104}$ & $\frac{22.174}{20813}$ & $\begin{array}{r}44.79 \\
44.682 \\
\end{array}$ & $\begin{array}{r}46.135 \\
46.26\end{array}$ & \begin{tabular}{|c|}
9.971 \\
9.963
\end{tabular} & $\begin{array}{l}35.636 \\
35.33\end{array}$ & $\begin{array}{l}-1.218 \\
-1.1218\end{array}$ & -586 & $\frac{48.141}{50.513}$ & $\begin{array}{l}0.177 \\
0.185\end{array}$ & $\frac{14.643}{14.643}$ & & 218.4 & 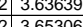 & $\frac{44.7}{44.8}$ & $\frac{11.8}{124}$ & 40. & 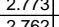 & & & & 6.652 & 0.56 \\
\hline & $4 / 19 / 2004$ & $\begin{array}{l}1.4 .1 .10 \mathrm{PM} \\
1.22 \cdot 18 \mathrm{PM}\end{array}$ & $\frac{25.342}{25.346}$ & 22877 & \begin{tabular}{|l|}
25.100 \\
25204 \\
\end{tabular} & $\frac{25.104}{25.117}$ & $\frac{2.1015}{22867}$ & 45.168 & $\begin{array}{r}40.20 \\
46.653\end{array}$ & 10.011 & $\begin{array}{l}3.4 .48 \\
35.8 \varepsilon\end{array}$ & -1.218 & & $\begin{array}{l}0.013 \\
49.184\end{array}$ & $\begin{array}{l}0.105 \\
0.181\end{array}$ & $\begin{array}{l}14.045 \\
14.643\end{array}$ & & 220. & & . & $\frac{1.4}{121}$ & & 794 & & & & $\mid$ & \\
\hline & 4/19/2004 & $\begin{array}{l}1.23: 18 \mathrm{PM} \\
\end{array}$ & $\frac{2.040}{25.356}$ & 22.882 & \begin{tabular}{|l|}
25.204 \\
25.209
\end{tabular} & 25.177 & & $\begin{array}{l}45.080 \\
45.085\end{array}$ & 4 & $\begin{array}{l}9.931 \\
\end{array}$ & 36.003 & -1.218 & -5.95 & $\begin{aligned} 49.104 \\
49.662\end{aligned}$ & $\begin{array}{l}.101 \\
0.182\end{array}$ & \begin{tabular}{l|l}
14.045 \\
14.643
\end{tabular} & & 221.2 & 23.68699 & 年 & $\frac{12.1}{12.2}$ & $\frac{10 .}{40 .}$ & 795 & & & & & $\frac{0.56}{0.57}$ \\
\hline & $4 / 19 / 2004$ & 1:24:18 PM & 25.361 & 22.892 & 25.255 & & & & & \begin{tabular}{|l|l|l|}
10.033 \\
\end{tabular} & $35.75 \mathrm{~s}$ & & & & 0.181 & & & 222 & 3.70306 & 45.0 & & 40.4 & & & & & 663 & \\
\hline 241 & $4 / 191 / 2004$ & 1::25:18 PM & 25.373 & 22.899 & \begin{tabular}{|l|}
25.282 \\
\end{tabular} & $\frac{25.18}{25.18}$ & & 44.884 & 46.32 & \begin{tabular}{|l|l|}
9.98 \\
\end{tabular} & $35.60 \mathrm{~s}$ & -1.218 & -5.9 & 50.528 & 0.161 & $\begin{array}{l}14.045 \\
14.643\end{array}$ & & 223.2 & $\frac{3.71972}{3.71972}$ & 44.8 & $\frac{11 .}{12 .}$ & 40.2 & & $\overline{024}$ & & 0.001 & - & \\
\hline $248 \mathrm{~S}$ & $4 / 19 / 2004$ & 1:26:18 PM & 25.381 & 22.892 & \begin{tabular}{|l|}
25.289 \\
\end{tabular} & 25.237 & $23.14 \varepsilon$ & \begin{tabular}{|l|l|l|}
44.966 \\
\end{tabular} & \begin{tabular}{l|l|}
46.398 \\
\end{tabular} & $\begin{array}{l}9.956 \\
\end{array}$ & 35.724 & -1.218 & & 46.875 & $\begin{array}{c}0.18 \\
0.18\end{array}$ & 14.643 & & 224 & 3.73639 & 44.9 & $\frac{x 1.5}{11 .}$ & & & & & & & \\
\hline & $4 / 19 / 2004$ & $1: 27: 18 \mathrm{PM}$ & 25.383 & 22.904 & \begin{tabular}{|l|}
25.286 \\
\end{tabular} & 25.314 & & & 46.295 & 9.938 & 35.636 & -1.218 & & 47.872 & 0.17 & 14.643 & & & & 448 & & & & & & & & \\
\hline & 4/19/2004 & 1:28:18 PM & 25.391 & 22.912 & 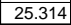 & 25.348 & 23.163 & 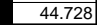 & 46.289 & 9.894 & 35.562 & -1.218 & & 50.632 & 0.178 & \begin{tabular}{|l|l|l|l|l|}
14.643 \\
\end{tabular} & & $\begin{array}{l}226.2 \\
\end{array}$ & \begin{tabular}{l|l|l|}
23.76972 \\
\end{tabular} & $\begin{array}{ll}44.8 \\
\end{array}$ & 12.4 & 40.1 & 2.768 & & 0.026 & 0.001 & \begin{tabular}{|c|c|c|}
0.655 \\
\end{tabular} & $\begin{array}{ll}0.56 \\
\end{array}$ \\
\hline & 4/119/2004 & 1:29:18 PM & 25.413 & 22.954 & \begin{tabular}{|l|}
25.281 \\
\end{tabular} & 20.305 & & 45.201 & 46.647 & 9.896 & 36.096 & -1.215 & & 48.809 & 0.175 & \begin{tabular}{|l|l|}
14.643 \\
\end{tabular} & & 227.2 & 3.78639 & 45.2 & & $\pi$ & & & & 0.00 & 0.637 & \\
\hline & $4 / 19 / 2$ & 1:30:18 PM & 25.415 & 22.941 & \begin{tabular}{|l|l|}
25.278 \\
\end{tabular} & 25.452 & & 44.71 & & 9.877 & & & & 49.451 & 0.173 & & & 228.2 & & 44.8 & 12.1 & 40 & 2.764 & & & & 0.638 & \\
\hline 20 & 4/11992004 & $8 \mathrm{PMM}$ & 25.414 & 22.95 & \begin{tabular}{|l|l|}
25.292 \\
\end{tabular} & 25.396 & & 45.06 & 46.465 & & 36.153 & -1.218 & & 49.1 & 0.18 & 14.643 & & 22 & & 45. & $2 .{ }^{2 .} \mathrm{s}$ & . & 80 & & & 0.00 & 0.655 & \\
\hline & & & & & & $\frac{25.429}{25.46}$ & & & $\begin{array}{l}46.645 \\
6.684\end{array}$ & & & & & 49. & 0.171 & & & & & & & & & & & & & \\
\hline 250 & $\begin{array}{l}4 / 1 / 19 / 2004 \\
/ 19 / 204\end{array}$ & $\begin{array}{l}1.35 .18 \mathrm{PM} \\
1: 34: 18 \mathrm{PM}\end{array}$ & $\frac{25.435}{25.42}$ & $\frac{22.960}{22967}$ & $\begin{array}{l}25.353 \\
25309\end{array}$ & $\frac{25.440}{25418}$ & & $\frac{45.219}{45.172}$ & 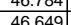 & 年 & $\begin{array}{l}36.18 \\
36.155\end{array}$ & - & -5.99 & $\frac{50.200}{44966}$ & $\begin{array}{l}0.178 \\
0.178\end{array}$ & $\begin{array}{l}14.643 \\
14.643\end{array}$ & & $\frac{231.2}{232.2}$ & $\begin{array}{l}\frac{2}{3.85306} \\
2.386972\end{array}$ & $\frac{4.5}{452}$ & $\frac{12.3}{122}$ & 40.7 & 2804 & & & 001 & $\begin{array}{l}0.6446 \\
0.647\end{array}$ & \\
\hline 251 & $4 / 19 / 2004$ & $1: 35: 18 \mathrm{PM}$ & 25.453 & 22.994 & 25.356 & $\begin{array}{r}25.410 \\
\end{array}$ & & 45.205 & 46.838 & & 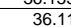 & $-1.21 \varepsilon$ & & 50.555 & $\begin{array}{l}.1170 \\
.179\end{array}$ & $\begin{array}{ll}14.045 \\
14.643\end{array}$ & & 233.2 & & & $\frac{1.4}{12.4}$ & 40.1 & 2.004 & & & & & \\
\hline 252 & $4 / 19 / 2004$ & $1: 36: 18 \mathrm{PM}$ & 25.449 & 22.996 & \begin{tabular}{|l|l|}
25.373 \\
\end{tabular} & 25.291 & & 45.23 & 46.784 & 9.767 & 36.258 & -1.215 & & 47.65 & 0.179 & 14.643 & & 234 & \begin{tabular}{|l|l}
3.9030606 \\
\end{tabular} & 45 & 117 & 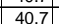 & & & & 000 & $\mid 0.648$ & \\
\hline & $4 / 19 / 2004$ & & 25.46 & & & & & & 46.73 & & 36.36 & -1.218 & & 48.6 & & & & & & & & & & & & & & \\
\hline & 4/19/2004 & 1:38:18 PM & 25.455 & 22.992 & 25.239 & 25.167 & & 45.018 & 46.401 & $\begin{array}{l}9.718 \\
\end{array}$ & 35.985 & $-1.21 \varepsilon$ & & 50.6 & 0.18 & & & & & & & & & & & & 0.658 & \\
\hline & 4/19/2004 & 1:39:18 PM & 25.45 & 22.977 & 25.234 & 25.132 & & 45.352 & $\begin{array}{l}46.807 \\
\end{array}$ & 9.7 & 36.426 & -1.22 & & 48. & 0.176 & 14 & & 237.2 & & & 11.9 & 409 & 2811 & & & 0. & \begin{tabular}{|c|}
0.638 \\
\end{tabular} & \\
\hline & 4/19/2004 & $18 \mathrm{PM}$ & 25.465 & 22.992 & 25.304 & 25.177 & & & 46.977 & 9.814 & & -1.21 & & 47. & 0.178 & & & 23 & & & 11.6 & & & & & & 0.644 & \\
\hline 257 & $4 / 199 / 2004$ & $\begin{array}{l}1: 11: 18 \mathrm{MM} \\
\mathrm{M}\end{array}$ & 25.455 & 22.971 & 25.273 & 25.086 & & 45.584 & 47.285 & $\begin{array}{l}9.806 \\
\end{array}$ & 36.467 & -1.21 & & 48.3 & 0.178 & 14 & & & & & 11.9 & & & & & & 542 & \\
\hline 258 & 4/19/2004 & 1:42:18 PM & 25.464 & 22.971 & 25.308 & 25.041 & & 45.24 & 46.74 & 10.039 & 36.098 & -1.221 & & 50. & 0.176 & 14. & & & & & 12. & & & & & & 640 & \\
\hline & $4 / 19 / 2004$ & & 25.459 & 22.96 & 25.347 & 255.01 & & & 46.1566 & & & -1.2. & & 50.6 & 0.177 & & & & & & & & & & & & & \\
\hline 20 & 411992004 & & 25.463 & 22.954 & \begin{tabular}{|l|l|}
25.346 \\
\end{tabular} & 25.004 & & 44.803 & 46.521 & 10.2377 & $\frac{3.221}{35.21}$ & -1.218 & & 48. & 0.163 & & & 24 & & 45. & 11.5 & 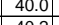 & 277 & & & & S011 & \\
\hline & $\frac{41912004}{4192014}$ & $\frac{14.4618}{1.48 \mathrm{PM}}$ & $\frac{25.40}{2546}$ & $\frac{26.945}{22955}$ & $\frac{2.351}{25407}$ & $\frac{2505}{251}$ & & & $\frac{4.0 .11}{46.44}$ & $\frac{10.1}{101}$ & & $-1211 \quad$ & & & & & & & & & $\overline{121}$ & & & & & & (.010) & \\
\hline$\sqrt{26}$ & $\frac{4}{41 / 912004}$ & $1.477 .18 \mathrm{PM}$ & 25.494 & 22961 & \begin{tabular}{|l|l|l|} 
\\
5428
\end{tabular} & 25176 & & $\frac{47.96}{44.96}$ & $\mid 46.598$ & 10.048 & & & & 48. & & & & & & & 120 & & & & & & & \\
\hline & $\frac{4 / 19 / 2004}{4}$ & 1:48:18 PM & $\frac{25.5}{25.5}$ & $\frac{22.952}{22.952}$ & 25.429 & $\frac{25.142}{25.142}$ & & & 46.282 & 10.076 & & -121 & & 47.6 & 0.169 & & & $\frac{24}{24}$ & & 44.8 & 115 & & & & & & 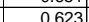 & \\
\hline & & & & & & & & & & & & & & & & & & & & & & & & & & & & \\
\hline & $4 / 19 / 2004$ & $1: 50: 18$ PM & 25.482 & 22953 & 25335 & 25208 & & & 46743 & 10083 & 35698 & -121 & & 49 & 0166 & & & 24 & & & 12 & & & & & & & \\
\hline & $4 / 199$ & 1:51:18 & 25.5 & 22.97 & \begin{tabular}{|l|}
25.337 \\
\end{tabular} & 25.3 & & & 46.328 & 10.0 & & & & & 0.162 & & & 2. & & & $\frac{11.5}{11.5}>$ & & & & & & & \\
\hline 268 & $4 / 19 / 2004$ & 1:52:18 PM & 25.5 & 22.966 & 25.363 & 25.387 & & 44.75 & 46.318 & 9.9 & 35.576 & \begin{tabular}{|l|l|}
6 & $-1.21 \varepsilon$ \\
\end{tabular} & & 51.4 & 0.141 & $\begin{array}{ll}14.643 \\
\end{array}$ & & 250.2 & 24.16 & 44 & $12.6 \mathrm{r}>\mathrm{l}$ & $40.8>$ & & 0.021 & & & 0.518 & \\
\hline $26-26>0$ & $4 / 191 / 2$ & 1:53:18 PM & 25 & 22. & 25.41 & 25.419 & & 45.178 & 46.767 & 10.1 & $35.73 \varepsilon$ & -1.218 & & 50. & 0.159 & & & 25 & $2 \mid 4.18 \quad \longrightarrow \quad$ & 45. & 12.4 & & & & & & $\begin{array}{l}0.579 \\
\end{array}$ & \\
\hline & & 1:54:18 PM & & 22.984 & 25.411 & 25.49 & & 44.89 & 46.504 & 10.008 & 35.677 & -1.212 & & 49. & 0.167 & & & 252.2 & & 45.0 & 12.2. & 40. & & & & & 0.611 & \\
\hline$\frac{271}{272}>>19$ & 4/119/20004 & 1:55:18 PM & 25.515 & 23.001 & 25.433 & 25.482 & & 44.94 & 46.39 & 10.109 & $35.55 \varepsilon$ & -1.218 & & 49. & 0.169 & 14.6 & & 20 & & 44 & 12.0 & & & & & & 0.618 & \\
\hline$\frac{21}{37}$ & 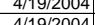 & $\begin{array}{l}1.55: 343 \mathrm{PM} \\
1.5614 \mathrm{PM}\end{array}$ & $\frac{25.52}{25.52}>0$ & 望2.9991 32014 & 255429 & 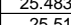 & & 年44.911 & 46.558 & $\frac{10.08}{0.915}$ & 33.52 .4 & -1.2219 & & 年 & 0.161 & 14.6 & & 253.6 & 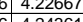 & 4 & 12.19 & no & & & & & 0.50 & \\
\hline 274 & 年 & $1.00 .44 \mathrm{r}$ & 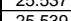 & $\frac{2.014}{23021}>-1$ & $\mid$\begin{tabular}{|l|l|}
25.421 \\
5200
\end{tabular} & $\frac{35.51}{25.57}$ & & 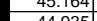 & (40.001/ & & 年 & - & & & & & & & & & & & & & & & (2) & \\
\hline$\frac{75}{275}>3$ & 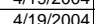 & $1.5 .84 \mathrm{PM}$ & & & 25 & 256 & & 45108 & 4 & & & -121 & & & 0.118 & & & 256 & & & 117 & & & & & & 烈 & \\
\hline 27 & & 1:59:44 PM & 25.6. & $\frac{23.045}{23.045}$ & & 25 & & & & & & -121 & & $48.2>>3$ & & & & & & $4533>>$ & 118 & & & & & & 0628 & \\
\hline 27 & & & & & & & & & 46.962 & & & & & & & & & & & 55 & 117 & & & & & & & \\
\hline & $4 / 19 / 2$ & 2:01:44 PM & & & 25.123 & 25.582 & & & 46.84 & 10. & 35 & .218 & & & 0. & & & & & 45.4 & 12.4 & 40. & 6.19 & & & & & \\
\hline & A1/01/2 & $2.02 .44 \mathrm{PM}$ & & & 20 & & & & 47097 & 918 & $36,2>$ & 221 & & 489 & 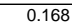 & 14.643 & & 26 & & 456 & 120 & & & & & 01 & 608 & \\
\hline 280 & $4 / 19 / 2004$ & $2: 03: 44 \mathrm{PM}$ & 25.507 & 23.039 & 25.09 & 25.364 & & 45.228 & 46.993 & 9.983 & 35.882 & \begin{tabular}{|l|l|l|}
2 & -1.224 \\
\end{tabular} & & 49.155 & 0.169 & 14.643 & & & 64.36028 & 45.5 & 12.0 & & 2.796 & 0.025 & 0.025 & 0.001 & 0.620 & 0.5 \\
\hline
\end{tabular}


WSRC-TR-2005-00105, REVISION 0

SRNL-RPP-2005-00012, REVISION 0

RUN \# 2.03A AND B; FIRST AND SECOND HALF OF SLURRY DEWATERING - CONT.

\begin{tabular}{|c|c|c|c|c|c|c|c|c|c|c|c|c|c|c|c|c|c|c|c|c|c|c|c|c|c|c|c|c|}
\hline & & & & & & & & & & & & & & & & & & & & & & & & & & & & \\
\hline & A & B & $\frac{\mathrm{D}}{\mathrm{N}}$ & $E$ & $F$ & \begin{tabular}{l|l}
$G$ \\
\end{tabular} & $\mathrm{H}$ & $\mathrm{J}$ & $\frac{K}{K}$ & $\mathrm{~L}$ & \begin{tabular}{l|l}
$\mathrm{M}$ \\
\end{tabular} & $\mathrm{N}$ & $\begin{array}{l}\mathrm{O} \\
\end{array}$ & Q & \begin{tabular}{l|l|l}
$R$ \\
\end{tabular} & 5 & \begin{tabular}{l|l|}
$\mathrm{T}$ \\
\end{tabular} & & W & $x$ & $\frac{1}{112}$ & & AA & $\mathrm{AB}$ & $A C$ & $A D$ & $A E$ & \\
\hline & $4 / 19 / 2004$ & $\begin{array}{l}2: 04: 44 \mathrm{PM} \\
2005: 44 \mathrm{PM}\end{array}$ & $\begin{array}{l}25.4777 \\
25.462\end{array}$ & \begin{tabular}{l|l|}
23.054 \\
23.044
\end{tabular} & \begin{tabular}{r|}
25.086 \\
25.07 \\
\end{tabular} & \begin{tabular}{|l|l|}
25.339 \\
25.339
\end{tabular} & \begin{tabular}{|l|}
22.438 \\
22.788
\end{tabular} & \begin{tabular}{r|}
45.031 \\
45.22
\end{tabular} & \begin{tabular}{|l|l|}
46.801 \\
46.626
\end{tabular} & $\begin{array}{r}9.893 \\
9.9\end{array}$ & \begin{tabular}{|l|l|}
35.999 \\
36.159
\end{tabular} & \begin{tabular}{|l|}
-1.218 \\
-1218 \\
\end{tabular} & $\begin{array}{l}-5.841 \\
-6.047\end{array}$ & \begin{tabular}{r|}
45.53 \\
50.404 \\
\end{tabular} & $\begin{array}{l}0.165 \\
0.169 \\
\end{array}$ & $\begin{array}{l}14.643 \\
14.643 \\
\end{array}$ & $\begin{array}{l}0.003 \\
0.003\end{array}$ & \begin{tabular}{l|}
262.6 \\
263.6
\end{tabular} & \begin{tabular}{|l|}
4.376944 \\
4.39361 \\
\end{tabular} & $\begin{array}{l}45.3 \\
45.2 \\
\end{array}$ & \begin{tabular}{|l|l|}
11.2 \\
12.4
\end{tabular} & $\begin{array}{l}40.5 \\
40.7 \\
\end{array}$ & \begin{tabular}{|l|}
2.793 \\
2805 \\
\end{tabular} & $\begin{array}{l}0.025 \\
0.025\end{array}$ & \begin{tabular}{l|l|}
0.025 \\
0.025
\end{tabular} & 0.001 & \begin{tabular}{l|l|}
0.606 \\
0.618
\end{tabular} & $\begin{array}{l}0.52 \\
0.53\end{array}$ \\
\hline 283 & 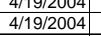 & & $\frac{25.462}{25.437}$ & 23.044 & \begin{tabular}{|l|}
25.01 \\
25.096 \\
\end{tabular} & \begin{tabular}{|}
25.3399 \\
25.389
\end{tabular} & $\begin{array}{l}22.188 \\
22.608 \\
\end{array}$ & & & $\begin{array}{r}9.943 \\
9.94\end{array}$ & $\begin{array}{l}36.159 \\
35.702\end{array}$ & & & & \begin{tabular}{|c|c|c|} 
\\
\end{tabular} & $\begin{array}{l}14.643 \\
14.643 \\
\end{array}$ & & & & & & & & & & & & \\
\hline 284 & $4 / 19 / 2004$ & 2:07:44 PM & 25.407 & 23.049 & 25.075 & 25.424 & 22.743 & 45.528 & 46.877 & 9.933 & 36.518 & $\begin{array}{l}-1.218 \\
-1.218\end{array}$ & & 47.776 & 0.171 & 14.643 & 0.003 & 265.6 & $\begin{array}{l}4.442694 \\
4.420\end{array}$ & 45.4 & & & \begin{tabular}{|l|}
2.828 \\
\end{tabular} & 0.025 & 0.025 & 0.001 & 0.620 & \\
\hline & $4 / 199 / 2004$ & 2:08:44 PM & 25.381 & 23.033 & 24.984 & \begin{tabular}{|l|}
25.373 \\
\end{tabular} & 23.008 & 45.363 & \begin{tabular}{|l|l|}
46.846 \\
\end{tabular} & 9.979 & 36.229 & -1.215 & & 48.444 & $\begin{array}{l}.111 \\
0.163 \\
\end{array}$ & 14.643 & & 266.6 & 4.44361 & 45.4 & \begin{tabular}{|l|l|}
11.9 \\
\end{tabular} & & 2.813 & & 0.024 & 0.001 & & \\
\hline & & & & 23.017 & & \begin{tabular}{|l|l|}
25.393 \\
\end{tabular} & & & 46.616 & & & & & & 0.166 & & & & & 45.1 & & 40.6 & & & & & & \\
\hline 287 & $4 / 19 / 2004$ & 2:10:44 PM & 25.362 & 23.029 & $\begin{array}{l}24.951 \\
\end{array}$ & \begin{tabular}{|l|}
25.399 \\
\end{tabular} & 22.979 & $\begin{array}{l}45.255 \\
\end{array}$ & 46.921 & 9.986 & 35.985 & -1.218 & -6.116 & \begin{tabular}{|l|l|l}
48.473 \\
\end{tabular} & $\begin{array}{l}0.164 \\
\end{array}$ & 14.643 & 0.003 & 268.6 & 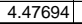 & 45.4 & \begin{tabular}{|l|}
11.9 \\
\end{tabular} & & \begin{tabular}{|l|}
2.801 \\
\end{tabular} & 0.024 & $\mid$ & 0.001 & & \\
\hline 28 & $\begin{array}{l}4 / 19 / 2004 \\
4 / 1 / 2004\end{array}$ & $\begin{array}{l}2: 11: 44 \mathrm{PM} \\
2 \cdot 12: 44 \mathrm{PM}\end{array}$ & $\begin{array}{l}25.348 \\
25.349 \\
2\end{array}$ & \begin{tabular}{|l|l|l|}
23.025 \\
230255
\end{tabular} & $\begin{array}{l}24.931 \\
24.917\end{array}$ & \begin{tabular}{|r|r|r|}
25.42 \\
25.496
\end{tabular} & $\begin{array}{l}23.025 \\
23.071 \\
2\end{array}$ & \begin{tabular}{|l|l|}
45.321 \\
45.246
\end{tabular} & \begin{tabular}{r|r|r|}
46.89 \\
46.925
\end{tabular} & $\begin{array}{l}10.001 \\
10.026\end{array}$ & $\begin{array}{r}36.11 \\
35.913 \\
\end{array}$ & \begin{tabular}{|c|c|}
-1.218 \\
-1.1218
\end{tabular} & $\begin{array}{l}-6.096 \\
-6058\end{array}$ & \begin{tabular}{r|r|r|}
47.954 \\
4836
\end{tabular} & \begin{tabular}{|l|l|}
0.167 \\
0.161
\end{tabular} & $\begin{array}{l}14.643 \\
14.643\end{array}$ & $\begin{array}{l}0.003 \\
0.03\end{array}$ & 269.6 & \begin{tabular}{|l|}
4.49361 \\
4.51028
\end{tabular} & $\begin{array}{l}45.4 \\
45.5\end{array}$ & $\begin{array}{ll}11.8 \\
11.9\end{array}$ & $\begin{array}{l}40.7 \\
40.6\end{array}$ & \begin{tabular}{|l|}
2.807 \\
2.798
\end{tabular} & $\begin{array}{l}0.025 \\
0.024\end{array}$ & $\mid \begin{array}{l}0.025 \\
0.024\end{array}$ & \begin{tabular}{|l}
0.001 \\
0.001
\end{tabular} & $\begin{array}{l}0.613 \\
0.59\end{array}$ & \\
\hline 290 & $4 / 19 / 2004$ & $\begin{array}{l}2: 12: 44 \mathrm{PM} \\
\text { 2:13:44 }\end{array}$ & $\frac{25.549}{25.324}$ & 23.011 & 24.902 & 25.441 & 23.077 & $\begin{array}{l}43.240 \\
45.232\end{array}$ & $\begin{array}{l}40.950 \\
46.751\end{array}$ & $\begin{array}{l}010.020 \\
9.969\end{array}$ & & $\begin{array}{l}-1.210 \\
-1.221\end{array}$ & & & $\begin{array}{l}.101 \\
0.158\end{array}$ & $\begin{array}{l}\frac{14.643}{14.643} \\
\end{array}$ & \begin{tabular}{|l}
0.003 \\
0.003
\end{tabular} & $\begin{array}{l}270.6 \\
271.6\end{array}$ & $\begin{array}{l}4.51082 \\
4.52694\end{array}$ & $\frac{45.5}{45.3}$ & $\begin{array}{l}11.9 \\
12.7\end{array}$ & \begin{tabular}{|c|c|}
40.6 \\
40.6
\end{tabular} & \begin{tabular}{|l|}
2.798 \\
2.800
\end{tabular} & $\begin{array}{l}0.024 \\
0.024 \\
\end{array}$ & \begin{tabular}{|c|}
0.024 \\
0.024
\end{tabular} & $\frac{0.001}{0.001}$ & 0.582 & 0.50 \\
\hline & $4 / 19 / 2004$ & 2:14:44 PM & 25.326 & & 24.929 & 25.508 & 23.238 & 45.236 & 46.736 & & 36.003 & -1.218 & & 47.586 & 0.163 & 14.643 & & & 4.54361 & 45.3 & & & & & & & & $\frac{0.49}{0.51}$ \\
\hline & $4 / 19 / 2004$ & 2:15:44 PM & 25.322 & \begin{tabular}{|l|l|}
23.034 \\
\end{tabular} & 24.925 & 25.574 & 23.215 & 45.578 & 47.232 & 10.048 & 36.13 & -1.218 & -6.08 & $\begin{array}{l}49.374 \\
\end{array}$ & 0.159 & 14.643 & & 273.6 & \begin{tabular}{|l|l|}
4.56028 \\
\end{tabular} & 45.8 & 12.1 & 40.9 & \begin{tabular}{|l|}
2.817 \\
\end{tabular} & & 0.024 & 0.001 & & $\frac{0.51}{0.49}$ \\
\hline & $4 / 19 / 2004$ & 2:16:44 PM & 25.308 & & & & & & $\begin{array}{l}46.948 \\
\end{array}$ & & & & & 47.885 & 0.17 & & & & 1.57694 & 45.5 & & & & & & & & \\
\hline 205 & & 2:17:44 PM & & & 24.795 & 255 & & $\begin{array}{l}45.489 \\
\end{array}$ & \begin{tabular}{|l|l|}
46.993 \\
\end{tabular} & 9.88 & 36.42 & -1.218 & & & 0.16 & 14.643 & & 275. & 4.59361 & & $\frac{{ }^{11} .6}{11.6}$ & & (n) & .024 & 0.024 & & & \\
\hline & $4 / 19 / 2004$ & 2:18:44 PM & & & 24.776 & 25.641 & & & 47.2 & 9.967 & & & & 1802 & 0.156 & 14.643 & & 276.6 & 4.61028 & 45.7 & & & & & & & & \\
\hline & $4 / 19 / 2004$ & 2:19:44 PM & 25.284 & 23.066 & 24.802 & 25.707 & 23.232 & \begin{tabular}{|l|l|l|l|l}
45.363 \\
\end{tabular} & \begin{tabular}{|l|l|l|l|l|}
47.107 \\
\end{tabular} & 10.015 & 36.046 & $\mid-1.218$ & & $\begin{array}{l}49.316 \\
\end{array}$ & 0.155 & 14.643 & & 277.6 & 4.62694 & 45.6 & 12.1 & 40.7 & 2.806 & 0.023 & 0.023 & 0.001 & 0.571 & \\
\hline & $4 / 19 / 2004$ & $2: 20: 44$ PM & 25.261 & 23.063 & 24.794 & 25.738 & 23.294 & 45.257 & 46.854 & 9.946 & 36.116 & -1.224 & & 46.56 & 0.159 & 14.643 & & 278. & 4.64361 & 45.4 & 11.4 & & & & 0.024 & 0.001 & 586 & \\
\hline & & 2::21:44 PM & & & & 25.845 & & & & & 35.076 & & & .494 & 0.16 & 14.643 & & & & 44.6. & & & & & & & & \\
\hline & 4/19/2004 & 2:22:44 PM & 5.231 & 23.073 & 24.794 & 25.793 & & 44.94 & 46.324 & 10.411 & 35.344 & .224 & & 313 & 0.149 & 14.643 & & & . 1.676944 & 44. & & & & & & 0.001 & 557] & \\
\hline 301 & $\frac{4 / 9 / 9004}{4 / 19 / 2004}$ & $2.23 .44 \mathrm{PM}$ & 5238 & $\frac{23.076}{23079}$ & 24.8271 & $\frac{25.1606}{25775}$ & 2000 & $\begin{array}{r}44.66 \\
44.07\end{array}$ & 46.49 & $\frac{10.353}{10236}$ & 每5.0.035 & 121 & & 894 & 0.0 .1024 & $\frac{14.643}{1.642}$ & & & 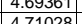 & & & & 7 & & & & & \\
\hline & $4 / 19 / 192004$ & $\begin{array}{l}2: 24.44 \mathrm{PM} \\
2: 25: 44\end{array}$ & $\begin{array}{l}25.256 \\
25.236\end{array}$ & $\frac{23.079}{23.088}$ & $\begin{array}{l}2.835 \\
24.834 \\
\end{array}$ & $\frac{25.715}{25.718}$ & $\frac{22.904}{22.757}$ & $\begin{array}{l}\frac{44.807}{45.045} \\
\end{array}$ & $\begin{array}{l}40.490 \\
46.711\end{array}$ & $\frac{1.350}{10.398}$ & $\begin{array}{l}35.184 \\
35.476\end{array}$ & $\begin{array}{l}-\frac{-1.210}{-1.218} \\
\end{array}$ & & $\begin{array}{l}40.864 \\
48.463\end{array}$ & \begin{tabular}{|c|c|}
0.166 \\
0.166
\end{tabular} & $\begin{array}{l}\frac{14.043}{14.643} \\
\end{array}$ & & 2836 & 年. & 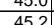 & $\frac{11.5}{119}$ & & $\frac{2.758}{2.776}$ & 025 & 0.025 & $\frac{0.001}{0.001}$ & 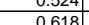 & \\
\hline & $4 / 19 / 2004$ & 2:26:44 PM & 25.233 & 23.09 & 24.776 & 25.635 & 22.614 & 45.066 & $\begin{array}{l}4.111 \\
46.61 \\
\end{array}$ & 10.384 & 35.404 & -1.215 & & 48.681 & 0.165 & 14.643 & 0.003 & 284.6 & 4.74361 & 45.1 & 11.9 & 40.2 & \begin{tabular}{|l|}
2.774 \\
\end{tabular} & & & 0.001 & 0.615 & \\
\hline & $4 / 19 / 2004$ & $2: 27: 44 \mathrm{PM}$ & 25.236 & 23.098 & 24.753 & 25.528 & 22.616 & $\begin{array}{l}44.825 \\
\end{array}$ & $\begin{array}{l}46.581 \\
\end{array}$ & 10.321 & 35.191 & \begin{tabular}{|l|l|l|}
-1.218 \\
\end{tabular} & & 49.184 & 0.144 & 14.643 & 0.003 & 285.6 & \begin{tabular}{|l|l|}
4.76028 \\
\end{tabular} & 45.1 & 12.1 & 40.0 & 2.758 & & & 0.001 & 0.540 & \\
\hline & $4 / 19 / 2 C$ & 2:28:44 PM & & 23.089| & $\begin{array}{r}24.75 \\
24.779\end{array}$ & $\frac{25.439}{25.212}$ & & 45.141 & 46.73 & & $\begin{array}{l}35.685 \\
35.077\end{array}$ & & & 49.437 & 0.155 & 14.643 & & & & & & & & & & & 76 & \\
\hline & $\frac{4 / 19 / 2004}{4 / 19 / 2004}$ & $2.29 .44 \mathrm{PM}$ & $\frac{253}{203}$ & $\frac{23.082}{23075}$ & 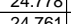 & $\frac{25.3512}{2524}$ & 22398 & $\frac{45.195}{45.356}$ & $\frac{46.6 / 6}{46.937}$ & $\frac{10.266}{10.078}$ & $\frac{35.697}{36.02}$ & -1.218 & & $\begin{array}{l}48.839 \\
48306\end{array}$ & $\begin{array}{l}149 \\
155 \\
\end{array}$ & $\frac{14.643}{14.643}$ & & 年 28.6 & 4810 & . & 18 & & 805 & & & & & 0.47 \\
\hline & $4 / 19 / 2004$ & $2 \cdot 31: 44$ PM & 25.195 & $\frac{23.070}{23.052}$ & $\begin{array}{l}24.101 \\
24768\end{array}$ & $\frac{25.24}{25.207}$ & 22.090 & $\begin{array}{l}45.350 \\
44.896\end{array}$ & 46.531 & $\frac{10.047}{10.047}$ & $\begin{array}{c}35.021 \\
35.621\end{array}$ & $\begin{array}{l}-\frac{-1.210}{-1.218} \\
\end{array}$ & & $\begin{array}{l}40.300 \\
55.947\end{array}$ & $\begin{array}{l}.1146 \\
0.146\end{array}$ & $\begin{array}{l}14.045 \\
114643\end{array}$ & & 280.0 & 4 & & 11.0 & & & & & & 544 & 4.49 \\
\hline & $4 / 19 / 2004$ & 2:32:44 PM & 25.204 & 23.06 & 24.811 & 25.24 & 22.534 & 45.394 & 47.051 & 10.183 & 35.899 & -1.215 & -6.128 & 49.118 & 0.161 & 14.643 & 0.003 & 290.6 & \begin{tabular}{|l|l|l} 
\\
\end{tabular} & 45.6 & 12.0 & $\mid 40.6$ & \begin{tabular}{|l|}
2.802 \\
\end{tabular} & 0.024 & 0.024 & 0.001 & $\begin{array}{l}0.594 \\
0.594 \\
0.04\end{array}$ & $\frac{4}{4}$ \\
\hline & $4 / 199 / 2004$ & 2:33:44 PM & 25.213 & \begin{tabular}{|l|l|}
23.06 \\
\end{tabular} & 24.806 & 25.31 & 22.609 & $\begin{array}{l}45.139 \\
\end{array}$ & $\begin{array}{l}46.865 \\
\end{array}$ & 10.121 & 35.763 & & & $\begin{array}{l}47.805 \\
\end{array}$ & \begin{tabular}{|l|l|l|l|l|} 
\\
\end{tabular} & 14.643 & & 291.6 & & 45. & 11.7 & & 2.789 & & $.0<2$ & & 548 & \\
\hline 31. & $4 / 199 / 2004$ & 2:34:44 PM & 25.197| & 23.039| & 24.825 & 25.334 & & 45.11 & 46.7033 & 10.08 & & -1.218 & & 3.696 & 0.172 & 14.643 & & 292.6 & 4.87694 & & 11.9 & & .788 & 026 & 0.026 & 0.001 & 0.637 & \\
\hline$\overline{313}$ & $\frac{4 / 1912004}{419200}$ & $2: 35: 44 \mathrm{PM}$ & 25.198 & $\frac{23.035}{23035}$ & 24.821 & 25.365 & $\frac{22.764}{20.896}$ & $\begin{array}{l}45.143 \\
45.302\end{array}$ & $\begin{array}{l}46.896 \\
46.095\end{array}$ & $\frac{10.136}{10.121}$ & $\begin{array}{l}35.673 \\
35.005\end{array}$ & $\begin{array}{l}-1.221 \\
1.218\end{array}$ & & $\frac{49.78}{48.15}$ & 0.16 & $\frac{14.643}{14643}$ & & 293.6 & $\frac{61}{28}$ & 45. & $\frac{12.2}{118}$ & & & & .024 & 0.001 & & 0.50 \\
\hline 314 & $\frac{4 / 19 / 19004}{4 / 192004}$ & $\begin{array}{l}2.50 .44 \mathrm{PM} \\
2: 37: 44\end{array}$ & $\frac{20.204}{25.201}$ & $\frac{20.000}{23.033}$ & $\begin{array}{l}2.032 \\
24.729 \\
\end{array}$ & 25.4523 & $\frac{22.000}{22.938}$ & 45..3021 & $\begin{array}{l}40.950 \\
47.356\end{array}$ & $\frac{1.121}{10.115}$ & 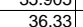 & $\begin{array}{l}-\frac{-1.210}{-1.218} \\
\end{array}$ & & 252 & 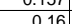 & $\begin{array}{l}14.045 \\
114643\end{array}$ & & $\begin{array}{l}294.0 \\
2956\end{array}$ & | & $\frac{45.6}{45 .}$ & $\frac{11.0}{123}$ & & 829 & 0.224 & 势 0.0244 & 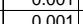 & 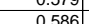 & \\
\hline & $4 / 19 / 2004$ & 2:38:44 PM & 25.207 & 23.034 & 24.715 & 25.61 & 23.0 & 45.555 & 47.064 & $\begin{array}{l}10.07 \\
10.07\end{array}$ & 36.223 & -1.218 & & 367 & 0 & 14643 & & 2066 & & & 11.9 & & 2819 & & & & & \\
\hline & 4/19/2004 & 2:39:44 PM & 25.189 & $\frac{23.026}{23.026}$ & 24.717 & $\frac{25.587}{25.57}$ & 230 & 45.668 & 47.279 & 10.057 & 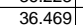 & $\frac{-1.218}{-1.218}$ & & $\frac{47.766}{4766}$ & $\frac{1.3 .16}{0.16}$ & 14.643 & & $\frac{207.6}{297.6}$ & | & $\frac{75.8}{45.8}$ & $\frac{\frac{x}{11.7}}{11.7}$ & & & & 024 & & 55 & \\
\hline & $4 / 19 / 2004$ & $2: 40: 44 \mathrm{PM}$ & 25.181 & 23.023 & 24.714 & 25.588 & & & 47.542 & 10.013 & 36.834 & & & & $\mid 0.155$ & 14.643 & & & & 46.1 & $\frac{11.4}{11.4}$ & & & & & & 563 & \\
\hline & $4 / 19 / 20$ & $2: 41: 44 \mathrm{PM}$ & 25.183 & 23.025 & 24.736 & $\mid 25.66$ & & 45.65 & 47.263 & 10.034 & 36.428 & -1.218 & & & \begin{tabular}{|l|l|l|} 
\\
\end{tabular} & & & & & 45. & 122 & & 52 & & 024 & & & \\
\hline 19 & $4 / 19 / 2004$ & 2:42:44 PM & 25.179 & 23.031 & 24.787 & 25.647 & & \begin{tabular}{|l|l|l|l|}
448 \\
\end{tabular} & \begin{tabular}{|l|l|}
46.293 \\
\end{tabular} & .023 & & & & 31. & & & & & & & 12.7 & & 2.107 & & & & & \\
\hline 326 & $4 / 19 / 2004$ & 2:43:44 PM & 25.181 & 23.033 & 24.799 & 25.679 & 23.1 & $\begin{array}{l}44.577 \\
\end{array}$ & 46.185 & 10.913 & 34.351 & \begin{tabular}{|c|}
-1.218 \\
\end{tabular} & & ).106 & 0.156 & 14.643 & & 301.6 & & 44.7 & $\begin{array}{ll}12.3 \\
\end{array}$ & 39.5 & & & 0.023 & & 0.593 & \\
\hline 32. & $4 / 19 / 2004$ & 2:44:44 PM & 25.178 & 23.05 & 24.826 & 25.681 & 23.1 & 44.18 & 45.793 & 10.793 & 34.181 & -1.218 & & 51.858 & $\begin{array}{ll}0.138 \\
\end{array}$ & 14.643 & & & & 44. & 12.7 & & & & & & & \\
\hline & 4/191/2004 & 2:45:44 PM & & 23.046 & 24.847] & 25.687 & & 44.57 & 46.166 & 10.584 & 34.751 & -1.218 & & .6.693 & 0.143 & & & 30 & & 44. & 12.2 & & & & & & 40 & \\
\hline & $4 / 191 / 2004$ & 2:46:4 & 5.184 & 2.3.045 & 24.792 & \begin{tabular}{|l|l}
25.726 \\
25721
\end{tabular} & & 44.475 & 46.409| & 10 & 34.4 & & & & 0.14 & 14. & & 30 & & 44. & 等 & & 75 & & & & & \\
\hline & $\frac{4419 / 2004}{419200}$ & $2: 47444 \mathrm{PM}$ & 25.114 & $\frac{23.055}{3205}$ & 年4. 24.424 & | & & $\begin{array}{l}44.966 \\
4689\end{array}$ & $\begin{array}{l}46.13 \\
46.309\end{array}$ & $\frac{10.682}{1066}$ & $\frac{34.928}{3477}$ & $\begin{array}{l}-\frac{1.215}{1212} \\
-12\end{array}$ & & 15 & | & & & 3066 & & 4 & $\frac{11.5}{12.5}$ & & 2739 & & & & & \\
\hline & 4 & $2.404 .445 \mathrm{PM}$ & 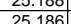 & $\frac{2.003}{23033}$ & $\frac{24.151}{24749}$ & 2558 & & $\frac{44.06}{447}$ & (46.30506 & $\frac{1.000}{10.481}$ & 35.143 & $\frac{1.42}{-1218}$ & & 51.5 & 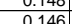 & & & & & & $\frac{1.0 .0}{120}$ & & 2.159 & & & & & \\
\hline 327 & $4 / 19 / 2004$ & $2 \cdot 45.444 \mathrm{PM}$ & 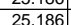 & 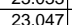 & 24,78 & $\mid$ & & $\frac{4.4}{44 .}$ & 46.552 & $\begin{array}{l}10.408 \\
10.498\end{array}$ & $\begin{array}{l}35.063 \\
35.063\end{array}$ & -1.218 & & 48.87 & 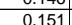 & $\frac{14.6}{14.6}$ & & $\frac{50}{305}$ & & 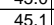 & $\frac{1.0}{120}$ & & 754 & & & & 5677 & \\
\hline 328 & $4 / 1 / 1 / 2004$ & 2:51:44 PM & 25.169 & 23.03 & & 25.346 & & & & 10.593 & & & & & & & & & & & $\frac{12.4}{12.4}$ & & & & & & & \\
\hline $2=$ & $4 / 19 / 2004$ & $2: 52: 44 \mathrm{P}$ & 25.157 & & 24795 & 25.309| & & 44.5 & 46.212 & 10.486 & 34.8 & $\frac{-1.218}{-1.218}$ & & 50.16 & 0.1 & 14.643 & & & & 44.7 & 123 & & & & & & 0.548 & \\
\hline & & 2.53 .4 & & $\begin{array}{l}23.018 \\
\end{array}$ & & \begin{tabular}{|l|l|}
25.243 \\
\end{tabular} & & & & 10.665 & & & & & & & & & & 445 & $\frac{128}{128}$ & & & & & & & \\
\hline 331 & $4 / 19 / 2004$ & 2:54:44 PM & 25.186 & 23.023 & 24.854 & 25.208 & & $\begin{array}{l}44.606 \\
\end{array}$ & 46.231 & 10.605 & 34.836 & -1.215 & & & 0.148 & 14.643 & & & & 44.8 & 12.6 & & & & & & 558 & \\
\hline 32 & $4 / 19 / 20$ & $2: 55: 44$ F & 25.176 & & 24.874 & \begin{tabular}{|l|}
25.253 \\
\end{tabular} & & 44.5 & & 10.578 & 34.8 & -1.218 & & .3 & \begin{tabular}{|l|l|}
0.14 \\
\end{tabular} & & & & & 44.. & 11.8 & & & & & & .527 & \\
\hline & 4/19/2004 & 2:56:44 PM & 25.198 & 23.02 & 24.926 & 25.375 & & 44.6 & 46. & 10.667 & 34.7 & -1.215 & & & 0.1 & 14. & & 3 & 5.24 & & 12.4 & & & & & & 04 & \\
\hline 334 & $4 / 199 / 26$ & 2:57:44 PM & 25.193 & 23 & 24.911 & 25.47 & & 44.834 & 46. & 10.746 & & -1.218 & & 5 & & & & 31. & & 45. & 12.0 & & $2.746 \mid$ & & & & & \\
\hline & $4 / 191 / 2004$ & 2:58:44 PM & 25.199 & 23.005 & 24.912 & 25.576 & & 44.672 & 46.61 & 10.733 & 34.7 & -1.215 & & 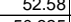 & 0.144 & 14.643 & & & & 45. & $\begin{array}{ll}12.8 \\
102\end{array}$ & & & & & & 42 & \\
\hline & & $2.59: 44 \mathrm{~F}$ & & 2.011 & $\frac{2.98}{2.78}$ & 25.62 & & & & 1.5 & & & & & & & & & & & & & & & & & & \\
\hline 年 & 4 & $\frac{3.004 .44}{3: 19}$ & $\frac{25.200}{25207}$ & $\frac{23.002}{23008}$ & 24.18005 & 25.0006 & & $\frac{44.55}{44.5}$ & 40.4 & $\begin{array}{ll}10.6 .6 \\
10.5\end{array}$ & & $\frac{-1.218}{-121}$ & & & & & & & & 44 & $\frac{1.2 .5}{123}$ & & & & & & & \\
\hline 339 & 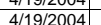 & $\frac{3.01 .444}{3.20}$ & 25209 & 2300 & 24817 & 25776 & & $\frac{47.56}{4496}$ & $\begin{array}{l}40.458 \\
46788\end{array}$ & $\frac{10.539}{10.519}$ & & & & & & & & & & & $\frac{125}{121}$ & & & & & & & \\
\hline$\frac{340}{340}$ & $4 / 1 / 1 / 2004$ & 3:03:44 PM & $\frac{25.209}{25}$ & $\frac{23.036}{23.036}$ & 24.842 & 25.837 & & 44.686 & 46.572 & $\frac{10.55}{10.5}$ & 34.9 & -1.218 & & 48.6 & & & & & & 45 & 119 & & & & & & & \\
\hline 341 & 4/19/2004 & 3:04:44 PM & 25.211 & 23.042 & 24.859] & 25.888 & & 44.38 & 46.228 & 10.56 & 34.6 & -1.218 & & & 0.142 & 14.643 & & & & $44 .{ }^{2}$ & 12.4 & & & & & & & \\
\hline & & & & 3069 & 24.876 & 25.895 & & 44.504 & $\begin{array}{l}46.386 \\
\end{array}$ & 10.686 & 34.5 & -1.218 & & & & & & & & & 12.9 & & & & & & 38 & 8 \\
\hline & 4/19/2004 & 3:06:44 PM & 25.224 & 23.076 & 24.898 & 25.85 & & 44.604 & 46.487 & 10.592 & 34.831 & -1.218 & & & 0.1 & 14. & & 32 & 5.41 & $\overline{45.0}$ & 12.9 & & 2738 & & & & 31 & \\
\hline 344 & $4 / 19 / 2004$ & 3:07:44 P & 231 & 23.068 & 24.894 & 25.894 & & $\begin{array}{l}44.564 \\
\end{array}$ & & $10.3 \mathrm{C}$ & 35. & -1.211 & & & & & & & & 44.8 & 12.0 & & & & & & & \\
\hline 345 & $4 / 19 / 20$ & 3:08:44 PM & & 23.0 & 24.936 & 25.965 & & 44.564 & $\begin{array}{lll}46.6 \\
\end{array}$ & 10. & 34.7 & -1.218 & & & & 14. & & & & 45.1 & 12.8 & & & & & & 39 & \\
\hline 346 & & & 25. & & $24.9 \mathrm{C}$ & 25.8 & & 44.703 & & 10.48 & & -1.218 & & & & & & & & 45. & 12.3 & & & & & & 0.536 & \\
\hline 347 & $4 / 19 / 2004$ & 3:10:44 PM & 25.257 & 23.094 & 24.915 & 25.88 & & 44.371 & 46.289 & 10. & 34.626 & -1.218 & & & & 14. & & & & 44.8 & 12.3 & & & & & & & \\
\hline (3) & $4 / 191 / 2004$ & 3:11:44 PM & 2.256 & 23.093 & 24.929 & 25.873 & & $\begin{array}{l}44.587 \\
44.877\end{array}$ & $46.347 ?$ & 10.438 & 34.983 & $=-1.218$ & & (4.3966 & 0.142 & 14.643 & & 32 & & 44.9 & 12.1 & & 2.14 & 0.021 & 0.021 & 0.001 & 0.533 & \\
\hline 3490 & $\frac{\mid 4 / 19 / 20004}{4 / 19 / 2004}$ & $\begin{array}{l}3: 12: 44 \mathrm{PM} \\
3: 13: 44 \mathrm{PM}\end{array}$ & $\mid \frac{25.264}{25.268}$ & $\begin{array}{l}23.086 \\
23.079\end{array}$ & $\frac{24.912}{22.941}$ & \begin{tabular}{|l|}
25.746 \\
25.775
\end{tabular} & $\begin{array}{r}22.449 \\
2.483 \\
\end{array}$ & $\begin{array}{l}44.407 \\
45.3 \\
\end{array}$ & $\mid \frac{46.6322}{46.842}$ & $\begin{array}{l}10.368 \\
10.487\end{array}$ & $\mid$ & $\frac{-1.221}{-1.221}$ & -6.93 & $\frac{50.058}{49.829}$ & $\mid \frac{0.145}{0.136}$ & $\frac{14.643}{14.643}$ & & & \begin{tabular}{|l|}
5.55028 \\
5.52694 \\
\end{tabular} & $\begin{array}{l}44.8 \\
45.4 \\
\end{array}$ & \begin{tabular}{|l|}
12.2 \\
\end{tabular} & & \begin{tabular}{|l|}
2.731 \\
2.785 \\
\end{tabular} & 0.020 & \begin{tabular}{|c|}
0.022 \\
0.020
\end{tabular} & $\mid \frac{0.001}{0.001}$ & $\frac{0.547}{0.503}$ & $\frac{0.47}{0.43}$ \\
\hline
\end{tabular}


WSRC-TR-2005-00105, REVISION 0

SRNL-RPP-2005-00012, REVISION 0

RUN \# 2.03A AND B; FIRST AND SECOND HALF OF SLURRY DEWATERING - CONT.

\begin{tabular}{|c|c|c|c|c|c|c|c|c|c|c|c|c|c|c|c|c|c|c|c|c|c|c|c|c|c|c|c|c|}
\hline & & & & & & & & & & & & & & & & & & & & & & & & & & & & \\
\hline & A & B & D & $E$ & $F$ & G & $\mathrm{H}$ & $\mathrm{J}$ & $\mathrm{K}$ & $\mathrm{L}$ & M & $\mathrm{N}$ & $\begin{array}{ll}0 \\
\end{array}$ & Q & $\mathrm{R}$ & 5 & $T$ & 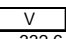 & $w$ & $x$ & 1 & 2 & AA & $\frac{A B}{A B}$ & $A C$ & $A D$ & AE & AF $A$ \\
\hline$\frac{351}{352}$ & \begin{tabular}{r|r|}
$4 / 19 / 2004$ \\
$411 / 2004$
\end{tabular} & $\begin{array}{l}3: 14: 44 \mathrm{PM} \\
3: 15: 44 \mathrm{PM}\end{array}$ & $\frac{25.281}{25.278}$ & $\begin{aligned} 23.083 \\
2.306\end{aligned}$ & $\begin{array}{l}24.944 \\
2456\end{array}$ & $\begin{array}{l}25.713 \\
25.701\end{array}$ & \begin{tabular}{|l|}
22.401 \\
22.443 \\
\end{tabular} & $\begin{array}{l}45.172 \\
45.217\end{array}$ & \begin{tabular}{r|}
46.68 \\
46.711 \\
\end{tabular} & $\begin{array}{l}10.476 \\
10.439\end{array}$ & \begin{tabular}{|c|}
35.433 \\
35.521
\end{tabular} & \begin{tabular}{|c|}
-1.221 \\
-1.1218 \\
\end{tabular} & $\begin{array}{r}-7.09 \\
-7.108 \\
\end{array}$ & $\begin{array}{l}49.347 \\
50.071\end{array}$ & 0.144 & $\begin{array}{l}14.643 \\
14.643 \\
\end{array}$ & $\begin{array}{l}0.003 \\
0.003\end{array}$ & $\begin{array}{l}332.6 \\
333.6\end{array}$ & \begin{tabular}{|l|l|}
5.54361 \\
5.56028
\end{tabular} & $\frac{45.2}{45.2}$ & $\frac{12.1}{12.3}$ & $\begin{array}{l}40.3 \\
40.4 \\
\end{array}$ & 2.779 & 0.021 & 0.022 & 0.001 & $\begin{array}{l}0.534 \\
0.532\end{array}$ & $\begin{array}{l}0.45 \\
0.45\end{array}$ \\
\hline 每553 & $\begin{array}{l}4 / 1912004 \\
/ 199 / 2004\end{array}$ & & $\frac{25.278}{25.291}$ & $\frac{23.066}{23.043}$ & $\begin{array}{l}24.956 \\
24.954\end{array}$ & $\begin{array}{l}25.101 \\
25.679\end{array}$ & \begin{tabular}{|l|}
22.4435 \\
22.647 \\
\end{tabular} & & & $\begin{array}{r}10.459 \\
10.43 \\
\end{array}$ & $\begin{array}{l}35.521 \\
35.441\end{array}$ & & & $\frac{50.011}{50.413}$ & 0.144 & $\frac{14.643}{14.643}$ & & & & & & & & & & & & \\
\hline 354 & $4 / 19 / 2004$ & 3:17:44 PM & 25.295 & 23.047 & 24.983 & $\begin{array}{l}25.647 \\
\end{array}$ & 22.576 & 45.319 & 46..423 & 10.363 & 35.767 & \begin{tabular}{|l|}
-1.218 \\
\end{tabular} & -6.069 & \begin{tabular}{|l|l|}
49.916 \\
\end{tabular} & 0.146 & 14.643 & 0.003 & 335.6 & \begin{tabular}{|l|l|}
6 & 5.59361 \\
\end{tabular} & $\begin{array}{l}4.5 \\
45.0\end{array}$ & $\begin{array}{l}12.4 \\
12.2 \\
\end{array}$ & & \begin{tabular}{|l|}
.1 .71 \\
2.795 \\
\end{tabular} & 0.022 & 0.022 & 0.001 & 0.537 & $\begin{array}{l}0.40 \\
0.46 \\
\end{array}$ \\
\hline & $4 / 19 / 2004$ & 3:18:44 PM & 25.305 & 23.052 & 24.988 & 25.697 & 22.696 & 45.126 & 46.401 & 10.398 & 35.515 & \begin{tabular}{|l|}
-1.218 \\
\end{tabular} & $\begin{array}{l}-6.0871 \\
-6.871\end{array}$ & $\begin{array}{l}48.365 \\
\end{array}$ & 0.144 & 14.643 & & & \begin{tabular}{|l|l|l|}
6 & 5.61028 \\
\end{tabular} & 44.9 & 11.9 & 40.3 & & & & 0.001 & & 0.45 \\
\hline & & & & & & & & 45.406 & 46.535 & & $\begin{array}{l}5.79 \\
\end{array}$ & & & & & & & & \begin{tabular}{|l|l|}
6 & 5.62694 \\
\end{tabular} & 45.1 & & 40.6 & & & & & & 0.44 \\
\hline 357| & 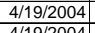 & $3: 20: 44 \mathrm{PM}$ & 25.33 & $\begin{array}{l}23.037 \\
2312\end{array}$ & \begin{tabular}{|l|}
25.0233 \\
\end{tabular} & 25.772 & $\begin{array}{l}22.957 \\
2.97\end{array}$ & $\begin{array}{l}45.396 \\
\end{array}$ & $\begin{array}{l}46.85 \\
\end{array}$ & 10.458 & $\begin{array}{l}35.652 \\
\end{array}$ & \begin{tabular}{|c|}
-1.218 \\
\end{tabular} & $\begin{array}{l}-7.073 \\
\end{array}$ & $\begin{array}{l}50.365 \\
\end{array}$ & 0.143 & 14.643 & 0.003 & 338.6 & 5.64361 & 45.4 & 12.3 & 40.5 & \begin{tabular}{|l|l|}
2.794 \\
\end{tabular} & 0.021 & 0.021 & 0.001 & $\begin{array}{l}0.526 \\
\end{array}$ & 0.45 \\
\hline$\frac{358}{359}$ & \begin{tabular}{|l|}
$4 / 19 / 2004$ \\
$41 / 292004$
\end{tabular} & $\begin{array}{l}3: 21: 44 \mathrm{PM} \\
3 \\
3.22: 44 \mathrm{PM}\end{array}$ & $\begin{array}{l}25.336 \\
25.336\end{array}$ & $\begin{array}{l}23.042 \\
23.033\end{array}$ & \begin{tabular}{|l|l|}
25.029 \\
248899
\end{tabular} & $\begin{array}{r}25.773 \\
25.729\end{array}$ & $\begin{array}{l}23.138 \\
22.998\end{array}$ & \begin{tabular}{|l|}
45.334 \\
45118
\end{tabular} & $\begin{array}{l}46.823 \\
46.492 \\
\end{array}$ & $\begin{array}{l}10.526 \\
1039 \\
\end{array}$ & $\begin{array}{r}35.63 \\
35.453\end{array}$ & \begin{tabular}{|l|l|}
-1.221 \\
-1.221
\end{tabular} & $\begin{array}{l}-7.056 \\
-7.05 \\
\end{array}$ & $\begin{array}{l}499.522 \\
46802\end{array}$ & $\begin{array}{l}0.142 \\
0.142\end{array}$ & $\begin{array}{l}14.643 \\
14643\end{array}$ & $\begin{array}{l}0.003 \\
003\end{array}$ & $\begin{array}{l}339.6 \\
3406\end{array}$ & $\begin{array}{l}65.66028 \\
6567694\end{array}$ & $\begin{array}{ll}45.4 \\
450\end{array}$ & $\frac{12.1}{11.5}$ & $\begin{array}{l}40.5 \\
40.3\end{array}$ & \begin{tabular}{|l|}
2.791 \\
2.778
\end{tabular} & $\frac{0.021}{0.021}$ & $\begin{array}{l}0.021 \\
0.021\end{array}$ & 0.001 & $\begin{array}{l}0.523 \\
0.572\end{array}$ & 0.44 \\
\hline 360 & $4 / 119 / 2004$ & $\begin{array}{l}3: 22: 44 \mathrm{PM} \\
3: 23: 44 \mathrm{PM}\end{array}$ & $\frac{25.350}{25.337}$ & 23.039 & $\begin{array}{l}24.8899 \\
24.895\end{array}$ & $\begin{array}{l}25.729 \\
25.775\end{array}$ & $\frac{22.998}{22.989}$ & 45..110 & $\begin{array}{l}40.492 \\
46.703\end{array}$ & 10.45 & $\begin{array}{l}35.453 \\
35.492 \\
\end{array}$ & \begin{tabular}{|}
$\mid-1.221$ \\
-1.218
\end{tabular} & & $\begin{array}{l}46.802 \\
49.491\end{array}$ & $\begin{array}{l}.142 \\
0.142\end{array}$ & $\frac{14.643}{14.643}$ & 0.003 & $\begin{array}{l}340.6 \\
341.6\end{array}$ & $\begin{array}{l}6 \\
6 \\
6\end{array}$ & $\frac{45.0}{45.2}$ & $\frac{11.5}{12.1}$ & . & \begin{tabular}{|l|}
2.778 \\
2.783 \\
\end{tabular} & $\frac{0.021}{0.021}$ & $\frac{0.021}{0.021}$ & $\frac{0.001}{0.001}$ & 0.526 & $\begin{array}{l}0.45 \\
0.45\end{array}$ \\
\hline 361. & & 3:24:44 PM & 25.333 & & 24.931 & & 23.196 & & 47.01 & & & \begin{tabular}{|l|}
-1.218 \\
\end{tabular} & & 49.881 & 0.142 & 14.643 & & 342.6 & \begin{tabular}{l|l}
6 & 5.71028 \\
\end{tabular} & 45.5 & & & & & & & & $\begin{array}{l}0.45 \\
0.44\end{array}$ \\
\hline 362 & $4 / 19 / 2004$ & 3:25:44 PM & 25.324 & 23.035 & 24.932 & 25.821 & 23.231 & \begin{tabular}{|l|l}
45.07 \\
\end{tabular} & $\begin{array}{l}46.566 \\
\end{array}$ & 10.41 & \begin{tabular}{|l|l|l}
35.344 \\
\end{tabular} & -1.218 & -7.01 & 46.725 & 0.137 & $\begin{array}{ll}14.643 \\
\end{array}$ & 0.003 & 343.6 & \begin{tabular}{|l|l|}
6 & 5.72694 \\
\end{tabular} & 45.1 & 11.5 & 40.2 & \begin{tabular}{|l|l|}
2.772 \\
\end{tabular} & & 0.020 & 0.001 & & $\frac{0.44}{0.43}$ \\
\hline 363 & $4 / 191 / 2004$ & 3:26:44 PM & 25.325 & & 24.958 & 25.863 & 23.243 & & & 10.361 & & & & 50.89 & 0.142 & & & & & & & & & & & & & $\begin{array}{l}0.43 \\
0.45\end{array}$ \\
\hline 364 & $6 / 10 / 2000$ & 3:27:44 PM & & 23.042 & 24.944 & 25802 & & & 47.114 & 10.484 & 35.767 & | & & 48.646 & 0.141 & 14.643 & & & & & & & & & & & & \\
\hline 365 & $4 / 19 / 2004$ & 3:28:44 PM & 25.467 & 23.049 & 24.95 & 25.945 & & \begin{tabular}{|l|l|l|}
45.219 \\
\end{tabular} & 46.722 & 10.318 & 35.706 & & & & 0.141 & 14.643 & & 346. & 5.77694 & 45.2 & & 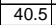 & 2.790 & & & & & $\begin{array}{l}0.44 \\
0.44\end{array}$ \\
\hline & $4 / 19 / 2004$ & 3:29:44 PM & 25.423 & 23.05 & 24.936 & 25.941 & 23.381 & 45.388 & |47.037 & 10.462 & 35.605 & $\mid-1.218$ & -6 & 47.443 & 0.137 & 14.643 & & 347.6 & \begin{tabular}{l|l|}
6 & 5.79361 \\
\end{tabular} & 45.6 & 11.6 & $\begin{array}{lll}40.5 \\
\end{array}$ & 2.792 & 0.020 & 0.020 & 0.001 & 0.505 & 0.43 \\
\hline 36 & $4 / 19 / 2004$ & 3:30:44 PM & 25.405 & 23.062 & 24.958 & 25.963 & 23.42 & $45.02 \mathrm{C}$ & 46.579 & 10.404 & 35.363 & -1.218 & & .758 & 0.14 & 14.643 & & 348.6 & & 45. & 11.7 & & & & & 0.001 & & 0.44 \\
\hline & $4 / 19 / 2004$ & & & & & & & & 46.467 & & 35.408 & & & & 0.14 & & & & & & & & & & & & & \\
\hline 365 & $4 / 19 / 2004$ & 3:32:44 PM & 25.352 & 23.079 & 24.86 & 25.995 & 23.0 & 45.263 & 46.927 & 10.381 & 35.527 & \begin{tabular}{|c|}
-1.218 \\
\end{tabular} & & .072 & 0.139 & 14.643 & & & & 45.5 & & 40. & & 0.021 & & .001 & 0.515 & 0.44 \\
\hline & $\frac{4 / 19 / 2004}{4 / 1 / 2004}$ & $\begin{array}{l}3: 33: 44 \mathrm{PM} \\
3.34444 \mathrm{P}\end{array}$ & 5291 & $\begin{array}{r}23.09 \\
23.073\end{array}$ & $\frac{24.806}{24779}$ & $\frac{26.025}{25.909}$ & & 45.60 & 47016 & $\frac{10.46}{10.421}$ & $\frac{35.829}{35.691}$ & $\frac{-1.221}{1.215}$ & & 556 & 0.14 & $\frac{14.643}{14643}$ & & & 587694 & & & & & & & & & 0.44 \\
\hline & $4 / 1 / 19 / 2004$ & $\begin{array}{l}3.54444 \mathrm{PM} \\
3: 35: 44\end{array}$ & $\begin{array}{l}25.291 \\
25.274\end{array}$ & $\frac{23.075}{23.066}$ & $\begin{array}{l}24.7179 \\
24.797\end{array}$ & $\begin{array}{l}25.909 \\
25.832\end{array}$ & $\frac{2.193}{22.48}$ & \begin{tabular}{|l|}
45.3699 \\
45.367
\end{tabular} & $\begin{array}{l}47.010 \\
46.964\end{array}$ & $\frac{10.421}{10.406}$ & 35.0.745 & $\mid$\begin{tabular}{|c|}
$\mid-1.215$ \\
-1.221 \\
\end{tabular} & & $\begin{array}{l}51.550 \\
50.552\end{array}$ & $\frac{0.139}{0.138}$ & $\begin{array}{l}\frac{14.045}{14.643} \\
\end{array}$ & & 353.6 & 5.893641 & & $\begin{array}{l}\frac{12.6}{12.4} \\
\end{array}$ & & & & 0.021 & $\frac{0.001}{0.001}$ & 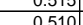 & 0.44 \\
\hline & $4 / 19 / 2004$ & 3:36:44 PM & 25.268 & 23.075 & 24.816 & 25.721 & 22.428 & 45.166 & 46.79 & 10.429 & 35.507 & \begin{tabular}{|l|}
-1.221 \\
\end{tabular} & -7.041 & 48.014 & 0.138 & 14.643 & 0.003 & 354.6 & \begin{tabular}{|l|l|}
6 & 5.91028 \\
\end{tabular} & 45 & $\begin{array}{ll}11.44 \\
11.8\end{array}$ & 40.3 & 2.781 & 0.021 & & 0.001 & & $\begin{array}{l}0.43 \\
0.44 \\
\end{array}$ \\
\hline & $4 / 19 / 2004$ & 3:37:44 PM & 25.251 & 23.057 & 24.794 & 25.598 & 22.36 & 44.958 & 46.572 & 10.388 & 35.248 & & & 51.132 & 0.138 & 14.643 & & 355.6 & $\begin{array}{ll}65.92694 \\
6\end{array}$ & 45.1 & 12.5 & 40.1 & 2.765 & & & 0.001 & & 0.44 \\
\hline & $4 / 19 / 2004$ & $3: 38: 44 \mathrm{PM}$ & 25.248 & 23.06 & $\begin{array}{l}24.841 \\
2.952\end{array}$ & $\begin{array}{l}25.545 \\
25562\end{array}$ & & $\begin{array}{l}45.247 \\
5.211\end{array}$ & \begin{tabular}{|l|l|}
46.97 \\
6.927
\end{tabular} & $\begin{array}{l}10.484 \\
10.67\end{array}$ & $\begin{array}{l}35.459 \\
25.621\end{array}$ & & -6. & 50.298 & 0.138 & 14.643 & 0.004 & & 5.94361 & 45. & 12.3 & 40.4 & & 0.02 & 0.021 & $\begin{array}{ll}0.001 \\
\end{array}$ & 512 & 0.44 \\
\hline & 4/19/2004 & $\begin{array}{l}3: 39: 44 \text { PM } \\
3.40 .44 P\end{array}$ & $\begin{array}{r}25.249 \\
25.242\end{array}$ & $\frac{23.046}{23.043}$ & 24.852 & $\frac{25.562}{25.564}$ & $\begin{array}{r}22.53 \\
22.552\end{array}$ & $\frac{45.311}{45.018}$ & $\frac{46.927}{46.726}$ & $\frac{10.467}{10.419}$ & $\begin{array}{l}35.624 \\
35305\end{array}$ & $\begin{array}{l}-1.215 \\
-1.1218\end{array}$ & & $\begin{array}{r}50.3 \\
50.684 \\
\end{array}$ & $\frac{0.138}{0.138}$ & $\frac{14.643}{14.643}$ & & 357.6 & 5.96028 & & \begin{tabular}{l|l}
12.3 \\
12.4
\end{tabular} & 40.5 & \begin{tabular}{|l|l|}
2.790 \\
2769
\end{tabular} & $\frac{0.021}{0.021}$ & 0.021 & 0.001 & 0.511 & 0.43 \\
\hline & $4 / 19 / 2004$ & $\begin{array}{l}3: 40: 44 \mathrm{PM} \\
3.41: 44 \mathrm{PM}\end{array}$ & $\begin{array}{l}25.242 \\
25.251\end{array}$ & $\frac{23.045}{23.052}$ & 24.835 & $\frac{25.564}{25.603}$ & $\frac{22.552}{22.596}$ & $\frac{45.018}{45.027}$ & $\begin{array}{l}46.726 \\
46.575\end{array}$ & $\frac{10.419}{10.378}$ & $\begin{array}{l}35.305 \\
35.449\end{array}$ & $\begin{array}{l}-1.2218 \\
-1.221\end{array}$ & & $\frac{50.684}{47.451}$ & 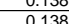 & 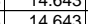 & & $\frac{358.6}{3596}$ & & $\frac{45 .}{45}$ & $\frac{12.4}{11.6}$ & & & & & & & 0.44 \\
\hline & $4 / 19 / 2004$ & 3:42:44 PM & 25.254 & 23.04 & 24.862 & $\frac{3.000}{25.641}$ & 22.715 & 45.51 & 47.279 & 10.519 & 35.794 & $\begin{array}{l}-1.212 \\
-1.215 \\
\end{array}$ & & $\frac{4.451}{48.946}$ & 0 & $\frac{14.045}{14.643}$ & & 360. & 6.011 & $\frac{45 .}{45}$ & $\frac{11.6}{12.0}$ & & 803 & & 0.021 & 0.001 & & $\frac{0.44}{0.44}$ \\
\hline 380 & $4 / 19 / 2004$ & 3:43:44 PM & & 23.05 & 24.841 & 25.61 & 22.87 & 45.352 & \begin{tabular}{|l|l|l|l|l|}
46.948 \\
\end{tabular} & 10.465 & 35.644 & & & & 0.137 & 14.643 & & & & 45 & & & & & & & & $\begin{array}{l}0.44 \\
0.43\end{array}$ \\
\hline 381. & $4 / 19 / 2004$ & 3:44:44 PM & 25.264 & 23.04 & 24.726 & 25.656 & & & & 10.403 & 35.125 & -1.218 & & & 0.137 & 14.643 & & 36 & $\frac{6.04361}{6.0 .04361}$ & 45 & $\frac{\pi .19}{11.9}$ & & . & 告 & 0.021 & 0.001 & $\frac{0.515}{0.515}$ & $\frac{0.43}{0.44}$ \\
\hline $\begin{array}{l}382 \\
383 \\
3\end{array}$ & $4 / 19 / 2004$ & $3: 45: 44 \mathrm{PM}$ & 25.249 & 23.036 & 24.752 & 25.682 & \begin{tabular}{|l|l|}
23.187 \\
\end{tabular} & \begin{tabular}{|l|l|l|l|} 
\\
\end{tabular} & $\begin{array}{l}46.871 \\
\end{array}$ & 10.391 & 35.548 & -1.218 & & 0.755 & 0.138 & 14.643 & & & & & 12.4 & & & & 0.021 & & 0.513 & 0.44 \\
\hline 383 & $4 / 19 / 2004$ & 3:46:44 PM & & & 24.788 & 25.717 & 23.233 & $\begin{array}{l}45.356 \\
155286\end{array}$ & 47.099 & 10.482 & 35.574 & -1.218 & $\begin{array}{l}-7.01 \\
702\end{array}$ & 9.376 & & 14.643 & & & 6.07694 & & 12.1 & & .790 & 0.020 & 0.021 & 0.001 & $\begin{array}{l}0.508 \\
\end{array}$ & \\
\hline & $4 / 19 / 2004$ & 3:47:44 PM & 25.251 & 23.053 & 24.834 & 25.824 & 23.354 & 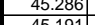 & 4 & 10.524 & 35.443 & -1.215 & $\begin{array}{l}-7.01 \\
\end{array}$ & 47.384 & 0.136 & 14.643 & & 365 & & 45.6 & 11.66 & & 2.783 & 0.020 & 0.020 & 0.001 & & \\
\hline & 4/19/2004 & 3:48:44 PM & 25.247 & 23.044 & 24.86 & 25.845 & & $\frac{4.191}{45251}$ & $\begin{array}{l}46.136 \\
47057\end{array}$ & 10.408 & $\begin{array}{r}35.621 \\
25.57\end{array}$ & -1. & & .587 & 0. & 14.643 & & 366 & & 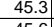 & 12.2 & & & 0.020 & & & & 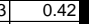 \\
\hline & $4 / 19 / 2004$ & 3:49:44 PM & & & 24.902 & & & & & $10-710$ & $\begin{array}{r}35.57 \\
25.992\end{array}$ & & & & 0. & & & & 6.12694 & & & & 2.786 & & & & & \\
\hline & $4 / 19 / 2004$ & 3:50:44 PM & 56.256 & $\frac{23.057}{22059}$ & 24.914 & $\frac{25.873}{25875}$ & & $45.3 t$ & 47.122 & 10.414 & & $\frac{218}{215}$ & & 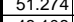 & & 14.643 & & & & 45. & $\frac{12.6}{12.6}$ & & 070 & & & & & \\
\hline & & & & & & & & & & & & & & & & & & & & & & & & & & & & \\
\hline & $4 / 19 / 2004$ & 3:52:44: PM & $\frac{2.279}{25.29}$ & $\frac{23.082}{23}$ & $\mid \begin{array}{l}2.45978 \\
\end{array}$ & $\begin{array}{l}25.901 \\
25.998\end{array}$ & $\frac{2.2 .273}{23.273}$ & \begin{tabular}{|l|}
45.215 \\
45.193
\end{tabular} & $\begin{array}{l}47.020 \\
47.097\end{array}$ & $\begin{array}{l}10.273 \\
10.425\end{array}$ & $\begin{array}{r}35.0 .056 \\
35.5 \\
\end{array}$ & $\mid$\begin{tabular}{|c|c|c|}
$\mid-1.215$ \\
-15
\end{tabular} & $\begin{array}{l}-0.9 \\
-6.98\end{array}$ & $\begin{array}{l}50.139 \\
49.063\end{array}$ & 0.137 & $\begin{array}{l}\frac{14.045}{14.643} \\
\end{array}$ & 0.003 & 371.6 & \begin{tabular}{ll|l}
$6.1 .1 / 6944$ \\
6 & 6.19361
\end{tabular} & $\frac{43.6}{45.6}$ & $\begin{array}{l}\frac{12.3}{12.0} \\
\end{array}$ & 40.3 & \begin{tabular}{|l|}
2.187 \\
2.782
\end{tabular} & $\begin{array}{l}0.020 \\
0.020\end{array}$ & $\begin{array}{l}0.020 \\
0.020\end{array}$ & $\begin{array}{l}0.001 \\
0.001\end{array}$ & 0.4907 & \\
\hline & $4 / 191 / 2004$ & 3:54:44 PM & 25.296 & & 24.959 & 26.004 & & 45.20 & 47.064 & $\begin{array}{ll}10.528 \\
\end{array}$ & 35.488 & $\mid$\begin{tabular}{|l|l|}
$\mid 1.215$ \\
\end{tabular} & & 48 & & \begin{tabular}{|l|l|l|l|}
14.643 \\
\end{tabular} & & 372.6 & \begin{tabular}{l|l}
6 & 6.21 \\
\end{tabular} & {$[F$} & 11.9 & & & & & 0.001 & 0.507 & 0.43 \\
\hline & $4 / 19 / 2004$ & 3:55:44 PM & & 23.104 & 24.86 & 25.935 & & 45.10 & & 10.479 & 35.445 & -1.218 & & & 0.135 & & & & & & & & & & & & & \\
\hline & $4 / 191 / 2004$ & 3:56:4 & 5.317 & 23.1044 & 24.865| & 25.935 & & & 46.68 & 10.482 & 35.191 & & & & 0.135 & & & & & & . & & & & & & & \\
\hline & $\begin{array}{l}4 / 19 / 2 / 204 \\
4 / 1 / 2004\end{array}$ & $\begin{array}{l}3.57 .44 \mathrm{PM} \\
3.5 .44 \mathrm{PM}\end{array}$ & $\frac{25.316}{25205}$ & $\begin{array}{l}23.093 \\
23087\end{array}$ & 2.8.899 & $\begin{array}{r}25.844 \\
25892\end{array}$ & & & $\begin{array}{l}47.033 \\
4651\end{array}$ & 10.569 & & $\mid-1.218$ & & 196 & & & & & & & $\frac{1.1}{120}$ & & 0707 & & & & & \\
\hline 396 & 4 & & & 23074 & & 25.84 & & & 40.59 & 10.446 & 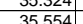 & $\frac{-1.40}{-1218}$ & & 49.0 & 然 & & & & & & $\frac{\frac{1.2 .2}{123}}{12}$ & & 2.10r & & & & & a \\
\hline & $4 / 19 / 2004$ & 4:0 & $\frac{20.004}{25.306}$ & 23.063 & 24.944 & $\begin{array}{r}25.04 \\
25.804\end{array}$ & 22.4 & $\frac{4.1}{45}$ & 4. 40.064 & 10.450 & 35..312 & $\frac{-1.210}{-1.221}$ & & 46.6 & 0. & $\begin{array}{l}14.045 \\
14.643\end{array}$ & & 378 & $\frac{0.2055}{6.315}$ & 45.6 & $\begin{array}{l}12.0 \\
11.4\end{array}$ & 40.2 & \begin{tabular}{|l|l|}
2.771 \\
\end{tabular} & & & & & 0.4 \\
\hline 398 & $4 / 19 / 2004$ & 4:01:44 F & 25.326 & 23.077 & 24.999 & 25.793 & & 45.188 & 47.238 & 10.529 & & & & 48.3 & & 14.643 & & $37 !$ & & 45.8 & 111.9 & 40 & \begin{tabular}{|l|l|}
2.777 \\
\end{tabular} & 0.020 & 0.020 & & 03 & \\
\hline & 4/19/2004 & & 25.319 & 23.066 & 25.012 & 25.777 & & 45.215 & 47.051 & 10.478 & 35.4 & -1.218 & & 50.38 & & 14.643 & & 38 & $\begin{array}{l}96.34 \\
9\end{array}$ & 45.6 & 12.3 & 40. & 2.781 & & & & $\frac{88}{88}$ & 0.4 \\
\hline & $4 / 19 / 20$ & 4:03:44 PM & $\frac{2.535}{25320}$ & $\begin{array}{l}23.061 \\
23055\end{array}$ & \begin{tabular}{|l|l|}
25.048 \\
25071
\end{tabular} & $\begin{array}{l}25.757 \\
25701\end{array}$ & & & 46.981 & $\begin{array}{r}10.54 \\
\end{array}$ & \begin{tabular}{|c|}
35.211 \\
25.289
\end{tabular} & \begin{tabular}{|c|}
-1.218 \\
-1.218
\end{tabular} & & & 0.125 & 14.643 & & $\frac{38}{38}$ & & 45. & $\frac{11.7}{123}$ & & 070 & & & & & 0.39 \\
\hline & $\frac{419120204}{41 / 2004}$ & & $\frac{2.330}{25359}$ & & 25.071 & $\frac{23.901}{25781}$ & & & $\frac{47.049}{46.776}$ & $\frac{1.0 .464}{10401}$ & 告35.460 & & & & & & & & & & $\frac{1 \frac{1.3}{128}}{128}$ & & & & & & & \\
\hline & $4 / 19 / 20$ & & 25364 & & 25.112 & 25.826 & & & & 10392 & $\begin{array}{l}35.259 \\
35.199\end{array}$ & -1218 & & & & & & & & & $\frac{126}{126}$ & & & & & & & \\
\hline & $4 / 19 / 20$ & & 25.374 & $\frac{23.056}{23.056}$ & 25.058 & 25.847 & & 45.1 & $\begin{array}{l}47.047 \\
\end{array}$ & 10.369 & 35.4 & $\frac{-1.221}{-1.21}$ & & $\frac{d 7.76}{44.76}$ & & & & & & 45.6 & $\frac{\frac{21.7}{11.7}}{4}$ & & & & & & & \\
\hline & & & & & & & & & & & & & & & & & & & & & & & & & & & & \\
\hline & $4 / 19 / 2004$ & & 25.381 & 23.067 & 25.029 & 25.828 & & & 47.279 & 10.418 & 35.56 & -1.218 & & & & & & & & 45. & 12.3 & & & & & & & \\
\hline & $4 / 19 / 2004$ & $0: 28 \mathrm{P}$ & 25.397 & 23.079 & 25.066 & 25. & & 44. & 46.5 & 10.32 & 35. & -1.218 & & & & & & & & & $\frac{12.1}{12.1}$ & & & & & & & \\
\hline & $4 / 19 / 2004$ & 4:11:28 PM & 25.388 & 23.07 & 25.076 & 25.941 & & 45.035 & 47.182 & 10.504 & 35.291 & -1.218 & & 45. & & 14. & & & & 45, & 11.1 & & & & & & 89 & \\
\hline & $4 / 199 / 26$ & $4: 12$ & 25.405 & 23.092 & 25.108 & 25.95 & & 44.996 & & 10.439 & 35.3 & -1.218 & & 48.3. & & & & & & & $\overline{11.8}$ & & & & & & & \\
\hline & 4/19/2004 & 4:13:28 $\mathrm{P}$ & 25.401 & & 25.12 & 25.969 & & 44.713 & 46.89 & 10.358 & 35.088 & -1.218 & & & & & & 39 & & 45. & 11.7 & & & & & & & \\
\hline & $4 / 191 / 2004$ & $8 \mathrm{PM}$ & 5.418 & 23.11 & 25.142 & 26.011 & & 44.738 & 46.689 & 10.371 & 35.256 & -1.221 & & 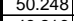 & & $14.6 \mathrm{C}$ & & & & 45. & 12.3 & & & & & & & \\
\hline & 4 & $\begin{array}{l}4.15: 28 \mathrm{PM} \\
415.28 \mathrm{PM}\end{array}$ & 5446 & 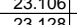 & 25.158 & 26.022 & & 每55.166 & 4.232 & 10.44 & 3.56 & - -1.215 & & & & & & & & 告5.8 & $\frac{11.8}{100}$ & & & & & & & \\
\hline & 4 & & 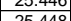 & $\frac{2.1 .120}{2213}$ & 25.100 & $\frac{2.029}{25065}$ & & 45. & - 41.31 .51 & $\begin{array}{l}1.503 \\
1.203\end{array}$ & & 1.210 & & & & & & & & & 些. & & & & & & & \\
\hline 41 & $4 \frac{41 / 19 / 24}{4 / 19 / 2}$ & 4:18:28 PM & $\begin{array}{l}55.448 \\
52548 \\
\end{array}$ & $\frac{20.1}{23.12}$ & | & 25 & & 45. & & $\frac{10.3}{10.3}$ & 35. & $\begin{array}{l}-1.2 \\
-1.2\end{array}$ & & & & & & & & & $\frac{11.0}{11.3}$ & & & & & & & \\
\hline & & & 25.4 & & 25.065 & 25.8 & & & & 10. & & -1.215 & & & & & & & & 45. & 12.2 & & & & & & & \\
\hline & $4 / 199 / 2004$ & & 25.44 & 23.11 & 25.088 & 25.763 & & & 47.294 & 10.53 & & -1.218 & & 48. & & & & & & 45.8 & 11.9 & & & & & & & \\
\hline & & 4:21:28 PM & & & 25.117 & & & & & 10.561 & & & & & & & & & & 45. & & & & & & & & \\
\hline & & $4 \cdot 22.28 \mathrm{PM}$ & & & & & & & 46.991 & 10.47 & 34003 & -1.218 & & 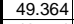 & & 14.643 & & (n) & & 45.5 & 12.1 & & & 0.020 & 0.020 & 0.000 & 0.492 & $\overline{0.42}$ \\
\hline & $4 / 19 / 2004$ & 4:23:28 PM & 25.469 & 23.136 & 25.177 & 25.761 & & \begin{tabular}{|l|l|}
44.848 \\
\end{tabular} & $\begin{array}{l}47.082 \\
\end{array}$ & 10.468 & 35.012 & & & & 0.13 & & & & \begin{tabular}{|l|l|}
6 & 6.69389 \\
\end{tabular} & & & & & & 0.019 & & & \\
\hline
\end{tabular}


WSRC-TR-2005-00105, REVISION 0

SRNL-RPP-2005-00012, REVISION 0

RUN \# 2.03A AND B; FIRST AND SECOND HALF OF SLURRY DEWATERING - CONT.

\begin{tabular}{|c|c|c|c|c|c|c|c|c|c|c|c|c|c|c|c|c|c|c|c|c|c|c|c|c|c|c|c|c|}
\hline & & & & & & & & & & & & & & & & & & & & & & & & & & & & \\
\hline & A & B & D & $E$ & \begin{tabular}{|l|}
$F$ \\
2518
\end{tabular} & G & $\mathrm{H}$ & $\mathrm{J}$ & $\mathrm{K}$ & $\mathrm{L}$ & \begin{tabular}{|l|} 
M \\
3507
\end{tabular} & $\mathrm{~N}$ & 0 & $\mathrm{Q}$ & $R$ & $\mathrm{~s}$ & $\mathrm{~T}$ & $\mathrm{~V}$ & w & $x$ & $r$ & $z$ & AA & $\frac{A B}{A B}$ & $A C$ & $A D$ & $\mathrm{AE}$ & AF $A$ \\
\hline$\frac{441}{422}$ & $\begin{array}{l}4 / 1 / 9 / 2004 \\
4 / 192004\end{array}$ & $\begin{array}{l}\text { 4:24:28 PM } \\
4: 25: 28 \text { PM }\end{array}$ & $\begin{array}{r}25.47 \\
25.481\end{array}$ & $\begin{array}{r}23.142 \\
23.169\end{array}$ & \begin{tabular}{|r|}
25.188 \\
25.24 \\
\end{tabular} & \begin{tabular}{|l|}
25.793 \\
25.914 \\
\end{tabular} & $\begin{array}{r}23.368 \\
23.49 \\
\end{array}$ & \begin{tabular}{|l|}
44.884 \\
44.877
\end{tabular} & \begin{tabular}{|c|}
47.149 \\
47018
\end{tabular} & $\begin{array}{l}10.448 \\
10.552\end{array}$ & \begin{tabular}{|l|}
35.197 \\
35024
\end{tabular} & \begin{tabular}{|l|l|}
-1.221 \\
-1.215 \\
\end{tabular} & $\begin{array}{l}-6.873 \\
-6.899 \\
-1\end{array}$ & $\begin{array}{l}49.357 \\
49.898 \\
\end{array}$ & $\begin{array}{l}0.125 \\
0.132\end{array}$ & \begin{tabular}{|c|} 
\\
5 \\
5
\end{tabular} & $\begin{array}{l}0.003 \\
0.003\end{array}$ & $\begin{array}{l}402.6 \\
403.6\end{array}$ & 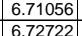 & $\begin{array}{l}45.7 \\
44.5\end{array}$ & \begin{tabular}{|l|l|}
12.1 \\
122
\end{tabular} & $\begin{array}{l}40.0 \\
40.0\end{array}$ & $\frac{2.761}{2.754}$ & 0.015 & $\frac{0.019}{0.020}$ & $\begin{array}{l}0.000 \\
0.000\end{array}$ & \begin{tabular}{|l|l|l}
0.463 \\
0.489
\end{tabular} & $\begin{array}{l}0.39 \\
0.42\end{array}$ \\
\hline$\frac{4223}{423}$ & & & $\frac{25.481}{25.478}$ & & & \begin{tabular}{|r}
55.914 \\
25.97 \\
\end{tabular} & & & & $\begin{array}{l}1.532 \\
10.436 \\
\end{array}$ & & & & $\begin{array}{l}49.898 \\
49.301\end{array}$ & & \begin{tabular}{|l|}
14.643 \\
\end{tabular} & & & & & & & & & & 0.0000 & & \\
\hline 424 & $4 / 19 / 2004$ & 4:27:28 PM & 25.51 & 23.203 & \begin{tabular}{|l|}
25.249 \\
\end{tabular} & 26.053 & 23.684 & \begin{tabular}{|l|}
44.767 \\
\end{tabular} & $\begin{array}{l}4.047 \\
\end{array}$ & 10.522 & $\begin{array}{r}34.95 \\
\end{array}$ & \begin{tabular}{|l|}
-1.218 \\
\end{tabular} & $\begin{array}{r}-6.839 \\
-6.839\end{array}$ & 48.994 & $\begin{array}{l}0.129 \\
\end{array}$ & \begin{tabular}{|l|l}
14.643 \\
\end{tabular} & & 405.6 & \begin{tabular}{|l|}
6.76056 \\
6.7505
\end{tabular} & 45.6 & \begin{tabular}{|l|l|}
12.0 \\
\end{tabular} & 39.9 & \begin{tabular}{|l|l|}
2.748 \\
\end{tabular} & 0.019 & 0.019 & 0.000 & 0.479 & 0.41 \\
\hline & $4 / 199 / 2004$ & 4:28:28 PM & & 23.21 & \begin{tabular}{|l|}
25.146 \\
\end{tabular} & \begin{tabular}{|l|}
26.131 \\
\end{tabular} & & \begin{tabular}{|l|l|}
44.796 \\
\end{tabular} & $\begin{array}{l}47.051 \\
\end{array}$ & 10.519 & \begin{tabular}{|l|}
34.938 \\
\end{tabular} & \begin{tabular}{|l|l|}
-1.221 \\
\end{tabular} & $\begin{array}{l}-6.902 \\
\end{array}$ & 50.782 & 0.131 & \begin{tabular}{|l|}
14.643 \\
\end{tabular} & & 406.6 & \begin{tabular}{|l|l|}
6.77722 \\
\end{tabular} & 45.6 & \begin{tabular}{|l|l|}
12.4 \\
\end{tabular} & & & & & 0.000 & $\begin{array}{l}.488 \\
\end{array}$ & 0.42 \\
\hline 426 & $4 / 19 / 2004$ & & & & \begin{tabular}{|r|}
25.17 \\
\end{tabular} & 26.26 & & & 47.443 & & & & & 49.695 & & 14.643 & & & \begin{tabular}{|l|l|}
6.79389 \\
\end{tabular} & 46.0 & \begin{tabular}{|l|l|}
12.2 \\
\end{tabular} & 40.2 & & & & & 0.484 & \\
\hline 427 & $4 / 19 / 2004$ & 4:30:28 PM & 25.525 & 23.258 & 25.189 & 26.333 & 23.985 & 44.969 & $\begin{array}{l}47.188 \\
\end{array}$ & $\begin{array}{l}10.397 \\
\end{array}$ & $\begin{array}{l}35.357 \\
3.27\end{array}$ & \begin{tabular}{|l|l|}
-1.218 \\
\end{tabular} & $\begin{array}{r}-6.833 \\
\end{array}$ & 48.786 & 0.117 & 14.643 & & $\begin{array}{l}408.6 \\
\end{array}$ & \begin{tabular}{|l|l|}
6.81056 \\
\end{tabular} & 45.7 & 12.0 & 40.2 & \begin{tabular}{|l|}
2.769 \\
\end{tabular} & 0.017 & 0.017 & 0.000 & $\begin{array}{l}0.432 \\
\end{array}$ & 0.37 \\
\hline$\frac{428}{429}$ & \begin{tabular}{|l|}
$/ 119 / 2004$ \\
$4 / 192004$
\end{tabular} & $\begin{array}{l}4: 31: 28 \text { PM } \\
4: 3228 \text { PM }\end{array}$ & $\begin{array}{l}25.518 \\
25.547\end{array}$ & $\begin{array}{r}23.271 \\
23.31 \\
\end{array}$ & $\begin{array}{l}25.182 \\
25.245\end{array}$ & $\begin{array}{l}26.356 \\
26.445\end{array}$ & $\begin{array}{l}24.047 \\
2441 \\
\end{array}$ & \begin{tabular}{|l|}
44.993 \\
44.929 \\
\end{tabular} & $\begin{array}{r}47.25 \\
47.242 \\
\end{array}$ & $\begin{array}{l}10.396 \\
10.353\end{array}$ & $\begin{array}{l}35.375 \\
35322 \\
\end{array}$ & \begin{tabular}{|c|}
-1.221 \\
-1.218 \\
\end{tabular} & $\begin{array}{l}-6.879 \\
-6.882 \\
\end{array}$ & $\begin{array}{l}50.233 \\
47025\end{array}$ & \begin{tabular}{|l|l|l}
0.129 \\
0.129
\end{tabular} & \begin{tabular}{l|l|l|l|l|l|l}
14.643 \\
14643
\end{tabular} & & $\begin{array}{l}409.6 \\
4106\end{array}$ & \begin{tabular}{|l|l|}
6.82722 \\
683289
\end{tabular} & $\begin{array}{l}45.8 \\
45.8\end{array}$ & \begin{tabular}{|l|l|}
12.3 \\
117
\end{tabular} & $\begin{array}{l}40.2 \\
40.1\end{array}$ & \begin{tabular}{|l|}
2.771 \\
2767 \\
\end{tabular} & 0.019 & 0.019 & 0.000 & $\begin{array}{l}0.476 \\
0.476\end{array}$ & 0.41 \\
\hline 430 & $4 / 19 / 2004$ & & 25.55 & 23.323 & $\begin{array}{l}25.245 \\
25.254\end{array}$ & \begin{tabular}{|l|}
26.445 \\
26.458 \\
\end{tabular} & & & $\begin{array}{l}41.242 \\
47.397\end{array}$ & $\begin{array}{l}10.553 \\
10.441\end{array}$ & $\begin{array}{l}50.022 \\
35.18\end{array}$ & & $\begin{array}{l}-6.882 \\
-6.845 \\
\end{array}$ & $\frac{44.935}{49.416}$ & $\begin{array}{l}0.129 \\
0.129\end{array}$ & $\begin{array}{l}14.045 \\
14.643\end{array}$ & $\begin{array}{l}0.000 \\
0.003\end{array}$ & \begin{tabular}{|l|}
4110.6 \\
411.6
\end{tabular} & $\mid$\begin{tabular}{|l|l}
6.883859 \\
6.86056
\end{tabular} & $\frac{44.8}{45.9}$ & \begin{tabular}{|l|l|}
12.1 \\
\end{tabular} & $\begin{array}{l}40.1 \\
40.1\end{array}$ & \begin{tabular}{|l|}
2.767 \\
2.762 \\
\end{tabular} & $\frac{0.019}{0.019}$ & $\frac{0.019}{0.019}$ & $\begin{array}{l}0.000 \\
0.000\end{array}$ & $\begin{array}{l}0.476 \\
0.477\end{array}$ & $\frac{0.41}{0.411}$ \\
\hline 431 & $4 / 19 / 2004$ & 4:34:28 PM & & & & & & 44.985 & $\begin{array}{l}47.58 \\
\end{array}$ & 10.456 & & $\mid-1.215$ & -6.836 & 47.952 & & 14.643 & & & \begin{tabular}{|l|}
6.87722 \\
6.005
\end{tabular} & 46.1 & 11.8 & & & & & 0.000 & & $\frac{0.41}{0.411}$ \\
\hline 432 & $4 / 19 / 2004$ & 4:35:28 PM & 25.592 & 23.39 & 25.305 & 26.565 & 24.206 & 44.854 & 47.114 & 10.39 & 35.26 & -1.218 & -6.833 & 47.695 & 0.13 & \begin{tabular}{|l|l} 
& 14.643 \\
\end{tabular} & & & & 45.6 & \begin{tabular}{|l|l|}
11.7 \\
\end{tabular} & 40.1 & \begin{tabular}{|l|l|}
2.762 \\
\end{tabular} & 0.019 & & & & $\frac{0.41}{0.41}$ \\
\hline 433 & $4 / 19 / 2004$ & 4:36:28 PM & & & & & & & & & & -1.218 & & & & 14.643 & & 4146 & & 46.0 & & & & & & & $\begin{array}{l}.400 \\
0.482 \\
\end{array}$ & $\frac{0.41}{0.41}$ \\
\hline 434 & $4 / 19 / 2004$ & 4:37:28 PM & & 23.435 & 2523 & $\frac{26.665}{20.65}$ & & 44.687 & $\begin{array}{l}46.989 \\
\end{array}$ & 10.478 & $\begin{array}{l}34.934 \\
\end{array}$ & -1.212 & & & $\begin{array}{ll}0.126 \\
\end{array}$ & 14.643 & & & & 45. & & & & & & & & \\
\hline 435 & $4 / 19 / 2004$ & 4:38:28 PM & 25.609 & 23.458 & \begin{tabular}{|l|}
25.228 \\
\end{tabular} & 26.697 & 24.894 & 44.5 & 46.935 & 10.345 & 34.932 & -1.221 & & 504 & 0.129 & $\begin{array}{l}14.643 \\
\end{array}$ & & 6.6 & \begin{tabular}{|l|l|}
6.94389 \\
\end{tabular} & & & 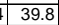 & & & & & 0.481 & \\
\hline & $4 / 19 / 2004$ & 4:39:28 PM & 25.63 & 23.499 & \begin{tabular}{|l|l|}
25.263 \\
\end{tabular} & 26.898 & 24.82 & 44.85 & $\begin{array}{l}47.221 \\
\end{array}$ & \begin{tabular}{l|l}
10.456 \\
\end{tabular} & 35.119 & -1.215 & $\begin{array}{ll}-6.856 \\
\end{array}$ & 49.524 & 0.129 & \begin{tabular}{|l|l|l|}
14.643 \\
\end{tabular} & & 417.6 & \begin{tabular}{|l|l|}
6.96056 \\
\end{tabular} & 45.7 & 12.1 & 40.0 & 2.757 & 0.019 & 0.019 & 0.000 & \begin{tabular}{|l|l|l|l|l} 
\\
\end{tabular} & \\
\hline & $4 / 19 / 2004$ & 4:40:28 PM & 25.639 & 23.523 & 25.283 & 27.037 & 24.665 & 44.896 & 47.302 & 10.508 & 35.166 & -1.218 & -6.87 & 49.944 & 0.129 & 14.643 & & 418.6 & 6.97722 & 45. & 12.2 & 40.6 & 2.760 & & & & 0.477 & 0.4 \\
\hline & & 4:41:28 PM & & & & & & & & & & & & 49.447 & & 14.643 & & & & & & & & & & & & \\
\hline r & $4 / 19 / 2004$ & 4:42:28 PM & 25.667 & 23.571 & 25.326 & 27.05 & 24.587 & 44.83 & 47.142 & 0.414 & 35.264 & -1.22 & -6.839 & 48.054 & 0.126 & 14.643 & & & & 45. & & & & & & 0.000 & 0.465 & \\
\hline$\frac{4+40}{441}$ & $\frac{4 / 1992004}{4}$ & 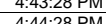 & $\frac{2.051}{2571}$ & $\frac{23.616}{23625}$ & $\frac{25.353}{25389}$ & $\frac{27.029}{27.138}$ & & $\begin{array}{l}44.608 \\
4.690\end{array}$ & 40.9586 & $\frac{10.405}{10407}$ & $\frac{35.03}{3501}$ & $\frac{-1.212}{-1214}$ & & 5 & $\begin{array}{l}0.133 \\
0125\end{array}$ & $\frac{14.645}{14643}$ & & & (70380 & & 123 & & & & & & & \\
\hline 412 & $\begin{array}{l}4 / 1 / 19 / 2004 \\
/ / 1 / 204\end{array}$ & $\begin{array}{l}4.44: 28 \mathrm{PP} \\
4: 45: 28 \mathrm{PM}\end{array}$ & $\frac{25.11}{25.724}$ & $\begin{array}{l}23.035 \\
23.664\end{array}$ & \begin{tabular}{|l|}
25.389 \\
25.408 \\
\end{tabular} & $\frac{27.156}{27.312}$ & 24.929 & $\begin{array}{l}44.099 \\
44.871\end{array}$ & $\begin{array}{l}47.250 \\
47.294\end{array}$ & $\begin{array}{l}10.447 \\
10.532\end{array}$ & $\begin{array}{l}35.01 \\
35.043\end{array}$ & & $\begin{array}{l}-0.841 \\
-6.911\end{array}$ & \begin{tabular}{|l}
50.1 \\
47.6
\end{tabular} & 0.125 & $\begin{array}{l}14.045 \\
14643\end{array}$ & & 4236 & $\mid$ & & $\frac{12.3}{117}$ & $\frac{39.9}{400}$ & $\frac{2.146}{2755}$ & & & 然 0.0000 & $\mid$ & 0.39 \\
\hline & $4 / 19 / 2004$ & 4:46.28 PM & 25.749 & 23.694 & \begin{tabular}{|l|}
25.423 \\
\end{tabular} & 27.326 & 24.934 & 44.763 & 47.196 & 10.418 & 35.211 & -1.218 & -6.879 & 49.272 & 0.125 & $\begin{array}{l}14.045 \\
14.643\end{array}$ & & $\begin{array}{l}424.6 \\
424\end{array}$ & \begin{tabular}{|l|l|}
7.07722 \\
\end{tabular} & 45.7 & 12.1 & 40.0 & 2.757 & 0.019 & & 0.000 & $\begin{array}{l}.461 \\
0.461\end{array}$ & 0.41 \\
\hline & & 4:47:28 PM & 25.773 & 23.723 & \begin{tabular}{|l|}
25.307 \\
\end{tabular} & 27.301 & 24.843 & 44.96 & 47.426 & 10.516 & 35.17 & -1.221 & & & 0.127 & \begin{tabular}{l|l}
14.643 \\
\end{tabular} & & 425.6 & & 46.0 & 12.4 & 40.1 & & & & & 0.469 & \\
\hline 445 & $4 / 19 / 2004$ & :28 PM & 25.796 & 23.761 & 25.265 & 27.219 & 24.581 & & 47.151 & 10.448 & 34.91 & & & & 0.126 & 14.643 & & 426.6 & 1056 & & & & & & & & 0.469 & \\
\hline 447 & $\begin{array}{l}4 / 1 / 1 / 2004 \\
/ 1 / 2004\end{array}$ & $\begin{array}{l}4.4 .28 \mathrm{PM} \\
4.528 \mathrm{PM}\end{array}$ & 25705 & 23801 & $\begin{array}{l}25.257 \\
25209\end{array}$ & 27.050 & $\begin{array}{l}24.445 \\
24215\end{array}$ & $\frac{44.918}{448}$ & $\begin{array}{l}47.455 \\
47321\end{array}$ & $\begin{array}{l}10.488 \\
10.97\end{array}$ & $\frac{35.115}{25026}$ & $\frac{-1.21}{-1210}$ & -689 & 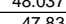 & $\begin{array}{l}0.121 \\
0.124\end{array}$ & $\frac{14.043}{14643}$ & & $\frac{477.6}{428}$ & $\begin{array}{l}\mid 1.12122 \\
71289\end{array}$ & 46. & $\frac{1.10}{117}$ & & & & & & 4 & \\
\hline 448 & $\begin{array}{l}4 / 1 / 19<0404 \\
4 / 1 / 204\end{array}$ & $4: 5.28 \mathrm{PM}$ & 25.792 & 23.817 & 25.321 & 26.77 & $\frac{24.215}{24066}$ & 44.894 & 年77.051 & $\frac{10.491}{10.409}$ & $\begin{array}{l}35.020 \\
35.268\end{array}$ & $\frac{-1.210}{-1.218}$ & -6.014 & $\frac{41.03}{49.199}$ & $\begin{array}{l}0.124 \\
0.127\end{array}$ & $\begin{array}{l}14.045 \\
114643\end{array}$ & & 4 & 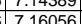 & 年5.5 & $\frac{1.1 .1}{121}$ & 401 & 6763 & & & 0 & $\mid$ & \\
\hline | & $4 / 19 / 2004$ & 4.51.20 Pि & 25.787 & 23.827 & & $\frac{0.11}{26.62}$ & $\frac{24.000}{24.051}$ & $\begin{array}{l}44.054 \\
44.633\end{array}$ & 47.161 & $\frac{10.405}{10.458}$ & $\frac{30.200}{34.922}$ & $\frac{-1.21}{-1.21 \varepsilon}$ & -6.879 & 50.413 & $\begin{array}{l}0.121 \\
0.126\end{array}$ & $\begin{array}{l}14.045 \\
14.643\end{array}$ & & $\begin{array}{l}430.6 \\
30.6\end{array}$ & 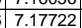 & 45.7 & $\frac{12.1}{12.4}$ & $\frac{40.1}{39.8}$ & 2.743 & 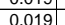 & & & $\mid$ & \\
\hline & $4 / 19 / 2004$ & $4: 53: 28 \mathrm{PM}$ & & 23.852 & 25.351 & 26.585 & 24.096 & 44.709 & 47.362 & \begin{tabular}{|l|l|l|}
10.473 \\
\end{tabular} & 34.911 & -1.21 & -6.87. & 49.3 & & & & 431.6 & & 45.9 & 12. & 39.8 & & & & & 0.471 & \\
\hline $\begin{array}{ll}4451 \\
\end{array}$ & $4 / 191 / 2004$ & 4:54:28 PM & 797 & 23.872 & 25.351 & 26.615 & 24.091 & 444.684 & 47.327 & 10.444 & 34.932 & -1.221 & & 47.075 & 0.129 & $\begin{array}{l}14.045 \\
14.643\end{array}$ & & 432.6 & $\frac{1.21056}{7.21056}$ & 45.9 & $\frac{16.5}{11.5}$ & $\frac{59.8}{39.8}$ & & 019 & & 0.000 & 0.478 & \\
\hline & $4 / 19 / 2004$ & $4: 55: 28 \mathrm{PM}$ & 25.797 & 23.887 & 25.376 & 26.615 & 24.111 & 44.79 & $\begin{array}{l}47.449 \\
\end{array}$ & 10.503 & 35.004 & -1.221 & -6.9 & 48.729 & 0 & 14.643 & & & $22 / 22$ & 46.6 & $\frac{11 .}{11 .}$ & 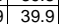 & & & & & 0.470 & \\
\hline & $4 / 19 / 2004$ & 4:56:28 PM & 25.822 & 23.917 & \begin{tabular}{|l|}
25.401 \\
\end{tabular} & 26.63 & 24156 & & & 10.386 & & -1.218 & & 48.707 & 0.133 & 14.643 & & 434.6 & 24389 & & & 39.8 & & & & & & \\
\hline & 4/19/2004 & 4:57:28 PM & 25.823 & 23.944 & 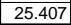 & 26.686 & 24.408 & 44.545 & 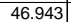 & 10.433 & 34.922 & -1.221 & -6.865 & 49.491 & 0.139 & \begin{tabular}{|l|l|l|l|l|}
14.33 \\
\end{tabular} & & 435.6 & 7.26056 & 45.5 & 12.1 & 39.7 & 2.739 & .021 & 0.020 & 0.001 & \begin{tabular}{|c|c|c|}
0.516 \\
\end{tabular} & \\
\hline & 4/119/2004 & 4:58:28 PM & 25.834 & 23.96 & 25.423 & 26.687 & 24 & 44.68 & 47.294 & 10.438 & 34.996 & -1.22 & & 48.619 & 0.128 & 14.643 & & 43 & & 45. & 11.9 & . & & & & & 0.475 & \\
\hline & 4/199/2004 & 4:59:28 PM & 25.845 & 23.981 & 25.434 & 26.698 & .375 & 44.6. & 47.259 & 10.527 & 34.796 & & & 52.507 & 0.12 & & & & & 45.8 & & 39. & & & & & 0.445 & \\
\hline 15 & 4/11992004 & & 25.856 & 24.002 & 25.471 & 26.709 & & 44.7 & 47.352 & 10.601 & 34.805 & -1.218 & & 0 & 0.124 & 14.643 & & & & 45.9 & 12.4 & 39 & & & & 0.00 & 459| & \\
\hline & & & & & & & & & & & 35.1 & & & 48. & 0.126 & & & & & & & & & & & & 4 & \\
\hline$\frac{30}{460}$ & $\begin{array}{l}4 / 1 / 19 / 2004 \\
/ 19 / 204\end{array}$ & 5.0 .2308 & $\frac{25.012}{25868}$ & 24.026 & \begin{tabular}{|l|}
25.500 \\
2497
\end{tabular} & $\frac{2.05}{26.626}$ & $\frac{24.52}{2432}$ & $\frac{44.049}{44657}$ & $\mid$ & $\begin{array}{r}10.48 \\
10.538\end{array}$ & $\begin{array}{l}34.932 \\
34807\end{array}$ & $\frac{-1.21}{-121}$ & $\begin{array}{l}-0.053 \\
-686\end{array}$ & $\frac{49.595}{47342}$ & $=0.125$ & $\begin{array}{l}14.643 \\
14.643\end{array}$ & & & 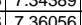 & 45.60 & $\frac{12.2}{116}$ & 3397 & & & & 0.000 & $\mid 0.495$ & \\
\hline 461 & $4 / 19 / 2004$ & 5:04:28 PM & 25.888 & & \begin{tabular}{|l|l|l|}
25.518 \\
\end{tabular} & \begin{tabular}{|l|l|l}
26.607 \\
\end{tabular} & & 44.867 & \begin{tabular}{|l|}
47.455 \\
\end{tabular} & 10.511 & 35.195 & $\frac{1.218}{-1218}$ & & 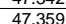 & $\frac{.0 .199}{0.119}$ & $\begin{array}{ll}14.045 \\
14.643\end{array}$ & & & & $\frac{4.00}{460}$ & $\frac{1.5}{116}$ & 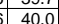 & & & & & 0.436 & \\
\hline & $4 / 19 / 2004$ & $5: 005: 28 \mathrm{PM}$ & 25.909 & 24.075 & \begin{tabular}{|l|l|}
25.553 \\
\end{tabular} & 26.662 & 24.475 & 44.054 & 46.883 & 10.468 & 34.363 & -1.212 & & 52.081 & 0.118 & 14.643 & & 44 & & 45.4 & $\frac{11.8}{12.8}$ & 30 & & & & & 0.442 & \\
\hline 40 & $4 / 19 / 2004$ & 5:006:28 PM & 25005 & & \begin{tabular}{|l|l|}
25.549 \\
\end{tabular} & 26.703 & & 44.236 & $\mid 47.14$ & & & & & & & & & & & & & & & & & & 0.440 & \\
\hline & 4/119/2004 & 5:07:28 PM & & & 25.58 & & & $\begin{array}{l}44.492 \\
\end{array}$ & & & & -1.21 & & 47. & & 14.6 & & & & & & & & & & & & \\
\hline & 4/19/2004 & 5:08:28 PM & 25.93 & 24.106 & \begin{tabular}{|l|}
25.579 \\
\end{tabular} & 26.678 & 24.164 & 44.5 & $\mid 47.503$ & 10.568 & 34.677 & $-1.21 \varepsilon$ & -6. & 47.9 & $\begin{array}{ll}0.123 \\
\end{array}$ & 14.6 & & & \begin{tabular}{|l|l|}
7.44389 \\
\end{tabular} & 46. & 11.8 & 396 & 2720 & & & & \begin{tabular}{|c|c|c|}
0.456 \\
\end{tabular} & \\
\hline & 4/19/2004 & 5:09:28 PM & & 24.109 & \begin{tabular}{|l|}
25.567 \\
\end{tabular} & 26.551 & & & 47.762 & 10.672 & & -1.22 & & 48. & & & & & & 46 & 11.8 & 39. & & & & & $\begin{array}{ll}0.460 \\
\end{array}$ & \\
\hline 467 & 4/19/2004 & 5:10:28 PM & 26.195 & 24.117 & 25.525 & 26.529 & & & \begin{tabular}{|l|l|l|l|} 
\\
7788
\end{tabular} & 10.588 & & -1.224 & & 50.9 & 0.135 & 14 & & & & 45.7 & 12.5 & 39. & 272 & & & & 502 & \\
\hline 4668 & 4/19/2004 & 11:28 PM & 154 & 24.156 & \begin{tabular}{|l|}
25.393 \\
\end{tabular} & 26.532 & & & \begin{tabular}{|l|l|}
47.074 \\
\end{tabular} & \begin{tabular}{|l|l|l} 
\\
\end{tabular} & & -1.218 & & 48. & 0 & & & & & 45 & 11.9 & & & & & & 0.465 & \\
\hline & $4 / 19 / 2004$ & 5:12:2 & & 24.155 & $3 / 2$ & 26.536 & & & & & & & & & & & & & & & 12.2 & & & & & & 53 & \\
\hline 78 & 411992004 & 5:13:28 PM & 25.991 & 24.158 & $\frac{25.38}{25.38}$ & 2.6 .499 & & & 47.555 & & & -1.221 & & 51.1 & 0.123 & & & & & 46. & 12.8 & 10 & & & & & 0.454 & \\
\hline & $\frac{41912004}{419204}$ & $\frac{5.4 .46}{5.1528 \mathrm{PM}}$ & & $\frac{24.182}{24201}$ & $\begin{array}{l}25.444 \\
2544\end{array}$ & $\frac{20.300}{26.623}$ & & & $\frac{4.1 .044}{47.16}$ & & & -1221 & & & & & & & & & $\frac{11.5}{125}$ & & & & & & & \\
\hline & $\frac{4}{41 / 912004}$ & 5 & 25884 & 24211 & \begin{tabular}{|l|l|l|l|} 
\\
\end{tabular} & 26.668 & & & 47.495 & & & & & 49 & & & & & & & 120 & & & & & & 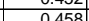 & \\
\hline 474 & $\frac{4 / 19 / 2004}{4}$ & 5:17:28 PM & 25.84 & 24.221 & 25.479 & $\frac{26.693}{26.693}$ & & & 46.904 & \begin{tabular}{|l|l|}
10.487 \\
\end{tabular} & 34.745 & -1221 & & 51. & 0.122 & & & & & 45.4 & 126 & & & & & & $\begin{array}{l}0.453 \\
0.45\end{array}$ & \\
\hline & $4 / 19 / 2004$ & & & & & & & & & & & & & & & & & & & & & & & & & & & \\
\hline 476 & $4 / 19 / 2004$ & $5 \cdot 19.2$ & 25.866 & 24243 & 2552 & 26824 & & 446 & 47414 & 1057 & 3477 & -1221 & & 48.7 & 012 & 14. & & & & & 110 & & & & & & & \\
\hline & $4 / 199 / 2$ & $5: 20: 28 \mathrm{PM}$ & & 24.259 & 25 & 26.8 & & & $\begin{array}{l}47.451 \\
\end{array}$ & 10.4 & 34.778 & -1.221 & & 49. & 0.1 & & & & & & 12.1 & 398 & & & & & 54 & \\
\hline $\begin{array}{lll}478 & \\
\end{array}$ & 4/19/2004 & $5: 21: 28 \mathrm{~F}$ & 25.88 & 24.274 & 25 & 26.895 & & 45.15 & 47.789 & \begin{tabular}{|l|l|}
10.594 \\
\end{tabular} & 35.318 & $-1.21 \varepsilon$ & -7. & 48.7 & 0.122 & \begin{tabular}{|l|l|l|l|l|}
1433 \\
\end{tabular} & & $\begin{array}{l}459.6 \\
\end{array}$ & 5) 7.66 & 46 & 11.9 & 40.2 & 2.774 & 0.018 & & & 0.445 & \\
\hline 4779 & 4/1/9/2004 & 5:22:28 PM & 25.88 & 24.285 & & 26.941 & & 44.78 & \begin{tabular}{|l|l|l|l}
47.389 \\
\end{tabular} & 10. & 34 & $-1.21 \varepsilon$ & & 49.5 & & & & & & 4 & 12.1 & & & & & & $\begin{array}{l}0.446 \\
\end{array}$ & \\
\hline 480 & & 5:23:28 F & 25.9 & 24.306 & 25.564 & 26.952 & & 44 & & & 34.948 & -1.218 & & 50. & 0.124 & & & 4 & & 45 & 12.3 & 39. & 2.12 & & & & 0.457 & \\
\hline 4811 & 4/19/20004 & 5:24:28 PM & 25.925 & 24.322 & 25.579 & 27.028 & 24. & 44.49 & 47.002 & 10.441 & 34 & -1.221. & & 45.3 & 0.122 & $14.6 \mathrm{C}$ & & & & & 11. & 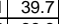 & & & & & 51 & \\
\hline & 4 & 5:25:28 PM & 2505 & 24.344 & \begin{tabular}{|l|l|}
25.591 \\
\end{tabular} & 20.7034 & 24.4 & $\begin{array}{l}44.163 \\
4.135\end{array}$ & $\begin{array}{lll}47.528 \\
47.78\end{array}$ & 10.548 & 34.903 & -1.218 & & 48.6 & 0.124 & 14.6 & & 463.6 & 077020 & & $\frac{11.9}{1.1}$ & 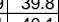 & 277 & & & & 0.456 & \\
\hline & 4 & $\begin{array}{l}0.20 .201 \\
5.27 .20\end{array}$ & 2 & 24.501 & 20.093 & 27.041 & & 年 & 4.101 & & 30.1064 & - & & (25.4 & & & & & & & $\frac{1.1}{122}$ & & & & & & & \\
\hline & 告/191/2 & $\begin{array}{l}5.42 .28 \\
5.28\end{array}$ & 25.99 & 24.393 & $\frac{2.0044}{2564}$ & & & & & $\begin{array}{l}10.450 \\
10.494\end{array}$ & & -1215 & & $\frac{4.56}{47}$ & $\frac{1.1}{01}$ & 14 & & $\frac{40}{46}$ & & & $\begin{array}{l}1.2 \\
1177\end{array}$ & & & & & & (1) & \\
\hline 486 & & $5 ; 29: 28$ & & & & & & 44.898 & $\mid 47.659$ & & & -122 & & & & & & & & & 128 & & & & & & & \\
\hline 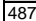 & $4 / 19 / 2004$ & & & 24.442 & & 271 & & & & & & & & 48.7 & & & & & & & 110 & & & & & & 446 & \\
\hline & $4 / 19 / 2004$ & 5:31:28 PM & & & & & & & & & & -1.221 & & & & & & & & 46.2 & 11.8 & 40. & & & 018 & & 743 & \\
\hline & $4 / 19 / 2004$ & $5 \cdot 32.2$ & & 481 & 588 & & & & & 10.406 & & 221 & & $\overline{91}$ & $\begin{array}{ll}0.118 \\
\end{array}$ & 14.643 & & & 84389 & 46.4 & 120 & & & & & 2000 & 0.429 & $f$ \\
\hline 490 & $4 / 19 / 2004$ & 5:33:28 PM & 26.05 & 24.498 & \begin{tabular}{|l|l|}
25.324 \\
\end{tabular} & 27.188 & & 45.052 & 477.845 & \begin{tabular}{|l|l|}
10.275 \\
\end{tabular} & 35.554 & \begin{tabular}{|l|l|} 
\\
\end{tabular} & -7.48 & 47.774 & 0.12 & $\begin{array}{l}14.643 \\
\end{array}$ & & 471.6 & 87.86056 & 46.4 & 11.7 & & \begin{tabular}{|l|l|}
2.779 \\
\end{tabular} & 0.018 & 0.018 & 0.000 & 0.440 & 0.3 \\
\hline
\end{tabular}


WSRC-TR-2005-00105, REVISION 0

SRNL-RPP-2005-00012, REVISION 0

RUN \# 2.03A AND B; FIRST AND SECOND HALF OF SLURRY DEWATERING - CONT.

\begin{tabular}{|c|c|c|c|c|c|c|c|c|c|c|c|c|c|c|c|c|c|c|c|c|c|c|c|c|c|c|c|c|}
\hline & & & & & & & & & & & & & & & & & & & & & & & & & & & & \\
\hline & A & B & D & $E$ & $F$ & $G$ & $\mathrm{H}$ & $\mathrm{J}$ & $\mathrm{K}$ & $\mathrm{L}$ & M & $\mathrm{N}$ & $\begin{array}{l}0 \\
\end{array}$ & Q & $\mathrm{R}$ & \begin{tabular}{|c|}
$\mathrm{s}$ \\
\end{tabular} & $\mathrm{T}$ & $\mathrm{V}$ & $\frac{W}{10772}$ & $\mathrm{X}$ & 1 & $z$ & AA & $\frac{A B}{A B}$ & $A C$ & $A D$ & $\mathrm{AE}$ & $\mathrm{AF} A$ \\
\hline$\frac{49}{49}$ & $\begin{array}{l}4 / 1 / 9 / 2004 \\
4 / 192004\end{array}$ & $\begin{array}{l}5: 34: 28 \mathrm{PM} \\
\text { 5:35:28 PM }\end{array}$ & 26.056 & 24.5345 & \begin{tabular}{|r|}
25.33 \\
25.351 \\
\end{tabular} & \begin{tabular}{|l|}
27.169 \\
2711
\end{tabular} & $\begin{array}{r}24.521 \\
24.397\end{array}$ & $\begin{array}{l}44.883 \\
45.072 \\
\end{array}$ & \begin{tabular}{|c|}
47.669 \\
47663
\end{tabular} & \begin{tabular}{|l|}
10.293 \\
10.363
\end{tabular} & \begin{tabular}{|c|}
35.285 \\
35.447
\end{tabular} & \begin{tabular}{|l|l|} 
& -1.221 \\
\end{tabular} & $\begin{array}{l}-7.501 \\
-7.492 \\
\end{array}$ & $\begin{array}{l}48.173 \\
49.257\end{array}$ & $\begin{array}{l}0.119 \\
0.12\end{array}$ & \begin{tabular}{|l|}
14.643 \\
14.643
\end{tabular} & $\begin{array}{l}0.003 \\
0.003\end{array}$ & $\begin{array}{l}472.6 \\
437.6\end{array}$ & $\begin{array}{c}5 \\
5\end{array}$ & $\frac{46.2}{46.2}$ & \begin{tabular}{|l|l|}
11.8 \\
12.1 \\
\end{tabular} & \begin{tabular}{|l|l|}
40.1 \\
40.3
\end{tabular} & $2.764 \mid$ & 0.018 & $\frac{0.018}{0.018}$ & $\begin{array}{l}0.000 \\
0.000\end{array}$ & $\begin{array}{c}0.439 \\
0.440\end{array}$ & \begin{tabular}{|l|}
0.37 \\
0.37
\end{tabular} \\
\hline$\frac{49}{49}$ & & & & & & & & $\begin{array}{l}45.012 \\
45.139 \\
\end{array}$ & & $\begin{array}{r}1.303 \\
10.27 \\
\end{array}$ & & & & $\begin{array}{l}49.251 \\
48.504\end{array}$ & & \begin{tabular}{|l|}
14.6433 \\
14.643
\end{tabular} & & & & & & & & & & & & \\
\hline 49 & $4 / 19 / 2004$ & 5:37:28 PM & 26.018 & 24.571 & \begin{tabular}{|l|}
25.393 \\
\end{tabular} & \begin{tabular}{|l|l|} 
& 26.966 \\
\end{tabular} & 24.038 & 45.11 & 47.851 & $\begin{array}{l}10.19 \\
\end{array}$ & 35.769 & \begin{tabular}{|l|l|} 
& -1.218 \\
\end{tabular} & -7.483 & 47.465 & 0.118 & \begin{tabular}{|l|}
14.643 \\
\end{tabular} & 0.004 & 475.6 & \begin{tabular}{|l|l|l}
6.92722 \\
\end{tabular} & 46.4 & \begin{tabular}{|l|l|}
11.6 \\
\end{tabular} & 40.4 & 2.788 & 0.018 & & 0.000 & $\begin{array}{l}0.434 \\
0.430 \\
\end{array}$ & 0.37 \\
\hline & $4 / 191 / 2004$ & 5:38:28 PM & 26.003 & 24.586 & \begin{tabular}{|l|}
25.413 \\
\end{tabular} & \begin{tabular}{|l|}
26.931 \\
\end{tabular} & 23.898 & $\begin{array}{r}45.197 \\
\end{array}$ & 47.909 & \begin{tabular}{|l|}
10.172 \\
\end{tabular} & \begin{tabular}{|l|l|}
35.714 \\
\end{tabular} & \begin{tabular}{|l|l|}
-1.215 \\
\end{tabular} & $\begin{array}{r}-7.466 \\
-1466\end{array}$ & 46.366 & 0.116 & \begin{tabular}{|l|}
14.643 \\
\end{tabular} & 0.004 & & \begin{tabular}{|l|l|}
7.94389 \\
\end{tabular} & 46.4 & \begin{tabular}{|l|l|}
11.4 \\
\end{tabular} & & & & & 0.000 & $\begin{array}{l}0.423 \\
\end{array}$ & 0.36 \\
\hline 496 & 4/19/2004 & & & & & & & 44.483 & 47.275 & 10.827 & & & & 49.624 & & 14.643 & & & \begin{tabular}{|c|c|}
6 & 7.96056 \\
\end{tabular} & 45.8 & & 39.4 & & & & & $\begin{array}{l}0.449 \\
\end{array}$ & \\
\hline 497 & $4 / 19 / 2004$ & 5:40:28 PM & 25.975 & \begin{tabular}{|l|l|}
24.583 \\
\end{tabular} & \begin{tabular}{|l|l|}
25.439 \\
\end{tabular} & 26.833 & \begin{tabular}{|l|l|}
24.044 \\
2
\end{tabular} & 44.485 & & \begin{tabular}{ll|}
10.827 \\
\end{tabular} & $\begin{array}{l}34.355 \\
\end{array}$ & \begin{tabular}{|l|}
-1.221 \\
\end{tabular} & $\begin{array}{r}-7.521 \\
\end{array}$ & 48.206 & 0.116 & \begin{tabular}{|l|l|}
14.643 \\
\end{tabular} & & & 7.97722 & 45.9 & $\begin{array}{ll}11.8 \\
\end{array}$ & 39.4 & 2.718 & 0.017 & & $\begin{array}{l}0.000 \\
\end{array}$ & \begin{tabular}{|l|l|l|l|}
0.33 \\
\end{tabular} & 0.37 \\
\hline$\frac{498}{499}$ & \begin{tabular}{|l|}
$/ 119 / 2004$ \\
$4 / 192004$
\end{tabular} & $\begin{array}{l}5: 41: 28 \mathrm{PM} \\
5 \cdot 4 \cdot 28 \mathrm{PM}\end{array}$ & $\begin{array}{l}25.985 \\
25.987\end{array}$ & $\begin{array}{l}24.598 \\
24601\end{array}$ & \begin{tabular}{|l|}
25.485 \\
25.507 \\
\end{tabular} & \begin{tabular}{|l|l|}
26.879 \\
26891
\end{tabular} & $\begin{array}{l}24.085 \\
22.207 \\
\end{array}$ & 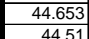 & \begin{tabular}{r|}
47.58 \\
47.507
\end{tabular} & \begin{tabular}{|l|l|}
10.758 \\
10778
\end{tabular} & \begin{tabular}{|l|}
34.651 \\
3409
\end{tabular} & \begin{tabular}{|l|l|}
-1.218 \\
-1221
\end{tabular} & $\begin{array}{r}-7.521 \\
-7.475\end{array}$ & $\begin{array}{l}49.622 \\
4382 \\
\end{array}$ & \begin{tabular}{|l|l|}
0.117 \\
0.117
\end{tabular} & \begin{tabular}{|l|}
14.643 \\
14643
\end{tabular} & & \begin{tabular}{|l|l|}
479.6 \\
48.6
\end{tabular} & \begin{tabular}{|l|l|} 
& 7.99389 \\
& 8001059
\end{tabular} & $\frac{46.1}{460}$ & \begin{tabular}{|l|l|}
12.2 \\
119
\end{tabular} & $\begin{array}{l}39.7 \\
39.5 \\
\end{array}$ & 2.734 & $\begin{array}{l}0.017 \\
0.017\end{array}$ & $\begin{array}{l}0.017 \\
0.017\end{array}$ & $\begin{array}{l}0.000 \\
0001\end{array}$ & $\begin{array}{l}0.434 \\
0.436\end{array}$ & 0.37 \\
\hline & $4 / 19 / 2004$ & & 25.989 & 24.623 & $\begin{array}{l}25.501 \\
25.534\end{array}$ & \begin{tabular}{|l|}
20.091 \\
26.968 \\
\end{tabular} & $\begin{array}{l}24.207 \\
24.324\end{array}$ & $\begin{array}{r}44.51 \\
44.463 \\
\end{array}$ & & $\begin{array}{r}10.7 / 78 \\
10.74\end{array}$ & $\begin{array}{r}33.4499 \\
3.495\end{array}$ & & & $\frac{48.392}{49.799}$ & 0.117 & $\frac{14.643}{14.643}$ & 0.003 & \begin{tabular}{|l|}
480.6 \\
481.6
\end{tabular} & \begin{tabular}{|c|} 
\\
68.01056 \\
6
\end{tabular} & $\frac{46.0}{45.8}$ & \begin{tabular}{|l|l|}
11.9 \\
12.2
\end{tabular} & & 2.721 & $\frac{0.017}{0.018}$ & $\frac{0.017}{0.017}$ & $\frac{0.000}{0.000}$ & $\begin{array}{l}0.436 \\
0.439\end{array}$ & 0.37 \\
\hline & $4 / 19 / 2004$ & 5:44:28 PM & & & & & & & \begin{tabular}{|l|}
47.501 \\
\end{tabular} & 10.725 & 34.515 & \begin{tabular}{|l|}
-1.221 \\
\end{tabular} & -7.518 & 48.636 & & 14.643 & & & \begin{tabular}{|l|l|}
$6.0 \angle Z 2 Z$ \\
5 & 8.04389 \\
\end{tabular} & 46.0 & & & & & & 0.000 & & $\frac{0.37}{0.37}$ \\
\hline 502 & $4 / 19 / 2004$ & 5:45:28 PM & 25.997 & 24.63 & \begin{tabular}{|l|}
25.542 \\
\end{tabular} & 27.02 & 24.292 & 44.61 & 47.65 & $\begin{array}{l}10.757 \\
\end{array}$ & 34.513 & -1.221 & -7.52 & 49.045 & 0.116 & \begin{tabular}{|l|l|}
14.643 \\
\end{tabular} & & 48 & 8.06056 & & \begin{tabular}{|l|l|}
12.0 \\
\end{tabular} & 39.6 & & & & & & 0.37 \\
\hline & & 5:46:28 PM & & & & & 24.379 & & & & & -1.218 & & & 0.116 & & & 484 & 07722 & 46.0 & & & & & & 0.000 & $\mid 0.429$ & $\begin{array}{l}0.37 \\
0.37\end{array}$ \\
\hline & $4 / 19 / 2004$ & & & & & 27.109 & & $\begin{array}{l}44.624 \\
\end{array}$ & $\begin{array}{ll}47.688 \\
\end{array}$ & 10.745 & $\begin{array}{l}34.513 \\
\end{array}$ & -1.221 & & 50.486 & $\begin{array}{ll}0.116 \\
\end{array}$ & $\begin{array}{l}14.643 \\
\end{array}$ & & & & & & & & & & & 0.430 & \\
\hline 505 & $4 / 19 / 2004$ & 5:48:28 PM & & 24.67 & \begin{tabular}{|l|}
25.597 \\
\end{tabular} & 27.12 & 24.587 & \begin{tabular}{|l|l}
44.689 \\
\end{tabular} & 47.451 & \begin{tabular}{|l|l|}
10.647 \\
\end{tabular} & 34.868 & -1.221 & & 47.985 & 0.114 & $\begin{array}{l}14.643 \\
\end{array}$ & & 486.6 & 8.11056 & 46.0 & & 39. & .743 & & & & & \\
\hline 506 & $4 / 19 / 2004$ & 5:49:28 PM & 26.048 & 24.682 & \begin{tabular}{|l|l|}
25.618 \\
\end{tabular} & 27.191 & 24.649 & $\begin{array}{l}44.369 \\
\end{array}$ & \begin{tabular}{|l|}
47.298 \\
\end{tabular} & \begin{tabular}{|l|l|l|}
10.588 \\
\end{tabular} & 34.548 & -1.221 & & 48.4 & 0.116 & \begin{tabular}{|l|l|l|}
14.643 \\
\end{tabular} & & 487.6 & 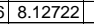 & 45.8 & 11.9 & 39.5 & 2.721 & .017 & 0.017 & 0.000 & $\begin{array}{l}0.431 \\
\end{array}$ & \\
\hline 50 & $4 / 19 / 2004$ & 5:50:28 PM & 26.05 & 24.678 & \begin{tabular}{|l|l|}
25.609 \\
\end{tabular} & 27.178 & 24.66 & 44.63 & 47.737 & 10.652 & 34.63 & -1.221 & & 48.969 & 0.114 & \begin{tabular}{|l|l|}
14.643 \\
\end{tabular} & & 488.6 & \begin{tabular}{l|l|l|}
6 & 8.14389
\end{tabular} & 46.3 & 12.8 & 39. & & & & & 0.422 & 0.36 \\
\hline & 411992004 & 5:51:28 PM & & & & & & & & & & & & 49.914 & & 14.643 & & 489.6 & \begin{tabular}{ll|l}
6 & 8.16056 \\
\end{tabular} & 46. & & & & & & & & \\
\hline & $4 / 199 / 2004$ & $\begin{array}{l}5: 52: 28 \mathrm{PM} \\
5.528 \mathrm{O}\end{array}$ & 26.081 & 24.735 & $\frac{25.661}{25692}$ & $\frac{27.224}{27221}$ & 24.646 & 44.597 & - 47.569 & 10.716 & $\begin{array}{l}34.563 \\
3.237\end{array}$ & & & $\begin{array}{l}49.368 \\
5.705\end{array}$ & & 14.643 & & & & 46. & 12. & & & & & 0.000 & 0.433 & \\
\hline & $\frac{4 / 1992004}{4}$ & $\frac{5.35 .28 \mathrm{PM}}{5.548}$ & & $\frac{24.746}{24.768}$ & $\frac{25.682}{25694}$ & & & $\begin{array}{r}44.467 \\
44.45\end{array}$ & $\begin{array}{l}47.065 \\
47550\end{array}$ & 10.826 & $\frac{34.44}{34.509}$ & $\frac{-1.24}{1.22}$ & & $\frac{50.105}{51.102}$ & $\frac{0.118}{0.115}$ & $\frac{14.645}{14643}$ & & & . & & & & & & & & & \\
\hline & $4 / 19 / 2004$ & 5:55:58 PM & 26.131 & 24.795 & $\begin{array}{l}25.926 \\
25.726\end{array}$ & $\mid 27.304$ & 22.626 & |44.579 & $\mid$\begin{tabular}{|l|l|}
$\mid 47.694$ \\
\end{tabular} & 10.728 & $\frac{34.54}{34.54}$ & -1.218 & & 48.074 & 0.1116 & $\begin{array}{l}\frac{14.045}{14.643} \\
\end{array}$ & & $\begin{array}{l}4.43 .6 \\
49.6\end{array}$ & 6 & & $\frac{11.6}{11.8}$ & 39.6 & & & & 0.000 & $\mid$ & 0.36 \\
\hline & $4 / 19 / 2004$ & 5:56:28 PM & 26.127 & \begin{tabular}{|l|l|}
24.791 \\
\end{tabular} & \begin{tabular}{|l|l|}
25.522 \\
\end{tabular} & \begin{tabular}{|l|}
27.245 \\
\end{tabular} & 24.658 & \begin{tabular}{|l|l|l|l}
44.546 \\
\end{tabular} & $\begin{array}{l}47.706 \\
\end{array}$ & 10.694 & 34.521 & -1.218 & -7.478 & $\begin{array}{l}48.982 \\
\end{array}$ & $\begin{array}{l}0.116 \\
\end{array}$ & 14.643 & & 4994.6 & \begin{tabular}{|ll}
6 & 8.24389 \\
\end{tabular} & 46.2 & 12.0 & 39.5 & 2.726 & & & 0.000 & 0.431 & 0.37 \\
\hline & & 7:28 PM & 26.123 & & 25.523 & 27.266 & & 44.518 & 47.509 & 10.497 & 34.821. & -1.22 & & 49.28 & 0.116 & \begin{tabular}{l|l}
14.643 \\
\end{tabular} & & 495.6 & & 46.0 & & & & & & & & \\
\hline & 4119920004 & 5:58:28 PM & & 24.834 & 25.55 & 27.268 & 24.661 & 44.593 & 47.893 & 10.516 & 34.645 & & & 0.106 & & 14.643 & & 496 & & 46.4 & & & & & & & 0.434 & \\
\hline & $\begin{array}{l}4 / 1 / 1 / 2004 \\
/ 1 / 2004\end{array}$ & $\frac{5.59 .28}{6.028}$ & $\frac{20.122}{26.235}$ & 24.851 & $\frac{25.561}{2561}$ & $\frac{27.215}{27202}$ & $\frac{24.628}{3468}$ & $\begin{array}{l}44.514 \\
44054\end{array}$ & $\begin{array}{l}47.596 \\
1821\end{array}$ & $\begin{array}{l}1.582 \\
1059\end{array}$ & $\begin{array}{l}34.634 \\
35076\end{array}$ & $\frac{-1.21}{-121}$ & -75 & 年 & $\begin{array}{l}0.115 \\
0117\end{array}$ & $\frac{14.043}{14643}$ & & $\begin{array}{l}49.6 \\
1906\end{array}$ & $\begin{array}{l}0.29389 \\
\end{array}$ & 年 & & 400 & 575 & & & 0 & $\mid$ & 3.36 \\
\hline$\frac{118}{518}$ & $4 / 1912004$ & $6.00128 \mathrm{PM}$ & 26106 & 24.87 & $\begin{array}{r}25.01 \\
25.596\end{array}$ & 27.294 & $\frac{24.00}{24712}$ & $\begin{array}{l}44.954 \\
44.444\end{array}$ & $\begin{array}{l}40.21 \\
47.59 \\
\end{array}$ & $\frac{10.59}{10.565}$ & $\begin{array}{l}35.070 \\
34.599\end{array}$ & $\frac{-1.40}{-1.218}$ & & $\begin{array}{l}40.990 \\
47.947\end{array}$ & $\frac{0.117}{0.117}$ & $\begin{array}{l}\frac{14.045}{14.643} \\
\end{array}$ & & \begin{tabular}{|l|}
490.0 \\
499.6
\end{tabular} & $\frac{0.35030}{6}$ & $\frac{40.1}{46.1}$ & 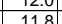 & & & & & 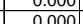 & $\mid$ & \\
\hline & $4 / 19 / 2004$ & 6:02:28 PM & 26.124 & 24.893 & \begin{tabular}{|l|}
25.629 \\
\end{tabular} & 27.312 & 24.615 & 44.396 & \begin{tabular}{|l|}
47.596 \\
\end{tabular} & 10.661 & $\mid 34.349$ & -1.218 & -7. & 48.135 & 0.113 & 14.643 & & 500.6 & \begin{tabular}{|l|l|}
68.34389 \\
\end{tabular} & 46. & $\frac{11.8}{11 .}$ & 39.4 & & 0.017 & & & 0.420 & \\
\hline & $4 / 19 / 2004$ & 6:03:28 PM & 26.135 & 24.914 & 25.64 & 27.263 & 24.4 & 44.477 & 477.654 & $\begin{array}{l}10.611 \\
\end{array}$ & 34.532 & -1.221 & & 46.448 & 0.11 & & & 501.6 & 8.36056 & 46.2 & 11.4 & 39.5 & & & & & 0.426 & \\
\hline 152 & $4 / 191 / 2004$ & 6:03:39 PM & 26.13 & 24.909 & 25.63 & 27.218 & $\begin{array}{l}24.44 \\
\end{array}$ & 44.713 & 47.856 & 10.654 & 34.817 & -1.221 & -7.53 & 47.38 & 0.115 & 14.643 & & 501.8 & 88.36361 & 46.4 & 11.6 & $\frac{59.8}{39.8}$ & & & & 0.000 & 0.424 & $\frac{0.36}{0.36}$ \\
\hline & $4 / 19 / 2004$ & 6:04:39 PM & 26.135 & \begin{tabular}{|l|l|}
24.909 \\
\end{tabular} & 25.64 & 27.153 & 24.37 & 44.674 & \begin{tabular}{|l|l}
47.719 \\
\end{tabular} & $\begin{array}{l}10.525 \\
\end{array}$ & 34.87 & -1.218 & & $\begin{array}{l}48.487 \\
\end{array}$ & 0.118 & $\begin{array}{l}14.643 \\
\end{array}$ & & & & 46.2 & 11.9 & & & & & & 0.434 & \\
\hline & $4 / 19 / 2004$ & & & & & & 24.209 & & 47.81 & & 34.544 & & & & & 14.643 & & 503 & 8.39694 & 46.3 & & 39 & & & & & & \\
\hline & 4/19/2004 & 6:06:39 PM & 26.153 & 24.938 & \begin{tabular}{|l|l|} 
& 25.663 \\
\end{tabular} & 26.972 & 24.064 & 44.62 & 47.845 & $\begin{array}{l}10.442 \\
\end{array}$ & 34.942 & -1.218 & & 49.099 & 0.115 & \begin{tabular}{|l|l|l|l|l|}
14.33 \\
\end{tabular} & & 504.8 & \begin{tabular}{|c|c|}
8 & 8.41331 \\
\end{tabular} & 46.4 & 12.0 & 39.8 & 2.743 & 017 & 0.017 & 0.000 & \begin{tabular}{|l|l|l|l|} 
\\
\end{tabular} & \\
\hline & 4/119/2004 & 7:39 PM & & 24.931 & \begin{tabular}{|l|l|}
25.672 \\
\end{tabular} & 26.86 & 20.911 & 44.622 & \begin{tabular}{|l|l|}
47.86 \\
\end{tabular} & 10.537 & 34.743 & -1.221 & & 47.962 & 0.116 & $\begin{array}{l}14.643 \\
\end{array}$ & & & & & 11.8 & . & & & & & 0.428 & \\
\hline 52 & 4/119/2004 & 6:08:39 PM & 26.161 & 24.94 & 25.68 & & 24.016 & 44.649 & 47.99 & 10.568 & & -1.221 & & 47.253 & 0.117 & 14.643 & & 506.8 & \begin{tabular}{ll|l}
8 & 8.44694 \\
\end{tabular} & 46.5 & 11.6 & & 2.734 & & & & 0.432 & \\
\hline & 4/11992004 & & 26.171 & 24.955 & 25.665 & 26.849 & 24. & 44.722. & 48.042 & 10.549 & & -1.221 & & 48.1 & 0.117 & 14.643 & & 507 & 8.46 & 46.6 & 11. & 39 & .74 & & & 0.000 & 0.430 & \\
\hline & & $\begin{array}{ll}0.10 .39 \mathrm{PM} \\
6.1 .120\end{array}$ & & & & & & & & & & & & & 0.117 & & & & & & & & & & & & & \\
\hline & $\begin{array}{l}4 / 1 / 19 / 2004 \\
/ 19 / 204\end{array}$ & $\frac{0.159}{6.123 \mathrm{PM}}$ & $\frac{20.109}{26164}$ & 24.9599 & \begin{tabular}{|l|l|}
25.053 \\
2654
\end{tabular} & $\frac{2.0630}{26868}$ & $\begin{array}{l}24.254 \\
22.305\end{array}$ & $\begin{array}{l}44.051 \\
44686\end{array}$ & 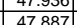 & $\begin{array}{r}10.634 \\
10644\end{array}$ & $\begin{array}{l}34.714 \\
3477\end{array}$ & $\frac{-1.10}{-1221}$ & & 4 & $\begin{array}{l}0.144 \\
0.115\end{array}$ & $\begin{array}{l}14.643 \\
14.643\end{array}$ & & \begin{tabular}{|l|l|}
509.8 \\
508
\end{tabular} & $\begin{array}{l}8.49694 \\
8\end{array}$ & $\frac{46.5}{46.4}$ & $\frac{11.9}{120}$ & $\frac{39.7}{39.7}$ & & & & 0.000 & 0.420 & \\
\hline & $4 / 19 / 2004$ & $6.1 .139 \mathrm{PM}$ & 26.169 & & \begin{tabular}{|l|l|l|}
25634 \\
\end{tabular} & \begin{tabular}{|l|l|}
26943 \\
\end{tabular} & 24.54 & 44803 & \begin{tabular}{|l|l|}
48.027 \\
\end{tabular} & 10.006 & 34.93 & -1.1218 & & 48.069 & $\frac{0.116}{0.116}$ & 14643 & & & I & $\frac{7.46}{466}$ & 119.9 & . & & & & & $\mid 0.426$ & \\
\hline 532 & $4 / 19 / 2004$ & $6: 14: 39 \mathrm{PM}$ & 26.164 & 24.959 & 25.484 & 26.993 & 24.495 & 44.662 & 47.972 & 10.605 & 34.694 & $\frac{-1.221}{-1.21}$ & & 49.676 & 0.113 & 14 & & 512 & \begin{tabular}{|l|}
8.54694 \\
\end{tabular} & 46 & 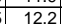 & $\frac{39.7}{39.7}$ & & & & 0.000 & 0.419 & \\
\hline & & 6:15:39 PM & 26.159 & & \begin{tabular}{|l|l|}
25.443 \\
\end{tabular} & & 24. & 44.574 & & & 247 & -1.221 & & 46.4 & & & & 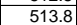 & & & & & & & & & 0.423 & \\
\hline & $4 / 19 / 2004$ & $6.16 \cdot 39 \mathrm{PM}$ & 26.149 & 24.974 & 25.449 & 27.073 & & 44.838 & 48.042 & & 34.938 & & & & 0.111 & & & & & & & & & & & & & \\
\hline & $4 / 19 / 2004$ & 6:17:39 PM & 26.134 & 24.969 & \begin{tabular}{|l|}
25.454 \\
\end{tabular} & 27.103 & 24.47 & 44.3 & 47.509 & 10.468 & 34 & -1.221 & & 49.126 & 0.112 & 14. & & $51 !$ & (594 & & 12.9 & 39.5 & & & & 0. & 0.417 & 0.36 \\
\hline & 4/19/2004 & $6: 18: 3$ & 26.141 & & 25.5 & 27.149 & & 44.614 & \begin{tabular}{|l|}
47.843 \\
\end{tabular} & 10.509 & & -1.21 & & 49.1 & & & & & & & $12.6 \mathrm{C}$ & 39 . & & & & & & \\
\hline 537 & 4/19/2004 & 6:19:39 PM & 26.116 & 24.991 & 25.466 & 27.12 & 24. & 44.5 & \begin{tabular}{|l|l|}
47.835 \\
\end{tabular} & \begin{tabular}{|c|}
10.517 \\
\end{tabular} & 34.657 & -1.221 & & 47.951 & 0.114 & 14 & & 517 & & $\begin{array}{lll}46.4 \\
\end{array}$ & 11.8 & & & & & & $\begin{array}{l}0.424 \\
\end{array}$ & \\
\hline 538 & 4/19/2004 & 6:20:39 PM & 26.112 & 24.996 & 25.472 & 27.12 & & 44.745 & & 10.542 & 34.92 & -1.218 & & 49. & 0.114 & & & & & 46 & 12.4 & 39.8 & & & & & 0.421 & \\
\hline & $4 / 19 / 2004$ & & 26.104 & 24.998 & & & & 44.3966 & 47.65 & & & & & & 0.1 & & & & & & 2 & & & & & & 439 & \\
\hline 5 & 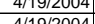 & $9 \mathrm{PM}$ & 26.1 & 25.014 & 25 & 27.183 & & & $\mid$\begin{tabular}{|l|}
$\mid 48.063$ \\
\end{tabular} & & & -1.218 & & 44 & 0.114 & & & & & 46. & 12 & 35. & & & & & 0.422 & \\
\hline & $\frac{41912004}{419204}$ & $\frac{0.23 .39}{6.24 .39}$ & $\frac{20.090}{26101}$ & $\frac{25.024}{25025}$ & \begin{tabular}{|l|l|}
25.450 \\
\end{tabular} & \begin{tabular}{|l|l|l|l|} 
\\
\end{tabular} & & & \begin{tabular}{|l|l|}
48.059 \\
\end{tabular} & & & $\frac{-1.22}{-1.22}$ & & & & & & & & & & & & & & & & \\
\hline & $\frac{4}{41 / 912004}$ & $6.4539 \mathrm{PM}$ & 26.096 & 25 & \begin{tabular}{|l}
25.53 \\
553
\end{tabular} & \begin{tabular}{|l|l|l|} 
& 27.129 \\
\end{tabular} & & $\mid$ & & & & $\frac{1.21}{-121}$ & & 528 & & & & 523 & & & 12 & & & & & & $\mid 0.424$ & \\
\hline 54 & $\frac{4 / 19 / 2004}{4}$ & $6: 26: 39 \mathrm{PM}$ & $\frac{26.106}{26.106}$ & 25.046 & 25.551 & 27.12 & & 44.6977 & $\mid$\begin{tabular}{|l|l|}
48.034 \\
\end{tabular} & 11. & & -1221 & & $\frac{39.9}{49.9}$ & 0.113 & & & 52 & & & 12 & 39. & & & & & 0.420 & \\
\hline & $4 / 19 / 2004$ & & & & & & & & & & & & & & & & & & & & & & & & & & & \\
\hline 54 & $4 / 19 / 2004$ & $6 \cdot 28.39 \mathrm{PM}$ & 26.118 & 25062 & 25572 & 27156 & & & 47.909 & 11.057 & 34.3 & -122 & & 52.159 & 0.114 & 14.6 & & 526 & & 464 & 12 & & & & & & 0.423 & \\
\hline & $4 / 19 / 2$ & $6: 29: 3$ & \begin{tabular}{l|l}
26.123 \\
\end{tabular} & \begin{tabular}{|l|l|}
25.078 \\
\end{tabular} & \begin{tabular}{|l|l|}
25.603 \\
\end{tabular} & 27.162 & & & & 10.8 & & -1.22 & & 49 . & & & & 5 & & & 12.8 & 39 & 2.7 & & & & 425 & \\
\hline & 4/19/2004 & 6:30:39 & 26.12 & 25.089 & \begin{tabular}{|l|}
25.524 \\
\end{tabular} & 27.102 & & \begin{tabular}{|c|}
44.807 \\
\end{tabular} & 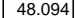 & 10.955 & 34.552 & -1.221 & & 48.4 & 0.1 & \begin{tabular}{|l|l|l|l|l|}
1433 \\
\end{tabular} & & 528 & 8.81 & & 11.9 & \begin{tabular}{|c|}
39.7 \\
\end{tabular} & 2.736 & 0.016 & & & $\begin{array}{l}0.404 \\
\end{array}$ & \\
\hline & $4 / 199 / 2004$ & 6:31:39 PM & 26.128 & 25.083 & \begin{tabular}{|l|}
25.483 \\
\end{tabular} & 27.152 & & 44.9 & \begin{tabular}{|l|l|l|l} 
\\
\end{tabular} & & & -1.22 & & 50.4 & 0.114 & & & 52 & & 4 & 12. & 35 & & 017 & & & 0.422 & \\
\hline 55 & $4 / 19 / 2004$ & $6: 32: 39$ & 26.129 & 25.109 & \begin{tabular}{|l|}
25.504 \\
\end{tabular} & 137 & & 44.908 & 48.268 & 10.8 & & -1.221 & & 50. & 0.113 & & & 530 & & $\frac{46}{46}$ & 12. & 39. & & & & & 0.417 & \\
\hline & 4/19/20004 & 6:33:39 PM & 26.129 & 25.124 & 25.529 & 27.137 & & 44.913 & 48.301 & 10. & 34.762 & -1.22 & & 49.9 & 0 & 14.6 & & & & 46 & 12. & 列 & & & & & 0.406 & \\
\hline & 4 & $6: 34: 39 \mathrm{PM}$ & 20.120 & 25.119 & \begin{tabular}{|l|l|}
25.524 \\
\end{tabular} & 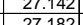 & & $\mid$ & $\mid 4.353$ & 10 & 34.106 & -1.221 & & 48. & 0.11 & 14.6 & & 532.8 & 0.000 & & 11.9 & 300 & 27 & 0.010 & & & (4) & \\
\hline & $4 / 191200^{4}$ & $\frac{0.35 .35}{6.3 .20}$ & 20.119 & 2.144 & \begin{tabular}{|l|}
2.5344 \\
\end{tabular} & 27.104 & & 44.150 & | 40.1 & & & -1.21 & & 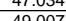 & & & & & & & . & & & & & & & \\
\hline & 告/191/2 & & 2613 & 25.176 & $\frac{25.536}{25.596}$ & 27.174 & & 45.151 & 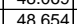 & & & -1.22 & & 48. & $\frac{.11}{0114}$ & 14 & & & & 47 & 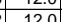 & & & & & & 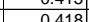 & \\
\hline $55 \mathrm{c}$ & & & 26.13 & & 25 & & & & 48.264 & $\begin{array}{c}10.896 \\
\end{array}$ & & -1.221 & & & 0.115 & & & & & & 13. & & & & & & 0.424 & \\
\hline & & & & 25.193 & & 27.192 & & & & & & & & & & & & & & & & & & & & & & \\
\hline & & 6.4 & 26.139 & 25.184 & 619 & & & & 48.589 & 10. & & & & 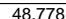 & & & & & & 471 & 121 & & & & & & & \\
\hline & 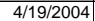 & & & & & 188 & & & 48.685 & & & 218 & & 47.599 & & 14.643 & & & 8.99694 & 472 & 117 & & & & & 2000 & 0.419 & \\
\hline 60 & 4/19/2004 & 6::22:39 PM & 26.156 & 25.216 & \begin{tabular}{|l|}
25.661 \\
\end{tabular} & \begin{tabular}{|l|}
27.184 \\
\end{tabular} & 24. & 44.981 & \begin{tabular}{|l|l|}
48.743 \\
\end{tabular} & \begin{tabular}{|l|l|}
10.869 \\
\end{tabular} & $\begin{array}{l}34.723 \\
\end{array}$ & \begin{tabular}{|l|l|} 
\\
\end{tabular} & & 48.9 & 0.114 & 14.643 & & 540.8 & 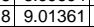 & 47.3 & 12.0 & $\begin{array}{l}39.9 \\
\end{array}$ & 2.748 & 0.017 & 0.017 & 0.000 & 0.419 & 0.3 \\
\hline
\end{tabular}


WSRC-TR-2005-00105, REVISION 0

SRNL-RPP-2005-00012, REVISION 0

RUN \# 2.03A AND B; FIRST AND SECOND HALF OF SLURRY DEWATERING - CONT.

\begin{tabular}{|c|c|c|c|c|c|c|c|c|c|c|c|c|c|c|c|c|c|c|c|c|c|c|c|c|c|c|c|c|}
\hline & $\mathrm{A}$ & B & D & E & $\mathrm{F}$ & G & $\mathrm{H}$ & $\mathrm{J}$ & $\mathrm{K}$ & \begin{tabular}{l|l}
$\mathrm{L}$ \\
\end{tabular} & $\mathrm{M}$ & $\mathrm{N}$ & $\mathrm{O}$ & Q & $R$ & $\mathrm{~s}$ & T & $\mathrm{V}$ & W & $x$ & $\mathrm{Y}$ & $z$ & $\mathrm{AA}$ & $A B$ & $A C$ & $\mathrm{AD}$ & $\mathrm{AE}$ & \\
\hline & $4 / 19 / 2004$ & 6:43:39 PM & $\begin{array}{l}26.166 \\
26.171\end{array}$ & & $\begin{array}{l}25.681 \\
2569\end{array}$ & $\begin{array}{l}27.179 \\
271969\end{array}$ & $\begin{array}{l}24.642 \\
24582\end{array}$ & $\begin{array}{l}44.977 \\
45.111\end{array}$ & $\begin{array}{l}48.49 \\
8.678\end{array}$ & 10.949 & $\begin{array}{r}34.739 \\
3487\end{array}$ & & $\begin{array}{l}-7.518 \\
-7521 \\
\end{array}$ & $\begin{array}{l}48.673 \\
5.955\end{array}$ & 0.112 & \begin{tabular}{|l|l|}
14.643 \\
14643
\end{tabular} & 0.0 & & 9.03028 & 47.0 & & & $\frac{2.748}{2.757}$ & & $\begin{array}{l}0.016 \\
0017\end{array}$ & 0.000 & $\begin{array}{l}0.411 \\
\end{array}$ & \\
\hline & & & & & & & & & & & & & & & & & & & & & & & & & & & & 0.35 \\
\hline 564 & $4 / 19 / 2004$ & $\begin{array}{l}\frac{6: 45}{6: 39} \mathrm{PM} \\
\text { 6:46:39 PM }\end{array}$ & $\frac{26.186}{26.196}$ & $\frac{25.241}{25.241}$ & 25.541 & $\frac{27.224}{27.204}$ & $\begin{array}{r}\frac{24.632}{24.662} \\
\end{array}$ & $\begin{array}{l}45.097 \\
44.931\end{array}$ & $\begin{array}{l}48.4 / 1 \\
48.562\end{array}$ & $\begin{array}{l}10.956 \\
10.963\end{array}$ & $\frac{34.928}{34.612}$ & -1.218 & $\begin{array}{l}-7.562 \\
-7.567\end{array}$ & $\frac{51.01}{52.215}$ & $\begin{array}{l}0.112 \\
0.108\end{array}$ & $\begin{array}{l}\frac{14.643}{14.643} \\
\end{array}$ & & 543.8 & $\begin{array}{r}89.06361 \\
39.08028\end{array}$ & $\begin{array}{l}47.0 \\
47.1\end{array}$ & \begin{tabular}{l|l|l|}
12.8 & \\
12.8
\end{tabular} & \begin{tabular}{|l|}
39.8 \\
\end{tabular} & \begin{tabular}{|l|}
2.742 \\
\end{tabular} & & $\frac{0.016}{0.016}$ & $\frac{0.000}{0.000}$ & $\frac{0.410}{0.399}$ & $\frac{0.35}{0.34}$ \\
\hline & $4 / 19 / 2004$ & 6:47:39 PM & 26.206 & 25.256 & 25.561 & 27.254 & $\begin{array}{l}24.692 \\
\end{array}$ & 44.832 & \begin{tabular}{|l|l|l}
48.357 \\
\end{tabular} & & $\begin{array}{l}34.528 \\
\end{array}$ & & $\begin{array}{l}-7.53 \\
\end{array}$ & 50.169 & 0.11 & 14.643 & 0.003 & & \begin{tabular}{|l|}
9.09694 \\
\end{tabular} & & & & & & & & & $\begin{array}{l}0.34 \\
0.35 \\
\end{array}$ \\
\hline & $4 / 19 / 2004$ & 6:48:39 PM & 26.196 & 25.261 & 25.571 & 27.214 & 24.647 & 44.873 & \begin{tabular}{|l|l|}
48.312 \\
\end{tabular} & 10.855 & 34.712 & \begin{tabular}{|l|l|}
-1.224 \\
\end{tabular} & & 48.936 & 0.111 & \begin{tabular}{|l|l|}
14.643 \\
\end{tabular} & 0.003 & & \begin{tabular}{|c|} 
\\
\end{tabular} & 46.8 & & & & & 0.016 & & & \\
\hline & $4 / 19 / 2004$ & 6:49:39 PM & 26.191 & 25.256 & 25.586 & 27.189 & 24.682 & $\begin{array}{l}44.877 \\
\end{array}$ & \begin{tabular}{|l|l|}
48.272 \\
\end{tabular} & 10.795 & 34.811 & \begin{tabular}{|l|}
-1.224 \\
\end{tabular} & -7.553 & 49.689 & 0.112 & \begin{tabular}{|l|l|}
14.643 \\
\end{tabular} & & 547.8 & \begin{tabular}{|c|}
9.13028 \\
\end{tabular} & 46.8 & & & \begin{tabular}{|l|}
2.747 \\
\end{tabular} & & & & & \\
\hline 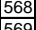 & $4 / 19 / 2004$ & 6:50:39 PM & $\begin{array}{l}26.181 \\
261.196\end{array}$ & $\frac{25.251}{25271}$ & $\begin{array}{l}25.586 \\
25626\end{array}$ & 27.124 & $\begin{array}{l}24.682 \\
2.572\end{array}$ & $\begin{array}{l}44.83 \\
\end{array}$ & \begin{tabular}{|l|l|}
48.386 \\
\end{tabular} & 10.884 & $\begin{array}{l}34.601 \\
2.702\end{array}$ & $\begin{array}{l}-1.224 \\
1221\end{array}$ & $\begin{array}{r}-7.501 \\
7.556 \\
\end{array}$ & 48.243 & $\begin{array}{l}0.108 \\
0.11\end{array}$ & $\begin{array}{l}14.643 \\
1.643\end{array}$ & 0.003 & $\begin{array}{l}548.8 \\
51.9\end{array}$ & \begin{tabular}{|l|l|l|l|} 
\\
\end{tabular} & 46.9 & 11.8 & \begin{tabular}{|l|}
39.7 \\
\end{tabular} & \begin{tabular}{|l|l|}
2.738 \\
\end{tabular} & $\begin{array}{l}0.016 \\
\end{array}$ & $\begin{array}{l}0.016 \\
\end{array}$ & 0.000 & $\begin{array}{l}0.399 \\
\end{array}$ & \\
\hline & 4/19/2004 & 6:51:39 PM & 26.196] & 25.271 & 25.626 & 27.1499 & & 44.786 & 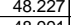 & 10.783 & & & $\begin{array}{r}-7.556 \\
\end{array}$ & 48.784 & 0.111 & 14.643 & & 549.8 & $\begin{array}{l}3.16361 \\
\end{array}$ & 46.8 & & & & & & & 0.409 & 0.35 \\
\hline$\frac{5 / 0}{571}$ & $4 / 19 / 2004$ & $6: 52: 39 \mathrm{PM}$ & 26.196 & $\frac{25.281}{25.286}$ & $\frac{25.631}{25641}$ & $\frac{27.169}{27114}$ & $\frac{24.532}{24.682}$ & $\begin{array}{l}45.408 \\
44718\end{array}$ & \begin{tabular}{|l|}
48.991 \\
48.173 \\
\end{tabular} & 10.863 & 35.312 & $\begin{array}{c}-1.218 \\
-1.218\end{array}$ & $\begin{array}{r}-7.483 \\
-7483 \\
\end{array}$ & $\begin{array}{r}48.83 \\
49.034\end{array}$ & 0.111 & $\begin{array}{l}14.643 \\
1.632\end{array}$ & 0.003 & $\frac{50.8}{550.8}$ & $\begin{array}{l}3.18028 \\
\end{array}$ & 47.5 & $\begin{array}{l}12.0 \\
10\end{array}$ & \begin{tabular}{|l|}
40.4 \\
\end{tabular} & \begin{tabular}{|l|}
2.783 \\
2.720
\end{tabular} & $0.017 \mid$ & \begin{tabular}{|r|}
$\mid$ \\
$\mid$
\end{tabular} & 0.000 & 0.403 & 0.34 \\
\hline 572 & 4/19/2004 & 6:54:39 PM & $\frac{\frac{2.201}{26.201}}{2}$ & $\frac{2.200}{25.286}$ & $\frac{2.044}{25.666}$ & $\frac{2.1044}{27.134}$ & 24.652 & 4 & \begin{tabular}{|l|}
48.772 \\
\end{tabular} & 10.857 & $\begin{array}{l}34.054 \\
34.831\end{array}$ & $\begin{array}{l}-1.210 \\
-1.218 \\
\end{array}$ & $\begin{array}{l}-1.400 \\
-7.547 \\
\end{array}$ & $\begin{array}{l}45.054 \\
48.972 \\
\end{array}$ & $\begin{array}{l}0.1113 \\
0.113\end{array}$ & $\begin{array}{l}\frac{1.045}{14.643} \\
\end{array}$ & $\begin{array}{l}0.003 \\
0.00\end{array}$ & 552.8 & 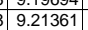 & $\frac{40.1}{47.3}$ & & & & & 0.017 & $\frac{0.000}{0.000}$ & $\begin{array}{l}0.414 \\
0.414\end{array}$ & $\frac{0.35}{0.35}$ \\
\hline & & & & & & & & & & & & & & & & & & & & & & & & & & & & \\
\hline & $4 / 19 / 2004$ & 6:56:39 PM & 26.218 & 25.323 & 25.697 & 27.171 & 24.593 & 44.799 & \begin{tabular}{|l|l|l}
48.465 \\
\end{tabular} & 10.855 & 34.56 & .221 & $\frac{7.535}{-7.535}$ & 47.499 & 0 & 14.643 & & & $\begin{array}{l}24694 \\
2469\end{array}$ & $\frac{47.0}{47.0}$ & 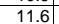 & & $\frac{736}{736}$ & & 8.017 & D00 & $\frac{124}{424}$ & \\
\hline & & & & & & & & & & & & & & & & & & & & 47.1 & & & & & & & & \\
\hline & $4 / 19 / 2004$ & 6:58:39 PM & 26.215 & 25.335 & 25.71 & 27.233 & 24.64 & 45.153 & 48.794 & 10.919 & 34.887 & & & 49.405 & 0.112 & 14.643 & & 556.8 & 928028 & 47.3 & $\overline{12.1}$ & & 1.759 & & & & 409 & \\
\hline & 4/19/2004 & 6:59:39 PM & 26.227 & 25.342 & 25.727 & 27.24 & 24.682 & 45.072 & 48.519 & 10.781 & 35.172 & 1.221 & -7.521 & 50.991 & 0.105 & 14.643 & 0.003 & 557.8 & \begin{tabular}{|l|l|} 
& 9.29694 \\
\end{tabular} & 47.0 & 12.5 & 40.1 & 2.766 & 516 & 0.015 & 0.000 & 0.382 & \\
\hline & $4 / 19 / 2004$ & 7:00:39 PM & 26.232 & 25.358 & 25.742 & 27.206 & 24.663 & 44.749 & \begin{tabular}{|l|l} 
\\
\end{tabular} & 10.809 & 34.723 & 1.22 & -7.55 & 50.075 & 0.11 & 14.643 & 0.003 & 558.8 & \begin{tabular}{|l|l|}
9.31361 \\
\end{tabular} & 46.8 & 12.3 & & 2.740 & & & 0.000 & & \\
\hline 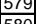 & $4 / 191 / 2004$ & 7:01:39 PM & 26.254 & & 25.789 & 20.257 & 24.625 & 44.863 & 48.49 & 10.869 & 34.721 & & -7.45 & & 0.112 & 14.643 & & & 9.33028 & 47.0 & & & 2.744 & & & 0.000 & & \\
\hline & $4 / 191 / 2004$ & T.02:39PM & & & 25.17 & & 24.596 & 44.8619 & 4.6864 & & 34.5867 & & -1.535 & & & & & & & & & & & & & & & \\
\hline & $\frac{4 / 19 / 2004}{4 / 19 / 2004}$ & $\begin{array}{l}7: 03: 39 \mathrm{PM} \\
7.04393\end{array}$ & $\frac{26.251}{26.272}$ & $\begin{array}{l}25.376 \\
25397\end{array}$ & $\frac{25.726}{25612}$ & $\frac{27.229}{27275}$ & $\frac{24.5 / 2}{24697}$ & $\frac{44.821}{44.707}$ & \begin{tabular}{|l|l}
48.589 \\
48.44
\end{tabular} & $\begin{array}{l}10.908 \\
1089\end{array}$ & $\frac{34.567}{34.404}$ & 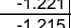 & & 51.007 & $\frac{0.11}{0.107}$ & $\frac{14.643}{14.643}$ & 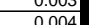 & $\frac{56 .}{56}$ & .36361 & $\frac{47.1}{470}$ & & & 727 & & & 0.000 & $\frac{405}{396}$ & \\
\hline & $4 / 19 / 2004$ & 7:05:39 PM & 26.262 & 25..037 2502 & $\frac{25.014}{25.602}$ & 27.19 & 24.652 & 45.201 & 48.925 & 10.025 & 35012 & 1.22 & 7.55 & $\frac{1.244}{48.444}$ & 0.11 & $\frac{14543}{14643}$ & 0.003 & 5638 & $\mid$ & 475 & 119 & & 2765 & & & 0.000 & $\frac{0.042}{0.402}$ & \\
\hline & 4/19/2004 & 7:06:39 PM & 26.262 & 25.417 & 25.637 & 27.23 & 24.657 & 44.958 & 48.535 & 10.825 & 34.821 & -1.218 & -7.5 & 48.11 & 0.111 & $\begin{array}{l}14.643 \\
14.04\end{array}$ & & 564.8 & 9.41361 & $\frac{47.1}{47.1}$ & 11.8 & 39.9 & 2.750 & & & 0.000 & 0.402 & \\
\hline & $4 / 19 / 2004$ & 7:07:39 PM & 26.251 & 25.406 & 25.646 & 27.164 & 24.612 & |44.828 & 48.506 & 10.855 & 34.688 & & -7.562 & & 0.108 & 14.643 & 0.003 & 565.8 & 9.43028 & 47.0 & $\overline{12.1}$ & $\overline{39.8}$ & .741 & & 0.016 & & & \\
\hline & 4/19/2004 & 7:08:39 PM & 26.266 & 25.431 & 25.676 & 27.194 & 24.552 & 45.079 & \begin{tabular}{|l|l|}
48.734 \\
\end{tabular} & 10.892 & 34.895 & 1.218 & & 48.148 & 0.107 & 14.643 & & 566.8 & 9.44694 & 47.3 & 11. & & & & & 0.000 & 0.391 & \\
\hline & $4 / 19 / 2004$ & 7:09:39 PM & & & & & & & 48.819 & & & & & & & & & & 9.46361 & 47.3 & & & & & & & & \\
\hline & $4 / 19 / 2004$ & 7:10:39 PM & $26.247 \mid$ & 25.443 & 25.687 & 27.236 & 24.488 & 45.01 & 48.711 & 10.9 & 34.854 & -1.218 & & 48.7 & 0.108 & 14.643 & & 568 & 9.48028 & 47.2 & & & & & & & & \\
\hline & $4 / 19 / 2004$ & 7:11:39 PM & 26.253 & 25.443 & 25.703 & 27.216 & 24.619 & 45.172 & 49.112 & 10.97 & 34.758 & 1.224 & & & 0.106 & 14.643 & & $56 !$ & 9.49694 & 47.6 & 12.1 & & & & & 0.000 & 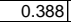 & \\
\hline 59 & $4 / 19 / 2004$ & $\begin{array}{l}7: 12: 39 \text { PM } \\
71030\end{array}$ & 26.259 & $\frac{25.444}{25.191}$ & 25.734 & 27.232 & 24.685 & $\begin{array}{r}44.84 \\
\end{array}$ & 48.591 & 10.957 & 34.515 & -1.218 & -7.54 & 48.344 & 0.1 & 14.643 & & 57( & 9.51361 & 47.1 & $11.8 \mathrm{\gamma}$ & & 736 & & 0.017 & 000 & 0.416 & \\
\hline & $4 / 191 / 2004$ & 7:13:39 PM & 26.265 & 25.461 & 25.75 & 27.199| & 24.686 & 44.678 & 48.452 & 10.856 & 34.556 & & & .95 & 0.10 & & & & & & & & & & & & & \\
\hline & $\frac{4 / 1912004}{419200}$ & 7:741:39 PM & $\frac{26.211}{26262}$ & 25.461 & 5.761 & 27.219 & $\frac{24.687}{24862}$ & 44.81 & 4.683 & 10.908 & $\frac{34.651}{3425}$ & & & 1.98 & 0.11 & 14.643 & & & & 47.22 & & & & & 016 & 000 & 404 & \\
\hline & 4 & $7.10 .39 \mathrm{PM}$ & $\frac{2.0204}{26277}$ & $\begin{array}{l}20.4645 \\
22577\end{array}$ & . & 27.1003 & $\begin{array}{l}24.064 \\
24647\end{array}$ & (45.506 & $\frac{14.178}{4868}$ & | & 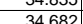 & 1218 & & 40 & 0 & $\mid$ & & 57.0 & $\mid$ & 告, & 115 & 398 & 743 & & 0.016 & & 0400 & \\
\hline & $4 / 1920004$ & 7.1717.39 PM & 26287 & 20.5477 & 25802 & 2719 & 24637 & 45.147 & 49.051 & 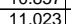 & 34776 & $\frac{1.218}{-1218}$ & 750 & 49.55 & 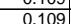 & 1464 & & 5758 & 50694 & 476 & 121 & & & & & & & \\
\hline & $\frac{d / 2004}{9604}$ & 7:18:39 PM & $\frac{\mid}{26.2887}$ & 年. 25.483 & $|25.803|$ & $\frac{27.201}{27.201}$ & 24.573 & 年4.794 & \begin{tabular}{|l|}
48.707 \\
\end{tabular} & $\frac{\mid}{10.969}$ & 34.448 & $\frac{1.221}{1.221}$ & & $\frac{4.50}{47.92}$ & o. 0 & $\begin{array}{l}\frac{1.0445}{14.643} \\
\end{array}$ & & 5756. & $\begin{array}{l}9.59044 \\
9.61361\end{array}$ & $\frac{4.0}{47.2}$ & 11.7. & $\frac{\pi 9}{39}$ & & & & & $\begin{array}{l}0.396 \\
0.397\end{array}$ & \\
\hline & g/2004 & 7:19:39 PM & & & \begin{tabular}{|l|}
25.793 \\
\end{tabular} & & & 45.083 & \begin{tabular}{|l|l|}
48.886 \\
\end{tabular} & 10.976 & & & & 46.7 & & & & & & 47.4 & -2 & & & & & & & \\
\hline 59 & $4 / 19 / 2004$ & 7:20:39 PM & 26.297 & 25.483 & 25.798 & 27.166 & 24.493 & 44.828 & \begin{tabular}{|l|l|}
48.479 \\
\end{tabular} & 10.923 & 34.6 & & & 478 & 0.109 & 14.643 & & 578.8 & $\begin{array}{l}99.64 \\
\end{array}$ & 47.6 & 11.7 & 39.7 & 740 & & 0.016 & 500 & 0.400 & \\
\hline & $4 / 19 / 2004$ & $7: 21: 39 \mathrm{PM}$ & & 25.497 & 25.697 & 27.165 & 24.5 & 46.10 & \begin{tabular}{|l|l|l|}
48.616 \\
\end{tabular} & 10.817 & 36.073 & -1.218 & & 8.6 & & 14.643 & & 579 & & 47.1 & $\frac{11 . .}{11.5}$ & & & & & & & \\
\hline & & 7:22:39 PM & & & \begin{tabular}{|l|l|}
25.67 \\
\end{tabular} & & & 43.988 & & & & & & 1788 & & 14.643 & & & & 47.3 & 11.7 & 38.5 & 2.685 & & & & & \\
\hline & $4 / 19 / 2004$ & & 26.299 & 25.494 & 25.699 & & & & \begin{tabular}{|l|l|l|} 
\\
\end{tabular} & & & & & & & 14.643 & & & & 47. & $12 .$. & & & & & & & \\
\hline & $4 / 19 / 2004$ & 7:24:39 PM & 26.307 & 25.503 & 25.707 & 27.061 & 24.743 & 46.18 & 49.126 & 10.862 & 35.954 & 1.22 & & 50.089 & & 14.643 & & & 9.71361 & 47.7 & $12.3 \mathrm{r}$ & & & & 0.017 & & 416 & \\
\hline 60 & 9/2004 & 7:25: & 26.301 & 25.491 & 25.726 & 27.069 & 24.822 & 46.167 & 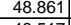 & 10.872 & 36.028 & 21 & & 49.549 & & 14.6 & & 583.8 & & 47.4 & 12. & & & & 0.010 & 0.000 & 0.398 & \\
\hline & 告/1 & $\frac{1.266 .39}{7.2730}$ & 2 & $\frac{25.5}{25.54}$ & 25.73 & $\frac{2.173}{2713}$ & 24.655 & 45.99 & 40510 & $\frac{11.1134}{1.125}$ & & & & & & & & & & & $\frac{12.1}{1.2}$ & & & & & & & \\
\hline & $41 / 910$ & & $\frac{2.0 .40}{2643}$ & $\frac{20.5044}{2502}$ & 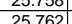 & 年. & $\frac{24.5}{245}$ & & | & & & & & & 0.10 & & & & & 41 & 127 & & & & & & & \\
\hline$\frac{60}{60}$ & $\frac{151 / 2004}{4}$ & $7 \cdot 29.39 \mathrm{PM}$ & $\frac{2.4}{26.3}$ & 25.511 & $|25.73|$ & 27.009 & 24.631 & $\begin{array}{l}45.053 \\
45.522\end{array}$ & & 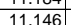 & & -1.218 & & $49.8 \mathrm{c}$ & & $\frac{1}{14.643}$ & & & & $\begin{array}{ll}4.0 .0 \\
46.9\end{array}$ & $\frac{1}{122}$ & & & & & & & \\
\hline & $4 / 1 / 1 / 2004$ & 7:30:39 PM & $\frac{26.354}{2.635}$ & 25.505 & 25.685 & $\mid$ & & | & & 11.182 & & $\frac{-1.218}{-1.218}$ & & 49.6 & & & & & & & $\frac{12.2}{12.2}$ & & & & 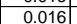 & & & \\
\hline & 4/19/2004 & 7:31:39 PM & 26.32 & 25.515 & 25.68 & 27.123 & 24.46 & 46.041 & $\begin{array}{l}48.896 \\
\end{array}$ & 11.256 & 35.4 & $\frac{1.12}{-1.2}$ & & (7) & & & & 589 & & & 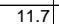 & & & & & & 0.384 & \\
\hline & $4 / 19 / 2004$ & $7: 32: 39 \mathrm{PM}$ & \begin{tabular}{|l|l|}
26.27 \\
\end{tabular} & & & & & 45.506 & 40255 & 1117 & & & & & & & & & & & $\overline{15}$ & & & & & & & \\
\hline & & $9 \mathrm{PM}$ & 26.225 & 25.5 & 25.595 & 27.078 & 24.56 & \begin{tabular}{|l|l|}
45.56 \\
\end{tabular} & 48.324 & 11.243 & 35.088 & -1.221 & & & & & & & & 46. & 12. & & & & & & & \\
\hline & $4 / 19 / 2004$ & 7:34:39 PM & 26.215 & 25.501 & 25.48 & 27.034 & 24.591 & 45.697 & \begin{tabular}{|l|}
48.537 \\
\end{tabular} & 11.281 & 35.18 & -1.2 & & 48.6 & & & & 592.8 & & 47 & 11.9 & 40.4 & 2.788 & & 0.015 & & & \\
\hline & $4 / 19 / 2004$ & 7:35:39 F & 26.195 & 25.505 & $25.409 \mid$ & $27.068 \mid$ & 24 & 45.56 & 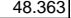 & 11.24 & & & & & & & & 593 & & 46 & 12.2 & & 78 & & 0.016 & & & \\
\hline 61 & $4 / 191200$ & 7:36:39 PM & 26.1 & 25.51 & 25.404 & & 24. & 45.348| & 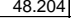 & 11.292 & & -1.22 & & & & & & 594 & & 46. & 12.2 & & & & & & & \\
\hline$\frac{61}{61}$ & 9/2004 & 7:37:3 & 26.1 & & 25.389 & & 24.454 & 45.728 & \begin{tabular}{|l|l|}
48.662 \\
\end{tabular} & 11.281 & & & & & & & & 595. & & $4 t 5$ & 12.6 & & & & & & & \\
\hline & & & 20.115 & 25.49 & 25.369 & 26.998 & & 45.194 & 48.689 & 11.344 & & & & & & & & & & $4 t$ & 12.5 & & & & & & & \\
\hline$\frac{6}{61}$ & 4 & $\frac{353.39}{7.39}$ & $\begin{array}{r}26.11 \\
26115\end{array}$ & 25.485 & $\mid$ & $\mid$ & $\frac{24.49}{24.5}$ & 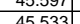 & 4 & 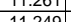 & $\begin{array}{l}355.174 \\
35067\end{array}$ & $\begin{array}{l}-1.221 \\
-1.215\end{array}$ & & & 0.1 & & & 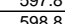 & & 46. & $\frac{11.6}{11.5}$ & & & & & & 71 & \\
\hline & 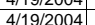 & $7 \cdot 4 \cdot 43.39 \mathrm{~F}$ & $\frac{2.1 .50}{26086}$ & $\frac{2.36}{55476}$ & $\frac{2.5035}{25353}$ & 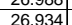 & & $\frac{4}{4}$ & \begin{tabular}{|l|l|}
48517 \\
\end{tabular} & & & & & & & & & & & & & & & & & & & \\
\hline & $4 / 1 / 1 / 2000$ & & 26.08 & 25.485 & $\frac{25.314}{25.314}$ & 26. & 24 & $\frac{15.682}{45682}$ & & $\frac{1 . .219}{11.21}$ & $\frac{25.1}{35.1}$ & -1.218 & & & & & & & & & 11. & & & & & & & \\
\hline 62 & $4 / 19 / 2004$ & $7: 43:$ & $\frac{26.076}{20.6}$ & 年.486 & 25.326 & $\frac{26 .}{26 .}$ & $\frac{24: 3}{24}$ & 46.252 & \begin{tabular}{|l|}
48.407 \\
\end{tabular} & 11.205 & 35.884 & -1.218 & & & & & & & & & & & & & 0.016 & & 89 & \\
\hline & & & & & & & & 46.035 & & 11.214 ] & & & & & & & & & & & & & & & & & & \\
\hline 62 & & $7: 45: 39$ & 26.051 & 25.486 & 25.295 & 26.889 & 24. & \begin{tabular}{|l|l|}
46.286 \\
\end{tabular} & \begin{tabular}{|l|l|}
48.67 \\
\end{tabular} & 11.206 & 35. & -1.218 & & & & & & & & & & & & & & & & \\
\hline & $4 / 19 / 200$ & $7: 46: 39 \mathrm{P}$ & 26.04 & 25. & 25.2 & 26 . & 24. & 46.2 & \begin{tabular}{|l|l|}
48.678 \\
\end{tabular} & 11.155 & & & & & & & & & & $\overline{47} \mathrm{C}$ & 12 & & $28 \quad 2$ & & & & & \\
\hline 62 & 4/19/2004 & 7:47:39 P & 26.046 | & 25.496 & 25.286 & 26.935 & 24. & $46.493 \mid$ & 48.78 & 11.232 & 36.007 & $-1.2 \quad \mathrm{C}$ & & & 0.106 & 14.64 & & 605.8 & 10.0 & & & & & & & & 80 & \\
\hline 62 & & 7:48: & & 25.495 & & & & 46.474| & 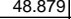 & 11.249 & & -1.218 & & & & & & 60 & & 47.4 & 12.1 & & & & & & & \\
\hline 627 & 4/19/2004 & 7:499:39! & 26.018 & 25.499 & 25.123 & 26.857 & 24.349 & 46.354 & \begin{tabular}{|l|l|} 
\\
\end{tabular} & 11.174 & 35.88 & -1.2211 & & 48.5 & 0.106 & 14.643 & & 607.8 & $3 \mid 10.1303$ & 47.2 & 11.9 & 4 & 2.8 & 0.6 & 0.016 & 0.0 & 0.383 & 0.35 \\
\hline$\frac{628}{629}$ & $4 / 19 / 2004$ & $\begin{array}{l}1: 503: 39 \mathrm{PM} \\
7.5139 .39 \mathrm{MM}\end{array}$ & 26.007 & $\frac{25.502}{25.505}$ & \begin{tabular}{|l|}
25.1214 \\
25149
\end{tabular} & $\frac{26.185}{26808}$ & $\begin{array}{l}24.352 \\
24.335\end{array}$ & \begin{tabular}{|l}
46.534 \\
46.107
\end{tabular} & \begin{tabular}{|l|}
48.923 \\
48323 \\
\end{tabular} & $\frac{11.139}{10957}$ & $\begin{array}{r}36.03 \\
35.948 \\
\end{array}$ & $\begin{array}{c}-1.218 \\
-1.218\end{array}$ & $\begin{array}{l}-7.4 \\
-7.4\end{array}$ & $\begin{array}{l}47.307 \\
49.049 \\
\end{array}$ & $\begin{array}{l}0.107 \\
0.155\end{array}$ & $\begin{array}{l}14.643 \\
14.643\end{array}$ & 0.00 & $\frac{608.8}{609.8}$ & \begin{tabular}{|l|l|}
30.1469 \\
3
\end{tabular} & \begin{tabular}{|l|}
47.5 \\
46.8 \\
\end{tabular} & $\frac{11.6}{120}$ & \begin{tabular}{|l|}
41.3 \\
41.0
\end{tabular} & \begin{tabular}{|l|}
2.846 \\
2829
\end{tabular} & 0.0 & $\begin{array}{c}0.016 \\
0.016\end{array}$ & 0.000 & $\begin{array}{l}0.385 \\
0.38\end{array}$ & $\frac{0.3}{0.3}$ \\
\hline & & & & & & & & & & & & & & & & 14.643 & & & & & & & & & 0.016 & 0.000 & 0.382 & 0.3 \\
\hline
\end{tabular}


WSRC-TR-2005-00105, REVISION 0

SRNL-RPP-2005-00012, REVISION 0

RUN \# 2.03A AND B; FIRST AND SECOND HALF OF SLURRY DEWATERING - CONT.

\begin{tabular}{|c|c|c|c|c|c|c|c|c|c|c|c|c|c|c|c|c|c|c|c|c|c|c|c|c|c|c|c|c|}
\hline & & & & & & & & & & & & & & & & & & & & & & & & & & & & \\
\hline & A & B & $\frac{D}{D}$ & $E$ & & $G$ & $\mathrm{H}$ & $\mathrm{J}$ & $\mathrm{K}$ & $\mathrm{L}$ & $\mathrm{M}$ & $\mathrm{N}$ & 0 & $\mathrm{Q}$ & $\mathrm{R}$ & $\mathrm{s}$ & $\mathrm{T}$ & $\mathrm{V}$ & W & $x$ & 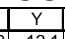 & $z$ & AA & $\frac{A B}{A B}$ & $A C$ & $A D$ & $\mathrm{AE}$ & AF $A$ \\
\hline $6 \frac{63}{63}$ & $\begin{array}{l}4 / 1 / 9 / 2004 \\
4 / 192004\end{array}$ & $\begin{array}{l}7: 53: 39 \text { PM } \\
775: 539 \text { PM }\end{array}$ & $\begin{array}{r}25.961 \\
25.94 \\
\end{array}$ & $\begin{array}{r}25.497 \\
25.48 \\
\end{array}$ & \begin{tabular}{|l|}
25.126 \\
25.114 \\
\end{tabular} & $\begin{array}{r}26.82 \\
26.768 \\
\end{array}$ & $\begin{array}{r}24.261 \\
24.19\end{array}$ & \begin{tabular}{|l|}
46.342 \\
46.342
\end{tabular} & \begin{tabular}{|c|}
48.678 \\
48.736 \\
\end{tabular} & $\begin{array}{r}11.094 \\
1.09\end{array}$ & \begin{tabular}{|r|}
36.05 \\
36.022
\end{tabular} & \begin{tabular}{|c|}
-1.221 \\
-1.221 \\
\end{tabular} & \begin{tabular}{|l|}
-7.483 \\
-7.492
\end{tabular} & $\begin{array}{l}49.339 \\
47.426\end{array}$ & $\begin{array}{l}0.105 \\
0.105\end{array}$ & \begin{tabular}{|l|}
14.643 \\
14.643 \\
\end{tabular} & $\begin{array}{l}0.003 \\
0.03\end{array}$ & $\begin{array}{l}611.8 \\
612.8 \\
\end{array}$ & \begin{tabular}{|c|}
10.1969 \\
10.2136
\end{tabular} & $\begin{array}{l}47.2 \\
4.3\end{array}$ & \begin{tabular}{l|l}
3 & 12.1 \\
3 & 11.6
\end{tabular} & 41. & $\frac{2.840}{2.839}$ & 0.016 & $\begin{array}{l}0.016 \\
0.016\end{array}$ & $\begin{array}{l}0.000 \\
0.000\end{array}$ & $\begin{array}{l}0.379 \\
0.379\end{array}$ & $\begin{array}{l}0.32 \\
0.32\end{array}$ \\
\hline $\begin{array}{ll}\frac{632}{633} \\
63\end{array}$ & & & $\begin{array}{r}25.924 \\
25.925 \\
\end{array}$ & & & & $\begin{array}{r}24.19 \\
24.135 \\
\end{array}$ & \begin{tabular}{|l|}
46.44449 \\
4
\end{tabular} & & $\begin{array}{r}11.0972 \\
1.07\end{array}$ & & \begin{tabular}{|l|l|}
-1.2218 \\
-1.218
\end{tabular} & & & & \begin{tabular}{|l|}
14.6433 \\
14.643
\end{tabular} & & & & & & & & & & & & \\
\hline 634 & $4 / 19 / 2004$ & 7:56:39 PM & 25.909 & 25.469 & \begin{tabular}{|l|}
25.109 \\
\end{tabular} & \begin{tabular}{|l|l|}
26.713 \\
\end{tabular} & 24.099 & 46.37 & 48.886 & $\begin{array}{l}11.016 \\
\end{array}$ & 36.102 & \begin{tabular}{|l|}
-1.218 \\
\end{tabular} & $\begin{array}{r}-7.481 \\
-7.451\end{array}$ & 49.53 & 0.105 & \begin{tabular}{|l|}
14.643 \\
\end{tabular} & 0.003 & & 10.2469 & 47.4 & \begin{tabular}{|l|l|}
4 & 12.1 \\
\end{tabular} & & 2.843 & 0.016 & 0.016 & 0.000 & 0.378 & 0.32 \\
\hline & $4 / 199 / 2004$ & 7:57:39 PM & 25.899 & 25.464 & \begin{tabular}{|l|}
25.113 \\
\end{tabular} & \begin{tabular}{|l|l|}
26.652 \\
\end{tabular} & 24.134 & $\begin{array}{r}46.217 \\
\end{array}$ & $\begin{array}{l}48.612 \\
\end{array}$ & $\begin{array}{l}11.112 \\
\end{array}$ & 35.868 & \begin{tabular}{|l|l|}
-1.218 \\
\end{tabular} & $\begin{array}{r}-7.457 \\
-7.457\end{array}$ & 50.953 & 0.105 & \begin{tabular}{|l|}
14.643 \\
\end{tabular} & & $\begin{array}{l}615.8 \\
\end{array}$ & $\begin{array}{l}10.2636 \\
\end{array}$ & 47.1 & 1212.5 & & & & & 0.000 & & 0.32 \\
\hline 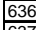 & $4 / 19 / 2004$ & & & & & & & & & & & & & 47.985 & & \begin{tabular}{|r|}
14.643 \\
\end{tabular} & & & \begin{tabular}{|l|}
10.2803 \\
\end{tabular} & 47.5 & & & & & & & & 0.32 \\
\hline 637 & $4 / 19 / 2004$ & 7:59:39 PM & $\begin{array}{l}25.887 \\
\end{array}$ & 25.452 & 25.101 & 26.63 & \begin{tabular}{|l|l|}
24.377 \\
\end{tabular} & 46.456 & $\begin{array}{l}48.939 \\
\end{array}$ & \begin{tabular}{ll|}
11.107 \\
\end{tabular} & \begin{tabular}{|l|l|}
36.118 \\
2.03
\end{tabular} & \begin{tabular}{|l|l|}
-1.221 \\
\end{tabular} & $\begin{array}{r}-7.46 \\
\end{array}$ & 49.971 & 0.104 & \begin{tabular}{|l|}
14.643 \\
\end{tabular} & & $\begin{array}{l}617.8 \\
\end{array}$ & 10.2969 & 47.5 & \begin{tabular}{|l|l}
5 & 12.2 \\
\end{tabular} & 41.3 & 2.847 & 0.016 & & 0.000 & 0.374 & 0.32 \\
\hline$\frac{638}{639}$ & \begin{tabular}{|l|}
$/ 119 / 2004$ \\
$4 / 192004$
\end{tabular} & $\begin{array}{l}7: 59: 43 \text { PM } \\
800043 \text { PM }\end{array}$ & $\begin{array}{l}25.892 \\
25881\end{array}$ & $\begin{array}{l}25.452 \\
25.436\end{array}$ & \begin{tabular}{|l|}
25.106 \\
25.105
\end{tabular} & \begin{tabular}{|l|}
26.635 \\
26.624 \\
\end{tabular} & \begin{tabular}{|l|l|}
24.362 \\
1316 \\
1316
\end{tabular} & \begin{tabular}{|l|l|}
46.424 \\
46.271 \\
\end{tabular} & \begin{tabular}{|l|}
48.942 \\
48873 \\
\end{tabular} & \begin{tabular}{|c|}
11.164 \\
11.099
\end{tabular} & \begin{tabular}{|l|}
35.929 \\
35874
\end{tabular} & \begin{tabular}{|c|}
-1.221 \\
-1.218 \\
\end{tabular} & $\begin{array}{r}-7.466 \\
-7.486 \\
\end{array}$ & $\begin{array}{l}50.031 \\
48112\end{array}$ & \begin{tabular}{|l|l|l|l}
0.104 \\
0.104
\end{tabular} & \begin{tabular}{|l|}
14.643 \\
14643
\end{tabular} & & \begin{tabular}{|l|l|}
617.9 \\
618.9
\end{tabular} & \begin{tabular}{|l|l|}
10.2981 \\
103147
\end{tabular} & $\begin{array}{l}47.5 \\
474\end{array}$ & $\frac{12.3}{11.8}$ & $\frac{41.2}{41.1}$ & $\begin{array}{l}2.839 \\
2832\end{array}$ & 0.016 & $\begin{array}{l}0.015 \\
0.015\end{array}$ & 0.000 & 0.375 & 0.32 \\
\hline & $4 / 19 / 2004$ & & 25.875 & 25.43 & \begin{tabular}{|l|}
25.134 \\
\end{tabular} & 26.613 & \begin{tabular}{|l|l}
3.410 \\
4.42
\end{tabular} & & & \begin{tabular}{|l|l|}
11.194 \\
1
\end{tabular} & 36.091 & & $\begin{array}{r}--1.486 \\
-7.469\end{array}$ & $\frac{48.112}{49.14}$ & $\begin{array}{l}0.104 \\
0.102\end{array}$ & $\begin{array}{l}14.045 \\
14.643\end{array}$ & $\begin{array}{l}0.003 \\
0.003\end{array}$ & $\begin{array}{l}618.9 \\
619.9\end{array}$ & $\left|\begin{array}{|l|}10.3147 \\
10.3314\end{array}\right|$ & $\begin{array}{l}44.4 \\
47.5 \\
\end{array}$ & \begin{tabular}{l|l}
4 & 11.8 \\
5 & 12.
\end{tabular} & $\frac{41.1}{41.3}$ & 2.832 & 0.016 & $\frac{0.015}{0.015}$ & $\begin{array}{l}0.000 \\
0.000\end{array}$ & $\begin{array}{l}0.376 \\
0.367\end{array}$ & 0.32 \\
\hline 641 & $4 / 19 / 2004$ & 8:02:43 PM & 25.859 & & & & & & 48.728 & 11.166 & 35.905 & & $\begin{array}{r}-7.481 \\
\end{array}$ & 49.553 & & 14.643 & & & & 47.3 & & & & & & & & $\frac{0.31}{0.32}$ \\
\hline (642 & $4 / 19 / 2004$ & 8:03:43 PM & 25.865 & 25.44 & \begin{tabular}{|l|l|}
25.139 \\
\end{tabular} & 26.708 & 24.7 & 46.055 & $\begin{array}{l}48.548 \\
\end{array}$ & 11.125 & 35.665 & -1.218 & -7.486 & $\begin{array}{l}47.626 \\
\end{array}$ & 0.102 & \begin{tabular}{|l|l|}
14.643 \\
\end{tabular} & & & 10.3647 & 47.1 & \begin{tabular}{l|l|}
11 & 11.7
\end{tabular} & 40.9 & & & & & & $\begin{array}{l}0.32 \\
0.32\end{array}$ \\
\hline 643 & $4 / 19 / 2004$ & 8:04:43 PM & 25.852 & 25.432 & 25.146 & & & & 49.248 & & 35.886 & -1.218 & & & 0.103 & 14.643 & & 622.9 & $\frac{10.3814}{10.314}$ & 47.8 & & & & & & & 0.371 & $\begin{array}{l}0.32 \\
0.32 \\
\end{array}$ \\
\hline 644 & $4 / 19 / 2004$ & 8:05:43 PM & & & 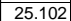 & 26.871 & & $\begin{array}{ll}46.416 \\
\end{array}$ & 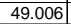 & \begin{tabular}{|l|l|}
11.14 \\
\end{tabular} & & 1221 & & & & & & 623 & & 47.5 & & & & & & & 0.371 & $\frac{0.32}{0.32}$ \\
\hline 645 & $4 / 19 / 2004$ & 8:06:43 PM & 25.844 & 25.434 & & & 24.945 & 46.449 & 48.962 & & 35.948 & -1.221 & & 49.568 & 0.104 & 14.643 & & 624.9 & \begin{tabular}{|l|l|}
10.4147 \\
\end{tabular} & 47.5 & & & 2.840 & & & & & \\
\hline & $4 / 19 / 2004$ & 8:07:43 PM & 25.824 & 25.429 & \begin{tabular}{|l|l|}
24.983 \\
\end{tabular} & 26.903 & 24.81 & $\begin{array}{l}46.331 \\
\end{array}$ & \begin{tabular}{|l|l|}
49.004 \\
\end{tabular} & 11.252 & 35.716 & -1.221 & -7.475 & 49.46 & 0.104 & \begin{tabular}{|l|l|l|}
14.643 \\
\end{tabular} & & 625.9 & \begin{tabular}{|l|l|}
10.4314 \\
\end{tabular} & 47.5 & 12.1 & 41.0 & 2.828 & 0.016 & 0.016 & 0.000 & 0.378 & 0.32 \\
\hline 64 & $4 / 19 / 2004$ & 8:08:43 PM & 25.826 & 25.446 & 25.015 & 26.914 & 24.686 & 46.308 & 48.989 & 11.234 & 35.73 & -1.22 & -7.48 & 49.339 & 0.102 & 14.643 & & 626.9 & 10.4481 & 47.5 & 12. & & & & & & 0.371 & 0.32 \\
\hline 164 & $4 / 19 / 2004$ & 09:43 PM & & & & & & & & & & & & & & & & & 0.4647 & & & & & & & & & \\
\hline & $4 / 19 / 2004$ & 8:10:43 PM & & $\frac{25.457}{25.92}$ & 25.016 & 26.83 & 24.457 & 45.902 & 48.386 & $\begin{array}{l}11.149 \\
11.105\end{array}$ & .468 & & & 9.904 & 0.103 & 14.643 & & & .4814 & 46.9 & & & & & & 0.000 & 0.377 & 0.32 \\
\hline 6 & 411912004 & $8.1 .45 \mathrm{PM}$ & $\frac{25.818}{25809}$ & $\frac{25.483}{25.844}$ & $\frac{25.052}{25028}$ & $\frac{2.6811}{26817}$ & $\frac{24.415}{2.360}$ & $\frac{46.483}{46.10}$ & $\begin{array}{l}49.122 \\
49812\end{array}$ & 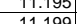 & & 122 & & 5 & 0.1 & $\frac{14.643}{14642}$ & & 630 & & 4.7 .6 & & & & & & & & \\
\hline 652 & $\begin{array}{l}4 / 1 / 19 / 2004 \\
/ / 1 / 204\end{array}$ & $8: 2: 13: 43 \mathrm{PM}$ & $\begin{array}{l}25.809 \\
25.789\end{array}$ & $\begin{array}{l}25.464 \\
25.484\end{array}$ & \begin{tabular}{|l|}
25.028 \\
25.043 \\
\end{tabular} & $\frac{20.811}{26.773}$ & $\frac{24.309}{22.344}$ & \begin{tabular}{|}
46.19 \\
46.099
\end{tabular} & \begin{tabular}{|l|}
48.88674 \\
48.674
\end{tabular} & $\frac{11.199}{11.209}$ & 35.1554 & $\frac{-1.221}{-1.218}$ & & $\begin{array}{l}49.483 \\
48.114\end{array}$ & $\begin{array}{l}0.102 \\
0.102\end{array}$ & $\begin{array}{l}14.045 \\
14643\end{array}$ & & $\frac{50.9}{6319}$ & & 44.4 & & & & 0.015 & & 0.000 & $\frac{.351}{0.372}$ & 0.32 \\
\hline & $4 / 19 / 2004$ & $8: 14: 43 \mathrm{PM}$ & 25.79 & 25.49 & \begin{tabular}{|l|}
25.059 \\
\end{tabular} & 26.723 & 24.24 & 46.047 & \begin{tabular}{|l|}
48.78 \\
\end{tabular} & \begin{tabular}{|l|l|}
11.177 \\
\end{tabular} & 35.574 & \begin{tabular}{|l|}
-1.2210 \\
\end{tabular} & -7.478 & 48.871 & 0.101 & $\begin{array}{l}14.045 \\
14.643\end{array}$ & & 632.9 & \begin{tabular}{|l|l|}
10.5481 \\
\end{tabular} & 47.3 & $\frac{11.0}{12.0}$ & 40.8 & 2.814 & 0.015 & & 0.000 & 0.368 & $\begin{array}{l}0.32 \\
0.31\end{array}$ \\
\hline 65 & & 8:15:43 PM & 25.775 & 25.475 & \begin{tabular}{|l|}
25.049 \\
\end{tabular} & 26.673 & 24.275 & 45.9 & 48.546 & 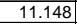 & 35.57 & & -7.475 & & 0.101 & \begin{tabular}{l|l}
14.643 \\
\end{tabular} & & 633.9 & 10.5647 & 47.1 & 12.2 & & & & & & 0.369 & 0.31 \\
\hline & $4 / 19 / 2004$ & 8:16:43 PM & 25.759 & 25.464 & 25.058 & 26.643 & 24.234 & & 48.946 & 11.23 & & & & & & 14.643 & & 634 & .5814 & 47.5 & & & & & & & 371 & \\
\hline & $\begin{array}{l}4 / 1 / 1 / 2004 \\
/ 1 / 2004\end{array}$ & $8: 1.434$ & 25..759 & $\frac{25.459}{25.458}$ & \begin{tabular}{|l|l|}
25.035 \\
25017
\end{tabular} & 20.608 & $\frac{24.124}{24118}$ & $\frac{40.11}{40.618}$ & $\begin{array}{l}48.913 \\
48805\end{array}$ & $\frac{11.248}{11214}$ & $\frac{35.042}{35.9}$ & 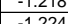 & -7.429 & $\frac{49.016}{5516}$ & 0.103 & $\frac{14.043}{14643}$ & & 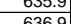 & 6.59814 & 47.4. & 126 & 40 & & & & & 375 & 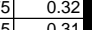 \\
\hline 然 & $\begin{array}{l}4 / 1 / 19<0404 \\
4 / 1 / 204\end{array}$ & 8.0 .4454 & $\frac{25.145}{25747}$ & $\frac{20.450}{25,457}$ & 250.041 & 20.300 & $\frac{24.110}{24042}$ & $\begin{array}{l}40.010 \\
46157\end{array}$ & 年 & $\mid$ & 35.45 & $\frac{1.242}{-1.215}$ & & 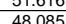 & 0.1 & $\begin{array}{l}14.045 \\
114643\end{array}$ & & (637. & $\mid$ & 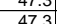 & $\frac{12.0}{118}$ & & & & & & 0.305 & 0.31 \\
\hline & $4 / 19 / 2004$ & $\frac{1.545 \mathrm{PM}}{8.20 .43}$ & 25.736 & 25 & $\begin{array}{l}2.0 .015 \\
25.05\end{array}$ & $\frac{20.54}{26.514}$ & 24.066 & 年 45.949 & 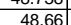 & $\begin{array}{ll}11.290 \\
11.166\end{array}$ & 3.011 & -1.21 & $\frac{-1.44}{-7.46}$ & $\begin{array}{l}48.061 \\
4.681\end{array}$ & 0.1 & $\begin{array}{l}14.045 \\
14.643\end{array}$ & & $\frac{53.9}{638.9}$ & \begin{tabular}{|l|l|l|}
10.04881 \\
\end{tabular} & 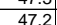 & $\frac{1.9}{11.9}$ & . & 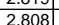 & & & & o. 0.365 & $\frac{0.31}{0.31}$ \\
\hline & $4 / 19 / 2004$ & 8:21:43 PM & 25.756 & 25.456 & 25.08 & & 24.096 & & 488.977 & & & & & & & & & $\begin{array}{l}639.9 \\
63\end{array}$ & & 47.5 & 12.8 & & & & & & & $\begin{array}{l}0.31 \\
0.311\end{array}$ \\
\hline 661 & $4 / 191 / 2004$ & $22: 43 \mathrm{PM}$ & 25.744 & 25.429 & \begin{tabular}{|l|}
25.073 \\
\end{tabular} & 26.502 & 23.988 & 46.05 & 49.018 & 11.299 & 35.404 & -1.218 & $\frac{-1.457}{-7.457}$ & 48.915 & 0.0999 & 14.643 & & $\frac{040.9}{6040}$ & $\mid 1.06814$ & 47.5 & 告. & 40. & 2.808 & $\frac{015}{015}$ & & $\frac{0.000}{0.000}$ & 0.362 & $\begin{array}{l}0.31 \\
0.31\end{array}$ \\
\hline & $4 / 19 / 2004$ & $8: 23: 43 \mathrm{PM}$ & 25.748 & $\begin{array}{l}25.423 \\
\end{array}$ & $\begin{array}{l}25.087 \\
\end{array}$ & 26.456 & 24.05. & 46.207 & 49.101 & 11.36 & 35.42 & 1.218 & $-7.4 \mathrm{r}$ & $\begin{array}{l}49.46 \\
\end{array}$ & 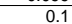 & 14.643 & & 6411.9 & & 47.6 & & & & & & & 0.364 & \\
\hline & $4 / 19 / 2004$ & 8:24:43 PM & 25.742 & 25.421 & 25.081 & 26.405 & 24.03 & 46.142 & \begin{tabular}{|l|l|}
48.79 \\
\end{tabular} & & 35.644 & -1.218 & -7.46 & 47.651 & 0.1 & 14.643 & & 642.9 & .7147 & 47.3 & & & & & & & & \\
\hline & 4/19/2004 & 8:25:43 PM & 25.73 & 25.395 & \begin{tabular}{|l|l|} 
& 24.959 \\
\end{tabular} & 26.423 & 23.98 & 46.064 & 48.819 & \begin{tabular}{|c|c|}
11.408 \\
\end{tabular} & 35.39 & -1.224 & & 49.364 & $\begin{array}{ll}0.1 \\
\end{array}$ & \begin{tabular}{|l|l|l|l|l|}
14.643 \\
\end{tabular} & & 643.9 & \begin{tabular}{|l|l|}
10.7314 \\
\end{tabular} & 47.3 & 12.1 & 40.7 & 2.808 & 0.015 & 0.015 & 0.000 & 0.366 & 0.31 \\
\hline & 4/119/2004 & 8:26:43 PM & 25.729 & 25.389 & 24.963 & 26.422 & 24.134 & 45.954 & \begin{tabular}{|l|l|}
48.79 \\
\end{tabular} & 11.332 & 35.266 & -1.22 & -7 & 49.057 & 0.1 & 14.643 & & & & 47.3 & & & & & & & $\begin{array}{l}0.368 \\
\end{array}$ & \\
\hline 66 & 4/119/2004 & 8:27:43 PM & 25.723 & 25.393 & \begin{tabular}{|l}
24.992 \\
\end{tabular} & & & 46.066 & 48.96 & 11.242 & 35.5 & & & 48.131 & 0.098 & & & 64 & \begin{tabular}{|l|l|l|l|} 
\\
\end{tabular} & 47.5 & 11.8 & & & & & & 0.358 & \\
\hline 年 & 4/11992004 & & 25.713 & 25.388 & 24.982 & 26.551 & & & 48.774 & 11.306 & & 100 & & 49 & 0.097 & $14.6 \mathrm{C}$ & & & & 47.3 & 2. & 40 & 80 & 014 & & 0.00 & 0.356 & \\
\hline & & $\frac{3 P \mathrm{M}}{3 \mathrm{DM}}$ & & 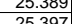 & \begin{tabular}{|l}
25.003 \\
35036
\end{tabular} & $\frac{20.580}{3669}$ & & & & & & & & (48. & & & & & & & & & & & & & & \\
\hline $\begin{array}{l}60 \\
670\end{array}$ & $\begin{array}{l}4 / 1 / 19 / 2004 \\
/ 19 / 204\end{array}$ & $\begin{array}{l}0.03 .4543 \\
8\end{array}$ & $\frac{25.114}{25704}$ & 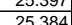 & 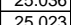 & $\frac{2.00}{26.737}$ & $\frac{24.648}{24.949}$ & $\frac{45.921}{45881}$ & 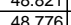 & 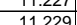 & $\begin{array}{l}35.451 \\
35371\end{array}$ & - & & $\begin{array}{l}47.949 \\
48239\end{array}$ & 0.11 & $\begin{array}{l}14.045 \\
14.643\end{array}$ & & $\begin{array}{l}648.9 \\
699.9\end{array}$ & 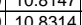 & 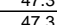 & $\frac{11.8}{118}$ & $\frac{40.1}{40.6}$ & 2001 & & & 0.000 & 0.306 & \\
\hline 671 & $4 / 19 / 2004$ & $\frac{0 \cdot 3 \cdot 43 \mathrm{PM}}{8.32}$ & 25701 & 25381 & 22504 & 26.754 & 24806 & & 4933 & 11.347 & & 1.24 & & 48.568 & & $\begin{array}{ll}14.045 \\
14.643\end{array}$ & & 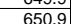 & $\mid 10.08481$ & 478 & 11. & 40.0 & & & & & 0.0354 & \\
\hline 672 & $4 / 19 / 2004$ & 8: $83: 43 \mathrm{PM}$ & 25.702 & 25.386 & 25.041 & 26.75 & 24.592 & 45.885 & $\begin{array}{l}48.898 \\
8.898\end{array}$ & 11.305 & 35.219 & -1.221 & & 49.23 & 0.098 & 14. & & 65 & $\mid 10.8647$ & 47.4 & $\frac{11}{12}$ & r & & & & & 0.360 & \\
\hline 673 & $4 / 19 / 2004$ & 8:34:43 PM & 25.702 & 25.407 & \begin{tabular}{|l|}
25.051 \\
\end{tabular} & 26555 & & & & 11.349 & & & & & & & & 65 & & & & & & & & & & \\
\hline & 4/119/2004 & $8: 35: 43 \mathrm{PM}$ & 25.697 & & 25.036 & 26.6 & & & 48.879 & & 35.55 & & & 49.5 & & 14.6 & & & & 47.4 & & & & & & & $\begin{array}{l}0.358 \\
\end{array}$ & \\
\hline por & $4 / 19 / 2004$ & $8: 36: 43 \mathrm{PM}$ & 25.696 & 25.411 & \begin{tabular}{|l|}
25.035 \\
\end{tabular} & 26.519 & 24.346 & 45.703 & \begin{tabular}{|l|l|l|}
4849 \\
\end{tabular} & 11.297 & 35.133 & -1.221 & -7.3 & 49 & \begin{tabular}{|l|l|}
0.097 \\
\end{tabular} & 14. & & & \begin{tabular}{|l|}
10.9147 \\
\end{tabular} & 47.2 & 12.2 & 40. & . 1.87 & 014 & 014 & & 0.357 & \\
\hline & $4 / 19 / 2004$ & $8: 37: 4$ & 25.705 & 25.425 & 25.079 & 26.543 & 24.0 & & 49.246 & 11.417 & 35.498 & -1.21 & & 51.9 & & & & & 10.9 & 47.8 & 12. & & .81 & & & & 0.357 & \\
\hline 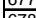 & $4 / 19 / 2$ & $8: 38: 43 \mathrm{PM}$ & 25.704 & 25.429 & 24.978 & 26.562 & 24.26 & 46.0 & 48.942 & 11.337 & 35.406 & -1.21 & -7.41 & 49.147 & & & & & & 47.5 & 12. & & & & & & 0.374 & \\
\hline 678 & 4/199/2004 & 8:39:43 PM & 25. & 25.432 & \begin{tabular}{|l|l|}
4.976 \\
\end{tabular} & 26.555 & & 46.068 & 49.145 & 11.371 & & -1.218 & -7 & 50.2 & & & & & & 47.7 & 12. & & & & & & 0.352 & \\
\hline & $4 / 19 / 2004$ & & & & & 26.54 & & & & 11.258 & & & & 49.7 & & & & & & & 12. & & & & & & 0.346 & \\
\hline 680 & 411992004 & & $\frac{25}{25}$ & 25.441 & 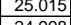 & 26.454 & & $\begin{array}{l}45.761 \\
4.752\end{array}$ & 48.902 & $\frac{11.259}{11.250}$ & & -1.241 & & 48. & & & & 659 & 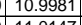 & 47.4 & 11.8 & (1) & & 2) & & & 0.361 & \\
\hline & $\frac{41912004}{4 / 19204}$ & & $\frac{23.065}{25678}$ & $\frac{25.424}{25418}$ & \begin{tabular}{|l|}
24.950 \\
25017
\end{tabular} & $\frac{2.048}{26401}$ & & 年 & - & $\frac{11.367}{11378}$ & 等 & -1.221 & & & & & & & & & 势 & & & & & & & \\
\hline & $\frac{4}{41 / 912004}$ & & 25.677 & 25.427 & \begin{tabular}{|l|l|l|} 
\\
5036
\end{tabular} & 2605 & & & 49.145 & 11.321 & & & & & & & & & & & 12 & & & & & & 0.0354 & \\
\hline 68 & $\frac{4 / 19 / 2004}{4}$ & & 25.67 & 25.414 & \begin{tabular}{|l|l|l|l|l|} 
\\
\end{tabular} & $\frac{26.373}{26.373}$ & & 45. & 48.977 & $\frac{11.25}{11.25}$ & 35.435 & -1218 & & 48. & & & & & & 47.5 & 118 & & & & & & 0.362 & \\
\hline & & & & & & & & & & & & & & & & & & & & & & & & & & & & \\
\hline & 4/19/2004 & & 25,67 & 25.402 & 25.056 & 26.315 & & 45 & 48.917 & 11.278 & 35.269 & -1.218 & & 48.3 & & 14. & & & & 47.4 & 11.8 & & & & & & 60 & \\
\hline \begin{tabular}{|l|l|}
68 \\
\end{tabular} & $4 / 19 / 2$ & & 25 & $\begin{array}{l}25.39 \\
\end{array}$ & \begin{tabular}{|l|}
25.054 \\
\end{tabular} & 26.283 & & & 49.10 & 11.371 & 35.316 & -1.221 & & 47. & & & & & 911.1 & & $\begin{array}{ll}11.6 \\
\end{array}$ & & & $\overline{1014}$ & & & 355 & \\
\hline $\begin{array}{ll}688 \\
\end{array}$ & 4/19/2004 & $8: 49: 43 \mathrm{PM}$ & 25.66 & 25. & \begin{tabular}{|l|}
25.088 \\
\end{tabular} & 26.347 & & 46.04 & 49.01 & 11.295 & 35.566 & -1.21 & & 47.5 & & 14.6 & & $\begin{array}{ll}667.9 \\
\end{array}$ & (11.1. & 47.5 & 11.7 & & 2.813 & 0.014 & .014 & & 0.354 & \\
\hline & $4 / 19 / 2004$ & $8: 50: 43$ & 25.674 & 25.39 & 25. & 26.437 & & 45.9 & 49.7 & 11.302 & 35 & -1.22 & & 48 & & & & 6 & 11.1. & 47.6 & 11. & & & 14 & & & 0.352 & \\
\hline 69 & $4 / 19 / 2004$ & 8:51:43 PM & & 25.368 & 25. & 26.496 & & 45.692 & 48.966 & 11.374 & 34.907 & $-1.21 \varepsilon$ & & 48.0 & & & & & & 47.5 & 11.8 & & & \begin{tabular}{|l|l|}
0.014 \\
\end{tabular} & 114 & & 0.358 & \\
\hline 691 & 4.119922004 & 8:52:43 PM & 25.675 & $\begin{array}{r}25.399 \\
25.391\end{array}$ & 25.119 & 26.548 & 24.715 & 45.94 & 49.172 & 11.352 & 35.269 & -1.218 & & 52. & 0.0 & 14.6 & & & 11.1814 & 41.1. & $12.8 \mathrm{r}$ & & & 114 & & & & \\
\hline & 411992004 & $8: 03: 43 \mathrm{PM}$ & 2567 & 25.381 & \begin{tabular}{|l|l|} 
\\
\end{tabular} & $\frac{2.659}{26.675}$ & $\frac{2.0 .0}{2.7}$ & $\begin{array}{l}45.883 \\
45765\end{array}$ & 49.047 & 11.259 & 35.33 & -1.218 & & 47.8 & & 14.6 & & & & 41.6 & 11.8 & & & & & & & \\
\hline & 4 & $\frac{0.04 .435}{0.55 .42}$ & & 25.301 & $\mid 2.111$ & 20.050 & & 45.105 & 40.0004 & $\frac{11.20}{1.201}$ & 30.444 & -1.216 & & $\frac{4}{1}$ & & & & & & & $\frac{11.5}{110}$ & & & & & & & \\
\hline 169 & 告/191/2 & 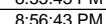 & 2569 & & & 26 & & $\frac{4.5}{45}$ & 年 48.108 & 11.219 & & -1.22 & & 年 & 0.096 & & & & & & 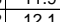 & & & & & & & \\
\hline 696 & & & 25. & 25.378 & 24 & 26. & & 45 & & & 35.31 & -1.224 & & & & & & & & & 117 & & & & & & & \\
\hline & & & & & & & & & & 11213 & & & & & & & & & & & & & & & & & & \\
\hline & & & & & & & & & & & & & & & & & & & & 477 & 116 & & & & & & & \\
\hline & $410 / 201$ & 9.00 .4 & & & 986 & & & & 49.068 & & & 221 & & 71 & & & & & & 47.6 & 116 & & & & 14 & & 0.348 & \\
\hline 00 & $4 / 19 / 2004$ & 9::01:43 PM & 25.676 & 25.391 & 25.01 & 26.509 & 24.246 & 45.777 & \begin{tabular}{|l|l}
49.06 \\
\end{tabular} & \begin{tabular}{|l|l|}
11.202 \\
\end{tabular} & 35.277 & \begin{tabular}{|l|l|} 
\\
\end{tabular} & & $\overline{46.302}$ & 0.095 & 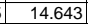 & & 679.9 & 911.3314 & 47.6 & 11.3 & & 2.794 & \begin{tabular}{|l|l|}
0.014 \\
\end{tabular} & 0.014 & 0.000 & 0.349 & 49 \\
\hline
\end{tabular}


WSRC-TR-2005-00105, REVISION 0

SRNL-RPP-2005-00012, REVISION 0

RUN \# 2.03A AND B; FIRST AND SECOND HALF OF SLURRY DEWATERING - CONT.

\begin{tabular}{|c|c|c|c|c|c|c|c|c|c|c|c|c|c|c|c|c|c|c|c|c|c|c|c|c|c|c|c|c|}
\hline & $\mathrm{A}$ & B & $\mathrm{D}$ & E & $\mathrm{F}$ & G & $\mathrm{H}$ & $\mathrm{J}$ & $\mathrm{K}$ & \begin{tabular}{ll|} 
\\
\end{tabular} & $\mathrm{M}$ & $\mathrm{N}$ & $\mathrm{O}$ & $Q$ & \begin{tabular}{l|l} 
\\
\end{tabular} & $\mathrm{s}$ & T & $\mathrm{v}$ & w & $x$ & $Y$ & $z$ & $\mathrm{AA}$ & $A B$ & $A C$ & $\mathrm{AD}$ & AE & AF $A$ \\
\hline & $4 / 19 / 2004$ & $\begin{array}{c}9: 02: 43 \text { PM } \\
9: 03: 43 \text { PM }\end{array}$ & \begin{tabular}{|l|}
25.67 \\
25644
\end{tabular} & $\begin{array}{l}25.38 \\
25379\end{array}$ & 25.034 & $\begin{array}{l}26.444 \\
26392\end{array}$ & $\begin{aligned} 24.165 \\
24.124\end{aligned}$ & $\begin{array}{l}45.545 \\
45804\end{array}$ & $\begin{array}{l}48.682 \\
.012\end{array}$ & \begin{tabular}{|c|}
11.15 \\
111.133 \\
\end{tabular} & $\begin{array}{l}35.106 \\
35271\end{array}$ & -1.218 & \begin{tabular}{|l|l|}
-7.397 \\
-7376
\end{tabular} & 45.90 & $\begin{array}{l}0.095 \\
\end{array}$ & 14.643 & $\begin{array}{l}0.003 \\
\end{array}$ & & \begin{tabular}{|l|l|}
11.3481 \\
\end{tabular} & 47.2 & & & $\frac{2.780}{2.795}$ & & 0.014 & 0.000 & 0.3. & \\
\hline & $4 / 19 / 2004$ & & & & & & & & & & & & & & & & & & & & & & & & & & & 0.30 \\
\hline 704 & 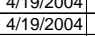 & $\begin{array}{l}9.044: 43 \mathrm{PM} \\
\text { 9:05:43 PM }\end{array}$ & $\frac{25.63}{25.661}$ & $\frac{25.367}{25.366}$ & \begin{tabular}{|l|}
25.045 \\
\end{tabular} & $\begin{array}{l}26.36 \\
26.379 \\
\end{array}$ & $\begin{array}{r}24.121 \\
24.136 \\
\end{array}$ & $\begin{array}{l}45.132 \\
45.862 \\
\end{array}$ & \begin{tabular}{|l|}
48.9448 \\
49.087 \\
\end{tabular} & $\frac{11.214}{11.288}$ & $\begin{array}{l}35.269 \\
35.285 \\
\end{array}$ & \begin{tabular}{|l|l|}
-1.221 \\
-1.218 \\
\end{tabular} & $\begin{array}{l}-7.359 \\
-7.391 \\
\end{array}$ & $\frac{48.219}{48.923}$ & $\mid \begin{array}{l}0.093 \\
0.094\end{array}$ & $\frac{14.643}{14.643}$ & $\begin{array}{l}0.003 \\
0.003\end{array}$ & $\frac{682.9}{683.9}$ & 111.38144 & $\begin{array}{l}477.5 \\
47.6\end{array}$ & $\begin{array}{l}11.8 \\
12.0\end{array}$ & \begin{tabular}{|l|l|}
40.6 \\
\end{tabular} & \begin{tabular}{|l|l|}
2.797 \\
\end{tabular} & $\mid$ & \begin{tabular}{|c|}
0.014 \\
0.014 \\
\end{tabular} & $\begin{array}{l}0.000 \\
0.000\end{array}$ & 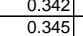 & 0.29 \\
\hline & $4 / 19 / 2004$ & 9:06:43 PM & $\begin{array}{l}25.66 \\
25\end{array}$ & 25.355 & \begin{tabular}{|l|}
25.034 \\
\end{tabular} & 26.363 & $\begin{array}{r}24.13 \\
\end{array}$ & 46.062 & \begin{tabular}{|l|l|}
49.393 \\
\end{tabular} & $\begin{array}{l}11.299 \\
\end{array}$ & 35.443 & & & 48.84 & & 14.643 & 0.003 & & $\mid 11.4147$ & & & & & & & & & \\
\hline & $4 / 19 / 2004$ & & & & & & & 45.962 & \begin{tabular}{|l|}
49.2533 \\
\end{tabular} & 11.254 & 35.408 & & & 46.988 & \begin{tabular}{ll|}
0.094 \\
\end{tabular} & 14.643 & & & 11.4314 & $\begin{array}{l}47.8 \\
\end{array}$ & & & & & & & & \\
\hline & $4 / 19 / 2004$ & 9:08:43 PM & 25.656 & & 25.05 & 26.349 & $\begin{array}{l}23.98 \\
\end{array}$ & 46.103 & \begin{tabular}{|l|}
49.223 \\
\end{tabular} & 11.27 & 35.671 & $\begin{array}{l}-1.224 \\
\end{array}$ & & 50.482 & $\begin{array}{l}0.092 \\
\end{array}$ & 14.643 & & 686.9 & 11.4481 & 47.8 & $\begin{array}{l}12.4 \\
\end{array}$ & & \begin{tabular}{|l|l|}
2.819 \\
\end{tabular} & 0.014 & \begin{tabular}{|l|l|}
0.014 \\
\end{tabular} & & & \\
\hline & 4/19/2004 & 9:09:43 PM & $25.653 \mid$ & $\begin{aligned} 25.318 \\
25.327\end{aligned}$ & 25.057 & $\frac{26.321}{26325}$ & $\begin{array}{l}24.007 \\
24072\end{array}$ & 45.862 & \begin{tabular}{|l|}
49.178 \\
\end{tabular} & \begin{tabular}{|c|}
11.241 \\
.1125 \\
\end{tabular} & $\begin{array}{r}35.297 \\
25.1202\end{array}$ & $\begin{array}{r}-1.218 \\
.215\end{array}$ & $\begin{array}{r}-7.385 \\
7.270\end{array}$ & 50.989 & 0.094 & 14.643 & 0.003 & 687.9 & 11.4647 & 47.7 & 12.5 & $\mid 40.6$ & \begin{tabular}{|l|}
2.798 \\
.702
\end{tabular} & & 0.014 & 0.000 & 0.345 & \\
\hline & $\begin{array}{l}4 / 19 / 12004 \\
4 / 1 / 2004\end{array}$ & $\begin{array}{l}9: 10: 43 \text { PM } \\
9 \cdot 11.43 \mathrm{PM}\end{array}$ & \begin{tabular}{|l|l|}
25.667 \\
25655
\end{tabular} & $\frac{25.327}{25.3}$ & 25.096 & $\begin{array}{l}26.335 \\
26.25\end{array}$ & $\begin{array}{l}24.072 \\
24.165\end{array}$ & $\begin{array}{r}45.616 \\
4564 \\
\end{array}$ & \begin{tabular}{|l|}
48.995 \\
49.149 \\
\end{tabular} & $\begin{array}{r}11.25 \\
11225\end{array}$ & $\begin{array}{l}35.123 \\
35002\end{array}$ & \begin{tabular}{|l}
-1.215 \\
-1.224
\end{tabular} & $\begin{array}{r}-7.376 \\
-7.391\end{array}$ & $\begin{array}{l}49.126 \\
47.345\end{array}$ & $\mid \begin{array}{l}0.093 \\
0.033\end{array}$ & $\frac{14.643}{14.643}$ & $\begin{array}{l}0.004 \\
0\end{array}$ & $\frac{688.5}{689.5}$ & $11.4818 \mid$ & $\begin{array}{l}47.5 \\
47.7\end{array}$ & 12.0 & & \begin{tabular}{|l|}
2.783 \\
2780
\end{tabular} & 0.014 & 0.014 & 0.000 & $\begin{array}{l}0.343 \\
\end{array}$ & 0.29 \\
\hline 711 & $4 / 19 / 19 / 2004$ & 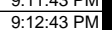 & $\frac{25.505}{25.66}$ & $\begin{array}{r}25.305 \\
25.305\end{array}$ & $\mid$\begin{tabular}{|l|l|l|l|}
$\mid 25.074$ \\
\end{tabular} & 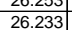 & $\begin{array}{l}24.1 .365 \\
24.375\end{array}$ & $\begin{array}{l}4.5 .044 \\
46.026\end{array}$ & $\begin{array}{l}\frac{49.149}{49.398} \\
\end{array}$ & $\begin{array}{l}\frac{11.229}{11.361} \\
\end{array}$ & $\begin{array}{l}35.002 \\
35.307 \\
\end{array}$ & $\begin{array}{l}-1.224 \\
-1.224\end{array}$ & $\begin{array}{l}-7.391 \\
-7.382\end{array}$ & $\frac{47.345}{48.246}$ & $\mid \begin{array}{l}0.093 \\
0.092\end{array}$ & $\frac{14.643}{14.643}$ & \begin{tabular}{|l}
0.003 \\
0.003 \\
\end{tabular} & $\frac{689 .}{690 .}$ & $\begin{array}{l}\frac{11.4981}{11.5147} \\
\end{array}$ & $\frac{47.7}{47.9}$ & $\begin{array}{l}11.6 \\
11.8 \\
\end{array}$ & $\begin{array}{l}40.3 \\
40.7 \\
\end{array}$ & \begin{tabular}{|l|}
2.180 \\
2.804
\end{tabular} \mid & $\mid$ & $\mid$ & $\begin{array}{l}0.000 \\
0.000\end{array}$ & $\begin{array}{l}0.343 \\
0.337\end{array}$ & $\frac{0.29}{0.29}$ \\
\hline & $4 / 19 / 2004$ & 9:13:43 PM & 25.65 & 25.289 & \begin{tabular}{|l|}
24.998 \\
\end{tabular} & 26.318 & 24.509 & 45.875 & 49.393 & 111.276 & 35.219 & -1.221 & & $\frac{40.240}{47.063}$ & 0.094 & 14.643 & 0.003 & & 11.5314 & 47.9 & & & & & & & & $\begin{array}{l}0.29 \\
0.29\end{array}$ \\
\hline & & & & & & & & & & & & & & & & & & & & & & & & & & & & \\
\hline & $4 / 19 / 2004$ & 9:15:43 PM & 25.656 & 25.29 & 25.035 & 26.434 & 24.716 & 45.842 & 49.304 & 11.282 & 35.246 & 1.221 & & .274 & $\mid 0.092$ & 14.643 & & & 5647 & 47.8 & & & 79 & & .014 & .000 & 338 & \\
\hline & & & & & & & & & 49.17 & & & & & & & & & & & & & & & & & & & \\
\hline & $4 / 19 / 2004$ & 9:17:43 PM & 25.689 & 25.283 & (2507) & 26.617 & 24.884 & $\begin{array}{l}44.069 \\
\end{array}$ & $\begin{array}{l}49.36 \\
\end{array}$ & 11.228 & 33.53 & -0.65 & & 47.824 & \begin{tabular}{|l|l|}
0.003 \\
\end{tabular} & 14.643 & & 695 & 1.5981 & 47.9 & & & 2.675 & & & 0.000 & & \\
\hline 71 & $4 / 19 / 2004$ & 9:18:43 PM & 25.534 & 25.294 & $25.068 \mid$ & 26.612 & 24.709 & 33.958 & 49.004 & 11.223 & 23.21 & 9.274 & & 46.785 & 0.004 & 14.643 & 0.003 & 696 & 1.6147 & 47.5 & 11.5 & 28.6 & 1.971 & 0.001 & 0.001 & 0.000 & 0.021 & \\
\hline & $4 / 19 / 2004$ & 9:19:43 PM & 25.55 & 25.29 & \begin{tabular}{|l|}
25.049 \\
\end{tabular} & 26.6288 & 24.675 & 17.605 & 49.188 & 11.256 & 6.925 & 26.071 & & 46.656 & 0.003 & 14.643 & 0.003 & 69 & 1.6314 & $47.7 \mathrm{r}$ & 11.4 & 12. & 0.846 & 0.000 & & 0.000 & & \\
\hline$\frac{11}{72}$ & $4 / 19 / 2004$ & 9:20:43 PM & 25.57 & 25.2.295 & 25.064 & 26.598 & 24.655 & 8.175 & 48.745 & 11..149 & -2.412 & 35.19 & & & 0.003 & 14.643 & 0.004 & 698 & 1.6481 & 47.3 & & & .199 & & 0.000 & 0.000 & & \\
\hline & $4 / 19 / 2004$ & $9.21 .432 \mathrm{PM}$ & $\frac{2.549}{20.59}$ & & & & & 0.931 & 4.8065 & & $\frac{-2.538}{2.371}$ & & & & & & & & & & & & & & & & & \\
\hline$\frac{12}{72}$ & $4 / 1 / 9 / 2004$ & $\begin{array}{l}9: 22: 43 \mathrm{PM} \\
9.23 \cdot 43 \mathrm{PM}\end{array}$ & $\frac{25.534}{25.532}$ & 25.299 & 50.003 & $\frac{26.507}{26.46}$ & $\begin{array}{l}24.494 \\
24.332\end{array}$ & $\frac{41.233}{42.376}$ & $\frac{48.465}{49.443}$ & $\frac{10.841}{12011}$ & $\frac{31.074}{30.858}$ & $\begin{array}{l}1.648 \\
1.098 \\
\end{array}$ & & 48.8 & 0.366 & $\frac{14.643}{14.643}$ & & & .68614 & $\frac{47.0}{480}$ & & & 49 & & & & $\frac{511}{612}$ & \\
\hline & $4 / 19 / 2004$ & $9: 24: 43$ PM & 25.531 & 25.295 & 25.055 & $\frac{20.406}{26309}$ & 24.326 & 42629 & \begin{tabular}{|l|l|}
49.684 \\
49.4
\end{tabular} & 12.291 & $\frac{3.050}{30.862}$ & 1.046 & & 45809 & $\frac{3.152}{0.122}$ & $\frac{14.043}{14643}$ & 0.004 & & 17147 & 482 & 112 & & 2533 & & 0.018 & 000 & 0.0494 & \\
\hline 72 & 4/19/2004 & 9:25:43 PM & 25.534 & 25.288 & \begin{tabular}{|l|}
25.063 \\
\end{tabular} & 26.347 & 24.083 & 42.494 & 49.342 & 12.338 & 30.776 & 1.049 & & 47.987 & 0.118 & 14.643 & & & $\frac{1.7314}{11.7314}$ & $\frac{47.9}{47.9}$ & $\frac{11.8}{11.8}$ & $\frac{36.6}{36.6}$ & & & 0.018 & 0.000 & 0.479 & \\
\hline & $4 / 19 / 2004$ & 9:26:43 PM & 25.542 & $\frac{25.292}{25}$ & 25.056 & 26.34 & 24.127 & 42.187 & $\begin{array}{l}49.068 \\
\end{array}$ & 12.323 & 30.571 & 1.116 & & 48.283 & \begin{tabular}{|c|}
0.11 \\
\end{tabular} & 14.643 & 0.004 & & 11.7481 & 47.6 & $\frac{11.8}{11.8}$ & $\overline{36.4}$ & 2.508 & $\frac{016}{016}$ & & 0.000 & & \\
\hline & $4 / 19 / 2004$ & 9:27:43 PM & 25.541 & 25.276 & 25.055 & 26.269 & 24.121 & 42.119 & 49.234 & 12.351 & 30.347 & & & & & 14.643 & 0.003 & & 1.7647 & & & 36. & 2.498 & & & 0.000 & 0.370 & \\
\hline & $4 / 19 / 2004$ & 9:28:43 PM & & & & & & & & 12.483 & 36.652 & & & & $\begin{array}{l}0.126 \\
\end{array}$ & & & & 1.7814 & 48.1 & & & & & & & 0.440 & \\
\hline & 4/19/2004 & 9:29:43 PM & 25.618 & 25.272 & 25.066 & 26.231 & 24.127 & 48.673 & 49.393 & $\mid$ & 36.888 & -1.22 & & 49.75 & \begin{tabular}{|l|l|l|}
0.113 \\
\end{tabular} & 14.643 & & & 1.7981 & 47. & & & .950 & & & & & \\
\hline 72 & $4 / 19 / 2004$ & 9:30:43 PM & 25.59 & 25.249 & 25.064 & 26.218 & 24.019 & 48.671 & 298 & 12.646 & 36.744 & -1.221 & & 48.204 & 0.109 & 14.643 & 0.003 & $70 €$ & 1.8147 & & 11.8 & & & & & 0.000 & 0.380 & \\
\hline 73 & $4 / 19 / 2004$ & 9:31:43 PM & $\begin{array}{l}25.594 \\
2.5-59\end{array}$ & 25.263 & \begin{tabular}{|l|}
25.132 \\
2.125 \\
\end{tabular} & 26.221 & 24.113 & 46.292 & 46.906 & 13.813 & 33.278 & $\frac{1.22}{1.22}$ & & 51.789 & 0.101 & 14.643 & & & 1.8314 & 45.4 & 12.7 & & .743 & & & 000 & & \\
\hline & $4 / 191 / 2004$ & 9:32:43 PM & 25.586] & $\frac{25}{5}$ & 25.115 & 26.194 & & 46.368 & 46.972 & 13.753 & 33.3 & & & 1.497 & 0.1 & 14.643 & & & 1.8481 & & & & & & & & & \\
\hline & $\frac{4 / 1912004}{419200}$ & $\begin{array}{l}9: 33543 \mathrm{PM} \\
0.24 .43 \mathrm{M}\end{array}$ & 2.5.5960 & & 25.15 & 26.224 & 24.141 & $\begin{array}{l}46.626 \\
46526\end{array}$ & 4.1756 & & & & & .359 & 0.098 & 14.643 & & & .8647 & (45. & 12.3 & & 166 & & & & & \\
\hline & 4 & 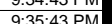 & | & 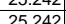 & (51018 & 26.311 & $\frac{24.251}{24.478}$ & 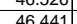 & 47099 & $\mid \frac{1.174}{1363}$ & $\begin{array}{l}3.455 \\
33526\end{array}$ & - & & $\frac{10.0067}{4092}$ & | & $\begin{array}{l}14.045 \\
114643\end{array}$ & & $\frac{11}{71}$ & 1..0014 & $\frac{43.0}{456}$ & 122 & 400 & 5757 . & & & & 364 & \\
\hline 73 & $4 / 41 / 2004$ & $\begin{array}{ll}9 \cdot 36.43 \\
9.36\end{array}$ & 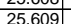 & 25299 & $\mid$\begin{tabular}{|c|c|c|c|}
$\mid 25178$ \\
\end{tabular} & 26.377 & 24.604 & 46684 & 4727 & 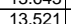 & 33898 & $\frac{1.251}{-1.221}$ & & $\frac{49.962}{49255}$ & | & $\frac{14.645}{14.643}$ & & & $\frac{1.8981}{1.9147}$ & & $\frac{12.4}{121}$ & & & & & & $\frac{0.364}{0.361}$ & \\
\hline & $\frac{d / 2004}{9604}$ & $9: 37: 43$ PM & $\frac{25.616}{25.616}$ & 25.235 & $\mid$\begin{tabular}{|l|}
$\mid 25.159$ \\
25.15
\end{tabular} & 26.444 & 24.75 & 46.595 & \begin{tabular}{|l|l|}
47.433 \\
\end{tabular} & $\frac{1.385}{13.385}$ & 33.887 & $\frac{1.224}{1.224}$ & & 50.473 & \begin{tabular}{|c|}
0.090 \\
0.098
\end{tabular} & $\frac{14.045}{14.643}$ & & $\frac{71}{71}$ & $\begin{array}{l}1.9141 \\
1.9314\end{array}$ & $\frac{43.0}{46.0}$ & $\frac{1.1 .1}{12.4}$ & 40. & .774 & & & & $\begin{array}{l}0.001 \\
0.361\end{array}$ & \\
\hline & g/2004 & & & 25.233 & \begin{tabular}{|l|}
25.097 \\
\end{tabular} & & 24.8 & 46.619 & 47.456 & 13.341 & 34.017 & & & & $\mid$ & 14.643 & & & & 46.8 & 10 & . & & & & & & \\
\hline 738 & g/2004 & $9: 39: 4$ & 25.625 & 25.229 & 25.094 & 26.538 & 24.7 & 46.607 & 47.501 & 13.391 & 33.885 & -1.221 & & 49.612 & \begin{tabular}{|c|}
0.097 \\
\end{tabular} & 14.643 & & & 11.9647 & 46.0 & 12.2 & $\frac{40}{40}$ & 2.775 & $\overline{014}$ & 0.014 & 0.000 & 0.358 & \\
\hline & $4 / 19 / 2004$ & 9:40:43 PM & 25.635 & 25.235 & 5.089 & 26.563 & 24.6 & & 47.292 & 13.274 & 34.0 & -1.224 & & 22.10 & |0.097 & 14.643 & & & $\begin{array}{l}1.9814 \\
\end{array}$ & 45. & 12. & & & & & & & \\
\hline 746 & 4/19/2004 & 9:41:43 PM & 25.646 & 25.251 & 25.105 & 26.534 & & & & 13.04 & & & & 1041 & $\mid 0.097$ & 14.643 & & & 11.9981 & 45.9 & 12.1 & & 2.796 & 0.014 & & & & \\
\hline 144. & $4 / 19 / 2004$ & & \begin{tabular}{|l|l|}
25.63 \\
\end{tabular} & 25.235 & & & & & 47.71 & & & -1.218 & & & \begin{tabular}{|c|c|}
0.098 \\
\end{tabular} & 14.643 & & & & 46. & 12. & & & & & 0.000 & & \\
\hline 742 & $4 / 19 / 2004$ & $9: 43: 43 \mathrm{PM}$ & 25.64 & 25.245 & $25.129 \mid$ & 26.443 & 24.245 & 46.5955 & \begin{tabular}{|l|l|} 
& 47.656 \\
\end{tabular} & 13.07 & 34.14 & -1.218 & & 48.9 & 0.095 & 14.643 & & & 12.0314 & 46. & 12.8 & 40.4 & 1.783 & 0.014 & 0.014 & 0.000 & 550 & \\
\hline$\frac{14.4}{710}$ & 9/2004 & 9:44: & 25.64 & 25.239 & 25.089 & 26.378 & 24.1 & 46.673 & \begin{tabular}{|l|l|l} 
\\
\end{tabular} & 13.002 & 34.415 & -1.227 & & 48.024 & 0.098 & 14.643 & & 722 & 12.0481 & 46. & $11 . \varepsilon$ & 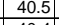 & & 0.015 & - & 0.000 & 0.359 & \\
\hline$\frac{14}{74}$ & 4119920 & $9: 45: 43$ & 2 & $\frac{25.238}{2.238}$ & 25.112 & $\frac{2.286}{20275}$ & & & 4.453 & 13.073 & & & & & 0.097 & & & & & & . & & & & & & & \\
\hline & & & 25.04 & 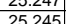 & 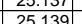 & $\mid$ & & & (4).1964 & $\frac{15.14}{13063}$ & & & & & & & & & 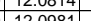 & 40. & 11. & & & & & & & \\
\hline 747 & 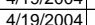 & $\begin{array}{l}3.46 .4 \\
9.48\end{array}$ & $\frac{2.0515}{25648}$ & $\frac{2.4358}{25.283}$ & $\mid$ & $\frac{2.0 .246}{26.236}$ & & & & 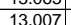 & & - & & (1) & & $\frac{14.043}{14.643}$ & & $\frac{125}{726}$ & 12.1147 & 460 & 125 & & & & & & 0358 & \\
\hline 74 & $4 / 1 / 1 / 2004$ & $9: 49: 43 \mathrm{PI}$ & 25.646| & 25.231 & $\mid$\begin{tabular}{|r|}
$\mid 25.14$ \\
\end{tabular} & $\frac{26.204}{26.20}$ & & & 47 & , & & $\frac{-1.218}{-1.218}$ & & & & & & & 12.1314 & 46.2 & 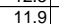 & & & & & & & \\
\hline & 4/19/2004 & 9:50:43 PM & $\begin{array}{l}25.655 \\
2\end{array}$ & 25.225 & $\mid 25.089$ & 26.153 & 23.9 & 46.7 & \begin{tabular}{|l|l|l|}
47.694 \\
\end{tabular} & 12.927 & 34.589 & $\frac{1.221}{-1.21}$ & & 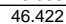 & $\mid$ & & & $\frac{728}{728}$ & 2.1481 & 46. & 1 & & & & & & & \\
\hline & $4 / 19 / 2004$ & & & 25.222 & & & & & & & & & & & & & & & & & & & & & & & & \\
\hline 75 & $4 / 19 / 2004$ & $9: 52: 43 \mathrm{PM}$ & 25.642 & 25.226 & 5075 & 26.14 & & & \begin{tabular}{|l|l|}
47.922 \\
\end{tabular} & 12.912 & 34.601 & -1.218 & & 48.64 & & & & & 2.1814 & & $\overline{11.9}$ & & & & & & & \\
\hline & $4 / 19 / 2004$ & $9: 53: 43 \mathrm{PI}$ & 25.631 & 25.21 & \begin{tabular}{|l|}
25.09 \\
\end{tabular} & 26.154 & 24.2 & & \begin{tabular}{|l|l|}
48.09 \\
\end{tabular} & 12.92 & 34.7 & -1.2 & & 51.1. & $0.0 \leqq$ & & & 731. & 12.1981 & 46. & 12.5 & 40 & 2.813 & & 0.014 & & & \\
\hline 75 & 4/19/2004 & 9:54:43 F & 25.641 & 25. & 25.135 & $26.184 \mid$ & & 46.8 & 48.001 & 12.889 ] & 34.651 & & & & 0.05 & 14.6 & & 732 & & 46 & 11.9 & & 808 & 014 & 0.014 & & & \\
\hline 75 & 4/19/2004 & 9:55:43। & 25.6 & 25.211 & 25.1 & 26 & & 46.277] & 47.536 & 12.8 & 34.15 & -1.218 & & & & $14.6 \mathrm{C}$ & & & 12.2. & 46. & 12.1 & $4 c+2$ & & & 0.01 & & & \\
\hline 155 & $4 / 19 / 2004$ & 9:56:43 & & & & 26.34 & & 46.6 & 47.82 & 12.907 & & & & & & & & & 12.2. & 46. & 11.8 & & & & & & & \\
\hline 15 & & & 25.6 & 25.21 & 25.133 & 20.427 & & & 48.084 & 12.99 & & & & & & & & & & 46. & 12. & & & & & & 0.348 & \\
\hline$\frac{15}{75}$ & 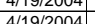 & & $\frac{2.3 .035}{2567 \mid}$ & 25,232 & 25166 & & & & (4l.928 & 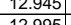 & & $\begin{array}{l}-1.224 \\
-1218\end{array}$ & & & 0 & & & $\frac{734}{733}$ & $\frac{1}{10.20914}$ & 46. & $\frac{1.1}{11 .}$ & & & & & & & \\
\hline & 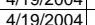 & 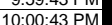 & $\frac{2.0436}{25638}$ & 20.20 & & & & & & & & & & & $\frac{0.09}{0.09}$ & & & & & & & & & & & & & \\
\hline & $4 / 19 / 2000$ & $10: 01: 43$ & 25.659 & 25.22 & $\frac{58}{68}$ & 26.462 & & & & & & -1.218 & & & & & & & & & 1.1. & & & & & & 48 & \\
\hline 76 & $4 / 199 / 200$ & 10:02:43! & | & $\frac{25.224}{25}$ & 25.153 & $\frac{26.402}{26}$ & & 46.736 & \begin{tabular}{|l|l|}
48.005 \\
\end{tabular} & 12.981 & 34.46 & -1.221 & & 49.898 & 0.095 & & & & & & & & & & & & & \\
\hline & & & & & & 26.357 & & & & 12.966 & & & & & & & & & & & & & & & & & & \\
\hline & & 10:04:43 PM & 25.654 & 5.224 & 5.148 & 26.322 & & 46.738 & 47.856 & $\mid 12.98$ & & -1.221 & & & & 14. & & 742 & 12.3 & 46.4 & & & & & & & & \\
\hline & $4 / 1$ & 10:05:43 F & 25.65 & 25.223 & 25 & 26.8 & 24. & 46.408 & 47.70 & 12.899 & & & & 49 & 0.0 & & & 743 & 1238 & 46 & & & 2 & & & & 6 & \\
\hline 76 & 4/19/2004 & 10:06:4 & $25.658 \mid$ & 25.217 & & 26.2 & 24. & 46.24 & 47.38 & 12.856 ] & 34.183 & $-1.2 \quad \mathrm{C}$ & & $48.3 \mathrm{H}$ & $0.0 \mathrm{~s}$ & 14.643 & & 744.9 & 12.4 & & & & & & & & 47 & \\
\hline & 4/19/2 & 10:07:43 F & 25.6 & 25.206 & & & & 46 & 47.7 & $12.891 \mid$ & & & & & 0.05 & & & & & 46 & 12. & & & & & & 5 & \\
\hline 767 & 4/19/2004 & 10:08:43 & 25.636] & 25.2 & 25.05 & 26.154 & 23.9 & 46.729 & \begin{tabular}{|l|l|} 
\\
\end{tabular} & 12.9999 & 34.441 & -1.218 & & 47.749 & 0.05 & 14.643 & & 746. & 12.4 & 46.6 & $11.7 \mathrm{r}$ & & 2.1 & 0.014 & 0.014 & 0.00 & 0.341 & 0.2 \\
\hline$\frac{768}{769}$ & $4 / 19 / 2004$ & $\begin{array}{l}10: 09: 43 \mathrm{PM} \\
01010.43\end{array}$ & $\begin{array}{l}25.6635 \\
25628\end{array}$ & $\frac{25.199}{25.182}$ & \begin{tabular}{|l|}
25.0044 \\
25077
\end{tabular} & 26.0968 & $\frac{23.964}{23892}$ & $\begin{array}{l}46.4 / 4 \\
462155\end{array}$ & \begin{tabular}{|l|}
47.988 \\
47368 \\
\end{tabular} & $\frac{12.9 / 4}{12839}$ & $\begin{array}{l}34.173 \\
34.205\end{array}$ & $\begin{array}{l}-1.218 \\
-1.211\end{array}$ & & $\frac{48.41}{49.3}$ & $\begin{array}{l}0.094 \\
0.093\end{array}$ & $\frac{14.643}{14643}$ & $\frac{0.0}{0.0}$ & 747 & \begin{tabular}{|l|}
$\mid 12.4647$ \\
12.4814
\end{tabular} & $\begin{array}{l}46.5 \\
45.9\end{array}$ & $\frac{11.9}{12.1}$ & $\begin{array}{ll}40.3 \\
40.2\end{array}$ & $\frac{2.180}{2.772}$ & 0.014 & \begin{tabular}{|c|}
0.014 \\
0.014 \\
\end{tabular} & $\mid$ & \begin{tabular}{|c|c|c|}
0.347 \\
0.0344
\end{tabular} & $\frac{0.30}{0.20}$ \\
\hline & & & & & & & & & & & & & & & 0.094 & 14.643 & & & & & & & & & 0.014 & & 0.346 & 0.2 \\
\hline
\end{tabular}


WSRC-TR-2005-00105, REVISION 0

SRNL-RPP-2005-00012, REVISION 0

RUN \# 2.03A AND B; FIRST AND SECOND HALF OF SLURRY DEWATERING - CONT.

\begin{tabular}{|c|c|c|c|c|c|c|c|c|c|c|c|c|c|c|c|c|c|c|c|c|c|c|c|c|c|c|c|c|}
\hline & & & & & & & & & & & & & & & & & & & & & & & & & & & & \\
\hline & A & \begin{tabular}{|c|} 
B \\
\end{tabular} & $\frac{\mathrm{D}}{20}$ & $E$ & & G & $\mathrm{H}$ & $\mathrm{J}$ & $\mathrm{K}$ & $\mathrm{L}$ & $\mathrm{M}$ & $\mathrm{N}$ & 0 & Q & $\mathrm{R}$ & $\mathrm{s}$ & $\mathrm{T}$ & $\mathrm{V}$ & w & $x$ & 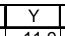 & $z$ & AA & $\frac{A B}{A B}$ & $\mathrm{AC}$ & $A D$ & $\mathrm{AE}$ & AF $\quad$ H \\
\hline & \begin{tabular}{|l|l|}
$4 / 19 / 2004$ \\
$4 / 19 / 2004$
\end{tabular} & \begin{tabular}{|c|}
$10: 12: 43$ PM \\
$10: 13: 22 ~ P M$ \\
\end{tabular} & $\begin{array}{l}25.635 \\
25.625\end{array}$ & $\begin{array}{r}25.195 \\
25.18 \\
\end{array}$ & \begin{tabular}{|l|}
25.119 \\
25.119 \\
\end{tabular} & \begin{tabular}{|l|}
26.078 \\
26.073 \\
\end{tabular} & $\begin{array}{l}23.98 \\
24.13\end{array}$ & \begin{tabular}{|l|}
46.13 \\
46.29 \\
\end{tabular} & $\begin{array}{l}47.592 \\
47.818 \\
\end{array}$ & \begin{tabular}{|r|}
12.88 \\
12.927
\end{tabular} & $\begin{array}{l}33.971 \\
34.152 \\
\end{array}$ & \begin{tabular}{|l|}
-1.215 \\
-1.218
\end{tabular} & $\begin{array}{r}-7.29 \\
-7.293 \\
\end{array}$ & $\begin{array}{l}48.584 \\
55.145 \\
\end{array}$ & $\begin{array}{l}0.093 \\
0.093\end{array}$ & \begin{tabular}{|l|}
14.643 \\
14.643 \\
\end{tabular} & \begin{tabular}{|l|l|}
0.004 \\
0.003
\end{tabular} & $\begin{array}{l}750.9 \\
751.5\end{array}$ & 12.5147 & $\frac{46.1}{46.3}$ & \begin{tabular}{|l|l|}
11.9 \\
126 \\
\end{tabular} & \begin{tabular}{|l|l|}
40.1 \\
40.2
\end{tabular} & $\begin{array}{l}2.761 \\
2.773 \\
\end{array}$ & $\frac{0.014}{0.014}$ & $\begin{array}{l}0.014 \\
0.014 \\
\end{array}$ & $\begin{array}{l}0.000 \\
0.000\end{array}$ & $\begin{array}{l}0.345 \\
0.344 \\
\end{array}$ & $\begin{array}{l}0.29 \\
0.29 \\
\end{array}$ \\
\hline$\frac{1 / 21}{773}$ & & & $2 \frac{25.625}{25.619}$ & & & \begin{tabular}{|l|}
26.013 \\
26.127 \\
\end{tabular} & & & & $\begin{array}{l}1.2 .924 \\
13.006 \\
\end{array}$ & $\frac{34.152}{34.347}$ & \begin{tabular}{|l|}
-1.218 \\
-1.218 \\
\end{tabular} & & & $\begin{array}{l}0.093 \\
0.093 \\
\end{array}$ & \begin{tabular}{|l|}
14.643 \\
14.643 \\
\end{tabular} & & & & & & & & & & & & \\
\hline 774 & $4 / 19 / 2004$ & \begin{tabular}{|l|l}
$10: 15: 22 \mathrm{PM}$ \\
\end{tabular} & 25.625 & 25.164 & 25.118 & \begin{tabular}{|l|l|}
26.188 \\
\end{tabular} & 24.484 & 46.157 & 47.64 & 12.872 & 34.084 & 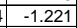 & -7.287 & 49.407 & 0.093 & 14.643 & & & 12.5589 & 46.2 & 12.1 & 40.1 & 2.766 & .014 & & 0.000 & 0.344 & 0.29 \\
\hline 775 & $4 / 19 / 2004$ & \begin{tabular}{|l|l|}
$10: 16: 22 \mathrm{PM}$ \\
\end{tabular} & 25.631 & 25.17 & \begin{tabular}{|l}
25.14 \\
\end{tabular} & \begin{tabular}{|l}
26.289 \\
\end{tabular} & 24.626 & 46.594 & 48.229 & 13.133 & 34.138 & \begin{tabular}{|l|l|}
$3-1.218$ \\
\end{tabular} & & 50.275 & 0.092 & 14.643 & 0.004 & 754.5 & $\begin{array}{l}12.5756 \\
12.576\end{array}$ & 46.8 & \begin{tabular}{|l|l}
12.3 \\
\end{tabular} & 40.4 & & & & & & 0.29 \\
\hline & & & & & & 26.35 & & & 47.947 & & & & & & 0.09 & 14.643 & & & & 46.5 & & 40.0 & & & & & & \\
\hline 777 & $4 / 19 / 2004$ & \begin{tabular}{|l|}
$10: 18: 22 ~ P M$ \\
\end{tabular} & 25.634 & 25.173 & 25.163 & 26.437 & 25.029 & $\begin{array}{l}46.344 \\
\end{array}$ & 48.164 & $\begin{array}{l}13.06 \\
\end{array}$ & 33.965 & -1.218 & -7.278 & 47.428 & 0.092 & 14.643 & & 756.5 & \begin{tabular}{|l|l|}
12.6089 \\
\end{tabular} & 46.7 & 11.6 & 40.2 & 2.769 & 0.014 & 0.014 & 0.000 & 0.340 & \\
\hline$\frac{778}{779}$ & \begin{tabular}{|l|}
$4 / 19 / 2004$ \\
$4 / 192004$ \\
\end{tabular} & $\begin{array}{l}\text { 10:19:22 PM } \\
10.202 \text { PM }\end{array}$ & $\frac{25.641}{25.643}$ & $\begin{array}{l}25.175 \\
25.182\end{array}$ & \begin{tabular}{|l|}
25.115 \\
25.072 \\
\end{tabular} & $\begin{array}{l}26.474 \\
26.491\end{array}$ & $\begin{array}{l}24.936 \\
22813 \\
\end{array}$ & $\begin{array}{l}46.319 \\
4.623 \\
\end{array}$ & \begin{tabular}{|l|l|}
47.866 \\
47.755 \\
\end{tabular} & \begin{tabular}{|l|l|}
13.042 \\
12.988
\end{tabular} & $\begin{array}{l}33.963 \\
33947\end{array}$ & \begin{tabular}{|c|c|} 
& -1.221 \\
& -1218
\end{tabular} & $\begin{array}{l}-7.272 \\
-7.272 \\
\end{array}$ & \begin{tabular}{|l|l|}
49.528 \\
47976 \\
\end{tabular} & $\begin{array}{l}0.092 \\
0.092 \\
\end{array}$ & \begin{tabular}{|l|l|}
14.643 \\
14643
\end{tabular} & 0000 & $\begin{array}{l}757.5 \\
7755.5 \\
\end{array}$ & \begin{tabular}{|l|l|}
12.6256 \\
126422
\end{tabular} & $\begin{array}{l}46.4 \\
46.3\end{array}$ & $\frac{12.1}{11 .}$ & $\begin{array}{l}40.1 \\
40.1 \\
\end{array}$ & 2.768 & $\frac{0.014}{0.014}$ & 0.014 & 0.000 & 0.341 & 0.29 \\
\hline 780 & $\begin{array}{l}4 / 19 / 2004 \\
4 / 19 / 2004\end{array}$ & $\begin{array}{l}10: 20: 22 \mathrm{PM} \\
10: 21: 22 \mathrm{PM}\end{array}$ & 25.028 & 25.177 & & \begin{tabular}{|l|}
20.491 \\
26.446 \\
\end{tabular} & 24.593 & & $\begin{array}{r}4.150 \\
47.86 \\
\end{array}$ & & 33.943 & & & $\begin{array}{l}4.9 / 6 \\
50.354\end{array}$ & $\begin{array}{l}.092 \\
0.092\end{array}$ & $\begin{array}{l}14.643 \\
14.643 \\
\end{array}$ & & $\begin{array}{r}5.58 .5 \\
759.5 \\
\end{array}$ & $\left|\begin{array}{|c|}12.6422 \\
12.6589\end{array}\right|$ & $\frac{46.3}{46.4}$ & \begin{tabular}{|l|l|l}
11.8 \\
123
\end{tabular} & & $2.764 \mid$ & $\frac{0.014}{0.014}$ & $\frac{0.014}{0.014}$ & $\begin{array}{l}0.000 \\
0.000\end{array}$ & $\begin{array}{l}0.341 \\
0.342\end{array}$ & 0.29 \\
\hline 781 & 4/19/2004 & & 25.63 & 25.184 & & & & 46.188 & & 13.007 & 33.846 & & & & 0.092 & 14.643 & & $\begin{array}{l}159.5 \\
7760.5\end{array}$ & \begin{tabular}{|l|}
12.65095 \\
12.6756 \\
\end{tabular} & 46.4 & & $\frac{40.1}{40.0}$ & & & & 0.000 & $\frac{0.342}{0.342}$ & $\frac{0.29}{0.299}$ \\
\hline 782 & $4 / 19 / 2004$ & 10:23:22 PM & 25.625 & 25.184 & 25.069 & 26.358 & 24.34 & 45.92 & 47.723 & 12.921 & 33.639 & 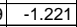 & -7.278 & 499.428 & 0.092 & 14.643 & & 761.5 & 12.6922 & 46.3 & 12. & & & & & & & \\
\hline 783 & $4 / 19 / 2004$ & 10:24:22 PM & & & & 26.307 & & & & & 34.076 & -1.218 & & & 0.092 & & & 762.5 & 2.7089 & 46.3 & & 2027 & & & & 0.000 & 0.344 & 0.29 \\
\hline 784 & $4 / 19 / 2004$ & 10:25:22 PM & & & & $263277>$ & & 46.271 & 47.951 & 13.009 & 33.988 & -1.218 & & & & $\begin{array}{ll}14.643 \\
\end{array}$ & & 763 & & 465 & & & & & & & 0.341 & \\
\hline 7875 & $4 / 19 / 2004$ & 10:26:22 PM & 25.606 & 25.176 & 25.075 & 26.269 & & 46.105 & & 12.958 & 33.805 & -1.221 & & 50.1 & 0.091 & 14.643 & & 764.5 & 12.7422 & 46. & & & & & & & & \\
\hline 786 & $4 / 19 / 2004$ & 10:27:22 PM & 25.596 & 25.17 & 25.075 & 26.179 & 23.895 & 46.439 & 48.289 & 13.032 & 34.082 & -1.221 & -7 & 49.973 & 0.091 & 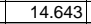 & & 765.5 & $\begin{array}{ll}12.7589 \\
\end{array}$ & $\begin{array}{lll}46.8 \\
\end{array}$ & 12.2 & 40.3 & 2.776 & 0.014 & 0.014 & 0.000 & 0.336 & \\
\hline & $4 / 19 / 2004$ & 10:28:22 PM & & 25.169 & 25.118 & 26.137 & 23.83 & 46.341 & 48.098 & 13.063 & 34.027 & -1.22 & & 47.931. & 0.091 & 14.643 & & 766.5 & 12.7756 & 46.6 & & 40. & 2.771 & & & & 0.337 & \\
\hline & 年 & 10:29:22 PM & & & & & & & & 12.975 & 33.748 & & & & 0.091 & 14.643 & & & 1922 & & & & & & & & & \\
\hline 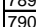 & $4 / 199 / 2004$ & $\begin{array}{l}10: 30: 22 \mathrm{PM} \\
0.22 \mathrm{MM}\end{array}$ & $\frac{25.62}{25}$ & 25.17 & 25.124 & 26.053 & 23.809 & 46.248 & 47.926 & 12.905 & 34.095 & $-\frac{1.215}{120}>0$ & & $\frac{49.17}{50.72}$ & 0.092 & 14.643 & & & . 80899 & 46.5 & & & & & & & 0.340 & \\
\hline & $\frac{4 / 1 / 9 / 2004}{4 / 19 / 2004}$ & $\begin{array}{l}10.31 .27 \mathrm{PM} \\
0.320 \mathrm{M}\end{array}$ & $\frac{2.0034}{25612}$ & 25.148 & 25.118 & $\frac{2.051}{26025}$ & 23037 & 46.11 & & 12.84 & $\frac{34.086}{34066}$ & $\frac{-1.22}{1.22}>>2$ & & 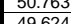 & & $\frac{14.643}{14642}$ & & $\frac{169}{770}$ & 8120 & 46.5 & & & & & & & & \\
\hline & $4 / 19 / 2004$ & $\begin{array}{l}10: 33: 2 \mathrm{PM} \\
10,33\end{array}$ & 25 & $\frac{25.142}{25.132}$ & 25 & $\frac{2.0601}{26.01}$ & & 46.474 & 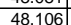 & 12.954 & $\frac{3.247}{33.247}$ & & & 477.97 & 0.091 & 14.643 & & 771.5 & 12.8589 & & 118 & 404 & 2783 & 014 & & 0.000 & 0.0355 & 0.29 \\
\hline & 4/19/2004 & 10:34:22 PM & 25.618 & $\begin{array}{ll}25.137 \\
\end{array}$ & \begin{tabular}{|l|l|}
25.161 \\
\end{tabular} & 26.075 & 24.247 & 45.985 & 47.65 & 12.92 & 33.78 & \begin{tabular}{|l|l|}
9 & -1.218 \\
\end{tabular} & -7.2 & 48.957 & 0.091 & 14.643 & & 772.5 & 12.8756 & 46.2 & 12.0 & 39.9 & & 0.014 & & 0.000 & 0.339 & s) \\
\hline & & 10:35:22 PM & 25.619 & 25.138 & 25.168 & 26.107 & & 46.00 & 48.077 & 12.826 & 33.893 & -1.224 & & 47.447 & 0.091 & 14.643 & & 773.5 & 12.8922 & 46.6 & & & & & & & 0.338 & \\
\hline & 41119/20004 & 10:36:22 PM & 25.61 & 25.129 & 25.178 & 26.163 & 24.404 & 46.144 & 48.108 & 12.884 & 33.914 & & & 47.899 & 0.09 & 14.643 & & 7774.5 & 9089 & 46.6 & & & & & & & & \\
\hline & $\begin{array}{l}4 / 1 / 19 / 2004 \\
4 / 1904 \\
\end{array}$ & $\begin{array}{l}10.37 .2 \mathrm{PMM} \\
10.38 \mathrm{PM}\end{array}$ & 告. & $\frac{25.135}{25138}$ & 25.13 & $\frac{20.274}{26320}$ & $\frac{24.526}{24.549}$ & 告5.918 & 年 47.6067 & 12.849 & $\frac{3.375}{32071}$ & 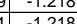 & & 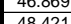 & - & 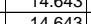 & & 717.5 & 年 & 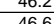 & 110 & 401 & & & & & & 0.25 \\
\hline 798 & 4 & $10.39 .2 \mathrm{PM}$ & 25615 & 25.20 & \begin{tabular}{|l|}
25129 \\
25129
\end{tabular} & 26048 & & & $\begin{array}{l}40.050 \\
48.067\end{array}$ & 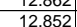 & 33.988 & $\begin{array}{l}-1.21 \mathrm{c} \\
-1.221 \\
\end{array}$ & & $\frac{20.421}{49918}$ & 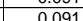 & (14.045 & & & 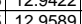 & & $\frac{1.1 .}{122}>>3$ & 401 & & & & & & 0.29 \\
\hline & $4 / 19 / 2004$ & 10.40.2 PM & $\frac{2.0 .016}{2561}$ & 25.136 & \begin{tabular}{|l|}
25.129 \\
25.125 \\
\end{tabular} & 26.414 & $\frac{24.05}{24.581}$ & 46.124 & $\begin{array}{l}40.001 \\
47.847\end{array}$ & $\begin{array}{l}12.056 \\
12.816\end{array}$ & $\frac{30.06}{34.125}$ & -1.221 & & $\begin{array}{l}45.940 \\
48.988 \\
\end{array}$ & $\frac{0.051}{0.09}$ & $\begin{array}{l}14.045 \\
14.643\end{array}$ & & 778.5 & 2.9756 & $\frac{70.0}{46.4}$ & $\frac{12.4}{12 .}$ & 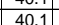 & & & & & 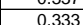 & 0.29 \\
\hline & $4 / 19 / 2004$ & 10:41:22 PM & 25.618 & 25.147 & 25.142 & & 24.438 & 45.893 & 47.742 & 12.868 & $33.80 \mathrm{~S}$ & $-1.218 \quad \mathrm{C}$ & & & 0.09 & & & 779.5 & 2.9922 & 46.3 & $12.3 \quad>>$ & 39.9 & & & & & 335 & \\
\hline 801 _ & 4/19/20004 & 10:42:22 PM & 25.623 & 25.162 & 25.142 & 26.226 & 24.253 & 45.831 & 47.932 & 12.978 & 33.524 & -1.221 & & 48.281 & 0.09 & $\begin{array}{l}14.045 \\
14.643\end{array}$ & & 780.5 & | 1.30089 & 46.5 & 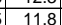 & $\begin{array}{ll}39.7 \\
39.7\end{array}$ & & 势1 & & $\frac{0.000}{0.000}$ & 0.337 & \\
\hline & $4 / 19 / 2004$ & 10:43:22 PM & 25.628 & 25.157 & 25.142 & 26.206 & 24.16 & 46.03 & 47.924 & 13.028 & 33.783 & $\frac{-1.218}{-1.218}$ & & 48.221 & $\begin{array}{ll}0.089 \\
\end{array}$ & 14.643 & & 781 & , & 46.5 & & . & & & & & 0.331 & \\
\hline & $4 / 19 / 2004$ & 10:44:22 PM & 25.616 & 25.166 & 25.14 & 26.099 & 2309 & 45.916 & & & & & & & 0.09 & 14.643 & & 782.5 & 30422 & 46.2 & & 39 & & & & & & \\
\hline & $4 / 19 / 2004$ & 10:45:22 PM & 25.609 & 25.174 & 25.158 & 26.082 & 23.904 & 45.862 & 47.882 & 12.944 & 33.64 & -1.218 & & 49.255 & 0.089 & \begin{tabular}{ll|l|}
14.643 \\
\end{tabular} & & 783.5 & 13.0589 & 46.4 & 12.1 & $39.8 \mathrm{C} \quad \mathrm{C}$ & & 013 & 0.013 & 0.000 & 0.332 & \\
\hline & 4/19/2004 & 10:46:22 PM & & 25.182 & 25.182 & 20.000 & & 45.877 & 40.075 & 12.987 & 33.594 & -1.221 & & $\begin{array}{lll}48.346 \\
\end{array}$ & 0.09 & 14.643 & & 78 & 3.0756 & 46. & 11 & . & & & & & 0.336 & \\
\hline 80 & 4/19/2004 & 10:47:22 PM & 25.617 & 25.181 & 25.166 & 25.964 & 23.85 & 46.147 & & 13.046 & & & & 49.854 & 0.09 & & & & & 46.6 & $12.2 \mathrm{r}-\mathrm{C}$ & 40.0 & 2.75 & & & & 334 & \\
\hline 80 & 4/11992/2004 & $2 \mathrm{PM}$ & 25.62 & 25.174 & 25.183 & 25.937 & 23.74 & 45.829 & 48.052 & 13.04 & 33.395 & -1.221. & & 47.8 & 0.089 & 14.643 & & 786 & 3.1089 & 46.6 & 11.7. & 39 & & & & 0.00 & & \\
\hline & & & & & & & & & & 年 12.997 & & & & 49.0 & & & & & & & & & & & & & & \\
\hline & $\begin{array}{l}4 / 19 / 2004 \\
4 / 19 / 2004 \\
\end{array}$ & $10.50 .25 \mathrm{M}$ & 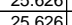 & $\frac{25.171}{2516}$ & 251.45 & 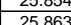 & $\frac{23.110}{2382}$ & 年 46.9982 & $\mid \begin{array}{l}4.1 .610 \\
4829\end{array}$ & - & 年. & $\frac{-1.210}{-1224}$ & & $\begin{array}{l}46.024 \\
550173\end{array}$ & $=0.088$ & 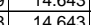 & & $\frac{788.5}{789.5}$ & 势. & 年0.4 467 & $\frac{11.8}{123}$ & 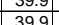 & & & & 0.000 & 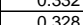 & \\
\hline 811 & $4 / 19 / 2004$ & $10: 52: 22 \mathrm{PM}$ & 25.626 & 25.17 & \begin{tabular}{|l|}
25.134 \\
2.134
\end{tabular} & 25.928 & & $\frac{4.006}{45.46}$ & 47.412 & \begin{tabular}{|l|l|l|}
12.923 \\
1.04
\end{tabular} & 33.28 & -1.218 & & 47.814 & & $\begin{array}{ll}14.045 \\
14.643 \\
\end{array}$ & & & 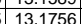 & & 117 & ( & & & & & & \\
\hline & $4 / 19 / 2004$ & $\begin{array}{l}10: 53: 22 \text { PM } \\
10.53\end{array}$ & $\frac{25.606}{25}$ & 25.145 & 25.144 & 25.928 & 24.2 & 45.553 & & 12.919 & $\frac{2.43}{3.342}$ & $\frac{-1.218}{-1.218}$ & & 48.892 & 0.088 & 14.643 & & 79 & $\frac{6.1922}{3.1922}$ & 46 & 12. & $\frac{59.5}{39.5}-2$ & & & & & 0.331 & \\
\hline & $4 / 19 / 2004$ & 10:54:22 $\mathrm{F}$ & 25.612 & 25.146 & 25.141 & 26.04 & & 45.767 & $\begin{array}{l}47.679 \\
\end{array}$ & & 33.647 & -1.218 & & & & 14.6 & & & & & & & & & & & & \\
\hline & $4 / 19 / 2004$ & 10:55:22 PM & 25.598 & 25.147 & 25.141 & 26.045 & 24.587 & & 47.92 & 13.01 & 33.471 & -1.224 & & 4 & & & & & & & 12.1 & ( & & & & & & \\
\hline & 4/199/2004 & 10:56:22 PM & 25.604 & $\begin{aligned} 25.158 \\
25.18\end{aligned}$ & \begin{tabular}{|l|}
25.178 \\
25.179
\end{tabular} & $\frac{26.102}{26.243}$ & $\frac{24.689}{2478}$ & $\begin{array}{r}45.543 \\
45636\end{array}$ & $\begin{array}{r}47.7 \\
\end{array}$ & & 33.46 & -1.24 & & 50.5 & 0.089 & $\begin{array}{r}14.643 \\
1.643\end{array}$ & & 794.5 & 13.2422 & 46.2 & 12.4 & $\begin{array}{l}39.5 \\
30.5 \\
\end{array}$ & \begin{tabular}{|l|l|}
2.724 \\
\end{tabular} & & & 0.000 & 0.334 & 0.28 \\
\hline 817 & | $4 / 19 / 2004$ & $\begin{array}{l}10: 5: 58.22 \mathrm{PM} \\
10: 58: 2 \mathrm{PM}\end{array}$ & $\frac{25.615}{25.608}$ & 25.187 & \begin{tabular}{|l|}
25.182 \\
252
\end{tabular} & \begin{tabular}{|l|}
20.245 \\
26.261 \\
\end{tabular} & 24.76. & $\begin{array}{l}45.636 \\
45.869 \\
\end{array}$ & $\begin{array}{l}48.123 \\
48.034 \\
\end{array}$ & \begin{tabular}{|c|}
13.022 \\
13.5
\end{tabular} & 33.598 & \begin{tabular}{|l|l|} 
& -1.212 \\
8 & -1.221
\end{tabular} & & 48.4 & $\begin{array}{l}0.089 \\
0.089\end{array}$ & 14. & & 7966.5 & 13.27 & $\frac{46.3}{46.6}$ & $\begin{array}{l}12.1 \\
12.0 \\
\end{array}$ & $\begin{array}{l}39.5 \\
39.7 \\
\end{array}$ & \begin{tabular}{|l|}
2.726 \\
2.739 \\
\end{tabular} & $\frac{0.013}{0.013}$ & & 0.000 & $\begin{array}{l}.334 \\
0.332\end{array}$ & 0. \\
\hline 818 & $4 / 19 / 2004$ & 10:59:22 PM & 25.609 & 25.184 & \begin{tabular}{|l|l|} 
& 25.198 \\
\end{tabular} & 26.277 & 24.664 & $\begin{array}{l}45.699 \\
\end{array}$ & & 13.065 & 33.265 & 218 & & 49. & & & & 797.5 & 133.2922 & 46.5 & 12.2. & 39.5 & & 0. & & & 0.330 & \\
\hline & $4 / 19 / 2004$ & & 25.621 & 25.195 & 25.185 & 26.269 & 24.396 & 45.47 & 47.696 & 12.958 & 33.315 & -1.218 & & 51.8 & & & & 798.5 & 13.3 & 46.2 & $12.7 \mathrm{r}-\mathrm{x}$ & 39.4 & & & & & 0.331 & \\
\hline & 4/19/2004 & 11:01: & 25.615 & 25.194 & 25.194 & 26.218 & & 45.416 & $\begin{array}{r}47.501 \\
48.39 \\
\end{array}$ & $\frac{12.923}{13187}$ & 33.5 & $-\frac{-1.221}{1213}$ & & 47. & & & & 799 & 13.3256 & 46.0 & 11.7 & 39.4 & & & & & 0.332 & 0. \\
\hline & $\frac{41912004}{4192004}$ & M & $\frac{25.03}{25.61}$ & $\frac{25.298}{25218}$ & \begin{tabular}{|l|l|l|l|}
25198 \\
\end{tabular} & $\frac{20.100}{25.977}$ & & $\frac{40.04}{4562}$ & 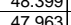 & $\frac{15.14}{130}>$ & $\frac{3.4424}{3325}$ & $\frac{-1.21}{-121}$ & & & & & & & & & $\frac{12.1}{125}>0$ & & & & & & & \\
\hline & 4 & $11.04 .2 \mathrm{PM}$ & 25.009 & 25.233 & 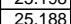 & 25.936 & & 45.093 & & 13.171 & $\frac{1.254}{3544}$ & & & & & & & & & & . & - & & & & & & 0.28 \\
\hline & $\frac{4 / 199 / 2004}{4}$ & $\begin{array}{l}\frac{11: 05: 22 \mathrm{PM}}{11} \\
\end{array}$ & 25.619 & $\frac{25.263}{25.263}$ & 25.213 & $\frac{25.901}{25.901}$ & $\frac{24.01}{24.01}$ & 45.879 & $\mid \begin{array}{l}48.108 \\
48.108\end{array}$ & 13.026 & 33.586 & $\frac{-1.224}{-1.24}$ & & 48.6 & & & & & $\frac{13.3}{13 .}$ & & 118 118 & 39. & & & & & 0.325 & \\
\hline & & & & & & & & & & & & & & & & & & & & & & & & & & & & \\
\hline & $4 / 19 / 2004$ & 11:07:22 PM & 25.622 & 25.317 & 25.091 & 25.8 & & 45.65 & 47.826 & 12948 & 33.424 & $-1.218 \quad>$ & & 49.8 & & & & & & 46.4 & 12. & & & & & & 335 & \\
\hline & $4 / 19 / 2$ & 11:08: & & 25.358 & \begin{tabular}{|l|l|}
25.107 \\
\end{tabular} & 25.756 & & 45.59 & 47.889 & 12.9 & 33.3 & -1.22 & & & & & & 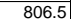 & & 46 & 11.8 & & & & & & 28 & \\
\hline & 4/19/2004 & 11:09: & 25 . & 25.367 & 25 . & & & 46.032 & 48.409 & \begin{tabular}{|l|l|}
12.959 \\
\end{tabular} & 33.848 & -1.221. & & $49.8 \mathrm{C}$ & 0.0 & \begin{tabular}{|c|c|}
14.643 \\
\end{tabular} & & 80 & 13.4 & 46 & $12.2 \mathrm{r}-\mathrm{l}$ & 39.9 & $2.7>$ & & & & 0.327 & \\
\hline & $4 / 199 / 2004$ & 11:10:22 F & 25.59 & 25.367 & \begin{tabular}{|l|l|}
25.111 \\
\end{tabular} & 25.67 & & 45.65 & 47.982 & 12.8 & 33.576 & -1.21 & & & & & & 80 & 13.4 & 46 & 11.9 & 39 & & & & & 0.326 & \\
\hline 85 & & 11:11:22 & 25.592 & 25.376 & 25.12 & 25.659 & & 45.808 & 47.9 & 12.967 & 33.658 & -1.221. & & 49.4 & & & & 8 & 513.4 & 46.5 & 12. $\mathrm{c}$ & 39.7 & 2.1. & & & & 0.329 & \\
\hline & 4/119/20004 & 11:12:22 PM & $\begin{array}{l}2.5592 \\
2.592\end{array}$ & 25.392 & 25.141 & 25.705 & & 45.53 & 48.025 & $12.893 \mathrm{r}$ & 33.245 & -1.218 & & 48. & 0.087 & $14.6 \mathrm{C}$ & & 81 & & 46.6 & 11. & 39. & & & & & & \\
\hline & 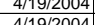 & 11:13:22 PM & 2558 & 255381 & \begin{tabular}{|l|l|l|l|l|} 
\\
25.152
\end{tabular} & 25.11 & 24.1 & (45.361 & 4.802 & $\frac{12.9}{12.9}+3$ & 33.188 & -1.218 & & & & 14.6 & & 811 & 1.3 .2566 & 46.3 & $\frac{12.0}{12.1} 1 \mathrm{C}$ & 300 & & & & & (2) & \\
\hline & 4 & $1.14 .25 \mathrm{MM}$ & 2.50 & $\frac{23.310}{25305}$ & 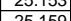 & $\frac{23.051}{25012}$ & & (35.1799 & 40.270 & & 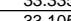 & -1.210 & & (a) & & & & $\frac{11}{91}$ & 10.35 & & $\frac{12.1}{122}>>2$ & & & & & & & \\
\hline & $\frac{4151204}{41 / 92004}$ & 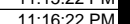 & 25.58 & 25,204 & $\frac{153}{193}+2$ & $\frac{25.545}{26012}$ & $24.7-3$ & 46.18 & 年 48.67 & 1311 & (3303 & -1218 & & & & 14 & & 81 & & & 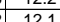 & & & & & & & \\
\hline 836 & & & & 25.407 & & & & $\mid 45.69$ & & $\frac{1.29}{12.9}>$ & 33.541 & -1221 & & & & & & & & & 122 & & & & & & & \\
\hline & $4 / 19 / 2004$ & & 25.585 & 25.399 & 25.174 & 26083 & & 45.491 & & & & & & & & & & & & & & & & & & & & \\
\hline & & & & 25381 & & & & & & & 33.372 & & & & & & & & & & & & & & & & & \\
\hline & 411910004 & $11.20 .22 \mathrm{PM}$ & & & 2510 & & & & & & 32327 & -1221 & & 87 & & 14.643 & & & 35152 & 466 & 120 & & & 213 & & & 0328 & \\
\hline 840 & $4 / 19 / 2004$ & \begin{tabular}{|l|}
$11: 21: 22$ \\
\end{tabular} & $25.584 \mathrm{I}$ & 25.384 & 25.188 & 25.972 & 24.16 & $\mid 45.846$ & \begin{tabular}{|l|l|}
48.227 \\
\end{tabular} & \begin{tabular}{|l|l|}
13.015 \\
\end{tabular} & 33.625 & -1.221 & & 48.936 & & 14.643 & & 819.5 & 136589 & & & & 2.740 & 0.013 & 0.013 & 0.00 & 0.325 & \\
\hline
\end{tabular}


WSRC-TR-2005-00105, REVISION 0

SRNL-RPP-2005-00012, REVISION 0

RUN \# 2.03A AND B; FIRST AND SECOND HALF OF SLURRY DEWATERING - CONT.

\begin{tabular}{|c|c|c|c|c|c|c|c|c|c|c|c|c|c|c|c|c|c|c|c|c|c|c|c|c|c|c|c|c|}
\hline & $\mathrm{A}$ & 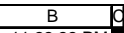 & D & $\mathrm{E}$ & $\mathrm{F}$ & $\mathrm{G}^{-1}$ & $\mathrm{H}$ & $\mathrm{J}$ & $\mathrm{K}$ & $\mathrm{L}$ & $\mathrm{M}$ & $\mathrm{N}$ & $\mathrm{O}$ & $Q$ & \begin{tabular}{l|l}
$\mathrm{R}$ & \\
\end{tabular} & \begin{tabular}{l|l}
$\mathrm{s}$ \\
\end{tabular} & $\mathrm{T}$ & $\mathrm{V}$ & w & $\mathrm{x}$ & $\mathrm{Y}$ & $\mathrm{z}$ & $\mathrm{AA}$ & $A B$ & $A C$ & $A D$ & $\mathrm{AE}$ & AF $A$ \\
\hline & $4 / 199 / 2004$ & $\begin{array}{l}1: 22: 22 \mathrm{PM} \\
11 \cdot 23: 22 \mathrm{PM}\end{array}$ & 25.595 & 25.389 & 25.209 & $\begin{array}{l}25.897 \\
25826 \\
\end{array}$ & $\begin{array}{l}24.079 \\
23989\end{array}$ & $\frac{45.636}{45.52}$ & 48.369 & $\begin{array}{l}13.049 \\
13062\end{array}$ & $\begin{array}{l}33.107 \\
332012 \\
\end{array}$ & -1.21 & $\begin{array}{l}-7.215 \\
-7217 \\
-217\end{array}$ & & $\begin{array}{l}0.087 \\
0.07 \\
\end{array}$ & $\begin{array}{ll}14.643 \\
1.63\end{array}$ & $\begin{array}{l}0.003 \\
.003\end{array}$ & & $\begin{array}{l}53.6756 \\
12602\end{array}$ & 46.9 & 12.6 & & & 0.013 & 0.013 & 0.000 & 0.3 & \\
\hline & & & & & & & & & & & & & & & & & & & & & & & & & & & & \\
\hline 844 & $\begin{array}{l}/ 19922004 \\
4 / 19 / 2004 \\
\end{array}$ & $\begin{array}{l}11: 2.22 .2 \mathrm{PM} \\
11: 25: 22 \mathrm{PM}\end{array}$ & $\begin{array}{l}25.5932 \\
25.592 \\
\end{array}$ & $\frac{25.363}{25.356}$ & \begin{tabular}{|l|}
25.1916 \\
25.216 \\
\end{tabular} & \begin{tabular}{|l|}
25.1966 \\
25.774 \\
\end{tabular} & $\begin{array}{l}23.841 \\
23.736 \\
\end{array}$ & $45 . .323$ & \begin{tabular}{|l|}
48.285 \\
47.866 \\
\end{tabular} & $\begin{array}{l}\frac{13.021}{12.952} \\
\end{array}$ & $\begin{array}{l}33.397 \\
33.023 \\
\end{array}$ & \begin{tabular}{|l|l|}
-1.21 \\
\end{tabular} & $\begin{array}{l}-7.217 \\
-7.215 \\
\end{array}$ & $\begin{array}{l}48.748 \\
51.283 \\
\end{array}$ & $|0.087|$ & \begin{tabular}{|l}
14.643 \\
14.643 \\
\end{tabular} & $\begin{array}{l}0.003 \\
0.004\end{array}$ & $\frac{822.5}{823.5}$ & \begin{tabular}{|l|l|}
13.009 \\
13.7256 \\
\end{tabular} & $\begin{array}{l}-46.8 \\
46.4 \\
\end{array}$ & $\frac{11.9}{12.6}$ & 39.2 & 2.701 & $\frac{0.01}{0.01}$ & $\frac{0.013}{0.013}$ & $\begin{array}{l}0.000 \\
0.000 \\
\end{array}$ & 0.326 & 0.28 \\
\hline & $4 / 19 / 2004$ & 11:26:22 PM & 25.585 & 25.34 & 25.219 & \begin{tabular}{|l|}
25.708 \\
\end{tabular} & 23.714 & 45.609 & \begin{tabular}{|l|l|}
48.1444 \\
\end{tabular} & 13 & 33.304 & -1.22 & -7.223 & 52.033 & $\begin{array}{l}0.086 \\
\end{array}$ & 14.643 & 0.003 & 824.5 & 13.7422 & 46.7 & & \begin{tabular}{|l|}
39.5 \\
\end{tabular} & & & 0.013 & 0.000 & 0.323 & \\
\hline & & & & & & & & & & & & & & & & 14.643 & & & 13.7589 & 46.9 & & & & & & & & \\
\hline & 4/19/2004 & 1:28:22 PM & 25.578 & 25.327 & 25.147 & 25.615 & 23.747 & 45.5. & 48.226 & 13.006 & & & $\begin{array}{l}-7.215 \\
-717 \\
-717\end{array}$ & $3.47:$ & 0.087 & 14.643 & 0.0 & 826 & 3.7756 & 46.8. & 11. & & & & & & & \\
\hline & 些04 & 11:29:22 PM & 25.553 & 20.517 & 25.11 & 25.64 & 23.8 & 45.275 & 17.773 & $\frac{12.932}{12923}$ & & & & & 0.086 & 14.643 & & & 3.7922 & 46. & 12. & & & & & .000 & $\begin{array}{l}0.326 \\
0.205 \\
0.05\end{array}$ & \\
\hline & 4 & 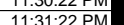 & 25563 & 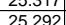 & 25106 & 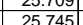 & $\frac{24.050}{24232}$ & 年55.440 & 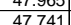 & 12865 & & & -72 & 17566 & & 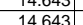 & & & & . & & & & & & & & \\
\hline 851 & $4 / 19 / 2004$ & $11: 32: 22$ PM & 25.559 & 25.294 & \begin{tabular}{|l|l|} 
& 25.128 \\
\end{tabular} & 25.817 & 24.244 & 4..1448 & 48.158 & 12.968 & 33.224 & $-1.218 \mathrm{~S}$ & --7.217 & 50.217 & 0.086 & $\begin{array}{l}14.643 \\
14.643\end{array}$ & 0.003 & $\frac{50.5}{830.5}$ & $\frac{13.8422}{13.42}$ & 46.7 & 123 & & & & 0.013 & 0.000 & 0.325 & $\frac{0.28}{0.28}$ \\
\hline & 4/19/2004 & 11:33:22 PM & 25.555 & & \begin{tabular}{|l|}
25.159 \\
\end{tabular} & 25.858 & 24.31 & & \begin{tabular}{|l|l|}
47.677 \\
\end{tabular} & 12.972 & 33.025 & -1.21 & -7.212 & 50.281 & 0.083 & 14.643 & 0.004 & & 13.8589 & & & & & & & & & \\
\hline & & & & & & & & & & & & & & & 0.086 & & & & & & & & & & & & & \\
\hline 854 & 4/19/2004 & 11:35:22 PM & 25.548 | & 25.298 & 25.162 & 25.931 & 24.458 & 45.607 & 48.394 & 13.056 & 33.206 & .218 & -7215 & 88.097 & 0.085 & 14.643 & 0.003 & & 3.8922 & 46.9 & 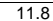 & & 717 & 013 & .013 13 & $\overline{000}$ & 20 & \\
\hline & & & & & 25.164 & & & & & & 33.044 & & & & & & & & & 46.1 & & & & & & & & \\
\hline & 4/19/2004 & 11:37:22 PM & 25.552 & 25.301 & 25.171 & 25.999 & 24.462 & 45.336 & 488.027 & 12.944 & 33.089 & 1.218 & & 49.042 & $\begin{array}{l}0.086 \\
\end{array}$ & 14.643 & & & 3.9256 & 46.6 & $120+3$ & & .704 & & & 0.000 & & \\
\hline 85 & 4/19/2004 & 11:38:22 PM & 25.553 & 25.308 & 25.157 & 26.016 & 24.258 & 45.446 & 48.222 & 13.026 & 33.103 & 1.22 & -7.203 & 51.072 & 0.085 & 14.643 & 0.003 & 836.5 & 13.9422 & 46.7 & 12.5 & 39.3 & 2.708 & . & 0.013 & 0.000 & 0.321 & \\
\hline & $4 / 19 / 2004$ & 11:39:22 PM & 25.554 & 25.319 & 25.178 & 25.962 & 24.104 & 45.421 & 48.208 & 12..982 & 33.163 & 1.22 & -7.194 & 49.622 & 0.086 & 14.643 & 0.003 & 837.5 & 13.9589 & 46.7 & 12.2 & & 2.709 & & & 0.000 & & \\
\hline & 4/19/2004 & 11:40:22 PM & 25.56 & 25.32 & 25.209 & 25.943 & 24.0355 & 45.151 & 47.94 & 12.938 & 32.874 & & -7.19 & 49.981 & 0.086 & 14.643 & 0.003 & & 13.9756 & 46.5 & 12.2 & & 2.690 & & & 0.000 & & \\
\hline & $4 / 19 / 2004$ & 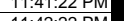 & & & & & & & 48.45 & & & & & & & & & & & & & & & & & & & \\
\hline 年 & $\frac{41912004}{41 / 2004}$ & $\frac{11: 42: 22 \mathrm{PM}}{111: 33 \cdot 23 \mathrm{PM}}$ & $\begin{array}{r}25.56 \\
25564\end{array}$ & $\frac{25.305}{25309}$ & $\begin{array}{l}25.194 \\
25203 \\
\end{array}$ & 先. & $\frac{23.894}{23.89}$ & $\begin{array}{l}45.164 \\
45508\end{array}$ & $\begin{array}{l}47.984 \\
8.121\end{array}$ & $\frac{12.886}{12.957}$ & 作 & 25 & & 部.558 & 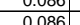 & 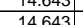 & & $\frac{84}{84} \cdot x-1$ & 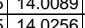 & $\frac{46.5}{46 .}$ & & & 717 & & & 0.000 & & \\
\hline & $4 / 19 / 2004$ & 1.4.4.22 PM & $\frac{2.5057}{25.74}$ & 25.313 & \begin{tabular}{|l|}
25.243 \\
\end{tabular} & $\frac{2.3716}{257616}$ & $\frac{23.053}{23.793}$ & 45.555 & 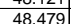 & 年. & 33.305 & $-1.218 \mathrm{~S}$ & 719 & 48.496 & 0.006 & 1464 & & & 140420 & $\frac{100}{470}$ & 119 & $\frac{3.94}{394}$ & & & $0.01:$ & 0.000 & 032 & \\
\hline 864 & $4 / 19 / 2004$ & 11:45:22 PM & 25.568 & 25.293 & \begin{tabular}{|l|}
25.217 \\
\end{tabular} & 25.67 & 23.807 & 45.329 & 48.115 & & 33.153 & $\frac{1.21}{-1.218}$ & -7.212 & 49.253 & 0.085 & 14.643 & 0.003 & 843 & $\begin{array}{l}14.0589 \\
\end{array}$ & 46.6 & $\begin{array}{ll}\frac{11.9}{12.1} \\
\end{array}$ & 39.2 & \begin{tabular}{|l|}
2.119 \\
2.706 \\
\end{tabular} & & 0.013 & 0.000 & & \\
\hline 86 & $4 / 19 / 2004$ & 11:46:22 PM & 25.561 & 25.275 & 25.225 & 25.618 & 23.655 & 45.5 & 48.208 & 12.902 & 33.376 & $\frac{1.21}{4}$ & & 48.41 & 0.086 & 14.643 & 0.003 & 844 & 14.0756 & 46.7 & $\frac{11.9}{11.9}>>$ & & $\begin{array}{l}.719 \\
\end{array}$ & $\overline{013}$ & 0.01 & 0.000 & & \\
\hline & $4 / 199 / 2004$ & 11:47:22 PM & 25.57 & 25.279 & 25.214 & 25.582 & 23.749 & 45.784 & 48.759 & & 33.339 & 1.218 & & 49.134 & $\begin{array}{ll}0.085 \\
\end{array}$ & 14.643 & 0.003 & 845 . & 14.0922 & 47.3 & 12.0 & 39.6 & & & 0.01 & 0.000 & 0.318 & \\
\hline & $4 / 199 / 2004$ & 11:48:22 PM & & & & & & & 47.963 & & & & & & & & & & & 46.5 & & & & & & & & \\
\hline & 4/19/2004 & 11:49:22 PM & 25.554 & 25.263 & 25.103 & 25.701 & 24.133 & 45.618 & 48.345 & 12.881 . & 33.46 & & & 48.725 & $\begin{array}{l}0.086 \\
\end{array}$ & 14.643 & & & 14.1256 & 46.9 & 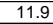 & & & & & & & \\
\hline 86 & 4/19/2004 & 11:50:22 PM & 25.555 & 25.264 & 25.113 & 25.717 & 24.229 & 45.667 & 48.629 & 12.859 & 33.499 & 1.21 & & 48.55 & 0.085 & 14.643 & 0.003 & & 14.1422 & & 11.9 & & & & & 0.000 & 0.319 & \\
\hline$\frac{87}{72}>2 x$ & 4/19/2004 & 11:51:22 PM & 25.545[ & 25.265 & 25.134 & 25.753 & 24.33 & 45.331 & 48.374 & 12.853 & 33.196 & 122. & -7.21 & 46.20 & 0.085 & 14.643 & & 849 & 14.1589 & 46.9 & 11.3 & & 707 & & 0.01 & 0.000 & & \\
\hline & 4/19/2004 & 11:52:22 PM & 25.546] & 25.26 & 25.125 & 25.849 & 24.481 & 45.188 & 48.253 & $12.861 \mid$ & 32.952 & & & & 0.086 & 14.643 & & & & & & & & & & & & \\
\hline & 4/19/2004 & 11:53:22 PM & $23.54 \pi$ & 25.271 & 25.146 & 25.869 & 24.527 & 45.63 & 48.56 & 12.95 & 33.276 & & & 46.266 & 0.085 & 14.643 & 0.003 & & & 47.1 & 1. & & & & 01. & 000 & & \\
\hline & $\frac{41512004}{41 / 9204}$ & 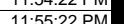 & 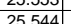 & 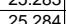 & 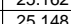 & $\frac{2.051}{25.96}$ & $\frac{24.590}{24.579}$ & 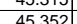 & 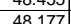 & 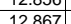 & 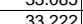 & & & 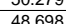 & 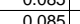 & 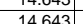 & & 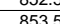 & 14225 & 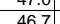 & 119 & 39.3 & 7799 & & 0.01 & 年 0.000 & & \\
\hline 87 & $4 / 11 / 2004$ & $\begin{array}{l}11.56 .25 \mathrm{PM} \\
11.56\end{array}$ & $\frac{25.541}{25.54}$ & 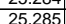 & 25.14 & (25.909 & & 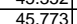 & \begin{tabular}{|c|c|}
48776 \\
\end{tabular} & 13.11 & (33.339 & & & $\frac{48.096}{4924}$ & $\begin{array}{l}0.085 \\
0.084 \\
\end{array}$ & $\begin{array}{l}14.645 \\
11.643 \\
\end{array}$ & & & $\begin{array}{l}14.22500 \\
14.2422 \\
\end{array}$ & $\frac{46.1}{473}$ & $\frac{11.9}{12.1}$ & & & & $\frac{0.01}{0.01}$ & & & \\
\hline$\frac{876}{876}$ & $\frac{|c| 2004}{9 / 204}$ & $\begin{array}{l}11: 57: 22 \mathrm{PM} \\
\end{array}$ & $\frac{25.557}{25.57}$ & $\frac{25.292}{25.292}$ & \begin{tabular}{|l|l|}
25.171 \\
\end{tabular} & 25.965 & $\frac{14.367}{24.367}$ & 45.182 & 48.104 & 12.825 & 33.169 & $\frac{1.21:}{10}$ & & $\begin{array}{r}48.24 \\
48.448 \\
\end{array}$ & $\begin{array}{l}0.004 \\
0.085 \\
\end{array}$ & $\begin{array}{l}\frac{1.0445}{14.643} \\
\end{array}$ & & $\frac{654}{855}$ & $\begin{array}{l}14.24<2 \\
14.2589\end{array}$ & $\frac{4.3}{46.6}$ & $\frac{1.1 .}{11.9}$ & & .701 & & & & & \\
\hline & g/2004 & $22 \mathrm{PM}$ & 25.547 & 25.292 & \begin{tabular}{|l|l|}
25.146 \\
\end{tabular} & 25.93 & & 45.541 & 48.763 & 12.989 & 23027 & & & & 0.084 & 14.643 & & 856 & 142756 & 473 & 120 & 39.4 & & & 然 & 0.000 & & \\
\hline 878 & g/2004 & 11:59.22 PM & 25.553 & 25.292 & 25.177 & 25.881 & 24.097 & 45.234 & 48.243 & 12896 & 32.968 & 1.218 & & 45.932 & 0.085 & 14.643 & & 857 & 14.2922 & 46.8 & 113 & 391 & 2.696 & & 0.013 & 0.000 & 0.322 & \\
\hline & $4 / 20 / 2004$ & 12:00:22 AM & 25.563 & 25.297 & 25.197 & 25.821 & 23.947 & 45.282 & 48.345 & $12.898 \mid$ & 33.161 & $.218 \mathrm{~S}>\mathrm{x}$ & & 50.47 & |0.084 & 14.643 & & 858 & 4.3089 & 46.9 & 12.4 & & 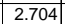 & & & & & \\
\hline & 4/20/2004 & 12:00:26 AM & 25.552 & & \begin{tabular}{|l|}
25.181 \\
\end{tabular} & 25.8 & & 45.396 & \begin{tabular}{|l|l|}
48.413 \\
\end{tabular} & 12.867 & 33.261 & 122 & & 47.90 & $\begin{array}{l}0.085 \\
\end{array}$ & 14.643 & & & 14.31 & 46.9 & 11.7 & $\overline{39.3}$ & 271 & & 0.01 & & & \\
\hline & 0/2004] & 2:01:26 AM & 25.568 & 25.302 & & & & & \begin{tabular}{|l|l|}
48.548 \\
\end{tabular} & & & & & & & & & & & 47. & 12.3 & & & & & & & \\
\hline & 4/20/2004 & 12:02:26 AM & 25.561 & 25.2955 & 25.195 & 25.743 & 23. & 45.124 & 48.137 & 12.912 & 32.97 & -1.21 & & 48.88 & 0.084 & 14.643 & & 866 & 14.3433 & 46.7 & 12.0 & $39->>>$ & & & 0.012 & 0.000 & 119 & \\
\hline $888^{8}$ & D/2004 & 12:03:26 AM & 25.565 & 25.295 & 25.189 & 25.698 & 23.8044 & 45.24 & \begin{tabular}{|l|l|}
48.216 \\
\end{tabular} & 12.89 & 33.132 & $1.2+4$ & & 49.81 & 0.084 & 14.643 & & 861.6 & $\begin{array}{r}14.36 \\
\end{array}$ & 46.7 & 12.2 & & & & 0.014 & 0.000 & 0.318 & \\
\hline 88 & 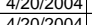 & $\begin{array}{l}12.042020 \mathrm{AM} \\
12.05 \mathrm{AM}\end{array}$ & | 255653 & 告5.288 & 25.217 & 25.655 & & 年55.122 & 4.425 & 12.899 & & 121 & & 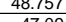 & 0.08 & & & & $\begin{array}{ll}14.3767 \\
1432\end{array}$ & & 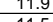 & & & & & & & \\
\hline & (1) & & 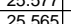 & 年. & $\frac{2.211}{25224}$ & $\frac{\mid 3.04 t}{2553}$ & & 年 & (4.2645 & & & 1.21 & & 189 & & & & 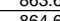 & & 年 & $\frac{1.15}{120}$ & & 7072 & & & & & \\
\hline 887 & 4 & $12: 07: 26$ AM & 25.57 & 25.28 & 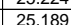 & $\frac{25.50}{25.57}$ & & 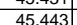 & $\frac{4.056}{48.56}$ & 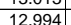 & & & & $\frac{1.05 .2}{48.2}$ & & $\frac{1.4643}{14634}$ & & & & 471 & 118 & & & & $0.12-3$ & & & \\
\hline & $\mid 4 / 20 / 2004$ & 12:08:226 AM & 25.571 & $\frac{25.285}{25}$ & 25.14 & 25.653 & $\frac{24.0}{24.0}-2$ & 44.944 & \begin{tabular}{|l|l|}
47.681 \\
\end{tabular} & 12.817 & 32.986 & $\frac{-1.218}{-1.218}$ & & 49.455 & & & & & 14.4433 & 462 & 12.1 & & Th & & $\frac{0.012}{0.012}$ & & & \\
\hline & 4/201/2004 & 12:09:26 AM & $\frac{25.541}{25}$ & 25.255 & 25.115 & 25.698 & 24.23 & 45.112 & \begin{tabular}{|l|l|}
48.11 \\
\end{tabular} & 12.854 & 33.003 & & & 舟 & 0.083 & & & 867.6 & 14.46 & $x_{1}$ & 123 & & & & 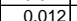 & & 0,0 & \\
\hline & 0 & $12 \cdot 10 \cdot 26 \mathrm{AM}$ & 25.543 & 25.272 & & 25.741 & & 45.369 & 48.44 & 12.876 & & & & 47.70 & & & & 868.6 & & & 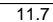 & & & & & & & \\
\hline & D/2004 & 12:11:26 AM & 25.544 & 25.279 & 25.163 & 25.822 & & 44.935 & 48.011 & 12.904 & 32.728 & 1.218 & & & & 14.643 & & & 14.4933 & & 12.6 & & & & 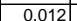 & & & \\
\hline & $4 / 20 / 2004$ & 12:12:26 AM & 25.546 & 25.28 & 25.165 & 25.893 & 24. & 45.315 & 48.513 & 12.904 & $33.6 \mathrm{r}-\mathrm{s}$ & $-1.25<>3$ & & 48.2. & 0.08 & & & 870 & & 47.0 & 11.8 & \begin{tabular}{|l|}
39.2 \\
\end{tabular} & \begin{tabular}{|l|l|l|}
2.703 \\
\end{tabular} & & 0.01. & & & \\
\hline & D/2004 & $12: 13$ & $25.527 \mid$ & 25.282 & 25.161 & 25.911 & & $45.2 .2>$ & 48.357 & & & & & & & & & 871.6 & 14.5267 & 46 & 11.7 & 39.1 & 998 & & 0.0 & & & \\
\hline 894 & 4/20/2004 & 12:14:26 A & 25.52 & 25.274 & 25.138 & 25.962 & & 44.83 & & 12.76 & 32.894 & & & & & 14.6 & & $872.6 \mathrm{Y}$ & 14.54 & 46. & 11.7 & & .679 & & 0.0 & & 17 & \\
\hline 895 & 4/20/2004 & 12::15: & 25.536] & 25.295 & 25.165 & & & 45.203 & 48.235 & 12.908 & & & & 48.18 & & 14.643 & & 873 & & (40.0 & 11.8 & & & & . & & & \\
\hline 89 & $4 / 2$ & $\frac{12: 16}{1.17}>>>$ & 25.536 & 25.296 & 25.16 & 2.5.9609 & & 44.985 & $48.2<4$ & $\frac{1.9 .91}{12.03}$ & & 1.214 & & & & & & & & 46.8 & 12.0 & & & & & & & \\
\hline 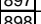 & $\mid$ & 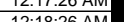 & $\frac{25.541}{25541}$ & 25.226 & 2517 & 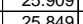 & 24.2 & $\frac{45.145}{45068}$ & \begin{tabular}{|l|}
48.488 \\
427 \\
\end{tabular} & 年2.896 & 年2.888 & -1.21 & & 40.904 & 0 & $\frac{1.4645}{1.643}$ & & $\frac{615}{876}+2$ & 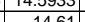 & 4.0 & $\frac{11.5}{11.9}$ & 380 & 2685 & & sonst & & & \\
\hline & & 2:19:26 & $\frac{2.5451}{25.51}$ & $\frac{25.503}{2531}$ & & & & $\frac{4.03}{448}$ & & & & & & & & & & & & & & & & & & & & \\
\hline & $4 / 2 / 2 / 2004$ & 12:20:26 AN & 25.55 & 25.31 & 年.5.179 & 25.732 & & 44.79 & & $\frac{12.85}{12.85}$ & & 1.22 & & & & $\frac{14.6}{14.6}-2$ & & & & 46 & 11. & & & & $\frac{1}{01}$ & & & \\
\hline 907 & $4 / 20120$ & $12: 21: 26$ & 25.549 & $\frac{25.323}{25}$ & 25.208 & 25.701 & $\frac{23.8}{23.8}$ & 444.981 & & 12.915 & $\frac{32.8}{32.8}$ & -1.218 & & 47.069 & & & & 879.6 & & & & & & & & & 0.320 & \\
\hline & & & 25.546 & & & 25.634 & & & \begin{tabular}{|l|l|}
48.268 \\
\end{tabular} & & & & & & & & & & & & & & & & & & & \\
\hline$\overline{9 c}$ & $4 / 20 / 2004$ & 12:23:26 & 25.544 & 25.309 & 25.228 & 25.577 & & 46.068 & 49.416 & 13.182 & 33.547 & 1.218 & & 49.1 & $0.0 \varepsilon$ & 14.6 & & 881.6 & & 47.9 & 2.1 & & & & & & & \\
\hline & & $12: 24: 26 \mathrm{Al}$ & 25.56 & 25.328 & & & & 45.85 & 49.22 & 13.173 & & & & 16. & & & & 882 & & & & & $\sqrt{2}+3$ & & & & & \\
\hline 905 & 4/20/2004 & 12:25:26 & 25.563 & 25.318 & 25.297 & 25.59 & & 45.987 & 49.373 & 13.204] & & 1.21: & & $30.7-7=0$ & 0.08 & 14.64 & & 883.6 > & & 9 & 12.4 & & 36 & & $0.8-8-8$ & & 0.309 & \\
\hline & 4/20/2004 & $12: 26: 26 \mathrm{f}$ & 25.5 & 25.303 & 25.3 & 25 . $\mathrm{x}$ & & & | 48.86 & 13.094] & 33.218 & & & 47.7 & 0.08 & & & 88 & 14.7 & 47.4 & & & & & & & 5 & \\
\hline 907 & 4/20/2004 & $12: 27: 26 \mathrm{AM}$ & 25.558 & 25.298 & 25.252 & 25.68 & 24.238 & 45.1866 & \begin{tabular}{|l|l|} 
& 49.068 \\
\end{tabular} & $13.067 \mathrm{t}$ & 33.442 & -1.22 & -7.1 .13 & 50.023 & 0.084 & 14.643 & & 885.6 & 14.76 & 47.6 & 12.3 & 39.6 & 2.131 & 0.013 & 0.012 & 0.000 & 0.314 & $0.2-2+3$ \\
\hline$\frac{908}{909}$ & 4/20/2004 & $\begin{array}{l}12: 28: 26 \mathrm{AM} \\
12 \cdot 29 \cdot 26 \mathrm{AM}\end{array}$ & 25.5595 & $\frac{25.298}{25.29}$ & \begin{tabular}{|l|l|}
25.218 \\
25189
\end{tabular} & $\begin{array}{l}25.156 \\
25763 \\
\end{array}$ & $\frac{24.288}{24.355}$ & $\begin{array}{ll}45.462 \\
45.916\end{array}$ & \begin{tabular}{|l|}
48.854 \\
49.053 \\
\end{tabular} & $\frac{13.0 / 3}{13018}$ & 32.997 & $\frac{-1.22}{-1.22}$ & $\frac{-7.1}{-7.1}$ & 49.82 & 0.082 & $\frac{14.663}{14643}$ & 0.00 & 886.6 & \begin{tabular}{|l|}
$\mid 14.7 / 673$ \\
14.7933
\end{tabular} & $\begin{array}{r}47.4 \\
476 \\
\end{array}$ & 12.2 & \begin{tabular}{|l|}
39.2 \\
39.8 \\
\end{tabular} & \begin{tabular}{|l|l|}
2.105 \\
2.746
\end{tabular} & 0.012 & $\frac{0.012}{0.012}$ & 0.000 & 0.310 & $\frac{0.22 .7}{0.27}$ \\
\hline & & & & & & & & & & & & & & & & & & & & & & & & & & & 0.314 & 0.2 \\
\hline
\end{tabular}


WSRC-TR-2005-00105, REVISION 0

SRNL-RPP-2005-00012, REVISION 0

RUN \# 2.03A AND B; FIRST AND SECOND HALF OF SLURRY DEWATERING - CONT.

\begin{tabular}{|c|c|c|c|c|c|c|c|c|c|c|c|c|c|c|c|c|c|c|c|c|c|c|c|c|c|c|c|c|}
\hline & & & & & & & & & & & & & & & & & & & & & & & & & & & & \\
\hline & A & \begin{tabular}{|c|} 
B \\
\end{tabular} & $\frac{\mathrm{D}}{\mathrm{N}}$ & $E$ & $r$ & G & $\mathrm{H}$ & $\mathrm{J}$ & $\frac{K}{K}$ & $\mathrm{~L}$ & $\mathrm{M}$ & $\mathrm{N}$ & 0 & $Q$ & $\mathrm{R}$ & $\mathrm{s}$ & $T$ & $\mathrm{v}$ & W & $x$ & S & $z$ & AA & $A B$ & $A C$ & $A D$ & $\mathrm{AE}$ & \\
\hline & $\begin{array}{l}4 / 20 / 2004 \\
4 / 2012004 \\
\end{array}$ & \begin{tabular}{|l|}
$12: 31: 26 \mathrm{AM}$ \\
$12.32 \cdot 2 \mathrm{GM}$
\end{tabular} & $\begin{array}{l}25.559 \\
25.556\end{array}$ & $\begin{array}{l}25.309 \\
25.295 \\
\end{array}$ & \begin{tabular}{|l|}
25.243 \\
25.24
\end{tabular} & \begin{tabular}{|l|}
25.922 \\
25.943 \\
\end{tabular} & $\begin{array}{l}24.489 \\
24.476 \\
\end{array}$ & $\begin{array}{l}46.016 \\
45.713 \\
\end{array}$ & \begin{tabular}{|r|}
49.425 \\
49.12 \\
\end{tabular} & \begin{tabular}{|l|}
13.163 \\
13.071
\end{tabular} & $\begin{array}{l}33.465 \\
33.348\end{array}$ & \begin{tabular}{|l|l|}
-1.218 \\
$3-1.221$
\end{tabular} & $\begin{array}{r}-7.151 \\
-7.151 \\
\end{array}$ & $\begin{array}{r}\frac{48.21}{45.578} \\
\end{array}$ & $\begin{array}{l}0.083 \\
0.084 \\
\end{array}$ & \begin{tabular}{|c|}
14.643 \\
14.633
\end{tabular} & $\begin{array}{l}0.003 \\
0.003 \\
\end{array}$ & $\begin{array}{l}889.6 \\
89.6 \\
\end{array}$ & \begin{tabular}{|l|l|}
14.8267 \\
1.8433
\end{tabular} & $\begin{array}{l}48.0 \\
4.6 \\
\end{array}$ & $\begin{array}{l}p \mid 11.8 \\
5 \\
511.2\end{array}$ & 39.7 & & 0.012 & $\frac{0.012}{0.012}$ & $\begin{array}{l}0.000 \\
0.000\end{array}$ & $\begin{array}{l}0.309 \\
0.315 \\
\end{array}$ & $\begin{array}{l}0.26 \\
0.27\end{array}$ \\
\hline & & & 25.5563 & $\begin{array}{l}25.295 \\
25.302 \\
\end{array}$ & \begin{tabular}{|l|}
25.267 \\
25.26 \\
\end{tabular} & \begin{tabular}{|l|}
25.943 \\
25.951 \\
\end{tabular} & $\begin{array}{l}24.4748 \\
24.448 \\
\end{array}$ & $\begin{array}{l}45.13 \\
45.674 \\
\end{array}$ & & $\begin{array}{l}13.011 \\
13.112 \\
\end{array}$ & $\begin{array}{r}33.348 \\
33.307 \\
\end{array}$ & & & 45.518 & $\begin{array}{l}0.084 \\
0.084 \\
\end{array}$ & \begin{tabular}{|l|}
14.6433 \\
14.643
\end{tabular} & & & & $\begin{array}{l}47.6 \\
47.6 \\
\end{array}$ & & & & & & $\begin{array}{l}0.000 \\
0.000 \\
\end{array}$ & & \\
\hline 914 & $4 / 20 / 2004$ & 12:34:26 AM & 25.564 & 25.294 & \begin{tabular}{|l|}
25.253 \\
\end{tabular} & \begin{tabular}{|l|l|}
25.912 \\
\end{tabular} & 24.309 & 45.707 & 49.199 & 13.102 & 33.208 & 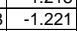 & -7.139 & 49.86 & 0.083 & \begin{tabular}{|l|}
14.643 \\
\end{tabular} & 0.003 & 892.6 & 14.8767 & 47.7 & $\begin{array}{l}12.2 \\
7\end{array}$ & 39.5 & 2.720 & 0.012 & & 0.000 & 0.311 & 0.27 \\
\hline & $4 / 20 / 2004$ & \begin{tabular}{|l|l|}
$12: 35: 26 \mathrm{AM}$ \\
\end{tabular} & 25.591 & 25.31 & \begin{tabular}{|l|l|}
25.31 \\
\end{tabular} & 25.873 & & 45.73 & $\begin{array}{l}49.308 \\
\end{array}$ & $\begin{array}{l}13.151 \\
\end{array}$ & 33.11 & \begin{tabular}{|l|}
-1.218 \\
\end{tabular} & -7.145 & 49.172 & 0.083 & 14.643 & & & \begin{tabular}{|l|l|}
14.8933 \\
\end{tabular} & 47.8 & & 39.4 & & & & & & 0.26 \\
\hline & 4/20/2004 & & & & & & & & 48.989 & 13.119 & & & & 51.821 & 0.084 & \begin{tabular}{|l|}
14.643 \\
\end{tabular} & & & 14.91 & 47.5 & & 39.07 & & & & & & \\
\hline$\frac{917}{019}$ & $4 / 20 / 2004$ & 12:37:26 AM & 25.58 & 25.304 & 25.309 & 25.717 & 24.054 & 45.628 & 48.91 & $\begin{array}{l}13.148 \\
\end{array}$ & 33.227 & \begin{tabular}{|c|} 
\\
\end{tabular} & $\begin{array}{r}-7.145 \\
\end{array}$ & 47.828 & 0.082 & \begin{tabular}{|l|}
14.643 \\
\end{tabular} & & 895.6 & \begin{tabular}{|l|l|}
14.9267 \\
\end{tabular} & 47.4 & \begin{tabular}{l|l}
4 & 11.7 \\
\end{tabular} & 39.4 & 2.718 & 0.012 & 0.012 & 0.000 & 0.307 & \\
\hline$\frac{918}{919}$ & $\begin{array}{l}4 / 20 / 2004 \\
4 / 2012004 \\
\end{array}$ & \begin{tabular}{|l|}
$12: 38: 26 \mathrm{AM}$ \\
$12: 39: 2 \mathrm{AM}$
\end{tabular} & $\begin{array}{l}25.594 \\
25.592\end{array}$ & $\begin{array}{l}25.314 \\
25.302 \\
\end{array}$ & \begin{tabular}{|l|}
25.338 \\
25.36 \\
\end{tabular} & \begin{tabular}{|l|l|}
25.677 \\
25.629 \\
\end{tabular} & $\begin{array}{l}23.893 \\
23.871 \\
\end{array}$ & \begin{tabular}{|c|}
45.9114 \\
46.117 \\
\end{tabular} & \begin{tabular}{|c|}
49.284 \\
49.688
\end{tabular} & \begin{tabular}{|l|l|}
13.183 \\
13.135 \\
\end{tabular} & $\begin{array}{l}33.442 \\
33569 \\
356\end{array}$ & \begin{tabular}{|l|l|}
-1.218 \\
-1218
\end{tabular} & $\begin{array}{l}-7.134 \\
-7128 \\
\end{array}$ & $\begin{array}{l}51.278 \\
48.93 \\
\end{array}$ & $\begin{array}{l}0.084 \\
0.084\end{array}$ & \begin{tabular}{|l|l|}
14.643 \\
14643
\end{tabular} & & $\begin{array}{l}896.6 \\
8966\end{array}$ & \begin{tabular}{r|}
14.9433 \\
1496
\end{tabular} & $\begin{array}{l}47.8 \\
482 \\
\end{array}$ & \begin{tabular}{l|l}
8 & 12.6 \\
2 & 120
\end{tabular} & \begin{tabular}{|l|}
39.7 \\
398 \\
\end{tabular} & 2.736 & $\frac{0.013}{0.013}$ & $\frac{0.012}{0.012}$ & 0.000 & 0.313 & 0.27 \\
\hline 920 & $\begin{array}{l}4 / 20 / 2004 \\
4 / 20 / 204\end{array}$ & \begin{tabular}{|l|}
$12: 39: 26 \mathrm{AM}$ \\
$12: 40: 26 \mathrm{AM}$
\end{tabular} & $\begin{array}{r}25.536 \\
25.6\end{array}$ & 25.294 & \begin{tabular}{|l|}
25.364 \\
\end{tabular} & \begin{tabular}{|l|}
25.029 \\
25.562
\end{tabular} & $\begin{array}{l}23.871 \\
23.814\end{array}$ & \begin{tabular}{|l|}
46.111 \\
45.809 \\
\end{tabular} & $\begin{array}{l}49.468 \\
49.468 \\
\end{array}$ & $\begin{array}{l}13.135 \\
13.088\end{array}$ & $\begin{array}{l}33.209 \\
33.276 \\
\end{array}$ & \begin{tabular}{|l|l|}
-1.210 \\
\end{tabular} & $\begin{array}{c}-1.128 \\
-7.139 \\
\end{array}$ & $\begin{array}{r}48.93 \\
48.606 \\
\end{array}$ & $\begin{array}{l}0.084 \\
0.082\end{array}$ & $\begin{array}{l}14.643 \\
14.643\end{array}$ & $\begin{array}{l}0.003 \\
0.003 \\
\end{array}$ & $\begin{array}{r}89.6 .6 \\
898.6\end{array}$ & \begin{tabular}{|r|r|}
14.96 \\
14.9767
\end{tabular} & $\begin{array}{l}48.2 \\
48.0\end{array}$ & 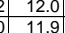 & & $\frac{2.747}{2.726}$ & $\frac{0.013}{0.012}$ & $\frac{0.012}{0.012}$ & $\begin{array}{l}0.000 \\
0.000\end{array}$ & $\begin{array}{l}0.311 \\
0.306\end{array}$ & 0.26 \\
\hline 921 & 4/20/2004 & \begin{tabular}{|l|}
$12: 41: 26$ AM \\
\end{tabular} & & & & & 23.748 & & 49.141 & & 33.302 & & -7.128 & 49.764 & 0.083 & 14.643 & & & & 47.7 & & & & & & 0.000 & & $\frac{0.26}{0.26}$ \\
\hline 922 & $4 / 20 / 2004$ & 12:42:26 AM & 25.602 & 25.282 & 25.386 & 25.489 & 23.756 & 45.464 & 49.101 & $\begin{array}{l}13.007 \\
\end{array}$ & 33.099 & & -7.093 & 49.42 & 0.084 & \begin{tabular}{|l|l|}
14.643 \\
\end{tabular} & & 900.6 & \begin{tabular}{|l|}
15.01 \\
\end{tabular} & 47.6 & \begin{tabular}{|l|l|} 
f) & 12.1 \\
\end{tabular} & 39.3 & & & & 0.000 & & $\frac{0.26}{0.27}$ \\
\hline 923 & $4 / 20 / 2004$ & & 25.592 & 25.267 & 25.381 & 25.474 & 23.982 & & 49.197 & & 33.442 & -1.224 & & & 0.082 & & & & & 47.7 & & & & & & & 0.305 & \\
\hline 524 & & 12:44:26 AM & & & 25,112 & 25575 & & 45.891 & $\mid 49.308$ & 13.087 & 33.481 & $\frac{-1.218}{-1218}$ & & & 00092 & & & 902.6 & & 47.8 & & & & & & & & \\
\hline & $4 / 20 / 2004$ & 12:45:26 AM & 25.614 & 25.263 & \begin{tabular}{|l|l|}
25.398 \\
\end{tabular} & 25.666 & 24.223 & 45.67 & 49.215 & 13.028 & 33.374 & & & 49.843 & & 14.643 & & 903.6 & 15.06 & 47.7 & & & & & & & & \\
\hline 926 & $4 / 20 / 2004$ & 12:46:26 AM & 25.614 & 25.259 & 25.428 & 25.692 & 24.239 & 45.761 & 49.288 & 13.042 & 33.434 & $\begin{array}{ll}-1.218 \\
\end{array}$ & -7.122 & 48.567 & 0.083 & 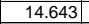 & & 904.6 & 15.0767 & $\begin{array}{ll}47.8 \\
\end{array}$ & 11.9 & 39.6 & 2.730 & 0.012 & 0.012 & 0.000 & 0.309 & \\
\hline & $4 / 20 / 2004$ & 12:47:26 AM & & 25.254 & 25.409 & 25.753 & 24.455 & 45.645 & 49.161 & 13.044 & 33.409 & -1.218 & -7.12 & 49.261 & 0.082 & 14.643 & & 905.6 & 15.0933 & 47.7 & & & & & & & 0.306 & \\
\hline & & 8:26 AM & & & & & & & & & & & & & 0.084 & 14.643 & & 906.6 & 15.11 & & & & & & & & & \\
\hline & $4 / 20 / 2004$ & 12:49:26 AM & 25.628 & 25.243 & 25.298 & 25.871 & 24.518 & 45.759 & 49.344 & 13.058 & 33.337 & -1.224 & -7.116 & 4 & 0.082 & 14.643 & & & & 47.9 & & & & 012 & & & & \\
\hline & $\frac{4 / 2 / 20 / 2004}{4 / 20 / 204}$ & 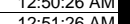 & $\frac{25.636}{25632}$ & $\frac{25.260}{25262}$ & \begin{tabular}{|l|l|}
2520707 \\
2507
\end{tabular} & 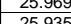 & 24.486 & 45.15 & 49.018 & $\frac{13.02}{13021}$ & 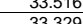 & $\frac{-1.21}{1.21}$ & & 40.1 & 0 & 14.643 & & & 15.1433 & 47.6 & & & & & & & & \\
\hline 932 & $\begin{array}{l}4 / 2012004 \\
4 / 20 / 2004 \\
\end{array}$ & $\begin{array}{l}12.51 .20 \mathrm{AM} \\
12: 52: 26 \mathrm{AM}\end{array}$ & $\begin{array}{l}25.035 \\
25.629\end{array}$ & $\begin{array}{l}25.202 \\
25.258\end{array}$ & 25298 & $\frac{25.955}{25.917}$ & $\begin{array}{l}24.301 \\
24219\end{array}$ & \begin{tabular}{|l|}
45.062 \\
45.416 \\
\end{tabular} & $\begin{array}{l}49.355 \\
49.064 \\
\end{array}$ & $\begin{array}{l}13.021 \\
12.989 \\
\end{array}$ & $\begin{array}{l}35.329 \\
33.071\end{array}$ & & $\frac{-1.111}{-7.113}$ & 年65.307 & $\begin{array}{l}0.063 \\
0.082\end{array}$ & 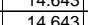 & & $\frac{910.0}{910}$ & : 153.10 & 44.96 & (12) & 392 & 270 & 012 & & 然0000 & $=0.314$ & 0.26 \\
\hline & $4 / 20 / 2004$ & $12: 53: 26$ AM & 25.635 & 25.269 & \begin{tabular}{|l|}
25.309 \\
\end{tabular} & 25.862 & 24.154 & 45.641 & 49.412 & 13.029 & 33.305 & -1.221 & -7.111 & 48.842 & 0.082 & $\begin{array}{ll}14.045 \\
14.643\end{array}$ & & 911.6 & \begin{tabular}{|l|l|}
15.1933 \\
\end{tabular} & 47.9 & 12.0 & & & & & & 0.307 & 0.26 \\
\hline & 4/20/2004 & 12:54:26 AM & 25.635 & 25.264 & 25.309 & 25.822 & & 45.458 & 49.014 & 13.004 & 33.183 & & -7.108 & 50.819 & 0.084 & 14.643 & & 912.6 & 15.21 & 47.5 & \begin{tabular}{|l|l|}
5 & 12.5 \\
\end{tabular} & \begin{tabular}{|l|l|}
39.3 \\
\end{tabular} & & & & & 0.316 & \\
\hline & $4 / 20 / 2004$ & 12:55:26 AM & $\begin{array}{r}25.63 \\
25.619\end{array}$ & 25.264 & \begin{tabular}{|l|l|}
25.324 \\
3.24
\end{tabular} & 25.737 & 23.984 & 45.678 & 49.574 & 13.077 & 33.21 & -1.218 & -7.11 & 47.887 & 0.08 & 14.643 & & 913.6 & 15.2267 & 48.1 & 11.7 & 39.4 & 2.72 & 0.012 & & 0.000 & 0.300 & \\
\hline & $\begin{array}{l}4 / 200 / 2004 \\
4 / 20 / 204 \\
\end{array}$ & $\begin{array}{l}\frac{12: 56: 26 \mathrm{AM}}{1257 \cdot 26 \mathrm{AM}} \\
\end{array}$ & $\frac{25.618}{25612}$ & $\frac{25.253}{25251}$ & $\begin{array}{l}25.312 \\
25.321\end{array}$ & $\frac{25.696}{25.574}$ & $\frac{23.853}{23.771}$ & $\begin{array}{r}45.56 \\
45.838 \\
\end{array}$ & $\begin{array}{l}49.196 \\
4956 \\
\end{array}$ & $\begin{array}{ll}\frac{13.029}{13133} & -1\end{array}$ & $\frac{33.265}{33.434}$ & $\begin{array}{l}-1.218 \\
-1.1218\end{array}$ & $\frac{-7.10}{-710}$ & $\frac{49.278}{49718}$ & $\frac{0.002}{0.083}$ & $\frac{14.643}{14643}$ & & $\begin{array}{l}914.6 \\
915.6\end{array}$ & 15.4233 & 47. & 12 & 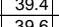 & & & & & 0.307 & 0.26 \\
\hline 938 & $4 / 20 / 2004$ & $\begin{array}{l}12.07 .20 \mathrm{AMT} \\
1258: 26 \mathrm{AM}\end{array}$ & $\frac{2.0 .014}{25626}$ & $\begin{array}{l}25.251 \\
25.27\end{array}$ & 25.37 & 25.548 & $\frac{20.111}{2373}$ & 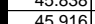 & 年 & $\begin{array}{l}13.150 \\
13.154\end{array}$ & $\begin{array}{l}30.434 \\
33.411 \\
\end{array}$ & $\begin{array}{l}-\frac{-1.21}{-1.218} \\
\end{array}$ & & $\frac{4.1}{501}$ & - 0.003 & 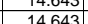 & & & $\begin{array}{l}15.20 \\
15767\end{array}$ & 年 & 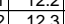 & $3977>$ & & & & & 年 & 0.26 \\
\hline & $4 / 20 / 2004$ & 12:59:26 AM & 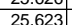 & 25.257 & $\frac{25.07}{25.357}$ & $\frac{2.340}{25.51}$ & 23.667 & 45.838 & $\begin{array}{l}45.020 \\
49.702\end{array}$ & $\begin{array}{l}1.1074 \\
13.079\end{array}$ & 3..411 & $-\frac{1.210}{-1.218}$ & -7.111 & 48.319 & 0.083 & 14.643 & & $\begin{array}{l}910.0 \\
917.6\end{array}$ & 15.2933 & 年 & $\frac{12.8}{11.8}$ & $\frac{3.4 .6}{39.6}$ & & & & & - & 0.266 \\
\hline 940 & $4 / 20 / 2004$ & 1:00:26 AM & 25.626 & 25.271 & 25.365 & 25.489 & 23.745 & 45.539 & 49.167 & 13.101 & 33.103 & -1.221 & -7.116 & 48.867 & 0.081 & & & 918.6 & 15.31 & 47.7 & & $39.3 \mathrm{r}>\mathrm{C}$ & & & & & 0.304 & \\
\hline & 4/20/2004 & 1:01:26 AM & 25.631 & 25.27 & 25.39 & 25.473 & 23.935 & 45.75 & $\mid 49.447$ & 13.115 & 33.294 & -1.218 & -7.111 & 47.728 & 0.083 & $\begin{array}{l}14.045 \\
14.643\end{array}$ & & $\frac{10.6}{919.6}$ & 15.3267 & 48.0 & $11.7 \quad>\quad>3$ & 239.5 & & 10 & & 0.000 & 0.031 & \\
\hline 942 & $4 / 20 / 2004$ & 1:02:26 AM & 25.63 & 25.274 & $\frac{25.419}{25}$ & 25.522 & 24.034 & 45.668 & $\mid 49.348$ & 13.092 & 33.305 & -1.221 & $-1.08+2>0$ & 49.155 & 0.083 & $\begin{array}{l}14.045 \\
14.643 \\
\end{array}$ & & 92 & 15.3433 & 47.9 & & & & & & & 0.310 & \\
\hline & $4 / 20 / 2004$ & 1:03:26 AM & 25.634 & 25.269 & \begin{tabular}{|l|l|}
25.403 \\
\end{tabular} & 25.607 & 24.164 & 45.427 & & 13.049 & & -1.218 & & 48.531 & 0.082 & 14.643 & & 921.6 & 15.36 & 47.8 & & 39 & & & & & & \\
\hline 94 & $4 / 20 / 2004$ & 1:04:26 AM & 25.644 & 25.273 & 25.438 & 25.651 & 24.418 & 45.541 & 49.257 & $\begin{array}{ll}3.056 \\
\end{array}$ & 33.146 & -1.218 & -7.108 & 48.755 & 0.081 & \begin{tabular}{ll|l|}
14.643 \\
\end{tabular} & & 922.6 & 15.3767 & \begin{tabular}{ll|l}
47.8 \\
\end{tabular} & 11.9 & 39.3 & 2.713 & 0.012 & 0.012 & 0.000 & \begin{tabular}{|c|c|c|}
0.303 \\
\end{tabular} & \\
\hline & $4 / 20 / 2$ & & 25.644 & & 25.433 & 25.716 & 24. & 45.75 & 49.713 & 13.063 & 33.259 & -1.221 & -7.07 & 48.54 & & 14.643 & & 923.6 & 15.3933 & 48.2 & . & 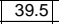 & & & & & & \\
\hline 1946 & 4/20/2 & 1:06:26 AM & $25.649 \mid$ & 25.274 & 25.443 & 25.757 & 24. & 45.665 & 49.4399 & 13.066 & 33.259 & -1.221 & -7.09 & 49. & 082 & & & 924.6 & 15.41 & 48.0 & $2.1 \quad 2-3$ & 39 & & & & & 0.306 & \\
\hline$\frac{947}{40}>>2$ & $4 / 2012004$ & & 25.651 & 25.275 & 25.445 & 25.833 & & 45.4977 & 49.17 & 12.952 & 33.309 & -1.221 & & 48.7 & 0.081 & 14. & & 925.6 & & 47. & 12.0 & 39. & & 112 & & 0.00 & & \\
\hline & & $\begin{array}{l}1.08206 \mathrm{AM} \\
10.06\end{array}$ & & & \begin{tabular}{|l|l|}
25.442 \\
\end{tabular} & & & & & & & & & & & & & & 15.4433 & & & & & & & & & \\
\hline 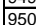 & $\begin{array}{l}4 / 2 / 20 / 2004 \\
4 / 20 / 204 \\
\end{array}$ & $\begin{array}{l}1.0 .2 .26 \mathrm{AM} \\
1.102\end{array}$ & 年3.0.060 & $\begin{array}{l}25.210 \\
25289\end{array}$ & \begin{tabular}{|l}
25.446 \\
2543
\end{tabular} & $\frac{23.901}{25892}$ & $\frac{24.440}{24549}$ & 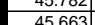 & $\mid \begin{array}{r}49.663 \\
\end{array}$ & $\frac{13.049}{13021}$ & $\frac{3.315}{33.35}$ & $\frac{-1.21}{-1218}$ & -70 & $\begin{array}{r}-41.91 \\
50292\end{array}$ & $=0.082$ & $\frac{14.643}{14.643}$ & & 922.6 & \begin{tabular}{|l|}
15.46 \\
15.4767 \\
\end{tabular} & 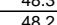 & $\frac{11.8}{12.4}$ & 39. & & & & 0.000 & 0.305 & \\
\hline 951 & $4 / 20 / 2004$ & $\begin{array}{l}1.1 .1 .0 \mathrm{~mm} \\
1: 11: 26 \mathrm{AM}\end{array}$ & 25.68 & 25.309 & \begin{tabular}{|l|l|}
25.469 \\
\end{tabular} & 25.822 & & $\frac{45009}{45.69}$ & $\begin{array}{l}4.617 \\
49.60\end{array}$ & 13.005 & 33.294 & $\frac{-.21}{-1.21 \varepsilon}$ & & 47.4 & & $\begin{array}{l}14.045 \\
14.643 \\
\end{array}$ & & & 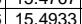 & 48. & $\frac{12.6}{11.6}$ & & & & & & $\mid 0.302$ & \\
\hline & $4 / 20 / 2004$ & $1: 12: 26 \mathrm{AM}$ & 25.664 & 25.299 & \begin{tabular}{|l|l|}
25.403 \\
\end{tabular} & 25.782 & & 45.39 & 49.066 & 12.882 & 33.265 & -1.218 & & 49.568 & 0.08 & 14.643 & & $\begin{array}{l}9.30 .6 \\
930.6\end{array}$ & $\frac{15.51}{15}$ & 47.6 & 12 & 39. & & & & & 0.300 & \\
\hline & $4 / 20 / 2004$ & $\begin{array}{l}1: 13: 26 \\
\end{array}$ & 25.664 & & \begin{tabular}{|l|}
25.418 \\
\end{tabular} & & & 45.701 & 49.634 & & 33.343 & -1.218 & & 47.55 & $\begin{array}{l}0.08 \\
\end{array}$ & & & & & & & & & & & & & \\
\hline & $4 / 20 / 2004$ & 1:14:26 AM & 25.663 & & 25.423 & & & & 49.725 & 13.114 & 33.257 & & & 50,05 & & & & & & & & & & & & & & \\
\hline 195 & 4/20/2004 & 1:15:26 AM & 25.666 & 25.306 & 25.436 & 25.584 & 23.645 & 45. & 49.275 & \begin{tabular}{|l|l|}
13.058 \\
\end{tabular} & 33.066 & -1.221 & -7.0 & 47.6 & 0.081 & 14. & & 933.6 & \begin{tabular}{|l|l|} 
& 15.56 \\
\end{tabular} & & 11. & 39.3 & & & & & 0.304 & \\
\hline & 4/20/2004 & 1:16: & 669 & 25.309 & 25.468 & 25.496 & & 246 & \begin{tabular}{|l|l|}
49.389 \\
\end{tabular} & 13.022 & & -1.22 & & 47.8 & & & & & 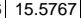 & 47.9 & $11.7 \mathrm{r}$ & 39 & & & & & 0.309 & \\
\hline 957 & 4/20/2004 & 1:17 & 25.671 & 25.311 & \begin{tabular}{|l|}
25.481 \\
\end{tabular} & 25.429 & & 45.178 & \begin{tabular}{|l|l|}
49.456 \\
\end{tabular} & $\begin{array}{l}13.083 \\
\end{array}$ & 32.668 & -1.22 & & 49.8 & 0.08 & 14 & & & 15.593 & 48.9 & 12. & & & & & & 302 & \\
\hline 1958 & 4/201/2004 & 1:18:26 & 25.664 & 25.309 & \begin{tabular}{|l|l|}
25.479 \\
\end{tabular} & 25.396 & & 45.24 & \begin{tabular}{|l|l}
49.443 \\
\end{tabular} & 13.044 & 32.793 & -1.224 & & 49. & & & & & & 48 & 14 & & & & & & 0.305 & \\
\hline & $4 / 201 / 2$ & & & 25.308 & \begin{tabular}{|l|}
25.507 \\
\end{tabular} & 25.46 & & 45.464 & & & & & & 49. & & & & & & & . & & & & & & & \\
\hline 960 & 4.212012004 & & 20.658 & 25.313 & & $\frac{25.5}{25.5}$ & & 45.251 & 49.624 & & $\frac{32.794}{326}$ & -1.24 & & 48. & & & & 938.6 & 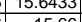 & 48. & 12.0 & (20. & & & & & 0.297 & \\
\hline & 年 & $\frac{1.4 .2 .2}{1.22 .2}$ & $\frac{23.079}{25694}$ & $\frac{25.306}{25324}$ & \begin{tabular}{|l}
25.546 \\
2544
\end{tabular} & $\frac{23.301}{25.57}$ & & - -5.363 & 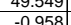 & $\begin{array}{l}13.104 \\
1227\end{array}$ & & $\frac{-1.24}{-121}$ & & 48. & & & & & $\frac{15}{156}$ & $\frac{40.1}{0.4}$ & 12.0 & & & & & & & \\
\hline 96 & $4 / 2012$ & & 25.989 & 25319 & \begin{tabular}{|l|l|l|}
25.19 \\
\end{tabular} & 25.642 & & -4.59 & $\mid-0.092$ & 124 & -507 & & & -0.0 & & & & 9946 & 15693 & -24 & & & & & & & & \\
\hline 96 & $4 / 201 / 2$ & $\begin{array}{l}1: 24: 26 \\
14: 26\end{array}$ & 25 & 25.31 & 25.48 & $\frac{25.493}{25.493}$ & & $\frac{-3.6}{-3.6}$ & $\mid-0.935$ & 1.23 & $\frac{-4.101}{-4.10}$ & -1218 & & $\frac{-0.6}{-0.6}$ & & & & $\frac{942.6}{992.6}$ & 15 & -24 & & & & 000 & & & & \\
\hline & & & & & & & & & & & & & & & & & & & & & & & & & & & & \\
\hline$\sqrt{96}$ & $4 / 20 / 2004$ & $1.26: 26 \mathrm{AM}$ & 25.669 & 25.299 & \begin{tabular}{|l|l|}
25.413 \\
\end{tabular} & 25.091 & & $-2,366$ & -0.956 & 1197 & -2897 & -1.218 & & 00 & & & & 944.6 & \begin{tabular}{|l|l|l}
15.7433 \\
\end{tabular} & -24 & & & & & & & 68 & \\
\hline & $4 / 20 / 2$ & $1: 27: 26 \mathrm{~A}$ & 25.5 & 25.299 & \begin{tabular}{|l|l|}
25.379 \\
\end{tabular} & & & $\mid-1.96$ & -0.94 & 1.185 & -2.501 & -1 & & & & & & 9 & & & & & & & & & & \\
\hline & $4 / 20 / 2004$ & $1: 28: 2$ & 25 & & 25.339 & 24.836 & & \begin{tabular}{|c|c|}
-1.647 \\
\end{tabular} & \begin{tabular}{|c|} 
\\
\end{tabular} & 1.172 & -2.193 & -1.21 & & -0.6 & 0.0 & \begin{tabular}{|l|l|l|l|l|}
1433 \\
\end{tabular} & & $\begin{array}{l}946.6 \\
\end{array}$ & 15.77 & -2.4 & 0.0 & -1.5 & -0.1 & 0.000 & 50 & & -0.231 & \\
\hline & $4 / 20 / 2$ & 1:29:26 & 25.63 & 25 & 25. & \begin{tabular}{|l|l}
24.729 \\
\end{tabular} & & -1.381 & -0.9 & 1.1 & -1.92 & -1.21 & & -0. & & & & & \begin{tabular}{|l|l|l}
15.7933 \\
\end{tabular} & -2. & & & & & & & (68) & \\
\hline 977 & $4 / 20 / 2$ & & 22.862 & 23.069 & \begin{tabular}{|l|l|}
23.717 \\
\end{tabular} & 23.865 & 23. & 13. & 14.446 & 3.785 & 10.186 & -1.215 & & & 0.0 & & & 1654.3 & & 13.0 & 3. & 11 & & 000 & & & 0.039 & \\
\hline |971 & $4 / 2012004$ & 1:17:10 PM & 22.91 & 23.09 & 24.184 & 23.84 & & 47.885 & 51.163 & 18.503 & 29.787 & -1.218 & & 46.12 & 0.0 & 14.6 & & & 27.5 & $49.7 \mathrm{r}$ & 11. & so. & & & & & 0.130 & \\
\hline & $\begin{array}{l}4212012004 \\
1202004\end{array}$ & $\frac{118.10 \mathrm{PM}}{1 \cdot 1 \cdot 10 \mathrm{PM}}$ & 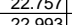 & $\frac{23.059}{23211}$ & $\begin{array}{l}24.655 \\
2.961\end{array}$ & 23.915 & 22000 & $\begin{array}{l}47.065 \\
47372\end{array}$ & 5.1 .08 & $\begin{array}{l}18.343 \\
18395\end{array}$ & 29.192 & -1.218 & & 告6.8 & (1) & 14.6 & & 16573 & 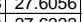 & 49.6 & $\frac{11.5}{1.6}$ & 20.1 & & & & & & \\
\hline & 41202004 & 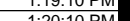 & 2..99 & $\frac{2.11}{2312}$ & \begin{tabular}{|l|}
24.9001 \\
\end{tabular} & 20.1400 & & 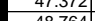 & $\begin{array}{l}51.400 \\
5.277\end{array}$ & $\begin{array}{l}10.200 \\
1.552\end{array}$ & $\frac{2.52}{30.57}$ & & & & & & & & & & & & & & & & & \\
\hline $97:$ & 4 & 1.1.21:10F & 23.406 & $\frac{23.5}{23.13}$ & & 24. & & 48 & 552762 & 18334 & & -1.4 & & & & & & & & 5 & $\frac{11 .}{120}$ & & & & & & & \\
\hline 97 & & $1: 2: 2: 1$ & 23.5 & 23.152 & 25.443 & $24 . \subseteq$ & & 49 & $\frac{23.3}{5.3}$ & 18.5 & & -121 & & & & & & & & 519 & 118 & & & & & & & \\
\hline & $4 / 20 / 2004$ & & & & & & & & & 18.466 & & & & & & & & & & 522 & 114 & & & & & & & \\
\hline & $4 / 20 / 2$ & 1:24:1 & & & 25.516 & 89 & & & 53.454 & & 31 & -1 & & & & & & & & 52.0 & 12.1 & & & & & & & \\
\hline & 412012 & & & & & & & & & 18325 & 343 & 218 & & 60 & & 14.643 & & & 27.7222 & 51.9 & 113 & & & & & & 84 & 3 \\
\hline 980 & $4 / 20 / 2004$ & \begin{tabular}{|l|}
$1: 26: 10 ~ P M$ \\
\end{tabular} & 24.065 & 23.173 & 25.535 & 25.408 & 23.584 & 49.262 & 53.298 & 18.276 & 31.328 & -1.221 & & 49.168 & 0.078 & 14.643 & & 6643 & 27.7389 & 51.8 & & 40.3 & 2.778 & 0.012 & 0.011 & 0.000 & 0.284 & 34! \\
\hline
\end{tabular}


WSRC-TR-2005-00105, REVISION 0

SRNL-RPP-2005-00012, REVISION 0

RUN \# 2.03A AND B; FIRST AND SECOND HALF OF SLURRY DEWATERING - CONT.

\begin{tabular}{|c|c|c|c|c|c|c|c|c|c|c|c|c|c|c|c|c|c|c|c|c|c|c|c|c|c|c|c|c|c|}
\hline & A & $\begin{array}{lll}B & 10\end{array}$ & D & $E$ & $F$ & G & $\mathrm{H}$ & $\mathrm{J}$ & $\mathrm{K}$ & $\mathrm{L}$ & $\mathrm{M}$ & $\mathrm{N}$ & 0 & $\mathrm{Q}$ & $\mathrm{R}$ & $\mathrm{s}$ & $\mathrm{T}$ & $\mathrm{V}$ & W & $x$ & $\mathrm{Y}$ & 2 & $z$ & $\mathrm{AA}$ & $A B$ & $A C$ & $\mathrm{AD}$ & $\mathrm{AE}$ & \\
\hline & $4 / 20 / 2004$ & $\begin{array}{l}1: 27: 10 \mathrm{PM} \\
1.28 \cdot 10 \mathrm{PM}\end{array}$ & $\frac{24.162}{2262}$ & & $\begin{array}{l}25.537 \\
25.7\end{array}$ & $\begin{array}{l}25.456 \\
25.443\end{array}$ & \begin{tabular}{|l|l|}
23.647 \\
2363
\end{tabular} & $\begin{array}{l}49.048 \\
49467\end{array}$ & $\begin{array}{l}52.853 \\
53.456\end{array}$ & $\begin{array}{l}18.153 \\
18.163\end{array}$ & \begin{tabular}{|l|l|}
31.363 \\
31646
\end{tabular} & \begin{tabular}{|c|}
-1.218 \\
-1218
\end{tabular} & \begin{tabular}{r|}
-7.585 \\
\\
-7579
\end{tabular} & $\begin{array}{l}49.113 \\
47011\end{array}$ & $\begin{array}{l}0.076 \\
0.076\end{array}$ & $\begin{array}{l}14.643 \\
14634\end{array}$ & \begin{tabular}{|l|l|}
0.003 \\
0.003
\end{tabular} & $\frac{1665.3}{166.3}$ & $\begin{array}{l}27.7556 \\
277722\end{array}$ & 51. & & & & $\frac{2.772}{2.706}$ & & 0.011 & $\begin{array}{l}0.000 \\
0000\end{array}$ & \begin{tabular}{|l|l|}
0.278 \\
.275
\end{tabular} & \\
\hline & & & & & $\frac{25.58}{25.57}$ & $\begin{array}{r}25.443 \\
25.192\end{array}$ & 23.63 & 49.467 & 53.456 & 18.163 & 31.646 & \begin{tabular}{|l|l|} 
& -1.218 \\
\end{tabular} & \begin{tabular}{|l|l|}
-7.579 \\
7.599
\end{tabular} & 47.011 & 0.076 & 14.643 & 0.003 & & 27.7722 & 52.4 & & & & 2.796 & & 0.011 & 0.000 & 0.275 & \\
\hline \begin{tabular}{|l|l|}
984 \\
\end{tabular} & $\begin{array}{r}44 / 2012004 \\
4 / 20 / 2004\end{array}$ & $\begin{array}{l}\frac{129}{12}: 10 \mathrm{PM} \\
13010 \mathrm{P}\end{array}$ & $\frac{24.34}{24.483}$ & $\frac{23.22}{23.24}$ & $\begin{array}{l}\frac{23}{46} 2525.579 \\
46\end{array}$ & \begin{tabular}{r|r}
25.482 \\
25.52
\end{tabular} & \begin{tabular}{|l|}
23.689 \\
23.752 \\
\end{tabular} & $\begin{array}{l}49.162 \\
49.121 \\
\end{array}$ & \begin{tabular}{|l|}
52.938 \\
53.045 \\
\end{tabular} & $\frac{18.084}{18.089}$ & $\begin{array}{l}31.67 \\
31.488 \\
\end{array}$ & \begin{tabular}{|l|}
-1.221 \\
-1.221
\end{tabular} & $\begin{array}{r}-7.588 \\
-7.59\end{array}$ & $\begin{array}{l}\mid 48.704 \\
47.684\end{array}$ & \begin{tabular}{|l}
0.077 \\
0.076
\end{tabular} & $\begin{array}{l}14.643 \\
14.643 \\
\end{array}$ & $\begin{array}{l}0.003 \\
0.003\end{array}$ & $\begin{array}{l}\frac{1667.3}{166.3} \\
166.3\end{array}$ & \begin{tabular}{|l|}
27.7889 \\
278056
\end{tabular} & \begin{tabular}{|l|l|}
$51 .$. \\
51.6 \\
\end{tabular} & 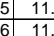 & & & $\begin{array}{l}2.784 \\
2.779\end{array}$ & & \begin{tabular}{|l}
0.011 \\
0.011 \\
\end{tabular} & $\begin{array}{l}0.000 \\
0.000\end{array}$ & $\begin{array}{l}0.280 \\
0.276\end{array}$ & \begin{tabular}{|l|}
0.24 \\
0.24 \\
\end{tabular} \\
\hline \begin{tabular}{|l|}
985 \\
\end{tabular} & $4 / 20 / 2004$ & $1: 31: 10 \mathrm{PM}$ & 24.586 & & \begin{tabular}{c|c|c|}
59 & 25.645 \\
\end{tabular} & 25.524 & & & 53.485 & & & & & & & 14.643 & & & & & & & & & & 0.011 & $\begin{array}{l}0.000 \\
0.000\end{array}$ & $\begin{array}{l}0.270 \\
0.273\end{array}$ & $\begin{array}{l}0.24 \\
0.23\end{array}$ \\
\hline & $4 / 20 / 2004$ & 1:32:10 PM & $\frac{24.668}{2.775}$ & & \begin{tabular}{ll|l|}
1 & 25.642 \\
\end{tabular} & $\begin{array}{r}25.576 \\
\end{array}$ & 23.857 & 49.262 & 53.338 & $\begin{array}{l}18.113 \\
18.12\end{array}$ & $\begin{array}{l}31.503 \\
2.020\end{array}$ & $\begin{array}{l}-1.218 \\
.221\end{array}$ & $\begin{array}{r}-7.588 \\
7.750\end{array}$ & \begin{tabular}{|l|l|}
48.661 \\
\end{tabular} & 0.075 & 14.643 & 0.003 & & 27.8389 & & & & & & & & & & \\
\hline & $4 / 20 / 2004$ & & 24.757 & & \begin{tabular}{|l|}
25.671 \\
\end{tabular} & 25.66 & 23.931 & 49.465 & \begin{tabular}{|l|l|}
53.524 \\
\end{tabular} & 18.194 & & \begin{tabular}{|c|} 
\\
\end{tabular} & & 48.106 & 0.074 & 14.643 & & & 27.8556 & & & & & 2.795 & & 0.011 & & & \\
\hline \begin{tabular}{|l|l|}
988 \\
989
\end{tabular} & $4 / 20 / 2004$ & $\begin{array}{l}1: 34: 10 \mathrm{PM} \\
1\end{array}$ & $\frac{24.855}{2.932}$ & $\begin{array}{l}23.31 \\
23.32\end{array}$ & $\begin{array}{l}25.704 \\
2.560 .\end{array}$ & $\begin{array}{r}25.697 \\
\\
-5.71\end{array}$ & $\begin{array}{l}23.894 \\
2 ? 027\end{array}$ & $\begin{array}{r}49.313 \\
48.977\end{array}$ & \begin{tabular}{|l|}
53.269 \\
5.021 \\
\end{tabular} & 18.021 & \begin{tabular}{|l|}
31.73 \\
31.50
\end{tabular} & $\begin{array}{r}-1.218 \\
.221\end{array}$ & $\begin{array}{r}-7.59 \\
7.58 \\
\end{array}$ & $\begin{array}{r}48.523 \\
1777\end{array}$ & 0.074 & $\begin{array}{l}14.643 \\
1.92\end{array}$ & $\begin{array}{l}0.003 \\
\end{array}$ & $\begin{array}{l}1672.3 \\
16702\end{array}$ & $\begin{array}{l}27.8722 \\
27800\end{array}$ & 51.8 & & & & 2.794 & & 0.011 & 0.000 & 0.267 & \\
\hline \begin{tabular}{|l|}
9890 \\
990 \\
\end{tabular} & $\begin{array}{r}4 / 2 / 20 / 2004 \\
4 / 20 / 2004\end{array}$ & $\begin{array}{l}\frac{1: 35: 10}{1} \\
1.36 \mathrm{PM}\end{array}$ & $\frac{24.932}{25.016}$ & $\frac{23.32}{23.33}$ & $\begin{array}{l}25.696 \\
25.745\end{array}$ & $\begin{array}{r}25.71 \\
25.753\end{array}$ & $\begin{aligned} \frac{23.927}{23.98} \\
23\end{aligned}$ & $\begin{array}{r}48.977 \\
49.315\end{array}$ & \begin{tabular}{|l|}
52.834 \\
53.193
\end{tabular} & $\begin{array}{l}17.868 \\
17.976\end{array}$ & $\begin{array}{r}31.529 \\
31.79\end{array}$ & $\begin{array}{l}-1.221 \\
-1.218\end{array}$ & $\begin{array}{l}-7.588 \\
-7.588 \\
\end{array}$ & \begin{tabular}{|l|}
47.774 \\
48.87
\end{tabular} & $\begin{array}{l}0.076 \\
0.074\end{array}$ & $\begin{array}{l}14.643 \\
14643\end{array}$ & \begin{tabular}{|l|l|}
0.003 \\
0.003
\end{tabular} & $\frac{1673.3}{1674.3}$ & $\begin{array}{l}27.8889 \\
279056\end{array}$ & & & & & & & 0.011 & $\begin{array}{l}0.000 \\
0.000\end{array}$ & $\begin{array}{l}0.276 \\
0.266\end{array}$ & \\
\hline \begin{tabular}{|l|}
991 \\
\end{tabular} & $4 / 20 / 2004$ & $\begin{array}{l}1.36 .10 \\
1: 37: 10 \mathrm{PM}\end{array}$ & 25.089 & $\frac{23.35}{23.35}$ & \begin{tabular}{l|l|}
57 & 23.145 \\
7 & 25.758
\end{tabular} & $\begin{array}{l}25.753 \\
25.717\end{array}$ & 24.023 & \begin{tabular}{|l}
49.515 \\
49.33 \\
\end{tabular} & \begin{tabular}{|l|}
53.343 \\
53.348
\end{tabular} & 17.916 & $\begin{array}{r}31.79 \\
31.776 \\
\end{array}$ & $\frac{-1.218}{-1.218}$ & $\begin{array}{l}-1.580 \\
-7.588 \\
\end{array}$ & \begin{tabular}{|l|}
48.035 \\
48.035
\end{tabular} & $\begin{array}{l}0.074 \\
0.075\end{array}$ & $\begin{array}{l}\frac{14.643}{14.643} \\
\end{array}$ & \begin{tabular}{|l}
.0033 \\
0.003
\end{tabular} & $\begin{array}{l}\frac{1674.3}{1675.3} \\
\end{array}$ & \begin{tabular}{|l|l|}
27.9956 \\
27.9222
\end{tabular} & & 11. & & & $\frac{2.796}{2.796}$ & 0.01 & $\begin{array}{l}0.01 \\
0.01\end{array}$ & \begin{tabular}{|l}
0.000 \\
0.000 \\
\end{tabular} & $\begin{array}{l}0.266 \\
0.270\end{array}$ & $\frac{0.23}{0.23}$ \\
\hline \begin{tabular}{|l|}
992 \\
\end{tabular} & $4 / 20 / 2004$ & $1: 38: 10 \mathrm{PM}$ & & & $\begin{array}{lll}71 & 25.721 \\
\end{array}$ & 25.72 & $\begin{array}{l}24.002 \\
\end{array}$ & & \begin{tabular}{|l|}
52.985 \\
\end{tabular} & 17.877 & 31.49 & $\begin{array}{l}-1.218 \\
\end{array}$ & & 48.546 & 0.076 & 14.643 & 0.003 & & & & & & & & & & & & \\
\hline & & 1:39:10 PM & & & \begin{tabular}{ll|}
55 & 25.745
\end{tabular} & & 24.016 & & & & & & & & & & & & & & & & & & & & & & \\
\hline & $4 / 2012004$ & 1:40:10 PM & 25.29 & & 25.764 & 25.758 & 24.05 & $\begin{array}{l}48.998 \\
\end{array}$ & 52.979 & 17.847 & & -1.218 & -7.593 & (48.081 & 0.074 & 14.643 & & 1678 & $\frac{19722}{.9722}$ & & & & & & & .01 & $\frac{0.000}{.000}$ & $\frac{268}{268}$ & \\
\hline & & & & 23.4 & & & & & & 17.875 & & & & & & & & & & & & & & & & & & & \\
\hline & 4/2012004 & 1:42:10 PM & 25.355 & 23.43 & 25.754 & 25.917 & 24.034 & 49.139 & 52.956 & 17.768 & 31.759 & 12 & -7.593 & $\begin{array}{l}47.609 \\
\end{array}$ & 0.075 & 14.643 & & 1680 & 8.0056 & & & & & 2.789 & & & 0.000 & & \\
\hline & 4/20/2004 & 1:43:10 PM & 25.381 & 23.4 & 25.74 & 25.964 & 24.096 & 49.009 & 52.871 & $17.766 \mathrm{C}$ & 31.638 & -1.218 & -7.585 & 47.296 & 0.075 & 14.643 & 0.003 & 1681. & 28.0222 & 51. & 11. & & 0.3 & 2.780 & & 0.01 & 0.000 & 0.272 & \\
\hline & 4/201/2004 & 1:44:10 PM & 25.429 & 23.47 & 25.768 & 26.021 & 24.123 & 49.423 & 53.334 & 17.899 & 31.923 & -1.218 & -7.585 .5 & 48.604 & 0.075 & 14.643 & 0.003 & 1682. & 8.0389 & & & & & 2.804 & & & 0.000 & & \\
\hline 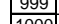 & $4 / 2012004$ & 1:45:10 PM & $\frac{25.466}{250}$ & 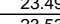 & 25.78 & 26.079 & 24.155 & 49.326 & 3.174 & 17.834 & 31.934 & -1.221 & -7.5880 & 46.285 & 0.074 & 14.643 & & 1683 & & & & & & 80 & & & 0.000 & 266 & \\
\hline & $4 / 2012004$ & $1.46 .10 \mathrm{PM}$ & $\frac{2.5099}{2050}$ & & & & & & & & & & & & & & & & & & & & & & & & & & \\
\hline & 年 & $\begin{array}{l}\frac{147: 10 \mathrm{PM}}{1: 48: 10 \mathrm{PM}} \\
\end{array}$ & $\frac{25.51}{25.566}$ & $\frac{23.56}{23.5}$ & $\begin{array}{r}25.799 \\
25.789\end{array}$ & $\frac{26.149}{26.038}$ & $\frac{23.835}{23.734}$ & $\begin{array}{l}48.758 \\
49.094 \\
\end{array}$ & 53.027 & 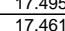 & $\begin{array}{l}31.735 \\
32.096\end{array}$ & $\frac{-1.215}{-1.218}$ & & | & $\frac{0.075}{0.074}$ & $\frac{14.664}{14643}$ & & & & & & & & 279 & & & & $\frac{272}{266}$ & \\
\hline 100 & $4 / 20 / 2004$ & $\frac{1.40 .10 \mathrm{PM}}{1: 49: 10}$ & 25.6 & 23.58 & 25.789 & $\frac{2.000}{26.017}$ & 23.698 & 48.921 & 52.846 & $\frac{1.7 .41}{17.414}$ & 31.905 & $\frac{1.210}{-1.221}$ & -7.55 & $\frac{4.119}{48.438}$ & 0.075 & $\frac{14.643}{1463}$ & & 1687 & 28.1 & $\overline{512}$ & & & & 2786 & & & 0.000 & & \\
\hline & $4 / 20 / 2004$ & 1:50:10 PM & 25.623 & 23.60 & 25.782 & 26.021 & 23.672 & 49.021 & 52.948 & 17.762 & 31.63 & $\begin{array}{l}-1.218 \\
-1.218\end{array}$ & -7.60 & 47.417 & 0.073 & 14.643 & & 1688. & 8.1389 & 51. & $\frac{11}{11}$ & & 0.3 & 2.780 & & 0.01 & 0.000 & & \\
\hline & $4 / 2012004$ & 1:51:10 PM & 25.657 & 23.62 & 25.811 & 26.015 & $\begin{array}{l}23.641 \\
\end{array}$ & 48.814 & 52.803 & 17.734 & 31.494 & -1.218 & -7.605 & 49.13 & 0.072 & 14.643 & 0.003 & 1689 & 8.1556 & & & & & 2.768 & & & 0.000 & 261 & \\
\hline & $4 / 20 / 2004$ & 1:52:10 PM & 25.661 & 23.62 & 25.815 & 25.994 & 23.685 & & 53.309 & 17.739 & 31.802 & -1.218 & & 50.169 & 0.074 & 14.643 & & 1690 & & & & & & & & & 0.000 & 0.266 & \\
\hline & $4 / 2012004$ & & & 23.64 & 25.196 & 26.025 & & 48.967 & 52.78 & & & & & & & & & & & & & & & & & & & & \\
\hline 年 & 4/20/2004 & 1:54:10 PM & 25.733 & & $25.777 \mid$ & 26.051 & 23.707 & 49.091 & 2.946 & 17.54 & 31.999 & -1.218 & & 47.758 & 1078 & 14.643 & & 166 & & & & & & & & & & 273 & \\
\hline & $4 / 2012004$ & 1:55:10 PM & 25.735 & 23.64 & 25.774 & 25.963 & 23.689 & 48.924 & .875 & 17.535 & 31.854 & -1.221 & & 48.525 & & 14.643 & & 16 & & 51.2 & 11 & & & & & & & 0.260 & \\
\hline & $4 / 20 / 2004$ & 1:56:10 PM & 25.768 & 23.67 & 25.797 & 26.001 & 23.737 & 48.99 & 年 & 17.429 & 32.047 & -1.221 & & 48.69 & & 14.643 & & 1694 & 28.2389 & 51. & 11 & & & 2.794 & & 0.01 & 1.000 & .263 & \\
\hline & $00 / 2004$ & 1:57:10 PM & 25.775 & & 25.7999 & 26.063 & 23.754 & & 2.946 & 17.449 & 31.969 & 218 & & 48.462 & 0.074 & & 0.004 & 16 & & & & & & & & & & & \\
\hline & 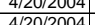 & $\begin{array}{l}1.588 .10 \mathrm{MM} \\
0.50 .10 \mathrm{~T}\end{array}$ & $\begin{array}{l}2.5084 \\
25796\end{array}$ & $\begin{array}{ll}2.609 \\
2367\end{array}$ & 25.807 & 26.092 & $\frac{23.77}{237}$ & $\begin{array}{l}49.133 \\
140212\end{array}$ & $\begin{array}{l}3.068 \\
3288\end{array}$ & $\frac{17.558}{1750^{2}}$ & & -1.218 & & 8.748 & 0.073 & 14.643 & & 169 & & & & & & & & & & 262 & \\
\hline & 4 & 1.50.10 & $\frac{2.500}{25819}$ & 年 & $\mid$ & $\frac{2.0 .047}{26.127}$ & 23.75 & $\frac{49.445}{4371}$ & 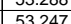 & 11.586 & 32,588 & -1.221 & & 17057 & 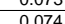 & $\frac{1.4645}{1463}$ & & 1698 & 830 & & & & 108 & 2814 & & & & $\frac{204}{264}$ & \\
\hline 1015 & $4 / 20 / 2004$ & 2:01:10 PM & 25.837 & 23.70 & 25.816 & 26.155 & & $\begin{array}{l}48.95 \\
\end{array}$ & 786 & 17.416 & 31.897 & -1218 & & 47.634 & & 14643 & & & & 513 & $\frac{\frac{1}{11}}{11}$ & & & & & & & & \\
\hline 101 & $0 / 2004$ & 2:02:10 PM & 25.862 & 23.72 & 25.825 & 26.21 & 23.84 & 499.123 & 53.164 & 17.588 & 31.938 & -1.218 & & 49.921 & 0.075 & 14.643 & & & & & & & & 2.794 & & 0.01 & & 270 & \\
\hline 1017 & | & 2:03:10 PM & 25.971 & 23.73 & 25.84 & 26.255 & & 45.0 & & 17.361 & & \begin{tabular}{l|l|l|l}
1.796 \\
\end{tabular} & & & & & & & & & 7 & & & & & & & & \\
\hline & |l/2004 & $\begin{array}{l}2: 04: 10 \mathrm{PM} \\
\end{array}$ & 25.893 & 23.74 & 25.872 & 26.257 & 24.0 & \begin{tabular}{|l|l|l|l}
48.223 \\
\end{tabular} & \begin{tabular}{|l|l}
52.699 \\
\end{tabular} & 17.501 & 31.059 & -0.969 & & 47.833 & 0.4 & $\overline{14.643}$ & & 170 & 8.3722 & 51. & 11 & & & 2.73 & & 0.01 & 0.000 & 0.356 & \\
\hline & 0.12004 & 2:05:10 PM & 25.889 & 23.74 & 25.868 & 26.272 & 23.9 & 48.565 & 52.666 & 17.345 & 31.517 & -1.218 & & 18.004 & & 14.643 & & & & & & & & & & & & & \\
\hline & D/2004 & 2:06:10 PM & 25.903 & & & 26.262 & 23.92 & & & & & & & 7153 & & 14.643 & & & 84056 & & 1 & & & 2.79 & & & & 262 & \\
\hline & $4 / 2$ & 2:07:10 PM & 25.914 & 23.76 & & & & & & & 31.944 & & & & & 14.643 & & & & & $1 \pm$ & & & & & & & & \\
\hline & $4 / 20 / 2004$ & 2:08:10 PM & 25.92 & 23.75 & 25.7922 & 26.282 & 23.68 & 49.16 & \begin{tabular}{|l|l|} 
\\
\end{tabular} & $17.335 \mathrm{~s}$ & 32.194 & -1.218 & & 46.967| & & 14.643 & & 170 & 8.44389 & 51. & 11 & & & & & 0.01 & & 269 & \\
\hline 104 & 0/2004 & 2:09:10 PM & 25.923 & 23.74 & 25.737 & 26.226 & 23.5 & 48.8 & 851 & 17.308 & 31. & -1.218 & & 48. & & $14.6 \mathrm{C}$ & & 170 & 28.4556 & . & $1 \pm$ & & & .884 & & 0.01 & 0.000 & 0.264 & \\
\hline & & $2: 10: 10 \mathrm{PM}$ & $\frac{25.921}{25023}$ & 年 23.15 & 25.1066 & 26.1115 & & 49.1871 & 255 & 1.371 & 32.1. & 1.215 & & & & & & & & & $\frac{12}{11}$ & & & & & & & & \\
\hline & & 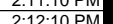 & & 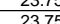 & 25.0969 & & 23 & & 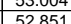 & 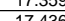 & & - & & & & & & & & & $\frac{11}{11}$ & & & & & & & & \\
\hline$\frac{10}{10}$ & 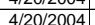 & $\frac{2 \cdot 1}{2 \cdot 13: 10 \mathrm{PM}}$ & 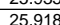 & 23.74 & 25.632 & 26.026 & & & & $\begin{array}{l}11.456 \\
17.281\end{array}$ & & $\begin{array}{l}-1.218 \\
-1.218\end{array}$ & & & & & & & & 51. & 11 & & & & & & & 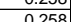 & \\
\hline 10 & 442012004 & $2: 14: 10 \mathrm{PM}$ & 25.926 & 23.74 & \begin{tabular}{c|c|}
47 & 25.625 \\
\end{tabular} & 26.07 & & 49.2 & 53.18 & 17.346 & & & & & & & & 11 & 8.5389 & . & 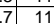 & & & & & & & & \\
\hline 102 & 4/20/2004 & 2:15:10 PM & 25.925 & 23.74 & 25.614 & 26.143 & 23.6 & 48.971 & 53.045 & 17.321 & $\begin{array}{l}32.046 \\
\end{array}$ & $\frac{-1.218}{-1218}$ & & 47.34 & 0.074 & & & 171 & 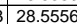 & 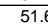 & 11 & 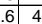 & & & & & & 0,268 & \\
\hline & & 2:16:10 PM & 25012 & & $25=07$ & & & & & 17.275 & & & & & & & & & & & & & & & & & & & \\
\hline & & 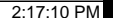 & 25.918 & 23.74 & 25.582 & 26.241 & 23.7 & 48.861 & 52.789 & 17.298 & & -1.221 & & 18506 & & & & & & & & & & & & & & & \\
\hline & $4 / 20 / 200$ & 2:18:10 PM & 25.92 & 23.74 & 25.567 & 26.277 & 23. & 49.158 & \begin{tabular}{|l|}
53.201 \\
\end{tabular} & 17.287 & & -1.218 & & 48 & & & & 171 & 28.6 & 51.8 & 11 & .9 & & 805 & & & & & \\
\hline & 0/2004 & 2:19:10 PM & 25.908 & 23.74 & \begin{tabular}{ll|}
49 & 25.547
\end{tabular} & & & 49.6 & 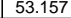 & 17.3 & & & & & & & & 17 & & 51 & 11 & & & & & 0. & & & \\
\hline 10 & $4 / 20 / 200$ & 2:20:10 PM & 25.8 & 23.75 & 25.492 & 26.261 & & 48.6 & & 17.2 & 31. & -1.221 & & & & & & 17 & 28.6: & 51 & 12 & 4 & & & & & & & \\
\hline$\frac{1035}{1036}$ & 0.21004 & 2:21:10 PM & 25.8 & 23.74 & 25.466 & & & 48.7 & \begin{tabular}{|l|l|}
52.764 \\
\end{tabular} & 17.246 & & & & & & & & 171 & & Nit & 12 & & & & & & & 6263 & \\
\hline & & $2: 2<: 1$ & $\frac{25.903}{2.500}$ & 23.176 & $25.48<4$ & & & & & & & -1.218 & & & & & & & & 51. & 11 & & & & & & & & \\
\hline 103 & $\frac{416}{412}$ & $\frac{2.25 .1 .10}{2 \cdot 24 \cdot 10 \mathrm{MM}}$ & $\frac{2.3908}{2588}$ & $\frac{23.11}{2378}$ & 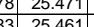 & & $\frac{23 .}{23}$ & (46.014) & 53074 & $\begin{array}{l}1.1 .0011 \\
1.6095\end{array}$ & $\frac{32.16}{320}$ & $-\frac{-1.18}{1.215}$ & & $\frac{11.0}{470}$ & & & & the & 287 & & $\frac{11}{11}$ & & & 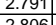 & & & & 60 & \\
\hline & & 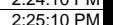 & & & 20.401 & & & | & & & & & & & & & & & & & & & & & & & & & \\
\hline 1046 & $4 / 20 / 200$ & $\frac{2: 26: 10 \mathrm{PM}}{2: 26,}$ & & $\frac{23.79}{23.79}$ & 25.442 & & & 48.548 & 668 & $\frac{17.405}{17405}$ & & -1.218 & & & & & & & & & & & & & & & & 6. & \\
\hline 104 & $4 / 2$ & $2: 27: 10$ PM & 25.874 & 23.80 & 25.433 & & & 48.737 & 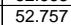 & 17.443 & & -1.218 & & 48.348 & 0.6 & 14.6 & & $1 / 2$ & 28.7 & & & & & & & & & $\frac{253}{253}$ & \\
\hline & & & 25.876 & 23.82 & 25.44 & & & & 52.743 & & & & & & & & & & & & & & & & & & & & \\
\hline & $\mid$ & 2:29:10 PM & 25.883 & 23.84 & 25.457 & 26.536 & & \begin{tabular}{|l|l|l|l|}
48.913 \\
\end{tabular} & 52.89 & 17.188 & 32.046 & -1.212 & & & & & & & & 51. & & & & & & & & & \\
\hline & & 2:30:10 PM & 25. & 23.84 & & & & 49. & 52.99 & & & & & & & & & 172 & & & 12 & & & & & & & & \\
\hline 1045 & $4 / 20 / 200$ & 2:31:10 P & 25.87 & 23.85 & 25.384 & 26.559 & 23 & 48.847 & 52.7 & 17.19 & & $-1.2 \quad 2$ & & 45.3 & & 14.64 & & 172 & & $51 \quad$ & 111 & & & & & & & 56 & \\
\hline & $4 / 20 / 200$ & 2:32:10 PM & & & & & & 48.6 & & 17.005 & & -1.218 & & & & & & 17 & & & 11 & .8 & & & & & & 33 & \\
\hline 1044 & $4 / 20 / 2004$ & $2: 33: 10$ & $25.88 \mathrm{~g}$ & 23.86 & 25.362 & 26.541 & 23.6 & 48.814 & 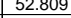 & 17.008 & 32.182 & -1.215 & & 46.742 & & 14.643 & & 173 & 328.8 & 51. & $\begin{array}{ll}3 & 11 \\
\end{array}$ & .5 & & 2.192 & 0.6 & 0.011 & 0.0 & 0.262 & 0.2 \\
\hline$\frac{1048}{1040}$ & | & $2.34 .10 \mathrm{PM}$ & $\frac{25.863}{25867}$ & $\frac{23.86}{2385}$ & $\begin{array}{c}59 \\
=7 \\
7\end{array}$ & $\begin{array}{l}26.491 \\
20.395\end{array}$ & $\frac{23.581}{.3217}$ & 48.8044 & \begin{tabular}{|l|}
52.903 \\
53204
\end{tabular} & $\frac{16.98}{1.016}$ & $\begin{array}{l}32.326 \\
32553\end{array}$ & $\frac{-1.218}{-1218}$ & & $\begin{array}{l}4.163 \\
5.99\end{array}$ & 0.011 & $\frac{14.643}{1063}$ & 0.0 & $\frac{113}{172}$ & $\frac{28.822}{28}$ & \begin{tabular}{|l}
51. \\
51.
\end{tabular} & $\begin{array}{ll}4 \\
4\end{array}$ & & & $\frac{2.800}{2820}$ & | & 0.010 & 0.000 & $\begin{array}{l}0.258 \\
.0253\end{array}$ & 0.2 \\
\hline & & $2: 36: 10$ PM & & & & & & & \begin{tabular}{|l|}
53.079 \\
\end{tabular} & & & & & & & 14.643 & & & & & & & & & & 0.011 & & 0.267 & 0.2 \\
\hline
\end{tabular}


WSRC-TR-2005-00105, REVISION 0

SRNL-RPP-2005-00012, REVISION 0

RUN \# 2.03A AND B; FIRST AND SECOND HALF OF SLURRY DEWATERING - CONT.

\begin{tabular}{|c|c|c|c|c|c|c|c|c|c|c|c|c|c|c|c|c|c|c|c|c|c|c|c|c|c|c|c|c|}
\hline & A & $\begin{array}{lll}\text { B } & \text { C }\end{array}$ & D & $E$ & $F$ & $\mathrm{G}$ & $\mathrm{H}$ & $\mathrm{J}$ & $\mathrm{K}$ & $\mathrm{L}$ & $\mathrm{M}$ & $\mathrm{N}$ & 0 & $\mathrm{Q}$ & $\mathrm{R}$ & $\mathrm{s}$ & $\mathrm{T}$ & $\mathrm{v}$ & w & $x$ & $\mathrm{Y}$ & $z$ & $\mathrm{AA}$ & $A B$ & $A C$ & $\mathrm{AD}$ & $\mathrm{AE}$ & AF \\
\hline & $4 / 20 / 2004$ & $\begin{array}{l}2: 37: 10 \mathrm{PM} \\
2.38 \cdot 10 \mathrm{PM}\end{array}$ & & & $\begin{array}{r}25.322 \\
25.3\end{array}$ & \begin{tabular}{|l|l|}
26.307 \\
2628
\end{tabular} & $\begin{array}{l}23.602 \\
23665\end{array}$ & 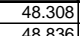 & $\begin{array}{r}52.33 \\
52747\end{array}$ & \begin{tabular}{|c|c|}
17.71 \\
17.538
\end{tabular} & $\begin{array}{l}30.955 \\
3.1595\end{array}$ & 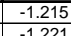 & \begin{tabular}{|l|l|}
-7.426 \\
-755
\end{tabular} & 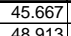 & $\begin{array}{l}0.071 \\
0.072\end{array}$ & $\begin{array}{l}14.643 \\
14634\end{array}$ & \begin{tabular}{|l|l|}
0.004 \\
0.023
\end{tabular} & $\begin{array}{l}1735.3 \\
17363\end{array}$ & \begin{tabular}{|l|l}
28.92222 \\
2283899
\end{tabular} & 50. & & 39.6 & $\frac{2.732}{2.772}$ & & $\begin{array}{l}0.010 \\
0.11\end{array}$ & $\begin{array}{l}0.000 \\
0000\end{array}$ & 0.265 & \\
\hline & & $\begin{array}{l}2: 38: 10 \mathrm{PM} \\
2.39 \cdot 10 \mathrm{PM}\end{array}$ & & $\frac{23.84}{23.85}$ & 25.3 & $\begin{array}{r}26.28 \\
25.200\end{array}$ & \begin{tabular}{|l|}
23.665 \\
\end{tabular} & $\begin{array}{l}48.836 \\
\end{array}$ & \begin{tabular}{|l|l|}
52.747 \\
\end{tabular} & 17.538 & $\begin{array}{l}31.595 \\
21201\end{array}$ & \begin{tabular}{|l|}
-1.221 \\
\end{tabular} & $\begin{array}{r}-7.55 \\
\end{array}$ & 48.913 & 0.072 & 14.643 & 0.003 & & \begin{tabular}{l|l|l|}
3 & 28.9389 \\
\end{tabular} & 51. & & & & & 0.011 & \begin{tabular}{|l|}
0.000 \\
\end{tabular} & & 0.23 \\
\hline $\begin{array}{ll}1054 \\
1054\end{array}$ & $\begin{array}{r}44 / 2012004 \\
4 / 20 / 2004\end{array}$ & $\begin{array}{l}2: 39: 10 \mathrm{PM} \\
2: 40: 10 \mathrm{PM}\end{array}$ & $\frac{25.841}{25.84}$ & $\frac{23.85}{23.85}$ & $\begin{array}{r}25.3 \\
25.304\end{array}$ & \begin{tabular}{|l|}
26.309 \\
26.393 \\
\end{tabular} & \begin{tabular}{|r|}
233.77 \\
23.824 \\
\end{tabular} & $\begin{array}{l}48.585 \\
48.699 \\
\end{array}$ & \begin{tabular}{|l|}
52.743 \\
52.658 \\
\end{tabular} & $\begin{array}{l}17.549 \\
17.521\end{array}$ & $\begin{array}{r}31.361 \\
31.54 \\
\end{array}$ & \begin{tabular}{|l|}
-1.221 \\
-1.218
\end{tabular} & $\begin{array}{l}-7.246 \\
-7.492\end{array}$ & $\mid \frac{45.857}{55.522}$ & 0.0677 & $\frac{14.643}{14.643}$ & $\begin{array}{l}0.003 \\
0.003\end{array}$ & $\begin{array}{l}1737.3 \\
1738.3\end{array}$ & $\begin{array}{l}328.9556 \\
3 \\
3\end{array}$ & \begin{tabular}{|l|}
51. \\
51.2 \\
\end{tabular} & \begin{tabular}{l|l}
3 & 11.2 \\
2 & 12.6 \\
\end{tabular} & 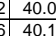 & $\begin{array}{l}\frac{0}{0} 2.756 \\
1 \\
1\end{array}$ & & \begin{tabular}{|l}
0.010 \\
0.011
\end{tabular} & \begin{tabular}{|l|} 
\\
\end{tabular} & $\begin{array}{l}0.248 \\
0.265\end{array}$ & \begin{tabular}{|l}
0.21 \\
0.23 \\
\end{tabular} \\
\hline 1055 & $4 / 20 / 2004$ & & 25.82 & & 25.298 & \begin{tabular}{|l|l|}
26.383 \\
\end{tabular} & & & 52.88 & & & & & & & 14.643 & 0.003 & & & 51. & & & & & 0.011 & $\begin{array}{l}0.000 \\
0.000\end{array}$ & $\begin{array}{l}.265 \\
0.257\end{array}$ & $\begin{array}{l}0.23 \\
0.22\end{array}$ \\
\hline & $4 / 20 / 2004$ & & 25.841 & & & & & $\begin{array}{l}48.691 \\
\end{array}$ & & \begin{tabular}{|l|l|}
17.048 \\
\end{tabular} & $\begin{array}{r}32.09 \\
30.090\end{array}$ & -1.218 & & \begin{tabular}{|l|l|}
49.253 \\
\end{tabular} & 0.068 & 14.643 & 0.003 & & & & & & & & & & & \\
\hline & $4 / 20 / 2004$ & & & & 25.26 & 26.505 & 23.936 & 48.214 & \begin{tabular}{|l|l|}
52.303 \\
\end{tabular} & 17.6 & & \begin{tabular}{|c|}
-1.218 \\
\end{tabular} & & $\begin{array}{l}48.569 \\
\end{array}$ & 0.069 & 14.643 & & & \begin{tabular}{l|l|}
3 & 29.0222 \\
\end{tabular} & & & $\begin{array}{l}939.6 \\
9\end{array}$ & & & & & & \\
\hline$\frac{1058}{1059}$ & $4 / 20 / 2004$ & $\begin{array}{l}2: 44: 10 \mathrm{PM} \\
2 \cdot 2 \cdot 5 \cdot 10 \mathrm{P}\end{array}$ & $\frac{25.828}{25.833}$ & & 25.246 & 26.541 & 23.977 & \begin{tabular}{|l|l|l|l|l|}
48.587 \\
48.677
\end{tabular} & \begin{tabular}{|l|l|}
52.573 \\
5.172 \\
\end{tabular} & $\begin{array}{l}17.499 \\
17.162\end{array}$ & 31.484 & $\begin{array}{r}-1.218 \\
1.218\end{array}$ & $\begin{array}{r}-7.588 \\
7.6\end{array}$ & 50.171 & 0.07 & 14.643 & 0.003 & 1742.3 & \begin{tabular}{|l|l|}
3 & 29.0389 \\
\end{tabular} & 51. & & $\begin{array}{l}340.0 \\
3\end{array}$ & $\begin{array}{l}0.2 .760 \\
0.7201\end{array}$ & & $\begin{array}{l}0.010 \\
0.011\end{array}$ & 0.000 & 0.259 & \\
\hline$\frac{1059}{1060}$ & $4 / 20 / 2004$ & & $\frac{25.833}{25.825}$ & $\begin{array}{r}23.859 \\
23.865\end{array}$ & $\begin{array}{l}25.252 \\
25.253\end{array}$ & $\frac{26.552}{26.618}$ & $\begin{aligned} 24.043 \\
24.054\end{aligned}$ & $\begin{array}{l}48.167 \\
48.447\end{array}$ & \begin{tabular}{|l|}
52.173 \\
52.494
\end{tabular} & $\begin{array}{l}17.468 \\
17.456\end{array}$ & & & & $\begin{array}{l}48.052 \\
48.145\end{array}$ & 0.071 & $\begin{array}{l}14.643 \\
14643\end{array}$ & $\begin{array}{l}0.003 \\
0.003\end{array}$ & $\begin{array}{l}1743.3 \\
1744.3\end{array}$ & \begin{tabular}{|c|c|} 
& 29.0556 \\
3 & 29.075
\end{tabular} & & & & 2.731 & & $\begin{array}{l}0.011 \\
0.010\end{array}$ & 0.000 & 0.265 & 0.21 \\
\hline 1061 & $4 / 20 / 2004$ & $\begin{array}{l}2: 46.10 \mathrm{PM} \\
2: 47: 10 \mathrm{PM}\end{array}$ & $\frac{25.825}{25.832}$ & & $\frac{25.253}{25.25}$ & $\begin{array}{r}20.010 \\
26.62\end{array}$ & $\begin{array}{l}24.054 \\
24.086\end{array}$ & $\begin{aligned} 488.447 \\
48.42\end{aligned}$ & \begin{tabular}{|l|}
52.494 \\
52.434 \\
\end{tabular} & $\begin{array}{l}17.450 \\
17.365\end{array}$ & $\begin{array}{l}31.281 \\
31.439\end{array}$ & $\frac{-1.218}{-1.218}$ & $\begin{array}{l}-1.550 \\
-7.602 \\
\end{array}$ & $\begin{array}{l}48.145 \\
50.559\end{array}$ & $\frac{0.069}{0.07}$ & $\begin{array}{l}\frac{14.643}{14.643} \\
\end{array}$ & \begin{tabular}{|l}
.0033 \\
0.003
\end{tabular} & $\begin{array}{l}\frac{1744.3}{1745.3} \\
\end{array}$ & 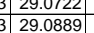 & \begin{tabular}{|l}
51. \\
51.
\end{tabular} & $\frac{12.4}{12.4}$ & & $\frac{2.749}{2.753}$ & $\frac{0.010}{0.010}$ & $\frac{0.011}{0.010}$ & $\begin{array}{l}0.000 \\
0.000\end{array}$ & $\frac{0.256}{0.260}$ & $\frac{0.22}{0.22}$ \\
\hline & $4 / 20 / 2004$ & $2: 48: 10 \mathrm{PM}$ & 25.817 & 23.873 & 25.236 & $\begin{array}{l}26.67 \\
\end{array}$ & 24.037 & \begin{tabular}{|l|l|l}
48.254 \\
\end{tabular} & \begin{tabular}{|l|l|}
52.113 \\
\end{tabular} & 17.36 & 31.25 & $\begin{array}{l}-1.218 \\
\end{array}$ & $\begin{array}{l}-7.478 \\
\end{array}$ & 47.476 & 0.065 & 14.643 & 0.003 & 1746.3 & & & & & & & & & & $\begin{array}{l}0.22 \\
0.21\end{array}$ \\
\hline & & & & & & & & & & & & & & & & & & & & & & 40. & & & & & & \\
\hline & $4 / 201 / 2004$ & 2:50:10 PM & 25.825 & 23.891 & 25.243 & 26.698 & 24.099 & $\begin{array}{l}48.868 \\
\end{array}$ & 52.799 & 17.526 & 31.718 & -1.218 & 99 & 7.175 & 0.069 & 14.643 & 003 & 1198 & & & & & 2.77 & & .011 & $\frac{000}{.000}$ & 254 & \\
\hline & & & & & & 26.719 & & & & 17.475 & 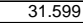 & & & & & & & & & & & & & & & & & \\
\hline & 4/2012004 & 2:52:10 PM & 25.827 & 23.918 & 25.255 & 26.705 & 23.896 & 499.245 & 53.149 & & $\begin{array}{l}31.977 \\
\end{array}$ & -1.215 & & $\begin{array}{ll}47.668 \\
\end{array}$ & 0.072 & 14.643 & .004 & 1750 & & & . & 40. & 2.800 & & & 0.000 & & \\
\hline 110 & 4/20/2004 & 2:53:10 PM & 25.943 & 23.929 & 25.277 & 26.757 & 24.083 & 48.403 & 52.482 & 17.394 & 31.377 & -1.215 & -7.128 & 49.581 & 0.061 & 14.643 & 0.004 & 1751.3 & \begin{tabular}{|l|l|}
29.1889 \\
\end{tabular} & 51. & 12.2 & 39.9 & 2.750 & .009 & $0.00 \mathrm{~s}$ & 0.000 & 0.226 & \\
\hline & $4 / 2012004$ & 2:54:10 PM & 25.953 & 23.954 & 25.277 & 26.736 & 24.308 & 48.565 & 52.728 & 17.3988 & 31.48 & -1.218 & -7.43 & 46.375 & 0.071 & 14.643 & & 1752.3 & 29.2056 & 51 & 11. & 40.0 & & & 0.01 & 0.000 & & \\
\hline & $4 / 2012004$ & 2:55:10 PM & 25.902 & $\frac{2.3699}{23209}$ & 25.296 & 26.846 & 24.287 & 48.563 & 2.486 & 17.382 & 31.622 & -1.218 & & $49.44 \pi$ & 0.073 & 14.643 & & & & & & & 2.164 & & & 0.000 & 6269 & \\
\hline & $4 / 2012004$ & $2.36 \cdot 10 \mathrm{PM}$ & 2.5 .8272 & & & & & & & & & & & 0.04 & & & & & & & & & & & & & & \\
\hline & 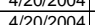 & 2:5:5:10 PM $2: 58 \cdot 10 \mathrm{P}$ & $\frac{25.716}{2575}$ & $\frac{23.967}{23.996}$ & $\frac{25.259}{25284}$ & $\frac{26.799}{26878}$ & $\frac{24.361}{24.415}$ & 年6.0259 & 20.519 & $\frac{17.415}{17417}$ & $\begin{array}{l}31.503 \\
31.607\end{array}$ & $\begin{array}{l}-1.224 \\
-1218\end{array}$ & & 4 & 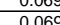 & $\frac{14.664}{14643}$ & & 175 & & & & & 768 & & & & & \\
\hline 1073 & $4 / 20 / 2004$ & $2: 59: 10 \mathrm{PM}$ & $\frac{25.725}{25.725}$ & 24.021 & 25.299 & $\frac{2.0918}{26.918}$ & 24,465 & 48.679 & 52.662 & 17.339 & 31.737 & $\frac{-1.218}{-1.218}$ & -7.611 & 47.317 & 0.069 & $\frac{1.4043}{14.643}$ & & 17573 & 92889 & 512 & 11 & & & & & 000 & & \\
\hline & 4/2012004 & 3:00:10 PM & 25.683 & 24.03 & 25.297 & 26.891 & 24.583 & \begin{tabular}{|l|l|l|l|l|}
48.643 \\
\end{tabular} & 52.614 & 17.428 & 31.579 & -1.218 & & 47.855 & 0.069 & 14.643 & & 1758.3 & 29.3056 & 51. & 11. & 40.1 & 2.766 & & 0.01 & 0.000 & & \\
\hline 107 & $4 / 2012004$ & 3:01:10 PM & 25.678 & 24.059 & 25.311 & 26.861 & 24.63 & \begin{tabular}{|l|l|l}
48.434 \\
\end{tabular} & 62.486 & 17.368 & 31.384 & -1.218 & -7.605 & $\begin{array}{l}48.487 \\
\end{array}$ & 0.069 & 14.643 & 0.003 & 1759.3 & & & 11. & 39.9 & 2.752 & & 0.01 & & & \\
\hline & 4/20/2004 & 3:02:10 PM & 25.662 & 24.068 & 25.31 & 26.96 & 24.726 & 48.641 & 52.467 & 17.413 & & & & 49.802 & 0.07 & 14.643 & 0.003 & & 29.3389 & & & & & & & 0 & 0.258 & \\
\hline & & & & & 25.3 & 27.019 & & & & & & -1.218 & & & 0.069 & & & & & & & & & & & & & \\
\hline & 4/20/2004 & 3:04:10 PM & 25.651 & & 25.244 & 27.084 & 24.836 & \begin{tabular}{l|l|l}
48.702 \\
\end{tabular} & 2.679 & 17.361 & 31.655 & -1.2 .24 & & 49.403 & & 14.643 & & & & & & & & & & & & \\
\hline & $0 / 2004$ & 3:05:10 PM & 25.64 & 24.141 & 25.258 & 27.132 & 24 & 48.617 & 6.695 & 17.324 & & -1.221 & & & & 14.643 & & 176 & 9.3889 & & & 40 & & & & & 0.262 & \\
\hline & $4 / 20 / 2004$ & $\begin{array}{l}3: 06: 10 \text { PM } \\
30710\end{array}$ & 25.64 & 24.172 & 25.259 & 27.188 & 24.8 & & 52.645 & 17.339 & 31.636 & -1.221 & -7.48 & 49.854 & & 14.643 & & 1764.3 & 29.4056 & 51. & 12.2 & 44 & .767 & & & .0000 & 0.266 & \\
\hline & $4 / 20 / 2004$ & 3:07:10 PM & 25.64 & 24.196 & 25.268 & 27.217| & 24.8 & 48 & .359 & 17.326 & 31.384 & & & 0.137 & 0.069 & & & & & & & & & & & & & \\
\hline & 4212012004 & 3:08:10 PM & $\frac{25.634}{25642}$ & & $\frac{2.251}{25207}$ & 20.260 & & & 2.793 & $\begin{array}{l}17.354 \\
117073\end{array}$ & $\frac{31.673}{32016}$ & & & & 0.072 & 14.643 & & 116 & & & 12. & & & & & & & \\
\hline & 4 & 3.05.10 PM & $\frac{2.0434}{2568}$ & 24245 & $\frac{25.261}{25302}$ & 273 & $\frac{24.97}{24.97}$ & 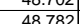 & 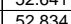 & 17181 & $\begin{array}{l}32.010 \\
31.989\end{array}$ & $\begin{array}{l}-1.241 \\
-1.221\end{array}$ & & 928 & 0.07 & 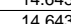 & & 17683 & 29.4722 & & 120 & 404 & 2784 & & & & & \\
\hline & $4 / 20 / 2004$ & 3:11:10 PM & 25.648 & 24.31 & 25.307 & 27331 & & 48.793 & 52778 & 17293 & 3188 & -1.121 & & & & 1464 & & 176 & 20.4889 & 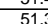 & 11 & & & & & & & \\
\hline & .0/2004 & 3:12:10 PM & 25.647 & 24.33 & 25.331 & \begin{tabular}{l|l}
27.32 \\
\end{tabular} & 24.9 & 48.556 & 623 & 17.171 & 31.722 & -1.218 & & 46.402 & 0.07 & 14.643 & & 177 & & & 11 & 4 & $2.767 \mathrm{C}$ & & & & & \\
\hline 100 & D/2004 & 3:13:10 PM & 25.641 & 24.354 & & 27.314 & & & 679 & & & & & & & & & & & 51 & (1) & & & & & & & \\
\hline & 0.12004 & 3:14:10 PM & 25.645 & 24.378 & 25.339 & 27.313 & 25.01 & \begin{tabular}{|l|l|}
48.65 \\
\end{tabular} & 52.811 & \begin{tabular}{|l|l|l|l|l|}
1733 \\
\end{tabular} & 31.841 & -1.221 & -7.2 & $\begin{array}{l}49.339 \\
\end{array}$ & 0.056 & 14.643 & $\begin{array}{l}0.003 \\
\end{array}$ & 1772. & 29.5389 & 51.3 & 12.1 & $4 c$ & 2.775 & & $0.00 \varepsilon$ & 0.000 & 0.205 & \\
\hline & 4/201/2004 & 3:15:10 PM & 25.634 & 24.397 & 25.323 & 27.302 & 25.029 & 48.459 & 52.579 & $\begin{array}{l}17.296 \\
\end{array}$ & 31.51 & -1.224 & -7. & 48.354 & 0.07 & 14.643 & & 1773.3 & \begin{tabular}{|l|}
29.5556 \\
\end{tabular} & 51. & 11.8 & & 2.757 & & 0.01 & 0.000 & 0.259 & 0. \\
\hline & | & $\begin{array}{l}3: 16: 10 \mathrm{PM} \\
3 \cdot 17 \cdot 10 \mathrm{PM}\end{array}$ & & & \begin{tabular}{|l|}
25.368 \\
25375 \\
\end{tabular} & & & \begin{tabular}{|c|}
48.693 \\
\end{tabular} & & & $\frac{31.831}{21.32}$ & & & \begin{tabular}{|l|}
4.5988 \\
5.20
\end{tabular} & 0.073 & 14.643 & & 1774.3 & $\begin{array}{l}3 \quad 29.5 / 22 \\
\end{array}$ & & & 40.3 & & & & & & 0.2. \\
\hline & 4/20/2004 & 3:19:10 PM & 25.671 & 24.484 & 25.38 & 27.363 & & 48.818 & 52.903 & 17.349 & & -1.221 & & & & & & 777 & & 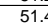 & & & & & & & & \\
\hline & 0.02004 & 3:20:10 PM & & 24.511 & 25.387 & 27.306 & & & 52.341 & 17.288 & 31.338 & 1221 & & & & & & 1778.3 & & & 1 & & & & 001 & & 0.256 & \\
\hline & & 3:21:10 PM & 25.694 & 24.53 & 25.418 & 27.247 & & 48.836 & 52.782 & 17.401 & & -1.2 & & & & & & & & 51 & & & & & & & & \\
\hline & $4 / 2$ & 3:22:10 PM & 25.6 & & 25.393 & 27.192 & & & & & & & & & & & & 178 & & & & & & & & & & \\
\hline & $4 / 20 / 2004$ & 3:23:10 PM & 25.67 & 24.528 & 25.389 & 27.173 & & 48.662 & 52.73 & 17.201 & & -1.2 & & 49. & & 14.643 & & 178. & 29.6889 & 51. & $12.1 \mathrm{C}$ & & & & 0.01 & & 0.267 & \\
\hline & 4/201/2004 & 3:24:10 PM & 25.696 & 24.574 & 25.456 & 27.244 & & 48.668 & 52.6 & 17.299 & 31. & -1.218 & & & & 14.643 & & 1782. & 29.1050 & 51. & 12. & & & & . & 0.0 & 0.256 & \\
\hline & $\mid 0,2004$ & 3:25:10 PM & 25.683 & 24.576 & 25.417 & 27.166 & 24.9 & 48.6 & 52.6 & 17.273 & 31. & -1.224 & & 48.924 & & & & 178 & & 51 & 12.0 & & 2.1000 & & & & & \\
\hline & $4 / 2$ & 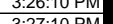 & 257.11 & 24.598 & $\frac{2.5499}{2.737}$ & $\frac{21.250}{2723}$ & 24. & 年8.764 & $\begin{array}{l}5.501 \\
52025\end{array}$ & 1.17 & & -1.221 & & 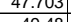 & 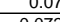 & 14.6 & & 188 & & 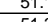 & & & & & & & & \\
\hline & & $\frac{3.4 .10 \mathrm{PM}}{3 \cdot 2 \cdot 10 \mathrm{PM}}$ & $\frac{23.60}{2570}$ & $\frac{24.00}{2462}$ & $\frac{25.370}{25.373}$ & $\frac{2.33}{27.347}$ & & & $\frac{52.025}{52471}$ & $\frac{1.25}{17.257}$ & & $\frac{-1.24}{-1221}$ & & & & & & 17863 & 3297722 & & 124 & & 768 & & & & & \\
\hline & 4212000 & 3.2.2.1. PM & 2570 & 24649 & 2538 & 27339 & & & $\frac{5.47}{52575}$ & 17178 & & & & & & & & 1700.0 & & & & & & & & & & \\
\hline 1104 & $4 / 20 / 200$ & 3:30:10 PM & $\frac{25.714}{25.74}$ & 24.663 & $\frac{25.399}{25.399}$ & 27.417 & & 48. & 52.699 & & & $\frac{-1.221}{-1.221}$ & & & & & & & 29.8056 & 51 & 12. & & & & & & & \\
\hline 1105 & $4 / 20 / 200$ & 3:31:10 PM & 25.712 & & 25.426 & 27.489 & & 48.7 & & & & & & & & & & $1 / 8$ & & & & & & & & & & \\
\hline & $4 / 2$ & & 25 & 24.698 & 25.414 & 27.492 & 25.2 & & & 17.071 & 31.905 & -1.218 & & 50.64 & & 14.643 & & 179 & 29.83 & 51 & 12. & & & & & & 0.260 & \\
\hline & & & 25. & 24.696 & 20.397 & 27.51 & & & & $\begin{array}{l}17.21 \\
\end{array}$ & & -1.224 & & & & & & & & & $1+$ & & & & & & & \\
\hline & $4 / 2$ & 3:34:10 PM & 25.731 & 24.739 & 25.435 & 27.523 & & 48.482 & 52.335 & 17.07 & 31.845 & -1.221 & & & & & & 1792 & & 50 & 12. & 4 & 27 & & & & & \\
\hline & $4 / 20 / 2004$ & 3:35:10 PM & 25.7 & 24.75 & 25.453 & 27.566 & & 48. & 52.544 & & 31.7 & -1.210 & & & & 14. & & & & & 1. & & & & & & & \\
\hline & $4 / 20 / 2004$ & 3:36:10 PM & 25.7. & 24.77: & 25.455 & 27.583 & & 48.7 & \begin{tabular}{|l|} 
\\
\end{tabular} 2.7477 & & & -1.218 & & & & & & & & 51 & & & & & & & & \\
\hline & 4/201/2004 & 3:37:10 PM & 25.748 & 24.797 & 25.473 & 27.581 & 25. & 48.648 & 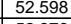 & 16.976 & & -1.221 & & 48.6 & & $14.6 \mathrm{C}$ & & 179 & & $\sigma_{1}$ & & & & & & & 0.263 & \\
\hline & 412 & $3: 38: 10 \mathrm{PM}$ & $\frac{2.566}{2.562}$ & 24.8915 & 2.4 .45 & 27.028 & 25. & 48.897 & 52.816 & $\begin{array}{l}16.968 \\
1.7017\end{array}$ & 32. & & & & & & & 175 & & & & & & & & & & \\
\hline 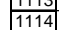 & & 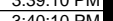 & 250.103 & & 25.4100 & $\frac{21.0}{2751}$ & & (4.5894 & & & & -1.221 & & & & & & 17 & & 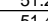 & . & & & & & & & \\
\hline & $4 / 20 / 200$ & $3 \cdot 461 \cdot 10 \mathrm{PM}$ & 2576 & 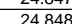 & 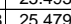 & 27 2737 & & $\begin{aligned} 4.0 .054 \\
491\end{aligned}$ & & $\begin{array}{l}1682 \\
1682\end{array}$ & & 1224 & & & & 146 & & 179 & & 51 & & & & & & & 5 & \\
\hline 111 & $4 / 20 / 20$ & 3:42:10 PM & 25.77 & 24.8 & $\frac{25.509}{25.09}$ & $\begin{array}{l}27.467 \\
22.767\end{array}$ & & 49.054 & 53108 & & & -1.218 & & & & & & & & & 11. & & & & & & & \\
\hline & $4 / 20 / 2004$ & & 25.77 & 24.868 & 25.509 & 27.422 & & 49.048 & 53.01 & $\begin{array}{l}16.867 \\
\end{array}$ & 32.44 & -1.221 & & 47.426 & 0.073 & 14.643 & & 18013 & \begin{tabular}{l|l|}
33 & 30.0222 \\
\end{tabular} & 51.5 & 11.6 & & & & 0.0 & & 0.263 & \\
\hline & & & & & & & 24.864 & & & & & & & & & & & & & & & & & & & & & \\
\hline & & & 25.755 & & & 27.392 & 24.9 & 49.056 & 3.033 & 16.867 & & 120 & & & & 14.643 & & & & & & & & & & & 252 & \\
\hline & & & & & & & & & & & & & & & & & & & & & & & & & & & 0.256 & \\
\hline
\end{tabular}


WSRC-TR-2005-00105, REVISION 0

SRNL-RPP-2005-00012, REVISION 0

RUN \# 2.03A AND B; FIRST AND SECOND HALF OF SLURRY DEWATERING - CONT.

\begin{tabular}{|c|c|c|c|c|c|c|c|c|c|c|c|c|c|c|c|c|c|c|c|c|c|c|c|c|c|c|c|c|}
\hline & A & B & D & $E$ & $F$ & $G$ & $\mathrm{H}$ & $\mathrm{J}$ & $\mathrm{K}$ & $\mathrm{L}$ & $\mathrm{M}$ & $\mathrm{N}$ & $\begin{array}{l}0 \\
\end{array}$ & Q & $R$ & 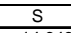 & \begin{tabular}{l|l|l|} 
\\
\end{tabular} & & w & $x$ & & $z$ & AA & $A B$ & $A C$ & $A D$ & AE & \\
\hline$\frac{1121}{1122}$ & \begin{tabular}{r|r|}
$4 / 20 / 2004$ \\
42012004
\end{tabular} & $\begin{array}{l}3: 47: 10 \mathrm{PM} \\
3: 47 \cdot 13 \mathrm{PM}\end{array}$ & $\begin{array}{l}25.772 \\
25.757\end{array}$ & $\begin{array}{r}\frac{24.905}{24.88} \\
\end{array}$ & $\begin{array}{l}25.446 \\
325.416\end{array}$ & \begin{tabular}{l|l|}
5 & 27.459 \\
5 & 27.42
\end{tabular} & \begin{tabular}{|l|}
24.972 \\
24.992 \\
\end{tabular} & $\begin{array}{l}48.571 \\
48.575\end{array}$ & \begin{tabular}{|l|}
52.614 \\
52.633 \\
\end{tabular} & \begin{tabular}{|l|}
16.742 \\
16.813
\end{tabular} & $\begin{array}{r}32.155 \\
31.969 \\
\end{array}$ & $\begin{array}{l}-1.218 \\
-1.224 \\
\end{array}$ & $\begin{array}{r}-7.588 \\
-7.59 \\
\end{array}$ & \begin{tabular}{|l|l|l}
48.325 \\
47.505
\end{tabular} & 0.071 & $\begin{array}{l}14.643 \\
14643 \\
\end{array}$ & $\begin{array}{l}0.003 \\
0.03\end{array}$ & \begin{tabular}{l|l}
1805.3 \\
1805.5 \\
\end{tabular} & \begin{tabular}{|l|}
30.0889 \\
30.08977 \\
\end{tabular} & \begin{tabular}{|l|}
51.1 \\
512
\end{tabular} & $\frac{11.8}{11.6}$ & & $\begin{array}{l}\frac{4}{4} 2.783 \\
3 \\
2.7777\end{array}$ & 0.011 & $\begin{array}{l}0.010 \\
0.010\end{array}$ & 0.000 & $\begin{array}{l}0.259 \\
0.252\end{array}$ & 0.22 \\
\hline & & & & & \begin{tabular}{|l|l|}
25.4410 \\
\end{tabular} & & \begin{tabular}{|l|l|}
25.123 \\
25
\end{tabular} & & & & & & & & & $\begin{array}{l}14.445 \\
14.643\end{array}$ & & & & & & & & & & & & \\
\hline \begin{tabular}{|l|l|}
1124 \\
\end{tabular} & $4 / 20 / 2004$ & 3:49:13 PM & 25.759 & 24.913 & 25.449 & 27.392 & 25.17 & 48.811 & \begin{tabular}{|l|}
52.805 \\
\end{tabular} & 16.905 & 32.256 & -1.224 & $\begin{array}{l}-7.596 \\
\end{array}$ & 50.34 & 0.069 & 14.643 & 0.003 & 1807.4 & 30.1231 & & 12.3 & 40.5 & \begin{tabular}{|l|l|}
5 & 2.795 \\
\end{tabular} & 0.010 & 0.010 & 0.000 & 0.251 & \\
\hline & $4 / 2012004$ & & 25.777 & 24.93 & 25.466 & 27.469 & 25.237 & 48.868 & \begin{tabular}{|l|}
52.855 \\
\end{tabular} & 16.843 & 32.381 & -1.221 & -7.472 & \begin{tabular}{|l|}
46.033 \\
\end{tabular} & 0.071 & 14.643 & & 1808.4 & \begin{tabular}{|l|l|}
30.1397 \\
\end{tabular} & & & & & & & & $\begin{array}{l}0.257 \\
0.257\end{array}$ & \\
\hline & & & & & 25.483 & & 25.309 & & & $\begin{array}{l}16.629 \\
1\end{array}$ & & & & & & 14.643 & 0.004 & & & & 11.9 & & & & & & & \\
\hline$\frac{1127}{1128}$ & $4 / 20 / 2004$ & $3: 52: 13 \mathrm{PM}$ & $\begin{array}{l}25.782 \\
25770\end{array}$ & $\begin{array}{r}24.96 \\
2.059\end{array}$ & 25.486 & $\begin{array}{ll}27.594 \\
27602\end{array}$ & $\begin{array}{l}25.332 \\
2539\end{array}$ & \begin{tabular}{|l|l|}
48.789 \\
8.896
\end{tabular} & \begin{tabular}{|l|l|}
52.805 \\
5.865
\end{tabular} & $\begin{array}{l}16.739 \\
16.69\end{array}$ & 32.42 & $\begin{array}{l}-1.221 \\
.221\end{array}$ & $\begin{array}{l}-7.446 \\
7.27 \\
\end{array}$ & \begin{tabular}{|r|}
48.98 \\
\end{tabular} & $\begin{array}{l}0.072 \\
0.072\end{array}$ & 14.643 & $\begin{array}{l}0.003 \\
0.023\end{array}$ & \begin{tabular}{|l|l|l|}
1810.4 \\
\end{tabular} & \begin{tabular}{|l|}
30.1731 \\
\end{tabular} & & 12.0 & & 2.800 & 0.011 & 0.011 & 0.000 & $\begin{array}{l}0.261 \\
\end{array}$ & \\
\hline $\begin{array}{l}1128 \\
1129\end{array}$ & $\begin{array}{l}4 / 2012004 \\
4 / 2012004\end{array}$ & 3:5:13:13 PM & $\begin{array}{l}25.779 \\
25.787\end{array}$ & $\begin{array}{l}24.958 \\
24.986\end{array}$ & $\begin{array}{l}25.469 \\
3\end{array}$ & $\begin{array}{l}27.602 \\
27.565 \\
\end{array}$ & \begin{tabular}{|l|}
25.284 \\
25.357 \\
\end{tabular} & $\begin{array}{l}48.826 \\
49.089\end{array}$ & \begin{tabular}{|l|}
52.865 \\
53.168 \\
\end{tabular} & $\begin{array}{l}16.619 \\
16.699\end{array}$ & $\begin{array}{l}32.543 \\
32.266 \\
\end{array}$ & $\begin{array}{l}-1.224 \\
-1.218 \\
\end{array}$ & $\begin{array}{l}-7.437 \\
-7.625\end{array}$ & \begin{tabular}{|r|}
48.83 \\
46.54
\end{tabular} & $\begin{array}{l}0.072 \\
0.072\end{array}$ & $\begin{array}{l}14.643 \\
16463\end{array}$ & \begin{tabular}{|l|l|}
0.003 \\
0.003
\end{tabular} & \begin{tabular}{|l|l|l|l|l|}
1811.4 \\
1812
\end{tabular} & \begin{tabular}{|l|l|}
30.1897 \\
302064
\end{tabular} & $\frac{51 .}{51 .}$ & $\frac{12.0}{111.4}$ & & $\begin{array}{ll}7 & 2.805 \\
9 & 2818\end{array}$ & 0.01 & \begin{tabular}{|l|l|}
0.011 \\
0.011
\end{tabular} & $\begin{array}{l}0.000 \\
0.000\end{array}$ & $\begin{array}{l}0.260 \\
0.259\end{array}$ & $\frac{0.42}{0.22}$ \\
\hline $\begin{array}{l}1130 \\
1130\end{array}$ & $4 / 20 / 2004$ & 3:55:13 PM & 25.795 & & 25.515 & 27.602 & \begin{tabular}{|r|}
25.050 \\
25.41 \\
\end{tabular} & 48.87 & \begin{tabular}{|l|}
52.768 \\
\end{tabular} & 16.6 & $\begin{array}{l}32.5000 \\
32.531\end{array}$ & $\begin{array}{l}-1.210 \\
-1.221\end{array}$ & $\begin{array}{l}-7.625 \\
-7.492\end{array}$ & \begin{tabular}{|l|}
47.3544 \\
47.301
\end{tabular} & 0.072 & $\begin{array}{l}14.643 \\
14.643 \\
\end{array}$ & 0.003 & $\frac{1812.4}{1813.4}$ & & $\begin{array}{l}51.1 \\
51.3\end{array}$ & 111.6 & \begin{tabular}{|l|l|}
40.7 \\
\end{tabular} & & & $\frac{0.011}{0.011}$ & $\begin{array}{l}0.000 \\
0.000\end{array}$ & $\begin{array}{l}0.259 \\
0.260\end{array}$ & 0.22 \\
\hline 1131 & $4 / 20 / 2004$ & & & & & 27.605 & 25.478 & & & 16.724 & 32.679 & -1.224 & & 49.535 & 0.076 & 14.643 & & & & & & & & & & & & $\begin{array}{l}0.22 \\
0.23\end{array}$ \\
\hline & $4 / 20 / 2004$ & 3:57:13 PM & 25.921 & & 25.515 & \begin{tabular}{|l|l|}
27.698 \\
\end{tabular} & 25.541 & 48.965 & 52.88 & 16.591 & 32.627 & -1.221 & -7.562 & 47.774 & 0.065 & 14.643 & 0.003 & \begin{tabular}{|l|l|l|}
1815.4 \\
\end{tabular} & \begin{tabular}{|l|}
30.2564 \\
\end{tabular} & & 11.7 & & & & & 0.000 & & \\
\hline 1133 & $4 / 20 / 2004$ & 3:58:13 PM & 25.904 & & 25.533 & & & & & & & & & & & & & & & & & & & & & & & \\
\hline 1134 & & & & & 25.515 & & 25.366 & 49.425 & 53.344 & 16.656 & 33.163 & -1.221 & & 15205 & 0.057 & & & & & & & & & & & & & \\
\hline & $4 / 20 / 2004$ & $4: 00: 13 \mathrm{PM}$ & & & 25.563 & \begin{tabular}{|l|l|}
27.69 \\
\end{tabular} & 25.238 & 48.863 & & & & -1.218 & & 50.308 & 0.104 & 14.643 & 0.004 & 318.4 & 1.3064 & & & 40 & & & & & & \\
\hline \begin{tabular}{|l|l|}
1136 \\
\end{tabular} & 4/2012004 & 4:01:13 PM & 26.023 & 25.097 & 25.553 & 27.705 & 25.143 & 48.951 & 52.788 & 16.674 & 32.656 & $\begin{array}{l}-1.221 \\
\end{array}$ & $\begin{array}{l}-7.443 \\
\end{array}$ & 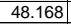 & 0.073 & 14.643 & $\begin{array}{l}0.003 \\
\end{array}$ & \begin{tabular}{|l|l|l|l|}
1819.4 \\
\end{tabular} & \begin{tabular}{|l} 
\\
0.3231 \\
\end{tabular} & 51.3 & 11.8 & 40.8 & 2.813 & .011 & 0.011 & 0.000 & 0.263 & \\
\hline & 0.2004 & 2:13 PM & 25.989 & 25.104 & 25.554 & 27.662 & 25.11 & 48.9 & 52.84 & 16.705 & 32.547 & -1.218 & -7.49 & 48.148 & 0.07 & 14.643 & & & & & 11. & & & & & & & \\
\hline & $4 / 22012004$ & 4:03:13 PM & & & & & & & & 16.576 & 32.99 & 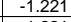 & & 6.992 & & 14.643 & & & & & & & & & & & & \\
\hline & 0/2004 & 4:04:13 PM & 25.924 & 25.114 & 25.554 & 27.592| & 25.185 & & .332 & 16.5899 & 33.151 & -1.221 & -7.562 & 9.889 & 0.072 & & & & & & & & & & & & & \\
\hline 1144 & $\frac{4 / 2 / 2012004}{4}$ & $\frac{5.13 \mathrm{PM}}{6.13 \mathrm{PM}}$ & $\frac{25.916}{25897}$ & $\frac{25.135}{25.162}$ & $\frac{25.49}{25.512}$ & $\frac{27.638}{27.679}$ & 255.34 & $\frac{49.394}{48.623}$ & $\frac{5.249}{0.18}$ & & 3180 & $\frac{-1.221}{1.219}$ & & 843 & & $\frac{14.664}{14643}$ & & 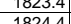 & 201064 & & & & & & & & & \\
\hline 114 & $4 / 20 / 2004$ & $\begin{array}{l}4: 0.015 \mathrm{PM} \\
\text { 4:07:13 PM }\end{array}$ & $\begin{array}{r}25.897 \\
25.88\end{array}$ & $\begin{array}{r}\frac{25.102}{25.17} \\
\end{array}$ & $\frac{25.512}{25.52}$ & $\begin{array}{l}27.019 \\
27.772\end{array}$ & $\begin{array}{l}25.417 \\
25.375\end{array}$ & $\begin{array}{l}48.023 \\
48.679\end{array}$ & $\frac{52.418}{52.558}$ & $\begin{array}{l}17.191 \\
17.219\end{array}$ & $\begin{array}{l}31.1 .004 \\
31.862\end{array}$ & $\frac{-1.218}{-1.224}$ & -7.512 & \begin{tabular}{|l|}
47.4518 \\
47.818
\end{tabular} & 0.069 & $\begin{array}{l}4.645 \\
14.643 \\
\end{array}$ & 0.003 & $\frac{1024.4}{1825}$ & $\mid \begin{array}{l}30.4064 \\
30.431\end{array}$ & & & 403 & & & & 0.000 & 0.0252 & \\
\hline & $4 / 201 / 2004$ & 4:08:13 PM & 25.842 & 25.176 & 25.531 & 27.779 & 25.332 & 48.868 & 52.78 & 17.35 & 31.876 & -1.221 & -7.56 & 49.518 & 0.068 & 14.643 & 0.003 & 1826.4 & \begin{tabular}{|l|}
30.4397 \\
\end{tabular} & 51.3 & 12.1 & 40.4 & \begin{tabular}{|l|l|}
4 & 2.784 \\
\end{tabular} & & 0.010 & 0.000 & 0.247 & 0. \\
\hline & $4 / 20 / 2004$ & 4:09:13 PM & 25.814 & 25.18 & 25.543 & 27.776 & 25.384 & $48.40=$ & 52.312 & & 31.572 & & -7.56 & 53.193 & 0.06 & 14.643 & & 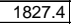 & & & & & & & & & & \\
\hline & 4212012004 & $0: 13$ PM & 25.785 & $\begin{array}{r}25.2 \\
\end{array}$ & 5.565 & $27.727 \mid$ & 25. & & 2.515 & $\frac{17.124}{17202}$ & 31.85 & & 7.5! & .479 & 0.07 & 14.643 & & & & & & & & & & & & \\
\hline & $\frac{4212012004}{412010}$ & $4.11 .13 \mathrm{MM}$ & $\frac{25.142}{2575}$ & 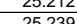 & $\frac{2.552}{25592}$ & 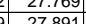 & 255.49 & 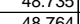 & 52.699 & $\frac{17.226}{17141}$ & $\frac{13.824}{2092}$ & 1218 & & 年9.606 & $\frac{0.069}{0.7}$ & $\begin{array}{l}\frac{1.4643}{1463} \\
\end{array}$ & & 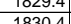 & 5064 & & $\frac{\frac{1.2 .2}{116}}{116}$ & 404 & 784 & & & & & \\
\hline$\frac{1171}{1148}$ & 4 & $4 \cdot 13: 13 \mathrm{PM}$ & 25.767 & 25.241 & 25581 & 27834 & & & 52.96 & $\begin{array}{l}17.141 \\
17.166\end{array}$ & $\begin{array}{l}31.995 \\
32.016\end{array}$ & $\begin{array}{l}-\frac{-1.110}{-1.221} \\
-\end{array}$ & .756 & $\begin{array}{ll}47.34161 \\
51746\end{array}$ & 0.069 & $\frac{1.4043}{1463}$ & 0.0003 & 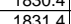 & 5 5.31 & 51.5 & 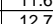 & & & & & & & \\
\hline 1145 & $4 / 20 / 2004$ & 4:14:13 PM & 25.81 & 25.274 & 25.609 & 27.0526 & $\frac{25.57}{25.65}$ & 4 & 52.262 & 17.025 & 31.706 & -1.221 & -7.56 & 48.6 & 0.07 & $\frac{14.043}{14.643}$ & & & \begin{tabular}{|l|l|}
0.02501 \\
0.5397
\end{tabular} & & $\frac{12.9}{11.5}$ & 40 & 2.762 & & & & 256 & \\
\hline & 4/2012004 & 15:13 PM & 25.862 & 25.286 & 25.637 & 27.963 & & 48.671 & & & & -1.218 & 7.5 & 48.381 & 0.069 & 14.643 & 0.004 & 1833.4 & & & & 40 & & & & & & \\
\hline 115 & |/2004] & 4:16:13 PM & 25.879 & 25.294 & 25.624 & 27.896 & & 488.604 & 52.54 & 16.981 & 32.014 & -1.221 & -7.5 & \begin{tabular}{|l|l|}
49.918 \\
\end{tabular} & 0.07 & 14.643 & & 1834.4 & 0.5731 & 51 & 12.2 & 40. & 770 & & .011 & 0.000 & 0.254 & \\
\hline & $4 / 20 / 2004$ & 4:17:13 PM & 25.898 & 25.288 & 25.603 & \begin{tabular}{|l|l|}
27.94 \\
\end{tabular} & 25.49 & $\begin{array}{l}48.747 \\
\end{array}$ & 52.66 & 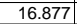 & 32.319 & -1.224 & & 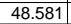 & 0.0 & 14.643 & & 5.4 & & & $\overline{11 . .}$ & & & & & & & \\
\hline & $4 / 20 / 2004$ & 4:18:13 PM & 25.934 & 25.324 & 25.564 & & & 48.72 & & 16.88 & & & & & & & & & 6064 & & & & & & & & & \\
\hline & 4/2012004 & 4:19:13 PM & 25.954 & 25.329 & 25.574 & 27.946 & 25 & \begin{tabular}{|l|l|l|l|l}
48.783 \\
\end{tabular} & .851 & $\begin{array}{l}16.917 \\
\end{array}$ & 32.28 & -1.221 & & 50.169 & 0.07 & 14.643 & & .4 & \begin{tabular}{|l}
30.6231 \\
\end{tabular} & 51.4 & 12.3 & 40.5 & & & 0.010 & 0.000 & 0.257 & \\
\hline & :0/2004 & 4:20:13 PM & & 25.338 & 25.573 & & 25.209 & 48.646 & 52.648 & 16.905 & 32.092 & -1.22 & & 46.118 & 0.065 & 14.643 & & & & & 11.3 & & & & & & & \\
\hline & $4 / 2012004$ & 4:21:13 PM & 25.967 & 25.342 & 25.577 & 27.854 & & 48.627 & 2.544 & 16.823 & 32.268 & .221 & & 47.914 & 0.07 & 14.643 & & 183 & & & 11. & & & & & & & \\
\hline & & 2:13 PM & 25.966 & 25.341 & 25.576 & 27.778 & & & 3.164 & 16.95 & 然 & -1.221 & & 年 & 0.6 & 14.643 & & & & & 13.2. & & & & & .000 & & \\
\hline & & & & & & & & & & & & $-\frac{1.2121}{-1.212}$ & & & & & & & & & & & & & & & & \\
\hline 1166 & $0.0 / 2004$ & $\begin{array}{l}\text { 4.4.1.13 } \\
4: 25: 13 \mathrm{PM}\end{array}$ & $\begin{array}{l}25.959 \\
25.949\end{array}$ & $\begin{array}{l}25.359 \\
25.349\end{array}$ & $\begin{array}{l}25.309 \\
25594\end{array}$ & 27.0060 & 25.02. & \begin{tabular}{|l|l|}
48.6777 \\
4877
\end{tabular} & \begin{tabular}{|l|l|l|l|}
52805 \\
520
\end{tabular} & $\begin{array}{l}10.095 \\
16.878\end{array}$ & $\begin{array}{l}32.231 \\
32.176\end{array}$ & $\begin{array}{l}-1.221 \\
-1.224\end{array}$ & & \begin{tabular}{|}
4.158 \\
47.491 \\
\end{tabular} & 0.061 & $\begin{array}{l}\frac{14.643}{14.643} \\
\end{array}$ & 0.003 & & $\mid \begin{array}{ll}3 \\
3\end{array}$ & 513 & $\frac{11.0}{116}$ & 40 & 4.2787 & & 0.18 & 0000 & 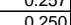 & \\
\hline 116 & $4 / 20 / 2004$ & 4:26:13 PM & 25.948 & 25.338 & 25.593 & 27.73 & 25.2 & & \begin{tabular}{|l|}
52.8260 \\
\end{tabular} & 16.852 & 32.307 & -1.224 & -7.4 & \begin{tabular}{|c|}
48.773 \\
\end{tabular} & 0.07 & 14.643 & 0.0 & 18 & \begin{tabular}{|l|l|}
30.7397 \\
\end{tabular} & \begin{tabular}{|l}
51.4 \\
\end{tabular} & $\begin{array}{l}1.0 .0 \\
12.0\end{array}$ & \begin{tabular}{|l|l}
40.5 \\
\end{tabular} & 522795 & & 0.01 & & & \\
\hline & 4/2012004 & 4:27:13 PM & & & \begin{tabular}{|l|}
25.613 \\
\end{tabular} & 27.795 & 25.303 & 48. & 52.892 & 16.875 & & & & 48.456 & & & & 1845.4 & \begin{tabular}{|l|l|l} 
\\
\end{tabular} & & & & & & & & & \\
\hline & $0.0 / 2004$ & 4:28:13 PM & 25.949 & 25.344 & 25.609 & 27.816 & 25.324 & 48.675 & 52.801 & 16.808 & 32.254 & $\begin{array}{l}-1.221 \\
\end{array}$ & & 46.923 & 0.0 & 14.643 & 0.0 & 184 & \begin{tabular}{|l|} 
\\
\end{tabular} & & 11.5 & & & & 0.010 & & 254 & \\
\hline & $\begin{array}{l}4 / 20 / 2004 \\
40\end{array}$ & $\begin{array}{l}4: 29: 13 \mathrm{PM} \\
4 \cdot 30 \cdot 13 \mathrm{P}\end{array}$ & $\begin{array}{r}25.96 \\
25.967\end{array}$ & $\begin{array}{r}25.35 \\
25.362\end{array}$ & $\frac{25.625}{25.632}$ & 27.9074 & & & & & $\begin{array}{l}32.272 \\
33208\end{array}$ & $\begin{array}{l}-1.221 \\
1.221\end{array}$ & & & & & & & & & $\frac{11.8}{11 .}$ & 40 & & & & & & \\
\hline & 4 & $4.31 .13 \mathrm{PM}$ & 25968 & $\frac{23.302}{25358}$ & $\frac{25.054}{25.23}$ & & $\frac{25.4}{25}$ & & . & & & -1.21 & & & & & & & & & 11. & & & & & & & \\
\hline 116 & 0 & . & $\frac{2.300}{25.98}$ & 25.375 & $\frac{2.050}{25.58}$ & 27.887 & 25 & 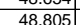 & 5282 & 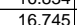 & $\begin{array}{l}32.450 \\
33.492\end{array}$ & -1.221 & & & 0.06 & 14. & & & & 告1 & $\frac{1.1 .}{11.5}$ & $\frac{44}{44}$ & 80 & & & & $\frac{0.254}{0.245}$ & \\
\hline & 442012004 & $4: 33: 13 \mathrm{PM}$ & 25.987 & & & & & & & & & -1.218 & & & & & & & & & 然 & & & & & & & \\
\hline 116 & & 4:34:13 PM & 25.984 & 25,384 & 25.594 & 27.941 & & & 53.075 & 16.887 & & -1221 & & & & 14.643 & & & & 51 & 124 & & & & & & & \\
\hline & & & $\begin{array}{l}25.996 \\
\end{array}$ & 25.406 & & & & & & & & -1.221 & & & & & & & & & $\frac{\frac{\pi 1.8}{11.8}}{4}$ & & & & & & & \\
\hline & $4 / 20 / 2004$ & 4:36:13 PM & 25.993 & 25.398 & & 28.014 & & & & & & & & & & & & & & 51.4 & 110 & & 279 & & & & & \\
\hline & & $4: 37: 13 \mathrm{~F}$ & 25.97 & & 25.598 & 28.01 & & & 888 & 16.714 & & -1.221 & & & & & & & & 51.4 & $\overline{11.9}$ & & & & & & & \\
\hline & $4 / 20 / 2004$ & 4:38:13 PM & 25.989 & 25.404 & & & & & .979 & & & -1.224 & & & & & & & & & 12.1 & & & & & & & \\
\hline $1 \pm$ & & $13 \mathrm{PM}$ & 25.99 & 25.42 & 25.625 & $27.8 \mathrm{P}$ & 25. & 48.766 & & & & -1.221 & & & & & & & & or & 11.9 & & & & & & & \\
\hline 117 & & 4:40:13 PM & 25.98 & 25.415 & 25.62 & 27.757 & 24. & 48.824 & $52.84 t$ & 16.774 & & -1.2211 & & & 0.0 & 14. & & & & & 12.2 & & & & & & & \\
\hline & & 4.41 .131 & 25.973 & 25.41 & & & & & 53.014 & & & -1.221 & & & & & & & & & 12. & & & & & & & \\
\hline & 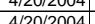 & $\frac{4.42 .43 \mathrm{M}}{4 \cdot 4 \cdot 3 \mathrm{PM}}$ & $\frac{25.940}{25948}$ & 25.389 & 256.0 & $\frac{27.545}{27476}$ & 24.6 & & $\frac{52.90}{52.955}$ & $\mid \frac{1.031}{16699}$ & & & & & & $\frac{14.045}{11643}$ & & & & 51 & 1 & & & & & & & \\
\hline 11 & $\frac{442012004 \mid}{4}$ & $4 \cdot 4.45 \cdot 13 \mathrm{PM}$ & $\frac{25.340}{25967}$ & $\frac{25.000}{25.407}$ & $\frac{25.004}{25.647}$ & $\frac{2.140}{2759}$ & & & 0 & & & -1218 & & & & & & & & 51 & 11.4 & & & & & & & \\
\hline & $4 / 201200$ & 4:45:13 PM & 25.957 & 25.402 & & 27.4 & & 48.5 & 52.989 & 16.784 & & -1.224 & & 47.9 & & & & & & 51 & 11.8 & 46 & & & & & & \\
\hline 111 & $4 / 20 / 2004$ & 4:46:13 PM & 25.956 & 25.406 & 25.646 & 27.554 & 25.241 & 48.733 & 52.679 & 16.758 & 32.556 & -1.221 & & & 0.06 & $14.6 \mathrm{rt}$ & & & & & & & & & & & & \\
\hline & & & 25.951 & 25.401 & & 27.584 & 25.2 & 48.865 & & 16.789 & 32.428 & $\begin{array}{c}-1.224 \\
\end{array}$ & & & & & & & & & 11.9 & & & & & & & \\
\hline & $4 / 20 / 2004$ & $4: 48: 13 \mathrm{PM}$ & 25.94 & 25.395 & 25.575 & 27.643 & 25.3 & 48.739 & 52.766 & 16.707 & 32.42 & -1.224 & & 49.2 & 0.0 & 14.6 & & 186 & 31.10 & 512 & 12.1 & 46 & 2 & & & & & \\
\hline & & $4: 49: 13 \mathrm{P}$ & 25.946 & 25.39 & 25.586 & 27.673 & & 48.644 & 52 & & 32. & -1.8 & & & & 14. & & & & & & & & & & & & \\
\hline & & 4:50:13 PM & & 25.402 & & 27.69 & 25 & 48.88 & & 16.499 & & -1.224 & & 48. & & & & & & & 11. & & & & & & & \\
\hline & & 4:51: & 25. & 25. & & & & 49.1 & & 16.752 & & -1. & & $47 .$. & & & & & & $51.7 \mathrm{r}$ & 11 & & & & & & & \\
\hline 1118 & $4 / 20 / 2004$ & 4:52: & 25.964 & 25.404 & 25.614 & $27 . .51$ & & 49.164 & 53.286 & 16.592 & & -1.221 & & & & 14.643 & & & & & 12.1 & & & & & & & \\
\hline$\frac{1188}{1189}$ & $4 / 20 / 2004$ & 4:53:13 PM & 25.96 & 25.415 & 25.605 & 27.824 & 25.465 & 48.984 & 52.979 & 16.658 & 32.101 & -1.221 & & 50.68 & 0.001 & 14.643 & & & 32.1897 & 51.5 & \begin{tabular}{|l|l|}
12.4 \\
\end{tabular} & 40. & 2.0 & 0.0 & 0.010 & 0.000 & 0.255 & \\
\hline$\frac{1189}{1190}$ & $\mid 4 / 2012004$ & $\begin{array}{l}4.45 .13: 13 \mathrm{PM} \\
\end{array}$ & $\frac{25.951}{25.967}$ & $\begin{array}{l}25.41 \\
25.432\end{array}$ & \begin{tabular}{|l}
$\angle 3.61$ \\
25.652
\end{tabular} & \begin{tabular}{|l|}
27.819 \\
279
\end{tabular} & \begin{tabular}{|l|}
25.451 \\
25.457 \\
\end{tabular} & $\begin{array}{r}47.692 \\
47.692\end{array}$ & \begin{tabular}{|l|}
51.105 \\
51.767 \\
\end{tabular} & \begin{tabular}{|l|l|}
17.832 \\
\end{tabular} & $\begin{array}{l}30.259 \\
30.255\end{array}$ & \begin{tabular}{|l|}
-1.224 \\
\end{tabular} & & $\frac{51.971}{49.71}$ & 0.068 & $\begin{array}{l}14.645 \\
14.643\end{array}$ & 0.003 & $\frac{16 / 2.4}{1873.4}$ & \begin{tabular}{|l|}
1.12004 \\
11.2231 \\
\end{tabular} & \begin{tabular}{|l|}
50.3 \\
50.3
\end{tabular} & 12.2 & & \begin{tabular}{|l|l|}
0 & 2.0817 \\
0
\end{tabular} & \begin{tabular}{|l|l|}
0.010 \\
\end{tabular} & \begin{tabular}{|c|}
0.010 \\
\end{tabular} & 0.000 & $\begin{array}{l}0.263 \\
0.255\end{array}$ & \\
\hline
\end{tabular}


WSRC-TR-2005-00105, REVISION 0

SRNL-RPP-2005-00012, REVISION 0

RUN \# 2.03A AND B; FIRST AND SECOND HALF OF SLURRY DEWATERING - CONT.

\begin{tabular}{|c|c|c|c|c|c|c|c|c|c|c|c|c|c|c|c|c|c|c|c|c|c|c|c|c|c|c|c|c|}
\hline & A & $\mathrm{B}$ & D & $E$ & $\mathrm{~F}$ & G & $\mathrm{H}$ & $\mathrm{J}$ & $\mathrm{K}$ & \begin{tabular}{ll|l}
$\mathrm{L}$ \\
\end{tabular} & $M$ & $\mathrm{~N}$ & 0 & $\mathrm{Q}$ & $\begin{array}{ll}\mathrm{R} \\
\end{array}$ & $\mathrm{s}$ & $\mathrm{T}$ & $\mathrm{v}$ & w & $x$ & $\mathrm{Y}$ & $z$ & AA & $A B$ & $A C$ & $\mathrm{AD}$ & $\mathrm{AE}$ & AF \\
\hline$\frac{1191}{1192}$ & $4 / 20 / 2004$ & 4:56:13 PM & $\frac{25.979}{2.957}$ & 25.439 & $\begin{array}{l}25.684 \\
25695\end{array}$ & $\begin{array}{l}27.731 \\
27.752\end{array}$ & $\begin{array}{l}25.449 \\
25.255\end{array}$ & 48.061 & 52.152 & $\begin{array}{l}17.815 \\
17591\end{array}$ & 30.51 & $-\frac{1.221}{-1.201}$ & \begin{tabular}{l|l|}
1 & -7.562 \\
\end{tabular} & 49.662 & 0.068 & 14.643 & $\begin{array}{l}0.003 \\
0.03\end{array}$ & 1874.4 & 31.2397 & 50. & 12. & 39.3 & 2.709 & & 0.010 & 0.000 & 0.253 & \\
\hline$\frac{1192}{1193}$ & & & & & & & & 47.64 & & & & & & & & & & & & & & & & & & & & \\
\hline$\frac{\mid 1193}{1194}$ & $\begin{array}{r}4 / 212004 \\
4 / 20 / 2004\end{array}$ & $\begin{array}{l}\text { 4:58::13 PM } \\
4: 59: 13 \mathrm{P}\end{array}$ & $\frac{25.966}{25.978}$ & $\frac{25.451}{25.448}$ & \begin{tabular}{|l|l|}
25.723 \\
\end{tabular} & $\begin{array}{r}21.183 \\
27.75 \\
\end{array}$ & \begin{tabular}{|l|}
25.482 \\
25.458
\end{tabular} & $\begin{array}{l}48.042 \\
48.053\end{array}$ & \begin{tabular}{|r|}
52.14 \\
52.049
\end{tabular} & $\begin{array}{l}17.691 \\
17.663 \\
\end{array}$ & $\begin{array}{l}30.682 \\
30.752\end{array}$ & $\begin{array}{l}-1.221 \\
-1.221 \\
\end{array}$ & $\begin{array}{c}-7.527 \\
-7.544 \\
\end{array}$ & $\begin{array}{l}55.402 \\
52.618 \\
\end{array}$ & $\begin{array}{l}0.071 \\
0.069\end{array}$ & $\begin{array}{l}14.643 \\
14.643 \\
\end{array}$ & $\begin{array}{l}0.003 \\
0.003\end{array}$ & $\begin{array}{l}1876.4 \\
1877.4\end{array}$ & \begin{tabular}{l|l}
4 & 31.231 .281 \\
4 & 31.299
\end{tabular} & 50. & $\begin{array}{l}13.0 \\
12.9\end{array}$ & \begin{tabular}{|l|}
39.4 \\
39.4 \\
\end{tabular} & $\mid \begin{array}{l}2.717 \\
\end{array}$ & 0.010 & 0.010 & $\begin{array}{l}0.000 \\
0.000\end{array}$ & $\begin{array}{l}0.264 \\
0.256\end{array}$ & $\begin{array}{l}0.22 \\
0.22\end{array}$ \\
\hline & $4 / 20 / 2004$ & 5:00:13 PM & 25.984 & 25.454 & 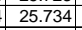 & 27.796 & 25.535 & $\begin{array}{ll}48.154 \\
\end{array}$ & 52.245 & 17.616 & 30.899 & -1.227 & -7.541 & 50.93 & 0.068 & 14.643 & 0.003 & 1878.4 & \begin{tabular}{|l|l}
4 & 31.3064 \\
\end{tabular} & & & 39.5 & & & 0.010 & 0.000 & $\begin{array}{l}0.251 \\
0.251\end{array}$ & \\
\hline & $4 / 20 / 2004$ & & & & & & & & & & & & & & & 14.643 & & 1879.4 & & & & & & & & & & \\
\hline & 4/201/2004 & $13 \mathrm{PM}$ & & 25.472 & 25.767 & 27.844 & & 48.1 & .196 & 17.478 & 31.16 & -1.2. & -7.486 & & 0.067 & 14.643 & & 1880. & & & 12 & & & & & & & \\
\hline & & & .002 & 25.477 & 25.787 & 27.829 & & 47.90 & 1038 & 17.505 & & & & 124 & $0.06 r$ & & & & & & & & & & & & 248 & \\
\hline 1200 & $\begin{array}{r}4 / 2012004 \\
\end{array}$ & & 20.004 & $\begin{array}{l}25.487 \\
25.481\end{array}$ & 25704 & 27.1579 & $\frac{25.0}{240}$ & 48.339 & 资.488 & 17.524 & & & & (45.049 & 0.0069 & 14.643 & & 18934 & & & & & & & & & & \\
\hline$\frac{1200}{1201}$ & $\begin{array}{r}4 / 201204 \\
/ 20 / 2004\end{array}$ & $5.06: 13 \mathrm{PM}$ & $\frac{25.996}{25.99}$ & $\begin{array}{r}25.481 \\
25.47\end{array}$ & $\begin{array}{l}25.1440 \\
25.74\end{array}$ & $\begin{array}{l}27.018 \\
27.553\end{array}$ & $\begin{array}{l}24.970 \\
24.906\end{array}$ & $\begin{array}{l}48.364 \\
48.104\end{array}$ & $\frac{52.509}{52.219}$ & $\begin{array}{l}17.469 \\
17.599\end{array}$ & $\begin{array}{l}1.1 .859 \\
30.881\end{array}$ & -1.124 & -7.478 & 51.992 & \begin{tabular}{|c|c|} 
\\
0.068
\end{tabular} & $\frac{14.043}{14.643}$ & 0.003 & 1080.4 & \begin{tabular}{l|l}
41.3897 \\
4 & 31.4064
\end{tabular} & $\frac{50}{50}$ & $\frac{1 . .1}{123}$ & & & 010 & & $\frac{0.000}{0.000}$ & 0.249 & 0.21 \\
\hline & $4 / 20 / 2004$ & 5:07:13 PM & 25.989 & $\begin{array}{r}25.464 \\
25.464\end{array}$ & 25.759 & 27.521 & $\frac{24.900}{24.964}$ & $\begin{array}{l}40.104 \\
47.901\end{array}$ & 52.042 & 17.055 & $\frac{0.001}{30.752}$ & $\frac{-1.224}{-1.218}$ & $\frac{-7.410}{-7.481}$ & 50.067 & 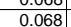 & $\frac{14.045}{14.643}$ & 0.0003 & $\frac{100.4 .4}{1885.4}$ & \begin{tabular}{l|l|l}
4 & 31.4231 \\
\end{tabular} & 50 & & & & & & & & \\
\hline & & & & & & & & & & & & & & & & & & & & & & & & & & & & \\
\hline & $4 / 20 / 2004$ & 5:09:13 PM & 25.992 & 25.462 & 25.762 & 27.49 & 25.203 & 48.03 & 52.218 & 17.468 & 30.877 & -1.224 & $\frac{-7.443}{-7.4}$ & .226 & 0.064 & 14.643 & & 188 & 13.4564 & & 2.5 & & 72 & & 0.09 & 0.000 & 237 & \\
\hline & & & & 25.446 & 25.776 & 27.559 & & & 52.150 & 17.416 & & 4 & & thes & & 14.643 & & & & & 12. & & & & & & & \\
\hline & 4/20/2004 & 5:11:13 PM & 25.975 & 25.43 & 25.75 & 27.597 & 25.225 & $\begin{array}{l}47.882 \\
\end{array}$ & 52.019 & 17.457 & 30.805 & -1.224 & & 52.081 & $\begin{array}{ll}0.072 \\
\end{array}$ & 14.643 & & 1889.4 & 31.4897 & & 12.8 & 39.3 & 2.713 & & & & 0.267 & \\
\hline & 4/20/2004 & 5:12:13 PM & 25.995 & 25.44 & 25.785 & 27.632 & 25.315 & 48.308 & 52.357 & 17.48 & 31.201 & -1.224 & -7.353 & 47.76 & 0.071 & 14.643 & $\begin{array}{l}0.003 \\
\end{array}$ & 1890.4 & $\begin{array}{ll}431.5064 \\
\end{array}$ & 50 & 11.7 & 39.8 & 2.741 & 011. & 0.010 & 0.000 & 0.260 & \\
\hline & $4 / 20 / 2004$ & 5:13:13 PM & 25.999 & 25.439 & 25.7944 & 27.716 & $25.388^{\circ}$ & 48.262 & 52.536 & 17.545 & 31.072 & -1.224 & -7.18 & 51.919 & 0.069 & 14.643 & 0.003 & 1891.4 & 4 4 31.5231 & 51. & 12. & & & & & 0.000 & & \\
\hline & $4 / 2012004$ & 5:14:13 PM & 26.001 & 25.441 & 25.786 & 27.6944 & 25.427 & 47.951 & 2.229 & 17.482 & 30.811 & -1.224 & -7.307 & 0.861 & 0.068 & 14.643 & & 1892. & 1.5397 & & 15 & 39.4 & & & 0.016 & 0.000 & & \\
\hline & 4422012004 & 5:15:13 PM & 2.0 .012 & & & & & 47.996 & & & & $-\frac{1.221}{1.210}$ & & & & & & 1893. & & & & & & & & & & \\
\hline & $\begin{array}{r}4 / 2012004 \\
42004\end{array}$ & $\begin{array}{l}5: 16: 13 \mathrm{PM} \\
5 \cdot 17 \cdot 13 \mathrm{P}\end{array}$ & $\frac{26.029}{26014}$ & 25.469 & $\frac{25.849}{25834}$ & 2.866 & 25.43 & $\begin{array}{r}47.93 \\
48001\end{array}$ & $\frac{52.063}{5034}$ & $\begin{array}{l}17.431 \\
17.90\end{array}$ & $\frac{30.893}{3096}$ & $\begin{array}{l}-1.218 \\
-1.221 \\
\end{array}$ & - & $\frac{50.014}{5191}$ & 0.068 & $\frac{14.643}{1.643}$ & & 1894.5 & .5 & & & & $\frac{2.11}{271}$ & & & 0.000 & & \\
\hline & $4 / 20 / 2004$ & 5:18:13 PM & $\frac{2.012}{26.031}$ & 25.456 & \begin{tabular}{|l|l|}
25.841 \\
\end{tabular} & 27.813 & 25.521 & $\frac{40.001}{48.25}$ & 52.393 & 17.4567 & 31.006 & -1.221 & $\begin{array}{l}-7.4507 \\
-7.507\end{array}$ & 50.938 & 0.069 & $\frac{14.045}{14.643}$ & $\begin{array}{l}0.003 \\
0.003\end{array}$ & 1896.4 & \begin{tabular}{|l|l|}
4 & 31.6064 \\
\end{tabular} & 50. & 12.5 & 39.6 & 2.732 & $\frac{0.010}{0.010}$ & 0.010 & 0.000 & 0.254 & \\
\hline & $4 / 20 / 2004$ & 5:19:13 PM & 26.042 & 25.477 & 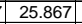 & 27.904 & 25.602 & $\begin{array}{l}48.306 \\
\end{array}$ & 52.548 & 17.584 & 31.041 & -1.221 & -7.426 & 51.21 & 0.066 & 14.643 & & 1897.4 & \begin{tabular}{|l|l|l}
4 & 31.6231 \\
\end{tabular} & 51. & 12.5 & 39.7 & & & & & 0.242 & \\
\hline & 4/201/2004 & 5:20:13 PM & 26.064 & 25.479 & 25.809 & 27.926 & 25.684 & 48.289 & 20.418 & 17.568 & 31.063 & -1.221 & -7.512 & 50.851 & 0.068 & 14.643 & 0.003 & 1898.4 & 1.6397 & & 12. & 39.7 & 2.736 & . 010 & 0.010 & 0.000 & & \\
\hline & $4 / 2012004$ & 5:21:13 PM & 26.076 & 25.491 & 25.801 & 27.963 & 25.661 & 48.081 & 52.167 & 17.453 & $\begin{array}{l}30.977 \\
30.700\end{array}$ & & & 0.488 & 0.065 & 14.643 & 0.003 & 1899. & 1.6564 & & 12. & & & & 0.009 & 0.000 & 0.240 & \\
\hline & 41212012004 & 5:22:13 PM & 26.081 & 25.508 & 25.812 & 27.979 & 25.593 & 4.8971) & 告.986 & 17.467 & $\frac{30.129}{2027}$ & -1.221 & & t5.4.409 & 0.069 & $\frac{14.643}{1.62}$ & & 1900.4 & $\begin{array}{l}4.31 .6 / 31 \\
3\end{array}$ & & 12 & & & & & & & \\
\hline$\frac{1210}{1219}$ & $\frac{4}{4 / 2012004}$ & $\begin{array}{l}5.3 .13 \mathrm{PM} \\
5 \cdot 24: 13 \mathrm{PM}\end{array}$ & $\frac{26.105}{26.105}$ & $\frac{2.525}{25.526}$ & $\begin{array}{r}25.855 \\
25.84\end{array}$ & $\frac{28.041}{27.897}$ & $\begin{array}{r}25.53 \\
25.296\end{array}$ & $\begin{array}{l}47.893 \\
48.154\end{array}$ & $\begin{array}{l}52.113 \\
52.301\end{array}$ & $\frac{17.479}{17.455}$ & $\begin{array}{l}30.123 \\
31.033\end{array}$ & $\frac{-1.218}{-1.221}$ & -7.457 & $\begin{array}{l}4.996 \\
48.354\end{array}$ & $\begin{array}{c}0.0068 \\
0.068\end{array}$ & $\frac{14.643}{14.643}$ & 0.003 & $\frac{1901.4}{1902.4}$ & \begin{tabular}{|l|l|}
4 & 3.6087 \\
4 & 31.7066 \\
\end{tabular} & & $\frac{12.3}{11.8}$ & $\begin{array}{l}39.3 \\
3.96\end{array}$ & & & & 年 & $=0.252$ & \\
\hline & 4/2012004 & 5:25:13 PM & 26.1 & 25.515 & \begin{tabular}{|l|}
25.825 \\
\end{tabular} & 27.763 & 25.096 & 48.25 & 52.438 & 17.492 & 31.072 & -1.224 & -7.46 & 51.614 & 0.067 & 14.643 & 0.003 & 1903.4 & \begin{tabular}{|l|l}
4 & 31.7231
\end{tabular} & 51. & 12.6 & 39.7 & 2.734 & \begin{tabular}{|l|}
0.010 \\
\end{tabular} & 0.010 & 0.000 & 0.246 & 0. \\
\hline & $4 / 2012004$ & 5:26:13 PM & 26.114 & & & & 24.864 & & 51.717 & 17.364 & 30.583 & & & & 0.068 & 14.643 & & $\begin{array}{l}1904.4 \\
\end{array}$ & & & & & & & & & & \\
\hline & $4 / 22012004$ & 5:27:13 PM & 26.117 & & & 27.514 & 24.7 & 48.071 & 2.293 & 17.458 & 30.947 & 1.218 & -7.4 & 1.339 & 0.066 & 14.643 & & 1905. & 1.7564 & & 12. & & & & & 000 & 243 & \\
\hline & 4121202004 & 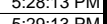 & $\frac{26.124}{26.123}$ & 25.5 & $\frac{25.844}{25829}$ & $\frac{2.461}{27.471}$ & 24.9 & $\begin{array}{ll}47.982 \\
48324\end{array}$ & $\frac{2.051}{2502}$ & $\frac{1.746}{17.455}$ & $\frac{30.881}{21214}$ & $-\frac{1.218}{1.201}$ & -73 & 50.00 & 0.069 & $\frac{14.643}{1.642}$ & & 10074 & (4) 31789 & & 124 & 398 & 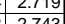 & & 5000 & 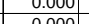 & & \\
\hline & 4 & 5:53:13 PM & 26131 & 25,482 & 25816 & 27,489 & & $\begin{array}{l}48.324 \\
478399\end{array}$ & \begin{tabular}{|l|l|}
52129 \\
\end{tabular} & $\begin{array}{l}1.45 \\
17348\end{array}$ & $\begin{array}{l}31.244 \\
30742\end{array}$ & - & & 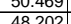 & 0.04 & $\begin{array}{l}\frac{14.045}{14642} \\
\end{array}$ & & 1900.4 & & & 12.4 & 9.0 & & & & & $\frac{254}{260}$ & \\
\hline & 0.12004 & 5:31:13 PM & $\frac{2.1 .146}{26.16}$ & 25.487 & 25.836 & 27,464 & $\frac{25.19}{25.19}$ & 48.198 & .295 & 17.326 & 31.222 & -1221 & -7.5 & 5 & 0.061 & 14.643 & & 19094 & 431.8 & & 12 & & 2738 & & & & & \\
\hline & 0 & 5:32:13 PM & 26.15 & 25.485 & 25.85 & & & & & 17.395 & & & & & & & & & & & 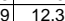 & & 2.73 & & & & & \\
\hline & 4/20/2004 & 5:33:13 PM & 26.15 & 25.475 & 25.825 & 27.453 & 25.2 & 48.189 & $\begin{array}{l}52.478 \\
\end{array}$ & 17.334 & 31.15 & $\frac{-1.218}{-1.218}$ & & $\frac{47.292}{47}$ & 0.067 & 14.643 & & 19114 & 431.8 & 51 & 11.6 & 397 & 2.735 & & 0.010 & 0.000 & 0.246 & \\
\hline & $4 / 20 / 2004$ & $5: 34: 13 \mathrm{PM}$ & 26.161 & 25.481 & 25.796 & 27.463 & 25.2 & & 52.167 & 17.318 & 31.055 & -1.221 & & 50.286 & & 14.643 & & 191 & & & & & & & & & & \\
\hline & $4 / 2012004$ & & & & 25.77 & & & 48.146 & & 17.195 & 31.265 & -1.224 & & 5009 & 0.067 & & & 19134 & \begin{tabular}{l|l}
4 & 31.8897
\end{tabular} & 50 & 123 & 39.7 & 2.73 & & & & 246 & \\
\hline & & 5:36:13 PM & 26.16 & 25.47 & 25.795 & & & & & & & & & 47.806 & & 14.643 & & & & & $11.1 \mathrm{r}$ & & & & & & & \\
\hline & $4 / 20 / 2004$ & 5:37:13 PM & 26.166 & 25.477 & 25.791 & 27.689 & 25.36 & 48.216 & \begin{tabular}{|l|l} 
\\
\end{tabular} & 17.236 & 31.365 & \begin{tabular}{|c|}
-1.224 \\
\end{tabular} & & 49.95 & $\begin{array}{l}0.069 \\
\end{array}$ & 14.643 & & 191 & $\begin{array}{l}431.92 \\
\end{array}$ & 50 & 12.2 & 39.8 & 2.743 & & 0.010 & 000 & 253 & \\
\hline & :0/2004 & 5:38:13 PM & 26.172 & 25.472 & 25.792 & 27.734 & 25 & 48.3 & \begin{tabular}{|l|}
52.504 \\
\end{tabular} & 17.282 & 31. & -1.221 & & 49.453 & 0.6 & 14.643 & & 191 & & 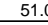 & 12. & 39.9 & & & 0.009 & 0.000 & 0.223 & \\
\hline & & 5:39:13 PM & 26.173 & 25.478 & 25.803 & 27.76 & & 48.2776 & & 17.286 & 31 & -1.218 & & 0.1 .202 & & & & & & $x_{4}$ & 12.6 & & & & & & & \\
\hline$\frac{1150}{1236}$ & & 5:401:13 PM & & 25.474 & 25.179 & $\frac{27.716}{27722}$ & 25. & & 52.44 & 17.35? & 31.2 & -1.221 & & 50.54 & & & & & & & 12. & & & & & & & \\
\hline & $4 / 20 / 2004$ & 5:42:1.13 PM & $\frac{2.14}{26.18}$ & 25.495 & $\mid$\begin{tabular}{|l|}
$\mid$ \\
$\mid$
\end{tabular} & 27.772 & 25. & $\begin{array}{l}40.4 .1 \\
48.1\end{array}$ & & 17.065 & & -1.224 & -7.4 & 47.078 & $\mid 0.069$ & 14.643 & & 1920.4 & $\frac{1}{44} 320006$ & 50.5 & $\frac{1.45}{11.5}$ & $\begin{array}{l}539.7 \\
5\end{array}$ & 2.737 & 010 & 0.010 & 0.000 & $\begin{array}{l}0.253 \\
0.253\end{array}$ & \\
\hline & 4/20/2004 & 5:43:13 PM & 26.201 & 25.507 & 25.817 & 27.819 & 25. & 48.063 & 52.235 & 17.342 & & -1.218 & & 50.198 & 0.06 & 14.643 & & 1921.4 & \begin{tabular}{|l|l|}
4 & 32.0231 \\
\end{tabular} & 50.8 & n. & 39 & 2.72 & & .00 & 0.000 & 0.236 & \\
\hline & 0/2004 & 5:44:13 PM & 26.197 & 25.49 & 25.797 & 27.785 & 25.5 & 48.053 & 52.173 & $\begin{array}{l}17.3 \\
\end{array}$ & & -1.224 & & 48.246 & 0.06 & & & 192 & & 50 & 11.8 & 399.6 & 2.150 & & & & & \\
\hline & $4 / 2$ & 5:45:13 PM & 26.219 & $\frac{25.514}{2514}$ & 25.829 & 27.851 & 25.564 & 48.274 & 52.476 & 17.276 & 31.3 & -1.221 & & 10.132 & 0.075 & 14.64 & & 1923.4 & & & & & & & 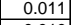 & & & \\
\hline & & $\begin{array}{l}5: 64: 13 \mathrm{PM} \\
5.54 .19\end{array}$ & $\frac{26.219}{26210}$ & $\frac{25.524}{2520}$ & $\frac{25.834}{2520}$ & $\frac{27.881}{27891}$ & & & $\frac{2.295}{2.296}$ & $\begin{array}{l}17.337 \\
17306\end{array}$ & & $\frac{-1.221}{-1224}$ & & & & & & & & & 126 & (n) & & & & & & \\
\hline 12 & 4 & $\begin{array}{l}5.46 .19 \\
5 \cdot 4 \cdot 19 \mathrm{PM}\end{array}$ & $\frac{20.219}{3624}$ & 235.524 & 23.654 & 27.081 & 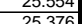 & 40.1 & \begin{tabular}{|l|}
52,500 \\
52701
\end{tabular} & $\begin{array}{l}1.400 \\
1.7394\end{array}$ & & $\frac{-1.24}{1201}$ & & & & & & & & 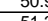 & & & & & & & & \\
\hline 124 & $\begin{array}{r}4 / 20120404 \\
/ 20 / 2004\end{array}$ & $5.44 .1 .19 \mathrm{PM}$ & $\frac{10.64}{2624}$ & $\frac{25.530}{25.526}$ & \begin{tabular}{|l|}
$\mid 25.04$ \\
2535
\end{tabular} & & 25. & & \begin{tabular}{|l|l|}
52.291 \\
5297
\end{tabular} & 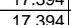 & & $\frac{-1.211}{-1221}$ & & 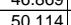 & & & & 192 & & & & & & & & & $\frac{0.440}{0.250}$ & \\
\hline 124 & $4 / 20 / 20$ & $5 \cdot 49 \cdot 19 \mathrm{P}$ & & & 25.77 & & & & & & & & & & & & & & & 50 & 12. & & 2.73 & & & & & \\
\hline 1246 & $4 / 2$ & 5.50.19 PM & 26249 & 25.529 & 25764 & 27681 & 249 & 48.1 & 52306 & 17.174 & 31.287 & -1221 & & 510 & & 14,643 & & 192 & & 50 & 12 & & & & & & 02 & \\
\hline & & & 26.242 & & & & & & & 17.347 & & -1.224 & & & & & & & & 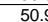 & $12.2 \mathrm{Y}$ & & & & & & & \\
\hline & 0.12004 & $5: 52: 19 \mathrm{PM}$ & 26.22 & 25.51 & 25.755 & 27.628 & 25 & 48.117 & 52.407 & 17.31 & 31.13 & -1.221 & & & & 14.643 & & 102 & 32.1 & 50 & 122 & 20 & 2 & & & & 554 & \\
\hline & $4 / 20 / 2004$ & $5: 53: 19$ म & 26.234 & 25.52 & 25.779 & & & 47.99 & & & 31.1 & -1.218 & & & & 14. & & & & & & & & & & & & \\
\hline & $4 / 20 / 2004$ & 5:54:19 PM & 26.213 & 25.508 & 25.768 & 27.62 & 25.1 & 47.895 & \begin{tabular}{|l|}
51.984 \\
\end{tabular} & 17.26 & 31.096 & -1.221 & & & & & & & & 50 & & & & & & & 44 & \\
\hline & 4/201/2004 & 5:55:19 P & 26.211 & 25.497 & 25.766 & 27.699 & 25.8 & 47.258 & 51.474 & 18.242 & & -1.218 & & & 0.0 & 14.643 & & 193 & 32.2 & & 12. & & & 0.010 & 0.010 & & 0.263 & \\
\hline$\sqrt{125}$ & $4 / 22012004$ & 5:56:19 PM & 26.216 & 25.496 & 25.766 & 27.703 & 25.22 & 48.65 & 52.768 & 16.916 & 32.139 & -1.221 & & & 0.6 & & & & & 51.2 & 12. & & & & & & & \\
\hline 1125 & & 5:57:19 PM & 26.226 & $25.49 \%$ & & 2.7 .54 & & 48.664 & & & & -1.22 & & & & & & & & 51. & 12.0 & & 2.15 & & & & & \\
\hline 125 & 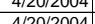 & $\begin{array}{l}5.58 .197 \\
5.59 \cdot 19\end{array}$ & 26. & $\frac{25.49}{25.95}$ & $\frac{2.5153}{2576}$ & $\frac{2.8}{277}$ & & 年 & & $\begin{array}{l}1.630 \\
1.6304\end{array}$ & & & & & 0. & 14.64 & & & & 告1. & 11 & & & & & & 然 & \\
\hline$\frac{125}{125}$ & $4 / 201200$ & $6.05 \cdot 19 \mathrm{PM}$ & & $\frac{2.3 .450}{25.49}$ & 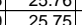 & & & $\mid$ & & & & & & & & & & & & & & & & & & & & \\
\hline 125 & 4 & 6.0119 & 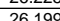 & 25.474 & 25.724 & 27766 & & 49.064 & 53,305 & 16428 & 3397 & -1224 & & 5178 & & 14643 & & 193 & & 518 & 127 & & & & 0.01 & & 0.256 & \\
\hline & & & & 25.484 & & & & & & & & & & & & & & & & & & & & & & & & \\
\hline & & 6:03: & 26.22 & 25.5 & 25.675 & 27.812 & 25. & 49.322 & 539 & 15.906 & 33.651 & & & 0.84 & 0.0 & 14.643 & & & & & & & & & & & & \\
\hline & & & & & & & & & & & & & & & & & & & & & & & & & & & 0.251 & \\
\hline
\end{tabular}


WSRC-TR-2005-00105, REVISION 0

SRNL-RPP-2005-00012, REVISION 0

RUN \# 2.03A AND B; FIRST AND SECOND HALF OF SLURRY DEWATERING - CONT.

\begin{tabular}{|c|c|c|c|c|c|c|c|c|c|c|c|c|c|c|c|c|c|c|c|c|c|c|c|c|c|c|c|c|}
\hline & & & & & & & & & & & & & & & & & & & & & & & & & & & & \\
\hline & $\begin{array}{ll}A \\
\end{array}$ & $\begin{array}{lll} \\
\end{array}$ & $D$ & $E$ & $\mathrm{~F}$ & $\mathrm{G}$ & $\mathrm{H}$ & $\mathrm{J}$ & $\mathrm{K}$ & $\mathrm{L}$ & M & $\mathrm{N}$ & 0 & Q & \begin{tabular}{l|l|l|}
$R$ & \\
\end{tabular} & $\mathrm{~S}$ & $\mathrm{~T}$ & $\mathrm{v}$ & $\mathrm{w}$ & $x$ & \begin{tabular}{|l|} 
\\
\end{tabular} & 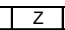 & AA & $A B$ & $A C$ & $A D$ & $\mathrm{AE}$ & \\
\hline & $4 / 20 / 2004$ & 6:0:19 PM & 26.216 & \begin{tabular}{|l|l|}
25.501 \\
3.571
\end{tabular} & 25.646 & \begin{tabular}{|l|l|}
27.728 \\
7771
\end{tabular} & \begin{tabular}{|l|}
25.381 \\
25307
\end{tabular} & $\begin{array}{l}49.961 \\
9.911\end{array}$ & $\begin{array}{l}54.173 \\
5.997\end{array}$ & $\begin{array}{l}15.726 \\
15655\end{array}$ & \begin{tabular}{|r|}
34.561 \\
\end{tabular} & -1.221 & \begin{tabular}{|r|r|} 
& -7.371 \\
\end{tabular} & 47.716 & 0.073 & 14.643 & $\begin{array}{l}.003 \\
\end{array}$ & 1943.5 & \begin{tabular}{|l|l|}
52.3914 \\
\end{tabular} & \begin{tabular}{|l|}
52.7 \\
\end{tabular} & & & 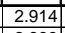 & & 0.011 & 0.000 & 0.253 & \\
\hline 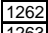 & 4/20/2004 & & & & 25.646 & 27.714 & & & 53.887 & 15.655 & \begin{tabular}{|l|}
34.396 \\
\end{tabular} & & & & 0.07 & 14.643 & 0.003 & 1944.5 & & & & & 2.09 & & & 0.000 & & \\
\hline$\frac{\mid 1263}{1264}$ & $\begin{array}{c}4 / 20 / 2004 \\
4 / 20 / 2004\end{array}$ & $\begin{array}{l}6: 07: 19 \mathrm{PM} \\
6608.19 \mathrm{P}\end{array}$ & $\begin{array}{l}26.201 \\
26.207\end{array}$ & $\begin{array}{l}25.497 \\
25512\end{array}$ & $\begin{array}{l}25.636 \\
25655\end{array}$ & \begin{tabular}{|l|}
27.634 \\
276055
\end{tabular} & $\begin{array}{l}25.327 \\
25.373\end{array}$ & 48967 & $\begin{array}{r}53.143 \\
52.99\end{array}$ & & $\begin{array}{r}32.666 \\
33.22 \\
3\end{array}$ & $\begin{array}{l}-1.224 \\
-1.221 \\
\end{array}$ & & $\begin{array}{l}50.152 \\
47207\end{array}$ & $\begin{array}{l}0.069 \\
0.07\end{array}$ & $\frac{14.643}{14643}$ & & & 32.4247 & \begin{tabular}{|l|l|}
51.7 \\
51.5
\end{tabular} & 12.3 & $\begin{array}{l}3 \\
\begin{array}{l}3 \\
6\end{array}\end{array}$ & $\mid$ & & \begin{tabular}{|l|} 
\\
\end{tabular} & 0.000 & 0.247 & 0.21 \\
\hline $\begin{array}{ll}1265 \\
\end{array}$ & $4 / 20 / 2004$ & & & & & $\begin{array}{r}27.605 \\
27.6\end{array}$ & & $\begin{array}{l}48.961 \\
49.195\end{array}$ & $\begin{array}{l}52.99 \\
53.369\end{array}$ & $\frac{16.133}{16.123}$ & & & & & & $\frac{14.643}{11.643}$ & & $\frac{1946.5}{19475}$ & 32.4414 & & & & \begin{tabular}{|l|}
2.833 \\
2.846
\end{tabular} & & & & & \\
\hline 1266 & $4 / 20 / 2004$ & 6:10:19 PM & 26.203 & 25.513 & $\mid$ & 27.571 & \begin{tabular}{|l|l|}
25.448 \\
\end{tabular} & 49.386 & \begin{tabular}{|l|}
53.674 \\
\end{tabular} & \begin{tabular}{|l|}
16.108 \\
16.108
\end{tabular} & $\begin{array}{l}33.370 \\
33.606\end{array}$ & $\begin{array}{l}-1.2<4 \\
-1.224\end{array}$ & $\begin{array}{r}-7.365 \\
-7.362 \\
\end{array}$ & 47.388 & $\begin{array}{l}0.07 \\
0.07\end{array}$ & $\begin{array}{l}14.643 \\
14.643 \\
\end{array}$ & $\frac{0.003}{0.003}$ & $\frac{1947.5}{1948.5}$ & \begin{tabular}{|l|}
32.4581 \\
532.4747 \\
\end{tabular} & \begin{tabular}{|l|}
51.9 \\
52.2
\end{tabular} & $\begin{array}{ll}11.9 \\
21.6\end{array}$ & \begin{tabular}{|l|l|l}
9 & 41.3 \\
6 & 41.5 \\
\end{tabular} & \begin{tabular}{|l|}
2.846 \\
2.861 \\
\end{tabular} & \begin{tabular}{|c|}
0.010 \\
0.010 \\
\end{tabular} & \begin{tabular}{|l|} 
\\
0.010 \\
\end{tabular} & $\frac{0.000}{0.000}$ & $\frac{0.248}{0.247}$ & 0.21 \\
\hline \begin{tabular}{|l|}
1267 \\
\end{tabular} & & & 26.193 & 25.523 & \begin{tabular}{|l|}
25.663 \\
\end{tabular} & 27.536 & 25.373 & 49.311 & 53.452 & 16.161 & 33.506 & -1.221 & -7.353 & 48.982 & 0.071 & 14.643 & 0.00 & 1949.5 & 32.4914 & & & & & & & & 0.251 & \\
\hline 1268 & $4 / 20 / 2004$ & 6:12:19 PM & 26.184 & 25.514 & 25.649 & 27.516 & 25.409 & & & 16.24 & & -1.224 & -7.359 & & & & & & & & & & & & & & & \\
\hline & & & & & & & & & 52.789 & & & & & 4017 & $\begin{array}{l}0.069 \\
\end{array}$ & 14.643 & & 1951. & 2.5247 & & & & & & & & & \\
\hline & $4 / 20 / 2004$ & 6:14:19 PM & 26.164 & 25.525 & 25.669 & 27.472 & 25. & & 53.201 & 16.555 & & & & & 0.07 & & & & .5414 & & & 0.8 & & & & & & \\
\hline & $4 / 20 / 2004$ & 6:15:19 PM & 26.17 & & 25.685 & 27.528 & 25.376 & 49.062 & 53.112 & 16.253 & 33.186 & -1.215 & -7.348 & 46.594 & 0.071 & 14.643 & & 1953.5 & 32.5581 & & & 41. & & & & & & \\
\hline & 4/2012004 & $6: 16: 19 \mathrm{PM}$ & 26.35 & 25.53 & \begin{tabular}{|l|l|}
25.68 \\
\end{tabular} & 27.493 & 25.326 & 49.036 & 53.242 & $\begin{array}{ll}16.344 \\
\end{array}$ & 33.034 & -1.224 & -7.362 & $\begin{array}{ll}48.792 \\
\end{array}$ & 0.069 & 14.643 & 0.003 & 1954.5 & 32.5747 & 51.8 & 12.0 & 41. & 2.829 & & 0.010 & 0.000 & 0.246 & 0.21 \\
\hline & $4 / 201 / 2004$ & 6:17:19 PM & 26.315 & 25.52 & 25.675 & 27.468 & 25.346 & 49.289 & 53.491 & 16.258 & 33.413 & -1.224 & & 48.846 & 0.07 & 14.643 & 0.003 & 1955.5 & 32.5914 & & 12.0 & 41. & & 0.010 & & 0.000 & & \\
\hline & 4/20/2004 & $6: 18: 19 \mathrm{PM}$ & & & & 27.519 & 25.3 & & 53.431 & 16.091 & & -1.221 & & .242 & 0.071 & 14.643 & & & & & & & & & & 0.000 & & \\
\hline & 412012004 & $6: 19: 19 \mathrm{PM}$ & 26.281 & 25.536 & 20.1014 & $\frac{27.533}{27517}$ & 25.426 & 48.853 & 53.039 & 16.738 & 32.519 & -1.221 & & 48.525 & 0.069 & 14.643 & & 150 & 32.0247 & & 11.9 & & & & & 0.000 & 248 & \\
\hline & 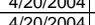 & $\begin{array}{l}0.199 \mathrm{M} \\
1.10 \mathrm{M}\end{array}$ & 20.249 & $\frac{25.525}{25.2}$ & | & 27.549 & & $\begin{array}{l}48.1855 \\
48026\end{array}$ & & & $\begin{array}{r}32.75 \\
32.927\end{array}$ & -1.221 & & 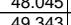 & 0.07 & & & 1958.5 & & & $\frac{11.8}{11.8}$ & & .811 & .010 & & & $\frac{251}{250}$ & \\
\hline 12 & $4 / 2012004$ & 6.2:22:19 PM & $\frac{2.205}{26.205}$ & $\frac{25.03}{25.545}$ & 25.71 & 27.4548 & 25.530 & $\begin{array}{r}40.920 \\
49.226\end{array}$ & 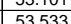 & $\begin{array}{l}\frac{10.020}{16.147} \\
\end{array}$ & $\begin{array}{l}32.921 \\
33.444\end{array}$ & $\begin{array}{l}-1.2<4 \\
-1.221\end{array}$ & $\begin{array}{l}-7.3 \\
\end{array}$ & $\begin{array}{l}49.545 \\
47.378\end{array}$ & $\frac{0.009}{0.07}$ & $\frac{14.043}{14.643}$ & $\overline{003}$ & 19505 & $\begin{array}{l}32.0501 \\
32.6747\end{array}$ & 52. & $\frac{12.1}{11.6}$ & $4 \frac{40.5}{41.3}$ & 2.850 & 0.010 & 0.010 & 0.000 & $\frac{0.246}{0.047}$ & \\
\hline 127 & $4 / 20 / 2004$ & 6:23:19 PM & $\frac{20.200}{26.184}$ & 25.535 & $\frac{25.119}{25.719}$ & 27.472 & 25.305 & 49.369 & 53.050 & $\begin{array}{l}16.144 \\
16.34\end{array}$ & 33.382 & -1.215 & -7.336 & 50.092 & 0.07 & $\frac{14.045}{14.643}$ & .004 & $\frac{19061.5}{1961.5}$ & 32.6914 & & & $\frac{45.4}{414}$ & 853 & $\frac{0.011}{0.010}$ & $\frac{0.011}{0.010}$ & 0.000 & 247 & \\
\hline & 4/20/2004 & & & & & & & & & & & & & & & & & & & & & & & & & & & \\
\hline & $4 / 20 / 2004$ & $6 \cdot 25 \cdot 19 \mathrm{PM}$ & 26.144 & 25.545 & 25.739 & 27.46 & 25.325 & 49.384 & 53.729 & 16.222 & 33.512 & -1.218 & & $\frac{0.335}{64.335}$ & 0.069 & 14.643 & & 1963. & 27247 & & 11 & & & & & & & \\
\hline & $4 / 20 / 2004$ & $6 \cdot 26 \cdot 19 \mathrm{PM}$ & 26.095 & 25.52 & 25.7 & 27.413 & 25.356 & 49398 & 53.709 & 16.177 & 33.551 & -1.224 & & 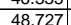 & 0.006 & $\frac{14.643}{14.643}$ & 003 & $\begin{array}{l}1964.5 \\
1964.5\end{array}$ & 32.7414 & 522 & $\frac{\frac{11}{11}}{11}$ & & 2.86 & .016 & & & 243 & \\
\hline & $4 / 20 / 2004$ & 6:27:19 PM & 26.064 & & 25.694 & 27.377 & & 49.114 & Jo.Juo & 10.939 & 33.504 & -1.224 & & & 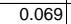 & 14.643 & & 1965.5 & & & 11. & & & & & & & \\
\hline & 4/2012004 & 6:28:19 PM & 26.066 & 25.546 & 25.741 & 27.424 & 25.351 & 48.716 & \begin{tabular}{|l|l|} 
& 53.104 \\
\end{tabular} & 16.599 & 32.324 & -1.221 & & 499.896 & 0.067 & 14.643 & & 1966.5 & \begin{tabular}{|l|l|}
32.7747 \\
\end{tabular} & 51 & 12.2 & 40.5 & 794 & & & 0.000 & 0.241 & \\
\hline & 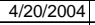 & 8:19 PM & 26.026 & 25.531 & 25.741 & 27.414 & 25.396 & 488.886 & 53.247 & 16.664 & 32.62 & -1.224 & & 50 & 0.069 & 14.643 & & 1967.4 & 32.7914 & & & & & 0.01 & 0.01 & 0.000 & 247 & \\
\hline & $4 / 20 / 2004$ & 6:30:19 PM & 26.016 & $25.536 \mathrm{C}$ & 25.751 & 27.449 & 25.401 & 48.882 & 33.197 & 16.498 & 32.683 & -1.221 & & & 0.067 & 14.643 & & & & & & & & & & & & \\
\hline & $4 / 20 / 2004$ & 6:31:19 PM & & & 25.745 & & 25.3 & & & & & & & & 0.067 & 14.643 & & & 32.8247 & & & & & & & & & \\
\hline & $\frac{4212102004}{412004}$ & $6: 03: 19 \mathrm{PM}$ & & 255.545 & 25.68 & 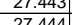 & 25.4 & $\begin{array}{l}48.239 \\
485252\end{array}$ & $\frac{52.679}{5.784}$ & $\frac{1.2 t}{17.202}$ & $\begin{array}{l}31.25 \\
31847\end{array}$ & $-\frac{1.221}{1.221}$ & & 51.848 & 0.068 & $\frac{14.643}{14643}$ & & $\frac{1970.5}{10715}$ & \begin{tabular}{|l|l|l|}
32.8414 \\
3285851
\end{tabular} & & $\frac{12.7}{121}$ & $40-2$ & & & & & & \\
\hline & 4 & $0.35 .449 \mathrm{PM}$ & $\frac{20.001}{26086}$ & $\frac{23.351}{25531}$ & 25.500 & 27.424 & $\frac{25.30}{25.37}$ & 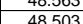 & 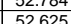 & 1672 & $\frac{31.041}{33096}$ & $\frac{1.212}{-1224}$ & & $\begin{array}{l}4.5020 \\
50327\end{array}$ & 年 & $\frac{14.045}{11643}$ & & 1972 & 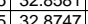 & 51.3 & $\frac{1.1}{123}$ & 40. & & & & . & 247 & \\
\hline & $\frac{4}{42 / 2102004}$ & $6.5319 \mathrm{PM}$ & 26.097 & 25.537 & 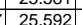 & 27.456 & 25.25 & $\frac{40.305}{48.326}$ & 52.871 & 16709 & $\begin{array}{l}31.987 \\
31.987\end{array}$ & $\begin{array}{l}-1.888 \\
-0.888\end{array}$ & & 51.199 & $\mid 0.003$ & $\frac{14.043}{14.643}$ & 0.003 & 1973. & 32.8914 & 势 & $\begin{array}{ll}12.3 \\
125\end{array}$ & $\frac{40.5}{40.2}$ & 年 & 01 & $\frac{0.014}{0.011}$ & 0.000 & 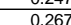 & \\
\hline & $4 / 2120 / 2000$ & 6:36:19 PM & & & & $\frac{17.489}{27}$ & 25.3 & & & $\frac{16.586}{16.56}$ & 32.523 & -1.224 & & 50.396 & 0.075 & 14.643 & & 19745 & & & 122 & 46 & & & & & & \\
\hline & 4 & $6 \cdot 37.19 \mathrm{PM}$ & & & 25592 & 27544 & & 485 & & 16583 & 32301 & -1.127 & & 17303 & & 14643 & & 1075 & 200 & 51 & 116 & 40 & & & . & & 0.71 & \\
\hline & $4 / 20 / 2004$ & $6 \cdot 38 \cdot 19 \mathrm{PM}$ & 26.07 & 25.543 & 25.612 & 27.57 & 25.4 & $\begin{array}{l}48.724 \\
\end{array}$ & 52.834 & 16.523 & 32.558 & -1.221 & & 48.742 & 0.074 & & & 1976.5 & $5 \quad 32.9414$ & 51.4 & 11.9 & 40. & & 011 & 0.011 & 000 & 0.267 & \\
\hline & $4 / 20 / 2004$ & 6:39:19 PM & & 25.548 & 25.607 & & & 48.747 & 52.948 & & 32.609 & -1.224 & & & 0 & & & 197 & & & 12.2 & & & & & & & \\
\hline & 4/2012004 & 6:40:19 PM & 26.102 & 25.537 & \begin{tabular}{|l|l|}
25.602 \\
\end{tabular} & 27.519 & 25.3 & 48.944 & 53.131 & 16.515 & 32.754 & -1.224 & & \begin{tabular}{|l|l|}
46.94 \\
\end{tabular} & $\begin{array}{l}0.068 \\
\end{array}$ & 14.643 & & 1978.5 & \begin{tabular}{|l|l|}
5 & 32.9747
\end{tabular} & 51.7 & 11.5 & 40.8 & $2.816 \mathrm{C}$ & 0.016 & 0.010 & 000 & 0.244 & \\
\hline & :0/2004 & 6:41:19 PM & 26.117 & 25.543 & 25.622 & 27.495 & & 49.056 & 53.282 & 16.492 & 32.904 & -1.221 & & 48.281 & 0.069 & 14.643 & & 1979.5 & 32.9914 & & $x$ & & & 0.01 & & 00 & 0.247 & \\
\hline & $4 / 20 / 2004$ & & & & & & & & & & & & & & & & & & & & & & & & & & & \\
\hline & $4 / 2012004$ & 6:43:19 PM & 26.122 & 25.553 & 25.6277 & $\begin{array}{r}27.51 \\
\end{array}$ & 25.3 & 48. & 53.054 & 389 & 32.951 & -1.218 & & 48.606 & 0.0 & & & & 33.0247 & & 11.9 & & & & & & & \\
\hline & $4 / 20 / 2004$ & $6.44 .19 \mathrm{PI}$ & & 25.548 & & & & & & & & -1.221 & & & & & & & & & 11.8 & & & & & & & \\
\hline & $=4$ & $6.4 .46 .9 \mathrm{PM}$ & $\frac{20.105}{26117}$ & $\frac{2.356}{2555}$ & 25.010 & 2.456 & 25.34 & $\begin{array}{l}40.141 \\
48478\end{array}$ & $\begin{array}{l}53.11 \\
52828\end{array}$ & $\begin{array}{l}16.450 \\
1.6365\end{array}$ & $\frac{32.607}{32.44}$ & $\frac{-1.24}{-1221}$ & & 年 49.1844 & 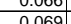 & & & 1984 & $\mid 30.0574$ & 51.6 & 12.1 & . & 2798 & & 0.01 & 0000 & 0.250 & \\
\hline & $\frac{4}{42120120004}$ & $6.44 .47 .19 \mathrm{PM}$ & $\frac{20.111}{26.129}$ & 20.000 & 20.007 & & 20.0 & $\begin{array}{l}40.410 \\
48.602\end{array}$ & & 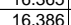 & & $\frac{-1.221}{-1221}$ & & & & & & & 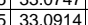 & 0.1 .4 & 12.0 & & & & & & & \\
\hline & 4 & 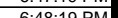 & 2610 & 2548 & 25629 & 2749 & 25329 & 4889 & 53250 & 16.329 & 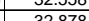 & 120 & & $\begin{array}{l}40.052 \\
48656\end{array}$ & م.068 & 1464 & & 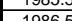 & 00.0914 & & 11. & & & & & 0.000 & & \\
\hline & 4/20/2004 & 6:49:19 PM & 26.128 & 25.563 & 25.648 & 27.456 & 25.3 & & 53.157 & 16.329 & & -1.221 & & 52.044 & & & & & 33.227 & 51.7 & 12.8 & & & & & & & \\
\hline & $4 / 20 / 2004$ & 0:19 PM & 26.114 & 25.554 & 25.624 & 27.422 & 25.3 & & & & & -1.224 & & 48 & & & & & & & 119 & & & & & & & \\
\hline & $4 / 20 / 2004$ & 6:51:19 PM & 26.124 & 25.549 & 25.629 & 27.462 & 25.3 & 48.561 & \begin{tabular}{|l|l|}
53.016 \\
\end{tabular} & 16.154 & 32.707 & -1.218 & & 48.85 & & 14.6 & & $19 \varepsilon$ & 33.1581 & 51.5 & 12. & & & & 0.01 & & 245 & \\
\hline & $4 / 20 / 2004$ & 6:52:19 PM & 26.129 & 25.549 & 25.624 & 27.457 & & & & 16.167 & 32.767 & -1.224 & & 46.448 & & & & & & 51.2 & 11.4 & & & & & & & \\
\hline & 4/20/2004 & 6:53:19 PM & 26.13 & 25.55 & $\mid 25.63$ & 27.472 & 25. & 48. & 52.963 & & & -1.221 & & 47.6 & & 14.643 & & 1991 & \begin{tabular}{|l|l|} 
& 33.1914
\end{tabular} & 51.5 & 11.7 & $\begin{array}{l}744 \\
4\end{array}$ & & & $0 . c_{0}$ & & 0.245 & \\
\hline & $4 / 2012004$ & 6:54:19 PM & 26.129 & 25.544 & 25.619 & 27.437 & 25.3 & & 52.946 & 16.414 & & -1.221 & & 49.712 & & & & & & 51. & 12. & & & & 0.0 & & & \\
\hline & $4 / 20 / 2004$ & 6:55:19 PM & 26.135 & & 25.635 & & & & & & & & & & & & & & & & & & & & & & & \\
\hline & $4 / 202100$ & $0.56: 19 \mathrm{PM}$ & 20.135 & $\frac{25.555}{2554}$ & 20.64 & 2.524 & 25 & & $\begin{array}{r}52.82 \\
5220\end{array}$ & & & -1.221 & & 48.62 & & & & & $\begin{array}{l}533.2414 \\
\end{array}$ & 51.3 & 11.9 & $\begin{array}{l}9 \\
7\end{array}$ & & & & & & \\
\hline & $\frac{4}{4212004}$ & $6.5 .19 .19 \mathrm{PM}$ & $\frac{26.1}{26.1}$ & $\begin{array}{l}25.54 \\
25.522\end{array}$ & $\begin{array}{l}25.644 \\
25.617\end{array}$ & 27.415 & 254 & & \begin{tabular}{|l|l|}
52,410 \\
53209
\end{tabular} & $\frac{100}{16}$ & & $\frac{-1.211}{-1224}$ & & 44.167 & & & & & & 51.0 & $\frac{11.1}{116}$ & & & & & & 0.245 & \\
\hline & 4 & 6.5.5.19 PM & 26131 & 25.546 & 25.571 & 27.519 & 20.54 & & \begin{tabular}{|l|l|l|l|}
52.923 \\
\end{tabular} & 1632 & 32.488 & -1221 & & 46.906 & 0.06 & 14643 & & & $\begin{array}{l}33.2914 \\
332914\end{array}$ & & 11.0 & & & & & & & \\
\hline & $4 / 2012004$ & 7.00.19 PM & 26.36 & 25.546 & 25.571 & 27.499 & 253 & & 52998 & 1648 & & -1221 & & & 0.06 & & & & & & & & & & & & & \\
\hline & & & & & & & & & 53.18 & 16.48 & & & & & & & & & & & & & & & & & & \\
\hline & $420 / 2004$ & $7 \cdot 02 \cdot 19 \mathrm{PM}$ & 26.135 & 25555 & 2558 & 27512 & 254 & 48851 & 53.11 & 16411 & 33837 & -1218 & & 468 & 0.06 & 14. & & & 333 & 516 & 115 & & & & & & 488 & \\
\hline & $4 / 20 / 200$ & 7:03:19 P & 26.145 & & 25.595 & 27.492 & 25. & & 52 & & 32.4 & -1.221 & & & & & & & 33.3 & 51.4 & \begin{tabular}{|l|l|l|}
4 & 11.8 \\
\end{tabular} & 14 & & & & & & \\
\hline & $4 / 20 / 2004$ & 7:04:19 PM & 26.128 & 25.553 & 25.583 & 27.471 & 25.3 & 48.737 & 53.131 & 16.482 & 32.576 & -1.221 & & 49.38 & 0.06 & 14. & & 2002.5 & \begin{tabular}{l|l}
5 & 33.374
\end{tabular} & 51.7 & $\begin{array}{l}7 \\
7 \\
121\end{array}$ & \begin{tabular}{l|l|}
1140.7 \\
\end{tabular} & & 10 & 0.010 & & 0.242 & \\
\hline & $4 / 20 / 2004$ & $19 \mathrm{PM}$ & 26.12 & $25.56 \mathrm{~s}$ & 25.594 & 27.442 & 25.329 & $\begin{array}{l}48.457 \\
\end{array}$ & 52.822 & 16.467 & 32.309 & -1.22 & & 48.6 & 0.06 & 14.643 & & & 3.3914 & & 11.9 & & & & & & & \\
\hline & $4 / 201200$ & 7:06:19 PM & & & & 27.361 & 25.2 & & 52.84 & 16.423 & & & & & & & & & & & & & & & & & & 0 \\
\hline & & $7: 0 \%$ & 26.112 & 25.548 & 25.587 & 27.325 & 25.3 & 48.5 & 52.805 & 16 & 32.492 & -1.221 & & & & & & & & 51.3 & 11.4 & & & & & & & \\
\hline & $4 / 212102004$ & :08:19 PM & 26. & $\frac{25.562}{2555}$ & 25.5 & $\frac{27.364}{27250}$ & . & 4.68 & 53.112 & $\frac{16.332}{16.57}$ & $\begin{array}{l}32.562 \\
32672\end{array}$ & -1.22 & & $A 64$ & & $\frac{14.6}{1.6}$ & & & 33.4 & 51.6 & $\frac{11.6}{11.6}$ & $6 \int^{6} 4$ & & & & & & \\
\hline & 4 & $7.0519 \mathrm{PM}$ & 260 & & & | & & 40.020 & 52786 & 10.45 & 32.012 & -1.241 & & & & & & & & 51.6 & 1.4 & 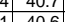 & & & & & $\frac{41}{12}$ & \\
\hline & 4 & $7.10 .11 \cdot 1.9 \mathrm{PM}$ & $\frac{20.001}{2609}$ & 25.535 & | & 20.525 & 25265 & 4 & 52884 & 16403 & 32.050 & 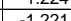 & & 48358 & & 14643 & & 200 & 33.4914 & 51.4 & $\frac{1.1}{119}$ & 46 & & 0016 & 0010 & & 0.044 & \\
\hline & 4 & 7:12.19 PM & 26069 & 25.544 & 25599 & 27267 & & 48.37 & & 16449 & 32. & & & & & & & & & & & & & & & & & \\
\hline & & & 26.074 & & 25.604 & 27.312 & & 48.417 & 52.791 & 16.463 & 32.268 & -1.218 & & 49.4 & & 14. & & & & & 12 & & & & & & 243 & \\
\hline & & $7 \cdot 14419 \mathrm{PM}$ & 26049 & & & & & & & & & & & & & $\frac{\frac{1.4043}{1463}}{1463}$ & & & & & $\frac{12.1}{11.8}$ & & & 0.010 & $\frac{1010}{010}$ & & $\frac{.245}{.235}$ & $\overline{0.2}$ \\
\hline
\end{tabular}


WSRC-TR-2005-00105, REVISION 0

SRNL-RPP-2005-00012, REVISION 0

RUN \# 2.03A AND B; FIRST AND SECOND HALF OF SLURRY DEWATERING - CONT.

\begin{tabular}{|c|c|c|c|c|c|c|c|c|c|c|c|c|c|c|c|c|c|c|c|c|c|c|c|c|c|c|c|c|c|}
\hline & A & B & $D$ & $E$ & \begin{tabular}{l|l|} 
\\
\end{tabular} & G & $\mathrm{H}$ & $\mathrm{J}$ & $\mathrm{K}$ & L & $\mathrm{M}$ & $\mathrm{N}$ & 0 & Q & $\mathrm{R}$ & $\mathrm{s}$ & $\mathrm{T}$ & $\mathrm{v}$ & w & $x$ & $\mathrm{Y}$ & 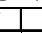 & $z$ & AA & $A B$ & $A C$ & $\mathrm{AD}$ & & AF \\
\hline$\frac{1331}{1322}$ & $4 / 20 / 2004$ & $7: 15: 19 \mathrm{PM}$ & 26.049 & & $\begin{array}{l}25.549 \\
2554\end{array}$ & $\begin{array}{l}27.342 \\
27307\end{array}$ & $\begin{array}{l}25.279 \\
25265\end{array}$ & 48.6 & 52.973 & \begin{tabular}{|l|}
16.429 \\
\end{tabular} & & -1.221 & \begin{tabular}{|l|l|}
-7.298 \\
7020
\end{tabular} & $\begin{array}{l}48.948 \\
47.6161\end{array}$ & 0.068 & $\begin{array}{l}14.643 \\
14.6\end{array}$ & $\begin{array}{l}0.003 \\
0.03\end{array}$ & 2013.5 & 33.5581 & 51. & & & & 2000 & 0.010 & 0.010 & 0.000 & 0.246 & \\
\hline & & & & $\begin{array}{r}25.5 \\
25.52\end{array}$ & & & & & & & & & & & & & & & & & & & & & & & & & 0.19 \\
\hline$\frac{1535}{1324}$ & $4 / 20 / 20004$ & $\begin{array}{l}7: 17: 19 \text { PM } \\
7: 18: 19 \text { M }\end{array}$ & $\frac{26.048}{26.043}$ & $\frac{25.52}{25.52}$ & \begin{tabular}{|l|l|}
25.553 \\
\end{tabular} & 27.3561 & $\begin{array}{l}25.198 \\
25.193\end{array}$ & $\begin{array}{l}\frac{48.426}{48.751} \\
\end{array}$ & \begin{tabular}{|l|}
5.2 .95 \\
53.157
\end{tabular} & \begin{tabular}{|l|}
16.442 \\
16.181
\end{tabular} & $\begin{array}{l}32.285 \\
32.923\end{array}$ & $\begin{array}{r}-1.154 \\
-1.154 \\
\end{array}$ & $\begin{array}{r}-7.171 \\
-7.258 \\
\end{array}$ & $\begin{array}{r}49.86 \\
50.515 \\
\end{array}$ & $\begin{array}{l}0.067 \\
0.072\end{array}$ & $\begin{array}{r}14.643 \\
14.643 \\
\end{array}$ & $\begin{array}{l}0.003 \\
0.003\end{array}$ & $\begin{array}{r}2015.5 \\
2015.5\end{array}$ & $\begin{array}{l}533.5914 \\
5\end{array}$ & \begin{tabular}{|l|}
51. \\
51.
\end{tabular} & 12 & & $\frac{40.4}{44.8}$ & 2.816 & $\mid 0.011$ & 0.010 & $\frac{0.000}{0.000}$ & $\begin{array}{l}0.244 \\
0.259 \\
\end{array}$ & 0.21 \\
\hline & $4 / 20 / 2004$ & & 26.037 & 25.52 & \begin{tabular}{|l|}
25.562 \\
\end{tabular} & 27.31 & 25.198 & 48.867 & \begin{tabular}{|l|l|}
53.128 \\
\end{tabular} & 16.258 & 32.935 & -1.221 & $\begin{array}{r}-7.209 \\
\end{array}$ & 49.501 & 0.062 & 14.643 & 0.004 & 2017.5 & $5 \quad 33.6247$ & & & & & & \begin{tabular}{|l|}
0.009 \\
\end{tabular} & $\begin{array}{l}0.011 \\
0.009\end{array}$ & 0.000 & & \\
\hline & $4 / 20 / 2004$ & & & & & & & & & & & $\begin{array}{r}-1.03 \\
\end{array}$ & & 48.204 & & 14.643 & & & 33.6414 & & & & & & & & & 0.249 & \\
\hline & $4 / 20 / 2004$ & 1:19 PM & & 25.51 & 25.551 & 27.314 & 25.217 & 48.7 & 53.05 & 16.041 & 33.056 & -1.157 & & & 0.073 & 14.643 & & 2019 & & & & & & & & & & & \\
\hline & $4 / 20 / 2004$ & $7: 22: 19$ PM & 26.015 & 25.50 & 25.545 & 27.288 & 25.185 & 48. & 3.22 & 16.107 & 33.027 & $\begin{array}{l}-1.221 \\
\end{array}$ & & 9.161 & 0.076 & 14.643 & & & .0747 & & & & & & & & 1.000 & 0.273 & \\
\hline 1340 & $4 / 20 / 2004$ & $\begin{array}{l}7: 23: 19 \text { PM } \\
7 \cdot 24 \cdot 19 P M\end{array}$ & $\frac{20.010}{26004}$ & $\begin{array}{r}25.5 \\
25.48\end{array}$ & 25.530 & 27.200 & 25184 & 49.1 & 年 & & $\begin{array}{r}33.409 \\
33.389\end{array}$ & -1.21 & & 8448 & & 14.643 & & & $\begin{array}{l}33.6914 \\
3327081\end{array}$ & & & & & & & & & 238 & \\
\hline 1341 & $\begin{array}{l}4 / 20 / 2004 \\
4 / 2004\end{array}$ & $\begin{array}{l}7.4 .19 \mathrm{P} \\
7.25 .19 \mathrm{PM}\end{array}$ & $\frac{20.004}{26.013}$ & $\frac{25.40}{25.50}$ & $\begin{array}{l}25.5356 \\
25.548\end{array}$ & $\begin{array}{l}27.251 \\
27.261\end{array}$ & 25.164 & $\begin{array}{l}49.0 / 3 \\
49.007\end{array}$ & $\begin{array}{l}53.392 \\
53.468\end{array}$ & $\begin{array}{l}\frac{15.976}{16.003} \\
\end{array}$ & $\begin{array}{l}35.369 \\
33.317\end{array}$ & $\frac{-1.221}{-1.221}$ & -7.33 & $\begin{array}{l}48.446 \\
47.622 \\
\end{array}$ & $\begin{array}{l}0.069 \\
0.068\end{array}$ & $\begin{array}{l}14.645 \\
14.643 \\
\end{array}$ & 0.0003 & 20235 & 5.33 .7247 & & 11 & & & & & 0.010 & $\frac{0.000}{0.000}$ & 0.246 & 0.21 \\
\hline & $4 / 2012004$ & & 26.011 & 25.49 & 25.571 & 27.259 & 25.201 & 48.729 & 52.936 & $\begin{array}{ll}16.017 \\
\end{array}$ & 33.038 & -1.218 & -7.241 & 46.642 & 0.062 & 14.643 & & & $5 \longdiv { 3 3 . 7 4 1 4 }$ & & & & & & & 0.009 & & $\frac{0.425}{0.222}$ & $\frac{0.21}{0.19}$ \\
\hline & & 7:27:19 PM & & 25.48 & 25.535 & & 25.156 & & & & & -1.16 & & & & 14.643 & & 2025.5 & 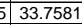 & & & & & & & & 0.000 & & \\
\hline & 4/201/2004 & 7:28:19 PM & 26.011 & & 25.481 & 27.214 & & 48.785 & 53.124 & 15.938 & 33.13 & & & 9.672 & 0.067 & & & 2026 & 3.7747 & & & & & 82 & & .010 & & 2411 & \\
\hline 134 & $\begin{array}{l}4 / 2 / 2 / 2004 \\
4 / 20 / 2004\end{array}$ & 7:29:19 PM & $\frac{26.005}{26.009}$ & $\frac{25.4 t}{25.45}$ & 25.475 & $\frac{27.163}{27.172}$ & 25.1 & $\begin{array}{l}48.948 \\
490099\end{array}$ & 年 & 15050 & $\begin{array}{l}33.235 \\
33.395 \\
\end{array}$ & $-\frac{-1.224}{1.224}$ & & 10168 & 0.000 & $\frac{14.643}{14643}$ & & 2028 & 33.7914 & & & & & 581 & & 0 & 0.000 & 243 & 0.21 \\
\hline$\frac{1340}{1347}$ & 4 & $7.3119 \mathrm{PM}$ & 26.003 & 25.45 & 25.478 & 27.151 & 25.159 & 48.561 & $\begin{array}{l}52.905 \\
52.905\end{array}$ & $\begin{array}{l}13.957 \\
15.957\end{array}$ & $\begin{array}{r}35.395 \\
3289\end{array}$ & $\frac{-1.224}{-1.154}$ & $\frac{-1.203}{-7.113}$ & $\frac{49.100}{46.012}$ & $\frac{0.064}{0.069}$ & $\frac{14.045}{14643}$ & $\frac{0.003}{0.003}$ & $\frac{2028}{2029}$ & $\frac{5.3247}{332847}$ & 51. & $\frac{15}{11}$ & & 407 & $\frac{2.041}{2808}$ & 然 & 0.009 & $\frac{0.000}{0.000}$ & $\frac{0.249}{0.249}$ & \\
\hline & 4/201/2004 & 7:32:19 PM & 25.993 & 25.46 & 25.478 & 27.186 & 25.184 & 48.809 & 53.151 & 15.932 & 33.196 & -1.183 & $\begin{array}{l}-7.226 \\
\end{array}$ & 47.92 & 0.068 & $\frac{14.643}{14.64}$ & 0.003 & 2030 & $\frac{33.8414}{33.84}$ & & & & & & & & & & \\
\hline & $4 / 20 / 2004$ & 7:33:19 PM & 25.978 & 25.45 & 25.467 & 27.151 & $\begin{array}{l}25.178 \\
\end{array}$ & & 53.081 & 15.901 & 33.044 & -1.221 & & $\begin{array}{l}48.652 \\
\end{array}$ & 0.066 & 14.643 & 0.003 & 2031 & & & & & & & & 0.010 & & & \\
\hline & 4/20/2004 & & 25.983 & & & & & $\begin{array}{ll}48.836 \\
\end{array}$ & 53.139 & & & & & & 0.063 & 14.643 & 0.003 & 2032 & 3.8747 & 51 & 12 & & & 2829 & & 0.009 & 0.000 & & \\
\hline & 0/2004 & 7:35:19 PM & & 25.43 & 25.469 & 27.147 & 25.17 & $\begin{array}{l}48.938 \\
\end{array}$ & 53.276 & 16.037 & 33.202 & -1.154 & & 47.111 & $\begin{array}{l}0.067 \\
\end{array}$ & 14.643 & & & 3.8914 & & & & & .85 & & & 0.000 & 240 & \\
\hline & J/2004 & 7:36:19 PM & & & & & & & & & & & & & & & & 203 & & & & & & & & & & & \\
\hline & :0/2004 & 7:37:19 PM & 25.938 & 25.43 & 25.483 & 27.191 & 25.179 & \begin{tabular}{|l|l|l}
49.204 \\
\end{tabular} & 53.603 & 15.916 & 33.701 & -1.177 & $-7.2 \mathrm{C}$ & $\begin{array}{l}47.618 \\
\end{array}$ & 0.068 & 14.643 & & 2035 & $\overline{33.9247}$ & 52. & & & & 2.858 & & 0.010 & 0.000 & 0.241 & \\
\hline & $4 / 20 / 2004$ & 7:38:19 PM & 25.938 & 25.42 & 25.478 & 27.186 & 25.169 & 48.841 & 3.058 & 15.907 & 33.327 & -1.163 & -7. & 46.502 & 0.069 & 14.643 & 0.003 & 2036. & 33.9414 & 51. & & 1.4 & 41. & 2.833 & & 0.010 & 0.000 & & \\
\hline & 4/201/2004 & 7:39:19 PM & 25.928 & 25.42 & 25.488 & 27.181| & 25.124 & 49.12 & 53.545 & 15.989 & 33.424 & -1.163 & & 47.549 & 0.066 & 14.643 & 0.003 & 2037. & 33.9581 & & & & & 2.846 & .010 & 0.010 & 0.000 & 235 & \\
\hline & 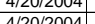 & $7.40 .19 \mathrm{PM}$ & 25.921 & 25.44 & 25.4924 & 27.145 & 25.0 & 48.868 & 53.346 & 15.988 & 33.185 & -1.137 & & 48.442 & 0.067 & 14.643 & 0.003 & 2038 & 33.9747 & & & & & 2.829 & & 0.010 & 0.000 & & \\
\hline & 4 & $\frac{1.4 .19}{7.19 \mathrm{PM}}$ & $\frac{25.911}{25916}$ & 25.45 & 20.455 & 27.059 & & 年 & 50.39 & 10.0303 & 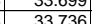 & $\begin{array}{l}-1.16 \\
-1154\end{array}$ & & 40.10 & 0.005 & $\begin{array}{ll}1.4045 \\
1.0612\end{array}$ & & 201 & 3.9014 & & & & & & & & & & \\
\hline & $4 / 20 / 2004$ & $7.42: 19 \mathrm{PM}$ & $\frac{2.5191}{25.91}$ & $\frac{20.45}{25}$ & $\mid$ & 27.003 & 25.015 & 49.143 & 53.553 & $\begin{array}{l}15.0608 \\
15.678\end{array}$ & $\begin{array}{l}33.1502 \\
33.822\end{array}$ & -1.154 & -7.2 & $\begin{array}{l}49.017 \\
49.017\end{array}$ & 0.066 & $\frac{14.643}{14.643}$ & 0.003 & 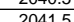 & $\frac{34.0001}{30247}$ & 52 & $\frac{11}{12}$ & & 41.5 & 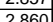 & 0.010 & & 0.000 & 0.254 & \\
\hline & $4 / 20 / 2004$ & 7:44:19 PM & 25.915 & 25.40 & 25.445 & 27.033 & 25.295 & 49.326 & \begin{tabular}{|l|}
53.835 \\
\end{tabular} & 15.809 & 33.865 & -1.154 & -7.22 & 47.401 & $\begin{array}{l}0.069 \\
\end{array}$ & 14.643 & 0.003 & 2042.5 & $5 \longdiv { 3 4 . 0 4 1 4 }$ & 52. & 11 & 1.6 & & 2.868 & $\mid 0.010$ & 0.010 & 0.000 & 0.244 & \\
\hline & $4 / 2012004$ & 7:45:19 PM & 25.91 & 25 & $\begin{array}{r}\mid \\
\end{array} 25.45$ & 27.063 & 25.435 & \begin{tabular}{|l|l|l|l|l}
48.374 \\
\end{tabular} & \begin{tabular}{|l|l|}
52.815 \\
\end{tabular} & 16.398 & 32.33 & -1.099 & & & 0.062 & 14.643 & & & & & & & & & & & & & \\
\hline & $4 / 2012004$ & 7:46:19 PM & & 25.40 & 25.455 & 27.173 & & 48.656 & & $\frac{16.125}{1.1155}$ & & -1.154 & & & 0.066 & 14.643 & & 204 & :0747 & & & & & & & & & & \\
\hline 136 & 4212012004 & $=0.4: 19 \mathrm{PP}$ & 25.902 & 年 25.39 & 2554524 & $\frac{2.2055}{27025}$ & $\frac{25.62}{2562}$ & 年8.6859 & 年 & $\frac{16.115}{1.6196}$ & $\begin{array}{l}32.945 \\
32025\end{array}$ & $\begin{array}{l}-1.186 \\
-1.105\end{array}$ & & t5.924 & 0.016 & $\frac{1.4643}{1063}$ & & 2045 & 年. & & $\frac{11}{11}$ & & 40.0 & & 0010 & 0010 & 0.000 & & \\
\hline$\frac{1365}{1365}$ & 4 & $7 \cdot 4 \cdot 4 \cdot 23 \mathrm{PM}$ & 25.908 & 25.42 & 25,478 & 年 & 25 & 4875 & 5 & 16063 & 33036 & 1.154 & & (46.104 & 0 & 1464 & & $\frac{204}{201}$ & $\frac{34.9425}{34102}$ & . & $\frac{11}{11}$ & & & 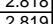 & & 0.010 & & & \\
\hline & 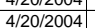 & $\begin{array}{l}7.0 .25 \mathrm{PM} \\
7: 49: 23 \mathrm{PM}\end{array}$ & 25.932 & $\frac{25.44}{25.44}$ & 25.450 & $\frac{21.512}{2742}$ & & 4 & 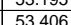 & 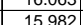 & $\begin{array}{l}33.050 \\
33.175\end{array}$ & $\begin{array}{l}-1.154 \\
-1.157\end{array}$ & & 年58.154 & - & $\frac{14.453}{1463}$ & & 200 & 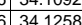 & & & & & & & 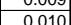 & & & \\
\hline & J/2004 & $7 \cdot 5 \cdot 5 \cdot 23 \mathrm{PM}$ & 25.918 & 25.44 & 25.48 & 27.701 & & 48.71 & & 10.04 & 32.896 & & & 47.755 & & 14.643 & & & & 然 & $x$ & & & (2) & & & & 0.252 & \\
\hline & |0/2004 & 7:51:23 PM & 25.915 & 25.43 & 25.47 & 27.323 & 25. & \begin{tabular}{|l|l|}
48.7 \\
\end{tabular} & 53.209 & 15.959 & 33. & -1.099 & & 499.733 & 0.069 & 14.643 & & 20496 & 34.1592 & 51 & 12 & & $\frac{40.9}{40.9}$ & $\frac{2.819}{2819}$ & 010 & 0.010 & 0.000 & 0.248 & \\
\hline & $4 / 20 / 2004$ & 7:52:23 PM & 25.916 & 25.44 & 25.496 & 27.324 & 25.3 & \begin{tabular}{|l|l}
48.643 \\
\end{tabular} & 53.103 & \begin{tabular}{|l|l}
15.93 \\
\end{tabular} & 33.044 & -1.172 & & 47.007 & \begin{tabular}{|l|}
0.067 \\
\end{tabular} & 14.643 & & & oncen & & $\frac{11}{11}$ & & & & & & & & \\
\hline & $4 / 20 / 2004$ & $7 \cdot 55 \cdot 23 \mathrm{PM}$ & 25.931 & 25.44 & 25.506 & & & 48.94 & & & & -1.221 & & 45.083 & 0.068 & 14.643 & & & 34.1925 & & 11 & & 411 & 283 & 0.010 & & & 243 & \\
\hline & 4/201/2004 & 7:54:23 PM & 25.915 & 25.4 & \begin{tabular}{|l|l|}
25.49 \\
\end{tabular} & 27.188 & & & & 16.742 & 31.63 & -1.221 & & & 0.064 & 14.643 & & & & 51 & & & & & & & & & \\
\hline & $4 / 20 / 2004$ & 7:55:23 PM & 25.919 & 25.44 & 25.509 & 27.127 & 25.1 & 48.299 & 52.672 & 16.666 & 31.938 & \begin{tabular}{|c|c|}
-1.218 \\
\end{tabular} & & & 0.066 & 14.643 & & & 34.2258 & 51. & 12 & & 40.1 & 2.766 & 0.010 & 0.010 & 0.000 & 0.242 & \\
\hline 4 & 0/2004 & 7:56:23 PM & 25.919 & 25.44 & 25.529 & 27.087 & & 48.198 & 52.618 & 16.573 & 31.942 & -1.189 & & 48.156 & 0.6 & 14.6 & & & 34.2425 & 1 & 11 & & 40.1 & 763 & & 0.009 & 0.000 & 0.221 & \\
\hline & & 7:57:23 PM & 25.924 & 25.45 & 25.5399 & 27.077 & 25. & 48.295 & 52.639 & 16.671 & & -1.157 & & $\begin{array}{ll}4.5 .50 \\
4.57\end{array}$ & 0.064 & & & & 34.2592 & 51 & 12 & & & 766 & & & & & \\
\hline$\frac{135}{1376}$ & & $=5.52 .23 \mathrm{PM}$ & & 25.44 & 25.513 & & & & & & & -1.151 & & 49.8 & & & & & & & & & 39.9 & .150 & & & & & \\
\hline 1377 & $\frac{4 / 2 / 2 / 2004}{4 / 20 / 2004}$ & $8.0 .023 \mathrm{PM}$ & $\frac{25.922}{25.916}$ & $\frac{25.44}{25.44}$ & 2551 & 26.599 & & $\frac{48 .}{48 .}$ & 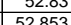 & 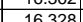 & & -1.16 & & $\frac{50.35}{4708}$ & 0.06 & $\frac{14.045}{11643}$ & & 205 & $\begin{array}{l}34.2425 \\
34302\end{array}$ & 51. & $\frac{12}{11}$ & & & & & 0010 & & & \\
\hline & $4 / 20 / 2004$ & 8:01:23 PM & 25.915 & 25.4 & $|25.495|$ & 26.968 & 25.0 & $\mid$\begin{tabular}{|c|}
48.455 \\
\end{tabular} & \begin{tabular}{|l|l|}
52.704 \\
\end{tabular} & 16.249 & & -1.221 & & $\mid 49.241$ & 0.062 & 14.643 & & 2059.6 & \begin{tabular}{l|l|l}
6 & 34.3258
\end{tabular} & 51. & 12 & & 40.5 & 2.794 & 0.009 & 0.009 & 0.000 & 0.225 & \\
\hline & 4/201/2004 & 8:02:23 PM & 25.914 & 25.43 & 25.514 & 26.967 & 25 & 48.639 & \begin{tabular}{|l|l|}
53.218 \\
\end{tabular} & 16.492 & 32.477 & -1.099 & & 47.044 & 0.062 & 14.643 & & 2060.6 & \begin{tabular}{l|l}
6 & 34.3425 \\
\end{tabular} & 51. & 11 & 2 & 40.6 & 90 & \begin{tabular}{|l|l|}
0.009 \\
\end{tabular} & & & 0.225 & \\
\hline & & 8:03:23 PM & 25.915 & 25.4 & 25.52 & 26.948 & 25.0 & 48.312 & 20.122 & 16.395 & 32.215 & -1.18 & & 47.338 & 0.063 & 14.643 & & & & 51 & 11 & & & & & 0.00 & & & \\
\hline & $\frac{4 / 202 / 200}{4 / 200}$ & $8: 04: 23 \mathrm{PM}$ & $\frac{25.909}{25094}$ & $\frac{25.43}{2540}$ & $\frac{25.529}{2559}$ & $\frac{26.992}{26907}$ & $\frac{25}{25}$ & & & & & $\frac{-1.221}{1.22 A}$ & & & & & & & & . & $\frac{11}{12}$ & & & & & & & & \\
\hline & 4 & $\frac{0.03 .25}{8.0201}$ & $\frac{25.094}{25898}$ & 250.44 & \begin{tabular}{|l|l|}
25.5180 \\
\end{tabular} & $\frac{2.9991}{26991}$ & & 48.1565 & \begin{tabular}{|l|}
53.201 \\
5.812
\end{tabular} & $\frac{10.514}{1647}$ & & - & & & & & & & & & $\frac{12}{12}$ & & & & & & & & \\
\hline & . & $\frac{0.0 .65}{8.023 \mathrm{PM}}$ & 25.050 & $\frac{20.45}{25.3}$ & \begin{tabular}{|l|l|}
25.535 \\
2535
\end{tabular} & & & 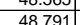 & & 16 & & - & & & & & & & & 热 & $\frac{12}{11}$ & 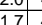 & & & & & & & \\
\hline 1385 & $4 / 2$ & $8: 08: 23 \mathrm{PM}$ & 25. & & & & & 48. & & 10.25 & & -1.183 & & & & & & & & $x_{1}$ & & & & & & & & & \\
\hline & $4 / 2$ & & & 25 & 25.51 & & 24 & 48.836 & & & & -1.224 & & $48 \mathrm{~s}$ & & & & & & 51 & 12 & & & & & & & 0.234 & \\
\hline & & $8: 10:$ & 25.88 & & & & & & & & & & & & & & & & & & $12 \quad$ & & & & & & & & \\
\hline & $4 / 2$ & $8: 11 \cdot 23 \mathrm{PM}$ & 25.877 & 25.35 & 25516 & & & 48. & 53.016 & 16.204 & 32.927 & 1221 & & 49.357 & 0.0 & & & 20 & & & 10 & & & 200 & & & & 516 & \\
\hline & $4 / 20 / 2004$ & $8: 12: 23 \mathrm{P}$ & $25.8 \varepsilon$ & 25.35 & \begin{tabular}{|c|}
25.54 \\
\end{tabular} & & & 48. & & & & & & 48.7 & 0.0 & & & & & & & & & & & & & & \\
\hline & $4 / 20 / 2004$ & 23 PM & & 25.35 & 25.549 & & & 48.7 & & & & -1.2 & & & & & & & & & & 1. & & & & & & & \\
\hline & $4 / 20 / 2004$ & $8: 14: 23 \mathrm{PM}$ & 25.876 & 25.35 & 25.486 & 26.974 & 25. & \begin{tabular}{|l|l|}
48.685 \\
\end{tabular} & 53.033 & 16.312 & & -1.183 & & 48.04 & 0.0 & 14.643 & & 207 & & 51. & 11 & & & 05 & 0.010 & 0.010 & & 0.235 & \\
\hline & $4 / 212$ & 8:15:23 PM & 25.888 & 25.36 & 25.502 & 27.066 & 25.5 & 48.563 & 52.902 & 16.25 & 32.586 & -1.195 & & 46.224 & 0.067 & 14. & & 201 & & 51. & 11 & & & & & & & & \\
\hline & & 8:16:23 PM & 25.884 & 25.36 & & $27.05 \pi$ & & 48.451 & 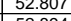 & & & -1.16 & & & & & & & & 51. & 12 & 2 & & 2.992 & & & & 30 & \\
\hline$\frac{109}{139}$ & 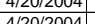 & 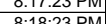 & $\frac{2.3 .592}{20588}$ & $\frac{25.36}{2538}$ & $\frac{25.524}{25517}$ & $\frac{2}{27-1}$ & & $\begin{array}{r}48.59 \\
18.51\end{array}$ & $\frac{52.894}{5.012}$ & 1615 | & & -1.1. & & & 0.06 & 116 & & 207 & & 51. & 11 & 10 & & & & & & 0 & \\
\hline & (1/201010 & $\frac{0.0 .25}{8.23 P M}$ & $\frac{25.000}{25878}$ & & & & & 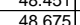 & & & & -1.1 & & & & & & & & & 11 & & & & & & & & \\
\hline 139 & $4 / 201 / 2004$ & $8.120 .23 \mathrm{PM}$ & 25.88 & 25.3 & 25535 & 27083 & 25 & 48.75 & 53191 & 16.334 & 33. & -1.157 & & 46806 & & 14643 & & 207 & 34625 & 51 & 11 & 15 & & $28 \quad$ & 8010 & 0010 & & 0.234 & \\
\hline & & & & & 25.54 & & & & & & & & & & & & & & & & & & & & & & & & \\
\hline & $4 / 2$ & & 25.871 & 25.39 & 25.546 & 26.904 & & 48.544 & 52965 & 16.287 & & 1006 & & 4812 & 0.064 & 14643 & & & & & & & & & & & 000 & & n \\
\hline & & & & & & & & & & & & & & & & & & & & & & & & & & & & 0.245 & \\
\hline
\end{tabular}


WSRC-TR-2005-00105, REVISION 0

SRNL-RPP-2005-00012, REVISION 0

RUN \# 2.03A AND B; FIRST AND SECOND HALF OF SLURRY DEWATERING - CONT.

\begin{tabular}{|c|c|c|c|c|c|c|c|c|c|c|c|c|c|c|c|c|c|c|c|c|c|c|c|c|c|c|c|c|}
\hline & A & B & D & $E$ & $\frac{1}{25}$ & $G$ & $\mathrm{H}$ & $\mathrm{J}$ & $\mathrm{K}$ & $\mathrm{L}$ & $\mathrm{M}$ & $\mathrm{N}$ & 0 & $a_{1}$ & $R$ & 5 & \begin{tabular}{l|l|l|} 
\\
\end{tabular} & $v$ & w & $x$ & & 2 & $\mathrm{AA}$ & $A B$ & $A C$ & $A D$ & $\mathrm{AE}$ & \\
\hline \begin{tabular}{|l|l|l|l|l|}
1402 \\
\end{tabular} & \begin{tabular}{r|r|}
$4 / 20 / 2004$ \\
42012004
\end{tabular} & $\begin{array}{l}8: 24: 23 \mathrm{PM} \\
8: 25 \cdot 23 \mathrm{PM}\end{array}$ & $\begin{array}{l}25.864 \\
25.874\end{array}$ & $\begin{array}{r}25.389 \\
25.389\end{array}$ & 25.549 & $\begin{array}{l}26.917 \\
26.937\end{array}$ & \begin{tabular}{|l|}
25.069 \\
25.111 \\
\end{tabular} & $\begin{array}{l}48.783 \\
48.598\end{array}$ & \begin{tabular}{|l|}
53.199 \\
53.122 \\
\end{tabular} & $\frac{16.267}{11.229}$ & \begin{tabular}{|l|}
32.867 \\
32709
\end{tabular} & $\begin{array}{l}-1.221 \\
-1.099\end{array}$ & $\begin{array}{l}-6.995 \\
-6.954\end{array}$ & \begin{tabular}{|l|l}
48.863 \\
46.656
\end{tabular} & 0.057 & $\begin{array}{l}14.643 \\
14643 \\
\end{array}$ & $\begin{array}{l}0.003 \\
0.003 \\
\end{array}$ & $\begin{array}{l}2082.6 \\
2083.6\end{array}$ & \begin{tabular}{|l|}
34.7092 \\
3.7258
\end{tabular} & \begin{tabular}{|l|l|}
51.7 \\
51.6
\end{tabular} & $\frac{12.6}{11.4}$ & \begin{tabular}{|l|}
40.8 \\
40.7 \\
\end{tabular} & 2.815 & $\mid$ & $\begin{array}{l}0.008 \\
0.010\end{array}$ & 0.000 & $\begin{array}{l}0.205 \\
0.238 \\
\end{array}$ & $\begin{array}{l}0.17 \\
0.20\end{array}$ \\
\hline \begin{tabular}{|l|l|l|l|l|l|l|}
1403 \\
\end{tabular} & & & \begin{tabular}{|l}
25.8634 \\
2563
\end{tabular} & & \begin{tabular}{|l|l|}
25.567 \\
\end{tabular} & & & & & $\frac{10.269}{16.279}$ & & & & & & $\begin{array}{l}14.045 \\
14.643\end{array}$ & & & & & & & & & & & & \\
\hline 1404 & $4 / 20 / 2004$ & 8:27:23 PM & 25.861 & 25.371 & 25.566 & 26.834 & 25.062 & 48.513 & \begin{tabular}{|l|}
52.907 \\
\end{tabular} & 16.273 & 32.498 & -1.157 & & 47.401 & 0.068 & 14.643 & 0.003 & 2085.6 & \begin{tabular}{|l|l|}
34.7592 \\
\end{tabular} & & & & & 0.010 & 0.010 & 0.000 & 0.246 & \\
\hline \begin{tabular}{|l|l|l|l|}
1405 \\
\end{tabular} & $4 / 2012004$ & & 25.871 & 25.381 & 25.556 & 26.879 & 25.077 & 48.511 & \begin{tabular}{|l|}
52.805 \\
\end{tabular} & 16.316 & & $\begin{array}{l}.1 .18 \\
\end{array}$ & & 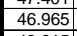 & 0.067 & $\begin{array}{l}14.643 \\
\end{array}$ & & 2086.6 & & & & & & & & & $\begin{array}{l}0.243 \\
0.243\end{array}$ & 0.21 \\
\hline$\frac{1400}{1407}$ & & & & & & & 25.019 & & \begin{tabular}{|l|}
52.967 \\
\end{tabular} & & $\begin{array}{l}32.757 \\
\end{array}$ & & & & & & & & & & & & & & & & & \\
\hline \begin{tabular}{|l|l|}
1407 \\
108
\end{tabular} & $4 / 20 / 2004$ & $\begin{array}{l}8: 30: 23 \mathrm{PM} \\
0: 202 \mathrm{P}\end{array}$ & $\begin{array}{l}25.848 \\
25.851\end{array}$ & 25.347 & \begin{tabular}{|l|l|}
25.517 \\
2551
\end{tabular} & \begin{tabular}{|l|l|}
26.801 \\
26.720
\end{tabular} & $\begin{array}{l}24.938 \\
2.026\end{array}$ & $\begin{array}{l}48.561 \\
\end{array}$ & \begin{tabular}{|l|l|}
52.894 \\
5207
\end{tabular} & $\begin{array}{l}16.192 \\
1607\end{array}$ & \begin{tabular}{|l|}
32.783 \\
3.087
\end{tabular} & $\begin{array}{l}-1.157 \\
\end{array}$ & \begin{tabular}{|l|l|}
-7.209 \\
\end{tabular} & \begin{tabular}{|l|l|}
47.778 \\
\end{tabular} & 0.074 & 14.643 & $\begin{array}{l}0.003 \\
\end{array}$ & 2088.6 & \begin{tabular}{|l|}
34.8092 \\
4.82
\end{tabular} & 51. & 11.7 & & \begin{tabular}{|l|l|}
2.804 \\
\end{tabular} & 0.011 & $\begin{array}{ll}0.011 \\
\end{array}$ & 0.000 & 0.267 & \\
\hline$\frac{1408}{1409}$ & $\begin{array}{l}4 / 2012004 \\
4 / 2012004\end{array}$ & $\begin{array}{l}8: 31: 23 \text { PM } \\
8: 22: 23 \text { PM }\end{array}$ & $\begin{array}{l}25.851 \\
25.855\end{array}$ & $\begin{array}{l}25.361 \\
25.349 \\
\end{array}$ & $\begin{array}{l}25.521 \\
25.529\end{array}$ & \begin{tabular}{|l|}
26.739 \\
26.723 \\
\end{tabular} & \begin{tabular}{|l|}
24.936 \\
24.915 \\
\end{tabular} & 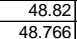 & \begin{tabular}{|l|}
53.197 \\
53.191
\end{tabular} & $\begin{array}{c}\frac{16.07}{15.964} \\
\end{array}$ & \begin{tabular}{|r|}
33.087 \\
33.198
\end{tabular} & $\begin{array}{l}-1.157 \\
-1.154 \\
\end{array}$ & $\begin{array}{l}-7.203 \\
-7.203\end{array}$ & \begin{tabular}{|l|l|}
49.172 \\
46.966 \\
\end{tabular} & $\begin{array}{l}0.067 \\
0.066\end{array}$ & $\begin{array}{l}144.643 \\
14643\end{array}$ & \begin{tabular}{|l|}
0.003 \\
0.003
\end{tabular} & $\begin{array}{l}2089.6 \\
2090.6\end{array}$ & \begin{tabular}{|l|l|}
34.8258 \\
344825
\end{tabular} & $\frac{51.7}{51.7}$ & $\frac{12.1}{11.5}$ & \begin{tabular}{|l|l|}
41.0 \\
41.0
\end{tabular} & 2.824 & 0.010 & \begin{tabular}{l|l|}
0.010 \\
0.010
\end{tabular} & $\begin{array}{l}0.000 \\
0.000\end{array}$ & $\begin{array}{l}0.240 \\
0.237\end{array}$ & $\begin{array}{l}0.20 \\
0.20\end{array}$ \\
\hline 1410 & $4 / 20 / 2004$ & 8:33:23 PM & 25.848 & 25.343 & 25.533 & & 24.910 & 48.851 & \begin{tabular}{|l|}
53.288 \\
\end{tabular} & 16.183 & 32.941 & -1.151 & & \begin{tabular}{|l|}
48.7800 \\
48.742
\end{tabular} & 0.060 & $\begin{array}{l}14.643 \\
14.643\end{array}$ & $\begin{array}{l}.0003 \\
0.003 \\
\end{array}$ & $\frac{2090.6}{20916}$ & & & & $\frac{41.0}{40.9}$ & & $\frac{0.010}{0.010}$ & $\begin{array}{l}0.010 \\
0.010\end{array}$ & $\begin{array}{l}0.000 \\
0.000\end{array}$ & $\begin{array}{l}0.237 \\
0.237\end{array}$ & 0.20 \\
\hline 1411 & $4 / 2012004$ & 8:34:23 PM & & & & 26.679 & 24.936 & \begin{tabular}{|l|l|l|l}
48.845 \\
\end{tabular} & \begin{tabular}{|l|l|} 
\\
\end{tabular} & & 33.058 & & & $\begin{array}{l}48.408 \\
\end{array}$ & 0.066 & 14.643 & & & & & & & & & & & & $\begin{array}{l}0.20 \\
0.20\end{array}$ \\
\hline 1412 & $4 / 20 / 2004$ & $8: 35: 23 \mathrm{PM}$ & 25.84 & 25.334 & 25.539 & $\begin{array}{l}26.653 \\
\end{array}$ & 24.935 & $\begin{array}{l}48.704 \\
\end{array}$ & \begin{tabular}{|l|l|}
53.234 \\
\end{tabular} & 16.038 & $\begin{array}{l}32.958 \\
\end{array}$ & $\begin{array}{l}-1.108 \\
\end{array}$ & -7.206 & 48.179 & 0.065 & 14.643 & 0.003 & 2093.6 & \begin{tabular}{|l|}
34.8925 \\
\end{tabular} & 51.8 & 11.8 & & & & & 0.000 & & \\
\hline 1413 & $4 / 20 / 2004$ & $8: 36: 23$ PM & & & & & & & & & & & & & & 14.643 & & & & & & & & & & & & \\
\hline 1414 & 4/2012004 & 0.72 .030 & & & 2 & 26.717 & & $\begin{array}{l}48.542 \\
\end{array}$ & 52.896 & 15.961 & & $\begin{array}{l}-1.099 \\
\end{array}$ & & 50031 & 0.067 & 14.643 & & & & & & & & & & & & \\
\hline & 4/20/2004 & $8: 38: 23 \mathrm{PM}$ & 25.824 & 25.318 & 25.543 & 26.792 & 25.384 & 48.863 & 55.323 & 16.101 & 33.128 & -1.099 & & 48.41 & 807 & 14.643 & & 2096.6 & 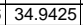 & & & & & & & & & \\
\hline 1416 & $4 / 20 / 2004$ & 8:39:23 PM & 25.82 & 25.3 & 25.535 & 26.878 & 25.45 & \begin{tabular}{|l|l|l|l}
48.706 \\
\end{tabular} & 53.188 & 16.189 & 32.83 & $\begin{array}{l}-1.033 \\
\end{array}$ & & | 45.803 & 0.069 & 14.643 & $\begin{array}{l}0.003 \\
\end{array}$ & 2097.6 & \begin{tabular}{|l|l|}
34.9592 \\
\end{tabular} & 51.7 & 11.2 & 40.8 & 2.811 & 0.010 & 0.010 & 0.000 & 0.249 & \\
\hline & 0.02004 & $8: 40: 23 \mathrm{PM}$ & 25.842 & 25.336 & 25.576 & \begin{tabular}{|l|l|}
27.04 \\
\end{tabular} & 25.532 & 48.98 & 53.437 & 15.961 & 33.341 & -1.096 & & 47.374 & 0.064 & 14.643 & & 2098.6 & 34.9758 & & 11. & & & & & & & \\
\hline & $0 / 2004$ & $8: 41: 23 \mathrm{PM}$ & & & & & 25.443 & & & & 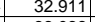 & -1.096 & & 48.266 & & 14.643 & & & & & & & & & & & & \\
\hline & 0/2004 & 8:42:23 PM & 25.835 & 25.334 & 25.564 & 27.023 & $\begin{array}{r}25.37 \\
25.320\end{array}$ & & .323 & & . & & & 48.36 & 0.066 & 14.643 & & & & & & & & & & & & \\
\hline$\frac{144}{142}$ & 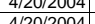 & $8.43 .23 \mathrm{PM}$ & 25.846 & 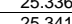 & $\frac{2.531}{25516}$ & $\frac{2.9994}{26074}$ & $\frac{25.320}{25206}$ & 480.18 & 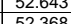 & $\frac{1.607}{1.6817}$ & $\frac{31.174}{32.155}$ & $-\frac{1.1993}{1.906}$ & & 8754 & & $\frac{1.4645}{1.643}$ & & & 5025 & & & & & & & & & \\
\hline 142 & 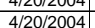 & 8:44:23:23 PM & $\begin{array}{r}25.830 \\
25.837\end{array}$ & $\begin{array}{r}25.341 \\
25.326\end{array}$ & $\begin{array}{l}25.510 \\
25.516\end{array}$ & $\begin{array}{r}20.9 / 4 \\
26.96\end{array}$ & $\begin{array}{l}\frac{35.220}{25.202} \\
25\end{array}$ & $\begin{array}{l}47.952 \\
48.446\end{array}$ & $\frac{52.368}{52.954}$ & $\frac{10.814}{16.649}$ & $\frac{11.455}{32.131}$ & $\begin{array}{l}-1.096 \\
-1.096\end{array}$ & & $\begin{array}{l}48.154 \\
47.557\end{array}$ & $\begin{array}{l}0.002 \\
0.064\end{array}$ & $\begin{array}{l}\frac{14.645}{14.643} \\
\end{array}$ & 0.003 & 21 & 5.0592 & 51.5 & & 403 & & & 0.09 & & & \\
\hline & 4/20/2004 & 8:46:23 PM & 25.848 & 25.342 & \begin{tabular}{|l|l|l|l}
25.532 \\
\end{tabular} & 26.931 & 25.098 & 48.081 & 52.579 & 16.43 & 31.999 & -1.099 & & 46.014 & 0.063 & 14.643 & & 2104.6 & 5.0758 & 51. & $\frac{11 .}{11.3}$ & & 2.761 & & .009 & 0.00 & & \\
\hline & $4 / 2012004$ & 8:47:23 PM & 25.838 & 25.342 & 25.532 & 26.861 & 25.098 & 48.249 & 52.675 & 16.444 & 32.166 & -1.143 & & 48.196 & 0.064 & & & $\frac{2105.6}{2105.6}$ & & & & & & & & & & \\
\hline & 20/2004 & & 25.842 & 25.33 & 25.557 & $\begin{array}{l}26.745 \\
\end{array}$ & $\begin{array}{l}25.092 \\
25\end{array}$ & 48.345 & 52.824 & 16.512 & & -0.995 & & 48.364 & 0.059 & 14.643 & & & & & & & & & & & & \\
\hline & & & & & & & & & & & & & & & & & & & & & & & & & & & & \\
\hline & 4/20/2004 & 8:50:23 PM & 25.804 & 25.309 & 25.529 & 26.687 & 25.084 & $\begin{array}{l}48.364 \\
\end{array}$ & 52.84 & 16.605 & 32.083 & \begin{tabular}{|c|}
-1.03 \\
\end{tabular} & & 49.914 & 0.066 & 14.643 & & 2108.6 & 35.1425 & & 12.2 & 46 & & & & & & \\
\hline & & $8: 51: 23 \mathrm{PM}$ & 25.814 & 25.319 & 25.559 & 26.697 & 24.989 & & 52.855 & 16.48 & 32.188 & -1.007 & & $\begin{array}{l}46.679 \\
\end{array}$ & 0.065 & 14.643 & 0.003 & 2109.6 & 35.1592 & 51. & 11.4 & & & & & & & \\
\hline 142 & $4 / 2012004$ & 52:23 PM & 25.813 & 25.313 & 25.573 & 26.721 & 24.954 & 48.179 & 52.741 & 16.475 & 31.962 & $\begin{array}{l}-0.998 \\
\end{array}$ & & 50.548 & 0.065 & 14.643 & & 2110.6 & 35.1758 & & 12.4 & 40. & 2.763 & & & & & \\
\hline 10 & $4 / 2012004$ & 8:53:23 PM & 25.829 & 25.339 & 25.609 & 26.722 & 24.944 & 48.465 & 53.07 & 16.57 & 32.194 & $1.02 \pm$ & & 4.271 & 0.064 & 14.643 & & 2111.6 & 35.1925 & & $11.8 \mathrm{~g}$ & 40. & & & & & 233 & \\
\hline & 0.12004 & $8: 54: 23 \mathrm{PM}$ & 25.803 & 25.308 & 25.583 & & 24. & & 53.162 & & & -0.998 & & 48.798 & 0.064 & & & & & & $12.6 \mathrm{C}$ & & & & & & & \\
\hline 14 & 4212012004 & $8.55: 23 \mathrm{PM}$ & 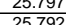 & 252.291 & $\begin{array}{l}25.586 \\
25506\end{array}$ & $\begin{array}{l}2.53 \\
26.515\end{array}$ & $\frac{24.8}{2.8}$ & $\begin{array}{ll}48.467 \\
48352\end{array}$ & 5 & $\begin{array}{l}1.638 \\
1.627\end{array}$ & $\frac{32.432}{32.424}$ & $\begin{array}{l}-0.066 \\
-0.092\end{array}$ & & (49.111 & 0.065 & $\begin{array}{l}1.4643 \\
11463\end{array}$ & & 211 & 352425 & & 2. & & & & & & & \\
\hline & $4 / 2012004$ & $\begin{array}{l}0.00 .20 \mathrm{PM} \\
8: 57: 23 \mathrm{PM}\end{array}$ & 25.78 & 25.27 & 25585 & $\frac{20.515}{26443}$ & $\frac{24.012}{24.921}$ & 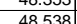 & 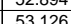 & 16.409 & $\begin{array}{l}3<.454 \\
32.373\end{array}$ & $=0.095$ & & 46.112 & $\frac{0.004}{0.064}$ & $\frac{1.4645}{1463}$ & & 21156 & $\begin{array}{l}35.2450 \\
352592\end{array}$ & 517 & $\frac{1.0}{11.3}$ & 40 & 2789 & 0,010 & 009 & 0,000 & o. & \\
\hline 143 & $4 / 20 / 2004$ & 8:58:23 PM & 25.795 & $\frac{25.24}{25.285}$ & 25.63 & 26.493 & & & 52.774 & 16.371 & 32.163 & -0.992 & & & & & & $\frac{2.1}{211}$ & $\frac{3.575}{352758}$ & . & 1.0 & & & & & & & \\
\hline 143 & 0.12004 & 8:59:23 PM & 25.775 & $\frac{25.254}{25.254}$ & 25.559 & 26.453 & $\frac{25.245}{25.245}$ & 48.38 & 55.089 & $\frac{16.399}{16399}$ & $\frac{25.299}{32.299}$ & $\frac{-0.94}{-0.94}$ & & 48.794 & 0.066 & $\frac{14.643}{14.64}$ & & $\frac{211}{211}$ & $\frac{35.2925}{35.2925}$ & & t12. & $\frac{14}{46}$ & .781 & & & & $\frac{250}{240}$ & \\
\hline & & & & & 25.586 & 26.395 & & & 4.938 & 16.384 & & & & & & 14.643 & & & & & & & & & & & & \\
\hline & & $3 \mathrm{PM}$ & 25.767 & 25.246 & & 226.42 & & & 317 & 478 & & $\mid-0.937$ & & 48.972 & & 14.643 & & & & & 12 & & 2801 & & & & & \\
\hline & $4 / 20 / 2004$ & 9:02:23 PM & 25.788 & & 25.598 & \begin{tabular}{|l|l|}
26.666 \\
\end{tabular} & & & & & & -0.934 & & & & & & & & & 12.6 & & & & & & & \\
\hline 1446 & 4/20/2004 & 9:03:23 PM & 25.806 & 25.291 & 25.616 & 26.834 & & . & 52.983 & 16.449 & .114 & \begin{tabular}{|l|l|}
-0.937 \\
\end{tabular} & & \begin{tabular}{|l|l|l|}
49.0699 \\
\end{tabular} & 0.063 & 14.643 & & 2121.6 & 35.3592 & 51.5 & 12.0 & 40 & & \begin{tabular}{|c|}
0.009 \\
\end{tabular} & 0.009 & 0,000 & 0.230 & \\
\hline & 4/2/20/2004 & 9:04:23 PM & & 25.29 & 25.627 & 26.941 & 25.4 & $\begin{array}{lll}48.2 \\
\end{array}$ & 52.956 & 16.434 & 32.151 & $\begin{array}{l}-0.937 \\
\end{array}$ & & 46.6 & & 14.643 & & 212 & 30.3 & 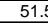 & 11.4 & & & & & & $0 . \angle 20$ & \\
\hline & | & 9:05:23 PM & 25.814 & 25.294 & 25.629 & 27.017 & 25.3 & 48.476 & 3.126 & & 32.379 & -0.937 & & 49.5 & & & & & $35.33^{\circ}$ & & 12.4 & & & & & & & \\
\hline $1 \frac{1445}{141}$ & & $32 \mathrm{PM}$ & $\begin{array}{l}25.82 \\
252.82\end{array}$ & 25.30 & $2 \frac{2.635}{2034}$ & 26.998 & 年 & $\begin{array}{l}48.38 \\
1937\end{array}$ & 2.987 & 16.367 & 2015 & -0.894 & & 6.016 & 0.07 & & & & & & & & & & & & & \\
\hline & 4212012004 & $\begin{array}{l}9: 01: 233 \mathrm{M} \\
0.02020\end{array}$ & & $\frac{25.306}{25312}$ & $\begin{array}{l}25.641 \\
25667\end{array}$ & 26.904 & & (48.137) & & $\frac{16.35}{16200}$ & $\begin{array}{l}32.151 \\
32002\end{array}$ & $=0.928$ & & & & & & & & & 120 & 39 & & & & & & \\
\hline & & & $\frac{20.034}{25818}$ & 25.30 & 25.0017 & $\frac{2.07}{26.676}$ & & (17.060 & & $\frac{1.259}{1642}$ & & $=0.004$ & & 49.049 & & & & & & & $\frac{1.20}{120}$ & & & & & & & \\
\hline $\begin{array}{ll}1447 \\
\end{array}$ & $\frac{4}{4 / 2012004 \mid}$ & $\begin{array}{l}9.53 .25 \\
9 \cdot 10.23\end{array}$ & $\frac{25.010}{25.807}$ & 25.29 & $\frac{2.0411}{25.632}$ & 26.705 & 25.6 & & 074 & $\frac{1.442}{16.48}$ & $\frac{52.34}{32.24}$ & $\begin{array}{l}-0.002 \\
-0.902\end{array}$ & & (4).801 & & 14.6. & & $\frac{212}{212}$ & 35.4. & $\frac{31}{51}$ & $\frac{\pi}{11 .}$ & & & & & & & \\
\hline 1448 & & 9:11:23 & & & & & & & & & & & & & & & & & & & & & & & & & & \\
\hline$\left.144\right|^{144}$ & & 9:12:23 PM & 25. & 25.31 & 25.684 & & & & 53.056 & 16.479 & 32.14 & -0.888 & & 49.27 & & 14.643 & & & & - & 121 & & & & & & & \\
\hline & & $9: 13$ & 25. & & & 26.682 & $\frac{24}{24}$ & & & & & & & & & & & & & , & 119 & & & & & & & \\
\hline & $4 / 20 / 2004$ & $9: 14$ & 25.8 & & & & & & 53.155 & 16.44 & & $\begin{array}{c}-0.937 \\
\end{array}$ & & & & & & & & & 11 & & & & & & & \\
\hline & $4 / 20 / 200$ & $9: 15: 23 \mathrm{P}$ & 25.821 & 25.29 & 25.696 & & & & & 16.516 & & \begin{tabular}{c|c|c|}
-0.93 \\
\end{tabular} & & & & & & 21 & & 51.6 & 11.9 & & & & & & & \\
\hline & $4 / 20 / 2004$ & 9:16:23 PM & 25.82 & 25.275 & 25.7 & & & & & & & -0.995 & & & & & & & & & 11. & & & & & & & \\
\hline 145 & & 9:17:23 PM & 25.814 & 25.269 & 25.699 & 26.587 & & & & 16 & & -1. & & & & & & 2 & 35.5925 & 51 & 11.9 & & & & & & & \\
\hline & & 9:18:23 PM & 25.813 & 25.262 & 25.647 & 26.581 & 24.8 & 48.8 & 53.356 & 16.547 & 32.576 & -0.995 & & & 0.0 & 14.643 & & & & 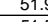 & 11. & & & & & & & \\
\hline & & $9.19 .23 \mathrm{PM}$ & 25.81 & & & 20.48 & & & 3.010 & 1.0305 & & -1.099 & & & & & & & & & & & & & & & & \\
\hline$\frac{145}{145}$ & 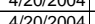 & $\frac{9.20 .23}{9 \cdot 23.25 M}$ & $\frac{2.6 .6}{25.8}$ & 23.251 & & $\begin{array}{ll}2.4554 \\
26309\end{array}$ & . & 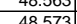 & $\frac{53.009}{52.104}$ & 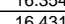 & & $\frac{-1.154}{-1.24}$ & & & 0.05 & $\begin{array}{l}\frac{1.4645}{1.663} \\
\end{array}$ & & & & 516 & $\frac{12.2}{12.2}$ & & & & & & $\frac{13}{25}$ & \\
\hline & $\frac{442012004 \mid}{4}$ & $\frac{2.25}{9 \cdot 23.23 \mathrm{PM}}$ & $\frac{25.007}{25807}$ & $\frac{15.250}{25246}$ & & & & & 53133 & & & -1.137 & & & & & & & & 51.7 & 117 & & & & & & 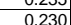 & \\
\hline 1460 & $4 / 20 / 200$ & $9: 93223 \mathrm{PM}$ & 25.802 & 25.246 & 25.667 & 2.6 .55 & 25 & 48.366 & $\frac{25.902}{52.902}$ & 16.384 & 32.246 & -1.151 & & & & & & & 35.6 & 51.4 & 11.4 & 4 & & & & & 232 & \\
\hline 1461 & $4 / 20 / 2004$ & 9:24:23 PM & 25.813 & 25.258 & 25.688 & 26.631 & 25.5 & & 52.9 & $16.334 \mathrm{r}$ & 32.461 & -1.093 & & & 0.064 & 14.643 & & & & & . & & & & & & & \\
\hline & & & 25.82 & & & 26.823 & & 48 & 53.147 & 16.347 & 32.592 & -1.096 & & & & & & & & & & & & & & & & \\
\hline 146 & $4 / 20 / 2004$ & 9:26:23 PI & 25.832 & 25.266 & 25.702 & 26.885 & 25.3 & 48.532 & 53.002 & 16.4 & 32.389 & \begin{tabular}{|c|}
-1.099 \\
\end{tabular} & & 49.9 & 0.06 & 14.6 & & 2144.6 & 35.74 & 515 & 12.2 & 4 & 27. & & & & 35 & \\
\hline & & 9:27: & 25.8 & 25.26 & 25.698 & 26. & & 48.575 & 53.046 & 16.434 & 32. & \begin{tabular}{|c|c|}
-1.099 \\
\end{tabular} & & & 0.0 & 14. & & & & $168 \mathrm{r}$ & $\overline{12 .}$ & & & & & & & \\
\hline & $4 / 20 / 2004$ & 9:28:23 PM & & 25.2 & & & & 48.583 & & 16.466 & 32.42 & -1.0 & & & & 14. & & 21. & & 51. & 12.6 & & & & & & & \\
\hline 146 & & 9:29:2 & 25. & 25. & & 26.887 & & & 52.888 & 16.36 & & -1.0 & & & & & & & & 51.2 & 12.4 & & & & & & 34 & \\
\hline 146 & $4 / 20 / 2004$ & & 25.844 & 25.269 & 25.109 & 26.802 & & 48.565 & 53.184 & 16.4 & & -1.059 & & & & 14.643 & & & & & 1.6 & & & & & & & \\
\hline$\frac{1440}{1006}$ & $4 / 20 / 2004$ & 9:31:23 PM & 25.84 & 25.269 & \begin{tabular}{|l|l|}
25.705 \\
5.725
\end{tabular} & $26.5 / 3$ & 24.54 & 48.426 & 52.967 & 16.411 & 32.36 & -1.035 & & 48.156 & 0.064 & 14.643 & & 21 & & 51. & $\begin{array}{l}5 \\
50\end{array}$ & 40.4 & 2.1 & 0.010 & 0.009 & 0.000 & 0.232 & \\
\hline $\begin{array}{l}\frac{1469}{1470} \\
140\end{array}$ & $\frac{4 / 2 / 2012004}{4 / 2012004}$ & $\begin{array}{l}9: 32: 23 \mathrm{PM} \\
9: 23 \mathrm{PM}\end{array}$ & $\frac{25.855}{25.838}$ & $\begin{array}{r}25.269 \\
25.253\end{array}$ & \begin{tabular}{|l|}
25.725 \\
25.683 \\
\end{tabular} & \begin{tabular}{|l|}
26.463 \\
26.071 \\
\end{tabular} & \begin{tabular}{|l|}
24.545 \\
24.439 \\
\end{tabular} & \begin{tabular}{r|r|}
48.587 \\
48.58
\end{tabular} & \begin{tabular}{|l|}
53.259 \\
53.095 \\
\end{tabular} & $\begin{array}{l}16.509 \\
16.489\end{array}$ & $\frac{32.441}{32.44}$ & \begin{tabular}{|l|}
-1.096 \\
-0.995 \\
\end{tabular} & & $\begin{array}{l}48.485 \\
47.426\end{array}$ & 0.067 & $\frac{14.4643}{14.643}$ & $\frac{0.003}{0.003}$ & & \begin{tabular}{|l|}
35.842525 \\
53.8592 \\
\end{tabular} & \begin{tabular}{|l}
51.6 \\
51.6
\end{tabular} & \begin{tabular}{c|c}
6 & $11 .$. \\
6 & 11.6
\end{tabular} & \begin{tabular}{|l|}
440.5 \\
\end{tabular} & \begin{tabular}{|l|}
2.793 \\
\end{tabular} & \begin{tabular}{|l|}
0.010 \\
\end{tabular} & \begin{tabular}{|l|}
0.010 \\
\end{tabular} & $\begin{array}{l}0.0000 \\
0.000\end{array}$ & $\frac{0.241}{0.235}$ & 0.2 \\
\hline
\end{tabular}


WSRC-TR-2005-00105, REVISION 0

SRNL-RPP-2005-00012, REVISION 0

RUN \# 2.03A AND B; FIRST AND SECOND HALF OF SLURRY DEWATERING - CONT.

\begin{tabular}{|c|c|c|c|c|c|c|c|c|c|c|c|c|c|c|c|c|c|c|c|c|c|c|c|c|c|c|c|c|}
\hline & A & $\mathrm{B} \quad \mathrm{C}$ & D & $\mathrm{E}$ & $\mathrm{F}$ & $\mathrm{G}$ & $\mathrm{H}$ & $\mathrm{J}$ & $\mathrm{K}$ & $\mathrm{L}$ & $\mathrm{M}$ & $\mathrm{N}$ & 0 & $Q^{Q}$ & $R$ & 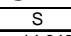 & $\mathrm{T}$ & & w & $x$ & & $z$ & $\mathrm{AA}$ & $A B$ & $A C$ & $A D$ & & \\
\hline$\frac{1471}{1472}$ & $\begin{array}{l}4 / 20 / 2004 \\
4 / 20 / 2004 \\
\end{array}$ & $\begin{array}{l}\text { 9:34:23 PM } \\
9.35 .23 \mathrm{PM}\end{array}$ & $\frac{25.835}{25.813}$ & $\begin{array}{l}25.235 \\
25.212\end{array}$ & \begin{tabular}{|r|}
25.65 \\
25.642 \\
\end{tabular} & $\begin{array}{l}25.838 \\
25.65 \\
\end{array}$ & \begin{tabular}{|l|}
24.461 \\
24.373 \\
\end{tabular} & $\begin{array}{l}48.571 \\
48.361\end{array}$ & $\begin{array}{l}53.112 \\
52.975 \\
\end{array}$ & $\frac{16.487}{16.384}$ & $\begin{array}{l}32.385 \\
32.229\end{array}$ & $\begin{array}{r}-1.09 \\
-1.033\end{array}$ & $\begin{array}{l}-7.073 \\
-7.111 \\
\end{array}$ & $\begin{array}{ll}48.696 \\
49.937\end{array}$ & 0.057 & $\begin{array}{l}14.643 \\
1.643\end{array}$ & $\begin{array}{l}0.003 \\
0.003\end{array}$ & $\begin{array}{l}2152.6 \\
2153.6 \\
\end{array}$ & \begin{tabular}{|l|}
35.8758 \\
3.5925 \\
\end{tabular} & \begin{tabular}{|l|l|}
51.6 \\
51.5
\end{tabular} & $\frac{11}{12}$ & \begin{tabular}{|l|l|}
40.5 \\
40.3 \\
\end{tabular} & $\frac{2.791}{2.78}$ & $\mid 0.008$ & $\begin{array}{l}0.008 \\
0.009\end{array}$ & 0.000 & $\begin{array}{l}0.206 \\
0.233 \\
\end{array}$ & $\begin{array}{l}0.18 \\
0.20 \\
\end{array}$ \\
\hline$\frac{1412}{1473}$ & & & & & & & & & \begin{tabular}{|l|l|}
52.938 \\
5.935
\end{tabular} & & & & & & & $\frac{14.045}{14.643}$ & & & & & & & & & & & & \\
\hline 1474 & $4 / 20 / 2004$ & 9:37:23 PM & 25.78 & 25.175 & 25.66 & 25.433 & 24.22 & 48.579 & \begin{tabular}{|l|}
53.048 \\
\end{tabular} & 16.466 & 32.436 & -1.044 & -7.139 & 48.748 & 0.065 & 14.643 & 0.003 & 2155.6 & 35.9258 & & & & $5 \quad 2.793$ & 0.010 & 0.010 & 0.000 & 0.235 & \\
\hline & $4 / 2012004$ & & 25.757 & 25.146 & \begin{tabular}{|l|}
25.656 \\
\end{tabular} & 25.324 & 24.212 & 48.334 & \begin{tabular}{|l|l|}
52.915 \\
\end{tabular} & 16.313 & 32.379 & -1.082 & & 47.924 & 0.066 & 14.643 & & & 35.9425 & & & & & & 0.010 & & & 0.20 \\
\hline & & & & & 25.661 & & & \begin{tabular}{|l|l|l|l|l}
48.144 \\
\end{tabular} & & 16.245 & & & & & & & & & & & & & & & & & & \\
\hline 1477 & $4 / 20 / 2004$ & 9:40:23 PM & 25.702 & $\begin{array}{l}25.096 \\
25077\end{array}$ & 25.657 & \begin{tabular}{|l|l|}
25.219 \\
2519
\end{tabular} & 24.137 & $\begin{array}{ll}48.403 \\
8.690\end{array}$ & \begin{tabular}{|l|l|}
52.892 \\
5.307
\end{tabular} & 16.266 & $\begin{array}{l}32.486 \\
2025 \\
\end{array}$ & \begin{tabular}{|c|}
-1.018 \\
.027
\end{tabular} & $\begin{array}{l}-7.056 \\
7009\end{array}$ & $\begin{array}{l}46.406 \\
4.57\end{array}$ & 0.064 & $\begin{array}{ll}14.643 \\
1.612\end{array}$ & $\begin{array}{l}0.003 \\
0.003\end{array}$ & 2158.6 & 35.9758 & & & & $\begin{array}{ll}4 & 2.789 \\
7\end{array}$ & 0.010 & 0.009 & 0.000 & 0.232 & \\
\hline$\frac{1478}{1479}$ & $\begin{array}{l}4 / 20 / 2004 \\
4 / 201204 \\
\end{array}$ & $\begin{array}{l}9: 41: 23 \text { PM } \\
9022: 23 \text { PM }\end{array}$ & $\begin{array}{l}25.698 \\
25.685\end{array}$ & $\begin{array}{l}25.077 \\
2554\end{array}$ & \begin{tabular}{|l|}
25.668 \\
25.675 \\
\end{tabular} & \begin{tabular}{r|r|}
25.18 \\
25.162
\end{tabular} & \begin{tabular}{|l|}
24.083 \\
24 \\
24
\end{tabular} & \begin{tabular}{|l|l|l|l|l|l}
48.689 & 48.936 \\
\end{tabular} & \begin{tabular}{|l|}
53.307 \\
53.464
\end{tabular} & $\begin{array}{l}16.354 \\
16.406\end{array}$ & $\begin{array}{l}32.625 \\
332835 \\
\end{array}$ & $\begin{array}{l}-1.027 \\
-1.027 \\
\end{array}$ & $\begin{array}{l}-7.099 \\
-7.024\end{array}$ & \begin{tabular}{|r|}
47.57 \\
48.262 \\
\end{tabular} & $\begin{array}{l}0.063 \\
0.065 \\
\end{array}$ & $\begin{array}{l}14.643 \\
1643 \\
\end{array}$ & \begin{tabular}{|l}
0.003 \\
0.003 \\
\end{tabular} & $\begin{array}{l}2159.6 \\
2160.6\end{array}$ & \begin{tabular}{|l|}
35.9925 \\
360092
\end{tabular} & $\frac{51.8}{52 .}$ & $\frac{11.7}{11.8}$ & & $\begin{array}{l}7.2 .03 \\
9 \\
9\end{array}$ & \begin{tabular}{|c|}
0.009 \\
0.010
\end{tabular} & 0.009 & 0.000 & \begin{tabular}{|l|l|}
0.227 \\
0.233
\end{tabular} & $\begin{array}{l}0.19 \\
0.20\end{array}$ \\
\hline 1480 & $4 / 20 / 2004$ & 9:43:23 PM & 25.667 & 25.031 & \begin{tabular}{|l|}
25.681 \\
\end{tabular} & 25.208 & \begin{tabular}{|r|}
24.402 \\
\end{tabular} & 48.969 & \begin{tabular}{|l|}
53.566 \\
\end{tabular} & 16.384 & & & & 46.9 & 0.063 & $\frac{14.043}{14.643}$ & $\begin{array}{l}0.0003 \\
0.003\end{array}$ & & \begin{tabular}{|l|l|}
5.00258 \\
36.0258
\end{tabular} & 52.1 & & & & \begin{tabular}{|l|l|} 
& 0.010 \\
\end{tabular} & $\begin{array}{l}0.010 \\
0.009 \\
\end{array}$ & $\begin{array}{l}0.000 \\
0.000 \\
\end{array}$ & 0.233 & 0.20 \\
\hline 1481 & 4/20/2004 & 9:44:23 PM & & & 25.693 & & 24.509 & 48.787 & & 16.393 & 32.73 & -1.099 & & 48.533 & 0.066 & 14.643 & & & 36.0425 & & & & & & & & & $\begin{array}{l}0.19 \\
0.20\end{array}$ \\
\hline 1482 & 4/20/2004 & 9:45:23 PM & 25.667 & 24.99 & 25.701 & \begin{tabular}{|l|l|}
25.354 \\
\end{tabular} & 24.632 & & 53.211 & 16.276 & 32.859 & -1.027 & -7.073 & 49.036 & 0.064 & 14.643 & 0.003 & 2163.6 & \begin{tabular}{|l|}
36.0592 \\
\end{tabular} & & & & & 0.010 & 0.009 & 0.000 & & \\
\hline 1483 & $4 / 20 / 2004$ & 9:46:23 PM & 25.661 & & & & & & & & & & & & & & & & & & & & & & & & & 0.20 \\
\hline 1484 & $4 / 20 / 2004$ & 9:46:43 PM & 20.070 & & & 25.467 & & & 53.164 & 16.475 & 32.58 & -1.125 & & 48.648 & 0.064 & & & & 5.0814 & & & & & & & & & \\
\hline & 4/20/2004 & 9:47:43 PM & 25.697 & 24.98 & 25.741 & & 24.767 & 48.482 & & 16.494 & 32.226 & -0.975 & & 45.845 & 0.064 & 14.643 & & & & & & 40 & 4.784 & & & & & \\
\hline & 4/20/2004 & 9:48:43 PM & 25.703 & 24.977 & 25.747 & 25.565 & 24.683 & 48.573 & 53.253 & 16.389 & 32.449 & -1.096 & & 49.503 & 0.061 & 14.643 & 0.003 & 2166.6 & 36.1147 & 51.8 & 12.1 & 40.5 & 2.793 & 0.009 & 0.009 & 0.000 & 0.220 & \\
\hline & 4/20/2004 & 9:49:43 PM & 25.714 & 24.963 & 25.724 & 25.557 & 24.545 & 48.706 & 53.267 & 16.519 & 32.41 & -1.03 & & $\begin{array}{ll}48.248 \\
\end{array}$ & 0 & 14.643 & & 2167. & 36.1314 & & & & .796 & & .009 & & & \\
\hline & $4 / 2012004$ & 9:50:43 PM & & & & & & & & & 32.7055 & -1.062 & & & 0.067 & 14.643 & & & & & & & & & & & & \\
\hline & 0 & 9:51:43 PM & 25.734 & 24.933 & 25.679 & 25.527 & 24.254 & & .211 & 16.422 & 32.627 & -1.09 & & 48.656 & 0.064 & 14.643 & & & & & & & & & & & & \\
\hline & 412012004 & $\frac{9.525 .43 \mathrm{PM}}{0.5 \cdot 32}$ & $\frac{25.124}{25755}$ & & 25.058 & $2 \frac{25.530}{25609}$ & $\frac{24.38}{2.425}$ & 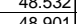 & (3) & $\frac{10.418}{1.672}>$ & 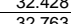 & - & & 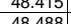 & & 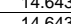 & & & 6108 & & & & & & & & & \\
\hline$\frac{1491}{1492}$ & $\begin{array}{l}/ 2 / 201 / 2004 \\
4 / 2004 \\
\end{array}$ & 9:54:43 PM & 25.756 & $\begin{array}{r}24.919 \\
24.89\end{array}$ & $\begin{aligned} 25.085 \\
25.67\end{aligned}$ & 25.0048 & $\begin{array}{l}24.455 \\
24.346\end{array}$ & $\begin{array}{l}48.901 \\
48.391 \\
\end{array}$ & $\begin{array}{l}53.309 \\
53.016\end{array}$ & $\begin{array}{l}10.475 \\
16.455\end{array}$ & $\begin{array}{l}32.103 \\
32.194 \\
\end{array}$ & $\begin{array}{l}-1.006 \\
-1.09 \\
\end{array}$ & & $\begin{aligned} 48.480 \\
48.98\end{aligned}$ & $\frac{0.006}{0.06}$ & $\begin{array}{l}\frac{14.645}{14.643} \\
\end{array}$ & 0.003 & & & & & & & (1) & .009 & 0.000 & 0.218 & \\
\hline & $4 / 20 / 2004$ & 9:55:43 PM & 25.752 & 24.855 & 25.666 & 25.509 & 24.236 & 48.502 & 53.166 & $\begin{array}{l}1.456 \\
16.436\end{array}$ & 32.404 & 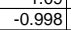 & -7.01 & 47.297 & 0.066 & 14.643 & & $\frac{11}{217}$ & 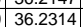 & & $\frac{1.6}{11.6}$ & & 2.78 & & .010 & 0.00 & $\frac{210}{239}$ & \\
\hline & 4/20/2004 & 9:56:43 PM & 25.764 & 24.84 & 25.668 & 25.496 & 24.199 & & 52.998 & 16.471 & 32.41 & -1.024 & & 47.814 & 0.064 & 14.643 & & & & & & & & & & & & \\
\hline & 4/20/2004 & 9:57:43 PM & 25.766 & & 25.645 & 25.448 & 24.126 & & & 16.359 & 38 & -1.024 & & 177.709 & 0.063 & 14.643 & & & & & & & & & & & & \\
\hline & & & 25.772 & 24.78 & 25.601 & 25.459 & 24.166 & 48.349 & & 10.334 & & & & 40.024 & 0.066 & 14.643 & & & & & & & & & & & & \\
\hline & 4/20/2004 & 9:59:43 PM & & 24.72 & 25.607 & 25.36 & 24.263 & 48.575 & 33.186 & & 32.572 & -0.937 & & 48.275 & 0.066 & 14.643 & & 217 & 36.2981 & & & & & & & & & \\
\hline & 20/2004 & $43 \mathrm{PM}$ & 25.774 & 24.718 & 25.619 & 25.381 & 24.329 & & 53.147 & 16.399 & 32.477 & -0.978 & & 49.28 & 0.062 & 14.643 & 0.003 & 2178 & & 51.7 - & 12.1 & & & & & & & \\
\hline 1499 & 4/20/2004 & 10:01:43 PM & 25.769 & 24.688 & 25.614 & 25.381 & 24.434 & 48.724 & 53.46 & 16.41 & 32.615 & $\begin{array}{ll}-0.992 \\
\end{array}$ & & 48.558 & 0.063 & 14.643 & & 217 & 36.3314 & & 11. & 40. & 2.804 & & & & & \\
\hline 1500 & 4/20/2004 & 10:02:43 PM & 25.776 & 24.664 & 25.595 & 25.408 & 24.4 & 48.585 & 0.027 & 16.442 & 32.529 & -1.009 & & 49.893 & 0.062 & 14.643 & & & 36.3481 & & $\bar{m}+2>$ & 40.6 & 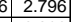 & & & & & \\
\hline 1501 & $4 / 2012004$ & 10:03:43 PM & 25.784 & 24.673 & 25.544 & & 24.4 & & 3.224 & & & -0.998 & & 46.325 & 0.063 & & & & 36.3647 & & 11. & & .806 & & & & & \\
\hline & 412012004 & $\begin{array}{ll}31 \mathrm{PM} \\
3 \mathrm{PM}\end{array}$ & $\begin{array}{r}25.79 \\
25792\end{array}$ & 24.668 & 25.555 & 25.537 & 24.595 & $\begin{array}{l}48.438 \\
48.397\end{array}$ & 2.977 & $\frac{16.409}{16324}$ & $\begin{array}{ll}32.297 \\
32326\end{array}$ & -0.995 & & 49.351 & 0.064 & $\frac{14.643}{14643} \mathrm{rar}$ & & 218 & & & & & & & & & & \\
\hline & $\begin{array}{l}4 / 201 / 2044 \\
4 / 20 / 2004 \\
\end{array}$ & 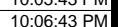 & $\frac{23.194}{25.785}$ & 24.0053 & 25.545 & 年3.0035 & $\frac{24.12}{247}$ & $\begin{aligned} 40.391 \\
48.27 \\
\end{aligned}$ & $\begin{array}{l}5.005 \\
52.844\end{array}$ & $\frac{10.324}{16.406}$ & $\begin{array}{l}32.320 \\
32.205 \\
\end{array}$ & $\begin{array}{l}-0.912 \\
-0.995 \\
\end{array}$ & & 47.632 & 0.004 & 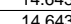 & & $218 \quad->$ & $\mid$ & 512 & 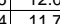 & 40 & 8774 & 0009 & 0009 & 0000 & 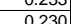 & \\
\hline & |01/2004 & $33 \mathrm{PM}$ & 25.781 & 24.655 & \begin{tabular}{|l|}
25.536 \\
25.54
\end{tabular} & 25.689 & $\frac{24.67}{24.62}$ & & 5 & & 32.377 & $=0.096$ & & & & & & & & & 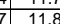 & & & & & & & \\
\hline & $4 / 20 / 2004$ & $\begin{array}{l}10: 08: 43 \text { PM } \\
10.03\end{array}$ & $\frac{25.789}{25.79}$ & 24.662 & 25.543 & 25.666 & $\frac{2.5}{24.5}$ & 48.494 & 53.062 & 16.485 & 32.36 & -0.937 & & $\frac{18.909}{48.909}$ & & $\frac{14.6}{14.6}$ & & $\frac{2186}{2186}$ & $\frac{36.4481}{3.641}$ & & 12 & $\frac{40}{40}$ & & & & & $\frac{0.229}{0.229}$ & \\
\hline & & & 25.786 & 24.639 & 25.545 & 25.623 & & 48.6 & 3.186 & 16.332 & 32. & -0.937 & & & & 14.643 & & & & & & & & & & 0.000 & & \\
\hline & & & 25.766 & & 25.515 & 25.523 & & 48.49 & & 16.225 & 32.49 & $\mid-0.937$ & & $49.2>>3$ & & & & & & & 12. & & & & & & & \\
\hline & $4 / 20 / 2004$ & 10:11:43 PM & 25.766 & 24.614 & 25.5 & 25.453 & $24.3>>3$ & & 182 & & & -0.94 & & & & 14. & & $218>-18$ & & & & & & & & & & \\
\hline & 0.0004 & 10:12:43 PM & 25.752 & 24.58 & \begin{tabular}{|l|l|}
25.492 \\
\end{tabular} & 25.374 & $24.8>>3$ & 48.465 & 53.103 & 16.243 & 32.453 & -0.957 & & 48.39 & 0.065 & 14.643 & & 2190 & \begin{tabular}{|l|l|}
36.5147 \\
\end{tabular} & 51.6 & $11 . .6$ & 40. & & & 0.010 & & 0.236 & \\
\hline & $4 / 20 / 2004$ & 10:13:43 PM & 25.747 & 24.56 & 25.477. & 25.354 & 24.2 & 48. & & 16.294 & 32.592 & $\begin{array}{ll}-0.902 \\
\end{array}$ & & & & & & 219 & 50.5 & 政 & 12. & $4 \mathrm{t}-2+3$ & & & & & & \\
\hline & (1/2004 & 10:14:43 PM & 25.765 & & 25.495 & 25.342 & 24.1 & 48. & 53.363 & & 32.849 & -0.972 & & 48.786 & 0.064 & & & & & & & & & & & & & \\
\hline & & PM & 25.764 & 24.5 & 25.484 & 25.266 & $24.0 .7>>$ & 48.536 & 22.996 & 16.218 & 32.674 & -0.98 & & (7) & 0.06 & & & & & & & & & & & & & \\
\hline & & $\begin{array}{l}10: 160.43 \mathrm{MM} \\
0.17 .43 \mathrm{M}\end{array}$ & 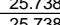 & 24.52 & 25.446] 250 & 25.1.155 & $\frac{24.1}{241}>-1$ & (48.525 & 53433 & & & & & & & & & & & & 年1.6 & 40 & & & & & & \\
\hline & & $10.1 .48 \mathrm{PP}$ & 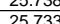 & 24.321 & 25.467 & 年. & & & $\frac{53.435}{5306}$ & & 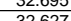 & - -0.934 & & & & & & $\frac{21}{210}$ & & & 11.428 & & & & & & & \\
\hline 51 & $4+21 / 2012004$ & 10:19:43 & 25.73 & 224.481 & 25.457 & $\frac{2.2531}{2531}$ & 24. & 48.0404 & $\frac{53.05}{53.45}$ & 16.296 & 32 & -0.937 & & & $\frac{0.0}{0.0}$ & $\frac{14 .}{14 .}$ & & $\frac{219}{219}$ & $\begin{array}{l}\frac{36.61}{36.63} \\
363\end{array}$ & 51 & $\frac{11.6}{12.3}$ & & & & & & 0.232 & \\
\hline & $4 / 20 / 2004$ & $10: 20: 43$ & 25.704 & 24.466 & 25.453 & 25.366 & & 48.841 & & & & -1.007 & & & & & & & & & & & & & & & & \\
\hline 151 & & & 25.722 & 24.484 & 25.396 & 25.424 & & 48.953 & & 299 & & -0.98 & & & & & & 21 & & 我 & 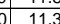 & & & & & & & \\
\hline & & 10.22 & 25703 & & & & & & & 16.319 & & & & 7. & & & & & & & 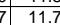 & & & & & & & \\
\hline & $4 / 20 / 200$ & $10: 23$ & 25.7 & 24.48 - & 25.374 & 25.542 & 24 & & & 16.24 & & $\frac{-1.027}{-1.027}$ & & & & & & & & & 11 & & & & & & & \\
\hline & 4 & 10:24: & 25.7 & 24.49 & 25.382 & 25.55 & 24.7 & & 53.398 & 16.214 & & -1.0 & & & 0.06 & & & 220 & & 51. & $12.4 \quad \mathrm{Y}$ & & & & & & & \\
\hline & 4/20/200 & 10:25:43 & 25.704 & 24.50 & 25.393 & 25.511 & 24. & 48.793 & 4.495 & & & -0.96 & & & & & & $2 \angle C$ & & & $11 .$. & & & & & & & \\
\hline & & 10:2 & 25.703 & 24.521. & 25.382 & 25.48 & & 48.6 & 53.178 & 16.292 & 32. & -0.954 & & & & & & 226 & & 51. & 11.5 & & & & & & 0.225 & \\
\hline & & $10: 27$ & 25.708 & $24.536 \mathrm{C}$ & 25.382 & 25.51 & $24.8 \mathrm{~g} \quad \mathrm{C}$ & 48.594 & 53.081 & 16.302 & 32.594 & -0.952 & & 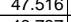 & 0.0 & 14.6 & & & & Nit & . 1 . & & & & & & & \\
\hline & & & $\frac{25.108}{270}$ & & 25.381 & & & 48.843 & & 16.228 & & $-0.98>0$ & & & & & & & & & & & & & & & & \\
\hline & 4 & & 年 & 24.375 & 25.3816 & 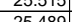 & $\frac{24.6}{24.5}>0$ & 48760 & $\frac{53.251}{5241}$ & $\frac{10.250}{16.329}$ & & - & & & 0.004 & $\frac{14.6}{1.6}>0$ & & & & $51.6-3$ & 1.0 & & & & & & & \\
\hline & 4 & $\frac{10.30 .43}{10.31 .43}$ & $\frac{2.019}{25698}$ & $\frac{24.501}{24601}$ & & $\frac{2.5}{25}>>$ & & 年 48.791 & & 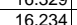 & & $\mid-0.092$ & & $47.13-3$ & & & & & & 52. & 116 & & & & & & & \\
\hline & $\mid \frac{4 / 20 / 2004}{4}$ & $10 \cdot 32 \cdot 43$ & $\frac{25.706}{25.706}$ & 24.624 & 25.365 & 25.398 & $\frac{24.7}{24.7}$ & 48.72 & & $\frac{16.276}{16.276}$ & & -0.937| & & & & & & $221+3$ & & $51->2>$ & 12 & & & & & & 0.224 & \\
\hline & & & & & & & & & & & & -0.937 & & & & & & & & & & & & & & & & \\
\hline & & & 25709 & $24,65 \mathrm{~F} \quad \mathrm{C}$ & 25373 & 25.346 & $2477>>$ & 48.644 & 52050 & 16.514 & & -0.946 & & 48 & & & & & & & & & & & & & & \\
\hline & $4 / 20 / 200$ & 10:35:43 & 25.696 & 24.654 & 25.355 & 25.353 & 24.7 & \begin{tabular}{|c|c|}
48.606 \\
\end{tabular} & 53.141 & 16.40 & 32.519 & -0.957 & & 48.29 & & $14.6>>>$ & & 2213.9 & 36.8 & $517.7-3$ & 118 & 4 & & & & & & \\
\hline & & & 25.7 & 24.678 & 25.364 & & 24. & 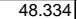 & & 16.3 & 32.36 & 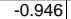 & & & & 14. & & & & & 1. & & & & & & & \\
\hline & & $10: 37$ & & 24.6 & 25.307 & 25.475 & & $48.6 \mathrm{r}-\mathrm{s}$ & 53.199 & & & -0.9 & & & & 14. & & & & & $11.6 \mathrm{11.6}$ & & & & & & & \\
\hline & & & 25. & 24. & 5.3 & 25.5 & & 48.438 & & 16.346 & & -0.966 & & & & & & & & $51.6-6$ & 12.0 & & & & & & & \\
\hline & & & 25.695 & 24.719 & 25.279 & 25.648 & 25. & 48.6 & 53.305 & & & -0.94 & & & & $14.6 \mathrm{r}-\mathrm{s}$ & & & & & 11.6 & & & & & & & \\
\hline 年 & $4 / 2012004$ & 10:40:43 PM & 25.695 & 24. 2444 & 25.294 & $25 . / 278$ & 25 & 48.145 & 53.387 & 16.387 & 32.61 & -0.937 & & 50.096 & 0.0611 & 14.643 & & 2218 & $36.8>30$. & 51.04 & $\begin{array}{ll} & 12.3 \\
\end{array}$ & 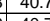 & 2.001 & 0.009 & 0.009 & 0.000 & 0.222 & . \\
\hline$\frac{13540}{1540}$ & $\begin{array}{l}/ 2012004 \\
4 / 20 / 2004 \\
\end{array}$ & $\frac{10.4: 42 \mathrm{SPM}}{10: 42: 43 \mathrm{PM}}$ & $\frac{23.101}{25.712}$ & \begin{tabular}{|c|}
24.769 \\
24.79
\end{tabular} & \begin{tabular}{|l|}
25.283 \\
25.296 \\
\end{tabular} & $\begin{array}{l}25.717 \\
25.809 \\
\end{array}$ & \begin{tabular}{|l|}
25.306 \\
25.287 \\
\end{tabular} & $\begin{array}{l}48.68 \\
48.57 \\
\end{array}$ & \begin{tabular}{|l|}
53.414 \\
53.093 \\
\end{tabular} & $\frac{\mid 1.309}{16.31}$ & $\frac{32.695}{32.644}$ & $\begin{array}{l}-0.957 \\
-0.937 \\
\end{array}$ & & 499.687 & $\frac{0.062}{0.06}$ & $\frac{1.4045}{14.643}$ & 0.003 & & \begin{tabular}{|l|l|}
37.0147 \\
\end{tabular} & 51.6 & \begin{tabular}{l|l|}
6 & 12.2 \\
\end{tabular} & 40. & 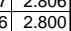 & 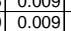 & \begin{tabular}{|l|}
0.009 \\
0.009 \\
\end{tabular} & 0.000 & $\begin{array}{l}0.225 \\
0.218\end{array}$ & 0.1 \\
\hline
\end{tabular}


WSRC-TR-2005-00105, REVISION 0

SRNL-RPP-2005-00012, REVISION 0

RUN \# 2.03A AND B; FIRST AND SECOND HALF OF SLURRY DEWATERING - CONT.

\begin{tabular}{|c|c|c|c|c|c|c|c|c|c|c|c|c|c|c|c|c|c|c|c|c|c|c|c|c|c|c|c|c|c|}
\hline & A & $\mathrm{B}$ & $D$ & $E$ & $F$ & G & $\mathrm{H}$ & $\mathrm{J}$ & $\mathrm{K}$ & $\begin{array}{ll}\mathrm{L} \\
\end{array}$ & $M$ & $\mathrm{~N}$ & $\mathrm{O}$ & $\mathrm{Q}$ & $\mathrm{R}$ & $\mathrm{s}$ & $\mathrm{T}$ & $\mathrm{v}$ & w & $\mathrm{x}$ & $\begin{array}{r}Y \\
\end{array}$ & 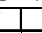 & $z$ & AA & $A B$ & $A C$ & $\mathrm{AD}$ & $\mathrm{AE}$ & AF $\mathbb{A}$ \\
\hline & 4/20/2004 & 10:43:43 PM & 25.722 & & $\frac{25.312}{25202}$ & $\begin{array}{ll}25.82 \\
2571\end{array}$ & $\begin{array}{l}25.207 \\
25.97 \\
250\end{array}$ & 48.72 & 53.282 & 16.279 & 32.76 & -0.931 & & 47.538 & 0.058 & \begin{tabular}{|l|l|}
8 & 14.643 \\
2
\end{tabular} & 0.004 & & 37.0314 & 51. & & & & 2.80 & 0.009 & 0.009 & 0.000 & 0.210 & \\
\hline$\frac{1542}{1543}$ & & & & & & & & & 53.421 & & & & & & & & & & & & & & & & & & & & \\
\hline$\frac{1543}{1544}$ & $\begin{array}{l}4 / 2012004 \\
/ 20 / 2004\end{array}$ & $\begin{array}{l}\text { 10:45:43 PM } \\
1046: 43 \text { PM }\end{array}$ & $\frac{25.121}{25.72}$ & $\begin{array}{r}24.86 \\
24.873\end{array}$ & $\frac{25.275}{25.274}$ & \begin{tabular}{|l|}
$\mid 25.664$ \\
25.602 \\
\end{tabular} & $\begin{array}{l}25.046 \\
24.955 \\
\end{array}$ & $\begin{array}{l}48.766 \\
48.629\end{array}$ & \begin{tabular}{|r|}
53.301 \\
53.28
\end{tabular} & $\frac{16.173}{16.248}$ & $\begin{array}{l}32.923 \\
32.668 \\
\end{array}$ & $\begin{array}{l}-0.882 \\
-0.934\end{array}$ & $\begin{array}{l}-6.937 \\
-6.966 \\
\end{array}$ & $\begin{array}{l}49.009 \\
48.462 \\
\end{array}$ & $\frac{0.062}{0.062}$ & $\begin{array}{l}14.643 \\
14.643 \\
\end{array}$ & $\begin{array}{l}0.003 \\
0.003 \\
\end{array}$ & $\frac{2 \angle 23.9}{2224.9}$ & 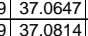 & \begin{tabular}{|l|l}
51.8 \\
51.8
\end{tabular} & 11 & & $\frac{40.8}{40.6}$ & 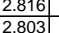 & 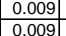 & \begin{tabular}{|l|}
0.0099 \\
0.009 \\
\end{tabular} & $\frac{0.000}{0.000}$ & 0.225 & $\begin{array}{l}0.19 \\
0.19\end{array}$ \\
\hline & $4 / 20 / 2004$ & 10:47:43 PM & 25.722 & 24.861 & 25.277 & 25.54 & 24.838 & 48.737 & 53.358 & 16.253 & 32.83 & -0.882 & & 47.401 & 0.064 & 14.643 & 0.003 & 2225.9 & \begin{tabular}{|l|l|}
9 & 37.0981 \\
\end{tabular} & & & & & & 0.010 & \begin{tabular}{|l|}
0.009 \\
\end{tabular} & 0.000 & 0.232 & \\
\hline & & & & & 25.245 & & & & & & & -0.888 & & & & 14.643 & & & & & & & & & & & & & \\
\hline & $\sqrt{12004}$ & $43 \mathrm{PM}$ & 25.71 & & 25.272 & $\begin{array}{ll}25.48 \\
\end{array}$ & & 48 & $\begin{array}{l}3.344 \\
\end{array}$ & 16.212 & & -0.885 & & & 0.061 & 14.643 & & & & & & & & & & & & & \\
\hline & & & 25.711 & 24.895 & 25.275 & 25.428 & & & .205 & 16.23 & & 0.882 & & & 0.062 & 14.643 & & & 7.1481 & & & & & & & & & & \\
\hline & 4120122004 & $20.50 .43 \mathrm{PM}$ & 256906 & 24.897 & (25.251 & 25.345 & $\frac{24.1}{2.7}$ & 48.961 & 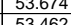 & 10.3606 & 32830 & $=0.8828$ & & & 0.06 & 14.643 & & 2030 & 371814 & & & & & & & & & & \\
\hline$\frac{1050}{1551}$ & $\begin{array}{l}\frac{4 / 201<004}{4 / 20 / 2004} \\
\end{array}$ & $\begin{array}{l}10.52 .43 \mathrm{M} \\
10: 53: 43 \mathrm{PM}\end{array}$ & $\begin{array}{l}25.096 \\
25.699\end{array}$ & (24.063 & $\mid \begin{array}{l}25.253 \\
2537\end{array}$ & 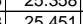 & 24.100 & $\begin{array}{l}48.809 \\
48.677\end{array}$ & $\begin{array}{ll}53.404 \\
55493\end{array}$ & $\frac{10.281}{16.204}$ & $\begin{array}{ll}32.039 \\
3274\end{array}$ & $\begin{array}{l}-0.0035 \\
-0885\end{array}$ & & (47.386 & 0.001 & $\frac{14.045}{14643}$ & 0.003 & $\frac{2230.9}{22319}$ & 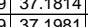 & 52 & $\frac{11}{11}>>$ & & & $\frac{2.017}{2807}$ & 0.009 & 0.009 & 0.000 & & 0.19 \\
\hline & $4 / 20 / 2004$ & $10: 54: 43$ PM & & $\frac{24.050}{24.876}$ & $\frac{25.240}{25.242}$ & $\frac{20.401}{25.44}$ & $\begin{array}{ll}24.005 \\
24.988\end{array}$ & 48.826 & 53.4526 & $\frac{10.204}{16.364}$ & 32.765 & $\frac{-.000}{-0.879}$ & & $\begin{array}{l}47.500 \\
45.951 \\
\end{array}$ & 0.062 & $\frac{14.045}{14.643}$ & 0.005 & & & & & & & & & 0.009 & & & \\
\hline & & & & & & & & & & & & & & & & & & & & & & & & & & & & & \\
\hline & $4 / 20 / 2004$ & 10:56:43 PM & 25.681 & 24.905 & 25.2 & 25.579 & 25.126 & 48.708 & $\frac{53.448}{53}$ & 16.377 & $\frac{32.611}{32.611}$ & -0.818 & & $\frac{7.038}{7038}$ & 0.06 & 14.643 & & & 7.2481 & & & & & $80:$ & 009 & 0.09 & & 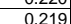 & \\
\hline & & & & & 25.186 & 25.645 & & & & 16.18 & & & & 47.110 & 0.061 & & & & & & & & & & & & & & \\
\hline & 4/20/2004 & 10:58:43 PM & 25.699 & 24.938 & 25.228 & 25.702 & 25.369 & 48. & 53.211 & 16.221 & 32.607 & -0.815 & & 47.928 & 0.061 & $\begin{array}{l}14.643 \\
\end{array}$ & & 2236.6 & 37.2814 & & & & 40.6 & 2.797 & 0.009 & 0.009 & 0.000 & & \\
\hline & 4/20/2004 & 10:59:43 PM & 25.67 & 24.929 & 25.174 & 25.722 & 25.285 & 48.637 & 53.331 & 16.118 & 32.793 & -0.839 & & 48.216 & 0.062 & 14.643 & 0.003 & 2237.9 & 937.2981 & $51 .$. & 11 & & 40.7 & 2.807 & 0.009 & 0.009 & 0.000 & 0.226 & \\
\hline & 4/20/2004 & 11:00:43 PM & 25.687 & 24.961 & 25.211 & 25.77 & $25.28 \mathrm{P}$ & 48.357 & 52.994 & 16.1499 & 32.594 & -0.804 & & 48.705 & 0.062 & 14.643 & 0.003 & 2238.9 & 937.31 & 51.5 & & & 40.5 & & & 0.009 & 0.000 & & \\
\hline & $4 / 220 / 2004$ & 11:01:43 PM & 25.683 & 24.977 & 25.212 & 25.7711 & 25.253 & 48.826 & 53.632 & $16.261 \mid$ & 32.886 & -0.815 & & 48.291 & 0.061 & 14.643 & & 2239.6 & 37.3314 & & & & & 2.81 & 0.009 & 0.009 & 0.000 & & \\
\hline & $4 / 22012004$ & $11.02 .43 \mathrm{PM}$ & & & & & & & & & & & & & & & & & & & & & & & & & & & \\
\hline & $4 / 2012044$ & $\begin{array}{l}11: 03: 43 \mathrm{PM} \\
11 \cdot 04.43 \mathrm{PM}\end{array}$ & $\frac{25.674}{25.683}$ & 24.98 & $\frac{25.183}{25.212}$ & 25.627 & $\frac{25.059}{25043}$ & 48.697 & $\frac{53.595}{53.288}$ & $\frac{16.309}{16.302}$ & 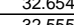 & $\begin{array}{l}-0.821 \\
-0.815\end{array}$ & & (48.531 & 0.0612 & 14.643 & & $\frac{2241}{2212}$ & 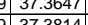 & & & & & & - & 0.009 & & & \\
\hline & $4 / 20 / 2004$ & $11: 05: 43 \mathrm{PM}$ & 25.677 & 25.001 & 25.201 & 25.544 & 24.947 & 48.513 & 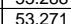 & $\frac{10.004}{16.111}$ & 32.693 & -0.784 & -6.902 & 4 & 0.061 & $\frac{14.045}{14.643}$ & 0.003 & 22439 & \begin{tabular}{|l|l|}
9 & 37.3981 \\
\end{tabular} & $\frac{01.6}{51.8}$ & & & & & $\mid \begin{array}{ll}\mid 0.009 \\
0.09\end{array}$ & 0.009 & 0.000 & 0.223 & 0 \\
\hline & $4 / 20 / 2004$ & 11:06:43 PM & 25.676 & 25.005 & 25.2 & 25.523 & 24.871 & 48.814 & \begin{tabular}{|l|l|}
53.609 \\
\end{tabular} & 16.311 & 32.869 & -0.778 & & 48.212 & 0.062 & 14.643 & & 2244.9 & $\begin{array}{l}937.4147 \\
\end{array}$ & $52.7 \quad>$ & 11 & & 40.8 & & 0.009 & 0.009 & & & 0. \\
\hline & 4/20/2004 & 11:07:43 PM & 25.679 & $25.018 \quad>\quad>$ & 25.213 & 25.481. & 24.804 & 48.76 & 53.431 & 16.319 & 32.748 & - -0.792 & & 48.07 & 0.06 & 14.643 & 0.003 & 2245.9 & 37.4314 & & & & 40.8 & 2.810 & 0.009 & 0.009 & 0.000 & 0.218 & \\
\hline & $4 / 2012004$ & 11:08:43 PM & 25.672 & 25.011 & 25.191 & 25.4594 & 24.84 & 48.278 & 35.016 & 16.252 & 32.34 & -0.772 & & 年7.23 & 0.063 & 14.643 & .003 & 2246. & 37.4481 & & & & & & 0.009 & 0.009 & 0.000 & 0.232 & \\
\hline & 4120122004 & $\begin{array}{l}11.094 .43 \mathrm{MM} \\
1.1032 \mathrm{M}\end{array}$ & 25.665 & 25.0044 & 25.204 & 25.387 & 24.16 & 48.86. & 年.5/4 320 & $\frac{16.272}{1.215} \mathrm{P}$ & 32.921 & - 0.818 & & 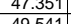 & 0.06 & 14.643 & & $2018-19$ & 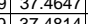 & & & & t.9. & & .009 & & & & \\
\hline$\frac{1506}{156 !}$ & $\begin{array}{l}4 / 201 / 2004 \\
4 / 20 / 2004\end{array}$ & $\begin{array}{l}11: 11: 43 \text { PM } \\
11: 11: 43 \text { PM }\end{array}$ & $\begin{array}{l}\frac{25.662}{25.644} \\
\end{array}$ & $\frac{25.001}{24.968}$ & 年5.226 & 年5.3064 & 24.712 & $\begin{array}{ll}48.471 \\
48.764\end{array}$ & $\begin{array}{l}53.302 \\
55.499\end{array}$ & (16.345 & $\begin{array}{ll}32.406 \\
3.208\end{array}$ & 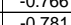 & & 4.54 .13 & 0.0025 & 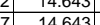 & 0.0003 & 2248.9 & 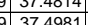 & 55 & $\frac{12}{11}$ & & 40 & 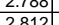 & 0.009 & & - & 0.221 & \\
\hline 1570 & $4 / 20 / 2004$ & $11: 12: 43 \mathrm{PM}$ & 25.641 & 24.97 & 25.205 & 25.338 & 24.821 & $\begin{array}{l}40.104 \\
48.573\end{array}$ & \begin{tabular}{|l|}
53.4956 \\
53.396
\end{tabular} & $\begin{array}{ll}10.549 \\
16.217\end{array}$ & $\begin{array}{l}32.000 \\
32.736 \\
\end{array}$ & $\begin{array}{l}-0.101 \\
-0.772 \\
\end{array}$ & & $\begin{array}{l}4.03 \\
46.247 \\
\end{array}$ & 0.061 & $\begin{array}{l}14.045 \\
14.643 \\
\end{array}$ & 0.003 & & \begin{tabular}{|l|l|}
9 & 37.5147 \\
\end{tabular} & 51. & $\frac{11}{11}$ & & & $\frac{2.014}{2.803}$ & \begin{tabular}{|l|}
.0 .009 \\
0.009
\end{tabular} & 0.009 & 0.000 & 0.222 & 0.18 \\
\hline & $4 / 20 / 2004$ & 11:13:43 PM & 25.638 & 24.957 & & 25.415 & 24.858 & 48.753 & & 16.248 & 32.906 & -0.772 & & 46.506 & 0.061 & & & 2251.9 & $\begin{array}{l}937.5314 \\
9\end{array}$ & & & & & & & & & & \\
\hline & $4 / 20 / 2004$ & 11:14:43 PM & 25.647 & 24.976 & 25.221 & 25.464 & 25.002 & $\begin{array}{ll}48.847 \\
4\end{array}$ & 53.636 & 16.378 & 32.779 & -0.772 & & 48.037 & 0.061 & 14.643 & 0.003 & 2252.9 & 37.5481 & 52.8 & & & 40.8 & 2.814 & $\begin{array}{ll}0.009 \\
\end{array}$ & 0.009 & 0.000 & 0.221 & \\
\hline & $\begin{array}{l}4 / 20 / 2004 \\
4 / 20 / 2004\end{array}$ & 11:15:43 PM & 25.637 & $\frac{24.966}{24.987}$ & $\frac{25.206}{25.177}$ & 25.544 & 25.177 & $\begin{array}{ll}48.648 \\
48.488 \\
\end{array}$ & $\begin{array}{l}53.466 \\
552206\end{array}$ & $\frac{16.325}{16346}$ & $\begin{array}{l}32.578 \\
32.461\end{array}$ & -0.772 & & 48.644 & $\frac{0.061}{0.06}$ & $\frac{14.643}{14643}$ & & 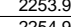 & 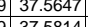 & 51 & $\frac{11}{2}$ & & . & 2.800 & 0.009 & 0.009 & 0.000 & & 0.19 \\
\hline$\frac{1575}{1575}$ & $4 / 2012004$ & & 25.043 & 24977 & 25.152 & 25636 & & $\begin{array}{lll}48.48007 & 4857 \\
48\end{array}$ & \begin{tabular}{|l|}
53.280 \\
53.373
\end{tabular} & $\frac{10.340}{16.412}$ & 32.401 & $\begin{array}{l}-0.169 \\
-0.769\end{array}$ & & $\frac{49.228}{47.522}$ & 0.060 & 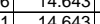 & & 2025.4 & 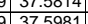 & $\frac{51}{51}$ & $\frac{12}{11}$ & & & & & & & & \\
\hline 1576 & 0 & $11: 18: 43 \mathrm{PM}$ & 25.65 & 24.971 & (25.159 & 25.0007 & 25.2 & $\begin{array}{l}40.006 \\
48.496\end{array}$ & 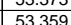 & $\frac{10.414}{16.393}$ & $\begin{array}{r}32.424 \\
32.42\end{array}$ & $\begin{array}{l}-0.109 \\
-0.717\end{array}$ & & 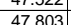 & $\frac{0.001}{0.06}$ & 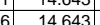 & & 年 & 9 376147 & 热 & $\frac{\frac{11}{11}}{11}$ & & & 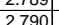 & & 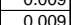 & & & \\
\hline $150 / 4$ & 0/2004 & 11:19:43 PM & 25.641 & 25.005 & 25.16 & 25.759 & $25.3 \times 3$ & 48.6 & 599 & & & & & & & & & & & & & & & & & & & & \\
\hline & :0/2004 & 11:20:43 PM & 25.643 & 25.022 & 25.172 & 25.791 & 25.2 & 48.731 & \begin{tabular}{|l|l|}
53.593 \\
\end{tabular} & 16.32 & 32.73 & -0.772 & & 48.387 & 0.059 & 14.643 & & 22589 & 37.6481 & 52 & 11 & & $\frac{74}{46}-2$ & 2.808 & \begin{tabular}{|l|l|l|l|}
0.009 \\
\end{tabular} & 0.009 & 0.000 & 0.215 & \\
\hline 157 & $4 / 20 / 2004$ & 11:21:43 PM & 25.644 & 25.0 & 25.168 & 25.771 & 25.0 & & 53.209 & 16.39 & & -0.769 & & 47.449 & 0.4 & $\begin{array}{ll}14.643 \\
\end{array}$ & & 2259 & & & & & & & & & & & \\
\hline & & 11:22:43 PM & & 25.03 & & & & & & & 32.769 & & & 47.424 & & & & 22609 & \begin{tabular}{l|l|}
9 & 37.6814 \\
\end{tabular} & & & & 407 & 2808 & 0.009 & 0.009 & & 219 & \\
\hline & 0/2004 & 11:23:43 PM & 25.643 & 25.04 & 25.167 & 25.621 & $24.8 \mathrm{r}$ & $\mid 44$ & & 16.358 & & -0.705 & & 49.714 & 0.061 & 14.643 & & 2261. & & 51 & & & & & & & & & \\
\hline & 4/20/2004 & 11:24:43 PM & 25.642 & 25.02 & 25.156 & 25.534 & 24.78 & & 33.499 & 16.369 & 32.4 & -0.705 & & 48.99 & 0.058 & 14.643 & & 226 & 37.7147 & 52 & 12 & & & & & 0.009 & & 212 & \\
\hline 10 & 0/2004 & 11:25:43 & 25.656 & 25.04 & 25.18 & 25.478 & 24.8 & 48.745 & 53.483 & 16.335 & 32. & -0.772 & & 48.717 & 0.064 & 14.6 & & 2263.9 & 9 9 37.7 & Ji & $I+$ & & & & 0.010 & 0.009 & 0.000 & 0.233 & \\
\hline & & 11:26:43 & $\frac{25.65}{25.61}$ & $25.0 ;$ & 25.169 & 25.427 & 24 & 48.961 & 53.872 & 16.405 & 32 & -0.708 & & & $0.055>>5$ & & & & & & & & & & & & & & \\
\hline & & & & & & 25.363 & & & & 16.34 & & -0.1606 & & & & & & 226 & & & 12 & & & & & & & & \\
\hline$\frac{1006}{158}$ & (1) & $\begin{array}{l}11.28 .45 \mathrm{MM} \\
11: 20.43 \mathrm{MM}\end{array}$ & $\begin{array}{l}25.646 \\
25635\end{array}$ & & 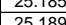 & & 24. & & & $\frac{1.3 .32}{16398}$ & & & & 46531 & 0.002 & 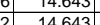 & & & & 52 & $\frac{11}{11}$ & & & & & & & & \\
\hline $158 \varepsilon$ & $4 / 20 / 2004$ & $11: 30: 43 \mathrm{PM}$ & 25.625 & 24.999 & 25174 & 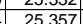 & & $\begin{array}{l}4.0006 \\
48.596\end{array}$ & \begin{tabular}{|l|}
53.421 \\
53.404 \\
\end{tabular} & $\begin{array}{l}10.050 \\
16.288 \\
\end{array}$ & & $\begin{array}{l}-.172 \\
-0.778\end{array}$ & & $\begin{array}{l}40.001 \\
47.399 \\
\end{array}$ & $\begin{array}{l}0.002 \\
0.061\end{array}$ & & & 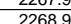 & 37801 & & $1 \pm>1 \pm$ & & & & 0.0009 & 0.000 & & & \\
\hline & $4 / 20 / 2004$ & 11.31.43 PM & 25.636 & & 25.19 & 25.428 & 25.0 & 48.915 & \begin{tabular}{|l|l|}
53.727 \\
\end{tabular} & 10.296 & & -0.807 & & (1.005 & 0.06 & & & 2269.9 & & 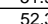 & $\pm x$ & & & & & 0.000 & & & \\
\hline & & & & $\overline{25.01}$ & 25.201 & \begin{tabular}{|l|l|}
25.54 \\
\end{tabular} & & 48.614 & & & & & & & & & & & & & & & & & & & & & \\
\hline & $4 / 2$ & 11:3: & 25.628 & 25.01 & 25.202 & 25.575 & & 48.7 & 53.421 & 16.4 & & -0.769 & & & 0.062 & 14.643 & & & & 51 & & & & & & & & & \\
\hline & $4 / 20 / 2004$ & 11:34:43 PM & 25.624 & 25.00 & 25.188 & 25.636 & 25. & 48.415 & \begin{tabular}{|l|l|}
53.104 \\
\end{tabular} & 16.346 & 32.3 & -0.7 & & 45. & $\frac{0.06}{0.06}$ & & & 2272.9 & \begin{tabular}{l|l|}
937.8814 \\
\end{tabular} & 51. & 11 & 1.3 .3 & 4 & 2.784 & & & & & \\
\hline & 0/2004 & $11: 35$ & 25.63 & 25.014 & 25.209 & 25.678 & 25 & 48.312 & \begin{tabular}{|l|l|l|l|l|}
53.168 \\
\end{tabular} & 16.287 & $32.2 \mathrm{C} \quad \mathrm{C}$ & -0.8 & & & 0.06 & 14.6 & & 227 & & 51 & 11 & $1.8 \mathrm{~g}-\mathrm{p}$ & & & & & & & \\
\hline 159 & 4/20/2004 & 11:36:43 PM & 25.6. & & 25.227 & 25.721 & & 48.372 & 53.2 & 16.497 & & $\begin{array}{c}-0.708 \\
\end{array}$ & & & 0.061 & & & & & 51 & 11 & 1. & & & & & & & \\
\hline $15 x$ & (1/2004 & & 25.634 & 25.038 & 25.208 & & & 48.5 & 53.475 & 16.38 & & -0.653 & & & & & & $2 \angle 1$ & & 34 & 11 & & & & & & & & \\
\hline & & & 25. & 25.066 & 25.251 & 25.729 & & 48.5 & 53.454 & & & & & & & & & 22 & & 52. & 11 & & & & & & & & \\
\hline & & $11: 39$ & 25.6 & $25.65>0$ & & & & 48. & 53.358 & 16. & & -0.7 & & & & & & 227.9 & & & 11 & & & & & & & 224 & \\
\hline & & & $\frac{25.641}{25644}$ & & & & & & & & & & & & & & & & & & & & & & & & & & \\
\hline & $4 / 202004$ & & 25.044 & 25071 & & 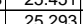 & & & & & & -0.5 & & & 0.055 & & & & & & $\frac{1}{11}$ & t. & & & & & & 16 & \\
\hline 160 & $4 / 2012004$ & $\frac{11.43}{1114:}$ & $\frac{25.043}{25.643}$ & 25.067 & $\frac{2.53}{25.32}$ & $\frac{2.255}{25.155}$ & $\frac{2.4 .5}{24.5}>0$ & 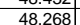 & \begin{tabular}{|l|l|}
53.319 \\
5319
\end{tabular} & $\frac{1.0 .046}{16.24}$ & & $\frac{-0.581}{-0.581}$ & & 48.229 & & & & 228 & & & & & & & & & & & \\
\hline & & & 25.645 & & & & & & & 16356 & & & & & & & & & & & & & & & & & & & \\
\hline & & $11: 45$ & 25.626 & 25.02 & & 24.912 & 24 & 48.428 & 53.611 & 16.355 & & -0.55 & & $48.6>-3$ & & 14. & & & & & & 1.9 & & & & & & & \\
\hline & & 11:46:43 & 25 . & 25.016 & & & & 48.5 & 53.659 & & & & & & & & & & & & & & & 28,50 & & & & & \\
\hline 160 & $4 / 20 / 2004$ & 11:44 & 25. & 25 & 25 & & 24. & 50.875 & & 16.24 & & $-1.22 \mathrm{C}>\mathrm{C}$ & & $\overline{46.94}>2$ & & 14.6. & & $2 ? 8 \mathrm{P}$ & 380 & $52+>$ & 11 & 1. & & & & & & 07 & \\
\hline & 4/20/2004 & 11:48 & & 25 & & & 24 & 50. & 53.466 & & & & & 47. & & & & & & & & 1. & & & & & & 50 & \\
\hline 1607 & 4/20/2004 & 11:49:01 & 25.626 & 24.99 & 25.245 & 25.308 & 24.916 & $48.025 \mid$ & 53.572 & 16.285 & 32.046 & -0.112 & & 47.3. & 0.037 & 14.643 & & 2287.2 & $\begin{array}{ll}2 & 38.1 \\
\end{array}$ & 52. & 11 & 1.6 & & 2.7 & 0.006 & $0 . c$ & & 0.137 & 0.12 \\
\hline $1 \frac{1608}{1160}$ & $4 / 2012004$ & 11:50:01 PM & 25.617 & 24.976 & 25.251 & 25.394 & 25.077 & 49.029 & \begin{tabular}{|l|l|}
53.684 \\
\end{tabular} & 16.331 & 32.988 & -0.818 & & 47.515 & 0.17 & 14.643 & 0.0 & 2288 & $2|38.1364|$ & 52.2 & $\begin{array}{l}211 \\
2\end{array}$ & 1.6 & & 2.827 & 0.025 & 0.025 & 0.001 & 0.614 & 0.5 \\
\hline & & $\frac{11.51 .01 \mathrm{PM}}{11: 52: 01 \mathrm{PM}}$ & & & & & & & & & & -1.221 & & & & $\frac{14.045}{14.643}$ & & & & & & & & & & & & 0.201 & 0.1 \\
\hline
\end{tabular}


WSRC-TR-2005-00105, REVISION 0

SRNL-RPP-2005-00012, REVISION 0

RUN \# 2.03A AND B; FIRST AND SECOND HALF OF SLURRY DEWATERING - CONT.

\begin{tabular}{|c|c|c|c|c|c|c|c|c|c|c|c|c|c|c|c|c|c|c|c|c|c|c|c|c|c|c|c|c|}
\hline & A & $\mathrm{B}$ & D & $E$ & $\mathrm{~F}$ & G & $\mathrm{H}$ & $\mathrm{J}$ & $\mathrm{K}$ & $\begin{array}{ll}\mathrm{L} \\
\end{array}$ & $M$ & $\mathrm{~N}$ & $\mathrm{O}$ & $Q$ & $\mathrm{R}$ & $\mathrm{s}$ & $\mathrm{T}$ & $\mathrm{v}$ & w & $x$ & $\mathrm{Y}$ & $z$ & AA & $A B$ & $A C$ & $\mathrm{AD}$ & $\mathrm{AE}$ & AF $\mathbb{A}$ \\
\hline & $4 / 20 / 2004$ & 11:53:01 PM & 25.62 & $24.97 \mathrm{9}$ & $\frac{25.164}{25.151}$ & $\begin{array}{l}25.657 \\
257\end{array}$ & 25.23 & 50.041 & $\begin{array}{r}53.45 \\
53.57\end{array}$ & 16.276 & 34.091 & -1.224 & \begin{tabular}{|l|l|}
-7.666 \\
7719
\end{tabular} & 47.294 & 0.064 & 14.643 & 0.003 & 2291.2 & $\frac{38.1864}{382021}$ & 52. & & 42. & 2.900 & 0.010 & 0.009 & 0.000 & 0.226 & \\
\hline$\frac{1612}{161}$ & & & & & & & & 50.157 & 53.557 & $\begin{array}{ll}16.274 \\
1.067 \\
\end{array}$ & 34.119 & -1.221 & \begin{tabular}{rl|l}
-7.718 \\
77
\end{tabular} & & 0.062 & 14.643 & 0.003 & & & 52.1 & & & & & 0.009 & 0.000 & 0.218 & 0.19 \\
\hline$\frac{\frac{1613}{1614}}{1614}$ & \begin{tabular}{|l}
$4 / 2012004$ \\
$/ 20 / 2004$
\end{tabular} & $\begin{array}{l}\text { 11:55:01 PM } \\
11: 5: 01 \text { PM }\end{array}$ & $\frac{25.635}{25.632}$ & $\begin{array}{l}\frac{25.004}{25.001} \\
\end{array}$ & $\begin{array}{l}25.149 \\
25.141 \\
\end{array}$ & $\begin{array}{l}25.738 \\
25.675 \\
\end{array}$ & $\begin{array}{r}25.18 \\
25.102 \\
\end{array}$ & $\begin{array}{l}50.452 \\
50.278 \\
\end{array}$ & \begin{tabular}{|l|}
53.844 \\
53.543 \\
\end{tabular} & $\begin{array}{l}16.067 \\
16.325 \\
\end{array}$ & $\begin{array}{r}34.636 \\
34.29\end{array}$ & \begin{tabular}{|l|l|}
-1.218 \\
-1.221 \\
\end{tabular} & $\begin{array}{r}-7.7 \\
-7.732 \\
\end{array}$ & $\begin{array}{l}46.352 \\
47.62\end{array}$ & $\begin{array}{l}0.06 \\
0.06\end{array}$ & $\frac{14.643}{14.643}$ & 0.003 & $\frac{2293.2}{2294.2}$ & \begin{tabular}{|l|}
38.2197 \\
38.2364 \\
\end{tabular} & \begin{tabular}{|l|}
52. \\
52.2 \\
\end{tabular} & 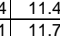 & $\begin{array}{lll} & 4 & 42.5 \\
7 & 42.3 & \end{array}$ & 2.915 & $\mid$\begin{tabular}{|c|}
0.009 \\
0.009
\end{tabular} & $\begin{array}{l}0.009 \\
0.009\end{array}$ & $\frac{0.000}{0.000}$ & 0.209 & 0.18 \\
\hline & $4 / 20 / 2004$ & 11:57:01 PM & 25.644 & 24.998 & 25.168 & 25.612 & 25.049 & 49.201 & 52.484 & 17.265 & 32.233 & -1.218 & -7.738 & 50.828 & 0.059 & 14.643 & 0.003 & 2295.2 & 38.2531 & & & 40.7 & \begin{tabular}{|l|}
2.807 \\
\end{tabular} & \begin{tabular}{|l|}
0.009 \\
\end{tabular} & 0.009 & & & \\
\hline & $4 / 20 / 2004$ & & & & & & & & & 17.443 & & -1.224 & & & & 14.643 & & & 38.2697 & & & & & & & & & \\
\hline & $4 / 20 / 2004$ & & & 25.0 & 25.185 & 25.484 & 24.907 & 49.25 & 52.59 & 17.207 & & & $\begin{array}{l}-7.747 \\
\end{array}$ & & 0.058 & 14.643 & & 229 & 38.2864 & & & & $\overline{814}$ & & 0.005 & & & \\
\hline & $4 / 21 / 2004$ & $01 \mathrm{AM}$ & 25.636 & 25.0 & 25.195 & 25.383 & & 49.342 & 52.66 & 17.162 & $32.46 \mathrm{C}$ & -1.218 & -7.744 & & 0.06 & 14.643 & & & 38.3031 & & & & & & & & & \\
\hline$\frac{1050}{1620}$ & $\begin{array}{l}4 / 21 / 2004 \\
4 / 21204\end{array}$ & 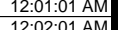 & $\frac{20.024}{25618}$ & 24.998 & $\frac{25.193}{25.212}$ & 25.3606 & 24.709 & 18.689 & 51078 & 17.113 & 31.815 & -1.2218 & 7740 & & 0.058 & 14.643 & & 2300 & (383264 & & 12 & 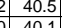 & 7763 & 0000 & & & & \\
\hline 162 & $\mid \begin{array}{l}\mid 4 / 21 / 2004 \\
4 / 21 / 2004\end{array}$ & $\begin{array}{l}12.02 .01 \mathrm{AM} \\
12: 03: 01 \mathrm{AM}\end{array}$ & $\begin{array}{l}25.018 \\
25.617\end{array}$ & $\begin{array}{l}\frac{24.994}{24.991} \\
\end{array}$ & \begin{tabular}{|l|l|l|l}
25.214 \\
25.226
\end{tabular} & $\begin{array}{l}25.305 \\
25.279\end{array}$ & $\begin{array}{l}24.130 \\
24.687 \\
\end{array}$ & $\begin{array}{l}48.069 \\
49.214\end{array}$ & $\begin{array}{l}51.978 \\
52.554\end{array}$ & $\begin{array}{l}17.506 \\
17.747\end{array}$ & $\frac{31.447}{31.804}$ & $\frac{-1.218}{-1.218}$ & $\begin{array}{l}-1.149 \\
-7.752 \\
\end{array}$ & 年 & $\begin{array}{l}0.058 \\
0.059\end{array}$ & $\frac{14.043}{14.643}$ & 0.003 & $\frac{250.4}{23012}$ & 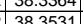 & 511 & 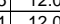 & 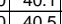 & 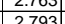 & 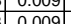 & 0.0099 & 0.000 & 0.215 & \\
\hline & $4 / 21 / 2004$ & & 25.622 & & 25.236 & $\frac{25.349}{25.349}$ & 24.857 & 49.243 & \begin{tabular}{|c|c|}
52.59 \\
\end{tabular} & 17.754 & 31.774 & -1.221 & -7.706 & 49.933 & 0.059 & 14.643 & & & 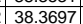 & & & & & & 0.009 & & & \\
\hline & & & & & & & & & & & & & & & & & & & & & & & & & & & & \\
\hline & $4 / 21 / 2004$ & 12:06:01 AM & 25.641 & 24.98 & 25.28 & 25.448 & 25.051 & 48.886 & 52.14 & 17.733 & 31.453 & -1.221 & & .899 & 0.058 & 14.643 & & now & 8.4031 & & & & & 0.009 & 0.009 & 000 & $\frac{114}{214}$ & \\
\hline & & & & & & & & & & & 31.482 & & & & 8059 & 14.643 & & & & & & & & & & & & \\
\hline & $\mid$ & 12:08:01 AM & 25.652 & 24.981 & 25.246 & 25.549 & 25.227 & 49.195 & 52.691 & 17.798 & 31.626 & -1.221 & -7.761 & 49.969 & 0.056 & 14.643 & & $2306.2 \mathrm{C}$ & 38.4364 & 51.2 & 12.2 & 40.4 & 2.786 & 0.008 & 0.008 & & & \\
\hline & 4/21/2004 & 12:09:01 AM & 25.653 & 24.992 & 25.252 & 25.595 & 25.178 & 49.023 & 52.523 & 17.738 & 31.482 & -1.221 & -7.764 & 51.954 & 0.056 & 14.643 & 0.003 & 2307.2 & 38.4531 & 51.1 & $12.7 \mathrm{r}-\mathrm{C}$ & 40.3 & 2.775 & 0.008 & 0.008 & 0.000 & 0.206 & \\
\hline & $4 / 21 / 2004$ & 12:10:01 AM & 25.659 & 24.998 & 25.263 & 25.676 & 25.294 & 48.783 & 52.204 & 17.7 & 31.341 & -1.218 & -7.7. & 49.074 & 0.059 & 14.643 & & 2308.2 & 8.4697 & & 12. $2>$ & & & 0.009 & & 0.000 & & \\
\hline$\frac{10}{16}>2>3$ & 4/21/2004 & 12:11:01 AM & 25.671 & 25.0 & 25.285 & 25.103 & 25.226 & 49.141 & 2.585 & 17.802 & 31.671 & -1.215 & -7.7522 & 49.676 & 0.059 & 14.643 & & 2309 & 38.48664 & & 12. & & 2.786 & 0.009 & 0.009 & 0.000 & & \\
\hline & $4 / 21 / 12004$ & 12:12:01 AM & & & & & & & & & & & & & & & & & & & & & & & & & & \\
\hline & $\begin{array}{l}4 / 2 / 1 / 2004 \\
4 / 212004\end{array}$ & $\begin{array}{l}\text { 12:13:01 AM } \\
\text { 12:14:01 AM }\end{array}$ & $\frac{25.689}{25.674}$ & $\frac{25.03}{2502}$ & $\begin{array}{ll}2.5328 \\
25208\end{array}$ & 25.652 & 25.039 & $\begin{array}{l}49.154 \\
49.015\end{array}$ & 年2.598 & $\begin{array}{lll}17.598 \\
17207\end{array}$ & $\frac{31.841}{32.926}$ & -1.218 & 7 & $\begin{array}{l}5.0 .087 \\
1075\end{array}$ & 0.059 & 14.643 & & $\frac{2511}{2312}$ & 8.5974 & & 2. & & (3) & (2) & 0.009 & & & 0.16 \\
\hline & $\frac{4 / 21 / 2004}{4}$ & $12: 15: 01 \mathrm{AM}$ & 25.674 & 25.03 & 25.293 & 25.471 & 24.924 & 49.164 & \begin{tabular}{|l|l|l|l|}
52.573 \\
\end{tabular} & 17431 & 32.063 & -1.221 & -7.764 & 49.547 & $\begin{array}{l}0.058 \\
0.058\end{array}$ & $\frac{14.045}{14.643}$ & 0.003 & 2313.2 & \begin{tabular}{|l|l|}
38.5531 \\
\end{tabular} & 51.1 & $\frac{1.2 .}{12.1}$ & & \begin{tabular}{|l|l|}
2.800 \\
\end{tabular} & 0.009 & 0.009 & 0.000 & 0.211 & $0.1 \quad>3$ \\
\hline & $\frac{1 / 2004}{11}$ & $12: 16: 01 \mathrm{AM}$ & 25.678 & 25.03 & 25.312 & 25.39 & 24.818 & 49.345 & \begin{tabular}{|l|l|}
52.693 \\
\end{tabular} & 17.429 & 32.26 & -1.221 & -7.761 & 52.505 & 0.058 & 14.643 & & 2314.2 & 38.5697 & 51.2 & 12. & 40.8 & & 0.009 & 0.009 & & & \\
\hline 163 & $4 / 21 / 2004$ & 12:17:01 AM & 25.672 & 25.02 & 25.306 & 25.359 & 24.762 & $\begin{array}{ll}49.013 \\
\end{array}$ & 52.463 & 17.307 & 32.03 & -1.221 & -7.758 & 49.628 & 0.058 & 14.643 & 0.003 & $2315.8 \mathrm{r}$ & 38.5864 & & 12. $\mathrm{r}$ & & 2.794 & 0.009 & 0.009 & 0.000 & & \\
\hline & $4 / 21212004$ & 12:18:01 AM & 25.676 & 25.02 & $\begin{array}{l}2.5325 \\
2.325\end{array}$ & 25.303 & 24.736 & 49.1855 & 52.61 & 17.363 & 32.12 & -1.215 & & 49.939 & 0.06 & 14.643 & & 2316 & 38.6031 & & 12. & & & & & 0.000 & & \\
\hline & 11:2004 & 1:2:19:01 AM & 25.664 & 24.993 20. & 25.313 2532 & 25.245 & 24.104 & 49.135 & 20... & 17.302 & 32.028 & & & $078-3$ & 0.058 & 告.643 & & 218 & 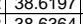 & & & & & & & & & \\
\hline$\frac{1050}{1639}$ & $\frac{142121204}{4 / 21204}$ & 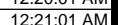 & $\frac{25.6 / 3}{25.672}$ & 24.991 & $\frac{25.334}{25.336}$ & (25.399 & $\begin{array}{l}24.838 \\
24.892 \\
\end{array}$ & $\begin{array}{l}49.1606 \\
49.311\end{array}$ & $\begin{array}{l}52.726 \\
52.898\end{array}$ & $\frac{1.39}{17.322}$ & $\begin{array}{r}32.005 \\
32.276 \\
\end{array}$ & $\frac{-1.211}{-1218}$ & & (49.171 & $\begin{array}{l}0.038 \\
0.059\end{array}$ & 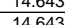 & & $\frac{2518}{2310}$ & $\begin{array}{l}38.6364 \\
336531\end{array}$ & 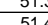 & $\frac{12.2}{121}>\mathrm{r}$ & 408 & & 0.009 & & 0.0000 & & \\
\hline & 4/21/2004 & $12: 22: 01 \mathrm{AM}$ & 25.672 & 24.996 & 25.341 & 25.404 & 24.97 & 49.282 & \begin{tabular}{|l|}
52.687 \\
\end{tabular} & 17.247 & 32.393 & -1.221 & -7.775 & 50.601 & 0.058 & 14.643 & 0.003 & 2320.2 & 38.6697 & 51.2 & 12.4 & \begin{tabular}{|l|l|}
4 & 40.8 \\
\end{tabular} & \begin{tabular}{|l|l|}
2.816 \\
\end{tabular} & \begin{tabular}{|l|}
0.009 \\
\end{tabular} & 0.009 & 0.000 & 0.210 & 0.18 \\
\hline & :1/2004 & 12:23:01 AM & 25.678 & 24.997 & 25.352 & 25.46 & & 49.243 & 52.639 & 17.226 & 32.438 & & & & 0.059 & 14.643 & & & 38.6864 & & & & & & & & & \\
\hline & $4 / 21 / 12004$ & 12:24:01 AM & 25.678 & $24.9828 \mathrm{rat}$ & 20.5327 & $\frac{25.51}{25.51}$ & 25.0 & & 2.633 & $\frac{17.135}{1720} \mathrm{rl}$ & 32.12 & & & 9.756 & 0.055 & 14.643 & & & & & & & & . 008 & 0.008 & & & \\
\hline$\frac{1643}{1644}$ & $\begin{array}{l}4 / 21 / 21204 \\
4 / 212004\end{array}$ & (1) & 望5.684 & 24.9989 & 25.318 & 255.602 & 25.106 & $\frac{49.152}{49245}$ & 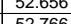 & $\frac{17.249}{17.251}$ & $\frac{32.209}{32.43}$ & $\frac{1.221}{1.210}$ & -774 & 每.43 & 0.061 & 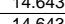 & & 23242 & 387364 & & 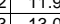 & 408 & 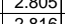 & 00009 & (20109 & 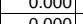 & & \\
\hline & 4 & $12.27 .01 \mathrm{AM}$ & 25.096 & 25005 & $\frac{15.050}{2531}$ & 25649 & & & 5271 & $\frac{11.151}{17120}$ & $\frac{3.45}{32.307}$ & 1224 & & 0.835 & 0.059 & $\frac{14045}{14643}$ & & 势 & 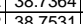 & (51. & 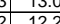 & & & & & & & \\
\hline 164 & $1 / 2004$ & 12:28:01 AM & $\frac{25.718}{25.718}$ & & 25.368 & 25.696 & 25378 & 49.154 & 52793 & 17.279 & 32.194 & -1.221 & $\frac{-7.74}{-7.74}$ & 49691 & 0.059 & 14643 & & 2326 & & & 12 & & 2804 & & 0.009 & & $\frac{214}{214}$ & . \\
\hline 1647 & 1/2004 & 12:29:01 AM & 25.706 & 250 & & 25.678 & & & 52.942 & 17.316 & & & & 50.498 & & & & & & & 50 & & & & & & & \\
\hline & 4/21/2004 & 12:30:01 AM & 25.707 & 25.041 & 25.356 & 25.594 & 25.6 & 49.029 & 52.683 & 17.193 & 32.196 & -1.218 & & 49.906 & 0.059 & 14.643 & & 2328.2 & 38.8031 & $\frac{25.12}{51 .}$ & $12.2 \mathrm{C}$ & 40.6 & 2800 & 0.009 & 0.009 & 0.000 & 0.214 & 18 \\
\hline & 4/21/2004 & $12: 31: 01 \mathrm{AM}$ & 25.713 & 25.037 & 25.358 & 25.511 & $\frac{24.9}{24.9}-3$ & $48.8 \mathrm{\gamma}>$ & 52.565 & $\frac{17.19}{17.19}$ & 31.948 & -1.224 & & 49.56 & 0.05 & 14.643 & & 2329 & & & 12.1 & & & & & & & \\
\hline & & $12 \cdot 32 \cdot 01 \mathrm{AM}$ & 25.713 & 25.05 & 25.367 & 25.45 & & & & & 32.264 & -1.221 & & (2) & 0.06 & 14.643 & & 2330.2 & 38.8364 & 51. & 122 & $408-2$ & 281 & 0.009 & 0.009 & & & 0.18 \\
\hline & |2004] & $12: 33: 01 \mathrm{AM}$ & 25.707 & 25.041 & 25.361 & & & & & 17.244 & 32.40 & & & 47.90 & & 14.643 & & & & $51.2>$ & 11. & & & & & & & \\
\hline & 1/2004 & 12:34:01 AM & 25.711 & & 25.37 & 25.263 & 24.8 & & \begin{tabular}{|l|l|}
52.619 \\
\end{tabular} & 17.137 & 32.22 & -1.218 & & 49.145 & 0.06 & 14.643 & & & 38.8697 & 51 & 12. & $40,8-8$ & $8027>2$ & 0.009 & 0.009 & 000 & 218 & \\
\hline 100 & $4 / 21 / 2004$ & 12:35:01 AM & 25.701 & 25.03 & 25.365 & 25.227 & 24. & 49. & 52.691 & 17.22 & 32.2 & -1.221 & & 48.517 & 0.06 & 14.643 & & 2333.2 & 38.8 & & 11. & & 803 & 0.009 & 0.009 & 0.000 & & \\
\hline & & $12: 36$ & 25.695 & 25.01 & 25.364 & 25.157 & & & & 17.215 & & & & & & & & & & & 12. & & & & & & & \\
\hline & & & & 25.01 & 望.5358 250 & 25.155 & & & & $17.187 \mathrm{r}=0$ & & -1.218 & & & & & & 20 & & & 12. & & & & & & & \\
\hline & 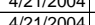 & $\begin{array}{l}12.380 .0 \mathrm{AM} \\
12.09 \mathrm{M}\end{array}$ & 年5.097 & (24.996 & 年5.326 & 年5.1.44 & & & & 17.166 & & & & & & & & & & 51. & 121 & & & & & & & \\
\hline $165 \varepsilon$ & $\mid$ & $12: 40: 01$ AM & 25.685 & 24.989 & 年 25.334 & 25357 & & & 52641 & 17049 & & -1221 & & 49.4 & & & & 200 & & S1. & 123 & & & & & & & \\
\hline 165 & $4 / 21 / 2004$ & $12.41 .01 \mathrm{AM}$ & 25.691 & 24.965 & 25.331 & 25.399 & & 49. & 5277 & 17.069 & 32.496 & -1.224 & & - & 0.06 & & & 2339 & 38.9 & 准 & 12. & & & & 0.000 & & 0.211 & \\
\hline 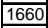 & $4 / 2$ & 12:42:01 AM & & & > & & & & & & & -1221 & & & & & & , & & & & & & & & & & \\
\hline & & & 25.686 & 249 & 25365 & 25578 & 250 & & 52921 & 17185 & 32.46 & -1.218 & & & 0.058 & & & 20 & & & & & & & & & & \\
\hline & $4 / 21 / 2004$ & 12:44:01 AM & 25.6 & 24.98 & 25.361 & 25.594 & & & .519 & 16.986 & & -1.221 & & 50.3 & 0.19 & & & 2342.2 & 39.0 & 51.6 & 12.3 & $\frac{74}{46}-2+3$ & 2.798 & & & & & \\
\hline & $\mid$ & :01 AM & 25. & 24.4 & 25.366 & 25.639 & & & 53.162 & 17.169 & & -1.218 & & & 0.0 & & & 23. & & 51. & 12. & & & & & & & \\
\hline 1664 & $4 / 21 / 200$ & 12:46:01 AM & 25.718 & & 25.408 & 25.696 & & & & & 32 & -1.215 & & 49.1 & & & & & & 51 & 12. & & & & & & & \\
\hline 1665.5 & $4 / 2$ & $12: 4$ & 25. & 25. & 25.373 & & & & & 17.109 & & & & & & & & 232 & & 51 & 12.1 & & & & & & & \\
\hline & & & & 25 & 25.4 & 25.558 & & & & 17.142 & & -1.218 & & & & & & & & 51. & 12. & & & & & & & \\
\hline & & NIAN & 25. 25 & 25. & 20.45025 & 25.513 & & & $\mid 53.218$ & 1.17.199 & & -1.218 & & & & & & $\frac{2041.4}{220}$ & 39.1 & 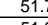 & . & & & & & & 116 & \\
\hline & & 12. & . & & & & & & & & & & & & & & & 2340 & & & & & & & & & & \\
\hline $167 \mathrm{c}$ & $\mid 2004$ & (1) & $\frac{20.110}{25713}$ & $\frac{25.12}{2502}$ & $\frac{25.405}{25.412}$ & $\frac{25.305}{25265}$ & & & 5.809 & 17048 & & $\frac{-1.22}{-122}$ & & & & & & & & & 2 & & & & & & & \\
\hline 1671 & & $01 \mathrm{AM}$ & 25.707 & 25.001 & 25.406 & & & 49.00 & \begin{tabular}{|l|l|}
52.7 \\
5.7
\end{tabular} & $\begin{array}{ll}17.040 \\
17.089\end{array}$ & & $\begin{array}{l}-1.221 \\
\end{array}$ & & \begin{tabular}{|l|l|l|l|}
503 \\
\end{tabular} & 0. & $\frac{14.045}{14.643}$ & & 235 & 39.1864 & 1.2 .8 & 12. & & & 0.009 & & & $\frac{218}{218}$ & \\
\hline & $4 / 2$ & 12:54:01 AM & 25.7 & 24.999 & 25.379 & 25.182 & & 49.085 & \begin{tabular}{|l}
52.851 \\
\end{tabular} & 17.201 & & -1.221 & & & & & & & & & & & & & & & & \\
\hline & & & & & & & & & & & & -1.218 & & & & & & & & 51.7 & & & & & & & & \\
\hline & & 12:56:01 AM & 25.7 & & & & & & & & & & & & & & & 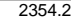 & & & & & & & & & & \\
\hline 1675 & $4 / 21 / 20$ & 12:57:01 AN & 25.7 & 24.98 & 25.351 & 252 & & 49.6 & 52838 & 17.062 & & & & & 0.8 & 14.6 & & 235 & & $\sqrt{51.2}$ & & & & & & & 8 & \\
\hline 1676 & $4 / 21 / 20$ & $12: 58$ & & & & 25. & & 49.438 & & & & -1.224 & & & & & & 235 & & & 12. & & & & & & 16 & \\
\hline 1677 & 4/21/2004 & 12:59:01 & 25.702 & 24.981 & 25.376 & $25.399 \mid$ & 25. & 49.143 & 53.046 & 16.979 & 32.555 & -1.221 & & 50.552 & 0.057 & 14.643 & & 2357.2 & 39.2 & 51.6 & \begin{tabular}{l|l}
6 & 12.4
\end{tabular} & & & 0.008 & 0.008 & & 0.206 & \\
\hline$\frac{1678}{1670}$ & 2004 & 1:00:01 AM & 25.687 & 24.976 & $\frac{25.371}{25.352}$ & $\begin{aligned} 25.424 \\
25.71\end{aligned}$ & $\frac{25.112}{25.093}$ & 49.038 & $\frac{52.836}{53.122}$ & $\frac{16.936}{17099}$ & 32.307 & -1.221 & $\begin{array}{l}1 \\
1\end{array}$ & 372 & 0.058 & 14.643 & 0.0 & 2358.2 & \begin{tabular}{|l|l|}
39.3031 \\
392107
\end{tabular} & \begin{tabular}{|l|l}
51. \\
51.
\end{tabular} & $\begin{array}{l}4 \quad 12.0 \\
4 \\
6.12 .1\end{array}$ & & \begin{tabular}{|l|l|}
2.804 \\
2816
\end{tabular} & \begin{tabular}{|l|l|}
0.009 \\
\end{tabular} & \begin{tabular}{|l|}
0.009 \\
\end{tabular} & 0.000 & 0.210 & \\
\hline & & $1: 02: 01 \mathrm{AM}$ & & & & $\frac{25.46}{25.526}$ & & & \begin{tabular}{|l|l|}
52.913 \\
52.913
\end{tabular} & $\frac{1.096}{16.991}$ & $\frac{32.441}{32.49}$ & & & & & $\frac{14.045}{14.643}$ & & & & & & & & & & & 0.210 & $\frac{0.1}{0.1}$ \\
\hline
\end{tabular}


WSRC-TR-2005-00105, REVISION 0

SRNL-RPP-2005-00012, REVISION 0

RUN \# 2.03A AND B; FIRST AND SECOND HALF OF SLURRY DEWATERING - CONT.

\begin{tabular}{|c|c|c|c|c|c|c|c|c|c|c|c|c|c|c|c|c|c|c|c|c|c|c|c|c|c|c|c|c|}
\hline & A & B & D & $E$ & $\mathrm{~F}$ & $\mathrm{G}$ & $\mathrm{H}$ & $\mathrm{J}$ & $\mathrm{K}$ & $\mathrm{L}$ & $\mathrm{M}$ & $\mathrm{N}$ & 0 & Q & $R$ & 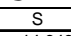 & $\mathrm{T}$ & $\mathrm{v}$ & w & $x$ & & 2 & $\mathrm{AA}$ & $A B$ & $\mathrm{AC}$ & $A D$ & $\mathrm{AE}$ & \\
\hline$\frac{1681}{1682}$ & $\begin{array}{l}4 / 21 / 2004 \\
4 / 21 / 12004\end{array}$ & $\begin{array}{l}1: 03: 01 \mathrm{AM} \\
1 \cdot 10401 \mathrm{AM}\end{array}$ & $\begin{array}{r}25.694 \\
25.696\end{array}$ & $\begin{array}{r}24.993 \\
22.995\end{array}$ & \begin{tabular}{|l|}
25.398 \\
25385 \\
\end{tabular} & $\begin{array}{l}25.546 \\
25.518 \\
\end{array}$ & \begin{tabular}{|l|}
25.184 \\
25.241 \\
\end{tabular} & $\begin{array}{l}49.197 \\
49.174\end{array}$ & \begin{tabular}{|l|}
52.992 \\
53.139 \\
\end{tabular} & \begin{tabular}{|l|}
17.048 \\
16.933
\end{tabular} & $\begin{array}{r}32.432 \\
32.461 \\
\end{array}$ & $\begin{array}{l}-1.221 \\
-1.224 \\
\end{array}$ & $\begin{array}{r}-7.732 \\
-7.744 \\
\end{array}$ & \begin{tabular}{|c|}
49.866 \\
48886 \\
\end{tabular} & $\begin{array}{r}0.06 \\
0.057 \\
\end{array}$ & $\begin{array}{l}14.643 \\
14.643 \\
\end{array}$ & $\begin{array}{l}0.003 \\
0.03\end{array}$ & $\frac{2361.2}{2362}$ & \begin{tabular}{|l|}
39.3531 \\
3.36967 \\
\end{tabular} & $\begin{array}{l}51.5 \\
51.7\end{array}$ & $\frac{12.2}{120}$ & \begin{tabular}{|l|l|}
40.8 \\
40.8
\end{tabular} & $\frac{2.814}{2.814}$ & \begin{tabular}{|c|}
0.009 \\
0.008
\end{tabular} & \begin{tabular}{|l|}
0.009 \\
0.008 \\
\end{tabular} & 0.000 & $\begin{array}{l}0.217 \\
0.206\end{array}$ & $\begin{array}{l}0.18 \\
0.18\end{array}$ \\
\hline & & & 25.706 & & & & & & & & & & & & & $\frac{14.045}{14.643}$ & & & & & & & & & \begin{tabular}{|l|}
0.0008 \\
\end{tabular} & & & \\
\hline 1684 & $4 / 21 / 2004$ & 1:06:01 AM & 25.706 & 25.02 & 25.426 & 25.459 & 24.907 & 49.259 & 53.18 & 17.004 & 32.519 & -1.215 & -7.735 & $\begin{array}{r}49.795 \\
\end{array}$ & 0.059 & 14.643 & 0.003 & 2364.2 & & & & & \begin{tabular}{|l|}
2.819 \\
\end{tabular} & 0.009 & 0.009 & 0.000 & 0.213 & 0.18 \\
\hline & $4 / 21 / 2004$ & 1:07:01 AM & 25.701 & 25.005 & 25.41 & 25.383 & 24.821 & 49.129 & 52.96 & 17.069 & $\frac{32.44}{32.44}$ & -1.221 & $\begin{array}{l}-7.738 \\
\end{array}$ & 50.213 & 0.058 & 14.643 & 0.003 & 2365.2 & \begin{tabular}{|l|l|}
39.4197 \\
\end{tabular} & & & & \begin{tabular}{|l|l|}
2.812 \\
\end{tabular} & & 0.009 & & & 0.18 \\
\hline & & & & & & & 24.745 & \begin{tabular}{|l|}
49.16 \\
\end{tabular} & & $\begin{array}{l}17.045 \\
\end{array}$ & & & & \begin{tabular}{|l|l|}
49.174 \\
\end{tabular} & & 14.643 & & & & & & & & & & & & \\
\hline \begin{tabular}{|l|}
1687 \\
\end{tabular} & $4 / 21 / 2004$ & 1:09:01 AM & 25.699 & 24.998 & 25.413 & \begin{tabular}{|l|l|}
25.21 \\
25.955
\end{tabular} & $\begin{array}{l}24.694 \\
2.602\end{array}$ & 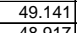 & \begin{tabular}{|l|l|}
52.958 \\
5.811
\end{tabular} & $\begin{array}{l}17.08 \\
16.067\end{array}$ & 32.373 & $\begin{array}{l}-1.221 \\
\end{array}$ & \begin{tabular}{|l|l|}
-7.738 \\
-7.70
\end{tabular} & \begin{tabular}{|l|l|}
48.283 \\
\end{tabular} & 0.059 & 14.643 & $\begin{array}{l}0.003 \\
\end{array}$ & 2367.2 & \begin{tabular}{|l|}
39.4531 \\
900
\end{tabular} & 51.4 & $\begin{array}{l}11.8 \\
1.7\end{array}$ & & $\begin{array}{l}82.810 \\
\end{array}$ & \begin{tabular}{|l|l|}
0.009 \\
\end{tabular} & 0.009 & 0.000 & 0.213 & \\
\hline \begin{tabular}{|c|c|c|}
1689 \\
\end{tabular} & $4 / 21 / 2004$ & $\begin{array}{l}1: 10: 01 \mathrm{AM} \\
1 \cdot 11.01 \mathrm{AM}\end{array}$ & $\begin{array}{l}25.703 \\
25.707\end{array}$ & $\begin{array}{l}24.997 \\
22.491\end{array}$ & \begin{tabular}{|l|}
$\frac{25.422}{25411}$ \\
\end{tabular} & $\begin{array}{l}25.185 \\
25.189 \\
25\end{array}$ & \begin{tabular}{|l|}
24.693 \\
24678
\end{tabular} & \begin{tabular}{|l|l|}
48.917 \\
49307 \\
\end{tabular} & \begin{tabular}{|l|}
52.811 \\
53232 \\
\end{tabular} & 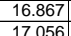 & $\begin{array}{l}32.356 \\
32518 \\
3518\end{array}$ & $\begin{array}{l}-1.218 \\
-1.215\end{array}$ & \begin{tabular}{|l|l|}
-7.738 \\
-7.735 \\
\end{tabular} & \begin{tabular}{|l|}
51.946 \\
48.577
\end{tabular} & $\begin{array}{l}0.06 \\
0.057\end{array}$ & $\begin{array}{l}14.643 \\
14.643\end{array}$ & $\begin{array}{l}0.003 \\
0.04\end{array}$ & \begin{tabular}{l|l}
2368.2 \\
23692
\end{tabular} & \begin{tabular}{|l|}
39.4697 \\
394864 \\
\end{tabular} & $\frac{51.3}{51.8}$ & $\begin{array}{l}12.7 \\
11.9\end{array}$ & \begin{tabular}{|l|l|}
40.6 \\
0.9
\end{tabular} & $\begin{array}{l}62.802 \\
9 \\
9 \\
2821\end{array}$ & \begin{tabular}{|c|}
0.009 \\
0.008
\end{tabular} & $\begin{array}{l}0.009 \\
0.008\end{array}$ & $\begin{array}{l}0.000 \\
0.000\end{array}$ & \begin{tabular}{|l|l}
0.218 \\
0.205
\end{tabular} & $\begin{array}{l}0.19 \\
0.17\end{array}$ \\
\hline 1690 & $4 / 21 / 2004$ & $\begin{array}{l}1: 11.01 \mathrm{AM} \\
1: 2201 \mathrm{Am}\end{array}$ & 25.701 & & 25.44 & 25.1093 & 24.007 & 499.289 & \begin{tabular}{|l|}
53.147 \\
\end{tabular} & & & $\begin{array}{l}-1.215 \\
-1.218\end{array}$ & $\begin{array}{l}-1.135 \\
-7.735 \\
\end{array}$ & \begin{tabular}{|l|}
47.57705 \\
\end{tabular} & 0.059 & $\frac{14.643}{14.643}$ & $\begin{array}{l}0.004 \\
0.003\end{array}$ & $\begin{array}{l}2369.2 \\
2370.2\end{array}$ & & $\begin{array}{l}51.8 \\
51.7\end{array}$ & & & $\frac{2.821}{2.820}$ & \begin{tabular}{|c|c|}
0.008 \\
0.009
\end{tabular} & $\begin{array}{l}0.008 \\
0.009\end{array}$ & & $\frac{0.205}{0.212}$ & 0.17 \\
\hline $16 \mathrm{~S}$ & $4 / 21 / 2004$ & 1:13: $\mathrm{AM}$ & & 24.968 & & 25.256 & 24.83 & \begin{tabular}{|l|l|l|l|}
48.899 \\
\end{tabular} & \begin{tabular}{|l|}
52.836 \\
\end{tabular} & 17.05 & & & -7.735 & 48.333 & 0.059 & 14.643 & & & $\begin{array}{l}39.5197 \\
\end{array}$ & & & & $\frac{2.820}{2.792}$ & 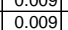 & & & & $\frac{0.18}{0.18}$ \\
\hline & $4 / 21 / 2004$ & $1: 14: 01 \mathrm{AM}$ & 25.704 & & 25.434 & 25.336 & & 49.031 & \begin{tabular}{|l|l|}
52.882 \\
\end{tabular} & $\begin{array}{ll}16.966 \\
\end{array}$ & 32.414 & -1.221 & -7.755 & 48.55 & 0.056 & 14.643 & & 2372.2 & 39.5364 & & 11.9 & & & & \begin{tabular}{|l|}
0.008 \\
\end{tabular} & 0.000 & & $\frac{0.18}{0.17}$ \\
\hline 1693 & $4 / 21 / 2004$ & 1:15:01 AM & 25.699 & & 25.359 & 25.331 & 24.96 & 48.95 & 52.876 & & & & & & & & & & & & & & 80 & & & & & $\frac{0.17}{0.19}$ \\
\hline 1694 & $4 / 21 / 2004$ & 1:16:01 AM & & & 252200 & & & & & 16.802 & 2351 & -1.221 & & 50.621 & & & & & & & & & & & & & & \\
\hline & $4 / 21 / 2004$ & 1:17:01 AM & 25.696 & 24.95 & 25.355 & 25.448 & 25.001 & \begin{tabular}{|l|l|}
49.027 \\
\end{tabular} & 52.983 & 16.836 & 32.434 & & -7.614 & & 0055 & 14.643 & & & 39.5864 & & 11. & & & & & & & \\
\hline & $4 / 21 / 2004$ & 1:18:01 AM & 25.707 & 24.97 & 25.386 & 25.514 & 25.062 & \begin{tabular}{|l|l|}
49.282 \\
\end{tabular} & 53.296 & 16.885 & 32.656 & -1.215 & -7.703 & \begin{tabular}{|l|l|l|l}
47.6999 \\
\end{tabular} & 0.058 & 14.643 & 0.004 & 2376.2 & 39.6031 & 51.8 & 11.7 & & 2.825 & 0.009 & 0.009 & 0.000 & 0.209 & \\
\hline & $11 / 2004$ & 1:19:01 AM & 25.703 & 24.962 & 25.377 & 25.53 & 25.138 & 49.303 & 53.207 & 16.927 & 32.674 & -1.221 & -7.694 & 50.039 & 0.06 & 14.643 & & 2377.4 & & & 12. & & & & 0.009 & & & \\
\hline & $4 / 21 / 2004$ & & & & & & & & & & 32.56 & -1.218 & & & & 14.643 & & & & & & & & & & & & \\
\hline & $4 / 21 / 2004$ & 1:21:01 AM & $\begin{array}{r}25.71 \\
25.705\end{array}$ & 24.984 & 25.394 & 25.562 & 25.07 & & 53.05 & 16.871 & 32.391 & -1.218 & -7.645 & 3.878 & 0.059 & 14.643 & & & & & 12. & & & & & .000 & & \\
\hline & $\frac{442112004}{4121204}$ & 1.22.01 AM & & $\frac{24.979}{3490}$ & $\frac{2.3859}{2530}$ & $\frac{25.492}{25309}$ & 24.955 & $\begin{array}{l}49.284 \\
19809\end{array}$ & & & & $\frac{-1.21}{-1212}$ & & 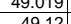 & & $\frac{1.4645}{1.643}$ & & 238 & 068 & & & & & & & & & \\
\hline 170 & $\frac{4 / 2 / 212004}{4}$ & $\begin{array}{l}1.23 .01 \mathrm{AM} \\
1: 24: 01 \mathrm{Am}\end{array}$ & $\frac{25.096}{25.695}$ & $\begin{array}{r}24.99 \\
24.989\end{array}$ & $\frac{2.35}{25.394}$ & $\begin{array}{l}25.350 \\
25322\end{array}$ & 24.921 & $\begin{array}{l}48.996 \\
49.048\end{array}$ & $\frac{5.250}{5306}$ & $\begin{array}{l}16.945 \\
16991\end{array}$ & $\begin{array}{l}32.320 \\
32363\end{array}$ & $\begin{array}{l}-1.221 \\
-1.221\end{array}$ & -7.666 & $\begin{array}{r}49.12 \\
48.577\end{array}$ & 0.030 & $\begin{array}{l}4.645 \\
14.643 \\
\end{array}$ & 0.003 & $\frac{250.4}{23822}$ & \begin{tabular}{|l|}
3.90604 \\
397031
\end{tabular} & & & & 2807 & & & 0.000 & & \\
\hline & $4 / 21 / 2004$ & $1: 25: 01 \mathrm{AM}$ & 25.689 & 24.978 & 25.374 & 25.226 & 24.759 & 49.058 & 52.896 & 16.889 & 32.482 & -1.224 & -7.671 & 49.349 & 0.057 & 14.643 & 0.003 & 2383.2 & \begin{tabular}{|l|} 
\\
\end{tabular} & 51.4 & 12.1 & & 2.811 & & 0.008 & 0.000 & 0.206 & 0.18 \\
\hline & $1: 12004$ & 1:26:01 $\mathrm{AM}$ & 25.692 & 24.981 & 25.396 & 25.159 & 24.647 & 49.096 & 53.052 & 16.864 & 32.568 & & -7.616 & 48.064 & 0.061 & 14.643 & & 2384.2 & 39.7364 & & & & & & & & & \\
\hline & $1 / 2004$ & 77:01 AM & 25.706 & 24.98 & $\frac{25.43}{25.11}$ & 25.117 & 24.65 & & .317 & $\frac{16.923}{17701}$ & 32.75 & -1.215 & -7.66 & .082 & 0.059 & 14.643 & & & & & & & & & & & & \\
\hline & $\frac{4 / 2 / 21 / 2004}{4}$ & $\frac{1.28 .01 \mathrm{AM}}{1.29 .01 \mathrm{~A}}$ & $\frac{25.689}{25692}$ & $\frac{24.968}{24.962}$ & $\frac{25.414}{25.22}$ & $\frac{25.146}{25.95}$ & $\frac{24.645}{2080}$ & $\begin{array}{l}49.264 \\
49212\end{array}$ & 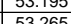 & $\begin{array}{r}17.004 \\
16.92\end{array}$ & $\begin{array}{r}32.57 \\
32.584\end{array}$ & - & $\begin{array}{l}-1.666 \\
7507\end{array}$ & $\begin{array}{r}48.1 \\
48.556\end{array}$ & $\frac{0.0}{0.0}$ & 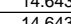 & & 2387 & 397864 & & $\frac{11.9}{11.9}$ & & & & (2) & & & \\
\hline & 4 & $1: 30.01 \mathrm{AM}$ & 25.688 & 24.962 & 25.422 & 2524 & & & 5004 & 16.896 & 32.354 & $\frac{1.241}{-1.201}$ & -761 & $\begin{array}{l}40.350 \\
48583\end{array}$ & 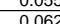 & $\frac{1.4043}{1463}$ & & $\frac{250}{238}$ & & 51 & $\frac{11.5}{110}$ & & & & & & & \\
\hline & $4 / 21 / 2004$ & 1:31:01 AM & 25.687 & 24.956 & 25.437 & 25.329 & 24.97 & $49.23 !$ & & $\begin{array}{l}16.923 \\
16.93\end{array}$ & 32.677 & $\frac{-1.218}{-1.218}$ & -7.6 & 51.752 & 0.06 & 14.643 & & & 39.8197 & & $\frac{12.7}{12.7}$ & & 2824 & & 009 & & $\frac{E 16}{216}$ & \\
\hline & $1: 1 / 2004$ & 1:32:1 $\mathrm{AM}$ & 25.698 & 24.947 & 25.437 & 25.34 & & 49.204 & & 17.006 & 32.49 & & -7.645 & & 0.062 & 14.643 & & 239 & & & 12.1 & & & & & & & \\
\hline 1771 & 4/21/2004 & $11 \mathrm{AM}$ & 25.689 & 24.958 & 25.439 & 25.397 & 25 & 49.27 & 53.166 & 16.869 & 32.73 & -1.224 & -7.66 & 499.683 & 0.06 & 14.643 & 0.003 & 2391.2 & 39.8531 & 51. & 12.2 & & 2.827 & 0.009 & 0.009 & 0.000 & 0.216 & \\
\hline$\frac{171}{171}$ & $4 / 21 / 2004$ & 1:34: & 25.699 & 24.958 & \begin{tabular}{|l|l|}
25.398 \\
\end{tabular} & 25.461 & 25.064 & 49.226 & 53.251 & 17.083 & 32.455 & -1.218 & & 49.533 & 0.06 & 14.643 & & & \begin{tabular}{|l|}
39.8697 \\
\end{tabular} & 51.8 & 12.1 & 40 & 2.816 & & 0.009 & 0.000 & 0 & 0.18 \\
\hline & $4 / 21 / 2004$ & & $\begin{array}{l}25.694 \\
25.711\end{array}$ & 24.963 & 25.374 & $25.467 \mid$ & $\begin{array}{r}25.105 \\
25.101\end{array}$ & 48.874 & $\begin{array}{r}52.83 \\
\end{array}$ & \begin{tabular}{|l|l|}
16.984 \\
\end{tabular} & $\begin{array}{r}32.155 \\
.20\end{array}$ & $\begin{array}{l}-1.221 \\
-1.215\end{array}$ & $\begin{array}{r}-7.616 \\
.71\end{array}$ & \begin{tabular}{|l|l|}
49.359 \\
\end{tabular} & 0.058 & 14.643 & & 2393.2 & 39.8864 & 51.4 & 12.1 & 40.5 & 2.793 & 0.009 & 0.009 & 0.000 & 0.211 & 0.18 \\
\hline & $4 / 2 / 21 / 2004$ & $\begin{array}{l}1: 36: 01 \mathrm{AM} \\
1.37 .01 \mathrm{~A}\end{array}$ & $\frac{25.111}{25.707}$ & 24.981 & $\frac{25.4}{25.396}$ & $\begin{array}{r}20.535 \\
25.54\end{array}$ & & $\begin{array}{l}49.1144 \\
49092\end{array}$ & $\frac{53.151}{53.006}$ & $\begin{array}{l}1.9683 \\
17\end{array}$ & $\begin{array}{r}32.43 \\
32.361 \\
\end{array}$ & $\frac{-1.215}{-1218}$ & & 47.054 & & $\frac{14.064}{14643}$ & & 2394.2 & 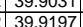 & & & 40.8 & & & & & & 0.18 \\
\hline & $\frac{4 / 21 / 2004}{4}$ & $1: 38: 01 \mathrm{AM}$ & $\frac{25.704}{25}$ & 24.988 & $\frac{25.398}{25.93}$ & 25.511 & $\frac{25.11}{25.11}$ & 48.897 & 2.882 & 16.875 & $\frac{25.389}{32.389}$ & $\frac{-1.218}{-1.218}$ & --7.61 & 48.419 & $\frac{0.059}{0.059}$ & $\frac{14.6}{14.6}$ & & 2396 & 39.9364 & & $\frac{1}{1 .}$ & & & & & & & \\
\hline & $1 / 2004$ & & & & & 25.447 & & & 2.894 & & & -1.224 & & & 0.059 & 14.643 & & & & & 11. & & & & .06 & 1.00 & & \\
\hline 171 & & & 25.7 & 24.994 & 25.409 & 25.362 & 24.8 & 48.998 & & 17.231 & & -1.221 & & & & & & 20 & & & 12 & & & & & 年 & & \\
\hline & & $1 \mathrm{AM}$ & 25.68. & 24.98 & 25.409 & 25.311 & & & & 17.186 & & -1.221 & & & & & & & & & & & & & & & & \\
\hline 1772 & $4 / 21 / 2004$ & 1:42:01 AM & 25.689 & 24.973 & 25.409 & 25.226 & 24.7 & 48.961 & 52.861 & 17.199 & 32.077 & -1.221 & & 51.427 & 0.059 & 14.643 & & 2400 & & 51.4 & 12.6 & & & & 0.009 & & & \\
\hline & $4 / 21 / 2004$ & 1:43:01 AM & 25.704 & 24.983 & 25.433 & 25.19 & 24.6 & 48 & $\begin{array}{lll}52.92 T \\
\end{array}$ & 17.138 & 32. & $\begin{array}{l}1.218 \\
\end{array}$ & -7.4 & 49.8 & & 14.6 & & 24 & 40.0 & $5+1$ & 12.2 & 4 & & & & & & \\
\hline & 12004 & 1:44:01 AM & 25.67 & 24.944 & 25.409 & 25.087 & 24.5 & 48.8 & 52.936 & 17.086 & 32.11 & -1.221 & & 49.628 & 0.056 & & & 24 & & & & & & & & & & \\
\hline & . & & 25.684 & 24.96 & 25.439 & 20.101 & & 48.951 & .979 & 17.105 & 20.1 & -1.215 & & 49. & 0.05 & & & & & & 12. & & & & n & & & \\
\hline & $\frac{42112004}{412104}$ & $\begin{array}{l}1: 40.01 \mathrm{AM} \\
14: 01 \mathrm{~A}\end{array}$ & 255618 & $\begin{array}{ll}24.944 \\
2.926\end{array}$ & 25.434 & $\begin{aligned} 25.133 \\
25.154\end{aligned}$ & & & & $\begin{array}{l}17.1055 \\
17062\end{array}$ & & $\frac{-1.218}{-1201}$ & 7. & & & & & & & & 126 & & & & & & & \\
\hline & 4 & 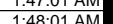 & $\frac{20.002}{2567}$ & 24.950 & 20.441 & 年, & & & 年 & & & $\frac{-1.241}{-1221}$ & & & & & & & & & $\frac{1.2 .0}{11.1}$ & & & & & & & \\
\hline & 4 & $1 \mathrm{AM}$ & 25.68 & 24.9 & $\frac{1.45}{25.45}$ & 25.262 & $\frac{24}{24}$ & (4) & 298 & 17.141 & & -1.221 & & 49.284 & & 势. & & 24 & $\frac{40.155}{40.1197}$ & $\frac{51}{51}$ & $\frac{1.4}{121}$ & & & & & & & \\
\hline & $4 / 21212004$ & $1: 50: \mathrm{C}$ & 25.675 & 24.90 & 25.454 & & & & 898 & $\mid 17.14$ & & & & & & & & & & & & & & & & & & \\
\hline & $4 / 212 / 2004$ & 1.51: & 25.685 & 24.919 & 25.46 & 25.367 & 24.5 & 48.82 & & 17.105 & & -1.221 & & 49.543 & & & & & & 51 & 121 & & & & & & & \\
\hline & & & $\begin{array}{l}25.696 \\
\end{array}$ & & & & & & & & & -1.221 & & & & & & & & & 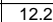 & & & & & & & \\
\hline 172 & $4 / 21 / 2004$ & & & 24. & 25.405 & 25.473 & & & & 17.138 & & -1.221 & & & & & & & & & 12 & & & & & & & \\
\hline & $4 / 21 / 2004$ & 1:54:01 AM & 25.703 & 24.9 & 25.417 & 25.49 & & 48.6 & 52.759 & 17.178 & & -1.218 & & & & & & & & 51 & $11.2 \mathrm{r}$ & & & & & & & \\
\hline & 4/21/2004 & 1:55 & 25.689 & 24.9 & 25.413 & 25.476 & 25.0 & 48.5 & 52.548 & 17.069 & 31.759 & -1.221 & & & & & & & & 51 & $12.2 \mathrm{r}$ & & & & & & & \\
\hline 17. & $4 / 21 / 2004$ & & 25.704 & 24. & 25.434 & 25.487 & & 48. & & 17.222 & 32.059 & -1.218 & & 48.7 & & & & & & $5 \pm$ & 11.9 & & & & & & & \\
\hline & & 1:57: & 25.706 & 24. & 25.43 & 25.413 & 24. & & 0 & 17.281 & 31.909 & -1.221 & & 50.96 & & & & & & & 12.2 & & & & & & & \\
\hline & & & & & & & & & & $\frac{17.17}{17.0}$ & & $\frac{-1.218}{-1.21}$ & & & & & & & & & & & & & & & & \\
\hline & $4421 / 12004$ & $1.55 .007 \mathrm{AM}$ & $\frac{25}{256}$ & 24. & $\frac{25.429}{25439}$ & & & & & $\frac{1.035}{172}$ & & $-\frac{1.24}{1.20}$ & & & & $\begin{array}{l}14.045 \\
1.643\end{array}$ & & & & 51 & $12 ?$ & & & & & & & \\
\hline 173 & $\frac{4}{42(21 / 20044}$ & $\frac{2.001 \cdot 01}{2 \cdot 0 M}$ & 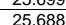 & 24.91 & $\frac{25.435}{25.432}$ & $\frac{25.215}{25.125}$ & & 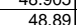 & 53.13 & & & -1.221 & & & & & & & & 51. & 11.8 & & & & & & & \\
\hline & $4 / 2121 / 2004$ & $2: 02: 01 \mathrm{AM}$ & 25.692 & $\frac{24.9}{24.9}$ & 25.447 & 25.104 & 24 & \begin{tabular}{|l|l|l}
48.724 \\
\end{tabular} & \begin{tabular}{|l|l|}
52.975 \\
\end{tabular} & $\frac{17.262}{17.26}$ & 31.716 & -1.221 & & 48 & & & & 242 & & 51 & 11.8 & & & & & & 090 & \\
\hline 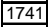 & $4 / 21212004$ & 2:03:01 AM & 25.675 & & 25.42 & & 24.74 & & & & & -1.221 & & & & & & & & & & & & & & & & \\
\hline & & & 25.695 & 24904 & 25.465 & 25152 & & 48.648 & & & 31886 & -1.218 & & & & & & & & 5 & $\frac{54}{24}$ & & & & & & & \\
\hline 174 & $4 / 21 / 2004$ & $2: 05: 01 \mathrm{AM}$ & 25.71 & 24.909 & 25.495 & 25.262 & 24.7 & $\begin{array}{l}48.507 \\
\end{array}$ & 52.577 & 17.066 & 31.8 & -1.218 & & 49.4 & 0.0 & 14.6 & & 2423. & 40.3 & 51.1 & 12.1 & & & & & & & \\
\hline & $21 / 200$ & 2:06:01 & 25. & 24.8 & 25.468 & & & 49.16 & 53.4 & 17.357 & 32.4 & -1.21 & & 50 & 0.0 & 14. & & & & & 12.4 & & & & & & & \\
\hline 174 & $4 / 21 / 2004$ & & & & & & & 48.82 & 53 & 17.2. & & -1.218 & & & & 14. & & & & 51 & $11.7 \mathrm{r}$ & & & & & & 34 & \\
\hline 174 & & & 25. & 24. & 25. & 25. & & 48.602 & & & & -1. & & & & & & & & 51. & 11.7 & & & & & & & \\
\hline .74 & $4 / 21 / 2004$ & 2:09: & & 24.895 & 25.51 & 25.433 & 25. & & 53.464 & 17.241 & & -1.218 & & 49. & & 14.643 & & & & & 12.2 & & & & & & & \\
\hline 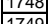 & $4 / 21 / 2004$ & 2:10:01 AM & 25.712 & 24.9 & 25.506 & 25.434 & $\frac{25.117}{25072}$ & $\begin{array}{l}48.94 \\
8.569\end{array}$ & 53.151 & 17.102 & 32.151 & $\frac{-1.221}{-1.210}$ & & 49.030 & 0 & 14.643 & & 244 & & 51.1 & 12.2 & 4 & 2.8 & (1) & 0.6 & 0.000 & 0.210 & $p$ \\
\hline 750 & $4 / 21 / 21 / 2004$ & 2:11.12:01 AM & $\frac{25.111}{25.711}$ & $\frac{24.895}{24.89}$ & \begin{tabular}{|l|l|}
25.461 \\
25.425 \\
\end{tabular} & 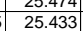 & \begin{tabular}{|l|}
23.072 \\
24.951 \\
\end{tabular} & $\begin{array}{l}\frac{48.569}{48.617} \\
\end{array}$ & \begin{tabular}{|l|}
52.151 \\
52.834 \\
\end{tabular} & $\frac{17.164}{17.072}$ & $\frac{31.155}{31.835}$ & \begin{tabular}{|l|}
-1.218 \\
\end{tabular} & \begin{tabular}{c|c|c|}
1 & -7.306 \\
1
\end{tabular} & $\frac{50.104}{49.24}$ & 0.058 & $\frac{1.4643}{14.643}$ & & & \begin{tabular}{|l|l|}
40.5031 \\
\end{tabular} & 51.4 & 12.1 & 40.2 & \begin{tabular}{|l|l|}
2 & 2.773 \\
\end{tabular} & \begin{tabular}{|l|}
0.0099 \\
\end{tabular} & \begin{tabular}{|l|}
0.000 \\
\end{tabular} & 0.000 & $\begin{array}{l}0.209 \\
0.212\end{array}$ & 0.1 \\
\hline
\end{tabular}


WSRC-TR-2005-00105, REVISION 0

SRNL-RPP-2005-00012, REVISION 0

RUN \# 2.03A AND B; FIRST AND SECOND HALF OF SLURRY DEWATERING - CONT.

\begin{tabular}{|c|c|c|c|c|c|c|c|c|c|c|c|c|c|c|c|c|c|c|c|c|c|c|c|c|c|c|c|c|}
\hline & A & B & D & $E$ & $F$ & $G$ & $\mathrm{H}$ & $\mathrm{J}$ & $\mathrm{K}$ & $\begin{array}{ll}\mathrm{L} \\
\end{array}$ & $\mathrm{M}$ & $\mathrm{N}$ & 0 & $\mathrm{Q}$ & $R$ & 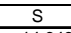 & \begin{tabular}{l|l|l|} 
\\
\end{tabular} & $\mathrm{v}$ & W & $x$ & & 2 & AA & $A B$ & $\mathrm{AC}$ & $A D$ & $\mathrm{AE}$ & AF $A$ \\
\hline $\begin{array}{l}1751 \\
1752 \\
\end{array}$ & $\begin{array}{l}4 / 21 / 2004 \\
4 / 2121204\end{array}$ & $2: 13: 01 \mathrm{AM}$ & $\frac{25.716}{25.72}$ & $\begin{array}{r}24.91 \\
24.903 \\
\end{array}$ & $\frac{25.42}{25.29}$ & $\begin{array}{l}25.358 \\
25.327\end{array}$ & \begin{tabular}{|r|}
24.871 \\
2475 \\
\end{tabular} & $\begin{array}{l}48.909 \\
48.951\end{array}$ & 53.116 & \begin{tabular}{|c|}
17.063 \\
177099
\end{tabular} & $\begin{array}{r}32.161 \\
32.243\end{array}$ & $\begin{array}{l}-1.221 \\
-1.221\end{array}$ & $\begin{array}{l}-7.376 \\
-7.374 \\
\end{array}$ & \begin{tabular}{|c|}
50.609 \\
49881
\end{tabular} & 0.057 & $\begin{array}{l}14.643 \\
14.643\end{array}$ & $\begin{array}{l}0.003 \\
0.03\end{array}$ & $\frac{2431.2}{2432.2}$ & \begin{tabular}{|l|}
40.5197 \\
0.5364
\end{tabular} & \begin{tabular}{|l|l|}
51.6 \\
51.8
\end{tabular} & $\frac{12.4}{12.2}$ & 40. & $\frac{2.795}{2.799}$ & \begin{tabular}{|c|c|}
0.008 \\
0.008
\end{tabular} & \begin{tabular}{|l|}
0.008 \\
0.008 \\
\end{tabular} & 0.000 & $\begin{array}{l}0.207 \\
0.207\end{array}$ & $\begin{array}{l}0.18 \\
0.18\end{array}$ \\
\hline & & & & & $\frac{20.425}{25.432}$ & & & & \begin{tabular}{|l|}
53.400 \\
53.417 \\
\end{tabular} & & & & & & & $\frac{14.043}{14.643}$ & & & & & & & & & & & & \\
\hline 1754 & $4 / 21 / 2004$ & 2:16:01 AM & 25.713 & 24.907 & 25.437 & 25.165 & 24.658 & 48.811 & \begin{tabular}{|l|l|}
53.174 \\
\end{tabular} & 16.904 & 32.196 & -1.221 & $\begin{array}{l}-7.362 \\
-10\end{array}$ & $\begin{array}{r}48.905 \\
\end{array}$ & 0.059 & 14.643 & 0.003 & 2434.2 & \begin{tabular}{|l|l|} 
\\
\end{tabular} & & 12.0 & & \begin{tabular}{|l|}
2.793 \\
\end{tabular} & \begin{tabular}{|l|l|} 
\\
\end{tabular} & \begin{tabular}{|l|}
0.009 \\
\end{tabular} & 0.000 & 0.215 & 0.18 \\
\hline $\begin{array}{ll}1755 \\
1754\end{array}$ & $4 / 21 / 2004$ & $2: 17: 01 \mathrm{AM}$ & 25.701 & & 25.43 & 25.108 & 24.541 & 48.527 & \begin{tabular}{|l|}
52.784 \\
\end{tabular} & \begin{tabular}{|l|l|}
16.938 \\
\end{tabular} & & $\frac{-1.221}{-1.21}$ & $\frac{-7.362}{-.362}$ & 49.455 & 0.059 & $\begin{array}{l}14.643 \\
\end{array}$ & & 2435.2 & \begin{tabular}{|l|l|}
40.5864 \\
\end{tabular} & & & & \begin{tabular}{|l|l|}
2.774 \\
\end{tabular} & & \begin{tabular}{|l|}
0.009 \\
\end{tabular} & & & 0.18 \\
\hline & & & & & & & & 48.755 & & 16.885 & & -1.218 & & & & 14.643 & & 2436.2 & & 51.6 & & 40.5 & & & & & & \\
\hline \begin{tabular}{|l|l|}
1757 \\
1759
\end{tabular} & $4 / 21 / 2004$ & 2:19:01 AM & $\begin{array}{l}25.694 \\
25690\end{array}$ & 24.873 & 25.443 & $\begin{array}{l}25.106 \\
25.06\end{array}$ & $\begin{array}{l}24.624 \\
2.770\end{array}$ & \begin{tabular}{|l|l|}
48.853 \\
.075
\end{tabular} & \begin{tabular}{|l|l|}
52.938 \\
5.20
\end{tabular} & \begin{tabular}{ll|}
16.983 \\
19.06
\end{tabular} & $\begin{array}{l}32.225 \\
20157\end{array}$ & $\begin{array}{l}-1.218 \\
1.221\end{array}$ & $\begin{array}{l}-7.342 \\
\end{array}$ & $\begin{array}{l}49.362 \\
\end{array}$ & $\begin{array}{l}0.062 \\
0056\end{array}$ & 14.643 & $\begin{array}{l}0.003 \\
\end{array}$ & 2437.2 & \begin{tabular}{|l|}
40.6197 \\
\end{tabular} & 51.5 & 12.1 & & 2.795 & \begin{tabular}{|l|l|}
0.009 \\
\end{tabular} & 0.009 & 0.000 & & \\
\hline$\frac{1758}{1759}$ & $\begin{array}{l}4 / 21 / 2004 \\
4 / 21204\end{array}$ & $\begin{array}{l}2: 20: 01 \mathrm{AM} \\
2 \cdot 21 \cdot 01 \mathrm{AM}\end{array}$ & $\begin{array}{l}25.689 \\
25.679\end{array}$ & $\begin{array}{l}24.863 \\
22853\end{array}$ & \begin{tabular}{|l|}
25.443 \\
25383 \\
\end{tabular} & $\begin{array}{l}25.126 \\
25.191\end{array}$ & \begin{tabular}{|l|}
24.779 \\
24864 \\
\end{tabular} & \begin{tabular}{|l|l|l|l|l|}
48.675 \\
88.637
\end{tabular} & \begin{tabular}{|r|}
53.029 \\
52.94
\end{tabular} & \begin{tabular}{|l|l|}
16.9066 \\
116922
\end{tabular} & $\begin{array}{l}32.157 \\
32053 \\
\end{array}$ & $\begin{array}{l}-1.221 \\
-1.221 \\
\end{array}$ & $\begin{array}{r}-7.18 \\
-.7264\end{array}$ & \begin{tabular}{|l|l|}
48.827 \\
49845 \\
\end{tabular} & $\begin{array}{l}0.056 \\
0.057\end{array}$ & $\begin{array}{l}144.643 \\
14643\end{array}$ & \begin{tabular}{|l|}
0.003 \\
0.003
\end{tabular} & $\begin{array}{l}2438.2 \\
243392\end{array}$ & \begin{tabular}{|l|}
40.6364 \\
40.6531 \\
\end{tabular} & \begin{tabular}{|l|l|}
51.6 \\
51.1
\end{tabular} & $\frac{12.0}{12.2}$ & $\frac{40 .}{40 .}$ & $\begin{array}{l}2.787 \\
2.782 \\
\end{array}$ & \begin{tabular}{|c|}
0.008 \\
0.008 \\
\end{tabular} & $\begin{array}{l}0.008 \\
0.008\end{array}$ & $\begin{array}{l}0.000 \\
0.000\end{array}$ & \begin{tabular}{|l|l|}
0.204 \\
0.208
\end{tabular} & $\begin{array}{l}0.17 \\
0.18\end{array}$ \\
\hline 1760 & $4 / 21 / 2004$ & $\begin{array}{l}2.21 .01 \mathrm{AM} \\
2: 22: 01 \mathrm{AM}\end{array}$ & 25.684 & & 25.453 & 25.1956 & \begin{tabular}{|l|}
24.004 \\
24.824 \\
\end{tabular} & & \begin{tabular}{|r|}
52.913 \\
\end{tabular} & & & $\begin{array}{l}-1.221 \\
-1.221\end{array}$ & $\begin{array}{l}-7.264 \\
-7.267\end{array}$ & \begin{tabular}{|}
4.045 \\
47.294 \\
\end{tabular} & 0.059 & $\begin{array}{l}14.643 \\
14.643 \\
\end{array}$ & 0.003 & $\frac{2439.2}{2440.2}$ & \begin{tabular}{|l|}
40.6531 \\
0.6697
\end{tabular} & $\frac{51 .}{51.2}$ & $\frac{12.2}{11.6}$ & $\frac{40 .}{40 .}$ & $\frac{2.782}{2.782}$ & \begin{tabular}{|c|c|}
0.008 \\
0.009
\end{tabular} & $\begin{array}{l}0.008 \\
0.009\end{array}$ & $\begin{array}{l}0.000 \\
0.000 \\
\end{array}$ & $\frac{0.208}{0.215}$ & 0.18 \\
\hline & $4 / 21 / 2004$ & $2: 23: 01 \mathrm{AM}$ & & & & & 24.894 & 48.774 & \begin{tabular}{|l|}
53.043 \\
\end{tabular} & 16.975 & & -1.218 & & 49.458 & 0.057 & & 0.003 & 2441.2 & \begin{tabular}{|l|l|}
40.6864 \\
\end{tabular} & & & & & & & & & $\begin{array}{l}0.18 \\
0.18\end{array}$ \\
\hline 1762 & $4 / 21 / 2004$ & $2: 24: 01 \mathrm{AM}$ & & & 25.458 & 25.335 & 24.959 & 48.422 & \begin{tabular}{|l|l|}
52.726 \\
\end{tabular} & 16.924 & 31.739 & -1.224 & -7.296 & 48.673 & 0.055 & 14.643 & 0.6 & 2442.2 & \begin{tabular}{|l|l|l|}
40.7031 \\
\end{tabular} & 51.3 & 11. & & & & \begin{tabular}{|l|}
0.008 \\
\end{tabular} & 0.000 & & \\
\hline 17763 & $4 / 21 / 2004$ & $2: 25: 01 \mathrm{AM}$ & 25.684 & & & & & & & & & & & & & & 0.003 & 2443 & & & 12. & & & & & & 207 & $\begin{array}{l}0.11 \\
0.18\end{array}$ \\
\hline 1764 & $4 / 21 / 2004$ & $2 \cdot 250109$ & & & 251819 & & & & 52067 & 16.963 & & -1.218 & & 7 & 0.056 & & & & $\frac{7364}{7364}$ & & & & & & & & & \\
\hline & $4 / 21 / 2004$ & 2:27:01 AM & 25.691 & 24.854 & 25.465 & 25.388 & & 48.942 & 53.247 & 16.991 & 32.213 & -1.227 & & & 0.057 & 14.643 & & & & & 12 & & 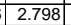 & D.008 & 0.008 & & & \\
\hline & 4/21/2004 & 2:28:01 AM & 25.716 & 24.87 & 25.5 & \begin{tabular}{|l|l|}
25.463 \\
\end{tabular} & 24.956 & \begin{tabular}{|l|l|l|l}
48.778 \\
\end{tabular} & 53.178 & 17.009 & 32.112 & $\begin{array}{l}-1.221 \\
\end{array}$ & -7.28 & \begin{tabular}{|l|l|l|l|}
48.867 \\
\end{tabular} & 0.057 & 14.643 & $\begin{array}{l}0.003 \\
\end{array}$ & 2446.2 & \begin{tabular}{|l|l|l|l|l|}
4697 \\
\end{tabular} & 51. & 12.0 & 40. & 2.789 & 0.008 & 0.008 & 0.000 & 0.207 & \\
\hline 1776 & $11 / 2004$ & $2: 29: 01 \mathrm{AM}$ & 25.716 & 24.885 & 25.505 & 25.388 & 24.831 & 48.955 & 53.352 & 16.989 & 32.29 & -1.218 & & 49.209 & 0.058 & 14.643 & & 2447.2 & 40.7864 & & 12. & & & & 0.009 & & & 0.18 \\
\hline & $11 / 2004$ & & & & & & & & & & 32.291 & -1.218 & & & 0.056 & 14.643 & & 2448. & & & & & & & & & & \\
\hline & $21 / 2004$ & $2: 31: 01 \mathrm{AM}$ & 25.71 & 24.869 & 25.509 & 25.217 & 24.665 & 48.619 & 2.907 & 16.988 & 31.948 & -1.215 & & 8.362 & 0.054 & 14.643 & & & & & & & & 008 & 0.008 & .000 & 197 & \\
\hline & & $2.320 .01 \mathrm{AM}$ & $\frac{25.109}{2572}$ & & & $\frac{25.151}{25059}$ & $\frac{24.099}{2.548}$ & 48.95 & $\frac{5.265}{2178}$ & $\frac{16.982}{16807}$ & $\frac{32.205}{22072}$ & $\frac{-1.1 .69}{12}$ & & $\frac{0.154}{10.147}$ & 年 & $\frac{1.4645}{1.643}$ & & (15) & & & & & & & & & & \\
\hline$\frac{1712}{1772}$ & $\begin{array}{l}4 / 21 / 21 / 204 \\
4 / 21 / 204\end{array}$ & $\begin{array}{l}2.35 .01 \mathrm{AM} \\
2: 34: 01 \mathrm{AM}\end{array}$ & $\begin{array}{l}25.113 \\
25.696\end{array}$ & $\frac{24.801}{24.844}$ & $\frac{25.512}{25.49}$ & $\begin{array}{l}25.059 \\
25.032\end{array}$ & $\begin{array}{l}24.546 \\
24.546\end{array}$ & $\begin{array}{l}48.828 \\
48.807\end{array}$ & \begin{tabular}{|l|}
53.118 \\
53.064
\end{tabular} & $\mid \begin{array}{l}10.097 \\
17.044\end{array}$ & $\begin{array}{l}32.281 \\
32.141 \\
\end{array}$ & $\begin{array}{r}-1.2 \\
-1.227 \\
\end{array}$ & & $\begin{array}{r}\frac{49.14 t}{50.11} \\
\end{array}$ & 0.057 & $\begin{array}{l}4.645 \\
14.643 \\
\end{array}$ & 0.003 & 24522 & \begin{tabular}{|l}
40.0697 \\
4
\end{tabular} & 51, & & & & & 0.008 & 0.000 & 0.207 & 0.18 \\
\hline & $4 / 21 / 2004$ & $2: 35: 01 \mathrm{AM}$ & 25.704 & 24.842 & 25.453 & 25.095 & 24.559 & 48.654 & \begin{tabular}{|l|}
52.984 \\
\end{tabular} & 17.065 & 31.923 & -1.195 & -7.264 & 48.256 & 0.056 & 14.643 & 0.003 & 2453.2 & \begin{tabular}{|l|l|}
40.8864 \\
\end{tabular} & 51.5 & 11.8 & 40. & 2.778 & & 0.008 & 0.000 & 0.205 & $\begin{array}{l}0.18 \\
0.17\end{array}$ \\
\hline & $4 / 21 / 2004$ & 2:36:01 AM & 25.703 & & 25.422 & 25.115 & & 48.687 & 53.012 & 17.026 & & -1.151 & & $\begin{array}{l}49.472 \\
\end{array}$ & 0.055 & 14.643 & & 2454.2 & & & & & & & & & & \\
\hline & $11 / 2004$ & 2:37:01 AM & 25.697 & 24.8 & 25.411 & 25.133 & 24.747 & & 3.516 & 17.126 & & -1.189 & & 19.149 & 0.059 & 14.643 & & & & & & & & & & & & \\
\hline & $\frac{421212004}{4121204}$ & 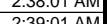 & 25.690 & $\frac{4.84}{24.83}$ & $\frac{25.43}{25.45}$ & $\frac{25.233}{25268}$ & 24.696 & $\frac{48.05}{4.525}$ & .0.054 & $\mid$ & $\begin{array}{l}31.854 \\
31842\end{array}$ & $\begin{array}{l}-1.154 \\
-1148\end{array}$ & & 1 & 年 0.051 & 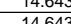 & 0004 & 2457.4 & 295 & & t. & 4 & & & & & & \\
\hline 1778 & $4 / 212004$ & $2: 40.01 \mathrm{AM}$ & 25.681 & 24.809 & 25.435 & 25.298 & 24.030 & 48.706 & 5083 & $\begin{array}{l}\frac{10.900}{17.077} \\
\end{array}$ & $\begin{array}{l}31.045 \\
31.938\end{array}$ & $\begin{array}{l}-1.140 \\
-1.154\end{array}$ & & 0.425 & $\begin{array}{l}0.050 \\
0.056\end{array}$ & $\frac{14.043}{14.643}$ & & 245.6 & & 516 & $\frac{12.0}{124}$ & & 8780 & 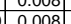 & 0.008 & & & \\
\hline & $4 / 21 / 2004$ & 2:4001:A1 AM & $\frac{20.001}{25.687}$ & $\frac{2.05}{24.825}$ & 25.451 & 25.354 & $\frac{24.642}{24.842}$ & 488.604 & 2.052 & 17.127 & 31.700 & $\begin{array}{l}-1.154 \\
-1.154 \\
\end{array}$ & & $\begin{array}{l}0.420 \\
49.067\end{array}$ & 0.057 & $\frac{14.043}{14.643}$ & & 2459.2 & 40.9864 & & $\frac{12.4}{12.0}$ & $4 \mathrm{4c}$ & & & & & 209 & \\
\hline & 4/21/2004 & $2: 42: 01 \mathrm{AM}$ & 25.682 & & 25.446 & 25.374 & 24.962 & 48.979 & & & & & & 49.499 & 0.043 & 14.643 & & 2460. & & & 12. & & 2.79 & & & & & \\
\hline \begin{tabular}{|l|l|l|l|l|}
1781 \\
\end{tabular} & 4/21/2004 & 2:43:01 AM & 25.689 & 24.817 & 25.448 & 25.376 & 25.044 & \begin{tabular}{|l|l|}
48.687 \\
\end{tabular} & 53.245 & 17.108 & 31.897 & -1.027 & & 48.634 & 0.054 & 14.643 & & 2461.2 & 41.0197 & & $11 . .5$ & 44 & & 0.008 & 0.008 & 0.000 & 0.197 & \\
\hline & $4 / 21 / 2004$ & 2:44: & 25.69 & 24.825 & 25.454 & \begin{tabular}{l|l|}
25.392 \\
\end{tabular} & 25.07 & 48.625 & 53.041 & 16.996 & 31.968 & -1.16 & & & & 14.643 & & & & & & & & & & & & \\
\hline 1783 & $4 / 21 / 2004$ & & 25.702 & 24.856 & 25.481 & 25.459 & 24.967 & 48.834 & 53.238 & 17.052 & 32.085 & & & 50479 & 0.5 & $\frac{14.643}{14.64}$ & & 2463.2 & & & 12.4 & & 2798 & & & & & 0.18 \\
\hline & 4/21/2004 & $\overline{D 1}$ AM & 25.698 & 24.841 & 25.477 & 25.415 & 24.888 & \begin{tabular}{|l|l|l|l|l}
48.324 \\
\end{tabular} & .722 & 17.001 & 31.62 & -1.154 & & 47.515 & 0.057 & 14.643 & & 2464.2 & 1.0697 & 51.2 & 11.6 & & & D.008 & 0.008 & 0.000 & 0.210 & 0.18 \\
\hline & 4/21/2004 & 2:47:01 AM & 25.688 & 24.842 & 25.468 & 25.325 & 24.7 & & .714 & 17.022 & 31.533 & -1.137 & & & & 14. & & & & & 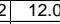 & & & & & & & \\
\hline & $4 / 21 / 2004$ & 2::48:01 AM & 25.703 & & 25.502 & 25.23 & & 48.785 & 1.99 & 17.093 & & -1.154 & & 48.969 & 0.057 & & & 2466. & & & 12.0 & 40. & & & & & & \\
\hline & $4 / 21 / 2004$ & 2:49:01 AM & 25.681 & 24.84 & 25.466 & 25.153 & & & 3.141 & 17.014 & 31.843 & -1.108 & & & & 14.643 & & 246 & & & 12.3 & & 7 & & 0.00 & 0.000 & & \\
\hline & & & & 24.83 & $\frac{15.48}{25.40}$ & & & & & & & -1.14 & & & & & & & & & & & & & & & & \\
\hline & 4 & $\frac{2.501 .01 \mathrm{AM}}{20.50}$ & $\begin{array}{l}23.064 \\
25.684\end{array}$ & $\frac{24.065}{24817}$ & $\frac{25.419}{25483}$ & $\frac{23.011}{2505}$ & $\frac{24.32}{24.52}$ & 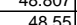 & 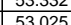 & $\mid$ & $\begin{array}{l}32.030 \\
31.774\end{array}$ & $\frac{1.0090}{-1.105}$ & & & 然 0.057 & $\frac{1.4645}{1463}$ & & $\frac{2409.2}{2402}$ & \begin{tabular}{|l}
41.1531 \\
41.697
\end{tabular} & 516 & $\frac{1.1}{122}$ & & & & 0 & 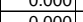 & $\frac{201}{212}$ & \\
\hline & $\frac{14}{4 / 212004}$ & $2 \cdot 5.01 .01 \mathrm{AM}$ & 25.683 & 24.812 & | 20.400 & 25084 & & & $\frac{153}{313}$ & & & -1.114 & & & & & & $\frac{24}{24}$ & 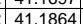 & 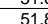 & 12.4 & & & & & & & \\
\hline & $4 / 21 / 2004$ & $2: 54: 01 \mathrm{AM}$ & 25.676 & 24.799 & 25.495 & 25.143 & 24.666 & 48.569 & 53.052 & 16.954 & 31.942 & -1.099 & & 48.581 & 0.05 & 14.6 & & 247 & & - & 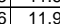 & & & & & & & \\
\hline & $1 / 2004$ & $2: 55: 01 \mathrm{AM}$ & & & 25.48 & & & & & 16.977 & & $\begin{array}{c}-1.099 \\
-1099\end{array}$ & & & & & & & & & & & & & & & & \\
\hline & & & 25.681 & 24.789 & 255 & 25.248 & & 48.69 & & 17108 & 31.92 & -1099 & & & 0.05 & & & & & & 12 & & & & 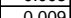 & & & \\
\hline & 4/21/2004 & 2:57:01 AM & 25.687 & 24.79 & 25.521 & 25.304 & & & 265 & 16.992 & & $\begin{array}{l}-1.099 \\
\end{array}$ & & & & & & & 41.2 & 51 & 12.1 & 40 & & & $0.6 \quad-6$ & & 0.207 & \\
\hline & 21/2004 & 2:58:C & & 24.79 & 25.491 & 25.299 & & & & 16.98 & & -1.096 & & & & & & & & & 11.8 & & & & & & & \\
\hline 179 & 4/21/2004 & 2:59:01 AM & 25.693 & 24.781 & 25.442 & 25.32 & & $\begin{array}{l}48.699 \\
\end{array}$ & 3.186 & & 31.975 & -1.1111 & & & & 14. & & & 41.2 & 51 & 12.4 & 4 & & & & & & \\
\hline 1779 & 4/21/2004 & 3:00: & 25.706 & 24.799 & 25.455 & 25.3 & & 48.523 & & & & -1.096 & & & & & & & 41.3 & 51 & $11 . .2$ & & & & & & 0.1 & \\
\hline & & & 25.706 & 24.794 & & 25.393 & & & & 17.185 & & -1.099 & & & & & & & & 52. & 11. & & & & & & & \\
\hline & $4 / 212121204$ & & 25.696 & 24. & $\frac{25.44}{25.45}$ & 25.408 & 25.0 & 48 & 52,1 & 16.869 & 31.16 & -1.102 & & & & & & & & 51 & 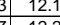 & & & & & & 100 & \\
\hline & 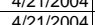 & & $\frac{25.090}{25703}$ & $\frac{24.6}{24.81}$ & $\frac{25.450}{25.462}$ & $\frac{25.404}{25425}$ & & & & & & $\frac{-1.099}{-1099}$ & & & & & & & & & & & & & & & & \\
\hline & 4 & & 25688 & 24812 & 25.443 & $\frac{2.425}{253}$ & & & & & & -109 & & & & & & & & & & & & & & & & \\
\hline 180 & $4 / 21 / 2004$ & & 25.693 & 24.826 & 25 & 25.23 & 24.6 & & \begin{tabular}{|l|l|}
52.905 \\
\end{tabular} & $\frac{17.046}{17.046}$ & & $\begin{array}{c}-1.079 \\
\end{array}$ & & & & & & & & & 12. & & & & & & 0.206 & \\
\hline & & & & & & & & & & & & & & & & & & & & & & & & & & & & \\
\hline & & & 25.686 & 24814 & 2546 & 25042 & 24.5 & & 5027 & 16.973 & 31.845 & -1076 & & & & & & & & & 15 & & & & & & & \\
\hline & $4 / 21 / 200$ & 3:08: & 25.6 & 24.8 & 25.483 & 25.01 & 24 & & 209 & & 31. & -1.02 & & & & & & & & 51 & & & & & & & & \\
\hline & $4 / 21 / 2004$ & 3:09:24 AM & 25.682 & 24.796 & 25. & 24.974 & 24. & 48.5 & 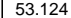 & & & -1.004 & & & 0.057 & 14.643 & & 248 & 41.4 & 51 & 11. & & & & & & 08 & \\
\hline & $4 / 21 / 2004$ & 3:10:24 AM & 25.6 & 24.791 & 25.471 & 25.059 & 24. & $\begin{array}{ll}48.4 \\
\end{array}$ & 52.936 & & & $-1.0 !$ & & 49.2 & & & & & & 51. & 12.1 & & & & & & 05 & \\
\hline & $4 / 21 / 200$ & & 25.6 & 24.784 & & & 24. & 48.654 & \begin{tabular}{|l|} 
\\
\end{tabular} & 16.894 & & -0.995 & & 47.82 & 0.055 & & & 24 & & 51 & 11. & & & & & & 200 & \\
\hline & $4 / 21212004$ & 3:12: & 25.675 & 24.779 & 25.484 & 25.147 & 24.73 & 48.602 & 53.101 & 16.939 & 32.001 & -0.995 & & $0 . \angle T T$ & & 14. & & & & & & & & & & & & \\
\hline & 4121212004 & 3:13:24 AM & $\frac{25.675}{2568}$ & 24.173 & 2.2554 & 25.256 & 24.17 & $\begin{array}{l}48.824 \\
48867\end{array}$ & 53423 & $\begin{array}{l}1.042 \\
1.7096\end{array}$ & 32.118 & -0.992 & & 179 & & & & $\frac{245}{240}$ & & & $\frac{12.3}{1.2}$ & & & & & & & \\
\hline & $412121200^{4}$ & $\frac{3.14 .24}{3.15}$ & $\frac{13.00}{25600}$ & 2.179 & 20.0549 & 25.201 & & $\begin{array}{ll}40.007 \\
18.053\end{array}$ & 0 & 17.090 & & $=1.012$ & & & 0 & & & & & & & & & & & & & \\
\hline & $\frac{4}{421212004}$ & $\begin{array}{l}0.1 .254 \\
3.162 .24\end{array}$ & 256 & $\frac{24}{24}$ & 25 & $\frac{25.470}{25312}$ & $\frac{24.6}{24}$ & 488 & & & 321 & -1018 & & & & & & & & $5 ?$ & $\frac{12.0}{122}$ & & & & & & & \\
\hline 1816 & & & & & 25 & $\frac{25.343}{25.343}$ & & & 52844 & & & & & & & & & & & 512. & 121 & & & & & & & \\
\hline & $4 / 21 / 2004$ & & & & 25.456 & & 24.9 & & & 16.997 & & -0.94 & & & & & & & & & & & & & & & & \\
\hline & & & & & & & & & & 17.043 & & -0.937 & & & & & & & & & & & & & & & & \\
\hline & $4 / 21 / 2004$ & $3.20 \%$ & 25.695 & 24.804 & 25469 & 25.382 & 24 & 48.492 & 53.162 & \begin{tabular}{|l|l|}
17.08 \\
\end{tabular} & & -0.937 & & & & 4.643 & & $24 \mathrm{C}$ & & & 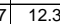 & & & & & & 206 & \\
\hline & $4 / 21 / 2004$ & 3:21:24 AM & & & & & 24.774 & & & 17.134 & & \begin{tabular}{|c|c|c|}
-0.937 \\
\end{tabular} & & & 0.057 & 14.643 & & & 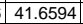 & & 12.0 & & 2.762 & & 0.008 & 0.000 & 0.209 & 0.1 \\
\hline
\end{tabular}


WSRC-TR-2005-00105, REVISION 0

SRNL-RPP-2005-00012, REVISION 0

RUN \# 2.03A AND B; FIRST AND SECOND HALF OF SLURRY DEWATERING - CONT.

\begin{tabular}{|c|c|c|c|c|c|c|c|c|c|c|c|c|c|c|c|c|c|c|c|c|c|c|c|c|c|c|c|c|}
\hline & A & B & D & $E$ & $F$ & $G$ & $\mathrm{H}$ & $\mathrm{J}$ & $\mathrm{K}$ & $\mathrm{L}$ & $\mathrm{M}$ & $\mathrm{N}$ & 0 & $Q$ & $R$ & 5 & \begin{tabular}{l|l|l|} 
\\
\end{tabular} & & w & $x$ & & 2 & AA & $A B$ & $\mathrm{AC}$ & $A D$ & & \\
\hline $\begin{array}{ll}1821 \\
1822 \\
\end{array}$ & $\begin{array}{l}4 / 21 / 212004 \\
421 / 2004\end{array}$ & $\begin{array}{l}3: 22: 24 \mathrm{AM} \\
3.23 \cdot 24 \mathrm{MM}\end{array}$ & $\begin{array}{l}25.679 \\
25.677\end{array}$ & $\begin{array}{l}24.792 \\
22.785 \\
\end{array}$ & \begin{tabular}{|l|}
25.458 \\
25.666 \\
\end{tabular} & $\begin{array}{l}25.205 \\
25.128\end{array}$ & \begin{tabular}{|l|}
24.659 \\
24.627 \\
\end{tabular} & $\begin{array}{l}48.822 \\
48.245 \\
\end{array}$ & \begin{tabular}{|l|}
53.367 \\
52.861 \\
\end{tabular} & \begin{tabular}{|c|}
17.071 \\
16.96
\end{tabular} & $\begin{array}{l}32.108 \\
3.1585 \\
\end{array}$ & $\begin{array}{r}-0.969 \\
-0.989 \\
\end{array}$ & $\begin{array}{r}-7.053 \\
-7.053 \\
\end{array}$ & $\begin{array}{l}48.915 \\
48.859 \\
4\end{array}$ & 0.055 & $\begin{array}{l}14.643 \\
14.643\end{array}$ & $\begin{array}{l}0.003 \\
0.03\end{array}$ & $\begin{array}{l}2500.6 \\
2501.6\end{array}$ & \begin{tabular}{|l|l|}
41.6761 \\
41.9928
\end{tabular} & $\begin{array}{l}51.9 \\
51.4\end{array}$ & $\frac{12}{12}$ & $\begin{array}{l}40.5 \\
339.9\end{array}$ & \begin{tabular}{|l|}
2.790 \\
2.752
\end{tabular} & \begin{tabular}{|c|c|}
0.008 \\
0.008
\end{tabular} & $\begin{array}{l}0.008 \\
0.008\end{array}$ & 0.000 & $\begin{array}{l}0.200 \\
0.203\end{array}$ & $\begin{array}{l}0.17 \\
0.17\end{array}$ \\
\hline & & & 25.677 & & & & & & & $\begin{array}{l}16.952 \\
16.92\end{array}$ & & & & & & $\frac{14.043}{14.643}$ & & & & & & & & & & & & \\
\hline 1824 & $4 / 21 / 2004$ & 3:25:24 AM & 25.676 & 24.784 & 25.48 & \begin{tabular}{|l|l|}
25.007 \\
\end{tabular} & 24.476 & $\begin{array}{l}48.453 \\
\end{array}$ & 53.077 & 17.094 & 31.616 & -0.934 & -6.937 & 49.812 & 0.057 & 14.643 & 0.003 & 2503.6 & 41.7261 & & 12.2 & 40.0 & 2.760 & 0.008 & 0.008 & 0.000 & 0.209 & \\
\hline \begin{tabular}{|l|l|}
1825 \\
\end{tabular} & $4 / 21 / 2004$ & 3:26:24 AM & 25.66 & 24.768 & 25.474 & 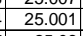 & 24.499 & 48.484 & 53.025 & 17.037 & 31.749 & $\begin{array}{l}-0.937 \\
\end{array}$ & $\begin{array}{l}-7.041 \\
-1.54\end{array}$ & 50.225 & 0.056 & 14.643 & & 2504.6 & \begin{tabular}{|l|l|}
41.7428 \\
\end{tabular} & & & 40.1 & & & 0.008 & & & 0.17 \\
\hline & & & & & & & & & \begin{tabular}{|l|l|}
53.046 \\
\end{tabular} & $\begin{array}{l}16.931 \\
\end{array}$ & & $\begin{array}{l}-0.96 \\
\end{array}$ & & & & & & & & & & & & & & & & \\
\hline \begin{tabular}{|l|l|}
1827 \\
1829
\end{tabular} & $4 / 21 / 2004$ & 3:28:24 AM & 25.662 & 24.76 & $\begin{array}{l}25.496 \\
25.512\end{array}$ & $\begin{array}{l}25.128 \\
25.00\end{array}$ & $\begin{array}{l}24.612 \\
2172\end{array}$ & $\begin{array}{l}48.695 \\
8.295\end{array}$ & \begin{tabular}{|l|l|}
53.211 \\
5.80
\end{tabular} & $\begin{array}{l}16.986 \\
16.089\end{array}$ & $\begin{array}{l}32.028 \\
21055\end{array}$ & $\begin{array}{l}-0.992 \\
.0 .91\end{array}$ & $\begin{array}{r}-7.038 \\
.9028\end{array}$ & $\begin{array}{l}48.654 \\
5.256\end{array}$ & 0.055 & 14.643 & $\begin{array}{l}0.003 \\
\end{array}$ & $\begin{array}{l}2506.6 \\
500 .\end{array}$ & \begin{tabular}{|l|l|}
41.7761 \\
\end{tabular} & 51.7 & 11. & & 2.783 & \begin{tabular}{|l|l|}
0.008 \\
\end{tabular} & 0.008 & 0.000 & 0.200 & \\
\hline $\begin{array}{l}1828 \\
1829\end{array}$ & $4 / 21 / 2004$ & $\begin{array}{l}3: 29: 24 \mathrm{AM} \\
3.3024 \mathrm{AM}\end{array}$ & $\begin{array}{l}25.668 \\
25.657\end{array}$ & $\begin{array}{l}24.766 \\
22.745\end{array}$ & $\begin{array}{l}25.512 \\
25.506\end{array}$ & \begin{tabular}{|l|l|}
25.199 \\
25.174
\end{tabular} & \begin{tabular}{|l|}
24.728 \\
24778
\end{tabular} & \begin{tabular}{|l|l|l|l|l}
48.285 \\
88.407
\end{tabular} & \begin{tabular}{|l|}
52.809 \\
53072
\end{tabular} & \begin{tabular}{|l|l|l|l|l|}
16.988 \\
16.985
\end{tabular} & $\begin{array}{l}31.655 \\
31172 \\
\end{array}$ & \begin{tabular}{|l|}
-0.934 \\
-0.934
\end{tabular} & $\begin{array}{l}-6.928 \\
-6.983\end{array}$ & $\begin{array}{l}51.256 \\
49.257\end{array}$ & $\begin{array}{l}0.053 \\
0.058\end{array}$ & $\begin{array}{l}14.643 \\
14.643\end{array}$ & \begin{tabular}{|l|l}
0.003 \\
0.003
\end{tabular} & $\begin{array}{l}2507.6 \\
2508\end{array}$ & \begin{tabular}{|l|l|}
41.7928 \\
418094
\end{tabular} & \begin{tabular}{|l|l|}
51.3 \\
516
\end{tabular} & $\begin{array}{ll}12.6 \\
12.1\end{array}$ & $\begin{array}{l}40.0 \\
40.1\end{array}$ & $\begin{array}{l}2.756 \\
2.763\end{array}$ & \begin{tabular}{|c|}
0.008 \\
0.099 \\
\end{tabular} & $\begin{array}{l}0.008 \\
0.009\end{array}$ & $\begin{array}{l}0.000 \\
0.000\end{array}$ & \begin{tabular}{|l}
0.195 \\
0.213
\end{tabular} & $\begin{array}{l}0.17 \\
0.18\end{array}$ \\
\hline 1830 & $4 / 21 / 2004$ & 3:31:24 AM & 25.663 & & 25.517 & 25.194 & 24.788 & & \begin{tabular}{|l|l|}
53.396 \\
\end{tabular} & & & \begin{tabular}{|l|}
-0.934 \\
\end{tabular} & $\begin{array}{l}-0.903 \\
-7.012 \\
\end{array}$ & $\begin{array}{l}49.257 \\
48.354\end{array}$ & 0.055 & $\frac{14.643}{14.643}$ & 0.003 & $\begin{array}{l}2500.6 \\
2509.6\end{array}$ & & & & $\frac{4.1}{40.3}$ & $\frac{2.763}{2.782}$ & \begin{tabular}{|c|}
0.009 \\
0.008
\end{tabular} & $\begin{array}{l}0.009 \\
0.008\end{array}$ & $\begin{array}{l}0.000 \\
0.000 \\
\end{array}$ & $\frac{0.213}{0.200}$ & 0.18 \\
\hline & $4 / 21 / 2004$ & $3: 32$ & 25.668 & 24.752 & 25.527 & & 24.868 & \begin{tabular}{|l|l|l|l|l|}
48.384 \\
\end{tabular} & \begin{tabular}{|l|l|} 
\\
53.112
\end{tabular} & 16.967 & 31.628 & -0.937 & & 48.567 & & 14.643 & & & \begin{tabular}{|l|l|}
41.8428 \\
\end{tabular} & & & & & & & & & $\frac{0.17}{0.17}$ \\
\hline 1832 & $4 / 21 / 2004$ & 3:33:24 AM & 25.669 & 24.747 & 25.523 & \begin{tabular}{l|l|}
25.231 \\
\end{tabular} & & 48.532 & 53.089 & \begin{tabular}{|l|l}
17.04 \\
\end{tabular} & 31.878 & -0.937 & & 48.669 & 0.057 & 14.643 & 0.003 & 2511.6 & \begin{tabular}{|l|l|}
41.8594 \\
\end{tabular} & 51.6 & & 40.2 & & & 0.008 & 0.000 & & 0.17 \\
\hline 1833 & $4 / 21 / 2004$ & 3:34:24 AM & 25.664 & & 25.523 & & & & 53.62 & & & & & & & & & & & & & & & & & & 203 & $\begin{array}{l}0.18 \\
0.17\end{array}$ \\
\hline 1834 & $4 / 21 / 2004$ & $2.25 \% 291$ & & 24.76 & 25181 & תर & & 48.312 & 52.944 & 16.934 & 31.7 & \begin{tabular}{l|l|}
-0.937 \\
\end{tabular} & & 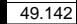 & & & & & & & & & & & & & & \\
\hline & $4 / 21 / 2004$ & 3:36:24 AM & 25.687 & 24.776 & 25.466 & \begin{tabular}{|l|l|}
25.344 \\
\end{tabular} & 24.9 & 48.617 & 53.207 & 16.952 & 32.036 & -0.931 & & 48.5 & 0.058 & 14.643 & 0.004 & & 41.9094 & & $1+x$ & 40 & 2.780 & 0.009 & 年 & & & \\
\hline 1836 & $4 / 21 / 2004$ & 3:37:24 AM & 25.678 & 24.761 & 25.437 & 25.325 & 24.963 & $\begin{array}{l}48.364 \\
\end{array}$ & 52.948 & \begin{tabular}{l|l|l|l|}
17.016 \\
\end{tabular} & 31.64 & -0.902 & -6 . & 46.76 & 0.056 & 14.643 & $\begin{array}{l}0.003 \\
\end{array}$ & \begin{tabular}{|l|l|}
2515.6 \\
\end{tabular} & \begin{tabular}{|l|l|}
41.9261 \\
\end{tabular} & 51.5 & 11.5 & 40.0 & 2.758 & 0.008 & 0.008 & 0.000 & 0.206 & \\
\hline & $4 / 21 / 2004$ & 3:38:24 AM & 25.699 & 24.792 & 25.468 & 25.381 & 24.879 & 48.476 & 53.058 & 17.084 & 31.677 & -0.885 & & 49.733 & 0.057 & 14.643 & & & 41.9428 & & & & & & .00 & & & \\
\hline & $4 / 21212004$ & & & & 25.487 & & & 48.411 & & & 31.737 & .934 & & & & 14.643 & & & & & & & & & & & & \\
\hline & $4 / 21 / 2004$ & 3:40:24 AM & 25.672 & 24.776 & 5.456 & & & & 3.099 & 17.034 & 13.739 & .934 & & 8.855 & 0.055 & 14.643 & & & & & & & & & .000 & & & \\
\hline & $\frac{421212004}{4121204}$ & $\begin{array}{l}3.44 .24 \mathrm{AM} \\
20.24\end{array}$ & & & $\frac{2.4 .41}{20.20}$ & & 24.547 & 48.2 & 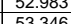 & & $\frac{31.497}{3190}$ & - 0.934 & & 1812 & & $\frac{1.4645}{1.643}$ & & & & & & & & & & & & \\
\hline$\frac{1041}{1842}$ & 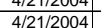 & $\begin{array}{l}3.242 .24 \mathrm{AM} \\
3: 43: 24 \mathrm{AM}\end{array}$ & $\begin{array}{r}25.00 \\
25.673 \\
\end{array}$ & $\begin{array}{l}\frac{24.108}{24.766} \\
\end{array}$ & $\frac{25.439}{25.472}$ & $\begin{array}{l}25.050 \\
25.019\end{array}$ & $\begin{array}{l}24.464 \\
24.367\end{array}$ & $\begin{array}{r}46.054 \\
48.83\end{array}$ & $\begin{array}{l}53.340 \\
53.516\end{array}$ & $\begin{array}{l}17.000 \\
17.095\end{array}$ & $\begin{array}{l}31.649 \\
32.051\end{array}$ & $\begin{aligned}-0.925 \\
-0.92\end{aligned}$ & & \begin{tabular}{|l|}
4.125 \\
50.356
\end{tabular} & 0.056 & $\begin{array}{l}4.645 \\
14.643 \\
\end{array}$ & 0.003 & 25216 & \begin{tabular}{|l|l|}
42.0094 \\
42.0261
\end{tabular} & & $\frac{1.2}{12.3}$ & 404 & & & 0.008 & 0.000 & 0.1904 & \\
\hline & $4 / 21 / 2004$ & 3:44:24 AM & 25.649 & 24.753 & 25.458 & 24.975 & 24.354 & 48.532 & 53.162 & 117.019 & 31.813 & -0.905 & & \begin{tabular}{|l|}
49.157 \\
\end{tabular} & 0.055 & 14.643 & 0.003 & 2522.6 & \begin{tabular}{|l|l|}
42.0428 \\
\end{tabular} & 51.7 & & & \begin{tabular}{|l|} 
\\
\end{tabular} & & 0.008 & & & $\begin{array}{l}0.17 \\
0.17\end{array}$ \\
\hline & $21 / 2004$ & 3:45:24 AM & 25.646 & 24.73 & 25.46 & 24.943 & 24.396 & 48.49 & & 17.055 & & -0.888 & & $\begin{array}{l}48.636 \\
\end{array}$ & 0.058 & 14.643 & & & & & & & & & & & & \\
\hline & $4 / 21212004$ & 3:46:24 AM & $\frac{25.651}{25.611}$ & 24.735 & 25.486 & 24.973 & 24.45 & & 53.348 & $\begin{array}{l}17.077 \\
17122\end{array}$ & 31.849 & -0.894 & & 8.859 & 0.056 & 14.643 & & & 0761 & & & & & & & & & \\
\hline & $\frac{421212004}{4121204}$ & $3.47 .24 \mathrm{AM}$ & 25.641 & 24.714 & $\frac{25.485}{2584}$ & $\frac{25.006}{25064}$ & 24.586 & $\begin{array}{r}48.60 \\
18.72\end{array}$ & $\frac{53.282}{53448}$ & $\frac{17.124}{17005}$ & $\begin{array}{l}31.808 \\
32092\end{array}$ & 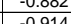 & & $\frac{49.276}{49.526}$ & 0 & $\begin{array}{l}\frac{1.4645}{1463} \\
\end{array}$ & & 2526 & 421094 & & 12 & 40.64 & (2) & & & & & \\
\hline \begin{tabular}{ll|}
1848 \\
\end{tabular} & $4 / 2 / 212004$ & & 25.639 & 24.698 & 25093 & 25106 & & $\begin{array}{l}40.140 \\
48.349\end{array}$ & 53.13 & $\frac{17.005}{16.965}$ & $\frac{32.092}{31.679}$ & 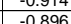 & & 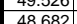 & 0 & $\frac{1.4045}{1463}$ & 0.0003 & & & 517 & $\frac{112 .}{11 .}$ & & & & & & & \\
\hline 1845 & $4 / 21 / 2004$ & 3:450:24 AM & 25.639 & $\frac{24.050}{24.693}$ & 25.498 & 25.156 & 24.044 & 4 & $\begin{array}{l}53.18 \\
53\end{array}$ & 1..904 & 31.927 & $\begin{array}{l}-0.0582 \\
-0.882\end{array}$ & & $\begin{array}{l}40.002 \\
50.457\end{array}$ & 0.055 & $\frac{14.043}{14.643}$ & & 2582.6 & \begin{tabular}{|l|l|}
42.14201 \\
428
\end{tabular} & & $\frac{11.4}{12.4}$ & 40. & $\frac{2.154}{2.774}$ & & . & & 201 & \\
\hline & $4 / 21 / 2004$ & 3:51:24 AM & 25.65 & & & 25.182 & 24.745 & 48.496 & 53.288 & & & -0.882 & & 49.983 & 0.056 & 14.643 & & & & & & & 6768 & & & & & \\
\hline 185. & $4 / 21 / 2004$ & 3:52:24 AM & 25.646 & 24.695 & 25.521 & 25.208 & & 48.565 & 53.211 & 16.96 & 31.934 & -0.882 & & 47.374 & 0.051 & $\frac{14.045}{14.643}$ & 0.004 & 2530. & 42.1761 & 51 & 11.6 & 40 & 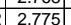 & 0.008 & 0.007 & 0.000 & 0 & \\
\hline & $4 / 21 / 2004$ & 3:53:24 AM & 25.646 & 24.68 & 25.516 & \begin{tabular}{|l|l|}
25.223 \\
\end{tabular} & 24.8 & \begin{tabular}{|l|l|l|l|}
48.357 \\
\end{tabular} & 53.155 & $\begin{array}{ll}16.909 \\
\end{array}$ & 31.591 & -0.882 & & 49.591 & & 14.643 & & & & & & & & & & & & \\
\hline & $4 / 21 / 2004$ & 3:54:24 AM & 25.663 & 24.701 & 25.527 & 25.275 & 24.748 & 48.585 & 53.28 & 17.146 & 31.741 & -0.882 & & & 0.05 & $\frac{14.643}{14.64}$ & & & & & 12 & & $\frac{6.769}{.769}$ & & & & & \\
\hline & $4 / 21 / 2004$ & 3:55:24 AM & 25.658 & 24.691 & 25.522 & 25.295 & 24.7 & 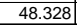 & \begin{tabular}{|l|l|} 
& 53.083 \\
\end{tabular} & 17.019 & 31.564 & -0.885 & & 49.409 & 0.056 & 14.643 & 0.6 & 2533.6 & 42.2261 & 51.6 & 12.1 & 39.9 & 4.754 & 0.008 & 0.008 & 0.000 & 0.206 & \\
\hline & $4 / 21 / 2004$ & 3:56:24 AM & & 24.696 & 20.027 & 25.3 & 24. & & 53.358 & 17.034 & 31.88 & -0.879 & & 48.427 & 0.0 & 14.643 & & & & & $t$. & & & & & & & \\
\hline & 4/21/2004 & 3:57:24 AM & 25.679 & 24.712 & 25.548 & 25.336 & 24.8 & 48.484 & 3.234 & 17.065 & 31.751 & -0.873 & & 46.3 & 0.055 & & & & 42.2594 & & 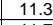 & & & & & & & \\
\hline & $4 / 21 / 2004$ & & 25.68 & 24.713 & 25.544 & 25.327 & & 48.88 & 562 & 17.019 & 32.18 & -0.882 & & 47 & 0.056 & 14.643 & & 25 & 12.2761 & & 11. & & 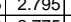 & 0.008 & 0.00 & 0.000 & & \\
\hline & & & & 24.719 & & & & & & $\frac{16.972}{17049}$ & & -0.8 & & & & & & & & & & & & & & & & \\
\hline$\frac{106}{186}$ & $\frac{42121204}{4 \mid 21204}$ & $\begin{array}{l}4.0 .2424 \mathrm{AM} \\
4\end{array}$ & $\begin{array}{l}\frac{23.001}{25695} \\
2.06\end{array}$ & $\frac{24.124}{24.729}$ & 25.35 & 25.190 & $\frac{24.64}{24 .}$ & 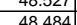 & 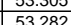 & $\frac{11.049}{16962}$ & $\frac{15.128}{3.1215}$ & $\begin{array}{l}-.0 .010 \\
-0.813\end{array}$ & & 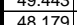 & 然 0.055 & $\frac{1.4645}{1463}$ & & 253 & $\frac{43.5034}{423261}$ & 518 & 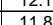 & 40 & & & 0.008 & & 0 & \\
\hline & 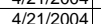 & $4.402 .24 \mathrm{Am}$ & 25.689 & 24,722 & $\frac{25.44}{2548}$ & & 244 & & $\frac{5.254}{53.34}$ & 17.011 & . & $\frac{-0.018}{-0.818}$ & & & & & & 200 & $\frac{14.53}{423}$ & 0 & $1+.6$ & & & & & & & \\
\hline & 4 & $4: 03: 24 \mathrm{AM}$ & 25.683 & 24.717 & 25.438 & 24.97 & 24.3 & 48.376 & 53.139 & 16.869 & 31.817 & -0.859 & & 10 & 0.055 & & & & & 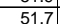 & 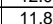 & $40, \quad$ & & & & & 201 & \\
\hline & $1 / 2004$ & & 25.661 & 24.69 & 25.435 & & & & 52.96 & & & -0.818 & & & & & & & & & t.t. & & & & & & & \\
\hline & & & 25.66 & 24.688 & 5.439 & 24.971 & & 48714 & 53.493 & 17057 & 31.96 & -0.818 & & & & & & & & & & & & & & & & \\
\hline & 4/21/2004 & 4:06:24 AM & 25.649 & 24.682 & 25.443 & 25.035 & 24.9 & 48. & 53.106 & 16.898 & & \begin{tabular}{|c|c|}
-0.83 \\
\end{tabular} & & 48. & & 14. & & 254 & \begin{tabular}{|l|l|}
42.4094 \\
\end{tabular} & 51 & $11 . .9$ & 40. & 755 & & $0.6 \quad-6$ & & 210 & \\
\hline & 4/21/2004 & & 25.649 & 24.682 & 25.463 & 25.105 & & & 53.195 & 16.963 & 31. & -0.815 & & & & & & & & 51 & 12. & & & & & & & \\
\hline 1866 & $4 / 21 / 2004$ & 4:08:24 AM & 25.644 & 24.667 & 25.453 & 25.115 & 24. & & 3.682 & 17.048 & & -0.818 & & & & 14. & & 25 & & 52 & 11. & & & & & & & \\
\hline 186 & 4/21/2004 & 4:09:24 & 25.644 & 24.662 & 25.463 & 25.2 & & 48.7 & 53.497 & 17.016 & & -0.818 & & & 0.6 & 14.643 & & 254 & & $J_{2}$ & 11. & & & & & & & \\
\hline & & & & 24.673 & 25.489 & 25.217 & & & & 16.982 & & -0.8 & & & & & & & & 51.6 & 12. & & & & & & & \\
\hline & $4 / 21122004$ & & $\begin{array}{l}25.63 \\
\end{array}$ & 24.6685 & 20.4646 & 25.207 & & & $\frac{53.222}{5.221}$ & 1.96 & 31.9 & -0.821 & & & & & & & & & 12. & & & & & & & \\
\hline & $\frac{4-4121204}{4 / 21204}$ & & $\frac{23.03 t}{25648}$ & & $\frac{2.4 .490}{2582}$ & $\frac{25.235}{25265}$ & & & & & & -0.1 & & & & & & & & 517 & & & & & & & & \\
\hline & 4 & 4.1424 & 25.043 & 2468 & 25487 & 2532 & & & \begin{tabular}{|l|l|l|l|}
53.396 \\
\end{tabular} & & & -0.8 & & & & & & & & & & & & & & & & \\
\hline $\begin{array}{l}187 \\
187\end{array}$ & $4 / 21 / 2004$ & & 25.655 & 24.703 & $\frac{25.504}{25.504}$ & 25.317 & 24. & & \begin{tabular}{|l|l|}
53.588 \\
\end{tabular} & 16.959 & 32. & $\frac{-0.772}{-0.772}$ & & & & & & 255 & & & \pm & & & & & & & \\
\hline & & & & & & & & & & & & & & & & & & & & & & & & & & & & \\
\hline 187 & & & 25.65 & 24704 & 255 & 25317 & 24.8 & 48.521 & 53,408 & 17009 & $318 \mathrm{r}$ & -0815 & & & & 14.6 & & & & & & & & & & & & \\
\hline & $4 / 21 / 2$ & & 25.6 & 24.7 & & 25.2 & 24. & & 53.402 & 16.999 & & -0.78 & & & 0.0 & & & 255 & & & & & & & & & & \\
\hline 187 & $4 / 21 / 2004$ & 4:19:24 AM & 25.6 & 24.725 & 25.5 & 25.163 & 24. & 48.654 & 53.369 & 17.073 & & -0.815 & & 49 & 0.05 & 14.643 & & 255 & & 51. & 12. & 46 & & \begin{tabular}{|l|l|} 
\\
\end{tabular} & 0.0 & & 82 & \\
\hline 187 & $4 / 21 / 2004$ & 4:20:24 AM & 25.661 & 24.709 & 25.5 & 25.078 & 24. & $\begin{array}{l}48.606 \\
\end{array}$ & 53.431 & 16. & & -0.7 & & & & 14. & & 25 & & 52. & 12.6 & 4 & & & & & & \\
\hline & $4 / 21 / 2004$ & & 25.66 & 24.719 & 25.52 & 25.0 & 24 & 48.494 & 53.431 & 17.033 & & -0.769 & & & 0.055 & & & 255 & & 52 & 11.6 & 4 & & & & & & \\
\hline & $4 / 21 / 2004$ & 4:22:24 AM & 25.65 & 24.703 & 25.514 & 24.931 & 24.485 & 48.614 & 53.4 & 17.08 & 31.90 & -0.769 & & $\begin{array}{ll}48.404 \\
\end{array}$ & 0.05 & 14.643 & & & & & & & & & & & & \\
\hline & $4 / 21 / 2004$ & 4:23:24 AM & $\frac{25.66}{25.649}$ & 24.608 & 2552 & 24.956 & 24.5 & (48.529 & 53.388 & 16.991 & & -0.016 & & 88 & 0.0 & 14.6 & & 256 & 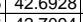 & & 12.14 & & & & & & & \\
\hline & $4121 / 2004$ & & $\frac{23.045}{2569}$ & 24.092 & $\frac{20.020}{25528}$ & $\frac{24.905}{25015}$ & & (20.201 & $\frac{5.03}{53.06}$ & $\begin{array}{l}1.9 .940 \\
1.6961\end{array}$ & & 0.0109 & & & & & & & & & & & & & & & & \\
\hline & 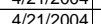 & 4.20 .44 & 25648 & & & $\frac{2.015}{2507}$ & & 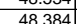 & 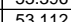 & 17.015 & 31. & -0.0 & & & & & & & & 51 & 些1 & & & & & & & \\
\hline 188 & & & & & 25. & & & & 53.5 & 17.048 & & & & & & & & & & 519 & 122 & & & & & & & \\
\hline $18 \varepsilon$ & $4 / 21 / 2004$ & & 25. & 24.687 & 25.443 & 25.165 & & & & & & -0.769 & & & & & & & & & & & & & & & & \\
\hline & & & & & & & & 48.689 & & & & -0.7 & & & & & & & & 523 & $118 \mathrm{~g}$ & & & & & & & \\
\hline & $4 / 21 / 2004$ & & & & 546 & & & 48.484 & 336 & 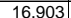 & & -0.726 & & & & 14.643 & & 2568 & & & 2 & & & & & & 201 & \\
\hline 1890 & $4 / 21 / 2004$ & 4:31:24 AM & 25.656 & 24.689 & 25.455 & & $\begin{array}{l}24.886 \\
\end{array}$ & & & $\begin{array}{ll}16.793 \\
\end{array}$ & 8036 & -0.755 & & 48715 & 0.053 & 14.643 & & & 438261 & & $11 . \mathrm{s}$ & & 2.777 & 0.0 & 0.008 & 0.000 & 0.194 & 0.1 \\
\hline
\end{tabular}


WSRC-TR-2005-00105, REVISION 0

SRNL-RPP-2005-00012, REVISION 0

RUN \# 2.03A AND B; FIRST AND SECOND HALF OF SLURRY DEWATERING - CONT.

\begin{tabular}{|c|c|c|c|c|c|c|c|c|c|c|c|c|c|c|c|c|c|c|c|c|c|c|c|c|c|c|c|c|}
\hline & A & B & D & $E$ & $\mathrm{~F}$ & $G$ & $\mathrm{H}$ & $\mathrm{J}$ & $\mathrm{K}$ & $\mathrm{L}$ & $\mathrm{M}$ & $\mathrm{N}$ & $\mathrm{O}$ & $\mathrm{Q}^{\mathrm{Q}}$ & \begin{tabular}{ll|}
$\mathrm{R}$ \\
\end{tabular} & 5 & \begin{tabular}{l|l|}
$\mathrm{T}$ \\
\end{tabular} & & w & $x$ & & $L_{2}$ & AA & $A B$ & $\mathrm{AC}$ & $A D$ & AE & \\
\hline $\begin{array}{l}1891 \\
1892 \\
\end{array}$ & $\begin{array}{l}4 / 21 / 2004 \\
4 / 2121204\end{array}$ & $\begin{array}{l}4: 32: 24 \mathrm{Am} \\
4.3224 \mathrm{M}\end{array}$ & $\begin{array}{r}25.653 \\
25.664\end{array}$ & $\begin{array}{l}24.706 \\
22.717\end{array}$ & $\begin{array}{l}25.462 \\
25.473\end{array}$ & $\begin{array}{l}25.304 \\
25.306\end{array}$ & \begin{tabular}{|l|}
24.863 \\
24.909 \\
\end{tabular} & $\begin{array}{l}48.341 \\
48.644 \\
4\end{array}$ & \begin{tabular}{|l|}
53.276 \\
53.489 \\
\end{tabular} & $\begin{array}{l}\frac{16.761}{16.875} \\
\end{array}$ & $\begin{array}{l}31.852 \\
332137 \\
\end{array}$ & $\begin{array}{l}-0.717 \\
-0.705 \\
\end{array}$ & $\begin{array}{l}-6.798 \\
-6.804\end{array}$ & \begin{tabular}{|l|}
48.342 \\
48.861
\end{tabular} & $\begin{array}{l}0.056 \\
0.056\end{array}$ & $\begin{array}{l}14.643 \\
14.643 \\
\end{array}$ & \begin{tabular}{|c|}
0.003 \\
0.003
\end{tabular} & 2570.6 & \begin{tabular}{|l|l|}
42.8428 \\
42.8594
\end{tabular} & $\frac{51.8}{52 .}$ & $\frac{11.8}{12.8}$ & 40. & $\frac{2.765}{2.785}$ & \begin{tabular}{|c|}
0.008 \\
0.008
\end{tabular} & \begin{tabular}{|l|}
0.008 \\
0.008 \\
\end{tabular} & 0.000 & $\begin{array}{l}0.206 \\
0.204\end{array}$ & $\begin{array}{l}0.17 \\
0.17\end{array}$ \\
\hline & & & & & \begin{tabular}{|l}
25.4759 \\
25.49
\end{tabular} & & & & & & & & & & & & & & & & & & & & & & & \\
\hline 1894 & $4 / 21 / 2004$ & 4:35:24 AM & 25.65 & 24.724 & 25.464 & 25.287 & 24.845 & 48.664 & \begin{tabular}{|l|}
53.578 \\
\end{tabular} & 16.795 & 32.159 & -0.705 & & \begin{tabular}{|l|}
47.837 \\
\end{tabular} & 0.057 & 14.643 & 0.003 & 2573.6 & 42.8928 & 52. & & 40.4 & \begin{tabular}{|l|}
2.786 \\
\end{tabular} & 0.008 & \begin{tabular}{|l|}
0.008 \\
\end{tabular} & 0.000 & 0.208 & \\
\hline & $4 / 21 / 2004$ & 4:36:24 AM & 25.655 & 24.734 & 25.474 & 25.257 & 24.66 & 48.712 & \begin{tabular}{|l|}
53.605 \\
\end{tabular} & 16.869 & 32.133 & \begin{tabular}{|c|}
-0.708 \\
\end{tabular} & -6.7 & $\begin{array}{l}47.303 \\
\end{array}$ & 0.057 & 14.643 & & 2574.6 & 42.9094 & 52. & & 80.4 & & & \begin{tabular}{|l|}
0.008 \\
\end{tabular} & & & \\
\hline & & & & & & & & & & & & & & 48.354 & & $\begin{array}{l}14.643 \\
\end{array}$ & & & & 52.2 & & & & & & & & \\
\hline \begin{tabular}{|l|l|}
1897 \\
\end{tabular} & $4 / 21 / 2004$ & 4:38:24 AM & 25.643 & 24.706 & \begin{tabular}{|l|l|}
25.467 \\
\end{tabular} & $\begin{array}{l}25.075 \\
\end{array}$ & $\begin{array}{l}24.483 \\
2.73\end{array}$ & $\begin{array}{l}48.668 \\
\end{array}$ & 53.458 & $\begin{array}{l}16.769 \\
6.79\end{array}$ & 32.264 & \begin{tabular}{|l|l|}
-0.772 \\
\end{tabular} & $\begin{array}{r}-6.81 \\
\end{array}$ & $\begin{array}{l}48.596 \\
\end{array}$ & \begin{tabular}{|l|l|}
0.057 \\
\end{tabular} & $\begin{array}{l}4.643 \\
\end{array}$ & $\begin{array}{l}0.003 \\
\end{array}$ & 2576.6 & 42.9428 & $a_{2}$ & 11.9 & & 2.790 & \begin{tabular}{|l|l|}
0.008 \\
\end{tabular} & 0.008 & 0.000 & 0.207 & \\
\hline $\begin{array}{l}1898 \\
1899 \\
\end{array}$ & $\begin{array}{l}4 / 21 / 2004 \\
4 / 21204\end{array}$ & $\begin{array}{l}4: 39: 24 \mathrm{AM} \\
44024 \mathrm{AM}\end{array}$ & $\begin{array}{l}25.653 \\
25.651\end{array}$ & $\begin{array}{l}24.742 \\
227724\end{array}$ & \begin{tabular}{|l|l|}
25.487 \\
25.485
\end{tabular} & \begin{tabular}{|l|l|}
24.989 \\
24.957
\end{tabular} & $\begin{array}{l}24.478 \\
24.46 \\
\end{array}$ & $\begin{array}{l}48.523 \\
45833 \\
\end{array}$ & \begin{tabular}{|l|}
53.553 \\
53.526 \\
\end{tabular} & 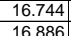 & \begin{tabular}{|l|l|}
32.083 \\
31.96
\end{tabular} & \begin{tabular}{|c|c|}
-0.691 \\
-0.705
\end{tabular} & $\begin{array}{l}-6.596 \\
-6.703\end{array}$ & \begin{tabular}{|l|}
52.476 \\
48.999 \\
\end{tabular} & $\begin{array}{l}0.052 \\
0.053\end{array}$ & $\begin{array}{l}14.643 \\
14.643 \\
\end{array}$ & \begin{tabular}{|l|}
0.003 \\
0.003
\end{tabular} & $\begin{array}{l}2577.6 \\
2578.6\end{array}$ & \begin{tabular}{|l|l|}
42.9594 \\
42.9761
\end{tabular} & $\frac{52}{52}$ & $\begin{array}{l}12.9 \\
120\end{array}$ & 40.3 & $\begin{array}{l}2.779 \\
2.777\end{array}$ & \begin{tabular}{|c|}
0.008 \\
0.008 \\
\end{tabular} & $\begin{array}{l}0.008 \\
0.008\end{array}$ & $\begin{array}{l}0.000 \\
0.000\end{array}$ & \begin{tabular}{|l|l}
0.190 \\
0.194
\end{tabular} & $\begin{array}{l}0.16 \\
0.16\end{array}$ \\
\hline 1900 & $4 / 21 / 2004$ & 4:41:24 AM & & & 25.495 & & $\begin{array}{r}24.440 \\
24.56\end{array}$ & & 53.653 & & 32.063 & $\begin{array}{l}-0.105 \\
-0.705\end{array}$ & & \begin{tabular}{|l|}
48.08493 \\
48.03
\end{tabular} & $\begin{array}{l}.053 \\
0.058\end{array}$ & $\frac{14.643}{14.643}$ & 0.003 & $\frac{25 / 8.6}{25796}$ & $\begin{array}{l}\frac{42.9761}{42.9928} \\
\end{array}$ & 52.1. & $\begin{array}{l}12.0 \\
11.8\end{array}$ & & $\frac{2.777}{2.782}$ & \begin{tabular}{|c|}
0.008 \\
0.009
\end{tabular} & \begin{tabular}{|c|}
0.008 \\
0.009
\end{tabular} & 0.000 & $\frac{0.194}{0.211}$ & 0.16 \\
\hline & $4 / 21 / 2004$ & 4::22:24 AM & 25.64 & & 25.49 & 24.947 & 24.66 & 48.714 & \begin{tabular}{|l|}
53.504 \\
\end{tabular} & 16.859 & 32.205 & -0.769 & & $\begin{array}{l}49.349 \\
\end{array}$ & & & $\begin{array}{l}0.003 \\
0.003\end{array}$ & 2580.6 & \begin{tabular}{|l|l|}
43.092894 \\
3.0094
\end{tabular} & & & & & $\begin{array}{l}0.009 \\
0.008\end{array}$ & $\begin{array}{l}0.009 \\
0.008 \\
\end{array}$ & & & $\frac{0.18}{0.18}$ \\
\hline & $4 / 21 / 2004$ & 4:43:24 AM & 25.655 & 24.714 & & 25.017 & 24.63 & 48.741 & 53.661 & \begin{tabular}{l|l}
16.874 \\
\end{tabular} & 32.186 & -0.711 & & 488.456 & 0.056 & $\begin{array}{l}14.643 \\
\end{array}$ & 0.0 & & & & & & & & & & & 0.18 \\
\hline 1903 & $4 / 21 / 2004$ & 4:44:24 AM & 25.666 & & 25.53 & 25.108 & & & 53.421 & & & & & & & 14.643 & 0.003 & & .0428 & & & & 78 & & & & & 0.17 \\
\hline 190 & $4 / 21 / 2004$ & 4:45:24 AM & & & 25.511 & & & 48.677 & & 16.567 & & & & \begin{tabular}{|c|}
48.108 \\
\end{tabular} & 0.054 & & & & $\frac{0.0594}{.059}$ & & & & & & & & & \\
\hline & $4 / 21 / 2004$ & 4:46:24 AM & 25.637 & 24.7 & 25506 & \begin{tabular}{|l|l|l|}
25.169 \\
\end{tabular} & 24.682 & 48.471 & 53.253 & 16.798 & 31978 & -0.743 & & T.001 & 0.054 & $\begin{array}{ll}14.643 \\
\end{array}$ & & & & & & 40.2 & & & & & & \\
\hline 1906 & 4/21/2004 & 4:47:24 AM & 25.643 & 24.686 & 25.522 & 25.169 & 24.728 & 48.592 & 53.504 & 16.836 & 32.067 & \begin{tabular}{|c|}
-0.749 \\
\end{tabular} & & $\begin{array}{l}48.097 \\
\end{array}$ & \begin{tabular}{|c|}
0.054 \\
\end{tabular} & 14.643 & $\begin{array}{l}0.003 \\
\end{array}$ & 2585.6 & \begin{tabular}{|l|l|l}
43.0928 \\
\end{tabular} & 52.0 & 11.8 & 40.3 & 2.781 & 0.008 & 0.008 & 0.000 & 0.197 & \\
\hline 199 & 4/21/2004 & 4:48:24 AM & 25.648 & 24.696 & 25.522 & 25.185 & 24.873 & $48.5 \mathrm{~K}$ & 53.402 & 16.917 & 31.973 & -0.763 & & 48.759 & 0.057 & 14.643 & & 2586.6 & 43.1094 & & & & 2.777 & & 0.008 & & & \\
\hline & $4 / 21 / 2004$ & $4: 49: 24 \mathrm{AM}$ & & & & & 24.92 & & & & 32.098 & & & & 0.056 & 14.643 & & & & & & & & & & & & \\
\hline & 4/21/2004 & 4:50:24 AM & $\begin{array}{r}25.66 \\
2562\end{array}$ & 24.719 & 25.544 & & 24.925 & & 3.437 & 16.937 & & 0.708 & & 9.288 & 0.056 & 14.643 & & & & & 12. & & & & .000 & & & \\
\hline & $41 / 21212004$ & $\begin{array}{l}4.5 .24 \mathrm{AM} \\
4.5 \% 2\end{array}$ & 25.602 & 24.715 & 25.551 & $\frac{25.29}{25316}$ & $\frac{24.922}{2090}$ & $\frac{48.10}{487}$ & 6715 & $\frac{16.929}{17030}$ & & - & & 4892 & & $\frac{14.643}{1.642}$ & & & & & & & & & & & & \\
\hline$\frac{1911}{1912}$ & $\begin{array}{l}4 / 21 / 21 / 204 \\
4 / 21 / 204\end{array}$ & $\begin{array}{l}4.25: 24 \mathrm{AM} \\
4: 53: 24 \mathrm{AM}\end{array}$ & $\begin{array}{l}25.014 \\
25.675\end{array}$ & $\begin{array}{l}24.751 \\
24.743\end{array}$ & 25.303 & $\frac{25.310}{25312}$ & $\begin{array}{r}\quad 4.939 \\
24.93\end{array}$ & $\begin{array}{l}48.10 \\
48.72\end{array}$ & 53.663 & $\frac{17.039}{16.954}$ & $\begin{array}{r}\frac{31.901}{32.04} \\
\end{array}$ & $\begin{array}{l}-0.155 \\
-0.705 \\
-10\end{array}$ & & \begin{tabular}{|l|}
47.9686 \\
47.563
\end{tabular} & $\begin{array}{l}0.055 \\
0.054\end{array}$ & $\begin{array}{l}\frac{14.043}{14.643} \\
\end{array}$ & 0.003 & 25916 & $\mid \frac{34.4171}{43928}$ & & 1117 & 40.4 & & $\mid$ & 0.008 & 0.000 & 0.020 & \\
\hline & $4 / 21 / 2004$ & 4:54:24 AM & 25.676 & 24.75 & 25.576 & 25.263 & 24.861 & 48.372 & 53.282 & 16.744 & 31.971 & -0.705 & -6.77 & \begin{tabular}{|l|}
47.916 \\
\end{tabular} & 0.056 & $\begin{array}{l}14.045 \\
14.643\end{array}$ & 0.003 & 2592.6 & $\begin{array}{l}3.13094 \\
43.209\end{array}$ & 51.8 & 11.7 & 40.2 & \begin{tabular}{|l|}
2.770 \\
\end{tabular} & & 0.008 & 0.000 & 0.205 & $\begin{array}{l}0.17 \\
0.17\end{array}$ \\
\hline 19 & $11 / 2004$ & 4:55:24 AM & 25.677 & 24.75 & 25.566 & 25.174 & 24.717 & $\begin{array}{ll}48.78 \\
\end{array}$ & 53.736 & 17.132 & 31.93 & -0.705 & & $\begin{array}{ll}46.652 \\
\end{array}$ & 0.055 & 14.643 & & & & & & 40.4 & & & & & & \\
\hline & $4 / 21 / 2004$ & 4:56:24 AM & 25.681 & $\frac{24.75}{24.70}$ & 25.576 & 25.088 & 24.651 & 48.4 & $\begin{array}{l}53.539 \\
55501\end{array}$ & $\frac{17.01}{17600}$ & 31.706 & 0.705 & & & 0.055 & 14.643 & & & & & & & & & & & & \\
\hline & $\frac{421212004}{4121204}$ & $\begin{aligned} 4.5 .24 \mathrm{AMM} \\
4.5 \% 2 \mathrm{AM}\end{aligned}$ & $\frac{25.6 / 4}{25.674}$ & $\frac{24.14}{2.732}$ & $\frac{2.5353}{2543}$ & $\begin{aligned} 25.035 \\
24.95\end{aligned}$ & $\begin{array}{r}24.484 \\
24.534\end{array}$ & $\begin{array}{r}48.55 \\
48.397\end{array}$ & $\frac{53.501}{53.34}$ & $\frac{16.909}{16.935}$ & $\begin{array}{l}31.952 \\
31.679\end{array}$ & $\begin{array}{r}-0.7 \\
-0.708\end{array}$ & & $\frac{47.868}{48356}$ & $\frac{0.05}{0.05}$ & $\frac{14.643}{14.643}$ & & 2596 & 432761 & & 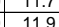 & 400 & 770 & & 008 & & & \\
\hline 191 & $4 / 212004$ & $4: 5 \cdot 24 \mathrm{AM}$ & 25.683 & 24741 & 25.467 & 24.989 & 24,83 & 48.422 & 53.265 & $\frac{16.819}{16.89}$ & $\begin{array}{l}31.079 \\
31.925\end{array}$ & -0.705 & & $\begin{array}{l}4.350 \\
4864\end{array}$ & & $\frac{14.045}{14643}$ & 0.0003 & 250 & 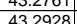 & 518 & $\frac{11.9}{11.9}$ & & & & & & & \\
\hline 191 & $4 / 21 / 2004$ & 5:00:04 AM & 25.683 & 24.736 & 25.477 & 25.009 & 24.558 & 48.791 & 53.808 & 16.937 & 32.149 & $\begin{array}{c}-0.703 \\
-\frac{1}{2}\end{array}$ & & 48.673 & 0.054 & 14.643 & & 2598. & $\begin{array}{l}43.3039 \\
43.30\end{array}$ & & $\frac{11.6}{11.6}$ & 40.5 & 2790 & & & & 196 & \\
\hline & $4 / 21 / 2004$ & 5:01:04 AM & 25.672 & 24.731 & 25.476 & 25.054 & 24.622 & 48.573 & 53.4 & $\begin{array}{ll}16.929 \\
\end{array}$ & & $\mid-0.705$ & & & 0.055 & 14.643 & & & 43.3206 & & & & & & & & & \\
\hline 1921 & $4 / 21 / 2004$ & 5:02:04 AM & 25.671 & 24.729 & 25.495 & 25.138 & 24.636 & 48.627 & & 16.953 & 31.946 & 103 & & 49.163 & 0.056 & 14.643 & & 2600 & 43.3372 & 52 & 12.6 & 40. & 2.778 & 0 & $\frac{0.008}{0.008}$ & 0.000 & $\frac{0.204}{0.204}$ & \\
\hline & $4 / 21 / 2004$ & 5:03:04 AM & 25.665 & 24.724 & 25.494 & \begin{tabular}{l|l}
25.122 \\
\end{tabular} & 24.72 & 48.809 & 53.767 & 16.879 & 32.252 & \begin{tabular}{|c|c|c|}
-0.648 \\
\end{tabular} & & \begin{tabular}{|l|l|}
48.982 \\
\end{tabular} & & 14.643 & & & & & & & & & & & & \\
\hline 192 & $4 / 21 / 2004$ & $5: 04: 04 \mathrm{AM}$ & 25.655 & 24.714 & 25.489 & 25.177 & & 48.337 & 53.387 & 16.882 & 31.685 & & & & & 14.643 & & & $\frac{13.3706}{433706}$ & & 1.2. & & & & & & & \\
\hline 19 & 4/21/2004 & 5:05:04 AM & 25.66 & 24.713 & 25.504 & 25.161 & 24.745 & $\begin{array}{l}48.592 \\
\end{array}$ & 53.603 & 16.853 & 32.03 & -0.703 & & 48.168 & $\mid 0.054$ & 14.643 & & 2603.2 & 43.3872 & 52.1 & 11.8 & 40.3 & & 0.008 & 0.008 & 0.000 & 0.197 & 0.17 \\
\hline & 4/21/2004 & 5:06:04 AM & 25.665 & 24.713 & 25.504 & 25.216 & 24.8 & 48.357 & .292 & 859 & 31.831 & -0.648 & & 49.264 & & 14.643 & & & & & 12.1 & & & & & & & \\
\hline & 4/21/2004 & 5:07:04 AM & 25.67 & 24.709 & 25.504 & 25.237 & 24.7 & 48.444 & 3.429 & 16.881 & 31.872 & & & $\begin{array}{r}48.2 \\
\end{array}$ & 0.054 & & & & 43.4206 & & 11. & & & & & & & \\
\hline & $4 / 21 / 2004$ & 5:08: & 25.671 & 24.719 & 25.51 & 25.303 & & 48.345 & 286 & 16.735 & 31. & -0.65 & & (2) & 0.05 & 14.643 & & & & & 12. & $4 c+2$ & 2.76 & .008 & 0.00 & 0.000 & 198 & \\
\hline & & & & 24.126 & & & & $\begin{array}{l}48.803 \\
48.909\end{array}$ & & & & & & & & & & & & & & & & & & & & \\
\hline$\frac{196}{1936}$ & $\begin{array}{l}4 / 21 / 2004 \\
4 / 21 / 2004\end{array}$ & $\begin{array}{l}5.10 .04 \mathrm{AM} \\
5: 11: 04 \mathrm{AM}\end{array}$ & $\begin{array}{l}25.011 \\
25.677\end{array}$ & $\frac{24.140}{24.741}$ & $\begin{array}{l}23.351 \\
2531\end{array}$ & 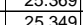 & $\frac{24.624}{24.907}$ & 40.496 & 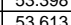 & $\frac{16.915}{16807}$ & $\begin{array}{l}31.540 \\
32046\end{array}$ & 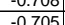 & & 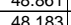 & $\mid$ & 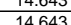 & & 266 & & 52. & $\frac{12.6}{118}$ & 40 & & & 0.008 & & 0.204 & \\
\hline & $4 / 21 / 2004$ & 5:12:04 AM & 25.688 & 24.746 & & 25.345 & 247 & 48.577 & & & & $\frac{-1.65}{-0.65}$ & & 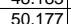 & & & & & & $J$ & 1.0 & 40.0 & & & & & & \\
\hline 193 & $4 / 21 / 2004$ & $5: 5: 13: 04 \mathrm{AM}$ & 25.693 & 24.756 & 25.547 & 25.26 & 24.748 & 48.447 & 53.427 & $\frac{16.866}{16.86}$ & 31.858 & $\mid-0.705$ & & |47.612 & 0.054 & 14.643 & & & & 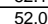 & 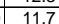 & 40 & & & & & & \\
\hline & $1 / 2004$ & & 25.699 & 24.75 & 25559 & 25.18 & & & & 17.004 & & & & & & & & & & & & & & & & & & \\
\hline & & & 25.687 & 24746 & 25556 & 25,104 & & 48.459 & 53.359 & 16907 & 31.975 & -0.705 & & 8.859 & 0.05 & & & & & & 12. & & & & & & & \\
\hline & $4 / 21 / 2004$ & $5: 16: 04 \mathrm{AM}$ & 25.681 & 24.729 & 25.55 & 24.972 & 24. & 48.415 & 53.396 & & & $\begin{array}{c}-0.653 \\
\end{array}$ & & 48.3 & & 14.6 & & 26 & 43.5 & 51 & 11.5 & 40 & & & & & & \\
\hline & $4 / 21 / 2004$ & 5:17:04 AM & 25.68 & 24.71 & 25.544 & & & 48 & 53.45 & & & -0.65 & & & & & & & & & 12. & & & & & & & \\
\hline 193 & $4 / 21 / 2004$ & 5:18:04 AM & 25.683 & 24.706 & 25.557 & 24.934 & 24.4 & 48.2 & 53.242 & 775 & 31.864 & -0.65 & & 47. & & 14. & & & & & 11. & 44 & & & & & & \\
\hline 1938 & 4/21/2004 & 5:19:04 AM & 25.6 & 24.71 & 25.571 & & & 48.386 & & 16 & & -0.705 & & 48. & & 14.643 & & & & 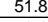 & 11.6 & 40 & 2.1 & & 0.0 & & & \\
\hline & & & 25.68 & 24.699 & & 24.967 & 24 & & & & & & & & & & & & & $J_{2}$ & 12.0 & & & & & & & \\
\hline 1944 & 1 & 5:21: & 25.685 & 24.688 & 255.58 & 25.032 & 24.6 & $\begin{array}{l}48.108 \\
4877\end{array}$ & 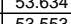 & $\frac{16.917}{16.829}$ & $\begin{array}{l}32.157 \\
32067\end{array}$ & $\begin{array}{r}-0.65 \\
\end{array}$ & & 47 & & 14.643 & & & & & $\frac{11.7}{1.7}$ & & & & & & & \\
\hline 1942 & 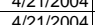 & $\begin{array}{l}\frac{3.22 .0}{5 \cdot 230} \\
5\end{array}$ & $\frac{23.075}{25685}$ & $\frac{2.0}{24.6}$ & $\frac{25.364}{25.585}$ & & & & & 16.918 & & $\frac{-0.040}{-0.65}$ & & & & & & & & & 界1. & & & & & & & \\
\hline 194 & 4 & $\begin{array}{l}5.25 .04 \\
5.04\end{array}$ & 25.69 & 226. & & & & 48648 & \begin{tabular}{|l|l|l|}
5344 \\
\end{tabular} & & & -0.65 & & & & & & & & & 11. & & & & & & & \\
\hline 194 & $4 / 21 / 2004$ & $\begin{array}{l}5: 25: 04 \\
5: 25\end{array}$ & 25.681 & 24.664 & $\frac{25.495}{25.495}$ & 25.193 & 24.7 & 48.6 & 53.52 & $\begin{array}{l}16.897 \\
\end{array}$ & & & & & & & & & & & & & & & & & & \\
\hline & & & & & & & & 48.341 & & & & & & & & & & & & & & & & & & & & \\
\hline 1946 & & & 25.688 & 24606 & 25517 & 25264 & 24.8 & & 53707 & 16964 & & -0.648 & & 48 & & 14.6 & & & & & & & & & & & & \\
\hline & $4 / 21 / 2$ & 5:28: & 25.6 & 24.6 & & 25.224 & 24.8 & & 53.98 & & & -0.8 & & & & & & & & & & & & & & & & \\
\hline 1948 & $4 / 21 / 2004$ & 5:29:04 AM & 25.675 & 24.683 & & & 24 & 48.279 & 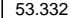 & 16.75 & & \begin{tabular}{|c|c|} 
& -0.65 \\
\end{tabular} & & 48.2 & 0.05 & 14.643 & & & \begin{tabular}{|l|l|}
433.7 \\
\end{tabular} & 51. & 11.8 & 40. & 2.663 & 0.008 & $0.0^{2}$ & & 0.194 & \\
\hline 194 & $4 / 21 / 2004$ & 5:30:04 & 25.69 & 24.70 & 25.534 & & 24 & 48.405 & 53.396 & 17.014 & 31. & -0. & & 48 & & 14. & & & & 51. & 11. & 4 & & & & & 98 & \\
\hline & $4 / 2$ & 5:31:0 & 25.693 & 24.726 & 25.547 & 25.3 & 24.5 & 48.621 & 53.584 & 16.966 & & -0.65 & & 48. & 0.05 & & & 262 & & 52 & 12.0 & 4 & & & & & 200 & \\
\hline 195 & 4/21/2004 & 5:32:04 AM & 25.693 & $24.736 \mathrm{C}$ & 20.0062 & 25.365 & 24.7 & 48.598 & 53.667 & 16.978 & 31.93 & -0.572 & & & 0.05 & 14.643 & & & & & & & & & & & & \\
\hline & $\frac{421212004}{4121204}$ & $\begin{array}{l}5: 33504 \mathrm{AM} \\
5.3204\end{array}$ & $\frac{25.005}{25672}$ & 24.131 & $\frac{25.547}{2521}$ & $\begin{array}{l}2.26 \\
25.20\end{array}$ & 24.5 & $\begin{array}{l}48.308 \\
4857\end{array}$ & 53.334 & $\begin{array}{l}1.005 \\
1.094\end{array}$ & 31.622 & $\begin{array}{l}-0.613 \\
=0.894\end{array}$ & & & 0.05 & & & 263 & 4 & & 11.0 & & & & & & & \\
\hline & 4421212004 & $\begin{array}{l}0.34 .04 \\
5.350 .01\end{array}$ & $\frac{23.012}{2567}$ & 24.110 & 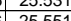 & | & & (40.501 & 52.003 & 年 & & -0.56 & & (40. & & & & & & & 2.0 & & & & & & & \\
\hline & $\frac{4}{421212004}$ & $\begin{array}{l}5.03 .04 \\
5.04\end{array}$ & 25 & 2472 & 25 & & & & 341 & & & & & & & & & & & & 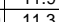 & & & & & & 37 & \\
\hline & & & & & & & & 48.438 & 53.485 & 16.931 & & & & & & & & & & & 11 & & & & & & & \\
\hline 1955 & $4 / 21 / 2004$ & & & 24.689 & 25.555 & 24.841 & & & & & 31.8 & & & & & & & & & & & & & & & & & \\
\hline & & & 25 & & & & & 48.266 & & & & & & & & & & & & & & & & & & & & \\
\hline & $4 / 21 / /$ & & 25.688 & 24.692 & & 24.965 & & 4831 & 53,23 & & & & & 49094 & & 14.643 & & 267 & & & & & & & & & 191 & \\
\hline 1960 & $4 / 21 / 2004$ & 5:41:04 AM & 25.667 & 24.65 & 25561 & $\begin{array}{l}24.953 \\
\end{array}$ & \begin{tabular}{|l|l|}
24.597 \\
\end{tabular} & & & 16.928 & 31542 & -0.546 & & 48202 & 0.052 & 14.643 & & & 43.9872 & & 118 & & 2.746 & & 0.008 & 0.000 & 0.192 & 0.1 \\
\hline
\end{tabular}


WSRC-TR-2005-00105, REVISION 0

SRNL-RPP-2005-00012, REVISION 0

RUN \# 2.03A AND B; FIRST AND SECOND HALF OF SLURRY DEWATERING - CONT.

\begin{tabular}{|c|c|c|c|c|c|c|c|c|c|c|c|c|c|c|c|c|c|c|c|c|c|c|c|c|c|c|c|c|}
\hline & A & B & D & $E$ & $F$ & $G$ & $\mathrm{H}$ & $\mathrm{J}$ & $\mathrm{K}$ & $\frac{L}{1 S}$ & M & $\mathrm{N}$ & 0 & $\mathrm{Q}$ & \begin{tabular}{l|l|}
$R$ \\
\end{tabular} & 5 & $\mathrm{~T}$ & $v$ & W & $x$ & & $\mathrm{z}$ & $\mathrm{AA}$ & $A B$ & $A C$ & $A D$ & & \\
\hline $\begin{array}{l}\frac{1961}{1962} \\
1962\end{array}$ & $\begin{array}{r}4 / 21 / 2004 \\
4 / 212004\end{array}$ & $\begin{array}{l}5: 22: 04 \mathrm{AM} \\
5.0304 \mathrm{AM}\end{array}$ & $\begin{array}{l}25.686 \\
25.671\end{array}$ & $\begin{array}{l}24.675 \\
22.645\end{array}$ & \begin{tabular}{|l|}
25.611 \\
25566
\end{tabular} & \begin{tabular}{rl|}
25.103 \\
25.088
\end{tabular} & \begin{tabular}{|l|}
24.566 \\
24.686 \\
\end{tabular} & $\begin{array}{l}48.324 \\
48.807\end{array}$ & $\begin{array}{l}53.516 \\
53.835\end{array}$ & $\begin{array}{l}\frac{16.937}{17.101} \\
17\end{array}$ & $\begin{array}{l}31.624 \\
3302626\end{array}$ & $\begin{array}{r}-0.54 \\
-0.543\end{array}$ & $\begin{array}{l}-6.608 \\
-6.61\end{array}$ & $\begin{array}{r}48.12 \\
49.361 \\
\end{array}$ & $\mid \begin{array}{l}0.054 \\
0.0 .04\end{array}$ & $\begin{array}{l}14.643 \\
14.643 \\
\end{array}$ & \begin{tabular}{|c|}
0.003 \\
0.003
\end{tabular} & $\frac{2640.2}{2641.2}$ & \begin{tabular}{|l|}
44.0039 \\
4.0206
\end{tabular} & \begin{tabular}{|l|}
52. \\
52.2
\end{tabular} & $\frac{11.8}{12.1}$ & \begin{tabular}{|l|}
40. \\
40.2
\end{tabular} & \begin{tabular}{|l|}
2.756 \\
2.787
\end{tabular} & \begin{tabular}{|l|}
0.008 \\
0.008 \\
\end{tabular} & $\begin{array}{l}0.008 \\
0.008\end{array}$ & 0.000 & $\begin{array}{l}0.198 \\
0.196\end{array}$ & $\begin{array}{ll}0.17 \\
0.17\end{array}$ \\
\hline & & & $\frac{25.671}{25.686}$ & & \begin{tabular}{|l|}
25.6806 \\
25.606 \\
\end{tabular} & $\frac{25.088}{25.173}$ & & & $\begin{array}{r}53.835 \\
53.427\end{array}$ & & & $\begin{array}{l}-0.543 \\
-0.54 \\
\end{array}$ & & & & $\frac{14.643}{14.643}$ & & & & & & & & & & & & \\
\hline 1964 & $4 / 21 / 2004$ & 5:45:04 AM & 25.678 & 24.651 & 25.582 & 25.149 & 24.828 & 48.37 & 53.487 & 16.977 & 31.646 & -0.546 & -6.605 & \begin{tabular}{|l|}
49.297 \\
\end{tabular} & 0.056 & 14.643 & 0.003 & 2643.2 & \begin{tabular}{|l|l|}
4.0539 \\
\end{tabular} & & & & 2.758 & \begin{tabular}{|l|}
0.008 \\
\end{tabular} & 0.008 & 0.000 & 0.205 & \\
\hline 1965 & $4 / 21 / 2004$ & $5: 46: 04 \mathrm{AM}$ & 25.689 & 24.652 & 25.558 & 25.176 & 24.884 & 48.349 & 53.497 & 17.029 & 31.655 & -0.511 & -6.486 & 48.613 & 0.053 & 14.643 & & 2644.2 & \begin{tabular}{|l|l|}
44.0706 \\
\end{tabular} & & & & & & 0.008 & & 0.194 & 0.17 \\
\hline & & & & & & $\begin{array}{l}25.181 \\
2\end{array}$ & 24.805 & & & \begin{tabular}{|l|l|}
17.014 \\
\end{tabular} & 31.486 & -0.546 & & $\begin{array}{r}48.398 \\
\end{array}$ & & 14.643 & & & & 51.7 & & & & & & & & \\
\hline 1967 & $4 / 21 / 2004$ & $5: 48: 04 \mathrm{AM}$ & 25.69 & $\begin{array}{l}24.679 \\
2.70\end{array}$ & \begin{tabular}{|l|}
25.565 \\
25.575 \\
\end{tabular} & $\begin{array}{l}25.232 \\
25.257\end{array}$ & $\begin{array}{l}24.831 \\
\end{array}$ & $\begin{array}{l}48.77 \\
\end{array}$ & 53.812 & \begin{tabular}{|l|l|l|l|l|}
17.017 \\
16.0923
\end{tabular} & $\begin{array}{l}32.003 \\
2121\end{array}$ & \begin{tabular}{|c|}
-0.653 \\
\end{tabular} & -6.7 & \begin{tabular}{|l|l|}
48.771 \\
\end{tabular} & $\begin{array}{l}0.055 \\
\end{array}$ & 14.643 & $\begin{array}{l}0.003 \\
\end{array}$ & 2646.2 & \begin{tabular}{|l|l|}
44.1039 \\
\end{tabular} & 52. & 12.0 & & 2.785 & $\begin{array}{ll}0.008 \\
\end{array}$ & $\begin{array}{l}0.008 \\
\end{array}$ & 0.000 & $\frac{200}{200}$ & \\
\hline$\frac{1968}{1969}$ & $\begin{array}{l}4 / 21 / 2004 \\
4 / 212004\end{array}$ & $\begin{array}{l}5: 49: 04 \mathrm{AM} \\
\text { 5:50:04 } \mathrm{AM}\end{array}$ & $\begin{array}{r}25.7 \\
25.685\end{array}$ & $\begin{array}{l}24.679 \\
22673\end{array}$ & \begin{tabular}{|l|}
25.575 \\
25.545
\end{tabular} & $\begin{array}{l}25.257 \\
25.272\end{array}$ & \begin{tabular}{|r|r|}
24.846 \\
2474
\end{tabular} & $\begin{array}{r}48.1 \\
4393 \\
\end{array}$ & $\begin{array}{l}53.265 \\
53.508\end{array}$ & $\begin{array}{l}16.983 \\
17.049\end{array}$ & $\frac{31.421}{31603}$ & $\begin{array}{l}-0.483 \\
-0.483\end{array}$ & $\begin{array}{l}-6.145 \\
-6.157\end{array}$ & \begin{tabular}{|l|}
51.126 \\
48.832
\end{tabular} & \begin{tabular}{|c|c|}
0.047 \\
0.052
\end{tabular} & $\begin{array}{l}14.643 \\
14.643\end{array}$ & \begin{tabular}{|l|}
0.003 \\
0.003
\end{tabular} & \begin{tabular}{l|l}
2647.2 \\
2648.2
\end{tabular} & \begin{tabular}{|l|}
44.1206 \\
441372
\end{tabular} & \begin{tabular}{|l|l|}
51.8 \\
52. \\
\end{tabular} & $\frac{12.5}{120}$ & $\begin{array}{l}39.8 \\
40 .\end{array}$ & $\begin{array}{l}2.741 \\
2.758 \\
\end{array}$ & \begin{tabular}{|c|}
0.007 \\
0.008 \\
\end{tabular} & $\begin{array}{l}0.007 \\
0.008\end{array}$ & $\begin{array}{l}0.000 \\
0.000\end{array}$ & \begin{tabular}{|l|l|}
0.173 \\
0.191
\end{tabular} & $\begin{array}{l}0.15 \\
0.16\end{array}$ \\
\hline 1970 & $4 / 21 / 2004$ & 5:51:04 AM & & & \begin{tabular}{|l|l|} 
& 25.575 \\
\end{tabular} & 25.268 & \begin{tabular}{|r|}
24.856 \\
\end{tabular} & 48.729 & 53.638 & & & -0.653 & & $\begin{array}{l}4.0 .025 \\
49.453\end{array}$ & -.055 & $\frac{14.045}{14.643}$ & $\begin{array}{l}0.0003 \\
0.003\end{array}$ & $\frac{2648.2}{26492}$ & \begin{tabular}{|l|}
44.13539 \\
44.1539
\end{tabular} & \begin{tabular}{|l}
52.2 \\
52.2
\end{tabular} & & $\frac{40 .}{40 .}$ & $\frac{2.158}{2.779}$ & \begin{tabular}{|l|}
0.0008 \\
0.008 \\
\end{tabular} & $\begin{array}{l}0.008 \\
0.008\end{array}$ & 0.000 & 0.191 & 0.16 \\
\hline 1971 & $4 / 21 / 2004$ & $5: 52: 04 \mathrm{AM}$ & 25.702 & & & 25.234 & & 48.596 & 53.599 & & 31.884 & & & $\begin{array}{l}47.459 \\
\end{array}$ & & 14.643 & & 2650.2 & \begin{tabular}{|l|}
44.1706 \\
\end{tabular} & & & & & & & & & $\begin{array}{l}0.17 \\
0.16\end{array}$ \\
\hline & $4 / 21 / 2004$ & 5:53:04 AM & 25.697 & & 25.596 & \begin{tabular}{|l|l|l}
25.148 \\
\end{tabular} & 24.622 & 48.316 & 53.344 & 17.119 & 31.535 & -0.639 & -6.599 & $\begin{array}{l}47.943 \\
\end{array}$ & 0.054 & 14.643 & 0.003 & 2651.2 & \begin{tabular}{|l|l|}
44.1872 \\
\end{tabular} & & & & & & 0.008 & 0.000 & & $\begin{array}{l}0.16 \\
0.17\end{array}$ \\
\hline 1973 & $4 / 21 / 2004$ & 5:54:04 AM & 25.691 & & 25.59 & & & & 53.765 & & 31.813 & & & & & & & & & & & & & & & & & 0.17 \\
\hline 1974 & $4 / 21 / 2004$ & & & & & 24.982 & & 48.809 & 52770 & 17065 & & \begin{tabular}{|c|c|}
-0.65 \\
\end{tabular} & & & 8053 & & & 2653.2 & 44.2206 & & & & & & & & & \\
\hline & $4 / 21 / 2004$ & 5:56:04 AM & 25.684 & & 25.593 & 24.905 & 24.374 & \begin{tabular}{|l|l|}
48.534 \\
\end{tabular} & 53557 & 16.913 & 31.958 & -0.65 & & 5 & & 14.643 & & 2654.2 & & & & & & & & & & \\
\hline 1976 & $4 / 21 / 2004$ & 5:57:04 AM & 25.672 & 24.665 & 25.591 & \begin{tabular}{|l|l|l|l|l|}
2483 \\
\end{tabular} & 24.382 & $\begin{array}{l}48.473 \\
\end{array}$ & 53.52 & 17.009 & 31.823 & \begin{tabular}{|c|c|c|}
-0.648 \\
\end{tabular} & -6.654 & $\begin{array}{l}49.387 \\
\end{array}$ & $\begin{array}{l}0.052 \\
\end{array}$ & 14.643 & $\begin{array}{l}0.003 \\
\end{array}$ & 2655.2 & \begin{tabular}{|l|l|l|}
44.2539 \\
\end{tabular} & 52.8 & 12.1 & 40. & 2.768 & 0.008 & 0.008 & 0.000 & 0.190 & \\
\hline & $1,1 / 2004$ & $5: 58: 04 \mathrm{AM}$ & 25.686 & 24.67 & 25.626 & 24.902 & 24.501 & 48.515 & 53.611 & 17.032 & 31.751 & -0.65 & & 48.2 & $\begin{array}{l}0.054 \\
\end{array}$ & 14.643 & & 2656.8 & 44.2706 & & & & & & .00 & & & \\
\hline & $4 / 21 / 2004$ & 5:59:04 AM & & & & & & & & & & -0.648 & & & & 14.643 & & & & & & & & & & & & \\
\hline 197 & $4 / 21 / 2004$ & 6:00:04 AM & 25.679 & 24.647 & 25.618 & 25.015 & 24.494 & & 51.601 & 17.024 & 31.847 & -0.624 & -6.645 & 9.658 & 0.054 & 14.643 & & 2658.2 & 3039 & & 12 & & & & .008 & & & \\
\hline & $\frac{421212004}{4121204}$ & $6.01 .04 \mathrm{AM}$ & $\frac{25.673}{25674}$ & 24.63 & 25.011 & $\frac{25.044}{25065}$ & & $\frac{48.534}{14570}$ & 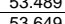 & $\frac{16.871}{17084}$ & 3177 & $\frac{-0.581}{0.616}$ & & $\frac{4.9600}{4890}$ & 告. & $\frac{11.643}{10.642}$ & & & & & & & & & & & & \\
\hline$\frac{1901}{1982}$ & 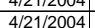 & $\begin{array}{l}0.020 .04 \mathrm{AM} \\
6: 03: 04 \mathrm{AM}\end{array}$ & $\begin{array}{l}25.074 \\
25.684\end{array}$ & $\frac{24.032}{24.637}$ & \begin{tabular}{|}
2.023 \\
25.638 \\
\end{tabular} & 2.0605 & $\begin{array}{l}24.584 \\
24.684\end{array}$ & $\frac{48.5 / 9}{48.256}$ & & $\begin{array}{l}\frac{17.064}{16.917} \\
\end{array}$ & $\frac{31.141}{31.624}$ & $\begin{array}{c}-0.010 \\
-0.584\end{array}$ & $\begin{array}{l}-0.648 \\
-6.645\end{array}$ & 48.961 & $\begin{array}{l}0.054 \\
0.054\end{array}$ & $\frac{14.045}{14643}$ & 0.003 & 26612 & 44,3539 & & & 39. & 2754 & & 0.008 & 0000 & $\begin{array}{l}0.197 \\
0.198\end{array}$ & \\
\hline & $4 / 21 / 2004$ & $6: 04: 04 \mathrm{AM}$ & 25.688 & 24.626 & \begin{tabular}{|l|}
25.057 \\
\end{tabular} & 25.13 & 24.698 & 49.585 & 54.739 & 17.082 & 32.794 & -0.569 & -6.634 & 49.541 & 0.061 & 14.643 & 0.004 & 2662.2 & \begin{tabular}{|l|l|}
44.3706 \\
\end{tabular} & 53. & 12.1 & & 2.840 & & \begin{tabular}{|l|l|}
0.000 \\
\end{tabular} & & 0.217 & 0.18 \\
\hline & $4 / 21 / 2004$ & 6:05:04 AM & 25.689 & & \begin{tabular}{|l|}
25.688 \\
\end{tabular} & 25.19 & & 49.452 & 54.48 & 17.607 & & -0.607 & & 49.47 & 0.056 & 14.643 & 0.004 & 2663.2 & 44.3872 & & & & & & & & & \\
\hline & $4 / 21 / 2004$ & 6:06:04 AM & 25.705 & 24.638 & \begin{tabular}{|l|}
25.704 \\
\end{tabular} & 25.187 & 24.765 & 49.102 & 54.148 & 17.675 & 31.72 & -0.543 & & 48.573 & $\begin{array}{l}0.053 \\
\end{array}$ & $\begin{array}{l}14.643 \\
\end{array}$ & 0.003 & 2664.2 & 44.4039 & 52. & 11.9 & & 2.786 & \begin{tabular}{|l|l|}
0.008 \\
\end{tabular} & 0.008 & & & \\
\hline & $4 / 21 / 2004$ & 6:07:04 AM & $\begin{array}{l}25.711 \\
257711\end{array}$ & $\frac{24.644}{24.645}$ & $\begin{array}{r}25.73 \\
25751\end{array}$ & $\begin{array}{l}25.197 \\
25.193\end{array}$ & $\begin{aligned} 24.816 \\
24.856\end{aligned}$ & $\begin{array}{l}49.278 \\
48.876\end{array}$ & $\frac{54.372}{53.908}$ & $\begin{array}{l}17.544 \\
17.658 \\
\end{array}$ & $\begin{array}{l}32.075 \\
31.523 \\
\end{array}$ & $\begin{array}{l}-0.569 \\
\end{array}$ & $\begin{array}{l}-6.602 \\
-6.605 \\
-10\end{array}$ & $\begin{array}{r}49.683 \\
40.53 \\
\end{array}$ & 0.055 & $\begin{array}{l}14.643 \\
114643\end{array}$ & 0.003 & $\frac{2665.2}{26662}$ & \begin{tabular}{|}
44.4206 \\
4.4372
\end{tabular} & $\frac{52 .}{52}$ & $\frac{12.2}{12.1}$ & 40. & 2.805 & 0.008 & 0.008 & 0.000 & & $\frac{17}{17}$ \\
\hline & \begin{tabular}{|l|}
$4 / 21 / 21 / 2004$ \\
\end{tabular} & & $\frac{25.111}{25.728}$ & $\frac{24.045}{24.661}$ & \begin{tabular}{|l|}
25.151 \\
25.762 \\
\end{tabular} & | & $\begin{array}{l}24.856 \\
24.788\end{array}$ & $\begin{array}{l}48.876 \\
49.434\end{array}$ & $\begin{array}{l}53.908 \\
54.573\end{array}$ & $\begin{array}{l}17.050 \\
17.681\end{array}$ & $\begin{array}{l}1.520 \\
32.088\end{array}$ & \begin{tabular}{|c|c|c|c|}
-0.54 \\
-54
\end{tabular} & $\frac{-6.6}{-6.6}$ & $\begin{array}{r}49.53 \\
48.025 \\
\end{array}$ & $\begin{array}{l}0.0044 \\
0.054\end{array}$ & $\frac{14.643}{14.643}$ & 0.003 & $\frac{2666.2}{26672}$ & $\begin{array}{l}44.43 / 2 \\
44.4539\end{array}$ & 52.4 & $\frac{12.1}{11.8}$ & & & & & & & \\
\hline 198 & $4 / 21 / 2004$ & $6: 10: 04 \mathrm{AM}$ & 25.718 & 24.6012 & 25.693 & 25.175 & 24.7 & $\begin{array}{l}45.404 \\
49.089\end{array}$ & 54.273 & 17.755 & 31.659 & -0.561 & -6.61 & 4 & | & $\frac{14.045}{14.643}$ & & 2668.2 & 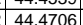 & & $\frac{11.6}{12.2}$ & 40. & $\frac{2.010}{2784}$ & & . & & $\frac{150}{192}$ & \\
\hline & $44 / 21 / 2004$ & 6:11:04 AM & 25.728 & & 25.703 & 25.18 & 24.5 & 48.673 & 53.765 & 17.59 & 31.429 & $\mid-0.543$ & & 48.235 & & 14.643 & & 2669.2 & \begin{tabular}{|l|l|}
44.4872 \\
\end{tabular} & & 11.2 & & & & & & & \\
\hline 1991 & 4/21/2004 & 6:12:04 AM & 25.744 & 24.662 & 25.733 & 25.096 & & $\begin{array}{l}499.289 \\
\end{array}$ & 54.335 & 17.692 & & -0.538 & & 49.581 & 0.054 & $\frac{14.045}{14.643}$ & 0.004 & 2670.2 & 44.5039 & & $\frac{15}{12 .}$ & 40 & 801 & 0 & 0.008 & 0.000 & $\frac{0.194}{0.194}$ & \\
\hline 195 & $4 / 21 / 2004$ & 6:13:04 AM & 25.738 & 24.651 & 25.732 & \begin{tabular}{|l|l|}
25.019 \\
\end{tabular} & 24. & 49.212 & 54.428 & 17.709 & 31.815 & \begin{tabular}{|c|}
-0.54 \\
\end{tabular} & & 48.865 & & 14.643 & & 2671.2 & 44.5206 & & & & & & & & & \\
\hline & $4 / 21 / 2004$ & $6: 14: 04 \mathrm{AM}$ & 25.731 & 24.639 & 25.745 & & & 49.199 & 54.304 & & 31.874 & -0.54 & & 49.518 & 0.054 & 14.643 & & 2672.2 & & & & & & & & & & \\
\hline 199 & $4 / 21 / 2004$ & 6:15:04 AM & 25.739 & 24.627 & 25.769 & 24.845 & 24.3 & $\begin{array}{l}49.042 \\
\end{array}$ & 54.217 & $\begin{array}{l}17.547 \\
\end{array}$ & 31.78 & -0.54 & & \begin{tabular}{|l|l|l|l|}
48.913 \\
\end{tabular} & \begin{tabular}{|c|}
0.056 \\
\end{tabular} & 14.643 & & 2673.2 & 4.5539 & 52.7 & 12.0 & 40. & 2.786 & & 0.008 & 0.000 & 0.202 & \\
\hline & $4 / 21 / 2004$ & 6:16:04 AM & 25.742 & 24.64 & 25.787 & 24.833 & 24 & 48.8 & 953 & & 31 & \begin{tabular}{|l|l|} 
\\
\end{tabular} & & $\begin{array}{l}47.903 \\
\end{array}$ & 0.061 & 14.6 & & 2674.2 & 44.5706 & & 11. & & & & & & & \\
\hline & 4/21/2004 & & 25.72 & 24.603 & 25.774 & 24.846 & & & & 17.533 & 32. & & & 49.664 & & & & & 44.5 & & & & & & & & & \\
\hline & $4 / 21 / 2004$ & 8:04 AM & 25.734 & 24.602 & 25.798 & 24.895 & & & 54.158 & 17.554 & & & & 49.455 & & 14.643 & & & & & 12. & & & & .0 & . & & \\
\hline & & $\frac{6.1}{6.2}$ & & & & & & & $\frac{239}{\frac{23}{40}}$ & $\frac{17.554}{17.720}$ & & 些.517 & & & & & & & & & & & & & & & & \\
\hline 20 & $\frac{42121204}{4 \mid 21204}$ & $\begin{array}{l}6.20 .04 \mathrm{Am} \\
6\end{array}$ & $\frac{25.13}{25.42}$ & $\frac{24.506}{2.595}$ & 25.0.64| & 25.9033 & $\frac{24.0}{246}$ & (46.050 & 21045 & 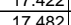 & $\begin{array}{l}31.003 \\
33.936\end{array}$ & 年 & & (45.455 & $\mid$ & $\begin{array}{l}\frac{14.045}{14643} \\
1.62\end{array}$ & & $\frac{2678.2}{26792}$ & 4.6539 & 52.7 & $\frac{12 .}{12}$ & 40 & & & 0 & & 190 & \\
\hline 200 & $4 / 21 / 12000$ & 6:22:04 AM & 25.749 & 24.592 & & & 24.6 & & 54.034 & & 31.646 & $\begin{array}{l}-.4474 \\
-0.474\end{array}$ & & 50.678 & & & & & & & 12. & & & & & & & \\
\hline & 4/21/2004 & 6:23:04 AM & 25.751 & 24.57 & 25.881 & 25.038 & 24.7 & 49.098 & 54.358 & 17.53 & 21.070 & $\mid-0.477$ & & 50.824 & $\mid$ & & & 268 & & & 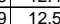 & & & & & & & \\
\hline & $\frac{1 / 2004}{11204}$ & & & 24.5 & & & & & 53.951 & 17.561 & & $\frac{0.474}{-0.47}$ & & & & & & & & & & & & & & & & \\
\hline & & & 2576 & 24.58 & 25.905 & 25.072 & 24 & 48.828 & 53.984 & 17.458 & 31 & -0.48 & & 48.999 & 0.049 & & & & & & & & & & & & & \\
\hline & 4/21/2004 & 6:26:04 AM & 25.762 & 24.58 & 25.912 & 25.079 & 24.7 & \begin{tabular}{|l|l|}
48.87 \\
\end{tabular} & 989 & 17.542 & & -0.425 & & 49 & & 14. & & 26 & & & 12. & $4 \mathrm{c}$ & & & & & & \\
\hline & $4 / 21 / 2004$ & $6: 27: 04 \mathrm{AM}$ & 25.77 & 24.58 & 25.934 & & & & .409 & $17.52 \varepsilon$ & & -0.474 & & & & & & & & & 12 & & & & & & & \\
\hline & 4/21/2004 & $6: 28: 04 \mathrm{AM}$ & 25.779 & 24.597 & 25.948 & 25.145 & 24. & 49.056 & 54.275 & 17.505 & 31.878 & -0.448 & & & & 14. & & 268 & & & & & & & & & & \\
\hline 200 & $4 / 21 / 2004$ & 6:29:04 & 25.775 & 24.598 & 25.949 & & & 49.1 & 341 & 17. & & -0.425 & & & 0.6 & & & & \begin{tabular}{|l|l|l}
44.7872 \\
\end{tabular} & & 1. & & & & & & & \\
\hline & & & 25.783 & 24.606 & & & & & & & & & & & & & & & & & & & & & & & & \\
\hline & $4 / 21122004$ & & 25.19 & 24.603 & 25.979 & & 24. & 48.961 & 54.010 & 17.47 & 31.819 & -0.433 & & & & 14. & & & & & 12. & & & & & & & \\
\hline & $\frac{4-4121204}{4 / 21204}$ & & $\frac{25.6}{25793}$ & $\frac{24.06}{24.60}$ & $\frac{25.994}{25983}$ & $\frac{23.030}{24945}$ & $\frac{24}{243}$ & & $\frac{54.331}{54.53}$ & $\frac{11.464}{1.543}$ & & $-0,427$ & & 492 & & & & & & & & & & & & & & \\
\hline & 4 & & 25797 & 24.59 & 25.096 & & 24. & & & & & $=0.0425$ & & & & & & & & & & & & & & & & \\
\hline 201 & $\frac{4 / 21 / 2004}{4}$ & & $\frac{25.806}{25.806}$ & 24.589 & 25.97 & 24.741 & 24.8 & & 461 & 17.57 & & $\frac{-0.425}{-0.45}$ & & & & & & & & & +1 & & & & & & & \\
\hline & & & & & & & & & & & & & & & & & & & & & & & & & & & & \\
\hline & & & 25.804 & 24567 & 25898 & 24734 & 24.1 & & 54256 & 17539 & & -0.425 & & & & & & & & & 12 & & & & & & & \\
\hline & $4 / 21 / 2$ & & 25.797 & 24.55 & & 24.728 & 24. & & 54.1 & & & & & & & & & $26 \subseteq$ & & & & & & & & & & \\
\hline & $4 / 21 / 2004$ & 6:39:04 AM & 25. & 24.544 & & 24.7 & 24. & 49. & 54.316 & 17.4 & 31. & -0.4 & & 50. & 0.05 & 14.643 & & 268 & & 52 & 12. & & & & & & 94 & \\
\hline & $4 / 21 / 2004$ & 6:40:04 & 25.796 & 24.52 & 25.93 & 24.872 & 24. & 48.77 & 54.074 & 17.51 & 31. & - & & & & 14. & & 26 & & 52. & 11. & 4 & & & & & & \\
\hline & $4 / 21 / 2004$ & & 25.78 & 24.518 & 25.929| & 24.916 & & 48.953 & 54.177 & 17.545 & & -0.425 & & 49.7 & & & & 265 & & 52. & 12. & 4 & & & & & & \\
\hline & $4 / 21 / 2004$ & 6:42:04 AM & 25.8 & 24.53 & 25.964 & 24.981 & 24.4 & 49.123 & 54.308 & 17.474 & 31.91 & -0.425 & & & 0.054 & 14. & & & & & 12. & & & & & & & \\
\hline \begin{tabular}{l|l}
202 \\
\end{tabular} & $\frac{421212004}{41212004}$ & $\begin{array}{l}6: 230.04 \mathrm{AM} \\
\end{array}$ & 25. 25795 & 24.508 & | & $\frac{20.021}{20561}$ & $\frac{24.4}{24}$ & $\begin{array}{l}48.886 \\
49062\end{array}$ & $54.1 / 9$ & $\begin{array}{l}17.528 \\
17622\end{array}$ & 3.6 & -0.4 & & $\frac{49.6}{50.2}$ & & & & 270 & & & $\frac{1.2}{102}$ & & & & & & & \\
\hline , & 4 & & 25795 & & 25.974 & 25001 & & $\frac{45.006}{48919}$ & 54.454 & $\begin{array}{l}17.04 \\
17.9\end{array}$ & & & & & & & & & & & $2: .3$ & & & & & & & \\
\hline & $\frac{4}{421212004 \mid}$ & (6.4.0.0 & 2579 & 24. & 25.959 & 25076 & 24. & 487 & & $\begin{array}{l}\frac{11.45}{175} \\
\end{array}$ & & 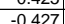 & & & & & & & & & $11 \mathrm{t}$ & & & & & & & \\
\hline & & & 25.806 & 24. & 25.99 & & & & & & & & & & & & & & & & 111 & & & & & & & \\
\hline & $4 / 21 / 2004$ & & & 24.515 & & 25.154 & & & & & & -0.4 & & & & 14.643 & & & & & & & & & & & & \\
\hline & & & 25.8 & & & & & & & & & -0.3 & & & & & & & & & & & & & & & & \\
\hline & $4 / 21 / 2004$ & & 25.816 & 1523 & 2602 & 102 & 245 & 48839 & 54.086 & 625 & & -0.358 & & 4977 & & 14.643 & & & & & & & & & & & 98 & \\
\hline & $4 / 21 / 2004$ & $6: 51: 04 \mathrm{AM}$ & & 24.501 & & & 24.073 & & & 17.511 & 31.936 & \begin{tabular}{|c|}
-0.407 \\
\end{tabular} & & 49.825 & $\begin{array}{l}0.052 \\
\end{array}$ & 14.643 & & & 451539 & & 12.2 & & $2.7 \quad$ & 0.00 & 0.008 & 0.000 & 0.186 & 0.1 \\
\hline
\end{tabular}


WSRC-TR-2005-00105, REVISION 0

SRNL-RPP-2005-00012, REVISION 0

RUN \# 2.03A AND B; FIRST AND SECOND HALF OF SLURRY DEWATERING - CONT.

\begin{tabular}{|c|c|c|c|c|c|c|c|c|c|c|c|c|c|c|c|c|c|c|c|c|c|c|c|c|c|c|c|c|}
\hline & & B & D & $E$ & $\mathrm{~F}$ & G & $\mathrm{H}$ & $\mathrm{J}$ & K & \begin{tabular}{|l|l|}
$\mathrm{L}$ \\
\end{tabular} & $\mathrm{M}$ & $\mathrm{N}$ & 0 & $Q$ & \begin{tabular}{l|l}
$R$ & \\
\end{tabular} & \begin{tabular}{l|l|} 
\\
\end{tabular} & $\mathrm{T}$ & $\mathrm{v}$ & w & $x$ & $\mathrm{Y}$ & $z$ & AA & $\mathrm{AB}$ & & $\overline{A D}$ & & AF \\
\hline 0.031 & $\begin{array}{l}4 / 21 / 2004 \\
4 / 21 / 2004\end{array}$ & $\begin{array}{l}6: 52: 04 \mathrm{AM} \\
6553: 04 \mathrm{AM}\end{array}$ & \begin{tabular}{|l|l|}
25.804 \\
2578
\end{tabular} & \begin{tabular}{|l|l|l|}
24.472 \\
24.441
\end{tabular} & $\begin{array}{l}26.019 \\
26013\end{array}$ & \begin{tabular}{l|l}
24.264 \\
23866
\end{tabular} & $\begin{array}{l}23.724 \\
23.517\end{array}$ & $\begin{array}{l}49.104 \\
9075\end{array}$ & \begin{tabular}{|l|}
54.447 \\
5.436 \\
\end{tabular} & \begin{tabular}{|l|l|}
17.615 \\
1766 \\
\end{tabular} & \begin{tabular}{|l|}
31.712 \\
31702
\end{tabular} & \begin{tabular}{|c|}
-0.358 \\
-0352
\end{tabular} & \begin{tabular}{|l|l|}
-6.385 \\
-6391 \\
\end{tabular} & \begin{tabular}{|c|}
48.74 \\
491272
\end{tabular} & $\begin{array}{l}0.055 \\
0.054\end{array}$ & \begin{tabular}{|l|}
14.643 \\
14643
\end{tabular} & $\begin{array}{l}0.003 \\
003\end{array}$ & $\begin{array}{l}2710.2 \\
27112 \\
\end{array}$ & \begin{tabular}{|l|l|}
45.1706 \\
45872
\end{tabular} & \begin{tabular}{|l|l|}
53.0 \\
5.9 \\
\end{tabular} & \begin{tabular}{|c|}
11.9 \\
1.9
\end{tabular} & \begin{tabular}{|l|}
40.4 \\
40.4 \\
\end{tabular} & \begin{tabular}{|l|l|}
2.786 \\
2785
\end{tabular} & \begin{tabular}{|l|l}
0.008 \\
0.008
\end{tabular} & \begin{tabular}{|c|}
0.008 \\
008
\end{tabular} & 0 & $\begin{array}{l}0.1977 \\
0.194\end{array}$ & 0.17 \\
\hline & $\frac{4 / 2 / 2 / 2004}{4 / / 2004}$ & $\frac{6: 53: 04 \mathrm{AM}}{6: 54: 04 \mathrm{AM}}$ & $\frac{25.788}{25.77}$ & $\frac{24.441}{24.388}$ & $\frac{26.013}{25.999}$ & $\begin{array}{l}23.866 \\
23.662\end{array}$ & $\frac{23.517}{23.293}$ & $\begin{array}{l}49.075 \\
48.753\end{array}$ & \begin{tabular}{|l|}
54.386 \\
54.101
\end{tabular} & \begin{tabular}{|r|}
17.62 \\
17499
\end{tabular} & $\begin{array}{l}31.702 \\
31.511\end{array}$ & 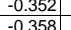 & $\begin{array}{l}-6.3911 \\
-6388 \\
-10\end{array}$ & $\frac{49.122}{49729}$ & $\frac{0.054}{0.054}$ & \begin{tabular}{|l|l|}
14.643 \\
14643
\end{tabular} & $\begin{array}{l}0.003 \\
0.003\end{array}$ & $\frac{2711.2}{2712.2}$ & \begin{tabular}{|l}
45.1872 \\
45.2039
\end{tabular} & \begin{tabular}{|l|}
52.9 \\
52.6 \\
\end{tabular} & $\frac{12.0}{12 .}$ & $\frac{40.4}{40.1}$ & \begin{tabular}{|l|}
2.785 \\
2767 \\
\end{tabular} & \begin{tabular}{|c|c|}
0.008 \\
0.008
\end{tabular} & \begin{tabular}{|c|c|}
0.008 \\
0.008
\end{tabular} & & & \\
\hline & $4 / 21 / 12004 \mid$ & 6:55:04 AM & 25.757 & 24.344 & 26.006 & 23.498 & 23.245 & 49.013 & 54277 & 11.4563 & $\frac{\mid}{31.79}$ & - -0.355 & $\begin{array}{l}-0.006 \\
-6388\end{array}$ & 50559 & 0.055 & 14.643 & $\begin{array}{l}0.003 \\
\end{array}$ & 2713.2 & 45.2206 & 52.8 & \begin{tabular}{|l|l|l|l|}
3 & 12.4 \\
\end{tabular} & 年 & 2.786 & $\mid .008$ & 0.008 & 0.000 & 0.197 & 0.17 \\
\hline & & $6: 04 \mathrm{AM}$ & 25.734 & 24.281 & 25.998 & 23.309 & & & 54.575 & 17.628 & & -0.358 & & 50.603 & 0.051 & 14.643 & 0.003 & 2714.2 & 45.2372 & 53.1 & 12.4 & & 2.797 & 0.008 & 0.007 & 0.000 & 0.182 & \\
\hline & $4 / 21 / 2004$ & & & 24.228 & & 23.236 & 23.179 & & & 17.511 & 31.743 & - 0.355 & & 49.539 & & 14.643 & & & & & & & & & 0.008 & 0.000 & & \\
\hline & $4 / 21 / 2004$ & 6:58:04 AM & 25.704 & 24.186 & 26.018 & \begin{tabular}{|l|l|}
23.244 \\
\end{tabular} & 23.197 & $\begin{array}{l}49.067 \\
\end{array}$ & \begin{tabular}{|l|l|}
54.273 \\
\end{tabular} & 17.6 & 31.689 & -0.355 & -6.376 & 50.196 & 0.053 & 14.643 & 0.003 & 2716.2 & \begin{tabular}{|l|l|}
45.2706 \\
\end{tabular} & 52.8 & \begin{tabular}{|l|l|}
3 & 12.3 \\
\end{tabular} & 40.4 & \begin{tabular}{|l|l|}
2.784 \\
\end{tabular} & 0.008 & & 0.000 & & 0.16 \\
\hline 03 & $4 / 21 / 2004$ & 6:59:04 AM & & 24.109 & 26.011 & 23.247 & 23.25 & 49.147 & \begin{tabular}{|l|}
54.179 \\
\end{tabular} & 17.512 & \begin{tabular}{|c|}
31.968 \\
\end{tabular} & -0.54 & -6.602 & 50.828 & 0.068 & 14.643 & 0.003 & & \begin{tabular}{|l|}
45.2872 \\
\end{tabular} & & & & \begin{tabular}{|l|l|}
2.796 \\
\end{tabular} & 0.010 & 0.010 & 0.000 & 0.243 & \\
\hline & $4 / 21 / 2004$ & 7:00:04 AM & & 24.037 & & & & & \begin{tabular}{|l|}
54.293 \\
5.90 \\
\end{tabular} & & \begin{tabular}{r|}
31.86 \\
\end{tabular} & - -0.471 & & & 0.03 & 14.643 & & & \begin{tabular}{|l|} 
\\
\end{tabular} & & & & \begin{tabular}{|l|}
2.790 \\
\end{tabular} & 0.004 & & & & \\
\hline & $4 / 21 / 2004$ & 7:00:06 AM & 25.6611 & $24.047 \mid$ & 26.015 | 25. & $\begin{array}{r}23.296 \\
23.277\end{array}$ & 22.933 & $\begin{array}{l}48.741 \\
48737\end{array}$ & \begin{tabular}{|c|}
53.866 \\
54453 \\
\end{tabular} & \begin{tabular}{|c|}
17.511 \\
17653
\end{tabular} & \begin{tabular}{|l|l|}
31.614 \\
3336
\end{tabular} & 122 & $\begin{array}{l}-5.734 \\
-5.577 \\
\end{array}$ & $\begin{array}{l}49.797 \\
49635\end{array}$ & $\begin{array}{l}0.014 \\
0\end{array}$ & \begin{tabular}{|l|l|}
14.643 \\
14643
\end{tabular} & 0.003 & $\begin{array}{l}2718.3 \\
27193\end{array}$ & \begin{tabular}{|l|}
45.3044 \\
453211 \\
\end{tabular} & \begin{tabular}{|l|}
52.4 \\
530 \\
\end{tabular} & $\begin{array}{l}12.2 \\
12.2\end{array}$ & 40.2 & \begin{tabular}{|l|}
2.770 \\
2760
\end{tabular} & $\begin{array}{ll}0.002 \\
0.008\end{array}$ & \begin{tabular}{l|l|l|l|}
0.002 \\
0.008
\end{tabular} & $\begin{array}{l}0.000 \\
0000\end{array}$ & 0.050 & \\
\hline $04 c^{2}$ & $\frac{4 / 21 / 2004}{4 / 21 / 2004}$ & 7:01:06 AM & & & & & & & & & & & & & & & $\frac{0.003}{0.003}$ & $\frac{2719.3}{27203}$ & 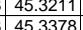 & 53.0 & & 40.0 & \begin{tabular}{|l|}
2.760 \\
2804 \\
\end{tabular} & \begin{tabular}{|c|c|}
0.008 \\
0.008
\end{tabular} & & & & \\
\hline 2043 & $4 / 21 / 2004$ & 7:03:06 AM & 26.067 & 23.857 & $\frac{2.9 .92}{25.896}$ & 23.317 & $\begin{array}{l}23.251 \\
23.195\end{array}$ & $\begin{array}{l}49.344 \\
49.235\end{array}$ & \begin{tabular}{|r|}
54.544 \\
54.13 \\
\end{tabular} & $\mid 17.586$ & $\begin{array}{l}31.965 \\
31.901\end{array}$ & $\begin{array}{l}-0.48 \\
-0.708\end{array}$ & $\begin{array}{l}-0.171 \\
-6.726\end{array}$ & $\begin{array}{l}\frac{49.204}{49.774} \\
\end{array}$ & \begin{tabular}{|c|} 
\\
0.058 \\
0.058
\end{tabular} & $\begin{array}{l}14.0454 \\
14.643\end{array}$ & $\begin{array}{l}0.003 \\
0.003\end{array}$ & 2721.3 & $\begin{array}{l}45.35318 \\
45.3544\end{array}$ & $\begin{array}{l}53.1 \\
52.7\end{array}$ & $\begin{array}{l}12.1 \\
12.2 \\
\end{array}$ & - 40.7 & \begin{tabular}{|l|}
2.804 \\
2.797
\end{tabular} & $\begin{array}{l}0.008 \\
0.009 \\
\end{array}$ & 0.008 & $\begin{array}{l}0.000 \\
0.000\end{array}$ & $\begin{array}{l}0.182 \\
0.208\end{array}$ & $\begin{array}{l}0.16 \\
0.18\end{array}$ \\
\hline 2044 & $4 / 21 / 2004$ & $7: 04: 06 \mathrm{AM}$ & & 23.808 & 25.906 & 23.358 & $\begin{array}{l}23.24 \\
\end{array}$ & & \begin{tabular}{|l|l|}
54.275 \\
\end{tabular} & $\begin{array}{l}17.537 \\
\end{array}$ & 31.946 & $\begin{array}{l}-0.766 \\
\end{array}$ & -6.78 & 49.457 & 0.054 & 14.643 & 0.003 & 2722.3 & \begin{tabular}{|l|l|}
45.3711 \\
\end{tabular} & 52.8 & \begin{tabular}{|l|l|}
3 & 12.1 \\
\end{tabular} & & \begin{tabular}{|l|l|}
2.799 \\
\end{tabular} & 0.008 & 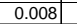 & 0.000 & 0.193 & \\
\hline 045 & $4 / 21 / 2004$ & 7:05:06 AM & 25.873 & 23.764 & 25.897] & 23.369 & 23.281 & 49.577 & \begin{tabular}{|l|l|}
54.48 \\
\end{tabular} & 17.621 & 32.196 & -0.766 & & \begin{tabular}{|l|l}
49.72 \\
\end{tabular} & 0.053 & 14.643 & 0.003 & 2723.3 & 40.5010 & 53.0 & & & 819 & & & & & \\
\hline & & & & & & & & & & & & & & & & & & 2724. & 45..4044 & & & & & & & & & \\
\hline & $4 / 21 / 2004$ & 7:07:06 AM & & 23.674 & 25.898 & 23.32 & 23.287 & 49.081 & 54.14 & 17.463 & 31.93 & & & .666 & 0.053 & 14.643 & & & 45.4211 & 27 & & & 793 & 0.008 & 0.008 & & 90 & \\
\hline & $4 / 21 / 200$ & 7:08:06 AM & & & & & & & & & 31.642 & & & 18258 & & 14.643 & & 2726.3 & 45.4378 & & & & & & & & & \\
\hline 044 & $4 / 21 / 2004$ & 7:09:06 AM & 25.626 & 23.63 & 25.919 & 23.291 & 23.329 & 49.276 & 54.308 & 17.569 & 31.964 & -0.645 & -6.42 & 50.114 & 0.05 & 14.643 & 0.004 & 2727.3 & 45.4544 & 52.8 & $12.3 \mathrm{~S}$ & 40.6 & 2.801 & 0.007 & 0.007 & 0.000 & 0.179 & 0.15 \\
\hline & $4 / 21 / 2004$ & 7:10:06 AM & 25.568 & 23.582 & 25.901 & 23.192 & 23.2 & 49.672 & 54.614 & 17.566 & 32.391| & -0.705 & -6.66 & 49.814 & 0.052 & 14.643] & 0.003 & 2728.3 & 45.4711 & 53.1. & 12.2 & & 2.829 & 0.008 & 0.008 & 0.000 & 84 & \\
\hline 05 & $4 / 21 / 2004$ & 7:11:06 AM & 25.518 & 23.547] & 25.902 & 23.142 & & 49.13 & 53.901 & 17.454 & 31.993| & -0.708 & & 375 & 50 & 14.643 & & 2729 & 年.48878 & & & & 91 & & 0 & .000 & & \\
\hline & & $\frac{7.12 .06 \mathrm{AM}}{7.12}$ & & & & & & 49.2 & & & & 0.108 & & & & & & & & & & & & & & & & \\
\hline & $11 / 2$ & $\frac{1.150 .06 \mathrm{AM}}{7.14 .06}$ & 5.4416 & 告. & 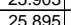 & 年 & 108 & & 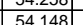 & 17474 & $\frac{31.9 / 1}{32.168}$ & & & . & 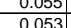 & 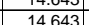 & & 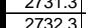 & & & & & & & & & & \\
\hline 2055 & $4 / 21 / 2004$ & 7:15:06 AM & 25.371 & 23.42 & 25.89 & 23.13 & $\frac{2.1088}{22.988}$ & 49.274 & 54.138 & 17.503 & 32.055 & 0.711 & & 50.204 & 0.052 & 14.643 & 0.003 & 2733.3 & 45.5544 & $\begin{array}{l}52.7 \\
52.7\end{array}$ & $\frac{1.4 .}{12.3}$ & & 2804 & 0.008 & $\mid$ & 0.000 & 0.186 & \\
\hline & $4 / 21 / 200$ & & 25.335 & 23.373 & 25.873 & 23.074 & 22.58 & 49.102 & 54.119 & 17.578 & 31.852 & $\begin{array}{l}0.705 \\
-0.75\end{array}$ & & 49.62 & 0.054 & 14.643 & & $\frac{2730.0}{2734.3}$ & 45.55711 & 526 & & & 2.0.794 & 0.008 & $\mid$ & 0.000 & & \\
\hline 2057 & $4 / 21 / 2004$ & 7:17:06 AM & 25.329 & & 25.882. & 23.002 & 404 & & 54.059 & 17.607 & 31.88 & & & & 0.054 & 14.643 & & & & & & & & & & & & \\
\hline & & & 25.307 & 23.315 & 25.876 & 22.92 & & & 54.2 & 17.574 & 31.977 & & & 788 & & 14.643 & & 2736.3 & \begin{tabular}{c|c|}
55.6044 \\
\end{tabular} & $\frac{2.7}{2.7}$ & & & & & & & & \\
\hline & 4/21/2004 & 7:19:06 & & & & 22.836 & 22.614 & 49.374 & 54.273 & 17.603 & 32.046 & & & 004 & & 14.643 & & 2737 & 45.6211 & 2.8 & & & 807 & $\mid$ & D08 & & & \\
\hline & $4 / 21 / 200$ & & & 23.228 & & 22.798 & & 49.1 & 54.018 & & 31.833 & & & 7.04 & & 14.643 & & 2100. & & & & & & & & & & \\
\hline 206 & $4 / 21 / 2004$ & 7:21:06 AM & 25.212 & 23.18 & 25.876 & 22.795 & 22.578 & 49.288 & 54.132 & $\begin{array}{l}17.62 \\
\end{array}$ & 31.997 & -0.703 & & |49.147 & 0.052 & 14.643 & & 2739.3 & 45.6544 & 52.7 & 2.0 & & & D.008 & 008 & & 86 & 0.16 \\
\hline & & & & 23.136 & & 816 & & & 54.179 & 17.499 & 32.25 & -0.703 & & & & 14.643 & & note & 45.6711 & & & & & & & & & \\
\hline & 4.121212006 & OAl & t5.114 & 23.0866 & 20.167 & 22.826 & & 49 & 54.256 & 1.546 & . .157 & & & 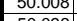 & & 14.643 & & & 然 & & 27 & & & & (2) & & & \\
\hline & & & 2 & - & 2 & 2.173 & 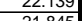 & 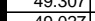 & & & & & & & & & & & & & & & & & & & & \\
\hline & 4 & & $\frac{2.044}{2504}$ & $\mid \frac{|c .304|}{22926}$ & 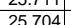 & $\begin{array}{l}22.0 .0 \\
22.752\end{array}$ & & 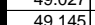 & | & 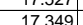 & $\frac{31.825}{32.112}$ & $\begin{array}{r}-0.1 \\
-0.688\end{array}$ & & $\frac{5260}{266}$ & & 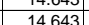 & & 2074.5 & 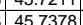 & $t$ & $\frac{2.1}{21}$ & & & & (1) & & & \\
\hline 2067 & $4 / 21 / 2004$ & $7 \cdot 27.06 \mathrm{AM}$ & $\frac{25.047}{25}$ & 22868 & 25.686 & 22724 & & & $\mid 54.1$ & 17.562 & 31.968 & & & 年3 & & 14.643 & & 2745 & 45.7544 & & & & & & & & & \\
\hline 2068 & $4 / 21 / 2004$ & $\frac{1}{7: 28: 06} \mathrm{Am}$ & 25.103 & 2 & 25.736 & 22.785 & $\frac{2.182}{22.182}$ & & 54.146 & $\begin{array}{l}17.527 \\
\end{array}$ & 31.805 & -0.703 & & 50.041 & 0.054 & $\begin{array}{l}\frac{1.0445}{14.643} \\
\end{array}$ & & 2746.3 & 45.77 & 2.7. & $\frac{1.2}{12.3}$ & & . & 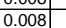 & 年 & & 95 & \\
\hline & & & & & & \begin{tabular}{|l|}
22.754 \\
\end{tabular} & & & & & & & & & & & & & & & & & & & & & & \\
\hline 2070 & $4 / 21 / 2004$ & 7:30:06 & 25.12 & 22.715 & 25.668 & 22.757 & & & 54.235 & 17.529 & 32.04 & & & .432 & $\mid 0.054$ & 14.643 & & 2748.3 & \begin{tabular}{c|}
55.8044 \\
\end{tabular} & 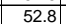 & 2.4 & & 1007 & $\mid$ & 5.008 & 0.000 & 5.194 & \\
\hline & 4/21/200 & & & 22.667 & 25.681 & 22.82 & & & 54.101 & 17.578 & 31.732 & & & .500 & 502 & 14.643 & & $2 / 49$ & & & & & & & & & & \\
\hline 2072 & $4 / 21 / 2004$ & 7:32:06 AM & 25.152 & 22.637 & $\mid 25.68$ & & & & 54.594] & $17.548 \mid$ & 32.426 & & & 864 & & 14.643 & & & & 53.7 & & & & & & & & \\
\hline 207 & $4 / 21 / 200$ & 7:33:06 & 25.163 & 22.602 & 25.691 & 22.911 & & & \begin{tabular}{|l|}
54.192 \\
\end{tabular} & 17.593 & 32.0 & & & 0.69 & & 14.643 & & & 5544 & 2.7 & 2. & & & & & & & \\
\hline & $4 / 21 / 2$ & & 25.148 & & 25.667 & 22.886 & & & 54.183 & 17.55 & 31.9 & & & .00 & & & & & 45.87 & & & & & & & & & \\
\hline 2015 & $4 / 2 / 2$ & & & 22.479 & 2.638 & $8 / 3$ & & 49.1 & 54.03 & 17.5 & & & & & & & & & & 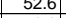 & 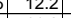 & & & & & & & \\
\hline 207 & $\frac{421212004}{4121004}$ & & & 2.259 & 20.538 & $\frac{2.2544}{20955}$ & & & $\begin{array}{l}54.0360 \\
5503\end{array}$ & $\begin{array}{l}1.6 \\
175\end{array}$ & 32. & & & & & & & & & 528 & 21 & & & & & & & \\
\hline & 年 & & & & & & & & 530 & & & & & $\begin{array}{lll}45.44 \\
\end{array}$ & & & & & & & & & & & & & & \\
\hline 2079 & $4 / 21200$ & & & 22.357 & 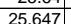 & 22943 & & & 53.316 & 17.536 & 31.6 & & & 48 & & & & 275 & 459544 & 523 & 1118 & & & & & & & \\
\hline & $\frac{1 / 21 / 200}{401 / 20}$ & & & non & & 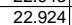 & & & | & 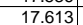 & 3 & & & 40. & & $\frac{1.4045}{14.643}$ & & 270 & 40.90 & & & & & & & & & \\
\hline & $4 / 21 / 2$ & & 5.078 & 22.296 & \begin{tabular}{|l|l|}
25.646 \\
\end{tabular} & 021 & & & $\frac{1.252}{5.4252}$ & 17.595 & 31.921 & & & 1002 & & & & & & & & & & & & & & \\
\hline 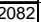 & & & 25. & 22.273 & 25.643 & \begin{tabular}{|l|l|}
22.848 \\
\end{tabular} & & & 53.843 & 17.52 & 31.5 & & & .0 & & & & & & & & & & & & & & \\
\hline & & & & & 25.616 & 22.76 & & & & & 32.0 & & & & & & & & & & & & & & & & & \\
\hline 2084 & $4 / 21 / 20 \mathrm{C}$ & & 25.027 & 22.21 & 25.63 & 22.759 & & 49.064 & \begin{tabular}{|l|}
54.173 \\
\end{tabular} & $17.688 \mid$ & 31. & & & & & & & & & & 21 & & & & & & & \\
\hline 2085 & $4 / 21 / 200$ & & & 22.192 & 25.618 & 22.717 & & 49. & 54.1 & 17.5 & & & & $8 \mathrm{cc}$ & & & & & & & & & & & & & & \\
\hline 2086 & $4 / 21 / 2$ & & 25.009 & 22.186 & 25.642 & 22.756 & & 49.19 & 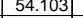 & 17.53 & & & & & & 14.6 & & & & . & $12.3 \mathrm{H}$ & & & & & & 88 & \\
\hline 2087 & $4 / 21 / 2004$ & & 2. & 22.175 & $2 \frac{2.666}{20.69}$ & 年 & & 48.8. & 53.904 & & & & & & & & & & & & 12. & & & & & & & \\
\hline & & & & $\frac{2.115}{2.159}$ & 5.696 & & & & & & & & & & & & & & & & & & & & & & & \\
\hline 2090 & 势 & & & 年 & 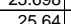 & 资.545 & & & 54005 & $\mid \frac{17478}{17628}$ & $31.7 / 4$ & & & it & & & & & & & 12.4 & & & & & & 5 & \\
\hline$\frac{205}{205}$ & $4 / 21 / 200$ & $7.51 .06 \mathrm{~A}$ & | & $\frac{2503}{22203}$ & $\frac{2.034}{5.634}$ & $\frac{2.375}{23.175}$ & & 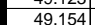 & $\mid$\begin{tabular}{|l|l|l|l|}
54.199 \\
\end{tabular} & & $\frac{\frac{310}{317}}{317}$ & & & if & & & & & & & & & & & & & & \\
\hline 0 & & & 25 & 22.23 & 年.643 & 23.336 & & & 54.272 & 17.64 & $\frac{3.1 .1}{31.7}$ & & & & & & & & & & & & & & & & & \\
\hline 2093 & $4 / 21 / 2004$ & & & 22.253 & 25.644 & & & & 54.31 & & & & & & & & & & & & & & & & & & & \\
\hline & & & & & 25.66 & & & & 54.393 & & & & & & & & & & & & & & & & & & & \\
\hline 2095 & $4 / 21 / 200$ & 7:55: & 24008 & 2232 & & 23.825 & & 49.1 & 5415 & & 31.9 & & & & & 146 & & & & & & & & & & & & \\
\hline 2096 & $4 / 21 / 2$ & 7:56:0 & 24.9 & $22.37 \mathrm{C}$ & 25.6 & & & 49.16 & 54.1 & 17.6 & 31.7 & -0.5 & & 10 & & & & 277. & & & & & & & & & & \\
\hline 2097 & $4 / 21 / 2004$ & 7:57:06 A & 25.01 & 22.419 & 25.689| & 24.179 & & 49.264] & 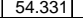 & 17.707 | & $31.788 \mid$ & -0.54 & & 0.84 & 0.05 & 14.6 & & 2775.3 & 46.25 & 52.9 & 12.5 & & & & & & 0.180 & \\
\hline 2098 & $4 / 21 / 2004$ & 7:58:06 AM & 25.018] & 22.4777 & 5.7011 & 24.307 & 24.0 & 49.21 & 54.181 & 17.502 & 32.083 & -0.54 & -6.54 & 49.99 & 0.054 & 14.643 & & 2776.3 & 46.27. & 52.1 & 12.3 & & 2.80 & .00 & 0.00 & 00 & 0.194 & 0.1 \\
\hline $20 \mathrm{~s}$ & $\frac{4 / 21 / 2004}{4 / 21 / 2004}$ & $\begin{array}{l}7: 59: 06 \mathrm{AM} \\
8.0006 \mathrm{AM}\end{array}$ & $\frac{25.014}{250.02}$ & $\frac{22.513}{22.56}$ & $\frac{25.703}{25716}$ & \begin{tabular}{|l|}
24.429 \\
24.45
\end{tabular} & $\frac{24.184}{24.136}$ & $\begin{array}{l}49.282 \\
48.971 \\
\end{array}$ & \begin{tabular}{|l|}
54.243 \\
54.02 \\
\end{tabular} & \begin{tabular}{|c|}
17.6 \\
17.45 \\
\end{tabular} & \begin{tabular}{|c|c|}
31.983 \\
31.88
\end{tabular} & \begin{tabular}{|c|c|}
-0.54 \\
-0.54
\end{tabular} & -6.5 & 49.23 & 0.053 & $\begin{array}{c}\frac{14.643}{14.643} \\
1\end{array}$ & & & \begin{tabular}{|l|l|}
46.28 \\
46.30
\end{tabular} & & & & $\frac{80}{78}$ & 0.008 & \begin{tabular}{|c|}
0.008 \\
0.008
\end{tabular} & $\frac{0.000}{0.000}$ & \begin{tabular}{|c|c|}
0.191 \\
0.192
\end{tabular} & $\frac{0.1}{0.1}$ \\
\hline
\end{tabular}


WSRC-TR-2005-00105, REVISION 0

SRNL-RPP-2005-00012, REVISION 0

RUN \# 2.03A AND B; FIRST AND SECOND HALF OF SLURRY DEWATERING - CONT.

\begin{tabular}{|c|c|c|c|c|c|c|c|c|c|c|c|c|c|c|c|c|c|c|c|c|c|c|c|c|c|c|c|c|}
\hline & A & B & D & $\mathrm{E}$ & $\mathrm{F}$ & $\mathrm{G}$ & $\mathrm{H}$ & $\mathrm{J}$ & $\mathrm{K}$ & $\mathrm{L}$ & $\mathrm{M}$ & $\mathrm{N}$ & 0 & Q & $R$ & 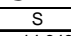 & \begin{tabular}{l|l|l|} 
\\
\end{tabular} & $\mathrm{V}$ & w & $x$ & & 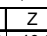 & AA & $A B$ & $\mathrm{AC}$ & $A D$ & & \\
\hline \begin{tabular}{|l|l|l|l|}
2102 \\
2102
\end{tabular} & $\begin{array}{r}4 / 21 / 2004 \\
4 / 212004\end{array}$ & $\begin{array}{l}8: 01: 06 \mathrm{AM} \\
802006 \mathrm{AM}\end{array}$ & $\begin{array}{r}25.051 \\
25.052\end{array}$ & $\frac{22.626}{22.662}$ & $\begin{array}{l}25.725 \\
25.731\end{array}$ & $\begin{array}{l}24.476 \\
24.467\end{array}$ & $\begin{array}{r}24.12 \\
24.026\end{array}$ & $\begin{array}{l}49.446 \\
49.006\end{array}$ & \begin{tabular}{|l|}
54.428 \\
54.105 \\
\end{tabular} & \begin{tabular}{|c|}
17.612 \\
17.641
\end{tabular} & $\begin{array}{l}32.168 \\
31.628 \\
\end{array}$ & $\begin{array}{c}-0.54 \\
-0.54 \\
-0.0\end{array}$ & $\begin{array}{l}-6.529 \\
-6.529\end{array}$ & $\begin{array}{l}49.028 \\
50.256\end{array}$ & 0.052 & $\begin{array}{l}14.643 \\
14.643\end{array}$ & $\begin{array}{l}0.003 \\
0.03\end{array}$ & $\begin{array}{r}2779.3 \\
2780.3\end{array}$ & \begin{tabular}{|l|l|}
46.3211 \\
46.3378 \\
\end{tabular} & $\begin{array}{l}53.0 \\
52.6\end{array}$ & $\frac{12 .}{12 .}$ & \begin{tabular}{|l|}
40.8 \\
0.3
\end{tabular} & $\begin{array}{l}82.813 \\
3 \\
3 \\
2.780\end{array}$ & $\begin{array}{l}0.008 \\
0.008\end{array}$ & \begin{tabular}{|l|}
0.008 \\
0.007 \\
\end{tabular} & 0.000 & $\begin{array}{l}0.186 \\
0.185\end{array}$ & $\begin{array}{l}0.16 \\
0.16\end{array}$ \\
\hline & & & $\frac{20.006}{25.068}$ & & & & & & & & & & & & & $\frac{14.043}{14.643}$ & & & & & & & & & & & & \\
\hline 2104 & $4 / 21 / 2004$ & 8:04:06 AM & 25.087 & 22.763 & 25.746 & 24.407 & 24.072 & 49.189 & \begin{tabular}{|l|}
54.221 \\
\end{tabular} & 17.634 & 31.901 & $\begin{array}{r}-0.517 \\
\end{array}$ & -6.518 & 48.598 & 0.053 & 14.643 & 0.003 & & 346.3711 & & & & 2.795 & 0.008 & 0.008 & 0.000 & 0.191 & \\
\hline$\frac{2104}{2105}$ & $4 / 221 / 2004$ & $8: 05: 06 \mathrm{AM}$ & 25.106 & 22.802 & 25.755 & \begin{tabular}{|l|l|}
24.386 \\
\end{tabular} & 24.025 & 49.247 & \begin{tabular}{|l|}
54.328 \\
\end{tabular} & 17.636 & 31.862 & $\begin{array}{l}-0.497 \\
-0.45\end{array}$ & -6.529 & 50.734 & 0.051 & $\begin{array}{l}14.643 \\
\end{array}$ & & & 346.3878 & & & & \begin{tabular}{|l|l|}
2.796 \\
\end{tabular} & & 0.007 & & & 0.16 \\
\hline & & & 25.12 & & & & & & \begin{tabular}{|l|l|}
54.735 \\
\end{tabular} & \begin{tabular}{|l|l|l|l|}
17.674 \\
\end{tabular} & & & & & & 14.643 & & & \begin{tabular}{|l|l|}
46.4044 \\
\end{tabular} & & & & & & & & & \\
\hline \begin{tabular}{|l|}
2107 \\
2109
\end{tabular} & $4 / 21 / 2004$ & 8:07:06 AM & 25.135 & 22.886 & \begin{tabular}{|l|l|}
25.778 \\
\end{tabular} & \begin{tabular}{|l|l|}
24.404 \\
\end{tabular} & $\begin{array}{l}24.184 \\
2130\end{array}$ & \begin{tabular}{|l|l}
48.946 \\
\end{tabular} & \begin{tabular}{|l|l|}
54.092 \\
5.25
\end{tabular} & $\begin{array}{ll}17.553 \\
17560\end{array}$ & $\begin{array}{l}31.685 \\
21.02\end{array}$ & \begin{tabular}{|l|l|}
-0.483 \\
\end{tabular} & $\begin{array}{l}-6.518 \\
6.521\end{array}$ & \begin{tabular}{|l|l|}
49.572 \\
50.12 \\
\end{tabular} & 0.051 & 14.643 & $\begin{array}{l}0.003 \\
\end{array}$ & 2785.3 & 46.4211 & 52.6 & \begin{tabular}{l|l}
6 & 12.1 \\
\end{tabular} & & $\begin{array}{l}3 \quad 2.780 \\
\end{array}$ & \begin{tabular}{|l|l|}
0.008 \\
\end{tabular} & $\begin{array}{l}0.007 \\
\end{array}$ & 0.000 & & \\
\hline$\frac{2108}{2109}$ & $\begin{array}{l}4 / 21 / 2004 \\
4 / 21 / 2004\end{array}$ & $\begin{array}{l}8: 08: 06 \mathrm{AM} \\
8: 09: 06 \mathrm{AM}\end{array}$ & $\begin{array}{l}25.164 \\
25.169\end{array}$ & $\begin{array}{l}22.941 \\
22.965 \\
\end{array}$ & $\begin{array}{l}25.798 \\
25.792 \\
\end{array}$ & \begin{tabular}{|l|}
24.494 \\
24.594 \\
\end{tabular} & \begin{tabular}{l|}
24.204 \\
24.298 \\
\end{tabular} & \begin{tabular}{|c|}
49.191 \\
49.154 \\
\end{tabular} & \begin{tabular}{|l|}
54.345 \\
54.302 \\
\end{tabular} & $\begin{array}{l}17.569 \\
17.465\end{array}$ & $\begin{array}{l}31.893 \\
31.936\end{array}$ & \begin{tabular}{|l|l|}
-0.477 \\
-0.52
\end{tabular} & $\begin{array}{l}-6.521 \\
-6.521\end{array}$ & \begin{tabular}{|c|}
50.114 \\
49174
\end{tabular} & $\begin{array}{l}0.053 \\
0.053\end{array}$ & $\begin{array}{l}144.643 \\
14643\end{array}$ & \begin{tabular}{|l}
0.003 \\
0.003
\end{tabular} & 2786.3 & $\begin{array}{l}346.4378 \\
3.46 .454\end{array}$ & $\begin{array}{l}52.9 \\
528\end{array}$ & $\frac{12.3}{12.1}$ & $\begin{array}{l}340.5 \\
140 .\end{array}$ & $\begin{array}{l}5 \\
5\end{array}$ & \begin{tabular}{|l|}
0.008 \\
0.008 \\
\end{tabular} & $\begin{array}{l}0.008 \\
0.008\end{array}$ & $\begin{array}{l}0.000 \\
0.000\end{array}$ & \begin{tabular}{|l|l|}
0.191 \\
0.191
\end{tabular} & $\begin{array}{l}0.16 \\
0.16\end{array}$ \\
\hline 2110 & $4 / 21 / 2004$ & 8:10:06 AM & 25.188 & 23.01 & \begin{tabular}{|l|l|}
25.817 \\
\end{tabular} & & $\begin{array}{l}24.290 \\
24.368\end{array}$ & & \begin{tabular}{|l|l|}
54.275 \\
\end{tabular} & 17.575 & & & & 50.63 & 0.053 & $\begin{array}{l}14.643 \\
14.643\end{array}$ & $\begin{array}{l}0.0003 \\
0.03\end{array}$ & $\frac{2187.3}{2788.3}$ & & $\frac{52.8}{52.8}$ & $\frac{12.1}{12.4}$ & & $\frac{2.795}{2.798}$ & $\begin{array}{l}0.008 \\
0.008\end{array}$ & $\begin{array}{l}0.008 \\
0.008\end{array}$ & $\begin{array}{l}0.000 \\
0.000 \\
\end{array}$ & $\begin{array}{l}0.191 \\
0.190\end{array}$ & 0.16 \\
\hline 2111 & $4 / 21 / 2004$ & 8:11:06 AM & & & & \begin{tabular}{|l|} 
\\
\end{tabular} 4.729 & 24.473 & 48.834 & & 17.629 & 31.474 & -0.48 & & 50.626 & & 14.643 & & & & & & & & & & & & $\begin{array}{l}0.16 \\
0.16\end{array}$ \\
\hline 2112 & $4 / 21 / 2004$ & $8: 12: 06 \mathrm{AM}$ & 25.214 & & 25.827 & \begin{tabular}{|l|l|}
24.774 \\
\end{tabular} & 24.503 & 49.125 & 54.185 & $\begin{array}{l}17.539 \\
\end{array}$ & 31.899 & $\begin{array}{l}-0.48 \\
\end{array}$ & -6.509 & 50.815 & 0.053 & 14.643 & 0.003 & 2790. & 46.5044 & & 12.5 & & \begin{tabular}{|l|l|}
2.793 \\
\end{tabular} & & \begin{tabular}{|l|}
0.008 \\
\end{tabular} & 0.000 & & $\frac{0.16}{0.16}$ \\
\hline 2113 & $4 / 21 / 2004$ & $8: 13: 06 \mathrm{AM}$ & 25.244 & & & & & & & & & & & & & & & & & & & & & & & & 184 & $\begin{array}{l}0.16 \\
0.16\end{array}$ \\
\hline $2+14$ & $4 / 21 / 2004$ & 8:14:06 AM & & 2318 & 25897 & \begin{tabular}{|l|l|}
24.934 \\
\end{tabular} & & & 54.347 & 17.582 & 31.934 & 0.477 & & & 0.056 & & & & & & & & & & & & & \\
\hline & $4 / 21 / 2004$ & 8:15:06 AM & 25.284 & & 25.808 & & & 49.036 & 54.198 & & 31.804 & -0.48 & & 8.377 & & 14.643 & & & 5544 & & & & & & & & & \\
\hline 21116 & $4 / 21 / 2004$ & 8:16:06 AM & 25.3 & 23.258 & 25.794 & \begin{tabular}{|l|l|}
24.961 \\
\end{tabular} & 24.875 & \begin{tabular}{|l|l|l|}
49.28 \\
\end{tabular} & 54.37 & 17.619 & 31.989 & $\begin{array}{l}-0.483 \\
\end{array}$ & -6. & $\begin{array}{l}49.061 \\
\end{array}$ & 0.052 & 14.643 & $\begin{array}{l}0.003 \\
\end{array}$ & 2794.3 & 46.5711 & 52.9 & 12.0 & 40. & 2.802 & 0.008 & 0.008 & 0.000 & 0.187 & \\
\hline & $1,1 / 2004$ & 8:17:06 AM & 25.326 & 23.304 & 25.814 & \begin{tabular}{|l|l|}
25.037 \\
\end{tabular} & 24.865 & & 54.154 & 17.6 & 31.743 & -0.48 & & 49.793 & 0.052 & 14.643 & & 2795.3 & 5.5878 & & 12. & & & & 0.008 & & & \\
\hline & $21 / 2004$ & $8: 18: 06 \mathrm{AM}$ & & & & & & & & & & -0.477 & & & & & & & & & & & & & & & & \\
\hline & $4 / 21 / 2004$ & 8:19:06 AM & 25.366 & $\begin{array}{r}23.39 \\
\end{array}$ & 25.85 & 25.113 & 24.801 & 48.797 & 44.007 & 17.4499 & 31.54 & -0.425 & & .418 & 0.052 & 14.643 & & & & & 12. & & & & .008 & & & \\
\hline$\frac{212}{212}$ & $\frac{\mid 4 / 21 / 2004}{4}$ & 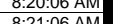 & $\frac{25.311}{25.20}$ & $\frac{23.419}{23450}$ & $\frac{25.85}{2584}$ & $\frac{25.082}{25027}$ & $\frac{24.141}{2469}$ & 年 & & $\begin{array}{l}17.553 \\
17584\end{array}$ & $\frac{31.962}{32657}$ & $\begin{array}{l}-0.425 \\
-048\end{array}$ & & 8786 & 然 & $\frac{14.643}{14642}$ & & & 465544 & & & & & & & & & \\
\hline & 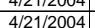 & $\begin{array}{l}\text { 8.12.00 AM } \\
\text { 8:22:06 AM }\end{array}$ & $\begin{array}{r}25.38 \\
25.399 \\
\end{array}$ & $\begin{array}{r}23.459 \\
23.498\end{array}$ & $\frac{25.054}{25.863}$ & $\begin{array}{l}25.027 \\
24.955\end{array}$ & $\begin{array}{r}24.09 \\
24.594\end{array}$ & $\begin{array}{r}48.920 \\
49.112\end{array}$ & 54.225 & $\begin{array}{l}17.564 \\
17.671\end{array}$ & $\frac{31.651}{31.72}$ & $\begin{array}{r}-0.48 \\
-0.425 \\
\end{array}$ & & \begin{tabular}{|l|}
40.140 \\
50.148
\end{tabular} & $\begin{array}{l}0.052 \\
0.051\end{array}$ & $\begin{array}{l}4.645 \\
14.643 \\
\end{array}$ & 0.003 & & $\begin{array}{l}46.0344 \\
46671\end{array}$ & & 123 & & & 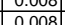 & 0.007 & 0.000 & $\begin{array}{l}0.160 \\
0.184\end{array}$ & \\
\hline & $4 / 21 / 2004$ & 8:23:06 AM & 25.408 & 23.527 & 25.872 & 24.894 & 24.423 & 48.868 & 53.945 & 17.505 & 31.679 & -0.427 & & 50.872 & 0.051 & 14.643 & 0.003 & 2801.3 & 46.6878 & 52.5 & & & \begin{tabular}{|l|l|}
3 & 2.777 \\
\end{tabular} & & 0.007 & & 0.184 & 0.16 \\
\hline & $1: 12004$ & $8: 24: 06 \mathrm{AM}$ & 25.426 & 23.565 & 25.885 & 24.837 & 24.351 & 48.7. & 8.866 & 17.594 & 31.418 & -0.442 & & 49.42 & 0.05 & 14.643 & & 2802.3 & 46.7044 & & & & & & & & & \\
\hline & $1 / 2004$ & :06 AM & 25.449 & & 25.90 & $\begin{array}{r}24.84 \\
24.957\end{array}$ & & & & $\frac{17.646}{17621}$ & 1.607 & -0.42 & & 9.766 & 0.053 & 14.643 & & & & & 12 & & & & & & & \\
\hline$\frac{\frac{25}{212}}{212}$ & $\frac{421212004}{4121204}$ & $\begin{array}{l}8.20 .06 \mathrm{AMM} \\
8.200 \mathrm{AM}\end{array}$ & 25.456 & $\frac{23.62}{23.644}$ & $\frac{25.915}{25092}$ & $\begin{array}{l}24.857 \\
2.8985\end{array}$ & $\begin{array}{l}24.601 \\
2.475\end{array}$ & $\frac{48.975}{14012}$ & $\frac{54.132}{54.316}$ & 17.021 & $\frac{31.694}{31.622}$ & $\begin{array}{l}-0.425 \\
0.025\end{array}$ & & 年9.154 & 0 & $\begin{array}{l}\frac{1.4645}{1463} \\
\end{array}$ & & 2805 & 46754 & & 122 & & & & & & & \\
\hline & $4 / 2 / 212004$ & $8: 28.06 \mathrm{AM}$ & 25.477 & 23.666 & 25.916 & 24.0004 & & 48.965 & 54.5194 & $\begin{array}{l}17.042 \\
17.605\end{array}$ & $\frac{31.024}{31.614}$ & $\begin{array}{l}-0.425 \\
-0.27\end{array}$ & & 年 & 然 & $\frac{1.4045}{1463}$ & & 2806 & & 527 & $\frac{12.4}{11.9}$ & & & & & & & \\
\hline & $4 / 21 / 2004$ & $9: 06 \mathrm{AM}$ & 25.505 & 23.705 & \begin{tabular}{|l|l|}
25.949 \\
\end{tabular} & 25.031 & 24.975 & 48.988 & 54.341 & $\frac{17.619}{17}$ & 31.63 & -0.425 & & & 0.053 & 14.643 & & 280 & \begin{tabular}{|l|l|}
46.7878 \\
\end{tabular} & & 12. & 40 & . & & 008 & & 191 & \\
\hline & $4 / 21 / 2004$ & 8:30:06 AM & 25.533 & 23.753 & & \begin{tabular}{|l|l|}
25.13 \\
\end{tabular} & & 49.05 & & 17.674 & 31.622 & -0.422 & & 49.307 & 0.052 & 14.643 & & 2808.3 & & & 12. & & & & & & & \\
\hline [213]. & $4 / 21 / 2004$ & $8: 31: 0$ & 25.526 & 23.751 & 25.96 & 25.123 & & 49.1 & 54.219 & 17.537 & 31.913 & -0.427 & & \begin{tabular}{|l|l|}
49.13 \\
\end{tabular} & 0.053 & 14.643 & & 2809.3 & 46.8211 & 52 & $\frac{12.0}{12.0}$ & 46 & & 0.008 & 0.008 & 0.000 & 0.190 & \\
\hline$\frac{2134}{2120}$ & $4 / 21 / 2004$ & & 25.555 & 23.796 & 25.994 & $\begin{array}{l}25.192 \\
\end{array}$ & $\begin{array}{l}24.955 \\
\end{array}$ & & 54.154 & 17.678 & 31.595 & -0.425 & -6.3 & 48.548 & 0.048 & 14.643 & & 2810.3 & & & 11.5 & & 2.77 & & & 0.06 & 0.173 & \\
\hline & $4 / 21 / 2004$ & & & & 25.983 & & & 48.965 & & & 31.546 & & & & 0.054 & & & & 46.8544 & & & & & & & & & \\
\hline & $4 / 21 / 2004$ & 8:34:0 & 25.593 & 23.869 & 26.047 & 25.34 & 25.19 & & 54.19 & 17.573 & 31.648 & -0.422 & & 49.044 & 0.053 & 14.643 & & 28. & 46.8711 & 52.7 & 12.0 & 40. & & 0.008 & 0.008 & 0.000 & 0.191 & \\
\hline & $4 / 21 / 2004$ & $8: 35: 06 \mathrm{AM}$ & & 23.878 & 26.031 & 25.359 & 20.20 & 48.708 & & 17.478 & 31.453 & & & & & 14.6 & & & & & 11. & & & & & & & \\
\hline 213 & 11/2004 & $8: 36: 06 \mathrm{AM}$ & 25.612 & & 26.046 & 25.409 & 25.257 & 48.517 & .736 & 17.502 & 31. & -0.425 & & & 0.052 & & & 2814.3 & 46.9044 & & & & & & & & & \\
\hline$\overline{D i 12}$ & . & & 25.641 & 23.952 & 26.07 & 25.433 & 25. & 49.237 & .453 & 17.58 & & - -0.361 & & 8.967 & & 14.643 & & & .2011 & & 12. & & 79 & .008 & 0 & .000 & & \\
\hline & & & & & & & & & .586 & & & & & & & & & & & & & & & & & & & \\
\hline$\frac{2.510}{2140}$ & $\frac{42121204}{4 \mid 21204}$ & $\begin{array}{l}6.35 .00 \\
8: 0.06\end{array}$ & $\frac{23.072}{25.69}$ & $\frac{24.003}{22.036}$ & $\frac{20.011}{25.974}$ & $\begin{array}{l}25.344 \\
25296\end{array}$ & $\frac{2.00}{248}$ & 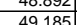 & $\frac{54.054}{5444}$ & 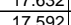 & $\begin{array}{l}31.529 \\
331921\end{array}$ & $\begin{array}{l}-0.350 \\
-0.0401\end{array}$ & & $\frac{49.17}{48523}$ & 然 0.053 & $\frac{14.045}{14643}$ & & $\frac{201.5}{28183}$ & 346.974 & 530 & $\frac{12.6}{116}$ & & & & . & & & \\
\hline & 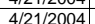 & $8: 41: 06 \mathrm{AM}$ & 25.707 & 24.058 & 25.991 & 25263 & & 49 & 54.244 & & 31.798 & $\frac{-0.455}{-0.355}$ & & & & & & & 846.978 & & & & & & & & & \\
\hline & 4 & 8:42:06 AM & 25.708 & 24.07 & 25.987 & 25.2 & 24.8 & 48.95 & 54.243 & 17.62 & 31.622 & -0.399 & & 49.898 & 0.05 & & & 288 & 47.0044 & 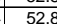 & 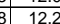 & & & & & & & \\
\hline & | & $8: 43: 06$ & 25.709 & & 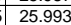 & 25.16 & & & & & & & & & & & & & & & & & & & & & & \\
\hline 2144 & & & 25725 & 24.12 & 26000 & 25121 & & 4889 & & 17,69 & 31.462 & -0.306 & & 1890 & 0.05 & & & & & & 11 & & & & & & & \\
\hline 2145 & $4 / 21 / 2004$ & 8:45:06 AM & 25.736 & $24.13 \varepsilon$ & 26.025 & 25.123 & 24.8 & 48. & 54.047 & 17.60 & 31. & -0.335 & & & & 14. & & 28 & 347.0544 & 52 & 12.2 & 40. & & & & & & \\
\hline & $4 / 21 / 2004$ & & 25.749 & 24.156 & 26.038 & & & & & 17.53 & & -0.3 & & & & & & & & 52 & 12.1 & & & & & & & \\
\hline 2147 & 4/21/2004 & & 25.751 & 24.16 & 26.045 & 25.198 & 25.0 & & 54.285 & 17.555 & 31.568 & -0.309 & & & & 14. & & & 47.0878 & 52 & $12 . \mathrm{C}$ & 4 & & & & & 184 & \\
\hline 2148 & $4 / 21 / 2004$ & & 25.752 & 24.184 & 26.066 & 25.269 & & 48.69 & 9976 & & & -0.268 & & & 0.05 & & & 288 & $\begin{array}{l}387.1044 \\
\end{array}$ & $J$ & 12.5 & & & & & & & \\
\hline & & & & 24.2 & 26.074 & 25.357 & & & 54.465 & 17.647 & & -0.274 & & & & & & & & . & 12.1 & & & & & & & \\
\hline & 4421212004 & & 25.183 & 24.2 & & 25.451] & & & 5.4509 & 17.591 & & -0.2 & & & & & & $\frac{28}{12}$ & & & 11.2 & & & & & & 年 & \\
\hline & $\frac{4-4121204}{4 / 21204}$ & 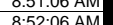 & $\frac{2.1}{25}$ & & & & & & & & & & & & & & & & & 528 & & & & & & & & \\
\hline & 4 & & 25.514 & 24296 & & 25647 & & & 54.3 & & & -0.2 & & & & & & & & & 124 & & & & & & & \\
\hline 215 & $4 / 21 / 2004$ & $8: 54: 00 \mathrm{AM}$ & $\frac{25.828}{25.828}$ & 24.32 & $\frac{20.137}{26.137}$ & 25.66 & & & 54.109 & & 31. & -0.28 & & & & & & & & & 12.0 & & & & & & & \\
\hline & & & & & & & & & & & & & & & & & & & & & & & & & & & & \\
\hline & & & 25.853 & 24376 & 26.172 & 25726 & 25.3 & 48.9 & 54,401 & 17516 & 31.728 & 0219 & & & & 14.6 & & & & 52 & 12 & & & & & & & \\
\hline & $4 / 21 / 2$ & 8:57: & 25.8 & 24.39 & & 25.709 & & & 4.215 & 17.6 & & -0.367 & & & & & & 283 & 347.2 & 52.7 & 12.2 & 4 & & & & & & \\
\hline 215 & $4 / 21 / 2004$ & 8:58:06 AM & 25.889 & 24.416 & 26.183 & 25.621 & & 48.8 & \begin{tabular}{|l|l|}
54.277 \\
\end{tabular} & 17.514 & 31.638 & -0.19 & & & 0.0 & 14.643 & & 2836.3 & 347.2 & 52.8 & 12.2 & 40 & & & & & 33 & \\
\hline & $4 / 21 / 2004$ & 8:59:06 AM & 26.129 & 24.432 & $26.1 \mathrm{~s}$ & 25. & 25. & 48.64 & 54.3 & 17.528 & 31. & -0.069 & & 49.2 & & 14. & & & & 52.8 & 12.1 & 4 & & & & & & \\
\hline & $4 / 2121 / 200$ & 9:00:06 AM & & 24.453 & & 25.548 & 25. & 49. & 54.66 & 17.606 & & -0.112 & & & & & & 2838.3 & & 53. & 12.3 & & & & & & & \\
\hline 216 & $4 / 21 / 2004$ & 9:00:13 AM & 26.111 & 24.458 & 26.215 & 25.543 & 25.11 & 48.7 & 54.235 & 17.59 & 31.548 & -0.112 & & & 0.04 & 14. & & & & & . & & & & & & & \\
\hline & $4 / 21 / 2004$ & $901: 13 \mathrm{AM}$ & $\frac{20.111}{2609}$ & $\begin{array}{r}24.49 \\
20.491\end{array}$ & $\frac{2.2 .246}{20237}$ & 25.535 & & $\begin{array}{l}48.129 \\
48092\end{array}$ & 5.201 & 17.56 & & -0.10 & & & & & & 28404 & 4.4 .3 & $\frac{52.8}{5.28}$ & $\frac{12.1}{12.1}$ & & & & & & 54 & \\
\hline & $4121 / 2004$ & & 20.090 & 24.491 & 20.201 & 20.405 & & 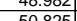 & 54.500 & $\begin{array}{l}1.40 \\
1750\end{array}$ & & -1.1 & & & & & & & & & 10 & & & & & & & \\
\hline & $\frac{4}{421212004 \mid}$ & & & 24506 & & 年. & & & 54.575 & & & & & & & & & & & & 12. & & & & & & & \\
\hline 216 & & & 261 & & & & & & & 17.654 & & & & & & & & & & 52 & 120 & & & & & & & \\
\hline $2167 \mathrm{P}$ & $4 / 21 / 2004$ & & & & 26.15 & 25.458 & & & & 17.61 & & & & & & & & & & & & & & & & & & \\
\hline & & & & & & & & & & & & & & & & & & & & & & & & & & & & \\
\hline & $4 / 21 / 2004$ & & 26048 & & & 611 & & & 231 & 612 & & 1.218 & & & & 4.643 & & & & & & & & & & & 84 & if \\
\hline $2170 \mid$ & $4 / 21 / 2004$ & 9:09:13 AM & & 24.574 & 26.181 & \begin{tabular}{|l|l} 
& 25.724 \\
\end{tabular} & 25.421 & $\begin{array}{l}50.102 \\
\end{array}$ & 54,154 & 17.487 & 32.91 & $\begin{array}{l}-1.218 \\
\end{array}$ & & 46.679 & & 14.643 & & 8474 & 47.4564 & & 11.4 & & 2.862 & 0.0 & 0.008 & 0.000 & 0.191 & 0.1 \\
\hline
\end{tabular}


WSRC-TR-2005-00105, REVISION 0

SRNL-RPP-2005-00012, REVISION 0

RUN \# 2.03A AND B; FIRST AND SECOND HALF OF SLURRY DEWATERING - CONT.

\begin{tabular}{|c|c|c|c|c|c|c|c|c|c|c|c|c|c|c|c|c|c|c|c|c|c|c|c|c|c|c|c|c|}
\hline & A & B & D & $\mathrm{E}$ & $\mathrm{F}$ & $\mathrm{G}$ & $\mathrm{H}$ & $\mathrm{J}$ & $\mathrm{K}$ & $\mathrm{L}_{\mathrm{L}}$ & $\mathrm{M}$ & $\mathrm{N}$ & 0 & Q & $R$ & $\mathrm{~s}$ & \begin{tabular}{l|l|}
$\mathrm{T}$ \\
\end{tabular} & & w & $x$ & & & $\mathrm{AA}$ & $A B$ & $\mathrm{AC}$ & $A D$ & & \\
\hline \begin{tabular}{|l|l|l|}
2171 \\
2172
\end{tabular} & $\begin{array}{r}4 / 21 / 2004 \\
4 / 212004\end{array}$ & $\begin{array}{l}9: 10: 13 \mathrm{AM} \\
911 \cdot 13 \mathrm{AM}\end{array}$ & $\begin{array}{r}26.064 \\
26.038\end{array}$ & $\frac{24.613}{22.631}$ & $\frac{26.209}{26.208}$ & $\begin{array}{l}25.787 \\
25.836\end{array}$ & \begin{tabular}{|l|}
25.475 \\
25.563 \\
\end{tabular} & $\begin{array}{l}50.622 \\
50.371\end{array}$ & \begin{tabular}{|l|}
54.656 \\
54.382 \\
\end{tabular} & $\begin{array}{l}17.492 \\
17.524\end{array}$ & $\begin{array}{r}33.463 \\
33.21 \\
\end{array}$ & $\begin{array}{l}-1.218 \\
-1.218\end{array}$ & $\begin{array}{r}-7.058 \\
-7.082 \\
\end{array}$ & $\begin{array}{l}48.112 \\
48.924 \\
\end{array}$ & 0.058 & \begin{tabular}{|l|}
14.643 \\
14.633
\end{tabular} & \begin{tabular}{|c|}
0.003 \\
0.003
\end{tabular} & $\begin{array}{l}2848.4 \\
2849.4\end{array}$ & \begin{tabular}{|l|l|}
47.4731 \\
47.4897
\end{tabular} & $\begin{array}{l}53.2 \\
52.9\end{array}$ & $\frac{11.8}{12.8}$ & \begin{tabular}{|l|l|}
42 \\
41
\end{tabular} & $\frac{2.899}{2.881}$ & \begin{tabular}{|c|}
0.009 \\
0.008
\end{tabular} & \begin{tabular}{|l|}
0.008 \\
0.008 \\
\end{tabular} & 0.000 & $\begin{array}{l}0.199 \\
0.183\end{array}$ & $\begin{array}{l}0.17 \\
0.16\end{array}$ \\
\hline & & & $\frac{20.000}{26.022}$ & & & & \begin{tabular}{|l|l|}
25.640 \\
\end{tabular} & & & & $\begin{array}{l}33.21 \\
33.081\end{array}$ & & & & & & & & & & & & & & & & & \\
\hline 2174 & $4 / 21 / 2004$ & 9:13:13 AM & 26.04 & 24.678 & 26.255 & 25.983 & 25.71 & 50.597 & \begin{tabular}{|l|}
54.675 \\
\end{tabular} & 17.614 & 33.313 & -1.218 & $\begin{array}{l}-7.009 \\
\end{array}$ & 49.195 & 0.054 & 14.643 & 0.003 & 2851.4 & 47.5231 & & 12.1 & & \begin{tabular}{|l|l|} 
\\
\end{tabular} & 0.008 & 0.008 & 0.000 & 0.185 & 0. \\
\hline & $4 / 221 / 2004$ & & 26.008 & 24.681 & \begin{tabular}{|l|l|}
26.227 \\
\end{tabular} & 25.951 & 25.793 & 50.234 & \begin{tabular}{|r|}
54.32 \\
\end{tabular} & 17.513 & 33.054 & -1.224 & $\begin{array}{r}-7.09 \\
\end{array}$ & 48.999 & 0.054 & 14.643 & & 2852.4 & & & & & & & 0.008 & & & 0.16 \\
\hline & & & & & & & & & \begin{tabular}{|l|l|}
54.716 \\
\end{tabular} & & & -1.218 & & 50.44 & & & & & & & & & & & & & & \\
\hline \begin{tabular}{|l|l|l|l|}
2177 \\
1798
\end{tabular} & $4 / 21 / 2004$ & 9:16:13 AM & $\begin{array}{l}26.007 \\
25080\end{array}$ & $\begin{array}{l}24.721 \\
2172\end{array}$ & \begin{tabular}{|l|l|}
26.272 \\
\end{tabular} & \begin{tabular}{|l|l|}
25.89 \\
25.821
\end{tabular} & $\begin{array}{l}25.657 \\
25.507\end{array}$ & \begin{tabular}{|l|l|}
50.717 \\
5.24
\end{tabular} & \begin{tabular}{|l|l|}
54.685 \\
5.503
\end{tabular} & $\begin{array}{l}17.543 \\
17541\end{array}$ & $\begin{array}{l}33.532 \\
2216\end{array}$ & \begin{tabular}{|l}
-1.221 \\
.221
\end{tabular} & $\begin{array}{l}-7.085 \\
\end{array}$ & \begin{tabular}{|l|l|}
49.633 \\
4.711
\end{tabular} & 0.055 & $\begin{array}{l}14.643 \\
1.643\end{array}$ & $\begin{array}{l}0.003 \\
0.023\end{array}$ & 2854.4 & \begin{tabular}{|l|l|}
47.5731 \\
\end{tabular} & 53.2 & 12.2 & & 2.904 & $\begin{array}{ll}0.008 \\
\end{array}$ & 0.008 & 0.000 & 0.188 & \\
\hline \begin{tabular}{|l}
2178 \\
2179 \\
\end{tabular} & $\begin{array}{l}4 / 21 / 2004 \\
4 / 21 / 2004\end{array}$ & $\begin{array}{l}9: 17: 13 \mathrm{AM} \\
\mathbf{2}\end{array}$ & $\begin{array}{l}25.989 \\
25.989\end{array}$ & $\begin{array}{l}24.742 \\
22.763 \\
\end{array}$ & \begin{tabular}{|l|l|}
26.258 \\
22.284 \\
\end{tabular} & $\begin{array}{l}25.821 \\
25.782 \\
\end{array}$ & \begin{tabular}{|l|}
25.509 \\
25.374 \\
\end{tabular} & \begin{tabular}{|c|}
50.344 \\
50.566
\end{tabular} & \begin{tabular}{|l|}
54.503 \\
54.625 \\
\end{tabular} & \begin{tabular}{|c|}
17.541 \\
17579
\end{tabular} & $\begin{array}{l}33.116 \\
33.354 \\
\end{array}$ & $\begin{array}{l}-1.224 \\
-1.218 \\
\end{array}$ & $\begin{array}{l}-7.038 \\
-7.105\end{array}$ & \begin{tabular}{|l|}
49.712 \\
49604
\end{tabular} & $\begin{array}{l}0.054 \\
0.055\end{array}$ & $\begin{array}{l}14.643 \\
14.643\end{array}$ & \begin{tabular}{|l}
0.003 \\
0.003
\end{tabular} & $\begin{array}{l}2855.4 \\
28564 \\
2854\end{array}$ & $\begin{array}{l}47.5897 \\
476064\end{array}$ & $\frac{53.0}{53.2}$ & $\frac{12.2}{122}$ & & $\begin{array}{l}2.877 \\
2.893 \\
\end{array}$ & \begin{tabular}{|c|}
0.008 \\
0.088
\end{tabular} & $\begin{array}{l}0.008 \\
0.008\end{array}$ & $\begin{array}{l}0.000 \\
0.000\end{array}$ & \begin{tabular}{|l|l|}
0.186 \\
0.189
\end{tabular} & $\begin{array}{l}0.16 \\
0.16\end{array}$ \\
\hline 2180 & $4 / 21 / 2004$ & 9:19:13 AM & & & 26.29 & 25.713 & 25.35 & 50.502 & \begin{tabular}{|l|l|}
54.774 \\
\end{tabular} & & & -1.221 & & & 0.055 & $\begin{array}{l}1.044 \\
14.643 \\
\end{array}$ & $\begin{array}{l}0.0003 \\
0.003\end{array}$ & $\begin{array}{l}2855.4 \\
2857.4\end{array}$ & & $\begin{array}{l}35.2 \\
53.3\end{array}$ & $\frac{12.2}{12.0}$ & & & \begin{tabular}{|c|} 
\\
0.0008 \\
\end{tabular} & $\begin{array}{l}.008 \\
0.008\end{array}$ & $\begin{array}{l}0.000 \\
0.000 \\
\end{array}$ & $\begin{array}{l}0.189 \\
0.189\end{array}$ & 0.16 \\
\hline & $4 / 21 / 2004$ & 9:20:13 AM & 25.945 & 24.784 & 26.294 & 25.648 & & & 54.445 & 17.589 & & -1.221 & & 49.142 & & 14.643 & & & & & & & & & & & & $\begin{array}{l}0.16 \\
0.16\end{array}$ \\
\hline 2182 & $4 / 21 / 2004$ & $9: 21: 13 \mathrm{AM}$ & & & & \begin{tabular}{|l|l|}
25.608 \\
\end{tabular} & 25.286 & 50.236 & 54.449 & \begin{tabular}{|l|l|l}
17.514 \\
\end{tabular} & 33.153 & -1.221 & -7.105 & 48.846 & 0.053 & $\begin{array}{l}14.643 \\
\end{array}$ & 0.003 & \begin{tabular}{|l|l|}
2859.4 \\
\end{tabular} & 47.6564 & & & & & & \begin{tabular}{|l|}
0.008 \\
\end{tabular} & 0.000 & & $\frac{0.16}{0.16}$ \\
\hline 2183 & $4 / 21 / 2004$ & $9: 22: 13 \mathrm{AM}$ & 25.961 & & 26.33 & & & & 03.071 & & & & & & 0.051 & 14.643 & & & & & & & & & & & 181 & $\begin{array}{l}0.16 \\
0.15\end{array}$ \\
\hline 2184 & $4 / 21 / 2004$ & $0.22 .12 \mathrm{Am}$ & & 298 & & 25530 & & & $=2$ & 18.355 & 21250 & -1.221 & & & & & & 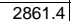 & & & 12 & & & & & & & \\
\hline & $4 / 21 / 2004$ & $9: 24: 13 \mathrm{Am}$ & & & 26.35 & \begin{tabular}{|l|l|}
25.493 \\
\end{tabular} & 25.106 & 49.533 & 53.644 & 18.269 & 31.704 & & & 53.61 & & $\begin{array}{l}14.643 \\
\end{array}$ & & 2862.4 & \begin{tabular}{|l|l|}
47.7064 \\
\end{tabular} & & 10 & & & & & & & \\
\hline 2186 & $4 / 21 / 2004$ & 9:25:13 AM & 26.056 & 24.844 & 26.375 & 25.513 & 25.206 & 50.006 & 54.142 & $\begin{array}{l}18.073 \\
\end{array}$ & 32.246 & $\begin{array}{l}-1.218 \\
\end{array}$ & -7.1 & 50.313 & 0.053 & $\begin{array}{l}14.643 \\
\end{array}$ & $\begin{array}{l}0.003 \\
\end{array}$ & 2863.4 & 47.7231 & 52.7 & 12.3 & & 2.835 & 0.008 & 0.008 & 0.000 & 185 & \\
\hline 21 & $4 / 21 / 2004$ & 9:26:13 AM & 26.076 & 24.844 & 26.385 & 25.568 & 25.386 & 49.91: & 54.113 & 18.063 & 32.13 & -1.218 & & 49.001 & 0.05 & 14.643 & .004 & 2864.4 & & & & & & & 0.007 & & & \\
\hline & $4 / 21 / 2004$ & $9: 27: 13 \mathrm{AM}$ & & 24.8 & & & & 50.124 & & 18.141 & 32.336 & -1.218 & & & & 14.643 & & & & & & & & & & & & \\
\hline & $4 / 21 / 2004$ & 9:28:13 AM & 26.148 & 24.87 & & 25.726 & 25.493 & 49.788 & 3.964 & 18.014 & 32.083 & -1.2211 & & .291 & 0.053 & 14.643 & & 2866.4 & & & 12. & & & 008 & 0.008 & .000 & 186 & \\
\hline$\frac{2191}{2191}$ & $41 / 21212004$ & $\begin{array}{l}9.29 .13 \mathrm{AM} \\
0.2013\end{array}$ & & & $\frac{2.044}{2.643}$ & $\frac{25.808}{25075}$ & & $\frac{50.085}{14080}$ & 年 & 10.020 & 322 & $\frac{-1.241}{1.201}$ & & 0.059 & 然 & $\frac{14.643}{1.643}$ & & 28691 & 47.1091 & & 124 & & & & & & & \\
\hline & 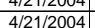 & $\begin{array}{l}9.00 .13 \mathrm{AM} \\
9: 31: 13 \mathrm{AM}\end{array}$ & $\frac{20.17 / 2}{26.194}$ & $\begin{array}{l}24.080 \\
24.893\end{array}$ & $\frac{20.431}{26.449}$ & $\begin{array}{l}25.810 \\
25.927\end{array}$ & $\begin{array}{l}25.057 \\
25.714\end{array}$ & $\begin{array}{l}49.8099 \\
49.925\end{array}$ & $\frac{53.964}{54.03}$ & $\begin{array}{l}17.910 \\
17.954\end{array}$ & $\begin{array}{l}32.25 \\
32.369 \\
\end{array}$ & $\frac{-1.211}{-1221}$ & & $\frac{50.490}{4971}$ & 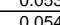 & $\begin{array}{l}\frac{14.043}{14.643} \\
\end{array}$ & 0.003 & $\frac{2060.4}{28694}$ & $\begin{array}{l}44.50064 \\
47831\end{array}$ & & $\frac{11.4}{12.2}$ & & & & & 0.000 & $\begin{array}{l}0.165 \\
0.188\end{array}$ & \\
\hline & $4 / 21 / 2004$ & $9: 32: 13 \mathrm{AM}$ & 26.202 & 24.906 & 26.402 & 25.96 & 25.777 & 49.73 & 53.926 & 17.99 & 32.106 & -1.224 & -7.09 & 49.074 & 0.054 & 14.643 & 0.003 & 2870.4 & 47.8397 & 52.5 & & 40.9 & 2.821 & & 0.008 & 0.000 & 0.189 & 0.16 \\
\hline & $4 / 21 / 2004$ & 9:33:13 AM & 26.225 & & 26.349 & 26.098 & 25.76 & & 53.862 & 17.999 & 32.196 & & & & 0.05 & 14.643 & & 2871.4 & & & & & & & & & & \\
\hline & $4 / 21212004$ & $\begin{array}{l}34: 13 \mathrm{AM} \\
50.12\end{array}$ & & $\frac{24.94}{24.94}$ & 6.326 & 26.089 & $\frac{25.741}{2577}$ & & 4.003 & & 32.18 & & & & 0.05 & 14.643 & & & & & & & & & & & & \\
\hline$\frac{1250}{2197}$ & $\frac{421212004}{4121204}$ & $\begin{array}{l}9.355 .15 \mathrm{AM} \\
0.32 \cdot 12\end{array}$ & $\frac{26.25}{26.266}$ & $\begin{array}{r}\frac{24.964}{24.99} \\
\end{array}$ & $\frac{26.354}{26.366}$ & $\frac{26.068}{26029}$ & $\frac{25.14}{25.56}$ & $\frac{49.132}{49.919}$ & 5.187 & $\frac{17.979}{17894}$ & $\begin{array}{l}32.011 \\
32323\end{array}$ & 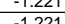 & & 年.4.15 & $\frac{0.05}{0.05}$ & $\begin{array}{l}14.643 \\
14643\end{array}$ & & 2873.4 & \begin{tabular}{|l}
4.50006 \\
479064
\end{tabular} & & 124 & & & & (1) & & & \\
\hline & $4 / 2 / 212004$ & 9:37:13 AM & 26.262 & 24.992 & 20.387 & 25.981 & 25.5 & $\begin{array}{l}49.919 \\
49.612\end{array}$ & 53.825 & $\begin{array}{l}17.094 \\
17.925 \\
\end{array}$ & $\begin{array}{l}32.003 \\
32.018\end{array}$ & $\begin{array}{l}-\frac{-1.241}{-1.215} \\
-\end{array}$ & & $\frac{5.4042}{49.27}$ & $\begin{array}{l}0.052 \\
0.051\end{array}$ & $\begin{array}{l}14.045 \\
14643\end{array}$ & 0.003 & 20.67 .4 & $\begin{array}{l}44.50034 \\
470231\end{array}$ & 524 & $\frac{1.4 .4}{121}$ & & & & & & & \\
\hline 219 & 4/21/2004 & 9:38:13 AM & 26.268 & 24.998 & 26.373 & 25.897 & 25.444 & $\begin{array}{l}49.99 \\
49.99\end{array}$ & 54.281 & 17.955 & 32.373 & -1.224 & & 49.883 & $\frac{0.0}{0.0}$ & 14.643 & & 2876.4 & 47.9 & & 12.2 & & 2839 & & 0.007 & & 78 & \\
\hline & $4 / 21 / 2004$ & 9:39:13 AM & 26.264 & & 26.384 & 25.827 & & 49.61 & 53.738 & 18.005 & & -1.224 & & 49.879 & & 14.643 & & 287 & \begin{tabular}{|l|l|}
47.9564 \\
\end{tabular} & & & & & & & & & \\
\hline & $4 / 21 / 2004$ & $9: 40: 13$ AM & 26.269 & 25.0 & 26.389 & 25.757 & & 49.892 & 54.165 & 17.982 & 32.157 & & & 51.422 & 0.049 & 14.643 & & 2878.4 & 47.9731 & 52 & $\frac{12.6}{12.6}$ & & .828 & 0.007 & 0.007 & 0.000 & & \\
\hline & $4 / 21 / 2004$ & 9:41:13 AM & 26.285 & 25.035 & 26.405 & 25.718 & 25.44 & 49.772 & 53.926 & \begin{tabular}{|l|l|l|l}
17.87 \\
\end{tabular} & 32.254 & -1.221 & & 50.694 & & $\begin{array}{l}14.643 \\
\end{array}$ & & \begin{tabular}{|l|l|}
2879.4 \\
\end{tabular} & & & 12.4 & & & & 0.001 & & & \\
\hline & $4 / 21 / 2004$ & $9: 42: 13 \mathrm{AM}$ & 26.28 & 25.024 & & & & 49.996 & 54.355 & & 32.523 & & & & & 14.643 & & 2880.4 & 48.0064 & & & & .84 & & & & & 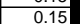 \\
\hline & $4 / 21 / 2004$ & 9:43:13 AM & 26.29 & 25.05 & 26.415 & 25.653 & 25.281 & \begin{tabular}{|l|l|l|}
49.695 \\
\end{tabular} & & 18.026 & 31.993 & -1.218 & & 49.908 & 0.054 & 14.643 & & 2881.4 & 48.0231 & 52.6 & 12.2 & 40.8 & & & 0.008 & 0.000 & 0.189 & \\
\hline & $4 / 21 / 2004$ & $9: 44: 13 \mathrm{AM}$ & 26.29 & 25.049 & 26.424 & 25.648 & 25 & 49.977 & 54.26 & & 32.299 & -1.218 & & 49.1. & & 14.643 & & & & & 12. & & & & & & & \\
\hline & 4/21/2004 & $13 \mathrm{AM}$ & 26.294 & & 26.424 & 25.642 & 25.22 & 49.579 & & 18.008 & 31.87 & -1.218 & & 1.435 & 0.05 & & & 288 & 48.0564 & & $12.6 \mathrm{C}$ & & & & & & & \\
\hline & $4 / 21 / 2004$ & $3 \mathrm{AM}$ & 26.294 & 25.05 & 26.429 & 25.682 & 25. & 49.85. & 239 & 18.062 & 32.127 & -1.221 & & & 0. & 14.643 & & & & & 12. & & on & 0.007 & 0.00 & 0.000 & & \\
\hline & & & & & $\frac{26.439}{3645}$ & & & & & & & $\frac{-1.218}{-1204}$ & & & & & & & & & & & & & & & & \\
\hline$\frac{2210}{2210}$ & 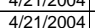 & $\begin{array}{l}9.84 .13 \mathrm{AM} \\
9: 49: 13 \mathrm{AM}\end{array}$ & $\begin{array}{l}20.31 \\
26.302\end{array}$ & $\begin{array}{l}25.005 \\
25.056\end{array}$ & $\frac{2.45}{26.432}$ & $\frac{25.644}{25.1}$ & & $\begin{array}{l}49.653 \\
4977\end{array}$ & (1) & 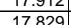 & $\begin{array}{l}32.216 \\
32246\end{array}$ & $\frac{-1.24}{-1221}$ & & 势 & 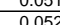 & $\begin{array}{l}\frac{14.045}{14643} \\
\end{array}$ & & $\frac{260}{288}$ & & 52.5 & $\frac{12.3}{121}$ & & & & 管 007 & 000 & 0 & \\
\hline & $4 / 21 / 2004$ & $9: 50: 13 \mathrm{AM}$ & $\frac{20.006}{26.324}$ & & \begin{tabular}{|l|l|l} 
\\
\end{tabular} & 25.982 & 20 & 49.904 & 54.254 & 17.958 & & -1.224 & & & & & & & 年 & $J_{2}$ & 1L. & & & & & & & \\
\hline & $4 / 21 / 2004$ & 9:51:13 AM & 26.335 & 25.09 & 26.47 & 26.059 & 25.8 & $\begin{array}{l}49.884 \\
\end{array}$ & 54.206 & 17.856 & 32.389 & -1.221 & & 49.41 & 0.0 & & & 2889.4 & & 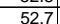 & 12. & 78 & & & & & & \\
\hline & 1/2004 & $13 \mathrm{AM}$ & & & & & & & & & & \begin{tabular}{|c|}
-1.224 \\
\end{tabular} & & & & 14. & & & & & & & & & & & & \\
\hline & & $13 \mathrm{AM}$ & & & & 26.189 & 25.80 & 49.25 & 3.702 & 18.25 & 31.291 & -1.218 & & 51216 & & & & & & & 12. & & & & & & & \\
\hline & 4/21/2004 & 9:54:13 AM & 26.3 & 25.158 & 26.548 & 26.177 & 25. & & 53.846 & & 31.445 & \begin{tabular}{|c|}
-1.218 \\
\end{tabular} & & & & 14. & & 286 & \begin{tabular}{|l|l|}
48.2064 \\
\end{tabular} & 52.4 & 12.5 & 40 & & & 0.6 & & & \\
\hline & $4 / 21 / 2004$ & 9:55:13 AM & 26. & 25.16 & 26.536 & 26.125 & & & 54.036 & 18.434 & 31.64 & -1.218 & & & & & & & & 52. & 12.7 & 4 & 60 & & & & & \\
\hline & 4/21/2004 & 9:56:13 AM & & 25.163 & 26.543 & 26.041 & 25. & 49.28 & 53.56 & 18.39 & 31.203 & -1.221 & & 50. & & 14. & & $28 \mathrm{~S}$ & 48.2 & 52 & 12.3 & 4 & & & & & & \\
\hline & $4 / 21 / 2004$ & 9:57:13 & & 25.164 & 26.539 & 25. & & 49.305 & & & & -1.221 & & & & 14.643 & & 289 & & $J_{2}$ & 12.2 & $4 \mathrm{c}$ & & & 0.6 & & & \\
\hline & & & 26. & 25.174 & 26.554 & & & 49.446 & 53.748 & & & -1.218 & & & & & & & & 52. & $12.6 \mathrm{r}$ & & & & & & & \\
\hline & $4 / 21122004$ & & & 25.194 & $2 \frac{26.564}{2062}$ & & & 49. & 年 & $18.297]$ & $\begin{array}{l}31.636 \\
31.631\end{array}$ & -1.224 & & & & 14. & & & & 52 & . & & & & & & 101 & \\
\hline & $\frac{4-4121204}{4 / 21204}$ & $\frac{10.00 .}{10.011}$ & $\frac{20.405}{26417}$ & $\frac{25.14}{2520}$ & & $\frac{25.052}{25881}$ & & & 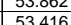 & & & $\frac{-1.244}{-1218}$ & & & & & & & & $\frac{52}{51}$ & 12 & & & & & & & \\
\hline & 4 & 10 & & 25.21 & 26.442 & & & 492 & & & 3126 & -1.221 & & & & & & & & & 12. & & & & & & & \\
\hline & $\frac{4 / 21 / 2004}{4}$ & $10: 03: 13 \mathrm{AM}$ & 26. & $\frac{25.211}{25.211}$ & $\frac{20.441}{26.441}$ & 25.784 & 25. & 49.2 & 53.686 & 18.343 & 31. & -1.221 & & & & & & & & $\frac{25}{52}$ & 124 & & & & & & & \\
\hline & & & & & & & & & & & & & & & & & & & & & & & & & & & & \\
\hline & & & 26.4 & 25.235 & 26,485 & 25758 & 25 & 49.1 & 53545 & 18.317 & 31.164 & -1.221 & & 503 & & 14.643 & & & & $\frac{5}{5}$ & 12 & & & & & & & \\
\hline & $4 / 21 / / 2$ & 10:06:13 AM & $26.2>>3$ & 25.2. & $26.4 \mathrm{r}-\mathrm{s}$ & & & & 3.611 & & & \begin{tabular}{c|c|c|}
-1.203 \\
\end{tabular} & & 49. & & & & & & & $\overline{2.2}$ & & & & & & & \\
\hline & $4 / 21 / 2004$ & 10:07:13 AM & 26.414 & 25.224 & 26.484 & 25.847 & 25. & 49. & 53.698 & 18.288 & 31. & -1.215 & & 74 & & 14.643 & & 290 & 48. & 52 & $12.6 \mathrm{r}-\mathrm{l}$ & 46 & & $\mid 0.008$ & 0.6 & & & \\
\hline & $4 / 21 / 2004$ & 10:08:13 AM & 26.425 & 25.24 & 26.51 & 25.923 & & 49.177 & 528 & 18.132 & & -1.215 & & 50.8 & & 14. & & & & 52. & 12.5 & 4 & & & & & 34 & \\
\hline & 1/2004 & 10:09:13 AM & 26.4 & $25.24 \mathrm{~F}$ & 26.507 & & 25. & 49.2 & 53. & 18.279 & & -1.169 & & 50 & & & & & & 52. & 12.4 & 4 & & & & & & \\
\hline & 4/21/2004 & 10:10:13 AM & 26.43 & $\frac{25.25}{25.25} \mathrm{Pl}$ & 26.53 & 26.093 & 25. & 49 & 54.069 & 18.293 & 31. & -1.195 & & 0.415 & 0.05 & 14.643 & & & & & 2.. & & & & & & & \\
\hline & 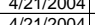 & $\begin{array}{l}10.11: 1.1 \mathrm{AM} \\
10.12 \mathrm{MM}\end{array}$ & 20.432 & 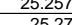 & 26.531 & 26.1666 & 2.0 .072 & - & (53.661 & $\frac{18.25}{18207}$ & & -1.154 & & 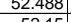 & & & & 20104 & $\sin ^{2}$ & $\frac{52.2}{5.1}>>0$ & $\frac{12.9}{120}>>$ & & & & & & & \\
\hline & & & 20.44 & $\frac{25.27}{25272}+2$ & $\frac{20.50}{26.52}$ & & & 年 & & 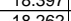 & 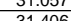 & - & & & & & & & & & & & & & & & & \\
\hline & & 10.14.13 & $\frac{20.455}{26.45}$ & 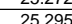 & & $\frac{2.4}{26.2}$ & & 年 49.5129 & & & $\begin{array}{ll}31.400 \\
31205\end{array}$ & -1.104 & & & & & & 29 & & & 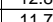 & & & & & & 31 & \\
\hline 223 & & & $\frac{26.4}{26.4}-4$ & & & & & & & & & & & & & & & & & & 124 & & & & & & & \\
\hline & $\mid 4 / 21 / 2004$ & & & 25.314 & 26.569 & & 25 & 49.585 & & 18.426 & & -1.154 & & & & & & & & & & & & & & & & \\
\hline & & & 26.48 & & & & & 49.048 & & & & & & & & & & & & 519 & 123 & & & & & & & \\
\hline & $4 / 21 / 200$ & 10.18 & 26.481 & 25 & & 095 & & 10250 & 53.756 & & 31.406 & $-1,154$ & & 0.018 & & 4.643 & & & & & & & & & & & 84 & Th \\
\hline 240 & $4 / 21 / 2004$ & 10:19:13 AM & & 25.348 & & & & & & 18.367 & 31.585 & -1.105 & & 51.005 & 0.051 & 14.643 & & 17.4 & 48.6231 & 52.9 & 12.5 & & $2.802 \mathrm{r}-\mathrm{C}$ & & 0.00 & 0.000 & 0.179 & 0.1 \\
\hline
\end{tabular}


WSRC-TR-2005-00105, REVISION 0

SRNL-RPP-2005-00012, REVISION 0

RUN \# 2.03A AND B; FIRST AND SECOND HALF OF SLURRY DEWATERING - CONT.

\begin{tabular}{|c|c|c|c|c|c|c|c|c|c|c|c|c|c|c|c|c|c|c|c|c|c|c|c|c|c|c|c|c|}
\hline & A & B & D & $\begin{array}{ll}\mathrm{E} \\
\end{array}$ & $\mathrm{F}$ & $\mathrm{G}$ & $\mathrm{H}$ & $\mathrm{J}$ & $\mathrm{K}$ & $\mathrm{L}$ & $\mathrm{M}$ & $\mathrm{N}$ & $\mathrm{O}$ & $Q^{-1}$ & $R$ & $\mathrm{~s}$ & $\mathrm{~T}$ & $v$ & w & $x$ & & 2 & $A A$ & $A B$ & $\mathrm{AC}$ & $A D$ & $\mathrm{AE}$ & \\
\hline$\frac{2244}{2242}$ & \begin{tabular}{|l|l|}
$4 / 21 / 2004$ \\
$4 / 212004$
\end{tabular} & $\begin{array}{l}10: 20: 13 \mathrm{AM} \\
10.2113 \mathrm{AM}\end{array}$ & $\frac{26.492}{26.509}$ & $\begin{array}{l}25.348 \\
25369\end{array}$ & \begin{tabular}{|l|}
26.612 \\
26.624 \\
\end{tabular} & $\begin{array}{l}25.981 \\
25.952 \\
\end{array}$ & \begin{tabular}{|l|}
25.613 \\
25.549 \\
\end{tabular} & $\begin{array}{l}49.241 \\
49.544\end{array}$ & \begin{tabular}{|l|}
53.713 \\
54.013
\end{tabular} & $\begin{array}{l}18.174 \\
18.349\end{array}$ & $\begin{array}{l}31.441 \\
33.579 \\
\end{array}$ & $\begin{array}{r}-1.14 \\
-1.096 \\
\end{array}$ & $\begin{array}{l}-7.058 \\
-7.038 \\
\end{array}$ & $\begin{array}{l}51.585 \\
50.613\end{array}$ & 0.049 & $\begin{array}{l}14.643 \\
1.643 \\
\end{array}$ & $\begin{array}{l}0.003 \\
0.003\end{array}$ & 2918.4 & 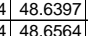 & $\frac{52.2}{52.5}$ & $\begin{array}{ll}12.6 \\
12.4\end{array}$ & $\begin{array}{l}40.3 \\
40.6 \\
\end{array}$ & \begin{tabular}{|l|}
2.781 \\
2.797
\end{tabular} & \begin{tabular}{|l|}
0.007 \\
0.008
\end{tabular} & \begin{tabular}{|l|}
0.007 \\
0.007 \\
\end{tabular} & 0.000 & \begin{tabular}{|l}
0.173 \\
0.183 \\
\end{tabular} & 0.15 \\
\hline$\frac{2<44}{2243}$ & & & 26.503 & & & & & & & $\frac{10.045}{18.381}$ & & & & & & $\frac{14.045}{14.643}$ & & & & & & & & & & & & \\
\hline 2244 & $4 / 21 / 2004$ & $10: 23: 13 \mathrm{AM}$ & 26.501 & 25.357 & 26.612 & 25.905 & 25.512 & 49.344 & \begin{tabular}{|l|}
53.939 \\
\end{tabular} & 18.348 & 31.273 & -1.099 & -7.041 & 50.425 & 0.052 & 14.643 & 0.003 & 2921.4 & \begin{tabular}{|l|l|}
4 & 48.6897 \\
\end{tabular} & & & & \begin{tabular}{|l|}
2.779 \\
\end{tabular} & \begin{tabular}{|l|l|}
0.008 \\
\end{tabular} & 0.007 & 0.000 & 0.184 & \\
\hline 2245 & & & 26.52 & 25.36 & 26.63 & 25.909 & 25.451 & 49.293 & \begin{tabular}{|l|l|}
53.758 \\
\end{tabular} & $\frac{18.311}{18.311}$ & 31.349 & -1.096 & & 50.703 & 0.051 & 14.643 & 0.003 & 2922.4 & 448.7064 & & & & 2.780 & & & & 0.180 & 0.15 \\
\hline & & & & & 26.644 & & 25.549 & & & & & & & 50.446 & & 14.643 & & & & 52.3 & & & & & & & & \\
\hline 2244 & $4 / 21 / 2004$ & 10:26:13 AM & 26.523 & 25.353 & 26.633 & 25.866 & 25.474 & 49.641 & 54.136 & 18.34 & 31.552 & $\begin{array}{l}-1.099 \\
\end{array}$ & $\begin{array}{l}-7.047 \\
7.070\end{array}$ & 48.511 & 0.051 & 14.643 & 0.003 & 2924.4 & \begin{tabular}{ll|l|}
4 & 48.7397 \\
\end{tabular} & 52.7 & \begin{tabular}{l|l}
7 & 11.9 \\
\end{tabular} & 406 & 2.799 & \begin{tabular}{|l|l|}
0.008 \\
\end{tabular} & $\begin{array}{ll}0.007 \\
\end{array}$ & 0.000 & 0.179 & \\
\hline $\begin{array}{l}\frac{2248}{2249} \\
\end{array}$ & $\begin{array}{l}/ / 21 / 2004 \\
4 / 21 / 2004\end{array}$ & $\begin{array}{l}10: 27: 13 \mathrm{AM} \\
10: 28,1 \mathrm{AM}\end{array}$ & $\frac{26.536}{26.54}$ & \begin{tabular}{|l|}
25.357 \\
25.536
\end{tabular} & \begin{tabular}{|l|}
26.655 \\
26.656 \\
\end{tabular} & \begin{tabular}{|r|r|}
25.92 \\
25.924
\end{tabular} & $\begin{array}{l}25.502 \\
25.066\end{array}$ & $\begin{array}{l}49.318 \\
49.398\end{array}$ & \begin{tabular}{|l|}
53.885 \\
53.879 \\
\end{tabular} & $\frac{18.216}{18.315}$ & \begin{tabular}{|l|l|}
31.478 \\
31408 \\
\end{tabular} & \begin{tabular}{|l|l|}
-1.093 \\
-1.096
\end{tabular} & $\frac{-7.035}{-7.058}$ & $\begin{array}{l}50.215 \\
49.883\end{array}$ & $\begin{array}{l}0.054 \\
0.048 \\
\end{array}$ & $\begin{array}{l}14.643 \\
1643 \\
\end{array}$ & $\begin{array}{l}0.004 \\
0.003 \\
\end{array}$ & 2925.4 & $\begin{array}{l}448.7564 \\
4487731\end{array}$ & $\frac{52 .}{52 .}$ & $\frac{12.3}{122}$ & $\begin{array}{l}40.4 \\
40.4 \\
\end{array}$ & \begin{tabular}{|l}
2.785 \\
2786
\end{tabular} & \begin{tabular}{|l|}
0.008 \\
0.07 \\
\end{tabular} & $\begin{array}{l}0.008 \\
0.007\end{array}$ & 0.000 & $\begin{array}{l}0.190 \\
0.169\end{array}$ & 0.16 \\
\hline$\frac{2250}{2250}$ & $4 / 21 / 2004$ & 10:29:13 AM & 26.551 & 25.357 & \begin{tabular}{|l|}
26.0502 \\
\end{tabular} & 25.99 & 25.732 & & \begin{tabular}{|l|}
54.109 \\
\end{tabular} & 18.386 & 31.484 & -1.099 & $\begin{array}{l}-7.050 \\
-7.047\end{array}$ & $\begin{array}{l}49.000 \\
50.899\end{array}$ & 0.05 & $\frac{14.043}{14.643}$ & $\begin{array}{l}0.003 \\
0.003 \\
\end{array}$ & 29262.4 & 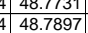 & 52. & $\frac{12.4}{12.5}$ & $\begin{array}{c}40.4 \\
40.5 \\
\end{array}$ & \begin{tabular}{|l|}
2.786 \\
2.792 \\
\end{tabular} & \begin{tabular}{|l|}
$\mid 0.000$ \\
0.007
\end{tabular} & $\begin{array}{l}0.001 \\
0.007\end{array}$ & $\begin{array}{l}0.000 \\
0.000 \\
\end{array}$ & $\begin{array}{l}0.169 \\
0.177 \\
\end{array}$ & $\begin{array}{l}0.14 \\
0.15\end{array}$ \\
\hline & & & & & \begin{tabular}{|l|l|} 
& 26.512 \\
\end{tabular} & 26.066 & 25.947 & & & & & -1.096 & & 49.954 & & 14.643 & & & \begin{tabular}{|l|l|}
4 & 48.8064 \\
\end{tabular} & & & & & & & & & 0.15 \\
\hline 225 & $4 / 21 / 2004$ & $10: 31: 13 \mathrm{AM}$ & 26.543 & 25.359 & 26.499 & 26.102 & 25.939 & 49.04 & 53.481 & 18.215 & 31.176 & -1.038 & -7.044 & 47.814 & 0.051 & 14.643 & 0.003 & 2929.4 & \begin{tabular}{|l|l|}
4 & 48.8231 \\
\end{tabular} & & 11.7 & 40.1 & 2.765 & 0.008 & \begin{tabular}{|l|}
0.007 \\
\end{tabular} & 0.000 & & \\
\hline 2253 & $4 / 21 / 2004$ & 10:32:13 AM & 26.541 & & & 26.2 & & 49.407 & & & & & & 51.504 & & 14.643 & & & & & & & & & & & & \\
\hline$\frac{225}{225}$ & $4 / 21 / 2004$ & $10.22 \cdot 13.91$ & & 25.383 & 26.542 & & & 48.857 & 53,159 & 18.178 & 31.014 & -1.067 & & & 0.053 & & & & 8.8564 & & & & & & & & & \\
\hline & $4 / 21 / 2004$ & 10:34:13 AM & 26.54 & & 26.55 & 26.349 & & & 53.844 & 18.204 & 31.423 & -1.047 & & 50.738 & & 14.643 & & 2932.4 & & & 12.4 & & & 0.007 & 0.007 & & & \\
\hline & 4/21/2004 & 10:35:13 AM & 26.543 & 25.414 & 26.573 & 26.422 & 26.158 & $\begin{array}{lll}49.438 \\
\end{array}$ & 54.038 & 18.241 & 31.486 & -1.015 & & 51.109 & 0.052 & 14.643 & 0.003 & 2933.4 & $\begin{array}{l}448.8897 \\
\end{array}$ & 52.6 & 12.5 & 40.5 & 2.790 & 0.008 & 0.007 & 0.000 & 0.183 & \\
\hline & 1/2004 & 10:36:13 AM & 26.552 & 25.422 & 26.572 & 26.396 & 26.022 & 49.311 & 53.868 & 18.325 & 31.234 & -1.03 & & 50.65 & 0.05 & 14.643 & & 2934.4 & 448.9064 & & & & & & & & & \\
\hline & 11/2004 & 10:37:13 AM & & & & & 25.974 & 49.102 & & & 31.201 & - & & 49.66 & & 14.643 & & & & & & & & & & & & \\
\hline & $4 / 21 / 120004$ & 10:38:13 AM & 26.561 & 25.457 & 26.606 & $\begin{array}{r}26.33 \\
26.251 \\
\end{array}$ & $\frac{26.061}{25.952}$ & 49.04 & 53.665 & 18.331 & 1.024 & -0.992 & & . & 0.05 & 14.643 & & & & & 12. & & & & & & & \\
\hline & $\mid \frac{\mid 4 / 21 / 2004}{4 / 21 / 2004}$ & $\frac{10.50 .13 \mathrm{AM}}{10.40 .13}$ & $\frac{26.542}{26.578}$ & $\frac{25.433}{25.474}$ & $\frac{26.5 / 2}{26.639}$ & $\frac{20.251}{26272}$ & $\frac{25.956}{25864}$ & $\frac{49.106}{49.411}$ & $\frac{53.62}{54.005}$ & $\frac{18.123}{18.253}$ & $\frac{31.446}{31.613}$ & - 1.001 & & 1. & & 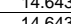 & & $\overline{0384}$ & 4 & & & & & & & & & \\
\hline & $\begin{array}{l}\mid 4 / 21 / 2004 \\
4 / 21 / 2004\end{array}$ & 10.41 .13 AM & $\frac{20.5 / 6}{26.569}$ & $\begin{array}{l}2.444 \\
25.475 \\
\end{array}$ & $\frac{20.059}{26.619}$ & $\begin{array}{l}20.27 / 2 \\
26.203\end{array}$ & $\begin{array}{r}25.804 \\
25.89 \\
\end{array}$ & $\begin{aligned} \frac{49.411}{49.26} \\
\end{aligned}$ & \begin{tabular}{|l|}
54.005 \\
53.729 \\
\end{tabular} & $\frac{18.253}{18.177}$ & $\frac{31.613}{31.548}$ & $\begin{array}{l}-0.995 \\
-0.995 \\
\end{array}$ & & $\begin{array}{l}31.302 \\
49.699\end{array}$ & $\begin{array}{l}0.051 \\
0.051 \\
\end{array}$ & $\frac{14.045}{14643}$ & 0.003 & 2930.4 & $\begin{array}{l}4 \\
4\end{array}$ & & 122 & 40.4 & & 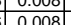 & 0.07 & 0.000 & 0.180 & \\
\hline & $4 / 21 / 2004$ & 10:42:13 AM & 26.573 & 25.474 & \begin{tabular}{|l|}
26.614 \\
\end{tabular} & 26.142 & 25.799 & 49.288 & 53.953 & 18.175 & 31.458 & -0.998 & & 51.612 & 0.051 & 14.643 & 0.003 & 2940.4 & \begin{tabular}{|l|l|}
44 & 49.0064 \\
\end{tabular} & 52.5 & 12.6 & 40.4 & \begin{tabular}{|l|l|}
2.784 \\
\end{tabular} & & 0.007 & & 0.180 & 0.15 \\
\hline 22 & 1/2004 & 10:43:13 AM & 26.572 & 25.483 & & 26.141 & & 49.36 & 53.943 & 18.093 & 31.614 & -0.992 & & 49.829 & 0.051 & 14.643 & & 2941.4 & & & & & & & & & & \\
\hline & $1: 12004$ & 10:44:13 AM & 26.592 & 25.498 & & 26.141 & & & 5 55.918 & 18.065 & 31.68. & 0.937 & & .824 & 0.05 & 144.643 & & 2942. & & & & & & & & & & \\
\hline$\frac{256}{226}>>$ & $\mid \begin{array}{l}\mid / 2 / 21 / 204 \\
4 / 21 / 2004\end{array}$ & $\begin{array}{l}10.45 .13 \text { AM } \\
10 \cdot 46 \cdot 13 \mathrm{M}\end{array}$ & $\frac{26.586}{26.586}$ & $\frac{25.494}{25.497}$ & $\frac{20.026}{26.616}$ & $\frac{26.115}{26.1}$ & 255.80 & $\frac{49.58}{49558}$ & $\begin{array}{l}54.262 \\
54.231\end{array}$ & $\frac{18.13}{18.161}$ & $\begin{array}{l}31.1288 \\
31.648\end{array}$ & $\begin{array}{l}-0.037 \\
-0.94 \\
-0.04\end{array}$ & & 0.0 & $\frac{0.054}{0.052}$ & $\frac{14.643}{14.643}$ & & 29434.4 & $4 \quad 49.0731$ & & 124 & & & & & & & \\
\hline & $4 / 21 / 2004$ & $10.47 .13 \mathrm{sM}$ & 26.598 & 25.494 & 26.638 & 26132 & 25.8 & $\begin{array}{l}49.530 \\
49.691 \\
\end{array}$ & 54.273 & 18.06 & $\begin{array}{l}11.040 \\
32.038 \\
\end{array}$ & $\begin{array}{r}-0.947 \\
-0.937\end{array}$ & & 48.362 & $\begin{array}{l}0.052 \\
0.051 \\
\end{array}$ & $\frac{14.045}{11643}$ & 003 & 2944.4 & & $52.8>-1$ & $\frac{12.4}{11.9}$ & & & & & & & \\
\hline & $4 / 21 / 2004$ & $10: 48: 13$ AM & 26.613 & 25.504 & \begin{tabular}{|l|l|}
26.658 \\
\end{tabular} & 26.122 & 25.72 & 49.42 & 54.121 & 18.054 & 31.737 & -0.937 & & 50.963 & 0.051 & 14.643 & & 2946.4 & $\begin{array}{l}449.1064 \\
\end{array}$ & $52.6>-1$ & 12. & 40.6 & 2.798 & & 007 & & 179 & \\
\hline & $4 / 21 / 2004$ & 10:49:13 AM & 26.617 & 25.513 & 26.667 & 26.141 & 25.7 & & 54.018 & 18.135 & & -0.937 & & & & 14.643 & & 2947.4 & & & & & & & & & & \\
\hline & 4/21/2004] & $13 \mathrm{AM}$ & & 25.522 & 26.686 & 26.125 & & 49.475 & 54.183 & 17.958 & 31.827 & -0.934 & & 51.061 & 0.052 & 14.643 & & 2948.4 & $\begin{array}{ll}4 & 49.1397 \\
\end{array}$ & 52 & 12.5 & 40 & 2.803 & 0.008 & 0.007 & 0.000 & 0.182 & \\
\hline & $4 / 21 / 2004$ & $10: 51: 13 \mathrm{AM}$ & 26.626 & 25.512 & 26.681 & 26.145 & 25.842 & 49.367 & 54.011 & 18.153 & 31.542 & -0.934 & & 51.694 & 0.051 & 14.643 & & 2949.4 & 49.1564 & & 12.7 & & 2.789 & & 0.007 & 0.000 & 0.179 & \\
\hline & & 10:52:13 AM & & & & & & 49.583 & 54.32 & 17.986 & & & & 49.624 & & & & & & & & & & & & & & \\
\hline & 4/21/2004 & 10:53:13 AM & 26.646 & 25.523 & & 26.281 & & 49.606 & 54.285 & 17.977 & 32.01 & -0.937 & & 49.641 & 0.052 & 14.643 & & 2951.4 & \begin{tabular}{|l|l|}
49.1897 \\
\end{tabular} & $52.8 \mathrm{~s}$ & 12.2 & $40.8 \mathrm{r}>\mathrm{s}$ & 2.814 & D.008 & 0.007 & 0.000 & 0.181 & \\
\hline & 4/21/2004 & 10:54:13 AM & 26.634 & 25.525 & 20.684 & 26.353 & 26.1 & 49.475 & 54.293 & 17.958 & 31.8 & -0.937 & & & & 14. & & 2952.4 & & & 12. & & & & & & & \\
\hline & 4/21/2004 & $10: 55$ & & 25.542 & 26.4777 & 26.47 & & 49.678 & 54.202 & 17.971 & 32.116 & -0.937 & & .194 & 0.051 & & & 29 & & & 12.3 & & & & & & & \\
\hline & & $\begin{array}{l}10: 566.13 \mathrm{AM} \\
0.572\end{array}$ & $\frac{26.65}{20.68}$ & 25.556 & 26.48 & 26.549 & & & 54.275 & 17.893 & & & & & & 14.643 & & & & & 12.2. & & & & $0.06-5$ & .000 & & \\
\hline 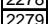 & & & & & & & & 年 & & & & & & & & & & & & & & & & & & & & \\
\hline 228 & $4 / 21 / 2004$ & $10: 59: 13 \mathrm{AM}$ & 26.649 & 25.59 & \begin{tabular}{|l|}
26.0509 \\
2.509
\end{tabular} & 26.523 & $\frac{2.2 .24}{26.144}$ & 49.542 & \begin{tabular}{|l|l|l|}
54.302 \\
\end{tabular} & $\frac{1.364}{17.884}$ & 31.93 & $\frac{-0.911}{-0.911}$ & & 49.019 & 0.054 & $\frac{1.4 .43}{14.43}$ & 0.003 & $\frac{209.7}{29574}$ & 4499897 & $\frac{2.8}{52.8}$ & 12.6 & 40.7 & 2.809 & & \begin{tabular}{|l|l|}
0.008 \\
\end{tabular} & 0.000 & 0.189 & \\
\hline & $4 / 21 / 2004$ & $11: 00: 13 \mathrm{AM}$ & 26.641 & 25.602 & \begin{tabular}{|l|}
26.522 \\
\end{tabular} & 26.495 & 26.0 & \begin{tabular}{|c|c|c|}
49.513 \\
\end{tabular} & \begin{tabular}{|l|l|l|l}
54.208 \\
\end{tabular} & 17.842 & 32.051 & \begin{tabular}{|c|c|c|}
-0.937 \\
\end{tabular} & & 48.352 & 0.05 & 14.643 & & 295 & 449.3064 & 52. & 11. & & 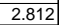 & & 0.007 & 0.000 & & \\
\hline & | & $11: 01: 13 \mathrm{AM}$ & 26.63 & 25.606 & & 26.499 & & 49 & 54.391 & 17.901 & 32.147 & -0.934 & & 9.731 & 0.05 & & & 295 & & & 12. & & & & & & & \\
\hline & & $13 \mathrm{AM}$ & & 61 & & 26.466 & & $49.446 \mathrm{r}$ & 54.308 & 17.762 & ( & -0.815 & & & 0.054 & 14. $\mathrm{C}$ & & & & & 20 & & & & & & & \\
\hline & 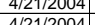 & 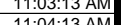 & & 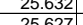 & (26.55? 2657 & $\frac{26.42}{26.325}$ & 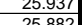 & (49.432 & $\begin{array}{l}54.088 \\
54272\end{array}$ & & & $=0.882$ & & & & & & & 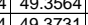 & 52 & 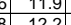 & 10 & $\sqrt{1808}+3$ & & & & & \\
\hline & & $\frac{1.04}{104}$ & & $\frac{23.04}{2564}$ & & $\frac{20.325}{26315}$ & & & \begin{tabular}{|l|l|}
54612 \\
54.210
\end{tabular} & 11.017 & & $=0.000$ & & & & & & & & & & & & & & & & \\
\hline & $\frac{142121204}{4 / 21204}$ & $\frac{11.05}{11.05}$ & 26.637 & 25.648 & 20.562 & 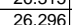 & 25.8 & (49.241 & $\mid$ & $\frac{1.547}{17.827}$ & 31 & $\begin{array}{l}-0.005 \\
-0.885\end{array}$ & & & & 14.6 & & & 9 & $\frac{52}{52}$ & $\frac{12.4}{121}>0$ & & & & & & & \\
\hline $228 \varepsilon$ & $\mid 4 / 21 / 2004$ & $\frac{11: 06: 46}{1106}$ & & & & & & 49.4 & & & & & & & & & & & & & & & & & & & & \\
\hline & $\mid 4 / 21 / 2004$ & & & 25.642 & 26.567 & 26.25 & 26.7 & 49.28 & \begin{tabular}{|l|l|}
54.065 \\
\end{tabular} & & & -0.8 & & & & & & & & $52-2>$ & 123 & & & & & & & \\
\hline & & & & & & & & & & & & & & & & & & & & s. & th & & & & & & & \\
\hline & $4 / 21 / 2$ & & 26.646 & & 26.5 & & & 49.407 & 54.159 & 17.994 & & -0.8 & & & & & & & & & T2 & & & & & & & \\
\hline & $4 / 21 / 2004$ & 11:10:46 & 26.651 & 25.66 & & 26.236 & & $49.4>>3$ & & 18.272 & & $\begin{array}{c}-0.865 \\
\end{array}$ & & & & & & & & 52. & 12.3 & & & & & & & \\
\hline & 4/21/2004 & 11:11:46 & & 25.667 & & 26.22 & & & & 18.111 & & $-0.8-8$ & & & & & & & & & 12. & & & & & & & \\
\hline 229 & & $11: 12$ & & 25. & & 26.205 & $25.7>>>$ & 49. & & 17.949 & & -0. & & & & & & & & 32 & 12.5 & & & & & & 87 & \\
\hline & & $111: 13$ & 26.6611 & 25.678 & 20.637 & 26.231 & 25. & 49. & 53.862 & $18.026 \mathrm{rat}$ & 31.3 & -0.8 & & & 0. & 14. & & & & & 12. & & & & & & & \\
\hline & & & & & & 20.19 & & & & & & & & & & & & & & & (2) & & & & & & & \\
\hline & $\mid$ & $\begin{array}{ll}11.153 .40 \\
11 \cdot 16.46\end{array}$ & $\begin{array}{ll}\frac{2.001}{2661} \\
2665\end{array}$ & $\frac{25.06}{2564}$ & & 20.4 & & & \begin{tabular}{|l|l|l|l|l|} 
\\
5.076
\end{tabular} & 年 18.041 & & -0.08 & & & & 14.60 & & & & $52+>3$ & 127 & & & & & & & \\
\hline & $\frac{4+21 / 2004}{4 / 212004}$ & $\frac{11.17}{11.17}$ & $\frac{2.06}{266}>0$ & 256. & & $\frac{2.1}{261}$ & & 49483 & 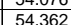 & $\frac{10.006}{18.223}$ & & 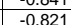 & & & & & & & & $52>2>$ & 123 & & & & & & & \\
\hline & $4 / 21 / 200$ & $\frac{11: 18}{11: 18}$ & & 25.6 & 20.64 & 26.149 & & 49.2 & & 17.999 & & $\frac{-0.8}{-0.8}$ & & & & & & & & & 12. & & & & & & & \\
\hline & $4 / 21 / 2004$ & $11: 19: 46 \mathrm{AM}$ & 26.671 & & & & & & 54.109 & & & & & & & & & & & & & & & & & & & \\
\hline & & $11 \cdot 20 \cdot 46$ & 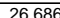 & & & 26301 & & 49 & 54.244 & 18.132 & & -0.818 & & & & & & & & & & & & & & & & \\
\hline $230>->$ & $\mid 4 / 21 / 2004$ & $11: 21: 46 \mathrm{AM}$ & 26.684 & 25.661 & 26.68 & 26.414 & 26.1 & 49.32 & 54.096 & 17.887 & 31. & -0.8 & & $955-5$ & 0.0 & 14.6 & & 297 & 949.6 & 5 & 12.1 & & & & & & & \\
\hline & 4 & $11: 2$ & 26.6 & 25 . 25 & & & & 49. & 54.14 & 17.846 & & & & & & 14. & & & & $52-2>$ & & & & & & & & \\
\hline & & 11:23:46 & 26.6 - & 25.665 & & & & 49.434] & 542. & 17.93 & & -0.7 & & & & 14. & & & & & 12.3 & & & & & & & \\
\hline $230>-2>$ & & $11: 2$ & 2 & 25. & & & & 49.38 & & 17.981 & & -0.8 & & & 0.0 & & & & & & 12. & & & & & & & \\
\hline & $4 / 21 / 2004$ & $11: 25$ & 26.6 & 25. & 26.543 & 26.101 & 26. & 49.328 & 54.14 & & & -0.818 & & & 0.0 & 14.643 & & & & & 12.5 & & & & & & & \\
\hline 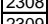 & $4 / 21 / 2004$ & $11: 26: 46 \mathrm{AM}$ & 26.19 & 25.696 & 26.551 & 26.694 & $\frac{26.19}{20.16}$ & 48.928 & $53.7 / 1$ & 11.909 & 31 & $=0.818$ & & 50.943 & 0.05 & 14.643 & & 2989 & 49.4889 & 52. & 12.5 & 40 & $2.1 .7-3$ & 0.000 & 0.001 & 0.0 & $\frac{11 / 8}{180}$ & i \\
\hline$\frac{2309}{2310}$ & $\begin{array}{l}|4| 21 / 21 / 2004 \\
4 / 2004\end{array}$ & $11: 28.46 \mathrm{AM}$ & $\frac{26.101}{26.707}$ & $|25.724|$ & \begin{tabular}{|l|}
26.5010 \\
26.583 \\
\end{tabular} & $\begin{array}{l}26.0353 \\
26.581 \\
\end{array}$ & \begin{tabular}{|l|}
20.050 \\
26.148 \\
\end{tabular} & $\begin{array}{ll}49.212 \\
49.579\end{array}$ & \begin{tabular}{|l|l|l|l}
54.443 \\
\end{tabular} & $\frac{10.041}{17.958}$ & $\frac{31.458}{31.895}$ & \begin{tabular}{|l|}
-0.715 \\
-0.792 \\
\end{tabular} & & 51.785 & 0.053 & $\frac{14.643}{14.643}$ & 0.003 & & \begin{tabular}{|l|l|}
94.178502 \\
9 & 49.7822 \\
\end{tabular} & 53.6 & 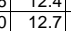 & $\begin{array}{ll}40.7 \\
\end{array}$ & 2.809 & $\mid$ & \begin{tabular}{|l|}
0.000 \\
0.007 \\
\end{tabular} & 0.000 & $\begin{array}{l}0.180 \\
0.179\end{array}$ & 0.1 \\
\hline
\end{tabular}


WSRC-TR-2005-00105, REVISION 0

SRNL-RPP-2005-00012, REVISION 0

RUN \# 2.03A AND B; FIRST AND SECOND HALF OF SLURRY DEWATERING - CONT.

\begin{tabular}{|c|c|c|c|c|c|c|c|c|c|c|c|c|c|c|c|c|c|c|c|c|c|c|c|c|c|c|c|c|}
\hline & A & B & D & E & $\mathrm{F}^{\mathrm{F}}$ & $\mathrm{G}$ & $\mathrm{H}$ & $\mathrm{J}$ & $\mathrm{K}$ & $\mathrm{L}$ & $\mathrm{M}$ & $\mathrm{N}$ & $\mathrm{O}$ & $Q^{Q}$ & $R$ & 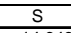 & $\mathrm{T}$ & $v$ & w & $x$ & & $z$ & $\mathrm{AA}$ & $A B$ & $\mathrm{AC}$ & $A D$ & & \\
\hline 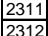 & \begin{tabular}{|l|l|}
$4 / 21 / 2004$ \\
$4 / 212004$
\end{tabular} & $\begin{array}{l}11: 29: 46 \text { AM } \\
1113044 \mathrm{AM}\end{array}$ & $\frac{26.704}{26.715}$ & \begin{tabular}{|r|}
25.73 \\
25.72
\end{tabular} & \begin{tabular}{|l|}
26.589 \\
26.01
\end{tabular} & 26.538 & \begin{tabular}{|l|}
26.149 \\
26.126
\end{tabular} & $\begin{array}{l}49.353 \\
48.951 \\
4\end{array}$ & \begin{tabular}{|l|}
54.208 \\
53.833 \\
\end{tabular} & $\begin{array}{l}17.796 \\
17.85 \\
\end{array}$ & $\begin{array}{l}31.934 \\
31.505 \\
\end{array}$ & $\begin{array}{l}-0.818 \\
-0.775 \\
-1\end{array}$ & $\begin{array}{l}-6.894 \\
-6.926\end{array}$ & \begin{tabular}{|c|}
48.189 \\
50.54
\end{tabular} & 0.052 & $\begin{array}{l}14.643 \\
1.643 \\
\end{array}$ & $\begin{array}{l}0.003 \\
0.003\end{array}$ & 2987.6 & $\begin{array}{l}949.7989 \\
999856\end{array}$ & \begin{tabular}{|l|}
52.2 \\
52.2
\end{tabular} & $\frac{11 .}{12 .}$ & \begin{tabular}{|l|}
40.6 \\
40.2
\end{tabular} & \begin{tabular}{|l|l|}
2.802 \\
2.774
\end{tabular} & $\begin{array}{ll}0.008 \\
0.07\end{array}$ & \begin{tabular}{|l|l|} 
& 0.007 \\
& 0.007 \\
\end{tabular} & 0.000 & \begin{tabular}{|l|l|}
0.182 \\
0.174
\end{tabular} & $\begin{array}{l}0.16 \\
0.15 \\
\end{array}$ \\
\hline $\begin{array}{l}\frac{2314}{2313} \\
\end{array}$ & & & $\frac{26.115}{26.711}$ & & & & & & \begin{tabular}{|l|}
53.833 \\
54.37 \\
\end{tabular} & & & & & & & $\frac{14.643}{14.643}$ & & & & & & & & & & & & \\
\hline 2314 & $4 / 21 / 2004$ & $11: 32: 46 \mathrm{AM}$ & 26.697 & 25.738 & 26.567 & 26.446 & 26.102 & 49.407 & 54.215 & 17.95 & 31.847 & -0.772 & -6.865 & 51.287 & 0.052 & 14.643 & 0.003 & 2990.5 & 9.49 .8489 & & 12.6 & & 2.801 & 0.008 & 0.007 & 0.000 & 0.183 & \\
\hline$\frac{25+4}{2315}$ & & $11: 33: 46 \mathrm{AM}$ & 26.712 & 25.743 & 26.567 & 26.446 & 25.997 & 49.235 & 54.011 & 17.953 & 31.611 & -0.781 & -6.923 & 48.777 & 0.047 & 14.643 & & 2991. & 9 9. 49.8656 & & & & & & & & & 0.14 \\
\hline & & & & & & 26.401 & 25.947 & 49.247 & & 17.976 & & & & \begin{tabular}{|l|}
51.47 \\
\end{tabular} & & & & & & 52.8 & & & & & & & & \\
\hline \begin{tabular}{|l|l|}
2317 \\
2319
\end{tabular} & $4 / 21 / 2004$ & 11:35:46 AM & 26.712 & $\begin{array}{l}25.758 \\
25755\end{array}$ & 26.508 & 26.406 & 25.977 & 49.396 & \begin{tabular}{|l|l|}
54.329 \\
5.203
\end{tabular} & $\begin{array}{l}18.04 \\
18.070\end{array}$ & $\begin{array}{l}31.589 \\
21.71\end{array}$ & \begin{tabular}{|c|c|}
-0.786 \\
\end{tabular} & $\begin{array}{l}-6.885 \\
6.907\end{array}$ & 48.748 & 0.052 & 14.643 & 0.003 & 2993.5 & 949.8989 & 52.4 & 11.9 & & 2.792 & 0.008 & 0.007 & 0.000 & 0.184 & \\
\hline$\frac{2318}{2319}$ & \begin{tabular}{|l|l|}
$4 / 21 / 2004$ \\
$4 / 212004$
\end{tabular} & $\begin{array}{l}11: 36: 46 \mathrm{AM} \\
1137.46 \mathrm{AM}\end{array}$ & 26.708 & $\begin{array}{l}25.765 \\
25.775\end{array}$ & \begin{tabular}{|l|}
26.499 \\
26495 \\
\end{tabular} & $\begin{array}{l}26.348 \\
26.378 \\
\end{array}$ & \begin{tabular}{|r|}
25.884 \\
25.91
\end{tabular} & \begin{tabular}{|l|l|}
49.428 \\
49.206 \\
\end{tabular} & \begin{tabular}{|l|}
54.293 \\
54065
\end{tabular} & $\begin{array}{l}18.078 \\
17865 \\
\end{array}$ & $\begin{array}{l}31.71 \\
31683 \\
\end{array}$ & \begin{tabular}{|c|c|c|c|}
-0.813 \\
-0.799
\end{tabular} & -6.897 & \begin{tabular}{|c|}
48.608 \\
50578 \\
\end{tabular} & $\begin{array}{l}0.05 \\
0.053 \\
\end{array}$ & $\begin{array}{l}14.643 \\
14.643\end{array}$ & \begin{tabular}{|l}
0.003 \\
0.003 \\
\end{tabular} & 2994.4 & $\begin{array}{l}949.9156 \\
994932 \\
9\end{array}$ & $\begin{array}{l}52.8 \\
52.6\end{array}$ & $\frac{11.9}{12.4}$ & \begin{tabular}{|l|}
40.6 \\
40.4 \\
4
\end{tabular} & \begin{tabular}{|l|}
2.797 \\
2.789
\end{tabular} & \begin{tabular}{|l|l|}
0.007 \\
0.008 \\
\end{tabular} & $\begin{array}{l}0.007 \\
0.008 \\
\end{array}$ & 0.000 & \begin{tabular}{|l|l|}
0.176 \\
0.187
\end{tabular} & 0.15 \\
\hline 2320 & $4 / 21 / 2004$ & 11:38:46 AM & 26.694 & 25.76 & \begin{tabular}{|l|}
26.44 \\
\end{tabular} & 26.328 & \begin{tabular}{|l|}
25.91 \\
\end{tabular} & 49.512 & \begin{tabular}{|l|}
54.358 \\
\end{tabular} & 18.139 & 31.667 & -0.784 & $\begin{array}{l}-0.015 \\
-6.894 \\
\end{array}$ & | 50.265 & & $\frac{14.643}{14.643}$ & 0.003 & 2999.6 & & & $\begin{array}{c}11.4 \\
12.3 \\
\end{array}$ & \begin{tabular}{|l}
$\mid 40.4$ \\
40.6 \\
\end{tabular} & \begin{tabular}{|l|l|}
2.789 \\
2.799
\end{tabular} & \begin{tabular}{|l|}
0.0008 \\
0.008 \\
\end{tabular} & $\begin{array}{l}.008 \\
0.007 \\
\end{array}$ & $\begin{array}{l}0.000 \\
0.000 \\
\end{array}$ & 0.181 & $\begin{array}{l}0.16 \\
0.15\end{array}$ \\
\hline & $4 / 21 / 2004$ & 11:39:46 AM & & 25.756 & 26.441 & 26.284 & 25.896 & 49.318 & 54.21 & & 31.704 & $\mid-0.798$ & & 48.661 & 0.048 & 14.643 & & 2997. & 949.9656 & & & & & & & & & $\begin{array}{l}0.15 \\
0.14\end{array}$ \\
\hline 2322 & $4 / 21 / 2004$ & $11: 40: 46 \mathrm{AM}$ & 26.674 & 25.76 & 26.34 & 26.328 & & 49.266 & 54.159 & 18.045 & 31.54 & -0.775 & -6.845 & 51.535 & 0.048 & 14.643 & 0.003 & 2998. & $9 \longdiv { 4 9 . 9 8 2 2 }$ & & 12.6 & \begin{tabular}{|l|l|}
5 & 40.4 \\
\end{tabular} & & & \begin{tabular}{|l|}
0.007 \\
\end{tabular} & 0.000 & & 0.14 \\
\hline 2323 & $4 / 21 / 2004$ & 11:41:46 AM & 26.669 & & 26.32 & 26.368 & & & 53.995 & 17.935 & & & & & & 14.643 & & & & & 12.3 & & & & & & 182 & \\
\hline 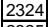 & $4 / 21 / 2004$ & 11:42:46 AM & 26505 & & 26.281 & & & 49.249 & Fin & 170572 & & -0.769 & & 50.267 & 0.052 & & & & & & & & & & & & & \\
\hline & $4 / 21 / 2004$ & 11:43:46 AM & 26.649 & 25.75 & 26.285 & & 25.86 & 49.452 & 54.297 & 18.013 & 31.794 & & & & & 14.643 & & & & & & & & & & & & \\
\hline & 4/21/2004 & 11:44:46 AM & 26.634 & 25.745 & 26.265 & 26.243 & 25.875 & 49.361 & 54.3 & 17.951 & 31.724 & \begin{tabular}{|c|c|c|}
-0.769 \\
\end{tabular} & & 50.719 & 0.049 & 14.643 & 0.003 & 3002.6 & 950.0489 & $52.8 \quad-5$ & 12.4 & 40.5 & 2.795 & 0.007 & 0.007 & 0.000 & 174 & \\
\hline & $1,1 / 2004$ & 11:45:46 AM & 26.618 & 25.755 & 26.264 & 26.227 & 25.909 & & 53.891 & 17.973 & 31.462 & -0.772 & & 50.676 & 0.051 & 14.643 & & & 0.0656 & & & & & & & & & \\
\hline & $4 / 21 / 2004$ & 11:46:46 AM & & & & & & & 54.194 & & 31.515 & $-0.769 \mid$ & & & & 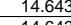 & & & & & & & & & & & & \\
\hline & $4 / 212122004$ & $\begin{array}{l}11: 4: 4: 4 \mathrm{AM} \\
1: 4 \mathrm{M}\end{array}$ & 26.5811 & 25.73 & & 26.1955 & 25.77 & 49.137 & 54.047 & 17.971 & 31.47 & - & & 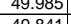 & 0.051 & 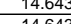 & & & & & & & & & & & & \\
\hline & $\mid \frac{\mid 4 / 21 / 2004}{4 / 21 / 2004}$ & $\frac{11.488 .46 \mathrm{AM}}{11: 49 \cdot 4 \mathrm{AM}}$ & $\frac{2.000}{2650}$ & & $\frac{26.21}{26.209}$ & $\frac{20.249}{26236}$ & $\frac{25.805}{25792}$ & $\frac{49.13}{492}$ & $\frac{54.041}{5413}$ & 17.1853 & $\frac{31.62}{31.636}$ & 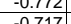 & & & & 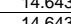 & & & & & & & & & & & & \\
\hline & $4 / 21 / 2004$ & 11:50:46 AM & $\frac{12.56}{26.567}$ & 25.75 & 26.237 & & $\frac{25.842}{25.842}$ & 49.475 & & 17.989 & $\frac{1.1 .72}{31.72}$ & $\mid-0.729$ & & 49.587 & $\frac{0.05}{0.05}$ & $\frac{1.4 .643}{14.643}$ & 0.003 & $3008 \mathrm{~s}$ & 501489 & 528 & & 406 & & & 0.007 & 0.000 & & \\
\hline & $4 / 21 / 2004$ & 11:51:46 AM & 26.545 & 25.741 & 26.206 & 26.244 & 25.911 & 49.427 & \begin{tabular}{|l|l|}
54.378 \\
\end{tabular} & 18.072 & 31.657 & -0.731 & & 51.011 & 0.05 & 14.643 & 0.003 & 3009. & $9 \longdiv { 5 0 . 1 6 5 6 }$ & & 12.5 & & 2.795 & & 0.007 & 0.000 & 0.178 & 0.15 \\
\hline & 1/2004 & 11:52:46 AM & 26.534 & 25.73 & & 26.233 & 25.725 & 49.22 & 54.235 & $\begin{array}{l}17.96 \\
\end{array}$ & 31.566 & -0.726 & & 49.956 & 0.052 & 14.643 & & 3010.6 & & & & & & & & & & \\
\hline & 1/2004 & 11:53:46 AM & $\frac{26.52}{2.521}$ & $\frac{25.7}{25.72}$ & 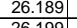 & & 25.749 & & 54.293 & $\begin{array}{r}17.93 \\
18.021\end{array}$ & 31.808 & 0.1 & & 9.766 & 0.049 & $\frac{14.643}{11.62}$ & & & & & & & & & & & & \\
\hline & $\mid$ & 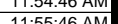 & $\frac{26.515}{26518}$ & $\frac{25 . / 14}{25.729}$ & $\frac{26.199}{26204}$ & 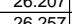 & 年5.8.14 & $\begin{array}{ll}49.40 \\
49.55\end{array}$ & $\begin{array}{l}54.328 \\
54.488\end{array}$ & $\frac{18.021}{18.128}$ & 31.742 & 0.0319 & & (45.051 & 年 & 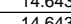 & & & & & 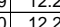 & & & & & & & \\
\hline & $4 / 21 / 2004$ & $\begin{array}{l}11 \cdot 56: 46 \mathrm{AM} \\
11.5\end{array}$ & $\frac{2.0516}{26.516}$ & 25.732 & 26.067 & $\begin{array}{c}20.251 \\
26.29\end{array}$ & & 48.998 & 53.866 & $\begin{array}{l}10.120 \\
18.008\end{array}$ & 31.314 & $\begin{array}{l}-0.149 \\
-0.752 \\
\end{array}$ & & & $\frac{0.051}{0.05}$ & $\frac{1.4043}{14.643}$ & & 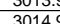 & 2489 & 52. & $\frac{12.4}{124}$ & & & & & & & \\
\hline & 4/21/2004 & 11:57:46 AM & 26.5 & 25.721 & 26.071 & 26.269 & 25.91 & 49.228 & 54.279 & 17.988 & 31.535 & -0.755 & & 48.848 & 0.048 & 14.643 & 0.004 & 3015.6 & 950.2656 & 52.8 & $\frac{12.6}{12 .}>-1$ & 40.4 & 2.784 & & 007 & & $\frac{172}{172}>2>$ & \\
\hline 234 & $1: 1 / 2004$ & 11:58:46 AM & 26.49 & 25.726 & & 26.264 & 25.86 & 49.04 & & 18.025 & 31.355 & \begin{tabular}{|c|c|c|c|}
-0.772 \\
\end{tabular} & & 0.648 & 0.048 & 14.643 & & 3016 & & & $12.4>2>$ & & & & & & & \\
\hline & 4/21/2004 & $11: 5$ & $26.485 \mathrm{~s}$ & 25.716 & 26.041 & 26.224 & 25.741 & 49.201 & 54.15 & 18.087 & & & & 50.452 & 0.051 & 14.643 & & & 50.2989 & 52 & 12.4 & 40.3 & & 0.008 & 0.007 & 0.000 & 0.183 & \\
\hline$\frac{234}{230}>2$ & 4/21/2004 & 12:00:46 PM & 26.455 & 25.716 & 26.036 & 26.129 & 25.691 & 49.257 & 54.175 & 17.978 & 31.665 & -0.743 & -6.8 & $\begin{array}{l}49.397 \\
\end{array}$ & 0.05 & 14.643 & 0.003 & 3018.5 & $9 \longdiv { 5 0 . 3 1 5 6 }$ & 52.7 & 12.1 & 40.5 & 2.790 & 0.007 & 0.007 & 0.000 & 179 & \\
\hline & 4/21/2004 & 12:01:46 PM & 26.461 & 25.1224 & $\mid 2.046$ & 26.124 & 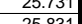 & $\begin{array}{l}48.984 \\
49.141\end{array}$ & 53.966 & 17.992 & 31.308 & -0.766 & & S0.304 & 0.051 & $\frac{1.4643}{1063}>>1$ & & 3019.6 & 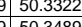 & $\frac{52.5}{52.5}$ & $\frac{12.3}{12 .}$ & 40.1 & & 0.008 & 0.007 & 0.000 & & 0.16 \\
\hline & 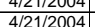 & $\frac{12.02 .46 \mathrm{PMM}}{12 \cdot 03 \cdot 46 \mathrm{PM}}$ & 26.44727 & 25717 & & 26.21 & & & $\begin{array}{l}53.981 \\
54022\end{array}$ & $\begin{array}{l}18.054 \\
18.009\end{array}$ & $\frac{31.44 t}{31.295}$ & $\begin{array}{l}-0.111 \\
-0.772 \\
\end{array}$ & & 51084 & 0.051 & & & $\frac{3020.4}{3021 .}$ & & & 12.1. & & & & & & & 0.16 \\
\hline & $\frac{7 / 21 / 2004}{4}$ & 12:04:46 & $\frac{2.643}{26.423}$ & $\frac{25.729}{25.729}$ & 26.054 & $\frac{26.312}{26.312}$ & $\frac{26.09}{26.09}$ & 49.044 & 54.034 & 18.05 & 31.341 & $\frac{-0.65}{-0.65}$ & & 50.623 & $\frac{0.05}{0.05}$ & $\frac{14.643}{14.643}$ & & 302 & & & 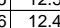 & $\frac{4}{4}$ & & & 20 & & & \\
\hline & $4 / 21 / 2004$ & & 26.42 & & & & & & 54.324 & & & -0.705 & & 0.799 & 0.054 & 14.643 & & & & & $12.4 \times \mathrm{P}$ & & & & & 0.000 & & \\
\hline 34 & & & & 25.737 & 26.067 & & & & & 17.845 & 31.858 & -0.708 & & & & 14.643 & & & & & 120 & & 80 & & & 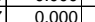 & & \\
\hline$\overline{234}$ & 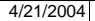 & 12:0 & 26.408 & 25.739 & 26.059 & 26.582 & & 49.479 & 54.542 & 17.862 & & -0.708 & & & & & & & & & $12.3 \mathrm{r}-\mathrm{l}$ & & & & & & & \\
\hline & 4/21/2004 & 12:08:46 PM & 26.41 & 25.746 & 26.071 & 26.599 & & & 54.27 & 17.807 & 31.743 & -0.705 & & & 0.05 & 14.643 & & & 950.4489 & 52.8 & 12.3 & 40. & & & 0.00 & 000 & & \\
\hline & $4 / 21 / 2004$ & 12:09:46 & 26.413 & 25.769 & 26.059 & 26.607 & 26.1 & & 54.476 & 17.787 & & $\mid-0.708$ & & 49.848 & 0.0 & 14.643 & & & 950.4 & & 12.2 & 4 & & & 0.0 & & & \\
\hline 23: & & 12:10:46 & & 25.771 & 26.07 & 26.554 & & & 54.637 & 17.83 & 32.137 & -0.694 & & & 0.05 & & & 30 & & & 12. & & & & & & & \\
\hline & 1120004 & 10.120 & 26.411 & 25.7711 & (1) & 26.519 & 26.16 & & 54.386 & 17.836 & 31.811 & -0. & & & 0.05 & & & & & & 2. & & & & & & & \\
\hline & 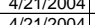 & $\begin{array}{l}12: 12: 46 \\
12 \cdot 12.46\end{array}$ & 26.401 & 年25.113 & 26.0486 & 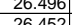 & & & 54.362 54.23 & 171.148 & & $\begin{array}{l}-0.65 \\
0.676\end{array}$ & & & & & & & & & 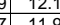 & ro & & & & & & \\
\hline & & & $\frac{20.414}{26419}$ & (25 & & & & & & (17.014 & & -0.0618 & & & & & & & & & 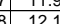 & & & & & & & \\
\hline 235 & 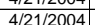 & $\frac{1.14 .46}{12 \cdot 15 \cdot 46}$ & $\frac{20 .}{26}$ & $\frac{2.005}{25.8}$ & $\frac{2.0 .004}{20.04}$ & 26.4 & $\frac{20.0 .1}{26.1}$ & & 54.685 & 17.963 & 32.046 & $\begin{array}{l}-0.053 \\
-0.653\end{array}$ & & $49.2>>3$ & $\frac{0.049}{0.049}$ & $\frac{14}{14}$ & & & 50.5 & & 年 & & & & & & & \\
\hline 235 & $\mid 4 / 21 / 2004$ & & & 25.805 & 26.065 & & & & & & & -0.653 & & & & & & & & & 项 & & & & & & & \\
\hline & $4 / 2$ & & 26. & 25.811 & 26.066 & 26.409 & 25 & 49.363 & 54.275 & 17.786 & 31.864 & $=-0.65$ & & & 0.051 & 14.643 & & & & & $12.2 \mathrm{r}$ & & & & & & & \\
\hline & & & 26. & 25 & & & & & & & & & & & 0.049 & & & & & & & & & & & & & \\
\hline & $4 / 21 / 2$ & & 26. & & & & & 49.044 & 53.987 & 17.724 & & & & & & & & & & & 124 & & & & & & & \\
\hline & $4 / 21 / 2004$ & 12:20:4 & 26.414 & 25.805 & & & & & \begin{tabular}{|l|l|l|l}
54.129 \\
\end{tabular} & & & \begin{tabular}{c|c|c|}
-0.65 \\
\end{tabular} & & 48.2 & 0.05 & & & & & & $11.8 \mathrm{z}-\mathrm{s}$ & & & & & & & \\
\hline & 4/21/2004 & 12:21:46 & & 25.805 & 25.979| & & & 49. & 54.225 & 17.894 & & -0.653 & & & 0.0 & & & & 50.6 & & 12. & & & & & & & \\
\hline 2364 & & & 26. & 25.81 & 25.9999 & & & & & 17.82 & & -0.65 & & & 0.05 & & & & & 52 & 12.3 & & & & & & & \\
\hline & & & 26.38 & 25.799 & $25.969 \mid$ & 26.357 & $25.8 \mathrm{r}-\mathrm{r}$ & & 54.198 & 17.88 & 31.2 & -0.653 & & 5.105 & 0.0 & 14. & & & & & 12. & & & & & & & \\
\hline & & & & & & 26.321 & & & & & & & & & & & & & & & & & & & & & & \\
\hline$\frac{15}{3268}$ & $\mid$ & & 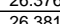 & & 25986 & $\frac{20.344}{26334}$ & & & \begin{tabular}{|l|l|}
54.012 \\
5.240
\end{tabular} & 1 & & $=0.053$ & & & 0.05 & & & & & $52+>3$ & 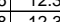 & & & & & & 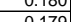 & \\
\hline$\frac{236}{236}>2$ & 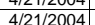 & 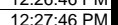 & $\frac{2.03}{26.3}>0$ & $\frac{25.6}{258}>3$ & & & & & 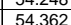 & 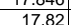 & & $=0.045$ & & & & & & & & 52. & $126 \mathrm{C}>\mathrm{C}$ & & & & & & & \\
\hline & $4 / 21 / 200$ & & $\frac{2.6 .379}{26.39}$ & 25.79 & & 26.353 & 255 & & 54.463 & & & -0.621 & & & 0.049 & & & & & & t2. & & & & & & & \\
\hline 237 & $4 / 21 / 2004$ & & & & & & & & & 17.844 & & & & & & & & & & & & & & & & & & \\
\hline & & & 2638 & 25.804 & & 26.387 & & 49264 & 54,308 & 17782 & 31.83 & $-0,6>-5$ & & & & & & & & & & & & & & & & \\
\hline 237 & $\mid 4 / 21 / 2004$ & $12: 31: 46$ & 26.383 & 25.808 & 26.018 & 26.436 & 25.8 & 49.091 & 54.217 & 17.724 & 31.6 & -0. & & 499 & 0.04 & $14.6>>>$ & & 304 & $50 . \varepsilon$ & 52 & 2.0 & & & & & & 52 & \\
\hline & $4 / 21 / 2$ & & 26. & 25.798 & 26.043 & 26.4 & & 49. & 54.194 & 17.8 & & 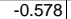 & & & & & & & 50.8 & $52->3$ & 12.4 & & & & & & & \\
\hline 23 & & \begin{tabular}{ll|l}
$12: 33: 46$ \\
\end{tabular} & & 25.792 & & 26.46 & & 49. & 54.4 & 17.804 & & $-0.648 \mid$ & & & & & & & & & $12.2 \mathrm{r}>\mathrm{l}$ & & & & & & 88 & \\
\hline 2376 & & & & 25. & & & & 49. & & & & -0. & & & & & & & & & 12.3 & & & & & & & \\
\hline & $4 / 21 / 2004$ & & & 25.786 & 26.041 & 26.419 & & 49.4 & 54.546 & 17.83 & & & & & & 14.643 & & & & & 1.8 & & & & & & & \\
\hline 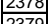 & $4 / 21 / 2004$ & $12: 306446 \mathrm{PM}$ & $\frac{26.38}{26.38}$ & 25.79 & 26.035 & $26.3 / 3$ & 25.82 & 49.186 & 54.89 & 17.906 & 32.125 & -0.581 & & 0.235 & & 14.643 & & & & 53. & $\begin{array}{ll}4 & 12.3 \\
& 12.3\end{array}$ & 4 & 2.0 & 0.007 & $0.00 \%$ & & 1.166 & \\
\hline$\frac{2379}{2380}$ & $\frac{4 / 2 / 1 / 2004}{4 / 21 / 2004}$ & $\frac{12: 37: 48 \text { PM }}{123: 46 \text { PM }}$ & $\frac{26.385}{26.385}$ & \begin{tabular}{r|}
25.796 \\
25.79
\end{tabular} & \begin{tabular}{|l|}
26.036 \\
26.055 \\
\end{tabular} & \begin{tabular}{|l|}
26.344 \\
26.303 \\
\end{tabular} & \begin{tabular}{|l|}
25.821 \\
25.965 \\
\end{tabular} & $\begin{array}{l}49.042 \\
49.376\end{array}$ & \begin{tabular}{|l|}
54.117 \\
54.465 \\
\end{tabular} & $\mid \begin{array}{l}77.85 \\
17.935 \\
\end{array}$ & $\begin{array}{l}31.574 \\
31.728 \\
\end{array}$ & $\begin{array}{l}-0.5811 \\
-\frac{-0.607}{1}\end{array}$ & & $\mid \frac{51.47}{44.556}$ & 0.049 & $\frac{14.643}{14.643}$ & $\frac{0.003}{0.003}$ & & 950.9489 & 53.4 & \begin{tabular}{l|l}
0 & 12.1 \\
\end{tabular} & 40.6 & 2.796 & $\mid \begin{array}{l}0.008 \\
\end{array}$ & \begin{tabular}{|l|l|}
0.007 \\
0.008
\end{tabular} & $\mid 0.000$ & $\frac{0.176}{0.186}$ & 0.16 \\
\hline
\end{tabular}


WSRC-TR-2005-00105, REVISION 0

SRNL-RPP-2005-00012, REVISION 0

RUN \# 2.03A AND B; FIRST AND SECOND HALF OF SLURRY DEWATERING - CONT.

\begin{tabular}{|c|c|c|c|c|c|c|c|c|c|c|c|c|c|c|c|c|c|c|c|c|c|c|c|c|c|c|c|c|}
\hline & A & B & $\mathrm{D}$ & $E$ & $\mathrm{~F}$ & $\mathrm{G}$ & $\mathrm{H}$ & $\mathrm{J}$ & $\mathrm{K}$ & $\mathrm{L}$ & $\mathrm{M}$ & $\mathrm{N}$ & $\mathrm{O}$ & $\mathrm{Q}^{\mathrm{Q}}$ & $R$ & 5 & $\mathrm{~T}$ & & w & $x$ & & $L$ & $\mathrm{AA}$ & $\mathrm{AB}$ & $\mathrm{AC}$ & $A D$ & $\mathrm{AE}$ & \\
\hline \begin{tabular}{|l|l|l|l|l|l|}
2381 & \\
2382 &
\end{tabular} & \begin{tabular}{|l|l|}
$4 / 21 / 2004$ \\
$4 / 212004$
\end{tabular} & $\begin{array}{l}12: 39: 46 \text { PM } \\
12.40 .46 \text { PM }\end{array}$ & $\begin{array}{r}26.39 \\
26.407 \\
\end{array}$ & $\begin{array}{l}25.796 \\
25.808\end{array}$ & $\begin{array}{l}26.046 \\
36.087 \\
26\end{array}$ & 26.319 & \begin{tabular}{|l|}
26.005 \\
26.072 \\
\end{tabular} & $\begin{array}{ll}49.372 \\
49.291\end{array}$ & $\begin{array}{l}54.501 \\
54.413 \\
\end{array}$ & \begin{tabular}{|l|l}
17.77 \\
17.93
\end{tabular} & $\begin{array}{l}31.897 \\
331.59 \\
\end{array}$ & $\begin{array}{l}-0.543 \\
-0.552 \\
\end{array}$ & $\begin{array}{l}-6.535 \\
-6.657\end{array}$ & \begin{tabular}{|l|}
49.597 \\
51.162
\end{tabular} & $\frac{0.038}{0.05}$ & $\begin{array}{l}14.643 \\
14.643 \\
\end{array}$ & $\begin{array}{l}0.003 \\
0.003 \\
\end{array}$ & $\begin{array}{l}3057.9 \\
3058.9\end{array}$ & \begin{tabular}{|l|}
50.9656 \\
5.0822 \\
\end{tabular} & 53.6 & $\frac{12}{12}$ & $\begin{array}{l}40 . \\
40\end{array}$ & $\frac{2.802}{2.791}$ & $\begin{array}{ll}0.006 \\
0.07\end{array}$ & \begin{tabular}{|l|}
0.006 \\
0.007 \\
\end{tabular} & 0.000 & $\begin{array}{l}0.135 \\
0.179 \\
\end{array}$ & 0.12 \\
\hline 年 2004 & & & $\frac{20.401}{26.392}$ & & $\begin{array}{l}26.067 \\
26.067\end{array}$ & & & & & 17.835 & & $\begin{array}{l}-0.0046 \\
-0.546\end{array}$ & & $\frac{11.104}{50.34}$ & & $\frac{14.045}{14.643}$ & & & & & & & & & & & & \\
\hline 2384 & $4 / 21 / 2004$ & $12: 42: 46 \mathrm{PM}$ & 26.391 & 25.782 & 26.052 & 26.35 & 25.986 & 49.243 & 54.366 & 17.7 & 31.87 & -0.555 & -6.665 & 50.212 & 0.05 & 14.643 & 0.003 & 3060.9 & 51.0156 & 52. & & & \begin{tabular}{|l|l|}
2.796 \\
\end{tabular} & 0.007 & \begin{tabular}{|l|}
0.007 \\
\end{tabular} & 0.000 & 0.178 & \\
\hline & $4 / 221 / 2004$ & $12: 43: 46 \mathrm{PM}$ & 26.39 & 25.791 & 26.081 & 26.359 & 25.931 & $\begin{array}{l}49.206 \\
\end{array}$ & \begin{tabular}{|l|l|}
54.333 \\
\end{tabular} & 17.794 & 31.661 & -0.543 & -6.561 & 50.692 & 0.038 & 14.643 & & 3061.9 & & & & & & & & & & 0.12 \\
\hline & & & & & & & & 49.409 & & & & -0.543 & & & & 14.643 & & & & & & & & & & & & \\
\hline \begin{tabular}{|l|}
2387 \\
2389
\end{tabular} & $4 / 21 / 2004$ & 12:45:46 PM & 26.396 & 25.787 & 26.072 & $\begin{array}{l}26.34 \\
26.273 \\
\end{array}$ & $\begin{array}{ll}26.081 \\
25005\end{array}$ & 49.309 & \begin{tabular}{|l|l|}
54.413 \\
5.211
\end{tabular} & $\begin{array}{l}17.74 \\
17.712\end{array}$ & 31.938 & -0.549 & $\begin{array}{l}-6.686 \\
-559 \\
5\end{array}$ & $\begin{array}{l}49.449 \\
4971\end{array}$ & 0.052 & $\begin{array}{l}14.643 \\
1.612\end{array}$ & $\begin{array}{l}0.003 \\
0.003\end{array}$ & 3063.9 & \begin{tabular}{|l|l|}
51.0656 \\
5060
\end{tabular} & 52.5 & 12.1 & & 2.801 & 0.008 & $\begin{array}{ll}0.008 \\
\end{array}$ & 0.000 & 0.185 & \\
\hline $\begin{array}{l}2388 \\
2389 \\
\end{array}$ & \begin{tabular}{|l|l|}
$4 / 21 / 2004$ \\
$4 / 212004$
\end{tabular} & $\begin{array}{l}12: 46: 46 \text { PM } \\
12.47 .46 \mathrm{PM}\end{array}$ & $\begin{array}{l}26.399 \\
26.402\end{array}$ & $\begin{array}{l}25.785 \\
25783\end{array}$ & \begin{tabular}{|l|}
26.085 \\
26078 \\
\end{tabular} & $\begin{array}{l}26.373 \\
26.376 \\
\end{array}$ & $\begin{array}{l}25.995 \\
2583 \\
\end{array}$ & \begin{tabular}{|l|}
49.206 \\
49.152 \\
\end{tabular} & \begin{tabular}{|l|}
54.241 \\
54.368
\end{tabular} & $\begin{array}{l}17.743 \\
17.789\end{array}$ & $\begin{array}{l}31.804 \\
331652\end{array}$ & $\begin{array}{l}-0.65 \\
-0.48 \\
\end{array}$ & -6.558 & \begin{tabular}{|l|}
49.714 \\
49.04 \\
\end{tabular} & 0.047 & $\begin{array}{l}14.643 \\
14.643 \\
\end{array}$ & \begin{tabular}{|l}
0.003 \\
0.003 \\
\end{tabular} & $\begin{array}{l}3064.9 \\
3065.9\end{array}$ & \begin{tabular}{|l|}
51.0822 \\
5109899
\end{tabular} & $\begin{array}{l}52.8 \\
52.8\end{array}$ & $\frac{12.2}{120}$ & 40.5 & \begin{tabular}{|l|}
2.793 \\
2.786
\end{tabular} & \begin{tabular}{|l|l|}
0.007 \\
0.008 \\
\end{tabular} & $\begin{array}{l}0.007 \\
0.008 \\
\end{array}$ & $\begin{array}{l}0.000 \\
0.000 \\
\end{array}$ & $\begin{array}{ll}0.168 \\
0.193\end{array}$ & $\begin{array}{l}0.14 \\
0.16\end{array}$ \\
\hline 2390 & $4 / 21 / 2004$ & $12: 48: 46 \mathrm{PM}$ & 26.411 & 25.782 & 26.101 & 26.334 & 25.816 & 49.378 & \begin{tabular}{|l|}
54.554 \\
\end{tabular} & & & -0.477 & & $\begin{array}{l}49.04 \\
50.797 \\
\end{array}$ & 0.047 & $\begin{array}{l}14.045 \\
14.643 \\
\end{array}$ & $\begin{array}{l}0.0033 \\
0.003 \\
\end{array}$ & 30656.9 & 51.1156 & & $\frac{12.4}{12.4}$ & & & \begin{tabular}{|l|}
0.0008 \\
0.007 \\
\end{tabular} & $\begin{array}{l}.008 \\
0.007 \\
\end{array}$ & 0.000 & $\begin{array}{l}0.193 \\
0.167\end{array}$ & 0.16 \\
\hline & $4 / 21 / 2004$ & 12:49:46 PM & & & & 26.161 & & 49.255 & 54.467 & 17.741 & 31.929 & & & 49.263 & & 14.643 & & & & & & & & & & & & 0.14 \\
\hline 2392 & $4 / 21 / 2004$ & $12: 50: 46$ PM & 26.383 & 25.754 & 26.049 & 26.032 & 25.534 & 49.16 & 54.405 & 17.829 & 31.661 & -0.483 & & 49.512 & 0.05 & 14.643 & 0.003 & 3068.9 & \begin{tabular}{|l|l|}
51.1489 \\
\end{tabular} & 52. & 12.1 & & 2.786 & & 0.007 & 0.000 & & \\
\hline 2393 & $4 / 21 / 2004$ & 12:51:46 PM & 26.39 & & & & & & & & & -0.483 & & 47.849 & & 14.643 & & & & & & & & & & & 168 & \\
\hline 2394 & & 12:52:46 PM & 26.391 & & & 25.89 & & $\begin{array}{ll}49.141 \\
\end{array}$ & & & & 0.1927 & & 50.104 & & & & & & & & & & & & & & \\
\hline & $4 / 21 / 2004$ & 12:53:46 PM & 26.383 & 25.744 & 26.079 & 25.947 & 25.799 & 50.848 & 54.581 & 17.814 & & -1.221 & & & 0.06 & 14.643 & & & l.1989 & & & & $\bar{n}$ & & & & & \\
\hline 2396 & 4/21/2004 & 12:54:46 PM & 26.407 & 25.738 & 26.022 & 26.101 & 25.832 & 49.832 & 54.53 & 17.917 & 32.196 & $\begin{array}{ll}-0.94 \\
\end{array}$ & -4.8 & $\begin{array}{ll}47.628 \\
\end{array}$ & 0.008 & 14.643 & 0.003 & 3072.9 & 51.2156 & 53. & 11.7 & 4 & 2.828 & 0.001 & 0.001 & 0.000 & 0.028 & \\
\hline & $4 / 21 / 2004$ & 12:55:46 PM & 26.411 & 25.742 & & 26.215 & 25.916 & 48.393 & 54.509 & 17.867 & 30.69 & & & 50.373 & 0.03 & 14.643 & & & & & & & $.726 \mathrm{~s}-1$ & & 0.004 & & & \\
\hline & $4 / 21 / 2004$ & & & & & & & 47.679 & & & 30.241 & & & & & 14.643 & & & & & & & & & & & & \\
\hline & 4/21/2004 & 12:57:46 PM & 26.37 & 25.73 & & 26.204 & & 50.357 & .436 & 17.879 & 32.806 & & & $8197 \mathrm{Y}>\mathrm{s}$ & 0.053 & 14.643 & & & .2656 & & & & .86 & & & & 185 & \\
\hline$\frac{240}{2401}$ & $\mid \frac{\mid 4 / 21 / 2004}{4 / 21 / 2004}$ & $\frac{12.589 .46 \mathrm{PMM}}{12.59 .46 \mathrm{PM}}$ & & $\frac{25.11}{25728}$ & & $\frac{20.235}{26206}$ & 25.844 & $\frac{50.831}{50.384}$ & 54.824 & $\frac{17.941}{17.934}$ & $\frac{33.194}{32.761}$ & -1227 & & & 0 & 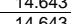 & & & & & & & & & & & & \\
\hline 2402 & $4 / 21 / 2004$ & 1:00:46 PM & $\frac{20.372}{26.367}$ & $\begin{array}{l}25.128 \\
25.728\end{array}$ & 26.017 & $\frac{20.200}{26.236}$ & 25.877 & $\begin{array}{c}50.364 \\
49.38\end{array}$ & 54.314 & $\begin{array}{l}\frac{17.954}{17.472} \\
\end{array}$ & 32.319 & $\begin{array}{l}-1.2218 \\
-1.218\end{array}$ & & 49.222 & 0.052 & $\frac{14.045}{14643}$ & 0.003 & 3078.9 & 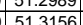 & & $\frac{12.4}{12.1}$ & & & & & 0000 & - & \\
\hline & $4 / 21 / 2004$ & 1:01:46 PM & 26.368 & 25.733 & 26.018 & 26.206 & 25.883 & $\begin{array}{lll}49.361 & \\
\end{array}$ & 53.164 & 17.555 & 32.157 & -1.221 & & 53.197 & 0.051 & 14.643 & 0.003 & 3079.9 & 51.3322 & 51.7 & 13.0 & & 2.810 & & 0.007 & 0.000 & 0.181 & 0.15 \\
\hline & 1/2004 & 1:02:46 PM & 26.363 & 25.7 & 25.989 & 26.262 & 25.919 & 49.307 & 53.058 & 17.38 & 32.354 & & & $\begin{array}{lll}48.414 \\
\end{array}$ & 0.051 & 14.643 & & & & & & & & & & & & \\
\hline & $4 / 21212004$ & 1:03:46 PM & 26.3599 & $\begin{array}{r}25.7 \\
25.71\end{array}$ & 25.97 & 26.283 & 25.8 & 49.344 & 管.095 & 17.598 & 32.05. & -1.218 & & 8.767 & 0.05 & 14.643 & & & 36 & & & & & & & & & \\
\hline$\frac{2406}{2407}$ & $\begin{array}{l}/ 21 / 2004 \\
4 / 21 / 2004 \\
\end{array}$ & $\begin{array}{l}\frac{1.04 .46 \mathrm{PM}}{1.05 .46 \mathrm{PM}} \\
\end{array}$ & $\frac{26.358}{26.343}$ & $\frac{25.19}{25.714}$ & 2.5.589 2509 & $\frac{26.277}{26257}$ & 年 & $\frac{49.336}{49.544}$ & $\frac{52.979}{53.28}$ & $\begin{array}{l}17.506 \\
17555\end{array}$ & 32.219 & 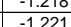 & & 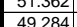 & 0.05 & 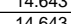 & & 308 & 3989 & & & & & & & & & \\
\hline & $4 / 21 / 2004$ & 1.06.46 PM & 26.348 & 25.719 & 25.954 & 26.247 & $25.7 \mathrm{ra}$ & $\begin{array}{l}49.544 \\
49.456\end{array}$ & 53.153 & 17.468 & 32.445 & $\frac{-1.221}{-1.221}$ & $-7.3 .3-3$ & $\begin{array}{l}49.204 \\
50.623\end{array}$ & 0.05 & $\frac{14.045}{14.643}$ & $\begin{array}{l}0.003 \\
0.003 \\
\end{array}$ & 3084 & - & 517 & $\frac{1.1}{12.4}$ & & & & & & & \\
\hline $24 \mathrm{C}$ & $4 / 21 / 2004$ & 1:07:46 PM & & 25.703 & \begin{tabular}{|l|l|}
25.917 \\
\end{tabular} & 26.276 & 25.867 & 49.413 & 3.033 & 17.431 & 32.385 & -1.224 & -7.59 & 49.276 & 0.053 & 14.643 & & 3085. & 51.4322 & 51. & $\frac{12.1}{12.1}>2$ & 40.9 & 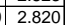 & & $\frac{0}{008}$ & & $188 \quad-3$ & \\
\hline & $1 / 2004$ & 1:08:46 PM & 26.347 & 25.718 & & 26.286 & 25.6 & 49.71 & 53.359 & 17.449 & 32.705 & & & 51.62 & 0.05 & 14.643 & & 3086 & & & & & & & & & & \\
\hline 241 & 4/21/2004 & 1:09: & 26.335 & 25.726 & 25.926 & 26.264 & 25.9 & 49.519 & 53.141 & 17.473 & & & & & 0.051 & 14.643 & 0.003 & & 51.4656 & & $\frac{12.0}{12.0}$ & & 2.828 & 0.008 & 0.007 & 0.000 & 0.181 & \\
\hline$\frac{2-1}{201}$ & 4/21/2004 & 1:10:46 PM & 26.32 & 25.715 & 25.91 & 26.308 & 25.935 & 49.681 & 53.39 & 17.554 & 32.461 & -1.221 & & 49.589 & 0.05 & 14.643 & 0.003 & 3088 & 51.4822 & 51.6 & 12.2 & 41.1 .1 & 2.832 & \begin{tabular}{|l|l|}
0.007 \\
\end{tabular} & 0.007 & 0.000 & 0.177 & \\
\hline & 4/21/2004 & 1:11:46 PM & & 25.709 & 25.909 & $\begin{array}{ll}26.302 \\
26.322 \\
202\end{array}$ & 26.008 & $\begin{array}{l}49.836 \\
40367\end{array}$ & 53.487 & 17.521 & 32.757 & -1.221 & -7.64 & 49.482 & 0.049 & 14.643 & & 3089.9 & 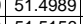 & 52.6 & $\frac{12.1}{12}$ & 41.3 & & & 0.007 & 0.000 & 0.172 & 0 \\
\hline & $\begin{array}{l}\quad 4 / 2 / 21 / 2004 \\
4 / 2004\end{array}$ & $\begin{array}{l}\text { 1:1:12:64 PM } \\
1: 13: 66 \text { PM }\end{array}$ & $\frac{26.318}{26.317}$ & 25.714 & \begin{tabular}{|l|}
25.859 \\
25.833 \\
\end{tabular} & & & $\begin{array}{l}49.301 \\
49.587\end{array}$ & 53.035 & $\begin{array}{l}17.505 \\
17.615\end{array}$ & $\begin{array}{l}32.256 \\
32.299 \\
\end{array}$ & 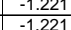 & $-\frac{-.61}{-1.61}$ & $\begin{array}{l}49.656 \\
47.645 \\
\end{array}$ & 0.049 & $\frac{14.645}{14.643}$ & & $\begin{array}{l}3090.9 \\
30919\end{array}$ & 51.5150 & & 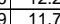 & & & & & & & 0.15 \\
\hline & $\frac{4 / 21 / 2004}{4.2004}$ & 1:14:46 PM & 20.317 & 25.707 & \begin{tabular}{|l|l|}
25.032 \\
2532
\end{tabular} & 26.305 & 25.9 & 49.709 & 53.414 & 17.612 & 32.576 & & & 48.85 & $\frac{0.05}{0.05}$ & 14.643 & & $\frac{309}{309}$ & .5489 & & $\frac{x}{12}$ & 4 & 837 & & 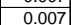 & & & \\
\hline & 4/21/2004 & & & & & 26.34 & & & 52.5 & & & -1.224 & & & 0.049 & 14.643 & & & & & & & & & $0.06-8>$ & & & \\
\hline 241 & & $1 \cdot 16:$ & 26.296 & 25.71 & 25.811 & 26.37 & & & 52.5 & 17.401 & & -1.221 & & & & & & & & & & & & & & & & \\
\hline & $\mid 4 / 21 / 2004$ & 1:17:46 PM & 26.302 & 25.732 & & 26.415 & & & & 17.348 & & -1.221 & & & 0.049 & & & & & & & & & & & & & \\
\hline 242 & $4 / 21 / 2004$ & 1:18:46 PM & 26.292 & 25.722 & 25.762 & 26.385 & & 48.617 & 52.27 & 17.553 & 31.47 & -1.224 & & & 0.048 & 14.643 & & & 51.6156 & $50.8 \mathrm{C}$ & 12.6 & 40.0 & & & 0.00 & 000 & 0.175 & \\
\hline 244 & $4 / 21 / 2004$ & 1:19:46 PM & 26.29 & 25.73 & 25.193 & 26.406 & & & 52.252 & 17.521 & 31.451 & -1.218 & & & & & & & & $x$ & 12. & $4 \mathrm{c}-2>3$ & 100 & & 0 & & & \\
\hline & 12004 & 1:20:46 PM & 26.273 & & & 26.386 & & 48.863 & 675 & 17.478 & 31.7 & & & & 0.048 & & & 309 & & 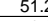 & $1 \pm$ & & & & & & & \\
\hline & $1 / 2004$ & 泣:46 & 26.268 & 25.73 & 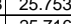 & 26.381 & 250. & 48.644 & 2.418 & 17.447 & 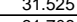 & 1.210 & & & 0.048 & & & & & & 5 & & & & & & & \\
\hline & $\frac{421212004}{41212004}$ & $\begin{array}{ll}1: 22.46 \mathrm{PM} \\
1: 206\end{array}$ & $\frac{26.245}{2625}$ & 25571 & 25.1616 & 26.4144 & & $\frac{4}{483}$ & $\begin{array}{l}52.35 ! \\
5.3086\end{array}$ & $\begin{array}{l}17.348 \\
17409\end{array}$ & & $\frac{-1.244}{-1201}$ & & & 0.048 & $\frac{14.6}{1.4}$ & & & 516989 & & 123 & 398 & 270 & & & & & \\
\hline & 4 & 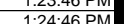 & $\frac{2.25}{26.236}$ & 25.14 & $\frac{20.110}{25696}$ & $\frac{20.304}{26355}$ & & & 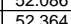 & & & & & & 0.045 & & & & & & 12. & & & & & & & \\
\hline 242 & $\frac{4}{4 / 2120004}$ & $\frac{1454}{1.2541 \mathrm{PM}}$ & 26. & $\frac{2.571}{25.743}$ & 25.708 & 26.376 & & 48.745 & 52,496 & 17.482 & $\frac{1.106}{31.65}$ & -1.221 & & & & 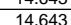 & & & & $\frac{51}{51}$ & 势 & $\frac{4}{4}$ & & & & & & \\
\hline & $4 / 21 / 2000$ & 1:26:41 PM & & & & & & & & & & & & & & & & & & & & & & & & & & \\
\hline 242 & $4 / 212004$ & $1.27 \cdot 41 \mathrm{P}$ & 26218 & 25739 & 25684 & & & & 52283 & 17491 & 31.4 & -1218 & & & 00 & 146 & & & & & 12 & & & & 0.0 & & & \\
\hline & & 1.28 & & & 256 & & & & & & & -1221 & & & & & & & & & & & & & & & & \\
\hline & $4 / 21 / 2004$ & & & 25.737 & & & & & & 17.472 & & -1.227 & & & & & & & & 5 & & & & & & & 179 & \\
\hline & $4 / 21 / 2004$ & 1:30:41 & 26.212 & 25.743 & 25.00 & 26.346 & & & 411 & \begin{tabular}{ll|}
17.412 \\
\end{tabular} & & -1.221 & & & & & & & & 50 & & 4 & & & & & & \\
\hline & $4 / 21 / 2004$ & 1:31:41 PM & & & 25.647 & & & & 399 & 17.587 & & -1.224 & & & & & & & & & 12 & & & & & & & \\
\hline 243 & - & 1:32: & 26. & 25.743 & 25.598 & 26.306 & 25. & 48. & 355 & 17.49 & & -1.221 & & & & & & & & & 12. & & & & & & 0.176 & \\
\hline & & 1:33: & 20.188 & 25.743 & 20.623 & 26.331 & 25.9 & 48. & 52.359 & 17.513 & 31.37 & -1.218 & & & & 14. & & & 0.1 .9 & 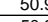 & tx & & & & & & 76 & \\
\hline & & & & & & 26.3 & & & & & & $\frac{-1.224}{-1.24}$ & & & & & & & & & & & & & & & & \\
\hline 243 & 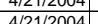 & $\begin{array}{l}1.353 .441 \mathrm{MM} \\
1.3 .61\end{array}$ & & $\frac{23.140}{25748}$ & & & & & & 1.6524 & & $-\frac{1.241}{1.224}$ & & & & $\begin{array}{l}\frac{14.043}{14643} \\
\end{array}$ & & & & & & & & & & & $\frac{19}{72}$ & \\
\hline & $\frac{4}{42 / 12004}$ & 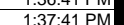 & $\frac{20 .}{26}$ & $\frac{25 .}{25 .}$ & & 26.361 & & & & 17451 & & -1.218 & & & & & & & & & 11 & & & & & & & \\
\hline & $4 / 2121 / 200$ & & & 25.748 & 25.587 & & & 48. & 52.548 & $\mid 17.52$ & & -1.221 & & & & & & & & & & & & & & & & \\
\hline 2441 & 4/21/2004 & & & & & 26.296 & & & & 17.494 & & $-1.227 \mathrm{P}$ & & & & & & & & & & & & & & & & \\
\hline & & $1.40 \%$ & & & & 26311 & & 486 & 5254 & 17466 & 31.646 & -1221 & & & & & & & & & & & & & & & & \\
\hline 2443 & $4 / 21 / 2004$ & $1: 41: 41 \mathrm{P}$ & 26.138 & 25.75 & 25.578 & 26.371 & 25.9 & 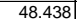 & 52.214 & 17.467 & 31.4 & -1.224 & & 49.8 & 0.04 & 14.6 & & 311 & 51.8 & & 2.0 & & & & & & 76 & \\
\hline & $4 / 21 / 200$ & $1: 42$ & 26.1 & 25.7 & & & & 48. & 52.25 & 17.41 & & 221 & & & & & & & & & 11.9 & & & & & & & \\
\hline & $4 / 21 / 2004$ & 1:43:41 & 26.13 & 25. & & 26.442 & & 48. & & & 31.42 & $-1.22 \quad$ & & & & 14. & & & & & 11 & & & & & & 72 & \\
\hline 24 & & & & 25. & & & & 48. & 52.38 & 17.548 & & & & & & & & & & 50 & 12 & & & & & & & \\
\hline 244 & $4 / 21 / 2004$ & & & 25 & 25.59 & 26.428 & & & 4222 & 17.62 & & & & & & 14.643 & & & & & 12.2 & & & & & & 176 & \\
\hline & $4 / 21 / 2004$ & $1: 46: 41 \mathrm{PM}$ & 26.124 & 25.165 & $\mid$ & 26.433 & 25.6 & 48.420 & 52.285 & 1.4.403 & 31.341 & -1.221 & & 49.6 & 0.048 & 14.643 & & & 52.0 & 50.8 & $\begin{array}{l}8 \\
0.12 .2\end{array}$ & 39. & 2.10 & 0.0017 & 0.007 & 0.000 & .177 & \\
\hline 150 & \begin{tabular}{|l|}
$4 / 21 / 20004$ \\
$4 / 2004$
\end{tabular} & $1: 48: 41 \mathrm{PM}$ & $\frac{20.124}{26.129}$ & 25.775 & 25.58 & $\begin{array}{l}20.400 \\
26.493 \\
\end{array}$ & \begin{tabular}{|l|}
25.979 \\
\end{tabular} & $\begin{array}{l}48.410 \\
48.683\end{array}$ & \begin{tabular}{|l|}
52.471 \\
52.471 \\
\end{tabular} & $\mid \begin{array}{l}1.530 \\
17.517\end{array}$ & $\begin{array}{ll}31.279 \\
31.654\end{array}$ & \begin{tabular}{|l|}
-1.224 \\
-1.221 \\
\end{tabular} & & \begin{tabular}{|l|l|}
47.428 \\
\end{tabular} & $\frac{0.048}{0.048}$ & $\frac{14.643}{14.643}$ & 0.003 & & 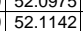 & 51.6 & 11.6 & & 2.769 & \begin{tabular}{|l|}
0.007 \\
\end{tabular} & \begin{tabular}{|l|}
0.007 \\
0.007 \\
\end{tabular} & 0.000 & $\begin{array}{l}0.16 \\
0.175 \\
\end{array}$ & 0.1 \\
\hline
\end{tabular}


WSRC-TR-2005-00105, REVISION 0

SRNL-RPP-2005-00012, REVISION 0

RUN \# 2.03A AND B; FIRST AND SECOND HALF OF SLURRY DEWATERING - CONT.

\begin{tabular}{|c|c|c|c|c|c|c|c|c|c|c|c|c|c|c|c|c|c|c|c|c|c|c|c|c|c|c|c|c|c|}
\hline & A & B & D & $\mathrm{E}$ & $\mathrm{F}$ & G & $\mathrm{H}$ & $\mathrm{J}$ & $\mathrm{K}$ & $\mathrm{L}_{\mathrm{L}}$ & $\mathrm{M}$ & $\mathrm{N}$ & 0 & $a_{1}$ & \begin{tabular}{l|l}
$R$ \\
\end{tabular} & $\mathrm{~s}$ & \begin{tabular}{l|l|l|} 
\\
\end{tabular} & & w & $x$ & & & 2 & $\mathrm{AA}$ & $A B$ & $\mathrm{AC}$ & $A D$ & $\mathrm{AE}$ & \\
\hline \begin{tabular}{|l|l|l|}
2451 \\
2452
\end{tabular} & $\begin{array}{r}4 / 21 / 2004 \\
4 / 212004\end{array}$ & $\begin{array}{l}1: 49: 41 \mathrm{PM} \\
1.50: 41 \mathrm{PM}\end{array}$ & $\frac{26.129}{26.12}$ & $\begin{array}{l}25.765 \\
25.781\end{array}$ & $\begin{array}{r}25.59 \\
25.585 \\
\end{array}$ & $\begin{array}{ll}026.478 \\
5 \\
5\end{array}$ & \begin{tabular}{|r|}
25.969 \\
26.02
\end{tabular} & $\begin{array}{l}48.397 \\
48.418\end{array}$ & \begin{tabular}{|l|}
52.237 \\
52.432 \\
\end{tabular} & $\begin{array}{r}17.533 \\
17.57 \\
\end{array}$ & $\begin{array}{l}31.205 \\
33.295 \\
\end{array}$ & $\begin{array}{l}-1.221 \\
-1.221\end{array}$ & $\begin{array}{r}-7.541 \\
-7.582 \\
\end{array}$ & \begin{tabular}{|c|}
49.62 \\
49.712
\end{tabular} & \begin{tabular}{|c|c|}
0.049 \\
0.048
\end{tabular} & $\begin{array}{l}14.643 \\
14.643 \\
\end{array}$ & $\begin{array}{l}0.003 \\
0.03\end{array}$ & $\begin{array}{l}3127.6 \\
3128.6\end{array}$ & \begin{tabular}{|l|}
52.1308 \\
52.475 \\
\end{tabular} & & & & $\begin{array}{l}39.8 \\
39.9 \\
\end{array}$ & \begin{tabular}{|c|}
2.744 \\
2778 \\
\end{tabular} & \begin{tabular}{|c|}
$\mid 0.007$ \\
\\
0.007
\end{tabular} \mid & \begin{tabular}{|l|}
0.007 \\
\end{tabular} & 0.000 & \begin{tabular}{|l}
0.181 \\
0.177
\end{tabular} & $\begin{array}{l}0.15 \\
0.15\end{array}$ \\
\hline & & & & & $\frac{20.000}{25.565}$ & & & & & & & & & & & $\frac{14.045}{14.643}$ & & & & & & & & & & & & & \\
\hline$\frac{2454}{2454}$ & $4 / 21 / 2004$ & $\begin{array}{l}1: 52: 41 \text { PM } \\
\text { 1 }\end{array}$ & 26.131 & 25.777 & 25.601 & 26.504 & 25.976 & 48.473 & \begin{tabular}{|l|}
52.502 \\
\end{tabular} & 17.472 & 31.394 & -1.221 & $\begin{array}{r}-7.579 \\
\end{array}$ & $\begin{array}{l}49.393 \\
\end{array}$ & 0.048 & 14.643 & 0.003 & 3130.6 & \begin{tabular}{|l|l|}
52.1808 \\
\end{tabular} & 51 & & 12.1 & $\begin{array}{l}30.9 \\
39.9 \\
\end{array}$ & 2.753 & \begin{tabular}{|l|l|} 
& 0.007 \\
\end{tabular} & \begin{tabular}{|l|l|}
0.007 \\
\end{tabular} & 0.000 & 0.176 & \\
\hline 2455 & $4 / 221 / 2004$ & 1:53:41 PM & 26.127 & 25.762 & 25.572 & 26.53 & 26.106 & $\begin{array}{l}40.415 \\
48.65 \\
\end{array}$ & \begin{tabular}{|l|}
52.656 \\
\end{tabular} & 17.518 & 31.556 & -1.221 & $\begin{array}{l}-7.588 \\
-1.58\end{array}$ & $\begin{array}{l}48.369 \\
\end{array}$ & 0.047 & 14.643 & & 3131. & \begin{tabular}{|l|l|}
52.1975 \\
\end{tabular} & 51 & & & & & & & & & 0.15 \\
\hline & & & & & & & & & & $\begin{array}{ll}17.362 \\
1.701\end{array}$ & & & & \begin{tabular}{|l|l|}
49.167 \\
\end{tabular} & $\begin{array}{l}0.048 \\
\end{array}$ & 14.643 & & & & & & & & & & & & & \\
\hline \begin{tabular}{|l|l|}
2457 \\
159
\end{tabular} & $4 / 21 / 2004$ & 1:55:41 PM & $\begin{array}{l}26.132 \\
26.127\end{array}$ & 25.787 & $\begin{array}{l}25.587 \\
25557\end{array}$ & 26.54 & $\begin{array}{l}25.992 \\
36001\end{array}$ & $\begin{array}{l}48.741 \\
\end{array}$ & $\begin{array}{r}52.6 \\
\end{array}$ & $\begin{array}{l}17.491 \\
\end{array}$ & $\begin{array}{l}31.661 \\
21169\end{array}$ & $\begin{array}{l}-1.221 \\
\end{array}$ & \begin{tabular}{|l|l|}
-7.579 \\
7570
\end{tabular} & $\begin{array}{l}47.411 \\
\end{array}$ & $\begin{array}{l}0.048 \\
\end{array}$ & 14.643 & $\begin{array}{l}0.003 \\
\end{array}$ & 3133.4 & \begin{tabular}{|l|l|}
52.2308 \\
\end{tabular} & & & & & 2.772 & \begin{tabular}{|l|l|}
0.007 \\
\end{tabular} & $\begin{array}{l}0.007 \\
\end{array}$ & 0.000 & & \\
\hline $\begin{array}{l}2458 \\
2459\end{array}$ & $\begin{array}{l}4 / 21 / 2004 \\
4 / 212004\end{array}$ & $\begin{array}{l}\text { 1:56:41 PM } \\
\text { 1.57:41 PM }\end{array}$ & $\begin{array}{l}26.127 \\
26.126\end{array}$ & $\begin{array}{l}25.787 \\
25782\end{array}$ & 25.557 & $\begin{array}{r}26.52 \\
26.419 \\
\end{array}$ & $\begin{array}{l}26.091 \\
26116 \\
\end{array}$ & \begin{tabular}{|l|l|l|l|l|}
48.652 \\
88.681
\end{tabular} & \begin{tabular}{|l|}
52.625 \\
55.608 \\
\end{tabular} & \begin{tabular}{|l|}
17.436 \\
17638
\end{tabular} & $\begin{array}{l}31.568 \\
331.198 \\
\end{array}$ & \begin{tabular}{|l|}
-1.221 \\
-1.224
\end{tabular} & $\begin{array}{r}-7.579 \\
-7.582 \\
\end{array}$ & $\begin{array}{l}49.405 \\
51041\end{array}$ & \begin{tabular}{|c|c|}
0.047 \\
0.048
\end{tabular} & $\begin{array}{l}14.643 \\
14.643\end{array}$ & \begin{tabular}{|l|}
0.003 \\
0.003
\end{tabular} & $\begin{array}{l}3134.6 \\
3135.5\end{array}$ & \begin{tabular}{|l|}
52.2475 \\
52642
\end{tabular} & 5 & & $\frac{12.1}{12.5}$ & 40.1 & $\begin{array}{l}2.765 \\
22761 \\
\end{array}$ & \begin{tabular}{|c|}
0.007 \\
0
\end{tabular} & $\begin{array}{l}0.007 \\
0.07\end{array}$ & $\begin{array}{l}0.000 \\
0.000\end{array}$ & \begin{tabular}{|l|l}
0.172 \\
0.176
\end{tabular} & $\begin{array}{l}0.15 \\
0.15\end{array}$ \\
\hline 2460 & $4 / 21 / 2004$ & $1: 58: 41 \mathrm{PM}$ & 26.13 & 25.786 & 25.55 & 26.384 & $\frac{20.110}{26.11}$ & $\begin{array}{l}40.001 \\
48.446\end{array}$ & \begin{tabular}{|l|}
52.359 \\
\end{tabular} & & 31.531 & -1.221 & $\begin{array}{l}-1.502 \\
-7.582 \\
\end{array}$ & 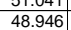 & $\begin{array}{l}0.040 \\
0.047\end{array}$ & $\begin{array}{l}\frac{14.045}{14.643} \\
\end{array}$ & $\begin{array}{l}0.0003 \\
0.003\end{array}$ & $\frac{3135.4}{3136.5}$ & & 5 & & $\frac{\frac{12.5}{12 .}}{12}$ & $\frac{40.0}{40.0}$ & & $\mid$\begin{tabular}{|c|c|}
$\mid 0.007$ \\
0.007
\end{tabular} & $\begin{array}{l}0.007 \\
0.007\end{array}$ & $\begin{array}{l}0.000 \\
0.000 \\
\end{array}$ & $\frac{0.116}{0.173}$ & 0.15 \\
\hline 246. & $4 / 21 / 2004$ & 1:59:41 PM & & & & \begin{tabular}{|l|l|}
26.337 \\
\end{tabular} & & \begin{tabular}{|l|l|l|l}
48.476 \\
\end{tabular} & \begin{tabular}{|l|}
52.494 \\
\end{tabular} & 17.496 & 31.312 & -1.218 & & 48.982 & 0.048 & 14.643 & & & 52.2975 & & & & & & & & & & $\frac{0.15}{0.15}$ \\
\hline 2462 & $4 / 21 / 2004$ & 2:00:41 PM & 26.118 & 25.789 & 25.543 & 26.291 & $\begin{array}{l}25.883 \\
\end{array}$ & $\begin{array}{l}48.498 \\
\end{array}$ & \begin{tabular}{|l|}
52.426 \\
\end{tabular} & $\begin{array}{ll}17.381 \\
\end{array}$ & 31.523 & -1.221 & -7.582 & 47.743 & $\begin{array}{l}0.047 \\
\end{array}$ & 14.643 & 0.003 & 3138.6 & \begin{tabular}{|l|l|}
52.3142 \\
\end{tabular} & & & & & $2.759 \mid$ & & \begin{tabular}{|l|}
0.007 \\
\end{tabular} & 0.000 & & \\
\hline 2463 & $4 / 21 / 2004$ & $2: 01: 41 \mathrm{PM}$ & & & & & & & & & & -1.218 & & & & 14.643 & & & & & & & & & & & & & $\begin{array}{l}0.15 \\
0.15\end{array}$ \\
\hline 2464 & $4 / 21 / 2004$ & 2:02:41PM & & 25.775 & 25.534 & 26.118 & & \begin{tabular}{|l|l|l|l}
48.54 \\
\end{tabular} & Fin & 17.414 & 21455 & -1.224 & & 49.161 & $\mid$ & & & & & & & & & & & & & $\frac{176}{176}$ & \\
\hline 2465 & 4/21/2004 & 2:03:41 PM & & & & 26.101 & 25.613 & 48.503 & 52.405 & & 31.418 & -1.224 & & & $\mid 0.048$ & 14.643 & & $3141 . \mathrm{s}$ & 52.3642 & & & 12. & 40 & 775 & & & & & \\
\hline 2466 & $4 / 21 / 2004$ & 2:04:41 PM & 26.101 & 25.766 & 25.536 & 26.059 & 25.426 & $\begin{array}{ll}48.662 \\
\end{array}$ & 52.695 & 17.467 & 31.564 & $\begin{array}{l}-1.221 \\
\end{array}$ & -7.582 & 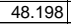 & $\begin{array}{l}0.047 \\
\end{array}$ & 14.643 & $\begin{array}{l}0.003 \\
\end{array}$ & 3142.6 & \begin{tabular}{|l|l|}
52.3808 \\
\end{tabular} & 51 & & 111.8 & 40.1 & 2.766 & 0.007 & 0.007 & 0.000 & 0.172 & \\
\hline $\mid 2467$ & $4 / 21 / 2004$ & 2:05:41 PM & 26.087 & 25.752 & 25.527 & 26.025 & 25.442 & 48.254 & 52.239 & 17.42 & 31.226 & -1.221 & -7.58 & 49.057 & 0.048 & 14.643 & & 3143.6 & & & & & & 2.740 & & & & & \\
\hline & $4 / 21212004$ & & & & $\frac{2.5 .515}{25.512}$ & & $\frac{25.45}{25.42}$ & 48.476 & & & 31.574 & 1020 & & & 0.048 & $\frac{11.643}{42}$ & & 3144. & & & & & & & & & & & \\
\hline & 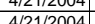 & $2.07 .41 P M$ & 20.001 & 25.142 & & & 25.3 & 48.1064 & 5 & & & -1.224 & & & 0.048 & & & & & & & & & & & & & & \\
\hline & $4 / 21 / 2004$ & $2: 0904: 41 \mathrm{PM}$ & $\frac{20.001}{26.066}$ & 25.716 & 25.521 & 26.00 & 25.021 & $\begin{array}{l}40.054 \\
48.376\end{array}$ & \begin{tabular}{|l|}
52.3240 \\
\end{tabular} & $\frac{17.244}{17.408}$ & 31.392 & $\frac{-1.2<4}{-1.224}$ & -7.585 & $\begin{array}{l}\frac{45.105}{49.211} \\
\end{array}$ & $\begin{array}{l}0.0440 \\
0.047\end{array}$ & $\frac{14.045}{14.643}$ & & $\frac{5140.6}{3147 .}$ & \begin{tabular}{|l|l|}
52.4647 \\
5
\end{tabular} & & & 12. & $\frac{40.1}{39.9}$ & $\frac{2.100}{2.750}$ & 0.07 & 0.007 & & & \\
\hline 247. & $4 / 21 / 2004$ & 2:10:41 PM & 26.059 & 25.705 & & \begin{tabular}{|l|l|}
26.118 \\
\end{tabular} & 25.66 & & 52.235 & \begin{tabular}{|l|l|l|l|l}
17.393 \\
\end{tabular} & & -1.224 & $\begin{array}{l}-7.582 \\
\end{array}$ & $\begin{array}{l}49.41 \\
\end{array}$ & $\begin{array}{l}0.048 \\
\end{array}$ & 14.643 & 0.003 & $3148 \mathrm{c}$ & \begin{tabular}{|l|l|}
52.4808 \\
\end{tabular} & & & 12.1 & & & & & & & \\
\hline & $4 / 21 / 2004$ & 2:11:41 PM & 26.064 & 25.715 & 25.529 & 26.168 & 25.67 & 48.76 & 52.871 & 17.578 & 31.484 & -1.221 & & 3.056 & 0.048 & 14.643 & & 3149. & 52.4975 & & & 11. & 4 & 2.76 & & 0.00 & 0.00 & 176 & \\
\hline & $21 / 2004$ & 2:12:41 PM & 26.055 & 25.706 & & 26.218 & 25.73 & 48.67. & .716 & 17.472 & 31.60 & -1.224 & & 9.687 & 0.048 & 14.643 & & & & & & & & & & & & & \\
\hline & $1 / 2004$ & 2:13:41 PM & $\frac{26.05}{26.05}$ & 25.70 & 5.465 & 26.263 & 25.74 & 48.386 & $\frac{2.426}{2727}$ & $\frac{17.507}{1701}$ & $\frac{31.32}{21.5}$ & -1.224 & & .7119 & 0.048 & 14.643 & & & & & & & & & & & & & \\
\hline & \begin{tabular}{|c|}
$4 / 21 / 21 / 204$ \\
$\mid 2004$
\end{tabular} & $\frac{2.14 .41 \mathrm{PM}}{2 \cdot 15 \cdot 41}$ & $\frac{26.041}{26.047}$ & $\frac{25.691}{25698}$ & 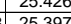 & $\frac{20.344}{26391}$ & 25.806 & (48.654 & 52.878 & $\begin{array}{l}17.491 \\
17512\end{array}$ & $\begin{array}{r}31.51 \\
31.735\end{array}$ & & -7 & $\begin{array}{l}49.247 \\
49093\end{array}$ & 年 & $\frac{14.643}{14643}$ & & 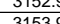 & 25642 & & & 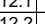 & 4 & & & & & & \\
\hline$\frac{2478}{247}$ & $4 / 2 / 212004$ & $2 \cdot 16 \cdot 41 \mathrm{PM}$ & 26.037 & 25.683 & 25.347 & 26381 & 25.7 & $\frac{40.012}{48.643}$ & 52552 & $\begin{array}{l}17.010 \\
17.467\end{array}$ & 31.587 & $\frac{1.212}{-1218}$ & -7.58 & (45.953 & $\begin{array}{l}.047 \\
0.047 \\
\end{array}$ & $\frac{14.045}{14643}$ & 0 & 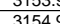 & 5250.44 & 5 & & $\frac{1.2}{117}$ & & & & & & & \\
\hline 247 & 4/21/2004 & 2:17:41 PM & 26.027 & 25.683 & 25.312 & 26.411 & 25.907 & 48.521 & 52.527 & 17.487 & 31.412 & $\frac{-1.221}{-1.221}$ & -7.58 & 46.537 & 0.047 & 14.643 & & 3155.6 & \begin{tabular}{|l|l|}
52.5975 \\
\end{tabular} & & & $\frac{d 1.4}{11.4}$ & 40 & 275 & & .007 & 000 & $\frac{174}{174}$ & \\
\hline & $4 / 21 / 2004$ & $41 \mathrm{PM}$ & 26.033 & 25.689 & 25.288 & 26.462 & & 48 & & 17.595 & 31.546 & & & & & 14.643 & & 315 & & & & & & & & & & & \\
\hline 248: & $4 / 21 / 2004$ & & 26.039 & 25.709 & 25.274 & 26.507 & & 488.749 & 2.751 & 17.438 & 31.741 & -1.218 & & 48.269 & 0.046 & 14.643 & & 3157.4 & $\frac{2.638}{5.26308}$ & 5 & & 11. & 40 & & 007 & 0.007 & 0.000 & 169 & \\
\hline & $4 / 21 / 2004$ & $2: 20: 41 \mathrm{PM}$ & 26.009 & 25.684 & & 26.472 & $\begin{array}{l}25.944 \\
\end{array}$ & $\begin{array}{l}48.513 \\
\end{array}$ & 20.471 & 17.557 & 31.324 & -1.224 & & 49.635 & $\begin{array}{l}0.046 \\
\end{array}$ & 14.643 & & & & & & & & & & & & & \\
\hline $24 \varepsilon$ & $4 / 21 / 2004$ & 2:21:41 PM & 25.995 & & 25.199 & 26.498 & & 48.778 & & 17.474 & 31.796 & & & & 0.046 & & & & & & & 12 & & & & & & & \\
\hline 248 & $4 / 21 / 2004$ & 2:22:41 PM & 25.975 & 25.691 & 25.16 & 26.479 & & \begin{tabular}{|l|l|l|l|l}
48.559 \\
\end{tabular} & 52.619 & 17.464 & 31.54 & -1.221 & & 48.218 & $\begin{array}{l}0.047 \\
\end{array}$ & 14.643 & & $3160 . \mathrm{s}$ & \begin{tabular}{|l|}
52.6808 \\
\end{tabular} & 5 & & 11.8 & 40. & 2.761 & & 0.007 & 0.000 & $\begin{array}{ll}0.174 \\
\end{array}$ & \\
\hline & $4 / 21 / 2004$ & 2:23:41 PM & 25.977 & 25.697 & 25.142 & 26.475 & 26.1 & & & 17.59 & 31.334 & -1.224 & & & & 14.643 & & 316 & & & & 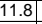 & & & & & & & \\
\hline & 4/21/2004 & 2:24:41 PM & 25.973 & & 25.123 & 26.477 & 26.0 & 48.687 & 2.737 & 17.468 & 31.654 & -1.218 & & 47.626 & 0.046 & & 0.004 & 3162 & 52.7142 & & & & & & & & & & \\
\hline $2 \pi$ & $4 / 21 / 2004$ & 2:25: & 25.939 & 25.704 & 25.049 & 26.452 & & & 523 & 17.476 & 31. & -1.224 & & & 0.047 & 14.643 & & 31 & & & & 11.4. & & . & & .00 & 0.000 & 177 & \\
\hline & & & & & $\frac{25.09}{2507}$ & & & & & & & -1.221 & & & & & & & & & & & & & & & & & \\
\hline$\frac{\frac{140}{249}}{249}$ & $\frac{42121204}{4 \mid 21204}$ & $2.27 .4 .41 \mathrm{PM}$ & $\frac{23.932}{25.923}$ & $\frac{23.117}{25728}$ & $\frac{25.007}{25062}$ & $\frac{26.455}{26.56}$ & & 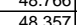 & $\frac{5.17}{52.494}$ & 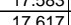 & 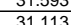 & $\frac{1.244}{-1224}$ & & 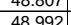 & $\mid$ & $\begin{array}{l}\frac{14.045}{14643} \\
1.62\end{array}$ & & $\frac{5150.6}{316.6}$ & 527808 & 5 & & $\frac{12.0}{120}$ & 397 & 270 & & 0.07 & 0,000 & $\frac{174}{176}$ & \\
\hline $24=$ & $4 / 21 / 2004$ & $2: 29: 41 \mathrm{PM}$ & 25.907 & 25.722 & 25.017 & 26.38 & 25.6 & & \begin{tabular}{|r|}
52.49 \\
52.49
\end{tabular} & 17516 & & -1218 & & & & & & $\frac{3}{31}$ & & & & & & & & & & & \\
\hline & 4 & $2: 30: 41$ PM & 25.896 & 25.727 & 25.006 & 2.6 .29 & & 48.353 & $\frac{12.345}{52.34}$ & 17.647 & & -1.218 & & 48.379 & $\mid$ & & & 316 & $\frac{12}{42}$ & the & & ta. & 35 & & & & & & \\
\hline 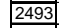 & $\frac{1 / 2004}{11204}$ & $2: 31: 1$ & 25.874 & 25.709 & & $\frac{26.182}{26.182}$ & & & & 17.432 & & -1.224 & & & $\mid 0.046$ & & & & & & & & & & & & & & \\
\hline & & 2.32. & 25.872 & 25712 & & 26.18 & & 48.509 & 2546 & 17.651 & 31 & -1218 & & & 0.046 & & & & & & & & & & & & & & \\
\hline & 4/21/2004 & 2:33:41 PM & 25.823 & 25.678 & 24.937 & 26.051 & 25.3 & $\begin{array}{l}48.666 \\
\end{array}$ & 52.67 & 17.499 & & \begin{tabular}{|c|}
-1.224 \\
\end{tabular} & & 49. & & & & & 52.864 & 5 & & 12.2 & 40. & & & 0.00 & & & \\
\hline & 4/21/2004 & 2:34: & 25.81 & 25.681 & 24.91 & 25.978 & & & & 17.681 & & -1.224 & & & & & & & & & & 12. 1 & & & & & & & \\
\hline 249 & 4/21/2004 & 2:35:41 PM & 25.813 & 25.683 & 24.957 & 25.971 & 25.4 & 48 & 52.268 & 17.525 & 31.078 & -1.221 & & & & 14. & & 317 & 52.8975 & & & 12. & & 2.73 & & & & 0.173 & \\
\hline 249 & $4 / 21 / 2004$ & 2:36:41 PM & 25.802 & 25.677 & 24.941 & 26.055 & & 48 & 538 & 17.654 & & -1.218 & & & & & & & 0.0 & - & & 11.. & 35 & & & 0.6 & & & \\
\hline & & & 25.785 & 25.665 & 24.919 & 26.128 & & & & 17.59 & & & & & & & & & & 5. & & 12.1 & & & & & & & \\
\hline & $4 / 21122004$ & & 25.773 & 25.658 & 24.904 & 26.196 & & & 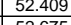 & $\begin{array}{l}17.377 \\
17.6\end{array}$ & $\begin{array}{l}31.275 \\
31.277\end{array}$ & -1.218 & & 年 & & & & 317 & 50210 & & & 16. & & & & & & 777 & \\
\hline & $\frac{4-4121204}{4 / 21204}$ & & $\frac{23.56}{2575}$ & $\frac{23.044}{25.647}$ & & & & & $\frac{2.075}{525}$ & $\begin{array}{l}11.403 \\
17563\end{array}$ & & $\frac{-1.244}{-1224}$ & & & & & & & & & & 111 & & & & & & & \\
\hline & 4 & $\frac{2 \cdot 4 \cdot 4 \cdot 41}{2 \cdot 41}$ & 25.742 & 25657 & & 26.265 & & & & & & -1.221 & & & & & & & & & & & & & & & & & \\
\hline 250 & $\frac{4 / 21 / 2004}{4}$ & $\frac{2: 42: 41 \mathrm{PM}}{2: 2}$ & $\frac{25.742}{25.742}$ & $\frac{25.647}{25.647}$ & 24.816 & 20.26 & 25. & 48.7 & 52.88 & 17.612 & & -1.218 & & 47 & & & & $\frac{318}{318}$ & & & & 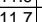 & & & & & & 72 & \\
\hline & & & & & & & & & & & & & & & & & & & & & & & & & & & & & \\
\hline & & $2: 44$ & 25,709 & 25.654 & 24758 & 26262 & & & 52434 & $\begin{array}{ll}17.528 \\
\end{array}$ & 31.174 & -1.221 & & & & & & & & & & & & & & & & & \\
\hline & $4 / 21 / 2$ & 2:45: & 25.6 & 25. & 24.7 & 26.347 & & & & & & \begin{tabular}{|c|}
-1.224 \\
\end{tabular} & & & & & & & & & & & & & & & & & \\
\hline 250 & $4 / 21 / 2004$ & $2: 46: 41 \mathrm{~F}$ & 25.675 & 25.63 & 24.668 & 26.333 & & 48.635 & \begin{tabular}{|l|l|}
52.658 \\
\end{tabular} & 17.59 & & -1.224 & & & $\mid 0.046$ & 14.643 & & & 53.0 & 5 & & 12.1 & 40. & & 0.0 & 0.6 & & & \\
\hline & $4 / 21 / 2004$ & 2:47: & 25.686 & 25.656 & 24. & 26.344 & & & 52.718 & & & \begin{tabular}{|c|}
-1.218 \\
\end{tabular} & & & & & & & & 5. & & $12.0 \mathrm{C}$ & & & & & & 170 & \\
\hline & $4 / 2$ & & 25.666 & 25.646 & 24.64 & & & 48. & 52.156 & 17.444 & & -1.221 & & & & & & 31 & 53.1 & 5 & & 12.0 & & & & & & & \\
\hline & $4 / 21 / 2004$ & 2:49:41 PM & 25.662 & 25.652 & 24.63 & 26.365 & 25.906 & 48.5 & 52.614 & 17.582 & & -1.221 & & & 0.04 & 14.6 & & & & & & & & & & & & & \\
\hline 251 & $\frac{42112004}{412104}$ & $2.500 .41 \mathrm{PM}$ & $\frac{25.642}{25642}$ & $\begin{array}{l}25.64 \\
2567\end{array}$ & 24.300 & $\begin{array}{l}26.385 \\
26605\end{array}$ & & 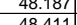 & $\frac{5.31}{5.520}$ & $\begin{array}{l}1.588 \\
17.479\end{array}$ & & -1.221 & & 487 & 0.06 & & & 31896 & 52170 & & & 11.1 & & & & & & & \\
\hline & $4121 / 2004$ & 2.01414 & $\frac{23.044}{25627}$ & 25.076 & & 2.005 & & & & 1017.47 & & -1.224 & & & & & & & & & & & & & & & & & \\
\hline 25 & $\frac{4}{421212004 \mid}$ & $\frac{2.55 .417}{2.53}$ & & 2562 & 24.5 & 26.645 & 26. & & 50418 & & & -1.221 & & & & & & & & & & & & & & & & $\frac{10}{72}$ & \\
\hline & & & & & & & & & & & & & & & & & & & & & & & & & & & & & \\
\hline & $4 / 21 / 2004$ & & & 25.676 & 24.479 & & & & & & & & & & & & & & & & & & & & & & & 176 & \\
\hline & & & 25.596 & & & & & & & & & & & & & & & & & & & & & & & & & & \\
\hline & & & 25.586 & 25.686 & .444 & 699 & & & & 648 & & \begin{tabular}{|c|c|}
-1.224 \\
\end{tabular} & & & & 14.643 & & & & & & & & & & & & & 011 \\
\hline & $4 / 21 / 2004$ & 2:58:41 PM & 25.58 & 25.69 & 24.433 & 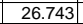 & & 48534 & 5266 & 17.647 & 31.226 & \begin{tabular}{|c|}
-1.221 \\
\end{tabular} & & 48788 & \begin{tabular}{l|l}
0.046 \\
\end{tabular} & 14.643 & & & 532808 & & & 12.0 & & 2.75 & 0.007 & 0.00 & 0.000 & 0.175 & 0.1 \\
\hline
\end{tabular}


WSRC-TR-2005-00105, REVISION 0

SRNL-RPP-2005-00012, REVISION 0

RUN \# 2.03A AND B; FIRST AND SECOND HALF OF SLURRY DEWATERING - CONT.

\begin{tabular}{|c|c|c|c|c|c|c|c|c|c|c|c|c|c|c|c|c|c|c|c|c|c|c|c|c|c|c|c|c|c|}
\hline & A & B & D & $\mathrm{E}$ & & $\mathrm{F}$ & G & $\mathrm{H}$ & $\mathrm{J}$ & $\mathrm{K}$ & $\mathrm{L}$ & $M$ & $\mathrm{~N}$ & 0 & Q & \begin{tabular}{l|l}
$R$ \\
\end{tabular} & 5 & \begin{tabular}{l|l|}
$\mathrm{T}$ \\
\end{tabular} & & w & $x$ & & z & AA & $\mathrm{AB}$ & $\mathrm{AC}$ & $A D$ & $\mathrm{AE}$ & \\
\hline$\frac{2521}{2522}$ & $\begin{array}{l}4 / 21 / 2004 \\
4 / 21 / 12004\end{array}$ & $\begin{array}{l}2: 59: 41 \mathrm{PM} \\
3.00 \cdot 41 \mathrm{PM}\end{array}$ & $\begin{array}{l}25.581 \\
25.577\end{array}$ & $\frac{25 .}{25 .}$ & & $\begin{array}{l}24.414 \\
22.415\end{array}$ & $\frac{26.729}{26.72}$ & $\begin{array}{r}26.04 \\
26086 \\
\end{array}$ & $\begin{array}{l}48.436 \\
48.274 \\
\end{array}$ & \begin{tabular}{|l|}
52.618 \\
52.457 \\
\end{tabular} & \begin{tabular}{|l|}
17.513 \\
17.52 \\
\end{tabular} & $\begin{array}{r}31.33 \\
31115\end{array}$ & $\begin{array}{l}-1.221 \\
-1.218\end{array}$ & $\begin{array}{r}-7.553 \\
-7.547 \\
\end{array}$ & $\begin{array}{l}49.286 \\
48.118\end{array}$ & $\begin{array}{l}0.045 \\
0.046\end{array}$ & $\begin{array}{l}14.643 \\
14.643 \\
\end{array}$ & \begin{tabular}{|c|}
0.003 \\
0.003
\end{tabular} & $\begin{array}{l}3197.6 \\
3198.6\end{array}$ & \begin{tabular}{|l|}
53.2975 \\
5.3142 \\
\end{tabular} & \begin{tabular}{|l|l}
51.1 \\
51.
\end{tabular} & $\frac{12.1}{11.8}$ & $\begin{array}{r}39.9 \\
339.7 \\
\end{array}$ & 2.750 & $\begin{array}{l}0.007 \\
0.07\end{array}$ & \begin{tabular}{|l|}
0.007 \\
0.007 \\
\end{tabular} & 0.000 & $\begin{array}{l}0.171 \\
0.176\end{array}$ & $\begin{array}{l}0.15 \\
0.15\end{array}$ \\
\hline$\frac{2524}{2523}$ & & & & 25. & & $\frac{24.410}{24.38}$ & & & & & & & & & & & $\frac{14.045}{14.643}$ & & & & & & & & & & & & \\
\hline 2524 & $4 / 21 / 2004$ & 3:02:41 PM & 25.552 & 25. & & 24.36 & 26.635 & 25.926 & 48.395 & \begin{tabular}{|l|}
52.639 \\
\end{tabular} & 17.64 & 31.014 & -1.221 & $\begin{array}{l}-7.547 \\
\end{array}$ & $\begin{array}{l}48.965 \\
\end{array}$ & 0.046 & 14.643 & 0.003 & & \begin{tabular}{|l|l|}
53.3475 \\
\end{tabular} & 51.2 & \begin{tabular}{l|l} 
\\
2 & 12.0 \\
\end{tabular} & 39.7 & \begin{tabular}{|l|l|} 
& 2.737 \\
\end{tabular} & 0.007 & \begin{tabular}{|l|}
0.007 \\
\end{tabular} & 0.000 & 0.176 & \\
\hline & $4 / 21 / 2004$ & 3:03:41 PM & 25.551 & 25. & & 24.354 & 26.594 & 25.756 & $\begin{array}{l}40.43 \\
48.43\end{array}$ & \begin{tabular}{|l|}
52.571 \\
\end{tabular} & 17.619 & 31.172 & $\begin{array}{l}-1.218 \\
-1.218\end{array}$ & -7.535 & $\begin{array}{l}49.543 \\
\end{array}$ & 0.047 & 14.643 & & 3201. & \begin{tabular}{|l|l|}
53.3642 \\
\end{tabular} & 51. & & & 2.744 & & & & & 0.15 \\
\hline & & & & & & & & & & & & & & & & $\begin{array}{l}0.045 \\
\end{array}$ & 14.643 & & & & & & & & & & & & \\
\hline 2527 & $4 / 21 / 2004$ & 3:05:41 PM & $\begin{array}{l}25.502 \\
5.52\end{array}$ & 25.6 & & $\begin{array}{l}24.27 \\
22.271\end{array}$ & $\begin{array}{l}26.36 \\
\end{array}$ & 25.552 & $\begin{array}{l}48.415 \\
8.155\end{array}$ & \begin{tabular}{|l|l|}
52.616 \\
5.528
\end{tabular} & $\begin{array}{l}17.475 \\
17595\end{array}$ & $\begin{array}{l}31.291 \\
21.25\end{array}$ & \begin{tabular}{|l|l|}
-1.221 \\
.221
\end{tabular} & $\begin{array}{r}-7.55 \\
7.52\end{array}$ & $\begin{array}{l}48.246 \\
4965\end{array}$ & $\begin{array}{l}0.045 \\
\end{array}$ & 14.643 & $\begin{array}{l}0.003 \\
0.023\end{array}$ & 3203.5 & \begin{tabular}{|l|l|}
53.3975 \\
\end{tabular} & 51.1 & \begin{tabular}{l|l}
11 & 11.8 \\
\end{tabular} & 39.9 & \begin{tabular}{|l|l|}
2.748 \\
\end{tabular} & \begin{tabular}{|l|l|}
0.007 \\
\end{tabular} & $\begin{array}{l}0.007 \\
\end{array}$ & $\begin{array}{l}0.000 \\
\end{array}$ & 0.172 & \\
\hline$\frac{250}{2529}$ & $4 / 21 / 2004$ & $\begin{array}{l}3: 06: 41 \mathrm{PM} \\
3.07 \cdot 41 \mathrm{PM}\end{array}$ & $\begin{array}{l}25.522 \\
25.489\end{array}$ & 25.6 & & $\begin{array}{l}24.304 \\
22.247\end{array}$ & $\begin{array}{r}26.3 \\
26217 \\
\end{array}$ & $\begin{array}{l}25.606 \\
25444 \\
\end{array}$ & \begin{tabular}{|r|r|}
48.415 \\
4871 \\
\end{tabular} & \begin{tabular}{|l|}
52.598 \\
52.902 \\
\end{tabular} & $\begin{array}{l}17.585 \\
17.629\end{array}$ & $\begin{array}{l}31.125 \\
331419 \\
3\end{array}$ & $\begin{array}{l}-1.221 \\
-1.227\end{array}$ & $\begin{array}{r}-7.553 \\
-7.562\end{array}$ & \begin{tabular}{|l|}
49.658 \\
48.23 \\
\end{tabular} & \begin{tabular}{|c|c|c|}
0.045 \\
0.044
\end{tabular} & $\begin{array}{l}14.643 \\
14.643\end{array}$ & \begin{tabular}{|l|}
0.003 \\
0.003
\end{tabular} & $\begin{array}{l}3204.5 \\
3205.5\end{array}$ & \begin{tabular}{|l|l|}
53.4142 \\
533408
\end{tabular} & \begin{tabular}{|l|l}
51.1 \\
51.
\end{tabular} & $\frac{12.2}{11.8}$ & \begin{tabular}{l|l|l|l|}
39.8 \\
40.1
\end{tabular} & \begin{tabular}{|l}
2.742 \\
2.762
\end{tabular} & \begin{tabular}{|l|l|}
0.007 \\
0.07
\end{tabular} & $\begin{array}{l}0.007 \\
0.007\end{array}$ & $\begin{array}{l}0.000 \\
0.000\end{array}$ & \begin{tabular}{|l|l|}
0.172 \\
0.167
\end{tabular} & $\begin{array}{l}0.15 \\
0.14\end{array}$ \\
\hline 2530 & $4 / 21 / 2004$ & 3:08:41 PM & 25.478 & 25. & & 24.246 & 26.251 & 25.4523 & & 52.426 & & & -1.221 & $\begin{array}{l}-7.502 \\
-7.556\end{array}$ & $\begin{array}{r}4.45 \\
47.42 \\
\end{array}$ & $\begin{array}{l}0.0444 \\
0.044\end{array}$ & $\frac{14.045}{14.643}$ & $\begin{array}{l}0.0003 \\
0.03\end{array}$ & $\frac{3206.6}{3206.5}$ & & & $\frac{11.8}{11.6}$ & & $\frac{2.762}{2.733}$ & $\begin{array}{l}0.007 \\
0.007\end{array}$ & $\begin{array}{l}0.007 \\
0.007\end{array}$ & 0.0000 & 0.167 & $\begin{array}{l}0.14 \\
0.14\end{array}$ \\
\hline & $4 / 21 / 2004$ & 3:09:41 PM & & & & 24.214 & 26.234 & & 48.469 & & & 31.144 & -1.221 & & 48.558 & 0.044 & 14.643 & & 3207. & \begin{tabular}{|l|l|}
53.4642 \\
\end{tabular} & & & & & & & & & $\begin{array}{l}0.14 \\
0.14\end{array}$ \\
\hline 2532 & $4 / 21 / 2004$ & 3:10:41 PM & 25.43 & & & 24.173 & 26.243 & 25.795 & 48.092 & 52.258 & $\begin{array}{l}17.565 \\
\end{array}$ & & -1.224 & -7.553 & 48.757 & 0.045 & 14.643 & & $3208 . \mathrm{s}$ & \begin{tabular}{|l|l|}
53.4808 \\
\end{tabular} & & 11.9 & & & & \begin{tabular}{|l|}
0.007 \\
\end{tabular} & 0.000 & & $\frac{0.14}{0.15}$ \\
\hline 2533 & $4 / 21 / 2004$ & 3:11:41 PM & 25.443 & & & & & & \begin{tabular}{|l|l}
48.316 \\
\end{tabular} & & & 31.242 & & & & & & & & & & & & & & & & & 0. \\
\hline 2534 & & $2 \cdot 12 \cdot 1918$ & & & & 24.165 & & & & & \begin{tabular}{l|l}
17.688 \\
\end{tabular} & 31.082 & -1.221 & & 80021 & $\begin{array}{l}0.047 \\
\end{array}$ & & & & & & & & & & & & & \\
\hline & $4 / 21 / 2004$ & 3:13:41 PM & 25.412 & & & 24.155 & 26.44 & 25.792 & & 2007 & & 31.369 & & -7.556 & \begin{tabular}{|l}
45.39 \\
\end{tabular} & 0.045 & 14.643 & & & & & .1. & & $.756 \mathrm{C}$ & & 0.007 & & & \\
\hline 2536 & $4 / 21 / 2004$ & 3:14:41 PM & 25.407 & 25. & & 24.155 & 26.475 & 25.822 & $\begin{array}{l}48.142 \\
\end{array}$ & 52.386 & 17.559 & 30.985 & $\begin{array}{l}-1.218 \\
\end{array}$ & -7.544 & 49.401 & \begin{tabular}{|l|l|l|} 
\\
\end{tabular} & 14.643 & $\begin{array}{l}0.003 \\
\end{array}$ & 3212.6 & 53.5475 & 50.9 & 12.1 & 39. & .728 & | & 0.007 & 0.000 & 0.166 & \\
\hline & $11 / 2004$ & 3:15:41 PM & 25.398 & 25. & & 24.13 & 26.491 & 25.87 & \begin{tabular}{|l|l|}
48.14 \\
\end{tabular} & 52.256 & 17.52. & 30.986 & -1.221 & -7.5 & 49.118 & 0.045 & 14.643 & & & 53.5642 & & 12. & & & & & & & \\
\hline & $4 / 21 / 2004$ & & & & & & & & & & & & 1.224 & & & & 14.643 & & & & & & & & & & & & \\
\hline & $4 / 21 / 2004$ & 3:17:41 PM & 25.374 & 25. & & $\begin{array}{l}24.106 \\
21112\end{array}$ & 26.547 & $\begin{aligned} 25.983 \\
25.034\end{aligned}$ & 48.249 & 2.507 & 17.458 & 31.123 & -1.218 & -7.541 & 49.153 & 0.046 & 14.643 & & & 3.5975 & & 12. & & & & & .000 & & \\
\hline & $\frac{442112004}{4121204}$ & 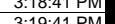 & 25000 & & & 24.1142 & $\frac{26.580}{2650}$ & $\frac{25.93}{36 \sqrt{2}}$ & $\begin{array}{l}48.424 \\
18237\end{array}$ & & $\begin{array}{l}17.699 \\
17550\end{array}$ & 31.08 & $\frac{-1.218}{-1201}$ & & $\frac{49.647}{101213}$ & 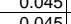 & $\frac{11.643}{10.642}$ & & & 36309 & & & & & & & & & \\
\hline 2542 & $4 / 21 / 21 / 2004$ & 3:19.44 PMM & $\frac{23.356}{25.357}$ & $\frac{25 .}{25 .}$ & & $\begin{array}{l}24.4085 \\
24.079\end{array}$ & $\frac{20.595}{26585}$ & $\frac{26 .}{26.0}$ & 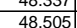 & $\begin{array}{l}52.451 \\
52762\end{array}$ & $\begin{array}{l}17.559 \\
17.706\end{array}$ & $\begin{array}{l}31.189 \\
31.189\end{array}$ & $\begin{array}{l}-1.221 \\
-1.221\end{array}$ & & $\begin{array}{l}49.145 \\
48.679\end{array}$ & \begin{tabular}{|c|} 
\\
0.048 \\
\end{tabular} & $\frac{14.643}{14.643}$ & 0.003 & 32 & 势. & & 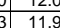 & 39.8 & & & 0.07 & 0.000 & 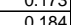 & \\
\hline & $4 / 21 / 2004$ & 3:21:02 PM & 25.364 & 25. & & 24.091 24.091 & $\frac{2.00}{26.632}$ & 26.06 & 4 & 52.646 & 17.674 & 31.047 & $\frac{-1.241}{-1.221}$ & -7.44 & 48.419 & 0.039 & $\frac{14.045}{14.643}$ & & $\frac{3210.4}{3219.2}$ & \begin{tabular}{|l|l|}
53.65333 \\
\end{tabular} & 51. & $\frac{11 .}{11 .}$ & & & & & & & \\
\hline & $4 / 21 / 2004$ & 3:22:02 PM & 25.339 & & & & 26.607 & 26.09 & 48.432 & 52.674 & 17.683 & 31.158 & & & 48.623 & $\mid 0.044$ & & & & & & & & & & & & & \\
\hline & $21 / 2004$ & & 25.337 & 25. & & 24.044 & 26.635 & & 48.561 & 52.751 & 17.597 & & & -7.446 & 88.529 & 0.045 & 14.643 & & & & & & & & & & & & \\
\hline & & & & 25. & & 24.035 & & & & 2.583 & & & & & & & & & & & & & & & & & & & \\
\hline & $4 / 21 / 2004$ & 3:25:02 PM & & & & 24.036 & 26.587 & 26.04 & $\begin{array}{l}48.449 \\
\end{array}$ & 2.637 & & 31.4 & -1.215 & $\begin{array}{l}-7.443 \\
\end{array}$ & 49.435 & $\mid$ & 14.643 & & 3223 & 53.72 & $v_{1}$ & & & & & & & & \\
\hline & $4 / 21 / 2004$ & 3:26:02 PM & 25.315 & 25 & & 24.022 & 26.578 & & 48.366 & 52.695 & 17.688 & 30.986 & -1.221 & & 49.299 & $\begin{array}{ll}0.045 \\
\end{array}$ & 14.643 & 0.003 & 322 & 53.7367 & 51 & 12.1 & & & & & & & \\
\hline 254 & $4 / 21 / 2004$ & 3:27:02 PM & 25.316 & 25. & & 24.013 & 26.574 & 25.881 & \begin{tabular}{|l|l|}
48.31 \\
\end{tabular} & 2.561 & 17.568 & 31.129 & -1.221 & -7.446 & 48.721 & $\mid 0.044$ & 14.643 & & 3225.8 & 53.7533 & 51 & $11 .$. & 39.7 & 2.73 & & 0.007 & .000 & 0.170 & \\
\hline 25 & 4/21/2004 & 3:28:02 PM & 25.29 & 25 & & 23.982 & 26.498 & & 48.278 & 577 & 17.666 & 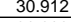 & -1.224 & -7.449 & 49.286 & 0.044 & 14.643 & & 3226.2 & & & 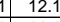 & & & & & & & \\
\hline 255 & $4 / 21 / 2004$ & 3:29:02 PM & 25.299 & & & 23.991 & $26.40 \pi$ & & 48.131 & 22.362 & 17.633 & 30.903 & & & 48.584 & 0.045 & & & & 53.7867 & & 11. & & & & & & & \\
\hline & $4 / 21 / 2004$ & 3:30:02 PM & $\frac{25.292}{25205}$ & $\frac{25 .}{25 .}$ & & $\begin{array}{l}23.984 \\
23072 \\
2072\end{array}$ & $\frac{26.26}{26.138}$ & $\frac{25.5}{254}$ & 48 & 2.457 & $\frac{17.688}{1772}$ & $\begin{array}{l}30.912 \\
30.899 \\
\end{array}$ & $-\frac{-1.218}{1.224}$ & & $\begin{array}{l}48.152 \\
49124\end{array}$ & 0.044 & $\frac{14.643}{14643}$ & & 32 & $\frac{3.8033}{5382}$ & & 1. & & & & & & & \\
\hline & $4 / 2 / 21 / 2004$ & $\begin{array}{l}3.15 .02 \mathrm{PM} \\
3: 32: 02 \mathrm{PM}\end{array}$ & $\frac{23.205}{25.257}$ & 25.8 & & 势.974 & $\frac{2.100}{26045}$ & $\frac{25.44}{2530}$ & $\begin{array}{r}48.27 \\
48.053\end{array}$ & 5 & $\begin{array}{ll}1.122 \\
17.588\end{array}$ & 30.774 & $\begin{array}{l}-\frac{1.2<4}{-1.224} \\
-1\end{array}$ & & 48.487 & $|0.044|$ & $\frac{14.045}{14643}$ & & 323 & 53.064 & 50.8 & 119 & 39.4 & & 2007 & 0007 & 0,000 & 0171 & \\
\hline & $4 / 21 / 2004$ & 3:33:02 PM & 25.254 & & & 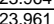 & 26057 & & 48.596 & & & & -1218 & & & & & & 323 & 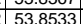 & & 121 & & & & & & & \\
\hline & $\frac{4 / 21 / 2004}{4}$ & 3:34:02 PM & $\frac{25.246}{25.246}$ & 25.6 & & 告. & $\frac{2.6089}{26.099}$ & 25.4 & 48.239 & 2.475 & $\begin{array}{l}17.703 \\
\end{array}$ & 30.94 & $\frac{-1.215}{-1.215}$ & & 4871 & 0.044 & 14.643 & & $\frac{223}{323}$ & $\frac{53.87}{537}$ & & 11. & & & & (2) & 0.000 & & \\
\hline & $4 / 21 / 2004$ & & & & & 23.95 & & & 48 & & & & -1.218 & & 48.496 & & 14.643 & & & 3.886 & & & & & & 0.06 & .000 & & \\
\hline & & $3: 36$ & 25.212 & 25.5 & & 23.924 & 26.12 & 25 & 48.2 & 52.5 & 17.6 & 3108 & -1.221 & & & & & & (2) & & & $\frac{12}{12}$ & & & & & & & \\
\hline & & $\overline{D 2 \mathrm{PM}}$ & 25.201 & 25.5 & & 23.918 & 26.174 & & 48.21. & & & & -1.215 & & 49.6 & & & & 32 & & & & & & & & & & \\
\hline 256 & $4 / 21 / 2004$ & 3:38:02 PM & 25.191 & 25 & & 23.897 & 26.218 & 25 & 48.038 & \begin{tabular}{|l|} 
\\
\end{tabular} & 17.528 & 30.903 & -1.221 & & 49.222 & 0.044 & 14.643 & & 3236.2 & 53.9367 & 50 & 12.1 & 39.5 & & & 0.00 & 000 & 0.171 & \\
\hline 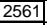 & $4 / 21 / 2004$ & 3:39:02 PM & 25.185 & 25. & & 23.891 & 26.268 & & 48. & 52.55 & 17.518 & 31.148 & -1.221 & & 50.094 & 0.0 & $\frac{14.643}{14}$ & & 323 & 53.9533 & 51 & $12 .$. & & & & 0.00 & & 170 & \\
\hline & |2004 & 3:40:02 PM & 25.17 & 25 & & 23.881 & 26.283 & 25. & 48.39 & \begin{tabular}{|l|}
52.704 \\
\end{tabular} & 17.528 & 31.209 & -1.221 & & 48.25 & & & & 3238 & & & & & 2.744 & & & & & \\
\hline 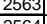 & 年2004 & & $\frac{25.16}{25.16}$ & 25 & & 20.876 & 26.293 & 25.7 & 48.335 & 然. & 17.618 & & -1.218 & & 48.717 & 0.04 & & & nat & & & & & & & & & & \\
\hline & $\frac{42112004}{412104}$ & $\begin{array}{l}3: 24: 02 \mathrm{PM} \\
3: 2020\end{array}$ & 25.155 & $\frac{25}{25}$ & & $\frac{2}{20.8606}$ & $\frac{26.35}{2638}$ & & & 527 & 10.64 & $\begin{array}{l}30.957 \\
32926\end{array}$ & $=\frac{-1.218}{-1218}$ & & & & & & 324 & 5402 & 51 & 121 & 396 & & & & & & \\
\hline & 4 & $3.450 .02 \mathrm{PM}$ & $\frac{25.150}{25.141}$ & $\frac{25}{256}$ & & $\frac{20.001}{23847}$ & $\frac{2.030}{26350}$ & & & & & & -1.210 & & & & & & & & & & & & & & & & \\
\hline 256 & $\frac{4}{42 / 12004}$ & PM & $\frac{2.414}{25.147}$ & $\frac{25 .}{25 .}$ & & 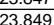 & $\frac{2.03}{2.62}$ & & 年 48.079 & 376 & 17.582 & 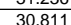 & -1.224 & & . & & $\frac{14 .}{14 .}$ & & $\frac{324}{324}$ & $\begin{array}{l}54.036 \\
54.0533\end{array}$ & 51 & 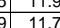 & 39. & & & & & $\frac{0.170}{0.172}$ & \\
\hline & $4 / 21 / 2004$ & $3: 46$ & & & & 23.823 & & & & & & & -1.224 & & & & & & & & & & & & & & & & \\
\hline 2565 & $4 / 21 / 2004$ & & 25.133 & $25:$ & & 23.85 & 26.486 & & 48 & 52.403 & & 30.788 & -1221 & & 48. & $\mid 0.043$ & 146 & & 32 & 54.0 & - & 120 & & & & 0.0 & & 168 & \\
\hline 25 & & & & & & 23.836 & & & & & & & \begin{tabular}{|c|}
-1.218 \\
\end{tabular} & & & & & & & & Fe & $\overline{11.8}$ & & & & & & & \\
\hline & $4 / 21 / 2004$ & 3:49: & 25.131 & 25.5 & & 23.827 & & & & & & & -1.221 & & & & & & & & 51 & 2127 & & & & & & & \\
\hline & $4 / 21 / 2004$ & 3:50:C & 25.137 & 25. & & 23.834 & 26.515 & 26. & & 2.625 & 17. & & -1.221 & & & & & & 32 & 54.136 & 51. & 11. & 35 & & & & & & \\
\hline 25 & $4 / 21 / 2004$ & 3:51:02 PM & & 25. & & 23.824 & 26.525 & & 48. & 52.712 & 17.5 & & -1.221 & & & & & & 32 & 54.1 & 51 & 11. & & & & & & & \\
\hline 257 & $4 / 21 / 2004$ & 3:52: & 25.123 & 25.5 & & 23.819 & 26.501 & & $4 \varepsilon_{2}$ & & 17.722 & & -1.221 & & & & & & 325 & & & & & & & & & 72 & \\
\hline 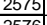 & & 3:5: & 25.135 & 25. & & 20.832 & 26.528 & & 48.4 & 52.716 & 17.7 & 31.121 & -1.224 & & & 0.0 & & & 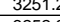 & 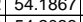 & Fit & & & & & & & & \\
\hline & & & 25.12 & & & 20.807 & $\frac{2.6514}{2.50}$ & & & & 17.65 & & -1.221 & & & & & & & & & & & & & & & & \\
\hline 2578 & $4421 / 12004$ & 3.530 .020 & $\frac{2.1}{251}$ & $\frac{25.8}{256}$ & & $\frac{2.0253}{23810}$ & & & & 52.06 & $\frac{1.172}{177}$ & & $\frac{-1.221}{1.204}$ & & & & $\frac{14.043}{1.643}$ & & $\frac{32}{32 x}$ & $\frac{54}{542}$ & 51 & & & & & & & $\frac{175}{162}$ & \\
\hline 257 & $\frac{4}{42(21 / 20044}$ & $\frac{3.5 .6}{3.57 .}$ & $\frac{2.525}{25124}$ & & & $\frac{2.013}{23831}$ & & & & 52529 & 17.5 & & -1218 & & & & & & & $\mid \frac{34.563}{542533}$ & 51 & $\frac{1.0}{117}$ & & & & & & & \\
\hline & $4 / 2121 / 2004$ & 3:58: & $\frac{2.1 .127}{25.17}$ & 25.6 & & $\frac{2.38}{23.828}$ & 26.59 & 25 & & \begin{tabular}{|l|l|}
52.776 \\
\end{tabular} & 17.539 & & -1.221 & & & & & & 325 & & & 117 & 73 & & & & & 174 & \\
\hline$\angle 58$ & $4 / 21 / 2004$ & 3:59.02 PM & & & & & & & & & & & & & & & & & & & & & & & & & & & \\
\hline & & & 25107 & & & 23838 & 26.45 & & 4838 & & 17700 & & -1.224 & & & & & & & & & & & & & & & & \\
\hline & $4 / 21 / 2004$ & $4: 01: 02 \mathrm{PM}$ & 25.116 & 25.6 & & 23.832 & 26.404 & 25. & $\begin{array}{l}48.181 \\
\end{array}$ & 52.451 & 17.606 & 30.9 & -1.218 & & 48.6 & 0.0 & & & 325 & 54.32 & & 110 & & 2 & & & & & \\
\hline & $4 / 21 / 200$ & $4: 02$ & 25.099 & 25.5 & & & 26.342 & & 48. & & & & -1.221 & & & 0.0 & & & & & & & & & & & & & \\
\hline & $4 / 21 / 2$ & 4:03 & 25.1 & 25. & & & & & 47.97 & 52.42 & 17.54 & & -1.22 & & & & & & & & & & & & & & & & \\
\hline$\angle 3$ & & 4:04 & 25. & & & 23.796 & 26.068 & & 48.4 & & & & & & & & & & $32 t$ & & & 12 & & & & & & & \\
\hline 258 & $4 / 21 / 2004$ & & & 25.5 & & 23.195 & 26.071 & & 48.14 & 52.525 & 17.47 & & -1.22 & & & 0.04 & 14.643 & & & & & & & & & & & & \\
\hline 年58 & $4 / 21 / 2004$ & 4:06:02 PM & 25.083 & 25.4 & & 20.3784 & 26.1269 & 25.538 & 48.193 & \begin{tabular}{|l|l}
52.569 \\
5
\end{tabular} & 17.541 & 30.918 & -1.224 & & 47.808 & 0.043 & 14.643 & & 320 & 54.4033 & 51. & 11.1 & 39 & 2.1. & | & 0.001 & 0.000 & 0.168 & i \\
\hline$\frac{25089}{2590}$ & $4 / 21 / 21 / 2004$ & $\begin{array}{l}4000.02: 02 \mathrm{PM} \\
\end{array}$ & $\frac{25.017}{25.076}$ & 25.5 & & 年. 23.777 & $\frac{20.109}{26.244}$ & \begin{tabular}{|r|}
3.040 \\
25.72
\end{tabular} & $\begin{array}{l}\frac{48.135}{47.625} \\
4\end{array}$ & \begin{tabular}{|r|}
52.501 \\
52.04 \\
\end{tabular} & $\begin{array}{l}17.527 \\
17.527\end{array}$ & $\begin{array}{l}30.806 \\
30.468\end{array}$ & $\frac{-1.221}{-1.221}$ & $\begin{array}{l}-1.2 \\
-7.2\end{array}$ & $\begin{array}{r}49.565 \\
49.124\end{array}$ & $\begin{array}{l}0.045 \\
0.043\end{array}$ & $\frac{14.643}{14.643}$ & 0.005 & & 54.4367 & $\begin{array}{l}51.6 \\
50.6\end{array}$ & 12.0 & & 2.692 & \begin{tabular}{|l|l|}
0.006 \\
\end{tabular} & \begin{tabular}{|l|}
0.007 \\
0.007
\end{tabular} & $\begin{array}{l}0.000 \\
0.000\end{array}$ & $\begin{array}{l}0.168 \\
0.170\end{array}$ & 0.12 \\
\hline
\end{tabular}


WSRC-TR-2005-00105, REVISION 0

SRNL-RPP-2005-00012, REVISION 0

RUN \# 2.03A AND B; FIRST AND SECOND HALF OF SLURRY DEWATERING - CONT.

\begin{tabular}{|c|c|c|c|c|c|c|c|c|c|c|c|c|c|c|c|c|c|c|c|c|c|c|c|c|c|c|c|c|}
\hline & A & B & $\frac{\mathrm{D}}{2007}$ & $E$ & $\mathrm{~F}$ & $\mathrm{G}$ & $\mathrm{H}$ & $\mathrm{J}$ & $\frac{K}{K K}$ & $\mathrm{~L}$ & $\begin{array}{ll} \\
\end{array}$ & $\mathrm{N}$ & 0 & $\mathrm{Q}_{1}$ & $R$ & $\mathrm{~s}$ & $\begin{array}{lll}\mathrm{T} \\
\end{array}$ & & w & $x$ & & 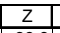 & $\mathrm{AA}$ & $\mathrm{AB}$ & $A C$ & $\mathrm{AD}$ & $A E$ & \\
\hline 2591 & $\begin{array}{l}4 / 21 / 2004 \\
4 / 21 / 2004\end{array}$ & $\begin{array}{l}4: 09: 02 \mathrm{PM} \\
4: 10.02 \mathrm{PM}\end{array}$ & $\begin{array}{l}25.076 \\
25.076\end{array}$ & $\frac{25.581}{25.576}$ & $\frac{23.772}{23.772}$ & $\begin{array}{l}26.284 \\
26.329 \\
\end{array}$ & $\begin{array}{l}25.76 \\
25.76\end{array}$ & $\begin{array}{l}48.146 \\
48.042\end{array}$ & \begin{tabular}{|l|}
52.604 \\
52.446 \\
\end{tabular} & \begin{tabular}{|l|}
17.451 \\
17.46
\end{tabular} & \begin{tabular}{|c|}
31.109 \\
30.971
\end{tabular} & $\begin{array}{l}-1.224 \\
-1.221\end{array}$ & $\begin{array}{l}-7.212 \\
-7209\end{array}$ & $\begin{array}{l}48.287 \\
48.488\end{array}$ & 0.043 & $\frac{14.643}{14.643}$ & $\begin{array}{l}0.003 \\
0.003\end{array}$ & $\begin{array}{l}3267.2 \\
3268.2\end{array}$ & 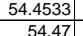 & $\frac{51.1}{51.0}$ & $\begin{array}{l}11.8 \\
11.9\end{array}$ & $\begin{array}{l}39.6 \\
39.5\end{array}$ & \begin{tabular}{|l|}
2.732 \\
2.724 \\
\end{tabular} & 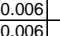 & \begin{tabular}{|c|c|}
0.007 \\
0.007
\end{tabular} & $\begin{array}{l}0.000 \\
0.000\end{array}$ & \begin{tabular}{|l|l|l|l|l|}
0.167 & 0.168 \\
\end{tabular} & $\begin{array}{l}0.14 \\
0.14\end{array}$ \\
\hline 2593 & $\frac{4 / 21 / 12004}{4 / 21 / 2004}$ & & $\frac{25.016}{25.071}$ & $\frac{25.566}{25.571}$ & $\frac{23.112}{23.772}$ & $\frac{26.329}{26.374}$ & & & & \begin{tabular}{|r|}
17.646 \\
\end{tabular} & & & & & & $\frac{14.643}{14.643}$ & & & & & & & & & & & $\mid$ & \\
\hline 2594 & $4 / 21 / 2004$ & 4:12:02 PM & 25.061 & 25.571 & 23.757 & 26.434 & 25.815 & \begin{tabular}{|l|l|}
48.069 \\
\end{tabular} & \begin{tabular}{|l|}
52.438 \\
\end{tabular} & 17.654 & 30.768 & -1.221 & $\begin{array}{l}-7.212 \\
\end{array}$ & 48.604 & 0.043 & 14.643 & 0.003 & & \begin{tabular}{|c|c|c|c|}
54.5033 \\
\end{tabular} & 51.0 & & & \begin{tabular}{|l|}
2.718 \\
\end{tabular} & 0.006 & 0.007 & 0.000 & 0.168 & \\
\hline & $4 / 21 / 2004$ & 4:13:02 PM & $\frac{25.046}{25.046}$ & 25.55 & 23.732 & 26.379 & 25.85 & 48.237 & \begin{tabular}{|l|}
52.674 \\
\end{tabular} & 17.652 & 30.932 & -1.224 & $\begin{array}{r}-7.212 \\
-1.212\end{array}$ & 48.027 & 0.044 & 14.643 & & 3271.2 & 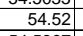 & 51.2 & \begin{tabular}{|l|}
11.8 \\
\end{tabular} & & 2.729 & & $\begin{array}{l}0.007 \\
\end{array}$ & & 0.172 & \\
\hline & & 4:14:02 PM & & & 23.763 & 26.43 & 25.852 & \begin{tabular}{|l|l|}
48.142 \\
\end{tabular} & & & \begin{tabular}{|l|l|}
30.879 \\
\end{tabular} & & & & & 14.643 & & & & 51.1 & & 39.5 & & & & & & \\
\hline 25977 & $4 / 21 / 2004$ & 4:15:02 PM & 25.063 & $\frac{25.568}{25.59}$ & $\frac{23.759}{32.70}$ & $\begin{array}{l}26.426 \\
2.436\end{array}$ & 25.907 & $\begin{array}{l}48.173 \\
\end{array}$ & \begin{tabular}{|l|l|}
52.618 \\
\end{tabular} & $\begin{array}{ll}17.664 \\
\end{array}$ & $\begin{array}{l}30.94 \\
\end{array}$ & $\begin{array}{l}-1.221 \\
\end{array}$ & \begin{tabular}{|c|}
-7.209 \\
7.72
\end{tabular} & $\begin{array}{l}48.268 \\
\end{array}$ & 0.043 & 14.643 & 0.003 & 3273.2 & \begin{tabular}{|l|l|}
54.5533 \\
53
\end{tabular} & 51.1 & \begin{tabular}{|l|l|}
11.8 \\
\end{tabular} & 39.6 & \begin{tabular}{|l|l|}
2.727 \\
\end{tabular} & 0.006 & $\begin{array}{l}0.007 \\
\end{array}$ & 0 & $\begin{array}{l}0.168 \\
\end{array}$ & \\
\hline 2598 & $\frac{4 / 21 / 2004}{4 / 21 / 2004}$ & $\begin{array}{ll}4: 16: 02 \mathrm{PM} \\
\text { 4:17:02 PM }\end{array}$ & 25.048 & $\frac{25.558}{25.569}$ & $\frac{23.739}{23.746}$ & \begin{tabular}{|l|}
26.436 \\
26.427
\end{tabular} & $\begin{array}{l}25.857 \\
25.944\end{array}$ & \begin{tabular}{|l|l|l|l}
48.299 & 48.279 \\
\end{tabular} & \begin{tabular}{|l|}
52.706 \\
52.646
\end{tabular} & \begin{tabular}{|r|}
17.74 \\
17.717
\end{tabular} & \begin{tabular}{|l|l|}
30.854 \\
30.864
\end{tabular} & $\begin{array}{l}-1.224 \\
-1.224 \\
\end{array}$ & $\begin{array}{l}-7.209 \\
-7.209\end{array}$ & \begin{tabular}{|l|}
48.803 \\
48544
\end{tabular} & 0.043 & $\begin{array}{l}14.643 \\
14.643\end{array}$ & 0.003 & $\begin{array}{l}3274.2 \\
3277.2 \\
327\end{array}$ & \begin{tabular}{|r|r|r|}
54.57 \\
54.5867
\end{tabular} & $\begin{array}{ll}51.2 \\
51.2\end{array}$ & $\frac{12.0}{11.9}$ & \begin{tabular}{|l|}
39.6 \\
396 \\
\end{tabular} & \begin{tabular}{|l|l|}
2.729 \\
2728
\end{tabular} & $\begin{array}{l}0.006 \\
0.07 \\
0.07\end{array}$ & \begin{tabular}{|c|}
0.007 \\
0.007
\end{tabular} & $\begin{array}{l}0.000 \\
0.000\end{array}$ & \begin{tabular}{|l|l|}
0.168 \\
0.172
\end{tabular} & \\
\hline & $4 / 21 / 2004$ & 4:18:02 PM & $\frac{25.049}{25.066}$ & 25.571 & $\frac{2.140}{23.757}$ & 26.464 & $\begin{array}{r}25.944 \\
25.9\end{array}$ & & \begin{tabular}{|l|}
52.040 \\
52.612
\end{tabular} & & $\begin{array}{l}30.004 \\
30.825\end{array}$ & $\begin{array}{l}-1.2<4 \\
-1.177\end{array}$ & & $\begin{array}{l}48.544 \\
48.175\end{array}$ & 0.044 & $\begin{array}{l}14.045 \\
14.643\end{array}$ & 0.003 & & $\begin{array}{l}54.5866 \\
54.6033\end{array}$ & $\begin{array}{l}51.2 \\
51.1 \\
\end{array}$ & $\frac{11.9}{11.8}$ & & \begin{tabular}{|l|}
2.728 \\
2.721
\end{tabular} & 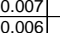 & $\frac{0.007}{0.006}$ & $\mid \begin{array}{l}0.000 \\
0.000\end{array}$ & 0.172 & 0.15 \\
\hline & $4 / 21 / 2004$ & 4:19:02 PM & & & & & & \begin{tabular}{|l|}
48.046 \\
\end{tabular} & \begin{tabular}{|l|}
52.457 \\
\end{tabular} & 17.613 & \begin{tabular}{|c|}
30.803 \\
\end{tabular} & -1.221 & -7.194 & 47.611 & & 14.643 & & & 54.62 & 51.0 & & & & $\begin{array}{l}0.006 \\
0.006\end{array}$ & $\begin{array}{l}0.0007 \\
0.007\end{array}$ & 0.000 & & $\frac{0.14}{0.14}$ \\
\hline 2602 & $4 / 21 / 2004$ & 4:20:02 PM & & 25.577 & 23.754 & 26.45 & 25.912 & \begin{tabular}{l|l|}
48.285 \\
\end{tabular} & \begin{tabular}{|l|}
52.884 \\
\end{tabular} & $\begin{array}{ll}17.693 \\
\end{array}$ & \begin{tabular}{|l|}
30.947 \\
\end{tabular} & -1.195 & $\begin{array}{l}-7.197 \\
\end{array}$ & 48.957 & 0.043 & 14.643 & & 3278.2 & \begin{tabular}{|l|l|}
54.6367 \\
\end{tabular} & 51.4 & 12.0 & & & & & & & \\
\hline 2603 & $4 / 21 / 2004$ & 4:21:02 PM & 25.058 & & & & & & & & & & & 47.117 & 0.043 & & & & & 51.5 & & & & & .007 & 0.000 & 0.167 & \\
\hline 2604 & & 4:22:02 PM & & & & & & & 52.461 & $\begin{array}{ll}17.66 \\
\end{array}$ & 3077 & 1105 & & 4851 & 0.043 & 14.643 & & & $54.67 \mid$ & 510 & & & & & & & & \\
\hline 200 & $4 / 21 / 2004$ & 4:23:02 PM & 25.069 & 25.594 & 23.76 & & & & & & 30.637 & & & 470 & 843 & 14.643 & & & 54.6867 & & & & .708 & & & & & \\
\hline 2606 & $4 / 21 / 2004$ & 4:24:02 PM & 25.054 & 25.599 & 23.751 & 26.597 & 26.069 & 48.173 & 52.616 & \begin{tabular}{l|l|l|l|}
17.68 \\
\end{tabular} & 30.852 & -1.154 & -7.19 & 48.054 & 0.043 & 14.643 & & 3282.2 & 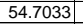 & 51.1. & 11.8 & 39.5 & 2.724 & 0.006 & 0.007 & 0.000 & 0.168 & \\
\hline & $4 / 21 / 2004$ & 4:25:02 PM & 25.067 & 25.602 & 23.768 & 26.59 & & 48.154 & 52.66 & 17.715 & 30.704 & -1.157 & & 49.812 & 0.042 & 14.643 & & & 54.72 & 51.2 & 12.2 & & 2.718 & & 5.006 & 0.000 & 0.164 & \\
\hline & & 4:26:02 PM & & & & & & & & & 30.817 & & & 49.034 & & & & & 54.73677 & & & & & & & & & \\
\hline & $4 / 21 / 2004$ & 7:02 PM & 25.058 & $\frac{25.608}{25.598}$ & $\begin{array}{l}23.759 \\
23270\end{array}$ & 26.456 & & & $\begin{array}{l}2.411 \\
2.597\end{array}$ & 17.679 & 30.723 & 1.157 & & 3.219 & 0.043 & 14.643 & & & 4.7533 & & & & & 006 & 0.007 & 0.000 & & \\
\hline & 年1/121/2 & 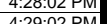 & & $\frac{25.5980}{25677}$ & $\frac{2.3 .49}{2270}$ & $\frac{2.301}{3.626}$ & & & & $\frac{\mid 1.604}{17441}$ & 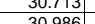 & - & & . & & $\frac{14.645}{11642}$ & & & 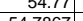 & & & & & & & & & \\
\hline$\frac{261}{261}$ & $\begin{array}{l}4 / 21 / 2004 \\
4 / 21 / 2004\end{array}$ & $0.02 \mathrm{PM}$ & $\frac{2.0507}{25057}$ & $\frac{2.007}{2567}$ & $\frac{2.475}{23753}$ & $\frac{20.66}{26.7}$ & & 年 48.071 & (52.53) & 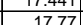 & 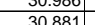 & - & & 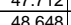 & 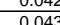 & 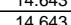 & & & (5).18013 & 1.1. & 然1 & & 730 & 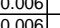 & 007 & 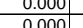 & & \\
\hline & $4 / 21 / 2004$ & 4:31:02 PM & 25.034 & 25.584 & 23.74 & $\frac{20.14}{26.097}$ & & & 52.846 & 17.797 & 30.947 & - & & 47.699 & 0.042 & $\frac{14.045}{14.643}$ & & $\frac{300.4}{3289.2}$ & $\frac{4.0050}{54.82}$ & 51.4 & 11.7. & & & & 006 & 0 & $\frac{604}{164}$ & \\
\hline & $4 / 21 / 2004$ & 4:32:02 PM & & $\frac{25.581}{20.581}$ & 23.738 & $\frac{2.0 .094}{26.04}$ & & 48.5 & & 17.684 & & & & 48.164 & & & & & & & & & & & & & & \\
\hline & $1 / 12004$ & 4:33:02 PM & $\begin{array}{l}5.036 \\
5036\end{array}$ & 25.576 & 23.752 & 26.109 & & 48.1 & 62.618 & 17.789 & 30.554 & & & | & 0.044 & 14.643 & & & & & & & & & & & & \\
\hline & & & & & & & & & & & & & & 48.064 & & 14.643 & & & 54.87 & & & & & & & & & \\
\hline & 4/21/2004 & $802 \mathrm{PM}$ & 25.018 & & 23.734 & 26.141 & & 48.3 & & 17.758 & 30.868 & & & 47.503 & 0.043 & 14.643 & & 3293.2 & 54.8867 & & $1.6 \quad-6$ & & .729 & & 007 & & & \\
\hline & & PM & & 25.582 & 23.768 & 26.23 & & 48.314 & 52.892 & 17.84 & 30.86 & -1.134 & & 48.412 & 0.043 & 14.643 & & 3294.2 & 54.90 & 51.4 & 11.9 & & & & & & & \\
\hline 61 & $4 / 21 / 2004$ & 7:02 PM & 25.021 & 25.566 & 23.773 & 26.234 & & 48. & 52.944 & 17.782 & 30.86 & -1.102 & -7.191 & 48.356 & 0.044 & 14.643 & & 3295.2 & 54.92 & 51.5 & 11.9 & 39.6 & .730 & $\mid 07$ & 0.007 & & 172 & \\
\hline 202 & $4 / 21 / 2004$ & 4:38:02 PM & 25.026 & 25.561 & 23.838 & 26.284 & & 48.071 & 6006 & $17.871 \mid$ & 30.499 & -1.137 & & 49.305 & 443 & 14.643 & & 329 & 54.9367| & 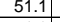 & 2.1 & & 2.709 & & & & & \\
\hline & $4 / 21 / 2004$ & & 25.023 & & 23.849 & 26.316 & & 47.957 & 52.502 & & 30.626 & & & 49.2666 & 0.041 & & & & 54.9533 & & & 39.3 & .109 & & .006 & & & \\
\hline 62 & 4 & 4:40:02 PM & 25.023 & $\frac{25.563}{2578}$ & $\begin{array}{l}23.839 \\
232355\end{array}$ & 26.361 & & $\frac{47.936}{47.92}$ & 52.531 & $17.766 \mid$ & $\begin{array}{l}30.571 \\
30.622\end{array}$ & $\begin{array}{c}-1.099 \\
1.099 \\
\end{array}$ & & 48.1911 & 0.041 & $\frac{14.643}{14643}$ & & 3298.2 & $\begin{array}{r}54.97 \\
458967\end{array}$ & & L. & 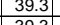 & . & & & 0.000 & & \\
\hline$\frac{2624}{2024}$ & $\begin{array}{l}4 / 21 / 2004 \\
4 / 21 / 2004\end{array}$ & $12: 02 \mathrm{PM}$ & 25.034 & 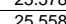 & 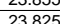 & 年 & & 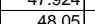 & .577 & \begin{tabular}{|c|c|c|c|c|}
17.77 \\
\end{tabular} & 30.0573 & - & & 48.25 & & 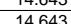 & & 33002 & (5).5003 & 511 & 118 & 39.3 & 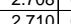 & $0006 \mid$ & 2007 & | & - & \\
\hline & $4 / 21 / 2$ & & & & 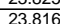 & 502 & & 47.945 & 52556 & 17699 & & -1093 & & 48.934 & & 14643 & & & $\frac{505}{55.02}$ & & 10. & & 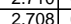 & & & & & \\
\hline & $4 / 21 / 2$ & 4:44:02 PM & 年. 25.031 & $\frac{25.561}{20.561}$ & $\frac{23.802}{20.302}$ & $\frac{2.6 .509}{26.509}$ & & 48.138 & & 17.793 & 30.594 & & & 48.181 & $\frac{0.6}{0.6}$ & 14.643 & & $\frac{330}{330}$ & 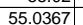 & t. & $\frac{1.8}{1.8}$ & 39.4 & $\frac{6}{.714}$ & & .007 & & 76 & \\
\hline & $4 / 21 / 2004$ & & 25.036 & & 23.798 & & & & 2563 & 17.604 & & & & 47,6 & & 14.643 & & & 5.0 & 51. & & & 2754 & & & & & \\
\hline 2628 & $4 / 2$ & & 25.043 & 25.568 & 23.789 & 26.526 & & & 52.552 & 17.84 & & & & 494 & 0.0 & 14.643 & & & 7 & 5 & 21 & & 2656 & & & & & \\
\hline & $4 / 21 / 2004$ & & & & 23.801 & 532 & & & & & 30.834 & & & 48.4 & & & & & & & 1.9 & & 2.725 & & & & & \\
\hline 2630 & $4 / 21 / 2004$ & PM & 25.051 & 25.576 & 23.782 & 26.524 & & & 52.631 & 17.792 & 30.717 & -1.221 & & 48.22 & & 643 & & 3306.2 & |55.1033 & 51.2 & 11.8 & 39.5 & & & & & & \\
\hline 2631 & $4 / 21 / 2004$ & 4:49:02 PM & 25.042 & 25.582 & & & & & 52.050 & 17.811 & 31.096 & -1.218 & & 48.992 & & 14.643 & & & 55.12 & $1 \pm$. & 2.0 & 39 & .745 & & & & & \\
\hline & & 4:50:02 PM & 25.054 & 25.599 & 23.795 & 26.562 & & 48.739 & \begin{tabular}{|l|}
52.766 \\
\end{tabular} & 17.745 & 31.421 & -1.2 & & 47.872 & & & & 3308 & 55.13 & t. & 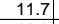 & & 2.763 [2. & & & & & \\
\hline & $4 / 22$ & & 20.05 & 25.574 & 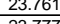 & $\frac{26.583}{2258}$ & & 48.532 & 20.007 & 17 & 31.302 & & & 43.010 & 0.044 & & & 5 & 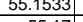 & & 10 & 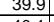 & s. & & & & & \\
\hline & 442112004 & & $\frac{2.2 .051}{25052}$ & $\frac{25.601}{25617}$ & $\frac{2.3111}{23792}$ & $2 \frac{2.559}{2.648}$ & 2584 & & & $\begin{array}{l}1.675 \\
17639\end{array}$ & $\begin{array}{l}3.1 .48 \\
31.176\end{array}$ & $\begin{array}{l}-1.221 \\
.1215\end{array}$ & & 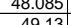 & & & & & \begin{tabular}{|c|c|}
5.1867 \\
55186
\end{tabular} & & 20 & & 8705 & & & & & \\
\hline & 4 & & & $\frac{2.011}{25.55}$ & 势 & 20.400 & & & 势 & | & | & & & 49.1 & & & & & $\mid$ & & . & & . & & & & & \\
\hline & $\frac{4}{421 / 12004}$ & & 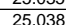 & $\frac{2.558}{2558}$ & 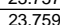 & $\frac{2.0501}{26.251}$ & & 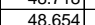 & 52.639 & 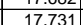 & | & -1.221 & & $\begin{array}{l}48.723 \\
47816\end{array}$ & $\frac{0.6}{0.6}$ & $\frac{14.643}{14.643}$ & & & $\frac{5.2033}{55.22}$ & 51.2 & $\frac{11.9}{11.7}$ & & . & & & & $\frac{13}{70}$ & \\
\hline 2638 & $4 / 21 / 2004$ & & & & 23.742 & & & & 52.679 & & & & & & & & & & & & 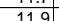 & & & & & & & \\
\hline 2639 & $4 / 21 / 2004$ & & 25.024 & 25.58 & 23.745 & 26 & & & 52.902 & 17.81 & & & & 48.1 & & 14.643 & & & $\frac{1.25}{55.25}$ & 51.4 & 11.18 & & & & & & 66 & \\
\hline 2640 & $4 / 21 / 2$ & & & & 23.754 & & & & & & & & & & & & & & & & & & & & & & & \\
\hline & $4 / 21 / 2004$ & 4:59:0 & & & 23.731 & 26. & & & 52.674 & 17.658 & 31.232 & & & 48.6 & & & & & & 51.2 & 110 & & & & & & 62 & \\
\hline 2642 & $4 / 21 / 200$ & 5:00:02 & 25.018 & 25.573 & 23.74 & 26.101 & & & 52.546 & 17. & 31.06 & -1.22 & & 48.9 & & & & & 55.30 & 51. & 2.0 & & & & & & 0.163 & \\
\hline 264 & $4 / 21 / 2004$ & 5:01:02 & & 25.562 & 23.738 & 26.115 & & & 52.788 & 17.71 & 31.263 & & & 48.1 & & $14.6 \mathrm{C}$ & & & & & .8 & & & & & & & \\
\hline 2644 & $4 / 21 / 2$ & 5:02:0 & 25. & 25.566 & 23.758 & 26.194 & & 48.6. & 52.77 & $17.8 \mathrm{C}$ & & -1.218 & & 48.7 & & & & & 55.3. & 11.3 & 1.9 & & 2.7 & & & & & \\
\hline 2045 & $4 / 21 / 2004$ & 5:03:02 $\mathrm{Pl}$ & t5. 50.016 & 25.566 & 23.748 & 26.244 & & 48. & $5=488$ & 17.844 & 31.018 & -1.218 & & 48.35 & 0.04 & 14.64 & & & . & . & 1.9 & & & & & & & \\
\hline & & & & & 30.162 & 20.324 & & & & & & & & 189 & & & & & & & & & & & & & (n) & \\
\hline 20478 & $\frac{442112004}{421004}$ & $\begin{array}{l}5.050 .02 \\
5.06 .02\end{array}$ & & $\frac{2.3 .545}{2551}$ & $\frac{2.3152}{23.73}$ & $\frac{2.299}{26319}$ & & & $\frac{53.000}{5.664}$ & $\begin{array}{l}17.84 \\
1768\end{array}$ & 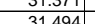 & & & 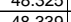 & & & & 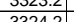 & 年55.30 & 512 & 18 & & & & & & 0 & \\
\hline$\frac{2649}{2649}$ & 4 & 5.0.07.02 & 25011 & 20.554 & $\frac{2.0 .45}{2353}$ & 26349 & & & \begin{tabular}{|l|l|}
52863 \\
\end{tabular} & 1..365 & $\frac{1.44}{3144}$ & & & & & & & & & 01.4 & 22 & & & & & & $\frac{0.162}{0.162}$ & \\
\hline & $4 / 21 / 200$ & $\begin{array}{l}5: 08: 02 \\
5\end{array}$ & 20.501 & 告.5.535 & 党3.757 & $\frac{2.354}{26.34}$ & & 48.476 & \begin{tabular}{|l|l|}
52.573 \\
\end{tabular} & 17.6 & 31.18 & $\frac{1.215}{-1.215}$ & & & & & & $\frac{5326.2}{3326.2}$ & 55.43 & 51.1 & 1118 & & & & & & 67 & \\
\hline 2651 & $4 / 21 / 2004$ & & & & & & & & $\frac{12.449}{52}$ & 17.611 & & -1.1221 & & & & & & & & & & & & & & & & \\
\hline & 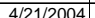 & & & 25.522 & 23824 & 26.34 & & 485 & & 17.609 & 31261 & & & 490 & & & & & 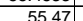 & 511 & & & & & & & & \\
\hline 65 & $4 / 21 / 2004$ & $5: 11: 02 \mathrm{PM}$ & 5.001 & 25.521 & 23.813 & 26.329 & 25.82 & 48.409 & 52.548 & 17.733 & 31.121 & -1.221 & & 48.07 & & 146 & & $\overline{3329.2}$ & \begin{tabular}{|l|l|}
55.4867 \\
\end{tabular} & & 18 & & 2 & & & & 苟 & \\
\hline & $4 / 21 / 200$ & $5: 12: 02$ & & 25.5 & 23.794 & 26.375 & & 48.3 & \begin{tabular}{|l|l|}
52.428 \\
\end{tabular} & 17.60 & 31.1 & -1.224 & & 48.644 & & & & 333 & & & & & 74 & & & & & \\
\hline 2655 & $4 / 21 / 200$ & & & 25.52 & 23.783 & 26.3 & & 48. & $52.96 \mathrm{C}$ & 17. & 31.164 & -1.224 & & 48.6 & & 14.6 & & & & 515 & & & & & & & 66 & \\
\hline & $4 / 21 /$ & & & & 23.783 & $26.35^{2}$ & & & 52.799 & 17.894 & & -1.224 & & 48.6 & & & & & & 51.3 & & & & & & & 0.162 & \\
\hline 2657 & $4 / 21 / 2004$ & & & & 23.783 & 26.3. & & & 52.6 & & & & & 48.3 & & & & & & & & & & & & & & \\
\hline$\frac{6568}{6.656}$ & $4 / 2121 / 2004$ & 5:16:02 PM & 25.006 & 20.516 & 23.158 & 26.294 & & 48.154 & \begin{tabular}{|l|}
52.299 \\
\end{tabular} & 11.14 & 30.854 & -1.224 & & 4.3699 & & 14.643 & & 333 & $55.5 ?$ & 50.8 & 11.6 & & 2.12 & (1) & $0.00 \%$ & 0.000 & $0.1 / 27$ & \\
\hline 2660 & $\frac{4 / 21 / 212004}{4 / 21 / 2004}$ & $\begin{array}{l}5: 17: 02 \mathrm{PM} \\
5: 17: 23 \mathrm{PM}\end{array}$ & $\frac{25.011}{25.006}$ & $\frac{25.516}{25.521}$ & $\frac{23.758}{23.763}$ & $\begin{array}{l}26.299 \\
26.294\end{array}$ & $\frac{25.736}{25.711}$ & $\left|\frac{48.554}{48.349}\right|$ & \begin{tabular}{|l|}
52.704 \\
52.583 \\
\end{tabular} & $\frac{17.825}{17.721}$ & \begin{tabular}{|c|}
31.156 \\
30.955
\end{tabular} & $\frac{-1.221}{-1.224}$ & -7.371 & $\frac{50.945}{44.052}$ & $\overline{0.041}$ & 14.643 & & & \begin{tabular}{|l|l|}
55.5867 \\
55.5925
\end{tabular} & 51.1 & \begin{tabular}{|c|}
11.8 \\
\end{tabular} & & \begin{tabular}{|l|l|}
2.734 \\
\end{tabular} & 0.006 & $\mid$ & \begin{tabular}{|}
$\mid 0.000$ \\
0.000
\end{tabular} & $\mid \frac{0.163}{0.160}$ & $\overline{0.14}$ \\
\hline
\end{tabular}


WSRC-TR-2005-00105, REVISION 0

SRNL-RPP-2005-00012, REVISION 0

RUN \# 2.03A AND B; FIRST AND SECOND HALF OF SLURRY DEWATERING - CONT.

\begin{tabular}{|c|c|c|c|c|c|c|c|c|c|c|c|c|c|c|c|c|c|c|c|c|c|c|c|c|c|c|c|c|}
\hline & A & \begin{tabular}{c|c|}
$\mathrm{B}$ \\
\end{tabular} & $D$ & $E$ & $F$ & G & $\mathrm{H}$ & $\mathrm{J}$ & $\mathrm{K}$ & \begin{tabular}{ll|l}
$\mathrm{L}$ \\
\end{tabular} & $\mathrm{M}$ & $\mathrm{N}$ & $\mathrm{O}$ & $Q$ & $\mathrm{R}$ & $\mathrm{s}$ & $\mathrm{T}$ & $\mathrm{v}$ & w & $x$ & $Y$ & $z$ & AA & $A B$ & $A C$ & $\mathrm{AD}$ & $\mathrm{AE}$ & AF $\mathbb{A A}$ \\
\hline & $4 / 21 / 2004$ & \begin{tabular}{ll|}
$5: 18: 23 \mathrm{PM}$ \\
$5: 12: 23$
\end{tabular} & $\frac{25.011}{25011}$ & $\begin{array}{l}25.525 \\
550\end{array}$ & 23.762 & \begin{tabular}{|l|l|}
26.274 \\
26301
\end{tabular} & \begin{tabular}{|l|}
25.67 \\
2581
\end{tabular} & 48.256 & $\begin{array}{r}52.44 \\
5.905\end{array}$ & 17.731 & 30.897 & -1.221 & \begin{tabular}{|l|l|}
-7.362 \\
-7350
\end{tabular} & 48.688 & 0.043 & $\begin{array}{l}14.643 \\
14.6\end{array}$ & 0.003 & 3336.6 & & 51. & & & 2.729 & 0.006 & 0.007 & 0.000 & 0.168 & \\
\hline & & 5:19:23 PM & 25.011 & & 23.757 & $\begin{array}{l}26.304 \\
\end{array}$ & $\begin{array}{r}25.81 \\
\end{array}$ & \begin{tabular}{|l|l|}
48.641 \\
\end{tabular} & \begin{tabular}{|l|l|}
52.805 \\
\end{tabular} & 17.868 & 31.244 & \begin{tabular}{|l|}
-1.218 \\
\end{tabular} & $\begin{array}{r}-7.359 \\
\end{array}$ & 48.671 & 0.042 & 144.643 & 0.003 & & & 51.3 & & & \begin{tabular}{|l|l|}
2.754 \\
\end{tabular} & 0.006 & \begin{tabular}{|l|}
0.006 \\
\end{tabular} & 0.000 & 0.162 & \\
\hline$\frac{2064}{2664}$ & $\frac{4 / 21 / 2004}{4 / 212004}$ & $\begin{array}{l}-122: 23 \mathrm{PM} \\
5: 2123 \mathrm{PM}\end{array}$ & 24.999 & $\begin{array}{l}25.514 \\
25.52 \\
\end{array}$ & $\begin{array}{r}23.74 \\
23.746 \\
\end{array}$ & \begin{tabular}{|l|}
26.292 \\
26.308 \\
\end{tabular} & \begin{tabular}{|l|}
25.779 \\
25.744 \\
\end{tabular} & \begin{tabular}{|c|c|}
48.534 \\
48.55 \\
\end{tabular} & \begin{tabular}{|r|}
52.78 \\
52.728
\end{tabular} & \begin{tabular}{|c|}
17.777 \\
17887
\end{tabular} & $\begin{array}{l}31.166 \\
31.107\end{array}$ & \begin{tabular}{|l|}
-1.224 \\
-1.224 \\
\end{tabular} & $\begin{array}{l}-7.356 \\
-7.362\end{array}$ & $\begin{array}{l}48.456 \\
49366\end{array}$ & $\begin{array}{l}0.042 \\
0.042\end{array}$ & $\begin{array}{l}14.643 \\
14643\end{array}$ & \begin{tabular}{|l|l|}
0.003 \\
0.003
\end{tabular} & 3338.6 & 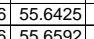 & \begin{tabular}{|l|l|}
51.3 \\
513
\end{tabular} & $\begin{array}{l}11.9 \\
121\end{array}$ & \begin{tabular}{|l|}
39.9 \\
39.8 \\
\end{tabular} & \begin{tabular}{|l|}
2.748 \\
2746
\end{tabular} & \begin{tabular}{|c|}
0.006 \\
0.060 \\
\end{tabular} & \begin{tabular}{|l|}
0.006 \\
0.006 \\
\end{tabular} & $\begin{array}{l}0.000 \\
0.000\end{array}$ & $\begin{array}{l}0.163 \\
0.163\end{array}$ & $\begin{array}{l}0.14 \\
014\end{array}$ \\
\hline 2665 & & 5:222:23 PM & 25.016 & & & & & & \begin{tabular}{|l|}
52.919 \\
\end{tabular} & & & & -7.397 & $\begin{array}{l}\frac{49.300}{47.916} \\
\end{array}$ & & 14.643 & $\begin{array}{l}.0003 \\
0.003\end{array}$ & & & \begin{tabular}{|l|}
51.3 \\
51.4
\end{tabular} & & & & \begin{tabular}{|l|}
0.0006 \\
0.006
\end{tabular} & 0.006 & & $\begin{array}{l}0.163 \\
0.162\end{array}$ & $\frac{0.14}{0.14}$ \\
\hline & & & 25.001 & & 23.747 & & & \begin{tabular}{|l|l|l|l|l}
48.484 \\
\end{tabular} & \begin{tabular}{|l|l|}
52.714 \\
\end{tabular} & 17.872 & 31.031 & \begin{tabular}{|l|}
-1.221 \\
\end{tabular} & -7.388 & 47.447 & 0.043 & 14.643 & 0.003 & & & & & & & & \begin{tabular}{|l|}
0.007 \\
\end{tabular} & & & $\frac{0.14}{0.14}$ \\
\hline & $4 / 21 / 2004$ & 5:24:23 PM & 24.996 & & & \begin{tabular}{|l|l|l|}
26.239 \\
\end{tabular} & & \begin{tabular}{|l|l}
48.554 \\
\end{tabular} & & 17.705 & 31.123 & -1.218 & -7.38 & $\begin{array}{l}48.079 \\
\end{array}$ & 0.043 & 144.643 & & & & & & & 2.747 & & \begin{tabular}{|l|l|}
0.007 \\
\end{tabular} & & & \\
\hline 2668 & $4 / 21 / 2004$ & 5:25:23 PM & 24.996 & 25.516 & 23.738 & \begin{tabular}{|l|l|}
26.214 \\
\end{tabular} & 25.761 & 48.546 & 52.82 & \begin{tabular}{l|l}
17.802 \\
\end{tabular} & & -1.224 & -7.385 & & 0.043 & & & & & & & & & & & & & \\
\hline 2669 & & & & & & & & & & & & & & & 0.042 & & & 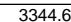 & & & & & & & & & & \\
\hline 2670 & $4 / 21 / 2004$ & 5:27:23 PM & 24.981 & 25.505 & 23.727 & 26.254 & 25.795 & 48.527 & 52.693 & 17.806 & 31.127 & -1.224 & -7.388 & 7.805 & 0.043 & 14.643 & & 3345.6 & 55.7592 & & & & 2.746 & 0.006 & 0.007 & & 0.167 & \\
\hline 2671 & $4 / 21 / 2004$ & 5:28:23 PM & 25.001 & 25.511 & 23.748 & 26.264 & 25.736 & \begin{tabular}{|l|l|l|l|l|}
48.324 \\
\end{tabular} & 52.453 & 17.852 & 30.903 & -1.218 & -7.379 & 48.241 & 0.044 & 14.643 & 0.003 & 3346.6 & 655.7758 & 51 & 11.8 & & & 0.007 & 0.007 & 0.000 & & \\
\hline 26 & $4 / 21 / 2004$ & 5:29:23 PM & 24.991 & 25.505 & 23.737 & 26.229 & 25.76 & $\begin{array}{l}48.469 \\
\end{array}$ & 52.627 & 17.687 & 31.158 & -1.221 & -7.38 .2 & 477.778 & 0.043 & 14.643 & & 3347.6 & 655.7925 & & & & & & 0.00 & 0.00 & 0.167 & 0.14 \\
\hline 2673 & $4 / 21 / 2004$ & 5:30:23 PM & & & & & 25.705 & 48.463 & 52.616 & 17.701 & 31.144 & & & 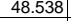 & & 14.643 & & & & & & & & & & & & \\
\hline$\frac{206 / 2}{2675}$ & $4 / 21212004$ & 5:312:23 PM & 24.999 & & 23.74 & 26.2377 & & 48.575 & 52.828 & $\begin{array}{l}17.736 \\
1.756\end{array}$ & 31.197 & -1.218 & & 9.382 & & & & & 1.8258 & & & & 2.750 & .006 & .006 & & 159 & \\
\hline$\frac{2017}{2676}$ & 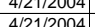 & $\begin{array}{l}5: 23: 23 \mathrm{PM} \\
5 \cdot 33.23 \mathrm{P}\end{array}$ & 24.989 & $\begin{array}{r}25.504 \\
25.499\end{array}$ & $\begin{array}{r}23.14 \\
23.735\end{array}$ & $\frac{26.222}{26.237}$ & $\frac{25.199}{25732}$ & $\begin{array}{l}48.469 \\
48.569\end{array}$ & 5 & $\begin{array}{l}17.591 \\
17.678 \\
\end{array}$ & $\begin{array}{r}31.275 \\
31.31\end{array}$ & $\frac{-1.21}{-1.21}$ & & 48584 & $\begin{array}{l}0.04 \\
0.043\end{array}$ & $\frac{14.643}{14.643}$ & & & 5 & & 11 & 390 & 2.149 & 0.06 & o. 0007 & 0.000 & & \\
\hline 2677 & $4 / 21 / 2004$ & 5:34:23 PM & 24.978 & 25.493 & 23.73 & 26.196 & 25718 & 48.571 & \begin{tabular}{|l|l|}
52759 \\
\end{tabular} & 17.646 & 31.314 & $\frac{-1.221}{-1.221}$ & -7.374 & 48.596 & 0.044 & $\frac{14.643}{14.643}$ & 0.003 & 33526 & $\begin{array}{l}053.5392 \\
\end{array}$ & 51 & $\frac{11 .}{11 .}$ & 35.90 & 2..154 & 0 & 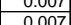 & $\frac{0.000}{0.000}$ & $\frac{160}{170}$ & \\
\hline & $\mid 1 / 2004$ & 5:35:23 PM & 24.973 & 25.497 & 23.739 & 26.211 & 25.692 & & 52.759 & 17.888 & 31.076 & -1.221 & & $\begin{array}{ll}477.678 \\
\end{array}$ & 0.042 & 14.643 & & $\frac{2353.6}{335.6}$ & $6 \quad 55.8925$ & & & & & & 0.006 & & & \\
\hline 267 & $4 / 21 / 2004$ & 5:36:23 PM & 24.978 & 25.492 & 23.744 & 26.171 & 25.697 & 48.749 & & 17.786 & 31.316 & -1.221 & -7.408 & 1.504 & & 14.643 & & 3354.6 & & & & & & & 0.007 & & & \\
\hline & $4 / 21 / 2004$ & 5:37:23 PM & 24.962 & & 23.713 & & & & & & & -1.224 & & & & 14.643 & & & & & & & & & 0.007 & 0.000 & 168 & \\
\hline 268 & $4 / 21 / 2004$ & $5: 38: 23 \mathrm{PM}$ & 24.972 & 25.482 & 23.733 & 26.165 & 25.572 & $\begin{array}{l}48.548 \\
\end{array}$ & 52.712 & 17.787 & 31.178 & -1.221 & & 48.56: & 0.044 & 14.643 & 0.003 & 3356.6 & 55.9425 & & 11.9 & 39.9 & 2.748 & 0.007 & 0.007 & 0.000 & 0.171 & 0.15 \\
\hline & $4 / 21 / 2004$ & 5:39:23 PM & $\frac{24.955}{2010}$ & 25.465 & 23.722 & 26.173 & 25.52 & & 52.766 & 17.814 & 31.129 & -1.221 & $\begin{array}{r}-7.403 \\
\end{array}$ & 47.301 & 0.043 & $\frac{14.643}{1.6210}$ & 0.003 & 3357.6 & $\begin{array}{c}655.9592 \\
\end{array}$ & $\frac{51}{51}$ & & 39.8 & 2.746 & 0.006 & 0.007 & 0.000 & & 0.14 \\
\hline$\frac{200}{268}$ & $\begin{array}{r}4 / 21 / 21204 \\
4 / 21 / 2004\end{array}$ & $\begin{array}{l}5: 4: 23 \mathrm{PM} \\
5: 41: 23 \mathrm{PM}\end{array}$ & $\frac{24.90}{24.955}$ & $\frac{25.465}{25.465}$ & $\frac{23 . / 72}{23.826}$ & $\frac{26.138}{26.113}$ & $\begin{array}{r}25.56 \\
25.605\end{array}$ & $\begin{array}{l}48.548 \\
48.256\end{array}$ & $\begin{array}{l}52.764 \\
52.529\end{array}$ & $\frac{1 . / 49}{17.912}$ & $\begin{array}{l}31.045 \\
30.727 \\
\end{array}$ & $\begin{array}{l}-\frac{1.221}{-1.224} \\
\end{array}$ & $\begin{array}{l}-.3 .39 \\
-7.408\end{array}$ & $\frac{48.075}{49.138}$ & 0.045 & $\frac{14.643}{14.643}$ & & $\frac{3358.6}{3359.6}$ & $\begin{array}{l}65.958 \\
6 \\
65.9925\end{array}$ & 51. & $\frac{11}{12 .}$ & $\begin{array}{l}39.8 \\
395\end{array}$ & $\frac{2.444}{2.723}$ & $\frac{0.007}{0.007}$ & $\begin{array}{l}0.007 \\
0.007\end{array}$ & $\frac{0.000}{0.000}$ & $\frac{175}{176}$ & 0.15 \\
\hline 2685 & 4/21/2004 & 5:42:23 PM & 24.954 & 25.464 & 23.821 & 26.132 & 25.659 & 48.218 & 52.457 & 17.765 & 30.879 & -1.221 & -7.397 & 49.094 & 0.043 & 14.643 & & 3360. & & & & & & & 0.007 & & & \\
\hline 2686 & 4/21/2004 & 5:43:23 PM & 24.959 & 25.464 & 23.821 & 26.147 & 25.709 & 48.235 & 52.475 & 17.679 & 30.963 & -1.221 & -7.4 & 48.154 & 0.041 & $\frac{14.040}{14.643}$ & & 336 & & & & & & 0.006 & 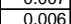 & 0.000 & 柴0 & \\
\hline 268 & & 5:44:23 PM & & & & & & \begin{tabular}{|l|l|l}
48.48 \\
\end{tabular} & & 17.847 & & & & & & & & & & & & & & & & & & \\
\hline 268 & $4 / 21 / 2004$ & 5:45:23 PM & 24.953 & 25.448 & 23.795 & 26.141 & 25.718 & 48.334 & 52.648 & 17.783 & 30.897 & -1.221 & & $\frac{18.696}{48.6}$ & 0127 & 14.643 & & 3363 & & & & & & .006 & & & & \\
\hline 268 & $4 / 21 / 2004$ & $5: 46: 23 \mathrm{Pl}$ & 24.947 & 25.432 & 23.773 & 26.125 & & $\begin{array}{l}48.399 \\
\end{array}$ & 52.6 & 17.808 & & -1.221 & & 47.9 & .042 & .643 & & & 5.0758 & 51.1 & 11.8 & & & & & & 0.163 & \\
\hline & $4 / 21 / 2004$ & 5:47:23 PM & 24.951 & 25.436 & 23.767 & 26.124 & 25.64 & & 52.449 & 17.708 & 31.051 & -1.224 & -7.4 & 50.194 & 0.042 & 14.643 & & 3365.6 & 656.0925 & 51. & 12.3 & & 736 & 006 & 0.006 & .000 & & \\
\hline & $4 / 21 / 2004$ & 5:48:23 PM & & 25.436 & 23.762 & 26.114 & 25.6 & 48.2 & 22.467 & 17.703 & 30.93 & & & t8.7 & 0.042 & 14.643 & & & & & & & & & & & & \\
\hline & $4 / 21 / 12004$ & 5:49:23 PM & & 25.445 & & 26.143 & 25 & & . 7333 & 17.74 & & & & 03 & 0.041 & 14.643 & & 336 & & & & & & 0.006 & 0.006 & & & \\
\hline & 4121212004 & $\begin{array}{l}5.50 .23 \\
5.5 \mathrm{PM}\end{array}$ & $\frac{24.95}{24943}$ & $\frac{25.434}{25418}$ & $\frac{23.161}{23.749}$ & $\frac{26.123}{2.121}$ & $\frac{25.644}{25499}$ & $\frac{48.417}{48.281}$ & $\frac{52.12}{2565}$ & $\begin{aligned} 17.929 \\
17.8\end{aligned}$ & $\begin{array}{l}30.799 \\
30.754\end{array}$ & $\begin{array}{l}-1.218 \\
1.271\end{array}$ & & $\frac{1.424}{18.46}$ & & $\frac{14.643}{1063}$ & & 3269 & & & & 39.5 & 2725 & 0006 & 0006 & & & \\
\hline 2695 & 4 & 5.55.23 PM & 24948 & 25.433 & 23.749 & 26.101 & & $\begin{aligned} 48.281 \\
48.53\end{aligned}$ & 871 & 17869 & & $\frac{-1.1241}{-1221}$ & & & 0.041 & 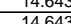 & & 337 & | & S1.1. & 薄1. & & & & & & & \\
\hline & $4 / 21 / 2004$ & 5:55:23 PM & 24.937 & $\frac{25.416}{25.416}$ & $\frac{23.728}{23.728}$ & 26.1 & $\frac{25.6}{25.6}$ & 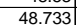 & 52.996 & 17.791 & $\frac{31.24}{31.24}$ & $\frac{-1.224}{-1.224}$ & & 1858 & $\frac{0.042}{0.042}$ & $\frac{14.643}{14.64}$ & & 3371. & 56.1925 & & $\frac{21 .}{11 .}$ & & 2757 & & $\frac{.006}{0.006}$ & & & \\
\hline $2699^{-1}$ & & $3 \mathrm{PM}$ & & & 23.733 & & & & 52.641 & & & & & & & & & & & & & & & & & & & \\
\hline & $4 / 21 / 2004$ & $3 \mathrm{PM}$ & 24.936 & 25.416 & 23.727 & 26.059 & & 48.3 & 52.527 & 17.839 & 30. & -1.221 & & 48.765 & 0.042 & 14.643 & & 3373.6 & 258 & 51 & 12. & 39.6 & 2.728 & 0.006 & 0.006 & 0.000 & 0.164 & \\
\hline & $4 / 21 / 2004$ & 5:56:23 PM & 24.925 & 25.405 & 23.721 & \begin{tabular}{|l|l|}
26.048 \\
\end{tabular} & 25.5 & \begin{tabular}{|l|l|l|l|l|}
48.77 \\
\end{tabular} & 20.716 & 17.686 & 31.024 & -1.221 & & 49.16 & 0.0 & 14.643 & & 337 & & & & & & & & & & \\
\hline & $4 / 21 / 2004$ & 5:57:23 PM & 24.93 & & 23.737 & & & & & 17.869 & & & & 48.469 & & & & & 5625 & & 119 & & 272 & 0.006 & & & & \\
\hline & 4/21/12004 & 5:58:23 PM & 24.929 & 25.394 & 23.726 & & & & .417 & & & -1.224 & & & & & & & & & 11.8 & & & & & & & \\
\hline & $4 / 21 / 2004$ & 5:59:23 PM & 24.914 & 25.394 & 23.715 & 26.072 & 25.48 & \begin{tabular}{l|l}
48.722 \\
\end{tabular} & 3.016 & 17.895 & 31.078 & -1.218 & & 48.54 & & 14.643 & & 337 & 56.2925 & 51. & 11.9 & 39.9 & & & 0006 & 000 & 163 & \\
\hline 210 & $4 / 21 / 2004$ & 6:00:23 PM & 24.918 & 25.393 & 23.714 & 26.081 & & $\begin{array}{l}48.451 \\
\end{array}$ & 52.706 & 17.794 & & -1.221 & & 46.8. & & 14. & & 3378 & & & 11. & & & & 0.000 & 0.000 & 163 & \\
\hline & .0.04 & $33 \mathrm{PM}$ & 24.912 & $\frac{25.387}{22.78}$ & 23.709 & 26.035 & & 48.3 & 52.793 & $17.8 \mathrm{~g}$ & & -1.224 & & & & & & 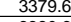 & & & 12. & & & & & & & \\
\hline & & S & 24.901 & $\frac{25.3}{253}$ & $\frac{2.3 .23}{2.23}$ & 26.015 & & & & & & -1.221 & & & & & & & & & (E) & & & & & & & \\
\hline & 4 & 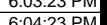 & $2 \frac{2.801}{240}$ & $\frac{25.306}{2538}$ & $\begin{array}{l}23.147 \\
23756\end{array}$ & 25.974 & & & & 17.1969 & & $\frac{-1.24}{-1221}$ & & & & & & 年 & & 51. & 115 & & & & & & 0 & \\
\hline 2708 & 4 & $6.045 .23 \mathrm{PM}$ & 24.89 & $\begin{array}{l}25.00 \\
25.36\end{array}$ & 23736 & 25.980 & & & .102 & 17668 & & -1221 & & & & & & 300 & 56 & 政 & 11.0 & & & \begin{tabular}{|l|l|l} 
\\
\end{tabular} & & & & \\
\hline 2709 & 4 & 6:06.23 PM & 24.894 & 25.355 & 23.746 & 25.997 & 25. & & $\begin{array}{l}52.446 \\
52.446\end{array}$ & 17.679 & 30.887 & -1.221 & & 40.06 & 0.04 & & & 338 & & J1 & & & & & & & 0.100 & \\
\hline & $\frac{4 / 21 / 2004}{4}$ & 6:07:23 PM & $\frac{24.894}{24.96}$ & 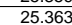 & 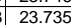 & 25.977 & & & & & & & & & & & & & & & & & & & & & & \\
\hline & & & 24.888 & 25.35 & 23714 & 25.946 & & & 2762 & 17866 & & -1.224 & & & & & & & & & & & & & & & & \\
\hline & $4 / 21 / 2004$ & 6:09:23 PM & 24.87 & 25. & 23.703 & 25.96 & 25 & \begin{tabular}{|l|l|l|}
48.167 \\
\end{tabular} & \begin{tabular}{|l|l|}
52.513 \\
\end{tabular} & 17.728 & & -1.221 & & $\frac{48.0}{48.0}$ & & & & 3387.6 & & 51. & 11.8 & 39.5 & 2.724 & 0 & 0. & & & \\
\hline & $4 / 21 / 2004$ & 6:10:23 PM & 24.8 & 25.3 & 23.708 & 25.944 & & & 882 & 17.733 & & & & 48.8 & & & & 338 & & 51. & 12.6 & & 749 & & & & & \\
\hline 271 & $4 / 21 / 2004$ & $23 \mathrm{PM}$ & 24.87 & 25.3 & 23.701 & 25. & & & & 17.53 & & \begin{tabular}{|c|c|}
-1.218 \\
\end{tabular} & & & & & & 33 & 56.4 & 51 & 12. & & & & & & 60 & \\
\hline 27. & $4 / 21 / 200$ & & 24.885 & 25.345 & 23.721 & & & & & & & -1.218 & & & & & & $33 \mathrm{~s}$ & & 51 & 11.9 & & & & & & & \\
\hline 227. & $4 / 21 / 2$ & & 24.869 & 25.318 & 23.68 & & & & & & & & & & & & & $33 \mathrm{~s}^{-1}$ & & 51. & $12.0 \mathrm{C}$ & & & & & & & \\
\hline & $4 / 21212004$ & $6: 14$ & $\frac{24.858}{2089}$ & $\frac{25.318}{2525}$ & $\frac{23.679}{23.77}$ & 25.866 & & 48.2 & . & 17.768 & & -1.221 & & 41. & & & & 年 & 5655 & 51 & 11.1 & & & & & & 108 & \\
\hline & $\frac{442112004}{4121004}$ & & $\frac{24.8}{24}$ & $\frac{2.303}{253}$ & & & & & & & & $\frac{-1.218}{-1224}$ & & $\frac{4.5}{48.5}$ & & & & & & & & & & & & & & \\
\hline 272 & $4 / 21 / 21 / 2004$ & & $\frac{24}{24}$ & 25.35 & 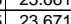 & 25.918 & & & & 1782 & & -1.221 & & & & & & & & 51 & & & & & & & 168 & \\
\hline 272 & $4 / 21 / 2004$ & $\frac{6.6}{6618.23 \mathrm{PM}}$ & $\frac{14.849}{24.89}$ & $\frac{2.5094}{25294}$ & 23.67 & $\frac{25.942}{25.942}$ & $\frac{25.4}{25.4}$ & 4 & \begin{tabular}{|l|l|}
52861 \\
\end{tabular} & 17.72) & 31. & -1.215 & & 48.5 & 0.044 & $\frac{1.6}{146}$ & & 339 & & & 111 & & & 0.007 & 0.0 & & & \\
\hline & & & 24839 & & 23655 & & & 48.384 & 5267 & 17.693 & & -1.221 & & & & & & & & & & & & & & & & \\
\hline & & $6: 20: 23 \mathrm{PM}$ & 24.83 & 25.28 & 23.66 & 26.057 & & 48.208 & & 17.657 & 30.893 & -1.221 & & & & & & & & 51.0 & & & & & & & & \\
\hline & & 3 PM & 24.844 & $25.28 \subseteq$ & & 26.132 & & & & & & & & & & & & & & & & & & & & & & \\
\hline 272 & $4 / 21 / 200$ & 6.22 & 24.861 & 25.32 & 23692 & 26.234 & & 48.436 & 849 & 17.774 & 30.944 & -1.218 & & 48.4 & 0.0 & $\overline{14.643}$ & & 340 & & 514 & 119 & & & & & & 60 & \\
\hline 272 & $4 / 21 / 200$ & 6:23: & 24. & 25. & 23. & & 25.9 & 48.5 & & & & -1. & & & & & & 34 & & & & & & & & & & \\
\hline 2727 & 4/21/2004 & 6:24:23 P & 24.855 & 25.31 & 23.666 & 26.348 & 25.9 & 47.98 & 52.266 & 17.676 & 30.663 & -1.227 & & 48.4 & 0.0 & 14.643 & & 3402.6 & \begin{tabular}{l|l|l} 
& 56.7092
\end{tabular} & 50.8 & 11.9 & 3 & 2.71 & \begin{tabular}{|l|l|} 
\\
\end{tabular} .0066 & 0.007 & 0.0 & 65 & \\
\hline$\frac{2728}{2720}$ & $4 / 21 / 2004$ & 6:25:23 PM & 24.852 & $\begin{array}{r}25.322 \\
25.310\end{array}$ & $\frac{23.673}{23.675}$ & $\frac{26.365}{26.329}$ & 25.827 & $\begin{array}{r}48.415 \\
18201\end{array}$ & \begin{tabular}{|l|}
52.844 \\
5259
\end{tabular} & 17.795 & $\begin{array}{l}31.025 \\
31.059\end{array}$ & -1.218 & & $\begin{array}{l}48.008 \\
48.129\end{array}$ & 0.042 & $\frac{14.643}{14643}$ & 0.0 & $\begin{array}{l}3403.6 \\
32046\end{array}$ & 656.7258 & \begin{tabular}{|l|}
51.4 \\
51.
\end{tabular} & \begin{tabular}{|l|l|}
11.8 \\
11.8
\end{tabular} & 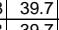 & 2.739 & 0.006 & \begin{tabular}{|l|}
0.007 \\
\end{tabular} & 0.000 & 0.164 & 0.14 \\
\hline & & & & & & & & & \begin{tabular}{|c|c|c|}
52.672 \\
\end{tabular} & $\frac{11.073}{1783}$ & & & & & & $\frac{14.643}{14643}$ & & & & & & & & & & & $\frac{.1 .94}{0.164}$ & \\
\hline
\end{tabular}


WSRC-TR-2005-00105, REVISION 0

SRNL-RPP-2005-00012, REVISION 0

RUN \# 2.03A AND B; FIRST AND SECOND HALF OF SLURRY DEWATERING - CONT.

\begin{tabular}{|c|c|c|c|c|c|c|c|c|c|c|c|c|c|c|c|c|c|c|c|c|c|c|c|c|c|c|c|c|}
\hline & A & B & D & $E$ & $\mathrm{~F}$ & $\mathrm{G}$ & $\mathrm{H}$ & $\mathrm{J}$ & $\mathrm{K}$ & $\mathrm{L}$ & M & $\mathrm{N}$ & 0 & $Q$ & $R$ & $\mathrm{~s}$ & $\mathrm{~T}$ & & w & $x$ & & $z$ & $\mathrm{AA}$ & $\mathrm{AB}$ & $\mathrm{AC}$ & $A D$ & & \\
\hline \begin{tabular}{|l}
$\frac{2731}{2732}$ \\
27
\end{tabular} & $\begin{array}{r}4 / 21 / 2004 \\
4 / 212004\end{array}$ & $\begin{array}{ll}6: 28: 24 \mathrm{PM} \\
6 \cdot 22 \cdot 24 \mathrm{PM}\end{array}$ & $\begin{array}{r}24.862 \\
24.867\end{array}$ & $\frac{25.336}{25.347}$ & $\begin{array}{l}23.678 \\
23.693 \\
\end{array}$ & $\begin{array}{l}26.28 \\
26.26\end{array}$ & \begin{tabular}{|l|}
25.771 \\
25.717 \\
\end{tabular} & $\begin{array}{l}48.409 \\
48.646\end{array}$ & \begin{tabular}{|l|}
52.851 \\
53.081 \\
\end{tabular} & \begin{tabular}{|l|}
17.604 \\
17.792
\end{tabular} & $\begin{array}{l}31.088 \\
31.16\end{array}$ & $\begin{array}{l}-1.221 \\
-1.221\end{array}$ & $\begin{array}{l}-7.272 \\
-7.281 \\
\end{array}$ & $\begin{array}{l}47.645 \\
48.208 \\
\end{array}$ & $\frac{0.043}{0.04}$ & $\begin{array}{l}14.643 \\
14.643\end{array}$ & $\begin{array}{l}0.003 \\
0.03\end{array}$ & $\begin{array}{l}3406.6 \\
34076\end{array}$ & \begin{tabular}{|l|}
56.7761 \\
56928
\end{tabular} & $\frac{51 .}{51 .}$ & & $\begin{array}{l}39.7 \\
39.9\end{array}$ & $\frac{2.741}{2.751}$ & $\begin{array}{l}0.006 \\
0.066\end{array}$ & \begin{tabular}{|l|}
0.007 \\
0.006 \\
\end{tabular} & 0.000 & $\begin{array}{l}0.167 \\
0.155\end{array}$ & \begin{tabular}{|l|}
0.14 \\
0.13
\end{tabular} \\
\hline & & & & & & & & & & & & & & & & $\frac{14.043}{14.643}$ & & & & & & & & & & & & \\
\hline 2734 & $4 / 21 / 2004$ & 6:31:24 PM & 24.857 & 25.341 & 23.668 & 26.16 & 25.676 & 48.529 & \begin{tabular}{|l|}
52.938 \\
\end{tabular} & 17.802 & 30.977 & -1.224 & $\begin{array}{r}-7.203 \\
-1.203\end{array}$ & 47.593 & 0.042 & 14.643 & 0.003 & 3409.6 & \begin{tabular}{|l|l|}
56.8261 \\
\end{tabular} & 51. & & \begin{tabular}{|l|}
39.8 \\
\end{tabular} & 2.741 & \begin{tabular}{|l|l|}
0.006 \\
\end{tabular} & \begin{tabular}{|l|}
0.007 \\
\end{tabular} & 0.000 & 0.164 & \\
\hline 2735 & $4 / 221 / 2004$ & 6:32:24 PM & 24.856 & 25.331 & 23.732 & 26.109 & 25.566 & 48.509 & \begin{tabular}{|r|}
52.84 \\
\end{tabular} & 17.774 & 31.111 & -1.224 & & 47.63 & 0.042 & 14.643 & & & 56.8428 & 51. & & & & & \begin{tabular}{|l|}
0.006 \\
\end{tabular} & & & \\
\hline & & & & & & & & & & & & & & & & & & & & & & & & & & & & \\
\hline \begin{tabular}{|l|l|}
2737 \\
2730
\end{tabular} & $4 / 21 / 2004$ & $6: 34: 24 \mathrm{PM}$ & $\begin{array}{r}24.86 \\
24.965\end{array}$ & 25.34 & \begin{tabular}{|l|l|}
23.746 \\
3276
\end{tabular} & $\begin{array}{ll}26.068 \\
20059\end{array}$ & $\begin{array}{l}25.55 \\
25.5\end{array}$ & \begin{tabular}{|l|l|}
48.258 \\
\end{tabular} & \begin{tabular}{|l|l|}
52.805 \\
5.821
\end{tabular} & \begin{tabular}{l|}
17.729 \\
1797
\end{tabular} & $\begin{array}{l}30.846 \\
32.92 \\
\end{array}$ & $\begin{array}{l}-1.221 \\
.229\end{array}$ & $\begin{array}{r}-7.235 \\
.7221\end{array}$ & 48.402 & 0.043 & 14.643 & $\begin{array}{l}0.003 \\
\end{array}$ & 3412.6 & \begin{tabular}{|l|}
56.8761 \\
\end{tabular} & & & \begin{tabular}{|l|l|}
39.6 \\
\end{tabular} & \begin{tabular}{|l|}
2.727 \\
\end{tabular} & $\begin{array}{l}0.006 \\
\end{array}$ & $\begin{array}{l}0.007 \\
\end{array}$ & 0.000 & 0.168 & \\
\hline $\begin{array}{l}2738 \\
2739 \\
\end{array}$ & $\begin{array}{l}4 / 21 / 2004 \\
4 / 212004\end{array}$ & $\begin{array}{l}6: 35: 24 \mathrm{PM} \\
6 \cdot 36 \cdot 24 \mathrm{PM}\end{array}$ & $\begin{array}{l}24.865 \\
24.869\end{array}$ & $\begin{array}{l}25.345 \\
25354\end{array}$ & \begin{tabular}{|l|l|}
23.746 \\
123.756
\end{tabular} & $\begin{array}{l}26.058 \\
26.047\end{array}$ & \begin{tabular}{r|}
25.45 \\
25444
\end{tabular} & \begin{tabular}{|l|l|}
48.357 \\
48.353 \\
\end{tabular} & \begin{tabular}{|l|}
52.824 \\
52.735 \\
\end{tabular} & \begin{tabular}{|l|l|}
17.867 \\
17773 \\
\end{tabular} & $\begin{array}{r}30.83 \\
30.983 \\
\end{array}$ & $\begin{array}{l}-1.218 \\
-1218\end{array}$ & $\begin{array}{l}-7.244 \\
-7.241 \\
\end{array}$ & $\begin{array}{l}49.531 \\
48.045\end{array}$ & $\begin{array}{l}0.042 \\
0.043\end{array}$ & $\begin{array}{l}144.643 \\
14643\end{array}$ & \begin{tabular}{|l|}
0.003 \\
0.003
\end{tabular} & \begin{tabular}{l|l}
3413.6 \\
34146
\end{tabular} & \begin{tabular}{|l|}
56.8928 \\
569094
\end{tabular} & $\frac{51 .}{51 .}$ & $\frac{12.1}{11.8}$ & $\mid \begin{array}{l}39.6 \\
397\end{array}$ & $\begin{array}{l}2.730 \\
2.734\end{array}$ & \begin{tabular}{|l|}
0.006 \\
0.006
\end{tabular} & $\begin{array}{l}0.006 \\
0.007\end{array}$ & $\begin{array}{l}0.000 \\
0.000\end{array}$ & \begin{tabular}{|l|l|}
0.164 \\
0.167
\end{tabular} & $\begin{array}{l}0.14 \\
0.14\end{array}$ \\
\hline 2740 & $4 / 21 / 2004$ & $6.00 .24 \mathrm{PM}$ & 24.858 & 25.333 & \begin{tabular}{|l|l} 
& 23.719 \\
\end{tabular} & & \begin{tabular}{|l|}
2.4544 \\
25.483
\end{tabular} & 48.581 & \begin{tabular}{|l|}
52.983 \\
\end{tabular} & & $\begin{array}{l}30.903 \\
31.176\end{array}$ & & $\begin{array}{l}-7.241 \\
-7.246\end{array}$ & $\begin{array}{l}40.045 \\
48.487\end{array}$ & 0.041 & $\begin{array}{l}14.643 \\
14.643 \\
\end{array}$ & 0.003 & $\begin{array}{l}34414.6 \\
3415.6\end{array}$ & & & & $\begin{array}{l}39.7 \\
39.9 \\
\end{array}$ & \begin{tabular}{|l|l|l} 
& 2.734 \\
\end{tabular} & \begin{tabular}{|l|}
0.0006 \\
0.006
\end{tabular} & 0.006 & 0.0000 & $\frac{0.161}{0.159}$ & $\begin{array}{l}0.14 \\
0.14\end{array}$ \\
\hline & $4 / 21 / 2004$ & 6:38:24 PM & 24.857 & & & 25.98 & 25.487 & 48.21 & 52.681 & 17.64 & 30.852 & -1.218 & & & 0.04 & 14.643 & & 3416.6 & \begin{tabular}{|l|l|}
56.9428 \\
\end{tabular} & & & & & $\begin{array}{l}0.006 \\
0.006\end{array}$ & 0.006 & 0.000 & & $\begin{array}{l}0.14 \\
0.13\end{array}$ \\
\hline \begin{tabular}{|l|l|}
2742 \\
\end{tabular} & $4 / 21 / 2004$ & 6:39:24 PM & 24.862 & 25.326 & 23.718 & \begin{tabular}{|l|l|}
25.954 \\
\end{tabular} & & 48.602 & 53.046 & 17.945 & 30.951 & -1.221 & -7.244 & 447.764 & 0.042 & 14.643 & 0.003 & 341 & \begin{tabular}{|l|l|}
56.9594 \\
\end{tabular} & & & & & & & 0.000 & & 0.13 \\
\hline 2743 & $4 / 21 / 2004$ & $6: 40: 24 \mathrm{PM}$ & 24.86 & & & 25.963 & 25.45 & 48.397 & & & & & & & & & & & & & & & & & & 0.000 & 164 & \\
\hline 2744 & $4 / 21 / 2004$ & 6:41:24 $\mathrm{PM}$ & & 25,311 & 23.695 & & 25.464 & & 52803 & 17.789 & & -1.215 & & & & & & & & & & & & & & & & \\
\hline \begin{tabular}{|l|l|}
2745 \\
\end{tabular} & $4 / 21 / 2004$ & 6:42:24 PM & 24.849 & & 23.7 & \begin{tabular}{|l|l|}
25.927 \\
\end{tabular} & 25.459 & 48.226 & 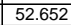 & & 30.905 & -1.18 & & 178 & 0.043 & 14.643 & & & 7.0094 & & & & & D. & 0.007 & & & \\
\hline 2746 & $4 / 21 / 2004$ & 6:43:24 PM & 24.852 & 25.307 & 23.693 & \begin{tabular}{|l|l|}
25.93 \\
\end{tabular} & $\begin{array}{l}25.397 \\
\end{array}$ & \begin{tabular}{|l|l|l|l|l}
48.393 \\
\end{tabular} & 52.824 & \begin{tabular}{|l|l|l|l|l|} 
\\
\end{tabular} & 30.971 & $\begin{array}{l}-1.183 \\
\end{array}$ & & $\begin{array}{l}48.054 \\
\end{array}$ & 0.042 & 14.643 & $\begin{array}{l}0.003 \\
\end{array}$ & 3421.6 & 57.0261 & 51. & 11.8 & 39.7 & 2.736 & 0.006 & 0.006 & 0.000 & 0.164 & \\
\hline 274 & $4 / 21 / 2004$ & 6:44:24 PM & 24.846 & 25.296 & 23.688 & 25.909 & 25.39 & 48.326 & 52.749 & 17.762 & 30.895 & -1.192 & & 48.7 & 0.042 & 14.643 & & & 57.0428 & & & & & & 0.006 & & & \\
\hline & $4 / 21 / 2004$ & 6:45:24 PM & & & & & & & & & & -1.163 & & & & & & & & & & & & & & & & \\
\hline \begin{tabular}{|l}
2744 \\
275
\end{tabular} & $4 / 21 / 2004$ & $6: 46: 24 \mathrm{PM}$ & 24.851 & 25.29 & 23.688 & 25.899 & 25.442 & 48.262 & 2.842 & 17.658 & 31.02 & -1.154 & & & 0.038 & 14.643 & & & & & & & & 006 & & 000 & & 0.13 \\
\hline & $\frac{421212004}{4121204}$ & $6.24 .24 \mathrm{FM}$ & $\frac{24.835}{24824}$ & 25. & $\frac{2.6061}{23675}$ & $\frac{25.808}{25846}$ & & $\begin{array}{l}48.314 \\
8.94\end{array}$ & 告, & & 30.951 & $\frac{-1.102}{-1096}$ & & 17947 & 0.044 & $\frac{1.4645}{1.643}$ & & & & & & & & & & & & \\
\hline & 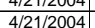 & $\begin{array}{l}0.48 .24 \mathrm{PM} \\
\text { 6:49:24 PM }\end{array}$ & $\begin{aligned} 24.854 \\
24.827\end{aligned}$ & $\frac{23.275}{25.267}$ & $\frac{23.615}{23.668}$ & $\begin{array}{r}\quad 3.640 \\
25.82\end{array}$ & $\frac{25.459}{25.412}$ & 48.1948 & $\frac{52.180}{52.818}$ & $\mid$\begin{tabular}{|c|}
17.688 \\
\end{tabular} & $\begin{array}{r}30.104 \\
31.09\end{array}$ & $\begin{array}{l}-1.096 \\
-1.099\end{array}$ & & \begin{tabular}{|l|}
$48.214 !$ \\
48.214
\end{tabular} & 0.042 & $\begin{array}{l}4.645 \\
14.643 \\
\end{array}$ & 0.0003 & 34276 & $\left\{\begin{array}{l}3.1794 \\
57261\end{array}\right.$ & & & 398 & & & & 0.000 & & 0.13 \\
\hline & $4 / 21 / 2004$ & 6:50:24 PM & 24.826 & 25.261 & \begin{tabular}{|l|l|}
23.672 \\
\end{tabular} & 25.809 & 25.392 & 48.467 & \begin{tabular}{|l|}
53.052 \\
\end{tabular} & 17.764 & 31.076 & -1.099 & & 48.254 & 0.041 & 14.643 & 0.003 & 3428.6 & \begin{tabular}{|l|l}
57.1428 \\
\end{tabular} & 51.6 & 11.8 & & 2.742 & & 0.006 & 0.000 & 0.160 & $\begin{array}{l}0.14 \\
0.14\end{array}$ \\
\hline 275 & $4 / 21 / 2004$ & 6:51:24 PM & & & 23.662 & 25.834 & 25.426 & 48.48 & & 17.903 & 30.959 & -1.096 & & & 0.042 & 14.643 & & & & & & & & & & & & \\
\hline & $4 / 21 / 2004$ & $24 \mathrm{PM}$ & & 25.2 & 23.667 & 25.909 & 25.536 & 48.38 & 2.853 & 17.871 & 30.864 & -1.099 & & .22 & 0.041 & 14.643 & & & & & & & & & & & & \\
\hline$\frac{2156}{275}$ & $\frac{421212004}{4121204}$ & $6.53 .24 \mathrm{FM}$ & & $\frac{25.255}{25256}$ & $\frac{23.671}{23.667}$ & 26.019 & 25.110 & $\frac{48.453}{48.067}$ & 2.969 & 11.108 & $\begin{array}{l}31.03 \\
30.81 \\
\end{array}$ & $\frac{-1.096}{-1.099}$ & & 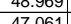 & 0.041 & $\frac{14.645}{14643}$ & & 3432 & 72094 & & & 394 & & & & & & \\
\hline \begin{tabular}{|l|l|}
2758 \\
\end{tabular} & $4 / 2 / 212004$ & 6:55:24 PM & 24.818 & 25.25 & 23.669 & 26186 & & $\begin{array}{l}40.001 \\
48.183\end{array}$ & 52733 & $\frac{17.024}{17.661}$ & $\frac{30.010}{30788}$ & - & & 4788 & 0.030 & $\frac{14.043}{14.643}$ & 003 & 343 & & 513 & & & & & & & & \\
\hline & $4 / 21 / 2004$ & 6:56:24 PM & $\frac{24.010}{24.814}$ & 25.259 & 23.68 & $\frac{20.100}{26.287}$ & 25.784 & $\frac{40.100}{48.33}$ & 52.871 & $\begin{array}{l}17.001 \\
17.787\end{array}$ & 30.817 & -1.096 & & 4 & 0.04 & $\frac{14.043}{14.643}$ & & $\begin{array}{l}3435.64 \\
34346\end{array}$ & \begin{tabular}{|l|l|}
57.2428 \\
\end{tabular} & & $\frac{11 .}{11 .}$ & 39.6 & 2728 & & 0.006 & & & \\
\hline & 4/21/2004 & 6:57:24 PM & 24.832 & & 23.698 & 26.35 & & & 793 & 17.748 & & -1.099 & & & 0.043 & 14.643 & & & & & & & & & & & & \\
\hline 2761 & 4/21/2004 & 6:58:24 PM & 24.829 & 25.269 & 23.726 & 26.327 & 25.8 & 48.266 & 52.869 & 17.75 & 30.823 & -1.102 & & 48.258 & 0.042 & 14.643 & & & 7..27.761 & & 11. & 39.5 & & .006 & 0.006 & 0.000 & $\frac{0.164}{0.164}$ & \\
\hline & $4 / 21 / 2004$ & $6: 59: 24 \mathrm{PM}$ & 24.821 & 25.271 & $\overline{23.743}$ & \begin{tabular}{|l|l|}
26.28 \\
\end{tabular} & & 48.337 & 52.998 & 17.772 & 30.858 & $\begin{array}{c}-1.073 \\
\end{array}$ & & & & 14.643 & & & & & & & & & & & & \\
\hline 年 & $4 / 21 / 2004$ & 7:00:24 PM & 24.848 & 25.288 & 23.764 & 26.256 & 25.7 & 48.34 & 2.834 & 17.682 & 31.031 & -1.099 & & 1700 & 0.04 & $\frac{14.643}{14.64}$ & & & .3094 & & & 30 & & & & & & \\
\hline 276 & $4 / 21 / 2004$ & 7:01:24 PM & 24.844 & 25.294 & 23.756 & \begin{tabular}{|c|c|}
26.223 \\
\end{tabular} & 25.7 & \begin{tabular}{|l|l|l|l|l|}
48.198 \\
\end{tabular} & 52.892 & 17.669 & 30.834 & -1.096 & & 49.278 & 0.041 & 14.643 & $\begin{array}{ll}0.003 \\
\end{array}$ & 343 & 57.3261 & 51. & 12.1 & & & & 0.006 & 0.000 & 0.160 & \\
\hline & $4 / 21 / 2004$ & 7:02:24 PM & 24.845 & 25.3 & 23.751 & & 25 & & 52.942 & 17.773 & 31.008 & -1.099 & & & 0.042 & 14.643 & & & & & & & & & & & & \\
\hline & 4/21/2004 & 7:03:24 PM & 24.841 & 25.295 & 23.742 & 26.144 & 25.72 & 48.38 & 2.902 & 17.791 & 30.97 & -1.088 & & & 0.04 & & & 34 & 57.3594 & & & 39 & & & & & & \\
\hline$\frac{276}{27 \pi}$ & $4 / 21 / 2004$ & $4 \mathrm{PM}$ & 24.851 & 25.305 & 23.737 & 26.109 & 25. & 48. & 2.855 & 17.635 & & -1.073 & & 8.0 & 0.041 & 14.643 & & & & & & & & 0.006 & 0.00 & 0.000 & & \\
\hline & & & & & $\frac{23.131}{23726}$ & & & & & $\mid \frac{1.144}{17098}$ & & -1.096 & & & & & & & & & & & & & & & & \\
\hline$\frac{\mid 77}{277}$ & $\frac{42121204}{4 \mid 21204}$ & $=0.024 \mathrm{PM}$ & 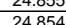 & $\frac{25.305}{25303}$ & $\frac{20.120}{2372}$ & $\frac{26.045}{26007}$ & $\frac{25}{256}$ & 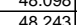 & 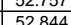 & 17.906 & $\begin{array}{l}30.440 \\
30.791\end{array}$ & $\frac{1.021}{-1027}$ & & 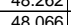 & 0.044 & $\frac{14.045}{14643}$ & & & & 51. & $\frac{11.6}{118}$ & & & & 0.0006 & & 156 & \\
\hline & 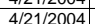 & $770.024 \mathrm{PM}$ & 24869 & 25.313 & $\frac{0.12}{23.73}$ & & 25.5 & & 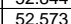 & 17.04 & & -1076 & & 40.000 & 0.039 & 14,643 & & & & - & 11.0 & & & & & & & \\
\hline & 4 & 7:00:24 PM & 24.867 & 25.306 & $\frac{23.728}{23.728}$ & 25.984 & & 48.442 & $\frac{25.952}{52.952}$ & $\frac{17.658}{17}$ & 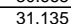 & -1.027 & & 50 & 0.041 & 14.6 & & 34 & & 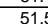 & 12. & & & & & & & \\
\hline & $\frac{1 / 2004}{11204}$ & & & & 23772 & 25.979 & & & & 17.802 & & -1027 & & $\frac{47.56}{4.75}$ & 0.04 & & & & & & & & & & & & & \\
\hline 2774 & & $7 \cdot 11.24$ & 24.854 & 25294 & 23.701 & 25.942 & 25.5 & & 53.12 & 17.672 & & -1027 & & & 0.041 & & & & & & & & & & & & & \\
\hline 2779 & 4/21/2004 & 7:12:24 PM & 24.848 & 25.298 & 23.704 & 25.946 & 25.5 & & 52.679 & 17.769 & 30. & -1.015 & & & & & & & 094 & 51. & 11. & & & & & & & \\
\hline 2776 & 4/21/2004 & 7:13:: & 24.858 & 25.29. & 23.709 & 25.951 & & & & & & -1.024 & & & 0.04 & & & & & 51. & 12. & & & & & & & \\
\hline 277 & 4/21/2004 & 7:14:24 PM & 24.842 & 25.272 & 23.683 & 25.905 & & & 52.836 & 17.749 & & -1.03 & & & 0.04 & 14. & & $34 !$ & 57.4 & 51. & 11. & & & & & & 0.157 & \\
\hline 27778 & $4 / 21 / 2004$ & 7:15: & 24.846 & 25.285 & 23.692 & 25.904 & & 48. & 52.012 & & & -1.024 & & & 0.041 & & & & & - & 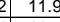 & & & & & & & \\
\hline 277 & & & 24.8 & $25.28 \mathrm{~m}$ & & 25.913 & & & & & & -1.0 & & & 0.0 & & & & & 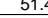 & 11.1 & & & & & & & \\
\hline$\frac{270}{278}$ & $4 / 21122004$ & $1: 11: 24 \mathrm{PM}$ & 24.829 & 25.269 & $\frac{2.2 .655}{2.265}$ & 25.902 & & 48. & $\frac{52 .}{52}$ & 17.664 & $30.79 \mathrm{~s}$ & -1.012 & & & 0.039 & & & & & & 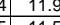 & 20 & 7 & & & & & \\
\hline 2782 & $\frac{4-4121204}{4 / 21204}$ & & $\frac{24.034}{24828}$ & $\frac{23.21}{2526}$ & $\frac{23.063}{23684}$ & $\frac{25.06}{25891}$ & & & & & & $\begin{array}{l}=-.9908 \\
-0.995\end{array}$ & & & & & & & & $\frac{51 .}{51 .}$ & & & & & & & & \\
\hline 278 & 4 & 7.2024 & 24837 & 25262 & 23698 & 2589 & & & & & & -0.952 & & & & & & & & & & & & & & & & \\
\hline$\frac{178}{278}$ & $\frac{4 / 21 / 2004}{4}$ & $\begin{array}{l}7: 21: 24 \\
\end{array}$ & 24.831 & 25.2 & $\frac{23.687}{23.687}$ & $\mid 25.879$ & 25. & 48.2 & \begin{tabular}{|l|}
52.774 \\
\end{tabular} & 17.778 & & $\mid-0.972$ & & & & & & & & & . & & & & & & & \\
\hline & & & & & & & & & & & & & & & & & & & & & & & & & & & & \\
\hline 278 & & 7:23: & 24.826 & 25.26 & 23747 & 25.984 & 25.6 & 48.196 & 52934 & 17.671 & & -0.952 & & & & & & & & & & & & & & & & \\
\hline & $4 / 21 / 2004$ & $7: 24: 24 \mathrm{P}$ & 24.821 & 25.2 & 23.758 & 26.115 & & & & & & -0.9 & & & & & & & & 51 & & & & & & & & \\
\hline 2788 & $4 / 21 / 2004$ & 7:25:24 PM & 24.827 & 25.252 & 23.748 & 26.205 & & 48.13 & 52.851 & $17.784 \mid$ & & -0.954 & & $49.7 \quad$ & 0.042 & 14.643 & & & & 51. & 12.2 & \begin{tabular}{l|l|}
2 & 39.3 \\
\end{tabular} & $<113$ & \begin{tabular}{|l|}
0.006 \\
\end{tabular} & 0.00 & & 65 & \\
\hline 278 & $4 / 21 / 2004$ & 7:26:24 PM & 24.829 & 25.8 & 23.746 & 26.292 & & $\begin{array}{l}48.142 \\
\end{array}$ & 52.832 & 17.771 & & -0.9 .9 & & & & 14. & & & & 51.2 & 11.7 & & & & & & 69 & \\
\hline 279 & $4 / 21 / 200$ & 7:27: & 24.8 & 25.2 & 23.763 & 26.38 & & 47. & 52.606 & 692 & & -0.966 & & 47.6 & 0.6 & & & 34 & & 51. & 11.7 & \begin{tabular}{l|l|l|}
7 & 39 \\
\end{tabular} & & & & & 0.169 & \\
\hline 279 & $4 / 21 / 2004$ & 7:28:24 PM & 24.849 & $\begin{array}{l}25.274 \\
2274\end{array}$ & 23.746 & 26.448 & 26. & 48.15 & 52.766 & 17.725 & 30.749 & -0.937 & & 48.2 & & 14. & & & & & & 35. & & & & & & \\
\hline 年 & $\frac{42112004}{412104}$ & $\frac{1}{729.24 \mathrm{FM}}$ & 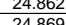 & 25.287 & 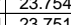 & 2.47 & & $\begin{array}{l}44.826 \\
14801\end{array}$ & $\frac{52.478}{5206}$ & 17.176 & $\begin{array}{l}30.485 \\
31059\end{array}$ & -0.946 & & 189 & & & & & & 01. & 12.0 & 207 & & & & & 02 & \\
\hline $\mid \frac{1794}{279}$ & $4121 / 2004$ & & th. 2.009 & 20.0344 & 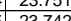 & 20.453 & & 40.251 & 52.502 & 11.00 & & $=0.934$ & & & & & & & & & 180 & & & & & & & \\
\hline$\frac{279}{279}$ & $\frac{4}{421212004 \mid}$ & $7.532 .24 \mathrm{PM}$ & & 25 & 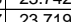 & $\frac{20.404}{26.31}$ & & 48.154 & & 1788 & & 烈 & & & & & & & & 51 & & & & & & & 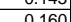 & \\
\hline 2796 & & & 24.8 & & 23.735 & & & & & 17.744 & & -0.899 & & & & & & & & 51.3 & & & & & & & & \\
\hline & $4 / 21 / 2004$ & & & 25308 & & & & & & & & -0.943 & & & & & & & & & & & & & & & & \\
\hline & & & 24.8 & & & & & & & & & & & & & & & & & $51: 3$ & & & & & & & & \\
\hline & $4 / 21 / 4$ & 720 & 24809 & & 2275 & 26167 & & & 52.467 & 176866 & & \begin{tabular}{|c|c|c|}
-0.937 \\
\end{tabular} & & 8.984 & & 14.643 & & & & & & & & & & & 158 & 0 \\
\hline 2800 & $4 / 21 / 2004$ & $7: 37: 24 \mathrm{PM}$ & 24.878 & 25.328 & 23.714 & 26.111 & 25.673 & 48156 & 52942 & 17.876 & & \begin{tabular}{l|l|}
-0.94 \\
\end{tabular} & & 47263 & 8043 & 14.643 & & & & 51.5 & 11.6 & & 2716 & 0.006 & 0.007 & 0.000 & 0.169 & 0.1 \\
\hline
\end{tabular}


WSRC-TR-2005-00105, REVISION 0

SRNL-RPP-2005-00012, REVISION 0

RUN \# 2.03A AND B; FIRST AND SECOND HALF OF SLURRY DEWATERING - CONT.

\begin{tabular}{|c|c|c|c|c|c|c|c|c|c|c|c|c|c|c|c|c|c|c|c|c|c|c|c|c|c|c|c|c|}
\hline & A & B & D & E & $\mathrm{F}$ & G & $\mathrm{H}$ & $\mathrm{J}$ & K & $\mathrm{L}$ & $\mathrm{M}$ & $\mathrm{N}$ & 0 & $a_{1}$ & $R$ & 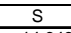 & \begin{tabular}{l|l|l|} 
\\
\end{tabular} & & w & $x$ & & 2 & AA & $\mathrm{AB}$ & $\mathrm{AC}$ & $A D$ & $\mathrm{AE}$ & \\
\hline \begin{tabular}{|l|l|}
2801 \\
2802
\end{tabular} & $\begin{array}{r}4 / 21 / 2004 \\
4 / 212004\end{array}$ & $\begin{array}{l}7: 38: 24 \mathrm{PM} \\
7 \cdot 39 \cdot 24 \mathrm{MM}\end{array}$ & $\begin{array}{r}24.882 \\
24.887\end{array}$ & $\begin{array}{r}25.332 \\
25.331 \\
\end{array}$ & $\begin{array}{l}23.724 \\
23.728\end{array}$ & \begin{tabular}{r|}
$\frac{26.085}{26.05}$ \\
26
\end{tabular} & \begin{tabular}{|l|}
25.687 \\
25.611 \\
\end{tabular} & $\begin{array}{r}48.16 \\
47.822 \\
\end{array}$ & $\begin{array}{r}52.9 \\
52.473\end{array}$ & \begin{tabular}{|c|}
17.772 \\
17.85 \\
\end{tabular} & $\begin{array}{l}30.729 \\
30.386\end{array}$ & \begin{tabular}{|l|}
-0.934 \\
-0.934
\end{tabular} & $\begin{array}{r}-7.006 \\
-7.004 \\
\end{array}$ & \begin{tabular}{|l|l}
48.404 \\
48.189
\end{tabular} & $\frac{0.041}{0.04}$ & $\begin{array}{l}14.643 \\
14.643\end{array}$ & $\begin{array}{l}0.003 \\
0.004\end{array}$ & $\begin{array}{l}3476.6 \\
3477.6\end{array}$ & \begin{tabular}{|c|}
57.9428 \\
57.9594
\end{tabular} & 51. & $\frac{11}{11}$ & 39.4 & 2.720 & \begin{tabular}{|c|c|}
0.006 \\
0.006
\end{tabular} & \begin{tabular}{|l|}
0.006 \\
0.006 \\
\end{tabular} & 0.000 & $\begin{array}{l}0.161 \\
0.158\end{array}$ & $\begin{array}{l}0.14 \\
0.13\end{array}$ \\
\hline \begin{tabular}{|l|l|l|l|l|l|}
2803 \\
\end{tabular} & & & $\frac{24.007}{24.887}$ & & $\frac{20.120}{23.723}$ & & & & & \begin{tabular}{|l|l|}
17.748 \\
\end{tabular} & & \begin{tabular}{|l|}
-0.504 \\
\end{tabular} & & & 0.04 & $\begin{array}{l}14.045 \\
14.643\end{array}$ & & & & & & & & & & & & \\
\hline 2804 & $4 / 21 / 2004$ & 7:41:24 PM & 24.88 & 25.315 & 23.711 & 26.003 & 25.5 & 48.391 & \begin{tabular}{|l|}
53.083 \\
\end{tabular} & 17.759 & 30.947 & $\begin{array}{r}-0.888 \\
\end{array}$ & & 48.348 & 0.04 & 14.643 & 0.003 & 3479.6 & 57.9928 & & 11.8 & 39.7 & \begin{tabular}{|l|l|}
2.735 \\
\end{tabular} & 0.006 & \begin{tabular}{|l|}
0.006 \\
\end{tabular} & 0.000 & 0.156 & 0.13 \\
\hline & $4 / 221 / 2004$ & 7:42:24 PM & 24.879 & 25.319 & 23.716 & 25.987 & 25.534 & 48.057 & \begin{tabular}{|l|}
52.824 \\
\end{tabular} & 17.754 & 30.589 & -0.931 & & 48.285 & 0.04 & 14.643 & & 3480.6 & 58.0094 & 51.4 & & & & & \begin{tabular}{|l|}
0.006 \\
\end{tabular} & & & \\
\hline & & & & & & & & & & & & & & 47.163 & & & & & & & & & & & & & & \\
\hline \begin{tabular}{|l|}
2807 \\
2809
\end{tabular} & $4 / 21 / 2004$ & $7: 44: 24 \mathrm{PM}$ & $\begin{array}{l}24.868 \\
2.873\end{array}$ & $\begin{array}{l}25.303 \\
5.212\end{array}$ & 23.704 & $\begin{array}{l}25.936 \\
25.091\end{array}$ & $\begin{array}{l}25.463 \\
25.023\end{array}$ & \begin{tabular}{ll|}
48.169 \\
8.007
\end{tabular} & \begin{tabular}{|l|l|}
52.834 \\
\end{tabular} & $\begin{array}{l}17.755 \\
\end{array}$ & $\begin{array}{l}30.731 \\
0.72\end{array}$ & \begin{tabular}{|l|l|}
-0.896 \\
\end{tabular} & -6.998 & $\begin{array}{l}9.047 \\
\end{array}$ & 0.039 & 14.643 & $\begin{array}{l}0.003 \\
\end{array}$ & 3482.6 & 58.0428 & 51. & 12.0 & ( & $\begin{array}{l}2.720 \\
\end{array}$ & $\begin{array}{ll}0.006 \\
\end{array}$ & 0.006 & 0.000 & 0.153 & 0.13 \\
\hline \begin{tabular}{|l|l|}
2808 \\
2809
\end{tabular} & $\begin{array}{l}4 / 21 / 2004 \\
4 / 212004\end{array}$ & $\begin{array}{l}7: 45: 24 \mathrm{PM} \\
7 \cdot 46.24 \mathrm{PM}\end{array}$ & $\begin{array}{l}24.873 \\
24.872\end{array}$ & $\begin{array}{l}25.313 \\
25301\end{array}$ & \begin{tabular}{|l|}
23.724 \\
23718 \\
\end{tabular} & \begin{tabular}{|l|}
25.941 \\
25.909
\end{tabular} & $\begin{array}{l}25.403 \\
25.452\end{array}$ & \begin{tabular}{|l|l|l|l|l|}
48.007 \\
8.256
\end{tabular} & \begin{tabular}{|l|}
52.697 \\
52.967 \\
\end{tabular} & $\begin{array}{l}17.685 \\
17.761\end{array}$ & $\begin{array}{l}30.782 \\
330766\end{array}$ & \begin{tabular}{|l|}
-0.894 \\
-0.885
\end{tabular} & $\begin{array}{l}-6.995 \\
-7.001\end{array}$ & \begin{tabular}{|l|}
48.141 \\
48.202 \\
\end{tabular} & $\begin{array}{l}0.04 \\
0.04\end{array}$ & $\begin{array}{l}14.643 \\
14643\end{array}$ & $\begin{array}{l}0.003 \\
0.003\end{array}$ & \begin{tabular}{l|l|l|l|l}
3483.6 \\
34846
\end{tabular} & $\begin{array}{l}58.0594 \\
58.0761\end{array}$ & $\frac{51}{51}$ & $\begin{array}{l}11.8 \\
11.8\end{array}$ & $\begin{array}{l}39.4 \\
39.5 \\
\end{array}$ & \begin{tabular}{|l|}
2.716 \\
2724 \\
\end{tabular} & \begin{tabular}{|l|l|} 
& 0.006 \\
0.006
\end{tabular} & $\begin{array}{l}0.006 \\
0.006\end{array}$ & $\begin{array}{l}0.000 \\
0.000\end{array}$ & \begin{tabular}{|l|l|}
0.157 \\
0.157
\end{tabular} & $\begin{array}{l}0.13 \\
0.13\end{array}$ \\
\hline 2810 & $4 / 21 / 2004$ & 7:47:24 PM & 24.881 & & 23.752 & 25.904 & 25.454 & $\begin{array}{l}40.250 \\
48.137\end{array}$ & \begin{tabular}{|l|}
52.921 \\
\end{tabular} & & $\begin{array}{l}30.600 \\
30.663\end{array}$ & \begin{tabular}{|l|}
-0.005 \\
\end{tabular} & $\begin{array}{l}-7.001 \\
-7.004\end{array}$ & & 0.041 & $\begin{array}{l}\frac{14.643}{14.643} \\
\end{array}$ & $\begin{array}{l}0.003 \\
0.003 \\
\end{array}$ & $\begin{array}{l}3484.6 \\
3485.6\end{array}$ & $\begin{array}{l}58.0761 \\
58.0928\end{array}$ & $\frac{51.5}{51.4}$ & & $\begin{array}{l}39.5 \\
39.4\end{array}$ & & \begin{tabular}{|c|}
0.006 \\
0.006
\end{tabular} & $\begin{array}{l}0.006 \\
0.006\end{array}$ & 0.0000 & $\frac{0.15 t}{0.161}$ & $\frac{0.13}{0.14}$ \\
\hline 2811 & $4 / 21 / 2004$ & 7:48:24 PM & 24.864 & & & & 25.454 & \begin{tabular}{|l|l|l|l|l|} 
\\
\end{tabular} & \begin{tabular}{|l|l|} 
& 52.875 \\
\end{tabular} & $\begin{array}{ll}17.616 \\
\end{array}$ & & -0.896 & & 48.475 & 0.041 & 14.643 & & 3486.6 & & & & & & $\begin{array}{l}0.006 \\
0.006\end{array}$ & $\begin{array}{l}0.000 \\
0.006\end{array}$ & & 0 & $\frac{0.14}{0.14}$ \\
\hline 28812 & $4 / 21 / 2004$ & 7:49:24 $\mathrm{PM}$ & 24.857 & 25.287 & 23.764 & $\begin{array}{l}25.835 \\
\end{array}$ & $\begin{array}{l}25.437 \\
\end{array}$ & \begin{tabular}{|l|l|l}
48.247 \\
\end{tabular} & 53.021 & \begin{tabular}{|l|l|l}
17.844 \\
\end{tabular} & 30.778 & -0.885 & & 48.237 & 0.04 & 14.643 & 0.003 & \begin{tabular}{|l|l|}
3487.6 \\
\end{tabular} & 58.1261 & & 11.8 & & & & & 0.000 & & 0.14 \\
\hline 2813 & $4 / 21 / 2004$ & 7:50:24 PM & 24.865 & & & 25.838 & 25.355 & \begin{tabular}{|l|l|}
47.999 \\
\end{tabular} & 52.675 & & 30.583 & -0.882 & & & & & & & & & & & & & & & & $\begin{array}{l}0.13 \\
0.14 \\
\end{array}$ \\
\hline 2814 & & 7:51:24 PM & & & & & & & & 178055 & & -0.894 & & & 0.04 & & & & 8.1594 & & & & & & & & & $\begin{array}{l}0.14 \\
0.13\end{array}$ \\
\hline & $4 / 21 / 2004$ & 7:52:24 PM & & & 23.774 & 25.89 & 25.457 & 48.16 & (2025 & 17.694 & 30.743 & -0.891 & & & 0.037 & 14.643 & & 349 & & & t1. & & & 0.006 & 8006 & & & \\
\hline 2816 & $4 / 21 / 2004$ & 7:53:24 PM & 24.862 & 25.272 & 23.758 & 25.945 & $\begin{array}{l}25.617 \\
\end{array}$ & $\begin{array}{ll}48.138 \\
\end{array}$ & 52.905 & 17.657 & 30.793 & -0.847 & & 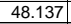 & 0.04 & 14.643 & $\begin{array}{l}0.003 \\
\end{array}$ & 3491.6 & 58.1928 & 51.4 & 11.8 & 39.5 & 2.721 & 0.006 & 0.006 & 0.000 & 0.157 & \\
\hline & $4 / 21 / 2004$ & 7:54:24 PM & 24.858 & 25.262 & 23.744 & 26.056 & 25.683 & $\begin{array}{l}47.997 \\
\end{array}$ & 52.855 & 17.794 & 30.495 & -0.865 & & 18.081 & 0.039 & 14.643 & & 3492.6 & 58.2094 & & 11. & & & & 0.006 & & & 0.13 \\
\hline & $4 / 21 / 2004$ & 7:55:24 PM & & & & & 25.774 & & & & 30.791 & & & & 0.04 & 14.643 & & & & & & & & & & & & \\
\hline & $4 / 21 / 2004$ & 7:56:24 PM & 24.86 & 25.26 & 23.756 & 26.218 & 25.89 & 47.961 & . & 17.638 & 30.784 & -0.856 & & 3.433 & 0.041 & 14.643 & & & & & & & & & .006 & & & 0.14 \\
\hline & $\frac{421212004}{4121204}$ & $\frac{T .57 .24 \mathrm{FM}}{7.5 \cdot 24}$ & 24.867 & $\frac{25.21}{2527}$ & $\frac{2.354}{23751}$ & $\frac{20.285}{26319}$ & 25.96 & 年 & 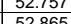 & $\frac{17.106}{17615}$ & 30743 & 些.879 & & & 0.041 & 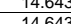 & & & & & & & & & & & & \\
\hline & 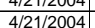 & $\begin{array}{l}1.50 .24 \mathrm{PM} \\
\text { 7:59:24 PM }\end{array}$ & $\begin{array}{l}24.805 \\
24.877\end{array}$ & $\begin{array}{l}25.275 \\
25.287\end{array}$ & $\frac{23.151}{23.749}$ & 20.348 & 25.999 & $\begin{array}{l}48.050 \\
48.196\end{array}$ & $\frac{52.805}{53.008}$ & $\begin{array}{l}17.015 \\
17.657\end{array}$ & $\begin{array}{l}30.143 \\
30.731\end{array}$ & $\frac{-0.050}{-0.818}$ & & 48.191 & $\begin{array}{l}0.04 \\
0.041\end{array}$ & $\begin{array}{l}4.643 \\
14.643 \\
\end{array}$ & 0.003 & & & & 11.8 & 39 & & & & 0.000 & & 0.13 \\
\hline & $4 / 21 / 2004$ & 8:00:24 PM & 24.874 & 25.294 & \begin{tabular}{|l|}
23.746 \\
\end{tabular} & 26.348 & 25.844 & 48.133 & 52.94 & 17.733 & 30.616 & -0.021 & & 477.947 & 0.04 & 14.643 & & 3498.6 & \begin{tabular}{|l|l|}
58.3094 \\
\end{tabular} & 51.5 & \begin{tabular}{|l|l|}
11.8 \\
11.8
\end{tabular} & 39.4 & & \begin{tabular}{|l|}
0.006 \\
\end{tabular} & \begin{tabular}{|l|l|}
0.000 \\
\end{tabular} & & 0.157 & $\begin{array}{l}0.14 \\
0.13\end{array}$ \\
\hline & $4 / 21 / 2004$ & 8:01:24 PM & 24.88 & 25.295 & 23.751 & 26.298 & 25.79 & 48.117 & 53.033 & 17.656 & 30.784 & -0.818 & & 48.139 & 0.04 & 14.643 & & 3499.6 & 58.3261 & & & 39.5 & & & & & & \\
\hline & $4 / 21 / 2004$ & 8:02:24 PM & 24.876 & & $\begin{array}{l}23.742 \\
23.721\end{array}$ & 26.219 & & & $\begin{array}{c}2.674 \\
-2.850\end{array}$ & $\frac{17.748}{17702}$ & 30.448 & & & & 0.041 & $\frac{14.643}{11.62}$ & & & & & & & & & & & & 0.14 \\
\hline & $\frac{421212004}{4121204}$ & $8.53 .24 \mathrm{FM}$ & $\frac{24.875}{24886}$ & $\frac{2.29}{25}$ & $\frac{23.1 / 11}{23.742}$ & $\frac{26.158}{26.119}$ & $\frac{25.625}{25606}$ & $\frac{48.025}{48.104}$ & $\frac{2.859}{52.985}$ & $\frac{17.02}{17844}$ & 30.569 & 0 & & 8.525 & 041 & $\frac{14.643}{14.643}$ & & & 83761 & & & & & & (1) & & & \\
\hline & $4 / 2 / 212004$ & 8:05:24 PM & 24.875 & 25 & $\frac{20.144}{23.726}$ & 26.038 & & & 5301 & $\begin{array}{l}17.044 \\
17.766\end{array}$ & 30.721 & $\begin{array}{l}-0.015 \\
-0.821\end{array}$ & & & $\frac{0.041}{0.041}$ & $\frac{1 \frac{1.645}{14643}}{104}$ & 0.0003 & 3503 & & 51 & $\frac{1.1}{11}$ & & & (1) & & & & \\
\hline & $4 / 21 / 2004$ & 8:06:24 PM & 24.889 & 25.30 & 23.741 & 25.992 & 25.564 & 48.108 & 52.859 & 17.796 & 30.6 & - -0.818 & & 47.094 & 0.039 & 14.643 & & 3504.6 & 58.4094 & & $\frac{11 .}{11 .}$ & 39.4 & 271 & & 006 & & 153 & \\
\hline & $4 / 21 / 2004$ & 8:07:24 PM & 24.873 & 25.293 & 23.709 & 25.931 & 25.4 & & 2.884 & 17.767 & 30. & & & 48.308 & & 14.643 & & & & & & & & & & & & \\
\hline 283 & $4 / 21 / 2004$ & 8:08:24 PM & 24.871 & 25.2 & 23.727 & 25.909 & 25.5 & 47.903 & & 17.637 & 30.616 & -0.818 & & 48.734 & 0.039 & 14.643 & 0.003 & & 58.4428 & & 11. & 39. & & 0.006 & 0.006 & 0.000 & 0.154 & \\
\hline & $4 / 21 / 2004$ & 8:09:24 PM & 24.864 & 25.289 & 23.721 & $\begin{array}{ll}25.862 \\
\end{array}$ & 25.325 & 48.386 & 3.188 & 17.794 & 30.86 & -0.815 & & 48.25 & 0.041 & 144.643 & & & 8.4594 & 51 & 11.8 & 39.6 & & & 0.006 & 0.000 & 0.160 & 0.14 \\
\hline & & 8:11:24 PM & 24.866 & 25.276 & 23.748 & 25.829 & & 47.822 & 52.538 & 17.755 & 30.495 & -0.818 & & 48.331 & 0.041 & 14.643 & & 350 & 58.4928 & 51.1 & 11.8 & 39.2 & .700 & & & 0.000 & 0.162 & 0.14 \\
\hline & $4 / 21 / 2004$ & 8:11:45 PM & 24.861 & 25.2 & 23.772 & 25.834 & 25.4 & 47.91 & & 17.676 & & -0.815 & & & 0.04 & 14.643 & & & 58.4986 & & $t$. & & & & & & & \\
\hline & $4 / 21 / 2004$ & 8:12: & 24.854 & 25.27 & 23.785 & 25.826 & 25.364 & 48.2 & 3.135 & 17.771 & 30.848 & -0.815 & & 8.575 & 0.039 & & & & & & & & & & & & & \\
\hline & $\frac{1.2004}{11204}$ & & 24.846 & 25.25 & $\frac{2.3772}{2.720}$ & 25.778 & 25. & 47. & .805 & 17.737 & & - 0.818 & & (1) & 0.043 & 14.643 & & & & & 12 & & & .006 & 0.00 & 0.000 & & \\
\hline 2839 & & & & & & & & & & & & & & & & & & & & & & & & & & & & \\
\hline 2840 & $4 / 21 / 2004$ & $8: 16: 45 \mathrm{PM}$ & 24.846 & 25.235 & | 23.757 & $\mid 25.743$ & 25.246 & 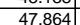 & \begin{tabular}{|l|l|}
52.753 \\
\end{tabular} & $\begin{array}{l}17.872 \\
17.872\end{array}$ & 30.316 & -0.807 & & 48.058 & 0.04 & 14.643 & & 2351 & $\mid$\begin{tabular}{|l|l|}
58.5819 \\
\end{tabular} & 51.3 & 11.8 & 39.1 & 2.695 & & \begin{tabular}{|l|l|}
0.006 \\
\end{tabular} & 0.000 & $\begin{array}{l}0.151 \\
0.158\end{array}$ & \\
\hline 204 & $4 / 21 / 2004$ & 8:17:45 PM & & 25.24 & 23.771 & \begin{tabular}{|l|l|}
25.808 \\
\end{tabular} & 25.4 & 47.918 & \begin{tabular}{|l|l|}
52.753 \\
\end{tabular} & \begin{tabular}{ll|l|l|}
17.879 \\
\end{tabular} & & -0.804 & & 48.634 & 0.04 & 14.643 & & & & 51. & 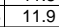 & & & & 0.00 & 0.000 & & 0. \\
\hline & 1/2004 & $8: 18: 45 \mathrm{PM}$ & 24.84 & 25.22 & 23.746 & 25.853 & & 48. & 1977 & & & -0.818 & & 49.295 & 0.042 & & & & & & 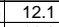 & & & & & & & \\
\hline 年 & $1 / 2004$ & 8:19:45 PM & 24.85 & $\frac{25.7}{25.2}$ & $\begin{array}{l}23.766 \\
22.76\end{array}$ & 25.968 & $\frac{25 .}{257}$ & 47.853 & 2.677 & $\begin{array}{l}17.745 \\
17.92\end{array}$ & & 年 & & 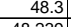 & 0.038 & & & & & & & & & & & & & \\
\hline 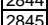 & $\frac{421212004}{41212004}$ & $\begin{array}{l}8020.45 \mathrm{PM} \\
0.20145\end{array}$ & $\frac{24.84}{24.841}$ & 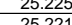 & $\begin{array}{l}23.146 \\
23.728\end{array}$ & 26.043 & 25.14 & 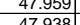 & & $\begin{array}{l}1.83 \\
17.736\end{array}$ & & $\begin{array}{l}-0.699 \\
0.072\end{array}$ & & & 0.041 & & & & & 51 & 116 & 392 & & & & & & \\
\hline 2846 & 41212004 & $8.2 \cdot 5 \cdot 15 \mathrm{PM}$ & $\frac{24.041}{24834}$ & $\frac{25.24}{2521}$ & $\frac{20.150}{2372}$ & $\frac{20.079}{26.147}$ & & & & 17.120 & & -0.18 & & & 0.04 & & & & & 51.4 & & & & & & & & \\
\hline 2847 & $\frac{4}{4 / 2120004}$ & $8.2 .345 \mathrm{PM}$ & 24.856 & $\frac{2.25}{25.245}$ & $\frac{2.76}{23762}$ & 26.229 & 25.78 & 47. & . & 17.797 & & -0.769 & & & $\frac{0.039}{0.039}$ & 站. & & 352 & 58.69866 & $\frac{v_{1}}{51}$ & 11.9 & 39 & & & & & & \\
\hline 2848 & $44 / 21 / 2004$ & $8: 24: 45$ & 24.842 & & & & & 47,8 & & & & & & & 0.039 & & & & & - & & & & & & & & \\
\hline 2849 & 4/21/2004 & $8.25:$ & 24.85 & 25.24 & 23.741 & & & 47.65 & 575 & 17.652 & & -0.7 & & & 0.039 & & & & & Fit & 12.1 & & & & & & & \\
\hline & & & 24846 & & & $\frac{26.175}{26.175}$ & 25. & & 52753 & & & & & & & & & & & 512 & . & & & & & & & \\
\hline & $4 / 21 / 2004$ & 8:27: & 24. & 25.232 & 23.733 & & & 47.961 & 52.88 & 17.761 & & -0.7 & & & & & & & & $\overline{51.4}$ & 12. & & & & & & & \\
\hline 85 & $4 / 21 / 2004$ & $8: 28: 45$ & 24.838 & 25.2. & 23.709 & 26.0 & & 47.7 & 600 & $\begin{array}{l}17.647 \\
\end{array}$ & & $-0.7 \quad$ & & So.1 & 0.0 & & & & & 51.1 & $\begin{array}{ll}12.3 \\
\end{array}$ & 39 & & & & & & \\
\hline & $4 / 21 / 2004$ & 8:29:45 PM & 24.846 & 25.2 & 23.728 & 25.994 & 25. & $47 . .5$ & 1894 & & & -0.7 & & & 0.0 & & & & & & 11.1. & & & & & & & \\
\hline 285 & & $8: 30$ & 24.8 & 25.25 & 23.736 & 25.958 & 25 & 47. & & 17.645 & & -0.7 & & & 0.04 & & & & & $\partial_{1}$ & 11.0 & & & & & & & \\
\hline & & 8:31: & 24.844 & 25.225 & 23.721 & 25.872 & 25.4 & 47.99 & 52.956 & 17.717 & 30.4 & -0.74 & & & 0.03 & & & & & & & & & & & & & \\
\hline & & & & & & 2.8 .811 & & & & & & & & & & & & & & & & & & & & & & \\
\hline 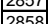 & 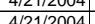 & 8.3 .445 & $\frac{24.041}{24829}$ & 25.220 & $\frac{23.123}{23726}$ & $\frac{20.1 / 44}{2575}$ & . & 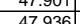 & $\frac{52.041}{5.054}$ & 1.84. & & -0.1 .0 & & & & & & & & & 11 & & & & & & & \\
\hline & $\frac{14}{4 / 2120004}$ & $\frac{8.34 .45}{8.35}$ & $\frac{24.053}{24842}$ & & 23.743 & 25.74 & & 48 & 53.118 & & & -0.711 & & & & & & & & 51.6 & & & & & & & & \\
\hline 286 & 4 & $8: 36: 45$ & 24.83 & & 23.727 & 25.6 & 25.4 & 48.196 & 53.118 & 17.854 & & -0.734 & & & & & & & & & 11.8 & 39.4 & & & & & & \\
\hline 286 & 4 & & & & & & & & 53.174 & & & & & & & & & & & & & & & & & & & \\
\hline & & 8.38 & & 25191 & & 25.614 & & 48.01 & 53.002 & 1767 & & -0.694 & & & 0.039 & & & & & & & & & & & & & \\
\hline 286 & $4 / 21 / 2004$ & 8:39: & 24.824 & 25.194 & 23.771 & 25.632 & 25.2 & 48.07 & 53.074 & 17.691 & 30. & -0.676 & & 18.4 & 0.04 & 14.6 & & 353 & 58.9 & & 119 & 36 & & & & & & \\
\hline & $4 / 21 / 2004$ & $8: 40$ & 24.8 & 25.17 & 23.789 & 25. & & 48.6 & 963 & 17.776 & & & & & & & & & & 515 & 120 & $38>$ & & & & & & \\
\hline & $4 / 21 / 2004$ & 8:41:45 & 24.7 & 25. & 23. & 25.6 & & 47.5 & & 17.6 & & -0.6 & & & & 14. & & & & 51 & & & & & & & & \\
\hline 286 & & & 24.7 & 25. & & & & 47.9 & .975 & 17.738 & & -0. & & & & & & & & 51.5 & & & & & & & 54 & \\
\hline 286 & $4 / 21 / 2004$ & & & 25.153 & 23.18 & 25.741 & 25. & 48.079 & 53.112 & 17.845 & & -0.4 & & & & 14.643 & & & & & & & & & & & & \\
\hline$\frac{2868}{8060}$ & $4 / 21 / 2004$ & 8:44:2 & 24.794 & 25.133 & 23.165 & 25.801 & 25.499 & $47 . .85$ & 52.731 & 17.856 & 30.245 & -0.65 & & 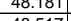 & 0.038 & 14.643 & & & & 51.3 & 11.8 & & 2.009 & $\mid$ & 0.0 & 0.000 & 0.150 & 0. \\
\hline$\frac{2869}{2870}$ & $\frac{4 / 21 / 121204}{4 / 21 / 2004}$ & 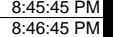 & $\frac{24.799}{24.801}$ & $\frac{25.134}{25.13}$ & \begin{tabular}{|l|l|}
23.761 \\
23.747
\end{tabular} & \begin{tabular}{|l|}
25.882 \\
25.939 \\
\end{tabular} & \begin{tabular}{|l|}
25.564 \\
25.661 \\
\end{tabular} & $\begin{array}{r}\mid 47.6 \\
47.652\end{array}$ & \begin{tabular}{|l|}
52.641 \\
52.699 \\
\end{tabular} & $\mid$\begin{tabular}{|}
$\mid 177.761$ \\
17.683
\end{tabular} & $\frac{30.16}{30.23}$ & $\begin{array}{c}-0.648 \\
-0.653 \\
\end{array}$ & & 48.235 & $\begin{array}{l}0.04 \\
0.041\end{array}$ & $\frac{14.643}{14.643}$ & $\frac{0.003}{0.003}$ & $\begin{array}{l}3543.9 \\
3544.9 \\
\end{array}$ & $\begin{array}{l}59.06533 \\
59.0819\end{array}$ & 51.2 & 111.8 & & \begin{tabular}{|l|l|}
2.685 \\
\end{tabular} & \begin{tabular}{|c|}
0.006 \\
\end{tabular} & \begin{tabular}{|l|}
0.006 \\
\end{tabular} & $\begin{array}{l}0.0000 \\
0.000\end{array}$ & 0.159 & 0.12 \\
\hline
\end{tabular}


WSRC-TR-2005-00105, REVISION 0

SRNL-RPP-2005-00012, REVISION 0

RUN \# 2.03A AND B; FIRST AND SECOND HALF OF SLURRY DEWATERING - CONT.

\begin{tabular}{|c|c|c|c|c|c|c|c|c|c|c|c|c|c|c|c|c|c|c|c|c|c|c|c|c|c|c|c|c|}
\hline & A & B & D & \begin{tabular}{ll|} 
\\
\end{tabular} & $\mathrm{F}$ & G & $\mathrm{H}$ & $\mathrm{J}$ & $\mathrm{K}$ & $\mathrm{L}_{\mathrm{L}}$ & $M$ & $\mathrm{~N}$ & 0 & Q & $R$ & $\mathrm{~s}$ & \begin{tabular}{l|l|l|} 
\\
\end{tabular} & & $\mathrm{w}$ & $x$ & & 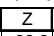 & AA & $A B$ & $\mathrm{AC}$ & $A D$ & $\mathrm{AE}$ & \\
\hline \begin{tabular}{|l|l|}
28871 \\
2872
\end{tabular} & $\begin{array}{r}4 / 21 / 2004 \\
4 / 212004\end{array}$ & $\begin{array}{l}8: 47: 45 \mathrm{PM} \\
8.48 .45 \mathrm{PM}\end{array}$ & $\frac{24.801}{22.803}$ & \begin{tabular}{|l|}
25.136 \\
25.137
\end{tabular} & \begin{tabular}{|c|}
23.758 \\
23.754
\end{tabular} & $\frac{26.011}{26.07}$ & \begin{tabular}{|l|}
25.571 \\
25.698 \\
\end{tabular} & $\begin{array}{l}47.847 \\
47.648\end{array}$ & \begin{tabular}{|l|}
52.818 \\
52.708 \\
\end{tabular} & \begin{tabular}{|l|}
17.655 \\
17.734
\end{tabular} & $\frac{30.52}{30.20}$ & $\begin{array}{l}-0.65 \\
-0.65\end{array}$ & $\begin{array}{l}-6.616 \\
-6.622\end{array}$ & $\begin{array}{l}48.479 \\
47.733\end{array}$ & 0.033 & $\begin{array}{l}14.643 \\
14.643\end{array}$ & $\begin{array}{l}0.003 \\
0.03\end{array}$ & $\begin{array}{l}3545.9 \\
3544.9 \\
3\end{array}$ & \begin{tabular}{|l|}
59.0986 \\
591153 \\
\end{tabular} & $\frac{51.3}{51.2}$ & $\frac{11}{11}$ & 39.8 & $\frac{2.702}{2.684}$ & \begin{tabular}{l|l|l|l|l|l}
0.006 & 0.05 \\
\end{tabular} & \begin{tabular}{|l|}
0.006 \\
0.005 \\
\end{tabular} & 0.000 & $\begin{array}{l}0.154 \\
0.135\end{array}$ & $\begin{array}{l}0.13 \\
0.11\end{array}$ \\
\hline & & & & & & 26.13 & & & & & & & & & & $\frac{14.043}{14.643}$ & & & & & & & & & & & & \\
\hline 2874 & $4 / 21 / 2004$ & 8:50:45 PM & 24.806 & 25.146 & \begin{tabular}{|l|}
23.743 \\
\end{tabular} & 26.1 & 25.751 & 47.885 & \begin{tabular}{|l|}
52.875 \\
\end{tabular} & 17.728 & 30.54 & -0.65 & -6.605 & 47.939 & 0.041 & 14.643 & 0.003 & 3548.9 & \begin{tabular}{|l|l|}
59.1486 \\
\end{tabular} & 51.4 & 11.7 & & 2.704 & \begin{tabular}{|l|}
0.006 \\
\end{tabular} & 0.006 & 0.000 & 0.162 & 0.14 \\
\hline 2875 & $4 / 21 / 2004$ & 8:51:45 PM & 24.818 & \begin{tabular}{|l|}
25.152 \\
\end{tabular} & \begin{tabular}{|l|}
23.754 \\
\end{tabular} & 26.13 & 25.663 & 47.694 & \begin{tabular}{|l|}
52.791 \\
\end{tabular} & 17.672 & 30.32 & $\begin{array}{c}-0.65 \\
-0.05\end{array}$ & -6.599 & 48.342 & 0.04 & 14.643 & & 3549.9 & & 51.3 & & & & & 0.006 & & & 0.13 \\
\hline & & & & & & & & & & & 30.49 & & & & & & & & & & & & & & & & & \\
\hline \begin{tabular}{|l|}
2877 \\
3879
\end{tabular} & $4 / 21 / 2004$ & $8: 53: 45 \mathrm{PM}$ & $\begin{array}{l}24.809 \\
28911\end{array}$ & $\begin{array}{l}25.149 \\
25159\end{array}$ & \begin{tabular}{|l|l|}
23.726 \\
3271
\end{tabular} & 26.00 & 25.489 & $\begin{array}{l}47.868 \\
\end{array}$ & \begin{tabular}{|l|l|}
52.954 \\
5350
\end{tabular} & $\begin{array}{l}17.641 \\
\end{array}$ & 30.4 & -0.65 & -6.593 & 48.46 & 0.04 & 14.643 & $\begin{array}{l}0.003 \\
\end{array}$ & $\begin{array}{l}3551.9 \\
\end{array}$ & \begin{tabular}{|l|l|l|}
59.1986 \\
\end{tabular} & 51. & 11.5 & & \begin{tabular}{|l|}
2.701 \\
\end{tabular} & $\begin{array}{ll}0.006 \\
\end{array}$ & 0.006 & 0.000 & 0.158 & 0.13 \\
\hline \begin{tabular}{|l|l|}
2878 \\
2879
\end{tabular} & $\begin{array}{l}4 / 21 / 2004 \\
4 / 212004\end{array}$ & $\begin{array}{l}8: 54: 45 \mathrm{PM} \\
8.55 .45 \mathrm{PM}\end{array}$ & $\begin{array}{l}24.815 \\
24.814\end{array}$ & \begin{tabular}{|l|}
25.159 \\
25.169
\end{tabular} & \begin{tabular}{|l|}
23.741 \\
23771 \\
\end{tabular} & $\begin{array}{l}25.948 \\
2589\end{array}$ & $\begin{array}{r}25.4 \\
25334 \\
\end{array}$ & \begin{tabular}{|l|}
48.561 \\
48.559 \\
\end{tabular} & \begin{tabular}{|l|}
53.659 \\
53647 \\
\end{tabular} & \begin{tabular}{|l|l|l|l|l}
17.906 \\
18.04
\end{tabular} & $\begin{array}{l}31.01 \\
3081\end{array}$ & $\begin{array}{r}-0.65 \\
-0.648 \\
\end{array}$ & $\begin{array}{l}-6.593 \\
-6.587\end{array}$ & $\begin{array}{l}488.442 \\
84.492 \\
\end{array}$ & $\begin{array}{l}0.041 \\
0.041\end{array}$ & $\begin{array}{l}144.643 \\
14643\end{array}$ & \begin{tabular}{|c|}
0.003 \\
0.003
\end{tabular} & \begin{tabular}{|l|l|l|l|l}
3552.9 & 3553.9 \\
35
\end{tabular} & \begin{tabular}{|l|}
59.2153 \\
592319
\end{tabular} & $\frac{52 .}{5.2}$ & $\begin{array}{l}11.9 \\
11.9\end{array}$ & \begin{tabular}{l|l|l|l|l|}
39.8 \\
397
\end{tabular} & 2.743 & \begin{tabular}{|c|}
0.006 \\
0.006 \\
\end{tabular} & $\begin{array}{l}0.006 \\
0.006\end{array}$ & $\begin{array}{l}0.000 \\
0.000\end{array}$ & $\begin{array}{l}0.159 \\
0.160\end{array}$ & $\begin{array}{l}0.14 \\
0.14\end{array}$ \\
\hline 2880 & $4 / 21 / 2004$ & $8.556: 45 \mathrm{PM}$ & & 25.163 & \begin{tabular}{|l|}
23.775 \\
\end{tabular} & 25.836 & 25.339 & & \begin{tabular}{|l|}
53.866 \\
\end{tabular} & 18.005 & 31.13 & $\begin{array}{l}-0.040 \\
-0.642\end{array}$ & $\begin{array}{l}-0.501 \\
-6.587\end{array}$ & $\begin{array}{l}40.492 \\
48.239\end{array}$ & 0.041 & $\begin{array}{l}14.643 \\
14.643 \\
\end{array}$ & $\begin{array}{l}0.003 \\
0.003\end{array}$ & $\begin{array}{l}35353.9 \\
3554.9\end{array}$ & \begin{tabular}{|l|}
59.2319 \\
59.2486 \\
\end{tabular} & 52.4 & & 39.7 & \begin{tabular}{|l|l|}
2.736 \\
2.756
\end{tabular} & \begin{tabular}{|c|}
0.006 \\
0.006
\end{tabular} & 0.006 & $\begin{array}{l}0.000 \\
0.000 \\
\end{array}$ & $\begin{array}{l}0.160 \\
0.158\end{array}$ & $\frac{0.14}{0.13}$ \\
\hline & $4 / 21 / 2004$ & 8:57:45 PM & 24.812 & & 23.768 & & & & & 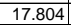 & 31.1 & -0.63 & & & 0.041 & 14.643 & & & & & & & & 0.006 & & & & $\begin{array}{l}0.13 \\
0.13\end{array}$ \\
\hline 2882 & $4 / 21 / 2004$ & $8: 58: 45$ PM & 24.805 & 25.144 & 23.786 & 25.69 & 25.22 & 48.529 & 53.597 & 17.992 & & -0.581 & -6.584 & $\begin{array}{l}48.953 \\
\end{array}$ & 0.042 & 14.643 & 0.003 & $\begin{array}{l}3556.9 \\
\end{array}$ & \begin{tabular}{|l|l|}
59.2819 \\
\end{tabular} & 52.1 & 12. & & & & \begin{tabular}{|l|}
0.006 \\
\end{tabular} & 0.000 & & 0.13 \\
\hline \begin{tabular}{|l|}
28883 \\
83
\end{tabular} & $4 / 21 / 2004$ & 8: & 24.809 & & 23.8 & 25.66 & & & 53.713 & & 31.03 & & & & & & & & & & & & & & & & & 0.14 \\
\hline 2884 & & 9:00:45 PM & & & & & & & & 18.064 & & \begin{tabular}{l|l|}
-0.627 \\
\end{tabular} & & & & & & & & & & & & & & & & \\
\hline & $4 / 21 / 2004$ & 9:01:45 PM & & & 23.82 & 25.60 & 25.149 & 48.67 & 53.81 & 17.935 & 31.09 & -0.598 & & 48.485 & 0.041 & 14.643 & .004 & & & & & & 2.750 & 0.006 & 0.006 & & & \\
\hline 2886 & $4 / 21 / 2004$ & 9:02:45 PM & 24.786 & 25.106 & 23.803 & 25.54 & $\begin{array}{l}25.067 \\
\end{array}$ & 48.5 & 53.589 & 17.819 & 31.01 & \begin{tabular}{l|l|l|}
-0.587 \\
\end{tabular} & -6.593 & $\begin{array}{l}477.125 \\
\end{array}$ & 0.041 & 14.643 & $\begin{array}{l}0.003 \\
\end{array}$ & $\begin{array}{l}3560.9 \\
3\end{array}$ & \begin{tabular}{|l|l|}
59.3486 \\
\end{tabular} & 52.1 & $11 . .5$ & 39.8 & & $\begin{array}{l}0.006 \\
\end{array}$ & 0.006 & 0.000 & 0.159 & 0.14 \\
\hline & $4 / 21 / 2004$ & 9:03:45 PM & 24.794 & 25.109 & 23.826 & 25.53 & 25.135 & 48.666 & 53.759 & 18.085 & 30.86 & -0.587 & -6. & 48.83 & 0.039 & 14.643 & & 3561.6 & & & & & 2.742 & & 0.006 & & & \\
\hline & $4 / 21 / 2004$ & 9:04:45 PM & & & & & & & & & 31.40 & & & & & & & & & & & & & & & & & \\
\hline & $4 / 21 / 2004$ & 9:05:45 PM & 24.791 & 25.085 & 23.852 & 25.544 & 25.136 & 48.299 & 3.394 & 17.905 & 30.64 & -0.569 & & . & 0.042 & 14.643 & & 3563.9 & & & & & & & & .000 & & 0.1 \\
\hline$\frac{2091}{2891}$ & $\frac{421212004}{4121204}$ & $\begin{array}{l}9.060 .45 \mathrm{PM} \\
0.075\end{array}$ & $\frac{24.19}{20.70}$ & $\frac{25.079}{2509}$ & $\frac{23.862}{2306}$ & $\frac{25.63}{2568}$ & 25.2 & $\frac{48.581}{18911}$ & 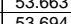 & $\frac{17.844}{17072}$ & & & & & 0 & $\frac{14.643}{14642}$ & & & & & & & & & & & & \\
\hline$\frac{2892}{2892}$ & 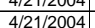 & $\begin{array}{l}9: 07: 45 P \\
9: 08: 45 \mathrm{PM}\end{array}$ & $\frac{24.719}{24.78}$ & $\begin{array}{l}25.069 \\
25.049\end{array}$ & $\begin{array}{l}\frac{23.000}{23.867} \\
\end{array}$ & $\frac{23.06}{25728}$ & $\frac{25.325}{25.42}$ & $\begin{array}{l}48.014 \\
48.787\end{array}$ & $\begin{array}{l}53.094 \\
53.879\end{array}$ & $\begin{array}{l}17.975 \\
18.089\end{array}$ & $\frac{30.92}{31.02}$ & $\frac{-0.535}{-0.566}$ & & 48.135 & 0.041 & $\begin{array}{l}14.045 \\
11643\end{array}$ & 0.0003 & 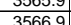 & 5.99486 & & $\frac{11.6}{11.8}$ & & & | & & 0.000 & 0 & \\
\hline & $4 / 21 / 2004$ & 9:09:45 PM & 24.792 & 25.056 & \begin{tabular}{|l|}
23.933 \\
\end{tabular} & 25.82 & 25.552 & 48.797 & 53.879 & 18.132 & 30.94 & -0.578 & -6.57 & 49.806 & 0.041 & 14.643 & 0.003 & 3567.9 & \begin{tabular}{|l|l|}
59.4653 \\
\end{tabular} & 52.4 & 12.2 & & & & 0.006 & 0.000 & 0.158 & 0.13 \\
\hline 28 & $1: 12004$ & 9:10:45 PM & 24.798 & & 23.94 & 25.88 & & 48.75 & 53.775 & 17.983 & 30.99 & -0.549 & & & 0.041 & 14.643 & & 3568.9 & & & & & & & & & & \\
\hline & $4 / 21212004$ & 9:11:45 PM & 24.799 & 25.05 & 3.961 & 25.93 & 25.5 & & 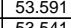 & 17.891 & 30.99 & -0.543 & & & 0.04 & 144.643 & & & & & & & & & & & & \\
\hline 2897 & $\frac{421212004}{4121204}$ & $\begin{array}{l}9.21 .45 \mathrm{PM} \\
0.12: 5 \mathrm{PM}\end{array}$ & $\frac{24.816}{24827}$ & $\begin{array}{l}25.075 \\
25061\end{array}$ & $\frac{23.973}{23974}$ & $\frac{26.00}{26.02}$ & $\frac{25.55}{25.5}$ & 48 & $\begin{array}{l}3.541 \\
3.505 \\
\end{array}$ & $\frac{17.965}{18.052}$ & 30.77 & $\begin{array}{l}-0.543 \\
-0.543 \\
\end{array}$ & & 483 & 0.04 & $\frac{14.643}{14.643}$ & & , & & & 11 & 39. & & & 2006 & & & \\
\hline & $4 / 2 / 212004$ & 9.9.45 PM & 24833 & 25.067 & 23.975 & 26.046 & 25 & 48.478 & 53.698 & $\begin{array}{l}18.007 \\
\end{array}$ & 30.74 & 然. & & 49.708 & 0.04 & $\frac{14.043}{14.643}$ & 0.0003 & & | & 52 & $\frac{\frac{11}{12}}{2}$ & & & (1) & & & & \\
\hline & $4 / 21 / 2004$ & 9:15:45 PM & 24.844 & 25.074 & 23.981 & 26.01 & 25.55 & 48.529 & 3.611 & 18.065 & 30.76 & -0.543 & -6.54 & 50.757 & 0.041 & 14.643 & & 3573.9 & 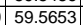 & 52 & 12.4 & 39.6 & 2.734 & & 0.006 & 000 & & $\sqrt{14}$ \\
\hline & $4 / 21 / 2004$ & 9:16:45 PM & & 25.084 & & 25.948 & & 48.681 & & 18.22 & & -0.54 & & & & 14.643 & & 357 & & & & & & & & & & \\
\hline 2901 & $4 / 21 / 2004$ & 9:17:45 PM & 24.861 & 25.085 & \begin{tabular}{|l|}
23.988 \\
\end{tabular} & 25.88. & 25.3 & \begin{tabular}{|l|l|}
48.534 \\
\end{tabular} & 53.636 & 17.927 & 31.03 & $\begin{array}{l}-0.54 \\
\end{array}$ & & & 0.041 & 14.643 & & 3575.9 & 59.5986 & & 11. & 39.8 & 2.743 & $\mid 0.006$ & 0.006 & 0.000 & 0.158 & \\
\hline 290 & $4 / 21 / 2004$ & 9:18:45 PM & 24.855 & 25.089 & 23.992 & 25.80 & 25.28 & 48.482 & 53.684 & $\begin{array}{ll}18.166 \\
\end{array}$ & 30.63 & $\begin{array}{l}-0.54 \\
\end{array}$ & & 48.7 & 0.041 & 14.643 & & 357 & & & & & & & & & & \\
\hline & $4 / 21 / 2004$ & $9: 19: 45 \mathrm{PM}$ & 24.885 & 25.094 & & 25.78 & & & 53.58 & & & & & & 0.04 & 14.643 & & & & & & & & & & & & \\
\hline 29 & $4 / 21 / 2004$ & 9:20:45 PM & 24.868 & 25.072 & 23.98 & 25.69 & 25.1 & \begin{tabular}{l|l|l}
48.756 \\
\end{tabular} & 806 & 17.968 & 31.18 & -0.546 & & 48.803 & 0.041 & 14.643 & & $\begin{array}{l}3578.9 \\
\end{array}$ & \begin{tabular}{|l|}
59.6486 \\
\end{tabular} & 52.3 & 12.0 & 40.0 & 4.756 & & 0.006 & 0.000 & 0.157 & 0.13 \\
\hline & $4 / 21 / 2004$ & & 24.856 & 25.07 & 23.978 & 25.628 & 25.1 & $\begin{array}{l}48.399 \\
\end{array}$ & 53.479 & 17.951 & 30.72 & $\begin{array}{ll}-0.5 \\
-1\end{array}$ & & & & 14. & & 3579.9 & & & & & & & & & & \\
\hline & 4/21/2004 & 9:22:45 PM & 24.865 & 25.069 & 23.987] & 25.613 & & 48.534 & & 18.048 & & -0.538 & & & 0.041 & & & & & & & & & & & & & \\
\hline & .1.1/2004 & 9:23 & 24.874 & 25.068 & 24.001 & 25.58 & 25.1 & & 53.75 & 18.104 & & -0.54 & & & 0.041 & 14.643 & & 358 & 866 & & 7 & & 2.73 & 0.006 & 0.00 & 0.000 & & \\
\hline & & & & & $\frac{23.989}{23096}$ & $\frac{25.52}{2548}$ & & & & & & & & & & & & & & & & & & & & & & \\
\hline$\frac{2910}{2910}$ & 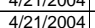 & $\begin{array}{l}9.25 .45 \mathrm{PM} \\
9: 26: 45 \mathrm{PM}\end{array}$ & $\begin{array}{l}24.859 \\
24.847\end{array}$ & $\frac{25.034}{25021}$ & 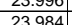 & $\frac{25.46}{2543}$ & . & $\begin{array}{l}48.000 \\
48.465\end{array}$ & 607 & 17.0035 & $\begin{array}{l}30.94 \\
30.88\end{array}$ & $\begin{array}{l}-0.530 \\
-0.509\end{array}$ & & & $\frac{0.04}{0.041}$ & $\frac{14.045}{14643}$ & & | & 599786 & 521 & $\frac{11.4}{12 .}$ & & & & 0.006 & 000 & 159 & \\
\hline 2911 & $4 / 21 / 2004$ & 9:27:45 PM & $\frac{24.041}{24.85}$ & & & 25.44 & 24. & & \begin{tabular}{|l|}
53.016 \\
\end{tabular} & 18.059 & & -0.543 & & & & 14.643 & & 358 & & $U_{2}$ & 12. & & & & & & & \\
\hline & $4 / 21 / 2004$ & 9:28:45 PM & 24.847 & 24.997 & $\frac{23.984}{23.94}$ & 25.42. & & 48.778 & 54.03 & & $\begin{array}{l}30.99 \\
30.99\end{array}$ & $\begin{array}{l}-0.497 \\
-0.497\end{array}$ & & & 0.037 & & & 358 & 59. & 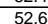 & Lte. & & & & 0.00 & & 0.06 & \\
\hline & & 9:29:45 PM & 24.856 & & & 25.498 & 25.1 & $\begin{array}{l}48.503 \\
\end{array}$ & $\begin{array}{l}53.647 \\
\end{array}$ & 17.872 & & -0.514 & & .6 & 80 & & & & & & 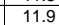 & & & & & & & \\
\hline & & 9:30:45 PM & 24.856 & & & & & 48.42 & 53.584 & & & -0.535 & & & 20 & & & & 58 & & & & & & & & & \\
\hline 291 & 4/21/2004 & 9:31:45 PM & 24.86 & $24.984]$ & 24.007 & 25.65 & 25 & & 33.703 & 17.982 & & -0.514 & & & 0.041 & 14. & & 358 & 59.8 & 52 & 12. & 397 & 4.736 & & & & 158 & \\
\hline & $4 / 21 / 2004$ & 9:32:45 PM & 24.856 & 24.98 & & 25.70 & & & & & & -0.491 & & & 0.6 & & & & & & 11. & ay & & & & & & \\
\hline 2917 & 4/21/2004 & 9:33:45 PM & 24.862 & 24.976 & 24.019 & 25.77: & 25.4 & 48. & .7.709 & & & $\begin{array}{l}-0.483 \\
\end{array}$ & & & 0.04 & 14. & & 359 & 59.8 & 52 & 11. & 39.6 & & & & & & \\
\hline 2918 & $4 / 21 / 2004$ & 9:34 & 24.868 & 24.982 & 24.03 & & & 48.9 & & & & -0.483 & & & & & & & & -2 & 11. & & & & & & & \\
\hline & & & 24.875 & 24.98 & 24.107 & & & & & & & & & & & & & & & $J_{2}$ & $12.2 \mathrm{CH}$ & & & & & & & \\
\hline & $4 / 21122004$ & & 24.817 & 24.986 & 2.0996 & & $\frac{25.8}{25.6}$ & & 5 & & & -0.5 & & & & 14. & & & & & $\begin{array}{l}12.5 \\
11.7\end{array}$ & & & & & & & \\
\hline & $\frac{4-4121204}{4 / 21204}$ & 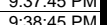 & $\frac{24.064}{24911}$ & $\frac{24.9}{250}$ & $\frac{24.0506}{24.128}$ & & & $\begin{array}{l}46.034 \\
4838\end{array}$ & & & & $\frac{-.054}{-0.569}$ & & & & & & & & & & & & & & & & \\
\hline & 4 & $9 \cdot 9 \cdot 3 \cdot 45$ & 25.132 & 2500 & 24.104 & & & 50.379 & & & & -1221 & & & 0.0 & & & & & & 12.0 & & & & & & 0 & \\
\hline & $4 / 21 / 2004$ & 9:4::45 PM & $\frac{25.382}{25382}$ & 25.011 & 24.1 & $25.90 !$ & 25 & $\mid \begin{array}{l}0.889 \\
48.89\end{array}$ & $\mid \begin{array}{l}\mid 53.41 \\
5\end{array}$ & 17.998 & & -1.096 & & & & & & & & & 11.9 & & & & & & & \\
\hline & & & & & & & & & & & & & & & & & & & & & & & & & & & & \\
\hline & & $9: 42: 45 \mathrm{PM}$ & 25.182 & 25.006 & 24084 & 2576 & 25.1 & 46.966 & 53709 & & 29.36 & 1035 & & & & 14.6 & & & & & & & & & & & & \\
\hline & $4 / 21 / 2$ & & 25.0 & & 24.079 & & & $\begin{array}{ll}48 . \subseteq \\
\end{array}$ & 53.883 & & & -0.7 & & & & & & & & & & & & & & & & \\
\hline 29 & $4 / 21 / 2004$ & 9:44:45 PM & 25.067 & 25.026 & $24.105 \mid$ & 25.65 & & 49.195 & \begin{tabular}{|l|l|}
53.568 \\
\end{tabular} & 17.965 & 31 & -1.218 & & & 0.0 & 14.643 & & 360 & 60.0 & 52. & 12. & 40. & 2.186 & \begin{tabular}{|l|}
0.006 \\
\end{tabular} & & & 51 & \\
\hline & $4 / 21 / 200$ & $9: 45: 45$ & 25.02 & 25.004 & 24.072 & 25.6 & 25.1 & $\begin{array}{l}49.342 \\
\end{array}$ & \begin{tabular}{|l|l|}
53.377 \\
\end{tabular} & & & -1.218 & & & & 14. & & & & 51 & $12.1 \mathrm{Cl}$ & $4 \mathrm{c}-2$ & & & & & 62 & \\
\hline & $4 / 21 / 200$ & & 25.004 & 24.998 & 24.066 & & & 50.066 & 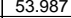 & 18.083 & & -1.224 & & & & & & 36 & 60.0 & 52. & 12.1 & & & & & & 156 & \\
\hline & $4 / 21 / 2004$ & 9:47:45 PM & 24.977 & 24.991 & 24.069 & 25.50 & 24.9 & 50.058 & 53.883 & 17.913 & 32.5 & -1.221 & & & 0.04 & 14.643 & & & & & 12. & & & & & & & \\
\hline & $\frac{421212004}{41212004}$ & $9: 48.45 \mathrm{PM}$ & 24.954 & 24.983 & 24074 & $2 \frac{2.45}{2.52}$ & & $\begin{array}{l}49.882 \\
5012\end{array}$ & $\frac{53.030}{5386}$ & $\frac{18.016}{18097}$ & $\frac{32.2}{32.2}$ & -1.218 & & 187 & & & & 360 & & 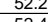 & 12.0 & & & & & & & \\
\hline & $4+21212004$ & 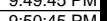 & 2.097 & 24.970 & (2.0.04 & 20.424 & & 50 & \begin{tabular}{|l}
53.00 \\
\end{tabular} & & & - & & & & & & & & & & & & & & & & \\
\hline & $\frac{4}{421212004 \mid}$ & 0.05 .45 & $\frac{24.51}{24.93}$ & 24.8 & $\frac{24.08}{248}$ & 25.44 & & & $530 \quad$ & & & -1218 & & & & & & & & 52 & 128 & & & & & & & \\
\hline & & & 248 & & & & & & & & & -1.221 & & & & & & & & & & & & & & & & \\
\hline & $4 / 21 / 2004$ & & 24.862 & 24.951 & 24.079 & 25.57 & & & & & & & & & & & & & & & & & & & & & 56 & \\
\hline & & & & & & & & 49.8 & & & & & & & & & & & & & & & & & & & & \\
\hline & $4 / 21 / 2004$ & & 24858 & 24032 & 085 & & & 40 & 53522 & & & -1.218 & & & & 14.643 & & & & & & & & & & & & 01 \\
\hline 2940 & $4 / 21 / 2004$ & 9:56:45 PM & 24.849 & 24.943 & 24.086 & 25.74 & 25.469 & & & 17.955 & & -1.224 & & 48621 & 0.041 & 14.643 & & & \begin{tabular}{|l|l|l|l}
6086 \\
\end{tabular} & & & & 2.858 & 0.006 & 0.006 & 0.000 & 0.151 & 0.1 \\
\hline
\end{tabular}


WSRC-TR-2005-00105, REVISION 0

SRNL-RPP-2005-00012, REVISION 0

RUN \# 2.03A AND B; FIRST AND SECOND HALF OF SLURRY DEWATERING - CONT.

\begin{tabular}{|c|c|c|c|c|c|c|c|c|c|c|c|c|c|c|c|c|c|c|c|c|c|c|c|c|c|c|c|c|}
\hline & A & B & D & $E$ & $F$ & $G$ & $\mathrm{H}$ & $\mathrm{J}$ & $\mathrm{K}$ & $\mathrm{L}$ & $\mathrm{M}$ & $\mathrm{N}$ & $\mathrm{O}$ & $Q$ & $\mathrm{R}$ & 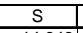 & $\mathrm{T}$ & 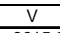 & w & $x$ & & $\mathrm{z}$ & $\mathrm{AA}$ & $A B$ & $\mathrm{AC}$ & $A D$ & & AF $A$ \\
\hline 2941 2942 & $\begin{array}{l}\mid 4 / 21 / 2004 \\
4 / 212004\end{array}$ & $\begin{array}{l}\text { 9:57:45 PM } \\
9.58: 4 \text { PM }\end{array}$ & $\begin{array}{r}24.83 \\
24.827 \\
\end{array}$ & $\frac{24.93}{24.94}$ & 24.098 & $\begin{aligned} 25.823 \\
25.83 \\
\end{aligned}$ & $\begin{array}{r}25.46 \\
25.57 \\
\end{array}$ & $\begin{array}{l}49.963 \\
49.961 \\
4\end{array}$ & \begin{tabular}{|l|}
53.719 \\
53.628 \\
\end{tabular} & $\begin{array}{l}17.954 \\
17.918 \\
\end{array}$ & $\frac{32.38}{32.4}$ & 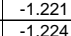 & $\begin{array}{c}-7.4 \\
-7.4\end{array}$ & $\begin{array}{l}48.536 \\
48.665 \\
\end{array}$ & 0.041 & \begin{tabular}{|l|l|}
14.643 \\
14.633 \\
\end{tabular} & $\begin{array}{l}0.003 \\
0.003\end{array}$ & $\frac{3615.6}{3616.5}$ & \begin{tabular}{|l|}
60.2653 \\
6.2819 \\
\end{tabular} & \begin{tabular}{|l|l|}
52.2 \\
52.2
\end{tabular} & $\frac{11}{11}$ & \begin{tabular}{|l|}
41.2 \\
41.2
\end{tabular} & 2.839 & $\mid$\begin{tabular}{|l|}
0.006 \\
0.006
\end{tabular} & \begin{tabular}{|l|}
0.006 \\
0.006 \\
\end{tabular} & 0.000 & $\begin{array}{l}0.152 \\
0.156 \\
\end{array}$ & $\begin{array}{l}0.13 \\
0.13 \\
\end{array}$ \\
\hline$\frac{2944}{2943}$ & & & & & $\frac{24.054}{24.11}$ & & & & & & & & & & & & & & & & & & & & & & & \\
\hline 2944 & $4 / 21 / 2004$ & 10:00:45 PM & 24.824 & 24.93 & 24.091 & 25.787 & 25.429 & 50.274 & \begin{tabular}{|l|}
54.109 \\
\end{tabular} & 18.144 & 32.46 & -1.224 & -7.394 & 48.828 & 0.042 & 14.643 & 0.003 & 3618.9 & 60.3153 & & & 41.4 & \begin{tabular}{|l|l|} 
& 2.852 \\
\end{tabular} & 0.006 & 0.006 & 0.000 & 0.155 & 0.13 \\
\hline 2945 & $4 / 21 / 2004$ & 10:01:45 PM & 24.839 & 24.95 & & 25.747 & 25.349 & 50.002 & \begin{tabular}{|l|l|}
53.723 \\
\end{tabular} & 18.172 & 32.14 & -1.221 & & 48.844 & 0.041 & 14.643 & 0.003 & 3619.9 & \begin{tabular}{|l|l|}
60.3319 \\
\end{tabular} & & & & & & 0.006 & 0.000 & & 0.13 \\
\hline & & & & & & & & & \begin{tabular}{|l|l|}
54.088 \\
\end{tabular} & 18.216 & & & & & & & & & & & & & & & & & & 0.13 \\
\hline \begin{tabular}{|l|l|}
2947 \\
2918
\end{tabular} & $4 / 21 / 2004$ & 10:03:45 PM & 24.855 & 24.94 & \begin{tabular}{|l|l|}
24.107 \\
2.121
\end{tabular} & $\begin{array}{l}25.637 \\
25.596 \\
\end{array}$ & 25.165 & \begin{tabular}{|l|l|}
49.844 \\
5.228
\end{tabular} & \begin{tabular}{|l|l|}
53.593 \\
53070
\end{tabular} & $\begin{array}{ll}18.066 \\
18072\end{array}$ & $\begin{array}{r}32.1 \\
3250\end{array}$ & -1.221 & -7.394 & 48.229 & 0.042 & $\begin{array}{ll}14.643 \\
\end{array}$ & 0.003 & 3621.6 & \begin{tabular}{|l|l|}
60.3653 \\
\end{tabular} & 52.1 & 11.8 & 41.0 & 2.827 & \begin{tabular}{|l|l|}
0.006 \\
\end{tabular} & 0.006 & 0.000 & 0.157 & 0.13 \\
\hline$\frac{2948}{2949}$ & \begin{tabular}{|l|}
$4 / 21 / 2004$ \\
$4 / 21204$ \\
\end{tabular} & $\begin{array}{l}10: 04: 45 \text { PM } \\
10.0554 \mathrm{PM}\end{array}$ & 24.864 & 24.95 & \begin{tabular}{|l|l|}
24.121 \\
24099 \\
\end{tabular} & \begin{tabular}{|l|}
25.586 \\
2514
\end{tabular} & $\begin{array}{l}25.169 \\
25.152 \\
\end{array}$ & \begin{tabular}{|l|}
50.228 \\
49.983 \\
\end{tabular} & \begin{tabular}{|l|}
53.978 \\
53742
\end{tabular} & $\begin{array}{ll}18.072 \\
18.013\end{array}$ & $\frac{32.59}{32.3}$ & \begin{tabular}{|l|}
-1.221 \\
-1.221
\end{tabular} & $\begin{array}{l}-7.394 \\
-7.388 \\
\end{array}$ & $\begin{array}{l}48.333 \\
8.584\end{array}$ & 0.042 & $\begin{array}{l}14.643 \\
11.643 \\
\end{array}$ & $\begin{array}{l}0.003 \\
0.003 \\
\end{array}$ & $\begin{array}{ll}3622.4 \\
362.9\end{array}$ & \begin{tabular}{|l|l|}
60.3819 \\
603986
\end{tabular} & \begin{tabular}{|l|l|}
52.5 \\
52.3
\end{tabular} & $\frac{11.8}{11.9}$ & 41.4 & \begin{tabular}{|l|}
2.855 \\
2838 \\
\end{tabular} & \begin{tabular}{|l|l|}
0.006 \\
0.066 \\
\end{tabular} & $\begin{array}{l}0.006 \\
0.007\end{array}$ & 0.000 & $\begin{array}{l}0.155 \\
0.160\end{array}$ & $\begin{array}{l}0.13 \\
0.14\end{array}$ \\
\hline 2950 & $4 / 21 / 2004$ & $10: 06: 45 \mathrm{PM}$ & & 24.94 & 24.138 & 25.488 & 25.031 & 49.394 & \begin{tabular}{|l|}
53.041 \\
\end{tabular} & 19.058 & 30.69 & -1.218 & & 49.22 & $\frac{0.049}{0.041}$ & $\begin{array}{l}\frac{14.045}{14.643} \\
\end{array}$ & $\begin{array}{l}0.003 \\
0.003\end{array}$ & & & & & $\frac{44.2}{40.0}$ & $\frac{2.838}{2.761}$ & $|0.006|$ & $\begin{array}{l}0.007 \\
0.006\end{array}$ & $\begin{array}{l}0.000 \\
0.000 \\
\end{array}$ & 0.160 & 0.14 \\
\hline 295. & $4 / 21 / 2004$ & 10:06:53 PM & & & & & & & & 18.993 & & & & 50.636 & & 14.643 & & & 60.4175 & & & & & & 0.006 & & & $\begin{array}{l}0.13 \\
0.13 \\
\end{array}$ \\
\hline 2952 & 4/21/2004 & 10:07:53 PM & 24.873 & & 24.196 & 25.436 & 25.048 & 49.266 & 52.948 & 19.126 & 30.54 & -1.224 & -7.403 & 50.083 & 0.04 & 14.643 & 0.003 & 3626.1 & 60.4342 & 51.5 & & 39.9 & 2.751 & 0.006 & 0.006 & 0.000 & & \\
\hline 2953 & $4 / 21 / 2004$ & 10:08:53 PM & 24.875 & 24.91 & 24.198 & & & & & & & & & & & & & & & & & & & & & & & $\begin{array}{l}0.13 \\
0.13 \\
\end{array}$ \\
\hline 2954 & $4 / 21 / 2004$ & (1) & & & & & & & & 18.887 & & -1.218 & & 50.74 & 0.04 & & & & & & & & & & & & & $\begin{array}{l}0.13 \\
0.13 \\
\end{array}$ \\
\hline & 4/21/2004 & 10:10:53 PM & 24.886 & 24.90 & 24.204 & 25.424 & & 49.664 & 53.402 & & 31.08 & -1.218 & & & 0.041 & 14.643 & & & .4842 & & & 40.4 & 2.784 & & $5006>$ & & & \\
\hline & 4/21/2004 & 10:11:53 PM & 24.9 & 24.91 & 24.217 & 25.507 & 25.215 & $\begin{array}{lll}49.092 \\
\end{array}$ & 52.888 & 18.824 & 30.65 & -1.221 & -7.391. & 50.659 & 0.041 & 14.643 & 0.003 & 3630.1 & 60.5008 & 51. & 12.4 & 39.9 & 2.749 & 0.006 & 0.006 & 0.000 & 0.157 & \\
\hline 29 & $1,1 / 2004$ & 10:12:53 PM & 24.896 & 24.89 & 24.218 & 25.568 & 25.296 & 49.35 & 53.164 & 19.03 & 30.72 & -1.218 & -7.39 & 51.316 & 0.041 & 14.643 & & 3631.1 & 0.5175 & & & & 2.761 & & 0.006 & & & 0.13 \\
\hline & $11 / 2004$ & & & & & & & & & & 30.79 & & & & & & & & & & & & & & & & & \\
\hline & $41 / 21212004$ & 10:14:53 PM & 24.8966 & 24 & 24.219 & 25.694 & 25.406 & 49.338 & 53.06 & 18.858 & .91 & -1.2211 & & 0.123 & 0.04 & 14.643 & & 3633. & & & & & & & .006 & 1000 & & \\
\hline & $41 / 21212004$ & $\begin{array}{l}1.1 .5 .53 \mathrm{MM} \\
10.53 \mathrm{MM}\end{array}$ & & & 24.219 & 年5.1/25 & & 年94.25 & $\frac{53.128}{5212}$ & & 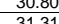 & $\frac{-1.218}{-1201}$ & & 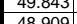 & 0.04 & 望4.643 & & & & & & & & & & & & \\
\hline 2962 & $\begin{array}{l}/ 21 / 2004 \\
4 / 21 / 2004 \\
\end{array}$ & $10: 17: 53 \mathrm{PM}$ & $\frac{24.903}{24.916}$ & $\frac{24.90}{24.90}$ & $\begin{array}{l}24.221 \\
24223\end{array}$ & 告3.001 & $\frac{25.445}{25.49}$ & 年45.352 & $\frac{5.13}{53228}$ & (18.476 & 31.31 3156 & $\frac{-1.211}{-1218}$ & & 年 & $\frac{0.041}{0.042}$ & 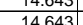 & 0.003 & & & & 121 & & & & & 0.000 & & 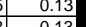 \\
\hline 296 & $4 / 21 / 2004$ & $10: 18: 53 \mathrm{PM}$ & $\frac{24.910}{24.917}$ & 24.91 & 24.204 & 25.895 & $\frac{25.54}{25.527}$ & & $\begin{array}{ll}0.220 \\
53.218\end{array}$ & 18.404 & 31.33 & -1.221 & -7.3 & 48.963 & 0.041 & $\frac{14.045}{14.643}$ & 0.00 & 3637. & 0.6175 & 51.7 & $\frac{12 .}{12 .}$ & $\frac{4 c}{44}$ & 8.784 & & 0.006 & 0.00 & & $\frac{0.13}{0.13}$ \\
\hline & $4 / 21 / 2004$ & 10:19:53 PM & 24.918 & 24.92 & 24.206 & 25.91 & 25.5 & 49.34 & 53.141 & 18.214 & 31.51 & & & & & 14.643 & & 3638. & & & & & & & & & & \\
\hline & $4 / 21 / 2004$ & 10:20:53 PM & 24.93 & & 24.212 & & $\overline{25.4}$ & & & 18.037 & 31.59 & -1.215 & & 9.754 & 0.041 & $\frac{14.643}{15}$ & & 3639 . & & & & & & & & & & \\
\hline & 4/21/2004 & & 24.932 & 24.93 & 24.194 & & & & 53.18 & & & -1.218 & & & & 14.643 & & & & & & & & & & & & \\
\hline & 4/21/2004 & 10:22:53 PM & & 24.94 & 24.18 & 25.82 & $25.3 !$ & 49.776 & & 18.064 & 31.99 & -1.221 & & $\begin{array}{ll}48.788 \\
\end{array}$ & & 14.643 & & 3641.1 & 1.6842 & & & $40.5-3$ & & & & & & \\
\hline & $4 / 21 / 2004$ & 0:23:53 PM & 24.932 & 24.94 & 24.169 & 25.729 & 25.292 & & 53.518 & 18.141 & 31.97 & -1.221 & & 47.768 & 0.041 & 14.643 & 0.003 & 3642 & 008 & $52.6-3$ & $11.7 \mathrm{r}-\mathrm{r}$ & & 1818 & D.006 & & & & \\
\hline 29 & 4/21/2004 & 10:24:53 PM & 24.927 & 24.93 & 24.144 & 25.649 & 25.2. & 49.45 & 53.238 & 18.113 & 31.68 & -1.224 & -7.37 & 48.784 & 0.041 & 14.643 & & 3643 & 60.7175 & 51. & 12. & 40.6 & 2.797 & & 006 & & 154 & 0.11 \\
\hline 2078 & $11 / 2004$ & 10:25:53 PM & 24.946 & 24.9 & 24.158 & 25.609 & 25.15 & 49.643 & 576 & 17.909 & 32.1 & -1.221 & & 48.951 & 0.04 & 14.643 & & 3644 & . & & & 40 & 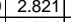 & & & & & \\
\hline 20971 & $4 / 21 / 2004$ & & 24.93 & 24.92 & & & & & & & & & & 48.5/5 & 0.043 & & & 3645 & & & & & & 0.006 & & & 0.162 & \\
\hline 207 & 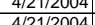 & $\begin{array}{l}10.253 \mathrm{PM} \\
10.28 \mathrm{PM}\end{array}$ & 2.4.453 2093 & 24.95 & 24.151 & 25.521 & 250.083 & 年49.475 & 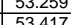 & $\frac{1.83}{17012}$ & 31.97 3108 & - 1.221 & & 年6.162 & 0.044 & 14.643 & & 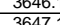 & 7842 & & & & 100 & & & & & 0.15 \\
\hline & 4 & $10: 29: 53 \mathrm{PM}$ & $\begin{array}{l}24.953 \\
24.931\end{array}$ & $\frac{24.94}{24.92}$ & 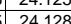 & (25.453 & 250006 & 年 49.5060 & (53.415 & 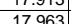 & $\frac{31.90}{320}$ & -1.244 & & 年45.251 & 0.041 & 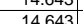 & & (3) 3648 & 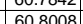 & $52.8-3$ & 119 & 40 & & & & 000 & 0 & 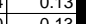 \\
\hline & $4 / 21 / 2004$ & $10.30 .53 \mathrm{PM}$ & 24.946 & 24.92 & 24.138 & 25.413 & & & 53.452 & 17.992 & 31.92 & -1218 & & & $\frac{0.042}{0.042}$ & & & 3649 & & & 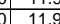 & & & & & & & \\
\hline 29 & $4 / 21 / 2004$ & 10:31:53 PM & $\frac{2.923}{24.923}$ & $\frac{24.89}{24.89}$ & 24.096 & 25.366 & $\frac{25.049}{25.049}$ & & 228 & $\frac{17.72}{11.72}$ & $\frac{21.99}{31.99}$ & -1.224 & & 47. & $\frac{0.042}{0.042}$ & 14.6 & & 365 & & & it. & 4 & 804 & & 0.006 & & & . \\
\hline & & & & 24.90 & 24.099 & & & & & 17.879 & & & & & & & & 365 & & & & & & 0.006 & 0.00 & & & \\
\hline & & & 24.916 & 24.88 & 24.103 & 25.469 & & & 487 & 18.015 & & -1.218 & & & & & & & & & & & & & & & & \\
\hline 297 & & $3 \mathrm{PM}$ & 24.916 & 24.88 & 24.108 & 25.529 & & & 53.329 & & & -1.218 & & & & & & & & & & & & & & & & \\
\hline 298 & $4 / 21 / 2004$ & 10:35:53 PM & 24.911 & 24.8 & 24.093 & 25.584 & 25.3 & 50.021 & 53.974 & 18.009 & 32.33 & \begin{tabular}{ll|l|}
-1.218 \\
\end{tabular} & & 47.891 & 0.04 & 14.643 & & 3654.1 & 0.9008 & 52 & $11.7 \mathrm{r}$ & & & & 0.006 & & 0.149 & 0.13 \\
\hline & 4/21/2004 & 10:36:53 PM & 24.918 & 24.88 & 24.1 & 25.665 & 25.4 & 49.61 & 50.543 & 17.713 & 32.14 & -1.218 & & 48.604 & 0.041 & 14.6 & & 365 & ( & 54 & 11. & $\frac{44}{46}+3$ & & & 0.00 & & & 0.15 \\
\hline & & 10:37:53 PM & 24.918 & & 24.1 & 25.706 & 25. & 49.637 & 53.601 & 17.768 & 32.29 & -1.221 & & 7.976 & 0.04 & & & 365 & & & $1+.6$ & & & & & & & \\
\hline 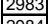 & 112004 & 53 PM & 24.52 & 24.88 & 24.1 & 25.758 & 25. & & 53.86 & $17.881 \mathrm{r}$ & , & -1.2. & & & 0.043 & & & 年 & & & & & & & & & & 0.1 \\
\hline & $4 \mid 4 / 212004$ & $\begin{array}{l}10.3953 \mathrm{PMM} \\
10.50 \mathrm{MMM}\end{array}$ & $\begin{array}{ll}2.4091 \\
2.923\end{array}$ & 24.86 & $\frac{24.113}{22.31}$ & $\frac{25.174 \mid}{25831}>3$ & & & & 1.944 & & $\frac{-1.221}{-1201}$ & & & & & & & & 52 & 11 & 40 & & & & & & 011 \\
\hline & & $10.40 .53 \mathrm{PM}$ & 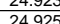 & 24.05 & 24.127 & 年.0.015 & & & & 17054 & & & & & & & & & & & & & & & & & & \\
\hline & $\frac{4 \pi / 1204}{4 / 212004}$ & $55 \mathrm{PM}$ & 24.93 & 24.90 & $\frac{2.42}{22.12}$ & 25.584 & $\frac{2.5}{25.3}$ & & 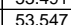 & 18.024 & & -1.218 & & & $\frac{0.041}{0.04}$ & & & $\frac{506}{366}+2$ & $\frac{010}{61.0}$ & & 势 & & & & & & & 21 \\
\hline $298 \varepsilon$ & $\mid 4 / 21 / 2004$ & & 24.953 & 24.91 & 24.145 & & & & & & & & & & & & & & & & & & & & & & & \\
\hline 2989 & $1 / 2004$ & & 24.932 & 24.91 & 24.104 & 25.744 & & 49. & 53.445 & 17.834 & & -1.221 & & 48. & & $14.6 \mathrm{C}>\mathrm{C}$ & & & & - & th: & & & & & & & \\
\hline & & & 24.942 & & 24.109 & & & & & 17.808 & & & & & & & & & & $512>-1$ & 争 & & & & & & & \\
\hline 299 & 4/21/2004 & & 24.935 & 24.90 & 24.103 & & & 49.398 & 53.404 & 17.83 & & -1.218 & & 48.604 & & & & 36 & & & 11 & & & & & & 154 & \\
\hline 299 & $4 / 21 / 2004$ & $10: 47: 53 \mathrm{~F}$ & 24.93 & 24.91 & 24.087 & 25.562 & 25 & & 53.468 & 17.855 & & \begin{tabular}{|c|c|}
-1.218 \\
\end{tabular} & & & & & & & & 52 & 11. & & & & & & ס.157 & $0.13>>$ \\
\hline 299 & 4/21/2004 & & 24.918 & 24.90 & 24.08 & & & 49. & & 17.98 & & -1.218 & & & & & & & & 36 & 12. & & & & & & & \\
\hline 299 & & $10: 49$ & 24. & 24.90 & 24.079 & 25.459 & & & & 17.842 & 31. & -1.218 & & & & & & 36 & & 51. & 11.9 & & & & & & 36 & \\
\hline 200 & & 10:50: & 24.91 & $24.8 \mathrm{~g}$ & 24.063 & 25.413 & $25.6 \mathrm{C}>\mathrm{C}$ & & 53.458 & 17.976 & 31.9 & -1.218 & & & 0.04 & 14. & & so & & ra $>2>0$ & 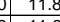 & & & & & & & \\
\hline & & & & & & & & & & & & -1.218 & & & & & & & & & & & & & & & & \\
\hline 20 & 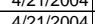 & $\begin{array}{l}10.52 .5 \mathrm{MPM} \\
10.533 \mathrm{MM}\end{array}$ & 20 & 24.060 & 24.004 & & & & & $\frac{1.1 .11}{17097}$ & & -1.24 & & & & & & & & & & & & & & & & \\
\hline & $\frac{421 / 204}{4 / 212004}$ & & 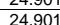 & 2486 & & & & & & & & $\frac{-1.24}{-1.224}$ & & & & & & & & 52 & 116 & & & & & & & \\
\hline & $4 / 21 / 200$ & 10:55:53 PM & 24.9 & 24.86 & 24.067 & 25.582 & 25.4 & 49.363 & 53.361 & 17.883 & & -1.215 & & 48.0 & 0.039 & & & 36 & & UL & $1+0$ & & & & & & 147 & \\
\hline 300 & $4 / 21 / 2004$ & $10: 56: 53 \mathrm{PM}$ & & & & & & & & & & & & & & & & & & & & & & & & & & \\
\hline & & $10: 57.53 \mathrm{PM}$ & & 24.8 & & 25.664 & & 49 & 53.674 & 17934 & & -1.22 & & & & & & & & & & & & & & & & \\
\hline 300 & $4 / 21 / 2004$ & 10:58:53 PM & 24.902 & 24.87 & 24.104 & 25.745 & 25.3 & 499.564 & 53.516 & 17.817 & & $\begin{array}{ll}-1.218 \\
\end{array}$ & & 48. & 0.0 & $14.6>>>$ & & 367 & $\overline{612}$ & & 119 & 408 & & & & & 54 & \\
\hline & $4 / 21 / 2$ & $10: 5$ & $24.8>>2$ & 24 & & & & $49.8 \quad>\quad>$ & & & & $-1.22<>3$ & & & & & & & & & & & & & & & & \\
\hline & $4 / 21 / 20$ & 11:00 & $24.8>>3$ & 24. & & & & 49. & 53.244 & & & -1.224 & & & & 14. & & & & & 1. & & & & & & & \\
\hline 300 & & & 24. & 24. & & & & 48.95 & 52.94 & & & & & & 0.04 & & & & & 51 & 12 & & & & & & 54 & \\
\hline 300 & 4/21/2004 & & 24.924 & 24. & 24.136 & $25.84 t$ & & 48. & 52.612 & & & -1.218 & & & 0.04 & $14.6 \mathrm{r}-\mathrm{s}$ & & & & & $2.3 .3>3$ & & & & & & 154 & \\
\hline 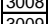 & $4 / 21 / 2004$ & 11:03:53 PM & 24.904 & 24.88 & 24.111 & $25.17 / 2$ & 25.384 & 49.1805 & 53.091 & 18.385 & 31.28 & -1.221 & & 49.16 & & 14.643 & & 368 & & $51.6>-4$ & $\begin{array}{ll}6 & 12.2 \\
\end{array}$ & 40. & 2.19 & 06 & 0.0 & 0.000 & 0.144 & 0.12 \\
\hline$\frac{509}{3010}$ & \begin{tabular}{|l|l|l|l|l|l|}
$4 / 21 / 2004$ \\
\end{tabular} & 11.04.05:53 PM & $\frac{24.905}{24.894}$ & 24.88 & \begin{tabular}{|l|l|}
24.101 \\
24.096 \\
\end{tabular} & \begin{tabular}{|l|l|}
25.101 \\
2522 \\
\end{tabular} & \begin{tabular}{|l|}
25.134 \\
25.134 \\
\end{tabular} & $\begin{array}{l}49.021 \\
49.262 \\
\end{array}$ & \begin{tabular}{|l|}
52.992 \\
53.137 \\
\end{tabular} & \begin{tabular}{|l|l|}
18.263 \\
18.263
\end{tabular} & $\frac{30.91}{31.39}$ & \begin{tabular}{|l|}
-1.224 \\
-1.224 \\
\end{tabular} & & $\begin{array}{l}49.505 \\
\end{array}$ & $\frac{0.041}{0.041}$ & $\begin{array}{l}\frac{14.045}{14.643} \\
\end{array}$ & 0.003 & & 61.4008 & \begin{tabular}{|l}
51.7 \\
51.7
\end{tabular} & 12.1 & 40.3 & 2.781 & $\mid$\begin{tabular}{|c|c|}
$\mid 0.006$ \\
\end{tabular} & 0.006 & 0.000 & 0.151 & 0.13 \\
\hline
\end{tabular}


WSRC-TR-2005-00105, REVISION 0

SRNL-RPP-2005-00012, REVISION 0

RUN \# 2.03A AND B; FIRST AND SECOND HALF OF SLURRY DEWATERING - CONT.

\begin{tabular}{|c|c|c|c|c|c|c|c|c|c|c|c|c|c|c|c|c|c|c|c|c|c|c|c|c|c|c|c|c|}
\hline & A & B & D & $\mathrm{E}$ & $\mathrm{F}$ & $\mathrm{G}$ & $\mathrm{H}$ & $\mathrm{J}$ & $\mathrm{K}$ & $\mathrm{L}$ & $\mathrm{M}$ & $\mathrm{N}$ & 0 & Q & $\begin{array}{ll}\mathrm{R} \\
\end{array}$ & $s$ & $\mathrm{~T}$ & $v$ & W & $x$ & & $\mathrm{z}$ & $A A$ & $A B$ & $\mathrm{AC}$ & $A D$ & $A E$ & AF $A$ \\
\hline $\begin{array}{l}\frac{3011}{3012} \\
012\end{array}$ & \begin{tabular}{|l|l|}
$4 / 21 / 2004$ \\
$4 / 212004$
\end{tabular} & $\begin{array}{l}11: 06: 53 \text { PM } \\
11.0755 \text { PM }\end{array}$ & $\frac{24.899}{24.893}$ & $\frac{24.883}{22.877}$ & $\begin{array}{l}24.111 \\
24.11\end{array}$ & $\begin{array}{l}25.576 \\
25.526 \\
\end{array}$ & \begin{tabular}{|l|}
25.289 \\
25.173 \\
\end{tabular} & $\begin{array}{l}49.243 \\
49.573 \\
\end{array}$ & \begin{tabular}{|l|}
53.184 \\
53.609 \\
\end{tabular} & $\frac{18.317}{18.249}$ & \begin{tabular}{|l|}
31.338 \\
31.59 \\
\end{tabular} & \begin{tabular}{|l|l|} 
& -1.218 \\
-1.221
\end{tabular} & $\begin{array}{l}-7.472 \\
-7.469 \\
\end{array}$ & $\begin{array}{l}48.523 \\
48.865\end{array}$ & \begin{tabular}{|c|}
0.04 \\
0.041
\end{tabular} & $\begin{array}{l}\frac{14.643}{14.643} \\
\end{array}$ & $\begin{array}{l}0.003 \\
0.003 \\
\end{array}$ & $\frac{3685.1}{368.5}$ & 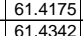 & \begin{tabular}{|l|}
51.2 \\
52.2
\end{tabular} & $\frac{11.0}{12.0}$ & \begin{tabular}{|l|}
40.3 \\
40.6
\end{tabular} & \begin{tabular}{|l|}
2.778 \\
2.798
\end{tabular} & \begin{tabular}{|l|l|}
0.006 \\
0.006 \\
\end{tabular} & \begin{tabular}{|l|}
0.006 \\
0.006 \\
\end{tabular} & 0.000 & $\begin{array}{l}0.152 \\
0.154\end{array}$ & \begin{tabular}{|l|l}
0.13 \\
0.13
\end{tabular} \\
\hline \begin{tabular}{|c|}
3013 \\
3013 \\
\end{tabular} & & & $\frac{24.050}{24.902}$ & & & & & & & $\frac{10.445}{18.103}$ & & & & & & & & & & & & & & & & & & \\
\hline 3014 & $4 / 21 / 2004$ & 11:09:53 PM & 24.894 & 24.873 & 24.091 & 25.406 & 24.934 & 49.428 & 53.416 & 18.096 & 31.751 & -1.224 & $\begin{array}{l}-7.429 \\
\end{array}$ & 48.513 & $\begin{array}{l}0.042 \\
0.04\end{array}$ & 14.643 & 0.003 & 3688.1 & $\begin{array}{l}61.4675 \\
61.450\end{array}$ & 51. & 11.9 & & \begin{tabular}{|l|l|}
2.799 \\
\end{tabular} & 0.006 & 0.006 & 0.000 & 0.158 & 0.13 \\
\hline 3015 & $4 / 221 / 2004$ & & 24.896 & 24.869 & 24.098 & 25.373 & 24.881 & 49.548 & \begin{tabular}{|l|}
53.477 \\
\end{tabular} & 18.244 & $\begin{array}{l}31.683 \\
31.62\end{array}$ & -1.218 & $\frac{-7.429}{-1.429}$ & 48.56 & $\begin{array}{l}0.039 \\
0.039\end{array}$ & 14.643 & & 3689.1 & 61.4842 & & & & & & 0.006 & & & 0.12 \\
\hline & & & & & & & 24.859 & 49.33 & & & 31.494 & & & & & 14.643 & & & & 51. & & & & & & & & \\
\hline 3017 & $4 / 21 / 2004$ & $\begin{array}{l}11: 12: 53 \mathrm{PM} \\
11: 152 \mathrm{PM}\end{array}$ & 24.881 & 24.845 & \begin{tabular}{|l|l|}
24.084 \\
2.075
\end{tabular} & $\begin{array}{l}25.283 \\
25205 \\
\end{array}$ & 24.887 & $\begin{array}{l}49.504 \\
\end{array}$ & \begin{tabular}{|l|l|}
53.462 \\
53.350
\end{tabular} & 18.305 & 31.501 & -1.218 & $\begin{array}{l}-7.426 \\
.717\end{array}$ & 49.022 & \begin{tabular}{|l|l|}
0.04 \\
\end{tabular} & 14.643 & 0.003 & 3691.1 & 61.5175 & 52.4 & \begin{tabular}{l|l}
0 & 12.0 \\
\end{tabular} & \begin{tabular}{l|l}
0 & 40.5 \\
0.7
\end{tabular} & 2.793 & \begin{tabular}{|l|l|l|l}
0.006 \\
\end{tabular} & 0.006 & 0.000 & 0.151 & 0.13 \\
\hline $\begin{array}{l}3018 \\
3019 \\
019\end{array}$ & $\begin{array}{l}/ / 21 / 2004 \\
4 / 21 / 2004\end{array}$ & $\begin{array}{l}\text { 11:13:53 PM } \\
\text { 11:14:53 PM }\end{array}$ & 24.873 & $\frac{24.832}{24.815}$ & \begin{tabular}{|l|}
24.075 \\
2.454 \\
\end{tabular} & $\begin{array}{l}25.305 \\
25.329 \\
\end{array}$ & \begin{tabular}{|l|}
24.913 \\
25.007 \\
\end{tabular} & \begin{tabular}{|r|}
49.481 \\
49
\end{tabular} & \begin{tabular}{|l|}
53.359 \\
52.934 \\
\end{tabular} & $\frac{18.058}{18.043}$ & \begin{tabular}{|l|}
31.841 \\
31.287 \\
\end{tabular} & \begin{tabular}{|l|l|}
-1.218 \\
-1.221
\end{tabular} & $\begin{array}{l}-7.417 \\
-7.417\end{array}$ & $\begin{array}{l}48.928 \\
49.952 \\
\end{array}$ & \begin{tabular}{|l|l|}
0.041 \\
0.041
\end{tabular} & $\begin{array}{l}14.643 \\
14.643\end{array}$ & \begin{tabular}{|l|l|}
0.003 \\
0.003
\end{tabular} & 3692.1 & \begin{tabular}{|l|l|}
61.5342 \\
61.508 \\
\end{tabular} & $\frac{51.6}{51.1}$ & $\frac{12.0}{122}$ & 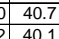 & \begin{tabular}{|l|l|}
2.803 \\
2768
\end{tabular} & \begin{tabular}{|l|l|}
0.006 \\
0.006
\end{tabular} & $\begin{array}{l}0.006 \\
0.006\end{array}$ & 0.000 & $\begin{array}{l}0.154 \\
0.156 \\
\end{array}$ & $\begin{array}{l}0.13 \\
0.13\end{array}$ \\
\hline 3020 & $4 / 21 / 2004$ & $11: 15: 53 \mathrm{PM}$ & 24.871 & & 24.093 & 25.408 & \begin{tabular}{|l|}
25.111 \\
25.11 \\
\end{tabular} & 49.396 & \begin{tabular}{|l|}
53.367 \\
\end{tabular} & 18.248 & 31.49 & -1.218 & & 48.89 & 0.041 & $\begin{array}{l}14.045 \\
14.643 \\
\end{array}$ & $\begin{array}{l}0.0003 \\
0.003\end{array}$ & 3694.1 & 61.5675 & 51. & & \begin{tabular}{|l|l|}
0 & 40.1 \\
\end{tabular} & \begin{tabular}{|l|l|} 
& 2.700 \\
\end{tabular} & \begin{tabular}{|l|}
0.0006 \\
0.006 \\
\end{tabular} & $\begin{array}{l}.006 \\
0.006 \\
\end{array}$ & 0.000 & 0.156 & 0.13 \\
\hline & $4 / 21 / 2004$ & 11:16:53 PM & & & & & & & 53.649 & 18.057 & 31.979 & & & 48.258 & & 14.643 & & 3695.1 & \begin{tabular}{|l|l|}
61.5842 \\
\end{tabular} & & & & & & & & & $\begin{array}{l}0.13 \\
0.12 \\
\end{array}$ \\
\hline 3022 & $4 / 21 / 2004$ & 11:17:53 PM & & & 24.093 & $\begin{array}{ll}25.543 \\
\end{array}$ & 25.201 & 49.222 & 53.234 & 18.03 & 31.581 & -1.215 & -7.411 & 47.653 & 0.041 & 14.643 & 0.003 & 3696.1 & \begin{tabular}{|l|l|}
61.6008 \\
\end{tabular} & & 11.7 & \begin{tabular}{l|l}
7 & 40.4 \\
\end{tabular} & & & 0.006 & 0.000 & & \\
\hline 3023 & $4 / 21 / 2004$ & & 24.862 & 24.811 & & 25.58 & & & & & & & & 47.301 & & 14.643 & & & & & 11. & & & & & & & $\frac{0.13}{0.13}$ \\
\hline 3024 & & 11:19:53 PM & & & 24.09 & & & 49.878 & 53.930 & & & -1.218 & & & 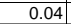 & & & 3698.7 & & & - 10 & & & & & & & \\
\hline & $4 / 21 / 2004$ & 11:20:53 PM & & 24.807 & & 25.646 & 25.378 & 49.459 & 53.458 & 18.234 & 31.499 & & & $\begin{array}{ll}47.297 \\
\end{array}$ & 0.04 & 14.643 & & & 61.6508 & & & 40 & $7070+3$ & 0.006 & 0.006 & & & \\
\hline 3026 & 4/21/2004 & 11:21:53 PM & 24.859 & 24.812 & 24.081 & 25.716 & 25.294 & 49.55 & 53.541 & 18.181 & 31.726 & -1.221 & -7.411 & 47.309 & 0.041 & $\begin{array}{ll}14.643 \\
\end{array}$ & 0.003 & 3700.1 & 61.6675 & 52. & 11.6 & 40.6 & 2.802 & 0.006 & 0.006 & 0.000 & 0.154 & 0.13 \\
\hline & $1,1 / 2004$ & 11:22:53 PM & 24.849 & 24.808 & 24.071 & 25.702 & 25.409 & 49.63 & 53.667 & 18.121 & 31.79 & -1.221 & -7.41 & 47.551 & 0.042 & 14.643 & & 3701.1 & 61.6842 & & 11. & & & & 0.006 & & & \\
\hline & 11/2004 & & & & & & 25.247 & & 3.416 & & 31.665 & -1.224 & & & 0.04 & 14.643 & & & & & & & & & & & & \\
\hline & 4/21/2004 & $\begin{array}{l}11: 24: 53 \mathrm{PM} \\
11.25 .52 \mathrm{PM}\end{array}$ & 24.867 & 24.82 & 24.089 & 25.655 & 25.1 & 49.456 & 53.466 & 18.187 & 31.648 & -1.224 & & 47.484 & 0.04 & 14.643 & & & 1.7175 & & & & & & .006 & & & \\
\hline 3031 & $\frac{|4| 21 / 2004}{1 / 21204}$ & $\begin{array}{l}11.2535 \mathrm{PM} \\
11.265 \mathrm{MM}\end{array}$ & & & & & & $\frac{49.64 t}{4043}$ & 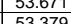 & $\frac{18.139}{1836}$ & $\frac{31.884}{32.151}$ & $\frac{-1.211}{-1210}$ & & & & 望4.643 & & & 17708 & & & & & & & & & \\
\hline $3033^{3}$ & $4 / 21 / 2004$ & $\begin{array}{l}11.27 .53 \mathrm{PM} \\
11.25\end{array}$ & 24.87 & $\frac{24.024}{24.824}$ & 24.097 & $\begin{array}{ll}25.487 \\
25.487\end{array}$ & 25.095 & 49.423 & $\frac{23.445}{53.445}$ & $\frac{10.29}{18.294}$ & 31.457 & $\frac{-1.221}{-1.221}$ & $\frac{-4.42}{-7.42}$ & 49.034 & 0 & 14.643 & 0.003 & 3706.1 & 每.1.7675 & & & 40. & & & & 0.000 & & \\
\hline & $4 / 21 / 2004$ & 11:28:53 PM & 24.869 & 24.818 & \begin{tabular}{|l|l|}
24.167 \\
\end{tabular} & $\begin{array}{ll}25.417 \\
\end{array}$ & 25.01 & 49.226 & 53.189 & 18.233 & 31.267 & -1.221 & -7.408 & 48.392 & 0.041 & 14.643 & 0.003 & 3707.1 & 61.7842 & 51.7 & 11.9 & & & & 0.006 & 0.000 & & 0.13 \\
\hline 30 & 1/2004 & 11:29:53 PM & 24.862 & & 24.145 & 25.379 & 24.87 & 49.4 & 53.402 & $18.28 \mathrm{~s}$ & 31.47 & -1.218 & -7.408 & & 0.04 & 14.643 & & 3708.1 & & & 12. & & & & & & & \\
\hline & $1: 12004$ & 11:30:53 PM & $\frac{24.865}{20.92}$ & 24.79 & & 25.322 & 24.8 & & $\begin{array}{l}3.323 \\
3.62 \\
3.62\end{array}$ & 18.13 & 31.614 & & & 22 & 0.041 & 14.643 & & & 8175 & & & & & & & & & \\
\hline & $\mid$ & 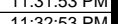 & $\frac{24.863}{24845}$ & $\frac{24.781}{24.769}$ & $\frac{24.12}{24.087}$ & 年5.255 & $\frac{24.646}{20896}$ & $\frac{49.345}{49.16}$ & $\begin{array}{ll}53.462 \\
53218\end{array}$ & $\frac{18.424}{18256}$ & $\frac{31.412}{31.18}$ & & -7.41 & 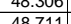 & $\begin{array}{r}0.039 \\
0.04 \\
\end{array}$ & $\frac{14.643}{14.643}$ & & 3711 & 10508 & & $\frac{11.6}{116}>>3$ & & & & & & & \\
\hline 303 & $4 / 21 / 2004$ & 11:33:53 PM & 24.865 & 24.774 & 22.413 & 25.277 & $\frac{24.090}{22.941}$ & $\begin{array}{r}49.10 \\
49.569\end{array}$ & 53.589 & $\frac{10.250}{18.276}$ & $\begin{array}{l}\text { S1.10 } \\
31.689 \\
\end{array}$ & $\frac{-1.221}{-1.218}$ & $\frac{-1.41}{-7.40}$ & $\begin{array}{r}40.111 \\
49.32 \\
\end{array}$ & 0.04 & $\frac{14.045}{14.643}$ & $\begin{array}{l}0.003 \\
0.003 \\
\end{array}$ & & 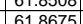 & 52 & 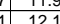 & & & (2) & & & & \\
\hline & $4 / 21 / 2004$ & 11:34:53 PM & 24.865 & 24.783 & 24.112 & 25.327 & & 49.55 & 53.576 & 18.299 & 31.572 & $\frac{-1.218}{-1.218}$ & -7.405 & 47.851 & 0.041 & 14.643 & & 3713. & $\frac{61.8842}{6.884}$ & $52,>>$ & $\frac{11.7}{11.7}$ & 40.6 & $\frac{2.097}{2.797}$ & & $\frac{006}{006}$ & & & 1 \\
\hline & 1/2004 & 11:35:53 PM & 24.86 & & & 25.387 & 25. & 49.519 & 53.56 & 18.303 & & $\begin{array}{ll}-1.218 \\
\end{array}$ & & 48.836 & 0.04 & 14.643 & & 3714 & & & & & 4794 & & & & & \\
\hline 304 & 4/21/2004 & 3 PM & 24.865 & 24.769 & 24.108 & 25.458 & 25.131 & 49.114 & 53.118 & 18.225 & 31.281 & -1.218 & & 48.26 & 0.04 & 14.643 & & 3715. & 61.9175 & 51 & $11.8 \mathrm{r} \quad \mathrm{s}$ & 40.2 & & 0.006 & 0.006 & 0.000 & 152 & \\
\hline 304 & $4 / 21 / 2004$ & 11:37:53 PM & 24.86 & 24.764 & 24.097 & 25.523 & 25.206 & 49.301 & 53.361 & 18.117 & 31.591 & -1.221 & & $\begin{array}{ll}49.178 \\
\end{array}$ & 0.04 & 14.643 & & & & & & & & & & & & \\
\hline & & & & & & & & 49.386 & 53.412 & & 31.474 & -1.218 & & & 0.04 & 14.643 & & & 1.9508 & & & 40 & & & & & & 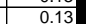 \\
\hline 304 & $4 / 21 / 2004$ & 11:39:53 PM & 24.857 & 24.755 & 24.084 & 25.614 & 25.312 & 499.542 & 53.713 & 18.165 & 31.681 & -1.221 & -7.403 & 48.725 & \begin{tabular}{|c|c|c|}
0.039 \\
\end{tabular} & 14.643 & & 3718.1 & 61.9675 & 52.8 & 11.9 & 40. & 2.800 & & 0.006 & 0.000 & 0.147 & \\
\hline & $4 / 21 / 2004$ & 11:40:53 PM & 24.842 & 24.751 & 24.064 & & 25.2 & 49.49 & 1559 & 18.101 & 31.849 & -1.221 & 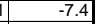 & 48.002 & 0.04 & 14.643 & & 3719.1 & 61.9 & & 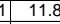 & & & & & & & \\
\hline & - 11/2004 & 11:41:53 PM & 24.875 & 24.788 & 24.107 & 25.712 & & 49.504 & & 18.275 & 31.691 & -1.218 & & 48.07 & 0.039 & & & & & & the & & 8798 & & & & & \\
\hline & & $33 \mathrm{PM}$ & 24.855 & 24.774 & 24.082 & 25.673 & 25 & 49.384 & 33.448 & & & -1.221 & $7.38-3$ & 48.73 & 0.04 & 14.643 & & & 2.0175 & & 11. & & & 0.006 & 0.00 & .000 & & \\
\hline & & & & & & & & & & & & & & & & & & & & & & & & & & & & \\
\hline$\frac{3056}{305}$ & 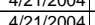 & 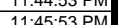 & 24.053 & $\frac{24.110}{24.782}$ & - & 25.594 & $\frac{25}{251}>>$ & $\frac{49.35}{49.363}$ & 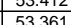 & $\frac{10.201}{18.353}$ & 年1.3944 & $\frac{-1.241}{-1221}$ & $\frac{-1.35}{-73}=0$ & $\begin{array}{ll}46.011 \\
99117\end{array}$ & $\mid 0.049$ & 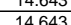 & & & 620675 & 51.9 & 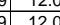 & 40 & & & 0.006 & & $\frac{152}{148}>>$ & \\
\hline (2) & $4 / 21 / 2004$ & $11: 46: 53 \mathrm{PM}$ & $\frac{24.049}{24.859}$ & 24.787 & & $\frac{31}{461}$ & 25.0 & 49.3 & - & & 31.349 & -1.218 & & 50.711 & & & & & & . & & & & & & & & \\
\hline & $4 / 21 / 2004$ & 11:47:53 PM & & 24.776 & 24.064 & 395 & 24.9 & 49.4 & $\frac{23.547}{53.54}$ & 18.138 & 31.591 & -1.221 & & 48.565 & 0.041 & 14.643 & & & $\frac{12.1008}{62.1008}$ & 我 & (2. & , & & & & & & \\
\hline soc & $\frac{1 / 2004}{1 / 204}$ & 11:48:53 PM & 24.845 & & & & & & & 18.149 & & -1.221 & & & 001 & & & & & & & & & & & & & \\
\hline & & $53 \mathrm{PM}$ & $24838 \quad \mathrm{C}$ & 2476 & 2405 & (25025 & & & 53761 & 18.314 & 31.698 & -1218 & & & 80 & & & & & & & & & & & & & \\
\hline & 4/21/2004 & 11:50:53 PM & 24.836 & 24.75 & 24.043 & 25.238 & $24.8 \mathrm{Y} \quad \mathrm{C}$ & \begin{tabular}{ll|l}
49.467 \\
\end{tabular} & 53.582 & 18 & 31. & -1.221 & & 48. & 0.039 & 14. & & & 62.1508 & & 11.9 & 44 & 2.794 & & & & & \\
\hline & & 11:51:53 PM & 24.834 & 24.758 & 24.061 & & & & 53.599 & & & -1.218 & & $4 \varepsilon$ & & & & & & 52 & 11. & & & & & & & \\
\hline 305 & 4/21/2004 & 11:52:53 PM & 24.822 & 24.746 & 24.049 & 25.184 & 24. & $49.47 !$ & 53.53 & & $\begin{array}{lll}31.486 \\
\end{array}$ & -1.218 & & 48. & 0.04 & 14. & & & 62.1 & 52 & 11. & & & & & & & \\
\hline 3058 & 4/21/2004 & $11: 53$ & 24.836 & 24.75 & 24.063 & 25.223 & 24.9 & 49.235 & 53.3 & & & & & & 0.041 & $14.6 \mathrm{r}-\mathrm{l}$ & & & & 秉 & 12. & & & & & & & \\
\hline & & & 24.841 & 24.749 & & & & & & & & -1.218 & & & & & & & & 52 & 11. & & & & & & & \\
\hline & 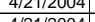 & $\frac{11.55}{1156}$ & 24.815 20.821 & 24.729 & 24.047 & 25.292 & $\frac{25}{250}$ & 49.291 & 53.383 & 18.317 & $\begin{array}{ll}31.326 \\
31.196\end{array}$ & -1.221 & & 48.3 & & 14.6 & & & & & . & - & & & & & & \\
\hline 306 & 年 & $\frac{11.50}{10.15:}$ & & $\frac{24.124}{24.72}>0$ & $\frac{24.000}{24.073}$ & $\frac{25.380}{25459}$ & & & $\frac{53.459}{55.516}$ & & & $\frac{-1.210}{-1218}$ & & & & & & & & & 120 & & & & & & & \\
\hline & 4 & 1.1:58: & 24. & 204701 & 24.049 & 20.455 & & 494 & & & & -1.221 & & & & & & & & & & & & & & & & \\
\hline 306 & $\frac{4 / 21 / 2004}{4.2004}$ & & 24.809 & 24.722 & & 25.546 & 25.2 & 49 & 53.895 & 18.215 & & $\frac{-1.221}{-1.221}$ & & 48 & & & & & & & tx. & & & & & & & \\
\hline & & & & & & & & & & & & & & & & & & & & & & & & & & & & \\
\hline 306 & & & 23.298 & 21.599 & $24373^{2}>$ & 22844 & 22.51 & 50.5 & 54722 & 19404 & $31.51:$ & -1.218 & & & & & & & & & & & & & & & & \\
\hline 306 & $4 / 22 / 2$ & 8:46: & $23.2>>$ & 21.636 & 24. & 22. & & & & $19.6>$ & & $-1.2 \times$ & & & & & & & & & & 4 & & & & & & \\
\hline 3068 & 4/22/2004 & 8:47:25 AM & 23.342 & 21.663 & 24.31 & 22.883 & 22. & 49.168 & 54.556 & 19.43 & & -0.106 & & $48.3>>>$ & & 14.643 & & & 71.0 & 53 & 11.9 & 39.6 & 2. & 0.004 & 0.00 & & & \\
\hline & 4/22/2004 & $8: 48: 25$ & 23. $\mathrm{Y}$ & 21.696 & 24.293 & 22.915 & 22.6 & 48.9 & 55.338 & 19.549 & & 0.939 & & & & 14. & & & & & 11.9 & 3 & & & & & & \\
\hline |3070 & 2/2004 & 8:49:25 & 23.513 & 21.7355 & & 22.994 & & 49. & 55.276 & & & 0.091 & & & & & & & & & $11.8 \mathrm{~s}$ & 4 & & & & & & \\
\hline 3071 & 4/22/2004 & 8:50:25 AM & 23.512 & 21.755 & 24.275 & 23.053 & $22.8 \mathrm{rg}$ & 51.02 & 55.539 & 19.406 & 31. & -0.995 & & & 0.01 & 14.643 & & & & & & & & & & & & \\
\hline $307:$ & $\frac{4.21210004}{4121004}$ & $\begin{array}{l}8.51 .251 \\
8.52 .25 A\end{array}$ & $\frac{23.400}{23.97}$ & 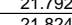 & 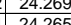 & 告3.112 & $\frac{22}{230}>0$ & 50.8033 & (54.1688 & 20.126 & & -1.212 & & & 0.046 & & & & & 52 & $\frac{12.1}{12.1}$ & 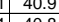 & & & & & & \\
\hline 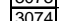 & 4 & $\frac{0.52 .4}{0.52}$ & (23.491 & 21.024 & & $\frac{23.174}{23270}+4$ & & 50.00 & 54.011 & 20.096 & & $=\frac{-1.215}{1.215}$ & & & & . & & & & & $\frac{1.2 .1}{120}>>0$ & & & & & & & \\
\hline 307 & $\frac{14521204 t}{4 / 212004}$ & - & 23. $>>$ & $\frac{21.054}{21.886}$ & & 年 & & $\frac{51.1}{51 .}$ & & & $\frac{1.060}{31.34}$ & -1218 & & & & & & & & & . & & & & & & & \\
\hline 3076 & & & & & & & & 50.1 & 54.138 & & & -1218 & & & & & & & & & $12.12-3$ & & & & & & & \\
\hline 3077 & $4 / 2 / 2 / 2004$ & & & 21.946 & & 23.487 & & & & & & & & & & & & & & & & & & & & & & \\
\hline & & & & & & & & 50.5 & & & & & & & & & & & & & & & & & & & & \\
\hline 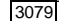 & $4 / 22120$ & & 23,469 & & & 503 & & & 596 & & & 218 & & & & 14.643 & & & & & & & & & & & 10 & 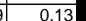 \\
\hline 280 & $4 / 22 / 2004$ & 8:59:25 AM & 23.512 & 22.045 & 24.195 & 23.631 & 23.522 & & 54.384 & 19.929 & & -1.215 & & 48.218 & 0.041 & $\begin{array}{ll}14.643 \\
\end{array}$ & & & 712031 & & & & 2.795 & 0.006 & 0.006 & 0.000 & 0.154 & 0.1 \\
\hline
\end{tabular}


WSRC-TR-2005-00105, REVISION 0

SRNL-RPP-2005-00012, REVISION 0

RUN \# 2.03A AND B; FIRST AND SECOND HALF OF SLURRY DEWATERING - CONT.

\begin{tabular}{|c|c|c|c|c|c|c|c|c|c|c|c|c|c|c|c|c|c|c|c|c|c|c|c|c|c|c|c|c|}
\hline & A & B & D & $E$ & $F$ & $G$ & $\mathrm{H}$ & $\mathrm{J}$ & $\mathrm{K}$ & $\mathrm{L}$ & M & $\mathrm{N}$ & 0 & $Q$ & $R$ & 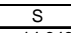 & \begin{tabular}{l|l|l|} 
\\
\end{tabular} & & w & $x$ & & $z$ & AA & $A B$ & $\mathrm{AC}$ & $A D$ & & $A F \quad A$ \\
\hline$\frac{3081}{3082}$ & $\begin{array}{l}4 / 221 / 2004 \\
4 / 212004\end{array}$ & $\begin{array}{l}9: 00: 25 \mathrm{AM} \\
900.25 \mathrm{AM}\end{array}$ & $\begin{array}{r}23.54 \\
23.573 \\
\end{array}$ & 22.078 & $\begin{array}{l}24.173 \\
24.091\end{array}$ & $\begin{array}{l}23.664 \\
23.702\end{array}$ & \begin{tabular}{|r|}
23.59 \\
23.653
\end{tabular} & \begin{tabular}{|l|}
50.39 \\
50.45
\end{tabular} & \begin{tabular}{|l|}
54.326 \\
54.457 \\
\end{tabular} & $\begin{array}{l}\frac{19.826}{19.902} \\
\end{array}$ & $\begin{array}{l}30.76 \\
30.71 \\
\end{array}$ & $\begin{array}{r}-1.215 \\
-1.218 \\
\end{array}$ & $\begin{array}{l}-6.532 \\
-6.655 \\
-x^{2}\end{array}$ & $\begin{array}{l}49.207 \\
49.168\end{array}$ & $\begin{array}{l}0.041 \\
0.041\end{array}$ & $\begin{array}{l}14.643 \\
14.643\end{array}$ & $\begin{array}{l}0.003 \\
0.03\end{array}$ & $\begin{array}{l}4278.6 \\
4279.6\end{array}$ & $\begin{array}{l}571.3097 \\
5\end{array}$ & \begin{tabular}{|l|l|}
52.6 \\
53.6
\end{tabular} & $\frac{12 .}{12 .}$ & \begin{tabular}{|l|}
40. \\
40. \\
\end{tabular} & $\frac{2.798}{2.798}$ & \begin{tabular}{|c|}
0.006 \\
0.006
\end{tabular} & \begin{tabular}{|l|}
0.006 \\
0.006 \\
\end{tabular} & 0.000 & $\begin{array}{l}0.154 \\
0.155\end{array}$ & $\begin{array}{l}0.13 \\
0.13\end{array}$ \\
\hline \begin{tabular}{|l|l|l|l|l}
3083 \\
\end{tabular} & & & $\frac{20.010}{23.606}$ & & \begin{tabular}{|l|l|}
24.051 \\
24.089
\end{tabular} & & \begin{tabular}{|l|l|}
23.640 \\
\end{tabular} & & & & & & & & & $\begin{array}{l}14.045 \\
14.643\end{array}$ & & & & & & & & & & & & \\
\hline 3084 & $4 / 22 / 2004$ & 9:03:25 AM & 23.638 & 22.187 & \begin{tabular}{|l|l|}
24.066 \\
\end{tabular} & 23.798 & 23.588 & 50.338 & \begin{tabular}{|l|l|}
54.289 \\
\end{tabular} & 20.034 & 30.59 & -1.215 & -6.532 & 49.117 & 0.04 & 14.643 & 0.003 & 4281.6 & \begin{tabular}{|l|l} 
& 71.3597 \\
\end{tabular} & 52.8 & & 40.5 & $\begin{array}{l}2.790 \\
\end{array}$ & 0.006 & 0.006 & 0.000 & 0.151 & 0.13 \\
\hline & $4 / 2212004$ & 9:04:25 AM & 23.667 & 22.231 & \begin{tabular}{|l|l|}
24.069 \\
\end{tabular} & 23.806 & \begin{tabular}{|l|l|}
23.482 \\
\end{tabular} & 50.379 & \begin{tabular}{|l|}
54.368 \\
\end{tabular} & 19.944 & 30.7 & -1.218 & & 48.809 & 0.04 & 14.643 & & 4282.6 & 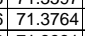 & & & & & & 0.006 & & & 0.13 \\
\hline & & & & & & & & & \begin{tabular}{|r|}
54.38 \\
\end{tabular} & \begin{tabular}{|l|l}
19.954 \\
\end{tabular} & 30.79 & & & & & 14.643 & & & & & & & & & & & & \\
\hline 3087 & $4 / 22 / 2004$ & $\begin{array}{l}9: 06: 25 \mathrm{AM} \\
9: 07: 250\end{array}$ & $\begin{array}{l}23.692 \\
23715\end{array}$ & $\begin{array}{l}22.276 \\
22.200\end{array}$ & 24.024 & \begin{tabular}{|l|}
23.796 \\
\end{tabular} & $\begin{array}{ll}23.422 \\
2.271\end{array}$ & 50.533 & \begin{tabular}{|l|l}
54.523 \\
\end{tabular} & $\begin{array}{l}19.942 \\
\end{array}$ & 30.95 & $\begin{array}{l}-1.218 \\
\end{array}$ & -6.535 & 49.305 & 0.04 & 14.643 & $\begin{array}{l}0.003 \\
\end{array}$ & $\begin{array}{l}4284.6 \\
\end{array}$ & \begin{tabular}{|l|l|}
71.4097 \\
\end{tabular} & 53. & 12.1 & & 2.809 & $\begin{array}{ll}0.006 \\
\end{array}$ & 0.006 & 0.000 & 0.150 & 0.13 \\
\hline 308 & $\begin{array}{l}4 / 22 / 2004 \\
4 / 212004\end{array}$ & $\begin{array}{l}9: 07: 25 \mathrm{AM} \\
90.025 \mathrm{AM}\end{array}$ & $\begin{array}{l}23.715 \\
23.722\end{array}$ & $\begin{array}{l}22.309 \\
22.337\end{array}$ & 24.017 & \begin{tabular}{|l|l|}
23.809 \\
23.776
\end{tabular} & \begin{tabular}{|l|}
23.374 \\
23342 \\
\end{tabular} & $\begin{array}{l}50.342 \\
5.0522\end{array}$ & \begin{tabular}{|l|}
54.341 \\
55476
\end{tabular} & \begin{tabular}{|l|l|l|l|l|}
19.788 \\
19719
\end{tabular} & $\begin{array}{l}30.87 \\
31.21 \\
\end{array}$ & $\begin{array}{l}-1.215 \\
-1.218\end{array}$ & & $\begin{array}{l}49.785 \\
49.848 \\
\end{array}$ & \begin{tabular}{|l|l}
0.04 \\
0.04
\end{tabular} & $\frac{14.643}{14.643}$ & \begin{tabular}{|l|}
0.003 \\
0.003
\end{tabular} & $\begin{array}{l}4285.6 \\
42866 .\end{array}$ & $\begin{array}{l}51.4264 \\
511431 \\
5\end{array}$ & \begin{tabular}{|l}
52.8 \\
53.0
\end{tabular} & $\frac{12.2}{12.2}$ & $\begin{array}{l}40.6 \\
40.9\end{array}$ & $\begin{array}{l}2.800 \\
2818\end{array}$ & \begin{tabular}{|c|}
0.006 \\
0.006 \\
\end{tabular} & $\begin{array}{l}0.006 \\
0.006\end{array}$ & $\begin{array}{l}0.000 \\
0.000\end{array}$ & & $\begin{array}{l}0.13 \\
0.13\end{array}$ \\
\hline 3090 & $4 / 22 / 2004$ & 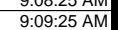 & 23.729 & 22.354 & 23.981 & \begin{tabular}{|l|}
23.75 \\
23.753 \\
\end{tabular} & 23.303 & & 54.428 & 19.914 & & -1.218 & & $\begin{array}{l}49.040 \\
49.658\end{array}$ & 0.042 & $\begin{array}{l}14.643 \\
14.643\end{array}$ & $\begin{array}{l}0.003 \\
0.003 \\
\end{array}$ & & & 53.6 & & 40.9 & $\frac{2.818}{2.795}$ & \begin{tabular}{|c|}
0.006 \\
0.006
\end{tabular} & 0.006 & 0.000 & $\begin{array}{l}0.150 \\
0.159\end{array}$ & $\frac{0.13}{0.14}$ \\
\hline & $4 / 22 / 2004$ & 9:10:25 AM & & & & & 23.355 & & 53.945 & 19.571 & & & & 48.143 & & 14.643 & & & 71.4764 & & & & & & & & & $\begin{array}{l}0.14 \\
0.13\end{array}$ \\
\hline 3092 & $4 / 22 / 2004$ & 9:11:25 AM & 23.752 & 22.402 & 23.955 & $\begin{array}{l}23.731 \\
\end{array}$ & \begin{tabular}{l|l|}
23.387 \\
\end{tabular} & 50.12 & 54.144 & \begin{tabular}{|l|l|}
19.634 \\
\end{tabular} & 30.6 & -1.215 & & 48.565 & 0.04 & 14.643 & 0.003 & $\begin{array}{l}4289.6 \\
\end{array}$ & 711.4931 & & & \begin{tabular}{l|l|}
40.4 \\
\end{tabular} & 2.786 & & \begin{tabular}{|l|}
0.006 \\
\end{tabular} & 0.000 & & $\begin{array}{l}0.13 \\
0.13\end{array}$ \\
\hline 3093 & $4 / 22 / 2004$ & 9:12:25 AM & & & & & & & & & & & & & & & & & & & & & & & & & & $\begin{array}{l}0.13 \\
0.13 \\
\end{array}$ \\
\hline & $4 / 22 / 2004$ & 9:13:25 AM & 20.101 & & 23.964 & 23816 & & 50.172 & 54.146 & 19.767 & 3072 & -1.215 & & \begin{tabular}{|l|l|l|l|l|}
48.88 \\
\end{tabular} & 0.039 & & & 12010 & & & & & & & & & & $\begin{array}{l}0.13 \\
0.13\end{array}$ \\
\hline & $4 / 22 / 2004$ & 9:14:25 AM & & 22.484 & & 23.793 & 23.714 & & 54.188 & 19.728 & 30.67 & & & & & 14.643 & & & 1.5431 & & & 40 & & & 8006 & & & \\
\hline & $4 / 22 / 2004$ & 9:15:25 AM & 23.801 & 22.502 & 23.928 & 23.886 & 23.726 & 50.315 & 54.277 & 19.614 & 31.04 & $\begin{array}{l}-1.218 \\
\end{array}$ & & $\begin{array}{l}49.493 \\
\end{array}$ & 0.04 & 14.643 & $\begin{array}{l}0.003 \\
\end{array}$ & 4293.6 & $\begin{array}{l}71.5597 \\
\end{array}$ & 52.8 & 12.1 & 40.7 & 2.806 & 0.006 & 0.006 & 0.000 & 0.151 & \\
\hline & 4/22/2004 & 9:16:25 AM & 23.798 & 22.524 & 23.911 & 23.983 & 23.779 & 50.11 & 54.086 & 19.63 & 30.85. & -1.218 & & 48.77 & 0.042 & 14.643 & & 4294.6 & 71.5764 & & & & 2.791 & & 0.006 & & & 0.14 \\
\hline & $4 / 22 / 2004$ & $55 \mathrm{AM}$ & & & & & & 50.48. & 54.42 & & & 5 & & & 0.04 & 14.643 & & & & & & & & & & & & \\
\hline & $4 / 221212004$ & 9:18:25 AM & $\frac{23.825}{23291}$ & $\frac{22.586}{201}$ & 23.907 & 24.11 & 23.91 & 50.201 & 54.119 & $\frac{19.807}{10.825}$ & 30.68 & -1.218 & & 48.158 & 0.039 & 14.643 & .003 & & & & & & & & .006 & .000 & 148 & \\
\hline 310 & $41 / 21212004$ & $\frac{9.19 .25 \mathrm{AM}}{9.202}$ & & $\frac{22.61}{22.601}$ & $\frac{2.3911}{2305}$ & $\frac{24.155}{2.918}$ & & $\frac{50.056}{5042}$ & 年.041 & & & $\frac{-1.218}{-1201}$ & & 48 & 0.04 & 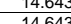 & & & 1642 & & & & & & & & & \\
\hline 32 & $\begin{array}{l}4 / 2421204 \\
4 / 22 / 2004\end{array}$ & $\begin{array}{l}9.20 .25 \mathrm{AM} \\
9: 21: 25 \mathrm{AM}\end{array}$ & $\frac{23.053}{23.856}$ & $\frac{22.639}{22.662}$ & $\frac{23.905}{23898}$ & $\begin{array}{l}24.210 \\
24.271\end{array}$ & 24.023 & $\begin{array}{l}50.444 \\
50.471\end{array}$ & S4.4513 & \begin{tabular}{|c|c|c|}
19.750 \\
19.759
\end{tabular} & 30.96 & $\frac{-1.241}{-1.215}$ & & $\begin{array}{l}48.15 \\
8.598\end{array}$ & 0.04 & $\begin{array}{l}4.645 \\
14.643 \\
\end{array}$ & 0.003 & 42996 & 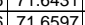 & & 111 & & & & & 0.000 & & 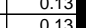 \\
\hline & $4 / 22 / 2004$ & $9: 22: 25 \mathrm{AM}$ & 23.88 & 22.696 & \begin{tabular}{|l|l|} 
& 23.902 \\
\end{tabular} & 24.386 & 24.175 & 50.581 & \begin{tabular}{|l|}
54.552 \\
\end{tabular} & 19.797 & 31.09 & -1.218 & & 47.924 & 0.039 & 14.643 & 0.003 & 4300.6 & 71.6764 & 53. & 11.7 & 40.8 & 2.816 & & 0.006 & 0.000 & 0.147 & 0.12 \\
\hline & 4/221/2004 & 9:23:25 AM & 23.899 & 22.736 & \begin{tabular}{|l|l|}
23.901 \\
\end{tabular} & 24.46 & & 50.34 & $\begin{array}{r}54.3 \\
\end{array}$ & 19.652 & 30.93 & -1.218 & & 48.732 & 0.041 & 14.643 & & & & & & & & & & & & \\
\hline & $2 / 2004$ & 24:25 AM & .919 & 22.766 & 3.906 & 24.465 & 24.254 & & 54.237 & 19.51 & 31.04 & 1.2 & & & 0.04 & 14.643 & & & & & & & & & & & & \\
\hline$\frac{5107}{3107}$ & $\begin{array}{l}4 / 21 / 21 / 2004 \\
/ 21204\end{array}$ & $\begin{array}{l}9.25 .25 \mathrm{AMM} \\
0.20 .52\end{array}$ & 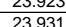 & $\frac{22.805}{2823}$ & $\frac{2.3095}{2309}$ & 24.484 & $\begin{array}{l}24.209 \\
22071\end{array}$ & $\frac{49.958}{49824}$ & 5 & $\frac{20.02}{20117}$ & $\frac{30.23}{30.01}$ & 1215 & & 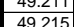 & 0.039 & $\begin{array}{l}\frac{1.4645}{1463} \\
\end{array}$ & & 43046 & 71.731 & & & & & & 1006 & & & 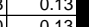 \\
\hline & 4 & $9 \cdot 27.25 \mathrm{AM}$ & 23.939 & 22552 & 23.901 & 24456 & 24.011 & 49.857 & 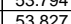 & $\frac{19.111}{19.911}$ & 30.31 & -1.218 & & $\begin{array}{r}\frac{49.215}{48.78} \\
\end{array}$ & 0.038 & $\frac{1.4045}{1463}$ & 0.003 & & $\begin{array}{l}71.14515 \\
717597\end{array}$ & 52 & 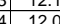 & & 2764 & & & & & 0.1. \\
\hline & $4 / 22 / 2004$ & $9: 28: 25 \mathrm{AM}$ & 23.964 & 22.886 & \begin{tabular}{|l|l|}
5.911 \\
5
\end{tabular} & 24.425 & 24.064 & 49.969 & 53.91 & 19.934 & 30.35 & -1.218 & & 50.382 & 0.041 & 14.643 & 0.003 & 4306.6 & 71.7764 & 52. & 12.3 & 40.2 & \begin{tabular}{|l|l|}
2.769 \\
\end{tabular} & .006 & 0.006 & 0.000 & 0.157 & 0.13 \\
\hline & $4 / 22 / 2004$ & 9:29:25 AM & 23.956 & 22.908 & 23.898 & 24.397 & 24.021 & 49.99 & 53.918 & 19.945 & & -1.218 & & 49.207 & 0.039 & 14.643 & & & 71.7931 & & 12 & & & & & & & 21 \\
\hline 311 & $4 / 2212004$ & 9:30:25 AM & 23.979 & 22.936 & 23.906 & 24.369 & & & 54.231 & 20.015 & & -1.218 & & 48.316 & 0.041 & & & & & 52.8 & & & .788 & .006 & 0.006 & & & \\
\hline 311 & 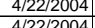 & $9: 31: 25 \mathrm{AM}$ & $\frac{23.985}{23087}$ & $\frac{22.963}{2208}$ & $\frac{23.922}{23014}$ & $24.361 \mid$ & 23.966 & $\frac{50.087}{50.074}$ & 54.061 & $\frac{19.832}{1987}$ & 30.62 & -1.212 & & 47.96. & 0.039 & $\frac{14.643}{14643}$ & & & 1.04 & & $\frac{1}{2}$ & & & & & & & 0.13 \\
\hline & 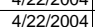 & $25 \mathrm{AM}$ & $\begin{array}{l}23.901 \\
23.993\end{array}$ & 22.986 & 年 238914 & $\begin{array}{l}24.345 \\
24294\end{array}$ & $\frac{23.919}{23854}$ & $\frac{50.074}{50.06}$ & 54065 & $\begin{array}{l}.071 \\
009 \\
\end{array}$ & 30.4 & $\frac{-1.218}{-1.218}$ & & 47359 & 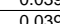 & 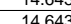 & & & & 526 & $\frac{1.4}{116}$ & 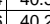 & 2774 & & 0.006 & 0.000 & 0 & 0.13 \\
\hline 311 & $4 / 2212004$ & 9:34:25 AM & 24 & 23.018 & 23.902 & 24.3 & & 50.162 & 54.187 & 19.974 & 304 & -1.218 & & & $\frac{0.004}{0.04}$ & 1464 & & & 71.58764 & & 11.6 & & & & & & & \\
\hline & $4 / 22 / 2004$ & 9:35:25 AM & 24.006 & & $\frac{23.903}{23.903}$ & 24.276 & $\frac{23.886}{23.886}$ & 50.052 & 54.082 & $\frac{19.819}{19819}$ & & $\frac{-1.215}{-1.215}$ & & 49.495 & 0.04 & $\frac{14.643}{14.64}$ & & & & & 12. & $\frac{4 c}{46}$ & & & 0.006 & & & 0.1 \\
\hline & 4/22/2004 & & 24.012 & 23.045 & & & & 50.128 & 54.208 & & 30.59 & -1.218 & & & 0.041 & 14.643 & & & & & & & & 006 & 0.00 & .000 & & \\
\hline & & & 24.019 & & 23.906 & 2429 & 24.0 & & 54.067 & & & -1218 & & & 0.1 & & & & 10 & & & & & & & & & \\
\hline & $4 / 22 / 2004$ & $5 \mathrm{AM}$ & 24.025 & & 23.912 & 24.326 & & $\begin{array}{l}49.884 \\
\end{array}$ & .032 & $\begin{array}{l}19.71 \\
\end{array}$ & & & & & 0.039 & & & & & & & & & & & & & \\
\hline 312 & 4/22/2004 & $25 \mathrm{AM}$ & 24.043 & 23.106 & 23.92 & 24.414 & 24.16 & 49.882 & 53.978 & 19.971 & & -1.218 & & 48. & 0.039 & 14.643 & & & 71.9597 & 52.5 & 11. & 40. & 2.765 & & 0.006 & 000 & 149 & $\overline{0.13}$ \\
\hline & 4/22/2004 & 9:40:25 AM & 24.056 & 23.124 & 23.933 & 24.507 & 24.2 & 50.29 & 54.299 & 19.361 & 31.23. & -1.218 & & 48. & 0.04 & 14. & & & 71.9 & $3_{2}$ & $1+$ & $4 \mathrm{te}$ & & & 0.00 & & & 0.1 \\
\hline & |l/2004 & $25 \mathrm{AM}$ & & 23.14 & 23.925 & 24.574 & 24.2 & 50.245 & 54.264 & 19.648 & & -1.218 & & 49.474 & 0.039 & & & & & & 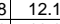 & & & & & & & \\
\hline & 年2004 & & 24.071 & $\frac{23.17}{230}$ & 20006 & 24.643 & 24. & 50.33. & & $\frac{19.652}{1928}$ & & -1.218 & & 49 & 0.037 & & & & & & & & & & & & & \\
\hline & $\frac{212004}{21204}$ & 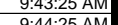 & $\begin{array}{l}24.084 \\
24.090\end{array}$ & 年 & $\begin{array}{l}23.936 \\
23046\end{array}$ & 24.1266 & 24. & $\frac{50.251}{50204}$ & 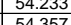 & & & $\frac{-1.211}{-1218}$ & & & 0.039 & & & & 72048 & & 11 & 40 & & & & & & 017 \\
\hline & & & $\frac{24.105}{24112}$ & $\frac{20.250}{23246}$ & $\frac{20.940}{23949}$ & 24.101 & & $\frac{50.404}{5013}$ & 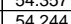 & & & & & & 0.039 & & & & 12.043 & & & & & & & & & 0.13 \\
\hline 312 & $\frac{4}{4 / 21212004}$ & $9.46 .525 \mathrm{AM}$ & $\frac{2.1 .14}{24.142}$ & 23.281 & $\frac{25.949}{23.979}$ & 24.874 & 24.6 & 50.054 & $\frac{54.244}{54.14}$ & & & -1.218 & & 49.1 & $\frac{0.00}{0.0}$ & 势. & & & $\begin{array}{l}7.050764 \\
72.076\end{array}$ & $\frac{32}{52}$ & $\perp$ & $\frac{44}{44}$ & & & & & & 11 \\
\hline $312 \varepsilon$ & $4 / 212004$ & & 24.147 & 23.301 & 23.899 & & & 50.30 & & & & & & & & & & & & & & & & & & & & \\
\hline & $4 / 2 / 2004$ & & 24.65 & 23.339 & 23.912 & 24.987 & & 50.26 & 54.401 & & & -1215 & & & & 14. & & & 721 & & & & & & & & & \\
\hline & $4 / 2$ & & 241 & 23.358 & & & & & & & & & & & & & & & & & & & & & & & & \\
\hline & 2/2004 & & 24.192 & 23.391 & 23.914 & 24.919 & & 50.26 & 54.434 & & & -1.218 & & & & & & & & & 12. & & & & & & 48 & \\
\hline 3132 & $4 / 22 / 2004$ & 9:51: & 24.189 & 23.34 & 23.896 & 24.8 & & 50.8 & & & & \begin{tabular}{|c|}
-1.218 \\
\end{tabular} & & & 0.0 & & & & & 52 & & 4 & & & & & & \\
\hline & $4 / 22 / 2004$ & 9:52:25 AM & 24.202 & 23.427 & 23.899 & 24.854 & & 50 & & & & -1.221 & & & & & & & & & & & & & & & & \\
\hline 313 & 4/22/2004 & 9:53: & 24.218 & 23.453 & 23.905 & 24 & & 50.3 & 54.434 & & & -1.221 & & & & & & & & 53 & 12.4 & & & & & & & \\
\hline & & & 24.21 & 23.479 & 23.906 & 24.816 & 24. & 50.39 & 54.463 & 19 & 31 & -1.218 & & & & & & & & rat & $x_{2}$ & & & & & & & \\
\hline & & & & & & & & & & & & -1.215 & & & & & & & & & & & & & & & & \\
\hline $\mid \frac{3 v 8}{3138}$ & 44242004 & & & $\frac{23.520}{23527}$ & $\frac{23.913}{23909}$ & 24.103 & & & & & & $\frac{-1.210}{1210}$ & & & & & & & & & 11 & & & & & & & \\
\hline 3139 & $\frac{4}{4 / 21212004}$ & $\frac{3.56 .5}{9.58 .25}$ & $\frac{24}{24}$ & & 239 & $\frac{24.134}{2475}$ & & $\frac{1.12}{502}$ & & & & -1.221 & & & & & & & & 52 & 11 & & & & & & & \\
\hline 3140 & $\frac{4 / 21 / 2004}{4}$ & 9:59: & 24.24 & 23.57 & $\frac{23.902}{23.902}$ & 24.737 & & 50.143 & 54.136 & 19 & & -1.218 & & & & & & & 72.2 & $52.7>-3$ & 11.9 & & & & & & & \\
\hline 3141 & $4 / 22 / 2004$ & 10:00: & 24.246 & 23.576 & 23.903 & 24.707 & 24.3 & 50.3 & 54.413 & 19.49 & 31.26 & -1.218 & & & 0.035 & & & & & & & & & & & & & \\
\hline & & & 24.24 & & $23.896 \mathrm{C}$ & $24.70 !$ & & & 54.215 & & & -1.224 & & & & & & & & & & & & & & & & \\
\hline 314 & $4 / 22 / 2004$ & 10:02:25 AM & 24.269 & 23.614 & 23.93 & 24.75 & 24.3 & 50.21 & 54.279 & 19.704 & & \begin{tabular}{|c|c|}
-1.218 \\
\end{tabular} & & $\overline{9}$ & 0.0 & 14. 2 & & & 72.34 & $528+3$ & 120 & & & & & & & \\
\hline & 22/200 & & & 23.621 & & 24.7 & & 50.8 & & & & $-1.2 .2>>3$ & & & & & & & & & 12. & & & & & & & \\
\hline 3145 & 4/22/2004 & 10:04 & 24. & & & 24. & 24. & 50.1 & & & & -1.218 & & & & 14. & & & & & 12 & & & & & & & \\
\hline 3146 & & & 24 & 23. & & & & 50. & 175 & & & -1.218 & & & & & & & & 52. & 11 & & & & & & 48 & .1 .1 \\
\hline 314 & 4/22/2004 & 10:06 & 24.2 & $23.6 \mathrm{r}-\mathrm{s}$ & 23.929 & 24.954 & & 50.143 & 54.237 & & & & & & 0.0 & $14.6 \mathrm{r}-\mathrm{s}$ & & & & & 12. & & & & & & 0.145 & \\
\hline$\frac{3148}{3140}$ & $4 / 2212004$ & 10:0:2:25 AM & 24.309 & 23.68 & 23.971 & 25.042 & 24.58 & 50.184 & 54.206 & 19.63 & 30.97 & -1.218 & & 40.00 & 0.03 & 14.643 & & $43^{43}$ & $|72.4264|$ & 52. & $\frac{11.8}{0.7}$ & 40 & t.t. & 年 0.006 & 0.0 & 0.000 & 0.147 & 0.13 \\
\hline$\frac{3149}{3150}$ & $\begin{array}{l}4 / 21 / 2004 \\
4 / 2212004 \\
\end{array}$ & 10:09:25 AM & $\frac{24.317}{24.319}$ & 23.71 & \begin{tabular}{|l|l|}
23.969 \\
23.961 \\
\end{tabular} & \begin{tabular}{|l|}
25.094 \\
25.156 \\
\end{tabular} & \begin{tabular}{|r|}
24.016 \\
24.82 \\
\end{tabular} & $\begin{array}{l}0.102 \\
50.321 \\
\end{array}$ & \begin{tabular}{|l|l|l|l}
54.343 \\
\end{tabular} & $\begin{array}{l}119.471 \\
19.521 \\
\end{array}$ & 31.21. & \begin{tabular}{|l|}
-1.218 \\
-1.218 \\
\end{tabular} & & $\begin{array}{l}47.950 \\
48.529 \\
\end{array}$ & 0.039 & $\frac{14.645}{14.643}$ & 0.003 & & 72.4597 & \begin{tabular}{|l|l}
52.2 \\
52.
\end{tabular} & \begin{tabular}{l|l}
9 & 11.1 \\
9 & 11.9 \\
\end{tabular} & & 2.811 & \begin{tabular}{|l|}
0.006 \\
\end{tabular} & \begin{tabular}{|l|l|}
0.006 \\
\end{tabular} & 0.000 & $\begin{array}{l}0.151 \\
0.147\end{array}$ & 0.1 \\
\hline
\end{tabular}


WSRC-TR-2005-00105, REVISION 0

SRNL-RPP-2005-00012, REVISION 0

RUN \# 2.03A AND B; FIRST AND SECOND HALF OF SLURRY DEWATERING - CONT.

\begin{tabular}{|c|c|c|c|c|c|c|c|c|c|c|c|c|c|c|c|c|c|c|c|c|c|c|c|c|c|c|c|c|}
\hline & A & B & D & $E$ & $\mathrm{~F}$ & G & $\mathrm{H}$ & $\mathrm{J}$ & $\mathrm{K}$ & $\mathrm{L}$ & $\mathrm{M}$ & $\mathrm{N}$ & \begin{tabular}{l|l|l}
0 & \\
\end{tabular} & $\mathrm{Q}_{\mathrm{O}}$ & \begin{tabular}{l|l|l|}
$R$ & \\
\end{tabular} & 5 & $\mathrm{~T}$ & & W & $x$ & & 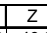 & $\mathrm{AA}$ & $A B$ & $A C$ & $A D$ & $\mathrm{AE}$ & $\mathrm{AF} A$ \\
\hline$\frac{3151}{3152}$ & \begin{tabular}{|l|l|}
$4 / 22 / 2004$ \\
$4 / 2 / 2004$
\end{tabular} & $\begin{array}{l}\text { 10:10:25 AM } \\
10.1125 \text { AM }\end{array}$ & $\frac{24.353}{24.339}$ & $\frac{23.754}{23.755}$ & \begin{tabular}{|r|}
23.99 \\
23.976 \\
\end{tabular} & $\begin{array}{l}25.235 \\
25.252 \\
\end{array}$ & \begin{tabular}{|l|}
24.873 \\
24.799 \\
\end{tabular} & $\begin{array}{l}49.919 \\
50.274\end{array}$ & $\begin{array}{l}54.011 \\
54.362\end{array}$ & $\begin{array}{l}19.545 \\
19.47\end{array}$ & $\begin{array}{l}30.77 \\
31.34\end{array}$ & $\begin{array}{l}-1.221 \\
-1.218\end{array}$ & $\begin{array}{l}-6.532 \\
-6.527\end{array}$ & \begin{tabular}{|l|l|}
48.262 \\
48.938
\end{tabular} & $\begin{array}{l}0.039 \\
0.039\end{array}$ & $\begin{array}{l}14.643 \\
14.643\end{array}$ & $\begin{array}{l}0.003 \\
0.003 \\
\end{array}$ & \begin{tabular}{ll|l}
4348.6 & 434.6 \\
4
\end{tabular} & $\begin{array}{l}72.4764 \\
72.4931\end{array}$ & \begin{tabular}{|l|l|}
52.5 \\
52.5 \\
\end{tabular} & $\frac{11.8}{12.8}$ & 40. & $\frac{2.782}{2.814}$ & \begin{tabular}{|l|l|}
0.006 \\
0.006 \\
\end{tabular} & \begin{tabular}{|l|}
0.006 \\
0.006 \\
\end{tabular} & 0.000 & $\begin{array}{l}0.148 \\
0.147\end{array}$ & $\begin{array}{l}0.13 \\
0.12\end{array}$ \\
\hline & & & & & \begin{tabular}{|l|}
23.979 \\
23.979 \\
\end{tabular} & & & & & & & & & & & $\frac{14.045}{14.643}$ & & & & & & & & & & & & \\
\hline 3154 & $4 / 22 / 2004$ & $10: 13: 25 \mathrm{AM}$ & 24.369 & 23.795 & 23.991 & 25.212 & 24.83 & 50.226 & 54.302 & 19.444 & 31.16 & -1.218 & -6.518 & 48.317 & 0.038 & 14.643 & 0.003 & 4351.6 & 72.5264 & 52.8 & \begin{tabular}{|l|l|l|}
8 & 11.8 \\
\end{tabular} & 40. & 2.806 & 0.006 & 0.006 & 0.000 & 0.143 & 0.12 \\
\hline & $4 / 2212004$ & & 24.391 & & 23.998 & 25.193 & 24.681 & & 54.438 & 19.616 & 31.14 & $\frac{-1.218}{-1.28}$ & -6.512 & 47.881 & 0.039 & 14.643 & & 4352.6 & 72.5431 & & & & & & 0.006 & & & 0.12 \\
\hline & & & & & 24.01 & & & & 54.362 & $\begin{array}{l}19.534 \\
\end{array}$ & & -1.218 & & & $\begin{array}{l}0.039 \\
\end{array}$ & & & & & & & & & & & & & \\
\hline $\begin{array}{ll}3157 \\
3158\end{array}$ & $4 / 22 / 2004$ & 10:16:25 AM & 24.405 & 23.856 & 24.012 & $\begin{array}{l}25.147 \\
2.17\end{array}$ & 24.695 & 50.253 & 54.329 & $\begin{array}{ll}19.469 \\
\end{array}$ & 31.17 & -1.221 & -6.515 & $\begin{array}{l}48.246 \\
\end{array}$ & $\begin{array}{l}0.039 \\
\end{array}$ & 14.643 & 0.003 & 4354.6 & \begin{tabular}{|l|l|}
72.5764 \\
72.63
\end{tabular} & 52.4 & \begin{tabular}{l|l}
9 & 11.8 \\
\end{tabular} & & 2.807 & 0.006 & 0.006 & 0.000 & $\begin{array}{ll}0.147 \\
\end{array}$ & 0.12 \\
\hline$\frac{3158}{3159}$ & $\begin{array}{l}/ / 22 / 22004 \\
4 / 2 / 2004\end{array}$ & $\begin{array}{l}10: 17: 25 \mathrm{AM} \\
10: 18.2 \mathrm{AM}\end{array}$ & 24.406 & $\begin{array}{l}23.862 \\
23.888 \\
\end{array}$ & 24.003 & \begin{tabular}{|l|}
25.118 \\
25.109 \\
\end{tabular} & \begin{tabular}{|l|}
24.656 \\
24.688 \\
\end{tabular} & $\begin{array}{l}50.508 \\
50.178 \\
\end{array}$ & 54.635 & $\begin{array}{l}\frac{19.569}{19.448} \\
\end{array}$ & $\frac{31.39}{30.98}$ & $\begin{array}{l}-1.218 \\
-1.218\end{array}$ & $\begin{array}{l}-6.515 \\
-6.518\end{array}$ & $\begin{array}{l}46.792 \\
4956\end{array}$ & \begin{tabular}{|l|l|}
0.039 \\
0.039
\end{tabular} & $\frac{14.643}{14.643}$ & $\begin{array}{l}0.003 \\
0.003\end{array}$ & $\begin{array}{ll}4355.6 \\
4356\end{array}$ & \begin{tabular}{|l|l|}
72.5931 \\
726097
\end{tabular} & $\frac{53.2}{52 .}$ & $\frac{11.5}{12.1}$ & 441. & $\frac{2.823}{2.798}$ & \begin{tabular}{|l|l|}
0.006 \\
0.006
\end{tabular} & $\begin{array}{l}0.006 \\
0.006\end{array}$ & 0.000 & $\begin{array}{l}0.146 \\
0.147\end{array}$ & $\begin{array}{l}0.12 \\
0.13\end{array}$ \\
\hline 3160 & $4 / 22 / 2004$ & $10: 19: 25 \mathrm{AM}$ & 24.417 & 23.893 & 24.024 & 25.089 & $\begin{array}{l}24.000 \\
24.712\end{array}$ & 50.311 & 54.434 & 19.627 & & $\frac{-1.24}{-1.221}$ & $\begin{array}{l}-0.510 \\
-6.509\end{array}$ & $\begin{array}{l}4.350 \\
48.327 \\
\end{array}$ & 0.039 & $\frac{14.045}{14.643}$ & $\begin{array}{l}0.003 \\
0.003\end{array}$ & $\begin{array}{l}4355.6 \\
4357.6\end{array}$ & $\mid \frac{72.0095}{72.6264}$ & 53. & & $\frac{40 .}{40 .}$ & $\frac{2.198}{2804}$ & \begin{tabular}{|l|}
0.0006 \\
0.006 \\
\end{tabular} & $\begin{array}{l}0.006 \\
0.006 \\
\end{array}$ & 0.0000 & $\frac{0.14 t}{0.147}$ & 0.13 \\
\hline 316 & $4 / 22 / 2004$ & 10:20:25 AM & & & & 25.114 & 24.638 & & 54.511 & & 31.31 & -1.218 & & 48.093 & 0.039 & 14.643 & & 4358.6 & 72.6431 & & & & & $\begin{array}{l}0.006 \\
0.006\end{array}$ & $\begin{array}{l}0.000 \\
0.006 \\
\end{array}$ & & & $\begin{array}{l}0.13 \\
0.12 \\
\end{array}$ \\
\hline 3162 & $4 / 22 / 2004$ & $10: 21: 25 \mathrm{AM}$ & 24.443 & 23.929 & 24.03 & 25.085 & 24.658 & 50.668 & 54.836 & 19.42 & 31.59 & -1.221 & -6.512 & 48.488 & 0.039 & 14.643 & & $\begin{array}{ll}4359.6 \\
\end{array}$ & \begin{tabular}{|l|l|}
72.6597 \\
\end{tabular} & & 11.9 & & & & & & & \\
\hline 3163 & $4 / 22 / 2004$ & 10:22:25 AM & 24.459 & & & & & 50.284 & 54.382 & & & & & & & & & & & & & & .808 & & & & & $\frac{0.12}{0.12}$ \\
\hline 3164 & $4 / 22 / 2004$ & (1) & 24.464 & 20.90 & 24.051 & & & & 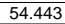 & 19.636 & & -1.218 & & & 0.039 & & & & & & 11. & & & & & & & 0.12 \\
\hline 3165 & 4/22/2004 & 10:24:25 AM & & & 24.062 & 25.077 & 24.72 & 50.477 & 54.621 & 19.736 & 31.05 & & & 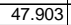 & 0.039 & 14.643 & & & & & & & & 0.006 & 0.006 & & & \\
\hline \begin{tabular}{|l|l|l|l|}
3166 \\
3167
\end{tabular} & 4/22/2004 & 10:25:25 AM & 24.486 & 23.978 & 24.083 & 25.078 & 24.672 & 50.338 & 54.43 & 19.724 & 30.99 & -1.218 & -6.503 & $\begin{array}{ll}48.583 \\
\end{array}$ & 0.038 & 14.643 & 0.003 & \begin{tabular}{ll|l}
4363.6 \\
\end{tabular} & 72.7264 & 53.6 & 11.9 & 40. & 2.804 & 0.006 & 0.006 & 0.000 & 0.143 & 0.12 \\
\hline 316 & 2/20004 & 10:26:25 AM & 24.481 & 23.978 & 24.018 & 25.058 & 24.672 & 50.25 & 54.372 & 19.553 & 31.17 & -1.224 & -6.50 & 48.556 & 0.038 & 14.643 & & & 72.7431 & & 11. & & 2.807 & & 0.006 & & & 0.12 \\
\hline & | & $55 \mathrm{AM}$ & 24.498 & & & & & & & 19.613 & 31.10 & & & & & 14.643 & & & & & & & & & & & & \\
\hline & 222004 & 10:28:25 AM & 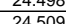 & 24.004 & 24.025 & $\frac{25.14}{25.191}$ & & & 54.254 572 & & $\frac{3.9}{30.9}$ & -1.215 & & 7.9.954 & 0.041 & 14.643 & & & & & & & & & & & & \\
\hline & $\mid$ & $10: 30: 25 \mathrm{AM}$ & 24.505 & 24.01 & $\begin{array}{ll}24.020 \\
24.032\end{array}$ & 25.101 & $\begin{array}{l}24.024 \\
24.961 \\
\end{array}$ & $\frac{0.31}{50.31 !}$ & 54.362 & $\frac{19.016}{19.495}$ & $\frac{0.0}{31.20}$ & $\frac{-1.24}{-1.218}$ & -6.501 & $\begin{array}{l}40.440 \\
47.801\end{array}$ & $\begin{array}{l}0.034 \\
0.034 \\
\end{array}$ & $\frac{14.045}{14.643}$ & & 43608.6 & 72.8097 & & 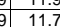 & $\frac{40}{40}$ & $\frac{2.000}{2.810}$ & & 0.005 & & 128 & \\
\hline & $4 / 22 / 2004$ & & & 24.055 & 24.035 & 25.33 & 25.068 & 49.979 & 54.115 & 19.434 & 30.90 & -1.218 & & 48.431 & 0.039 & 14.643 & & & 72.8264 & 52. & & & & & & & & 0.13 \\
\hline & $4 / 221 / 2004$ & 10:32:25 AM & 24.536 & 24.072 & 24.048 & 25.413 & 25.131 & 50.22 & 54.422 & 19.338 & 31.27 & -1.218 & -6.48 & 48.321 & .039 & 14.643 & & 4370 & 2.8431 & & $11.8 \mathrm{r}-\mathrm{r}$ & & & & 0.006 & 0.00 & 147 & 0.12 \\
\hline & 2/2004 & 10:33:25 AM & 24.538 & 24.074 & 24.024 & 25.445 & 25.348 & & 54.393 & 19.256 & & & & 48.2 & .041 & 14.643 & & & & & & & & & & & & \\
\hline & 2/2004] & 10:34:25 AM & $\begin{array}{l}24.56 \\
22.502\end{array}$ & 24.1 .4 & 24.057 & 25.528 & 25.28. & & 54.393 & 19.351 & 31.42 & 1.2 & & 48.341 & 0.04 & 14.643 & & & & & & & & & & & & \\
\hline 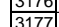 & |4/2/2/2004 & $10.3 .62 \mathrm{AM}$ & (24.582 & $\frac{24.134}{24.15}$ & 24.059 & 年 & 年 25.201 & $\frac{50.471}{50187}$ & (54.505 & $\frac{19.569}{19467}$ & $\frac{31.19}{3106}$ & 1.24 & & (4).900 & 0.037 & 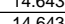 & & 43746 & 29097 & & 11. & 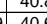 & & & 1006 & & & $\frac{0.12}{0.12}$ \\
\hline & $4 / 2 / 2004$ & $10.37 .25 \mathrm{AM}$ & 246 & 24.172 & 24.001 & 25528 & $\frac{25.194}{25.12}$ & 50.222 & 54.31 & $\frac{19.407}{19.366}$ & $\frac{31.00}{31.27}$ & $\frac{-1.210}{-1.215}$ & & $\begin{array}{ll}40.492 \\
48.266\end{array}$ & $\begin{array}{l}0.04 \\
0.039\end{array}$ & 1. 14.0453 & $\begin{array}{l}0.005 \\
0.003 \\
\end{array}$ & & 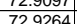 & 52.8 & $\frac{11.5}{118}$ & & & & & & & \\
\hline 317 & 4/22/2004 & $10: 38: 25$ AM & 24.613 & 24.195 & 24.075 & 25.51 & 24.97 & 50.21 & 54.299 & 19.639 & 31.04 & $\frac{-1.218}{-1.218}$ & & 48.21 & 0.039 & 14.643 & & 4376.6 & 72.9431 & 52 & $\frac{11.8}{11 .}$ & 40 & 880 & & & & 0.147 & $\frac{0.1}{0.13}$ \\
\hline & 4/221/2004 & 10:39:25 AM & 24.619 & 24.206 & 24.081 & 25.511 & 24.8 & & 54.482 & 19.547 & & & & 48.7 & 0.039 & 14.643 & & & & & & & & & & & & \\
\hline 3181 & 4/22/2004] & 10:40:25 AM & 24.62 & 24.217 & 24.077 & 25.482 & 24. & 50.187 & 54.331 & 19.545 & 31.03 & $-1.218 \quad$ & & 48.45 & 0.039 & 14.643 & 0.003 & & 72.9764 & 52. & 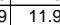 & 40.6 & 2.800 & 0.006 & 0.006 & 0.000 & 0.147 & 01 \\
\hline . & $4 / 22 / 2004$ & 10:41:25 AM & 24.62 & 24.227 & 24.077 & 25.463 & 24.775 & 50.18 & 54.258 & 19.382 & 31.12 & -1.218 & & 48.575 & 0.038 & 14.643 & 0.003 & & 72.9931 & 52.8 & 11.9 & 40.2 & 2.803 & 0.006 & 0.006 & 0.000 & 0.143 & 0.12 \\
\hline & 4/22/2004 & 10:42:25 AM & 24.641 & 24.243 & 24.108 & 255448 & 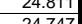 & $\frac{50.224}{50.473}$ & 54.241 & $\begin{array}{l}19.527 \\
10.615\end{array}$ & $\frac{31.11}{31.13}$ & -1.215 & -6.477 & 49.215 & 0.038 & 14.643 & & & \begin{tabular}{|l|l|}
73.0097 \\
730264
\end{tabular} & $\frac{52.8}{5.2}$ & $\frac{12.1}{11.9}$ & 40. & 2.804 & & 0.006 & 年 & 0.143 & 0.12 \\
\hline & $\mid \begin{array}{l}4 / 2 / 2 / 2004 \\
4 / 2 / 2004\end{array}$ & $\frac{10.35 .25 \mathrm{AMM}}{10 \cdot 44 \cdot 25 \mathrm{AM}}$ & $\frac{24.642}{24.662}$ & 24.275 & 24.119 & & & 50.545 & 54745 & $\begin{array}{l}19.010 \\
19.281\end{array}$ & $\frac{31.13}{31.64}$ & $\frac{-1.21}{-1.218}$ & & & $\begin{array}{l}0.039 \\
0.039 \\
\end{array}$ & $\frac{14.645}{14.643}$ & & & & & 11.8 & $\frac{40 .}{41 .}$ & & & & & & 0.12 \\
\hline & $\frac{2 / 2004}{2004}$ & $\begin{array}{l}\text { 10:44:27 AM } \\
04\end{array}$ & $\frac{24.652}{24.652}$ & 24.264 & 24.104 & 25.414 & 24. & 50.639 & 54.791 & $\mid \frac{19.39}{1093}$ & & $\frac{-1.218}{-1.218}$ & & 08 & $\mid 0.039$ & 14.643 & & & & & 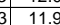 & & & & 006 & & & 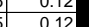 \\
\hline & $4 / 22 / 2004$ & & 24.667 & 24.289 & \begin{tabular}{|l|l|}
24.124 \\
\end{tabular} & 25.409 & & 50.454 & 54.61 & & 31.39 & -1.218 & & 314 & & 14.643 & & & & & $11:$ & & & 0.006 & 0.00 & 1.000 & & \\
\hline & & & 24.646 & 24.268 & 24.083 & 25.363 & & & 54.847 & 19.344 & & -1221 & & & 0.039 & 14.643 & & & & & 111: & & & & & & & \\
\hline 18 & & 10:47:28 AM & 24.682 & 24.304 & 24.134 & 25.424 & & 50.38 & & 19.383 & & -1.215 & & & & & & & & & & & & & & & & \\
\hline & 4/22/2004 & 10:48:28 AM & 24.676 & 24.303 & \begin{tabular}{|l|l|}
24.128 \\
\end{tabular} & 25.383 & 24. & 50. & 54.252 & 19.802 & 30.61 & -1.221. & & 48.815 & 0.039 & 14.643 & & & 73.1106 & 52.8 & 12.6 & 40. & & & 0.006 & & 0.148 & .13 \\
\hline & 4/22/2004 & 10:49:28 AM & 24.68 & 24.31 & 24.132 & & 24.7 & 49. & & 19.759 & (30.51 & -1.218 & & & & 14.643 & & & & & 11. & & & & & & & 0.1 \\
\hline & & 10:50:28 AM & 24.685 & & 24.142 & 25.3977 & 24 & & .119 & 19.752 & & -1.218 & & 18. & 0.039 & & & & & & & & & & & & & \\
\hline 10 & & $1: 28 \mathrm{AM}$ & 24.679 & 24.32 & 24.146 & 25.3811 & 24.7 & 50.0 & 54.161 & 19.753 & 30.6 & -1.224 & & & 0.038 & & & & & & 2.. & & & & & & & \\
\hline & & $\begin{array}{l}10.52 .28 \mathrm{AMM} \\
0.52 .28 \mathrm{M}\end{array}$ & 24.703 & $\frac{24.34}{24.320}$ & 24.185 & & & ${ }^{49.921}$ & 年4.096 & & & - & & & & & & & & & 118 & $40-2$ & & & & & & 012 \\
\hline & & & $\frac{24.102}{24701}$ & 24334 & & 25394 & & & & (19.006 & & - & & & & & & & & & & & & & & & & \\
\hline 319 & 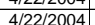 & $28 \mathrm{AM}$ & $\frac{2.7 .71}{24.711}$ & $\frac{1.354}{22.344}$ & $\mid 24.178$ & 年.5.354 & & 50.3 & 54.416 & 19.834 & & -1.21 & & & & 站. & & & & & . & & & & & & $\frac{1443}{143}-3$ & $\frac{0.14}{0.12}+2$ \\
\hline 319 & $|4 / 22 / 2004|$ & $10: 56$ & 24.727 & 24.365 & \begin{tabular}{|l|l|}
24.199 \\
\end{tabular} & & & & & & & & & & & & & & & & 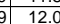 & & & & & & & \\
\hline$\sqrt{319}$ & 4/2/2/2004 & & 24,72 & 24.354 & 24.189 & 25.404 & & 49.786 & 53.885 & & & -1.221 & & & & 14.643 & & & & & 124 & & & & & & & \\
\hline & & & 24.731 & & & & & & & & & & & & & & & & & & then & & & & & & & \\
\hline & & 10:59:28 AM & 24.741 & 24.364 & 24.214 & & & 50. & 54.244 & & & -1.218 & & & & & & & & & 121 & & & & & & & \\
\hline & $4 / 22 / 20$ & $11: 00$ & 24.7. & $24.3>>>3$ & $24.8 \mathrm{P}>\mathrm{C}$ & 408 & 24. & & $54.4 \div-4$ & 19.992 & & -1.218 & & & & & & & & & $12.0 \mathrm{P}>\mathrm{l}$ & & & & & & & 7 \\
\hline & 4/22/2004 & 11:01 & 24.7 & 24 & 24.244 & 434 & & 50. & 54.264 & & & -1.2211 & & & & & & & & & 12.1 & & & & & & & \\
\hline 20 & & & 24.7 & 24 & 24.2 & & & & & 19.719 & & -1.221 & & & & & & & & 52 & 12.2. & & & & & & & \\
\hline & & & 24.762 & 24.39 & 24.254 & 25.449 & 24. & & 54.1360 & 19.843 & & -1.215 & & & & 14.643 & & & & & 12. & & & & & & 44 & \\
\hline & & & $\frac{24.1773}{170}$ & 24.406 & & & & & & & & -1.218 & & & & & & & & & & & & & & & 44 & \\
\hline $320 \varepsilon$ & 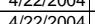 & $\frac{11.03 .28}{11.06}$ & $\frac{24.75}{24.796}$ & $\frac{24 .}{24}$ & $\frac{24.2}{22.2}$ & 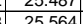 & & 年 & (54.2979 & $\mid \frac{19.979}{1974}$ & & $\frac{-1.21}{-1218}+8$ & & & & 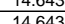 & & & & & 118 & & & & & & $\frac{144}{144}>>>$ & \\
\hline 3205 & $\mid 4 / 221 / 2004$ & 11:07: & $\frac{24.150}{24.798}$ & $\frac{24.44}{244}$ & 2424 & & & & & 19.814 & & $\frac{-1.42}{-1221}$ & & & & & & & & $52-2>$ & 120 & & & & & & & \\
\hline & & 11:08 & 24.806 & 24.4 & 24.23 & 25.708 & 25. & 49.9 & 54.188 & 19.852 & & -1.221 & & & & & & & & 52 & $11.8 \mathrm{\gamma}>\mathrm{l}$ & & & & & & & \\
\hline & $4 / 22 / 2004$ & 11:09: & 24.818 & 24.456 & 24.251 & 25.796 & 25.4 & 50.07 & & 19.826 & & -1.224 & & & & 14. & & & & & 12.4 & & & & & & & \\
\hline & & & & & & 25.844 & & & & & & -1.218 & & & & & & & & & & & & & & & & 12 \\
\hline & 4/22/2004 & 11:11:28 AM & & 24.487 & 24.266 & 25.837 & 25.3 & 49.784 & 53.899 & 19.80 & & -1.221 & & 48.5 & 0.03 & 14. & & & 73.4939 & 52. & 11.9 & 4 & 2. & & & & 48 & 0.13 \\
\hline & $4 / 22120004$ & $\frac{11: 12: 28}{11 \cdot 13208}$ & & 24.494 & \begin{tabular}{|l|}
24.253 \\
24.281 \\
\end{tabular} & & & 50.6 & 54.212 & $\begin{array}{l}19.896 \\
19759\end{array}$ & & 年.1.21 & & & 0.04 & $\frac{14 .}{14 .}$ & & $\frac{44}{44}$ & $\begin{array}{l}73.5106 \\
73.5272\end{array}$ & 52.7 & \begin{tabular}{l|l}
7 & 12.1 \\
7 & 125
\end{tabular} & 14 & 2.778 & 0.0 & 0.0 & & $\frac{51}{48}$ & \\
\hline & & $111: 14$ & 24.86 & & $\begin{array}{l}24.201 \\
24.267\end{array}$ & 年.7.768 & & & 4.25 & & & -1.1. & & & & & & & & & & & & & & & b144 & \\
\hline & & & 24.862 & 24.525 & 24.264 & 25.76 & & & 54.111 & & & & & & & & & & & & & & & & & & 144 & \\
\hline & & & 24889 & & & & & & & & & & & & & & & & & 52. & & & & & & & & \\
\hline & $4 / 2312 / 25$ & & 249 & & & 748 & & & & & & $.218 \mathrm{r}$ & & & & 14.643 & & & & & 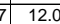 & & & & & & 44 & 01 \\
\hline & $4 / 22 / 2004$ & 11:18:28 AM & 24.89 & 24.573 & \begin{tabular}{|l|l|}
24.293 \\
\end{tabular} & 25.723 & 25.305 & & 54,445 & 19.639 & 30.91 & -1.221 & & 47.119 & 0.038 & 14.643 & & & & & & & 2.798 & 0.006 & 0.006 & 0.000 & 0.142 & 0.1 \\
\hline
\end{tabular}


WSRC-TR-2005-00105, REVISION 0

SRNL-RPP-2005-00012, REVISION 0

RUN \# 2.03A AND B; FIRST AND SECOND HALF OF SLURRY DEWATERING - CONT.

\begin{tabular}{|c|c|c|c|c|c|c|c|c|c|c|c|c|c|c|c|c|c|c|c|c|c|c|c|c|c|c|c|c|}
\hline & A & B & D & $\mathrm{E}$ & $\mathrm{F}$ & $\mathrm{G}$ & $\mathrm{H}$ & $\mathrm{J}$ & $\mathrm{K}$ & $\mathrm{L}$ & $\mathrm{M}$ & $\mathrm{N}$ & 0 & Q & \begin{tabular}{l|l|l|}
$R$ & \\
\end{tabular} & $\mathrm{~s}$ & $\mathrm{~T}$ & & w & $x$ & & 2 & $A A$ & $A B$ & $\mathrm{AC}$ & $A D$ & $\mathrm{AE}$ & AF \\
\hline$\frac{322}{320}$ & \begin{tabular}{|l|l|}
$4 / 22 / 2004$ \\
$4 / 2 / 2004$
\end{tabular} & $\begin{array}{l}11: 19: 28 \mathrm{AM} \\
11.2028 \mathrm{AM}\end{array}$ & 24.907 & \begin{tabular}{r|}
24.6 \\
24.605
\end{tabular} & $24.299 \mid$ & \begin{tabular}{|l|}
25.714 \\
25.709 \\
\end{tabular} & \begin{tabular}{|l|}
25.317 \\
25.287 \\
\end{tabular} & $50.365 \mid$ & \begin{tabular}{|r|}
54.65 \\
54.364
\end{tabular} & $\begin{array}{l}19.529 \\
19.395\end{array}$ & $\begin{array}{l}31.15 \\
31.08\end{array}$ & $\begin{array}{l}-1.218 \\
-1.221 \\
\end{array}$ & -6.417 & \begin{tabular}{|l}
47.855 \\
47.79 \\
\end{tabular} & \begin{tabular}{|l|}
0.04 \\
0.038
\end{tabular} & $\begin{array}{l}14.643 \\
14.643\end{array}$ & $\begin{array}{l}0.003 \\
0.003\end{array}$ & $\begin{array}{l}4417.6 \\
4418.6\end{array}$ & $\begin{array}{l}73.6272 \\
73.6439 \\
\end{array}$ & \begin{tabular}{|l|l|}
53.2 \\
52.5 \\
\end{tabular} & $\frac{11 .}{11 .}$ & \begin{tabular}{|l|}
40.8 \\
40.6 \\
\end{tabular} & $\frac{2.810}{2801}$ & \begin{tabular}{l|l|l}
0.006 & \\
0.006 &
\end{tabular} & \begin{tabular}{|l|}
0.006 \\
0.006 \\
\end{tabular} & 0.000 & $\begin{array}{l}0.149 \\
0.142 \\
\end{array}$ & $\begin{array}{l}0.13 \\
0.12\end{array}$ \\
\hline & & & & & & & & & & $\frac{19.030}{19.676}$ & & & & & & $\frac{14.045}{14.643}$ & & & & & & & & & & & & \\
\hline 3224 & $4 / 22 / 2004$ & 11:22:28 AM & 24.932 & 24.63 & 24.315 & 25.69 & 25.232 & 50.218 & \begin{tabular}{|l|}
54.53 \\
\end{tabular} & 19.614 & 30.992 & -1.221 & -6.411 & 48.834 & 0.039 & 14.643 & 0.003 & 4420.6 & 73.6772 & 53. & & & 2.800 & 0.006 & 0.006 & 0.000 & 0.146 & 0.12 \\
\hline & $4 / 2212004$ & $11: 23: 28 \mathrm{AM}$ & 24.938 & 24.646 & 24.325 & 25.701 & 25.168 & 50.296 & 54.476 & 19.593 & 31.063 & -1.218 & -6.414 & 48.145 & 0.039 & 14.643 & & 4421.6 & 73.6939 & & & & & & 0.006 & & 0.146 & 0.12 \\
\hline & & & & & 24.32 & 25.681 & 25.148 & & & & 30.895 & & & $\begin{array}{l}49.299 \\
\end{array}$ & & 14.643 & & 4422.6 & & & & & & & & & & 0.12 \\
\hline 3227 & $4 / 22 / 2004$ & 11:25:28 AM & 24.954 & $\begin{array}{l}24.662 \\
2.669 \\
\end{array}$ & 24.351 & 25.726 & 25.189 & 50.031 & \begin{tabular}{|l|l|}
54.214 \\
\end{tabular} & 19.642 & 30.71 & -1.218 & -6.405 & 49.101 & $\begin{array}{l}0.039 \\
\end{array}$ & 14.643 & 0.003 & 4423.6 & 73.7272 & 52.7 & 12.6 & 40.4 & 2.783 & 0.006 & 0.006 & 0.000 & $\begin{array}{ll}0.147 \\
\end{array}$ & 0.12 \\
\hline$\overline{322 .}$ & $\begin{array}{l}/ / 22 / 22004 \\
4 / 2 / 2004\end{array}$ & $\begin{array}{l}\frac{11: 26: 28 \mathrm{AM}}{11127: 28 \mathrm{AM}} \\
\end{array}$ & $\frac{24.954}{24.949}$ & 24.668 & $\begin{array}{ll}24.342 \\
24.332\end{array}$ & $\begin{array}{l}25.722 \\
25.717 \\
\end{array}$ & \begin{tabular}{|l|}
25.179 \\
25.229 \\
\end{tabular} & \begin{tabular}{|l|}
50.102 \\
50.091
\end{tabular} & \begin{tabular}{|l|}
54.306 \\
54.426 \\
\end{tabular} & $\frac{19.556}{19.533}$ & $\begin{array}{l}30.907 \\
30.864\end{array}$ & \begin{tabular}{|l|l|}
-1.218 \\
-1.221
\end{tabular} & $\begin{array}{l}-6.405 \\
-6.411\end{array}$ & \begin{tabular}{|l|}
48.525 \\
47601
\end{tabular} & \begin{tabular}{|l|l|}
0.039 \\
0.039
\end{tabular} & $\frac{14.643}{11.643}$ & \begin{tabular}{|l|l}
0.003 \\
0.003
\end{tabular} & $\begin{array}{l}4424.6 \\
44256\end{array}$ & $\begin{array}{l}73.7439 \\
77.7606\end{array}$ & $\frac{52.8}{53 .}$ & $\frac{11.9}{11.7}$ & $\begin{array}{l}40.5 \\
40.5\end{array}$ & $\begin{array}{l}2.793 \\
2791 \\
\end{array}$ & $\begin{array}{ll}0.006 \\
0.06\end{array}$ & $\begin{array}{l}0.006 \\
0.006\end{array}$ & 0.000 & $\begin{array}{l}0.146 \\
0.146\end{array}$ & 0.12 \\
\hline 3230 & $4 / 22 / 2004$ & $\begin{array}{l}11.7 .20 \mathrm{AMT} \\
11: 28: 28 \mathrm{AM}\end{array}$ & 24.955 & & 24.343 & 25.693 & \begin{tabular}{|l|}
25.229 \\
25.16 \\
\end{tabular} & & \begin{tabular}{|l|l|}
54.467 \\
\end{tabular} & 19.535 & 31.018 & & & \begin{tabular}{|l|}
49.626 \\
49.626
\end{tabular} & 年 & $\frac{14.045}{14.643}$ & $\begin{array}{l}0.003 \\
0.003 \\
\end{array}$ & $\frac{4425.6}{4426.6}$ & & 53.6 & $\frac{11.1}{12.2}$ & $\begin{array}{l}40.5 \\
40.6 \\
\end{array}$ & & $\begin{array}{l}0.006 \\
0.006\end{array}$ & $\begin{array}{l}.006 \\
0.006 \\
\end{array}$ & 0.000 & $\begin{array}{l}0.146 \\
0.142\end{array}$ & $\frac{0.12}{0.12}$ \\
\hline & $4 / 22 / 2004$ & 11:29:28 AM & 24.964 & 24.683 & 24.347 & & 25.199 & & 54.5 & & 31.146 & -1.218 & & 48.218 & 0.04 & 14.643 & & & 73.7939 & & & & & & 0.006 & & & \\
\hline 3232 & $4 / 22 / 2004$ & $11: 30: 28 \mathrm{AM}$ & & & 24.347 & \begin{tabular}{|l|l|}
25.707 \\
\end{tabular} & 25.204 & 50.35 & 54.604 & 19.519 & 31.17 & -1.221 & -6.405 & 47.284 & 0.039 & 14.643 & 0.003 & 4428.6 & \begin{tabular}{|l|l|l|l|l|}
73.8106 \\
\end{tabular} & 53. & & \begin{tabular}{|l|l|}
40.8 \\
\end{tabular} & 2.810 & \begin{tabular}{|l|l|}
0.006 \\
\end{tabular} & 0.006 & 0.000 & & \\
\hline 3233 & $4 / 22 / 2004$ & $11: 31: 28 \mathrm{AM}$ & 24.985 & & 24.368 & & & & 54.343 & & & & & & & & & & & & & & & & & & & 0.12 \\
\hline 3234 & & 11:32:28 AM & & 24.704 & 24.343 & $2577^{3}$ & & 50.166 & Fen & 19.458 & 31.053 & -1.218 & & 18904 & 0.039 & & & & 382020 & & & & & & & & & $\frac{0.12}{0.12}$ \\
\hline & $4 / 22 / 2004$ & 11:33:28 AM & 24.99 & & & 25.738 & 25.1 & 50.334 & 54623 & 19.598 & 31.09 & & & 48.919 & 0.04 & 14.643 & & & 3.8606 & & & & 807 & & & & & \\
\hline 3236 & 4/22/2004 & 11:34:28 AM & 24.989 & 24.698 & 24.327 & 25.722 & 25.139 & 50.037 & 54.324 & 19.452 & 30.885 & -1.221 & -6.405 & 50.231 & \begin{tabular}{|c|c|c|} 
& 0.039 \\
\end{tabular} & 14.643 & 0.003 & 4432.6 & 73.8772 & 52.9 & 12.3 & 40 & 2.790 & 0.006 & 0.006 & 0.000 & 0.146 & 0.12 \\
\hline & 2/2004 & 11:35:28 AM & 24.994 & 24.713 & 24.337 & 25.762 & 25.139 & 50.274 & 54.382 & 19.555 & 31.057 & -1.221 & -6.39 & 48.94 & 0.038 & 14.643 & & & 73.89399 & & & & 2.804 & 5.006 & 0.006 & & & 0.12 \\
\hline & 2/2/2004 & 11:36:28 AM & & & & 25.756 & 25.134 & 50.025 & & & & & & & 0.036 & & & & & & & & & & & & & \\
\hline & | & 11:37:28 AM & 24.993 & 24.72 & 24.33 & 25.741 & 25.11 & 50.056 & 4.405 & 19.601 & 30.75 & -1.218 & & 3.535 & 0.038 & 14.643 & 1003 & & & & & & & .006 & & & .143 & \\
\hline$\sqrt{324}$ & $\mid \frac{4 / 21212004}{4 / 2212004}$ & $\frac{11.38 .28 \mathrm{AM}}{11.20 .09 \mathrm{M}}$ & 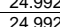 & $2 \frac{24.16}{2.725}$ & $\frac{24.34}{2434}$ & $\frac{25.1}{25709}$ & $\frac{25.15}{25.5}>>$ & $\frac{50.201}{50085}$ & 543 & 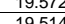 & 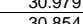 & $\frac{-1.218}{-1201}$ & & 19128 & 0.038 & 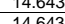 & & & 39606 & & & & & & & & & \\
\hline & $4 / 22 / 2004$ & 11:40.22 AM & 24.997 & & $\frac{2.4035}{22.45}$ & 25.745 & 25.17 & 50.089 & \begin{tabular}{|l|l|}
54.362 \\
\end{tabular} & & 30.908 & -1.218 & $\frac{-6.388}{-6.388}$ & $\begin{array}{l}4.57 .78 \\
47.78\end{array}$ & $\mid 0.039$ & 14.643 & 0.003 & 4438.6 & & & & & & & & 0.000 & 0.146 & $\frac{0.13}{0.12}$ \\
\hline & $4 / 22 / 2004$ & $11: 41: 28 \mathrm{AM}$ & 25.004 & 24.737 & 24.371 & 25.791 & 25.47 & 50.255 & \begin{tabular}{|l|}
54.567 \\
\end{tabular} & 19.641 & 30.959 & -1.218 & -6.382 & 48.773 & 0.038 & 14.643 & 0.003 & 4439.6 & 73.9939 & 53.1 & 12.0 & 40.6 & & 0.006 & 0.006 & 0.000 & 0.142 & 0.12 \\
\hline & 2/2004 & 11:42:28 AM & 25.006 & & 24.378 & 25.803 & & 50.13 & 54.391 & 19.572 & 36 & & & 49.18 & 0.039 & 14.643 & & 4440 & & & & & & & & & & \\
\hline & $4 / 22120004$ & 28 AM & 25.006 & $\frac{24.74}{21751}$ & 24.374 & 25.829 & 25.2 & 50.09 & 54.416 & & 30.90 & $\frac{1.21}{121}$ & & .2 & 0.038 & 14.643 & & & & & & & & & & & & 0.12 \\
\hline & 2 & 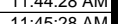 & 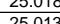 & 年4.151 & 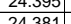 & $\begin{aligned} 25.85 \\
25.786\end{aligned}$ & 年5.2589 & 年 50.230 & 5 & $\frac{19.61}{19376}$ & $\frac{30.94 t}{31119}$ & - & & 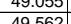 & | & 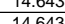 & & 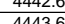 & $\frac{14.0439}{70606}$ & & & & & & & & & \\
\hline 3248 & $4 / 2 / 2004$ & 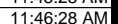 & 25.02 & 24.758 & 24.387 & 25787 & & 49.934 & 5.4394 & 19.310 & & 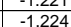 & & & | & 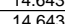 & 0.0003 & & & 52 & $\frac{12}{12}$ & & & & & & & 0.12 \\
\hline$\overline{3249}$ & 4/221/2004 & $\begin{array}{l}11.4 .20 \mathrm{AMT} \\
11: 47: 28 \mathrm{AM}\end{array}$ & $\frac{25.06}{25.036}$ & 24.775 & 24.414 & 25.799 & 25.266 & 499.878 & 54.198 & $\frac{19.0}{19.598}$ & $\frac{30.121}{30.762}$ & $\begin{array}{l}-1.2<4 \\
-1.218 \\
\end{array}$ & & 40.920 & 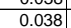 & $\frac{14.045}{14.643}$ & & (4445 & 74.0939 & & $\frac{12 .}{11 .}$ & $\frac{40}{40}$ & & & & & $\frac{145}{143}$ & $\frac{1.12}{0.12}-2$ \\
\hline & $4 / 22 / 2004$ & 11:48:28 AM & 25.047 & 24.78 & 24.425 & 25.825 & & & 54.36 & 19.522 & & & & 49.353 & 0.039 & 14.643 & & 44 & 74.1106 & & & & & & & & & \\
\hline 325 & 4/22/2004 & $28 \mathrm{AM}$ & 25.037 & 24.775 & 24.415 & 25.825 & & 50.122 & 54.478 & 19.606 & 30.856 & -1.218 & & 47.069 & 0.039 & 14.643 & 0.003 & & 74.1272 & 53 & 11. & 4 & & 0.006 & 0.006 & 0.000 & 0.146 & $0.1 .>3$ \\
\hline & 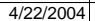 & 11:50:28 AM & 25.043 & 24.781 & 24.425 & 25.795 & 25.3 & 49.846 & 54.142 & 19.371 & 30.836 & $\frac{-1.221}{-1.221}$ & & 48.193 & 0.039 & 14.643 & & & 74.1439 & & & & & & & & & \\
\hline & 4/22/2004 & $11: 51: 28 \mathrm{AM}$ & 25.048 & 24.776 & 24.42 & & & & & & 30.986 & -1.224 & & & 0.039 & & & & & & & & & & & & & $\frac{x_{1}}{0.12}$ \\
\hline & $2 / 2004$ & 11:52:28 AM & 25.057 & 24.79 & 24.43 & 25.815 & & $\begin{array}{lll}49.989 \\
\end{array}$ & 54.335 & 19.529 & 1.754 & -1.221 & & 50.062 & $\mid 0.038$ & 14.643 & & 445 & 74.1772 & 52.4 & 12.3 & 40. & . 1.783 & & 0.006 & 0.000 & 0.143 & 0.12 \\
\hline & $2 / 2004$ & 11:53:28 AM & 25.063 & 24.801 & 24.445 & 25.825 & 25.3. & 50.166 & 54.445 & 19.512 & 31.016 & -1.221 & & & 0.038 & 14. & & & & & 11 & & & & & & & \\
\hline & 2/2004 & $11: 54: 28 \mathrm{AM}$ & 25.072 & & 24.45 & 25.825 & $25.28 \mathrm{~F}$ & 50.261 & . & 19.63 & & -1.221 & & 46.458 & 0.04 & & & & 74.2106 & & 10 & & & & & & & \\
\hline & |2:212004] & $11: 55$ & 25.078 & 24.806 & 24.45 & 25.835 & & & 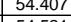 & 19.54 & 36 & 2 & & (8.0.018 & 0.03 & 14. & & & 74.2272 & & & & & 0.006 & 0.00 & .000 & 142 & \\
\hline & & & & & & & & & & & & & & & & & & & & & & & & & & & & \\
\hline 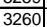 & $\mid \begin{array}{l}\mid 4 / 242 / 2004 \\
4 / 22 / 2004\end{array}$ & $11.57 .28 \mathrm{AM}$ & $\frac{23.094}{25.107}$ & 年4.025 & $\frac{2.48}{248}$ & 25.676 & $\frac{23 .}{25}$ & $\begin{array}{l}50.265 \\
55^{2} 224\end{array}$ & $\frac{54.021}{5455}$ & $\frac{19.475}{10652}$ & 年 & $\frac{1.210}{-1224}$ & & $\frac{40.035}{4757}$ & $\mid$ & 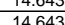 & & & The.2000 & 53. & $\frac{11.5}{117}>0$ & 40 & 2796 & & 0.00 & 0.000 & 0.142 & \\
\hline 326 & $4 / 22 / 2004$ & $11: 59: 28 \mathrm{AM}$ & 25.111 & 24.84 & 24.489 & 25.864 & 25.306 & 50.433 & \begin{tabular}{|l|}
54.714 \\
\end{tabular} & 19.551 & & -1.218 & -6.47 & 48.404 & & 14.643 & 0.0 & & 74.2939 & 53 & $\frac{11.6}{11 .}$ & & 2.816 & & \begin{tabular}{|l|l|}
0.006 \\
\end{tabular} & & & 0.16 \\
\hline & 4/22/2004 & 12:00:28 PM & & 24.834 & & 25.863 & 25.2 & 50.211 & 54.606 & 19.567 & & & & & 0.038 & & & 445 & 74.3106 & 5 & & & & & & & & \\
\hline 26 & $4 / 22 / 2004$ & 12:01:28 PM & 25.116 & 24.83 & $\begin{array}{ll}24.504 \\
\end{array}$ & 25.889 & 25.276 & 50 & 54.3 & 19.458 & 30.901 & $\begin{array}{l}-1.218 \\
.221\end{array}$ & -6.47 & 50.684 & 0.038 & 14.643 & 0.00 & 445 & & 5. & 12. & & & & 0.4 & & & 0. \\
\hline & $\begin{array}{r}4 / 22 / 2004 \\
4 / 2 / 2004\end{array}$ & 12:02:28 PM & $\frac{25.132}{25.138}$ & $\begin{aligned} 24.85 \\
24856\end{aligned}$ & $\begin{array}{r}24.51 \\
24515 \\
\end{array}$ & $\frac{25.885}{25.91}$ & & 50.286 & $\frac{54.592}{54716}$ & $\begin{array}{l}\frac{19.536}{19.53} \\
\end{array}$ & & $\begin{array}{l}-1.221 \\
-1.212 \\
-12\end{array}$ & & & & & & & & & 11. & 40 & & & & & 144 & \\
\hline & & 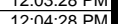 & 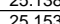 & 24.050 & 24.5156 & 25.94 & & S 50.45009 & \begin{tabular}{|l|l|l|l|}
54442 \\
54
\end{tabular} & 19482 & & - & & & & & & & & & & & & & & & & \\
\hline 326 & 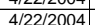 & 12.05.22 PM & 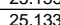 & $\begin{array}{l}24.0057 \\
24.857\end{array}$ & 24.506 & $\frac{25.591}{25.891}$ & 25.4 & 50.452 & \begin{tabular}{|l|l|}
54.778 \\
547
\end{tabular} & 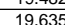 & 31.127 & -1.221 & & 年 48.097 & & 站. & & & & & 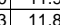 & & & & & & & 1 \\
\hline 326 & $\mid 4 / 22 / 2004$ & $12: 06: 28$ & 25.154 & 24.868 & $\frac{24.542}{24}$ & & & 50.045 & & & & & & & & & & & & & & & & & & & & J.1 \\
\hline & $\mid 4 / 22 / 2004$ & $12.07 \cdot 28$ & 25 & 24.873 & 24.558 & 25.937 & 25. & & 54,343 & 19.484 & 30.8 & -1.221 & & & & & & & & & 11.8 & & & & & & 0.142 & \\
\hline & & & $25, \quad>>$ & & & & & & & & & $-1,218$ & & & & & & & & & & & & & & & & \\
\hline & & 12:09: & 25 . & 24.885 & 24.559 & & & & 54.49 & 19.529 & & -1.221 & & & & & & & & & & & & & & & 38 & \\
\hline & $4 / 22 / 2004$ & $\begin{array}{ll}12: 10: 28 \\
\end{array}$ & 25.197 & 24 & 24.595 & 26 & & & & 19.586 & & \begin{tabular}{l|l|l|}
-1.218 \\
\end{tabular} & & & & & & & & 53 & & & & & & & 0.137 & \\
\hline & 4/221/2004 & 12:11 & 25. & 24.8955 & 24.575 & 26 & & 50. & 54.486 & & & -1.221 & & & & & & & & & 12. & & & & & & & \\
\hline 227 & & 12:12: & 25.192 & 24.916 & 24.5855 & & & 50 & 54.66 & 19.558 & & -1.221 & & & & & & & & 53 & 11.9 & & & & & & & \\
\hline & & & 25.208 & 24.92 & 24.5966 & 26.031 & 25.5 & 50. & 54.708 & 19.004 & 31.139 & -1.218 & & & & 14. & & & & & & & & & & & & \\
\hline & & & & & & & & & & & & -1.221 & & & & & & & & & & & & & & & & \\
\hline & 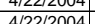 & $\frac{1.13 .28 \mathrm{MM}}{12 \cdot 26 \mathrm{PM}}$ & & 24.929 & $\frac{24.000}{24614}$ & 年.0.060 & & & S4.104 & 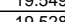 & & $\frac{-1.210}{-201}$ & & & & & & & & 5 & & & & & & & $\frac{14+1}{142}>3$ & \\
\hline & 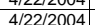 & $\frac{12.10 .25 \mathrm{PM}}{12.172 \mathrm{PM}}$ & 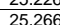 & 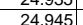 & $\frac{24.0414}{24611}$ & & & & & & & & & & & & & & & & & & & & & & & \\
\hline & $4 / 22 / 20$ & $12: 59: 34$ & $\frac{2.5007}{25.507}$ & 25.19 & 24.926 & 26.04 & & 50.4 & 54.789 & $\frac{19.866}{19.86}$ & & $\mid-0.937$ & & & & & & & & & $11.8 \mathrm{\gamma}>\mathrm{l}$ & & & & & & 134 & \\
\hline & $4 / 2 / 2004$ & $1: 00 \cdot 34 \mathrm{PM}$ & 25.5 & 25.184 & 24.92 & & & & & & & -0.934 & & & & & & & & & & & & & & & & \\
\hline & & $1.01: 34 \mathrm{PM}$ & & & & & & & 5010 & 19785 & & -0.937 & & & & & & & & & & & & & & & & \\
\hline & $\mid 4 / 22 / 2004$ & 1:02:34 PM & 25.526 & 25.225 & 24.959 & 26.109 & 26.6 & 49.8 & 54.648 & 19.75 & & -0.975 & & 8.1 & 0.0 & & & & 75.3 & $57+3$ & $118 \mathrm{118}+\mathrm{C}$ & 4 & & & & & 34 & \\
\hline & $4 / 22 / 2 / 2$ & & 25.504 & 25.213 & & 26 & & & & & & -0.9 & & & & & & & & & & & & & & & & \\
\hline & $4 / 22 / 20$ & 1:04: & 25. & 25. & & 26. & & & & & & -0.9 & & & & 14. & & & & & & & & & & & 34 & \\
\hline & & & & 25.25 & & & & 49. & & 19.908 & & & & & & & & & & & 11. & & & & & & 35 & \\
\hline & $4 / 22 / 20$ & & 25. & 25.258 & 24.927 & 26.142 & & 49 & 54.648 & .82 & & -0.934 & & & & 14.643 & & & & & & & & & & & & \\
\hline (320 & $4 / 2272004$ & 1:0:34 PM & 25.53 & 25.2644 & 24.993 & $26.1 / 3$ & 25.97 & 49.88 & $54.569 \mathrm{r}$ & $\frac{942}{920}>2$ & 30.245 & $=-0.937$ & & 40.527 & 0.03 & 14.643 & & 45 & 15.4 & 3 & 1 1) 11.9 & 40 & 2.1 & 0.45 & 0.6 & 0.0 & 0.138 & 0.1. \\
\hline 3290 & $\begin{array}{l}\mid 4 / 21212004 \\
4 / 22 / 2004\end{array}$ & 1:00.3:34 PM & $\frac{23.55}{25.53}$ & $\begin{array}{l}25.264 \\
25.275 \\
\end{array}$ & \begin{tabular}{|l|}
24.8984 \\
24.884
\end{tabular} & $\begin{array}{l}26.133 \\
26.118 \\
\end{array}$ & $\frac{25.894}{25.71}$ & $\begin{array}{ll}49 . / 10 \\
49.917\end{array}$ & \begin{tabular}{|l|l|l|l|l|}
546 \\
\end{tabular} & $\begin{array}{l}19.832 \\
19.865 \\
\end{array}$ & $\frac{30.044}{30.427}$ & \begin{tabular}{|l|}
-0.934 \\
-0.934 \\
\end{tabular} & & $\begin{array}{ll}48.209 \\
48.448\end{array}$ & $\begin{array}{l}0.0350 \\
0.037\end{array}$ & $\frac{14.643}{14.643}$ & 0.003 & & 75.4622 & 53.2 & 11.9 & 40.2 & 2.770 & $\mid$ & \begin{tabular}{|l|l|}
0.006 \\
\end{tabular} & 0.000 & $\begin{array}{l}0.135 \\
0.138\end{array}$ & 0.1 \\
\hline
\end{tabular}


WSRC-TR-2005-00105, REVISION 0

SRNL-RPP-2005-00012, REVISION 0

RUN \# 2.03A AND B; FIRST AND SECOND HALF OF SLURRY DEWATERING - CONT.

\begin{tabular}{|c|c|c|c|c|c|c|c|c|c|c|c|c|c|c|c|c|c|c|c|c|c|c|c|c|c|c|c|c|}
\hline & A & B & D & $E$ & $\mathrm{~F}$ & G & $\mathrm{H}$ & $\mathrm{J}$ & $\mathrm{K}$ & $\mathrm{L}$ & $\mathrm{M}$ & $\mathrm{N}$ & $\mathrm{O}$ & Q & $R$ & $\mathrm{~s}$ & \begin{tabular}{l|l|}
$\mathrm{T}$ \\
\end{tabular} & & $w$ & $x$ & & $z$ & AA & $A B$ & $\mathrm{AC}$ & $A D$ & & AF \\
\hline$\frac{329}{329}$ & $\begin{array}{r}4 / 22 / 2004 \\
4 / 2 / 2004\end{array}$ & $\begin{array}{l}1: 10: 34 \mathrm{PM} \\
1 \cdot 11 \cdot 34 \mathrm{PM}\end{array}$ & $\begin{array}{r}25.53 \\
25.541 \\
\end{array}$ & $\begin{array}{r}25.27 \\
25.286 \\
\end{array}$ & 24.874 & $\begin{array}{r}26.148 \\
26.184\end{array}$ & \begin{tabular}{|l|}
25.575 \\
25.586 \\
\end{tabular} & $\begin{array}{l}49.882 \\
49.589 \\
\end{array}$ & \begin{tabular}{|l|}
54.529 \\
54.293 \\
\end{tabular} & \begin{tabular}{|c|}
19.933 \\
19.818
\end{tabular} & $\begin{array}{l}30.208 \\
30.07\end{array}$ & $\begin{array}{r}-0.94 \\
-0.937\end{array}$ & $\begin{array}{l}-5.896 \\
-5.893\end{array}$ & \begin{tabular}{|c|}
49.478 \\
47906
\end{tabular} & $\frac{0.036}{0.036}$ & $\begin{array}{l}14.643 \\
14.643 \\
\end{array}$ & \begin{tabular}{|c|}
0.003 \\
0.003
\end{tabular} & $\begin{array}{l}4528.7 \\
4529.7\end{array}$ & \begin{tabular}{|l|l|}
75.4789 \\
75.4956
\end{tabular} & \begin{tabular}{|l|l}
53. \\
52.8
\end{tabular} & $\frac{12.1}{11.7}$ & \begin{tabular}{|l|}
40.0 \\
39.8 \\
\end{tabular} & \begin{tabular}{|l|}
2.761 \\
2.746
\end{tabular} & 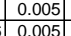 & \begin{tabular}{|l|}
0.005 \\
0.005 \\
\end{tabular} & 0.000 & $\begin{array}{l}0.135 \\
0.135\end{array}$ & $\begin{array}{l}0.11 \\
0.12\end{array}$ \\
\hline \begin{tabular}{|l|l|l|}
3293 \\
3
\end{tabular} & & & & & & & & & & & & & & & & & & & & & & & & & & & & \\
\hline 3294 & $4 / 22 / 2004$ & 1:13:34 PM & 25.55 & 25.295 & \begin{tabular}{|l|}
24.884 \\
\end{tabular} & 26.208 & 25.58 & 49.863 & \begin{tabular}{|l|}
54.567 \\
\end{tabular} & 19.733 & 30.487 & $\begin{array}{l}-0.934 \\
\end{array}$ & -5.893 & 48.077 & 0.036 & 14.643 & 0.003 & 4531.7 & 75.5289 & 53. & \begin{tabular}{l|l|l}
1 & 11.8 \\
\end{tabular} & & 2.770 & 0.005 & \begin{tabular}{|l|}
0.005 \\
\end{tabular} & 0.000 & 0.134 & \\
\hline & $4 / 221 / 2004$ & 1:14:34 PM & 25.556 & 25.306 & \begin{tabular}{|l|l|}
24.884 \\
\end{tabular} & 26.199 & 25.576 & 50.251 & \begin{tabular}{|l|l|}
55.048 \\
\end{tabular} & 20.134 & 30.417 & $\begin{array}{c}-0.94 \\
\end{array}$ & -5.891 & 48.396 & 0.036 & 14.643 & & 4532.7 & 75.5456 & & & & 2.781 & & \begin{tabular}{|l|}
0.005 \\
\end{tabular} & & & \\
\hline & & & & & & & & & & & & \begin{tabular}{|l|l|} 
& -0.94 \\
\end{tabular} & & & & & & & & 53. & & & & & & & & \\
\hline \begin{tabular}{|l|l|}
3297 \\
3298
\end{tabular} & $4 / 22 / 2004$ & 1:16:34 PM & 25.554 & 25.299 & 24.882 & 26.232 & $\begin{array}{l}25.559 \\
25.523\end{array}$ & \begin{tabular}{|l|l|l|}
50.074 \\
\end{tabular} & \begin{tabular}{|l|l}
54.842 \\
\end{tabular} & $\begin{array}{l}19.975 \\
\end{array}$ & 30.292 & \begin{tabular}{|c|}
-0.934 \\
\end{tabular} & $\begin{array}{l}-5.891 \\
\end{array}$ & $\begin{array}{l}48.321 \\
\end{array}$ & 0.037 & 14.643 & $\begin{array}{l}0.003 \\
\end{array}$ & 4534.7 & \begin{tabular}{|l|l|}
75.5789 \\
77. \\
\end{tabular} & & & & 2.770 & \begin{tabular}{|l|l|}
0.006 \\
\end{tabular} & 0.006 & 0.000 & 0.138 & 0.12 \\
\hline $\begin{array}{l}3298 \\
3299 \\
\end{array}$ & $\begin{array}{l}4 / 22121204 \\
4 / 212004\end{array}$ & $\begin{array}{l}1: 17: 34 \mathrm{PM} \\
1: 18: 34 \mathrm{PM}\end{array}$ & $\begin{array}{l}25.568 \\
25.568\end{array}$ & $\begin{array}{l}25.303 \\
25.292 \\
\end{array}$ & \begin{tabular}{|l|}
24.902 \\
24.991 \\
\end{tabular} & $\begin{array}{l}26.241 \\
26.191\end{array}$ & \begin{tabular}{|l|}
25.533 \\
25.527 \\
\end{tabular} & \begin{tabular}{|l|l|}
50.089 \\
49.819
\end{tabular} & \begin{tabular}{|l|}
54.8766 \\
54.486
\end{tabular} & \begin{tabular}{|l|l|l|l}
19.978 \\
19.924
\end{tabular} & $\begin{array}{r}30.44 \\
30.206 \\
\end{array}$ & \begin{tabular}{|c|c|c|}
-0.934 \\
-0.931
\end{tabular} & $\begin{array}{l}-5.891 \\
-5.893\end{array}$ & \begin{tabular}{|l|}
47.822 \\
47.47
\end{tabular} & $\begin{array}{l}0.036 \\
0.036\end{array}$ & $\begin{array}{l}14.643 \\
14.643\end{array}$ & \begin{tabular}{|l}
0.003 \\
0.003
\end{tabular} & $\begin{array}{l}4535.7 \\
4536.7\end{array}$ & \begin{tabular}{|l|l|}
75.5956 \\
756122
\end{tabular} & $\begin{array}{l}53 . \\
530\end{array}$ & $\frac{11.7}{11.6}$ & $\begin{array}{l}40.3 \\
40.0\end{array}$ & \begin{tabular}{|l}
2.776 \\
2.759
\end{tabular} & \begin{tabular}{|c|}
0.005 \\
0.05 \\
\end{tabular} & $\begin{array}{l}0.005 \\
0.005\end{array}$ & $\begin{array}{l}0.000 \\
0.000\end{array}$ & $\begin{array}{l}0.134 \\
0.135\end{array}$ & 0.11 \\
\hline 3300 & $4 / 22 / 2004$ & $\begin{array}{l}1.10 .34 \mathrm{PM} \\
1: 1934\end{array}$ & 25.558 & & \begin{tabular}{|l|}
24.876 \\
\end{tabular} & 26.16 & 25.577 & 49.566 & \begin{tabular}{|l|l|}
54.229 \\
\end{tabular} & & $\begin{array}{l}30.200 \\
30.113\end{array}$ & \begin{tabular}{|c|}
-0.94 \\
\end{tabular} & & $\begin{array}{r}41.491 \\
49.08\end{array}$ & 0.037 & $\begin{array}{l}\frac{14.045}{14.643} \\
\end{array}$ & $\begin{array}{l}0.0003 \\
0.003\end{array}$ & $\frac{4536.1}{4537.7}$ & \begin{tabular}{|l|}
15.512122 \\
75.6289
\end{tabular} & \begin{tabular}{|l}
53. \\
52.8
\end{tabular} & $\frac{11.6}{12.0}$ & $\frac{40.0}{39.8}$ & & $|0.005|$ & $\begin{array}{l}0.005 \\
0.006\end{array}$ & 0.000 & $\frac{135}{139}$ & 0.11 \\
\hline & $4 / 22 / 2004$ & 1:20:34 PM & & & 24.895 & 26.204 & 25.516 & & \begin{tabular}{|l|l|} 
& 54.652 \\
\end{tabular} & $\begin{array}{ll}19.849 \\
\end{array}$ & 30.505 & \begin{tabular}{|c|c|c|} 
\\
\end{tabular} & & 47.764 & 0.037 & 14.643 & & 4538.7 & \begin{tabular}{|l|l|}
75.6456 \\
\end{tabular} & & & & & & & & & $\begin{array}{l}0.12 \\
0.12\end{array}$ \\
\hline 3302 & $4 / 22 / 2004$ & 1:21:34 PM & 25.555 & 25.274 & \begin{tabular}{|l|} 
\\
\end{tabular} 4.878 & 26.193 & \begin{tabular}{|l|l|}
25.634 \\
\end{tabular} & 49.834 & $\begin{array}{l}54.53 \\
\end{array}$ & 20.079 & 30.005 & -0.899 & & 47.805 & 0.036 & 14.643 & 0.003 & 4539.7 & \begin{tabular}{|l|l|}
75.6622 \\
\end{tabular} & 53. & & \begin{tabular}{|l|l|}
39.9 \\
\end{tabular} & 2.752 & 0.005 & \begin{tabular}{|l|}
0.005 \\
\end{tabular} & 0.000 & & \\
\hline 3303 & $4 / 22 / 2004$ & $1: 22: 34 \mathrm{PM}$ & 25.558 & & 24.867 & & & 49.705 & 54.536 & 19.775 & 30.335 & & & & & & & & & & & & & & & & & $\begin{array}{l}0.1 \\
0.1\end{array}$ \\
\hline 3304 & $4 / 22 / 2004$ & 1:23:34 PM & 25.000 & 25.302 & & & & & 54.876 & 1000 & & \begin{tabular}{|c|c|c|}
-0.914 \\
\end{tabular} & & & 0.037 & & & & & & & & & & & & & \\
\hline & 4/22/2004 & 1:24:34 PM & & 25.296 & & 26.209 & 25.556 & 49.817 & & 19999 & 30.308 & -0.92 & & $\begin{array}{l}48.767 \\
\end{array}$ & 0.037 & 14.643 & & 4542.7 & 5.7122 & & & & 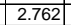 & 5.006 & 0.006 & & & \\
\hline 3306 & 4/22/2004 & 1:25:34 PM & 25.607 & 25.302 & 24.916 & 26.2 & 25.592 & 50.072 & 54.892 & 19.821 & 30.54 & \begin{tabular}{|l|l|}
-0.92 \\
\end{tabular} & -5 & $\begin{array}{l}48.707 \\
\end{array}$ & 0.036 & 14.643 & $\begin{array}{l}0.003 \\
\end{array}$ & 4543.7 & \begin{tabular}{|l|l|}
75.7289 \\
\end{tabular} & 53. & 11.9 & 40.3 & 2.779 & 0.005 & 0.005 & 0.000 & 133 & \\
\hline & $4 / 22 / 2004$ & 1:26:34 PM & 25.603 & 25.307 & 24.921 & 26.286 & 25.65 & 49.98 & 54.671 & 19.905 & 30.43 & -0.885 & & 47.628 & 0.036 & 14.643 & & 4544.7 & 75.7456 & & & & 2.772 & & & & & \\
\hline & $4 / 22 / 2004$ & & & & & & & & & & & & & & & & & & & & & & & & & & & \\
\hline & $4 / 22 / 2004$ & 1:28:34 PM & 25.613 & 25.307 & 24.921 & 26.291 & 25.687 & 49.94 & 4.747 & $\begin{array}{r}19.99 \\
10.952\end{array}$ & $\begin{array}{l}30.224 \\
32.126\end{array}$ & -0.902 & & 48.544 & 0.037 & 14.643 & 1003 & 4546.7 & & & & & .764 & 006 & .006 & .000 & & \\
\hline$\sqrt{3311}$ & 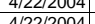 & $\frac{1.29 .34 \mathrm{FM}}{2.32 .03}$ & $\frac{25.618}{25619}$ & $\frac{25.30}{2532}$ & $\frac{24.520}{21926}$ & $\frac{20.290}{26271}$ & $\frac{25.60}{2574}$ & 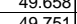 & & $\frac{1.9825}{10925}$ & & $\begin{array}{l}-0.085 \\
0.017\end{array}$ & & & 0.036 & $\frac{11.643}{10.642}$ & & 548 & & & & & 275 & & & & & \\
\hline$\overline{3312}$ & $4 / 22 / 2004$ & $1: 31: 34 \mathrm{PM}$ & $\frac{25.618}{25.618}$ & $\frac{25.32}{25.312}$ & 24.921 & 26.256 & 25.677 & & $\begin{array}{l}54.279 \\
54.279\end{array}$ & 19.957 & 30.039 & $\begin{array}{l}-0.888 \\
-0.88\end{array}$ & & $\mid 48.164$ & 0.036 & $\begin{array}{l}14.643 \\
4.643\end{array}$ & 0.003 & 4549.7 & T. & & & 398 & & & 0.005 & 0.000 & & \\
\hline & $4 / 22 / 2004$ & 1:32:34 PM & 25.638 & 25.358 & \begin{tabular}{|l|l|}
24.967 \\
\end{tabular} & 26.296 & 25.683 & 49.824 & 54.592 & 19.771 & 30.444 & -0.882 & -5.888 & 48.49 & 0.036 & 14.643 & 0.003 & 4550.7 & 755.8456 & 53. & 11.9 & & \begin{tabular}{|l|}
2.767 \\
\end{tabular} & & 0.005 & 0.000 & 134 & 0.1 \\
\hline 3 & R/2004 & 1:33:34 PM & 25.63 & 25.32 & 24.947 & 26.261 & 25.688 & 49.64 & & 19.768 & 30.206 & -0.882 & & $\begin{array}{l}47.966 \\
\end{array}$ & 0.036 & 14.643 & & 4551.7 & & & & & & & & & & \\
\hline & $2 / 2004$ & 1:34:34 PM & $\frac{25.63}{25.632}$ & $\frac{25.433}{2.529}$ & 24.937 & 26.266 & 25. & 49.834 & 54.55 & $\begin{array}{r}19.84 \\
10.804\end{array}$ & .27 & -0.882 & & 1.945 & 0.036 & 14.643 & & & & & & & & & & & & \\
\hline 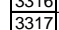 & 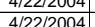 & $\begin{array}{l}1.353 .34 \mathrm{PM} \\
132.34 \mathrm{MM}\end{array}$ & $\frac{25.633}{25.643}$ & $\frac{25.348}{25.357}$ & $\frac{24.931}{24.946}$ & $\frac{26.216}{26316}$ & 25.12 & $\begin{array}{l}50.006 \\
49753\end{array}$ & 54.178 & $\frac{19.804}{20.13}$ & $\frac{30.563}{29.935}$ & 0.005 & & 4857 & 0.031 & $\frac{14.043}{14643}$ & & $\frac{4535.1}{455.7}$ & 759122 & & & $36>$ & 2747 & & & & & \\
\hline & 4 & $1 \cdot 37 \cdot 34 \mathrm{PM}$ & 25.643 & 25.357 & 22.951 & $\begin{array}{l}20.310 \\
26.356\end{array}$ & & $\begin{array}{l}49.150 \\
49.579\end{array}$ & $\begin{array}{r}54.530 \\
54.31\end{array}$ & $\mid \begin{array}{c}2.1 .10 \\
19.813\end{array}$ & $\begin{array}{l}39.905 \\
30.146\end{array}$ & $\begin{array}{c}-0.000 \\
-0.882 \\
-10\end{array}$ & & $\begin{array}{l}40.951 \\
48.392\end{array}$ & $\frac{0.030}{0.036}$ & $\frac{14.045}{14.643}$ & $\frac{0.005}{0.003}$ & & & 52 & & & & 0005 & 0.005 & & & \\
\hline & 4/22/2004 & 1:38:34 PM & 25.653 & 25.378 & \begin{tabular}{|l|}
24.967 \\
\end{tabular} & 26.382 & 25.7 & 49. & 54.36 & $\begin{array}{l}19.866 \\
19.86\end{array}$ & 29.953 & $\mid-0.885$ & & 48 & 0.036 & 14.643 & & 4556.7 & $\begin{array}{l}775.9456 \\
\end{array}$ & & 12. & 39.8 & 2741 & & & & & 0.1 \\
\hline & $4 / 221 / 2004$ & 1:39:34 PM & 25.653 & 25.368 & 24.957 & 26.376 & 25.5 & 49.53 & 54.254 & 19.838 & & -0.885 & & 49.85 & 0.036 & 14.643 & & 4557.7 & & & & & & & & & & \\
\hline 332 & $4 / 22 / 2004$ & 1:40:34 PM & 25.642 & 25.377 & 24.951 & 26.325 & & 49.92 & 54.621 & 19.838 & 30.396 & $\begin{array}{c}-0.882 \\
\end{array}$ & & 48.212 & 0.036 & 14.643 & & 4558.7 & $\frac{75.9789}{77}$ & 53 & 11. & & 2.769 & .005 & 0.005 & 0.000 & & \\
\hline & $4 / 22 / 2004$ & 1:41:34 PM & 25.656 & 25.38 & 24.969 & \begin{tabular}{|l|l|}
26.344 \\
\end{tabular} & 25.6 & 49.6 & 54.463 & $\begin{array}{ll}19.768 \\
\end{array}$ & 30.259 & -0.879 & & \begin{tabular}{|l|l|l|l|l}
48.784 \\
\end{tabular} & 0.036 & 14.643 & & & & & & & & & & & & \\
\hline & & $1: 42: 34 \mathrm{PM}$ & 25.655 & 25.385 & 24.944 & & & 49.86 & & & 30.148 & & & & & & & & & & & & & & & & & \\
\hline & $4 / 22 / 2004$ & 1:43:34 PM & 25.658 & 25.373 & \begin{tabular}{l|l}
24.947 \\
\end{tabular} & 26.291 & 25.4 & 49.805 & 54.648 & 19.936 & 30.144 & $\mid-0.876$ & & 46.932 & 0.036 & 14.643 & & 4561.7 & \begin{tabular}{|l|l|}
76.0289 \\
\end{tabular} & 53. & 11.5 & 40.0 & 2.756 & $\mid$ & 0.005 & 0.000 & 0.134 & 0.1 \\
\hline & 4/22/2004 & 1:44:34 PM & 25.656 & 25.376 & 24.925 & 26.189 & 25. & & 54.407 & 19.796 & 30.128 & -0.888 & & & 0.0 & 14. & & & 76.0 & & 11. & & & & & & & 0. \\
\hline & & & 25.663 & & 24.937 & 26.116 & 25.2 & & 54.471 & 19.941 & .056 & -0.882 & & 48.485 & 0.036 & & & & & & 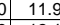 & & 749 & & & & & \\
\hline & $4 / 22 / 2004$ & & 25.666 & 25.385 & 24.939 & 26.028 & 25. & & 54.333 & 19.803 & 30.068 & -0.882 & & 99.314 & & 14.643 & & & .0789 & & 12. & & 2.744 & 0.005 & 0.005 & 0.000 & & \\
\hline & & & & $\frac{25.373}{3530}$ & & & & & & & & & & & & & & & & & & & & & & & & \\
\hline 3330 & 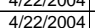 & $\begin{array}{l}1.48 .34 \mathrm{PM} \\
1: 49: 34 \mathrm{PM}\end{array}$ & $\begin{array}{l}25.075 \\
25.683\end{array}$ & $\begin{array}{r}25.39 \\
25.378\end{array}$ & $\begin{array}{l}24.944 \\
324.937\end{array}$ & $\frac{23.976}{26026}$ & $\frac{25.4}{2536}$ & $\begin{array}{l}49.105 \\
49.494\end{array}$ & $\frac{521}{3222}$ & $\frac{19.01}{19702}$ & $\frac{3 x}{20}$ & $\mid=0.087$ & & 48316 & 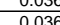 & $\begin{array}{l}\frac{14.045}{14643} \\
1.62\end{array}$ & & $\frac{430.6}{4567}$ & 776289 & 528 & $\frac{11.6}{11.8}$ & & 2740 & 0005 & 0.005 & 000 & & \\
\hline & $4 / 22 / 2004$ & 1:50:34 PM & 25.676 & 25.376 & \begin{tabular}{|l|l|}
54.93 \\
5
\end{tabular} & 26.029 & 25.4 & & & 19.861 & 30.318 & $\mid-0.85$ & & 50.444 & & 14.643 & & & & 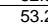 & 10 & & & & & & & \\
\hline & $4 / 22 / 2004$ & 1:51:34 PM & 25.691 & 25.39 & 24.964 & 26.068 & 25 & & 1.443 & 19.607 & & -0.865 & & & 0.036 & & & & & & 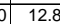 & & 2.754 & & & & & 0. \\
\hline & |2/2004 & & 25.686 & 25.381 & 24.95 & & 25.6 & & & 19.769 & & & & & & & & & & & & & & & & & & \\
\hline & & & & & & & & & & & 30.2 & -0.833 & & 8256 & & & & & & & & & & & & & & \\
\hline & $4 / 22 / 2004$ & 1:54:34 PM & 25.6 & 25.382 & 24.956 & 26.16 & & & 54.416 & & 30.6 & -0.847 & & 47.5 & 0.0 & & & & 76.2122 & 52 & 11. & $\overline{39.9}$ & & & & & & \\
\hline & .2/2004 & 1:55:34 PM & 25.674 & 25.379 & \begin{tabular}{|l|l|} 
& 24.947 \\
\end{tabular} & 26.157 & & & & & & -0.8 & & & & & & & & 53 & 11 & & & & & & & \\
\hline & 4/221/2004 & 1:56:34 PM & 25.659 & 25.369 & 24.932 & 26.157 & 25.6 & 49.97. & 54.714 & 19.969 & & -0.815 & & 48. & & 14. & & & 766.2456 & 53 & 11 & 40 & & & & & 34 & \\
\hline 3338 & $4 / 221 / 2004$ & 1::57:34 PM & 25.66 & 25.369 & 24.933 & 26.178 & & & 54.349 & 19.765 & & -0.8. & & & & & & & & Jat & 11. & & & & & & 35 & \\
\hline & & & 25.666 & 25.381 & 24.939 & 26.254 & & & & & & -0.815 & & 48.84 & & & & & & $J_{c}$ & $12.6 \mathrm{C}$ & & & & & & & \\
\hline 34 & 2 & $1: 593.34$ & & $25.3 \pi$ & 24.859 & & & & 54.015 & 20.32 & & -0.8 & & 10 & & & & & & 5 & 12. & & & & & & 104 & \\
\hline 342 & $\frac{44242004}{4 / 212004}$ & $\frac{2.300 .34}{2 \cdot 34}$ & $\frac{25.072}{25673}$ & 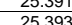 & $\begin{array}{l}24.04 \\
24.796\end{array}$ & & & & & & & $\frac{-0.0}{-0.8}$ & & & & & & & & & 121 & & & & & & & \\
\hline & 4 & 2.02 .34 & 25.659 & 2538 & 24.747 & & & & 53.748 & & & -0.8 & & & & & & & & $J_{2}$ & 12.1. & & & & & & & \\
\hline 3 & 4 & $\frac{2: 03: 34}{2034}$ & 25.656 & $\frac{25.395}{25.395}$ & 24.724 & 26.294 & 25 & & 53.702 & $\frac{20.296}{20.296}$ & & $\frac{-0.8}{-0.8}$ & & & & & & & & & $\frac{12.4}{12.4}$ & & & & & & & \\
\hline & & & & & & & & & & & & & & & & & & & & & & & & & & & & \\
\hline 334 & & & 25.638 & 25,398 & 24.656 & 26.306 & 25.7 & & 53792 & & & -0.818 & & & & 14.6 & & & & & & & & & & & & \\
\hline & $4 / 22 / 2$ & 2:06:34 PM & 25. & 25 . & 24.658 & 26.338 & 25.8 & & 1 & & & -0.8 & & & & & & & & & 12.3 & & & & & & & \\
\hline 3348 & 4/221/2004 & 2:07:34 PM & 25.6 & 25.4 & 24.611 & 26.296 & & 48.6 & 53.5 & 20.245 & 28.6 & -0.818 & & 50.14 & 0.03 & 14.643 & & & 76.4 & 52 & 12.3 & 38 & 2.66 & & & & 136 & \\
\hline & $4 / 22 / 2004$ & 2:08:34 & 25.62 & 25.405 & 24.5 & & & 48. & 53.719 & 20 & & -0.8 & & & & & & & & 52. & 12.6 & & & & & & 136 & \\
\hline & $4 / 22 / 20$ & 2:09:3 & 25.616 & 25. & 24.554 & & 25.8 & & 53.839 & 20.156 & & -0.818 & & 49.138 & 0.05 & & & & & 52. & 12.0 & 39 & & & & & 35 & \\
\hline & $4 / 22 / 2004$ & 2:10:34 PM & 25.613 & 25.423 & 24.531 & 26.371 & 25.8 & 49.054 & 53.902 & 20.243 & 29.114 & -0.8 & & & & 14.6 & & & & & & & & & & & & \\
\hline & $\frac{421212004}{41210}$ & $2: 11.34 \mathrm{PM}$ & $\frac{25.614}{25616}$ & 25.4395 & 24.517 & $\frac{26.412}{26379}$ & $\frac{25.1}{25}$ & $\begin{array}{l}48.803 \\
48.843\end{array}$ & $\begin{array}{l}5.3 .68 \\
5.74\end{array}$ & $\frac{2.202}{20201}$ & $\frac{28.9}{200}$ & $\begin{array}{l}-0.013 \\
-0.039\end{array}$ & & & & & & & & & 120 & & & & & & . & \\
\hline & 41212004 & & $\frac{23.010}{2567}$ & 20.445 & $\begin{array}{l}2.4 .40 \\
20.75\end{array}$ & 2.0074 & $\frac{25}{257}$ & 40.045 & $\frac{5.174}{53.43}$ & 20.201 & & -0.0 & & & & & & & & & 2.0 & & & & & & & \\
\hline & $\frac{4}{42 / 2120004}$ & $\frac{2.1034}{2 \cdot 14.34}$ & & 党. & $\frac{24.410}{24466}$ & $\frac{20.4}{2016}$ & & & 5374 & & & & & & & & & & & & 步 & & & & & & 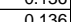 & \\
\hline 335 & & & & & & & & & & 19.964 & & -0.8 & & & & & & & & & 120 & & & & & & & \\
\hline & $4 / 2 / 2004$ & & 25.584 & & & & & & & & & & & & & & & & & & & & & & & & $\frac{5136}{136}$ & \\
\hline & & & & 25.49 & & & & & & & & & & & & & & & & 523 & & & & & & & & \\
\hline & $4 / 22 / 2004$ & & 25. & & & 26.253 & & 48.594 & 387 & 902 & $2 \varepsilon$ & & & & & 14.643 & & & & & & & & & & & 137 & 0 \\
\hline 360 & $4 / 22 / 2004$ & 2:19:34 PM & 25.545 & 25.49 & 24.373 & \begin{tabular}{|l|l|}
26.203 \\
\end{tabular} & 2553 & & \begin{tabular}{|l|}
53.674 \\
\end{tabular} & 20.094 & & -0.818 & & 49.933 & $\begin{array}{ll}0.035 \\
\end{array}$ & 14.643 & & & 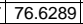 & & 12.2 & & 2.684 & 0.005 & 0.005 & 0.000 & 0.136 & 0.1 \\
\hline
\end{tabular}


WSRC-TR-2005-00105, REVISION 0

SRNL-RPP-2005-00012, REVISION 0

RUN \# 2.03A AND B; FIRST AND SECOND HALF OF SLURRY DEWATERING - CONT.

\begin{tabular}{|c|c|c|c|c|c|c|c|c|c|c|c|c|c|c|c|c|c|c|c|c|c|c|c|c|c|c|c|c|}
\hline & & & & & & & & & & & & & & & & & & & & & & & & & & & & \\
\hline & $\mathrm{A}$ & \begin{tabular}{l|l} 
\\
\end{tabular} & D & $E$ & $\mathrm{~F}$ & \begin{tabular}{l|l} 
\\
\end{tabular} & $\mathrm{H}$ & $\mathrm{J}$ & $\mathrm{K}$ & $\mathrm{L}$ & $\mathrm{M}$ & $\mathrm{N}$ & \begin{tabular}{l|l} 
\\
\end{tabular} & Q & \begin{tabular}{l|l|}
$R$ \\
\end{tabular} & $\mathrm{~s}$ & \begin{tabular}{l|l} 
\\
\end{tabular} & $\mathrm{v}$ & w & $|x|$ & \begin{tabular}{|l|} 
\\
\end{tabular} & $\mathrm{z}$ & AA & $\mathrm{AB}$ & AC & $\mathrm{AD}$ & $\mathrm{AE}$ & AF \\
\hline & $4 / 22 / 2004$ & 2:20:34 PM & $\begin{array}{l}25.519 \\
25.517\end{array}$ & $\begin{array}{l}25.469 \\
2.572\end{array}$ & 24.346 & \begin{tabular}{|l|l|}
26.097 \\
609
\end{tabular} & 25.329 & 48.897 & 53.831 & \begin{tabular}{|l|}
20.098 \\
\end{tabular} & $\begin{array}{l}29.052 \\
3020\end{array}$ & \begin{tabular}{|l|l|}
-0.818 \\
\end{tabular} & \begin{tabular}{|l|l|}
-5.879 \\
5.870
\end{tabular} & 50.046 & $\begin{array}{l}0.035 \\
\end{array}$ & 14.643 & 0.003 & 4598.7 & 766.6456 & \begin{tabular}{|l|l|}
6 & 52.4 \\
\end{tabular} & 12.3 & 39.0 & \begin{tabular}{l|l}
0 & 2.687 \\
\end{tabular} & 0.005 & \begin{tabular}{|l|}
0.005 \\
\end{tabular} & 0.000 & 0.136 & 0.12 \\
\hline $\begin{array}{l}3362 \\
3363 \\
336\end{array}$ & $\frac{4 / 22 / 2004}{4 / 23 / 2004}$ & $\begin{array}{ll}2: 21: 34 \mathrm{PM} \\
2: 22: 34 \mathrm{PM}\end{array}$ & $\begin{array}{l}25.517 \\
25496\end{array}$ & $\begin{array}{l}25.472 \\
25.466\end{array}$ & 24.334 & \begin{tabular}{|l|l|}
26.08 \\
26.074
\end{tabular} & $\begin{array}{l}25.212 \\
25201\end{array}$ & 48.913 & $\begin{array}{l}53.742 \\
5601\end{array}$ & \begin{tabular}{|r|}
19.97 \\
\end{tabular} & $\begin{array}{r}29.288 \\
30103 \\
\end{array}$ & $\begin{array}{r}-0.804 \\
-0804\end{array}$ & \begin{tabular}{|l|}
-5.879 \\
5876
\end{tabular} & & $\begin{array}{l}0.035 \\
0025\end{array}$ & 14.643 & $\begin{array}{l}0.003 \\
\end{array}$ & 4599.7 & & & & & 20.090 & & \begin{tabular}{|l|}
0.005 \\
\end{tabular} & 0.000 & & 0.12 \\
\hline $\begin{array}{l}3503 \\
3364 \\
\end{array}$ & $\begin{array}{l}4 / 252 / 2004 \\
44 / 21 / 2004\end{array}$ & $\begin{array}{l}2: 22: 34 \mathrm{PM} \\
2 \cdot 23: 34 \mathrm{P}\end{array}$ & $\begin{array}{l}25.496 \\
25.498\end{array}$ & $\begin{array}{r}25.466 \\
25.473\end{array}$ & $\frac{24.319}{24.321}$ & \begin{tabular}{r|}
26.074 \\
26106
\end{tabular} & $\begin{array}{l}25.291 \\
25.363\end{array}$ & $\begin{array}{l}48.772 \\
49.087\end{array}$ & $\begin{array}{l}53.601 \\
53.972\end{array}$ & $\begin{array}{l}\frac{19.997}{20.092} \\
\end{array}$ & $\begin{array}{r}29.063 \\
29257 \\
\end{array}$ & $\begin{array}{l}-0.804 \\
-0.071\end{array}$ & $\begin{array}{l}-5.876 \\
-5876\end{array}$ & $\begin{array}{r}49.603 \\
49.856\end{array}$ & $\begin{array}{l}0.035 \\
0.035\end{array}$ & $\frac{14.643}{14643}$ & 0.003 & & \begin{tabular}{|l|l|}
76.6789 \\
766956
\end{tabular} & $\begin{array}{l}52.1 \\
5.5\end{array}$ & $\begin{array}{l}12.2 \\
122\end{array}$ & 38.9 & $\begin{array}{l}92.683 \\
2.2701\end{array}$ & 0.005 & \begin{tabular}{|l|} 
\\
\end{tabular} & 0.000 & 0.137 & 0.12 \\
\hline 3365 & $4 / 22 / 2004$ & $2: 24: 34 \mathrm{PM}$ & 25.478 & & \begin{tabular}{|l|l|}
24.321 \\
24.296
\end{tabular} & \begin{tabular}{|l|}
20.100 \\
26.156
\end{tabular} & $\frac{25.363}{25.503}$ & $\begin{aligned} \frac{49.081}{49.21} \\
\end{aligned}$ & \begin{tabular}{|l|}
3.9712 \\
54.047
\end{tabular} & $\begin{array}{l}20.137 \\
\end{array}$ & $\frac{29.257}{29.352}$ & $\begin{array}{l}-0.807 \\
-0.799 \\
-10\end{array}$ & $\frac{-5.876}{-5873}$ & $\begin{array}{c}49.856 \\
49.668 \\
\end{array}$ & $\begin{array}{l}0.0335 \\
0.035\end{array}$ & $\begin{array}{l}14.643 \\
14.463\end{array}$ & 0.003 & & \begin{tabular}{|l|l|}
76.6956 \\
7767122
\end{tabular} & & $\frac{12.2}{122}$ & \begin{tabular}{|l|}
39.2 \\
39.3
\end{tabular} & $\begin{array}{l}\frac{2}{2} 2.701 \\
3\end{array}$ & $\begin{array}{l}0.005 \\
0.05 \\
\end{array}$ & \begin{tabular}{|l|}
0.005 \\
0005 \\
\end{tabular} & 0.000 & 0.136 & 0.12 \\
\hline 3366 & $4 / 22 / 2004$ & 2:25:34 PM & 25.458 & 25.453 & \begin{tabular}{|l|l|} 
& 24.281 \\
\end{tabular} & 26.236 & 25.483 & 48.851 & \begin{tabular}{|l|}
53.682 \\
\end{tabular} & 19.949 & 29.184 & -0.795 & $\begin{array}{l}-3.010 \\
-5.876 \\
\end{array}$ & 51.481 & 0.035 & $\begin{array}{l}14.045 \\
14.643\end{array}$ & $\begin{array}{l}.003 \\
0.003\end{array}$ & $\begin{array}{l}\frac{4602.1}{4603.7} \\
\end{array}$ & \begin{tabular}{|l|l|l|l|l|}
76.7289 \\
\end{tabular} & \begin{tabular}{|l|l|}
9 & 52.6 \\
\end{tabular} & $\begin{array}{l}12.2 \\
12.6\end{array}$ & \begin{tabular}{|l|}
39.3 \\
39.0 \\
\end{tabular} & 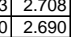 & $\begin{array}{l}0.005 \\
0.005\end{array}$ & \begin{tabular}{|l|l|}
0.005 \\
0.005 \\
\end{tabular} & $\begin{array}{c}0.000 \\
0.000\end{array}$ & $\frac{0.136}{0.136}$ & $\frac{0.12}{0.12}$ \\
\hline \begin{tabular}{|l|}
3367 \\
\end{tabular} & $4 / 22 / 2004$ & 2:26:34 PM & 25.444 & 25.444 & 24.256 & 26.267 & 25.604 & 48.61 & \begin{tabular}{|l|}
53.47 \\
\end{tabular} & $\begin{array}{l}19.912 \\
\end{array}$ & 28.962 & -0.789 & -5.882 & 50.004 & 0.035 & 14.643 & 0.003 & 4604.7 & \begin{tabular}{|l|l|}
76.7456 \\
\end{tabular} & \begin{tabular}{|l|l|}
6 & 52.0 \\
\end{tabular} & 12.3 & \begin{tabular}{|l|l|}
388.8 \\
\end{tabular} & \begin{tabular}{|l|}
2.674 \\
\end{tabular} & 0.005 & \begin{tabular}{|l|} 
\\
\end{tabular} & 0.000 & 0.137 & $\begin{array}{l}0.12 \\
0.12\end{array}$ \\
\hline 3368 & $4 / 22 / 2004$ & 2:27:34 PM & 25.44 & 25.449 & 24.247 & 26.303 & 25.764 & & 53.825 & 19.966 & 29.2 & -0.795 & -5.876 & $\begin{array}{l}48.717 \\
\end{array}$ & & 14.643 & 0.003 & & & & & & & & & & & \\
\hline 3369 & $4 / 22 / 2004$ & 2:28:34 PM & 25.431 & & 24.243 & 26.324 & 25.715 & $\begin{array}{ll}48.782 \\
\end{array}$ & 53.615 & 19.978 & 29.206 & -0.798 & & 48.335 & 0.035 & 14.643 & & 4606.7 & 5.7789 & 521 & $\frac{11 .}{11 .}$ & 39.6 & 2.688 & 0.005 & 0.005 & 0.000 & 137 & $\begin{array}{l}0.12 \\
0.12\end{array}$ \\
\hline 3370 & $4 / 22 / 2004$ & 2:29:34 PM & 25.427 & 25.452 & 24.23 & & & & & 19.839 & & $\mid-0.789$ & & & & & & & 76.7956 & & & & & & 0.005 & & & \\
\hline & $4 / 22 / 2004$ & 2:30:34 PM & 25.423 & 25.458 & 24.205 & 26.446 & 26.252 & 48.662 & \begin{tabular}{|l|l|} 
& 53.537 \\
\end{tabular} & $\begin{array}{ll}19.936 \\
\end{array}$ & 29.022 & $\mid-0.818$ & -5.879 & 47.297 & 0.036 & 14.643 & 0 & 4608.7 & \begin{tabular}{|l|l|}
76.8122 \\
\end{tabular} & 52.1 & & 38.8 & $\begin{array}{ll}2.678 \\
\end{array}$ & 0.005 & 0.005 & 0.000 & & 0.12 \\
\hline 3372 & $4 / 22 / 2004$ & 2:31:34 PM & 25.449 & 25.484 & 24.227 & $\begin{array}{l}26.657 \\
\end{array}$ & 25.744 & |48.517 & \begin{tabular}{|l|l|}
53.404 \\
\end{tabular} & 19.889 & 28.952 & \begin{tabular}{|c|c|}
-0.772 \\
\end{tabular} & -5.873 & $\begin{array}{l}49.167 \\
\end{array}$ & 0.035 & 14.643 & 0.003 & $\begin{array}{l}4609.7 \\
\end{array}$ & \begin{tabular}{|l|l|}
76.8289 \\
\end{tabular} & 51.9 & 12.0 & 38.7 & 2.671 & 0.005 & 0.005 & 0.000 & 0.138 & 0.12 \\
\hline 3377 & 4/22/2004 & $2: 32: 34$ PM & 25.434 & 25.484 & 24.197 & 26.512 & 25.319 & 48.612 & 53.576 & 19.911 & 28.993 & -0.766 & -5.873 & 49.747 & 0.035 & 14.643 & 0.003 & 4610.7 & 76.8456 & 52. & 12. & 38.8 & 2.675 & 0.005 & 0.005 & 0.000 & & 0.12 \\
\hline & 4/22/2004 & $2: 33: 34 \mathrm{PM}$ & 25.419 & 25.484 & 24.166 & 26.372 & 25.379 & 48.942 & 53.902 & 20.002 & 29.204 & -0.769 & -5.87 & 0.0 & 0.035 & 14.643 & & 4611. & & & & & & 0.005 & 0.00 & 0.000 & & \\
\hline 年 & $4 / 22 / 2004$ & 2:34:34 PM & 25.414 & 25.499 & 24.156 & 26.322 & 25.364 & 48.486 & 53.313 & 19.783 & 29.044 & -0.772 & -5.8 & 49.109 & 0.035 & 14.643 & 0.003 & 4612.7 & 76.8789 & & $12.6 \mathrm{C}$ & 38.8 & 2.673 & .005 & 0.005 & 0.000 & 138 & 0.12 \\
\hline 3310 & 412512004 & $2: 35: 34 \mathrm{PM}$ & 25.419 & $\frac{25.514}{25.534}$ & $\frac{24.156}{24.156}$ & 26.312 & 25.409 & $\frac{48.801}{48.619}$ & \begin{tabular}{|l|}
53.779 \\
55.539
\end{tabular} & 19.861 & 29.159 & -0.769 & & 46.986 & 0.036 & $\frac{14.643}{14643}$ & 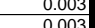 & $\frac{4613.7}{46147}$ & \begin{tabular}{|l|l|}
76.8956 \\
769122
\end{tabular} & & 11.5 & 38 & 2.688 & $\frac{0.005}{0.005}$ & 0.005 & 0.000 & & $\frac{0.12}{0.12}$ \\
\hline 3378 & $4 / 22 / 2004$ & 2:.37:34 PM & $\frac{2.414}{25.41}$ & 25.54 & $\frac{24.100}{24.127}$ & 20.0538 & $\frac{20.454}{25.56}$ & 4 & \begin{tabular}{|l|}
53.7659 \\
\end{tabular} & $\begin{array}{l}19.055 \\
19.928\end{array}$ & 29.157 & -0.772 & -5.87 & 49.683 & 0.036 & $\frac{14.040}{14.643}$ & 0.003 & $\frac{4614.1}{46157}$ & $\mid$\begin{tabular}{|l|l|}
76.9282 \\
76289
\end{tabular} & 52 & $\frac{12.2}{12.2}$ & $\frac{38.6}{390}$ & $\frac{2.671}{2689}$ & $\frac{0.005}{0.005}$ & $\frac{0.005}{0.006}$ & $\frac{0.000}{0.000}$ & 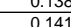 & $\frac{0.12}{0.12}$ \\
\hline$\overline{3379}$ & $4 / 22 / 2004$ & 2:38:34 PM & 25.416 & 25.556 & 24.133 & & 25.585 & 48.845 & \begin{tabular}{|l|l|}
53.777 \\
\end{tabular} & 19.894 & & -0.769 & -5.87 & 49.122 & 0.035 & 14.643 & 0.00 & 4616.7 & $\begin{array}{l}76.9456 \\
\end{array}$ & & & & & & & & & $\frac{0.12}{0.12}$ \\
\hline & $4 / 22 / 2004$ & 2:39:34 PM & 25.406 & & & & 25.576 & 48.843 & & 19.748 & 29.446 & $\mid-0.769$ & & $\begin{array}{r}45.124 \\
49.76\end{array}$ & 0.036 & 14.643 & & & & & & & & & & & & $\frac{0.12}{0.12}$ \\
\hline & $4 / 22 / 2004$ & 2:40:34 PM & 25.396 & 25.551 & 24.103 & 26.109 & 25.481 & 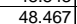 & 53.296 & 19.857 & 28.857 & -0.772 & -5.8 & 49.505 & 0.035 & 14.643 & 0.003 & 4618.7 & 76.9789 & & 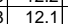 & & 36 & 0.005 & $\frac{0.005}{0.005}$ & $\frac{0.000}{0.000}$ & 138 & 0.12 \\
\hline & $4 / 22 / 2004$ & 2:41:34 PM & 25.376 & 25.551 & 24.078 & & & 48.704 & & 19.787 & & $-0.769 \mid$ & & 40 & 0.036 & 14.643 & & & 76.9956 & & & & & & & 0.000 & & \\
\hline & $4 / 22 / 2004$ & 2:42:34 PM & \begin{tabular}{|l|l|}
25.37 \\
\end{tabular} & 25.55 & 24.077 & 26.028 & 25.15 & \begin{tabular}{|l|l|l|}
48.697 \\
\end{tabular} & \begin{tabular}{|l|l|}
53.616 \\
\end{tabular} & 19.767 & 29.204 & $\mid-0.769$ & & & 0.036 & 14.643 & & 4620.7 & & 52 & 12.4 & 39 & 2.686 & & 0.006 & & & 0.12 \\
\hline 3384 & 4/22/2004 & 2:43:34 PM & 25.357 & 25.542 & 24.049 & 25.995 & 24.907 & $\begin{array}{l}48.559 \\
\end{array}$ & \begin{tabular}{|l|}
53.417 \\
\end{tabular} & 19.738 & 29.186 & \begin{tabular}{|c|c|}
-0.772 \\
\end{tabular} & & $\begin{array}{l}49.618 \\
\end{array}$ & 0.035 & 14.643 & $\begin{array}{l}0.003 \\
\end{array}$ & 4621.7 & \begin{tabular}{|l|l|}
77.0289 \\
\end{tabular} & 51.9 & 12.2 & 38.9 & 2.680 & D.005 & & 0.000 & 138 & 0.12 \\
\hline 3385 & 4/22/2004 & 2:44:34 PM & 25.354 & 25.539 & 24.041 & 25.917 & 24.839 & 48.648 & \begin{tabular}{|l}
53.603 \\
\end{tabular} & 19.787 & 29.169 & -0.769 & & 49.328 & 0.035 & 14.643 & 0.003 & 4622.7 & 77.0456 & 52 & 12.1 & 38.9 & 2.683 & 0.005 & 0.005 & 0.000 & 38 & 0.12 \\
\hline 20 & $4 / 22 / 2004$ & 2:45:34 PM & 25.323 & 25.523 & 24.014 & 25.805 & 24.763 & 48.903 & 53.835 & 19.929 & 29.149 & -0.772 & & 48.873 & 0.035 & 14.643 & & 4623.7 & 77.0622 & & 12.0 & 39. & 707 & 0.005 & 0.005 & 0.000 & & 0.14 \\
\hline 3387 & $4 / 22 / 2004$ & 2:46:34 PM & 25.312 & & & 25.779| & 24.917 & 48.505 & 53.417 & 19.848 & & -0.769 & & 51.906 & 0.036 & & & & 77.0789 & & $12.7 \mathrm{r}$ & 38. & & & & 0.000 & & 0.12 \\
\hline & $4 / 22 / 2004$ & 2::47:34 PM & 25.32 & 25.54 & \begin{tabular}{|l|}
24.016 \\
\end{tabular} & 25.7777 & 25.175 & 48.621 & 53.568 & 19.812 & 28.956 & -0.729 & & 48.867 & 0.0 & 14.643 & & & 17.0956 & & & & 2.674 & & & 0.000 & & 0.12 \\
\hline & & & & & & & & 48.617 & & & & & & & & & & & & & 12.1 & 38.9 & & & & & & \\
\hline 39 & $4 / 22 / 2004$ & 2:49:34 PM & 25.296 & 25.516 & 24.008 & 25.874 & 25.4 & 48.641 & \begin{tabular}{|l|}
53.564 \\
\end{tabular} & 19.712 & 29.18 & -0.726 & & 49.161 & 0.05 & 14.643 & 0.003 & 4627.7 & 77.1289 & $\begin{array}{l}9 \\
9\end{array}$ & 12.0 & 38.9 & 2.683 & 0.005 & 0.005 & 0.000 & 0.138 & $\overline{0.12}$ \\
\hline & $4 / 22 / 2004$ & 2:50:34 PM & 25.271 & 25.491 & 23.992 & 25.933 & 25.43 & 48.469 & \begin{tabular}{|l}
53.425 \\
\end{tabular} & 19.67 & 29.122 & -0.769 & & 50.527 & 0.0 & 14.6 & & & 77.1456 & & $12.4 \mathrm{C}$ & 38.8 & & & 0.006 & 0.000 & 0.142 & $\overline{0.12}$ \\
\hline 3392 & $4 / 22 / 2004$ & 2:51:34 PM & 25.271 & 25.481 & 23.997 & 25.998 & 25.485 & 48.629 & 53.535 & 19.79 & 29.173 & -0.752 & & 50.845 & 0.05 & 14.643 & & & & & & & 2.006 & & & & & \\
\hline 3393 & $4 / 22 / 2004$ & 2:52:34 PM & 25.261 & 25.491 & 23.997 & 26.069 & 25.58 & 48.656 & 53.582 & 19.745 & 29.106 & -0.758 & -5.86 & 49.981 & 0.036 & 14.643 & 0.003 & & \begin{tabular}{|l|l|}
77.1789 \\
\end{tabular} & \begin{tabular}{l|l}
9 & 52.1 \\
\end{tabular} & \begin{tabular}{|l|l|}
12.2 \\
\end{tabular} & 38.9 & \begin{tabular}{l|l}
9 & 2.681 \\
\end{tabular} & \begin{tabular}{|l|l|}
0.005 \\
\end{tabular} & \begin{tabular}{|l|l|} 
\\
\end{tabular} & 0.000 & 0.142 & \\
\hline$\frac{3549}{3395}$ & $\operatorname{Vash} 1 \& 2$ & & & & & & & & & & & & & & & & & & & & & & & & & & & \\
\hline 3396 & asin $1<2$ & Averages & 25.526 & 24.690 & 25.117 & 25.880 & 24.739 & 47.754 & 51.606 & 15.759 & 32.436 & -1.037 & $-6.842 \quad>>$ & 48.809 & 0.076 & 14.643 & 0.003 & & & 50.13 & 1196 & 4010 & 2764 & 0.011 & 0011 & 0.000 & & \\
\hline 3397 & & Maximum & 26.715 & 25.816 & 26.702 & 28.041 & 26.414 & 51.190 & \begin{tabular}{|l|}
55.539 \\
\end{tabular} & 20.351 & $\begin{array}{l}37.877 \\
\end{array}$ & 36.412 & & 55.402 & 0.426 & 14.643 & 0.006 & & & 54.07 & 713.58 & 42.93 & & \begin{tabular}{|l|l|}
0.064 \\
\end{tabular} & 0.063 & 0.002 & 1.823 & \\
\hline & & Media & 25.647 & & 25.285 & 25.902 & & 48.561 & $52.897 \mathrm{r}$ & 17.127 & & -1.218 & & 48.849 & 0.058 & & & & & 51.42 & I..ST & & & & & & & \\
\hline 10 & & Minimum & $22.757 \mathrm{r}$ & 21.578 & 23.655 & 22.717 & & -5.637 & -0.958 & 1.15 & -6.0 & -1.2 & & -0.07 & & 14.643 & & & & 2.43 & -0.02 & $-5.8 \mathrm{~s}-2$ & & 0.00 & 0.000 & 0.000 & -0.268 & \\
\hline & Number & 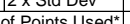 & $\frac{1.19}{3370}$ & 1..951 & $\frac{1.481}{330}$ & $\begin{array}{l}1.941 \\
370\end{array}$ & $\frac{1.675}{337}$ & $\frac{0.011}{3370}$ & $\frac{1.009}{3270}$ & 0.517 & $\begin{array}{c}3.440 \\
3.370\end{array}$ & $\frac{2.131}{3270}$ & & (5) & & & & & & 2370 & & & & & & & & \\
\hline 3402 & Nurniver & 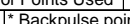 & ts & are notive & thed & S & & & & & & Soru & & & & & & & & & & & & & & & & \\
\hline & & & S & & & & & & & & & & & & & & & & & & & & & & & & & \\
\hline 3404 & Wash 1 & & & & & & & & & & & & & & & & & & & & & & & & & & & \\
\hline 3405 & & Averages & 25.556 & 24.136 & 25.196 & 25.810 & 23.835 & 44.738 & 47.126 & 10.882 & 34.579 & -1.079 & -6.46 & $\begin{array}{ll}48.667 \\
\end{array}$ & 0.138 & 14.643 & 0.003 & & & 45.653 & \#\#\#\#| & 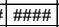 & 2.734 & .021 & 0.020 & 0.001 & 0.508 & 0.43 \\
\hline 3406 & & & 26.499 & 25.521 & 20.000 & 27.326 & 25.448 & \begin{tabular}{|l|l|}
48.673 \\
\end{tabular} & 49.800 & 13.813 & 37.877 & 36.412 & & 53.454 & & 14. & & & & 48.328 & & 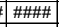 & & 0.064 & 0.063 & 0.002 & 1.823 & \\
\hline & & Median & 25.573 & 24.585 & 25.148 & 25.870 & 24.04 & 45.178 & 47.454 & 10.588 & 35.191 & -1.218 & & 49.03. & & & & & & 45.982 & \#\#\#\#| & 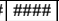 & & & & 0.000 & 0.440 & \\
\hline 3408 & & Minimum & 24.784 & 22.156 & 24.714 & 23.206 & 22.260 & -5.637 & -0.958 & 1.158 & -6.078 & -1.227 & -7.5 & -0.079 & $\mid 0.003$ & 14.643 & & & & $\mid-2.430$ & |-0.019 & A \#\#\# & 0.404 & 0.000 & 0.000 & 0.000 & $-0.268 \mathrm{C} \quad \mathrm{C}$ & -0.228 \\
\hline 340 & & $2 \times \operatorname{Std}$ Dev & 0.710 & 2.382 & 0.471 & 2.013 & 1.561 & 9.788 & 9.128 & 3.207 & 8.416 & 3.917 & & 9.450 & 0.117 & 0.000 & 0.001 & & & 9.128 & 82.316 & 8.983 & 0.619 & 0.017 & 0.017 & 0.000 & 0.438 & \\
\hline & Number & of Points Used* & 946 & 946 & & 946 & 946 & 946 & 946 & 946 & 946 & 946 & & 946 & 946 & 946 & 946 & & & 946 & 946 & 946 & 946 & 946 & 946 & 946 & 946 & 946 \\
\hline 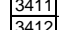 & & * Backpulse poi & tits in box & are not inc & cluded & & & & & & & & & & & & & & & & & & & & & & & \\
\hline 3413 & Wash 2 & & & & & & & & & & & & & & & & & & & & & & & & & & & \\
\hline 3414 & & Averages & 25.516 & & \begin{tabular}{|l|l|}
3 & 25.103 \\
\end{tabular} & 25.901 & 25.082 & 48.935 & \begin{tabular}{|l|l|}
53.348 \\
\end{tabular} & 17.615 & $\begin{array}{ll}31.652 \\
\end{array}$ & -1.025 & -7.01 & 48.845 & 然.053 & 14.643 & $\begin{array}{ll}0.003 \\
\end{array}$ & & & 51.876 & 6 6\#\#\#1 & 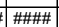 & 2.778 & \begin{tabular}{|l|l|l|l|}
0.008 \\
\end{tabular} & 0.008 & 0.000 & 0.193 & 0.16 \\
\hline & & Maximum & 26.715 & 25.816 & 26.702 & 28.041 & 26.414 & 51.190 & 55.539 & 20.347 & 34.989 & 1.796 & 0.2 & 55.402 & 0.170 & 14. & & & & 54.066 & & \#\#\# & & 0.025 & 0.025 & 0.001 & 0.614 & 0.5 \\
\hline & & Median & 25.678 & 25.007 & 25.445 & 25.903 & 25.2 & 48.8111 & 53.224 & 17.578 & 31.683 & -1.218 & -7.0 & 48.748 & 0.6 & 14. & & & & 51.751 & 11\#\#\#壮 & \#\#\# & 2.78 & 0.008 & 0.008 & 0.000 & 0.186 & \\
\hline 341 & & Minimum & 22.757 & 21.578 & 23.655 & 22.717 & 21.6 & 13.619 & 14.446 & 3.7 & $\begin{array}{lll}10.186 \\
757\end{array}$ & $-1.227 \mid$ & & 12.671 & 0.003 & 14.643 & 0.6 & & & 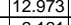 & 3. 3.105 & 5 & 0.0 & 0.000 & 0.000 & 0.000 & 0.011 & \\
\hline & & I x s stavev & $\frac{1.255}{2374}$ & 1.561 & $\frac{1.118}{1.271}$ & $\frac{1.927}{227}$ & $\frac{1.48}{237}$ & 1.952 & 2.121 & $\frac{1.998}{1.237}$ & 1.775 & 0.570 & & 2.833 & 0.024 & 0.000 & & & & 2.121 & & & 0.109 & 0.004 & 0.003 & 0.000 & (. & \\
\hline & Number & & $23 / 4$ & 2374 & $23 / 4$ & $23 / 4$ & $23 / 4$ & $23 / 4$ & 2374 & 2374 & 2374 & $23 / 4$ & $23 / 4$ & 2374 & 2314 & 2374 & $23 / 4$ & & & 2374 & 4. 2374 & 2374 & \begin{tabular}{l|l|l|}
4 & 2374 \\
\end{tabular} & $23 / 4$ & 2374 & $23 / 4$ & 2374 & 2374 \\
\hline & & & - & & & & & & & & & & & & & & & & & & & & & & & & & \\
\hline & & & & & & & & & & & & & & & & & & & & & & & & & & & & \\
\hline 3424 & & & & & & & & & & & & & & & & & & & & & & & & & 0.018 & & & \\
\hline & & & & & & & & & & & & & & & & & & & & & Avera & age flux & ux to $20^{\circ}$ C & UDSS $=$ & 0.015 & & & \\
\hline
\end{tabular}


RUN \# WASH1 \& WASH2; CAMPAIGN II SLURRY WASHING

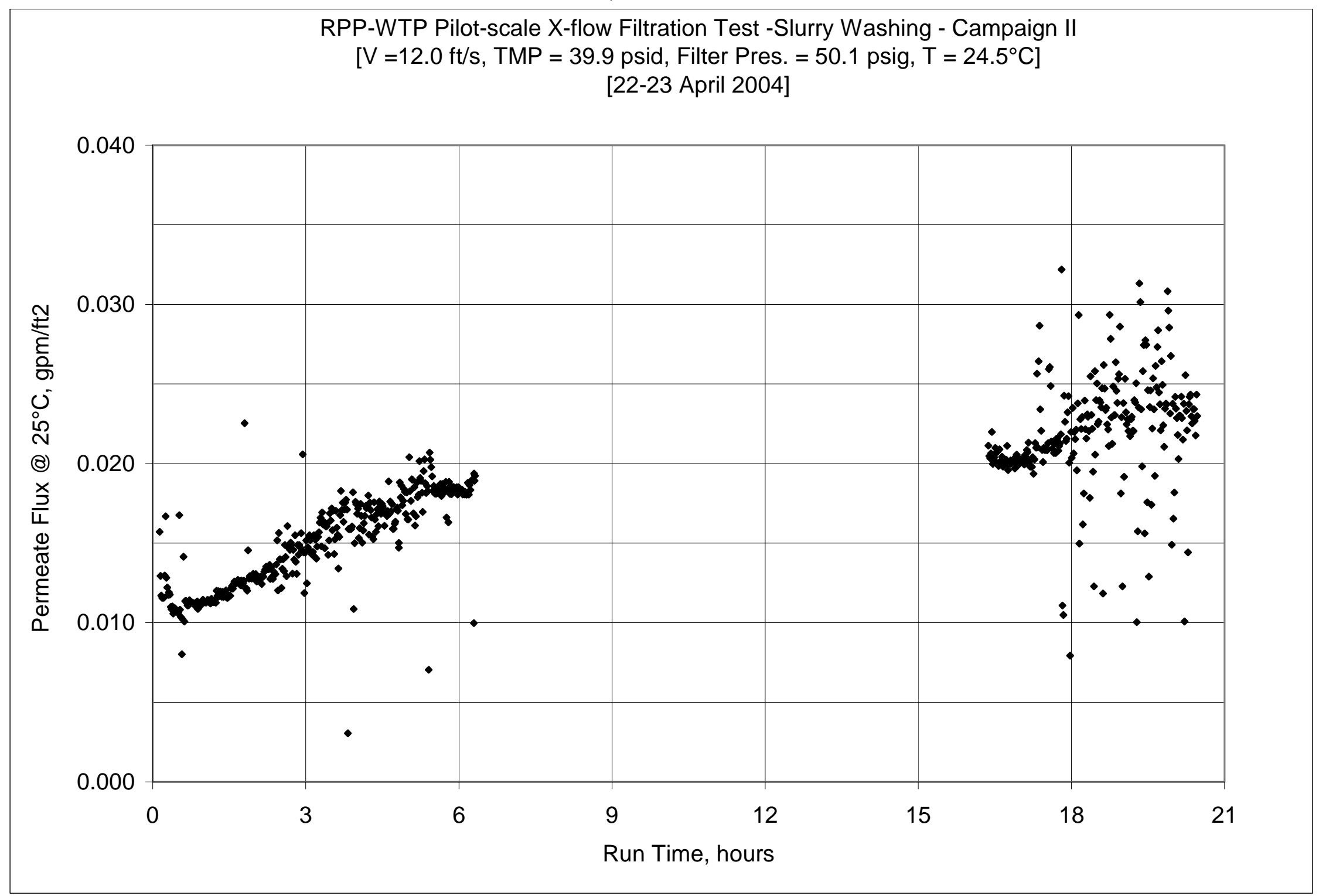


WSRC-TR-2005-00105, REVISION 0

SRNL-RPP-2005-00012, REVISION 0

RUN \# WASH1 \& WASH2; CAMPAIGN II SLURRY WASHING - CONT.

\begin{tabular}{|c|c|c|c|c|c|c|c|c|c|c|c|c|c|c|c|c|c|c|c|c|c|c|c|c|c|c|c|c|}
\hline & $\mathrm{A}$ & B & $\mathrm{D}$ & E & $\mathrm{F}$ & G & $\mathrm{H}$ & $\mathrm{J}$ & $\mathrm{K}$ & $\mathrm{L}$ & $\mathrm{M}$ & $\mathrm{N}$ & 0 & \begin{tabular}{l|l} 
\\
\end{tabular} & $\mathrm{R}$ & $\mathrm{s}$ & $T$ & $\mathrm{v}$ & w & $x$ & $\begin{array}{r}Y \\
\end{array}$ & $z$ & AA & $A B$ & $A C$ & $A D$ & $\mathrm{AE}$ & AF \\
\hline & & & & & & & & & & & & & & & & & & & & & & & & & & & & \\
\hline & DATE & TIME & $\begin{array}{l}\text { Filtrate } \\
\text { deace }\end{array}$ & \begin{tabular}{|l|} 
Cleaning \\
deag
\end{tabular} & \begin{tabular}{|l|} 
Slurry \\
deac
\end{tabular} & \begin{tabular}{|l|} 
Hi Amb. \\
degc.
\end{tabular} & Lo Amb. & $\frac{B \text { BotTMP }}{\text { nsid }}$ & $\begin{array}{l}\text { Filter } \\
\text { nsian }\end{array}$ & Filter dP & \begin{tabular}{|l|} 
TopTMP \\
\end{tabular} & \begin{tabular}{|l|} 
Filtrate \\
\end{tabular} & Pulsepot & Slurry & Filtrate & Hi Filtate & Backpulse & & & & & & & & & & & \\
\hline 4 & & & $\frac{\operatorname{deg} C}{\mathrm{~T} 2}$ & \begin{tabular}{|l|}
$\operatorname{deg} \mathrm{C}$ \\
\end{tabular} & $\operatorname{deg} \mathrm{C}$ & \begin{tabular}{|c|}
$\operatorname{deg} \mathrm{C}$ \\
$\mathrm{TA}$
\end{tabular} & $\frac{\operatorname{deg} C}{15}$ & $\begin{array}{l}\text { psid } \\
\text { PP? }\end{array}$ & $\begin{array}{l}\text { psig } \\
\text { P1 }\end{array}$ & $\begin{array}{l}\text { psid } \\
\text { PD1 }\end{array}$ & \begin{tabular}{|c|} 
psid \\
\end{tabular} & \begin{tabular}{|l|} 
psig \\
P?
\end{tabular} & \begin{tabular}{|c|} 
\\
psig
\end{tabular} & gpm & $\begin{array}{ll}\mathrm{gpm} \\
\end{array}$ & gpm & gpm & & & & & & & & & & & \\
\hline & & & & T3 & $\mathrm{T1}$ & & & $\mathrm{dP2}$ & P1 & $\mathrm{dP1}$ & $\mathrm{dP3}$ & $\mathrm{P} 2$ & & Q1 & & Q3 & Qbp & & & & & & & & & & & \\
\hline & eros- $01 / 26$ & 2004 & & & & & & & & & & & & & & & & & & & & & & & & & & \\
\hline & $4 / 14 / 2004$ & 1:30:58 PM & 24.687 & 21.862 & \begin{tabular}{|l|}
24.835 \\
\end{tabular} & 24.226 & 22.583 & \begin{tabular}{|l|l|}
0.054 \\
\end{tabular} & 0.02 & -0.021 & -0.005 & -0.179 & 0.099 & $\begin{array}{l}-0.08 \\
\end{array}$ & $\begin{array}{l}0.003 \\
\end{array}$ & 0.086 & 0.0 & & & & face $A$ & A 6.707 & & & & & & \\
\hline 0 & $4 / 19 / 2004$ & 7:37:13 AM & & $\begin{aligned} 22.228 \\
2.232\end{aligned}$ & \begin{tabular}{|l|}
23.926 \\
2220
\end{tabular} & $\begin{array}{l}21.772 \\
22.191\end{array}$ & $\begin{array}{l}22.175 \\
22.109\end{array}$ & 0.046 & $\begin{array}{l}0.082 \\
\end{array}$ & $\begin{array}{l}-0.025 \\
-0.021\end{array}$ & \begin{tabular}{|c|c|}
-0.009 \\
\end{tabular} & \begin{tabular}{|c|c|c|}
-0.179 \\
-0.202
\end{tabular} & 0.143 & -0.086 & 0.003 & 14.643 & & & & Conver: & & & & daylbarc & $\mathrm{rg} / \mathrm{gpm}$ & lbarg & & \\
\hline 9 & 4/19/2004 & 8:21:39 АМ & 22.248 & 22.333 & 22.324 & 22.194 & $\begin{array}{l}22.199 \\
2.710\end{array}$ & -0.008 & 0.059 & 0.002 & $\begin{array}{l}0.007 \\
\end{array}$ & -0.132 & 0.131 & -0.084 & 0.003 & $\begin{array}{r}14.643 \\
1.64\end{array}$ & 0.00 & & & & & & & & & & & \\
\hline$\frac{1}{1}$ & $4 / 20 / 2004$ & 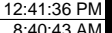 & $\frac{22.772}{22558}$ & & \begin{tabular}{|l|}
22.049 \\
2.171 \\
\end{tabular} & & $\begin{aligned} 24.746 \\
22489\end{aligned}$ & \begin{tabular}{r|r|}
-0.004 \\
\end{tabular} & $\begin{array}{l}0.045 \\
0.028\end{array}$ & $\begin{array}{l}-0.009 \\
0.011\end{array}$ & .007 & -0.176 & 0.108 & $\begin{array}{r}-0.084 \\
\end{array}$ & & \begin{tabular}{|l|l|}
14.643 \\
1.62 \\
\end{tabular} & & & & Note: & & & & & ely & & & \\
\hline$\frac{11}{12}$ & 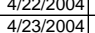 & $\begin{array}{l}8: 40: 43 \mathrm{AM} \\
8: 53: 20 \mathrm{AM}\end{array}$ & $\frac{22.558}{21.548}$ & $\frac{21.446}{21.737}$ & \begin{tabular}{|l|}
24.471 \\
22.041 \\
\end{tabular} & $\begin{array}{r}22.716 \\
21.89\end{array}$ & $\frac{22.489}{21.369}$ & $\begin{array}{r}0.01 \\
-0.004\end{array}$ & $\begin{array}{l}0.028 \\
0.026\end{array}$ & $\begin{array}{l}0.001 \\
0.003\end{array}$ & $\begin{array}{c}0.001 \\
-0.005\end{array}$ & $\begin{array}{c}-0.205 \\
-0.196\end{array}$ & $\begin{array}{l}0.096 \\
0.082\end{array}$ & $\begin{array}{r}46.562 \\
-0.082 \\
\end{array}$ & $\begin{array}{l}0.043 \\
0.003\end{array}$ & $\begin{array}{l}y_{14.643} \\
14.643\end{array}$ & $\frac{0.00}{0.00}$ & & & SSSt & eplis & recte & 1 for 40 & $0.88 \mathrm{incl}$ & sof we & It tubind & & \\
\hline & $4 / 26 / 2004$ & 9:00:47 AM & 22.379 & 22.907 & \begin{tabular}{|l|l|}
23.455 \\
\end{tabular} & 22.416 & \begin{tabular}{|l|l|}
5 & 21.819 \\
\end{tabular} & -0.012 & 0.08 & 0 & -0.009 & \begin{tabular}{|l|}
-0.205 \\
\end{tabular} & 0.044 & -0.08 & 0.003 & \begin{tabular}{|l|l|}
14.643 \\
\end{tabular} & 0.00 & & & VWVV & & & & < Filtrat & & $\geqslant<<$ PERN & $\begin{array}{l}\text { MIABILIT } \\
\end{array}$ & $\gg$ \\
\hline & $4 / 27 / 2004$ & $9: 14: 59 \mathrm{AM}$ & 20.374 & 19.305 & \begin{tabular}{|l|l|} 
& 19.368 \\
\end{tabular} & 20.841 & \begin{tabular}{l|l|l}
1 & 19.777
\end{tabular} & -0.002 & 0.02 & -0.001 & 0.005 & \begin{tabular}{|l|l|l|}
5 & -0.193 \\
\end{tabular} & 0.111 & -0.084 & 0.003 & \begin{tabular}{|l|l|}
14.643 \\
\end{tabular} & 0.00 & & & & & & & & \begin{tabular}{|l|} 
at $25 \mathrm{C}$ \\
\end{tabular} & & & \\
\hline 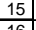 & & & & & & & & & & & & & & & & & & fime & Time & Press & Vel. & TMP & TMP & $\mathrm{gpm}$ & & $m$ & pm & \\
\hline$\frac{16}{17}$ & ata - Per Mi & & & & & & & & & & & & & & & & & & tour & & & & & 12 & & Ipsi & & $\begin{array}{c}y y b a r \\
b a\end{array}$ \\
\hline$\frac{17}{18}$ & $\begin{array}{l}4 / 2 / 2 / 212004 \\
4 / 21 / 2004\end{array}$ & $\begin{array}{l}4: 4: 04 \mathrm{PM} \\
4: 46: 15 \mathrm{PM}\end{array}$ & $\begin{aligned} 24.927 \\
24.927\end{aligned}$ & $\begin{array}{l}25.572 \\
25.572\end{array}$ & \begin{tabular}{|l|}
23.883 \\
23.883 \\
\end{tabular} & $\frac{26.605}{26.605}$ & $\begin{array}{l}27.278 \\
27.278\end{array}$ & \begin{tabular}{|l}
48.625 \\
48.625
\end{tabular} & $\begin{array}{l}54.032 \\
54.032\end{array}$ & $\mid$\begin{tabular}{|}
$\mid 18.901$ \\
18.901
\end{tabular} & \begin{tabular}{|}
$\mid 30.013$ \\
30.013
\end{tabular} & $\begin{array}{l}-0.184 \\
-0.184\end{array}$ & $\begin{array}{l}-5.44 \\
-5.44 \\
\end{array}$ & \begin{tabular}{|l|}
48.095 \\
48.895 \\
\end{tabular} & \begin{tabular}{|l}
0.036 \\
0.036
\end{tabular} & $\begin{array}{l}14.643 \\
14.643 \\
\end{array}$ & & $\begin{array}{l}0.0 \\
3.2\end{array}$ & .05306 & $\frac{52.6}{52.6}$ & $\frac{11.8}{11.8}$ & $\frac{39.3}{39.3}$ & $\frac{2.711}{2.711}$ & $\frac{0.005}{0.005}$ & 0.006 & $\frac{0.000}{0.000}$ & 0.141 & $\frac{0.12}{0.12}$ \\
\hline 19 & $4 / 22 / 2004$ & 4:47:15 PM & 24.939 & 25.569 & 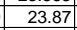 & 26.532 & 27.095 & 46.368 & 53.816 & 18.891 & 27.671 & 1.903 & $\begin{array}{r}-50.457 \\
20.45\end{array}$ & 51.195 & $\begin{array}{l}0.003 \\
\end{array}$ & 14.643 & & $\begin{array}{l}.5 \\
4.2 \\
\end{array}$ & 0.06972 & 52.3 & 12.5 & 37.0 & 2.552 & 0.000 & 0.000 & 0.000 & 0.141 & \\
\hline 20 & $4 / 22 / 2004$ & 4:48:15 PM & 24.97 & 25.575 & \begin{tabular}{|l|}
23.897 \\
\end{tabular} & 26.433 & $\begin{array}{l}27.057 \\
\end{array}$ & 43.401 & 53.796 & \begin{tabular}{|l|l|}
18.816 \\
\end{tabular} & 24.726 & 4.865 & 20.24 & 49.597 & $\begin{array}{ll}0.003 \\
\end{array}$ & \begin{tabular}{|l|l|l|l|}
14.33 \\
\end{tabular} & & 5.8 & 0.08639 & 52.3 & 12.2 & 34.1 & 2.349 & 0.000 & 0.000 & 0.000 & 0.014 & \\
\hline 21 & $4 / 22 / 2004$ & 4:49:15 PM & 25.184 & 25.574 & \begin{tabular}{|r|}
23.88 \\
\end{tabular} & 26.367 & 26.741 & $\begin{array}{r}-7.265 \\
\end{array}$ & 52.612 & 11.331 & -18.543 & 59.7 & 61.348 & \begin{tabular}{|l|}
52.25 \\
\end{tabular} & $\begin{array}{ll}0.003 \\
\end{array}$ & 14.643 & 1.069 & 6.2 & 0.10306 & 51.1 & 12.8 & -12.9 & -0.890 & 0.000 & 0.000 & 0.000 & -0.036 & -0.03 \\
\hline & $4 / 22 / 2004$ & 4:50:15 PM & 24.854 & 25.579 & \begin{tabular}{|r|}
23.89 \\
\end{tabular} & 26.372 & 26.776 & 46.615 & \begin{tabular}{|l|l|}
52.971 \\
5
\end{tabular} & & & $\begin{array}{l}0.609 \\
\end{array}$ & 0.394 & $\begin{array}{l}51.028 \\
\end{array}$ & $\begin{array}{l}0.158 \\
\end{array}$ & $\begin{array}{l}14.643 \\
\end{array}$ & & 7.2 & 0.11972 & 51.5 & $\begin{array}{l}12.5 \\
12 . \\
\end{array}$ & & 2.741 & & & .001 & & \\
\hline$\frac{23}{24}$ & $4 / 22 / 2004$ & $\begin{array}{l}4: 51: 15 \text { PM } \\
4: 52 \cdot 15 \mathrm{PM}\end{array}$ & $\begin{array}{l}24.447 \\
24.292\end{array}$ & $\begin{array}{l}25.552 \\
25.562\end{array}$ & \begin{tabular}{|l|}
23.859 \\
\end{tabular} & $\begin{array}{r}26.28 \\
26.255\end{array}$ & \begin{tabular}{c|c}
8 & 26.865 \\
\end{tabular} & $\begin{array}{r}47.03 \\
47.229 \\
\end{array}$ & \begin{tabular}{|l|}
53.182 \\
53288
\end{tabular} & $\begin{array}{l}15.748 \\
16691\end{array}$ & \begin{tabular}{|l|}
31.648 \\
3085
\end{tabular} & 0.38 & & $\begin{array}{r}50.15 \\
5\end{array}$ & 0.102 & \begin{tabular}{|l|l|}
14.643 \\
1.62
\end{tabular} & & 8.2 & 0.13639 & & $\begin{array}{l}12.3 \\
12\end{array}$ & 39.3 & $\frac{2.712}{2.600}$ & & 0.016 & 0.000 & 0.399 & 0.34 \\
\hline $\begin{array}{l}24 \\
25\end{array}$ & $4 / 2 / 2 / 2004$ & $\begin{array}{ll}\frac{4}{4: 52: 15} \mathrm{PM} \\
4: 53: 15\end{array}$ & $\begin{array}{l}24.292 \\
24.296\end{array}$ & $\frac{25.562}{25.582}$ & \begin{tabular}{|l|}
23.839 \\
23.878 \\
\end{tabular} & $\frac{26.255}{26.355}$ & $\frac{26.904}{26.944}$ & $\begin{array}{r}47.229 \\
47.196\end{array}$ & $\begin{array}{r}53.288 \\
53.34 \\
\end{array}$ & $\begin{array}{l}\frac{16.691}{17.122} \\
\end{array}$ & $\begin{array}{l}30.805 \\
30.436 \\
\end{array}$ & \begin{tabular}{|c|c|c|} 
\\
\end{tabular} & $\begin{array}{l}0.498 \\
0.518\end{array}$ & \begin{tabular}{|l|}
51.452 \\
48.659
\end{tabular} & $\frac{0.084}{0.076}$ & $\begin{array}{r}4.643 \\
14643\end{array}$ & & & 0.15306 & $\frac{51.8}{51.9}$ & $\begin{array}{l}-12.6 \\
11.9 \\
\end{array}$ & $\begin{array}{l}39.0 \\
38.8\end{array}$ & $2.690 \mid$ & & & 0.000 & 0.332 & \\
\hline & $4 / 22 / 2004$ & 4:53:.5:15 PM & $\frac{24.290}{24.267}$ & $\frac{25.582}{25.567}$ & \begin{tabular}{|l|l|}
23.829 \\
\end{tabular} & $\frac{20.359}{26.31}$ & $\frac{20.944}{27.004}$ & $\begin{aligned}-41.196 \\
46.8\end{aligned}$ & 52.985 & 16.1776 & $\begin{array}{l}30.345 \\
3\end{array}$ & 0.467 & 0.544 & |50.156 & 0.075 & \begin{tabular}{|l|l|}
14.643 \\
\end{tabular} & & $\frac{10.2}{11.2}$ & & $\frac{51.5}{51.5}$ & $\frac{11.9}{1.9}$ & & & & & & $\frac{0.301}{0.300}$ & \\
\hline & $4 / 22 / 2004$ & & 24.255 & 25.551 & & 26.329 & & 47.063 & 53.178 & 16.901 & 30.495 & 0.476 & & 52.96 & 0.075 & \begin{tabular}{|l|l|}
14.643 \\
\end{tabular} & & 12. & & 51. & 13.0 & 38.8 & 2.674 & & & & 0.298 & \\
\hline & $4 / 22 / 2004$ & $4: 56: 15 \mathrm{PM}$ & 24.201 & 25.562 & 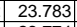 & $\begin{array}{l}26.37 \\
\end{array}$ & 27.048 & 46.866 & 53.01 & \begin{tabular}{|l|l|}
16.984 \\
\end{tabular} & 30.238 & 0.479 & 0.594 & 49.316 & 0.075 & 14.643 & & 13. & & 51. & 12.1 & 38.6 & \begin{tabular}{|l|l|}
5 & 2.658 \\
\end{tabular} & & & & 0.300 & \\
\hline$E$ & $4 / 22 / 2004$ & 4:57:15 PM & 24.056 & 25.562 & 23.774 & 26.445 & 27.044 & 47.183 & \begin{tabular}{|l|l|}
53.414 \\
\end{tabular} & 16.902 & 30.596 & 0.545 & 0.585 & 49.914 & 0.084 & 14.643 & & 14. & 0.23639 & 51.9 & 12.2 & 38.9 & 2.681 & & & 000 & 0.333 & \\
\hline & $4 / 22 / 2004$ & $\begin{array}{ll}4: 58: 15 ~ P M \\
4: 59 \cdot 15 \mathrm{PM}\end{array}$ & $\begin{aligned} 24.508 \\
24639\end{aligned}$ & 25.553 & \begin{tabular}{|l|}
23.744 \\
\end{tabular} & $26.461 \mid$ & $\begin{array}{l}27.09 \\
2712\end{array}$ & $\begin{array}{r}50.178 \\
5.720\end{array}$ & 53.114 & $\begin{array}{l}16.948 \\
1710\end{array}$ & $\begin{array}{r}33.655 \\
23.2 \\
2.03\end{array}$ & $\frac{-1.221}{1.221}$ & & $\begin{array}{r}49.82 \\
\end{array}$ & 0.108 & $\begin{array}{r}14.643 \\
1.42\end{array}$ & & 15. & $\begin{array}{l}0.25306 \\
02072\end{array}$ & 51.6 & 12.2 & 41.9 & 2.890 & & & 0.000 & 0.398 & \\
\hline & $4 / 22 / 2004$ & & & & \begin{tabular}{|r|}
23.73 \\
\end{tabular} & & & & \begin{tabular}{|r|}
53.099 \\
52152
\end{tabular} & & & -1.221 & & 50.411 & 0.083 & $\begin{array}{c}14.643 \\
1.32\end{array}$ & & 16. & 0.26972 & 51.6 & $\begin{array}{l}12.4 \\
\end{array}$ & 42.4 & 2.920 & & & & 0.303 & 0.2 \\
\hline & $4 / 22 / 2004$ & 5:00:15 PM & $\frac{24.569}{24.531}$ & $\frac{25.544}{25.562}$ & \begin{tabular}{|l|}
23.711 \\
23708 \\
\end{tabular} & $\begin{aligned} 26.518 \\
265.2\end{aligned}$ & $\frac{27.151}{27.208}$ & $\begin{array}{l}51.209 \\
51.224\end{array}$ & \begin{tabular}{|l|}
53.452 \\
53.574 \\
\end{tabular} & $\begin{array}{r}17.313 \\
17.34\end{array}$ & $\begin{array}{r}34.181 \\
34.173\end{array}$ & \begin{tabular}{|l|l|} 
& -1.221 \\
3 & -1.221 \\
\end{tabular} & $\begin{array}{r}-7.614 \\
-7.625\end{array}$ & \begin{tabular}{|l|}
49.706 \\
49.476
\end{tabular} & 0.079 & $\begin{array}{r}14.643 \\
14643\end{array}$ & 3 & $\frac{17.2}{18.2}$ & 0.28639 & $\frac{52.0}{52.1}$ & $\begin{array}{r}12.2 \\
121\end{array}$ & 42.7 & 2.944 & 0.012 & 0.012 & 0.000 & 0.286 & 0.2 \\
\hline 34 & $\begin{array}{l}4 / 21 / 212004 \\
4 / 2004\end{array}$ & $\begin{array}{l}\text { 5:01:15 PM } \\
\text { 5:02:15 }\end{array}$ & $\begin{array}{l}24.531 \\
24.528\end{array}$ & 25.562 & \begin{tabular}{|l|}
23.1 .699 \\
2399
\end{tabular} & $\begin{array}{r}26.52 \\
26.561 \\
\end{array}$ & $\begin{array}{l}27.208 \\
27.239\end{array}$ & $\begin{array}{r}51.224 \\
51.35 \\
\end{array}$ & \begin{tabular}{|l|}
$53.55 / 49$ \\
53.591 \\
\end{tabular} & $\begin{array}{l}\frac{17.34}{17.448} \\
\end{array}$ & $\begin{array}{l}34.173 \\
34.191 \\
\end{array}$ & $\begin{array}{l}-1.221 \\
-1.218\end{array}$ & $\begin{array}{l}-7.625 \\
-7.57 \\
\end{array}$ & $\begin{array}{l}9.476 \\
51.794 \\
\end{array}$ & $\begin{array}{l}0.016 \\
0.077\end{array}$ & $\begin{array}{l}14.6433 \\
14.643\end{array}$ & & $\frac{18.2}{19.2}$ & $\frac{0.330306}{0.31972}$ & 52.1 & $\begin{array}{l}\frac{12.1}{12.7} \\
\end{array}$ & $\frac{42.7}{42.8}$ & $\frac{2.944}{2.949}$ & 011 & 0.012 & $\begin{array}{l}0.000 \\
0.000\end{array}$ & $\begin{array}{l}0.275 \\
0.278\end{array}$ & $\begin{array}{l}0.2 \\
0.2\end{array}$ \\
\hline 35 & $4 / 22 / 2004$ & 5:03:1 & 24.513 & 25.569 & 23.685 & 26.602 & 27.255 & $\begin{array}{l}51.178 \\
\end{array}$ & \begin{tabular}{|l|}
53.305 \\
\end{tabular} & 17.396 & 34.13 & -1.221 & -7.62 & $\begin{array}{l}49.86 \\
\end{array}$ & 0.076 & 14.643 & & 20.2 & & 51.8 & 12.2 & 42 & 2.941 & & 0.012 & & 0.276 & \\
\hline 36 & $4 / 22 / 2004$ & & 24.508 & 25.564 & \begin{tabular}{|l|}
23.665 \\
\end{tabular} & 26.627 & 27.25 & 50.135 & \begin{tabular}{|l|}
52.361 \\
\end{tabular} & 17.204 & 33.216 & & & 50.34 & 0.071 & 14.643 & & 21. & & 50.9 & 12.3 & s. & 20.070 & & & & 0.264 & \\
\hline \begin{tabular}{l|l|}
37 \\
38
\end{tabular} & $4 / 22 / 2004$ & & $\begin{array}{r}24.51 \\
\end{array}$ & 25.57 & \begin{tabular}{|l|}
23.626 \\
22572
\end{tabular} & 26.658 & & & \begin{tabular}{|l|}
51.423 \\
51.151
\end{tabular} & 16.842 & 32.629 & & & $\begin{array}{r}48.882 \\
1.5522\end{array}$ & $\begin{array}{r}0.07 \\
\end{array}$ & & & 22. & & 49.9 & 12.0 & 40. & 2.819 & & & 000 & 0.265 & \\
\hline $\begin{array}{l}38 \\
39 \\
\end{array}$ & $4 / 22 / 2004$ & $\begin{array}{l}5: 06: 1: \\
5 \cdot 2 \cdot 0 \cdot 1 .\end{array}$ & $\begin{array}{r}24.496 \\
24.498\end{array}$ & $\begin{array}{r}25.592 \\
25599\end{array}$ & \begin{tabular}{|l|}
23.572 \\
23529 \\
\end{tabular} & & 27.3 & & \begin{tabular}{|l|}
51.151 \\
51.076
\end{tabular} & $\begin{array}{l}16.898 \\
16.929\end{array}$ & $\begin{array}{l}32.399 \\
32.282\end{array}$ & -1.221 & $\begin{array}{r}-7.63 \\
\end{array}$ & \begin{tabular}{|c|}
49.533 \\
4908
\end{tabular} & $\begin{array}{l}0.071 \\
0.690\end{array}$ & \begin{tabular}{|l|l|}
14.643 \\
1.42
\end{tabular} & & 23. & & 49.7 & $\begin{array}{l}12.1 \\
\end{array}$ & 40. & 27090 & & & & 0.271 & 0.2 \\
\hline$\frac{39}{40}$ & 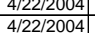 & $\begin{array}{l}5: 7: 15 \text { PM } \\
\text { 5:08:15 PM }\end{array}$ & $\frac{24.498}{24.495}$ & $\frac{25.599}{25.616}$ & \begin{tabular}{|l|}
23.529 \\
23506
\end{tabular} & $\frac{26.722}{26809}$ & $\frac{27.374}{26.474}$ & & \begin{tabular}{|c|}
$\mid 51.076$ \\
51249
\end{tabular} & $\frac{16.929}{16.855}$ & $\begin{array}{l}32.282 \\
32.555\end{array}$ & $\begin{array}{l}2-1.2 \\
-1.2 \\
-12\end{array}$ & -76 & $\begin{array}{l}49.508 \\
46.283\end{array}$ & $\begin{array}{l}0.068 \\
0.069 \\
\end{array}$ & & & 24.2 & & $\frac{49.6}{49.8}$ & $\begin{array}{c}12.1 \\
113 \\
\end{array}$ & $\frac{40.6}{40.8}$ & 2.7988 & & & 0.0000 & 260 & 0.2. \\
\hline 41 & $4 / 22 / 2004$ & 5:09:15 PM & 24.472 & 25.607 & \begin{tabular}{|l|}
23.437 \\
2.437 \\
\end{tabular} & 26.0965 & \begin{tabular}{|l|l|l|}
5 & 25.757 \\
\end{tabular} & 48.6 & \begin{tabular}{|l|}
51.126 \\
\end{tabular} & $\begin{array}{l}1.000 \\
16.921 \\
\end{array}$ & \begin{tabular}{|l|}
32.21950 \\
\end{tabular} & \begin{tabular}{|l|l|} 
& -1.224 \\
\end{tabular} & $\begin{array}{r}-1.031 \\
-7.631 \\
\end{array}$ & \begin{tabular}{|l|}
47.60501 \\
47.601
\end{tabular} & \begin{tabular}{|l|l|}
0.07 \\
\end{tabular} & \begin{tabular}{|l|}
14.643 \\
14.643
\end{tabular} & & 26.2 & & 年 49.9 .7 & $\frac{11.5}{11.7}$ & $\begin{array}{l}40.0 \\
40.5 \\
\end{array}$ & & 510 & 0.011 & & 0.263 & 0.2. \\
\hline & $4 / 22 / 2004$ & $5: 10: 1$ & 24.468 & 25.624 & \begin{tabular}{|l|}
23.419 \\
\end{tabular} & 26.697 & 25.4 & & \begin{tabular}{|l|}
51.201 \\
50
\end{tabular} & 17.038 & 32.241 & $\mid-1.221$ & & \begin{tabular}{|l|l|}
49.138 \\
\end{tabular} & $\begin{array}{l}0.069 \\
\end{array}$ & 14.643 & & 27.2 & 0.45306 & 49. & 12.0 & 40.6 & \begin{tabular}{|l|l|}
5.801 \\
\end{tabular} & 810 & 0.011 & 0.000 & 0.265 & \\
\hline & $4 / 221 / 2004$ & & 24.448 & 25.624 & & & & & \begin{tabular}{|l|l|}
51.246 \\
\end{tabular} & & 32.2899 & & & 49.155 & 0.069 & & & 28. & & 49.8 & 12.0 & & & & & & & \\
\hline & $4 / 22 / 2004$ & 5:12:15 PM & 24.428 & 25.624 & \begin{tabular}{|l|}
23.338 \\
22007
\end{tabular} & 26.567 & 25.2 & 49. & \begin{tabular}{|l|l|}
51.379 \\
51.20
\end{tabular} & \begin{tabular}{ll|}
17.07 \\
\end{tabular} & $\begin{array}{l}32.299 \\
2250\end{array}$ & \begin{tabular}{|c|} 
\\
\end{tabular} & & \begin{tabular}{|l|l|}
48.723 \\
1202
\end{tabular} & 0.068 & 14.6 & & 29. & 0.48639 & 49.9 & $\begin{array}{l}11.9 \\
\end{array}$ & & 2.804 & 10 & 0.011 & 000 & 0.261 & 0.2 \\
\hline 46 & $4 / 22 / 2004$ & $\begin{array}{l}5: 13: 15 \mathrm{PM} \\
5 \cdot 14 \cdot 15 \mathrm{PM}\end{array}$ & $\begin{aligned} 24.402 \\
2369 \\
3\end{aligned}$ & $\frac{25.617}{25.622}$ & \begin{tabular}{|l|}
23.297 \\
23262
\end{tabular} & $\begin{aligned} 26.485 \\
26.455\end{aligned}$ & $\frac{25.16}{25.16}$ & $\begin{array}{r}49.002 \\
45.22\end{array}$ & \begin{tabular}{|r|}
51.248 \\
55.139
\end{tabular} & 17.004 & $\begin{array}{r}32.358 \\
28.371\end{array}$ & \begin{tabular}{|c|c|} 
& -1.218 \\
& 0.594
\end{tabular} & & \begin{tabular}{|c|}
49.2222 \\
47.584
\end{tabular} & $\begin{array}{l}0.068 \\
0.108\end{array}$ & $\begin{array}{r}14.643 \\
14643\end{array}$ & & 30. & & 49.8 & $\frac{12.1}{1177}$ & 40. & $\frac{2.805}{2.537}$ & 010 & 0.011 & 0.000 & 0.262 & 0.2 \\
\hline 47 & $4 / 2 / 2 / 20040$ & & & & & & & & 11.439 & & & & & & & & & & & & $\frac{11.7}{11.7}$ & & & & & & & \\
\hline 48 & $4 / 22 / 2004$ & & 24.293 & 25.609 & \begin{tabular}{|l|}
23.193 \\
\end{tabular} & 26.482 & & & 51.429 & 17.1 & & & & 47. & & & & & & & $\frac{11.7}{11.7}$ & & & & & & & \\
\hline 49 & $4 / 221 / 2004$ & 5:17: & 24.241 & 25.607 & 23.161 & 26.515 & 25.0 & & 51.344 & 17.175 & & $\begin{array}{l}3 \\
\end{array}$ & & \begin{tabular}{|l|l|l|l|}
483 \\
\end{tabular} & 0.051 & & & & & & 11.8 & & & & & & & \\
\hline (2) & $4 / 22 / 2004$ & 5:18:1 & & 25.615 & \begin{tabular}{|l|}
23.129 \\
\end{tabular} & 26.513 & & & \begin{tabular}{l|l|}
51.058 \\
\end{tabular} & 17.04 & 31.462 & -1.2 & & \begin{tabular}{|l|l|}
48.319 \\
\end{tabular} & 0.00 & 14.64 & & & & . & 11.0 & & & & & & & \\
\hline & & & 24.269 & 25.62 & 23.139 & 26.503 & & 48.741 & 51.485| & 16.959 & 32.196 & -1.221 & & 48.402 & 0.09 & & & & & & 11.9 & & & & & & 0.356 & \\
\hline & & & 24.26 & & \begin{tabular}{|l|l|}
23.083 \\
\end{tabular} & 26.51 & & & 50.4 & & & & & 48 & & & & & & & 11.9 & & & & & & & \\
\hline & & & 24.21 & 25.6 & \begin{tabular}{|l|}
23.024 \\
\end{tabular} & 26.54 & & & 50.5 & & & & & 49.9 & & & & & & & & & & & & & & \\
\hline & $4 / 221 / 2004$ & & 24.18 & 25.6 & \begin{tabular}{|l|} 
\\
$\mid 22.974$
\end{tabular} & & & & & 16 & 31.958 & -1. & & & & & & & & & & & & & & & & \\
\hline & $4 / 22 / 2004$ & & 24.167 & 25.61 & 22.941 & 26. & 25. & 48.0 & 50.685 & 16. & 31.995 & -1.2 & & 48.7 & & 14.6 & & & & & 11.9 & & & & & & 0.281 & \\
\hline 56 & 4/221/2004 & & 24.149 & 25.625 & \begin{tabular}{|l|l|} 
& 22.903 \\
\end{tabular} & 26.638 & & 47. & 50.459 & & 31.759 & -1.22 & & 49.134 & 0 & & & (41. & & & 12.0 & & & & & & 0.218 & \\
\hline & & & 24.12 & 25.6 & 22.864 & & & 48 & & & & & & & & & & & & & 11.6 & & & & & & & \\
\hline 58 & & & 24.096 & 25.607 & $\begin{array}{r}2.885 \\
\end{array}$ & & & 48.1 & 50.124 & & & & & & & & & & & & 11.9 & & & & & & & \\
\hline & 4 & & 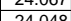 & 23.04 & \begin{tabular}{|l|l|}
2.0400 \\
\end{tabular} & & & & & & 31.901 & & & & & & & & & & 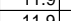 & & & & & & & \\
\hline & $4 / 2121200$ & & 24.05 & 2563 & $\frac{2.25}{228}$ & 26.08 & & & 50. & 16.51 & 31.88 & & & (46.49. & & & & & & & 11.5. & & & & & & & \\
\hline & $4 / 2 / 21200$ & 5.:30:1 & 24.047 & 25.6 & 22.866 & & & & 50.579 & 16.50 & 31.936 & & & 48.85 & & & & & & & 120 & & & & & & & \\
\hline & $4 / 21212004$ & & 24.025 & 25.646 & $\mid 22.924$ & 26.684 & & 48.057 & 50.772 & 16.5 & 31.891 & $\frac{-1.2}{-1.2}$ & & 50.1 & 0.07 & 14.6 & & & & & & & & & & & & \\
\hline & & & 24.032 & 25.658 & 22.976 & & & & 50.55 & & & -1.2 & & 47.07 & & & & & & & 11.5 & & & & & & & \\
\hline & & & 24.032 & 25.648 & 22.996 & 26.5 & & 47.99 & 50.656 & & & & & & & & & & & & 11.2 & & & & & & & \\
\hline & & & & 25.643 & & & & & & & & & & & & & & & & & & & & & & & & \\
\hline & $4 / 22 / 2004$ & 5:35:1! & 24.042 & 25.653 & \begin{tabular}{|l|}
23.072 \\
\end{tabular} & 26.391 & 25. & 47.97 & 50.664 & 16.45 & 31.819 & -1.2. & & $48.6 \mathrm{C}$ & 0.0 & 14.6 & & 52 & & $9.2 \mathrm{C}$ & 11.9 & & & & & & & \\
\hline & $4 / 22 / 2004$ & 5:36:15 P & 24.045 & 25.636 & 23.075 & 26.304 & 25. & 47.94 & 50.654 & 16.429 & 31.905 & -1.224 & & 48.8 & 0.0 & 14.6. & & & & & 2.0 & & & & & & & \\
\hline & $4 / 22 / 2004$ & & 24.083 & 25.62 & 23.087 & 26.187 & 24. & 47.992 & 50.679 & 16.488 & 31.866 & -1.221 & & 48.193 & 0.07 & 14.643 & & 54 & & & 11.8 & & & & 0.0 & & & \\
\hline & $4 / 22 / 2004$ & & 24.14 & 25.626 & 23.125 & \begin{tabular}{|l|}
26.074 \\
\end{tabular} & & 47.93 & \begin{tabular}{|l|l|} 
\\
\end{tabular} & \begin{tabular}{|l|}
16.6222 \\
\end{tabular} & 31.661 & \begin{tabular}{|l|l|}
$\mid$ & -1.221 \\
\end{tabular} & & 48.586 & 0.07 & \begin{tabular}{|l|l|}
14.643 \\
\end{tabular} & & 55.2 & 0.91972 & \begin{tabular}{l|l|}
2 & 49.2 \\
\end{tabular} & \begin{tabular}{|l|}
11.9 \\
\end{tabular} & \begin{tabular}{|l|}
39.8 \\
\end{tabular} & \begin{tabular}{|l|l|l|}
$\mid$ & 2.744 \\
\end{tabular} & 0.010 & \begin{tabular}{|l|l|} 
& 0.011 \\
\end{tabular} & 0.000 & \begin{tabular}{|l|l|}
$\mid$ & 0.277 \\
\end{tabular} & $\begin{array}{ll}7 \mid & 0.2\end{array}$ \\
\hline
\end{tabular}


WSRC-TR-2005-00105, REVISION 0

SRNL-RPP-2005-00012, REVISION 0

\begin{tabular}{|c|c|c|c|c|c|c|c|c|c|c|c|c|c|c|c|c|c|c|c|c|c|c|}
\hline \multicolumn{23}{|c|}{ RUN \# WASH1 \& WASH2; CAMPAIGN II SLURRY WASHING - CONT. } \\
\hline & & & & & 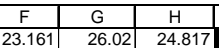 & & 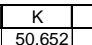 & & & & & 48.9 & $\frac{R}{0.07}$ & & & 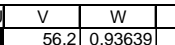 & & 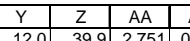 & & & & 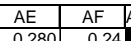 \\
\hline & & & & & 23161 - > & & 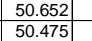 & $\frac{116.5}{16.348}$ & $\begin{array}{lll}31.75 & -1 \\
31.728 & -1 & -1\end{array}$ & & $\begin{array}{c}-7.555 \\
-7.579\end{array}$ & 8.285 & 0.07. & $\frac{144643}{14.643}$ & 0.003 & 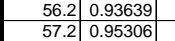 & & 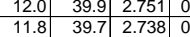 & & & & 280 \\
\hline & 212004 & & & & 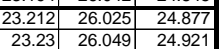 & $\frac{47.6}{47.8}$ & 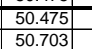 & $\begin{array}{lll}16.306 \\
11.353\end{array}$ & $\frac{31.73}{31.89}$ & & 7570 & $\begin{array}{lll}0.528 \\
804\end{array}$ & $\frac{0.07}{0.07}$ & $\frac{14.643}{1.643}$ & $\frac{0 .}{0.0}$ & & & & & & & 85 \\
\hline & & & & & & & & 10.3.3 & 31.7499 & & \begin{tabular}{|c|c|c|c|}
-7.55 \\
-7.55
\end{tabular} & & 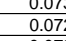 & & & & & & & & & 284 \\
\hline & & 5:44:15:15 PM & & & & 47.515 & .0.536 & 16.3918 & 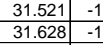 & & $\begin{array}{c}-7.55 \\
-7.553 \\
\end{array}$ & 18.729 & $\frac{0.07}{0.07}$ & 14.643 & 0.6. & 1.101972 & & & & & & 0.285 \\
\hline & & & & & & & & 16.358 & & & $\begin{array}{r}-7.5 \\
-7.54 \\
\end{array}$ & & & & & & & & & & & \\
\hline & & : $15 \mathrm{PM}$ & & 25.5253 & 3..2389 & 47.6384 & 50.734 & 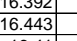 & 31.733 & & -7. & \begin{tabular}{|c|c|c|c|c|}
40.337 \\
\end{tabular} & 0.07 & 年.643 & 0 & 1.086 & & & & & & 2.287 \\
\hline & & & & .548. & ( & 47..5636 47.576 & $\frac{5.983 .48}{50.48}$ & $\frac{16.41}{16.42}$ & .486] & & -7. & & 0.077 & 4.6433 & & & & & & & & the \\
\hline & & & & & $\frac{55}{5}$ & 47.77 & & & 1..632 & & & & & 4.643 & & & & & & & & 2 \\
\hline & 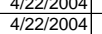 & & & & 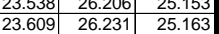 & & 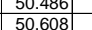 & 6.6398 & 31.,4507 & & & & & 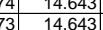 & & & & & & & & \\
\hline & & & & & 染5 & 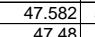 & $\frac{50.542}{50.5}$ & $\begin{array}{l}16.369 \\
11.477\end{array}$ & \begin{tabular}{ll|l}
31.657 & -1 \\
312.287 & -1 & -1
\end{tabular} & & 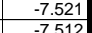 & 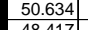 & 0 & 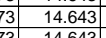 & 0.0 & 1.18639 & & $4_{4}$ & 0.011 & & 000 & 2.285 \\
\hline & & $5: 56: 15 \mathrm{PM}$ & & & . & 47.044 & 50.034 & $\frac{1.402}{16.02}$ & 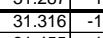 & & $\frac{-.7 .51 .1}{-7.5}$ & 477.799 & 0.07 & 14.6453 & $\frac{0.00}{0.00}$ & \begin{tabular}{|l|l|}
73.2 & 1.219792 \\
\end{tabular} & & \begin{tabular}{l|l}
$11.7 \quad 39.22$ & 2. \\
\end{tabular} & 0.011 & & & 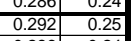 \\
\hline & & & & & & & & 16.0.056 & $\begin{array}{l}31.45 \\
31.279 \\
3\end{array}$ & & & & & 14.643 & & & & & & & & \\
\hline & & & & & & 477.1 & & 6.007 & 31.392 & & & & 0.077 & (4.643 & & & & & & & & \\
\hline & & & & & & $\begin{array}{ll}47.003 \\
47.202\end{array}$ & 50.0888 & 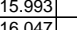 & 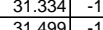 & & & & 0.078 & 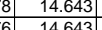 & & & & & & & & $f(x)+2$ \\
\hline & & 6:02:15 PM & & 25.564 & \begin{tabular}{l|l}
24.011 & 2 \\
2
\end{tabular} & 47.352 & 50.513 & 5.885 & \begin{tabular}{ll|l}
31.778 & -1 \\
\end{tabular} & & $\begin{array}{c}-7.527 \\
\end{array}$ & 99.136. & 0.07 & .643 & 3 & 1.31 & & 12.0 & 0.012 & & & .302 \\
\hline & & & & & 217[ & 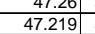 & 50.307 & 5.7986 & $\begin{array}{l}31.65 \\
31.753-1 \\
3\end{array}$ & & & 7.839 & & & & & & & & & & 299 \\
\hline & & & & & & $\begin{array}{lll}47.304 \\
\end{array}$ & & 5.944 & (31.681 & & & & 0.078 & & & & & & & & & \\
\hline & & & & & 5.84 & $\frac{47.341}{47.071}$ & $\begin{array}{l}5.0 .504 \\
50.81\end{array}$ & $1 \frac{1.921}{15217}$ & $\begin{array}{l}31.794 \\
3.529\end{array}$ & & $\frac{-7}{7}$ & 7.772 & $\frac{0.0}{0.0}$ & 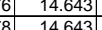 & & & & & & & & \\
\hline & & & & & 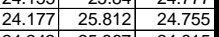 & $\frac{47.061}{47.061}$ & & & 31.525 & & & & & & & & & & & & & \\
\hline & & & & & $\begin{array}{ll}5.867 \\
25.9\end{array}$ & $\begin{array}{l}47.047 \\
4759\end{array}$ & \begin{tabular}{|l|l|}
50.229 \\
55.378 \\
\end{tabular} & 5.888 & $31.451-1-1$ & & & . 1118 & 0.07. & 4.643 & & .43 & & & & & & \\
\hline & & $1: 15 \mathrm{PM}$ & & $\frac{25.51}{2552}$ & $\begin{array}{ll}24.4535 & 25.9 \\
24.304 & 25.965 \\
\end{array}$ & $\begin{array}{ll}44.159 \\
47.32 \\
\end{array}$ & \begin{tabular}{|l|l|}
50.578 \\
50.546 \\
\end{tabular} & $\frac{15.971}{15.91}$ & 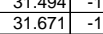 & & $\begin{array}{l}-. .564 \\
-7.521 \\
\end{array}$ & 50.484 & $\frac{0.07}{0.07}$ & . & & $\frac{45}{46}$ & & 12 & & & & 0.0 .293 \\
\hline & & & & & 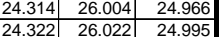 & & & 15.886 & 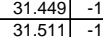 & & & $\begin{array}{ll}7.491 \\
9.224\end{array}$ & & & & & & & & & & 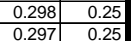 \\
\hline & 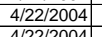 & 6:14:15 PM & 4.784 & 5.494 & \begin{tabular}{|l|l|l|}
24.347 & 26.098 & 25.035 \\
\end{tabular} & $\begin{array}{ll}46.802 \\
4.672\end{array}$ & 50.061 & $\frac{15.829}{15.557}$ & \begin{tabular}{ll|l}
31.252 & -1 \\
\end{tabular} & $\begin{array}{c}-1.218 \\
-1.219 \\
\end{array}$ & $\begin{aligned}-7.541 \\
\end{aligned}$ & $\begin{array}{l}46.74 \\
18.96 \\
\end{array}$ & 0.077 & 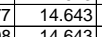 & 3 & 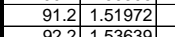 & & 6.691 & .011 & & & 0.300 \\
\hline & 20212004 & 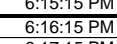 & & 5 5.944. & 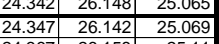 & 46.253 & 年9.9689 & $\frac{15.55 / 7}{15.608}$ & $\frac{31.285}{31.285}-1$ & & $\begin{array}{r}-7.53 \\
-7.486 \\
\end{array}$ & $4 \frac{48.865}{47.935}$ & & $\frac{14.643}{14.643}$ & & 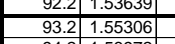 & & $\frac{11.7}{11.7}$ & 0.0121 & & & 0.311 \\
\hline & & & & & 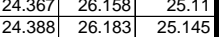 & $\begin{array}{l}46.555 \\
47.885\end{array}$ & $\begin{array}{l}49.851 \\
51.224\end{array}$ & 15.9622 & 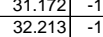 & & & & & & & & & & & & & \\
\hline & & $5 \mathrm{PM}$ & & & $\frac{4.392}{4.92}-2$ & & & & 31.97 & & & & & .643 & & & & & & & & \\
\hline & |/20004 & $\begin{array}{l}: 15 \mathrm{PM} \\
15 \mathrm{PM}\end{array}$ & & (4.466] & $\begin{array}{ll}4.424 & 26.6239 \\
4.465 & 26.29 \\
\end{array}$ & 47.845 & 51.246 & 15.962.987 & 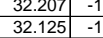 & & $\begin{array}{l}-7.7 \\
-7\end{array}$ & 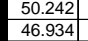 & 0.6 & $\begin{array}{l}4.643 \\
4643 \\
\mid\end{array}$ & & .616369 & & & & & & 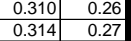 \\
\hline & & & & & & $\begin{array}{l}47.806 \\
47.87\end{array}$ & 51.199 & $\frac{15.88}{15.998}$ & $\begin{array}{ll}32.2685 \\
32.155\end{array}$ & & & 50.144 & & & & & & & & & & \\
\hline & 212004 & $4: 15 \mathrm{PM}$ & & & 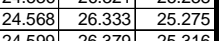 & 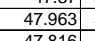 & 51.404 & $\begin{array}{l}15.8933 \\
15.893 \\
\end{array}$ & $\begin{array}{ll}32.328 & -1 \\
3\end{array}$ & & -7.5 & 49.835 & 0.6 & 4.643 & & 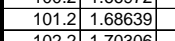 & & 12.2 & & & & 0.312 \\
\hline & $\begin{array}{lll}21212004 \\
22004\end{array}$ & $\begin{array}{l}0.26 .15 \mathrm{PM} \\
6: 26: 15 \mathrm{PM} \\
\end{array}$ & & & $\begin{array}{ll}24.599 & 26.39 \\
24.606 & 26.376 \\
\end{array}$ & (47.804 & 51.228 & 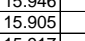 & 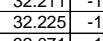 & & $\begin{array}{l}-1.551 \\
-7.521 \\
\end{array}$ & 42.1057 & & $\begin{array}{lll}4.64343 \\
4.643\end{array}$ & & $\begin{array}{ll}2 & 1.7 .7507 \\
2 & 1.71972 \\
\end{array}$ & & $\frac{\frac{12.0}{12.2}}{4}$ & & & & 0.0 .309 \\
\hline & & & & & 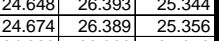 & & 11.2084 & & 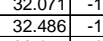 & & $\frac{-1 .}{-7 .}$ & $\begin{array}{l}8.671 \\
77.655 \\
\end{array}$ & & & & & & & & & & \\
\hline & 12004| & $\begin{array}{l}6: 29: 15 \mathrm{MM} \\
6: 30: 15 \mathrm{PM} \\
\end{array}$ & & & $\begin{array}{l}24.666 \\
24.703 \\
26.36 .428 \\
26.48\end{array}$ & $\begin{array}{l}47.808 \\
77.77 \\
\end{array}$ & 年1.2055 & 15.952. & 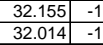 & & $\begin{aligned}-7.552 \\
-7.509 \\
\end{aligned}$ & $\begin{array}{l}51.42 \\
47.895 \\
\end{array}$ & \begin{tabular}{|l}
0.083 \\
0.084 \\
\end{tabular} & 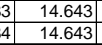 & & $\begin{array}{l}.21 .76 \\
1.78\end{array}$ & & & & & & .0.312 \\
\hline & $\mid 2004$ & $\frac{15 \mathrm{PM}}{15 \mathrm{PM}}$ & & & $\begin{array}{l}\frac{24.724}{26.4429} \\
24.739 \\
26.444\end{array}$ & $\begin{array}{r}44.324 \\
47.24 \\
\end{array}$ & $\begin{array}{ll}51.228 \\
51.325 \\
\end{array}$ & $\frac{15.966}{15.948}$ & 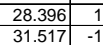 & & \begin{tabular}{|l|l|}
-0.106 \\
-6.1977 \\
\end{tabular} & & $\begin{array}{l}0.15 \\
0.018 \\
\end{array}$ & & & 108.21 .805 & & & & & & \\
\hline & & $\begin{array}{l}\text { 6:33:15 PM } \\
6: 34: 15 \mathrm{PM} \\
\end{array}$ & & & 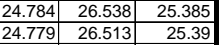 & \begin{tabular}{|c|c|}
47.37 \\
4775
\end{tabular} & 51.449 & $\frac{15.998}{110007}$ & \begin{tabular}{ll|l}
31.614 & -1 \\
310404 & -1
\end{tabular} & & \begin{tabular}{|l|l|}
-6.339 \\
-6116
\end{tabular} & & & 4.643 & & $\begin{array}{l}0.2 \\
0.83\end{array}$ & & & & & & \\
\hline & & 6:35:15 PM & & & \begin{tabular}{l|ll}
24.8 & 26.46 & 25.292 \\
4.75
\end{tabular} & $\begin{array}{lll}47.306 \\
\end{array}$ & 51.118 & & $\begin{array}{ll}31.654-1 \\
31.654-1\end{array}$ & & $\begin{array}{r}-7.07 \\
-707\end{array}$ & & & 14.643 & & 4.36097 & & & & & & 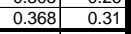 \\
\hline & 1221212004 & $\begin{array}{l}\text { 6:366:15M } \\
\text { 6:37:15 PM } \\
\end{array}$ & & & 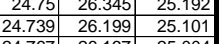 & 47.3.162 & $\begin{array}{l}50.625 \\
50.927 \\
\end{array}$ & 15.5696 & $\begin{array}{ll}31.81 & -1 \\
31.78 & -1 \\
\end{array}$ & & \begin{tabular}{|l|}
-7.384 \\
-7.32 \\
\end{tabular} & $\begin{array}{l}48.61 \\
49.973 \\
\end{array}$ & $\begin{array}{l}0.085 \\
0.086 \\
\end{array}$ & $\begin{array}{l}\frac{14.643}{14.643} \\
\end{array}$ & & $\begin{array}{l}\frac{1112.21 .86839}{114.2} 1.90306 \\
\end{array}$ & & $\begin{array}{ll}11.9 & 3 \\
12.2 & 3 \\
\end{array}$ & & & & 年.324 \\
\hline & & $1: 15 \mathrm{PM}$ & & & $\begin{array}{l}24.677726 .137 \\
24.76 \\
26.065\end{array}$ & $\begin{array}{r}47.253 \\
47.053 \\
\end{array}$ & $\begin{array}{l}50.938 \\
50.749 \\
\end{array}$ & 15.6977 & $\begin{array}{l}31.81-1 \\
31.726-1\end{array}$ & & \begin{tabular}{|l|l|}
-7.469 \\
-7.475 \\
\end{tabular} & 327 & $\begin{array}{l}0.006 \\
0.085 \\
\end{array}$ & $\begin{array}{ll}4.6 .633 \\
4.643 \\
\end{array}$ & & $\begin{array}{l}115.21 .919 \\
\frac{116.2}{1.936} \\
1.932\end{array}$ & & $\begin{array}{l}12.3 \\
12.0 \\
\end{array}$ & & & & . 0.327 \\
\hline & & : $15 \mathrm{PM}$ & & & \begin{tabular}{|l|l|}
24.748 & 26.002 \\
24.757 & 26.016 \\
\end{tabular} & $\begin{array}{l}47.244 \\
47.281 \\
7.281\end{array}$ & & $\begin{array}{l}15.663 \\
15.762 \\
15.72\end{array}$ & \begin{tabular}{ll|l}
31.905 & -1 \\
31.813 & -1 \\
3.1
\end{tabular} & $\begin{array}{l}-1.221 \\
-1.221 \\
\end{array}$ & \begin{tabular}{|l|l|}
-7.472 \\
-7.466 \\
\end{tabular} & \begin{tabular}{|l|}
349 \\
195
\end{tabular} & & 4.4 .643 & & \begin{tabular}{l|l}
117.2 & 1.95 \\
118.2 & 1.966 \\
\end{tabular} & & $\frac{12.1}{11.8}$ & & & & $\begin{array}{l}0.326 \\
0.330 \\
\end{array}$ \\
\hline & 20044 & : 15 PM & & & \begin{tabular}{l|l|l|}
1 & 26.07 & 24.997 \\
8 & 26.074 & 25.031 \\
\end{tabular} & \begin{tabular}{ll|l}
47.302 & 4.072 \\
\end{tabular} & $\begin{array}{l}50.987 \\
50.792\end{array}$ & 15.631 & 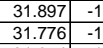 & & \begin{tabular}{|l|}
-7.405 \\
-7.452 \\
\end{tabular} & $\begin{array}{l}48.928 \\
48.442 \\
\end{array}$ & \begin{tabular}{|l}
0.086 \\
0.086 \\
\end{tabular} & 4.643 & $\frac{3}{3}$ & $\begin{array}{ll}\frac{119.2 .2}{1.9863939} \\
1120.2 & 2.030306\end{array}$ & & 11.9 & & & & 3266 \\
\hline 136 & & $\begin{array}{l}6: 44: 15 \mathrm{PM} \\
6: 45: 15 \mathrm{PM} \\
\end{array}$ & & & $\begin{array}{cccc}24.782 & 26.082 & 25.049 \\
244.808 & 26.127 & 25.11 \\
\end{array}$ & $\begin{array}{r}47.343 \\
47.25 \\
\end{array}$ & \begin{tabular}{|r|}
51.087 \\
51.05 \\
\end{tabular} & $\begin{array}{l}15.682 \\
15.729\end{array}$ & 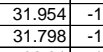 & & \begin{tabular}{|l|}
-7.457 \\
-7.472 \\
\end{tabular} & & 0.087 & 4.6. & 0.0 & $\begin{array}{l}\frac{121.2}{12.01972} \\
122.22 \\
2.036399\end{array}$ & & \begin{tabular}{r|r|c}
12.3 & 39.6 & 2.734 \\
11.9 & 39.5 & 2.725 \\
\end{tabular} & 013 & 013 & $\begin{array}{l}.000 \\
.000 \\
\end{array}$ & 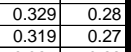 \\
\hline & & & & & 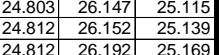 & & $\begin{array}{ll}521 \\
396 \\
105\end{array}$ & & 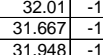 & & & & & & & & & & & & & \\
\hline
\end{tabular}


WSRC-TR-2005-00105, REVISION 0

SRNL-RPP-2005-00012, REVISION 0

RUN \# WASH1 \& WASH2; CAMPAIGN II SLURRY WASHING - CONT.

\begin{tabular}{|c|c|c|c|c|c|c|c|c|c|c|c|c|c|c|c|c|c|c|c|c|c|c|c|c|c|c|c|c|}
\hline & $\begin{array}{ll}A \\
\end{array}$ & $\begin{array}{lll}\text { B } & \text { C } \\
\end{array}$ & D & $E$ & $\mathrm{~F}$ & $\begin{array}{ll} \\
\end{array}$ & \begin{tabular}{ll|}
$\mathrm{H}$ \\
\end{tabular} & $\mathrm{J}$ & $\begin{array}{ll} \\
\end{array}$ & \begin{tabular}{l|l|}
$\mathrm{L}$ & \\
\end{tabular} & \begin{tabular}{|l|}
$\mathrm{M}$ \\
\end{tabular} & $\mathrm{N}$ & 0 & \begin{tabular}{l|l|}
$P \mid$ & $Q$
\end{tabular} & \begin{tabular}{l|l}
$R$ \\
\end{tabular} & \begin{tabular}{l|l} 
\\
\end{tabular} & $\begin{array}{ll} \\
\end{array}$ & \begin{tabular}{ll|}
$U$ & $v$
\end{tabular} & w & $x$ & & & & & & & & AF $\quad A$ \\
\hline 141 & $\begin{array}{l}4 / 22 / 2004 \\
4 / 22 / 2004\end{array}$ & $\begin{array}{l}6: 49: 15 \mathrm{PM} \\
6 \\
6.50 \cdot 115 \mathrm{PM}\end{array}$ & $\frac{25.215}{25.22}$ & $\begin{array}{r}25.555 \\
25.56 \\
\end{array}$ & \begin{tabular}{|l|}
24.833 \\
24894 \\
\end{tabular} & \begin{tabular}{|l|}
26.243 \\
26232 \\
\end{tabular} & $\begin{array}{l}25.195 \\
25 .\end{array}$ & \begin{tabular}{|l|l|l|}
46.173 \\
4612
\end{tabular} & \begin{tabular}{|l|l|}
51.002 \\
5021
\end{tabular} & \begin{tabular}{|l|l}
15.757 \\
1566
\end{tabular} & \begin{tabular}{|l|}
31.671 \\
3151
\end{tabular} & \begin{tabular}{|l|l|}
1 & -1.218 \\
1.221
\end{tabular} & \begin{tabular}{|l|}
-7.466 \\
7446 \\
\end{tabular} & \begin{tabular}{|l|l|}
51.831 \\
\end{tabular} & $\begin{array}{l}0.086 \\
0086\end{array}$ & \begin{tabular}{|l|}
14.643 \\
1.3
\end{tabular} & $\begin{array}{l}0.003 \\
003\end{array}$ & 126.2 & $\begin{array}{l}22 \\
2\end{array} 2.10306$ & 49.5 & $\begin{array}{l}12.7 \\
121\end{array}$ & $\begin{array}{l}39.4 \\
392 \\
39\end{array}$ & \begin{tabular}{|l|}
2.718 \\
2703
\end{tabular} & \begin{tabular}{|l|l|}
0.013 \\
0.013
\end{tabular} & \begin{tabular}{|l|}
0.013 \\
\end{tabular} & 0.000 & 0.327 & \\
\hline 144 & $\frac{4 / 2 / 2 / 2004}{4 / 22 / 2004}$ & $\begin{array}{l}\frac{6.50: 15 \mathrm{SPM}}{6: 51: 15 \mathrm{PM}} \\
6\end{array}$ & $\frac{25.22}{25.23}$ & $\frac{25.56}{25.56}$ & \begin{tabular}{|l|}
24.84949 \\
2489 \\
\end{tabular} & \begin{tabular}{|l|}
26.2633 \\
2.303 \\
\end{tabular} & $\begin{array}{l}25.24 \\
25.23\end{array}$ & $\begin{array}{l}46.912 \\
46.941\end{array}$ & \begin{tabular}{|l|}
50.112 \\
50.871
\end{tabular} & $\frac{\mid c 5.66}{15.709}$ & $\frac{31.511}{31.455}$ & $\frac{-1.221}{-1.221}$ & $\begin{array}{l}--7.446 \\
-7.394\end{array}$ & \begin{tabular}{|c|}
48.2608 \\
48.26
\end{tabular} & \begin{tabular}{|c|c|}
0.083 \\
0.033
\end{tabular} & $\mid \frac{11.643}{14.643}$ & $\begin{array}{l}0.003 \\
0.003\end{array}$ & $\frac{127.2}{128.2}$ & \begin{tabular}{|l|l|}
2 & 2.11972 \\
2 & 2.13639
\end{tabular} & $\frac{49.24}{49.4}$ & $\frac{12.1}{11.9}$ & \begin{tabular}{|l|}
39.2 \\
39.2
\end{tabular} & \begin{tabular}{|l|l|l|}
2.03 \\
\end{tabular} & $\mid$\begin{tabular}{|c|c|}
0.013 \\
0.12
\end{tabular} & \begin{tabular}{|l|}
0.013 \\
0.012
\end{tabular} & 0.000 & $\frac{0.328}{0.317}$ & 0.28 \\
\hline 144] & $4 / 22 / 2004$ & 2:15 PM & 25.237 & & 24.825 & $\frac{26.29}{26.29}$ & & 46.978 & 年.0.917 & & & & & & & \begin{tabular}{|l|l|}
14.643 \\
\end{tabular} & & & & 49.4 & & & & & & 0.0004 & & $\begin{array}{l}0.27 \\
0.28\end{array}$ \\
\hline 145] & & & & & 24.8 & & & & & & & & & & & & & & & & & & & & & & & 0.28 \\
\hline 146 146 & & & 25.24 & & & & & & & & & & & & & & & & & & & & & & & & & \\
\hline 1478 & $4 / 22 / 2004$ & $5: 15$ PM & 25.238 & 25.563 & 24.787 | & 26.251 & 25.253 & 46.671 & 50.645 & 15.492 & 31.414 & \begin{tabular}{|l|l|l|}
4 & -1.221 \\
\end{tabular} & -7. & 48.483 & 0.089 & 14.643 & & 132.2 & .22 .20306 & 49.2 & 11.9 & & 2.692 & & .010 & & 0.342 & 29 \\
\hline$\frac{1489}{149}$ & $4 / 22 / 2004$ & 6:56:15 PM & 25.238 & 25.563 & 24.772 & 26.306 & 25.263 & 46.792 & 50.753 & 15.561 & 31.445 & 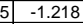 & -7.44 & 50.208 & 0.089 & \begin{tabular}{|l|l|l|l|}
1443 \\
\end{tabular} & 0.003 & 133.2 & $\begin{array}{l}.22 .21972 \\
\end{array}$ & |49.3 & & & & 0.013 & \begin{tabular}{|l|l|}
0.013 \\
\end{tabular} & 0.000 & 0.341 & \\
\hline & $4 / 22 / 2004$ & 6:56:25 PM & & 25.558 & 24.757 & 26.286 & 25.258 & & 50.44 & 15.455] & 31.195 & -1.221 & -7.437 & 50.546 & 0.09 & 14.643 & & 133.4 & & 49.0 & 12.4 & 38.8 & .676 & & 0.014 & 0.000 & 0.348 & 30 \\
\hline & 4/222/2004 & $: 25 \mathrm{PM}$ & 25.244 & 25.564 & 24.777 & 26.327 & 25.2599 & 46.657 & 5. 50.705 & 15.53 & 31.32 & -1.218 & -7.443 & 47.096 & 0.089 & 14.643 & & 134.4 & $\begin{array}{l}4 \\
4 \\
2.23917\end{array}$ & 49.2 & 11.5 & 39.0 & 2.688 & 013 & & 0.000 & 0.342 & 29 \\
\hline & & 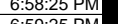 & & & $\frac{2.487}{2.792}$ & $\frac{26.357}{203}$ & & & $\frac{50.13}{60.69}$ & & . & & & & & 14.643 & & & & & & & & & & & 343 & \\
\hline & $\frac{4 / 222 / 2004}{4 / 22 / 2004}$ & $\frac{6.59: 25 \mathrm{PM}}{7.00 .25 \mathrm{PM}}$ & $\begin{array}{r}25.24 \\
25.255\end{array}$ & $\frac{25.584}{25.59}$ & $\frac{24.183}{2814}$ & $\frac{26.239}{2.633}$ & $\frac{25.255}{25.3}$ & & 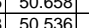 & 542 & $\frac{31.31}{31.248}$ & $\frac{1.21}{-1215}$ & $\begin{array}{l}-7.388 \\
-7.403 \\
\end{array}$ & 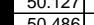 & | & $\frac{14.643}{14643}$ & & & & & 12.34 & & (. 6018 & 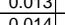 & . & & . & \\
\hline & |2004 & 7:01:25 PM & 25.246 & 25.591 & 24.785 & 26.294 & 25.301 & 46.507 & \begin{tabular}{|l|l|} 
\\
\end{tabular} & 15.509 & \begin{tabular}{|}
$\mid 1.4 .10$ \\
31.193
\end{tabular} & $\begin{array}{l}0 \\
\end{array}$ & $\begin{array}{r}-7.405 \\
-7.33 \\
\end{array}$ & 49.687 & & $\begin{array}{l}1.040 \\
14.643 \\
\end{array}$ & 0.003 & 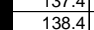 & \begin{tabular}{ll|l|}
4 & 2.2 .3058 \\
4 & 2
\end{tabular} & - 49.14 & $\frac{1.4}{12.2}$ & 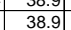 & \begin{tabular}{|l|l|} 
\\
\end{tabular} & \begin{tabular}{l|l|l|}
013 \\
013
\end{tabular} & $\mid \begin{array}{l}0.014 \\
0.013\end{array}$ & 0.000 & 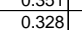 & \\
\hline & $4 / 22 / 2004$ & 7:02:25 PM & 25.246 & 25.596 & 24.779 & 26.359 & 25.286 & 46.468 & 50.542 & 15.541 & 31.135 & \begin{tabular}{|l|l|}
5 & -1.221 \\
\end{tabular} & $\begin{array}{l}-7.348 \\
\end{array}$ & 499.791 & 0.09 & $\begin{array}{l}14.643 \\
\end{array}$ & & 139.4 & \begin{tabular}{l|l|}
4 & 2.3222 \\
\end{tabular} & 49.1 & & & & 0.013 & 0.014 & & 0.348 & 0.30 \\
\hline & $4 / 222 / 2004$ & :25 PM & 25.246 & 25.596 & 24.79 & 26.319 & 25.266 & 46 & 50.96 & 15.376 & 31.774 & -1.221 & -7.252 & 51.139 & 0.086 & 14.643 & & 140.4 & $\begin{array}{l}442.33917 \\
\end{array}$ & 49.5 & $12.5 \mathrm{~S}$ & 39.4 & \begin{tabular}{|l} 
\\
\end{tabular} .714 & & & 0.000 & & 0.28 \\
\hline & $4 / 222 / 2004$ & 7:04:25 PM & 25.236 & 25.596] & 24.785 & 26.314 & 25.246 & 46. & .8711 & 15.484 & 31.453 & & -7.209 & 49.645 & & 14.643 & & 141.4 & & 49.4 & & & & & & & & \\
\hline & 4/22/2004 & 7:05:25 PM & 25.247 & $\frac{25.607}{2560}$ & \begin{tabular}{ll|l}
24.796 & \\
20195
\end{tabular} & 26.275 & $\begin{array}{l}25.292 \\
25.231\end{array}$ & & $529 \mid$ & 15.487 & 31.158 & $\begin{array}{l}8 \\
8\end{array}$ & $\begin{array}{l}-7.223 \\
-72 .\end{array}$ & $\begin{array}{r}49.03 \\
0103\end{array}$ & 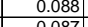 & 14.643 & & & & 49.1 & . & & 67 & & & & 40 & \\
\hline 160 & $\frac{4 / 2 \angle 2 / 2004 \mid}{4 / 22 / 2004}$ & $\frac{7.06 .25 \mathrm{PM}}{7.07 .25 \mathrm{P}}$ & 25246 & 25.0014 & $\frac{24.85}{24.779}$ & $\frac{2.0304}{26304}$ & & $\frac{46}{46}$ & $\frac{0.1571}{0.697}$ & 15.4515 & 每.1435| & $\frac{3.1 .224}{0.1204}$ & & $\frac{49.1353}{555}$ & $\frac{0.087}{0.087}$ & $\frac{14.643}{1.642}$ & & & & & & & & & & & & \\
\hline & $4 / 22 / 2004$ & $7: 08: 25$ PM & 25.251 & $\frac{2.001}{25616}$ & 24734 & $\frac{2.037 \mid}{26279}$ & & & t5.097/ & 1519 & $\frac{31.419}{31232}$ & \begin{tabular}{|l|l|}
2 & -1.221 \\
\end{tabular} & & 50.423 & $\begin{array}{l}0.08 \\
0.091\end{array}$ & $\frac{14.045}{14643}$ & & & & & $\frac{12.4}{124}$ & & & & & & . & \\
\hline & & 7:09:25 PM & 25.25 & 25.62 & 24.739 & 26.278 & 25.28 & $\frac{7}{4}$ & 50.334 & $\mid 15.267$ & $\frac{31.17}{31.1}$ & -1.221 & -7.307 & 49.629 & 0.101 & 14.643 & & 146.4 & 42.43917 & 48.9 & 12.2 & 38.7 & 2670 & 0.015 & 0.015 & 0.000 & 0.392 & \\
\hline & $4 / 22 / 2004$ & :25 PM & 25.255 & 25.62 & 24.733 & & & & 50.419 & 15.204 & 31.398 & & -6.96 & 50.569 & & \begin{tabular}{|l|l|}
14.643 \\
\end{tabular} & & $\begin{array}{l}147.4 \\
\end{array}$ & & & 12.4 & & 2.680 & 0.012 & 0.012 & & 0.309 & 0.26 \\
\hline 64 & 4/22/2004 & 7:11:25 PM & 25.24 & 25.625 & 24.708 & 26.283 & 25.285 & 46.306 & 50.436 & 15.249 & 31.314 & -1.218 & \begin{tabular}{|l}
-7.142 \\
7207
\end{tabular} & 49.591 & 0.104 & 14.643 & & 148.4 & 4725 & 49.0 & & 38.8 & 2.676 & 0.016 & $\begin{array}{ll}0.016 \\
\end{array}$ & 0.000 & $\begin{array}{ll}0.403 \\
\end{array}$ & 0.34 \\
\hline & $4 / 22212004$ & :25 PM & 25.24 & 25.625 & 24.698 & 26.293 & 25.295 & & 50.334 & 15.297] & . & -1.221 & -7.32 & 49.403| & 0.093 & 14.643 & & 149.4 & & 48.9 & & & 67 & & & & 0.361 & \\
\hline & 4/22/2004 & :25 P & $\frac{25.25}{25.25}$ & 25.64 & 24.729 & $\frac{2.6338}{2}$ & & & 069 & 15.233 & 31.105 & & & $51.3 / 80$ & & 14.643 & & & & & & & & & & & & \\
\hline 160 & $\frac{4 / 2 \angle 2 / 2004}{4 / 22 / 2004}$ & $\begin{array}{l}7.14 .25 \mathrm{P} \\
7.15 .25 \mathrm{PM}\end{array}$ & $\frac{25.225}{25.215}$ & $\begin{array}{l}25.02 \\
25.63 \\
\end{array}$ & $\frac{24.099}{24.694}$ & $\frac{26.333}{26.313}$ & $\frac{25.295}{25.29}$ & & | & $\begin{array}{c}\frac{1.3551}{15.31} \\
\end{array}$ & $\frac{31.275}{31209}$ & 年.2.21 & $\frac{-7.15}{-7.25}$ & $\begin{array}{l}51.009 \\
51.001\end{array}$ & $\frac{0.081}{0.089}$ & $\frac{14.643}{14643}$ & & $\begin{array}{ll}51.4 \\
5.24\end{array}$ & $\frac{4}{4}$ & $\frac{49}{48}$ & & & 673 & & & & & 21 \\
\hline & $4 / 22 / 2004$ & 7:16:25 PM & $\frac{25.210}{25.225}$ & 25.625 & 24.6094 & 26.323 & 25.299 & 4 & 51.112 & $\begin{array}{l}15.01 \\
15.53 \\
\end{array}$ & 31..694 & $\frac{-1.224}{-1.218}$ & -7.319 & | & 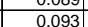 & $\begin{array}{l}14.045 \\
14.643\end{array}$ & & 153.4 & $\frac{2.05558}{2.5583}$ & 49.6 & 11.9 & 39.4 & & 0.014 & 0.014 & 0.000 & 0 & \\
\hline & 4/222/2004 & & 25.21 & 25.63 & \begin{tabular}{|l|}
24.688 \\
\end{tabular} & $\begin{array}{l}26.283 \\
263\end{array}$ & 25.304 & & & & 31.897 & & & & & 14.643 & & & & 49.6 & 11.8 & & & & & & & 29 \\
\hline 171 & 4/22/2004 & 7:18:25 PM & 25.215 & 25.645 & 24.724 & 26.258 & 25.28 & 47.038 & 51.105 & 15.481 & 31.772 & -1.224 & -7.278 & 49.806 & 0.099 & 14.643 & & 155.4 & \begin{tabular}{l|l|}
42.58917 \\
\end{tabular} & 49.6 & $\frac{12.2}{12.2}$ & 39. & 6.717 & 015 & & 0.000 & 0.377 & 32 \\
\hline & 4/22/2004 & 7:19:2 & & 25.64 & 24.734 & & & & 51.244 & & 31.7 & & & 51.147 & 0.094 & 14.643 & & & & & & & & & & & & \\
\hline & $4 / 22 / 2004$ & $7: 2$ & 25.21 & & 24.738 & 26.247 & & & & \begin{tabular}{|l|l|}
15.48 \\
\end{tabular} & 31.966 & & & & & 14.64 & & & & & {$[2.3$} & & & & .013 & & & \\
\hline & $4 / 22 / 2004$ & $7: 2$ & 25.21 & 25.64 & 24.739 & 26.288 & & & & & .079 & & & & & 14.6 & & & & & 12.2 & $39.7 \mathrm{C}$ & & & & & 405 & \\
\hline & $4 / 22 / 2004$ & 7:22:2: & 25.225 & 25.645 & \begin{tabular}{|l|}
24.743 \\
\end{tabular} & & & & 50.983 & 15.438 & 31.133 & -0.978 & & 47.85 & & 14.6. & & & & 49. & 11.7 & & & & & & & \\
\hline & $4 / 22 / 2004$ & & & & & 26.293 & & & 50.631 & & & & & & & $\overline{14.6}$ & & & & & & & & & & & & \\
\hline & $4 / 22 / 2004$ & 7:24:25 PM & 25.225 & 25.65 & 24.724 & 26.313 & 25.26 & & 50 & & 31.847 & -1.218 & -7.2 & 49.7. & 0.097 & 14.6. & & & & & 12.2 & & & & & & & \\
\hline & 4/222/2004 & & 25.22 & 25.65 & 24.719 & 26.283 & 25.2 & & 51.085 & 15.283 & 31.96 & -1.221 & -7.3 & 50.99 & 0.1 & 14.643 & & & 2.70583 & 49 & 12.5 & & 72. & & 0.015 & & 0.381 & \\
\hline 1 & $4 / 22 / 20$ & $7: 26$ & 25.225 & 25.66 & 24.709 & 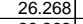 & & & & & 31.8 & 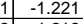 & & & & 14.6. & & & & & $\bar{T}$ & -5 & & & 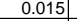 & & 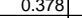 & \\
\hline & $4 / 22212004$ & & & & & & & & & & & & & & & & & & & & & & & & & & & \\
\hline & 4 & $\frac{7.28 .24}{7.2020}$ & $\frac{23.214}{25209}$ & 20.039 & 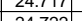 & $\frac{2.201}{2625}$ & $\frac{23.24}{2521}$ & $\begin{array}{l}40.012 \\
467\end{array}$ & | & 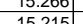 & $\frac{3.1 .18}{31657}$ & 1.1221 & $\frac{-1.2}{7.1}$ & | & $\frac{0.091}{0.092}$ & 14.645 & & & & & 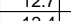 & (20 & 170 & niv & (4) & & 2077 & \\
\hline & 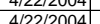 & & 23.209 & 20.044 & & & & 468.17 & 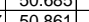 & & & & & & & & & & & & 12.4 & & & & & & & \\
\hline 184 & $4 / 22 / 2004$ & $7 \cdot 31.25 \mathrm{PM}$ & 年. 25.517 & 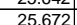 & 24.72 & $\frac{20.25}{26.255}$ & 20 & & 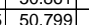 & 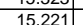 & 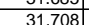 & -1.224 & .724 & 50.613 & $\frac{0.4}{0.6}$ & $\frac{1.6}{14.6}$ & & & & $\frac{49}{49}$ & $\frac{1.6}{124}$ & 39 & 70 & 势 & . & & 552 & 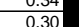 \\
\hline & $4 / 22 / 2004$ & & 25.21 & 25.666 & 24.719 & $\frac{26.284}{26.284}$ & $\frac{25.2}{25.2}$ & & 50.85 & \begin{tabular}{|l|l|}
155.207 \\
\end{tabular} & 31.794 & -1.221 & & 49.74 & & 14.6 & & & & & & & & & & & 0.332 & \\
\hline & $4 / 22 / 20$ & & & & 24.724 & & & & & & & & & & & & & & & & & & & & & & & \\
\hline 87 & $4 / 22 / 20$ & & 25.199 & 25.659 & \begin{tabular}{|l|l|}
24.732 \\
\end{tabular} & 26.272 & 25.2 & & 51.02 & 15.402 & 31. & -1.221 & & 51.12 & & 14.6 & & & & & 12. & 39 & & & 0.014 & & 0.364 & \\
\hline & $4 / 22 / 2$ & & 25.199 & 25.664 & \begin{tabular}{|l|l|}
24.722 \\
\end{tabular} & 26.227 & & & $51.0 \varsigma$ & & $31.9 !$ & -1.2 .2 & & 51.6 & & 14.6 & & & & & & & & & & & 0.376 & \\
\hline & $4 / 22 / 26$ & & 25.15 & & \begin{tabular}{|l|}
24.722 \\
\end{tabular} & 26.286 & & & & & & & & & & & & & & & & & & & & & & \\
\hline & $4 / 22 / 20$ & & 25.19 & & 24.725 & 26. & & & 50 & & & \begin{tabular}{|l|l|}
2 & -1.221 \\
\end{tabular} & & & & & & & & & 12. & 39 & & & & & 0.397 & \\
\hline & $4 / 22 / 2004$ & & 25.207 & 25.657 & 24.7 & 26.2 & & & & 15.472 & & & & & & & & & & & & & & & & & & \\
\hline & $4 / 22 / 20$ & & 25.22 & 25 & 24.7 & 213 & & & & & & -0.5 & & & & & & & & & 12. & & & & & & & \\
\hline & & & 25.214 & 25.644 & 24.1 & 26.242 & & & & & & & & & & & & & & & & & & & & & & \\
\hline 05 & $4 / 24212004$ & & 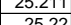 & 年5.63 & 24.74 & $\frac{2.234}{20.32}$ & & & & & & & & & & & & & & & & & & & & & & \\
\hline & & & $\frac{25.22}{20215}$ & & & & & & & & & & & 51 & & & & & & & & & & & & & & \\
\hline & 年 & & $\frac{25.25}{25214}$ & $\frac{25.04}{25.63}$ & $\mid$ & $\frac{2.003}{26.17}$ & & & & & & $\frac{-1.2}{-12}$ & & & & & & & & & & & & & & & (3) & \\
\hline 98 & $44 / 22 / 20$ & & $\frac{2.21}{25.19}$ & & 2466 & & & & & & & & & & & & & & & & & & & & & & & \\
\hline 99 & $4 / 22 / 2004$ & & 25.192 & 25.6 & $24.69 \mid$ & 26.095 & & & & $\frac{15.1}{15.1}$ & & -1.221 & & & & 14. & & & & & $13.6 \mathrm{C}$ & & & & & & 0 & \\
\hline & & & & & & & & & & & & & & & & & & & & & & & & & & & & \\
\hline & $4 / 22 / 2004$ & & 25.1 & & 24.6 & 26.6 & & & 50.374 & & & & & & & & & & & & & & & & & & & \\
\hline & & & 25.16 & 25.607 & & & & & 50.3 & & & -1.224 & & & & & & & & & & & & & & & & \\
\hline & 4/22/20 & 7:50:25 P & 25.1 & 5.60 & 24.68 & & & 46.4 & 50.41 & 15.2 & 31.304 & -1.224 & & 489 & & 14.6 & & 187 & 3.12 & 48 & & & & $\begin{array}{lll}4 & \end{array}$ & 0.014 & & 68 & \\
\hline & 4/22/2004 & 7:51:2 & & 25.66 & & & & 46.3 & 50. & & 31.21 & -1. & & & & 14.6 & & & & & & & & & & & & \\
\hline & $4 / 22 / 26$ & & & 25.5 & 24.67 & & & & & & & 1.2 & & & & & & & & & & & & & & & & \\
\hline 06 & & & 25.146 & 25.6 & 24.695] & 26.034 & & & & & & $-1.2 \quad$ & & & & & & & & 48.8 & $12.3 \mathrm{~S}$ & 38.8 & & & & & 0.364 & \\
\hline & & & 25.1 & & & 26.044 & & 45.8 & & & & 1.218 & & & & & & & & 48 & & & & & & & 0.395 & 0.34 \\
\hline & $4 / 22120004$ & 7.55:25MM & 25.129 & $\frac{25.589}{2567 \mid}$ & $\frac{24.608}{2069}$ & $\frac{25.977}{2007}$ & 24.989 & 46 & 50.154 & 15.0994 & $\begin{array}{l}31.047 \\
32.071\end{array}$ & $\frac{-1.211}{-1218}$ & -6.8 & 50.85 & 0.093 & 14.64 & & 192 & 4. 3.20 & 48.7 & 1.5 & 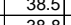 & 2.656 & 0.014 & 0.014 & 0.000 & 0.364 & 0. \\
\hline 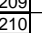 & 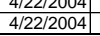 & $\begin{array}{l}7.56 .25 \mathrm{PM} \\
7: 57: 25 \mathrm{PM}\end{array}$ & \begin{tabular}{|l|}
25.108 \\
25.102 \\
\end{tabular} & 25.582 & $\begin{array}{l}\mid 24.596 \\
24.585 \\
\end{array}$ & \begin{tabular}{|l|}
25.936 \\
25.939 \\
\end{tabular} & $\begin{array}{l}24.918 \\
24.977 \\
\end{array}$ & & $\mid$\begin{tabular}{|l}
50.229 \\
50.229
\end{tabular} & \begin{tabular}{|c|}
14.97 \\
15.093 \\
\end{tabular} & 31.267 & $\begin{array}{l}-1.218 \\
-1.221 \\
\end{array}$ & $\begin{array}{l}-7.053 \\
-7.085 \\
-10\end{array}$ & $\begin{array}{l}49.49 r \\
51.395 \\
\end{array}$ & $\begin{array}{l}0.098 \\
0.102 \\
\end{array}$ & \begin{tabular}{|l|}
14.6433 \\
14.643
\end{tabular} & & $\begin{array}{l}193.4 \\
194.4 \\
\end{array}$ & \begin{tabular}{|l|l|} 
& 3.2225 \\
4 & 3.23917 \\
\end{tabular} & 48.8 & \begin{tabular}{l|l|}
12.1 \\
12.6
\end{tabular} & & $\mid$\begin{tabular}{|l|}
$\mid 2.673$ \\
2.671 \\
\end{tabular} & $\begin{array}{l}0.015 \\
0.015 \\
\end{array}$ & \begin{tabular}{|l|}
0.015 \\
0.015 \\
\end{tabular} & $\begin{array}{l}0.000 \\
0.000\end{array}$ & \begin{tabular}{|l|}
0.381 \\
0.397 \\
\end{tabular} & 0.34 \\
\hline
\end{tabular}


WSRC-TR-2005-00105, REVISION 0

SRNL-RPP-2005-00012, REVISION 0

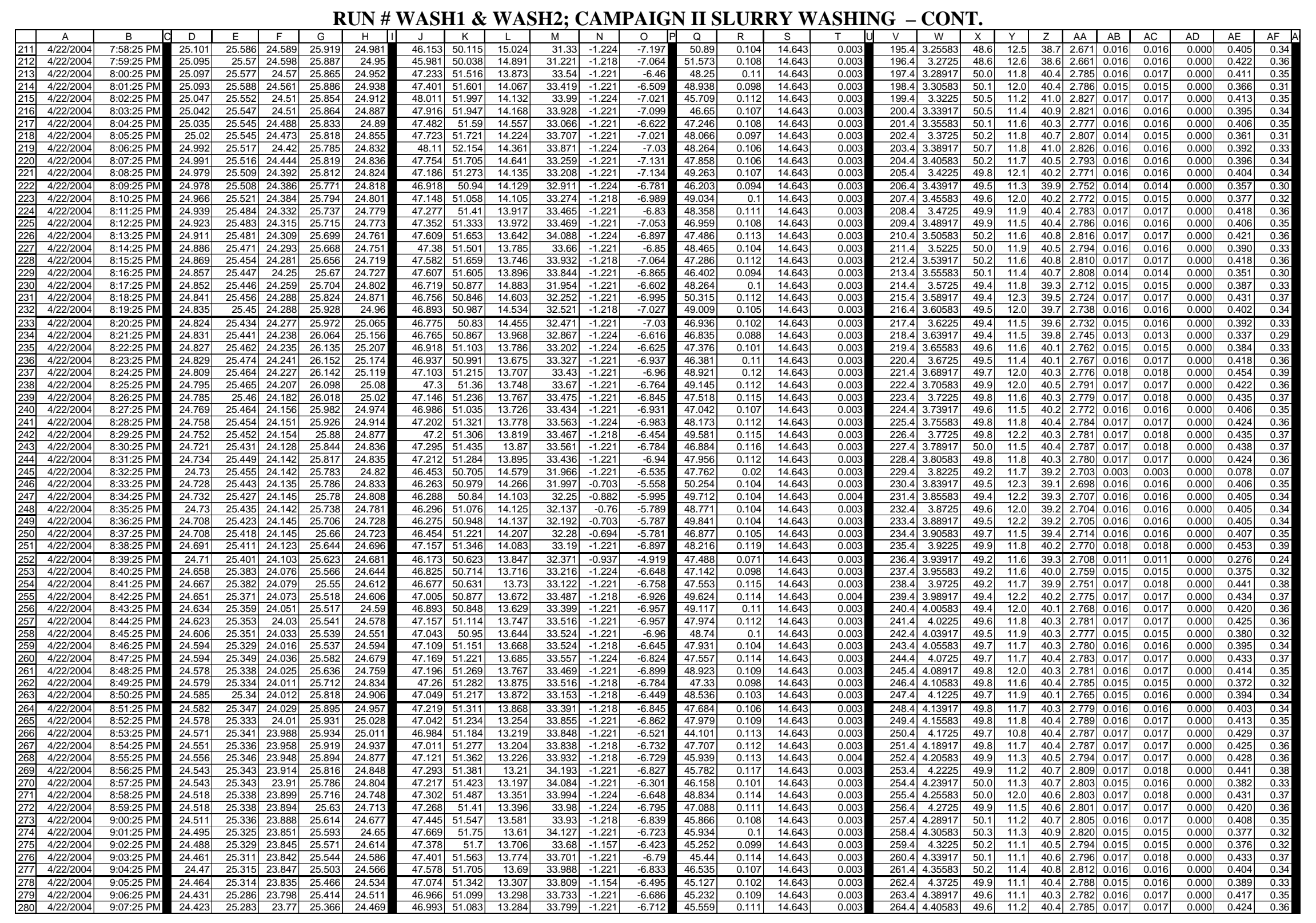


WSRC-TR-2005-00105, REVISION 0

SRNL-RPP-2005-00012, REVISION 0

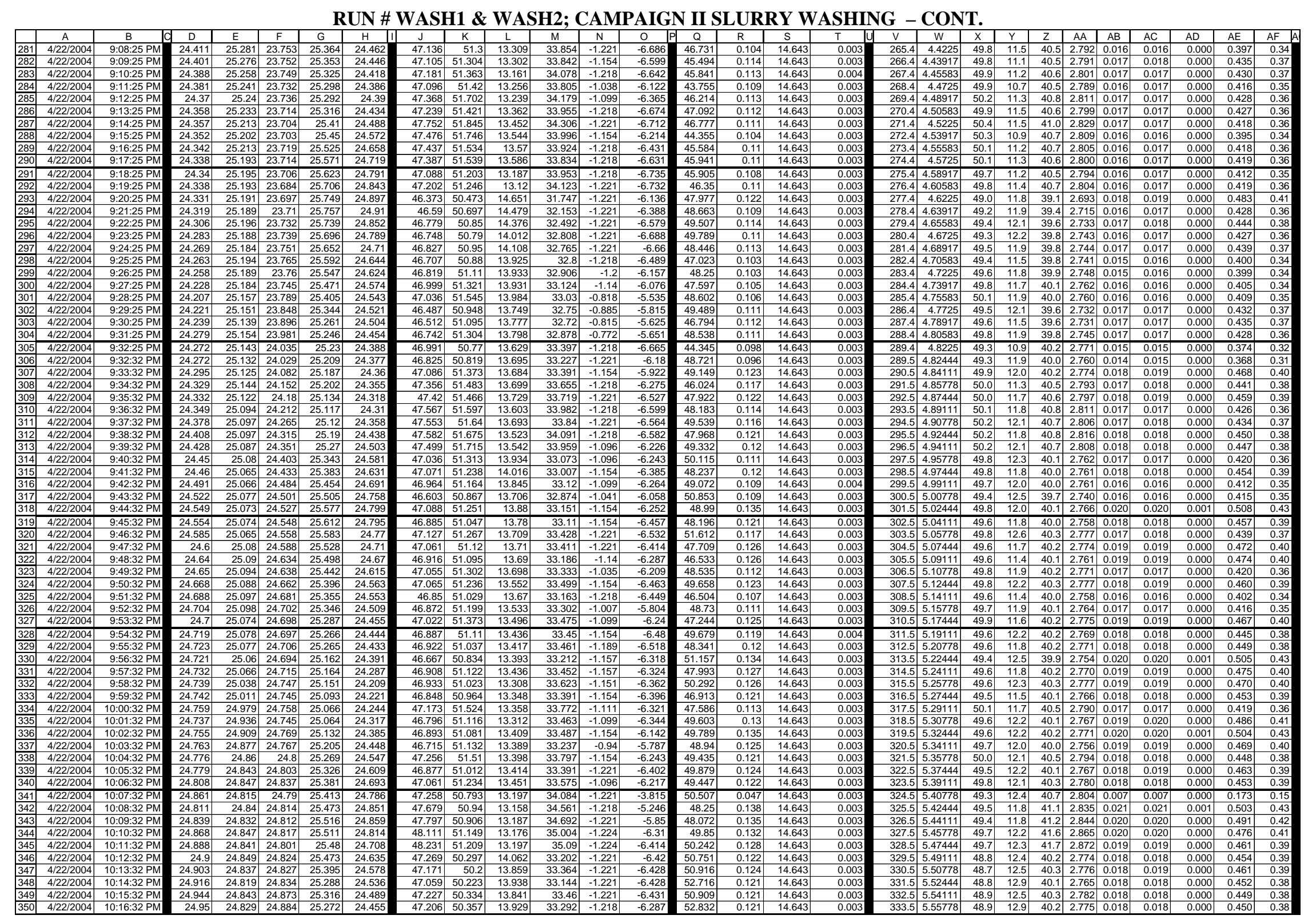


WSRC-TR-2005-00105, REVISION 0

SRNL-RPP-2005-00012, REVISION 0

RUN \# WASH1 \& WASH2; CAMPAIGN II SLURRY WASHING - CONT.

\begin{tabular}{|c|c|c|c|c|c|c|c|c|c|c|c|c|c|c|c|c|c|c|c|c|c|c|c|c|c|c|c|c|}
\hline & A & $B$ & D & $E$ & $\mathrm{~F}$ & G & \begin{tabular}{ll|}
$\mathrm{H}$ \\
\end{tabular} & $\mathrm{J}$ & $\mathrm{K}$ & \begin{tabular}{l|l}
$\mathrm{L}$ \\
\end{tabular} & M & $\mathrm{N}$ & \begin{tabular}{l|l}
$\mathrm{O}$ & $\mathrm{IP}$ \\
\end{tabular} & \begin{tabular}{ll|}
$P$ & $Q$
\end{tabular} & $\begin{array}{ll}R \\
\end{array}$ & $\mathrm{~s}$ & $\begin{array}{ll}T \\
\end{array}$ & $\mathrm{~V}$ & \begin{tabular}{l|l} 
w \\
\end{tabular} & $x$ & $\mathrm{Y}$ & & AA & $\mathrm{AB}$ & AC & & & AF $\quad A$ \\
\hline & $4 / 22 / 2004$ & $10: 17: 32$ PM & 24.958 & 24.821 & \begin{tabular}{|l|}
24.856 \\
\end{tabular} & 25.24 & 24.363 & 46.762 & 50.1 & \begin{tabular}{|l|}
13.611 \\
\end{tabular} & \begin{tabular}{|l|}
33.177 \\
\end{tabular} & -1.215 & -6.278 & 48.734 & \begin{tabular}{|l|l|}
0.123 \\
\end{tabular} & \begin{tabular}{|l|l|}
14.643 \\
\end{tabular} & 0.004 & 334.5 & \begin{tabular}{|l|l|}
5.57444 \\
\end{tabular} & 48.6 & 11.9 & 40.0 & 2.756 & 0.018 & 0.018 & 0.000 & 0.461 & 0.39 \\
\hline & $4 / 22 / 2004$ & $\begin{array}{l}10: 18: 32 \mathrm{PM} \\
10.1903\end{array}$ & $\begin{array}{l}24.965 \\
24.966\end{array}$ & \begin{tabular}{|l|l}
24.803 \\
24794
\end{tabular} & \begin{tabular}{|l|}
24.859 \\
24544 \\
\end{tabular} & $\begin{array}{l}25.197 \\
25.67\end{array}$ & $\begin{array}{r}24.365 \\
24.32\end{array}$ & $\begin{array}{l}46.711 \\
46738 \\
\end{array}$ & \begin{tabular}{|l|}
50.023 \\
503 \\
\end{tabular} & \begin{tabular}{|l|l|}
13.683 \\
1593
\end{tabular} & \begin{tabular}{|r|}
33.068 \\
33194
\end{tabular} & \begin{tabular}{ll|}
68 & -1.221 \\
4 & -1221
\end{tabular} & $\begin{array}{l}-6.284 \\
-6202\end{array}$ & \begin{tabular}{|l|l|}
51.379 \\
53306
\end{tabular} & $\begin{array}{l}0.124 \\
0123\end{array}$ & \begin{tabular}{|l|l|}
14.643 \\
1463
\end{tabular} & $\begin{array}{l}0.003 \\
0.03\end{array}$ & $\begin{array}{l}335.5 \\
365\end{array}$ & \begin{tabular}{|l|l|}
5.59111 \\
556078
\end{tabular} & 48.6 & $\begin{array}{l}12.6 \\
131\end{array}$ & $\begin{array}{l}39.9 \\
40.9\end{array}$ & \begin{tabular}{|l|l|}
2.750 \\
2756
\end{tabular} & $\frac{0.018}{0.018}$ & 0.019 & 0.000 & $\begin{array}{l}0.465 \\
0461\end{array}$ & 0.40 \\
\hline 35 & $\begin{array}{l}4 / 2 / 2 / 2 / 2004 \\
4 / 22004\end{array}$ & $\begin{array}{l}10: 19523 \mathrm{PM} \\
1020: 32 \mathrm{PM}\end{array}$ & $\begin{array}{l}24.966 \\
24.961\end{array}$ & $\begin{array}{l}24.794 \\
24.759\end{array}$ & $\mid$\begin{tabular}{|}
$\mid$\begin{tabular}{|l}
$\mid 24.584$ \\
24.849
\end{tabular} \\
\end{tabular} & $\frac{25.167}{25.127}$ & $\begin{array}{r}24.34 \\
24.3 \\
\end{array}$ & $\begin{array}{l}46.738 \\
46.659\end{array}$ & \begin{tabular}{|r|}
50.003 \\
50.009
\end{tabular} & \begin{tabular}{|l|}
3.593 \\
13.638
\end{tabular} & $\begin{array}{l}33.194 \\
33.083\end{array}$ & $\frac{1.222}{1.22}$ & $\begin{array}{l}-6.294 \\
-6.266\end{array}$ & $\begin{array}{r}53.306 \\
49.66\end{array}$ & $\begin{array}{l}.123 \\
0.121\end{array}$ & $\begin{array}{r}14.6643 \\
14.643\end{array}$ & & $\begin{array}{l}336.5 \\
337.5 \\
\end{array}$ & 5.602474 & $\frac{48.6}{48.5}$ & $\frac{13.1}{122}$ & $\frac{40.0}{39.9}$ & .756 & $\frac{0.018}{0.018}$ & $\frac{0.018}{0.018}$ & 0.000 & \begin{tabular}{|c|c|} 
\\
\\
\end{tabular} & \\
\hline & & & & & & & & 46.675 & \begin{tabular}{|l|}
49.903 \\
\end{tabular} & 13.647 & 33.015 & & & 50.88 & 0.125 & 14.643 & & 338.5 & 5.64111 & 48.4 & 12.5 & & & & 0.019 & 0.000 & & \\
\hline & $4 / 22 / 2004$ & $\begin{array}{l}10: 22: 32 \mathrm{PM} \\
\end{array}$ & 24.973 & 24.731 & \begin{tabular}{|l|}
24.041 \\
\end{tabular} & 25.084 & $\frac{2.200}{24.207}$ & 46.495 & \begin{tabular}{|l|}
49.904 \\
\end{tabular} & $\begin{array}{l}10.041 \\
13.695\end{array}$ & $\frac{50.010}{32.8}$ & \begin{tabular}{|l|l}
8 & -1.218
\end{tabular} & $\begin{array}{l}-0.250 \\
-6.252\end{array}$ & 51.904 & 0.12 & $\mid \begin{array}{l}14.645 \\
14.643\end{array}$ & & 339.5 & 5 5.65771 & 488.4 & 12.7 & 39.6 & 2.734 & 0.018 & 0.018 & 0.000 & 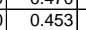 & \\
\hline 35 & $4 / 22 / 2004$ & & 24.964 & 24.697 & \begin{tabular}{|l|}
24.858 \\
\end{tabular} & 25.045 & 24.199 & 46.735 & \begin{tabular}{|l|}
50.216 \\
\end{tabular} & 13.62 & & & & & & \begin{tabular}{|l|l|}
14.643 \\
\end{tabular} & & & 5.67444 & & & & & & & & & \\
\hline & $4 / 22 / 2004$ & 10:24:32 PM & 24.987 & 24.705 & \begin{tabular}{|l|}
24.886 \\
\end{tabular} & 25.093 & 24.322 & 46.843 & \begin{tabular}{|l|l|}
50.279 \\
\end{tabular} & 13.697 & 33.175 & -1.218 & -6.261 & 52.918 & 0.121 & 14.643 & & 341.5 & 5.69111 & 48.8 & 13.0 & 40.0 & 2.758 & 0.018 & 0.018 & 0.000 & 0.452 & \\
\hline 359 & $4 / 22 / 2004$ & 10:25:32 PM & 24.98 & 24.683 & \begin{tabular}{|l|}
24.878 \\
\end{tabular} & 25.121 & 24.395 & 46.709 & \begin{tabular}{|l|}
50.15 \\
\end{tabular} & 13.609 & 33.093 & -1.224 & -6.264 & 50.598 & 0.122 & 14.643 & & 342.5 & \begin{tabular}{|l|l|}
5.70778 \\
\end{tabular} & 48.7 & 12.4 & 39.9 & 2.751 & \begin{tabular}{|l|l}
0.018 \\
\end{tabular} & 0.018 & 0.000 & 0.457 & \\
\hline & $4 / 22 / 2004$ & & & & & 25.241 & 24.444 & & & & & & & & & $\begin{array}{l}14.643 \\
\end{array}$ & & & & & & & & & & & & \\
\hline$\frac{361}{362}$ & $4 / 22 / 2004$ & $\begin{array}{ll}10: 27: 32 \mathrm{PM} \\
10: 28: 282\end{array}$ & $\begin{array}{l}24.964 \\
24.076\end{array}$ & \begin{tabular}{|l|l|}
24.637 \\
24629
\end{tabular} & \begin{tabular}{|l|l|}
24.883 \\
24857 \\
\end{tabular} & $\frac{25.231}{25.243}$ & $\begin{array}{l}24.469 \\
22586\end{array}$ & \begin{tabular}{|l|l|}
46.777 \\
6395
\end{tabular} & \begin{tabular}{|l|l|}
50.301 \\
5032 \\
\end{tabular} & \begin{tabular}{l|l|}
13.785 \\
1659
\end{tabular} & \begin{tabular}{|l|l|}
33.003 \\
3264
\end{tabular} & 1.218 & $\begin{array}{l}-6.235 \\
\end{array}$ & $\begin{array}{l}51.669 \\
5.20\end{array}$ & $\begin{array}{l}0.126 \\
\end{array}$ & $\begin{array}{l}14.643 \\
\end{array}$ & & 344.5 & \begin{tabular}{|l|l|}
5.74111 \\
\end{tabular} & 48.8 & $\begin{array}{l}12.7 \\
\end{array}$ & 39.9 & 7.75 & 0.019 & 0.019 & 0.000 & $\begin{array}{l}0.472 \\
\end{array}$ & \\
\hline$\frac{362}{363}$ & 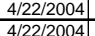 & $\begin{array}{ll}\frac{10: 28: 32 \mathrm{PM}}{10} \\
0.2932 \mathrm{PM}\end{array}$ & $\frac{24.976}{24.977}$ & $\frac{24.629}{24.63}$ & \begin{tabular}{|l|}
24.8785 \\
24.855 \\
\end{tabular} & $\frac{25.243}{25.294}$ & $\begin{array}{r}24.586 \\
24.682\end{array}$ & & \begin{tabular}{|l|}
50.336 \\
50.32 \\
\end{tabular} & \begin{tabular}{|l|l|}
13.658 \\
13.529
\end{tabular} & \begin{tabular}{|l|}
32.664 \\
33.298
\end{tabular} & & $\begin{array}{l}-5.396 \\
-5.5966\end{array}$ & \begin{tabular}{|c|}
51.264 \\
52013
\end{tabular} & $\begin{array}{l}0.111 \\
0.123\end{array}$ & \begin{tabular}{|c|}
14.643 \\
1.4633 \\
\end{tabular} & & $\begin{array}{l}345.5 \\
346.5 \\
\end{array}$ & \begin{tabular}{|c|}
55.75778 \\
5
\end{tabular} & 48.9 & \begin{tabular}{ll|l}
12.6 \\
127
\end{tabular} & & & 0.017 & & 0.000 & $\frac{0.420}{0.464}$ & 0.36 \\
\hline 364 & $4 / 22 / 2004$ & $\begin{array}{l}10: 29.3 \mathrm{PMM} \\
10: 30: 32 \mathrm{PM}\end{array}$ & $\begin{array}{l}24.971 \\
24.987\end{array}$ & $\begin{array}{r}24.63 \\
24.63 \\
\end{array}$ & \begin{tabular}{|r|}
24.8 .85 \\
\end{tabular} & $\frac{25.294}{25.389}$ & $\begin{array}{l}\frac{24.682}{24.732} \\
\end{array}$ & $\begin{array}{l}46.476 \\
46.696\end{array}$ & \begin{tabular}{|l|}
50.332 \\
50.535 \\
\end{tabular} & \begin{tabular}{r|r|}
3.529 & 13.5
\end{tabular} & \begin{tabular}{|l|l|l|}
898 \\
\end{tabular} & $\begin{array}{l}-1.218 \\
-1.221 \\
\end{array}$ & $\begin{array}{l}-5.596 \\
-5.466\end{array}$ & \begin{tabular}{|}
52.013 \\
50.371
\end{tabular} & $\mid \begin{array}{l}0.123 \\
0.109 \\
\end{array}$ & $\begin{array}{r}14.643 \\
14.643\end{array}$ & & $\begin{array}{l}346.5 \\
347.5\end{array}$ & \begin{tabular}{|c|}
5.774444 \\
5
\end{tabular} & $\frac{48.9}{49.1}$ & & & \begin{tabular}{|l|}
2.736 \\
2.751 \\
\end{tabular} & $\frac{0.016}{0.016}$ & $\frac{0.018}{0.016}$ & $\begin{array}{l}0.000 \\
0.000\end{array}$ & $0.464 \mid 0.409$ & 0.39 \\
\hline & 4/221/2004 & 10:31:32 PM & 24.997 & 24.64 & \begin{tabular}{|c|}
24.88 \\
\end{tabular} & 25.429 & 24.672 & 46.675 & \begin{tabular}{|l|}
50.61 \\
\end{tabular} & 13.562 & 33.062 & -1.221 & $\begin{array}{l}-5.4092 \\
-\end{array}$ & 499.948 & 0.126 & 14.643 & & $\begin{array}{l}347.5 \\
348.5\end{array}$ & $\frac{5.19111}{5.80778}$ & $\frac{49.1}{49.1}$ & 12.2 & & \begin{tabular}{|l|}
2.751 \\
2.749 \\
\end{tabular} & & 0.010 & & & $\frac{0.35}{0.40}$ \\
\hline 366 & 4/221/2004 & 10:32:32 PM & 25.009 & 24.657 & \begin{tabular}{|l|}
24.908 \\
\end{tabular} & 25.486 & 24.759 & 46.3 & 50.258 & 13.544 & 32.754 & -1.218 & -5.469 & 49.136 & 0.121 & \begin{tabular}{|l|l|}
14.643 \\
\end{tabular} & & 349.5 & 55.82444 & 48.8 & & 39.5 & 2.725 & 0.018 & & & 0.058 & 0 \\
\hline 367 & $4 / 221 / 2004$ & 10:33:32 PM & 24.998 & 24.661 & 24.887 & 25.45 & 24.778 & 46.298 & 50.237 & 13.486 & & & & & & 14.643 & & & & & & & & & & & & $\frac{39}{40}$ \\
\hline & & & & 24.683 & & & & & & & \begin{tabular}{|l|l|}
32.693 \\
\end{tabular} & & & $\begin{array}{ll}48.462 \\
\end{array}$ & 0.121 & $\begin{array}{l}14.643 \\
\end{array}$ & & & 55778 & & & & & & & & 458 & \\
\hline & $\mid$ & 10:35:32 PM & 25.036 & 24.704 & 24.935 & 25.418 & & & 50.405 & 13.513 & 32.839 & & & 49.658 & & $\begin{array}{l}14.643 \\
\end{array}$ & & & 5.87444 & & & & & & & & 467 & \\
\hline & 4/22/2004 & 10:36:32 PM & & 24.695 & 24.935 & 25.364 & & & & 13.514 & 32.746 & -1.2 .1 & & & & \begin{tabular}{|l|l|l|}
14.643 \\
\end{tabular} & & & & & & & & & & & & \\
\hline 371 & $4 / 221 / 2004$ & 10:37:32 PM & 25.054 & 24.712 & 24.948 & 25.346 & 24.449 & 46.211 & 50.278 & 13.535 & 32.601 & -1.221 & & 51.581 & 0.122 & $\begin{array}{l}14.643 \\
\end{array}$ & & 354. & 5.90778 & \begin{tabular}{l|l|}
48.8 \\
\end{tabular} & \begin{tabular}{|l|l|}
12.6 \\
\end{tabular} & 39. & 2.717 & $\mid$ & 0.018 & 0.000 & 0.462 & \\
\hline & & 10:38:32 PM & 25.054 & 24.697 & 24.948 & 25.266 & 24.404 & 46.21: & 50.279 & 13.533 & 32.555 & -1.221 & -5 & 49.351 & 0.122 & 14.643 & & 355. & 5.92444 & 48. & 12.1 & 39. & 2.71. & & $0.010^{0}$ & 0.00 & 0.463 & \\
\hline$\frac{373}{373}$ & $4 / 22122004$ & $10: 39: 32 \mathrm{PM}$ & 25.053 & 24.696 & 24.942 & 25.21 & 24.3833 & 46.238 & 50.285 & 13.463 & 32.703 & -1.2 & & 50.375 & 0.123 & 14.643 & & 356 & 5.941111 & 48.8 & 12.3 & & & & & & 0.465 & \\
\hline $3 / 4$ & $4 / 2212004$ & $2 \mathrm{PM}$ & 25.053 & 24.696 & 24.952 & 25.185 & 24.36 & & 5.504 & | & 32.715 & -1.157 & & 51.137 & 0.124 & 14.643 & & & & & & & & & & & 0.468 & \\
\hline & 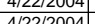 & $10.41 .32 \mathrm{PM}$ & 25.062 & 24.686 & 24.976 & 25.174 & & & 50.484] & 13.583 & 32.672 & -1.151 & & 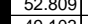 & 0.12 & 14.643 & & & & & 12. & & & & & & 0.457 & \\
\hline $\begin{array}{ll}310 \\
377\end{array}$ & $\frac{42512004}{4212004}$ & PM & $\frac{23.041}{2505}$ & $\begin{array}{l}24.0035 \\
24633\end{array}$ & $\frac{\mid 24.95}{24.98}$ & & & $\frac{46.02}{46.21}$ & (5.1436) & $\frac{13.643}{13.533}$ & $\frac{32.27}{32.633}$ & $\begin{array}{l}-1.1 \\
-1.1 \\
\end{array}$ & & 49.103 & & $\frac{14.643}{14.643}$ & & & .999111 & & & & & & & & & \\
\hline 378 & $4 / 22 / 2004$ & 10:44:32 PM & $\frac{25.06}{25.065}$ & 24.638 & \begin{tabular}{|l|}
24.953 \\
\end{tabular} & 25.066 & 24,194 & $\frac{40.215}{46.395}$ & 50.533 & 13.54 & 32.707 & -1.128 & & 49.528 & 0.122 & $\frac{14.045}{14.643}$ & & 361 & 6.02444 & (4) & 121 & & & & 0.018 & & $\frac{160}{1460}$ & \\
\hline 379 & & & 25.047 & 24.615 & 24.951 & 25.013 & & 46.229 & 50.457 & 13.486 & 32.679 & & & & & 14.643 & & & 604411 & & & & & & & & 465 & \\
\hline & & $2 \mathrm{PM}$ & 25.05 & 24.598 & 24.954 & 24.992 & & & 50.467 & 13.572 & & -1.6 & & $\mid$ & 0.123 & 14.643 & & 363 & 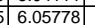 & 49 & 121 & 20 & 77 & & & & 0.465 & \\
\hline 38 & $4 / 22 / 2004$ & 47:32 PM & \begin{tabular}{|l|l|}
25.064 \\
\end{tabular} & \begin{tabular}{l|l}
24.582 \\
\end{tabular} & \begin{tabular}{|l|}
24.968 \\
\end{tabular} & 24.975 & 24.1 & $\begin{array}{l}46.163 \\
\end{array}$ & 50.351 & 13.543 & 32.508 & -1.056 & & 50.167 & & 14.643 & & & 7444 & & $\overline{12.3}$ & & & & & & D.459 & \\
\hline & |2004 & 10:48 & 25.057 & 24.565 & \begin{tabular}{|l|}
24.971 \\
\end{tabular} & 24.959 & 24.0 & 46.064 & 50.33 & 13.495 & 32.471 & -0.995 & & 51.798 & 0.12 & \begin{tabular}{|l|l|l|l|l|}
1433 \\
\end{tabular} & & & 09111 & & -7 & & & & & & 0.464 & \\
\hline 38 & & 10:49:32 PM & 25.055 & 24.528 & 24.964 & 24.977 & 24. & 45.945 & 50.26 & 13.476 & 32.356 & -0.937 & & 50.469 & & $\begin{array}{l}14.643 \\
\end{array}$ & & 366 & 6.10778 & & 12.4 & 39 & 69 & & & & 461 & 39 \\
\hline 38 & $\mid$ & & 25.0 & 24.5 & 24.969 & & & & 50.486 & 13.557 & .455 & & & 48.463 & & 14.6 & & & 2444 & & 11.2 & & & & & & (463 & \\
\hline & $4 / 221 / 2004$ & 10:51:32 PM & $25.07 \mathrm{e}$ & 24.5 & 24.989 & 25.111 & & & 50.343 & 13.54 & 32.369 & 59 & & 50.263 & 0.12 & 14.643 & & & 6.141111 & & $\overline{123}$ & & 70 & & & & & \\
\hline 386 & P/2004 & 10:52:32 PM & 25.063 & 24.511 & 24.977 & 25.15 & 24.458 & 45.966 & 50.395 & 13.5 & 32.297 & -0.885 & & 48.016 & 0.12 & 14.643 & & & & 48 & 11.8 & & 6 & 18 & & 0.00 & 0.461 & \\
\hline$\pi$ & & 10:53:3 & 25.075 & 24.5 & 24.994 & 25.236 & & & 50.413 & 13.506 & 32.422 & -0 & & 年 & & & & & & & , & & & & & & 0.479 & \\
\hline & & & & & & & & & & & & & & & & & & & & & & & & & & & & \\
\hline & & & 23.00 & 24.8 & & $\frac{23.325}{25307}$ & & & $\frac{50.712}{5.12}$ & 1.300 & & & & 49. & & 14. & & & & & 11.2. & & & & & & $0.4 / 3$ & \\
\hline$\frac{291}{391}$ & & $\begin{array}{l}10.57 \\
10.57\end{array}$ & 25.087 & 24585 & $\mid$\begin{tabular}{|c|}
$\mid 24.0030$ \\
2496
\end{tabular} & 25.301 & & & 年 & $\frac{13.44}{1396}$ & $\begin{array}{l}4.45 \\
139\end{array}$ & & & & & & & & & & 124 & & 68 & & & & 0.467 & \\
\hline & & $10: 58: 3$ & $25.08 \varepsilon$ & 24.5 & $|25007|$ & 25.5 & & & \begin{tabular}{|l|}
50.256 \\
\end{tabular} & 13.417 & 32.305 & & & 48.177 & & 14. & & & & & & & & & & & & \\
\hline 393 & & $10: 59$ & 25.05 & 24.7 & & $\begin{array}{l}25.497 \\
5.497\end{array}$ & & 45 & $\mid$ & & & & & 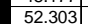 & & & & & & & 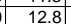 & & & & & & & \\
\hline 394 & & & 25.03 & & & 25.525 & & & 50.33 & & & & & 51.616 & & & & & & & 126 & & & & & & & \\
\hline & & & 25.02 & & & 25.482 & & & 50.349 & & & & & & & & & & & & 13. & & & & & & & \\
\hline & & & 25.03 & 24.6 & \begin{tabular}{|l|}
25.024 \\
\end{tabular} & 25.437 & & & & 13.585 & & & & & & & & & & & & & & & & & & \\
\hline & & 11:03 & 25.067 & 24.6 & \begin{tabular}{|l|}
25.026 \\
\end{tabular} & 25.404 & 24.5 & 47.2 & 50.633 & 13.457 & & & & 48.244 & 0.125 & 14.6 & & & 6.315 & $4 \mathrm{sc}$ & 11.8 & & & & & & 0.475 & 0.40 \\
\hline & & & $\overline{22.7}$ & 21. & 24.0 & 22.929 & & & 50 & & & & & 50.7 & & & & & & & 12 & & & & & & $\overline{0.540}$ & \\
\hline & & & 22.8 & 21.94 & \begin{tabular}{|l|}
24.144 \\
\end{tabular} & 22.947 & & & \begin{tabular}{|l|l|l|}
50.70 \\
\end{tabular} & 14.4 & $32.0 \mathrm{~s}$ & & & & & 14.6 & & & & & 12. & &. .16 & & & & & \\
\hline & & & 22.946 & 21.966 & 24.121 & 22.999 & & & 50.54 & & & & & & & & & & & & 12. & & & & & & & \\
\hline 401 & & & 22.994 & 21.994 & | 24.119 & & & & 50.32 & & & & & & & & & & & & 12.5 & & & & & & 0.529 & \\
\hline 402 & & & & & & 23.1. & & & & & & & & 50.229 & & & & & & & 12 & & & & & & 0.557 & \\
\hline & & & & & & & & & & & & & & & & & & & & & & & & & & & & \\
\hline & & & 23.19 & & & & & & & & & & & & & & & & & & & & & & & & & \\
\hline & & & $\frac{2.42}{2327}$ & 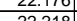 & $\mid$ & 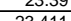 & & & & & & & & & & & & & & & & & & & & & & \\
\hline 407 & & & $\frac{2.24}{233}$ & $\frac{2.21}{222}$ & & & & & & & & & & & & & & & & & & & & & & & & \\
\hline 408 & & & $\frac{23.37}{23.37}$ & & & & & & & & & & & & & & & & & & & & & & & & & \\
\hline 409 & & & & & & & & & & & & & & & & & & & & & & & & & & & & \\
\hline 41 & & & & & & & & & & & & & & & & & & & & & & & & & & & & \\
\hline & & & 23.46 & 22.413 & 24.4 & 23.496 & & & 50.2 & & & & & & & & & & & & & & & & & & & \\
\hline & & & & 222.46 & $\overline{24.4}$ & & & $\overline{46.4}$ & $\overline{4 !}$ & & & & & & & & & & & & & & & & & & & \\
\hline & & & & 22.46 & 24.445 & 23.609 & & 46. & 49.86 & & & -1. & & & & & & & & & & & & & & & & \\
\hline 414 & $4 / 23 / 2$ & & 3.52 & 22.493 & 24.476 & & 22. & 46. & 49.6 & & 33.177 & -1.2 & & & & & & & 166. & & & & & & & & & \\
\hline 415 & & & 3.5 & 22.536 & 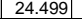 & & & & 50.0 & & & -1.2 & & & & & & & & & & & & & & & & \\
\hline 416 & & & 23.57 & 22.557 & & 23.569 & 22.7 & & & & & -1.2 & & 50.8 & & & & & 16.6744 & & 2.5 & & & & & & & \\
\hline 41 & & & & 22.58 & \begin{tabular}{|l|}
24.547 \\
\end{tabular} & 33.622 & 22.7 & & 49.93 & 13.494 & & -1.2 & & & & & & & & & & & & & & & & \\
\hline & & $\begin{array}{l}9: 2:: 00 \\
\end{array}$ & 23.6211 & 22.608 & \begin{tabular}{|l|}
24.57 \\
\end{tabular} & 23.66 & $\frac{2.2804}{2.204}$ & 46.146 & $50.07 / 7$ & $\frac{1.3644}{3.157}$ & 33.329 & \begin{tabular}{ll|l|}
9 & -1.218 \\
\end{tabular} & & 48.953 & 0.134 & $\frac{14.643}{14}$ & & & 88 & 48.6 & 12.0 & & 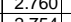 & 0.020 & & 0.001 & 0.505 & L \\
\hline & 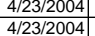 & $\begin{array}{c}\text { 9:28:06 AM } \\
\text { 9:29:06 AM }\end{array}$ & 3.676 & \begin{tabular}{r|r|}
22.62 \\
22.658 \\
\end{tabular} & \begin{tabular}{|l|}
$\mid 24.5 .77$ \\
24.61 \\
\end{tabular} & $\begin{array}{r}23.677 \\
23.72\end{array}$ & \begin{tabular}{|l|}
22.840 \\
22874 \\
\end{tabular} & $\begin{array}{l}46.607 \\
46.723\end{array}$ & \begin{tabular}{|l|}
$\mid 49.957$ \\
50.079 \\
\end{tabular} & $\mid$\begin{tabular}{|l|}
$\mid 13.537$ \\
13.668
\end{tabular} & \begin{tabular}{|r|}
33.28 \\
33.261 \\
\end{tabular} & 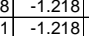 & -6.466 & (51.564 & $\begin{array}{l}0.1344 \\
0.14\end{array}$ & $\begin{array}{l}4 \\
\begin{array}{l}4 \\
4\end{array} \mid 14.643 \\
\end{array}$ & & 1004.5 & \begin{tabular}{|}
5 \\
5 \\
5 & 16.72444 \\
\end{tabular} & \begin{tabular}{|l|}
$\mid 48.5$ \\
48.6 \\
\end{tabular} & $\mid$\begin{tabular}{|l|l|}
11.6 \\
12.6
\end{tabular} & \begin{tabular}{|l|}
$\mid 39.9$ \\
40.0 \\
\end{tabular} & \begin{tabular}{|l|}
$\mid 2.754$ \\
2.757
\end{tabular} \mid & & $\begin{array}{l}0.020 \\
0.021\end{array}$ & 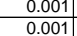 & $\begin{array}{l}0.556 \\
0.528\end{array}$ & \begin{tabular}{|l|l|}
0.44 \\
0.4
\end{tabular} \\
\hline
\end{tabular}


WSRC-TR-2005-00105, REVISION 0

SRNL-RPP-2005-00012, REVISION 0

RUN \# WASH1 \& WASH2; CAMPAIGN II SLURRY WASHING - CONT.

\begin{tabular}{|c|c|c|c|c|c|c|c|c|c|c|c|c|c|c|c|c|c|c|c|c|c|c|c|c|c|c|c|c|}
\hline & A & B & D & E & $\mathrm{F}$ & G & $\mathrm{H}$ & $\mathrm{J}$ & $\mathrm{K}$ & $\mathrm{L}$ & $\mathrm{M}$ & $\mathrm{N}$ & $\mathrm{O}$ & Q & $\mathrm{R}$ & $\mathrm{s}$ & $T$ & $\mathrm{v}$ & w & $x$ & $\mathrm{Y}$ & $z$ & $\mathrm{AA}$ & $A B$ & AC & $A D$ & $\mathrm{AE}$ & \\
\hline & $4 / 23 / 2004$ & $9: 30: 06 \mathrm{AM}$ & 23.709 & $\begin{array}{l}22.676 \\
\end{array}$ & \begin{tabular}{|l|}
24.633 \\
\end{tabular} & 23.763 & 22.877 & 46.512 & \begin{tabular}{|l|}
49.982 \\
\end{tabular} & 13.534 & 33.146 & -1.21 & $\begin{array}{l}-6.437 \\
\end{array}$ & 48.809 & 0.13 & 14.643 & 0.003 & 1005.5 & 16.7578 & & 12.0 & 39.8 & 2.746 & & 0.020 & 0.000 & & \\
\hline & & 9:31:06 AM & 23.736 & \begin{tabular}{|l|}
22.698 \\
\end{tabular} & 24.59 & $\begin{array}{l}23.795 \\
\end{array}$ & 22.889 & 46.246 & \begin{tabular}{|l|}
49.617 \\
\end{tabular} & 13.498 & 32.952 & & -6.483 & 51.547 & 0.132 & 14.643 & 0.003 & & & 48.1 & 12.6 & & & & 0.020 & 0.001 & & 0.43 \\
\hline & 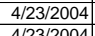 & 9:32:06 AM & $\begin{array}{l}23.794 \\
23855\end{array}$ & \begin{tabular}{|l|}
22.731 \\
27777
\end{tabular} & \begin{tabular}{|l|}
24.618 \\
2614
\end{tabular} & \begin{tabular}{|l|}
23.783 \\
23855 \\
\end{tabular} & $\begin{array}{l}22.942 \\
23019\end{array}$ & \begin{tabular}{|l|l|}
46.08 \\
46103
\end{tabular} & \begin{tabular}{|l|}
49.528 \\
4958 \\
\end{tabular} & $\begin{array}{l}13.505 \\
13417\end{array}$ & $\begin{array}{l}32.757 \\
32.88\end{array}$ & $\frac{-1.218}{1.221}$ & $\begin{array}{r}-6.46 \\
6.37\end{array}$ & \begin{tabular}{|l|}
50.261 \\
48924 \\
\end{tabular} & & \begin{tabular}{|l|l|}
14.643 \\
14613
\end{tabular} & \begin{tabular}{|l}
0.004 \\
0.003
\end{tabular} & $\begin{array}{l}1007.5 \\
1\end{array}$ & \begin{tabular}{|l|l|}
16.7911 \\
1.8078
\end{tabular} & \begin{tabular}{|l|l|}
48.1 \\
481
\end{tabular} & $\begin{array}{l}12.3 \\
120\end{array}$ & & $\frac{2.11}{2.72}$ & & $\begin{array}{l}0.020 \\
0020\end{array}$ & 0.001 & $\begin{array}{l}0.508 \\
0511\end{array}$ & $\begin{array}{l}0.43 \\
0.44\end{array}$ \\
\hline & 4/23/2004 & 9:33:06 AM & 23.855 & \begin{tabular}{|l|l|}
22.737 \\
20791
\end{tabular} & 24.614 & $\begin{array}{r}23.855 \\
2.05 \\
\end{array}$ & $\begin{aligned} 23.019 \\
22.232\end{aligned}$ & $\begin{array}{l}46.103 \\
6.241\end{array}$ & \begin{tabular}{|l|}
49.584 \\
\end{tabular} & $\begin{array}{l}13.417 \\
13.60\end{array}$ & $\begin{array}{r}32.88 \\
3061\end{array}$ & -1.221 & \begin{tabular}{|l|l|}
-6.437 \\
.5309
\end{tabular} & \begin{tabular}{|l|l|} 
\\
50.724 \\
\end{tabular} & 0.134 & 14.643 & $\begin{array}{l}0.003 \\
0.03\end{array}$ & 1008.5 & \begin{tabular}{|l|l|}
16.8078 \\
\end{tabular} & 48.1 & 12.0 & & 2.12 & \begin{tabular}{|l|} 
\\
\end{tabular} & \begin{tabular}{|l|} 
\\
\end{tabular} & 0.001 & $\begin{array}{l}0.511 \\
\end{array}$ & 0.44 \\
\hline$\frac{425}{426}$ & $\begin{array}{l}4 / / 23 / 2004 \\
4 / 23 / 2004\end{array}$ & $\begin{array}{l}9: 34: 06 \mathrm{AM} \\
9.3506\end{array}$ & $\frac{23.904}{23.922}$ & $\mid$\begin{tabular}{l|}
22.781 \\
22804
\end{tabular} & $\begin{array}{l}24.648 \\
2461\end{array}$ & $\begin{array}{r}23.954 \\
23966 \\
\end{array}$ & $\frac{23.123}{23.191}$ & $\begin{array}{l}46.344 \\
46.437\end{array}$ & \begin{tabular}{|l|}
49.847 \\
49891 \\
\end{tabular} & $\begin{array}{l}13.468 \\
13.476\end{array}$ & $\begin{array}{r}33.064 \\
33.155 \\
\end{array}$ & \begin{tabular}{|l|l|} 
& -1.218 \\
5.218
\end{tabular} & $\begin{array}{l}-6.399 \\
-6.437\end{array}$ & \begin{tabular}{|r|}
50.709 \\
50.97
\end{tabular} & $\frac{0.133}{0.134}$ & \begin{tabular}{|l|l}
14.643 \\
14.643
\end{tabular} & & $\frac{1009.5}{1010.5}$ & $\frac{16.8244}{166411}$ & $\begin{array}{l}48.4 \\
48.4\end{array}$ & & & $\frac{2.737}{2.744}$ & \begin{tabular}{|c|c|}
0.020 \\
0.020
\end{tabular} & \begin{tabular}{|l|}
0.020 \\
\end{tabular} & 0.001 & & $\begin{array}{l}0.43 \\
0.43 \\
\end{array}$ \\
\hline 427 & $4 / 23 / 2004$ & 9:36:06 AM & 23.955 & 22.843 & 24.694 & 24.126 & 23.249 & 46.035 & \begin{tabular}{|l|}
49.613 \\
\end{tabular} & 13.494 & & & & 48.604 & 0.134 & 14.643 & 0.003 & 1011.5 & & $\begin{array}{l}-40.4 \\
48.1 \\
\end{array}$ & $\frac{12.5}{11.9}$ & 39.4 & $\frac{2.244}{2.713}$ & $\mid \begin{array}{l}0.020 \\
0.020 \\
\end{array}$ & $\begin{array}{l}0.0220 \\
0.020\end{array}$ & $\begin{array}{l}0.001 \\
0.001\end{array}$ & $\begin{array}{l}0.507 \\
0.512\end{array}$ & $\begin{array}{l}0.43 \\
0.44\end{array}$ \\
\hline & & & & & & & & 46.155 & \begin{tabular}{|l|l|}
49.677 \\
\end{tabular} & 13.53 & & & & 50.06 & 0.134 & 14.643 & & & & 48.2 & & & & & & & & $\begin{array}{l}0.44 \\
0.43 \\
\end{array}$ \\
\hline 425 & $4 / 23 / 2004$ & $9: 38: 06 \mathrm{AM}$ & 23.992 & 22.904 & 24.721 & 24.157 & 23.311 & 46.18 & \begin{tabular}{|l|}
49.719 \\
\end{tabular} & 13.496 & 32.867 & -1.218 & & 50.801 & 0.131 & 14.643 & 0.003 & 1013.5 & \begin{tabular}{|l|l|}
16.8911 \\
\end{tabular} & 48.2 & 12.4 & & 2.725 & & 0.020 & 0.000 & 0.498 & \\
\hline 430 & $4 / 23 / 2004$ & & & 22.943 & 24.749 & 24.186 & & & & & & & & & & $\begin{array}{l}14.643 \\
\end{array}$ & & & & & & & & & 0.020 & 0.001 & & \\
\hline & & & & 22.971 & & 24.184 & & & 49.988 & 13.46 & 33.095 & & & & & $\begin{array}{l}14.643 \\
\end{array}$ & & & & & & & & & & & & $\begin{array}{l}0.44 \\
0.44 \\
\end{array}$ \\
\hline & 4/23/2004 & 9:41:06 AM & 24.09 & 23.001 & 24.711 & $\mid 24.148$ & & & 49.445 & $\begin{array}{l}13.341 \\
\end{array}$ & 2.521 & & & $.88 !$ & & 146 & & & & & & & & & & & & \\
\hline & $4 / 23 / 2004$ & & & & & & & & & & & & & & & & & & & & & & & & & & & \\
\hline 434 & $4 / 23 / 2004$ & 9:43:06 AM & 24.135 & 23.058 & 24.743 & 24.195 & 23.264 & & 499.273 & 13.315 & 32.48 & -1.218 & & 49.808 & & 14.643 & & & 16.9744 & 47.8 & & & & 8.020 & 0.020 & 0.001 & & .44 \\
\hline & $4 / 23 / 2004$ & 9:44:06 AM & 24.154 & 23.097 & 24.752 & 24.178 & 23.307 & 45.83. & $\begin{array}{l}49.458 \\
\end{array}$ & 13.307 & 32.701 & -1.221 & & \begin{tabular}{|l|l|}
49.088 \\
\end{tabular} & 0.135 & 14.643 & & 1019! & 16.9911 & 48.0 & 12.0 & 39 & 2.70 & $\overline{0.02}$ & $\begin{array}{l}0.020 \\
\end{array}$ & 0.001 & 0.516 & 0.44 \\
\hline 43 & $4 / 23 / 2004$ & 9:45:06 AM & 24.171 & 23.114 & 24.769 & 24.206 & 23.3 & 46.329 & 50.055 & 13.353 & 33.179 & -1.218 & -6. & \begin{tabular}{|l|l|}
49.249 \\
\end{tabular} & 0.135 & 14.643 & & 1020.5 & $\begin{array}{l}7.0078 \\
\end{array}$ & 48.6 & 12.1 & & 2.74 & 8.020 & & 0.001 & & .43 \\
\hline & $4 / 23212004$ & $6 \mathrm{AM}$ & 24.173 & & 24.7577 & & & 45.842 & 49.58 & 13.246 & 2.769 & & & 0.943 & 0.134 & 14.643 & & & 7.0244 & & & & & & & & & \\
\hline 438 & $4 / 23 / 2004$ & 9:47:06 AM & 24.191 & 23.155 & & & & 46.215 & 49.97 & 13.329 & 33.107 & -1.221 & & 50.851 & & 14.643 & & & & 48.5 & 12.5 & & & & 0.020 & & 0.507 & \\
\hline 43 & $\begin{array}{r}4 / 23 / 2004 \\
423 / 2004\end{array}$ & 9:48:06 AM & 24.219 & $\frac{23.172}{23208}$ & 24.787 & 24.244 & $\begin{array}{l}23.343 \\
23243\end{array}$ & $\begin{array}{r}44.77 \\
46.346\end{array}$ & $\begin{array}{l}48.529 \\
503116\end{array}$ & 14.361 & . & $-\frac{-1.2 .}{1.21}$ & & $\begin{array}{l}50.134 \\
50957\end{array}$ & 0.134 & $\frac{14.643}{14643}$ & & 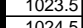 & 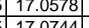 & 47.1 & & & 2. & 0 & 020 & 0.00 & 334 & \\
\hline & 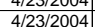 & $\begin{array}{l}9.49 .00 \mathrm{AM} \\
9 \cdot 50: 06 \mathrm{AM}\end{array}$ & $\frac{24.244}{24.247}$ & $\frac{23.200}{23.225}$ & $\frac{24.010}{24.815}$ & 24.352 & $\frac{20.045}{23.345}$ & $\frac{40.540}{46.881}$ & 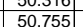 & $\frac{10.079}{12.684}$ & $\frac{30.039}{34.285}$ & $\frac{-1.21}{-1.218}$ & $\frac{-0.4}{-6.4}$ & 50.671 & 0.137 & $\frac{14.045}{14.643}$ & & 1025 & $\begin{array}{l}\frac{1.71744}{17.0911} \\
\end{array}$ & $\frac{40.0}{493}$ & 124 & & & 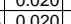 & 021 & & 0.050 & \\
\hline 442 & $4 / 23 / 2004$ & 9:51:06 AM & 24.262 & 23.236 & 24.821 & 24.367 & 23.316 & 46.812 & 50.734 & 12.771 & 34.136 & \begin{tabular}{|c|c|}
-1.222 \\
\end{tabular} & -6.512 & \begin{tabular}{|l|}
47.945 \\
\end{tabular} & 0.136 & 14.643 & & 1026.5 & 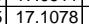 & 49.3 & 11.7 & & $\frac{2.79}{2.79}$ & $\frac{0.020}{0.020}$ & 0 & 0.001 & 0.504 & 0.43 \\
\hline & $4 / 23 / 2004$ & 9:52:06 AM & 24.291 & \begin{tabular}{|l|}
23.27 \\
\end{tabular} & 24.79 & $\mid 24.457$ & 23.375 & 46.603 & 50.411 & $\begin{array}{l}12.441 \\
\end{array}$ & 34.232 & -1.218 & & \begin{tabular}{|l|}
47.856 \\
\end{tabular} & 0.139 & $\begin{array}{l}14.643 \\
\end{array}$ & & 1027.5 & 5 & 48.9 & $\begin{array}{l}11.7 \\
\end{array}$ & & 2.787 & 0.021 & 0.021 & 0.001 & 0.516 & $\frac{43}{44}$ \\
\hline 444 & $4 / 23 / 2004$ & $6 \mathrm{AM}$ & 24.29 & 23.269 & 24.769 & 24.486 & $23.40 \mathrm{~s}$ & 46.972 & 51.004 & 12.169 & 34.909 & -1.2. & & 47.115 & 0.138 & 14.643 & & & & 49.5 & & & & & & & & \\
\hline & 3/2004 & $9: 5$ & 24.309 & 23.288 & 24.767| & 24.484] & & & 50.902 & 11.997 & 5.045 & & & 5.406 & 0.142 & 14.643 & & & & & & & & & & & & \\
\hline & & & & & & & & & & & & & & & & 14.643 & & & .1744 & & 11. & & & & & & & \\
\hline . & $4 / 23 / 2004$ & 9:56:0 & 24.334 & $23.348 \mid$ & 24.783 & 24.48 & 23.5 & 46. & 50.509 & 12.624 & 34.033 & -1.22 & & 4 & 0.132 & 14.6 & & 1031. & & & 111.8 & & & & 0.020 & & & \\
\hline 440 & $4 / 23 / 2004$ & & & & & & & & 50.525 & 12.55 & & & & & 0.132 & 14.64 & & & & $4 !$ & & & & & & & 0.491 & \\
\hline & $4 / 23 / 2004$ & 9:58:C & 24.342 & 23.381 & 24.796 & 24.638 & 23.687 & & Fo.78 & 12.565 & 34.263 & -1.21 & & .121 & 0.132 & 14.643 & & 1033. & 17.2244 & 49 & .1 & & 2.79 & & 0.020 & 0.000 & \begin{tabular}{|c|}
0.489 \\
\end{tabular} & \\
\hline & 3i/2004 & 9:59:06 AM & 24.357 & 23.406 & 24.785 & 24.643 & 23.731 & 46.702 & 50.834 & 12.459 & 34.384 & -1.218 & & .312 & 0.136 & 14.643 & & 1034. & 17.2411 & 49. & 11.8 & & 79 & 0.026 & 0.020 & 0.00 & 503 & 0.43 \\
\hline 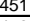 & $4 / 23 / 2004$ & & & 23.446 & 24.79 & 24.713 & & & 50.654 & 12.729 & 33.906 & -1.218 & & & & 14.643 & & & & 49. & 12.0 & & & & & & 0.481 & \\
\hline & $4 / 23232004$ & & 24.365 & 23.459 & 24.83 & & & & 50.647 & & & & & & & & & & & & & & & & & & & \\
\hline & & & & & & & & & 50.807 & 12.896 & 33.452 & -0.882 & & & 0.142 & 14.6 & & & & $4 s^{4}$ & 11.8 & & & & & & (534 & \\
\hline 4 & 4,212322004 & & 24. & 23.520 & 24.1950 & 24.813 & & & 994 & 12.954 & 33.619 & & & & & 14.64 & & & $.30 / 8$ & & 119 & & & & & & 0.524 & \\
\hline & 4 & 10 & 24.410 & 20.0459 & $\mid$ & $\mid \begin{array}{ll}24.081 \\
24835\end{array}$ & 237 & 46 & 5049 & 12559 & 34.095 & -1.128 & & 5 & $\frac{0.171}{0.14}$ & 14.6 & & & & $\frac{45}{46}$ & 11.4 & & 877 & & & & & 044 \\
\hline 457 & $4 / 23 / 2004$ & \begin{tabular}{|l|}
$10: 06:$ \\
\end{tabular} & 24.422 & $\frac{2.3597}{2597}$ & 22456 & 2484 & $\frac{25 .}{23 .}$ & & $\frac{1.404}{50.42}$ & 12465 & 33.629 & & & & 0.176 & $\frac{146}{146}$ & & & & $\frac{44}{44}$ & $\frac{1.40}{110}$ & & & & & & & 0.44 \\
\hline & $4 / 23 / 2004$ & 10 & 24.436 & 23.621 & 24.769 & 24.847 & & 46.42 & 50.318 & 1248 & $\frac{3.9}{33.9}$ & & & & 0.191 & 14.6 & & & & & & & & & & & & \\
\hline & $4 / 23 / 2004$ & & 24.433 & & & & & & & & 34.0 & & & & & & & & & 48 & & & & & & & & \\
\hline & $4 / 23 / 2004$ & 10 & 24.4 & 23.662 & \begin{tabular}{|l|l|}
24.77 \\
\end{tabular} & \begin{tabular}{|c|}
24.914 \\
\end{tabular} & 23.8 & & 50.54 & 12.516 & 34.519 & -1.218 & & & $\overline{0.1}$ & 14.6 & & & & 49 & 11. & & & & & & & \\
\hline & & & 24.4 & $\begin{array}{l}23.691 \\
\end{array}$ & 24.784 & & & & & & & & & & & & & & & & & & & & & & & \\
\hline & 4/23/2004 & & 24.41 & & \begin{tabular}{|l|l|}
24.786 \\
\end{tabular} & & & & 50.579 & & 34.22 & -1.221 & & & & 14.6 & & & & 49 & 11 & & & & & & 0.495 & \\
\hline & $4 / 23 / 2004$ & & $24.43 !$ & 23.726 & \begin{tabular}{|l|l|}
24.809 \\
\end{tabular} & & & & \begin{tabular}{|l|l|}
50.66 \\
\end{tabular} & & & -1.2 & & & 0 & 14. & & & & & 11 & & & & & & & \\
\hline & $4 / 23$ & & 24.452 & 23.743 & & & & & & 12.962 & 33.68 & -1.221 & & & & 14.6 & & & & & 11.6 & & & & & 0001 & 0.520 & 244 \\
\hline & $3 / 2004$ & & 24.469 & 23.76 & 24.7 & 24.906 & & & 50.063 & & 33.8 & 210 & & 4 & 0.139 & 14. & & & & 48 & & & & & & & & \\
\hline & & & & & & & & & & & & & & & & & & & & & & & & & & & & \\
\hline & $4 / 23 / 2004$ & 10:16:0 & 24.47 & 23.798 & 24.801 & 24.869 & & & 50.077 & 12.566 & 33.785 & -1.22 & & 50.36 & & 14.6 & & & 17.5244 & 48. & $12.3 \mathrm{r}$ & & & & & & 0.521 & \\
\hline & 4421352004 & 10017 & 24.469 & $\begin{array}{l}23.815 \\
23027\end{array}$ & 20.9.98 & 24.911 & 23.85 & & S5.1506 & $\frac{12.586}{12501}$ & $\frac{33.121}{3232}$ & $-\frac{1.221}{1.216}$ & & \begin{tabular}{|l|}
4.8002 \\
4.94
\end{tabular} & 0.144 & $\frac{14.64}{1.64}$ & & & & 48. & 1. & & & & (2) & & 年 & \\
\hline & & & $\frac{24.4}{2.4}$ & & & & & & & & & & & & & & & & & & & & & & & & & \\
\hline & $\begin{array}{l}4 / 2012004 \\
4 / 23 / 2004\end{array}$ & & 24.4 & 23. & & & & & & $\frac{12.56}{126}$ & & & & & & & & & & & & & & & & & & \\
\hline & $4 / 23 / 200$ & & 24. & 23.881 & 24.879 & 24.952 & & & & & & -1.218 & & & & 144 & & & & & 11.9 & & & & & & 0525 & \\
\hline $4 \pi$ & & & & & & & & & & & & & & & & & & & & & & & & & & & & \\
\hline & $4 / 23 / 2004$ & & 24.4 & 23.916 & 24.888 & 24.976 & & & 50.521 & 12.699 & 34.643 & -1.221 & & & 0.1 & 14.6 & & & & 49 & 11.8 & & & & & & 0.523 & \\
\hline & & & & & & & & & 49.8 & 12.5 & 34.4 & & & & & & & & & & & & & & & & & \\
\hline & $4 / 23 /$ & & 24. & 23.9 & 24.8 & 25.006 & & & & & 34.3 & & & & & 14. & & & & & 11.6 & & & & & & & \\
\hline & & & & 23. & 24.844 & 24. & & & 49.7 & 12. & & -1. & & & & & & & & & & & & & & & & \\
\hline & $4 / 23 / 2004$ & & 24.3 & 23.964 & 24.856 & 25.0 & & & 50.384 & 12.4 & 34.4 & -1.2 & & & & 14. & & & & & 12.1 & & & & & & 0.531 & \\
\hline 476 & 4/23/2004 & & 24. & 23.964 & 24.847 & 25.06 & & & & $\begin{array}{l}12.447 \\
\end{array}$ & 33.8 & -1.2 & & & 0.1 & 14.6 & & & & $4 \varepsilon$ & II & & & & & & 0.539 & \\
\hline & 4/23//2 & & 24. & & & & & & & & & -1.2 & & & & & & & & & 12.4 & & & & & & & \\
\hline 48 & $4 / 23 / 2004$ & & 24. & 24.008 & 24.865 & & & & & & & -1.215 & & & & & & & & 48 & $12.2 \mathrm{r}$ & & & & & & 0.526 & \\
\hline & $41 / 23212004$ & & 24.433 & 24.025 & 24.867 & $\frac{25.1153}{25.92}$ & & & $\begin{array}{l}49.956 \\
5.159\end{array}$ & $\frac{12.506}{12559}$ & $\begin{array}{r}33.621 \\
32820\end{array}$ & $\frac{-1.218}{-1215}$ & & & & 年 14.6 & & & & 40 & 11.6 & & & & & & & \\
\hline & & & & $\mid 24.4056$ & 24.88 & $\begin{array}{l}2.193 \\
25169\end{array}$ & & & & & & & & & & $\frac{14 .}{14 .}$ & & & & & $\frac{12.4}{11.2}$ & & & & & & & \\
\hline & 4/23/2004 & & 24 & 24.077 & 24.849 & 25.20 & & & 49.781 & & 30.8 & $\begin{array}{ll}3 . & 1 .\end{array}$ & & & & 14.6 & & & & 4 & 12.5 & 37 & & & & & 0.299 & \\
\hline & $4 / 23 / 2$ & & 24.8 & \begin{tabular}{|l|l|}
24.09 \\
\end{tabular} & & 25 & & 46 & & & & & & & & & & & & & 12 & & & & & & & \\
\hline & $4 / 23 / 2004$ & & 24.475 & 24.107 & 24.844 & 25.217 & & 48. & 50.1 & 12.23 & 36.8 & -1.218 & & & 0.1 & 14.6 & & & & $48 \quad-1$ & 11.5 & & & & & & 0.565 & \\
\hline & $4 / 23 / 2004$ & & 24.517 & 24.104 & 24.8 & & & & 50.5 & & & & & 48.4 & & $14.6 \mathrm{C}$ & & & & & 11.9 & 4 & & & & & & \\
\hline & $\begin{array}{r}4 / 233 / 2004 \\
/ 23 / 2004\end{array}$ & \begin{tabular}{|l|l|}
$10: 38: 06$ AM \\
4 & $10: 39: 06$.
\end{tabular} & \begin{tabular}{|l|l|}
24.549 \\
24.54
\end{tabular} & $\begin{array}{l}24.126 \\
24.137 \\
\end{array}$ & $\begin{array}{r}24.838 \\
24.849\end{array}$ & $\begin{array}{l}25.241 \\
25.253 \\
\end{array}$ & $\frac{24.204}{24.175}$ & $\begin{array}{l}47.553 \\
47.281 \\
\end{array}$ & \begin{tabular}{|l|}
49.002 \\
48925 \\
\end{tabular} & $\begin{array}{l}13.464 \\
13.169 \\
\end{array}$ & $\begin{array}{l}34.357 \\
34.283 \\
\end{array}$ & \begin{tabular}{|l|l|}
-1.221 \\
-1.221 \\
\end{tabular} & -6.174 & $\begin{array}{l}52.1 .245 \\
52.248\end{array}$ & $\frac{0.143}{0.144}$ & $\begin{array}{l}14.643 \\
14.643 \\
\end{array}$ & & $\frac{10 / 3.5}{1074.5}$ & \begin{tabular}{|c|c|}
5 & 17.8911 \\
5 & 17.9078 \\
\end{tabular} & \begin{tabular}{|l|}
47.5 \\
47.5 \\
\end{tabular} & $\begin{array}{l}12.5 \\
12.8\end{array}$ & \begin{tabular}{|l|}
41.0 \\
40.8
\end{tabular} & $\begin{array}{ll} & 2.824 \\
8 & 2.812 \\
\end{array}$ & \begin{tabular}{|l|l|}
0.021 \\
0.021
\end{tabular} & \begin{tabular}{|l|}
0.021 \\
0.022 \\
\end{tabular} & $\begin{array}{l}0.001 \\
0.001\end{array}$ & $\begin{array}{l}0.523 \\
0.529 \\
\end{array}$ & $\frac{0.4}{0.4}$ \\
\hline
\end{tabular}


WSRC-TR-2005-00105, REVISION 0

SRNL-RPP-2005-00012, REVISION 0

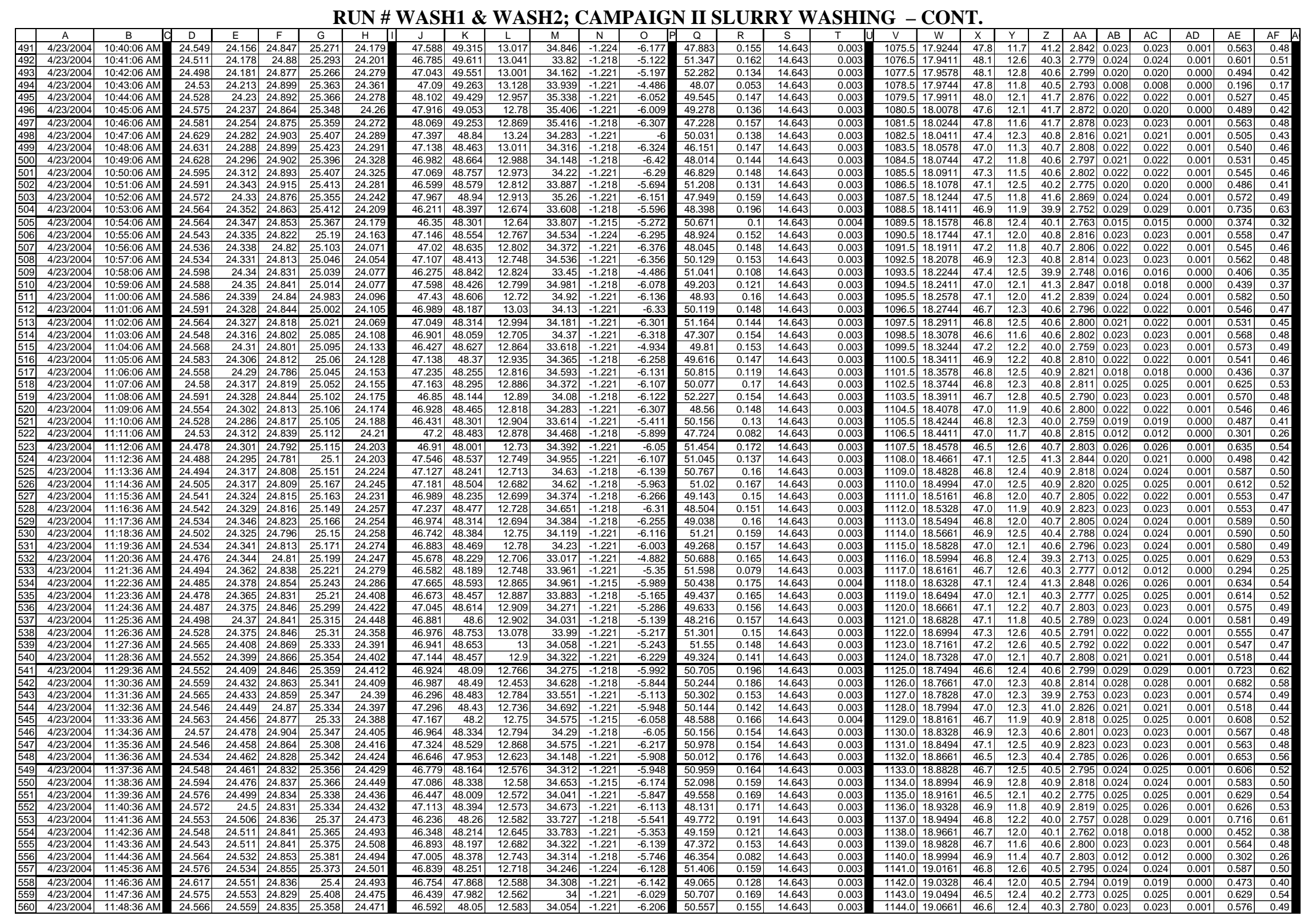


WSRC-TR-2005-00105, REVISION 0

SRNL-RPP-2005-00012, REVISION 0

RUN \# WASH1 \& WASH2; CAMPAIGN II SLURRY WASHING - CONT.

\begin{tabular}{|c|c|c|c|c|c|c|c|c|c|c|c|c|c|c|c|c|c|c|c|c|c|c|c|c|c|c|c|c|}
\hline & A & $B$ & $\mathrm{D}$ & $E$ & \begin{tabular}{|l|}
$F$ \\
\end{tabular} & \begin{tabular}{l|l|l|}
$G$ &
\end{tabular} & $\mathrm{H}$ & $\mathrm{J}$ & $\mathrm{K}$ & \begin{tabular}{ll|}
$\mathrm{L}$ \\
\end{tabular} & $M$ & $\mathrm{~N}$ & 0 & Q & $R$ & \begin{tabular}{l|l|} 
&
\end{tabular} & $T$ & $v^{\prime}$ & w & $x$ & Y & z & AA & $\mathrm{AB}$ & \begin{tabular}{ll|}
$\mathrm{AC}$ & \\
\end{tabular} & & $\mathrm{AE}$ & \\
\hline & $4 / 23 / 2004$ & 11:49:36 AM & 24.548 & 24.566 & \begin{tabular}{|l|}
24.837 \\
\end{tabular} & 25.405 & 24.523 & 46.209 & 47.926 & 12.532 & 33.736 & -1.215 & -5.914 & 49.495 & 0.15 & 14.643 & 0.003 & 1145.0 & 9.0828 & 46.5 & 12.1 & & 2.756 & & 0.022 & 0.001 & 0.562 & \\
\hline$\frac{562}{562}$ & $4 / 23 / 2004$ & 11:50:36 AM & 24.554 & 24.573 & \begin{tabular}{|l|}
24.823 \\
\end{tabular} & 25.422 & 24.505 & 46.485 & \begin{tabular}{|l|l|}
48.204 \\
\end{tabular} & 12.619 & 33.916 & \begin{tabular}{|l|} 
\\
\end{tabular} & -6.096 & 49.209 & 0.152 & 14.643 & & 1146.0 & 19.0994 & 46.7 & & 40.2 & & & & 0.001 & 0.567 & \\
\hline 563 & $4 / 23 / 2004$ & 11:51:36 AM & 24.54 & 24.563 & \begin{tabular}{|l|l|}
24.809 \\
\end{tabular} & 25.392 & 24.5 & 46.46 & \begin{tabular}{|l|l|}
48.226 \\
\end{tabular} & 12.607 & 33.896 & \begin{tabular}{|l|} 
\\
\end{tabular} & $\begin{array}{l}-6.136 \\
-.16\end{array}$ & $\begin{array}{l}49.925 \\
\end{array}$ & 0.147 & 14.643 & & $\begin{array}{l}1147.0 \\
\end{array}$ & | 19.1161 & 46.8 & 12.2 & 40.2 & 2.770 & 0.022 & \begin{tabular}{l|l}
0.022 \\
\end{tabular} & 0.001 & 0.548 & \\
\hline & 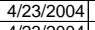 & 11:52:36 AM & 24.521 & 24.575 & \begin{tabular}{|l|}
24.82 \\
21.07 \\
\end{tabular} & 25.384 & $\begin{array}{l}24.527 \\
2.53 \\
\end{array}$ & 46.242 & \begin{tabular}{|l|l|}
48.034 \\
\end{tabular} & $\begin{array}{l}12.629 \\
\end{array}$ & 33.668 & & -6.148 & 49.12 & 0.152 & \begin{tabular}{ll|l}
14.643 \\
\end{tabular} & & \begin{tabular}{ll|}
1148.0 \\
\end{tabular} & 19.1328 & 46.6 & 12.0 & & 2.755 & 0.023 & $\begin{array}{l}0.023 \\
\end{array}$ & 0.001 & 0.570 & \\
\hline$\frac{303}{566}$ & $\begin{array}{l}\mid 4 / 23 / 2004 \\
4 / 23 / 2004\end{array}$ & $\begin{array}{l}\frac{11: 53: 3 \mathrm{AM}}{11} \\
1154 ; 36 \mathrm{AM}\end{array}$ & $\frac{24.518}{24.483}$ & $\begin{array}{l}24.586 \\
24.577\end{array}$ & $\mid$\begin{tabular}{|l|}
$\mid 24.827$ \\
2.807
\end{tabular} & $\frac{25.415}{25.431}$ & $\begin{array}{l}24.538 \\
24544\end{array}$ & $\frac{46.412}{45.84}$ & \begin{tabular}{|l|}
48.359 \\
47.746 \\
\end{tabular} & \begin{tabular}{|l|l}
12.606 \\
12.517
\end{tabular} & $\begin{array}{l}33.887 \\
33.313 \\
\end{array}$ & \begin{tabular}{|l|}
-1.221 \\
-1.221 \\
\end{tabular} & $\begin{array}{l}-6.084 \\
-6.136\end{array}$ & $\begin{array}{l}48.266 \\
50.3 \\
\end{array}$ & $\frac{0.145}{0.152}$ & $\begin{array}{l}14.643 \\
14.643\end{array}$ & 0.00 & $\begin{array}{ll}\frac{11449.0}{1150.0} & \\
\end{array}$ & | 19.1494 & $\begin{array}{l}46.9 \\
46.3 \\
\end{array}$ & $\begin{array}{l}11.8 \\
12.3 \\
\end{array}$ & $\begin{array}{l}40.1 \\
39.6\end{array}$ & 2.7629 & 0.022 & $\begin{array}{l}0.022 \\
0.023\end{array}$ & $\frac{0.001}{0.001}$ & 0.541 & 0.46 \\
\hline 567 & $4 / 23 / 2004$ & & & $\begin{array}{l}24.603 \\
\end{array}$ & \begin{tabular}{|l|}
24.799 \\
\end{tabular} & 25.467 & 24.54 & 455.696 & \begin{tabular}{|l|}
47.858 \\
\end{tabular} & 12.519 & 33.247 & -1.221 & $\begin{array}{c}-6.044 \\
\end{array}$ & 49.065 & 0.153 & 14.643 & & & & 46.4 & 12.0 & & & & & & & \\
\hline & $4 / 23 / 2004$ & $11: 56: 36 \mathrm{AM}$ & 24.49 & 24.593 & \begin{tabular}{|l|}
24.784 \\
\end{tabular} & 25.492 & 24.575 & 45.79 & 47.918 & 12.52 & 33.27 & \begin{tabular}{|c|} 
\\
\end{tabular} & -6.0 & 48.268 & 0.147 & $\mid$\begin{tabular}{|l|l|}
14.643 \\
\end{tabular} & & & & 46.4 & 11.8 & & & & & & & $\begin{array}{l}0.49 \\
0.47\end{array}$ \\
\hline & $4 / 23 / 2004$ & 11:55:36 AM & & & \begin{tabular}{|l|l|}
24.809 \\
\end{tabular} & 25.543 & 24.575 & 45.653 & $\begin{array}{l}47.804 \\
\end{array}$ & 12.523 & 33.11 & \begin{tabular}{|l|l|} 
\\
\end{tabular} & -6.1 & 49.96 & 0.147 & \begin{tabular}{|l|l|l}
14.643 \\
\end{tabular} & & 1153.0 & 19.2161 & $\begin{array}{l}46.3 \\
\end{array}$ & 12.2 & 39.4 & & $\overline{0.022}$ & $\begin{array}{l}0.022 \\
\end{array}$ & & & \\
\hline 570 & & & 24.491 & 24.62 & & & & & \begin{tabular}{|l|l|l|l|l}
47.988 \\
\end{tabular} & 12.543 & & & & & & \begin{tabular}{|l|l|l|l|}
14.643 \\
\end{tabular} & & & & 46.5 & 12.3 & & & .024 & & & 0.608 & \\
\hline & & & 24.497 & 24.626 & \begin{tabular}{|l|}
24.806 \\
\end{tabular} & 25.53 & & & & 12.283 & & & & & & & & & & & & & & & & & & \\
\hline & $4 / 23 / 2004$ & $12: 00: 36 \mathrm{PM}$ & 24.379 & 24.638 & \begin{tabular}{|l|l|l|}
24.799 \\
\end{tabular} & 25.527 & & & & & 33.998 & & & & & & & & 2661 & & & & & & & & & \\
\hline & 4/23/2004 & 12:01:36 PM & 24.452 & 24.651 & \begin{tabular}{|l|l|}
24.826 \\
\end{tabular} & 25.555 & 24.603 & & 48.558 & 12.314 & 31.679 & 0.888 & & & 0.0 & $\mid 14.643$ & & 1157.0 & & 47.1 & 11.9 & 37. & 2.61. & & 0010 & & 0.265 & \\
\hline $57 \quad$ & $4 / 23 / 2004$ & 12:02:36 PM & 24.404 & 24.653 & \begin{tabular}{|l|l|}
24.813 \\
\end{tabular} & 25.537 & 24.625 & 45.709 & 48.027 & 12.651 & 33.007 & -1.221 & & 49.261 & 0.105 & 144.643 & & 1158.0 & 19.2994 & $\begin{array}{lll}46.6 & \end{array}$ & 12.1 & 39.4 & 2.71 & 0.016 & 0.016 & 0.00 & 0.400 & \\
\hline 57: & 3/2004 & 12:03:36 PM & 24.396 & 24.674 & 24.835 & 25.614 & 24.686 & & 48.042 & 12.735 & 33.056 & 1.22. & & 48.473 & 0.157 & 14.643 & & 1159.0 & 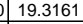 & 46.6 & 11.9 & & & & 0.024 & & & \\
\hline 578 & $4 / 23 / 2004$ & 12:04:36 PM & & 24.666 & \begin{tabular}{|l|}
24.8212 \\
\end{tabular} & $\begin{aligned} 255.61 \\
25.61\end{aligned}$ & & 46.754 & 48.2977 & 12.687| & 34.142 & & & 50.094 & & $\mid \begin{array}{l}14.643 \\
\end{array}$ & & & & 46. & & & & & & & 0.774 & \\
\hline & $4 / 23 / 2004$ & & 24.403 & & 24.787 & & & & 48.123 & 12.554 & & & & & & 14.643 & & & .3494 & & & & & .030 & 0.030 & & & \\
\hline 578 & $4 / 23 / 2004$ & 12:06:36 PM & 24.439 & 24.673 & \begin{tabular}{|l|}
24.788 \\
\end{tabular} & 25.547 & 24.595 & 45.688 & 47.853 & 12.546 & 33.134 & -1.221 & -4.83 & 49.766 & 0.156 & 14.643 & & 1162.0 & 19.3661 & 46.4 & 12.2 & 39.4 & & 0.023 & 0.023 & & 0.594 & \\
\hline 57 & 4/23/2004 & 12:07:36 PM & 24.46 & 24.674 & \begin{tabular}{|l|}
24.759 \\
\end{tabular} & 25.488 & 24.641 & 46.864 & 47.837 & $\begin{array}{r}12.482 \\
1.570\end{array}$ & 34.499 & -1.224 & & 50.834 & 0.132 & 14.643 & & 1163.0 & 19.3828 & 46.4 & 2.0 .8 & 40. & & 0.026 & 0.020 & 0.00 & 0.487 & 0.41 \\
\hline$\frac{251}{581}$ & $\begin{array}{l}4 / 23 / 22004 \\
4 / 23 / 2004 \\
\end{array}$ & $\frac{12: 08.36 \mathrm{PM}}{12: 09 \cdot 36 \mathrm{PM}}$ & $\frac{24.491}{24.476}$ & $\frac{24.694}{24.689}$ & \begin{tabular}{|r|}
24.48 \\
24.8
\end{tabular} & $\frac{25.539}{25.523}$ & $\frac{24.631}{24.621}$ & $\frac{46.52}{46.402}$ & $\frac{47.953}{48.096}$ & $\mid \frac{\mid 12.5 / 8}{12.554}$ & $\frac{34.251}{33.889}$ & 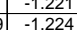 & & $\begin{array}{l}5.198 \\
50.559 \\
\end{array}$ & 0.183 & $\begin{array}{l}14.643 \\
14.643 \\
\end{array}$ & & $\begin{array}{l}1164.0 \\
1165.0\end{array}$ & $\begin{array}{l}19.3994 \\
19461 \\
1941\end{array}$ & $\frac{46.5}{46.6}$ & 124 & $\frac{40 .}{40 .}$ & 768 & & $\frac{0.027}{0.027}$ & 0.00 & $\frac{0.637}{0.683}$ & 0.54 \\
\hline & 4/23/2004 & 12:10:36 PM & 24.469 & 24.688 & \begin{tabular}{|l|}
24.773 \\
\end{tabular} & 25.532 & 24.645 & 46.219 & 48.075 & 12.521 & 33.77 & \begin{tabular}{|l|l|} 
& -1.221 \\
\end{tabular} & & 51.059 & 0.104 & $\mid$\begin{tabular}{|c|}
14.643 \\
\end{tabular} & & 1166.0 & \begin{tabular}{|l|l|l}
19.4328 \\
\end{tabular} & 46.6 & $\frac{12.4}{12.5}$ & $\frac{40}{40}$ & 2.757 & 0.016 & $\begin{array}{l}0.016 \\
0.016\end{array}$ & & & 0.58 \\
\hline & $4 / 23 / 2004$ & 12:11:36 PM & 24.459 & 24.703 & \begin{tabular}{|l|l|l|} 
\\
\end{tabular} & 25.512 & 24.66 & 46.881 & $\begin{array}{l}48.092 \\
\end{array}$ & 12.563 & 34.392 & \begin{tabular}{|l|l|} 
& -1.221
\end{tabular} & -5.8 & 49.215 & 0.185 & 14.643 & & 1167.0 & \begin{tabular}{|l|l|l} 
\\
\end{tabular} & 46.6 & 12.1 & 40. & & 028 & 0.028 & & 0.083 & $\begin{array}{l}0.33 \\
0.58 \\
\end{array}$ \\
\hline 584 & $33 / 2004$ & 12:12:36 PM & 24.473 & 24.717 & 24.782 & 25.561 & & & 47.835 & 12.523 & 33.715 & -1.2 & & 50.402 & 0.18 & 14.643 & & 1168.0 & & 46.4 & $\overline{12.4}$ & & & & & & & \\
\hline & & & & & & & & & .936 & & & & & & & & & & & & 12.0 & & & .017 & D.018 & 00 & 0.438 & \\
\hline & $4 / 23 / 2004$ & $12: 14: 3$ & 24.492 & 24.726 & \begin{tabular}{|l|l|}
24.771 \\
\end{tabular} & 25.575 & & & 7.696 & 12.598 & 33.748 & & & 48.765 & 0.1 & $\begin{array}{l}14.643 \\
\end{array}$ & & 1170 . & & 46. & & & & & & & 0.614 & \\
\hline & $4 / 23 / 2004$ & 12:15:3 & 24.543 & 24.752 & \begin{tabular}{|l|}
24.797 \\
\end{tabular} & 25.691 & & 45 & 7.549 & 12.458 & 33.405 & -1.2 & & 75 & 0.0 & $\begin{array}{l}14.643 \\
\end{array}$ & & 117. & 5161 & & $12.3 \mathrm{r}$ & & & .013 & & & & \\
\hline & $4 / 23 / 2004$ & 12:16:3 & 24.514 & 24.7 & \begin{tabular}{|l|l|l|}
2483 \\
\end{tabular} & 25.642 & & & 47.715 & $\begin{array}{l}12.52 \\
\end{array}$ & & & & & 0.1 & 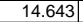 & & & & & 12.0 & & & & & & & \\
\hline & 3/2004 & $12: 17: 36$ PM & 24.546 & 24.77 & $|24.795|$ & 25.659 & 24.64. & 46 & 47.723 & $12.449 \mid$ & 33.982 & -1.221 & & 49.401] & 0.16 & 14.643 & & 1173.6 & 19.5494 & 46.3 & 12.1 & 40 & & & & & 0.612 & \\
\hline & 3/2004 & 12:18:36 PM & 24.561 & 24.765 & 24.78 & 25.604 & 24.546 & 46.526 & 47.858 & 12.5 & 34.146 & $\mid-1.22$ & & 49.328 & 0.11 & 14.643 & & 1174.6 & 19.5661 & 46.4 & 12.1 & 40 & .781 & .017 & 0.017 & & 0.431 & \\
\hline 592 & 4/23/2004 & $12: 13$ & 24.571 & 24 & \begin{tabular}{|l|}
$\mid 24.78$ \\
\end{tabular} & 25.539] & & 46.4 & 47.741 & 12.4955 & 34.006 & & & 51.1. & 0.1 & 14.643 & & 1175.0 & & 46.3 & 12.0 & 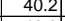 & & & & & 0 & \\
\hline & $\begin{array}{l}4 / 2 / 2 / 2004 \\
4 / 23 / 2004\end{array}$ & & 24.588 & & $|24.776|$ & 25.445 & & & 年 & 1.2.549 & & & & 51.1 & 0.16 & 14.643 & & & & & & & & 025 & .025 & & 0.630 & \\
\hline & $4 \frac{4 / 23 / 2}{4 / 23 / 2}$ & & & & & & & & 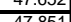 & $\mid$ & & & & & & $\begin{array}{l}14.045 \\
1.60\end{array}$ & & & & & & & & & & & & \\
\hline & & & & & & & & & 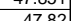 & 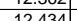 & $\frac{34.226}{32848}$ & & & & & 14.64 & & & & & & & & & 019 & & (450 & \\
\hline 596 & $4 / 23 / 2004$ & $12 \cdot 24 \cdot 3$ & 24.479 & 24.703 & \begin{tabular}{|l|}
2.743 \\
\end{tabular} & 25.417 & 24 & & 47.741 & 12,441 & 34.078 & -1.2 & & 51.689 & 0.16 & $\mid 1,4643$ & & 118 & & & 127 & 40 & 77 & 0025 & 0.25 & & 0.061 & \\
\hline & & 12:25:36 PM & 24.464 & 24.698 & \begin{tabular}{|l|}
24.748 \\
\end{tabular} & 25.422 & 24.475 & 46.43 & 47.913 & 12.528 & 33.943 & \begin{tabular}{|l|l|}
3 & -1.218 \\
\end{tabular} & & 51.241 & 0.1 & $\mid 14.643$ & & 1181.0 & 19.6828 & 46. & 12.6 & 40 & & & $\frac{0.027}{0.027}$ & & 0.680 & \\
\hline 598 & $4 / 23 / 2004$ & 12:26:36 P & 24.491 & 24.705 & 24.75 & 25.444 & 24.4 & 45. & 47.746 & 12.366 & 33.497 & \begin{tabular}{|l|l|} 
& -1.218 \\
\end{tabular} & & 50.35 & 0.1 & 14.6 & & \begin{tabular}{|l|l|l|l|l}
1182 \\
\end{tabular} & \begin{tabular}{|l|l|}
19.6994 \\
\end{tabular} & 46. & 12.3 & 39. & .734 & & 0.0 & & 0.716 & \\
\hline & & & & & $24.762 \mid$ & & & & & & & & & & 0.1 & & & & & & & & & & & & 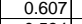 & \\
\hline & $4 \mid 232 / 2 / 2$ & 12:28:36 F & 24.45 & 24.6 & \begin{tabular}{|l|}
$24 . / 48$ \\
\end{tabular} & 25.517 & & & 47.77 & 12.405 & 33.742 & -1.2 & & & & 14.6 & & & & 46 & $12.4 \mathrm{r}$ & & & & & & 0.594 & \\
\hline & 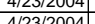 & & 告年.5. & $\frac{24.6}{2.6 .6}$ & $\mid$\begin{tabular}{|}
$\mid 24.74$ \\
2475
\end{tabular} & $\frac{2.5368}{2557}$ & 24.56 & & $\begin{array}{r}47.93 \\
47797 \\
\end{array}$ & 12.459 & 34.3 & $-\frac{1.2}{12}$ & & & 0.14 & $\begin{array}{r}14.643 \\
14643 \\
\end{array}$ & & $\frac{118}{118} \mathrm{r} \cdot \mathrm{x}$ & & & 120 & & & & & & & \\
\hline 603 & 4 & & $\frac{24.502}{24.502}$ & $\frac{24.0}{24.6}$ & \begin{tabular}{|l|}
24.731 \\
24.731
\end{tabular} & 25.58 & 24.0 & & $\frac{44.191}{47.694}$ & \begin{tabular}{|c|}
2.390 \\
12.407
\end{tabular} & & 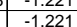 & & 49. & & \begin{tabular}{|l|l|l}
14.643 \\
\end{tabular} & & & & & & & & & & & & \\
\hline & $4 / 23 / 2004$ & $12: 32: 36$ PM & $\frac{24.006}{24.485}$ & $\frac{24.051}{24.679}$ & 24.704 & 25.628 & 24.65 & & 47.53 & $\mid \frac{1.357}{12.357}$ & & \begin{tabular}{|l|l|}
$5-1.221$ \\
5 & -1.221
\end{tabular} & & $\frac{45.050}{49.676}$ & 0.1 & \begin{tabular}{|l|l|l}
14.643 \\
\end{tabular} & & & & & $\frac{12.2}{12.2}$ & & & & & & $\frac{0.0647}{0.52}$ & \\
\hline 60 & $4 / 23 / 2004$ & 12:33: & 24.489 & 24.683 & \begin{tabular}{|l|}
24.698 \\
\end{tabular} & 25.637 & 24.675 & 46.171 & $\begin{array}{l}47.677 \\
\end{array}$ & & 33.746 & & & & & & & & & & & & & & & & & \\
\hline & & & 24.49 & & & & & & & & & -1.22 & & & & & & & & & 11.9 & & & & & & & \\
\hline & 3/2004 & 12:35:36 PM & 24.5 & 24.7 & 24.709 & 25.663 & 24.6 & 46.10 & & 12.374 & 33.705 & $\begin{array}{l}-1.22 \\
\end{array}$ & & $\begin{array}{l}49.466 \\
\end{array}$ & 0.15 & 14.6 & & 119 & 9.8494 & & $\frac{12.1}{12.1}$ & & & 0.024 & & & 0.595 & \\
\hline & & & 24.52 & 24. & & & & & 47.9 & & & & & & & & & & & & 5 & & & & & & & \\
\hline & $4 / 23 / 2004$ & $12: 37:$ & 24.488 & 24.7 & $|24.707|$ & 25.706 & 24.72 & & 47.866 & 12.376 & & -1.2 & & & 0.26 & 14.643 & & & 9.8828 & 46. & 12.5 & & 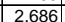 & & & & 077 & \\
\hline & $4 / 23 / 2$ & & 24.45 & 24.75 & \begin{tabular}{|l|l|}
24.7 \\
\end{tabular} & & 24 & & & \begin{tabular}{|l|l|l|}
12.346 \\
\end{tabular} & & -1.2 & & & 0.1 & 14.6 & & & & & 12.1 & & & & & & & \\
\hline 61 & $4 / 23 / 2004$ & & 24.45 & 24.77 & 24.7 & & 24.7 & & $\begin{array}{l}47.327 \\
\end{array}$ & 12.284 & & -1.2 & & & & & & & & & 12.0 & & & & & & & \\
\hline 612 & & & 24.457 & 24.7 & 24.716 & & & & \begin{tabular}{|l|l|l|l}
47.76 \\
\end{tabular} & 12.411 & & \begin{tabular}{|c|c|} 
& -1.2 \\
\end{tabular} & & & 0.1 & 14.64 & & & & & 12.3 & & & & & & 578 & \\
\hline & & & 24.444 & & 24. & & & & 47.46 & 12.302 & & 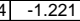 & & & 0.17 & & & & & & 12.0 & & & & & & 0.679 & \\
\hline OI & $4 / 23$ & & 24.48 & 24.77 & \begin{tabular}{|l|}
24.689 \\
\end{tabular} & 25.714 & & & $4 . .383$ & 12.257 & & -1.2 & & & & 14.6 & & & & $45-2$ & 12.0 & & & & & & & \\
\hline & & & 24.49 & 24.79 & $24.107 \mid$ & & & & 77 & 12.33 & & & & & & & & & & & & & & & & & & \\
\hline & & & 2. & 24.198 & \begin{tabular}{|l|}
2.6988 \\
\end{tabular} & & & & 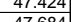 & $\frac{1.2 .362}{1.3227}$ & & -1.2 & & & & & & & & 46.6 & $\frac{11.4}{12 .}$ & & & & & & 19 & \\
\hline$\frac{218}{618}$ & $\frac{42512004}{4232004}$ & $\frac{12.45}{12.46: 3}$ & 24.48 & $\begin{array}{l}24.034 \\
24841\end{array}$ & 24.11 & $\frac{25.0445}{2585}$ & & & \begin{tabular}{|l|l|}
47.044 \\
4763
\end{tabular} & $\mid \frac{1.303}{1236}$ & & -1.12 & & & & & & & & & $\frac{1.4 .4}{123}$ & & & & & & 0.460 & \\
\hline 619 & $4 / 23 / 2004$ & & 24.442 & 24842 & $\mid$\begin{tabular}{|}
$\mid 24707$ \\
24707
\end{tabular} & & & & \begin{tabular}{|l|l|}
47899 \\
\end{tabular} & 12423 & 33.674 & -1.22 & & & 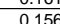 & & & & & & 121 & & & & & & & \\
\hline & & & $\frac{2.45}{24.45}$ & $\frac{24.8}{24.8}$ & & & & & & & & & & & & & & & & & $\frac{21.9}{11.9}$ & & & & & & & \\
\hline 62 & & & 24. & 24.8 & & & & & & & & -1.224 & & & & & & & & & 118 & & & & & & & \\
\hline & & & 24.49 & 248 & 24.7 & & & & & & & -1221 & & & & & & & & & & & & & & & & \\
\hline & $23 / 200$ & & 24.48 & $\overline{24.8}$ & \begin{tabular}{|l|l|l|}
24.6 \\
\end{tabular} & & 24. & & & $\overline{12.42}$ & 32.8 & -1.2 & & & & & & & & & $\overline{12.1}$ & & & & & & & \\
\hline & $4 / 23 / 200$ & & 24.56 & 24.864 & 24.69 & 25.59 & 24.5 & & & 12.49 & & -1.2 & & & & 14.6 & & & & & & & & & & & & \\
\hline & $4 / 23 / 2004$ & 12:53:3 & 24.5 & 24.864 & 24.76 & 25.548 & 24. & 45.3 & 477.799 & 12.396 & 32.931 & -1.224 & & 49.36 & 0.1 & 14.6 & & $12 \mathrm{C}$ & & & 12.1 & & & & & & 0.618 & \\
\hline & $4 / 23 / 2$ & & 24.497 & 24.831 & 24.701 & 25.49 & 24. & 45.197 & & 12.387| & & -1.22 & & & .15 & 14.6 & & & & & 12.4 & & & & & & & \\
\hline & & & 24.514 & & & & 24.4 & & & $12.439]$ & & -1.22 & & & 0.143 & & & & & & & & & & & & & \\
\hline & $4 / 23 / 2004$ & 12:56:3 & 24.527 & 24.816 & \begin{tabular}{|l|}
24.696 \\
\end{tabular} & 25.535 & 24.53: & 45.626 & 48.106 & $12.329]$ & 33.294 & 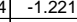 & & 49.385 & 0.158 & 14.643 & & 1212 & 20.1994 & 46.6 & 12.1 & 35 & 2.121 & & 0.024 & 0.001 & 0.602 & \\
\hline & 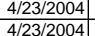 & $\begin{array}{l}12: 57: 36 \mathrm{PM} \\
12: 58: 36 \mathrm{PM}\end{array}$ & $\begin{array}{l}24.454 \\
24.538\end{array}$ & 24.823 & \begin{tabular}{|l|}
24.698 \\
24.707 \\
\end{tabular} & \begin{tabular}{|l|l|}
25.587 \\
25.606
\end{tabular} & $\begin{array}{r}24.59 \\
24.624 \\
\end{array}$ & $\begin{array}{l}44.772 \\
45.979 \\
\end{array}$ & \begin{tabular}{|l|}
47.99 \\
4.794 \\
\end{tabular} & \begin{tabular}{|r|r|}
12.502 \\
12.48
\end{tabular} & \begin{tabular}{|l}
32.151 \\
33.522 \\
\end{tabular} & $\begin{array}{l}1 \\
1\end{array}$ & $\begin{array}{l}-3.572 \\
-4.948 \\
\end{array}$ & $51.669 \mid$ & $\frac{0.067}{0.17}$ & $\begin{array}{l}14.643 \\
14.643 \\
\end{array}$ & & & D) 20.2161 & \begin{tabular}{|l|}
46.5 \\
46.5 \\
\end{tabular} & $\begin{array}{ll}\frac{12.7}{12.2} & \\
\end{array}$ & \begin{tabular}{|l|}
38.5 \\
39.8
\end{tabular} & $\left|\begin{array}{|l|}2.652 \\
2.741\end{array}\right|$ & $\begin{array}{l}0.010 \\
0.025\end{array}$ & \begin{tabular}{|l|}
0.010 \\
0.026
\end{tabular} & $\begin{array}{l}0.000 \\
0.001 \\
\end{array}$ & \begin{tabular}{|l|}
0.262 \\
0.643
\end{tabular} & \\
\hline
\end{tabular}


WSRC-TR-2005-00105, REVISION 0 SRNL-RPP-2005-00012, REVISION 0

RUN \# WASH1 \& WASH2; CAMPAIGN II SLURRY WASHING - CONT.

\begin{tabular}{|c|c|c|c|c|c|c|c|c|c|c|c|c|c|c|c|c|c|c|c|c|c|c|c|c|c|c|c|c|}
\hline & A & B & D & $E$ & \begin{tabular}{l|l}
$F$ \\
\end{tabular} & $G$ & $\mathrm{H}$ & $\mathrm{J}$ & $\mathrm{K}$ & 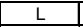 & M & $\mathrm{N}$ & 0 & $Q$ & R & $\mathrm{s}$ & T & v & w & $x$ & 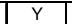 & $z$ & $\mathrm{AA}$ & $A B$ & $\begin{array}{ll}A C \\
\end{array}$ & $A D$ & $\mathrm{AE}$ & AF \\
\hline 631 & $4 / 23 / 2004$ & 12:59:36 PM & 24.517 & 24.806 & 24.706 & 25.65 & $\begin{array}{ll}24.678 \\
\end{array}$ & 45.676 & 47.858 & 12.556 & $\begin{array}{ll}33.097 \\
\end{array}$ & -1.221 & -5.075 & 51.954 & 0.155 & 14.643 & 0.003 & 1215.0 & 20.2494 & 46.4 & 12.7 & \begin{tabular}{l|l}
7 & 39.4 \\
\end{tabular} & 2.716 & 0.023 & 0.023 & 0.001 & 0.592 & 0.50 \\
\hline 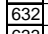 & 4/23/2004 & 1:00:36 PM & & $\begin{array}{l}24.832 \\
2937 \\
\end{array}$ & 24.742 & 25.766 & 24.653 & 45.927 & 48.183 & 12.548 & 33.313 & -1.218 & -4.937 & 50.298 & 0.147 & 14.643 & 0.003 & 1216.0 & $20.2661 \mid$ & 46.7 & 12.3 & 39.6 & 2.732 & 0.024 & 0.022 & 0.001 & 0.557 & 0.47 \\
\hline 633 & 4/23/2004 & 1:01:36 PM & 24.563 & 24.817 & 7. 24.737 & 25.706 & 24.669 & 45.294 & 47.659 & \begin{tabular}{|l|l|}
12.403 \\
\end{tabular} & \begin{tabular}{ll|}
32.828 \\
\end{tabular} & & $\begin{array}{l}-4.607 \\
\end{array}$ & 48.702 & 0.096 & $\begin{array}{l}14.643 \\
\end{array}$ & 0.003 & & 20.2828 & 46.2 & $\begin{array}{ll}11.9 \\
\end{array}$ & 39.1 & 2.693 & 0.014 & \begin{tabular}{|c|c|c|} 
\\
\end{tabular} & 0.000 & $\begin{array}{ll}0.369 \\
\end{array}$ & 0.31 \\
\hline $6 \frac{634}{635}$ & $4 / 23 / 2004$ & $\begin{array}{l}: 02: 36 \mathrm{PM} \\
102: 36 \mathrm{PM}\end{array}$ & 24.573 & \begin{tabular}{|l|l|}
24.817 \\
2.813
\end{tabular} & $\begin{array}{l}724.752 \\
7.720 \\
\end{array}$ & \begin{tabular}{|l|}
25.716 \\
25692
\end{tabular} & 24.689 & 46.07 & \begin{tabular}{|l|}
47.967 \\
\end{tabular} & $\begin{array}{l}2.453 \\
1.450 \\
\end{array}$ & \begin{tabular}{|l|}
33.543 \\
3227
\end{tabular} & \begin{tabular}{|l|l|}
-1.218 \\
\end{tabular} & $\begin{array}{l}-5.096 \\
-5006\end{array}$ & 51.558 & 0.158 & 14.643 & 0.003 & 1218.0 & \begin{tabular}{l|l|}
0 & 20.2994 \\
\end{tabular} & 46.5 & $\begin{array}{ll}12.6 \\
\end{array}$ & \begin{tabular}{l|l|}
6 & 39.8 \\
\end{tabular} & \begin{tabular}{ll|l|}
8.745 \\
\end{tabular} & \begin{tabular}{|l|l|} 
\\
\end{tabular} & 0.024 & & 0.596 & $\begin{array}{l}0.51 \\
0.52 \\
\end{array}$ \\
\hline \begin{tabular}{|c|}
6356 \\
636 \\
\end{tabular} & $\begin{array}{l}4 / 23 / 2004 \\
4 / 23 / 2004 \\
\end{array}$ & \begin{tabular}{|l} 
1:03:36 PM \\
$104: 36$ PM
\end{tabular} & $\frac{24.534}{24.524}$ & \begin{tabular}{r|}
24.813 \\
24.808 \\
\end{tabular} & \begin{tabular}{|c|c|}
3 & 24.728 \\
3 & 24.733 \\
\end{tabular} & \begin{tabular}{|l|}
25.692 \\
25.697 \\
\end{tabular} & $\begin{array}{r}24.675 \\
24.67\end{array}$ & $\begin{array}{r}45.775 \\
45.431\end{array}$ & \begin{tabular}{|l|}
$\mid 48.025$ \\
47.764 \\
\end{tabular} & \begin{tabular}{|l|}
12.459 \\
12.531 \\
\end{tabular} & \begin{tabular}{|c|}
33.227 \\
32.88
\end{tabular} & \begin{tabular}{|c|}
$\mid-1.222$ \\
-1.221 \\
\end{tabular} & $\begin{array}{l}-5.006 \\
-5.075 \\
\end{array}$ & \begin{tabular}{|l|}
52.15 \\
50.797 \\
\end{tabular} & $\begin{array}{l}0.161 \\
0.162 \\
\end{array}$ & \begin{tabular}{|c|}
14.643 \\
14.643
\end{tabular} & $\begin{array}{l}0.003 \\
0.003\end{array}$ & $\begin{array}{l}\frac{1219.0}{1220.0} \\
\end{array}$ & \begin{tabular}{|c|c|}
0 & 20.3161 \\
0 & 20.3328
\end{tabular} & $\frac{46.6}{44.3}$ & \begin{tabular}{l|l}
12.8 \\
12.4
\end{tabular} & \begin{tabular}{|l|l|}
8 & 39.5 \\
4 & 39.2 \\
\end{tabular} & 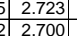 & \begin{tabular}{|l|}
0.024 \\
0.024 \\
\end{tabular} & $\begin{array}{l}0.024 \\
0.024\end{array}$ & 0.001 & 0.612 & \begin{tabular}{|c|}
0.52 \\
0.53
\end{tabular} \\
\hline |637 & $4 / 23 / 2004$ & 1:05:36 PM & 24.515 & 24.819 & \begin{tabular}{|l|l|}
9 & 24.744 \\
\end{tabular} & 25.698 & 24.686 & 45.51 & 47.947 & \begin{tabular}{|l|l|}
12.4699 \\
\end{tabular} & 32.993 & \begin{tabular}{|l|}
-1.221 \\
\end{tabular} & -5.191 & 49.937 & 0.153 & $\begin{array}{l}14.643 \\
14.643 \\
\end{array}$ & 0.003 & & \begin{tabular}{|c|c|} 
& 20.3348 \\
0 & 20.3494 \\
\end{tabular} & $\begin{array}{l}40.3 \\
46.5\end{array}$ & $\frac{12.4}{12.2}$ & \begin{tabular}{l|l|l}
$\begin{array}{c}4 \\
2\end{array}$ & 39.4 .3 \\
\end{tabular} & & \begin{tabular}{|l|}
0.024 \\
0.023 \\
\end{tabular} & & & & 0.53 \\
\hline 638 & $4 / 23 / 2004$ & 1:06:36 PM & 24.518 & 24.822 & \begin{tabular}{|l|}
24.747 \\
\end{tabular} & 25.751 & 24.743 & 45.612 & 48.135 & 12.443 & 33.12 & -1.224 & -5.197 & 48.392 & 0.15 & 14.643 & 0.00 & 1222.0 & \begin{tabular}{|l|l|}
0 & 20.3661 \\
\end{tabular} & 46.7 & 11.9 & 39.4 & 2.714 & \begin{tabular}{|l|l|} 
\\
\end{tabular} & 0.023 & 0.001 & & $\frac{0.50}{0.49}$ \\
\hline 639 & $4 / 23 / 2004$ & & & & & & & & & & & & & & 0.156 & & & & & & & & & & & & & 0.49 \\
\hline 640 & 4/23/2004 & $36 \mathrm{PM}$ & 24.546 & 24.845 & 24.76 & 25.839 & 24.847 & 45.383 & 48.013 & 12.245 & 33.064 & -1.221 & -5044 & 49.537 & 0.156 & 14.643 & & & 39 & & & & & & $\frac{0.023}{0.023}$ & & & 0.51 \\
\hline 6 & & & & & & & & & & & & & & & & & & & & & & & & & & & & $\frac{0.51}{0.49}$ \\
\hline 642 & $4 / 23 / 2004$ & 1:10:36 PM & 24.561 & 24.865 & \begin{tabular}{|l|l|}
5 & 24.75 \\
\end{tabular} & 25.894 & 24.942 & 45.502 & 48.208 & 12.243 & 33.159 & \begin{tabular}{|c|c|}
-1.221 \\
\end{tabular} & -5.142 & 50.402 & 0.145 & 14.643 & & 1226.0 & 20.432 & $\frac{40.5}{46.7}$ & 12.4 & 39.3 & 2.712 & & & 0.001 & $\begin{array}{l}0.575 \\
0.554\end{array}$ & 0.49 \\
\hline 643 & $4 / 23 / 2004$ & $1: 11: 36 \mathrm{PM}$ & 24.56 & 24.865 & \begin{tabular}{|l|}
5 \\
\end{tabular} & 25.964 & 24.966 & 45.331 & 48.083 & 12.335 & 32.8 & -1.221 & -5.127 & 48.298 & 0.162 & 14.643 & & & 20.4494 & 46.6 & 11.8 & 39.1 & 2.693 & \begin{tabular}{|c|c|c|}
0.024 \\
\end{tabular} & 0.024 & 0.001 & 0.623 & 0.53 \\
\hline 6444 & $4 / 23 / 2004$ & 1:12:36 PM & 24.57 & 24.884 & \begin{tabular}{|l|l|}
4 & 24.734 \\
\end{tabular} & \begin{tabular}{|l|}
25.988 \\
\end{tabular} & 24.88 & 45.188 & \begin{tabular}{|l|l|}
48.017 \\
\end{tabular} & \begin{tabular}{|l|l|}
12.384 \\
\end{tabular} & 32.687 & \begin{tabular}{|l|l|}
-1.221 \\
\end{tabular} & -5.07 & 48.803 & 0.153 & \begin{tabular}{ll|}
14.643 \\
\end{tabular} & 0.003 & 1228.0 & 20.4661 & 46.5 & 12.0 & 38.9 & 2.685 & \begin{tabular}{|l|l|}
0.023 \\
\end{tabular} & 0.023 & 0.001 & 0.590 & 0.50 \\
\hline 645 & & & & & & & & & & & & & & & & & & & & & & & & & & & & \\
\hline 647 & $\operatorname{asn} 1$ ana & Averages & & & 24.473 & & 24,620 & & & 023 & 2063 & & & 49244 & 0.117 & 14643 & & & & $4867+2$ & $207-2+2$ & & & $00^{\circ}$ & 01018 & & 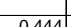 & \\
\hline & & Maximum & 25.255 & 25.672 & 25.031 & 26.809 & & 51.350 & & $\begin{array}{ll}17.448 \\
17\end{array}$ & & & $\begin{array}{l}-0.294 \\
0.594\end{array}$ & $\begin{array}{l}49.244 \\
53.306\end{array}$ & & $\begin{array}{l}14.045 \\
14.643\end{array}$ & & & & $\begin{array}{l}48.00 \\
52.12\end{array}$ & $\frac{12.07}{13.06}$ & 43.911 & $\frac{2.152}{2.981}$ & \begin{tabular}{|l|}
0.032 \\
0.032 \\
\end{tabular} & $\begin{array}{ll}0.018 \\
0.032 \\
\end{array}$ & 0.001 & $\begin{array}{l}0.444 \\
0.791 \\
\end{array}$ & 0.67 \\
\hline 649 & & Median & 24.548 & 24.995 & 24.723 & 25.548 & 24.663 & 46.827 & 0.469 & 13.610 & 33.121 & -1.221 & & 49.262 & & 14.643 & & & & 49.00 & $\begin{array}{l}12.07 \\
12.07\end{array}$ & & & & & & & \\
\hline 650 & & Minimum & 22.761 & 21.901 & 122.820 & 22.929 & 22.175 & 43.253 & 47.327 & 11.997 & 28.371 & \begin{tabular}{|l|}
-1.227 \\
\end{tabular} & -7.640 & 43.755 & & 14.643 & & & & 45.85 & 10.72 & 36.360 & 2.507 & \begin{tabular}{|l|l|}
.003 \\
\end{tabular} & 0.003 & & & \\
\hline 651 & & $2 \times \operatorname{Std}$ Dev & 0.836 & 1.684 & 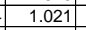 & 1.414 & 1.497 & 1.699 & 2.594 & 2.837 & 2.276 & \begin{tabular}{|l|l|}
0.504 \\
\end{tabular} & 2.369 & 3.322 & 0.063 & 0.000 & & & & 2.59 & 0.81 & 1.512 & 0.104 & \begin{tabular}{|l|l|}
0.009 \\
\end{tabular} & 0.009 & 0.000 & & \\
\hline 652 & Number & of Points Used* & \begin{tabular}{|r|}
622 \\
\end{tabular} & 622 & 622 & 622 & 622 & 622 & 622 & 622 & 622 & $\begin{array}{r}622 \\
\end{array}$ & $5 ?$ & 622 & 622 & 622 & 622 & & & 622 & 622 & 622 & 622 & 622 & 622 & 622 & 622 & 62 \\
\hline 653 & & * Backpulse poil & ts in box & are not incli & Iluded & & & & & & & & & & & & & & & & & & & & & & & \\
\hline 654 & & & & & & & & & & & & & & & & & & & & & & & & & & & & \\
\hline$\left|\frac{655}{656}\right|$ & Wash 1 & Averac & & 25.343 & & 25891 & 24.991 & 47.136 & 50.944 & 14835 & 32451 & 1138 & -6.596 & 49,024 & 0.099 & 14643 & & & & 49.47 & & 9793 & $2744 \mathrm{f}$ & & & 0000 & & \\
\hline \begin{tabular}{|c|}
657 \\
\end{tabular} & & Maximum & $\begin{array}{l}24.166 \\
25.255 \\
\end{array}$ & \begin{tabular}{|l}
25.643 \\
25.672
\end{tabular} & $\begin{array}{l}24.290 \\
25.031 \\
\end{array}$ & $\begin{array}{l}25.891 \\
26.809\end{array}$ & 24.991 & $\frac{44.136}{51.350}$ & \begin{tabular}{|l|}
50.944 \\
53.591 \\
\end{tabular} & $\begin{array}{l}14.835 \\
17.448\end{array}$ & $\begin{array}{l}32.451 \\
35.090\end{array}$ & \begin{tabular}{|r|}
-1.138 \\
1.501 \\
\end{tabular} & $\begin{array}{l}-6.596 \\
0.594 \\
\end{array}$ & $\begin{array}{l}49.024 \\
53.306\end{array}$ & $\begin{array}{l}0.099 \\
0.150\end{array}$ & 14.643 & & & & $\begin{array}{l}49.4 ! \\
52.12 \\
\end{array}$ & $\frac{12.01}{13.06}$ & & 2.2 .449 & \begin{tabular}{|l|}
0.015 \\
0.022 \\
\end{tabular} & 0.015 & $\begin{array}{l}0.000 \\
0.001\end{array}$ & 0.0 .360 & 0.528 \\
\hline \begin{tabular}{|c|}
658 \\
\end{tabular} & & Median & 24.808 & 25.509 & 24.465 & 25.976 & 24.950 & 47.059 & 50.877 & 14.970 & 32.289 & \begin{tabular}{|l|}
-1.221 \\
\end{tabular} & -6.891 & 49.076 & 0.100 & 14.643 & & & & & 12.03 & & 2.745 & 0.015 & 0.015 & 0.000 & 0.381 & 0.3 \\
\hline 659 & & Minimum & 23.690 & 24.511 & 22.820 & 24.959 & & 44.324 & \begin{tabular}{|l|l}
49.843 \\
\end{tabular} & 13.120 & & & & 43.755 & & 14.643 & & & & 48.37 & 10.72 & & & & & & & \\
\hline 660 & & $2 \times \mathrm{Std}$ & 0.726 & 0.670 & 1.156 & 0.940 & 1.158 & 1.667 & 1.280 & 2.475 & 2.054 & 0.590 & 2.66 & 3.456 & 0.040 & 0.000 & 0.0 & & & $\begin{array}{l}1.28 \\
\end{array}$ & 0.85 & \begin{tabular}{|l|l|}
5.509 \\
\end{tabular} & $\begin{array}{lll}9 & 0.104 \\
\end{array}$ & 0.006 & 0.006 & 0.000 & 0.144 & \\
\hline \begin{tabular}{|l|l|}
661 \\
695
\end{tabular} & Number & of Points Used* & 375 & 375 & 375 & 375 & 375 & 375 & 375 & 375 & 375 & 375 & 37 & 375 & 375 & 375 & 375 & & & 375 & 375 & 375 & 375 & 375 & 375 & 375 & 375 & 37 \\
\hline & & * Backpulse poi & ts in box & re not incli & luded & & & & & & & & & & & & & & & & & & & & & & & \\
\hline$\left|\frac{663}{664}\right|$ & Wash 2 & & & & & & & & & & & & & & & & & & & & & & & & & & & \\
\hline \begin{tabular}{|c|}
604 \\
665 \\
\end{tabular} & & Ave & 24.348 & 24.058 & 24.751 & 25.034 & 24.082 & 46.441 & 906 & $\begin{array}{ll}12.789 \\
\end{array}$ & 33.739 & \begin{tabular}{|c|c|}
-1.201 \\
\end{tabular} & & 49.579 & $\begin{array}{ll}0.146 \\
\end{array}$ & 14.643 & & & & $\begin{array}{l}47.43 \\
\end{array}$ & $\mid 22.15$ & & 2.764 & & 0.022 & 0.001 & 0.548 & 0.46 \\
\hline 6666 & & & 24.631 & & 24. & 25.988 & & 49.216 & & 14.4 & & \begin{tabular}{|l|l|}
1.116 \\
\end{tabular} & & & & 14. & & & & 49.53 & 86 & & & & & & 0.791 & \\
\hline 667 & & Media & 24.485 & 24.331 & 24.787 & 25.266 & & 46.437 & 48.483 & 12.623 & 33.838 & 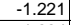 & & 49.6 & 0.147 & 14. & & & & 47.01 & 12.18: & B 340.079 & $\begin{array}{l}9 \\
9\end{array} 2.763$ & 0.022 & 0.022 & 0.001 & 0.545 & 5 \\
\hline 668 & & & 22.761 & 21.901 & 24.057 & 22.929 & 22.175 & 43.253 & $\frac{47.327}{2.107}$ & 11.997 & 30.507 & \begin{tabular}{|c|}
-1.224 \\
\end{tabular} & -6.625 & $\frac{44.821}{.097}$ & 0.053 & 14.643 & & & & 45.85 & 10.98 & 37.046 & | 2.554 & \begin{tabular}{|l|l|} 
& 0.008 \\
\end{tabular} & 0.008 & 0.000 & 0.196 & \begin{tabular}{|c|c|}
50.166 \\
1
\end{tabular} \\
\hline 6669 & Number & $\frac{12 \times \text { Std Dev }}{2}$ & 0.734 & $\frac{1.573}{227}$ & 0.292 & 1.387 & 1.271 & 1.374 & 2.107 & 0.935 & $\begin{array}{r}1.624 \\
271\end{array}$ & \begin{tabular}{|l|}
0.317 \\
\end{tabular} & $\frac{1.406}{206}$ & 2.987 & 0.047 & 0.000 & 0.000 & & & 2.11 & 0.73 & B| $1.444 \mid$ & 4] $0.100 \mid$ & $\mid$\begin{tabular}{|c|}
0.007 \\
27
\end{tabular} & 0.007 | & 0.000 & 0.174 & \begin{tabular}{rl|l}
0.148 & \\
247
\end{tabular} \\
\hline$\left|\frac{670}{671}\right|$ & Number & $\begin{array}{l}\text { of Points Used | } \\
\text { |* Backpulse pi }\end{array}$ & $\begin{array}{r}247 \\
t \sin \text { box }\end{array}$ & $\begin{array}{rl}247 & 247 \\
\text { are not tincli }\end{array}$ & 247 & 247 & 247 & 247 & 247 & 247 & 247 & 247 & 24 & 247 & 247 & 247 & 24 & & & 247 & 247 & 247 & 247 & 247 & 247 & 247 & & 247[ \\
\hline
\end{tabular}


WSRC-TR-2005-00105, REVISION 0

SRNL-RPP-2005-00012, REVISION 0

RUN \# 2.18 \& 2.19; ACID CLEANING AND CAUSTIC RINSING

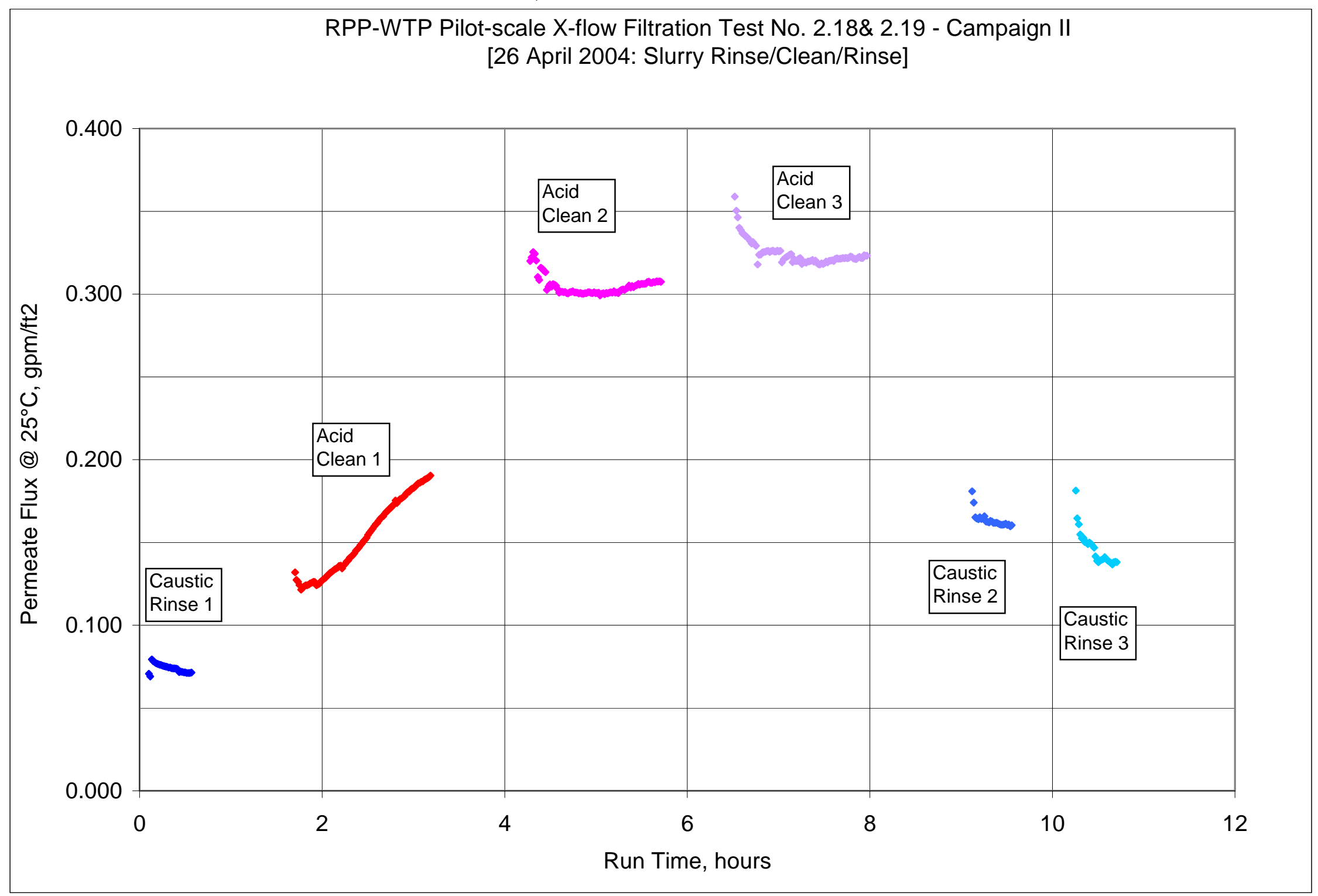


WSRC-TR-2005-00105, REVISION 0

SRNL-RPP-2005-00012, REVISION 0

RUN \# 2.18 \& 2.19; ACID CLEANING AND CAUSTIC RINSING - CONT.

\begin{tabular}{|c|c|c|c|c|c|c|c|c|c|c|c|c|c|c|c|c|c|c|c|c|c|c|c|c|c|c|c|c|}
\hline & $\mathrm{A}$ & B & $\frac{\mathrm{D}}{1<<<<}$ & $\begin{array}{l}\mathrm{E} \quad \mathrm{V} \\
<\mathrm{STemen}\end{array}$ & $F$ & G & I H & \begin{tabular}{l|l}
$\mathrm{J}$ & 1 \\
\end{tabular} & $\frac{K}{\ll<\ll<C}$ & L L & $\frac{M}{\text { assere }}$ & $\mathrm{N}$ & 10 & $\frac{Q}{Q<\ll \ll \ll}$ & $\begin{array}{ll}\mathrm{R} \\
\mathrm{R}\end{array}$ & \begin{tabular}{|c|}
$s$ \\
s
\end{tabular} & $T$ & $\frac{v}{\ll \ll \ll<}$ & $\frac{\mid w}{<\ll<\ll<\ll<}$ & $\frac{|x|}{<\ll<\ll c}$ & 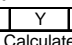 & $\frac{\mathrm{z}}{\mathrm{z}}$ & $\frac{\mid A A}{A B}$ & $\frac{|A B|}{|A|}$ & \begin{tabular}{|l|l|}
$A C$ \\
$\gg \gg \gg \gg \geqslant$
\end{tabular} & $\frac{\mid A D}{\mid A_{\lambda \gg \gg \gg \gg \geqslant}}$ & $\mathrm{AE}$ & AF \\
\hline & DATE & TIME & Filtrate & Cleaning & $\begin{array}{l}\text { enature vit } \\
\text { Slury }\end{array}$ & Hi Amb. & Lo Amb. & 30tTMP & Filter & Filter dP & TopTMP & I Filtrate & Pulsepot & & & $\begin{array}{l}\text { Measureme| } \\
\mid \text { Hi Filtate| }\end{array}$ & & & & & & & & & & & & \\
\hline & & & $\operatorname{deg} C$ & \begin{tabular}{|l|l|}
$\operatorname{deg} C$ & \\
\end{tabular} & \begin{tabular}{|l|l|}
$\operatorname{deg} C$ \\
\end{tabular} & $\operatorname{deg} C$ & $\operatorname{deg} C$ & psid & psig & psid & psid & psig & psig & $\mathrm{gpm}$ & gpm & & & & & & & & & & & & & \\
\hline & & & & & & & & dP2 & & $\mathrm{dP1}$ & $\mathrm{dP3}$ & & & Q1 & $\mathrm{Q}^{2}$ & $\mathrm{Q}^{3}$ & & & & & & & & & & & & \\
\hline & & & & & & & & & & & & & & & & & & & & & & & & & & & & \\
\hline 1 & $4 / 14 / 2004$ & 1:30:58 PM & 24.687 & 21.862 & 24.835 & 24.226 & 22.583 & 0.054 & 0.02 & -0.021 & -0.005 & \begin{tabular}{|l|l|} 
& -0.179 \\
\end{tabular} & 0.099 & -0.08 & 0.003 & \begin{tabular}{|l|}
0.086 \\
\end{tabular} & 0.005 & & & Filter St & Surface $A$ & A 6.707 & FT2 & & & & & \\
\hline 9 & 4/19/2004 & 7:37:13 AM & $22.595 \mid$ & 22.228 & \begin{tabular}{|l|}
23.926 \\
2324 \\
\end{tabular} & $\begin{array}{r}21.772 \\
22.194 \\
\end{array}$ & 22.175 & 0.046 & 0.082 & $-\frac{-0.025}{0.022}$ & $\begin{array}{l}-0.009 \\
\end{array}$ & -0.179 & 0.143 & $\begin{array}{r}-0.086 \\
0.084 \\
\end{array}$ & 0.003 & $\begin{array}{r}14.643 \\
14643 \\
\end{array}$ & 0.003 & & & Convers & ersion & 851 & $\mathrm{~m} 3 / \mathrm{m} 2$ & dday/barg & $\mathrm{g} / \mathrm{gpm} / \mathrm{ft}$ & libarg & & \\
\hline 10 & 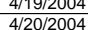 & $\begin{array}{l}0 . \angle 2.1 .59 \mathrm{AMV} \\
12: 36 \mathrm{PM}\end{array}$ & & 22.333 & $\left|\begin{array}{|l|}\mid 22.3234 \\
2049\end{array}\right|$ & $\begin{array}{l}22.194 \\
23603 \\
\end{array}$ & $\frac{22.199}{24.746}$ & $\begin{array}{l}-0.008 \\
-\frac{-0.004}{4}\end{array}$ & $\frac{0.059}{0.045}$ & $\begin{array}{r}-0.002 \\
-0.009\end{array}$ & $\begin{array}{r}-0.007 \\
-0.007\end{array}$ & $=\frac{-0.132}{-0.176}$ & $\begin{array}{l}0.131 \\
0.108 \\
\end{array}$ & $\begin{array}{l}-0.084 \\
-0.084 \\
\end{array}$ & $\begin{array}{l}0.003 \\
0.003 \\
\end{array}$ & $\frac{14.643}{14.643}$ & 0.00 & & & Note: & unge t & & 1 is a & onroximat & ately 14 & ia & & \\
\hline & 4/22/2004 & 8:40:43 AM & 22.558 & 21.446 & $\mid$\begin{tabular}{|l|}
24.471 \\
\end{tabular} & 22.716 & 22.489 & & 0.028 & 0.001 & 0.001 & -0.205 & 0.096 & $\frac{1.054}{46.52}$ & 0.043 & 14.643 & 0.00 & & & & & & & & & & & \\
\hline & $4 / 23 / 2004$ & $8: 53: 20 \mathrm{AM}$ & & & \begin{tabular}{|l|}
22.041 \\
\end{tabular} & 21.89 & & & & 0.003 & -0.005 & -0.196 & $\begin{array}{ll}0.082 \\
\end{array}$ & -0.082 & $\mid 0.003$ & 14.643 & & & & & Plis o & 50 & A & & & & & \\
\hline & $4 / 26 / 2004$ & & & & & 22.416 & & -0.012 & 0.08 & & & & 0.044 & $\mid$\begin{tabular}{|c|}
-0.08 \\
\end{tabular} & 0.003 & 14.643 & & & & WVW & & & & & & & & \\
\hline 14 & $4 / 27 / 2004$ & 9:14:59 AM & 20.374 & 19.305 & \begin{tabular}{l|l|}
19.368 \\
\end{tabular} & 20.841 & 19.777 & -0.002 & 0.02 & -0.001 & 0.005 & -0.193 & 0.111 & -0.084 & $\begin{array}{l}0.003 \\
\end{array}$ & 14.643 & & & & & & & & & & & & \\
\hline \begin{tabular}{l|l}
16 \\
\end{tabular} & $2 a t a-P r M$ & & & & & & & & & & & & & & & & & Time & Time & Press. & Vel. & TMP & TMP & $\mathrm{gpm}$ & gpm & $\mathrm{pm}$ & $\mathrm{pm}$ & eter \\
\hline 17 & $4 / 26 / 2004$ & $12: 36: 30 \mathrm{PM}$ & & & 22.179 & 23.658 & 22.6 & 23.558 & 26.778 & 5.483 & 18.137 & -1.218 & -2.925 & \begin{tabular}{|l|l|}
45.06 \\
\end{tabular} & $|0.457|$ & 14.643 & 0.003 & & & $\frac{p 19}{25.3}$ & & & & 0.068 & 0.074 & 0.004 & $\frac{p S 1}{3.541}$ & $\frac{y y b a r}{3.0}$ \\
\hline & & 12:37:31 PM & & & & & & & & & & & & 44.257 & 0.455 & 14.643 & & & 0.01694 & 25.4 & & 21.0 & & & & & 3.486 & \\
\hline 19 & 4/26/2004 & 12:38:31 PM & $21.434 \mid$ & 23.178 & \begin{tabular}{|l|}
22.405 \\
$2 ? .09$ \\
\end{tabular} & 23.767 & 22.7 & 3.859 & 26.671 & 5.479 & -1.641 & 17.898 & $\begin{array}{r}3.967 \\
10.27\end{array}$ & 43.947| & 0.003 & 14.643 & & & 0.03361 & 25.2 & & & & & & & & \\
\hline & 4120121004 & 12.35 .315 & & $\frac{2.11 t}{23.171}$ & & 告. & & & $\frac{26.814}{26092}$ & 5.453 & $\frac{-1.43}{1.421}$ & $\begin{array}{l}17.843 \\
17802\end{array}$ & & 4 & & 14.643 & & & & & & & & & & & & \\
\hline$\frac{21}{22}$ & $\begin{array}{l}4 / 201 / 2004 \\
4 / 26 / 2004\end{array}$ & 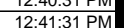 & \begin{tabular}{|c|c|c|c|}
21.536 \\
21.83
\end{tabular} & $\begin{array}{l}\frac{23.1 / 1}{23.187} \\
\end{array}$ & $\mid$\begin{tabular}{|l|}
$\mid 22.534$ \\
22.666 \\
\end{tabular} & $\frac{23.820}{23.913}$ & & $\begin{array}{l}4.102 \\
4.484 \\
\end{array}$ & $\begin{array}{l}20.992 \\
26.899\end{array}$ & $\begin{array}{l}5.486 \\
5.468\end{array}$ & $\frac{-1.421}{-0.949}$ & $\begin{array}{l}17.095 \\
17.502\end{array}$ & $\begin{array}{l}64.175 \\
30.323 \\
\end{array}$ & $\begin{array}{l}44.627 \\
40.905\end{array}$ & $\begin{array}{l} \\
0.003 \\
0.003\end{array}$ & $\begin{array}{l}14.045 \\
14.643\end{array}$ & & & $\begin{array}{l}0.06094 \\
0.08361 \\
0.01\end{array}$ & $\frac{25.5}{25.4}$ & $\frac{11.0}{10.0}$ & 1.8 & & & & & $\frac{4.6}{0.2}$ & \\
\hline & $4 / 26 / 2004$ & $12: 42: 31 \mathrm{PM}$ & 21.837 & 23.194 & \begin{tabular}{|l|}
22.768 \\
\end{tabular} & 23.979 & 23. & 20.403 & 26.911 & 5.26 & 15.187 & 1.133 & 0.475 & 44.994 & 0.445 & 14.643 & 0.00 & 6. & 0.10028 & 25.4 & 11.0 & $\frac{1.0}{17.8}$ & & 0.066 & & 0.004 & 3.972 & \\
\hline & 5/2004 & 12:43:31 PM & & 23.211 & 22.85 & & & 20.496 & 27.029 & 5.282 & 15.222 & 1.24 & 0.585 & & $\begin{array}{l}0.436 \\
\end{array}$ & 14.643 & & & 0.11694 & 25.6 & & 17.9 & 1.231 & & & & 3.869 & \\
\hline & 4/26/2004 & 12:44:31 PM & \begin{tabular}{r|r|}
22.02 \\
22027
\end{tabular} & 23.21 & $22.921 \mid$ & 24.037 & & 22.931 & 26.712 & 5.182 & $\frac{17.83}{180 ?}$ & -1.218 & -2.5 & 924 & 0.502 & 14.643 & & & 0.13361 & 25.2 & & 0. & & & & & & \\
\hline \begin{tabular}{l|l}
$\frac{26}{27}$ \\
\end{tabular} & $\frac{4 / 26 / 2004}{4 / 2612004}$ & $\begin{array}{l}\frac{12: 45: 31 \text { PM }}{12: 46: 31 ~ P M} \\
\end{array}$ & $22.087 \mid$ & 23.223 & \begin{tabular}{|l|}
22.983 \\
23.024 \\
\end{tabular} & $\begin{aligned} 24.078 \\
24.07 \\
\end{aligned}$ & $\frac{23.002}{23.043}$ & $23.159 \mid$ & $\frac{26.861}{26.747}$ & $\begin{array}{l}5.172 \\
5.093 \\
\end{array}$ & $\begin{array}{l}\frac{18.022}{18.095} \\
\end{array}$ & $\frac{-1.218}{-1.224}$ & $\begin{array}{l}-2.531 \\
-2.572 \\
\end{array}$ & $\begin{array}{l}44.351 \\
43.83\end{array}$ & $\begin{array}{l}0.496 \\
0.092\end{array}$ & $\begin{array}{l}14.663 \\
14.643 \\
\end{array}$ & 0.00 & $\frac{3.0}{10 .}$ & 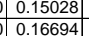 & \begin{tabular}{|l|l|}
8 & 25.4 \\
4 & 25.3 \\
\end{tabular} & 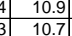 & 20.6 & 1.420 & \begin{tabular}{|l|l|}
0 & 0.073 \\
\end{tabular} & $\frac{0.078}{0.078}$ & $\begin{array}{l}0.004 \\
0.004 \\
\end{array}$ & $\begin{array}{l}3.805 \\
3.766\end{array}$ & \\
\hline & 4/26/2004 & & 22.236 & 23.241 & \begin{tabular}{|l|}
23.101 \\
\end{tabular} & & & 23.178 & 26.801 & 5.098 & 18.175 & -1.218 & -2.604 & 45.086 & 0.489 & & & & 0.18361 & 25.3 & $\frac{11.0}{11.0}$ & 20.7 & 1.426 & & & & 3.721 & \\
\hline & $4 / 26 / 2004$ & 12:48:31 PM & 22.302 & 23.257 & \begin{tabular}{|l|l|}
23.178 \\
\end{tabular} & 24.148 & & 23.253 & 26.791 & 5.008 & 18.329 & -1.215 & -2.609 & 45.968 & 0.488 & 14.643 & & 12.0 & & 25.3 & 11.3 & 0.8 & 1.433 & 0.073 & 0.077 & 0.004 & & \\
\hline 30 & 6/2004 & 12:49:31 PM & 22.375 & 23.284 & 23.255 & 24.175 & & 23.319| & & 5.044 & 18.334 & -1.218 & -2.633 & 45.28 & 0.486 & 14.6 & & $13.0 \mathrm{C}$ & 0.21694 & 25.4 & 11.1 & 20.8 & 1.436 & & & & & \\
\hline & $4 / 26 / 2004$ & 12:50:31 PM & & $23.281 \mid$ & & & & 23.033 & 26.461 & & & -1.218 & -2.673 & 46.646 & 0.486 & & & & 0.23361 & 25.0 & 11.4 & 20.6 & 1.417 & 0.072 & & 0.004 & 3.698 & \\
\hline 32 & 4/26/2004 & 12:51:31 PM & $22.464 \mid$ & 23.283 & $\frac{23.37}{23.41}$ & 24.179 & $\frac{23.2}{23}$ & \begin{tabular}{r|r|r|}
23.17 \\
23.47
\end{tabular} & $\frac{26.602}{27013}$ & 4.916 & $\frac{18.329}{1859}$ & $-\frac{-1.215}{1.15}$ & $\begin{array}{l}-2.662 \\
2664\end{array}$ & 43.742 & 0.484 & $\frac{14.64}{14.64} \mathrm{r}=$ & & 15.6 & 0 & 25.1 & $\frac{10.1}{108}$ & 10 & .431 & & & 004] & & \\
\hline 34 & $\begin{array}{l}4 / 201 / 2004 \\
4 / 26 / 2004\end{array}$ & $31 \mathrm{PM}$ & $\frac{22.010}{22.573}$ & $\begin{array}{ll}20.295 \\
23.312\end{array}$ & $\frac{2.4154}{23454}$ & 24.100 & & 23.387 & $\frac{2.013}{26.847}$ & | & 18.533 & $\frac{1.45}{-1218}$ & & 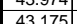 & $\mid$ & 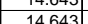 & & 17. & $\mid$ & 254 & 势. & 210 & 1.445 & 0.072 & 0.075 & 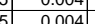 & 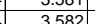 & \\
\hline 35 & $6 / 2004$ & $\frac{1250.431 \mathrm{PM}}{12.53}$ & 22.62 & 23.324 & $\mid$\begin{tabular}{|r|}
2 \\
$\mid 23.439$ \\
\end{tabular} & $\begin{array}{r}24.24 \\
2424\end{array}$ & & 23.481 & 26.907 & & 18623 & -1218 & -2 & 44627 & 0.481 & $\frac{1.96}{1466}$ & & $18 \mathrm{c}$ & 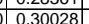 & 25.4 & 100 & $t .0$ & & & & & & \\
\hline 36 & 4/26/2004 & 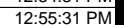 & $\frac{22.656}{22.65}$ & 23.32 & $\frac{23.522}{23.52}$ & 24.241 & & 23.311 & 26.71 & $\frac{3.929}{4.929}$ & 18.471 & -1.221 & $\frac{-2.708}{-2.708}$ & 42.983 & $\mid$ & $\frac{14.6}{14.6}$ & & $\frac{19}{19}$ & $\frac{0.31694}{0.31694}$ & $\frac{25.2}{25.2}$ & $\frac{x-5}{10.5}$ & 50.9 & .440 & & 774 & $\frac{0.004}{0.004}$ & . & \\
\hline & & 12:56: & & & & 24.263 & & 23.344 & 26.791 & 4.865 & 18.541 & -1.221 & -2.7 & \begin{tabular}{|l|l|}
43.6 \\
\end{tabular} & \begin{tabular}{|l|l|l|l} 
\\
\end{tabular} & 14.6 & & & & 25.3 & & & .444 & & & 0.004 & & \\
\hline & $5 / 2004$ & 12:57: & 22.756 & 23.354 & \begin{tabular}{|l|l|}
23.636 \\
\end{tabular} & 24.28 & & 23.599 & 27.062 & 4.902 & 18.756 & -1.215 & -2.7 & 43.988 & \begin{tabular}{|c|}
0.478 \\
\end{tabular} & & & 21. & & 25.6 & 10.8 & 21.2 & 1460 & & & & & \\
\hline & & 12:58: & 22.786 & 23.35 & & 24.19 & & & 27.087 & & & -1.218 & & & & & & $\overline{22}$ & & $\mid 25.6$ & $\frac{10.8}{10.8}$ & 21. & & & & & & \\
\hline 40 & 4/26/2004 & 12:59:31 PM & 22.813 & 23.361 & \begin{tabular}{|l|}
23.663 \\
\end{tabular} & 24.151 & & 23.632 & 27.029 & 4.921 & 18.754 & -1.221 & -2.7 & 43.936 & \begin{tabular}{|c|}
0.477 \\
\end{tabular} & 14.64 & & 23.6 & 0.38361 & 25.6 & 10.8 & 21.2 & 1.461 & $\begin{array}{lll}1 & 0.071\end{array}$ & & 0.003 & 3.485 & \\
\hline & $4 / 26 / 2004$ & 1:00:31 PM & 22.859 & 23.372 & \begin{tabular}{|l|}
23.724 \\
\end{tabular} & 24.142 & & & 26.973 & & 18.791 & -1.218 & -2.1 & 44.779 & $0.4 / 7$ & & & 24. & & 25.5 & $\overline{11.0}$ & 21.2 & 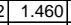 & & & & 483 & \\
\hline 42 & $4 / 26 / 2004$ & 1:01:31 PM & 22.884 & 23.372 & \begin{tabular}{|l|}
23.734 \\
\end{tabular} & 24.117 & & 23.443 & 26.814 & 4.793| & 18.736 & -1.218 & & 44.316 & 0.476 & & & 25. & 0.41694 & 25.3 & 10.9 & 21. & .454 & & & & & \\
\hline 43 & 6/2004 & $1: 02: 311 \mathrm{P}$ & 22.935 & 23.382 & \begin{tabular}{|l|}
23.79 \\
\end{tabular} & 24.183 & & 年. & 26.582 & 5.087 & 18.286 & -1.218 & $=0.0$ & 44.818 & 0.465 & 14.6 & & 20. & 0.43361 & 25.1 & 1.0 & 20 & . & - & & & & \\
\hline & & & & & & & & & & & & $\frac{-1.218}{-1218}$ & & & & & & & & & & 20.9 & & & & & & \\
\hline & 421262004 & & $\frac{2.004}{23033}$ & 20.044 & \begin{tabular}{|l|l|} 
\\
\end{tabular} & 24.250 & & $\frac{2.0350}{2324}$ & 2.04 & - & 18309 & $=1.210$ & & | & 0.040 & & & 20.6 & 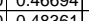 & 25.4 & $\frac{1.1 .1}{110}$ & 2 & 1.440 & | & & & & \\
\hline 47 & $4 / 26 / 2004$ & 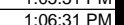 & 年 & $\mid$ & \begin{tabular}{|l|}
23.0003 \\
23.93
\end{tabular} & 24.241 & & 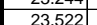 & $\frac{20.450}{26.731}$ & $\mid$ & 18.551 & -1.215 & -2. & 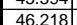 & | & & & 30.6 & $\frac{0.960018}{0.5002}$ & $\frac{2.03}{25.3}$ & $\frac{1.4}{11.3}$ & $\frac{2.0 .0}{21.0}$ & 1.456 & \begin{tabular}{l|l}
$0 \mid$ \\
0
\end{tabular} & & & 999 & \\
\hline $\begin{array}{ll}48 \\
\end{array}$ & $4 / 26 / 2004$ & $1: 07: 31 \mathrm{P}$ & 23.085 & $\frac{23.422}{23}$ & \begin{tabular}{|l|}
23.905 \\
\end{tabular} & & & & & & 18. & -1.218 & & & & & & & & 25.3 & 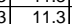 & -1. & & & & & & \\
\hline 49 & $4 / 2612004$ & $1: 08: 31$ & 23.116 & 23.429 & & 24.289 & & & & & 18.6 & -1.218 & & 44.862 & & & & 32 & & 253 & 110 & 21. & .453 & & & & & \\
\hline & & & 23.142 & 23.4 & \begin{tabular}{|l|l|}
23.9 \\
\end{tabular} & & & & & & 18.508 & -1.218 & & & & & & & & 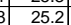 & 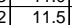 & $\frac{\frac{11.0}{21.0}}{4}$ & & & & & & \\
\hline $\begin{array}{l}51 \\
5\end{array}$ & 4/26/2004 & & 23.173 & 23.451 & \begin{tabular}{|l|}
23.998 \\
\end{tabular} & 24.346 & & 23.833 & 27 & & & -1.221 & & & $\begin{array}{l}0.466 \\
\end{array}$ & 14.6 & & 34 & 694 & 25.6 & $\overline{11.1}$ & 21.5 & 1.480 & 269 & & & 29 & \\
\hline & 4/26/2004 & & 24.908 & $\mid 23.04$ & \begin{tabular}{|l|}
25.686 \\
\end{tabular} & & & & & & 38.308 & & & & $\begin{array}{l}0.003 \\
\end{array}$ & & & & & |41.4 | & & 40.3 & \begin{tabular}{ll|l|}
$3 \mid$ & 2.782
\end{tabular} & \begin{tabular}{l|l|} 
& 0.119 \\
\end{tabular} & & & & \\
\hline & $4 / 26 / 2004$ & 2:13:39 & 24.944 & 23.061 & 25.732 & 24.108 & & 37.88 & 42.748 & \begin{tabular}{|l|l|}
4.19 \\
\end{tabular} & 33.822 & -0.477 & & 44.6 & 0.533 & -0.0 & & 97.2 & & 41.3 & 10.9 & 35.9 & & \begin{tabular}{l|l|l|}
2 & -0.002 \\
\end{tabular} & & & & \\
\hline & 4/26/2004 & $2: 14: 39 \mathrm{P}$ & 24.911 & 23.058 & 25.734 & 24.095 & & 2.684 & 43.202 & 4.11 & -1.36 & 35.61 & & 43.594 & $\begin{array}{l}0.003 \\
\end{array}$ & -0.013 & & 98.2 & 1.63583 & 41.7 & & 0 & & & & .005 & 668 & \\
\hline & $4 / 26 / 200$ & & & & & & & & & & & & & & & & & & & & & 0 & & & & & & \\
\hline 56 & $4 / 26 / 2004$ & $2: 16: 39$ & 24.375 & 23.118 & $\mid 25.804$ & 24.164 & & 2.663 & 43.372 & 4.002 & -1.233 & 36.036 & & & 0.003 & 14.64 & & & & 41.9 & 10.2 & 0. & & 2.183 & 2.134 & 2.985 & 85.196] & \\
\hline & $\begin{array}{l}4 / 201 / 200 \\
1 / 2620\end{array}$ & $\begin{array}{l}2.17 .39 \\
2.18 .39\end{array}$ & 2 & $\frac{2.1 .131}{23177}$ & \begin{tabular}{|c|c|c|c|} 
\\
\end{tabular} & $\frac{24.2}{2.3}$ & & $\frac{3.111}{2019}$ & $\frac{43.291}{4256}$ & 4.001 & $\frac{32.541}{37891}$ & 4.2. & & & & & & & & $\frac{4.18}{41.1}$ & & 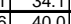 & & & & & & \\
\hline 59 & $4 / 2612004$ & & 25.142 & 23159 & \begin{tabular}{|l|}
1 \\
\end{tabular} & 24204 & & $\begin{array}{l}4.2 .40 \\
42.254\end{array}$ & & & & & & & & & & & & $\frac{4.1 .1}{412}$ & 108 & 40 & & & & & & \\
\hline 60 & $4 / 26 / 2004$ & $2: 20: 39$ & 25.153 & 23. & 25.846 & 24.151 & 23 & 42.5 & 42. & 3.988 & 38.776 & -1.218 & & & $\mid$ & 0.86 & & 104.2 & & $\frac{\pi 1.5}{41.5}$ & 10.3 & 40. & .80 & \begin{tabular}{|c|c|}
50.129 \\
\end{tabular} & & & 3.103 & \\
\hline & $4 / 26 / 2004$ & & 25.173 & 23.155 & & & & 42.949 & & & 39 & -1.2. & & & & & & & & 42.0 & 10.4 & & & & & & & \\
\hline & 4/26/200 & & 25.199 & $23.171]$ & 25.877 & & & & & & & & & & & & & & \begin{tabular}{|l|l|}
1.76917 \\
\end{tabular} & 41.5 & & 40 & & & & & & \\
\hline os & 4/26/2004 & $2.23 .39 \mathrm{PMM}$ & 25.208 & 23.181 & 25.887 & 24.182 & \begin{tabular}{l|l}
2 & 23.231. \\
\end{tabular} & 42.078 & 42.46 & 4.093 & 38.176 & -1.218 & & 42.989 & 0.003 & 0.842 & & 107.2 & 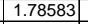 & \begin{tabular}{ll|}
411.0 \\
\end{tabular} & $\begin{array}{l}0 \\
0\end{array}$ & \begin{tabular}{|l|l}
40.1 \\
\end{tabular} & \begin{tabular}{|l|l|}
12.767 \\
\end{tabular} & $\begin{array}{ll}7 & 0.126 \\
\end{array}$ & \begin{tabular}{|l|l|} 
\\
\end{tabular} & 0.003 & 3.052 & 2. \\
\hline & $\frac{4 / 26 / 22004}{4 / 262004}$ & $\frac{2: 24: 399}{2 \cdot 25 \cdot 39}$ & $\frac{25.228}{25248}$ & $\frac{23.196}{23.2}$ & \begin{tabular}{|l|}
25.907 \\
25.916
\end{tabular} & $\frac{24.2}{24.1}$ & & $\frac{42.592}{42611}$ & $\frac{43.097}{43068}$ & $\begin{array}{l}4.108 \\
4056\end{array}$ & $\begin{array}{ll}38.644 \\
38.687\end{array}$ & $\frac{-1.218}{1.2121}$ & & $\frac{43.95}{41.98}$ & 0.003 & 0.849 & & 108.2 & 17 & $\frac{4.6}{416}$ & $\frac{10.8}{10.3}$ & 406 & 5802 & $\begin{array}{l}0 \mid 0.127 \\
\end{array}$ & 24 & (3) & $\frac{3.038}{3.53}$ & \\
\hline & $4 / 26 / 2004$ & $\frac{2.2539}{2.26 .39}$ & 25.257 & 23.21 & $\frac{25.94}{25.94}$ & 24141 & & & & 3.961 & $\begin{array}{l}38.924 \\
38.924\end{array}$ & - 1.218 & & & $\mid$ & 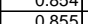 & & 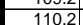 & & 418 & a. & 40. & & & & & 304 & \\
\hline & $4 / 2612004$ & & 25.277 & 23219 & \begin{tabular}{|l|l|} 
& 25.95 \\
\end{tabular} & 24 & & & & & & -1218 & & & & & & 112 & & 41.8 & & & & & & & & \\
\hline 68 & $4 / 26 / 2004$ & $2: 28: 39$ & 25.282 & 23.219 & 25.955 & 24.16 & & 42.7 & & 3.911 & 39.098 & -1.218 & & \begin{tabular}{|l|l|l|}
42.489 \\
\end{tabular} & 0.0 & 0.863 & & $\frac{112.2}{112.2}$ & & $\frac{41.9}{41.9}$ & 10.4 & & & & & & . & \\
\hline & & & & & & 24.151 & & & & 3.86 & & -1218 & & & & 0.866 & & 113.2 & 88583 & & & & & & & & 3061 & \\
\hline & $4 / 26 / 2004$ & $2: 30: 39 \mathrm{PM}$ & 25.292 & 23.229 & 25.95 & 24.12 & & 42.737 & 43.432 & 3.831 & 39.129 & -1.221 & & 41.106 & $\mid 0.003$ & 0.869 & & 114.2 & & 420 & & & & 0.130 & 26 & 0.003 & 3.082 & \\
\hline
\end{tabular}


WSRC-TR-2005-00105, REVISION 0

SRNL-RPP-2005-00012, REVISION 0

RUN \# 2.18 \& 2.19; ACID CLEANING AND CAUSTIC RINSING - CONT.

\begin{tabular}{|c|c|c|c|c|c|c|c|c|c|c|c|c|c|c|c|c|c|c|c|c|c|c|c|c|c|c|c|c|}
\hline & A & $\begin{array}{ll} \\
\end{array}$ & $\mathrm{D}$ & $\mathrm{E}$ & $\mathrm{F}$ & G & $\mathrm{H}$ & $\mathrm{J}$ & $\mathrm{K}$ & $\mathrm{L}$ & $\mathrm{M}$ & $\mathrm{N}$ & 0 & $\mathrm{Q}$ & $\mathrm{R}$ & $\mathrm{s}$ & $\mathrm{T}$ & $\mathrm{v}$ & w & $x$ & $Y$ & $z$ & AA & $\mathrm{AB}$ & AC & $\mathrm{AD}$ & $\overline{A E}$ & AF $\mathbb{A}$ \\
\hline & $4 / 26 / 2004$ & \begin{tabular}{|l|l|}
$2: 31: 39 \mathrm{PM}$ \\
$2 \cdot 32 \cdot 39 \mathrm{P}$
\end{tabular} & $\frac{25.322}{25325}$ & $\begin{array}{l}23.235 \\
23263\end{array}$ & $\begin{array}{l}25.965 \\
25.978\end{array}$ & $\frac{24.171}{2.199}$ & $\begin{array}{r}23.26 \\
23.207\end{array}$ & 43.094 & 43.77 & 3.822 & $\begin{array}{r}39.5 \\
39.9\end{array}$ & -1.218 & $\begin{array}{l}0.729 \\
\end{array}$ & 41.663 & 0.003 & $\begin{array}{l}0.869 \\
\end{array}$ & 0.005 & 115. & 1.91917 & 42.3 & 10.2 & 41.3 & 2847 & \begin{tabular}{|l|l|} 
\\
\end{tabular} & 0.126 & 0.003 & 3.053 & \\
\hline & & & & 23.263 & \begin{tabular}{|l|}
25.978 \\
3.931 \\
\end{tabular} & \begin{tabular}{|l|l|}
24.193 \\
\end{tabular} & & & 42.804 & & & \begin{tabular}{|l|}
-1.218 \\
\end{tabular} & & 43.967 & 0.003 & 0.856 & & & .47 .95000 & \begin{tabular}{|l|}
41.3 \\
\end{tabular} & & & & & 0.124 & & & \\
\hline & \begin{tabular}{r|r|}
$4 / 26 / 2004$ \\
$426 / 2004$
\end{tabular} & \begin{tabular}{l|l|l}
$2: 33: 39 \mathrm{PM}$ \\
$2 \cdot 34: 39 \mathrm{PM}$
\end{tabular} & $\begin{array}{l}25.343 \\
25.354 \\
\end{array}$ & $\begin{array}{l}23.276 \\
23287\end{array}$ & \begin{tabular}{|l|}
26.001 \\
26012 \\
\end{tabular} & \begin{tabular}{|l|l|}
24.216 \\
24278 \\
\end{tabular} & $\begin{array}{l}23.325 \\
23241\end{array}$ & 41.777 & \begin{tabular}{|l|l|}
42.398 \\
2827
\end{tabular} & $\begin{array}{l}4.255 \\
\end{array}$ & $\begin{array}{l}37.715 \\
38259\end{array}$ & \begin{tabular}{|l|l|}
-1.218 \\
\end{tabular} & $\begin{array}{l}0.732 \\
\end{array}$ & $\begin{array}{l}42.993 \\
20022\end{array}$ & $\begin{array}{l}0.003 \\
0.032\end{array}$ & \begin{tabular}{|l}
0.859 \\
.862
\end{tabular} & $\begin{array}{l}0.005 \\
0.005\end{array}$ & $\begin{array}{l}117.2 \\
11.9\end{array}$ & 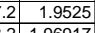 & \begin{tabular}{|l|}
40.9 \\
\end{tabular} & \begin{tabular}{|l|}
10.5 \\
10.5
\end{tabular} & 39.7 & \begin{tabular}{|l|l|}
7 & 2.740 \\
& 2.777 \\
\end{tabular} & \begin{tabular}{|l|l|}
0.128 \\
0120
\end{tabular} & $\begin{array}{l}0.125 \\
0125\end{array}$ & $\begin{array}{l}0.003 \\
\end{array}$ & 3.133 & \\
\hline & & & $\frac{25.354}{25.345}$ & $\begin{array}{l}23.287 \\
23.273\end{array}$ & \begin{tabular}{|l|}
26.012 \\
26.003 \\
\end{tabular} & \begin{tabular}{|l|}
24.278 \\
24.244 \\
\end{tabular} & $\begin{array}{r}23.241 \\
23262\end{array}$ & $\begin{array}{r}42.268 \\
42.111\end{array}$ & \begin{tabular}{|l|}
42.827 \\
42821
\end{tabular} & $\begin{array}{r}4.219 \\
4.239\end{array}$ & $\begin{array}{l}38.285 \\
38.7 \\
\end{array}$ & $\frac{-1.218}{-1.218}$ & $\begin{array}{l}0.738 \\
0.735\end{array}$ & $\begin{array}{r}42.922 \\
4.011\end{array}$ & 0.003 & $\begin{array}{r}0.863 \\
0.82\end{array}$ & $\begin{array}{l}0.005 \\
0.005\end{array}$ & $\frac{118.2}{1192}$ & & $\begin{array}{l}41.4 \\
41.3 \\
\end{array}$ & $\frac{10.5}{10.8}$ & 402 & & \begin{tabular}{|l|l|} 
\\
0.130
\end{tabular} & & 0.003 & $\begin{array}{l}3.105 \\
3.146\end{array}$ & \\
\hline & $4 / 26 / 2004$ & 2:36:39 PM & 25.352 & 23.285 & \begin{tabular}{|l|}
25.986 \\
\end{tabular} & \begin{tabular}{|l|}
24.322 \\
\end{tabular} & 23.375 & & $\begin{array}{l}42.821 \\
42.533\end{array}$ & $\begin{array}{l}4.239 \\
4.173 \\
\end{array}$ & \begin{tabular}{|c|}
30.11 \\
37.961 \\
\end{tabular} & \begin{tabular}{|l|}
-1.218 \\
-1.218 \\
\end{tabular} & $\begin{array}{l}0.135 \\
0.741\end{array}$ & \begin{tabular}{|l|}
43.011 \\
43.851 \\
\end{tabular} & $\begin{array}{l}0.003 \\
0.003\end{array}$ & \begin{tabular}{|l|}
0.872 \\
0.877 \\
\end{tabular} & $\begin{array}{l}0.005 \\
0.006\end{array}$ & $\frac{119.2}{120.2}$ & $\begin{array}{r}1.98583 \\
2.0025 \\
\end{array}$ & \begin{tabular}{|l|}
41.3 \\
41.1
\end{tabular} & $\begin{array}{l}10.8 \\
10.7 \\
\end{array}$ & $\frac{40.2}{39.9}$ & \begin{tabular}{|c|c|}
. & 2.770 \\
& 2.753 \\
\end{tabular} & \begin{tabular}{|l|}
0.130 \\
0.131 \\
\end{tabular} & $\begin{array}{l}0.126 \\
0.127\end{array}$ & $\mid \begin{array}{c}0.003 \\
0.003\end{array}$ & $\begin{array}{l}3.146 \\
.185\end{array}$ & $\frac{2.68}{2.71}$ \\
\hline & & & 25.364 & 23.317 & 25.962 & \begin{tabular}{|l|}
24.323 \\
\end{tabular} & 23.507 & 41.746 & 42.334 & 4.176 & 37.828 & \begin{tabular}{|r|}
-1.218 \\
\end{tabular} & 0.741 & \begin{tabular}{|r|}
43 \\
\end{tabular} & 0.003 & 0.882 & & 121.2 & $\frac{\frac{2.0027}{2.01917}}{2}$ & \begin{tabular}{|l|}
40.9 \\
\end{tabular} & 10.5 & & \begin{tabular}{|l|}
2.743 \\
\end{tabular} & & 0.128 & 0.003 & & \\
\hline & $4 / 26 / 2004$ & $2: 38: 39$ PM & $\begin{array}{r}25.32 \\
\end{array}$ & & \begin{tabular}{|l|}
25.914 \\
2.94
\end{tabular} & & 23.549 & $\begin{array}{l}42.007 \\
\end{array}$ & \begin{tabular}{|l|l|}
42.568 \\
\end{tabular} & 4.187 & 38.043 & & 0.15 & 44.721 & & $\begin{array}{l}0.885 \\
\end{array}$ & & & & 41.1 & 11.0 & & $2.760 \mid$ & $\mid 0.132$ & 0.129 & & & \\
\hline & $4 / 26 / 2004$ & $\begin{array}{ll}2: 39: 39 \text { PM } \\
2: 020 P\end{array}$ & $\begin{array}{l}25.266 \\
25.221\end{array}$ & $\begin{array}{r}23.32 \\
22.202\end{array}$ & $\begin{array}{r}25.845 \\
25.702\end{array}$ & $\begin{array}{r}24.26 \\
\end{array}$ & $\begin{array}{l}23.565 \\
22577\end{array}$ & 42.395 & 42.985 & 4.153 & $\begin{array}{r}38.49 \\
38.211\end{array}$ & -1.218 & \begin{tabular}{|l|l|l|}
0.757 \\
0.755
\end{tabular} & \begin{tabular}{|l|l|}
4.691 \\
\end{tabular} & 0.003 & \begin{tabular}{|l|l|}
0.892 \\
\end{tabular} & & 123.2 & 2.0525 & 41.5 & 11.0 & & \begin{tabular}{ll|l|l|l|l|} 
& 2.788 \\
\end{tabular} & \begin{tabular}{|l|} 
\\
\end{tabular} & 0.130 & $\begin{array}{l}0.003 \\
\end{array}$ & 3.211 & \\
\hline & $4 / 26 / 2004$ & 2:40:39 PM & \begin{tabular}{r|r}
25.224 \\
25.15
\end{tabular} & $\begin{aligned} 23.322 \\
23.303\end{aligned}$ & $\begin{array}{r}25.792 \\
25.684\end{array}$ & $\frac{24.288}{24.244}$ & $\begin{array}{l}23.577 \\
23.619\end{array}$ & $\frac{42.179}{42009}$ & \begin{tabular}{|l|}
42.846 \\
42.678
\end{tabular} & \begin{tabular}{|l|l|}
4.189 \\
4.124
\end{tabular} & $\begin{array}{r}38.211 \\
38.129 \\
\end{array}$ & $\frac{-1.212}{-1.224}$ & $\begin{array}{l}0.755 \\
0.747\end{array}$ & \begin{tabular}{|l|}
43.413 \\
42.318
\end{tabular} & 0.003 & \begin{tabular}{r|r}
0.894 \\
0.9
\end{tabular} & 0.005 & $\frac{124.2}{125.2}$ & $\begin{array}{l}2.06917 \\
208583 \\
\end{array}$ & $\begin{array}{l}41.4 \\
41.2\end{array}$ & $\frac{10.6}{10.4}$ & $\frac{40.2}{40.1}$ & $\frac{2.771}{2.763 \mid}$ & \begin{tabular}{|l|}
0.133 \\
0.134 \\
\end{tabular} & \begin{tabular}{|c|c|}
0.130 \\
0.132
\end{tabular} & $\begin{array}{l}0.003 \\
0.03\end{array}$ & $\begin{array}{l}3.243 \\
3.285 \\
\end{array}$ & $\begin{array}{l}2.76 \\
28\end{array}$ \\
\hline 82 & $4 / 26 / 2004$ & 2:42:39 PM & 25.101 & 23.315 & 25.65 & \begin{tabular}{|l|}
24.245 \\
\end{tabular} & & 42.017 & \begin{tabular}{|l|}
42.010 \\
42.657 \\
\end{tabular} & \begin{tabular}{|l|}
4.127 \\
.127
\end{tabular} & 38.103 & -1.218 & & \begin{tabular}{|l|}
42.310 \\
42.249 \\
\end{tabular} & 0.003 & \begin{tabular}{r|}
0.902 \\
\end{tabular} & & $\frac{120.4}{126.2}$ & \begin{tabular}{|l|}
2.00303 \\
2.1025 \\
\end{tabular} & $\begin{array}{l}41.2 \\
41.2 \\
\end{array}$ & $\frac{10.4}{10.4}$ & $\frac{40.1}{40.1}$ & \begin{tabular}{|l|}
2.763 \\
2.762 \\
\end{tabular} & \begin{tabular}{|l|}
0.134 \\
0.134 \\
\end{tabular} & $\begin{array}{l}0.132 \\
0.132\end{array}$ & \begin{tabular}{|c|}
0.003 \\
0.003
\end{tabular} & $\begin{array}{l}3.285 \\
3.296\end{array}$ & $\frac{2.80}{281}$ \\
\hline 83 & $4 / 26 / 2004$ & 2:43:39 PM & 25.068 & 23.341 & 25.621 & \begin{tabular}{|l|}
24.216 \\
\end{tabular} & 23.556 & 42.053 & 42.672 & 4.081 & 38.197 & \begin{tabular}{|l|} 
\\
\end{tabular} & & \begin{tabular}{|l|l|}
42.543 \\
\end{tabular} & 0.003 & 0.907 & 0.00 & 127.2 & $\begin{array}{l}2.11917 \\
\end{array}$ & 41.2 & 10.4 & 40.1 & \begin{tabular}{|l|}
2.766 \\
\end{tabular} & 0.135 & 0.133 & 0.003 & 3.312 & $\begin{array}{l}2.81 \\
2.82\end{array}$ \\
\hline 84 & $4 / 26 / 2004$ & $2: 44: 39 \mathrm{PM}$ & \begin{tabular}{|l|l|}
24.997 \\
\end{tabular} & 23.316 & 25.551 & 24.146 & 23.581 & & 43.016 & & 38.64 & & & \begin{tabular}{|l|l|}
43.949 \\
\end{tabular} & & & & & 2.13583 & 41.5 & \begin{tabular}{ll|}
10.8 \\
\end{tabular} & 40.5 & 2.796 & \begin{tabular}{|l|l|}
0.136 \\
\end{tabular} & 0.134 & \begin{tabular}{|c|c|} 
\\
\end{tabular} & 3.298 & \\
\hline & $4 / 266 / 2004$ & 2:45:39 PM & 24.963 & 23.331 & 25.517 & 24.177 & 23.541 & & 43.107 & & 38.593 & -1.218 & & \begin{tabular}{|l|l|}
43.753 \\
\end{tabular} & & 0.913 & & 129. & 2.1525 & 41.6 & 10.7 & 40. & 2.793 & 0.136 & 0.134 & 0.003 & 3.312 & \\
\hline$\frac{8}{8}$ & 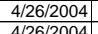 & $\begin{array}{l}2: 46: 39 \mathrm{PM} \\
2 \cdot 47 \cdot 39 \mathrm{PM}\end{array}$ & \begin{tabular}{l|l}
24.922 \\
24.882
\end{tabular} & $\begin{aligned} 23.336 \\
23.345\end{aligned}$ & $\begin{array}{l}25.471 \\
25.255\end{array}$ & $\begin{array}{l}24.196 \\
24145\end{array}$ & $\begin{array}{r}23.551 \\
23545\end{array}$ & $\begin{array}{l}42.216 \\
4268\end{array}$ & $\begin{array}{l}42.686 \\
2089\end{array}$ & $\begin{array}{l}3.998 \\
404\end{array}$ & $\begin{array}{l}38.447 \\
38.445\end{array}$ & \begin{tabular}{|c|}
-1.218 \\
-1218
\end{tabular} & $\begin{array}{l}0.761 \\
0764\end{array}$ & \begin{tabular}{|l|l|}
43.851 \\
43517 \\
\end{tabular} & \begin{tabular}{|l|l|}
0.003 \\
0.023
\end{tabular} & \begin{tabular}{|l}
0.916 \\
0.922
\end{tabular} & & 130.2 & $\begin{array}{l}2.16917 \\
2.18583\end{array}$ & $\begin{array}{l}11.2 \\
1.2\end{array}$ & $\begin{array}{ll}10.7 \\
107\end{array}$ & & $\begin{array}{l}2.781 \\
2789\end{array}$ & \begin{tabular}{|l|} 
\\
\end{tabular} & 0.135 & $\begin{array}{l}0.003 \\
\end{array}$ & $\begin{array}{l}3.342 \\
33\end{array}$ & \\
\hline$\frac{8}{8}$ & $\begin{array}{r}4 / 2 / 2 / 2004 \\
4 / 26 / 2004\end{array}$ & $\begin{array}{l}2: 47: 39 \mathrm{PM} \\
22: 48: 39 \mathrm{PM}\end{array}$ & $\begin{array}{l}24.882 \\
24.862\end{array}$ & $\frac{23.345}{23.355}$ & \begin{tabular}{|}
25.425 \\
25.4 \\
\end{tabular} & $\begin{array}{r}24.145 \\
24.14 \\
\end{array}$ & \begin{tabular}{|l|}
23.545 \\
\end{tabular} & $\frac{42.258}{42.513}$ & \begin{tabular}{|l|}
42.989 \\
43.238 \\
\end{tabular} & $\begin{array}{r}4.04 \\
4.043 \\
\end{array}$ & $\begin{array}{l}38.445 \\
38.722\end{array}$ & \begin{tabular}{|c|}
-1.218 \\
-1.218
\end{tabular} & $\begin{array}{l}0.764 \\
0.767\end{array}$ & \begin{tabular}{|l|}
43.517 \\
42.895 \\
\end{tabular} & $\begin{array}{l}0.003 \\
0.003\end{array}$ & \begin{tabular}{|c|c|c|c|}
0.922 \\
\end{tabular} & $\begin{array}{l}0.005 \\
0.005\end{array}$ & $\frac{131.2}{132.2}$ & $\frac{2.18583}{2.2025}$ & $\begin{array}{l}41.5 \\
41.8\end{array}$ & $\begin{array}{l}10.7 \\
10.5\end{array}$ & $\frac{40.4}{40.6}$ & 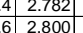 & \begin{tabular}{|l|}
0.137 \\
0.137 \\
\end{tabular} & $\begin{array}{l}0.136 \\
0.136 \\
\end{array}$ & \begin{tabular}{|c|c|}
0.003 \\
0.003
\end{tabular} & $\begin{array}{l}3.366 \\
3.346 \\
\end{array}$ & $\frac{2.86}{2.85}$ \\
\hline & $4 / 26 / 2004$ & $2: 49: 39 \mathrm{PM}$ & 24.825 & 23.359 & \begin{tabular}{|r|}
25.369 \\
\end{tabular} & 24.114 & 23.504 & 41.889 & \begin{tabular}{|l|}
42.624 \\
\end{tabular} & 4.293 & 37.813 & -1.218 & 0.764 & \begin{tabular}{|l|}
43.392 \\
\end{tabular} & 0.003 & 0.91 & 0.00 & 133.2 & $\frac{2.20<5}{2.21917}$ & 41..2 & 10.6 & $\frac{40.6}{39.9}$ & $\frac{2.800}{2.748}$ & \begin{tabular}{|l|l|}
0.137 \\
0.136
\end{tabular} & \begin{tabular}{|l|l|l|l|l|l|}
0.134 \\
\end{tabular} & $\begin{array}{l}.0003 \\
0.03\end{array}$ & $\frac{3.340}{3.369}$ & \\
\hline & $4 / 26 / 2004$ & & & & & 24.179 & 23.543 & & & & & & & 44.585 & & & & & & \begin{tabular}{|l|l|}
41.3 \\
\end{tabular} & $\begin{array}{ll}10.9 \\
\end{array}$ & & & & & & & \\
\hline & $4 / 26 / 2004$ & 2:51:39 PM & 24.758 & 23.367 & 25.342 & 24.192 & & 42.105 & 42.97 & 4.191 & 38.172 & 218 & 0.7 & 45.154 & & 0.928 & & 135.2 & 2.2525 & 41.5 & 11.1 & & 2.7. & $\begin{array}{l}0.138 \\
0.0\end{array}$ & .137 & & & \\
\hline & $4 / 26 / 2004$ & & 24.732 & & 25.306 & 24.196 & & 41.839 & 42.587 & 4.11 & 37.953 & -1.218 & & $\begin{array}{l}44.351 \\
\end{array}$ & & & & 136.2 & & & & & & & & Do3 & & \\
\hline & $4 / 26 / 2004$ & 2:53:39 PM & 24.7 & 23.364 & 25.209 & 24.164 & & & 42.956 & 4.10 & 38.433 & & & 45.30 & & & & & 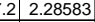 & 41. & & & & & & & & \\
\hline & $4 / 26 / 2004$ & 2:54:39 PM & 24.668 & 23.361 & 25.227 & 24.162 & 23.52 & 42.214 & \begin{tabular}{|l|l|}
42.767 \\
\end{tabular} & 4.13 & 38.343 & -1.218 & & $\begin{array}{l}42.268 \\
\end{array}$ & & 0.95 & & 138. & 2.3025 & & 10.4 & & & & 0.141 & & & \\
\hline & 4/26/2004 & & 24.635 & 23.359 & 25.189 & 24.164 & & 42.442 & 43.248 & 4.091 & 38.564 & & 0.77 & 43.194 & & 0.954 & & & 19 & 41. & 0.6 & & & & 0.141 & & & \\
\hline & 4/26/2004 & :39 PM & 24.628 & 23.377 & 25.187 & 24.153 & 23.51 & 42.021 & $\begin{array}{ll}42.726 \\
-125\end{array}$ & 4.094 & 38.148 & & & 1002 & & 0.959 & & 140. & 25 & 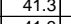 & 0.5 & & & 143 & .142 & & & \\
\hline & 4420120004 & & & & & & & 42.1300 & & & 30.310 & & & & & & & & & & & & & & & & & \\
\hline & 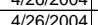 & $39 \mathrm{PM}$ & 24.575 & $\frac{2.374}{23294}$ & 25.169 & 24.1612 & $\frac{23.51}{23.16}$ & $\begin{array}{l}42.084 \\
4197\end{array}$ & $\begin{array}{l}42.852 \\
4.875\end{array}$ & $\begin{array}{l}4.0044 \\
4089\end{array}$ & $\begin{array}{l}38.281 \\
38.092\end{array}$ & & & $\begin{array}{l}42.38 \\
2.34\end{array}$ & & 0 & & & & 告1. & & & & & 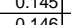 & & & \\
\hline & $4 / 26 / 2004$ & .39 PM & 24.552 & 23.391 & \begin{tabular}{|l|}
25.131 \\
\end{tabular} & 24.182 & 22.3516 & 42.046 & 43.049 & & 38.215 & -1.218 & & 42.459 & & 0.99 & & 144 & 24025 & 416 & 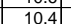 & & 2767 & 2118 & 1147 & & 34 & \\
\hline 10 & $4 / 26 / 2004$ & 3:01:39 PM & 24.529 & 23.378 & 25.108 & 24.123 & 23.5 & 42.312 & 43.219 & 4.183 & 38.378 & & & $\begin{array}{l}42.4593 \\
44.593\end{array}$ & 0.00 & 0.997 & & 145 & & $\frac{11 .}{41 .}$ & 10.9 & & 2.782 & 0.149 & 0.148 & & $\begin{array}{l}0.004 \\
3.673 \\
\end{array}$ & \\
\hline & $4 / 26 / 2004$ & & 24.512 & & 25.101 & & 23.53 & 42.571 & & & 38.67 & & & & & 1.06 & & 1146. & & 41.5 & 10.4 & & 30 & & 149 & & & \\
\hline & & & & 23.378 & 25.078 & 24.052 & & & 3.036 & & 38.259 & & & 42.791 & & 013 & & 147 & 2.45 & 41. & 105 & & 277 & 151 & 151 & & 3.751 & \\
\hline & $4 / 26 / 2004$ & & 24.486 & 23.375 & \begin{tabular}{|l|}
25.07 \\
\end{tabular} & 24.08 & 23.485 & 42.194 & \begin{tabular}{|l|l|l|}
43.121 \\
\end{tabular} & 4.098 & 38.343 & $\begin{array}{l}-1.215 \\
-1.215\end{array}$ & & 43.365 & & & & 148.2 & 2016 & 41. & $\frac{10.6}{10.6}$ & & & 0.152 & & & 3.765 & \\
\hline & $4 / 26 / 2004$ & & & & & & & & 43.575 & & 38.765 & -1.215 & & & & & & 149 & & & 10.6 & & & & & & & \\
\hline & 4/26/2004 & : $: 39$ PM & 24.47 & 23.379 & 25.084 & 24.104 & 23.5 & 42.376 & 43.105 & $\begin{array}{l}4.083 \\
\end{array}$ & 38.587 & -1.221 & & $\begin{array}{l}42.739 \\
\end{array}$ & & 103 & & 150. & 2.5025 & 41.6 & 10.5 & & 2.791 & 0.155 & 155 & & 3.818 & \\
\hline & 4/266/2004 & & 24.458 & 23.366 & 25.042 & 24.102 & & 42.067 & 43.227 & 4.022 & 38.269 & 224 & & 42.624 & 0.00 & 1.04 & & 151. & & |41.8 & 0.4 & & & 0.156 & 0.156 & & 3.882 & \\
\hline & $4 / 26 / 2004$ & & 24.4599 & & 23.050 & & & & 43.171 & 4.048 & & & & & & & & & & & 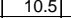 & & & & & & & \\
\hline & $42126 / 2004$ & & 24.455 & 23.364 & \begin{tabular}{|l|}
25.034 \\
\end{tabular} & 24.134 & & 42.302 & 43.349 & 4.048 & 38.493 & -1.218 & & 42.3. & & 1.0 & & 踏 & 2.5 & & & & & 0.158 & & & & \\
\hline & $\frac{1421212004}{112604}$ & & & 23.376 & \begin{tabular}{|l|l|}
25.020 \\
\end{tabular} & 24.161 & & & 43.339 & 3.965 & 38.614 & & & & & & & 154 & & 41. & & & & . 160 & & & 3.949 & \\
\hline & $\begin{array}{l}4 / 2 / 2 / 2004 \\
/ 26 / 2004\end{array}$ & $39 \mathrm{PM}$ & $\frac{24.435}{2449}$ & $\frac{2.376}{23.348}$ & 25078 & 24.140 & & $\frac{42.505}{42.301}$ & \begin{tabular}{|l|l|}
43503 \\
4351
\end{tabular} & $\frac{4.001}{309}$ & 38.6 & $\frac{-1.21}{-122}$ & & $\begin{array}{l}42.350 \\
42968\end{array}$ & & 10 & & 势 & 2602 & 42. & 105 & & & & & & & \\
\hline & $4 / 26 / 2004$ & 3:13:39 PM & $\frac{24.419}{24.42}$ & 20.040 & & 24.074 & & $\frac{42.001}{42.16}$ & $\frac{45.021}{43.298}$ & 4.015 & $\frac{30.091}{38.412}$ & & & & & $\frac{1.004}{1.094}$ & & 100. & & $\frac{42.0}{41.8}$ & & & & & & & & \\
\hline & $4 / 26 / 2004$ & & & 23.35 & 24.995 & 24.045 & & 42.133 & 43.242 & 3.999 & 38.388 & & & 45.718 & & 1102 & & the & 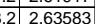 & 41.8 & . & & & & $\frac{0.164}{0.164}$ & & & \\
\hline & $4 / 26 / 2004$ & & 24.406 & 23.355 & 24.99 & 24.04 & & 42.223 & 43.4 & 3.976 & 38.517 & 5 & & & & 1.108 & & 159 & 26 & 420 & 101 & & & & & & & \\
\hline & $4 / 26 / 2004$ & & 24.39 & 23.356 & 24.981 & & & 42.14 & 43. & 4.009 & & & & & & & & & & & & & & & & & & \\
\hline & $4 / 26 / 2004$ & & 24.392 & & 24.971 & 24.046 & & & \begin{tabular}{|l|l|}
43.374 \\
\end{tabular} & & & -1.221 & & & 0.00 & & & 161. & & 41. & 10 & & & & & & 43 & \\
\hline & $4 / 26 / 2004$ & :39 PM & 24.383 & 23.342 & $\begin{array}{l}24.947 \\
\end{array}$ & 23.992 & & & $\begin{array}{l}43.028 \\
\end{array}$ & & & & & & & & & 162 & & & 10.6 & & & & & & \begin{tabular}{|l|l|}
4.198 \\
\end{tabular} & \\
\hline & $4 / 26 / 2004$ & & 24.379| & 23.347 & $\begin{array}{l}24.963 \\
\end{array}$ & 23.947 & & 42.119 & 43.124 & 3.972 & 38.452 & $\begin{array}{l}-1.218 \\
\end{array}$ & & $\begin{array}{l}43.538 \\
\end{array}$ & 0.0 & 1.1 & & 163 & & $\begin{array}{lll}4.7 \\
\end{array}$ & 10. & & & 0.169 & 0.169 & & 4.201 & \\
\hline & 4/26/2004 & & 24.37 & 23.333 & 24.963 & 23.998 & & 42.239 & 43.387 & 3.984 & & -1 & & 43.4 & & 1.1. & & 106 & & 41. & . & & 2.1 & & & & & \\
\hline & $4 / 2612004$ & & 24.3 & 23.349 & 24.979 & 23.98 & & & & 3.97 & & & & & & & & & & & & & & & & & & \\
\hline & 412120004 & & & 23.334 & 24.964 & & & & & 3. & & -1.218 & & & & & & & & & & & & & & & & \\
\hline & $\begin{array}{r}4 / 2 / 26 / 2004 \\
4 / 26 / 2004\end{array}$ & & $\frac{24.36}{2436}$ & $\frac{2.359}{23.33}$ & 24.945 & $\frac{23.909}{2401}$ & & $\frac{4.1}{423}$ & $\begin{array}{l}43.414 \\
43445\end{array}$ & $\frac{3.90}{4.02}$ & $\begin{array}{l}30.451 \\
38626\end{array}$ & $\frac{-1.2}{-1.2}$ & & & & & & 16. & & & & & & & & & & \\
\hline & $4 / 26 / 2004$ & & 2435 & $\frac{25.56}{2316}$ & 24.946 & 24.006 & & & 43.271 & & & & & & & 1.1 & & & 2.6 & 41,8 & & & & & & & & \\
\hline & 4/26/2004 & & & & & & & & & & & & & & & & & & & & & & & & & & & \\
\hline & & & 24.35 & 23.317 & 24.962 & & & 41.885 & \begin{tabular}{|l|l}
43.285 \\
\end{tabular} & & & -1.218 & & & & & & 17. & & & & & & & & & & \\
\hline & $4 / 26 / 2004$ & & 24.374 & 23.312 & \begin{tabular}{|l|l|}
24.988 \\
\end{tabular} & 23.998 & & 42.04 & \begin{tabular}{|l|}
43.546 \\
\end{tabular} & 4.1 & 38.156 & -1.212 & & 44.4 & & 11. & & & & & & & & & & & & \\
\hline & $4 / 26 / 2004$ & $3: 2$ & 24.3 & 23.304 & \begin{tabular}{|l|l|}
24.97 \\
\end{tabular} & \begin{tabular}{|l|}
23.964 \\
\end{tabular} & & 2.042 & 43. & & 38.201 & -1.224 & & & & & & & & & & & & & & & & \\
\hline & $4 / 262 / 2004$ & 3:30:39 PN & 24.376 & 23.315 & 24.99 & \begin{tabular}{|l|l|} 
& 23.965 \\
\end{tabular} & & 42.424 & 43.72 & 4.1 & 38.571 & -1.221 & & 44.46 & & 1.1 & & 174.2 & 2.90 & 42.2 & & & 2.7 & 0.178 & 0.178 & & 93. & \\
\hline & $4 / 26 / 2004$ & & 24.373 & 23.311 & 24.992 & $\begin{array}{l}24.007 \\
\end{array}$ & & 42.204 & $\begin{array}{l}43.486 \\
\end{array}$ & 4.14 & 38.365 & & & 44.1 & & 1.2 & & 175 & .91 & 42 & & & & & & & 4.4 & \\
\hline & 4/26/2004 & & & & 24.994 & 24.109 & & & & & & & & & & & & 176. & & 41.9 & & 40 & & & & & & \\
\hline & & & 24.382 & 23.32 & 24.986 & 24.166 & & 42.131 & & & 38.3 & -1.218 & & & & & & 177. & & 42.2 & & & & & & & & \\
\hline & 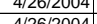 & & 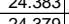 & 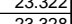 & 20.4971 & 24.2 & & 42.061 & 43. & & $\frac{38.226}{38290}$ & -1.2 & & & & 1.42 & & & & 4 & & & & & & & & \\
\hline & 4 & & 24.37 & 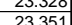 & 2.4 & 24.26 & & 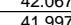 & 年 & & | & - & & & & 12 & & 1802 & & 12 & & & & & & & & \\
\hline & 4 & & 24.39, & 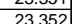 & $\frac{25012}{25012}$ & 24288 & & 44.756 & 年 & 40064 & & -1218 & & 43.1 & & & & (181. & & 417 & & & & & & & 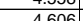 & \\
\hline & $4 / 26 / 2004$ & & 24.39 & & 25015 & 24291 & & & 43,366 & & & -1 & & & & & & 182.2 & & $419 \quad$ & & & & & & & & \\
\hline & 4/26/2004 & & 21.20 & & & & & & $\begin{array}{l}43.37 \\
\end{array}$ & 4.08 & & & & 28 & & & & & & & & & & & & & 58 & \\
\hline & & & & & & & & & & & & & & & & & & & & & & & & & & & $\frac{657}{657}$ & \\
\hline
\end{tabular}


WSRC-TR-2005-00105, REVISION 0

SRNL-RPP-2005-00012, REVISION 0

RUN \# 2.18 \& 2.19; ACID CLEANING AND CAUSTIC RINSING - CONT.

\begin{tabular}{|c|c|c|c|c|c|c|c|c|c|c|c|c|c|c|c|c|c|c|c|c|c|c|c|c|c|c|c|c|}
\hline & $\mathrm{A}$ & B & D & \begin{tabular}{|l|l|} 
E & \\
\end{tabular} & $F$ & $G$ & $\mathrm{H}$ & $\mathrm{J}$ & \begin{tabular}{l|l}
$\mathrm{K}$ & \\
\end{tabular} & L & $\mathrm{M}$ & $\mathrm{N}$ & 0 & $\mathrm{Q}$ & \begin{tabular}{ll|}
$\mathrm{R}$ \\
\end{tabular} & $\mathrm{s}$ & $\mathrm{T}$ & v & w & \begin{tabular}{l|l|}
$x$ \\
\end{tabular} & $Y$ & z & AA & $A B$ & $A C$ & $A D$ & AE & AF \\
\hline & $4 / 26 / 2004$ & 3:41:39 PM & 24.405 & \begin{tabular}{|l|l|}
23.384 \\
\end{tabular} & \begin{tabular}{|l|}
24.9999 \\
2.90 \\
\end{tabular} & $\begin{array}{l}24.23 \\
24.23\end{array}$ & 23.604 & 42.302 & \begin{tabular}{|l|}
43.969 \\
\end{tabular} & 4.077 & 38.51 & -1.221 & 0.86 & 42.693 & & 1.252 & 0.005 & 185.2 & \begin{tabular}{|l|l|}
3.08583 \\
\end{tabular} & 42.5 & 10.5 & & 2.786 & \begin{tabular}{|l|}
$A B$ \\
0.187 \\
\end{tabular} & $\begin{array}{l}\mathrm{Ac} \\
0.187\end{array}$ & $\frac{A D}{0.005}$ & $\frac{A E}{4.620}$ & 3.93 \\
\hline$\frac{14}{14}$ & $4 / 26 / 2004$ & $\begin{array}{ll}3: 42: 39 \text { PM } \\
3: 32 \mathrm{P}\end{array}$ & 24.406 & \begin{tabular}{|r|}
23.39 \\
23.29
\end{tabular} & \begin{tabular}{|l|}
25.005 \\
3500 \\
\end{tabular} & \begin{tabular}{|l|}
24.2266 \\
2.176
\end{tabular} & \begin{tabular}{|l|}
23.586 \\
3251
\end{tabular} & 41.932 & \begin{tabular}{|l|l|}
43.358 \\
3232
\end{tabular} & 4.05 & $38.20 \mathrm{C}$ & -1.22 & $\begin{array}{l}0.862 \\
\end{array}$ & & & 1.254 & & 18 & \begin{tabular}{|l|}
3.1025 \\
3.101 \\
\end{tabular} & 41.9 & 10.7 & 40. & 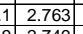 & 0.107 & 0.187 & & 4.665 & 3.07 \\
\hline$\frac{143}{144}$ & $\begin{array}{l}4 / 26 / 2004 \\
4 / 262004\end{array}$ & $\begin{array}{l}3: 4: 3: 39 \mathrm{PM} \\
\text { 3:44:39 }\end{array}$ & 24.3973 & \begin{tabular}{r|}
23.386 \\
23.397
\end{tabular} & \begin{tabular}{|l|}
25.006 \\
25027 \\
\end{tabular} & \begin{tabular}{|l|}
24.176 \\
24.173 \\
\end{tabular} & $\begin{array}{r}23.541 \\
23547\end{array}$ & \begin{tabular}{|l|l|}
41.756 \\
4156
\end{tabular} & \begin{tabular}{|l|}
43.362 \\
43115 \\
\end{tabular} & $\begin{array}{r}4.06 \\
4063\end{array}$ & & $\begin{array}{l}-1.221 \\
-1.218 \\
\end{array}$ & & & 0 & $\begin{array}{r}1.26 \\
1246 \\
\end{array}$ & & & & & & & & \begin{tabular}{|l|l|}
0.188 \\
\end{tabular} & 0.188 & & 4.711 & 4.01 \\
\hline 145 & $4 / 26 / 2004$ & 3:45:39 PM & 24.414 & 23.403 & \begin{tabular}{|l|}
25.028 \\
\end{tabular} & \begin{tabular}{|l|}
24.178 \\
\end{tabular} & 23.548 & $\mid \begin{array}{l}41.775 \\
\end{array}$ & \begin{tabular}{|l|}
43.492 \\
\end{tabular} & $\frac{4.403}{4.122}$ & $\frac{37.754}{37.941}$ & $\frac{-1.218}{-1.221}$ & & .536 & & $\frac{1.264}{1.268}$ & & $\frac{188}{180}$ & $\begin{array}{l}3.13553 \\
3.1525 \\
\end{array}$ & $\frac{41.6}{42.0}$ & $\frac{\frac{10.7}{10.7}}{10 .}$ & $\frac{39 .}{39 .}$ & $\frac{2.733}{2.748}$ & & $\mid 0.188$ & $\frac{0.005}{0.005}$ & $\begin{array}{l}\frac{4.750}{4.739} \\
4\end{array}$ & $\begin{array}{l}4.04 \\
4.03 \\
\end{array}$ \\
\hline 146 & $4 / 26 / 2004$ & $3: 46: 39 \mathrm{PM}$ & 24.409 & 23.393 & \begin{tabular}{|l|}
25.023 \\
\end{tabular} & \begin{tabular}{|l|}
24.143 \\
\end{tabular} & 23.538 & 41.868 & \begin{tabular}{|l|}
43.452 \\
43.54
\end{tabular} & 4.065 & 38.103 & -1.221 & 0.871 & 44.614 & 0.003 & 1.271 & 0.00 & 190.2 & \begin{tabular}{c|c|c|c|} 
& 3.16917 \\
\end{tabular} & 42.1 & 10.9 & 40.0 & 2.757 & \begin{tabular}{|l|l|} 
& 0.189 \\
\end{tabular} & 0.189 & 0.005 & 4.736 & \\
\hline 147 & $4 / 26 / 2004$ & $3: 47: 39 \mathrm{PM}$ & 24.425 & 23.409 & \begin{tabular}{|l|}
25.034 \\
\end{tabular} & \begin{tabular}{|l|l|}
24.184 \\
\end{tabular} & 23.544 & 41.916 & \begin{tabular}{|l|}
43.49 \\
\end{tabular} & 4.057 & 38.207 & -1.218 & 0.874 & 42.086 & 0.003 & 1.278 & & 191.2 & $\begin{array}{ll}2 & 3.18583 \\
\end{array}$ & 42.0 & 10.3 & & 2.762 & & & 0.005 & & 4.04 \\
\hline 148 & $4 / 26 / 2004$ & 4:44:13 PM & 23.43 & 23.039 & \begin{tabular}{|l|}
24.159 \\
\end{tabular} & \begin{tabular}{|r|}
23.84 \\
\end{tabular} & 23.36 & 41.918 & 45.215 & 4.26 & 38.123 & -1.215 & $\begin{array}{l}-4.798 \\
\end{array}$ & 42.076 & & 1.766 & & & & 43.7 & 10.3 & & 2.759 & & & & & \\
\hline$\frac{149}{156}$ & 4/26/2004 & 4:45:13 PM & $\begin{array}{l}23.551 \\
23.609\end{array}$ & \begin{tabular}{|l|}
23.05 \\
2.072
\end{tabular} & \begin{tabular}{|l|}
24.301 \\
2.447 \\
\end{tabular} & \begin{tabular}{|l|l|}
23.806 \\
2308 \\
\end{tabular} & \begin{tabular}{|l|}
23.352 \\
23363
\end{tabular} & \begin{tabular}{|l|l|}
31.716 \\
302
\end{tabular} & \begin{tabular}{|l|}
45.039 \\
45149
\end{tabular} & $\begin{array}{r}4.23 \\
4.11\end{array}$ & $\begin{array}{l}27.741 \\
.1565\end{array}$ & \begin{tabular}{|l|l|}
7.346 \\
3746
\end{tabular} & $\begin{array}{l}2.808 \\
3.633\end{array}$ & \begin{tabular}{|l|}
44.205 \\
4.602 \\
\end{tabular} & 0.942 & \begin{tabular}{|c|c|c|}
-0.009 \\
\end{tabular} & & 248.7 & \begin{tabular}{|l|l|}
4.14528 \\
.161904
\end{tabular} & $\begin{array}{ll}43.6 \\
337\end{array}$ & $\begin{array}{ll}10.8 \\
10.7\end{array}$ & & 2.050 & & -0.001 & & & \\
\hline & $\begin{array}{r}4 / 26 / 2004 \\
426 / 204\end{array}$ & $\begin{array}{l}4: 46: 13 \mathrm{PM} \\
4: 77 \cdot 13 \mathrm{PM}\end{array}$ & $\begin{array}{l}23.608 \\
23.588\end{array}$ & \begin{tabular}{|l|l}
23.072 \\
23067
\end{tabular} & \begin{tabular}{|l|}
24.447 \\
24.593
\end{tabular} & \begin{tabular}{|l|}
23.848 \\
23888 \\
\end{tabular} & $\begin{array}{r}23.363 \\
23299 \\
\end{array}$ & $\begin{array}{r}3.022 \\
334 \\
3\end{array}$ & \begin{tabular}{|c|}
45.184 \\
45768 \\
\end{tabular} & \begin{tabular}{|l}
4.211 \\
4243
\end{tabular} & $\begin{array}{l}-1.056 \\
-0.787\end{array}$ & \begin{tabular}{|r|}
37.46 \\
37.567
\end{tabular} & $\begin{array}{r}-3.633 \\
20249 \\
2020\end{array}$ & \begin{tabular}{|l|}
43.602 \\
43.273 \\
\end{tabular} & $\begin{array}{l}0.003 \\
0.003\end{array}$ & \begin{tabular}{|c|c|c|}
-0.013 \\
-0.012
\end{tabular} & 0 & 249.7 & \begin{tabular}{ll|}
7 & 4.16194 \\
7 & 4.17861
\end{tabular} & $\begin{array}{l}43.7 \\
44.3\end{array}$ & \begin{tabular}{|c|c|}
10.7 \\
106
\end{tabular} & $\frac{1.0}{1.3}$ & | & $\begin{array}{l}-0.002 \\
-0.002\end{array}$ & $\begin{array}{l}-0.002 \\
-0.002\end{array}$ & $\begin{array}{l}-0.002 \\
-001\end{array}$ & $\begin{array}{l}-2.003 \\
-1.432\end{array}$ & $\begin{array}{l}-1.70 \\
\end{array}$ \\
\hline & $4 / 26 / 2004$ & $\begin{array}{l}4.47 .13 \mathrm{PM} \\
4: 48: 13 \mathrm{PM}\end{array}$ & & 23.084 & \begin{tabular}{|l|}
24.705 \\
\end{tabular} & & & \begin{tabular}{|c|}
.3 .344 \\
3.306
\end{tabular} & \begin{tabular}{|l|}
45.7406 \\
45.746
\end{tabular} & 4.181 & $\begin{array}{l}-0.181 \\
-0.711\end{array}$ & & $\begin{array}{l}20.249 \\
21.087\end{array}$ & $\begin{array}{l}43.275 \\
43.093\end{array}$ & $\begin{array}{l}0.003 \\
0.003\end{array}$ & $\mid \begin{array}{r}-0.012 \\
-0.013\end{array}$ & & $\frac{250.7}{251.7}$ & \begin{tabular}{|l|l|}
7 & 4.18081 \\
7
\end{tabular} & $\begin{array}{l}44.3 \\
44.3 \\
\end{array}$ & $\begin{array}{l}10.6 \\
10.6\end{array}$ & & $\begin{array}{l}0.0879 \\
0.089\end{array}$ & & $\begin{array}{l}-0.002 \\
-0.002 \\
-0.02\end{array}$ & $\begin{array}{l}-0.001 \\
-0.002\end{array}$ & $\begin{array}{l}-\frac{1.432}{-1.506} \\
\end{array}$ & $\begin{array}{l}-1.22 \\
-1.28\end{array}$ \\
\hline & $4 / 26 / 2004$ & 4:49:13 PM & 23.56 & & \begin{tabular}{|l|}
24.826 \\
\end{tabular} & & 23.311 & 2.937 & 45.176 & 4.17 & -1.079 & \begin{tabular}{|l|}
37.437 \\
\end{tabular} & & \begin{tabular}{|l|}
43.471 \\
\end{tabular} & & \begin{tabular}{|l|l|}
14.643 \\
\end{tabular} & & 252.7 & $\begin{array}{lll}7 & 4.21194 \\
\end{array}$ & 43.7 & 10.7 & 0.0 & 0.064 & $\begin{array}{l}.183 \\
\end{array}$ & $\begin{array}{c}-2.0064 \\
2.194\end{array}$ & & & \\
\hline 154 & $4 / 26 / 2004$ & 4:50:13 PM & 23.586 & \begin{tabular}{|l|}
23.11 \\
\end{tabular} & \begin{tabular}{|l|}
24.952 \\
\end{tabular} & 23.861 & 23.311 & $\begin{array}{l}2.941 \\
\end{array}$ & 45.59 & 4.222 & -1.14 & 37.88 & & 43.281 & 0.003 & \begin{tabular}{|l|l|}
14.643 \\
\end{tabular} & & & 4.22861 & 44.1 & 10.6 & 0. & $\begin{array}{ll}0.062 \\
\end{array}$ & 2.183 & 2.186 & & & 665.96 \\
\hline 155 & $4 / 26 / 2004$ & 4:51:13 PM & 23.566 & 23.105 & \begin{tabular}{|l|}
25.037 \\
\end{tabular} & 23.846 & 23.311 & $\begin{array}{r}2.924 \\
\end{array}$ & 45.81 & 4.174 & & 37.871 & & 42.86 & 0.003 & $\begin{array}{r}-0.009 \\
\end{array}$ & & 254. & \begin{tabular}{|l|l|}
4.24528 \\
\end{tabular} & 44.3 & 10.5 & 0. & 0.062 & \begin{tabular}{|c|} 
\\
\end{tabular} & -0.001 & -0.001 & -1.488 & \\
\hline$\frac{156}{157}$ & $4 / 26 / 2004$ & $4: 52: 13 \mathrm{PM}$ & $\begin{array}{l}24.148 \\
2.309\end{array}$ & \begin{tabular}{l|l}
23.126 \\
23.2121
\end{tabular} & \begin{tabular}{|l|l|}
25.148 \\
25203
\end{tabular} & $\begin{array}{l}23.857 \\
23836\end{array}$ & $\begin{array}{l}23.332 \\
23207\end{array}$ & 25.096 & \begin{tabular}{|l|l|}
44.869 \\
\end{tabular} & 4.211 & $\frac{21.315}{36.512}$ & 14.731 & $\begin{array}{l}0.61 \\
361\end{array}$ & $\begin{array}{r}45.49 \\
5.355 \\
5\end{array}$ & 1.205 & \begin{tabular}{r|}
$\frac{14.643}{2.159}$ \\
\end{tabular} & & 255.7 & \begin{tabular}{|l|l|}
4.261949 \\
4727661 \\
\end{tabular} & 43.4 & \begin{tabular}{ll|l}
11.1 \\
11.1
\end{tabular} & $\frac{23.2}{2.2}$ & $\frac{1.600}{2601}$ & $\begin{array}{l}2.183 \\
0.322 \\
\end{array}$ & $\begin{array}{l}2.174 \\
0320\end{array}$ & 0.094 & $\begin{array}{c}93.688 \\
8349\end{array}$ & 79.73 \\
\hline & $\frac{4 / 266 / 2004}{4426 / 2004}$ & $\frac{4: 53: 13 \mathrm{PM}}{4 \cdot 54 \cdot 13 \mathrm{PM}}$ & $\frac{24.398}{24.543}$ & $\frac{23.121}{23.131}$ & \begin{tabular}{|l|}
25.203 \\
25.293
\end{tabular} & $\frac{23.826}{23867}$ & & $\begin{array}{l}\frac{40.132}{41308} \\
4\end{array}$ & $\mid \frac{45.057}{46.044}$ & $\begin{array}{l}\frac{4.254}{3.916} \\
\end{array}$ & $\frac{36.512}{33.817}$ & $\begin{array}{r}-0.882 \\
-0.87 \\
-0.8\end{array}$ & & $\begin{array}{r}45.355 \\
33.692 \\
\end{array}$ & & $\frac{2.158}{2179}$ & & & \begin{tabular}{|l|}
4.27861 \\
429528
\end{tabular} & 43.6 & $\frac{11.1}{107}$ & 势 & & & & & & $\begin{array}{l}7.10 \\
6.95\end{array}$ \\
\hline 1 & $4 / 26 / 2004$ & 4:55:13 PM & 24.644 & 23.126 & \begin{tabular}{|l|}
25.348 \\
\end{tabular} & 23.841 & 23.317 & \begin{tabular}{|}
41.00 \\
42.109
\end{tabular} & \begin{tabular}{|l|}
47.194 \\
\end{tabular} & 3.873 & 38.8911 & $\begin{array}{r}-0.01 \\
-0.763 \\
\end{array}$ & $\begin{array}{l}-3.532 \\
-3.491 \\
\end{array}$ & 42.9 & 0.00 & 2.204 & & $\frac{257.1}{258.7}$ & \begin{tabular}{|l|}
4.2 .3528 \\
.31194 \\
\end{tabular} & $\begin{array}{l}44.6 \\
45.7\end{array}$ & $\frac{10.7}{10.5}$ & 40.5 & \begin{tabular}{|l|l|l|l|l|l|l|l|}
5 & 2.793
\end{tabular} & $\frac{0.329}{0.329}$ & 0.325 & & .034 & $\begin{array}{l}6.95 \\
6.84\end{array}$ \\
\hline & $4 / 26 / 2004$ & 4:56:13 PM & 24.764 & 23.136 & \begin{tabular}{|l|}
25.468 \\
\end{tabular} & 23.851 & 23.277 & 42.716 & \begin{tabular}{|l|l|}
47.582 \\
\end{tabular} & 4.044 & 39.385 & $\begin{array}{c}-0.74 \\
\end{array}$ & -3.468 & 44.418 & & 2.204 & & 259.7 & \begin{tabular}{|l|l|}
4.32861 \\
\end{tabular} & 46.1 & 10.9 & 41.1 & & & & & & $\frac{6.84}{6.72}$ \\
\hline & 4/26/2004 & 4:57:13 PM & 24.864 & 23.141 & \begin{tabular}{|l|}
25.563 \\
\end{tabular} & 23.791 & \begin{tabular}{|l|l|}
23.237 \\
\end{tabular} & 42.086 & \begin{tabular}{|l|}
46.958 \\
\end{tabular} & 4.445 & & -0.841 & -3.462 & & 0.003 & \begin{tabular}{l|l}
2.182 \\
\end{tabular} & & & & & 10.9 & 40.2 & & & & & & $\frac{6.72}{6.78}$ \\
\hline & & & & & & & & & & & 3.101 & -1.096 & & $\begin{array}{l}46.448 \\
\end{array}$ & & & & & 4.36194 & & 11.4 & & & & & & & $\frac{.18}{62}$ \\
\hline$\frac{100}{160}$ & $4 / 26 / 2004$ & 9:13 PM & 25.033 & 23.14 & \begin{tabular}{|l|l|} 
& 25.742 \\
\end{tabular} & 23.75 & 23.221 & $\begin{array}{l}42.086 \\
\end{array}$ & \begin{tabular}{|l|l|l|}
4603 \\
\end{tabular} & 4.195 & & -1.096 & & & & & & 262 & & & & & & & & & & \\
\hline & $4 / 26 / 2004$ & 5:00:13 PM & 25.128 & 23.15 & \begin{tabular}{|l|}
25.832 \\
\end{tabular} & 23.755 & 23.246 & 43.1 & \begin{tabular}{|l|l|}
48.05 \\
\end{tabular} & 4.227 & 562 & -0.882 & -3.45 & $\begin{array}{l}45.056 \\
\end{array}$ & 0.003 & 2.169 & & 263. & $\begin{array}{l}4.39528 \\
\end{array}$ & $\begin{array}{lll}46.6 \\
\end{array}$ & 11.0 & 41. & 850 & & 0.316 & 0.008 & & \\
\hline & $4 / 26 / 2004$ & 5:01:13 PM & 25.289 & 23.151 & 25.948 & 23.816 & 23.297 & 43.571 & 48.343 & 4.176 & 40.028 & -0.882 & -3.44 & 6.657 & & 2.171 & & 264. & 4.41194 & 46.9 & 11.2 & & & 324 & 315 & & 540 & .42 \\
\hline & $4 / 26 / 2004$ & 2:13 PM & 25.389 & 23.151 & 26.028 & 23.821 & 23.3 & 43.019 & 47.706 & 4.128 & 39.552 & -0.882 & -3.4 & & & 2.168 & & 265 & 4.42861 & 46.2 & 1.2 & & 846 & & 314 & & & \\
\hline & $4 / 26 / 2004$ & 5:03:13 PM & $\frac{25.484}{25.549}$ & $\frac{23.156}{23.161}$ & 26.108 & $23.897 \mid$ & & 43.608 & 48.428 & 4.172 & 40.101 & -0.885 & & & & .167 & & 26 & & & & & & & & & & \\
\hline & $\begin{array}{r}4 / 2612004 \\
/ 26 / 2004\end{array}$ & $\begin{array}{l}5.04 .13 \mathrm{P} \\
5: 05: 13 \mathrm{PM}\end{array}$ & $\begin{array}{l}25.549 \\
25.609\end{array}$ & $\frac{23.101}{23.166}$ & \begin{tabular}{|l|}
20.173 \\
26.233 \\
\end{tabular} & $\begin{array}{l}\frac{23.904}{23.947} \\
\end{array}$ & $\begin{array}{l}23.351 \\
23.357\end{array}$ & 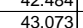 & \begin{tabular}{|l|}
47.143 \\
47.542 \\
\end{tabular} & $\begin{array}{l}4.059 \\
4.472\end{array}$ & $\begin{array}{l}38.456 \\
39.276\end{array}$ & \begin{tabular}{|l|}
-1.154 \\
-1.111
\end{tabular} & $\begin{array}{r}-3.454 \\
-3.347\end{array}$ & \begin{tabular}{|l|}
48.223 \\
46.986 \\
\end{tabular} & 0.00 & 2.097 & & $\frac{267.7}{268.7}$ & \begin{tabular}{l|l|}
7 & 4.46194 \\
7 & 4.47861
\end{tabular} & $\frac{45.7}{46.1}$ & $\frac{11.8}{11.5}$ & 41.2 & 2.839 & & $\begin{array}{l}0.303 \\
0.304 \\
\end{array}$ & $\frac{0.007}{0.007}$ & $\begin{array}{l}7.475 \\
7.395 \\
\end{array}$ & $\frac{0.30}{6.29}$ \\
\hline & 4/26/2004 & 5:06:13 PM & 25.68 & & \begin{tabular}{|l|l|}
26.288 \\
\end{tabular} & 23.958 & & & \begin{tabular}{|l||}
47.603 \\
\end{tabular} & 4.432 & & \begin{tabular}{|l|}
-1.096 \\
\end{tabular} & & \begin{tabular}{|l|}
47.62 \\
\end{tabular} & & & & 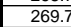 & & 46.1 & & & & & & & & \\
\hline $1 / 1$ & 4/26/2004 & 5:07:13 PM & 25.719 & 23.171 & 26.338 & 23.962 & 23.32 & 43.146 & \begin{tabular}{|l|l|l|} 
\\
\end{tabular} & $\begin{array}{l}4.424 \\
\end{array}$ & 39.404 & -1.096 & & $\begin{array}{l}46.558 \\
\end{array}$ & & 2.12 & & 270. & 4.51194 & 46.4 & 11.4 & & 846 & & 0.304 & & 7.376 & \\
\hline & $4 / 26 / 2004$ & 5:08:13 PM & 25.774 & 23.176 & 26.398 & 23.962 & 23.3 & 43.054 & \begin{tabular}{|l|} 
\\
\end{tabular} & 4.39 & 39.318 & -1.09 & -3. & 46.529 & .00 & 2.134 & & 211. & .52861 & 46. & 1.4 & & 2.840 & 0.318 & & & 7.428 & \\
\hline & $4 / 226 / 2004$ & :13 PM & 25.828 & 23.171 & 26.442 & 23.931 & & 42.725 & & 4.345 & & -1.009 & & .702 & & 2.134 & & & & 46. & 11.4 & & & 0.318 & 306 & & & \\
\hline & $4 / 2062004$ & 5:10:13 PM & 25.882 & 23.184 & \begin{tabular}{|l|}
26.466 \\
\end{tabular} & $\begin{array}{l}23.965 \\
22.9655\end{array}$ & 23.375 & 43.013 & 47.829 & 4.382 & 39.3 & -1.024 & & & & & & 273. & 年. & 46. & 11.1 & & & 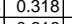 & & & & \\
\hline & $4 / 20 / 2004$ & 5:11:13 PM & & & & & & 42.934 & & & & & & & & & & & & & & & & & & & & 6.27 \\
\hline$\frac{117}{177}$ & $4 / 2612004$ & 5..4..4 & 25.975 & 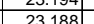 & 26.53 & $\frac{2.5044}{23.959}$ & $\frac{2.009}{2336}$ & 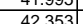 & 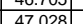 & 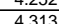 & $\begin{array}{l}\frac{30.415}{3869} \\
3864\end{array}$ & $\frac{-1.11}{-1.13}$ & & $\begin{array}{l}40.100 \\
44793\end{array}$ & & 2112 & & 276 & 619194 & 年 45.6 & 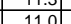 & & & & & & $\frac{1.401}{7.436}$ & \\
\hline 178 & $4 / 26 / 2004$ & 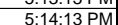 & 26.006 & 23.199 & \begin{tabular}{|l|}
26.605 \\
\end{tabular} & | & $\frac{23.015}{23.45}$ & 4 & \begin{tabular}{|l|l|}
46.993 \\
\end{tabular} & $\frac{7.52}{4.282}$ & 38.607 & $\frac{1.1125}{-1.25}$ & & 44.96 & 100 & $\overline{2.114}$ & & 277 & & $\frac{75}{45}$ & & & 2.787 & 0.315 & 0.301 & & 7.455 & \\
\hline & $4 / 26 / 2004$ & 5:15:13 PM & & & & & & & & & & & & & & & & & & & & & & & & & & \\
\hline & 4/26/2004 & 5:16:13 PM & 26.061 & 23.209 & $\frac{1.6 .65}{22.65}$ & 24.045 & 23.3 & 42.416 & 47.024 & 4.252 & $\frac{38.813}{38.813}$ & $\frac{-1.137}{-1.37}$ & & 45.62 & & 21 & & $\frac{27}{27}$ & 4.66194 & 45.6 & 11 & & 89 & $\pi$ & & & $\frac{17}{17}$ & \\
\hline & $4 / 26 / 2004$ & $13 \mathrm{PM}$ & 26.071 & 23.204 & 26.64 & \begin{tabular}{|l|l|}
24.02 \\
\end{tabular} & & & & & & & & & & & & & & & & & & & & & & \\
\hline & $4 / 26 / 2004$ & 5:18:13 PM & 26.086 & $23.204 \mid$ & \begin{tabular}{|l|l|}
26.665 \\
\end{tabular} & 23.999 & & 42.299 & & 4.2 & 38.704 & -1.099 & & & & & & 28 & & 45. & $\overline{11.1}$ & & & & & & & \\
\hline & 4/26/2004 & 5:19:13 PM & 26.106 & 23.214 & \begin{tabular}{|l|l|}
26.685 \\
\end{tabular} & 23.979 & & 42.337 & 46.944 & 4.19 & 38.8 & -1.12 & & & & & & 282 & & 45. & & & & & & & & \\
\hline & $4 / 26 / 2004$ & 5:20:13 PM & 26.105 & 23.203 & \begin{tabular}{|l|}
26.689 \\
\end{tabular} & 23.979 & 23.4 & 42.277 & 47. & 4.221 & 38.735 & -1.143 & & & & 2.118 & & 283 & & 45 & & & & & & & 7.436 & \\
\hline & $4 / 226 / 2004$ & 5:21:13 PM & 26.131 & 23.209 & 26.675 & 23.974 & & 42. & 46.954 & $4.19 \mathrm{c}$ & 38.78 & -1.099 & & & & 2.14 & & 284 & & & & & & & & & 142 & \\
\hline & 4212620004 & 5:22:13 PM & $\frac{26.146}{26.161}$ & & & 24.009 & & & & & & & & & & & & & & & 1 & & & & & & & \\
\hline & 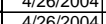 & $0.23 .13 \mathrm{P}$ & $\frac{2.101}{26.61}$ & 2030.239 & \begin{tabular}{|l|l|l|l|} 
\\
\end{tabular} & 24.034 & & & & & & -1.09 & & & & & & & & & & & & & & & & \\
\hline & 4 & 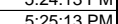 & $\frac{2.10101}{26.171}$ & $\frac{2.4213}{2322}$ & \begin{tabular}{|l|l|}
2675 \\
\end{tabular} & $\frac{24.014}{24065}$ & & $\begin{array}{ll}42,4 \\
423\end{array}$ & & & & -1.009 & & & & & & 20 & & $\frac{45}{45}$ & & & & & & & & \\
\hline & $\frac{4}{4 / 2612004}$ & 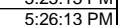 & 26.19 & $\frac{23.241}{23.241}$ & 26.767 & 24.167 & & & & & & & & & & & & & & & & & & & & & & \\
\hline & $4 / 26 / 2000$ & $5 \cdot 27 \cdot 13 \mathrm{PM}$ & 26.19 & & & & & & & & & -1.13 & & & & & & & & & & & & & & & & \\
\hline & & & & & & & & & & & & & & & & & & & & & & & & & & & & \\
\hline & $4 / 26 / 2004$ & $5: 29: 13 \mathrm{~F}$ & 26.23 & 23.289 & \begin{tabular}{|l|}
26.799 \\
\end{tabular} & 24.33 & & 424 & & & 38. & -1.0 & & & & & & & & & & & & & & & & \\
\hline & $4 / 26 / 2004$ & $5: 30: 13 \mathrm{~F}$ & & 305 & 26. & 24.3 & & & & & & -1.0 & & & & & & 29 & & & & & & & & & & \\
\hline & $4 / 26 / 2004$ & 5:31:13 PM & 26.253 & & & 24. & & & & & & -1.08 & & & & & & & & & & & & & & & & \\
\hline 19 & $4 / 26 / 2004$ & 5:32:13 PM & 26.254 & 23.332 & 26.823 & 24.374 & & 42.693 & 47.3 & $4.0 \mathrm{~s}$ & & -1.095 & & & & & & $29 !$ & & 45.9 & & & & & & & 7.346 & \\
\hline & $4 / 226 / 2004$ & 5:33:13 PM & 26.265 & 23.354 & \begin{tabular}{|l|l}
26.834 \\
\end{tabular} & 24.4 & & & 47.516 & 4.14 & & -1.0 & & & & & & & & & & & & & & & & \\
\hline & & & 26.25 & 23.364 & & & & & & & & & & & & & & & & & & & & & & & & \\
\hline & 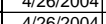 & 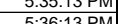 & & 23.386 & 20.851 & & & 42 & 4.2 & & & -1.08 & & & & & & & & & & & & & & & & \\
\hline & 4 & 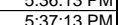 & 26284 & & & & & & & & & -1.06 & & & & & & & & & & & & & & & & \\
\hline & 4 & $5: 38: 13 \mathrm{P}$ & 26.295 & 23.429 & \begin{tabular}{|l|l|}
26.864 \\
\end{tabular} & 24,315 & & 42. & & $\frac{4.4}{4}$ & & \begin{tabular}{|l|l|} 
& -1.05 \\
\end{tabular} & & $\frac{4.34}{44}$ & 0.0 & & & 30 & & 46 & & & & & & & 7340 & \\
\hline & $4 / 26 / 2004$ & & & & & 24.266 & & & & & & & & & & & & & & & & & & & & & & \\
\hline & & $13 \mathrm{P}$ & 2630 & 2343 & 26896 & & & 426 & 474 & & & -1.096 & & 443 & & & & & & & & & & & & & & \\
\hline & $4 / 26 / 2004$ & $5: 41: 13 \mathrm{~F}$ & 26.309 & 23.443 & 26.882 & 24.273 & 23.68 & 42.231 & \begin{tabular}{|l|l|}
47.101 \\
\end{tabular} & 4093 & 38.8 & -1.09 & & 44.5 & & $\overline{2.1}$ & & & & 456 & 100 & & 27 & & & & & \\
\hline & $4 / 26 / 2004$ & $5: 42: 13 \mathrm{~F}$ & 26300 & 23.444 & \begin{tabular}{|l|l|}
26.878 \\
\end{tabular} & 24.284 & & 42.6 & 47.2 & 4.0 & & $-1.0 \subseteq$ & & & & & & & & & & & & & & & & \\
\hline & 4/26/2004 & 5:43:13 PI & & 23.455 & 26.8 & & & 42.511 & & 4.0 & & -1.09 & & & & & & & & 45.7 & & & & & & & & \\
\hline & 4/26/2004 & 5:44:13 PM & 26.311 & 23.451 & 26.89 & 24.241 & & 42.5 & 47.354 & 4.116 & 39.051 & -1.096 & & 44.2 & 0.0 & 2.1 & & 307. & 5.12 & 45.9 & 10.8 & & & & 0.300 & 077 & 7.366 & \\
\hline & \begin{tabular}{l|l|}
$4 / 26 / 2004$ \\
$4 / 26 / 2004$
\end{tabular} & $\begin{array}{l}5: 45: 13 \mathrm{PM} \\
5: 4: 4: 3 \mathrm{PM}\end{array}$ & $\frac{26.327}{26316}$ & \begin{tabular}{|l|l|}
23.451 \\
23.446
\end{tabular} & \begin{tabular}{|l|}
26.911 \\
26895 \\
\end{tabular} & $\begin{array}{l}24.262 \\
24221 \\
\end{array}$ & & \begin{tabular}{r|}
42.75 \\
42571
\end{tabular} & \begin{tabular}{|l|}
47.509 \\
47006 \\
\end{tabular} & $\begin{array}{r}4.09 \\
4.085\end{array}$ & $\begin{array}{l}39.371 \\
339.150\end{array}$ & \begin{tabular}{|l|l|} 
& -1.056 \\
& -1.053 \\
\end{tabular} & & $\begin{array}{l}44.332 \\
43.909 \\
\end{array}$ & \begin{tabular}{|l|l|}
0.003 \\
0.03
\end{tabular} & \begin{tabular}{|l|l|}
2.129 \\
2129
\end{tabular} & & $\begin{array}{l}308 \\
309 \\
309\end{array}$ & 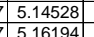 & \begin{tabular}{ll|}
46.0 \\
456
\end{tabular} & \begin{tabular}{|l|}
10.9 \\
108
\end{tabular} & & 年. & $\mid$\begin{tabular}{|l|l|l|l|l|l|l|} 
\\
\end{tabular} & $\begin{array}{l}0.301 \\
0.301\end{array}$ & $\begin{array}{l}0.007 \\
0.007\end{array}$ & \begin{tabular}{|c|}
7.328 \\
7338
\end{tabular} & \\
\hline
\end{tabular}


WSRC-TR-2005-00105, REVISION 0

SRNL-RPP-2005-00012, REVISION 0

RUN \# 2.18 \& 2.19; ACID CLEANING AND CAUSTIC RINSING - CONT.

\begin{tabular}{|c|c|c|c|c|c|c|c|c|c|c|c|c|c|c|c|c|c|c|c|c|c|c|c|c|c|c|c|c|}
\hline & A & B & 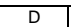 & \begin{tabular}{|l|l|}
$E$ &
\end{tabular} & $F$ & $G$ & $\mathrm{H}$ & $\mathrm{J}$ & $\mathrm{K}$ & $\mathrm{L}$ & M & $\mathrm{N}$ & 0 & \begin{tabular}{l|l} 
\\
\end{tabular} & $R$ & s & $T_{T}$ & $\mathrm{v}$ & w & \begin{tabular}{|l|}
$x$ \\
\end{tabular} & \begin{tabular}{|l|l|} 
\\
\end{tabular} & $z_{z}$ & AA & $A B$ & AC & $\mathrm{AD}$ & $\mathrm{AE}$ & \\
\hline & $4 / 26 / 2004$ & 5:47:13 PM & 26.333 & 23.457 & 26.887 & 24.262 & 23.672 & 42.482 & 47.055 & 4.066 & \begin{tabular}{l|l}
6 & 39.092 \\
\end{tabular} & -1.096 & & 43.59 & $\begin{array}{l}.003 \\
\end{array}$ & \begin{tabular}{|l|l|}
3 & 2.127 \\
\end{tabular} & 0.006 & 310.7 & 5.17861 & \begin{tabular}{|l|}
45.6 \\
\end{tabular} & \begin{tabular}{|l|}
50.7 \\
5
\end{tabular} & 40.8 & 2.812 & 0.317 & 0.301 & 0.007 & 7.375 & $\frac{A r .28}{6.28}$ \\
\hline & 4/26/2004 & $5: 48: 13$ PM & & 23.447 & $\begin{array}{l}26.872 \\
2.97\end{array}$ & $\begin{array}{l}24.232 \\
2.283\end{array}$ & 23.642 & 42.841 & $\begin{array}{r}47.59 \\
\end{array}$ & 4.085 & 39.398 & & & 45.156 & & & 0.005 & 311.7 & 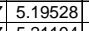 & 46.1 & \begin{tabular}{|l|}
11.1 \\
\end{tabular} & & & D... & & 0.007 & & \\
\hline$\frac{213}{214}$ & $\begin{array}{l}4 / 26 / 2004 \\
4 / 261 / 204\end{array}$ & $\begin{array}{l}5: 49: 13 \mathrm{PM} \\
5: 50.13 \mathrm{PM}\end{array}$ & $\begin{array}{l}26.338 \\
26.323\end{array}$ & $\begin{array}{ll}23.463 \\
23.448\end{array}$ & \begin{tabular}{|l|}
26.907 \\
26862
\end{tabular} & $\begin{array}{r}24.283 \\
24.308\end{array}$ & & $\begin{array}{r}42.496 \\
259 \\
\end{array}$ & \begin{tabular}{|l|l|}
47.424 \\
\end{tabular} & $\begin{array}{l}4.08 \\
406\end{array}$ & $\begin{array}{l}39.057 \\
39.194\end{array}$ & -1.096 & & & $\begin{array}{l}0.003 \\
0.003\end{array}$ & $\begin{array}{l}2.128 \\
2.27\end{array}$ & $\begin{array}{l}0.006 \\
0.005\end{array}$ & & & 46.0 & 10.6 & 40.8 & $\begin{array}{l}2.811 \\
2.819 \\
\end{array}$ & 0.317 & 0.301 & & 7.376 & $\begin{array}{l}6.28 \\
6.26\end{array}$ \\
\hline 215 & $4 / 2612004$ & $5: 51: 13 \mathrm{PM}$ & & 23.468 & $\frac{\mid 26.757}{22.757}$ & $\begin{array}{l}24.000 \\
24.449\end{array}$ & $\frac{23.673}{23.688}$ & $\begin{array}{r}42.59 \\
42.337\end{array}$ & & & $\begin{array}{l}39.194 \\
38.999 \\
\end{array}$ & $\frac{-1.096}{-1.122}$ & & $\begin{array}{l}44.2899 \\
44.454\end{array}$ & & $\frac{2.1218}{2.118}$ & $\begin{array}{l}0.005 \\
0.005\end{array}$ & $\begin{array}{l}313.7 \\
314.7\end{array}$ & & $\frac{45.9}{45.5}$ & $\frac{10.9}{10.9}$ & & & $\frac{0.317}{0.316}$ & 0.301 & 0.007 & & \\
\hline 216 & $4 / 26 / 2004$ & 5:52:13 PM & 26.189 & 23.463 & 26.633 & 24.439 & \begin{tabular}{|l|}
23.713 \\
\end{tabular} & 42.581 & \begin{tabular}{|l|l|}
47.143 \\
\end{tabular} & 4.05 & 39.213 & -1.102 & & $\begin{array}{l}43.869 \\
43.09\end{array}$ & 0.003 & $\frac{2.112}{2.12}$ & $\begin{array}{l}0.005 \\
0.004\end{array}$ & $\begin{array}{l}314.7 \\
315.7\end{array}$ & \begin{tabular}{|l|l|l}
5.24528 \\
5.26194
\end{tabular} & \begin{tabular}{|l|}
45.5 \\
45.7 \\
\end{tabular} & \begin{tabular}{|c|}
10.9 \\
10.8
\end{tabular} & \begin{tabular}{l|l} 
& 40.6 \\
& 40.9 \\
\end{tabular} & $\frac{2.801}{2.820}$ & $\mid$ & $\frac{0.301}{0.302}$ & $\begin{array}{l}0.007 \\
0.007\end{array}$ & $\begin{array}{r}7.399 \\
7.383 \\
\end{array}$ & $\frac{6.30}{6.28}$ \\
\hline 217 & & & 26.075 & 23.469 & 26.519 & 24.455 & 23.754 & 42.613 & 47.207 & 4.052 & 39.238 & -1.137 & & 43.926 & 0.003 & & & 316.7 & 5.27861 & \begin{tabular}{|l|}
45.7 \\
\end{tabular} & 10.8 & & & 0.316 & 0.302 & & & $\frac{6.28}{6.29}$ \\
\hline & $4 / 26 / 2004$ & 5:54:13 PM & 25.971 & 23.47 & 26.415 & 24.451 & $\begin{array}{l}23.69 \\
\end{array}$ & 42.776 & & 4.044 & & -1.154 & -3.066 & 43.999 & 0.003 & 2.113 & 0.004 & & & & & & & & & & & $\frac{6.29}{6.27}$ \\
\hline 219 & & 5:55:13 PM & & & & & & $\begin{array}{l}42.874 \\
\end{array}$ & 47 47.441 & & 39.496 & & & & & & & 318.7 & 5.31194 & 46.0 & 11.0 & & & 0.314 & .302 & & $\begin{array}{l}7.311 \\
7.344\end{array}$ & $\frac{6.27}{6.25}$ \\
\hline$\frac{23}{3}$ & & 5:56:13 PM & 25.808 & 23.482 & $\begin{array}{l}26.242 \\
\end{array}$ & 24.468 & & 42.492 & 47.308 & 4.099 & 39.057 & -1.154 & & \begin{tabular}{|l|l|}
43.692 \\
\end{tabular} & 0.00 & 2.106 & & 31 & 5.32861 & 45.8 & 10.7 & & 2.811 & 0.314 & 0.303 & & 7.437 & $\begin{array}{l}0.25 \\
6.33 \\
\end{array}$ \\
\hline & $4 / 26 / 2004$ & 5:57:13 PM & 25.714 & 23.492 & 26.158 & 24.463 & & $\begin{array}{ll}42.498 \\
\end{array}$ & 346.904 & $4.03 \varepsilon$ & 39.096 & -1.154 & & 44.476 & 0.003 & 2.105 & & 320.7 & 5.34528 & 45.4 & 10.9 & & 2.813 & 0.314 & & & & \\
\hline & $4 / 26 / 2004$ & 5:58:13 PM & 25.634 & 23.488 & \begin{tabular}{|l|l|}
26.078 \\
\end{tabular} & 24.444 & 23.668 & \begin{tabular}{|l|l|l|l|}
42.787 \\
\end{tabular} & 47.362 & 4.03 & 39.424 & -1.154 & & 45.34 & 0.003 & 2.11 & & 321.7 & 5.36194 & 45.9 & $\begin{array}{l}11.1 \\
\end{array}$ & 41. & 2.834 & | 0.315 & 0.305 & 0.007 & 7.425 & 6.32 \\
\hline & $4 / 26 / 2004$ & 5:59:13 PM & 25.56 & 23.494 & 26.014 & 24.46 & 23.734 & 43.085 & \begin{tabular}{|l|l|}
47.889 \\
\end{tabular} & 4.06 & 39.697 & -1.154 & & $\begin{array}{l}44.011 \\
\end{array}$ & 0.003 & 2.097 & & 322.7 & 5.37861 & 46.4 & & & 2.854 & 0.313 & 0.304 & 0.007 & 7.342 & \\
\hline & & 6:00:13 PM & & & 25.955 & & & & 47.273 & & 39.406 & 1.209 & & & & 2.1 & & & . & 45. & 11.0 & & & & & & & \\
\hline & 4/26/2004 & 6:01:13 PM & 25.4166 & 23.505 & 25.87 & 24.451 & 23.745 & 42.532 & $\begin{array}{r}47.19 \\
\end{array}$ & 4.022 & 39.188 & -1.177 & & 44.495 & 0.0 & 2.091 & & 32 & ..41194 & 45.7 & 10.9 & & 20.011 & 0.312 & 0.304 & 007 & 7.446 & \\
\hline$\frac{\frac{250}{227}}{227}$ & $\frac{4 / 26 / 2004}{4}$ & 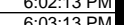 & & | & 25.814 & 24.422 & & $\begin{array}{l}43.013 \\
42608\end{array}$ & (47.594 & 年 & & $\begin{array}{l}-1.218 \\
1.105\end{array}$ & & $\begin{array}{l}44.122 \\
44272\end{array}$ & & $\begin{array}{l}2.093 \\
2092\end{array}$ & & 325.7 & & 46.1 & $\frac{10.8}{10.8}$ & 41.3 & 2.846 & 0.312 & 0.305 & & & 6.29 \\
\hline 228 & $4 / 26 / 2004$ & $\begin{array}{l}6.00 .13 \\
6: 04: 13 \mathrm{PM}\end{array}$ & $\begin{array}{l}25.290 \\
25.243\end{array}$ & $\frac{20.017}{23.517}$ & $\begin{array}{l}25.7121 \\
25.712\end{array}$ & $\begin{array}{r}24.090 \\
24.413\end{array}$ & 23.707 & $\begin{array}{r}42.000 \\
42.65 \\
\end{array}$ & $\begin{array}{r}47.200 \\
47.12 \\
\end{array}$ & 4.016 & 39.19 & $\begin{array}{l}-1.195 \\
-1.218\end{array}$ & & \begin{tabular}{|c|}
44.112 \\
44.18
\end{tabular} & $\frac{0.003}{0.003}$ & 2.095 & & $\begin{array}{l}32.1 .1 \\
327.7\end{array}$ & 5.461928 & $\frac{43.0}{45.6}$ & \begin{tabular}{l|}
10.0 \\
10.8
\end{tabular} & $\frac{71}{41}$ & $\frac{2.526}{2.826}$ & 0.312 & 0 & $\begin{array}{l}0.007 \\
0.007\end{array}$ & $\begin{array}{l}7.464 \\
7.468 \\
\end{array}$ & $\frac{6.35}{6.36}$ \\
\hline & $4 / 26 / 2004$ & 6:05:13 PM & 25.189 & 23.518 & 25.648 & $\begin{array}{l}24.4114 \\
2.434\end{array}$ & 23.73 & \begin{tabular}{|l|l|l|l}
43.009 \\
\end{tabular} & 447.688 & 4.08 & 39.572 & -1.218 & -3.04 & 44.159 & 0.003 & 2.088 & & 328.7 & 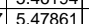 & 46.2 & $\frac{10.8}{10.8}$ & & $\frac{2.020}{2.847}$ & $\frac{1.511}{0.311}$ & 0.306 & 0.007 & 7.403 & $\frac{6.36}{6.30}$ \\
\hline & $4 / 26 / 2004$ & $6: 06: 13 \mathrm{PM}$ & & & & & & & & & & & & & & & & & & & & & & & & & & \\
\hline 231 & 4/26/2004 & 6:07:13 PM & 25.116 & 23.54 & 25.595 & 24.431 & 23. & 42.743 & 47.163 & 4.072 & 39.338 & -1.203 & & 45.063 & & 2.088 & & 330.7 & 51194 & 45.7 & 11.0 & & 2.83 & 0.311 & 0.306 & 0.007 & 7.460 & \\
\hline & $4 / 26 / 2004$ & 6:08:13 PM & & & & & & 43.063 & 47.733 & 4.087 & & & & & & & & 33 & 52861 & 46.3 & & & & 0.311 & 0.306 & & 7.402 & \\
\hline 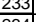 & 4/26/2004 & 6:09:13 PM & & 23.526 & 25.496 & 24.416 & & 42.776 & & & 39.587 & $-1.2 \angle 1$ & & & & & & 33 & & 45. & & & & & 0.300 & & & \\
\hline 234 & $4 / 26 / 2004$ & 6:10:13 & 24.987 & 23.541 & 25.451 & 24.426 & 23.746 & $\begin{array}{l}42.772 \\
\end{array}$ & 47.269 & 4.07 & 39.342 & -1.218 & & 43.137 & 0.00 & 2.087 & & 333 & 5.56194 & 45.8 & 10.6 & & 283 & & 0.307 & & 7.483 & 20 \\
\hline & $4 / 26 / 2004$ & 6:11:13 PM & 24.947 & 23.541 & 25.411 & 24.431 & 23.7 & $\begin{array}{l}42.874 \\
\end{array}$ & 47.723 & 4.072 & 39.441 & -1.177 & & $\begin{array}{l}44.5899 \\
\end{array}$ & $0.0-2$ & 2.087 & & 334.7 & 5.57861 & 46.3 & 10.9 & & .838 & 0.311 & 0.308 & .007 & 7.473 & \\
\hline & $4 / 26 / 20004$ & 6:12:13 PM & 24.917 & 23.546 & 25.401 & 24.487 & & 42.778 & $\begin{array}{l}37.039 \\
\end{array}$ & 4.075 & 39.357 & -1.221 & & 44.212 & & 2.081 & & & 59528 & 45.6 & 10.8 & & & & 0.307 & & 470 & \\
\hline & $4 / 26212004$ & 6:13:13 PM & & 23.551 & $25.3 / 1$ & 24.537 & & 43.087 & 47.65 & 4.100 & 39.619 & & & 44.1399 & & 2.079 & & & 5.61194 & 46.2 & & & & & & & & \\
\hline$\frac{23}{3}$ & 42126212004 & $6: 14.13 \mathrm{MM}$ & 24.852 & $\frac{23.567}{23579}$ & $\begin{array}{l}25.360 \\
25312\end{array}$ & $\begin{array}{l}24.628 \\
2.675\end{array}$ & . & $\begin{array}{r}42.77 \\
40.965\end{array}$ & $\begin{array}{l}47.298 \\
47.431\end{array}$ & 4.108 & $\begin{array}{l}39.299 \\
30498\end{array}$ & $-\frac{1.218}{1.201}$ & & (43.9599 & 0.0 & $\frac{2.08}{2078}$ & & & $\begin{array}{l}5.62861 \\
564528\end{array}$ & 45.8 & 10.8 & & & 0.310 & & & 7.486 & \\
\hline 240 & $\begin{array}{ll}/ 26 / 2004 \\
/ 26 / 204\end{array}$ & $\begin{array}{l}0.15 .15 \mathrm{PM} \\
6.16: 13 \mathrm{PM}\end{array}$ & $\frac{24.029}{24.816}$ & $\frac{23.57}{236}$ & $\begin{array}{r}25.15 \\
25.3\end{array}$ & $\begin{array}{l}24.015 \\
24716\end{array}$ & & $\begin{array}{r}\frac{42.900}{43.11} \\
\end{array}$ & \begin{tabular}{|l|l|}
47.451 \\
47.683
\end{tabular} & 4.08 & $\begin{array}{l}53.400 \\
39703\end{array}$ & $\frac{1.241}{-1.201}$ & & 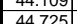 & & $\begin{array}{l}2.0810 \\
2.081\end{array}$ & & 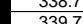 & $\begin{array}{l}5.004520 \\
566194\end{array}$ & 460 & 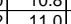 & & & 0.310 & 年 & . & 1.4430 & \\
\hline & $4 / 26 / 2004$ & $6.1717 \cdot 13 \mathrm{PM}$ & 24.792 & $\begin{array}{c}23.622 \\
2362\end{array}$ & $\begin{array}{r}25.286 \\
\end{array}$ & 24,763 & 24.1 & 年.5.15 & 47.489 & $\frac{4.072}{4.072}$ & 39.449 & 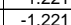 & & 44.249 & 0.003 & $\frac{2.001}{2.079}$ & & 340 & $\begin{array}{l}5.07867 \\
5\end{array}$ & $\frac{40.6}{46.0}$ & 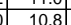 & $\frac{41}{41}$ & 2838 & 0 & 307 & 0.007 & $\begin{array}{l}7.450 \\
7.470\end{array}$ & $\frac{32}{36}$ \\
\hline 242 & 6/2004 & $6: 18: 13 \mathrm{PM}$ & & 23.638 & 25.262 & $\begin{array}{l}24.774 \\
\end{array}$ & & & 47.542 & & & -1.218 & & & & 2.079 & & & & 46.1 & 10.8 & & & 310 & & & & \\
\hline & & 6:19:13 PM & 24.735 & 23.649 & 25.234 & 24.745 & & \begin{tabular}{|l|l|l|l|l}
42.484 \\
\end{tabular} & 447.02 & 4.085 & 39.034 & -1.221 & & 43.884 & 0.00 & 2.075 & & 34 & $\frac{71194}{77194}$ & 45.5 & 10.8 & 40. & 2810 & 0.309 & 0.307 & 0.008 & 7540 & 6.42 \\
\hline 244 & $4 / 26 / 2004$ & 7:00:42 PM & 22.796 & 23.415 & 23.401 & 24.165 & 23. & 41.992 & 47.041 & 4.245 & 38.369 & -0.723 & & 44.631 & & 2.1 & & & & 45.6 & 10.9 & & & & 0.343 & & 8.529 & \\
\hline 245 & $4 / 26 / 2004$ & 7:01:42 PM & & & & & & 24.816 & & 4.14 & 20.888 & 17.701 & & 43.753 & & & & & 6.42 & 46.3 & & & 1.576 & & & & & \\
\hline 246 & 6 & 7:02:42 PM & 22.898 & 23.406 & \begin{tabular}{|l|}
23.578 \\
\end{tabular} & 24.146 & & 3.115 & \begin{tabular}{|l|l|} 
\\
\end{tabular} & 4.10 & & 39.924 & & 43.204 & & & & & 6.43667 & \begin{tabular}{l|l|l|l|l}
46.4 \\
\end{tabular} & 10.6 & & & & & & & \\
\hline 247 & $4 / 26 / 2004$ & 7:03:42 PM & 22.923 & 23.421 & \begin{tabular}{|l|l|}
23.673 \\
\end{tabular} & 24.171 & 23.4 & 2.626 & 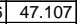 & 4.072 & -1.257 & 40.118 & & 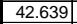 & 0.0 & -0.012 & & & 6.45333 & 45.6 & \begin{tabular}{l|l|}
10.4 \\
\end{tabular} & 0 & 0.047 & & $\overline{002}$ & $\overline{003}$ & 714 & -2.31 \\
\hline 248 & $4 / 26 / 2004$ & 7:04:42 PM & 22.938 & 23.401 & 23.708 & 24.156 & 23.4 & 2.926 & \begin{tabular}{|l|l|l|}
47.949 \\
\end{tabular} & 4.065 & & 40.019 & & 42.359 & 0.00 & $\begin{array}{l}-0.013 \\
\end{array}$ & & 388.2 & 6.47 & 46.5 & 10.4 & & 0.067 & \begin{tabular}{|l|l|}
-0.002 \\
\end{tabular} & -0.002 & 002 & & -1.76 \\
\hline$\frac{24}{25}$ & $4 / 26 / 2004$ & 7:05:42 PM & 23.228 & 23.39 & 23.732 & 24.145 & & 2.543 & 47.69 & 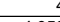 & -1.214 & 40.477 & & 42.000 & & 14.64 & & 38 & tont & 40.6 & 10.4 & & 0 & 2.105 & 2.200 & & & \\
\hline & & & & & & & & & 46.894 & 4.05 & & 3.162 & & & & & & & & & & & & & & & & \\
\hline$\frac{201}{252}$ & $\frac{4 / 266 / 2004}{4}$ & 0.00 .4240 & 年3.263 & 23.3899 & 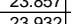 & $\frac{24.155}{2417}$ & & & $\begin{array}{l}4.47 .445 \\
147425\end{array}$ & 先 4.065 & $\begin{array}{l}38.402 \\
38665\end{array}$ & 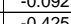 & & $\begin{array}{l}44.011 \\
4408\end{array}$ & 0 & & & & 6.524 & 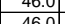 & 10.8 & 404 & & & 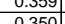 & & & \\
\hline$\frac{253}{253}$ & $4 / 26 / 20004$ & 1.0 .454 & $\frac{20.050}{23.393}$ & 23.509 & $\begin{array}{l}2.952 \\
23.988 \\
\end{array}$ & $\frac{2.11}{24.7}$ & & & $\begin{array}{l}\mid 41.450 \\
548081\end{array}$ & 年. & $\begin{array}{l}30.0005 \\
33.344\end{array}$ & $\begin{array}{l}-0.425 \\
-0.506\end{array}$ & & 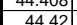 & & $=$ & & & & 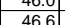 & 109 & & & & & & & $7.3 \mathrm{E}$ \\
\hline 254 & $4 / 26 / 2004$ & 7:10:43 PM & 23.443 & 23.384 & 24.043 & 24.175 & 23. & $\begin{array}{l}42.239 \\
\end{array}$ & \begin{tabular}{|l|l|}
477.263 \\
\end{tabular} & 4.32. & 38.581 & -0.691 & & $\begin{array}{l}455.492 \\
\end{array}$ & 0.00 & 2.22 & & 394 & 6.57028 & 45.8 & 111.1 & 40 & 2786 & & 0.340 & & 8.415 & 1.1 \\
\hline & $4 / 26 / 2004$ & 7:11:43 PM & 23.489 & \begin{tabular}{l|l|}
23.37 \\
\end{tabular} & 24.063 & 24.14 & & 42.806 & \begin{tabular}{|l|l|}
477.901 \\
\end{tabular} & 4.3 & 39.157 & -0.705 & & $\begin{array}{l}44.731 \\
\end{array}$ & & 2.211 & & & & $\begin{array}{l}46.4 \\
\end{array}$ & $\begin{array}{l}411.0 \\
4\end{array}$ & & & & & & & \\
\hline & & & & & & & & 42.206 & & 4.215 & 38.722 & & & & & & & & & 45.9 & 11.0 & & & & & & & 7.08 \\
\hline & $4 / 2012004$ & $1.158 .45 \mathrm{PM}^{-1}$ & 23.604 & 20.379 & 24.213 & & & 42.098 & 41.00 & $4.19 \mathrm{c}$ & 39.155 & -0. & & & & 2.20 & & 39 & \begin{tabular}{|l|}
6.62028 \\
\end{tabular} & 46.2 & 11.0 & 40.9 & 2.822 & $\begin{array}{ll}0.329 \\
\end{array}$ & 0.336 & & 8.213 & 6.99 \\
\hline$\frac{250}{259}$ & $\frac{4 / 2012004}{4 / 26 / 2004}$ & $\frac{1.4 .4}{7.15:}$ & $\frac{23.035}{23678}$ & $\frac{2.3044}{23.358}$ & $\mid \begin{array}{ll}24.252 \\
2452\end{array}$ & $\frac{24.159}{24.143}$ & & 年 4284 & 5 & & $\frac{30.951}{39256}$ & -0 & & & & | & & & 6.63694 & $\frac{46.1}{463}$ & $\frac{11.0}{110}$ & $\begin{array}{ll}40.8 \\
41.0\end{array}$ & $\frac{2.811}{2829}$ & & & & 告14 & 6.99 \\
\hline & $4 / 26 / 2004$ & $7: 1: 4: 4$ & $\frac{23 .}{23 .}$ & 23.352 & $\frac{24.42}{22.25}$ & 241 & & 42.928 & 34.953 & 4.995 & & & & & & & & & & 46.5 & 10.9 & & & & & & & \\
\hline 261 & & & 23.741 & & & 24.102 & & & 247.259 & & 38.8 & & & & & 2.18 & & & & 458 & (and & & & & & & & \\
\hline & & & 23.78 & 23. & 24.354 & 24.111 & & & \begin{tabular}{|l|l|}
47.953 \\
\end{tabular} & 4.195 & & & & & & & & & & & & & & & & & & \\
\hline 263 & 4/26/2004 & & 23815 & 23.339 & 24.384 & 24.16 & & 42.756 & 477.887 & & 39.22 & -0.818 & & 44 & & 2.18 & & & & 46.4 & 110 & & & & & & & \\
\hline 264 & 4/26/2004 & $7: 20:$ & 23.844 & 23.334 & 24.398 & $\begin{array}{l}24.21 \\
\end{array}$ & & & 47. & $4.14 !$ & 39.276 & & & & & 2.1 & & & & 46 & 10.3 & & & & & & & \\
\hline & $4 / 26 / 2004$ & $7: 21: 43$ & 23.88 & 23.344 & \begin{tabular}{|l|}
24.429 \\
\end{tabular} & 24.26 & & 43. & 48. & & 39.689 & -0. & & & & 2.17 & & & & 46. & 10.9 & & & & & & & \\
\hline & $4 / 26 / 2004$ & $7: 22: 43$ & 23.899 & 23.354 & 24.463 & 24.295 & & $\frac{41 .}{41 .}$ & \begin{tabular}{|l|l|l|}
46.108 \\
\end{tabular} & 4.212 & 37.955 & -1.154 & & 44.19 & 0.0 & 2.1 & & & & 44.6 & 10.8 & & 2.739 & & 0.318 & .0008 & 8.002 & \\
\hline & $4 / 26 / 2004$ & 7:23: & 23.911 & 23.355 & 24.47 & 24.366 & & 42.449 & $\begin{array}{l}37.116 \\
\end{array}$ & 4.165 & 38. & -1. & & & 0.0 & 2.139 & & & & 45. & & & & & & & & \\
\hline & & & 23.926 & 23.311 & 24.485 & 24.41 & & 42.46 & 47.34 & 4.152 & & & & & & 2.14 & & & & 45 & . & & & & & & & \\
\hline & 4212012004 & 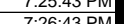 & 年3.953 & $\frac{2.3936}{2340}$ & 24.4517 & 24.44 & & $\frac{4.2 .}{12.09}$ & 47.134 & & 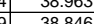 & & & & & 2.14 & & & & & 105 & & & & & & & \\
\hline$\frac{\pi}{2}$ & 42126004 & $\frac{1.26 .43}{7 \cdot 72.43}$ & 20.500 & $\frac{20.405}{2345}$ & 24544 & & & 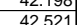 & & & & & & & & & & & & & & & & & & & & \\
\hline$\frac{172}{272}$ & $4 / 2662004$ & $772: 43$ & 2 2.3.99 & 23.3 & 24. & 24.4 & & 42,499 & 47.275 & 3.921 & 39.26 & -0.8 & & 43.22 & 0.00 & 22158 & & 41 & & 458 & 106 & & & & 0.020 & & 7973 & \\
\hline & $4 / 26 / 2004$ & & 24.012 & 23.437 & 24.546 & 24.413 & & 42.26 & 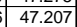 & 3.94 & & -0.92 & & 43.5 & & 2.15 & & & & 45.1 & 10.6 & & & & & & & \\
\hline & & & & 23.447 & 24.557 & 24.418 & & 42.355 & $\mid$ & 3.903 & 39.127 & & & 42.80 & & 2.15 & & & & 45.7 & & & & & & & & \\
\hline & $4 / 26 / 2004$ & $7: 31: 43 \mathrm{~F}$ & 24.022 & 23.442 & 24.562 & 24.358 & 23. & 42.581 & \begin{tabular}{|l|l|}
47281 \\
\end{tabular} & $3.90 \mathrm{~s}$ & 39.363 & -0.888 & & 43.141 & & 2.159 & & $\overline{41}$ & 8 & 45.8 & 10.6 & & & & & & 54 & \\
\hline & $4 / 26 / 2004$ & 7:32:43 & 24.012 & 23.437 & 24.547 & 24.3 & & 42.472 & 47. & 3.89 & & $-0 . \varepsilon_{-1}$ & & & & 2.1 & & & & 458 & 15 & & & & & & & \\
\hline & $4 / 26 / 2004$ & 7:33:43 P| & 24.037 & 23.452 & 24.567 & 24.31 & & 42.561 & 47.464 & 3.8 & 39.3 & -0.8 & & 42 & & 2.15 & & 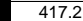 & & & & & & & & & 49 & \\
\hline $278 \mathrm{CP}$ & 4/26/2004 & 7:34:43 PM & 24.047 & 23.447 & 24.557| & 24.288 & & 43.102 & 48.069 & 3.877 & 39.909 & -0.934 & & 42.966 & 0.00 & 2.157 & & 41 & \begin{tabular}{|c|c|c|}
6.97028 \\
\end{tabular} & \begin{tabular}{|l|l|}
46.6 \\
\end{tabular} & 10.5 & $\begin{array}{l}5 \\
5\end{array}$ & 2.862 & 0.32 & 0.326 & 0.008 & 7.846 & 6.68 \\
\hline 279 & $\begin{array}{l}/ 26 / 2004 \\
4 / 26 / 2004\end{array}$ & $\begin{array}{l}7: 35: 43 \mathrm{PM} \\
7 \cdot 36: 43 \mathrm{PM}\end{array}$ & \begin{tabular}{|l|l}
24.047 \\
24057 &
\end{tabular} & $\begin{array}{l}23.452 \\
23447\end{array}$ & \begin{tabular}{|l|}
24.571 \\
2451 \\
\end{tabular} & $\begin{array}{l}24.247 \\
24227 \\
\end{array}$ & & $\begin{array}{ll}42.552 \\
42.416\end{array}$ & \begin{tabular}{|l|l|}
47.443 \\
47.153
\end{tabular} & $\begin{array}{l}3.86 \\
3.352 \\
\end{array}$ & $\begin{array}{l}39.377 \\
39281 \\
3928\end{array}$ & $\begin{array}{l}-0.934 \\
-0.894 \\
\end{array}$ & & \begin{tabular}{r|r|}
42.9 \\
42678
\end{tabular} & 0.003 & $\begin{array}{ll}2.162 \\
216 \\
\end{array}$ & & $\begin{array}{ll}419.2 \\
4202\end{array}$ & \begin{tabular}{|l|}
6.98694 \\
70361
\end{tabular} & $\begin{array}{l}46.0 \\
457\end{array}$ & $\begin{array}{l}10.5 \\
10.5 \\
\end{array}$ & & $\frac{2.824}{2816}$ & 0.322 & $\begin{array}{l}0.326 \\
0326\end{array}$ & \begin{tabular}{|c|}
0.008 \\
0008
\end{tabular} & $\begin{array}{l}7.964 \\
77977 \\
\end{array}$ & $\begin{array}{l}6.78 \\
6.79\end{array}$ \\
\hline
\end{tabular}


WSRC-TR-2005-00105, REVISION 0

SRNL-RPP-2005-00012, REVISION 0

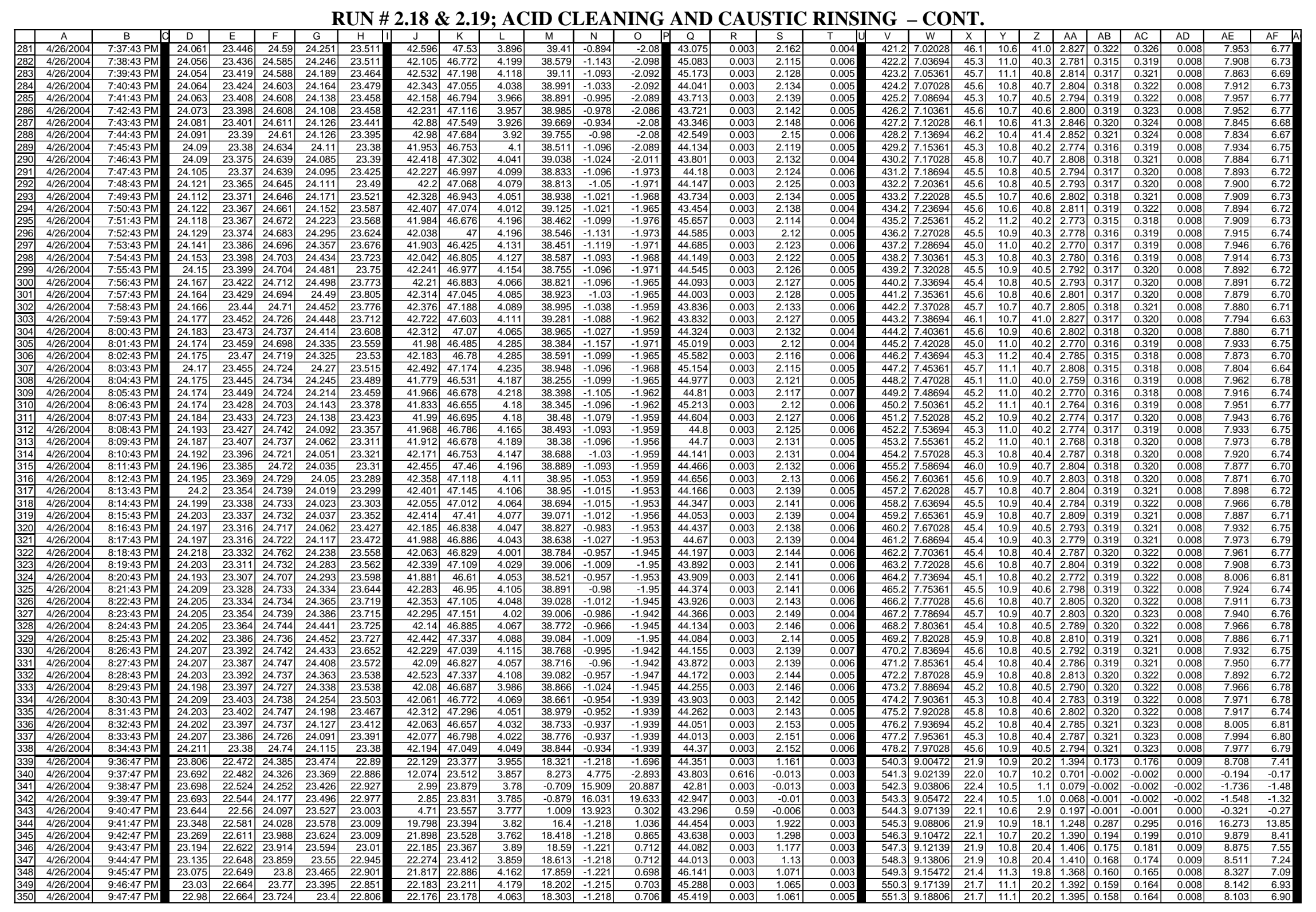


WSRC-TR-2005-00105, REVISION 0

SRNL-RPP-2005-00012, REVISION 0

RUN \# 2.18 \& 2.19; ACID CLEANING AND CAUSTIC RINSING - CONT.

\begin{tabular}{|c|c|c|c|c|c|c|c|c|c|c|c|c|c|c|c|c|c|c|c|c|c|c|c|c|c|c|c|c|}
\hline & A & B $\quad$ IC & D & $E$ & $\mathrm{~F}$ & G & $\mathrm{H}$ & $\mathrm{J}$ & $\mathrm{K}$ & L & $\mathrm{M}$ & $\mathrm{N}$ & 0 & \begin{tabular}{l|l} 
Q \\
\end{tabular} & $\begin{array}{ll}\mathrm{R} \\
\end{array}$ & $\mathrm{s}$ & T $\quad$ T & $\mathrm{V}$ & w & \begin{tabular}{c|c|}
$x$ & \\
\end{tabular} & $\mathrm{Y}$ & $z$ & & & & $A D$ & & AF \\
\hline$\frac{351}{352}$ & $\begin{array}{l}4 / 26 / 2004 \\
4 / 26 / 2004\end{array}$ & $\begin{array}{l}\text { 9:48:47 PM } \\
\text { 9:49:47 PM }\end{array}$ & $\begin{array}{l}22.935 \\
22904\end{array}$ & $\begin{array}{l}22.684 \\
22.689 \\
\end{array}$ & \begin{tabular}{|l|}
23.684 \\
2369 \\
\end{tabular} & \begin{tabular}{|l|}
23.395 \\
234005 \\
\end{tabular} & $\begin{array}{l}22.816 \\
2278 \\
\end{array}$ & \begin{tabular}{|l|}
22.18 \\
223636 \\
\end{tabular} & $\begin{array}{l}23.253 \\
23.373 \\
23\end{array}$ & \begin{tabular}{|l}
3.988 \\
3.984
\end{tabular} & \begin{tabular}{|l|}
18.373 \\
18.576 \\
\end{tabular} & \begin{tabular}{|c|}
-1.218 \\
-1215 \\
\end{tabular} & \begin{tabular}{|l|l|}
5 & 0.703 \\
503
\end{tabular} & \begin{tabular}{l|l}
44.639 \\
44.962
\end{tabular} & \begin{tabular}{l|}
0.003 \\
0.003
\end{tabular} & \begin{tabular}{|l|l|}
1.068 \\
106
\end{tabular} & \begin{tabular}{|l|l|}
0.003 \\
0.005
\end{tabular} & \begin{tabular}{|l|}
552.3 \\
553.3
\end{tabular} & \begin{tabular}{|l|}
9.20472 \\
922139 \\
\end{tabular} & 21.8 & 10.9 & 20.3 & \begin{tabular}{|l|l|}
1.398 \\
1.310
\end{tabular} & \begin{tabular}{|l|l|}
0.159 \\
0.158
\end{tabular} & 0.165 & 0.008 & 8.151 & $\begin{array}{l}6.94 \\
683\end{array}$ \\
\hline & $\begin{array}{l}4 / 26 / 2004 \\
4 / 264\end{array}$ & $\begin{array}{l}\text { 9:499:4/PM } \\
\text { 9:50:47 PMM }\end{array}$ & & $\frac{22.689}{22.684}$ & \begin{tabular}{|l|l|}
23.659 \\
23.619 \\
\end{tabular} & \begin{tabular}{|l|}
23.4045 \\
23.344 \\
\end{tabular} & 22.78 & $\frac{22.36}{22.214}$ & \begin{tabular}{|l|l|}
$23.3 / 3$ \\
23211
\end{tabular} & \begin{tabular}{|l}
3.984 \\
3.957 \\
\end{tabular} & $\begin{array}{r}\frac{18.576}{18.45} \\
\end{array}$ & $\begin{array}{l}-1.2118 \\
-1.218 \\
\end{array}$ & 0.703 & $\begin{array}{l}44.9646 \\
44.946\end{array}$ & $\begin{array}{l}0.0033 \\
0.003 \\
\end{array}$ & $\begin{array}{l}1.06 \\
1.061 \\
\end{array}$ & $\begin{array}{l}0.005 \\
0.004\end{array}$ & 5554.3 & $\frac{9.22139}{9.23806}$ & & $\frac{11.0}{11.0}$ & $\frac{20.5}{20.3}$ & $\begin{array}{l}1.4100 \\
1.402\end{array}$ & & $\begin{array}{l}0.1644 \\
0.164 \\
\end{array}$ & 0.008 & & $\begin{array}{l}6.83 \\
6.88 \\
\end{array}$ \\
\hline & & & 22.818 & & & 23.303 & 22.745 & & 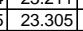 & $\begin{array}{l}0.907 \\
3.862 \\
\end{array}$ & $\begin{array}{l}18.495 \\
18.695 \\
\end{array}$ & $\begin{array}{l}-1.218 \\
-1.218 \\
\end{array}$ & 0.70 & 44.5400 & 0.00 & \begin{tabular}{l|l}
1.001 \\
1.068
\end{tabular} & 0.005 & 53.4 & 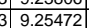 & 21.8 & $\frac{11.0}{10.8}$ & $\frac{2.5}{20.5}$ & . & & & 0.008 & 8.078 & $\begin{array}{l}6.88 \\
6.87 \\
\end{array}$ \\
\hline & & 9:52:47 म & 22.789 & 22.684 & 23.554 & 23.314 & & 22.444 & \begin{tabular}{|l|}
423.472 \\
\end{tabular} & 4 & 18.645 & -1.218 & & & $\begin{array}{ll}0.003 \\
\end{array}$ & 1.047 & & 556.3 & 27139 & & $\frac{10.9}{10.9}$ & 20.5 & 416 & 156 & & 0.008 & 7.915 & $\begin{array}{l}0.014 \\
6.74\end{array}$ \\
\hline & | & 9:53:47 PM & 22.764 & 22.669 & 23.514 & 23.329 & 22.66 & 22.253 & 23.199 & 3.967 & 18.489 & -1.21 & & .577 & 0.003 & 1.045 & & 557. & 28806 & & & 20.4 & .405 & & & & 7.977 & 6.79 \\
\hline & 6/2004 & 9:54:47 PM & 22.712 & 22.652 & 23.467 & 23.283 & 22.649 & 22.373 & 23.416 & 3.995 & 18.557 & -1.218 & & 44.627 & 0. & 1.041 & & 558. & S.30472 & 21.9 & 10.9 & 20.5 & 1.411 & & 0.162 & 0.008 & 7.920 & 6.74 \\
\hline & & 9:55:47 PM & 22..692 & 22.652 & 23.442 & 23.237 & 22.644 & 22.386 & | 23.383 & 3.905 & 18.666 & $-1.22>0$ & & & 0. & 1.046 & & 559. & 32139 & 21.9 & 10.9 & 20.5 & 1.415 & & & 0.008 & .940 & 6.76 \\
\hline & & & & & 23.432 & & & & 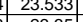 & 3.961 & & & & & & 1.043 & & 560. & & & & & & & & & & \\
\hline & 2004 & $47 \mathrm{PM}$ & 22.631 & 年2.031 & 每.3806 & $\frac{2.2110}{23242}$ & & $\frac{2.35}{2032}$ & $=23.35$ & 年.951 & & $\frac{1.4}{10}$ & & 1149 & & 1036 & & 争 & & $138>-18$ & 然. & 204 & & 盺 & 1.162 & & .904 & .73 \\
\hline & & $\begin{array}{l}9.50 .41 \mathrm{~F} \\
9.59: 47 \mathrm{P}\end{array}$ & & & $\mid$ & $\frac{2.4244}{23261}$ & & & & & & & & . .149 & & & & & & & & 20.4 & & & & & & 6.74 \\
\hline & $4 / 26 / 2004$ & \begin{tabular}{|l|}
$10: 00: 47$ PM \\
\end{tabular} & 22.571 & 22.626 & 23.315 & \begin{tabular}{|l|}
23.286 \\
23.286 \\
\end{tabular} & $\frac{22.728}{22.728}$ & 22..354 & 23.354 & 3.959 & $\frac{10.051}{18.592}$ & $\begin{array}{l}-1.210 \\
-1.218 \\
\end{array}$ & 0.6 & 44.111 & 0.003 & 1.033 & & 564. & 9.40472 & 21.9 & $\frac{10.8}{10.8}-8$ & 20.5 & 4.412 & 0.154 & 0.162 & 0.008 & 7.890 & $\frac{6.73}{6.71}$ \\
\hline & & 10:01:47 PM & 22.535 & 22.61 & 23.28 & 23.291 & 22.742 & 22.552 & 23.512 & 3.964 & 18.81 & -1.221 & 0.69 & 44.101 & 0.003 & 1.028 & & 565.3 & 9.42139 & 22.0 & 10.8 & 20.7 & 1.426 & 153 & 0.161 & 0.008 & .781 & $\frac{6.11}{6.62}$ \\
\hline & 4/26/2004 & 10:02:47 PM & 22.529 & 22.614 & 23.264 & 23.355 & 22.797 & 22.531 & 23.533 & 3.945 & 18.773 & -1.218 & & 44.122 & 0.003 & 1.026 & & & & & & & & & & & & 0.02 \\
\hline & & & & & & & & & & & & -1.21 & & & & & & 567. & 5472 & 21.9 & & & & & & & & 5.67 \\
\hline & 6/2004 & 10:04:47 PM & 22.509 & 22.645 & 23.244 & 23.451 & 86 & 22.357 & 23.342 & 3.93 & & -1.21 & & $.05-3-5$ & & 1.026 & & 568. & & & $0.8 \mathrm{0.8}-2$ & 20.4 & & & & & 869 & 5.70 \\
\hline & 4/26/2004 & & & 22.644 & & & 22.861 & 22.545 & 23.489 & & 847 & $-1.218 \mathrm{C}>\mathrm{C}$ & & -1 & 0.0 & 1.029 & & & 1.48806 & & & & & & & & & $x_{0}$ \\
\hline & 4/26/2004 & 10:06:47 PM & 22.469 & 22.66 & 23.214 & 23.511 & 22.907 & 22.579 & 23.553 & 3.997 & 18.762 & -1.218 & & 43.986] & 0.003 & 1.024 & & 570. & .50472 & 22.11 & 10.8 & 20.7 & 1.425 & 0.153 & 0.161 & 0.008 & 7.769 & 6.61 \\
\hline & & 10:07:47 PM & 22.453] & 22.674 & $\mid 23.203$ & 23.551 & 22.977 & 22.666] & 23.512 & 3.964 & 18.937 & -1.215 & & 44.445 & 0.003 & 1.025 & & 571. & .52139| & & 10.9 & 20.8 & 1.434 & 153 & 161 & 0.008 & 7300 & 6.58 \\
\hline & $\frac{4 / 26 / 2004}{4 / 26 / 2004}$ & \begin{tabular}{|l|}
$10: 08: 47$ PM \\
$10.09: 47$ PM
\end{tabular} & $\frac{22.449}{22.424}$ & $\begin{array}{r}22.69 \\
22.706\end{array}$ & \begin{tabular}{|l|}
23.199 \\
23.184
\end{tabular} & \begin{tabular}{|r|}
23.566 \\
23.587
\end{tabular} & $\begin{array}{l}23.002 \\
23.033 \\
\end{array}$ & $\frac{22.344}{22.483}$ & $\begin{array}{l}4 \\
3 \\
3\end{array} 23.2053$ & $\begin{array}{l}3.909 \\
3.918 \\
\end{array}$ & $\begin{array}{l}18.604 \\
18.787\end{array}$ & $\begin{array}{l}-1.218 \\
-1.218 \\
\end{array}$ & & $\begin{array}{ll}44.368 \\
44.074\end{array}$ & $\begin{array}{l}0.0003 \\
0.003 \\
\end{array}$ & $\begin{array}{l}1.018 \\
1.022\end{array}$ & 05 & $\frac{572.3}{573.3}$ & $\begin{array}{l}9.53806 \\
9.55472 \\
\end{array}$ & $\frac{21.7}{21.9}$ & $\begin{array}{l}10.9 \\
10.8\end{array}$ & $\frac{20.5}{20.6}$ & $\frac{1.414}{1.423}$ & 0.152 & 0.160 & $\begin{array}{l}0.008 \\
0.008\end{array}$ & $\begin{array}{l}7.801 \\
7.774 \\
\end{array}$ & $\frac{6.64}{6.62}$ \\
\hline & |/2004 & & 3.853 & 22.685 & 24.502 & 23.144 & 22.15 & 21.801 & 22.693 & 3.912 & 18.075 & -1.224 & & (n) & & 0.984 & & & & & & & & & & & & 6.34 \\
\hline & & & & & & & & 2.569 & 23.429 & & & & & & & & & & & & & & & & & & & -2.27 \\
\hline & II2004 & & 23.788 & 22.69 & 24.362 & 23.144 & 666 & 2.686 & \begin{tabular}{|l|l|}
23.522 \\
\end{tabular} & 3.534 & -0.857 & 15.967 & & 41.617 & 0.003 & 0.013 & & 609.3 & (0.1553 & 2.0 & 0.2 & & . 063 & & & & 158 & -1.84 \\
\hline & $4 / 26 / 2004$ & & 3.779 & 22.7 & 24.277 & $23.22 \mathrm{P}$ & & 2.503 & 23.649 & 3.421 & -0.904 & 16.28 & & 40.695 & & -0.013 & & 610. & . & & & & & & & & 474 & -2.11 \\
\hline & 4/26/2004 & $10: 47: 4$ & 23.739 [2) & 22.691 & 24.188 & 23.226 & 22.707 & 2.443 & $\begin{array}{l}3 \\
3\end{array}$ & 3.439 & -1.052 & 16.245 & & 41.018 & 0.003 & -0.013 & & 611. & 10.1886 & 5 & 10.1 & 0. & 0.048 & & & & & -2.43 \\
\hline & & $10: 48: 4$ & 23.714 & 22.686 & 24.123 & 23.241 & 22.707 & $\begin{array}{r}2.603 \\
764\end{array}$ & 23.827 & 3.376 & -0.773 & 16.352 & & 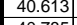 & 0.003 & 14.643 & & & 0.2053 & 22.4 & 10.0 & & 0.063 & .183 & 2.238 & 2.446 & | & 31.38 \\
\hline 380 & $\begin{array}{ll}0.2004 \\
6 / 2004\end{array}$ & 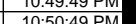 & 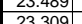 & 24.070 & $\begin{array}{l}2.0 .08 \\
2303 \\
\end{array}$ & $\begin{array}{l}23.297 \\
23277 \\
\end{array}$ & 22768 & 1.04 & 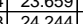 & 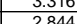 & $\frac{4.5}{1.94}$ & $\frac{1.916}{121} 5$ & & 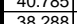 & & 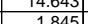 & & 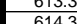 & 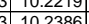 & 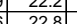 & & & & & & & & 1.4.099 109 \\
\hline & $\begin{array}{l}4 / 26 / 2004 \\
4 / 26 / 2\end{array}$ & 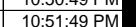 & 23.244 & $\begin{array}{l}22.000 \\
22.667\end{array}$ & $\mid$\begin{tabular}{|l|l|}
23.94903 \\
\end{tabular} & $\begin{array}{l}23.2748 \\
23.348\end{array}$ & & $\begin{array}{ll}21.950 \\
21.678\end{array}$ & $\begin{array}{l}24.444 \\
22.963\end{array}$ & $\frac{2.044}{3.615}$ & & $\begin{array}{l}-1.21 \\
-1.218 \\
\end{array}$ & & \begin{tabular}{|l|}
52.6001 \\
42.601
\end{tabular} & & 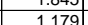 & & $\frac{014 .}{615 .}$ & 10.4503 & 2..0) & $\frac{9.4}{10.4}$ & $\frac{1.1}{200} \mathrm{C}>\mathrm{C}$ & & & 势 & | & & 12.24 \\
\hline & $4 / 26 / 2004$ & $10: 52: 49$ PM & 23.174 & 22.678 & 23.864 & 23.363 & 22.845 & 21.805 & $\mid \begin{array}{ll}\mid 22.894 \\
5\end{array}$ & 3.814 & 18.134 & -1.221 & & 43.807 & & 1.069 & & 616.3 & 10.2719 & 21.4 & 10.7 & 20.0 & 377 & .159 & 0.165 & 0.008 & 8.241 & 7.01 \\
\hline & 4/266/2004 & 10:53:49 PM & 23.125 & & 23.809 & 23.384 & & 22.035 & \begin{tabular}{|l|l|}
5 & 23.01 \\
\end{tabular} & & & -1.218 & & 43.821 & & 1.044 & & 617. & 10.2886 & 21.5 & 10.7 & 20.2 & 1.393 & & 0. & & 7.968 & $\begin{array}{ll}6.78 \\
\end{array}$ \\
\hline & & 10:54:4 & 23.091 & $\begin{array}{r}22.69 \\
22.675\end{array}$ & 23.811 & 23.456 & $\frac{22.902}{2002}$ & $\begin{array}{l}22.002 \\
205 \\
\end{array}$ & 22.911 & 3.974 & $\frac{18.167}{1.272}$ & -1.215 & & 44. & 0.00 & 1.004 & & 618. & 10.3053 & 21.4 & 11.0 & 20.1 & . & 0.150 & 0.1. & 0.008 & 7.708 & 6.56 \\
\hline & & & $\frac{23.026}{22091}$ & & & 23.466 & & $\frac{22.05}{22324}$ & & & & & & $\frac{44.2}{430}-1$ & & & & & & & & & & & & & & 6.42 \\
\hline 年87 & 6.6004 & & $\begin{array}{ll}22.919 \\
22.952\end{array}$ & $\frac{2.306}{22696}$ & $\mid 2366$ & 23.47 & & $\frac{22.324}{22085}$ & 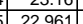 & 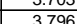 & & $\frac{-1.21}{-122}$ & & 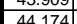 & & $\frac{0.900}{0.97}$ & & & & 215 & 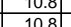 & 203 & 398 & 145 & 0.150 & $\frac{01}{07}$ & . & 6.32 \\
\hline & $4 / 26 / 2004$ & 10:58: & 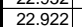 & & 23.647 & 23.473 & & 22.359 & & & & $\frac{-1.24}{-1.21}$ & & (3).482 & & & & & & t.t. & & & & & & & & $\frac{6.30}{6.20}$ \\
\hline 385 & & & 22.883 & & $\mid 23.618$ & 23.4 & & 22.365 & $\mid 23.164$ & & & -1.2 .20 & & 126 & & & & & & s & & & & & $\frac{0.159}{0.149}$ & & & 0.20 \\
\hline & $4 / 26 / 2004$ & 11:00:4 & $\begin{array}{l}22.848 \\
\end{array}$ & 22.718 & $\mid 23.583$ & 23.39 & & $\frac{22.56}{20}-1$ & $\mid$ & 3.674 & 19.0 & $-1.218 \mathrm{~g}$ & & 43.2 & & & & 624.3 & 0.4053 & 22.0 & 10.6 & $20 . \varepsilon$ & & 1.144 & 0.150 & & 702 & 6.13 \\
\hline & $4 / 26 / 2004$ & & 22.824 & 22.719 & 23.559 & 23.344 & & 22.409 & 23.278 & 3.656 & & -1.22 & & 43.0 & & & & 625. & & 1.8 & 10.0 & & & & & & & \\
\hline & $4 / 26 / 2004$ & & 22.784 | & 22.704 & 23.514 & 23.264 & & 22.78 & 23.626 & & & -1.22 & & 43.1 ; & & & & ST & 10.4386 & $222+3$ & 1060 & 21.0 & 1.449 & 0.142 & 48 & 0.007 & 7.050 & 6.00 \\
\hline & | & & 22.754 & 22.69 & 23.493 & 23.249 & & 22.4 & 23.205 & & & -1.2. & & 43.0 & & & & 627 & & 1.7 & & 20 & $4<0$ & 141 & & & 7.104 & 4 \\
\hline 年 & | & & 22.729 & 22.694 & 23.488 & 23.203 & & 21.8 & 22.633 & & & & & 45 & & & & & & at & & & & & 142 & & & 6.0 \\
\hline & | 12004 & & & 22.668 & 23.453 & 23.142 & & 22.137 & & & & -1.2. & & 45.5 & & & & & & 21.4 & & & & & & & & \\
\hline 996 & & & $22.667 \mid$ & 22.65 & 23.442 & 23.076 & & $\frac{22.029}{22030}$ & 22.667] & & & & & $45.77>3$ & & & & & & 21.2 & & & & & & & & \\
\hline & 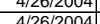 & & & $\frac{2.06}{202}$ & & 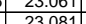 & & 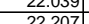 & $\frac{22.501}{2009}$ & & & & & 年 & & & & & & $\frac{1.1}{514}$ & & & & & & & & \\
\hline & $\frac{4 / 20 / 2604}{4 / 26 / 2004}$ & $\mid \begin{array}{ll}11.0 .4 \\
11.09 .4\end{array}$ & 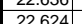 & $\frac{2.04}{2262}$ & 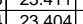 & $\frac{2.001}{23119}$ & & 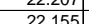 & $\mid$ & & & & & 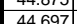 & & & & & & $\frac{2.4}{21.5}-2$ & & 202 & & & & & & \\
\hline 400 & $4 / 26 / 2004$ & t1:10:4 & 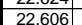 & (2) & 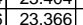 & $\frac{23.151}{23.111}$ & & 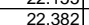 & 23.04 & & & $\frac{1.21}{-1.21}>>$ & & 43.976 & & & & 634 & 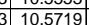 & & & 20.2 & 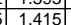 & & & & & \\
\hline & 4/26/2004 & th 11:11: & 22.597 & $\frac{22.602}{22.602}$ & 23.347 & 23.132 & & $\frac{22.355}{22.35}$ & 23.019 & & & $\frac{-1.21}{-1.21}>0$ & & 43.502 & & & & & & st & & & & & & & & \\
\hline & 04 & & $\frac{22.589}{20}$ & 22.61 & \begin{tabular}{|l|l|}
23.329 \\
\end{tabular} & & & 22.348 & 23.064 & & & & & & & & & & & & & & & & & & & \\
\hline & 4/26/26 & & 2.583 & 22.623 & 23.33 & 23.224 & & 22.12 & 22.871 & & & -1.21 & & 45.1 & & & & & & 21.4 & & & & & & & & \\
\hline & $4 / 26 / 2004$ & & 22.5 & 22.628 & 323.3 & 23.283 & & 22.278 & \begin{tabular}{|l|} 
\\
\end{tabular} & & & $-1.22>0$ & & & & & & & & 21.4 & & & & & & & & \\
\hline & 4/26/2004 & 11:15:4 & 22.55 & 22.63 & 23.308 & 23.304 & & 22.33 & 22.927 & & & -1.21 & & 45.0 & & & & 639.3 & & 21.5 & $1.0-0=3$ & 20.4 & & 0.130 & 0.137 & & 6.694 & \\
\hline & 4/26/2004 & 11:16:4 & 22.543 & 22.644 & 23.313 & 23.369 & & 22.342 & 23.021 & & & -1.21 & & 44.4. & & & & 64 & & & & & & & & & 5.757 & .15 \\
\hline & & & 08 & 22.624 & 23.263 & 23.3855 & & 22.57 & | 23.303 & & & & & 44 & & & & 641. & & 52 & & & & & & & & \\
\hline & 4/26/2004 & 11:18:49 & & 645 & 23.269] & & & 22.344 & 22.961 & 3.756 & 18.73 & -1.221 & & 43.945 & 0.003 & 0.882 & & & .7053 & 21.5 & 0.8 & 20.5 & 1.416 & 0.131 & 138 & & & 5.72 \\
\hline$\frac{7}{41}$ & Ise/Clean/l & & & & & & & & & & & & & & & & & & & & & & & & & & & \\
\hline & & Average & 24.209 & 23.216 & $\mid 24.849$ & & & 34.934 & 40.082 & 4.148 & & & & 44.0 & & & & & & & & & 2.280 & \begin{tabular}{|l|l|} 
\\
\end{tabular} & 0.261 & 0.051 & 0.780 & 43.214 \\
\hline & & Maxin & 26.338 & 23.649 & 26.91 & $24.7 \mathrm{r}-\mathrm{s}$ & & 43.60 & 48.428 & & & 40.4 & & & & & & & & & & & & & 2.344 & 3.405 & 405.308 & 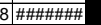 \\
\hline & & Medi & 24.2 & 23.334 & 24.739 & 24.1 & & 42.104 & & & 38.4 & -1.2 & & 44. & & & & & & & 313 & & & 0.187 & 0.187 & & & \\
\hline 41 & & Minimum & 21.273 & 22.472 & 22.19 & 23.061 & 22.5 & $\frac{2.443}{20}$ & $\begin{array}{l}32.581 \\
3\end{array}$ & 2.844 & $\frac{-1.641}{2.678}$ & $\frac{-1.224}{1-1.224}$ & & 38.288 & 0.003 & $\frac{-0.01}{0.01}$ & & & & 21.108 & 9.383 & 0.66 & $\begin{array}{l}50.046 \\
\end{array}$ & $\mid-0.002$ & 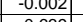 & $\frac{-0.003}{0.003}$ & 2.868 & 2.441 \\
\hline 416 & Number 0 & $\frac{\mid 2 \times \text { Std Dev }}{\text { of Points Used }}$ & $\begin{array}{r}2.325 \\
392 \\
\end{array}$ & $\begin{array}{r}0.570 \\
392 \\
\end{array}$ & \begin{tabular}{|l|}
2.180 \\
392
\end{tabular} & $\mid 0.739$ & & \begin{tabular}{rl|}
23.828 & \\
392 &
\end{tabular} & $\begin{array}{l}39.282 \\
332\end{array}$ & \begin{tabular}{|r|}
0.684 \\
392 \\
\end{tabular} & $24.276 \mid$ & \begin{tabular}{|r|}
$\mid 15.305$ \\
392
\end{tabular} \mid & & $\frac{2.411}{392}$ & $\begin{array}{r}0.359 \\
392 \\
\end{array}$ & $\begin{array}{c}8.206 \\
392\end{array}$ & & & & $\begin{array}{r}19.283 \\
392 \\
\end{array}$ & \begin{tabular}{|l|}
0.591 \\
392 \\
\end{tabular} & $\frac{24.05}{392}$ & \begin{tabular}{|c|}
1.658 \\
392
\end{tabular} & \begin{tabular}{|l|}
0.625 \\
392 \\
\end{tabular} & $\begin{array}{r}0.639 \\
392\end{array}$ & . 0665 & 5.094 & $\frac{4}{2}$ \\
\hline
\end{tabular}


WSRC-TR-2005-00105, REVISION 0 SRNL-RPP-2005-00012, REVISION 0

RUN \# 2.18 \& 2.19; ACID CLEANING AND CAUSTIC RINSING - CONT.

\begin{tabular}{|c|c|c|c|c|c|c|c|c|c|c|c|c|c|c|c|c|c|c|c|c|c|c|c|c|c|c|c|c|}
\hline & A & B & $\mathrm{D}$ & $E$ & $\mathrm{~F}$ & G & $\mathrm{H}$ & $\mathrm{J}$ & $\mathrm{K}$ & $\mathrm{L}$ & $\mathrm{M}$ & $\mathrm{N}$ & $\mathrm{O}$ & $\mathrm{Q}$ & $\mathrm{R}$ & \begin{tabular}{|c|}
$\mathrm{s}$ \\
\end{tabular} & $\begin{array}{lll} & \mathrm{T} & \mathrm{L} \\
\mathrm{L}\end{array}$ & $\mathrm{v}$ & w & $\mathrm{x}$ & \begin{tabular}{|l|} 
\\
\end{tabular} & $\mathrm{z}$ & AA & $A B$ & \begin{tabular}{l|l}
$\mathrm{AC}$ \\
\end{tabular} & $\mathrm{AD}$ & $\mathrm{AE}$ & $\mathrm{AF}$ \\
\hline$\frac{419}{420}$ & Caustic Rins & ise 1 & & & & & & & & & & & & & & & & & & & & & & & & & & \\
\hline$\frac{420}{421}$ & & Averages & & & $\begin{array}{l}923.503 \\
102009 \\
1020\end{array}$ & 24.184 & & & \begin{tabular}{|l|}
26.809 \\
27097
\end{tabular} & 4.999 & & $-\frac{1.052}{1.20}$ & $\begin{array}{l}-2.499 \\
0.595 \\
\end{array}$ & 44.842 & 0.475 & 14.643 & & & & & & & & & & & & 3.040 \\
\hline$\frac{421}{422}$ & & \begin{tabular}{|l|} 
Maximum \\
Median
\end{tabular} & $\begin{array}{l}23.173 \\
22.693 \\
\end{array}$ & $\begin{array}{l}3 \\
3 \\
3\end{array}$ & 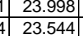 & \begin{tabular}{|l|}
24.346 \\
24.186 \\
\end{tabular} & $\begin{array}{l}23.399 \\
23.208\end{array}$ & $\begin{array}{l}23.833 \\
23.375 \\
\end{array}$ & \begin{tabular}{|l|}
27.087 \\
26.797 \\
\end{tabular} & $\begin{array}{l}5.282 \\
5.002\end{array}$ & $\begin{array}{l}19.107 \\
18.508 \\
\end{array}$ & $\frac{1.240}{-1.218}$ & $\begin{array}{l}0.585 \\
-2.714 \\
\end{array}$ & $\begin{array}{l}47.059 \\
44.818\end{array}$ & \begin{tabular}{l|l}
0.502 \\
0.477
\end{tabular} & \begin{tabular}{|l}
14.643 \\
14.643
\end{tabular} & $\begin{array}{l}0.003 \\
0.003 \\
\end{array}$ & & & $\begin{array}{l}25.61 \\
2532 \\
\end{array}$ & \begin{tabular}{ll|l}
1 & 11.53 \\
2 & 10.98
\end{tabular} & \begin{tabular}{|l|l|}
3 & 21.47 \\
8 & 20.94 \\
\end{tabular} & \begin{tabular}{|l|l|}
1.480 \\
1.444 \\
\end{tabular} & \begin{tabular}{|l|}
0.075 \\
0.071
\end{tabular} & $\begin{array}{l}0.079 \\
0.074\end{array}$ & 0.004 & 3.972 & 3.380 \\
\hline 423 & & Minimum & 21.837 & $7 \quad 23.194$ & 422.768 & \begin{tabular}{|l|}
23.979 \\
\end{tabular} & 22.996 & 20.403 & 26.461 & 4.793 & 15.187 & & -2.873 & 42.983 & 0.436 & 14.643 & 0.003 & & & 24.99 & & \begin{tabular}{|c|c|}
3 & 17.80 \\
\end{tabular} & \begin{tabular}{|l|}
1.227 \\
\end{tabular} & \begin{tabular}{|l|l|}
0.065 \\
\end{tabular} & 0.069 & & & \\
\hline$\frac{765}{424}$ & & $2 \times$ Std Dev & 0.770 & 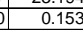 & \begin{tabular}{|l|l|}
3 & 0.720 \\
\end{tabular} & 0.177 & 0.227 & 1.541 & \begin{tabular}{|r|} 
\\
\end{tabular} & 0.240 & 1.747 & 1.219 & 1.662 & 2.074 & 0.028 & \begin{tabular}{|r|}
0.000 \\
\end{tabular} & 0.000 & & & 0.34 & & \begin{tabular}{|l|l|}
1 & 1.64 \\
\end{tabular} & \begin{tabular}{|l|}
1.2613 \\
\end{tabular} & 0.004 & 0.005 & 0.000 & 0.336 & 0.286 \\
\hline$\frac{425}{425}$ & Number & rof Points Used* & 29 & \begin{tabular}{|l|l|l|l|}
9 & 29 \\
\end{tabular} & $\begin{array}{ll}9 & 29 \\
\end{array}$ & 29 & $\begin{array}{r}29.29 \\
\end{array}$ & 29 & 29 & $\begin{array}{r}29 \\
29 \\
\end{array}$ & 29 & $\frac{2.29}{29}$ & 29 & 29 & $\begin{array}{r}29 \\
29 \\
\end{array}$ & $\begin{array}{r}29 \\
\end{array}$ & 29 & & & 29 & \begin{tabular}{|l|l|}
9 & 29 \\
\end{tabular} & \begin{tabular}{|l|l|}
9 & 29 \\
\end{tabular} & \begin{tabular}{|r|}
29.15 \\
\end{tabular} & \begin{tabular}{|l|l|}
29 \\
\end{tabular} & $\begin{array}{r}29 \\
\end{array}$ & $\frac{0.09}{29}$ & 29 & 29 \\
\hline $\begin{array}{l}426 \\
27\end{array}$ & & $F^{*}$ Backpulse poir & sin box & tare not inc & & & & & & & & & & & & & & & & & & & & & & & & \\
\hline \begin{tabular}{|l|l|l}
427 \\
28
\end{tabular} & & & & & & & & & & & & & & & & & & & & & & & & & & & & \\
\hline$\frac{428}{429}$ & Acid Clean & $\frac{1,2 \text {, and } 3}{1 \text { Averages }}$ & & & & & & & & & & -1094 & -1.442 & 43.988 & & & 0.005 & & & 4436 & & & & & & & & \\
\hline 430 & & Maximum & 26.338 & 23.649 & 26.911 & 24.774 & 24.102 & 43.608 & \begin{tabular}{|l|l|}
48.428 \\
\end{tabular} & 4.659 & 40.101 & -0.092 & 0.874 & \begin{tabular}{|l|l|}
48.223 \\
\end{tabular} & 0.004 & 2.330 & 0.007 & & & 46.96 & \begin{tabular}{|l|l|}
6 & 11.82 \\
\end{tabular} & 241.855 & \begin{tabular}{|l|}
2.886 \\
\end{tabular} & \begin{tabular}{|l|l|} 
& 0.347 \\
\end{tabular} & 0.359 & 0.009 & $\begin{array}{l}8.391 \\
8.935 \\
\end{array}$ & $\begin{array}{l}\frac{3.439}{7.604} \\
\end{array}$ \\
\hline 431 & & Median & 24.493 & 23.367 & 25.097 & 24.182 & 23.521 & 42.333 & 46.958 & 4.093 & 38.802 & -1.111 & -1.971 & \begin{tabular}{|l|l|}
44.134 \\
\end{tabular} & 0.003 & 2.116 & 0.005 & & & 45.49 & \begin{tabular}{|l|l|}
9 & 10.82 \\
\end{tabular} & 240.547 & \begin{tabular}{|l|}
2.796 \\
\end{tabular} & 0.315 & 0.302 & 0.007 & 7.418 & 6.313 \\
\hline 432 & & Minimum & 23.263 & 23.121 & 23.857 & 23.750 & 23.189 & 40.132 & 42.334 & 3.822 & 36.512 & -1.224 & & 39.750 & $\begin{array}{ll}0.003 \\
\end{array}$ & 0.835 & 0.003 & & & 40.86 & & 438.322 & \begin{tabular}{|l|}
2.642 \\
\end{tabular} & 0.124 & 0.121 & 0.003 & 3.010 & \\
\hline 433 & & $2 \times \operatorname{Std}$ Dev & 1.595 & 0.210 & 1.605 & $\begin{array}{ll}0.367 \\
\end{array}$ & 0.347 & 0.790 & 3.919 & 0.229 & 1.003 & 0.307 & 3.342 & 2.273 & 0.000 & 1.058 & 0.002 & & & 3.92 & & & & \begin{tabular}{|l|l|}
0.158 \\
\end{tabular} & 0.157 & 0.004 & 3.817 & 3.249 \\
\hline 434 & Number & rof Points Used ${ }^{*}$ & 265 & 5 & $\begin{array}{l}5 \quad 265 \\
\end{array}$ & 265 & 265 & 265 & 265 & 265 & 265 & 265 & & 265 & 265 & 265 & 265 & & & 265 & 265 & \begin{tabular}{l|l|}
5 & 265 \\
\end{tabular} & 265 & 265 & 265 & 265 & 265 & 265 \\
\hline$\frac{435}{436}$ & & ${ }^{*}$ Backpulse poi & & & & & & & & & & & & & & & & & & & & & & & & & & \\
\hline 437 & Acid Clean & & & & & & & & & & & & & & & & & & & & & & & & & & & \\
\hline 438 & & Averages & 24.715 & & \begin{tabular}{|l|l|}
3 & 25.319 \\
\end{tabular} & \begin{tabular}{|l|}
24.1477 \\
\end{tabular} & 23.463 & 42.186 & \begin{tabular}{|l|l|}
43.157 \\
\end{tabular} & 4.076 & 38.360 & -1.219 & 0.791 & 43.095 & & & & & & & & & & & & 0.004 & 3.795 & \\
\hline 439 & & Maximum & 25.364 & $\begin{array}{l}4 \quad 23.409 \\
4\end{array}$ & $\begin{array}{l}926.012 \\
9\end{array}$ & \begin{tabular}{|l|}
24.323 \\
\end{tabular} & 23.706 & 43.094 & 43.969 & 4.300 & 39.500 & -1.212 & 0.874 & 45.718 & 0.004 & 1.278 & 0.007 & & & 42.50 & \begin{tabular}{l|l|}
0 & 11.20 \\
\end{tabular} & & \begin{tabular}{|l|l|}
2.847 \\
\end{tabular} & 0.191 & 0.190 & 0.005 & 4.752 & 4.044 \\
\hline$\frac{440}{141}$ & & Median & 24.506 & $\begin{array}{l}6 \quad 23.344 \\
23.17 \\
5\end{array}$ & $\begin{array}{l}4 \quad 25.099 \\
7\end{array}$ & $24.161 \mid$ & 23.504 & $\begin{array}{l}42.137 \\
1.526\end{array}$ & \begin{tabular}{|l|}
43.230 \\
42334
\end{tabular} & $\begin{array}{r}4.079 \\
3.820 \\
\end{array}$ & $\begin{array}{r}38.312 \\
37715 \\
\end{array}$ & -1.218 & 0.795 & \begin{tabular}{|l|}
43.061 \\
39750
\end{tabular} & $\begin{array}{l}0.003 \\
0.023\end{array}$ & $\begin{array}{l}1.009 \\
0.85 \\
\end{array}$ & 0.005 & & & 41.76 & $\begin{array}{ll}6 \quad 10.55 \\
\end{array}$ & & \begin{tabular}{|l|}
2.774 \\
2.702 \\
\end{tabular} & 0.150 & 0.150 & 0.004 & 3.715 & 3.161 \\
\hline$\frac{441}{442}$ & & $\frac{\mid \text { Minimum }}{2 x \text { Std Dev }}$ & $\begin{array}{r}24.352 \\
0.741 \\
\end{array}$ & $\begin{array}{l}2 \quad 23.127 \\
1\end{array}$ & \begin{tabular}{|l|l|l|}
7 & 24.945 \\
1 & 0.782 \\
\end{tabular} & $\begin{array}{r}23.947 \\
0.189 \\
\end{array}$ & $\begin{aligned} 23.189 \\
0.258 \\
\end{aligned}$ & $\begin{array}{l}41.526 \\
0.582 \\
\end{array}$ & \begin{tabular}{|r|}
42.334 \\
0.702 \\
\end{tabular} & \begin{tabular}{|l}
3.822 \\
0.183 \\
\end{tabular} & $\frac{37.715}{0.664}$ & $\frac{-1.224}{0.005}$ & \begin{tabular}{|l|l|}
0.683 \\
0.104
\end{tabular} & \begin{tabular}{|r|}
39.750 \\
2.094
\end{tabular} & \begin{tabular}{|l|}
0.003 \\
\end{tabular} & $\begin{array}{l}0.835 \\
0.287\end{array}$ & $\begin{array}{l}0.003 \\
0.002 \\
\end{array}$ & & & $\begin{array}{r}40.86 \\
0.70\end{array}$ & \begin{tabular}{|c|c|} 
& 9.74 \\
0 & 0.51 \\
\end{tabular} & $\begin{array}{l}439.640 \\
10.615\end{array}$ & \begin{tabular}{|l|}
$\mid 2.733$ \\
0.042
\end{tabular} & \begin{tabular}{|l|l|} 
\\
\end{tabular} & $\begin{array}{l}0.121 \\
0.045\end{array}$ & 0.003 & $\begin{array}{l}3.010 \\
1199\end{array}$ & 2.562 \\
\hline 443 & Number & rof Points Used & 90 & 90 & 0 & 90 & 90 & 90 & 90 & 90 & 90 & 90 & 90 & $\begin{array}{r}2.044 \\
90\end{array}$ & $\begin{array}{r}0.000 \\
90\end{array}$ & $\frac{0.28}{90}$ & 9.06 & & & $\begin{array}{c}.1 .10 \\
90\end{array}$ & \begin{tabular}{|c|c|} 
& 0.51 \\
0 & 90
\end{tabular} & $\begin{array}{l}1 \\
0 \\
0.0150\end{array}$ & \begin{tabular}{|l|}
0.042 \\
90
\end{tabular} & \begin{tabular}{|c|}
0.045 \\
90
\end{tabular} & $\begin{array}{c}0.045 \\
90\end{array}$ & $\frac{0.001}{90}$ & $\frac{1.149}{90}$ & \\
\hline 444 & & * Backpulse poin & is in box & are not inc & cluded & & & & & & & & & & & & & & & & & & & & & & & \\
\hline 446 & & & & & & & & & & & & & & & & & & & & & & & & & & & & \\
\hline$\frac{446}{447}$ & Acid Clean & & & & & & & & & & & & & & & & & & & & & & & & & & & \\
\hline 448 & & Averages & 25.708 & 23.356 & 26.266 & 24.224 & 23.605 & $\begin{array}{ll}42.583 \\
\end{array}$ & 47.270 & 4.152 & 39.096 & -1.102 & $\begin{array}{l}-3.167 \\
\end{array}$ & 44.731 & 0.003 & 2.117 & 0.0 & & & 45.80 & 10.96 & 640.840 & .816 & 0.316 & 0.305 & 0.007 & 7.463 & \\
\hline$\frac{249}{449}$ & & Maximum & 26.338 & 23.649 & 26.911 & 24.774 & 24.102 & 43.608 & 48.428 & 4.659 & $\begin{array}{lll}40.101 \\
\end{array}$ & -0.740 & -3.006 & 48.223 & 0.004 & 2.204 & & & & 466.96 & 11.82 & 1.855 & 2.886 & 0.329 & 0.325 & 0.008 & 8.348 & \\
\hline & & & 25.932 & 23.397 & 26.486 & 24.273 & 23.672 & 42.602 & 47.281 & 4.108 & 39.188 & -1.099 & -3.124 & 44.629 & 0.003 & 2.118 & .00 & & & 45.81 & 10.94 & $4 \mid 40.898$ & 2.820 & & & 0.007 & 7.419 & \\
\hline 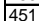 & & Minimum & 24.398 & 23.121 & 25.203 & $23.750 \mid$ & 23.217 & 40.132 & 45.057 & 3.873 & 36.512 & -1.2219 & & 42.645 & 0.003 & 2.075 & & & & 43.587 & 10.45 & & 2.642 & 0.309 & 0.299 & & 1.02 & \\
\hline 452 & Number & $\frac{12 \times \mathrm{SSd} \text { Dev }}{\mathrm{rof} \text { Points Us }}$ & & 0.316 & $\frac{1.150}{87}$ & $\begin{array}{r}0.525 \\
87\end{array}$ & $\begin{array}{r}0.429 \\
87\end{array}$ & $\frac{0.910}{87}$ & $\frac{0.874}{87}$ & 0.251 & 0.949 & 0.203 & 0.290 & 1.862 & 0.000 & 0.053 & & & & 0.87 & 0.46 & $\begin{array}{l}6.921 \\
6\end{array}$ & 0.063 & 0.008 & 0.011 & 0.000 & 0.348 & \\
\hline & & * Backpulse po & $\frac{81}{\sin b x}$ & are not ind & cluded or & 87 & 81 & & 81 & 81 & 81 & 81 & 81 & 81 & 87 & 81 & & & & 81 & 87 & 81 & 87 & 87 & 81 & 87 & 87 & \\
\hline 454 & & & & & & & & & & & & & & & & & & & & & & & & & & & & \\
\hline$\frac{455}{456}$ & Acid Clean: & & & & & & & & & & & & & & & & & & & & & & & & & & & \\
\hline & & Averages & 24.039 & & 24.585 & 24.244 & 23.531 & 42.319 & 47.145 & 4.087 & 38.922 & & & 44.166 & 0.003 & 2.150 & & & & & 0.82 & & & & 0.324 & 0.008 & 7.986 & \\
\hline$\frac{457}{458}$ & & Maximum & 24.218 & 23.473 & 24.762 & 24.525 & 23.805 & 43.158 & $48.168 \mathrm{r}$ & 4.329 & 39.909 & -0.092 & -1.939 & 45.657 & 0.003 & 2.330 & & & & 46.70 & 11.19 & 941.506 & 2.862 & .347 & 0.359 & 0.009 & 8.935 & \\
\hline$\frac{450}{459}$ & & Mealan & 24.124 & 年3.390 & 24.6067 & 24.2253 & 23.518 & 年 42.304 & 47.110 & (4.089 & 38.930 & 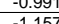 & -1.97 & 44.1808 & 0.003 & 2.141 & & & & 45.64 & & & & & & & & \\
\hline 460 & & $2 \times$ Std Dev & $\begin{array}{l}20.203 \\
0.445 \\
\end{array}$ & $\frac{23.301}{0.082}$ & $\frac{23.057}{2} 0.411$ & $\begin{array}{ll}24.019 \\
0.276\end{array}$ & $\begin{array}{r}23.289 \\
0.267 \\
\end{array}$ & $\begin{aligned} 41.495 \\
0.621\end{aligned}$ & $\begin{array}{l}40.106 \\
0.803 \\
\end{array}$ & 0.052 & $\begin{array}{r}37.955 \\
0.701 \\
\end{array}$ & $\frac{-1.1 .34}{0.324}$ & $\frac{-2.104}{0.148}$ & $\begin{array}{l}42.034 \\
1.444\end{array}$ & 0.000 & 0.071 & & & & $\begin{array}{ll}44.640 \\
0.80\end{array}$ & $\frac{10.50}{0.35}$ & & 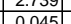 & $\mid$ & $\begin{array}{l}.318 \\
0.014 \\
\end{array}$ & $\frac{0.000}{0.000}$ & $\frac{1.944}{0.341}$ & \\
\hline 461 & Number & rof Points Used & 88 & 88 & 88 & 88 & 88 & 88 & 8.005 & 8.220 & 88 & $\frac{0.04}{88}$ & 0.148 & $\begin{array}{r}1.444 \\
\end{array}$ & 88 & 88 & & & & 88 & 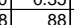 & $\mid \frac{\mid .005}{8.88}$ & \begin{tabular}{|l|}
0.045 \\
88
\end{tabular} & \begin{tabular}{|l|l|l|}
88 \\
\end{tabular} & $\frac{.014 \mid}{88}$ & 0.000 & $\frac{0.341}{88}$ & \\
\hline 462 & & * Backpulse poir & ts in box & are not ind & cluded & & & & & & & & & & & & & & & & & & & & & & & \\
\hline$\frac{463}{464}$ & & & & & & & & & & & & & & & & & & & & & & & & & & & & \\
\hline$\frac{464}{465}$ & |Caustic Rins & ise 2 and 3 & & & & & & & & & & & & & & & & & & & & & & & & & & \\
\hline$\frac{465}{466}$ & & Averages & 22.745 & 22.659 & 23.488 & 23.339 & 22.761 & 22.290 & 23.177 & 3.913 & 18.553 & -1.218 & 0.642 & 44.318 & 0.003 & 0.994 & & & & 21.70 & \begin{tabular}{l|l}
0 & 10.86 \\
\end{tabular} & 620.421 & 1.408 & 0.148 & 0.155 & 0.008 & 7.577 & \\
\hline 466 & & Maximum & 23.244 & 22.719 & 23.914 & 23.594 & 23.6 & 22.780 & 23.626 & $\begin{array}{ll}4.192 \\
\end{array}$ & 19.259 & -1.212 & 0.71 & 46.141 & 0.003 & 1.179 & & & & 22.15 & \begin{tabular}{l|l|l}
5 & 11.31 \\
\end{tabular} & $1 \mid 21.020$ & \begin{tabular}{|l|l|} 
& 1.449 \\
\end{tabular} & 176 & 0.181 & & $\begin{array}{l}9.069 \\
\end{array}$ & \\
\hline 4667 & & Median & 22.692 & 22.652 & 23.442 & 23.348 & 22.745 & 22.342 & 23.205 & 3.945 & 18.578 & $-1.218 \mathrm{~S}$ & 0.602 & $\begin{array}{lll}44.234 \\
\end{array}$ & 0.003 & 1.024 & & & & 21.73 & $\begin{array}{l}33 \\
3\end{array}$ & $\begin{array}{l}420.456 \\
\end{array}$ & 1.410 & 153 & 0.161 & 0.008 & 7.774 & \\
\hline 4688 & & Minimum & 22.424 & 22.602 & 23.184 & 23.061 & 22.5 & 21.678 & 22.581 & 3.615 & 17.859 & -1.221 & 0.5 & 42.601 & 0.003 & 0.874 & & & & 21.11 & 10.44 & 4|19.838 & 1.368 & 130 & 0.137 & 0.001 & 6.686 & \\
\hline 469 & & $2 \times$ Std Dev & 0.444 & 0.064 & 0.414 & 0.271 & 0.265 & 0.434 & 0.500 & 0.261 & 0.549 & 0.004 & 0.118 & 1.386 & 0.000 & 0.155 & & & & 0.50 & 0.34 & $\begin{array}{l}4 \mid 0.477] \\
\end{array}$ & 0.033 & 0.023 & 0.023 & 0.001 & 1.156 & \\
\hline$\frac{470}{471}$ & Tumber & rof Points Used ${ }^{*}$ & 50 & 55 & 55 & 55 & 55 & 55 & 55 & 55 & 55 & 55 & 5 & 55 & 55 & 55 & & & & 55 & 55 & 55 & 55 & 55 & 55 & 55 & 55 & \\
\hline$\frac{471}{472}$ & & * Backpulse pc & $\sin$ box & & & & & & & & & & & & & & & & & & & & & & & & & \\
\hline 473 & Caustic Rins & & & & & & & & & & & & & & & & & & & & & & & & & & & \\
\hline 474 & & Averages & 22.715 & 22.655 & 23.462 & 23.387 & & 22.352 & 23.346 & 3.965 & 18.588 & $-1.218 \mathrm{P} \quad \mathrm{C}$ & 0.702 & 44.421 & 0.003 & 1.050 & & & & 21.87 & 10.89 & 920.470 & 411 & 0.157 & 0.163 & 0.008 & & \\
\hline 475 & & Maximum & 23.194 & 22.706 & 23.914 & 23.594 & 23.6 & 22.666 & 23.553 & 4.179 & 18.937 & -1.215 & 0.1 & 46.141 & 0.003 & 1.177 & & & & 22.08 & $\frac{11.31}{11 .}>0$ & & 1.434 & \begin{tabular}{|l|l|}
0.175 \\
\end{tabular} & 0.181 & & & \\
\hline & & Median & 22.667 & 22.652 & 23.432 & 23.385 & & 22.357 & 23.354 & 3.957 & $\begin{array}{ll}18.604 \\
\end{array}$ & $-1.218 \mathrm{C} \quad \mathrm{C}$ & 0.7 & 44.234 & & 1.041 & & & & 21.88 & 10.84 & & & & & & & \\
\hline 4777 & & Minimum & 22.424 & 22.610 & 23.184 & 23.217 & & 21.817 & 22.886 & 3.859 & 17.859 & -1.2211 & 0.6 & 43.711 & 0.003 & 1.0 & & & & 21.41 & 10.71 & 119.838 & & & & & & ot \\
\hline 478 & & $2 \times \operatorname{Std} \mathrm{Dev}$ & 0.452 & 0.051 & 0.443 & 0.233 & 0.25 & 0.338 & 0.286 & 0.145 & 0.438 & 0.003 & 0.0 & 1.053 & 0.000 & 0.068 & & & & 0.29 & \begin{tabular}{|l|l|}
0.26 \\
\end{tabular} & \begin{tabular}{l|l}
6 & 0.382 \\
\end{tabular} & $\mid$\begin{tabular}{|l|}
0.026 \\
\end{tabular} & 0.010 & 0.009 & 0.000 & 0.498 & 0.48 \\
\hline 479 & Number & rof Points Used ${ }^{*}$ & 27 & 27 & 27 & 27 & 27 & 27 & 27 & 27 & 27 & 27 & 27 & 27 & 27 & 27 & & & & 27 & 27 & \begin{tabular}{l|l|}
7 & 27 \\
\end{tabular} & 27 & 27 & 27 & 27 & 27 & 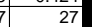 \\
\hline & & * Backpulse poi & in box & tare not ing & & & & & & & & & & & & & & & & & & & & & & & & \\
\hline$\frac{481}{482}$ & & & & & & & & & & & & & & & & & & & & & & & & & & & & \\
\hline$\frac{482}{483}$ & austic Rins & $\frac{\text { sie } 3}{\text { Averagt }}$ & 22.774 & 22.663 & $3 \quad 23.514$ & 23.291 & 22.7 & 22.229 & \begin{tabular}{|l|l|}
23.015 \\
\end{tabular} & 3.862 & $\mid 18.518$ & -1.217 & 0.5 & 44.218 & 0.003 & 0.941 & & & & 21.54 & \begin{tabular}{l|l|l|}
4 & 10.84
\end{tabular} & 420.374 & 1.405 & 0.140 & 0.146 & 0.007 & 7.182 & 6.112 \\
\hline 484 & & Maximum & 23.244 & 22.719 & 23.903 & 23.477 & 22.9 & 22.780 & 23.626 & 4.192 & 19.259 & -1.212 & 0.60 & 45.797 & 0.003 & 1.179 & & & & 22.15 & \begin{tabular}{l|l|l|}
5 & 11.22
\end{tabular} & $\frac{121.6}{221 .}$ & $\mid$\begin{tabular}{|l|l|}
1.449 \\
\end{tabular} & & 0.181 & 0.0 & 9.069 & 7.718 \\
\hline & & Median & 22.711 & 22.668 & 23.471 & 23.324 & 22.72 & 22.301 & 22.966 & 3.865 & 18.493 & -1.218 & 0.5 & $\begin{array}{l}44.214 \\
\end{array}$ & 0.003 & 0.9 & & & & 21.49 & 10.84 & $4|20.408|$ & 1.407| & & 0.141 & $0.0>>-2$ & 85 & \\
\hline 486 & & & 22.508 & 22.602 & 23.263 & 23.061 & & 21.678 & 581 & 3.615 & 17.976 & -1.221 & & 42.601 & 0.003 & 0.8 & & & & 21.11 & | 10.44 & 4[19.966 & & 0.130 & 0.137 & 0.007 & 6.686 & \\
\hline 4887| & & $2 \times$ Std Dev & 0.429 & 0.074 & 0.377 & 0.272 & 0.253 & 0.479 & 0.443 & 0.304 & 0.630 & 0.005 & 0.010 & 1.620 & 0.000 & 0.139 & & & & 0.44 & 0.40 & $0[0.536$ & $\mid 0.037$ & 0.021 & 0.020 & 0.001 & 1.054 & \\
\hline$\left|\frac{488}{489}\right|$ & Number & 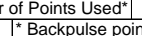 & $\begin{array}{l}1 \\
t \sin b o x \\
\end{array}$ & are not inc & $\begin{array}{l}8 \quad 28 \\
\text { cluded }\end{array}$ & & & 28 & 28 & & & 28 & & & & & & & & 28 & 28 & 28 & 28 & 28 & 28 & & 28 & \\
\hline
\end{tabular}


WSRC-TR-2005-00105, REVISION 0 SRNL-RPP-2005-00012, REVISION 0

RUN \# 2.21A, B, AND C; POST-TEST BASELINE, CAUSTIC RINSE 3 SOLUTION AND 0.1 M NAOH

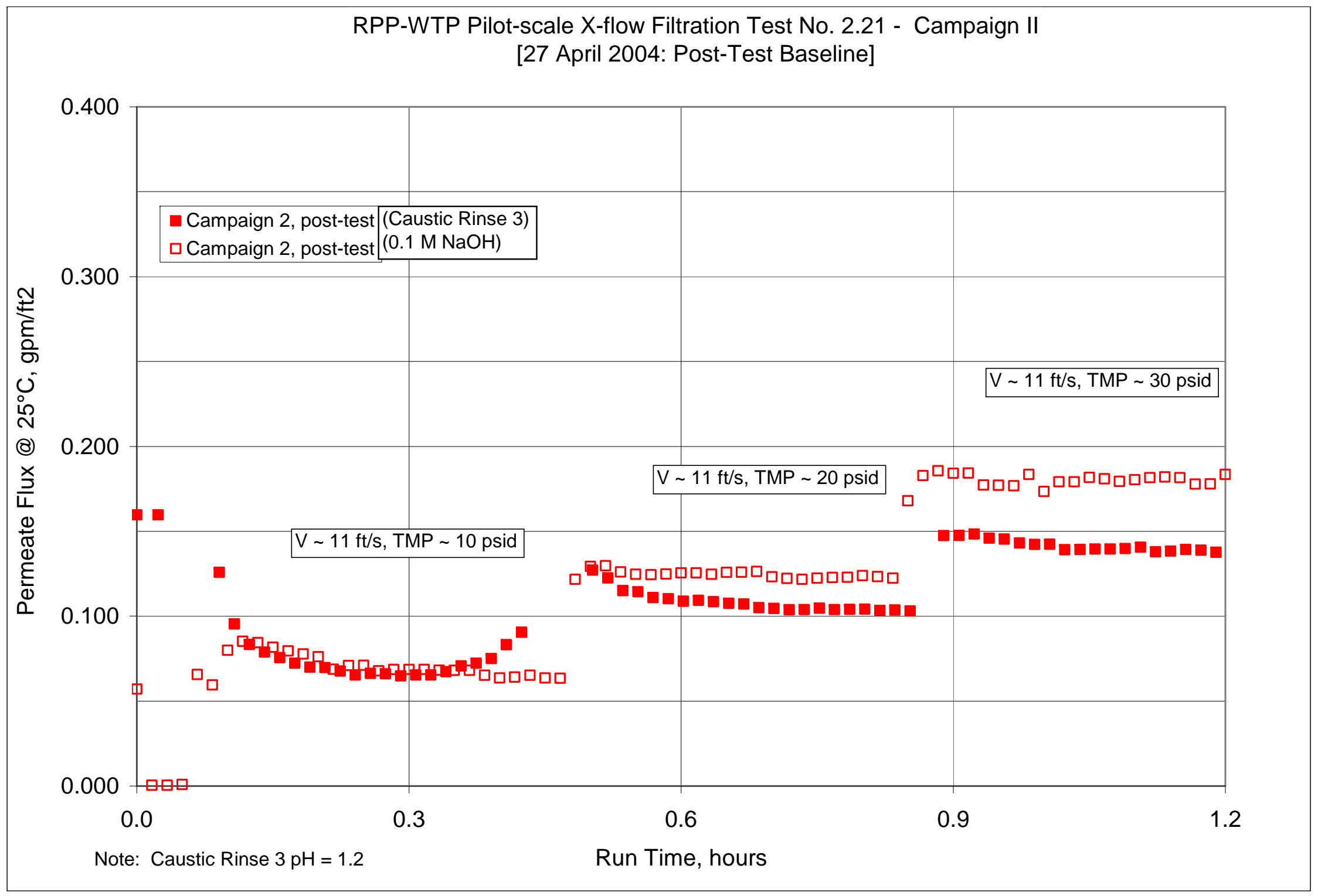


WSRC-TR-2005-00105, REVISION 0

SRNL-RPP-2005-00012, REVISION 0

RUN \# 2.21A, B, AND C; POST-TEST BASELINE WITH CAUSTIC RINSE 3 SOLUTION - CONT.

\begin{tabular}{|c|c|c|c|c|c|c|c|c|c|c|c|c|c|c|c|c|c|c|c|c|c|c|c|c|c|c|c|c|}
\hline & $\mathrm{A}$ & B & D & $\frac{\mathrm{E}}{\mathrm{Ten}}$ & $F$ & G & $\frac{H}{4 t 5 \gg>}$ & $\mathrm{J}$ & $\frac{K}{\ll \ll \ll<}$ & $\frac{L}{\mathrm{ressut}}$ & $M$ & $\mathrm{~N}$ & 0 & $\frac{Q}{<\ll \ll<}$ & $\begin{array}{ll}\mathrm{R} \\
\mathrm{SEl}\end{array}$ & \begin{tabular}{c|}
$\mathrm{s}$ \\
asurem
\end{tabular} & & v & $w$ & $\frac{x}{x}$ & $\begin{array}{r}Y \\
\text { alculats }\end{array}$ & $\frac{1}{z}$ & AA & $\frac{A B}{D a t a}$ & $A C$ & $A D$ & $\mathrm{AE}$ & $\mathrm{AF}$ \\
\hline$\frac{1}{2}$ & DATE & TIME & Filtrate & Cleaning & 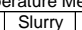 & $\begin{array}{l}\text { elasurem } \\
\text { Hi Amb. }\end{array}$ & Lo Amb. & BotTMP & Filter & $\begin{array}{l}\text { Plessuret } \\
\text { Fiter dP }\end{array}$ & 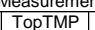 & Filtrate & Pulsepot & Slurry & Filtrate & Hi Filtate & Backpulse & & & & & & & & & & & \\
\hline 3 & & & $\operatorname{deg} C$ & $\operatorname{deg} C$ & deg $\mathrm{C}$ & $\operatorname{deg} C$ & $\operatorname{deg} \mathrm{C}$ & psid & psig & psid & psid & psig & psig & $\mathrm{gpm}$ & gpm & $\mathrm{gpm}$ & & & & & & & & & & & & \\
\hline & & & & & & & & & & $\frac{d P 1}{d P 1}$ & & & & & & & & & & & & & & & & & & \\
\hline & $\overline{s-01 / /}$ & & & & & & & & & & & & & & & & & & & & & & & & & & & \\
\hline 7 & $4 / 14 / 2004$ & 1:30:58 PM & 24.687 & 21.862 & \begin{tabular}{|l|}
24.835 \\
\end{tabular} & 24.226 & 22.583 & 0.054 & 0.02 & -0.021 & -0.005 & -0.179 & 0.099 & -0.08 & 0.003 & 0.086 & 0.005 & & & Filter $\mathrm{S}$ & urface & & FT2 & & & & & \\
\hline & $4 / 19 / 2004$ & 7:37:13 AM & 22.595 & 22.228 & 23.926 & 21.772 & 22.175 & $\begin{array}{l}0.046 \\
\end{array}$ & 0.082 & -0.025 & -0.009 & -0.179 & 0.143 & & 0.003 & $\begin{array}{l}14.643 \\
\end{array}$ & 0.003 & & & Conver & & 851 & $\mathrm{~m} 3 / \mathrm{m}$ & & g gpm/tit & barg & & \\
\hline & 4/19/2004 & $\begin{array}{r}\text { 8:21:39 AM } \\
12.41 .36 \mathrm{PM}\end{array}$ & 22.248 & $\begin{aligned} 22.333 \\
22767\end{aligned}$ & 22.324 & $\begin{aligned} 22.194 \\
2363\end{aligned}$ & 22.199 & $\begin{array}{ll}-0.008 \\
-0.04\end{array}$ & 0.059 & 0.002 & 0.007 & -0.132 & 0.131 & $\begin{array}{l}-0.084 \\
\end{array}$ & 0.003 & \begin{tabular}{|l|l|}
14.643 \\
14632 \\
\end{tabular} & 0.005 & & & & & & & & & & & \\
\hline$\frac{11}{11}$ & $\frac{4 / 202 / 2004}{4 / 22 / 2004}$ & $\begin{array}{c}12: 41: 36 \text { PM } \\
84: 43 \mathrm{AM}\end{array}$ & $\frac{22.772}{22.558}$ & $\frac{22.767}{21.446}$ & \begin{tabular}{|l|}
22.049 \\
24.471 \\
\end{tabular} & $\frac{23.603}{22.716}$ & $\begin{array}{l}24.746 \\
22.489 \\
\end{array}$ & $\begin{array}{l}-0.004 \\
0.01\end{array}$ & 0.045 & $\begin{array}{l}-0.009 \\
0.001\end{array}$ & $\begin{array}{l}-0.007 \\
0.001\end{array}$ & $\begin{array}{l}-0.176 \\
-0.205\end{array}$ & $\begin{array}{l}0.108 \\
0.096\end{array}$ & \begin{tabular}{|l|l|}
-0.084 \\
46.562 \\
\end{tabular} & 0.003 & \begin{tabular}{|l|}
14.6643 \\
14.643 \\
\end{tabular} & 0.005 & & & & & & $P 1$ & oroy & ately 1 & sig & & \\
\hline & $4 / 23 / 2004$ & 8:53:20 AM & 21.548 & 21.737 & \begin{tabular}{|l|}
22.041 \\
\end{tabular} & 21.89 & 21.369 & -0.004 & 0.026 & 0.003 & -0.005 & -0.196 & 0.082 & -0.082 & 0.003 & 14.643 & 0.005 & & & \begin{tabular}{|l|l|} 
Pressur \\
\end{tabular} & re Plis & scorrect & ted for 40 & & & vater tub & & \\
\hline & $4 / 26 / 2004$ & 9:00:47 AM & 22.379 & 22.907 & 23.455 & 22.416 & 21.819 & -0.012 & 0.08 & 0 & $\mid-0.009$ & -0.205 & 0.044 & -0.08 & 0.003 & \begin{tabular}{|l|l|l|l|}
1443 \\
\end{tabular} & 0.005 & & & 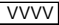 & & & & < F Filtra & ate Flux & $\ll P E$ & & p> \\
\hline & 4/27/2004 & 9:14:59 AM & 20.374 & 19.305 & 19.368 & 20.841 & 19.777 & -0.002 & 0.02 & $\begin{array}{l}-0.001 \\
-x^{-}\end{array}$ & 0.005 & -0.193 & 0.111 & $\begin{array}{l}-0.084 \\
\end{array}$ & 0.00 & $\begin{array}{l}14.643 \\
\end{array}$ & 0.006 & & & & & & & & at $25 \mathrm{C}$ & & 要1000 & \\
\hline 16 & seline & & & & & & & & & & & & & & & & & Time & Time & Press. & Vel. & $\mathrm{MP}$ & TMP & $\mathrm{gpm}$ & $\mathrm{gpm}$ & $\mathrm{gpm}$ & om & neter \\
\hline & & $\bar{B}$ & $\mathrm{D}$ & $F$ & $F$ & $\mathrm{G}^{\prime}$ & H & J & K & $=$ & $\bar{M}$ & 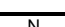 & 0 & 0 & 8 & & & & Hour & $\frac{p s i g}{x}$ & & & & & & & & \\
\hline & aseline 2A & & & & & & & & & & & & & Q & R & $\mathrm{S}$ & & V & w & $x$ & $\mathrm{Y}$ & z & $\mathrm{AA}$ & $A B$ & $A C$ & $A D$ & $\mathrm{AE}$ & $\mathrm{AF}$ \\
\hline & $4 / 27 / 2004$ & 9:23:51 AM & 21.162 & 19.444 & 22.273 & 20.97 & 20.192 & 11.904 & 11.834 & 4.008 & 8.046 & -1.215 & 0.631 & 44.988 & 0.003 & 0.991 & 0.005 & 0.00 & & 10.4 & 11.0 & 10. & 0.688 & 0.148 & 0.160 & 0.016 & 16.005 & 13.62 \\
\hline & $4 / 27 / 2004$ & $9: 25: 16 \mathrm{AM}$ & 21.162 & 19.444 & \begin{tabular}{|l|}
22.273 \\
\end{tabular} & 20.97 & 20.192 & 11.904 & 11.834 & 4.008 & 8.046 & -1.215 & 0.631 & 44.988 & & & & 1.42 & 0.02361 & 10.4 & 11.0 & & \begin{tabular}{|l|l|}
0.688 \\
\end{tabular} & 0.148 & 0.160 & 0.016 & 16.005 & \\
\hline & $4 / 27 / 2004$ & 9:25:18 AM & & 19.456 & 22.34 & 21.032 & 20.214 & $\begin{array}{l}2.974 \\
\end{array}$ & 11.816 & 4.065 & -1.14 & 3.961 & 13.672 & 44.458 & 0.003 & -0.013 & 0.003 & 1.45 & \begin{tabular}{|l|l|}
0.02417 \\
\end{tabular} & 10.3 & 10.9 & 0.9 & \begin{tabular}{|l|l|}
0.063 \\
\end{tabular} & -0.002 & -0.002 & -0.002 & -2.280 & -1.94 \\
\hline & $4 / 27 / 2004$ & 9:26:18 AM & 21.171 & 19.473 & \begin{tabular}{|l|}
22.428 \\
\end{tabular} & 21.06 & 20.257 & 3.256 & $\begin{array}{l}12.123 \\
\end{array}$ & 4.163 & -0.955 & 3.892 & $\begin{array}{l}19.763 \\
\end{array}$ & $\begin{array}{l}44.683 \\
\end{array}$ & 0.003 & 14.643 & 0.003 & 2.45 & \begin{tabular}{|l|l|l|} 
\\
\end{tabular} & 10.6 & 11.0 & 1.2 & \begin{tabular}{|l|} 
\\
\end{tabular} & 2.183 & 2.349 & 2.041 & 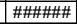 & \#\#\#\#\#" \\
\hline & $4 / 2772004$ & 9:27:18 AM & 21.144 & $\begin{array}{l}19.481 \\
\end{array}$ & \begin{tabular}{|l|}
22.441 \\
\end{tabular} & 21.103 & 20.491 & \begin{tabular}{|l|}
3.204 \\
\end{tabular} & 11.992 & 4.208 & $\begin{array}{l}-1.042 \\
\end{array}$ & \begin{tabular}{|l}
3.889 \\
\end{tabular} & 19.636 & 44.527 & $\begin{array}{l}0.003 \\
\end{array}$ & 14.643 & 0.003 & 3.45 & \begin{tabular}{|l|l}
0.0575 \\
0.0717
\end{tabular} & 10.5 & 10.9 & 1.1 & \begin{tabular}{|l|l|} 
& 0.075 \\
\end{tabular} & 2.183 & 2.348 & 2.172 & man! & 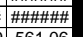 \\
\hline & $\begin{array}{l}4 / 27 / 2004 \\
4\end{array}$ & $\begin{array}{l}9: 28: 18 \mathrm{AM} \\
9 \cdot 29 \cdot 18 \mathrm{AM}\end{array}$ & 21.087 & $\begin{array}{r}19.515 \\
19575\end{array}$ & $\begin{array}{r}22.46 \\
22554\end{array}$ & $\frac{21.147}{21.156}$ & $\begin{array}{l}20.707 \\
20.852\end{array}$ & & $\begin{array}{l}11.677 \\
11.743\end{array}$ & $\begin{array}{l}3.935 \\
4.047\end{array}$ & $\begin{array}{l}1.624 \\
77677\end{array}$ & $\begin{array}{r}1.13 \\
-1.215\end{array}$ & $\begin{array}{l}0.307 \\
0.432\end{array}$ & $\begin{array}{l}45.033 \\
45.158\end{array}$ & 0.485 & $\begin{array}{c}14.643 \\
0787\end{array}$ & & & 0.0741 & $\frac{10.2}{10^{2}}$ & & 3.6 & & & 2.346 & & & $\frac{561.06}{1126}$ \\
\hline \begin{tabular}{|l|}
129 \\
129
\end{tabular} & $\begin{array}{l}4 / 27 / 20004 \\
/ 27 / 204\end{array}$ & $9: 30: 18 \mathrm{AM}$ & $\frac{21.413}{21.478}$ & $\begin{array}{l}19.6 / 5 \\
19.615\end{array}$ & \begin{tabular}{|l|}
22.5373 \\
22.573 \\
\end{tabular} & 21.186 & $\frac{20.052}{20.926}$ & $\begin{array}{r}11.925 \\
11.925\end{array}$ & $\frac{11.745}{11.988}$ & $\frac{4.041}{4.082}$ & 8.039 & -1.215 & $\begin{array}{l}0.432 \\
0.437\end{array}$ & $\begin{array}{l}45.150 \\
45.033\end{array}$ & & 0.1897 & $\frac{0.003}{0.003}$ & $\frac{3.45}{6.45}$ & \begin{tabular}{|l|l|}
0.09603 \\
0.1075
\end{tabular} & 10.5 & $\frac{11.1}{11.0}$ & $\begin{array}{r}\frac{9.5}{10.0} \\
\end{array}$ & \begin{tabular}{|l|l|}
0.658 \\
\end{tabular} & 0.0111 & $\frac{0.120}{0.095}$ & $\frac{0.013}{0.010}$ & $\frac{15.221}{9.553}$ & 11.26 \\
\hline & $4 / 27 / 2004$ & $9: 31: 18 \mathrm{AM}$ & 21.553 & 19.67 & \begin{tabular}{|l|l|} 
& 22.589 \\
\end{tabular} & 21.221 & 20.982 & 12.016 & 11.975 & 4. & 8.224 & -1.215 & 0.435 & 44.723 & & & & 7.45 & \begin{tabular}{|l|l|}
0.12417 \\
\end{tabular} & 10.5 & 11.0 & 10.1 & \begin{tabular}{|l|l|} 
\\
\end{tabular} & 0.078 & 0.083 & 0.008 & & \\
\hline & $4 / 27 / 2004$ & 9:32:18 AM & \begin{tabular}{|l|l|}
21.578 \\
\end{tabular} & 19.736 & \begin{tabular}{|l|}
22.639 \\
\end{tabular} & & & \begin{tabular}{|l|l|}
11.973 \\
\end{tabular} & 11.926 & & 8.081 & -1.215 & 0.435 & 44.831 & & 0.494 & & 8.45 & 0.14083 & 10.5 & 11.0 & 10.0 & & 0.074 & & 0.008 & 7.854 & \\
\hline & $4 / 27 / 2004$ & 9:33:18 & 21.593 & 19.796 & \begin{tabular}{|l|}
22.683 \\
\end{tabular} & 21.311 & 20.971 & \begin{tabular}{|c|}
12.08 \\
\end{tabular} & 11.952 & & 8.3 & & 0.435 & \begin{tabular}{|l|l}
4.535 \\
\end{tabular} & & & 0.005 & 9.45 & \begin{tabular}{|l|l|} 
\\
\end{tabular} & 10.5 & 10.9 & 10.2 & & & 0.075 & 0.007 & 7.406 & 6.30 \\
\hline & $4 / 27 / 2004$ & 9:34:18 AM & 21.628 & $\begin{array}{l}19.862 \\
1021\end{array}$ & \begin{tabular}{|l|l|}
22.694 \\
\end{tabular} & 21.331 & $\begin{array}{l}21.097 \\
2.1260\end{array}$ & \begin{tabular}{|l|l|}
12.103 \\
12.25
\end{tabular} & $\begin{array}{l}12.048 \\
12.07\end{array}$ & & $\begin{array}{l}8.085 \\
.8020\end{array}$ & $\begin{array}{l}-1.218 \\
1.215 \\
\end{array}$ & $\begin{array}{l}0.435 \\
0.25\end{array}$ & 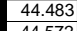 & & 0.454 & & 10.45 & \begin{tabular}{|l|}
0.17417 \\
\end{tabular} & 10.6 & 10.9 & & & & 0.072 & 0.007 & 7.159 & 6.0 \\
\hline & 4/27/2004 & $\begin{array}{l}9: 35: 18 \mathrm{AM} \\
9 \cdot 3 \cdot 18\end{array}$ & $\frac{21.673}{21.687}$ & 19.931 & \begin{tabular}{|l|}
22.738 \\
\end{tabular} & 21.381 & $\begin{array}{l}21.136 \\
21.196\end{array}$ & 12.265 & $\begin{array}{l}12.197 \\
12.120\end{array}$ & $\begin{array}{l}4.063 \\
.2070\end{array}$ & $\begin{array}{l}8.302 \\
8.20\end{array}$ & -1.215 & 0.435 & 44.5 & 0.0 & $\begin{array}{l}0.44 \\
\end{array}$ & 0.005 & & 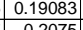 & 10.7 & & & 0.709 & 0.066 & & & & 5.7. \\
\hline 136 & $\begin{array}{l}4 / 27 / 2004 \\
4 / 27 / 2004\end{array}$ & $\begin{array}{l}9: 36: 18 \mathrm{AM} \\
9: 37: 18 \mathrm{~A}\end{array}$ & $\frac{21.687}{21.697}$ & $\frac{19.982}{20.032}$ & \begin{tabular}{|l|}
22.733 \\
22.768
\end{tabular} & 21.395 & $\frac{21.186}{21.292}$ & \begin{tabular}{|l|}
$\frac{12.333}{12.244}$ \\
\end{tabular} & $\frac{12.162}{12.098}$ & $\begin{array}{l}3.879 \\
4.04\end{array}$ & $\begin{array}{r}8.62 \\
8.308\end{array}$ & $\frac{-1.210}{-1.215}$ & $\begin{array}{l}0.435 \\
0.435\end{array}$ & $\frac{44 .}{44 .}$ & & & 0.005 & $\frac{12.45}{13.45}$ & 0.22 & $\frac{10.7}{10.6}$ & $\begin{array}{l}10.9 \\
10.9\end{array}$ & & & 0.064 & 0. & & & $\frac{5.6}{56}$ \\
\hline & $4 / 27 / 2004$ & 9:38:18 & 21.732 & 20.092 & 22.797 & 21.415 & 21. & \begin{tabular}{|l|l|}
12.149 \\
\end{tabular} & & & 8.189 & & & 44.6 & 0.003 & & & 14.45 & \begin{tabular}{|l|l|}
0.24083 \\
\end{tabular} & 10.6 & 10.9 & 10.2 & 0.7 & 0.061 & 0.065 & 0.006 & 6.430 & 5.4 \\
\hline 138 & $4 / 27 / 2004$ & 9:39:18 & 21.772 & 20.167 & & 21.44 & 21.4 & 12.114 & & & & & & 44.3 & & & & 15.45 & 0.2 & 10.6 & 10.9 & & & & & & & 5.5 \\
\hline 139 & $4 / 27 / 2004$ & 9:40:18 & 21.781 & 20.212 & \begin{tabular}{|l|}
22.831 \\
\end{tabular} & 21.439 & 21.4 & \begin{tabular}{|l|l|}
12.234 \\
\end{tabular} & 12.085 & & 399 & \begin{tabular}{|l|l|}
-1.215 \\
\end{tabular} & & 44.1 & & & & 16.45 & & 10.6 & $\begin{array}{l}10.8 \\
\end{array}$ & \begin{tabular}{l|l}
8 & 10.3 \\
\end{tabular} & & & 66 & 06 & 6.393 & \\
\hline$\frac{140}{141}$ & $4 / 27 / 2004$ & 9:41:18 AM & 21.805 & $\begin{array}{l}20.261 \\
2020\end{array}$ & \begin{tabular}{|l|l|}
22.861 \\
\end{tabular} & 21.418 & 21.324 & \begin{tabular}{|l|l|}
12.288 \\
12231
\end{tabular} & $\begin{array}{l}12.127 \\
12112\end{array}$ & & 8.243 & \begin{tabular}{|c|c|}
-1.218 \\
-1210
\end{tabular} & 0.435 & 44 & & & & 17.45 & $\begin{array}{l}0.29083 \\
\end{array}$ & 10.7 & 10.9 & \begin{tabular}{l|l}
9 & 10.3 \\
\end{tabular} & & $\begin{array}{l}30.061 \\
\end{array}$ & 0.065 & 0.006 & 6.312 & 5.3 \\
\hline 141 & $4 / 27 / 2004$ & $\begin{array}{l}9: 42: 18 \\
9.43 \cdot 18\end{array}$ & $21.804 \mid$ & $\begin{array}{r}20.295 \\
20.33\end{array}$ & $22.884 \mid$ & $\begin{array}{l}21.366 \\
21305\end{array}$ & $\frac{21.167}{21.161}$ & $\frac{12 .}{12.3}$ & $\frac{12.112}{12.116}$ & & $\begin{array}{r}8.37 \\
8.432\end{array}$ & $\begin{array}{r}-1.218 \\
-1.215\end{array}$ & \begin{tabular}{|l}
0.435 \\
0.435
\end{tabular} & $\begin{array}{l}44 . \\
45 .\end{array}$ & 0.1 & & & $\begin{array}{l}18.45 \\
19.55\end{array}$ & \begin{tabular}{|l|l|} 
\\
\end{tabular} & 10.6 & 11.0 & 10.3 & & & 0.065 & 0.006 & $\begin{array}{l}6.320 \\
6.302 \\
\end{array}$ & $5.3 \varepsilon$ \\
\hline \begin{tabular}{|l|}
143 \\
143
\end{tabular} & $4 \frac{4 / 27 / 2004}{4 / 27 / 2004}$ & $\begin{array}{l}9: 43: 18 \mathrm{AM} \\
9: 44: 18 \mathrm{AM}\end{array}$ & 21.833 & $\begin{array}{r}20.33 \\
20.353 \\
\end{array}$ & \begin{tabular}{|l}
22.884 \\
22.887
\end{tabular} & $\frac{21.1305}{21.283}$ & $\begin{array}{r}21.161 \\
21.059\end{array}$ & \begin{tabular}{|l|}
12.317 \\
12.018
\end{tabular} & $\begin{array}{l}12.116 \\
11.878\end{array}$ & & & $\frac{-1.215}{-1.218}$ & & $\frac{45 .}{44 .}$ & & & & $\begin{array}{l}19.45 \\
20.45 \\
\end{array}$ & \begin{tabular}{|l|}
0.32417 \\
0.34083 \\
\end{tabular} & $\begin{array}{l}10.6 \\
10.4\end{array}$ & $\begin{array}{l}11.0 \\
11.0\end{array}$ & $\begin{array}{ll}0 & 10.4 \\
0 & 10.1\end{array}$ & & \begin{tabular}{|l|l|l|}
0.062 \\
5063
\end{tabular} & & $\begin{array}{l}0.006 \\
0.007\end{array}$ & $\frac{6.302}{6.661}$ & $\frac{5.3}{5.6}$ \\
\hline 144 & $4 / 27 / 2$ & 9:45:1 & 21.88 & 20.387 & 22.931 & 21.246 & 20.9 & & 12.141 & 4.056 & 8.436 & -1 & & 44.8 & 0. & 0.447 & & 21.45 & 0.3575 & 10.7 & 11.0 & $\frac{10.4}{10.4}$ & 0.7 & 0.067 & 0.0 & 0.007 & & 5. \\
\hline \begin{tabular}{|l|l|}
145 \\
\end{tabular} & $4 / 27 / 2004$ & 9:46:1 & 21.894 & 20.411 & 22.954 & 21.219 & & 12.1 & 11.979 & & & & & 44. & & & & & & 10.5 & 11.0 & & & & & & 148 & 6. \\
\hline 146 & $4 / 27 / 2004$ & 9:47: & 21.902 & 20.429 & \begin{tabular}{|l|}
22.967 \\
\end{tabular} & 21.248 & 21.1 & 12.1 & 12.031 & & & & & 44. & & & & 23.45 & 0.39083 & 10.6 & 10.9 & 10.2 & & & & & 7.363 & \\
\hline 147 & $4 / 27 / 2004$ & 9:48:18 AM & 21.915 & 20.438 & 22.951 & 21.246 & 21.118 & $\begin{array}{l}12.147 \\
\end{array}$ & 11.982 & & 8.208 & -1.215 & & 44.6 & & 0.526 & 0.00 & 24.45 & \begin{tabular}{|l|l|}
0.4075 \\
\end{tabular} & 10.5 & 11.0 & 10.2 & 0.7 & 0.078 & 0.083 & 0.008 & 8.166 & 6.9 \\
\hline & 4/27/2004 & 9:49:18 AM & 21.934 & 20.462 & \begin{tabular}{|l|}
22.975 \\
\end{tabular} & 21.25 & 21.187 & \begin{tabular}{|l|l|}
12.28 \\
\end{tabular} & 12.112 & 4.032 & 8.356 & -1.218 & & 44. & 0.003 & 0.573 & & 25.45 & 0.42417 & 10.6 & 11.0 & \begin{tabular}{l|l|}
0 & 10.3 \\
\end{tabular} & \begin{tabular}{|l|} 
\\
\end{tabular} & 0.085 & 0.090 & 0.009 & 8.769 & \\
\hline 50] & & & & & & & & & & & & & & & & & & & & & & & & & & & & \\
\hline & & Averages & \begin{tabular}{|l|}
21.7 \\
\end{tabular} & 20.1 & 22.8 & 21.3 & 21.2 & $\begin{array}{l}12.2 \\
\end{array}$ & 12.1 & 4.0 & 8.3 & -1.2 & 0.4 & 4 & 0.0 & 0.5 & 0.0 & & & 10.6 & $\begin{array}{ll}10.9 \\
\end{array}$ & & & & 0.073 & 0.007 & 7.2 & 6 \\
\hline & & Maximum & 21.9 & 20.5 & 23.0 & 21.4 & & & & 4 & 8.6 & -1.2 & 0.4 & 45 & 0.0 & & 0.6 & & & 10.7 & 11.0 & & & & & & 9.6 & 8 \\
\hline & & Median & 21.8 & 20.2 & 22.8 & 21.3 & \begin{tabular}{l|l}
21.1 \\
\end{tabular} & 12.2 & 12.1 & 4.0 & 8.2 & -1.2 & 0.8 & 4 & 0.6 & 0.4 & & & & 10.6 & 10.9 & $\begin{array}{l}9 \\
9\end{array}$ & $\mid 0.703$ & & & & 6. & \\
\hline & & $\begin{array}{l}\text { Minimum } \\
2 \text { cats }\end{array}$ & 21.5 & 19.6 & 22.6 & 21.2 & $\begin{array}{l}20.9 \\
021\end{array}$ & 11.9 & 11.9 & $\begin{array}{r}3.9 \\
0.122 \\
\end{array}$ & 8.0 & -1.2 & 0.4 & 44. & 0.0 & 0.4 & 0.0 & & & 10.4 & 10.8 & \begin{tabular}{l|l|}
8 & 10.0 \\
\end{tabular} & \begin{tabular}{|l|l|} 
& 0.688 \\
\end{tabular} & 0.061 & 0.065 & 0.006 & 6.3 & 5.4 \\
\hline 56 & Number 0 & $\frac{2 \times \text { Std Dev Dev }}{\text { of Points Used }}$ & $\begin{array}{r}0.262 \\
20 \\
\end{array}$ & & & & $\frac{0.315}{20}$ & & $\frac{0.166}{20}$ & $\frac{0.122}{20}$ & & $\frac{0.004}{20}$ & $\frac{0.003}{20}$ & $\begin{array}{r}0.425 \\
20\end{array}$ & 0.00 & $\begin{array}{r}0.107 \\
20\end{array}$ & $\frac{0.002}{20}$ & & & $\frac{0.166}{20}$ & $\frac{0.104}{20}$ & $\begin{array}{l}\frac{4}{4} 0.256 \\
0.20\end{array}$ & $\mid$\begin{tabular}{|r|}
0.018 \\
\end{tabular} & $\begin{array}{l}30.016 \\
20\end{array}$ & $\frac{0.017}{20}$ & $\frac{0.002}{20}$ & $\frac{1.777}{20}$ & \\
\hline 157 & & Backpulse poin & ts in box & are not inc & cluded & & & & & & & & & & & & & & & 20 & & & & & & & & \\
\hline
\end{tabular}


WSRC-TR-2005-00105, REVISION 0

SRNL-RPP-2005-00012, REVISION 0

RUN \# 2.21A, B, AND C; POST-TEST BASELINE WITH CAUSTIC RINSE 3 SOLUTION - CONT.

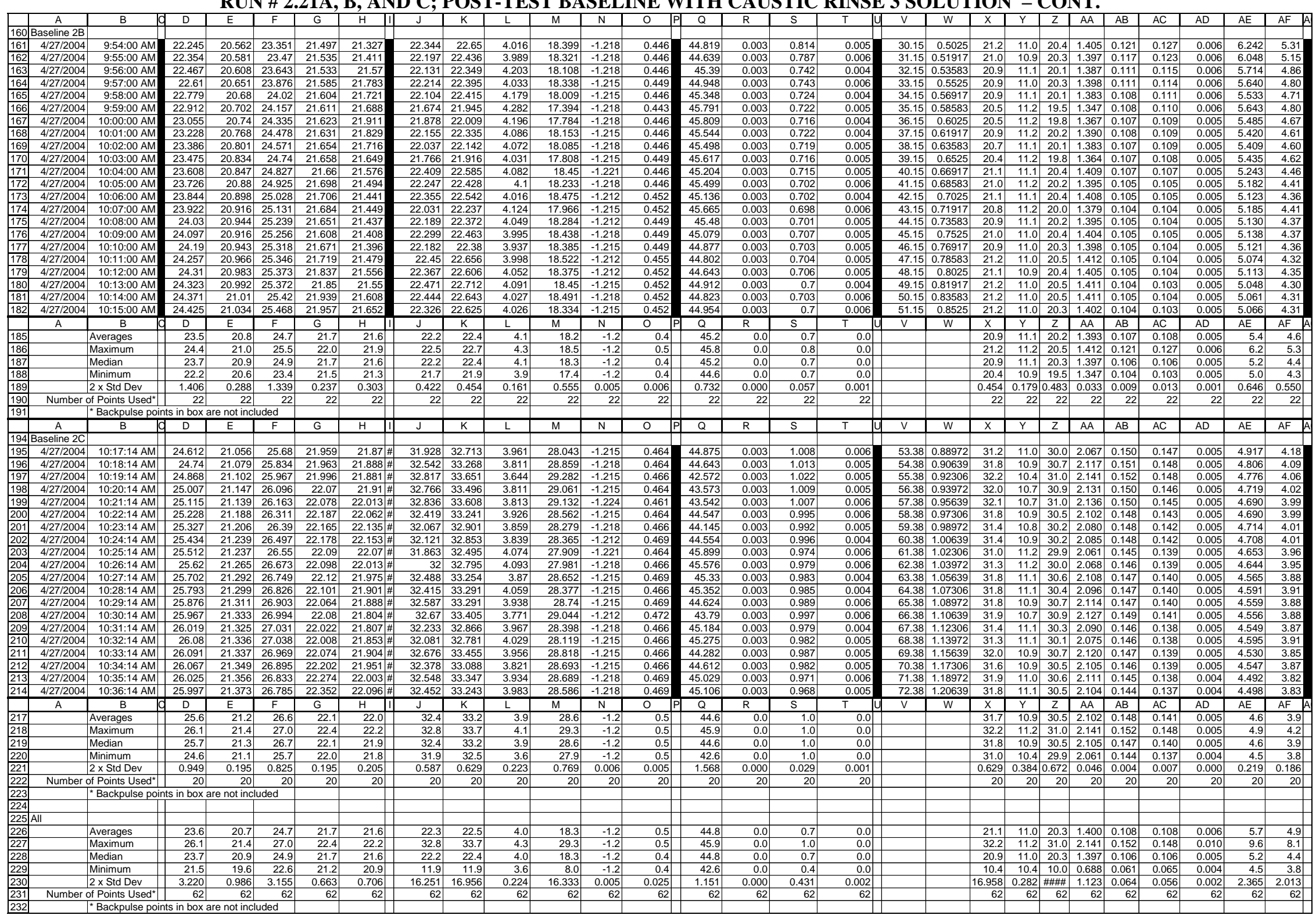


WSRC-TR-2005-00105, REVISION 0

SRNL-RPP-2005-00012, REVISION 0

RUN \# 2.21A, B, AND C; POST-TEST BASELINE WITH 0.1 M NAOH

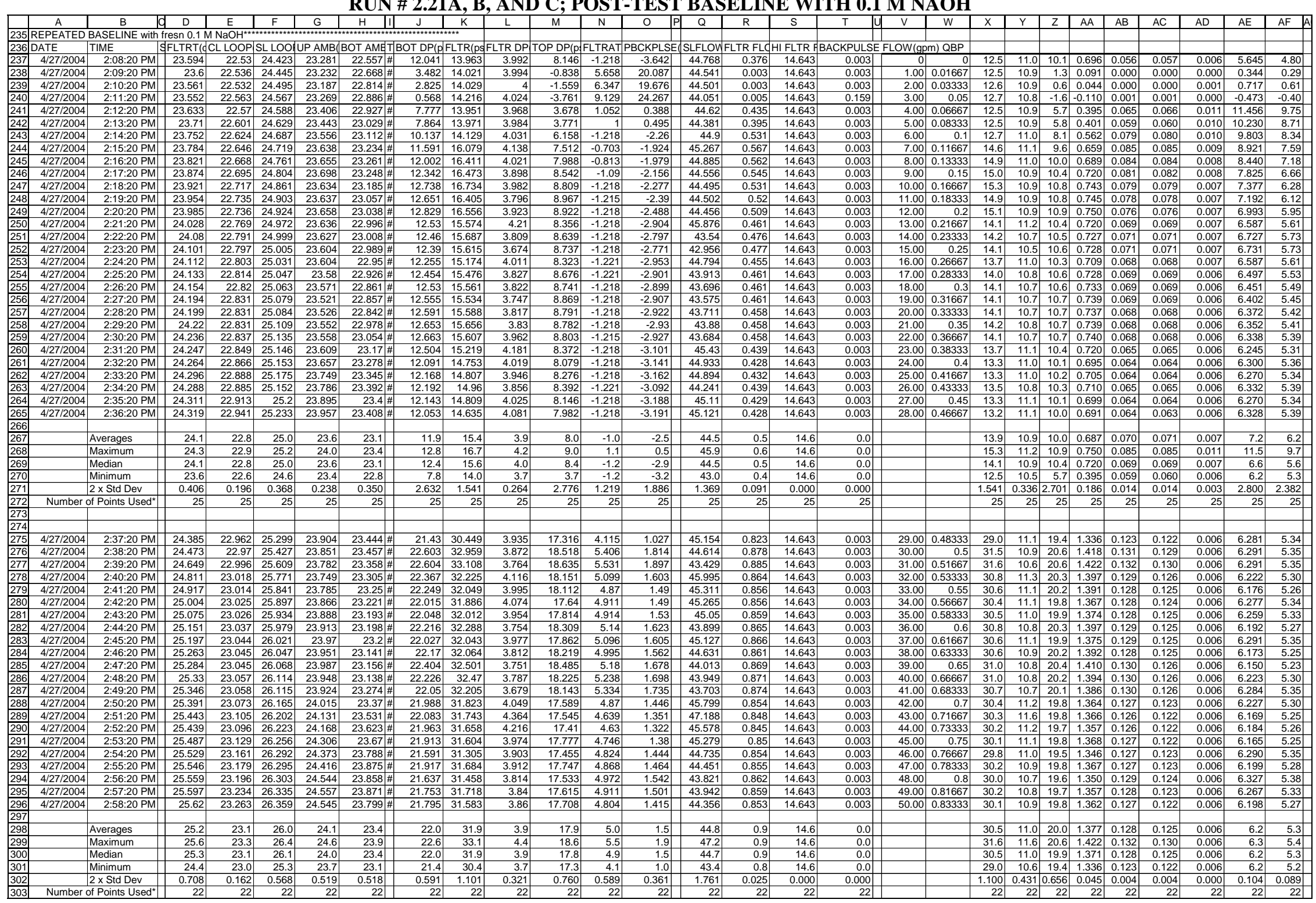


WSRC-TR-2005-00105, REVISION 0

SRNL-RPP-2005-00012, REVISION 0

RUN \# 2.21A, B, AND C; POST-TEST BASELINE WITH 0.1 M NAOH - CONT.

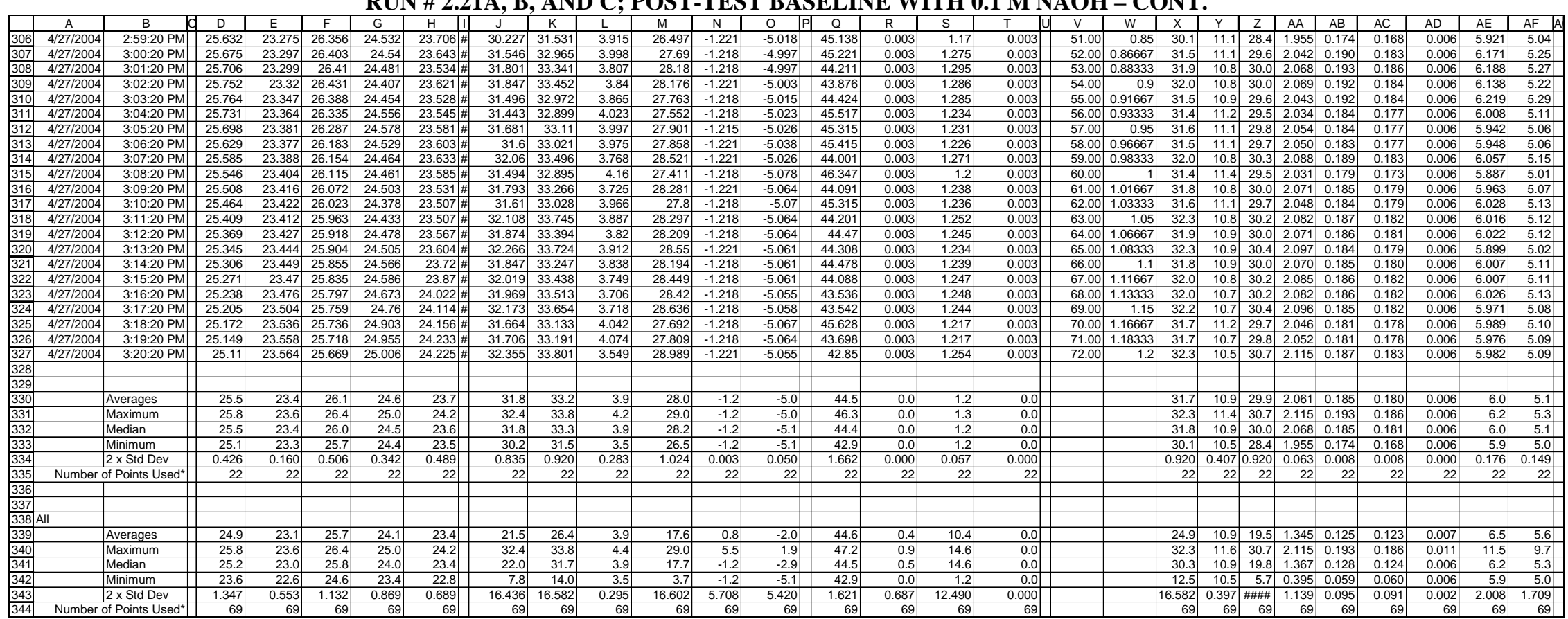


WSRC-TR-2005-00105, REVISION 0 SRNL-RPP-2005-00012, REVISION 0

PRE-TEST BASELINE WITH 0.1 M NAOH, BEFORE ACID CLEANING

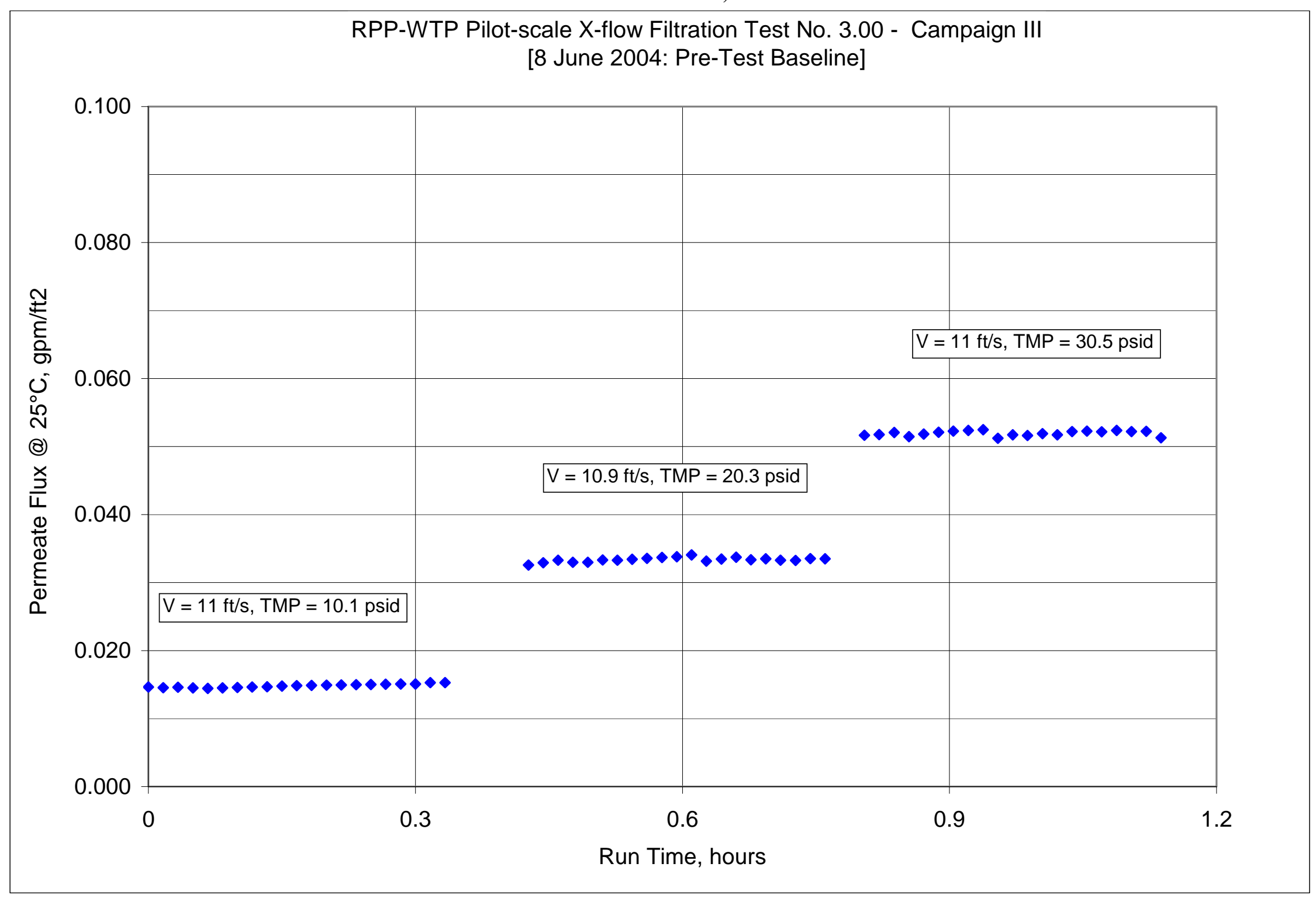


WSRC-TR-2005-00105, REVISION 0

SRNL-RPP-2005-00012, REVISION 0

PRE-TEST BASELINE WITH 0.1 M NAOH, BEFORE ACID CLEANING - CONT.

\begin{tabular}{|c|c|c|c|c|c|c|c|c|c|c|c|c|c|c|c|c|c|c|c|c|c|c|c|c|c|c|c|c|}
\hline 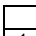 & A & B & $\mathrm{D}$ & \begin{tabular}{|l|l|l|}
$E$ & \\
\end{tabular} & $F$ & $\mathrm{G}$ & $\mathrm{H}$ & $\mathrm{J}$ & $\mathrm{K}$ & $\mathrm{L}$ & $\mathrm{M}$ & \begin{tabular}{|l|}
$\mathrm{N}$ \\
\end{tabular} & 0 & $Q$ & $\mathrm{R}$ & $\mathrm{s}$ & $\mathrm{T}$ & $\mathrm{V}$ & w & \begin{tabular}{|l|l|}
$x$ \\
\end{tabular} & \begin{tabular}{|l|}
$\mathrm{Y} \mid$ \\
\end{tabular} & $1 z$ & AA & $A B$ & \begin{tabular}{|l|l|} 
& $A C$ \\
\end{tabular} & $A D$ & $\mathrm{AE}$ & AF \\
\hline 1 & DATE & TIME & 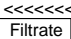 & $\begin{array}{l}\text { 《< Tempe } \\
\text { Cleaning }\end{array}$ & $\begin{array}{l}\text { erature M } \\
\text { Slurry }\end{array}$ & $\begin{array}{l}\text { easureme } \\
\text { Hi Amb. }\end{array}$ & $\begin{array}{l}\text { ents } \gg>> \\
\text { iLo Amb. }\end{array}$ & $\begin{array}{l}\text { BotTMP } \\
\text { Bos< }\end{array}$ & $\begin{array}{l}\text { Fiter } \\
\text { Filter }\end{array}$ & $\begin{array}{l}\text { Pressure I } \\
\text { Filter dP }\end{array}$ & $\begin{array}{l}\text { Measureme } \\
\text { TopTMP }\end{array}$ & \begin{tabular}{|c|}
$n_{n+15} \gg \gg>$ \\
Filtrate
\end{tabular} & Pulsepot & 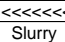 & $\begin{array}{l}<<\text { Flown } \\
\text { Filtrate }\end{array}$ & $\begin{array}{l}\text { Measure } \\
\text { IHi Filtat }\end{array}$ & 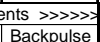 & & & & & & & & & & & \\
\hline & DAIE & & $\operatorname{deg} C$ & $\operatorname{deg} C$ & \begin{tabular}{|l|l|}
$\operatorname{deg} C$ \\
\end{tabular} & $\operatorname{deg} \mathrm{C}$ & $\operatorname{deg} C$ & psid & psig & psid & psid & \begin{tabular}{|l|l|} 
psigat \\
\end{tabular} & psig & $\mathrm{gpm}$ & $\begin{array}{l}\text { Filluate } \\
\text { gpm }\end{array}$ & gpm & $\begin{array}{l}\text { Backpulse } \\
\text { gpm }\end{array}$ & & & & & & & & & & & \\
\hline 4 & & & $\mathrm{~T} 2$ & & T1 & & & $\mathrm{dP2}$ & P1 & dP1 & $\mathrm{dP3}$ & $P^{2}$ & P3 & Q1 & $\mathrm{Q} 2$ & $\mathrm{Q} 3$ & Opbo & & & & & & & & & & & \\
\hline & 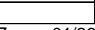 & 2000 & & & & & & & & & & & & & & & & & & & & & & & & & & \\
\hline \begin{tabular}{|l|}
6 \\
7 \\
\end{tabular} & $\frac{e r o s-01 / 26}{6 / 8 / 2004}$ & $\frac{2004}{9 \cdot 09 \cdot 09 \mathrm{AM}}$ & 22.545 & 24,705 & \begin{tabular}{|l|}
27.987 \\
\end{tabular} & 26.054 & 24.911 & -0.004 & 0.128 & -0.01 & -0.003 & -0.129 & 0.111 & 45,741 & 0.293 & - -0.013 & 0.00 & & & Filter Su & iufface $A$ & A6.707 & FT2 & & & & & \\
\hline & 6/10/2004 & 1:47:47 PM & 25.596 & 26.216 & 26.68 & 26.834 & & -0.019 & 0.196 & -0.012 & $\begin{array}{l}-0.02 \\
\end{array}$ & -0.106 & 0.073 & $\frac{-0.071}{-0.071}$ & 0.003 & 14.643 & 0.003 & & & Convers & rsion & 851 & & Iday/lbart & $\mathrm{rg} / \mathrm{gpm} / \mathrm{tt} \mathrm{t}$ & (ftr2/barg & & \\
\hline & $6 / 14 / 2004$ & $7: 41: 41 \mathrm{AM}$ & & & & 24.174 & 24.204 & & 0.12 & & -0.016 & \begin{tabular}{|l|l|l|l|l|} 
& -0.163 \\
\end{tabular} & & -0.083 & & 14.643 & & & & & & & & & & & & \\
\hline & $6 / 15 / 2004$ & 12:06:06 PM & 25.175 & 24.315 & 23.747 & 26.127 & & -0.012 & 0.139 & -0.002 & -0.012 & -0.177 & 0.043 & -0.073 & 0.003 & 14.643 & & & & Note: $G_{c}$ & Sauge $\mathrm{Pr}$ & ressure & & approxin & mately 1.4 & 4 psig & & \\
\hline 11 & 6/17/2004 & 7:28:28 AM & 24.492 & 23.951 & 23.799 & 24.225 & 23.743 & -0.01 & 0.164 & -0.001 & -0.014 & -0.18 & & -0.075 & 0.003 & $\begin{array}{l}14.643 \\
\end{array}$ & & & & & & & thor & & & & & \\
\hline & $\frac{6 / 17 / 2004}{6 / 21 / 2004}$ & $\begin{array}{l}4: 57: 57 \mathrm{PM} \\
6.525 \mathrm{AM} \\
\end{array}$ & $\begin{array}{l}26.259 \\
23.109\end{array}$ & \begin{tabular}{|l|}
26.737 \\
23008 \\
\end{tabular} & \begin{tabular}{|l|}
24.765 \\
22621 \\
\end{tabular} & $\frac{27.805}{23.418}$ & \begin{tabular}{|l|}
26.456 \\
22.888 \\
\end{tabular} & $\begin{array}{r}-0.002 \\
-0.01 \\
\end{array}$ & $\begin{array}{l}0.197 \\
0.075\end{array}$ & 0 & $\begin{array}{l}-0.012 \\
-0.01 \\
\end{array}$ & \begin{tabular}{|l|}
-0.183 \\
-0.197
\end{tabular} & $\begin{array}{l}-0.009 \\
0.055\end{array}$ & $\begin{array}{l}47.043 \\
-0.079 \\
\end{array}$ & $\begin{array}{l}0.098 \\
0.003 \\
\end{array}$ & \begin{tabular}{|l|l|}
14.643 \\
14.643 \\
\end{tabular} & 0.004 & & & $\begin{array}{l}\text { Pressur } \\
\text { vylyl }\end{array}$ & & & & $\begin{array}{l}10.88 \text { inc } \\
\text { K F Fittra }\end{array}$ & $\begin{array}{l}\text { ches of } w \\
\text { ate Flux : }\end{array}$ & K PERN & MAPBUTY & \\
\hline $\begin{array}{ll}14 \\
14 \\
\end{array}$ & $\begin{array}{l}0 / 21 / 2004 \\
6 / 22 / 2004\end{array}$ & 8:12:12 AM & $\frac{20.109}{23.36}$ & 23.555 & \begin{tabular}{|r|}
2.021 \\
23.63 \\
\end{tabular} & $\begin{array}{l}23.410 \\
23829\end{array}$ & \begin{tabular}{|r|}
22.000 \\
24.45 \\
\end{tabular} & $\begin{array}{c}-0.01 \\
-0.008\end{array}$ & $\begin{array}{l}0.070 \\
0.108 \\
\end{array}$ & $\begin{array}{r}0 \\
-0.004\end{array}$ & $\frac{-0.012}{-0.012}$ & $\begin{array}{l}-0.191 \\
-0.194\end{array}$ & 0.06 & $\begin{array}{l}-0.075 \\
-0.083 \\
-10\end{array}$ & 0.003 & $\begin{array}{l}14.045 \\
14.643\end{array}$ & 0.000 & & & & & & & & tall & & 1. & \\
\hline & & & & & & & & & & & & & & & & & & Time & Time & $\begin{array}{l}\text { Press. } \\
\end{array}$ & Jel. & TMP & TMP & gpm & gpm & gpm & $\begin{array}{ll}\mathrm{gpm} \\
\mathrm{gpm}\end{array}$ & meter \\
\hline 16 & aseline $1 \mathrm{~A}$ & & & & & & & & & & & & & & & & & & Hour & psig & & psi & bar & & & t2/psi & & day/bar \\
\hline 17 & $6 / 8 / 2004$ & 12:44:40 PM & 23.04 & 25.389 & 25.033 & 26.807 & 25.504 & 12.242 & 12.628 & 4.059 & 8.21 & -1.212 & -5.052 & 45.14 & $\begin{array}{ll}0.093 \\
\end{array}$ & $\begin{array}{l}14.643 \\
\end{array}$ & 0.003 & & & 11.2 & 11.1 & 10.2 & 0.705 & \begin{tabular}{|l|l|}
0.014 \\
\end{tabular} & 0.014 & 0.001 & 1.355 & 1.15 \\
\hline & $6 / 8 / 2004$ & 12:45:40 PM & $\begin{array}{l}22.921 \\
22705\end{array}$ & $\begin{array}{l}25.405 \\
25.12\end{array}$ & 24.558 & $\begin{array}{l}26.808 \\
2750 \\
2750\end{array}$ & 25.45 & $\begin{array}{ll}11.767 \\
1.205\end{array}$ & $\begin{array}{l}12.054 \\
12576\end{array}$ & 4.1 & 7.617 & \begin{tabular}{|r|}
-1.215 \\
\end{tabular} & $\begin{array}{l}-5.058 \\
5050\end{array}$ & 45.152 & $\begin{array}{ll}0.092 \\
0002\end{array}$ & $\begin{array}{l}14.643 \\
1.612\end{array}$ & 0.003 & 1.00 & \begin{tabular}{l|l|}
0 & 0.01667 \\
\end{tabular} & $\begin{array}{ll}10.6 \\
1.1\end{array}$ & 11.1 & 9.7 & 0.668 & \begin{tabular}{|l|l|}
0.014 \\
\end{tabular} & 0.014 & 0.001 & 1.433 & 1.22 \\
\hline$\frac{19}{20}$ & $\frac{6 / 8 / 2004}{6 / 8 / 2004}$ & $\begin{array}{l}12: 46: 40 \mathrm{PM} \\
12 \cdot 47.40 \mathrm{PM}\end{array}$ & $\frac{22.795}{22.628}$ & $\begin{array}{r}25.43 \\
25.444 \\
\end{array}$ & $\frac{24.223}{23855}$ & $\frac{26.758}{26.667}$ & $\begin{array}{r}25.38 \\
25.349 \\
\end{array}$ & $\begin{array}{r}12.25 \\
12.151\end{array}$ & $\frac{12.576}{12421}$ & $\begin{array}{l}4.026 \\
4.041\end{array}$ & \begin{tabular}{|c|c|}
8.239 & 8148 \\
8
\end{tabular} & $\begin{array}{l}-1.212 \\
-1.212\end{array}$ & $\begin{array}{l}-5.055 \\
-5.055\end{array}$ & 44.969 & $\begin{array}{l}0.092 \\
0.091\end{array}$ & $\begin{array}{l}14.643 \\
14.643 \\
\end{array}$ & 0.003 & $\frac{2.00}{3300}$ & \begin{tabular}{|l|l|}
0 & 0.03333 \\
0 & 0.05 \\
\end{tabular} & $\begin{array}{ll}11.1 \\
10.9\end{array}$ & $\frac{11.0}{11.0}$ & & $\begin{array}{l}0.706 \\
0.700\end{array}$ & & & & & 1.16 \\
\hline 21 & $6 / 8 / 2004$ & $12: 48: 40$ PM & 22.44 & 25.461 & \begin{tabular}{|l|}
23.643 \\
\end{tabular} & 26.63 & \begin{tabular}{|l|}
25.317 \\
\end{tabular} & 12.151 & $\begin{array}{l}12.4 \angle 1 \\
12.636\end{array}$ & 4.1 & 8.261 & $\mid-1.212$ & -5.058 & $\begin{array}{r}44.969 \\
44.99\end{array}$ & 0.09 & 14.643 & 0.003 & 4.00 & 0.06667 & $\mid 11.2$ & 110 & 10.3 & 0.709 & \begin{tabular}{|l|}
0.014 \\
0.013
\end{tabular} & $\begin{array}{l}0.0144 \\
0.014 \\
\end{array}$ & 0.001 & 1..301 & 1.18 \\
\hline & $6 / 8 / 2004$ & 12:49:40 PM & 22.263 & 25.455 & 23.44 & 26.523 & 25.28 & 12.11 & 12.359 & 4.122 & 7.992 & -1.215 & -5.061 & 44.99 & 0.09 & 14.643 & 0.003 & 5.00 & \begin{tabular}{l|l|l|l|}
0 & 0.08333 \\
\end{tabular} & 10.9 & 11.0 & 0.1 & 0.693 & $\begin{array}{l}0.013 \\
0.013 \\
\end{array}$ & $\begin{array}{l}0.014 \\
0.014 \\
\end{array}$ & & 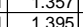 & 1.15 \\
\hline 23 & $6 / 8 / 2004$ & 12:50:40 PM & 22.12 & 25.478 & 23.423 & 26.596 & 25.293 & 12.116 & 12.408 & 4.118 & 8.029 & $\mid-1.206$ & -5.0 & & 0.09 & & & 6.00 & $\begin{array}{l}0 \\
0\end{array}$ & 10.9 & 110 & 0.1 & 0.694 & & & & 1.393 & 1.19 \\
\hline & $6 / 8 / 2004$ & 12:51:40 PM & 21.962 & 25.49 & 23.33 & 26.618 & 25.28 & 12.221 & & & 8.214 & -1.215 & -50 & 44.816 & & 14.643 & & & \begin{tabular}{l|l|}
0 & 0.11667 \\
\end{tabular} & & 11.0 & $\overline{0.2} \mathrm{P}-\mathrm{s}$ & 0.704 & 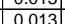 & 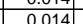 & 0.001 & 1377 & $\frac{3}{7}$ \\
\hline 25 & & 12:52:40 PM & & 25.499 & \begin{tabular}{|l|}
23.264 \\
\end{tabular} & 26.627 & & 12.155 & 12.299 & 4.08 & 8.072 & -1.212 & & 44.923 & 0.09 & 14.643 & & 8.00 & $\begin{array}{ll}0 & 0.13333 \\
\end{array}$ & 10.8 & & 20.1 & & $\frac{013}{013}$ & & & 1.394 & $\frac{1}{4}$ \\
\hline & $6 / 8 / 2004$ & 12:53:40 PM & 21.611 & 25.502 & 23.196 & 26.605 & 25.382 & 12.076 & 12.305 & 4.113 & 8.009 & -1.215 & -5.05 & 44.85 & 0.09 & 14.643 & & 9.00 & & $\frac{10.8}{10.8}>$ & 11 & & 0.692 & .013 & & & 1.406 & $\frac{4}{6}$ \\
\hline & $6 / 8 / 2004$ & 12:54:40 PM & 21.482 & 25.518 & & 26.646 & & & 12.286 & & 8.066 & -1.215 & & 44.731 & 0.09 & 14.643 & & 10.00 & 0.16667 & 10.8 & $11.6 \mathrm{C}>\mathrm{s}$ & 10.1 & 0.694 & & & & 1.401 & 1.1 .1 \\
\hline 28 & $6 / 8 / 2004$ & 12:55:40 PM & 21.363 & 25.515 & 23.285 & 26.653 & 25.455 & 12.008 & 12.185 & 4.046 & 7.959 & -1.212 & $-5.05 !$ & 44.787 & 0.09 & 14.643 & 0.0 & 11.00 & $\begin{array}{ll}0 & 0.18333 \\
\end{array}$ & 10.7 & 11.0 & 10.0 & & & 0.014 & 0.001 & 1.411 & $\frac{1}{1}$ \\
\hline & $6 / 8 / 2004$ & 12:56:40 PM & 21.285 & 25.548 & 23.343 & 26.641 & 25.458 & 11.906 & 12.21 & 4.095 & 7.765 & -1.212 & -5.05 & 44.864 & 0.09 & 14.6 & $\overline{0.0}$ & 12.00 & 0.2 & $\begin{array}{ll}10.7 \\
\end{array}$ & 11. & 9.8 & & & ||$_{14}$ & & 1.430 & 1.22 \\
\hline 30 & $6 / 8 / 2004$ & 12:57:40 PM & 21.198 & 25.566 & 23.382 & 26.665 & 25.516 & 12.29 & 12.554 & 4.175 & 8.097 & -1.212 & -5.0 & 44.912 & 0.09 & 14.6 & 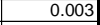 & 13.00 & \begin{tabular}{l|l|l|l|}
0 & 0.21667 \\
\end{tabular} & 11.1 & 11 & 10.2 & 703 & 0.013 & D14 & & 1.378 & 1.17 \\
\hline 31 & $6 / 8 / 2004$ & 12::58: & 21.116 & 25.569 & 23.415 & & & & & & 7.98 & -1.215 & & & & & & 14.00 & & & $11.0 \mathrm{~s}$ & & & & & & & 1.19 \\
\hline 32 & $6 / 8 / 2004$ & 12:59: & 21.055 & 25.599 & \begin{tabular}{|l|l|}
23.444 \\
\end{tabular} & 26.127 & 25.56 & 12.221 & 12.485 & 4.066 & 8.156 & -1.215 & & 44.82 & 0.09 & 14.643 & & 15.00 & & 11.0 & 11. & & & 0.013 & & & $3 / 6$ & 1.1 \\
\hline$\frac{35}{34}$ & $6 / 882004$ & $\begin{array}{l}1: 00: 40 \mathrm{PM} \\
1.110 \mathrm{PM}\end{array}$ & 20.998 & 25.612 & 望3.483 & 26.186 & 25.5 & 12.197 & 12.502 & 4.053 & & -1.215 & & 44.9 & & & & 16.00 & $\begin{array}{l}0.26067 \\
0\end{array}$ & 11.0 & & 0.2 & & 13 & 14 & & & 5 \\
\hline \begin{tabular}{|l|l|}
35 \\
35
\end{tabular} & (1) & 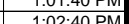 & 20.931 & $\frac{2.035}{25.644}$ & \begin{tabular}{|l|l|}
2.500 \\
2015
\end{tabular} & 20.198 & $\frac{23.06}{2565}$ & 10.105 & 12.3514 & $\frac{4.13}{4052}$ & $\frac{1.954}{7094}$ & & & 44.9 & & & & 1000 & & & $\frac{11 .}{1 .}$ & 10.0 & & 13 & & & 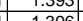 & \\
\hline$\frac{35}{36}>2$ & $6 / 182004$ & 1.04 .4010 & 20.032 & 25.044 & 23.510 & 20.011 & 2560 & $=12008$ & 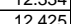 & 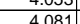 & 7951 & $\frac{1.410}{-1218}$ & & & 0.0 & & & 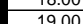 & 0.56 & 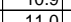 & 110 & 100 & & & 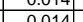 & & 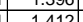 & $127+2$ \\
\hline 37 & $\frac{618 / 2004}{6 / 82004}$ & $\frac{1: 0040}{1: 04: 40}$ & 20.791 & 25.662 & 23.552 & 26.79 & & 11.981 & 12.284 & $\frac{4.059}{4.059}$ & 7.914 & $\frac{1.215}{-1.215}$ & & 44.9 & 0.011 & & & 20.00 & 0 & 10.8 & $\frac{110}{110}$ & & & & & & & \\
\hline & & 1.05 .40 & & & & & & & 12489 & & & & & & & & & $\frac{2.00}{2100}$ & 0.00000 & & 11.6 & & & & & & & \\
\hline 39 & $6 / 812004$ & $1.06 .40 \mathrm{PM}$ & 20746 & 25697 & 23.568 & 26705 & 25.43 & 12205 & 12467 & 4052 & 8138 & -1212 & & & 0091 & 14643 & & 2200 & 0.36667 & 110 & 110 & 102 & 701 & 014 & 14 & & 1389 & $\frac{1.1}{11}$ \\
\hline 40 & $6 / 8 / 2004$ & 1:07:40 PM & 20.713 & 25.684 & 23.6 & 26.672 & 25.37 & 12.203 & 12.551 & 4.021 & 8.193 & -1.218 & -5.05 & 44.885 & 0.091 & \begin{tabular}{|c|c|}
14.643 \\
\end{tabular} & & 23.00 & 0.38333 & 11.1 & & & 0.703 & $\mid \begin{array}{ll}0.014 \\
\end{array}$ & 0.014 & & 1.384 & \\
\hline & & & & & & & & & & & & & & & & & & & & & & & & & & & & \\
\hline 42 & & Averac & 21.6 & 25.5 & 23.6 & 26.7 & & 12 & 12.4 & & 8.1 & & & & 0 & & & & & & & & & & & & 392 & \\
\hline 43 & & Maximum & 23.0 & 25.7 & 25.0 & 26.9 & & 12.3 & 12.6 & $4.2 \quad y$ & 8.3 & -1.2 & & 45 & 0.1 & 14 & & & & 11.2 & 11.1 & 0.3 & & & & & & \\
\hline 44 & & Median & 21.3 & 25.5 & 23.5 & 26.7 & & 12.1 & 12.4 & 4.1 & 8.1 & -1.2 & & 44.9 & 0.1 & 14.6 & & & & 10.9 & 11.0 & 10.1 . & $\mid 0.696$ & $\mid 0.013$ & 0.014 & 0.001 & 1.393 & 1.18: \\
\hline 45 & & & 20.7 & 25.4 & 23.2 & 26.5 & 2 & 11.8 & 12.1 & $\begin{array}{l}4.0 \\
\end{array}$ & 7.6 & -1.2 & & 44.4 & 0.1 & 14.6 & & & & 10.6 & 10.9 & 9.7 & 0.668 & & 114 & & & \\
\hline 46 & & $22 \times \operatorname{sid} \mathrm{Dev}$ & 1.501 & 0.187 & 0.844 & 0.169 & & 0.242 & 0.283 & 0.076 & 0.296 & 0.005 & & 0.280 & 0.002 & 0.000 & & & & 0.284 & 0.069 & 0.266 & 0.018 & .000 & .000 & 0.000 & 0.040 & p] \\
\hline \begin{tabular}{|l|}
47 \\
48 \\
\end{tabular} & Number & 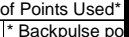 & \begin{tabular}{|l}
24 \\
ts in box
\end{tabular} & $\begin{array}{l}24 \\
\text { are not incl }\end{array}$ & 24 & & & & & & & & & & & & & & & 24 & & & & & & & & \\
\hline
\end{tabular}


WSRC-TR-2005-00105, REVISION 0

SRNL-RPP-2005-00012, REVISION 0

PRE-TEST BASELINE WITH 0.1 M NAOH, BEFORE ACID CLEANING - CONT.

\begin{tabular}{|c|c|c|c|c|c|c|c|c|c|c|c|c|c|c|c|c|c|c|c|c|c|c|c|c|c|c|c|c|}
\hline & A & B & D & $E_{1}$ & $F$ & $G$ & $\mathrm{H}$ & $\mathrm{J}$ & $\mathrm{K}$ & L & $M$ & $\mathrm{~N}$ & 0 & Q & $R$ & $\mathrm{~s}$ & T & $\mathrm{v}$ & $w$ & $x$ & Y & 7 & AA & $A B$ & $\mathrm{AC}$ & AD & $\mathrm{AE}$ & \begin{tabular}{l|l} 
AF & $A$
\end{tabular} \\
\hline & Baseline 1B & & & & & & & & & & & & & & & & & & & & & & & & & & & \\
\hline & $6 / 8 / 2004$ & 1:10:17 PM & 20.134 & 25.699 & \begin{tabular}{|l|}
23.997 \\
\end{tabular} & 26.56 & 25.26 & 22.29 & \begin{tabular}{|l|}
22.946 \\
\end{tabular} & 4.108 & \begin{tabular}{|l|l|}
18.147 \\
\end{tabular} & -1.215 & -4.781 & 45.144 & 0.19 & 14.643 & 0.003 & 25.62 & 0.42694 & 21.5 & $\begin{array}{ll}11.1 \\
\end{array}$ & 20.2 & 1.394 & $\mid .028$ & 0.029 & 0.001 & 1.441 & \\
\hline & $\begin{array}{l}0 / 8 / 2004 \\
6\end{array}$ & $1: 11: 17 \mathrm{PM}$ & 20.307 & \begin{tabular}{|l|}
25.697 \\
\end{tabular} & \begin{tabular}{|l|}
24.154 \\
\end{tabular} & 26.55 & \begin{tabular}{|l|}
25.277 \\
25
\end{tabular} & 22.386 & \begin{tabular}{|l|l|}
23.193 \\
\end{tabular} & 4.064 & $\begin{array}{l}18.292 \\
\end{array}$ & \begin{tabular}{|l|}
-1.212 \\
\end{tabular} & $\begin{array}{r}-4.761 \\
-4.766\end{array}$ & \begin{tabular}{|l|}
44.806 \\
\end{tabular} & $\begin{array}{l}0.193 \\
\end{array}$ & \begin{tabular}{|l|}
14.643 \\
14.643
\end{tabular} & 0.003 & 26.62 & 0.44361 & 21.7 & 11.0 & $\frac{2.4}{20.3}$ & 1.054 & 0.029 & $\begin{array}{l}.029 \\
0.029\end{array}$ & & $\begin{array}{l}1.441 \\
1.449 \\
\end{array}$ & 1.23 \\
\hline 54 & $6 / 8 / 2004$ & $\begin{array}{l}1: 12: 17 \mathrm{PM} \\
\end{array}$ & $\begin{array}{r}20.255 \\
2023\end{array}$ & 25.695 & 24.303 & 26.53 & $\begin{array}{r}25.26 \\
2529 \\
\end{array}$ & $\frac{22.444}{2.20}$ & \begin{tabular}{|l|l}
23.269 \\
2309
\end{tabular} & $\begin{array}{l}4.036 \\
.302\end{array}$ & \begin{tabular}{|l|l|}
18.377 \\
18.925
\end{tabular} & \begin{tabular}{|r|}
-1.212 \\
\end{tabular} & $\begin{array}{r}-4.754 \\
\end{array}$ & $\begin{array}{l}44.376 \\
\end{array}$ & 0.195 & 14.643 & 0.003 & 27.62 & \begin{tabular}{|l|l|}
2 & 0.46028 \\
\end{tabular} & 21.8 & 10.9 & 20.4 & 1.407 & 0.029 & 0.030 & 0.001 & 1.453 & 1.24 \\
\hline $\begin{array}{l}555 \\
56\end{array}$ & 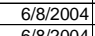 & 1:13:17 PM & 20.23 & 25.69 & 24.414 & 26.51 & 25.226 & 22.28 & \begin{tabular}{|l|l|}
23.019 \\
202
\end{tabular} & 3.983 & $\begin{array}{l}18.305 \\
\end{array}$ & \begin{tabular}{|l|l|} 
& -1.212 \\
\end{tabular} & \begin{tabular}{|r|r|} 
\\
\end{tabular} & \begin{tabular}{|l|l|}
44.629 \\
\end{tabular} & 0.193 & \begin{tabular}{|l|l|}
14.643 \\
\end{tabular} & 0.003 & 28.62 & \begin{tabular}{|l|l|}
2 & 0.47694 \\
\end{tabular} & 21.5 & \begin{tabular}{l|l|}
5 & 10.9 \\
\end{tabular} & 20.3 & $\begin{array}{l}3.399 \\
\end{array}$ & 0.029 & \begin{tabular}{|l|}
0.029 \\
\end{tabular} & 0.001 & 1.442 & 1.23 \\
\hline $\begin{array}{l}566 \\
57 \\
\end{array}$ & $\frac{6 / 8 / 2004}{6 / 8 / 2004}$ & 1:14:17 PM & $\frac{20.225}{20235}$ & \begin{tabular}{|l|}
25.68 \\
25.66
\end{tabular} & \begin{tabular}{l|l}
24.534 \\
24624
\end{tabular} & 26.48 & $\begin{array}{l}25.221 \\
2511 .\end{array}$ & 22.07 & \begin{tabular}{|l|l|}
22.913 \\
2302
\end{tabular} & \begin{tabular}{|l|l|}
4.097 \\
4027
\end{tabular} & \begin{tabular}{|l|}
17.933 \\
18495 \\
\end{tabular} & \begin{tabular}{|r|}
-1.212 \\
1312 \\
\end{tabular} & $\begin{array}{r}-4.763 \\
4.454 \\
\end{array}$ & \begin{tabular}{|l|l|}
45.142 \\
1.777
\end{tabular} & $\begin{array}{l}0.193 \\
0.155\end{array}$ & \begin{tabular}{|l|l|}
14.643 \\
1.62
\end{tabular} & 0.003 & 29.62 & \begin{tabular}{|c|c|}
2 & 0.49361 \\
\end{tabular} & \begin{tabular}{|l|}
21.4 \\
\end{tabular} & \begin{tabular}{l|l|}
4 & 11.1 \\
9
\end{tabular} & 20.0 & 1.379 & $\begin{array}{l}0.029 \\
023\end{array}$ & \begin{tabular}{|l|}
0.029 \\
\end{tabular} & $\begin{array}{l}0.001 \\
\end{array}$ & 1.458 & 1.24 \\
\hline 58 & $6 / 8 / 82004$ & $\begin{array}{l}\frac{1}{1: 15: 17 \mathrm{PM}} \\
1: 1617 \mathrm{P}\end{array}$ & $\frac{20.235}{20.266}$ & $\begin{array}{r}25.66 \\
25.661\end{array}$ & \begin{tabular}{rl|}
24.624 \\
24.74
\end{tabular} & $\begin{array}{l}26.41 \\
26.41 \\
\end{array}$ & $\begin{array}{r}25.141 \\
25166\end{array}$ & $\begin{array}{l}22.514 \\
22.178\end{array}$ & \begin{tabular}{|l|}
23.309 \\
23.031 \\
\end{tabular} & \begin{tabular}{|l|l|}
4.027 \\
4.008
\end{tabular} & \begin{tabular}{|c|}
18.459 \\
18.108
\end{tabular} & \begin{tabular}{|}
$\mid-1.215$ \\
-1.212 \\
\end{tabular} & $\begin{array}{l}-4.754 \\
-4.754 \\
\end{array}$ & \begin{tabular}{|l|}
44.777 \\
44756
\end{tabular} & $\begin{array}{l}0.195 \\
0.195 \\
\end{array}$ & $\begin{array}{c}14.643 \\
14643 \\
\end{array}$ & $\begin{array}{l}0.003 \\
0.003\end{array}$ & $\begin{array}{l}30.62 \\
3162\end{array}$ & $\begin{array}{l}20.51028 \\
2\end{array}$ & $\begin{array}{l}21.8 \\
211.6\end{array}$ & $\frac{11.0}{110}$ & \begin{tabular}{|l|}
20.5 \\
20.1 \\
\end{tabular} & \begin{tabular}{|c|c|}
$\mid 1.412$ \\
1 \\
\end{tabular} & \begin{tabular}{|c|c|} 
\\
9 & 0.029 \\
\end{tabular} & $\begin{array}{l}0.029 \\
0.029\end{array}$ & 0.001 & $\begin{array}{l}1.434 \\
1.454\end{array}$ & \\
\hline 59 & $6 / 8 / 2004$ & 1:17:17 PM & 20.287 & 25.642 & 24.811 & & \begin{tabular}{|l|}
25.197 \\
\end{tabular} & & & $\begin{array}{r}.000 \\
3.93\end{array}$ & 18.383 & \begin{tabular}{|c|}
-1.212 \\
-1.212
\end{tabular} & -4.749 & \begin{tabular}{|l|l|}
44.664 \\
\end{tabular} & 0.196 & $\begin{array}{l}4.640 \\
14.643\end{array}$ & 0.003 & & \begin{tabular}{|l|l|}
2 & 0.54361 \\
\end{tabular} & 21.6 & & & & & & & & $\begin{array}{l}\frac{1.24}{1.23} \\
\end{array}$ \\
\hline 60 & $6 / 8 / 2004$ & 1:18:17 PM & 20.344 & 25.643 & 24.908 & 26.44 & 25.289 & 22.463 & 23.257 & 4.008 & 18.413 & \begin{tabular}{|l|}
-1.212 \\
\end{tabular} & -4.746 & 44.428 & $\begin{array}{l}0.197 \\
\end{array}$ & 14.643 & & 33.62 & 0.56028 & 21.8 & 10.9 & 20.4 & $\begin{array}{ll}4 & 1.409\end{array}$ & 0.029 & 0.029 & & $\begin{array}{l}1.440 \\
1.441\end{array}$ & $\frac{1.23}{1.23}$ \\
\hline 61 & $6 / 8 / 2004$ & 1:19:17 PM & 20.388 & 25.627 & 24.976 & 26.4 & 25.297 & 22.593 & & 3.997 & 18.592 & -1.212 & $\begin{array}{l}-4.743 \\
\end{array}$ & 44.412 & 0.198 & & & & & & & & & 030 & 0.030 & & & \\
\hline & & 1:20:17 PM & & & & & & & 23.34 & & & & & & & $\begin{array}{l}14.045 \\
14.643\end{array}$ & & & & & 10.5 & & & & & & $\frac{1.435}{1.439}$ & $\frac{1.22}{1.22}$ \\
\hline 63 & 12004 & 1.21.17 PM & 20.516 & 25.628 & 25.203 & 26.516 & 25.389 & 22.477 & 23.253 & 3.94 & 18.557 & -1.21 & & 44.366 & 0.201 & 14.6 & & 36 & 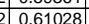 & 218 & 10.9 & 20.5 & 415 & 030 & 0.030 & 0.001 & $\frac{1.459}{1.452}$ & $\frac{1.22}{1.24}$ \\
\hline 64 & $\frac{6 / 8 / 2004}{6 / 200}$ & 1:22:17 PM & 20.588 & $\frac{25.641}{25}$ & 25.31 & 26.59 & 25.426 & 22.214 & 23.066 & 4.202 & 17.966 & $\frac{-1.21}{-1.2}>0$ & & 45.288 & 0.196 & 14.643 & & $\frac{37.62}{37.62}$ & 2) 0.62694 & $\frac{21.6}{21.6}$ & $\frac{11.1}{11.1}$ & $\frac{20.1}{20.1}-2$ & (c) & .029 & 0.029 & & $\begin{array}{l}\frac{1.452}{1.442} \\
\end{array}$ & $\frac{1.24}{1.23}$ \\
\hline 65 & $3 / 2004$ & 1:23:17 PM & 20.635 & 25.637 & 25.322 & 26.6 & 25.457 & 22.268 & 23.135 & 4.051 & 18.202 & \begin{tabular}{|c|}
-1.215 \\
\end{tabular} & -4.743 & \begin{tabular}{|c|c|}
45.094 \\
\end{tabular} & 0.198 & 14.643 & & 38.62 & \begin{tabular}{|l|l}
2 & 0.64361 \\
\end{tabular} & & $\frac{1.1}{11.1}$ & & 1.395 & 0.030 & & 0.001 & 1.446 & $\frac{1.50}{1.23}$ \\
\hline 66 & $6 / 8 / 2004$ & 1:24:17 PM & 20.677 & 25.629 & 25.313 & 26.67 & 25.499 & 22.149 & 23.031 & 3.969 & 18.159 & -1.212 & -4.728 & 444.677 & 0.2 & 14.643 & 0.00 & 39.62 & $\begin{array}{ll}20.66028 \\
\end{array}$ & $\frac{21.6}{21.6}$ & $\frac{\frac{10.9}{10.9}}{2}$ & $\frac{20.2}{20.2}-2$ & 1.390 & 0.030 & 0.030 & 0.001 & 1.467 & $\frac{1.25}{1.25}$ \\
\hline 67 & $6 / 8 / 2004$ & 1:25:17 PM & 20.736 & 25.637 & 25.266 & 26.74 & 25.567 & 22.373 & 23.263 & 3.957 & 18.411 & -1.215 & -4.743 & 44.643 & 0.198 & 14.643 & 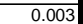 & 40.62 & \begin{tabular}{|l|l|}
2 & 0.67694 \\
\end{tabular} & 21.8 & $\overline{10.9}$ & 20.4 & 1.406 & 0.030 & 0.029 & 0.001 & 1.437 & 1.22 \\
\hline & & 1:26:17 PM & 20.762 & 25.648 & 5.248 & 26.75 & 25.663 & 22.247 & 23.068 & 4.044 & 18.169 & -1.209 & -4.737 & 44.624 & 0.199 & 14.643 & & & & & & & & & & & 1.458 & 1.24 \\
\hline 69 & & 1:27:17 PM & 20.785 & 25.656 & 25.1955 & 26.72 & 25.7 & 22.442 & 23.23 & 4.004 & 18.381 & -1.2 & & 44.741 & 0.198 & 14.643 & & & & 21. & 1.0 & & 407 & 030 & & & 1.438 & 1.22 \\
\hline \begin{tabular}{|l|l}
71 \\
71
\end{tabular} & & 1:28:17 PM & & 25.668 & 25.166 & 26.1664 & 25.138 & 22.375 & 23.188 & 3.9981 & 18.348 & -1.22 & & 44.143 & 0.198 & 14.6 & & & & 21. & 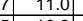 & & 1.404 & & & & 1.443 & 1.23 \\
\hline 72 & $6 / 8720044$ & 1:29:17 PM & 2.004 & 25.694 & 25.1024 & 26.8. & 25.167 & 22.268 & 23.016 & 3.921 & 18.282 & -1.1218 & & 44.406 & 0. & 14.643 & (2000 & 44.62 & $\mid=0.14361$ & 21.5 & 10.9 & 20.3 & 1.398 & 0.030 & 0.000 & 0.001 & 1.466 & 1.25 \\
\hline & & 1.30:17 PM & & 25.110 & & & 25.73 & 22.587 & 23.535 & 3.953 & 18.613 & -1.212 & -4.74 & 44.266 & & 14.643 & 0.003 & 45.62 & $\begin{array}{l}20.76028 \\
\end{array}$ & 22.1 & $10.8 \mathrm{P}$ & 20.6 & 1.420 & 0.030 & 0.030 & & 1.443 & 1.23 \\
\hline 74 & & & & & & & & & & & & & & & & & & & & & & & & & & & & \\
\hline 75 & & Averages & & & & 26. & 25.4 & 22.4 & & 4.0 & 18.3 & -1.2 & & & & & & & & & 10.9 & & 402 & . 029 & . 029 & & & \\
\hline 76 & & Maximum & 20.9 & & 25.3 & 26.8 & 25.8 & 22.6 & 23.5 & 4.2 & 18.6 & -1.2 & & 45.3 & 0.2 & & & & & $\frac{22.1}{22.1}+2$ & & & & & & & .467 & .248 \\
\hline & & Median & & & & & & 22.4 & & & & & & 447 & & 146 & & & & & & & & & & & & \\
\hline & & Minimum & 20.1 & 25.6 & 24.0 & 26. & & 22.1 & 22.9 & 3.9 & 17.9 & $-\frac{-1.2}{-1.2} \quad \mathrm{C}$ & -4.8 & 442 & 0.2 & 146 & & & & 214 & 108 & & & & & & & \\
\hline 79 & & $2 \times \mathrm{Std}$ Dev & 0.495 & 0.055 & 0.805 & 0.27 & 0.404 & 0.279 & 0.310 & 0.139 & 0.371 & 0.004 & 0.026 & 0.587 & 0.006 & 0.000 & 0.000 & & & 0.310 & $0.144 d c$ & 0.319 & 0.022 & 0.001 & 0.000 & 0.000 & 0.019 & 0.016 \\
\hline 30 & Number c & of Points Used* & 21 & 21 & 21 & 2 & 21 & 21 & 21 & 21 & 21 & 21 & 2. & 21 & 21 & 21 & & & & 21 & 21 & 21 & 21 & 21 & 21 & 21 & 21 & 2 \\
\hline ( & & қpulse pa & in box & re not incl & luded & & & & & & & & & & & & & & & & & & & & & & & \\
\hline 82 & & & & & & & & & & & & & & & & & & & & & & & & & & & & \\
\hline 84 & Baseline 10 & $1.20 .56 \mathrm{DP}$ & & & & & & 2201 & 33006 & 4003 & 28015 & 1210 & & 15031 & & & & & 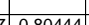 & & & & & & & & & \\
\hline & & & & & & 2687 & 25.8448 & $\frac{52.454}{32621}$ & $\frac{35.550}{34.269}$ & $\begin{array}{l}4.003 \\
4008\end{array}$ & $\frac{28.445}{28552}$ & $|-1.218|$ & $\frac{-4 .}{-4 .}$ & $\frac{45.031}{45.038}$ & 0.303 & \begin{tabular}{|l|}
14.6643 \\
14643
\end{tabular} & & (4) & & $\frac{32.5}{328}$ & $\frac{11.0}{110}$ & & & & $\begin{array}{l}0.045 \\
0.045\end{array}$ & & $\frac{1.4 / 2}{1.467}$ & 25 \\
\hline 86 & & $1: 34: 5$ & 20.376 & 25.78 & 25.425 & 26.84 & 25.7 & 32.668 & 34.248 & 4.0 & 28.586 & $\frac{-1.212}{-1.21}$ & & 44.921 & 0.306 & 14.6 & & 50.27 & & 32.8 & $\frac{11.0}{11.0}$ & $\frac{30.6}{30.6}$ & 2.112 & 0.046 & 0.045 & 0.001 & $\begin{array}{l}\frac{1.40}{1.472} \\
\end{array}$ & 1.25 \\
\hline & & & 20.445 & 25.808 & 25.493 & 26.82 & 25.6 & 32.386 & 34.002 & $3.9 \subseteq$ & 28.328 & -1.21 & & 44.389 & 0.3 & 14.6 & & 51.27 & $\begin{array}{l}7 \\
0.85444\end{array}$ & 32. & 10.9 & & & & 0.045 & & 1.468 & 1.2 \\
\hline 88 & & 1:36:56 PM & 20.541 & 25.799 & 25.539 & 26.77 & 25.54 & 32.681 & 34.238 & 3.98 & 28.634 & -1.2 & & \begin{tabular}{|l|l|l|l|l|}
447 \\
\end{tabular} & 0.3 & 14. & & 52.27 & & 32.8 & 11.0 & & $\mid 2.114$ & & 0.045 & & $\begin{array}{l}1.466 \\
\end{array}$ & 1.25 \\
\hline (2) & & 1:37:56 PM & 20.57 & 25.827 & 25.612 & 26.7 & 25.5 & 32.703 & 34.221 & 4.000 & 28.613 & -1.21 & & $44.72 !$ & 0.3 & & & 53.27 & & 32. & 11.0 & 00.1 & 2.114 & 0.046 & 0.045 & & & \\
\hline & & & & & & & & & & & & & & & & & & & & & & & & & & & & $12 \quad$ \\
\hline & & & 20.634 & 25.811 & 25.661 & $26.70 \mathrm{~s}$ & & 32.724 & 34.126 & & 28 & -1.2 & & 44.3 & & & & & & 32. & $10.6 \mathrm{r}$ & & 2.118 & & & & 1.477 & 1.4 \\
\hline & & & 20.677 & & $\frac{25.1 .03}{2.73}$ & 26.66 & & $\frac{32.664}{32.605}$ & 34.30 & & $\frac{28.6}{20}$ & -1.2 & & & & & & & & & & & & & & & & \\
\hline \begin{tabular}{|l|l|}
94 \\
5
\end{tabular} & & & $\frac{20.701}{20.713}$ & 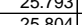 & 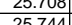 & $\frac{2.694}{26.67}$ & 25. & $\begin{array}{l}32.405 \\
32015\end{array}$ & $\frac{33.921}{3406}$ & & 28.8 & $\frac{-1.21}{-121}$ & & 45.18 & & . & & & & 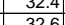 & 11.2. & & & & & & 1.467 & 1.2 \\
\hline \begin{tabular}{|l|l|}
95 \\
\end{tabular} & & & $\frac{20.110}{20.775}$ & $\frac{23.00}{2580}$ & $\frac{2.1 .744}{25771}$ & $\frac{2.067}{26635}$ & & $\frac{52.415}{3226}$ & $\frac{34.00}{33.62}$ & 40 & & $\frac{1.24}{-12}$ & & & & & & & & & $1 \pm$ & & & & & & 10.410 & \\
\hline \begin{tabular}{|l|l|}
96 \\
\end{tabular} & & & 207 & & & & & $\frac{2.20}{3243}$ & & & & & & 年 45. & & & & & & & & & & & & & & \\
\hline \begin{tabular}{|l|}
97 \\
\end{tabular} & & & 20.81 & & & 26 & & & & & & & & & & & & & & & & & & & & & & \\
\hline \begin{tabular}{|l|l|}
98 \\
\end{tabular} & & & 2073 & 25789 & 25824 & 26.65 & & $\frac{52.450}{32351}$ & $\frac{5.0}{33}$ & & 28.2 & -1.2 & & $\frac{45.5}{45}$ & & & & & & & $\frac{1.1}{111}$ & & & & & & $\frac{7102}{492}$ & \\
\hline 99 & & & 20.92 & 25. & 258 & 26.6 & & 324 & 340 & & 28328 & -121 & & 45 & & & & 63 & & & & & & & & & 1.495 & \\
\hline & $6 / 8 / 2004$ & & 20.977 & & 25.85 & $26.72 !$ & & 326 & $\sqrt{342}$ & & 28.472 & \begin{tabular}{|l|l|}
-1.2. \\
\end{tabular} & & 45.2 & & 14. & & & & & $\overline{111}$ & & 2.1 & & & & $\begin{array}{ll}877 \\
877\end{array}$ & \\
\hline & & & 20.847 & 25.788 & 25.873 & & & 32.378 & & & & -1.2 & & & & & & & & & & & & & & & 88 & \\
\hline & & & 20.84 & 25.79 & 25.885 & 26.808 & & 32.639 & 34.172 & 4.029 & 28.547 & $\frac{-1.215}{-1.215}$ & & 45.1 & & & & & & & & & & & & & 1.478 & \\
\hline 10 & & & 20.926 & & & $26.87 \mathrm{t}$ & & 32.4 & & & & & & & & & & & & & & & & & & & & \\
\hline Th & & & 20.903 & 25.803 & 25.898 & $26.90 \mathrm{f}$ & & & 33 & & & -1.2 & & 45 & & & & & & & & & & & & & & \\
\hline & & & 20.92 & & 25.914 & 26.92 & & 32.7 & 34.2 & & 28.56 & -1.215 & & & & & & & & & & & & & & & 79 & \\
\hline 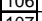 & & & 20.942 & & 25.937 & 26.994 & & $\begin{array}{l}32.494 \\
32.572\end{array}$ & 33.966 & & 28.424 & -1.212 & & 45.077 & & & & 70.27 & & & & & & & & & , & . \\
\hline & & $\frac{155: 56 \mathrm{PP}}{1.5650}$ & $\frac{2.098}{20096}$ & $\frac{25.843}{25851}$ & $\frac{25.988}{25051}$ & 2.03. & 255.95 & $\frac{32.5 / 3}{3250}$ & $\frac{34.081}{34015}$ & 告.032 & 28.4898 & $\frac{-1.215}{-1215}$ & & 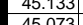 & 8315 & $\begin{array}{l}14.643 \\
1.643\end{array}$ & & $\mid \frac{\mid 1.21}{7207}$ & $1.18 / 18$ & 230 & 110 & 305 & 2.105 & 8 & 80 & 0.001 & 1.493 & 1.27 \\
\hline & & & & & & & & & & & & -1.215 & & & & & & & & & & & & & & & & 1.27 \\
\hline & & & 207 & & & & & & & & & & & & & & & & & & & & & 2046 & 2045 & 8001 & 1480 & \\
\hline & & & 210 & 259 & 260 & & & 329 & 34 & 41 & 288 & & & & & & & & & & & & & & & & & \\
\hline & & & 20.7 & 25.8 & 25.8 & 26.8 & & & 34.1 & & 28.5 & -1.2 & & 45.1 & & & & & & 32.6 & & & & & & & 478 & \\
\hline & & Minimum & 20.3 & 25.8 & 25.3 & 26.6 & & 32. & 33.6 & 3 & 28.1 & $\mid-1.2$ & & 44.3 & & & & & & & & 0.2 & & & 44 & & 1464 & $\frac{\pi}{46}$ \\
\hline & & Dev & 0.407 & 0.043 & 0.376 & 0.25 & $0.30 \mathrm{~s}$ & 0.287 & 0.418 & 0.096 & 0.355 & $\mid 0.004$ & 0.056 & 0.738 & 0.007 & 0.000 & 0.00 & & & 0.418 & 0.181 & 317 & $\mid 0.022$ & 0.001 & 0.001 & 0.000 & 0.022 & 0018 \\
\hline & mberc & sused $\mathrm{d}^{*} \mid$ & 25 & 25 & 25 & $\frac{2}{2}$ & 4. & 25 & 25 & 25 & 25 & 25 & & 25 & 25 & 25 & & & & $\frac{15}{25}$ & 25 & 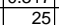 & 25 & 25 & 25 & 25 & 25 & \\
\hline 16 & & epoir & ts in box & are not incll & & & & & & & & & & & & & & & & & & & & & & & & \\
\hline
\end{tabular}


WSRC-TR-2005-00105, REVISION 0

SRNL-RPP-2005-00012, REVISION 0

PRE-TEST ACID CLEAN WITH 2 M HNO3, FOLLOWING PUMP REPAIR

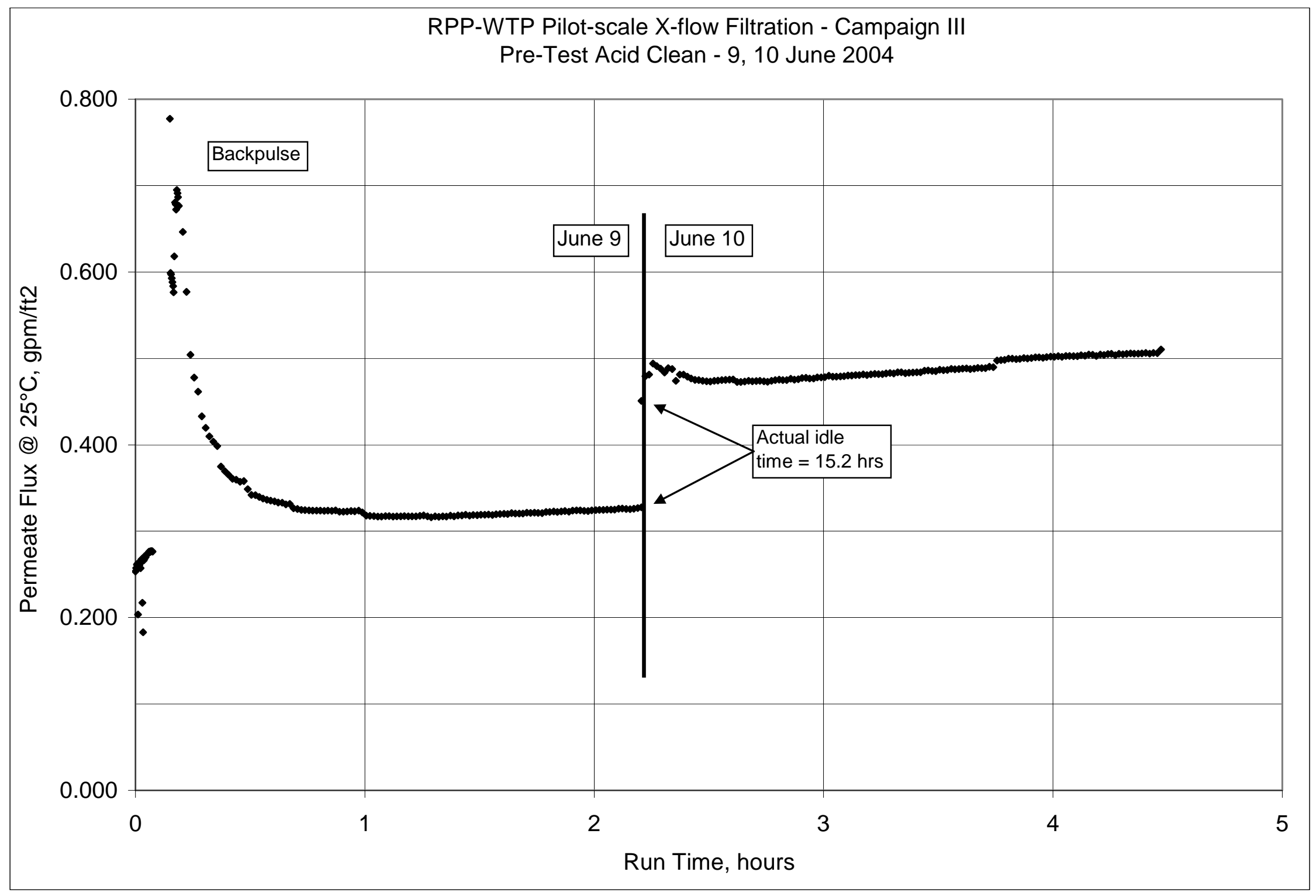


WSRC-TR-2005-00105, REVISION 0

SRNL-RPP-2005-00012, REVISION 0

PRE-TEST ACID CLEAN WITH 2 M HNO3, FOLLOWING PUMP REPAIR - CONT.

\begin{tabular}{|c|c|c|c|c|c|c|c|c|c|c|c|c|c|c|c|c|c|c|c|c|c|c|c|c|c|c|c|c|}
\hline & A & $B$ & \begin{tabular}{l|l} 
\\
\end{tabular} & \begin{tabular}{|l|l|}
$E$ \\
\end{tabular} & $\mathrm{~F}$ & G & $\mathrm{H}$ & \begin{tabular}{l|l|l|}
$\mathrm{J}$ & $\mathrm{s}$ \\
\end{tabular} & $\mathrm{K}$ & $\mathrm{L}$ & $\mathrm{M}$ & \begin{tabular}{|l|} 
\\
$N$
\end{tabular} & 0 & \begin{tabular}{l|l} 
& Q
\end{tabular} & $\begin{array}{ll}\mathrm{R} \\
\end{array}$ & \begin{tabular}{|c|}
$\mathrm{s}$ \\
\end{tabular} & $T$ & $\mathrm{~V}$ & w & $|x|$ & \begin{tabular}{|l|l|} 
\\
\end{tabular} & $\mathrm{z}$ & AA & & $\begin{array}{ll}A C \\
\end{array}$ & $A D$ & $\mathrm{AE}$ & AF \\
\hline & & TIME & & 宛 Tempe & & teasureme & ents > >> & & & Pressure 1 & Measuremen & & & & « Flow M & Measuremer & & & & & & & & & & & & \\
\hline & DAIE & & $\frac{\mid \text { Filtrate }}{\text { deg } C}$ & \begin{tabular}{|l|} 
Cleaning \\
deg $\mathrm{s}$
\end{tabular} & \begin{tabular}{|l|} 
Slurry \\
denc
\end{tabular} & Hi Amb. & Lo Amb. & $\begin{array}{l}\text { BotTMP } \\
n \text { nsid }\end{array}$ & $\begin{array}{l}\text { Filter } \\
\text { nsig }\end{array}$ & Filter $\mathrm{dP}$ & TopTMP & \begin{tabular}{|l|l|} 
Filtrate \\
sciph
\end{tabular} & Pulsepot & & Filtrate & $\begin{array}{l}\text { Hi Filtate } \\
\end{array}$ & Backpulse & & & & & & & & & & & \\
\hline & & & T2 & $\frac{\text { degC }}{\mathrm{T} 3}$ & $\frac{d e g C}{\text { T1 }}$ & $\frac{\mathrm{deg}}{\mathrm{T} 4}$ & $\frac{\mathrm{deg}}{\mathrm{T} 5}$ & dP2 & $\frac{\frac{P S I 9}{\text { S19 }}}{\mathrm{P} 1}$ & $\begin{array}{l}\text { psid } \\
\text { dP1 }\end{array}$ & $\begin{array}{l}\text { PSId } \\
\mathrm{dP3}\end{array}$ & $\frac{\mathrm{psig}}{\mathrm{P} 2}$ & $\begin{array}{l}\text { pssg } \\
\text { P3 }\end{array}$ & $\frac{\mathrm{gpm}}{\mathrm{Q} 1}$ & $\frac{\mathrm{gpm}}{\mathrm{Q} 2}$ & $\begin{array}{l}\mathrm{gpm} \\
03 \\
\end{array}$ & gpm & & & & & & & & & & & \\
\hline & & & & & & & & & & & & & & & & & & & & & & & & & & & & \\
\hline & eros- $01 / 26$ & & & & & & & & & & & & & & & & & & & & & & & & & & & \\
\hline 7 & $6 / / 8 / 2004$ & 9:00:09 AM & 22.545 & 24.705 & \begin{tabular}{|l|}
27.987 \\
\end{tabular} & 26.054 & 24.911 & $\begin{array}{c}-0.004 \\
\end{array}$ & \begin{tabular}{|l|l|}
0.128 \\
\end{tabular} & $\begin{array}{l} \\
\end{array}$ & -0.003 & \begin{tabular}{|c|c|}
-0.129 \\
\end{tabular} & 0.111 & 45.741 & 0.293 & -0.013 & 0.00 & & & Filter Su & Surface $t$ & |6.707 & & & & & & \\
\hline & 6/10/2004 & 1:47:47 PM & 25.596 & 26.216 & \begin{tabular}{|l|l|}
$j$ & 26.68 \\
\end{tabular} & 26.834 & & \begin{tabular}{|c|c|}
-0.019 \\
\end{tabular} & 0.196 & -0.012 & -0.02 & \begin{tabular}{|l|l|}
-0.106 \\
\end{tabular} & 0.073 & -0.071 & $\mid 0.003$ & \begin{tabular}{|l|l|}
14.643 \\
\end{tabular} & 0.00 & & & Convers & rsion & 851 & $\mathrm{~m} 3 / \mathrm{m} 2$ & |day/bat & $/ \mathrm{gpm} / \mathrm{tt}$ & $/ / \mathrm{t} 2 / \mathrm{barg}$ & & \\
\hline & 6/114/2004 & 7::41:41 AM & & 23.885 & \begin{tabular}{|l|l|} 
& 66.603 \\
\end{tabular} & 24.174 & 24.204 & -0.012 & 0.12 & -0.01 & -0.016 & -0.163 & 0.107 & -0.083 & $\begin{array}{ll}0.003 \\
\end{array}$ & $\begin{array}{l}14.643 \\
\end{array}$ & 0.00 & & & & & & & & & & & \\
\hline 10 & & 12:06:06 PM & & 24.315 & 23.747 & 26.127 & & -0.012 & & -0.002 & -0.012 & & 0.043 & & 0.003 & & & & & Note: & & & P1 is & ppro & tely : & psig & & \\
\hline 11 & $6 / 1 / 72004$ & 7:28:28 AM & & & 23.199 & 24.225 & 23.143 & -0.01 & & -0.001 & -0.014 & -0.18 & 0.032 & $-0.0 / 5$ & 0.003 & 14.643 & & & & & & & & & & & & \\
\hline & & 4:57:57 PM & 26.259 & $26.737 \mid$ & 24.765 & 27.805 & 26.456 & -0.002 & 0.197 & 0 & -0.012 & -0.183 & -0.009 & 47.043 & 0.098 & $\begin{array}{r}14.643 \\
1.643 \\
\end{array}$ & & & & & P1is & orrec & ed for 4 & & & & & \\
\hline 13 & 60121212004 & 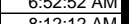 & $\frac{23.199}{3236}$ & & & $\begin{array}{l}23.418 \\
23800\end{array}$ & & . & 0.075 & 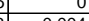 & -0.012 & & & & 0.0007 & & & & & wov & & & & Filtre & & PEF & & \\
\hline & $0 / 2 \angle I \angle U 04$ & 8.12:12 AMI & 23.30 & 25.530 & 23.03 & 23.829 & 24.45 & -0.000 & 0.108 & & & -0.194 & 0.00 & - 0.003 & 0.003 & 14.643 & & & & & & TAP & & & 250 & & $\begin{array}{r}\times 1000 \\
\times 1000 \\
\end{array}$ & \\
\hline & Cid Clean 1 & & & & & & & & & & & & & & & & & rime & Time & \begin{tabular}{|l} 
Press. \\
nsig.
\end{tabular} & $\begin{array}{lll}\text { vel. } \\
\text { vas }\end{array}$ & & & (1) & (1) & 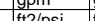 & $\mathrm{gpm}$ & meter \\
\hline $\begin{array}{ll}17 \\
\end{array}$ & $6 / / 9 / 2004$ & 1:17:48 PM & 21.939 & 24.651 & 32.266 & 25.33 & 24.758 & 43.658 & $\begin{array}{l}46.999 \\
\end{array}$ & 4.158 & 39.437 & -1.218 & -2.575 & 46.285 & 0.486 & \begin{tabular}{|l|l|}
14.643 \\
\end{tabular} & 0.0 & & Hour & $\frac{19}{45.5}$ & & $\frac{51.5}{41.5}$ & & & 0.059 & $\frac{0.01}{0.01}$ & & $\frac{a y / b a r}{1.22}$ \\
\hline \begin{tabular}{|l|l|}
18 & \\
\end{tabular} & $6 / / 9 / 2004$ & & 21.939 & 24.651 & 32.266 & 25.33 & 24.758 & 43.658 & 46.999 & $\begin{array}{l}4.158 \\
\end{array}$ & 39.437 & -1.218 & -2.575 & 46.285 & 0.486 & $\begin{array}{l}14.643 \\
\end{array}$ & 0.0 & 0.2 & 0.0047 & 45.5 & & & 2.865 & 0.072 & 0.059 & $\frac{0.001}{0.001}$ & 1.428 & $\frac{1.2}{1.2}$ \\
\hline 19 & & 1:18:16 PM & 21.934 & \begin{tabular}{l|l}
24.666 \\
\end{tabular} & 32.192 & 25.375 & & \begin{tabular}{|l|l|}
43.262 \\
\end{tabular} & \begin{tabular}{|l|l|}
46.519 \\
\end{tabular} & $\begin{array}{l}4.167 \\
\end{array}$ & 39.001 & -1.215 & -2.581 & $|46.418|$ & \begin{tabular}{l|l}
0.487 \\
\end{tabular} & $\begin{array}{l}14.643 \\
\end{array}$ & & & 0.00778 & $\overline{45.0}$ & 11. & & & 0.073 & 0.060 & & $\frac{1.449}{1.9}$ & \\
\hline & $6 / 9 / 2004$ & 1:18:26 PM & 21.929 & 24.666 & 32.187 & 25.36 & 24.698 & 43.502 & 47.035 & 4.142 & 39.268 & $\begin{array}{l}-1.215 \\
\end{array}$ & -2.586 & 46.341 & 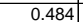 & \begin{tabular}{l|l|}
14.643 \\
\end{tabular} & & 0.6 & .01056 & 45.6 & & 41.4 & .853 & 0.072 & & & & \\
\hline 21 & $6 / 9 / 2004$ & $1: 18: 36$ & 21.903 & 24.665 & 32.226 & 25.354 & 24.687 & 43.569 & 47.13 & $\begin{array}{l}4.171 \\
\end{array}$ & \begin{tabular}{|l|l|l|l|}
39.318 \\
\end{tabular} & -1.212 & -2.604 & 46.431 & \begin{tabular}{|c|c|}
0.484 \\
\end{tabular} & \begin{tabular}{|l|l|}
14.643 \\
\end{tabular} & & & 1333 & & 11. & & 8.857 & & & & & \\
\hline 22 & $6 / 9 / 2004$ & 1:18:46 PM & 21.929 & 24.651 & 34.254 & 25.315 & 24.678 & 44.813 & 48.03 & 4.22 & 40.518 & -1.215 & -2.792 & 46.181 & 0.461 & \begin{tabular}{|l|l|l}
14.643 \\
\end{tabular} & 0.00 & 0.9 & \begin{tabular}{|l|l|l|l|l|} 
\\
\end{tabular} & $\begin{array}{l}46.6 \\
\end{array}$ & 11.3 & \begin{tabular}{|l|l|}
42.7 \\
\end{tabular} & 2.942 & \begin{tabular}{|l|l|l|l|} 
\\
\end{tabular} & 0.053 & 0.001 & 1.251 & 1.06 \\
\hline & 6/9/2004 & 1:18:56 PM & 21.949 & 24.666 & $\begin{array}{l}54.175 \\
\end{array}$ & 25.34 & 24.673 & 44.232 & 47.897 & 4.236 & 39.888 & -1.218 & -2.546 & 46.128 & 0.493 & 14.643 & & 1.1 & 0.01889 & 46.4 & & 42.1 & .900 & 0.074 & 0.057 & 0.001 & 1.360 & 1.16 \\
\hline 24 & 6/9/2004 & 1:19:06 PM & & 24.665 & 34.924 & & 24.69 & & 48.357 & & 38.63 & 0.062 & & 46.445 & 0.58 & 14.643 & & & & 46.9 & & 40.8 & 814 & .086 & 0.066 & & & \\
\hline 25 & $6 / 9 / 2004$ & 1:19:16 PM & 22.215 & & 34.49 & 25.324 & & 40.311 & 48.094 & & 35.97 & 2.653 & & 47.032 & 0.14 & 14.643 & & & 0.02444 & 46.6 & & & & & 0.085 & & 2.233 & \\
\hline & $\begin{array}{ll}6 / 912004 \\
6 / 2004\end{array}$ & $\begin{array}{l}1: 19: 26 \mathrm{PM} \\
1 \cdot 19 \cdot 36 \mathrm{PM}\end{array}$ & $\frac{22.556}{22807}$ & $\begin{array}{ll}24.675 \\
246\end{array}$ & 35.057 & $\begin{array}{r}25.344 \\
25339\end{array}$ & 24.712 & $\begin{array}{ll}38.994 \\
37.588\end{array}$ & 48.656 & $\frac{4.328}{4.35}$ & 34.581 & $\begin{array}{r}4.445 \\
5765 \\
\end{array}$ & $\frac{1.316}{2097}$ & $\begin{array}{l}46.587 \\
46777\end{array}$ & 0.834 & $\begin{array}{l}14.643 \\
14.643 \\
\end{array}$ & & $\frac{1.6}{1.8} \mathrm{rat}$ & 0.003 & $\frac{47.2}{471}$ & 111 & 5.4 & 吕30 & 0.124 & 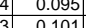 & 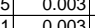 & $\frac{2.570}{2.07}$ & 20 \\
\hline 28 & 6 & 1:19:46 PM & 23.053 & 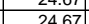 & 35614 & 25339 & 2473 & 36582 & 4 & 4.576 & $\frac{50.105}{32.12}$ & 6.173 & 271 & 4685 & $\mid$ & 1464 & 0,00 & & 0.0378 & 年 & 111 & $\frac{3.4 .4}{34.4}$ & 2368 & & 0 & $\frac{0.003}{0.003}$ & & 2.42 \\
\hline 29 & $6 / 9 / 2004$ & $1 \cdot 1.956$ PM & 23.243 & 24664 & 134.726 & 25298 & 2247 & 35.597 & 48.295 & 441 & 31.146 & 7.58 & 320 & 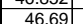 & $\mid$ & 1464 & & 211 & \begin{tabular}{|l|l|l|l|}
0.03556 \\
\end{tabular} & 468 & 11 & 33.4 & 301 & 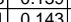 & 0.110 & 0.003 & 3293 & 2.57 \\
\hline & & $\frac{1: 20: 06 \mathrm{PM}}{10.0}$ & $\frac{23.514}{23.514}$ & & $\begin{array}{l}5.43 .79 \\
54.79\end{array}$ & & & 35.651 & 48.765 & 4.392 & $\frac{3.244}{3.1244}$ & 7.881 & $\frac{1.234}{3.334}$ & & & & & 2.1 & & 47.3 & & & & & & & & $\frac{2.80}{282}$ \\
\hline & 61912004 & 1.20. & 23624 & 2467 & 35899 & 25313 & 24742 & 3551 & 48854 & 4423 & 31084 & 8269 & 3.034 & 46982 & 0.095 & 14643 & & & & 474 & & & 296 & 147 & 00 & 0003 & & $\frac{2.82}{279}$ \\
\hline 32 & $6 / 9 / 2004$ & 1:20:26 PM & 23.804 & 24.674 & 34.928 & 25.308 & & 34.495 & 48.434 & $\begin{array}{l}4.337 \\
\end{array}$ & $\mid 30.249$ & 8.877 & & 46.831 & 1.005 & \begin{tabular}{|l|l|l|l|l}
14.643 \\
\end{tabular} & & 2.63 & \begin{tabular}{|l|l|}
0.04389 \\
\end{tabular} & 47.0 & 11. & & 232 & 0.150 & 0.114 & & 3.532 & $\frac{2.10}{301}$ \\
\hline & & & & & & & & & & & 30.417 & & & 46.827 & & 14.643 & & & & & & & & & & & & 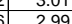 \\
\hline 34 & $6 / / 9 / 2004$ & 1:20:46 PM & 24.005 & \begin{tabular}{|l|}
24.679 \\
\end{tabular} & 34.849 & 25.303 & 24.736 & 34.379 & $\begin{array}{l}48.622 \\
\end{array}$ & 4.394 & \begin{tabular}{|c|}
30.023 \\
\end{tabular} & 8.987 & 4.01 & 46.846 & 1.01 & \begin{tabular}{|l|l|l|l|l|}
14.33 \\
\end{tabular} & & & 0.04944 & 47.2 & 11 & 322 & 2.220 & & 0.115 & 0.004 & 3.576 & 3.04 \\
\hline 35 & $6 / 9 / 2004$ & $1: 20: 56$ & 24.05 & 24.674 & 34.76 & 25.298 & 24.7 & 34.41 & 48.579 & 4.415 & 30.039 & 9.057 & 4.05 & 46.802 & \begin{tabular}{|l|l|}
1.012 \\
\end{tabular} & \begin{tabular}{|c|}
14.643 \\
\end{tabular} & 0.00 & 3.1 & \begin{tabular}{|l|l|}
0.05222 \\
\end{tabular} & 47.1 & 11. & 32.2 & .222 & 0.151 & 0.116 & 0.004 & 3.588 & 3.05 \\
\hline 36 & $6 / 9 / 2004$ & 1:21:06 PM & 24.08 & 24.669 & 35.677 & 25.263 & 24.7 & 34.329 & 48.571 & 4.375 & 29.992 & 9.12 & & 46.756 & 1.015 & $\begin{array}{l}14.643 \\
\end{array}$ & & 3.3 & 0.055 & $\begin{array}{ll}47.1 \\
\end{array}$ & 11. & & 217 & 0.15 & 0.113 & & 3.520 & \\
\hline 37 & 6/9/2004 & $1: 21: 16$ & 24.115 & 24.68 & $\begin{array}{l}34.859 \\
3\end{array}$ & 25.278 & 24.7 & 34.276 & 49.024 & 4.44 & 29.906 & 9.491 & & 46.616 & 1.027 & 14.6 & & 3.4 & 0.05778 & 47.6 & 11.4 & JL.I & 213 & & & & 3.647] & \\
\hline 38 & 6/9/2004 & $1: 21: 22$ & 24.2 & 24.684 & 34.79 & 25.293 & 24.7 & 34.205 & & 4.43 & 29.83 & 9.508 & & 46.644 & 1.029 & & & 3.63 & $\begin{array}{ll}63 & 0.06056\end{array}$ & 47.6 & 11.4 .4 & & & & & & 3.669 & \\
\hline 39 & & $1: 21: 36 \mathrm{PM}$ & 24.214 & $24.6 / 3 \mid$ & 34.725 & 25.287 & & 34.041 & 48.803 & 4.361 & 29.771 & 9.526 & & 46.608 & 1.028 & 14.6 & & 3.80 & 0.06333 & 47.3 & 11.4 & 31.9 & 2.200 & 0.15 & 0.118 & & 3.685 & 5 \\
\hline$\frac{40}{41}$ & $\frac{19192004}{669204}$ & $\begin{array}{l}1.2 .46 \\
1.21 .56\end{array}$ & $\begin{array}{l}24.234 \\
2.2204\end{array}$ & 24.678 & $\begin{array}{l}34.066 \\
3.587\end{array}$ & 25.292 & $\frac{24.135}{.177}$ & $\begin{array}{l}33.998 \\
32023\end{array}$ & 48.854 & 4.397 & 29.689 & 9.534 & & $\begin{array}{l}46.648 \\
46387\end{array}$ & - 1.029 & $\begin{array}{l}14.643 \\
1.633\end{array}$ & & 3.91 & 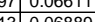 & 47.4 & 114 & 2318 & $\frac{2.195}{2101}$ & & 0.118 & 0.004 & 3. & 3.16 \\
\hline 42 & $6 / 9 / 2004$ & $\frac{1.4506}{1.2006}$ & 24208 & 24.6063 & $3.34,472$ & 25281 & 24. & 年 & | & 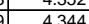 & 29711 & $\frac{3.044}{9.618}$ & & 4 & 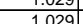 & $\mid \begin{array}{l}14.4543 \\
1463\end{array}$ & & 告. & & 告17.1 & 然. & & & & & & $\mid$ & \\
\hline 43 & $6 / 1 / 20204$ & $1.42 \cdot 16$ & 24.909 & 2069 & 04,567 & 25.201 & 24.17 & (3) & 年 & 40409 & 20.114 & 0.010 & & 40.021 & 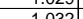 & & & 400 & . & 4 & $\frac{1.44}{114}$ & , & \begin{tabular}{|l|l|l|l|l|}
2100 \\
\end{tabular} & 0.150 & 0 & & 3.170 & 3.. \\
\hline 44 & $6 / / 2 / 2004$ & 1.22 .26 & 24188 & 2468 & 3456 & 25286 & 247 & 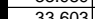 & 48306 & 4266 & 290044 & 9697 & & 40.4647 & 1.034 & 14.6 & & 167 & 0.00729 & 469 & 114 & 315 & 2,173 & 154 & | & 0.004 & 3765 & 占 \\
\hline & & 1.22 .3 & & & 34.941 & & & & & & & & & & & & & 480 & & & . & & & & & & & \\
\hline 46 & 6//9/2004 & 1.22 .4 & 24183 & 24678 & 3466 & 25266 & & 33844 & 48869 & 4335 & 29.649 & 996 & & 46733 & 1041 & 14 & & 4.97 & 00878 & 474 & 11.5 & & 2189 & & 0119 & & 3757 & 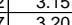 \\
\hline $\begin{array}{ll}47 \\
\end{array}$ & $\begin{array}{ll}6 / 9 / 2004 \\
\end{array}$ & & 24.193 & 24.683 & 34.616 & 25.271 & 24.7 & 33.703 & 48.635 & 4.376 & 29.461 & 9.96 & & & & & & & & & & & & & & & & \\
\hline 48 & $6 / / 9 / 2004$ & 1:23:06 PM & 24.188 & 24.693 & 34.512 & 25.276 & 24.78 & 33.807 & 48.778 & 4.309 & 29.635 & 9.96 & & 46.462 & $\begin{array}{l}1.042 \\
\end{array}$ & \begin{tabular}{|l|l|l|}
14.643 \\
\end{tabular} & & 5.30 & & 47.3 & 114 & 31.7 & 2.187 & & 0120 & 0.004 & $\mid 3.778$ & $\frac{5.45}{3.22}$ \\
\hline 49 & $6 / 9 / 2004$ & 1:23:16 & 24.163 & 24.677 & 34.467 & 25.266 & 24. & 33.63 & $\begin{array}{l}48.593 \\
\end{array}$ & $\begin{array}{l}4.289 \\
\end{array}$ & \begin{tabular}{|c|}
29.493 \\
\end{tabular} & 10.029 & 4.55 & \begin{tabular}{l|l|}
46.395 \\
\end{tabular} & \begin{tabular}{l|l}
1.042 \\
\end{tabular} & 14.6 & & 5.47 & \begin{tabular}{|l|l|}
47 & 0.09111
\end{tabular} & $\begin{array}{ll}47.1 \\
\end{array}$ & 11.4 & $\begin{array}{ll}4 & 41.6 \\
\end{array}$ & 2.176 & \begin{tabular}{|l|l|l|l|} 
\\
\end{tabular} & 0.120 & 0.004 & 3.802 & 3.2 \\
\hline & $6 / 9 / 2004$ & 1:23:2 & 24.148 & 24.688 & 34.398 & 25.266 & & 33.672 & 48. & 4.345 & 29.467 & 10.029 & & 46.433 & 1.042 & & & 5.6 & & 47.5 & 11.4 & 1.6] & 177 & & & & & \\
\hline 51 & 6//20004 & 1:23: & 24.112 & 24.681 & 34.308 & 25.255 & 24.8 & 33.852 & 49.17 & 4.336 & 29.627 & 10.027 & & 46.393 & 1.041 & 14. & & 5.80 & $\begin{array}{lll}80 & 0.09667\end{array}$ & 47.71 & 11.4 & 31.7| & 188 & & 0.120 & 0 & 3.793 & \\
\hline 52 & 6/9/2004 & & 24.093 & 24. & 34.269 & 25.246 & & 33.66 & & & & 9.957 & & & 1.04 & & & 0.5 & & 47.4 & & & & & & & & \\
\hline 53 & & & $24.04 \pi$ & $24.6 / 1$ & 34.254 & 25.245 & & 33.512 & 48.558 & 4.3 & 29.27 & 10.027 & & 46.425 & 1.042 & & & 6.15 & 130.10222 & 47.1 & & & 164 & & 0.121 & & & \\
\hline 55 & 6 & $\begin{array}{l}1.24 .06 \\
1.24 \cdot 16\end{array}$ & $\begin{array}{l}24.022 \\
24003\end{array}$ & 2 & $\begin{array}{l}54.214 \\
324181\end{array}$ & 25.245 & 24.86 & $\begin{array}{l}3.358 \\
33548\end{array}$ & 48.483 & $\begin{array}{l}4.356 \\
4.339\end{array}$ & $\frac{29.01}{2036}$ & 10.029 & & $\mid \begin{array}{l}46.189 \\
4625\end{array}$ & $\mid 1.044$ & $\mid \begin{array}{l}1.4645 \\
1463\end{array}$ & & $\frac{6.30}{6.47}$ & $\begin{array}{ll}30 & 0.105 \\
17 & 0.17\end{array}$ & 4.0 & $\begin{array}{ll}\frac{1.150}{114} \\
\end{array}$ & 215 & 2.151 & & 0.12 & 0.004 & 3.8.806 & 3 \\
\hline 56 & $6 / 9 / 2004$ & & 23.072 & 24707 & 34.047 & 25261 & & 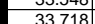 & 40.50 & 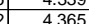 & 29.044 & 10.50 & & 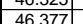 & $\frac{1.041}{1.041}$ & 146 & & 0.417 & 0 & 4 & 11.44 & 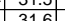 & \begin{tabular}{|l|l|}
2.177 \\
\end{tabular} & & 0.121 & & | & \\
\hline & & & 年 & & 30.041 & & & 年 & & & & & & & 1.041 & & & & & 47.0 & 1.4 & & & & & & & \\
\hline 58 & 61602004 & & 23864 & 24685 & 33067 & 25214 & & 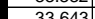 & 48765 & 4352 & 20943 & 10027 & & 464 & 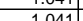 & & & 609 & & 年 & 114 & 31.5 & \begin{tabular}{|l|l|l|}
2173 \\
\end{tabular} & & & & & \\
\hline & & & & & & & & & & & & & & & & & & & & & & & & & & & & \\
\hline 60 & & & 23785 & & & 25203 & & 33593 & 48.923 & & & & & & & & & & & & & & & & & & & \\
\hline 62 & $6 / 9 / 2004$ & $1 \cdot 25 \cdot 26$ & 23.715 & 24695 & & 25.214 & 24 & 33.378 & & 4314 & 22.994 & 10.076 & & $\frac{4.26}{46.36}$ & $\mid$ & 14.6 & & 7.46 & 0.12722 & 47.0 & 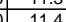 & 313 & 157 & 年 & & & $\begin{array}{l}3.908 \\
3.905 \\
\end{array}$ & 5 \\
\hline 63 & $6 / 9 / 2004$ & 1:25:36 & \begin{tabular}{|l|l|}
23.699 \\
\end{tabular} & \begin{tabular}{|l|l|}
24.69 \\
\end{tabular} & 33.784 & 25.219 & 24.7 & 33.311 & \begin{tabular}{|l|l|}
48.645 \\
\end{tabular} & 4.338 & $\frac{29.1}{29}$ & \begin{tabular}{|l|l|}
10.139 \\
\end{tabular} & 4.6 & $\frac{26.324}{46.32}$ & 1.043 & 14.64 & & 7.80 & $\frac{1.13}{0.13}$ & 47.2 & 11.4 & 31.2 & 2.152 & 56 & & & 3.919 & 9 \\
\hline 64 & & 1:25:4 & 23.645 & 24.705 & $\begin{array}{l}533.739 \\
\end{array}$ & \begin{tabular}{|l|l|}
25.229 \\
\end{tabular} & & 33.446 & 48. & 4.328 & 29.2 & & & & 1.04 & 14. & & 7.9 & \begin{tabular}{|l|l|l|l|} 
\\
\end{tabular} & 47.1 & 11. & 4 & .162 & & & & 13 & 3. \\
\hline 65 & 6/9/2004 & 1:25:5 & 23.615 & 24.695 & 33.695 & 25.224 & & 33.409 & 48.747] & 4.315 & 29.241 & 10.139 & & 46.281 & 1.045 & 14. & & 8.13 & $\begin{array}{cc}13 & 0.13556\end{array}$ & & 11. & 31.3 & 2.160 & 56 & 0.123 & & 3.921 & 3. \\
\hline 66 & 6/9/2004 & & 23.57 & 24.695 & 33.646 & 25.234 & 24. & 33.492 & 48.83 & & & & & 46.27 & 1.046 & & & 0.0 & & 47.4 & & 1.4 & \begin{tabular}{|l|l|}
2.166 \\
\end{tabular} & & & & & \\
\hline 61 & $6 / 9 / 2004$ & $1.26:$ & 23.529 & 24.6955 & $\begin{array}{l}33.61 \\
32.691\end{array}$ & 25.238 & 24.17 & 33.367 & 48.4/9 & 4.291 & 29.2255 & 10.194 & & 46.216 & 1.046 & 14.62 & & 8.44 & & 47.0 & $11 . .5$ & 31.3 & 2.158 & 0.156 & 0.120 & & 3.937 & \\
\hline 60 & 年 & 1.26.26 P & 2.3.450 & 24.1 & $\begin{array}{l}3.581 \\
32551\end{array}$ & $\begin{array}{ll}4 \\
\end{array}$ & 4.107 & 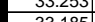 & 48.14 & 4.333 & 29.052 & $\begin{array}{l}1.191 \\
10201\end{array}$ & 4.1 .7 & 46.191 & 1.046 & $\begin{array}{l}14.643 \\
\end{array}$ & & 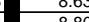 & & 47.3 & $\frac{11.3}{112}$ & 31.2 & 2.148 & \begin{tabular}{|l|l|}
0.156 \\
\end{tabular} & 0.123 & 0.004 & 3.958 & 年 \\
\hline$\frac{69}{70}$ & $\frac{6 / 6 / 2004}{6 / 9 / 2004}$ & \begin{tabular}{|l} 
1::26:36 PM \\
$1: 26: 46 \mathrm{PM}$
\end{tabular} & $\mid$\begin{tabular}{|c|}
23.4490 \\
23.40
\end{tabular} & \begin{tabular}{|}
$\quad 24.695$ \\
24.684 \\
\end{tabular} & \begin{tabular}{|l|l|}
33.551 \\
33.501
\end{tabular} & \begin{tabular}{|l|}
25.248 \\
15.218 \\
\end{tabular} & 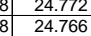 & $\begin{array}{l}33.185 \\
33.037 \\
\end{array}$ & \begin{tabular}{|l|}
48.712 \\
48.504 \\
\end{tabular} & $\frac{4.349}{4.331}$ & $\begin{array}{l}28.8831 \\
28.831\end{array}$ & \begin{tabular}{|l|}
10.212 \\
10.302 \\
\end{tabular} & $\frac{4.733}{4.751}$ & $\mid \frac{46.247}{46.26}$ & $\begin{array}{l}1.048 \\
1.049 \\
\end{array}$ & \begin{tabular}{|l|l|}
14.643 \\
14.643 \\
\end{tabular} & 0.003 & $\frac{8.80}{8.97}$ & 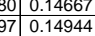 & \begin{tabular}{|l|l|}
4 & 47.2 \\
4 & 47.0
\end{tabular} & \begin{tabular}{|l|l|}
11.3 \\
11.3
\end{tabular} & \begin{tabular}{|l|}
31.1 \\
30.9 \\
\end{tabular} & \begin{tabular}{|l|}
2.143 \\
2.133 \\
\end{tabular} & 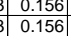 & $\mid \frac{0.124}{0.124}$ & $\mid \begin{array}{l}0.004 \\
0.004 \\
\end{array}$ & $\begin{array}{l}3.998 \\
4.006 \\
\end{array}$ & $\frac{3.39}{3.41}$ \\
\hline
\end{tabular}


WSRC-TR-2005-00105, REVISION 0

SRNL-RPP-2005-00012, REVISION 0

PRE-TEST ACID CLEAN WITH 2 M HNO3, FOLLOWING PUMP REPAIR - CONT.

\begin{tabular}{|c|c|c|c|c|c|c|c|c|c|c|c|c|c|c|c|c|c|c|c|c|c|c|c|c|c|c|c|c|}
\hline & A & $B$ & $\mathrm{D}$ & $E$ & $\mathrm{~F}$ & $\mathrm{G}$ & $\mathrm{H}$ & $\mathrm{J}$ & $\mathrm{K}$ & $\mathrm{L}$ & M & $\mathrm{N}$ & 0 & \begin{tabular}{l|l} 
\\
\end{tabular} & $R$ & $\mathrm{~s}$ & $\mathrm{~T}$ & $\mathrm{~V}$ & w & $x$ & \begin{tabular}{l|l} 
\\
\end{tabular} & $z$ & AA & $A B$ & AC & & AE & $\begin{array}{ll}A F & A \\
\end{array}$ \\
\hline 71 & $6 / 9 / 2004$ & $1: 26: 56 \mathrm{PM}$ & 23.403 & 24.694 & \begin{tabular}{|l|}
33.461 \\
\end{tabular} & 25.248 & 24.746 & 33.166 & 48.544 & 4.333 & 29.001 & \begin{tabular}{|l|}
10.302 \\
\end{tabular} & 4.754 & 46.176 & 1.05 & $\begin{array}{ll}14.643 \\
\end{array}$ & 0.0 & 9.13 & \begin{tabular}{c|c|c|}
13 & 0.15222 \\
\end{tabular} & 47.1 & 11.3 & 31.1 & 2.143 & 0.157 & 0.124 & 0.004 & 3.995 & 3.40 \\
\hline & $\begin{array}{l}6 / 9 / 2004 \\
\end{array}$ & & 23.358 & 24.694 & \begin{tabular}{|l|}
33.422 \\
\end{tabular} & 25.248 & 24.736 & 33.249 & 48.828 & 4.298 & 29.11 & \begin{tabular}{|l|}
10.368 \\
\end{tabular} & 4.797 & 46.149 & 1.051 & 14.643 & & 9.30 & \begin{tabular}{c|c|}
30 & 0.155 \\
\end{tabular} & 47.4 & \begin{tabular}{|l|l|} 
& 11.3 \\
\end{tabular} & & & 0.157 & & 0.004 & & \\
\hline 73 & $\begin{array}{l}6 / 9 / 2004 \\
\end{array}$ & $1: 27: 16 \mathrm{PM}$ & 23.312 & 24.703 & \begin{tabular}{|l|}
33.372 \\
3.321 \\
\end{tabular} & 25.257 & 24.71 & 32.892 & 48.438 & 4.285 & \begin{tabular}{|l|}
28.759 \\
2897
\end{tabular} & \begin{tabular}{|l|l|}
10.365 \\
\end{tabular} & $\begin{array}{l}4.826 \\
\end{array}$ & 46.166 & & 14.643 & & 9.47 & \begin{tabular}{ll|}
47 & 0.15778 \\
\end{tabular} & 47.0 & $\begin{array}{l}11.3 \\
1.2\end{array}$ & & 210 & 0.157 & 0 & 0.004 & 4.046 & \\
\hline 74 & 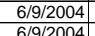 & $\begin{array}{l}1: 27: 26 \mathrm{PM} \\
1 \\
1.27 .36 \mathrm{PM}\end{array}$ & $\begin{array}{l}23.276 \\
23241\end{array}$ & $\begin{array}{l}24.688 \\
24698 \\
2469\end{array}$ & \begin{tabular}{|l|l|l}
33.331 \\
3307
\end{tabular} & $\begin{array}{l}25.241 \\
25.236 \\
\end{array}$ & $\begin{array}{l}24.665 \\
2.615\end{array}$ & $\begin{array}{l}33.172 \\
2052\end{array}$ & \begin{tabular}{|l|l|}
48.533 \\
8.282
\end{tabular} & \begin{tabular}{|l|l|}
4.354 \\
\end{tabular} & \begin{tabular}{l|l|}
28.962 \\
28.969
\end{tabular} & \begin{tabular}{|l|}
10.406 \\
\end{tabular} & $\begin{array}{l}4.788 \\
\end{array}$ & \begin{tabular}{|l|l|}
46.243 \\
\end{tabular} & $\begin{array}{l}1.051 \\
105\end{array}$ & \begin{tabular}{|l|l|}
14.643 \\
1.643
\end{tabular} & & 9.63 & $\begin{array}{c}63 \\
3\end{array}$ & 47.1 & 11.3 & 31.1 & 2.142 & 0.157 & $\begin{array}{l}0.125 \\
\end{array}$ & 0.004 & 4.015 & 3.42 \\
\hline $\begin{array}{l}75 \\
76 \\
\end{array}$ & $\frac{6 / 9 / 2004}{6 / 9 / 2004}$ & $\begin{array}{l}1: 27: 36 \mathrm{PM} \\
1.27 .46 \mathrm{PM}\end{array}$ & $\begin{array}{l}\frac{23.241}{23231} \\
232\end{array}$ & $\begin{array}{l}24.698 \\
24708\end{array}$ & \begin{tabular}{|l|}
33.307 \\
33287 \\
\end{tabular} & $\begin{array}{l}25.236 \\
25256\end{array}$ & & $\begin{array}{l}32.952 \\
3204 \\
3204\end{array}$ & \begin{tabular}{|l|l|}
48.282 \\
49.0028
\end{tabular} & \begin{tabular}{|l|l|}
4.257 \\
4358
\end{tabular} & \begin{tabular}{|l|l|}
28.896 \\
2075
\end{tabular} & \begin{tabular}{|l|}
10.417 \\
10434 \\
\end{tabular} & $\begin{array}{l}4.814 \\
4881\end{array}$ & \begin{tabular}{|l|l|}
46.158 \\
\end{tabular} & $\begin{array}{l}1.052 \\
1055\end{array}$ & $\begin{array}{l}14.643 \\
14643\end{array}$ & & 9.80 & & $\begin{array}{l}46.8 \\
7.5\end{array}$ & 11.3 & $\begin{array}{l}30.9 \\
\end{array}$ & \begin{tabular}{|l|}
2.132 \\
.155 \\
\end{tabular} & 0.157 & 0.125 & 0.004 & 4.040 & 3.44 \\
\hline 70 & $\frac{6 / 9 / 2004}{6 / 9 / 2004}$ & $\begin{array}{l}1: 27: 46 \text { PM } \\
127756 \text { T }\end{array}$ & $\frac{23.231}{23.182}$ & $\begin{array}{l}24.708 \\
24.703\end{array}$ & \begin{tabular}{|l|}
33.287 \\
33.228 \\
\end{tabular} & $\begin{array}{l}25.256 \\
252.247\end{array}$ & $\begin{array}{l}24.65 \\
24.64 \\
\end{array}$ & $\begin{array}{l}33.294 \\
32.894 \\
\end{array}$ & \begin{tabular}{|l|}
49.008 \\
48.283 \\
\end{tabular} & $\begin{array}{r}4.358 \\
4.35 \\
\end{array}$ & \begin{tabular}{r|}
29.075 \\
28.693
\end{tabular} & \begin{tabular}{|l|}
10.444 \\
10.487 \\
\end{tabular} & $\begin{array}{l}4.881 \\
4.881 \\
\end{array}$ & \begin{tabular}{|}
$\mid 46.354$ \\
46.356 \\
\end{tabular} & $\begin{array}{l}1.055 \\
1.055\end{array}$ & $\begin{array}{l}\frac{14.643}{14.643} \\
\end{array}$ & & $\begin{array}{r}9.97 \\
10.13 \\
\end{array}$ & $\begin{array}{l}97 \\
13\end{array}$ & \begin{tabular}{|c|c|}
47.5 \\
46.8
\end{tabular} & \begin{tabular}{|l|l|}
11.4 \\
\end{tabular} & $\begin{array}{l}4 \\
4 \\
4 \\
\end{array}$ & \begin{tabular}{|l|l|}
2.150 \\
2123
\end{tabular} & 0.157 & $\begin{array}{l}0.125 \\
0.126\end{array}$ & $\begin{array}{l}0.004 \\
0.04\end{array}$ & $\begin{array}{l}4.020 \\
4.077\end{array}$ & $\begin{array}{l}3.42 \\
3.34\end{array}$ \\
\hline 78 & $6 / 9 / 2004$ & 1:28:06 PM & 23.141 & & & & 24.625 & 33.226 & \begin{tabular}{|l|}
48.925 \\
\end{tabular} & & & 10.533 & 4.933 & & $\mid$ & $\begin{array}{l}\frac{14.045}{14.643} \\
\end{array}$ & & 10.30 & $\begin{array}{cc}1 & 0.1688 \\
0 & 0.17167\end{array}$ & \begin{tabular}{|l|}
4.8 \\
47.5
\end{tabular} & $\begin{array}{l}11.4 \\
5\end{array}$ & & & $\frac{0.157}{0.158}$ & $\begin{array}{l}0.126 \\
0.126\end{array}$ & \begin{tabular}{|c|}
0.004 \\
0.004
\end{tabular} & & $\begin{array}{l}3.47 \\
3.44\end{array}$ \\
\hline 79 & & 1:28:16 PM & & 24.697 & \begin{tabular}{|l|}
33.217 \\
\end{tabular} & 25.246 & 24.629 & 32.998 & 48.915 & 4.315 & 28.825 & \begin{tabular}{|l|}
10.655 \\
\end{tabular} & 4.979 & 45.939 & 1.061 & 14.643 & & & $47 \quad 0.17444$ & 47.4 & 11.3 & & & & & 0.004 & & \\
\hline 80 & $6 / 9 / 2004$ & $1: 28: 26 \mathrm{PM}$ & & 24.697 & \begin{tabular}{|l|l|}
33.128 \\
\end{tabular} & 25.231 & & 32.925 & \begin{tabular}{|l|l|l}
48.753 \\
\end{tabular} & 4.361 & & \begin{tabular}{|l|}
10.597 \\
\end{tabular} & & 46.364 & & \begin{tabular}{|l|l|}
14.643 \\
\end{tabular} & & 10.63 & \begin{tabular}{l|l|l|l|l|l|}
53 & 0.1772
\end{tabular} & \begin{tabular}{|l|l|}
47.3 \\
\end{tabular} & 11.4 & & & & & & & \\
\hline 81 & & & 23.05 & & 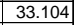 & 25.216 & 24.625 & 32.822 & \begin{tabular}{|l|l}
48.66 \\
\end{tabular} & 4.392 & 3.541 & 10.6 & & 46.481 & 1.061 & $\begin{array}{l}14.643 \\
\end{array}$ & & 10.80 & 0.18 & & & & & 158 & 0.127 & 0.004 & 4.129 & $\begin{array}{l}49 \\
51\end{array}$ \\
\hline 82 & & $5 \mathrm{PM}$ & & & & & & & 48.813 & & & & & & & 14.6 & & & 0.18278 & & & & & & 127 & & & \\
\hline 83 & 2004 & $5 \mathrm{PM}$ & & & 33.049 & 25.231 & & 32.687 & 48.5 & & & & & & & & & & 1955 & & & & & & 0127 & & & \\
\hline 84 & $6 / 9 / 2004$ & 1:29:06 PM & 22.949 & 24.711 & 33.009 & 25.24 & 24.618 & 32.745 & \begin{tabular}{|l|l|l|l|l|}
48.649 \\
\end{tabular} & & 3.502 & 10.724 & & $\begin{array}{ll}46.366 \\
\end{array}$ & 1.063 & \begin{tabular}{l|l}
14.643 \\
\end{tabular} & & 11.30 & \begin{tabular}{l|l|l|l}
30 & 0.18833
\end{tabular} & 47.2 & & & 2.111 & 0.158 & 0.127 & 0.004 & & \\
\hline 85 & $6 / 9 / 2004$ & 1:29:16 PM & 22.944 & 24.712 & 33.034 & 25.251 & 24.574 & 32.77 & 48.506 & 4.331 & 28.634 & 10.745 & 5.04 & 46.433 & 1.064 & $\begin{array}{l}14.643 \\
\end{array}$ & & 11.47 & $\begin{array}{lll}77 & 0.19111 \\
\end{array}$ & 47.0 & 11.4 & & 2.11 & 0.159 & 0.127 & 0.004 & & 3.53 \\
\hline 86 & $6 / 9 / 2004$ & 1:29:26 PM & $\frac{22.924}{2.074}$ & 24.712 & 32.975 & 25.251 & 24.565 & 32.654 & 48.511 & 4.376 & 28.41 & 10.733 & 5.034 & 46.393 & 1.064 & $\begin{array}{l}14.643 \\
1.62\end{array}$ & & 11.63 & $\begin{array}{lll}63 & 0.19389 \\
\end{array}$ & & & & & & & & & \\
\hline & 699/2004 & : 36 PM & & & 2.934 & 25.234 & & 32.48 & $48.471 \mid$ & 4.376 & & & & 46.385 & 1.066 & 14.643 & & & 19667 & 47.0 & & & & & & .004 & & \\
\hline 88 & & & 22.863] & & & 25.245 & .563 & 32.511 & 48.442 & 4.402 & .223 & 0.811 & & & 1.065 & 14.643 & & & 0.19944 & & & & & & .128 & & & 3.58 \\
\hline & & $5 \mathrm{PM}$ & 22.813 & 24.716 & 32.905 & $\begin{array}{r}25.25 \\
\end{array}$ & 24.568 & 32.751 & $\begin{array}{l}48.776 \\
1.575\end{array}$ & 4.386 & .547 & 10.863 & & 46.458 & 1.068 & 14.643 & & 12. & 130.20222 & 47.3 & 11.4. & & 2.11 & .159 & 0.128 & 004 & & 3.56 \\
\hline & & & $\frac{22.1686}{22.769}$ & $\frac{24.716}{24.712}$ & $\begin{array}{l}32.85 \\
32826\end{array}$ & $\begin{array}{l}25.245 \\
25.236\end{array}$ & $\frac{24.5 / 6}{24580}$ & $\frac{32.508}{32.604}$ & $\frac{5 / 5}{813}$ & $\frac{336}{345}$ & 28.414 & $\begin{array}{l}\frac{10.866}{10.933} \\
\end{array}$ & & 3 & $\begin{array}{l}1.068 \\
1.071\end{array}$ & $\frac{14.643}{14643}$ & & & \begin{tabular}{|r|}
0.205 \\
0.20778
\end{tabular} & & & & & & & 0.004 & & $\begin{array}{l}3.59 \\
3.59 \\
3\end{array}$ \\
\hline 92 & 6/9/2004 & 1:30:26 PM & 22.713 & 24.717 & \begin{tabular}{|l|}
32.777 \\
\end{tabular} & 25.256 & 24.614 & 32.396 & 48.533 & 4.376 & 28.186 & \begin{tabular}{|l|l|}
10.976 \\
\end{tabular} & 5.20 & 46.458 & 1.072 & $\begin{array}{ll}14.0453 \\
14.643\end{array}$ & & 12.63 & \begin{tabular}{|l|l}
3 & 0.21056
\end{tabular} & 47.1 & $\overline{11.4}$ & & 8088 & 0.160 & 0.129 & 0.004 & 4.263 & $\begin{array}{l}3.59 \\
3.63 \\
\end{array}$ \\
\hline & & 1:30:36 PM & 22.683 & 24.707 & 32.767 & 25.236 & 24.619 & 32.662 & 48.678 & 4.363 & 28.486 & \begin{tabular}{|l|}
10.976 \\
\end{tabular} & & 46.291 & 1.072 & 14.643 & & $\frac{12.80}{12.80}$ & $\begin{array}{l}30.21333 \\
0\end{array}$ & 47.2 & & & & & & 0.004 & & $\begin{array}{l}3.65 \\
3.59 \\
\end{array}$ \\
\hline & $6 / 9 / 2004$ & $5 \mathrm{PM}$ & 22.665 & 24.728 & & 25.267 & 24.6 & & 48.483 & & 28.19 & & 5.242 & 46.187 & 1.073 & 14.643 & & & & 47.0 & & & & & & 0.004 & & $\begin{array}{l}\frac{3.59}{3.64} \\
\end{array}$ \\
\hline & & PM & 22.64 & & 32.744 & 25.287 & 24.595 & 32.291 & \begin{tabular}{|l|l|l|}
48.498 \\
\end{tabular} & \begin{tabular}{|l|l|}
4.318 \\
\end{tabular} & 28.135 & 11.101 & & i.281 & 1.076 & 14.643 & & 13.13 & $\frac{21889}{21889}$ & & & & & & $\frac{0.130}{0.130}$ & & & \\
\hline & & & & & & & & & & & & & & & & & & & & & & & & & & & & \\
\hline & $6 / 9 / 2004$ & 1:31:16 PM & 22.548 & 24.717 & \begin{tabular}{|l|}
32.638 \\
\end{tabular} & 25.251 & 24.559 & $\begin{array}{l}32.240 \\
32.266\end{array}$ & 4 & 318 & 20.135 & \begin{tabular}{|l|l|}
1.10 \\
11.19
\end{tabular} & $\begin{array}{r}0.335 \\
5.335\end{array}$ & \begin{tabular}{|l|}
46.09109 \\
46
\end{tabular} & 1.079 & $\begin{array}{l}14.045 \\
14.643\end{array}$ & & $\begin{array}{l}1.50 \\
13.47\end{array}$ & $\begin{array}{lll}17 & 0.22444\end{array}$ & $\begin{array}{l}47.1 \\
47.0\end{array}$ & $\begin{array}{l}11.3 \\
11.3\end{array}$ & 30 & . & 0.161 & $\begin{array}{l}0.130 \\
0.130\end{array}$ & $\begin{array}{l}0.004 \\
0.004\end{array}$ & $\begin{array}{l}4.312 \\
4.319\end{array}$ & \\
\hline 98 & $6 / 9 / 2004$ & 1:31:26 PM & 22.538 & \begin{tabular}{|l|l|}
24.713 \\
\end{tabular} & \begin{tabular}{|c|}
32.609 \\
\end{tabular} & \begin{tabular}{|l|}
25.241 \\
\end{tabular} & 24.565 & 31.864 & 48.214 & 4.28 & 27.694 & \begin{tabular}{|l|}
11.202 \\
\end{tabular} & 5.311 & & 1.079 & 14.643 & & 13.63 & \begin{tabular}{|l|l|}
33 & 0.22722 \\
\end{tabular} & 46.7 & 11.3 & & 2.053 & 0.161 & 0.131 & 0.004 & 4.384 & \\
\hline 99 & $6 / 9 / 2004$ & $1: 31: 36 \mathrm{PM}$ & 22.503 & 24.728 & \begin{tabular}{|l|}
32.589 \\
\end{tabular} & \begin{tabular}{|l|}
25.241 \\
\end{tabular} & 24.575 & 32.195 & 48.502 & 4.279 & 28.094 & \begin{tabular}{|l|}
11.219 \\
\end{tabular} & & 46.057 & 1.08 & 14.643 & & 13.80 & \begin{tabular}{|l|l}
30 & 0.23 \\
\end{tabular} & 47.0 & 11.3 & & 2.078 & \begin{tabular}{|l|}
0.161 \\
\end{tabular} & 0.131 & 0.004 & & \\
\hline & & $1: 31: 46 \mathrm{P}$ & 22.472 & & & & & 32.32 & $\begin{array}{l}48.857 \\
\end{array}$ & 4.321 & 28.201 & & & & 1.081 & & & & & 47.4 & & & & & & & & \\
\hline & & 1:31:56 PM & 22.447 & 24.716 & 32.543 & 25.225 & 24.563 & 32.139 & \begin{tabular}{|l|l|}
48.629 \\
\end{tabular} & $\begin{array}{r}4.3 \\
\end{array}$ & $\begin{array}{l}27.985 \\
\end{array}$ & \begin{tabular}{|l|l|}
11.269 \\
\end{tabular} & & \begin{tabular}{|l|l|}
46.099 \\
\end{tabular} & 1.081 & \begin{tabular}{|l|l|}
14.643 \\
\end{tabular} & & 14.13 & \begin{tabular}{l|l}
3 & 0.23556 \\
\end{tabular} & 47.2 & 11.3 & & 2.073 & & 0.131 & 0.004 & 4.358 & \\
\hline 102 & & 1:32:06 PM & 22.412 & 24.702 & 32.49 & \begin{tabular}{|l|l|}
25.18 \\
\end{tabular} & 24.579 & 32.065 & \begin{tabular}{|l|l|}
48.37 \\
\end{tabular} & $\begin{array}{l}4.284 \\
\end{array}$ & .942 & $\begin{array}{l}11.266 \\
\end{array}$ & & \begin{tabular}{|l|l|}
46.089 \\
\end{tabular} & 1.082 & & & 14.30 & $\begin{array}{lll}30 & 0.23833\end{array}$ & 46.9 & 11.3 & & 2.069 & 0.161 & 0.131 & . 004 & & \\
\hline & $\frac{6 / 9 / 2004}{66 / 1 / 2004}$ & $\begin{array}{l}1: 32: 16 \text { PM } \\
1 \text { 1322.26 PM }\end{array}$ & $\frac{22.382}{22357}$ & $\begin{array}{l}24.722 \\
24722\end{array}$ & $\begin{array}{r}32.5 \\
32475\end{array}$ & $\begin{array}{r}25.21 \\
25.215\end{array}$ & $\begin{array}{l}24.579 \\
24.569\end{array}$ & $\begin{array}{l}32.164 \\
32.11\end{array}$ & 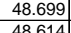 & $\begin{array}{l}4.302 \\
4.34 \\
\end{array}$ & $\begin{array}{r}28.034 \\
27.94\end{array}$ & \begin{tabular}{|l|}
11.295 \\
11.298 \\
\end{tabular} & $\begin{array}{r}5.41 \\
5.418 \\
\end{array}$ & \begin{tabular}{|l|l|}
46.093 \\
45895 \\
\end{tabular} & $\begin{array}{l}1.083 \\
108 \\
\end{array}$ & $\begin{array}{l}14.643 \\
14643\end{array}$ & & $\frac{14.47}{1463}$ & \begin{tabular}{c|c|}
7 & 0.24111 \\
3 & 024389
\end{tabular} & $\begin{array}{ll}47.2 \\
471\end{array}$ & $\frac{11.3}{11.2}$ & & 2.075 & \begin{tabular}{|l|l|}
0.161 \\
0.161 \\
\end{tabular} & $\begin{array}{l}0.131 \\
0.131\end{array}$ & 0.004 & \begin{tabular}{ll|l}
4.366 & 4376 \\
437
\end{tabular} & $\begin{array}{l}3.72 \\
3.72\end{array}$ \\
\hline$\frac{104}{105}$ & $\frac{\mid 6 / 9 / 2004}{6 / 92004}$ & $\begin{array}{l}\frac{132.26 \mathrm{PM}}{1: 32: 36 \mathrm{PM}} \\
\end{array}$ & $\frac{22.35}{22.332}$ & $\begin{array}{l}24.722 \\
24.707\end{array}$ & \begin{tabular}{|l|}
$32.4 / 5$ \\
32.455 \\
\end{tabular} & $\begin{array}{l}25.2155 \\
25.205\end{array}$ & $\begin{array}{l}24.569 \\
24.549\end{array}$ & $\begin{array}{r}32.11 \\
31.774 \\
\end{array}$ & \begin{tabular}{|l|}
48.611 \\
48.187 \\
\end{tabular} & & $\begin{array}{r}27.94 \\
27.663\end{array}$ & \begin{tabular}{|l|}
11.289 \\
11.324 \\
\end{tabular} & & $\begin{array}{r}45.895 \\
46.12\end{array}$ & $\begin{array}{l}1.082 \\
1.083 \\
\end{array}$ & $\frac{14.6}{14.6}$ & & $\frac{14.63}{14.80}$ & \begin{tabular}{rl|l|}
3 & 0.24389 \\
0 & 0.24667
\end{tabular} & & $\frac{11.2}{11.3}$ & & $\begin{array}{l}.070 \\
0.49 \\
\end{array}$ & & & & & $\begin{array}{l}3.72 \\
3.77 \\
\end{array}$ \\
\hline 106 & & 1:32:46 PM & 22.316 & 24.731 & \begin{tabular}{|l|}
32.459 \\
\end{tabular} & 25.23 & 24.53 & 32.071 & 48.709 & 4.351 & 27.872 & 11.39 & 5.42 & \begin{tabular}{|c|}
46.1216 \\
46.016
\end{tabular} & $\begin{array}{l}.083 \\
1.083\end{array}$ & 14.6 & & $\frac{14.0}{14.9}$ & 70.24944 & $\frac{46}{47}$ & 11.3 & & & & & & & $\frac{3.77}{3.74}$ \\
\hline 107 & & $1: 32: 5$ & 22.271 & & \begin{tabular}{|r|}
32.37 \\
\end{tabular} & 25.205 & & 31.818 & 48.048 & 274 & & \begin{tabular}{|l|}
11.379 \\
\end{tabular} & & \begin{tabular}{|l|}
46.093 \\
\end{tabular} & 1.082 & & & 15.1 & & 46 & 11.3 & & & & & & & $\begin{array}{l}3.74 \\
3.77 \\
\end{array}$ \\
\hline & & 1:33:0 & & 24.726 & 32.345 & \begin{tabular}{|l|}
25.21 \\
\end{tabular} & 24.5 & 31.888 & $\begin{array}{l}48.241 \\
\end{array}$ & 4.286 & & \begin{tabular}{|l|l}
11.39 \\
\end{tabular} & & & 1.084 & & & $15 \%$ & & & 11.3 & & & & & & & $\begin{array}{l}3.77 \\
3.77 \\
\end{array}$ \\
\hline 109 & & 1:33:1 & 22.252 & 24.733 & 32.337 & 25.206 & 24.56 & 32.299 & \begin{tabular}{ll|}
48.952 \\
\end{tabular} & 4.32 & 28 & 11.387 & 5.4 & & 1.084 & & & 15.47 & \begin{tabular}{l|l|}
77 & 0.25778
\end{tabular} & 47.5 & 11.3 & & & & & & & 3.71 \\
\hline$\frac{110}{111}$ & & & & & & & & & & & & & & 46.1 & & & & & & & & & & & & & & $\frac{3.12}{3.76}$ \\
\hline & & $1: 33: 3$ & 22.181 & 24.717 & \begin{tabular}{|l|}
32.272 \\
\end{tabular} & 25.19 & 24.58 & 32.399 & & & & \begin{tabular}{l|l|l|l|}
11.387 \\
\end{tabular} & & & 1.085 & & & & 263 & & 11.3 & & & & & D04 & & 3.72 \\
\hline & 6/9/2004 & $1: 33: 4$ & 22.166 & 24.737 & \begin{tabular}{|l|l} 
& 32.257 \\
\end{tabular} & 25.22 & 24.5 & 31.849 & & & & 11.39 & & 46.0 & 1.085 & & & 15. & & & 11.3 & & & & & & 4.447 & \\
\hline & $6 / 9 / 2004$ & & 22.126 & 24.732 & \begin{tabular}{|l|}
32.227 \\
\end{tabular} & 25.226 & 24.5 & 31.845 & & & & & & & & & & & & & 11.3 & & & & & & & \\
\hline & & & 22.137 & 24.728 & \begin{tabular}{|l|}
32.203 \\
\end{tabular} & 25.216 & 24.60 & 32.013 & \begin{tabular}{|l|l|l|} 
\\
\end{tabular} & & 27.934 & 11.387 & & & 1.085 & & & 16.30 & & & 11.3 & & 2.06 & & & & & \\
\hline 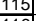 & & & C.2.101 & & & & & 32.146 & & & & & & & & & & & & & 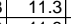 & & & & & & & \\
\hline & & & & & & & & & & & & & & & & & & 16. & & & & & & & & & & \\
\hline & & & $\frac{2.063}{202032}$ & 24.734 & 32.125 & $\frac{25.223}{2523}$ & 24.54 & $\begin{array}{l}32.17 \\
32.17\end{array}$ & 48.817 & & & 11.41 & & 5.1 & 1.085 & & & & & & 110 & & & & & & & \\
\hline & & & & & & & & & & & & & & & & & & & & & & & & & & & & \\
\hline & 6/9/2004 & 1:34:5 & 22.007] & 24.73 & 32.09 & 25.2 & & 32.1 & & & & 11.434 & & 46.0 & 1.0 & & & 17.1 & & & 11.3 & & & & & 0.004 & 4.421 & \\
\hline & & & 22.012 & & \begin{tabular}{|l|}
32.116 \\
\end{tabular} & & & 31.9 & & & & & & 45. & & & & & & & 11.3 & & & & & & & \\
\hline & & 1:35: & 21.961 & 24.738 & 32.06 & 25.242 & & 31.756 & & 4.2 & & 11.393 & & 46 & 1.0 & & & 17. & & & 11.3 & & & & & & & \\
\hline & & & & & & & & & & & & & & & & & & & & & & & & & & & & \\
\hline$\frac{12}{12}$ & & & 21.927 & & 32.041 & 25.253 & & 32.218 & & & 2 & & & & & & & & & & & & & & & & & \\
\hline & & 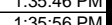 & 10.808 & $\begin{array}{l}2.153 \\
24733\end{array}$ & \begin{tabular}{|l|l|}
320.026 \\
32011
\end{tabular} & $\begin{array}{l}25.247 \\
25242\end{array}$ & & $\frac{32.027}{32.15}$ & 48 & $\frac{4.30}{4.33}$ & & $\begin{array}{r}11.39 \\
11.324\end{array}$ & & & $\frac{1.0}{10}$ & $\frac{14 .}{14 .}$ & & $\frac{17.6}{18.4}$ & $\frac{7.29}{30.30}$ & 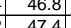 & $\begin{array}{ll}\frac{11.3}{113} \\
1.3\end{array}$ & & & & & & (146) & \\
\hline & 6. & & 21.856 & 2474 & | & 25247 & & $\frac{32.10}{32.098}$ & & & & $1138 \mathrm{~g}$ & & 50 & & & & 1830 & & & 111 & & & & & & & \\
\hline & & & & & & & & & & & & & & & & & & & & & & & & & & & & \\
\hline & & & $\mid 21.816$ & 24. & & 25.238 & & 31.8 & & & & 11.326 & & 45.8 & & & & 18.6 & & & $\frac{11.2}{11.2}$ & & & & & & & \\
\hline & & & 21.78 & & & & & & & & & & & & & & & & & & & & & & & & & \\
\hline & & & & & & & & & & & & & & & & & & & & & & & & & & & & \\
\hline & & & 21.745 & 24.743 & \begin{tabular}{|c|}
31.985 \\
\end{tabular} & & & $\overline{32.3}$ & & & & $\frac{11.2}{11.2}$ & & & & 14. & & & & & 1 & & & & & & & \\
\hline & $6 / 9 / 2$ & 1:37:06 & 1.714 & 24.72 & 31.93 & 25.195 & & 45.29 & & & & -1.21. & & $6.4 \mathrm{r}$ & 0.0 & 7 & & 19.3 & & & & & & & & & & \\
\hline & $6 / 9 / 200$ & 1:37: & 41.643 & 24.726 & 31.939 & 25. & & 45.095 & 48.44. & & & -1.211 & & 46.4 & 0.0 & 1.7 & & 19.4 & 0.32 & & 11.4 & & & & & & & \\
\hline & & & & & 31.9 & & & 44.9 & 48.4 & & & & & & & & & & & & 11.4 & & & & & & & \\
\hline & & & 21.536 & & 31.909 & 25.259 & & 44.85 & 48.299 & & & -1.215 & & & & & & $19.8 \mathrm{Cl}$ & .33 & 46.8 & 11.4 & & & & & & 887 & \\
\hline 136 & & & 21.50 & 24.72 & & & & 44.664 & & & & & & & & & & & & & & & & & & & & \\
\hline & & & |. 1.439 & $24 . / 79$ & \begin{tabular}{|c|}
31.866 \\
\end{tabular} & 25.298 & & 44.388 & 48.16 & & & & & & & & & & & & & & & & & & & \\
\hline & & $\begin{array}{l}1.38 .06 \\
1.30 .19\end{array}$ & $\frac{2.144}{21.34}$ & 20.749 & | & $\begin{array}{l}25.3008 \\
25317\end{array}$ & & (43.094 & 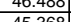 & & & $\mid$\begin{tabular}{|l|l|}
$\mid-1.215$ \\
$\mid$
\end{tabular} & & & 0.00 & 1.857 & & 20. & & 3 & 1111 & & 2.0 & & & 06 & 5.580 & \\
\hline$\frac{150}{140}$ & \begin{tabular}{|c|}
$6 / 9 / 2004$ \\
\end{tabular} & $1: 38.26 \mathrm{PM}$ & \begin{tabular}{|l|}
21.404 \\
21.397
\end{tabular} & \begin{tabular}{|l|l|}
24.722 \\
\end{tabular} & \begin{tabular}{|l|}
51.002 \\
31.806 \\
\end{tabular} & 25.331 & \begin{tabular}{|l|}
24.060 \\
\end{tabular} & $\frac{41.574}{42.169}$ & \begin{tabular}{|l|}
45.022 \\
\end{tabular} & 4.096 & $\frac{30.107}{38.374}$ & \begin{tabular}{|l|}
$\mid .1 .212$ \\
-1.212
\end{tabular} & -4.899 & $45.146 \mid$ & $\begin{array}{l}0.003 \\
0.03\end{array}$ & $\begin{array}{l}.0771 \\
.771\end{array}$ & & $\begin{array}{l}20.63 \\
\end{array}$ & $\begin{array}{ll}3 & 0.34389 \\
\end{array}$ & \begin{tabular}{|l|}
43.5 \\
\end{tabular} & \begin{tabular}{|l|l|}
1.1 \\
11.1
\end{tabular} & & \begin{tabular}{|l|}
2.777 \\
\end{tabular} & \begin{tabular}{|l|}
0.264 \\
0.264 \\
\end{tabular} & \begin{tabular}{|l|}
0.219 \\
0.219
\end{tabular} & 0.005 & \begin{tabular}{|l|}
5.437 \\
\end{tabular} & 4.63 \\
\hline
\end{tabular}


WSRC-TR-2005-00105, REVISION 0

SRNL-RPP-2005-00012, REVISION 0

PRE-TEST ACID CLEAN WITH 2 M HNO3, FOLLOWING PUMP REPAIR - CONT.

\begin{tabular}{|c|c|c|c|c|c|c|c|c|c|c|c|c|c|c|c|c|c|c|c|c|c|c|c|c|c|c|c|c|}
\hline & A & $B$ & $\mathrm{D}$ & $E$ & $\mathrm{~F}$ & G & $\mathrm{H}$ & $\mathrm{J}$ & \begin{tabular}{l|l|}
$\mathrm{K}$ & \\
\end{tabular} & $\mathrm{L}$ & M & $\mathrm{N}$ & 0 & \begin{tabular}{l|l} 
\\
\end{tabular} & $\mathrm{R}$ & $\mathrm{s}$ & $\begin{array}{ll}T \\
\end{array}$ & $\mathrm{~V}$ & w & $x$ & \begin{tabular}{|l|l|} 
& \\
\end{tabular} & $z$ & & $A B$ & & & & $\begin{array}{l}A F \\
A A\end{array}$ \\
\hline & $6 / 9 / 2004$ & 1:38:36 PM & 21.371 & 24.706 & \begin{tabular}{|l|l|}
31.811 \\
\end{tabular} & 25.34 & 24.698 & 42.235 & \begin{tabular}{|l|l|}
45.462 \\
\end{tabular} & 4.104 & 38.464 & -1.215 & -4.931 & \begin{tabular}{l|l|}
45.092 \\
\end{tabular} & 0.003 & 1.759 & 0.00 & 20.80 & \begin{tabular}{|l|l|}
0.34667 \\
\end{tabular} & 44.0 & \begin{tabular}{|l|l|}
0 & 11.1 \\
\end{tabular} & $\begin{array}{l}\frac{1}{1} 40.3 \\
\frac{1}{1}\end{array}$ & 2.782 & 0.262 & 0.217 & 0.005 & 5.389 & \\
\hline & $\begin{array}{l}6 / 9 / 2004 \\
\end{array}$ & 1:38:46 PM & 21.344 & & \begin{tabular}{|l|l}
31.804 \\
\end{tabular} & 25.384 & \begin{tabular}{|l|}
24.652 \\
\end{tabular} & $\begin{array}{l}42.148 \\
\end{array}$ & \begin{tabular}{|l|l|}
45.176 \\
\end{tabular} & 4.1 & \begin{tabular}{|c|}
38.339 \\
\end{tabular} & \begin{tabular}{|l|l|}
-1.215 \\
\end{tabular} & & \begin{tabular}{|l|l}
45.075 \\
\end{tabular} & $\begin{array}{l}0.003 \\
\end{array}$ & 1.756 & & 20.97 & \begin{tabular}{|ll}
7 & 0.34944 \\
\end{tabular} & 43.7 & 11.0 & & & \begin{tabular}{|l|l|}
0.262 \\
\end{tabular} & & 0.005 & 5.394 & \\
\hline 143 & $\begin{array}{l}6 / 9 / 2004 \\
\end{array}$ & $1: 38: 56 \mathrm{PM}$ & 21.309 & 24.709 & \begin{tabular}{|l|l|}
31.774 \\
\end{tabular} & 25.423 & 24.596 & \begin{tabular}{|l|}
42.378 \\
\end{tabular} & \begin{tabular}{|r|}
45.42 \\
\end{tabular} & 4.131 & \begin{tabular}{|l|}
38.53 \\
32.78
\end{tabular} & \begin{tabular}{|c|}
-1.212 \\
\end{tabular} & -4.91 & \begin{tabular}{|l|}
45.01 \\
\end{tabular} & $\begin{array}{l}0.003 \\
\end{array}$ & $\begin{array}{l}1.744 \\
\end{array}$ & & 21.13 & $\begin{array}{ll}30.35222 \\
\end{array}$ & $\begin{array}{l}44.0 \\
\end{array}$ & 11.0 & & $\begin{array}{l}2.789 \\
.779 \\
\end{array}$ & \begin{tabular}{|l|l|}
0.260 \\
\end{tabular} & 0.216 & 0.005 & 5.334 & \\
\hline \begin{tabular}{|l|l|}
144 \\
145
\end{tabular} & $6 / 9 / 2004$ & 1:39:06 PM & $\begin{array}{l}21.298 \\
21276\end{array}$ & 24.698 & \begin{tabular}{|l|}
1.748 \\
\end{tabular} & $\begin{array}{l}25.467 \\
25.517\end{array}$ & $\begin{array}{r}24.61 \\
\end{array}$ & $\begin{array}{r}42.248 \\
\end{array}$ & \begin{tabular}{|l|l|}
45.279 \\
4555 \\
\end{tabular} & $\begin{array}{l}4.076 \\
.130\end{array}$ & \begin{tabular}{|l|l|l|l|l|}
38.98 \\
\end{tabular} & \begin{tabular}{|l|l|} 
& -1.218 \\
\end{tabular} & \begin{tabular}{|l|l|} 
& -4.931 \\
\end{tabular} & \begin{tabular}{|l|l|}
45.023 \\
\end{tabular} & $\begin{array}{l}0.003 \\
\end{array}$ & $\begin{array}{l}1.737 \\
\end{array}$ & & 21.30 & \begin{tabular}{l|l}
0 & 0.355 \\
\end{tabular} & 43.8 & 11.0 & 40.4 & 2.783 & 0.259 & $\begin{array}{l}0.215 \\
\end{array}$ & 0.005 & $\begin{array}{l}5.328 \\
\end{array}$ & \\
\hline $\begin{array}{l}145 \\
146 \\
\end{array}$ & $\frac{6 / 9 / 2004}{6 / 9 / 2004}$ & $\begin{array}{l}1: 39: 16 \mathrm{PM} \\
1139 \cdot 26 \mathrm{PM}\end{array}$ & $\begin{array}{l}21.276 \\
2126\end{array}$ & $\begin{aligned} 24.712 \\
24711\end{aligned}$ & \begin{tabular}{|l|}
31.796 \\
31766 \\
\end{tabular} & $\begin{array}{l}25.541 \\
25.555\end{array}$ & $\begin{aligned} 24.609 \\
24598\end{aligned}$ & \begin{tabular}{|l|}
42.347 \\
42.453
\end{tabular} & \begin{tabular}{|l|}
45.553 \\
45.47 \\
\end{tabular} & \begin{tabular}{|l|l|}
4.103 \\
407
\end{tabular} & \begin{tabular}{|l|}
38.495 \\
38677
\end{tabular} & \begin{tabular}{|l|l|} 
& -1.212 \\
\end{tabular} & $\begin{array}{l}-4.954 \\
\end{array}$ & & 0.003 & $\begin{array}{l}1.725 \\
1702\end{array}$ & & 21.47 & $\begin{array}{ll}7 & 0.35778 \\
\end{array}$ & 44.1 & $\begin{array}{l}1 \\
11\end{array}$ & & \begin{tabular}{|l|}
2.787 \\
2707 \\
\end{tabular} & \begin{tabular}{|l|}
0.257 \\
0.257 \\
\end{tabular} & \begin{tabular}{|l|l|}
0.213 \\
\end{tabular} & 0.005 & $\begin{array}{l}5.277 \\
5.525\end{array}$ & 4.49 \\
\hline $\begin{array}{l}\frac{140}{147} \\
\end{array}$ & $\frac{6 / 9 / 2004}{6 / 9 / 2004}$ & $\begin{array}{l}1: 29: 26 \mathrm{PM} \\
1\end{array}$ & $\begin{array}{r}21.266 \\
21.219\end{array}$ & $\begin{array}{l}24.7111 \\
24.706\end{array}$ & \begin{tabular}{|l|}
31.766 \\
31.691 \\
\end{tabular} & \begin{tabular}{|l|}
$\mid 25.555$ \\
25.545 \\
\end{tabular} & $\begin{array}{r}24.598 \\
24.602 \\
\end{array}$ & $\begin{array}{l}42.453 \\
42.519\end{array}$ & $\mid$\begin{tabular}{|}
$\mid 45.5574$ \\
55.994
\end{tabular} & $\begin{array}{l}4.057 \\
4.107 \\
\end{array}$ & $\begin{array}{l}38.677 \\
38.696\end{array}$ & \begin{tabular}{|c|}
-1.212 \\
-1.215 \\
\end{tabular} & $\begin{array}{l}-4.937 \\
-4.951\end{array}$ & \begin{tabular}{|l|}
45.002 \\
5.5055
\end{tabular} & $\begin{array}{l}0.003 \\
0.003\end{array}$ & \begin{tabular}{|c|}
1.722 \\
1779
\end{tabular} & & 21.63 & \begin{tabular}{ll|}
3 & 0.36056 \\
0 & 0
\end{tabular} & $\begin{array}{l}44.1 \\
44.5 \\
\end{array}$ & $\begin{array}{l}1 \\
5 \\
5\end{array}$ & $\begin{array}{l}50.6 \\
5 \\
50.6\end{array}$ & \begin{tabular}{|l|}
$\mid 2.797$ \\
2.800
\end{tabular} & \begin{tabular}{|l|}
0.257 \\
0.255 \\
\end{tabular} & \begin{tabular}{|l|}
0.213 \\
0.212
\end{tabular} & $\begin{array}{l}0.005 \\
0.005\end{array}$ & \begin{tabular}{|c|}
5.253 \\
52219
\end{tabular} & 4.47 \\
\hline & & & 21.184 & & & & 24.638 & 42.401 & 45.225 & 4.053 & & & $\begin{array}{l}-4.966 \\
\end{array}$ & & 0.003 & 1.701 & $\frac{1}{1}$ & $\begin{array}{l}1.1 .90 \\
21.97\end{array}$ & \begin{tabular}{|c|c|}
7 & 0.363531 \\
\end{tabular} & \begin{tabular}{|l|}
43.5 \\
43.8
\end{tabular} & 11.0 & & $\frac{2.2800}{2.794}$ & $\frac{0.255}{0.254}$ & $\mid \begin{array}{l}0.2121 \\
0.211\end{array}$ & $\begin{array}{l}0.005 \\
0.005\end{array}$ & $\begin{array}{l}3.204 \\
5.204\end{array}$ & $\begin{array}{l}4.44 \\
4.43 \\
\end{array}$ \\
\hline 149 & $6 / 9 / 2004$ & 1:39:56 PM & & 24.699 & & & 24.636 & 42.49 & 45.238 & 4.11 & \begin{tabular}{|l|}
38.675 \\
\end{tabular} & \begin{tabular}{|l|}
-1.212 \\
\end{tabular} & -4.963 & \begin{tabular}{|l|}
45.037 \\
\end{tabular} & 0.003 & 1.699 & & & \begin{tabular}{|l|l}
3 & 0.36889 \\
\end{tabular} & 43.8 & \begin{tabular}{|l|l|}
8 & 11.0 \\
\end{tabular} & & 2.798 & 0.253 & 0.211 & & & \\
\hline & $6 / 9 / 2004$ & $1: 40: 06$ PM & 21.158 & 24.709 & \begin{tabular}{|r|}
31.66 \\
\end{tabular} & 25.588 & 24.661 & 42.604 & 45.464 & 4.087 & \begin{tabular}{|l|l|}
38.804 \\
\end{tabular} & \begin{tabular}{|l|l|} 
& -1.212 \\
\end{tabular} & -4.96 & & 0.003 & & & & & 44.0 & 11.0 & & & & & & & \\
\hline 151 & & & 21.117 & & & 25.583 & & 42.341 & 45.302 & 4.032 & .608 & -1.218 & & 444.946 & & 1.684 & & 22.47 & $\begin{array}{lll}7 & 0.37444\end{array}$ & 43.8 & & & 791 & .251 & 0.209 & 0.005 & 5.167 & $\frac{4.40}{4.40}$ \\
\hline 152 & & & & 24.709 & 31.644 & & 24.745 & 42.526 & & 4.083 & & & & 44.86 & & & & & & & & & & & 209 & & i.143 & \\
\hline 153 & $/ 2004$ & & 21.066 & 24.698 & 31589 & 25602 & 24.755 & 42.63 & 45.745 & & & & & & & 1.678 & & & 0.38 & 44.3 & & & .808 & & & & 123 & \\
\hline 154 & 6/9/2004 & 1:40:46 PM & 21.039 & 24.706 & \begin{tabular}{|l|l|}
31.578 \\
\end{tabular} & 25.636 & 24.77. & 42.627 & 45.551 & 4.074 & 38.846 & -1.218 & & |44.996 & 0.003 & 1.668 & & 22.97 & $\begin{array}{ll}77 & 0.38278 \\
\end{array}$ & $\begin{array}{l}44.1 \\
\end{array}$ & 11.0 & & 2.809 & 0.249 & 0.207 & 0.005 & 5.093 & \\
\hline 155 & $6 / 9 / 2004$ & 1:40:56 PM & 21.043 & 24.705 & 31.542 & 25.634 & 24.752 & 42.743 & 45.397 & 4.053 & 38.965 & -1.215 & & 45.038 & 0.003 & 1.661 & & 23.13 & 0.38556 & 43.9 & 11.0 & 40.9 & 2.817 & 0.248 & 0.207 & 0.005 & 5.062 & \\
\hline & 6/9/2004 & 1:41:06 PM & 20.987 & 24.704 & 31.541 & 25.644 & 24.70 & 42.552 & 45.418 & 4.064 & 38.804 & & & 44.86 & & 1.66 & & & $\begin{array}{lll}0 & 0.38833 \\
\end{array}$ & 43.9 & & & & 0.247 & & & .081 & 4.32 \\
\hline$\frac{157}{158}$ & 6/9/2004 & & 20.97 & & 31.5 & 25.667 & 24.705 .5 & 42.68 & 45.544 & 4.037 & 38 & & & & & 1.655 & & 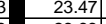 & 3 & 44.1 & & & & & & & 5.054 & \\
\hline 158 & & & & & & & 24.718 & & 46.002 & 4.099 & .155 & & & & 0.03 & & & & 39389 & & & & & & & & & \\
\hline & & $\begin{array}{l}1: 41: 3 \\
1.41 .4\end{array}$ & 20.924 & 24.124 & $\begin{array}{l}31.474 \\
31.48\end{array}$ & $\frac{25.682}{25705}$ & 24.784 & $\begin{array}{l}42.579 \\
42.845\end{array}$ & 45.393 & 4. & .809 & .218 & & 44.864 & & 1.641 & & & 39667 & 43.9 & & & 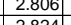 & .245 & & & 然3 & 4.28 \\
\hline 161 & & $\frac{1.44 .45}{1.56}$ & 20.9296 & 247009 & 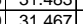 & $\frac{2.105}{25.719}$ & $\frac{24.066}{24786}$ & $\begin{array}{l}42.045 \\
42.347\end{array}$ & 年 4502024 & $\begin{array}{l}4.001 \\
4.011\end{array}$ & 38.601 & & & 44.846 & & 1633 & & $\frac{23.97}{24.13}$ & \begin{tabular}{|l}
.39944 \\
0.00222
\end{tabular} & $\frac{44.3}{43 .}$ & & & 791 & 243 & & 0.005 & & 4.24 \\
\hline & $6 / 9 / 2004$ & 1:42:06 PM & 20.864 & 24.697 & \begin{tabular}{|l|l|} 
& 31.425 \\
\end{tabular} & 25.697 & 24.784 & 42.745 & \begin{tabular}{|l|}
4.042 \\
4.422
\end{tabular} & 4.071 & 38.9019 & \begin{tabular}{|l|l|} 
& 1.212 \\
\end{tabular} & -5.00 & \begin{tabular}{|l|}
44.885 \\
\end{tabular} & 0.003 & 1.626 & & 24.30 & \begin{tabular}{|c|}
.4042 \\
0.405 \\
\end{tabular} & 44.0 & 11.0 & & 2.817 & 0.242 & 0.03 & 0.005 & 4.970 & $\begin{array}{l}4.28 \\
4.23 \\
\end{array}$ \\
\hline & 6/9/2004 & 1:42:16 PM & 20.844 & 24.712 & 31.435 & 25.722 & 24.839 & 42.834 & 45.814 & 4.103 & 39.001 & -1.215 & & 44.825 & 0.003 & 1.621 & & 24.47 & \begin{tabular}{|l|l|}
7 & 0.40778 \\
\end{tabular} & \begin{tabular}{|l|l|}
44.3 \\
\end{tabular} & \begin{tabular}{l|l}
3 & 11.0 \\
\end{tabular} & & 821 & 0.242 & & 0.005 & 4.947 & \\
\hline 164 & 6/9/2004 & & & 24.727 & 31.415 & 25.737 & 24.865 & & 45.66 & & 39.248 & & & & & 1.618 & & 24.63 & 0.41056 & & & & & & & 0.005 & & \\
\hline & & 1:42: & 20.808 & & & 25.736 & .878 & 42.722 & 45.447 & 4.055 & 38.928 & -1.215 & & 44.837 & $\begin{array}{l}0.003 \\
\end{array}$ & \begin{tabular}{|l|l|}
1.612 \\
\end{tabular} & & 24.80 & & 44.0 & & & & & 202 & & $\frac{936}{936}$ & \\
\hline & & & & & & & & & & & & & & & & & & & & & & & & & & & & \\
\hline 167 & $6 / 9 / 2004$ & 1:42:56 PM & 20.797 & 24.736 & $\mid 31.359$ & 25.765 & 24.858 & 41.447 & \begin{tabular}{|l|}
43.994 \\
\end{tabular} & 3.955 & $\mid 37.75$ & $\frac{-1.218}{-1.218}$ & & $\mid 44.36$ & $\mid 0.003$ & 1.582 & & 25.13 & 30.41889 & $\begin{array}{l}42.0 \\
42.5\end{array}$ & 10.9 & 939. & $\frac{2.730}{2.70}$ & 0.236 & $\mid$ & 0.005 & $\begin{array}{l}4.006 \\
4.999\end{array}$ & $\frac{14}{25}$ \\
\hline 168 & $6 / 9 / 2004$ & 1:43:06 PM & 20.796 & 24.73 & 31.358 & 25.785 & \begin{tabular}{|l|}
24.812 \\
\end{tabular} & \begin{tabular}{|l|l|}
32.384 \\
\end{tabular} & \begin{tabular}{|l|}
33.666 \\
\end{tabular} & 4.718 & 27.864 & -1.215 & -5.1 & 50.901 & 0.003 & 1.083 & & 25.30 & $\begin{array}{lll}0 & 0.42167\end{array}$ & 32.2 & 12.5 & 30. & 2.077 & 0.161 & 0.136 & 0.004 & 4.498 & \\
\hline 169 & 6/9/2004 & 1:43:16 PM & 20.781 & 24.72 & 31.299 & 25.725 & \begin{tabular}{|l|}
24.77 \\
\end{tabular} & 32.925 & \begin{tabular}{|l|}
34.358 \\
\end{tabular} & 4.444 & \begin{tabular}{|l|}
28.63 \\
\end{tabular} & \begin{tabular}{|l|} 
\\
\end{tabular} & -5.17 & 46.792 & 0.003 & 1.125 & & 25.47 & $\begin{array}{ll}77 & 0.42444 \\
\end{array}$ & 32.9 & 11.5 & & .122 & 0.168 & & 0.005 & & \\
\hline & & & 20.755 & 24.735 & 31.258 & & 24.706 & 32.108 & \begin{tabular}{|l|l|}
33.108 \\
\end{tabular} & 4.389 & & & & 46.226 & 0.003 & 1.084 & & 25.63 & \begin{tabular}{|l|l|}
3 & 0.42722 \\
\end{tabular} & 31.6 & \begin{tabular}{|l|l|}
6 & 11.3 \\
\end{tabular} & & & 0.162 & 0.136 & & & \\
\hline 171 & $6 / 9 / 2004$ & 1:43:36 PM & 20.755 & $\begin{array}{l}24.72 \\
\end{array}$ & 31.248 & 25.714 & 24.686 & 31.65 & \begin{tabular}{|l|}
32.754 \\
\end{tabular} & & \begin{tabular}{|l|}
27.468 \\
\end{tabular} & & & 45.953 & & 1.041 & & 25.80 & \begin{tabular}{|l|l|}
0.43 \\
\end{tabular} & 31.3 & 11.3 & & & & & 0.004 & 4.420 & \\
\hline & & 1:43:46 & 20.745 & 24.735 & \begin{tabular}{|l|}
31.209 \\
\end{tabular} & 25.709 & 24.726 & \begin{tabular}{|l|l|}
31.978 \\
\end{tabular} & \begin{tabular}{|l|}
32.831 \\
\end{tabular} & 4.135 & \begin{tabular}{|l|l|}
28.032 \\
\end{tabular} & \begin{tabular}{|c|} 
\\
\end{tabular} & & \begin{tabular}{|l|l|}
44.906 \\
\end{tabular} & 003 & $\begin{array}{l}1.041 \\
\end{array}$ & & 25.97 & 70.43278 & 31.4 & 11.0 & & 2.069 & & & 0.004 & 4.359 & \\
\hline \begin{tabular}{ll|l}
173 \\
174
\end{tabular} & $\frac{6 / 9 / 2004}{66 / 2004}$ & $\begin{array}{l}1: 43: 56 \text { PM } \\
1.4406 \mathrm{PM}\end{array}$ & 20.745 & $\begin{array}{l}24.745 \\
24.749\end{array}$ & \begin{tabular}{|l|}
31.174 \\
31.14 \\
\end{tabular} & $\frac{25.724}{25.698}$ & $\frac{24.726}{24.716}$ & $\begin{array}{l}31.982 \\
31.992 \\
\end{array}$ & \begin{tabular}{|c|}
32.943 \\
32.5999
\end{tabular} & \begin{tabular}{|l|l|}
4.175 \\
4.1
\end{tabular} & $\begin{array}{l}27.981 \\
28.018\end{array}$ & $\begin{array}{l}-1.212 \\
-1.215 \\
\end{array}$ & $\begin{array}{l}-5.1911 \\
-5.197\end{array}$ & \begin{tabular}{|l|}
44.862 \\
44.923 \\
\end{tabular} & $\begin{array}{l}0.003 \\
0.03\end{array}$ & $\begin{array}{l}1.033 \\
1.017 \\
\end{array}$ & & 26.13 & 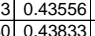 & $\begin{array}{l}31.5 \\
31.5 \\
\end{array}$ & $\frac{11.0}{11.0}$ & & 2.067 2.069 & $\begin{array}{l}0.154 \\
0.152\end{array}$ & \begin{tabular}{|l|l|}
0.130 \\
0.128
\end{tabular} & $\mid \begin{array}{l}0.004 \\
0.004\end{array}$ & \begin{tabular}{|l|l}
4.333 \\
4.269
\end{tabular} & $\begin{array}{l}3.69 \\
3.63 \\
\end{array}$ \\
\hline 175 & $\frac{\mid 6 / 9 / 2004}{6 / 92004}$ & $\begin{array}{l}\frac{144.06}{1: 44} 16 \mathrm{PM} \\
\end{array}$ & 20.679 & $\begin{array}{l}24.749 \\
24.754\end{array}$ & $\begin{array}{l}31.114 \\
31.089\end{array}$ & $\begin{array}{r}25.6089 \\
25.698\end{array}$ & $\begin{array}{r}24.6416 \\
24.641\end{array}$ & $\frac{31.992}{32.141}$ & 32.959 & \begin{tabular}{|l|l|}
4.166 \\
.166
\end{tabular} & $\mid \begin{array}{l}28.018 \\
28.131\end{array}$ & \begin{tabular}{|l|l|l|l|l|} 
\\
-1.212
\end{tabular} & & \begin{tabular}{|l|}
44.933 \\
4.835 \\
\end{tabular} & 0.0 & $\begin{array}{l}\frac{1.011}{1.017} \\
\end{array}$ & & $\begin{array}{l}\frac{26.30}{26.47} \\
\end{array}$ & \begin{tabular}{|c|c|c|c|}
7 & 0.438311 \\
\end{tabular} & $\begin{array}{l}31.5 \\
31.5 \\
\end{array}$ & $\frac{11.0}{11.0}$ & & . 069 & .152 & & \begin{tabular}{|c|c|}
0.004 \\
0.004
\end{tabular} & & $\begin{array}{l}3.63 \\
3.62 \\
6\end{array}$ \\
\hline 176 & $6 / 9 / 2004$ & & 20.669 & 24.759 & \begin{tabular}{|l|l|}
31.054 \\
\end{tabular} & 25.688 & 24.616 & 32.312 & \begin{tabular}{|l|}
33.251 \\
\end{tabular} & & 28.316 & -1. & & \begin{tabular}{|l|l|}
44.812 \\
\end{tabular} & 0.0 & $\frac{1.011}{1.004}$ & & $\frac{20.47}{26.63}$ & & $\frac{31.5}{31.8}$ & $\frac{11.0}{11.0}$ & & & & & & & $\frac{3.62}{3.56}$ \\
\hline 177 & & & 20.658 & 24.728 & \begin{tabular}{|l|}
30.949 \\
\end{tabular} & 25.642 & 24.565 & & \begin{tabular}{|l|}
33.396 \\
\end{tabular} & 186 & 28.467 & & & \begin{tabular}{|l|}
44.86 \\
\end{tabular} & & 0.994 & & 26.8 & & & & & & & & & & $\begin{array}{l}3.56 \\
3.51\end{array}$ \\
\hline 178 & $6 / 9 / 2$ & & 20.606 & 24.747 & 30.898 & 25.611 & 24.544 & 32.417 & \begin{tabular}{|l|}
33.347 \\
\end{tabular} & \begin{tabular}{|l|l|}
4.207 \\
\end{tabular} & & & & & & & & 26. & $\begin{array}{lll}7 & 0.44 \\
\end{array}$ & 31.9 & & & & & & & & $\begin{array}{l}3.11 \\
3.49 \\
\end{array}$ \\
\hline 179 & & & 20.581 & 24.742 & 30.879 & 25.576 & 24.59 & 32.338 & \begin{tabular}{|l|}
33.202 \\
\end{tabular} & 4.212 & & \begin{tabular}{|c|} 
\\
\end{tabular} & & 44.7 & & 0.9 & & 27.13 & & 31.7 & $\frac{\frac{11.0}{11.0}}{4}$ & & & & & & 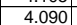 & $\begin{array}{l}\frac{3.49}{3.48} \\
\end{array}$ \\
\hline$\frac{180}{181}$ & & & 20.575 & 24.741 & & & & 32.1 & & & & & & $\overline{4}$ & & & & 27.30 & & & & & & & & & & \\
\hline 18. & & & & 24.741 & 803 & 25.535 & 24.64 & 32.519 & \begin{tabular}{|l|l|}
33.475 \\
\end{tabular} & 4.157 & & & & 4 & & 0.9 & & $27.47 \mid$ & & 320 & 11.0 & & & & & & & $\frac{2.48}{3.41}$ \\
\hline & 6/9/2004 & & 20.48 & 24.746 & 759 & 25.535 & 24.70 & 32.284 & 33.065 & & & & & 42 & 0.0 & 0.955 & & 27. & $\mid$ & \begin{tabular}{|c|}
31.6 \\
\end{tabular} & 11. & & & & & .004 & 4.012 & \\
\hline & & & 20.444 & & & & & 32.34 & \begin{tabular}{|l|}
33.212 \\
\end{tabular} & & & & & & & & & & & 31.7 & & & & & & & & \\
\hline 184 & & & 20.444 & 24.746 & 30.684 & 25.565 & 24.71 & 32.341 & \begin{tabular}{|l|}
33.046 \\
\end{tabular} & 236 & & -1.215 & & 4.988 & & 0.94 & & 27.9 & & 31.6 & 11.0 & & 508 & & 0.1 & & 3.959 & \\
\hline & & & 20.43 & & & 25.62 & & 32.299 & & & & & & & & & & & & 31. & & & & & & & & \\
\hline & & & & & & & & & & & & & & & & & & & & & & & & & & & & \\
\hline & & & & 24.761 & 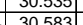 & $\begin{array}{r}25.65 \\
25663\end{array}$ & 24.6 & $\begin{array}{l}32.314 \\
32.488 \\
\end{array}$ & $\frac{32.926}{33133}$ & 4.168 & 28.313 & $\frac{-1.21}{-121}$ & & . & & & & 28.47 & 40 & 31. & 110 & & & & & 04 & $\begin{array}{l}3.896 \\
3.897\end{array}$ & \\
\hline 189 & & & & & & & & $\frac{3.2 .406}{32,44}$ & & & & & & & & & & & & 31.8 & & & & & & & & \\
\hline & & & & & & & & $\frac{252727}{3227}$ & & & & & & & & & & & & 0 & & & & & & & & \\
\hline 19 & & & 20.234 & & & 25.646 & & 32.276 & \begin{tabular}{|l|l|}
33.046 \\
\end{tabular} & & 28.129 & -1.215 & & $\overline{9}$ & & $0.9 c$ & & $\frac{29}{29}$ & & $\frac{31.6}{31.6}$ & 11. & & & & & & & \\
\hline & & & & & & & & & & & & & & & & & & & & & & & & & & & & \\
\hline & & & & & & & & 32.436 & 33.0 & & & & & & & & & & & & & & & & & & & \\
\hline & & & 20.15 & & & & & 32.44 & & & & & & & & & & & & & & & & & & & & \\
\hline & $6 / 9 / 200$ & & & 24.751 & 30.118 & & & 32.146 & \begin{tabular}{|l|l|}
32.723 \\
\end{tabular} & 4.19 & 28.1 & \begin{tabular}{|l|l|} 
& -1.212 \\
\end{tabular} & & & & & & & & \begin{tabular}{|l|l|}
31.3 \\
\end{tabular} & 11.1 & & & & & & & \\
\hline & 6/9/2004 & & 20.0 & 24.75 & & 25.64 & & 32.527 & $33.2 \mathrm{G}$ & & & \begin{tabular}{|c|} 
\\
\end{tabular} & & 45.2 & & & & & 10 & 31.8 & 11. & & & & & & & \\
\hline & & & & & & & & 32.216 & & & & & & & & & & & & & 11 & & & & & & & \\
\hline 198 & & & 20.046 & 24.751 & 30. & 25.635 & & 32.239 & 32.76 & 4.215 & & -1.2 & & 45.1 & & & & 30. & & 31.3 & 11.1 & & & & & & & \\
\hline & & & & & & & & & & & & -1. & & & & & & & & & & & & & & & & \\
\hline & & & & 24. & & 25 & & $\begin{array}{l}32.324 \\
32537\end{array}$ & 33.05 & 4. & & & & & & & & & & & $\frac{I 1.1}{11.1}$ & & & & & & & \\
\hline & & & 年 & 24.14 & & & & 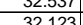 & S3.109 & 4.19 & & -1.2 & & & & & & & & & & & & & & & & \\
\hline & $\frac{0.15 / 2044}{6 / 92004}$ & & $\mid \frac{19584}{19884}$ & 24.751 & & $\frac{2.00}{256}$ & & $\frac{3.25}{32,444}$ & 3 & 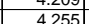 & & $\frac{-1.4}{12}$ & & 51 & & & & 然.0.5 & & & 111 & & & & & & & \\
\hline & & & & & & & & & 41,64 & & & & & & & & & & & & & & & & & & & \\
\hline & & & 19.71 & 24.745 & $\mid 29.864$ & $\frac{25.664}{25.64}$ & & 44.411 & 46.007 & 5.624 & & \begin{tabular}{|c|c|} 
& -1215 \\
\end{tabular} & & 53.5 & & & & $\frac{51.4}{31.4}$ & & & 13.1 & & & & & & & \\
\hline 0 & & & & 24.74 & & & & 43.73 & & & & & & & & & & & & & & & & & & & & \\
\hline & & & & & & & & & & & & & & & & & & & & & & & & & & & & \\
\hline & & & 19.63 & 2474 & 30.013 & $25.68 \mathrm{c}$ & & 41.65 & 43.179 & 5.30 & & -1.218 & & 1.9 & & & & 31.9 & & & & & & & & & & \\
\hline & & & 10.5 & 73 & & & & & & & & & & & & & & & & & & & & & & & & \\
\hline 210 & $6 / 9 / 2004$ & 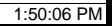 & $\mid$ & 24.739 & \begin{tabular}{|l|}
30.036 \\
\end{tabular} & \begin{tabular}{|l|}
25.678 \\
\end{tabular} & & 42.449 & \begin{tabular}{|l|l|} 
& 44.002 \\
\end{tabular} & 4.879 & 37.719 & $\begin{array}{ll}-1.215 \\
\end{array}$ & & 48.519 & 0.003 & 1.346 & & 32.30 & \begin{tabular}{l|l|l|}
0 & 0.53833
\end{tabular} & 42.5 & 11.9 & & & 0.201 & $\mid$ & $\mid 0.004$ & 4.355 & \\
\hline
\end{tabular}


WSRC-TR-2005-00105, REVISION 0

SRNL-RPP-2005-00012, REVISION 0

PRE-TEST ACID CLEAN WITH 2 M HNO3, FOLLOWING PUMP REPAIR - CONT.

\begin{tabular}{|c|c|c|c|c|c|c|c|c|c|c|c|c|c|c|c|c|c|c|c|c|c|c|c|c|c|c|c|c|}
\hline & A & $B$ & $\mathrm{D}$ & $E$ & $\mathrm{~F}$ & $G^{-1}$ & \begin{tabular}{ll|} 
\\
\end{tabular} & $\mathrm{J}$ & $\mathrm{K}$ & $\mathrm{L}$ & $\mathrm{M}$ & $\mathrm{N}$ & 0 & \begin{tabular}{l|l} 
\\
\end{tabular} & $\mathrm{R}$ & $\mathrm{s}$ & $T$ & $\mathrm{v}$ & w & $x$ & $\mathrm{Y}$ & & & & & & & $\begin{array}{ll}A F & A \\
\end{array}$ \\
\hline & $6 / 9 / 2004$ & 1:50:16 PM & $\begin{array}{l}19.51 \\
\end{array}$ & 24.729 & \begin{tabular}{|l|}
30.027 \\
\end{tabular} & 25.688 & 24.556 & 42.185 & 43.882 & 4.976 & 37.454 & \begin{tabular}{|c|} 
\\
\end{tabular} & -5.096 & \begin{tabular}{|l|l|}
50.017 \\
\end{tabular} & 0.003 & 1.331 & 0.003 & 32.47 & \begin{tabular}{|l|l|}
7 & 0.54111 \\
\end{tabular} & 42.4 & \begin{tabular}{|l|}
12.3 \\
\end{tabular} & \begin{tabular}{|l|}
39.8 \\
\end{tabular} & \begin{tabular}{|l|}
2.745 \\
\end{tabular} & 0.198 & 0.173 & 0.004 & 4.336 & 3.69 \\
\hline & $\begin{array}{l}6 / 9 / 2004 \\
\end{array}$ & 1:50:26 PM & $\begin{array}{l}19.499 \\
\end{array}$ & 24.744 & \begin{tabular}{|l|}
29.967 \\
\end{tabular} & 25.688 & \begin{tabular}{|l|}
24.515 \\
\end{tabular} & 42.355 & 44.234 & 4.987 & 37.581 & \begin{tabular}{|l|}
-1.215 \\
\end{tabular} & & 50.004 & 0.003 & 1.336 & & 32.63 & \begin{tabular}{c|c|c|c|}
3 & 0.54389 \\
\end{tabular} & 42.8 & & & & \begin{tabular}{|l|}
0.199 \\
\end{tabular} & & 0.004 & & 3.70 \\
\hline & $\begin{array}{l}6 / 9 / 2004 \\
\end{array}$ & $1: 50: 36 \mathrm{PM}$ & 19.484 & 24.739 & \begin{tabular}{|l|}
29.937 \\
2.967 \\
\end{tabular} & 25.683 & 24.555 & \begin{tabular}{|l|l|}
42.428 \\
\end{tabular} & \begin{tabular}{|l|l|}
44.398 \\
\end{tabular} & 5.047 & \begin{tabular}{|l|}
37.596 \\
2790
\end{tabular} & \begin{tabular}{|r|}
-1.215 \\
\end{tabular} & & 50.025 & 0.003 & 1.34 & & 32.80 & \begin{tabular}{ll|l}
0 & 0.54667 \\
0 & 0
\end{tabular} & $\begin{array}{l}42.9 \\
\end{array}$ & 112.3 & 40.0 & 告, & \begin{tabular}{|l|l|}
0.200 \\
\end{tabular} & 0.174 & 0.004 & 4.355 & 3.71 \\
\hline $\begin{array}{l}214 \\
215 \\
215\end{array}$ & 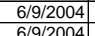 & $\begin{array}{l}1: 50: 46 \mathrm{PM} \\
1.50: 56 \mathrm{PM}\end{array}$ & \begin{tabular}{|l|l|l|}
19.474 \\
19.468
\end{tabular} & $\begin{array}{l}24.739 \\
24738\end{array}$ & \begin{tabular}{|l|}
29.967 \\
29966 \\
\end{tabular} & $\begin{array}{l}25.713 \\
25720\end{array}$ & \begin{tabular}{|r|}
24.51 \\
\end{tabular} & $\begin{array}{r}42.333 \\
\end{array}$ & \begin{tabular}{|l|l|}
43.907 \\
\end{tabular} & $\begin{array}{l}4.944 \\
\end{array}$ & \begin{tabular}{|l|l|}
37.639 \\
2778
\end{tabular} & \begin{tabular}{|c|}
-1.218 \\
\end{tabular} & $\begin{array}{r}-5.09 \\
\end{array}$ & 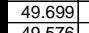 & 0.003 & $\begin{array}{l}1.351 \\
1.351\end{array}$ & 0.00 & $\begin{array}{l}32.97 \\
\end{array}$ & $\begin{array}{ll}77 & 0.54944 \\
\end{array}$ & 42.4 & 12.2 & 40.0 & $\begin{array}{l}2.757 \\
.777\end{array}$ & \begin{tabular}{|l|l|}
0.201 \\
\end{tabular} & $\begin{array}{l}0.176 \\
\end{array}$ & 0.004 & 4.390 & 3.74 \\
\hline$\frac{215}{216}$ & $\frac{6 / 9 / 2004}{6 / 9 / 2004}$ & $\begin{array}{l}1: 50: 56 \mathrm{PM} \\
1.51 .06 \mathrm{PM}\end{array}$ & $\begin{array}{l}19.468 \\
19.402\end{array}$ & $\frac{24.738}{24772}$ & & & \begin{tabular}{l|}
24.534 \\
24519
\end{tabular} & $\begin{array}{l}42.474 \\
42.096\end{array}$ & & $\begin{array}{l}4.945 \\
4927\end{array}$ & & & & \begin{tabular}{|l|l|}
49.576 \\
4951
\end{tabular} & \begin{tabular}{|l|l}
0.003 \\
0.003
\end{tabular} & $\begin{array}{l}1.354 \\
1355\end{array}$ & & 33.13 & & \begin{tabular}{|l|}
43.0 \\
42.4
\end{tabular} & \begin{tabular}{|l|}
12.1 \\
121
\end{tabular} & & & & \begin{tabular}{|l|l|}
0.176 \\
0.176
\end{tabular} & $\begin{array}{l}0.004 \\
\end{array}$ & 4.385 & 3.73 \\
\hline 217 & $\frac{6 / 9 / 2004}{6 / 9 / 2004}$ & & $\frac{19.402}{19.442}$ & $\begin{array}{l}24.727 \\
24.727\end{array}$ & \begin{tabular}{|l|}
29.965 \\
29.916 \\
\end{tabular} & \begin{tabular}{|l|}
25.711 \\
25.731 \\
\end{tabular} & $\begin{array}{r}24.519 \\
24.524\end{array}$ & $\begin{array}{r}42.096 \\
42.14\end{array}$ & \begin{tabular}{rl|}
43.832 & 43.87 \\
4
\end{tabular} & $\begin{array}{l}4.926 \\
4.953 \\
\end{array}$ & \begin{tabular}{|}
377.31 \\
37.409 \\
\end{tabular} & \begin{tabular}{|c|}
$\mid-1.218$ \\
-1.215 \\
\end{tabular} & $\begin{array}{r}-5.09 \\
-5.084\end{array}$ & \begin{tabular}{|}
$\mid 49.541$ \\
50.067
\end{tabular} & $\begin{array}{l}0.003 \\
0.003\end{array}$ & $\begin{array}{r}1.355 \\
1.36\end{array}$ & $\frac{0.005}{0.005}$ & $\begin{array}{l}33.30 \\
33.47\end{array}$ & \begin{tabular}{c|c|}
0 & 0.5555 \\
7
\end{tabular} & \begin{tabular}{|l|}
42.4 \\
42.4 \\
\end{tabular} & \begin{tabular}{|l|}
12.1 \\
12.3
\end{tabular} & 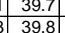 & $\mid$\begin{tabular}{|l|}
2.7399 \\
2.742
\end{tabular} & \begin{tabular}{|l|}
0.202 \\
0.203 \\
\end{tabular} & $\mid$\begin{tabular}{|l}
0.176 \\
0.177
\end{tabular} & \begin{tabular}{|c|}
0.004 \\
0.004
\end{tabular} & \begin{tabular}{|c|}
4.432 \\
4.449
\end{tabular} & $\begin{array}{l}3.77 \\
3.79 \\
\end{array}$ \\
\hline 218 & $6 / 9 / 2004$ & 1:51:26 PM & 19.416 & 24.726 & & & 24.468 & 42.459 & 44.324 & 4.906 & & & & \begin{tabular}{|l|l|}
49.553 \\
\end{tabular} & 0.003 & 1.359 & 0.005 & 33.63 & & $\begin{array}{l}42.9 \\
\end{array}$ & & & & $\begin{array}{l}0.203 \\
0.203 \\
\end{array}$ & & $\begin{array}{l}0.004 \\
0.004 \\
\end{array}$ & & $\begin{array}{l}3.79 \\
3.76\end{array}$ \\
\hline 219 & $6 / 9 / 2004$ & 1:51:36 PM & 19.386 & 24.726 & & 25.731 & 24.438 & 42.248 & $\begin{array}{l}43.834 \\
\end{array}$ & 4.879 & 37.631 & \begin{tabular}{|l|} 
\\
\end{tabular} & -5.084 & 49.566 & 0.003 & 1.361 & & & \begin{tabular}{l|l}
0 & 0.56333 \\
\end{tabular} & 42.4 & \begin{tabular}{|l|}
12.1 \\
\end{tabular} & & 2.754 & \begin{tabular}{|l|}
0.203 \\
\end{tabular} & 0.177 & 0.004 & & $\frac{3.60}{3.77}$ \\
\hline & $6 / 9 / 2004$ & 1:51:46 PM & & 24.721 & 29.82 & 25.716 & 24.448 & & $\begin{array}{l}44.212 \\
\end{array}$ & & & -1.218 & & $\begin{array}{l}49.756 \\
\end{array}$ & 0.003 & 1.364 & & & \begin{tabular}{l|l}
7 & 0.56611 \\
\end{tabular} & 42.7 & 12.2 & 40.0 & 2.758 & & & .004 & & \\
\hline 221 & & & $\begin{array}{l}19.39 \\
\end{array}$ & & & & 24.512 & $\begin{array}{ll}42.443 \\
\end{array}$ & & & 37.69 & & & \begin{tabular}{|l|l|l|}
491 \\
\end{tabular} & & 1.366 & & 34.13 & & \begin{tabular}{l|l|}
42.8 \\
\end{tabular} & & & & 0.204 & 0.178 & 0.004 & 4.441 & $\begin{array}{l}3.19 \\
3.78\end{array}$ \\
\hline$\frac{222}{202}$ & & & & 24.716 & 20.75 & & & 42.102 & \begin{tabular}{|l|l|l|l}
43.843 \\
\end{tabular} & 4.897 & & & & & & 1.364 & & & & 42.4 & & & & & & & & \\
\hline 223 & 2004 & 1:52:16 PM & 19.345 & 24.731 & & 25.66 & & 42.378 & & & & & & & & 1.359 & & & 0.57444 & \begin{tabular}{ll|}
42.8 \\
\end{tabular} & & & 776 & 0.203 & & & & $\begin{array}{l}3.01 \\
3.76\end{array}$ \\
\hline 224 & $6 / 9 / 2004$ & 1:52:26 PM & \begin{tabular}{|l|l|}
19.33 \\
\end{tabular} & 24.731 & 29.85 & 25.66 & 24.72. & 42.289 & $\begin{array}{l}44.002 \\
\end{array}$ & 4.945 & \begin{tabular}{|l|l|}
37.569 \\
\end{tabular} & -1.215 & & |49.583 & 0.003 & $\begin{array}{l}1.36 \\
\end{array}$ & & 34.63 & \begin{tabular}{|l|l|}
0.57722 \\
\end{tabular} & 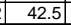 & & 39.9 & 2.75 & 0.203 & \begin{tabular}{|c|}
0.177 \\
\end{tabular} & 0.004 & & 3.78 \\
\hline & 6/9/2004 & $1: 52: 36 \mathrm{PM}$ & 19.314 & 24.725 & 29.834 & 25.639 & 24.712 & 42.434 & 44.158 & 4.909 & 37.744 & -1.215 & & 49.443 & 0.003 & 1.362 & & 34.80 & 0.58 & 42.7 & 12.1 & 40.1 & 2.764 & 0.203 & \begin{tabular}{|c|c|}
0.178 \\
\end{tabular} & 0.004 & 4.430 & 3.77 \\
\hline & & 1:52:46 PM & 19.309 & 24.725 & 29.744 & $\frac{25.644}{2.030}$ & 24.712 & 42.416 & $\begin{array}{r}44.38 \\
\end{array}$ & 4.951 & \begin{tabular}{|l|}
37.67 \\
\end{tabular} & & & 49.551 & 0.003 & 1.36 & & 34.97 & $\frac{.58278}{5.0278}$ & 42.9 & & & & & 0.178 & 0.004 & & 3.78 \\
\hline & 6/9/2004 & 1:52:56 PM & & 24.719 & 29.813 & 25.639| & 24.671 & 41.949 & & 4.905 & 37.263 & & & . 407 & 0. & 1.363 & & & 58556 & & & & & 203 & & 0.004 & 4.490 & \\
\hline 22 & $6 / 9 / 2004$ & 53:06 PM & 19.282 & & 29.757 & 25.642 & 24.68 & & 44.342 & 9931 & 37.824 & -1.212 & & $49.441]$ & 0.003 & 1.365 & & 35.30 & 58833 & 42.9 & & & & & 0.178 & & 4.440 & \\
\hline & & 1:53:16 PM & 19.28 & 24.712 & & & & 42.581 & 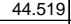 & & 3 & -1.215 & & 49.443 & & 1.365 & & 35.4 & 59111 & 43.0 & 12. & & & 0.204 & & & 4.434 & 3.77 \\
\hline & 6/9/2004 & $\begin{array}{l}1: 53: 26 \mathrm{PM} \\
1.53 \cdot 36 \mathrm{PM}\end{array}$ & $\frac{19.265}{10235}$ & 24.717 & 29.156 & 25.646 & 24.398 & 42.308 & \begin{tabular}{|l|l}
4.054 \\
\end{tabular} & 4.929 & \begin{tabular}{|c|}
37.598 \\
\end{tabular} & \begin{tabular}{|r|} 
\\
\end{tabular} & & 49.474 & 0.003 & & & 35.6. & 0.59389 & $\begin{array}{l}42.6 \\
\end{array}$ & $\frac{1.1}{121}$ & 40.0 & 2.755 & 0.203 & $\begin{array}{ll}0.178 \\
\end{array}$ & 0.004 & 458 & 3.79 \\
\hline 232 & $6 / 9 / 9 / 2004$ & $\begin{array}{l}1.553 .50 \mathrm{PM} \\
1: 5346 \mathrm{P}\end{array}$ & $\begin{array}{l}\frac{19.250}{19.223} \\
\end{array}$ & 24.7 & 29.675 & 25.639 & 24.307 & $\begin{array}{l}42.350 \\
42.214\end{array}$ & \begin{tabular}{|l|}
44.272 \\
44.135 \\
\end{tabular} & $\begin{array}{l}4.901 \\
4.993 \\
\end{array}$ & $\begin{array}{l}37.113 \\
37.444\end{array}$ & \begin{tabular}{|l|}
-1.210 \\
-1.212 \\
\end{tabular} & & $\begin{array}{l}49.4200 \\
49.412\end{array}$ & $\begin{array}{l}.0033 \\
0.003\end{array}$ & $\begin{array}{l}1.302 \\
1.364\end{array}$ & & $\begin{array}{l}35.80 \\
35.97\end{array}$ & \begin{tabular}{|c|c|}
7 & 0.596966 \\
\end{tabular} & $\begin{array}{l}42.8 \\
42.7 \\
\end{array}$ & $\begin{array}{ll}12.1 \\
12.1\end{array}$ & $\begin{array}{l}40.0 \\
39.8 \\
\end{array}$ & $\mid$\begin{tabular}{|l|}
2.1 .740 \\
2.746
\end{tabular} & $\begin{array}{l}0.203 \\
0.203 \\
\end{array}$ & $\begin{array}{l}0.118 \\
0.179\end{array}$ & $\frac{0.004}{0.004}$ & $\begin{array}{l}4.449 \\
4.485\end{array}$ & $\frac{3.9}{3.82}$ \\
\hline & 6/9/2004 & 1:53:56 PM & 19.243 & 24.71 & 29.715 & 25.639 & 24.377 & 42.586 & 44.319 & 4.892 & \begin{tabular}{|l|l|}
37.979 \\
\end{tabular} & \begin{tabular}{|l|l|} 
& -1.215 \\
\end{tabular} & & 49.33 & 0.003 & 1.361 & & 36.13 & $\begin{array}{ll}3 & 0.60222 \\
\end{array}$ & \begin{tabular}{|c|}
42.8 \\
\end{tabular} & & \begin{tabular}{|l|l|}
40.3 \\
\end{tabular} & \begin{tabular}{|l|}
2.777 \\
\end{tabular} & 0.203 & & & & $\begin{array}{l}3.82 \\
3.76\end{array}$ \\
\hline 2344 & 6/9/2004 & & 19.243 & 24.71 & 29.789 & 25.584 & 24.427 & 42.569 & & $\begin{array}{l}4.904 \\
\end{array}$ & 37.877 & & & 49.334 & & & & 36.3 & $\frac{0.605}{0.605}$ & & & & & & & 0.004 & & $\begin{array}{l}3.67 \\
3.77 \\
\end{array}$ \\
\hline 235 & & 1:54:1 & 19.233 & 24.72 & 29.834 & 25.539 & 24.462 & 42.343 & 44.235 & 4.904 & 37.663 & -1.212 & & 49.414 & 003 & 1.36 & & 36.47 & 0.60778 & 42.8 & & & & 203 & b.177 & & & 3.17 \\
\hline 236] & & & & & & & & & \begin{tabular}{|l|l|}
43.712 \\
\end{tabular} & 4.859 & 37.429 & & & 49.288 & 0.003 & & & & & & & & & & & & & \\
\hline & $6 / 9 / 2004$ & 1:54:36 PM & 19.202 & 24.699 & 29.754 & 25.529 & & 42.337 & \begin{tabular}{|c|c|}
44.284 \\
\end{tabular} & 4.89 & 37.655 & -1.218 & & \begin{tabular}{|l|l|}
49.37 \\
\end{tabular} & & 1.357 & & & & & & & & & & & & \\
\hline 238 & $6 / 9 / 2004$ & 1:54:46 PM & \begin{tabular}{|c|}
19.207 \\
\end{tabular} & 24.709 & \begin{tabular}{|l|}
29.843 \\
\end{tabular} & 25.554 & 24.426 & 42.749 & 44.587 & 4.908 & 38.082 & -1.212 & & 49.341 & 0.003 & 1.359 & & 36.97 & & 43.1 & 12.1 & & $.787 \mathrm{Cl}$ & .203 & & & & \\
\hline 239 & 6/9/2004 & $1: 54: 56 \mathrm{PM}$ & 19.166 & 24.704 & \begin{tabular}{|l|l|}
29.818 \\
\end{tabular} & 25.538 & 24.435 & 42.451 & $\begin{array}{l}44.363 \\
\end{array}$ & \begin{tabular}{|l|l}
4.843 \\
\end{tabular} & 37.828 & -1.215 & & 48.938 & 0.003 & 1.361 & & 37.13 & 0.61889 & 42.9 & 12. & 40 & & 203 & 0.178 & 0.004 & 424 & 3.76 \\
\hline 27 & & & 19.156 & 24.698 & 29.847 & 25.507 & 24.445 & 42.378 & 44.185 & & & & & 48.865 & & 1.36 & & & & 42.7 & & & & & & & & \\
\hline$\frac{241}{212}$ & $6 / 9 / 2004$ & 55:16 $\mathrm{P}$ & 19.176 & 24.713 & 99.862 & 25.522 & 24.47 & 42.621 & 4.55 & & & & & 48.8 & & 1.36 & & & $\begin{array}{l}70 \\
0.62444 \\
\end{array}$ & 43.1 & 2. & & & & & & & \\
\hline & & 1:55:2 & 19.16 & 24.692 & 29.831 & 25.451 & & 42.364 & 257] & & 37.842 & -1.218 & & 48.152 & 0.003 & 1.365 & & 37.63 & $\begin{array}{ll}3 & 0.62 \\
\end{array}$ & 42.8 & & & & .204 & & & 139 & \\
\hline 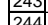 & $691 / 2004$ & $1: 55: 36 \mathrm{PM}$ & $\frac{19.149}{1.94}$ & 24.6887 & $\frac{29.816}{27.16}$ & 25.441 & 24.528 & 42.594 & 44.541 & & $\begin{array}{l}38.035 \\
39057\end{array}$ & 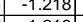 & & 48.75 & & 1.355 & & & & & & & & & & & & 74 \\
\hline 245 & $6 / 1 / 2004$ & $1555.56 \mathrm{PM}$ & 19.134 & 年 & 年 & 25.456 & 24.05 & 42.050 & 先, & 4.179 & $\begin{array}{l}38.051 \\
37895\end{array}$ & & & 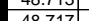 & 0 & 1.550 & & & 0.63278 & 10 & & & & & & & & 3.75 \\
\hline 246 & $\begin{array}{l}6 / 9 / 2004 \\
\end{array}$ & 1:56:06 PM & $\frac{1.104}{19.113}$ & 24686 & \begin{tabular}{|l|}
29.775 \\
\end{tabular} & 25.475 & 24.60 & 4 & \begin{tabular}{|l|}
44.405 \\
\end{tabular} & 4.773 & 38.6 & -1.21 & & $\frac{4.11}{48.661}$ & & $\begin{array}{l}.0558 \\
1.358\end{array}$ & & 38 & \begin{tabular}{|l|l|l|}
0.68333 \\
\end{tabular} & $\begin{array}{l}42.8 \\
42.9\end{array}$ & $\frac{11.9}{11.9}$ & & & & $\begin{array}{l}0.177 \\
0.177\end{array}$ & & & $\frac{3.6}{3.74}$ \\
\hline 247 & & & 19.118 & 24.691 & \begin{tabular}{|l|}
29.795 \\
\end{tabular} & 25.47 & & 42.629 & & & & & & 48.769 & 0.003 & 1.35 & & 38. & & 43.0 & & & & & & & & \\
\hline & & & 19.092 & 24.69 & 29.804 & 25.444 & & 42.511 & & & & & & 48. & & 1.35 & & & & 42.8 & & & & & & & & 74 \\
\hline 249 & & $1: 56: 3$ & 19.076 & 24.6 & \begin{tabular}{|l|}
29.709 \\
\end{tabular} & 25.489 & & 42.76 & 44.662 & 4.78 & & -1.2. & & 48.5 & & 1.35 & & 38. & $\mid 0.64$ & 43.2 & & & & & & & & \\
\hline & & & & & & & & & & & & & & 48.429 & & & & & 44 & & & & & & & & & \\
\hline & 6/9/2004 & & 19.092 & 24.695 & \begin{tabular}{|l|}
29.784 \\
\end{tabular} & 25.489 & & 42.552 & 44.299 & 4.687 & 38.072 & -1.215 & & & & 1.35 & & & & 42.8 & $\frac{11.8}{11.8}$ & & & & & & & 3.73 \\
\hline & 6/9/2004 & & 19.096 & 24.694 & \begin{tabular}{|l|}
30.414 \\
\end{tabular} & 25.488 & \begin{tabular}{|l|}
24.50 \\
\end{tabular} & 42.922 & & & & -1.2 & & 48.1 & & 1.362 & & & 0.655 & 43. & 11. & & 04 & & & 0.004 & & 3.66 \\
\hline & $6 / 9 / 2004$ & & 19.101 & 24.699 & & 25.498 & 24.48 & 43.463 & & & & & & 48.546 & & & & & & & & & & & & & & \\
\hline 254 & 6/9/2004 & 1:57:2 & 19.258 & 24.69 & \begin{tabular}{|l|}
32.423 \\
\end{tabular} & $25.499 \mid$ & 24.516 & 43.598 & & & 38.993 & -1.215 & & 48.498 & 0.0 & 1.4 & & & 0.66056 & 44.3. & 11.9 & & & & & & & \\
\hline & & & 19.611 & & & & & 43.6 & & & & & & & & & & & & & 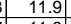 & & & & & & & \\
\hline$\frac{25}{25}$ & & & & & & 25.528 & & & & & & & & & & & & & & & & & & & & & & \\
\hline & & & 20.467 & 24.693 & 33.628 & $\frac{25.537}{25577}$ & 24. & 42.455 & & & & -1.21 & & 48.0 & & & & & & 40.4 & 100 & & & & & & & 0.0 \\
\hline & & & & & & & & & & & & & & & & & & & & & & & & & & & & 3.96 \\
\hline 260 & & & 21.18 & & & & & & & & & & & 484 & & & & & & & & & & & & & & 3.83 \\
\hline 261 & $6 / 9 / 2004$ & & 21.257 & (698 & \begin{tabular}{|l|}
32.525 \\
\end{tabular} & 25.512 & & 43.488 & & & & & & 48.6 & & & & & & $\begin{array}{l}44.4 \\
44.6\end{array}$ & $\frac{71.9}{11.9}$ & & & & & & & $.97 \quad-97$ \\
\hline & & & & & & & & & & & & & & & & & & & & & & & & & & & & \\
\hline 263 & & & 21.362 & & & & & 43.32 & & & & & & & & & & & & & & & & & & & & \\
\hline & & & 21.443 & & \begin{tabular}{|l|l|}
32.649 \\
\end{tabular} & & & 43.023 & & & & & & & & & & & & & & & & & & & & \\
\hline & 6/9/2004 & & 21.494 & 24.703 & \begin{tabular}{|l|}
32.605 \\
\end{tabular} & 25.457 & & 32.005 & 33.498 & 3.6 & & -1.215 & & & & 1.3 & & 41 & & 320 & 5 & & & & & & & 4.47 \\
\hline & 6/9/2004 & & 21.504 & 24.698 & 32 & 25.457 & & 38.8 & $40.4 \mathrm{C}$ & & & -1.2. & & 41.5 & & & & & & & & & & & & & & \\
\hline $267 \mid$ & $6 / 9 / 26$ & & 21.52 & & & 25.487 & & 43.3 & & & & & & & & & & & & & & & & & & & & \\
\hline 268 & $6 / 9$ & 1:59: & 21.534 & & \begin{tabular}{|l|l|}
32.412 \\
\end{tabular} & 25.502 & & 43.647 & & & & -1.215 & & 48.5 & & 1.6 & & & & & 11.9 & & & & & 005 & 4.786 & \\
\hline & $6 / 9$ & & 21.5 & & & & & & & & & & & & & & & & & & & & & & & & & \\
\hline$\frac{2 \pi}{271}$ & & & 21.508 & 24. & \begin{tabular}{|l|l|}
32.381 \\
\end{tabular} & 25.5. & & 43.552 & & & & & & 48. & & & & & & & 1.0 & & & & & & & \\
\hline$\frac{21}{27}$ & 6 & & $\frac{14.441}{20142}$ & 24.6801 & \begin{tabular}{|l|l|l|}
32.30 \\
3219
\end{tabular} & $\frac{2.3}{25.3}$ & & 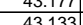 & & & & \begin{tabular}{|c|c|}
-1.2 \\
4
\end{tabular} & & & & & & & & & & & & & & & & \\
\hline & $6 / 9 / 2004$ & & 01.437 & 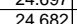 & 3 & 25.096 & & 4 & 4586 & & & $\frac{-1.4}{-12}$ & & 485 & & & & & & & 1119 & & & & & & & \\
\hline 274 & & & 21,43 & & & 25.515 & & 43.32 & & & & $\mid-12$ & & 486 & & & & & & & & & & & & & & \\
\hline 275 & & & 21.39 & & \begin{tabular}{|l|}
32.052 \\
\end{tabular} & $=25.5$ & & 43.038 & 45.733 & & & -1.215 & & 48. & & & & & & 44.3 & 11.6 & & & & & & & \\
\hline 276 & & & 21.349 & 24.674 & & & & & & & & -1.2 & & & & & & & & & & & & & & & & \\
\hline & & & & & & & & & & & & & & & & & & & & & & & & & & & & \\
\hline 278 & 2004 & & 21.329 & 24.689 & \begin{tabular}{|l|l|}
32.145 \\
\end{tabular} & 25.523 & & 43.034 & 45.961 & 4.9 & 38.4 & -1.215 & & 48. & & 1.6 & & 43 & \begin{tabular}{|l|l|l|} 
\\
\end{tabular} & 5 & & & & & & 05 & & \\
\hline & & $\mathrm{P}$ & 1293 & 689 & & 25552 & & 43.245 & & & & & & $\overrightarrow{B E}$ & & & & & 0.73 & 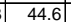 & & & & & & & | & \\
\hline 280 & $6 / 9 / 2004$ & 2 $2: 11: 46$ & 21.288 & 24.699 & \begin{tabular}{|l|}
32.035 \\
\end{tabular} & \begin{tabular}{|l|}
25.583 \\
\end{tabular} & & 43.318 & \begin{tabular}{|l|l|}
46.239 \\
\end{tabular} & 4.902 & 38.796 & \begin{tabular}{|l|}
$\mid-1.212$ \\
\end{tabular} & & \begin{tabular}{|l|l|}
48.579 \\
\end{tabular} & 0.003 & 1.671 & & 43.97] & 70.73278 & 44.8 & 11. & & \begin{tabular}{|l|}
2.831 \\
\end{tabular} & \begin{tabular}{|l|l|l|l|} 
\\
\end{tabular} & $\mid$ & 0.005 & 5.001 & 4.2 \\
\hline
\end{tabular}


WSRC-TR-2005-00105, REVISION 0

SRNL-RPP-2005-00012, REVISION 0

PRE-TEST ACID CLEAN WITH 2 M HNO3, FOLLOWING PUMP REPAIR - CONT.

\begin{tabular}{|c|c|c|c|c|c|c|c|c|c|c|c|c|c|c|c|c|c|c|c|c|c|c|c|c|c|c|c|c|}
\hline & A & $B$ & $\mathrm{D}$ & $E$ & $F$ & $\mathrm{G}$ & $\mathrm{H}$ & $\mathrm{J}$ & $\mathrm{K}$ & $\mathrm{L}$ & $\mathrm{M}$ & $\mathrm{N}$ & 0 & \begin{tabular}{l|l} 
\\
\end{tabular} & $\mathrm{R}$ & $\mathrm{s}$ & $\begin{array}{ll}T \\
\end{array}$ & $\mathrm{~V}$ & w & $x \mid$ & \begin{tabular}{l|l} 
\\
\end{tabular} & $\mathrm{z} \mid$ & $A A$ & $A B$ & $\begin{array}{ll}A C \\
\end{array}$ & AD & AE & $\begin{array}{ll}A F & A \\
\end{array}$ \\
\hline 28 & $6 / 9 / 2004$ & 2:01:56 PM & 21.242 & $\begin{array}{l}24.683 \\
\end{array}$ & \begin{tabular}{|l|}
31.971 \\
\end{tabular} & 25.578 & 24.62 & 42.816 & 45.607 & 4.815 & $\begin{array}{l}38.382 \\
\end{array}$ & -1.218 & -4.954 & 48.513 & 0.003 & 1.665 & 0.005 & 44.13 & 0.73556 & 44.1 & 11.9 & 40.6 & \begin{tabular}{|l|}
2.799 \\
\end{tabular} & 0.248 & 0.205 & 0.005 & 5.047 & 4.30 \\
\hline & $\begin{array}{l}6 / 9 / 2004 \\
\end{array}$ & & & 24.698 & \begin{tabular}{|l|}
31.911 \\
\end{tabular} & 25.572 & 24.545 & 35.895 & 46.204 & $\begin{array}{r}4.87 \\
\end{array}$ & \begin{tabular}{|l|}
31.222 \\
11.22
\end{tabular} & \begin{tabular}{|l|}
2.37 \\
\end{tabular} & 1.186 & 48.333 & 0.831 & $\begin{array}{l}-0.01 \\
-0.01\end{array}$ & & 44.30 & \begin{tabular}{|l|l|}
0.73833 \\
\end{tabular} & 44.7 & 11.8 & & & & & & & \\
\hline & $\begin{array}{l}6 / 9 / 2004 \\
\end{array}$ & $2: 02: 16 \mathrm{PM}$ & $\frac{11.216}{21.216}$ & 24.682 & \begin{tabular}{|l|}
31.955 \\
3.190 \\
\end{tabular} & 25.591 & 24.549 & 29.993 & \begin{tabular}{|l|l|}
46.286 \\
507
\end{tabular} & 4.903 & \begin{tabular}{|l|}
25.344 \\
2.344
\end{tabular} & 11.164 & & \begin{tabular}{|l|l|}
47.878 \\
7.890
\end{tabular} & $\begin{array}{l}1.072 \\
\end{array}$ & -0.012 & & 44.47 & $\begin{array}{ll}7 & 0.741111 \\
& \end{array}$ & 44.8 & 11.7 & & & & -0.001 & 0.000 & $\begin{array}{l}-0.053 \\
-055\end{array}$ & \\
\hline 284 & 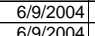 & $\begin{array}{l}2: 02: 26 \mathrm{PM} \\
20.02 \cdot 36 \mathrm{PM}\end{array}$ & $\begin{array}{l}21.191 \\
21.19\end{array}$ & \begin{tabular}{|l|l|l|l|}
24.687 \\
24.686
\end{tabular} & \begin{tabular}{|l|}
31.989 \\
31.934 \\
\end{tabular} & $\begin{array}{l}25.596 \\
25641\end{array}$ & $\begin{array}{l}24.519 \\
2.519\end{array}$ & $\begin{array}{l}29.835 \\
3020\end{array}$ & \begin{tabular}{|l|l|}
45.967 \\
\end{tabular} & $\begin{array}{l}4.944 \\
\end{array}$ & 25.114 & $\begin{array}{l}11.04 \\
1071\end{array}$ & $\begin{array}{r}5.15 \\
\end{array}$ & \begin{tabular}{|l|l|}
47.968 \\
17702
\end{tabular} & $\begin{array}{l}1.067 \\
\end{array}$ & -0.012 & & \begin{tabular}{|l|l|l|}
4.63 \\
49
\end{tabular} & $\begin{array}{lll}3 & 0.74389 \\
\end{array}$ & 44.5 & 11.8 & 27.5 & 1.894 & 0.002 & -0.001 & 0.000 & -0.054 & -0.05 \\
\hline & $\frac{6 / 9 / 2004}{6 / 9 / 2004}$ & $2: 02: 36 \mathrm{PM}$ & $\frac{21.19}{21.19}$ & $\begin{array}{l}24.686 \\
24.681\end{array}$ & \begin{tabular}{|l|}
31.934 \\
31.898 \\
\end{tabular} & 25.641 & $\frac{24.518}{24.673}$ & \begin{tabular}{|r|}
30.289 \\
344
\end{tabular} & \begin{tabular}{|r|}
46.4 \\
46.479 \\
\end{tabular} & $\begin{array}{l}4.924 \\
4.858 \\
\end{array}$ & \begin{tabular}{|l|}
25.619 \\
-1.267
\end{tabular} & & & \begin{tabular}{|l|}
47.962 \\
4705 \\
\end{tabular} & $\begin{array}{l}1.059 \\
0.004\end{array}$ & $\begin{array}{l}-0.012 \\
0.011\end{array}$ & & 44.80 & 0.74667 & 44.9 & 11.8 & 28.0 & & & \begin{tabular}{|c|}
-0.001 \\
0.01
\end{tabular} & & $\begin{array}{l}-0.053 \\
.061\end{array}$ & $\frac{-0.05}{10}$ \\
\hline 287 & $\frac{6 / 91 / 2004}{6 / 9 / 2004}$ & $\begin{array}{l}2: 02: 46 \mathrm{PM} \\
202: 5 \mathrm{PM}\end{array}$ & & $\begin{array}{r}24.681 \\
24.69\end{array}$ & \begin{tabular}{|l|}
31.8829 \\
31.829 \\
\end{tabular} & $\begin{array}{l}25.645 \\
25.629\end{array}$ & $\begin{array}{l}24.4673 \\
24.557\end{array}$ & $\begin{array}{l}3.414 \\
3.246\end{array}$ & \begin{tabular}{|}
$\mid 46.479$ \\
46.506 \\
\end{tabular} & $\begin{array}{l}4.858 \\
4.847 \\
\end{array}$ & \begin{tabular}{r|}
-1.267 \\
-1.464
\end{tabular} & $\begin{array}{r}38.161 \\
38.3\end{array}$ & $\begin{array}{l}26.715 \\
18.635 \\
\end{array}$ & \begin{tabular}{|r|}
$\mid 47.05$ \\
47.052 \\
\end{tabular} & $\begin{array}{l}0.004 \\
0.004\end{array}$ & $\frac{-0.011}{-0.013}$ & $\frac{1}{3}$ & \begin{tabular}{|l|}
44.97 \\
45.13
\end{tabular} & $\begin{array}{l}0.77944 \\
0.75222\end{array}$ & $\begin{array}{l}45.0 \\
45.0 \\
\end{array}$ & \begin{tabular}{|l|}
11.5 \\
11.5
\end{tabular} & & $\frac{0.074}{0.061}$ & .002 & \begin{tabular}{|c|c|}
-0.001 \\
-0.002
\end{tabular} & $\begin{array}{l}-0.001 \\
-0.002 \\
\end{array}$ & $\begin{array}{l}-1.264 \\
-1803\end{array}$ & \begin{tabular}{|l|l|l|}
-1.08 \\
-1.53
\end{tabular} \\
\hline 288 & $6 / 9 / 2004$ & 2:03:06 PM & 21.253 & 24.684 & & 25.619 & 24.656 & 3.196 & 46.454 & 4.836 & \begin{tabular}{|c|}
-1.4049 \\
\end{tabular} & 38.294 & 18.508 & & 0.003 & $\begin{array}{l}-0.013 \\
-0.013\end{array}$ & 0.0 & $\begin{array}{l}45.13 \\
5.30\end{array}$ & $\frac{0.15242}{0.755}$ & 45.0 & 11.6 & & & $\begin{array}{l}-0.002 \\
-0.002\end{array}$ & $\begin{array}{l}-0.002 \\
-0.002\end{array}$ & $\begin{array}{l}-\frac{-0.002}{-0.002} \\
\end{array}$ & $\begin{array}{l}-1.803 \\
-1.884 \\
\end{array}$ & $\begin{array}{l}-1.53 \\
-1.60 \\
\end{array}$ \\
\hline & $6 / 9 / 2004$ & 2:03:16 PM & & 24.669 & \begin{tabular}{|l|}
31.748 \\
\end{tabular} & 25.618 & 24.55 & & 46.49 & 4.881 & -1.448 & 38.303 & 18.419 & 46.986 & 0.003 & -0.013 & & 45.47 & \begin{tabular}{l|l|l}
7 & 0.75778 \\
\end{tabular} & 45.0 & 11.5 & & & & -0.002 & & -1.777 & \\
\hline & $6 / 9 / 2004$ & 2:03:26 PM & 21.358 & 24.689 & \begin{tabular}{|l|}
31.743 \\
\end{tabular} & 25.598 & 24.465 & 3.047 & & & $\begin{array}{l}-1.622 \\
\end{array}$ & 38.401 & & 46.99 & & -0.009 & & & & \begin{tabular}{|l|l|}
44.7 \\
\end{tabular} & 11.5 & & & & & & & \\
\hline 291 & & & 21.429 & & 31.739 & 25.604 & & 3.32 & $\begin{array}{l}46.384 \\
\end{array}$ & 4.825 & & 38.213 & & \begin{tabular}{|l|l|l|}
47.105 \\
\end{tabular} & 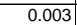 & $\begin{array}{l}-0.013 \\
\end{array}$ & & & 76333 & & & & & 002 & 0.002 & & $\frac{627}{6.627}$ & \\
\hline 292 & & & & 24.709 & & 2501 & 24.41 & 3.08 & 257 & & -1.577 & & & 46.896 & & & & & & & & & & & & & & \\
\hline 293 & 12004 & SPM & 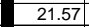 & 24.694 & 31.719 & 25.589 & & 3.237 & .533 & & $\begin{array}{l}-1.407 \\
\end{array}$ & & & & & & & & & & & & & & & & & \\
\hline 294 & 12004 & 2:04:06 PM & 21.621 & 24.699 & \begin{tabular}{|l|}
31.714 \\
\end{tabular} & 25.543 & 24.436 & 3.474 & 46.829 & 866 & $\mid-1.245$ & 38.236 & & |47.073 & & -0.01 & & & 77167 & 45.4 & & & & 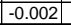 & -0.002 & & .446 & \\
\hline 295 & $6 / 9 / 2004$ & 2:04:16 PM & 21.682 & 24.7 & 31.685 & 25.529 & 24.442 & 3.215 & 46.5 & 4.838 & -1.51 & 38.3 & 18.45 & 47.036 & $\begin{array}{l}0.003 \\
\end{array}$ & -0.011 & & 4.47 & 70.77444 & 45.0 & 11.5 & & & .00 & -0.001 & & & \\
\hline & $6 / 9 / 2004$ & 2:04:26 PM & $\frac{21.722}{12.151}$ & 24.715 & 31.72 & 25.534 & 24.472 & 3.281 & 46.523 & 4.864 & $\begin{array}{r}-1.45 \\
\end{array}$ & 38.236 & & 46.984 & & -0.01 & & 5.63 & $\begin{array}{ll}3 & 0.77722 \\
\end{array}$ & & & & & & & & & \\
\hline$\frac{297}{298}$ & 699/2004 & & $21.751 \mid$ & 24.694 & & 25.498 & 24.446 & 3.161 & 46.1333 & 4.806 & -1.503 & 38.19 & & 46.994 & & & & & 0.78 & 44.7 & & & & & & & & \\
\hline 2999 & & 20.044 & $\frac{2.1 .92}{21921}$ & & & $\frac{25.444}{25.859}$ & 24.462 & $\frac{3.434}{3.307}$ & 46.483 & 4.803 & -1.146 & $\frac{38.118}{307}$ & & 47.017 & & & & & 0.18278 & & & & & & & & & \\
\hline$\frac{250}{300}$ & & $2: 04: 54$ & 21.822 & 2.0.699 & $\frac{13.65}{31595}$ & $\begin{array}{l}25.458 \\
25.442\end{array}$ & 24.450 & $\frac{3.021}{3144}$ & $\begin{array}{l}46.1442 \\
16.191\end{array}$ & $\begin{array}{l}4.846 \\
4797\end{array}$ & -1.017 & \begin{tabular}{|c|c|c|}
38.187 \\
38.184
\end{tabular} & & $\begin{array}{ll}47.013 \\
47.136\end{array}$ & & 0.013 & & & 85833 & & & & & & & & & \\
\hline & $6 / 9 / 2004$ & $2: 055: 1$ & $\frac{21.001}{21.901}$ & $\frac{24.094}{24.704}$ & 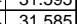 & 25.463 & $\frac{24.45}{24.525}$ & $\begin{array}{l}3.144 \\
3.329\end{array}$ & 539 & & $\begin{array}{l}-1.491 \\
-1.384\end{array}$ & & & & & -0.01 & & & (1)035 & & & & & & & & & \\
\hline & 6/9/2004 & 2:05:26 PM & 21.926 & 24.693 & 31.599 & 25.452 & 24.53 & 3.322 & 46.388 & 4.853 & -1.356 & $\mid 38.23$ & 86.38 & 46.998 & 0.003 & $\begin{array}{l}14.643 \\
\end{array}$ & & 47.63 & \begin{tabular}{|c|c|}
3 & 0.79389
\end{tabular} & 44.9 & 11.5 & & 0.068 & 2.183 & 1.820 & 1.852 & N\#\# & \\
\hline & & 5:36 PM & 21.972 & 24.694 & 31.605 & 25.438 & 24.58 & 3.524 & 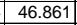 & 4.872 & -1.157 & 38.227 & 86.273 & \begin{tabular}{|l|}
46.952 \\
\end{tabular} & 0.003 & 14.643 & & 47.80 & $\begin{array}{ll}0 & 0.79667\end{array}$ & \begin{tabular}{|l|l|l|l|}
45.4 \\
\end{tabular} & 11.5 & & 0.082 & 2.183 & & & & \\
\hline 304 & 6/9/2004 & 2:05:4 & & & 31.568 & 25.431 & $24.53 \mathrm{G}$ & -3.746 & & & & & & & & 14.643 & & & 79944 & & & & & & & & mm!n & \\
\hline & 6/9/2004 & $2: 05: 5$ & 22.894 & 24.697 & 31.538 & & 24.60 & 3.171 & $\begin{array}{l}46.077 \\
\end{array}$ & \begin{tabular}{|l|l|}
4.763 \\
\end{tabular} & & 38.091 & & 46.961 & 303 & 14.643 & & & & & & & & & .823 & & & \\
\hline & & & & & & & & & \begin{tabular}{|l|l|l|l|}
46.467 \\
\end{tabular} & & & & & \begin{tabular}{|l|}
48.437 \\
\end{tabular} & & & & & 0.805 & & & & & & & & & \\
\hline 307 & $6 / 9 / 2004$ & 2:06:16 PM & 21.441 & 24.706 & 31.532 & \begin{tabular}{|l|}
25.435 \\
\end{tabular} & 24.543 & 20.024 & \begin{tabular}{|l|}
45.777 \\
\end{tabular} & $\begin{array}{l}4.500 \\
4.874 \\
\end{array}$ & \begin{tabular}{|c|c|}
15.623 \\
\end{tabular} & 20.376 & 0.70 & $\mid 48.56$ & 1.205 & $\begin{array}{l}14.040 \\
14.643\end{array}$ & & 48.47 & \begin{tabular}{|l|l|l|}
7 & 0.80778 \\
\end{tabular} & 44.3 & $\begin{array}{ll}11.5 \\
11.9\end{array}$ & & $.22 \quad$ & & $\frac{1.024}{1.824}$ & & 0 & \\
\hline 308 & $6 / 9 / 2004$ & 2:06:26 PM & 21.029 & 24.686 & \begin{tabular}{|l|l|}
31.458 \\
\end{tabular} & 25.4 & 24.513 & 32.436 & 44.987 & 4.885 & 28.849 & \begin{tabular}{|l|}
6.338 \\
\end{tabular} & 1.2 & \begin{tabular}{|l|}
49.887 \\
\end{tabular} & & 4.655 & & 48.63 & 0.81056 & 43.5 & 12.2 & 30.6 & 2.113 & 0.694 & 0.581 & & 18.957 & \\
\hline & $6 / 9 / 2004$ & 2:06:36 PM & 20.793 & 24.707 & 31.469 & 25.421 & 24.52 & 32.465 & 45.403 & 4.938 & 28.841 & \begin{tabular}{|l|}
6.709 \\
\end{tabular} & 1.296 & \begin{tabular}{|l|}
49.86 \\
\end{tabular} & & 3.562 & & & \begin{tabular}{c|c|c|c|}
0 & 0.81333 \\
\end{tabular} & 43.9 & 12.2 & & 2.11 & & 0.444 & & 14.496 & \\
\hline & & $2: 06: 4$ & & \begin{tabular}{|l|l|}
24.707 \\
2.717
\end{tabular} & 31.444 & & & 32.367 & 45.153 & & & $\begin{array}{l}6.683 \\
\end{array}$ & & \begin{tabular}{|l|l|l|}
50.183 \\
\end{tabular} & & 3.536 & & & & 43.7 & & & & & & & & \\
\hline 311 & 6/9/2004 & 2:06:5 & 20.572 & 24.717 & 31.504 & 25.491 & 24.579 & 32.647 & & 4.914 & . & 6.399 & & \begin{tabular}{|l|l|}
49.925 \\
\end{tabular} & & 3.503 & & 49.13 & & 43.8 & & & & & & & 14.142 & \\
\hline 312 & & $2: 07: 06 \mathrm{P}$ & 20.522 & \begin{tabular}{|l|l|}
24.718 \\
\end{tabular} & $\begin{array}{l}31.44 \\
11.4\end{array}$ & 25.517 & 24.585 & 33.149 & \begin{tabular}{|l|l|}
45.631 \\
\end{tabular} & 4.947 & 9.481 & $\begin{array}{ll}6.2222 \\
\end{array}$ & & \begin{tabular}{|l|l|}
49.827 \\
\end{tabular} & 03 & 3.472 & & 49.30 & \begin{tabular}{l|l}
0 & 0.82167 \\
\end{tabular} & 44.2 & 12.2 & 31.3 & 2.159 & 0.518 & & & 13.842 & \\
\hline$\frac{313}{314}$ & $\frac{6 / 9 / 2004}{66 / 1 / 2004}$ & $2: 07: 16$ PM & $\frac{20.472}{20.46}$ & $\begin{array}{l}24.709 \\
24.724 \\
\end{array}$ & \begin{tabular}{|l|}
31.381 \\
\end{tabular} & $\begin{array}{l}25.538 \\
25.584\end{array}$ & $\begin{array}{l}24.625 \\
24631\end{array}$ & $\begin{array}{l}32.965 \\
34.176\end{array}$ & \begin{tabular}{|l|}
45.192 \\
46.156 \\
\end{tabular} & $\begin{array}{r}4.933 \\
4.564 \\
\end{array}$ & $\begin{array}{l}29.301 \\
30.979\end{array}$ & \begin{tabular}{|l|l|}
5.976 \\
5704
\end{tabular} & $\frac{1.29}{1.279}$ & \begin{tabular}{|l|}
49.812 \\
49.747 \\
\end{tabular} & $\begin{array}{l}0.003 \\
0.03\end{array}$ & $\begin{array}{l}3.439 \\
3.396\end{array}$ & & \begin{tabular}{|l|l|l|}
49.47 \\
49.63
\end{tabular} & \begin{tabular}{ll|}
7 & 0.82444 \\
3 & 0.82722
\end{tabular} & \begin{tabular}{|l|l|}
43.7 \\
44.7
\end{tabular} & $\frac{12.2}{12.2}$ & 31. & $\frac{2.147}{2.246}$ & \begin{tabular}{|l|l|}
0.513 \\
0.506
\end{tabular} & $\begin{array}{l}0.430 \\
0.424\end{array}$ & & \begin{tabular}{|c|c|}
13.813 \\
133014
\end{tabular} & $\frac{11.75}{11.08}$ \\
\hline 315 & $\frac{\mid 6 / 9 / 2004}{6 / 92004}$ & $\begin{array}{l}\frac{207: 26 \mathrm{PM}}{2: 07: 36 \mathrm{PM}} \\
\end{array}$ & $\frac{20.466}{20.413}$ & 24.724 & \begin{tabular}{|l|l|}
31.442 \\
31.362 \\
\end{tabular} & $\begin{array}{l}25.5584 \\
25.589\end{array}$ & 24.626 & & \begin{tabular}{|l|}
48.156 \\
48.421 \\
\end{tabular} & $\begin{array}{l}4.564 \\
3.526 \\
\end{array}$ & 30.979 & $\begin{array}{l}5.104 \\
7.227\end{array}$ & & \begin{tabular}{|l|l|}
$41.318 !$ \\
418
\end{tabular} & & $\begin{array}{l}3.3 \\
3.6\end{array}$ & & & \begin{tabular}{|l|l|}
0 & $0.8272 \angle$ \\
\end{tabular} & $\begin{array}{l}44.7 \\
47.0\end{array}$ & $\begin{array}{l}12.2 \\
10.1\end{array}$ & & & \begin{tabular}{|l|}
0.506 \\
0.542
\end{tabular} & & & \begin{tabular}{|l|l|l|l|}
13.014 \\
13366
\end{tabular} & $\frac{11.08}{1140}$ \\
\hline 316 & $6 / 9 / 2004$ & 2:07:46 PM & 20.399 & 24.721 & 31.349 & 25.595 & 24.628 & 39.164 & \begin{tabular}{|l|}
55.386 \\
\end{tabular} & 3.942 & 36.937 & 9.812 & 1.4 & \begin{tabular}{|}
44.08 \\
4.10 \\
\end{tabular} & 0.004 & & & & $\begin{array}{r}0.83278 \\
0.8328\end{array}$ & 53.9 & 10.8 & & & & $\begin{array}{l}0.4501 \\
0.501 \\
\end{array}$ & & & $\frac{11.40}{11.20}$ \\
\hline 317 & $6 / 9 / 2$ & 2:07:5 & 20.4 & 24.727 & 31.3 & 25.611 & & 38.722 & 54.646 & 3.875 & 36.535 & 9.743 & & \begin{tabular}{|l|l|}
44.105 \\
\end{tabular} & 0.004 & 3.989 & & & \begin{tabular}{|l|}
0.83556 \\
\end{tabular} & 53.2 & 10.8 & & & & & & & $\begin{array}{ll}11.20 \\
11.31\end{array}$ \\
\hline & 6/9/2004 & & & & 31.345 & 25.616 & 24.644 & 39.348 & & & & $\begin{array}{l}9.462 \\
\end{array}$ & 1.4 & & & & & & & 53.8 & & & & & 0.4 & & & \\
\hline 319 & & & 20.381 & 24.718 & 31.331 & 25.622 & 24. & \begin{tabular}{|l|l|l}
41.194 \\
\end{tabular} & \begin{tabular}{|l|l|} 
\\
\end{tabular} & 4.07 & 38.8 & & & & & & & 50. & & 56.7 & & & & & & & & \\
\hline & & & & & & 25.658 & & 41.551 & 58.389 & & & & & 44. & & & & & & & & & & & & & & \\
\hline & & & & 24.719 & 31.317 & 25.663 & & 41.692 & 58.08 & 4.042 & 39.276 & 10.038 & & & & & & & .84611 & 566 & $\begin{array}{ll}11.0 \\
\end{array}$ & & & & & & & \\
\hline & $6 / 9 / 2004$ & 2:08: & 20.383 & 24.736 & 31.363 & 25.71 & 24.6 & 442.163 & \begin{tabular}{|l|l|} 
\\
\end{tabular} & & & 9.633 & & 45.0 & & & & & 8.85 & 56. & 11. & & & & & & & \\
\hline & $6 / 9 / 2004$ & 2:09: & 20.353 & & \begin{tabular}{|l|l}
31.398 \\
\end{tabular} & & & 43.668 & 58.198 & & & & & 43.5 & & & & & & & & & & & & & & \\
\hline 324 & 6/9/2004 & 2:10:4 & 20.318 & 24.6955 & 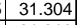 & 25.7955 & 24.7 & 43.027 & 54.677 & 4.095 & 40.161 & 5.638 & & 45.106 & & 3.3 & & 53 & 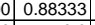 & 53.2 & 11.1 & & & & & & 10.198 & \\
\hline & & & 20.268 & & & & & & & & & & & & & & & & & & & & & & & & & \\
\hline$\frac{32}{20}$ & & & 20.18 & $24.6 / 8$ & 31.168 & 25.843 & & 42.499 & & & & & & & & & & & & & & & & & & & & \\
\hline 328 & & $2: 13: 4$ & $\frac{20.093}{20.011}$ & $\begin{array}{l}\frac{2}{2.46062} \\
24651\end{array}$ & $\begin{array}{l}31.067 \\
30.962\end{array}$ & $\frac{25.841}{25.831}$ & & $\frac{42.53}{42.12}$ & $\begin{array}{l}49.854 \\
48.838\end{array}$ & 4.272 & $\begin{array}{l}39.145 \\
38714 \\
\end{array}$ & $\frac{1.715}{0.829}$ & & $\frac{15}{18}$ & & 25 & & & 0.95 & 40.44 & 110 & & & & & & E76 & \\
\hline & $6 / 6 / 9 / 20$ & & 19.904 & & & 25.9 & & & & & & & & 45.4 & & & & & & & & & & & & & & \\
\hline & & $2: 16: 4$ & 19.81 & & & & & & & & & & & & & & & & 0.98333 & & & & & & & & & \\
\hline 33 & & 2:17: & 19.69 & 597 & & 25.972 & & 42.8 & & & & & & 45.5 & & & & & & & 11.2 & & & & & & & \\
\hline & & & & & & & & & & & & & & & & & & & & & & & & & & & & \\
\hline 333 & & & 19.50 & & & & & & & & & & & & & & & & & & & & & & & & & \\
\hline & & & 19.413 & 24.542 & & 25.987 & & 41.903 & & 4.355 & & & 1. & & & & & & & & 11.2 & & 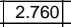 & & & & & \\
\hline & 6/9/200 & 2:21:4 & 19.296 & 24.507] & & 26.0 & & & 46.5 & $\begin{array}{r}4.4 \\
\end{array}$ & 37.9 & & & 45.4 & & & & 64 & & & 11.1 & & & & & & & \\
\hline & $6 / 9 / 2004$ & 2:22:4 & $19.199^{6}$ & 24.487 & & & & 41.876 & & 4.355 & 38.0 & -1.2 & & 45.4 & & 2.04 & & 65 & 1.0833 & & 11 & & & & & & 6.654 & \\
\hline & & & & & & & & 42. & & & & & & 45.6 & & & & & & & & & & & & & & \\
\hline & & & 18.986 & 24.444 & & & & 42.385 & & 4.3. & 38.595 & -1.22 & & 45.0 & & & & & 1.11667 & 45.3 & 11.0 & & & & & & & \\
\hline & & & & & & & & & & & & & & & & & & & & & & & & & & & & \\
\hline & & & & & & 20.0 & & $\frac{42.4}{420}$ & & & & & & 40.14 & & & & & & & & & & & & & & \\
\hline & $6 / 9 / 2004$ & $\frac{2.67 \cdot 48 \mathrm{PM}}{2 \cdot 28.0}$ & 18.6 & 24,384 & 429.148 & 220.095 & & 4 & 45.87 & 4.274 & 38.234 & \begin{tabular}{|l|l|}
$\mid-1.212$ \\
\end{tabular} & & $\frac{14.4}{44}$ & & & & 77.0 & of 1.18333 & $\begin{array}{r}44.0 \\
44.4\end{array}$ & 110 & & 2.7 & & 0.2 & & & \\
\hline & $6 / 9 / 2004$ & 2:29:4 & 18.541 & 24.384 & $\begin{array}{l}429.088 \\
\end{array}$ & 26.12 & & \begin{tabular}{|l|l|l|}
42.231 \\
\end{tabular} & \begin{tabular}{|l|l|}
46.301 \\
\end{tabular} & \begin{tabular}{|l|l|}
4.294 \\
\end{tabular} & 38.3 & -1.2 & & 4 & & & & 72 & \begin{tabular}{l|l}
0 & 1.2
\end{tabular} & $\sqrt{44} \quad 2$ & 11.0 & & & & $\overline{0.2}$ & & $\begin{array}{l}6.245 \\
\end{array}$ & \\
\hline & & & 18.4 & & & & & 42. & & 4.294 & & & & 447. & & & & & 21 & & & & & & & & & \\
\hline 345 & & & 18.373 & 24.388 & 28.968 & 26.168 & & 42.469 & 46.367 & 4.3 & & -1.2 & & $44.7 \mathrm{r}$ & & & & 74 & 1.23333 & & 11.0 & & & & & & 6.138 & \\
\hline 346 & & & 18.29 & & & & & 42. & & & & & & & & & & & & & & & & & & & & \\
\hline$\frac{3 x}{2}$ & & & 18.22 & 24.386 & & 26.242 & & 42.68 & 46.58 & & & & & 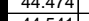 & & & & & 20007 & & & & & & & & & \\
\hline 势 & & $2.34 .46 \mathrm{PM}$ & 18.1489 & 24.395 & $\frac{28.150}{2002}$ & 2 & & 42.710 & $\begin{array}{r}46.49 \\
6.192\end{array}$ & & & $|-1.209|$ & & 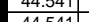 & & 1.86 & & & $\mid 1.283333$ & 43.0 & 100 & & & & & & & \\
\hline $\begin{array}{l}349 \\
350\end{array}$ & $\frac{6 / 9 / 2004}{6 / 9 / 2004}$ & $\begin{array}{l}2: 33: 58 \mathrm{PM} \\
2: 36: 48 \mathrm{PM}\end{array}$ & $\frac{18.083}{18.032}$ & $\begin{array}{l}24.405 \\
24.421\end{array}$ & $\begin{array}{l}528.682 \\
1128.587\end{array}$ & \begin{tabular}{|l|}
26.2414 \\
26.266 \\
\end{tabular} & 24.959 & $\frac{42.413}{42.316}$ & \begin{tabular}{|l|l|}
46.143 \\
54.812
\end{tabular} & $\begin{array}{r}.333 \\
4.208 \\
\end{array}$ & 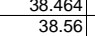 & \begin{tabular}{|c|}
-1.212 \\
-1.212 \\
\end{tabular} & 1.079 & $\mid$ & \begin{tabular}{|l|l|}
0.003 \\
\end{tabular} & 1.816 & & $\begin{array}{c}\frac{78.00}{79.00} \\
\end{array}$ & \begin{tabular}{l|r|}
0 & 1.31667 \\
0
\end{tabular} & 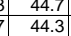 & $\begin{array}{l}10.9 \\
30.9\end{array}$ & 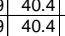 & \begin{tabular}{|l|}
2.788 \\
\end{tabular} & \begin{tabular}{|l|}
0.273 \\
0.271 \\
\end{tabular} & \begin{tabular}{|l|}
0.246 \\
0.245
\end{tabular} & \begin{tabular}{|c|}
0.006 \\
\end{tabular} & \begin{tabular}{|l|l|}
6.060 \\
\end{tabular} & 5.1 \\
\hline
\end{tabular}


WSRC-TR-2005-00105, REVISION 0

SRNL-RPP-2005-00012, REVISION 0

PRE-TEST ACID CLEAN WITH 2 M HNO3, FOLLOWING PUMP REPAIR - CONT.

\begin{tabular}{|c|c|c|c|c|c|c|c|c|c|c|c|c|c|c|c|c|c|c|c|c|c|c|c|c|c|c|c|c|}
\hline & A & & $D_{1}$ & $E$ & $F$ & $G_{G}$ & $\mathrm{H}$ & & $\mathrm{K}$ & 1 & $M$ & $N$ & & 0 & & & & & & & & & & & & & & \\
\hline & 6/9/2004 & $2: 37: 48 \mathrm{PM}$ & 17.945 & 24.429 & 28.521 & 26.285 & 24.967 & 42.944 & $\begin{array}{l}4.548 \\
44.548\end{array}$ & 4.269 & $\frac{M}{39.141}$ & \begin{tabular}{|l|l|}
1 & -1.215 \\
\end{tabular} & 1.08 & $\frac{Q}{44.41}$ & $\frac{R}{0.003}$ & $\begin{array}{ll} \\
1.814\end{array}$ & $\frac{T}{0.006}$ & $\frac{V}{80.00}$ & $\begin{array}{l}w \\
0.3333 \\
\end{array}$ & $\begin{array}{l}45.1 \\
4\end{array}$ & $\frac{Y}{10.9}$ & & AA & $\frac{A B}{0.270}$ & $\frac{\mathrm{AC}}{0.245}$ & $\frac{A D}{0.006}$ & $\begin{array}{l}\mathrm{AE} \\
5.975 \\
\end{array}$ & \begin{tabular}{l|l}
$A \mathrm{~A}$ \\
5.08
\end{tabular} \\
\hline 352 & $6 / 9 / 2004$ & 2:38:48 PM & 17.929 & 24.439 & 28.431 & 26.334 & 24.997 & 42.302 & $\begin{array}{l}2.5 .828 \\
\end{array}$ & 4.388 & 38.374 & \begin{tabular}{|l|l|l|}
4 & -1.215 \\
\end{tabular} & & 45.534 & 0.003 & 1.786 & 0.004 & 81.00 & & 44.4 & 11.2 & & 2.781 & 0.266 & 0.242 & & & \\
\hline & 6/9/2004 & 2:39:48 PM & 17.883 & 24.448 & 28.286 & 26.294 & 25.031 & 42.447 & 75.988 & 4.424 & 38.449 & \begin{tabular}{|l|l|}
9 & -1.215 \\
\end{tabular} & & 45.492 & 0.003 & 1.779 & 0.005 & 82.00 & $\begin{array}{ll}01.36667 \\
\end{array}$ & 44.5 & 11.1 & & & & 0.242 & & & 5.09 \\
\hline 354 & & $2: 40: 48 \mathrm{PM}$ & 17.847 & 24.468 & 28.166 & 26.353 & 25.011 & 42.453 & $\begin{array}{l}35.745 \\
\end{array}$ & 4.43 & 38.482 & \begin{tabular}{|l|l|}
2 & -1.200 \\
\end{tabular} & & 45.344 & 0.003 & 1.771 & 0.004 & 83.00 & $\begin{array}{ll} & 1.38333 \\
\end{array}$ & 44.3 & $\begin{array}{ll}11.1 \\
11.1 \\
\end{array}$ & & 2.790 & 0.264 & 0.242 & 0.006 & 5.974 & 5.08 \\
\hline 355 & 6/9/2004 & $2: 41: 48 \mathrm{PM}$ & & & 27.996 & 26.308 & 25.055 & & \begin{tabular}{|l|l|}
45.644 \\
\end{tabular} & 4.388 & 38.386 & & & 45.444 & & 1.768 & & 84.00 & & 44.2 & 11.1 & & & & & & & \\
\hline 356 & $\begin{array}{l}6 / 9 / 2004 \\
6 / 2 / 204\end{array}$ & \begin{tabular}{|l|}
$2: 42: 48$ PM \\
\end{tabular} & $\begin{array}{l}17.77 \\
17.752\end{array}$ & 24.486 & $27.865 \mid$ & $\begin{array}{l}26.297 \\
2020\end{array}$ & 25.034 & & |46.233 & 4.423 & $\begin{array}{l}38.687 \\
2.67\end{array}$ & $\begin{array}{l}7.1 .215 \\
7.210\end{array}$ & & \begin{tabular}{|l|l|l|l}
45.486 \\
5.936
\end{tabular} & & $\begin{array}{l}1.765 \\
\end{array}$ & & 85.00 & \begin{tabular}{|l|l|}
0 & 1.41667 \\
\end{tabular} & 44.8 & 11.1 & & 2.805 & \begin{tabular}{|l|l|}
0.263 \\
\end{tabular} & $\begin{array}{ll}0.243 \\
\end{array}$ & 0.006 & 5.971 & 5.08 \\
\hline 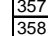 & 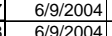 & \begin{tabular}{|l} 
2:43:48 PM \\
$2444.48 \mathrm{PM}$
\end{tabular} & $\begin{array}{l}17.753 \\
17.73 \\
\end{array}$ & $\begin{array}{l}24.489 \\
24.497\end{array}$ & \begin{tabular}{|l|}
27.709 \\
27.542 \\
\end{tabular} & \begin{tabular}{|l|l|}
26.335 \\
26398
\end{tabular} & \begin{tabular}{|l|}
25.037 \\
25.035 \\
\end{tabular} & \begin{tabular}{|l|}
42.393 \\
42.469 \\
\end{tabular} & \begin{tabular}{|l|l|}
3 & 45.712 \\
9 & 45.688 \\
\end{tabular} & $\begin{array}{l}4.394 \\
4.401 \\
\end{array}$ & \begin{tabular}{|c|}
38.421 \\
38.49
\end{tabular} & $\begin{array}{l}1 \\
\end{array}$ & $\begin{array}{l}1.082 \\
1082\end{array}$ & \begin{tabular}{|l|}
45.361 \\
45.355 \\
\end{tabular} & $\begin{array}{l}0.003 \\
0.003\end{array}$ & $\begin{array}{l}1.762 \\
1761\end{array}$ & 0.005 & $\begin{array}{l}86.00 \\
8700\end{array}$ & $\begin{array}{ll}0 & 1.43333 \\
\end{array}$ & 44.2 & 11.1 & & \begin{tabular}{|l|l|}
2.786 \\
2791
\end{tabular} & \begin{tabular}{|l|}
0.263 \\
0263
\end{tabular} & $\begin{array}{l}0.244 \\
0.255\end{array}$ & 0.006 & $\begin{array}{ll}6.028 \\
6042\end{array}$ & 5.13 \\
\hline 359 & 6/9/2004 & $\frac{2.44 .40 \mathrm{PM}}{2: 45: 48 \mathrm{PM}}$ & 17.699 & 24.516 & & $\frac{20.090}{26.391}$ & \begin{tabular}{|l|}
25.035 \\
25.049
\end{tabular} & $\frac{-42.469}{42.61}$ & \begin{tabular}{|l|l|}
145.6889 \\
14.889
\end{tabular} & $\begin{array}{l}4.401 \\
.361\end{array}$ & \begin{tabular}{|c|}
38.496 \\
38.696
\end{tabular} & 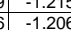 & $\frac{1.082}{1.088}$ & $\begin{array}{r}45.355 \\
45.15 \\
\end{array}$ & $\begin{array}{l}0.003 \\
0.003 \\
\end{array}$ & $\frac{1.761}{1.76}$ & $\begin{array}{l}0.006 \\
0.005 \\
\end{array}$ & 878.00 & \begin{tabular}{|c|} 
\\
0
\end{tabular} & - $\frac{44.2}{44.4}$ & $\frac{11.1}{11.1}$ & & & $\frac{0.263}{0.262}$ & $\frac{0.245}{0.245}$ & $\frac{0.006}{0.006}$ & $\frac{6.042}{6.031}$ & $\frac{5.14}{5.13}$ \\
\hline & $6 / 9 / 2004$ & & & & \begin{tabular}{|l|l|}
3 & 27.38 \\
\end{tabular} & & 25.048 & & 246.061 & 4.428 & & & & 45.159 & 0.003 & 1.756 & & & \begin{tabular}{|l|l|}
0 & 1.48333 \\
\end{tabular} & 44.6 & $\begin{array}{ll}11.1 \\
11.1\end{array}$ & & & & & & & $\frac{5.13}{5.11}$ \\
\hline 361 & $6 / 9 / 2004$ & 2:47:48 PM & 17.665 & 24.523 & 27.289 & 26.328 & \begin{tabular}{|l|}
25.031 \\
\end{tabular} & 42.579 & 95.822 & 4.333 & \begin{tabular}{|l|l|}
38.663 \\
\end{tabular} & \begin{tabular}{|l|l|}
3 & -1.215 \\
\end{tabular} & & 45.123 & 0.003 & 1.757 & & 90.00 & $\begin{array}{ll}0 & 1.5 \\
\end{array}$ & 44.4 & 11.1 & & 2.801 & 0.262 & 0.246 & 0.006 & 6.049 & 5.15 \\
\hline 362 & $6 / 9 / 2004$ & 2:48:48 PM & 17.634 & 24.547 & \begin{tabular}{|l|} 
\\
\end{tabular} & 26.337 & 25.044 & $\begin{array}{l}42.747 \\
\end{array}$ & & & & & & 45.11 & & $\begin{array}{l}1.754 \\
\end{array}$ & 0.004 & 91.00 & \begin{tabular}{|l|l|l}
0 & 1.51667 \\
\end{tabular} & 44.6 & 11.1 & & & 0262 & & & & $\begin{array}{l}\frac{5.15}{5.12} \\
\end{array}$ \\
\hline 363 & 6/9/2004 & & & & & & & & & & & & & & & & & & & & & & & & & & & $\frac{5.12}{5.15}$ \\
\hline 364 & $6 / 9 / 2004$ & 2:50:48 PM & 17.586 & 24.555 & \begin{tabular}{|l|l|}
27.176 \\
\end{tabular} & 26.315 & 25. & 42.658 & 45.81 & & & -1.212 & & $\begin{array}{l}45.058 \\
\end{array}$ & & 1.746 & & 93.00 & 1.55 & 44.3 & $\frac{11.0}{11.0}$ & & .80 & .260 & 0.245 & 0.006 & $\frac{021}{021}$ & 5.12 \\
\hline & $6 / 9 / 2004$ & 2:51:48 PM & 17.569 & 24.553 & 27.099 & 26.329 & 25.0 & $\begin{array}{l}42.806 \\
\end{array}$ & 45.965 & & & -1.21 & & 45.154 & & 1.746 & & & 1.56667 & 44.5 & & & & .260 & 0.245 & & & \\
\hline & $6 / 9 / 2004$ & 2:52:48 PM & 17.553 & 24.567 & 27.118 & 26.312 & 25.045 & $\begin{array}{l}42.731 \\
\end{array}$ & 145.855 & & 38.883 & -1.215 & & 44.954 & 0.003 & 1.746 & & 95.00 & \begin{tabular}{|l|l}
0 & 1.58333 \\
\end{tabular} & 44.4 & $\frac{11.0}{11.0}$ & & 2.81 & 0.260 & 0.245 & 0.006 & 6.012 & 5.12 \\
\hline & 6/9/2004 & 2:53:48 PM & 17.521 & 24.58 & 27.096 & 26.29 & 25.068 & 42.708 & 46.019 & 4.328 & 38.802 & -1.215 & & 44.965 & 003 & 1.747 & & 96.00 & & 44.5 & & & 2.810 & 0.260 & 0.246 & 0.006 & & 5.13 \\
\hline & 6/9/2004 & 2:54:48 PM & 17.534 & 24.583 & \begin{tabular}{|l|l|} 
& 27.094 \\
\end{tabular} & 26.319 & 25.031 & 42.741 & 45.945 & 4.36 & 38.817 & -1.218 & & 44.96 & .003 & 1.746 & & 97.0 & 1.61667 & 44.5 & & & 181 & .260 & 0.246 & & & 5.12 \\
\hline & 6/9/2004 & & & 24.586 & \begin{tabular}{|l|l|} 
& 27.092 \\
\end{tabular} & 26.331 & & 42.57: & 45.611 & 4.327 & & -1.212 & & 44.9355 & & 1.75 & & & 1.6333 & 44. & & & & & & & & \\
\hline$\frac{330}{371}$ & $6 / 9120044$ & $2: 56: 4$ & $\begin{array}{l}1.481 \\
1.491\end{array}$ & 24.591 & $\mid \begin{array}{l}\mid 27.042 \\
\end{array}$ & 26.386 & & & 46.015 & & & -1.2 .2 & & 45.353 & & 1.736 & & & 1.65 & & & & & 259 & 0.244 & & 0.022 & .12 \\
\hline & 0 & $2.57 .45 \mathrm{PM}$ & 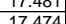 & 24.601 & \begin{tabular}{|l|l|}
27.082 \\
127055
\end{tabular} & 26.347 & & 42.223 & 45.424 & & 38.254 & $\frac{-1.2}{12}$ & & & & & & & 1.66667 & & & & & & 0.242 & & & 5.11 \\
\hline & $\begin{array}{l}6 / 9 / 9004 \\
6 / 204\end{array}$ & 2.50 .45 & $\frac{11.474}{1744}$ & $\begin{aligned} 24.004 \\
246\end{aligned}$ & 27061 & 26351 & 250 & $\begin{array}{l}42.454 \\
42113\end{array}$ & $\begin{array}{l}4 \\
3 \\
3\end{array}$ & & 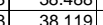 & $\frac{-1.214}{-1.212}$ & & 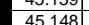 & & & & 10200 & 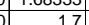 & 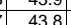 & & & & & $\frac{0.241}{0241}$ & 1006 & $\frac{3.301}{6003}$ & $\frac{5.08}{5.11}$ \\
\hline 374 & $6 / 9 / 2004$ & 3:00:48 PM & $\begin{array}{l}17.424 \\
17.422\end{array}$ & $\begin{array}{r}24.603 \\
\end{array}$ & 327.049 & 26.388 & 24.96 & & 45.553 & & 38.445 & -1.215 & & 45.05 & & 1.707 & & & 1716.67 & $\frac{74}{44}$ & 1.0 & & 78 & 254 & 240 & 1000 & 5.945 & $\frac{5.11}{5.06}$ \\
\hline 375 & $6 / 9 / 2004$ & 3:01:48 PM & 17.408 & 24.604 & $\begin{array}{l}27.025 \\
\end{array}$ & 26.344 & 25.002 & 42.451 & 45.64 & 4.408 & 38.497 & -1.212 & & 45.131 & 0.003 & 1.707 & & 104.06 & 1.73333 & 44.2 & & & 2.79 & 0.254 & 0.240 & & & .06 \\
\hline & $6 / 9 / 2004$ & 3:02:48 PM & 17.4 & 24.606 & & & & & \begin{tabular}{|l}
45.59 \\
\end{tabular} & & 38.39 & -1.215 & & & & 1.71 & & & 1.75 & 44.11 & & & & & $0.0+0$ & & 964 & \\
\hline & & 03.4 & 17.388 & 24.614 & 27.1 & 26.374 & & & 45.721 & & & -1.20 & & 992 & & & & & .76667 & & & & & & 0.240 & & & \\
\hline & 6/9/2004 & 3:04:4 & 17.379 & 24.61 & 27.106 & 26.356 & & 42.4 & 45.449 & & & -1.21 & & & & & & & 1.78333 & & 11. & & & & & & & \\
\hline & 6/9/2004 & 3:05:4 & 17.36 & & 27.098 & \begin{tabular}{l|l|l|}
26.377 \\
\end{tabular} & & 42. & $7 \quad 45.681$ & & & & & & & & & & & & & & & & & & & SO \\
\hline & $6 / 9 / 2004$ & $3: 06: 4$ & 17.341 & 24.608 & 27.119 & $\begin{array}{ll}26.363 \\
\end{array}$ & 24.97 & $\begin{array}{l}42.416 \\
\end{array}$ & \begin{tabular}{|l|l|}
5 & 45.46 \\
\end{tabular} & & 38.499 & -1.21 & & 45.117 & & 1.705 & & & 1.81667 & & $\overline{111.1}$ & & & & & & & \\
\hline & 6/9/2004 & 3:07:4 & & 24.599 & 27.125 & 26.32 & 25. & 42.37 & 45.372 & 4.366 & 38.443 & -1.215 & & 45 & & 1.706 & & 110 & 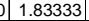 & 43. & & & 78 & & 0.240 & & & 505 \\
\hline$\sqrt{38}$ & 6/9/2004 & 3:08:48 PM & 17.334 & 24.606 & \begin{tabular}{|l|l|} 
& 27.157 \\
\end{tabular} & 26.296 & 24.959 & 42.436 & 45.518 & 4.379 & 38.482 & -1.212 & & 44.971 & 0.003 & 1.705 & & 111.00 & 1.85 & 44.0 & 1.0 & 4 & 2.790 & 0.254 & 0.239 & & & \\
\hline 89 & 1/2004 & $\begin{array}{l}3: 09: 4 \\
2: 0\end{array}$ & $\frac{17.33}{17211}$ & & 27.198 & 26.332 & 24. & 42.3 & 40.012 & 4.36 & $\begin{array}{l}38.445 \\
3.501\end{array}$ & & & 44.967 & & & & 190 & 1.86667 & & 100 & & $\sqrt{7}+2$ & $n=1$ & , & & 5.917 & \\
\hline & 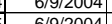 & & & & & & & & & & & & & & & & & & & & & & & & & & & \\
\hline & $\frac{0}{619 / 20040}$ & & $\frac{1.2 .290}{17207}$ & & 27.24 & 26.3380 & & & 45.36 & & & & & & & & & & & & $\frac{11.6}{11.6}$ & & 17 & 254 & 0.239 & & & .05 \\
\hline & $\begin{array}{l}6 / 9 / 9004 \\
6 / 204\end{array}$ & & 17283 & $\frac{24}{24}$ & 27.25 & 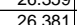 & & & 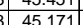 & 4. & 38.5566 & $\frac{-1.2 .2}{-12}$ & & & & & & & $\begin{array}{l}1.91067 \\
1.9232\end{array}$ & & $\frac{11 .}{11 .}$ & & & & 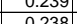 & & $\frac{5.900}{5.945}$ & \\
\hline & $6 / 9 / 2004$ & 3:14:4 & 17264 & 24.566 & 27.257 & 26.312 & 24.9 & 42 & 45.154 & 4.388 & 38.18 & -12 & & & & & & $\frac{x_{1}}{11}$ & 1.95 & & 1.1. & & & & 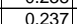 & & & \\
\hline 389 & $6 / 9 / 2004$ & 3:15: & 17.268 & 24.585 & 5 & 26.331 & 24.9 & & 45.422 & 4.36 & & -1.21 & & & & & & & 1.96667 & & & & & 254 & 238 & & & \\
\hline & $6 / 5$ & & & & & & & & 45.3 & & & & & & & & & & & & 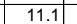 & & & & & & & \\
\hline & $\mid 1 / 2004$ & 3:1 & 17.253 & 24.561 & \begin{tabular}{|l|}
27.257 \\
\end{tabular} & 26.287 & 24.8 & & 45.787 & & 38.46 & $-\frac{1.2}{-1.2}$ & & 45.142 & & 1.7 & & 120.00 & 2 & 44 & 11. & 40 & $2.7 \mathrm{G}$ & 253 & 0.238 & & 5.880 & .00 \\
\hline & 6/9/2004 & & 17.23 & & 27.231 & & & & 45.3 & & & & & & & & & & 01667 & & $\overline{11}$ & & & & 0.238 & & 5.906 & \\
\hline & $6 / 9 / 2 / 2$ & & 17.237 & & & & & & & & & -1.2 & & & & & & & \begin{tabular}{|l|l|}
.03333 \\
\end{tabular} & & & 74 & & & 0.238 & & & \\
\hline & 6/9/2004 & & 17.237 & & \begin{tabular}{|l|l|}
27.286 \\
\end{tabular} & & & & 45 & & & -1.2 & & & & & & & 2.05 & & $110 \mathrm{C}$ & 44 & & & 0.238 & & 5.885 & \\
\hline & 6/9/2004 & & 17.241 & & & & & & 294 & & & -1.2 & & & & & & & 2.06 & & & & & & & & & \\
\hline & $6 / 9 / 22$ & 3:22:4 & 17.22 & & 27.239 & & & & 5. 4 & & & -1.2 & & & & & & 12 & \begin{tabular}{|l|l|} 
\\
\end{tabular} .08333 & & $x .0$ & & & & 0.239 & & 5.938 & \\
\hline 年 & $6 / 9 / 2004$ & & 17.229 & & 27.253 & 26.233 & 24 & & 45.345 & & & & & & & & & & 2.1 & & 11.6 & & & 200 & 0.205 & & r. & \\
\hline & & & & & & & & & & & & & & & & & & & & & & & & & & & & \\
\hline & $\frac{0}{619 / 20040}$ & & $\frac{1.24}{1.22}$ & & 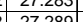 & & & & (45.40 & & & -1.2 & & & & & & & $\frac{2.15333}{215}$ & & & & & & 0.2 & & 50.924 & \\
\hline & 6/1/212 & & 17.23 & & & & & & & & & & & & & & & & & & & & & & 0.4 & & S.910 & \\
\hline 402 & 6/9/2004 & & 17.23 & & & & & & & & & -1.2 & & & & & & & 218333 & & & & & & & & & \\
\hline & $6 / 9 / 2004$ & $3: 29: 4$ & 17.244 & & $\mid$ & $\frac{26.1}{20.1}$ & 24 & & $\begin{array}{ll}9 & 45 . \\
\end{array}$ & & & \begin{tabular}{|l|l|}
5 & -1.215 \\
\end{tabular} & & & & & & & 10.2. & & 11. & & & & 0.240 & & $\frac{5.980}{5.980}$ & \\
\hline 404 & $6 / 9 / 2$ & & & & & & & & & & & & & & & & & & & & & & & & & & & \\
\hline & $6 / 9 / 2 / 2$ & & 17.235 & & $|27.264|$ & 26.223 & & & 45 & & & -1.2 & & & & & & & .23333 & & & & & $\sqrt{0^{2}}+2$ & 0.244 & & 5.941 & \\
\hline & $6 / 5$ & & 17.2 & 24. & 27.24 & & 24. & & $4 !$ & & & -1.2 & & & & & & & & & & & & & & & & \\
\hline & $6 / 9 / 2$ & & $17.23 \mathrm{C}$ & & $\begin{array}{ll}3 & 27.2\end{array}$ & & & & & & & -1.2 & & & & & & & 2.26667 & & & & & & & & & \\
\hline & $6 / 9 / 20$ & & 17.23 & & & & 24. & & & & & -1.2 & & & & & & & 2.28333 & & 11.0 & & & & & & 5.969 & \\
\hline & 6/9/2004 & & 17.22 & & 27.248 & & 24 & & & 4 & & -1.2. & & & & & & & 2.3 & & & & & & 41 & & 5.977 & \\
\hline & $6 / 9 / 2 \mid$ & & 17.22 & & & & 24. & & & 4.3 & & -1.21 & & & & & & & 2.31667 & & & & & & & & & \\
\hline & & & & & & & & & & & & & & & & & & & & & & & & & & & & \\
\hline & & & 17. & & & & & & & & & & & & & & & & & & & & & & & & & \\
\hline & & & $\frac{1.1 .24}{1070}$ & 24.341 & $\frac{2.325}{27207}$ & & 24 & & 45.2 & & & $-\frac{1.215}{121}$ & & & & & & & $\frac{2.36667}{232023}$ & & & & & & & & & \\
\hline & 6 & & 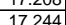 & $\frac{24}{24}$ & & & $\frac{24 .}{24}$ & & $\frac{43.16}{45.1}$ & & & $\frac{-1.24}{121}$ & & & & & & & 2 & & & & & & & & (a) & \\
\hline & $6 / 9 / 2004$ & & 172 & & & & & & 45 & 4 & & -1.21 & & & & & & & 41667 & & & & & & & & & \\
\hline & 6/9/2004 & & $\frac{17.235}{17.235}$ & & 27289[ & & & & 45.169 & & & -1215 & & & & & & & & & & & & & & & & \\
\hline & $6 / 9 / 2004$ & & & & $27.299 \mid$ & & & & & & & & & & & & & & & & & & & & & & & \\
\hline & & & 17.22 & & & & & & & & & -1 & & & & & & & & & & & & & & & & \\
\hline & $6 / 9 / 2004$ & $3: 46: 48 \mathrm{PM}$ & & 24.324 & & & & & & 4.312 & & -1.215 & & & & $\frac{729}{729}$ & & & & 33.7 & & & 2.167 & 0.25 & 0.242 & & & \\
\hline
\end{tabular}


WSRC-TR-2005-00105, REVISION 0 SRNL-RPP-2005-00012, REVISION 0

PRE-TEST ACID CLEAN WITH 2 M HNO3, FOLLOWING PUMP REPAIR - CONT.

\begin{tabular}{|c|c|c|c|c|c|c|c|c|c|c|c|c|c|c|c|c|c|c|c|c|c|c|c|c|c|c|c|c|}
\hline & A & B & D & $E$ & $\mathrm{~F}$ & G & \begin{tabular}{ll|}
$\mathrm{H}$ \\
\end{tabular} & $\mathrm{J}$ & $\mathrm{K}$ & $\mathrm{L}$ & $\mathrm{M}$ & $\mathrm{N}$ & 0 & Q & $R$ & $\mathrm{~s}$ & $\mathrm{~T}$ & $\mathrm{v}$ & w & $x$ & $Y$ & $z$ & AA & $A B$ & AC & $A D$ & $\mathrm{AE}$ & AF \\
\hline 421 & $6 / 9 / 2004$ & $3: 47: 48$ PM & 17.244 & 24.336 & \begin{tabular}{|l|}
27.323 \\
\end{tabular} & 26.372 & 24.865 & \begin{tabular}{|l|l|}
42.131 \\
\end{tabular} & 45.331 & 4.351 & 38.191 & -1.209 & 1.143 & 44.998 & 0.003 & 1.728 & 0.006 & 150.6 & 2.5 & 43.9 & 11.0 & 40.2 & 2.769 & 0.258 & 0.241 & 0.006 & 6.012 & \\
\hline \begin{tabular}{|l|l|}
422 \\
\end{tabular} & $6 / 9 / 2004$ & 3:48:48 PM & $\frac{17.226}{17.226}$ & 24.354 & \begin{tabular}{|l|}
27.325 \\
\end{tabular} & 26.32 & 24.812 & 42.131 & 45.343 & 4.31 & 38.281 & $\frac{-1.212}{-1.212}$ & 1.14 & 44.842 & 0.003 & 1.729 & 0.005 & 151.6 & & 43.9 & 11.0 & & & 0.258 & 0.242 & 0.006 & & 5.11 \\
\hline 423 & $6 / 9 / 2004$ & $3: 49: 48 \mathrm{PM}$ & $\frac{17.234}{1724}$ & $\frac{24.361}{2.361}$ & \begin{tabular}{|l|}
27.337 \\
2731 \\
\end{tabular} & 26.362 & 24.9 & \begin{tabular}{|c|}
42.059 \\
\end{tabular} & 45.375 & 4.328 & \begin{tabular}{|c|}
38.158 \\
3807
\end{tabular} & $\frac{-1.212}{1.212}$ & 1.146 & $\begin{array}{l}44.841 \\
4.921\end{array}$ & $\begin{array}{l}0.003 \\
\end{array}$ & 1.733 & & $\begin{array}{l}152.00 \\
15.00\end{array}$ & \begin{tabular}{|r|}
2.53333 \\
255
\end{tabular} & 43.9 & 11.0 & 40.1 & $\begin{array}{l}2.765 \\
2.762\end{array}$ & & 0.242 & 0.006 & 6.035 & 5.14 \\
\hline \begin{tabular}{|l|l|}
424 \\
255
\end{tabular} & $6 / 9 / 2004$ & $\begin{array}{ll}3: 50: 48 \mathrm{PM} \\
3.51: 19 \mathrm{P}\end{array}$ & $\begin{array}{l}17.24 \\
17221\end{array}$ & 24.347 & \begin{tabular}{|l|}
27.313 \\
27213 \\
\end{tabular} & $\begin{array}{l}26.318 \\
26395\end{array}$ & $\begin{array}{l}24.68 \\
2312\end{array}$ & \begin{tabular}{|r|}
42.08 \\
42180
\end{tabular} & $\begin{array}{l}45.354 \\
45.209\end{array}$ & \begin{tabular}{|l|l|}
4.377 \\
.324
\end{tabular} & $\begin{array}{l}38.07 \\
383\end{array}$ & \begin{tabular}{|l|l|}
-1.212 \\
1.215
\end{tabular} & $\begin{array}{l}1.143 \\
1.34\end{array}$ & \begin{tabular}{|l|l|}
44.921 \\
4946
\end{tabular} & $\begin{array}{l}0.003 \\
\end{array}$ & $\begin{array}{l}1.728 \\
\end{array}$ & 0.005 & 153.00 & $\begin{array}{r}2.55 \\
\end{array}$ & $\begin{array}{l}43.9 \\
\end{array}$ & \begin{tabular}{ll|}
11.0 \\
\end{tabular} & 40.1 & $\begin{array}{l}2.763 \\
2775\end{array}$ & \begin{tabular}{|l|l|}
0.258 \\
\end{tabular} & $\begin{array}{l}0.242 \\
\end{array}$ & 0.006 & $\begin{array}{l}6.026 \\
\end{array}$ & 5.13 \\
\hline 每25 & $6 / 9 / 2004$ & $\begin{array}{l}3: 51: 48 \mathrm{PM} \\
33: 52.48 \mathrm{PM}\end{array}$ & $\begin{array}{l}17.212 \\
17249\end{array}$ & $\frac{24.349}{24.371}$ & & $\begin{array}{l}26.395 \\
26.427\end{array}$ & $\begin{array}{r}23.12 \\
23.53 \\
\end{array}$ & \begin{tabular}{|c|}
42.189 \\
42.49
\end{tabular} & \begin{tabular}{|l|l|}
45.298 \\
4564
\end{tabular} & \begin{tabular}{|l|l|}
4.334 \\
4.342
\end{tabular} & $\begin{array}{r}38.3 \\
38.587 \\
\end{array}$ & \begin{tabular}{r|r|}
-1.215 \\
-1.215
\end{tabular} & $\begin{array}{l}1.143 \\
11143\end{array}$ & \begin{tabular}{|l|r|}
44.946 \\
44844
\end{tabular} & $\begin{array}{l}0.003 \\
0.03\end{array}$ & $\begin{array}{l}1.734 \\
1737\end{array}$ & & $\begin{array}{l}154.00 \\
11500\end{array}$ & & 43.8 & & & $\begin{array}{l}2.775 \\
2795 \\
\end{array}$ & & $\frac{0.242}{0.243}$ & & & 5.12 \\
\hline $\begin{array}{l}4427 \\
427 \\
\end{array}$ & $\frac{6 / 9 / 2004}{6 / 9 / 2004}$ & $\begin{array}{l}3: 52: 48 \mathrm{PM} \\
33: 53: 48 \mathrm{PM}\end{array}$ & 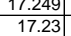 & $\begin{array}{l}24.371 \\
24.362\end{array}$ & 27.347 & $\begin{array}{l}26.427 \\
26.398\end{array}$ & $\begin{array}{l}23.533 \\
23.795\end{array}$ & \begin{tabular}{|}
42.49 \\
42.237
\end{tabular} & $\begin{array}{l}45.6944 \\
45.505\end{array}$ & $\begin{array}{r}4.342 \\
4.331 \\
\end{array}$ & $\begin{array}{l}38.587 \\
38.339\end{array}$ & $\left|\begin{array}{|c|}\mid-1.25 \\
-1.212\end{array}\right|$ & $\begin{array}{l}1.1 .143 \\
1.466\end{array}$ & \begin{tabular}{|l|l|}
44.844 \\
44.837
\end{tabular} & $\begin{array}{l}0.003 \\
0.003\end{array}$ & $\begin{array}{l}1.737 \\
1.737\end{array}$ & 0.004 & $\begin{array}{l}\frac{155.00}{156.00} \\
150\end{array}$ & $\begin{array}{r}\mid \begin{array}{r}2.58333 \\
2.6\end{array} \\
\end{array}$ & $\begin{array}{l}44.2 \\
44.0\end{array}$ & & & \begin{tabular}{|l|l|}
2.7775 \\
2.778
\end{tabular} & \begin{tabular}{|l|l|}
0.259 \\
0.259 \\
\end{tabular} & $\mid \begin{array}{l}0.243 \\
0.243\end{array}$ & $\frac{0.006}{0.006}$ & $\begin{array}{l}5.983 \\
6.024 \\
\end{array}$ & $\frac{5.09}{5.13}$ \\
\hline | & $6 / 9 / 2004$ & 3:54:48 PM & 17.242 & 24.379 & 27.37 & 26.46 & 24.017 & \begin{tabular}{|l|l|}
42.142 \\
\end{tabular} & 45.321 & 4.294 & 38.306 & \begin{tabular}{|l|}
-1.212 \\
\end{tabular} & $\begin{array}{l}1.140 \\
1.152\end{array}$ & 44.896 & 0.003 & 1.735 & 0.004 & $\begin{array}{l}150.00 \\
157.00\end{array}$ & \begin{tabular}{|r|}
2.61667 \\
\end{tabular} & $\begin{array}{ll}44.0 \\
43.8\end{array}$ & 11.0 & \begin{tabular}{|l|}
40.5 \\
40.2
\end{tabular} & 2.773 & \begin{tabular}{|l|}
0.259 \\
0.259
\end{tabular} & & $\begin{array}{l}0.006 \\
0.006 \\
\end{array}$ & $\begin{array}{l}6.024 \\
6.019 \\
\end{array}$ & $\frac{5.13}{5.12}$ \\
\hline (429. & $6 / 9 / 2004$ & 3:55:48 PM & 17.238 & 24.376 & \begin{tabular}{|l|}
27.352 \\
\end{tabular} & 26.502 & 24.349 & \begin{tabular}{|l|l|}
42.357 \\
\end{tabular} & \begin{tabular}{|l|l}
45.603 \\
\end{tabular} & 4.354 & \begin{tabular}{|l|l|l|}
38.454 \\
\end{tabular} & \begin{tabular}{|l|}
-1.215 \\
\end{tabular} & 1.149 & $\begin{array}{l}44.835 \\
\end{array}$ & & $\begin{array}{l}1.733 \\
\end{array}$ & & 158.00 & \begin{tabular}{|l|l|}
2.63333 \\
\end{tabular} & 44.1 & 11.0 & 40.4 & \begin{tabular}{|l|}
2.786 \\
\end{tabular} & 0.258 & 0.242 & 0.006 & 5.988 & \\
\hline 430 & $6 / 9 / 2004$ & 3:56:48 PM & 17.225 & 24.387 & \begin{tabular}{|l|} 
\\
\end{tabular} & 26.508 & 24.696 & \begin{tabular}{|l|l|}
42.113 \\
\end{tabular} & $\begin{array}{l}45.341 \\
\end{array}$ & 4.275 & $\begin{array}{l}38.296 \\
\end{array}$ & & 1.152 & $\begin{array}{l}44.912 \\
\end{array}$ & $\begin{array}{l}0.003 \\
\end{array}$ & \begin{tabular}{|l|l|}
1.737 \\
\end{tabular} & & $\begin{array}{l}159.00 \\
\end{array}$ & \begin{tabular}{|l|}
2.65 \\
\end{tabular} & \begin{tabular}{|l|l|}
43.9 \\
\end{tabular} & 11.0 & 40.2 & \begin{tabular}{|l|}
2.772 \\
\end{tabular} & $\begin{array}{ll}0.259 \\
\end{array}$ & & & & \\
\hline \begin{tabular}{|l|l|}
431 \\
\end{tabular} & 6/9/2004 & 3:57:48 PM & 17.233 & 24.39 & & \begin{tabular}{|l}
26.531 \\
\end{tabular} & 25.039 & 42.088 & & 4.32 & & -1.215 & & 44.879 & 0.003 & & & & 6.66667 & 43.8 & & & 2.769 & \begin{tabular}{|l|l|l|} 
& 0.259 \\
\end{tabular} & 0.242 & & 6.035 & 5.14 \\
\hline & 6/9/2004 & 3:58:48 PM & & 24.403 & 27.384 & & & \begin{tabular}{|l|}
42.14 \\
\end{tabular} & 45.327 & 4.379 & 38.213 & & 1.154 & 44.818 & & 1.739 & & & & & & & & & & & & \\
\hline & & 3:59:48 PM & & & & & 25.204 & \begin{tabular}{|l|l|}
42.389 \\
\end{tabular} & 45.783 & 4.399 & 38.394 & -1.212 & & 44.864 & 0.0 & 1.739 & & 162.00 & & 44.3 & 11.0 & 40.4 & 2.785 & \begin{tabular}{|l|}
0.259 \\
\end{tabular} & 0.242 & & 6.003 & \\
\hline 434 & $6 / 9 / 2004$ & 4:00:48 PM & 17.235 & 24.423 & 27.389 & 26.614 & 25.186 & 42.243 & 45.52 & \begin{tabular}{|l|l|}
4.35 \\
\end{tabular} & & -1.215 & 1.15 & 44.869 & 0.0 & 1.741 & & & \begin{tabular}{|l|l|}
2.71667 \\
\end{tabular} & 44.0 & 11.0 & & & & & & 6.028 & 5.13 \\
\hline \begin{tabular}{|l|l|}
435 \\
\end{tabular} & $6 / 9 / 2004$ & 4:01:48 PM & 17.21 & 24.418 & \begin{tabular}{|l|l|} 
\\
\end{tabular} & 26.559 & 25.231 & \begin{tabular}{|l|l|}
42.308 \\
\end{tabular} & 45.619 & 4.36 & 38.386 & \begin{tabular}{|l|}
-1.212 \\
\end{tabular} & 1.16 & 44.904 & & 1.74 & & 164.00 & 2.73333 & 44.1 & 11.0 & 40.3 & 2.782 & 0.259 & 0.243 & & 6.016 & 5.12 \\
\hline \begin{tabular}{|l|l|}
436 \\
\end{tabular} & $6 / 9 / 2004$ & 4:02:48 PM & & 24.436 & \begin{tabular}{|l|}
27.402 \\
\end{tabular} & & 25.229 & & \begin{tabular}{|l|l|}
45.418 \\
5.58
\end{tabular} & & & \begin{tabular}{|l|l|}
-1.215 \\
\end{tabular} & 1.157 & 44.875 & & 1.74 & & & & 43.9 & 11.0 & & & & 0.243 & & $\begin{array}{ll}6.031 \\
\end{array}$ & \\
\hline 437 & $6 / 9 / 2004$ & 4:03:48 PM & 17.231 & 24.468 & \begin{tabular}{|l|}
27.404 \\
\end{tabular} & 26.649 & & $\begin{array}{l}42.299 \\
\end{array}$ & & $\begin{array}{l}4.368 \\
\end{array}$ & & $\begin{array}{l}-1.209 \\
\end{array}$ & 1.166 & \begin{tabular}{|l|l|l|}
44.891 \\
\end{tabular} & $\begin{array}{l}0.003 \\
\end{array}$ & $\begin{array}{l}1.747 \\
\end{array}$ & & 166.00 & & 44.2 & 11.0 & 40.3 & 2.779 & 0.260 & 0.244 & & 6.042 & 5.14 \\
\hline 438 & & 4:04:48 PM & 17.198 & 24.491 & \begin{tabular}{|l|}
27.387 \\
\end{tabular} & 26.652 & 23.813 & $\begin{array}{l}42.163 \\
\end{array}$ & 45.457 & & 38.298 & -1.215 & 1.16 & 44.75 & 0.003 & 1.746 & (0) & 167.00 & 2.78333 & 44.0 & 11.0 & 40.2 & \begin{tabular}{|l|l|}
2.774 \\
\end{tabular} & 0.260 & 0.244 & & 6.053 & 5.15 \\
\hline 439| & 6/9/2004 & 4:05:48 PM & \begin{tabular}{|c|}
17.21 \\
17201
\end{tabular} & 24.478 & \begin{tabular}{|l|}
27.374 \\
27.273
\end{tabular} & $\begin{array}{l}26.714 \\
26707\end{array}$ & 23.931 & $\begin{array}{l}42.256 \\
2.01\end{array}$ & \begin{tabular}{|l|l|}
45.607 \\
\end{tabular} & 4.338 & $\begin{array}{r}38.341 \\
28.16 \\
\end{array}$ & \begin{tabular}{|l|}
-1.215 \\
\end{tabular} & 1.163 & \begin{tabular}{|l|l|}
44.892 \\
$14.72 ?$
\end{tabular} & 0.003 & 1.744 & 0.004 & 168.00 & \begin{tabular}{|r|}
2.8 \\
\end{tabular} & 44.1 & 11.0 & 40.3 & \begin{tabular}{|l|l|}
2.778 \\
2.766
\end{tabular} & 0.260 & 0.243 & 0.006 & 6.038 & 5.14 \\
\hline \begin{tabular}{|l|}
440 \\
441 \\
\end{tabular} & $6 / 6 / 2004$ & $\begin{array}{l}4: 06: 48 \mathrm{PM} \\
\text { 4:07:48 PM }\end{array}$ & $\frac{17.214}{17206}$ & 24.481 & \begin{tabular}{|l|}
27.373 \\
27405 \\
\end{tabular} & $\begin{array}{l}26.707 \\
26705\end{array}$ & $\begin{array}{l}24.169 \\
22.472\end{array}$ & \begin{tabular}{|l|l|}
42.084 \\
42.161
\end{tabular} & \begin{tabular}{|l|}
45.433 \\
45426 \\
\end{tabular} & \begin{tabular}{r|r|}
4.328 \\
4367
\end{tabular} & $\begin{array}{r}38.16 \\
38.259 \\
\end{array}$ & \begin{tabular}{|c|}
-1.215 \\
-1.212 \\
\end{tabular} & & \begin{tabular}{|r|}
44.723 \\
44.9 \\
\end{tabular} & $\begin{array}{l}0.003 \\
0.03 \\
\end{array}$ & $\begin{array}{l}1.743 \\
1.75 \\
\end{array}$ & $\begin{array}{l}0.004 \\
0.005\end{array}$ & & \begin{tabular}{|l|l|}
2.81667 \\
283333 \\
\end{tabular} & $\begin{array}{ll}44.0 \\
44.0\end{array}$ & \begin{tabular}{l|l|l|l|l|}
11.0 \\
11.0
\end{tabular} & & \begin{tabular}{|l|l|}
2.766 \\
2.772
\end{tabular} & \begin{tabular}{|l|l|}
0.260 \\
0260
\end{tabular} & $\begin{array}{l}0.243 \\
0.223 \\
\end{array}$ & 0.006 & $\begin{array}{ll}6.062 \\
6.050 \\
\end{array}$ & $\begin{array}{l}5.16 \\
5.15 \\
\end{array}$ \\
\hline $\mid \frac{441}{442}$ & $\frac{6 / 9 / 2004}{6 / 9 / 2004}$ & $\begin{array}{l}\text { 4:06:48 PM } \\
\text { 4:08:48 PM }\end{array}$ & $\frac{\mid \frac{1.206}{17.213}}{4}$ & 24.481 & \begin{tabular}{|l|}
27.4006 \\
19306 \\
\end{tabular} & $\begin{array}{l}26.105 \\
26.666\end{array}$ & 24.5242 & $\begin{array}{l}42.161 \\
42.154\end{array}$ & $\begin{array}{l}45.366 \\
45.364 \\
\end{array}$ & $\begin{array}{l}4.366 \\
4.279 \\
\end{array}$ & $\begin{array}{ll}38.259 \\
38.324\end{array}$ & \begin{tabular}{|l|}
$\mid-1.212$ \\
-1.215 \\
\end{tabular} & $\begin{array}{l}1.1 .66 \\
1.169\end{array}$ & $\begin{array}{r}44.91 \\
44\end{array}$ & $\begin{array}{l}0.003 \\
0.003 \\
\end{array}$ & $\begin{array}{r}\mid .7 .75 \\
1.75 \\
\end{array}$ & $\begin{array}{ll}0.005 \\
0.004\end{array}$ & $\frac{110.00}{171.00}$ & \begin{tabular}{|l|l|}
2.833333 \\
2.85 \\
\end{tabular} & $\begin{array}{l}44.0 \\
43.9 \\
\end{array}$ & $\frac{11.0}{11.0}$ & $\frac{40.2}{40.2}$ & 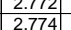 & \begin{tabular}{|l|}
0.260 \\
0.261 \\
\end{tabular} & $\frac{0.243}{0.307}$ & $\begin{array}{l}0.006 \\
0.008 \\
\end{array}$ & $\frac{6.050}{7.635}$ & $\frac{5.15}{6.50}$ \\
\hline 443 & $6 / 9 / 2004$ & 4:09:48 PM & 17.266 & 24.479 & 18.966 & 26.604 & 25.127 & $\begin{array}{ll}42.565 \\
\end{array}$ & 46.03 & 4.368 & 38.614 & \begin{tabular}{|l|}
-1.215 \\
\end{tabular} & 1.169 & 44.929 & 0.003 & 1.757 & 0.005 & 172.00 & 2.86667 & 44.6 & 11.0 & 40.6 & 2.799 & \begin{tabular}{|l|}
0.262 \\
\end{tabular} & 0.312 & 0.008 & 7.676 & 6.53 \\
\hline \multirow{2}{*}{\multicolumn{29}{|c|}{$\mid$}} \\
\hline & & & & & & & & & & & & & & & & & & & & & & & & & & & & \\
\hline \begin{tabular}{|l|}
447 \\
\end{tabular} & & Averages & 20.4 & 24.6 & 30.8 & 25.7 & & 37.0 & 45.5 & 4.4 & 32.9 & $\begin{array}{l}4.1 \\
\end{array}$ & & $\begin{array}{ll}46.3 \\
\end{array}$ & 03 & & & & & 44.1 & 113 & 350 & 24 & $\mid 0243$ & 0209 & & 17.4 & \\
\hline $\mid$ & & Maximum & 24.2 & 24.8 & & 26.7 & & 45.3 & 58.4 & 5.6 & 41.3 & 46.0 & $\begin{aligned} 1.4 \\
86.4 \\
\end{aligned}$ & 53.5 & 1.2 & 14.6 & & & & 56.9 & 13.1 & 43.3 & $\frac{2.4}{3.0}$ & \begin{tabular}{|l|}
2.2183 \\
\end{tabular} & 1.824 & 2.079 & 2079.0 & 1769.3 \\
\hline |449 & & Median & \begin{tabular}{|l|l|}
20.6 \\
\end{tabular} & 24.7 & 31.4 & 25.6 & 24 & \begin{tabular}{l|l}
42.1 \\
\end{tabular} & \begin{tabular}{|l|l|}
45.9 \\
\end{tabular} & \begin{tabular}{ll|l}
4.4 \\
\end{tabular} & 37.8 & -1.2 & 1 & 46.1 & $\begin{array}{ll} \\
\end{array}$ & 1.7 & & & & |44.4 & \begin{tabular}{|l|l|}
11.3 \\
\end{tabular} & 40.1 & 2.8 & \begin{tabular}{|l|l|l|l|} 
\\
\end{tabular} & \begin{tabular}{|c|}
0.178 \\
\end{tabular} & 0.004 & 4.5 & 3.8 \\
\hline 450 & & Minimum & 17.2 & 24.3 & 19.0 & 25.2 & 23.1 & $\begin{array}{r}-3.7 \\
\end{array}$ & 32.6 & 3.5 & -8.5 & -1.2 & -5 & 41.3 & 0.0 & 0.0 & & & & 31.1 & \begin{tabular}{|l|l|} 
& 10.1 \\
\end{tabular} & -6.1 & -0.4 & \begin{tabular}{|l|}
-0.002 \\
\end{tabular} & - & -0.299 & $\mid-298.8$ & \begin{tabular}{|c|c|} 
& -254.3 \\
\end{tabular} \\
\hline 451 & & $2 \times$ Std Dev & 4.249 & 0.231 & 4.832 & 0.829 & 0.430 & 17.949 & 8.873 & 0.604 & 18.191 & 18.578 & 18.21 & 3.465 & 0.924 & 11.824 & 0.11 & & & 8.873 & \begin{tabular}{|l|l|} 
\\
\end{tabular} & m\#! & 1.246 & \begin{tabular}{|l|l|l|l} 
\\
\end{tabular} & 0.430 & 0.308 & 307.734 & 4 4\#\#\#州 \\
\hline & Number & of Points Used ${ }^{*}$ & 427 & 427 & 427 & 427 & & 427 & 427 & 427 & 427 & 427] & & 427 & 427 & 4277 & & & & 427 & 427 & 427 & 427 & 427 & 427| & 427 & 427. & 27 \\
\hline 453] & & * Backpulse poir & $s$ in box a & are not inc & cluded & & & & & & & & & & & & & & & & & & & & & & & \\
\hline
\end{tabular}


WSRC-TR-2005-00105, REVISION 0

SRNL-RPP-2005-00012, REVISION 0

PRE-TEST ACID CLEAN WITH 2 M HNO3, FOLLOWING PUMP REPAIR - CONT.

\begin{tabular}{|c|c|c|c|c|c|c|c|c|c|c|c|c|c|c|c|c|c|c|c|c|c|c|c|c|c|c|c|c|}
\hline & & & & & & & & & & & & & & & & & & & & & & & & & & & & \\
\hline & A & B & D & $E$ & $F$ & G & $\mathrm{H}$ & $\mathrm{J}$ & $\mathrm{K}$ & $\mathrm{L}$ & M & $\mathrm{N}$ & 0 & $Q$ & $\mathrm{R}$ & $\mathrm{s}$ & $T$ & $\mathrm{v}$ & W & $x$ & $\mathrm{Y}$ & $\mathrm{z}$ & AA & $A B$ & $\mathrm{AC}$ & $A D$ & $\mathrm{AE}$ & AF \\
\hline & Acid Cleaa 2 & & & & & & & & & & & & & & & & & & & & & & & & & & & \\
\hline & & 7:20:45 AM & & 23.73 & 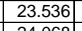 & & 23.514 & 37.648 & \begin{tabular}{|l|l|}
3 & 45.92 \\
\end{tabular} & 4.119 & \begin{tabular}{|l|l|}
34.376 \\
3790
\end{tabular} & & & & & & & & & 44.4 & 11. & & & & 0.447 & & & \\
\hline & $\frac{6 / 110 / 2004}{66 / 10204}$ & $\frac{21: 45 \mathrm{AM}}{22.45} \mathrm{AM}$ & $\frac{23.734}{24.335}$ & $\begin{array}{l}23.736 \\
23.736\end{array}$ & \begin{tabular}{|l|}
24.068 \\
24.799 \\
\end{tabular} & $\begin{array}{l}23.819 \\
23.808 \\
\end{array}$ & $\begin{array}{r}23.55 \\
23.635\end{array}$ & & \begin{tabular}{|l|}
50.809 \\
250.784
\end{tabular} & $\begin{array}{l}4.056 \\
4.207\end{array}$ & \begin{tabular}{|c|}
37.895 \\
30.706
\end{tabular} & \begin{tabular}{|c|}
3.848 \\
4.161
\end{tabular} & $\begin{array}{l}.073 \\
1.079\end{array}$ & $\begin{array}{l}45.601 \\
45.872\end{array}$ & $\begin{array}{l}0.003 \\
0.003\end{array}$ & $\begin{array}{l}3.102 \\
3.168\end{array}$ & $\begin{array}{l}0.006 \\
0.006\end{array}$ & & 8.0658 & $\begin{array}{l}49.3 \\
49.3 \\
\end{array}$ & $\frac{11 .}{11 .}$ & $\begin{array}{l}39.4 \\
38.9\end{array}$ & & 0.462 & $\begin{array}{l}0.475 \\
0.476\end{array}$ & $\frac{0.01}{0.01}$ & $\frac{12.044}{12.230}$ & $\frac{10.25}{10.41}$ \\
\hline$\frac{460}{461}$ & $\begin{array}{l}601 / 10 / 2044 \\
6 / 12004\end{array}$ & $\begin{array}{l}7: 222.45 \mathrm{AM} \\
7: 23: 45 \mathrm{Am}\end{array}$ & $\begin{array}{l}24.355 \\
24.797\end{array}$ & & \begin{tabular}{|l|}
24.099 \\
24.981 \\
\end{tabular} & 24.046 & $\begin{array}{l}3.335 \\
23.717 \\
\end{array}$ & $\begin{array}{l}40.5 / 2 \\
41.099 \\
\end{array}$ & \begin{tabular}{|l|l|}
50.184 \\
51.866
\end{tabular} & $\begin{array}{l}3.207 \\
.755\end{array}$ & $\begin{array}{r}38.306 \\
38.454 \\
\end{array}$ & & 1.079 & & 0.003 & $\begin{array}{l}3.168 \\
.294\end{array}$ & & & \begin{tabular}{|l|l|}
18.00292 \\
\end{tabular} & & & $\begin{array}{l}38.9 \\
39.8 \\
\end{array}$ & $\begin{array}{l}2.065 \\
2.742 \\
\end{array}$ & & $\begin{array}{l}.476 \\
0.491 \\
\end{array}$ & 0.012 & & \\
\hline & $6 / 10 / 2004$ & & & & & 24.434 & 23.818 & & & & 38.757 & & 1.102 & & 0.003 & & & & 58.1158 & & & & & & & 0.012 & & 10.40 \\
\hline 463 & $6 / 10 / 2004$ & 7:25:45 AM & 25.096 & 23.746 & \begin{tabular}{|l|}
25.005 \\
\end{tabular} & 24.637 & 23.986 & 41.544 & 452.241 & 3.701 & 38.952 & 4.818 & 1.099 & 43.361 & 0.003 & 3.284 & 0.004 & 87.95 & 18.1325 & 50.8 & 10.6 & 40.2 & 2.775 & 0.490 & 0.490 & 0.012 & 12.163 & 10.35 \\
\hline 464 & $6 / 10 / 2004$ & & 25.074 & 23.744 & \begin{tabular}{|l|}
24.902 \\
\end{tabular} & 24.774 & 24.128 & 41.404 & \begin{tabular}{|l|l|}
451.727 \\
\end{tabular} & 3.897 & 38.566 & 4.575 & 1.088 & 44.211 & 0.003 & 3.251 & 0.004 & 1088.95 & \begin{tabular}{|l|l|} 
& 18.1492 \\
\end{tabular} & 50.3 & 10.8 & & 2.757 & 0.485 & 0.486 & & 12.155 & 10.34 \\
\hline & & $27: 45 \mathrm{AM}$ & & & \begin{tabular}{|l|}
24.761 \\
\end{tabular} & 24.989 & 24.218 & 41.489 & \begin{tabular}{|l|l|}
52.517 \\
\end{tabular} & & 38.856 & 4.804 & & $\begin{array}{l}43.137 \\
\end{array}$ & & & & & \begin{tabular}{|l|l|}
18.1658 \\
\end{tabular} & 51.0 & 10.6 & & & 0.489 & 0.492 & & 12.248 & \\
\hline & $6 / 10 / 2004$ & $7: 28: 45 \mathrm{AM}$ & 24.869 & $\begin{array}{l}23.804 \\
2302\end{array}$ & 24.662 & $\begin{array}{r}25.06 \\
25.111\end{array}$ & 24.333 & $\begin{array}{l}41.708 \\
\end{array}$ & \begin{tabular}{|l|l|}
52.364 \\
51213
\end{tabular} & 3.688 & \begin{tabular}{|l|l|}
39.057 \\
3703
\end{tabular} & $\begin{array}{l}4.746 \\
\end{array}$ & $\begin{array}{l}1.099 \\
1071\end{array}$ & 43.033 & 0.003 & 3.259 & 0.004 & 90.95 & 18.1825 & 50.9 & 10.5 & 40.4 & 2.784 & \begin{tabular}{|l|l|}
0.486 \\
\end{tabular} & 0.491 & 0.012 & 12.147 & 10.34 \\
\hline $\begin{array}{ll}467 \\
468\end{array}$ & $6 / 10 / 2004$ & $\begin{array}{l}7: 29: 45 \mathrm{AM} \\
7 \cdot 30: 45 \mathrm{AM}\end{array}$ & & $\begin{array}{r}23.83 \\
23.866\end{array}$ & & $\begin{array}{l}25.111 \\
25.172\end{array}$ & $\begin{array}{l}24.404 \\
24481 \\
\end{array}$ & $\begin{array}{r}11.26 \\
4.905\end{array}$ & \begin{tabular}{|l|l|}
51.213 \\
552.032
\end{tabular} & \begin{tabular}{|l|l}
4.345 \\
3991
\end{tabular} & & & $\begin{array}{l}1.071 \\
1082\end{array}$ & $\begin{array}{l}46.982 \\
4499\end{array}$ & 0.003 & $\begin{array}{l}3.155 \\
3.95 \\
\end{array}$ & 0.005 & \begin{tabular}{|l|l|}
1091.95 \\
102059 \\
\end{tabular} & $\begin{array}{l}18.1992 \\
182159\end{array}$ & \begin{tabular}{|l|l|}
49.7 \\
506
\end{tabular} & 11. & & .730 & \begin{tabular}{|l|}
0.470 \\
\end{tabular} & \begin{tabular}{|l|l|l|}
0.477 \\
\end{tabular} & 0.012 & 12.050 & $\begin{array}{l}10.25 \\
1\end{array}$ \\
\hline$\frac{468}{469}$ & $6 / 10 / 2004$ & $7: 30: 45$ АM & 24.63 & 23.866 & 24.539 & 25.172 & 24.481 & 41.905 & 52.032 & 3.991 & 38.923 & & 1.082 & 44.99 & 0.003 & & 0.004 & \begin{tabular}{|l|l|}
1092.95 \\
\end{tabular} & 18.2158 & 50.6 & 11. & & 2.786 & 0.476 & 0.483 & 0.012 & 11.941 & 10.16 \\
\hline$\frac{469}{470}$ & 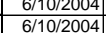 & $\begin{array}{l}\text { 7:31:45 AM } \\
\text { 7:32:45 A }\end{array}$ & $\begin{array}{l}24.602 \\
24.585 \\
\end{array}$ & $\frac{23.893}{23.936}$ & \begin{tabular}{|l|}
24.535 \\
24.48 \\
\end{tabular} & $\begin{array}{l}25.184 \\
25.292\end{array}$ & $\begin{aligned} 24.507 \\
24.55\end{aligned}$ & $\begin{array}{r}41.943 \\
42.301\end{array}$ & $\begin{array}{l}352.042 \\
1152.438\end{array}$ & $\begin{array}{r}3.948 \\
3.91 \\
\end{array}$ & $\begin{array}{r}38.96 \\
39.293\end{array}$ & $\begin{array}{r}4.219 \\
.187\end{array}$ & $\begin{array}{l}1.085 \\
1.079\end{array}$ & \begin{tabular}{|l|}
44.485 \\
44.468
\end{tabular} & \begin{tabular}{|l}
0.003 \\
0.003
\end{tabular} & $\begin{array}{l}3.192 \\
3.175\end{array}$ & $\frac{0.003}{0.004}$ & $\mid$\begin{tabular}{|l|}
$\mid 1093.95$ \\
1099.95
\end{tabular} & \begin{tabular}{|l|l|}
18.2325 \\
18.2492
\end{tabular} & \begin{tabular}{|c|}
50.6 \\
51.0
\end{tabular} & $\frac{10.9}{10.5}$ & $\frac{40.5}{40.8}$ & $\frac{2.789}{2813}$ & 0.476 & $\frac{0.482}{0.480}$ & $\frac{0.012}{0.012}$ & $\begin{array}{l}\frac{11.920}{11.768} \\
\end{array}$ & $\begin{array}{l}\frac{10.14}{10.02} \\
\end{array}$ \\
\hline & $6 / 10 / 2004$ & 7:33:45 AM & 24.577 & 23.973 & 24.52 & 25.299 & 24.607 & 42.237 & 52.393 & 3.962 & 39.274 & 4.176 & 1.079 & 44.387 & 0.003 & 3.16 & $\begin{array}{l}0.004 \\
0.004\end{array}$ & 1095.95 & \begin{tabular}{|l|l|}
18.2492 \\
18.2658
\end{tabular} & 50.9 & $\frac{10.9}{10.9}$ & t4.o.8 40.8 & $\frac{2.010}{2.810}$ & 0.471 & $\begin{array}{l}0.400 \\
0.478\end{array}$ & 0.012 & $\begin{array}{l}11.100 \\
11.717\end{array}$ & $\begin{array}{r}10.02 \\
9.97\end{array}$ \\
\hline & & & & & & & & & & & & & & & & & & & & & & & & & & & & \\
\hline & 6/10/2004 & & 24.558 & 24.044 & 24.531 & 25.435 & 24.673 & 42.368 & \begin{tabular}{|l|l|}
352.384 \\
\end{tabular} & 3.975 & 39.322 & 4.005 & 1.079 & 44.56 & 0.003 & $\frac{1.146}{3.146}$ & & & . & $\frac{20.9}{50.9}$ & 10. & 40 & 816 & 469 & 0.475 & 0.01 & 告.636 & $\begin{array}{l}9.91 \\
9.90\end{array}$ \\
\hline & $6 / 10 / 2004$ & & 24.54 & 24.077 & & & & & \begin{tabular}{|l|l|}
5 & 52.109
\end{tabular} & 3.947 & & 4.051 & & & & & & & & & & & & & & & & \\
\hline & 6/10/2004 & 7:37:45 AM & $\begin{array}{ll}24.538 \\
\end{array}$ & 24.11 & 24.476 & 25.406 & 24.613 & 42.405 & \begin{tabular}{|l|l|}
52.478 \\
\end{tabular} & 3.957 & \begin{tabular}{|l|l|}
39.437 \\
\end{tabular} & 3.999 & & & & & & & 3.3325 & & & & & 0.467 & 0.474 & & & \\
\hline 47 & 6/10/2004 & & 24.541 & 24.148 & 24.53 & & & 42.302 & \begin{tabular}{|l|l|} 
& 52.276 \\
\end{tabular} & 3.951 & & 4.048 & & 44.574 & & & & & & 50.8 & 10. & & & 0.467 & 0.473 & & $\begin{array}{l}11.607 \\
\end{array}$ & 9.88 \\
\hline & 6/110/2004 & 7:39:45 AM & 24.529 & 24.161 & 24.482 & 25.406 & 24.544 & 422.133 & 352.053 & 3.941 & 39.157 & 4.037 & 1.082 & 44.57 & 003 & 3.138 & & 101.95 & 18.3658 & 50.6 & 10. & & 802 & \begin{tabular}{|l|l|}
0.468 \\
\end{tabular} & 0.475 & .01 & 11.680 & 9.94 \\
\hline & 6/110/2004 & $7: 40: 45$ AM & 24.527 & 24.199 & 24.465 & 25.395 & 24.547 & 41.992 & \begin{tabular}{|l|l|l|} 
& 51.694 \\
\end{tabular} & 3.938 & 39.082 & 4.011 & 1.076 & 44.516 & 0.003 & 3.139 & & 02.95 & \begin{tabular}{|l|l|}
18.3825 \\
\end{tabular} & 50.2 & 10. & 40.5 & 2.795 & 0.468 & 0.475 & $\frac{5.0}{.0}$ & 11.720 & 9.97 \\
\hline & $6 / 11012004$ & $5 \mathrm{AM}$ & 24.514 & 24.221 & 24.452 & 25.422 & 24.539 & 42.254 & 4) 52.119 & 3.966 & 39.309 & 4 & 1.079 & 44.429 & .003 & & & & 3992 & & 10. & & & 0.468 & 0.476 & & & \\
\hline & $6 / 110 / 2004$ & & & & & & & & & & & & & & & & & & & & & & & & & & & \\
\hline & 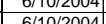 & & $\frac{24.509}{2.527}$ & $\frac{24.261}{2.205}$ & 20.437 & 25.401 & 24.529 & & 52.107 & 3.983 & 39.416 & 4.074 & & 44.071 & & & & & & & & & & & 0.476 & & 1.643 & 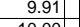 \\
\hline$\frac{753}{483}$ & $6 / 110 / 2004$ & & $\frac{24.527}{24.515}$ & $\frac{295}{313}$ & $\frac{24.400}{24.474}$ & & & & \begin{tabular}{|l|}
51.964 \\
51.953 \\
\end{tabular} & & 205 & $\begin{array}{l}4.009 \\
3.895 \\
\end{array}$ & & 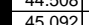 & & S.146 & & & & 50.5 & 11. & $4 \mathrm{C}$ & 势 & 4.409 & & & $\frac{11.154}{11625}$ & 89 \\
\hline & $6 / 10 / 2004$ & & 24.507 & 4.32 & 24.481 & 25.44 & 24.53 & $\frac{44}{42}$ & 51.644 & 4.047 & 38.923 & 3.915 & 1.091 & 45.121 & & & & & 18.4825 & No. & 11 11. & & 790 & \begin{tabular}{|l|l|}
0.466 \\
\end{tabular} & & & & 89 \\
\hline & 6/110/2004 & & 24.51 & 24.328 & \begin{tabular}{|l|}
24.478 \\
\end{tabular} & 25.453 & 24.53 & $\begin{array}{l}42.053 \\
\end{array}$ & \begin{tabular}{|l|l|}
351.974 \\
\end{tabular} & 4.013 & 38.989 & 3.976 & & 45.133 & 0.003 & $\begin{array}{l}3.13 \\
\end{array}$ & & & & $\overline{50.5}$ & & 40 & 794 & \begin{tabular}{|l|l|}
0.467 \\
\end{tabular} & 0.474 & & 6887 & \\
\hline & $6 / 10 / 2004$ & & & 24.34 & & & & & & & & & & & & & & & & & & & & & & & & \\
\hline & $6 / 10 / 2004$ & & 24.549 & 24.347 & \begin{tabular}{|l|}
24.528 \\
\end{tabular} & 25.477 & 24.62 & 42.31 & 52.355 & 4.079 & & \begin{tabular}{|l|l|}
4.022 \\
\end{tabular} & & $\frac{45.071}{45.07}$ & 0.003 & & & & & 50.9 & 11. & & .809 & 468 & $\overline{0.4}$ & & 629 & \\
\hline & & & & & & & & & & & & & & & & & & & & & & & & & & & & \\
\hline & 6/10/2004 & & 24.686 & 24.403 & \begin{tabular}{|l|}
24.649 \\
\end{tabular} & & 24.72 & \begin{tabular}{|l|l}
41.924 \\
\end{tabular} & 451.845 & & & 4.074 & & 45.038 & & & & & & & & & & & & & & \\
\hline & 6/10/2004 & & 24.734 & . 416 & \begin{tabular}{|l|}
24.722 \\
\end{tabular} & 25.561 & & & \begin{tabular}{l|l}
3 & 51.76
\end{tabular} & & 38.78 & 4.063 & & 44.981 & & & & & & 50.3 & 11 & 40 & & & & & 11.753 & \\
\hline & 6/110/2004 & & 24.786 & 24 & 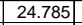 & 25.618 & 24.8 & & 51.835 & & 38.7 & 4.141 & & & & & & 5.9 & & & & & & & & & 11.752 & \\
\hline & $6 / 10 / 2$ & & 24.855 & 24.468 & 24.834 & 25.728 & 24.89 & 41.683 & 51.698 & 4.014 & 38.642 & 4.124 & & 44.946 & 003 & & & & & & & & & 472 & 0. & & 808 & \\
\hline & $6 / 10 / 2004$ & & 24.915 & & 24.904 & 25.753 & & & 51.914 & 3.976 & & 4.176 & & & & & & & & & & & & & & & 743 & \\
\hline & & & 24.939 & & & & & & & & & & & & & & & & & & & & & & & & & \\
\hline & 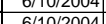 & & 24.99 & 24.535 & 24.951 & 25.88 & & & 51.8 & & 38.6 & 4.2 & & $44.8 \mathrm{r}$ & & & & & & & & & & & & & 势 & \\
\hline$\frac{x^{4}}{49}$ & $6 / 110 / 2004$ & & $\frac{23.011}{25074}$ & & $\mid$\begin{tabular}{|l|}
$\mid 25.95$ \\
$\mid 2503$
\end{tabular} & & & & \begin{tabular}{|l}
5.535 \\
52.23
\end{tabular} & & & & & & & & & & & 508 & & & 786 & & & & 11.804 & \\
\hline & $6 / 10 / 2004$ & & 25.113 & 24 & \begin{tabular}{|l|}
$\mid 25.007$ \\
\end{tabular} & 25826 & & & $\begin{array}{l}25.3518 \\
523\end{array}$ & & & 436 & & 44 & & & & & & & & & & & & & & . \\
\hline 49 & $6 / 10 / 2$ & & 25.152 & & & 25.804 & & & 52.022 & & & 4.41 & & & & & & & & & & & & & & & & \\
\hline & & & 25.19 & & & & & & 52.015 & & & & & & & & & & & & & & & & & & & \\
\hline & $6 / 10 / 2$ & & 25.209 & & 25.158 & 25.786 & 24.9 & 41.6 & 52.6 & & 38.71 & 4.4 & & & & & & & & 50 & & & & & & & 395 & \\
\hline & $\mid$ & & 25.243 & 24 & 50.207 & 25.91 & & & & 3.951 & & 4.4 & & & & & & & & & & & & & & & & \\
\hline & & & 25.271 & 24.704 & 25.25 & 25.904 & & 41.495 & 52.175 & 3.977 & 38.427 & & & & & & & & & 507 & & & & & & & & 51 \\
\hline 50 & 6/10/2004 & & 25.3 & 24.723 & \begin{tabular}{|l|}
25.274 \\
\end{tabular} & 25.903 & & & \begin{tabular}{|l|l|}
52.067 \\
\end{tabular} & 3.971 & & 4.569 & & & & 3.234 & & & & 50.6 & 11. & & & & 0.4 & & 11.991 & \\
\hline & $6 / 10 / 2$ & & & & & & & & & & & & & & & & & & & & & & & & & & 1.977 & \\
\hline 50 & 6/10/2004 & & 25.331 & 24.745 & 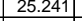 & 25.894 & & & $\begin{array}{l}51.932 \\
\end{array}$ & & & & & & & & & & & & & & & & & & 110 & \\
\hline & & & & & 25.284 & & & & & & & & & & & & & & & & & & & & & & & \\
\hline & & & 25.38 & & & & & & & & & & & & & & & & & & & & & & & & & \\
\hline & & & 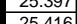 & $\frac{24.191}{24804}$ & $\mid$ & 年. & & & $\begin{array}{l}51.844 \\
51047\end{array}$ & 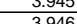 & 38.3 & & & & & & & & & & & & & & & & & \\
\hline & $6 / 10 / 2004$ & & $\frac{25.41}{25.43}$ & & \begin{tabular}{|l|}
25.3040 \\
\end{tabular} & & & & 651.544 & & & & & & & & & & & & & & & & & & & \\
\hline & $6 / 10 / 2004$ & & $\frac{2.4 .45}{25.45}$ & & & & & & & & & & & & & & & & & & & & & & & & & 0.32 \\
\hline & $6 / 10 / 2004$ & & 25.47 & 24.868 & & & & & & & & & & & & & & & & & & & & & & & & \\
\hline & & & 25.48 & & & & & & & & & & & & & & & & & & & & & & & & & \\
\hline & $6 / 1$ & & 25.50 & \begin{tabular}{|l|l|}
24.927 \\
\end{tabular} & \begin{tabular}{|l|}
25.463 \\
\end{tabular} & 26.231 & & $\overline{41.2}$ & 51.8 & & & & & & & & & & & & & & & & & & & \\
\hline & & & 25.516 & & 25.471 & & & & 5 & & & & & & & & & & & & & & & & & & & \\
\hline & & & & 24 & 25.51 & & & & & & 38.1 & & & & & & & & & & & & & & & & & \\
\hline & $6 / 10 / 2004$ & 8:20: & 25.548 & 25 & 25.508 & & & 40.829 & 51.4 & 3.92 & & & & & & & & & & & & & & & & & 236 & \\
\hline & & & & & 5 & & & 41. & & & & & & & & & & & & & & & & & & & & \\
\hline & $6 / 10 / 2$ & & 25.568 & 25 & & & & & 51.8 & & & & & & & & & & & & & & & & & & & \\
\hline & & & 25.5 & & & & & & & & & & & & & & & & & & & & & & & & & \\
\hline & $\frac{6 / 10 / 2}{61212}$ & & $\frac{25.59}{2.50}$ & $\frac{25}{25}$ & & 20.216 & & & - $5<.1$ & & & & & & & & & & & & & & & & & & & \\
\hline & 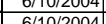 & 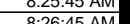 & $\frac{23.060}{25635}$ & 2 & 203560 & 20.234 & & $\begin{array}{l}41.101 \\
409047\end{array}$ & $\begin{array}{l}3.0 \\
1510\end{array}$ & 4. & & 4.8 & & & & & & & & & & & & & & & & \\
\hline & $110 / 2004$ & 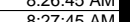 & 25.63 & 25.131 & 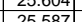 & 262015 & & $\frac{40.54 t}{41086}$ & 51007 & - 4.0000 & | & 1802 & & & & $\frac{5.250}{2304}$ & & & & & & & & & & & & \\
\hline & & & & & & & & 4078 & 814 & 3.099 & & 4014 & & 4.987 & & & & & & & & & & & & & & \\
\hline & & & & & & $\frac{20.100}{26.165}$ & & $\begin{array}{l}40.101 \\
41.111\end{array}$ & $\begin{array}{l}52.014 \\
52.102\end{array}$ & 4.01 & 215 & $\begin{array}{l}4.914 \\
4.923 \\
\end{array}$ & & $\begin{array}{l}4.9017 \\
4.967\end{array}$ & 003 & $\frac{0.005}{3.299}$ & & & & 50.6 & & & & \begin{tabular}{|l|}
0.493 \\
0.492 \\
\end{tabular} & $\frac{0.404}{0.483}$ & .01 & . 2.176 & \\
\hline
\end{tabular}


WSRC-TR-2005-00105, REVISION 0

SRNL-RPP-2005-00012, REVISION 0

PRE-TEST ACID CLEAN WITH 2 M HNO3, FOLLOWING PUMP REPAIR - CONT.

\begin{tabular}{|c|c|c|c|c|c|c|c|c|c|c|c|c|c|c|c|c|c|c|c|c|c|c|c|c|c|c|c|c|}
\hline & A & B & $\begin{array}{ll}D \\
\end{array}$ & $E$ & $F$ & $\mathrm{G}$ & $\mathrm{H}$ & $\mathrm{J}$ & \begin{tabular}{l|l} 
\\
\end{tabular} & $\mathrm{L}$ & M & $\mathrm{N}$ & 0 & Q & $R$ & $\mathrm{~s}$ & $T$ & $\mathrm{v}$ & w & \begin{tabular}{l|l}
$x$ \\
\end{tabular} & $\mathrm{Y} \mid$ & $z$ & AA & $A B$ & $A C$ & AD & $\mathrm{AE}$ & AF \\
\hline & $6 / 10 / 2004$ & $8: 30: 45 \mathrm{AM}$ & 25.67 & 25.159 & 25.68 & 26.123 & 25.245 & 40.908 & \begin{tabular}{|l|}
51.781 \\
\end{tabular} & 3.973 & 38.064 & 4.975 & 1.157 & 44.965 & 0.003 & 3.304 & 0.005 & 1152.95 & \begin{tabular}{l|l}
5 & 19.2158
\end{tabular} & 50.3 & & & 2.722 & \begin{tabular}{|l|}
0.493 \\
\end{tabular} & 0.483 & & 12.239 & \\
\hline & & & 25.672 & 25.162 & 25.597 & 26.22 & & 40.786 & \begin{tabular}{|l|}
51.505 \\
\end{tabular} & & & 4.975 & 1.16 & & & & & & & & & & & & & & & \\
\hline 53 & & & & & & & & & \begin{tabular}{|l|}
51.974 \\
\end{tabular} & & & & & & 003 & & & & & & & & & & & & & \\
\hline & & 8:33:45 AM & & & & & & & & & & & & & & & & & & & & & & & & & & \\
\hline 532 & $6 / 10 / 2004$ & 8:34:45 AM & 25.589 & 25.193 & 25.488 & 26.242 & 25.289 & 842 & \begin{tabular}{|l|l|}
51.603 \\
\end{tabular} & 3.976 & 901 & & & .939 & 003 & 3.312 & & 56.9 & 9.2825 & 50.1 & & 39.4 & & 0.494 & 0.487 & & 12.371 & \\
\hline & & 8:35:45 AM & & & 25.441 & $\begin{array}{l}26.194 \\
\end{array}$ & & & 51.804 & & 37.846 & 966 & & 44.987 & 0.003 & 3.31 & & 115 & .2994 & 50 & & & & & 0.487 & & & \\
\hline 53 & $6 / 10 / 2004$ & $8: 36: 45 \mathrm{AM}$ & 25.512 & 25.192 & 25.412 & 26.265 & 25.378 & 40.885 & 51.794 & $\begin{array}{l}4.013 \\
\end{array}$ & 37.992 & 975 & 1.16 & 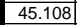 & 0.003 & 3.303 & & & 9.3158 & 50. & 11. & & & 0.492 & 0.487 & 0.0 & 12.343 & \\
\hline & $6 / 10 / 2004$ & 8:37:45 AM & 25.495 & 25.214 & 25.405 & 26.328 & 25.445 & 40.814 & \begin{tabular}{|l|l|l|} 
\\
\end{tabular} & 3.958 & 37.916 & 4.946 & 1.166 & 45.052 & 0.003 & 3.301 & & 1159.9 & 9.3325 & 50. & 11 & 39. & & 0.492 & 0.487 & & 361 & \\
\hline & & & 25.467 & 25.221 & 25.377 & 26.41 & & & & 4.009 & 37.789 & 4.92 & & & & & & & 7.3492 & & & & & & & & 12.429 & \\
\hline & & & 25.454 & 25.243 & 25.348 & 26.462 & & & 51.854| & $\begin{array}{l}4.013 \\
2.092 \\
2\end{array}$ & 117 & & & 1.915 & & & & & 3658 & 50.4 & & & & .493 & 488 & & & \\
\hline & $\frac{6 / 102}{612 \pi}$ & $8: 4: 45 \mathrm{AM}$ & 25.442 & 25.211 & 25.332 & $\frac{26.515}{2652}$ & & & 51.982 & 3.983 & . & & & 44.979 & & & & & & & & & & & & & & \\
\hline 5 & $6 / 110 / 2004$ & $\begin{array}{l}8: 4.425 \mathrm{AM} \\
8: 42.45 \mathrm{AM}\end{array}$ & $\frac{25.414}{25.401}$ & $\begin{array}{l}25.278 \\
25296\end{array}$ & & $\frac{26.534}{26.584}$ & & $\begin{array}{r}40.97 \\
40.86\end{array}$ & $\begin{array}{l}51.995 \\
51.877\end{array}$ & $\frac{999}{034}$ & $\begin{array}{r}38.076 \\
37.94\end{array}$ & & & $\begin{array}{l}44.99 \\
4073\end{array}$ & & $\begin{array}{r}3.31 \\
3.306\end{array}$ & & & 4158 & 504 & & & & & & & & \\
\hline & $6 / 10 / 2004$ & 8:43:45 AM & 25.385 & $\begin{array}{l}25.33 \\
\end{array}$ & 25.501 & $\begin{array}{l}20.5044 \\
26.648\end{array}$ & $\frac{25.151}{25.72}$ & 4 & \begin{tabular}{|l|l|}
51.975 \\
51.935
\end{tabular} & $\begin{array}{l}4.054 \\
4.024\end{array}$ & 38.016 & $\begin{array}{l}900 \\
972 \\
\end{array}$ & $\begin{array}{l}1.114 \\
1.172\end{array}$ & \begin{tabular}{|l|}
45.077 \\
45.07
\end{tabular} & 0.003 & $\begin{array}{l}0.000 \\
3.306\end{array}$ & & $\begin{array}{l}1104.95 \\
1165.95 \\
\end{array}$ & 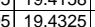 & $\begin{array}{l}50.4 \\
50.5\end{array}$ & 11.0 & & 2.723 & $\frac{0.495}{0.493}$ & $\begin{array}{l}0.409 \\
0.489\end{array}$ & {$[011$} & & \\
\hline & 6/10/2004 & 8:44:45 AM & 25.378 & 25.362 & 25.282 & 26.666 & 25.693 & 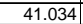 & \begin{tabular}{|l|l|} 
\\
\end{tabular} & 4.012 & 38.135 & $\begin{array}{l}4.94 \\
\end{array}$ & 1.178 & \begin{tabular}{|l|l|} 
& 44.9 \\
\end{tabular} & 0.003 & 3.309 & & 1166.95 & \begin{tabular}{|l|l|}
5 & 19.4492 \\
\end{tabular} & 50.5 & 11.0 & & 2.729 & 0.493 & 0.489 & 0.01 & 365 & \\
\hline 54 & $6 / 10 / 2$ & 8:45:45 AM & & 25.376 & 25.241 & & 25.701 & 40.711 & \begin{tabular}{|l|}
51.678 \\
\end{tabular} & 3.991 & 37.797 & 5.004 & & \begin{tabular}{|l|l|l|l}
45.008 \\
\end{tabular} & 0.003 & 3.31 & & & \begin{tabular}{|l|l|}
5 & 19.4658
\end{tabular} & 50.2 & & & & & 0.490 & & & \\
\hline & $6 / 10 / 2004$ & 8:46:4! & 25.36 & & & 26.628 & & & & 4.01 & 8.189 & & & \begin{tabular}{|l|l|} 
\\
\end{tabular} & & & & & & & & & 273 & 493 & 0.489 & 0.01 & 1234 & \\
\hline & & & & & & & & & \begin{tabular}{|l|l|}
51.659 \\
\end{tabular} & 3.952 & 37. & & & \begin{tabular}{|l|l|}
44.95 \\
\end{tabular} & & & & & & & & & & & & & & \\
\hline 154 & $6 / 10 / / 4$ & 8:48:45 AM & $\begin{array}{l}25.332 \\
\end{array}$ & 25.446 & \begin{tabular}{|l|}
25.302 \\
\end{tabular} & & & & \begin{tabular}{|l|}
51.829 \\
\end{tabular} & 4.03 & 37.83 & & & \begin{tabular}{|l|l|}
44.971 \\
\end{tabular} & & & & & $\begin{array}{l}9.5158 \\
9\end{array}$ & & & & & & & & & \\
\hline 54 & 6/10/2004 & $8: 49: 4$ & 25.326 & \begin{tabular}{|l|l|}
25.47 \\
\end{tabular} & 25.281 & 26.604 & & 40.848 & \begin{tabular}{|l|l|} 
& \\
1.837
\end{tabular} & 3.952 & 37.953 & 98 & & $\begin{array}{l}45.056 \\
\end{array}$ & & & & & 19.5325 & 50.4 & 11.0 & 39 & & D.493 & 0.489 & & 12.419 & \\
\hline & $6 / 10 / 2$ & 8:50:45 AM & 25.302 & 25.482 & 25.252 & 26.57 & & 40.841 & 51.951 & & 37.953 & & 1.18 & \begin{tabular}{|l|l|}
44.937 \\
\end{tabular} & & & & 2.95 & \begin{tabular}{|l|l|}
5 & 19.5492 \\
\end{tabular} & 50.5 & 11 & & 2.716 & & & 0.0 & 12.419 & \\
\hline & $6 / 10 / 2004$ & $8: 11: 45 \mathrm{AM}$ & 25.295 & 25.485 & 25.245 & 26.563 & & 40.881 & \begin{tabular}{|l|}
52.028 \\
\end{tabular} & 4.015 & 37.924 & 4.983 & 1.18 & 45.002 & 0.003 & 3.316 & & 173.95 & 19.5658 & 50.6 & | & & 2.71 & & 0.491 & 0.0 & 461 & \\
\hline 政 & 6 & $8: 52: 45$ AM & 25.294 & 25.513 & 25.198 & 26.572 & 25.70 & 40.974 & $\begin{array}{l}52.036 \\
\end{array}$ & 4.01 & 37.945 & & & \begin{tabular}{|l|l|l|l|} 
\\
\end{tabular} & & & & & 10.002 & 0.0 & & & & 0.494 & & & 12.448 & \\
\hline 55 & $66110 / 2004$ & 8:53:45 & 25.28 & & & & & & & & & & & & & & & & & & & & & & & & & \\
\hline 55 & $6 / 10 / 2$ & & 25.283 & 25.52 & 25.152 & 26.606 & & 41.18 & 52.77 & & 38.2 & & & 45.096 & & & & 17 & .6158 & $\frac{51}{52}$ & & & 2.74 & & & & & \\
\hline & & & & & & & & & & & & & & 44.985 & & & & & & & & & & & & & & \\
\hline 55 & $6 / 10 / 20$ & $8.50 .45 \mathrm{Am}$ & $\frac{25.294}{25294}$ & 25.3564 & $\frac{25.204}{25214}$ & $\frac{2.030}{26.612}$ & & & (52.951 & 4 & & 5 & & $\begin{array}{r}45.11 \\
45.008\end{array}$ & & & & & .6492 & $\frac{51.5}{512}$ & $\frac{11}{11}$ & & $\frac{2.65}{2.747}$ & & O..501 & $0.0 \quad-1$ & 2.514 & \\
\hline & $6 / 10 / 2004$ & $8: 58$ & 25.296 & 25.576 & 25201 & 26.599 & & & $\begin{array}{l}52.648 \\
\end{array}$ & 4.015 & 38.304 & 5.383 & & $\mid 45.06$ & & 3.376 & & & 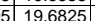 & 51. & & & & & 0.500 & 0.0 & & \\
\hline 5 & & & & & & & & & & & & & & & & & & & & & & & & & & & & \\
\hline & $6 / 10 / 2004$ & $9 \cdot 00: 45$ & 25.295 & 25.584 & $\mid 251.84$ & 26.638 & & 41.117 & 52.714 & 3.98 & 38.3 & 5.458 & & 每5.096 & & & & & & 51.2 & 11. & & & & 0.502 & 0.0 & 12.638 & \\
\hline & & & & & & 26.72 & & & & & & 5.4 & & 45.0 & & & & & & 51.2 & & & & & & & & \\
\hline 56 & & & 25.324 & & \begin{tabular}{|l|l|}
25.214 \\
\end{tabular} & & & & & & & & & & & & & & & 51.5 & & & & & & & & \\
\hline 56 & $6 / 10 / 2 C$ & & 25.316 & & 25.196 & & & & & 4.02 & & & & 45.121 & & & & & & 51.3 & 11 & & $2.7 \quad-2$ & & & & 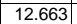 & \\
\hline & $6 / 10 / 2$ & & 25.323 & 25. & 25.197 & 26.711 & & & & 3.957 & & & & & & & & & & 51 & & & & & & & & \\
\hline 56 & $6 / 10 /$ & & 25.331 & 25.6 & 25.231 & 26.729 & & & 52.809 & 4.017 & & & & 45.175 & & & & & & 51 & & &. .1 & & 0.502 & & & \\
\hline 然 & $6 / 110 / 2004$ & & 25.323 & 25.668 & 25.213 & 26.831 & & & & & & & & 45.0 & & & & & & 51. & 11 & & & & & & & \\
\hline & & & 25.33 & & & 26.843 & & & & & & & & & & & & & & & & & & & & & & \\
\hline & $\frac{6 / 102}{6102}$ & & 25.343 & 25.718 & 25.243 & 26.906 & & 41.1 & 52.14 & & & & & 45.1 & & & & & & 51.3 & 11 & & 2.1 & & & & & \\
\hline 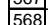 & $\frac{6 / 11 / 20}{6 / 10 / 20}$ & & $\begin{array}{r}25.34 \\
25.349\end{array}$ & & & 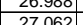 & & & & & & & & 4511 & & & & & & 517 & & & & & & & & \\
\hline 56 & 60 & & 25352 & & & & & & & & & & & & & & & & & & 11 & & & & & & & \\
\hline 57 & $6 / 10 / 2$ & & 25.34 & & & 26.993 & & & & & & & & & & & & & & 51.4 & & & & & & & & \\
\hline 571 & $6 / 10 / 2004$ & & & & 25.243 & 26.996 & & & & & & & & & & & & & & & & & & & & & & \\
\hline & $6 / 10 / 2$ & & 25.362 & 25.867 & \begin{tabular}{|l|l|}
25.252 \\
\end{tabular} & 27.005 & & 40.76 & B 52.507 & 3.99 & & & & & & & & & & & & & & & 0.504 & & & \\
\hline 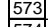 & $6 / 10 / 2004$ & & \begin{tabular}{|l|l|}
25.363 \\
\end{tabular} & 25.878 & \begin{tabular}{|l|}
25.268 \\
\end{tabular} & 27.031 & & 41.041 & \begin{tabular}{|l|l|}
52.511 \\
\end{tabular} & 3.984 & 38.261 & 5.562 & 1.24 & & & & & 197.95 & \begin{tabular}{l|l}
5 & 19.9658 \\
\end{tabular} & 51.0 & 11.0 & 0 & 2.734 & & 0.505 & 0.0 & 736 & 10.8 \\
\hline 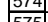 & $6 / 10 / 200$ & $9: 16: 45 \mathrm{AM}$ & & 255060 & & & & & $\begin{array}{l}45.722 \\
\end{array}$ & & & & & 45.1 & & & & & $\begin{array}{ll}5 & 19.9 \\
\end{array}$ & 51.2. & & & & & & & & \\
\hline 57 & $6 / 1020$ & & & & & 2701 & & & & & & & & & & & & & & 51.4 & & & & & & & & \\
\hline 57 & $6 / 10 / 2$ & & 25.369 & 25.949 & \begin{tabular}{|l|l|}
25.269 \\
\end{tabular} & 27.0 & & & 52.606 & $\begin{array}{l}3.963 \\
3.963\end{array}$ & & & & & & & & & & & 110 & & & & 0.504 & & 2723 & \\
\hline 157 & & & & & & 27.044 & & & & & & & & & & & & & & & & & & & & & & \\
\hline & & & 25.383 & & & 27.036 & & & 52.7 & 3.988 & & 583 & & & & & & & & & & & & 509 & 506 & & & \\
\hline & $\frac{26 / 10 / /}{6 / 2}$ & $\begin{array}{l}9: 22: 4 \\
9\end{array}$ & 25. & 5.98 & 25.245 & o28 & & 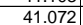 & 252.743 & & 3.293 & 5.641 & & & & & & & 5 & 51.3 & & & 2.736 & $\mid$ & $\frac{0.507}{0.507}$ & & 2.778 & \\
\hline
\end{tabular}


WSRC-TR-2005-00105, REVISION 0 SRNL-RPP-2005-00012, REVISION 0

PRE-TEST ACID CLEAN WITH 2 M HNO3, FOLLOWING PUMP REPAIR - CONT.

\begin{tabular}{|c|c|c|c|c|c|c|c|c|c|c|c|c|c|c|c|c|c|c|c|c|c|c|c|c|c|c|c|c|}
\hline & $\mathrm{A}$ & \begin{tabular}{|c|}
$\mathrm{B}$ \\
\end{tabular} & $\mathrm{D}$ & $E$ & $F$ & $G$ & $\mathrm{H}$ & $\mathrm{J}$ & $\mathrm{K}$ & $\mathrm{L}$ & $\mathrm{M}$ & $\mathrm{N}$ & $\mathrm{O}$ & Q & $\mathrm{R}$ & $\mathrm{s}$ & $T^{T}$ & $\mathrm{~V}$ & $\mathrm{~W}$ & $x$ & $\mathrm{Y}$ & $z$ & AA & $A B$ & $\mathrm{AC}$ & AD & AE & \begin{tabular}{l|l} 
AF & $A$ \\
\end{tabular} \\
\hline 581 & $6 / 10 / 2004$ & \begin{tabular}{|c|}
$9: 23: 40 \mathrm{AM}$ \\
\end{tabular} & 25.387 & 26.00 & 25.25 & 27.03 & 26.141 & 40.972 & 52.65 & 3.978 & 38.234 & 5.655 & 1.238 & 45.146 & 0.003 & 3.426 & 0.004 & 2058 & 0.0978 & 51.2 & 11.1 & & 2.730 & 0.511 & 0.507 & 0.01 & & \\
\hline & $6 / 10 / 2004$ & 9:24:40 AM & & & 25.25 & & 26.113 & 41.038 & 52.718 & 3.985 & & 5.6 & & 45.06 & 0.003 & & & & 20.1144 & & & & & & 0.505 & & 2.757 & \\
\hline 583 & 6/10/2004 & 9:25:40 AM & 25.373 & 26.003 & 25.23 & 27.03 & 26.123 & 40.814 & 52.627 & 3.962 & $\begin{array}{l}37.988 \\
7067\end{array}$ & 5.669 & 1.238 & 45.181 & 0.003 & 3.425 & 0.004 & 1207.87 & 20.1311 & 51.2 & 11.1 & 39.4 & 2.717 & 0.511 & 0.507 & 0.013 & 12.875 & $\begin{array}{l}10.96 \\
\end{array}$ \\
\hline 584 & $6 / 10 / 2004$ & 9:26:40 AM & $\begin{array}{l}25.401 \\
25.402\end{array}$ & $\frac{26.046}{22043}$ & $\frac{25.29}{25.29}$ & 27.064 & 26.135 & $\begin{array}{ll}40.788 \\
41.186\end{array}$ & $\begin{array}{r}52.61 \\
52.971 \\
\end{array}$ & $\frac{3.926}{4022}$ & $\begin{array}{l}37.957 \\
38347\end{array}$ & $\frac{5.646}{569}$ & $\begin{array}{ll}1.244 \\
1244\end{array}$ & $\begin{array}{l}45.156 \\
45.11 \\
\end{array}$ & 0.003 & 3.423 & $\begin{array}{l}0.004 \\
0.004\end{array}$ & $\frac{1208.87}{120987}$ & \begin{tabular}{|l|l|}
20.1478 \\
201644
\end{tabular} & $\begin{array}{l}51.1 \\
51.5\end{array}$ & $\begin{array}{ll}11.1 \\
1.1\end{array}$ & $\begin{array}{ll}39.4 \\
30.9\end{array}$ & 2.715 & 0.510 & 0.506 & 0.013 & 12.856 & $\begin{array}{ll}10.94 \\
1.91\end{array}$ \\
\hline 586 & $\begin{array}{l}6 / 110 / 2004 \\
6 / 10 / 2004\end{array}$ & 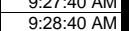 & $\frac{25.402}{25.388}$ & $\frac{26.043}{26.043}$ & $\frac{2.25}{25.26}$ & 27.11 & $\begin{array}{l}26.1 / 2 \\
26.167\end{array}$ & $\begin{array}{ll}41.186 \\
41.043\end{array}$ & $\begin{array}{l}52.9 / 1 \\
52.853\end{array}$ & $\frac{4.026}{3.994}$ & $\frac{38.34 t}{38.179}$ & 5.078 & $\frac{1.244}{1.244}$ & $\frac{45.11}{45.056}$ & $\frac{0.003}{0.003}$ & $\frac{3.427}{3.429}$ & 0.0004 & $\frac{1209.87}{121087}$ & \begin{tabular}{|l|}
2.0 .1644 \\
20.1811
\end{tabular} & $\frac{51.5}{51.4}$ & & $\begin{array}{ll}39.8 \\
396\end{array}$ & $\frac{2.742}{2.731}$ & $\frac{0.551}{0.511}$ & 0.507 & $\frac{0.013}{0.013}$ & \begin{tabular}{r|}
12.743 \\
12809 \\
\end{tabular} & $\begin{array}{l}10.84 \\
10.90\end{array}$ \\
\hline & 6/10/2004 & 9:29:40 AM & 25.4 & & 25.2 & 27.13 & 26.144 & 41.111 & 53.081 & & 38.24 & 5.704 & 1.244 & 45.098 & 0.003 & 3.429 & 0.004 & 211.87 & 20.1978 & 51.6 & 11.1 & & & 0.51 & 0.507 & & & \begin{tabular}{ll|l|}
10.90 \\
10.88
\end{tabular} \\
\hline & 6/10/2004 & 9:30:40 AM & 25.386 & 26.067 & 25.25 & 27.12 & 26.161 & 40.949 & 52.708 & 4.042 & 38.039 & 5.672 & 1.241 & 45.017 & 0.003 & 3.427 & 0.0 & 2.87 & 20.2144 & 51.2 & 11.0 & $\frac{29.5}{39.5}$ & 2.723 & 0.511 & 0.507 & & 844 & $\frac{10.88}{10.93}$ \\
\hline & $6 / 10 / 2004$ & & & & & 27.17 & 26.162 & 41.049 & & & & 5.684 & 1.244 & & 0.003 & 3.4 & & & & 51.3 & 11.0 & & & & & & 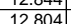 & 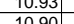 \\
\hline & & $2.00 \mathrm{AM}$ & & & & & & & & 308 & 38055 & & & & & 34 & & & & & 11.0 & & & & & & & $\frac{10.90}{100}$ \\
\hline & $\frac{6 / 10 / 2004}{6.004}$ & 9:3:4:40 AM & 25.4 & $\frac{20.105}{26.105}$ & 2526 & 27.17 & $\frac{20.209}{26.209}$ & 40.572 & 52.418 & 3.987 & 37.729 & 5.626 & & $\frac{45.07}{45}$ & $\frac{0.003}{0.003}$ & $\frac{3.4}{3.4}$ & & $\frac{1.67}{15} 87$ & & 50.9 & $\frac{1.1 .}{110}>>>$ & & 2699 & $\frac{0.51}{0.511}$ & 0.507 & & 12957 & $\frac{10.96}{11.03}$ \\
\hline & $6 / 10 / 2004$ & $9: 34: 40 \mathrm{AM}$ & 25.406 & 26.112 & 25.26 & 27.18 & 26.236 & 40.702 & 52.482 & 3.951 & 37.877 & 5.696 & & & & 3.4. & & & 20.2811 & 51.0 & 11.0 & 39.3 & 709 & 0.51 & & & 12.937 & \\
\hline 593 & $6 / 10 / 2004$ & 9:35:40 AM & 25.408 & 26.133 & \begin{tabular}{|l|l|}
25.26 \\
\end{tabular} & 27.21 & 26.242 & 40.862 & 52.629 & 3.977 & $\begin{array}{lll}37.953 \\
\end{array}$ & 5.681 & & 45.108 & & 3.4 & & & & 51.2 & 11.1 & $\overline{39.4}$ & & 0.512 & 0.508 & & 12.891 & $\frac{10.97}{10.97}$ \\
\hline & $6 / 10 / 2004$ & 9:36:40 AM & 25.404 & 26.134 & 25.25 & 27.26 & 26.213 & 41.051 & 52.631 & 4.015 & 38.189 & 5.568 & 1.178 & 45.148 & 0.003 & 3.461 & & & 20.3144 & 51.2 & 11.1 & 39.6 & 2.732 & 0.516 & 0.512 & 0.013 & 12.929 & 11.00 \\
\hline 595 & & & & & & & & & & & & & & & & & & & & & & & & & & & & \\
\hline 59 & & Averages & 25.2 & 25.0 & 25. & 26. & & 41.3 & 52.2 & 4.0 & 38.4 & 4.9 & & 44.9 & 0.0 & 3.3 & & & & 50.7 & 110 & $39.8 \mathrm{C}$ & 27 & 0.490 & 0.488 & 0.012 & 123 & $10.4 \mathrm{Y}>\mathrm{C}$ \\
\hline & & Maximum & 25.7 & 26.1 & 25. & 27. & 26.2 & 42.4 & 53.2 & 4.3 & 39.4 & 5.7 & & 47.0 & & & & & & & & & 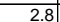 & & & & 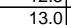 & 10.4 \\
\hline & & Median & 25.3 & 25.1 & $25.8>>2$ & 26.7 & 25.3 & 41.2 & & 4.0 & 38.3 & 4.9 & & 45 & & & & & & & & & & 0.49 & & & & \\
\hline & & Minimum & 23.3 & 23.7 & 23. & 23. & 23.5 & 37.6 & 45.9 & 3.7 & 34.4 & 2.6 & & $\begin{array}{ll}43.0 \\
\end{array}$ & 0.0 & 2.9 & & & & 44.4 & 10.5 & 36.0 & 2.5 & 0.42. & 0.447 & 0.012 & \begin{tabular}{ll|l}
11.6 \\
\end{tabular} & \\
\hline 601 & & $2 \times \mathrm{Std}$ & 0.810 & 1.422 & 0.74 & 1.51 & 1.325 & 1.121 & 1.399 & 0.142 & 1.111 & 1.194 & 0.112 & 0.836 & 0.000 & 0.209 & & & & 1.399 & 0.205 & 1.111 & 0.077 & 0.031 & 0.025 & 0.001 & 0.780 & 6.664 \\
\hline 602 & Number C & of Points Used & 137 & 137 & 13 & 13 & $\begin{array}{ll}137 \\
\end{array}$ & & $\begin{array}{ll}137 \\
\end{array}$ & & & 137 & & $\begin{array}{ll}137 \\
\end{array}$ & $137 \mathrm{r}$ & $137 \mathrm{r}$ & & & & $\begin{array}{l}137 \\
\end{array}$ & 137 & 137 & 137 & 137 & 137 & 137 & 137 & $\overline{137}$ \\
\hline
\end{tabular}


WSRC-TR-2005-00105, REVISION 0 SRNL-RPP-2005-00012, REVISION 0

RUN \# 3.00A, B, AND C; PRE-TEST BASELINE, DIF WATER AND 0.1 M NAOH

RPP-WTP Pilot-scale X-flow Filtration Test No. 3.00 - Campaign III

[10 June 2004, Pre-Test Baseline Comparison]

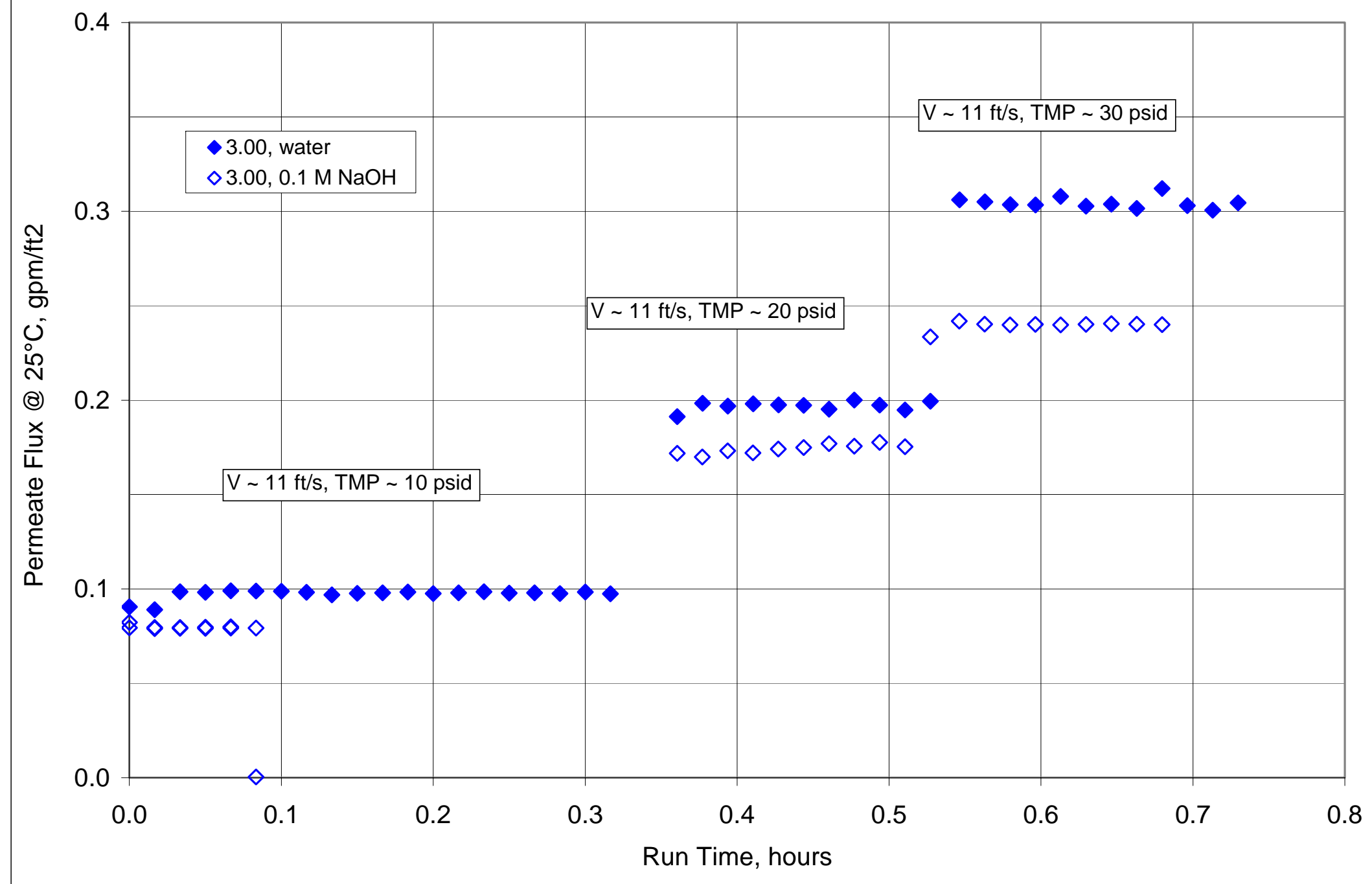


WSRC-TR-2005-00105, REVISION 0

SRNL-RPP-2005-00012, REVISION 0

RUN \# 3.00A, B, AND C; PRE-TEST BASELINE WITH DIF WATER - CONT.

\begin{tabular}{|c|c|c|c|c|c|c|c|c|c|c|c|c|c|c|c|c|c|c|c|c|c|c|c|c|c|c|c|c|}
\hline & $\mathrm{A}$ & $\mathrm{B}$ & $\mathrm{D}$ & E & $1 \mathrm{~F}$ & $G_{1}$ & I H & $\mathrm{J}$ & $\mathrm{K}$ & $\mathrm{L}$ & $\begin{array}{ll}\mathrm{M} \\
\end{array}$ & \begin{tabular}{|l|l|}
$N$ \\
\end{tabular} & 0 & Q & $\mathrm{R}$ & $\mathrm{s}$ & $T$ & $\begin{array}{lllll}v & & \end{array}$ & w & $\mathrm{x}$ & $\mathrm{Y} / \mathrm{T}$ & & $A A$ & $A B$ & $\mathrm{AC}$ & $\mathrm{AD}$ & $\mathrm{AE}$ & AF $A$ \\
\hline \begin{tabular}{|l|l|}
1 \\
2
\end{tabular} & & & & K⿺ Temp & erature $M$ & leasuremer & & & & $\begin{array}{l}\text { Pressure } 1 \\
\text { Pitterd }\end{array}$ & & & & & & & & & & & & & & & & & & \\
\hline \begin{tabular}{|l|}
2 \\
3 \\
\end{tabular} & DATE & TIME & $\begin{array}{l}\text { Fittrate } \\
\text { degc }\end{array}$ & $\begin{array}{l}\text { Cleaning } \\
\text { deg C }\end{array}$ & \begin{tabular}{|l|} 
Slurry \\
deg C \\
\end{tabular} & \begin{tabular}{|l|} 
Hi Amb. \\
$\operatorname{deg} C$.
\end{tabular} & \begin{tabular}{|l|} 
Lo Amb. \\
deg C
\end{tabular} & $\begin{array}{l}\text { BotTMP } \\
\text { nsid }\end{array}$ & \begin{tabular}{|l|} 
Filter \\
nsiga \\
\end{tabular} & \begin{tabular}{|l|l|} 
Filter dP \\
sidd
\end{tabular} & \begin{tabular}{|l|} 
TopTMP \\
nsid
\end{tabular} & \begin{tabular}{|l|} 
Filtrate \\
nsia
\end{tabular} & \begin{tabular}{|l|l|} 
Pulsepot \\
nsia
\end{tabular} & $\begin{array}{l}\text { Slurry } \\
\text { som }\end{array}$ & \begin{tabular}{|l|l|l} 
Filtrate \\
\end{tabular} & Hi Filtate & Backpulse & & & & & & & & & & & \\
\hline \begin{tabular}{|l|l|}
4 \\
\end{tabular} & & & $\mathrm{~T} 2$ & T3 & T1 & T4 & & $\begin{array}{l}\text { Psid } \\
\text { dP2 }\end{array}$ & $\frac{\mathrm{psig}}{\mathrm{P} 1}$ & $\frac{\text { PId }}{d P 1}$ & $\begin{array}{l}\text { psid } \\
d P 3\end{array}$ & \begin{tabular}{|l|l|} 
psigg \\
p2
\end{tabular} & $\frac{p s i g}{\text { P3 }}$ & $\frac{\mathrm{gpm}}{\mathrm{O} 1}$ & gpm & gpm & gpm & & & & & & & & & & & \\
\hline 5 & & & & & & & & & & & & & & $\mathrm{Q}_{1}$ & & & & & & & & & & & & & & \\
\hline $\begin{array}{ll}6 \\
\end{array}$ & Zeros & & & & & & & & & & & & & & & & & & & & & & & & & & & \\
\hline & & 9:09:09 AM & 22.545 & 24.705 & \begin{tabular}{|l|l|}
27.987 \\
\end{tabular} & \begin{tabular}{|l|}
26.054 \\
\end{tabular} & 24.911 & -0.004 & 0.128 & -0.01 & -0.003 & \begin{tabular}{|l|l|}
-0.129 \\
\end{tabular} & 0.111 & 45.741 & 0.293 & -0.013 & 0.003 & & & Filter Su & & & & & & & & \\
\hline \begin{tabular}{|l|l|}
8 \\
0
\end{tabular} & 6/10/2004 & 1:47:47 PM & 25.596 & 26.216 & 26.68 & $\begin{array}{l}26.834 \\
2.874\end{array}$ & 26 & $\begin{array}{ll}-0.019 \\
\end{array}$ & 0.196 & -0.012 & $\begin{array}{l}-0.02 \\
\end{array}$ & $\begin{array}{l}-0.106 \\
\end{array}$ & 0.073 & \begin{tabular}{|c|c|}
-0.071 \\
\end{tabular} & 0.003 & 14.643 & 0.003 & & & Convers & ision & 851 & $\mathrm{~m} 3 / \mathrm{m} 2$ & p/day/barts & $\mathrm{arg} / \mathrm{gpm} / \mathrm{tt}$. & fft/barg & & \\
\hline \begin{tabular}{|c|}
9 \\
10
\end{tabular} & 6/14/2004 & 7:41:41 AM & 23.83 & 23.885 & & $\begin{array}{l}24.174 \\
\end{array}$ & 24.204 & \begin{tabular}{|l|l|}
-0.012 \\
\end{tabular} & 0.12 & -0.01 & -0.016 & & 0.107 & \begin{tabular}{|c|c|c|}
-0.033 \\
\end{tabular} & 0.003 & \begin{tabular}{|l|l|}
34.643 \\
\end{tabular} & 0.005 & & & & & & & & & & & \\
\hline$\frac{10}{11}$ & $6 / 15 / 2004$ & \begin{tabular}{|l|}
$12: 06: 06 ~ P M$ \\
\end{tabular} & 25.175 & 24.315 & 23.747 & $26.127 \mid$ & \begin{tabular}{|l|}
23.997 \\
\end{tabular} & -0.012 & 0.139 & $\begin{array}{l}-0.002 \\
0.012\end{array}$ & -0.012 & \begin{tabular}{|c|}
-0.177 \\
\end{tabular} & 0.043 & -0.073 & 0.003 & \begin{tabular}{|l|}
14.643 \\
\end{tabular} & 0.006 & & & Note: $G$ & & & & & ately 1.4 & 4 psig & & \\
\hline$\frac{11}{12}$ & 6 & $\begin{array}{l}7: 28: 28 \mathrm{AM} \\
\end{array}$ & 24.492 & 233.951 & $\begin{array}{l}23.799 \\
17.795\end{array}$ & 24.225 & 23.743 & -0.01 & 0.164 & -0.001 & -0.014 & -0.18 & 0.032 & $\mid-0.075$ & 0.003 & $\begin{array}{l}\text { 3. } \\
\text { 3. } 14.643 \\
\end{array}$ & 0.006 & & & Drects & nengis & 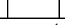 & & & & & & \\
\hline 13 & $\begin{array}{l}6 / 1 / 12004 \\
6 / 212004\end{array}$ & $\begin{array}{l}\text { 4:57:57 PM } \\
\text { 6:52:52 AM }\end{array}$ & 26.259 & $\frac{26.37}{23.008}$ & 24.655 & \begin{tabular}{|l|l|}
27.805 \\
23.418
\end{tabular} & 26.456 & $\begin{array}{l}-0.002 \\
-0.01 \\
\end{array}$ & \begin{tabular}{|l|l|}
0.197 \\
0.075
\end{tabular} & & $\begin{array}{r}-0.012 \\
-0.01 \\
\end{array}$ & $\begin{array}{l}-0.183 \\
-0.197 \\
-0.07\end{array}$ & $\begin{array}{r}-0.009 \\
0.055 \\
\end{array}$ & 47.043 & 0.098 & \begin{tabular}{|l|l|} 
& 14.643 \\
\end{tabular} & 0.004 & & & Pressur & ure Plis & orrec & ed for 4 & 0.88 incl & & rt tubir & & \\
\hline 14 & $6 / 22 / 2004$ & $\begin{array}{l}0.52 .5<\mathrm{AM} \\
8: 12: 12 \mathrm{AM}\end{array}$ & $\frac{23.109}{23.36}$ & 23.5005 & $\frac{22.021}{23.63}$ & $\frac{23.418}{23829}$ & $\frac{22.800}{24.45}$ & $\frac{-0.01}{-0.008}$ & 0.0708 & -0.004 & $\begin{array}{l}-0.01 \\
-0.012 \\
\end{array}$ & $\begin{array}{l}-0.197 \\
-0.194 \\
-0.03\end{array}$ & $\frac{0.055}{0.06}$ & \begin{tabular}{|c|c|}
-0.019 \\
0083
\end{tabular} & 0.003 & \begin{tabular}{|l|l|}
14.643 \\
314643
\end{tabular} & 0.006 & & & VWVW & 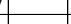 & & & $<$ Filtrat & rate Flux & $\ll<P E$ & $\| A B I L$ & \\
\hline & 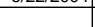 & & & & & & & & & & & -0.194 & 0.06 & & 0.003 & 14.643 & & Time & Time & Press. & Vel. & TMP & TMP & $\mathrm{anm}$ & $\frac{\text { at } 25}{\mathrm{gpm}}$ & pm & $\begin{array}{l}x 1000 \\
\text { apm }\end{array}$ & \\
\hline 16 & Baseline wA] & & & & & & & & & & & & & & & & & & & & & & & & & & $2 / \mathrm{psi}$ & \\
\hline 17 & $6 / 10 / 2004$ & 2:35:47 PM & 25.097 & 26.618 & 24.872 & 27.555 & 26.471 & $\begin{array}{ll}11.12 \\
\end{array}$ & 18.394 & 3.832 & 7.35 & 1.923 & 0.464 & 44.766 & 0.605 & $\begin{array}{l}14.643 \\
\end{array}$ & & & & 16.9 & & $9.2>>3$ & & & 0.091 & & & \\
\hline $\begin{array}{ll}18 \\
18\end{array}$ & 6/10/2004 & 2:36:47 PM & 24.802 & 26.613 & 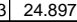 & 27.525 & 26.416 & 10.861 & 18.104 & 3.814 & 7.136 & 2.019 & 0.637 & 44.781 & 0.595 & 14.643 & 0.003 & 1.05 & 0.01667 & 16.6 & & 9.0 & 0.620 & $\begin{array}{ll}0.089 \\
\end{array}$ & 0.089 & 0.010 & 9.887 & 8.41 \\
\hline 19 & 6/10/2004 & 2:37:47 PM & 24.793 & 26.619 & $\begin{array}{ll}9 & 24.938 \\
\end{array}$ & 27.516 & 26.467 & 11.85 & 18.351 & 3.785 & 8.118 & 1.116 & -0.707 & 44.925 & 0.659 & 14.643 & & 2.0 & 0.03333 & 16.9 & 11. & 10.0 & 0.688 & 0.098 & & & 9.858 & \\
\hline 20 & 6/10/2004 & 2:38:47 PM & 24.8 & 26.61 & $\begin{array}{l}11 \\
11\end{array}$ & 27.542 & 26.519 & 11.873 & 18.224 & 3.904 & 8.025 & 1.098 & -0.716 & 44.862 & 0.658 & 14.643 & 0.00 & 3.00 & 0.05 & 16.8 & & 9.9 & 0.686 & 0.098 & 0.098 & 0.010 & 9.874 & \\
\hline & $6 / 11012004$ & 2:39:47 PM & 24.817 & 26.612 & 24.957 & 27.544 & 26.565 & 11.798 & 18.187 & 3.922 & 7.904 & 1.055 & -0.73 & 44.818 & 0.663 & 14.643 & & 4.00 & 0.06667 & 16.7 & & & & 0.099 & & & 10.046 & \\
\hline$\frac{22}{23}$ & 6/10/2004 & 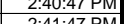 & 24.835 & 26.625 & 24.98 & 27.557 & 26.554 & 11.825 & 18.282 & 3.894 & .925 & 1.052 & -0.128 & 44.971 & 0.663 & 14.643 & & 5.00 & 0.08333 & 16.8 & & $9.9 .9>3$ & & (0.099 & 0.099 & & 0.016 & \\
\hline \begin{tabular}{|l|}
24 \\
24
\end{tabular} & 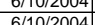 & $\begin{array}{l}2.244 .47 \mathrm{FM} \\
2.04 .07 \mathrm{PM}\end{array}$ & 24.852 & $\frac{20.028}{26605}$ & 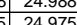 & 20.575 & 20.571 & 11.7151 & \begin{tabular}{|l|}
18.1 \\
18.492
\end{tabular} & $\begin{array}{l}3.905 \\
3.847\end{array}$ & $\begin{array}{r}7.875 \\
8294\end{array}$ & $\begin{array}{l}1.055 \\
1052\end{array}$ & -0.73 & 年44.718 & 0.662 & $\frac{14.643}{14.643}$ & 0.003 & 6.00 & $\begin{array}{r}0.1 \\
011667\end{array}$ & $\frac{16.6}{170}-x-3$ & & $\frac{9.8}{102}+x-1$ & & \begin{tabular}{|c|c|c|c|}
0.099 \\
0.098
\end{tabular} & $\begin{array}{l}0.099 \\
0.098 \\
|c| c \mid\end{array}$ & & $\frac{10.080}{9651}$ & \\
\hline 25 & $6 / 10 / 2004$ & 2:42.43:47 PM & 24.876 & 26.611 & 125.016 & 27.573 & 26.629 & $\begin{array}{ll}12.051 \\
11.798\end{array}$ & \begin{tabular}{|l|}
10.452 \\
18.185 \\
\end{tabular} & $\begin{array}{l}0.041 \\
3.788 \\
\end{array}$ & 8.054 & $\begin{array}{l}1.054 \\
1.043\end{array}$ & -0.745 & 444.773 & 0.65 & 14.045 & 0.003 & 8.00 & 0.13333 & $\begin{array}{ll}16.7 \\
16.7\end{array}$ & 11. & 0.4 & 0.684 & 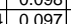 & 0.097 & 0.010 & 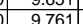 & \\
\hline 26 & $6 / 10 / 2004$ & 2:44:47 PM & 24.888 & 26.633 & 25038 & & 26.676 & 12.06 & \begin{tabular}{|l|l|}
18.508 \\
\end{tabular} & 3.87 & 8.22 & 1.055 & -0.745 & 44833 & 0.056 & 14.643 & & 900 & & 170 & & 101 & 0 & 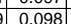 & 0.08 & & & 31 \\
\hline & $6 / 10 / 2004$ & 2:45:47 PM & 24.9 & $\frac{26.631}{26.631}$ & 25.05 & 27.642 & 26.693 & 11.954 & 18.226 & 3.828 & 8.236 & 1.014 & -0.745 & 45.094 & 0.658 & 14.643 & & $\frac{10.00}{10.00}$ & 0.16667 & $\begin{array}{l}11.0 \\
16.8\end{array}$ & $\frac{x_{11}}{11}$ & $\frac{10.1}{10.1}$ & 696 & 0.098 & $\frac{0.098}{0.098}$ & & $\begin{array}{l}9.050 \\
9.704\end{array}$ & 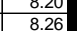 \\
\hline 28 & $6 / 10 / 2004$ & 2:46:47 PM & 24.908 & 26.648 & 25.068 & 27.655 & 26.721 & 11.803 & \begin{tabular}{|l|l|l|}
18.171 \\
\end{tabular} & 3.766 & 8.204 & 1.052 & -0.742 & 44.802 & 0.661 & 14.643 & & 11.00 & 0.18333 & 16.7 & $\frac{11}{11}$ & 100 & 6990 & 0.099 & 0.098 & 0.010 & 9.833 & \\
\hline & & & & & & & & & 18224 & 3.929 & 7867 & & & & & 14643 & & & & 168 & & 98 & & 0.098 & & & & \\
\hline 30 & $6 / 10 / 2004$ & $2: 48: 47$ PM & 24.932 & 26.662 & 25.077 & \begin{tabular}{|l|l|}
27.739 \\
\end{tabular} & 26.775 & 12.062 & \begin{tabular}{|l|l|}
18.446 \\
\end{tabular} & 3.822 & 8.327 & 1.032 & -0.745 & 44.889 & 0.658 & 14.643 & 0.003 & 13.00 & 0.21667 & 17.0 & 11.6 & 10.2 & & 0.098 & 0.098 & 0.010 & $\frac{0.602}{9.602}$ & 8.17 \\
\hline & 6/10/2004 & 2:49:47 PM & 24.945 & 26.67 & $\begin{array}{l}7 \\
7\end{array}$ & 27.762 & & 12.01 & 18.426 & 3.91 & 8 & 1.0 & & 45.1 & 0.662 & 14.6 & & 14.00 & 0.23333 & 17.0 & 11. & & & .099 & 0.098 & & 9.759 & \\
\hline 32 & 6/10/2004 & 2:50:47 PM & 24.973 & 26.703 & 25.113 & 27.795 & & 11.962 & 18.314 & 3.826 & 8.117 & 1.00 & & 44.942 & 0.6 & 14.6 & & 15.00 & 0.25 & 16.8 & 11. & $\begin{array}{lll}10.0 & \\
\end{array}$ & 692 & 0.098 & & & & 2 \\
\hline 33 & 6/10/2004 & 2:51:47 PM & 24.971 & 26.706 & 25.096 & 27.798 & 26.863 & 11.827 & 18.21 & 3.847 & 8.064 & 1.052 & & 44.697 & 0.659 & 14.643 & 0.003 & 16.00 & 0.26667 & $16.7 \mathrm{r}-\mathrm{l}$ & 11 & 9.9 & 0.686 & 0.098 & 0.098 & 0.010 & 9.852 & 8.38 \\
\hline 34 & 10/2004 & 2:52:47 PM & 24.979 & 26.719 & 25.119 & 27.781 & & 11.9 & 18.297 & & & & -0.74 & 44.99 & & 14. & & 17.00 & 0.28333 & $16.8 \mathrm{r}$ & 11 & 10.0 & & & & & 9.710 & \\
\hline 35 & 6/110/2004 & & 24.998 & 26.743 & 25.133 & 27.765 & & 11.917 & 18.312 & & 161 & 1.069 & & 44.406 & & & & 18.00 & 0.3 & 16.8 & & & & .099 & & & & \\
\hline 36 & $6 / 10 / 2004$ & 2:54:47 PM & 25.004 & 26.74 & 25.14 & 27.736 & 26.737 & 11.859 & 18.311 & 3.763 & 8.15 & 1.055 & -0.72 & 44.593 & 0.656 & 14.643 & & 19.0 & 0.31667 & 16.8 & 10. & 10.0 & 0.696 & 0.098 & 0.097 & 0.010 & 9.738 & 8.2. \\
\hline$\frac{31}{38}$ & & & & & & & & & & & & & & & & & & & & & & & & & & & & \\
\hline \begin{tabular}{|l|l|}
39 \\
\end{tabular} & & Averages & 24.9 & 26.7 & 25.0 & 27.6 & 26.7 & 11.8 & 18.3 & 3.8 & 8.0 & 1.1 & $-0.6>-6$ & 44.9 & 0.7 & 14.6 & & & & 168 & 11. & 99 & 0.7 & 01 & 01 & 0.0 & 98 & \\
\hline 40 & & Maximum & 25.1 & 26.7 & 25.1 & 27.8 & 26.9 & 12.1 & 18.5 & 3.9 & 8.3 & $\frac{1.1}{2.0}$ & & 45.1 & 0.7 & 14.6 & & & & 17.0 & 11.1 & 10.2 & 0.7 & $\frac{1.1}{0.1}$ & & & $\frac{.01}{10.1}$ & 8. \\
\hline 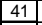 & & $\begin{array}{l}\text { Median } \\
\end{array}$ & 24.9 & 26.6 & & 27.6 & 26.7 & $\mid 11.9$ & 18.3 & 3.8 & 8.1 & 1.1 & & 44.8 & 0.7 & 14.6 & & & & $\frac{16.8}{16.8}$ & \begin{tabular}{|l|l|}
$B$ & 11.0 \\
\end{tabular} & 100 & 0.7 & 0.1 & & & 9.8 & $\frac{8 .}{8 .}$ \\
\hline 42 & & & 24.8 & 26.6 & 24.9 & 27.5 & 26.4 & 10.9 & 18.1 & 3.8 & 7.1 & 1.0 & & 44.4 & 0.6 & 14.6 & & & & 16.6 & 10.9 & 9.0 & 0.6 & 0.1 & 0.1 & 0.0 & 9.6 & $\frac{8 .}{8 .}$ \\
\hline 43 & & $2 \times \operatorname{Std} \mathrm{Dev}$ & 0.159 & 0.089 & 0.156 & 0.204 & & 0.580 & 0.234 & 0.106 & 0.582 & 0.554 & & 0.347 & 0.036 & 0.000 & & & & 0.234 & 0.085 & .5 & $\overline{044}$ & & 005 & 0.000 & 0.261 & \\
\hline 44 & Number 0 & of Points Used ${ }^{*}$ & 20 & & & 20 & & 20 & 20 & 20 & 20 & 20 & & 20 & 20 & 20 & & & & 20 & 26 & 20 & 2 & 20 & 20 & 20 & 20 & \\
\hline 45 & & & & & & & & & & & & & & & & & & & & & & & & & & & & \\
\hline 46 & & & & & & & & & & & & & & & & & & & & & & & & & & & & \\
\hline S & W & & & & & & & & & & & & & & & & & & & & & & & & & & & \\
\hline & Baseline wB] & & & & & & & & & & & & & & & & & & & & & & & & & & & \\
\hline$\frac{49}{50}$ & 6/10/2004 & 2:57:25 PM & 25.016 & 26.731 & 25.146 & 27.593 & 26.35 & 21.45 & 23.215 & 3.739 & 17.903 & -1.215 & & 44.785 & & & & 21.66 & & 21.7 & & 19.7 & 1.357 & | 0.192 & 0.191 & & 9.719 & \\
\hline$\frac{50}{51}$ & $\begin{array}{l}6 / 1012004 \\
6 / 10 / 2004 \\
\end{array}$ & \begin{tabular}{|l|}
$2: 58: 25$ PM \\
$259.25 \mathrm{PM}$ \\
\end{tabular} & $\begin{array}{l}25.051 \\
25.086\end{array}$ & $\frac{26.126}{26.711}$ & $\begin{array}{l}025.81 \\
11 \\
1125211\end{array}$ & $\begin{array}{l}27.558 \\
27.503\end{array}$ & 26.28 & $\frac{2.052}{21.452}$ & \begin{tabular}{|l|}
3.433 \\
23.28 \\
\end{tabular} & & & -1.221 & $\begin{array}{l}-4.895 \\
-4.89 \\
-109\end{array}$ & 44 & 0. & 1.3 & & 年 & & $\frac{|c|}{21.8}$ & \begin{tabular}{|l|l|}
11.0 \\
3
\end{tabular} & 19.6 & \begin{tabular}{|l|l|}
1.364 \\
1.356
\end{tabular} & & $\begin{array}{l}98 \\
97\end{array}$ & 0.010 & $\frac{10.026}{10.010}$ & 0.0 \\
\hline 52 & $6 / 10 / 2004$ & $3: 00: 25 \mathrm{~F}$ & 25.094 & & \begin{tabular}{|l|l|}
5 & 25.155 \\
\end{tabular} & 27.5 & 26.408 & & 23.282 & 3. & & & & 44.835 & & 1.334 & & 24.63 & 0.41056 & 21.8 & \begin{tabular}{|l|l|}
8 & 11.0 \\
\end{tabular} & 19.7 & 1.357 & 0.199 & 0.198 & 0.01 & \begin{tabular}{|l|}
10.063 \\
\end{tabular} & 8.5 \\
\hline 53 & $6 / 10 / 2004$ & 3:01: & \begin{tabular}{|l|l}
25.073 \\
\end{tabular} & & & 27.565 & 26.586 & 21.755 & 23.506 & 3.774 & 18.184 & -1.215 & & 45.035 & 0.003 & 1.328 & & 25.63 & 0.42722 & 22.0 & 11. & $20.6 \mathrm{C}$ & 1.377 & \begin{tabular}{|c|c|}
0.198 \\
\end{tabular} & 0.197 & 0.01 & \begin{tabular}{|l|l|} 
& 9.889 \\
\end{tabular} & 8.4 \\
\hline 54 & 6/10/2004 & & 25.038 & 26.7 & 25.048 & 27.64 & 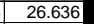 & 21.6 & 23.458 & & & & & 44.92 & & & & 26.6 & & 22.0 & 11.0 & 198 & & & & & \begin{tabular}{|l|l|} 
\\
\end{tabular} & 84 \\
\hline & $6 / 10 / 2004$ & 3:03:25 PM & 25.002 & 26.712 & $\begin{array}{ll}225.002 \\
\end{array}$ & \begin{tabular}{ll|}
27.694 \\
\end{tabular} & 26. & $\begin{array}{l}21.761 \\
2.70\end{array}$ & 23.558 & 3.801 & & -1.215 & & 45.081 & & $1.30 \mathrm{~s}$ & & $21.0 \mathrm{~s}$ & & 22.1 & 11.0 & 19.9 & & & & 0.010 & 9.791 & 8.3 \\
\hline & 6/10/2004 & $\begin{array}{l}3: 04: 25 \mathrm{PI} \\
23.05 \cdot 25 \mathrm{PI}\end{array}$ & 24.946 & 26.696 & $\begin{array}{lll}6 & 24.946 \\
1\end{array}$ & 27.718 & & 21.799 & \begin{tabular}{|l|l|}
23.589 \\
\end{tabular} & 3.8 & & & & 45.1133 & & & & 20 & & 22. & & & & & & & 10.004 & \\
\hline & $\frac{0.11012044}{612004}$ & & 24.901 & & $\frac{2.4901}{2.097}$ & 27.190 & & $\frac{2.1753}{21654}$ & & & & & & 45.292 & & & & & & ZZ. & & & & & & & & \\
\hline$\frac{25}{59}$ & $6 / 1012044$ & $3.007 .25 \mathrm{~F}$ & $\frac{24.000}{24822}$ & 26.733 & 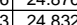 & 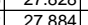 & & $\frac{2.0 .030}{21832}$ & 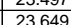 & 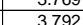 & & $\frac{1.25}{-1215}$ & & 年 & & & & 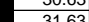 & & $\frac{2.0}{222}$ & 11. & $2018-4$ & 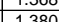 & & & 10 & $\frac{9.021}{9062}$ & \\
\hline \begin{tabular}{|l|l|l|l}
60 \\
\end{tabular} & $6 / 10 / 2004$ & 3:08.25 PM & 24.819 & 26.745 & $5 \quad 24.864$ & 27.901 & 26.862 & 32.034 & 36.222 & 3.672 & 28.724 & -1.215 & & 45.279 & 0.003 & 2048 & & $32.6:$ & 0 & $34.7 \mathrm{r}>\mathrm{C}$ & 11.1 & 30.4 & 2.095 & 0.305 & 0.307 & 0010 & 1009 & \\
\hline & & & & & & & & & & & & & & & & & & & & & & & & & & & & \\
\hline & & & & & & & & & & & & & & & & & & & & & & & & & & & & \\
\hline & & Averages & 25.01 & 26.1 & 25.1 & 27.6 & 26.6 & 21.6 & 23.4 & 3.8 & 18.6 & -1.2 & & 45.0 & 0.0 & 1.3 & & & & 22.0 & 11.0 & 19.8 & 1.4 & 0.197 & 0.197 & 0.010 & 9.9 & 8. \\
\hline 65 & & 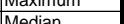 & $\frac{23.1}{250}$ & 267 & $\frac{25.4}{251}$ & 27.6 & & 21.6 & 23.0 & 3.0 & $\frac{10}{18}$ & -12 & & 告 45.4 & & 13 & & & & 220 & $\frac{1.1}{1.0}$ & 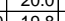 & 1.4 & & & 001 & trit. & \\
\hline & & & 24.9 & 26.7 & 24.9 & 27.5 & 26 & 21.5 & 23. & 3.0 & 179 & $\frac{1.2}{-1.2}$ & & 448 & 00 & 13 & & & & 217 & 110 & 197 & 1.4 & $\mid 0.190$ & 0191 & 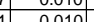 & 年 & \\
\hline \begin{tabular}{|l|}
67 \\
\end{tabular} & & $2 \times$ Std Dev & 0.150 & 0.022 & 0.228 & 0.210 & 0.369 & 0.267 & 0.255 & 0.069 & 0.238 & 0.004 & & 0.344 & 0.000 & 0.032 & & & & 0.255 & $5 \quad 0.084$ & $\mid 0.249$ & \begin{tabular}{|l|}
1.4 \\
0.017 \\
\end{tabular} & \begin{tabular}{|l|l|}
0.005 \\
\end{tabular} & 0.005 & 0.000 & $\begin{array}{r}9.16 \\
0.216\end{array}$ & $\begin{array}{r}8.4 \\
0.184\end{array}$ \\
\hline 68 & Number o & of Points Usedt & 10 & 10 & $\begin{array}{ll}0 & 10 \\
0\end{array}$ & 10 & 10 & 10 & $\frac{0.250}{10}$ & 10 & $\frac{1.200}{10}$ & $\begin{array}{r}10.4 \\
\end{array}$ & & $\frac{0.044}{10}$ & 10 & 10 & & & & $\frac{0.000}{10}$ & \begin{tabular}{|l|}
0.04 \\
\end{tabular} & 10 & & \begin{tabular}{|l|}
0.003 \\
\end{tabular} & 10 & 10 & $\frac{0.10}{10}$ & 1 \\
\hline & & & $\sin$ box & are not inc & & & & & & & & & & & & & & & & & & & & & & & & \\
\hline
\end{tabular}


WSRC-TR-2005-00105, REVISION 0 SRNL-RPP-2005-00012, REVISION 0

RUN \# 3.00A, B, AND C; PRE-TEST BASELINE WITH DIF WATER - CONT.

\begin{tabular}{|c|c|c|c|c|c|c|c|c|c|c|c|c|c|c|c|c|c|c|c|c|c|c|c|c|c|c|c|c|}
\hline & & & E & $\mathrm{F}$ & $G_{\text {G }}$ & $\mathrm{H}$ & J & $\mathrm{K}$ & 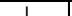 & $M$ & $N$ & 0 & 0 & $R_{1}$ & $\mathrm{~s}$ & & $\mathrm{v}$ & $W^{\prime}$ & $x$ & $Y^{\prime}$ & & & & & & & \\
\hline & $6 / 10 / 2004$ & 3:00:34 PM & & 26.75 & \begin{tabular}{|l|l|}
3 & 24.988 \\
\end{tabular} & $\begin{array}{l}27.879 \\
\end{array}$ & 26.915 & 32.278 & \begin{tabular}{|l|}
36.746 \\
\end{tabular} & 3.779 & 28.81 & -1.218 & $\begin{array}{l}-4.607 \\
-4.607\end{array}$ & 45.071 & 0.003 & 2.045 & 0.003 & 33.78 & \begin{tabular}{|l}
.5403039 \\
0.56306
\end{tabular} & 35.3 & 11.0 & & 2.106 & \begin{tabular}{|l|}
0.305 \\
0.305 \\
\end{tabular} & 0 & $\frac{0.010}{0.010}$ & $\begin{array}{l}9.983 \\
9.985 \\
\end{array}$ & $\begin{array}{l}8.50 \\
8.50 \\
\end{array}$ \\
\hline & $6 / 110 / 2004$ & 3:10:34 PM & 25.009 & 26.77 & 25.109 & 27.945 & 26.926 & 32.386 & 36.506 & 3.71 & 29.134 & -1.215 & -4.601 & \begin{tabular}{|l|l|l|} 
\\
\end{tabular} & 0.003 & $\begin{array}{l}2.042 \\
\end{array}$ & 0.005 & $\begin{array}{l}34.78 \\
\end{array}$ & 0.57972 & 35.0 & \begin{tabular}{|l|}
11.1 \\
\end{tabular} & 30.8 & 2.121 & 0.304 & 0.304 & 0.010 & $\begin{array}{l}9.867 \\
\end{array}$ & 8.40 \\
\hline & 6/10/2004 & & 25.1 & 26.79 & 25.201 & & & & $\begin{array}{l}36.709 \\
\end{array}$ & 3.682 & 29.044 & -1.209 & & & & & & & & & & & & & & & & 8.42 \\
\hline & 6 & $\frac{3.12 .34 \mathrm{PM}}{3.120}$ & & & & & & & & 3.67 & & -1.1 .15 & & & & & & 36.18 & & & & & & & & & & 8.55 \\
\hline & $\frac{011102004}{6120104}$ & $\frac{3.35 .34 \mathrm{PM}}{2.1 .210}$ & 20.244 & 2.00 & & & & & & & & -1.2 & & 44. & & & & & & & & & & 0.300 & & & $\begin{array}{ll}9.000 \\
0.00\end{array}$ & 8.39 \\
\hline 80 & 6 & 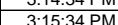 & $\frac{25.540}{25.417}$ & $\frac{2.01}{2680}$ & $\mid$ & 27.938 & & $\begin{array}{l}\frac{52.001}{32083} \\
32\end{array}$ & $\mid$ & 3.075 & & $\frac{-1.212}{-1212}$ & - 4.5359 & & & 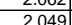 & & 年 30.10 & & 34.9 & $\frac{1.0}{110}$ & 步..04 & & 0.307 & & & 26 & 845 \\
\hline & 6 & $3 \cdot 1.1634$ & 25.471 & 26.78 & $\begin{array}{ll}25.506 \\
\end{array}$ & 27.783 & 26.584 & 32.316 & $\mid \begin{array}{l}\mid 36.539 \\
6\end{array}$ & & & -1.21 & -4.5 & & & 2.123 & & & & & 11.0 & & & & & & 10168 & 8.45 \\
\hline & $\frac{26 / 10 / 20}{6.10}$ & 3:117:3 & $\frac{25.527}{25.27}$ & $\frac{26.79}{26.79}$ & 25.583 & 27.784 & & 32.351 & \begin{tabular}{|l|}
36.767 \\
\end{tabular} & & 29.0 & & -4.584 & & & & & & & & & & & & & & & 8.6 \\
\hline 83 & $6 / 1012$ & & 25592 & & 25648 & 27759 & & 31878 & 36,13 & 3685 & & -1212 & -459 & & & & & & & & & & & & & & & 846 \\
\hline 84 & 6/10/2004 & 3:19:34 PM & 25.635 & 26.77 & 25.685 & 27.707 & 26.513 & 31.733 & \begin{tabular}{|l|l|}
35.923 \\
\end{tabular} & 3.75 & 28.369 & -1.215 & -4.598 & 45.012 & 0.00 & 2.082 & 0.00 & $\begin{array}{ll}43.78 \\
\end{array}$ & 0.72972 & 34. & 11.0 & 30. & 2.072 & $0.31 \mathrm{C}$ & 0.304 & 0.010 & 10.133 & 8.6 \\
\hline & & & & & & & & & & & & & & & & & & & & & & & & & & & & \\
\hline & & & & & & & & & & & & & & & & & & & & & & & & & & & & \\
\hline $8 t$ & & & 25.3 & & $\begin{array}{l}25.3 \\
\end{array}$ & 27.9 & & 32.3 & 36.6 & 3.7 & & -1.2 & & & & 2.1 & & & & & & & & & 0.3 & & & \\
\hline 88 & & & & 26 & & & & & & 3.8 & & -1.2 & -4 & & & 2.1 & & & & & & & & & & 0.0 & $10.2 \mathrm{Y}$ & \\
\hline 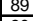 & & & 25.3 & 26 & 25 & 27. & & & & 3.7 & & -1.2 & & & & 2.1 & & & & 35.2 & 11.0 & 30 & & & 0.3 & .0 & 9.9 & \\
\hline$y$ & & & 24.8 & 26 & 24.9 & 2.7 .7 & & & 35.9 & 3.6 & 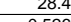 & -1.2 & -4.6 & 44.9 & & 2.0 & & & & 34.5 & 11. & 30.1 & 2.1 & 0.3 & 0.3 & 0.0 & 9.8 & \\
\hline & Nun & Soints Used & 0.512 & 0.03 & $\begin{array}{l}0.487 \\
2\end{array}$ & $\begin{array}{l}0.178 \\
12\end{array}$ & $\begin{array}{r}0.283 \\
12\end{array}$ & 0.484 & $\begin{array}{r}0.596 \\
12\end{array}$ & $\begin{array}{r}0.081 \\
12\end{array}$ & 0.526 & 0.006 & $\begin{array}{r}0.013 \\
12\end{array}$ & $\begin{array}{r}0.175 \\
12\end{array}$ & 12 & $\begin{array}{l}0.045 \\
12\end{array}$ & 11 & & & 0.596 & & & $\frac{.035}{12}$ & & & & $\frac{218}{12}$ & \\
\hline
\end{tabular}


WSRC-TR-2005-00105, REVISION 0

SRNL-RPP-2005-00012, REVISION 0

RUN \# 3.00A, B, AND C; PRE-TEST BASELINE WITH 0.1 M NAOH

\begin{tabular}{|c|c|c|c|c|c|c|c|c|c|c|c|c|c|c|c|c|c|c|c|c|c|c|c|c|c|c|c|c|}
\hline & $A$ & $B$ & $\mathrm{D}$ & $E$ & $F$ & G & $\mathrm{H}$ & $\mathrm{J}$ & K & L & $\mathrm{M}$ & $\mathrm{N}$ & $\mathrm{O}$ & \begin{tabular}{l|l} 
\\
\end{tabular} & $R$ & $\mathrm{~s}$ & $\mathrm{~T}$ & $\mathrm{v}$ & w & $x$ & Y & $z$ & $\mathrm{AA}$ & $A B$ & $\mathrm{AC}$ & $\mathrm{AD}$ & $\mathrm{AE}$ & $\mathrm{AF} \quad \mathrm{A}$ \\
\hline & & & & & & & & & & & & & & & & & & & & & & & & & & & & \\
\hline & $\frac{6 / 110 / 2004}{66110 / 2004}$ & $\begin{array}{l}\text { 3:30:13 PM } \\
\text { 3:31:13 PM }\end{array}$ & 25.937 & $\begin{array}{l}26.783 \\
26.805 \\
\end{array}$ & $25.388 \mid$ & $\begin{array}{l}27.914 \\
27.977\end{array}$ & $\begin{array}{l}26.87 \\
26.928 \\
\end{array}$ & $\begin{array}{l}11.187 \\
11.765\end{array}$ & $\frac{15.725}{15.916}$ & $\begin{array}{l}3.806 \\
3.842 \\
\end{array}$ & $\begin{array}{l}7.364 \\
7.97 \\
\end{array}$ & \begin{tabular}{|c|c|}
-0.786 \\
-1.218
\end{tabular} & $\begin{array}{r}-1.93 \\
-2.231 \\
\end{array}$ & $\begin{array}{ll}44.892 \\
44.892\end{array}$ & $\begin{array}{l}0.558 \\
0.54 \\
\end{array}$ & $\begin{array}{l}14.643 \\
14.63 \\
\end{array}$ & 0.003 & 0.00 & \begin{tabular}{r|}
0 \\
00167 \\
\end{tabular} & $\begin{array}{l}14.3 \\
14.4 \\
\end{array}$ & \begin{tabular}{ll|l}
11.0 \\
110
\end{tabular} & 9.3 & 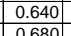 & \begin{tabular}{l|l}
0.083 \\
0080
\end{tabular} & $\begin{array}{l}0.082 \\
0.079 \\
\end{array}$ & 0.009 & 8.872 & 7.55 \\
\hline & $6 / 10 / 2004$ & 3:32:13 PM & 25.577 & 26.822 & 25.167 & 28.018 & 26.979 & $\begin{array}{l}1.100 \\
11.83 \\
\end{array}$ & \begin{tabular}{|l|}
15.510 \\
15.882 \\
\end{tabular} & 3.842 & 8.035 & $\begin{array}{l}-1.215 \\
-1.215 \\
\end{array}$ & $\begin{array}{l}-2.231 \\
-2.237\end{array}$ & $\begin{array}{l}44.8424 \\
44.842\end{array}$ & $\begin{array}{l}.534 \\
0.533 \\
\end{array}$ & $\begin{array}{l}14.643 \\
14.643 \\
\end{array}$ & $\begin{array}{l}0.003 \\
0.003 \\
\end{array}$ & $\frac{1.00}{2.00}$ & $\begin{array}{l}0.0166 / 33 \\
0.03333 \\
\end{array}$ & $\begin{array}{l}14.4 \\
14.4\end{array}$ & \begin{tabular}{|l|}
11.0 \\
11.0 \\
\end{tabular} & 9.9 & 685 & 079 & $\begin{array}{l}0.079 \\
0.079\end{array}$ & $\begin{array}{l}0.0008 \\
0.008 \\
\end{array}$ & $\begin{array}{l}8.004 \\
7.963\end{array}$ & $\begin{array}{l}6.81 \\
6.78\end{array}$ \\
\hline & $6 / 10 / 2004$ & 3:33:13 PM & & & & & & 11.836 & 15.971 & & & -1.215 & & 44.904 & & & & & & & & & & & & & & 6.79 \\
\hline & $6 / 101 / 2004$ & $\begin{array}{l}3: 34: 13 \mathrm{PM} \\
\end{array}$ & 25.294 & 26.839 & \begin{tabular}{|l|}
24.939 \\
\end{tabular} & 28.06 & 27.056 & $\frac{11.825}{11.825}$ & 155.94 & $\begin{array}{l}3.000 \\
3.861 \\
\end{array}$ & $\begin{array}{l}0.021 \\
8.007 \\
\end{array}$ & $\begin{array}{l}-1.218 \\
-1.218\end{array}$ & - 2.254 & 44.058 & 0.531 & 14.043 & 0.003 & 3.00 & \begin{tabular}{|l|l|} 
& 0.06667 \\
\end{tabular} & $\begin{array}{l}14.5 \\
14.5\end{array}$ & 11.0 & 9.9 & \begin{tabular}{|l|}
0.680 \\
\end{tabular} & 0 & $\begin{array}{l}0.079 \\
0.079\end{array}$ & $\begin{array}{l}0.008 \\
0.008 \\
0\end{array}$ & 7.998 & 6.81 \\
\hline & 6/10/2004 & 3:35:13 PM & 25.217 & 26.862 & 24.872 & 28.063 & 26.924 & 11.854 & 15.899 & 3.813 & 8.128 & -1.215 & -2.26 & 44.914 & 0.53 & 14.643 & 0.003 & 5.00 & 0.08333 & 14.4 & & & \begin{tabular}{|l|}
0.689 \\
\end{tabular} & \begin{tabular}{|l|l|}
0.079 \\
\end{tabular} & 0.079 & 0.008 & 7.938 & 6.75 \\
\hline 02 & 6/10/2004 & 3:36:13 PM & 25.094 & 26.879 & \begin{tabular}{|l|}
24.784 \\
\end{tabular} & 28.041 & 26.872 & 11.817 & 15.806 & 3.839 & 7.968 & -1.215 & -2.271 & 44.727 & 0.529 & 14.643 & 0.003 & 0.00 & & 14.3 & 11.0 & 9.9 & \begin{tabular}{|l|}
0.682 \\
\end{tabular} & 0.079 & 0.079 & 0.008 & 8.021 & 6.83 \\
\hline & $6 / 10 / 2004$ & 3:37:13 PM & 24.991 & 26.876 & \begin{tabular}{|l|}
24.686 \\
\end{tabular} & 27.993 & 26.754 & 11.811 & 15.996 & 3.923 & 7.957 & -1.215 & -2.265 & 44.745 & 0.529 & 14.643 & 0.003 & 1.00 & 0.01667 & 14.5 & 11.0 & 9.9 & 0.681 & 0.079 & 0.080 & 0.008 & 8.050 & 6.85 \\
\hline & $6 / 10 / 2004$ & 3:38:13 PM & 24.928 & 26.893 & \begin{tabular}{|l|}
24.617 \\
\end{tabular} & 27.924 & & 11.699 & \begin{tabular}{|l|l|}
15.617 \\
\end{tabular} & 3.757 & & -1.215 & -2.277 & 44.908 & & & & 2.00 & 0.03333 & 14.1 & 111.0 & & & & & & 8.059 & 6.86 \\
\hline 年 & 6/10/2004 & $\begin{array}{l}3: 39: 13 \text { PM } \\
\end{array}$ & 24.829 & $\begin{array}{l}26.895 \\
26.897 \\
\end{array}$ & \begin{tabular}{|l|}
24.544 \\
21401 \\
\end{tabular} & $\begin{array}{l}27.846 \\
27863\end{array}$ & 26.718 & 11.635 & 15.702 & 3.849 & & \begin{tabular}{|c|}
-1.215 \\
1.212
\end{tabular} & -2.277 & 44.816 & 0.528 & & & 3.00 & \begin{tabular}{|l|}
0.05 \\
\end{tabular} & 14.2 & 11.0 & 9.7 & & 0.079 & 0.080 & 0.008 & 8.220 & 7.00 \\
\hline & 6/10/2004 & 3:40:13 PM & 24.781 & 26.897 & \begin{tabular}{|l|}
24.481 \\
2.02 \\
\end{tabular} & \begin{tabular}{|l|l|}
27.863 \\
27.925
\end{tabular} & 26.65 & & & 3.885 & & & -2.283 & 44.743 & 0.528 & \begin{tabular}{|l|}
14.643 \\
\end{tabular} & & 4.00 & 0.06667 & 14.6 & 11.0 & & & 0.079 & & 0.008 & $\begin{array}{l}7.906 \\
\end{array}$ & 6.73 \\
\hline & & & & & & & & & $\begin{array}{l}24.069 \\
\end{array}$ & & & & & 45.845 & & 1.26 & & 5.00 & & 22.6 & 11.2 & & & & & & & 0.02 \\
\hline$\frac{\mid 11}{11 .}$ & & & & & & & & & & & & & & & & & & & & & & & & & & & & \\
\hline 1112 & & Averages & 25.2 & 26.9 & 24.9 & 28.0 & 26.8 & $\begin{array}{l}12.7 \\
\end{array}$ & 16.6 & 3.9 & 8.9 & -1.2 & & 44.9 & 0.5 & 13.5 & & & & 5.1 & (1. & 10.8 & & & & & & \\
\hline & & & & & & & & & & & & & & & & & & & & & & & & & & & & \\
\hline & & Median & 25.2 & 26.9 & 24.8 & 28.0 & & 11.8 & 15.9 & 3.8 & 8.0 & -1. & & $\frac{84.9}{44.9}$ & 0.5 & 14.6 & & & & 14.4. & 11.0 & 9.9 & 拉. & $\frac{0.1}{0.1}$ & & & & $\frac{7.6}{6.8}$ \\
\hline & & & & & \begin{tabular}{l|l|l|}
24.4 \\
\end{tabular} & 27.8 & & & & & & & & & 0.0 & & & & & & & & & & & & & \\
\hline 1 & & $2 \times S t d$ Dev & 0.787 & 0.075 & 0.618 & \begin{tabular}{|c|c|}
0.169 \\
\end{tabular} & 0.27 & 6 & $\frac{4.540}{4.540}$ & 0.104 & 6.144 & 0.237 & 1.606 & 0.577 & 0.293 & 7.398 & & & & 4.540 & \begin{tabular}{|l|}
1.141 \\
\end{tabular} & 16.131 & 0.423 & 0.044 & 0.044 & 0.004 & 4.488 & 3.820 \\
\hline & Number o & of Points Used* & 12 & & 12 & 12 & & 12 & 12 & 12 & 12 & 12 & & 12 & 12 & 12 & & & & 12 & 12 & 12 & 12 & \begin{tabular}{|r|} 
\\
\end{tabular} & 12 & 12 & 12 & 12 \\
\hline & & & in box & re not incl & Iluded & & & & & & & & & & & & & & & & & & & & & & & \\
\hline & A & B & $\mathrm{D}$ & $\mathrm{E}$ & $\mathrm{F}$ & G & & $\mathrm{J}$ & K & L & $M$ & $\mathrm{~N}$ & & Q & $\mathrm{R}$ & $\mathrm{s}$ & & $\mathrm{V}$ & w & $\mathrm{x}$ & $Y$ & z & $\mathrm{AA}$ & $A B$ & $\mathrm{AC}$ & $A D$ & $\mathrm{AE}$ & $\mathrm{AF}$ \\
\hline & aseline wB2 & & & & & & & & & & & & & & & & & & & & & & & & & & & \\
\hline & 6/10/2004 & 3:41:33 PM & 24.542 & 26.877 & 24.406 & 27.844 & 26.666 & 22.236 & 23.346 & 3.732 & 18.705 & -1.218 & -5.133 & 45.023 & 0.0 & 1.1 & & 21.63 & & 2.00 & & & .411 & .100 & & & 8.391 & .14 \\
\hline & $\frac{6 / 10 / 2004}{6 / 10 / 2004}$ & $\begin{array}{l}3: 42: 33 \mathrm{PM} \\
3.4333 \mathrm{PM}\end{array}$ & & $\frac{26.879}{26.869}$ & & $27.836 \mid$ & 26.6 & $\frac{2.0015}{21026}$ & & $\frac{3.799}{3.853}$ & $\frac{18.42}{18.177}$ & & & 444.871 & & & & & & 21.8 & & & 396 & & & & & 7.14 \\
\hline 127 & 6/10/2004 & $\begin{array}{l}3.45 .35 \mathrm{PMV} \\
3.4433 \mathrm{PM}\end{array}$ & $\begin{array}{l}24.448 \\
22413\end{array}$ & $\frac{26.869}{26.854}$ & 24.393 & $\mid$ & $\frac{2.03}{265}$ & $\frac{2.1900}{21857}$ & $\frac{2.1 .153}{23048}$ & 年. 3035 & 10.18041 & -1.212 & 5.1 & $\begin{array}{ll}44.622 \\
44.962\end{array}$ & 然 & & & $\frac{2.03}{2.63}$ & & $\frac{2.1}{215}$ & $\frac{10.9}{110}$ & 199 & .38 & & 0.17 & & 8.6364 & 7.35 \\
\hline 128 & $6 / 10 / 2004$ & & $\frac{24.410}{24.399}$ & $\begin{array}{l}\frac{20.034}{26.85} \\
\end{array}$ & 24.3.350 & 27.772 & & $\frac{21.051}{21.886}$ & \begin{tabular}{|l|}
22.940 \\
22.985 \\
\end{tabular} & 3.010 & & $\begin{array}{l}-1.218 \\
-1.218\end{array}$ & $\begin{array}{l}-3.127 \\
-5.125\end{array}$ & $\begin{array}{l}44.9034 \\
44.833\end{array}$ & & & & $\frac{24.05}{25.63}$ & & $\begin{array}{ll}21.5 \\
21.5\end{array}$ & 11.0 & 势, & & & 174 & & & $\begin{array}{l}7.34 \\
7.40 \\
-1\end{array}$ \\
\hline & $6 / 10 / 2004$ & $3: 46: 3$ & 24.375 & 26.836 & 24.365 & 27.823 & & 22.083 & \begin{tabular}{|l|}
23.269 \\
\end{tabular} & & & -1.218 & -5.1 & 44.821 & & & & 26.63 & & 21.8 & $\overline{11.0}$ & 20.3 & 2 & 0.172 & 0.175 & & & 7.32 \\
\hline 100 & $6 / 10 / 2 C$ & 3:47:3: & 24.391 & 26.852 & 24.436 & \begin{tabular}{|l|l|}
27.799 \\
\end{tabular} & & 21.963 & 23. & 3.751 & 18.3 & $\mid-1.215$ & -5.125 & 44.904 & & & & 27.63 & 0.46 & 21.6 & 11.0 & 20.2 & & & & & & \\
\hline & $6 / 10 / 2004$ & 3:48:3 & 24.4 & 26.841 & 24.535 & 27.813 & & & 23.346 & 3.858 & & -1.21 & & 4 & & & & & & 21.9 & 11. & & & & & & & 7. \\
\hline 132 & 6/10/2004 & $3: 49: 33 \mathrm{P}$ & $\frac{24.442}{2.472}$ & $\begin{array}{ll}26.853 \\
26854\end{array}$ & $\frac{24.6}{2.6}$ & $\begin{aligned} 27.859 \\
2799\end{aligned}$ & & $\frac{22.00}{21.97}$ & \begin{tabular}{|l|}
23.006 \\
23019
\end{tabular} & $\begin{array}{r}3.83 \\
.82\end{array}$ & 18. & \begin{tabular}{|c|c|}
-1.215 \\
1215
\end{tabular} & $\frac{-5.1}{5.1}$ & $\begin{array}{ll}44.929 \\
44709\end{array}$ & & & & $\begin{array}{l}29.63 \\
30.63 \\
30.5\end{array}$ & 0.49383 & 21.5 & 11.0 & 20.2 & \begin{tabular}{|l|}
1.391 \\
\end{tabular} & 0.176 & & & 305 & 7.49 \\
\hline & 6/10/2004 & $\begin{array}{l}3: 50: 33 \mathrm{PM} \\
3.51 .33 \mathrm{PM}\end{array}$ & 24.473 & 26.854 26.849 & $24.643 \mid$ & $\begin{array}{r}27.89 \\
27.965 \\
\end{array}$ & 26.861 & 21.878 & $\frac{23.019}{33.214}$ & $\begin{array}{r}3.82 \\
2.576 \\
\end{array}$ & 18.1675 & $\begin{array}{l}-1.215 \\
-1.125\end{array}$ & -4.939 & 44.768 & 0.003 & $\begin{array}{l}1.164 \\
1.52 \\
\end{array}$ & & $\begin{array}{ll}30.63 \\
21.62 \\
21\end{array}$ & $\begin{array}{r}0.5105 \\
0.52717 \\
\end{array}$ & $\begin{array}{r}21.5 \\
317 \\
\end{array}$ & \begin{tabular}{|l|}
11.0 \\
108
\end{tabular} & $\begin{array}{l}20.0 \\
3291\end{array}$ & $\begin{array}{l}1.380 \\
2007 \\
\end{array}$ & 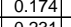 & 0.175 & 0.009 & 8.755 & 7.45 \\
\hline 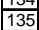 & $6 / 10 / 2004$ & $3.51 .33 \mathrm{PMN}$ & 4.493 & & & & & & & & & & & & & & & & & & & & & & & & & 6.83 \\
\hline & & & & & & & & & & & & & & & & & & & & & & & & & & & & \\
\hline & & & 24.4 & 26.9 & 24.5 & 27.8 & & 22 & 24 & 3.8 & 19.2 & -1.2 & & 44.8 & 0.0 & 1.2 & & & & 22.6 & 11.0 & 21.0 & 1.4 & 0.2 & 0.4 & & & \\
\hline & & & 24.5 & 26.9 & 24.7 & 28.0 & & 30.8 & 33.2 & 3.9 & & & & & & 1.6 & & & & 31.7 & 11.0 & 29.1 & 2.0 & 2 & 0.2 & 0.0 & 8.8 & \\
\hline 39 & & Median & 24.4 & 26.9 & 24.4 & 27.8 & 26 & $\begin{array}{r}22.0 \\
219 \\
\end{array}$ & 23.2 & $\begin{array}{l}3.8 \\
3\end{array}$ & 18.4 & $=-1.2$ & & 44.8 & 0.0 & 1.2 & & & & 21.7 & \begin{tabular}{|l|}
11.0 \\
\end{tabular} & 20.2 & 1.4 & \begin{tabular}{|l|}
0.2 \\
\end{tabular} & 0.2 & 0.0 & $\begin{array}{l}8.6 \\
.8 \\
0\end{array}$ & 7.3 \\
\hline$\frac{40}{41}$ & & $\frac{\text { Minimum }}{2 \times \text { Std De }}$ & $\begin{array}{r}24.4 \\
0.098\end{array}$ & $\frac{2.8 .8}{0.026}$ & $\begin{array}{r}24.4 \\
0.227\end{array}$ & \begin{tabular}{r|r}
27.8 \\
0.104
\end{tabular} & $\frac{26.6}{0.222}$ & $\frac{22.9}{5.050}$ & $\frac{22.9}{5.795}$ & $\begin{array}{r}3.6 \\
0.172 \\
\end{array}$ & $\begin{array}{r}18.0 \\
5.247\end{array}$ & $\mid$\begin{tabular}{|c|}
-1.2 \\
0.003
\end{tabular} & 0.107 & \begin{tabular}{|c|c|}
44.00 & 0.53 \\
\end{tabular} & $\begin{array}{r}0.0 \\
0.000\end{array}$ & $\begin{array}{r}1.1 \\
0.233\end{array}$ & & & & $\begin{array}{r}21.5 \\
5.795\end{array}$ & \begin{tabular}{|l|}
10.8 \\
0.133 \\
\end{tabular} & $\begin{array}{l}819.9 \\
35.148\end{array}$ & $\begin{array}{r}1.4 \\
0.355\end{array}$ & $\frac{0.2}{0.035}$ & $\frac{0.2}{0.034}$ & $\begin{array}{c}0.0 \\
0.000 \\
\end{array}$ & $\begin{array}{r}8.0 \\
0.439 \\
\end{array}$ & $\begin{array}{r}\quad 6.8 \\
0.374 \\
\end{array}$ \\
\hline & Number o & of Points Used & 11 & & & 11 & & 11 & 11 & 11 & & 11 & & 11 & 11 & 11 & & & & 11 & 11 & 11 & 11 & 11 & 11 & 11 & 11 & 11 \\
\hline & & $I^{*}$ Backpulse $\mathrm{pg}$ & in box & re not incl & luded & & & & & & & & & & & & & & & & & & & & & & & \\
\hline
\end{tabular}


WSRC-TR-2005-00105, REVISION 0 SRNL-RPP-2005-00012, REVISION 0

RUN \# 3.00A, B, AND C; PRE-TEST BASELINE WITH 0.1 M NAOH - CONT.

\begin{tabular}{|c|c|c|c|c|c|c|c|c|c|c|c|c|c|c|c|c|c|c|c|c|c|c|c|c|c|c|c|c|}
\hline \begin{tabular}{|l|l|l|}
147 \\
\end{tabular} & $\frac{A}{\text { Baseline wC }}$ & $B$ & D & $E$ & $\mathrm{~F}$ & G & $\mathrm{H}$ & $\mathrm{J}$ & $\mathrm{K}$ & L & M & $\mathrm{N}$ & 0 & Q & $\mathrm{R}$ & $\mathrm{s}$ & $T$ & $\mathrm{v}$ & w & $x$ & $Y$ & $z$ & $\mathrm{AA}$ & $A B$ & $A C$ & $A D$ & $\mathrm{AE}$ & $\mathrm{AF} \quad \mathrm{A}$ \\
\hline $\mid \frac{\mid 4 \pi}{148}$ & $\begin{array}{c}\text { Baseline we } \\
6 / 10 / 2004\end{array}$ & 3:52:04 PM & 24.554 & 26.85 & 24.734 & 28.026 & 26.962 & & 34.748 & & & -1.21 & $-4.91>-3$ & 44.914 & 0.003 & 1.61 & 0.006 & & & 33.3 & 11.0 & 30.3 & 2.090 & 0.240 & & & 7978 & 679 \\
\hline \begin{tabular}{|l|l|}
149 \\
\end{tabular} & 6/10/2004 & 3:53:04 PM & 24.669 & 26.865 & 24.889 & 28.056 & 26.982 & 31.984 & 34.652 & 3.721 & 28.508 & -1.215 & -4.908 & 44.996 & 0.003 & 1.606 & 0.004 & 33.78 & 0.563 & 33.2 & 11.0 & \begin{tabular}{|l|}
30.2 \\
\end{tabular} & 2.085 & 0.239 & 0.240 & 0.008 & 7.941 & 6.76 \\
\hline & $6 / 10 / 2004$ & 3:54:04 PM & 24.836 & 26.891 & 25.077 & 28.102 & 26.974 & 31.988 & 34.785 & 3.762 & $\begin{array}{l}28.467 \\
\end{array}$ & -1.215 & -4.905 & 44.819 & 0.003 & 1.612 & 0.004 & 34.78 & 0.57967 & $\begin{array}{l}33.3 \\
\end{array}$ & \begin{tabular}{|l|l|}
11.0 \\
\end{tabular} & 30.2 & 2.084 & $\begin{array}{l}0.240 \\
\end{array}$ & $\begin{array}{l}0.240 \\
\end{array}$ & 0.008 & 7.934 & 6.75 \\
\hline 151 & $6 / 10 / 2004$ & 3:55:04 PM & 24.963 & 26.893 & 25.188 & 28.064 & 27.025 & 31.853 & 34.462 & 3.73 & $\begin{array}{l}28.381 \\
2.307\end{array}$ & -1.218 & -4.908 & 44.908 & 0.003 & $\begin{array}{l}1.619 \\
\end{array}$ & 0.005 & 35.78 & 0.59633 & 33.0 & \begin{tabular}{|l|l|}
11.0 \\
\end{tabular} & $\begin{array}{l}30.1 \\
\end{array}$ & \begin{tabular}{|l|}
2.076 \\
\end{tabular} & 0.241 & 0.240 & 0.008 & 7.972 & 6.78 \\
\hline & $6 / 10 / 2004$ & 3:56:04 PM & 25.099 & 26.909 & 25.314 & 28.07 & 26.931 & \begin{tabular}{|l|l|}
31.893 \\
\end{tabular} & 34.677 & $\begin{array}{r}3.79 \\
3.907\end{array}$ & 28.307 & -1.215 & -4.908 & 44.965 & 0.003 & 1.623 & 0.004 & 36.78 & 0.613 & 33.2 & \begin{tabular}{|l|}
11.0 \\
1.0
\end{tabular} & 30.1 & 2.075 & 0.242 & 0.240 & 0.008 & 7.968 & 6.78 \\
\hline$\frac{153}{150}$ & $6 / 10 / 2004$ & 3:57:04 PM & $\frac{25.224}{25.21}$ & 26.924 & 25.435 & 28.075 & 26.892 & 31.808 & 34.518 & 3.787 & 28.209 & -1.215 & $-\frac{-4.905}{4}$ & 44.877 & 0.003 & 1.63 & 0.004 & 37.78 & 0.62967 & 33.0 & \begin{tabular}{|l|}
11.0 \\
\end{tabular} & \begin{tabular}{|l|l|}
30.0 \\
\end{tabular} & \begin{tabular}{|l|}
2.069 \\
\end{tabular} & 0.243 & 0.240 & 0.008 & 8.000 & 6.81 \\
\hline$\frac{154}{155}$ & $\begin{array}{l}6 / 101 / 2004 \\
6 / 10 / 2004\end{array}$ & $\begin{array}{l}3: 5: 5: 504 \mathrm{PM} \\
\text { 3:59:04 PM }\end{array}$ & $\begin{array}{r}\frac{25.34}{25.445} \\
\end{array}$ & $\frac{26.935}{26.93}$ & $\begin{array}{r}25.545 \\
25.631\end{array}$ & $\begin{array}{r}28.041 \\
28.011\end{array}$ & $\begin{array}{l}26.878 \\
26.838\end{array}$ & $\begin{array}{l}31.866 \\
32.056\end{array}$ & $\begin{array}{l}34.667 \\
34.692 \\
\end{array}$ & $\begin{array}{l}3.724 \\
3.768\end{array}$ & $\begin{array}{l}28.316 \\
28.584\end{array}$ & $\begin{array}{l}-1.1209 \\
-1.218 \\
\end{array}$ & $\begin{array}{l}-4.896 \\
-4.905\end{array}$ & $\begin{array}{l}44.944 \\
44.875\end{array}$ & 0.003 & $\begin{array}{r}1.638 \\
1.64 \\
\end{array}$ & $\begin{array}{l}0.004 \\
0.005\end{array}$ & $\begin{array}{l}38.78 \\
39.78 \\
\end{array}$ & $\begin{array}{c}0.64633 \\
0.663 \\
\end{array}$ & $\begin{array}{r}33.2 \\
33.2 \\
\end{array}$ & \begin{tabular}{|l|}
11.0 \\
11.0 \\
\end{tabular} & \begin{tabular}{|l|}
30.1 \\
30.3
\end{tabular} & \begin{tabular}{|l|}
2.075 \\
2.090
\end{tabular} & $\frac{0.244}{0.245}$ & $\frac{0.240}{0.240}$ & $\begin{array}{l}0.008 \\
0.008\end{array}$ & $\begin{array}{r}7.992 \\
7.923 \\
\end{array}$ & $\frac{6.80}{6.74}$ \\
\hline \begin{tabular}{|l|l|}
156 \\
\end{tabular} & $6 / 10 / 2004$ & 4:00:04 PM & 25.547 & 26.942 & 25.732 & 28.013 & 26.834 & 32.002 & 34.862 & 3.784 & 28.393 & -1.215 & -4.902 & 44.896 & 0.003 & 1.643 & 0.005 & 40.78 & 0.67967 & 33.4 & \begin{tabular}{|l|l|}
11.0 \\
\end{tabular} & \begin{tabular}{|l|}
30.2 \\
\end{tabular} & 2.082 & 0.245 & 0.240 & 0.008 & 7.947 & 6.76 \\
\hline \begin{tabular}{|l|}
157 \\
158
\end{tabular} & & & & & & & & & & & & & & & & & & & & & & & & & & & & \\
\hline \begin{tabular}{|l|}
1508 \\
159
\end{tabular} & & Averages & 25.1 & 26.9 & 25.3 & 28.1 & & 31.9 & 34.7 & 3.8 & 28.4 & -1.2 & -4.9 & 44.9 & 0.0 & 1.6 & & & & $33.2-2>0$ & 11.6 & & $2.1 .12=0$ & & & & & 6.8 \\
\hline 160 & & Maximum & 25.5 & 26.9 & 25.7 & 28.1 & 27.0 & 32.1 & 34.9 & 3.8 & 28.6 & -1.2 & -4.9 & 45.0 & 0.0 & 1.6 & & & & 33.4 & & 30.3 & 2.1 & 0.2 & 0.2 & 0.0 & 8.0 & 6.8 \\
\hline 161 & & Median & 25.1 & 26.9 & 25.3 & 28.1 & 26.9 & 32.0 & 34.7 & 3.8 & 28.4 & -1.2 & -4.9 & 44.9 & 0.0 & 1.6 & 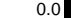 & & & 33.2 & 110 & 30.2 & 2.1. & 0.2 & 0.2 & 0.0 & 8.0 & 6.8 \\
\hline 162 & & Minimum & 24.6 & 26.9 & 24.7 & 28.0 & 26.8 & 31.8 & 34.5 & 3.7 & 28.2 & -1.2 & -4.9 & 44.8 & 0.0 & 1.6 & & & & 33.0 & & 30.0 & 2.1 & 0.2 & 0.2 & 0.0 & 7.9 & $\begin{array}{ll}6.7 \\
\end{array}$ \\
\hline & & $2 \times$ Std Dev & 0.653 & 0.059 & 0.641 & 0.058 & 0.127 & 0.179 & 0.234 & 0.054 & 0.236 & 0.005 & 0.008 & 0.100 & 0.000 & 0.026 & 0.001 & & & 0.234 & 0.024 & 0.203 & 0.014 & 0.004 & 0.001 & 0.000 & 0.051 & 0.043 \\
\hline & Number $\mathrm{c}$ & ff Points Used ${ }^{\star}$ & 9 & & & & & & & & & & & & & & & & & & & & 9 & 9 & & 9 & 9 & \\
\hline 1165 & & Backpulse p & $n$ box & not inc & & & & & & & & & & & & & & & & & & & & & & & & \\
\hline
\end{tabular}


RUN \# 3.03A AND B; FIRST AND SECOND HALF OF SLURRY DEWATERING

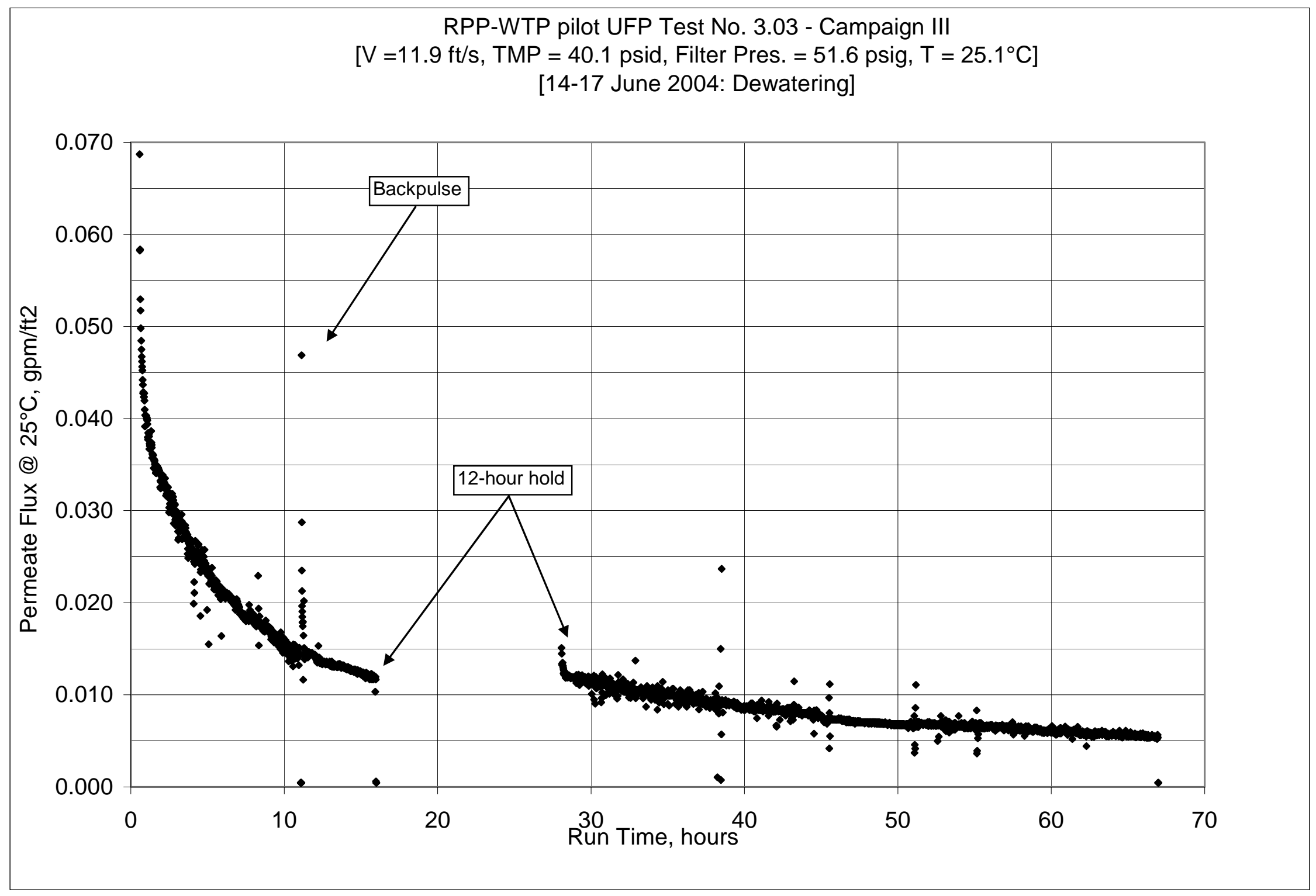


WSRC-TR-2005-00105, REVISION 0

SRNL-RPP-2005-00012, REVISION 0

RUN \# 3.03A AND B; FIRST AND SECOND HALF OF SLURRY DEWATERING - CONT.

\begin{tabular}{|c|c|c|c|c|c|c|c|c|c|c|c|c|c|c|c|c|c|c|c|c|c|c|c|c|c|c|c|c|}
\hline 1 & $\mathrm{~A}$ & $\mathrm{~B}$ & $\mathrm{D}$ & $\frac{E}{<T}$ & rature & $G$ & $\frac{H}{2}$ & $\mathrm{~J}$ & \begin{tabular}{l|l}
$K$ \\
$K \ll<\ll<E$
\end{tabular} & $\frac{1}{\text { Pressure }}$ & $\frac{M}{\text { easuren }}$ & $1 \mathrm{~N}$ & 0 & $\frac{Q}{Q<\ll \ll<}$ & $\begin{array}{l}\mathrm{R} \\
<\text { Elow }\end{array}$ & $\frac{\mathrm{s}}{\mathrm{s}}$ & $\mathrm{T}$ & $\mathrm{V}$ & 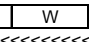 & $\frac{|x|}{<\ll<\ll c \mid}$ & $\begin{array}{ll}\mathrm{Y} \\
\text { Calculat }\end{array}$ & $\frac{1 z}{\text { lated }}$ & AA & $\mid A B$ & 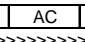 & $\frac{1 \text { AD } \mid}{\gg \gg \gg \gg \gg \gg}$ & 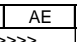 & AF \\
\hline$\frac{1}{2}$ & DATE & TIME & Filtrate & Cleaning & Slurry & Hi Amb. & Lo Amb. & BotTMP & Filter & $\begin{array}{l}\text { Pressure M } \\
\text { Fitter dP }\end{array}$ & $\begin{array}{l}\text { Measureme } \\
\text { TopTMP }\end{array}$ & | Filtrate & Sulsepot & & 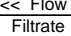 & $\begin{array}{l}\text { Measureme } \\
\text { | Hi Filtate }\end{array}$ & & & & & & & & & & & & \\
\hline & & & $\operatorname{deg} C$ & & & & & psid & & psid & & \begin{tabular}{|l|} 
\\
\end{tabular} & & & & $\mathrm{gpm}$ & & & & & & & & & & & & \\
\hline 4 & & & $\mathrm{~T} 2$ & & T1 & & & & $\mathrm{P}$ & $\mathrm{dP1}$ & $\mathrm{dP} 3$ & $P 2$ & & & & $\mathrm{Q} 3$ & & & & & & & & & & & & \\
\hline 5 & & & & & & & & & & & & & & & & & & & & & & & & & & & & \\
\hline & & & & & & & & & & & & & & & & & & & & & & & & & & & & \\
\hline 7 & $6 / 8 / 2004$ & 9:09:09 AM & 22.545 & 24.705 & 27.987 & \begin{tabular}{|l|l|}
26.054 \\
\end{tabular} & 24.911 & -0.004 & $\begin{array}{ll}0.128 \\
\end{array}$ & $\begin{array}{l}-0.01 \\
\end{array}$ & -0.003 & -0.129 & & 45.741 & 0.293 & \begin{tabular}{|c|c|c|}
-0.013 \\
\end{tabular} & 0.00 & & & Filter Su & Surface, & & T2 & & & & & \\
\hline & 6/10/2004 & & & & 26.68 & $\begin{array}{l}26.834 \\
2.174\end{array}$ & & -0.019 & 0.196 & -0.012 & $\begin{array}{l}-0.02 \\
\end{array}$ & -0.106 & & -0.071 & 0.003 & \begin{tabular}{|l|l|}
14.643 \\
\end{tabular} & & & & Convers & & & & $y / b=$ & & aarg & & \\
\hline 9 & 6/14/2004 & & 23.83 & 23.885 & 66.603 & $24.1 / 4$ & & -0.012 & 0.12 & -0.01 & & -0.163 & & -0.083 & 0.003 & 14.643 & & & & & & & & & & & & \\
\hline & 66170 & $12: 06$ & 25.175 & & 23.747 & 26.127 & & -0.0 & 0.139 & & & & & & & & & & & & & & & & & & & \\
\hline 12 & 611712004 & & 24.492 & 23.951 & 23.199 & 24.225 & 23.143 & 20 & 0.164 & -0.001 & & & & -0.075 & 0.003 & 14.643 & & & & & & & 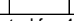 & & & & & \\
\hline 13 & $6 / 21 / 2004$ & 6:52:52 AM & $\begin{array}{l}20.259 \\
23.109\end{array}$ & 23.008 & 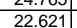 & \begin{tabular}{|l|}
27.005 \\
23.418 \\
\end{tabular} & $\frac{20.450}{22.888}$ & $\begin{array}{l}-0.004 \\
-0.01\end{array}$ & 0.1975 & & $\begin{array}{c}-0.012 \\
-0.01\end{array}$ & $\begin{array}{l}-0.103 \\
-0.197\end{array}$ & & $\begin{array}{l}-41.045 \\
-0.079\end{array}$ & 0.003 & $\begin{array}{l}14.045 \\
11.643\end{array}$ & 0.00 & & & WWYY & & & & $\leqslant$ F Filtra & ate Flux $=$ & $\forall<<$ PER & IABपI & $\bar{Y}\rangle \gg$ \\
\hline 14 & $6 / 22 / 2004$ & $8: 12: 12 \mathrm{AM}$ & 23.36 & 23.555 & 23.63 & 23.829 & 24.45 & -0.008 & 0.108 & -0.004 & -0.012 & -0.194 & 0.06 & -0.083 & 0.003 & 14.643 & 0.00 & & & & & & & & & & & \\
\hline & & & & & & & & & & & & & & & & & & Time & Time & Press. & vel. & TMP & TMP & gpm & $\mathrm{gpm}$ & gpm & $\mathrm{gpm}$ & meter \\
\hline & Data - Per M & & & & & & & & & & & & & & & & & & Hour & & & psi & & & & & & day/bar \\
\hline$\frac{17}{18}$ & $\begin{array}{l}6 / 144 / 2004 \\
6 / 142004\end{array}$ & $\begin{array}{l}\text { 9:11:414AM } \\
\text { 9:15:54 }\end{array}$ & \begin{tabular}{|}
26.805 \\
26.805 \\
\end{tabular} & $\begin{array}{l}24.883 \\
24.863\end{array}$ & $\begin{array}{l}79.051 \\
79.051\end{array}$ & \begin{tabular}{|}
26.343 \\
26.343 \\
\end{tabular} & $\begin{array}{r}25.614 \\
25.614 \\
\end{array}$ & $\begin{array}{r}45.463 \\
45.463 \\
\end{array}$ & $\begin{array}{l}47.021 \\
47.021\end{array}$ & $\begin{array}{l}8.649 \\
8.649\end{array}$ & $\begin{array}{r}37.7509 \\
37.509 \\
\end{array}$ & $\begin{array}{l}-1.219 \\
-1.219\end{array}$ & $\begin{array}{r}-4.955 \\
-4.955 \\
\end{array}$ & $\begin{array}{l}47.527 \\
47.527\end{array}$ & $\begin{array}{l}0.281 \\
0.281\end{array}$ & \begin{tabular}{|l|}
14.643 \\
14.643 \\
\end{tabular} & $\begin{array}{l}0.004 \\
0.004\end{array}$ & 1.8 & 20.02028 & 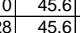 & \begin{tabular}{|l|l|}
11.6 \\
11.6
\end{tabular} & 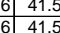 & \begin{tabular}{|l|l|}
5 & 2.860 \\
5 & 2.860
\end{tabular} & $\begin{array}{l}0.042 \\
0 \\
0.042\end{array}$ & $\begin{array}{l}2 \\
2 \\
2\end{array}$ & $\mid \begin{array}{l}0.001 \\
0.001\end{array}$ & $\begin{array}{l}0.960 \\
0.960\end{array}$ & $\begin{array}{l}0.82 \\
0.82\end{array}$ \\
\hline (10) & $6 / 14 / 2004$ & 9:16:54 AM & 27.274 & 24.914 & 80.155 & 26.419 & 25.659 & 45.68 & 47.222 & 8.445 & 37.993 & -1.222 & & 46.676 & 0.282 & \begin{tabular}{|l|}
14.643 \\
\end{tabular} & 0.004 & 2.22 & \begin{tabular}{|l|l|}
2 & 0.03694 \\
\end{tabular} & 45.8 & \begin{tabular}{|l|l|}
11.4 \\
\end{tabular} & $\begin{array}{lll}4 & 41.8 \\
\end{array}$ & 2.884 & 0.042 & 0.039 & 0.001 & 0.943 & 0.80 \\
\hline 201 & 6/14/2004 & 9:17:54 AM & & & 80.642 & 26.395 & & 45.116 & $\begin{array}{ll}46.662 \\
\end{array}$ & 8.996 & 36.823 & & & 48.638 & 0.274 & 14.643 & & & 0.05361 & 45.2 & 11.9 & 41.0 & & 0.041 & & & & 0.80 \\
\hline 21 & 6/14/2004 & 9:18:54 AM & 27.56 & 24.915 & 80.92 & 26.42 & 25.81 & 45.459 & 47.01 & 8.903 & 37.27 & -1.214 & -4.99 & 43.991 & 0.276 & 14.643 & 0.00 & 4. & . & 455.6 & 10.8 & 41.4 & 2.852 & 0.041 & 0.038 & 0.001 & 926 & 0.79 \\
\hline & $6 / 14 / 2004$ & 9:19:54 AM & 27.738 & 24.944 & 176.14 & 26.459 & 25.759 & 45.143 & 46.683 & 8.789 & 37.03 & -1.219 & & 45.299 & 0.276 & 14.643 & 0.00 & & 0.08694 & 45.4 & 11.1 & 41. & 2.83 & 0.041 & & & & 0.79 \\
\hline 23 & 6/114/2004 & 9:20:54 AM & 27.997| & 24.989 & 176.18 & 26.4999 & 25.784 & 45.318 & 46.938 & 8.576 & 37.453 & -1.219 & & 51.617 & 0.276 & 14.643 & & & 0.10361 & 45.7 & 12. 6 & 41. & 2.85 & .041 & .038 & & & 0.78 \\
\hline 24 & 60114212004 & 9:21:54 AM & 28.1321 & 24.989 & $1 / 6.21$ & 26.534 & 25.834 & 45.116 & 46.691 & & 37.162 & -1.219 & & 50.09 & 0.227 & & & & & 45.4 & & & & & & & & 0.78 \\
\hline$\frac{25}{26}$ & 6 & $9: 22: 54, \mathrm{AM}$ & 28.228 & 24.990 & 28.9808 & 26.521 & 25.806 & 45.305 & 4.785 & 8.688 & 37.336 & -1.222 & & 45.139 & 0.277 & 14.643 & & & | & 45.3. & 11.4 & 年1.3 & & 0.041 & 0.038 & & & 0.78 \\
\hline$\frac{20}{27}$ & 0 & 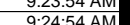 & $\frac{20.394}{28.476}$ & 24.950 & 17622 & 20.530 & 25.910 & (45.0164 & $\mid \begin{array}{l}4.1 .45 \\
4656\end{array}$ & & 37.101 & $=\frac{-1.219}{120}$ & & 45.652 & 0.21 & $\begin{array}{l}14.645 \\
1.643\end{array}$ & & & 0.15301 & & & & & & 0.038 & & & 0.77 \\
\hline 28 & $6 / 14 / 2004$ & $9: 25: 54 \mathrm{AM}$ & 28.593 & 25.043 & 28.815 & 26.573 & 25.997 & $\begin{array}{l}4.1 .34 \\
45.351\end{array}$ & \begin{tabular}{|l|}
47.147 \\
4.003
\end{tabular} & $\begin{array}{l}.0587 \\
8.58\end{array}$ & $\begin{array}{l}37.412 \\
37.45\end{array}$ & \begin{tabular}{|l|l|} 
& -1.219 \\
& -1.219 \\
\end{tabular} & $\begin{array}{l}-0.010 \\
-4.978\end{array}$ & \begin{tabular}{|l|}
50.426 \\
5
\end{tabular} & 0.273 & \begin{tabular}{|l|l|}
14.643 \\
14.643
\end{tabular} & 0.00 & $\frac{10.42}{11.22}$ & \begin{tabular}{|c|}
2 \\
0.18694
\end{tabular} & \begin{tabular}{|c|c|}
4 & 45.7 \\
4
\end{tabular} & 12.4 & 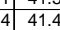 & \begin{tabular}{|l|l|}
4 & 2.0405 \\
\end{tabular} & \begin{tabular}{|l|l|}
30.041 \\
\end{tabular} & 0.037 & $\frac{0.001}{0.001}$ & 年 & $\frac{0.18}{0.75}$ \\
\hline & $6 / 14 / 2004$ & 9:26:54 AM & & $\begin{array}{l}25.06 \\
\end{array}$ & 28.837 & 26.67 & 26.024 & & 46.53 & 8.504 & 37.42 & $\begin{array}{l}-1.219 \\
\end{array}$ & & 49.419 & 0.278 & 14.643 & & & 0.20361 & 45.1 & 12.1 & $\begin{array}{ll}14 & 41.3 \\
\end{array}$ & 2.848 & 0.041 & 0.037 & 0.001 & 0.902 & 0.77 \\
\hline$\frac{30}{30}$ & $6 / 14 / 2004$ & 9:27:54 AM & 28.583 & 25.102 & 28.819 & 26.857 & 25.957 & \begin{tabular}{|l|l|}
43.559 \\
\end{tabular} & \begin{tabular}{|l|l|}
45.122 \\
\end{tabular} & 9.579 & 34.619 & -1.222 & & 48.966 & 0.261 & \begin{tabular}{|l|l|l|}
14.643 \\
\end{tabular} & 0.00 & 13. & 0.22028 & 433.7 & 12.0 & 39.1 & 2.695 & 0.039 & & 0.001 & 0.895 & 0.76 \\
\hline & $6 / 14 / 2004$ & & & 25.101 & 28.724 & 26.896 & & & & & & -1.222 & & 45.775 & & & & 14. & 0.23694 & 44. & 11.2 & & $2.74 \mathrm{f}$ & & 039 & & & 0.84 \\
\hline 32 & 6/14/2004 & 9:29:5 & 28.154 & 25.119 & 28.637 & 26.899 & & 44.264 & \begin{tabular}{|l|l|}
4 \\
\end{tabular} & 9.281 & 35.719 & -1.21 & & 52.212 & 0.256 & & & & .25361 & 43 & 12.8 & & 2.15 & .038 & . 034 & & 0.863 & 0.73 \\
\hline & $\frac{601412}{611212}$ & & 28.631 & & & 26.936 & & 44.231 & 45.155 & & 35.78 & $\frac{-1.21}{-12}$ & & 44.494] & 0.2 & 14. & & & 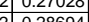 & & $\frac{10.9}{1.29}$ & $\begin{array}{ll}4.0 .0 \\
0.5\end{array}$ & & & . 036 & & . 8888 & 0.76 \\
\hline & $\frac{01141 / 2}{6 / 142}$ & 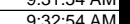 & (20.056 & 25.153 & $\frac{20.444}{28393}$ & 26.948 & & $\begin{array}{r}41.14 \\
42149\end{array}$ & $\begin{array}{r}45.13 \\
45419\end{array}$ & & 33.167 & -1.22 & & -50.3953 & 0. & & & & & & $\frac{1.3 .1}{127}$ & & & & & & & 0.58 \\
\hline$\frac{36}{36}$ & $6 / 14 / 2004$ & $9.5354 \mathrm{AM}$ & $\mid$ & 25.218 & 28342 & 26.968 & 266 & 42202 & $\begin{array}{l}40.458 \\
44.88\end{array}$ & 9 & 33.862 & -1219 & & 46365 & 0.125 & $\frac{14}{0.7}$ & & 19 & 232 & 43.4 & 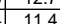 & $\begin{array}{ll}4 & 380 \\
\end{array}$ & 262 & (1) & 017 & & 0.446 & 0.89 \\
\hline 37 & $6 / 14 / 2004$ & 9:34:54 AM & 28.329 & $\mid$ & 28.242 & 26.923 & & 40.375 & 45.077 & 8.949 & 32.056 & -1.196 & & 53.25 & 0.004 & 14.643 & & $\frac{20}{20 .}$ & . & 43.6 & $\frac{13.0}{130}$ & & & & & 0.000 & 015 & 0.38 \\
\hline 38 & $6 / 14 / 2$ & & 28.589 & 25.204 & 28.164 & 26.914 & & 6.162 & 45.284 & & -2.344 & & & 45.08 & & & & & & & 11.0 & & & & & & & 18 \\
\hline & 6/14/2004 & $9: 36: 54$ & 28.525 & 25.25 & 28.125 & 27 & & 6.042 & 44.95 & & -2.245 & & & 50077 & 0.1 & 14. & & & & & $\frac{21.3}{12.3}$ & & & & & & & 0.10 \\
\hline & $6 / 7$ & & \begin{tabular}{|l|l|l|}
28.444 \\
\end{tabular} & 25.264 & \begin{tabular}{|l|}
28.068 \\
\end{tabular} & 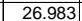 & & & & & $\frac{-2.10}{-2.10}$ & 33.6 & & & & & & & & & 116 & & & & & & & $\frac{0.16}{016}$ \\
\hline & & & \begin{tabular}{|l|l|}
28.38 \\
\end{tabular} & 25.274 & \begin{tabular}{|l|l|}
28.024 \\
\end{tabular} & \begin{tabular}{|l|}
26.979 \\
\end{tabular} & & & 45.216 & & & 33.6 & & $\begin{array}{l}48.589 \\
\end{array}$ & & & & 242 & & 43 & 11.9 & & & 000 & & & 0.191 & $\frac{1.16}{0.16}$ \\
\hline 42 & $6 / 14 / 2$ & & 28.313 & 25.287 & & \begin{tabular}{|l|}
27.007 \\
\end{tabular} & & 6.114 & & & & 33.6 & & & & & & 25. & & & 11.3 & 2. & .1 & & & & & 0.17 \\
\hline 43 & 6/14/2004 & 9:40: & 28.254 & 25.308 & 27.948 & \begin{tabular}{|l|}
27.057 \\
\end{tabular} & & \begin{tabular}{|c|}
6.239 \\
\end{tabular} & \begin{tabular}{|l|l|}
45.487 \\
\end{tabular} & & -1.907 & 33.837 & & \begin{tabular}{|l|l|l|}
48.148 \\
\end{tabular} & 0.0 & & & 26 & & 44. & \begin{tabular}{ll|}
11.8 \\
\end{tabular} & 2.2 & & 000 & .000 & .000 & 0.190 & 0.16 \\
\hline 44 & $6 / 14 / 2$ & & 28.205 & 25.339 & \begin{tabular}{|l|}
27.899 \\
\end{tabular} & \begin{tabular}{|l|l|l|l|} 
\\
\end{tabular} & & & 336 & & & 33.788 & & & & & & $2 t r$ & & & 11.8 & & & & & & 0.189 & 0.16 \\
\hline 45 & $6 / 14 / 2$ & 9:42: & 28.178 & 25.372 & 27.907 & 27.286 & & 6.347 & & 8. & -1.8 & 33.73 & & 52.3 & & & & 28. & & & $12.8 \mathrm{C}$ & 2. & & & & & 0.182 & 0.16 \\
\hline 46 & $6 / 14 / 2004$ & $9: 43: 54$ & 28.1115 & 25.389 & 27.859 & 27.288 & & 6.189 & \begin{tabular}{|l|l|} 
\\
\end{tabular} & 0.000 & -1.962 & 33.793 & & 40.680 & & & & 23. & & 44. & 11.4 & 2.1 & . & & 000 & .000 & 0.195 & 0.11 \\
\hline $4 r_{1}$ & & & 28.083 & 25.421 & 27.841 & 27.36 & & 6.228 & \begin{tabular}{|r|r|}
55.89 \\
\end{tabular} & & & 33.817 & & & & & & & & 43.9 & & & & & & & & 0.16 \\
\hline$\frac{40}{49}$ & & & $\frac{2.8}{27.8}$ & 25. & 27.818 & \begin{tabular}{|l|l|}
27.456 \\
\end{tabular} & & & & & & 34.2 & & & & & & & & 43 & 12.9 & 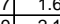 & & & & & & 0.20 \\
\hline 45 & & & 27.005 & 25.450 & 27.100 & 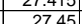 & & & $\mid$ & & & $\frac{34.5}{23.3}$ & & & & & & & & & 11.99 & & & & & & 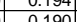 & 34 \\
\hline & $6 / 114 / 2$ & & 28.098 & 25.502 & 27.1717 & 27.46 & & 3...53 & $\mid$ & & 294 & 21 & & & & & & . & & & 108 & $\frac{2.4}{33.4}$ & 2300 & & 0.069 & & 2059 & 0.16 \\
\hline & $6 / 14 / 2 / 2$ & & $\frac{2.2005}{28.205}$ & 25.52 & 27.68 & $\mid 27.422$ & & 37.603 & & & & 20 & & . & & & & & & & 10.0 & & & & & & & 1.15 \\
\hline 53 & & & 28.205 & 25.5 & 27.605 & $\mid 27.437$ & & & 45.244 & & & & & & & & & & & & 然 & & & & & & & 1.4 \\
\hline 54 & & & & & 27.606 & & & & & & & & & & & & & & & & & & & & & & & \\
\hline & & & 28.195 & 25.5 & 276 & 27.47 & & & 45.6 & & & -1.2 & & & & & & & & 44 & 117 & & & & & & & 13 \\
\hline 56 & & & 28.1 & 25.6 & 27.587 & 27.445 & & & & & & & & & & & & & & & 13. & & & & & & & \\
\hline & $6 / 14 / 2$ & 9:54: & \begin{tabular}{|l|l|}
28.079 \\
\end{tabular} & 25.6 & 27.558 & 27.451 & & $\begin{array}{l}43.743 \\
\end{array}$ & \begin{tabular}{|l|l|}
45.489 \\
\end{tabular} & & & -1. & & 0.1 & & & & & & & 12.3 & & & 5 & & & & $\frac{1.0}{1.0}$ \\
\hline & $6 / 14 / 2004$ & 9:55: & \begin{tabular}{|l|l|}
28.12 \\
\end{tabular} & 25.6 & 27. & 27.47 & & 44.2 & 45.79 & & 36.4 & -1.2 & & & & & & & & & 10. & & & & & & 77 & 77 \\
\hline & 6/14/2004 & 9:56: & 28.0 & 25. & 27.486 & 27.468 & & 44.312 & 45.7 & & & -1.2 & & & & & & & & 44 & 11.5 & & & & & & & \\
\hline 60 & & 9:57: & 28.072 & 25.681 & 27.4 & 27.434 & & 44 & 45.688 & & & & & 46.6 & & & & & & 44 & $11.4 \mathrm{~s}$ & & & & & & 1.142 & 42 \\
\hline 61 & & & 28.0 & & 27.486 & 27. & & & & & & -1.22 & & & & & & & & & 11.6 & & & & & & 1.116 & 16 \\
\hline & & $9: 59$ & 27.986 & & 27.475 & 2.462 & & & & & & & & & & & & & & & 14 & & & & & & & \\
\hline & & & 27.944 & 25.1524 & 27.513 & 27.48 & & 44.56 & 45.7 & 8.304 & & -1.219 & & & 0.318 & & & & & 44.2 & & & & & & & & \\
\hline 65 & 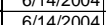 & $\frac{10.01}{10.02}$ & 27.01918 & 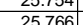 & 27.440 & $|27.45|$ & $\frac{2.6}{26.6}$ & $\begin{array}{l}44.155 \\
44.924\end{array}$ & 45. & $\frac{0.414}{84}$ & $\begin{array}{ll}36.9 \\
372\end{array}$ & $\frac{-1.2 .2}{-12}$ & & & 0.3 & $\frac{14.0}{14.6}$ & & & & 44.4 & & & & & & & $\frac{10}{41}$ & 70 \\
\hline 66 & 6 & 10.03: & 27.831 & 25779 & 27.455 & 27.482 & 266 & 45235 & \begin{tabular}{|l|}
46.0076 \\
46
\end{tabular} & 8.46 & 3747 & 12 & & 455 & 0.3 & 146 & & 49 & & 446 & 11. & & & & & & & \\
\hline 67 & $\frac{26 / 4 / 21}{6 / 21}$ & $\begin{array}{l}10: 04: 5 \\
\end{array}$ & 27.807 & 25789 & 27.44 & \begin{tabular}{|l|l|} 
\\
\end{tabular} & & 4.48 & 456 & 8.656 & 36.8 & -12 & & & 0.3 & & & 50 & & & 11.5 & & & & & & & \\
\hline 68 & $6 / 14 / 2004$ & 10:05:5 & 27.799 & 25.812 & 27.428 & $\begin{array}{l}27.47 \\
\end{array}$ & & 44.836 & 45.64 & & 37.00 & & & & 0.36 & & & $512 \quad 2$ & & 44.2 & 10. & & & 0.045 & & & 1035 & 5 \\
\hline & & & 27.794 & & 27.4 & 27.47 & & 45.747 & & & & -1.2 & & & & & & & & & & & & & & & 1019 & 19 \\
\hline & & $07: 5$ & .174 & & $4 \angle 0$ & 7.435 & & & & 8.278 & 37.942 & & & 578 & .301 & 4.643 & & & & t5. 6 (2) & & & 2.8 & 0.045 & & & 1.004 & \\
\hline
\end{tabular}


WSRC-TR-2005-00105, REVISION 0

SRNL-RPP-2005-00012, REVISION 0

RUN \# 3.03A AND B; FIRST AND SECOND HALF OF SLURRY DEWATERING - CONT.

\begin{tabular}{|c|c|c|c|c|c|c|c|c|c|c|c|c|c|c|c|c|c|c|c|c|c|c|c|c|c|c|c|c|}
\hline & A & & D & $\mathrm{E}$ & $\mathrm{F}$ & \begin{tabular}{l|l} 
\\
\end{tabular} & $\mathrm{H}$ & 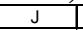 & $\mathrm{K}$ & $\mathrm{L}$ & \begin{tabular}{l|l}
$\mathrm{M}$ \\
\end{tabular} & $\mathrm{N}$ & $\mathrm{O}$ & Q & $\mathrm{R}$ & $\mathrm{s}$ & $\mathrm{T}$ & U $\quad v$ & w & $x \mid$ & $\mathrm{Y}$ & $z$ & & $A B$ & AC & & & AF $[A$ \\
\hline 71 & 6/14/2004 & $10: 08: 54 \mathrm{AM}$ & 27.758 & 25.851 & 27.422 & 27.409 & 26.693 & 45.237 & \begin{tabular}{|l|l|}
45.943 \\
\end{tabular} & 8.277 & 37.632 & -1.219 & -5.869 & 51.83 & $\begin{array}{ll}0.294 \\
\end{array}$ & \begin{tabular}{ll|}
14.643 \\
\end{tabular} & 0.004 & 54.22 & 0.90361 & 44.5 & \begin{tabular}{|l|}
12.7 \\
\end{tabular} & 41.4 & \begin{tabular}{|l|}
2.857 \\
\end{tabular} & 0.044 & 0.041 & 0.001 & 0.989 & 0.84 \\
\hline 72 & $6 / 14 / 2004$ & 10:09:54 AM & 27.744 & 25.872 & 27.433 & 27.41 & 26.714 & 44.343 & \begin{tabular}{|l|}
44.797 \\
\end{tabular} & 9.097 & 35.957 & -1.225 & & 51.598 & 0.281 & \begin{tabular}{|l|l|}
14.643 \\
\end{tabular} & 0.004 & 55.22 & & 43.3 & $\frac{12.6}{12.6}$ & & & 0.042 & 0.039 & & & \\
\hline 73 & 6/14/2004 & 10:10:54 AM & 27.751 & 25.908 & 27.469 & 27.466 & 26.71 & 44.892 & \begin{tabular}{|l|l|}
45.574 \\
\end{tabular} & 8.646 & 36.883 & -1.219 & & 45.789 & 0.29 & 14.643 & 0.004 & 56.22 & 0.93694 & 44.1 & 11.2 & & & & 0.040 & & & \\
\hline 74 & 6/144/2004 & 10:11:54 AM & & 25.909 & $\begin{aligned} 27.46 \\
27.067\end{aligned}$ & 27.552 & 26.731 & 44.996 & \begin{tabular}{|l|l|}
45.557 \\
5571
\end{tabular} & 8.591 & $\begin{array}{l}37.067 \\
2729\end{array}$ & -1.219 & & $\begin{array}{l}52.116 \\
4190\end{array}$ & & $\begin{array}{l}14.643 \\
14.3\end{array}$ & $\begin{array}{l}0.004 \\
0001\end{array}$ & $\begin{array}{ll}57.22 \\
5822\end{array}$ & & 44.1 & & & & & 0.040 & & 0.984 & \\
\hline$\frac{75}{76}$ & 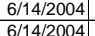 & $\begin{array}{l}10: 12: 54 \mathrm{AM} \\
10: 13: 54 \mathrm{AM}\end{array}$ & \begin{tabular}{rl|}
27.744 & \\
27.745 &
\end{tabular} & $\begin{array}{l}25.941 \\
25.952 \\
\end{array}$ & $27.462 \mid$ & $\begin{array}{l}27.604 \\
27.645\end{array}$ & 26.733 & \begin{tabular}{|l|l|}
45.249 \\
45.384
\end{tabular} & \begin{tabular}{|l|}
45.719 \\
45799 \\
\end{tabular} & $\begin{array}{l}8.523 \\
8.367\end{array}$ & $\begin{array}{l}37.396 \\
37.751\end{array}$ & 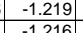 & $\begin{array}{r}-6.002 \\
599\end{array}$ & \begin{tabular}{|c|}
44.189 \\
43399
\end{tabular} & $\begin{array}{r}0.29 \\
\end{array}$ & $\begin{array}{l}14.643 \\
14634 \\
\end{array}$ & 0.004 & $\begin{array}{l}58.22 \\
59.22 \\
\end{array}$ & 0.97028 & \begin{tabular}{|l|}
44.2 \\
\end{tabular} & \begin{tabular}{|l|l|}
10.8 \\
106
\end{tabular} & 416 & \begin{tabular}{|l|}
2.849 \\
2866
\end{tabular} & \begin{tabular}{|l|l|}
0.043 \\
0.043
\end{tabular} & 0.040 & 0.001 & 0.977 & \\
\hline \begin{tabular}{l|l}
76 \\
77
\end{tabular} & $\begin{aligned} 6 / 11 / 42004 \\
6 / 1 / 2004\end{aligned}$ & $\begin{array}{l}\text { 10:13:54 AM } \\
10: 14: 54 \mathrm{AM}\end{array}$ & $\begin{array}{l}27.745 \\
27.749\end{array}$ & 25.952 & $27.493 \mid$ & $\begin{array}{l}27.645 \\
27.675\end{array}$ & 26.769 & \begin{tabular}{|l|l|}
45.384 \\
45.757
\end{tabular} & \begin{tabular}{|r|}
$\mid 45.879$ \\
46.3 \\
\end{tabular} & $\begin{array}{l}8.367 \\
8.372 \\
\end{array}$ & $\begin{array}{l}37.751 \\
38.089\end{array}$ & $\mid$\begin{tabular}{|l|}
$\mid-1.216$ \\
-1.219 \\
\end{tabular} & $\begin{array}{r}-5.99 \\
-5.973 \\
\end{array}$ & \begin{tabular}{|l|}
$\mid 43.349$ \\
50.272
\end{tabular} & $\begin{array}{r}0.29 \\
0.29\end{array}$ & $\begin{array}{r}14.643 \\
14643 \\
\end{array}$ & \begin{tabular}{|l|}
0.004 \\
0.004 \\
\end{tabular} & $\begin{array}{l}59.22 \\
60.22 \\
\end{array}$ & $\begin{array}{l}0.98694 \\
\end{array}$ & \begin{tabular}{|l|}
44.4 \\
\end{tabular} 4.8 & \begin{tabular}{|l|}
10.6 \\
12.3
\end{tabular} & \begin{tabular}{|l|l|}
41.6 \\
419
\end{tabular} & \begin{tabular}{|l|}
2.866 \\
2.890 \\
\end{tabular} & \begin{tabular}{|l|}
0.043 \\
0.043 \\
\end{tabular} & 0.040 & 0.001 & $\begin{array}{ll}0.970 \\
0.961\end{array}$ & 0.83 \\
\hline 78 & $6 / 14 / 2004$ & $10: 15: 54 \mathrm{AM}$ & 27.835 & 25.972 & 27.548 & 27.705 & 20.824 & | 45.5615 & \begin{tabular}{|r|}
45.947 \\
\end{tabular} & 8.365 & $\begin{array}{l}38.089 \\
37.882\end{array}$ & \begin{tabular}{|l|l|}
-1.219 \\
-1.222 \\
\end{tabular} & $\begin{array}{l}-5.975 \\
-6.013 \\
\end{array}$ & $\begin{array}{l}30.272 \\
39.004 \\
\end{array}$ & $\begin{array}{l}0.29 \\
0.288 \\
\end{array}$ & $\begin{array}{l}1.4 .643 \\
14.643 \\
\end{array}$ & $\begin{array}{l}0.004 \\
0.004 \\
\end{array}$ & $\begin{array}{l}00.22 \\
61.22 \\
\end{array}$ & 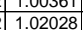 & 44.8.5 & $\begin{array}{ll}1.3 .5 \\
9.6\end{array}$ & & & \begin{tabular}{|l|}
0.043 \\
0.043 \\
\end{tabular} & $\begin{array}{l}0.040 \\
0.040\end{array}$ & $\frac{0.001}{0.001}$ & \begin{tabular}{|c|}
0.961 \\
0.958 \\
\end{tabular} & $\frac{0.82}{0.82}$ \\
\hline 79 & $6 / 14 / 2004$ & $10: 16: 54 \mathrm{AM}$ & 27.852 & 25.995 & 27.561 & 27.733 & 26.907 & 45.699 & 46.345 & 8.174 & 38.196 & -1.219 & -5.987 & 44.715 & 0.289 & 14.643 & 0.004 & 62.22 & 1.03694 & \begin{tabular}{|l|}
44.9 \\
\end{tabular} & 11.0 & & \begin{tabular}{|l|}
2.892 \\
\end{tabular} & 0.043 & 0.040 & & & 0.82 \\
\hline 80 & 6/14/2004 & 10:17:54 AM & 27.871 & 26.019 & 27.599 & 27.761 & 26.885 & 45.523 & 46.206 & 8.072 & 38.124 & -1.219 & & 44.337 & 0.288 & 14.643 & 0.004 & & 1.05361 & \begin{tabular}{|l|}
44.7 \\
\end{tabular} & & & & 0.043 & 0.040 & & & 0.81 \\
\hline 81 & $6 / 14 / 2004$ & $10: 18: 54 \mathrm{AM}$ & 27.934 & 26.027 & 27.597 & 27.764 & 26.883 & $\begin{array}{l}45.67 \\
\end{array}$ & \begin{tabular}{|l|}
46.293 \\
\end{tabular} & & $\begin{array}{l}38.276 \\
\end{array}$ & -1.219 & & 41.914 & $\begin{array}{ll}0.287 \\
\end{array}$ & $\begin{array}{l}14.643 \\
\end{array}$ & 0.00 & 64.22 & & \begin{tabular}{|l|}
44.8 \\
\end{tabular} & 10.3 & & \begin{tabular}{|l|}
2.894 \\
\end{tabular} & & & & 0.948 & 0.81 \\
\hline 82 & $6 / 14 / 2004$ & 10:19:54 AM & 27.924 & 26.032 & 27.593 & 27.785 & 26.904 & 45.687 & 46.273 & 8.134 & 38.276 & -1.219 & & 50.841 & 0.284 & 14.643 & & & 1.08694 & & & & & & 0.039 & & & \\
\hline 83 & & & & & & & & & & & & & & & & 14.643 & & & & 44.2 & & & & & 0000 & & & \\
\hline 84 & $6 / 14 / 2004$ & 10:21:54 AM & 27.959 & 26.068 & 27.608 & 27.915 & & 44.718 & 45.259 & 8.889 & 36.476 & -1.222 & & 46.847 & & 14.643 & & 67.2. & .1.12028 & 43.8 & 11 & & .79 & & 0.038 & & .929 & \\
\hline & 6/14/2004 & 10:22:54 AM & 27.975 & 26.083 & 27569 & 27.901 & & & 45.634 & 8549 & 37.193 & -1.222 & & & & 14.643 & & & 1.13694 & 44.2 & & & & & & & & \\
\hline 86 & $6 / 14 / 2004$ & 10:23:54 AM & 27.991 & 26.114 & 27.585 & 27.951 & 26.941 & 45.284 & 45.792 & 8.332 & 37.679 & -1.216 & & 46.336 & 0.277 & 14.643 & .004 & 69.22 & 1.15361 & 44.3 & 11.4 & 41 & 2.860 & 0.041 & 0.038 & & 0.926 & \\
\hline & 6/114/2004 & 10:22:54 AM & 28.014 & 26.133 & 27.564 & 27.935 & & 45.666 & 46.391 & 8.38 & 37.96 & -1.222 & & 45.462 & 0.276 & 14.643 & & & & 44.99 & 11.. & & & & & & & \\
\hline$\frac{88}{80}$ & 6/144/2004 & 10:25:54 AM & 28.008 & 26.152 & 27.527] & 27.984] & 26.858 & 45.68 & 46.474 & 8.319 & 38.02 & -1.222 & & 48.253 & 0.274 & 14.643 & & 71.22 & 1.18694 & $45.0]$ & 11.8 & & 2.885 & 0.041 & 0.038 & 0.001 & 0.910 & \\
\hline 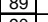 & 6/14/2004 & 10:26:54 AM & 27.984 & 26.162 & & 27.964 & & 44.482 & 45.1999 & & & -1.222 & & 52.844 & 0.264 & 14.643 & & & 1.20361 & 43.7 & & & & & 0.037 & & & \\
\hline 90 & 6/14/2004 & $\begin{array}{l}10: 2: 54: 54 \mathrm{AM} \\
\end{array}$ & 2.966 & 26.18 & $\frac{1}{2.791}$ & 2.942 & & 44.041 & 45.586 & 8.634 & 36.905 & -1.219 & & & & 14.643 & & 73.22 & .22028 & 44.1. & . & & & & 0.037 & & 0 & \\
\hline & $\frac{01442004}{6 / 1204}$ & $\frac{1.20 .34 \mathrm{AMM}}{10.29 \mathrm{AM}}$ & (2) & $\frac{26.2}{2027}$ & $\frac{27.4 / 1}{27.473}$ & $\frac{27.9089}{27099}$ & & $\frac{44.616}{44.520}$ & 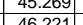 & $\frac{8.821}{87757}-2$ & 36.484 30 & $\frac{-1.2}{-12}>>2$ & & 54.437 & & 告14.643 & & & $\frac{1.23694}{10261}$ & 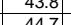 & & & & & (1) & & & \\
\hline & 6 & 10.3.5. & 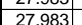 & $\frac{20.247}{26242}$ & $\frac{2.4710}{27,45}$ & 27.934 & & 45083 & $\frac{40.221}{45.746}$ & & & $\frac{-1.2}{-12}$ & & 46.455 & & $146 \mathrm{cos}$ & & & & $\frac{44 .}{44.3}$ & 114 & & & & & & & 0.17 \\
\hline 94 & $6 / 14 / 2004$ & 10:31:54 AM & 27.994 & 26.268 & 27.449 & 27.975 & 26.78 & 45.177 & 46.042 & 8.466 & 37.377 & -1.2. & & 48.712 & 0.268 & 14.643 & & 77.2 & 1.28694 & 44.6 & $\frac{51.9}{11.9}-5$ & & 8.846 & & 0.037 & $0.6 \quad-\quad>\quad-3$ & 0.904 & $\begin{array}{l}0.71 \\
0.77 \\
\end{array}$ \\
\hline 95 & $6 / 14 / 2004$ & $10: 32: 54 \mathrm{AM}$ & 27.98 & 26.283 & 27.424 & 27.881 & 26.691 & 45.38 & 46.333 & 8.508 & 37.541 & -1.2. & & 47.575 & 0.264 & 14.643 & & 78.2 & 1.30361 & 44.9 & $\frac{11.7}{11.7}>2$ & & 2.859 & 0.039 & 0.037 & 0.001 & 0.887 & 0.76 \\
\hline 96 & $6 / 14 / 2004$ & 10:33:54 AM & 27.976 & 26.299 & 27.385 & 27.871 & 26.686 & 45.233 & 46.339 & 8.545 & 37.332 & -1.222 & & 49.043 & 0.277 & 14.643 & & 79.2 & 1.32028 & 44.9 & $12.6 \mathrm{C}>\mathrm{l}$ & & $2.846 \mid$ & 0.041 & $\mid 0.039$ & 00 & 0.936 & \\
\hline & $6 / 14 / 2004$ & 10:34:54 AM & & & & & & & & & & & & & & & & & & & & & & & & & & \\
\hline 98 & $6 / 14 / 2$ & $10: 35: 5$ & 27.951 & 26.334 & 27.355 & 27.826 & & $45.18 \mathrm{~s}$ & $\begin{array}{ll}46.237 \\
\end{array}$ & 8.343 & 37.515 & -1.2 & & 49.335 & & 14.643 & & & & 44.8 & & & & & 0.037 & & 0.898 & \\
\hline & & & 27.947 & & & & & 45.425 & 46.499 & & & & & & & & & & & & & & & & & & & \\
\hline & 6/14/2004 & 10:37:54 AM & & 26.346 & 27.362 & 27.748 & & 44.67 & 45.725 & 8.967 & 36.419 & -1.21 & & & 0.256 & \begin{tabular}{|c|c|c|}
14.643 \\
\end{tabular} & & & 1.38694 _ & 44.3 & 10.8 & & & & & & & \\
\hline 101 & 6/114/2004 & 10:38:54 AM & 27.922 & 26.345 & 27.346 & 27.677 & 26.463 & 45.139 & 46.246 & 8.711 & 37.115 & -1.22 & & 47.593 & 0.259 & \begin{tabular}{ll|l}
14.643 \\
\end{tabular} & & 84.22 & 1.40361 & 44.8. & 11.7 & & 2.836 & .039 & 0.036 & 0.001 & 0.879 & \\
\hline & 6/14/12004 & 10:39:54 AM & 27.925 & 26.358 & 27.35 & 27.701 & & 45.019 & 46.028 & 8.745 & 36.983 & $-1.2 .24>0$ & & 48.1944 & & 14.643 & & & & 44.6 & 118 & & & & & & & \\
\hline & $6 / 144 / 26$ & 10:40:5 & 27.922 & 26.361 & 27.332 & 27.683] & & 44.7 & 45.74 & 8.633 & 36.743 & -1.2 & & 48.684 & 0.258 & 14. & & 86. & 1.43694 & 44.3 & 11. & & $2.80 \varepsilon$ & 038 & 0.036 & & 0.885 & \\
\hline$\frac{104}{100}$ & $6 / 14 / 200$ & 10:41:5 & 27.946 & 26.3 & & 27.7277 & & 44.926 & & 8.733 & & -1.2 & & 44.54 & & 14. & & & & 44.6 & & & & & & & & \\
\hline & $\frac{61 / 421}{61 / 12}$ & & 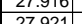 & $\frac{2.39}{26.409}$ & 年.346 & 20.1564 & & & & 8.877 & & & & 48.148 & & 14.6 & & & & 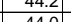 & $\frac{11}{12}$ & & & & & & 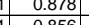 & \\
\hline 107 & 6 & & 27.91912 & 26.41 & 年. & 27763 & & (4) & 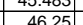 & & & & & $4511: 5$ & & 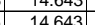 & & & & & 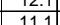 & & & & & & 0865 & \\
\hline & $6 / 14 / 2004$ & & 27.901 & 264 & 27.351 & 27,782 & & 44.741 & 46.013 & 8.046 & & & & 47 & & 146 & & & & & & & & & & & & \\
\hline & $6 / 14 / 2004$ & $10: 46: 54 \mathrm{AM}$ & 27.901 & 26.424 & 27.325 & 27.776 & 26.876 & 44.857 & \begin{tabular}{|l|}
46.214 \\
\end{tabular} & 8.732 & \begin{tabular}{|l|l|}
36.747 \\
\end{tabular} & -1.22 & & 47.702 & 0.253 & 14.643 & & 92.22 & 1.53694 & \begin{tabular}{|l|}
44.7 \\
\end{tabular} & 11.7 & 40 & 2.813 & 0.038 & $\begin{array}{l}0.035 \\
\end{array}$ & 0.001 & 0.866 & 0.74 \\
\hline & $6 / 144 / 20$ & $10: 47: 54 \mathrm{AM}$ & 27.095 & & & 27.825 & & 444.693 & 40.999 & 8.782 & & -1.222 & & 47.917 & & $\begin{array}{ll}14.643 \\
\end{array}$ & & & & 44.5. & & & & & & & & \\
\hline 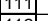 & & & 27.884 & 26.438 & 27.319 & 27.815 & & 44.664 & & 8.798 & & & & & & & & & & 44.4. & & & 2.79 & & & & & \\
\hline & $6.141 / 2$ & & & & & 2.7.853 & & 44.4 & 45.5 & & & & & & & 14. & & & & 44 & & & & & & & & \\
\hline & & & & & 年 & & & & & & & & & & & & & & & & & & & & & & & \\
\hline & $\frac{21 / 41}{6 / 4 / 2}$ & & $\frac{17.6}{278}$ & & $\frac{27.5}{2732}$ & & & & & & & & & & & & & & & & & & & & & & & \\
\hline & $\frac{1 / 41426}{6 / 42}$ & $10.53: 5$ & & & 2.00 & 年 27.889 & & & & & & & & & & & & & & & & & & & & & & \\
\hline 117 & $6 / 14 / 20$ & & & & 27.32 & 27.87 & & 44.0 & & & & & & & & 14. & & & & & & & & & & & & \\
\hline 118 & $6 / 14 / 2$ & & 27.8 & & 27.34 & 27.886 & & 44.422 & & 8.854 & & & & & & & & & & & & & & & & & 0.859 & \\
\hline & $6 / 14 / 2$ & & & & & & & 44.3 & & & & & & & & & & & & & & & & & & & & \\
\hline & $6 / 14 / 2$ & & & & 27.2 & 27. & & 44.7 & & & & & & & & 14. & & & & & & & & & & & & \\
\hline & & & & & & & & & & & & & & & & & & & & & & & & & & & & \\
\hline & $6 / 14 / 2)>20$ & $10: 5$ & 27.8 & 26.5 & 27.234 & 27.999 & & 44.5 & & & & & & & & & & & & & & & & & & & & \\
\hline & $6 / 14 / 2$ & & & & & 27.9 & & 44.9 & & & & & & & & & & & & 44 & & & & & & & & \\
\hline & $6 / 14 / 20$ & 11:01: & $27.8 \mathrm{r}>\mathrm{l}$ & & 27.1 & 27.984 & & \begin{tabular}{|c|}
44.596 \\
\end{tabular} & & 8.78 & & -1.2 & & 44. & & 14. & & 106 & & 44 & 0.5 & & & & & & 0.854 & \\
\hline & $6 / 14 / 2 / 2$ & & & 26.5 & 27.12 & 27.96 & & 44.739 & & 8.779 & & -1.2 & & & & 14. & & 107 & & & & & & & & & & \\
\hline & $6 / 14 / 2 / 2>3$ & & 27.8 & & 27.11 & 27.978 & & 44.571 & & 8.704 & & & & & & 14. & & 108 & & 44.4 & & & & & & & 0.846 & \\
\hline & $6 / 144$ & & & & & & & 44. & & & & & & & & & & & & & & & & & & & & \\
\hline & 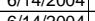 & & 27.194 & & 年. & (2) & & 44.004 & & 6. & & -1.2 & & & & & & & & & & & & & & & & \\
\hline & $\frac{01 / 420}{6 / 14 / 20}$ & 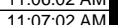 & 27766 & $\begin{array}{l}20.056 \\
26.683\end{array}$ & 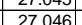 & 22.797 & & $\frac{14.0}{446}$ & & 然. & 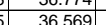 & -12 & & 490 & & 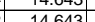 & & 11. & & 44.3 & $\frac{\frac{11.5}{120}}{120}+2$ & & & & & & 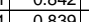 & \\
\hline & $6 / 14 / 2$ & & 27.754 & 26.6 & 27.074 & 27.989 & & 44.66 & & & & & & & & & & & & & & & & & & & & \\
\hline 132 & $6 / 14 / 20$ & $11: 0$. & 27.73 & & 27.033 & 28.003 & & 43.918 & 45.104 & 9.56 & & & & & & $14.6>$ & & & & & $14.2>>3$ & & & & & & 0.823 & \\
\hline & $6 / 14 / 2$ & 11:10:02 A & 27.7 & & & & & 44.119 & & 9.343 & & & & & & 14.6 & & 115 & & & & & & & & & & \\
\hline & & & $27.7+3$ & & & & & 44.07 & & & & & & & & & & & & & & & & & & & & \\
\hline & $6 / 14 / 2$ & & & & & & & 44.438 & & & & & & & & & & & & & 1.4 & & & & & & & \\
\hline & & & & & & 28.06 & & 44.409 & & & & & & & & & & & & & & & & & & & & \\
\hline & $6 / 14 / !$ & 11:14:02 AM & $27665>2$ & 26.754 & 95 & 28.06 & & 44.328 & 45.543 & 9. & 35.965 & $-1.22>3$ & & & & 14. & & & & 44.1. & & & & & & & & \\
\hline & /14/200 & 11:15:02 AM & 27.68 & 26.763 & $69-9$ & 28.06 & & 44.44 & 45.754 & 9.1 & $36056 \mathrm{C}$ & $-1.22 \mathrm{P}>\mathrm{C}$ & & & & 14.6 & & & & 44.3 & & & & & & & & \\
\hline & 6/14/200 & 11:16:02 AM & $77682-2$ & 788 & & 077077 & & 44.558 & 5.835 & $9.102 \mathrm{z}-\mathrm{s}$ & 36.16 & $1.22 \times-3$ & & & $0.2+2-3$ & 14.64 & & 121 & & 444 & & & & & & 0 & & $7+2$ \\
\hline 140| & 6/14/2004 & 11:17:02 AM & .671| & 26.798 & 26.981 & 28.085 & & 44.436 & 45.553 & 8.967 & 36.15 [ & $\mid-1.225$ & & 6.271 & 0.236 & 14.643 & & 122.35 & 2.03917 & $44.1]$ & 11.3 & & & & .033 & 0.001 & 0.826 & \\
\hline
\end{tabular}


WSRC-TR-2005-00105, REVISION 0

SRNL-RPP-2005-00012, REVISION 0

RUN \# 3.03A AND B; FIRST AND SECOND HALF OF SLURRY DEWATERING - CONT.

\begin{tabular}{|c|c|c|c|c|c|c|c|c|c|c|c|c|c|c|c|c|c|c|c|c|c|c|c|c|c|c|c|c|}
\hline & A & & $\mathrm{D}$ & $\mathrm{E}$ & $\mathrm{F}$ & G & $\mathrm{H}$ & $\begin{array}{lll} \\
\end{array}$ & $\mathrm{K}$ & $\mathrm{L}$ & M & $\mathrm{N}$ & 0 & $Q^{-1}$ & \begin{tabular}{l|l}
$\mathrm{R}$ & \\
\end{tabular} & $\mathrm{s}$ & $\mathrm{T}$ & $U$ U $\quad v$ & w & \begin{tabular}{|l|l|}
$x$ \\
\end{tabular} & $\mathrm{Y}$ & $z$ & $\mathrm{AA}$ & $A B$ & AC & & & AF $D A$ \\
\hline & 6/14/2004 & 11:18:02 AM & 27.67 & 26.822 & 26.98 & 28.099 & 27.178 & 44.886 & \begin{tabular}{|l|l|}
46.347 \\
\end{tabular} & 8.906 & 36.638 & -1.222 & $\begin{array}{ll}-6.227 \\
\end{array}$ & 51.602 & \begin{tabular}{l|l|}
0.238 \\
\end{tabular} & \begin{tabular}{ll|}
14.643 \\
\end{tabular} & 0.004 & 123.35 & \begin{tabular}{|l|}
2.05583 \\
\end{tabular} & \begin{tabular}{|l|}
44.9 \\
\end{tabular} & 12.6 & 40.8 & 2.810 & 0.035 & 0.034 & 0.001 & 0.824 & 0.70 \\
\hline$\frac{142}{142}$ & $6 / 14 / 2004$ & 11:19:02 AM & 27.669 & 26.826 & $\frac{26.974}{26.95}$ & 28.133 & 27.178 & 44.249 & $\begin{array}{r}45.4 \\
\end{array}$ & 8.792 & 36.119 & \begin{tabular}{|l|l|}
9 & -1.219 \\
\end{tabular} & & & $\begin{array}{l}0.24 \\
0.24\end{array}$ & \begin{tabular}{ll|}
14.643 \\
\end{tabular} & 0.004 & 124.35 & 2.0725 & 43.9 & 10.5 & & & & 0.034 & & & \\
\hline & $6 / 14 / 2004$ & 11:20:02 AM & 27.661 & 26.828 & $\frac{26.951}{2.01}$ & 28.115 & 27.174 & 44.556 & \begin{tabular}{|l|l|}
46.036 \\
\end{tabular} & 8.903 & 36.308 & \begin{tabular}{l|l}
8 & -1.222 \\
7 & 1.220 \\
\end{tabular} & & \begin{tabular}{|l|}
49.548 \\
5.179
\end{tabular} & 0.233 & \begin{tabular}{|l|l|}
14.643 \\
\end{tabular} & 0.004 & 125.35 & & 44.6 & $\frac{12.1}{127}$ & 40.4 & & .035 & 0.033 & & & \\
\hline 144 & 6/14/2004 & 11:21:02 AM & 27.654 & 26.841 & 26.944 & 28.128 & 27.197 & 44.811 & \begin{tabular}{|l|l|}
46.418 \\
\end{tabular} & & & -1.222 & & 51.786 & $\begin{array}{l}0.237 \\
0.236\end{array}$ & $\begin{array}{l}14.643 \\
14.3\end{array}$ & 0.003 & & 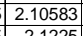 & 44.9 & & & & & 0.033 & & & \\
\hline$\frac{145}{146}$ & 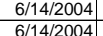 & $\begin{array}{l}11: 22: 02 \mathrm{AM} \\
12302 \mathrm{AM}\end{array}$ & 27.651 & $\frac{26.853}{26.866}$ & $\frac{26.931}{26.929}$ & $\begin{array}{l}28.155 \\
28.163 \\
\end{array}$ & 27.209 & $\begin{array}{r}44.84 \\
45004\end{array}$ & \begin{tabular}{|l|}
46.04 \\
46.2
\end{tabular} & $\begin{array}{r}8.84 \\
8.824 \\
\end{array}$ & $\begin{array}{l}36.692 \\
36.889 \\
\end{array}$ & 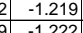 & $\begin{array}{l}-6.166 \\
-6.239\end{array}$ & $\begin{array}{r}48.83 \\
41179\end{array}$ & $\begin{array}{l}0.236 \\
0.236\end{array}$ & $\begin{array}{l}14.643 \\
14634 \\
\end{array}$ & $\begin{array}{l}0.004 \\
0.04\end{array}$ & $\begin{array}{l}127.35 \\
128.35 \\
\end{array}$ & $\begin{array}{l}2.1225 \\
2.1317 \\
\end{array}$ & \begin{tabular}{|l|}
44.6 \\
\end{tabular} & \begin{tabular}{|l|}
12.0 \\
10.1
\end{tabular} & 409 & \begin{tabular}{|l|}
2.811 \\
2823
\end{tabular} & 0.035 & 0.033 & $\begin{array}{l}0.001 \\
0.001\end{array}$ & 0.818 & \\
\hline$\frac{146}{147}$ & $\begin{array}{l}6 / 1 / 1 / 2004 \\
6 / 12004\end{array}$ & $\begin{array}{l}11: 23: 02 \mathrm{AM} \\
11: 02: 02 \mathrm{AM}\end{array}$ & 27.644 & $\frac{26.866}{26.873}$ & $26.929 \mid$ & $\begin{aligned} 28.163 \\
28.15 \\
\end{aligned}$ & 27.222 & \begin{tabular}{|l|r|}
45.004 \\
4.608
\end{tabular} & \begin{tabular}{|r|}
46.2 \\
45761 \\
\end{tabular} & $\begin{array}{l}8.824 \\
8.866 \\
\end{array}$ & $\begin{array}{l}36.889 \\
36.38 \\
\end{array}$ & \begin{tabular}{|l|l|}
9 & -1.222 \\
8 & -1.222 \\
\end{tabular} & $\begin{array}{r}-6.239 \\
-6.23 \\
\end{array}$ & $\begin{array}{l}41.179 \\
45.992\end{array}$ & $\begin{array}{l}0.236 \\
0.234\end{array}$ & $\begin{array}{r}14.643 \\
14643 \\
\end{array}$ & $\begin{array}{l}0.004 \\
0.003\end{array}$ & $\begin{array}{r}128.35 \\
129.35\end{array}$ & \begin{tabular}{|l|l|}
2.13917 \\
2.15583
\end{tabular} & \begin{tabular}{|l|}
44.7 \\
44.3
\end{tabular} & \begin{tabular}{|l|}
10.1 \\
11.3
\end{tabular} & $\begin{array}{l}40.9 \\
40.5\end{array}$ & \begin{tabular}{|l|}
2.823 \\
2.792 \\
\end{tabular} & $\begin{array}{l}0.035 \\
0.035\end{array}$ & $\begin{array}{l}0.033 \\
0.033\end{array}$ & $\begin{array}{r}0.001 \\
0.001 \\
\end{array}$ & 0.814 & 0.69 \\
\hline 148 & $6 / 14 / 2004$ & 11.24.25:02 AM & 27.649 & $\frac{20.873}{26.911}$ & \begin{tabular}{|l|}
20.911 \\
26.939 \\
\end{tabular} & $\begin{array}{rl}28.10 & 288 \\
28.22\end{array}$ & 27.249 & $\begin{array}{l}44.608 \\
44.643 \\
\end{array}$ & \begin{tabular}{|l|}
45.1918 \\
45.978 \\
\end{tabular} & 8.803 & $\begin{array}{l}36.385 \\
36.485 \\
\end{array}$ & \begin{tabular}{c|c}
5 & -1.2222 \\
5
\end{tabular} & $\begin{array}{l}-0.23 \\
-6.195 \\
\end{array}$ & 45.9923 & $\begin{array}{l}0.234 \\
0.237 \\
\end{array}$ & $\begin{array}{l}11.643 \\
14.643 \\
\end{array}$ & \begin{tabular}{|l|l|}
0.003 \\
0.003 \\
\end{tabular} & $\begin{array}{l}129.35 \\
130.35\end{array}$ & \begin{tabular}{|l|l|} 
\\
\end{tabular} & 44.5 & $\frac{11.3}{11.7}$ & & & \begin{tabular}{|l}
0.035 \\
0.035 \\
\end{tabular} & $\begin{array}{l}0.033 \\
0.033\end{array}$ & $\frac{0.001}{0.001}$ & $\begin{array}{l}0.825 \\
0.825 \\
\end{array}$ & $\frac{0.69}{0.70}$ \\
\hline 149 & 6/14/2004 & 11:26:02 AM & 27.647 & 26.909 & 26.932 & 28.245 & 27.27 & 44.785 & \begin{tabular}{|l|}
46.013 \\
\end{tabular} & 8.848 & 36.636 & \begin{tabular}{|l|l|}
6 & -1.219 \\
\end{tabular} & & 51.171 & 0.234 & 14.643 & 0.004 & 131.35 & 2.18917 & 44.5 & & & \begin{tabular}{|l|}
2.807 \\
\end{tabular} & 0.035 & & & & 0.69 \\
\hline & 6/14/2004 & 11:27:02 AM & 27.633 & 26.91 & 26.894 & 28.237 & 27.306 & 45.031 & \begin{tabular}{|l|l|} 
& 46.279 \\
\end{tabular} & 8.811 & 36.95 & \begin{tabular}{|l|l|}
5 & -1.225 \\
\end{tabular} & & 49.577 & 0.233 & 14.643 & 0.004 & & $2.20583 \mid$ & 44.8 & & & & 0.035 & & & & 0.68 \\
\hline 151 & $6 / 14 / 2004$ & $11: 28: 02 \mathrm{AM}$ & 27.641 & 26.933 & 26.906 & 28.274 & 27.344 & 44.942 & \begin{tabular}{|l|l|}
46.179 \\
\end{tabular} & & 36.757 & -1.222 & & $\begin{array}{l}48.816 \\
\end{array}$ & 0.237 & $\begin{array}{l}14.643 \\
\end{array}$ & 0.004 & 133.35 & 2.2225 & 44.7 & 12.0 & & & & 0.034 & & & $\frac{0.68}{0.70}$ \\
\hline$\frac{152}{152}$ & $6 / 14 / 2004$ & 11:29:02 AM & 27.633 & 26.945 & 26.873 & 28.296 & 27.355 & 44.536 & \begin{tabular}{|l|l|}
45.655 \\
\end{tabular} & 8.827 & 36.376 & -1.222 & & 52.51 & 0.229 & 14.643 & & & & & & & & & & & & \\
\hline & & 11:30:02 AM & & & & & & & & & & & & & & & & & & 44.4 & & & & & & & & \\
\hline 15 & 6/144/2004 & 11:31:02 AM & 27.632 & 26.984 & 26.792 & 28.465 & & 44.677 & 46.013 & 8.883 & 36.468 & -1.222 & & 41.242 & 0.229 & 14.643 & & $136.35 \mathrm{r}$ & 2.2725 & 44.5 & 10. & & 79 & .034 & 0.032 & & 0.800 & \\
\hline & $6 / 14 / 2004$ & 11:32:02 AM & & 27.001 & 26769 & 28512 & & 44.708 & 46.016 & 8.874 & 36.519 & -1.21 & & 50.167 & & 14.643 & & & & 44.5 & & & & & & & (0.779 & \\
\hline & 6/14/2004 & 11:33:02 AM & 27.611 & 27.017 & 26.736 & 28.518 & & $\begin{array}{ll}44.708 \\
\end{array}$ & 46.1177 & 8.9 & 36.482 & -1.222 & & 51.463 & 0.227 & 14.643 & . .003 & 138.35 & \begin{tabular}{|l|l|} 
\\
\end{tabular} & $\begin{array}{lll}44.6 \\
\end{array}$ & $12.6 \mathrm{C}-\mathrm{C}$ & & 2.799 & .034 & 0.032 & & 0.794 & \\
\hline$\frac{15}{2}$ & $6 / 14 / 2004$ & 11:34:02 AM & 27.592 & 27.029 & 26.703 & 28.565 & 27.479 & 44.836 & 46.078 & 8.899 & 36.643 & -1.222 & & 49.871 & 0.228 & 14.643 & & $139.3 \mathrm{~s}^{\mathrm{s}}$ & 2.3225 & 444.6 & & & & & & 0.001 & 0.796 & \\
\hline 15 & 6/144/2004 & 11:35:02 AM & 27.5811 & 27.048 & 26.686 & 28.573 & 27.518 & 44.301 & 45.4933 & 9.208 & 35.772 & -1.225 & & 46.25 & 0.224 & 14.643 & & 140.35 & 2.33917 & 44.00 & 11.3. & & 2.760 & .033 & 0.032 & 0.001 & 0.796 & \\
\hline & 6/14/2004 & 11:36:02 AM & & 27.06 & 26.649 & 28.586 & & & 45.858 & 9.099 & 36.132 & -1.2222 & & 每3.706 & 0.223 & 14.643 & & 141.3 & 2.35583 & 44.4 & 10. & & 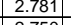 & & 0.032 & & & \\
\hline & $\frac{0.1142004}{6-1 / 2004}$ & 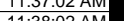 & $2 \frac{2.500}{27516}$ & 27.065 & $20.008 \mathrm{C}$ & 28.0 & & 44.1633 & 45.045 & 9.247 & 350.6 & 年.1.19 & & 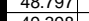 & & 14.0435 & & $142.3 \mathrm{~m}=\mathrm{r}$ & 2.3725 & & & & & & 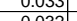 & & 0.817 & \\
\hline & $\frac{61412}{6 / 1212}$ & $\frac{1.50 .02 \mathrm{Min}}{11.30}$ & $\frac{27.510}{27514}$ & $\frac{21.031}{2711}$ & $\frac{2.0064}{26.14}$ & 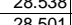 & & $\frac{44.39}{44.58}$ & $\frac{45.601}{4572}$ & 年. & $\frac{35.951}{36.77}$ & $\frac{-1.2}{-12}>>2$ & & & & 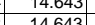 & & & 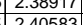 & 年 & & & & & & & . & \\
\hline$\frac{263}{163}+3$ & $6 / 14 / 2004$ & 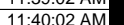 & (2) 27467 & 27078 & $\frac{2.0 .045}{26.52}$ & $\frac{28.501}{28.455}$ & & $\begin{aligned} 44.565 \\
44.7 \\
\end{aligned}$ & 46.13 & & 36.10 & & & 52476 & & 1464 & & & 2.424225 & $\begin{array}{l}44.3 \\
44.7 \\
\end{array}$ & & & & & & & 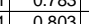 & \\
\hline 164 & $6 / 14 / 2004$ & 11:41:02 AM & 27.43 & 27.071 & 26.53 & 28.447 & & 44.575 & 45.891 & 8.903 & 36.329 & -1.22 & & 52.385 & 0.224 & 14.643 & & 146.35 & 2.43917 & 44.4 & $\frac{12 . \varepsilon}{12 .}>$ & & 2.789 & & 0.032 & & 0.791 & \\
\hline 165 & $6 / 14 / 2004$ & 11:42:02 AM & 27.431 & 27.087 & 26.516 & 28.448 & 27.18 & 44.882 & 46.157 & 8.866 & $\begin{array}{ll}36.647 \\
\end{array}$ & -1.22 & & 50.159 & 0.224 & 14.643 & & 147.35 & 2.45583 & 44.7 & & & 2.811 & 033 & 0.032 & & 0.785 & \\
\hline 16 & $6 / 14 / 2004$ & 11:43:02 AM & 27.427 & 27.098 & 26.437 & 28.484 & 27.1 & 42.045 & \begin{tabular}{|l|l|} 
& 43.317 \\
\end{tabular} & 10.446 & 32.247 & -1.219 & & 52.009 & 0.219 & 14.643 & & 148.35 & 2.4725 & $|41.8|$ & 12. & & 2.561 & & 0.031 & & 0.844 & \\
\hline & 6/14/2004 & 11:44:02 AM & & & & & & & & & & & & & & & & & & & & & & & & & & \\
\hline 168 & $6 / 14 / 2$ & 11:45 & 27.429 & 27.135 & $\frac{26.314}{26}$ & 28.541 & & 43.95 & 45.338 & 9.627 & & $-1.22 .2>$ & & 44.1299 & & 14.643 & & 150 & & 43.9 & & & & & .030 & & 0.768 & \\
\hline & & & & & & & & & & & & & & & & & & & & & & & & & & & & \\
\hline 170 & 6/14/2004 & 11:47:02 AM & 27.364 & 27.175 & 26.159 & 28.641 & & $\begin{array}{lll}44.548 \\
\end{array}$ & 45.924 & $\begin{array}{l}9.359 \\
\end{array}$ & 883 & -1.22 & & 46.482 & & \begin{tabular}{|c|c|c|}
14.643 \\
\end{tabular} & & & $\begin{array}{l}2.53917 \\
\end{array}$ & 44.5 & 11.4 & & & & & & 0.764 & 0.65 \\
\hline 171 & 6/114/2004 & 11:48: & 27.338 & 27.194 & 26.078 & 28.729 & & 44.784 & 46.128 & 9.09 & 36.341 & -1.22 & & 48.766 & 0.218 & \begin{tabular}{ll|l}
14.643 \\
\end{tabular} & & 153.35 & 2.55583 & 44.7. & 12.0 & 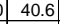 & 2.797 & 0.033 & 0.032 & & 0.777 & 0.66 \\
\hline 172 & 6/1/4/2004 & 11:49:02 AM & 27.312 & 27.198 & 26.017 & 28.763 & & 45.145 & 46.438 & 8.88 & 36.948 & $-1.2 .28>0$ & & 45.301 & 0.22 & 14.643 & & 15 & & 45.0 & 11.1. & & $28.5-3$ & & & & & \\
\hline & $6 / 14 / 2 / 2$ & 11:50: & 27.232 & 27.207 & 25.926 & 28.767 & & 44.181 & 45.456 & 9.655 & 35.192 & -1.2 .2 & & 49.7611 & 0.209 & 14.6 & & 155.3 & 2.58917 & 44.0. & & & 2.736 & 31 & 0.030 & & 0.765 & \\
\hline$\frac{1 / 4}{175}$ & $6 / 14 / 200$ & 11:51:02 AM & 27.1933 & 27.219 & & 28.804 & & 44.212 & 45. & & 35.56 & -1.22 & & & & 14. & & 15 & 2.6 & 44.0 & & & & & & & & \\
\hline$\frac{175}{176}$ & $\frac{6144 / 2}{6 / 142}$ & & $\frac{2.1 .153}{27123}$ & & | & 28.821 & & 44.654 & & & & -1.2 .2 & & & & 14.643 & & 15 & & 44.3 & . & & & & & & $\frac{175}{712}$ & \\
\hline & $6 / 141 / 2004$ & & 27066 & & 20.056 & 年.0.000 & & 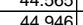 & 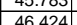 & 9.09 & & -1.2 & & 5127 & & . & & & & 4 & 126 & & 2808 & & & & & \\
\hline 178 & $6 / 14 / 2004$ & & 27.019 & 27.254 & $\frac{25.013}{2561}$ & 28.909 & & 44.903 & 46.049 & 8.094 & & -1. & & 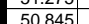 & & 146 & & & & 446 & & & & & & & 0.762 & \\
\hline 179 & $6 / 14 / 2004$ & $11: 56: 02 \mathrm{AM}$ & 27.023 & 27.267 & \begin{tabular}{|l|}
25.576 \\
\end{tabular} & 28.917 & 27.483 & 45.091 & 46.391 & 8.903 & 36.854 & \begin{tabular}{|l|l|}
4 & -1.225 \\
\end{tabular} & & $\begin{array}{lll}45.787 \\
\end{array}$ & 0.217 & \begin{tabular}{|l|l|}
14.643 \\
\end{tabular} & & 161.35 & \begin{tabular}{|l|}
2.68917 \\
\end{tabular} & 44.9 & 11.2 & 41 & 2.825 & 32 & 0.032 & 0.001 & 0.777 & 0.66 \\
\hline & $6 / 14 / 2 C$ & 11:57:02 AM & 26.931 & & 25.55 & $\begin{array}{ll}28.911 \\
\end{array}$ & & 43.66 & 44.869 & 9.911 & & \begin{tabular}{l|l|}
1 & -1.22 \\
\end{tabular} & & 47.998 & & \begin{tabular}{ll|l}
14.643 \\
\end{tabular} & & 100 & & \begin{tabular}{ll|}
43.4 \\
\end{tabular} & & & & & & & & \\
\hline & & & 26.9 & 27.284 & & 28.894 & & 44.37 & 45.862 & 9.618 & & -1.22 & & & & & & & & 44.4 & & & & & & & & \\
\hline & $0.1 / 4 / 2 / 2$ & & 2.800 & & 25.449 & 28.8 & & & & & & & & & & 14. & & & & 44.3 & 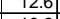 & & & & & & & \\
\hline & & & $\frac{2.024}{26702}$ & 27.292 & 25.4511 & 20.02 & & & & & & -1.2 & & & & & & & & & 10. & & & & & & & \\
\hline & $\frac{0141 / 2}{6 / 14 / 2}$ & & $\frac{2.1759}{26.9}$ & 27.304 & $\frac{2.037}{2525}$ & 20.010 & & & & & & & & & & . & & & & 44. & & & & & & & & \\
\hline & $6 / 141 / 20$ & $120002 \mathrm{P}$ & $\frac{26.666}{26.666}$ & 27.31 & 25.388 & 28.705 & & & & & & & & & & & & & & & & & & & & & & \\
\hline 187 & $6 / 14 / 200$ & $12: 04$ & 26.638 & 27.3 & 25.341 & 28.718 & & & 45.663 & 9.5 & & -1.2 & & & & 14.6 & & & & 44 & & & & & & & 0 & \\
\hline 188 & $6 / 14 / 2$ & & 26.626 & & 25.364 & 28.725 & & 44.6 & 45.9 & 9.46 & & -1.2 & & & & & & & & $\mid 44.4$ & & & & & & & 0.744 & \\
\hline & $6 / 14 / 2$ & & 26.60 & & & 28.708 & & & & & & & & & & & & & & $\mid 44.4$ & & & & & & & & \\
\hline & $6 / 14 / 2$ & & 26.58 & & 25.27 & & & & & & & & & & & 14. & & & & 44. & & & & & & & & \\
\hline & & & & & & & & & & & & & & & & & & & & & & & & & & & & \\
\hline & $6 / 14 / 2$ & 12:09: & 26.54 & 27.3 & 25.277 & 28.804 & & 44. & 45.7 & 9.79 & & & & & & & & & & 44 & & & & & & & & \\
\hline & $6 / 14 / 20$ & 12:10 & & 27. & 25.2 & 28. & & 44.1 & & & & & & & & 14. & & & & 44 & & & & & & & & \\
\hline 194 & $6 / 144 / 200$ & 12:11:02 F & 26.4 & 27.3 & 25.24 & $28 . \varepsilon$ & & 44.254 & 45. & 9.5 & & -1.2 & & 49. & & 14. & & 176 & & 43.9 & 12.2 & & & & & & 0.739 & \\
\hline & $6 / 14 / 2$ & & 26.4 & 27.5 & & 28.8 & & 44.4 & 45. & 9.4 & & -1.2 & & & & 14. & & & & 44 & 12.4 & & & & & & 0.724 & \\
\hline 196 & $6 / 14 / 4$ & & 26.4 & & 25.234 & 28.875 & & 44.7. & 45.97 & 9.52. & & & & & & & & 17 & & 44.5 & 12.4 & & & & & & 0.109 & \\
\hline & $6 / 14 / 4$ & & & & & & & & & & & & & & & & & & & & & & & & & & & \\
\hline & - & & $\frac{2.4441}{2645}$ & & 20.2535 & 20.94 & & & 45.013 & 9.4 & & & & & & & & & & & & & & & & & & \\
\hline & $\frac{6142 / 2}{6 / 42 / 2}$ & 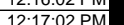 & $\frac{26.454}{26.424}$ & 27. & 年 & $\frac{2.54}{28.94}$ & & $\begin{array}{l}44.364 \\
43683\end{array}$ & 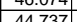 & $\frac{1.200}{10086}$ & & $\frac{-1.22}{121}$ & & & $\frac{.1}{0.1}$ & . & & & & $\begin{array}{l}44.6 \\
4.33\end{array}$ & 117 & & & & & & & \\
\hline & $6 / 141 / 20$ & & 26.44 & 27384 & 25 & 28.95 & & 44.4 & & 97 & & -12 & & & & & & & & 443 & & & & & & & & \\
\hline 202 & $6 / 14 / 20$ & $\begin{array}{l}12: 19: 02 \mathrm{~F} \\
\end{array}$ & 26.421 & 27.3 & 25.193 & 28.95 & & 44.2 & & 9.7 & & $\frac{-1.2}{-1.2}$ & & & & 14.6 & & & & 44.1 & 11.9 & & & & & & & \\
\hline & $6 / 14 / 2$ & $12: 20:$ & 26.40 & & 25.19 & 28. & & 44.4 & & 9.608 & & & & & & 14.6 & & & & 44.1 & $11.6 \mathrm{C}$ & & & & & & & \\
\hline$=04$ & $6 / 14 / 2$ & 12:21:0? & & & & & & 44.7 & & & & & & & & & & & & 44.4 & & & & & & & & \\
\hline 20 & $6 / 14 / 2$ & & 26.388 & & 25.165 & & & & & & & & & & & & & & & 4.6 & 1.6 & & & & & & & \\
\hline & & & & 27.2 & 25.165 & 28.9 & & 44. & 45.86 & & & & & & & & & & & 44.4 & & & & & & & & \\
\hline 20 & $6 / 14 / /$ & $12: 22$ & 26.378 & 27.426 & 25. & $28.8 \mathrm{C}$ & & 44.8 & 45.843 & 9.4 & $36.1 \varepsilon$ & -1.2 & & & & & & & & \begin{tabular}{ll|}
4.4 \\
\end{tabular} & & & & & & & & \\
\hline & /14/200 & 12:25:02 PM & 26.373 & 27.436 & 25.16 & 28.90 & & 44.96 & 46115 & 9.4 & 36.1 & -1.22 & & 49.95 & 0.1 & 14.64 & & $19 \mathrm{c}$ & 3.1 & \begin{tabular}{l|l|}
44.6 \\
\end{tabular} & & & & & & & & \\
\hline & 6/14/200 & 2:26:02 PM & 26.353 & 27.446 & 5.15 & 8.886 & & 44.62 & 6.01 & 9.446 & 35.86 & $-1.21 \mathrm{~g}$ & & 52.08 & & 14.643 & & & $3.18 \mathrm{~s}$ & 44.5 & & & & & & 0. & $\mid$ & \\
\hline $10 \mid$ & 6/14/2004 & 12:27:02 PM & 6.382 & 27.45 & 25.149 & 28.83| & & 45.28 & 46.347 & 9.412 & 36.665 & \begin{tabular}{|l|l|l|}
5 & -1.222 \\
\end{tabular} & & 7.7377 & $0.198]$ & 14.643 & & 192.35 & 3.20583 & 44.9] & \begin{tabular}{|l|l|} 
& 11.7 \\
\end{tabular} & & & & & 0.001 & 0.717 & \\
\hline
\end{tabular}


WSRC-TR-2005-00105, REVISION 0

SRNL-RPP-2005-00012, REVISION 0

RUN \# 3.03A AND B; FIRST AND SECOND HALF OF SLURRY DEWATERING - CONT.

\begin{tabular}{|c|c|c|c|c|c|c|c|c|c|c|c|c|c|c|c|c|c|c|c|c|c|c|c|c|c|c|c|c|}
\hline & & & & & & & & & & & & & & & & & & & & & & & & & & & & \\
\hline & $\mathrm{A}$ & $\mathrm{B}$ & D & $E$ & $\mathrm{~F}$ & \begin{tabular}{l|l} 
\\
\end{tabular} & $\mathrm{H}$ & $\mathrm{J}$ & \begin{tabular}{l|l}
$\mathrm{K}$ \\
\end{tabular} & $\mathrm{L}$ & M & $\mathrm{N}$ & 0 & Q & $\mathrm{R}$ & $\mathrm{s}$ & $\mathrm{T}$ & $\mathrm{v}$ & $\mathrm{w}$ & \begin{tabular}{l|l}
$x$ & \\
\end{tabular} & $\mathrm{Y}$ & $\mathrm{z}$ & $\mathrm{AA}$ & $A B$ & $\begin{array}{ll}A C \\
\end{array}$ & AD & $\mathrm{AE}$ & AF $[A$ \\
\hline & 6/14/2004 & 12:28:02 PM & $\frac{2.374}{26.374}$ & 27.443 & 25.176 & 28.773 & 27.304 & 44.955 & \begin{tabular}{|l|l|}
46.169 \\
\end{tabular} & 9.286 & 36.4 & -1.219 & -6.103 & $\frac{275}{46.275}$ & 0.187 & 14.643 & 0.004 & 193.35 & \begin{tabular}{|l|l|}
5.2225 \\
\end{tabular} & 44.7 & 11.3 & & & 0.028 & 0.028 & 0.001 & & \\
\hline & 6/14/2004 & 12:29:02 PM & 26.398 & 27.467 & 25.175 & 28.742 & 27.248 & 44.824 & 46.304 & 9.385 & 36.129 & -1.222 & & 43.985 & 0.194 & 14.643 & & 194.35 & 3.23917 & 44.8 & & 40.5 & & & 0.029 & 0.001 & & \\
\hline & $6 / 14 / 2004$ & 12:30:02 PM & 26.353 & 27.456 & 25.125 & 28.677 & & 44.614 & 45.688 & 9.299 & 36.037 & -1.222 & & 49.604 & 0.188 & 14.643 & & 195.35 & 3.25583 & 44.2 & & & & & 0.028 & 0.001 & & \\
\hline & 6/14/2004 & 12:31:02 PM & 26.365 & $\begin{array}{l}27.463 \\
2738\end{array}$ & $\begin{array}{ll}25.142 \\
25126\end{array}$ & $\begin{array}{l}28.574 \\
28.49 \\
\end{array}$ & $\begin{array}{l}27.184 \\
27.34 \\
\end{array}$ & 45.052 & $\begin{array}{l}46.287 \\
\end{array}$ & $\begin{array}{l}9.489 \\
\end{array}$ & $\begin{array}{ll}36.294 \\
3.19\end{array}$ & -1.219 & \begin{tabular}{|l|} 
\\
\end{tabular} & 52.032 & 0.193 & 14.643 & & $\begin{array}{l}196.35 \\
\end{array}$ & $\begin{array}{l}3.2725 \\
\end{array}$ & \begin{tabular}{ll|}
44.8 \\
\end{tabular} & \begin{tabular}{|l|}
12.8 \\
\end{tabular} & 4.10 & \begin{tabular}{|l|l|}
2.804 \\
\end{tabular} & & $\begin{array}{l}0.029 \\
\end{array}$ & 0.001 & 0.705 & \\
\hline 216 & 6/14/2004 & $\begin{array}{l}\text { 12:32:02 PM } \\
\text { 12:33:02 PM }\end{array}$ & $\frac{26.314}{26.305}$ & $\mid$\begin{tabular}{|l|}
27.438 \\
27.423
\end{tabular} & $\begin{array}{l}25.126 \\
25.117 \\
\end{array}$ & \begin{tabular}{|l|}
28.479 \\
28.435
\end{tabular} & 27.134 & $\begin{array}{l}44.878 \\
45.125\end{array}$ & $\begin{array}{r}46.14 \\
46.511\end{array}$ & 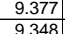 & \begin{tabular}{r|r|}
36.162 \\
36.48 \\
\end{tabular} & \begin{tabular}{|c|}
$\mid-1.219$ \\
-1.219 \\
\end{tabular} & & 51.625 & 0.192 & $\begin{array}{l}14.643 \\
14.643 \\
\end{array}$ & 0.004 & $\begin{array}{r}197.35 \\
198.35\end{array}$ & $\begin{array}{l}3.28917 \\
3.30583 \\
5\end{array}$ & $\begin{array}{l}44.7 \\
45\end{array}$ & \begin{tabular}{|l|}
12.7 \\
132
\end{tabular} & \begin{tabular}{|l|}
40.5 \\
40.8 \\
\end{tabular} & \begin{tabular}{|l|}
2.794 \\
813
\end{tabular} & $\frac{0.029}{0.030}$ & \begin{tabular}{|c|}
0.029 \\
0.030
\end{tabular} & $\begin{array}{l}0.001 \\
0.001 \\
\end{array}$ & $\begin{array}{l}0.704 \\
0.725 \\
\end{array}$ & 0.60 \\
\hline 217 & 6/14/2004 & $12: 34: 02 \mathrm{PM}$ & 26.377 & 27.425 & 25.109 & 28.456 & & & & & & & & & 0.194 & & & & & & & & & & & & & \\
\hline & & & 26.366 & 27.409 & 25.093 & 28.465 & 27.121 & 44.415 & 45.999 & 9.289 & 35.787 & -1.222 & -5.713 & 49.585 & 0.181 & 14.643 & & 200.35 & & 44.5 & 12.2 & & & & 0.027 & & & \\
\hline & $6 / 14 / 2004$ & 12:36:02 PM & 26.37 & 27.398 & 25.097 & 28.494 & 27.13 & 44.446 & 45.966 & 9.296 & 35.789 & -1.222 & -5.695 & 45.526 & 0.19 & 14.643 & & 201.35 & 5.35583 & 44.5 & 11.2 & 40.1 & 2.766 & \begin{tabular}{l|l|}
0.028 \\
\end{tabular} & 0.028 & 0.001 & 0.704 & \\
\hline & & 12:37:02 PM & & & & 28.513 & 27.139 & 44.795 & 46.449 & & & -1.222 & & & & & & & & & 13.8 & & & & & & & \\
\hline & 6/14/2004 & $12: 38: 02 \mathrm{PM}$ & 26.498 & 27.381 & 25.08 & 463 & & & & 235 & & & & 48.301 & & 14.643 & & & 3917 & $\frac{\mid 44.8}{4}$ & & & & & $\frac{128}{28}$ & 001 & $\frac{0.097}{0.697}$ & \\
\hline & & & & & & 28.403 & & 44.629 & & & & & & & 0 & & & & & 44.6 & $\frac{\pi 1.1}{11.1}$ & & & & & & & \\
\hline & $6 / 14 / 2004$ & 12:40:02 PM & 26.452 & 27.38 & 25.084 & 28.447 & & 44.61 & |46.243 & 9.273 & 35.996 & $\frac{1.222}{-1.22}$ & & 48.891 & 0.188 & 14.643 & & .35 & 3.4225 & 44.8 & $\frac{1.1}{12.0}$ & $\frac{40}{40}$ & 2.77 & 028 & 0.028 & $\begin{array}{l}0.001 \\
0.001\end{array}$ & 0.694 & \\
\hline & $6 / 14 / 2004$ & 12:41:02 PM & 26.438 & 27.362 & 25.091 & 28.498 | & $27.18 \mathrm{r}-\mathrm{l}$ & 44.677 & 46.297 & 9.28 & 36.127 & -1.222 & & 39.586 & 0.187 & $\begin{array}{ll}14.643 \\
\end{array}$ & & 206.35 & \begin{tabular}{|c|}
3.43917 \\
\end{tabular} & 44.8 & & & 2.786 & & & & 0.688 & \\
\hline & $6 / 14 / 2004$ & 12:42:02 PM & 26.437 & 27.37 & 25.094 & 28.566 & 27.237 & 44.811 & 46.471 & 9.375 & 36.099 & -1.222 & & 43.605 & 0.187 & 14.643 & & & $\begin{array}{l}5.45583 \\
\end{array}$ & 45.0 & 10.7 & & 2.789 & & $\begin{array}{ll}0.028 \\
\end{array}$ & 0.001 & 0.687 & \\
\hline & $6 / 14 / 2004$ & 12:43:02 PM & 26.43 & 27.373 & 25.082 & 28.589 & & 44.807 & 46.329 & 9.376 & 36.09 & -1.222 & & 51.386 & 0.187 & $\begin{array}{ll}14.643 \\
\end{array}$ & & & 3.4725 & 44.9 & & & & & & & & \\
\hline & 6/14/2004 & 12:44:02 PM & 26.536 & 27.37 & 25.0999 & 28.641 & & 45.1 & 46.339 & 9.237 & 36.552 & -1.22 & & 54.744 & 0.183 & 14.643 & & & \begin{tabular}{|l|l|}
3.48917 \\
\end{tabular} & 44.99 & $13.4 \mathrm{r}>\mathrm{C}$ & & & & & & 1.666 & \\
\hline & 6/14/20004 & 12:45:02 PM & 26.358 & 27.367] & & 28.648 & & & 46.689 & 9.369 & 36.876 & -1.222 & & & 0.187 & 14.643 & & & & & & & & & & & 0.675 & \\
\hline & $6 / 14 / 2$ & 12:46:02 PM & 26.357 & & & 28.676] & & 45.216 & 46.519 & 9.303 & 36.565 & -1.2. & & 44.206 & 0.185 & 14.6 & & & 3.5225 & 45.0 & $10.8 \mathrm{r}>\mathrm{r}$ & & & & & & 0.673 & \\
\hline & $0.1142004 \mid$ & 12:47.02 $\mathrm{PM}$ & 2.373 & 27.370 & 25.075 & $28.717 \mid$ & & 44.988 & $\begin{array}{ll}46.026 \\
46.353\end{array}$ & 9.24 & 36.427 & & & 466.79 & & 14.6 & & & 3.53917 & 44.6 & & & & & & & 0.698 & 0.59 \\
\hline & $\frac{0 / 14 / 2004}{6 / 14 / 2004}$ & $\frac{122.4 .62 \cdot \mathrm{PMI}}{12 \cdot 49 \cdot 02 \mathrm{PM}}$ & $\frac{20.294}{26297}$ & 27.530 & $\frac{25.101}{25089}$ & $\frac{28.180}{28.711}$ & & 年5..318 & $\mid \frac{\mid 4.5353}{46.604}$ & 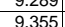 & $\frac{30.143}{36718}$ & -1.22 & & $\frac{39.903}{50.941}$ & $\frac{0.180}{0.189}$ & $\frac{14.045}{14643}$ & & 20135 & 35725 & 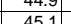 & & & & & & & & 57 \\
\hline & $6 / 14 / 2004$ & $\begin{array}{l}\text { 12.4.4.02: PMT } \\
\text { 12:50:02 PM }\end{array}$ & $\frac{20.291}{26.314}$ & 27.382 & 25.081 & 28.14 & 27.324 & $\begin{array}{l}43.031 \\
45.195 \\
\end{array}$ & \begin{tabular}{|l|}
40.0042 \\
46.212
\end{tabular} & $\begin{array}{r}.550 \\
9.33\end{array}$ & 30.1.51 & $\begin{array}{l}-1.225 \\
-1.225 \\
\end{array}$ & & $\begin{array}{l}5.941 \\
47.272\end{array}$ & 0.185 & $\frac{14.045}{14.643}$ & & 年 & 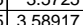 & $\begin{array}{l}45.1 \\
44.7 \\
\end{array}$ & $\frac{1.5}{11.6}$ & & 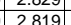 & & & 001 & 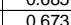 & $\frac{58}{57}$ \\
\hline & $6 / 14 / 2004$ & 12:51:02 PM & 26.275 & 27.373 & 25.062 & 28.759 & 27.324 & 45.369 & 46.586 & 9.341 & 36.741 & -1.222 & & 45.795 & 0.186 & 14.643 & & 216.35 & 3.60583 & 45.1 & 11.2 & $\frac{\overline{41}}{41}>2$ & 2.831 & 年 & 0.028 & 0.001 & 0.674 & \\
\hline & 6/14/2004 & 12:52:02 PM & 26.313 & 27.376 & 25.075 & 28.781 & & & 46.013 & 9.3 & 36.505 & $\begin{array}{l}-1.219 \\
\end{array}$ & & & 0.185 & $\begin{array}{ll}14.643 \\
\end{array}$ & & & & & & & & & & & & \\
\hline & $6 / 14 / 2004$ & 12:53:02 PM & 26.31 & 27.393 & 25.077 & 28.789 & & $\begin{array}{l}45.176 \\
\end{array}$ & 46.31 & 9.335 & 36.526 & $-1.22 \mathrm{P}-\mathrm{C}$ & & \begin{tabular}{|c|c|}
77.858 \\
\end{tabular} & & 14.643 & & & 3.62917 & 44.8 & 11.7 & & 81 & & & 001 & 0.666 & \\
\hline & & & & & & 28.815 & & & & & & & & & & 14.643 & & & & 44.9 & & & & & & & & \\
\hline & 6 & 12:55:02 PM & $\frac{26.304}{26}$ & $\frac{27.388}{2788}$ & 25.046 & $\frac{28.828}{28.8}$ & & 45.006 & 46.208 & 9.357 & 36.335 & $\frac{-1.2}{-1.2}<>$ & & 1.131 & 0.18 & 14.6 & & & 3.6725 & 44.7 & & & & & & & & \\
\hline & $6 / 14 / 2004$ & 12:56:02 PM & & & & 28.0 & & 45.442 & \begin{tabular}{|l|l|}
46.849 \\
\end{tabular} & 9.19 & 36.924 & -1.2. & & 40.178 & 0.184 & 14. & & & & $\mid 45.4$ & $\overline{9 . \varepsilon}$ & & & & & & & \\
\hline & 6/14/2004 & 12:57:02 PM & 26.345 & 27.373 & 25.072 & 28.714 & 27.215 & 43.945 & 45.095 & 10.189 & 34.502 & -1.222 & & 44.715 & 0.17 & $\begin{array}{ll}14.643 \\
\end{array}$ & & 222.35 & 5.70583 & \begin{tabular}{c|c|c|}
43.6 \\
\end{tabular} & 11.0 & 39. & 2.704 & & 0.025 & 0.001 & 0.645 & \\
\hline & 6/14/2004 & 12:58:02 PM & & 27.4 & 25.109 & 28.716 - & & 44.432 & 45.665 & 9.859 & 35.274 & $-1.21 \mathrm{~s}$ & & & 0.174 & 14. & & & & 44.2 & & & & & & & & \\
\hline & 6/14/2004 & 12:59:02 PM & 26.291 & 27.384 & 25.078 & 28.65 & & 44.305 & 45.502 & 9.726 & 35.245 & -1.219 & & 48.638 & 0.167 & 14.643 & & 224.35 & 3.73917 & 44.0 & 11.9 & & 2.74 & 25 & 0.025 & 0.001 & 0.625 & \\
\hline & 6/14/2004 & & & & & 28.624 & & 44.3 & & & & -1.22 & & 45.90r & & & & & & 44 & & & & & & & & \\
\hline & & :02 PM & 26.349 & 27.373 & 25.126 & 28.5899 & & 44.741 & 46.059] & 9.668 & 35.756 & & & 51.999] & 0.178 & & & & & 44 & & & & & & & 0.657 & \\
\hline & $\frac{601142004}{6.112004}$ & 1:02:02 PM & 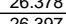 & 20.375 2725 & 25.1151 & 28.618 & & 45.085 & 46.285 & $\frac{9.668}{9.517}$ & $\begin{array}{l}36.154 \\
3615 \\
\end{array}$ & $-\frac{1.22}{12}$ & & (7).402 & 0.179 & 14.6 & & & 3.78 & 44.8 & $\frac{11.6}{11.3}$ & & 270 & & & & 554 & \\
\hline & $\frac{0.142004}{61 / 12004}$ & & & & 2520149 & | & & 年5.0 & & 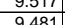 & & - & & 634 & & 14. & & & & 告4 & 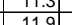 & & & & & & & \\
\hline & $6 / 14 / 2004$ & 5:02 PM & 26.411 & 27.35 & 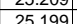 & $\begin{array}{l}28.000 \\
28.721 \\
\end{array}$ & $\frac{17.246}{27.246}$ & 44.8 & 年0.150 & $\begin{array}{c}0.401 \\
9.51\end{array}$ & $\begin{array}{l}35.961 \\
35.010 \\
3\end{array}$ & $\begin{array}{l}-1.222 \\
-1.222 \\
-1\end{array}$ & & 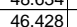 & 0.177 & $\frac{14.6}{14.6}$ & & $\frac{250}{230}$ & & 44.8 & $\frac{11.5}{11.4}$ & & & & & & 0.059 & \\
\hline & $6 / 14 / 2004$ & $1.06 .02 \mathrm{PM}$ & $\frac{20.463}{26.463}$ & $\frac{27.38}{2782}$ & & $\mid$ & & 44.9 & 4 & 9.427 & 36.242 & -1.21 & & & & $\frac{1.4}{14.4}$ & & & & 44.6 & $\frac{1.4}{102}$ & & & & & & & \\
\hline & & 2PM & 26.48 & 27.373 & & $\mid 28.779$ & & 44.635 & & 9.479 & & -1.2 & & 455.424 & & & & & & 44.5 & $\overline{11.1}$ & & & & & & & \\
\hline & $6 / 114 / 2 C$ & $2 \mathrm{PM}$ & $\frac{26.497}{26}$ & & 25.285 & 28.821 & & 44.05 & & 9.566 & 36.6 & -1.22 & & 47.114 & & & & & & 44.8 & $\frac{11.5}{11.5}$ & & & & & & $\frac{654}{654}$ & \\
\hline & $6 / 14 / 2$ & & & $\frac{\mid}{27.368}$ & 25.267 & & & 45.1 & & & & $\frac{-1.2}{-1.2}$ & & & & & & & & 45 & $\frac{11.4}{11.4}$ & & & & & & & \\
\hline & $6 / 114 / 2004$ & $1: 1$ & 26.526 & 27.379 & 25.288 & 28.864 & & & 46.181 & 9.474 & & -12 & & & & & & & & 44.7 & 118 & & & & & & & \\
\hline & 6/14/2004 & $1: 11: 02 \mathrm{PM}$ & & 27.39 & \begin{tabular}{|l|}
25.349 \\
\end{tabular} & & & & & & & -1.2 & & & & & & & & 44 & 11.5 & & & & & & & \\
\hline & $6 / 14 / 2004$ & 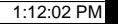 & 26.553 & 27.382 & 25.341 & 28.887 & & $\begin{array}{l}44.801 \\
\end{array}$ & & 9.581 & 35. & $-1.22 !$ & & 45.967 & & & & & & 44 & $\overline{11.3}$ & & & & & & 0.641 & \\
\hline & $6 / 14 / 2 C$ & 1:13:02 PM & 26.57 & 27.388 & 25.348 & 28.943 & & 44.832 & & 9.485 & & -1.22 & & 47..051 & & & & & & 44 & 11.5 & & & & & & & \\
\hline & $6 / 14 / 2$ & 1:14:02 PM & 26.587 & 27.401 & 25.375 & 28.966 & & 45.141 & 46.588 & 9.606 & 36.1 & -1.222 & & 50.505 & 0.1 & & & & & 45 & 12.4 & & $2.8 \mathrm{C}$ & & & & & \\
\hline & 6/14/1/2 & & & & 25.4 & & & 45.2 & & 9.56 & & -1.2 & & & & & & & & & 11.0 & & & & & & & \\
\hline & & & & & & & & 44.9 & & 9.4 & & & & & & & & & & & 10.5 & & & & & & & \\
\hline & & & & & 25.37 & & & & & & & -1.2 & & & & & & & & & t.t. & & & & & & & \\
\hline & & & & & & & & & & & & & & & & & & & & & & & & & & & & \\
\hline & 6 & & & & & & & $\begin{array}{l}44.1044 \\
45023\end{array}$ & & & & -1.4 & & & & & & & & & & & & & & & & \\
\hline & $6 / 41 / 2 c$ & & & 27.462 & & & & 43.895 & & & & & & & & & & & & & & & & & & & & \\
\hline & $6 / 14 / 2$ & & & $\frac{27.465}{27}$ & & & & & & 10.166 & & -1.2 & & & & & & & & 438 & & & & & & & & \\
\hline & & & & & & & & & & & & & & & & & & & & & & & & & & & & \\
\hline & & & 26.66 & & & & & & & & & & & & & & & & & 44 & & & & & & & & \\
\hline & $6 / 14 / 2$ & & 26.67 & 27.4 & & & & 44.6 & 45.98 & & & & & & & 14 & & & & 44. & & & & & & & & \\
\hline & $6 / 14 / 2$ & $1: 25$ & 26.65 & 27.45 & 25. & 28.76 & & & & & & -1.2 & & & & 14 & & & & 44. & 3.0 & & & & & & & \\
\hline & $6 / 14 / 2 C$ & $1: 26$ & 26.67 & $27.438 \mid$ & 25.338 & 28.699 & & 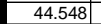 & 45.9 & & 35 & -1.2 & & 48.7 & & 14.6 & & & & 44.5 & 11.9 & & & & & & & \\
\hline & $6 / 14 / 2$ & $1: 27: 37 \mathrm{P}$ & 26.68 & 27.435 & 25.31 & 28.6 & & 44.6 & \begin{tabular}{|l|l|}
45.914 \\
\end{tabular} & & 35.489 & -1.2 & & 51.2 & & 14. & & & & \begin{tabular}{|l|l|}
44.4 \\
\end{tabular} & 12 & & & & & & & \\
\hline & $6 / 14 / 2$ & & 26.7 & 27.4 & 25.3 & & & 44. & & 9.882 & & $-1.8 \mathrm{~s}$ & & & & & & & & 44.7 & \begin{tabular}{ll|}
11.6 \\
\end{tabular} & & & & & & & \\
\hline & & & $26.7 \mathrm{r}$ & & & & & & & & & -1.2 & & & & & & & & & & & & & & & & \\
\hline & 6 & 1:30:3 & 26.757 & 27.46 & \begin{tabular}{|l|}
$\mid 25.35$ \\
\end{tabular} & 28.73 & & 44.465 & & & 35.335 & -1.225 & & & 0.1 & 14. & & & & 44.4 & & & & & & & & \\
\hline 776 & 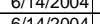 & $\begin{array}{l}1.31 .375 \\
1.32 .370\end{array}$ & $\frac{2.1}{26.72}$ & $\frac{2.764}{2.763}$ & $\mid$\begin{tabular}{|l|}
$\mid 25.3828$ \\
\end{tabular} & 28.824 & & $\begin{array}{l}44.114 \\
4.307\end{array}$ & 45.91 & $\begin{array}{l}9.86 \\
.87\end{array}$ & $\begin{array}{l}35.500 \\
3.520\end{array}$ & -1.24 & & 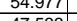 & 0.1 & & & 257 & & 44.4 & 13.5 & & & & & & & \\
\hline & $\frac{6}{61.142004}$ & 1.22 .378 & 20.12 & 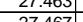 & 25.323 & 2 & & 444.391! & 45.049 & 年 & (35.315) & - 1.2 .2 & & & 0.14 & & & & & 44.24 & 11.6 & & & & & & & \\
\hline & 6 & 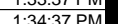 & $\frac{2.1100}{26700}$ & 27.4010 & 25307 & 28062 & & 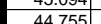 & 40.0150 & 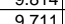 & 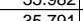 & 1210 & & 18232 & 0.105 & & & & & 43.6 & 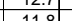 & & & & & & 0.010 & \\
\hline & $\frac{6}{661 / 2004}$ & $1.35 .37 \mathrm{PM}$ & 26.717 & 2776 & 25.28 & 28971 & & 44364 & 45657 & 9662 & 35.45 & $\frac{1.252}{-1.222}$ & & 4.59 & 16 & 14643 & & & & 442, & 134 & & & & & (1) & 1623 & \\
\hline 800 & & & & 7.481 & 266 & 20.966 & & & 032 & 9.776 & 35.569 & 1.22 & & .676 & 0.165 & $\begin{array}{l}\frac{14.045}{14.643} \\
\end{array}$ & & & & 44.6 & & & & & .024 & .001 & 0.609 & 09 \\
\hline
\end{tabular}


WSRC-TR-2005-00105, REVISION 0

SRNL-RPP-2005-00012, REVISION 0

RUN \# 3.03A AND B; FIRST AND SECOND HALF OF SLURRY DEWATERING - CONT.

\begin{tabular}{|c|c|c|c|c|c|c|c|c|c|c|c|c|c|c|c|c|c|c|c|c|c|c|c|c|c|c|c|c|}
\hline & A & B & D & $E$ & $F$ & G & $\mathrm{H}$ & $\mathrm{J}$ & $\mathrm{K}$ & $\mathrm{L}$ & $M$ & $\mathrm{~N}$ & 0 & 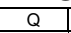 & $R$ & $\mathrm{~s}$ & $T$ & v & W & $x$ & $\mathrm{Y}$ & $z$ & AA & $A B$ & $\mathrm{AC}$ & $\mathrm{AD}$ & $\mathrm{AE}$ & AF $[A$ \\
\hline & $6 / 14 / 2004$ & 1:37:37 PM & 26.717 & 27.49 & 25.265 & 28.965 & 27.521 & 44.4 & 45.989 & 9.698 & 35.462 & -1.225 & $\begin{array}{l}-6.843 \\
\end{array}$ & & 0.17 & 14.643 & 0.004 & 262.9 & & 44.5 & & & 2.75 & & 0.026 & 0.001 & 0.656 & 0.56 \\
\hline & $6 / 14 / 2004$ & $\begin{array}{l}1: 38: 37 \mathrm{PM} \\
\end{array}$ & 26.73 & 27.494 & 25.249 & 28.939 & 27.465 & \begin{tabular}{|l|l|}
44.639 \\
\end{tabular} & \begin{tabular}{|l|l|}
46.117 \\
\end{tabular} & 9.594 & $\begin{array}{l}35.776 \\
\end{array}$ & $\begin{array}{l}-1.219 \\
\end{array}$ & $\begin{array}{l}-6.794 \\
-770\end{array}$ & 4.404 & 0.172 & 14.643 & 0.004 & 263.9 & 4. 4.95009 & 44.6 & & & 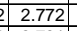 & & 0.025 & 0.001 & 0.633 & \\
\hline $\begin{array}{l}283 \\
284 \\
284\end{array}$ & $6 / 144 / 2004$ & $1: 39: 37 \mathrm{PM}$ & 26.719 & $\begin{array}{l}27.492 \\
275\end{array}$ & 25.232 & 28.923 & 27.449 & \begin{tabular}{|l|l|l|}
44.811 \\
4.785
\end{tabular} & \begin{tabular}{|l|l|}
46.018 \\
\end{tabular} & 9.702 & $\begin{array}{l}35.848 \\
5.565\end{array}$ & -1.222 & $\begin{array}{r}-7.008 \\
7.720\end{array}$ & 50.676 & 0.178 & 14.643 & 0.004 & 264.93 & 4.41556 & 44.5 & \begin{tabular}{|l|}
12.4 \\
\end{tabular} & 40.0 & $\begin{array}{l}3.781 \\
\end{array}$ & & $\begin{array}{l}0.026 \\
\end{array}$ & 0.001 & 0.654 & \\
\hline$\frac{204}{285}$ & 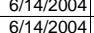 & $\begin{array}{l}1: 40: 37 \mathrm{PM} \\
1: 14: 37 \mathrm{PM}\end{array}$ & $\frac{26.702}{26668}$ & $\begin{array}{r}27.5 \\
27.487\end{array}$ & $\begin{array}{r}25.22 \\
25.182\end{array}$ & $\begin{array}{r}28.9 \\
28857\end{array}$ & 27.397 & $\begin{array}{l}44.785 \\
45.351\end{array}$ & $\begin{array}{r}45.943 \\
46.61\end{array}$ & $\begin{array}{r}9.601 \\
9656\end{array}$ & $\begin{array}{l}35.965 \\
3638\end{array}$ & $\begin{array}{r}-1.219 \\
-1.222\end{array}$ & $\begin{array}{r}-7.12 \\
-70 \\
\end{array}$ & \begin{tabular}{ll|l}
44.083 & \\
47.533
\end{tabular} & $\begin{array}{l}0.166 \\
0.169 \\
\end{array}$ & $\begin{array}{l}14.643 \\
14.643\end{array}$ & 0.004 & 266.93 & \begin{tabular}{|l|}
4.43222 \\
448889 \\
\end{tabular} & $\begin{array}{l}44.5 \\
45.1\end{array}$ & 116 & 409 & $\begin{array}{l}4.784 \\
2.818 \\
4\end{array}$ & & $\begin{array}{l}0.025 \\
0.025\end{array}$ & 0.001 & $\begin{array}{l}0.609 \\
0613\end{array}$ & \\
\hline 286 & 6 & $1: 42: 37 \mathrm{PM}$ & 26.000 & 27.401 & $\frac{2.104}{25.16}$ & 28.057 & 27.342 & 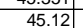 & 46.01018 & $\begin{array}{l}9.050 \\
9.675\end{array}$ & $\begin{array}{l}3.1000 \\
36.129\end{array}$ & & $\begin{array}{r}-7.00 \\
-7.097\end{array}$ & & 0.166 & $\begin{array}{l}14.045 \\
14.643\end{array}$ & & & $\begin{array}{l}4.4 .4005 \\
4.46556\end{array}$ & 45.1 & $\frac{11.6}{11.1}$ & 40.9 & 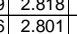 & 0.025 & $\begin{array}{l}0.025 \\
0.025\end{array}$ & $\frac{0.001}{0.001}$ & $\begin{array}{l}0.613 \\
0.606\end{array}$ & $\begin{array}{l}0.52 \\
0.52\end{array}$ \\
\hline 287 & $6 / 14 / 2004$ & 1:43:37 PM & 26.671 & 27.499 & 25.164 & 28.79 & 27.306 & 44.969 & $\begin{array}{l}46.387 \\
\end{array}$ & 9.58 & 36.088 & -1.225 & & 43.499 & 0.165 & 14.643 & 0.004 & 268.93 & & 44.9 & & & & & 0.024 & & & \\
\hline 288 & $6 / 14 / 2004$ & 1:44:37 PM & 26.663 & 27.497 & 25.172 & 28.768 & 27.305 & 45.212 & 46.615 & 9.658 & 36.209 & -1.219 & & 50.015 & 0.167 & 14.643 & 0.004 & 269.93 & 4.49889 & 45.1 & 12.3 & 40.7 & 72.807 & 25 & 0.025 & 0.001 & & \\
\hline 289 & $6 / 14 / 2004$ & 1:45:37 PM & 26.646 & & 25.164 & 28.735 & 27.281 & 44.886 & 46.185 & & $\begin{array}{l}35.93 \\
3.97\end{array}$ & -1.222 & -7.109 & 54.627 & 0.164 & 14.643 & 0.004 & 270.93 & 4.51556 & 44.7 & & & \begin{tabular}{|l|l|}
4 & 2.786 \\
\end{tabular} & & & & 0.602 & \\
\hline 290 & $6 / 14 / 2004$ & $\begin{array}{l}1: 46: 37 \mathrm{PM} \\
\end{array}$ & 26.633 & 27.491 & 25.146 & & & & 46.519 & & & & & 46.265 & & 14.643 & & & & & & & & & & & & \\
\hline 291 & $6 / 1 / 4 / 2004$ & 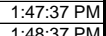 & $\begin{array}{l}26.655 \\
26.641\end{array}$ & $\begin{array}{l}27.503 \\
27.495\end{array}$ & $\begin{array}{r}25.148 \\
25.15\end{array}$ & 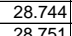 & $\begin{array}{l}27.235 \\
27.237\end{array}$ & \begin{tabular}{|l|l|}
44.93 \\
45.154
\end{tabular} & \begin{tabular}{ll|l}
46.322 \\
46608
\end{tabular} & $\begin{array}{l}9.565 \\
9.48\end{array}$ & $\begin{array}{l}36.062 \\
20.66\end{array}$ & $\begin{array}{l}-1.222 \\
\end{array}$ & $\begin{array}{l}-6.901 \\
\end{array}$ & $\begin{array}{l}46.088 \\
2020\end{array}$ & $\begin{array}{l}0.157 \\
0.150 \\
\end{array}$ & 14.643 & 0.004 & $\begin{array}{l}272.9 \\
2720\end{array}$ & 4.54889 & 44.8 & $\begin{array}{ll}11.3 \\
\end{array}$ & 40.5 & \begin{tabular}{|l|l|}
5 & 2.792 \\
\end{tabular} & & \begin{tabular}{|c|c|c|}
0.023 \\
\end{tabular} & 0.001 & 0.576 & \\
\hline$\frac{292}{293}$ & $\frac{61 / 1421204}{661 / 2004}$ & $\begin{array}{l}1: 4:: 37 \mathrm{PM} \\
1: 99: 37 \mathrm{PM}\end{array}$ & $\frac{26.641}{26.643}$ & $\begin{array}{l}27.495 \\
27.487\end{array}$ & $\begin{array}{r}25.15 \\
25.131\end{array}$ & $\begin{array}{l}28.751 \\
28.768\end{array}$ & 27.237 & \begin{tabular}{|l|}
45.154 \\
54.176
\end{tabular} & $\begin{array}{l}46.608 \\
46.368\end{array}$ & $\begin{array}{l}9.648 \\
9.597\end{array}$ & \begin{tabular}{|l|}
36.166 \\
36.1212
\end{tabular} & $\begin{array}{l}-1.219 \\
-1.219\end{array}$ & $\begin{array}{r}-7.048 \\
-7.123 \\
\end{array}$ & $\begin{array}{l}43.839 \\
50.428\end{array}$ & $\begin{array}{l}0.159 \\
0.162\end{array}$ & $\begin{array}{l}14.643 \\
14643\end{array}$ & \begin{tabular}{|l|}
0.004 \\
0.004
\end{tabular} & $\begin{array}{l}273.93 \\
279.93\end{array}$ & \begin{tabular}{|l|l|l}
4.56556 \\
458222
\end{tabular} & $\begin{array}{l}45.1 \\
44.9\end{array}$ & \begin{tabular}{|l|}
10.7 \\
124 \\
\end{tabular} & $\begin{array}{l}40.7 \\
4007\end{array}$ & $\begin{array}{l}7.803 \\
2.809\end{array}$ & 0.024 & $\begin{array}{l}0.024 \\
0.024\end{array}$ & 0.001 & $\begin{array}{l}0.581 \\
0.551\end{array}$ & 0.49 \\
\hline 294 & $6 / 14 / 2004$ & 1:50:37 PM & 26.61 & & 25.093 & 28.725 & & 45.106 & 46.341 & & 36.368 & & & 44.164 & & 14.643 & $\begin{array}{l}0.004 \\
0.003\end{array}$ & & \begin{tabular}{|l|}
4.59889 \\
\end{tabular} & 44.9 & & & & & 0.025 & 0.001 & $\begin{array}{l}.624 \\
0.624 \\
\end{array}$ & \\
\hline & $6 / 14 / 2004$ & 1:51:37 PM & 26.616 & 27.48 & 25.114 & 28.705 & 27.182 & 45.081 & \begin{tabular}{|l|l|}
46.37 \\
\end{tabular} & 9.605 & 36.138 & -1.222 & & 45.103 & 0.164 & 14.643 & 0.004 & 276.93 & 4.61556 & 44.9 & & & & & 0.024 & 0.001 & & $\frac{0.53}{0.51}$ \\
\hline 296 & $6 / 14 / 2004$ & $1: 52: 37 \mathrm{PM}$ & 26.623 & 27.496 & 25.121 & 28.737 & 27.179 & 45.261 & 46.453 & 9.627 & 36.366 & -1.219 & -7.106 & 47.429 & 0.163 & 14.643 & 0.004 & 277.93 & 4.63222 & 45.0 & 11.6 & 40.8 & \begin{tabular}{|l|l|}
8.814 \\
\end{tabular} & 0.024 & 0.024 & 0.001 & 0.593 & \\
\hline 297 & $6 / 14 / 2004$ & 1:53:37 PM & 26.594 & 27.467 & \begin{tabular}{|l|}
25.097 \\
\end{tabular} & \begin{tabular}{|l|l|}
28.673 \\
\end{tabular} & & 45.181 & 46.55 & 9.656 & $\begin{array}{l}36.183 \\
\end{array}$ & -1.222 & $\begin{array}{r}-7.04 \\
\end{array}$ & 50.843 & 0.173 & $\begin{array}{l}14.643 \\
\end{array}$ & & 278.9 & 4.64889 & 45.1 & 12.5 & 40.7 & & & $\begin{array}{l}0.026 \\
\end{array}$ & & & \\
\hline 298 & $6 / 14 / 2004$ & & 26.595 & & 25.083 & 28.594 & & & 46.461 & & & & & & & & & & & 45.0 & & & & & & & 0.634 & \\
\hline & $6 / 14 / 2004$ & & & & 25.094 & 28.556 & & 44.971 & & & 35.945 & & & & 0.163 & $\begin{array}{l}14.643 \\
\end{array}$ & & & & & & & & & & & & \\
\hline 300 & 6/14/2004 & 1:56:37 PM & 26.591 & 27.459 & 25.089 & 28.556 & & 45.378 & 46.619 & 9.695 & 36.382 & -1.22 & & \begin{tabular}{|l|l|}
45.67 \\
\end{tabular} & 0.161 & 14.643 & & 281.93 & 69889 & 45 & $\frac{11.2}{11.2}$ & & 2.819 & & .024 & & 0.586 & \\
\hline & 6/14/2004 & 1:57:37 PM & 26.58 & 27.448 & & \begin{tabular}{|l|l|}
28.44 \\
\end{tabular} & & $\begin{array}{l}44.894 \\
\end{array}$ & 40.035 & 9.708 & 35.88 & -1.22 & & & & $\begin{array}{l}14.643 \\
\end{array}$ & & & 4.71556 & $44 . \subseteq$ & & & & & & & & \\
\hline 302 & $6 / 14 / 2004$ & $1: 58: 37 \mathrm{PM}$ & 26.576 & 27.459 & \begin{tabular}{|l|}
25.094 \\
\end{tabular} & 28.446 & & $\begin{array}{l}45.077 \\
\end{array}$ & $\begin{array}{l}46.382 \\
\end{array}$ & 9.671 & 36.051 & -1.22 & & 54.894 & 0.163 & $\begin{array}{l}14.643 \\
\end{array}$ & & 283.93 & 4.73222 & $\frac{44.9}{4.9}$ & 13.5 & & 2.797 & & 0.024 & 0.001 & \begin{tabular}{|c|}
0.598 \\
\end{tabular} & \\
\hline$\pi$ & $6 / 144 / 2004$ & 1:59:37 PM & 26.561 & 27.449 & 25.084 & 28.381 & & 45.087 & 46.329 & 9.609 & 36.209 & -1.22 & & 49.974 & 0 & 14.643 & & 284.9 & 4.74889 & $44 .$. & & & & & 0.024 & & & \\
\hline & $6 / 14 / 2004$ & 2:00:37 PM & 26.567 & 27.45 & 25.07 & & & 45.197 & 46.447] & 9.615 & 36.333 & & & 57.439 & & 14.643 & & & 4.76556 & & & & & & & & & \\
\hline 305 & $6 / 1412004$ & & 26.542 & 27.44 & 25.07 & 28.262 & & 45.413 & 46.706 & 9.59 & 36.499 & -1.222 & & 48.248 & 0.165 & 14.643 & & & & & & & & & & & 0.599 & \\
\hline & $6 / 14 / 24$ & & 26.551 & & 25.049 & 28.286 & & 44.88 & 46.4611 & 9.442 & & -1.222 & & & & & & & 889 & & & & & & & & & \\
\hline & $\frac{6114212044}{61 / 21204}$ & $2.035 .5 \%$ & $\frac{26.540}{26525}$ & $\frac{27.454}{27.12}$ & $\frac{25.049}{25019}$ & $\frac{28.250}{2824}$ & & 455.583 & 年66.909 & & $\begin{array}{r}36.78 \\
36.762\end{array}$ & $-\frac{-1.2}{12}$ & & 50 & & $\frac{14.643}{1.642}$ & & & & & & & & & & & & \\
\hline 309 & $\frac{6 / 1 / 1 / 2004}{6 / 142004}$ & $2.04 .37 \mathrm{PM}$ & $\begin{aligned} 20.525 \\
26.51\end{aligned}$ & $\frac{27.413}{27.413}$ & $\frac{25.010}{25018}$ & $\begin{aligned} 28.24 \\
28.245\end{aligned}$ & & $\begin{aligned} \frac{45.5062}{45.78} \\
\end{aligned}$ & $\begin{array}{l}40.100 \\
47021\end{array}$ & $\frac{9.521}{9.504}$ & $\begin{array}{l}30.104 \\
36.975 \\
\end{array}$ & -1.22 & & $\frac{43.049}{41.19}$ & & $\begin{array}{l}14.045 \\
14643\end{array}$ & & & 4889 & & & & & & & & 0.0000 & \\
\hline & $6 / 14 / 2004$ & 2:06:37 PM & 26.51 & $\frac{27.413}{27.413}$ & $\frac{25.018}{25}$ & 28.221 & $\frac{26.8}{26.8}$ & 45.606 & 46.915 & $\frac{9.547}{9.547}$ & 36.757 & $\frac{-1.22}{-1.22}$ & & $\begin{array}{r}\frac{41.19}{52.791} \\
\end{array}$ & & 14.643 & & 291.9 & oftoos & & 129 & & 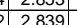 & & & & 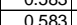 & \\
\hline 311 & 6/14/2004 & 2:07:37 PM & 26.51 & 27.413 & 25.048 & \begin{tabular}{|l|l|}
28.28 \\
\end{tabular} & 26.901 & 45.311 & 46.571 & 9.446 & 36.55 & -1.219 & -6.979 & 49.127 & 0.163 & 14.643 & & 29293 & 4.88222 & 45.1 & $\frac{12.0}{12.0}$ & & 2822 & 024 & 0.024 & 0.001 & 0.593 & \\
\hline & $6 / 14 / 2004$ & 2:08:37 PM & 26.489 & 27.392 & 25.012 & 28.265 & & 45.25 & $\begin{array}{l}46.604 \\
4\end{array}$ & 9.519 & 36.413 & -1.22 & & & & 14.643 & & 293.9 & 4.89889 & & 0.4 & & & & & & & \\
\hline 313 & $6 / 14 / 2$ & & 26.474 & 27.382 & & 28.379 & & 45.315 & 46.53 & & & & & 47.6 & 0.1 & $\begin{array}{l}14.643 \\
\end{array}$ & & & 4.91556 & 45.1 & $11.7 \quad$ & & & & & & 0.586 & \\
\hline & $6 / 14 / 2$ & $2: 10: 3$ & 26.47 & & 25.026 & 28.368 & & & & $\begin{array}{l}9.617 \\
\end{array}$ & 36.34 & -1.2 & & & & $\begin{array}{l}14.643 \\
\end{array}$ & & & & & & & & & & & & \\
\hline & & & 26.494 & & & 28.459 & & 45.062 & $\begin{array}{ll}46.239 \\
\end{array}$ & \begin{tabular}{|l|l|}
9.48 \\
\end{tabular} & & & & & & & & & 4889 & & & & & & & & & \\
\hline 316 & 6/14/2004 & & & 27.374 & \begin{tabular}{|l|}
25.034 \\
\end{tabular} & 28.506 & & \begin{tabular}{|l|l|}
45.307 \\
\end{tabular} & \begin{tabular}{|l|l|} 
& 46.7 \\
\end{tabular} & 9.635 & & -1.2 & & & & $\begin{array}{l}14.643 \\
\end{array}$ & & & & & 0.9 & & & & & & 0.471 & \\
\hline 317 & 6/14/2004 & & 26.471 & 27.359 & \begin{tabular}{|l|}
25.009 \\
\end{tabular} & \begin{tabular}{l|l|}
28.496 \\
\end{tabular} & & 45.235 & 46.561 & 9.583 & 36.314 & -1.225 & & 41.307 & 0.159 & $\begin{array}{l}14.643 \\
\end{array}$ & & 298.93 & 4.98222 & 45.1 & 10.1 & & & 024 & & & 0.581 & \\
\hline 318 & $6 / 14 / 20$ & & & & 25.045 & & & 45.338 & & & & -1.2. & & & & 14.6 & & 299. & & & & & & & & & & \\
\hline 31 & $6 / 14 / 2004$ & 2:15:37 PM & 26.52 & 27.384 & 25.038 & 28.525 & & 45.494 & 46.94 & 9.632 & 36.544 & -1.22 & & 42.387 & 0.156 & 14.643 & & 300 & 5.01556 & 45 & 1.4 & & & & & & 0.566 & \\
\hline & $6 / 14 / 2004$ & 2:16: & 26.517 & 27.001 & 25.04 & 28.502 & & 45.212 & 46.581 & 9.595 & 36.326 & -1.2 .2 & & 70.00 & 0.154 & $14.64 \mathrm{~s}$ & & & (1) & 45. & t & & 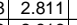 & & & & 0.003 & \\
\hline & & & & & & & & & & & & & & & & & & & & & & & & & & & & \\
\hline & $\frac{61 / 4 / 20}{6 / 1420}$ & & & 2 & 25.043 & 28.455 & & & & & & & & & & & & & & & & & & & & & 0.569 & \\
\hline 年 & $\frac{014420}{6 / 120}$ & & & 27.359 & 250.093 & 2 & & & & & & & & & & & & & & & & & & & & & & \\
\hline 325 & $\frac{01+4 / 140}{6 / 14 / 20}$ & $\frac{2.20 .01 \mathrm{PM}}{2: 21: 37 \mathrm{PM}}$ & $\frac{20.401}{26.487}$ & 27.345 & 25.025 & 20.054 & & $\frac{45.2}{45.4}$ & & & & -1.2 & & 43.1 .0 & & 14. & & 306 & 5.11 & 45. & $\frac{11.0}{10.6}$ & & & & & & 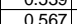 & \\
\hline & $6 / 144 / 20$ & $2: 22: 37 \mathrm{PM}$ & 26.495 & 27.343 & 25.042 & 28.315 & & 45.349 & & 9.498 & & & & & & & & & & & & & & & & & & \\
\hline & $6 / 14 / 21$ & & 26.554 & 27.348 & 25.032 & & & 45.396 & & & & -1.2 & & 46.402 & & 14.6 & & 308. & 514 & 45.3 & 1.4 & & & & & & & \\
\hline 328 & $6 / 14 / 20$ & & 26.493 & 27.316 & 25.01 & 28.288 & & 45.411 & 46.79 & 9.55 & & -1.2 & & 40.6 & 0.154 & & & & & 45.3 & 9.8 & & & & & & 0.560 & \\
\hline & $6 / 14 / 2 C$ & & & 27.326 & & & & & & & & & & & & 14 & & & & & & & & & & & & \\
\hline 330 & $6 / 14 / 20$ & & 26.4 & 27.31 & 25. & 28.2 & & & 47.126 & 9.56 & & & & & & & & & & & & & & & & & & \\
\hline & $6 / 14 / 2 C$ & 2:27: & 26.5 & 27.3. & & 28.234 & & 45 & & & & -1. & & & & 14. & & & & & & & & & & & & \\
\hline & $6 / 14 / 2004$ & & 26.4 & 27.3 & 25.016 & 28.269 & & 45. & 46. & 9.48 & & & & & & 14. & & & & 45 & $\overline{11.3}$ & & & & & & 0.5 & \\
\hline [333 & $6 / 14 / 20$ & 2:29:37 P & 26.499 & 27.317 & & 28.3 & & 45.394 & & & & & & & & 14. & & & & 45. & 1.5 & & & & & & & \\
\hline 334 & $6 / 14 / 2$ & & 26.479 & 27.302 & 25.026 & 28.369 & & 46.085 & 47. & 9.645 & & -1.2 & & 40.4 & 0.154 & 14. & & & & $\begin{array}{lll}46.2 \\
\end{array}$ & 9.9 & & & & & & & \\
\hline 2 & $6 / 14 / 20$ & & 26 & 27.30 & & & & & & & & & & & & 14. & & & & 47. & & & & & & & & \\
\hline 336 & & & & & & 28.41 & & & & & & & & & & & & & & & & & & & & & & \\
\hline & $\frac{6 / 4 / 2 / 2}{6 / 12 / 2}$ & & & & & 28.405 & & & & & & -1. & & & & & & & & & & & & & & & & \\
\hline & $\frac{01142004}{61 / 2004}$ & & & 年 & & $\frac{2.4}{28.40}$ & & & & & & & & & & & & & & & & & & & & & & \\
\hline 340 & $\frac{2 / 14 / 20}{6 / 10}$ & $\frac{2: 36: 37}{2: 37}$ & $\frac{20.5}{26.5}$ & 2730 & $\frac{25.279}{25}$ & $\frac{20.40}{2844}$ & & $\frac{44.0}{44.9}$ & 年 & 105 & & -1.2 & & & & 14.6 & & & & 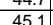 & $\frac{1 .}{46}$ & & & & & & & \\
\hline 341 & $6 / 14 / 2$ & & 26.5 & 27.3 & 25.8 & & & 45.1 & & & & & & & & 14. & & & & & & & & & & & & \\
\hline [342 & & $2: 38: 37$ & & & & 28.4 & & 44. & & & & -1.2 & & & & & & & & 45 & & & & & & & 0.549 & \\
\hline 343 & $6 / 14 / 20$ & & & & & 28.4 & & 45.0 & & & & & & & & 14. & & & & & & & & & & & & \\
\hline & & & & & & & & & & & & & & & & & & & & & & & & & & & & \\
\hline 345 & 6/14/2004 & & 26.6 & 27.314 & & 28.466 & & 44.366 & & 10.4 & & & & & & 14. & & & & \begin{tabular}{|l|l|}
44.6 \\
\end{tabular} & & & & & & & & \\
\hline 346 & $6 / 14 / 200$ & 2:42:37 P & 26.6 & 27.316 & 5.326 & 28.457 & & 44.16 & 45.8 & 10.3 & 34.4 & -1.22 & & 28, & 0.1 & 146 & & 327 & & 44.4 & & & & & & & & \\
\hline 347] & 6/14/2004 & & 26.65 & 27230 & 25.338 & 28.4 & & \begin{tabular}{|l|l|}
44.42 \\
\end{tabular} & 46.2. & 10.4 & 34.662 & -1.22 & & & 0. & 14.6 & & 328 & & 44.8 & & & & & & & & \\
\hline & $6 / 14 / 2004$ & 2:44:3 & 26.66 & 27.329 & 25.338 & 28.345 & & 44.299 & 45.972 & 10.188 & 34.8 & -1.225 & & 48.5 & 0.14 & 14.6 & & & & 44.5 & 1.5 & & & & & & 0.552 & \\
\hline & $6 / 14 / 20$ & & 26.676 & 27.31 & 25.324 & 28.311 & & 44.571 & 46.279 & 10.249 & 34.99 & -1.228 & & 0.485 & 0.148 & 14.643 & & 30 & 51556 & 44.8 & 2.45 & & & & & & 550 & \\
\hline & & 2:46:37 PM & & & & 8.277 & & $\begin{array}{l}44.664 \\
\end{array}$ & & & 5.136] & & & 3.23 & 0.145 & 14.643 & & & & & & & & & & 0.001 & 0.537 & \\
\hline
\end{tabular}


WSRC-TR-2005-00105, REVISION 0

SRNL-RPP-2005-00012, REVISION 0

RUN \# 3.03A AND B; FIRST AND SECOND HALF OF SLURRY DEWATERING - CONT.

\begin{tabular}{|c|c|c|c|c|c|c|c|c|c|c|c|c|c|c|c|c|c|c|c|c|c|c|c|c|c|c|c|c|}
\hline & A & B & $\mathrm{D}$ & $E$ & $\mathrm{~F}$ & $G$ & $\mathrm{H}$ & $\mathrm{J}$ & K & L & \begin{tabular}{l|l|}
$\mathrm{M}$ \\
\end{tabular} & $\mathrm{N}$ & 0 & $a_{1}$ & $\mathrm{R}$ & $\mathrm{s}$ & \begin{tabular}{l|l|l}
$T$ & \\
\end{tabular} & \begin{tabular}{l|l}
$\mathrm{V}$ \\
\end{tabular} & & $x$ & & $z$ & AA & $A B$ & $A C$ & $A D$ & $\mathrm{AE}$ & AF $A$ \\
\hline$\frac{351}{352}$ & 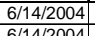 & \begin{tabular}{|l|l|}
$2: 47: 37 \mathrm{PM}$ \\
$2 \cdot 4 \cdot 87 \mathrm{P}$
\end{tabular} & $\begin{array}{l}26.695 \\
26674\end{array}$ & $\begin{array}{l}27.324 \\
27303\end{array}$ & \begin{tabular}{|l|}
25.353 \\
2532 \\
\end{tabular} & \begin{tabular}{|l|}
28.296 \\
2877
\end{tabular} & $\begin{array}{l}26.991 \\
26.945 \\
\end{array}$ & \begin{tabular}{|l|l|}
44.475 \\
4.24
\end{tabular} & \begin{tabular}{|l|}
46.476 \\
46181 \\
\end{tabular} & $\begin{array}{l}10.144 \\
10102\end{array}$ & \begin{tabular}{|l|}
34.988 \\
35105
\end{tabular} & \begin{tabular}{|c|}
-1.219 \\
1.20
\end{tabular} & -6.979 & 48.322 & 0.152 & $\begin{array}{l}14.643 \\
\end{array}$ & 0.004 & & 5.54889 & 45.0 & 11.8 & & 2.739 & & 0.022 & 0.001 & 0.565 & 0.48 \\
\hline 353 & $6 / 114 / 2004$ & 2:48:37 PM & 26.674 & $\begin{array}{r}27.303 \\
27.306\end{array}$ & \begin{tabular}{|l|}
25.322 \\
25316
\end{tabular} & $\begin{array}{r}28.27 \\
28.298\end{array}$ & $\begin{array}{l}26.945 \\
26899 \\
2689\end{array}$ & 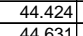 & \begin{tabular}{|l|}
46.181 \\
46.623
\end{tabular} & $\begin{array}{l}10.102 \\
10259\end{array}$ & $\begin{array}{l}35.105 \\
33503\end{array}$ & \begin{tabular}{|c|}
-1.225 \\
-1219 \\
\end{tabular} & $\begin{array}{r}-6.99 \\
-6.993\end{array}$ & \begin{tabular}{ll|l}
44.563 \\
47646
\end{tabular} & 0.151 & $\begin{array}{l}14.643 \\
14.643\end{array}$ & 0.004 & \begin{tabular}{|l|l|}
333.93 \\
32492
\end{tabular} & $\begin{array}{l}5.56556 \\
559202\end{array}$ & \begin{tabular}{|l|l|}
44.7 \\
45.
\end{tabular} & \begin{tabular}{|l|l|}
10.9 \\
\end{tabular} & $\begin{array}{l}39.8 \\
39.8\end{array}$ & \begin{tabular}{|l|}
2.742 \\
\end{tabular} & & $\begin{array}{ll}0.022 \\
0022\end{array}$ & $\begin{array}{l}0.001 \\
001\end{array}$ & $\begin{array}{l}0.561 \\
0.557\end{array}$ & \\
\hline 354 & $6 / 114 / 2004$ & $2: 59.57 \mathrm{PM}$ & & & $\frac{25.3616}{25.324}$ & & $\frac{26.899}{26.947}$ & \begin{tabular}{|l|}
44.631 \\
44.482
\end{tabular} & \begin{tabular}{|l|}
46.6233 \\
46.447 \\
\end{tabular} & & $\begin{array}{r}5.023 \\
35.03\end{array}$ & $\frac{-1.219}{-1.222}$ & & $\begin{aligned} 47.646 \\
5047\end{aligned}$ & $\begin{array}{r}0.15 \\
0.151\end{array}$ & $\frac{14.643}{14.643}$ & 0.004 & & $\frac{5.58222}{5.59889}$ & \begin{tabular}{ll|}
45.2 \\
45.0
\end{tabular} & & \begin{tabular}{|l|l|}
39.8 \\
39.8
\end{tabular} & & & $\begin{array}{l}0.022 \\
0.022\end{array}$ & 0.001 & 0.557 & \begin{tabular}{|l|l|}
0.47 \\
\end{tabular} \\
\hline & 6/14/2004 & $2: 51: 37 \mathrm{PM}$ & 26.674 & 27.293 & $|25.328|$ & 28.295 & 26.966 & 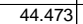 & \begin{tabular}{|l|l|}
46.38 \\
\end{tabular} & $\begin{array}{ll}10.188 \\
\end{array}$ & \begin{tabular}{|l|}
34.958 \\
\end{tabular} & -1.222 & & 46.544 & $\begin{array}{l}0.149 \\
\end{array}$ & 14.643 & & 336.93 & 5.61556 & 44.9 & 11.4 & & & & & & 0.561 & $\begin{array}{l}0.48 \\
0.47 \\
\end{array}$ \\
\hline & 6/14/2004 & 2:52:37 PM & 26.657 & 27.291 & 25.341 & 28.313 & 26.979 & 44.606 & \begin{tabular}{|l|l|}
46.488 \\
\end{tabular} & 10.283 & 35.079 & -1.222 & & 51.408 & 0.148 & $\begin{array}{l}14.643 \\
\end{array}$ & 0.004 & 337.93 & 5.63222 & 45.0 & 12.6 & & \begin{tabular}{|l|}
2.747 \\
\end{tabular} & & 0.022 & 0.001 & & 0.477 \\
\hline 357 & $6 / 14 / 2004$ & 2:53:37 PM & 26.652 & 27.285 & 25.335 & $\begin{array}{l}28.347 \\
\end{array}$ & 26.993 & 44.532 & 46.519 & $\begin{array}{l}10.227 \\
\end{array}$ & \begin{tabular}{|l|l|}
34.993 \\
\end{tabular} & -1.222 & & 48.951 & 0.144 & $\begin{array}{l}14.643 \\
\end{array}$ & 0.003 & \begin{tabular}{|l|}
338.93 \\
\end{tabular} & 5.64889 & 45.0 & 12.0 & \begin{tabular}{|l|l}
39.8 \\
\end{tabular} & \begin{tabular}{|l|}
2.741 \\
\end{tabular} & 0.021 & 0.021 & 0.001 & 0.535 & $\begin{array}{l}0.47 \\
0.46\end{array}$ \\
\hline & $6 / 14 / 2004$ & 2:54:37 PM & & 27.285 & & \begin{tabular}{l|l|}
28.257 \\
\end{tabular} & 26.998 & $\begin{array}{l}44.689 \\
\end{array}$ & .789 & & & -1.225 & & \begin{tabular}{|l|l}
44.143 \\
\end{tabular} & 0.147 & $\begin{array}{l}14.643 \\
\end{array}$ & 0.004 & 339.93 & & \begin{tabular}{|l|l|}
45.3 \\
\end{tabular} & & & & & & & & $\frac{0.46}{0.46}$ \\
\hline 359 & $6 / 14 / 2004$ & & 26.648 & 27.281 & 25.341 & \begin{tabular}{|l|l|}
28.263 \\
\end{tabular} & & 44.328 & $\begin{array}{l}46.374 \\
\end{array}$ & 10.194 & & -1.225 & & & & & & & & \begin{tabular}{|l|l|}
44.9 \\
\end{tabular} & 10.9 & & & & 0.021 & 0.001 & 0.538 & \\
\hline & & & & & & & & & & 10.198 & & & & & & & & & & & & & & & & & & \\
\hline 361 & $6 / 1 / 4 / 2004$ & $2: 57: 37 \mathrm{PM}$ & 26.633 & & 25.336 & 28.218 & & $\begin{array}{l}44.146 \\
\end{array}$ & 312 & 10.109 & 685 & -1.22 & & & & & & & & 44.8 & & & & & & & & \\
\hline & 6/14/2004 & $2: 58: 37 \mathrm{PM}$ & & & & 28.214 & & 44.552 & & $\begin{array}{ll}10.188 \\
\end{array}$ & 35.05 & -1.219 & & \begin{tabular}{|l|l|}
60.14 \\
\end{tabular} & 0.147 & \begin{tabular}{|l|l|l|l|l}
14.643 \\
\end{tabular} & & 343.9 & & & & & 2.744 & & & & & \\
\hline 363 & 6/114/2004 & $2: 59: 37 \mathrm{PM}$ & 26.628 & 27.251 & 25.336 & 28.223 & 26.98 & 44.285 & \begin{tabular}{|l|l|l|}
45.978 \\
\end{tabular} & 10.105 & 35.005 & -1.222 & & 50.195 & 0.146 & $\begin{array}{l}14.643 \\
\end{array}$ & 0.004 & 344.93 & 5.74889 & 44.5 & $12.3 \mathrm{C}$ & 39.6 & \begin{tabular}{|l|l|}
2.733 \\
\end{tabular} & 1.022 & 0.022 & 0.001 & 0.544 & \\
\hline 364 & $6 / 14 / 2004$ & 3:00:37 PM & 26.622 & 27.24 & 25.335 & 28.237 & & 44.65 & 46.801 & 10.191 & 35.112 & -1.22 & & 48.795 & 0.148 & 14.643 & & 345.9 & 5.76556 & 45.3 & 12.0 & & & & & & 0.548 & \\
\hline 365 & $6 / 14 / 2004$ & $\begin{array}{l}3: 01: 37 \mathrm{PM} \\
\end{array}$ & 26.63 & 27.244 & 25.333 & 28.261 & & 44.137 & 46.291 & 10.056 & 34.74 & -1.22. & & 53.35 & 0.146 & 14.643 & 004 & 346.9 & 5.78222 & 44.8 & 13.7. & 39. & 2.7 & & & & 0.547 & \\
\hline & $6 / 14 / 26$ & 3:02:37 PM & 26.624 & & 25.337| & 28.275 & & 44.809 & 000 & & 35.229 & -1.222 & & & 0.146 & 14.643 & & $34 \pi$. & & & & & & & & & & \\
\hline$\frac{367}{368}$ & 601142120404 & $30303.37 \mathrm{MM}$ & 2.6299 & 27.233 & $\frac{25.357}{25.35}$ & $\frac{28.314}{28.205}$ & 27.025 & $\begin{array}{l}44.233 \\
4.239 \\
4\end{array}$ & 3.355 & 10.126 & 34.802 & -1.222 & & 49.319 & 0.146 & 14.643 & & 348.9 & 81556 & & 2.1. & & .725 & & 0.022 & & 0.555 & \\
\hline & 0 & $\frac{3.04 .35}{3.25}$ & $\frac{2.035}{26.28}$ & $\frac{27.254}{27202}$ & 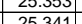 & 2 & & 44.339 & .3.34 & 10.012 & $\begin{array}{l}34.939 \\
34855\end{array}$ & -1.22 & & $\begin{array}{l}46.196 \\
53.075\end{array}$ & 0.144 & $\frac{14.643}{14.643}$ & & & & & & & & & & & & \\
\hline 370 & $6 / 14 / 2004$ & $3: 06: 37$ PM & $\begin{array}{l}20.020 \\
26.623\end{array}$ & 27.222 & $\begin{array}{l}\frac{25.341}{25.326} \\
\end{array}$ & 20.254 & 27.044 & $\begin{array}{c}\frac{44.212}{44.44} \\
\end{array}$ & $\frac{40.459}{46.525}$ & $\begin{array}{l}10.029 \\
10.098\end{array}$ & $\begin{array}{l}35.035 \\
35.032 \\
\end{array}$ & $\frac{-1.22}{-1.22}$ & & 年 & 0.144 & $\begin{array}{l}14.045 \\
14643\end{array}$ & & | & 年. & 年 & 121 & & 2740 & & 0.020 & $\frac{0.001}{0.001}$ & & \\
\hline & $6 / 14 / 2004$ & 3:07:37 PM & 26.628 & 27.222 & 25.331 & 28.259 & $27.02 \mathrm{~S}$ & 44.353 & $\begin{array}{l}46.527 \\
\end{array}$ & 10.101 & 34.923 & -1.222 & -6.95 & 48.755 & 0.141 & 14.643 & 0.004 & 352.93 & 5.88222 & 45.1 & $\frac{11.9}{11.9}$ & \begin{tabular}{|l|l|}
39.6 \\
\end{tabular} & 2.733 & & & 0.001 & & \\
\hline & $6 / 14 / 2004$ & 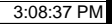 & 26.618 & & 25.336 & 28.249 & 27.029 & $\begin{array}{l}44.845 \\
\end{array}$ & 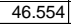 & 10.124 & 35.409 & \begin{tabular}{l|l|l|}
-1.22 \\
\end{tabular} & & 45.739 & 0.111 & $\begin{array}{l}14.643 \\
\end{array}$ & & 353.9 & 5.89889 & 45.1 & $\frac{11.2}{11.2}$ & 40. & $\frac{2.767}{2.767}$ & & 0.016 & & 0.409 & \\
\hline & $6 / 14 / 2004$ & 3:09:37 PM & 26.61 & 27.229 & $25.348 \mid$ & 28.271 & 27.046 & 44.718 & $\begin{array}{l}46.5699 \\
\end{array}$ & 10.109 & & -1.222 & & 50.887 & 0.14 & 14.643 & & 354.93 & 5.91556 & & & & & & & & & \\
\hline $3 / 4$ & 6/14/2004 & 3:10:37 PM & 26.62 & & 25.378 & 28.251 & & 45.017 & 46.639 & 10.102 & 35.662 & -1.219 & & 50.14 & 0.146 & 14.643 & & 355.93 & 5.93222 & 45.2 & 12.3 & & & & 0.022 & 0.001 & 0.534 & \\
\hline & & & & & & & & & & & & & & & & 14.6 & & & .94889 & & & & & & & & & \\
\hline 310 & $6 / 14 / 2004$ & 3:12:3 & 26.561 & 27.195 & 25.284] & 28.217 & & 44.982 & 46.718 & 10.15 & 35.553 & -1.22 & & 53.859 & 0.139 & 14.6 & & 357.93 & .96556 & 45.2 & 13.2 & & 2.776 & & 0.021 & & & \\
\hline & $6 / 14 / 2004$ & 3:13:37 PM & \begin{tabular}{|l|l|}
26.57 \\
\end{tabular} & 27.209 & $25.244 \mid$ & & & 44.861 & $\begin{array}{l}46.606 \\
\end{array}$ & 10.144 & 35.428 & -1.2 & & 51.767 & 0.145 & 14. & & & & & & & 2.76 & & & & & \\
\hline 378 & 6/14/2004 & 3:14:37 PM & 26.554 & 27.198 & 25.213 & 28.255 & 27.0 & 44.95 & 46.932 & 10.103 & 35.506 & -1.2 & & 46.098 & $\begin{array}{l}0.143 \\
\end{array}$ & $\begin{array}{l}14.643 \\
\end{array}$ & & 359.93 & 99889 & 45.5 & 11.3 & & 2.774 & & 0.021 & & 0.527. & \\
\hline & $6 / 14 / 2004$ & & 26.56 & & & & & 45.189 & & 10.129 & & -1.225 & & 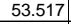 & 0.143 & 14.643 & & & 6.01556 & & 213 & & & & & & & \\
\hline 386 & 6/14/2004 & 3:16:37 PM & 26.535 & 27.194 & 25.163 & 28.231 & 27.0 & 44.853 & 46.774 & 10.086 & 35.456 & -1.225 & & 53.578 & 0.143 & 14.643 & & 361.93 & 6.03222 & 45.3. & 13.1 & 40. & 2.769 & & 0.021 & 0.001 & 529 & \\
\hline & $6 / 14 / 2004$ & & 26.519 & 27.202 & 25.1117 & 28.249 & & 44.596 & 46.364 & 10.086 & & -1.2 & & 47.9 & 0.1 & 14.643 & & 362.9 & & 44.9 & 11.7 & & 2.75 & & 0.021 & 0.00 & 229 & \\
\hline 382 & $6 / 1 / 242004$ & & 26.496 & 27.184 & $25.0 / 9$ & 28.187 & & 44.849 & the.59 & & & & & 49.485 & & $14 . \mathrm{C}$ & & & & & & & & & & & 0.526 & \\
\hline 384 & & & 26.4864 & $\frac{27.11}{27.178}$ & & 28.1877 & & $\begin{array}{l}44.683 \\
4.637\end{array}$ & $\frac{5.577}{5.97}$ & $\frac{10.073}{10.132}$ & 35 & & & $\frac{51.47}{1.72}$ & 0. & 14. & & $\begin{array}{ll}364 . \\
\frac{365}{405}\end{array}$ & & & & & 87 & & & & 20 & \\
\hline & 0 & $\frac{3.20 .35}{3.37}$ & $\frac{20.4068}{26468}$ & 27187 & 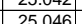 & 年. & & $\mid$ & $\begin{array}{l}4.0 .042 \\
468 ?\end{array}$ & & & $\frac{-1.2}{-12}$ & & 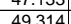 & 0.144 & 146 & & & 10056 & & & & & & & & & \\
\hline 386 & 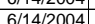 & $\frac{3.25}{3.32 .37 \mathrm{PM}}$ & $\begin{array}{l}2.460 \\
2634\end{array}$ & 27.159 & 年. & 28 & & 44884 & | 46.0699 & 1016 & 35.041 & -12 & & 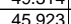 & $\frac{0.141}{0.14}$ & 14.643 & & 367.93 & 6 & 4554 & 望1.5 & & 2768 & & & & 0.520 & \\
\hline 387 & $6 / 14 / 2004$ & 3:23:37 PM & 26.425 & 27.153 & 24.952 & $\begin{array}{l}28.12 \\
\end{array}$ & & 44.88 & 46.899 & 10.161 & 35.393 & -1.22 & & 52.798 & 0.141 & 14.6 & & 368.9 & \begin{tabular}{|l|l|l|l|l|}
6.14889 \\
\end{tabular} & & & & .7 & & 0.021 & & 0.524 & \\
\hline & & & & & & 28.06 & & $\begin{array}{lll}44.73 \\
\end{array}$ & & & & -1.2 & & & & & & & & & & & & & & & & \\
\hline & $6 / 14 / 2 C$ & & 26.399 & 27.1 & 24.961 & 27.995 & & $\begin{array}{l}44.71 \\
\end{array}$ & & & & -1.2 & & 38556 & 0 & 14. & & 370 & & 45 & 9. & & 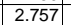 & & & & & \\
\hline & $6 / 14 / 20$ & $3: 26: 37 \mathrm{PM}$ & & 27.142 & 24.946 & 28.025 & & & & & & $\frac{-1.2}{-1.2}$ & & 49.7 & & 14 & & & & & & & & & & & & \\
\hline & $6 / 14 / 2004$ & & 26.356 & & & & & 44.683 & & 10.112 & & & & & 0.139 & & & & 6.215 & 45.3 & $\frac{11.5}{11.5}$ & & 2.756 & & & & 0.520 & \\
\hline & 6/14/2004 & & 26.34 & & 24.872 & 28.006 & & 44.59 & & 10.126 & & & & & & 14. & & & & 44 & 11.6 & & & & & & 0.525 & \\
\hline 3933 & 6/14/2004 & & 26.3 & 27.107 & 24.876 & 28.014 & & 44.5 & & 10.062 & & & & 48.367 & 0.1 & 14. & & 374. & .24889 & 45.0 & 11.9 & & 2.745 & & $\overline{021}$ & & 0.530 & \\
\hline & $6 / 14 / 2004$ & $3: 30: 3$ & & 27.102 & & 28.064 & & 44.745 & & 10.153 & & & & 48.85 & & 14. & & & & & & & & & & & 0.524 & \\
\hline 395 & $6 / 14 / 4$ & 3:31:3 & 26.327 & 27.106 & 24.865 & 28.093 & & & & 10.152 & & -1. & & 45.32 & 0.1 & & & & 6.28 & 45 & 11.1 & & & & & & & \\
\hline 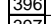 & $6 / 14 / 2004$ & 3:32:37 F & 20.000 & 27.096 & 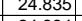 & 28.083 & & 44. & & 10.176 & & -1.21 & & 46.761 & 0.14 & 14. & & 377.90 & & 45. & 11.5 & & 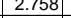 & & & & 0.524 & \\
\hline & & & & & & & & & & & & & & & & & & & & 45.6 & & & & & & & 0.521 & \\
\hline (399 & $\frac{0.14 / 2}{6 / 142}$ & & & & & & & & & & & & & & & & & & & & 102 & & 2.74 & & & & & \\
\hline 400 & 0 & & $\frac{2.2609}{2629}$ & & 24.049 & 20.0085 & & & & & & & & & & & & & & & & & & & & & & \\
\hline 401 & $\frac{1142004}{6142004}$ & & 262 & & $\mid$ & $\frac{2.0001}{281}$ & & & & $\frac{1.4}{10.16}$ & & & & & & 14. & & & & 年 & 107 & & & & & & 0 & \\
\hline 402 & $6 / 14 / 2004$ & & & & & 28.141 & & 44. & & 10.136 & & & & & & & & & & & & & & & & & & \\
\hline 403 & $6 / 14 / 2$ & & 26.2 & & & 28.135 & & & & & & & & & & & & & & & & & & & & & & \\
\hline 404 & & & & & & & & & & & & & & & & & & & & & & & & & & & & \\
\hline & $6 / 14 / /$ & & 26.251 & 27.0 & 24.798 & 28.1 & & & & & & & & & & & & & 6.4 & & & & & & & & & \\
\hline 406 & & & & & & 28.16 & & & & & & & & & & & & & & & & & & & & & & \\
\hline |407, & $6 / 14 / 2$ & 3:43:37 & 26.23 & 27.07 & 24.762 & 28.2 & & 44.42 & & & & & & & & & & & & & & & & & & & 18 & \\
\hline $\begin{array}{lll}408 \\
\end{array}$ & $6 / 14 / 2004$ & & 26.2 & 27.08 & & & & & & & & & & & & 14. & & & & & & & & & & & & \\
\hline 4009 & $6 / 14 / 2004$ & 3:45:37 PM & 26.2 & & & 28.26 & & 44.6 & & & & & & & & 14. & & & & 45 & & & & & & & & \\
\hline 410 & $6 / 144$ & & 26.2 & $27.08 \mathrm{~s}$ & & 28.279 & & 44.5 & & 10.176 & & & & 43.126 & 0.135 & & & & & 45. & 0.6 & & & & & & 0.508 & \\
\hline 每111 & $6 / 14 / 2$ & & 26.2 & & & & & & & & & & & & & & & & & & & & 6.1 & & & & & \\
\hline $4 \frac{412}{412}$ & $6 / 14 / 2$ & & 26.2 & & & & & & & & & & & & & & & & & 45.4 & & & & & & & & \\
\hline$\frac{45}{41}$ & & $\begin{array}{l}3.49 .3 \\
2.50 .3\end{array}$ & $\frac{20.24}{26.21}$ & 27.098 & 24. & 28.32 & & 44. & 46.5 & $\frac{10}{10}$ & 34. & -1.2 & & & & & & & 6.58 & 45.1 & & & & & & & & \\
\hline 告15 & 0 & $3.35 .51 .37 \mathrm{PM}$ & $\frac{2.2124}{26214}$ & 27.107 & $\frac{24.154 \mid}{24781}$ & $\frac{2.3 .5}{283}$ & & 年 & 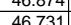 & $\frac{1.2}{101}$ & & & & & 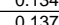 & $\frac{14.6}{146}$ & & $\begin{array}{ll}359.50 \\
39060\end{array}$ & $\frac{0.530}{66}$ & 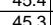 & & & 27 & & & & & \\
\hline \begin{tabular}{|l|l|}
416 \\
\end{tabular} & $6 / 14 / 2004$ & $3: 52: 3$ & 26.22 & & & 28.36 & & 44.496| & & 10.1 & & -1.21 & & 46.99 & 0.136 & 14.6 & & 397. & 6.63 & 45.7 & & & 27. & & & & 0.514 & \\
\hline \begin{tabular}{|l|l|}
417 & \\
\end{tabular} & 6/14/2004 & $3: 5$ & & 27.115 & \begin{tabular}{|l|l|}
24.7 \\
\end{tabular} & 28.3 & & 44. & \begin{tabular}{|l|}
46.861 \\
\end{tabular} & 10.126 & 35.12 & -1.219 & & 49.8 & & 14.643 & & 398. & \begin{tabular}{|l|}
6.64 \\
\end{tabular} & 45.4 & 12.2 & & & & & & 0.501 & \\
\hline $\mid 418$ & $6 / 14 / 2$ & 3:54:3 & 26.212 & 27.11 & 24.744 & 28.357 & & 44.764 & 47. & 10.137 & & -1.2 & & 45.92 & 0.134 & 14.6 & & 399.90 & $\begin{array}{l}6.665 \\
\end{array}$ & 45.9 & 111 & & 2.1. & 0.020 & 0.020 & & 0.503 & \\
\hline $4 \sqrt{13}$ & $661 / 142004$ & & 0.2077 & 27.105 & 24. & 28.347 & & 44. & & 10.106 & & & & & & & & & & & & & & & & & 0.501 & \\
\hline & & 3:56:37 PM & 6.202 & & .764 & 28.272 & & 44.577 & 46.998 & 0.106 & 35.198| & 1.228 & & 1.459| & 0.132 & 14.643 & & & 6.69833 & 5.5 & & & & 0.020 & 1020 & 0.000 & 0.497 & \\
\hline
\end{tabular}


WSRC-TR-2005-00105, REVISION 0

SRNL-RPP-2005-00012, REVISION 0

RUN \# 3.03A AND B; FIRST AND SECOND HALF OF SLURRY DEWATERING - CONT.

\begin{tabular}{|c|c|c|c|c|c|c|c|c|c|c|c|c|c|c|c|c|c|c|c|c|c|c|c|c|c|c|c|c|}
\hline & A & B & $\mathrm{D}$ & $E$ & $\mathrm{~F}$ & $\begin{array}{ll} \\
\end{array}$ & $\mathrm{H}$ & $\mathrm{J}$ & $\begin{array}{ll} \\
\end{array}$ & $\mathrm{L}$ & $M$ & $\mathrm{~N}$ & 0 & \begin{tabular}{l|l} 
\\
\end{tabular} & $\begin{array}{ll}R \\
\end{array}$ & $\mathrm{~s}$ & $\begin{array}{ll} \\
\end{array}$ & $\mathrm{v}$ & w & $x$ & \begin{tabular}{l|l} 
\\
\end{tabular} & & & & & & & AF $\mathbb{A}$ \\
\hline & $6 / 14 / 2004$ & 3:57:37 PM & 26.196 & 27.105 & 24.758 & 28.202 & 26.852 & 44.335 & 46.7 & 10.121 & 34.919 & $\begin{array}{l}-1.222 \\
\end{array}$ & -6.872 & 53.736 & 0.135 & 14.643 & 0.004 & $\begin{array}{l}402.90 \\
\end{array}$ & 6.715 & 45.2 & 13.2 & 39.6 & 2.732 & 0.020 & 0.020 & 0.001 & 0.511 & 0.44 \\
\hline & $6 / 14 / 2004$ & & 26.187 & $\begin{array}{r}27.09 \\
27.09\end{array}$ & 24.744 & $\frac{28.222}{28.22}$ & 26.823 & 44.616 & \begin{tabular}{|l|}
46.969 \\
\end{tabular} & 10.133 & 35.144 & $\frac{-1.222}{-1.22}$ & $\begin{array}{l}-6.774 \\
\end{array}$ & 47.875 & 0.132 & 14.643 & 0.004 & 403.90 & 6.73167 & 45.5 & & & & & & 0.000 & & 0.42 \\
\hline & $6 / 14 / 2004$ & & $\frac{26.194}{2.19}$ & 27.087 & 24.736 & $\frac{28.224}{2.21}$ & 26.805 & 44.434 & \begin{tabular}{|l|}
46.851 \\
18.819
\end{tabular} & $\frac{10.117}{10}$ & 35.036 & $\begin{array}{l}-1.219 \\
-2.225\end{array}$ & $\begin{array}{r}-6.883 \\
-.88\end{array}$ & 52.777 & 0.134 & \begin{tabular}{|l|}
14.643 \\
\end{tabular} & & 404.90 & & & & & $\begin{array}{l}2.740 \\
7.55\end{array}$ & & & & & \\
\hline & $6 / 14 / 2004$ & $4: 00: 37 \mathrm{PM}$ & 26.192 & 27.095 & & 28.212 & 26.778 & $\begin{array}{l}44.646 \\
\end{array}$ & \begin{tabular}{|l|l|}
47.199 \\
\end{tabular} & 10.13 & 35.243 & $\begin{array}{l}-1.225 \\
\end{array}$ & $\begin{array}{l}-6.849 \\
-.78 \\
\end{array}$ & & $\begin{array}{l}0.134 \\
\end{array}$ & \begin{tabular}{|l|l|}
14.643 \\
\end{tabular} & & & \begin{tabular}{l|l|}
0 & 6.765 \\
\end{tabular} & $\begin{array}{l}45.7 \\
\end{array}$ & & & & & & & & 0.43 \\
\hline$\frac{425}{426}$ & 6/14/12004 & $\begin{array}{l}4: 01: 37 \mathrm{PM} \\
\text { 4:02:37 }\end{array}$ & $\begin{array}{l}26.2 \\
26.2\end{array}$ & $27.099 \mid$ & \begin{tabular}{|c|}
24.727 \\
24.72
\end{tabular} & \begin{tabular}{|l|}
28.181 \\
28.21 \\
\end{tabular} & $\begin{array}{l}26.831 \\
26.901\end{array}$ & $\begin{array}{r}44.502 \\
44227\end{array}$ & $\begin{array}{r}46.95 \\
46.65\end{array}$ & $\begin{array}{l}10.149 \\
10066\end{array}$ & \begin{tabular}{|r|}
35.071 \\
34.908
\end{tabular} & $\begin{array}{l}-1.222 \\
-1.222\end{array}$ & $\begin{array}{l}-6.782 \\
-6866\end{array}$ & \begin{tabular}{|r|r|}
45.443 \\
4755
\end{tabular} & 0.1 & \begin{tabular}{|c|}
14.643 \\
14643
\end{tabular} & $\begin{array}{l}0.004 \\
0.004\end{array}$ & \begin{tabular}{|l|l|}
406.90 \\
407.90
\end{tabular} & o 6.78167 & $\begin{array}{l}45.5 \\
45.5\end{array}$ & & & & \begin{tabular}{|l|}
0.020 \\
\end{tabular} & 0.020 & 0.000 & 0.495 & 0.42 \\
\hline 告26] & $6 / 1 / 1 / 2004|6|$ & $\begin{array}{l}\frac{4: 2:: 37 \mathrm{PM}}{4} \\
4: 03: 37 \mathrm{PM}\end{array}$ & $\begin{array}{r}26.2 \\
26.26\end{array}$ & $27.114 \mid$ & \begin{tabular}{l|}
24.742 \\
24.788
\end{tabular} & \begin{tabular}{|l|}
28.221 \\
28.206 \\
\end{tabular} & $\begin{array}{l}26.901 \\
26.907\end{array}$ & $\begin{array}{r}44.227 \\
44.113\end{array}$ & 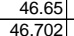 & $\begin{array}{r}10.066 \\
10.07\end{array}$ & $\begin{array}{l}34.908 \\
34.759 \\
\end{array}$ & $\begin{array}{l}-1.222 \\
-1.222\end{array}$ & $\begin{array}{c}-6.866 \\
-6.831 \\
-6.8\end{array}$ & \begin{tabular}{|r|}
$\mid 47.75$ \\
51.023
\end{tabular} & $\begin{array}{l}0.132 \\
0.128\end{array}$ & \begin{tabular}{|c|}
14.643 \\
14.643
\end{tabular} & $\begin{array}{c}0.004 \\
0.004\end{array}$ & \begin{tabular}{|c|}
407.90 \\
408.90
\end{tabular} & $\begin{array}{l}0.79833 \\
0\end{array}$ & $\begin{array}{l}45.2 \\
45.2 \\
\end{array}$ & & \begin{tabular}{|l|}
39.6 \\
394
\end{tabular} & \begin{tabular}{|c|c|}
2.728 \\
2719
\end{tabular} & $\mid$\begin{tabular}{|c|}
0.0220 \\
0.019
\end{tabular} & \begin{tabular}{|c|c|}
0.020 \\
0.019
\end{tabular} & $\begin{array}{l}0.001 \\
0.000\end{array}$ & $0.501 \mid$ & \begin{tabular}{|l|}
0.43 \\
0.41
\end{tabular} \\
\hline 428 & & 4:04:37 PM & & & 24.774 & & 26.938 & & & & $\begin{array}{l}34.1591 \\
34.941\end{array}$ & $\frac{-1.224}{-1.222}$ & $\begin{array}{l}-0.851 \\
-6.828\end{array}$ & \begin{tabular}{|l|l|}
44.874 \\
\end{tabular} & $\begin{array}{l}0.18 \\
0.135 \\
\end{array}$ & $\begin{array}{l}14.643 \\
14.643\end{array}$ & $\begin{array}{l}0.004 \\
0.004\end{array}$ & $\begin{array}{l}408.90 \\
409.90\end{array}$ & & $\begin{array}{l}45.2 \\
45.5 \\
\end{array}$ & & & & & & $\begin{array}{l}0.000 \\
0.001\end{array}$ & \begin{tabular}{|l|l|}
0.4511 \\
0.511
\end{tabular} & $\frac{0.41}{0.43}$ \\
\hline 429 & 6/14/2004 & & 26.203 & 27.106 & 24.79 & \begin{tabular}{|l|}
28.248 \\
\end{tabular} & 26.979 & 44.328 & 47.131 & 10.102 & 34.888 & -1.219 & -6.86 & 47.911 & 0.134 & 14.643 & 0.004 & 410.90 & 0.84833 & 45.7 & & & & & 0.020 & 0.001 & & $\begin{array}{l}0.433 \\
0.43\end{array}$ \\
\hline 430 & 6/14/2004 & 4:06:37 PM & 26.191 & & 24.758 & \begin{tabular}{|l|}
28.231 \\
\end{tabular} & 26.997 & $\begin{array}{l}44.523 \\
\end{array}$ & 47.166 & & 35.124 & -1.222 & & 49.888 & 0.133 & 14.643 & & 411.90 & & 45.7 & & & & 0.020 & 0.020 & & & $\begin{array}{l}0.43 \\
0.43 \\
\end{array}$ \\
\hline 431 & & $4: 07: 37 \mathrm{PM}$ & & & $\begin{array}{l}24.768 \\
\end{array}$ & \begin{tabular}{|l|l|}
28.246 \\
\end{tabular} & 27.037 & 44.561 & $\begin{array}{l}47.346 \\
4\end{array}$ & 10.101 & 35.132 & -1.222 & & \begin{tabular}{|l|l|}
47.347 \\
\end{tabular} & $\begin{array}{l}0.131 \\
\end{array}$ & $\begin{array}{l}14.643 \\
\end{array}$ & & 412.90 & 06.88167 & 45.9 & & & 2.747 & 0.020 & 0.020 & & 0.493 & 0.45 \\
\hline 432 & $6 / 14 / 2004$ & $4: 08: 37$ PM & 26.196 & 27.114 & 24.788 & 28.281 & 27.062 & 44.33 & \begin{tabular}{|l|l|}
46.973 \\
\end{tabular} & 10.083 & 34.937 & -1.219 & & 54.731 & 0.136 & 14.643 & & & & & & & & & & & & 0.44 \\
\hline 433 & & & & & & & & & & & & & & & & & & & & 45.8 & & & & & & & & 0.44 \\
\hline$\frac{43}{3}$ & $6 / 14 / 2004$ & $4: 10: 37 \mathrm{~F}$ & 26.178 & 27.086 & 24.72 & 28.273 & & 44.135 & \begin{tabular}{|l|l|}
46.588 \\
\end{tabular} & 10.087 & 34.796 & -1.22 & & 49.782 & 0.1 & 14.643 & & 415.90 & \begin{tabular}{l|l|}
0.93167 \\
\end{tabular} & 45 & & & & 019 & & 000 & 0.495 & 0.42 \\
\hline & $6 / 14 / 2004$ & $\begin{array}{l}4: 11: 37 \\
\end{array}$ & & 27.098 & & & & 44.391 & 46.884 & 10.168 & & & & & 0.13 & 14.643 & & & & & & & & & & & & 0.43 \\
\hline & $6 / 14 / 2004$ & 4:12:37 & 26.175 & 27.103 & $\begin{array}{l}24.707 \\
\end{array}$ & \begin{tabular}{|l|}
28.28 \\
\end{tabular} & 27.02 & 44.432 & 47.064 & 10.145 & 34.956 & -1.225 & & 54.372 & 0.131 & 14.643 & & 417.90 & 6.965 & 45.6 & 13.3 & 39 & 7.737 & 0.020 & & 0.000 & 0.496 & \\
\hline & 6/14/2004 & 4:13:37 PM & 26.187 & 27.11 & 24.719 & 28.307 & & 44.189 & 46.874 & 10.08 & 34.847 & -1.225 & & 49.606 & 0.131 & 14.643 & & 418.90 & 5.98167 & 45.4. & & & & & & & 0.498 & 0.42 \\
\hline & 6/144/2004 & 4:14:37 PM & 26.195 & 27.129 & 24.727 & 28.34 & & 44.345 & 47.126 & 10.151 & 34.837 & -1.222 & & 48.467] & 0.132 & 14.643 & & 419.90 & $\begin{array}{l}0.99833 \\
\end{array}$ & 45.7 & 118 & & & & & 0.001 & 0.501 & 0.43 \\
\hline & $6 / 144 / 2004$ & & 26.203 & 27.1366 & $\begin{array}{r}24.73 \\
\end{array}$ & 28.363 & & & 告6.988 & & 34.666 & & & 47.195 & 0.13 & 14.643 & & 42.90 & 1.010 & 45.5 & & & 714 & & & 000 & 0.500 & \\
\hline & $6 / 14 / 2004$ & & $\frac{20.25}{26201}$ & $\frac{27.163}{2765}$ & 24.52 & 28.425 & & 44.463 & 47.301 & $\frac{10.213}{1.215}$ & 34.917 & -1.22 & & $\frac{55.66}{17205}$ & 0.1 & 14.645 & & & 7.0310 & & & & & & & & 0.492 & \\
\hline & $6 / 14 / 2004$ & & $\frac{26.212}{2620}$ & & $\frac{24.159}{2475}$ & $\frac{28.41}{28211}$ & & & $\frac{4.516}{4609}$ & $\frac{10.215}{10202}$ & 34.960 & $\frac{-1.2}{1.2}$ & & . & 0. & $\frac{14.6}{14.6}$ & & & 7065 & & & & & & & & 4.491 & $\frac{42}{42}$ \\
\hline & 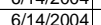 & & 年 & 27149 & & $\frac{2.0 .541}{28.271}$ & & & 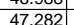 & $\frac{10.206}{10236}$ & $\frac{34.000}{34.744}$ & & & 48.751 & e & & & & $\begin{array}{r}.0065 \\
7.08167\end{array}$ & & & & & & & & & \\
\hline & $6 / 14 / 2004$ & $0: 37 \mathrm{~F}$ & 26.18 & 27.153 & $\mid 24.747$ & $\frac{28.25}{28.1}$ & 26. & 44.594 & 47.562 & 10.18 & 35.087 & -1.22 & & 51.607 & 0.127 & 14.643 & & 425.90 & \begin{tabular}{l|l|l} 
& 7.09833
\end{tabular} & 46.1 & 12.6 & & 747 & 019 & & $\frac{000}{000}$ & 0 & $\frac{0.42}{0.41}$ \\
\hline 445 & $6 / 14 / 2004$ & 4 & 26.189 & 27.157 & 24.736 & 28.234 & & 44.281 & 47.164 & 10.179 & 34.808 & -1.22 & & 51.778 & 0.12 & 14.643 & & 426.90 & \begin{tabular}{l|l}
0 & 7.115 \\
\end{tabular} & & & & 72 & 019 & 0.015 & 000 & 0.475 & 0.40 \\
\hline 446 & $6 / 14 / 2004$ & 2:37/PM & 26.195 & 27.168 & 24.732 & 28.235 & & 44.359 & \begin{tabular}{|l|l|}
47.166 \\
\end{tabular} & 10.204 & 34.882 & -1.222 & & 52.258 & 0.127 & 14.643 & & 427.9 & 7.13167 & 45.7 & & & th & 019 & & 0.000 & 0.482 & 0.41 \\
\hline & $6 / 14 / 2004$ & & & & & & & & & & & & & & & & & & & & & & & & & & & \\
\hline & $6 / 14 / 2004$ & & $\mid 26.19$ & 27.168 & & 28.225 & & 44.32 & 477.184 & D.207 & 34.841 & -1.2 & & 50.107 & 0.1 & 14.643 & & 429.9 & 7.165 & 45 & & & & & & & & $\frac{0.40}{0.40}$ \\
\hline & & & & & & & & & & & & & & 48.334 & & & & & & & & & & & & & & \\
\hline & 6/14/2004 & & 26.169 & 27.142 & \begin{tabular}{|l|}
24.761 \\
\end{tabular} & 28.124 & & $\begin{array}{l}44.206 \\
\end{array}$ & 46.988 & 10.202 & 34.783 & -1.2 & & 54.45 & 0.12 & 14.643 & & 431.90 & $07.19 \varepsilon$ & 45.5 & & & & & & & & 0.41 \\
\hline 451 & 6/14/2004 & & 26.171 & 27.144 & 24.743 & 28.091 & 26.8 & 44.633 & 47.616 & 10.344 & 34.966 & -1.222 & & 42.068 & 0.12 & 14.643 & & 432.90 & 7.215 & \begin{tabular}{ll|}
46.1 \\
\end{tabular} & 10. & & & & & 0.000 & & 0.40 \\
\hline & 6/14/2004 & & 26.184 & 27.157] & 24.755 & 28.134 & & 44.137 & 47.048 & 10.239 & 34.601 & -1.22 & & 46.596 & 1 & 14.643 & & & $\begin{array}{l}0 \\
0\end{array}$ & 45 & $11.4 \mathrm{H}$ & & $(14$ & & & & & 0.41 \\
\hline & & & 26.18 & 27.153 & 24.756 & 28.115 & & 44.187 & 47.004 & 10.227 & 34.699 & -1.21 & & 54.975 & 0.12 & 14.643 & & 434 & $\begin{array}{l}07.24833 \\
\end{array}$ & & 13.5 & & & & & 000 & 0.476 & 0.40 \\
\hline 45 & $6 / 14 / 2004$ & 4 & 26.1811 & $27.149 \mid$ & 24.788 & & & 44.073 & $46.957]$ & .233 & & -1.22 & & 50.009 & 1. & & & 435 & & 45 & 12 & & & & & & & 0.40 \\
\hline & 6/14/20004 & & $\frac{2.185}{26.191}$ & & & $\frac{28.105}{2.05}$ & & 44.121 & - & 10.245 & & & & & & $\frac{14.643}{14.6}$ & & & & & & & & & & & & 0.40 \\
\hline & & & $\mid$ & & & $\frac{2.141}{28.163}$ & & 年4.1. & 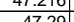 & 1 & & -1.4 & & 497 & & & & & 7315 & & 列 & & & & 0019 & & & 2010 \\
\hline & $6 / 14 / 212$ & & 26.50 & 27.144 & & 28156 & & & 年 & 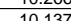 & & & & 45266 & & & & & & & & & & & & & & 0.40 \\
\hline 45 & $6 / 14 / 2004$ & & 26.205 & $\frac{27.134}{27.134}$ & $\frac{24.842}{24.82}$ & \begin{tabular}{|l|}
28.226 \\
\end{tabular} & & 43.889 & \begin{tabular}{|c|}
46.94 \\
\end{tabular} & $\frac{10.90 .1}{10.1}$ & $\frac{34.514}{34.514}$ & $\frac{1.1 .225}{-1.25}$ & & 46.306 & $\frac{1.12}{0.12}$ & 14.643 & & 440.90 & 07.34833 & $\frac{74.5}{45.5}$ & $\frac{21.3}{11.3}$ & & 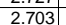 & \begin{tabular}{|l|l|l|l|l|} 
\\
\end{tabular} & $\frac{0.018}{0.018}$ & 0.000 & $\frac{1.466}{0.466}$ & $\frac{0.40}{0.40}$ \\
\hline . & $6 / 14 / 2004$ & & & & & & & 44.083 & \begin{tabular}{|l|l|}
47.359 \\
\end{tabular} & & & $\frac{-1.2}{-1.2}$ & & 20072 & & & & & 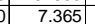 & & -1 & & & & & & & 0.40 \\
\hline & $6 / 14 / 2004$ & & 26.218 & 27.146 & 24.845 & $\mid 28.213$ & & 44.38 & 477.682 & 10.183 & & -1.219 & & 48.15 & 0.1 & 14.643 & & 442 & 7.381 & 46. & & & & & & 000 & 0.469 & $\frac{0.40}{0.40}$ \\
\hline & & & & & & & & & \begin{tabular}{|l|l|l|l|l|}
47. \\
\end{tabular} & & & & & & & & & & & & & & & & & & & 0.40 \\
\hline & & & 26.222 & & & 28.272 & & & & & & -1.2 & & 51.3. & & & & & & & & & & & & & & 0.40 \\
\hline & & & 26.23 & 27.16 & & 28.30 & & & & & & -1.2 & & & & & & & & & & & & & & & & 0.44 \\
\hline & $6 / 14 / 2$ & & & & & & & & & 10.1 & & & & & & & & & 7.44 & & & & & & & & & \\
\hline & 6/144/2004 & & 26.228 & 27.161 & & 28.3. & & & & & & -1.2 & & 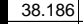 & & & & & & & 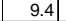 & & & & & & & 0.44 \\
\hline & $6 / 14 / 2$ & & & & 24.799 & & & & & 10.0 & & -1.2 & & & & & & & & & 12 & & & & & & & \\
\hline & & & 26.249 & 27.183 & 24.817 & $28.369 \mid$ & & 44.4 & & & & -1.2 & & & & & & & & & & & & & & & & $0.3 \varepsilon$ \\
\hline & & & & & 24 & & & & & 10.061 & & & & & & & & 450 & & & & & & & & & & \\
\hline & & & & & & & & & & 10.114 & & & & & & & & & & & & & & & & & & \\
\hline & & & & & & & & & $\mid 4.819$ & & & & & & & & & & & & & & & & & & & \\
\hline & & & & & $\mid$ & & & 439 & & $\frac{10.191}{0.972}$ & & & & & & & & & & & & & & & & & 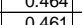 & \\
\hline & $6 / 14 / 26$ & & 26.23 & & 24.84 & 28.497 & & & \begin{tabular}{|l|l|}
47.806 \\
\end{tabular} & & & & & & & & & & & & & & & & & & & \\
\hline & $6 / 14 / 2$ & & 26.245 & & 24.842 & & & & & 10.147 & & -1.2 & & & & & & & & & & & & & & & & \\
\hline & & & 26.256 & & 24.8 & 28.495 & & & & & & & & & & & & & & & & & & & & & & \\
\hline & & & & & & & & & & & & & & & & & & & & & & & & & & & & \\
\hline & & & 26.24 & & & 28.4 & & 44.0 & 47.529 & & & & & & & & & & & & & & & & & & & \\
\hline & & & & & & & & 44. & & & & & & & & & & & & & & & & & & & & \\
\hline & $6 / 14 / 2$ & & 26.245 & 27.233 & 24.817 & 28.374 & & 44.5 & $\begin{array}{l}48.123 \\
\end{array}$ & 10.147 & 35.0 & -1.219 & & 530 & & 14. & & 461 & 07.698 & 46 & & & & & & & 59 & \\
\hline & $6 / 14 / 2$ & & & 27.23 & 24.84 & & & 48.4 & & 10.76 & 38.43 & -1.219 & & & & & & & & 5 & & & & & & & 5 & \\
\hline & $6 / 14 / 2$ & & 26.22 & 27.2. & 24.89 & 28.3 & & 48.0 & 51.456 & 10.644 & 38.1 & -1.222 & & & & & & & 7.73 & $x$ & & & & & & & 7 & U. \\
\hline & $6 / 144 / 2$ & & 26.265 & 27.248 & 24.9 & 28.315 & & 46.578 & 50.153 & 11.262 & & -1.2 .2 & & & & & & & & & & & & & & & 1 & .0 \\
\hline & & & & & & & & 46 & & & & -1.2 & & & & & & & & 48 & & & & & & & & \\
\hline & & & $\frac{2.2}{2.2}$ & 27.250 & th.1 & & & 400 & & & & - & & & & & & & & & & & & & & & & \\
\hline & & & $\frac{2.034}{2632}$ & $\frac{2.201}{2021}$ & & & & 年 & & 11.0 & & - & & & & & & & & & & & & & & & & \\
\hline 48 & 6 & & 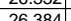 & 27.272 & 5 & 28.040 & & 46.0166 & 40.066 & 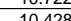 & 36698 & -1.25 & & 4920 & 0.14 & 146 & & 469 & 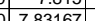 & (4t.80 & 12 & & & & & & 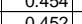 & \\
\hline & $6 / 14 / 2004$ & & 26.414 & 27.267 & 25.257 & \begin{tabular}{|l|}
28.449 \\
\end{tabular} & & 45.587 & \begin{tabular}{|l|}
49.015 \\
\end{tabular} & 10.893 & 35.399 & -1.222 & & 53.985 & 0.12 & 14.643 & 0.004 & 470.90 & 07.84833 & 47.5 & 13.2 & 40.5 & 2.792 & 0.018 & 0.018 & 0.000 & 0.450 & 0.38 \\
\hline & 6/14/2004 & 5:06:37 PM & 26.442 & 27.275 & 25.285 & 28.491 & 27.31 & 46.176 & \begin{tabular}{|l|l|l|} 
& \\
\end{tabular} & 10.651 & 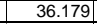 & $\mid-1.219$ & -6.73 & 53.726 & 0.126 & $\mid 14.643$ & 0.004 & 471.90 & $\begin{array}{l} \\
0\end{array} 7.865 \mid$ & 48.4 & 13.2 & \begin{tabular}{|l|l|} 
& 41.2 \\
\end{tabular} & 2.839 & \begin{tabular}{|l|l|}
0.019 \\
\end{tabular} & 0.019 & 0.000 & \begin{tabular}{|l|l|l|} 
& 0.453 \\
\end{tabular} & 0.3 \\
\hline
\end{tabular}


WSRC-TR-2005-00105, REVISION 0

SRNL-RPP-2005-00012, REVISION 0

RUN \# 3.03A AND B; FIRST AND SECOND HALF OF SLURRY DEWATERING - CONT.

\begin{tabular}{|c|c|c|c|c|c|c|c|c|c|c|c|c|c|c|c|c|c|c|c|c|c|c|c|c|c|c|c|c|}
\hline & $\mathrm{A}$ & B & $\mathrm{D}$ & $E$ & $\mathrm{~F}$ & $G$ & $\mathrm{H}$ & $\mathrm{J}$ & $\mathrm{K}$ & $\mathrm{L}$ & M & $\mathrm{N}$ & 0 & Q & $R$ & $\mathrm{~s}$ & $\begin{array}{ll} \\
\end{array}$ & $\mathrm{v}$ & w & $x$ & Y & & $\mathrm{AA}$ & $\mathrm{AB}$ & & & $\mathrm{AE}$ & \\
\hline & $6 / 14 / 2004$ & $5: 07: 37 \mathrm{PM}$ & 26.454 & 27.273 & 25.297 & 28.539 & 27.389 & 45.025 & $\begin{array}{l}48.517 \\
\end{array}$ & 11.059 & 34.674 & -1.222 & -6.736 & 52.597 & 0.126 & 14.643 & 0.004 & 472.90 & .88167 & 47.0 & 12.9 & 39.8 & 2.747 & 0.019 & 0.019 & 0.000 & $\begin{array}{ll}0.467 \\
\end{array}$ & \\
\hline & $6 / 14 / 2004$ & 5:08:37 PM & 26.483 & 27.281 & 25.296 & $\frac{28.563}{28.63}$ & 27.432 & 46.278 & 49.947 & 10.653 & 36.285 & -1.222 & $\begin{array}{l}-6.733 \\
\end{array}$ & 53.171 & 0.124 & 14.643 & & & \begin{tabular}{|l}
7.89833 \\
\end{tabular} & 48.5 & & . 41. & .040 & & & & 0.444 & \\
\hline & $6 / 14 / 2004$ & 5:09:37 PM & $\frac{26.512}{2.50}$ & 27.3 & $\begin{array}{r}25.33 \\
5.217\end{array}$ & 28.586 & 27.446 & 45.514 & 48.936 & \begin{tabular}{|l|l|}
10.769 \\
\end{tabular} & 35.485 & -1.222 & $\begin{array}{l}-6.745 \\
-6.970\end{array}$ & $\begin{array}{l}48.686 \\
5.8207\end{array}$ & & 14.643 & & 474.90 & $\begin{array}{ll}07.915 \\
\end{array}$ & 47.5 & & & & & & & 0.449 & \\
\hline & $6 / 14 / 2004$ & $5: 10: 37 \mathrm{PM}$ & $\begin{array}{l}26.508 \\
25517\end{array}$ & & & 28.553 & $\begin{array}{l}27.463 \\
27.777\end{array}$ & 45.875 & \begin{tabular}{|l|l|}
49.1355 \\
9.972
\end{tabular} & \begin{tabular}{|l|l|}
10.277 \\
10260
\end{tabular} & & $\begin{array}{l}-1.225 \\
1.22\end{array}$ & $\begin{array}{l}-6.678 \\
-6.79\end{array}$ & $\begin{array}{l}51.427 \\
1.72\end{array}$ & & \begin{tabular}{|l|l|}
14.643 \\
\end{tabular} & & & & $\begin{array}{l}47.7 \\
\end{array}$ & & & & & & & 0.449 & \\
\hline 496 & 6/14/12004 & $\begin{array}{l}5: 11: 37 \mathrm{PM} \\
5 \cdot 12 \cdot 37 \mathrm{PM}\end{array}$ & $\frac{26.547}{26.54}$ & $27.306 \mid$ & $\begin{array}{r}25.32 \\
25.317\end{array}$ & \begin{tabular}{|c|}
28.642 \\
28.658
\end{tabular} & & \begin{tabular}{|l|}
46.166 \\
46.21
\end{tabular} & \begin{tabular}{|c|}
49.763 \\
49747
\end{tabular} & $\begin{array}{l}10.268 \\
10.279\end{array}$ & & $\begin{array}{l}-1.222 \\
-1.222\end{array}$ & & \begin{tabular}{|c|}
48.497 \\
47.458 \\
\end{tabular} & 2 & $\begin{array}{r}14.643 \\
14643\end{array}$ & 0.0 & $\begin{array}{l}476.90 \\
477.90\end{array}$ & $\begin{array}{c}9.94833 \\
7965\end{array}$ & $\begin{array}{r}48.3 \\
485\end{array}$ & & & 2054 & & & 0.000 & & 0.38 \\
\hline$\frac{496}{497}$ & 6/14/1/2004 & $\begin{array}{l}5: 2: 277 \mathrm{PM} \\
5: 13: 37 \mathrm{PM}\end{array}$ & 26.5546 & $27.303 \mid$ & \begin{tabular}{|l|}
25.317 \\
25329
\end{tabular} & \begin{tabular}{|l|}
28.658 \\
28.645 \\
\end{tabular} & $\begin{array}{l}27.518 \\
27.505\end{array}$ & \begin{tabular}{|}
$\mid 46.211$ \\
46.599 \\
\end{tabular} & \begin{tabular}{|r|r|}
49.974 \\
50.383
\end{tabular} & $\begin{array}{r}10.279 \\
10.26\end{array}$ & \begin{tabular}{|}
36.583 \\
37.041 \\
\end{tabular} & $\begin{array}{l}-1.222 \\
-1.222\end{array}$ & $\begin{array}{r}-6.675 \\
-6.713\end{array}$ & \begin{tabular}{|c|}
$\mid 47.458$ \\
43.649 \\
\end{tabular} & $\begin{array}{l}0.125 \\
0.127\end{array}$ & \begin{tabular}{|c|}
14.643 \\
14.643
\end{tabular} & 0.0004 & $\begin{array}{l}477.90 \\
478.90\end{array}$ & $\begin{array}{r}7.965 \\
981676\end{array}$ & \begin{tabular}{|l|l|}
488.5 \\
48.9
\end{tabular} & \begin{tabular}{|l|l|}
11.6 & \\
107
\end{tabular} & \begin{tabular}{|l|l|}
41.5 \\
41.
\end{tabular} & $\begin{array}{l}2.854 \\
2.883 \\
\end{array}$ & \begin{tabular}{|c|}
0.019 \\
0.019
\end{tabular} & $\frac{0.018}{0.019}$ & $\frac{0.000}{0.000}$ & 0.446 & $\begin{array}{l}0.38 \\
038\end{array}$ \\
\hline 498 & & 5:14:37 PM & 26.557 & & 25.295 & $\mid 28.641$ & 27.471 & $\begin{array}{l}45.475 \\
\end{array}$ & 49.266 & \begin{tabular}{|l}
11.068 \\
\end{tabular} & $\begin{array}{l}37.041 \\
35.071\end{array}$ & $\begin{array}{l}-1.222 \\
-1.225\end{array}$ & $\begin{array}{l}-0.113 \\
-6.707\end{array}$ & \begin{tabular}{|l|}
44.657 \\
\end{tabular} & $\begin{array}{l}0.127 \\
0.123\end{array}$ & $\begin{array}{l}14.645 \\
14.643\end{array}$ & $\begin{array}{l}0.004 \\
0.004\end{array}$ & $\begin{array}{l}448.90 \\
479.90\end{array}$ & $\begin{array}{l}0 \\
0\end{array}$ & 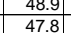 & & & & & & $\frac{0.000}{0.000}$ & $\begin{array}{l}0.449 \\
0.452\end{array}$ & $\frac{0.38}{0.38}$ \\
\hline 499 & $6 / 14 / 2004$ & & 26.589 & 27.312 & 25.322 & \begin{tabular}{|l|}
28.623 \\
\end{tabular} & 27.513 & 45.776 & 49.435 & 10.754 & 35.727 & -1.225 & -6.739 & 51.571 & 0.123 & 14.643 & & 480.90 & & 48.0 & 12.6 & & & & 0.018 & 0.000 & 0.446 & 0.38 \\
\hline & $6 / 14 / 2004$ & 5:16:37 PM & 26.594 & 27.328 & 25.358 & \begin{tabular}{|l|}
28.594 \\
2.55
\end{tabular} & 27.45 & 45.135 & 49.021 & \begin{tabular}{|l|l|}
11.059 \\
1190
\end{tabular} & 34.718 & -1.222 & -6.742 & 51.144 & 0.12 & 14.643 & & 481.90 & \begin{tabular}{|c|c|}
0 & 8.03167 \\
\end{tabular} & & & & & & & & 0.444 & \\
\hline 501 & & $5: 17: 37 \mathrm{PM}$ & & 27.319 & & \begin{tabular}{|l|l|}
28.545 \\
20527
\end{tabular} & $\begin{array}{l}27.425 \\
7.27\end{array}$ & $\begin{array}{l}45.687 \\
5.750\end{array}$ & \begin{tabular}{|l|}
49.239 \\
\end{tabular} & $\begin{array}{ll}10.802 \\
10.0\end{array}$ & $\begin{array}{l}35.619 \\
25.777\end{array}$ & $\begin{array}{l}-1.225 \\
1.225\end{array}$ & $\begin{array}{l}-6.724 \\
-6.707\end{array}$ & 48.123 & 0.122 & 14.643 & & 482.90 & $\begin{array}{ll}0.04833 \\
\end{array}$ & 47.8 & 11.8 & & & 0.018 & & & 0.443 & \\
\hline 502 & $6 / 1 / 4 / 2004$ & $\begin{array}{l}5: 18: 37 \mathrm{PM} \\
5 \cdot 19 \cdot 37 \mathrm{PM}\end{array}$ & $\frac{26.612}{26.18}$ & $\begin{array}{l}27.326 \\
27.327\end{array}$ & $\begin{array}{l}25.366 \\
25.412 \\
25\end{array}$ & $\begin{array}{l}28.537 \\
28518\end{array}$ & & \begin{tabular}{|l|}
45.759 \\
45.38 \\
\end{tabular} & \begin{tabular}{|l|l|l|}
49.516 \\
\end{tabular} & \begin{tabular}{|l|}
10.661 \\
1.016
\end{tabular} & $\begin{array}{l}35.777 \\
34078\end{array}$ & $\begin{array}{l}-1.225 \\
-1252 \\
-120\end{array}$ & \begin{tabular}{|l|l|}
-6.727 \\
67716
\end{tabular} & $\begin{array}{l}56.865 \\
51194\end{array}$ & & $\begin{array}{l}14.643 \\
14643\end{array}$ & & & \begin{tabular}{r|r}
8.0655 \\
80867
\end{tabular} & $\begin{array}{l}48.0 \\
47\end{array}$ & & $\frac{40}{40}$ & 2.811 & 0.018 & 0.018 & 0.000 & 0.445 & \\
\hline & $6 / 1 / 4 / 2004$ & 5:19:37 PM & 26.618 & $27.327 \mid$ & $\begin{array}{r}25.412 \\
25.446\end{array}$ & $\begin{array}{l}28.518 \\
28477\end{array}$ & $\begin{array}{r}27.279 \\
27.238\end{array}$ & $\begin{array}{l}45.382 \\
45.214\end{array}$ & \begin{tabular}{|l|l|}
49.199 \\
49.212
\end{tabular} & $\begin{array}{c}11.016 \\
1092\end{array}$ & $\begin{array}{r}34.978 \\
34.931\end{array}$ & $\begin{array}{l}-1.225 \\
-1.228\end{array}$ & & \begin{tabular}{|l|l|}
51.194 \\
49631
\end{tabular} & $\begin{array}{l}0.122 \\
0.12\end{array}$ & $\begin{array}{l}14.643 \\
14643\end{array}$ & & & $8.08167 \mid$ & \begin{tabular}{|l|l|l|l|}
47.7 \\
47.7
\end{tabular} & 12.5 & & $\begin{array}{l}2.770 \\
2.762\end{array}$ & 0.018 & 0.018 & & 0.447 & \\
\hline & $6 / 1 / 1 / 1 / 2004$ & $\begin{array}{l}5: 20: 37 \mathrm{PM} \\
5: 21: 37 \mathrm{PM}\end{array}$ & $\frac{26.622}{26.643}$ & $\frac{27.321}{27.327}$ & $\begin{array}{l}25.446 \\
25.452\end{array}$ & \begin{tabular}{|l|}
28.4777 \\
28.473
\end{tabular} & & \begin{tabular}{|l|}
45.2145 \\
45.588
\end{tabular} & \begin{tabular}{|r|}
49.212 \\
49.33
\end{tabular} & $\begin{array}{l}10.92 \\
10.85 \\
\end{array}$ & $\begin{array}{l}34.931 \\
35.356 \\
\end{array}$ & $\frac{-1.228}{-1.222}$ & $\begin{array}{l}-6.742 \\
-6.745\end{array}$ & \begin{tabular}{|c|}
49.631 \\
45.729
\end{tabular} & $\begin{array}{r}0.112 \\
0.119\end{array}$ & $\frac{14.643}{14.643}$ & & \begin{tabular}{|c|}
485.90 \\
486.90
\end{tabular} & $8.09833 \mid$ & & & & $\frac{2.763}{2.789}$ & $\mid \frac{0.018}{0.018}$ & $\frac{0.018}{0.018}$ & $\frac{0.000}{0.000}$ & 0.4411 & 0.38 \\
\hline 506 & $6 / 14 / 2004$ & 5:22:37 PM & 26.653 & 27.332 & 25.447 & \begin{tabular}{|l|}
28.508 \\
\end{tabular} & 27.308 & \begin{tabular}{|l|l|}
45.753 \\
\end{tabular} & 49.574 & 10.787 & 35.619 & -1.222 & $\begin{array}{l}-0.145 \\
-6.719\end{array}$ & 55.125 & 0.122 & $\begin{array}{l}\frac{14.045}{14.643} \\
\end{array}$ & & \begin{tabular}{|l|}
487.90 \\
\end{tabular} & \begin{tabular}{|c|c|c|}
0 & 8.13167 \\
\end{tabular} & $\begin{array}{l}48.1 \\
48.1\end{array}$ & $\frac{11.4}{13.5}$ & & & & 0.010 & 00000 & $\begin{array}{l}0.435 \\
0.441\end{array}$ & 0.38 \\
\hline & $6 / 14 / 2004$ & & & 27.326 & 25.431 & \begin{tabular}{|l|}
28.517 \\
\end{tabular} & & & $\begin{array}{l}49.195 \\
\end{array}$ & \begin{tabular}{l|l|}
10.622 \\
\end{tabular} & 35.528 & -1.222 & & & 0.122 & & & & & 47.7 & & & & & & & & \\
\hline & $6 / 14 / 2004$ & 5:24:03 PM & 26.652 & 27.326 & \begin{tabular}{|l|l|}
25.431 \\
\end{tabular} & 28.517 & 27.298 & $\begin{array}{l}45.44 \\
\end{array}$ & $\begin{array}{l}49.195 \\
\end{array}$ & 10.622 & 35.528 & $\begin{array}{l}-1.222 \\
\end{array}$ & -6.722 & $\begin{array}{l}51.5 \\
\end{array}$ & 0.122 & \begin{tabular}{|l|l|}
14.643 \\
\end{tabular} & & 489.33 & 8.15556 & $\begin{array}{l}47.7 \\
\end{array}$ & 12.6 & 40. & 2.791 & 0.018 & 0.018 & 0.000 & 0.444 & \\
\hline & $6 / 14 / 2004$ & & 26.666 & \begin{tabular}{|l|l|}
27.34 \\
\end{tabular} & & $\begin{array}{l}28.646 \\
\end{array}$ & & $\begin{array}{l}45.098 \\
\end{array}$ & & \begin{tabular}{|l|l|}
11.08 \\
\end{tabular} & & $\begin{array}{r}-1.225 \\
\end{array}$ & & & & & & & & 47.4 & & & & 0.018 & & & & \\
\hline 510 & $6 / 14 / 2004$ & $\begin{array}{l}5: 26: 03 \\
5 \\
5.27 .03\end{array}$ & 26.675 & $\begin{array}{l}27.334 \\
27345\end{array}$ & $\begin{array}{r}25.274 \\
2525\end{array}$ & $\begin{array}{r}28.71 \\
28771\end{array}$ & $\begin{array}{r}27.4 \\
27.441\end{array}$ & \begin{tabular}{|l|l|}
44.708 \\
4484
\end{tabular} & \begin{tabular}{|l|}
48.293 \\
48525 \\
\end{tabular} & \begin{tabular}{|l|}
10.881 \\
10666
\end{tabular} & $\begin{array}{r}34.539 \\
34.861\end{array}$ & $\begin{array}{c}-1.222 \\
1.222\end{array}$ & & $\begin{array}{l}45.201 \\
\end{array}$ & $\begin{array}{l}0.118 \\
\end{array}$ & $\begin{array}{l}14.643 \\
1.62\end{array}$ & & 491.33 & 8.18889 & 46.8 & 11.1 & & & 0.018 & 0.017 & 0.000 & 0.441 & 0.37 \\
\hline 511 & $6 / 14 / 2004$ & & $\begin{array}{r}26.691 \\
2661\end{array}$ & $\begin{aligned} 27.345 \\
2733 \\
\end{aligned}$ & $\begin{array}{r}25.25 \\
25205\end{array}$ & $\frac{28.721}{28716}$ & & & & \begin{tabular}{|l|l|}
10.666 \\
10626
\end{tabular} & & $\begin{array}{l}-1.222 \\
-1.225\end{array}$ & & \begin{tabular}{|l|l|}
48.574 \\
52001 \\
\end{tabular} & $\begin{array}{l}0.12 \\
0.19\end{array}$ & $\begin{array}{l}\frac{14.643}{14643} \\
1464\end{array}$ & & & & $\begin{array}{l}47.1 \\
46.8\end{array}$ & & & & & & & 0.446 & 0.38 \\
\hline & $6 / 1 / 1 / 2004$ & $\begin{array}{l}5: 22: 03 \mathrm{PM} \\
5: 29: 03 \mathrm{PM}\end{array}$ & $\begin{array}{r}26.691 \\
26.69\end{array}$ & $\begin{array}{r}27.36 \\
27.354\end{array}$ & $\begin{array}{l}25.205 \\
25.19\end{array}$ & $\begin{aligned} 28.116 \\
2876\end{aligned}$ & $\begin{array}{r}27.451 \\
27.45\end{array}$ & $\begin{array}{r}44.482 \\
45.04 \\
\end{array}$ & \begin{tabular}{|l|}
48.271 \\
49.079
\end{tabular} & \begin{tabular}{|l|}
10.626 \\
10.712
\end{tabular} & & $\frac{-1.225}{-1.222}$ & & \begin{tabular}{|c|}
52.001 \\
54627
\end{tabular} & & $\frac{14.643}{14.643}$ & & & & $\begin{array}{ll}46.8 \\
47.6 \\
\end{array}$ & & 39.4 & 2.724 & & 0.018 & $\frac{0.000}{0.000}$ & & 0.38 \\
\hline & $6 / 14 / 1 / 2004$ & $\begin{array}{l}5: 29: 03 \mathrm{PM} \\
5: 30: 03 \mathrm{PM}\end{array}$ & $\frac{26.69}{26.684}$ & $\frac{27.354}{27.363}$ & $\begin{array}{l}25.149 \\
25.098\end{array}$ & $\begin{array}{r}28.76 \\
28.759\end{array}$ & $\begin{array}{r}27.45 \\
27.514\end{array}$ & $\begin{array}{r}445.062 \\
44.62\end{array}$ & $\begin{array}{r}\quad 99.0 / 9 \\
48.77\end{array}$ & \begin{tabular}{|l|}
$\frac{10 .}{10.112}$ \\
0.676
\end{tabular} & $\begin{array}{l}35.019 \\
34.638\end{array}$ & $\frac{-1.222}{-1.222}$ & $\begin{array}{r}-6.73 \\
-6.724 \\
\end{array}$ & 51.627 & $\mid \begin{array}{c}0.119 \\
0.118\end{array}$ & $\begin{array}{l}14.643 \\
14.643\end{array}$ & & $\begin{array}{l}494.33 \\
995.33 \\
\end{array}$ & $\mid \begin{array}{c}8.23889 \\
8.25556\end{array}$ & $\begin{array}{l}\mid 47.6 \\
47.3\end{array}$ & \begin{tabular}{|c|c|}
$\frac{13.4}{12.6}$ \\
\end{tabular} & \begin{tabular}{|l|l|}
30.6 \\
39.7
\end{tabular} & $\frac{2.760}{2.734}$ & $\mid 0.018$ & $\frac{0.018}{0.018}$ & $\begin{array}{l}0.000 \\
0.000\end{array}$ & 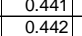 & $\begin{array}{l}0.38 \\
0.38\end{array}$ \\
\hline & $6 / 14 / 2004$ & 5:31:03 PM & 26.674 & 27.368 & 25.058 & 28.759 & 27.534 & 45.338 & 49.093 & 10.699 & 35.333 & -1.228 & & 45.81 & 0.119 & 14.643 & & 496.33 & & 47.6 & & & 2.7.784 & & .018 & & $\begin{array}{l}0.4399 \\
0.439\end{array}$ & 0 \\
\hline & $6 / 14 / 2004$ & 5:32:03 PM & 26.681 & 27.39 & 25.029 & \begin{tabular}{|l|l|}
28.83 \\
\end{tabular} & 27.6 & 45.05 & $\begin{array}{l}48.776 \\
\end{array}$ & 10.599 & 35.188 & -1.222 & -6.7. & 49.892 & 0.119 & 14.643 & & & 28889 & & & & & & & & 0.442 & \\
\hline & $6 / 14 / 2004$ & & & & & & & & & & & & & & & & & & & & & & & & & & & \\
\hline & & & 26.678 & 27.441 & & 28.94 & & 44.76 & & 11.026 & & -1.24 & & 5 & 0 & & & & & & & & & & & & & \\
\hline & & & & & & & & & & & & & & & & & & & & & & & & & & & & \\
\hline & $6 / 14 / 2004$ & $5: 36: 03$ & & 27.463 & & & & 44.7 & 48.83 & 10.964 & 34.369 & -1.222 & & \begin{tabular}{|c|}
44.49 \\
\end{tabular} & 0.121 & $\begin{array}{l}14.643 \\
\end{array}$ & & 501.3 & 8.35556 & |47.4 & & 39. & & & & & 0.457 & \\
\hline 21 & $6 / 14 / 2004$ & & 26.628 & 27.467 & 24.936 & 28.937 & & 45.571 & 49 & \begin{tabular}{|c|}
10.743 \\
\end{tabular} & & -1.222 & & 46.271 & $\mid 24$ & $\begin{array}{l}14.643 \\
\end{array}$ & & 502 & & $\mid 47.5$ & \begin{tabular}{|l|l|}
11.3 \\
\end{tabular} & 40. & & & & 0.000 & 0.457 & $\begin{array}{ll}0.39 \\
\end{array}$ \\
\hline & $6 / 14 / 2004$ & & 26.638 & 27.501 & 24.936 & \begin{tabular}{|l|}
28.907 \\
\end{tabular} & & 45.525 & \begin{tabular}{l|l|}
49.475 \\
\end{tabular} & 10.754 & 35.405 & -1.225 & -7. & \begin{tabular}{|l|l|}
44.949 \\
\end{tabular} & 24 & \begin{tabular}{|l|l|}
14.643 \\
\end{tabular} & & & & 48.0 & 11. & 40 & 2.194 & & & & & \\
\hline & & & 26.632 & 27.51 & 24.905 & 28.905 & & $\begin{array}{l}45.369 \\
\end{array}$ & \begin{tabular}{|l|}
49.162 \\
\end{tabular} & \begin{tabular}{|l|l|}
10.821 \\
\end{tabular} & 35.21 & -1.222 & & 50.017 & 0.118 & 14.643 & & 504.33 & 8.40556 & 47.7 & 12.3 & & 2.778 & 018 & 0.018 & & 0.438 & \\
\hline & $6 / 14 / 2004$ & & 26.616 & 27.514 & $\begin{array}{l}24.869 \\
\end{array}$ & & 27.4 & & \begin{tabular}{|l|}
49.11 \\
\end{tabular} & & & -1.2 & & & & & & & & 47.6 & 13.2 & & & & & & & \\
\hline 52: & $6 / 14 / 2004$ & & \begin{tabular}{r|r|}
26.597 \\
2659
\end{tabular} & \begin{tabular}{|l|}
27.521 \\
27539
\end{tabular} & $\begin{array}{r}24.86 \\
24838\end{array}$ & \begin{tabular}{|l|}
28.871 \\
28841
\end{tabular} & 27.4 & & \begin{tabular}{ll|l}
762 \\
907
\end{tabular} & $\begin{array}{l}10.716 \\
10733\end{array}$ & 35 & $-\frac{1.2 .2}{1.2}$ & & \begin{tabular}{|l|}
43.067 \\
4904
\end{tabular} & & $\begin{array}{l}14.643 \\
1.643\end{array}$ & & & & 47.3 & 10.6 & & $\frac{2.778}{2.778}$ & $\begin{array}{l}017 \\
017 \\
0.17\end{array}$ & 0.018 & 0.000 & & 0.3 \\
\hline & $6 / 14 / 2004$ & & $\begin{array}{r}26.59 \\
26.591\end{array}$ & 27.539 & $\begin{array}{l}24.828 \\
24.85 \\
\end{array}$ & \begin{tabular}{|l|}
28.844 \\
28.25 \\
\end{tabular} & $\begin{aligned} 27.604 \\
27.561\end{aligned}$ & & \begin{tabular}{|c|}
$\mid 48.917$ \\
48938
\end{tabular} & & & $\begin{array}{r}-1.225 \\
-1.228\end{array}$ & & \begin{tabular}{|c|}
49.064 \\
46202
\end{tabular} & & & & & & & & & & & 0.017 & 0.000 & & 0.37 \\
\hline 21 & $6 / 1 / 1 / 2004$ & $\begin{array}{l}5: 43: 0 \\
5: 44: 0\end{array}$ & $\frac{26.591}{26.589}$ & $\frac{27.554}{27.567}$ & $\begin{array}{r}24.859 \\
2482 \\
\end{array}$ & \begin{tabular}{|l|}
28.825 \\
28.863 \\
\end{tabular} & $\frac{27.561}{27.534}$ & $\begin{array}{r}45.266 \\
55.228 \\
\end{array}$ & \begin{tabular}{|l|}
48.938 \\
4864 \\
\end{tabular} & \begin{tabular}{|l|}
10.665 \\
10.617
\end{tabular} & $\begin{array}{l}35.2 / 8 \\
35.317\end{array}$ & $\begin{array}{l}-1.228 \\
-1.225 \\
\end{array}$ & $\frac{-1}{-7}$ & $\begin{array}{l}46.202 \\
41.945 \\
\end{array}$ & $\begin{array}{l}0.118 \\
0.119 \\
\end{array}$ & $\frac{14.643}{14.643}$ & & \begin{tabular}{|l|}
508.33 \\
509.33
\end{tabular} & $\frac{8.47222}{8.48899}$ & $\begin{array}{l}47.5 \\
47.3 \\
\end{array}$ & $\begin{array}{l}\frac{11.3}{103} \\
\end{array}$ & \begin{tabular}{|l|}
40.3 \\
40.3
\end{tabular} & $\frac{2.771}{2.777}$ & \begin{tabular}{|l|}
0.018 \\
0.018 \\
\end{tabular} & $\mid \begin{array}{c}0.018 \\
0.018\end{array}$ & $\frac{0.000}{0.000}$ & 0.439 & 0.37 \\
\hline 529 & $6 / 14 / 12004$ & & $\frac{20.509}{26.601}$ & 27.594 & \begin{tabular}{|l|}
24.029 \\
24.929
\end{tabular} & \begin{tabular}{|r|}
2.0000 \\
28.87 \\
\end{tabular} & 27.51 & $\begin{array}{l}4.260 \\
45.299 \\
\end{array}$ & \begin{tabular}{|l|}
49.01045 \\
4915
\end{tabular} & $\begin{array}{l}1.0119 \\
10.569\end{array}$ & $\frac{30.017}{35.44}$ & $\begin{array}{l}-1.240 \\
-1.219 \\
\end{array}$ & $\begin{array}{l}-1.4 \\
-7.4\end{array}$ & \begin{tabular}{|l|}
47.7753 \\
4.773
\end{tabular} & \begin{tabular}{|l|l|l|l|l|}
0.117 \\
\end{tabular} & $\frac{14.643}{14.643}$ & & $\frac{50.33}{510.33}$ & 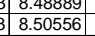 & $\begin{array}{l}47.35 \\
47.5 \\
\end{array}$ & $\begin{array}{l}10.9 \\
11.7 \\
\end{array}$ & & 2.783 & \begin{tabular}{|l|}
0.018 \\
0.017 \\
\end{tabular} & & & $\begin{array}{l}0.442 \\
0.433\end{array}$ & 0.3 \\
\hline & $6 / 14 / 2004$ & & & & & 28.781 & & & \begin{tabular}{|l|l|}
49.156 \\
\end{tabular} & & & -1.222 & & & & & & & & 47.7 & 11.2 & & & & & & & \\
\hline 531 & & & 26.594 & 27.607 & $\begin{array}{l}24.917 \\
2.23\end{array}$ & 28.798 & & $\begin{array}{r}45.56 \\
\end{array}$ & \begin{tabular}{|l|l|}
49.183 \\
\end{tabular} & $\begin{array}{l}10.449 \\
\end{array}$ & & & & $\begin{array}{l}46.442 \\
\end{array}$ & & & & & & 47.7 & & & & & & & 0.430 & \\
\hline & & & 26.5 & & & 28.7 & & & & & & $\frac{-1.2}{1.2}$ & & & & & & & & 47 & 11. & & & & & & 55] & \\
\hline 533 & & & 26.6 & & $\begin{array}{l}24.931 \\
24.897\end{array}$ & 28.8 & & & & & & -1.2 & & & & & & & & & & & & & 0.017 & & & 0. \\
\hline 534 & $6 / 14 / 2004$ & & $\frac{26.624}{26616}$ & $\frac{27.647}{27.654}$ & $\begin{array}{r}24.887 \\
24.890\end{array}$ & \begin{tabular}{|l}
28.89 \\
28.90
\end{tabular} & & & \begin{tabular}{|l|}
48.888 \\
48876
\end{tabular} & & & $\frac{-1.2}{-1.2}$ & & $\frac{52.9}{54}$ & & & & $\frac{51}{51}$ & & $\frac{47.4}{47.4}$ & $\frac{13.0}{13.4}$ & & & & 0.017 & & 0.437 & 0.37 \\
\hline & $6 / 1 / 1 / 2004$ & $\begin{array}{l}5: 51: 03 \mathrm{PM} \\
5.5203 \mathrm{PM}\end{array}$ & $\begin{array}{l}26.616 \\
26.617\end{array}$ & $\frac{27.654}{27.661}$ & $\begin{array}{r}24.849 \\
24805 \\
\end{array}$ & \begin{tabular}{|r|}
28.9044 \\
28.941
\end{tabular} & & & \begin{tabular}{|l|}
$\mid 48.80 .61$ \\
49011
\end{tabular} & $\begin{array}{l}\frac{10.465}{10.582} \\
\end{array}$ & & $\frac{-1.2}{-1.2}$ & & $\begin{array}{r}54 . \\
53.56\end{array}$ & & & & $\frac{511}{511}$ & 8.60556 & $\begin{array}{l}47.4 \\
47.5 \\
\end{array}$ & $\frac{13.4}{13 .}$ & 40. & & & 0.017 & & & 0.36 \\
\hline 37] & $6 / 14 / 2004$ & & $\frac{2.014}{26.624}$ & 27.682 & \begin{tabular}{|l|}
24.807 \\
\end{tabular} & \begin{tabular}{|l|}
2.3 .948 \\
28.988
\end{tabular} & & 45..349 & & 10.462 & & -1.2 & & 53.56 & & & & & & & & & & & & & & $\frac{0.3}{0.3}$ \\
\hline 538 & & & 26.622 & & & & & & & & & -1.2 & & & & & & & & & & & & & & & & \\
\hline 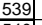 & & & & 27.701 & & & & & & & & & & & & & & & & & & & & & & & & \\
\hline 540 & & & 26.6 & & & & & & & & & & & & & & & & & & & & & & & & & \\
\hline & & & & 27.7. & & & & & & & & -1.2 & & & & & & & & & & & & & & & & \\
\hline & & & 26.595 & 27.758 & & 29.0 & & & & & & & & 38.4 & & & & & & & & & & & & & & \\
\hline & $6 / 14 / 2$ & & & 27.7 & & & & & 49. & & & -1.2 & & & & & & & & & & & & & & & & \\
\hline & $6 / 14 / 2$ & & 26.5 & 27.782 & 24.64 & & & & & 10.2 & & -1.2 & & 52. & & & & & & & & & & & & & & \\
\hline & & & & & & 29.1 & & & & & & -1.2 & & & & & & & & & & & & & & & & \\
\hline & & & & 27.8 & & 29.0 & & & & & & -1.2 & & & & & & & & & & & & & & & & \\
\hline & & & & & & & & & & & & & & & & & & & & & & & & & & & & \\
\hline & & & 26.555 & $\frac{2.8}{270}$ & & & & & & & & -1.2 & & & & & & & & & & & & & & & & \\
\hline & & & 26542 & 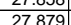 & 24.599 & 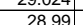 & & & & 10.24 & & 1.2, & & & & & & & & & & & & & & & in & \\
\hline & & & & & 245 & & & & & & & -12 & & & & & & & & & & & & & & & & \\
\hline & $6 / 14 / 2$ & & & & 24.53 & & & & & & & -12 & & & & & & & & & & & & & & & & \\
\hline & $6 / 1 / 4 / 2$ & & & & 24.5 & & & & & & & -1.22 & & & & & & & & & & & & & & & & \\
\hline & & & & & & & & & & & & & & & & & & & & & & & & & & & & \\
\hline & & & & & & & & & & & & & & & & & & & & & & & & & & & & \\
\hline & & & & & & & & & & & & -1.2 & & & & & & & & & & & & & & & & \\
\hline & & & 26. & & & & & 45. & & & & , & & & & & & & & & & & & & & & & \\
\hline & 6/14/2 & & 47 & 27.93 & & 88844 & & 46. & 50.46 & 101 & & -12 & & & & & & & & & & & & & & & & \\
\hline & & & & & & & & 45.6 & & & & 12 & & & 01 & & & & & & & & & & & & & \\
\hline & & & & & & 961 & & 46.2 & 549] & .059] & . & \begin{tabular}{|l|l|} 
& -1.222 \\
\end{tabular} & & 0.286 & & 4.64 & & & & . & & & & & & & 0.419 & \\
\hline
\end{tabular}


WSRC-TR-2005-00105, REVISION 0

SRNL-RPP-2005-00012, REVISION 0

RUN \# 3.03A AND B; FIRST AND SECOND HALF OF SLURRY DEWATERING - CONT.

\begin{tabular}{|c|c|c|c|c|c|c|c|c|c|c|c|c|c|c|c|c|c|c|c|c|c|c|c|c|c|c|c|c|}
\hline & A & & & $E$ & $\mathrm{~F}$ & G & $\mathrm{H}$ & $\mathrm{J}$ & $\mathrm{K}$ & $\mathrm{L}$ & $\mathrm{M}$ & $\mathrm{N}$ & 0 & Q & $\begin{array}{ll} \\
\end{array}$ & $\mathrm{s}$ & $\mathrm{T}$ & U $\quad \mathrm{V}$ & w & \begin{tabular}{|l|}
$x$ \\
\end{tabular} & $\mathrm{Y}$ & $z$ & $\mathrm{AA}$ & $A B$ & AC & & & AF $\mathbb{A}$ \\
\hline & $6 / 14 / 2004$ & $6: 17: 03$ PM & 26.448 & 27.965 & 24.515 & 28.966 & 27.921 & $\begin{array}{l}44.361 \\
\end{array}$ & \begin{tabular}{|l|l|} 
& 48.457 \\
\end{tabular} & \begin{tabular}{|l|l|}
11.133 \\
\end{tabular} & \begin{tabular}{|l|l|}
3 & 33.911 \\
\end{tabular} & -1.222 & \begin{tabular}{|r|}
-7.467 \\
\end{tabular} & 50.689 & \begin{tabular}{|c|}
0.114 \\
\end{tabular} & \begin{tabular}{|l|}
14.643 \\
\end{tabular} & 0.004 & 542.33 & \begin{tabular}{|l|}
9.03889 \\
\end{tabular} & \begin{tabular}{|l|}
47.0 \\
\end{tabular} & 12.4 & 39.1 & \begin{tabular}{|l|l|}
2.698 \\
\end{tabular} & 0.017 & 0.017 & 0.000 & 0.440 & \\
\hline & $6 / 14 / 2004$ & & 26.423 & $\begin{array}{r}27.96 \\
\end{array}$ & 24.475 & 28.946 & 27.916 & \begin{tabular}{|l|l|}
45.587 \\
\end{tabular} & \begin{tabular}{|l|l|}
49.726 \\
\end{tabular} & $\begin{array}{l}10.466 \\
\end{array}$ & 35.822 & $\begin{array}{l}-1.225 \\
-1.225\end{array}$ & & \begin{tabular}{|l|}
47.973 \\
\end{tabular} & $\begin{array}{l}0.112 \\
\end{array}$ & \begin{tabular}{|r|}
14.643 \\
\end{tabular} & 0.004 & \begin{tabular}{|l|l|}
543.33 \\
5
\end{tabular} & & \begin{tabular}{|l|}
48.3 \\
\end{tabular} & \begin{tabular}{|l|l}
11.8 \\
\end{tabular} & & \begin{tabular}{|l|l|}
2.806 \\
\end{tabular} & & 0.017 & 0.000 & & \\
\hline & $6 / 14 / 2004$ & 6:19:03 PM & 26.439 & 27.986 & 24.501 & 28.997 & 27.917 & \begin{tabular}{|l|}
45.701 \\
5.720
\end{tabular} & \begin{tabular}{|l|}
49.759 \\
\end{tabular} & $\begin{array}{l}10.314 \\
10.25\end{array}$ & 36.041 & -1.225 & & \begin{tabular}{|l|}
49.085 \\
\end{tabular} & 0.11 & \begin{tabular}{|l|}
14.643 \\
14
\end{tabular} & 0.004 & \begin{tabular}{|l|}
544.33 \\
5.32
\end{tabular} & \begin{tabular}{|l|l|}
.07222 \\
\end{tabular} & \begin{tabular}{|l|}
48.3 \\
\end{tabular} & \begin{tabular}{|l|l|}
12.0 \\
\end{tabular} & & & & & 0.000 & 0.407 & \\
\hline & 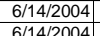 & $\begin{array}{ll}6: 20: 03 \mathrm{PM} \\
6 \\
6: 21: 03 \mathrm{PM}\end{array}$ & & & $\begin{array}{l}24.516 \\
24507 \\
2507\end{array}$ & & $\begin{array}{l}27.942 \\
27.097\end{array}$ & \begin{tabular}{|l|l|}
45.739 \\
5030
\end{tabular} & \begin{tabular}{|l|l|}
49.782 \\
\end{tabular} & $\begin{array}{l}10.205 \\
1.10\end{array}$ & & $\begin{array}{l}-1.225 \\
.222\end{array}$ & $\begin{array}{r}-7.493 \\
\end{array}$ & \begin{tabular}{|l|l|l|}
47.178 \\
5.501
\end{tabular} & \begin{tabular}{|c|c|}
0.114 \\
0.11
\end{tabular} & $\begin{array}{l}14.643 \\
1.63\end{array}$ & 0.004 & \begin{tabular}{|l|l|}
545.33 \\
5
\end{tabular} & & \begin{tabular}{|l|l|}
48.3 \\
19.1
\end{tabular} & 10.0 & & & & \begin{tabular}{|l|l|}
0.017 \\
\end{tabular} & 0.000 & 0.420 & \\
\hline & $6 / 14 / 2004$ & 6:21:03 PM & $\begin{array}{r}26.45 \\
26.437\end{array}$ & $\begin{aligned} 28.022 \\
28.024 \\
\end{aligned}$ & \begin{tabular}{|l|l|}
24.507 \\
24.499
\end{tabular} & $\begin{array}{r}29.103 \\
291\end{array}$ & 27.987 & \begin{tabular}{|l|}
45.838 \\
46.037
\end{tabular} & \begin{tabular}{|l|l|}
59.916 \\
5057
\end{tabular} & $\begin{array}{l}10.104 \\
10.14\end{array}$ & $\begin{array}{l}36.491 \\
335534\end{array}$ & $\begin{array}{l}\mid-1.222 \\
-1.225\end{array}$ & $\begin{array}{l}-7.482 \\
-7449\end{array}$ & $\begin{array}{l}45.051 \\
\end{array}$ & \begin{tabular}{|c|}
0.114 \\
\end{tabular} & \begin{tabular}{|l|l|}
14.643 \\
1.62
\end{tabular} & 0.004 & \begin{tabular}{|l|}
546.33 \\
517.22
\end{tabular} & $\begin{array}{l}9.10556 \\
010220\end{array}$ & \begin{tabular}{|r|}
48.4 \\
\end{tabular} & | & 14 & \begin{tabular}{|l|}
2.838 \\
2.916 \\
\end{tabular} & & 0.017 & 0.000 & 0.419 & \\
\hline 567 & $\frac{6 / 1 / 4 / 2004}{66 / 12004}$ & $\begin{array}{l}6: 22: 03 \mathrm{PM} \\
6: 23: 03 \mathrm{PM}\end{array}$ & 26.437 & $\begin{array}{l}28.024 \\
28.036\end{array}$ & $\frac{24.499}{24.511}$ & $\begin{array}{r}29.1 \\
29.151\end{array}$ & $\frac{28.049}{28.021}$ & 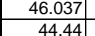 & \begin{tabular}{|l|}
50.507 \\
48.839 \\
\end{tabular} & $\begin{array}{l}0.124 \\
11.139 \\
\end{array}$ & $\begin{array}{l}36.534 \\
33.903 \\
\end{array}$ & 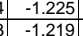 & $\begin{array}{l}-7.496 \\
-7.525 \\
\end{array}$ & \begin{tabular}{|l|}
46.549 \\
48.017
\end{tabular} & \begin{tabular}{|l|}
0.112 \\
0.106
\end{tabular} & \begin{tabular}{|c|}
14.643 \\
11.6433
\end{tabular} & \begin{tabular}{|l|}
0.003 \\
0.004
\end{tabular} & \begin{tabular}{|}
$\mid 547.33$ \\
558.33
\end{tabular} & \begin{tabular}{|l|l|}
9.122222 \\
9.13889
\end{tabular} & \begin{tabular}{|l|}
49.0 \\
474
\end{tabular} & \begin{tabular}{|l|}
11.4 \\
11.8 \\
\end{tabular} & \begin{tabular}{|c|c|}
4 & 41.3 \\
3 & 39.2
\end{tabular} & \begin{tabular}{|l|}
2.846 \\
2.701
\end{tabular} & \begin{tabular}{|l|}
0.017 \\
0.016
\end{tabular} & $\begin{array}{c}0.017 \\
0.016\end{array}$ & 0.000 & 0.410 & 0.35 \\
\hline 568 & $6 / 14 / 2004$ & 6:24:03 PM & 26.45 & 28.042 & \begin{tabular}{l|l|} 
& 24.511 \\
2 & 24.507
\end{tabular} & 29.151 & $\frac{28.021}{28.007}$ & & \begin{tabular}{|l|}
$\mid 4.0 .85$ \\
49.409 \\
\end{tabular} & $\begin{array}{r}11.139 \\
10.82\end{array}$ & $\begin{array}{l}33.903 \\
34.691 \\
\end{array}$ & $\begin{array}{l}-1.219 \\
-1.225\end{array}$ & & $\begin{array}{l}48.017 \\
47.725\end{array}$ & \begin{tabular}{|l|l|l|l|l|l|}
0.108 \\
\end{tabular} & $\begin{array}{l}14.643 \\
14.643 \\
\end{array}$ & $\begin{array}{l}0.004 \\
0.004\end{array}$ & \begin{tabular}{|}
548.33 \\
549.33
\end{tabular} & \begin{tabular}{|l|l|}
9.15009 \\
9.15556
\end{tabular} & $\begin{array}{l}44.4 \\
47.9\end{array}$ & $\frac{11.8}{11.7}$ & & & \begin{tabular}{|l|}
0.016 \\
0.016
\end{tabular} & \begin{tabular}{|c|}
0.016 \\
0.016
\end{tabular} & $\begin{array}{l}0.000 \\
0.000\end{array}$ & \begin{tabular}{|c|}
0.4109 \\
0.410
\end{tabular} & $\frac{0.35}{0.35}$ \\
\hline 569 & 6/14/2004 & 6:25:03 PM & 26.467 & 28.054 & 24.509 & 29.085 & 27.939 & \begin{tabular}{|l|l|}
45.033 \\
\end{tabular} & \begin{tabular}{|l|} 
\\
\end{tabular} & 10.578 & 35.148 & -1.225 & & 48.91 & 0.109 & $\begin{array}{l}14.643 \\
\end{array}$ & 0.003 & & 9.17222 & 47.9 & 12.0 & & \begin{tabular}{|l|}
2.764 \\
\end{tabular} & \begin{tabular}{|l|}
0.016 \\
\end{tabular} & 0.016 & & & $\begin{array}{l}0.35 \\
0.35\end{array}$ \\
\hline 57 & 6/14/2004 & 6:26:03 PM & 26.468 & & 24.515 & 29.071 & 27.866 & \begin{tabular}{|l|l|}
45.349 \\
\end{tabular} & \begin{tabular}{|l|l|}
49.719 \\
\end{tabular} & \begin{tabular}{|l|l|}
10.646 \\
\end{tabular} & 35.395 & -1.225 & & 47.279 & 0.112 & $\begin{array}{l}4.643 \\
\end{array}$ & 0.00 & & 9.18889 & & & & & & & & & $\frac{0.35}{0.36}$ \\
\hline 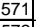 & $6 / 14 / 2004$ & $6: 27: 03$ PM & & 28.066 & 24.506 & $\begin{array}{l}29.062 \\
\end{array}$ & 27.782 & \begin{tabular}{|l|l|}
45.917 \\
\end{tabular} & 50.408 & \begin{tabular}{|l|l|}
10.494 \\
\end{tabular} & $\begin{array}{l}36.07 \\
\end{array}$ & -1.219 & & \begin{tabular}{|l|l|}
48.643 \\
\end{tabular} & $\begin{array}{l}0.107 \\
\end{array}$ & $\begin{array}{l}14.643 \\
\end{array}$ & $\begin{array}{l}0.004 \\
\end{array}$ & \begin{tabular}{|l|l|}
552.33 \\
\end{tabular} & \begin{tabular}{|l|}
9.20556 \\
\end{tabular} & 48.9 & 11.9 & & & & & 0.000 & & $\frac{0.36}{0.34}$ \\
\hline & $6 / 14 / 2004$ & 6:28:03 PM & 26.465 & 28.072 & 24.492 & 29.038 & 27.778 & 45.529 & 50.001 & 10.518 & 35.684 & -1.219 & & 42.022 & $\begin{array}{l}0.111 \\
\end{array}$ & 14.643 & 0.004 & & \begin{tabular}{|l|l|} 
\\
\end{tabular} & 48.5 & 10.3 & & \begin{tabular}{|l|l|} 
& 2.800 \\
\end{tabular} & & & & & $\begin{array}{l}0.34 \\
0.35 \\
\end{array}$ \\
\hline & & & & & & & & & & & & & & & & & & & & \begin{tabular}{|l|l|}
48.9 \\
\end{tabular} & & & & & & & & \\
\hline & 6/144/2004 & 6:30:03 PM & 26.47 & 28.077 & 24.492 & 28.968 & 27.738 & 45.913 & 50.461 & 10.482 & 36.035 & -1.219 & & 44.901 & 0.109 & 14.643 & & 555.33 & 9.25556 & 49.0 & 11 & & 2.825 & .016 & 0.016 & .000 & 0.402 & 34 \\
\hline & $6 / 114 / 2004$ & 6:31:03 PM & & & 24.508 & 28.884 & & & & 10.464 & 36.088 & -1.222 & & & & $\begin{array}{l}14.643 \\
\end{array}$ & & & & $48 . .9$ & 9 & & & & & & 0.398 & \\
\hline & $6 / 14 / 2004$ & 6:32:03 PM & 26.461 & 28.078 & 24.473 & 28.839 & 27.625 & 46.22 & 50.681 & 10.399 & 36.503 & -1.225 & & 49.913 & 0.107 & 14.643 & 0.004 & 557.33 & \begin{tabular}{|l|l|}
9.288899 \\
\end{tabular} & 49.2 & & 41.4 & 2.852 & \begin{tabular}{|l|l|}
0.016 \\
\end{tabular} & 0.016 & 0.000 & 0.391 & \\
\hline & $6 / 14 / 2004$ & 6:33:03 PM & 26.467 & 28.089 & 24.514 & 28.791 & & 46.245 & 50.814 & 10.458 & 36.404 & -1.216 & & 44.669 & 0.107 & 14.643 & & 558.3 & & 49.3 & & & & & & 0.000 & 0.391 & \\
\hline & 6/14/2004 & 6:34:03 PM & 26.473 & 28.085 & 24.505 & 28.802 & 27.756 & 45.029 & 49.51 & 11.365 & 34.305 & -1.219 & & 49.429 & 0.105 & 14.643 & & 559.33 & 9.32222 & 48.0 & 12.1 & & 2.735 & 016 & 0.016 & 0.000 & 0.400 & 0.34 \\
\hline & $6 / 1442004$ & : $: 03 \mathrm{PM}$ & 26.477 & 28.094 & 24.599] & 28.845 & & & & 11.088 & $\begin{array}{l}34.96 \\
25\end{array}$ & -1.222 & & 50.704 & 0.104 & 14.643 & & & & 48.6 & . & & & & 0.016 & 0.000 & 0.390 & \\
\hline & $6 / 14 / 2004$ & PMM & 26.468 & & 4.645 & 28.831 & & 45.481 & 50.03 & 10.83 & $\begin{array}{l}35.305 \\
3.302\end{array}$ & -1.222 & & & 0.106 & 14.643 & & 561.3 & 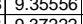 & 48.6 & & & .785 & & 0.016 & & 0.395 & \\
\hline & 0 & $\frac{7.03 \mathrm{PM}}{8: 3 \mathrm{PM}}$ & $\frac{20.474}{2648}$ & $\frac{26.090}{28097}$ & $\frac{2.40004}{2467}$ & 28051 & & $\begin{array}{l}45.093 \\
45360\end{array}$ & $\frac{4.45}{40706}$ & $\frac{10.656}{10721}$ & $\frac{35.183}{25232}$ & $\frac{-1.22}{-121}$ & & & & $\frac{14.643}{1.643}$ & & 5632 & & & & & & & 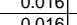 & & $\frac{0.405}{0.402}$ & \\
\hline & $\frac{1}{6 / 1442004}$ & $03 \mathrm{PM}$ & 26.486 & & 40703 & 28.953 & & $\begin{array}{l}45.309 \\
45367\end{array}$ & $\frac{49.150}{49746}$ & $\frac{10.131}{10.673}$ & $\begin{array}{l}35.353 \\
35.378 \\
\end{array}$ & $\frac{-1.21}{-1.21}$ & & $\frac{51.913}{46684}$ & $\begin{array}{l}0.100 \\
0.104\end{array}$ & $\frac{14.043}{14.643}$ & & S56.3 & 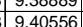 & 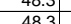 & 114 & & & & & & & \\
\hline & $6 / 14 / 2004$ & 6:40:03 PM & 26.477 & 28.103 & 24.654 & 28.945 & 27.91 & 45.454 & 5 & 10.608 & 35.489 & -1.22 & & 44.035 & 0.105 & 14.643 & & & $\frac{1.42222}{9}$ & 48 & $\frac{1.8}{10.8}$ & & 2.790 & & 016 & & 0.391 & \\
\hline 585 & $6 / 14 / 2004$ & 6:41:03 PM & 26.493 & 28.109 & 24.665 & 28.986 & 27.885 & 44.772 & 49.091 & 10.893 & 34.592 & -1.22 & & 44.459 & 0.104 & 14.643 & & 566.3 & 9.43889 & 47.6 & 10. & & 2.736 & 016 & 0.016 & 0.000 & 0.394 & \\
\hline & $6 / 14 / 2004$ & 6:42:03 PM & 26.499 & 28.125 & 24.661 & 29.007 & 6.91 & 45.28 & 49.701 & 10.609 & 35.356 & -1.219 & & 45.084 & 0.105 & \begin{tabular}{|l|l|}
14.643 \\
\end{tabular} & & 567.3 & 9.45556 & 48.2 & 11. & & 2.780 & 016 & 0.016 & .000 & 0.392 & \\
\hline & 6/14/2004 & & & & & & & & & & & & & & & & & & & & & & & & & & & \\
\hline & $6 / 14 / 2$ & उPM & 26.509 & 28.121 & $\begin{array}{l}24.637 \\
\end{array}$ & \begin{tabular}{|l|l|l|}
28.993 \\
\end{tabular} & & 45.65 & 0.298 & 10.285 & 36.015 & -1.22 & & 49.588 & 0.109 & $\begin{array}{l}14.643 \\
\end{array}$ & & 569.3 & & $48 . \varepsilon^{2}$ & 12. & & & & .016 & & 0.402 & \\
\hline & & & & 28.106 & & & & 45.581 & 50.296 & & & & & 488.374 & & & & & & & & & & & & & & \\
\hline & $6 / 14 / 2004$ & $6: 46: 03 \mathrm{PM}$ & 26.511 & 28.123 & 24.609 & 29.005 & & 45.002 & \begin{tabular}{|l|l}
49.583 \\
\end{tabular} & 10.759 & 34.908 & -1.22 & & 52787 & \begin{tabular}{|c|c|}
0.104 \\
\end{tabular} & \begin{tabular}{|l|l|l|l|l|}
1433 \\
\end{tabular} & & 571.3 & & 48. & & & & & & & & \\
\hline & $6 / 14 / 2004$ & PM & 26.512 & 28.129 & 24.655 & 28.976 & & 45.421 & 49.891 & 10.506 & 35.633 & -1.225 & & 52.923 & 0.106 & \begin{tabular}{l|l|l}
14.643 \\
\end{tabular} & & 572.33 & 9.53889 & $\begin{array}{ll}48.4 \\
\end{array}$ & 13.0 & & 2.794 & 0.016 & 0.016 & 0.000 & 0.394 & 0.34 \\
\hline & 6/14/2004 & 33 PM & 26.518 & 28.13 & 24.706 & 28.957 & & & 50.631 & 10.495 & 36.082 & -1.22 & & & & 14.643 & & & & & 103 & & & & & & 0.389 & \\
\hline & $6 / 14 / 2$ & 6:49:03 PM & 26.514 & 28.131 & 24.747] & 28.988 & & 45.56 & 50.445 & 10.433 & 35.733 & -1.2 & & 48.559 & 0.1 & 14.6 & & 574.3 & 9.57222 & & 11. & & 2.802 & & 0.016 & 000 & 0.392 & \\
\hline 9 & $6 / 14 / 2004$ & & & 28.136 & & & & & & $10.467]$ & & -1.21 & & & & 14. & & & & & & & & & & & & \\
\hline & $6 / 14 / 22$ & & $\frac{26.508}{26523}$ & $\frac{28.12}{28109}$ & 24.886 & 年 28.917 & & & 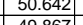 & 10.343 & & -1.2 & & & & $\frac{14.643}{1.642}$ & & & & & 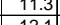 & & & & & & 0 & \\
\hline 597 & 6 & & $\frac{20.524}{26.546}$ & 资. & $\mid$ & 20.051 & & 年 45.6 & & $\begin{array}{l}1.175 \\
10528\end{array}$ & & & & & & 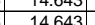 & & & & & 133 & & 2807 & & & & 02909 & \\
\hline & $6 / 14 / 2004$ & & $\frac{2.375}{26.575}$ & 28.127 & 24.99 & 28.759 & & & & 10.453 & & & & & & 14. & & & & & & & & & & & 0.000 & \\
\hline & $6 / 14 / 2004$ & $6: 5: 5$ & 26.574 & 28.116 & 25.003 & 28.679 & & 45.581 & 50.204 & 10.429 & 35.795 & -1.22 & & 44.917 & & 14.643 & & $\frac{580.33}{580}$ & \begin{tabular}{|l|l|}
9.672222 \\
\end{tabular} & 48. & $\frac{x_{11.0}}{11.0}$ & & 2.805 & & 0.015 & 0.000 & $\frac{0.374}{0.0374}$ & \\
\hline & $6 / 14 / 20$ & $6: 56: 03$ PM & 26.577 & 28.104 & & 28.652 & & & & & & & & & & & & & & & & & & & & & & \\
\hline & $6 / 14 / 20$ & 6:57:03 PM & 26.59 & 28.092 & 25.074 & 28.65 & & & 50.24 & 10.436 & & -1.22 & & 437 & & \begin{tabular}{|l|l|}
14.643 \\
\end{tabular} & & & & & & & & & & & 395 & \\
\hline & $6 / 14 / 2$ & & & & 25.133 & 28.669 & & 45.458 & & 10.644 & & & & & & 14. & & & & & & & & & & & & \\
\hline & $6 / 14 / 2$ & & 26.6 & & 25.18 & 28.66 & & & 49.112 & 11.0 & & -1.2 & & 50. & & & & & & & 12 & & & & & & 0.379 & \\
\hline & & & & 28.0 & 25.1 & & & & & 10.5 & & & & & & & & & & & & & & & & & & \\
\hline & $6 / 14 / 21$ & 7:01:C & 26.6 & 28.0 & 25.1 & 28.6 & & & & & & & & & & & & & & & & & & & & & $\overline{0.409}$ & \\
\hline & $6 / 14 / 200$ & & 26.6 & & 25.146 & 28.618 & & & & 11.109 & & & & & & & & & & & 11.6 & & & & & & .380 & \\
\hline & $6 / 14 / 2$ & & & & & 28.596 & & & & & & & & & & & & & & & & & & & & & & \\
\hline & $6 / 14 / / 2$ & & 26.684 & 28.007 & 25.138 & 28.609 & & 45. & & 10.686 & & & & 47.2. & & & & & & & 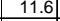 & & & & & & 373 & \\
\hline & $6 / 144 / 2$ & & 26.71 & 28.0 & 25.114 & & & & & & & -1.2 & & & & & & & & & & & & & & & & \\
\hline & $6 / 14 \mid$ & & 26.1260 & 28.009 & 25.153 & & & & & & & & & & & & & & & & & & & & & & & \\
\hline & & & $\frac{2.16}{267}$ & $\frac{2.0001}{27088}$ & $\frac{25.154}{25.21}$ & 20.1954 & & & & & & -1.2 & & & & 144 & & & & & & & & & & & & \\
\hline & $6 / 1 / 4 / 20$ & & & & 20.154 & $\mid$ & & & & & & & & & & & & & & & & & & & & & & \\
\hline & $6 / 141 / 20$ & & $\frac{26.7}{26.7}$ & 27.9 & 25.117 & 28.833 & & & & & & & & & & & & & & & 123 & & & & & & 0.367 & \\
\hline & $6 / 14 / 20$ & 7:10:15 PM & 26.728 & 27.951 & 25.132 & & & & & 10.8 & & & & & & 14. & & & & & 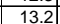 & & & & & & & \\
\hline & $6 / 14 / 2$ & & 26.729 & & 25.093 & 28.834 & & 45.261 & & 10.754 & & -1.2 & & 46.4 & & & & & & 48.4 & 11. & & & & & & 0.403 & \\
\hline & & & & & & & & & & & & & & & & & & & & & & & & & & & & \\
\hline & $6 / 144$ & & & 27.919 & 25.1 & & & & & $\overline{10}$ & & & & & & & & & & & & & & & & & & \\
\hline & & & & & & & & & & & & & & & & & & & & & & & & & & & & \\
\hline & $6 / 14 / 2$ & 7:15:1 & 26.722 & 27.93 & 25.091 & 28.792 & & $\begin{array}{l}45.448 \\
\end{array}$ & 50. & 10.6 & 35.532 & -1.22 & & 49.2 & & 14. & & & & \begin{tabular}{l|l|}
48.6 \\
\end{tabular} & 12.1 & & 27 & & & & & \\
\hline & $6 / 14 / 2$ & 7:16:1 & 26.724 & 27.99 & 25.098 & 28.73 & & 45.3 & & $10.6 \mathrm{r}$ & & -1.2 & & $47 .$. & & 14. & & & & & & & & & & & & \\
\hline & $6 / 14 / 200$ & & 26.729 & 27.9 & 25.138 & 28.754 & & 45.41 & 49.896 & 10.6 & & -1.2 & & & & 14. & & & & 48.4 & $12.7 \mathrm{P}$ & & & & & & 0.374 & \\
\hline & $6 / 14 / 2 / 2$ & 7:18:15 P & 26.7 & & 25. & & & $\begin{array}{r}45 \\
\end{array}$ & & 10.55 & & -1.2 & & 52.5 & & $14.6 \mathrm{C}$ & & & & & & & & & & & & \\
\hline & $6 / 14 / 4$ & & 26.7 & 27.9 & 25.1 & & & 45.68 & & 10.5 & & & & & & & & & & & 10.8 & & & & & & $3 / 9$ & \\
\hline & $0 / 11422004$ & & & 21.6 & 250.09 & & & 45.60 & & 1.5. & & & & & & & & & & 3.4 & 1.03 & & & & & & & \\
\hline & & & 20.00 & 27.01 & 25.047 & 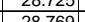 & & 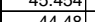 & & 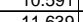 & & -1.2 .4 & & & & & & & & 48.4 & & & & & & & & \\
\hline & $6 / 141200$ & $7 \cdot 22 \cdot 15 \mathrm{PM}$ & 26.678 & 27861 & 25092 & 2873 & & 44.416 & 49178 & 11 & 3454 & 122 & & $\frac{4.4}{22}$ & & 146 & & & & 年17.3 & 104 & & & & & & (2) & \\
\hline & $6 / 14 / 200$ & $7 \cdot 24 \cdot 5 \mathrm{PM}$ & 26.678 & 7.86 & & 8.7 & & 45.15 & 49.84 & $8.85-2$ & 34.9 & -1.22 & & 51.69 & & $14.64 \mathrm{~s}$ & & & & & & & & & & & & \\
\hline 30 & 6/14/2004 & 7:25:15 PM & 6.681 & 27.853 & \begin{tabular}{|l|l|}
3 & 25.134 \\
\end{tabular} & 28.7 & & 45.255 & 49.95 & $\mid 10.928$ & 35.007 & 7] -1.222 & & 13.449 & 0.105 & 14.643 & & 610.53 & 10.1756 & 48.5 & 10.6 & & & 016 & .016 & 0.000 & 0.389 & \\
\hline
\end{tabular}


WSRC-TR-2005-00105, REVISION 0

SRNL-RPP-2005-00012, REVISION 0

RUN \# 3.03A AND B; FIRST AND SECOND HALF OF SLURRY DEWATERING - CONT.

\begin{tabular}{|c|c|c|c|c|c|c|c|c|c|c|c|c|c|c|c|c|c|c|c|c|c|c|c|c|c|c|c|c|}
\hline & A & & D & $E$ & $\mathrm{~F}$ & G & $\mathrm{H}$ & \begin{tabular}{l|l}
$\mathrm{J}$ \\
\end{tabular} & $\mathrm{K}$ & $\mathrm{L}$ & M & $\mathrm{N}$ & 0 & Q & $\mathrm{R}$ & $\mathrm{s}$ & \begin{tabular}{l|l}
$\mathrm{T}$ & $\mathrm{s}$
\end{tabular} & U $\quad \mathrm{V}$ & w & \begin{tabular}{|l|}
$|x|$ \\
\end{tabular} & $\mathrm{Y}$ & $z$ & AA & $A B$ & & & & AF \\
\hline & $6 / 14 / 2004$ & 7:26:15 PM & 26.663 & 27.816 & 25.106 & 28.638 & 27.248 & 45.033 & \begin{tabular}{|l|}
49.931 \\
\end{tabular} & 10.943 & 34.699 & -1.228 & $\begin{array}{l}-7.369 \\
\end{array}$ & 46.582 & \begin{tabular}{l|l|}
0.099 \\
\end{tabular} & \begin{tabular}{|l|l|}
14.643 \\
\end{tabular} & 0.003 & 611.53 & \begin{tabular}{|l|l|}
10.1922 \\
\end{tabular} & \begin{tabular}{|l|}
48.5 \\
\end{tabular} & \begin{tabular}{|l|l}
11.4 \\
\end{tabular} & 39.9 & 92.749 & 0.015 & 0.015 & 0.000 & 0.369 & \\
\hline & $6 / 14 / 2004$ & & 26.682 & 27.83 & 25.165 & 28.612 & 27.108 & \begin{tabular}{|l|l|}
45.145 \\
57
\end{tabular} & \begin{tabular}{|l|}
49.96 \\
\end{tabular} & \begin{tabular}{|l|l|}
10.833 \\
\end{tabular} & 34.935 & 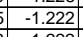 & & 43.084 & \begin{tabular}{|c|}
0.103 \\
\end{tabular} & \begin{tabular}{|l|}
14.643 \\
\end{tabular} & 0.004 & \begin{tabular}{l|l|}
612.53 \\
653
\end{tabular} & 10.2089 & 48.5 & 10.6 & & 2.761 & & 0.015 & 0.000 & & \\
\hline & $6 / 14 / 2004$ & $7: 28: 15 \mathrm{PM}$ & 26.681 & 27.819 & $\frac{25.154}{2.17}$ & 28.636 & 26.948 & 45.724 & 50.312 & \begin{tabular}{|l|}
10.879 \\
\end{tabular} & 35.448 & \begin{tabular}{|l|l|}
3.222 \\
\end{tabular} & & \begin{tabular}{|l|}
45.96 \\
\end{tabular} & $\begin{array}{l}0.1 \\
\end{array}$ & \begin{tabular}{|l|}
14.643 \\
493
\end{tabular} & 0.004 & \begin{tabular}{|l|l|}
613.53 \\
19.52
\end{tabular} & 10.2256 & 48.8 & 11.3 & 40.6 & & & & 0.000 & 0.366 & \\
\hline & $6 / 14 / 2004$ & $\begin{array}{l}7: 29: 15 \mathrm{PM} \\
7: 20: 15 \mathrm{P}\end{array}$ & 26.686 & \begin{tabular}{|l|}
27.819 \\
77709
\end{tabular} & $\begin{array}{l}25.174 \\
25171\end{array}$ & \begin{tabular}{|l|l|}
28.621 \\
2.5655
\end{tabular} & $\begin{array}{l}27.057 \\
27151\end{array}$ & \begin{tabular}{|l|l|}
45.295 \\
4511
\end{tabular} & 49.788 & $\begin{array}{r}10.8 \\
\end{array}$ & $\begin{array}{l}35.126 \\
35.00\end{array}$ & \begin{tabular}{|c|c|}
5.222 \\
\end{tabular} & \begin{tabular}{|l|l|}
7.569 \\
7500
\end{tabular} & \begin{tabular}{|l|l|}
51.584 \\
\end{tabular} & \begin{tabular}{|l|l|l|}
0.103 \\
0.101
\end{tabular} & \begin{tabular}{|l|l|}
14.643 \\
143
\end{tabular} & 0.004 & \begin{tabular}{|l|l|}
614.53 \\
\end{tabular} & \begin{tabular}{|l|l|}
10.2422 \\
\end{tabular} & 48.3 & 120 & & & & $\begin{array}{l}0.015 \\
0015\end{array}$ & 0.000 & & \\
\hline$\frac{635}{636}$ & $6 / 14 / 2004$ & $\begin{array}{l}7: 30: 15 \mathrm{PM} \\
7.31 .15 \mathrm{P}\end{array}$ & $\begin{array}{r}26.69 \\
26.689\end{array}$ & \begin{tabular}{|l|}
27.798 \\
27.792
\end{tabular} & $\begin{array}{l}25.174 \\
25138\end{array}$ & $\begin{array}{l}28.565 \\
28519\end{array}$ & $\begin{array}{l}27.151 \\
27.155\end{array}$ & $\begin{array}{r}45.11 \\
45.633\end{array}$ & \begin{tabular}{|l|}
49.827 \\
50.555 \\
\end{tabular} & $\begin{array}{l}10.665 \\
1079\end{array}$ & $\begin{array}{r}35.109 \\
35.325\end{array}$ & 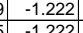 & $\begin{array}{r}-7.589 \\
\end{array}$ & $\begin{array}{r}48.88 \\
44073\end{array}$ & \begin{tabular}{|c|}
0.101 \\
\end{tabular} & $\begin{array}{l}14.643 \\
14634\end{array}$ & $\begin{array}{l}0.003 \\
004\end{array}$ & \begin{tabular}{|c|}
615.53 \\
615.52
\end{tabular} & \begin{tabular}{|l|l|}
10.2589 \\
102756
\end{tabular} & \begin{tabular}{|r|}
48.4 \\
\end{tabular} & \begin{tabular}{|l|}
12.0 \\
\end{tabular} & 40.1 & \begin{tabular}{|l|l|} 
& 2.765 \\
12 & 2775 \\
\end{tabular} & 0.015 & $\begin{array}{l}0.015 \\
\end{array}$ & 0.000 & 0.374 & \\
\hline$\frac{636}{637}$ & $6 / 1 / 142004$ & $\begin{array}{l}7: 31: 15 \mathrm{PM} \\
7: 32: 15 \mathrm{PM}\end{array}$ & $\frac{26.689}{26.684}$ & \begin{tabular}{l|l}
27.792 \\
27.777
\end{tabular} & 25.188 & $\begin{array}{l}28.519 \\
28.509\end{array}$ & $\frac{27.255}{27.215}$ & \begin{tabular}{|l|}
45.463 \\
45.456
\end{tabular} & \begin{tabular}{|l|}
50.555 \\
50.165 \\
\end{tabular} & \begin{tabular}{|c|}
10.779 \\
10.076
\end{tabular} & $\begin{array}{l}35.325 \\
35.348 \\
\end{array}$ & $\begin{array}{l}-1.222 \\
-1.222\end{array}$ & $\begin{array}{r}-7.36 \\
-7.349\end{array}$ & \begin{tabular}{|c|c|}
44.073 \\
46.371
\end{tabular} & \begin{tabular}{|l|}
0.102 \\
0.092
\end{tabular} & $\begin{array}{r}14.643 \\
14.643\end{array}$ & $\begin{array}{l}0.004 \\
0.004\end{array}$ & $\begin{array}{r}616.53 \\
67.53\end{array}$ & \begin{tabular}{|l|l|}
10.2756 \\
1029292
\end{tabular} & \begin{tabular}{|l|}
49.1 \\
\end{tabular} & \begin{tabular}{|l|}
10.8 \\
11.4 \\
\end{tabular} & \begin{tabular}{|l|l|}
$\mid 340.4$ \\
14
\end{tabular} & \begin{tabular}{|c|c|}
4 & 2.785 \\
4 & 2.786 \\
\end{tabular} & 0.015 & $\begin{array}{r}0.015 \\
0.014\end{array}$ & 0.000 & 0.374 & $\begin{array}{l}0.32 \\
0.29\end{array}$ \\
\hline 338 & $6 / 14 / 2004$ & $\begin{array}{l}7.32 .15 \\
7: 33: 15 \mathrm{PM}\end{array}$ & 26.689 & 27.767 & $\frac{25.193}{25.213}$ & \begin{tabular}{|}
28.5249 \\
28.524
\end{tabular} & 27.210 & \begin{tabular}{|}
45.450 \\
45.701
\end{tabular} & \begin{tabular}{|l|}
50.103 \\
50.227 \\
\end{tabular} & $\begin{array}{l}10.702 \\
10.702\end{array}$ & $\begin{array}{r}35.348 \\
35.69 \\
\end{array}$ & $\begin{array}{ll}-1.222 \\
\end{array}$ & $\begin{array}{l}-7.349 \\
-7.566\end{array}$ & \begin{tabular}{|c|}
46.3711 \\
46.778
\end{tabular} & 0.092 & $\begin{array}{l}14.643 \\
14.643 \\
\end{array}$ & $\begin{array}{l}0.004 \\
0.004\end{array}$ & $\begin{array}{c}617.53 \\
618.53\end{array}$ & \begin{tabular}{|l|}
10.2922 \\
10.3089 \\
\end{tabular} & $\begin{array}{l}48.1 \\
48.8\end{array}$ & \begin{tabular}{|l|}
11.4 \\
11.5 \\
\end{tabular} & & & \begin{tabular}{|l}
0.014 \\
0.015 \\
\end{tabular} & $\begin{array}{l}0.014 \\
0.015\end{array}$ & $\begin{array}{l}0.000 \\
0.000\end{array}$ & \begin{tabular}{|c|}
0.378 \\
0.375
\end{tabular} & $\frac{0.29}{0.32}$ \\
\hline & 6/14/2004 & 7:34:15 PM & & 27.763 & 25.224 & 28.495 & 27.285 & 45.512 & \begin{tabular}{|l|}
50.298 \\
\end{tabular} & 10.618 & 35.506 & -1.219 & & 45.243 & 0.101 & $\begin{array}{l}14.643 \\
\end{array}$ & 0.004 & 619.53 & 10.3256 & 48.8 & 11.1 & & \begin{tabular}{|l|l|}
5 & 2.793 \\
\end{tabular} & & 0.015 & & & $\begin{array}{l}0.32 \\
0.31 \\
\end{array}$ \\
\hline 640 & 6/14/2004 & & & 27.765 & 25.19 & 28.522 & 27.28. & 45.562 & 50.186 & 10.622 & 35.58 & & & 42.625 & 0.104 & \begin{tabular}{|l|l|}
14.643 \\
\end{tabular} & 0.004 & & 10.3422 & 48.7 & & & & 0.016 & 0.015 & & & $\begin{array}{l}0.31 \\
0.32\end{array}$ \\
\hline 641 & $6 / 14 / 2004$ & $7: 36: 15$ PM & 26.698 & 27.751 & 25.196 & \begin{tabular}{|l|l|}
28.488 \\
\end{tabular} & 27.28 & \begin{tabular}{|l|l|l}
45.264 \\
\end{tabular} & \begin{tabular}{|l|l}
49.978 \\
\end{tabular} & \begin{tabular}{|l|l|}
10.573 \\
\end{tabular} & 35.325 & -1.222 & -7. & 52.85 & 0.1 & $\begin{array}{l}14.643 \\
\end{array}$ & $\begin{array}{l}0.004 \\
\end{array}$ & \begin{tabular}{|l|l|}
621.53 \\
\end{tabular} & \begin{tabular}{|l|l|}
10.3589 \\
\end{tabular} & 48.5 & $\overline{13.0}$ & 40.3 & & & & 0.000 & 0.368 & \\
\hline 642 & $6 / 14 / 2004$ & 7:37:15 PM & 26.694 & 27.737 & 25.177 & 28.449 & 27.29 & & 50.731 & 10.638 & 35.688 & -1.222 & & 54.936 & \begin{tabular}{|l|l|} 
\\
\end{tabular} & \begin{tabular}{|l|l|l|}
14.643 \\
\end{tabular} & & & & & & & & & & & 0.361 & \\
\hline & & & & & & 28.455[ & & & & & & & & & & & & & & 48.9 & & & & & 0.014 & & 0.343 & \\
\hline 644 & 6/14/2004 & 7:39:15 PM & 26.68 & 27.728 & 25.128 & 28.48 & & 45.527 & 50.155 & 10.597 & 35.592 & -1.225 & & 47.934 & 0.101 & $\begin{array}{l}14.643 \\
\end{array}$ & & 624.53 & 10.4089 & 48.7 & & & 2.796 & 015 & .015 & 5.000 & 0.370 & \\
\hline & 6/14/2004 & 7:40:15 PM & 26.675 & 27.713 & 25.143 & 28.465 & & & .757 & & 34.516 & -1.219 & & & & $\begin{array}{l}14.643 \\
\end{array}$ & & & 101256 & 48.3 & & & & & $\mid$ & & & \\
\hline & $6 / 14 / 2004$ & 7:41:15 PM & 26.671 & 27.694 & 25.149 & \begin{tabular}{|l|}
28.431 \\
\end{tabular} & 27.16 & 45.27 & 49.833 & 10.95 & 35.009 & -1.225 & & 48.209 & 0.095 & $\begin{array}{ll}14.643 \\
\end{array}$ & 004 & 626.53 & $\begin{array}{ll}10.4422 \\
\end{array}$ & 48.4 & 11.8 & 40. & 2.767 & 0.014 & 0.014 & 0.000 & 0.351 & \\
\hline 447 & $6 / 14 / 2004$ & 7:42:15 PM & 26.66 & 27.673 & 25.108 & 28.44 & & 45.761 & 50.316 & 10.63 & 35.842 & -1.219 & & 51.724 & 0.099 & 14.643 & & & 10.4589 & & & & & & & 0.000 & & \\
\hline 48 & 6/14/2004 & 7:43:15 PM & $\begin{array}{r}26.65 \\
26.651\end{array}$ & 27.678 & 25.108 & 28.435 & 27.1 & 45.454 & $50.177]$ & 10.544 & 35.631 & -1.222 & & 46.695 & 0.101 & 14.643 & & 628.5 & 10.4756 & 48.7 & 11.4 & & 2.795 & & 0.015 & 0.000 & 0.370 & \\
\hline 4.9. & 6/14/2004 & 7:44:15 PM & 26.651 & 27.674 & 25.144 & 28.436] & & & 年.643 & 11.198 & & -1.222 & & 41.628 & 0.092 & 14.643 & & & 0.4922 & & & & 2.744 & & 0.014 & & & \\
\hline & 6 & $7: 45: 12$ & 20.040 & 20.6.69 & 20.5999 & 28.436 & & 45.141 & 4.9.516 & 10.949 & 34.912 & $\begin{array}{l}-1.228 \\
-1.227\end{array}$ & & 50.339 & (.099 & 14.643 & & & 年 & & & & & 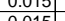 & . & 0.000 & 0.368 & \\
\hline & $\frac{18412}{661212}$ & $\frac{15}{1.5 \mathrm{PM}}$ & & & |2.5.149| & 28.451 & & $\frac{45.372}{15606}$ & 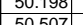 & 10.914 & $\frac{35.103}{35.42}$ & $\frac{-1.22}{-120}$ & & $\begin{array}{l}40.141 \\
5.414\end{array}$ & 0.090 & $\frac{14.643}{1.642}$ & & & & & & & & & . & & & \\
\hline 5 & $\frac{1}{6 / 1442004}$ & . & & & $\frac{25.108}{25.111}$ & $\begin{array}{l}28.415 \\
28.423\end{array}$ & & $\frac{45.000}{45.606}$ & (3) & $\frac{10.710}{10.551}$ & $\begin{array}{l}35.402 \\
35.686\end{array}$ & $\frac{-1.2}{-12}$ & & $\frac{5.4204}{5207}$ & & $\frac{14.045}{14.643}$ & & & & 48. & 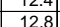 & & & & .015 & & & \\
\hline 654 & $6 / 14 / 2004$ & 7:49:15 PM & 26.627 & 27.625 & 25.105 & 28.427 & 27.19 & 45.909 & 50.656 & 10.588 & 36.006 & -1.216 & & 44.417 & 0.088 & 14.643 & & 634.5 & 10.5756 & 49.2 & 10.9 & 41 & 2.824 & & 0.013 & & 0.319 & \\
\hline 555 & $6 / 14 / 2004$ & 7:50:15 PM & 26.618 & 27.626 & 25.086 & 28.408 & 7.. & 45.817 & 50.395 & 10.588 & 35.934 & & & 50.954 & 0.104 & 14.643 & & 635.53 & 110.5922 & 48.9 & & & 2.818 & 016 & 0.015 & . 000 & 0.378 & $\frac{0.27}{0.32}$ \\
\hline & $6 / 14 / 2004$ & 7:51:15 PM & 26.622 & 27.615 & 25.115 & 28.402 & $27.2 \mathrm{Z}$ & 44.162 & 48.94 & 11.602 & 33.258 & -1.222 & & 47.272 & 0.099 & $\begin{array}{l}14.643 \\
\end{array}$ & & 030.5 & 10.6089 & 47. & 11 & & 2.665 & 015 & 0 & Doo & 0.380 & \\
\hline 65 & $6 / 14 / 2004$ & & & & & & & & & & & & & & & & & & & & & & & & & & & \\
\hline & $6 / 14 / 2$ & 3:15 PM & 26.61 & 27.598 & 25.133 & 28.405 & & 45.65 & 50.482 & 11.275 & 34.937 & -1.22 & & $\begin{array}{l}46.884 \\
\end{array}$ & \begin{tabular}{l|l}
0.098 \\
\end{tabular} & $\begin{array}{l}14.643 \\
\end{array}$ & & & & 49.1 & $\frac{\overline{11}}{11}$ & & & & .015 & & & \\
\hline & & & & & & & & & 50.493 & & & & & 45.349 & & & & & & & & & & & & & & \\
\hline & $6 / 14 / 2004$ & 7:55:15 PM & 26.607 & 27.581 & 25.236 & 28.393 & 27.1 & 45.112 & & 11.124 & $\begin{array}{l}34.601 \\
\end{array}$ & -1.22 & & 51.417 & $\begin{array}{l}0.097 \\
\end{array}$ & \begin{tabular}{|l|l|l|l|l|}
1433 \\
\end{tabular} & & 640.5 & \begin{tabular}{|l|l|}
10.6756 \\
\end{tabular} & & 12.6 & & 2.748 & & & & & \\
\hline 661 & 6/14/2004 & 7:56:15 PM & 26.594 & 27.568 & 25.203 & 28.375 & & 45.486 & 50.142 & 10.922 & 35.204 & -1.225 & & 45.854 & 0.104 & 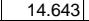 & & 641.53 & 922 & 48.7 & 11.2 & 40. & 2.782 & 0.016 & 0.015 & 0.000 & 0.382 & \\
\hline & 6/14/2004 & $15 \mathrm{PM}$ & & 27.555 & 25.22 & & 27.138 & 46.417 & , & 10.923 & 36.127 & -1.22. & & 46.158 & & 14.643 & & & & & 11.3 & & $288 \quad 2$ & & 0.014 & & & \\
\hline 663 & $6 / 14 / 2$ & 7:58: & 26.614 & 27.562 & 25.242 & 28.364 & & 45.676 & 50.528 & 11.325 & 34.995 & -1.22 & & 50.407 & 0.0 & 14.6 & & $643.5 \mathrm{~K}$ & 7256 & & 12.4 & & & & & 000 & 0.360 & \\
\hline 204 & $6 / 14 / 20$ & 5 PM & & & 25.218 & 28.355 & & 45.815 & 459 & 11.163 & 35 & -1.2 .2 & & & & 14. & & & 10.7422 & $49 . \mathrm{C}$ & & & & & & & & \\
\hline 6056 & $6 / 14 / 2$ & & $\frac{26.614}{26.31}$ & $\begin{array}{l}2.5424 \\
27539\end{array}$ & 20.5237 & $\mid \begin{array}{l}2.8544 \\
\end{array}$ & & 46.13 & 9222 & $\begin{array}{l}11.091 \\
11.502\end{array}$ & & $-\frac{1.1219}{1250}$ & & & & 14.643 & & & & & & & & & & & 3 & \\
\hline$\frac{267}{567}$ & $6 / 614200$ & & & & | & $|2.04118|$ & & $\frac{44.930}{45.45}$ & 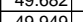 & 1.594 & & -1.24 & & & & . & & & & & 146 & & 27 & & & & 0370 & \\
\hline & $6 / 14 / 2004$ & & 26.6 & & 25203 & $\mid 28.45$ & & 45.751 & 50.374 & | & & & & & & 14. & & & & & & & & & & & 0.367 & \\
\hline (369 & $6 / 14 / 200$ & & 26.642 & 27.536 & 25.186 & 28.383 & & 45.989 & 50.849 & 11.249 & 35.393 & -1.222 & & 52.172 & 0.096 & 14.643 & & $\begin{array}{l}6499.53 \\
\end{array}$ & $\mid 10.8256$ & 49.4 & 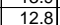 & & 2.806 & $\overline{114}$ & $\mid \frac{0.014}{0.014}$ & 0.000 & $\frac{0.350}{0.350}$ & \\
\hline & $6 / 14 / 20$ & & & 27.534 & 25150 & $\mid 28.336$ & & & & 11.114 & & -1.22 & & & & 14.6 & & & & & & & & & & & & \\
\hline & $6 / 14 / 2$ & 8:06:1 & 26.635 & 27.523 & 25.183 & 28.335 & & $\begin{array}{l}45.344 \\
\end{array}$ & 50.126 & 11.722 & 34.266 & -1.222 & & 51212 & & $\begin{array}{l}14.643 \\
\end{array}$ & & & 889 & & & & 2.74 & & & & & \\
\hline 012 & $6 / 14 / 2$ & & & & & & & & & 11.6 & & & & & & 14. & & & & & & & & & & & & \\
\hline & $6 / 14 / /$ & & 26.63 & 27.5 & 25.188 & 28.2 & & 45.3 & 49.7. & 11.6 & & -1.2 & & & & & & & & & 10. & & & & & & & \\
\hline 574 & & & & & 25.1 & 28.16 & & & & 11.5 & & & & & & 14. & & & & & & & & & & & & \\
\hline & $6 / 14 / 21$ & $8: 16$ & 26.6 & & 25.147 & \begin{tabular}{|l|l|l|}
28.13 \\
\end{tabular} & & & & 11.4 & & -1.8 & & & & & & & & & 11. & & & & & & .351 & \\
\hline & $6 / 14 / 20 \mathrm{C}$ & & 26.605 & 27.473 & 25.173 & 28.121 & & & & 11. & & -1.22 & & & & & & & & & 13.4 & & & & & & 0.335 & \\
\hline & $6 / 14 / 2$ & & 26.584 & 27.452 & 25.177 & 28.115 & & 45.143 & & 11.669 & & -1.22 & & & & 14. & & & & & 1.8 & & & & & & & \\
\hline 58 & $6 / 14 / 2 / 2$ & & 26.5 & & 25.239] & & & & & 11.5 & & -1.2 & & 51.429 & & 14. & & & & & 2.6 & & & & & & 380 & \\
\hline Col & $6 / 14 / 2$ & & 2.0 & & - & 28.066 & & 45.284 & 49.5004 & 111.4 & & -1. & & & & 14. & & & & & 12.4 .4 & & & & & & & \\
\hline & - & & & 27.3565 & 25.141 & 28.10 & & $\frac{1.983}{9.102}$ & th & 10.8 & & & & & & & & & & & 11.1 & & & & & & & \\
\hline & & & 20.354 & $\frac{21.0}{272}$ & $\frac{2.124}{25.129}$ & 2 & & 0.10 & S.1.101 & $\frac{10.06}{0.5}$ & & & & & & & & & & & & & & & & & & \\
\hline & $6 / 1 / 4 / 20$ & & & & 年 & & & & & & $\frac{-18.462}{-2.132}$ & & & & & & & & & & & & & & & & & \\
\hline 684 & $6 / 6 / 1 / 200$ & & 26.664 & 27.3 & 25.142 & 28.115 & & & 50.544 & $\frac{10.0}{10.0}$ & & & & & & & & & & & & & & & & & 0.177 & \\
\hline & $6 / 14 / 20$ & & & 27.347 & 25.117 & & & & 50.843 & 9.98 & & & & & & & & & & & & & & & & & 0.176 & \\
\hline 588 & & & & & & & & & 50.318 & 10.049 & -2.286 & & & & & & & & & & & & & & & & 0.185 & \\
\hline & & & & & & & & & & & & & & & & & & & & & & & & & & & & \\
\hline & $6 / 144$ & & & & & & & & & 11.6 & & & & & & & & & & & & & & & & & & \\
\hline & & & & & & & & $\begin{array}{l}44.249 \\
\end{array}$ & & & & & & & & 14. & & & & & & & & & & & & \\
\hline & $6 / 14 / 2$ & $8: 2$ & 26.3 & 27.314 & 25.063 & 28.03 & & 44.1 & 50.922 & 12. & 32. & 0.6 & & 47.7 & & 14. & & & 47 & 49.4 & 11.7 & & & & & & & \\
\hline & $6 / 14 / 20$ & & & 27.3 & 25.062 & 28.0 & & 44.5 & 51.32 & 12.4 & & & & & & 14. & & & & & & & & & & & & \\
\hline & $6 / 144 / 20$ & & 26.3 & 27.3 & 25.05 & 28.04 & & 44.612 & & 12. & & & & & & 14. & & & & 4 & 2.6 & & & & & & 491 & \\
\hline & $6 / 14 / 2 / 2$ & & 26.27 & 27.306 & 25.036 & 28.034 & & 44.3 & 51.23 & 12.276 & & 0.6 & & 43.1 & 0.1 & 14.6 & & & 11.1. & & 0. & & & & & & & \\
\hline (5945 & $6 / 14 / 4$ & & 26.2 & 27.2 & & & & 44.5 & & 12. & & & & & & & & & & & $\frac{12.9}{12.9}$ & & & & & & & \\
\hline & 0 & & & 27.3 & 250.04 & 27.90 & & 44. & S1.1.044 & $\frac{12.26}{12 x}$ & & & & & & 14 & & & & 7 & 1.0 & & & & & & & \\
\hline & & & $\frac{20.25}{36.20}$ & & $\frac{23.6}{25.6}$ & 2 & & 年4.4 & 51. & 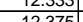 & & & & & & & & & & & & & & & & & & \\
\hline & 114200 & $8 \cdot 28 \cdot 10 \mathrm{PM}$ & 2615 & $272\}$ & 24052 & | & & 460 & 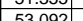 & 年 & 352 & 0.17 & & 1786 & 0 & t4. & & & & 年4.9 & 17 & & & & & & 41 & \\
\hline & $6 / 14 / 20$ & & 26.104 & & & 7.924 & & 44.85 & & 1.21 & & 178 & & & & 14.64 & & & & & 0 & & & & & & 294 & \\
\hline 00 & 6/14/2004] & 8:30:10 PM & 6.081 & 27.254 & 24.903 & 27.932 & 26.18 & 46.043 & |52.889 & \begin{tabular}{|l|}
$\mid 11.284$ \\
\end{tabular} & 35.448 & 0.778 & & 38.831 & 0.11 & 14.643 & & 675.45 & 11.2575 & 51.4 & 9.5 & & & 016 & .016 & 0.000 & 0.404 & \\
\hline
\end{tabular}


WSRC-TR-2005-00105, REVISION 0

SRNL-RPP-2005-00012, REVISION 0

RUN \# 3.03A AND B; FIRST AND SECOND HALF OF SLURRY DEWATERING - CONT.

\begin{tabular}{|c|c|c|c|c|c|c|c|c|c|c|c|c|c|c|c|c|c|c|c|c|c|c|c|c|c|c|c|c|}
\hline & & & D & $E$ & $F$ & $\begin{array}{ll} \\
\end{array}$ & $\mathrm{H}$ & $\mathrm{J}$ & $\mathrm{K}$ & $\mathrm{L}$ & $M$ & $\mathrm{~N}$ & 0 & Q & $\begin{array}{ll} \\
\end{array}$ & $\mathrm{s}$ & \begin{tabular}{l|l}
$\mathrm{T}$ & $\mathrm{s}$
\end{tabular} & $\mathrm{v}$ & w & \begin{tabular}{|l|}
$x$ \\
\end{tabular} & Y & $z$ & & $A B$ & & & & AF $\mathbb{A}$ \\
\hline 70 & $6 / 14 / 2004$ & $8: 31: 10 \mathrm{PM}$ & 26.673 & 27.252 & 24.896 & 27.95 & 26.76 & 52.031 & \begin{tabular}{|l|l|}
53.318 \\
\end{tabular} & $\begin{array}{l}11.206 \\
\end{array}$ & 41.521 & -1.225 & \begin{tabular}{|l|}
-6.372 \\
\end{tabular} & 40.612 & \begin{tabular}{l|l|}
0.135 \\
\end{tabular} & \begin{tabular}{|l|l|}
14.643 \\
\end{tabular} & 0.003 & 676.45 & \begin{tabular}{|l|l|}
11.2742 \\
\end{tabular} & \begin{tabular}{|l|}
51.8 \\
\end{tabular} & 10.0 & 46.8 & 3.225 & 0.020 & 0.020 & 0.000 & 0.432 & \\
\hline$\frac{70}{70}$ & $6 / 14 / 2004$ & & 26.586 & 27.234 & 24.908 & \begin{tabular}{|l|}
27.927 \\
27.92
\end{tabular} & 26.728 & 48.165 & \begin{tabular}{|l|}
49.066 \\
\end{tabular} & \begin{tabular}{|l|l|}
14.659 \\
\end{tabular} & 34.17 & -1.228 & & 55.09 & \begin{tabular}{|l|}
0.101 \\
\end{tabular} & \begin{tabular}{|l|}
14.643 \\
\end{tabular} & & 677.45 & \begin{tabular}{|l|l|}
11.2908 \\
\end{tabular} & $\begin{array}{l}47.6 \\
\end{array}$ & & 41.2 & & & 0.015 & & & \\
\hline & $6 / 14 / 2004$ & $8: 33: 10 \mathrm{PM}$ & 26.464 & $\frac{27.233}{27237}$ & 24.937 & \begin{tabular}{|l|}
27.935 \\
77050
\end{tabular} & 26.731 & \begin{tabular}{|l|}
47.315 \\
\end{tabular} & \begin{tabular}{|l|l|}
48.111 \\
\end{tabular} & \begin{tabular}{|l|}
15.299 \\
19.8
\end{tabular} & $\begin{array}{l}32.698 \\
2.650\end{array}$ & -1.222 & $\begin{array}{r}-7.441 \\
7.772\end{array}$ & $\begin{array}{ll}47.041 \\
.551\end{array}$ & $\begin{array}{l}0.1 \\
0.1\end{array}$ & \begin{tabular}{|l|}
14.643 \\
\end{tabular} & 0.004 & 678.45 & $\begin{array}{l}11.3075 \\
\end{array}$ & \begin{tabular}{|l|}
46.6 \\
\end{tabular} & 11.5 & & & & & 0.000 & & \\
\hline 704 & $6 / 14 / 2004$ & $8: 34: 10 \mathrm{PM}$ & & & 24.996 & \begin{tabular}{|l|}
27.959 \\
7799
\end{tabular} & $\begin{array}{r}26.76 \\
2769\end{array}$ & & & \begin{tabular}{|l|l|}
15.081 \\
1.21
\end{tabular} & $\begin{array}{l}31.459 \\
3235\end{array}$ & \begin{tabular}{|l|l|}
9 & -1.222 \\
\end{tabular} & \begin{tabular}{|l|l|}
-7.473 \\
\end{tabular} & \begin{tabular}{|l|}
55.47 \\
\end{tabular} & \begin{tabular}{|c|c|c|}
0.093 \\
\end{tabular} & \begin{tabular}{|l|l|}
14.643 \\
143
\end{tabular} & $\begin{array}{l}0.004 \\
0.04\end{array}$ & \begin{tabular}{|l|l|}
679.45 \\
69.5
\end{tabular} & 1.3242 & & & & & & 0.014 & 0.000 & 0.359 & \\
\hline $\mid \frac{705}{706}$ & $6 / 14 / 2004$ & $\begin{array}{l}8: 35: 10 \mathrm{PM} \\
8 \cdot 36 \cdot 10 \mathrm{PM}\end{array}$ & $\begin{array}{r}\frac{26.401}{26.38} \\
\frac{2}{2}\end{array}$ & $\begin{array}{r}27.22 \\
27.213\end{array}$ & $\begin{array}{l}25.004 \\
2502\end{array}$ & \begin{tabular}{|l|l|}
27.948 \\
27.936
\end{tabular} & $\begin{aligned} 26.768 \\
26796\end{aligned}$ & $\begin{array}{r}46.802 \\
46.734\end{array}$ & \begin{tabular}{|l|}
47.645 \\
476599 \\
\end{tabular} & \begin{tabular}{|l|l|}
14.219 \\
1378
\end{tabular} & $\begin{array}{r}33.345 \\
3372\end{array}$ & $\begin{array}{l}5.222 \\
5.1222\end{array}$ & $\begin{array}{r}-7.485 \\
7404\end{array}$ & \begin{tabular}{|c|}
47.498 \\
50.124
\end{tabular} & $\begin{array}{l}0.095 \\
0099\end{array}$ & $\begin{array}{l}14.643 \\
14634\end{array}$ & $\begin{array}{l}0.004 \\
\end{array}$ & $\begin{array}{l}680.45 \\
\end{array}$ & $\begin{array}{l}11.3408 \\
11.2575\end{array}$ & \begin{tabular}{|l|}
46.2 \\
\end{tabular} & \begin{tabular}{|l|}
11.6 \\
\end{tabular} & 40.1 & \begin{tabular}{|l|}
2.763 \\
2.774 \\
\end{tabular} & \begin{tabular}{|l|l|}
0.014 \\
015
\end{tabular} & $\begin{array}{l}0.014 \\
\end{array}$ & 0.000 & $\begin{array}{l}0.353 \\
\end{array}$ & \\
\hline 707 & 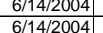 & $\begin{array}{l}8: 36: 10 \mathrm{PM} \\
8: 37: 10 \mathrm{PM}\end{array}$ & $\begin{array}{r}26.38 \\
26.378\end{array}$ & $\frac{27.213}{27.206}$ & $\begin{array}{l}255.022 \\
25.025\end{array}$ & \begin{tabular}{|l|}
27.936 \\
27.944
\end{tabular} & $\frac{26.796}{26.774}$ & $\mid$\begin{tabular}{|c|c}
46.734 \\
46.618
\end{tabular} & \begin{tabular}{|l|}
47.6599 \\
47.468 \\
\end{tabular} & \begin{tabular}{|l|}
13.788 \\
13.621
\end{tabular} & $\begin{array}{r}33.72 \\
33.751 \\
\end{array}$ & $\frac{-1.222}{-1.222}$ & $\begin{array}{r}-7.404 \\
-7.462 \\
\end{array}$ & 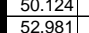 & $\begin{array}{l}0.098 \\
0.097 \\
\end{array}$ & $\begin{array}{l}14.643 \\
14.643\end{array}$ & $\begin{array}{l}0.004 \\
0.004\end{array}$ & $\begin{array}{l}681.45 \\
682.45\end{array}$ & 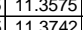 & \begin{tabular}{|l|}
46.2 \\
46.0
\end{tabular} & $\frac{12.3}{130}$ & 40.2 & \begin{tabular}{|l|}
2.774 \\
2.771
\end{tabular} & \begin{tabular}{|l|}
0.015 \\
0.014
\end{tabular} & $\begin{array}{r}0.015 \\
0.014\end{array}$ & 0.000 & $\begin{array}{l}0.363 \\
0.360\end{array}$ & 0.31 \\
\hline 708 & $6 / 14 / 2004$ & $8: 38: 10$ PM & 26.387 & 27.205 & 25.019 & 27.928 & 26.749 & & \begin{tabular}{|l|}
48.468 \\
48.111 \\
\end{tabular} & 13.365 & 34.576 & \begin{tabular}{|l|l|l|l|l}
5 & -1.222 \\
\end{tabular} & & $\begin{array}{l}32.981 \\
46.878\end{array}$ & o.0998 & \begin{tabular}{l|l}
$\frac{14.043}{14.643}$ &
\end{tabular} & $\begin{array}{l}0.004 \\
0.004\end{array}$ & $\frac{602<.45}{683.45}$ & \begin{tabular}{|l|}
1.1 .3342 \\
11.3908 \\
\end{tabular} & 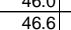 & $\begin{array}{l}11.0 \\
11.5 \\
\end{array}$ & & $\left|\begin{array}{|l|}2.771 \\
2.820\end{array}\right|$ & & $\begin{array}{l}0.014 \\
0.015\end{array}$ & $\begin{array}{l}0.000 \\
0.000\end{array}$ & \begin{tabular}{|l|l|}
0.350 \\
0.357
\end{tabular} & $\frac{0.31}{0.30}$ \\
\hline 709 & 6/14/2004 & $8: 39: 10 \mathrm{PM}$ & 26.384 & 27.213 & 25.012 & 27.935 & 26.771 & 46.753 & 48.076 & \begin{tabular}{|l|l|l|l|}
1393 \\
\end{tabular} & 33.472 & -1.222 & $\begin{array}{l}-7.476 \\
\end{array}$ & 61.45 & 0.096 & $\begin{array}{l}14.643 \\
\end{array}$ & 0.004 & 684.45 & \begin{tabular}{|l|l|}
11.4075 \\
\end{tabular} & 46.6 & 15.1 & 40.1 & & \begin{tabular}{|l|}
0.014 \\
\end{tabular} & & & & $\begin{array}{l}0.30 \\
0.30\end{array}$ \\
\hline & 6/14/2004 & $8: 40: 10 \mathrm{PM}$ & 26.367 & 27.185 & 25.019 & 27.908 & 26.758 & \begin{tabular}{|l|l}
47.057 \\
\end{tabular} & 48.422 & \begin{tabular}{|c|}
13.678 \\
\end{tabular} & 34.042 & \begin{tabular}{|l|l|} 
& -1.222 \\
\end{tabular} & & 53.086 & $\begin{array}{l}0.097 \\
\end{array}$ & $\begin{array}{l}14.643 \\
\end{array}$ & 0.004 & \begin{tabular}{|l|l|}
685.45 \\
\end{tabular} & 11.4242 & 46.9 & & & & & 0.014 & & & 0.30 \\
\hline 711 & $6 / 14 / 2004$ & $8: 41: 10 \mathrm{PM}$ & & & \begin{tabular}{|l|}
25.037 \\
\end{tabular} & \begin{tabular}{|l|}
27.896 \\
\end{tabular} & 26.721 & 47.109 & \begin{tabular}{|l|l|}
48.513 \\
\end{tabular} & & 34.044 & & -7.473 & 50.572 & \begin{tabular}{|l|l|}
0.097 \\
\end{tabular} & $\begin{array}{l}14.643 \\
\end{array}$ & $\begin{array}{l}0.004 \\
\end{array}$ & $\begin{array}{l}686.45 \\
\end{array}$ & 11.4408 & $\begin{array}{l}47.0 \\
\end{array}$ & $\overline{12.4}$ & & \begin{tabular}{|l|}
2.798 \\
\end{tabular} & & & & 0.356 & $\frac{0.30}{0.30}$ \\
\hline 712 & $6 / 14 / 2004$ & $8: 42: 10 \mathrm{PM}$ & 26.357 & 27.17 & 25.04 & 27.858 & 26.704 & 46.916 & 48.32 & 13.687 & 33.812 & -1.219 & & & 0.097 & & & & & & & & & & & & & \\
\hline & & & & & & & & & & & & & & & & \begin{tabular}{l|l}
14.643 \\
\end{tabular} & & & & 46.5 & & & & & 0014 & & 0.359 & \\
\hline & $6 / 14 / 2004$ & $8: 44: 10 \mathrm{PM}$ & 26.349 & 27.153 & 24.982 & 27.796 & & 47.34 & 48.619 & 13.282 & 34.783 & -1.222 & & 52.804 & 0.098 & $\begin{array}{l}14.643 \\
\end{array}$ & & 689.45 & 1.4908 & & & & 2.831 & . 015 & | & 500 & 0.356 & \\
\hline-2 & $6 / 114 / 2004$ & 8:45:10 PM & & 27.156 & 24.975 & 27.814 & 26.674 & \begin{tabular}{|l|l|}
47.18 \\
\end{tabular} & & 13.197 & 34.666 & -1.22 & & & & 14.643 & & 690.45 & & & & & & & & & & \\
\hline & $6 / 14 / 2004$ & 8:46:10 PM & 26.345 & 27.143 & 24.967 & 27.816 & 26.697 & 46.931 & 48.1199 & 13.158 & 34.535 & -1.222 & -7.464 & 49.187 & 0.097 & 14.643 & 0.004 & 691.45 & 11.5242 & $\begin{array}{ll}46.6 \\
\end{array}$ & 12.1 & & 2.808 & 0.014 & 0.014 & 0.000 & 0.355 & 30 \\
\hline 717 & $6 / 14 / 2004$ & 8:47:10 PM & & 27.143 & 24.957 & 27.811 & 26.697 & 46.869 & 48.412 & 13.227 & 34.338 & -1.222 & & 48.374 & 0.094 & 14.643 & & 692.45 & 11.5408 & 46.9 & & & & & 0.014 & & 0.346 & \\
\hline$\frac{718}{710}$ & 6/14/2004 & 8:48:10 PM & 26.353 & 27.156 & 24.955 & 27.819 & 26.675 & 47.136 & 48.463 & 13.294 & 34.594 & -1.216 & & 48.674 & 0.098 & 14.643 & & 693.45 & 11.5575 & 47.0 & 11.9 & & 2.817 & & 0.015 & 0.000 & 0.358 & \\
\hline$\frac{19}{720}$ & $6 / 14 / 2004$ & 8:49:10 PM & 26.337 & 27.136 & 24.945 & 27.769 & 26.6 & 47.196 & 年 48.642 & 13.24 & & -1.225 & & 324 & 0.097 & 14.643 & & 694.45 & .5742 & 47.2 & & & & & 0.014 & 000 & 0.354 & \\
\hline & $6 / 14 / 2004$ & 8:50:10 PM & 26.332 & 27.131 & 24.919 & $20.7 / 74$ & & 46.993 & 8.281 & $13.110 \mathrm{G}$ & 34.623 & -1.225 & & & 0.080 & 14.043 & & 695.45 & 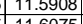 & 46.8 & $9.8 \mathrm{Cl}$ & & & & . & & 0.359 & \\
\hline & $\frac{6 / 14 / 20}{61 / 420}$ & $8: 51.10 \mathrm{PM}$ & $\frac{26.315}{26.31}$ & & $\frac{24.986}{2.907}$ & $\frac{2.1 .124}{2774}$ & & $\begin{array}{l}44.398 \\
17370\end{array}$ & 告.9.054 & $\frac{13.258}{1.2285}$ & $\begin{array}{l}34.804 \\
24621\end{array}$ & $\frac{-1.22}{-122}$ & & & & $\frac{14.643}{1.643}$ & & $\frac{696}{6907}$ & & & & & & & - & & (3) & \\
\hline$\frac{72:}{72:}$ & $\frac{1}{6 / 144 / 2004}$ & 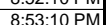 & & & $\mid$ & 27.74 & & & $\frac{48.004}{48528}$ & $\begin{array}{l}\frac{13.265}{13237} \\
132\end{array}$ & $\begin{array}{l}34.021 \\
34.469\end{array}$ & $-\frac{-1.24}{-122}$ & & & & 146 & & $\frac{6917.45}{698.45}$ & & & & & & & & & & \\
\hline & $6 / 14 / 2004$ & 8:54:10 PM & 26.312 & 27.085 & 24.889 & 27.758 & 26.6 & 47.387 & & 13.349 & 34.656 & -1.219 & -7.41 & 53.501 & 0.097 & 14.643 & & 699.45 & & 47.6 & 13.1 & & 2.828 & & 0.015 & & 0.354 & \\
\hline 72 & $6 / 14 / 2004$ & 8:55:10 PM & 26.322 & 27.09 & 24.899 & 27.778 & 26.5 & 47.041 & 48.691 & 13.283 & 34.408 & & & 53.127 & 0.096 & 14.643 & & 700.45 & 111.6742 & 47.2 & & & 2.808 & 014 & 0.014 & 000 & 0.352 & \\
\hline 72 & $6 / 14 / 2004$ & $8: 56: 10$ PM & 26.296 & 27.079 & 24.883 & 27.777 & 26.5 & 46.732 & \begin{tabular}{|l|l|l|l|}
48.61 \\
\end{tabular} & 13.203 & $\begin{array}{l}34.077 \\
\end{array}$ & -1.225 & & 50.213 & \begin{tabular}{|l|l|}
0.097 \\
\end{tabular} & 14.643 & & 701.45 & 11.6908 & 47.1 & & & 2.786 & & 0.015 & D00 & 0.359 & \\
\hline & 6/14/2004 & & & & & & & & & & & & & & & & & & & & & & & & & & & \\
\hline & $6 / 14 / 2$ & $8: 58: 10 \mathrm{PM}$ & 26.259 & 27.063 & 24.876 & 27.716 & & $\begin{array}{ll}47.03 \\
\end{array}$ & 49.071 & $\begin{array}{ll}13.196 \\
\end{array}$ & 34.42 & -1.22 & & 47.16 & 0.0 & 14.643 & & & 7242 & 47. & & & & & 101 & & 0.356 & \\
\hline & & & & & & & & & & & & & & & & & & & & & & & & & & & & \\
\hline & $6 / 1 / 4 / 2004$ & 9:00:10 PM & 26.237 & 27.05 & 24.874 & \begin{tabular}{|l|l|}
27.658 \\
\end{tabular} & & 47.319 & |49.104 & 12.988 & 34.968 & -1.22 & & & & \begin{tabular}{|l|l|l|l|l|}
1433 \\
\end{tabular} & & 705.45 & 111.7575 & $\begin{array}{lll}47.6 \\
\end{array}$ & & & & & & & & \\
\hline 7331 & $6 / 14 / 2004$ & 9:01. & 26.226 & 27.039 & 24.888 & 27.617 & & 47.031 & 48.737 & 12.973 & 34.748 & -1.22 & & 49.683 & & \begin{tabular}{l|l|l}
14.643 \\
\end{tabular} & & 706.45 & \begin{tabular}{|l|l|l|l|l|l|} 
\\
\end{tabular} & 47.3 & 12.2 & & 2.819 & 0.014 & 0.015 & 0.000 & 0.355 & \\
\hline & 6/14/2004 & 9:02:10 PM & 26.214 & 27.037 & 24.871 & 27.65 & & 47.257 & 49.118 & 12.998 & 34.921 & -1.22 & & 52.149 & & 14.643 & & 707.45 & & $47.6 \mathrm{~s}$ & $12.8 \mathrm{C}$ & & & & & & 0.353 & \\
\hline 733 & $6 / 14 / 2$ & & & 27.031 & 24.885] & 27.689 & & 47.358 & 49.208 & 13.007] & 35.01 & -1.2 & & 45.616 & & $14.6 \mathrm{C}$ & & 708.45 & 11.8075 & 47 & & & 2.84 & & 14 & 000 & 349 & \\
\hline (334) & $6 / 14 / 2004$ & & 26.1 & 27.022 & 2000 & 27.675 & & 47.464 & 9.8 & 13.014 & & -1.2 .2 & & & & 14. & & 70 & & 47.8 & & & & & & & & \\
\hline & $\frac{6144 / 2}{6 / 142}$ & & $\frac{20.197}{26207}$ & 27.016 & 24.809 & | & & $\begin{array}{l}47.124 \\
47601\end{array}$ & 9.054 & 12.953 & & & & & & 14.6 & & & & 47.6 & & & & & & & 354] & \\
\hline & $6 / 141 / 2004$ & & & 26.020 & 24897 & 27.075 & & 年17.001 & 516 & 1 & & -1.2 & & 498 & & 14. & & & & 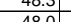 & 122 & & & & & & & \\
\hline 738 & $6 / 14 / 2004$ & & & 20.004 & 2488 & 2768 & & & & 12875 & & -12 & & & & 146 & & & & & & & & & & & & \\
\hline & $6 / 14 / 2004$ & $9: 09: 10 \mathrm{PM}$ & 26.191 & 26.995 & 24.903 & 27.633 & 26.573 & 46.952 & \begin{tabular}{|l|l|}
48.689 \\
\end{tabular} & 12.857 & 34.798 & \begin{tabular}{|l|l|}
3 & -1.222 \\
\end{tabular} & -7.436 & 47.852 & 0.096 & 14.643 & & \begin{tabular}{|l|l|}
714.45 \\
\end{tabular} & \begin{tabular}{|l|l|}
11.9075 \\
\end{tabular} & 47.2 & 11.7 & & 2.818 & .014 & 0.014 & 0.000 & 0.351 & 0.30 \\
\hline & $6 / 144 / 20$ & $9: 10: 10 \mathrm{PM}$ & 26.19 & 26.984 & 24.882 & 27.632 & & 47.192 & & $\begin{array}{l}12.931 \\
\end{array}$ & & \begin{tabular}{|l|l|}
4 & -1.222 \\
\end{tabular} & & 46.371 & & 14.6 & & & & 47.6 & & & & & & & & \\
\hline 741 & $6 / 11412004$ & & 26.179 & $26.9 / 2$ & & 2.7555 & & 46.943 & 48.8844 & 12.897] & & -1.21 & & & & & & & & 47.4 & & & & & & & & \\
\hline$\frac{144}{174}$ & $6 / 14 / 2$ & & & & 24.86 & & & & & 1.944 & & & & & & 14. & & & & 48. & 11.9 & & & & & & & \\
\hline & & & & & & & & & & $\frac{11 .}{120}$ & & & & & & & & & & & & & & & & & & \\
\hline & 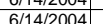 & & $\frac{20.1}{2611}$ & & $\frac{2.0}{24.8}$ & & & & & 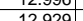 & & & & & & & & & & & & & & & & & & \\
\hline & $6 / 141 / 20$ & & & & & & & & & 13084 & & & & & & & & & & & 然1.0. & & & & & & & \\
\hline $\begin{array}{ll}747 \\
\end{array}$ & $6 / 14 / 20$ & 9:17: & 26.1 & & 24.889 & 27.588 & & & & 13.072 & tring & -1.2 & & & & 14. & & & & & 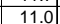 & & & & & & & \\
\hline 748 & $6 / 14 / 2$ & & 26.165 & 26.918 & 24.882 & 27.581 & & 46.854 & & 13.045 & 34.502 & -1.2 & & & & & & & & & 12.4 & & & & & & & \\
\hline 7449 & $6 / 14 / 2$ & & & & & & & & & & & & & & & & & & & & & & & & & & & \\
\hline & $6 / 14 / 2$ & & 26.1 & & 24.8 & & & & & 13.145 & & & & 52.4 & & & & & & & & & & & & & & \\
\hline & & & & & & & & & & 13.144 & & & & & & & & & & & & & & & & & & \\
\hline & $6 / 14 / 2$ & & 26. & 26.9 & 25.0 & 27.555 & & & & 13.071 & & -1.2 & & & & & & & & & & & & & & & & \\
\hline & $6 / 14 / 20$ & & & & & & & & & & & & & & & 14 & & 728 & & & & & & & & & & \\
\hline & $6 / 14 / 20$ & 9:24:1 & 26.2 & & 25.195 & 27.579 & & 46.6 & & 12.86 & & -1.22 & & 45.21 & & 14. & & & & 47.0 & 11.1 & & & & & & 0.332 & \\
\hline & $6 / 14 / 2$ & & 26.7 & & 25.148 & 27.567 & & 46.8 & & 12. & & -1.2 & & & & 14. & & 736 & & & 10 & & & & & & & \\
\hline & $6 / 14 / 4$ & & 26. & & 25.1288 & 27.552 & & 46.5 & & 12.923 & & & & & & & & & & & & & & & & & 0.335 & \\
\hline & $6 / 14 / 2$ & & & & & & & & & & & & & & & & & & & & & & & & & & & \\
\hline & & & & & 2.0 & $\frac{2.350}{2750}$ & & 41.04 & & $\frac{1.2,9}{127}$ & & & & & & & & & & & & & & & & & & \\
\hline & $\frac{6142 / 2}{6 / 42 / 2}$ & 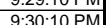 & $\begin{array}{l}\frac{2.2634}{2625} \\
\end{array}$ & $\begin{array}{l}20.06 \\
2685\end{array}$ & 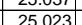 & 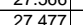 & & $\begin{array}{l}460.535 \\
46902\end{array}$ & 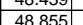 & 12714 & $\begin{array}{l}54.590 \\
34933\end{array}$ & -1.22 & & & & 1468 & & 735 & & 47.04 & $\frac{1.10}{116}$ & & & & & & 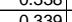 & \\
\hline 761 & $6 / 14 / 20$ & & 26.242 & & 24.984 & 27.48 & & 47.1 & & 12.75 & & & & & & & & & & 78 & & & & & & & & \\
\hline $\begin{array}{ll}762 \\
\end{array}$ & $6 / 14 / 20$ & $9: 32: 1$ & 26.2 & 26.8 & 24.96 & 27.525 & & 46.896 & 49.046 & 12.66 & & & & & & 14. & & & & 47.6 & 9.8 & & & & & & & \\
\hline & $6 / 14 / 2$ & $9: 33: 10 \mathrm{P}$ & 26.237 & & 24.944 & $\frac{27.524}{2754}$ & & 46.927 & & 12.578 & & -1.22 & & 49.3 & & 14.6 & & 138 & & & 12 & & & & & & & \\
\hline & & & & & & & & 46.5 & & & & & & & & & & & & & $10.7 \mathrm{C}$ & & & & & & & \\
\hline & & & & & & & & & & & & & & & & & & & & & 10.8 & & & & & & & \\
\hline & & & & & & 27.5 & & $\begin{array}{l}46.487 \\
\end{array}$ & & 13.0 & & & & & & & & & & & & & & & & & & \\
\hline & $114 /$ & & 26.19 & & 24.89 & 27.5 & & 46.6 & 49. & 13.1 & 34.194 & & & & & & & & & & & & & & & & & \\
\hline & $114 / 200$ & 9:38:10 PM & 26.18 & 26.79 & 24.894] & 27.569 & & 46.97 & 49.6 & 130 & 34.586 & -1.225 & & 51.064 & & 14.6 & & 743.4 & $12.3 \mathrm{~S}$ & 4 & & & & & & & 34 & \\
\hline & 6/14/200 & 9:39: & 26.179 & & 1.876 & 77.556 & & 46.5. & 48.98 & 190 & $34.27 \mathrm{r}$ & -1.22 & & 8.71 & & 14.62 & & & & & 1 & & & 114 & 4 & & 341 & \\
\hline 70 & 6/114/2004 & 9:40:10 PM & .176 & 26.79 & $|24.868|$ & 27.568 & & 46.51 & $|49.131|$ & 12.857 & 34.297 & \begin{tabular}{l|l|l|} 
& -1.222
\end{tabular} & & 2.886 & $0.091 \mid$ & 14.643| & & 745.45] & 12.4242 & $47.7]$ & 10.5 & & & |. & 0.14] & 0.000 & $0.337 \mid$ & \\
\hline
\end{tabular}


WSRC-TR-2005-00105, REVISION 0

SRNL-RPP-2005-00012, REVISION 0

RUN \# 3.03A AND B; FIRST AND SECOND HALF OF SLURRY DEWATERING - CONT.

\begin{tabular}{|c|c|c|c|c|c|c|c|c|c|c|c|c|c|c|c|c|c|c|c|c|c|c|c|c|c|c|c|c|}
\hline & A & & D & $\mathrm{E}$ & $\mathrm{F}$ & \begin{tabular}{l|l} 
\\
\end{tabular} & $\mathrm{H}$ & $\mathrm{J}$ & $\mathrm{K}$ & $\mathrm{L}$ & $M$ & $\mathrm{~N}$ & 0 & $Q^{-1}$ & $\mathrm{R}$ & $\mathrm{s}$ & \begin{tabular}{l|lll}
$\mathrm{T}$ & $\mathrm{s}$
\end{tabular} & $\mathrm{v}$ & w & \begin{tabular}{|l|l|}
$x$ & \\
\end{tabular} & $\mathrm{Y}$ & $\mathrm{z}$ & $\mathrm{AA}$ & $A B$ & AC & & & AF $D A$ \\
\hline 771 & $6 / 14 / 2004$ & 9:41:10 PM & 26.175 & 26.783 & 24.867 & \begin{tabular}{l|l|}
27.561 \\
\end{tabular} & 26.412 & $\begin{array}{l}46.336 \\
\end{array}$ & \begin{tabular}{|l|}
49.048 \\
\end{tabular} & \begin{tabular}{l|l|}
13.206 \\
\end{tabular} & 33.718 & -1.222 & $\begin{array}{l}-7.404 \\
\end{array}$ & 46.002 & \begin{tabular}{l|l|}
0.092 \\
\end{tabular} & \begin{tabular}{|l|l|}
14.643 \\
\end{tabular} & 0.004 & 746.45 & \begin{tabular}{|l|}
12.4408 \\
\end{tabular} & \begin{tabular}{|l|}
47.6 \\
\end{tabular} & 11.3 & & 2.760 & 0.014 & 0.014 & 0.000 & 0.344 & 0.29 \\
\hline 772 & $6 / 14 / 2004$ & 9:42:10 PM & 26.162 & 26.776 & 24.889 & 27.534 & 26.414 & \begin{tabular}{|l|l|}
46.207 \\
\end{tabular} & \begin{tabular}{|l|l|}
48.617 \\
\end{tabular} & \begin{tabular}{|l|l|}
13.227 \\
\end{tabular} & 33.661 & 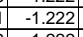 & & 49.944 & \begin{tabular}{|r|}
0.09 \\
\end{tabular} & \begin{tabular}{|l|}
14.643 \\
\end{tabular} & 0.004 & 747.45 & \begin{tabular}{|l|l|}
12.4575 \\
\end{tabular} & \begin{tabular}{|l|}
47.1 \\
\end{tabular} & $\frac{12.2}{12.2}$ & & & & 0.013 & 0.000 & & \\
\hline & $6 / 14 / 2004$ & 9:43:10 PM & 26.159 & $\begin{array}{l}26.768 \\
2.771\end{array}$ & 24.866 & 27.526 & 26.406 & $\begin{array}{l}46.172 \\
\end{array}$ & \begin{tabular}{|l|}
48.513 \\
\end{tabular} & $\begin{array}{r}3.15 \\
13.19\end{array}$ & 33.759 & \begin{tabular}{|l|l|}
$y^{2}$ & -1.222 \\
\end{tabular} & & \begin{tabular}{|l|}
50.007 \\
5015
\end{tabular} & $\begin{array}{l}0.0899 \\
\end{array}$ & \begin{tabular}{|l|}
14.643 \\
\end{tabular} & 0.004 & 748.45 & \begin{tabular}{|l|l|}
12.4742 \\
12010
\end{tabular} & 47.0 & 12.3 & & & & & 0.000 & 0.333 & \\
\hline 774 & $6 / 14 / 2004$ & 9:44:10 PM & & $\begin{array}{l}26.771 \\
20.771\end{array}$ & $\begin{array}{l}24.875 \\
2.890\end{array}$ & \begin{tabular}{|l|}
27.524 \\
7572
\end{tabular} & $\begin{array}{l}26.395 \\
26.309\end{array}$ & $\begin{array}{ll}46.272 \\
6.265\end{array}$ & \begin{tabular}{|l|l|}
48.538 \\
\end{tabular} & $\begin{array}{ll}13.082 \\
132\end{array}$ & & $\begin{array}{l}-1.222 \\
-1219\end{array}$ & $\begin{array}{r}.411 \\
\end{array}$ & \begin{tabular}{|l|l|}
58.15 \\
\end{tabular} & \begin{tabular}{|l|l|}
0.089 \\
\end{tabular} & \begin{tabular}{|l|l|}
14.643 \\
143
\end{tabular} & $\begin{array}{l}0.004 \\
004\end{array}$ & \begin{tabular}{|l|l|}
749.45 \\
75.5
\end{tabular} & & \begin{tabular}{|l|}
47.1 \\
\end{tabular} & 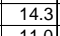 & & & & 0.013 & 0.000 & & \\
\hline 7756 & $6 / 1 / 1 / 2004$ & $\begin{array}{l}9: 45: 10 \mathrm{PM} \\
9 \cdot 46 \cdot 10 \mathrm{PM}\end{array}$ & $\begin{array}{l}26.161 \\
26.154\end{array}$ & $\frac{26.774}{26.773}$ & $\begin{array}{l}24.888 \\
24.868\end{array}$ & $\begin{array}{l}27.572 \\
27.52 \\
\end{array}$ & $\begin{array}{l}26.398 \\
26411\end{array}$ & $\begin{array}{l}46.265 \\
46502\end{array}$ & \begin{tabular}{|c|}
48.534 \\
48.959 \\
\end{tabular} & $\begin{array}{r}13.104 \\
1306\end{array}$ & $\begin{array}{r}33.899 \\
34.176\end{array}$ & \begin{tabular}{|l|l|} 
& -1.219 \\
& -1225 \\
\end{tabular} & $\begin{array}{r}-7.415 \\
-741\end{array}$ & \begin{tabular}{|l|}
45.053 \\
56663 \\
\end{tabular} & $\begin{array}{r}0.09 \\
0.91\end{array}$ & $\begin{array}{l}14.643 \\
14634\end{array}$ & $\begin{array}{l}0.004 \\
0.03\end{array}$ & $\begin{array}{l}750.45 \\
775.15\end{array}$ & \begin{tabular}{|l|l|}
12.5075 \\
12502
\end{tabular} & \begin{tabular}{|l|}
47.1 \\
\end{tabular} & 11.0 & 403 & \begin{tabular}{|l|}
2.764 \\
2781 \\
\end{tabular} & \begin{tabular}{|l|}
0.013 \\
0011
\end{tabular} & 0.013 & 0.000 & 0.336 & \\
\hline 7776 & 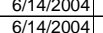 & $\begin{array}{l}9: 46: 10 \mathrm{PM} \\
9 \text { 9:47:10 PM }\end{array}$ & $\frac{26.154}{26.148}$ & $\frac{26.773}{26.762}$ & \begin{tabular}{r|r|}
24.866 \\
24.86
\end{tabular} & \begin{tabular}{|l|}
27.521 \\
27.495
\end{tabular} & $\frac{26.411}{26.405}$ & $\begin{array}{l}46.502 \\
46.605\end{array}$ & \begin{tabular}{|l|}
$\mid 48.855$ \\
49.187 \\
\end{tabular} & \begin{tabular}{|r|r|r|}
13.06 & \\
13.288
\end{tabular} & $\begin{array}{l}34.176 \\
34.231 \\
\end{array}$ & \begin{tabular}{|c|c|} 
& -1.225 \\
& -1.225 \\
\end{tabular} & & \begin{tabular}{|c|}
56.663 \\
49.929 \\
\end{tabular} & \begin{tabular}{|l|}
0.091 \\
0.091
\end{tabular} & $\begin{array}{r}14.643 \\
14643\end{array}$ & $\begin{array}{l}0.003 \\
0.003\end{array}$ & $\begin{array}{l}751.45 \\
752.45\end{array}$ & \begin{tabular}{|l|l|}
12.5242 \\
15408
\end{tabular} & \begin{tabular}{|l|}
47.4 \\
4477
\end{tabular} & \begin{tabular}{|l|}
13.9 \\
12.3
\end{tabular} & & \begin{tabular}{|l|}
2.781 \\
2.787
\end{tabular} & \begin{tabular}{|l|}
0.014 \\
0.014
\end{tabular} & $\begin{array}{l}0.014 \\
0.014\end{array}$ & 0.000 & $\begin{array}{l}0.337 \\
0.373\end{array}$ & $\begin{array}{l}0.29 \\
0.29\end{array}$ \\
\hline 778 & $6 / 14 / 2004$ & 9:48:10 PM & 26.142 & 26.765 & $\begin{array}{r}24.80 \\
24.883\end{array}$ & \begin{tabular}{|l}
27.5953 \\
27.533
\end{tabular} & & \begin{tabular}{|l}
40.005 \\
46.384
\end{tabular} & \begin{tabular}{|l|}
49.189 \\
48.992 \\
\end{tabular} & 13.002 & $\begin{array}{l}34.231 \\
34.087\end{array}$ & \begin{tabular}{|l|}
$-1.2<5$ \\
\end{tabular} & & \begin{tabular}{|}
4.992 \\
51.663 \\
\end{tabular} & $\begin{array}{l}0.019 \\
0.09 \\
\end{array}$ & $\begin{array}{l}14.643 \\
14.643 \\
\end{array}$ & \begin{tabular}{|c|}
0.003 \\
0.004 \\
\end{tabular} & $\begin{array}{r}552.45 \\
753.45\end{array}$ & 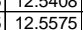 & \begin{tabular}{|l|}
47.1 \\
47.5 \\
\end{tabular} & $\frac{12.3}{12.7}$ & & & \begin{tabular}{|l|}
0.014 \\
0.013 \\
\end{tabular} & $\mid \begin{array}{c}0.014 \\
0.013\end{array}$ & $\begin{array}{l}0.000 \\
0.000\end{array}$ & $\begin{array}{l}0.33 \\
0.335 \\
\end{array}$ & $\begin{array}{l}0.29 \\
0.28\end{array}$ \\
\hline 779 & 6/14/2004 & 9:49:10 PM & 26.138 & 26.752 & 24.855 & 27.525 & 26.39 & \begin{tabular}{|l|l|}
46.425 \\
\end{tabular} & \begin{tabular}{|l|}
48.924 \\
\end{tabular} & 12.996 & 34.174 & -1.219 & & 40.777 & 0.09 & 14.643 & 0.004 & 754.45 & \begin{tabular}{|l|l|}
12.5742 \\
\end{tabular} & \begin{tabular}{|l|}
47.5 \\
\end{tabular} & 10.0 & 40.3 & & \begin{tabular}{|l|}
0.013 \\
\end{tabular} & & & & $\begin{array}{l}0.28 \\
0.28 \\
\end{array}$ \\
\hline 780 & 6/14/2004 & 9:50:10 PM & & 26.746 & 24.839 & 27.494 & 26.414 & 46.589 & $\begin{array}{lll} & 49.379 \\
\end{array}$ & \begin{tabular}{|l|l|}
13.014 \\
\end{tabular} & $\begin{array}{l}34.26 \\
\end{array}$ & \begin{tabular}{|l|l|}
5 & -1.225 \\
\end{tabular} & & 51.784 & 0.091 & \begin{tabular}{|l|l|}
14.643 \\
\end{tabular} & 0.004 & 755.45 & \begin{tabular}{|l|l|}
12.5908 \\
\end{tabular} & \begin{tabular}{|l|}
47.9 \\
\end{tabular} & & & & & 0.014 & & & $\begin{array}{l}0.28 \\
0.29\end{array}$ \\
\hline & $6 / 14 / 2004$ & 9:51:10 PM & 26.122 & 26.74 & 24.848 & \begin{tabular}{|l|}
27.538 \\
\end{tabular} & 26.428 & \begin{tabular}{|l|l|l}
46.433 \\
4
\end{tabular} & \begin{tabular}{|l|l|}
48.899 \\
\end{tabular} & $\begin{array}{l}12.955 \\
\end{array}$ & 34.239 & -1.225 & & $\begin{array}{l}52.72 \\
\end{array}$ & \begin{tabular}{|l|}
0.09 \\
\end{tabular} & $\begin{array}{l}14.643 \\
\end{array}$ & & 756.45 & \begin{tabular}{|l|}
12.6075 \\
\end{tabular} & \begin{tabular}{|l|}
47.4 \\
\end{tabular} & 12.9 & & & & & & & \\
\hline 78 & $6 / 14 / 2004$ & 9:52:10 PM & 26.127 & 26.745 & 24.868 & \begin{tabular}{|l|l|}
27.573 \\
\end{tabular} & 26.508 & 46.406 & 48.623 & 12.908 & & -1.222 & -7.4 & 57.679 & 0.09 & 14.643 & & & & & & & & & & & & \\
\hline & & & & & & & & & & & 34.346 & & & & & & & & & 47.4 & & & & & & & 0.333 & \\
\hline 784 & 6/144/2004 & 9:54:10 PM & 26.147 & 26.761 & 25.009 & 27.639 & 26.644 & 46.479 & 8.992 & 12.838 & 34.342 & -1.219 & & 46.609 & 0.09 & 14.643 & & 759.45 & 5575 & 47.5 & 11.4 & & $2.786 \mathrm{~s}-\mathrm{s}$ & 0.013 & . .013 & 500 & 0.332 & \\
\hline & 6/14/2004 & 9:55:10 PM & & 26.763 & $0.071>>1$ & 27.66 & & 46.182 & 48.644 & 1276 & 34.207 & -1.22 & & 41.98 & (2) & 14.643 & & ans. & 12.6742 & 47.2 & & & & & & & & \\
\hline 178 & $6 / 14 / 2004$ & 9:56:10 PM & 26.166 & 26.764 & 24.978 & 27.637 & 26.707 & 46.796 & 49.29 & 12.873 & 34.672 & -1.222 & & 52.583 & 0.089 & 14.643 & 0.004 & 761.45 & 12.6908 & 47.8 . & & & & 0.013 & 0.013 & 0.000 & 0.326 & \\
\hline 1787 & $6 / 14 / 2004$ & 9:57:10 PM & 26.173 & 26.777 & 24.96 & 27.655 & 26.6 & 46.367 & 48.838 & 12.758 & 34.369 & -1.219 & & 46.231 & 0.09 & 14.643 & & 762.45 & & 47.4. & & & & & & 0.000 & 0.333 & \\
\hline 188 & $6 / 14 / 2004$ & 9:58:10 PM & 26.175 & 26.764 & 24.922 & 27.612 & 26.637 & 46.301 & 48.816 & $12.731 \mid$ & 34.284 & -1.222 & & 48.186 & 0.09 & 14.643 & & 763.45 & 12.7242 & 47.3 & 11.8 & & & 0.013 & 0.013 & 0.000 & 0.334 & \\
\hline$\sqrt{70}+2>$ & $6 / 1442004$ & 9:59:10 PM & 26.178 & 26.776 & 24.925 & $27.604]$ & & & 49.294] & $12.828 \mathrm{~g}$ & 34.375 & -1.2222 & & & 0.09 & 14.643 & & 764.45 & & 47.88 & & & & & & .000 & & \\
\hline 190 & $\frac{0.1142004}{6-1 / 2004}$ & 10.00 & 20.184 & $26.7 / 2$ & 24.99 & 27.59 & & 40.360 & 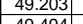 & 12.87 & 34.1399 & $\begin{array}{ll}-1.222 \\
1.220\end{array}$ & & 48.56 & 0.091 & 14.0435 & & & $\frac{12.25 / 5}{12.753}$ & & & & & & 0.014 & & 0 & \\
\hline & $\frac{0.141 / 20}{6 / 1420}$ & $\frac{1.001 .10}{10.0 \mathrm{PM}}$ & $\frac{20.175}{26.162}$ & 20.100 & & & & $\frac{46.643}{46138}$ & 告.9444 & $\frac{12.944}{12767}>0$ & $\frac{34.356}{34129}$ & 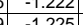 & & $\frac{45.42}{14.410}$ & & 告14.643 & & 67 & & 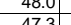 & & & & & 0.0 & & & \\
\hline$\frac{193}{793}$ & 6 & 10.0.0. & $\frac{20.105}{26165}$ & 26.759 & 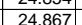 & 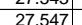 & & & & 12802 & $\begin{array}{l}\frac{34.129}{34.379} \\
\end{array}$ & $-\frac{-1.24}{-122}$ & & 年15.454 47074 & & $\frac{14.6}{146}>\mathrm{C}$ & & 768 & & $\frac{41 .}{47 .}$ & & & & & .013 & & & 0.28 \\
\hline 794 & $6 / 14 / 2004$ & 10:04:10 PM & 26.181 & 26.779 & 24.863 & 27.523 & & 46.238 & 48.853 & 12.875 & 34.075 & -1.219 & & 49.156 & 0.09 & 14.643 & & $\frac{769.44}{769}$ & 12.8242 & 47.4 & 12 & & 2.769 & & 0.013 & & 0.335 & $\frac{0.28}{0.29}$ \\
\hline 795 & $6 / 14 / 2004$ & 10:05:10 PM & 26.172 & 26.765 & 24.854 & 27.478 & 26.4 & 46.444 & 49.299 & 12.83 & 34.285 & -1.219 & & 46.357 & 0.089 & 14.643 & & 770.45 & 12.8408 & 47.8 & 11.4 & & 2.783 & 0.013 & 0.013 & 0.000 & 0.330 & \\
\hline 796 & $6 / 14 / 2004$ & 10:06:10 PM & 26.173 & 26.771 & 24.854 & 27.454 & 26.4 & 46.431 & 49.139 & 12.83 & 34.342 & -1.222 & & 49.039 & 0.089 & 14.643 & & 771.45 & 12.8575 & 47.7 & 12.0 & & 2.785 & 0.013 & 0.013 & .000 & 330 & \\
\hline & 6/144/2004 & & & 26.76 & & & & & & & & & & & 0.09 & & & & & & & & & & & & & \\
\hline 798 & $6 / 14 / 2$ & 10:07. & 26.157 & 26.755 & 24.849 & 27.404 & & 46.66 & 499.777 & 12.917 & 34.356 & $-1.22 \mathrm{~s}-2$ & & 48.64 & & 14.643 & & & 72 & $\overline{48.3}$ & & & 2.79 & & 10 & & 0.333 & \\
\hline 19 & & & & & 24.854 & & & $\begin{array}{lll}46.664 \\
\end{array}$ & & & 34.479 & & & & & & & & & & & & & & & & & \\
\hline & 6/114/2004 & 10:09:57 PM & 26.149 & 26.752 & & 27.38 & & 46.255 & 48.917 & 12.825 & 34.139 & -1.22 & & 48. & 09 & \begin{tabular}{|c|c|c|}
14.643 \\
\end{tabular} & & 775.2. & 12.9206 & 47.4 & $\frac{11.8}{11.8}$ & & & & & & & \\
\hline 801 & 6/114/2004 & 10:10:5 & 26.176 & 26.78 & 24.868 & 27.408 & 26.4 & 46.249 & 48.913 & 12.779 & 34.227 & -1.219 & & 45.433 & 0.089 & \begin{tabular}{ll|l}
14.643 \\
\end{tabular} & & 776.23 & 12.9372 & 47.4. & 11.1 & 40 & S.774 & 0.013 & 0.013 & 0.000 & 0.331 & \\
\hline & 6/14/12004 & 10:11:57 PM & 26.157 & 26.756 & 24.859 & 27.349 & & 46.618 & 49.469 & 12.848 & 34.473 & -1.21 & & & & 14.643 & & & & 48.0 & 122 & & 2.795 & & 0.014 & & & \\
\hline 803 & $6 / 144 / 26$ & 10:12:5 & 26.149 & 26.747 & 24.836] & 27.341 & & 46.672 & 49.7888 & 12.87 & 34.434 & & & 44.905 & 0.088 & $14.6 \mathrm{C}$ & & 7778. & 12.9706 & 48.3 & & & 796 & & & 000 & 0.325 & \\
\hline 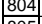 & $6 / 14 / 200$ & $10: 13: 5$ & 26.12 & 26.726 & & 27.304 & & & & 12.763 & & -1.2 .2 & & & & 14. & & & 12.9872 & 47.8 & & & & & & & & \\
\hline & $\frac{6144 / 2}{6 / 142}$ & & & 20.126 & 2 & $\mid$ & & & & $\begin{array}{l}12.862 \\
12771\end{array}$ & $\begin{array}{l}34.301 \\
32.305\end{array}$ & -1.129 & & 45.875 & & 14.6 & & & & 47.8 & & & 年 & & & & & \\
\hline 807 & 6 & & 26.141 & $\frac{20.11}{26705}$ & $\frac{24.454}{24.98}$ & 年 & & 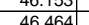 & 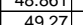 & 12756 & & -1.2 & & 55192 & & 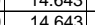 & & & & $\begin{array}{l}474.48 \\
478\end{array}$ & 135 & & 2786 & 0.013 & & & 0333 & \\
\hline & $6 / 14 / 2004$ & & & 26.7 & 24898 & $27288 \mid$ & & & 49.477 & 1276 & 34 & -12 & & & & 146 & & & & & & & & 0.010 & & & & \\
\hline 805 & $6 / 14 / 2004$ & $10: 18: 57 \mathrm{PM}$ & 26.157 & 26.711 & 24.909 & 27.304 & 26.359 & 46.607 & 49.487 & 12.743 & 34.557 & -1.225 & -7. & 50.301 & 0.089 & 14.643 & & 784.23 & 13.0706 & 48.0 & 12.3 & & 2.798 & 013 & 0.013 & 0.000 & 0.328 & \\
\hline & $6 / 14 / 2 C$ & 10:19:5 & 26.142 & 26.696 & 24.884 & $\begin{array}{l}27.314 \\
7214\end{array}$ & & 46.5 & $\begin{array}{l}49.62 \\
9.459\end{array}$ & \begin{tabular}{ll|}
12.841 \\
12.71
\end{tabular} & & -1.216 & & 41.801 & 0. & $\begin{array}{ll}14.643 \\
1\end{array}$ & & & & 48.1 & & & & & & & 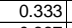 & \\
\hline 81 & & 10:20: & 26.132 & 26.686 & 24.869 & 27.244 & & & & 12.714 & & -1.222 & & 49.928 & & & & & & & & & & & & & & \\
\hline & $0.1 / 4 / 2 / 2$ & & & & 24.841 & & & & & & & & & 45.1 & & 14. & & & & 48. & & & & & & & & \\
\hline$\frac{81}{81}$ & & & $\frac{20.133}{26.11}$ & & 24.87 & 2.7.253 & & & - & $\frac{12.2}{127}$ & & & & & & & & & & & & & & & & & & \\
\hline & $\frac{0141 / 2}{6 / 14 / 2}$ & & & & & & & & & $\frac{14.616}{1278}$ & & & & & & & & & & & & & & & & & 230 & \\
\hline 816 & $6 / 1 / 14 / 20$ & 10.25 .5 & & 26. & 24.794 & 27.119 & & & & & & & & & & & & & & & & & & & & & & \\
\hline 817 & $6 / 14 / 20$ & 10:26:57 PM & 26.107 & & 24.803 & 27.224 & & & & 12.7 & & -1.2 & & & & 14. & & & & & & & & & & & & \\
\hline 818 & $6 / 14 / 2$ & & 26.09 & 26.6 & 24.822 & 27.193 & & & & 12.7 & & & & & & & & & & & & & & & & & & \\
\hline & $6 / 14 / 2$ & & & & & 27.167 & & & & \begin{tabular}{l|l|l|}
12.7 \\
\end{tabular} & & & & & & & & & & & & & & & & & & \\
\hline & $6 / 14 / 2$ & & & & 24.832 & 27.192 & & & & & & & & & & & & & & & & & & & & & & \\
\hline & & & & & & 27.156 & & & & 12.7 & & & & & & & & & & & & & & & & & & \\
\hline & $6 / 14 / 2$ & 10:31: & 26.05 & 26.597 & 24.795 & 27.17 & & 46.2 & 49. & 12.72 & & -1.22 & & & & & & & & & & & & & & & & \\
\hline & $6 / 14 / 2$ & 10:32:5 & & 26.5 & 24.79 & 27.27 & & & & 12.7 & & & & & & 14. & & & & & & & & & & & & \\
\hline 824 & 6/114/2004 & $10: 33: 5$ & 26.06 & 26.6 & 24.81 & 27.32 & & & & $12.8 \mathrm{~T}$ & & -1.2 & & 46. & & 14. & & & & 48.1 & 11.3 & & & & & & 0.328 & \\
\hline & $6 / 14 / 2$ & & & & 24.8 & 27.375 & & & & 12.7 & & -1.2 & & & & 14. & & & & & & & & & & & & \\
\hline 82 & $6 / 14 / 4$ & & 26.0 & & 24.772 & 27.422 & & & & 12.733 & & & & & & & & & & & & & & & & & 0.328 & \\
\hline & $6 / 141$ & & & & 2.00 & & & & & & & & & & & & & & & & & & & & & & & \\
\hline & $0 / 414$ & & & & 2.170 & & & & & $\begin{array}{l}12.134 \\
10771\end{array}$ & & & & & & & & & & & & & & & & & & \\
\hline 830 & $\frac{1141 / 20}{6 / 14 / 20}$ & & 2605 & $\begin{array}{l}2.0305 \\
26.59\end{array}$ & 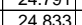 & | & & 46. & 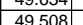 & 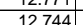 & $\begin{array}{l}34.3 / 9 \\
34.073\end{array}$ & $\frac{-1.2}{-12}$ & & 44 & & 146 & & & & 40.4 & 00 & & & & & & 年 & \\
\hline & $6 / 14 / 2$ & & & 26.58 & 24.928 & 27.373 & & & & 12.65 & & & & & & & & & & & & & & & & & & \\
\hline 832 & $6 / 14 / 20$ & 10:41:5 & 26.0 & 26.5 & 24.9 & 27.355 & & & & 12.76 & 34. & & & & & 14.6 & & & & 48.1 & & & & & & & 0.324 & \\
\hline & $6 / 14 / 2$ & & 26.0 & 26.5 & 24.89 & 27.34 & & & & \begin{tabular}{|l|l|l|l|}
126 \\
\end{tabular} & $34.5 \mathrm{k}$ & & & 41.2. & & 14. & & & & & & & & & & & & \\
\hline 834 & $6 / 14 / /$ & & & & 24.885 & & & & & $\overline{12.4}$ & & & & & & & & & & & & & & & & & & \\
\hline & & & & & & 27.305 & & & & $12.3 \mathrm{r}$ & & & & & & & & & & & & & & & & & & \\
\hline & & & & 26.581 & 24.844 & & & & & $12.3 \mathrm{~s}$ & & & & & & & & & & & & & & & & & & \\
\hline 83 & $6 / 14 /$ & & & & 24.83 & 27.2 & & 46. & & 12.3 & 34.8 & & & & & & & & & & & & & & & & & \\
\hline & $6 / 144 / 200$ & 10:47:57 PM & 26.069 & 26.572 & 24795[ & 27.295| & & & & 12.434 & 34.9 & -1.22 & & & & 14.6 & & & & \begin{tabular}{l|l|}
48.4 \\
\end{tabular} & & & & & & & & \\
\hline & $6 / 14 / 2$ & & 26.053 & & . & 27 & & 46.6 & & $2.407 \mathrm{Z}$ & 34.93 & -1.21 & & 15.13 & 0.0 & $14.64 \mathrm{~s}$ & & & & & & & & & & & & \\
\hline 340 & $6 / 14 / 20$ & 10:49:57 PM & .054 & 26.562 & $24.765 \mid$ & 27.275 & & $46.423 \mid$ & $|49.819|$ & 12.404 & 34.685 & \begin{tabular}{|l|l|l|} 
& -1.222 \\
\end{tabular} & & $9.164]$ & 0.088 & 14.643 & & 815.23 & 3.5872 & $48.3 \mid$ & 12.0 & & & . 013 & .013 & 0.000 & 0.326 & \\
\hline
\end{tabular}


WSRC-TR-2005-00105, REVISION 0

SRNL-RPP-2005-00012, REVISION 0

RUN \# 3.03A AND B; FIRST AND SECOND HALF OF SLURRY DEWATERING - CONT.

\begin{tabular}{|c|c|c|c|c|c|c|c|c|c|c|c|c|c|c|c|c|c|c|c|c|c|c|c|c|c|c|c|c|}
\hline & & & D & $\mathrm{E}$ & $\mathrm{F}$ & G & $\mathrm{H}$ & $\mathrm{J}$ & $\mathrm{K}$ & $\begin{array}{ll} \\
\end{array}$ & M & $\mathrm{N}$ & $\mathrm{O}$ & Q & $\begin{array}{ll} \\
\end{array}$ & $\mathrm{s}$ & $\mathrm{T}$ & $U$ U $\quad v$ & w & \begin{tabular}{|l|}
$x$ \\
\end{tabular} & $\mathrm{Y}$ & $z$ & $\mathrm{AA}$ & & & & & AF \\
\hline & $6 / 14 / 2004$ & 10:50:57 PM & 26.055 & 26.558 & 24.786 & 27.241 & 26.342 & 46.753 & 50.13 & \begin{tabular}{|l|l|}
12.414 \\
\end{tabular} & $\begin{array}{l}35.046 \\
\end{array}$ & -1.222 & $\begin{array}{l}-7.352 \\
\end{array}$ & 50.727 & \begin{tabular}{|c|}
0.088 \\
\end{tabular} & \begin{tabular}{|l|l|}
14.643 \\
\end{tabular} & 0.004 & 816.23 & \begin{tabular}{|l|l|}
3 & 13.6039 \\
\end{tabular} & \begin{tabular}{|l|}
48.7 \\
\end{tabular} & 12.4 & 40.9 & 2.820 & 0.013 & 0.013 & 0.000 & 0.323 & \\
\hline & $6 / 14 / 2004$ & 10:51:57 PM & 26.035 & 26.553 & 24.756 & 27.241 & 26.307 & 46.5 & \begin{tabular}{|l|}
49.862 \\
\end{tabular} & 12.339 & $\begin{array}{l}34.835 \\
\end{array}$ & -1.219 & & \begin{tabular}{|l|l|}
48.449 \\
\end{tabular} & \begin{tabular}{|c|}
0.089 \\
\end{tabular} & \begin{tabular}{|l|}
14.643 \\
\end{tabular} & 0.003 & 817.23 & \begin{tabular}{|l|l|}
3 & 13.6206 \\
\end{tabular} & 48.4 & 11.9 & & $\begin{array}{l}7.2804 \\
\end{array}$ & & & & & \\
\hline & $6 / 14 / 2004$ & 10:52:57 PM & 26.029 & 26.557 & 24.745 & $\begin{array}{l}27.211 \\
27171\end{array}$ & 26.301 & \begin{tabular}{|l|}
46.649 \\
\end{tabular} & \begin{tabular}{|l|}
49.931 \\
\end{tabular} & \begin{tabular}{|l|}
12.349 \\
327
\end{tabular} & 35.073 & -1.225 & & \begin{tabular}{|l|l|}
44.786 \\
\end{tabular} & \begin{tabular}{|l|l|}
0.088 \\
\end{tabular} & 14.643 & 0.004 & & \begin{tabular}{|l|l|l|}
3 & 13.6372 \\
\end{tabular} & 48.5 & 11.0 & & & & & 0.000 & & \\
\hline 344 & $6 / 14 / 2004$ & $\begin{array}{ll}0: 53: 57 \mathrm{PM} \\
0: 51: 57\end{array}$ & 26.024 & \begin{tabular}{l|}
26.542 \\
3550
\end{tabular} & \begin{tabular}{rl|}
24.73 \\
4.731
\end{tabular} & $\begin{array}{l}27.171 \\
27.96\end{array}$ & 26.296 & & \begin{tabular}{|l|l|}
49.761 \\
5.16 \\
\end{tabular} & & $\begin{array}{l}34.652 \\
3.969\end{array}$ & \begin{tabular}{|l|l|} 
& -1.225 \\
\end{tabular} & & & & \begin{tabular}{|l|l|}
14.643 \\
143
\end{tabular} & $\begin{array}{l}0.003 \\
003\end{array}$ & & \begin{tabular}{|l|l|}
3 & 13.6539 \\
12670
\end{tabular} & 48.3 & & & & & 0.013 & 0.000 & $\begin{array}{l}0.327 \\
0320\end{array}$ & \\
\hline 846 & $6 / 1 / 4 / 2004$ & $\begin{array}{ll}10: 54: 57 \mathrm{PM} \\
10.555\end{array}$ & $\frac{26.035}{26.026}$ & \begin{tabular}{l|}
26.538 \\
26.545
\end{tabular} & $\frac{24.731}{24733}$ & $27.186 \mid$ & $\begin{array}{l}26.312 \\
26289\end{array}$ & \begin{tabular}{|c|}
46.659 \\
46.338
\end{tabular} & \begin{tabular}{|l|}
50.163 \\
49.991 \\
\end{tabular} & $\begin{array}{r}12.377 \\
12.362\end{array}$ & \begin{tabular}{|l|l|}
34.966 \\
3466
\end{tabular} & $\begin{array}{l}-1.222 \\
-1.225\end{array}$ & & \begin{tabular}{|r|}
51.686 \\
45.914
\end{tabular} & \begin{tabular}{|c|}
0.087 \\
0.088
\end{tabular} & $\begin{array}{l}14.643 \\
14634\end{array}$ & $\begin{array}{l}0.003 \\
0.033\end{array}$ & $\begin{array}{r}820.23 \\
821.23\end{array}$ & \begin{tabular}{|l|l|} 
& 13.6706 \\
\end{tabular} & \begin{tabular}{|l|l|l|}
48.7 & \\
\end{tabular} & \begin{tabular}{|l|}
12.7 \\
113 \\
\end{tabular} & & 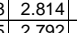 & \begin{tabular}{|l|}
0.013 \\
0013
\end{tabular} & 0.013 & 0.000 & 0.320 & \\
\hline 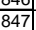 & $6 / 14 / 2 / 2004$ & $\begin{array}{l}10: 55557 \mathrm{PM} \\
10557 \mathrm{PM}\end{array}$ & $\frac{26.026}{26.026}$ & $\frac{26.545}{26.545}$ & \begin{tabular}{|}
24.733 \\
24.733 \\
\end{tabular} & $\begin{array}{l}27.178 \\
27.218\end{array}$ & 26.289 & $\begin{array}{r}46.338 \\
46.05\end{array}$ & \begin{tabular}{|l|}
49.9911 \\
49.493 \\
\end{tabular} & $\begin{array}{l}12.362 \\
12.272\end{array}$ & $\begin{array}{l}34.566 \\
34.506\end{array}$ & $\begin{array}{c}-1.225 \\
-1.219\end{array}$ & $\begin{array}{r}-7.291 \\
-7.279 \\
\end{array}$ & \begin{tabular}{|}
45.914 \\
47.933 \\
\end{tabular} & $\begin{array}{l}0.088 \\
0.088 \\
\end{array}$ & $\begin{array}{l}14.643 \\
14.643\end{array}$ & $\begin{array}{l}0.003 \\
0.004 \\
\end{array}$ & $\begin{array}{l}821.23 \\
822.23\end{array}$ & 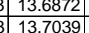 & \begin{tabular}{|c|}
48.5 \\
48.0
\end{tabular} & \begin{tabular}{|l|}
11.3 \\
117 \\
\end{tabular} & $\begin{array}{r}40.5 \\
40.3 \\
\end{array}$ & \begin{tabular}{|l|}
2.792 \\
3
\end{tabular} & \begin{tabular}{|l|}
0.013 \\
0.013
\end{tabular} & $\begin{array}{l}0.013 \\
0.013\end{array}$ & 0.000 & 0.326 & $\begin{array}{l}0.28 \\
0.28\end{array}$ \\
\hline 848 & $6 / 14 / 2004$ & 10:57:57 PM & 26.011 & 26.54 & 24.743 & 27.238 & & & \begin{tabular}{|l|}
50.453 \\
\end{tabular} & 12.406 & \begin{tabular}{|}
4.500 \\
34.57
\end{tabular} & & & $\begin{array}{l}4.9533 \\
55.338\end{array}$ & $\begin{array}{l}0.088 \\
0.086\end{array}$ & $\begin{array}{l}14.045 \\
14.643 \\
\end{array}$ & $\begin{array}{l}0.004 \\
0.004\end{array}$ & $\begin{array}{r}822.23 \\
823.23\end{array}$ & \begin{tabular}{|l|}
3.039 \\
\end{tabular} & $\begin{array}{l}48.0 \\
48.7\end{array}$ & $\frac{11.7}{13.6}$ & & \begin{tabular}{|l|}
2.777 \\
2.790 \\
\end{tabular} & \begin{tabular}{|l|}
0.013 \\
0.013
\end{tabular} & $\frac{0.013}{0.013}$ & $\begin{array}{l}0.000 \\
0.000\end{array}$ & \begin{tabular}{|c|}
0.328 \\
0.319
\end{tabular} & $\frac{0.28}{0.27}$ \\
\hline 849 & 6/14/2004 & 10:58:57 PM & 26.001 & 26.529 & 24.717 & 27.252 & 26.248 & 46.547 & \begin{tabular}{|l|}
50.252 \\
\end{tabular} & 12.425 & 34.763 & 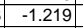 & & 48.132 & 0.088 & 14.643 & 0.004 & 824.23 & \begin{tabular}{|l|l}
3 & 13.7372 \\
\end{tabular} & 48.8 & 11.8 & & & \begin{tabular}{|l|}
0.013 \\
\end{tabular} & 0.013 & & & $\begin{array}{l}0.28 \\
0.28\end{array}$ \\
\hline & $6 / 14 / 2004$ & 10:59:57 PM & 25.996 & 26.52 & 24.713 & 27.238 & 26.249 & 46.259 & \begin{tabular}{|l|l|}
49.724 \\
\end{tabular} & $\begin{array}{l}12.389 \\
\end{array}$ & 34.539 & \begin{tabular}{|l|} 
\\
\end{tabular} & & $\begin{array}{l}47.982 \\
\end{array}$ & 0.087 & $\begin{array}{l}14.643 \\
\end{array}$ & & & \begin{tabular}{|l|l|}
3 & 13.7539 \\
\end{tabular} & $\begin{array}{l}48.3 \\
\end{array}$ & & & & 0.013 & & & & $\begin{array}{l}0.28 \\
0.28\end{array}$ \\
\hline & $6 / 14 / 2004$ & 11:00:57 PM & & 26.519 & 24.727 & \begin{tabular}{|l|l|}
27.242 \\
\end{tabular} & 26.203 & $\begin{array}{l}46.325 \\
\end{array}$ & \begin{tabular}{|l|}
49.583 \\
\end{tabular} & \begin{tabular}{|l|l|}
12.407 \\
\end{tabular} & \begin{tabular}{|l|}
34.693 \\
\end{tabular} & \begin{tabular}{|l|l|} 
& -1.222 \\
\end{tabular} & -7.2 & & 0.088 & $\begin{array}{l}14.643 \\
\end{array}$ & $\begin{array}{l}0.004 \\
\end{array}$ & \begin{tabular}{|l|l|}
826.23 \\
\end{tabular} & \begin{tabular}{|l|}
3.7706 \\
\end{tabular} & 48.1 & 12.2 & & & & & & & $\frac{0.28}{0.28}$ \\
\hline & $6 / 14 / 2004$ & 11:01:57 PM & 25.985 & 26.513 & 24.731 & 27.226 & 26.19 & 46.589 & 49.962 & $\begin{array}{ll}12.436 \\
\end{array}$ & 34.874 & -1.222 & & 44.617 & 0.088 & $\begin{array}{l}4.643 \\
\end{array}$ & 0.004 & & & & & & & & & & & \\
\hline & & & & & & & & & & & & & & & & & & & & \begin{tabular}{|l|l|}
48.4 \\
\end{tabular} & & & & & & & & \\
\hline 85 & $6 / 14 / 2004$ & 11:03:57 PM & 25.987 & 26.51 & 24.743 & 27.199 & & 46.355 & 50.144 & 12.424 & 34.551 & -1.222 & & 47.819 & 0.0 & \begin{tabular}{l|l}
14.643 \\
\end{tabular} & & 829.23 & 13.8206 & 48.7 & & & 2.78 & 0.013 & . .013 & 000 & 0.323 & \\
\hline & $6 / 114 / 2004$ & 11:04:57 PM & & & 24.758 & 27.189 & & & & $\begin{array}{ll}12.402 \\
\end{array}$ & 34.703 & -1.22. & & & & 14.643 & & & & 48.6 & & & 2.795 & & & & & \\
\hline & $6 / 14 / 2004$ & 11:05:57 PM & 25.986 & 26.505 & 24.753 & 27.208 & 26. & 46.464 & 50.119 & 12.461 & 34.664 & -1.219 & & 46.753 & 0.087 & 14.643 & 0.004 & 831.23 & 13.8539 & $\begin{array}{ll}48.6 \\
\end{array}$ & 11.5 & 40 & & 0.013 & 0.013 & 0.000 & 0.322 & \\
\hline & $6 / 14 / 2004$ & 11:06:57 PM & 25.986 & 26.49 & 24.758 & 27.178 & & 46.786 & 50.265 & 12.507 & 35.036 & -1.219 & & 49.079 & & 14.643 & & 832.23 & 13.8706 & 48.8 & & & 2.821 & & & 0.000 & 0.323 & \\
\hline & 6/14/2004 & 11:07:57 PM & 25.979 & 26.483 & 24.741 & 27.181 & & 46.558 & 50.229 & 12.525 & 34.716 & -1.219 & & 53.461 & 0.087 & 14.643 & & 833.23 & 13.8872 & 48.8 & 13.1 & & 2.802 & .013 & 0.013 & 0.000 & 0.322 & \\
\hline & 6/14/2004 & 11:08:5 & 25.974 & & 24.76 & 27.15 & & & & & 34.56 & -1.219 & & & 0.086 & 14.643 & & & & 48.5 & & & & & 0.013 & & $0.319 \mid$ & \\
\hline & $\frac{0.1142004}{6121204}$ & 10.093 & 25.979 & 20.4839 & 24./71 & 27.201 & & 46.412 & 49.8933 & 12.494 & $\begin{array}{l}34.668 \\
3.67\end{array}$ & -1.219 & & & & 14.043 & & & 131.9206 & & 12. & & & & 0.013 & & 0.318 & \\
\hline & 0 & 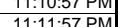 & $\frac{25.984}{25.987}$ & & $\frac{24.81}{2.9214}$ & $\frac{27.196}{27269}$ & & $\begin{array}{l}46.255 \\
46192\end{array}$ & $\frac{49.875}{4069}$ & $\frac{12.463}{1.2241}$ & $\frac{34.521}{32609}$ & $\frac{-1.21}{-121}$ & & 45081 & & $\frac{14.643}{1.643}$ & & & & $\begin{array}{lll}48.4 \\
14.2\end{array}$ & & & & & & & . & \\
\hline 863 & $\frac{1}{6 / 1442004}$ & $\begin{array}{ll}11.125 \\
11.25 \mathrm{PM}\end{array}$ & 25.991 & $\frac{20.47}{26.499}$ & $\mid$ & 27308 & & $\begin{array}{l}40.182 \\
46.603\end{array}$ & $\begin{array}{r}49.000 \\
50366\end{array}$ & $\frac{12.341}{12393}$ & $\begin{array}{l}34.009 \\
34.882 \\
\end{array}$ & $\frac{-1.21}{-122}$ & & $\frac{45.961}{49237}$ & & $\frac{14.0}{146}$ & & & & 48. & & & & & .013 & & & \\
\hline 864 & $6 / 14 / 2004$ & 11:13:57 PM & 26.006 & 26.464 & 24.898 & 27.343 & & 46.34 & 50.047 & 12.421 & 34.609 & -1.222 & & 45.966 & 0.086 & 14.643 & & 839.2 & $\frac{13.9872}{13.972}$ & 48.6 & $11.3 \mathrm{C}$ & & 2.791 & & 0.013 & & 0.318 & \\
\hline 865 & $6 / 14 / 2004$ & 11:14:57 PM & 26.01 & 26.458 & 24.852 & 27.377 & 26.4 & 46.485 & 50.047 & 12.428 & 34.765 & -1.22 & & 48.649 & 0.086 & 14.643 & & & & 48.6 & $1.9 \mathrm{C}$ & & 2.801 & 0.013 & 0.013 & 0.000 & & \\
\hline & $6 / 14 / 2004$ & 11:15:57 PM & 26.022 & 26.475 & $24.868 \mid$ & 27.358 & & 46.172 & 49.856 & 12.339 & 34.574 & -1.219 & & 47.85 & 0.087 & 14.643 & & 841.2 & 14.0206 & 48.4 & 11. & & 2.784 & $\overline{013}$ & 0.013 & .000 & & \\
\hline & 6/14/2004 & 11:16:57 PM & & & & & & & & & 34.699 & & & & & & & & & & & & & & & & & \\
\hline & $6 / 14 / 2$ & $11: 17: 57 \mathrm{PM}$ & 26.027 & 26.465 & $\begin{array}{l}24.843 \\
24\end{array}$ & 27.383 & & 46.16 & 50.18 & 12.388 & 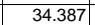 & -1.22 & & .736 & 0.6 & $\begin{array}{l}14.643 \\
\end{array}$ & & & 4.0539 & 48.7 & 12. & & & & 20 & & 320 & \\
\hline & & & & 26.46 & & & & & 49.918 & & & & & 50.199 & & & & & & & & & & & & & & \\
\hline & 6/14/2004 & 11:19:57 PM & 26.026 & 26.469 & 24.802 & 27.348 & & $\begin{array}{l}46.412 \\
\end{array}$ & 50.275 & 12.427 & $34.65 \varepsilon$ & -1.22 & & 45.831 & & \begin{tabular}{|l|l|l|l|l|}
1433 \\
\end{tabular} & & 845.23 & 14.08 & 48.8 & 11.2 & & & & & & j.314 & \\
\hline 8711 & 6/114/2004 & 11:20:5 & 26.015 & 26.453 & 24.777 & 27.347 & & 46.213 & 49.995 & 12.376 & 34.529 & -1.222 & & 46.336 & 0.086 & 14.643 & & 846.23 & 14.1039 & 48.5 & 11.4 & & & 0.013 & 0.013 & 0.000 & 0.320 & \\
\hline & 6/14/2004 & 11:21:57 PM & & 26.447 & 24.74 & 27.32 & & 46.431 & 50.13 & 12.422 & 34.728 & -1.22 & & 44.7966 & & 14.643 & & & 1206 & 48.7 & 11.0 & & 2.798 & & & & 0.315 & \\
\hline trit & $6 / 14 / 2$ & 11:22:5 & 26.007 & 26.466 & 24.764 & 27.329 & & & 49.5766 & 12.37 & 34.3 & -1.219 & & 51.928 & 0.085 & 14.6 & & 848.2 & 14.1372 & 48.1 & & & & & 013 & 000 & & \\
\hline (1) & $6 / 14 / 2004$ & 11:23:57 PM & 26.022 & 26.466 & 24.789 & 27.269 & & 46.363 & & 12.392 & 34.74 & -1.222 & & 53.1 & & 14. & & & & 48. & & & & & & & & \\
\hline & $\frac{61 / 421}{61 / 12}$ & & $\frac{20.011}{2600}$ & $\frac{20.444}{26499}$ & 2 & 27.248 & & 46.305 & 49.925 & $\begin{array}{l}1.2 .36 \\
12.373\end{array}$ & & -1.226 & & & & 14.643 & & & & $\frac{48.5}{4.98}$ & 11 & & & & & & 年199 & \\
\hline 877 & 6 & 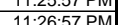 & $\frac{20.010}{26011}$ & $\frac{2.449}{26.459}$ & 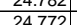 & 27208 & & $\begin{array}{l}46.44 \\
647\end{array}$ & 50425 & & & & & & & . & & & & 40.0 & 112 & & & & & & . 0.314 & \\
\hline 878 & $6 / 14 / 2004$ & 11.27.5 & $\frac{2011}{26}$ & 26.453 & 24761 & 27.157 & & 46 & 50.316 & 12398 & & -12 & & & & 146 & & & & 48.8 & & & & & & & 0.314 & \\
\hline & $6 / 14 / 2004$ & $11: 28: 5$ & 25.994 & 26.447 & $\mid 24.74$ & 27.161 & & 46.247 & 50.051 & 12.407 & 34.557 & -1.225 & & $\begin{array}{l}46.144 \\
\end{array}$ & 0.086 & 14.643 & & \begin{tabular}{|c|c|c|}
854.23 \\
\end{tabular} & \begin{tabular}{|l|l|}
314.2372 \\
\end{tabular} & 48.6 & $\frac{1.4}{11.3}$ & & 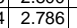 & 13 & 0.013 & 0.000 & $\frac{0.320}{0.0320}$ & \\
\hline & $6 / 14 / 2004$ & 11:29:5 & & & & 27.175 & & 46.454 & & \begin{tabular}{|l|l|}
12.445 \\
\end{tabular} & & & & & & & & & & & & & & & & & & \\
\hline & $6 / 14 / 2$ & $11: 30: 5$ & 26.007 & 26.456 & 24.789 & 27.199 & & $\begin{array}{lll}46.533 \\
\end{array}$ & 50.428 & 12.458 & & -1.219 & & 46.087 & & $\begin{array}{l}14.643 \\
\end{array}$ & & & & 49.0 & 1 & & & & & 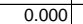 & 0317 & \\
\hline & $6 / 14 / 20$ & & & 26.434 & 24.767 & 27.142 & & & . 3.372 & & & & & & & 14.6 & & & & & & & & & & & & \\
\hline & $6 / 14 / 2$ & & 25.979 & 26.4 & 24.76 & 27.161 & & & 50.4 & 12.4 & & -1.22 & & & & 14. & & & & 49.0 & 12.4 & & & & & & & \\
\hline & $6 / 14 / /$ & & 25.99 & & 24.7 & 27.1 & & & & 12.4 & & & & & & & & & & & & & & & & & & \\
\hline & $6 / 14 / 2$ & 11:34:5 & 25.9 & 26. & 24.91 & 27.189 & & & 49.7 & & & & & & & & & & & $\frac{4 \varepsilon}{4 \varepsilon}$ & 11 & & & & & & .313 & \\
\hline & $6 / 14 / 200$ & 11:35: & 25.996 & 26.42 & 24.918 & 27.183 & & & 50.115 & 12.32 & & -1.2 & & & & 14. & & & & 48. & I0. & & & & & & & \\
\hline & $6 / 14 / 2$ & $11: 36: 5$ & & 26.419 & & 27.1177 & & & \begin{tabular}{|l|l|} 
\\
\end{tabular} & 12.323 & & -1.2 & & & & 14. & & & & & 11.2 & & & & & & & \\
\hline 888 & $6 / 14 / 2 / 2$ & & 26.015 & 26.419 & 24.8977 & 27.157 & & & 49.958 & 12.388 & & & & & & 14. & & & & 48.5 & & & & & & & 315 & \\
\hline & $6 / 14 / 2$ & 11:38: & & & 24.8 & 27.14 & & & & 12.3 & & -1.2 & & & & & & & & 48 & & & & & & & & \\
\hline & $6 / 14 \mid$ & & & & 24.8 & 27.14 & & & S0.312 & 12. & & & & & & & & & & & & & & & & & & \\
\hline & & & 25091 & 20 & & & & & & $\begin{array}{l}12.3017 \\
12220\end{array}$ & & -1.2 & & & & & & & & & & & & & & & & \\
\hline & $6 / 1426$ & & & & $\mid$ & 年, 27098 & & & 50. & $\frac{12.2 .2}{122}$ & & & & & & & & & & 4 & & & & & & & & \\
\hline 894 & 6/14/2004 & & 25.995 & 26.3 & 24.791 & 27.091 & & & 50.244 & 12.15 & & -1.2 & & & & 14.6 & & & & 48.8 & 10.8 & & & & & & 0 & \\
\hline & $6 / 14 / 2$ & & 25.989 & & & 27.10 & & & & 12.198 & & & & & & & & & & & & & & & & & & \\
\hline 896 & $6 / 14 / / 2$ & & 25.9 & 26. & 24.758 & 27.104 & & & 50.0 & 12.2 & & -1.2 & & & & & & & & 48.6 & 10. & & & & & & & \\
\hline & & & & & & & & & & & & & & & & & & & & & & & & & & & & \\
\hline & $6 / 14 / 2$ & & & & 24.742 & 27.137 & & & & $12.1: \mathrm{r}$ & & & & & & & & & & & & & & & & & & \\
\hline & & & 25.991 & 26.37 & & & & & & & & & & & & 14. & & & & & & & & & & & 0.315 & \\
\hline & $6 / 14 / 20$ & 11:49:5 & 25.98 & 26.369 & 24.752 & 27.217 & & 46. & 50.3 & 12.1 & 34. & -1.22 & & 43 & & 14. & & & & 48.9 & $10.7 \mathrm{Y} \quad \mathrm{l}$ & & 278 & & & & 0.312 & \\
\hline & $6 / 14 / 20$ & 11:50 & 25.99 & 26.37 & 24.73 & 27.2 & & 46. & 50.1 & 12.1 & & -1.2 & & & & 14. & & & & & 11 & & & & & & & \\
\hline 902 & $6 / 14 / 200$ & 11:51:5 & 26.007 & $26.33^{2}>$ & 24.768 & $27.28 \mathrm{~s}-\mathrm{r}$ & & & 50.2 & 12.1 & 34. & -1.2. & & & & 14. & & & & 48.8 & 11. & & & & & & 0.316 & \\
\hline & $6 / 14 / 2 / 2$ & 11:52:5 & 25.992 & 26.3 & 24.733 & 27.304 & & 46.184 & 50.296 & 12.203 & & $-1.2 .2>>3$ & & 41. & & 14.6 & & & & & & & & & & & & \\
\hline$\frac{904}{905}>2$ & $6 / 144 / 2$ & & $25.8 .7>3$ & 26.3 & 24. & 27.268 & & & & & & & & & & & & & & & 10.8 & & & & & & 08 & \\
\hline 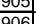 & $0 / 14 / 2$ & $\begin{array}{lll}11.54 \\
11.52\end{array}$ & & 20 & 24.104 & 27.228 & & 40.6 & & 12.248 & & & & & & & & & & & 10.0 & & & & & & 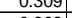 & \\
\hline$\overline{9 c}>-1$ & & & (25.905 & $\frac{2.3}{262}>0$ & 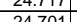 & 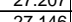 & & & 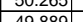 & 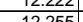 & & & & & & & & & & (40.6 & & & & & & & & \\
\hline 90 & $\frac{1}{61 / 4 / 200}$ & 11.57. PM & 25968 & $\frac{2.00}{2638}$ & 20 24694 & 20.148 & & 46.224 & 500 & 年 & 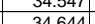 & 1210 & & $46.88>-2$ & & 146 & & & 1472 & 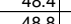 & & & & & & & (1) & \\
\hline & $6 / 14 / 200$ & $\frac{11: 58 \cdot 5}{11.58}$ & $\frac{25.956}{25.956}$ & (l) & $702-2+2$ & 193 & & 46.47. & 0.555 & 12.339 & 34.865 & 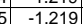 & & 48.24 & & 14.64 & & & & & & & & & & & 307 & \\
\hline 10] & 6/14/2004 & 11:59:57 PM & 5.961 & 26.384 & 24.732 & 27.208 & & \begin{tabular}{|l|l|}
46.074 \\
\end{tabular} & | 50.343 & 12.33 & 34.43 & $\begin{array}{l}3 \\
3\end{array}$ & & 49.527 & 0.082 & $\begin{array}{ll}14.643 \\
\end{array}$ & & 885.23 & 3) 14.7539 & 48.9 & $12.11+2$ & & & & 1012 & 0.000 & 0.306 & \\
\hline
\end{tabular}


WSRC-TR-2005-00105, REVISION 0

SRNL-RPP-2005-00012, REVISION 0

RUN \# 3.03A AND B; FIRST AND SECOND HALF OF SLURRY DEWATERING - CONT.

\begin{tabular}{|c|c|c|c|c|c|c|c|c|c|c|c|c|c|c|c|c|c|c|c|c|c|c|c|c|c|c|c|}
\hline & & & & & & & & & & & & & & & & & & & & & & & & & & & \\
\hline & $\mathrm{A}$ & $\mathrm{B} \quad \mathrm{C}$ & D & $E$ & $\mathrm{~F}$ & G & $\mathrm{H}$ & $\mathrm{J}$ & $\mathrm{K}$ & $\mathrm{L}$ & $\mathrm{M}$ & $\mathrm{N}$ & $\mathrm{O}$ & Q & $\mathrm{R}$ & $\mathrm{s}$ & $\begin{array}{lll}T \\
\end{array}$ & $\mathrm{v}$ & w & $x$ & $\mathrm{Y}$ & \begin{tabular}{l|l|}
$\mathrm{Z}$ & $\mathrm{AA}$ \\
\end{tabular} & $A B$ & $A C$ & AD & $\mathrm{AE}$ & AF $[A$ \\
\hline & 6/15/2004 & 12:00:57 AM & $\frac{25.954}{25}$ & 26.388 & 24.911 & 27.221 & 26.197 & 46.193 & 50.405 & 12.214 & \begin{tabular}{|l|l|}
4 & 34.722 \\
\end{tabular} & -1.222 & $\begin{array}{r}-7.251 \\
\end{array}$ & 51.569 & 0.082 & 14.643 & 0.003 & 886.23 & 14.7706 & 48.9 & 12.6 & \begin{tabular}{c|c}
40.5 & 2.789 \\
\end{tabular} & & 0.012 & & 0.303 & \\
\hline & $6 / 15 / 2004$ & 12:01:57 AM & 25.974 & 26.403 & 24.926 & 27.211 & 26.217 & 46.798 & 50.725 & 12.346 & 35.192 & -1.219 & & & 0.085 & 14.643 & 0.004 & & 14.7872 & 49.3 & 10.6 & & & & 0.000 & 0.310 & \\
\hline 91 & $6 / 15 / 2004$ & 12:02:57 AM & 25.983 & 26.397 & 24.905 & 27.195 & & 46.634 & 51.021 & 12.32 & 34.97 & -1.216 & & 46.323 & $\begin{array}{ll}0.083 \\
\end{array}$ & 14.643 & 0.004 & 888.23 & 14.8039 & 49.5 & \begin{tabular}{|l|l|}
11.4 \\
\end{tabular} & & & 0.012 & 0.000 & 0.304 & \\
\hline 514 & 6/15/2004 & 12:03:57 AM & 25.983 & 26.381 & 24.869 & 27.169 & 26.18 & $\begin{array}{l}46.58 \\
\end{array}$ & \begin{tabular}{|l|l|}
50.7999 \\
\end{tabular} & 12.321 & 34.97 & -1.222 & -7.236 & $\begin{array}{ll}45.67 \\
\end{array}$ & $\begin{array}{ll}0.084 \\
\end{array}$ & 14.643 & $\begin{array}{l}0.004 \\
\end{array}$ & 889.23 & 14.8206 & \begin{tabular}{|l|}
49.3 \\
\end{tabular} & \begin{tabular}{|l|l|}
11.2 \\
\end{tabular} & 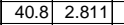 & & $\begin{array}{ll}0.013 \\
\end{array}$ & & 0.308 & \\
\hline 915 & 6/15/2004 & 12:04:57 AM & 26.002 & 26.39 & 24.898 & 27.149 & 26.179 & $\begin{array}{l}46.556 \\
\end{array}$ & 50.333 & 12.259 & 35.066 & -1.219 & -7.239 & 42.323 & 0.084 & 14.643 & 0.004 & 890.23 & 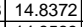 & 48.9 & 10.4 & \begin{tabular}{|l|l|}
40.8 & 2.814 \\
\end{tabular} & 0.013 & 0.013 & 0.000 & 0.308 & 0.26 \\
\hline $\begin{array}{ll}910 \\
917\end{array}$ & 6/15/2004 & $12: 05: 57 \mathrm{AM}$ & $\frac{25.991}{25.996}$ & 26.374 & $\begin{array}{l}24.847 \\
24857\end{array}$ & 27.103 & 26.143 & $\begin{array}{r}46.643 \\
\end{array}$ & 50.893 & $\frac{12.341}{12.231}$ & 35.001 & -1.219 & -7.233 & 52.224 & 0.084 & 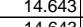 & 0.004 & 891.23 & \begin{tabular}{|l|l|}
14.8539 \\
10706
\end{tabular} & 49.4 & 12.8 & \begin{tabular}{ll|}
40.8 & 2.815 \\
\end{tabular} & 0.013 & 0.013 & 0.000 & 0.308 & 0.26 \\
\hline & & & & & $\begin{array}{l}24.85 \\
24.819\end{array}$ & 27.085 & 26.185 & $\begin{array}{r}46.56 \\
46.572\end{array}$ & 50.951 & $\begin{array}{l}\frac{12.354}{12.302} \\
\end{array}$ & 34.982 & $\frac{-1.2 \angle 2}{-1.222}$ & $\begin{array}{l}-7.236 \\
-7.236\end{array}$ & 48.194 & $\begin{array}{l}0.083 \\
0.083 \\
\end{array}$ & $\begin{array}{l}\frac{14.645}{14.643} \\
\end{array}$ & 0.004 & $\begin{array}{l}892.23 \\
893.23 \\
\end{array}$ & $\begin{array}{l}\frac{14.8}{14.8872} \\
\end{array}$ & $\begin{array}{l}49.5 \\
49.4 \\
\end{array}$ & $\frac{11.9}{11.3}$ & $\begin{array}{l}40.7 \\
40.82 .8091 \\
40.8\end{array}$ & 0.012 & $\frac{0.012}{0.012}$ & & $\frac{0.305}{0.305}$ & $\frac{0.26}{0.26}$ \\
\hline 919 & $6 / 15 / 2004$ & 12:08:57 AM & 25.986 & 26.355 & 24.827 & 27.078 & 26.148 & 46.446 & 50.634 & 12.276 & 34.954 & -1.225 & -7.242 & 49.936 & 0.083 & $\begin{array}{l}14.643 \\
14.643\end{array}$ & & 894.23 & \begin{tabular}{|l|l|l|l}
14.9039 \\
\end{tabular} & 49.2 & 12.2 & $\mid 2.806$ & 0.012 & 0.012 & 0.000 & $\frac{0.036}{0.306}$ & $\frac{0.26}{0.26}$ \\
\hline & & & & & & & 26.128 & & 51.274 & & & & & & & & & & & & & & & & & & 0.26 \\
\hline 92 & 6/15/2004 & 12:10:57 AM & 25.99 & 26.368 & |4.816 & 27.117 & & 46.666 & 51.087 & 12.352 & 34.999 & -1.219 & & 48.127 & 082 & $\begin{array}{l}14.045 \\
14.643 \\
\end{array}$ & & $\begin{array}{l}39.6 \\
896.2\end{array}$ & 1.9372 & 49.6 & & & 012 & 2.012 & 000 & $\frac{0.001}{0.301}$ & \\
\hline 这 & & & & & & & & & & & & & & & & 14.643 & & & & & & & & & & & \\
\hline & $6 / 155 / 2004$ & 12:12:57 AM & 25.984 & 26.352 & 24.79 & 27.141 & & 46.634 & 50.619 & 12.297 & 35.126 & $\frac{-1.219}{-1.219}$ & & 466.84 & 0.082 & 14.643 & & 898.23 & 14.9706 & 49.1 & $\frac{11.5}{11.5}>>$ & $\frac{2.819}{2.89}$ & 0.012 & 0.012 & 0.000 & 0.301 & \\
\hline & 6/15/2004 & 12:13:57 AM & 25.974 & $26.347 \mathrm{r}>\mathrm{C}$ & 24.805 & 27.101 & & 46.707 & 51.023 & & 35.077 & -1.219 & & 51.102 & & $\begin{array}{lll}14.643 \\
\end{array}$ & & 899.23 & \begin{tabular}{|l|l|}
14.9872 \\
\end{tabular} & 49.6 & & & & & 0.000 & & \\
\hline 925 & 6/15/2004 & 12:14:57 AM & 25.981 & 26.336 & 24.827 & 27.088 & 26.083 & $\begin{array}{lll}46.263 \\
\end{array}$ & 50.646 & 12.301 & 34.638 & -1.222 & & 49.246 & 0.083 & 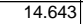 & 0.004 & 900.23 & \begin{tabular}{|l|l|}
15.0039 \\
\end{tabular} & 49.2 & 12.1 & \begin{tabular}{|l|l|}
2.789 \\
\end{tabular} & 0.012 & 0.012 & 0.000 & 0.307 & \\
\hline & $6 / 15 / 2004$ & $12: 15: 29 \mathrm{AM}$ & 25.975 & 26.338 & 24.816 & 27.077 & 26.082 & 46.415 & 50.696 & 12.353 & 34.752 & -1.222 & & 49.318 & 0.082 & $\begin{array}{lll}14.643 \\
\end{array}$ & 0.004 & 900.77 & 15.0128 & 49.2 & & 2.798 & & 0.012 & 0.000 & 0.303 & \\
\hline & $6 / 15 / 2004$ & 12:16:29 AM & 25.985 & 26.338 & 24.806 & 27.087 & & 46.643 & 50.706 & 12.334 & 35.034 & -1.22. & & 53.221 & 0.082 & 14.643 & & 901.77 & 15.0294 & 49.2. & & & & & & & \\
\hline & $6 / 15 / 2004$ & $12: 17: 29 \mathrm{AM}$ & & 26.342 & 24.815 & 27.021 & & 46.578 & 51.038 & 12.386 & 34.869 & -1.222 & & & 0.081 & 14.643 & & 902.77 & 15.0461 & 49.6 & & & & & & 0.298 & \\
\hline 92 & 6/15/2004 & 12:18:29 AM & $\begin{array}{r}25.98 \\
25.068\end{array}$ & 26.333 & $24.806 \mid$ & 27.062 & & 46.377 & 51 & 12.329 & 34.724 & -1.222 & & 515 & 0.083 & 14.643 & & 903.7 & 5.0628 & 49.5 & 10. & 2.796 & & 0.012 & 0.000 & 0.307 & \\
\hline 931 & 66115/2004] & $\frac{11: 19: 29 \mathrm{AM}}{12: 29 \mathrm{MM}}$ & 25.968 & 26.324 & 24.825 & 27.044 & & 46.599 & 50.808 & 12.348 & 34.956 & -1.219 & & 46.198 & 0.082 & 14.643 & & 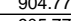 & 15.0/94 & 49.3 & & & & & & 0.301 & \\
\hline 932 & 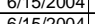 & $\begin{array}{l}12.2 .29 \mathrm{AM} / \\
12.219 \mathrm{AM}\end{array}$ & 25.98 & 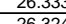 & 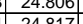 & 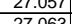 & & 46.614 & & 12.345 & 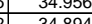 & - & & 48.814 & & 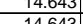 & & $\begin{array}{ll}905.11 \\
00677\end{array}$ & & 年, & & & & & & & \\
\hline 933 & - $6 / 115 / 2004$ & 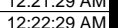 & $\begin{array}{l}25.970 \\
25.991\end{array}$ & 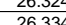 & $\begin{array}{l}\frac{24.011}{24.837} \\
\end{array}$ & 27.035 & & $\begin{array}{l}40.471 \\
46.744\end{array}$ & 51293 & $\frac{12.302}{12.427}$ & $\begin{array}{r}34.094 \\
34.97 \\
\end{array}$ & - & & $\frac{49.01}{4513}$ & 年 & (14.045 & & 900777 & 年 & 年 & 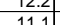 & 817 & 012 & & 年 & 0 & \\
\hline & $6 / 15 / 2004$ & $\frac{12: 23: 29 \mathrm{AM}}{1123}$ & $\frac{25.981}{25.981}$ & $\frac{26.329}{26.32}$ & 24.812 & 27.138 & $26.7-3$ & 46.603 & 51.063 & 12.43 & 34.81 & $\frac{-1.219}{-1.219}$ & & 46.092 & 0.083 & 14.643 & & 908.77 & 15.1461 & 49.6 & $\frac{21.3}{11.3}>3$ & $\frac{2.807}{2.807}$ & & $\frac{0.012}{0.012}$ & & $\frac{0.306}{0.306}$ & \\
\hline 935 & 6/15/2004 & 12:24:29 AM & 25.971 & 26.319 & 24.817 & 27.148 & 26.2 & 46.651 & 50.919 & 12.424 & 34.964 & -1.222 & & 46.962 & 0.08 & 14.643 & & 909.77 & 15.1628 & 49.4 & $\frac{11.5}{11.5}$ & 2.814 & .012 & 0.012 & 0.000 & 0.294 & \\
\hline & 6/15/2004 & $12: 25: 29 \mathrm{AM}$ & 25.987 & 26.321 & 24.819 & 27.179 & & 46.626 & 51.332 & & 34.89 & -1.216 & & 43.524 & & $\begin{array}{l}14.643 \\
\end{array}$ & & 910.77 & 15.1794 & 49.9 & & & & & 0.000 & & \\
\hline 937 & 6/15/2004 & 12:26:29 AM & 25.983 & 26.327 & 24.835[ & 27.21 & & 46.423 & 51.071 & 12.408 & 34.722 & -1.222 & & 44.744 & 0.081 & 14.643 & & 911.7 & 5.1961 & 49.6 & 11.0 & & .012 & & 000 & 0.299 & \\
\hline & & & & & & & & & & 12.433 & & -1.21 & & & & 14.643 & & & & 49.6 & & & & & & 0.298 & \\
\hline & $6 / 15 / 2004$ & 12:28:29 AM & 25.986 & 26.329 & 24.832 & 27.263 & & 46.338 & 50.606 & 12.433 & 34.6 & $-1.22 .2 \quad \mathrm{C}$ & & 46.826 & & & & 913.77 & 155.2294 & 49.1 & & & & & 100 & 0.300 & \\
\hline & 6/15/2004 & 12:29:2 & 25.976 & & 24.997 & 27.263 & & & 50.919 & 12.374 & & -1.22 .2 & & 46.119 & & $\begin{array}{l}14.643 \\
\end{array}$ & & 914.7. & 5.2461 & 49.4 & & & & & & 0.298 & \\
\hline 94 & 6/15/2004 & 12:30: & 26.008 & 26.331 & 24.989 & 27.279 & & 46. & . .776 & 12.314 & 34.763 & -1.222 & & 37.139 & & 14. & & 915 & 5.26 & 49.3 & 9.1 & & & & & 0.298 & \\
\hline 94 & 6/15/2004 & 12:31:2: & 26.008 & & 24.969 & 27.299 & & 46.164 & 522 & 12.365 & 34.52. & $-1.2 .2>0$ & & 48.545 & & 14.6 & & & & & 1.9 & $2.7>-7$ & & & & & \\
\hline 943 & 6/15/2004 & 12:32:29 AM & 26.028 & 26.337 & 24.96 & 27.31 & 26.2 & 45.807 & 50.727 & 12.332 & 34.122 & -1.216 & & 50.66 & 0.079 & 14.643 & & 917.7 & 15.2961 & 49.3 & $12.4 \mathrm{H}>\mathrm{l}$ & 2.75 & & & 0.000 & 0.295 & \\
\hline 944 & 6/15/2004 & AM & 26.036 & 344 & 24.963 & 27.268 & & & ( & $12.306 \mathrm{r}$ & 34.78 & $-1.22>0$ & & . & & 14.643 & & & & 49.5 & 11. & & & & & 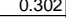 & \\
\hline & & & & & & & & & 51.688 & & & & & & & & & & & & & & & & & & \\
\hline & $\begin{array}{ll}6 / 15 / 200 \\
6 / 15200\end{array}$ & 12:35:29 AM & 26.029 & 26.338 & 24.911 & 27.216 & & & 0.996 & $12.388 \mathrm{rl}$ & 34.6 & -1.22 & & 44.104 & & 14.643 & & 021.7 & & 49.5 & $12+3$ & & & & & 0 & \\
\hline 948 & 0 & $\begin{array}{l}1.2 .30 \\
12.37\end{array}$ & & & $\frac{2.9514}{2.8909}$ & $\frac{21.243}{27179}$ & & & 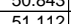 & $\frac{12.300}{1232}$ & 年4.0 345 & -1.2 & & & & & & & & & & & & & & & \\
\hline 949 & 6/115/2004 & 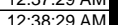 & $\frac{2.0 .042}{26.042}$ & 年 & 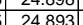 & 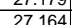 & & 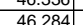 & 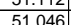 & 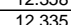 & $\frac{34.355}{3465}$ & $\frac{-1.41}{-122}$ & & 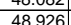 & & $\frac{14 .}{14}$ & & 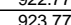 & | & 年 & 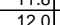 & 270 & & & & 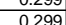 & \\
\hline 950 & $6 / 15 / 2004$ & $12: 39: 29 \mathrm{AM}$ & 26.036 & 26.324 & 24.852 & 27.158 & & 46.458 & 51.098 & 12.343 & 34.798 & -1.219 & & 44.824 & & 14.6 & & 924 & & 49.6 & & & & & & 0.298 & \\
\hline 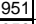 & & 12:40:29 AM & & & & 27.134 & & & & & & & & & & & & & & & & & & & & & \\
\hline & $6 / 15 / 2004$ & 12:41:29 AM & 26.036 & $\frac{26.314}{26.3}$ & 24.837 & 27.153 & & & 51023 & 12.349 & 34.7 & $\frac{-1.22}{-1.22}>$ & & $\frac{43.343}{434}$ & & $14.6 \mathrm{C}-\mathrm{s}$ & & 926.77 & & & & & & & & > & \\
\hline & & & & & 24.8 & & & & & 12.3 & & & & $\frac{49.4}{49.4}$ & & & & & & & & & & & & & \\
\hline 95 & $6 / 15 / 2004$ & & 26.0 & 26.3 & 24.837 & 27.183 & & & 51.3 & & & & & 44.6 & & & & 928.77 & & $49.8 \mathrm{r} \quad>$ & 10.9 & & & & & 0.301 & \\
\hline & 6/15/2004 & 12:44: & & & 24.835 & 27.18 & & & 50.648 & & & $-1.22>>3$ & & & & & & & & & 11. & & & & & & \\
\hline 956 & 6/15/2004 & 12:45: & 26.026 & 26.304 & 24.922 & 27.237 & & & 51.025 & 12.388 & & -1.2 & & 49.8 & & & & & & & 12.2 & & & & & 0.295 & \\
\hline & $6 / 15 / 20$ & $12: 46: 29 \mathrm{~A}$ & 26.028 & & 24.915 & 27.18 & & & & 12.381 & & -1.21 & & 45.879 & & & & & & & & & & & & .295 & \\
\hline 95 & 6/15/2004 & 12:47:2 & 26.024 & 26.282 & 24.89 & 27.116 & & 46. & & 12.357 & 34. & $-1.22>0$ & & 46.703 & & & & & 15.5 & 49. & $11.4 \mathrm{~s}-\mathrm{s}$ & & & & & 0.299 & \\
\hline$\frac{959}{92}$ & 6 & 12:48:2 & 20.034 & 26.296 & 24.879 & 27.114 & & & 51.1114 & 12.288 & 34.7 & -1.222 & & 47.623 & & $14.6 \mathrm{C}$ & & & & 49.6 & 11.1. & +5 & & & & 0.299 & \\
\hline & & & 26.047 & 26.311 & 24.8944 & & & & & & & & & & & & & & & & & & & & & & \\
\hline 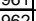 & $\frac{6 / 15 / 2}{6 / 1 / 2}$ & 12.50 .24 & & 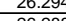 & 24.8679 & 2.0 & & & & 12.36 & & & & & & & & & & & 1.4 & & & & & 00 & \\
\hline & 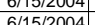 & & & & 2.8014 & & & & & & & & & 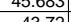 & & & & & & & & & & & & & \\
\hline 964 & $\frac{0110120}{6 / 15 / 20}$ & $12: 53: 29 \mathrm{AM}$ & $\frac{20.054}{26.048}$ & $\frac{20.20}{26.306}$ & $\begin{array}{l}24.05 \\
24.89 \\
\end{array}$ & 27.095 & & & 51.494 & $\frac{12.0}{12.3}$ & $\begin{array}{l}34.748 \\
34.748\end{array}$ & -121 & & 49.12 & & 14. & & (39877 & 15.0 & & 121 & & & & & $\frac{0.251}{0.291}$ & \\
\hline 965 & & $12: 54: 29 \mathrm{AM}$ & 26.03 & 26.273 & 24.836 & 27.052 & & 46.298 & 51.063 & 12348 & 34.621 & -121 & & 51092 & & 14643 & & & & & & & & & & 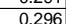 & \\
\hline & $6 / 15 / 24$ & 12:55:2 & 26.03 & 26.286 & 24.854 & 27.08 & & & & 12.42 & & -1.21 & & 43.426 & & 14.6 & & & 15.6 & & & & & & & 302 & \\
\hline 967 & 6/15/2004 & & & & & & & & & & & & & & & & & & & & & & & & & & \\
\hline & & & & & $24.86 \quad>\quad>$ & & & & & & & $-1.27>>>$ & & & & 14. & & & & & & & & & & & \\
\hline 969 & $6 / 15 / 20$ & & 26 & & 24.9 & 27.18 & & & 50.7 & 12.36 & & $-1.3 .2>0$ & & & & & & & & & & & & & & & \\
\hline & $6 / 15 / 2$ & $12: 59: 29 \mathrm{~A}$ & 26.03 & $26.2 \mathrm{C}>\mathrm{C}$ & $24.8 \mathrm{C}>\mathrm{T}$ & 27.20 & & 46.3 & 51.1 & 12.34 & & -1.2. & & & & 14 & & 94 & 15. & & & & & & & & \\
\hline 97. & $6 / 15 / 200$ & 1:00: & 26.0 & 26.304 & 24.907 & & & 46.274 & 51.1 & 12.3 & & -1.2. & & 44. & & 14. & & & & & & & & & & & \\
\hline & $6 / 15 / 2$ & & 26.0 & & $24.8 \mathrm{~s}$ & & & & & 12.2 & & -1.2 & & & & 14. & & & & & & & & & & & \\
\hline $9 / 3$ & $6 / 15 / 200$ & & 26.058 & & 24.88 & 27.325 & & & 50.882 & 12.267 & & -1.22 & & 44.2. & & & & & & 49.4 & $10.8>>$ & & & & & 0.289 & \\
\hline 974 & $6 / 15 / 2$ & & & & $24 . \varepsilon-8$ & & & 46. & & & & -1.2 & & & & 14. & & & & & & & & & & & \\
\hline & $6 / 15 / 2 / 2$ & & & & $24.8 / 5$ & 27.266 & & & & 12.2 & 34. & -1.159 & & & & & & & & .5 & & & & & & & \\
\hline & & & 26.6 & & 24.825 & 2.28 & & & 51.1 & 316 & 34.5 & -1.156 & & & & & & & & $49 .$. & & & & & & & \\
\hline & 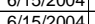 & $\begin{array}{l}1.0 .25 \mathrm{AM} \\
1.0 .09 \mathrm{AM}\end{array}$ & $\frac{2.00}{260}$ & 2520 & 2.000 & $\frac{2.2}{272}$ & & 40.2 & S1.9.94 & $\frac{1.3}{123}$ & 34 & -1.15 & & 4.18 & 0 & 14.6 & & (2050 & & 49.5 & $\frac{10.5}{120}$ & & & & & 200 & \\
\hline 070 & 6 & 108.04 & 26.046 & 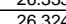 & 24857 & 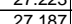 & & 年 40.2069 & 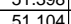 & $\frac{12.54}{12323}$ & $\begin{array}{l}34.525 \\
34289\end{array}$ & - & & 4716 & 00 & 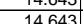 & & & & 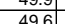 & . & & 012 & 12 & 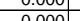 & 0 & \\
\hline & $6 / 15 / 2$ & $10.0929 \mathrm{AM}$ & $\frac{6.049}{6.049}$ & 26.337 & $\mid$ & 27.161 & & 45.689 & 50.44 & 12.234 & 34.182 & -1.156 & & 8.152 & 0.079 & 14.64 & & 54.7 & & 49.0 & & & & 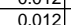 & 0.000 & $\begin{array}{l}0.295 \\
0.296 \\
\end{array}$ & \\
\hline
\end{tabular}


WSRC-TR-2005-00105, REVISION 0

SRNL-RPP-2005-00012, REVISION 0

RUN \# 3.03A AND B; FIRST AND SECOND HALF OF SLURRY DEWATERING - CONT.

\begin{tabular}{|c|c|c|c|c|c|c|c|c|c|c|c|c|c|c|c|c|c|c|c|c|c|c|c|c|c|c|c|c|}
\hline & A & B & D & $E$ & $\mathrm{~F}$ & G & $\mathrm{H}$ & & $n$ & $L_{L}$ & $\mathrm{M}$ & $\mathrm{N}$ & 0 & $Q_{1}$ & \begin{tabular}{l|l}
$R$ \\
\end{tabular} & $\mathrm{~s}$ & $\begin{array}{lll}T & \mathrm{u} \\
\end{array}$ & . & 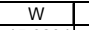 & \begin{tabular}{l|l|l|}
$x$ \\
\end{tabular} & 1 & & $\mathrm{AA}$ & $A B$ & $A C$ & $\mathrm{AD}$ & $\mathrm{AE}$ & \\
\hline 981 & $6 / 15 / 2004$ & $\begin{array}{l}1: 10: 29 \mathrm{AM} \\
1 \cdot 1 \cdot 29\end{array}$ & $\begin{array}{l}26.042 \\
26047\end{array}$ & $\begin{array}{l}26.331 \\
26.21\end{array}$ & 24.874 & 27.129 & 26.264 & \begin{tabular}{|l|l|}
46.081 \\
5.839
\end{tabular} & 51.405 & \begin{tabular}{ll|}
12.352 \\
12021
\end{tabular} & 34.399 & $\begin{array}{l}-0.776 \\
\end{array}$ & $\begin{array}{l}-6.328 \\
\end{array}$ & 48.417 & $\begin{array}{l}0.069 \\
\end{array}$ & 14.643 & $\begin{array}{l}0.004 \\
\end{array}$ & \begin{tabular}{|l|l|}
955.77 \\
956.77
\end{tabular} & \begin{tabular}{l|l|}
15.9294 \\
50944
\end{tabular} & 49.9 & 11.9 & 40.2 & $\begin{array}{l}2.774 \\
2.750\end{array}$ & 0.010 & 0.010 & 0.000 & 0.257 & 0.22 \\
\hline 883 & $6 / 15 / 2004$ & 1:11:29 AM & $\begin{array}{l}26.047 \\
26.15\end{array}$ & $\frac{26.341}{26.339}$ & 24.869 & 27.164 & $\frac{26.264}{262.13}$ & \begin{tabular}{|c|c|}
45.838 \\
4611
\end{tabular} & 51.141 & $\frac{12.294}{12326}$ & $\frac{34.204}{34.494}$ & $\begin{array}{r}-0.91 \\
-1005\end{array}$ & $\begin{array}{l}-6.733 \\
607 \\
\end{array}$ & 47.228 & $\begin{array}{r}0.08 \\
0078\end{array}$ & $\frac{14.643}{14613}$ & & \begin{tabular}{|l|l|}
956.77 \\
\end{tabular} & \begin{tabular}{|l|l|}
15.9461 \\
\end{tabular} & 49.7 & 11.6 & 40.0 & 2.759 & ...stent & 0.012 & & 0.299 & \\
\hline 984 & $6 / 15 / 12004$ & $\begin{array}{l}1: 12: 29 \mathrm{AM} \\
1: 13: 29 \mathrm{AM}\end{array}$ & $\begin{array}{l}26.145 \\
26.157\end{array}$ & $\frac{26.339}{26.341}$ & $\frac{24.882}{24.894}$ & 27.1729 & $\frac{26.213}{26.164}$ & $\begin{array}{r}446.11 \\
44.859 \\
\end{array}$ & $\begin{array}{l}51.127 \\
50.861\end{array}$ & $\begin{array}{l}12.326 \\
12.374\end{array}$ & $\begin{array}{l}\frac{34.494}{33.125} \\
\end{array}$ & $\begin{array}{r}-1.005 \\
-0.09 \\
\end{array}$ & $\begin{array}{r}-6.978 \\
-6.386\end{array}$ & $\begin{array}{l}43.007 \\
44.002\end{array}$ & $\mid \begin{array}{l}0.078 \\
0.004\end{array}$ & $\frac{14.643}{14.643}$ & $\frac{0.004}{0.004}$ & \begin{tabular}{|c|}
957.77 \\
958.77
\end{tabular} & $\begin{array}{l}y_{15.968} \\
15.9794\end{array}$ & $\begin{array}{l}49.7 \\
49.4 \\
\end{array}$ & & & & $\frac{0.012}{0.001}$ & $\mid \frac{0.012}{0.001}$ & $\begin{array}{l}0.000 \\
0.000\end{array}$ & $\mid \frac{0.290}{0.015}$ & $\begin{array}{l}0.25 \\
0.01\end{array}$ \\
\hline 985 & $6 / 15 / 2004$ & $1: 14: 29 \mathrm{AM}$ & 26.076 & 26.34 & 24.898 & 27.133 & 26.189 & -5.249 & -0.645 & 1.413 & -5.853 & -1.219 & -6.392 & -0.066 & 0.003 & 14.643 & & 959.77 & \begin{tabular}{|l|}
15.9961 \\
\end{tabular} & $\begin{array}{l}4.4 .1 \\
-2.1 \\
\end{array}$ & $\frac{1.0}{0.0}$ & & 0.383 & & 0.000 & & & \\
\hline 986 & $6 / 15 / 2004$ & $1: 15: 29 \mathrm{AM}$ & 26.09 & 26.349 & 24.907 & 27.027 & 26.143 & -4.089 & -0.794 & 1.274 & -4.609 & -1.219 & -6.392 & -0.072 & 0.003 & 14.643 & & & .0120 & -2.3 & & & & & & & & \\
\hline & & 1:19:09 PM & $\begin{array}{l}25.392 \\
2.207\end{array}$ & 24.853 & & 27.053 & & 46.848 & & & $\begin{array}{l}34.215 \\
2.205\end{array}$ & -1.219 & & \begin{tabular}{|l|l|}
49.016 \\
\end{tabular} & $\begin{array}{l}0.099 \\
\end{array}$ & 14.643 & & 1684.43 & 8.0739 & 49.9 & & 40.5 & & & & & 0.372 & \\
\hline 988 & $6 / 15 / 2004$ & 1:20:09 PM & 25.375 & 24.892 & 24.264 & 27.057 & 25.357 & 47.555 & 51.214 & 12.999 & 35.165 & -1.219 & -7.566 & 45.172 & 0.095 & 14.643 & 0.004 & 1685.43 & \begin{tabular}{|l|l|}
28.0906 \\
\end{tabular} & $\begin{array}{l}49.7 \\
\end{array}$ & 11.1 & 41.4 & 2.852 & 0.014 & 0.014 & 0.000 & 0.350 & \\
\hline $\begin{array}{l}989 \\
900\end{array}$ & $6 / 15 / 2004$ & 1:21:09 PM & $\begin{array}{l}25.373 \\
25405\end{array}$ & $\begin{array}{l}24.914 \\
24.936\end{array}$ & $\frac{24.321}{2.369}$ & \begin{tabular}{ll|}
27.084 \\
27051
\end{tabular} & $\begin{array}{l}25.385 \\
25307\end{array}$ & $\begin{array}{l}.041 \\
252 \\
\end{array}$ & $\begin{array}{l}50.551 \\
50955\end{array}$ & $\begin{array}{l}13.781 \\
12539\end{array}$ & $\begin{array}{l}33.864 \\
3232\end{array}$ & $\begin{array}{r}-1.225 \\
.214\end{array}$ & $\begin{array}{l}-7.664 \\
-7659\end{array}$ & \begin{tabular}{|l|}
51.609 \\
54815
\end{tabular} & & $\begin{array}{l}14.643 \\
1.643\end{array}$ & & \begin{tabular}{|l|}
1686.43 \\
68732
\end{tabular} & \begin{tabular}{|l|}
28.1072 \\
28.129
\end{tabular} & 49.1 & 12.6 & & \begin{tabular}{l|l|}
2.7899 \\
2809
\end{tabular} & $\begin{array}{l}0.013 \\
0.13\end{array}$ & \begin{tabular}{|l|l|}
0.013 \\
0.13
\end{tabular} & & $\begin{array}{l}0.331 \\
0.32\end{array}$ & \\
\hline$\frac{990}{991}$ & 6/15/2004 & $\begin{array}{l}1: 22: 09 \text { PM } \\
1.23 .09 \mathrm{PM}\end{array}$ & $\begin{array}{l}25.405 \\
25.47\end{array}$ & $\begin{array}{l}24.936 \\
24.949\end{array}$ & $\frac{24.369}{24.401}$ & $\begin{array}{l}27.051 \\
26.989\end{array}$ & $\begin{aligned} 25.387 \\
2537\end{aligned}$ & \begin{tabular}{ll|l}
47.252 & 47.982 \\
4
\end{tabular} & $\begin{array}{l}50.855 \\
51.61\end{array}$ & $\begin{array}{l}\frac{13.538}{13.283} \\
\end{array}$ & $\begin{array}{r}34.233 \\
35.237 \\
\end{array}$ & $\begin{array}{r}-1.214 \\
-1.216\end{array}$ & $\begin{array}{l}-7.658 \\
-7.661\end{array}$ & \begin{tabular}{|l|}
54.815 \\
54227
\end{tabular} & $\begin{array}{l}0.087 \\
0.089\end{array}$ & $\frac{14.643}{14.633}$ & $\frac{0.004}{0.004}$ & $\begin{array}{l}1687.43 \\
1688.43\end{array}$ & $28.1239 \mid$ & $\begin{array}{l}49.4 \\
50.2 \\
\end{array}$ & $\frac{13.4}{13.3}$ & $\frac{4.6}{416}$ & 2.809 & $\begin{array}{l}0.013 \\
0.013\end{array}$ & \begin{tabular}{|c|}
0.013 \\
0.013
\end{tabular} & 0.000 & \begin{tabular}{|l|l|l|l|l|l}
0.324 \\
0.324
\end{tabular} & 0.28 \\
\hline $\begin{array}{c}951 \\
992\end{array}$ & $6 / 15 / 1 / 2004$ & $\begin{array}{l}1: 23: 09 \mathrm{P} \\
1: 24: 09 \mathrm{P}\end{array}$ & 25.415 & $\frac{24.949}{24.967}$ & $\frac{24.401}{24.444}$ & 26.997 & $\begin{array}{r}25.37 \\
25.373\end{array}$ & $\begin{array}{l}4.98 .074 \\
48\end{array}$ & & 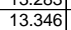 & 35.235 & & & $\begin{array}{l}4.221 \\
46.742 \\
\end{array}$ & & $\begin{array}{l}4.643 \\
14.643 \\
\end{array}$ & & $\begin{array}{l}10888.43 \\
1689.43\end{array}$ & $\begin{array}{l}28.1400 \\
28.1572\end{array}$ & $\begin{array}{l}50.4 \\
50.4\end{array}$ & $\begin{array}{l}11.3 \\
11.5 \\
\end{array}$ & & & & \begin{tabular}{|c|}
0.013 \\
0.013
\end{tabular} & & $\begin{array}{l}\mid 0.324 \\
0.316\end{array}$ & 0.28 \\
\hline 993 & $6 / 15 / 2004$ & 1:25:09 PM & 25.404 & 24.986 & 24.539 & 26.926 & 25.387 & 47.611 & 51.342 & 13.305 & $\begin{array}{l}34.777 \\
\end{array}$ & -1.225 & -7.661 & 44.273 & 0.086 & 14.643 & & 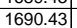 & 28.1739 & 49.9 & & & 2.840 & 0.013 & $\mid 0.013$ & & & 0.27 \\
\hline & $6 / 15 / 2004$ & 1:26:09 PM & & 24.999 & & 26.984 & & 47.798 & & & 35.181 & -1.219 & -7.661 & 51.646 & & 14.643 & & $\begin{array}{l}16991.43 \\
\end{array}$ & 28.1906 & 50.0 & $\begin{array}{l}12.0 \\
12.7\end{array}$ & & & & & & & $\frac{0.27}{0.26}$ \\
\hline 995 & & & & & 24.64 & & & & & $\begin{array}{l}14.32 \\
\end{array}$ & & & & & 0.082 & & & 69213 & 28.2072 & 48.5 & 10.6 & & & & & 000 & 0.314 & \\
\hline 950 & & & 25.434 & 25.046 & 24.683 & & & \begin{tabular}{|l|l|}
46.943 \\
\end{tabular} & (50.486 & & 33.677 & -1.219 & & & & 14.643 & & & & 100 & 15.5 & & & & & & & \\
\hline 997 & 504 & 1:29:09 PM & 25.437 & 25.064 & 24.697 & 27.164 & & 46.952 & 50.604 & 13.813 & 33.652 & -1.219 & & 44.308 & & 14.643 & & & 28.2406 & 101 & $\frac{10.9}{10}$ & 40. & & & & & 302 & \\
\hline & 6/115/2004 & 1:30:09 PM & 25.457 & 25.079 & 24.712 & 27.254 & & 47.008 & 50.644 & 13.801 & 33.798 & -1.222 & & 48.534 & 0.083 & 14.643 & & 1695.43 & 28.2572 & 49.2 & 11.9 & & 786 & & & & & \\
\hline 999 & $6 / 15 / 2004$ & 1:31:09 PM & 25.498 & 25.12 & 24.782 & 27.344 & 25.6 & 47.155 & 50.924 & \begin{tabular}{|l|l|l|}
13.7 \\
\end{tabular} & 33.964 & -1.216 & -7. & $\begin{array}{l}46.584 \\
\end{array}$ & $\begin{array}{l}0.083 \\
\end{array}$ & 14.643 & & 1696.43 & 28.2739 & 49.5 & 11.4 & 40. & 2.796 & 0.012 & 0.012 & & 0.307 & \\
\hline$\Gamma$ & $5 / 2004$ & 1:32:09 PM & 25.502 & 25.119 & 24.747 & 27.424 & 25.75 & 47.035 & 50.602 & 13.588 & & -1.219 & -7 & 48.395 & 0.082 & 14.643 & & 1697.43 & 28.2906 & 49.1 & 11.9 & & 2.794 & & & & 0.304 & \\
\hline & $6 / 15 / 2004$ & 1:33:09 PM & & 25.139 & & & & 47.203 & 50.961 & 558 & 34.174 & -1.219 & & .5334 & & 14.643 & & 98.43 & & 49.5 & & & & & & & & \\
\hline 00 & $6 / 151 / 2004$ & 1:34:09 PM & 25.568 & 25.19 & 4.828 & 27.524 & & 46.661 & & 14.057 & 33.158 & -1.222 & & & 0.083 & 14.643 & & & & 48. & & & & & & & & \\
\hline & $6 / 15 / 2004$ & $\begin{array}{l}1.350 .09 \mathrm{MM} \\
1.32 .09 \mathrm{MM}\end{array}$ & 年 25.568 & $\frac{25.1944}{2523}$ & $\begin{array}{ll}2.8 .812 \\
201942\end{array}$ & 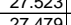 & 25.944 & 46.303 & 年. & $\mid \frac{14.2515}{1.297 \mid}$ & $\begin{array}{l}32.651 \\
32094\end{array}$ & $\begin{array}{l}-1.219 \\
-1202\end{array}$ & $\frac{1-1.0}{7.60}$ & 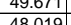 & 0.079 & 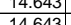 & & 0.43 & (8).34060 & 48.2 & $\frac{12.2}{11.2}$ & 20 & 706 & & | & & (3) & 26 \\
\hline & $6 / 515004$ & $\frac{1.30 .009}{1.09}$ & $\frac{2.0025}{2564 \mid}$ & $\frac{25.35}{25261}$ & 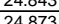 & $\frac{2.475}{27505}$ & $\frac{25}{25}$ & (4.5095 & | & $\mid \frac{10.392}{1386}$ & 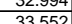 & $\frac{-1.242}{-1219}$ & & & & $\frac{1.4045}{14633}$ & & & & & & & & & & & & \\
\hline 1006 & $\frac{6 I 15 / 2004}{6 / 15 / 2004}$ & $\frac{1}{1: 38: 09 \mathrm{PM}}$ & 25.6040 & $\frac{25.284}{25286}$ & 告.675 & $\frac{27.050}{27.54}$ & & $\frac{40.505}{46.846}$ & 告. & 迹. & $\frac{0.052}{33.654}$ & $\begin{array}{l}-1.219 \\
-1.219\end{array}$ & & $\frac{40.271}{52.825}$ & $\begin{array}{l}0.001 \\
0.081\end{array}$ & $\frac{1.4045}{14.643}$ & & & $\frac{2.0509}{28306}$ & $\frac{4.4}{489}$ & $\frac{1.5}{129}$ & $\frac{40}{40}$ & & $\overline{712}$ & 0.012 & & 301 & $\frac{0.26}{0.26}$ \\
\hline 1007 & $6 / 15 / 2004$ & 1:39:09 PM & 25.699 & 25.311 & 24.984 & 27.535 & 25.9 & 46.016 & 50.517 & | & 33.655 & -1.219 & & 42.844 & 年 & 14.643 & & $\begin{array}{l}\frac{0.43}{0.43} \\
\end{array}$ & 28.4072 & 40.0 & $\frac{1.5}{10.5}$ & $\frac{40.3}{40.3}$ & 778 & 然21 & 0.012 & 0.000 & 0 & $\frac{26}{26}$ \\
\hline .008 & $6 / 15 / 2004$ & 1:40:09 PM & 25.734 & 25.336 & & 27.61 & & & & & 33.964 & $\begin{array}{r}-1.219 \\
\end{array}$ & & 54.456 & & 14.643 & & & 28.4239 & 49.2 & 13.3 & & & & & & & \\
\hline & $6 / 15 / 2$ & 1:41:09 PM & 25.768 & 25.355 & 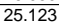 & 27.624 & & 46.796 & 582 & 13.589 & 33.714 & -1.216 & & 47.456 & 0.082 & $\begin{array}{l}14.643 \\
14.643\end{array}$ & & & & 49.1 & $\frac{11.6}{11.6}$ & & & & D12 & 0.000 & 0.303 & \\
\hline$\overline{011}$ & & & 25.791 & & 25.142 & 27.567 & & & & 14.167 & 32.682 & & & 51.657 & & 14.643 & & & & & $\frac{12.7}{12.7}$ & & & & & & & \\
\hline & $6 / 15 / 2$ & :09 PM & 25.829 & 25.397 & 25.175 & & & 46.415 & 50.007 & 13.845 & 33.139 & & & 44.792 & 0.08 & 14.643 & & & 28.4739 & & 11.0 & 39.8 & & & & & .298 & \\
\hline & 6/15/2004 & 1:44:09 PM & 25.874 & 25.432 & 25.2 & $\mid 27.66$ & & $\begin{array}{l}46.464 \\
\end{array}$ & $\mid 50.03$ & 708 & 33.365 & -1.216 & & & 0.081 & 14.643 & & & & 48 & 11.2 & & & & & & & \\
\hline 1013 & 6/15/2 & & 25.897 & 25.445 & 25.203 & 27.603 & & & 50.546 & 354 & & -1.225 & & & & 14.643 & & & & 49. & 11.6 & & & & | & & & \\
\hline 1014 & 6/15/2 & 1:46:0 & 25.936 & 25.459 & 25.227 & 27.597 & & 46.664 & 50.217 & 13.811 & 33.421 & -1.222 & & 54.523 & 0.081 & 14.643 & & 7711.43 & 28.5239 & 48. & 13.4 & & & D12 & 0.012 & 0.000 & 0.300 & \\
\hline $\bar{m}+2$ & & & & 25.467 & & & & & & & & & & & & 14.643 & & & & 48 & 11.5 & & & & & & & \\
\hline & & & 25.998 & & 年. & 27.615 & & & 0.19 & 13.801 & 33.258 & -1.219 & & & 0.081 & & & & & 48 & 10.4 & & & & & & & \\
\hline & $6 / 15 / 2004$ & $\begin{array}{l}1.49 .099 \mathrm{MM} \\
1.50 .09 \mathrm{M}\end{array}$ & $2 \frac{2.6027}{2659}$ & 25.52 & 25.298 & 年. & 25.91 & 46.6 & 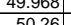 & $\frac{13.14}{13.64}$ & $\begin{array}{l}3.354 \\
3.54\end{array}$ & - 1.1 .1919 & & & 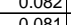 & 14.643 & & 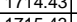 & $\frac{20.51}{2850}$ & $\begin{array}{l}48 . \\
8\end{array}$ & (11.06 & $\frac{40}{n}$ & & & 2013 & & 10200 & \\
\hline & $6 / 515004$ & & $\mid$ & 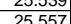 & $\frac{2.504}{25.33}$ & 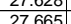 & & & 50.20 & $\mid \frac{10.034}{13693}$ & $\frac{3.354}{33297}$ & & & & & $\begin{array}{l}1.0453 \\
14643\end{array}$ & & & & & $\frac{11.5}{116}$ & & & & & & & \\
\hline 1020 & 6 & & 2609 & 25.555 & $\begin{array}{l}25.53 \\
2538\end{array}$ & 2762 & & & $\mid$ & | & & $\frac{-1.2}{-12}$ & & & & $\begin{array}{l}14.045 \\
114643\end{array}$ & & & & 然. & 121 & 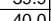 & & & & & 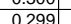 & \\
\hline$\frac{1021}{1021}$ & $\frac{1 / 1 / 1 / 2004}{6 / 2}$ & & $\frac{2.0 .126}{26.126}$ & 25.574 & 告. 25.367 & 27.672 & $\frac{25.9}{25.9}$ & 46.636 & $\frac{5.603}{50.53}$ & 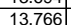 & $\frac{23.465}{33.365}$ & -1.219 & & & $\frac{0.081}{0.081}$ & $\frac{14.643}{14.63}$ & & 43 & & 49 & $\frac{\frac{c 1.1}{11.2}}{1.2}$ & & & & 0 & 0 & 0 & \\
\hline & & & & & & & & & & & & & & & & & & & & & 120 & & & & & & & \\
\hline 023 & & & \begin{tabular}{l|l|}
26.162 \\
\end{tabular} & & 25.394 & 27.724 & & & 50.509 & 13.767 & & & & & & $\frac{14.6}{14.6}$ & & & & $\frac{79}{49}$ & $\frac{12.0}{12.0}$ & & & & & & 0295 & \\
\hline$\overline{0^{2}}$ & & & 26.185 & & & 27.712 & & & 50.49 & & & & & & & 14.6 & & & & & & & & & & & & \\
\hline 1025 & $6 / 15 / 2$ & & 26.21 & 25.648 & 5.436 & 27.806 & & & 50.374 & & 33.3 & & & & & & & & & & 14.1 & & & & & & 0.298 & \\
\hline & $6 / 15 / 2$ & & & 25.647 & 25.425 & 27.7 & & 46.5 & 50.374 & & & & & & & 14.6. & & & & & 10.4 & 35 & & & & & 0.29 & \\
\hline 1027 & $6 / 15 / 2004$ & 1:59:09 PM & 26.242 & & 25.439 & 27.863 & & & 50.242 & 13.68 & & & & & & & & & & & 10.8 & $4 C$ & & & & & & \\
\hline 1028 & $6 / 15 / 2004$ & & 26.272 & 25.701 & 25.484 & 27.928 & & 46.282 & 50.13 & 13.723 & 33.074 & -1.2 & & & 0.081 & 14.64 & & & & 48. & 13.1 & 39 & & & & & 0.300 & \\
\hline 1029 & & & 26.283 & & 25.47 & 27.939| & & & & & & & & & & & & & & & 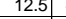 & & & & & & 0.29 & \\
\hline (1) & & & 26.308 & & 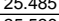 & 28.0399 & & & 50 & & 33.34 & & & & & & & & & 48 & & & & & & & & \\
\hline 1032 & & & $\frac{26.34}{26.34}$ & & & & & & $\frac{50.194 \mid}{50995}$ & & & & & & & & & & & & 1 & & & & & & 年 & \\
\hline 1002 & 6. & & $\frac{2.0454}{2639}$ & & 55 & $\frac{10.06}{28.03}$ & & & 49962 & & & & & & & & & & & & $\frac{11.4}{11.4}$ & & & & & & & \\
\hline 103 & & & 263 & & & 年 27.06 & & & & & & & & & & & & & & & $\frac{1.1}{118}$ & & & & & & & \\
\hline$\frac{1035}{1035}$ & $6 / 15 / 2004$ & $\frac{2: 07: 09 \mathrm{PM}}{2: 07}$ & $\frac{26.403}{26.403}$ & $\frac{2.0 .8}{25.8}$ & $\frac{25.545}{25.545}$ & 27.984 & & & 50.606 & 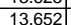 & $\begin{array}{l}33.442 \\
33.462\end{array}$ & $\frac{-1.2}{-1.2}$ & & & & $\frac{14.6}{14.6}$ & & & & 499. & $\begin{array}{ll}11.6 \\
11.2\end{array}$ & & & & & & & \\
\hline 1036 & & & & & & & & & & & & & & & & & & & & & & & & & & & & \\
\hline 1037 & & & & & & & & & & & & & & & & & & & & & & & & & & & & \\
\hline 1038 & $6 / 15 / 2004$ & & 26.45 & & 25.5 & 28.0 & & & 510 & & & -1.2 & & & & & & & & & 100 & & & & & & & \\
\hline 1039 & $6 / 15 / 2$ & $2: 11: 09 \mathrm{PM}$ & 26.47 & 25.929 & 25.55 & 27.97 & 26.3 & & 50.56 & 13.49 & 33.64 & -1.22 & & & & 14.6 & & & & 49 & 9 & 40 & & & & & $\begin{array}{ll}36 \\
6\end{array}$ & \\
\hline 104 & $6 / 15 / 2004$ & $2: 12$ & 26.508 & 25.94 & 25.565 & 28.085 & & & 49.966 & 13.428 & 33.414 & -1.222 & & & & 14.6 & & & & 48 & 4 & & & & & & & \\
\hline 1041 & 6/15/2 & & 26.5 & 25.9 & 25.559 & 28.08 & & & & 13.457| & 33.681 & & & & & & & & & & .8 & & & & & & & \\
\hline 1042 & & & 26.546 & & 25.588 & 28.122 & & & 50.4 & 13.541 & 33. & -1.2 & & & & & & & & 49. & 11.1 & & & & & & 0.296 & \\
\hline 1043 & & & & & & & & & & & & & & & & & & & & & & & & & & & & \\
\hline 1044 & $6 / 15 / 2004$ & & & & 25.602 & $28.191\}$ & & & & $13.22 / 4$ & & & & & & & & & & & 1.5 & & & & & & & \\
\hline 1046 & $6 / 151 / 2004$ & 2.1.1.09P1 & 5090 & & $\frac{5.6009}{20.595}$ & 8.171 & & & & $\frac{13.24}{1.22}$ & & -1.26 & & & & $\frac{11.6}{11.6}$ & & & & & & & & & & & & \\
\hline 1047 & $01515 / 200^{4}$ & 势 & | & & 20.5003 & $\frac{2.004}{2012}$ & & & & 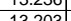 & & & & & & & & & & & 0.0 & & & & & & & \\
\hline 1048 & 6 & $\frac{1.40}{2 \cdot 20}$ & | & $\frac{2.0003}{26053}$ & 5576 & 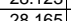 & & 468 & 5076 & 年 & $\begin{array}{l}34.540 \\
34227\end{array}$ & & & & & & & & & & & & & & & & (2) & \\
\hline 049 & & & 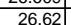 & 26.08 & 5.578 & 28.226 & & 46.8 & 50.774 & 13.174 & 34.133 & & & & 0.08 & $146 \mathrm{C}$ & & & & 193 & 107 & & & & & & 297 & \\
\hline 1050 & 6/15/2004 & 2:22:09 PM & $\frac{1.639}{6.639}$ & $\frac{26.099}{269}$ & $\frac{25.587}{25.587}$ & $\frac{20.220}{28.235}$ & & 466.811 & 839 [ & 13.162 & 34.194 & $\frac{1.262}{-1.219}$ & & & & 14.643 & & 43 & & 49.4! & 24 & & & 0.012 & 1012 & 000 & (1) & \\
\hline
\end{tabular}


WSRC-TR-2005-00105, REVISION 0

SRNL-RPP-2005-00012, REVISION 0

RUN \# 3.03A AND B; FIRST AND SECOND HALF OF SLURRY DEWATERING - CONT.

\begin{tabular}{|c|c|c|c|c|c|c|c|c|c|c|c|c|c|c|c|c|c|c|c|c|c|c|c|c|c|c|c|c|}
\hline & & & D & $E$ & $F$ & \begin{tabular}{l|l}
$G_{1}$ \\
\end{tabular} & $\begin{array}{lll}\mathrm{H} & \mathrm{H} \\
\end{array}$ & $\mathrm{J}$ & \begin{tabular}{l|l}
$\mathrm{K}$ \\
\end{tabular} & $\begin{array}{ll} \\
\end{array}$ & \begin{tabular}{l|l} 
\\
\end{tabular} & \begin{tabular}{|l|l|} 
\\
\end{tabular} & $\begin{array}{lll}0 \\
\end{array}$ & \begin{tabular}{l|l} 
\\
\end{tabular} & \begin{tabular}{l|l}
$R$ \\
\end{tabular} & \begin{tabular}{l|l}
$\mathrm{s}$ &
\end{tabular} & $T$ & U $\quad v$ & \begin{tabular}{l|l} 
w \\
\end{tabular} & \begin{tabular}{l|l}
$x$ \\
\end{tabular} & \begin{tabular}{l|l} 
&
\end{tabular} & & & & AC & & & 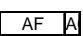 \\
\hline & $6 / 15 / 2004$ & 2:23:09 PM & 26.653 & 26.107 & 25.601 & 28.219 & 26.406 & 46.612 & 50.507 & \begin{tabular}{|c|}
13.103 \\
\end{tabular} & $\begin{array}{l}34.077 \\
\end{array}$ & \begin{tabular}{|l|}
-1.219 \\
\end{tabular} & \begin{tabular}{|l|l|}
-7.684 \\
\end{tabular} & \begin{tabular}{|l|}
48.303 \\
\end{tabular} & 0.082 & \begin{tabular}{|l|l|}
14.643 \\
\end{tabular} & 0.004 & 1748.43 & \begin{tabular}{|l|}
329.1406 \\
\end{tabular} & 49.0 & 11.8 & 40.3 & 2.782 & 0.012 & 0.012 & 0.000 & 0.298 & 0.25 \\
\hline & & $\begin{array}{l}2: 24: 09 \mathrm{PM} \\
20\end{array}$ & & 26.111 & 25.594 & 28.207 & 26.424 & 46.931 & $\mid$\begin{tabular}{|l|}
51.036 \\
\end{tabular} & \begin{tabular}{|l|l|}
13.207 \\
\end{tabular} & \begin{tabular}{|l|}
34.233 \\
\end{tabular} & \begin{tabular}{|l|r|}
-1.219 \\
\end{tabular} & & $\begin{array}{l}45.837 \\
\end{array}$ & 0.082 & \begin{tabular}{|l|l|}
14.643 \\
\end{tabular} & & & & 49.6 & $\frac{11.2}{11.2}$ & & & & & & & \\
\hline$\frac{1053}{103}$ & $6 / 15 / 2004$ & 2:25:09 PM & 26.67 & 26.14 & $\frac{25.608}{25.652}$ & 28.246 & 26.418 & 46.655 & \begin{tabular}{|l|}
50.549 \\
\end{tabular} & \begin{tabular}{|l|l|}
13.168 \\
3.162
\end{tabular} & $\begin{array}{l}34.044 \\
2.049\end{array}$ & \begin{tabular}{|l|l|}
-1.216 \\
\end{tabular} & $\begin{array}{l}-7.687 \\
-767\end{array}$ & $\begin{array}{l}48.91 \\
\end{array}$ & 0.081 & \begin{tabular}{|l|l|}
14.643 \\
\end{tabular} & & $\begin{array}{l}1750.43 \\
750.4\end{array}$ & 29.1739 & 49.1 & $\frac{12.0}{12.5}$ & 40.3 & $\begin{array}{l}2.782 \\
.782 \\
\end{array}$ & & 0.012 & 0.000 & 0.294 & \\
\hline 1054 & 6/15/2004 & 2:26:09 PM & 26.674 & 26.144 & 25.552 & 28.215 & 26.447 & 46.717 & 50.48 & $\begin{array}{l}13.152 \\
13.02\end{array}$ & \begin{tabular}{|l|l|l|}
34.149 \\
\end{tabular} & \begin{tabular}{|l|}
-1.225 \\
\end{tabular} & -7.696 & $\begin{array}{l}47.072 \\
\end{array}$ & $\begin{array}{l}0.083 \\
\end{array}$ & \begin{tabular}{|l|l|}
14.643 \\
\end{tabular} & 0.003 & 1751.43 & 9.1906 & 49.0 & 11.5 & $\begin{array}{l}40.4 \\
\end{array}$ & 2.788 & .012 & \begin{tabular}{l|l}
0.012 \\
\end{tabular} & 0.000 & 0.301 & 0.26 \\
\hline$\frac{1055}{1056}$ & $6 / 15 / 2004$ & $\begin{array}{l}2: 27: 09 \text { PM } \\
2.28 .09 \mathrm{PM}\end{array}$ & $\frac{26.683}{26.686}$ & \begin{tabular}{l|l|l|l|}
26.162 \\
26.176
\end{tabular} & & 28.244 & & & & & & \begin{tabular}{|l|}
-1.222 \\
1.222 \\
\end{tabular} & & \begin{tabular}{|r|}
52.55 \\
\end{tabular} & & $\begin{array}{l}14.643 \\
14643\end{array}$ & & $\begin{array}{l}1752.43 \\
1750.4\end{array}$ & & \begin{tabular}{|l|}
49.4 \\
\end{tabular} & $\begin{array}{l}12.9 \\
12.5\end{array}$ & $\begin{array}{l}40.6 \\
\end{array}$ & & & $\begin{array}{l}0.012 \\
\end{array}$ & & \begin{tabular}{|l|l|}
0.297 \\
\end{tabular} & \\
\hline 1057 & $6 / 6 / 15 / 2004$ & $\begin{array}{l}2: 28: 09 \mathrm{PM} \\
2: 29: 09 \mathrm{PM}\end{array}$ & $\frac{26.686}{26.685}$ & \begin{tabular}{r|r|}
26.176 \\
26.19
\end{tabular} & \begin{tabular}{|l|}
25.484 \\
25.423 \\
\end{tabular} & $\frac{28.267}{28.266}$ & $\begin{array}{r}26.499 \\
26888 \\
\end{array}$ & \begin{tabular}{rl|l}
46.786 & \\
46.74
\end{tabular} & \begin{tabular}{|l|}
$\mid 50.7979$ \\
50.671 \\
\end{tabular} & \begin{tabular}{r|}
13.141 \\
13.184
\end{tabular} & $\begin{array}{r}34.198 \\
34.12\end{array}$ & \begin{tabular}{|l|l|}
-1.222 \\
-1.222 \\
\end{tabular} & $\begin{array}{l}-7.702 \\
-7.67 \\
\end{array}$ & \begin{tabular}{|}
$\mid 42.946$ \\
46.221
\end{tabular} & $\begin{array}{l}0.082 \\
0.075\end{array}$ & \begin{tabular}{|l|}
14.643 \\
14.643
\end{tabular} & $\frac{0.004}{0.004}$ & 175.4.43 & $\mid$\begin{tabular}{|l|}
29.2239 \\
292406
\end{tabular} & $\begin{array}{l}49.3 \\
49.2 \\
\end{array}$ & $\begin{array}{l}\frac{10.5}{11.3} \\
\end{array}$ & $\begin{array}{r}40.5 \\
40.4\end{array}$ & \begin{tabular}{|l|l|}
2.792 \\
2.788
\end{tabular} & | 012 .011 & \begin{tabular}{|}
0.012 \\
0.011 \\
\end{tabular} & $\mid \frac{0.000}{0.000}$ & 0.298 & $\begin{array}{l}0.25 \\
0.23\end{array}$ \\
\hline & $6 / 15 / 2004$ & 2:30:09 PM & 26.668 & 26.193 & 25.351 & & & & & & & & & 45.645 & 0.081 & $\begin{array}{l}14.043 \\
14.643\end{array}$ & $\begin{array}{l}0.004 \\
0.003\end{array}$ & $\begin{array}{c}\frac{1}{1754.45} \\
1755.43\end{array}$ & \begin{tabular}{|l|l|l|l|l}
29.2400 \\
\end{tabular} & $\begin{array}{l}49.2 \\
49.2\end{array}$ & $\begin{array}{ll}\frac{11.3}{11.2} \\
\end{array}$ & & $\frac{2.180}{2.786}$ & & $\begin{array}{l}0.011 \\
0.012\end{array}$ & & & $\frac{0.23}{0.25}$ \\
\hline 1059 & $6 / 15 / 2004$ & 2:31:09 PM & 26.648 & 26.192 & 25.29 & 28.064 & 26.465 & 46.946 & \begin{tabular}{|l|}
51.226 \\
\end{tabular} & & 34.159 & \begin{tabular}{|r|}
-1.219 \\
\end{tabular} & -7.704 & 43.24 & 0.08 & 14.643 & & 1756.43 & & & & & & & & & & $\frac{0.25}{0.25}$ \\
\hline 1060 & $6 / 15 / 2004$ & 2:32:09 PM & & $\begin{array}{l}26.18 \\
\end{array}$ & 25.213 & 27.952 & & 46.769 & \begin{tabular}{|l|}
51.009 \\
\end{tabular} & \begin{tabular}{|l|}
13.18 \\
\end{tabular} & \begin{tabular}{|l|l|}
34.079 \\
\end{tabular} & \begin{tabular}{|l|}
-1.219 \\
\end{tabular} & -7.702 & \begin{tabular}{|l|l|}
44.454 \\
\end{tabular} & 0.081 & \begin{tabular}{|l|l|}
14.643 \\
\end{tabular} & & & \begin{tabular}{l|l|}
3 & 29.2906 \\
\end{tabular} & 49.5 & 10.9 & 40.4 & & & & & & $\frac{25}{25}$ \\
\hline 1061 & 6/15/2004 & & 26.618 & & 25.141 & 27.905 & & & $50.517 \mid$ & 13.151 & 34.04 & & & 46.321 & \begin{tabular}{|c|}
0.08 \\
\end{tabular} & 14.643 & & & & & & & & 012 & $\mid 012$ & 0.000 & & $\frac{25}{25}$ \\
\hline & & & & 26.205 & 25.078 & 27.862 & & & 50.486 & 13.123 & 34.061 & -1.22 & & & & 14.643 & & & & 49.0 & 9.5 & $\begin{array}{cc}40 . \\
0 .\end{array}$ & & & & & 291 & \\
\hline & & 2:35:09 PM & 26.598 & 26.227 & 25.035 & 27.889 & & & 50.878 & 13.189 & 34.108 & -1.219 & & & $\mid 0.078$ & 14.643 & & & & 494 & 12.8 & & 790 & & & & D.287 & \\
\hline & 6/15/2004 & :09 PM & 26.579 & 26.238 & 24.996 & 27.891 & & 446.636 & 50.718 & 13.164 & 34.034 & -1.219 & -7.71 & 56.654 & \begin{tabular}{|c|}
0.078 \\
\end{tabular} & 14.643 & & & & 49.2 & 13.9 & & 781 & & & & 0.288 & \\
\hline 1065 & $6 / 15 / 2004$ & 2:37:09 PM & 26.563 & 26.252 & 24.925 & 27.869 & 26.34 & 46.649 & \begin{tabular}{|l|}
50.617 \\
\end{tabular} & 13.109 & 34.092 & -1.222 & -7.713 & 45.681 & 0.079 & 14.643 & & & \begin{tabular}{l|l|}
3 & 29.3739
\end{tabular} & 49.1 & 11.2 & 40.4 & 2.783 & & 0.012 & & & 0.25 \\
\hline & & $\begin{array}{l}2: 38: 09 \text { PM } \\
23:\end{array}$ & 26.557 & 26.281 & 24.903 & $\begin{array}{l}27.908 \\
2705\end{array}$ & 26.34 & 46.726 & 50.704 & 13.176 & 34.106 & $\begin{array}{l}-1.219 \\
1230\end{array}$ & $\begin{array}{r}-7.713 \\
771 \\
\end{array}$ & 49.337 & 0.079 & 14.643 & & & & & & & & & & & & \\
\hline & $6 / 151 / 2004$ & 2:39:09 PM & & 26.28 & 24.847 & 27.852 & & 46.636 & 50.785 & $\begin{array}{ll}13.225 \\
1323\end{array}$ & & -1.222 & & 48.641 & 0.081 & 14.643 & & 764.43 & $.40 / 2$ & 49.3 & & & & & & & & \\
\hline & $6 / 15 / 2004$ & & & & 24.801 & 27.852 & & & & & 33.188 & & & 5.11 & 0.008 & & & & & & & & & & & & & \\
\hline & & $2: 41: 09$ PM & $\frac{26.486}{26.478}$ & $\begin{array}{l}26.301 \\
20.317\end{array}$ & $\frac{24.768}{24724}$ & 27.853 & & $\begin{array}{l}46.585 \\
46.688\end{array}$ & $\mid$\begin{tabular}{|l|}
50.577 \\
50374
\end{tabular} & $\begin{array}{l}13.102 \\
13137\end{array}$ & $\begin{array}{l}34.016 \\
34207\end{array}$ & -1.2222 & -1.12 & 48.079 & 0.4 & $\begin{array}{l}14.643 \\
14643\end{array}$ & & & 9. 9.4066 & 49.1 & 11.8 & & & & & 0.000 & & 25 \\
\hline .071 & 6 & & $\frac{20.410}{26449}$ & $\mid$ & 20.6475 & 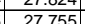 & & $\begin{array}{l}40.000 \\
46.479\end{array}$ & | & 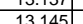 & 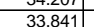 & $\begin{array}{l}-1.2<2 \\
-1.219\end{array}$ & & 47568 & 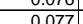 & 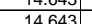 & & & & 49 & 117 & & 76 & & & 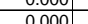 & & 24 \\
\hline 1072 & $6 / 15 / 2004$ & 2:44:09 PM & $\frac{2.4427}{26.427}$ & 26.326 & 24.638 & 27.703 & 26.33 & 46.628 & \begin{tabular}{|l|}
50.737 \\
\end{tabular} & 13.145 & 33.041 & \begin{tabular}{|l|}
-1.222 \\
\end{tabular} & & 42.9 & & $\begin{array}{l}\frac{1.045}{14.643} \\
\end{array}$ & & 1769.43 & $\mid 29.4906$ & 49.3 & $\frac{1.1}{10.5}$ & 40 & .777 & .011 & .012 & 0.000 & 0.288 & \\
\hline & $6 / 15 / 2004$ & 2:45:09 PM & 26.409 & 26.328 & 24.61 & 27.731 & & 46.468 & 50.451 & 13.124 & \begin{tabular}{|c|}
33.88 \\
\end{tabular} & \begin{tabular}{|l|}
-1.216 \\
\end{tabular} & & $\mid 48.993$ & 0.078 & 14.643 & & & & & $\frac{12.0}{12.0}$ & & 770 & & & 0.000 & & \\
\hline & $6 / 15 / 2004$ & 2:46:09 PM & 26.388 & & 24.564 & 27.764 & & 46.892 & 51.127 & & 34.18 & -1.222 & -7.70 & 477.164 & & 14.643 & & & & $\begin{array}{l}49.7 \\
\end{array}$ & $\frac{11.6}{11.6}$ & & & & & & & \\
\hline 775 & $6 / 15 / 2004$ & 2:47:09 PM & 26.357 & 26.336 & & 27.778 & & 46.726 & 50.762 & 13.165 & 34.1 & -1.222 & & 47.838 & 0.077 & 14.643 & & & $\mid .5406$ & 49.3 & $\frac{11.7}{11.7}$ & & 78 & & D12 & 5.000 & 0.288 & \\
\hline & & 2:48:09 PM & & & 24.496 & 27.777 & & \begin{tabular}{|c|}
46.815 \\
\end{tabular} & \begin{tabular}{|l|l|}
50.843 \\
\end{tabular} & & 34.165 & & & & & 14.643 & & & & 49.4 & $\begin{array}{l}9.7 \\
\end{array}$ & & & & & & & \\
\hline 077 & 5/2004 & 2:49:09 PM & 26.309 & 26.338 & 24.47 & 27.775 & & 47.369 & & 13.287 & 34.631 & -1.219 & -7 & 52.247 & 0.079 & 14.643 & & & & & 128 & & & & & & & \\
\hline 1078 & 6/15/2004 & :09 PM & 26.292 & 26.351 & 24.462 & 27.773 & 26.43 & 46.415 & \begin{tabular}{|l|}
50.509 \\
\end{tabular} & 13.984 & 32.953 & -1.219 & & $\begin{array}{l}44.404 \\
\end{array}$ & 0.077 & 14.643 & & & & 49. & & & & & & & & \\
\hline 1079 & $6 / 15 / 2004$ & 2:51:09 PM & 26.277 & 26.361 & 24.462 & 27.803 & & 46.182 & 50.169 & 13.918 | & 32.879 & \begin{tabular}{|l|l|} 
\\
\end{tabular} & -7.719 & 50.766 & 0.076 & 14.643 & & .43 & \begin{tabular}{|l|l}
3 & 29.6072 \\
\end{tabular} & 48.7 & 12.4 & & $.72 \mathrm{~s}$ & 011 & 0.012 & & & 25 \\
\hline 01 & $6 / 11$ & :09 PM & $\frac{26.235}{2.235}$ & & 24.431 & 27.866 & & & \begin{tabular}{|l|}
50.621 \\
\end{tabular} & 13.954 & 8.858 & $-\frac{-1.2}{1.2}$ & & 46.334 & & 14.643 & & & & 4.1 .14 & & & & & & & & \\
\hline & $6 / 15 / 2004$ & $009 \mathrm{PM}$ & 26.245 & 26.369 & 24.441 & $27.941]$ & & & 50.464 & 3.9366 & & -1.222 & & 56.8011 & & 14.643 & & & & 49.6 & & & & & & & & \\
\hline 082 & 6 & $2: 54: 09 \mathrm{PM}$ & 26.24 & 26.38 & 24 & 28.01 & & 46.614 & $|-50.98|$ & 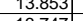 & 33.172 & -1.222 & & 51.4466 & & 14.643 & & & & & 12.6 & & 15 & & 012 & & & \\
\hline$\overline{082}$ & 6/15/2004 & $2: 55: 09$ PM & $\frac{26.229}{26.219}$ & $\frac{26.383}{26.398}$ & $\begin{array}{ll}24.404 \\
22304\end{array}$ & 28.015 & & \begin{tabular}{ll|l}
46.632 \\
46759
\end{tabular} & \begin{tabular}{|l|}
50.808 \\
51118
\end{tabular} & \begin{tabular}{|c|c|}
3.747 \\
1375
\end{tabular} & $\begin{array}{r}33.414 \\
3.36\end{array}$ & $\begin{array}{l}-1.222 \\
-1.222\end{array}$ & & 48. & & $\frac{14.643}{14643}$ & & & & $\frac{49}{49}$ & & & & & & & & \\
\hline 1085 & $6 / 15 / 2004$ & . & 26.21 & $\frac{20}{26}$ & 2 & 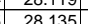 & & 467 & 5096 & 13792 & 33.417] & -1222 & & & & 14643 & & & & & 28 & & & & & & & \\
\hline 1086 & $6 / 15 / 2004$ & 2:58:09 PM & $\frac{2.24}{26.21}$ & $\frac{2.44}{26.429}$ & 24376 & $\frac{2.1006}{28.096}$ & & & \begin{tabular}{|l|}
50.806 \\
\end{tabular} & 13.774 & & \begin{tabular}{|l|}
-1.216 \\
\end{tabular} & & & 0.0 & $\frac{1.045}{14.643}$ & & & & $\frac{49}{49}$ & 137 & & & & 0.011 & & 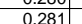 & \\
\hline 1087 & $6 / 15 / 2$ & 2:59:09 PM & 26.201 & $\frac{26.44}{22.44}$ & 24.351 & 28.057 & & 46.506 & \begin{tabular}{|l|l|}
50.735 \\
\end{tabular} & 13.776 & & -1.222 & & & & $\frac{14.643}{14}$ & & & & & A. & & & & & & & \\
\hline & 004 & 3:00:09 PM & 26.197 & 26.461 & 24.347 & 28.008 & & & \begin{tabular}{|l|}
50.573 \\
\end{tabular} & 13.704 & & -1.22 & & & & & & & & 49. & $\frac{11.9}{11.9}$ & & & & & & 0.294 & \\
\hline 1089 & $6 / 15 / 2$ & 3:01:09 PM & 26.191 & 26.465 & 24.316 & 28.022 & & 46.5 & \begin{tabular}{|l|}
50.901 \\
\end{tabular} & 13.741 & 33.25 & -1.222 & & 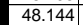 & & 14.643 & & & & 49. & 11.8 & & & 011 & .011 & & 0.286 & \\
\hline & 004 & & & & 24.316 & 28.042 & & & & & & & & & & 14.643 & & & & 49 & 107 & & & & & & & \\
\hline 091 & $6 / 15 / 2004$ & $09 \mathrm{PM}$ & 26.181 & 26.489 & 24.31 & 28.046 & & & \begin{tabular}{|l|l|}
51.245 \\
\end{tabular} & 13.846 & 33.433 & -1.2 & & & & 14.643 & & & & 49 & 125 & & & & & & & \\
\hline & 6/15/2004 & & & 26.474 & 24.285 & 28.021 & & & \begin{tabular}{|l|l|} 
\\
\end{tabular} & 13.772 & 33.2 & \begin{tabular}{|l|l|} 
\\
\end{tabular} & & & & 14.643 & & & & 49 & 12.6 & & & & 0.012 & & & \\
\hline & $6 / 15 / 2004$ & & & & 24.286 & 28.057 & & & \begin{tabular}{|l|l|l|l|} 
\\
\end{tabular} & 13.789 & & & & & & & & & & & 11.5 & & & & & & & \\
\hline 29 & $6 / 15 / 2004$ & & 26.156 & 26.494 & 24.29 & 27.976 & & & \begin{tabular}{|l|l|} 
& 50.762 \\
\end{tabular} & 13.7277 & 33.365 & -1.2 & & 44.68 & 0.072 & 14.643 & & & & & 10.9 & & & & & & & \\
\hline & 004 & & & & & 20.019 & & & 50.96 & 13.1008 & & -1.2 & & & & & & & & 49 & 12.4 & & & & & & & \\
\hline & & & 26.153 & & & & & & & & & & & & & & & & & & & & & & & & & \\
\hline & $6 / 15 / 2044$ & $\begin{array}{l}5.083 .51 \\
3.251 \mathrm{PM}\end{array}$ & $\frac{26.143}{26.138}$ & $\frac{26.517}{26522}$ & $\begin{array}{ll}24.258 \\
22.243\end{array}$ & 27.984 & & $\begin{array}{r}47.13 \\
46.483 \\
\end{array}$ & 51.384 & $\frac{13.501}{13.369}$ & $\begin{array}{l}34.139 \\
33667\end{array}$ & $\begin{array}{l}-1.222 \\
-1.219\end{array}$ & & & $\begin{array}{l}0.074 \\
0.074\end{array}$ & $\frac{14.6}{14.6}$ & & & & & $\frac{1.4 .4}{118}$ & & & & & & 81 & \\
\hline & $6 / 15 / 2004$ & & & & 2422 & 27.957 & & & $\frac{5.4}{51.41}$ & 1358 & & & & & & & & & & & & & & & & & & \\
\hline & 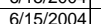 & & & & 24212 & $\frac{21.543}{27.943}$ & & & & & & -1.2 & & & & & & & & & 10.4 & & & & & & & \\
\hline 110 & $6 / 15 / 2004$ & & $\frac{26.16}{26.10}$ & & 24.186 & 27.997 & & 46.7 & $|51.152|$ & $\mid 13.674$ & & \begin{tabular}{|l|l|}
$\mid-1.2$ \\
\end{tabular} & & & & $\frac{14.6}{14.6}$ & & & & & $\frac{118}{118}$ & & & & & & & \\
\hline & & & & & & & & & & & & & & & & & & & & & & & & & & & & \\
\hline 10 & & & 26.11 & & 24.1 & 28.062 & & & 51.4 & 13.70 & & $\frac{-1.21}{-1.2}$ & & & & & & & & & & & & & & & & \\
\hline & & & & 26. & & & & & & $\mid 13.68$ & & & & & & & & & & 49. & & & & & & & & \\
\hline & $6 / 15 / 2$ & 3:16:51 PM & 26.0 & 26.535 & 24 & 27.967 & & & $\mid 51.514$ & 13.735 & 33.646 & -1.22 & & & & & & & & 50 & 109 & & & & & & 0.284 & \\
\hline & 6/15/2004 & 3:17 & 26.085 & 26.529 & 24.15 & 27.961 & & 46.6 & 50.86 & 13.719 & 33.4 & \begin{tabular}{|l|l|} 
\\
\end{tabular} & & 48.5 & & 14.6. & & & & 49. & 11.9 & & & & & & .251 & \\
\hline 110 & 6/15/2 & & 26.0 & 26.5 & 24.15 & & & & 51.4 & & & -1.2 & & & & & & & & & 2.8 & & & & & & 0 & \\
\hline 1108 & & & 26.0 & & 24.149 & 27.995 & & $46.7 \mathrm{~T}$ & 51 & 13.684 & & -1.22 & & & & & & & & 49. & 13.0 & & & & & & 0.292 & \\
\hline & & & 26.0 & & 24.14 & 27.9 & & & & & & & & & & & & & & 49 & & & & & & & & \\
\hline$\frac{ \pm}{2}$ & & & & & 24.1 & 2 & & & $\mid 51.05$ & 13.6 & & & & & & & & & & 49. & & & & & & & & \\
\hline 112 & (1) & $\frac{3.22 .5171}{3.22 .5101}$ & & $\frac{2.0300}{2650}$ & 24.14t & $\begin{array}{l}27.7480 \\
27908\end{array}$ & & & S & & & - & & & & & & & & . & & & & & & & 0.207 & \\
\hline 113 & 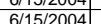 & $\frac{3.25 .5 .51 P M}{3.51}$ & $\frac{2.006}{2606}$ & $\frac{2.040}{2651}$ & $\frac{2.141}{2127}$ & 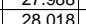 & & 46995 & $\frac{10.34 t}{5154}$ & 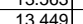 & & \begin{tabular}{|l|l|l|l|} 
\\
\end{tabular} & & & & $\frac{11.0}{146}$ & & & & 45: & $\frac{14}{2 \pi}$ & & & & & & 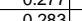 & \\
\hline 1114 & $6 / 15 / 21$ & $\frac{5.2 .55}{3.251 \mathrm{PM}}$ & & 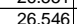 & 241 & | & & 46904 & & 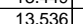 & & -1.22 & & & & 146 & & & & 497 & & & & & & & (1) & \\
\hline & & & & & 2207 & $\frac{27.942}{27}$ & & 46 & & & & -1.2 & & & & $\frac{14.6}{14.6}$ & & & & 49 & 102 & & & & & & 234 & \\
\hline 116 & & & & & & & & & & & & & & & & & & & & & & & & & & & & \\
\hline & & & & & & & & & & & & & & & & & & & & & & & & & & & & \\
\hline 118 & & & 26.05 & 26.5 & 24.085 & 28.161 & & 46.792 & 50.926 & 13.547 & & \begin{tabular}{|l|l|} 
& -1.2 \\
\end{tabular} & & 45 & & 14.6 & & & & 5 & 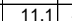 & & & & & & 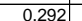 & \\
\hline 118 & $815 / 2$ & $3: 30: 51 \mathrm{PM}$ & & 2656 & 057 & 28.183 & & & & 617 & & -1.2 & & & & 46 & & & & 100 & & & & & 39 & & .224 & \\
\hline 1120 & $6 / 15 / 2004$ & 3:31:51 PM & 26.051 & 226.585 & \begin{tabular}{|l|}
24.071 \\
\end{tabular} & 28.256 & 26.897 & \begin{tabular}{|l|l|}
46.848 \\
\end{tabular} & \begin{tabular}{|l|l|}
50.839 \\
\end{tabular} & \begin{tabular}{|l|}
13.562 \\
\end{tabular} & \begin{tabular}{|l|l|}
33.93 \\
\end{tabular} & \begin{tabular}{|l|l|} 
\\
\end{tabular} & -7.595 & 45.212 & 0.073 & 14.643 & & 17.13 & 30.2856 & & 11.1 & & . 7855 & \begin{tabular}{|l|l|}
0.011 \\
\end{tabular} & 0.011 & 0.000 & 0.277 & \\
\hline
\end{tabular}


WSRC-TR-2005-00105, REVISION 0

SRNL-RPP-2005-00012, REVISION 0

RUN \# 3.03A AND B; FIRST AND SECOND HALF OF SLURRY DEWATERING - CONT.

\begin{tabular}{|c|c|c|c|c|c|c|c|c|c|c|c|c|c|c|c|c|c|c|c|c|c|c|c|c|c|c|c|c|}
\hline & A & $\mathrm{B}$ & D & $E$ & $\mathrm{~F}$ & $\begin{array}{ll}G \\
\end{array}$ & \begin{tabular}{ll|}
$\mathrm{H}$ \\
\end{tabular} & $\mathrm{J}$ & $\mathrm{K}$ & $\mathrm{L}$ & $M$ & $\mathrm{~N}$ & 0 & $\mathrm{Q}$ & R & s & $T^{T}$ & $\mathrm{v}$ & w & $|x|$ & $\mathrm{Y} \mid$ & & & $A B$ & $A C$ & & AE & AF $\mid A$ \\
\hline & $6 / 15 / 2004$ & 3:32:51 PM & 26.049 & 26.603 & 24.074 & 28.339 & 26.984 & 46.792 & 51.21 & 13.546 & 33.743 & -1.219 & $\begin{array}{l}-7.635 \\
\end{array}$ & \begin{tabular}{|l|l|}
51.087 \\
\end{tabular} & 0.075 & 14.643 & 0.004 & 1818.13 & \begin{tabular}{l|l}
330.3022 \\
3
\end{tabular} & \begin{tabular}{|l|} 
\\
\end{tabular} & 12.5 & 40.3 & 2.776 & 0.011 & 0.011 & 0.000 & 0.285 & 0.24 \\
\hline & $6 / 151 / 2004$ & & 26.08 & 26.633 & 24.099 & 28.424 & 26.99 & 46.657 & 50.727 & \begin{tabular}{|l|}
13.549 \\
\end{tabular} & 33.694 & \begin{tabular}{|l|l|l|}
4 & -1.219 \\
\end{tabular} & $\begin{array}{l}-7.655 \\
-.655\end{array}$ & 48.795 & 0.078 & \begin{tabular}{|l|l|}
14.643 \\
\end{tabular} & 0.004 & & 3.30 .3189 & 49.3 & & & & & & & & \\
\hline & $6 / 15 / 2004$ & 3:34:51 PM & 26.058 & 26.641 & \begin{tabular}{|l|l|}
24.072 \\
\end{tabular} & 28.387 & 26.958 & $\begin{array}{l}47.109 \\
\end{array}$ & \begin{tabular}{|l|l|}
51.473 \\
5.131
\end{tabular} & \begin{tabular}{|l|}
13.597 \\
\end{tabular} & $\begin{array}{l}34.034 \\
3037\end{array}$ & \begin{tabular}{|l|l|l|}
4 & -1.219 \\
\end{tabular} & $\begin{array}{l}-7.569 \\
-7.55\end{array}$ & \begin{tabular}{|l|}
53.336 \\
\end{tabular} & $\begin{array}{l}0.07 \\
0.075\end{array}$ & \begin{tabular}{|l|l|}
14.643 \\
\end{tabular} & & $\frac{1820.13}{120.13}$ & $\begin{array}{l}3.30 .3356 \\
2.3252\end{array}$ & & \begin{tabular}{|l|l|}
13.1 \\
\end{tabular} & 40.6 & $\begin{array}{l}.797 \\
.790\end{array}$ & 0.010 & 0.011 & 0.000 & 0.264 & \\
\hline 1125 & $6 / 15 / 2004$ & 3:35:51 PM & 26.065 & 26.649 & \begin{tabular}{|l|l|}
24.069 \\
\end{tabular} & $\begin{array}{l}28.365 \\
2.35\end{array}$ & 26.92 & 46.941 & \begin{tabular}{|l|l|}
51.141 \\
\end{tabular} & $\begin{array}{l}13.577 \\
\end{array}$ & 33.847 & \begin{tabular}{l|l|}
7 & -1.222 \\
\end{tabular} & $\begin{array}{r}7.58 \\
\end{array}$ & \begin{tabular}{|l|l|}
42.343 \\
\end{tabular} & 0.075 & 14.643 & 0.004 & 1821.13 & 30.3522 & \begin{tabular}{|l|l|}
49.7 \\
\end{tabular} & \begin{tabular}{|l|l|} 
& 10.4 \\
\end{tabular} & $\begin{array}{l}40.4 \\
\end{array}$ & 2.785 & 0.011 & 0.011 & 0.000 & 0.284 & \\
\hline$\frac{1125}{1126}$ & $\frac{6 / 15 / 2004}{6 / 15 / 204}$ & $\begin{array}{ll}3: 36: 51 \mathrm{PM} \\
3 \cdot 37 \cdot 51 \mathrm{PM}\end{array}$ & $\frac{26.057}{26.047}$ & 26.66 & \begin{tabular}{|l|}
24.061 \\
24057
\end{tabular} & $\begin{array}{r}28.351 \\
28.342\end{array}$ & & $\begin{array}{r}46.684 \\
47035 \\
\end{array}$ & \begin{tabular}{|r|}
50.99 \\
51371
\end{tabular} & \begin{tabular}{|l|}
13.506 \\
3625
\end{tabular} & & \begin{tabular}{l|l|}
4 & -1.219 \\
5 & 1220
\end{tabular} & $\begin{array}{l}-7.687 \\
7522\end{array}$ & \begin{tabular}{|l|l} 
\\
\end{tabular} & \begin{tabular}{|c|} 
\\
\end{tabular} & \begin{tabular}{|l|l|}
14.643 \\
11643
\end{tabular} & 0.004 & & $\begin{array}{l}330.3689 \\
2.02096\end{array}$ & 49.5 & \begin{tabular}{|l|l|}
12.0 \\
\end{tabular} & & \begin{tabular}{|l|}
2.771 \\
2701 \\
\end{tabular} & & 0.011 & & 0.267 & \\
\hline \begin{tabular}{|l|l|}
1127 \\
112
\end{tabular} & $\begin{array}{l}6 / 1 / 15 / 2004 \\
/ 2004\end{array}$ & $\begin{array}{l}3: 37: 51 \mathrm{PM} \\
3: 38: 51 \mathrm{PM}\end{array}$ & $\frac{26.047}{26.059}$ & $\frac{26.671}{26.678}$ & $\frac{24.057}{24.073}$ & $\begin{array}{l}28.342 \\
28.304\end{array}$ & \begin{tabular}{|l|}
26.868 \\
26.829 \\
\end{tabular} & $\begin{array}{r}47.035 \\
47.041 \\
\end{array}$ & \begin{tabular}{|l|}
51.371 \\
51.127 \\
\end{tabular} & \begin{tabular}{|}
$\mid \begin{array}{l}13.625 \\
13.571\end{array}$ \\
\end{tabular} & $\begin{array}{r}33.925 \\
34.042 \\
\end{array}$ & $\begin{array}{l}-1.222 \\
-1.225 \\
\end{array}$ & $\begin{array}{l}-7.522 \\
-7.719\end{array}$ & \begin{tabular}{|l|}
46.123 \\
47.053 \\
\end{tabular} & $\begin{array}{l}0.075 \\
0.075\end{array}$ & $\begin{array}{l}\frac{14.643}{14.643} \\
\end{array}$ & $\frac{0.002}{0.003}$ & $\begin{array}{l}\frac{1823.13}{1824.13} \\
13\end{array}$ & $\begin{array}{l}\frac{30.3856}{30} 3022 \\
3\end{array}$ & $\begin{array}{r}49.9 \\
49.7 \\
\end{array}$ & \begin{tabular}{|l|}
11.3 \\
11.5
\end{tabular} & & 2.791 & 0.011 & $\begin{array}{l}0.011 \\
0.011\end{array}$ & 0.000 & 0.284 & 0.24 \\
\hline \begin{tabular}{|l|}
1128 \\
\end{tabular} & & & 26.064 & 26.702 & 24.103 & 28.319 & \begin{tabular}{|l|}
26.829 \\
\end{tabular} & & \begin{tabular}{|l|l|}
50.795 \\
\end{tabular} & \begin{tabular}{|l|}
13.41 \\
13.469
\end{tabular} & 33.603 & & $\begin{array}{l}-7.499 \\
\end{array}$ & 56.356 & 0.072 & $\begin{array}{l}\frac{14.045}{14.643} \\
\end{array}$ & 0.004 & $\begin{array}{l}1824.13 \\
1825.13\end{array}$ & \begin{tabular}{|c|c|}
3 & 30.42422 \\
30.4189
\end{tabular} & $\begin{array}{ll}49.1 \\
49.3\end{array}$ & \begin{tabular}{|l|l|}
13.5 \\
\end{tabular} & $\begin{array}{l}40.5 \\
40.1\end{array}$ & 2.7.764 & 0.011 & 0.011 & & & $\begin{array}{l}0.24 \\
0.23 \\
\end{array}$ \\
\hline 1000 & $6 / 15 / 2004$ & 3:40:51 PM & & 26.719 & & 28.245 & 26.796 & 46.771 & \begin{tabular}{|l|}
50.917 \\
\end{tabular} & 13.483 & 33.852 & \begin{tabular}{|l|l|}
2 & -1.222 \\
\end{tabular} & -7.54 & & 0.076 & 14.643 & & 1826.13 & 330.4356 & 49.4 & 13.5 & & & & & & & \\
\hline & $6 / 15 / 2004$ & 3:41:51 PM & & 26.713 & 24.074 & & & 46.765 & 50.899 & $\begin{array}{l}13.476 \\
\end{array}$ & & -1.219 & & 44.997 & & \begin{tabular}{|l|l|}
14.643 \\
\end{tabular} & & & 30.4522 & 49.4 & \begin{tabular}{|l|l|} 
\\
\end{tabular} & & & 0.011 & 0.011 & 0.000 & 0.281 & \\
\hline 1131 & $6 / 15 / 2004$ & 3:42:51 PM & 26.085 & 26.734 & 24.085 & $\begin{array}{l}28.225 \\
2.20\end{array}$ & 26.761 & $\begin{array}{r}46.819 \\
\end{array}$ & 51.112 & \begin{tabular}{|l|l|l|}
13.498 \\
\end{tabular} & 33.808 & & $\begin{array}{r}-7.673 \\
\end{array}$ & \begin{tabular}{|l|l|}
46.87 \\
\end{tabular} & \begin{tabular}{|l|l|}
0.075 \\
\end{tabular} & \begin{tabular}{|l|l|}
14.643 \\
\end{tabular} & & 1828.13 & 30.4689 & $\begin{array}{l}49.6 \\
\end{array}$ & \begin{tabular}{|l|l|} 
& 11.5 \\
\end{tabular} & 40.3 & & 0.011 & 0.011 & 0.000 & 0.285 & \\
\hline$\frac{1132}{1133}$ & $6 / 15 / 2004$ & $\begin{array}{l}3: 43: 51 \text { PM } \\
3: 44: 51 \mathrm{P}\end{array}$ & $\begin{array}{r}26.07 \\
26.065\end{array}$ & $\begin{array}{l}26.728 \\
26733\end{array}$ & 24.054 & & & $\begin{array}{r}47.19 \\
\end{array}$ & \begin{tabular}{|l|l|}
51.461 \\
516
\end{tabular} & $\begin{array}{l}13.549 \\
1543\end{array}$ & $\begin{array}{l}34.176 \\
34.25 \\
\end{array}$ & $\begin{array}{l}-1.222 \\
122\end{array}$ & & 48.007 & $\begin{array}{l}0.074 \\
0.073\end{array}$ & $\begin{array}{l}14.643 \\
14643\end{array}$ & & & $\begin{array}{l}30.4856 \\
2.5022\end{array}$ & 50.0 & \begin{tabular}{|l|l|} 
\\
\end{tabular} & & 2007 & $\begin{array}{l}5.011 \\
5011\end{array}$ & $\begin{array}{l}0.011 \\
0.011\end{array}$ & 0.000 & 0.279 & \\
\hline$\frac{1133}{1134}$ & $\begin{array}{l}6611552004 \\
6 / 12512004\end{array}$ & $\begin{array}{l}3: 44: 51 \mathrm{PM} \\
3: 55: 51 \mathrm{PM}\end{array}$ & $\frac{26.065}{26.065}$ & $\frac{26.733}{26.734}$ & $\begin{array}{r}24.059 \\
24.04\end{array}$ & $\begin{array}{r}28.18 \\
28.196\end{array}$ & 26.785 & $\begin{array}{l}47.153 \\
47.275 \\
\end{array}$ & \begin{tabular}{|r|}
51.46 \\
51.661
\end{tabular} & $\begin{array}{l}\frac{13.543}{13.566} \\
\end{array}$ & & $\begin{array}{l}-1.222 \\
-\frac{1.2222}{2}\end{array}$ & $\begin{array}{l}-7.661 \\
-7.595 \\
\end{array}$ & \begin{tabular}{|l|}
46.526 \\
44.479
\end{tabular} & $\begin{array}{l}0.073 \\
0.072\end{array}$ & $\begin{array}{l}14.643 \\
14.643\end{array}$ & 0.004 & 1313 & $\begin{array}{l}30.5022 \\
30.5189\end{array}$ & 50.0 & \begin{tabular}{|l|}
11.4 \\
109
\end{tabular} & & 2.802 & 0.011 & 0.011 & $\begin{array}{l}0.000 \\
0.000\end{array}$ & & 0.23 \\
\hline \begin{tabular}{|l|l|}
1135 \\
\end{tabular} & $\begin{array}{l}0 / 113 / 204 \\
6 / 15 / 2004\end{array}$ & $\begin{array}{l}3: 45.51 \\
3: 46: 51 \mathrm{PM}\end{array}$ & $\frac{26.065}{26.074}$ & $\frac{26.154}{26.763}$ & $\begin{array}{r}24.04 \\
24.054\end{array}$ & $\frac{28.190}{28.204}$ & 26.78 & \begin{tabular}{|l|}
41.215 \\
47.024
\end{tabular} & \begin{tabular}{|l|}
51.001 \\
51.282 \\
\end{tabular} & $\begin{array}{l}13.500 \\
13.483 \\
\end{array}$ & $\begin{array}{r}34.204 \\
34.04 \\
\end{array}$ & -1.219 & $\begin{array}{l}-7.595 \\
-7.696 \\
\end{array}$ & \begin{tabular}{|l}
4.419 \\
51.991
\end{tabular} & $\begin{array}{l}0.072 \\
0.077\end{array}$ & \begin{tabular}{l|l}
$\frac{14.045}{14.643}$ \\
\end{tabular} & & $\frac{181.15}{1832.13}$ & \begin{tabular}{|l|l}
3 & 30.5359 \\
\end{tabular} & $\begin{array}{l}30.4 \\
49.8 \\
\end{array}$ & $\begin{array}{l}10.9 \\
12.7\end{array}$ & & 2.8099 & $\frac{0.011}{0.011}$ & $\frac{0.011}{0.012}$ & & & $\begin{array}{l}0.23 \\
0.25 \\
\end{array}$ \\
\hline & $6 / 15 / 2004$ & 3:47:51 PM & 26.061 & 26.754 & 24.035 & 28.216 & 26.737 & 47.172 & \begin{tabular}{|l|l|}
51.454 \\
\end{tabular} & 13.575 & 34.077 & & -7.603 & 44.999 & 0.071 & 14.643 & & & & & \begin{tabular}{|l|l|}
11.0 \\
\end{tabular} & & & & & & & $\begin{array}{l}0.25 \\
0.23\end{array}$ \\
\hline & 6/15/2004 & 3:48:51 PM & & 26.754 & 24.04 & 28.181 & & 47.265 & 51.672 & $\begin{array}{l}13.666 \\
\end{array}$ & 34.007 & -1.219 & -7.554 & \begin{tabular}{|l|l|}
40.948 \\
\end{tabular} & 0.075 & $\begin{array}{l}14.643 \\
\end{array}$ & & & & 50.2 & & & & & & & & $\frac{0.23}{0.24}$ \\
\hline 1138 & & & 26.066 & & & & & 47.13 & 51.423 & & & & & & & 14.643 & & & .5856 & & 10.5 & & & & & .000 & 0.279 & \\
\hline 139 & & 3:50:51 PM & 26.065 & 26.764 & 24.039 & \begin{tabular}{|l|l|}
28.16 \\
\end{tabular} & & 47.199 & 51.531 & $\begin{array}{ll}13.674 \\
\end{array}$ & 33.995 & 222 & & 40.739 & 0.073 & 14.643 & & & & & 10.0 & & & .011 & 0 & & & \\
\hline & 6/15/2004 & 3:51:51 PM & 26.069 & 26.778 & 24.049 & 28.204 & & $\begin{array}{l}47.141 \\
\end{array}$ & 51.521 & $\begin{array}{l}13.641 \\
\end{array}$ & & & & & & 14.643 & & & & & & & & & & & & \\
\hline 14. & 6/15/2004 & & 26.064 & 26.767 & 24.038 & 28.169 & & & 177 & 13.55 & .977 & & -7. & 48.457 & .074 & 14.643 & & & & & & & & & & & & \\
\hline & $6 / 15 / 2004$ & 3:53:51 PM & 26.053 & 26.756 & 24.022 & 28.168 & 26.719 & 47.143 & 51.624 & 13.551 & 34.059 & -1.222 & -7.661 & 51.947 & $\begin{array}{l}0.073 \\
\end{array}$ & 14.643 & & 1839.13 & 30.6522 & 50.2 & 12.7 & 40. & 2.799 & 0.011 & 0.011 & 0.000 & 0.276 & 0.23 \\
\hline & 6/15/2004 & 3:54:51 PM & 26.057 & 26.766 & 24.021 & 28.162 & 26.703 & 47.143 & 51.562 & 13.688 & $\begin{array}{l}33.888 \\
2017\end{array}$ & $\begin{array}{l}-1.219 \\
\end{array}$ & \begin{tabular}{|c|}
-7.331 \\
750
\end{tabular} & 45.739 & 0.06 & 14.643 & & 184. & 30.6689 & 50. & & & 793 & . 00.09 & .009 & & & \\
\hline 144 & $6 / 15 / 2004$ & & 26.065 & 26.164 & 24.09 & 28.211 & & 46.842 & 51.029 & 13.538 & .817 & & & 4.8 .8 & 0.063 & 14.643 & & & 30.6856 & 49. & & & & & & & & \\
\hline & & 3:56:51 PM & $26.0 / 8$ & 26.871 & 24.092 & 28.228 & & 46.883 & 51.206 & 13.554 & & & & & & 14.643 & & & 30.1022 & & 12.2 & & & & & & & \\
\hline & $601 / 15120404$ & 3:57:51 PM & $\begin{array}{l}26.015 \\
26067\end{array}$ & $\frac{20.179}{26767}$ & $\begin{array}{l}24.084 \\
24056\end{array}$ & $\begin{array}{l}28.285 \\
28.257\end{array}$ & & $\begin{array}{r}46.85 \\
46564\end{array}$ & $\frac{50.969}{50.824}$ & $\begin{array}{l}13.518 \\
13.486\end{array}$ & $\begin{array}{r}33.93 \\
33.628\end{array}$ & & & $\frac{57.084}{501165}$ & .067 & $\frac{14.643}{14.643}$ & & & & & & & & & & & & \\
\hline 1148 & $6 / 15 / 2004$ & 3.5:59:51 PM & 26.09 & 26.799 & $\frac{24.050}{24.08}$ & 28.255 & & $\begin{array}{l}40.504 \\
46.873\end{array}$ & $\begin{array}{c}50.024 \\
50.88\end{array}$ & \begin{tabular}{|}
13.400 \\
13.53 \\
\end{tabular} & $\begin{array}{l}30.020 \\
33.932 \\
\end{array}$ & $\begin{array}{l}-1.222 \\
-1.222 \\
\end{array}$ & -7.658 & 4518 & $\begin{array}{r}0.073 \\
0.073\end{array}$ & 14643 & & & & 494 & 116 & & & & & & & \\
\hline 1149 & $6 / 15 / 2004$ & 4:00:51 PM & 26.089 & 26.803 & 24.083 & 28.319 & 26.75 & 47.002 & 51.205 & 13.56 & 33.999 & -1.222 & -7.678 & 49.469 & 0.072 & 14.643 & & & 307689 & 49.7 & 12.1 & & & & & & & \\
\hline & & & & & & 28.337 & & & 51.193 & & & \begin{tabular}{|l|l|}
4 & -1.222 \\
\end{tabular} & & 48.849 & 0.075 & 14.643 & & & & & & & & & & & & \\
\hline & $6 / 15 / 2004$ & & 26.09 & 26.788 & 24.064 & 28.32 & & 47.149 & & 13.548 & & & & & 0.074 & 14.643 & & & & 49.8 & & & & & & & & \\
\hline & & & 26.082 & 26.79 & 24.046 & 28.272 & & 46.95 & 1.448 & 13.59 & & & & & & 14.643 & & & 8189 & & 11.9 & & & & & 000 & & \\
\hline & $6 / 15 / 200$ & & 26.081 & & 24.05 & \begin{tabular}{|l|l|}
28.301 \\
\end{tabular} & & & 51.208 & 13.597 & 34.071 & & & & & 14.643 & & & & 49 & & & & & & & & \\
\hline $15 \mathrm{~L}$ & 6/15/2004 & :51 PM & 26.075 & 26.774 & 24.014 & 28.285 & & $47.08 !$ & 51.461 & 13.579 & 3.973 & & & & & 14.643 & & & & & 12.3 & & 7.79 & & & & & \\
\hline & 6/15/2004 & $4: 0$ & 26.085 & 26.784 & 24.009 & 28.335 & & & .317 & \begin{tabular}{|c|c|}
13.574 \\
\end{tabular} & 33.942 & 222 & -7.5 & 49.308 & & 14.643 & & 185 & 30.8689 & 49.8 & $\begin{array}{ll}12.1 \\
\end{array}$ & & & & & & & \\
\hline & $6 / 15 / 2$ & $4: 0$ & 26.103 & 26.777 & 24.003 & 28.398 & & & 51.038 & 13.57 & & -1.222 & -7.6 & 49.29 & 0.074 & 14.643 & & & & 49 . & 12.1 & & & & & & 0.281 & \\
\hline & $6 / 15 / 2$ & & 26.093 & 26.782 & 23.998 & 28.318 & & & .757 & 13.615 & 4.094 & & -7.68 & & & & & & & & 13.0 & & & & & & & \\
\hline & $6 / 15 / 2004$ & & 26.108 & 26.18 & 23.998 & 8.258 & & & 168 & & & & & & & & & & & & 11.3 & & & & & & & \\
\hline & . & 4:10:51 PM & 26.111 & 26. & & 28.341 & & $4 ., 713$ & 51.599 & 13.532 & & -1.219 & & 52.01 & & 14.64 & & & 30.9356 & 50. & 12.7 & & & & 0.0 & & 0.283 & \\
\hline $16, \quad 2$ & 6 & $\begin{array}{l}4.15515 \mathrm{P} \\
4.1 .5 \mathrm{PM}\end{array}$ & 26 & $\frac{20.808}{2682}$ & 24.009 & 28.3 & & $\begin{array}{l}47.155 \\
47138\end{array}$ & 152 & 13.554 & & & & & & & & & & 50.1 & 12.1 & & & & & & & \\
\hline & 6 & & & 26.039 & $\frac{24.010}{2409}$ & 28.445 & & & & $\begin{array}{l}13.475 \\
13526\end{array}$ & & & & & & & & & & & 11.48 & & & & & & & \\
\hline & $6 / 15 / 2004$ & & 26.131 & & $\frac{24.091}{24091}$ & 28.406 & & & & 13.453 & & & & & & & & & & $\frac{39}{49}$ & 103 & & & & & & & \\
\hline$\overline{1164}$ & $\frac{6}{6 / 15 / 2004}$ & 4:15: & $\frac{26.132}{26.132}$ & 26.846 & 24.072 & 28.332 & & 47.232 & $\frac{15.446}{51.446}$ & 13.541 & & & & 42.535 & $\mid 0.072$ & 14.643 & & & 31.0189 & 50.0 & 10.4 & & & & $\frac{.0011}{0.011}$ & 0.000 & 0.270 & \\
\hline 110 & $15 / 2004$ & & & & & & & & & & & & & & & & & & & & 11.1 & & & & & & & \\
\hline & $6 / 15 / 2004$ & & 26.13 & 26.85 & 24.055 & 28.295 & & \begin{tabular}{|l|l|}
47.18 \\
\end{tabular} & .359 & 13 & & & & & & & & & & 49.9 & 13.1 & & & & & & & \\
\hline & $6 / 15 / 200$ & & & & 24.045 & & & & & 13.471 & & & & & & & & & & 49. & 11.6 & & & & & & & \\
\hline & $6 / 15 / 2004$ & & 26.125 & 26.85 & 24.045 & 28.265 & & & .836 & & & & & & & & & & & 501 & 11.1 & & & & & & 278 & \\
\hline & 6/15/2004 & & 26.119 & 26.848 & 24.034 & 28.234 & & \begin{tabular}{|l|l|l|}
46.968 \\
\end{tabular} & .365 & & & & & & & 14.6 & & & & & $\begin{array}{ll}12.4 \\
\end{array}$ & & & & 0.01 & 0.000 & 0.254 & \\
\hline & $6 / 15 / 2$ & 4:21:5 & 26.12 & & 24.047 & & & & & & & & & & & & & & & & 11.2 & & & & & & .2000 & \\
\hline 114 & $6 / 15 / 2004$ & $4: 22: 5$ & 26.11 & 26.863 & 24.039 & 28.215 & & 47. & 502 & & & & & & & & & & & & 10.9 & & & & & & 0.267 & \\
\hline 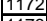 & 67 & & 26.12 & & & & & & & & & & & & & & & & & & 10.0 & & & & & & & \\
\hline & . & & 26.11 & & 24.04 & 28.262 & & $4 i$ & 51.732 & 13.5 & & & & & & & & & & & 12.0 & & & & & & & \\
\hline 7 & $\frac{61517}{66151}$ & & $\frac{26.115}{26129}$ & $\frac{20.814}{26888}$ & 24.049 & $\begin{array}{l}28.255 \\
28249\end{array}$ & & & 419 & & & & & & & & & & & & 11.5 & & & & & & $\frac{249}{270}$ & \\
\hline & $6611 / 200$ & & $\frac{2.12}{26.11}$ & & 24.036 & & & & y & & & & & & & & & & & & & & & & & & & \\
\hline 117 & $6 / 1 / 5200$ & & & & 2404 & & & & & & & & & & & & & & & & 116 & & & & & & & \\
\hline 117 & $6 / 6 / 15 / 200$ & & 26.1 & 26.884 & 24.04 & $\frac{2.28}{28.2}$ & & & \begin{tabular}{|l|l|}
51.498 \\
\end{tabular} & & & & & & & & & & & 500 & 124 & & & & & & 0.270 & \\
\hline & $6 / 15 / 200$ & & & & & & & & & & & & & & & & & & & & & & & & & & & \\
\hline & & & 26.11 & & & & & & & & & & & & & & & & & & & & & & & & & \\
\hline & $6 / 15 /$ & & 26.111 & 26 & $24.0 \varepsilon$ & 28.2 & & & & & & & & & & & & & & & 10.7 & & & & & & & \\
\hline & $6 / 15 / 2$ & 4:33:51 & 26.106 & 26.85 & 24.0 & 28.20 & & 47.6 & 51.45 & 13.48 & & & & & & & & & 31.3 & & 12.3 & & & & & & 264 & \\
\hline & $6 / 15 / 20$ & 4:34:5 & 26.136 & $26.89 \mathrm{~s}$ & 24.1 & 28.226 & & 47.178 & 51.562 & 13.486 & 34.1 & -1. & & & 00 & 14.6 & & & 31.3 & & \begin{tabular}{ll|}
11.4 \\
\end{tabular} & & & & & & 271 & \\
\hline 18 & $6 / 1$ & & 26.114 & 26.873 & 24.06 & 28.249 & & & 51.626 & & 34. & & & & & $14.6 \mathrm{C}$ & & & & & 12.2 & & & & & & & \\
\hline & $6 / 15 /$ & & 26.129 & 26.8 & 24.07 & 28.334 & & & & 13.472 & & $\begin{array}{l}8 \\
8\end{array}$ & & & & & & & & 50.2 & 13.4 & & & & & & 0.268 & \\
\hline 11 & $6 /$. & & 26.1 & & 24.0 & & & 47 & & & & & & & & & & & & 50.4 & 1.2 & & & & & & & \\
\hline 188 & & & 26.118 & 26.81 & $\begin{array}{l}24.063 \\
2003\end{array}$ & 28.592 & & 41. & & & & & & & & & & & & & 11.6 & & & & & & & \\
\hline 18. & 0115122004 & 4.3951 & 20.12 & $\frac{20.093}{36.8}$ & 24.000 & 28.693 & 20.6 & 4.06 & $\begin{aligned} 50.09 \\
507\end{aligned}$ & 13.022 & & 120 & -1.0 & 516 & & 14.64 & & & & 50.4 & 11.5 & & 26 & & 2 & & 0.201 & \\
\hline$\frac{1100}{1190}$ & \begin{tabular}{|l|l|}
$6 / 15 / 2004$ \\
\end{tabular} & 4:40.01 PM & $\frac{0.121}{26.127}$ & $\frac{20.05}{26.896}$ & \begin{tabular}{|l|l|}
24.000 \\
24.112 \\
\end{tabular} & 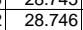 & \begin{tabular}{|l|l|}
20.093 \\
26.932
\end{tabular} & $\begin{array}{l}40.200 \\
46.624\end{array}$ & \begin{tabular}{|l|}
50.928 \\
\end{tabular} & \begin{tabular}{|l|}
14.150 \\
14.573
\end{tabular} & \begin{tabular}{|l}
1.1512 \\
32.536
\end{tabular} & \begin{tabular}{|l|l|}
6 & -1.222 \\
\end{tabular} & -7.667 & 43.781 & 0.072 & \begin{tabular}{|l|}
14.045 \\
14.643 \\
\end{tabular} & & 1887.13 & $\begin{array}{l}31.4500 \\
3\end{array}$ & \begin{tabular}{|l|}
49.5 \\
\end{tabular} & \begin{tabular}{|l|l|}
10.1 \\
10.7
\end{tabular} & & \begin{tabular}{|l|l}
2.729 \\
\end{tabular} & 0.011 & $\mid$\begin{tabular}{|c|}
0.011 \\
\end{tabular} & 0.000 & \begin{tabular}{|l|}
0.278 \\
\end{tabular} & $\begin{array}{lll}8 & 0.2 \\
\end{array}$ \\
\hline
\end{tabular}


WSRC-TR-2005-00105, REVISION 0

SRNL-RPP-2005-00012, REVISION 0

RUN \# 3.03A AND B; FIRST AND SECOND HALF OF SLURRY DEWATERING - CONT.

\begin{tabular}{|c|c|c|c|c|c|c|c|c|c|c|c|c|c|c|c|c|c|c|c|c|c|c|c|c|c|c|c|c|}
\hline & $\mathrm{A}$ & B & D & $E$ & $F$ & G & $\mathrm{H}$ & $\mathrm{J}$ & $\mathrm{K}$ & $\mathrm{L}$ & $M$ & $\mathrm{~N}$ & 0 & $Q$ & $\mathrm{R}$ & $\mathrm{s}$ & $\begin{array}{ll}T \\
\end{array}$ & v & w & $x$ & \begin{tabular}{|r|} 
\\
\end{tabular} & $z$ & $\mathrm{AA}$ & $A B$ & & $A D$ & AE & \\
\hline & $6 / 15 / 2004$ & 4:42:51 PM & 26.123 & 26.901 & 24.128 & 28.916 & 26.933 & 46.84 & 51.148 & \begin{tabular}{ll|l}
14.464 \\
\end{tabular} & 32.885 & -1.225 & -7.534 & 50.368 & 0.066 & $\begin{array}{l}14.643 \\
\end{array}$ & 0.004 & 1888.13 & 331.4689 & 49.7 & 12.3 & 39.9 & 2.748 & 0.010 & 0.010 & 0.000 & 0.253 & \\
\hline & $6 / 15 / 2004$ & & 26.109 & 26.887 & 24.123 & 28.852 & 26.949 & 46.699 & 50.996 & 14.39 & 32.811 & -1.225 & -7.511 & 46.186 & $\begin{array}{l}.067 \\
\end{array}$ & $\begin{array}{l}4.643 \\
\end{array}$ & 0.004 & & 31.4856 & 49.5 & 11.3 & & & & & & 0.258 & \\
\hline & $6 / 15 / 2004$ & 4:44:51 PM & & 26.919 & 24.17 & 28.868 & 26.97 & $\begin{array}{l}47.101 \\
\end{array}$ & \begin{tabular}{|l|}
51.261 \\
5.211 \\
\end{tabular} & $\begin{array}{l}14.461 \\
\end{array}$ & $\begin{array}{r}33.16 \\
33.0\end{array}$ & -1.222 & $\begin{array}{r}-7.543 \\
750\end{array}$ & 49.045 & \begin{tabular}{|l|}
0.069 \\
\end{tabular} & \begin{tabular}{|l|}
14.643 \\
\end{tabular} & 0.004 & 1890.13 & & 49.8 & $\begin{array}{l}12.0 \\
12.0\end{array}$ & 40 & 2.101 & & 0.011 & 0.000 & 0.262 & \\
\hline 1194 & $6 / 15 / 2004$ & 4:45:51 PM & \begin{tabular}{|l|l|}
26.14 \\
26.141
\end{tabular} & 26.919 & $\begin{array}{r}24.18 \\
\end{array}$ & 28.764 & 26.936 & $\begin{array}{l}47.028 \\
\end{array}$ & 51.145 & 14.484 & $\begin{array}{l}33.074 \\
\end{array}$ & -1.219 & $\begin{array}{r}-7.609 \\
7.760\end{array}$ & 48.76 & \begin{tabular}{l|l|}
0.07 \\
\end{tabular} & $\begin{array}{l}14.643 \\
\end{array}$ & 0.004 & 1891.13 & 31.5189 & 49.7 & 11.9 & 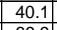 & 2.761 & 0.010 & 0.011 & 0.000 & 0.267 & \\
\hline & $\frac{6 / 115 / 2004}{6}$ & $\begin{array}{l}4: 46: 51 \mathrm{PM} \\
4 \cdot 47 \cdot 51 \mathrm{PM}\end{array}$ & $\frac{26.141}{2615}$ & $\begin{array}{r}26.92 \\
26.929\end{array}$ & & & & \begin{tabular}{|l|l|}
4685 \\
47132
\end{tabular} & \begin{tabular}{|l|}
51.141 \\
51.471 \\
\end{tabular} & $\begin{array}{r}14.418 \\
1441\end{array}$ & & \begin{tabular}{|l|}
-1.222 \\
-1252 \\
\end{tabular} & & 45.531 & 0.07 & \begin{tabular}{|l|l|l|}
14.643 \\
14643
\end{tabular} & & 1892.13 & $\frac{31.5356}{215522}$ & 49.7 & \begin{tabular}{|l|l|}
11.2 \\
\end{tabular} & $\begin{array}{l}39.9 \\
\end{array}$ & \begin{tabular}{|l|}
2.754 \\
2770 \\
\end{tabular} & & $\begin{array}{l}0.011 \\
\end{array}$ & & $\begin{array}{l}0.267 \\
0.207\end{array}$ & \\
\hline \begin{tabular}{|l|l|l|l|l}
1197 \\
\end{tabular} & $6 / 1 / 15 / 20404$ & $\begin{array}{l}4: 4: 51 \mathrm{PM} \\
4: 48: 51 \mathrm{PM}\end{array}$ & $\begin{array}{l}26.15 \\
2.17\end{array}$ & $\frac{26.929}{26.944}$ & $\begin{array}{r}24.2 \\
24.23 \\
\end{array}$ & $\begin{array}{l}28.529 \\
28.495\end{array}$ & $\begin{array}{l}26.901 \\
26.871\end{array}$ & $\begin{array}{l}47.132 \\
46.794 \\
\end{array}$ & 51.471. & \begin{tabular}{|r|}
14.41 \\
14.375 \\
\end{tabular} & $\begin{array}{r}33.219 \\
32.948\end{array}$ & \begin{tabular}{|l|}
-1.225 \\
-1.2222 \\
\end{tabular} & $\begin{array}{r}-7.644 \\
-7.612 \\
\end{array}$ & $\begin{array}{l}48.493 \\
50.107\end{array}$ & $\begin{array}{l}0.069 \\
0.072\end{array}$ & $\begin{array}{l}\frac{14.643}{14.643} \\
\end{array}$ & $\begin{array}{l}0.003 \\
0.004\end{array}$ & $\frac{1893.13}{1894.13}$ & $\begin{array}{l}331.522 \\
3 \\
3\end{array}$ & $\begin{array}{l}50.0 \\
49.7\end{array}$ & \begin{tabular}{|l|l|}
11.9 \\
12.3
\end{tabular} & $\begin{array}{l}40.2 \\
39.9\end{array}$ & \begin{tabular}{|l|}
2.770 \\
2.749 \\
\end{tabular} & $\frac{0.010}{0.011}$ & $\begin{array}{l}0.011 \\
0.011\end{array}$ & $\begin{array}{l}0.000 \\
0.000\end{array}$ & $\begin{array}{ll}0.262 \\
0.275\end{array}$ & 0.22 \\
\hline 1198 & & & & 26.939 & 24.285 & 28.405 & 26.871 & & & & 33.256 & & $\begin{array}{r}-7.43 \\
\end{array}$ & & 0.071 & $\begin{array}{l}14.643 \\
14.643\end{array}$ & 0.004 & $\frac{1044.15}{1895.13}$ & \begin{tabular}{|l|l|}
31.5089 \\
31.5856
\end{tabular} & $\begin{array}{l}50.1 \\
50.0\end{array}$ & $\begin{array}{l}11.3 \\
11.3\end{array}$ & \begin{tabular}{|}
39.9 \\
40.1 \\
\end{tabular} & \begin{tabular}{|l|}
2.7495 \\
2.765 \\
\end{tabular} & 0.011 & & 0.000 & 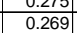 & $\begin{array}{l}0.23 \\
0.23 \\
\end{array}$ \\
\hline & $6 / 15 / 2004$ & & & 26.944 & 24.285 & 28.39 & 26.861 & 47.006 & 51.591 & \begin{tabular}{|l|l|}
14.238 \\
\end{tabular} & \begin{tabular}{|l|l|}
33.207 \\
\end{tabular} & \begin{tabular}{|l|}
-1.219 \\
\end{tabular} & -7.519 & 50.015 & 0.071 & 14.643 & 0.004 & 1896.13 & 331.6022 & & 12.3 & & & & & & & \\
\hline & $6 / 15 / 2004$ & 4:51:51 PM & & 26.922 & 24.264 & 28.393 & & & \begin{tabular}{|l|l|l|l|}
51.14 \\
\end{tabular} & & $\begin{array}{r}33.24 \\
\end{array}$ & \begin{tabular}{|l|}
-1.225 \\
\end{tabular} & $\begin{array}{l}-7.592 \\
\end{array}$ & 45.449 & & 14.643 & & 1897.13 & 31.6189 & 49.7 & 11.1 & 40.1 & 2.764 & & & 0.000 & 0.273 & \\
\hline 1201 & $6 / 15 / 2004$ & 4:52:51 PM & $\begin{array}{l}26.173 \\
2.102\end{array}$ & 26.931 & $\begin{array}{l}24.283 \\
2.207\end{array}$ & 28.358 & 26.853 & $\begin{array}{r}46.734 \\
6.703\end{array}$ & \begin{tabular}{|l|l|}
51.073 \\
\end{tabular} & $\begin{array}{l}14.152 \\
1.12\end{array}$ & 33.113 & $\begin{array}{l}-1.219 \\
\end{array}$ & \begin{tabular}{|l|l|}
-7.597 \\
7.53
\end{tabular} & 58.615 & \begin{tabular}{|l|l|}
0.069 \\
\end{tabular} & 14.643 & & $\begin{array}{l}1898.13 \\
102.12\end{array}$ & 31.6356 & 49.6 & $\begin{array}{l}14.4 \\
\end{array}$ & 39.6 & 2.753 & 0.010 & 0.010 & 0.000 & 0.263 & \\
\hline & $\frac{6 / 115 / 2004}{6}$ & $\begin{array}{l}4: 53: 51 \mathrm{PM} \\
4 \cdot 45.51 \mathrm{PM}\end{array}$ & $\begin{aligned} 26.192 \\
262\end{aligned}$ & $\begin{array}{l}26.936 \\
26.939\end{array}$ & $\begin{array}{l}24.287 \\
24.301\end{array}$ & \begin{tabular}{|l|}
28.302 \\
28266
\end{tabular} & $\frac{26.818}{26.786}$ & \begin{tabular}{|l|}
46.703 \\
46.522
\end{tabular} & $\begin{array}{l}50.876 \\
50.978\end{array}$ & \begin{tabular}{|l|}
14.037 \\
14.156
\end{tabular} & \begin{tabular}{|l|}
33.215 \\
32875 \\
\end{tabular} & $\begin{array}{l}-1.225 \\
-1.219\end{array}$ & $\begin{array}{l}-7.592 \\
7.822\end{array}$ & \begin{tabular}{|r|}
47.579 \\
47.89
\end{tabular} & \begin{tabular}{r|}
0.07 \\
0.071
\end{tabular} & $\begin{array}{l}14.643 \\
1.643\end{array}$ & 0.004 & 1899.13 & & $\begin{array}{l}49.4 \\
19.5\end{array}$ & \begin{tabular}{|l|l|}
11.7 \\
117
\end{tabular} & $\begin{array}{l}40.6 \\
397\end{array}$ & $\begin{array}{l}2.755 \\
2.77 \\
\end{array}$ & 0.010 & $\begin{array}{l}0.011 \\
0.011\end{array}$ & $\begin{array}{l}0.000 \\
0000\end{array}$ & $\frac{0.266}{0272}$ & \\
\hline$\frac{12203}{1204}$ & $6 / 1155 / 20404$ & $\begin{array}{l}4: 5: 51 \mathrm{PM} \\
4: 55: 51 \mathrm{PM}\end{array}$ & $\begin{array}{r}26.2 \\
26.2\end{array}$ & $\frac{26.939}{26.938}$ & $\begin{array}{l}24.301 \\
24.295\end{array}$ & $\begin{array}{l}28.266 \\
28.265\end{array}$ & $\frac{26.786}{26.8}$ & $\frac{46.552}{44.719}$ & $\begin{array}{r}50.978 \\
51.11\end{array}$ & $\begin{array}{r}14.156 \\
14.032 \\
\end{array}$ & .8.875 & 1.1.229 & $\begin{array}{r}-7.482 \\
-7.56 \\
\end{array}$ & $\begin{array}{r}47.89 \\
45.804\end{array}$ & & $\begin{array}{l}14.643 \\
14.643 \\
\end{array}$ & $\begin{array}{l}0.004 \\
0.004\end{array}$ & & $\begin{array}{l}31.6689 \\
331.8566\end{array}$ & 49.5 & 11.7 & 400 & \begin{tabular}{|l|}
$\mid 2.737$ \\
2.755
\end{tabular} & 0.011 & & 0.000 & D.272 & 0.23 \\
\hline 1205 & $6 / 15 / 2004$ & $\begin{array}{l}4.555 .51 \mathrm{PM} \\
4: 561 \mathrm{M}\end{array}$ & 26.209 & $\frac{20.938}{26.948}$ & $\begin{array}{r}24.295 \\
24.309\end{array}$ & \begin{tabular}{|l|}
28.234 \\
28.234
\end{tabular} & $\begin{array}{r}26.8 \\
26.795 \\
\end{array}$ & $\begin{array}{l}40.119 \\
47.257\end{array}$ & $\begin{array}{l}51.11 \\
51.705\end{array}$ & \begin{tabular}{|l|}
14.059 \\
\end{tabular} & $\begin{array}{l}33.169 \\
33.566\end{array}$ & \begin{tabular}{|l|l|}
-1.253 \\
-1.219 \\
\end{tabular} & $\begin{array}{l}-7.50 \\
-7.415\end{array}$ & $\begin{aligned} 45.804 \\
48.221\end{aligned}$ & $\begin{array}{l}0.071 \\
0.063\end{array}$ & $\begin{array}{l}14.645 \\
14.643 \\
\end{array}$ & $\begin{array}{l}0.004 \\
0.004\end{array}$ & \begin{tabular}{|l|l|l|l|}
1902.13 \\
\end{tabular} & 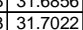 & 50.2 & $\begin{array}{l}11.2 \\
11.8\end{array}$ & & \begin{tabular}{|l|}
2.155 \\
2.786
\end{tabular} & $\frac{0.011}{0.009}$ & & & $\begin{array}{l}0.270 \\
0.237\end{array}$ & $\frac{0.23}{0.20}$ \\
\hline & 6/115/2004 & 4:57:51 PM & 26.212 & & 24.312 & 28.257 & & & 51.253 & 14.074 & 33.166 & & -7.574 & 41.501 & & 14.643 & & & & 49.8 & & & & & & & 0.278 & $\frac{0.20}{0.24}$ \\
\hline & $6 / 15 / 2004$ & 4:58:51 PM & & 26.94 & 24.322 & 28.277 & 26.797 & 47.317 & 51.566 & 14.155 & $\begin{array}{l}33.671 \\
\end{array}$ & & $\begin{array}{l}-7.464 \\
\end{array}$ & 52.962 & 0.065 & $\begin{array}{l}14.643 \\
\end{array}$ & & 04.1. & 31.7356 & 50.1 & 13.0 & $40 !$ & & & & & & 0.24 \\
\hline & & & & & & & & & & & & & & & & & & & & & & & & & & .000 & & \\
\hline & $6 / 15 / 2004$ & :04 PM & & 26.919 & 24.306 & \begin{tabular}{l|l|l|}
28.266 \\
\end{tabular} & & $\begin{array}{l}46.417 \\
\end{array}$ & 51.191 & $\begin{array}{l}14.081 \\
\end{array}$ & 32.762 & -1.031 & & 42.734 & $\begin{array}{l}0.08 \\
\end{array}$ & $\begin{array}{l}14.643 \\
\end{array}$ & & & & 49.7 & 10.5 & & & & & & & \\
\hline & & 5:01:04 PM & 26.231 & & 24.336 & 28.286 & & 47.196 & 51.45 & 14.135 & 33.587 & & & & & 14.643 & & & & & $\begin{array}{ll}9.6 \\
\end{array}$ & & & & & & & \\
\hline & 6/15/2004 & & & 26.9 & 24.332 & & & 47.3 & 51.684 & 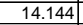 & 33.739 & -1.219 & & 54.141 & 0.075 & $\begin{array}{l}14.643 \\
\end{array}$ & & & & 50.2 & & & & & & & & \\
\hline & $6 / 15 / 2004$ & 5:03:04 PM & 26.237 & 26.93 & 24.342 & 28.192 & & 47.25 & 51.633 & 14.05 & \begin{tabular}{|l|l|}
33.667 \\
\end{tabular} & -1.219 & -7.519 & 47.425 & 0.07 & 14.643 & 0.004 & 1908.3 & 31.8058 & 50.2 & 11.6 & 40.5 & 2.790 & 0.01 & 0.011 & 0.000 & 0.263 & \\
\hline & 6/115/2004 & 5:04:04 PM & 26.247 & 26.925 & 24.352 & 28.157 & 26.757 & 47.192 & \begin{tabular}{|l|l|}
51.562 \\
\end{tabular} & $\begin{array}{l}13.965 \\
1.052\end{array}$ & \begin{tabular}{|l|l|l|}
33.774 \\
\end{tabular} & -1.222 & -7.519 & \begin{tabular}{|l|l|}
52.764 \\
\end{tabular} & 0.07 & 14.643 & 0.004 & 1909.35 & 31.8225 & 50.1 & 12.9 & 40.5 & 2.791 & & 0.011 & 0.000 & 0.263 & \\
\hline & $6 / 15 / 2004$ & 5:05:04 PM & 26.242 & 26.92 & 24.357 & 28.187 & 26.742 & 47.292 & \begin{tabular}{|l|l|}
51.744 \\
\end{tabular} & $\begin{array}{l}14.052 \\
\end{array}$ & 33.718 & $\begin{array}{r}-1.219 \\
\end{array}$ & & 53.138 & 0.071 & 14.643 & & & 31.8392 & 50.3 & 13.0 & 40.5 & \begin{tabular}{|l|}
2.793 \\
\end{tabular} & & & 0.000 & 0.266 & \\
\hline & $6 / 15 / 2004$ & 5:06:04 PM & 26.241 & & 24.346 & 28.146 & 26.712 & & 51.836 & \begin{tabular}{|l|l|}
14.084 \\
\end{tabular} & & & & & & 14.643 & & & & 50.4 & 12.3 & & 2.788 & 0.011 & & & .270 & \\
\hline & $6 / 115 / 2004$ & $\begin{array}{l}5: 07: 04 \mathrm{PM} \\
\text { 5:08:04 PM }\end{array}$ & $\frac{26.251}{26.254}$ & $\frac{26.909}{26.908}$ & $\begin{array}{r}24.371 \\
24.41\end{array}$ & $\frac{28.141}{28.125}$ & $\frac{26.712}{26.67}$ & $\begin{array}{l}47.236 \\
47.439\end{array}$ & \begin{tabular}{|r|}
51.608 \\
51.97 \\
\end{tabular} & \begin{tabular}{|c|}
14.006 \\
14.064 \\
\end{tabular} & \begin{tabular}{|l|}
33.673 \\
33.837 \\
\end{tabular} & \begin{tabular}{|l|}
-1.222 \\
-1.222 \\
\end{tabular} & $\begin{array}{r}-7.514 \\
-7.508 \\
\end{array}$ & $\begin{array}{l}50.847 \\
39.019\end{array}$ & \begin{tabular}{|c|}
0.07 \\
0.071
\end{tabular} & $\begin{array}{l}\frac{14.643}{14.643} \\
\end{array}$ & & $\frac{1912.35}{1913.35}$ & $\frac{31.8725}{3118892}$ & $\frac{50.1}{50.3}$ & $\begin{array}{r}12.5 \\
9.6\end{array}$ & & \begin{tabular}{|c|}
2.789 \\
2.02
\end{tabular} & 0.010 & 0.011 & 0.000 & $\begin{array}{l}0.263 \\
.265 \\
\end{array}$ & 0 \\
\hline 1218 & $6 / 1 / 15 / 2004$ & $\begin{array}{l}5: 00: 04 \mathrm{PM} \\
5: 09: 04 \mathrm{PM}\end{array}$ & $\frac{26.254}{26.248}$ & $\begin{array}{l}\frac{26.908}{26.897} \\
\end{array}$ & $\begin{array}{r}24.41 \\
24.414 \\
\end{array}$ & 28.1259 & $\begin{array}{l}26.61 \\
26.65\end{array}$ & $\begin{array}{r}4.439 \\
47.423\end{array}$ & $\begin{array}{r}51.19 \\
51.786\end{array}$ & \begin{tabular}{|}
14.064 \\
14.053 \\
\end{tabular} & \begin{tabular}{|}
33.831 \\
33.878 \\
\end{tabular} & \begin{tabular}{|l|}
-1.222 \\
-1.222 \\
\end{tabular} & $\begin{array}{l}-1.508 \\
-7.462\end{array}$ & $\begin{array}{l}39.019 \\
45.629\end{array}$ & 0.071 & $\begin{array}{l}\frac{14.643}{14.643} \\
\end{array}$ & & & $\frac{31.8892}{31.9058}$ & 50.3 & $\begin{array}{l}9.6 \\
11.2\end{array}$ & & & & & & & \\
\hline 121 & 6/15/2004 & 5:10:04 PM & 26.248 & 26.876 & 24.398 & \begin{tabular}{|l|}
28.048 \\
\end{tabular} & 26. & 47.205 & 51.711 & \begin{tabular}{|l|}
14.027 \\
\end{tabular} & $\begin{array}{l}33.64 \\
\end{array}$ & -1.222 & -7.511 & 45.741 & & 14.643 & & 1915 & $\frac{31.9058}{31.9225}$ & 50.2 & $\frac{11.2}{11.2}$ & & 2.78 & & 0.01 & & & 0.23 \\
\hline & 6/115/2004 & 5:11:04 PM & 26.257 & 26.881 & 24.397 & 28.072 & & & & \begin{tabular}{|l|}
14.027 \\
\end{tabular} & 33.445 & & & 41.499 & & 14.643 & & & & & 10.2 & & & & & & & 0.23 \\
\hline & $6 / 15 / 2004$ & 5:12:04 PM & 26.255 & 26.879 & 24.401 & 28.031 & 26. & 47.375 & \begin{tabular}{|l|}
51.74 \\
\end{tabular} & & & -1.222 & -7.56 & 52.403 & & $\begin{array}{l}14.643 \\
\end{array}$ & & & & & 12.8 & & & & & & & \\
\hline & $6 / 15 / 2004$ & 5:13:0 & 26.255 & 26.873 & 24.395 & 28.03 & & & \begin{tabular}{|l|l|} 
\\
\end{tabular} & $\begin{array}{ll}14.002 \\
\end{array}$ & \begin{tabular}{|l|l|}
33.54 \\
\end{tabular} & -1.222 & & $\begin{array}{l}41.14 \\
\end{array}$ & & 14.643 & & & & 50 & 10.1 & & 10 & & .01 & & & \\
\hline & $6 / 15 / 2004$ & 5:14:04 PM & 26.264 & 26.867 & 24.394 & 27.98 & & 47.045 & 51.311 & 13.962 & $\begin{array}{ll}33.562 \\
\end{array}$ & & & . & & 14.6 & & & & & & & & & & & & \\
\hline & $6 / 15 / 2004$ & 5:15:04 PM & & 26.85 & 24.377 & 27.998 & & 47.197 & 51.564 & 13.974 & 33.761 & -1.222 & & 262 & & 14.64 & & & & & 12.4 & & & & & & & \\
\hline & $6 / 15 / 2004$ & 5:16:0 & 26.246 & 26.8 & 24.382 & 27.952 & & 47.18 & 51.707 & 14.004 & $\begin{array}{l}33.704 \\
\end{array}$ & \begin{tabular}{|l|l|} 
\\
\end{tabular} & & $51.26 \mathrm{~s}$ & & 14.643 & & & & 50.2 & 12.6 & & & & & & & \\
\hline & $6 / 15 / 2$ & 5:17:04 PM & 26.246 & 26.84 & 24.381 & 27.971 & & 47.3 & 51.637 & 14.076 & 33.751 & \begin{tabular}{|l|l|} 
\\
\end{tabular} & & 54.32 & & 14.643 & & & & & 13.3 & & & & & & & \\
\hline & $6 / 15 / 2004$ & & 26.249 & 26.84 & 24.379 & 27.911 & & 47. & 51.811 & 13.98 & & 1210 & & & & 14. & & & & 50 & 11.5 & & & & & & & \\
\hline & 0 & & & & & & & & $51.8 / 1$ & & & & & & & & & & & & & & & & & & & \\
\hline & & $\begin{array}{ll}5: 20: 0 \\
5: 210\end{array}$ & $\frac{26.232}{26202}$ & & 24.382 & 21.883 & & 47.1 & 51.566 & $\frac{13.9}{120}$ & 33.696 & $\frac{-1.219}{-1.210}$ & & & & 14.6 & & & & 50 & 13.8 & & 2.1 & & & & & \\
\hline & $\frac{6 / 5 / 2}{6 / 5 / 2}$ & $\begin{array}{l}5.21 .1 \\
5: 22:\end{array}$ & 26.218 & $\frac{20.00}{26.79}$ & 24.373 & 27.804 & & 47.4 & $\frac{51.434}{5197]}$ & $\begin{array}{l}13.96 \\
13.99\end{array}$ & & $\frac{-1.219}{-1.222}$ & & & & . & & & & & $\frac{1.9}{11.1}$ & & & & & & & \\
\hline & $6 / 15 / 2004$ & 5:23:04 PM & 26.221 & 26.784 & 24.371 & 27.802 & 26. & 47. & 51.49 & \begin{tabular}{|l|l|}
14.013 \\
\end{tabular} & & \begin{tabular}{|l|}
-1.219 \\
\end{tabular} & & 42.625 & & 14.643 & & & 532.1392 & 50.0 & 10.4 & & $2.7 \quad$ & & & & 0.266 & \\
\hline & $6 / 15 / 2004$ & & & & & 27.804 & & & 51.471 & & & & & & & & & & & & $\begin{array}{ll}13.2 \\
\end{array}$ & & & & & & & \\
\hline & 6/115/2004 & & 26.206 & 26.76 & 24.376 & 27.827 & & 47.45 & 51.825 & 14.023 & 33.932 & & & 47.1 & & 14. & & & & 50 & 11.5 & & 8 & 0.010 & 0.010 & & & \\
\hline & 6/115/2004 & & & 26.75 & 24.394 & & & & & & & -1. & & & & & & & & & 10. & & & & & & & \\
\hline & & & & & & & & & & & 33.86 & & & & & & & & & & & & & & & & & \\
\hline & $\frac{011512}{615 / 2}$ & $\begin{array}{l}5: 28: 04 \mathrm{PN} \\
5\end{array}$ & 6201 & 2672 & 24.42 & 27.1880 & & 47. & $\begin{array}{l}51.688 \\
51.682\end{array}$ & $\begin{array}{l}13.928 \\
13.916\end{array}$ & $\begin{array}{l}33.975 \\
34003\end{array}$ & -1.420 & & & & $\frac{14 .}{14 .}$ & & & & & 1190 & & & & & & & \\
\hline & $6 / 15 / 2004$ & & & & & & & & & & & & & & & & & & & 50 & 11.8 & & & & & & & \\
\hline & $6 / 15 / 2004$ & & & & & & & & & & & & & & & & & & & 50 & 10.5 & & & & & & & \\
\hline & $6 / 15 / 2004$ & & 26.192 & 26.71 & 24.4 & 27.978 & & & & 13.8 & & \begin{tabular}{|l} 
\\
\end{tabular} & & & & & & & & 50.0 & 11.4 & & & & & & & \\
\hline & $6 / 15 / 2004$ & & & & & & & & & & & & & & & & & & & & 11. & & & & & & & \\
\hline & & $5: 34: 0$ & 26.199 & 26.722 & 24.4 & \begin{tabular}{|l|l|}
28.0399 \\
2
\end{tabular} & 26. & 47.325 & $\begin{array}{l}51.819 \\
5.719\end{array}$ & \begin{tabular}{|l|l}
13.858 \\
3.70
\end{tabular} & \begin{tabular}{|l|}
33.93 \\
\end{tabular} & -1.222 & & 55.282 & $\begin{array}{ll}0.07 \\
\end{array}$ & 14. & & & 32.3225 & 50.3 & \begin{tabular}{|l|l|}
13.5 \\
\end{tabular} & & & & 0.011 & & & \\
\hline & $6 / 15 / 2004$ & $\begin{array}{l}5: 35: 04 \text { PM } \\
\text { 5:35:04 PM }\end{array}$ & $\frac{26.191}{26.207}$ & 26.725 & $\begin{array}{r}24.401 \\
24.407\end{array}$ & $\begin{array}{l}28.097 \\
28107\end{array}$ & & $\begin{array}{r}47.275 \\
47.286 \\
\end{array}$ & $\begin{array}{r}51.715 \\
5173 \\
\end{array}$ & $\begin{array}{r}13.784 \\
1387\end{array}$ & $\begin{array}{r}33.95 \\
33.903\end{array}$ & \begin{tabular}{|l|}
-1.222 \\
-1220 \\
\end{tabular} & -7 & & $\begin{array}{l}0.072 \\
0.067\end{array}$ & $\begin{array}{l}14.643 \\
14.643\end{array}$ & & 194 & 32.34 & $\frac{50.2}{50.3}$ & 12.7 & & $\frac{1}{9}$ & \begin{tabular}{|l|}
0. \\
0.1
\end{tabular} & $\begin{array}{l}0.011 \\
0.010\end{array}$ & 500 & 269 & 0 \\
\hline & 60 & $\begin{array}{l}5: 35: 04 \mathrm{PM} \\
5: 37: 04 \mathrm{PM}\end{array}$ & & $\frac{26.126}{26.736}$ & $\begin{array}{l}\frac{24.401}{24.408} \\
\end{array}$ & 28.133 & & $\begin{array}{l}47.286 \\
47.576\end{array}$ & & $\begin{array}{l}13.867 \\
13.822 \\
\end{array}$ & & & & & & $\frac{14.6}{146}$ & & & & & $\frac{11.2}{11.8}$ & & & & & & & \\
\hline & & & & & & & & & & & & & & & & & & & & & 124 & & & & & & & \\
\hline & $6 / 15 / 2$ & & 26.223 & 26.74 & & $\frac{28.0}{28.0}$ & & 47. & 51.852 & $\frac{13.8}{13.8}$ & & -1.222 & & & & & & & & & 11.7 & & & & & & & \\
\hline & $6 / 15 / 20$ & & & & & & & & & & & & & & & & & & & & & & & & & & & \\
\hline & & & & & & & & & & & & & & & & & & & & & & & & & & & & \\
\hline & $6 / 15 / 200$ & & & & 24.46 & 28.0 & & 47. & & & & & & & & & & & & & & & & & & & & \\
\hline & $6 / 15 / 2$ & 5:43:C & 624 & & 24.42 & $27.98 \varepsilon$ & & $45 . \varsigma$ & 50.405 & 15.22 & 31.126 & -1.222 & & & & 14.6 & & & & & 11.5 & & & & & & & \\
\hline & $6 / 15 / 20$ & 5:44:C & & & 24.44 & & & $45 .$. & 50.36 & 15.19 & 31.2 & -1.22 & & & & 14.6 & & & & & 135 & & & & & & & \\
\hline & $6 / 15 / 2$ & & & & 24.4 & & & & & 14.864 & & -1. & & & & 14. & & & & & $0.8>$ & & & & & & & \\
\hline & & & 26.264 & & 24.47 & & & & & & & -1.219 & & & & & & & & & 14.1 & & & & & & & \\
\hline & $6 / 151 / 2$ & & & & & & & & & & & & & & & & & & & & & & & & & & & \\
\hline & & & & 26. & 24.52 & & & 46. & & & & & & & & & & & & & 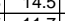 & & & & & & & \\
\hline & $0 / 15 / 2004$ & & 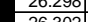 & 20.172 & 24.539 & 28.095 & & 45.042 & S0.198 & 14.96 & 31.405 & $\frac{-1.219}{1.202}$ & & $n e^{2}$ & & $\begin{array}{l}1.645 \\
1.612\end{array}$ & & & & 40.14 & $\begin{array}{ll}1.1 \\
1.0\end{array}$ & & & & 0100 & & & \\
\hline 126 & $\begin{array}{l}6 / 15 / 2004 \\
\end{array}$ & 5:51:04 PM & 26.322 & 26.771 & \begin{tabular}{|l|}
24.500 \\
24.583 \\
\end{tabular} & \begin{tabular}{|l|}
20.000 \\
28.073 \\
\end{tabular} & 26.658 & - 40.201 & \begin{tabular}{|l|}
50.439 \\
5
\end{tabular} & \begin{tabular}{|l|}
14.025 \\
14.796 \\
\end{tabular} & \begin{tabular}{|l|l|c|}
31.949 \\
\end{tabular} & \begin{tabular}{|l|l|}
-1.2222 \\
-1.222
\end{tabular} & -7.519 & 55.386 & 0.067 & \begin{tabular}{|c|}
14.045 \\
14.643
\end{tabular} & & 1956.35 & 532.6058 & \begin{tabular}{|l|}
49.0 \\
\end{tabular} & \begin{tabular}{|l|l|}
14.6 \\
13.6
\end{tabular} & & \begin{tabular}{|l|}
2.693 \\
\end{tabular} & \begin{tabular}{|l|}
0.010 \\
\end{tabular} & $\mid$\begin{tabular}{|c|}
0.010 \\
\end{tabular} & 0.000 & \begin{tabular}{|l|l|} 
& 0.259 \\
\end{tabular} & 0.2 \\
\hline
\end{tabular}


WSRC-TR-2005-00105, REVISION 0

SRNL-RPP-2005-00012, REVISION 0

RUN \# 3.03A AND B; FIRST AND SECOND HALF OF SLURRY DEWATERING - CONT.

\begin{tabular}{|c|c|c|c|c|c|c|c|c|c|c|c|c|c|c|c|c|c|c|c|c|c|c|c|c|c|c|c|c|}
\hline & A & B & D & $E$ & $\mathrm{~F}$ & $G^{G}$ & $\mathrm{H}$ & $\mathrm{J}$ & K & $\mathrm{L}$ & $\mathrm{M}$ & $\mathrm{N}$ & $\mathrm{O}$ & Q & \begin{tabular}{ll|}
$\mathrm{R}$ \\
\end{tabular} & 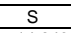 & T & & & $x$ & & & $\mathrm{AA}$ & & $A C$ & $A D$ & $\mathrm{AE}$ & \\
\hline & 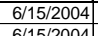 & $\begin{array}{ll}5: 52: 04 \mathrm{PM} \\
5: 5: 24 \mathrm{P}\end{array}$ & & $\begin{array}{l}26.77 \\
2677\end{array}$ & 24.578 & $\begin{array}{l}28.053 \\
2.030\end{array}$ & $\begin{array}{l}26.678 \\
5705\end{array}$ & 46.093 & \begin{tabular}{|l|l|}
50.602 \\
5107
\end{tabular} & $\begin{array}{l}14.747 \\
\end{array}$ & $\begin{array}{l}31.869 \\
2077\end{array}$ & $\begin{array}{r}-1.219 \\
1219\end{array}$ & \begin{tabular}{|l|l|}
-7.277 \\
7502
\end{tabular} & $\begin{array}{l}46.384 \\
1989\end{array}$ & $\begin{array}{l}0.069 \\
0\end{array}$ & $\begin{array}{l}14.643 \\
\end{array}$ & 0.004 & & 32.6225 & 49.1 & 11.4 & & 2.688 & 0.010 & 0.010 & 0.000 & $\begin{array}{l}0.267 \\
\end{array}$ & \\
\hline & $\frac{6 / 115 / 2004}{661 / 2004}$ & 5:5:53:04 PM & $\frac{26.33}{26.34}$ & $\frac{26.77}{26.77}$ & 24.574 & \begin{tabular}{|l|}
28.109 \\
2808
\end{tabular} & $\begin{array}{l}26.705 \\
26.877\end{array}$ & $\begin{array}{l}46.668 \\
48.842\end{array}$ & \begin{tabular}{|l|}
51.077 \\
51313 \\
\end{tabular} & $\begin{array}{r}14.428 \\
14329\end{array}$ & $\begin{array}{r}32.77 \\
33018\end{array}$ & \begin{tabular}{|c|}
-1.219 \\
-1219
\end{tabular} & $\begin{array}{l}-7.502 \\
-7.514\end{array}$ & $\begin{array}{l}48.488 \\
49371\end{array}$ & $\begin{array}{l}0.069 \\
0.065\end{array}$ & $\begin{array}{l}14.643 \\
14643\end{array}$ & $\begin{array}{l}0.004 \\
0.004\end{array}$ & $\begin{array}{l}1958.35 \\
1959.25\end{array}$ & \begin{tabular}{|l|l|}
32.6392 \\
326558
\end{tabular} & 49.6 & 11.9 & & $\frac{2.738}{2753}$ & 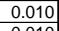 & 0.010 & & 0.262 & \\
\hline 1264 & $6 / 15 / 2004$ & $\begin{array}{l}5.540 .04 \mathrm{PM} \\
5: 55: 04 \mathrm{PM}\end{array}$ & $\frac{20.34}{26.36}$ & $\frac{20.11}{26.79}$ & $\frac{24.512}{24.592}$ & $\begin{array}{l}28.004 \\
28.117\end{array}$ & $\begin{array}{l}26.087 \\
26.707\end{array}$ & $\begin{array}{l}40.042 \\
46.724\end{array}$ & \begin{tabular}{|l|}
51.313 \\
51.454 \\
\end{tabular} & $\begin{array}{l}14.329 \\
14.348\end{array}$ & $\begin{array}{l}33.018 \\
32.786 \\
\end{array}$ & $\begin{array}{l}-1.219 \\
-1.219\end{array}$ & $\begin{array}{l}-7.514 \\
-7.516\end{array}$ & $\begin{array}{l}49.3 / 1 \\
50.885\end{array}$ & $\begin{array}{l}0.066 \\
0.068\end{array}$ & $\begin{array}{l}14.643 \\
14.643 \\
\end{array}$ & $\frac{0.004}{0.004}$ & $\frac{19599.35}{1960.35}$ & \begin{tabular}{|l|l|}
32.65588 \\
32.6725 \\
\end{tabular} & $\frac{49.8}{50.0}$ & $\frac{12.1}{12.5}$ & $\frac{39.6}{39.8}$ & $\mid$\begin{tabular}{|l|l|}
2.753 \\
2.741 \\
\end{tabular} & $\begin{array}{l}0.010 \\
0.010\end{array}$ & $\frac{0.010}{0.010}$ & 0.0000 & $\frac{0.249}{0.258}$ & $\frac{0.21}{0.22}$ \\
\hline 1265 & $6 / 15 / 2004$ & 5:56:04 PM & 26.35 & 26.79 & 24.582 & 28.087 & 26.728 & 46.773 & \begin{tabular}{|l|}
51.367 \\
\end{tabular} & 14.372 & 32.829 & -1.216 & -7.447 & 52.447 & 0.071 & 14.643 & & 1961.3 & 32.6892 & 49.9 & 12.9 & & \begin{tabular}{|l|}
2.744 \\
\end{tabular} & & & & & \\
\hline & $6 / 15 / 2004$ & 5:57:04 PM & & & 24.541 & 28.066 & 26.776 & 46.556 & 51.091 & 14.263 & 32.776 & -1.222 & -7.522 & 53.38 & 0.072 & 14.643 & & & & 49.6 & 13.1 & & & & & & & \\
\hline & $6 / 15 / 2004$ & 5:58:04 PM & 26.33 & 26.76 & 24.54 & 28.17 & 26.855 & $\begin{array}{l}47.145 \\
9.527\end{array}$ & \begin{tabular}{|l|l|}
51.614 \\
5512
\end{tabular} & $\begin{array}{l}14.408 \\
\end{array}$ & & -1.225 & & 50.758 & \begin{tabular}{|l|l|}
0.068 \\
\end{tabular} & 14.643 & & & & 50.1 & 12.4 & & & & & & & \\
\hline 1268 & $6 / 15 / 2004$ & 5:59:04 PM & 26.35 & 26.78 & 24.563 & 28.243 & 26.928 & & & 14.322 & 32.63 & -1.222 & $\begin{array}{l}-7.323 \\
\end{array}$ & 50.432 & 0.071 & 14.643 & & 1964. & 32.7392 & 49.7 & 12.4 & & 2.726 & & 0.011 & 0.000 & 0.271 & \\
\hline & 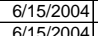 & 6:00:04 PM & & $\begin{array}{l}26.77 \\
2279\end{array}$ & $\begin{array}{l}24.546 \\
24579\end{array}$ & \begin{tabular}{|l|l|}
28.29 \\
28.359
\end{tabular} & $\begin{array}{l}26.985 \\
27068\end{array}$ & $\begin{array}{r}46.73 \\
4848\end{array}$ & \begin{tabular}{|l|}
51.249 \\
51.376 \\
\end{tabular} & $\begin{array}{l}14.371 \\
1.379\end{array}$ & $\begin{array}{r}32.823 \\
33\end{array}$ & $\begin{aligned}-1.225 \\
1202\end{aligned}$ & \begin{tabular}{|l|l|}
-7.525 \\
7.277
\end{tabular} & $\begin{array}{l}53.592 \\
7325\end{array}$ & $\begin{array}{l}0.075 \\
0.698\end{array}$ & $\begin{array}{l}14.643 \\
1.643\end{array}$ & & & \begin{tabular}{|l|l|}
32.7558 \\
327725
\end{tabular} & 49.8 & $\begin{array}{l}13.1 \\
116\end{array}$ & & \begin{tabular}{|l|l|}
2.742 \\
.775
\end{tabular} & 0.011 & $\begin{array}{l}0.011 \\
0.010\end{array}$ & & 0.285 & \\
\hline$\frac{1<70}{1271}$ & $\frac{661 / 15 / 2004}{6 / 12 / 2004}$ & 6:10:04 PM & $\frac{26.37}{26.37}$ & $\frac{26.79}{26.81}$ & $\begin{array}{r}24.579 \\
24.58 \\
\end{array}$ & $\mid$\begin{tabular}{|c|}
28.358 \\
22.399
\end{tabular} & $\begin{array}{r}27.068 \\
27.09\end{array}$ & $\begin{array}{r}46.848 \\
46.802\end{array}$ & \begin{tabular}{|c|}
$\mid 51.376$ \\
55.1548
\end{tabular} & $\begin{array}{l}14.379 \\
14.367\end{array}$ & $\begin{array}{r}33 \\
32.834 \\
\end{array}$ & $\begin{array}{l}-1.222 \\
-1.222\end{array}$ & $\begin{array}{l}-7.427 \\
-7.462 \\
\end{array}$ & $\begin{array}{r}47.335 \\
48.52 \\
\end{array}$ & \begin{tabular}{|c|}
0.068 \\
0.068
\end{tabular} & $\begin{array}{l}\frac{14.643}{14.643} \\
\end{array}$ & $\frac{0.004}{0.004}$ & 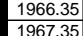 & \begin{tabular}{|l|}
32.7725 \\
33.7892
\end{tabular} & $\begin{array}{l}\frac{49.9}{50.1} \\
5\end{array}$ & $\frac{11.6}{11.9}$ & $\begin{array}{ll}39.9 \\
339.8\end{array}$ & \begin{tabular}{|l|}
2.753 \\
2745 \\
\end{tabular} & $\frac{0.010}{0.010}$ & $\begin{array}{l}0.010 \\
0.010\end{array}$ & $\begin{array}{l}0.000 \\
0.000\end{array}$ & 0.257 & \\
\hline \begin{tabular}{|l|}
1272 \\
\end{tabular} & $6 / 15 / 2004$ & 6:03:04 PM & 26.3 & 26.83 & 24.586 & 28.445 & 27.055 & 46.794 & \begin{tabular}{|l|}
51.311 \\
\end{tabular} & 14.385 & 32.907 & -1.222 & $\begin{array}{l}-7.406 \\
-7.462 \\
\end{array}$ & 42.944 & 0.068 & 14.643 & & $1968.3^{5}$ & $\begin{array}{l}32 . .892 \\
32.8058\end{array}$ & 49.8 & 10.5 & & \begin{tabular}{|l|}
2.744 \\
2.748 \\
\end{tabular} & 0 & $\begin{array}{l}0.010 \\
0.010\end{array}$ & $\begin{array}{l}0.000 \\
0.000\end{array}$ & $\begin{array}{l}0.258 \\
0.257\end{array}$ & 027 \\
\hline & $6 / 15 / 2004$ & 6:04:04 PM & 26.39 & 26.82 & 24.58 & 28.439 & & 46.562 & 51.063 & $\begin{array}{l}4.319 \\
\end{array}$ & 32.688 & -1.222 & -7.479 & 43.632 & \begin{tabular}{|l|}
0.07 \\
\end{tabular} & 14.643 & 800 & 1969.3 & 32.8225 & 49.6 & 10.7 & 39.6 & \begin{tabular}{|l|}
2.732 \\
\end{tabular} & & 0.011 & 0.000 & 0.267 & \\
\hline & $6 / 15 / 2004$ & 6:05:04 PM & & & & 28.374 & & 46.661 & 51.11 & $\begin{array}{l}14.296 \\
\end{array}$ & & -1.222 & & \begin{tabular}{|l|l|}
43.47 \\
\end{tabular} & \begin{tabular}{|l|l|}
0.066 \\
\end{tabular} & $\begin{array}{l}14.643 \\
\end{array}$ & & 1970.3 & 32.8392 & 49.6 & 10.7 & & 2.741 & & & 0.000 & 0.251 & \\
\hline 1275 & $6 / 15 / 2004$ & 6:06:04 PM & 26.42 & 26.85 & 24.6 & 28.379 & 26.954 & 46.904 & 51.533 & $\begin{array}{l}14.383 \\
\end{array}$ & 32.963 & -1.222 & $\begin{array}{r}-7.384 \\
\end{array}$ & 55.103 & \begin{tabular}{|l|l|}
0.067 \\
\end{tabular} & 14.643 & & & 32.8558 & 50.1 & 13.5 & & 2.753 & 0.010 & & 0.000 & 0.253 & \\
\hline & 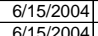 & 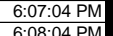 & $\frac{26.41 .}{26}$ & $\begin{array}{l}26.86 \\
26.87\end{array}$ & $\begin{array}{l}24.588 \\
24.602\end{array}$ & $\begin{array}{l}28.322 \\
28326\end{array}$ & $\begin{array}{l}26.923 \\
26.911\end{array}$ & $\begin{array}{l}466.645 \\
47.085\end{array}$ & \begin{tabular}{|l|}
51.197 \\
51.46 \\
\end{tabular} & $\begin{array}{r}14.37 \\
14.411\end{array}$ & $\begin{array}{l}32.768 \\
33.313\end{array}$ & $\begin{array}{l}-1.225 \\
-1.219\end{array}$ & $\begin{array}{r}-7.441 \\
-7.326\end{array}$ & $\begin{array}{l}52.112 \\
52.856\end{array}$ & \begin{tabular}{|l|l|}
0.067 \\
0.091
\end{tabular} & $\begin{array}{l}14.643 \\
14.643\end{array}$ & 0.00 & & $\begin{array}{r}32.8725 \\
38892\end{array}$ & \begin{tabular}{|l|l|}
49.7 \\
500
\end{tabular} & $\begin{array}{l}12.8 \\
130\end{array}$ & 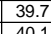 & $\begin{array}{l}2.738 \\
.2765 \\
\end{array}$ & $\begin{array}{l}0.010 \\
0.014\end{array}$ & $\begin{array}{l}0.010 \\
0014\end{array}$ & - & $\begin{array}{l}0.255 \\
0.342\end{array}$ & \\
\hline & $6 / 1 / 15 / 2004$ & 6:08:04 PM & $\frac{26 .}{26.78}$ & $\frac{26.87}{26.88}$ & $\frac{24.602}{24.602}$ & $\begin{array}{l}28.326 \\
28.345\end{array}$ & $\frac{26.911}{26.905}$ & $\begin{array}{r}47.085 \\
46.124 \\
\end{array}$ & \begin{tabular}{|c|}
51.456 \\
51118 \\
\end{tabular} & $\begin{array}{r}14.411 \\
143\end{array}$ & $\begin{array}{r}33.113 \\
32.275 \\
\end{array}$ & $\begin{array}{l}-1.219 \\
-0.739\end{array}$ & $\begin{array}{l}-7.326 \\
-6.097\end{array}$ & $52.856 \mid$ & $\begin{array}{l}0.091 \\
0.064\end{array}$ & $\begin{array}{l}14.643 \\
14643\end{array}$ & & $\frac{1973.3}{1074.3}$ & $\begin{array}{l}32.8892 \\
33.9058\end{array}$ & 50.0 & $\begin{array}{l}13.0 \\
11.5\end{array}$ & $\begin{array}{ll}40.1 \\
392\end{array}$ & \begin{tabular}{|l|}
2.765 \\
2703 \\
\end{tabular} & 0.014 & 0.014 & 0.000 & 0.342 & \\
\hline 127 & $6 / 15 / 2004$ & 6:030:04 PM & 26.74 & 26.90 & $\frac{24.011}{24.642}$ & 28.366 & $\frac{20.905}{26.931}$ & $\begin{array}{l}40.164 \\
46.769\end{array}$ & \begin{tabular}{|l|}
51.407 \\
\end{tabular} & $\begin{array}{r}14.439 \\
14.0\end{array}$ & $\frac{32.275}{32.756}$ & $\begin{array}{l}-.1 .216 \\
-1.216\end{array}$ & $\frac{-6.091}{-6.909}$ & $\begin{array}{l}46.974 \\
49.469\end{array}$ & $\begin{array}{l}0.064 \\
0.067\end{array}$ & $\frac{14.643}{14.643}$ & & 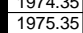 & $\begin{array}{l}32.9058 \\
32.9225\end{array}$ & $\frac{49.6}{49.9}$ & $\frac{11.5}{12.1}$ & $\frac{39.2}{39.8}$ & \begin{tabular}{|l|}
2.703 \\
2.741 \\
\end{tabular} & \begin{tabular}{|l}
0.010 \\
0.010
\end{tabular} & $\begin{array}{l}.010 \\
0.010\end{array}$ & 0 & $\begin{array}{l}0.256 \\
0.254\end{array}$ & 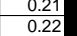 \\
\hline & $6 / 15 / 2004$ & 6:11:04 PM & 26.6 & 26.89 & 24.622 & 28.331 & 26.911 & 46.73 & 51.127 & 14.305 & & -1.222 & $\begin{array}{l}-7.187 \\
\end{array}$ & 48.782 & 0.066 & 14.643 & & 1976.35 & 32.9392 & 49.7 & 12.0 & & \begin{tabular}{|l|} 
\\
\end{tabular} & & 0.010 & 0.000 & 0.250 & \\
\hline & $6 / 115 / 2004$ & 6:12:04 PM & 26.56 & 26.90 & 24.621 & 28.335 & 26.9 & 47.161 & & $\begin{array}{l}14.348 \\
\end{array}$ & 33.334 & -1.222 & & 50.678 & \begin{tabular}{|l|l|} 
\\
\end{tabular} & 14.643 & & & 32.9558 & 50.1 & 12.4 & 40. & 2.775 & & & 0.000 & 0.251 & \\
\hline & $6 / 15 / 2004$ & 6:13:04 PM & 26.4 & 26.91 & 24.657 & 28.326 & & 46.688 & 51.065 & 14.366 & 32.848 & -1.222 & -7.415 & $\begin{array}{r}42.25 \\
\end{array}$ & & 14.643 & & & 32.9725 & 49.6 & 10.4 & & \begin{tabular}{|l|l|}
2.742 \\
\end{tabular} & 0.010 & 0.011 & & 0.265 & \\
\hline & $6 / 15 / 2004$ & $\begin{array}{l}6: 14: 04 \mathrm{PM} \\
6 \\
6\end{array}$ & $\frac{26.40 .}{2636}$ & 26.90 & $\begin{array}{r}24.705 \\
24.684\end{array}$ & 28.314 & $\begin{array}{r}26.87 \\
26.878\end{array}$ & $\begin{array}{l}46.724 \\
47068\end{array}$ & \begin{tabular}{|l|l|}
51.143 \\
51.378 \\
\end{tabular} & $\begin{array}{l}14.241 \\
1.277\end{array}$ & $\begin{array}{l}32.985 \\
33285 \\
3285\end{array}$ & $\begin{array}{r}-1.219 \\
.2222\end{array}$ & $\begin{array}{r}-7.412 \\
7.41\end{array}$ & $\begin{array}{r}45.729 \\
45.36\end{array}$ & $\begin{array}{l}0.069 \\
0069\end{array}$ & $\begin{array}{l}14.643 \\
1643\end{array}$ & & \begin{tabular}{|l|l|}
1979.3 \\
10803
\end{tabular} & $\begin{array}{l}32.9892 \\
32058\end{array}$ & $\begin{array}{l}49.7 \\
499 \\
\end{array}$ & 11.2 & $\frac{39.9}{40.9}$ & \begin{tabular}{|l|}
2.748 \\
\end{tabular} & 0.010 & 0.010 & 0.000 & 0.260 & \\
\hline & $\frac{6 / 11 / 2 / 204}{6 / 15 / 2004}$ & $\begin{array}{l}6: 15: 04 \mathrm{PM} \\
6: 16: 04 \mathrm{PM}\end{array}$ & $\frac{26.36}{26.36}$ & $\frac{26.91}{26.90}$ & $\begin{array}{l}24.684 \\
24.679 \\
\end{array}$ & $\frac{28.283}{28.273}$ & & $\begin{array}{r}47.068 \\
46.946\end{array}$ & \begin{tabular}{|r|}
51.3 .8 \\
51.27
\end{tabular} & $\begin{array}{l}14.277 \\
14.287\end{array}$ & $\begin{array}{r}33.285 \\
33.144 \\
\end{array}$ & $\frac{-1.222}{-1.222}$ & $\begin{array}{l}-1.4 \\
-7.4\end{array}$ & $\begin{array}{l}45.136 \\
50.804\end{array}$ & \begin{tabular}{|c|}
0.009 \\
0.069 \\
\end{tabular} & $\frac{14.643}{14.643}$ & & & & & & & 2.11 & & & & & 0 \\
\hline & $6 / 15 / 2004$ & 6.10.04 PM & 26.37 & $\frac{20.90}{26.92}$ & $\frac{24.015}{24.684}$ & $\frac{20.475}{28.293}$ & $\frac{20.000}{26.878}$ & $\begin{array}{l}40.940 \\
46.861\end{array}$ & $\begin{array}{l}51.299 \\
51.299\end{array}$ & $\frac{14.201}{14.193}$ & $\frac{50.144}{33.182}$ & -1.222 & $\begin{array}{l}-. .41 \\
-7.41\end{array}$ & $\begin{array}{c}0.004 \\
49.01\end{array}$ & $\begin{array}{l}.0068 \\
0.068\end{array}$ & $\frac{14.045}{14.643}$ & & $1982.3^{5}$ & $\frac{0.0020}{33.0392}$ & $\begin{array}{l}44.8 \\
49.8\end{array}$ & $\frac{12.0}{12.0}$ & & \begin{tabular}{|l}
2.161 \\
2.759 \\
\end{tabular} & 0.010 & 0.010 & 0.000 & 0.256 & \\
\hline & $6 / 15 / 2004$ & 6:18:04 PM & 26.38 & 26.91 & 24.674 & 28.248 & 26.848 & 46.885 & \begin{tabular}{|l|}
51.249 \\
\end{tabular} & 14.236 & 33.18 & -1.222 & -7.404 & 58.133 & $\begin{array}{l}0.07 \\
\end{array}$ & 14.643 & & 1983.35 & 33.0558 & 49.8 & 14.2 & 40.0 & \begin{tabular}{|l|}
2.760 \\
\end{tabular} & 0.010 & 0.011 & 0.000 & 0.263 & \\
\hline & $6 / 15 / 2004$ & 6:19:04 PM & & 26.91 & 24.674 & 28.288 & 26.843 & & \begin{tabular}{|l|}
51.241 \\
\end{tabular} & $\begin{array}{l}14.372 \\
\end{array}$ & 32.942 & -1.219 & -7.407 & & \begin{tabular}{|l|l|}
0.068 \\
\end{tabular} & $\begin{array}{l}14.643 \\
\end{array}$ & & & 33.0725 & 49.8 & 11.9 & & \begin{tabular}{|l|}
2.749 \\
\end{tabular} & & & & 0.257 & \\
\hline & $6 / 15 / 2004$ & 6:20:04 PM & 26.41 & 26.92 & 24.674 & 28.269 & & & \begin{tabular}{|l|l|}
51.346 \\
\end{tabular} & 14.281 & 32.987 & -1.219 & $\begin{array}{r}-7.401 \\
\end{array}$ & 56.926 & .067 & 14.643 & & & 33.0892 & 49.9 & 14.0 & & 2.750 & 0.010 & 0.010 & 0.000 & 0.253 & \\
\hline & 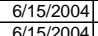 & \begin{tabular}{l|l}
$6: 21: 04 \mathrm{PM}$ \\
$6 \cdot 62.04 \mathrm{PM}$
\end{tabular} & $\frac{26.42}{26.4}$ & $\begin{array}{l}26.92 \\
26.92\end{array}$ & $\begin{array}{l}24.684 \\
24667\end{array}$ & $\begin{array}{l}28.298 \\
28.236\end{array}$ & $\begin{array}{l}26.814 \\
26802\end{array}$ & $\begin{array}{l}46.834 \\
46902\end{array}$ & \begin{tabular}{|l|}
51.461 \\
51388 \\
\end{tabular} & $\begin{array}{l}14.337 \\
14339\end{array}$ & $\begin{array}{l}32.961 \\
33022\end{array}$ & $\begin{array}{l}-1.216 \\
-1.225\end{array}$ & $\begin{array}{r}-7.398 \\
-7.41 \\
\end{array}$ & $\begin{array}{r}43.34 \\
49.331\end{array}$ & \begin{tabular}{|c|c|}
0.068 \\
0.068
\end{tabular} & $\begin{array}{l}14.643 \\
1.643\end{array}$ & & 1986.3 & $\begin{array}{l}33.1058 \\
33.225 \\
3.25\end{array}$ & $\begin{array}{l}50.0 \\
49.9\end{array}$ & $\begin{array}{l}10.6 \\
12.1\end{array}$ & & \begin{tabular}{|l|}
2.751 \\
2755 \\
\end{tabular} & $\begin{array}{l}0.010 \\
0.010\end{array}$ & $\begin{array}{l}0.010 \\
0.010\end{array}$ & $\begin{array}{l}0.000 \\
0.000\end{array}$ & 0.256 & \\
\hline & $\frac{6 / 11 / 2 / 204}{6 / 15 / 2004}$ & $\begin{array}{l}6: 22: 04 \mathrm{PM} \\
6: 23: 04 \mathrm{PM}\end{array}$ & $\frac{26.4}{26.45}$ & & $\begin{array}{l}24.66 / \\
24.675\end{array}$ & $\begin{array}{l}28.236 \\
28.299\end{array}$ & & & \begin{tabular}{|r|}
51.388 \\
51.32
\end{tabular} & $\begin{array}{l}14.339 \\
14.312 \\
\end{array}$ & 0.192 & $\begin{array}{l}-1.225 \\
-1.219\end{array}$ & & $\begin{array}{r}49.331 \\
52.13\end{array}$ & 0.068 & $\frac{14.643}{14.643}$ & & & & $\frac{49.9}{49.8}$ & & & & & & & & \\
\hline & $6 / 15 / 2004$ & 6:24:04 PM & 26.4 & $\frac{20.36}{26.91}$ & $\frac{24.075}{24.666}$ & 28.305 & $\frac{20.040}{26.816}$ & & $\begin{array}{l}0.1 .54 \\
51.51\end{array}$ & $\frac{14.012}{14.37}$ & 33.105 & -1.222 & $\frac{-1.4}{-7.3}$ & 54.239 & & $\frac{14.045}{14.643}$ & & & & $\frac{45}{50}$ & $\frac{13.3}{13.3}$ & & & & & & & \\
\hline & 6/15/2004 & 6:25:04 PM & 26.43 & 26.89 & 24.645 & 28.244 & 26.785 & 46.612 & 51.013 & 14.326 & 32.817 & -1.225 & -7.398 & 49.805 & 0.068 & 14.643 & & $\frac{1969.5}{1990.35}$ & 33.1725 & 49.5 & \begin{tabular}{|l|}
12.2 \\
\end{tabular} & & $\mid 2.738$ & 0 & 0.010 & 0.000 & $\begin{array}{l}0.250 \\
0.258 \\
0.0\end{array}$ & \\
\hline & $6 / 15 / 2004$ & 6:26:04 PM & 26.46 & 26.91 & & 28.236 & & & & & & & & & & & & & & 49.9 & 14.3 & & & & & & & \\
\hline & $6 / 15 / 2004$ & $6: 27: 04$ PM & 26.46. & 26.91 & 24.677 & 28.2 & & & \begin{tabular}{|l|l|}
51.543 \\
5
\end{tabular} & 14.358 & 33.154 & -1.216 & & 53.288 & & & & & 33.2058 & 50.1 & \begin{tabular}{|l|}
13.1 \\
\end{tabular} & 40.1 & 2.763 & 0.0 & & & 0.255 & \\
\hline & $6 / 15 / 2004$ & $\begin{array}{l}6: 28: 04 \mathrm{PM} \\
6 \\
6\end{array}$ & $\frac{26.46}{26.4}$ & 26.90 & $\begin{array}{l}24.664 \\
24661\end{array}$ & $\begin{aligned} 28.283 \\
28.25\end{aligned}$ & $\begin{array}{l}26.804 \\
26.821\end{array}$ & $\begin{array}{l}46.972 \\
48.848\end{array}$ & \begin{tabular}{|c|}
51.661 \\
51.349
\end{tabular} & $\begin{array}{r}14.445 \\
14.424\end{array}$ & $\begin{array}{r}32.948 \\
33.893 \\
3\end{array}$ & $\begin{array}{r}-1.222 \\
.225\end{array}$ & $\begin{array}{l}-7.389 \\
73292 \\
\end{array}$ & $\begin{array}{l}47.372 \\
55059\end{array}$ & 0.069 & $\begin{array}{l}14.643 \\
11.643\end{array}$ & & \begin{tabular}{|l|l|}
1993.3 \\
10943
\end{tabular} & $\begin{array}{r}33.2225 \\
332392\end{array}$ & 50.2 & $\begin{array}{l}11.6 \\
128\end{array}$ & & \begin{tabular}{|l|}
2.755 \\
2790
\end{tabular} & $\begin{array}{l}0.010 \\
0010\end{array}$ & 0.010 & 0.000 & 0.260 & 0 \\
\hline & $\frac{6 / 11 / 2 / 204}{6 / 15 / 2004}$ & & $\begin{array}{r}26.4 \\
26.48\end{array}$ & & $\frac{24.661}{24.669}$ & & & & \begin{tabular}{|r|}
51.349 \\
51.33
\end{tabular} & $\begin{array}{r}14.424 \\
14.384\end{array}$ & & & & & & $\frac{14.6}{14.6}$ & & & & & & & & & & & & 0 \\
\hline & $6 / 15 / 2004$ & 4 PM & 26.48 & & 24.605 & 20.200 & & & J1.250 & $\begin{array}{l}14.504 \\
14.334\end{array}$ & & $\frac{-1.22}{-1.219}$ & & & & 14. & & & & 45 & & & & & & & & \\
\hline & $6 / 15 / 2004$ & 6:32:04 PM & 26.49 & 26.93 & 24.687 & 28.4 & & 46.964 & 51.523 & 14.452 & 32.985 & $\frac{1.213}{-1.222}$ & & 49.905 & & 14.6 & & & & 50.1 & 2.2 & & & & 0.010 & & & \\
\hline & $6 / 15$ & & 26.48 & & & & & & & & & & & & & & & & & & & & & & & & & \\
\hline & & & & & 24.6 & & & & 51.448 & & & & & & & & & & & & & & & & & & & \\
\hline & & & & & & & & & & & & & & & & & & & & & & & & & & & & \\
\hline & $6 / 15 / 2$ & & & 26.95 & 24.6 & 28.5 & & & & 14.4 & & & & & & & & & & $4 !$ & & & & & & & & \\
\hline & $6 / 15 / 2004$ & & & & & & & & & $\begin{array}{l}14.448 \\
\end{array}$ & & & & & & $\begin{array}{l}14.643 \\
\end{array}$ & & & & 49 & 2.4 & & & & & & .261 & \\
\hline & $6 / 15 / 2$ & & & & 24. & & & & & 14.3 & & & & & & & & & & & & & & & & & & \\
\hline & 6/15/2004 & & 26.52 & & 24.7 & 28.4 & & & \begin{tabular}{|l|}
50.828 \\
\end{tabular} & 14.313 & 32.782 & -1.222 & & 49.296 & & 14. & & & & 49.4 & 2.1 & & & & & & & \\
\hline & $6 / 115 / 2004$ & & 20.52 & 26.98 & 24.7 & & & & & & & & & & & & & & & & & & & & & & & \\
\hline & & & & 27.0 & 24 & & & & 51.0 & 14. & & & & & & & & & & & & & & & & & & \\
\hline & & & & 270 & 24.721 & & & & & & & & & & & & & & & & & & & & & & & \\
\hline & $\frac{01515120041}{6}$ & & $\frac{2.535}{2653}$ & 2701 & $\frac{24.159}{2472}$ & & & & & $\frac{14.5}{143}$ & & & & & & & & & & & & & & & & & & \\
\hline 131 & $6 / 15 / 2004$ & & & & 247. & & & & & & & & & & & & & & & & & & & & & & & \\
\hline & $6 / 15 / 2$ & & $\frac{2.53}{26.53}$ & 27.01 & 24.711 & 28.3 & & $\frac{11.6}{46.6}$ & & 14.3 & & -1.1 & & & & & & & & $\frac{15.0}{50.0}$ & 4.3 & & & & & & & \\
\hline & $6 / 15 / 2004$ & & 26.54 & & & & & & & & & & & & & & & & & & & & & & & & & \\
\hline & & & & & & & & & & & & & & & & & & & & & & & & & & & & \\
\hline & $6 / 15 / 2$ & & & & 246 & 28.2 & & & & & & -1.222 & & & & & & & & & & & & & & & & \\
\hline & $6 / 15 / 2$ & 6:50 & & 27.0 & 24.724 & 28.25 & & 46.91 & 51.84 & 14.4 & & -1.092 & & & & 14.6 & & & & & & & & & & & & \\
\hline & $6 / 15 / 2$ & 6:51: & & & 24.6 & 28.2 & & 47.0 & 51.678 & 14.22 & 33. & -1.15 & & & & 14. & & & & & & & & & & & & \\
\hline & $6 / 15 /$ & & & & 24.7 & 28 & & & & & & & & & & & & & & & & & & & & & & \\
\hline & $6 / 15 / 2$ & & 26.54 & 27.0 & 24. & 28.4 & & & & 14.429 & & -1.2 & & & & & & & & 50 & & & & & & & $2 / 4$ & \\
\hline & $6 / 15 / 2 / 2$ & & 26.55 & & 24.7 & & & & & & & & & & & & & & & & & & & & & & & \\
\hline & $66115 / 2004$ & & 60.54 & 27.0 & 24.72 & 28.4 & & 46.8 & $4 / 3$ & 14.332 & & -1.2 & & & & & & & & & & & & & & & & \\
\hline & $\frac{61 / 151 / 2}{61 / 21}$ & $\frac{6.560 .04}{60.57 .04}$ & & & $\frac{24.116}{2.707}$ & $\frac{28.29}{2020}$ & & 40.802 & $\frac{51.21}{51.21}$ & 14.2. & 33.082 & -1.2 .25 & & 15.12 & & & & & & & & & & & & & & \\
\hline & 年 & & & & 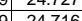 & $\frac{20.2}{2027}$ & & 40.00 & & & & $\frac{1.12}{120}$ & & & & & & & & & & & & & & & & \\
\hline & the & & & 26.98 & $\frac{2.170}{2471}$ & 283 & & 46 & & 1427 & (32749 & -1.22 & & & & & & & & & & & & & & & & \\
\hline & & & 54 & $\frac{26.99}{26.99}$ & 24799 & 28.392 & & $\frac{46.759}{46.75}$ & & 14.222 & $\frac{25.981}{32.981}$ & -1.222 & & & & & & & & 498 & 25 & & & & & & & \\
\hline & $6 / 15 / 2$ & & & & 24.814 & & & & & 14.23 & & -1.222 & & & 069 & $\frac{14.045}{14.643}$ & & & & & & & & & & & 126 & \\
\hline
\end{tabular}


WSRC-TR-2005-00105, REVISION 0

SRNL-RPP-2005-00012, REVISION 0

RUN \# 3.03A AND B; FIRST AND SECOND HALF OF SLURRY DEWATERING - CONT.

\begin{tabular}{|c|c|c|c|c|c|c|c|c|c|c|c|c|c|c|c|c|c|c|c|c|c|c|c|c|c|c|c|c|}
\hline & A & B & D & $E$ & $\mathrm{~F}$ & $G^{-1}$ & $\mathrm{H}$ & $\mathrm{J}$ & K & L & $\mathrm{M}$ & $\mathrm{N}$ & 0 & Q & \begin{tabular}{l|l} 
\\
\end{tabular} & $\mathrm{s}$ & T & 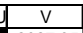 & & $x$ & & $z$ & $\mathrm{AA}$ & & $A C$ & $A D$ & $\mathrm{AE}$ & \\
\hline & 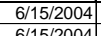 & 7:02:04 PM & 26.565 & 27.01 & $\begin{array}{l}24.80 \\
2.70\end{array}$ & 28.475 & 27.081 & 46.84 & $\begin{array}{l}51.342 \\
51002\end{array}$ & $\begin{array}{l}14.224 \\
1.40\end{array}$ & $\begin{array}{l}33.139 \\
2025\end{array}$ & $\frac{-1.222}{-1.220}$ & \begin{tabular}{|l|l|l|}
-73218 \\
7321
\end{tabular} & $\begin{array}{l}41.565 \\
1.866\end{array}$ & $\begin{array}{l}0.066 \\
\end{array}$ & $\begin{array}{l}14.643 \\
1.642\end{array}$ & 0.004 & & 33.7892 & 49.9 & 10.2 & & 2.757 & 0.010 & 0.010 & 0.000 & $\begin{array}{l}0.247 \\
\end{array}$ & \\
\hline & $6 / 15 / 2004$ & 7:03:04 PM & 26.564 & 27.01 & $\frac{24.78}{24.77}$ & 28.469 & 27.099 & $\begin{array}{r}46.68 \\
46763\end{array}$ & $\begin{array}{l}51.092 \\
51.315\end{array}$ & $\begin{array}{l}14.169 \\
14216\end{array}$ & $\begin{array}{l}33.035 \\
3.0311\end{array}$ & $\begin{array}{l}-1.222 \\
-1.159\end{array}$ & $\begin{array}{l}-7.334 \\
-7.96\end{array}$ & $\begin{array}{l}48.866 \\
42187\end{array}$ & $\begin{array}{l}0.068 \\
0069\end{array}$ & $\frac{14.643}{11643}$ & 0.004 & $\begin{array}{r}2028.35 \\
202935\end{array}$ & \begin{tabular}{|l|l|}
33.8058 \\
332252
\end{tabular} & 49.6 & $\frac{12.0}{10.3}$ & $\frac{39.5}{30.6}$ & \begin{tabular}{|c|c|}
2.748 \\
2751
\end{tabular} & 0 & 0.010 & & 0.256 & \\
\hline 1334 & $6 / 15 / 2004$ & & & 27.01 & 24.76 & $\begin{array}{l}28.489 \\
28.495\end{array}$ & 27.1056 & $\begin{array}{l}40.103 \\
46.552\end{array}$ & \begin{tabular}{|l|}
51.315 \\
50.986 \\
\end{tabular} & \begin{tabular}{|c|}
14.168 \\
14.168
\end{tabular} & $\begin{array}{l}35.041 \\
32.875\end{array}$ & $\begin{array}{l}-1.1 .19 \\
-1.159 \\
\end{array}$ & $\begin{array}{l}-7.196 \\
-7.256\end{array}$ & $\begin{array}{l}42.187 \\
46.937\end{array}$ & \begin{tabular}{|c|}
0.069 \\
0.068
\end{tabular} & $\begin{array}{l}14.643 \\
14.643 \\
\end{array}$ & $\frac{0.004}{0.004}$ & $\frac{2029.35}{2030.35}$ & $\begin{array}{l}33.8225 \\
33.8392 \\
\end{array}$ & $\frac{49.8}{49.5}$ & $\frac{10.3}{11.5}$ & \begin{tabular}{|l}
$\mid 39.9$ \\
39.7 \\
\end{tabular} & & $\begin{array}{l}0.010 \\
0.010\end{array}$ & $\frac{0.010}{0.010}$ & 0.0000 & $\begin{array}{l}0.259 \\
0.257\end{array}$ & $\frac{0.22}{0.22}$ \\
\hline 1335 & $6 / 15 / 2004$ & 7:06:04 PM & 26.555 & 27.00 & 24.74 & 28.39 & 26.996 & 46.983 & \begin{tabular}{|l|l|}
51.693 \\
\end{tabular} & 14.317 & 33.15 & -1.2222 & -7.358 & 55.974 & 0.07 & 14.643 & & 2031.35 & 33.8558 & 50.2 & 13.7 & & \begin{tabular}{|l|}
2.762 \\
\end{tabular} & & & & & \\
\hline & $6 / 15 / 2004$ & & 26.566 & 27.02 & 24.75 & 28.351 & 26.982 & $\begin{array}{l}46.904 \\
\end{array}$ & \begin{tabular}{|l|l|}
51.5799 \\
\end{tabular} & 14.25 & 33.119 & -1.199 & & 45.837 & 0.068 & 14.643 & & & & & & & & & & & & \\
\hline & $6 / 15 / 2004$ & 7:08:04 PM & 26.579 & 27.034 & 24.76 & \begin{tabular}{|l|}
28.31 \\
80.77
\end{tabular} & 26.94 & $\begin{array}{l}46.846 \\
\end{array}$ & \begin{tabular}{|l|l|}
51.535 \\
\end{tabular} & $\begin{array}{r}14.3 \\
\end{array}$ & 32.983 & $\begin{array}{l}-1.219 \\
\end{array}$ & $\begin{array}{r}-7.372 \\
-7320\end{array}$ & 60.82 & 0.065 & 14.643 & & & 33.8892 & 50.1 & 14.9 & & & & & & 0.244 & \\
\hline 1338 & $6 / 15 / 2004$ & 7:09:04 PM & 26.572 & 27.04 & 24.75 & 28.277 & 26.923 & $\begin{array}{l}46.964 \\
\end{array}$ & 51.695 & 14.336 & 33.01 & -1.222 & $\begin{array}{r}-7.398 \\
\end{array}$ & 43.096 & 0.068 & 14.643 & & \begin{tabular}{|l|l|}
2034.3 \\
\end{tabular} & 33.9058 & 50.2 & 10.6 & & 2.757 & 0.010 & 0.010 & 0.000 & 0.255 & \\
\hline 13 & 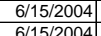 & $\begin{array}{l}7: 10: 04 \mathrm{PM} \\
7 \\
710104 \mathrm{P}\end{array}$ & $\begin{array}{l}26.562 \\
26561\end{array}$ & $\begin{array}{ll}27.02 \\
2702\end{array}$ & & $\begin{array}{l}28.268 \\
281977\end{array}$ & & \begin{tabular}{|l|l|}
46.796 \\
46.726 \\
\end{tabular} & \begin{tabular}{|l|l|}
51.562 \\
51.201 \\
\end{tabular} & 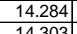 & $\begin{array}{l}32.949 \\
32.897\end{array}$ & $\begin{array}{c}-1.202 \\
1222\end{array}$ & \begin{tabular}{|l|l|}
-7.323 \\
7.04
\end{tabular} & $\begin{array}{l}45.756 \\
4045\end{array}$ & \begin{tabular}{|l|l|}
0.063 \\
0.067
\end{tabular} & $\begin{array}{l}14.643 \\
1.642\end{array}$ & & & \begin{tabular}{|l|}
33.9225 \\
3230202
\end{tabular} & 50.1 & 11.2 & & \begin{tabular}{|l|}
2.749 \\
\end{tabular} & & $\begin{array}{l}0.009 \\
\end{array}$ & $\begin{array}{l}0.000 \\
.000\end{array}$ & 0.237 & \\
\hline$\frac{\frac{1340}{1341}}{1341}$ & $\frac{6 / 115 / 2004}{6 / 15 / 2004}$ & $\begin{array}{l}\text { 7:11:04 PM } \\
7: 1204 \mathrm{PM}\end{array}$ & $\frac{26.561}{26.543}$ & $\frac{27.02}{27.00}$ & $\begin{aligned} \frac{24.74}{24.7} \\
\end{aligned}$ & $\frac{28.197}{28.129}$ & 1832 & $\begin{array}{l}46.726 \\
46.736\end{array}$ & \begin{tabular}{|l|}
51.421 \\
51.427
\end{tabular} & \begin{tabular}{|l|}
14.303 \\
14.267
\end{tabular} & $\begin{array}{l}32.897 \\
32.951 \\
\end{array}$ & $\begin{array}{l}-1.222 \\
-1.156\end{array}$ & $\begin{array}{r}-7.404 \\
-7.242 \\
\end{array}$ & $\begin{array}{r}\frac{44.945}{51.867} \\
567\end{array}$ & $\begin{array}{l}0.0667 \\
0.0068\end{array}$ & $\begin{array}{l}14.643 \\
14.643 \\
\end{array}$ & $\begin{array}{l}0.004 \\
0.004\end{array}$ & 336.35 & \begin{tabular}{|l|}
33.9392 \\
33.9558
\end{tabular} & $\begin{array}{l}49.9 \\
550 .\end{array}$ & \begin{tabular}{|l|}
11.0 \\
12.7 \\
\end{tabular} & $\frac{39.8}{39.8}$ & \begin{tabular}{|l|}
2.745 \\
2.747 \\
\end{tabular} & 0.010 & $\begin{array}{l}0.010 \\
\end{array}$ & $\begin{array}{r}0.000 \\
0.000\end{array}$ & 0.253 & \\
\hline 1342 & $6 / 15 / 2004$ & $\begin{array}{l}1.12 .04 \\
7: 12: 42 \mathrm{PM}\end{array}$ & & 27.00 & 24.71 & 28.099 & 26.774 & & \begin{tabular}{|l|}
51.4477 \\
51.74
\end{tabular} & & 33.191 & & & 44.006 & 0.071 & $\begin{array}{l}14.643 \\
14.643\end{array}$ & & $\begin{array}{l}2037.55 \\
2037.98\end{array}$ & \begin{tabular}{|l|l}
33.9558 \\
33.9664
\end{tabular} & 50.3 & $\frac{12.1}{10.8}$ & \begin{tabular}{|l|}
39.8 \\
40.1 \\
\end{tabular} & & & & & $\begin{array}{l}0.266 \\
0.266 \\
\end{array}$ & 0.23 \\
\hline & $6 / 15 / 2004$ & 7:13:42 PM & 26.543 & 27.01 & 24.7 & 28.075 & 26.74 & 46.719 & 51.091 & 14.314 & 32.965 & -1.153 & -7.277 & 53.874 & 0.068 & 14.643 & 0.004 & 2038.98 & 33.9831 & 49.6 & 13.2 & 39.8 & \begin{tabular}{|l|}
2.747 \\
\end{tabular} & & 0.010 & 0.000 & 0.256 & \\
\hline & $6 / 15 / 2004$ & 7:14:42 PM & & & 24.73 & & 26.698 & 46.518 & \begin{tabular}{|l|l|l|}
51.129 \\
\end{tabular} & \begin{tabular}{|l|l|}
14.239 \\
\end{tabular} & & -1.182 & & 49.986 & 0.072 & 14.643 & & & \begin{tabular}{|l|l|}
33.9997 \\
\end{tabular} & 49.7 & \begin{tabular}{|l|l|}
12.2 \\
\end{tabular} & & \begin{tabular}{|l|}
2.733 \\
\end{tabular} & 0.011 & 0.011 & 0.000 & 0.273 & \\
\hline 1345 & $6 / 15 / 2004$ & 7:15:42 PM & 26.537 & 27.00 & 24.74 & 27.998 & 26.689 & & 51.332 & & 33.154 & -1.222 & & 50.699 & 0.071 & 14.643 & & & \begin{tabular}{|l|l|}
34.0164 \\
\end{tabular} & 49.9 & 12.4 & & 2.762 & & & 0.000 & 0.266 & \\
\hline$\frac{1346}{1347}$ & 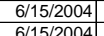 & $\begin{array}{l}7: 16: 42 \mathrm{PM} \\
7 \\
7\end{array}$ & $\begin{array}{r}26.54 \\
26.528\end{array}$ & $\begin{array}{l}26.99 \\
26.99 \\
2\end{array}$ & $\begin{array}{l}24.74 \\
24.74\end{array}$ & $\begin{array}{l}27.991 \\
27.984\end{array}$ & $\begin{array}{l}26.682 \\
26.664\end{array}$ & \begin{tabular}{|l|l|}
46.703 \\
46.954
\end{tabular} & \begin{tabular}{|l|}
51.525 \\
$51.64 \mid$ \\
\end{tabular} & \begin{tabular}{|l|l|}
14.246 \\
14269
\end{tabular} & $\begin{array}{r}32.905 \\
33.18\end{array}$ & $\begin{array}{l}-1.098 \\
-1.222\end{array}$ & $\begin{array}{r}-7.06 \\
-.3499\end{array}$ & $\begin{array}{l}56.728 \\
51.459\end{array}$ & \begin{tabular}{|c|c|}
0.065 \\
0.068
\end{tabular} & $\begin{array}{l}14.643 \\
1643\end{array}$ & 0.00 & & \begin{tabular}{|l|l|}
34.0331 \\
304907
\end{tabular} & 50.1 & $\begin{array}{l}13.9 \\
12 .\end{array}$ & 39.8 & \begin{tabular}{l|l|}
2.744 \\
2762
\end{tabular} & \begin{tabular}{|l|l|}
0.010 \\
0010
\end{tabular} & $\begin{array}{l}0.010 \\
0.010\end{array}$ & $\begin{array}{l}0.000 \\
\end{array}$ & 0.245 & 0.21 \\
\hline $\begin{array}{l}\frac{1344}{1348} \\
\end{array}$ & $6 / 15 / 2004$ & $\begin{array}{l}7: 17: 42 \mathrm{PM} \\
7: 18: 42 \mathrm{PM}\end{array}$ & 26.528 & 26.99 & 24.74 & $\begin{array}{l}27.984 \\
27.967\end{array}$ & $\frac{26.664}{26.628}$ & \begin{tabular}{|c|}
46.954 \\
47.19 \\
\end{tabular} & \begin{tabular}{|l|}
51.604 \\
51.836 \\
\end{tabular} & $\begin{array}{l}14.269 \\
14.337\end{array}$ & $\begin{array}{r}33.18 \\
33.258 \\
\end{array}$ & $\begin{array}{l}-1.222 \\
-1.222\end{array}$ & $\begin{array}{l}-7.349 \\
-7.386\end{array}$ & 51.459 & $\begin{array}{l}0.068 \\
0.06\end{array}$ & $\frac{14.643}{14643}$ & 0.00 & \begin{tabular}{|l}
2042.98 \\
2043.98
\end{tabular} & \begin{tabular}{|l|}
34.0497 \\
3306664
\end{tabular} & 50.1 & 12.6 & \begin{tabular}{|l|l|}
40.1 \\
40.2
\end{tabular} & 2.762 & 0.010 & 0.010 & 0.000 & 0.255 & \\
\hline & $6 / 15 / 2004$ & 7:1.0.4:42 PM & $\frac{20.450}{26.489}$ & $\frac{20.30}{26.95}$ & $\frac{24.16}{24.72}$ & 27.995 & $\frac{20.020}{26.655}$ & \begin{tabular}{|l|l|}
47.089 \\
4.09
\end{tabular} & \begin{tabular}{|r|}
51.00 \\
\end{tabular} & $\begin{array}{l}14.2808 \\
14.288\end{array}$ & $\begin{array}{l}30.250 \\
33.226\end{array}$ & $\frac{-1.242}{-1.222}$ & $\frac{-1.386}{-7.381}$ & $\frac{55.616}{46.148}$ & $\begin{array}{l}0.066 \\
0.067\end{array}$ & $\frac{14.643}{14.643}$ & & \begin{tabular}{|l|l|}
2044.98 \\
204.98
\end{tabular} & \begin{tabular}{|l|}
34.06064 \\
34.0831
\end{tabular} & $\frac{50.4}{50.4}$ & $\frac{13.6}{11.3}$ & \begin{tabular}{|l|}
40.2 \\
0.2
\end{tabular} & \begin{tabular}{|l|}
2.713 \\
2.769 \\
\end{tabular} & \begin{tabular}{|l}
0.010 \\
0.010
\end{tabular} & $\begin{array}{l}.010 \\
0.010\end{array}$ & 0 & $\begin{array}{l}0.247 \\
0.251\end{array}$ & 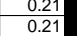 \\
\hline & $6 / 15 / 2004$ & 7:20:42 PM & 26.494 & 26.95 & 24.73 & 28.035 & 26.701 & 46.587 & \begin{tabular}{|l|}
51.176 \\
\end{tabular} & \begin{tabular}{|l|l|}
14.197 \\
\end{tabular} & 32.85 & -1.214 & -7.392 & 50.845 & 0.066 & 14.643 & & & \begin{tabular}{|l|}
34.0997 \\
\end{tabular} & 49.7 & 12.5 & & \begin{tabular}{|l|}
2.738 \\
\end{tabular} & & 0.010 & 0.000 & 0.250 & \\
\hline & 6/15/2004 & 7:21:42 PM & 26.489 & & 24.73 & 28.07 & 26.726 & $\begin{array}{l}46.804 \\
\end{array}$ & \begin{tabular}{|l|l|}
51.518 \\
\end{tabular} & 14.266 & 32.967 & -1.214 & & 49.909 & $\begin{array}{l}0.066 \\
\end{array}$ & 14.643 & & & \begin{tabular}{|l|l|}
34.1164 \\
\end{tabular} & & 12.2 & & \begin{tabular}{|l|}
2.750 \\
\end{tabular} & & & & 0.249 & \\
\hline 1352 & $6 / 15 / 2004$ & 7:22:42 PM & 26.485 & 26.93 & 24.72 & 28.041 & 26.782 & & 51.682 & $\begin{array}{l}14.235 \\
\end{array}$ & 32.998 & -1.156 & $\begin{array}{r}-7.381 \\
\end{array}$ & 53.142 & $\begin{array}{l}0.069 \\
\end{array}$ & 14.643 & & & 34.1331 & 50.2 & \begin{tabular}{|l|l}
13.0 \\
\end{tabular} & & \begin{tabular}{|l|}
2.752 \\
\end{tabular} & 0.010 & & & 0.260 & \\
\hline & $6 / 15 / 2004$ & $\begin{array}{l}\text { 7:23:42 PM } \\
7\end{array}$ & $\begin{aligned} 26.482 \\
26.478\end{aligned}$ & 26.93 & $\begin{aligned} 24.72 \\
24.72\end{aligned}$ & 28.118 & $\begin{aligned} 26.808 \\
26.829\end{aligned}$ & $\begin{array}{r}47.074 \\
46865\end{array}$ & \begin{tabular}{|l|}
51.655 \\
51587 \\
\end{tabular} & \begin{tabular}{|l|l|}
14.276 \\
1430
\end{tabular} & $\begin{array}{r}33.271 \\
33.887 \\
3\end{array}$ & $\begin{array}{r}-1.199 \\
1.156\end{array}$ & $\begin{array}{r}-7.381 \\
7.239\end{array}$ & \begin{tabular}{|l|l|l|l|l}
57.374 \\
51769
\end{tabular} & 0.067 & $\begin{array}{l}14.643 \\
1643\end{array}$ & 0.00 & & & 50.2 & $\begin{array}{l}11.6 \\
127\end{array}$ & 40.2 & \begin{tabular}{|l|l|}
2.770 \\
2.779
\end{tabular} & $\begin{array}{l}0.010 \\
0009\end{array}$ & 0.010 & 0.000 & 0.251 & \\
\hline & $6 / 15 / 2004$ & $\begin{array}{l}7: 24: 42 \mathrm{PM} \\
7 \cdot 25: 42 \mathrm{PM}\end{array}$ & 26.478 & $\frac{26.93}{26.94}$ & $\frac{24.72}{247}$ & 28.1699 & $\frac{26.829}{26865}$ & $\begin{array}{r}46.865 \\
440\end{array}$ & \begin{tabular}{|l|}
51.587 \\
51.608 \\
\end{tabular} & \begin{tabular}{l|l|}
14.398 \\
14235
\end{tabular} & $\begin{array}{r}32.887 \\
33.308 \\
\end{array}$ & $\begin{array}{l}-1.156 \\
-1.156\end{array}$ & $\begin{array}{r}-7.239 \\
-7323\end{array}$ & $\begin{array}{l}51.769 \\
37774\end{array}$ & $\begin{array}{l}0.063 \\
0.07\end{array}$ & $\frac{14.643}{14643}$ & & & \begin{tabular}{|l|l|}
34.1664 \\
33.1831
\end{tabular} & $\frac{50.1}{50.1}$ & $\frac{12.7}{9.3}$ & 39.9 & \begin{tabular}{|l|}
$\mid 2.749$ \\
2769
\end{tabular} & $\begin{array}{l}0.009 \\
0.010\end{array}$ & 0.009 & 0.000 & 0.237 & 0. \\
\hline 1356 & $6 / 115 / 2004$ & $\begin{array}{ll}.25: 542 \mathrm{PM} \\
7: 26: 42 \mathrm{PM}\end{array}$ & $\frac{26.489}{26.5}$ & $\frac{26.94}{26.94}$ & $\frac{24.1}{24.74}$ & $\frac{28.209}{28.196}$ & $\frac{26.865}{26.911}$ & \begin{tabular}{|l|l|}
46.82 \\
46.89
\end{tabular} & \begin{tabular}{|l|}
51.008 \\
51.541 \\
\end{tabular} & \begin{tabular}{|l|}
14.235 \\
14.267
\end{tabular} & $\begin{array}{l}33.508 \\
33.109 \\
\end{array}$ & $\begin{array}{l}-1.156 \\
-1.156\end{array}$ & $\begin{array}{l}-7.323 \\
-7.369 \\
\end{array}$ & $\frac{37.194}{48.128}$ & 0.066 & $\frac{14.643}{14.643}$ & & \begin{tabular}{|l|}
2050.98 \\
2051.98
\end{tabular} & \begin{tabular}{|l|}
34.1831 \\
34.1997 \\
\end{tabular} & 50.1. & $\begin{array}{l}9.3 \\
11.8\end{array}$ & $\frac{40.2}{40.0}$ & $\frac{2.669}{2.758}$ & \begin{tabular}{|l|l}
0.010 \\
0.010
\end{tabular} & $\frac{0.010}{0.010}$ & $\frac{0.000}{0.000}$ & $\frac{0.251}{0.255}$ & 0.21 \\
\hline 1357 & $6 / 15 / 2004$ & 7:27:42 PM & 26.504 & 26.95 & 24.74 & 28.224 & 26.934 & 46.948 & \begin{tabular}{|r|}
51.57 \\
\end{tabular} & 14.3 & 33.152 & -1.199 & -7.378 & 48.515 & 0.067 & 14.643 & & 2052.98 & 34.2164 & 50.1 & 11.9 & 40.1 & \begin{tabular}{|l|}
2.761 \\
\end{tabular} & 0.010 & 0.010 & 0.000 & 0.251 & \\
\hline & $6 / 15 / 2004$ & 7:28:42 PM & 26.489 & 26.94 & 24.7 & 28.234 & & 47.136 & \begin{tabular}{|l|l|}
51.935 \\
\end{tabular} & \begin{tabular}{|l|l|l|l}
14.327 \\
\end{tabular} & 33.219 & \begin{tabular}{|l|l|} 
\\
\end{tabular} & & 45.645 & & 144.643 & & 2053.98 & \begin{tabular}{|l|}
34.2331 \\
\end{tabular} & 50.5 & 11.2 & 40.2 & \begin{tabular}{|l|}
2.770 \\
\end{tabular} & & 0.010 & & & \\
\hline 1359 & $6 / 15 / 2004$ & & 26.496 & 26.9 & 24.74 & & & & & & 33.189 & & & 47.135 & & 14.643 & & & & & 11.6 & & & & & 0.000 & & \\
\hline 1360 & $6 / 15 / 2004$ & 7:30:42 PM & & & 24.82 & 28.173 & & & \begin{tabular}{|l|l|l|l|l|}
51 \\
\end{tabular} & \begin{tabular}{|l|l|}
14.146 \\
\end{tabular} & \begin{tabular}{|l|l|}
33.027 \\
\end{tabular} & -1.135 & & & & 14.643 & & & & & & & & & & & & \\
\hline & 6/15/2004 & 7::31:42 PM & & 26.95 & 24.82 & & & 46 & & & 33.098 & -1.153 & & & & 14.643 & & & & & & & & & & & & \\
\hline & 6/15/2004 & $7: 32: 42$ & 26.485 & 26.94 & 24.79 & 28.066 & & & 51.502 & 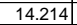 & .232 & -1.19 & & & 0.069 & 14.643 & & & & & 2.7 & & & & & & & \\
\hline & $6 / 15 / 2004$ & 7:33:42 PM & 26.498 & 26.96 & 24.80 & 28.059 & & & \begin{tabular}{|l|l|}
51.483 \\
\end{tabular} & 14.217 & 32.996 & & & 54.189 & 556 & 14.643 & & & & & 3.3 & & 877 & & & & & \\
\hline 364 & 6/15/2004 & 7:34: & 26.482 & 26.93 & 24.77 & 27.968 & 26 & 46.892 & 51.661 & 14.234 & 33.082 & -1.115 & & 45.779 & 0.073 & 14.643 & & 2059.98 & 34.3331 & 50.2 & 11.2 & & 2.757 & 0.011 & 011 & 0.000 & .274 & \\
\hline & 5/2004 & & 26.485 & & & & & & & & & & & & & & & & & & & & & & & & & \\
\hline & & & & 26.92 & 24.76 & 27.895 & & & 51.226 & 14.218 & & -1.159 & & 10.53 & & & & & & & & & & & & & & \\
\hline & 6 & $\begin{array}{ll}0.3: 42 \mathrm{PP} \\
7.38\end{array}$ & $\frac{26.416}{26452}$ & 26.92 & 24.18 & 27.8919 & & 414.126 & (51.1524 & $\frac{14.306}{14206}$ & $\begin{array}{l}33.269 \\
32258\end{array}$ & - & & 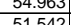 & 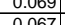 & $\frac{14.643}{14643}$ & & & & & 26 & & 278 & & & & 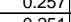 & \\
\hline & 6 & & $\frac{20.452}{26.439}$ & & 24.15 & - & & & S1.519 & & & & & & & 146 & & & & & & & & & & & & \\
\hline & $6 / 15 / 2004$ & $7: 40$ & 26.450 & $\frac{2.000}{26.8}$ & 24.13 & 27778 & & & 51.968 & 1439 & & -1.1 & & & & 146 & & & & & & & & & & & & \\
\hline & $6 / 15 / 2004$ & $7: 41$ & 26.424 & 26.85 & 24.73 & 27.825 & & 46.869 & $\mid$\begin{tabular}{|l|}
51.319 \\
\end{tabular} & $\begin{array}{ll}14.232 \\
14.03\end{array}$ & 33.16 & -1.156 & & & & $\frac{14.6}{14.6}$ & & & & 49.8 & 2.9 & & 2.759 & 0.009 & $\frac{0.009}{0.009}$ & $\frac{0.000}{0.000}$ & $\frac{0.229}{0.229}$ & \\
\hline & $6 / 15 / 2$ & & & & & & & & & & & & & & & & & & & & & & & & & & & \\
\hline & $5 / 2004$ & & 26.423 & 26.84 & 24.73 & 27.8 & & & 52.068 & 14.3 & & & & & & & & & & & & & & & & & & \\
\hline & $6 / 15 /$ & & 26.4 & 26.8 & & & & & & 14.21 & 32 & & & & & & & & & & & & & & & & & \\
\hline & $6 / 15 / 2$ & & 26.409 & 26.8 & 24.71 & & & & & 14.325 & & -1.1. & & & & & & & & & 1. & & & & & & & \\
\hline & $6 / 15 / 2004$ & & 26.423 & 26.83 & 24.71 & & & & & 14.23 & & & & & & 14.6 & & & & 49 & & & & & & & 0.254 & \\
\hline & $6 / 15 / 2$ & & 26.418 & & & & & & & $\begin{array}{l}14.373 \\
\end{array}$ & & & & & & & & & & & & & & & & & & \\
\hline 137 & $6 / 15 / 2004$ & & 26.411 & 26.81 & 24.71 & 28.032 & & & & 14.332 & & -1.127 & & & 0 & 14. & & & & 50.0 & 10.2 & & & & & & 0.255 & \\
\hline & $6 / 15 / 2004$ & & 26.421 & 26.8 & & & & & & & & & & & & & & & & & & & & & & & & \\
\hline & & & & & & & & & & & & & & & & & & & & & & & & & & & & \\
\hline & & & 2.488 & & 24.12 & & & & & 14. & & & & & & & & & & & & & & & & & & \\
\hline & $\frac{01515120041}{6}$ & & $\frac{20.44}{2644}$ & $\frac{2.044}{2686}$ & 24.15 & $\frac{20.1}{28.1}$ & & & & $\frac{14.21}{143}$ & & & & & & & & & & & & & & & & & & \\
\hline & 6 & & & 2684 & & & & & & & & & & & & & & & & & & & & & & & & \\
\hline & $\frac{6 / 15 / 2004}{6}$ & & $\frac{20.427}{26.427}$ & $\frac{20.04}{26.84}$ & 24.73 & 28.0 & & & & 14.275 & & -1.1. & & & & & & & & & 24 & & & & & & & \\
\hline & $6 / 15 / 2004$ & & & & & & & & & & & & & & & & & & & & & & & & & & & \\
\hline & & & & & & & & & & & & & & & & & & & & & & & & & & & & \\
\hline & $6 / 15 / 2004$ & & 26 & 26. & 248 & 27.9 & & & & & & -1. & & & & & & & & & & & & & & & & \\
\hline & $6 / 15 / 2$ & $7: 59: 2$ & 26.44 & 26.8 & 24.83 & 27.882 & & 47.00 & 51.7 & 14. & & $\begin{array}{c}-0.996 \\
\end{array}$ & & & 0.1 & 14.6 & & & & & & & & & & & 0.224 & \\
\hline & $6 / 15 / 2004$ & 8:00 & 439 & 26.8. & 24.80 & 27.8 & & & 51.655 & 14. & & -1.101 & & & 0.0 & 14. & & & & & & & & & & & & \\
\hline & $6 / 15 / 2$ & & & & 24.76 & 27.7 & & & & & & & & & & & & & & & & & & & & & & \\
\hline 3 & $6 / 15 / 2$ & & & & & 27. & & & & & & -1.156 & & & & & & & & & 10.3 & & & & & & 0.248 & \\
\hline & $6 / 15 / 2004$ & & & & & 27.6 & & & & & & -1.1 & & & & & & & & & & & & & & & & \\
\hline & $6 / 15 / 2004$ & & 411 & 26.8 & 24.75 & 27.713 & & $4 !$ & & 14. & 33. & -1. & & & & & & & & & & & & & & & & \\
\hline & 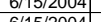 & 8.: & & 260 & $\frac{24.12}{2.76}$ & $\frac{21.14}{277 \pi}$ & & $46 . .11$ & 515 & 14.1 & $\frac{32.985}{32115}$ & -1.156 & & & & & & & & & & & & & & & & \\
\hline & 01572004 & & 121 & $\frac{2.00}{2600}$ & $\frac{2.10}{2.77}$ & 27.150 & & 40.9 & 51,34 & 14.213 & & -1.150 & & & & & & & & & & & & & & & & \\
\hline & 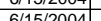 & & 2641 & & 2475 & 27.010 & & 47014 & & 14266 & & - -1.156 & & & & & & & & & & & & & & & & \\
\hline & & & 26.405 & 2678 & 24,75 & 2787 & & $\frac{47.1}{47.1}$ & 51788 & 14.284 & 33.353 & $-1.0 \leqq$ & & & & 14. & & & & & 120 & & & & & & & \\
\hline $40 \mathrm{CO}$ & $6 / 15 / 2004$ & $8: 10: 4$ & 26.4 & 26.77. & \begin{tabular}{|l|l|}
24.74 \\
\end{tabular} & \begin{tabular}{|l|l|}
27.861 \\
\end{tabular} & & 46.9 & 51.311 & 203 & 283 & -1.135 & & 45.074 & .066 & $\frac{14.045}{14.643}$ & & & & & 11.0 & & & 010 & & 1000 & 0.247 & \\
\hline
\end{tabular}


WSRC-TR-2005-00105, REVISION 0

SRNL-RPP-2005-00012, REVISION 0

RUN \# 3.03A AND B; FIRST AND SECOND HALF OF SLURRY DEWATERING - CONT.

\begin{tabular}{|c|c|c|c|c|c|c|c|c|c|c|c|c|c|c|c|c|c|c|c|c|c|c|c|c|c|c|c|c|}
\hline & A & $B$ & $\mathrm{D}$ & $E$ & $\mathrm{~F}$ & G & \begin{tabular}{ll|}
$\mathrm{H}$ \\
\end{tabular} & $\mathrm{J}$ & $\mathrm{K}$ & $\mathrm{L}$ & \begin{tabular}{l|} 
\\
\end{tabular} & $\mathrm{N}$ & 0 & $\mathrm{Q}$ & R & s & $\begin{array}{ll}T \\
\end{array}$ & v & w & \begin{tabular}{l|}
$x$ \\
\end{tabular} & $\mathrm{Y}$ & $z$ & $\mathrm{AA}$ & $A B$ & $A C$ & & & AF \\
\hline 140 & $6 / 15 / 2004$ & 8:11:42 PM & 26.392 & 26.776 & 24.748 & \begin{tabular}{|l|l|}
27.913 \\
\end{tabular} & 26.683 & \begin{tabular}{|l|l|l|l|}
47.134 \\
\end{tabular} & 51.765 & \begin{tabular}{ll|l}
14.207 \\
\end{tabular} & 33.466 & \begin{tabular}{|l|}
-1.156 \\
\end{tabular} & \begin{tabular}{|c|} 
\\
\end{tabular} & 49.715 & $\begin{array}{l}0.067 \\
\end{array}$ & 14.643 & 0.004 & 2096.98 & \begin{tabular}{l|l}
834.9497 \\
8
\end{tabular} & 50.3 & 12.2 & 40.3 & 2.779 & 0.010 & 0.010 & 0.000 & 0.250 & \\
\hline & & & 26.388 & 26.767 & 24.74 & \begin{tabular}{|l|}
27.91 \\
27
\end{tabular} & 26.69 & & 51.993 & 14.107 & $\begin{array}{l}33.696 \\
\end{array}$ & 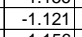 & $\begin{array}{l}-7.329 \\
\end{array}$ & 46.163 & 0.067 & \begin{tabular}{|l|l|}
14.643 \\
\end{tabular} & 0.000 & & 834.9664 & 50.5 & 11.3 & & & & & & & \\
\hline 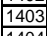 & 6/15/2004 & 8:13:42 PM & 26.382 & $\frac{26.761}{2.782}$ & 124.738 & \begin{tabular}{|l|}
27.898 \\
7790
\end{tabular} & 26.683 & 47.25 & \begin{tabular}{|l|l|}
51.975 \\
\end{tabular} & $\begin{array}{l}4.12 \\
\end{array}$ & $\begin{array}{l}33.562 \\
3267\end{array}$ & \begin{tabular}{|r|}
-1.156 \\
\end{tabular} & $\begin{array}{l}-7.355 \\
-7350\end{array}$ & \begin{tabular}{|l|}
45.176 \\
\end{tabular} & $\begin{array}{l}0.067 \\
\end{array}$ & 14.643 & 0.004 & $\begin{array}{l}0998.98 \\
0898 \\
\end{array}$ & $\begin{array}{l}834.9831 \\
8\end{array}$ & 50.5 & 11.1 & 40.4 & .786 & 0.010 & 0.010 & 0.000 & 0.249 & \\
\hline 1404 & $6 / 15 / 2004$ & 8:14:42 PM & 26.393 & 26.782 & \begin{tabular}{|l|l|}
2 & 24.764 \\
\end{tabular} & $\begin{array}{l}27.989 \\
27.95\end{array}$ & 26.699 & $\begin{array}{r}47.296 \\
\end{array}$ & \begin{tabular}{|l|l|}
52.136 \\
5170
\end{tabular} & \begin{tabular}{|l|l|}
14.202 \\
1.20
\end{tabular} & \begin{tabular}{|c|}
33.507 \\
\end{tabular} & -1.219 & $\begin{array}{l}-7.158 \\
-1007\end{array}$ & 48.566 & $\begin{array}{l}0.06 \\
\end{array}$ & 14.643 & 0.004 & 099.98 & 4.9997 & $\begin{array}{l}50.7 \\
\end{array}$ & 11.9 & 40.4 & 2.786 & 0.009 & \begin{tabular}{|l|l|l|}
0.009 \\
\end{tabular} & 0.000 & 0.223 & \\
\hline$\frac{1405}{1406}$ & $\frac{6 / 15 / 2004}{6 / 15 / 204}$ & $\begin{array}{l}8: 15: 42 \mathrm{PM} \\
8.16: 42 \mathrm{PM}\end{array}$ & $\frac{26.383}{26.373}$ & $\frac{26.762}{26.762}$ & \begin{tabular}{|l|l|}
24.735 \\
2
\end{tabular} & \begin{tabular}{|r|}
27.95 \\
27599
\end{tabular} & $\frac{26.715}{26.725}$ & \begin{tabular}{|l|}
46.861 \\
47.42 \\
\end{tabular} & \begin{tabular}{|r|}
51.79 \\
52.298 \\
\end{tabular} & $\begin{array}{r}14.18 \\
14255\end{array}$ & & $\begin{array}{l}-0.996 \\
1.111\end{array}$ & $\begin{array}{r}-6.907 \\
7.77 \\
\end{array}$ & \begin{tabular}{|l|l|}
46.403 \\
57635
\end{tabular} & $\begin{array}{l}0.07 \\
0067\end{array}$ & \begin{tabular}{|l|l|}
14.643 \\
14643
\end{tabular} & 0.004 & & 835.0164 & \begin{tabular}{l|l|}
50.3 \\
5.8
\end{tabular} & \begin{tabular}{|l|}
11.4 \\
141
\end{tabular} & & & & \begin{tabular}{|c|c|}
0.011 \\
\end{tabular} & & 0.263 & \\
\hline $\begin{array}{ll}1407 \\
1407\end{array}$ & $\begin{array}{l}6 / 1 / 15 / 2004 \\
/ 2004\end{array}$ & 8:11::42 PM & $\frac{26.373}{26.394}$ & $\frac{26.672}{26.778}$ & $\begin{aligned} 24.725 \\
24.756\end{aligned}$ & $\begin{array}{r}27.959 \\
27.98\end{array}$ & $\frac{26.725}{26.731}$ & $\begin{array}{l}47.7422 \\
7.437 \\
\end{array}$ & \begin{tabular}{|l|}
52.298 \\
52.238 \\
\end{tabular} & \begin{tabular}{|l|}
$\mid 14.255$ \\
14.236
\end{tabular} & $\begin{array}{r}33.55 \\
33.555\end{array}$ & \begin{tabular}{|l|} 
\\
\end{tabular} & $\begin{array}{r}-7.17 \\
-7.135 \\
\end{array}$ & \begin{tabular}{|}
57.635 \\
49.135 \\
\end{tabular} & $\begin{array}{l}0.066 \\
0.068\end{array}$ & $\begin{array}{l}\frac{14.643}{14.643} \\
\end{array}$ & $\frac{0.004}{0.004}$ & \begin{tabular}{|c|c|}
101.98 \\
102.98
\end{tabular} & $\begin{array}{l}835.0331 \\
88540497\end{array}$ & $\begin{array}{l}50.8 \\
50.8 \\
\end{array}$ & $\frac{14.1}{12.0}$ & \begin{tabular}{|l|}
40.5 \\
40.5
\end{tabular} & $2.791 \mid$ & $\frac{0.010}{0.010}$ & 0.0010 & $\frac{0.000}{0.000}$ & $\begin{array}{l}0.245 \\
0.225\end{array}$ & \\
\hline 1408 & & 8:18:42 PM & 26.391 & 26.785 & 24.787 & 27.922 & 26.692 & & \begin{tabular}{|l|}
51.97 \\
\end{tabular} & \begin{tabular}{|l|}
14.179 \\
\end{tabular} & 33.466 & & $\begin{array}{l}-7.265 \\
\end{array}$ & \begin{tabular}{|l|}
45.086 \\
\end{tabular} & 0.066 & $\begin{array}{l}14.045 \\
14.643\end{array}$ & 0.004 & $\frac{210<.98}{2103.98}$ & \begin{tabular}{|l}
835.0064 \\
8
\end{tabular} & $\begin{array}{r}50.8 \\
50.5 \\
\end{array}$ & $\frac{12.0}{11.0}$ & \begin{tabular}{|l|}
40.3 \\
40.3
\end{tabular} & & 0.010 & 0 & 0.000 & $\begin{array}{l}0.245 \\
0.245\end{array}$ & $\frac{0.21}{0.21}$ \\
\hline 1409 & $6 / 15 / 2004$ & 8:19:42 PM & 26.369 & 26.763 & 24.811 & 27.841 & 26.621 & 46.964 & 51.664 & 14.12 & & -1.167 & $\begin{array}{r}-7.32 \\
\end{array}$ & 48.472 & 0.059 & 14.643 & & & \begin{tabular}{l|l}
835.0831 \\
8
\end{tabular} & & 11.9 & & & & & & & \\
\hline 1410 & $6 / 15 / 2004$ & 8:20:42 PM & & & 24.808 & 27.813 & 26.564 & $\begin{array}{l}47.221 \\
\end{array}$ & 51.856 & \begin{tabular}{|l|l|}
14.129 \\
\end{tabular} & & -1.156 & & 57.157 & & 14.643 & & & 35.0997 & & 14.0 & & & & & 0.000 & 0.245 & \\
\hline$\frac{1411}{1412}$ & $6 / 15 / 2004$ & 8:21:42 PM & 26.393 & 26.762 & \begin{tabular}{|l|l|}
24.8 \\
\end{tabular} & 27.72 & 26.495 & 47.02 & 51.668 & \begin{tabular}{|l|l|}
14.069 \\
\end{tabular} & $\begin{array}{l}33.396 \\
\end{array}$ & -1.156 & & 51.292 & \begin{tabular}{|l|l|}
0.067 \\
\end{tabular} & \begin{tabular}{|l|l|}
14.643 \\
\end{tabular} & & & 35.1164 & 50.2 & 12.6 & 40.2 & & 0.010 & $\begin{array}{l}0.010 \\
\end{array}$ & 0.000 & 0.250 & \\
\hline$\frac{1412}{1413}$ & $\frac{6 / 15 / 2004}{6 / 15 / 204}$ & $\begin{array}{l}8: 22: 42 \mathrm{PM} \\
8.42 .42 \mathrm{PM}\end{array}$ & $\frac{26.378}{26.36}$ & $\begin{array}{l}26.762 \\
26.739 \\
\end{array}$ & $\begin{array}{r}24.79 \\
24.762\end{array}$ & \begin{tabular}{|l|}
27.685 \\
27622
\end{tabular} & $\begin{array}{r}26.45 \\
26.412\end{array}$ & \begin{tabular}{|l|}
47.157 \\
47.062 \\
\end{tabular} & \begin{tabular}{|l|}
51.867 \\
51838
\end{tabular} & $\begin{array}{l}14.109 \\
14.134\end{array}$ & 3.517| & $\begin{array}{r}-1.159 \\
-1.156\end{array}$ & $\begin{array}{l}-7.294 \\
7308\end{array}$ & \begin{tabular}{|l|}
48.203 \\
52202
\end{tabular} & \begin{tabular}{|l|l|}
0.066 \\
0.066
\end{tabular} & $\begin{array}{l}14.643 \\
14643\end{array}$ & & $\begin{array}{l}107.98 \\
10898\end{array}$ & 835.1331 & $\begin{array}{l}50.4 \\
55.4\end{array}$ & 11.8 & \begin{tabular}{|l|}
40.3 \\
40.2 \\
\end{tabular} & $\frac{2.781}{2.772}$ & 0.010 & $\begin{array}{l}0.010 \\
\end{array}$ & $\begin{array}{l}0.000 \\
0000\end{array}$ & $\begin{array}{l}0.245 \\
024\end{array}$ & \\
\hline$\frac{1413}{1414}$ & $\begin{array}{l}6611552004 \\
6 / 12512004\end{array}$ & $\begin{array}{l}8: 23: 42 \mathrm{PM} \\
8: 24: 42 \mathrm{PM}\end{array}$ & $\begin{array}{r}26.36 \\
26.367 \\
\end{array}$ & $\begin{array}{r}26.739 \\
26.736\end{array}$ & $\begin{array}{l}24.762 \\
24.773\end{array}$ & \begin{tabular}{|l|l|}
27.622 \\
27.604
\end{tabular} & $\frac{26.412}{26.389}$ & $\begin{array}{l}47.062 \\
4.702\end{array}$ & 51.838 & $\begin{array}{l}14.134 \\
14.142 \\
\end{array}$ & \begin{tabular}{|l|}
33.343 \\
33.708 \\
\end{tabular} & \begin{tabular}{rl|l}
1.156 \\
1.144
\end{tabular} & $\begin{array}{r}-7.308 \\
-7.337 \\
\end{array}$ & \begin{tabular}{|l|}
52.222 \\
45.241 \\
\end{tabular} & $\begin{array}{l}0.066 \\
0.066\end{array}$ & $\begin{array}{l}14.643 \\
14.643 \\
\end{array}$ & $\frac{0.00}{0.004}$ & & $\begin{array}{l}835.1497 \\
8.35 .164\end{array}$ & $\begin{array}{l}50.4 \\
50.5 \\
\end{array}$ & $\frac{12.8}{11.1}$ & $\begin{array}{l}40.2 \\
40.6\end{array}$ & 2796 & 0.010 & 0.010 & 0.000 & 0.246 & 0.21 \\
\hline 1415 & $6 / 15 / 2004$ & $8: 25: 42 \mathrm{PM}$ & 26.352 & 26.721 & $\frac{24.775}{24.74}$ & 27.594 & 26.409 & $\begin{array}{l}4.456 \\
4.358\end{array}$ & \begin{tabular}{|l|}
52.081 \\
52.086 \\
\end{tabular} & $\frac{1.142}{14.12}$ & 33.712 & -1.156 & $\begin{array}{r}-7.329 \\
\end{array}$ & 56.189 & 0.068 & $\begin{array}{l}14.045 \\
14.643\end{array}$ & 0.004 & $\frac{21199.98}{2110.98}$ & \begin{tabular}{|l|l}
855.1804 \\
8
\end{tabular} & $\begin{array}{l}30.5 \\
50.6 \\
\end{array}$ & $\begin{array}{ll}11.1 \\
13.8\end{array}$ & $\begin{array}{l}40.6 \\
40.5\end{array}$ & & & 0.010 & & & $\begin{array}{l}0.2 .2 \\
0.21\end{array}$ \\
\hline 1416 & $6 / 15 / 2004$ & 8:26:42 PM & 26.347 & 26.705 & 24.728 & 27.608 & 26.424 & $\begin{array}{l}47.076 \\
7.721\end{array}$ & 51.784 & $\begin{array}{l}14.03 \\
\end{array}$ & 33.478 & -1.156 & & 51.575 & 0.068 & 14.643 & & & & 50.3 & 12.6 & & & & & & 0.254 & \\
\hline 1417 & 6/15/2004 & $8: 27: 42 \mathrm{PM}$ & & 26.696 & 24.723 & 27.638 & & 47.211 & & 14.117 & $\begin{array}{l}33.423 \\
\end{array}$ & -1.156 & & \begin{tabular}{|l|l|} 
\\
\end{tabular} & \begin{tabular}{|l|l|}
0.067 \\
\end{tabular} & \begin{tabular}{|l|l|}
14.643 \\
\end{tabular} & & & $\begin{array}{l}835.2164 \\
\end{array}$ & & & & & 0.010 & 0.010 & 0.000 & 0.250 & \\
\hline 1418 & 6/15/2004 & 8:28:42 PM & 26.335 & & 24.722 & 27.687 & 26.452 & 47.14 & 51.89 & & & -1.098 & & 41.355 & 8.069 & $\begin{array}{r}14.643 \\
\end{array}$ & & & & 50.4 & 10.1 & & & & 0.010 & 0.000 & 0.257 & \\
\hline$\frac{1419}{1420}$ & $\frac{6 / 15 / 2004}{6 / 15 / 204}$ & $\begin{array}{l}8: 29: 42 \mathrm{PM} \\
8: 30.42 \mathrm{PM}\end{array}$ & $\frac{26.339}{26.352}$ & $\begin{array}{r}26.688 \\
26.696 \\
\end{array}$ & 24.715 & 27.721 & $\begin{array}{l}26.496 \\
26.543\end{array}$ & $\begin{array}{l}46.921 \\
44.445\end{array}$ & $\begin{array}{r}51.434 \\
5229 \\
\end{array}$ & $\frac{14.027}{14.137}$ & & & & \begin{tabular}{|l|}
52.078 \\
47727
\end{tabular} & $\begin{array}{l}0.066 \\
0.068\end{array}$ & $\begin{array}{l}14.643 \\
14643\end{array}$ & & & & $\begin{array}{l}50.0 \\
50.8\end{array}$ & 12.8 & 40.1 & 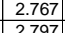 & 0.010 & $\begin{array}{l}0.010 \\
\end{array}$ & 000 & 0.247 & \\
\hline$\frac{1420}{1421}$ & $\begin{array}{l}61 / 1512004 \\
661 / 204\end{array}$ & $\begin{array}{l}30: 42 \mathrm{PM} \\
31: 42 \mathrm{PM}\end{array}$ & $\frac{26.352}{26.352}$ & $\frac{26.696}{26.691}$ & $\begin{array}{l}24.733 \\
24.718\end{array}$ & $\begin{array}{l}27.763 \\
27.788\end{array}$ & & \begin{tabular}{|}
47.445 \\
47.136
\end{tabular} & $\begin{array}{r}52.29 \\
51.718\end{array}$ & $\begin{array}{r}r 4.137 \\
11.09\end{array}$ & $\begin{array}{l}3.696 \\
3.544 \\
\end{array}$ & & & \begin{tabular}{|l|}
47.727 \\
47.354 \\
\end{tabular} & \begin{tabular}{|c|}
0.068 \\
0.067
\end{tabular} & $\begin{array}{l}14.643 \\
14.643\end{array}$ & & & $\begin{array}{l}835.2664 \\
8.3523831\end{array}$ & $\frac{50.8}{50.2}$ & $\frac{11.7}{116}$ & 40.0 & 2.781 & $\frac{0.010}{0.010}$ & 0.010 & 0.000 & 0.252 & 0.2. \\
\hline \begin{tabular}{l|l}
1422 \\
\end{tabular} & $6 / 15 / 2004$ & $8: 3: 32: 42 \mathrm{PM}$ & $\frac{20.352}{26.353}$ & $\frac{20.691}{26.701}$ & 24.724 & 27.824 & $\begin{array}{l}20.503 \\
26.594\end{array}$ & \begin{tabular}{|l|}
47.130 \\
47.294 \\
\end{tabular} & \begin{tabular}{|l|}
51.718 \\
51.854 \\
\end{tabular} & \begin{tabular}{|l|l|}
14.103 \\
\end{tabular} & $\begin{array}{l}33.544 \\
33.693 \\
\end{array}$ & -1.115 & $\begin{array}{l}-7.291 \\
-7.274 \\
\end{array}$ & \begin{tabular}{|r|}
4.5254 \\
52.94
\end{tabular} & 0.071 & \begin{tabular}{l|l}
$\frac{14.045}{14.643}$ \\
\end{tabular} & 0.004 & 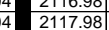 & $\begin{array}{l}355.2951 \\
8\end{array}$ & $\begin{array}{l}30.4 \\
50.4\end{array}$ & $\begin{array}{ll}11.6 \\
13.0\end{array}$ & 40.5 & $\frac{2.181}{2.792}$ & 0.010 & $\begin{array}{l}0.0010 \\
0.011\end{array}$ & & $\begin{array}{l}.260 \\
0.263 \\
\end{array}$ & $\frac{0.2 .2}{0.22}$ \\
\hline 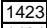 & $6 / 15 / 2004$ & 8:33:42 PM & 26.356 & 26.7 & 24.737 & 27.847 & 26.607 & 47.221 & \begin{tabular}{|l|}
51.935 \\
\end{tabular} & 14.09 & 33.527 & -1.077 & -7.164 & 46.486 & 0.067 & 14.643 & & 2118.98 & $\begin{array}{l}85.3164 \\
\end{array}$ & 50.5 & 11.4 & 40.4 & 2.784 & 0.010 & 0.010 & 0.000 & 0.249 & \\
\hline $\begin{array}{l}1424 \\
14\end{array}$ & 6/15/2004 & 8:34:42 PM & 26.376 & 26.72 & 24.757 & 27.892 & & 47.041 & 51.657 & 14.141 & & & & & 0.067 & 14.643 & & & & & & & & & & & & \\
\hline 1425 & & $8: 35: 42 \mathrm{PM}$ & 26.347 & 26.701 & 24.729 & 27.859 & & & 51.763 & 14.108 & .262 & & & & $\begin{array}{l}0.066 \\
\end{array}$ & 14.643 & & & & & 10.6 & & & .010 & 0.010 & & & \\
\hline 1426 & & . & 26.351 & 26.70 & 24.728 & 27.887 & & & & & & & & 48.434 & 0.071 & 14.643 & & & & & & & & & & & & \\
\hline & $6 / 15 / 2004$ & 77:42 PM & 26.35 & 26.70 & 24.737 & 27.896 & & 47.338 & 52.25 & & 3.613 & & & & & 14.643 & & & & & & & & & & & & \\
\hline 428 & 6/15/2004 & 38:42 PM & 26.32 & 26.67 & 24.701 & 27.896 & & 47.2 & 52.06 & 14.096 & 33.673 & -1.051 & & 52.182 & \begin{tabular}{|l|l|l|l|} 
\\
\end{tabular} & 14.643 & & & & 50.6 & 12.8 & & .79 & & & & & \\
\hline & 6/15/2004 & $99: 42 \mathrm{PM}$ & 26.325 & 26.67 & 24.712 & 27.891 & 26. & 46.97 & 51.861 & 14.145 & 33.224 & -1.034 & -7. & & 0.066 & 14.643 & & 1.124 .98 & 835.4164 & 0.4 & 13.1 & & 76 & 010 & 0.010 & & 0.247 & \\
\hline & & & 26.344 & & 24.736 & 2702 & & 47.186 & (19-5 & 14.192 & 3.394 & -1.034 & & & 1066 & 14.643 & & & & & & & & & & & & \\
\hline & $6 / 15 / 2004$ & & 26.334 & 26.7 & 24.726 & 27.93 & & 47.041 & 51.85 & 14.144 & & & & & & 14.643 & & & & & & & & & & & & \\
\hline & & 8:42: & 26.328 & & 24.729 & $27.8 / 4$ & & $47.15 \pi$ & $51 . / 8$ & 4.13 & 33.515 & & & & 0.06 & 14.643 & & & & & 13.4 & & & & .010 & & & \\
\hline & $6 / 15 / 2004$ & 8:43:42 PM & $\begin{array}{r}26.32 \\
26295\end{array}$ & $\frac{26.68}{26.67}$ & $\frac{24.121}{24706}$ & 27.831 & & $\begin{array}{l}47.342 \\
46.97\end{array}$ & 51.831 & $\frac{14.175}{14.173}$ & $\begin{array}{l}33.689 \\
332.258\end{array}$ & & & 5 & 0.066 & $\frac{14.643}{14.643}$ & & & & & & & & & & & & \\
\hline 1435 & $6 / 15 / 2004$ & & $\frac{20.295}{26.311}$ & 26.07 & 24.722 & 27.727 & & 47.103 & 5 & 194 & 33.384 & & & $\frac{5.24}{4463}$ & 0.066 & 14643 & & & & 504 & 109 & & & & & & & \\
\hline 1436 & $6 / 15 / 2004$ & $8: 46: 42 \mathrm{PM}$ & $\frac{20.011}{26.343}$ & & 24.774 & 27.679 & & & $\frac{31.066}{51.966}$ & 14217 & 33.537 & & & & 0.066 & 14643 & & 等3 & & & $\frac{1.0}{11.1}$ & & & & & & $\frac{0.245}{0.245}$ & \\
\hline & & & 26.311 & 26.69 & 24.828 & 27.598 & & & 1.682 & 158 & & & & & 0.065 & & & & & & & & & & & & & \\
\hline & 15/2004 & & 26.29 & & 24.814 & 27.579 & & & & & & & & & & & & & & & & & & & & & & \\
\hline & & 8:49:42 PM & 26.294 & 26.6 & 24.795 & 27.556 & 26.3 & 47.36 & 52.109 & 14.19 & 33.538 & & & & & 14.64 & & & 35.58 & 50.6 & 11.9 & & & & & & 249 & \\
\hline & $6 / 1$ & & 26.289 & & 24.775 & & & & 51.41 & & & & & & & & & & & & & & & & & & & \\
\hline 441 & $6 / 15 / 2004$ & & 26 & 26.6 & 24.787 & 27.577 & & & 51.587 & & & & & & & & & & 35.6164 & & 12.5 & & & & & & 0.244 & \\
\hline & 6/15/2004 & $8: 52: 4$ & 26.318 & & 24.784 & 27.59 & & & 52.12 & 14.176 & $\begin{array}{l}33.492 \\
\end{array}$ & & & 48.768 & \begin{tabular}{|c|c|}
0.066 \\
\end{tabular} & 14.643 & & & & 50.6 & 12.0 & & & & 0.010 & & 0.245 & \\
\hline $144 \mathrm{~s}$ & 6/15/2004 & $8: 5$ & 26.306 & & 24.772 & 27.587 & & & & & & & & & & & & & & & & & & & & & 0.240 & \\
\hline & $6 / 15 / 2004$ & & 26.31 & 26.634 & 24.776 & 27.672 & & 47.335 & 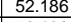 & 14.107 & 3.714 & & & $47.60=$ & 0.064 & & & & & 50.7 & 1 & & & & & & & \\
\hline 144 & $6 / 15 / 2004$ & & 26.28 & & 24.751 & & & & 年 & & & & & & & & & & & 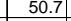 & 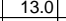 & & & & & & & \\
\hline & & & 26.262 & & & & & & & & & & & & & & & & & & & & & & & & & \\
\hline 1448 & $601 / 15120404$ & & 26 & 20.56 & 24.709 & 27757 & & & 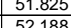 & & & & & & 2068 & & & & & $=7$ & & & & & & & 25 & \\
\hline 1449 & $6 / 15 / 2004$ & & 26.264 & & 2471 & & & & & & & & & & & & & & & & 130 & & & & & & & \\
\hline & & & & & 24,713 & & & & & & & & & & & & & & & & & & & & & & & \\
\hline & $6 / 15 / 2004$ & & 26.282 & 26.63 & 24.713 & 27.753 & & 47. & .805 & & & & & & 0.066 & & & & & 50.3 & 12.2 & & & & & & $\frac{5.246}{0.246}$ & \\
\hline & $6 / 15$ & & & & & & & & & & & & & & & & & & & & & & & & & & & \\
\hline & $6 /$ & & 26.30 & & 24.7 & 27.76 & & & 52.0 & & 3 & & & & & & & & & & & & & & & & & \\
\hline & & & 26.296 & & 24.7 & & & & & & & & & & & & & & & & & & & & & & & \\
\hline & $6 / 15 /$ & 9:05:4 & 26.308 & 26.6 & 24.769 & 27.769 & & & 51 & 14.134 & 33 & & & & 0.0 & & & & & & 12.0 & & & & & & 247 & \\
\hline & $6 / 15 / 200$ & & 6.30 & 26.6 & 24.774 & 27.7 & & & & 14.142 & 33.548 & -1.098 & & 42.2 & 0.0 & & & & & & 10.4 & & & & & & .234 & \\
\hline & 6/15/2 & & & & 24.7 & & & & 5 & & & & & & & & & & & & 12.8 & & & & & & & \\
\hline 14 & $6 / 15 / 2$ & & 26.28 & & 24.751 & 27.657] & & & & & 33.624 & & & & & & & & & & 11.7 & & & & & & .245 & \\
\hline & $6 / 151 / 2000$ & & 26.2 & & 24. & & & & & & & & & & & & & & & & & & & & & & & \\
\hline & $\frac{61512}{6.15 / 2}$ & & $\frac{26.21}{2625}$ & 26.6 & 24.72 & 27.55 & & & & & & & & & & & & & & & 1 & & & & & & & \\
\hline & $\frac{0.15120}{615 / 20}$ & $\frac{1.1 .44}{0.1 \cdot 2)}$ & 6264 & 20. & 24.150 & 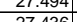 & & & (5) & & & & & & & & & & & & 11.3 & & & & & & & \\
\hline & $6 / 15 / 20$ & $\frac{3.1 .42}{9.13 .42}$ & 26.256 & 20.5 & $\frac{24.747}{2470}$ & 年. & & $\frac{4.573}{4722}$ & $\frac{52.24}{5214}$ & & 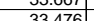 & - & & & 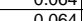 & & & & & & 127 & & & & & & & \\
\hline & & & & 2658 & 24724 & 27 & & & & & & & & & & & & & & & & & & & & & & \\
\hline & $\frac{2 !}{6 !}$ & & 26.244 & & 24.715 & & & & & & & & & & & & & & & & 104 & & & & & & & \\
\hline & $6 / 15 / 2$ & & 26.26 & & & & & & & & & & & & & & & & & & & & & & & & & \\
\hline & & & & & & & & & & & & & & & & & & & & & & & & & & & & \\
\hline & $6 / 15 / 2$ & & 26.23 & 26 & 24.783 & 27.523 & & 46.9 & & & & -0.5 & & & & & & & & & 0.2 & & & & & & & \\
\hline & 615 & & & & & 27.544 & & & & & & -0.9 & & & & 46. & & & & & 14 & & & & & & & \\
\hline 47 & $6 / 15 / 2004$ & 9:20:4 & 26.249 & \begin{tabular}{|l|l}
26.523 \\
\end{tabular} & $\begin{array}{l}3 \mid 24.775 \\
3\end{array}$ & 27.54 & 26.4 & \begin{tabular}{|l|l|}
47.228 \\
\end{tabular} & \begin{tabular}{|l|l|}
52.012 \\
\end{tabular} & \begin{tabular}{|l|l|}
14.242 \\
\end{tabular} & 33.453 & 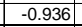 & 7.16 & 58.112 & 0.066 & 14.643 & & & 36.0997 & 00.5 & $\begin{array}{ll}14.2 \\
\end{array}$ & & 2.781 & 0.010 & 0.010 & 0.000 & 0.245 & \\
\hline
\end{tabular}


WSRC-TR-2005-00105, REVISION 0

SRNL-RPP-2005-00012, REVISION 0

RUN \# 3.03A AND B; FIRST AND SECOND HALF OF SLURRY DEWATERING - CONT.

\begin{tabular}{|c|c|c|c|c|c|c|c|c|c|c|c|c|c|c|c|c|c|c|c|c|c|c|c|c|c|c|c|c|}
\hline & A & & D & $E$ & $F$ & \begin{tabular}{l|l|l|} 
G &
\end{tabular} & $\mathrm{H}$ & $\mathrm{J}$ & \begin{tabular}{l|l}
$\mathrm{K}$ \\
\end{tabular} & \begin{tabular}{|l|l|}
$\mathrm{L}$ & \\
\end{tabular} & $\mathrm{M}$ & $\mathrm{N}$ & 0 & Q & R & \begin{tabular}{l|l} 
s \\
\end{tabular} & $\begin{array}{lll}T & \end{array}$ & $\mathrm{~V}$ & w & \begin{tabular}{|c|}
$x \mid$ \\
\end{tabular} & $\begin{array}{lll} & \end{array}$ & z & AA & $A B$ & $\overline{A C}$ & $\overline{A D}$ & $\overline{A E}$ & \\
\hline 1471 & $6 / 15 / 2004$ & 9:21:42 PM & 26.25 & 26.509 & 24.767 & 27.517 & 26.447 & 47.228 & 52.12 & 14.21 & 33.402 & -1.011 & $\begin{array}{l}-7.048 \\
\end{array}$ & 50.676 & 0.06 & 14.643 & 0.004 & 2166.98 & \begin{tabular}{l|l|}
36.1164 \\
\end{tabular} & 50.6 & 12.4 & 40.3 & 2.780 & 0.009 & 0.009 & 0.000 & 0.223 & \\
\hline & $6 / 15 / 2004$ & 9:22:42 PM & 26.262 & 26.516 & 24.778 & 27.544 & 26.449 & 46.927 & 51.86 & 14.193 & 33.205 & $\begin{array}{l}-0.82 \\
-0.82\end{array}$ & -6.75 & 47.473 & 0.065 & 14.643 & & & 36.1331 & 50.4 & $\begin{array}{l}11.6 \\
11.6\end{array}$ & 40.1 & & & & & & \\
\hline 1473 & $6 / 15 / 2004$ & $9: 23: 42 \mathrm{PM}$ & $\frac{26.244}{26.256}$ & 26.497 & 24.75 & 27.54 & 26.465 & 47.418 & \begin{tabular}{|c|}
52.333 \\
579 \\
\end{tabular} & $\frac{14.215}{1.10}$ & $\begin{array}{l}33.609 \\
3.2009\end{array}$ & $\begin{array}{l}-0.936 \\
-0.936\end{array}$ & -6.996 & $\begin{array}{l}47.879 \\
5.839\end{array}$ & 0.065 & 14.643 & 0.00 & 2168.98 & 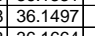 & 50.9 & $\frac{11.7}{11.7}$ & 40.5 & & 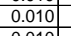 & $\begin{array}{l}0.010 \\
0.10\end{array}$ & 0.000 & 0.241 & \\
\hline$\frac{1474}{1475}$ & $6 / 15 / 2004$ & 9:24:42 PM & 26.256 & 26.505 & 24.762 & 27.548 & 26.488 & 47.026 & 51.78 & 14.192 & 33.297 & - 0.936 & $\begin{array}{l}-7.016 \\
\end{array}$ & $\begin{array}{l}52.929 \\
\end{array}$ & 0.064 & 14.643 & 0.004 & 2169.98 & 6.1664 & 50.3 & 13.0 & 40.2 & $\frac{.769}{7709}$ & .010 & 0.010 & 0.000 & 0.239 & \\
\hline$\frac{1475}{1476}$ & $\frac{6 / 115 / 2004}{66 / 12004}$ & $\begin{array}{l}9: 25: 42 \mathrm{PM} \\
9: 26: 42 \mathrm{PM}\end{array}$ & $\frac{26.261}{26.253}$ & $\frac{26.51}{26.501}$ & $\frac{24.772}{24.754}$ & $27.593 \mid$ & $\frac{26.478}{26.499}$ & $\begin{array}{rl}47.037 & 4736 \\
4736\end{array}$ & \begin{tabular}{|l|l|l|}
51.946 \\
52.161
\end{tabular} & \begin{tabular}{|c|c|}
14.19 \\
14232
\end{tabular} & $\begin{array}{l}33.308 \\
33618\end{array}$ & \begin{tabular}{|l|l|}
-0.933 \\
-0.904
\end{tabular} & $\begin{array}{l}-7.022 \\
-70025 \\
\end{array}$ & $\begin{array}{l}43.916 \\
44896\end{array}$ & 0.063 & $\frac{14.643}{14643}$ & & \begin{tabular}{|c|}
2170.98 \\
2171.98
\end{tabular} & \begin{tabular}{|l|l|}
3631 \\
8
\end{tabular} & 50.5 & $\frac{10.8}{110}$ & $\frac{40.2}{40.5}$ & & 009 & 0.009 & & $\frac{0.235}{0.271}$ & \\
\hline 1477 & $6 / 6 / 15 / 2004$ & $\begin{array}{l}9: 26: 42 \mathrm{PM} \\
9: 27: 42 \mathrm{PM}\end{array}$ & $\frac{26.253}{26.239}$ & $\frac{26.501}{26.478}$ & \begin{tabular}{|l|l|}
324.754 \\
3
\end{tabular} & 27.5699 & $\frac{26.489}{26.526}$ & $\begin{array}{r}47.36 \\
47.402\end{array}$ & \begin{tabular}{|l|l|}
52.1161 \\
52.157
\end{tabular} & \begin{tabular}{rl|}
14.223 \\
14.16
\end{tabular} & $\begin{array}{l}33.618 \\
33.735 \\
\end{array}$ & $\begin{array}{l}-.0904 \\
-0.901\end{array}$ & $\begin{array}{l}-7.0255 \\
-7.0222\end{array}$ & \begin{tabular}{|l|}
45.986 \\
50.505
\end{tabular} & $\mid \begin{array}{l}0.064 \\
0.064\end{array}$ & $\begin{array}{l}14.643 \\
1.4643\end{array}$ & $\frac{0.004}{0.004}$ & 2177.2.98 & \begin{tabular}{|c|}
36.1997 \\
3 \\
36.2164
\end{tabular} & $\begin{array}{l}50.7 \\
50.7 \\
\end{array}$ & $\frac{11.0}{12.4}$ & $\frac{40.5}{40.6}$ & $\begin{array}{l}2.792 \\
.2797 \\
\end{array}$ & \begin{tabular}{|c|c|}
.010 \\
.010
\end{tabular} & \begin{tabular}{|l|l|}
0.010 \\
0.010
\end{tabular} & 0.000 & & 0.20 \\
\hline 1478 & $6 / 15 / 2004$ & 9:28:42 PM & 26.25 & 26.484 & 24.741 & 27.557 & & & \begin{tabular}{|l|}
52.19 \\
\end{tabular} & 14.247 & 33.562 & -0.936 & -7.031 & & 0.058 & 14.643 & $\begin{array}{l}0.004 \\
0.004\end{array}$ & 2173.98 & & S0.7 & $\begin{array}{ll}\frac{112.4}{11.6} \\
\end{array}$ & $\begin{array}{l}40.6 \\
40.5\end{array}$ & $\frac{2.791}{2.789}$ & 0.009 & $\begin{array}{l}0.0109 \\
0.009\end{array}$ & $\begin{array}{l}0.000 \\
0.000\end{array}$ & & $\frac{0.20}{0.18}$ \\
\hline 1479 & & 9:29:42 PM & & 26.489 & 24.741 & 27.572 & 26.537 & 47.576 & \begin{tabular}{|l|}
52.574 \\
\end{tabular} & 14.167 & 33.802 & -0.895 & -7.013 & 49.218 & $\begin{array}{l}0.063 \\
\end{array}$ & 14.643 & 0.004 & 2174.98 & 36.2497 & 51.1 & 12.1 & & & & & & & $\frac{0.18}{0.20}$ \\
\hline 1480 & $6 / 15 / 2004$ & 9:30:42 PM & 26.248 & 26.492 & 24.76 & & 26.52 & & \begin{tabular}{|l|} 
\\
\end{tabular} & & 33.15 & -0.904 & $\begin{array}{r}-7.06 \\
\end{array}$ & $\begin{array}{l}45.332 \\
\end{array}$ & 0.063 & $\begin{array}{l}14.643 \\
\end{array}$ & & & & 50.3 & 11.1 & 40.0 & & & 0.009 & & & \\
\hline 1481 & $6 / 15 / 2004$ & & & & & 27.567 & & & 51.659 & 14.135 & & & & & $\begin{array}{l}0.065 \\
\end{array}$ & 14.643 & & & & 50.2 & 11.6 & & & & 010 & 000 & 244 & \\
\hline 1482 & & 9:32:42 PM & 26.25 & 26.499 & 24.741 & 27.592 & & & 51.981 & 14.156 & 281 & 0.886 & & & & 14.643 & & & & & $\frac{11.0}{11.0}$ & & & & & & & \\
\hline 1483 & & 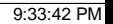 & 26.256 & 26.5 & 24.752 & 27.602 & & & 51817 & 14.182 & & & & & .07 & 14.643 & & & & & 12 & & & & & & & \\
\hline 484 & 6/15/2004 & 1:42 PM & 26.242 & 26.506 & 24.739 & 27.624 & 26.52 & 47.24 & \begin{tabular}{|l|} 
\\
\end{tabular} & 14.26 & 33.427 & 0.886 & -6.967 & 47.694 & 0.061 & 14.643 & & & \begin{tabular}{|l|l|}
36.3331 \\
\end{tabular} & 50.7 & & & & & 0.009 & & & \\
\hline 48 & $6 / 15 / 2004$ & 9:35:42 PM & 26.238 & 26.512 & 24.744 & 27.57 & 26.46 & 47.493 & \begin{tabular}{|l|}
52.646 \\
\end{tabular} & 14.227 & 33.663 & -0.939 & -7.109 & 39.782 & 0.063 & 14.643 & & $\mid 180.98$ & \begin{tabular}{l|l|}
36.3497 \\
\end{tabular} & 51.2 & 9.7 & \begin{tabular}{l|l}
40.6 \\
\end{tabular} & 2.798 & & 0.009 & & & \\
\hline & & 9:36:42 PM & 26.242 & 26.495 & 24.743 & \begin{tabular}{ll|}
27.528 \\
27087
\end{tabular} & $\begin{array}{l}26.423 \\
2.257\end{array}$ & $\begin{array}{l}47.161 \\
147\end{array}$ & \begin{tabular}{|l|}
52.103 \\
\end{tabular} & 14.224 & $\begin{array}{l}33.388 \\
32.58\end{array}$ & -0.936 & & 50.76 & 0.064 & 14.643 & & & 36.3664 & & 12.4 & & & & & & & \\
\hline & $6 / 15 / 2$ & 9:37:42 PM & & 26.489 & & 27.487 & & & & & & & & $\begin{array}{l}49.054 \\
\end{array}$ & & & & & 36.3831 & & & & & & 0.010 & & & \\
\hline & 6/15/200 & $\mathrm{PM}$ & 26.245 & & & 27.457 & & & & & & & & & & 14.643 & & & & & 12.0 & & & & & & & \\
\hline & & $\begin{array}{l}9: 39: 42 \mathrm{~F} \\
0.40 .42 \mathrm{~F}\end{array}$ & $\frac{26.232}{26244}$ & 26.466 & $\begin{array}{l}24.778 \\
247895\end{array}$ & 27.359 & $\frac{26.274}{26.251}$ & \begin{tabular}{|l|l|}
47.06 \\
46.95
\end{tabular} & \begin{tabular}{|l|}
51.805 \\
51.43
\end{tabular} & $\frac{14.113}{14207}$ & $\begin{array}{l}33.425 \\
33225\end{array}$ & \begin{tabular}{|c|c|}
-0.889 \\
-1005
\end{tabular} & & \begin{tabular}{|l|}
47.813 \\
5.20
\end{tabular} & \begin{tabular}{|c|c|}
0.064 \\
0.07
\end{tabular} & 14.643 & & 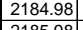 & & 50.3 & $\frac{11.7}{129}$ & 40.6 & 2.775 & .010 & 0.010 & 0.000 & $\begin{array}{l}0.239 \\
0.202\end{array}$ & \\
\hline 4911 & $\frac{01150 / 204}{6 / 15 / 2004}$ & & $\frac{26.244}{26.235}$ & $\frac{26.467}{26.459}$ & $\begin{array}{l}\frac{24.85}{24.777} \\
2\end{array}$ & $\begin{array}{l}27.355 \\
27.382\end{array}$ & & \begin{tabular}{ll|l}
46.956 & 46.827
\end{tabular} & \begin{tabular}{|l|}
$\mid 51.433$ \\
51.07 \\
\end{tabular} & $\begin{array}{l}\frac{14.207}{14.117} \\
\end{array}$ & $\begin{array}{l}33.226 \\
33.254\end{array}$ & $\begin{array}{l}-1.005 \\
-0.649 \\
\end{array}$ & & $\frac{56.248}{50.676}$ & $\begin{array}{r}0.07 \\
0.067\end{array}$ & $\begin{array}{l}\frac{14.643}{14.643} \\
\end{array}$ & & & & $\frac{50.3}{50.3}$ & $\frac{13.8}{12.4}$ & & & & & & & 22 \\
\hline 1492 & $6 / 15 / 2004$ & $9: 42: 42$ PM & 26.233 & 26.447 & 24.754 & 27.375 & 26.295 & 47.213 & 51.948 & 14.128 & 33.574 & -0.889 & -6.883 & 44.532 & 0.062 & $\begin{array}{l}14.043 \\
14.643\end{array}$ & & 2187.98 & \begin{tabular}{|l|}
30.4497 \\
36.4664
\end{tabular} & 50.5 & $\frac{1.4}{10.9}$ & 40. & $2.785 \mathrm{~S}$ & 0.009 & $\begin{array}{l}0.0109 \\
0.009\end{array}$ & .000 & 230 & $\frac{0.21}{0.20}$ \\
\hline & & 9:43:42 PM & 26.23 & 26.444 & 24.761 & 27.382 & & 47.238 & \begin{tabular}{|l|}
52.08 \\
\end{tabular} & 14.238 & 33.419 & -0.886 & -6.898 & 51.196 & 0.064 & 14.643 & & 2188.98 & & & 12.5 & & 2.781 & 0.010 & 0.010 & & & $\frac{0.20}{0.20}$ \\
\hline & $6 / 15 / 2$ & 9:44:42 PM & 26.227 & 26.426 & 24.754 & 27.424 & & 47.344 & \begin{tabular}{|l|} 
\\
\end{tabular} & 14.202 & & -0.884 & & & | & 14.643 & & & & & & & & & & & & $\frac{20}{20}$ \\
\hline 495 & $6 / 15 / 2004$ & & 26.236 & 26.44 & & & & & & 14.096 & 33.542 & - -0.881 & & 43.165 & $\mid 0.064$ & 14.643 & & & 6.5164 & & $\frac{10.6}{10.6}$ & & & & & & & \\
\hline & & & & & & & & & & & & & & & & & & & & & & & & & & & & \\
\hline & 004 & :42 PM & 26.246 & 26.44 & 24.762 & & 26.46 & 47.045 & \begin{tabular}{|l|}
51.863 \\
\end{tabular} & 14.104 & 33.458 & -0.884 & & $\begin{array}{r}53.197 \\
4.197\end{array}$ & 0.063 & $\begin{array}{l}14.045 \\
14.643\end{array}$ & & & tal & 504 & 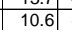 & 40. & .77 & & & & & \\
\hline 1498 & $6 / 15 / 2004$ & PM & 26.237 & 26.436 & 24.754 & 27.494 & 26.4 & 47.338 & 52.234 & 14.131 & 33.675 & -0.886 & $-6.9 !$ & 46.405 & 0.063 & 14.643 & & & 336.56 & 50.8 & 11.4 & 40. & & & & & & \\
\hline 149 & $6 / 15 / 2$ & 9:49:42 PM & 26.233 & 26.427 & 24.754 & 27.505 & & 46.977 & \begin{tabular}{|l|}
52.03 \\
\end{tabular} & 14.091 & 33.297 & -0.884 & -6.9 & 45.22 & 0.063 & 14.643 & & 2194 & & 50.6 & 11.1 & 40. & & 0.009 & 0.009 & & & \\
\hline & & 9:50:42 PM & & 26.423 & 24.745 & 27.516 & & 47.416 & \begin{tabular}{|l|}
52.132 \\
\end{tabular} & 14.15 & 33.8 & -0.884 & & & & 14.643 & & & & & 115 & & & & & & & \\
\hline & 6/15/2004 & 9:51:42 PM & 26.211 & 26.415 & 24.723 & 27.498 & & & \begin{tabular}{|l|l|l|l|} 
\\
\end{tabular} & 14.044 & 33.876 & & & \begin{tabular}{|l|l|}
48.361 \\
\end{tabular} & 0.067 & 14.643 & & & & 50.7 & & & & & & & & \\
\hline 50 & $6 / 15 / 2004$ & 9:52:42 PM & 26.217 & & 24.748 & 27.539 & & & & 14.121 & 8.533 & -0.884 & -7.0 & $\begin{array}{l}46.622 \\
\end{array}$ & 0.063 & 14.643 & & & & 50.6 & 11.4 & & 2.784 & 009 & 0.009 & 0.000 & 0.234 & \\
\hline$\frac{1503}{1504}$ & $6 / 15 / 2004$ & $\begin{array}{l}9: 53: 42 \mathrm{PM} \\
9.54: 42 \mathrm{PM}\end{array}$ & $\frac{26.213}{26.214}$ & $\frac{26.417}{26.428}$ & $\begin{array}{l}24.734 \\
24.75\end{array}$ & 27.535 & $\frac{26.45}{26.47}$ & $\begin{array}{l}47.364 \\
47.234\end{array}$ & \begin{tabular}{|l|}
52.352 \\
52.107 \\
\end{tabular} & $\begin{array}{l}14.126 \\
14.147\end{array}$ & $\begin{array}{l}33.646 \\
33.585\end{array}$ & $\begin{array}{l}-0.884 \\
-0.884\end{array}$ & $\begin{array}{r}-7.04 \\
-7.005\end{array}$ & \begin{tabular}{|l|}
53.912 \\
52109
\end{tabular} & $\begin{array}{c}0.064 \\
0.06\end{array}$ & $\frac{14.643}{14.633}$ & & & $\begin{array}{l}836.64 \\
3.36 .66\end{array}$ & $\begin{array}{l}50.9 \\
50.6 \\
\end{array}$ & $\frac{13.2}{128}$ & $\frac{40 .}{40 .}$ & \begin{tabular}{|l|l}
2.793 \\
2.786
\end{tabular} & |.010 & $\begin{array}{l}0.010 \\
0.009\end{array}$ & 0.000 & 237 & 0.20 \\
\hline 1505 & & & $\frac{26.214}{26.215}$ & $\frac{26.428}{26.429}$ & $\begin{array}{l}24.75 \\
24.737\end{array}$ & 27.555 & $\frac{26.4}{26.4}$ & $\begin{array}{l}47.254 \\
47.352\end{array}$ & $\mid$\begin{tabular}{|l|l|}
52.263 \\
52.263
\end{tabular} & $\begin{array}{l}\frac{14.14 !}{14.079} \\
\end{array}$ & $\begin{array}{r}35.585 \\
33.78\end{array}$ & $\frac{-0.884}{-0.826}$ & & $\begin{array}{l}52.109 \\
52.658\end{array}$ & \begin{tabular}{|c|}
0.06 \\
0.063
\end{tabular} & $\frac{14.645}{14.643}$ & & & & $\frac{5.6}{50.8}$ & & & & & & & & 0.19 \\
\hline 1506 & $6 / 15 / 2004$ & 9:56:42 PM & 26.229 & 26.447 & 24.775 & 27.565 & $\frac{2.4}{24}$ & 47.26 & \begin{tabular}{|l|}
52.062 \\
\end{tabular} & 14.1 & 33.622 & -0.86 & & 51.267 & & 14.643 & & & & $\frac{50.6}{50.6}$ & 12.6 & & & & & & & $\frac{0.20}{0.20}$ \\
\hline 1507 & $6 / 15 / 2004$ & 9:57:42 P & 26.214 & & 24.741 & 27.556 & 26.5 & & \begin{tabular}{|l|}
51.883 \\
\end{tabular} & 14.096 & 33.386 & & & & 0.064 & 14.643 & & & & & & & & & & & & $\frac{0.20}{0.20}$ \\
\hline 150 & 6/15/: & & 26.225 & 26.434 & 24.756 & 27.592 & & 47.7 & \begin{tabular}{|l|}
52.766 \\
\end{tabular} & & 33.845 & -0.8 & & & & 14.643 & & & & & & & & & & & & \\
\hline 50 & & & 26.217 & & 24.74 & 27.553 & & & 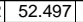 & 14.308 & \begin{tabular}{|l|l|}
33.8 \\
\end{tabular} & -0.8 & & & & 14.6 & & & & & 12.4 & & & & & & & \\
\hline & & & & 26.441 & & & & & & & & & & & & & & & & & & & & & & & & \\
\hline 51 & $6 / 15 / 2$ & 10:01:42 PM & & 26.437 & 24.75 & 27.535 & & 47.302 & 52.136 & 14.212 & 33.548 & -0.846 & & & 0.062 & $\begin{array}{l}14.643 \\
\end{array}$ & & & & 50 & $\frac{10.1}{10.1}$ & 40 & & & & & 20 & \\
\hline & 6/15/2004 & & 26.213 & & 24.75 & 27.535 & & & & \begin{tabular}{|l|l|l|}
14.183 \\
\end{tabular} & & -0. & & & & 14.643 & & & & 50.8 & 10.9 & 40 & & & & & & \\
\hline & & & & & 24.751 & 27.547 & & & & & & & & & & & & & & 50.8 & 12.5 & & & & & & & \\
\hline 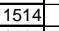 & $6 / 15 / 2004$ & 10:03: & 26.211 & 26.44 & 24.752 & 27.567 & & 47.2 & 52.113 & 14.32 & 33.382 & -0.884 & & 49.55 & 0.063 & 14.6 & & & & 50.6 & 12.1 & 40 & & & & & & \\
\hline & $6 / 1$ & & 26.217 & 26. & 24.824 & 27.569 & & & & & & & & & & & & & & 50.8 & 11.2 & & & & & & 0.22 & \\
\hline 1516 & & & 26.21 & & 24.82 & 27.557 & & & 51.5 & 14.12 & & & & & & & & & & 50.1 & 9.4 & & & & & & & \\
\hline$\frac{1517}{1518}$ & $6 / 15 / 2004$ & $\begin{array}{l}10: 06: 49 \text { PM } \\
10070\end{array}$ & 26.212 & 26.441 & $\begin{array}{l}24.809 \\
2.781\end{array}$ & 27.519 & 26.36 & $\begin{array}{l}47.456 \\
7026\end{array}$ & \begin{tabular}{|l|}
52.267 \\
\end{tabular} & 14.166 & $\begin{array}{l}33.804 \\
23.236\end{array}$ & $\begin{array}{l}-0.823 \\
-0.82\end{array}$ & -6.88 & 50.704 & $\begin{array}{ll}0.062 \\
0.061\end{array}$ & 14.643 & & $\frac{2212.10}{2210}$ & & 50.8 & $\frac{12.4}{1.2}$ & 40.6 & 2.801 & \begin{tabular}{|l|l|} 
\\
\end{tabular} & $\begin{array}{l} \\
\end{array}$ & 0.000 & 0.229 & \\
\hline 151 & 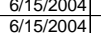 & $\begin{array}{l}10: 07: 499 \mathrm{PM} \\
1008\end{array}$ & $\frac{26.198}{26.204}$ & & & & & $\begin{array}{l}47.226 \\
47.184\end{array}$ & & \begin{tabular}{|c|c|c|}
14.128 \\
114.134
\end{tabular} & & \begin{tabular}{|l}
-0.82 \\
-0.814
\end{tabular} & & & & & & 2213.10| & & \begin{tabular}{|l|}
50.7 \\
50.5 \\
\end{tabular} & $\frac{12.2}{1115}$ & 40.4 & $2.788 \mid$ & & & & 0.226 & \\
\hline 1520 & & & & & & $\frac{27.400}{27.293}$ & & & & & & & & & & & & & & & 11.5 & & & & & & & \\
\hline 1521 & & & & & 24.744 & $\frac{27.265}{27.265}$ & & & & 14.124 & & & & & & & & & & & .0 & & & & & & & \\
\hline & & & & & & & & & & & & & & & & & & & & & & & & & & & & \\
\hline 523 & & & 26.15 & & & 27.303 & & & $\overline{52.3}$ & & & & & & & & & & & & & & & & & & & \\
\hline & & & & & 24.746 & & & 47.0 & & & & & & & & & & & & & & & & & & & & \\
\hline 52 & $6 / 15 /$ & 10:14: & & 26.3 & 24.706 & 27.292 & & 47.42 & 52.234 & 14.145 & & & & & & & & & & & & & & & & & & \\
\hline & 6/15/20 & & 26.1 & 26.3 & 24.7 & 27.311 & & 47.008 & 52.057 & 14.125 & & & & & & 14.6 & & & & & & & & & & & & \\
\hline 52 & & & & & & & & & & & & & & & & & & & & & & & & & & & & \\
\hline 1528 & & & & 26.4 & 24.736 & 27.367 & & 47. & 52.0 & 14.287 & & & & & & & & & & & .8 & & & & & & & \\
\hline & & & & & & & & & & & & & & & & & & & & & & & & & & & & \\
\hline 537 & & & & & 24.17 & & & & & & & & & & & & & & & & & & & & & & & \\
\hline 53. & & $\begin{array}{ll}10.20 .497 \\
10219\end{array}$ & & & $\frac{2.145}{2475}$ & 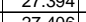 & & & ST.1.8. & & & & & & & & & & & & & & & & & & & \\
\hline 53. & 6 & & $\frac{20.104}{26.55}$ & & 24.15 & $\frac{2.4501}{27,47}$ & & 年17.50 & \begin{tabular}{|l|l|l|l|l|l|}
5190 \\
\end{tabular} & $\frac{\frac{14.20}{1437}}{102}$ & & $\frac{0.006}{0.086}$ & & & & & & & & & & & & & & & & \\
\hline 53. & & & & & 24759 & 274 & & & 517 & & & & & & & & & & & & & & & & & & & \\
\hline & & & & & 2074 & $\frac{2.44}{27.44}$ & & & & & & & & & & & & & & & & & & & & & & \\
\hline & & & & & & & & & & & & & & & & & & & & & & & & & & & & \\
\hline & & & & & & 27.48 & & & & & & & & & & & & & & & & & & & & & & \\
\hline 538 & & & 26.164 & 26.4 & 24.769 & 27.52 & & & 52.2 & 14.46 & & & & & & & & & & & & & & & & & & \\
\hline & & & 26148 & & 24.744 & 1749 & & & & & & & & & & & & & & & & & & & & & & \\
\hline 540 & $6 / 15 /$ & & 6.162 & 26.416 & 24.773 & \begin{tabular}{|l|}
27.499 \\
\end{tabular} & & \begin{tabular}{|l|l|}
47.298 \\
\end{tabular} & \begin{tabular}{|l|}
51.972 \\
\end{tabular} & 14.399 & $\begin{array}{l}33.382 \\
\end{array}$ & -0.892 & & 49.767 & 1.06 & .643 & & & & & 12.2 & & & .009 & 0.009 & . 0000 & 0.223 & \\
\hline
\end{tabular}


WSRC-TR-2005-00105, REVISION 0

SRNL-RPP-2005-00012, REVISION 0

RUN \# 3.03A AND B; FIRST AND SECOND HALF OF SLURRY DEWATERING - CONT.

\begin{tabular}{|c|c|c|c|c|c|c|c|c|c|c|c|c|c|c|c|c|c|c|c|c|c|c|c|c|c|c|c|c|}
\hline & $\mathrm{A}$ & $\mathrm{B}$ & D & $E$ & $F$ & G & $\mathrm{H}$ & $\mathrm{J}$ & K & $\mathrm{L}$ & M & $\mathrm{N}$ & 0 & Q & $R$ & $\mathrm{~s}$ & $\begin{array}{ll}T \\
\end{array}$ & $\mathrm{v}$ & w & $x$ & $\mathrm{Y}$ & $\mathrm{z}$ & $\mathrm{AA}$ & $A B$ & $A C$ & $\mathrm{AD}$ & $\mathrm{AE}$ & \\
\hline & $6 / 15 / 2004$ & 10:29:58 PM & 26.156 & 26.395 & 24.762 & 27.488 & 26.453 & 47.333 & 52.155 & 14.412 & 33.377 & -0.831 & -6.811 & $\begin{array}{ll}49.344 \\
\end{array}$ & 0.063 & 14.643 & 0.004 & 2235.25 & 37.2542 & 50.7 & 12.1 & 40.4 & \begin{tabular}{|l|l|}
2.782 \\
\end{tabular} & 0.009 & 0.009 & 0.000 & 0.234 & \\
\hline & $6 / 15 / 2004$ & & 26.169 & 26.413 & 24.865 & 27.531 & 26.476 & 46.649 & 51.454 & $\begin{array}{l}14.232 \\
\end{array}$ & 32.881 & $\begin{array}{r}-0.791 \\
\end{array}$ & -6.794 & 44.584 & 0.069 & $\begin{array}{l}14.643 \\
\end{array}$ & 0.004 & & & 50.0 & & & & & & & 0.260 & \\
\hline (1500 & $6 / 15 / 2004$ & $10: 31: 58 \mathrm{PM}$ & $\frac{26.176}{26.18}$ & 26.43 & 24.857 & 27.532 & 26.467 & 46.833 & \begin{tabular}{|l|}
51.726 \\
502
\end{tabular} & \begin{tabular}{|l|}
14.214 \\
\end{tabular} & 33.068 & $\begin{array}{c}-0.794 \\
-0.7927\end{array}$ & -6.837 & 51.156 & 0.061 & \begin{tabular}{|l|}
14.643 \\
\end{tabular} & 0.004 & & 5 & 50.3 & $\frac{12.5}{12.5}$ & & $\frac{2.754}{2.774}$ & \begin{tabular}{|l|l|} 
\\
\end{tabular} & 0.009 & 0.000 & 0.229 & \\
\hline 1544 & $6 / 15 / 2004$ & $\begin{array}{ll}10: 32: 58 \mathrm{PM} \\
10: 3250 \mathrm{P}\end{array}$ & $\begin{array}{ll}26.182 \\
26189\end{array}$ & 26.431 & 24.839 & 27.519 & 26.464 & 47.136 & \begin{tabular}{|l|}
52.003 \\
51709 \\
\end{tabular} & $\begin{array}{r}14.324 \\
\end{array}$ & \begin{tabular}{|l|l|}
33.256 \\
32115
\end{tabular} & $\begin{array}{r}-0.82 \\
\end{array}$ & $\begin{array}{l}-6.909 \\
6.027\end{array}$ & \begin{tabular}{|c|}
39.713 \\
\end{tabular} & \begin{tabular}{|l|l|}
0.062 \\
\end{tabular} & $\begin{array}{l}14.643 \\
\end{array}$ & 0.003 & & \begin{tabular}{l|l|}
5 & 37.3042 \\
\end{tabular} & 50.5 & 9.7 & 40.2 & 2.771 & \begin{tabular}{|l|l|} 
\\
\end{tabular} & 0.009 & 0.000 & 0.231 & \\
\hline & $6 / 115 / 2004$ & $\begin{array}{l}\frac{10: 33: 58 \mathrm{PM}}{10} \\
10.3458 \mathrm{PM}\end{array}$ & $\begin{array}{l}26.189 \\
26.176\end{array}$ & $\frac{26.433}{26.410}$ & & \begin{tabular}{l|}
27.545 \\
2757
\end{tabular} & & \begin{tabular}{|l|l|}
46.966 \\
47008
\end{tabular} & \begin{tabular}{|l|l|}
51.786 \\
5171 \\
\end{tabular} & $\begin{array}{r}14.3 \\
1432 \\
\end{array}$ & $\begin{array}{r}33.115 \\
33.144\end{array}$ & $\begin{array}{l}-0.82 \\
-0.83\end{array}$ & & \begin{tabular}{|r|}
48.97 \\
48.27 \\
\end{tabular} & \begin{tabular}{|l|l|}
0.061 \\
0.622
\end{tabular} & \begin{tabular}{|l|l|l|}
14.643 \\
14634 \\
\end{tabular} & \begin{tabular}{|l|l|}
0.004 \\
0.023
\end{tabular} & 2239.25 & \begin{tabular}{|l|l|}
5 & 37.3208 \\
\end{tabular} & \begin{tabular}{|l|}
50.3 \\
50.
\end{tabular} & \begin{tabular}{l|l}
3 & 12.0 \\
\end{tabular} & $\begin{array}{l}0 \quad 40.0 \\
\end{array}$ & \begin{tabular}{|l|}
2.761 \\
2762 \\
\end{tabular} & \begin{tabular}{|l|l|}
0.009 \\
000
\end{tabular} & $\begin{array}{l}0.009 \\
\end{array}$ & & $\begin{array}{l}0.228 \\
2.232\end{array}$ & 0.19 \\
\hline $\begin{array}{ll}1547 \\
154\end{array}$ & $6 / 1 / 15 / 20404$ & $\begin{array}{l}10: 34: 5 \mathrm{PM} \\
103558 \mathrm{PM}\end{array}$ & $\frac{26.176}{26.188}$ & $\frac{26.419}{26.432}$ & $\begin{array}{r}24.797 \\
24.814\end{array}$ & 27.527 & $\frac{26.467}{26.484}$ & $\begin{array}{r}47.008 \\
46.917 \\
\end{array}$ & \begin{tabular}{|l|}
51.71 .71 \\
525 \\
\end{tabular} & \begin{tabular}{|l|l|l}
4.322 \\
14.312
\end{tabular} & $\begin{array}{r}33.144 \\
33.006\end{array}$ & $\begin{aligned}-0.823 \\
-0.82 \\
\end{aligned}$ & $\frac{-6}{-6}$ & $\begin{array}{l}48.257 \\
455.624\end{array}$ & \begin{tabular}{|c|}
0.062 \\
0.063
\end{tabular} & \begin{tabular}{|l|}
14.643 \\
14643 \\
\end{tabular} & $\begin{array}{l}0.003 \\
0.004\end{array}$ & $\frac{22240.25}{2241.25}$ & $\begin{array}{c}537.3375 \\
5 \\
5\end{array}$ & $\frac{50.3}{50.4}$ & $\frac{11.8}{11.2}$ & $\begin{array}{l}8 \\
\begin{array}{l}8 \\
2\end{array} 40.10 .0\end{array}$ & \begin{tabular}{|l|}
2.763 \\
2.755 \\
\end{tabular} & \begin{tabular}{|l|l|} 
& 0.009 \\
\end{tabular} & $\begin{array}{l}0.009 \\
0.009\end{array}$ & $\begin{array}{l}0.000 \\
0.000\end{array}$ & 0.232 & 0.20 \\
\hline \begin{tabular}{|l|l|}
1548 \\
\end{tabular} & & & 26.205 & 26.444 & 24.826 & & 26.492 & & 52.136 & \begin{tabular}{|l|l|}
14.369 \\
\end{tabular} & 33.386 & -0.834 & $\begin{array}{l}-7.031 \\
\end{array}$ & 52.689 & $\begin{array}{l}0.063 \\
0.062\end{array}$ & $\begin{array}{l}14.645 \\
14.643 \\
\end{array}$ & $\begin{array}{l}0.004 \\
0.004\end{array}$ & $\frac{2441.25}{2242.25}$ & $\begin{array}{l}0 \\
5 \\
5\end{array}$ & \begin{tabular}{|l|l}
50.4 \\
50.7
\end{tabular} & $\frac{11.2}{12.9}$ & $\frac{40.0}{40.4}$ & & \begin{tabular}{|l|l|}
0.009 \\
\end{tabular} & $\begin{array}{l}0.0099 \\
0.009\end{array}$ & 0.000 & $\begin{array}{l}0.230 \\
0.230\end{array}$ & $\frac{0.20}{0.20}$ \\
\hline & $6 / 15 / 2004$ & $10: 37: 58 \mathrm{PM}$ & & & 24.803 & 27.578 & 26.493 & 47.076 & 51.981 & 14.385 & & -0.855 & -7.034 & 48.188 & 0.061 & 14.643 & 0.004 & & \begin{tabular}{|l|l|}
37.3875 \\
\end{tabular} & 50.5 & 11.8 & & & & & & & \\
\hline & $6 / 15 / 2004$ & & & 26.44 & 24.808 & 27.538 & 26.493 & 47.246 & \begin{tabular}{|l|}
51.91 \\
\end{tabular} & & 33.445 & -0.817 & & & .061 & $\begin{array}{l}14.643 \\
\end{array}$ & & & \begin{tabular}{|l|l|l}
5 & 37.4042 \\
\end{tabular} & 50.4 & & & 2.782 & & & & & \\
\hline 1551 & $6 / 15 / 2004$ & 10:39:58 PM & $\begin{array}{l}26.192 \\
26.102\end{array}$ & 26.435 & 24.808 & $\begin{array}{l}27.533 \\
27561\end{array}$ & 26.493 & $\begin{array}{r}47.105 \\
\end{array}$ & \begin{tabular}{|l|}
51.923 \\
\end{tabular} & $\begin{array}{l}14.318 \\
\end{array}$ & 33.314 & -0.817 & $\begin{array}{l}-7.02 \\
\end{array}$ & \begin{tabular}{|l|l}
41.363 \\
\end{tabular} & \begin{tabular}{|l|l|}
0.063 \\
\end{tabular} & 14.643 & & & \begin{tabular}{|l|l|}
37.4208 \\
\end{tabular} & 50.5 & 10.1 & & \begin{tabular}{l|l|}
2.772 \\
\end{tabular} & \begin{tabular}{|l|l|} 
\\
\end{tabular} & 0.009 & 0.000 & 0.235 & \\
\hline & $6 / 15 / 2004$ & $\begin{array}{ll}10: 40: 58 \mathrm{PM} \\
10.4155 \mathrm{PM}\end{array}$ & \begin{tabular}{|l|l}
26.193 \\
261999
\end{tabular} & $\frac{26.431}{2.442}$ & $\begin{array}{r}24.794 \\
2479\end{array}$ & \begin{tabular}{|l|l}
27.564 \\
27541
\end{tabular} & & \begin{tabular}{|l|l|l|}
4695 \\
\end{tabular} & \begin{tabular}{|l|}
51.875 \\
5161 \\
\end{tabular} & \begin{tabular}{|l|l|}
14.292 \\
1424
\end{tabular} & \begin{tabular}{|l|}
33.304 \\
33162
\end{tabular} & $\begin{array}{c}-0.82 \\
0832\end{array}$ & & \begin{tabular}{|l|l|}
43.215 \\
51.296
\end{tabular} & \begin{tabular}{|l|l|l|}
0.061 \\
0.662
\end{tabular} & \begin{tabular}{|l|l|}
14.643 \\
1.643
\end{tabular} & $\begin{array}{l}0.00 \\
0.00\end{array}$ & 246.25 & \begin{tabular}{|l|l|}
37.4375 \\
374542
\end{tabular} & 50.4 & $\frac{10.6}{126}$ & 40.2 & \begin{tabular}{|l|}
2.773 \\
2762 \\
\end{tabular} & \begin{tabular}{|l|l|} 
\\
\end{tabular} & $\begin{array}{l}0.009 \\
0009\end{array}$ & 0.000 & $\frac{0.227}{0.232}$ & 0.19 \\
\hline | & $6 / 1155 / 20404$ & $\begin{array}{l}10: 41: 5 \mathrm{PM} \\
1024: 58 \mathrm{PM}\end{array}$ & \begin{tabular}{|}
$\mid 26.199$ \\
26.211
\end{tabular} & $\frac{26.443}{26.46}$ & $\begin{array}{r}24.79 \\
24.803 \\
\end{array}$ & 27.541 & $\frac{451}{408}$ & $\begin{array}{r}46.95 \\
46.958\end{array}$ & \begin{tabular}{|c|}
51.614 \\
55.661
\end{tabular} & $\frac{14.244}{14.373}$ & 1.162 & $\begin{array}{r}-0.82 \\
-0.817\end{array}$ & & $\begin{array}{l}51.396 \\
47.752\end{array}$ & $\begin{array}{l}.062 \\
0.06\end{array}$ & \begin{tabular}{|l|}
14.643 \\
14.643 \\
\end{tabular} & \begin{tabular}{|l}
0.003 \\
0.004
\end{tabular} & 2248.2 & $\frac{37.4442}{3377408}$ & $\frac{50.2}{50.2}$ & $\frac{12.6}{11.7}$ & 40.0 & \begin{tabular}{l|l|}
2.762 \\
2.758
\end{tabular} & \begin{tabular}{|l|}
0.009 \\
0.009
\end{tabular} & $\begin{array}{l}0.009 \\
0.009\end{array}$ & $\begin{array}{l}0.000 \\
0.000\end{array}$ & 0.232 & 0.20 \\
\hline & $6 / 15 / 2004$ & 10:43:58 PM & 26.186 & 26.429 & 24.772 & 27.447 & 26.343 & $\begin{array}{l}4.057 \\
4.057\end{array}$ & 52.032 & \begin{tabular}{|l|}
14.307 \\
\end{tabular} & $\begin{array}{r}33.15 \\
\end{array}$ & -0.82 & & 46.386 & $\begin{array}{l}0.062 \\
0.0\end{array}$ & $\begin{array}{l}14.045 \\
14.643 \\
\end{array}$ & 0.004 & $\frac{2484.25}{2249.25}$ & \begin{tabular}{|l|l|}
37.48408 \\
37.45
\end{tabular} & 50.6 & $\frac{11.1}{11.4}$ & $\frac{4.0}{40.1}$ & \begin{tabular}{|l|}
2.7505 \\
2.765 \\
\end{tabular} & \begin{tabular}{|l|l|}
0.009 \\
\end{tabular} & $\begin{array}{l}0.0099 \\
0.009\end{array}$ & & 0 & $\frac{0.19}{0.20}$ \\
\hline & $6 / 15 / 2004$ & $10: 44: 58 \mathrm{PM}$ & 26.186 & 26.425 & 24.768 & 27.363 & & 47.261 & 52.283 & 14.388 & & -0.788 & & & & 14.643 & & & 37.5042 & & & & & & & & & \\
\hline & $6 / 15 / 2004$ & 10:45:58 PM & & 26.439 & 24.777 & 27.348 & & 47.114 & & & & & & 51.014 & \begin{tabular}{|l|l|}
0.064 \\
\end{tabular} & $\begin{array}{l}14.643 \\
\end{array}$ & & & 37.5208 & 50.7 & & 40.1 & & 0.010 & 0.010 & & 0.239 & \\
\hline & & 10:46:58 PM & & & 24.77 & 27.276 & & & 52.464 & & & -0.771 & & & & 14.643 & & & & 51.0 & $12.8 \mathrm{C}$ & & & & & 0.000 & 0.230 & \\
\hline & $6 / 15 / 2004$ & $\begin{array}{l}10: 47: 58 \mathrm{PM} \\
10.485\end{array}$ & 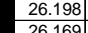 & $\frac{26.431}{26308}$ & $\frac{24.799}{2474}$ & $\begin{array}{ll}27.259 \\
27.221\end{array}$ & & $\begin{array}{l}46.997 \\
4682 \\
\end{array}$ & $\begin{array}{l}51.923 \\
5165 \mid\end{array}$ & $\begin{array}{l}14.356 \\
14264\end{array}$ & & & & $\begin{array}{r}50.92 \\
47596\end{array}$ & .061 & \begin{tabular}{|l|l|}
14.643 \\
14.643
\end{tabular} & & & $\begin{array}{l}57.5542 \\
5\end{array}$ & 50.5 & 12.5 & & \begin{tabular}{|l|}
2.759 \\
2751
\end{tabular} & 0.009 & 0.009 & 0.000 & 0.229 & 19 \\
\hline & $\begin{array}{c}6 / 115 / 2004 \\
6 / 15 / 2004\end{array}$ & $\begin{array}{l}\text { 10:44:5:5 PM } \\
\text { 10:49:58 PM }\end{array}$ & \begin{tabular}{r|}
26.169 \\
26.168
\end{tabular} & $\frac{26.398}{26.402}$ & $\begin{array}{r}24.74 \\
24.759\end{array}$ & 27.2215 & & & $\mid$\begin{tabular}{|l|l|}
51.645 \\
5.1466
\end{tabular} & $\begin{array}{l}14.264 \\
14.256 \\
\end{array}$ & & & & $\begin{array}{l}\frac{47.596}{50.284} \\
\end{array}$ & & \begin{tabular}{|l|}
14.643 \\
14.643 \\
\end{tabular} & & & 37.7508 & 50.4 & $\frac{11.7}{12.3}$ & & $\begin{array}{l}2.751 \\
2.754 \\
\end{array}$ & \begin{tabular}{|l|l|}
0.009 \\
0.009
\end{tabular} & 0.009 & 0.000 & .230 & 0.20 \\
\hline & $6 / 15 / 2004$ & 10:50:58 PM & 26.166 & 26.39 & 24.767 & 27.358 & 26.174 & 46.991 & \begin{tabular}{|l|}
51.753 \\
\end{tabular} & \begin{tabular}{|l|}
14.381 \\
14.381 \\
\end{tabular} & $\begin{array}{r}33.049 \\
33.104 \\
\end{array}$ & -0.785 & & 48.059 & 0.061 & $\begin{array}{l}14.045 \\
14.643 \\
\end{array}$ & 0.004 & $\frac{2233.25}{2256.25}$ & \begin{tabular}{|l|l|}
37.6042 \\
\end{tabular} & 50.3 & $\begin{array}{l}12.5 \\
11.8 \\
\end{array}$ & & \begin{tabular}{|l|}
2.7541 \\
2.761 \\
\end{tabular} & \begin{tabular}{|l|l|}
0.009 \\
\end{tabular} & $\begin{array}{l}0.0099 \\
0.009\end{array}$ & 0.000 & 0.0229 & $\frac{0.20}{0.19}$ \\
\hline & $6 / 15 / 2004$ & 10:51:58 PM & 26.17 & 26.388 & 24.766 & 27.372 & 26.237 & 47.002 & 51.997 & 14.393 & 33.074 & & & 48.68 & 0.06 & 14.643 & & & 37.6208 & 50.5 & 11.9 & $\begin{array}{ll}9 & 40.0 \\
\end{array}$ & \begin{tabular}{|l|}
2.760 \\
\end{tabular} & \begin{tabular}{|l|l|} 
\\
\end{tabular} & 0.009 & 0.000 & 0.225 & \\
\hline & 6/15/2004 & $10: 52: 58 \mathrm{PM}$ & 26.17 & 26.393 & 24.761 & 27.342 & & 47.045 & 51.863 & 14.254 & & & & & 0.06 & 14.643 & & & $\begin{array}{l}77.6375 \\
\end{array}$ & 50.4 & & 40.2 & \begin{tabular}{|l|} 
\\
\end{tabular} & \begin{tabular}{|l|l|} 
\\
\end{tabular} .0099 & 0.009 & 0.000 & 0.224 & \\
\hline & 6/15/2004 & 10:53:58 PM & 26.164 & 26.383 & 24.755 & 27.366 & & & & & 33.386 & & & & & 14.643 & & & 37.6542 & 50.6 & 11.9 & & 2.781 & 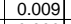 & 0.009 & 0.000 & & \\
\hline & $\frac{6 / 115 / 2004}{66 / 12504}$ & $\begin{array}{l}10: 54: 58 \mathrm{PM} \\
10.5558 \mathrm{PM}\end{array}$ & $26.164 \mid$ & $\frac{26.378}{26.378}$ & $\begin{array}{r}24.765 \\
24.77\end{array}$ & 27.376 & $\frac{26.291}{26.296}$ & $\begin{array}{r}46.85 \\
47.506 \\
\end{array}$ & $\begin{array}{l}51.844 \\
52.727\end{array}$ & \begin{tabular}{|l|}
14.311 \\
14437
\end{tabular} & $\begin{array}{l}33.002 \\
33.48 \\
\end{array}$ & $\begin{array}{l}-0.771 \\
-0.071\end{array}$ & $\begin{array}{l}-6.92 .1 \\
-6.995\end{array}$ & $\begin{array}{l}51.855 \\
48.444\end{array}$ & $\begin{array}{l}0.061 \\
0.059 \\
\end{array}$ & \begin{tabular}{|l}
14.643 \\
14.633 \\
\end{tabular} & & 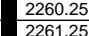 & \begin{tabular}{|l|l|}
37.6708 \\
37.6875 \\
\end{tabular} & $\frac{50.4}{55.3}$ & $\frac{12.7}{11.9}$ & $\frac{39.9}{40.5}$ & $\begin{array}{l}2.753 \\
2.792\end{array}$ & \begin{tabular}{|l|l|}
0.009 \\
0.009
\end{tabular} & $\begin{array}{l}0.009 \\
0.099\end{array}$ & 0.000 & 0.229 & $\frac{0.20}{0.19}$ \\
\hline 1568 & $\begin{array}{l}6 / 11 / 2 / 2004 \\
6 / 15 / 2004\end{array}$ & $\begin{array}{l}\frac{10.555 .58 \mathrm{PM}}{10: 56: 58 \mathrm{PM}} \\
\end{array}$ & 26.169 & $\frac{26.378}{26.363}$ & $\begin{array}{l}24.17 \\
24.745\end{array}$ & $\begin{array}{l}27.381 \\
27.356\end{array}$ & & $\begin{array}{l}47.506 \\
47.047 \\
\end{array}$ & $\begin{array}{l}52.272 \\
51.962\end{array}$ & $\begin{array}{l}14.437 \\
14.385 \\
\end{array}$ & 33.48 & $\begin{array}{l}-0.711 \\
-0.773 \\
\end{array}$ & & & $\begin{array}{r}0.059 \\
0.06\end{array}$ & $\begin{array}{l}\frac{14.643}{14.643} \\
\end{array}$ & & & & & $\frac{11.9}{11.4}$ & & 2.792 & $\begin{array}{l}0.009 \\
0.009\end{array}$ & $\begin{array}{l}0.009 \\
0.009\end{array}$ & & & \\
\hline & 6/15/2004 & 10:57:58 PM & 26.165 & 26.35 & 24.766 & 27.367 & & 47.21 & 52.277 & $\begin{array}{l}14.396 \\
14.00\end{array}$ & 33.193 & -0.739 & & & 0.06 & 14.643 & & & & & 11.4 & & & & 0.009 & & & $\frac{0.19}{0.19}$ \\
\hline & 6/15/2004 & $10: 58: 58 \mathrm{PM}$ & 26.165 & 26.36 & & & & & 52.053 & 14.408 & & -0.739 & & & & \begin{tabular}{|l|l|}
14.643 \\
\end{tabular} & & & & & & & & & & & & \\
\hline & 6/15/2004 & $10: 59: 58 \mathrm{PM}$ & 26.165 & & 24.771 & 27.426 & & 47. & 52.563 & 14.456 & 33.269 & & & $\frac{1.096}{4.096}-3$ & D.06 & 14.643 & & & & & & & & & & & & \\
\hline & & 11:00:58 & 26.166 & 26.354 & 24.782 & & & 47.238 & 52.229 & & 33.265 & & & & & 14.643 & & & & & & & & & & & & \\
\hline & 6/15/2004 & 11:01:58 PM & & 26.36 & 24.782 & 27.447 & & 47.302 & & 14.446 & & & & & & 14.6 & & & & & & & & & & & & \\
\hline & $6 / 15 / 2004$ & 11:02:58 PM & & 26.3 & 24.827 & & & 46.987 & 51.946 & \begin{tabular}{|l|l|}
14.4 \\
\end{tabular} & 33.066 & & & & & 146 & & & 8042 & & & & & & & & & \\
\hline & 6/15/2004 & 11:03 & 26.166 & 26.3 & 24.857 & 27.408 & & & $\begin{array}{l}51.807 \\
\end{array}$ & 14.328 & 32.842 & & & & & 14.643 & & & & 50.3 & 2.3 & & & 0.009 & . 0009 & & & \\
\hline & 6/15/2004 & 11:04:58 PM & 26.171 & 26.3 & 24.857 & 27.473 & & 47.12 & 52.115 & 14.351 & & & & 45.3 & 0.0 & 14.643 & & & & & 1.1 & & & & & & 0.227 & 7 \\
\hline & $6 / 15 / 2004$ & & 26.177 & 26.37. & 24.853 & 27.479 & & 47. & 52.06 & 14.312 & 33.402 & & & & & 14.6 & & & & 0 & 0.0 & & & & & & & \\
\hline & $6 / 15 / 2004$ & & & & & & & 47.1 & & & & & & & & & & & & & & & & & & & & \\
\hline & & 11:07:58 PM & 26.1877 & 26.381. & 24.838 & 2.7 .4 & & 47.2 & 20.146 & 14.26 & 33.464 & & & & & 14.6 & & & & 50 & 11.3 & & 2.14 & & & & .223 & \\
\hline & 0 & $1.0898 \mathrm{PP}$ & & 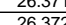 & $\begin{array}{l}24.823 \\
24829\end{array}$ & 27.444 & & 40.9 & 25.049 & 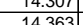 & $\begin{array}{l}33.086 \\
33.427\end{array}$ & & & & & 14. & & & & & 113 & & & & & & & \\
\hline & $6 / 15 / 2004$ & & 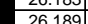 & 26378 & 24826 & & & & & 14.304 & & & & & & 14643 & & & & & & & & & & & & \\
\hline & $6 / 15 / 2004$ & 11:11 & 26.205 & 26.6399 & 24841 & 27.52 & & & 327 & $\begin{array}{l}14.004 \\
14.315 \\
\end{array}$ & & & & & & $\frac{14.045}{14.643}$ & & & & & $\frac{13}{30}$ & & & & & & & \\
\hline & $6 / 15 / 2004$ & 11:12:58 PM & 26.196 & 26.38 & 24.817 & $\frac{27.508}{27.508}$ & & 47. & $\frac{52.2}{52.2}$ & 14.346 & 33.252 & & & & 0 & & & & & & $\frac{0.1 .2}{11.2}$ & & & 0.009 & 0.009 & & & \\
\hline & $6 / 15 / 2004$ & & & & & & & & & & & & & & & & & & & & & & & & & & & \\
\hline & $6 / 15 / 2004$ & & 26.193 & 26.386 & 24.814 & 27.4 & & 47.043 & 52.188 & 14.315 & & & & & & & & & & & 40 & & & & & & 28 & \\
\hline & 6/15/2004 & 11:15:58 PM & & 26.37 & 24.19 & 27.411 & & 47.12 & 52.132 & 14.35 & & & & & & & & & & & & & & & & & & \\
\hline & $6 / 15 / 2004$ & 11:16:58 PN & & 26.3 & 24.797 & & & 46.7 & & 14.45 & & & & & & & & & & & & & & & & & 219 & \\
\hline & 6/15/2004 & 11:17 & 26.167 & 26.36 & 24.788 & & & & \begin{tabular}{|l|}
52.254 \\
\end{tabular} & 14.464 & & & & & & 14.6 & & & & 50 & {$[2$.} & & & & & & 220 & \\
\hline & 6/115/2004 & & 26.174 & & 24.79 & & & & & & & & & & & & & & & & & & & & & & & \\
\hline & $6 / 15 / 2004$ & & 26.178 & 26.38 & 24.809 & 27.215 & & 47. & 52.484 & 14.41 & 33.125 & & & & & & & & & & 1.6 & & & & & & & \\
\hline & $6 / 15 / 2004$ & & 20.170 & & & & & & & & & & & & & & & & & & & & & & & & & \\
\hline & & & & 26.33 & & 2.1 .17 & & $47 . . \mathrm{Cl}$ & 52. & 14 & 33.0 & & & & & & & & & & & & & & & & & \\
\hline & 6 & $\begin{array}{l}11: 22.58 \\
111.258\end{array}$ & & 年. & $\begin{array}{l}24.804 \\
24798\end{array}$ & 27.1809 & & 告17.4 & 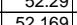 & $\begin{array}{r}14.51 \\
14.494\end{array}$ & $\frac{33.5}{33 .}$ & & & & & . & & & & & 52 & & & & & & & \\
\hline & $6 / 15 / 2004$ & 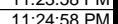 & 26173 & $\frac{2.34}{2635}$ & 24829 & 2724 & & & 552 & 14374 & & & & & & & & & & & & & & & & & & \\
\hline & & & & & & & & & & & & & & & & & & & & & & & & & & & & \\
\hline & $6 / 15 / 2004$ & & 26.153 & $\frac{26.32}{26.32}$ & 24.79 & 27.24 & & & $\frac{12.078}{52.078}$ & & 33.185 & & & & & & & & & & 12.1 & & & & & & & \\
\hline & $6 / 15 / 2004$ & & & & & & & & & & & & & & & & & & & & & & & & & & & \\
\hline & & & & & & & & & & & & & & & & & & & & & & & & & & & & \\
\hline & $6 / 15 / 200$ & & & 26. & 24.8 & 27.2 & & & & & & & & & & & & & & & & & & & & & & \\
\hline & $6 / 15 / 200$ & 11:30:58 & 26.142 & 26.3 & 24.798 & 27.239 & & 47.1 & 52.275 & 14.51 & 33.131 & & & & & 14.6 & & & & & 1.9 & & & & & & & \\
\hline & $6 / 15 / 2004$ & 31. & 6.144 & & 24.805 & 27.276 & & 47.11 & 52.59 & 14.5 & 32.9 & -0.4 & & & & 14.6 & & & & & 1311 & & & & & & 17 & \\
\hline & $6 / 15 / 2$ & $11: 32: 5$ & 145 & 26.308 & 24.8 & 27.252 & & & 51.9 & 14. & & & & & & $14.6 \mathrm{C}$ & & & & & & & & & & & 24 & \\
\hline & $6 / 15 / 2$ & & 26.134] & & 24 & 27.2. & & & & & & & & & & & & & & & 13.5 & & & & & & & \\
\hline & $6 / 15 / 2004$ & & 26.1 & & 24.8 & & & & & & & & & & & & & & & & & & & & & & & \\
\hline & 6 & & & 26.299 & 24.80 & 27.305 & & & S2.105 & 14. & & & & & & & & & & & & & & & & & & \\
\hline & 0151212004 & 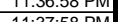 & 2 & 20.314 & 24.030 & 27.347 & & 40.001 & (51.193 & $\begin{array}{l}14.525 \\
1.573\end{array}$ & $\begin{array}{l}32.017 \\
320101\end{array}$ & $\begin{array}{l}-0.415 \\
-0.175\end{array}$ & & $\begin{array}{l}48.251 \\
35.176\end{array}$ & 0.0 & $\begin{array}{l}14.045 \\
1.643\end{array}$ & & & & 然 & 6 & & & & & & 226 & \\
\hline 1610 & \begin{tabular}{|l|}
$6 / 15 / 2004$ \\
\end{tabular} & 11:38:58 PM & $\begin{array}{l}26.141 \\
26.148\end{array}$ & 26.306 & \begin{tabular}{|l|l|}
24.000 \\
24.814
\end{tabular} & \begin{tabular}{|l|}
27.201 \\
\end{tabular} & 26.235 & 4 & \begin{tabular}{|l|}
52.236 \\
\end{tabular} & \begin{tabular}{|l|}
14.41728 \\
\end{tabular} & $\begin{array}{l}02.001 \\
32.881\end{array}$ & \begin{tabular}{|l|l|} 
& -.417 \\
\end{tabular} & \begin{tabular}{l|l|}
-6.34 \\
\end{tabular} & \begin{tabular}{|l|l|}
46.444 \\
\end{tabular} & $\mid \frac{0.06}{0.06}$ & \begin{tabular}{|l|}
14.045 \\
14.643 \\
\end{tabular} & & 2304.25 & \begin{tabular}{|l|l|}
58.4042 \\
\end{tabular} & \begin{tabular}{|l|l|} 
& 0.40 .8 \\
\end{tabular} & \begin{tabular}{c|c}
8 & 0.0 \\
8 & 11.4
\end{tabular} & & \begin{tabular}{|l|}
2.750 \\
\end{tabular} & \begin{tabular}{|l|}
0.009 \\
\end{tabular} & $\begin{array}{l}0.009 \\
0.009\end{array}$ & 0.000 & \begin{tabular}{|l|l|l|l|l}
0 & 0.225 \\
\end{tabular} & 0.1 \\
\hline
\end{tabular}


WSRC-TR-2005-00105, REVISION 0

SRNL-RPP-2005-00012, REVISION 0

RUN \# 3.03A AND B; FIRST AND SECOND HALF OF SLURRY DEWATERING - CONT.

\begin{tabular}{|c|c|c|c|c|c|c|c|c|c|c|c|c|c|c|c|c|c|c|c|c|c|c|c|c|c|c|c|c|}
\hline & & & D & $E$ & $F$ & \begin{tabular}{l|l|l}
$G$ & \\
\end{tabular} & $\begin{array}{ll}\mathrm{H} \\
\end{array}$ & $\mathrm{J}$ & $\begin{array}{ll}\mathrm{K} \\
\end{array}$ & $\mathrm{L} /$ & \begin{tabular}{l|l} 
\\
\end{tabular} & $\begin{array}{ll}\mathrm{N} \\
\end{array}$ & $\begin{array}{ll}0 & \\
\end{array}$ & \begin{tabular}{l|l} 
\\
\end{tabular} & \begin{tabular}{l|l}
$R$ & \\
\end{tabular} & \begin{tabular}{l|l} 
s \\
\end{tabular} & $\begin{array}{llll}T & \end{array}$ & $\mathrm{v}$ & w & $x \mid$ & $\begin{array}{llll} & & & \end{array}$ & $\mathrm{z}$ & & $\mathrm{AB}$ & $\overline{A C}$ & & $\overline{A E}$ & $\overline{A F}$ \\
\hline 611 & 6/15/2004 & 11:39:58 PM & 26.149 & 26.302 & 24.815 & 27.325 & 26.216 & 46.767 & 51.999 & 14.467 & 32.77 & -0.475 & -6.343 & 52.533 & 0.059 & 14.643 & 0.004 & 2305.25 & \begin{tabular}{|l|l|}
58.4208 \\
\end{tabular} & 50.5 & 12.9 & 39.8 & 2.742 & 0.009 & 0.009 & 0.000 & 0.222 & \\
\hline & & 11:40:58 PM & 26.144 & 26.293 & 24.805 & 27.261 & 26.241 & 46.964 & 52.244 & $\begin{array}{l}14.552 \\
\end{array}$ & 32.866 & \begin{tabular}{|l|l|}
-0.443 \\
\end{tabular} & -6.343 & 44.5 & 0.059 & \begin{tabular}{|l|l|}
14.643 \\
\end{tabular} & & & 38.4375 & 50.8 & $\begin{array}{l}10.9 \\
0\end{array}$ & & & & 0.009 & & & \\
\hline 613 & 6/15/2004 & $\begin{array}{l}11: 41: 58 \mathrm{PPM} \\
11: 59 \mathrm{PM}\end{array}$ & 26.149 & 26.288 & $\frac{24.815}{24.825}$ & $\frac{27.246}{272075}$ & 26.271 & 46.997 & \begin{tabular}{|l|l|}
52.095 \\
5.091
\end{tabular} & $\begin{array}{l}14.458 \\
\end{array}$ & $\begin{array}{l}32.987 \\
2087\end{array}$ & $\begin{array}{r}-0.58 \\
\end{array}$ & -6.828 & 48.395 & 0.1 & $\begin{array}{ll}14.643 \\
\end{array}$ & & $\begin{array}{l}2307.25 \\
202.25 \\
200\end{array}$ & \begin{tabular}{|l|l|}
38.4542 \\
39.470
\end{tabular} & 50.6 & $\frac{11.9}{11.9}$ & 40. & (1) & 管 & $\begin{array}{l}0.015 \\
0.01\end{array}$ & 0.000 & 0.375 & \\
\hline 615 & $6 / 15 / 2004$ & 11:42:58 PM & 26.178 & 26.302 & 24.835 & 27.275 & 26.251 & 48.02 & \begin{tabular}{|l|l|}
51.991 \\
\end{tabular} & $\begin{array}{l}14.517 \\
\end{array}$ & 33.977 & -1.216 & -4.932 & $\begin{array}{l}41.868 \\
4\end{array}$ & 0.005 & 14.643 & 0.004 & 2308.25 & \begin{tabular}{|l|l|}
38.4708 \\
\end{tabular} & 50.5 & 10.3 & 41.0 & 2.827 & 8.001 & 0.001 & 0.000 & 0.018 & \\
\hline $1 \frac{1615}{1616}$ & 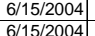 & $\begin{array}{l}11: 43: 58 \mathrm{PM} \\
11.44 .58 \mathrm{PM}\end{array}$ & 26.183 & $\frac{26.301}{26.306}$ & 24.824 & $\frac{27.28}{27.205}$ & & $\begin{array}{l}46.541 \\
47545\end{array}$ & \begin{tabular}{|l|}
52.414 \\
52246
\end{tabular} & \begin{tabular}{|l|l|l|l|l|l|}
14.523 \\
14.84
\end{tabular} & \begin{tabular}{|l|l|}
32.433 \\
33949 \\
\end{tabular} & \begin{tabular}{|l|}
0.075 \\
1083
\end{tabular} & $\begin{array}{l}-2.125 \\
5126 \\
\end{array}$ & \begin{tabular}{|r|}
45.48 \\
43998
\end{tabular} & 0.038 & $\begin{array}{l}14.643 \\
14643 \\
\end{array}$ & & & & 50.9 & 11.1 & & & \begin{tabular}{|l|l|l|l|l|}
0.006 \\
\end{tabular} & 0.006 & & 0.144 & \\
\hline 1617 & $\frac{6 / 15 / 2004}{6 / 15 / 2004}$ & $\begin{array}{l}\text { 11:44:55 PM } \\
\text { 11:45:58 PM }\end{array}$ & $26.193 \mid$ & $\frac{26.306}{26.296}$ & $\frac{24.834}{24.814}$ & 27.295 & $\frac{26.7}{26.29}$ & $\begin{array}{l}47.545 \\
48.401\end{array}$ & \begin{tabular}{|l|r|}
52.246 & 52.52 \\
\end{tabular} & $\begin{array}{l}14.484 \\
14.462 \\
\end{array}$ & \begin{tabular}{|l|l|l|l}
33.494 \\
34.336
\end{tabular} & \begin{tabular}{|l|}
-1.083 \\
-.219 \\
\end{tabular} & $\begin{array}{l}-5.126 \\
-6.889\end{array}$ & \begin{tabular}{|l|l|}
43.998 \\
47.107
\end{tabular} & $\begin{array}{l}0.158 \\
0.061 \\
\end{array}$ & $\begin{array}{l}14.643 \\
14.643\end{array}$ & $\frac{0.004}{0.004}$ & $\begin{array}{l}2330.25 \\
2311.25\end{array}$ & \begin{tabular}{|l|l|}
5 & 38.5042 \\
58.5208
\end{tabular} & 50.8 & $\frac{10.8}{11.5}$ & $\begin{array}{r}40.5 \\
41.4 \\
\end{array}$ & $\frac{2.794}{2852}$ & . 0.024 & \begin{tabular}{|c|c|}
0.024 \\
0.009
\end{tabular} & $\begin{array}{l}0.001 \\
0.000\end{array}$ & 0.584 & \\
\hline 618 & & & 26.149 & & 24.81 & 27.296 & 26.291 & & 52.08 & & & & -7.337 & 51.769 & $\begin{array}{l}0.001 \\
0.063 \\
\end{array}$ & $\begin{array}{l}14.043 \\
14.643 \\
\end{array}$ & $\begin{array}{l}0.004 \\
0.004\end{array}$ & 23112.25 & \begin{tabular}{|l|l|}
38.5208 \\
38.5375 \\
\end{tabular} & S1.0. & $\begin{array}{ll}\frac{11.5}{12.7} \\
\text {. }\end{array}$ & & 2.8560 & $\begin{array}{l}0.009 \\
0.009\end{array}$ & $\begin{array}{l}0.009 \\
0.009 \\
\end{array}$ & & & $\frac{0.19}{0.19}$ \\
\hline & & 11:47:58 PM & & 26.299 & 24.816 & 27.327 & 26.307 & 48.725 & \begin{tabular}{|l|l|}
52.499 \\
\end{tabular} & 14.669 & 34.508 & \begin{tabular}{|l|}
-1.222 \\
\end{tabular} & -7.259 & 44.811 & 0.061 & 14.643 & 0.004 & & 38.5542 & 51.0 & 11.0 & & & & & & & \\
\hline & $6 / 15 / 2004$ & 11:48:58 PM & 26.135 & 26.289 & & 27.332 & & & \begin{tabular}{|l|l|}
52.424 \\
\end{tabular} & & 34.477 & -1.222 & -7.355 & 48.628 & 0.061 & \begin{tabular}{ll|l}
14.643 \\
\end{tabular} & & 2314.25 & \begin{tabular}{|l|}
38.5708 \\
\end{tabular} & & 11.9 & & & $\begin{array}{l}.009 \\
\end{array}$ & 0.009 & & & \\
\hline 621 & $6 / 15 / 2004$ & $\begin{array}{l}\text { 11:49:58 PM } \\
11: 50: 58 M\end{array}$ & 26.14 & 26.299 & 24.826 & 27.377 & 26.307 & $\begin{array}{l}48.99 \\
\end{array}$ & \begin{tabular}{|l|l|}
52.654 \\
\end{tabular} & 14.749 & 34.765 & -1.222 & & 48.705 & 0.054 & 14.643 & 0.00 & 2315.25 & 38.5875 & 51.2 & 11.9 & & 2.887 & $\begin{array}{l}0.008 \\
.08\end{array}$ & 0.008 & 0.000 & 0.193 & \\
\hline$\frac{622}{623}$ & $\begin{array}{ll}6 / 15 / 2004 \\
6 / 15 / 2004\end{array}$ & $\begin{array}{l}11: 50: 58 \mathrm{PM} \\
11.51 .58 \mathrm{PM}\end{array}$ & $\begin{array}{l}26.415 \\
26.352 \\
\end{array}$ & $\begin{aligned} 26.31 \\
26.316\end{aligned}$ & $\frac{24.912}{24024}$ & 27.403 & & $\begin{array}{ll}48.455 \\
4.509\end{array}$ & \begin{tabular}{|l|l|}
52.252 \\
512
\end{tabular} & $\begin{array}{l}14.544 \\
14.95\end{array}$ & \begin{tabular}{|l|l|}
34.301 \\
34.94
\end{tabular} & \begin{tabular}{|l|l|}
-1.222 \\
-126
\end{tabular} & & $\begin{array}{l}47.105 \\
5.275 \\
\end{array}$ & 0.062 & \begin{tabular}{|l|l|}
14.643 \\
1.643
\end{tabular} & & $\begin{array}{l}2316.25 \\
231725\end{array}$ & \begin{tabular}{|l|l|}
38.6042 \\
386208
\end{tabular} & 50.8 & $\frac{11.5}{133}$ & & & .009 & $\begin{array}{l}0.009 \\
0009\end{array}$ & & 0.224 & \\
\hline$\frac{623}{624}$ & 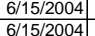 & $\begin{array}{l}11: 51: 58 \mathrm{PM} \\
11.55: 58 \mathrm{PM}\end{array}$ & $\begin{array}{l}26.352 \\
26.283\end{array}$ & $26.316 \mid$ & $\frac{24.924}{24.894}$ & $\begin{aligned} 27.414 & \\
22.77 & \end{aligned}$ & & .509 & \begin{tabular}{|r|r|}
52.12 & \\
552.107 &
\end{tabular} & $\begin{array}{l}\frac{14.495}{14.461} \\
\end{array}$ & \begin{tabular}{|l|l|}
.494 & 299 \\
\end{tabular} & 216 & $\begin{array}{l}-7.592 \\
-7.597 \\
\end{array}$ & 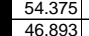 & $\begin{array}{l}0.061 \\
0.062\end{array}$ & $\begin{array}{l}14.643 \\
14.643\end{array}$ & $\begin{array}{l}0.004 \\
0.004\end{array}$ & $\begin{array}{l}7.25 \mid \\
8.25\end{array}$ & 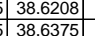 & 50.6 & $\frac{11.5}{11.5}$ & 413 & $\begin{array}{l}2.861 \\
849 \\
\end{array}$ & |.009 & $\begin{array}{l}0.009 \\
0.009\end{array}$ & 0.000 & & 0.19 \\
\hline 625 & 6/15/2004 & 11.25:53:58 PM & 26.259 & $\frac{20.301}{26.313}$ & $\begin{aligned} 24.894 \\
24.88\end{aligned}$ & 27.386 & $\frac{20.37}{26.366}$ & $\begin{array}{ll}48.353 \\
48.381\end{array}$ & \begin{tabular}{|l|}
52.101 \\
52.122 \\
\end{tabular} & $\begin{array}{l}\frac{14.41}{14.497} \\
\end{array}$ & 34.299| & \begin{tabular}{|l|l|}
-1.222 \\
-1.219 \\
\end{tabular} & $\begin{array}{l}-\frac{-1.5979}{-7.609} \\
\end{array}$ & $\begin{array}{l}40.0935 \\
51.098 \\
\end{array}$ & $\begin{array}{l}0.002 \\
0.061 \\
\end{array}$ & $\begin{array}{l}\frac{14.643}{14.643} \\
\end{array}$ & & $\begin{array}{ll}318.25 \\
319.25\end{array}$ & $\begin{array}{ll}38.65472 \\
88.654\end{array}$ & $\begin{array}{l}50.6 \\
50.6\end{array}$ & $\frac{11.5}{12.5}$ & & .8.851 & .009 & 0.009 & & & $\frac{0.19}{0.19}$ \\
\hline 626 & & 11:54:58 PM & 26.239 & & 24.865 & 27.386 & & 48.588 & 52.07 & 14.573 & 34.514 & \begin{tabular}{|l|}
-1.222 \\
\end{tabular} & -7.635 & 40.435 & 0.06 & 14.643 & & & & & & & & & & & & $\frac{0.19}{0.18}$ \\
\hline & & & 26.235 & 26.313 & 24.866 & 27.391 & & & 51.655 & 14.404 & 34.165 & -1.219 & & & 0.061 & 14.643 & & & & & & & & & & & & \\
\hline 628 & $6 / 15 / 2004$ & 11:56:58 PM & & & & & & & & & & & & 47.443 & & & & & $\frac{7042}{7042}$ & 50.5 & 11.6 & & & D09 & 009 & 000 & & \\
\hline & $6 / 15 / 2$ & 11:57:58 P & & & 24.876 & 27.396 & & 48.24 & 51.653 & 14.466 & 34.301 & -1.216 & & & & 14.643 & & & & r $>$ & 13.7 & & & & & & & \\
\hline & & $11: 58: 58 \mathrm{~F}$ & 26.176 & & 24.847 & 27.392 & & & 5213 & 14.489 & 34.488 & & & & & & & & & & & & & & & & & \\
\hline & 5/2004 & 11:59:5 & & & 24.857 & 27.382 & & & 51.995 & 14.473 & & & & 53.814 & & 14.643 & & & & 50.5 & 13.2 & & & & & & & \\
\hline 63 & 6/16/20 & 12:00:58 AM & 26.131 & 26.315 & 24.852 & 27.343 & & 48.397 & 52.086 & 14.528 & 34.334 & -1.216 & -7.635 & 42.404 & 0.063 & 14.643 & & 326.25 & \begin{tabular}{|l|l|}
58.7708 \\
\end{tabular} & 50.6 & 10.4 & & 2.852 & .009 & 0.009 & 0.000 & 0.228 & \\
\hline & 6/16/2004 & 12:01:58 AM & 26.121 & 26.315 & 24.842 & 27.313 & & 48.418 & 52.155 & 14.485 & 34.41 & -1.222 & 7.647 & 51.035 & 0.06 & 14.643 & & & \begin{tabular}{|l|l|}
38.7875 \\
\end{tabular} & 50.7 & 12.5 & & 2.855 & 009 & .009 & .000 & 0.217 & \\
\hline 25 & 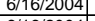 & $12: 02: 58$ AM & 26.095 & 26.298 & 24.831 & 27.252 & & 48.155 & 51.925 & 14.434 & & $\begin{array}{l}-1.225 \\
1.20\end{array}$ & & 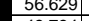 & 0.061 & 14.643 & & & & 0.5 & 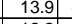 & & & 009 & & & & \\
\hline & $6 / 16 / 2004$ & 12:03:58 A & 26.092 & & 24.839 & 2.7 .224 & & & 5.1.518 & 15.194 & & & & & & & & & & & 12.2 & & & & & & & \\
\hline 37 & $\begin{array}{ll}6.6 / 2004 \\
6 / 2004\end{array}$ & $\begin{array}{l}12: 04: 12 \mathrm{AM} \\
1005 \cdot 12 \mathrm{AM}\end{array}$ & 26.086 & $\frac{26.29}{26.303}$ & 24.827 & $\frac{27.188}{27131}$ & $\frac{26.15}{2608}$ & \begin{tabular}{ll|l}
48.142 \\
47788
\end{tabular} & 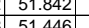 & $\frac{15.029}{1409}$ & $\begin{array}{ll}33.576 \\
33382\end{array}$ & $\begin{array}{l}-1.219 \\
-1219 \\
-1219\end{array}$ & & 年8.04 & 0.06 & $\frac{14.643}{14643}$ & & & & 0.4 & 11.8 & & & & & & & \\
\hline 638 & 6/16/2004 & 12:06:12 AM & 26.088 & $\frac{20.000}{26.286}$ & 248.06 & 27.07 & 26 & $\begin{array}{l}48.074 \\
48\end{array}$ & 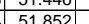 & 14.789 & $\begin{array}{l}0.029 \\
33.769\end{array}$ & $\begin{array}{l}-1.215 \\
-1.222 \\
\end{array}$ & & $427+2$ & 0.06 & 14.043 & & & & 504 & 104 & & & & & & & \\
\hline 1639 & $6 / 16 / 2004$ & $12: 07: 12 \mathrm{AM}$ & 26.086 & 26.275 & 22.642 & 27.093 & & 48.348 & \begin{tabular}{|l|l|}
52.01 \\
\end{tabular} & 14.678 & 34.075 & -1.222 & -7.647 & 54.433 & 0.06 & $\begin{array}{l}14.045 \\
14.643 \\
\end{array}$ & & 33248 ; & & $\frac{5.4}{50.5}$ & $\frac{1.45}{13.3}$ & & 84 & 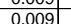 & .009 & & 年 & \\
\hline & & & & 26.28 & 24.851 & 27.112 & & & 51.703 & 14.504 & & & & 50.576 & 0.061 & 14.643 & & & & & & & & & & & & \\
\hline & $6 / 2$ & & 26.093 & & 24.849 & & & & 51.902 & 14.617 & & -1.2. & & & & 14.643 & & & & 50.4 & & & & & & & & \\
\hline & $6 / 20$ & 12:10:1 & 26.092 & & 24.853 & 27.169 & & & 51.831 & \begin{tabular}{|c|c|}
14.57 \\
\end{tabular} & 34.28 & -1.219 & & 49.4 & 0.06 & 14.643 & & & & 50.4 & 12.1 & & 34 & & & & & \\
\hline 643 & $6 / 200$ & & 26.082 & & 24.828 & 27.144 & 25.9 & 48.617 & 52.402 & 14.684 & 34.35 & & & 51.00 & $\begin{array}{ll}0.06 \\
\end{array}$ & 14.643 & & & & & & & & & & & & \\
\hline & $6 / 2004$ & 12:12:12 AM & 26.092 & 26.25 & 24.833 & 27.164 & & 48.65 & 52.478 & 14.533 & 34.53 & -1.222 & & & 0.062 & 14.643 & & & & 1 & 122 & & & & & & & \\
\hline 1645 & 6/16/2004 & AM & 26.097 & 26.245 & 24.843 & 27.179 & 26.1 & 48.352 & 51.946 & \begin{tabular}{|c|c|}
14.48 \\
\end{tabular} & 34.334 & $\mid-1.222$ & & & $\begin{array}{ll}0.06 \\
\end{array}$ & 14.643 & & & & 50.5 & 2.0 & & & & & & & \\
\hline & $6 / 2$ & 12:14: & 26.113 & 26.267 & 24.874 & 27.21 & & & 52.462 & 14.49 & 34.7. & -1.219 & & 48 & 0.06 & 14.643 & & & & 51.0 & 11.9 & & & & & & & \\
\hline 64 & 2004 & & 26.097] & & 24.843 & 27.249 & & & 52.694 & 14.555 & & -1.219 & & & 0.059 & 14.643 & & & & 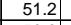 & .0 & & & & & & & \\
\hline & sin & & & & 24.855 & & & & & 14.499 & & -1.222 & & & 0.06 & & & & & & & & & & & & & \\
\hline & & 12:11:12 A & 26.109 & & 24.855 & 27.331 & & 48.13 & & 14.431 & $34.1 / 8$ & -1.222 & & 45.66 & & 14.64 & & & & 50.4 & 1.2 & & & & & & & \\
\hline & 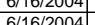 & $\begin{array}{l}12.18 .1 . \mathrm{AM} \\
12.19 \mathrm{MM}\end{array}$ & & 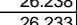 & $\frac{24.803}{24.55}$ & 年. & & & 52.7545 & 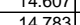 & 34. & $-\frac{1.2}{-1.2}$ r & & & 0.06 & 告4.6453 & & & & $\frac{51}{51}$ & 126 & & & & & & & \\
\hline & $6 / 16 / 2004$ & $12 \cdot 20$ & $\mid \frac{2.15}{2618}$ & & $\frac{24.04}{24844}$ & 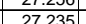 & & & & 1458 & $\frac{34.4}{34.3}$ & & & & 0.06 & 14643 & & & & 506 & & & & & & & & \\
\hline & 6/16/2004 & 12:21: & $\frac{2.150}{26113}$ & & 24859 & $\frac{21.635}{27235}$ & & & \begin{tabular}{|l|}
51983 \\
51983
\end{tabular} & 14531 & $\frac{34.28}{34.28}$ & & & & 0.061 & $\frac{1.4043}{14643}$ & & & & 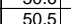 & $\frac{1.16}{22}$ & & & & & & 2.51 & \\
\hline & $\frac{6 / 16 / 2004}{6 / 12004}$ & 12:22:1 & $\frac{26.119}{26.119}$ & $\frac{2.2}{26.2}$ & 24.86 & $\frac{27.261}{27.261}$ & & & $\mid$\begin{tabular}{|l|}
$\mid 52.578$ \\
\end{tabular} & $\frac{14.645}{14.65}$ & $\frac{34.715}{34}$ & & & 51.45 & 0.06 & 14.643 & & & & 51. & $\frac{\frac{12.6}{12.6}}{2}$ & & & & & & & \\
\hline & & & & & & & & & & & & & & & & & & & & & & & & & & & & \\
\hline 656 & & & 26.13 & & 24.871 & 27.242 & & & 52.528 & 14.712 & & & & & 0.0 & & & & & 51.1 & 13.1 & & & & & & & \\
\hline & $6 / 200$ & & & & 24.911 & & & & 52.076 & 14.526 & & & & & & 14.6 & & & & & .4 & & & & & & & \\
\hline & $6 / 200$ & 12:26:1, & 26.124 & 26.233 & 24.94 & 27.321 & & 48.4 & \begin{tabular}{|l|}
52.325 \\
\end{tabular} & 14.51 & 34.4 & -1.216 & & & 0.06 & 14.6 & & & & & 15 & & & & & & & \\
\hline 165 & 6/16/200 & 12:27: & 26.129 & & 24.905 & 27.286 & & & & & 34.6 & \begin{tabular}{|l|} 
\\
\end{tabular} & & & 0.061 & 14.64 & & & & 50.6 & 13.0 & & & & & & .219 & \\
\hline & 6/16/2 & 12:28:1 & 26.14 & & 24.906 & 27.301 & & & & & & & & & & & & & & & $12.8 \mathrm{C}$ & & & & & & & \\
\hline 1661 & 6/2004 & & 26.15 & 26.243 & 24.906 & 27.316 & & & 52.44 & 14.493 & & -1.2 & & & 0.05 & & & & & & 12.9 & & & & & & & \\
\hline & & & & & & & & & & & & & & & & & & & & & & & & & & & & \\
\hline & & & 26.16 & & 24.88 & 2.3 & & & \begin{tabular}{|l|}
52.089 \\
\end{tabular} & & & & & & & & & & & & & & & & & & & \\
\hline 66 & 6 & 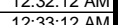 & & $\begin{array}{ll}2.6 \\
262\end{array}$ & $\begin{array}{l}24.088 \\
24869\end{array}$ & 20 & & & 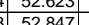 & $\begin{array}{c}14.48 \\
14489\end{array}$ & $\begin{array}{r}34.72 \\
34794\end{array}$ & & & & & $\frac{1.6}{146}$ & & & & 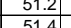 & 18 & & & & & & & \\
\hline 166 & 6 & & $\frac{2.1}{261}$ & 年 & 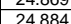 & 27.35 & & & \begin{tabular}{|l|l|}
51.04 \\
5
\end{tabular} & 1487 & 33.1549 & -1.21 & & & & & & & & & 128 & & & & & & & \\
\hline 1667 & & & & & 2488 & & & & & & & & & & & & & & & & & & & & & & & \\
\hline 1668 & & & & & 24.88 & $\frac{27.316}{27}$ & & 47.2 & 7) 51.048 & & & $\frac{-1.22}{-1.2}$ & & & & & & & & & 133 & & & & & & & \\
\hline & & & & & & & & & & & & & & & & & & & & & & & & & & & & \\
\hline 67 & & & & & & & & & & & & & & & & & & & & & & & & & & & & \\
\hline & & & & 26. & 24.8 & 27.323 & & & 53.0 & & & & & & & & & & & & & & & & & & & \\
\hline 167. & 6/16/2 & $12: 40: 12 \mathrm{~A}$ & $26.1:$ & 26.254 & 24.842 & 27.302 & & 49.5 & & 13 & 36.1. & -1.22 & & 51.0 & 0. & 14.6 & & & & 51 & .5 & & & & & & & \\
\hline 67 & 6/16/20 & $12: 41: 12$ & $26.158 \mid$ & & 24.859 & 27.345 & & 48.074 & 51.88 & 14.771 & 33. & -1.222 & & 50.5 & 0.05 & 14.6 & & & & 50 & 7.4 & & & & & & & \\
\hline 1674 & 6/16/2 & & 26.16 & & 24.872 & 27.328 & & 48.4 & 52.146 & 14.624 & 34.3 & -1.219 & & & & 14.6 & & & & & .1 & & & & & & & \\
\hline 67 & $6 / 16 / 2$ & & 26.16 & & 24.881 & 27.262 & & 47.848 & $\begin{array}{l}31.6 \\
31.6\end{array}$ & 14.896 & 33. & -1.22 & & & & & & & & & 12.8 & & & & & & & \\
\hline & & & & & 24.9 & & & & & 14. & & & & & & & & & & & & & & & & & & \\
\hline & & & $20.1 / 4$ & & 24.906 & 20.7261 & & & 52.58 & 14.311 & & & & & & & & & & & & & & & & & & \\
\hline$\frac{010}{670}$ & 011012004 & & 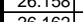 & 20.260 & 24.014 & 27.49 & & 4 & \begin{tabular}{|c|} 
\\
\\
\end{tabular} & 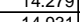 & S5.155 & 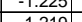 & & 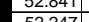 & 0.00 & 14.04 & & & & (5.5 & & & & & & & 0.2317 & 7 \\
\hline 1680 & 6/16/2004 & $12: 48: 12 \mathrm{AM}$ & 26.156 & 26.274 & \begin{tabular}{|l|}
24.008 \\
24.887 \\
\end{tabular} & \begin{tabular}{|l|}
27.217 \\
\end{tabular} & 26.312 & 40.217 & \begin{tabular}{|l|l|}
7 & 51.782 \\
\end{tabular} & \begin{tabular}{|l|l|}
15.238 \\
\end{tabular} & $\begin{array}{l}33.102 \\
3.102 \\
\end{array}$ & \begin{tabular}{|l|}
-1.219 \\
-1.219 \\
\end{tabular} & -7.667 & $\frac{2.24 !}{52.22}$ & 0.057 & 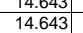 & & $\mid$ & \begin{tabular}{|l|}
39.5414 \\
39.5581 \\
\end{tabular} & \begin{tabular}{|l|}
50.1 \\
50.3 \\
\end{tabular} & \begin{tabular}{|l|}
12.8 \\
\end{tabular} & & 2.790 & \begin{tabular}{|l|}
0.0008 \\
0.008
\end{tabular} & \begin{tabular}{|l}
0.009 \\
0.009
\end{tabular} & 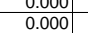 & $\begin{array}{l}0.210 \\
0.211 \\
\end{array}$ & $0.1 \varepsilon$ \\
\hline
\end{tabular}


WSRC-TR-2005-00105, REVISION 0

SRNL-RPP-2005-00012, REVISION 0

RUN \# 3.03A AND B; FIRST AND SECOND HALF OF SLURRY DEWATERING - CONT.

\begin{tabular}{|c|c|c|c|c|c|c|c|c|c|c|c|c|c|c|c|c|c|c|c|c|c|c|c|c|c|c|c|c|}
\hline & A & B & D & $E$ & $F$ & \begin{tabular}{ll|} 
G \\
\end{tabular} & $\mathrm{H}$ & $\mathrm{J}$ & K & $\mathrm{L}$ & M & $\mathrm{N}$ & 0 & Q & $R$ & $\mathrm{~s}$ & $\begin{array}{ll}T \\
\end{array}$ & $\mathrm{v}$ & w & $x$ & $\begin{array}{lll} & \end{array}$ & $\mathrm{z}$ & AA & $A B$ & $\overline{A C}$ & $\overline{A D}$ & $\overline{A E}$ & \\
\hline & 6/16/2004 & 12:49:12 AM & 26.16 & 26.284 & 24.90 & 27.242 & 26.322 & 47.97 & 51.601 & 15.067 & 33.423 & 1.222 & -7.661 & 51.35 & & 14.643 & 0.0 & 2374.48 & 9.5747 & 50.1 & 12.6 & 40.7 & 2.806 & 0.009 & 0.009 & 0.00 & 0.213 & \\
\hline & & & $\frac{26.164}{26.164}$ & 26.288 & 24.916 & 27.276 & 26.311 & & $\begin{array}{ll}51.682 \\
5\end{array}$ & 14.873 & 33.562 & \begin{tabular}{|l|l|}
-1.219 \\
\end{tabular} & $\begin{array}{l}-7.661 \\
-1.661 \\
\end{array}$ & 51.2 & & 14.643 & & & & 50.2 & $\frac{12.6}{12.6}$ & & & & & & & \\
\hline & 6/16/2004 & $\begin{array}{l}12: 51: 12 \mathrm{AM} \\
125: 12 \mathrm{M}\end{array}$ & $\frac{26.159}{26.152}$ & 26.293 & 24.92 & $\frac{27.246}{2720}$ & 26.306 & & \begin{tabular}{|l|l|}
51.676 \\
57.675
\end{tabular} & 14.773 & $\begin{array}{l}33.589 \\
2720\end{array}$ & \begin{tabular}{|l|l|}
-1.2222 \\
\end{tabular} & & 48.105 & & 14.643 & & $\begin{array}{l}2376.48 \\
3778\end{array}$ & & s..2 & $\frac{11.8}{11.8}$ & & & & 0.009 & 0.000 & & \\
\hline & $6 / 16 / 2004$ & 12:52:12 AM & 26.152 & 26.281 & 24.904 & 27.229 & 26.269 & 48.119 & \begin{tabular}{|l|l|}
51.975 \\
\end{tabular} & 14.804 & 33.732 & \begin{tabular}{|l|l|} 
\\
\end{tabular} & -7.664 & $\begin{array}{l}49.811 \\
\end{array}$ & \begin{tabular}{ll|l}
0.058 \\
\end{tabular} & 14.643 & & 2377.48 & 6247 & 50.5 & 12.2 & 40. & 822 & 009 & .009 & 0.000 & 0.212 & \\
\hline 686 & 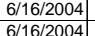 & $\begin{array}{l}12: 53: 12 \mathrm{AM} \\
12.5412 \mathrm{AM}\end{array}$ & $\begin{array}{l}26.143 \\
26.156 \\
\end{array}$ & 26.272 & 24.899 & 27.25 & & \begin{tabular}{|l|l|l|l|}
48.179 \\
8.097
\end{tabular} & & $\begin{array}{l}14.836 \\
14844 \\
\end{array}$ & & \begin{tabular}{|l|l|}
-1.222 \\
-1209 \\
\end{tabular} & & 54.078 & & $\begin{array}{l}14.643 \\
14.643 \\
\end{array}$ & & 2378.48 & & 50.6 & 13.3 & 41. & & 009 & \begin{tabular}{ll|l}
009 \\
000
\end{tabular} & & & \\
\hline $\begin{array}{l}680 \\
687 \\
\end{array}$ & 6/16/2004 & $\begin{array}{l}\text { 12:54:12 AM } \\
1255: 12 \mathrm{AM}\end{array}$ & $26.156 \mid$ & 26.285 & 24.993 & 27.273 & $\frac{26.278}{26.313}$ & $\begin{array}{l}48.097 \\
48.039\end{array}$ & \begin{tabular}{|l|}
51.983 \\
51.931 \\
\end{tabular} & $\begin{array}{l}\frac{14.844}{14.637} \\
\end{array}$ & $\begin{array}{l}33.759 \\
33.876\end{array}$ & \begin{tabular}{|l|}
-1.219 \\
-1.222 \\
\end{tabular} & $\begin{array}{c}-7.661 \\
-7.658 \\
\end{array}$ & \begin{tabular}{|l|l|}
45.216 \\
4.667
\end{tabular} & $\begin{array}{l}0.058 \\
0.059\end{array}$ & $\begin{array}{l}14.643 \\
14.433\end{array}$ & & $\begin{array}{l}379.48 \\
3.30 .48\end{array}$ & $\begin{array}{l}9.6581 \\
9.6747\end{array}$ & $\begin{array}{l}50.5 \\
50.5\end{array}$ & $\frac{11.1}{10.9}$ & 40. & .822 & |.009 & $\begin{array}{l}0.009 \\
0.009\end{array}$ & 0.000 & & \\
\hline 688 & 6/16/2004 & 12:56:12 AM & 26.157 & 26.286 & 24.979 & 27.199 & 26.334 & & \begin{tabular}{|l|}
51.879 \\
\end{tabular} & 14.817 & & & -7.655 & 51.569 & $\begin{array}{l}0.059 \\
0.059 \\
\end{array}$ & $\begin{array}{l}14.6434 \\
14.643 \\
\end{array}$ & 0.004 & $\begin{array}{l}380.48 \\
381.48\end{array}$ & \begin{tabular}{|l|l|} 
& 39.6141 \\
3 & 39.6914
\end{tabular} & 50.4 & $\frac{10.9}{12.6}$ & & & 0.009 & 0 & & & 0.0 .18 \\
\hline & & & 26.146 & 26.285 & 24.963 & & 26.348 & 48.043 & \begin{tabular}{|l|l|}
51.865 \\
\end{tabular} & 14.702 & 33.847 & \begin{tabular}{|l|l|}
-1.219 \\
\end{tabular} & -7.661 & 46.765 & 0.058 & 14.643 & & & & 50.4 & & & & & & & & \\
\hline & & 12:58:12 AM & & 26.285 & & & & & \begin{tabular}{|l|}
51.941 \\
\end{tabular} & & & \begin{tabular}{|l|l|}
-1.222 \\
\end{tabular} & & $\begin{array}{l}47.383 \\
\end{array}$ & & 14.643 & & & & & & & & & & & & \\
\hline 691 & 6/16/2004 & 12:59:12 AM & 26.149 & 26.278 & 24.93 & 27.241 & 26.351 & 47.873 & \begin{tabular}{|l|l|}
51.906 \\
\end{tabular} & 14.644 & 33.644 & \begin{tabular}{|l|l|} 
& -1.219 \\
\end{tabular} & & $\begin{array}{l}43.115 \\
\end{array}$ & 0.058 & 14.643 & & & 7414 & 50.4 & 10.6 & & 2.810 & .009 & ..009 & 0.000 & & \\
\hline & $\begin{array}{ll}6 / 16 / 2004 \\
6 / 1 / 6004\end{array}$ & 1:00:12 AM & \begin{tabular}{|l|l|}
26.14 \\
26.5 \\
\end{tabular} & \begin{tabular}{l|l|l|}
26.278 \\
26289
\end{tabular} & $\begin{array}{ll}24.926 \\
24031\end{array}$ & 27.282 & $\begin{array}{l}26.367 \\
26373\end{array}$ & \begin{tabular}{ll|l}
48.368 & 47839 \\
4
\end{tabular} & \begin{tabular}{|l|l|}
52.304 \\
51784 \\
\end{tabular} & $\begin{array}{l}14.832 \\
14705\end{array}$ & \begin{tabular}{|l|}
34.008 \\
3257 \\
\end{tabular} & -1.222 & $\begin{array}{r}-7.661 \\
7655 \\
\end{array}$ & \begin{tabular}{|l|}
52.71 \\
5024
\end{tabular} & 0.058 & $\begin{array}{l}14.643 \\
14643\end{array}$ & & \begin{tabular}{|l|l|}
2385.48 \\
2328.8
\end{tabular} & 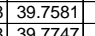 & $\begin{array}{ll}50.8 \\
503 \\
\end{array}$ & $\frac{12.9}{123}$ & 41. & $\frac{2.840}{2807}$ & $\begin{array}{l}.009 \\
009 \\
\end{array}$ & \begin{tabular}{|c|c|}
0.009 \\
0009
\end{tabular} & & $\begin{array}{ll}0.210 \\
2213 \\
\end{array}$ & \\
\hline$\overline{994}$ & 6/16/12004 & $\begin{array}{l}\frac{1: 01: 12 \mathrm{AM}}{1: 02: 12 \mathrm{AM}} \\
\end{array}$ & \begin{tabular}{r|r|}
26.15 & \\
2.157 &
\end{tabular} & 26.289 & $\frac{24.931}{24.928}$ & 27.282 & & & \begin{tabular}{|l|}
51.784 \\
5.1659 \\
\end{tabular} & $\begin{array}{l}14.7055 \\
15.095\end{array}$ & $\begin{array}{l}3.597 \mid \\
.988\end{array}$ & $\begin{array}{l}-1.216 \\
-1.219\end{array}$ & & $\begin{array}{r}50.24 \\
57068\end{array}$ & 0.058 & $\begin{array}{l}14.643 \\
14643\end{array}$ & & & & 50.3 & $\frac{12.3}{140}$ & & 2.807 & |009| & .009| & 0.000 & & \\
\hline 1695 & 6/16/2004 & $\begin{array}{l}\text { 1.0Z.12 AMI } \\
1: 03: 12 \text { AM }\end{array}$ & 资. 26.145 & 20.291 26.288 & 24.931 & $\begin{array}{l}27.24 \\
27.317 \\
\end{array}$ & $\frac{20.45}{26.44}$ & 47.684 & \begin{tabular}{|l|}
51.0099 \\
51.603
\end{tabular} & $\begin{array}{l}15.059 \\
14.932\end{array}$ & $\begin{array}{l}32.988 \\
33.271\end{array}$ & \begin{tabular}{|l|l|}
-1.219 \\
-1.219
\end{tabular} & $\begin{array}{l}-1.594 \\
-7.644 \\
\end{array}$ & 50.009 & $\begin{array}{l}0.050 \\
0.057 \\
\end{array}$ & $\frac{14.043}{14.643}$ & & 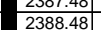 & 8081 & $\begin{array}{l}50.4 \\
50.1\end{array}$ & $\frac{1.0}{12.4}$ & & 791 & 0.008 & 0.000 & & & $\frac{0.18}{0.18}$ \\
\hline 696 & $6 / 16 / 2004$ & 1:04:12 AM & 26.158 & & 24.945 & 27.325 & 26.46 & 47.792 & \begin{tabular}{|l|}
51.69 \\
\end{tabular} & 14.985 & 33.26 & -1.219 & -7.649 & 499.842 & 0.057 & 14.643 & & & & & & & & & & & & $\frac{0.18}{0.18}$ \\
\hline & & 1:05:12 AM & & & 24.933 & 27.299 & 26.48 & 47.576 & & 14.984 & 32.996 & \begin{tabular}{|l|}
-1.222 \\
\end{tabular} & & & 557 & & & & & & & & & & & & & \\
\hline 698 & & & & & & & & & & & & & & 5.262 & & 14.643 & & & & & & & & & & & & \\
\hline & & & & 26.324 & & & & 47.703 & 51.651 & 14.964 & .148 & & & $70+2$ & & 14.64 & & & & & & & & & & & & \\
\hline & & $1: 08: 12 \mathrm{~A}$ & 26.169 & 26.333 & & 27.361 & & 47.9 & 51.975 & & & & & & & & & & & & & & & & & & & \\
\hline & 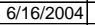 & & & & 24.93 & 27.36 & & 48.07 & & 14.956 & & & & & & 14.643 & & & & & & & & & & & & \\
\hline & $6 / 16 / 20$ & 1:10:12 AM & 26.175 & 26.338 & 24.956 & 27.386 & 26.48 & 47.624 & 51.718 & 14.86 & 33.203 & -1.219 & -7.649 & 52.449 & 057 & 14.643 & & \begin{tabular}{|c|}
395.48 \\
\end{tabular} & \begin{tabular}{|l|l|}
39.9247 \\
\end{tabular} & 50.2 & 12.9 & & 78 & . 008 & 609 & 00 & j.211 & \\
\hline & & & 26.165 & 26.344 & 24.951 & 27.357 & & 47.858 & 51.831 & 14.874 & 33.46 & & & & .057 & 14.643 & & & & & & & & 08 & & & & \\
\hline & & & 26.181 & 26.36 & 44.977 & 27.393 & & 47.88 & 51.858 & 14.91 & & & & 441 & 056 & 14.643 & & & & & & & & & & & & \\
\hline & & & & 26.356 & 4.958 & 2 & & & 51.63 & (4.863 & & & & & & 14.643 & & & & & & & & & & & & \\
\hline & & 1:14:1 & 26.169 & $\frac{26.358}{26.358}$ & 望.4975 & $\frac{27.331}{27.331}$ & $\frac{26.5}{26.4}$ & $\frac{47.9}{47 .}$ & 51.979 & $\begin{array}{l}14.912 \\
1485\end{array}$ & $\frac{33.513}{3220}$ & 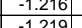 & & 514 & & $\frac{14.643}{14643}$ & & & & & & & & & & & & \\
\hline 708 & $6 / 16 / 2004$ & 1:16:12 AM & 26.182 & 26.376 & 24.978 & 27.299 & 26.4 & 47.481 & \begin{tabular}{|l|}
51.438 \\
\end{tabular} & 14.878 & 33.102 & \begin{tabular}{|l|l|}
-1.22 \\
\end{tabular} & & 36.119 & 0.058 & 14.643 & & 48 & & 50.0 & 8.9 & & & & & & & \\
\hline & 6/16/2004 & 1:17:12 AM & 26.181 & 26.3 & 24.993 & 27.333 & & 47.821 & 51.989 & 14.96 & 33.299 & -1.219 & & 52.6 & & 14.643 & & & & 50.5 & 12.9 & & & 0.008 & 0.008 & & & \\
\hline & & 1:18:12 & $\frac{26.186}{20.20}$ & 26.384 & 24.987 & & & 47.883 & 51.9 & 14.95 & 33.382 & \begin{tabular}{|l|l|}
-1.222 \\
\end{tabular} & & 55.964 & & 14.643 & & & & & & & & & & & & \\
\hline & $6 / 16 / 2004$ & 1:19:12 AM & 26.206 & 26.409 & 25.017 & 27.363 & 26.46 & & & & & & & & & 14.643 & & & & 50.4 & 13.2 & & & & & & & \\
\hline & 6/16/2004 & 1:20:12 AM & 26.188 & 26.402 & 25.015 & 27.36 & 26 & & \begin{tabular}{|l|l|}
51.686 \\
5.11
\end{tabular} & 14.934 & & & & & & 14.643 & & & & 50.2 & 12.1 & & $2.788 \mid$ & 009 & 0.009 & & & \\
\hline & 6/16/2004 & $\begin{array}{l}1: 21: 12 \mathrm{AM} \\
1.22112 \mathrm{AM}\end{array}$ & $\begin{array}{l}26.186 \\
26.181\end{array}$ & \begin{tabular}{r|}
26.4 \\
26.4 \\
\end{tabular} & 25.002 & 27.343 & 26.50 & $\begin{array}{l}47.819 \\
47.773\end{array}$ & \begin{tabular}{|l|}
51.641 \\
51846 \\
\end{tabular} & $\begin{array}{l}14.886 \\
14.855\end{array}$ & $\begin{array}{l}33.425 \\
33.365\end{array}$ & \begin{tabular}{|l|}
-1.219 \\
-1.222 \\
\end{tabular} & & \begin{tabular}{|l}
51.006 \\
53.35 \\
\end{tabular} & $\begin{array}{l}0.057 \\
0.058\end{array}$ & $\begin{array}{l}\frac{14.643}{14.633} \\
\end{array}$ & & $\begin{array}{l}48 \\
48\end{array}$ & $\begin{array}{l}081 \\
27 \\
\end{array}$ & 50.2 & $\frac{12.5}{13.1}$ & & 2.801 & 0 & $\begin{array}{l}0.008 \\
0.009\end{array}$ & & & 0.18 \\
\hline 715 & $\frac{6 / 16 / 12004}{6 / 16 / 2004}$ & $\begin{array}{l}\text { 1:22:12 AM } \\
\text { 1:23:12 AM }\end{array}$ & $\frac{26.181}{26.188}$ & \begin{tabular}{r|r|}
26.402 \\
26.4
\end{tabular} & $\begin{array}{l}24.979 \\
25.009\end{array}$ & $\begin{array}{l}27.323 \\
27.315\end{array}$ & & $\begin{array}{l}47.773 \\
48.088\end{array}$ & \begin{tabular}{|l|}
51.846 \\
52.057 \\
\end{tabular} & $\begin{array}{l}14.855 \\
14.919\end{array}$ & $\begin{array}{l}33.365 \\
33.644\end{array}$ & \begin{tabular}{|l|}
-1.222 \\
-1.216 \\
\end{tabular} & & $\begin{array}{r}53.35 \\
51.753 \\
\end{array}$ & & $\frac{14.643}{14.643}$ & & & & $\begin{array}{l}50.4 \\
50.6\end{array}$ & $\begin{array}{l}13.1 \\
\frac{12.7}{4}\end{array}$ & & & & & & & \\
\hline 716 & $6 / 16 / 2004$ & $1: 24: 12 \mathrm{AM}$ & 26.204 & 26.423 & 25.026 & 27.306 & & & \begin{tabular}{|l|l|}
52.074 \\
\end{tabular} & 14.951 & & -1.222 & & 46.7 & & 14.643 & & & & 50.6 & 11.5 & & & & & & & \\
\hline 717 & $6 / 16 / 2$ & & 26.197 & 26.4 & 25.018 & 27.313 & & & \begin{tabular}{|l|}
51.941 \\
5020
\end{tabular} & 15.024 & & & & 44.3 & 0.0 & 14.6. & & & & 50.5 & 10.9 & & & & & & & \\
\hline & $6 / 1$ & 1:26:12 AM & & & & 27.316 & & & \begin{tabular}{|l|l|}
52.035 \\
\end{tabular} & 14.964 & & & & & & & & & & & 12.7 & & & & & & & \\
\hline 719 & 6/16/2004 & 1:27:12 AM & 26.184 & 26. & 25.08 & 27.276 & 26 & 47.7. & \begin{tabular}{|l|l|}
51.948 \\
\end{tabular} & 14.8 & 33.291 & -1.219 & & 50.063 & & 14.6 & & 48 & 40.20 & 50.5 & 12.3 & & & & & & 0.209 & \\
\hline & 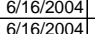 & $\begin{array}{l}1: 28: 12 \mathrm{AM} \\
1.29 .12 \mathrm{AM}\end{array}$ & $\frac{26.188}{26.192}$ & $\frac{26.4}{26.4}$ & 25.079 & $27.275 \mid$ & $\frac{26}{264}$ & 47.4 & \begin{tabular}{|l|}
51.456 \\
51.57
\end{tabular} & $\frac{14.815}{14819}$ & $\begin{array}{l}33.063 \\
3.129\end{array}$ & $\begin{array}{r}-1.222 \\
-1.222 \\
\end{array}$ & & 56.9 & $\begin{array}{l}0.059 \\
0.058\end{array}$ & 14.6 & & & & 50.0 & $\frac{13.9}{118}$ & $4 \mathrm{4C}$ & & & \begin{tabular}{l|l}
0.009 \\
0.009
\end{tabular} & . 000 & 0.218 & $\frac{19}{18}$ \\
\hline$\frac{21}{22}$ & 6/16/2004 & $\begin{array}{l}1.29912 \mathrm{AM} \\
1: 30: 12 \mathrm{AM}\end{array}$ & 26 & $\frac{26.4106}{26.406}$ & $\begin{array}{l}25.004 \\
25.058 \\
\end{array}$ & 27.2.24] & & & & & $\begin{array}{l}33.129 \\
33.297 \\
\end{array}$ & & & & & $\frac{14.6}{14.6}$ & & & & $\frac{50.1}{50.3}$ & & & & & & & & \\
\hline .123 & $6 / 16 / 2004$ & & 26.182 & 26.405 & 25.048 & 27.274 & & & & 14.799 & & & & & & & & & & & 11.8 & & & & & & & \\
\hline 724 & $6 / 16 / 2$ & & 26.191 & 26.404 & 25.047 & 27.278 & & & & 14.831 & 33.6 & & & & & 14.6 & & & & 50.5 & 12.3 & & & & & & 208 & \\
\hline 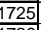 & 6/2004 & & & & 25.02 & 27.267 & & & & & & & & & & & & & & - & 11.0 & & & & & & & \\
\hline$\frac{726}{720}$ & & & 26.196 & & & & & & \begin{tabular}{|l|l|}
51.775 \\
\end{tabular} & & & & & & & & & & & 50.3 & 12.0 & & & & & & & \\
\hline & $\begin{array}{l}\text { 6/16/2004 } \\
6 / 16 / 2004\end{array}$ & $\begin{array}{l}1: 35: 12 \mathrm{AM} \\
136.12 \mathrm{AM}\end{array}$ & $\begin{array}{l}26.206 \\
26.208\end{array}$ & \begin{tabular}{r|r}
26.42 \\
26.427 \\
\end{tabular} & 25.033 & 27.253 & $\frac{26.4}{26.4}$ & $\begin{array}{l}48.123 \\
47.877\end{array}$ & \begin{tabular}{|r|}
52.3 \\
52066 \\
\end{tabular} & $\frac{14.931}{14.834}$ & \begin{tabular}{|c|}
33.581 \\
33.499
\end{tabular} & \begin{tabular}{|l|}
-1.219 \\
-1.219 \\
\end{tabular} & & $\begin{array}{r}56.05 \\
54.335 \\
\end{array}$ & $\begin{array}{l}0.057 \\
0.057\end{array}$ & $\frac{14.6}{14.62}$ & & $\frac{242}{242}$ & & $\begin{array}{l}50.8 \\
50.6 \\
\end{array}$ & $\frac{13.7}{13 .}$ & $\frac{4}{4}$ & 2.81 & & $\frac{58}{88}$ & $\begin{array}{ll}000 \\
000 \\
\end{array}$ & 0.208 & 18 \\
\hline 29 & $\frac{6 / 11 / 12004}{6 / 2004}$ & & & & $\begin{array}{ll}25.034 \\
25.035 \\
\end{array}$ & & & & & & & & & & & & & & & 50.4 & 13.3 & & & & & & & \\
\hline 73 & & & & & 25.041 & & & & & & & & & & & & & & & 50. & 12.5 & & & & & & & \\
\hline 731 & & & 26.1 & & 25.023 & 27.224 & & & \begin{tabular}{|l|}
51.948 \\
\end{tabular} & 14.7 & & -1.2 & & & & 14.6 & & & & 50.5 & 12.4 & & & & & & & \\
\hline & & & 26.194 & & 25.02 & & & & & & & & & & & & & & & & & & & & & & & \\
\hline 733 & & 1:41:12 AM & 26.203 & 26. & 25.029 & 27.265 & & 47.8 & 51.86 & 14.7 & $\begin{array}{l}33.615 \\
300\end{array}$ & -1.2 & & & & & & & & 50.4 & 11.9 & & & & & & & \\
\hline & 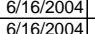 & $\begin{array}{l}1: 42: 12 \mathrm{AM} \\
1.13 \cdot 12 \mathrm{AM}\end{array}$ & \begin{tabular}{r|r}
26.2 & 26.208 \\
\end{tabular} & 26.4 & $\frac{25.031}{2505}$ & 27.242 & $\frac{26.4}{26}$ & 47.3 & \begin{tabular}{|l|}
51.448 \\
51.61 \\
\end{tabular} & $\frac{14.789}{14774}$ & $\begin{array}{l}33.0 \\
331 \\
3\end{array}$ & \begin{tabular}{|l|}
-1.219 \\
-12019 \\
\end{tabular} & -7 & & $\frac{0 .}{0.0}$ & $\frac{14.6}{14.6}$ & & & & 50.0 & $\frac{12.4}{139}$ & $\frac{4}{4}$ & & & & & & \\
\hline$\frac{735}{736}$ & $\frac{6 / 16 / 12004}{6 / 16 / 2004}$ & $\begin{array}{l}\text { 1:43:12 AM } \\
\text { 1:44:12 AM }\end{array}$ & $\frac{26.208}{26.213}$ & & $\begin{array}{r}25.05 \\
25.055\end{array}$ & 27.26 & & & & & & & & & & $\frac{14.6}{14.6}$ & & & & $\frac{50.2}{50.2}$ & $\frac{11.9}{11.6}$ & & & & & & 214 & \\
\hline & & & & & & & & & & & & & & & & & & & & & 13.1 & & & & & & & \\
\hline 1738 & & & 26.207 & 26.4 & 25.043 & 27.274 & & & \begin{tabular}{|l|}
52.151 \\
\end{tabular} & 14.7 & & $\mid-1.21$ & & & & 14.6 & & & & 50.7 & 13.0 & & & & & & 0.216 & \\
\hline & & & & & & & & & & & & & & & & & & & & 50.4 & & & & & & & & \\
\hline 74 & 6/16/2004 & & $\begin{array}{l}26.199 \\
26203 \\
\end{array}$ & & & 27.246 & & 47.5 & 52.1 & & & & & & & & & & & 50.7 & 13.5 & & & & & & & \\
\hline 741 & 6/16/2004 & $\begin{array}{l}1: 49: 12 \mathrm{AM} \\
1.50 \cdot 12 \mathrm{AM}\end{array}$ & 26.203 & $26.442 \mid$ & $\frac{25.034}{25018}$ & $\begin{array}{l}27.27 \\
27269\end{array}$ & & $\begin{array}{ll}47.846 \\
47.684\end{array}$ & \begin{tabular}{|l|}
52.088 \\
51324 \\
\end{tabular} & $\begin{array}{r}14.79 \\
14736\end{array}$ & & \begin{tabular}{|l|}
-1.219 \\
-1.222
\end{tabular} & & & & & & & & 50.6 & & & & & & & .205 & \\
\hline 743 & $6 / 16 / 2004$ & $\begin{array}{l}1.50 .12 \mathrm{AM} \\
1: 51: 12 \mathrm{AM}\end{array}$ & $\frac{2.6182}{26.186}$ & & 25.008 & |l. 27.309 & & $\begin{array}{l}48.684 \\
48.111\end{array}$ & \begin{tabular}{|l|}
51.884 \\
52.236 \\
\end{tabular} & $\frac{14.750}{14.814}$ & & \begin{tabular}{|l|}
-1.2219 \\
-1.219
\end{tabular} & & & & $\frac{14.6}{14.6}$ & & & & $\frac{50.4}{50.8}$ & $\frac{10.9}{12.4}$ & & & & & & & \\
\hline 14. & & & & & & 27.299 & & & & & & \begin{tabular}{|l|l|} 
\\
\end{tabular} & & & & 14.6 & & & & 50. & 11.4 & & & & & & & \\
\hline & & & & & & & & 47 & & & & & & & & & & & & & 9.9 & & & & & & & \\
\hline & & $1: 5$ & 26.2 & 26.4 & & 27.2 & & 47.9 & & 14 & & -1.2 & & & & 14. & & & & 50.4 & & & & & & & & \\
\hline & & 1:55 & 26.19 & & & 27.287 & & 47.5 & 51.7 & & & $\mid-1.22$ & & & & & & & & & .1 & & & & & & & \\
\hline 749 & & & 26.174 & | & 23.003 & $\frac{2.281}{27205}$ & & 47. & & & & -1.2 .2 & & & & & & & & & & & & & & & & \\
\hline 750 & $6 / 16 / 2004$ & 1:58:12 AM & 26.181 & 26.38 & \begin{tabular}{|l|}
25.017 \\
\end{tabular} & \begin{tabular}{|l|}
27.280 \\
\end{tabular} & 26.33 & 48.024 & \begin{tabular}{|l|}
52.254 \\
\end{tabular} & \begin{tabular}{|l|l|l|l|}
1493 \\
\end{tabular} & 33.661 & \begin{tabular}{|l|l|}
-1.2162 \\
-1.216
\end{tabular} & & $\mid 48.86$ & $\mid$ & \begin{tabular}{|l|l}
14.643 \\
\end{tabular} & & & $\mid \frac{40.1081}{340.7247}$ & \begin{tabular}{|l|}
0.1 \\
50.8 \\
\end{tabular} & \begin{tabular}{|l|l|}
12.0 \\
\end{tabular} & & 2.816 & $\mid$ & $\begin{array}{c}0.009 \\
\end{array}$ & \begin{tabular}{|c|}
0.000 \\
\end{tabular} & 0.212 & 0.18 \\
\hline
\end{tabular}


WSRC-TR-2005-00105, REVISION 0

SRNL-RPP-2005-00012, REVISION 0

RUN \# 3.03A AND B; FIRST AND SECOND HALF OF SLURRY DEWATERING - CONT.

\begin{tabular}{|c|c|c|c|c|c|c|c|c|c|c|c|c|c|c|c|c|c|c|c|c|c|c|c|c|c|c|c|c|}
\hline & A & & D & $E$ & $\mathrm{~F}$ & \begin{tabular}{l|l|l} 
G & \\
\end{tabular} & $\begin{array}{ll}\mathrm{H} \\
\end{array}$ & \begin{tabular}{l|l}
$\mathrm{J}$ \\
\end{tabular} & $\mathrm{K}$ & \begin{tabular}{|l|} 
\\
\end{tabular} & $\mathrm{M}$ & $\begin{array}{ll}\mathrm{N} \\
\end{array}$ & 0 & \begin{tabular}{l|l} 
Q \\
\end{tabular} & \begin{tabular}{l|l}
$R$ \\
\end{tabular} & \begin{tabular}{l|l} 
s \\
\end{tabular} & $\begin{array}{lll}T \\
\end{array}$ & $\mathrm{v}$ & w & \begin{tabular}{|c|}
$x \mid$ \\
\end{tabular} & \begin{tabular}{l|l} 
&
\end{tabular} & $z$ & & $\mathrm{AB}$ & $\overline{A C}$ & & $\overline{A E}$ & $\overline{\mathrm{AF}}$ \\
\hline & $6 / 16 / 2004$ & 1:59:12 AM & 26.178 & 26.377 & 25.014 & 27.285 & 26.325 & 48.225 & 52.343 & \begin{tabular}{|l|l|}
14.813 \\
\end{tabular} & 33.851 & -1.219 & -7.728 & 55.182 & 0.058 & 14.643 & 0.004 & 2444.48 & \begin{tabular}{|l|l|}
3 & 40.7414 \\
\end{tabular} & 50.9 & 13.5 & 41.0 & 2.829 & 0.009 & 0.009 & 0.000 & 0.211 & 0.18 \\
\hline & & $2: 00: 12 \mathrm{AM}$ & & 26.363 & 25.011 & 27.262 & 26.316 & 47.987 & \begin{tabular}{|l|}
51.977 \\
\end{tabular} & \begin{tabular}{|l|l|}
14.793 \\
\end{tabular} & \begin{tabular}{|l|}
33.63 \\
\end{tabular} & \begin{tabular}{|r|r|}
-1.219 \\
\end{tabular} & $\begin{array}{l}-1.702 \\
-7.702 \\
\end{array}$ & 44.344 & 0.057 & \begin{tabular}{|l}
14.643 \\
\end{tabular} & & & & & & & & & 0.008 & & & \\
\hline t75n & $6 / 16 / 2004$ & $2: 01: 13 \mathrm{AM}$ & $\frac{26.177}{26.172}$ & 26.366 & 25.008 & 27.274 & 26.334 & \begin{tabular}{|l|}
48.03 \\
8.03
\end{tabular} & \begin{tabular}{|l|}
52.225 \\
5.220 \\
\end{tabular} & \begin{tabular}{|r|}
14.786 \\
\end{tabular} & \begin{tabular}{|l|l|}
33.698 \\
325
\end{tabular} & \begin{tabular}{|l|l|}
-1.2222 \\
\end{tabular} & $\begin{array}{l}-7.647 \\
7772\end{array}$ & \begin{tabular}{|l|l|}
51.213 \\
5239
\end{tabular} & & 14.643 & & 2446.50 & \begin{tabular}{|l|l|}
50.775 \\
\end{tabular} & 50.8 & 12.6 & 40.9 & & 8009 & $\begin{array}{l}.009 \\
0.09\end{array}$ & 0.000 & 0.212 & \\
\hline 754 & $6 / 16 / 2004$ & $\begin{array}{l}2: 02: 13 \mathrm{AM} \\
202: 12\end{array}$ & $\begin{array}{l}26.173 \\
26.17\end{array}$ & 26.346 & 25.009 & 27.265 & $\begin{array}{r}26.32 \\
26.322\end{array}$ & 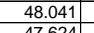 & \begin{tabular}{|l|}
52.236 \\
\end{tabular} & \begin{tabular}{|r|}
14.81 \\
\end{tabular} & \begin{tabular}{|l|l|}
33.65 \\
\end{tabular} & \begin{tabular}{|l|}
-1.222 \\
\end{tabular} & $\begin{array}{l}-7.725 \\
\end{array}$ & $\begin{array}{l}51.296 \\
\end{array}$ & $\begin{array}{l}0.057 \\
\end{array}$ & 14.643 & 0.004 & 2447.50 & $\mid 40.7917$ & 50.8 & 12.6 & 40.8 & 2.816 & . 0008 & $\begin{array}{l}.008 \\
\end{array}$ & 0.000 & 0.208 & \\
\hline 1755 & 6/16/12004 & $2: 03: 13 \mathrm{AM}$ & $\frac{26.17}{26.182}$ & $\frac{26.344}{26.346}$ & \begin{tabular}{|l|}
24.996 \\
\end{tabular} & 27.272 & & 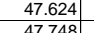 & & $\begin{array}{l}14.705 \\
14655\end{array}$ & \begin{tabular}{|l|l|}
33.326 \\
3519
\end{tabular} & \begin{tabular}{|l|}
-1.222 \\
-129 \\
\end{tabular} & $\begin{array}{l}-7.641 \\
7551 \\
\end{array}$ & $\begin{array}{r}50.351 \\
41.9\end{array}$ & $\begin{array}{l}0.05 \\
0.07\end{array}$ & $\begin{array}{l}14.643 \\
14643\end{array}$ & & & & 50.4 & 12.3 & 40.5 & 2.791 & & 0.007 & & 0.184 & \\
\hline 1757 & $6 / 6 / 16 / 2004$ & $\begin{array}{l}\text { 2:04:13 AM } \\
\text { 2:05:13 }\end{array}$ & $\frac{26.182}{26.185}$ & $\frac{26.346}{26.344}$ & \begin{tabular}{|l|}
25.054 \\
425.082 \\
\end{tabular} & $\begin{array}{l}27.299 \\
27.267\end{array}$ & $\frac{26.319}{26.322}$ & \begin{tabular}{|l}
477.748 \\
47.804
\end{tabular} & \begin{tabular}{|r|}
52.027 \\
52.024 \\
\end{tabular} & \begin{tabular}{|l|}
14.685 \\
14.761 \\
\end{tabular} & $\begin{array}{r}33.519 \\
33.484\end{array}$ & \begin{tabular}{|l|l|}
-1.219 \\
-1.222 \\
\end{tabular} & $\begin{array}{l}-7.531 \\
-7.7647 \\
\end{array}$ & $\begin{array}{r}\mid 41.49 \\
49.604\end{array}$ & $\begin{array}{c}0.057 \\
0.06\end{array}$ & $\begin{array}{l}\frac{14.643}{14.643} \\
\end{array}$ & $\frac{0.004}{0.003}$ & $\begin{array}{r}2449.50 \\
2450.50\end{array}$ & 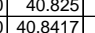 & \begin{tabular}{|l|l|}
50.6 \\
50.6
\end{tabular} & $\frac{10.2}{12.2}$ & \begin{tabular}{|l|}
40.6 \\
40.6
\end{tabular} & $\begin{array}{l}2.802 \\
2.802\end{array}$ & . 0008 & $\mid \begin{array}{l}0.008 \\
0.009\end{array}$ & $\begin{array}{l}0.000 \\
0.000\end{array}$ & & 0.18 \\
\hline 758 & & & 26.188 & & & & & & & & & & & & & & & & & & & & & & $\begin{array}{l}0.009 \\
0.009\end{array}$ & & & $\frac{0.19}{0.18}$ \\
\hline 759 & $6 / 16 / 2004$ & 2:07:13 AM & & & 25.066 & 27.187 & 26.352 & $\begin{array}{ll}47.92 \\
\end{array}$ & \begin{tabular}{|l|l|}
52.101 \\
\end{tabular} & 14.705 & 33.665 & \begin{tabular}{|r|}
-1.216 \\
\end{tabular} & -7.592 & 47.527 & 0.058 & 14.643 & 0.004 & & & 50.6 & 11.6 & & & & & & & $\frac{0.18}{0.18}$ \\
\hline & $6 / 16 / 2004$ & 2:07:41 AM & & 26.344 & & & & \begin{tabular}{|l|l|}
47.978 \\
\end{tabular} & \begin{tabular}{|l|}
52.08 \\
\end{tabular} & 14.764 & 33.691 & \begin{tabular}{|l|}
-1.219 \\
\end{tabular} & -7.595 & \begin{tabular}{|l|l|}
53.013 \\
\end{tabular} & & \begin{tabular}{|l|l|}
14.643 \\
\end{tabular} & & $2452.97 \mid$ & & 50.6 & 13.0 & 40.8 & 2815 & & 0.009 & & & $\begin{array}{l}0.18 \\
0.18\end{array}$ \\
\hline 1761 & & 8:41 AM & & & 25.062 & 27.188 & & & 52.059 & 14.67 & 3.759 & & & 50.716 & 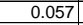 & 14.643 & & & & & 12.4 & & & 008 & .008 & D.000 & 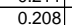 & \\
\hline 762 & & (1) & 26.186 & 26.314 & & & & \begin{tabular}{|l|l|l|}
47.696 \\
\end{tabular} & 51.908 & 14.705 & 476 & -1.225 & & & & 14.643 & & & & 50.4 & 13.1 & & & & & & | 206 & \\
\hline$\overline{71}$ & & 2:10:41 AM & & 26.315 & 2504 & 27.124 & & 47.999 & 52.323 & 14.816 & & 1.225 & & & & 14.643 & & & & & 108 & & & & & & & \\
\hline & $6 / 16 / 2004$ & $1: 41 \mathrm{AM}$ & 26.181 & 26.32 & 25.033 & 27.124 & & 47.922 & 52.217 & 14.853 & 33.414 & -1.219 & -7.615 & $\begin{array}{l}47.174 \\
\end{array}$ & 0.057 & 14.643 & & 2456.97 & 40.9494 & 50.7 & 11.6 & & & . & | & & & \\
\hline & $6 / 16 / 2004$ & 2:12:41 AM & 26.205 & 26.333 & 25.056 & 27.172 & 26.291 & 47.57 & 51.788 & 14.653 & 33.363 & -1.216 & -7.606 & 56.461 & 0.057 & 14.643 & & 2457.97 & 661 & 50.3 & 13.8 & & & .008 & 0.008 & & & 0.18 \\
\hline 766 & & 2:13:41 AM & & $\frac{26.342}{2.202}$ & 25.065 & 27.185 & & 47.926 & 52.074 & 14.778 & 33.62 & $\begin{array}{l}-1.219 \\
\end{array}$ & & & 0.057 & 14.643 & & & & & & & & & & & & \\
\hline 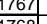 & $6 / 16 / 2004$ & 2:14:41 AM & & 26.325 & & & & & 20.542 & & 34.008 & -1.219 & & 54.46 & 0.057 & 14.643 & & & 0.9994 & p1.1.1 & & & & & & & & \\
\hline & $6 / 16 / 2004$ & $2: 15: 41 \mathrm{AM}$ & & & & & & & & & . & -1.219 & -1.626 & & & 14.643 & & & & & 11.8 & & & & & & & \\
\hline & & 2:16:41 AM & 26.173 & 26.311 & 25.014 & $\frac{27.175}{27165}$ & & 47.864 & $52.184 \mid$ & 14.793 & . 513 & -1.225 & -1.0260 & 50.482 & & 14.643 & & & & . & 12.4 & & & & 然8 & & & \\
\hline 771 & $6 / 10 / 16 / 2004$ & $4: 41 \mathrm{AM}$ & $\frac{20.100}{26.179}$ & & & 27.121 & & $\begin{array}{l}4.096 \\
47.456\end{array}$ & 151.547 & $\begin{array}{l}1.090 \\
14.989\end{array}$ & & $\begin{array}{l}-1.215 \\
-1.225 \\
\end{array}$ & & 年. & 0.056 & $\begin{array}{l}14.045 \\
114643\end{array}$ & & & & 501 & & & & & & & & \\
\hline 1772 & $6 / 16 / 2004$ & 2:19:41 AM & 26.197 & $\frac{2.010}{26.326}$ & 25.044 & 27.169 & $\frac{2.21}{26.26}$ & 4 & 51.728 & 15.065 & 32.897 & -1.219 & $\begin{array}{l}-7.020 \\
-7.629\end{array}$ & 49.148 & 0.057 & $\begin{array}{l}\frac{1.045}{14.643} \\
\end{array}$ & & 2464.97 & | & 50.3 & $\begin{array}{l}12.0 \\
12.0\end{array}$ & & $778 \mathrm{C}$ & . & .008 & 0.000 & & \\
\hline & $6 / 16 / 2004$ & 2:20:41 AM & 26.186 & 26.32 & 25.053 & 27.163 & & 47.846 & 51.962 & 15.075 & 33.207 & -1.216 & 1.020 & 54.235 & 0.056 & 14.643 & & & 41.0994 & & 13.3. & & 794 & & .008 & & & \\
\hline & & $: 41 \mathrm{AM}$ & 26.422 & 26.306 & & & & & 51.699 & 15.114 & & & & 43.864 & & & & & & & & & & & & & & \\
\hline 775 & $6 / 16 / 2004$ & :41 AM & 26.278 & 26.297 & 25.05 & 27.141 & & $\mid 47.603$ & & 15.055 & 32.981 & -1.219 & & 53.603 & 0.06 & 14.643 & & & $\mid$ & 50.6 & 13.1 & & & | & .009 & & & \\
\hline & & 2:23:41 AM & 26.199 & 26.278 & 25.001 & & & 47.705 & 51.981 & & & & & 1.636 & & 14.643 & & & & & & & & & & & & \\
\hline & 6/2004 & 2:24:41 AM & 26.183 & 26.301 & 25.024 & \begin{tabular}{|l|l|}
27.19 \\
\end{tabular} & & 47.85 & 52.339 & 15.045 & 33.232 & -1.216 & & 1.459 & & 14.643 & & & & & & & & & & & & \\
\hline 778 & 6/16/2004 & 5:41 AM & 26.157 & 26.296 & 25.009 & 27.199 & & 47.632 & 52.062 & 15.055 & 32.973 & -1.219 & & 44.085 & 0.056 & 14.643 & & & & 50.6 & 10.8 & & & & & & & \\
\hline 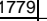 & 04 & 2:26:41 AM & 26.169 & 26.312 & 25.03 & 27.196 & 26.24 & 47.83 & 52.169 & 15.054 & 33.254 & \begin{tabular}{|l|l|} 
\\
\end{tabular} & -7.386 & 51.988 & & 14.643 & & $\begin{array}{l}4471.97 \\
\end{array}$ & 141.1994] & 50.7 & 12.7 & & 795 & .008 & 0.008 & & & \\
\hline & & & & & & 27.175 & & 477. & 1.871 & 15.009 & & -1.22 & & & & 14.643 & & & & & & & & & & & & \\
\hline & 04 & $A M$ & 26.14 & & & 27.167 & & 4.647? & 1.1944 & 14.962 & & & & 44.0000 & 0.057 & 14.643 & & & & & & & & & & & & \\
\hline 1182 & & AM & 26.149 & & & $27.171 \mid$ & & & 51.993 & 15.051 & 33.174 & -1.222 & & $49.044 \mathrm{~g}$ & & 14.643] & & & & 50.5 & & & & & & & & \\
\hline 784 & $6 / 16 / 1 / 2004$ & $2: 30: 41 \mathrm{AM}$ & $\frac{26.135}{26127}$ & $\frac{26.269}{26.255}$ & 5 & 27.162 & & $\frac{47.4}{47.3}$ & $\begin{array}{l}51.784 \\
51.57]\end{array}$ & $\frac{15.102}{14.904}$ & $\begin{array}{l}32.778 \\
32.96\end{array}$ & $\begin{array}{l}-1.216 \\
-1.1219\end{array}$ & & $\frac{51.434}{45028}$ & 057 & $\frac{14.643}{14643}$ & & & & & 10 & & & & & & & \\
\hline 1785 & $6 / 16 / 2004$ & $32: 41$ AM & 26.146 & 26.265 & 25.023 & 27.68 & 26.2 & 47.572 & 51.975 & 15.07 & \begin{tabular}{|l|}
32.907 \\
\end{tabular} & \begin{tabular}{|l|} 
\\
\end{tabular} & & 58.989 & 0.057 & 14.643 & & & \begin{tabular}{|c|}
41.2994 \\
\end{tabular} & 50.5 & 14.5 & & & $\begin{array}{l}\mid \\
0.008 \\
.008\end{array}$ & 0.008 & & & \\
\hline 1786 & 6/16/2004 & 2:33:41 AM & $\begin{array}{l}26.137 \\
\end{array}$ & 26.246 & & 27.199 & & & 51.979 & 14.956 & 33.15 & \begin{tabular}{|l|l|}
-1.216 \\
\end{tabular} & & 50.647 & & 14.643 & & & & 50.5 & 12.4 & & & & 0.008 & & & \\
\hline 1787 & $6 / 16 / 2004$ & 2:34:41 AM & & & 25.023 & 27.193 & & \begin{tabular}{|l|}
47.796 \\
\end{tabular} & 52.097 & 14.912 & 33.365 & \begin{tabular}{|l|}
-1.219 \\
\end{tabular} & & 53.613 & 0.057 & 14.643 & & & & 50.6 & 13.1 & & & & 0.008 & & & \\
\hline & $6 / 16 / 20$ & & 26.144 & & 25.02 & 27.196 & & & 52.151 & & 115 & $\begin{array}{l}-1.219 \\
\end{array}$ & & & & & & & & 50.7 & 13.1 & & & & & & & \\
\hline 1789 & 6/16/2004 & $2: 36: 41 \mathrm{AM}$ & 26.154 & 26.258 & 25.05 & 27.191 & & 47.387 & 52.016 & 14.911 & \begin{tabular}{|l|l|}
32.813 \\
\end{tabular} & \begin{tabular}{|l|l|}
-1.225 \\
\end{tabular} & $\begin{array}{r}7.41 \\
\end{array}$ & & 0.0 & 14.643 & & 2481.97 & \begin{tabular}{|l|}
41.3661 \\
\end{tabular} & 50.5 & 13.9 & & & \begin{tabular}{|l|l|l|l|}
0.008 \\
\end{tabular} & 88 & & 0.212 & 0.18 \\
\hline & 6/16/12004 & $2: 37: 41 \mathrm{AM}$ & $\frac{26.182}{26.186}$ & $\frac{26.276}{26.275}$ & $\frac{25.094}{2503}$ & $27.194 \mid$ & $\frac{26.254}{26.218}$ & $\begin{array}{l}47.649 \\
47.584\end{array}$ & \begin{tabular}{|r|}
52.072 \\
55195
\end{tabular} & \begin{tabular}{r|r|}
14.91 \\
14.972
\end{tabular} & \begin{tabular}{|l|}
33.162 \\
3.2996
\end{tabular} & $\begin{array}{r}-1.222 \\
-1.225\end{array}$ & $\begin{array}{l}-7.4 \\
-7.4\end{array}$ & \begin{tabular}{|l|l|}
48.488 \\
48054
\end{tabular} & $\begin{array}{l}0.056 \\
0.056\end{array}$ & $\frac{14.643}{14.63}$ & & $\frac{248}{248}$ & 41.3 & $\begin{array}{l}50.6 \\
50.5\end{array}$ & 11.9 & $\frac{40 .}{40}$ & 86 & & \begin{tabular}{ll|}
8 \\
28
\end{tabular} & 0.000 & 0.2067 & 0.18 \\
\hline$\frac{751}{792}$ & $6 / 16 / 1 / 2004$ & & $\frac{26.186}{26.196}$ & & $\begin{array}{l}25.0083 \\
25.092\end{array}$ & 27.183 & & & & $\frac{14.9 / 2}{14.868}$ & \begin{tabular}{|c|}
32.996 \\
32.869
\end{tabular} & \begin{tabular}{|l|l|}
-1.225 \\
-1.222
\end{tabular} & & & & $\frac{14.643}{14.643}$ & & & & $\frac{50.5}{50.2}$ & & & & & & & & \\
\hline 1793 & $6 / 16 / 2004$ & & 26.196 & & & 27.208 & & & & \begin{tabular}{|c|}
14.78 \\
14.78
\end{tabular} & & & & & & $\frac{14.045}{14.643}$ & & & & 00.4 & t2.t. & & & & & & & $\frac{0.18}{0.18}$ \\
\hline 1794 & $6 / 16 / 2004$ & & 26.176 & 26.244 & 25.052 & $\frac{27.148}{27.14}$ & & 47.3 & \begin{tabular}{|l|}
51.705 \\
\end{tabular} & 14.87 & & -1.2 & & $\frac{07.725}{47.75}$ & 0.058 & 14.643 & & & & 50 & $\frac{31.7}{11.7}$ & & & & & & & \\
\hline & & & & & & & & & & & & & & & & & & & & & & & & & & & & \\
\hline 1796 & & & 26.164 & & 25.05 & 27.136 & & & & 14.91 & & & & & & & & & & & 120 & & & & & & & \\
\hline & & & & $\frac{26.219}{26}$ & & 27.118 & & & 51.916 & 14.971 & 3.143 & -1.219 & & & & 14.6 & & & & & & & & & & & & \\
\hline & & & & 26.22 & 25.043 & 27.084 & & & 52.19 & 14.922 & 33.205 & -1.222 & & & & & & & & & 58 & & & & & & & \\
\hline & 6/16/2004 & & & 26.2 & 25.069 & 27.145 & & 47.6 & 52 & 14.864 & & & & & & 14.64 & & & & 50. & 14.1 & & & & & & & \\
\hline & & & & & & 27.132 & & & & & & & & & & & & & & & .9 & & & & & & & \\
\hline 801 & & & & & 25.065 & 27.16 & & & & & & -1.2 & & & & & & & & & 11.0 & & & & & & & \\
\hline 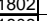 & & & & & & 27.114 & & & & & & & & & & & & & & & & & & & & & & \\
\hline 803 & & & 20.15 & & & 27.093 & & & 5.1 .9 & & & & & & & & & & & & & & & & & & & \\
\hline & & 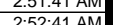 & & & 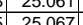 & 资, & & $\frac{47 .}{470}$ & \begin{tabular}{|l|}
51.975 \\
52.325 \\
\end{tabular} & & $\frac{33.2}{33.3}$ & $\begin{array}{l}-1.222 \\
-1.219 \\
-120\end{array}$ & & & & $\frac{1.6}{146}$ & & & & & & & & & & & & \\
\hline 80 & $6 / 16 / 20$ & & $\frac{2.1}{261 .}$ & & $\frac{2.4}{256}$ & 27.150 & & 478 & $\mid$\begin{tabular}{|l|}
5218 \\
521
\end{tabular} & & & \begin{tabular}{|l|l|l|} 
\\
-1.22
\end{tabular} & & & & & & & & & & & & & & & & \\
\hline & & & & & & 27065 & & & & & & & & & & & & & & & & & & & & & & \\
\hline 1808 & & & & & $\frac{25.066}{20.066}$ & 27.047 & & & & $\frac{14.92}{11.92}$ & & -1.2 & & & & & & & & & .4 & & & & & & & \\
\hline & & & & & & & & & & & & & & & & & & & & & & & & & & & & \\
\hline 816 & & & & & & & & & & & & & & & & & & & & & & & & & & & & \\
\hline & & & 26. & & & 27.072 & & 47.7 & 5 & & & -1.2 & & & & & & & & & & & & & & & & \\
\hline & $6 / 16 / 2$ & 2:59:41 A & 26.18 & & 25 & 27.09 & & \begin{tabular}{|l|l|}
47.74 \\
\end{tabular} & & 14.96 & 33.2 & -1.22 & & & & 14.6 & & & & & & & & & & & & \\
\hline 813 & 6/116/2 & & 26.198 & & & 27.145 & & 47.8 & 52.1 & 14.952 & 33.3 & -1.219 & & & & $14.6 \mathrm{r}$ & & & & & & & & & & & & \\
\hline 814 & & & 26.1 & 26. & & 27.13 & & & & 14.945[ & 33.4 & -1.219 & & & & 14.6 & & & & & & & & & & & & \\
\hline & & & 26. & & & 27.1 & & 47. & & & & -1.2 & & & & & & & & & & & & & & & & \\
\hline & & & & & & 27.12 & & & & & & & & & & & & & & & & & & & & & & \\
\hline & & & 26.203 & & & 27.125 & & & & & & & & & & & & & & & & & & & & & & \\
\hline 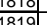 & & & 2 & $\frac{2.224}{26241}$ & 20.099 & 27.09 & & 471.1508 & & 14.979 & $\frac{33.2 \mathrm{c}}{3.20}$ & 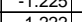 & & 年.1864 & & $\begin{array}{ll}1.662 \\
11.6\end{array}$ & & & & 50.0 & & & & & & & 0.213 & 3 \\
\hline 1820 & $\frac{6 / 16 / 12004}{6 / 16 / 2004}$ & $\begin{array}{l}3: 00: 071 \mathrm{AM} \\
3: 07: 11\end{array}$ & $\frac{26.222}{26.222}$ & \begin{tabular}{|l|}
26.241 \\
26.241 \\
\end{tabular} & 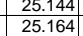 & \begin{tabular}{|l|}
27.154 \\
27.149 \\
\end{tabular} & $\frac{20.204}{26.224}$ & \begin{tabular}{|c|}
477.447 \\
47.418
\end{tabular} & \begin{tabular}{|l|}
51.92 \\
51.977 \\
\end{tabular} & \begin{tabular}{|l|l|}
14.919 \\
\end{tabular} & $\begin{array}{r}32.99 \\
32.914\end{array}$ & \begin{tabular}{|l|l|}
-1.222 \\
-1.222 \\
\end{tabular} & -7.496 & $\begin{array}{r}48.667 \\
51.6699 \\
\end{array}$ & $\mid 0.055$ & \begin{tabular}{|l|}
14.643 \\
\end{tabular} & & |512.97 & 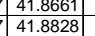 & \begin{tabular}{|l|}
50.5 \\
50.5 \\
\end{tabular} & \begin{tabular}{|l|}
12.7 \\
\end{tabular} & & \begin{tabular}{|l|l|}
2.7699 \\
\end{tabular} & \begin{tabular}{|l|}
0.008 \\
0.008 \\
\end{tabular} & \begin{tabular}{|l|}
0.008 \\
0.008
\end{tabular} & $\frac{0.000}{0.000}$ & \begin{tabular}{|l|l|}
0.207 \\
0.203
\end{tabular} & 0.17 \\
\hline
\end{tabular}


WSRC-TR-2005-00105, REVISION 0

SRNL-RPP-2005-00012, REVISION 0

RUN \# 3.03A AND B; FIRST AND SECOND HALF OF SLURRY DEWATERING - CONT.

\begin{tabular}{|c|c|c|c|c|c|c|c|c|c|c|c|c|c|c|c|c|c|c|c|c|c|c|c|c|c|c|c|c|}
\hline & A & B & $\mathrm{D}$ & $E$ & $\mathrm{r}=\mathrm{H}$ & $a_{0}$ & $n$ & & & 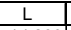 & $\mathrm{M}$ & $\mathrm{N}$ & $\begin{array}{l}0 \\
0\end{array}$ & Q & R & & $\begin{array}{c}T \\
T\end{array}$ & $\begin{aligned} 0 \\
\end{aligned}$ & w & $x$ & r & 2 & AA & $\mathrm{AB}$ & $A C$ & $\mathrm{AD}$ & $A E$ & \\
\hline 821 & 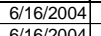 & \begin{tabular}{ll|}
$3: 08: 41 \mathrm{AM}$ \\
$3: 09: 41$
\end{tabular} & $\begin{array}{l}26.232 \\
26228\end{array}$ & $\frac{26.25}{2.21}$ & 25.183 & $\begin{array}{l}27.169 \\
27101\end{array}$ & 26.234 & & \begin{tabular}{|l|l|}
51.979 \\
57.760
\end{tabular} & \begin{tabular}{ll|l|}
14.8099 \\
\end{tabular} & 33.16 & $\frac{-1.222}{1220}$ & $\begin{array}{l}-7.499 \\
7.92\end{array}$ & 47.739 & 0.055 & 14.643 & 0.004 & $\begin{array}{l}2513.97 \\
251.97\end{array}$ & $\begin{array}{lll}41.8994 \\
\end{array}$ & 50.5 & 11.7 & 40.3 & 2.782 & 0.008 & 0.008 & 0.000 & 0.202 & \\
\hline $\begin{array}{ll}\frac{024}{823} \\
x^{2}\end{array}$ & $6 / 6 / 16 / 2004$ & $\begin{array}{l}3: 09: 41 \mathrm{AM} \\
3310411 \mathrm{~A}\end{array}$ & $\frac{26.228}{262313}$ & $\frac{26.24}{26.22}$ & $\frac{25.179}{25.164}$ & & & $\frac{47.367}{4734}$ & \begin{tabular}{|l|l|}
51.769 \\
5
\end{tabular} & & & & $\begin{array}{l}-7.493 \\
-7.735 \\
\end{array}$ & 5.612 & & $\frac{14.643}{14643}$ & & & \begin{tabular}{ll|}
41.9161 \\
41.928
\end{tabular} & 50.3 & & & & & & & & \\
\hline 1824 & $6 / 16 / 2004$ & 3:11:41 AM & 26.22 & $\frac{2 . .24}{26.21}$ & $\frac{2.104}{25.152}$ & $\frac{27.12}{27.072}$ & $\frac{20.253}{26.252}$ & 47.391 & 51.763 & $\begin{array}{l}14.80 \\
14.797\end{array}$ & $\frac{32.821}{33.041}$ & $\begin{array}{l}-1.219 \\
-1.222\end{array}$ & $\begin{array}{l}-7.355 \\
-7.395\end{array}$ & $\begin{array}{l}53.553 \\
48.132\end{array}$ & $\begin{array}{l}0.056 \\
0.055\end{array}$ & $\frac{14.643}{14.643}$ & 0.004 & 2515.97 & 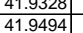 & $\begin{array}{l}50.4 \\
50.3\end{array}$ & $\frac{13.1}{11.8}$ & $\frac{40.1}{40.2}$ & 2.2 .763 & . & $\begin{array}{l}0.008 \\
0.008\end{array}$ & $\begin{array}{l}0.000 \\
0.000\end{array}$ & & \\
\hline 1825 & $6 / 16 / 2004$ & $3: 12: 41 \mathrm{AM}$ & 26.224 & 26.21 & 25.15 & 27.111 & 26.256 & 47.729 & 52.165 & 14.963 & 33.187 & \begin{tabular}{|r|}
-1.219 \\
\end{tabular} & -7.415 & 57.443 & 0.056 & 14.643 & & 2517.97 & 41.96451 & 50.7 & $\begin{array}{ll}\frac{1.6}{14.1} \\
\end{array}$ & 40.5 & & 0.008 & $\begin{array}{l}0.008 \\
\end{array}$ & & & \\
\hline 1826 & $6 / 16 / 2004$ & 3:13:41 AM & & & 25.149 & 27.104 & 26.234 & \begin{tabular}{|l|l|}
47.628 \\
\end{tabular} & 51.923 & 14.747 & 33.334 & \begin{tabular}{|c|}
-1.219 \\
\end{tabular} & $\begin{array}{r}-7.43 \\
\end{array}$ & 41.772 & 0.058 & 14.643 & & & 41.9828 & 50.5 & & & & & & & & \\
\hline 1827 & & 3:14:41 AM & 26.22 & 26.22. & 25.136 & 27.097 & & $\begin{array}{l}47.603 \\
7.750\end{array}$ & 51.86 & & & $\begin{array}{l}-1.219 \\
\end{array}$ & $\begin{array}{l}-7.436 \\
\end{array}$ & & 0.056 & 14.643 & & & 41.9994 & 50.4 & 12.3 & 40.5 & & & & & & \\
\hline 1828 & 6/16/2004 & $3: 15: 41 \mathrm{AM}$ & 26.231 & 26.23 & 25.147 & 27.108 & 26.203 & 47.759 & 52.049 & 14.754 & 33.505 & \begin{tabular}{|l|} 
\\
\end{tabular} & -7.436 & \begin{tabular}{|l|}
46.261 \\
\end{tabular} & 0.055 & 14.643 & 0.00 & 2520.97 & 42.0161 & 50.6 & 11.3 & 40.6 & 2.801 & 0.008 & 0.008 & 0.000 & 0.201 & \\
\hline 1829 & $6 / 16 / 2004$ & $\begin{array}{l}3: 16: 41 \mathrm{AM} \\
3: 17: 41 \mathrm{~A}\end{array}$ & $\begin{array}{l}26.231 \\
26232\end{array}$ & $\begin{array}{l}26.23 \\
26.23\end{array}$ & $\begin{array}{l}25.142 \\
25.134\end{array}$ & \begin{tabular}{|l|}
27.133 \\
27.144
\end{tabular} & & \begin{tabular}{ll|l}
47.493 \\
4759
\end{tabular} & \begin{tabular}{|l|l|}
52.026 \\
51807
\end{tabular} & $\begin{array}{l}14.744 \\
1.725\end{array}$ & $\begin{array}{l}33.166 \\
32.113\end{array}$ & \begin{tabular}{|l|l|}
-1.219 \\
\end{tabular} & $\begin{array}{r}-7.441 \\
7036 \\
\end{array}$ & \begin{tabular}{|l|l|}
47.994 \\
4.320
\end{tabular} & 0.056 & \begin{tabular}{|l|l|}
14.643 \\
1.643
\end{tabular} & & \begin{tabular}{|l|}
2521.97 \\
25207
\end{tabular} & & 50.6 & $\frac{11.8}{0.9}$ & & & & 0.008 & & & \\
\hline$\frac{1830}{183}$ & 6/16/2004 & $\begin{array}{l}\text { 7:41 AM } \\
8.41 \mathrm{AM}\end{array}$ & $\frac{26.232}{26.227}$ & $\frac{26.23}{26.22}$ & $\frac{25.134}{25.124}$ & \begin{tabular}{|l|r|}
27.144 \\
27.164
\end{tabular} & $\frac{26.234}{26.24}$ & $\begin{array}{ll}47.458 \\
47.983\end{array}$ & \begin{tabular}{|r|r|}
51.887 \\
52.36
\end{tabular} & $\begin{array}{l}\frac{14.735}{14.855} \\
\end{array}$ & $\begin{array}{r}33.143 \\
33.597 \\
\end{array}$ & \begin{tabular}{|r|}
-1.219 \\
-1.219 \\
\end{tabular} & $\begin{array}{l}-7.436 \\
-7.447\end{array}$ & \begin{tabular}{|l|}
40.329 \\
46.332
\end{tabular} & $\begin{array}{l}0.056 \\
0.056\end{array}$ & \begin{tabular}{l|l|}
14.643 \\
14.643
\end{tabular} & 0.004 & \begin{tabular}{|l|l|}
2522.97 \\
2523.97
\end{tabular} & \begin{tabular}{|l}
42.0494 \\
420061
\end{tabular} & $\begin{array}{l}50.4 \\
50.9\end{array}$ & $\frac{9.9}{11.4}$ & 40.5 & 2.779 & |.008 & $\begin{array}{l}0.008 \\
0.008\end{array}$ & $\begin{array}{l}0.000 \\
0.000\end{array}$ & $\begin{array}{l}0.206 \\
0.0204\end{array}$ & 0.18 \\
\hline 1832 & & 3:19:41 AM & 26.229 & $\frac{2.24}{26.22}$ & 25.126 & & & & & & & & & 4 & 0.045 & $\begin{array}{l}\frac{14.643}{14.643} \\
\end{array}$ & & $\frac{2532.97}{2524.97}$ & | $\begin{array}{l}42.061 \\
42.0828\end{array}$ & $\begin{array}{l}50.9 \\
50.7 \\
\end{array}$ & $\begin{array}{ll}11.4 \\
11.9\end{array}$ & $\begin{array}{l}40.7 \\
40.7\end{array}$ & $\frac{2.814}{2.804}$ & 0.007 & $\begin{array}{l}0.007 \\
0.007\end{array}$ & & & $\frac{0.17}{0.14}$ \\
\hline & $6 / 16 / 2004$ & 3:20:41 AM & 26.575 & 26.22 & 25.127 & 27.162 & 26.237 & 47.298 & 51.96 & 14.898 & 32.809 & -1.014 & & 56.34 & 0.044 & 14.643 & 0.004 & 2525.97 & 42.0994 & 50.5 & 13.8 & 40.1 & 2.762 & 0.007 & \begin{tabular}{l|l}
0.007 \\
\end{tabular} & 0.000 & 0.163 & \\
\hline & $6 / 16 / 2004$ & 3:21:41 AM & 26.441 & & & 27.129 & 26.249 & & 51.69 & & & -0.886 & -6.45 & & & \begin{tabular}{|l|l|}
14.643 \\
\end{tabular} & & 2526.97 & 42.1161 & 50.2 & 11.7 & & 2.743 & 0.009 & 0.009 & 0.000 & & \\
\hline 835 & $6 / 16 / 2004$ & $3: 22: 41$ AM & 26.324 & 26.23 & 25.131 & 27.131 & 26.261 & 47.425 & 52.146 & 14.914 & & -0.918 & -6.516 & 48.173 & & 14.643 & & 2527.97 & 42.1328 & 50.7 & 11.8 & & & 0.008 & 0.008 & 0.000 & & \\
\hline (1) & $6 / 16 / 2004$ & $\begin{array}{l}3: 23: 41 \mathrm{AM} \\
3.24: 41 \mathrm{~A}\end{array}$ & $\begin{array}{l}26.25 \\
2621\end{array}$ & $\frac{26.21}{26.919}$ & $\begin{array}{l}25.126 \\
251116\end{array}$ & $\begin{array}{l}27.132 \\
27.132\end{array}$ & $\begin{array}{l}26.247 \\
26227\end{array}$ & \begin{tabular}{ll|l}
47.982 & 47.292 \\
4
\end{tabular} & \begin{tabular}{|l|l|}
52.292 \\
51.726
\end{tabular} & $\begin{array}{l}14.884 \\
14799\end{array}$ & $\begin{array}{l}33.566 \\
32.969\end{array}$ & $\begin{array}{l}-1.222 \\
-1.219 \\
\end{array}$ & $\begin{array}{r}-7.135 \\
-7.19\end{array}$ & \begin{tabular}{|l|}
46.038 \\
52124 \\
\end{tabular} & $\begin{array}{l}0.057 \\
0.058\end{array}$ & $\begin{array}{l}14.643 \\
14663\end{array}$ & & \begin{tabular}{|l|l|}
8.97 \\
9.97
\end{tabular} & $\frac{1494}{1664}$ & 50.8 & $\frac{11.3}{128}$ & 40.5 & 2.811 & D.008 & $\begin{array}{l}0.008 \\
0.009\end{array}$ & 0.000 & & \\
\hline$\frac{1031}{1838}$ & $6 / 6 / 1 / 1 / 2004$ & $\begin{array}{l}3: 24: 41 \mathrm{AM} \\
3.25: 41 \mathrm{~A}\end{array}$ & $\begin{array}{r}26.121 \\
26.197\end{array}$ & $\frac{26.19}{26.21}$ & $\frac{25.116}{25.124}$ & $\frac{27.1324}{27154}$ & $\frac{26.221}{26.229}$ & $\frac{47.292}{47.472}$ & \begin{tabular}{|c|}
51.126 \\
51.892
\end{tabular} & $\frac{14.99}{14827}$ & $\frac{32.969}{33.098}$ & \begin{tabular}{|l|l|}
-1.219 \\
-1.219
\end{tabular} & $\begin{array}{r}-7.19 \\
-7.305\end{array}$ & $\frac{52.124}{45.297}$ & $\frac{0.058}{0.056}$ & $\frac{14.643}{14.643}$ & & 30.97 & 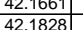 & $\begin{array}{l}\frac{50.3}{50.4} \\
\end{array}$ & $\frac{11.8}{11.1}$ & & & .009 & \begin{tabular}{|l}
0.009 \\
0.008
\end{tabular} & & & \\
\hline 1839 & $6 / 16 / 2004$ & 3 & 26.179 & $\frac{20.21}{26.21}$ & $\frac{2.124}{25.12}$ & $\frac{21.144}{27.121}$ & $\frac{2.253}{26.251}$ & 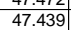 & $\frac{5.1 .034}{51.74}$ & 14.92 & 32.983 & \begin{tabular}{|l|}
-1.219 \\
\end{tabular} & $\begin{array}{l}-7.000 \\
-7.242\end{array}$ & 45.362 & 0 & $\frac{14.045}{14.643}$ & & 2531.97 & $\frac{42.1020}{42.1994}$ & 50.3 & $\frac{1.1}{11.1}$ & $\frac{40}{40}$ & $\frac{2.178}{2.772}$ & 年.008 & \begin{tabular}{|c|c|}
0.008 \\
0.008
\end{tabular} & & 0.207 & $\frac{0.18}{0.18}$ \\
\hline 1840 & $6 / 16 / 2004$ & 3:27:41 AM & 26.16 & 26.19 & 25.111 & 27.112 & & 47.441 & 51.765 & $\begin{array}{l}14.82 \\
\end{array}$ & 33.086 & \begin{tabular}{|l|}
1.222 \\
\end{tabular} & -7.262 & 40.99 & 0.055 & 14.643 & & 2532.97 & 42.2161 & 50.3 & 10.0 & 40. & 2.776 & 0.008 & 0.008 & & & \\
\hline & 6/16/2004 & 3:28:41 AM & & 26.19 & & & & \begin{tabular}{|l|l}
47.798 \\
\end{tabular} & 52.161 & 15.01 & 33.244 & \begin{tabular}{|l|}
-1.216 \\
\end{tabular} & & 50.904 & 0.056 & 14.643 & & 2533.97 & & 50.7 & 12.5 & $40 !$ & 2.794 & \begin{tabular}{|c|}
0.008 \\
\end{tabular} & & & & \\
\hline 1842 & $6 / 16 / 2004$ & $3: 29: 41 \mathrm{AM}$ & 26.151 & 26.1 & 25.113 & 27.118 & 26.228 & 47.744 & 52.126 & 14.835 & 33.408 & \begin{tabular}{|l|}
-1.222 \\
\end{tabular} & -7.213 & \begin{tabular}{|l|l|}
49.392 \\
\end{tabular} & 0.056 & 14.643 & & 2534.97 & 2494 & 50.7 & 12.1 & & 2.798 & 008 & .008 & & & \\
\hline & & 3:30:41 AM & & & & & & & & 14.816 & & & & & & & & & & & & & & & & & & \\
\hline 844 & $6 / 16 / 2004$ & 3:31:41 AM & 26.14 & 26.17 & 25.117 & 26.998 & 26.218 & 47.897 & 52.408 & \begin{tabular}{|l|l|l|l|}
14.96 \\
\end{tabular} & 33.338 & -1.222 & -7.236 & 44.304 & 0.057 & 14.643 & & 536.97 & & & 10.9 & & 800 & .008 & $\begin{array}{l}.008 \\
.008\end{array}$ & & & \\
\hline 345 & 6/16/2004 & & 26.145 & 26.19 & 25.121 & 26.992 & & 47.705 & 52.142 & 14.886 & 33.223 & \begin{tabular}{|l|l|} 
& -1.219 \\
\end{tabular} & & 52.804 & 0.049 & 14.643 & & & & 50.7 & 12.9 & & & & & & & \\
\hline & $6 / 16 / 2$ & $41 \mathrm{AM}$ & 26.143 & 26.17 & & 26.955 & & 47.727 & 52.153 & |14.873 & 33.31 & \begin{tabular}{|l|l|} 
\\
\end{tabular} & -7.447 & 52.297 & & 14.643 & & 2538.97 & & 50.7 & 12.8 & & & $\mid$ & .008 & & & \\
\hline 847 & $6 / 16 / 2004$ & $1 \mathrm{AM}$ & 26.158 & 26.18 & 25.109 & 26.995 & & 47.968 & 52.254 & 14.959 & 33.49 & -1.225 & 456 & 48.686 & 0.056 & 14.643 & & 2539.97 & 1.3328 & 50.8 & 11.9 & 40. & 808 & .008 & .008 & 5.000 & D.204 & \\
\hline 年 & $6 / 16 / 2004$ & $1 \mathrm{AM}$ & 26.163 & 26.18 & 25.12 & 27.005 & & 47.7611 & 52.113 & 931 & .289 & -1.225 & & & 0.056 & 14.643 & & 5540.97 & & & 11.6 & & & & & & & \\
\hline & $6 / 16 / 2$ & & 26.152 & & & 26.984 & & & & 712 & 33.634 & -1.219 & & 47.908 & & 14.643 & & & & & 11.7 & & & & & & & \\
\hline 85 & $\frac{61512004}{61 / 2004}$ & & 26.173 & $\frac{26.19}{26.19}$ & $\frac{25.15}{25.15}$ & 27.005 & $\frac{26.13}{26.12}$ & $\begin{array}{l}47.983 \\
47736\end{array}$ & 52.431 & 15.034 & $\begin{array}{l}33.301 \\
33.324\end{array}$ & -1.222 & & 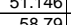 & 0 & 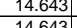 & & 54397 & & & 144 & & & & & & & \\
\hline & $6 / 1612004$ & & $\mid$ & & & | & & & $\mid$ & & 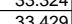 & & & 4818 & & $\frac{14.643}{14643}$ & & 5444.97 & & 50 & & & & & & & & \\
\hline 1853 & $6 / 16 / 2004$ & & $\frac{2.104}{26.18}$ & $\frac{2.25}{26.18}$ & $\frac{25}{251}$ & 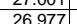 & & 年 & 52.391 & 势.014 & $\frac{3.34 .452}{33402}$ & \begin{tabular}{|l|l|}
-1.219 \\
\end{tabular} & & & & $\frac{14.045}{14643}$ & & & & 505 & $\frac{1.6}{131}$ & $\frac{40}{40}$ & & & & & & \\
\hline 1854 & $6 / 16 / 2004$ & & 26.169 & 26.17 & 25.155 & 26.966 & & 47.75 & 52.234 & 14.976 & 33.174 & \begin{tabular}{|l|}
-1.219 \\
\end{tabular} & 459 & 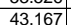 & $\frac{0.057}{0.057}$ & 14.643 & & $\begin{array}{l}546.97 \\
246.97\end{array}$ & 42.4494 & $\frac{50.8}{50.8}$ & $\frac{1.1}{10.6}$ & & 790 & | & 008 & .000 & . & \\
\hline & & & & & & & & & & & & & & & & & & & & & 12.2 & & & & & & & \\
\hline 856 & 6/16/ & & 26.185 & & & 26.972 & & & \begin{tabular}{|c|}
52.464 \\
\end{tabular} & $\frac{062}{062}$ & 33.406 & -1.2 & & 42.988 & & $\frac{14.643}{14.643}$ & & & & $\overline{51}$ & $\frac{2.5}{10.5}$ & & & & & & & \\
\hline & & & 26.178 & 26.16 & 25.21 & 26.995 & & & 52.234 & $\begin{array}{l}14.93 \\
\end{array}$ & 33.34 & -1.2. & & & & 14.643 & & & & & & & & & & & & \\
\hline & $6 / 16 / 2004$ & & 26.184 & 26.16 & 25.195 & 26.966 & & 47.537 & 51.894 & 14.862 & 33.15 & -1.222 & & 52.476 & 0.057 & 14.643 & & & & & 12.9 & & 789 & & & & & \\
\hline & 6/16/2004 & & 26.2 & 26.18 & 5.2.207 & 26.983 & & & \begin{tabular}{|l|l|l|l|} 
\\
\end{tabular} & 14.919 & 33.0 & & & 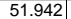 & & 14.643 & & & & 50.4 & 12.7 & & & & & & & \\
\hline 1860 & 6/16/2004 & & 26.197| & & 25.193 & 26.984] & & & 52.285 & & & & & & & 14.643 & & & & & 11.9 & & & & & & & \\
\hline 861 & $6 / 16 / 2004$ & & 26.193 & 26.15 & 25.18 & 26.951 & & 47..775 & 52.29 & 14.954 & 33.234 & \begin{tabular}{|l|l|} 
\\
\end{tabular} & & 44.175 & 0.056 & 14.643 & & 2553 & & 50. & 10.8 & 40 & & 008 & . & 0.000 & 0.205 & \\
\hline 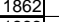 & & & 26.202 & & & & & & & & & & & & & & & & & & 12.4 & & & & & & & \\
\hline$\frac{863}{861}$ & & & 26.203 & & & & & & 5.1.639 & 158 & $\frac{32.9}{32}$ & & & & & & & & & & & & & & & & & \\
\hline & & & 26.2.24 & 2 & 25.1060 & 年. & & & & & & & & & & & & & & & 116 & & & & & & & \\
\hline 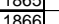 & $6 / 16 / 2$ & & $\frac{2.0 .244}{2619}$ & & $\frac{25.10}{25141}$ & $\mid$ & & & & & & & & & & & & & & & & & & & & & & \\
\hline 1867 & $\frac{6}{661 / 1 / 2004}$ & & & & & (2) & & & & & & & & & & & & & & & 5 & & & & & & & \\
\hline 1868 & $6 / 6 / 1 / 2004$ & & 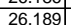 & $\frac{2.51}{26.11}$ & $\frac{25.131}{25.131}$ & $\frac{2.6 .971}{26.91}$ & & 47.423 & 51.834 & $\frac{14.071}{14.871}$ & $\frac{25.981}{32.981}$ & & & & & $\begin{array}{l}14.64 \\
14.64\end{array}$ & & & & & $\frac{12.9}{12.9}$ & & & & & & 0192 & \\
\hline & & & & & & & & & & & & & & & & & & & & & & & & & & & & \\
\hline & & & 26.1 & & & & & & 294 & & & & & & & & & & & & & & & & & & & \\
\hline 871 & $6 / 16 / 2004$ & & 26.202 & & 25.139 & 26.954 & & & \begin{tabular}{|l|l|}
51.97 \\
\end{tabular} & 14. & & & & & & & & & & & & & & & & & & \\
\hline & & & \begin{tabular}{|l|}
26.2 \\
\end{tabular} & 26.11 & 251 & 26.942 & & 47.4 & .014 & 14.79 & 33.1 & -1.2 & & & & 14.6 & & & & 80 & & & & & & & & \\
\hline & $6 / 16 / 2$ & & 26 & & & 26.932 & & 47.5 & 898 & 14.917 & & -1.2. & & & & 14.6 & & & & 50. & 2 & & & & & & & \\
\hline 1874 & $6 / 16 / 2$ & & 26.19 & & & 26.941 & & & 52.155 & & & & & & & $14.6 \mathrm{C}$ & & & & & 4 & & & & & & & \\
\hline 1875 & & & 26.196 & & 25.117 & 26.928 & & & 52.018 & 14.934 & & & & & & $14.6 \mathrm{r}$ & & & & & 0 & & & & & & & \\
\hline $18 / 6$ & & & & & & & & & .45 & & & & & & & & & & & & & & & & & & & \\
\hline$\frac{871}{870}$ & & & & & 25.1 & & & & & & & & & & & & & & & 50. & 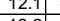 & & & & & & & \\
\hline 1870 & & & 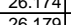 & & 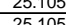 & & & & & & 年 & & & & & & & & & & & & & & & & & \\
\hline & $\frac{0110120}{6 / 120}$ & & $\frac{2.0179}{26184}$ & & $\frac{2.010}{2511}$ & $\frac{2.040}{2696}$ & & & & & 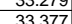 & -1.2 & & & & & & & & & & & & & & & & \\
\hline 881$]$ & & & & & & 26.95 & & & & & & & & & & & & & & & & & & & & & & \\
\hline (1882 & & & & & & $\frac{2.69}{26.9}$ & & & & & & $\frac{-1.2}{-1.2}$ & & & & & & & & & & & & & & & 4 & \\
\hline & & & & & & & & & & & & & & & & & & & & & & & & & & & & \\
\hline & & & & & & & & & & & & & & & & & & & & & & & & & & & & \\
\hline 885 & $6 / 16 / 2004$ & & 26.18 & & 251 & 26.92 & & 47.7 & 3 & & & -1.2 & & & & & & & & & & & & & & & & \\
\hline 886 & $6 / 16 / 2$ & $4: 12: 45 \mathrm{~A}$ & 26.18 & & & & & 48.1 & & & 8.646 & -1.2 & & & & $\overline{146}$ & & & & & & & & & & & & \\
\hline 887 & 6/16/2004 & & $6.17 \mathrm{r}$ & 26.04 & 5.1 & 26.935 & & 47.8 & 52.30 & & 33.41 & & & & & & & & & & & & & & & & & \\
\hline 1888 & $6 / 16 / 2004$ & & 26.158 & 26.0 & 25.09 & 26.905] & & 47.9 & & $14.889]$ & 33.484 & & & & & & & & & 51. & & & & & & & 175 & \\
\hline 1889 & & 4:15:45 AM & 26.176] & 26.03 & 25.117 & 26.933 & & 47.63 & 52.261 & 14.887 & 33.131 & & & & & 14.6 & & & & 50.8 & 12.6 & & 2.78 & & 0.008 & & & \\
\hline 1890| & 6/16/2004 & 4:16:45 AM & 26.166 & 26.0 & 25.108 & $\mid 26.914$ & & 47.893 & 52.366 & 14.894 & 33.498 & \begin{tabular}{|c|} 
\\
\end{tabular} & & 44.075 & 0.054 & \begin{tabular}{|l}
14.643 \\
\end{tabular} & & & 43.0339 & 50.9 & & & 2.806 & $\mid$ & $\begin{array}{l}0.008 \\
\end{array}$ & \begin{tabular}{|l|l|}
$0.000 \mid$ \\
\end{tabular} & 0.197 & \\
\hline
\end{tabular}


WSRC-TR-2005-00105, REVISION 0

SRNL-RPP-2005-00012, REVISION 0

RUN \# 3.03A AND B; FIRST AND SECOND HALF OF SLURRY DEWATERING - CONT.

\begin{tabular}{|c|c|c|c|c|c|c|c|c|c|c|c|c|c|c|c|c|c|c|c|c|c|c|c|c|c|c|c|c|}
\hline & A & & \begin{tabular}{l|l} 
D \\
\end{tabular} & $\mathrm{E}$ & $F$ & \begin{tabular}{l|l|l} 
G & \\
\end{tabular} & $\begin{array}{ll}\mathrm{H} \\
\end{array}$ & $\mathrm{J}$ & $\begin{array}{ll}\mathrm{K} \\
\end{array}$ & \begin{tabular}{|l|} 
\\
\end{tabular} & $\mathrm{M}$ & $\begin{array}{ll}\mathrm{N} \\
\end{array}$ & 0 & \begin{tabular}{l|l} 
Q \\
\end{tabular} & \begin{tabular}{l|l}
$R$ \\
\end{tabular} & \begin{tabular}{l|l} 
s \\
\end{tabular} & $\begin{array}{lll}T \\
\end{array}$ & $\mathrm{v}$ & w & \begin{tabular}{|c|}
$x \mid$ \\
\end{tabular} & \begin{tabular}{l|l} 
&
\end{tabular} & z & & AB & & & $\overline{\mathrm{AE}}$ & $\overline{\mathrm{AF}}$ \\
\hline 891 & $6 / 16 / 2004$ & 4:17:45 AM & 26.162 & 26.026 & 25.109 & 26.909 & 25.97 & 48.07 & 52.632 & \begin{tabular}{|l|l|}
14.898 \\
\end{tabular} & 33.616 & -1.199 & -7.337 & 50.222 & 0.055 & 14.643 & 0.004 & 2583.03 & \begin{tabular}{|l|}
43.0506 \\
\end{tabular} & 51.2 & 12.3 & 40.8 & 2.816 & 0.008 & 0.008 & 0.000 & 0.200 & \\
\hline & $6 / 16 / 2004$ & $4: 18: 45 \mathrm{AM}$ & 26.162 & 26.026 & 25.114 & 26.894 & & 47.705 & 5 & \begin{tabular}{|l|l|}
14.854 \\
\end{tabular} & 33.265 & \begin{tabular}{|l|l|}
-1.173 \\
\end{tabular} & $\begin{array}{l}-7.346 \\
\end{array}$ & 46.72 & & \begin{tabular}{|l}
14.643 \\
\end{tabular} & & & 43.0672 & 50.8 & 11.4 & & & & 0.008 & & & \\
\hline 1893] & 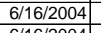 & 4:19:45 AM & \begin{tabular}{|l|l|}
26.157 \\
6.159
\end{tabular} & \begin{tabular}{|l|l|}
26.011 \\
6017
\end{tabular} & 25.099 & & 25.955 & $\begin{array}{l}47.599 \\
7.925\end{array}$ & \begin{tabular}{|l|}
52.321 \\
5.211 \\
\end{tabular} & \begin{tabular}{|l|l|}
14.9344 \\
\end{tabular} & \begin{tabular}{|l|l|}
33.047 \\
3208
\end{tabular} & \begin{tabular}{|l|l|}
-1.161 \\
\end{tabular} & $\begin{array}{r}-7.193 \\
7103\end{array}$ & $\begin{array}{l}46.04 \\
\end{array}$ & 0.05 & \begin{tabular}{|l|}
14.643 \\
\end{tabular} & & 2585.03 & \begin{tabular}{|l|l|}
43.0839 \\
\end{tabular} & 50.8 & 11.3 & 40.3 & 170 & 2.007 & 0.007 & 0.000 & 0.184 & \\
\hline 1894 & 6/16/2004 & 4:20:45 AM & 26.158 & 26.017 & 25.15 & 26.88 & 25.956 & 47.425 & \begin{tabular}{|l|}
52.113 \\
\end{tabular} & 14.845 & \begin{tabular}{|l|l|}
32.998 \\
\end{tabular} & \begin{tabular}{|l|l|}
-1.156 \\
\end{tabular} & $\begin{array}{l}-7.193 \\
\end{array}$ & $\begin{array}{r}47.7 \\
\end{array}$ & 0.056 & 14.643 & 0.004 & 2586.03 & \begin{tabular}{|l|}
43.1006 \\
\end{tabular} & 50.6 & 11.7 & $\begin{array}{l}40.2 \\
\end{array}$ & 2.772 & 0.008 & 0.008 & 0.000 & 0.207 & \\
\hline 1895| & $\frac{6 / 16 / 2004}{6 / 16 / 2004}$ & $\begin{array}{l}\frac{421: 45 \mathrm{AM}}{4 \cdot 22.45 \mathrm{AM}} \\
\end{array}$ & $\frac{26.164}{26.161}$ & 26.022 & & 26.876 & & $\begin{array}{l}48.254 \\
48020\end{array}$ & \begin{tabular}{|l|}
52.916 \\
52598 \\
\end{tabular} & \begin{tabular}{|l|}
14.516 \\
1456 \\
\end{tabular} & \begin{tabular}{|l|l|}
34.157 \\
33025
\end{tabular} & \begin{tabular}{|l|l|}
-1.156 \\
\end{tabular} & & $\begin{array}{l}51.479 \\
5.777\end{array}$ & $\begin{array}{l}0.054 \\
0.05\end{array}$ & $\begin{array}{l}14.643 \\
14643\end{array}$ & & & |li.1172 & \begin{tabular}{|l|}
51.4 \\
5.1
\end{tabular} & 12.6 & & \begin{tabular}{|l|}
2.841 \\
2955
\end{tabular} & & \begin{tabular}{|c|}
0.008 \\
\end{tabular} & & 0.195 & \\
\hline 1897 & $6 / 6 / 16 / 2004$ & $\begin{array}{l}4: 22: 45 \text { AM } \\
4: 23: 45 \text { A }\end{array}$ & $26.161 \mid$ & $\frac{26.005}{26.001}$ & $\frac{25.118}{25.109}$ & $\begin{array}{r}26.799 \\
26.85\end{array}$ & $\begin{array}{r}25.964 \\
25.91 \\
\end{array}$ & $\begin{array}{l}48.022 \\
48.385\end{array}$ & \begin{tabular}{|l|}
52.598. \\
52.6
\end{tabular} & \begin{tabular}{r|r}
14.56 \\
14.525
\end{tabular} & \begin{tabular}{|l}
33.925 \\
34.268
\end{tabular} & \begin{tabular}{|l|l|}
-1.156 \\
-1.222 \\
\end{tabular} & $\begin{array}{r}-7.32 \\
-6.635 \\
\end{array}$ & $\frac{50.777}{45.272}$ & $\begin{array}{l}0.058 \\
0.057\end{array}$ & $\begin{array}{l}14.643 \\
1.4643\end{array}$ & $\frac{0.003}{0.004}$ & \begin{tabular}{|l|}
2558.03 \\
2589.03
\end{tabular} & \begin{tabular}{|c|}
43.1339 \\
43.1506
\end{tabular} & 51.1 & $\frac{12.4}{11.1}$ & $\frac{41.0}{41.3}$ & $2.825 \mid$ & .0.009 & | 0.009 & 0.000 & \begin{tabular}{l|l|l|l|l|l}
0.210 & \\
0.205
\end{tabular} & 0.18 \\
\hline 1898 & $6 / 16 / 2004$ & & 26.173 & 26.007 & 25.115 & & 25.901 & & & 14.539 & & & -4.698 & 50.405 & $\begin{array}{l}0.051 \\
0.049\end{array}$ & $\mid \begin{array}{l}14.6434 \\
14.643\end{array}$ & 0.004 & 250990.03 & 年5.1.1000 & $\begin{array}{l}51.1 \\
51.3 \\
\end{array}$ & $\begin{array}{ll}\frac{11.1}{12.4} \\
\end{array}$ & \begin{tabular}{|l|}
40.3 \\
40.3
\end{tabular} & $\begin{array}{l}2.849 \\
2.778\end{array}$ & \begin{tabular}{|c|} 
\\
0.0008 \\
\end{tabular} & $\begin{array}{l}0.007 \\
0.007\end{array}$ & & & $\frac{0.17}{0.15}$ \\
\hline 1899 & 6/16/2004 & 25:45 AM & 26.149 & 25.993 & 25.071 & 26.872 & 25.897 & 46.983 & & 14.462 & & \begin{tabular}{|l|}
-0.545 \\
\end{tabular} & -5.305 & 46.663 & 0.052 & 14.643 & 0.004 & 2591.03 & 43.1839 & & & & & & 0.008 & & & \\
\hline 1900 & $6 / 16 / 2004$ & 26:45 AM & 26.146 & 25.98 & & & 25.889 & $\begin{array}{l}47.962 \\
\end{array}$ & \begin{tabular}{|l|} 
\\
\end{tabular} & & \begin{tabular}{|l|}
33.78 \\
\end{tabular} & \begin{tabular}{|l|}
-1.014 \\
\end{tabular} & & \begin{tabular}{|l|l|}
46.183 \\
\end{tabular} & & $\begin{array}{l}14.643 \\
\end{array}$ & & 2592.03 & 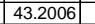 & 51.3 & 11.3 & 40.9 & & & 0.009 & & & \\
\hline 1901 & & & & & 25.07 & 26.87 & & & & 14.631 & & & & & \begin{tabular}{|l|l|}
0.06 \\
\end{tabular} & & & & & 51.4 & & & .831 & 009 & & D.000 & & \\
\hline & & :45 AM & 26.15 & & & 26.822 & & 47.924 & 52.211 & 14.867 & 502 & -1.21. & & 48.319 & & 14.643 & & & & 507 & $\frac{11.8}{11.8}$ & - & & & 0.011 & & 281 & \\
\hline & & $: 45 \mathrm{AM}$ & 26.141 & 25.974 & 25.082 & 26.808 & & 47.466 & 51.802 & 15124 & 758 & & & & & 14.643 & & & & $50-3$ & 117 & & & & & & & \\
\hline & 6/16/2004 & $45 \mathrm{AM}$ & 26.145 & 25.989 & 25.107 & 26.857 & 25.95 & 47.622 & 51.744 & 15.154 & 32.973 & \begin{tabular}{|l|l|} 
& -1.216 \\
\end{tabular} & & 53.766 & \begin{tabular}{|l|l|l|}
0.053 \\
\end{tabular} & 14.643 & & & 43.2672 & 50.3 & 13.2 & & 2.778 & . & \begin{tabular}{|c|}
0.008 \\
\end{tabular} & & & \\
\hline & $6 / 16 / 2004$ & $1: 45 \mathrm{AM}$ & 26.136 & 25.97 & 25.083 & 26.793 & 25.984 & 47.669 & 51.91 & 15.092 & 33.012 & $-1.225 \mid$ & -7.225 & \begin{tabular}{|l|l|}
47.614 \\
\end{tabular} & 0.053 & 14.643 & & 2597.03 & 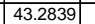 & 50.4 & 11.7 & 40.3 & & 0.008 & \begin{tabular}{|l|l|}
0.008 \\
\end{tabular} & & & \\
\hline 906 & & $32: 45 \mathrm{AM}$ & 26.132 & \begin{tabular}{|l|l|}
25.97 \\
25.97
\end{tabular} & 25.083 & 26.749 & 26.004 & 477.902 & 52.261 & $\begin{array}{l}15.17 \\
1507\end{array}$ & \begin{tabular}{|l|l|l|}
33.117 \\
220707
\end{tabular} & \begin{tabular}{|l|l|} 
& -1.219 \\
\end{tabular} & & \begin{tabular}{|l|l|l|l|}
51.13 \\
\end{tabular} & & 14.643 & & & & & & & & & & & & \\
\hline & 6/16/2004 & 4:33:45 AM & & 25.966 & & 26.72 & 25.95 & 47.887 & & & 33.297 & $\begin{array}{l}-1.222 \\
\end{array}$ & & \begin{tabular}{|l|l|}
49.79 \\
\end{tabular} & 0.054 & & & 2599.03 & 43.3172 & & & & 2.799 & 0.008 & $\begin{array}{ll}0.008 \\
\end{array}$ & & & \\
\hline & 6/16/2004 & 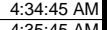 & 26.125 & & $\begin{array}{l}25.082 \\
2.02\end{array}$ & 26.747 & & 47.68 & \begin{tabular}{|l|}
52.12 \\
\end{tabular} & 15.056 & 051 & -1.219 & & 55.194 & 0.055 & 14.643 & & & 43.3339| & & 13.5 & & & 0.008 & 0.008 & 0.000 & & \\
\hline & $6 / 16 / 2004$ & $\begin{array}{ll}4: 35: 45 \mathrm{AM} \\
: 36: 45 \mathrm{~A}\end{array}$ & \begin{tabular}{|l|}
26.128 \\
26.255
\end{tabular} & \begin{tabular}{|l|l|}
25.971 \\
25.963
\end{tabular} & $\frac{25.084}{2508}$ & \begin{tabular}{l|l}
26.73 \\
26757
\end{tabular} & $\frac{25.926}{25.022}$ & $\begin{array}{r}47.578 \\
4758\end{array}$ & \begin{tabular}{|l|l|}
51.829 \\
51.075 \\
\end{tabular} & $\begin{array}{l}15.038 \\
15143\end{array}$ & .033 & $\begin{array}{l}-\frac{1.222}{1.222} \\
\end{array}$ & $\begin{array}{r}-7.225 \\
.723\end{array}$ & \begin{tabular}{|l|}
46.098 \\
5359 \\
\end{tabular} & $\begin{array}{l}0.055 \\
0.054\end{array}$ & $\begin{array}{l}14.643 \\
14643\end{array}$ & 0.004 & 2601.03 & \begin{tabular}{ll|}
43.3506 \\
13672
\end{tabular} & 50.4 & $\begin{array}{l}11.3 \\
131\end{array}$ & 40.3 & 2.779 & . & $\begin{array}{l}0.008 \\
0008\end{array}$ & 0.000 & 0.203 & 0.17 \\
\hline$\frac{1910}{1911}$ & $6 / 6 / 1 / 1 / 2004$ & $\frac{A M}{A M}$ & $\frac{26.125}{26.124}$ & $\frac{25.963}{25.977}$ & $\begin{array}{r}25.086 \\
25.1\end{array}$ & $\frac{26.757}{26.756}$ & & $\begin{array}{r}47.588 \\
47.688\end{array}$ & 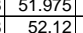 & $\begin{array}{l}\frac{15.143}{15.079} \\
\end{array}$ & \begin{tabular}{|l|l|l|}
844 \\
037
\end{tabular} & \begin{tabular}{|l|}
-1.222 \\
-1.219 \\
\end{tabular} & & $\begin{array}{l}53.5599 \\
49.389\end{array}$ & $\mid \begin{array}{l}0.054 \\
0.054\end{array}$ & $\begin{array}{l}14.643 \\
14.643\end{array}$ & & & & 50.6 & & & & |.008 & $\begin{array}{l}0.008 \\
0.008\end{array}$ & & & \\
\hline 1912 & $6 / 16 / 2004$ & 4:38:45 AM & 26.123 & 25.977 & 25.115 & 26.78 & 25.906 & 48.055 & \begin{tabular}{|l|}
52.408 \\
\end{tabular} & 15.112 & 33.402 & -1.216 & -7.227 & 50.704 & 0.054 & $\frac{14.045}{14.643}$ & & 2604.03 & 43.0006 & 50.9 & 12.4 & 40 & $\frac{2.183}{2.808}$ & . & 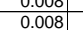 & 0.000 & $\begin{array}{l}0.199 \\
0.197\end{array}$ & 0.17 \\
\hline & 6/116/2004 & 9:45 AM & 26.123 & 25.977 & 25.115 & 26.79 & 25.901 & 47.962 & 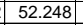 & 15.177 & \begin{tabular}{|l|}
33.248 \\
\end{tabular} & \begin{tabular}{|l|} 
\\
\end{tabular} & & 47.005 & 0.055 & 14.643 & & 2605.03 & \begin{tabular}{|l|l|}
43.4172 \\
\end{tabular} & 50.8 & 11.5 & & & & & & & $\frac{0.17}{0.17}$ \\
\hline 1914 & & :45 AM & 26.117 & 25.971 & 25.114 & 26.75 & & 47.792 & 52.215 & 15.166 & & \begin{tabular}{|l|}
-1.219 \\
\end{tabular} & & & & 14.643 & & & 4339| & & & & & & & & & $\frac{117}{17}$ \\
\hline 1915 & $6 / 16 / 2004$ & $45 \mathrm{AM}$ & 26.112 & & 25.104 & 26.775 & & 47.879 & & 15.071 & 33.24 & & & 50.036 & $\mid 0.054$ & 14.643 & & & 4506 & 50.8 & 12.3 & & 796 & Don & .008 & & 198 & \\
\hline 1916 & & & 26.113 & 25.967 & 25.105 & & 25.91 & 47.943 & 52.176 & 15.075 & 33.291 & & & 49.396 & 0.054 & 14.643 & & & & & & & & & & & & \\
\hline & $6 / 16 / 2004$ & $45 \mathrm{AM}$ & 26.092 & 25.951 & 25.089 & 26.775 & $25.8 \varepsilon$ & 48.074 & 52.547 & 15.116 & 33.392 & -1.222 & & & 0.054 & 14.643 & & & & & & & & & & & & \\
\hline 1918 & 6/116/2004 & & 26.103 & 25.947 & 25.09 & 26.8 & 25.88 & 47.705 & 52.07| & 15.155 & 32.967 & -1.219 & & 49.694 & 0.055 & 14.643 & & & & 50.6 & 12.2 & & & & .008 & & & \\
\hline 1919 & $6 / 1$ & & 26.109 & 25.957 & 25.1 & 26.846 & 25.9 & 47.951 & 52.505 & 15.245 & 33.104 & -1.222 & -7.245 & 53.386 & & 14.643 & & & $\mid$ & 51.0 & 13.1 & & 794 & .009 & 5.009 & & & \\
\hline 27 & & & \begin{tabular}{|l|}
26.1 \\
2.0
\end{tabular} & & & 26.887 & & 47. & .062 & 15.115 & & & & 49.51 & & 14.643 & & & & & & & & & & & & \\
\hline & 104 & & 26.106 & 25.964 & & 26.958 & & 47.615 & 51.954] & 15.175 & & -1.216 & & 43.16? & 5 & 14.643 & & & & & & & & & & & & \\
\hline 924 & & & 26.093 & 25.942 & $\frac{25.13}{20.13}$ & & & $4 . .102$ & 52.014 & 14.916 & 33.254 & -1.222 & -7.2 & 50.612 & & 14.643] & & & & & 12.4 & & & & & & & \\
\hline 922 & $6 / 16 / 2004$ & . & $\frac{26.107}{26111}$ & 25.951 & 25.134 & $27.119 \mid$ & $\frac{26}{26 ?}$ & $\frac{47.862}{47618}$ & $\begin{array}{r}52.18 \\
52028\end{array}$ & $\begin{array}{l}14.958 \\
1497\end{array}$ & $\begin{array}{l}33.351 \\
33.109\end{array}$ & $\begin{array}{l}-1.216 \\
-12222\end{array}$ & & 52.844 & $\begin{array}{l}0.054 \\
0.054\end{array}$ & $\frac{14.643}{14643}$ & & & & & 15 & & & & & & & \\
\hline 1925 & $6 / 16 / 2004$ & & $\frac{2.114}{26.146}$ & & 年 & 27.153 & 26.4 & 47.0 & 5 & $\mid$ & | & \begin{tabular}{|l|l|}
-1.2192 \\
-1.219
\end{tabular} & & 51.934 & 0.054 & 14643 & & & & 508 & & & & & & & & \\
\hline 1926 & $6 / 16 / 2004$ & & 26.15 & & 25.147 & 27.137 & & 47.748 & $\mid$\begin{tabular}{|l|l|}
$\mid 52.219$ \\
\end{tabular} & 14.919 & 254 & \begin{tabular}{|l|l|}
-1.225 \\
\end{tabular} & & 50.825 & & $\frac{14.045}{14.643}$ & & & & 50.7 & $\frac{\frac{1.1 .1}{12.5}}{12.5}$ & & & & 0 & & D. & \\
\hline 1927 & $6 / 16 / 2$ & & 26.14 & & 25.126 & 27.117 & & 48.192 & 52.644 & 14.975 & 33.634 & $\begin{array}{r}-1.219 \\
\end{array}$ & & $\frac{19.36}{49.36}$ & 0.6 & 14.643 & & & & & . & & & & & & 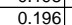 & \\
\hline & & & & & & 27.082 & & & & & & & & & & & & & & 500 & & & & & & & & \\
\hline .929 & & & 26.142 & & 25.119 & 27.054 & & 47.597 & 52.003 & 14.951 & 33.082 & -1.22 & & $\begin{array}{l}40.7 \\
40\end{array}$ & & 14.643 & & & & 50. & 10.0 & & & & & & 0.199 & \\
\hline & & & & & 25.138 & 26.984 & & & 51.956 & 14.914 & & & & & & 14.6 & & & & & 11.9 & & & & & & & \\
\hline 931 & $6 / 16 / 2$ & & 26.154 & 26.018 & 25.126 & 26.961 & & & $\mid 52.026$ & 14.881 & 33 & -1.222 & & 3.58 & $0 . c^{-1}$ & 14.643 & & & & & 107 & & & & & & 0.197 & \\
\hline & 6/16/2004 & & & & 25.116 & 26.982 & & & \begin{tabular}{|l|l|} 
& 52.244 \\
\end{tabular} & 14.923 & & $|-1.228|$ & & 43.2 & & 14.643 & & & & 50.8 & 10.6 & & & & & & | & \\
\hline $19 x$ & & & & & & & & & \begin{tabular}{|l|l|l|l|} 
\\
\end{tabular} & & & & & & & & & & & & 11.5 & & & & & & & \\
\hline 1934 & 6/2004 & & 26.133 & & 25.104 & 26.975 & & 47.862 & 52.422 & 14.905 & 33.359| & -1.22 & & 47.929 & 0.053 & 14.643 & & & & & 11.7 & & & & & & & \\
\hline & & & & & 2.10 & 26.966 & & & & & & -1.2 & & & & & & & & & 13.0 & & & & & & 00 & \\
\hline 1936 & & & & & & & & & & & & & & & & & & & & & & & & & & & & \\
\hline 38 & & & $\frac{26.174}{26162}$ & $\frac{26.043}{26.045}$ & 25.121 & $\frac{26.967}{26889}$ & & $\frac{47.665}{47.854}$ & $\begin{array}{l}52.341 \\
52.335\end{array}$ & 14.996 & .004 & \begin{tabular}{|c|c|}
-1.219 \\
-1.222
\end{tabular} & & 50.1313 & 然 0.054 & $\frac{14.64}{14.64}$ & & & & & 124 & & & & & & 98 & \\
\hline & $6 / 16 / 2004$ & & & & 25.123 & 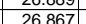 & & & & 1487 & & & & $\frac{30.49}{44.98}$ & & & & & & & & & & & & & & \\
\hline 940 & & & & & 25089 & & & & & & & & & & & & & & & & 10.5 & & & & & & & \\
\hline 1941 & & & $\frac{2.6 .156}{26.56}$ & & 25.103 & $\frac{26.909}{26.909}$ & & & $\mid 52.068$ & 14.899 & & & & 51.154 & & $\frac{14.6}{14.6}$ & & & & & $\frac{12.5}{11.5}$ & & & & & & 198 & \\
\hline & & & & & & & & & & & & & & & & & & & & & & & & & & & & \\
\hline$\overline{943}$ & & & \begin{tabular}{|l|l|}
26.13 \\
\end{tabular} & & $25.0 \varepsilon$ & 26.912 & & & 52.4 & & $\begin{array}{l}33.263 \\
\end{array}$ & $\frac{-1.2}{-1.2}$ & & & & & & & & & & & & & & & & \\
\hline 944 & & & & & 25.069 & & & & & & & & & & & & & & & & & & & & & & & \\
\hline 1945 & $6 / 16 / 2$ & 5:11:45 & & & 25.085 & 26. & & & \begin{tabular}{|l|}
52.178 \\
\end{tabular} & 14.9 & & -1.21 & & & & & & & & & 11.8 & & & & & & 198 & \\
\hline 194 & $6 / 16 / 2 C$ & & 26.1 & & 25.0 & 26.966 & & 47.4 & 51.82 & 14.88 & & -1.22 & & & & 14.6 & & & & 50 & & & & & & & & \\
\hline 1947 & & & & & 25.065 & & & & 52.613 & & & & & & & & & & & & & & & & & & & \\
\hline 1948 & & & 26.125 & & 25.066 & 26.912 & & & 52.059 & & & -1.2. & & & & & & & & & .5 & & & & & & D.199 & \\
\hline 94 & & & & & & & & & & & & & & & & & & & & & & & & & & & & \\
\hline 951 & & & 26.13 & & 25.6 & & & & & & & & & & & & & & & & 1.0 & & & & & & & \\
\hline 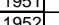 & & $\frac{5.17 .45}{5.18 .15}$ & $\frac{2.13}{26.14}$ & & $\frac{2.07}{2507}$ & & & & & & & $\begin{array}{l}-1.225 \\
1.190\end{array}$ & & & & & & & & 51 & & & & & & & & \\
\hline 95 & $\frac{1131 / 20}{6 / 6 / 20}$ & $\frac{3.1 .45}{5.19 .45}$ & $\frac{2.442}{26159}$ & 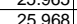 & 50906 & & & 年 & $\frac{5.03}{5.48}$ & 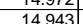 & & 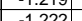 & & & & $\frac{11.0}{146}$ & & & & 51 & 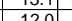 & & & & & & 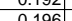 & \\
\hline 1954 & & $\frac{1.23 .45}{5.20 .45}$ & 26.16 & & 25116 & 26.93 & & 48 & 5284 & & 338 & - & & & & & & & & & & & & & & & 95 & \\
\hline & & & 26.16 & & & 268 & & 47.5 & & & 84 & & & & & & & & & & 0 & & & & & & 4 & \\
\hline & & & & & & & & & & & & & & & & & & & & & & & & & & & & \\
\hline & & & & & & & & & & & & & & & & & & & & & & & & & & & & \\
\hline 958 & & & 2615 & & $25.132 \mid$ & 26.733 & & & & 14.86 & & \begin{tabular}{|l|l|}
-1.22 \\
\end{tabular} & & & & 146 & & & & & & & & & & & 8 & \\
\hline 056 & & & 26155 & 5 & $25136 \mid$ & & & & & 89 & & 12 & & 50 & & 46.25 & & & & & & & & & & & 4 & \\
\hline $1960 \mid$ & 6/16/2004 & 5:26:45 AM & $\mid$ & \begin{tabular}{|l|l|}
25.937 \\
\end{tabular} & 25.12 & $|26.716|$ & & 47.983 & \begin{tabular}{|l|}
52.457 \\
\end{tabular} & \begin{tabular}{|l|l|}
14.864 \\
\end{tabular} & 33.538 & \begin{tabular}{|l|l|} 
\\
\end{tabular} & & 50.205 & 0.054 & 14.643 & & 2.03 & 444.2006 & 51.0 & 12.3 & & 2.810 & $\mid 0.008$ & $\mid$ & 0.000 & $|0.197|$ & \\
\hline
\end{tabular}


WSRC-TR-2005-00105, REVISION 0

SRNL-RPP-2005-00012, REVISION 0

RUN \# 3.03A AND B; FIRST AND SECOND HALF OF SLURRY DEWATERING - CONT.

\begin{tabular}{|c|c|c|c|c|c|c|c|c|c|c|c|c|c|c|c|c|c|c|c|c|c|c|c|c|c|c|c|c|}
\hline & & & \begin{tabular}{l|}
$\mathrm{D}$ \\
\end{tabular} & $\mathrm{E}$ & $\mathrm{F}$ & \begin{tabular}{l|l|l} 
G & \\
\end{tabular} & \begin{tabular}{l|l}
$\mathrm{H}$ \\
\end{tabular} & $\mathrm{J}$ & K & $\begin{array}{ll}\mathrm{L} \\
\end{array}$ & \begin{tabular}{l|l} 
\\
\end{tabular} & \begin{tabular}{l|l}
$\mathrm{N}$ \\
\end{tabular} & 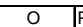 & $\begin{array}{ll} \\
\end{array}$ & \begin{tabular}{l|l}
$R$ & \\
\end{tabular} & \begin{tabular}{l|l}
$\mathrm{s}$ &
\end{tabular} & $T$ & $\begin{array}{lll} \\
\end{array}$ & \begin{tabular}{l|l} 
w \\
\end{tabular} & \begin{tabular}{|c|}
$x \mid$ \\
\end{tabular} & \begin{tabular}{l|l} 
&
\end{tabular} & & & & AC & & $\overline{\mathrm{AE}}$ & AF \\
\hline 961 & 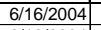 & 5:27:45 AM & 26.148 & 25.937 & 25.13 & 26.73 & 25.931 & 47.987 & \begin{tabular}{|l|l|}
52.418 \\
\end{tabular} & 14.96 & 33.484 & \begin{tabular}{|l|}
-1.159 \\
\end{tabular} & \begin{tabular}{|l|l|}
-7.314 \\
\end{tabular} & \begin{tabular}{|l|}
43.026 \\
\end{tabular} & 0.054 & \begin{tabular}{|l|}
14.643 \\
\end{tabular} & 0.004 & 2653.03 & \begin{tabular}{|l|l|}
34.2172 \\
\end{tabular} & 50.9 & 10.5 & 40.7 & 2.809 & 0.008 & 0.008 & 0.000 & 0.197 & \\
\hline & & $5: 28: 45 \mathrm{AM}$ & 26.135 & 25.928 & & 26.737 & 25.928 & & & \begin{tabular}{|l|l|}
14.874 \\
\end{tabular} & 33.324 & \begin{tabular}{|l|l|}
-1.156 \\
\end{tabular} & & 54.348 & 0.056 & $\begin{array}{l}14.643 \\
\end{array}$ & & & & & & & & & 0.008 & & & 0.17 \\
\hline 1963 & 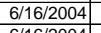 & $5: 29: 45 \mathrm{AM}$ & \begin{tabular}{l|l|}
26.154 \\
6.14
\end{tabular} & $\begin{array}{l}25.948 \\
25.08\end{array}$ & $\begin{array}{l}25.141 \\
2.117\end{array}$ & $\begin{array}{l}26.761 \\
2.702\end{array}$ & $\begin{array}{l}25.922 \\
2580\end{array}$ & 48.032 & \begin{tabular}{|l|l|}
52.592 \\
5530
\end{tabular} & \begin{tabular}{ll|}
14.928 \\
1920
\end{tabular} & \begin{tabular}{|l|l|}
33.589 \\
2.26
\end{tabular} & \begin{tabular}{|l|l|} 
& -1.167 \\
\end{tabular} & & & $\begin{array}{l}0.053 \\
\end{array}$ & \begin{tabular}{|l|l|}
14.643 \\
\end{tabular} & & 2655.03 & 344.2506 & 51.1 & 12.3 & & 814 & 2.008 & $\begin{array}{ll}0.008 \\
\end{array}$ & 0.000 & 0.193 & \\
\hline 1964 & 6/16/2004 & 5:30:45 AM & \begin{tabular}{|l|l|}
26.161 \\
\end{tabular} & 25.949 & 25.147 & 26.793 & 25.893 & $\begin{array}{l}47.644 \\
\end{array}$ & \begin{tabular}{|l|l|}
52.408 \\
5
\end{tabular} & $\begin{array}{l}14.868 \\
\end{array}$ & \begin{tabular}{|l|l|l|l|}
33.166 \\
3
\end{tabular} & \begin{tabular}{|l|l|}
-1.176 \\
\end{tabular} & \begin{tabular}{|l|l|}
-7.303 \\
7201
\end{tabular} & \begin{tabular}{|l|l|}
53.042 \\
\end{tabular} & \begin{tabular}{|c|}
0.054 \\
\end{tabular} & \begin{tabular}{|l|l|}
14.643 \\
\end{tabular} & 0.004 & 2656.03 & \begin{tabular}{|l|l|}
44.2672 \\
\end{tabular} & 50.9 & 13.0 & 40.4 & 2.786 & \begin{tabular}{|l|l|}
0.008 \\
\end{tabular} & $\begin{array}{ll}0.008 \\
\end{array}$ & 0.000 & 0.198 & \\
\hline $1965 \mid$ & $\frac{6 / 16 / 2004}{6 / 16 / 2004}$ & 5:31:45 AM & $\begin{array}{l}26.15 \\
26.155\end{array}$ & & & 26.747 & $\begin{array}{l}25.908 \\
25.928\end{array}$ & $\begin{array}{l}47.945 \\
47835\end{array}$ & \begin{tabular}{|l|}
52.58 \\
52.43 \\
\end{tabular} & \begin{tabular}{|l|}
14.984 \\
14958
\end{tabular} & & \begin{tabular}{|r|r|}
-1.156 \\
-1.59 \\
\end{tabular} & & \begin{tabular}{|l|}
51.991 \\
45109 \\
\end{tabular} & $\begin{array}{l}0.052 \\
0053\end{array}$ & \begin{tabular}{|l|}
14.643 \\
14643
\end{tabular} & & & 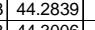 & \begin{tabular}{|l|}
51.1 \\
\end{tabular} & \begin{tabular}{|l|}
12.7 \\
1.1
\end{tabular} & & 2.804 & & \begin{tabular}{|c|}
0.008 \\
\end{tabular} & & $\begin{array}{l}0.190 \\
\end{array}$ & \\
\hline 1967 & $6 / 16 / 2004$ & $\begin{array}{l}5: 32: 45 \mathrm{AM} \\
\text { 5:33:45 }\end{array}$ & $\frac{26.145}{26.134}$ & $\begin{array}{l}25.938 \\
25.948\end{array}$ & \begin{tabular}{|l|}
25.132 \\
25.136 \\
\end{tabular} & $\frac{26.752}{26.776}$ & $\begin{array}{r}25.928 \\
25.922 \\
\end{array}$ & \begin{tabular}{|r|r}
47.835 \\
47.8
\end{tabular} & \begin{tabular}{|l|}
52.43 \\
52.35 \\
\end{tabular} & \begin{tabular}{|l|}
44.958 \\
14.899
\end{tabular} & \begin{tabular}{|l}
33.332 \\
33.316
\end{tabular} & \begin{tabular}{|l|}
-1.159 \\
-1.156
\end{tabular} & $\begin{array}{l}-7.308 \\
-7.705 \\
\end{array}$ & \begin{tabular}{|r|}
45.109 \\
54.66
\end{tabular} & $\mid \begin{array}{l}0.053 \\
0.054\end{array}$ & \begin{tabular}{|r|}
14.6463 \\
14.643
\end{tabular} & $\frac{0.004}{0.004}$ & $\frac{2658.03}{2659.03}$ & \begin{tabular}{|l|}
$\mid 44.3006$ \\
3
\end{tabular} & 51.0| & \begin{tabular}{|l|}
11.1 \\
13.4
\end{tabular} & $\frac{40.6}{40.6}$ & $\begin{array}{l}2.798 \\
7.796\end{array}$ & $\begin{array}{l}0.008 \\
0.088\end{array}$ & \begin{tabular}{|r|}
0.008 \\
0.008
\end{tabular} & $\begin{array}{l}0.000 \\
0.000\end{array}$ & \begin{tabular}{|l|}
0.194 \\
0.198
\end{tabular} & $\begin{array}{l}0.17 \\
0.17\end{array}$ \\
\hline 1968 & 6/16/2004 & 5:34:45 AM & 26.132 & & 25.128 & & 25.924 & & & & & & -7.305 & & 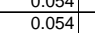 & $\mid \begin{array}{l}14.6434 \\
14.643\end{array}$ & $\begin{array}{l}0.004 \\
0.004\end{array}$ & $\frac{25390.03}{2660.03}$ & \begin{tabular}{|l|}
44.3172 \\
44.3339 \\
\end{tabular} & 50.9 & $\frac{15.4}{12.9}$ & \begin{tabular}{|}
40.6 \\
40.9
\end{tabular} & & $\begin{array}{l}0.008 \\
0.008\end{array}$ & $\mid$\begin{tabular}{|c}
0.008 \\
\end{tabular} & & & $\frac{0.17}{0.17}$ \\
\hline 1969 & 6/16/2004 & 5:35:45 AM & 26.133 & 25.936 & & 26.75 & 25.916 & 47.897 & 52.348 & 14.976 & $\begin{array}{l}33.34 \\
\end{array}$ & \begin{tabular}{|l|}
-1.153 \\
\end{tabular} & -7.305 & 52.287 & 0.054 & 14.643 & 0.004 & 2661.03 & 34.3506 & & & & & & & & & $\frac{0.17}{0.17}$ \\
\hline 1970 & $6 / 16 / 2004$ & 5:36:45 AM & 26.133 & 25.932 & 25.11 & 26.76 & & 47.989 & \begin{tabular}{|l|}
52.401 \\
\end{tabular} & \begin{tabular}{|l|}
14.94 \\
\end{tabular} & \begin{tabular}{|l|l|}
33.562 \\
\end{tabular} & \begin{tabular}{|l|}
-1.156 \\
\end{tabular} & -7.308 & 48.857 & $\begin{array}{l}0.053 \\
\end{array}$ & \begin{tabular}{|l|l|}
14.643 \\
\end{tabular} & & 2662.03 & \begin{tabular}{l|l|}
3 & 44.3672
\end{tabular} & 50.9 & 12.0 & 40.8 & & & 0.008 & & & $\frac{17}{16}$ \\
\hline 1971 & & & & 25.935 & & & & & & 14.882 & & & & \begin{tabular}{|l|l|}
48.91 \\
\end{tabular} & & & & & & & & & & 008 & 0.008 & 0.000 & & \\
\hline & & 8:45 AM & 26.131 & & 25.113 & 26.759 & & 47.9 & 52.615 & 14.939 & 402 & -1.1 & & 50.044 & & 14.643 & & & & 51.1 & $\frac{12.3}{12.3}$ & & & & & & . 1.197 & \\
\hline (1) & & :45 AM & 26.142 & 25.941 & 25.119 & 26.83 & & 48.072 & 52.609 & 14.929 & & & & \begin{tabular}{ll|l}
43.582 \\
\end{tabular} & & 14.643 & & & & 511 & & & 816 & & 0.008 & & & \\
\hline 1974 & 6/16/2004 & :45 AM & 26.154 & 25.953 & 25.131 & 26.911 & & 47.837 & 52.339 & | & 33.334 & \begin{tabular}{|l|l|} 
\\
\end{tabular} & -7.30 & 54.637 & $\mid$ & 14.643 & & 2666.03 & 44.4339 & 50.9 & 13.4 & & & . & \begin{tabular}{|l|l|}
0.008 \\
\end{tabular} & & & \\
\hline 1975 & $6 / 16 / 2004$ & 5:41:45 AM & 26.161 & 25.95 & 25.133 & 26.963 & 26.104 & 47.866 & 52.231 & 14.984 & 33.308 & -1.156 & $\begin{array}{r}-7.3 \\
\end{array}$ & $\begin{array}{l}44.924 \\
\end{array}$ & 0.054 & 14.643 & & 2667.03 & . & 50.8 & 11.0 & & 2.798 & 0.008 & 0.008 & & & \\
\hline 976 & $6 / 16 / 20$ & 5:42:45 AM & 26.158 & 25.962 & 25.145 & 26.98 & & $\begin{array}{r}47.69 \\
\end{array}$ & 52.192 & 14.97 & 33.207 & $\begin{array}{l}-1.118 \\
-1150\end{array}$ & & $\begin{array}{l}48.246 \\
2.01\end{array}$ & 0.052 & 14.643 & & 68.0 & & 50.7 & & & & & $\begin{array}{l}.008 \\
\end{array}$ & & & \\
\hline & & $: 45 \mathrm{AM}$ & & 25.97 & & & & 47.458 & 52.167 & 15.405 & 4.466 & -1.153 & & 48.81 & 0.049 & 14.643 & & & 年4.48399 & 50.1 & r & & & & & & & \\
\hline & $6 / 16 / 2004$ & : & 20.175 & 25.973 & & & & & 52.973 & & 999 & -1.106 & & & & $\frac{14.643}{14}$ & & & . & & 13.1 & & & & .008 & & & \\
\hline & & $5: 45: 45 \mathrm{AM}$ & & 25.982 & 255.14 & $\frac{26.926}{2028}$ & & 48.758 & 5.3.257] & 14.514 & 1.734 & $\begin{array}{ll}-1.101 \\
\end{array}$ & & 48.194 & & 14.643 & & & & $51.8 \mathrm{~s}$ & 11.8 & & & & 0.008 & . .000 & 0.196 & \\
\hline 981 & $6 / 16 / 2004$ & & 26.175 & 25.578 & 年. & $\frac{20.001}{26.882}$ & & 年 48.1035 & 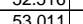 & 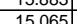 & .646 & & & $\begin{array}{r}50.0240 \\
50.04\end{array}$ & 0 & 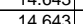 & & & & 515 & 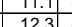 & & & (2) & 0.0006 & & & \\
\hline 1982 & $6 / 16 / 2004$ & $5: 48: 45 \mathrm{Am}$ & 26.169 & 25.988 & 25.171 & 26.891 & & 48.538 & 53.278 & $\begin{array}{l}14.692 \\
14.692\end{array}$ & 34.321 & -0.996 & -6.964 & 49.757 & & $\begin{array}{l}\frac{1.045}{14.643} \\
\end{array}$ & & 2674.03 & 44.5672 & 51.8 & 12.2 & 41. & 856 & .008 & 0.008 & 0.000 & 0.186 & \\
\hline & $6 / 16 / 2004$ & 5:49:45 AM & 26.173 & 25.997 & 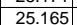 & 26.886 & & 48.252 & 52.839 & 14.555 & 34.194 & \begin{tabular}{|l|l|} 
& -0.944 \\
\end{tabular} & 6.007 & 45.48 & 0.054 & 14.643 & & $\frac{267.03}{2675.03}$ & 44.5839 & 51.4 & 11.1 & & $\frac{642}{842}$ & .008 & 0.008 & 0.000 & $\frac{\mid .1994}{0.194}$ & \\
\hline & $6 / 16 / 2004$ & 0:45 AM & 26.16 & 25.978 & & & & 48.623 & 53.264 & & 34.767 & & & 41.398 & 0.054 & & & & $\mid$ & 51.8 & & & & & & & & \\
\hline .985 & $6 / 16 / 2004$ & 5:51:45 AM & \begin{tabular}{|l|l|}
26.16 \\
\end{tabular} & 25.993 & 25.121 & 26.837 & & & 53.458 & 14.295 & 34.931 & -0.936 & & 50.147 & & 14.643 & & & 44.6172 & & $12.3 \mathrm{~S}$ & & 886 & 0.08 & $\mid$ & & 685 & \\
\hline & & 5:52:45 AM & & & 25.112 & $\mid 26.818$ & & \begin{tabular}{|l|l|}
48.717 \\
\end{tabular} & & 14.115 & 35.007 & -0.936 & & & & 14.643 & & & & & $\frac{12.9}{12.9}$ & & & & & & & \\
\hline 987 & $6 / 16 / 2004$ & 5:53:45 AM & 26.163 & 25.997 & 25.099 & 26.815 & 25.996 & 48.972 & 53.844 & 14.136 & 35.259 & -0.936 & 60 & 47.762 & & 14.643 & & & & & 117 & & & & & & & \\
\hline 988 & 6/16/2004 & & 26.167 & 26.015 & 25.088 & 26.794 & & |48.833 & \begin{tabular}{|l|l|l}
5324 \\
\end{tabular} & 14.001 & 35.261 & -0.936 & -6.9 & 47.982 & 0.053 & 14.643 & & 680 & 44.6672 & 52.3 & 11.8 & & .899 & .008 & | 0.008 & & & \\
\hline 1988 & $6 / 16 / 2004$ & 5:45 AM & 26.16 & 26.009 & 25.061 & 26.762 & & 49 & \begin{tabular}{|l|l|l}
54.142 \\
\end{tabular} & 14.094 & 35.684 & -0.939 & & 46.313 & & 14.643 & & 2681.03 & 44.6839 & 52.7. & 11.3 & & 93 & $\mid .008$ & 0.008 & & 0.196 & \\
\hline 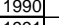 & & & & & 25.058 & 26.739 & & 49.474 & 54.143 & 828 & 36.111 & $\begin{array}{l}-0.939 \\
-0.39\end{array}$ & & & & 14.643 & & & & & & & & & & & & \\
\hline & 6 & & 26.142 & 26.0011 & . & 26.735 & & & 5.302 & 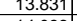 & & -0.936 & & 1.682 & & 14.643 & & & & & & & & & & & & \\
\hline 1992 & $6 / 14$ & $5: 58$ & 26.144 & 26.008 & 25.026 & 26.132 & & $49 . / 16$ & 54.4/4 & & 36.025 & - -0.936 & & 45.134] & & 14.643] & & & & & 11.1 & & & & & & & \\
\hline$\frac{99}{99}$ & 6 & . & 26.1414 & $\begin{array}{r}26.01 \\
26008\end{array}$ & $\frac{25.012}{25011}$ & 26.774 & & $\frac{49.638}{49.712}$ & \begin{tabular}{|l|l|l|}
54.279 \\
5455
\end{tabular} & $\begin{array}{l}14.242 \\
14187\end{array}$ & $\begin{array}{r}35.85 \\
35.967\end{array}$ & $\begin{array}{l}-0.936 \\
-0.036\end{array}$ & & $\begin{array}{l}4.011 \\
45147\end{array}$ & & $\frac{14.643}{14643}$ & & & & 2. & 10.8 & & & & & & & \\
\hline$\overline{995}$ & $6 / 6162004$ & & $\frac{20.14}{26.13}$ & 26017 & $\mid$ & 年 26805 & & & 年 & 1424 & 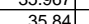 & $\begin{array}{l}-0.036 \\
-0.996\end{array}$ & & & 0.052 & 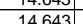 & & & & 530 & 115 & & & & & & & \\
\hline 1996 & $6 / 16 / 2004$ & & 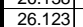 & 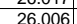 & 25.04 & 20.677 & & 49.581 & \begin{tabular}{|l|l|}
54.4599 \\
\end{tabular} & 势, & $\begin{array}{r}35.64 \\
35.9\end{array}$ & $=0.936$ & & $\frac{46}{46}$ & & 1464 & & & & 53. & $\frac{1.5}{114}$ & $\frac{42}{12}$ & & 0 & 0.008 & & 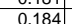 & \\
\hline 1997 & $6 / 16 / 2004$ & & 26.127 & $\frac{26.005}{26.00}$ & 25.033 & 26.799 & & & \begin{tabular}{|l|}
53.421 \\
\end{tabular} & 15.037 & 34.147 & $\frac{-0.953}{-0.953}$ & & 49.763 & 0.05 & $\frac{14.643}{14}$ & & & & & & & & & & & & \\
\hline & & & $\frac{26.122}{26.122}$ & & 25032 & $\begin{array}{l}26.759 \\
26\end{array}$ & & & \begin{tabular}{|l|}
53.475 \\
\end{tabular} & 14.776 & & -0.93 & & & & 14.643 & & & & 520 & {$[1.6$} & & & & & & & \\
\hline 199 & & 6: & 26.11 & 26.008 & 25.016 & 26.742 & & 48.829 & \begin{tabular}{|l|l|}
53.79 \\
\end{tabular} & & & -0.936 & & $\mid 49.99$ & & 14.643 & & & & $\overline{52.3}$ & $\frac{12.3}{12.3}$ & & & & & & 0.182 & \\
\hline & & & & & 25.021 & & & & 53.96 & & & & & & & 14.643 & & & & & & & & & & & & \\
\hline & & $13 \mathrm{AM}$ & 26.098 & 26.007 & 25.009 & $\mid 26.7$ & & 49.08 & \begin{tabular}{|l|}
53.749 \\
\end{tabular} & 635 & & & & & & 14.643 & & & & 52.3 & 116 & & & & & & 85 & \\
\hline & 6/16/2004 & & 26.104 & & 25.01 & 26.716 & & & \begin{tabular}{|l|}
53.989 \\
\end{tabular} & 14.793 & & -0.93 & & & & 14.643 & & & & 52.5 & 12.7 & & & & & & 0.184 & \\
\hline & 6/16/2004 & & 26.082 & & 25.003 & 26.724 & & & \begin{tabular}{|l|l|l|l|} 
\\
\end{tabular} & & & -0.9 & & & & & & & & 52. & 12.0 & & & & & & 0.183 & \\
\hline 000 & $6 / 16 / 2004$ & & 26.082 & 25.986 & 24.973 & 26.729 & & 49.5011 & 54.41 & 14.594 & 35.302 & -0.9 & & 48.361 & 0.052 & 14.643 & & & & $52 .$. & 11.9 & & & 008 | & & & & \\
\hline & & & & & 24.988| & 26.769 & & & \begin{tabular}{|l|}
54.012 \\
\end{tabular} & & & & & & $0.6-6$ & & & & & sen & 0.0 & & & & & & & \\
\hline 200 & & & & & & & & & & & & & & & & & & & & & 11.4 & & & & & & & \\
\hline & 6/16/2004 & $\frac{3 \mathrm{AM}}{3 \mathrm{Am}}$ & 26.074 & $\frac{25.988}{25.991}$ & 24.975 & $\frac{26.721}{26715}$ & & $\begin{array}{l}49.134 \\
49.409\end{array}$ & \begin{tabular}{|r|r|r|r|}
54.179 \\
5
\end{tabular} & $\begin{array}{ll}14.647 \\
117711\end{array}$ & $\begin{array}{l}34.925 \\
35149\end{array}$ & $\begin{array}{l}-0.936 \\
-0.936\end{array}$ & & & 0.0 & $\frac{14.643}{14643}$ & & & & 2 & $\frac{64}{4}$ & & & & & & 80 & \\
\hline & 6 & & & & 年 & 年 26703 & & & 5 & & & $=0.936$ & & & & 146 & & & & 526 & 118 & & & & & & & \\
\hline & & & & & 24.95 & 26.696 & & & \begin{tabular}{|l|l|}
53.74 \\
\end{tabular} & & & $\begin{array}{c}-0.933 \\
-0.933\end{array}$ & & & & & & & & & $\frac{1.50}{10.5}$ & & & & & & & \\
\hline 201 & & & $\frac{26.063}{20.603}$ & 25.961 & 24.923 & $\frac{26.715}{26.75}$ & & & 53.90 & $\frac{14.823}{14.83}$ & & -0.9 & & & & 14.6 & & & & 52. & 11. & & & & & & & \\
\hline & & & & & & & & & & & & & & & & & & & & & & & & & & & & \\
\hline & & & 26.08 & & 24.961 & 26.933 & & & & & & $\frac{-0.93}{-0.93}$ & & & & & & & & & & & & & & & & \\
\hline & & & & & 24.969 & 26.96 & & & & & & & & & 0.049 & & & & & & & & & & & & & \\
\hline & & 13 & & 25.974 & $24.977 \mid$ & 27.013 & & 49 & \begin{tabular}{|l|l|}
54.242 \\
\end{tabular} & 14.708 & 35. & & & & & 14.6 & & & & & & & & & & & 180 & \\
\hline & $6 / 16 / 2 C$ & & 26.08 & 25.96 & 24.96 & 27.051 & & 49.438 & 54.1 & 14.79 & & -0.85 & & & & 14.6. & & & & 52. & .1 & & & & & & D.180 & \\
\hline 201 & & & & 25.9 & 24.946 & 27.0 & & & 54.40 & & & & & & & & & & & & .6 & & & & & & 6 & \\
\hline 201 & & & 26.08 & & 24.937 & 27.058 & & & $53.9 \mathrm{C}$ & & & -0.8 & & & & & & & & 52. & 12.1 & & & & & & & \\
\hline 01 & & & & & 24.9 & & & & & & & & & & & & & & & & & & & & & & & \\
\hline & & & 20.04 & & 24.956 & 26.987 & & & 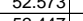 & & & & & & & & & & & J1. & 0 & & & & & & & \\
\hline & & & $\frac{2.07}{2608}$ & & 2 & $\frac{2.5900}{26061}$ & & & & & 31.992 & -0.9 & & & & & & & & 51 & 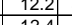 & & & & & & 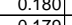 & \\
\hline & 6. & & $\frac{2.004 \mid}{26084}$ & 年 & 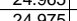 & $\frac{2.301}{20.911}$ & & 478 & 5 & 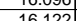 & $\frac{32.1}{322}$ & \begin{tabular}{|l|l|}
-0.039 \\
\end{tabular} & & & & $\frac{11.0}{146}$ & & & & 5.1.1 & 2 & & & & & & (1) & \\
\hline & & & & 25.942 & 249 & & & & 5244 & & & $\begin{array}{l}-0.8 \\
-0.8\end{array}$ & & & & 146 & & & & & & & & & & & & \\
\hline $02-2$ & & & 26.08 & & 20.984 & & & 47.644 & & & & & & & & & & & & 50. & & & & & & & & \\
\hline & & & & & & 26.88 & & & & & & & & & & & & & & & & & & & & & & \\
\hline & & & & & & & & & & & & & & & & & & & & & & & & & & & & \\
\hline 02 & & & 26.08 & \begin{tabular}{|l|l|}
25.93 \\
\end{tabular} & $24.988 \mid$ & 26.869 & & & 52.8 & & 32.4 & -0.8 & & & & 146 & & & & & & & & & & & \begin{tabular}{l|l|}
78 &
\end{tabular} & \\
\hline $2 x$ & & & 26097 & 25.945 & & 874 & & & 706 & 014 & & $-0.8 \varepsilon$ & & & & 4.6 & & & & $12=$ & & & & & & & & \\
\hline $2030 \mid$ & 6/16/2004 & 6:36:13 AM & |26.092 & 25.925 & \begin{tabular}{|l|}
24.993 \\
\end{tabular} & \begin{tabular}{|l|}
26.854 \\
\end{tabular} & 25.90 & 47.862 & \begin{tabular}{|l|}
52.663 \\
\end{tabular} & \begin{tabular}{|l|l|}
15.842 \\
\end{tabular} & \begin{tabular}{|l|l|l}
32.493 \\
\end{tabular} & \begin{tabular}{|l|l|}
-0.886 \\
\end{tabular} & & 56.283 & $\mid$ & 14.643 & & 21.50 & 45.3583 & $51.2]$ & 13.8 & & 2.770 & $\mid$ & | 0.007 & 0.000 & 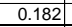 & 0.1 \\
\hline
\end{tabular}


WSRC-TR-2005-00105, REVISION 0

SRNL-RPP-2005-00012, REVISION 0

RUN \# 3.03A AND B; FIRST AND SECOND HALF OF SLURRY DEWATERING - CONT.

\begin{tabular}{|c|c|c|c|c|c|c|c|c|c|c|c|c|c|c|c|c|c|c|c|c|c|c|c|c|c|c|c|c|}
\hline & A & B & D & $E$ & $\mathrm{~F}$ & $\mathrm{G}^{\mathrm{S}}$ & $\mathrm{H}$ & $\mathrm{J}$ & $n$ & $\begin{array}{ll} \\
\end{array}$ & \begin{tabular}{l|l} 
\\
\end{tabular} & \begin{tabular}{l|l}
$\mathrm{N}$ \\
\end{tabular} & 0 & $Q$ & $R^{R}$ & & $\begin{array}{lll}T & \mathrm{~T} \\
\end{array}$ & & & \begin{tabular}{l|l|l|}
$x$ \\
\end{tabular} & & & $A A$ & $\mathrm{AB}$ & $A C$ & $\mathrm{AD}$ & $A E$ & \\
\hline :031 & 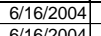 & $\begin{array}{c}6: 37: 13 \mathrm{AM} \\
6\end{array}$ & $\begin{array}{l}26.096 \\
26096\end{array}$ & $\frac{25.92}{25.91}$ & $\frac{24.99}{24.99}$ & \begin{tabular}{|l|}
26.853 \\
26.853
\end{tabular} & $\begin{array}{l}25.893 \\
25887\end{array}$ & \begin{tabular}{|l|l|l}
47.721 \\
8264
\end{tabular} & $\begin{array}{l}52.509 \\
55235\end{array}$ & \begin{tabular}{|r|}
15.76 \\
15812
\end{tabular} & \begin{tabular}{|l|l|}
32.386 \\
32819
\end{tabular} & \begin{tabular}{|l|}
-0.837 \\
0.846
\end{tabular} & $\begin{array}{l}-7.034 \\
7002 \\
\end{array}$ & $\begin{array}{l}48.745 \\
5798\end{array}$ & 0.046 & 14.643 & 0.004 & 2722.50 & 45.375 & 51.0 & $\frac{11.9}{11.9}$ & 40.1 & $\begin{array}{l}2.762 \\
2707 \\
2\end{array}$ & $\begin{array}{l}.007 \\
\end{array}$ & $\begin{array}{l}0.007 \\
\end{array}$ & 0.000 & 0.171 & \\
\hline (2) & $6 / 6 / 16 / 2004$ & $\frac{6: 38: 13 \mathrm{AM}}{6.39 \cdot 13 \mathrm{AM}}$ & 096 & $\frac{25.91}{25.91}$ & $\frac{24.99}{2500}$ & \begin{tabular}{|l|}
26.853 \\
26.858
\end{tabular} & $\frac{25.878}{25888}$ & \begin{tabular}{ll|l}
48.264 \\
47837
\end{tabular} & \begin{tabular}{|c|}
53.235 \\
52768 \\
\end{tabular} & $\frac{15.812}{15732}$ & $\begin{array}{l}32.819 \\
32511\end{array}$ & $\begin{array}{l}-0.846 \\
-0.866\end{array}$ & $\begin{array}{l}-7.002 \\
-6.996\end{array}$ & $\begin{array}{l}45.798 \\
55.823\end{array}$ & 0.048 & $\frac{14.643}{14643}$ & & 2723.50| & \begin{tabular}{|l|}
45.3917 \\
45.498 \\
\end{tabular} & \begin{tabular}{|c|}
51.8 \\
51.3
\end{tabular} & $\frac{11.2}{137}$ & 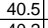 & 2.795 & \begin{tabular}{|c|c|}
0.007 \\
0.07
\end{tabular} & \begin{tabular}{|c|c|} 
\\
0.007
\end{tabular} & & $\frac{0.177}{0.02}$ & \\
\hline 034 & $6 / 16 / 2004$ & $6: 40: 13 \mathrm{AM}$ & $\frac{2.100}{26.11}$ & $\frac{2.51}{25.90}$ & $\frac{25.00}{25.02}$ & $\frac{26.858}{26.822}$ & $\frac{25.888}{25.883}$ & $\begin{array}{r}4.837 \\
47.85 \\
\end{array}$ & \begin{tabular}{|l|}
52.7684 \\
52.764
\end{tabular} & $\begin{array}{l}15.1233 \\
15.559\end{array}$ & $\frac{32.511}{32.719}$ & $\begin{array}{l}-0.866 \\
-0.86\end{array}$ & $\begin{array}{l}-6.966 \\
-6.99\end{array}$ & $\begin{array}{l}55.833 \\
47.654\end{array}$ & | 0.049 & $\frac{14.643}{14.643}$ & $\frac{0.004}{0.004}$ & $2724.50 \mid$ & \begin{tabular}{|r|}
45.4083 \\
45.425 \\
\end{tabular} & $\begin{array}{l}51.3 \\
51.3 \\
\end{array}$ & $\begin{array}{l}\frac{13.7}{11.7} \\
\end{array}$ & $\begin{array}{l}40.2 \\
40.3 \\
\end{array}$ & $\begin{array}{l}2.770 \\
2.777 \\
\end{array}$ & .007| 007 & $\mid \begin{array}{l}0.007 \\
0.007\end{array}$ & $\begin{array}{l}0.000 \\
0.000\end{array}$ & & $\frac{0.15}{0.15}$ \\
\hline 2035 & $6 / 16 / 2004$ & $6: 41: 13 \mathrm{AM}$ & 26.105 & 25.91 & 25.01 & 26.812 & 25.873 & 47.991 & 52.76 & 15.765 & 32.622 & -0.817 & $\begin{array}{l}-6.99 \\
\end{array}$ & 49.742 & 0.05 & 14.643 & & & \begin{tabular}{|l|}
45.4417 \\
\end{tabular} & & 12.2 & 40.3 & & .007 & 0.007 & & & \\
\hline & & $6: 42: 13 \mathrm{AM}$ & & & 25.01 & 26.827 & 25.873 & \begin{tabular}{|l|l|}
47.908 \\
\end{tabular} & & 15.617 & & -0.817 & & 52.199 & 0.048 & 14.643 & & & & 51.2 & 12.8 & & & & & & & \\
\hline 2037 & & $6: 43: 13 \mathrm{AM}$ & 26.099 & 25.88 & & 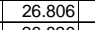 & 25.866 & 47.887 & \begin{tabular}{|l|l|}
52.783 \\
502
\end{tabular} & & & $\begin{array}{l}-0.82 \\
-0.77\end{array}$ & & 47.666 & 0.049 & \begin{tabular}{|l|l|}
14.643 \\
\end{tabular} & & & \begin{tabular}{|r|}
45.475 \\
\end{tabular} & 51.3 & & & & & & & & \\
\hline 2038 & $6 / 16 / 2004$ & $6: 44: 13 \mathrm{AM}$ & 26.109 & 25.88 & 25.01 & \begin{tabular}{|l|l|}
26.826 \\
\end{tabular} & 25.866 & 47.895 & \begin{tabular}{|l|l|}
52.804 \\
\end{tabular} & 15.464 & $\begin{array}{l}32.891 \\
\end{array}$ & -0.817 & -6.964 & 49.481 & 0.051 & 14.643 & 0.00 & & \begin{tabular}{|l|l|l|l|} 
\\
\end{tabular} & 51.3 & 12.1 & 40.4 & 2.785 & .008 & 0.008 & 0.000 & 0.188 & \\
\hline 0.039 & $6 / 16 / 2004$ & $\begin{array}{l}5: 13 \mathrm{AM} \\
6.13 \mathrm{AM}\end{array}$ & $\begin{array}{l}26.108 \\
26123\end{array}$ & $\frac{25.88}{25.87}$ & $\frac{25.00}{2502}$ & \begin{tabular}{|l|}
26.82 \\
26.76
\end{tabular} & $\begin{array}{r}25.875 \\
258\end{array}$ & \begin{tabular}{l|l|l|l|l}
48.451 \\
47282
\end{tabular} & \begin{tabular}{|l|}
52.822 \\
52404 \\
\end{tabular} & $\begin{array}{l}15.676 \\
15575\end{array}$ & \begin{tabular}{ll|l}
33.168 \\
32.165
\end{tabular} & $\begin{array}{l}-1.219 \\
.5519\end{array}$ & $\begin{array}{l}-6.889 \\
.521\end{array}$ & \begin{tabular}{|l|l|l|l|}
43.947 \\
7609
\end{tabular} & & $\begin{array}{l}14.643 \\
1.643\end{array}$ & 0.004 & $\begin{array}{l}2730.50 \\
272150\end{array}$ & & 51.3 & $\frac{10.8}{117}$ & $\frac{40.8}{20.7}$ & 2.814 & & 0.010 & & & \\
\hline 2041 & $6 / 6 / 16 / 12004$ & $\begin{array}{l}6: 46: 13 \mathrm{AM} \\
6 \text { 6:47:13 }\end{array}$ & $\frac{26.123}{26.132}$ & $\frac{25.87}{25.87}$ & $\frac{25.02}{25.02}$ & \begin{tabular}{r|}
26.76 \\
26.764
\end{tabular} & $\frac{25.8}{25.8}$ & $\begin{array}{ll}47.282 \\
46.912\end{array}$ & \begin{tabular}{|c|}
52.404 \\
55.791
\end{tabular} & $\frac{15.575}{15.584}$ & $\begin{array}{l}32.165 \\
31.688\end{array}$ & $\begin{array}{r}-0.542 \\
0.199\end{array}$ & $\begin{array}{r}-5.21 \\
-3.744 \\
\end{array}$ & $\begin{array}{l}47.698 \\
45.796 \\
\end{array}$ & \begin{tabular}{|c|c|}
0.028 \\
0.054
\end{tabular} & $\frac{14.643}{14.643}$ & 0.004 & 731.50 & \begin{tabular}{|r|}
$45.525 \mid$ \\
45.5417
\end{tabular} & \begin{tabular}{|l}
50.9 \\
51.3
\end{tabular} & $\frac{11.7}{11.2}$ & & . & \begin{tabular}{|l|l|l|}
.004 \\
008
\end{tabular} & \begin{tabular}{|l|l|}
0.004 \\
0.008
\end{tabular} & 0.000 & 0.105 & \\
\hline 2042 & $6 / 16 / 2004$ & $6: 48: 13 \mathrm{AM}$ & 26.128 & 25.86 & 25.02 & & 25.841 & 47.462 & \begin{tabular}{|l|}
52.967 \\
\end{tabular} & 15.664 & 32.15 & -0.238 & & 46.044 & & 14.643 & & & 45.5583 & 51.5 & & 39.8 & $\frac{2.110}{2.744}$ & & $\begin{array}{l}0.0081 \\
0.011\end{array}$ & & $\begin{array}{l}0.205 \\
0.281\end{array}$ & \\
\hline 2043 & $6 / 16 / 2004$ & $6: 49: 13 \mathrm{AM}$ & 26.099 & & 25.02 & 26.751 & 25.831 & 47.783 & \begin{tabular}{|l|}
52.712 \\
\end{tabular} & 15.527 & 32.747 & -0.817 & -6.146 & 47.264 & $\begin{array}{l}0.037 \\
\end{array}$ & 14.643 & 0.004 & 2734.50 & \begin{tabular}{|l|l|}
45.575 \\
\end{tabular} & 51.2 & 11.6 & 40.3 & 2.776 & 0.006 & 0.006 & 0.000 & & \\
\hline 2044 & 6/16/2004 & $6: 50: 13 \mathrm{AM}$ & 26.113 & & & & & & & & & -0.721 & -5.99 & & & \begin{tabular}{|l|l|}
14.643 \\
\end{tabular} & & 2735.50 & \begin{tabular}{|l|l|}
45.5917 \\
\end{tabular} & 51.2 & 11.4 & & & 0.007 & 0.007 & 0.000 & 0.185 & \\
\hline 2045 & 6/16/2004 & 6:51:13 AM & 26.092 & 25.8 & 25.00 & 26.719 & 25.84 & 48.02 & 52.98 & 15.443 & 33.037 & $\begin{array}{l}-0.759 \\
-7.79\end{array}$ & & 46.3 & $\begin{array}{l}0.049 \\
\end{array}$ & 14.643 & & $\begin{array}{l}2736.50 \\
2770\end{array}$ & \begin{tabular}{|l|l|}
45.6083 \\
\end{tabular} & 51.5 & 11.3 & & 2.794 & 0.007 & 0.007 & 0.000 & 0.180 & \\
\hline 2046 & $6 / 16 / 2004$ & $6: 52: 13 \mathrm{AM}$ & $\frac{26.101}{26.111}$ & $\begin{array}{l}25.8 \\
25.83 \\
\end{array}$ & $\frac{25.02}{25.01}$ & \begin{tabular}{|l|l|}
26.748 \\
26.763
\end{tabular} & $\begin{array}{l}25.839 \\
25864\end{array}$ & \begin{tabular}{|l|l|l|l|l}
47.997 \\
4713
\end{tabular} & \begin{tabular}{|c|}
52.967 \\
52752
\end{tabular} & \begin{tabular}{l|l|}
15.401 \\
15245
\end{tabular} & \begin{tabular}{|c|}
33.086 \\
32.91
\end{tabular} & $\begin{array}{l}-0.736 \\
-0.724\end{array}$ & $\begin{array}{r}-6.01 \\
-6007\end{array}$ & \begin{tabular}{|l|}
45.683 \\
51475 \\
\end{tabular} & \begin{tabular}{|c|}
0.049 \\
0.05 \\
\end{tabular} & $\begin{array}{l}14.643 \\
14663\end{array}$ & & \begin{tabular}{|l|l|}
2737.50 \\
273850
\end{tabular} & \begin{tabular}{|r|r|}
45.625 \\
55.6417 \\
\end{tabular} & $\begin{array}{l}51.5 \\
513 \\
\end{array}$ & $\frac{11.2}{126}$ & 403 & $\begin{array}{l}2.795 \\
2.7799\end{array}$ & \begin{tabular}{|c|c|}
0.007 & \\
0.07 &
\end{tabular} & \begin{tabular}{|l|l|}
0.007 \\
0.007
\end{tabular} & $\begin{array}{l}0.000 \\
0.000\end{array}$ & $\begin{array}{l}0.180 \\
0.185\end{array}$ & \\
\hline 2048 & $6 / 6 / 16 / 2004$ & 3:13 AM & $\frac{26.111}{26.117}$ & $\frac{25.83}{2584}$ & $\frac{25.01}{25.02}$ & 26.1639 & $\frac{25.864}{25.919}$ & $\frac{47.713}{47.995}$ & \begin{tabular}{|l|}
52.152 \\
53.162
\end{tabular} & & $\begin{array}{r}32.91 \\
32.985 \\
\end{array}$ & 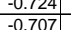 & $\frac{-6.00}{-6.0}$ & $\frac{51.4 / 5}{48.278}$ & $\begin{array}{l}0.05 \\
0.05\end{array}$ & $\frac{14.643}{14.643}$ & & 739.50 & \begin{tabular}{|l|}
45.64117 \\
45.6583
\end{tabular} & & & & & & & & & \\
\hline 2049 & $6 / 16 / 2004$ & 6:55:13 AM & 26.123 & $\frac{2.04}{25.84}$ & $\frac{2.04}{25.02}$ & \begin{tabular}{|l|}
26.87 \\
\end{tabular} & 25.975 & 47.703 & \begin{tabular}{|l|}
52.64 \\
\end{tabular} & 15.463 & 32.684 & -0.713 & $\begin{array}{r}-0.01 \\
-6.01\end{array}$ & $\begin{array}{l}40.270 \\
42.929\end{array}$ & \begin{tabular}{|l}
0.05 \\
0.05
\end{tabular} & $\frac{14.045}{14.643}$ & & 2740.50 & \begin{tabular}{|l|}
45.05000 \\
45.675 \\
\end{tabular} & 51.2 & $\frac{11.8}{10.5}$ & $\begin{array}{l}40.5 \\
40.2 \\
\end{array}$ & & $\begin{array}{l}0.007 \\
0.007\end{array}$ & $\begin{array}{l}0.007 \\
0.007\end{array}$ & & $\frac{0.184}{0.185}$ & 016 \\
\hline & $6 / 16 / 2004$ & 6:56:13 AM & 26.138 & & 25.04 & \begin{tabular}{|l|}
26.926 \\
\end{tabular} & 26.041 & 48.045 & \begin{tabular}{|l|l|l|}
53.011 \\
\end{tabular} & 15.453 & 33.051 & -0.704 & -6.013 & 44.529 & 0.049 & 14.643 & & 2741.50 & \begin{tabular}{|l|l|}
45.6917 \\
\end{tabular} & 51.5 & 10.9 & 40.5 & 2.796 & 0.007 & 0.007 & 0.000 & 0.180 & \\
\hline 0051 & 6/16/2004 & 6:57:13 AM & 26.135 & 25.84 & & 26.942 & 26.112 & 47.871 & & & 32.916 & & & 48.591 & $\begin{array}{l}0.049 \\
\end{array}$ & \begin{tabular}{|l|l|}
14.643 \\
\end{tabular} & & 2742.50 & \begin{tabular}{|l|l|}
45.7083 \\
\end{tabular} & \begin{tabular}{|c|}
51.6 \\
\end{tabular} & 11.9 & 40.4 & 2.785 & 0.007 & $\begin{array}{l}0.007 \\
\end{array}$ & & & \\
\hline 2052 & $6 / 16 / 2004$ & 6:58:13 AM & 26.141 & 25.85 & 25.03 & \begin{tabular}{|l|}
26.994 \\
\end{tabular} & 26.134 & 47.854 & 52.831 & 15.288 & 33.035 & -0.704 & & 47.491 & \begin{tabular}{|l|l|l|} 
\\
\end{tabular} & 14.643 & & & \begin{tabular}{|l|l|} 
\\
45.725 \\
\end{tabular} & 51.4 & 11.6 & & 2.789 & .007 & 0.007 & & 1.180 & \\
\hline & & 6:59:13 AM & & & & & & & & & & -0.704 & & 45.894 & & & & & & & 11.2 & & & & & & & \\
\hline 2054 & $6 / 16 / 2004$ & 7:00:13 AM & 26.154 & 25.87 & 25.04 & 26.982 & 26.08 & 47.899 & \begin{tabular}{|l|}
52.949 \\
\end{tabular} & 15.347 & 32.938 & -0.704 & & 47.477 & \begin{tabular}{|c|}
0.049 \\
\end{tabular} & 14.643 & & 745.50 & \begin{tabular}{|l|l|l|l|l}
4583 \\
\end{tabular} & 51.5 & $\frac{11.6}{11.6}$ & & & & & & & \\
\hline & 6/16/2004 & BAM & & 25.86 & 25.02 & 26.917 & 26.05 & \begin{tabular}{|l|l|} 
\\
\end{tabular} & \begin{tabular}{|l|}
52.973 \\
\end{tabular} & 395 & 32.854 & -0.71 & & 51.732 & 0.049 & 14.643 & & & & 51.5 & 12.7 & & & & & & & \\
\hline & $6 / 16 / 2004$ & $13 \mathrm{AM}$ & 26.147 & 25.8 & & 26.924 & & \begin{tabular}{|l|l}
48.009 \\
\end{tabular} & \begin{tabular}{|l|l|}
53.081 \\
\end{tabular} & 15.387 & & . & & 50.789 & & 14.643 & & 7747.50 & & 51.6 & 12.4 & & 793 & 07 & |0.007 & & & \\
\hline 205 & $6 / 16 / 2004$ & 7:03:13 AM & 26.142 & 25.87: & 25.02 & 26.969 & 26.00 & \begin{tabular}{|l|l|}
47.62 \\
\end{tabular} & 52.495 & 15.325 & 32.78 & -0.707 & & 49.348 & 0.049 & 14.643 & & 7748.50 & 45.8083 & 51.0 & 12.1 & 40. & 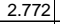 & .007 & 0.007 & . 0000 & 0.182 & \\
\hline 205 & $6 / 16 / 2004$ & & 26.148 & 25.88 & .03 & 26.92 & & 47.759 & 52.748 & 15.401 & 32.762 & -0.704 & & 51.571 & 0.049 & 14.643 & & 749.5 & & 51.3 & 12.6 & & & & & & & \\
\hline & $6 / 16 / 2$ & $: 13 \mathrm{AM}$ & 26.148 & 25.89 & & 26.94 & & 4.866 & 52.974 & 353 & 32.92 & 0.698 & & & & 14.643 & & & & 51.5 & 11.8 & & & & & & & \\
\hline & $6 / 16 / 2004$ & T:060.13 AM & $\frac{26.148}{26.154}$ & 25.899 & 5502 & 26.885 & $\frac{25.9}{25.9}$ & $\begin{array}{l}47.808 \\
48092\end{array}$ & 52.942 & $\frac{15.416}{15.43}$ & $\begin{array}{l}32.788 \\
33.043\end{array}$ & 0.052 & & 5019 & | & 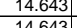 & & & 45875 & & $\frac{14.2}{11.8}$ & & & & & & & \\
\hline & $6 / 16 / 2004$ & & & 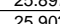 & $\overline{03}$ & $\mid$ & & (4.0.096 & & $\mid$ & 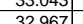 & 0.049 & & $\frac{40.01}{4470}$ & & 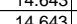 & & & & & & & & & & & & \\
\hline 206 & $\frac{6 / 16 / 2004}{6 / 16 / 2004}$ & SAM & & 2589 & & $\frac{2.064}{26877}$ & & & \begin{tabular}{|l|}
52802 \\
528
\end{tabular} & 捛. & & $\begin{array}{r}-0.05 \\
-0.652\end{array}$ & & 5517 & & $\frac{14.045}{14643}$ & & & & 5.1.4. & $\frac{1.0}{127}$ & $\frac{40}{40}$ & & & & & & \\
\hline 2064 & $6 / 16 / 2004$ & 7:10:13 AM & $\frac{26.155}{26.15}$ & 25.899 & $=5.04$ & $\frac{20.692}{26.892}$ & & 48.163 & $\mid$\begin{tabular}{|l|}
5.32 .291 \\
5
\end{tabular} & 年. & 3..53.15 & 年-.649 & & |c..851 & | & 14.643 & & 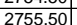 & 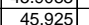 & 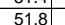 & $\frac{10.7}{10.7}$ & $\frac{40}{40}$ & 803 & & 0.007 & 0.000 & 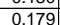 & \\
\hline & & & & & & 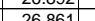 & & & & & & & & & & & & & & & & & & & & & & \\
\hline 06 & 6/16/ & & $\frac{26.143}{26}$ & 25.89 & .05 & 26.875 & & 48.209 & 53.175 & 5.44 & & $\frac{-.0649}{-0.649}$ & & 48.47 & & $\frac{14.643}{14.643}$ & & & & 51.7 & $\frac{x_{11.9}}{11.9}$ & & & & & & 179 & \\
\hline 2067 & & & 26.153 & 25.88 & 25.05 & 26.925 & & \begin{tabular}{l|l|}
47.657 \\
\end{tabular} & \begin{tabular}{|l|}
52.772 \\
\end{tabular} & \begin{tabular}{|l|l|l|}
15.34 \\
\end{tabular} & 32.684 & -0.649 & & 55.843 & \begin{tabular}{|l|l|}
0.049 \\
\end{tabular} & 14.643 & & & & & 10.7 & & & & & & & \\
\hline & $6 / 16 / 2004$ & 7:14:13 AM & 26.153 & 25.89 & 504 & 26.97 & 25.95 & \begin{tabular}{|l|l|}
48.07 \\
\end{tabular} & 53.237 & 15.406 & 33.086 & -0.652 & & 40.61 & 0.049 & 14.643 & & & & 51.8 & 10.0 & & & & & & 0.180 & \\
\hline & 6/16/2004 & & & 25.89 & 25.04 & 26.92 & & 47.702 & \begin{tabular}{|l|l|}
52.911 \\
\end{tabular} & 15.247 & & -0.646 & & & & 14.643 & & & & 51.4 & 13.7 & & & & & & & \\
\hline 2070 & 6/16/2004 & $B$ AM & & 25.88 & & 26.888 & & & 52.86 & 15.209 & & & & & & 14.643 & & & & 51.4 & 11.7 & & & & & & & \\
\hline 207 & $6 / 16 / 2004$ & & 26.145 & 25.87 & 25.01 & 26.872 & & 48.011 & 53.359 & 15.248 & 33.144 & -0.614 & & 47.176 & 0.049 & 14.643 & & 7762.50 & \begin{tabular}{|l|l|}
46.0417 \\
\end{tabular} & 51.9 & 11.6 & & 79 & & 0.00 & 0.000 & 0.180 & \\
\hline 0.72 & & & & & . & 20.917 & & & & $k+0$ & & & & & & & & & & - & 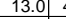 & & & & & & & \\
\hline nit & & & & & & 26.912 & & & & & & & & & & & & & & -4 & & & & & & & & \\
\hline & & & & & & 2.9 .914 & & & & & & & & & & & & & & & & & & & & & & \\
\hline 076 & $6 / 16 / 2$ & & & $\frac{2.00}{2507}$ & $\frac{25.05}{2498}$ & 26.911 & & & \begin{tabular}{|l|l|l|l|}
5305 \\
5
\end{tabular} & 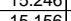 & & & & & & & & & & & & & & & & & & \\
\hline 2077 & $6 / 16 / 2$ & & & & & $\frac{2.0561}{26961}$ & & & & & & & & & & $\frac{1.46}{146}$ & & & & $\frac{3.6}{519}$ & $\frac{1.44}{128.25}$ & & & & & & & \\
\hline 年 2078 & $6 / 6 / 1 / 2004$ & $\frac{1: 2424: 13 \mathrm{AM}}{7}$ & $\frac{2.1 .144}{26.14}$ & $\frac{25.87}{25.87}$ & 24.98 & $\frac{2.0 .907}{26.907}$ & & 48.036 & $\mid$\begin{tabular}{|c|}
5.284 \\
5.324
\end{tabular} & $\mid$ & & & & & o. & 14.64 & & 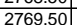 & & 告1.8 & $\frac{1.5}{12.5}$ & & & & & & 0184 & \\
\hline & & & & & & & & & & & & & & & & & & & & & & & & & & & & \\
\hline 0007 & & & $\frac{26.1}{26.1}$ & & & 26.972 & & & 53 & & & & & & & & & & & & & & & & & & & \\
\hline 2081 & $6 / 16 / 2$ & & & & 24.97 & 26.927 & & & & 15.202 & 33.185 & & & & & 14.6 & & & & & & & & & & & & \\
\hline$\overline{0.8}$ & $6 / 16 /$ & 7:28:13 AM & 26.136 & 25.86 & 24.9 & $26.95 \mathrm{~S}$ & & & 52.91 & & 33.1 & & & & & 14.6 & & & & 51 & 5 & & & & & & & \\
\hline 208 & $6 / 16 / 2$ & $7: 29: 13 \mathrm{AM}$ & & & & 26.934 & & $47 . .5$ & \begin{tabular}{|l|l|}
53.069 \\
\end{tabular} & 15 & & -0.542 & & 46.9 & & 14.6 & & & & 51.6 & 5 & & & & & & & \\
\hline 2084 & 6/16/2 & & & 25.8 & 24.95 & 26.919 & & 48.1 & \begin{tabular}{|l|}
53.258 \\
\end{tabular} & & & & & & & & & & & & 3 & & & & & & & \\
\hline 2085 & & & & & 24.94 & 26.934 & & 48.1 & 53.4 & 14.867 & & -0.542 & & & & 14.6 & & & & $51 .{ }_{5}$ & 3.4 & & & & & & & \\
\hline 2086 & & & & & & & & & & & & & & & & & & & & & 1 & & & & & & & \\
\hline 2087 & $6 / 16 / 2004$ & 7:33: & 26.128 & & 24.93 & 26.88 & & & & 15.22 & & & & & & & & & & & 1.9 & & & & & & & \\
\hline 5089 & $6 / 1012044$ & T.3.5.15 AM & $\frac{26.11}{2.10}$ & & 19 & $\mid \frac{2.873}{2.823}$ & & & 5 & 1 & $\begin{array}{l}32.809 \\
33041\end{array}$ & & & & & & & & & & 6 & & & & & & & \\
\hline 2010 & $\frac{0}{6} 6$ & 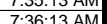 & $\frac{2.1424}{26118}$ & $\frac{2.04}{2585}$ & & $\frac{2.0044}{26951}$ & & & 年 & 15.024) & 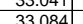 & $\begin{array}{l}-0.5435 \\
-0.533\end{array}$ & & & & & & & & & 1 & & & & & & & \\
\hline 2091 & & & & & & 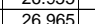 & & & & & & & & & & & & & & & & & & & & & & \\
\hline 2092 & & & & & & $\frac{26.926}{26.26}$ & & & 53.15 & $\mid$ & & $\frac{-.5548}{-0.548}$ & & & & & & & & & & & & & & & & \\
\hline 2093 & & & & & & & & & & & & & & & & & & & & & & & & & & & & \\
\hline & & & & & & & & & & & & & & & & & & & & & & & & & & & & \\
\hline 095 & $6 / 16 / 2004$ & & 2611 & & 24.93 & 27.009 & & 48. & 534 & & & & & & & & & & & & & & & & & & & \\
\hline 209 & $6 / 16 / 2$ & $7: 42: 13 \mathrm{AM}$ & $\overline{26.11}$ & 25.8 & $\overline{24}$ & 27.11 & & 47.748 & & & & & & & & $\overline{146}$ & & & & & 2 & 40 & & & & & & \\
\hline 209 & $6 / 16 / 2004$ & $7: 43$ & 26.12 & 25.86 & $4.93 \quad$ & 27.122 & & $47.7 \mathrm{C}$ & 52.91 & 15.19 & 33.014 & & & & & & & & & 514 & 17 & & & & & & & \\
\hline 2098 & $6 / 16 / 2004$ & 7:44:13 & 26.12 & 25.87 & 24.9 & 27.145 & & 47.8 & 52.996 & & 33.047 & -0.4 & & & & & & & & 1.5 & & & & & & & & \\
\hline 0999 & & 7:45:13 AM & 26.13 & 25.88 & \begin{tabular}{|l|l|}
24.95 \\
\end{tabular} & 27.222 & & 47.742 & & 15.497 & 32.624 & & -0.96 & 46.3 & & 14.6 & & 790.5 & & 51.6 & 11.4 & & 2.770 & & & & .182 & \\
\hline 100 & 6/16/2004 & 7:46:13 AM & 26.14 & 25.89 & \begin{tabular}{|l|}
24.96 \\
\end{tabular} & $\mid 27.202$ & & 47.57 & 52.849 & 15.755 & 32.212 & -0.481 & & 48.23 & 0.048 & \begin{tabular}{|l|l|}
14.643 \\
\end{tabular} & & & & $51.4 \mid$ & 11.8 & & 2.750 & 0.007 & $|0.007|$ & 0.000 & 0.180 & \\
\hline
\end{tabular}


WSRC-TR-2005-00105, REVISION 0

SRNL-RPP-2005-00012, REVISION 0

RUN \# 3.03A AND B; FIRST AND SECOND HALF OF SLURRY DEWATERING - CONT.

\begin{tabular}{|c|c|c|c|c|c|c|c|c|c|c|c|c|c|c|c|c|c|c|c|c|c|c|c|c|c|c|c|c|}
\hline & & & \begin{tabular}{l|l} 
D \\
\end{tabular} & $\mathrm{E}$ & $\mathrm{F}$ & \begin{tabular}{l|l}
$G_{1}$ \\
\end{tabular} & \begin{tabular}{ll|}
$\mathrm{H}$ \\
\end{tabular} & $\mathrm{J}$ & $\begin{array}{ll}\mathrm{K} \\
\end{array}$ & \begin{tabular}{|l|} 
\\
\end{tabular} & M & \begin{tabular}{|l|l|} 
\\
\end{tabular} & $\begin{array}{ll}0 \\
\end{array}$ & \begin{tabular}{l|l} 
\\
\end{tabular} & \begin{tabular}{l|l}
$\mathrm{R}$ & \\
\end{tabular} & \begin{tabular}{l|l} 
s \\
\end{tabular} & $T$ & $\mathrm{v}$ & \begin{tabular}{l|l} 
w \\
\end{tabular} & \begin{tabular}{l|l}
$x$ \\
\end{tabular} & \begin{tabular}{l|l} 
&
\end{tabular} & & & & AC & & $\overline{\mathrm{AE}}$ & \\
\hline 101 & \begin{tabular}{|c|}
$6 / 16 / 2004$ \\
\end{tabular} & $7: 47: 13 \mathrm{AM}$ & 26.151 & 25.924 & 24.982 & 27.163 & 26.288 & 47.852 & 53.067 & \begin{tabular}{|l|}
5.49 \\
\end{tabular} & \begin{tabular}{|l|l|}
32.776 \\
\end{tabular} & \begin{tabular}{|l|}
-0.481 \\
\end{tabular} & -5.961 & \begin{tabular}{|l|}
46.279 \\
\end{tabular} & 0.049 & \begin{tabular}{|l|l|}
14.643 \\
\end{tabular} & 0.004 & 2792.50 & \begin{tabular}{|l|l|}
46.5417 \\
\end{tabular} & 51.6 & 11.3 & 40.3 & 2.780 & 0.007 & 0.007 & 0.000 & 0.181 & 15 \\
\hline & & & 26.154 & 25.927 & 24.985 & 27.186 & 26.231 & 47.445 & \begin{tabular}{|l|}
52.698 \\
\end{tabular} & \begin{tabular}{|l|}
15.371 \\
\end{tabular} & 32.513 & \begin{tabular}{|l|l|}
-0.478 \\
\end{tabular} & $\begin{array}{r}-5.961 \\
-5.96\end{array}$ & 55.795 & 0.048 & \begin{tabular}{|l}
14.643 \\
\end{tabular} & & & & 51.2 & $\begin{array}{l}13.7 \\
13.7 \\
\end{array}$ & & & & & & & \\
\hline 2103 & $6 / 16 / 2004$ & $7: 49: 13 \mathrm{AM}$ & $\frac{26.139}{26.130}$ & 25.917 & 24.985 & 27.171 & $\begin{array}{l}26.186 \\
2.186\end{array}$ & 47.609 & \begin{tabular}{|l|}
52.967 \\
561
\end{tabular} & \begin{tabular}{|l|l|}
15.3799 \\
\end{tabular} & \begin{tabular}{|l|l|}
32.698 \\
2.26
\end{tabular} & \begin{tabular}{|l|l|}
-0.481 \\
\end{tabular} & $\begin{array}{l}-5.964 \\
-5969\end{array}$ & $\begin{array}{l}48.474 \\
1949\end{array}$ & 0.048 & \begin{tabular}{|l}
14.643 \\
\end{tabular} & & $\frac{2794.50}{2705}$ & \begin{tabular}{|l|l|}
56.575 \\
\end{tabular} & 51.5 & $\frac{11.9}{1.9}$ & 40.2 & . & . 007 & 0.007 & 0.000 & 0.178 & \\
\hline $\begin{array}{l}2104 \\
2105 \\
105\end{array}$ & 6/16/2004 & 7:50:13 AM & \begin{tabular}{|l|l|}
26.138 \\
\end{tabular} & 25.927 & 24.974 & 27.23 & 26.165 & $\begin{array}{l}47.47 \\
\end{array}$ & \begin{tabular}{|l|}
52.613 \\
\end{tabular} & \begin{tabular}{|l|l|}
15.415 \\
\end{tabular} & $\begin{array}{l}32.466 \\
\end{array}$ & -0.478 & $\begin{array}{l}-5.958 \\
-50 .\end{array}$ & \begin{tabular}{|l|l}
49.406 \\
\end{tabular} & 0.048 & 14.643 & 0.004 & 2795.50 & $\mid 46.5917$ & 51.1 & 12.1 & 40.0 & 2.756 & $\begin{array}{l}0.007 \\
\end{array}$ & $\begin{array}{l}0.007 \\
\end{array}$ & 0.000 & 0.179 & \\
\hline$\frac{2105}{2106}$ & $\frac{6 / 16 / 2004}{6 / 16 / 2004}$ & $7: 51: 13 \mathrm{AM}$ & \begin{tabular}{|l|l|}
26.153 \\
26.153
\end{tabular} & $\begin{array}{l}25.947 \\
25.952\end{array}$ & & & & & \begin{tabular}{|l|}
$\mid 53.247$ \\
52.85 \\
\end{tabular} & \begin{tabular}{|l|}
15.368 \\
15367 \\
\end{tabular} & & \begin{tabular}{|r|}
-0.461 \\
-0.788 \\
\end{tabular} & & & $\begin{array}{l}0.048 \\
00747\end{array}$ & \begin{tabular}{|l|}
14.643 \\
14643 \\
\end{tabular} & & & & \begin{tabular}{|l|}
51.8 \\
51.
\end{tabular} & \begin{tabular}{|l|l|}
11.5 \\
\end{tabular} & 40.6 & $\begin{array}{l}2.796 \\
2.771 \\
\end{array}$ & \begin{tabular}{|l|l|}
0.007 \\
\end{tabular} & \begin{tabular}{|c|}
0.007 \\
\end{tabular} & & \begin{tabular}{|l|l|}
0.177 \\
\end{tabular} & \\
\hline 2107 & $6 / 6 / 16 / 2004$ & $\begin{array}{r}7: 52.13 \mathrm{AM} \\
7: 53: 13 \mathrm{AM}\end{array}$ & $\frac{26.153}{26.146}$ & $\begin{array}{l}25.952 \\
25.954\end{array}$ & $\frac{24.9899}{24.962}$ & $\begin{array}{r}27.26 \\
27.293\end{array}$ & $\frac{26.155}{26.138}$ & \begin{tabular}{|l|l}
47.669 & \\
47.8
\end{tabular} & \begin{tabular}{|l|}
$\mid 52.855$ \\
53.906
\end{tabular} & \begin{tabular}{|l|}
15.367 \\
15.423
\end{tabular} & \begin{tabular}{|r|}
32.7715 \\
32.778
\end{tabular} & \begin{tabular}{|c|}
-0.448 \\
-0.47
\end{tabular} & $\begin{array}{l}-\frac{-5.958}{-5.953} \\
\end{array}$ & \begin{tabular}{|}
$\mid 48.501$ \\
49.752
\end{tabular} & $\frac{0.047}{0.048}$ & \begin{tabular}{|l|}
14.643 \\
14.643
\end{tabular} & $\frac{0.004}{0.004}$ & $\frac{2779.50}{2798.50}$ & $\mid \begin{array}{r}46.625 \\
\mid\end{array}$ & \begin{tabular}{|c|}
$\mid 51.4$ \\
51.6 \\
\end{tabular} & $\begin{array}{l}11.9 \\
12.2\end{array}$ & $\frac{40.2}{40.3}$ & 2.7771 & $0.007 \mid$ & \begin{tabular}{|}
$\mid 0.007$ \\
0.007
\end{tabular} & $\begin{array}{l}0.000 \\
0.000\end{array}$ & \begin{tabular}{|l|}
0.174 \\
0.178
\end{tabular} & 0.15 \\
\hline 2108 & $6 / 16 / 2004$ & 7:54:13 AM & 26.15 & 25.963 & & & & & & & & & -5.95 & & 0.049 & 14.643 & 0.004 & & & 51.4 & $\begin{array}{ll}\frac{11.2}{11.8} \\
1\end{array}$ & $\begin{array}{l}40.3 \\
40.2\end{array}$ & $\frac{2.770}{2.770}$ & $\begin{array}{l}0.007 \\
0.007\end{array}$ & \begin{tabular}{|l|}
0.007 \\
0.007
\end{tabular} & & & $\frac{0.15}{0.15}$ \\
\hline & & & & 25.974 & 24.976 & 27.272 & 26.142 & 47.595 & \begin{tabular}{|l|}
52.866 \\
\end{tabular} & 15.352 & & -0.435 & & 48.317 & 0.047 & 14.643 & & & 46.675 & 51.4 & 11.8 & & & & 0.007 & & & \\
\hline 2110 & $6 / 16 / 2004$ & $7: 56: 13 \mathrm{AM}$ & 26.175 & 25.984 & & 27.257 & 26.157 & 47.68 & \begin{tabular}{|l|}
53.05 \\
\end{tabular} & \begin{tabular}{|l|l|}
15.33 \\
\end{tabular} & \begin{tabular}{|l|l|}
32.799 \\
\end{tabular} & -0.426 & & & 0.048 & $\begin{array}{l}14.643 \\
\end{array}$ & & 2801.50 & & \begin{tabular}{|c|}
51.6 \\
\end{tabular} & 111.8 & 40. & & & $\mid 0.007$ & & & \\
\hline 2111 & $6 / 16 / 2004$ & & 26.165 & & 24.966 & 27.247 & & & 53.363 & 15.268 & & & & 49.362 & & 14.643 & & & | & & & & & 007 & 0.007 & 0.000 & 0.176 & \\
\hline 2112 & & 7:58:13 AM & & & & & & 47.889 & & & & -0.42 & & 40.612 & & 14.643 & & & & 51.9 & $\frac{10.0}{10.0}$ & & 789 & & 0.007 & & 市.181 & \\
\hline 2113 & & 7:59:13 AM & 26.161 & 25.984 & 24.962 & 27.193 & & & 53.09 & 15.314 & 32.84 & & & & & 14.643 & & & & 516 & 11.7 & & & & & & & \\
\hline 2114 & 6/16/2004 & :13 AM & 26.171 & & 24.968 & 27.198 & & $\begin{array}{l}47.57 \\
\end{array}$ & 52.793 & 15.235 & 32.813 & -0.426 & -5.95 & 51.596 & $\mid$\begin{tabular}{|c|c|}
0.048 \\
\end{tabular} & 14.643 & & 805.50 & \begin{tabular}{|l|l|}
46.7583 \\
\end{tabular} & 51.3 & 12.6 & & & & 0.007 & & & \\
\hline 2115 & 6/16/2004 & $8: 01: 13 \mathrm{AM}$ & 26.161 & 25.979 & 24.942 & 27.218 & 26.108 & 47.854 & 53.173 & 15.308 & 32.948 & -0.426 & -5.944 & 45.493 & 0.049 & 14.643 & & 8006.5 & 46.775 & 51.7 & 11.1 & 40. & 2.786 & .007 & 0.007 & & & \\
\hline & & :13 AM & 26.168 & 26.002 & 24.949 & 27.225 & & $\begin{array}{r}47.75 \\
70 ? 5\end{array}$ & 53.222 & $\begin{array}{l}15.284 \\
15225\end{array}$ & $\begin{array}{l}32.848 \\
207\end{array}$ & $\begin{array}{l}-0.426 \\
-0.26\end{array}$ & -5.941 & 46.567 & 0.047 & 14.643 & & & 46.7917| & 51.7 & & & & & & & & \\
\hline t14t & $6 / 16 / 2004$ & : $113 \mathrm{AM}$ & 26.17 & 2. 25.998 & 24.956 & & & 47.935 & 53.427 & 15.252 & 33.07] & -0.426 & & 50.591 & 0.048 & 14.643 & & & & 52.0 & 12.4 & & & & 007 & & & \\
\hline 118 & $6 / 16 / 2004$ & $324 \mathrm{AM}$ & 26.154 & & 24.93 & 27.236 & & & 5.3.311 & 15.321 & 33.059 & 0.426 & & 40.11 & & & & & 8124 & & & & & & & & & \\
\hline & & $24 \mathrm{AM}$ & $\frac{26.16}{26.16}$ & $\frac{26.003}{2600^{3}}$ & 24.9966 & $\frac{27.236}{27202}$ & & 47.947 & 53.286 & $\frac{15.321}{10.9}$ & .3.02 & $\begin{array}{l}-0.426 \\
0.423\end{array}$ & & & & 14.643 & & & & 5 & 212.0 & & & & 0.007 & & . & \\
\hline 2121 & 6 & A AM & $\frac{2.0 .00}{26.17}$ & 26013 & 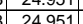 & 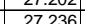 & & $\frac{41.003}{4819}$ & $\begin{array}{l}53.014 \\
53.496\end{array}$ & 势. & & & & 45643 & 2049 & 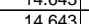 & & & & & 3.4 & & & & 0007 & 0.0000 & & \\
\hline 2122 & $6 / 16 / 2004$ & 7:24 AM & $\frac{20.11}{26.164}$ & $\frac{2.010}{25.997}$ & 年. & 27.186 & 26.126 & 47.644 & \begin{tabular}{|l|l|} 
& 52.816 \\
\end{tabular} & 15.85 & 32.169 & $\begin{array}{l}-0.426 \\
-0.426\end{array}$ & -5.941 & 50.743 & 0.047 & $\begin{array}{l}\frac{1.045}{14.643} \\
\end{array}$ & & 2812.68 & $\mid 46.8781$ & 51.3 & 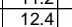 & & 75 & .007 & 0.007 & 0.000 & 0.176 & \\
\hline & $6 / 16 / 20$ & $88: 24 \mathrm{AM}$ & 26.164 & 26.002 & 24.955 & 27.196 & 26.12 & 47.447 & \begin{tabular}{|l|l|} 
& 52.768 \\
\end{tabular} & 15.738 & 32.113 & -0.426 & -5.941 & 50.401 & 0.048 & 14.643 & & $\begin{array}{l}2813.68 \\
213\end{array}$ & \begin{tabular}{|l|l|}
346.8947 \\
\end{tabular} & & $\frac{1.24}{12.4}$ & & $\frac{104}{744}$ & & 0.007 & 0.000 & & \\
\hline 2124 & $6 / 16 / 2004$ & :24 AM & 26.172 & 26.02 & 24.988 & & & 47.742 & 53.087 & 15.702 & 32.425 & -0.426 & & & & 14.643 & & 814.68 & 46.9114 & 51.6 & & & & & & & & \\
\hline 2125 & 6/16/2004 & $0: 24 \mathrm{AM}$ & 26.158 & 26.006 & 24.994 & 27.18 & 26. & 47.678 & 53.013 & 15.489 & .632 & -0.386 & & 49.318 & 0.048 & 14.643 & & & .9281 & & 12.1 & & 769 & 007 & 0.007 & & $\frac{178}{178}$ & \\
\hline & & .24 AMI & & & 24.985 & 27.17 & & & & & & -0.394 & & 46.947 & 0.047 & 14.643 & & & & & $\frac{11.5}{11.5}$ & & & & & & & \\
\hline ל127 & $6 / 16 / 2004$ & 8:12:24 AM & 26.164 & 26.013 & 24.975 & 27.206 & 26.12 & 47.416 & \begin{tabular}{|l|}
52.918 \\
\end{tabular} & 15.429 & 32.388 & -0.417 & & 49.836 & 0.048 & 14.643 & & & & & 12.2 & & & & & & & \\
\hline 2128 & 6/16/2004 & & 26.164 & 26.003 & 24.965 & 27.146 & 26.1 & 47.447] & \begin{tabular}{|l|l|} 
& 53.079 \\
\end{tabular} & 15.493 & 32.321 & -0.423 & & 488.622 & 0.048 & 14.643 & & 68 & \begin{tabular}{|l|l|}
3 & 46.9781
\end{tabular} & 51.6 & 11.9 & & & & & & & \\
\hline 2125 & $6 / 1$ & 4:24 AM & 26.179 & 26.013 & 24.97 & 27.176 & 26.1 & 47.605 & \begin{tabular}{|l|l|} 
\\
\end{tabular} & 15.463 & 32.485 & -0.426 & -5.93 & 54.166 & $\mid$ & 14.643 & & 819 & 46.9947 & 51.6 & 13.3 & 40. & .76. & 007 & 0.007 & & & \\
\hline & & & 26.169 & 26.013 & 24.96 & 27.236 & & 47.422 & 52.826 & 15.418 & & & & .238 & & 14.643 & & & & & 8 & & & & & & & \\
\hline & 04 & $24 \mathrm{AM}$ & 26.179 & & 24.96 & & & 47.464 & 52.69 & 15.45 & & -0.36 & & 47.349| & $0.04 \pi$ & 14.643 & & & & & 11.6 & & & & & & & \\
\hline 2132 & $6 / 16 / 22$ & 7:24 AM & 26.173 & & 24.959 & 27.25 & & 4.479 & 52.82 & 15.405 & 32.532 & $-0.3 / 1$ & & 43.013 & & & & & & & 10.5 & & & & & & & \\
\hline 2134 & $6 / 16 / 1 / 2004$ & $8: 18: 24 \mathrm{AM}$ & \begin{tabular}{|l|l|l|}
26.165 \\
226186
\end{tabular} & $\frac{26.014}{26.025}$ & $\begin{array}{ll}24.946 \\
24957\end{array}$ & $\frac{27.237}{27238}$ & $\frac{26.1}{261}$ & $\begin{array}{l}47.508 \\
47804\end{array}$ & 52.996 & 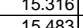 & 32.604 & 2357 & & & 0.047 & $\frac{14.643}{14643}$ & & & & 步 & 18 & & & & & & & \\
\hline 2135 & $6 / 16 / 2004$ & & & 26.02 & 24.928 & 27.254 & & $\begin{array}{l}47.004 \\
47.435\end{array}$ & $\mid$\begin{tabular}{|l|l|l|l|l|}
52.669 \\
\end{tabular} & 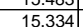 & 32.528 & $\frac{0.07}{-0.4}$ & & 50.668 & 0.0 & 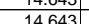 & & & & $\frac{5.1}{512}$ & 124 & & & & & & & \\
\hline 2136 & $6 / 16 / 2004$ & $1: 24 \mathrm{AM}$ & 26.187 & $\begin{array}{l}26.03 \\
26.03\end{array}$ & 年 & 27.294 & & 47.871 & \begin{tabular}{|l|}
53.208 \\
\end{tabular} & 15.418 & & -0.383 & & & & $\frac{1.045}{14.643}$ & & & & $\frac{U 1.2}{51.7}$ & $\frac{\frac{1}{13.4}}{13.1}$ & & & & 0.00 & & 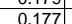 & \\
\hline 2137 & 6/16/2004 & $8: 2$ & 26.193 & 26.041 & 24.944 & 27.275 & & $\begin{array}{l}47.7 \\
\end{array}$ & $|53.172|$ & 15.397 & 32.684 & & & & 0.048 & 14.643 & & & & & 11.2 & & & & & & & \\
\hline & & & & & 24.96 & & & 47.236 & & 15.579 & & & & & & & & & & & 110 & & & & & & & \\
\hline 2139| & S/2004 & 4:24 A & 26.188 & & 24.9 & 27.33 & & 477.404 & 52.82 & 15.63 & 32.1 & -0.357 & & & 0.6 & 14.643 & & & 47.1614 & 51.3 & 12.3 & & & & & & 0.176 & \\
\hline 140 & & & & & 24.94 & 27.301 & & 47.2 & & & & & & & & 14.643 & & & & & 13.4 & & & & & & & \\
\hline ל141 & $6 / 16 / 2$ & $24 \mathrm{AM}$ & 26.196 & & 24.947 & $27.328 \mid$ & & 47.3 & \begin{tabular}{|l|l|}
52.76 \\
\end{tabular} & 15.789 & & \begin{tabular}{|l|l|} 
& -0.3 \\
\end{tabular} & & & $0 . c^{0}$ & 14.643 & & & & 51.3 & 11.7 & & & & & & 0.173 & 3 \\
\hline 2142 & 6/16/2004 & $8: 4$ & & & 24.953 & 27.369 & & 47.1 & \begin{tabular}{|l|l|l} 
\\
\end{tabular} & 15.732 & 31.8 & - -0.357 & & & & 14.643 & & & & 51.0 & 12.2 & & & & 0.001 & & 0.178 & \\
\hline 2143 & $6 / 16 / 2004$ & $8: 2$ & 26.212 & & 24.963 & 27.399 & & & \begin{tabular}{|l|l|} 
\\
\end{tabular} & & & -0.3 & & & & 14.643 & & & & 51.4 & 12.6 & & & & & & 0.179 & \\
\hline 1144 & 6/20004 & & 26.208 & & 24.959 & 27.405 & & 47.649 & \begin{tabular}{|l|} 
\\
\end{tabular} & 15.642 & 32.442 & -0.3 & & $33 . .5$ & 0.047 & 14.643 & & & & 51.6 & 8.3 & & & & 0.007 & & & \\
\hline 145 & $6 / 11$ & & & & 24.935 & 27.406 & & & & 15.62 & & & & & & & & & & 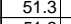 & 12.1 & & & & & & 0.176 & \\
\hline & & & & & & & & & & & & & & & & & & & & & 10.8 & & & & & & & \\
\hline$\frac{14 \pi}{148}$ & & 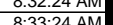 & $\frac{26.214}{26209}$ & $\frac{26.112}{26.27}$ & 24.955 & \begin{tabular}{|l|l|}
27.406 \\
27.45
\end{tabular} & & $\frac{47.6}{474}$ & \begin{tabular}{|r|}
53.22 \\
52.937 \\
\end{tabular} & $\frac{15}{15}$ & . & $\begin{array}{r}-0.36 \\
-0.357 \\
\end{array}$ & & & 0.047 & $\frac{14.643}{14643}$ & & & & 5.5 & $\frac{1.60}{132}$ & & & & & & 76 & t \\
\hline 514 & $6 / 16 / 2004$ & & & & 24.971 & 27.44 & & & $\mid$\begin{tabular}{|}
$\mid 5252$ \\
5225
\end{tabular} & 15694 & & & & & & 146 & & & & & & & & & & & & \\
\hline t2. & 6 & & & & 年 & 27040 & & & $\mid$ & & & & & & & $\frac{14.64}{1.61}$ & & & & & $\frac{11.4}{11.9}$ & & & & & & 0.1777 & \\
\hline 215 & & & $\frac{26.226}{20.626}$ & & 25.007 & $\frac{\mid c 7.428}{27.428}$ & & & $\mid 52.841$ & 15.599 & & & & & & & & & & & $\frac{23.1}{13.1}$ & & & & & & 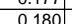 & \\
\hline & & & & & & & & & & & & & & & & & & & & & t. & & & & & & & \\
\hline 215 & & & $\frac{2.24}{26.24}$ & & 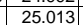 & 27.459 & & & 5.52. & 15.57 & & & & & & & & & & 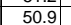 & & & & & & & & \\
\hline & & & & & 24.978 & 27.424 & & & 52.486 & & & & & & & & & & & & & & & & & & & \\
\hline & $6 / 16 / 2$ & $8: 40: 24$ & 26.2 & 26.1 & 24.99 & 27.44 & & 47.2 & \begin{tabular}{|l|}
52.596 \\
\end{tabular} & $\begin{array}{l}15.58 \\
\end{array}$ & & & & & & & & & & 51.1 & 10.9 & & & & & & & 7 \\
\hline 21 & $6 / 16 / 2 C$ & & 26.238 & 26.17 & 24.985 & 27.455 & & 47.3 & \begin{tabular}{|l|l|l|l|l|l|} 
\\
\end{tabular} & 15.588 & 32.1 & - 0.348 & & & & 14.6. & & & 347.44 & 51.3 & 11.5 & & & & & & $\overline{0.180}$ & \\
\hline 215 & & & 26 & & 24.976 & 27.442 & & & & 15.66 & & & & & & $14.6 \mathrm{C}$ & & & & 51. & 6 & & & & & & & 6 \\
\hline 2158 & & & 26.235 & & 24.946 & 27.402 & & 46.889 & \begin{tabular}{|l|} 
\\
\end{tabular} & 15.528 & & -0.36 & & & & & & & & 50.7 & 11.2 & & & & & & 0.178 & 8 \\
\hline$\frac{15}{16}$ & & & & & 24.97 & 27.47 & & & & & & & & & & & & & & & & & & & & & & \\
\hline 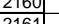 & & & & & 24.96 & 2.7 .452 & & & 53.6 & & & & & & & & & & & 51.6 & & & & & & & & \\
\hline & & & $\mid$ & $\frac{2.19}{26.19}$ & 2 & 27.447 & & & $\begin{array}{l}5.2 .095 \mid \\
5.67]\end{array}$ & | & & & & & & & & & & 512 & 5 & & & & & & & $\begin{array}{l}0 \mid \\
6\end{array}$ \\
\hline$\overline{516}$ & $\frac{1131 / 20}{6 / 6 / 20}$ & & $\frac{2.425 \mid}{26256}$ & $\frac{2.0344}{262.14}$ & 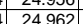 & $\frac{27.452}{27,48}$ & & 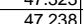 & 52.54 & 捛. & $\frac{3.254 \mid}{32107}$ & $\frac{0.055}{-0.316}$ & & 50.04 & & $\frac{1.46}{146}$ & & & & 51.4 & (4) & & & & & & & \\
\hline 10.64 & & 8.49 .24 & & $\frac{2.21}{26211}$ & 24 & 年, 27408 & & & & & & $\begin{array}{l}-\frac{0.313}{-0.313} \\
\end{array}$ & & 51961 & & 146 & & & & & 7 & & & & & & & \\
\hline 216 & & & $26.25 \mathrm{~S}$ & $\frac{2.6 .20}{26.20}$ & 24.952 & 27.492 & & 47.499 & & & & & & & & $\frac{14.6}{14.6}$ & & & & & 8 & & & & & & & \\
\hline 166 & & & & & 24.95 & & & & & & & & & & & & & & & & & & & & & & & \\
\hline & & & & & & 1750 & & & & & & & & & & & & & & & & & & & & & & \\
\hline 2168 & & $8: 53: 24 \mathrm{~A}$ & 26.245 & 26.21 & 24.956 & 27.446 & & 47.3 & 52.818 & & & -0.31 & & & & 146 & & & & & 8 & & & & & & & \\
\hline & & & 2625 & & $24.986 \mid$ & 27.436 & & & & 15.44 & & -0.3 & & 198. & & 4.62 & & & & & & & & & & & & \\
\hline 2170| & 6/16/2004 & $8: 55: 24 \mathrm{AM}$ & $26.25 \mid$ & $\mid 26.224$ & $\mid 24.962$ & $|27.447|$ & 26.352 & 47.23 & \begin{tabular}{|l|l|} 
\\
\end{tabular} & 15.442 & 32.173 & \begin{tabular}{|l|} 
\\
\end{tabular} & 5.889 & 55.355| & 0.047 & 14.643 & & & \begin{tabular}{|l|l|}
47.6781 \\
\end{tabular} & & 13.6 & & 2.737 & $\mid 0.007$ & 0.007 & 0.000 & $\mid$\begin{tabular}{|l|l|l|} 
\\
\end{tabular} & \\
\hline
\end{tabular}


WSRC-TR-2005-00105, REVISION 0

SRNL-RPP-2005-00012, REVISION 0

RUN \# 3.03A AND B; FIRST AND SECOND HALF OF SLURRY DEWATERING - CONT.

\begin{tabular}{|c|c|c|c|c|c|c|c|c|c|c|c|c|c|c|c|c|c|c|c|c|c|c|c|c|c|c|c|}
\hline & $\mathrm{A}$ & $\mathrm{B}$ & $\mathrm{D}$ & $E$ & $\mathrm{~F}$ & G & $\mathrm{H}$ & $\mathrm{J}$ & $\mathrm{K}$ & $\mathrm{L}$ & $M$ & $\mathrm{~N}$ & 0 & Q & $\mathrm{R}$ & $\mathrm{s}$ & $T$ & $\mathrm{v}$ & w & $x$ & \begin{tabular}{l|l} 
\\
\end{tabular} & \begin{tabular}{|l|l|}
$\mathrm{Z}$ & $\mathrm{AA}$ \\
\end{tabular} & $A B$ & $A C$ & $A D$ & $\mathrm{AE}$ & \\
\hline & 6/16/2004 & 8:56:24 AM & 26.256 & 26.23 & 24.972 & 27.493 & 26.368 & 47.429 & 52.845 & 15.412 & 32.423 & -0.296 & -5.88 & 48.319 & $\begin{array}{ll}0.047 \\
\end{array}$ & 14.643 & 0.004 & 2861.68 & $\begin{array}{l}47.6947 \\
\end{array}$ & 51.4 & 11.8 & \begin{tabular}{|l|l|}
39.9 & 2.753 \\
\end{tabular} & 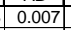 & 0.007 & 0.000 & 0.176 & \\
\hline & & 8:57:24 AM & 26.262 & 26.231 & 24.978 & 27.489 & 26.364 & 47.327 & 52.787 & 15.448 & & -0.307 & -5.88 & 47.79 & $\begin{array}{ll}0.047 \\
\end{array}$ & 14.643 & 0.004 & 2862.68 & 3.77 .7114 & 51.3 & 11.7 & \begin{tabular}{|l|l|}
39.8 & 2.744 \\
\end{tabular} & & & & 0.176 & \\
\hline & $6 / 116 / 2004$ & 8:58:24 AM & 26.258 & $\frac{26.236}{26227}$ & 24.954 & 27.449 & 26.389 & 47.205 & \begin{tabular}{|l|}
52.621 \\
\end{tabular} & 15.384 & $\begin{array}{l}32.253 \\
2.253 \\
\end{array}$ & $\begin{array}{l}-0.31 \\
0.31\end{array}$ & -5.883 & 48.924 & $\begin{array}{l}0.047 \\
\end{array}$ & $\begin{array}{l}14.643 \\
493\end{array}$ & & $\frac{2863.68}{280360}$ & 347.7281 & 51.1 & 12.0 & 39.7 & \begin{tabular}{|l|l|}
0.007 \\
\end{tabular} & 0.007 & 0.000 & 0.177 & \\
\hline 2174 & $6 / 16 / 2004$ & 8:59:24 AM & 26.263 & 26.237 & 24.945 & 27.485 & 26.375 & $\begin{array}{l}47.425 \\
\end{array}$ & \begin{tabular}{|l|l|}
52.88 \\
\end{tabular} & 15.444 & 32.433 & -0.249 & $\begin{array}{l}-5.88 \\
\end{array}$ & 48.661 & $\begin{array}{l}0.047 \\
\end{array}$ & 14.643 & 0.004 & 2864.68 & \begin{tabular}{|l|l|}
47.7447 \\
\end{tabular} & 51.4 & 11.9 & 2.753 & \begin{tabular}{|l|l|} 
\\
\end{tabular} & 0.007 & 0.000 & 0.176 & \\
\hline & $\frac{6 / 16 / 2004}{66162004}$ & $\begin{array}{l}9: 00: 24 \mathrm{AM} \\
\cdot 901 \cdot 24 \mathrm{AM}\end{array}$ & $\begin{array}{ll}26.248 \\
26264\end{array}$ & $\frac{26.227}{26.243}$ & 24.925 & 27.43 & & \begin{tabular}{|l|l}
47.358 \\
47.441
\end{tabular} & $\begin{array}{l}52.936 \\
5.911 \\
510\end{array}$ & $\begin{array}{l}15.505 \\
1554 \\
\end{array}$ & \begin{tabular}{|l|l|}
32.169 \\
32302
\end{tabular} & $\begin{array}{l}-0.307 \\
-0307 \\
-0.37\end{array}$ & -5.88 & $\begin{array}{l}49.598 \\
47715\end{array}$ & $\begin{array}{l}0.047 \\
0.047\end{array}$ & \begin{tabular}{|l|l|l|l|l|}
14.643 \\
14.643
\end{tabular} & $\begin{array}{ll}0.004 \\
0.004\end{array}$ & \begin{tabular}{|l|l|}
2865.68 \\
\end{tabular} & $\begin{array}{l}47.7614 \\
\end{array}$ & 51.5 & \begin{tabular}{|l|l|}
12.2 \\
\end{tabular} & \begin{tabular}{|l|l|}
39.8 & 2.742 \\
\end{tabular} & \begin{tabular}{|l|l|} 
& 0.007 \\
\end{tabular} & 0.007 & & 0.177 & 0.15 \\
\hline$\frac{2116}{2177}$ & $\begin{array}{l}661166 / 2004 \\
6 / 16 / 2004\end{array}$ & $\begin{array}{l}\text { 9:101:24 AM } \\
\text { 9:02:24 AM }\end{array}$ & 26.264 & $\frac{26.243}{26.239}$ & $\begin{array}{l}24.931 \\
24.921\end{array}$ & 27.4917 & $\frac{26.371}{26.377}$ & $\frac{47.441}{47.468}$ & 52.911 & \begin{tabular}{|l|}
15.554 \\
15.365 \\
\end{tabular} & $\begin{array}{r}32.302 \\
32.61 \\
\end{array}$ & $\begin{array}{l}-0.307 \\
-0.307\end{array}$ & $\begin{array}{l}-5.874 \\
-5.874 \\
\end{array}$ & $\begin{array}{l}47.715 \\
54.129\end{array}$ & $\begin{array}{l}0.047 \\
0.047 \\
\end{array}$ & \begin{tabular}{|l|}
14.643 \\
14.643 \\
\end{tabular} & 0.004 & \begin{tabular}{|l|}
2866.68 \\
2867.68
\end{tabular} & \begin{tabular}{|l|l|}
47.7781 \\
347947 \\
\end{tabular} & \begin{tabular}{|l|}
51.4 \\
51.5
\end{tabular} & \begin{tabular}{|l|}
11.7 \\
13.3
\end{tabular} & \begin{tabular}{|l|l|}
39.9 & 2.749 \\
40.0 & 2.761
\end{tabular} & \begin{tabular}{|l|}
0.007 \\
0.007
\end{tabular} & $\begin{array}{l}0.007 \\
0.007 \\
\end{array}$ & $\begin{array}{l}0.000 \\
0.000\end{array}$ & 0.176 & 0.15 \\
\hline 2178 & & & 26.266 & 26.25 & 24.923 & 27.458 & 26.388 & & & \begin{tabular}{|l|l|}
15.363 \\
\end{tabular} & 31.937 & -0.241 & -5.866 & & $\begin{array}{l}0.047 \\
0.047 \\
\end{array}$ & $\begin{array}{l}14.043 \\
14.643 \\
\end{array}$ & $\begin{array}{l}0.004 \\
0.004\end{array}$ & 2860.68 & \begin{tabular}{|l|l|}
4.1 .941 \\
47.8114 \\
\end{tabular} & \begin{tabular}{|l|}
50.5 \\
50.8
\end{tabular} & $\begin{array}{l}13.3 \\
12.0\end{array}$ & \begin{tabular}{|c|c|}
4.0 .0 & 2.101 \\
39.4 & 2.716 \\
\end{tabular} & \begin{tabular}{|l|}
0.007 \\
\end{tabular} & & 0.000 & 0.178 & $\begin{array}{l}0.15 \\
0.15\end{array}$ \\
\hline 2179 & $6 / 16 / 2004$ & 9:04:24 AM & & 26.256 & 24.934 & 27.484 & 26.384 & 47.279 & 52.766 & 15.445 & 32.277 & -0.241 & -5.872 & 47.679 & 0.046 & 14.643 & 0.004 & 2869.68 & 347.8281 & 51.3 & 11.7 & & & & & & \\
\hline & 6/16/2004 & 9:05:24 AM & & 26.258 & & & & 47.225 & 52.735 & $\begin{array}{l}15.397 \\
\end{array}$ & & -0.238 & & 53.302 & & 14.643 & & & \begin{tabular}{|l|l}
47.8447 \\
\end{tabular} & 51.3 & 13.1 & 2.738 & & & 0.000 & 0.177 & \\
\hline 2181 & $6 / 16 / 2004$ & 9:06:24 AM & 26.255 & 26.258 & 24.891 & 27.461 & 421 & 47.356 & $\begin{array}{l}52.841 \\
5.065\end{array}$ & 15.43 & 32.273 & & & $\begin{array}{l}43.29 \\
\end{array}$ & $\begin{array}{l}0.047 \\
\end{array}$ & 14.643 & & 2871.68 & $\begin{array}{l}37.8614 \\
1.701\end{array}$ & 51.4 & 10.6 & 2.745 & \begin{tabular}{|l|l|} 
\\
\end{tabular} & 0.007 & 0.000 & 0.177 & \\
\hline & 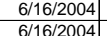 & $\begin{array}{l}9: 07: 24 \mathrm{AM} \\
\cdot 08: 24 \mathrm{AM}\end{array}$ & $\begin{array}{l}26.254 \\
26.239 \\
\end{array}$ & $\frac{26.253}{26.248}$ & $\begin{array}{r}24.885 \\
2486\end{array}$ & $\begin{array}{l}27.466 \\
27.446 \\
\end{array}$ & $\frac{396}{416}$ & \begin{tabular}{|l|l}
47.508 \\
47213 \\
\end{tabular} & $\begin{array}{l}52.965 \\
52745\end{array}$ & $\begin{array}{l}15.424 \\
1547 \\
\end{array}$ & \begin{tabular}{|l|}
32.524 \\
32185
\end{tabular} & $\begin{array}{l}-0.209 \\
-0203 \\
-20\end{array}$ & & $\begin{array}{l}52.589 \\
46367\end{array}$ & $\begin{array}{l}0.047 \\
0.047\end{array}$ & $\begin{array}{l}14.643 \\
1.643 \\
\end{array}$ & 0.004 & & 7.8781 & $\begin{array}{c}51.5 \\
51.3\end{array}$ & \begin{tabular}{|l|l|}
12.9 \\
114
\end{tabular} & \begin{tabular}{|l|l|}
2.759 \\
2.737
\end{tabular} & \begin{tabular}{|l|l|} 
& 0.007 \\
\end{tabular} & 0.007 & 0.000 & 0.176 & 15 \\
\hline \begin{tabular}{|l|l|l|}
2184 \\
\end{tabular} & $\begin{array}{c}6 / 116 / 2004 \\
6 / 16 / 2004\end{array}$ & 9:08:24 AM & $\frac{26.239}{26.265}$ & $\frac{26.248}{26.264}$ & $\begin{array}{r}24.86 \\
24.877 \\
\end{array}$ & $27.446 \mid$ & $\frac{4.427}{4.427}$ & $\frac{47.213}{44.082}$ & $\begin{array}{l}52.745 \\
52.677\end{array}$ & $\begin{array}{r}\mid 15.417 \\
15.35 \\
\end{array}$ & \begin{tabular}{|l|}
32.185 \\
32.157 \\
\end{tabular} & $\begin{array}{l}-0.203 \\
-0.183 \\
\end{array}$ & & $\begin{array}{l}46.367 \\
46.209 \\
\end{array}$ & & $\begin{array}{l}14.643 \\
14.643 \\
\end{array}$ & $\begin{array}{l}0.003 \\
0.004\end{array}$ & $\begin{array}{l}2873.68 \\
22784.68\end{array}$ & $\begin{array}{l}7.8947 \\
7.9114\end{array}$ & 51.3 & \begin{tabular}{|l|l|}
11.4 \\
1.3
\end{tabular} & $\frac{2.777}{2.730}$ & \begin{tabular}{|l|} 
\\
\end{tabular} & 0.007 & $\begin{array}{r}0.000 \\
0.000 \\
\end{array}$ & 0.177 & 0.15 \\
\hline & $6 / 16 / 2004$ & $9: 10: 24 \mathrm{AM}$ & 26.255 & 26.264 & 24.882 & 27.507 & $\frac{20.427}{26.457}$ & $\begin{array}{l}47.217 \\
4.02\end{array}$ & \begin{tabular}{|l|l|}
52.673 \\
\end{tabular} & 15.436 & 32.214 & $\begin{array}{l}-.1109 \\
-0.189\end{array}$ & & $\begin{array}{l}40.209 \\
44.373 \\
\end{array}$ & $\begin{array}{l}0.047 \\
0.047 \\
\end{array}$ & $\begin{array}{l}14.0454 \\
14.643 \\
\end{array}$ & $\begin{array}{l}0.004 \\
0.004\end{array}$ & $\begin{array}{l}\mid 284.606 \\
2875.68 \\
\end{array}$ & \begin{tabular}{|l|l|l|l|l|}
47.9281 \\
\end{tabular} & \begin{tabular}{|l|l|}
51.2 \\
\end{tabular} & 11.5 & \begin{tabular}{|c|c|}
39.6 & 2.150 \\
39.7 & 2.738 \\
\end{tabular} & & & & & 0.15 \\
\hline & $6 / 16 / 2004$ & 9:11:24 AM & & 26.271 & 24.878 & 27.519 & 26.454 & 47.126 & 52.611 & 15.37 & & & & 50.53 & & 14.643 & & & 347.9447 & & 12.4 & & & & & & \\
\hline & 6/16/2004 & 9:12:24 AM & & 26.266 & 24.858 & 27.504 & & 47.267 & 52.915 & 15.421 & 32.187 & -0.194 & & 45.382 & & 14.643 & & & 47.9614 & 51.4 & 11.1 & & \begin{tabular}{|l|l|}
0.007 \\
\end{tabular} & 0.007 & 0.000 & 0.177 & \\
\hline 2188 & & 9:13:24 AM & 26.246 & 26.265 & 24.862 & 27.518 & & & 52.928 & & & & & & & 14.643 & & & & 51.5 & 13.4 & 2.747 & & & 0.000 & 0.177 & \\
\hline & 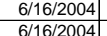 & $\begin{array}{l}9: 14: 24 \mathrm{AM} \\
9 \cdot 15 \cdot 24 \mathrm{AM}\end{array}$ & $\frac{26.257}{26261}$ & $\frac{26.271}{26.28}$ & 24.858 & 27.509 & & $\begin{array}{l}47.203 \\
47076 \\
\end{array}$ & $\begin{array}{l}52.712 \\
5.648\end{array}$ & $\begin{array}{l}15.433 \\
15407 \\
\end{array}$ & 2.169 & & -5. & 49.585 & & $\begin{array}{l}14.643 \\
1.643 \\
\end{array}$ & & & \begin{tabular}{|l|l|}
47.9947 \\
380114
\end{tabular} & 51.2 & 12.2 & 2.736 & \begin{tabular}{|l|l|} 
& 0.007 \\
\end{tabular} & 0.007 & 0.000 & 0.177 & 15 \\
\hline & $\begin{array}{c}6 / 116 / 2004 \\
6 / 16 / 2004\end{array}$ & $24 \mathrm{AM}$ & $\frac{26.261}{26.266}$ & $\begin{array}{r}26.28 \\
26.285 \\
\end{array}$ & 24.872 & 27.5633 & & $\begin{array}{l}47.076 \\
47.225 \\
\end{array}$ & $\begin{aligned} 52.648 \\
52.59\end{aligned}$ & \begin{tabular}{|l|}
15.407 \\
15.401
\end{tabular} & :054 & & & $\begin{array}{l}49.383 \\
47.715\end{array}$ & & $\begin{array}{l}14.643 \\
14.643 \\
\end{array}$ & & & 0.0114 & 51.2 & $\frac{12.1}{11.7}$ & $2.728 \mid$ & \begin{tabular}{|l|}
0.007 \\
0.007 \\
\end{tabular} & 0.007 & 0.000 & & $\frac{8}{7}$ \\
\hline 216 & $6 / 16 / 2004$ & 9:17:24 AM & 26.255 & 26.274 & 24.842 & 27.562 & 26.487 & 47.093 & 52.547 & 15.324 & 32.259 & -0.186 & -5.857 & 49.629 & 0.047 & $\begin{array}{l}\frac{14.645}{14.643} \\
\end{array}$ & 0.004 & $\begin{array}{l}\mid 8001.1060 \\
2882.68 \\
\end{array}$ & 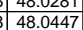 & 51.1 & 11.12 & $\frac{2.140}{2.736}$ & \begin{tabular}{|l|}
0.007 \\
0.007 \\
\end{tabular} & 0.007 & 0.000 & 0.177 & $\begin{array}{l}0.15 \\
0.15 \\
\end{array}$ \\
\hline & 6/16/2004 & 9:18:24 AM & 26.256 & 26.29 & 24.847 & 27.558 & & 47.06 & 52.671 & 15.423 & & -0.183 & -5.86 & 48.127 & 0.046 & 14.643 & & 2883.68 & 38 & 51.2 & 11.8 & 2.726 & \begin{tabular}{|l|l|} 
& 0.007 \\
\end{tabular} & 0.007 & 0.000 & 0.174 & \\
\hline 219 & $6 / 16 / 2004$ & 9:19:24 AM & 26.261 & 26.29 & 24.842 & 27.578 & 26.498 & 47.468 & 52.965 & 15.524 & 333 & & & & & 14.643 & & & & & 10.6 & 33. & & & & & \\
\hline 2195 & & 9:20:24 AM & 26.277 & 26.316 & 24.868 & 27.614 & & 47.391 & 52.949 & & & & & & & 14.643 & & & & & & & & $007 \mathrm{~T}$ & & & \\
\hline & & & 26.273 & 26.312 & 24.859 & & & & & & & -0. & & 47.94 & & 14.643 & & & & & & & & & & & \\
\hline & 6/16/2004 & 9:22:24 AM & & 26.307 & 24.849 & 27.709 & & 47.174 & 52.627 & 15.479 & & -0.17 & & & & 14.643 & & & & & & & & & & & \\
\hline & $6 / 16 / 2004$ & $9: 23$ & 26.264 & 26.31. & 24.846 & 27.825 & & 47.184 & 52.804 & 15.395 & 32.146 & -0.168 & & 47.984 & & 14.643 & & & . 1447 & 51.3 & 11.8 & & & & & & \\
\hline & $6 / 16 / 2$ & 9:24:24 AM & 26.261 & 26.325 & 24.847 & 27.737 & & 47.2 & 52.634 & 15.382 & 32.253 & -0.113 & & 52.598 & 0.046 & 14.643 & & 2889.6 & 48.1614 & 51.2 & 12.9 & 2.739 & \begin{tabular}{|l|l|} 
& 0.007 \\
\end{tabular} & 0.00 & & & \\
\hline & & & 26.248 & 26.307 & 24.834 & & & 47.1 & & $\begin{array}{l}15.47 \\
\end{array}$ & 2.06 & & & & & 14.643 & & & & & & & & & & & \\
\hline & 6.116622004 & $6: 24 \mathrm{AM}$ & & & 24.851 & 27.756 & & 47.2 & 22.741 & 5.3688 & 32.31 & -0.166 & & & & 14.643 & & & & & & & & & & & \\
\hline & & & 6.251 & 26.33 & 24.848 & 27.1878 & & & 52.83 & 15.416 & 32.3924 & & & & & 14.643 & & & & 51.4 & 11. & & & & & & \\
\hline & $6 / 16 / 2004$ & $\begin{array}{l}9: 28: 24 \mathrm{AM} \\
9.2024 \mathrm{AM}\end{array}$ & $\frac{254}{256}$ & $\frac{26.33}{26335}$ & $\begin{array}{r}24.85 \\
24857\end{array}$ & $\begin{array}{rl}27.71 & 27.989\end{array}$ & & $\frac{47.55}{4715}$ & $\begin{array}{l}53.173 \\
588\end{array}$ & $\begin{array}{r}15.475 \\
15395 \\
\end{array}$ & 32.479 & $\frac{-0.11}{-0.11}$ & & 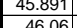 & & $\frac{14.6}{146}$ & & & & 514 & 113 & & & & & & \\
\hline & 6/16/2004 & & 26.200 & $\frac{20.000}{26.346}$ & 24.051 & 27.783 & & 47.002 & \begin{tabular}{r|r}
52.524 \\
52.524
\end{tabular} & 15.436 & $\begin{array}{ll}31.947 \\
31.940\end{array}$ & $\begin{array}{l}-0.11 \\
-0.11 \\
-1\end{array}$ & & 46.35 & 0.047 & $\begin{array}{l}14.045 \\
14.643 \\
\end{array}$ & & & & 5111 & 势1.5 & & & & & & \\
\hline & $6 / 16 / 2004$ & 9:31:24 AM & 26.253 & $\frac{20.040}{26.351}$ & 24854 & 27.849 & & 47.36 & 52.942 & $\begin{array}{l}1.4 .463 \\
15.463\end{array}$ & 32.247 & -0.11 & & 48.50 & & $\frac{14.045}{14.643}$ & & & & 51.5 & $\frac{1.49}{11.9}$ & 74 & & & & 0 & \\
\hline & & & 26.243 & 26.341 & 24.844 & $27.89 !$ & & 47.4 & 133 & 15.489 & 425 & & & .85 & & 14. & & & & & $127+2$ & & & & & & \\
\hline & 6/16/2004 & & & $26.367 \mathrm{r}>\mathrm{s}$ & & 27.91 & & & & & & & & & & & & & & & & & & & & & \\
\hline & & & 26.26 & 26.379 & $24.8 \mathrm{C}>\mathrm{l}$ & 27.93. & & 47.12 & 52.64 & 15.503 & 32.083 & $-0.0<>0$ & & 47.44 & & 14.6 & & & & 51.2 & 11.6 & & & & & $\frac{78}{78}$ & \\
\hline & $6 / 16 / / 2$ & & & & 24.867 & & & & & 15.442 & & & & & & & & & & & & & & & & & \\
\hline & $6 / 16 /$ & & 278 & 26.402 & 24.90 & 27.994 & & 47.21 & 52.899 & \begin{tabular}{|l|l|}
15.347 \\
\end{tabular} & & & & $\frac{44}{44}$ & & 14.6 & & & & 51.4 & 9 & 27 & & & & 177 & \\
\hline & 6/16/2004 & & 26.256 & 26.39 & 24.863 & 27.972 & & 47.1 & 638 & 15.329 & & & & & & 14.643 & & & & 51.2 & 11.5 & & & & & 0.177 & \\
\hline & $6 / 16 / 2$ & & & 26.398 & & 27.995 & & & & & & & & & & & & & & & 1.5 & & & & & & \\
\hline & & & 26.27 & 26.389 & 24.87 & 27.971 & & 47.0 & 52.698 & 15.46 & & & & & & 14. & & & & 51.2 & 11.9 & & & & & & \\
\hline & 6/16/2004 & & & 26.4 & & & & & & & & & & & & & & & & & 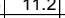 & & & & & & \\
\hline & & & 20.281 & & 24.88 & & & & & & & & & & & & & & & & & & & & & & \\
\hline & 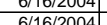 & & 6.309 & 26.454 & $\frac{24.90}{24.91}$ & $\begin{array}{ll}28.054 \\
28115\end{array}$ & & & 年 & $\begin{array}{r}15.449 \\
15548\end{array}$ & & $=0$ & & & & $\frac{14.6}{146}$ & & & & 513 & 121 & 274 & & & & & \\
\hline & $6 / 16 / 2004$ & & 26 & & & & & & & & & & & & & & & & & \begin{tabular}{|l|l|} 
& 51.4 \\
\end{tabular} & $\frac{11.2}{11.2}$ & & & & 500 & 0.173 & \\
\hline & 6/16/2004 & & & & & & & & & & & & & & & & & & & 51.7 & \begin{tabular}{|l|l|}
11.5 \\
\end{tabular} & & & & & 0.172 & \\
\hline & 6/16/2004 & & 26.32 & 26.469 & 24.921 & 28. & & 47. & \begin{tabular}{|l|}
52.94 \\
\end{tabular} & 15.512 & & & & & & 14. & & & & 51.5 & 12.2 & 2.748 & 0. & & 0. & 176 & \\
\hline & 6/16/2004 & & & & & & & & & & & & & & & & & & & 51.4 & 12.6 & & & & & & \\
\hline & $6 / 16 / 200$ & & 26.349 & 26.4 & 24.945 & 28.199 & & 47. & 53.255 & $\begin{array}{l}15.505 \\
\end{array}$ & $\begin{array}{l}32.423 \\
\end{array}$ & -0. & -5 & & & 14. & & & & \begin{tabular}{|l|l}
51.8 \\
\end{tabular} & 11.3 & & & & & 0.176 & \\
\hline & $6 / 16 / 2004$ & 9:49:24 AM & $\frac{26.365}{26367}$ & 26.514 & 24.957 & $\frac{28.221}{28212}$ & 26. & $\frac{47.101}{47306}$ & $\begin{array}{l}52.781 \\
5.851\end{array}$ & $\begin{array}{l}15.502 \\
15637 \\
\end{array}$ & \begin{tabular}{|r|}
32.017 \\
32095
\end{tabular} & $\begin{array}{l}-0.0 \\
-0.0\end{array}$ & -5.81 & $\frac{43.9}{501}$ & 0.4 & $\frac{14.643}{11.643}$ & & & 48.5 & \begin{tabular}{|l|}
51.3 \\
\end{tabular} & \begin{tabular}{|l|l|}
10.8 \\
103
\end{tabular} & $2.727 \mid$ & & 0.007 & 200 & 0.177 & 7 \\
\hline & $\frac{6 / 16 / 200}{6 / 16 / 200}$ & & & $\frac{26.516}{26.523}$ & $\frac{24.944}{24.956}$ & & & $\frac{4.35}{47.36}$ & & $\begin{array}{l}15.653 \\
1528\end{array}$ & 32.095 & & & & & $\frac{14.6}{14.6}$ & & & & & $\frac{12.3}{118}$ & & & & & & \\
\hline & & & & 26546 & & & & & & & & & & & & & & & & & & & & & & & \\
\hline & $6 / 16 / 2$ & & $\frac{26}{26}$ & 26.54 & 24.94 & 28.236 & & 47. & 53 & 15.5 & & & & & & $14.6 \mathrm{C}-\mathrm{C}$ & & & & & 12.3 & & & & & & \\
\hline & 6/16/2004 & & & 26.5 & & & & & & & & & & & & & & & & & & & & & & & \\
\hline & & & & & & & & & & & & & & & & & & & & & & & & & & & \\
\hline & 6/16/20 & & & $26.58 \mathrm{C}>\mathrm{s}$ & & 28.1 & & 47. & & & & & & & & 14. & & & & & & & & & & & \\
\hline & $6 / 16 / 2$ & & & 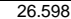 & 25.00 & 28.09 & & 47.23 & 53.058 & 15.546 & 32.154 & -0.0 & & 5 & & 14.6 & & & & & & & & & & 76 & \\
\hline & $6 / 16 / 200$ & & 426 & & $24.9->>$ & $27.98 \mathrm{r}$ & & 46.5 & 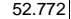 & 15.49 & 31.869 & -0.6 & & & & 14.6. & & & & & 12.6 & & & & & 178 & \\
\hline & 6/16/2 & & & 26.6 & 24.9 & 27.96 & & 47.1 & & 15.481 & & & & & & 14. & & & & & 11 & & & & & & \\
\hline & & & 26.444 & 26.608 & 25.6 & 27.96 & & 47. & & & & & & & & & & & & & 11.3 & & & & & & \\
\hline & $6 / 16 / 2 / 2$ & & 26.43 & & & & & & & & & & & & & & & & & & & & & & & & \\
\hline & & & 20 & 26.581 & 25.0 & 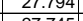 & & $41.38>3$ & & & & & & & & & & & & & & & & & & & \\
\hline & 然 & & 20.459 & 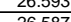 & 23.011 & 2.1.145 & & (17.5 & 53.421 & 15.544 & S2.415 & 0.0 & & 1800 & 0.040 & 14.045 & & & & & & & & & & & \\
\hline 2240 & \begin{tabular}{|l|}
$6 / 16 / 2004$ \\
6
\end{tabular} & $10: 05: 04 \mathrm{AM}$ & \begin{tabular}{|l|l|l}
26.463 \\
\end{tabular} & 26.587 & \begin{tabular}{|r|}
25.02 \\
\end{tabular} & \begin{tabular}{|l|}
27.7699 \\
\end{tabular} & 26.709 & 46.703 & \begin{tabular}{|l|}
52.147 \\
\end{tabular} & \begin{tabular}{|l|}
15.000 \\
15.456 \\
\end{tabular} & $\begin{array}{l}0.427 \\
31.764 \\
\end{array}$ & \begin{tabular}{|l|l|}
0.026 \\
\end{tabular} & -5.785 & $\frac{4.000}{54.32}$ & 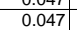 & \begin{tabular}{|l|}
14.645 \\
14.643 \\
\end{tabular} & & 9930.35 & 5 & \begin{tabular}{|l|l|} 
& 50.7 \\
\end{tabular} & 13.3 & \begin{tabular}{|l|l|}
39.2 & 2.705 \\
\end{tabular} & \begin{tabular}{|l|}
0.007 \\
\end{tabular} & $\begin{array}{l}.001 \\
0.007\end{array}$ & 0.000 & \begin{tabular}{|l|l|}
0.179 \\
\end{tabular} & \begin{tabular}{l|l}
9 & 0.1 \\
\end{tabular} \\
\hline
\end{tabular}


WSRC-TR-2005-00105, REVISION 0

SRNL-RPP-2005-00012, REVISION 0

RUN \# 3.03A AND B; FIRST AND SECOND HALF OF SLURRY DEWATERING - CONT.

\begin{tabular}{|c|c|c|c|c|c|c|c|c|c|c|c|c|c|c|c|c|c|c|c|c|c|c|c|c|c|c|c|c|}
\hline & A & & D & $\mathrm{E}$ & $\mathrm{F}$ & $\begin{array}{ll}G \\
\end{array}$ & $\begin{array}{lll}\mathrm{H} & \mathrm{I} \\
\end{array}$ & $\mathrm{J}$ & \begin{tabular}{|l|l}
$\mathrm{K}$ \\
\end{tabular} & $\mathrm{L}$ & \begin{tabular}{l|l} 
\\
\end{tabular} & $\begin{array}{ll}\mathrm{N} \\
\end{array}$ & $\begin{array}{lll}0 & \end{array}$ & Q & \begin{tabular}{l|l}
$R$ & \\
\end{tabular} & \begin{tabular}{l|l} 
\\
\end{tabular} & $T$ & $v$ & \begin{tabular}{l|l} 
w \\
\end{tabular} & \begin{tabular}{c|c|}
$x$ &
\end{tabular} & $\begin{array}{llll} & & & \end{array}$ & $z$ & & & AC & & & $\overline{A F} \mid A$ \\
\hline 241 & 6/16/2004 & $10: 06: 04 \mathrm{AM}$ & $\frac{6.469}{26.9}$ & 26.589 & 25.012 & 27.751 & 26.731 & 47.317 & \begin{tabular}{|l|}
53.228 \\
\end{tabular} & 15.505 & 32.214 & 0.02 & -5.791 & 48.589 & 0.046 & \begin{tabular}{|l|l|}
14.643 \\
\end{tabular} & 0.003 & 2931.35 & \begin{tabular}{|l|l|}
58.8558 \\
\end{tabular} & 51.8 & 11.9 & 39.8 & 2.742 & 0.007 & 0.007 & 0.000 & 0.172 & 0.15 \\
\hline & & 10:07:04 AM AM & 26.469 & 26.583 & 325.016 & & 26.745 & 47.138 & \begin{tabular}{|l|l|}
52.949 \\
\end{tabular} & $\begin{array}{l}15.451 \\
15.451\end{array}$ & 32.093 & 0.023 & -5.788 & 50.026 & 0.046 & \begin{tabular}{|l|l|}
14.643 \\
\end{tabular} & & & & & & & & & & & & \\
\hline 2243 & 6/16/2004 & 10:08:04 AM & 26.479 & 26.589 & & $\frac{27.791}{277987}$ & 26.741 & 47.085 & \begin{tabular}{|l|l|}
52.766 \\
52725
\end{tabular} & 15.562 & 31.926 & $\begin{array}{l}0.023 \\
\end{array}$ & $\begin{array}{l}-5.788 \\
-5701\end{array}$ & $\begin{array}{l}48.077 \\
8.077\end{array}$ & 0.046 & \begin{tabular}{|l|}
14.643 \\
1
\end{tabular} & & 2933.35 & \begin{tabular}{|l|l|l|l}
58992 \\
\end{tabular} & 51.3 & 11.8 & 39.5 & . & .007 & 0.007 & 0.000 & 0.173 & \\
\hline 2244 & $\begin{array}{l}6 / 11 / 2004 \\
6 / 16 / 2004 \\
\end{array}$ & 10:09:04 AM & $\begin{array}{l}26.475 \\
26.486 \\
\end{array}$ & $\begin{array}{ll}26.584 \\
26.59 \\
\end{array}$ & \begin{tabular}{|l|}
25.007 \\
25033
\end{tabular} & 27.787 & 26.767 & $\begin{array}{l}47.029 \\
6.009\end{array}$ & \begin{tabular}{|l|l|}
52.735 \\
5.692
\end{tabular} & $\begin{array}{l}15.364 \\
1555 \\
\end{array}$ & 32.101 & 0.02 & $\begin{array}{l}-5.791 \\
-5709\end{array}$ & 54.71 & 0.046 & 14.643 & 0.004 & 2934.35 & \begin{tabular}{|l|l|}
48.9058 \\
\end{tabular} & 51.3 & 13.4 & 39.6 & 2.728 & 0.007 & 0.007 & 0.000 & 0.173 & \\
\hline 2245 & 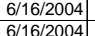 & $\begin{array}{l}10: 10: 04 \mathrm{AM} \\
10.11 .04 \mathrm{AM}\end{array}$ & $\begin{array}{l}26.486 \\
26.496 \\
\end{array}$ & $\frac{26.59}{26.605}$ & & & & $\begin{array}{l}46.908 \\
46.881 \\
4\end{array}$ & \begin{tabular}{|l|l|}
52.683 \\
5.443
\end{tabular} & & & 0.02 & $\begin{array}{l}-5.788 \\
-5775 .\end{array}$ & $\begin{array}{l}46.949 \\
48\end{array}$ & 0.046 & \begin{tabular}{|l|}
14.643 \\
14643 \\
\end{tabular} & & & & \begin{tabular}{|l|}
51.2 \\
5.12
\end{tabular} & \begin{tabular}{|l|l|}
11.5 \\
199
\end{tabular} & & & 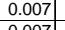 & \begin{tabular}{|l|}
0.007 \\
\end{tabular} & & 0.174 & \\
\hline 2247 & 6/16/2004 & $\begin{array}{l}\text { 10:11:04 AM } \\
1011: 04 \mathrm{AM}\end{array}$ & $\begin{array}{l}26.496 \\
26.485\end{array}$ & $\frac{26.605}{26.594}$ & \begin{tabular}{|l|}
25.048 \\
25.032 \\
\end{tabular} & 27.8432 & $\frac{26.802}{26.812}$ & $46.881 \mid$ & \begin{tabular}{|r|}
52.243 \\
53.214 \\
\end{tabular} & $\begin{array}{l}15.416 \\
15.664\end{array}$ & $\begin{array}{l}31.1941 \\
32.117 \\
\end{array}$ & $\begin{array}{l}0.026 \\
0.017 \\
\end{array}$ & $\begin{array}{l}-\frac{-5785}{-5.782} \\
\end{array}$ & \begin{tabular}{r|r}
41.027 \\
5
\end{tabular} & $\begin{array}{l}0.046 \\
0.047\end{array}$ & $\begin{array}{l}\frac{14.643}{14.643} \\
\end{array}$ & $\begin{array}{l}0.004 \\
0.003\end{array}$ & $\begin{array}{l}2936.35 \\
2237.35\end{array}$ & 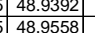 & 51.0 & $\frac{11.8}{12.5}$ & $\begin{array}{l}39.4 \\
39.8 \\
\end{array}$ & 2.7171 & $\mid \frac{0.007}{0.007}$ & \begin{tabular}{|l|}
$\mid 0.007$ \\
0.007 \\
\end{tabular} & $\begin{array}{l}0.000 \\
0.000\end{array}$ & & 0.15 \\
\hline 2248 & $6 / 16 / 2004$ & 10:13:04 AM & 26.479 & 26.594 & & & & & & & & & & & & & & & & & & & & & & & & 0.15 \\
\hline 2249 & & 10:14:04 AM & 26.474 & 26.583 & 25.021 & 27.835 & 26.84 & $\begin{array}{ll}47.364 \\
\end{array}$ & 53.098 & 15.62 & 32.16 & 0.046 & -5.782 & 48.689 & 0.046 & 14.643 & 0.00 & & 48.9892 & 51.6 & & & & & & & & $\frac{0.15}{0.15}$ \\
\hline 2250 & $6 / 16 / 2004$ & 10:15:04 AM & & 26.599 & & 27.846 & 26.841 & \begin{tabular}{ll|l}
47.118 \\
\end{tabular} & \begin{tabular}{|l|l|}
52.903 \\
\end{tabular} & \begin{tabular}{l|l|}
15.505 \\
\end{tabular} & $\begin{array}{l}32.005 \\
\end{array}$ & 0.083 & & & 0.046 & 14.643 & & 2940.35 & & 51.4 & 11.7 & 39.6 & & & 0.007 & & & \\
\hline 2251 & & & 26.51 & 26.619 & 25.067 & 27.882 & & & 52.934 & 15.529 & & 0.063 & & (45.695 & & 14.643 & & & 0225 ; & & 11.2 & & & 007 & 0.007 & 0.000 & 0.173 & \\
\hline 2252 & & & & & & & & 46.902 & 52557 & & .791 & 0.046 & & & & 14.643 & & & & 51.1 & $\frac{11.9}{11.9}$ & & & & 0077 & & ס.174 & \\
\hline 2253 & & 10:18:04 AM & 26.526 & 26.63 & 25.088 & 27.863 & & 47.066 & 2.895 & 15572 & & 0069 & & & & 14.643 & & & & 514 & 12.0 & & & & 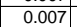 & & & \\
\hline & $6 / 16 / 2004$ & 10:19:04 AM & 26.521 & 26.625 & 25.073 & 27.927 & & (47.024 & 3.013 & 15.438 & 31.988 & 0.092 & & 477.641 & 0.046 & 14.643 & & 2944.35 & & 51.5 & 11.7 & & & 007 & 0.007 & & & \\
\hline 2255 & $6 / 16 / 2004$ & 10:20:04 AM & 26.512 & 26.616 & 25.064 & 27.883 & 26.7. & 46.966 & 52.841 & 15.507 & 31.91 & 0.112 & -5.776 & 45.287 & 0.047 & 14.643 & & 2945.35 & 9.0892 & 51.4 & 11.1 & 39.4 & 2.719 & .007 & 0.007 & 0.000 & & 0.15 \\
\hline & & 21:04 AM & & 26.617 & 25.07 & 27.844 & 26.6 & 47. & $\begin{array}{l}3.077 \mid \\
.027\end{array}$ & 15.582 & 189 & 0.095 & & 57.645 & 0.046 & 14.643 & & 946.3 & 1058 & 51.6 & 14.1 & & & & & & & \\
\hline$\frac{20}{25}$ & & $2: 04 \mathrm{AM}$ & 26.558 & 20.623 & t5.056 & $\frac{22.74}{27.67}$ & & 47.304 & $299 ?$ & $\frac{11.549}{11.559}$ & ?.105 & 0.112 & & 47.967] & 0.046 & 14.643 & & 9947.3 & 1225 & 51.8 & 11.8 & & & 007 & 007 & & & \\
\hline & $6 / 16 / 20$ & 10:23:04 AM & 20.519 & 26.613 & & & & & .932 & & 31.9474 & 0.124 & & 50.3999 & & 14.643 & & & 1392 & & & & & & & & & \\
\hline & & 10:22:04 AM & 26.499 & 26.593 & $\frac{25.036}{25.051}$ & 27.62 & & (47.084 & |.934 & $\frac{15.547}{1556}$ & 31.8777 & 0.13 & & 48.7877 & & 14.663 & & & & 51.5. & 12.0 & & & 007 & 0.007 & 0.000 & & \\
\hline 2261 & $\frac{16 / 16 / 2004}{6 / 2004}$ & $\frac{10}{10}$ & $\frac{20.019}{26.497}$ & $\frac{20.590}{26.581}$ & $\frac{2.0 .01}{25034}$ & $\begin{array}{r}27.60 \\
27.609\end{array}$ & & 4..194 & 53.088 & 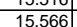 & & 0.133 & & & & 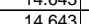 & & & & & 20 & & & & 0007 & 0.0000 & & \\
\hline 2262 & $6 / 16 / 2004$ & 10:27:04 AM & 26.502 & $\frac{2.001}{26.576}$ & 25.039 & 27.664 & 26.585 & 47.113 & 52.86 & 15.525 & 32.091 & 0.107 & -5.768 & 48.876 & 0.046 & $\begin{array}{l}14.043 \\
14.643\end{array}$ & & 2952.35 & 49.2058 & 51.4 & $\frac{12.0}{12.0}$ & 39. & $.730 \mathrm{~T}=\mathrm{s}$ & .007 & 0.007 & 0.000 & 0.173 & \\
\hline & & 10:28:04 AM & 26.507 & 26.581 & 25.044 & 27.699 & 26.62 & 47.051 & 52.953 & 15.441 & \begin{tabular}{|l|l|}
31.966 \\
\end{tabular} & 0.133 & & 48.482 & 0.046 & 14.643 & & 2053.35 & & 51.5 & 11.9 & & .724 & & 0.007 & 0.000 & & \\
\hline & & 10:29:04 AM & 26.507 & 26.576 & 25.044 & & & 47.062 & & 15.53 & 31.902 & & & & & & & 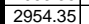 & & 51.6 & & & & & & & & \\
\hline 265 & $6 / 16 / 2$ & $10: 30: 04 \mathrm{AM}$ & 26.503 & 26.567 & & 27.734 & & & 963 & 15.418| & . & 0.13 & & 50.124 & 0.046 & 14.643 & & & & 51.5 & $\frac{12.3}{12.3}>$ & & & 007 & 0.007 & 5.000 & & \\
\hline & & 10:31:04 AM & & & & 27.819 & & 47.29 & 3.214 & & & 0.133 & & 49.11 & 0.046 & 14.643 & & & & & $\frac{12.0}{12.0}$ & & & & & & & \\
\hline 26 & 6/16/2004 & 10:32:04 AM & 26.518 & 26.572 & 25.06 & 27.859 & $26.7 \underbrace{2}$ & 47.062 & j2.866 & $15.437 \mid$ & 32.068 & $\begin{array}{l}0.138 \\
\end{array}$ & & & 0.046 & 14.643 & & & & 51.4 & 12.0 & & & & & & & \\
\hline 268 & 6/16/2004 & 10:33:04 AM & 26.508 & 26.562 & 25.035 & 27.844 & 26.8 & 47.211 & 52.743 & 15.562 & 32.183 & 0.133 & -5.765 & 50.286 & 0.046 & 14.643 & & & |3058 & 51.3. & 12.3 & & & & 007 & & & \\
\hline 2655 & & 10:34:04 AM & 26.509 & 26.573 & 25.041 & 27.866 & $26.8 \mathrm{r}$ & 47 & 53.061 & 15.498 & 31.861 & 0.13 & & 691 & 45 & 14.643 & & $2959.33^{5}->2$ & 49.3225 & 51.6 & 12.9 & 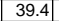 & 719 & .007 & 0.007 & & & \\
\hline & & & 26.52 & & 25.062 & 27.921 & & 47.124 & & 15.451 & & & & 48.453 & 346 & 14.643 & & & & - & & & & & & & & \\
\hline $2 \frac{271}{272}+2$ & $6 / 2004$ & 10:36:04 AM & 26.52 & 26.579 & t5.047 & 27.9211 & & & .423 & 15.57 & 1899 & 0.13 & & .673 & 0.046 & 14.643 & & & & & 1..5 & & & & & & & \\
\hline & & 10: & 26.535 & & t5.067 & 27.996 & & & & & & 0.173 & & 49.1980 & & 14.643] & & & & & & & & & & & & \\
\hline$\frac{213}{274}$ & $\begin{array}{l}6 / 2004 \\
62004\end{array}$ & $0.04 \mathrm{AM}$ & $\frac{26.529}{2654}$ & $\frac{26.598}{26.609}$ & 55.062 & $\frac{27.971}{28026}$ & & $\begin{array}{l}47.099 \\
47205\end{array}$ & 973 & $\frac{15.4199}{15592}$ & $\begin{array}{r}32.14 \\
31.959\end{array}$ & 0.162 & & $\begin{array}{l}44.8011 \\
46548\end{array}$ & 0.046 & $\frac{14.643}{14643}$ & & & & 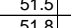 & $\frac{11.0}{11.4}$ & & & & & & & \\
\hline 275 & $6 / 16 / 2004$ & & $\frac{20.04}{26.558}$ & $\frac{20}{26}$ & 2508 & 年 & & (4) & (57) & 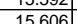 & 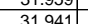 & 0.196 & & & 年 0.046 & 14.043 & & & & 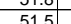 & & & & & & & & \\
\hline 2276 & $6 / 16 / 2004$ & $10: 41: 04 \mathrm{~A}$ & $\frac{2.050}{26.553}$ & $\frac{2.021}{26.633}$ & 25.081 & $\frac{2.00}{27.98}$ & & & 286 & 15.615 & & 0.199 & & 44.611 & & $\frac{1.045}{14.643}$ & & & & 518 & $\frac{\frac{1.1}{109}}{109}>-1$ & & & & 0.007 & & 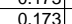 & \\
\hline 2277 & $6 / 2004$ & 10:42:04 & & 26.643 & 25.096 & 28.01 & & 47.066 & 073 & 15.499 & 32.007 & 0.202 & & 46.5 & 0.047 & 14.643 & & $\frac{2967}{2067}>2$ & & & $\frac{51.4}{11.4}$ & & & & & & & \\
\hline & & & 26.548 & & & & & 47.095 & & 15.514 & & 0.193 & & 51.6 & & & & & & $5177+7$ & & & & & & & & \\
\hline 2279 & & $10: 44$ & 26.582 & 26.651 & 25.099 & 28.008 & & 47.03 & .011 & 15.554 & 31.8 & 0.196 & & 47.08 & & 14.643 & & & & 51.5 & $\frac{11.5}{3}$ & & & & & & 0.173 & \\
\hline & & & & & 25.107 & 27.936 & & & 399 & & & 0.196 & & 5303 & & 14.643 & & & & & & & & & & & & \\
\hline 28. & $6 / 16 / 2$ & $10:$ & 26.581 & 26.665 & & 27.897 & & & \begin{tabular}{|l|l|}
828 \\
\end{tabular} & 15.494 & 31.994 & 0.199 & & & & 14.643 & & & & 51.4 & 11.4 & & & & & & 0.173 & \\
\hline & 6/16/2004 & & 26.571 & 26. & 25.123 & 27.867 & & 47.1 & 791 & 15.554 & 32.091 & 0.199 & & 45.5 & 46 & 14.643 & & & & 51.3 & 11.2 & & & & .007 & & 0.173 & \\
\hline 28 & & & & & & 27.792 & & & & & & 0.196 & & & & & & & & 51.8 & 11.9 & & & & & & & \\
\hline 2284 & 6/20004 & & 26.575 & 26.649 & 25.112 & 27.777 & & 47. & \begin{tabular}{|l|}
53.419 \\
\end{tabular} & $15.571]$ & 31.974 & 0.196 & & & 0.045 & 14.643 & & & & 51.9 & 12.9 & & & & 0.007 & & 0.169 & \\
\hline 2000 & & & 20.000 & & & 27.812 & & & & & & 0.225 & & & & & & & & 51.4 & 1.1 & & & & & & & \\
\hline 286 & & & $26.5 / 8$ & & & 27.915 & & & & $15.5 / 8$ & & & & & & & & & & & & & & & & & & \\
\hline$\frac{28}{8}$ & & $\frac{10:}{10:}$ & $\frac{26.582}{26552}$ & $\frac{26.646}{26.637}$ & 25.104 & 27.893 & & $\frac{47.311}{47155}$ & 4.423 & 15.635 & $\begin{array}{l}32.066 \\
32013\end{array}$ & 0.234 & & & 0.046 & $\frac{14.643}{14643}$ & & & & 515 & $\frac{11.0}{115}$ & & & & & & 173 & \\
\hline 228 & 6 & & 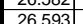 & & $\mid$ & $\frac{2.1034}{2789}$ & & & & & & $\mid$ & & & & 146 & & & & 516 & 112 & & & & & & & \\
\hline 2290 & & & $\frac{}{26.6}$ & & 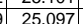 & 27.946 & & & & & & $\frac{0.2402}{0.22}$ & & & & & & & & $\frac{310}{514}$ & $\frac{1.6}{123}$ & & & & & & 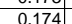 & \\
\hline 2291 & & & $\frac{26.601}{26.001}$ & & 25.094 & $\frac{|c| .98}{27988}$ & & $\begin{array}{c}46.8 \\
46.8\end{array}$ & $|22.723|$ & $\frac{15.434}{15.434}$ & & 0.237 & & & & $\frac{14.6}{14.6}$ & & & & $\frac{21.3}{5.3}$ & $\frac{1.7}{1.7}$ & & & & & & D.174 & \\
\hline & & & & & & & & & & & & & & & & & & & & & & & & & & & & \\
\hline 2293 & & & 26.61 & & & 28.05 & & & 53.193 & & & 0.257 & & 47.4 & & & & & & & & & & & & & & \\
\hline & & & & & & 28.081 & & & 53.056 & 15.551 & & 0.254 & & & & 14.6 & & & & 0.0 & 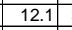 & & & & & & & \\
\hline 2295 & & & 26. & & & 28.081 & & & \begin{tabular}{|l|l|}
53.191 \\
\end{tabular} & 15.549 & 32.01 & 0.251 & & & & & & & & 51.7 & 11.0 & & & & & & 69 & \\
\hline 229 & $6 / 16 / 2 C$ & 11:01 & 26.6 & & 25.098| & 28.072 & & 46.68 & 52.7 & 15.40 & & 0.248 & & 50.0 & & 14.6. & & & & 51.2 & 3 & & & & & & .175 & \\
\hline 2297 & & & & & & & & & & & & 0.25 & & & & & & & & 51. & & & & & & & 169 & \\
\hline 2298| & & & & & 25.108 & 28.032 & & & \begin{tabular}{|l|}
52.741 \\
\end{tabular} & 15.4411 & & 0.251 & & & & & & & & 51.3 & 11.6 & & & & & & 0.174 & \\
\hline 25 & & & & & & & & & & & & & & & & & & & & 51.2 & & & & & & & & \\
\hline 先 & & & 26.6 & & $\frac{25}{25}$ & 2 & & & 5.2.129 & & & & & & & & & & & 5.1 .3 & & & & & & & & \\
\hline 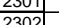 & & 11.00 .6 & & $\frac{2.124}{20.72}$ & 20.105 & 20.7.099 & & & & & ( & 0.248 & & & & & & & & the & & & & & & & 979 & \\
\hline 30 & 6 & 110.084 & $\frac{2.005}{26633}$ & $\frac{2.17}{20.7}$ & s. & 2.000 & & 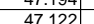 & 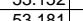 & 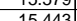 & 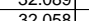 & 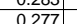 & & 5106 & & 然. & & & & 5.17 & & & & & & & & \\
\hline (30) & & 110.004 & $6.629]$ & & | & 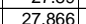 & & & 53.442 & 15606 & & 0.254 & & & & 146 & & & & & & & & & & & (1) & \\
\hline 2305 & & & 26.6 & & & $\frac{27.848}{27.8}$ & & 46.7 & $|52.549|$ & & & $\frac{0.303}{0.303}$ & & & & $\frac{14.6}{14.6}$ & & & & & & & & & & & 74 & \\
\hline & & & & & & 27.81 & & & & & & & & & & & & & & & & & & & & & & \\
\hline 230 & & & & & & 27.76 & & & & & & & & & & & & & & & & & & & & & & \\
\hline 2308 & & 11:13: & 26.606 & 26.6 & 25.103 & 27.713 & & 46. & & & & 0.306 & & & & 146 & & & & & & & & & & & & \\
\hline 309 & & $11 \cdot 1$ & 2661 & 2668 & & 27777 & & $4696 \mathrm{f}$ & 5297 & 475 & & 5.286 & & 0164 & & 464 & & & & & & & & & & & & \\
\hline 2310 & $6 / 16 / 2004$ & 11:15:04 AM & 26.615 & \begin{tabular}{|l|l|l|}
26.684 \\
\end{tabular} & $\mid$ & 27.851 & 26.756 & \begin{tabular}{|l}
47.246 \\
\end{tabular} & \begin{tabular}{|l|l|}
53.4 \\
\end{tabular} & \begin{tabular}{|l|l|}
15.646 \\
\end{tabular} & $\begin{array}{l}31.98 \\
\end{array}$ & $\begin{array}{ll}0.321 \\
\end{array}$ & 5.71 & 51.673 & 0.045 & 14.643 & & 00.35 & 50.0058 & & & & .7.731 & 0.007 & 0.007 & 0.000 & 0.169 & 0.1 \\
\hline
\end{tabular}


WSRC-TR-2005-00105, REVISION 0

SRNL-RPP-2005-00012, REVISION 0

RUN \# 3.03A AND B; FIRST AND SECOND HALF OF SLURRY DEWATERING - CONT.

\begin{tabular}{|c|c|c|c|c|c|c|c|c|c|c|c|c|c|c|c|c|c|c|c|c|c|c|c|c|c|c|c|c|}
\hline & A & & $\begin{array}{lll} & \\
\end{array}$ & $E$ & $F$ & \begin{tabular}{l|l|l}
$G$ & \\
\end{tabular} & $\begin{array}{ll}\mathrm{H} \\
\end{array}$ & $\mathrm{J}$ & \begin{tabular}{l|l}
$K$ \\
\end{tabular} & $\begin{array}{ll} \\
\end{array}$ & $\mathrm{M} \mid$ & \begin{tabular}{|l|l|}
$\mathrm{N}$ \\
\end{tabular} & 0 & \begin{tabular}{l|l} 
\\
\end{tabular} & $\begin{array}{lll}R & \\
\end{array}$ & \begin{tabular}{l|l} 
s \\
\end{tabular} & $\begin{array}{llll}T & \end{array}$ & $\mathrm{v}$ & w & $x \mid$ & $\begin{array}{lll} & \end{array}$ & $\mathrm{z}$ & & $\mathrm{AB}$ & AC & $\overline{A D}$ & $\overline{A E}$ & \\
\hline 3111 & 6/16/2004 & 11:16:04 AM & 26.62 & 26.679 & 25.117 & 27.926 & 26.826 & 47.116 & 53.295 & 15.569 & 31.896 & \begin{tabular}{|l|}
0.318 \\
\end{tabular} & -5.715 & $\begin{array}{l}44.058 \\
\end{array}$ & 0.046 & 14.643 & 0.004 & 3001.35 & 0.0225 & 51.8 & 10.8 & 39.5 & 2.724 & 0.007 & 0.007 & 0.000 & 0.173 & \\
\hline & & 11:17:04 AM & 26.626 & 26.675 & & 27.982 & 26.922 & 47.136 & \begin{tabular}{|l|l|} 
\\
53.224 \\
\end{tabular} & \begin{tabular}{|l|}
15.608 \\
\end{tabular} & 31.927 & 0.321 & -5.713 & 49.894 & 0.046 & 14.643 & & & & 51.8 & $\frac{12.2}{12.2}$ & & & & & & & \\
\hline (1) & 6/16/2004 & 11:18:04 AM & 26.642 & 26.691 & \begin{tabular}{|r|}
25.14 \\
25.19
\end{tabular} & & 26.983 & 47.267 & \begin{tabular}{|l|}
53.168 \\
\end{tabular} & \begin{tabular}{|l|l|}
15.6222 \\
1.620
\end{tabular} & $\begin{array}{l}32.138 \\
3.1391\end{array}$ & 0.321 & $\begin{array}{l}-5.71 \\
-572 \\
\end{array}$ & $\begin{array}{l}48.296 \\
8701\end{array}$ & 0.046 & 14.643 & & $\frac{3003.35}{30.32}$ & $\begin{array}{l}50.0558 \\
5.072\end{array}$ & $\begin{array}{l}51.7 \\
5.7 \\
\end{array}$ & $\frac{11.8}{11.0}$ & $\frac{39}{39 .}$ & (7) & 007 & 0.007 & 0.000 & 0.172 & \\
\hline (315 & $\begin{array}{l}6 / 11 / 2004 \\
6 / 162004 \\
\end{array}$ & 11:19:04 AM & $\begin{array}{l}26.659 \\
26.665 \\
\end{array}$ & $\begin{array}{ll}26.688 \\
26695\end{array}$ & $\begin{array}{l}25.136 \\
25134 \\
213\end{array}$ & \begin{tabular}{r|r|r|}
28.18 \\
28206
\end{tabular} & 27.045 & 47.103 & \begin{tabular}{|l|l|}
53.216 \\
5220
\end{tabular} & \begin{tabular}{|l|l|}
15.603 \\
15.6
\end{tabular} & $\begin{array}{l}31.931 \\
31.775 \\
\end{array}$ & 0.321 & $\begin{array}{l}-5.707 \\
-5.51 \\
\end{array}$ & $\begin{array}{l}48.791 \\
4260\end{array}$ & 0.046 & 14.643 & 0.004 & 3004.35 & $\begin{array}{l}50.0725 \\
500802\end{array}$ & 51.7 & 12.0 & 39.5 & .725 & .007 & 0.007 & 0.000 & 0.173 & \\
\hline 316 & 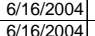 & $\begin{array}{l}\frac{11: 20: 04 \mathrm{AM}}{11 \cdot 21 \cdot 14 \mathrm{AM}} \\
\end{array}$ & $26.665 \mid$ & 26.695 & 25.143 & & & $\begin{array}{ll}47.045 \\
47236\end{array}$ & \begin{tabular}{|l|}
53.284 \\
52903 \\
\end{tabular} & $\begin{array}{l}15.64 \\
15.595 \\
\end{array}$ & & \begin{tabular}{|l|}
0.321 \\
0338
\end{tabular} & $\begin{array}{r}-5.71 \\
5707\end{array}$ & \begin{tabular}{|c|}
44.269 \\
47347
\end{tabular} & $\begin{array}{ll}0.046 \\
0.045\end{array}$ & $\frac{14.643}{14643}$ & & 3005.35 & & $\begin{array}{l}51.8 \\
5.15\end{array}$ & \begin{tabular}{|l|l|}
10.8 \\
1.6
\end{tabular} & 39.4 & $\frac{2.717}{2.725}$ & 0.007 & 0.007 & & 0.173 & \\
\hline$\frac{310}{317}$ & $\frac{6 / 11 / 12004}{6 / 16 / 2004}$ & $\begin{array}{l}\frac{11: 21: 04 \mathrm{AM}}{11: 22: 04 \mathrm{AM}} \\
\end{array}$ & $\frac{26.673}{26.68}$ & 26.7129 & \begin{tabular}{|l|}
25.1 .161 \\
25.162 \\
\end{tabular} & 28.249 & $\begin{array}{l}27.124 \\
27.115\end{array}$ & $\begin{array}{l}47.236 \\
47.064\end{array}$ & \begin{tabular}{|l|}
52.963 \\
5.129 \\
\end{tabular} & $\begin{array}{l}15.595 \\
15.659\end{array}$ & $\begin{array}{l}32.093 \\
31.818\end{array}$ & \begin{tabular}{|l|}
0.338 \\
0.327 \\
\end{tabular} & $\begin{array}{l}-5.707 \\
-5.704 \\
\end{array}$ & $\mid$\begin{tabular}{|l}
$\mid 47.347$ \\
5.271
\end{tabular} & $\begin{array}{l}0.045 \\
0.046\end{array}$ & $\begin{array}{l}14.643 \\
14.643\end{array}$ & 0.004 & $\begin{array}{l}3006.35 \\
3007.35\end{array}$ & \begin{tabular}{|l|}
50.1058 \\
50.1225 \\
\end{tabular} & \begin{tabular}{|l|l|}
51.5 & \\
51.7 &
\end{tabular} & \begin{tabular}{|l|}
11.6 \\
12.9
\end{tabular} & $\begin{array}{l}39.7 \\
39.4 \\
\end{array}$ & $2.735 \mid 2.719$ & $\begin{array}{l}0.007 \\
0.007\end{array}$ & \begin{tabular}{|l|}
0.007 \\
0.007
\end{tabular} & $\begin{array}{l}0.000 \\
0.000\end{array}$ & $\begin{array}{l}0.168 \\
0.173\end{array}$ & 0.14 \\
\hline 318 & $6 / 16 / 2004$ & & 26.686 & 26.726 & 25.169 & 28.162 & & & 53.108 & & & & & 44.229 & 0.045 & $\begin{array}{l}14.643 \\
\end{array}$ & 0.004 & & & & & & 2.724 & 0.007 & $\begin{array}{l}0.007 \\
0.007 \\
\end{array}$ & 0.000 & 0.169 & $\frac{15}{14}$ \\
\hline & & 11:24:04 AM & 26.677 & 26.706 & 25.14 & & 26.973 & 47.452 & 53.678 & 15.761 & 32.002 & \begin{tabular}{|l|}
0.318 \\
\end{tabular} & -5.707 & 48.855 & 0.045 & 14.643 & & & & & & & & & & & & \\
\hline & 6/16/2004 & 11:25:04 AM & 26.704 & 26.743 & & & & 47.155 & \begin{tabular}{|l|}
53.154 \\
5.250 \\
\end{tabular} & & $\begin{array}{l}31.859 \\
2.072\end{array}$ & \begin{tabular}{|l|l|}
0.338 \\
\end{tabular} & & $\begin{array}{r}46.5 \\
\end{array}$ & 0.046 & 14.643 & & 3010.35 & \begin{tabular}{|l|}
50.1725 \\
\end{tabular} & & 11.4 & & & & 0.007 & & & \\
\hline 321 & 6/16/2004 & 11:26:04 AM & 26.694 & 26.733 & 25.171 & 28.075 & 26.925 & 47.085 & \begin{tabular}{|l|}
53.258 \\
5211 \\
\end{tabular} & 15.618 & $\begin{array}{l}31.873 \\
31.936\end{array}$ & 0.327 & & $\begin{array}{l}47.606 \\
\end{array}$ & 0.046 & 14.643 & 0.00 & 3011.35 & \begin{tabular}{|l|l|}
50.1892 \\
\end{tabular} & 51.8 & 11.7 & & & $\begin{array}{l}0.007 \\
\end{array}$ & 0.007 & 0.000 & 0.173 & \\
\hline$\frac{322}{323}$ & 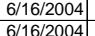 & $\begin{array}{l}11: 27: 04 \mathrm{AM} \\
1112004 \mathrm{AM}\end{array}$ & \begin{tabular}{|l|l|}
26.699 \\
26.689
\end{tabular} & $\begin{array}{l}26.748 \\
26.733 \\
\end{array}$ & $25.191 \mid$ & \begin{tabular}{r|r|}
28.01 \\
27.905 \\
\end{tabular} & & $\begin{array}{l}46.929 \\
47.093 \\
\end{array}$ & \begin{tabular}{|l|}
53.112 \\
53.033 \\
\end{tabular} & \begin{tabular}{r|r|}
15.49 \\
15.613
\end{tabular} & \begin{tabular}{|l|l|}
31.826 & \\
33.955
\end{tabular} & 0.332 & & \begin{tabular}{|l|l|}
40.397 \\
4703
\end{tabular} & 0.045 & $\begin{array}{l}14.643 \\
14663\end{array}$ & & $\begin{array}{l}3012.35 \\
30135\end{array}$ & 50.2058 & $\begin{array}{l}51.6 \\
51.6\end{array}$ & 9.9 & & 2.715 & $\begin{array}{l}0.007 \\
0.007\end{array}$ & \begin{tabular}{|c|c|}
0.007 & \\
0007 &
\end{tabular} & & 0.169 & 0.14 \\
\hline 323 & 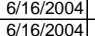 & $\begin{array}{l}\frac{11: 28: 04 \mathrm{AM}}{11} \\
\text { 11:20:04 AM }\end{array}$ & $26.689 \mid$ & 26.733 & 25.186 & \begin{tabular}{rl|}
27.905 & \\
22.87 &
\end{tabular} & $\begin{array}{r}26.83 \\
26.805\end{array}$ & |.093 & \begin{tabular}{|l|}
53.077 \\
53.649
\end{tabular} & $\frac{15.613}{15.696}$ & .955 & $\begin{array}{l}0.356 \\
0.35\end{array}$ & & \begin{tabular}{|l|l|}
47.035 \\
49.969
\end{tabular} & $\begin{array}{l}0.045 \\
0.045\end{array}$ & $\begin{array}{l}14.643 \\
14.643\end{array}$ & 03 & $\begin{array}{l}3013.35 \\
3014.35\end{array}$ & 50.22255 & \begin{tabular}{|c|c|}
51.6 \\
52.2
\end{tabular} & $\frac{11.5}{12.2}$ & $\frac{39.5}{39.8}$ & $\frac{5.742}{.742}$ & 007 & 0.007 & 0.000 & |0.169| & \\
\hline 325 & $6 / 16 / 2004$ & $11: 30: 04 \mathrm{AM}$ & 26.701 & 26.736 & 25.194 & 27.903 & 26.778 & 46.871 & 52.806 & 15.462 & \begin{tabular}{|c|}
31.83 \\
\end{tabular} & \begin{tabular}{|l|}
0.358 \\
\end{tabular} & 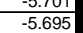 & $\begin{array}{l}4.969 \\
48.309 \\
\end{array}$ & $\begin{array}{l}0.045 \\
0.046 \\
\end{array}$ & $\begin{array}{l}14.643 \\
14.643 \\
\end{array}$ & $\begin{array}{l}0.003 \\
0.004\end{array}$ & $\begin{array}{l}3014.55 \\
3015.35 \\
\end{array}$ & $\begin{array}{l}30.2932 \\
50.2558\end{array}$ & $\begin{array}{ll}52.2 \\
51.3 \\
\end{array}$ & $\frac{12.2}{11.8}$ & $\begin{array}{l}3.9 .8 \\
39.4\end{array}$ & & $\begin{array}{l}0.007 \\
0.007\end{array}$ & $\begin{array}{l}0.007 \\
0.007 \\
\end{array}$ & & & $\frac{0.14}{0.15}$ \\
\hline & & 11:31:04 AM & 26.694 & 26.723 & 25.166 & 27.88 & 26.78 & & \begin{tabular}{|l|l|}
52.644 \\
\end{tabular} & 15.45 & 31.553 & 0.358 & & 45.992 & & 14.643 & & & & & 11.3 & & & 0.007 & & & & $\frac{0.15}{0.15}$ \\
\hline & 6/16/2004 & 11:32:04 AM & 26.708 & 26.732 & & 27.87 & 26.775 & 46.715 & 52.959 & 15.506 & 31.592 & 0.361 & -5.689 & & $\begin{array}{l}0.046 \\
\end{array}$ & 14.643 & & 3017.35 & 50.2892 & 51.5 & 12.1 & 39. & & & 0.007 & & & \\
\hline 328 & & & 26.69 & 26.72 & 25.163 & 27.847 & & & 52.675 & & & & & 52.764 & & & & & & & & & & & & 000 & & \\
\hline & & $11: 34: 04 \mathrm{~A}$ & & 26.717 & 25.161 & & & & 52.872 & 15.47 & 31.769 & 0.361 & & & & 14.643 & & & & 51.4 & 12.0 & & & & & & & \\
\hline & & $11: 35: 04$ & 26.692 & 26.706 & 25.16 & 27.744 & & 46.583 & 52.872 & 15.497 & & & & & & & & & & & & & & & & & & \\
\hline & 6/2004 & & 26.707 & 26.716 & 25.175 & 27.714 & & & & 15.51 & | & 0.358 & & $\begin{array}{ll}47.533 \\
\end{array}$ & 0.046 & 14.643 & & & & & & & & & & & & \\
\hline 332 & $6 / 2$ & 11::37:04 AM & 26.686 & 26.701 & 25.159 & 27.778 & 26.668 & 46.86 & 52.967 & 15.563 & 31.729 & 0.361 & 5.68 & 51.304 & 0.046 & 14.643 & & \begin{tabular}{|c|}
3022.35 \\
\end{tabular} & 50.3725 & 51.5 & 12.6 & 39.3 & 2.709 & 0.007 & 0.007 & 0.000 & 0.174 & \\
\hline & & 11:38:04 AM & 26.68 & 26.675 & 25.143 & 27.827 & 26.762 & 47.124 & 53.529 & 15.617 & 31.812 & 0.358 & -5.684 & \begin{tabular}{ll|l}
48.438 \\
\end{tabular} & 0.045 & 14.643 & & 3023.35 & 50.3892 & 52.1 & 11.9 & & & 0.007 & 0.007 & 0.000 & 0.169 & \\
\hline & & 11:39:04 AM & 26.681 & 26.676 & 25.139 & 27.868 & & 46.678 & \begin{tabular}{|l|}
52.706 \\
\end{tabular} & 15.437 & 31.705 & 0.37 & & 49.26 & 0.046 & 14.643 & & 3024.35 & 50.4058 & 51.2 & 12.1 & & 2.702 & \begin{tabular}{|l|l|}
0.007 \\
\end{tabular} & 0.007 & 0.000 & 0.174 & \\
\hline 33 & $6 / 1$ & 11:40:04 AM & 26.687 & 26.681 & 25.15 & 27.939 & & 46.749 & \begin{tabular}{|l|}
52.897 \\
\end{tabular} & 15.514 & 31.619 & & & 43.803 & 0.045 & 14.643 & & 3025.35 & & 51.4 & 10.7 & 39.2 & 2.702 & 0.007 & 0.007 & 0.000 & & \\
\hline 337 & $6 / 2004$ & $\begin{array}{l}\frac{11: 41: 04 \mathrm{AM}}{11.42: 04 \mathrm{AM}} \\
\end{array}$ & $\begin{array}{l}26.694 \\
26.695\end{array}$ & $\begin{array}{l}26.688 \\
26.679 \\
\end{array}$ & 25.161 & $28.015 \mid$ & $\begin{array}{r}26.96 \\
26.991 \\
\end{array}$ & $\begin{array}{l}47.058 \\
46.749 \\
\end{array}$ & \begin{tabular}{|l|}
53.353 \\
52.899 \\
\end{tabular} & $\frac{15.557}{15.528}$ & \begin{tabular}{l|l}
.922 \\
633
\end{tabular} & \begin{tabular}{|l|}
0.422 \\
0.419 \\
\end{tabular} & $\begin{array}{l}-5.672 \\
-5669 \\
\end{array}$ & \begin{tabular}{|c|}
48.918 \\
48.58 \\
\end{tabular} & $\begin{array}{l}0.045 \\
0.045 \\
\end{array}$ & $\frac{14.643}{14.633}$ & & $\begin{array}{l}3026.35 \\
302.35 \\
\end{array}$ & 392 & \begin{tabular}{|l|l|}
51.9 \\
51.4
\end{tabular} & $\frac{12.0}{11.9}$ & $\frac{39.5}{39.2}-x-1$ & $\begin{array}{l}2.723 \\
.702\end{array}$ & \begin{tabular}{|l|l|}
0.007 & 0.007
\end{tabular} & \begin{tabular}{|c|c|}
0.007 \\
0.007
\end{tabular} & 0.000 & 169 & 0.14 \\
\hline (338 & 6. & 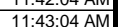 & $2 \frac{2.6055}{26.711}$ & 26.701 & 25.169 & 28.152 & 27.9 .0 & 46.871 & \begin{tabular}{|l|}
52.953 \\
\end{tabular} & 15.47 & | & 0.431 & $\begin{array}{l}-5.6 \\
-5.6 \\
\text { - }\end{array}$ & \begin{tabular}{|l|}
48.697 \\
48.697
\end{tabular} & 0.045 & $\begin{array}{l}14.0453 \\
14.643\end{array}$ & & $\begin{array}{l}3027.35 \\
3028.35\end{array}$ & $\begin{array}{l}50.456 \\
50.4725\end{array}$ & 51.5 & $\begin{array}{ll}11.9 \\
11.9\end{array}$ & $\begin{array}{l}39.4 \\
39.4\end{array}$ & & & & & & $\frac{0.15}{0.14}$ \\
\hline 2339 & $6 / 16 / 2004$ & 11:44:04 AM & 26.703 & 26.702 & 25.151 & 28.124 & & 46.952 & 53.09 & 15.54 & \begin{tabular}{|l|}
31.84 \\
\end{tabular} & \begin{tabular}{|l|l|} 
& 0.422 \\
\end{tabular} & & 44.75 & 45 & 14.643 & & 0029.35 & 50.48 & 51.6 & 11.0 & 39.4 & 2.716 & $\mid 0.007$ & 0.007 & & & 0.14 \\
\hline & $6 / 16 / 2$ & $11: 45: 04 \mathrm{AM}$ & 26.699 & 26.708 & & 28.11 & & & & & & & & & & 14.643 & & & & & 12.9 & & & & & & & $\frac{0.14}{0.14}$ \\
\hline 341 & $6 / 16 / 2$ & 11:46:04 AM & 26.689 & 26.698 & 25.136 & 28.1 & 26.98 & 47.6 & \begin{tabular}{|l|} 
\\
\end{tabular} & 15.598 & 31.81 & 0.4 & & \begin{tabular}{ll|l}
47.448 \\
\end{tabular} & 0.046 & 14.643 & & & & 51.9 & 11.6 & & & & & & & \\
\hline 342 & & 11:47:04 & 26.709 & 26.719 & 25.162 & 28.051 & & & 52.915 & 15.49 & 31.76 & & & 45.606 & & 14.643 & & & & & & & & & & & & \\
\hline & 0.12004 & 11:48:04 AM & 26.71 & 26.715 & 25.158 & 28.017 & & 47.319 & \begin{tabular}{|l|l|}
53.66 \\
\end{tabular} & 15.632 & 32.074 & 0.425 & & 45 & & 14.643 & & & & & & & & & & & & \\
\hline 344 & 6/2004 & 11:49:04 AM & 26.7 & 26.725 & .143 & 27.967 & & 46.97 & 53.069| & 15.546 & 31.855 & 0.422 & & 46.484 & 0.044 & 14.643 & & & & & 11.4 & & & & & & & \\
\hline 345 & 6/2004 & 11:50:04 & 26.704 & 26.734 & 25.162 & 27.931 & & 46.904 & 53.075 & 15.524 & 7777 & $\begin{array}{ll}0.448 \\
\end{array}$ & & & & 14.643 & & & & 51.6 & 13.1 & & & & & & & \\
\hline & t & 11:51:04A & 26.69 & 26.724 & 25.142 & 27.871 & & 46.7. & 53.108 & 15.474 & 627 & 0.4 & & $\begin{array}{ll}48.128 \\
\end{array}$ & & 14.643 & & & & 51.6 & 11.8 & & & & & & & \\
\hline 344 & i/2004 & 11:: & 26.68 & & & 27.826 & & 47.02 & 53.307 & 15.651 & 758 & 0.4 & & & 0.046 & 14.643 & & & & 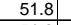 & & & & & & & & \\
\hline 344 & & & & & & & & & & 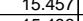 & & & & & & & & & & & & & & & & & & \\
\hline & & & 26.69 & & & 27.936] & & & & 15.496 & $31.791 \mid$ & & & 10.73. & & 14.643 & & & & & 12.7 & & & & & & & \\
\hline & & $\begin{array}{l}11.50 .4 \mathrm{AA} \\
11.56 \mathrm{~A}\end{array}$ & 年. & $\frac{2.1}{266}$ & $25.16-16$ & 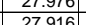 & & & $\begin{array}{l}53.313 \\
53305 \\
\end{array}$ & 509 & 808 & 0.419 & & 363 & & 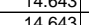 & & & & & 119 & & & & & & 67 & \\
\hline & 6/2004 & & 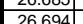 & & & 年 & & & 53231 & & & 0.0503 & & & & 14643 & & & & & 123 & & & & & & & \\
\hline & & & & & & $\frac{20.684}{2784}$ & & & & 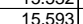 & & (0.593 & & & & $\frac{1.4043}{14643}$ & & & & 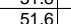 & thes & & & & & & (170) & \\
\hline $\begin{array}{ll}354 \\
354\end{array}$ & $\frac{0}{6 / 2004}$ & & $\frac{26.682}{26.682}$ & 26.686 & 年. & $\frac{27.664}{27.864}$ & & & 53.315 & 15.494 & 31.935 & 0.55 & & & 0.045 & $\begin{array}{l}\frac{14.045}{14.643} \\
\end{array}$ & & & & $51.8>-1$ & 1.3 & & & & & & & \\
\hline & & & & & & & & & & & & & & & & & & & & & & & & & & & & \\
\hline 2356 & & & 26.679 & & 25.15 & 27.875 . & & & 53.102 & 15.556 & & 0.63 & & & & & & & & 51. & 6 & & & & & & & \\
\hline & & & 26.694 & 26.683 & & 27.985 & & & & 15.601 & & 0.657 & & & & & & & & & & & & & & & & \\
\hline & & $23.03 \mathrm{PM}$ & 26.69 & 26.68 & & 28.012 & & & & 15.456 & & 0.622 & & & & & & & & & re & & & & & & & \\
\hline 3.5 & $6 / 16 / 2 \mathrm{C}$ & 12:04: & 26.7 & & 25.17 & 28.069 & & & 53.264 & 15.58 & & 0.645 & & & & 14.64 & & & & 51.8 & 12.1 & & & & & & .169 & \\
\hline & & & & & & & & & & & & 0.5 & & & & & & & & & & & & & & & & \\
\hline 3361 & & & 26.70 & & 25.1999 & 28.127 & & & & & & & & & & & & & & 1. & 9 & & & & & & & \\
\hline & & & & & & & & & & & & & & & & & & & & & & & & & & & & \\
\hline 3363 & & & 26.69 & 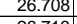 & 25.19 & 28.19 & & & 52.777 & 15.488 & 31. & & & & & & & & & & & & & & & & & \\
\hline$\overline{36}$ & & & 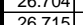 & $\frac{20.113}{2672}$ & $\frac{25.5}{25}$ & 28.18 & & & & $\begin{array}{l}10.524 \\
1559\end{array}$ & $\frac{31.592}{31.613}$ & 0.5 & & & & $\frac{1.6}{146}>>>$ & & & & 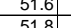 & 118 & & & & & & 70 & 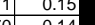 \\
\hline & & $\frac{12.10}{12 \cdot 1}$ & $\frac{2.1}{26.7}$ & 26.741 & | & 年 & & & 53087 & $\frac{15.53}{15.54}$ & 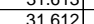 & 0.059 & & & & & & & & & & & & & & & & \\
\hline$\frac{56}{36}+2-3$ & & & & & & 2800 & & & & & & & & & & & & & & & & & & & & & & \\
\hline 年368 368 & & & $\frac{26.7}{26.7}$ & & $\frac{25.21}{25.21}$ & $\frac{28.029}{28}$ & & & $\frac{52.91}{52.91}$ & $\frac{15.525}{15.52}$ & & & & & & & & & & & & & & & & & & \\
\hline & & & & & & & & & & & & & & & & & & & & & & & & & & & & \\
\hline$\sqrt{37}+2-3$ & & & & & & & & & & & & & & & & & & & & & & & & & & & & \\
\hline & & & 26.7. & & 25.1 & 27.993 & & & & & & & & & & & & & & & & & & & & & & \\
\hline 2372 & $6 / 16 / 2$ & 12:17 & 26.7 & 26.7 & 25.198 & 27.977 & & 46.20 & 52.58 & 15.9 & & 0.65 & & & & $14.6 \mathrm{C}>\mathrm{C}$ & & & & & & & & & & & & $\frac{4}{4}$ \\
\hline 237 & 6/16/20 & 12:18 & 6.714 & 26.7 & 251 & 28 & & 47.7. & 54.25 & 14.95 & 6] & 0.715 & & & & 4.6 .6 & & & & & & & & & & & & \\
\hline 2374 & & 12:19 & 26.72 & 26.743 & 25.1 & 885] & & & 55.218 & & 34.664 & & & & & 14.6 & & & & & & & & & & & & \\
\hline 2375 & & & $26.7 \mathrm{C}$ & & & 27.981 & & 48. & 52.472 & 17.2 & & & & & & & & & & & 12.11 & & & & & & & \\
\hline 3 & & & & & & & & & & & & & & & & & & & & & & & & & & & & \\
\hline$\frac{371}{578}$ & & & 20.621 & & 25.26 & 20.708 & & & 52.2 & & & & & & & & & & & & & & & & & & & \\
\hline 6379 & & & $\frac{20.123}{26720}$ & 20.154 & 20.2000 & 27.913 & & 4.15 & 1.1725 & 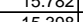 & & 1.11 & & & & & & & & 530 & & & & & & & & \\
\hline 2380 & $\frac{6 / 16 / 12004}{6 / 16 / 2004}$ & $\frac{12: 24: 03 \mathrm{MM}}{12: 25: 03 \mathrm{PM}}$ & $\frac{26.729}{26.717}$ & $\begin{array}{l}26.739 \\
26.716 \\
\end{array}$ & \begin{tabular}{|l|}
25.2297 \\
25.285 \\
\end{tabular} & \begin{tabular}{|l|}
27.931 \\
27.934 \\
\end{tabular} & \begin{tabular}{|l|l|}
26.839 \\
\end{tabular} & 49.1542 & \begin{tabular}{|l|}
54.43556 \\
53.396
\end{tabular} & \begin{tabular}{|l|l|}
16.879 \\
\end{tabular} & $\begin{array}{r}34.192 \\
32.591 \\
\end{array}$ & \begin{tabular}{|l|}
-0.1262 \\
-1.222 \\
\end{tabular} & -6.594 & \begin{tabular}{r|}
4.906 \\
54.906
\end{tabular} & 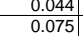 & \begin{tabular}{|l|}
14.643 \\
\end{tabular} & & 1070.33 & \begin{tabular}{|l|}
51.1756 \\
51.1722 \\
\end{tabular} & \begin{tabular}{|l|}
53.9 \\
51.9
\end{tabular} & \begin{tabular}{|l|l|}
13.5 \\
\end{tabular} & & \begin{tabular}{|l|l}
2.812 \\
\end{tabular} & \begin{tabular}{|l|}
0.0071 \\
0.011 \\
\end{tabular} & \begin{tabular}{|l|}
0.0071 \\
0.011
\end{tabular} & 0.000 & 0.272 & 2 \\
\hline
\end{tabular}


WSRC-TR-2005-00105, REVISION 0

SRNL-RPP-2005-00012, REVISION 0

RUN \# 3.03A AND B; FIRST AND SECOND HALF OF SLURRY DEWATERING - CONT.

\begin{tabular}{|c|c|c|c|c|c|c|c|c|c|c|c|c|c|c|c|c|c|c|c|c|c|c|c|c|c|c|c|c|}
\hline & A & & D & $\mathrm{E}$ & $\mathrm{F}$ & G & $\begin{array}{ll}\mathrm{H} \\
\end{array}$ & $\mathrm{J}$ & $\begin{array}{ll}\mathrm{K} & \mathrm{s} \\
\end{array}$ & \begin{tabular}{|l|} 
\\
\end{tabular} & $\begin{array}{ll}\mathrm{M} \\
\end{array}$ & \begin{tabular}{|l|l|}
$\mathrm{N}$ \\
\end{tabular} & 0 & \begin{tabular}{l|l} 
\\
\end{tabular} & \begin{tabular}{l|l}
$R$ & \\
\end{tabular} & \begin{tabular}{l|l} 
& $s$ \\
\end{tabular} & $T$ & $\mathrm{v}$ & w & \begin{tabular}{c|c|}
$x$ &
\end{tabular} & $\begin{array}{llll} & & & \end{array}$ & & & & AC & & & $\overline{A F} \quad \mid \mathbb{A}$ \\
\hline 2381 & 6/16/2004 & 12:26:03 PM & 26.733 & 26.737 & 25.336 & 27.989 & 26.894 & 48.603 & 53.156 & \begin{tabular}{|l|l|}
16.872 \\
\end{tabular} & \begin{tabular}{|l|}
32.093 \\
\end{tabular} & \begin{tabular}{|l|}
-1.222 \\
\end{tabular} & -6.632 & $\begin{array}{ll}48.47 \\
\end{array}$ & 0.047 & 14.643 & 0.003 & 3071.33 & 81.1889 & 51.7 & 11.9 & 40.3 & 2.782 & 0.007 & 0.007 & 0.000 & 0.172 & 0.15 \\
\hline & & & 26.739 & 26.728 & 25.352 & & \begin{tabular}{|l}
26.935 \\
\end{tabular} & & $\begin{array}{l}52.802 \\
5\end{array}$ & 17.15 & 31.602 & \begin{tabular}{|l|l|}
-1.219 \\
\end{tabular} & $\begin{array}{l}-6.635 \\
\end{array}$ & 54.529 & 0.046 & \begin{tabular}{|l|l|}
5 & 14.643 \\
\end{tabular} & & & 1.2056 & 51.3 & $\begin{array}{ll}13.4 \\
3.4\end{array}$ & & & & & & & 0.14 \\
\hline 2383 & 6/16/2004 & 12:28:03 PM & $\frac{26.745}{26.750}$ & 26.73 & $\frac{25.363}{25.303}$ & 28.072 & \begin{tabular}{|l}
26.987 \\
2707
\end{tabular} & 48.491 & \begin{tabular}{|l|}
53.096 \\
\end{tabular} & $\frac{16.833}{16.83}$ & 32.099 & \begin{tabular}{|l|l|}
-1.2222 \\
\end{tabular} & -6.632 & $\begin{array}{l}48.44 \\
4020\end{array}$ & 0.046 & \begin{tabular}{|l|l|}
5 & 14.643 \\
\end{tabular} & & $\begin{array}{l}3073.33 \\
27323\end{array}$ & 1.2222 & 51.6 & $\frac{11.9}{11.9}$ & 40.3 & $\begin{array}{l}2.778 \\
.78 \\
\end{array}$ & 0.007 & 0.007 & 0.000 & 0.168 & \\
\hline $\begin{array}{l}2384 \\
2325 \\
\end{array}$ & $6 / 16 / 2004$ & 12:29:03 PM & 26.759 & 26.739 & 25.393 & 28.121 & 27.006 & 48.534 & \begin{tabular}{|l|}
53.243 \\
\end{tabular} & 16.619 & $\begin{array}{l}32.331 \\
3.570\end{array}$ & \begin{tabular}{|l|l|} 
& -1.222 \\
\end{tabular} & -6.638 & $\begin{array}{l}48.338 \\
\end{array}$ & $\begin{array}{l}0.046 \\
\end{array}$ & 14.643 & 0.004 & 3074.33 & $\begin{array}{l}1.2389 \\
\end{array}$ & 51.8 & 11.8 & 40.4 & 2.788 & .007 & 0.007 & 0.000 & 0.168 & \\
\hline 2385 & 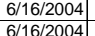 & $\begin{array}{l}12: 30: 03 \text { PM } \\
12.31 \cdot 03 \mathrm{PM}\end{array}$ & $\begin{array}{l}26.764 \\
26.776 \\
\end{array}$ & 26.744 & & \begin{tabular}{|l|l|}
28.121 \\
28.177 &
\end{tabular} & & $\begin{array}{l}48.843 \\
49274\end{array}$ & & \begin{tabular}{|l|l|}
16.692 \\
16647
\end{tabular} & \begin{tabular}{|l|}
32.579 \\
32323 \\
\end{tabular} & \begin{tabular}{|r|}
-1.219 \\
-125 \\
\end{tabular} & & \begin{tabular}{|l|l|}
48.157 & \\
6 & 5852 \\
\end{tabular} & $\begin{array}{ll}0.048 \\
0.047\end{array}$ & \begin{tabular}{|l|l|}
3 & 14.643 \\
\end{tabular} & & 3075.33 & & \begin{tabular}{|l|l|}
51.8 \\
5.2
\end{tabular} & 11.8 & & & 0.007 & 0.007 & & 0.174 & \\
\hline 2387 & 6/16/2004 & $\begin{array}{l}\text { 12:31:03 PM } \\
123203 \mathrm{PM}\end{array}$ & $\begin{array}{l}26.776 \\
26.806\end{array}$ & $\frac{26.746}{26.771}$ & $\frac{25.414}{25.449}$ & $28.177 \mid$ & \begin{tabular}{|l|}
27.112 \\
27.137 \\
\end{tabular} & $\begin{array}{l}49.274 \\
49.098\end{array}$ & \begin{tabular}{|l|}
53.838 \\
53.722 \\
\end{tabular} & \begin{tabular}{|l|}
16.647 \\
16.366 \\
\end{tabular} & $\begin{array}{r}33.033 \\
33.185 \\
\end{array}$ & \begin{tabular}{|l|}
-1.225 \\
-1.219 \\
\end{tabular} & $\begin{array}{l}-6.756 \\
-6.756 \\
\end{array}$ & $\frac{62.582}{48.566}$ & \begin{tabular}{|c|c|}
0.047 \\
0.048
\end{tabular} & $\begin{array}{l}14.643 \\
14.643\end{array}$ & $\frac{0.003}{0.004}$ & $\begin{array}{l}3076.33 \\
3077.33\end{array}$ & $\begin{array}{l}1.2722 \\
1.2889 \\
\end{array}$ & 52.4 & $\frac{15.3}{11.9}$ & $\frac{41.2}{41.1}$ & $2.837 \mid$ & 0.0007 & \begin{tabular}{|l|}
0.007 \\
0.007
\end{tabular} & $\begin{array}{l}0.000 \\
0.000\end{array}$ & $\begin{array}{l}0.168 \\
0.172\end{array}$ & $\begin{array}{l}0.14 \\
0.15\end{array}$ \\
\hline 2388 & $6 / 16 / 2004$ & 12:33:03 PM & 26.821 & 26.771 & 25.459 & & \begin{tabular}{|l|}
27.097 \\
\end{tabular} & & & & & & -6.765 & 51.498 & $\begin{array}{l}0.048 \\
0.047 \\
\end{array}$ & $\begin{array}{l}14.645 \\
14.643 \\
\end{array}$ & 0.004 & 3078.33 & 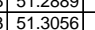 & $\begin{array}{ll}52.2 \\
52.3 \\
\end{array}$ & $\frac{11.9}{12.6}$ & 41.2 & 2.854 & . & $\begin{array}{l}0.007 \\
0.007\end{array}$ & & $\frac{0.1 / 2}{0.168}$ & $\frac{0.15}{0.14}$ \\
\hline & & 12:34:03 PM & 26.837 & 26.791 & 25.445 & 28.273 & 27.073 & 49.198 & \begin{tabular}{|l|}
53.749 \\
\end{tabular} & 16.366 & 33.287 & \begin{tabular}{|l|}
-1.222 \\
\end{tabular} & -6.805 & 43.929 & 0.044 & 14.643 & & 3079.33 & & & & & 2.844 & & 0.006 & & & 0.14 \\
\hline 2390 & $6 / 16 / 2004$ & 12:35:03 PM & 26.864 & 26.804 & 25.433 & 28.28 & 27.08 & 49.318 & 53.885 & 16.318 & 33.414 & \begin{tabular}{|l|}
-1.222 \\
\end{tabular} & & 49.636 & 0.047 & 14.643 & & 3080.33 & 1.3389 & 52.4 & 12.2 & 41. & & & 0.007 & & & \\
\hline 2391 & & & 26.875 & & & 28.241 & & & 53.687 & & & & & 44.575 & & & & & & & 10.9 & & .851 & 007 & 0.007 & 0.000 & 0.167 & \\
\hline 2392 & & & & & 25.398 & 28.177 & & 49.387 & 53.915 & 16.18 & 33.698 & & & & & 14.643 & & & & 52.4 & $\frac{12.0}{12}$ & & & & 0.007 & & .170 & \\
\hline 2393 & & 12:38:03 PM & 26.875 & 26.815 & 25.363 & 28.102 & & & 53.898 | & 16.062 & 33.644 & & & & & 14.643 & & & & $5 ?$ & 125 & & ra & & & & 1.167 & \\
\hline & $6 / 16 / 2004$ & 12:39:03 PM & 26.884 & 26.819 & 25.338 & 28.106 & & 49.391 & 53.883 & 16.129 & . & -1.222 & & 49.27 & 0.047 & 14.643 & & 3084.33 & .4056 & 52.4 & 12.1 & & .865 & 007 & 0.007 & & & \\
\hline 2395 & $6 / 16 / 2004$ & 12:40:03 PM & 26.889 & 26.819 & 25.328 & 28.111 & 26.94 & 48.939 & 53.568 & 16.438 & 32.92 & -1.222 & -6.768 & 51.05 & 0.046 & 14.643 & & 3085.33 & 4222 & 52.1 & 12.5 & 40. & .822 & .007 & 0.007 & & & \\
\hline & & 12:41:03 PM & 26.883 & 26.823 & $\frac{25.282}{20.275}$ & 28.07 & & 48.91 & 53.303 & 16.217 & 1.156 & $\begin{array}{ll}-1.222 \\
1.220\end{array}$ & & 48.384 & 0.047 & 14.643 & & 886.3 & & & & & & & & & & \\
\hline & & 12:42:03 PM & & 26.826 & $\begin{array}{l}25.275 \\
20.252 \\
-252\end{array}$ & 28.068 & & 48.555 & 53.073 & 16.744 & 32.241 & $\begin{array}{l}-1.222 \\
1.202 \\
\end{array}$ & & 49.919| & & 14.643 & & & 4556 & 51.6 & 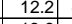 & & & & 007 & & & \\
\hline (3980 & & 12:43:03 $\mathrm{PM}$ & $26.8 / 5$ & & & & & & 53.459 & & & & & 50.62 & & & & & & & 13.6. & & & & & & & \\
\hline & & $\begin{array}{l}12: 44: 03 \mathrm{PM} \\
120.4 \cdot 53 \mathrm{PM}\end{array}$ & $\begin{array}{l}26.88 \\
268.86 \\
\end{array}$ & $\frac{26.845}{26.826}$ & $\frac{25.248}{25.184}$ & 28.082 & & $\frac{48.851}{48799}$ & 53.322 & $\frac{16.428}{16.361}$ & 32.9011 & -1.216 & & 50.762 & (4) & $\begin{array}{l}14.643 \\
14643\end{array}$ & & & & 51.8 & 12.4. & & & & . & 0.000 & & 14 \\
\hline 2401 & - & 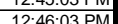 & 年. 26.800 & $\frac{20.020}{26.857}$ & (25.104 & 年.0.060 & & 4 & 5. 53.757 & $\begin{array}{l}\frac{10.001}{16.392} \\
\end{array}$ & 热. & $\begin{array}{l}-1.250 \\
-1.216 \\
\end{array}$ & & $\frac{47.224}{50.466}$ & 0.047 & 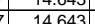 & & & & & 24 & & & & & & & \\
\hline 2402 & 6/16/2004 & 12:470:03 PM & 26.859 & 26.839 & 25.147 & 27.996 & 26.77 & 48.928 & 53.349 & 16.323 & 33.018 & \begin{tabular}{|l|}
-1.219 \\
\end{tabular} & -6.759 & 53.816 & 0.047 & $\begin{array}{l}14.043 \\
14.643 \\
\end{array}$ & & 3092.33 & 1.5389 & 51.9 & $\frac{x .4}{13.2}$ & & .825 & .007 & 0.007 & 0.000 & 0.170 & \\
\hline 2403 & $6 / 16 / 20$ & 12:48:03 PM & 26.853 & 26.842 & 25.131 & 27.979 & & 48.271 & 52.671 & 16.998 & 31.799 & \begin{tabular}{|l|}
-1.222 \\
\end{tabular} & -6.762 & $\mid 50.466$ & $\frac{0.046}{0.046}$ & $\frac{14.643}{14.64}$ & & $\frac{3093.33}{30933}$ & $\frac{1.5556}{1.556}$ & $\frac{51.2}{51.2}-2$ & $\frac{1.24}{12.4}$ & & $\frac{6}{7.60}$ & & 0.007 & 0.000 & & \\
\hline & & 12:49:03 PM & 26.834 & & 25.112 & & & 48.841 & 53.446 & 16.77 & & & & & $\begin{array}{ll}0.046 \\
\end{array}$ & 14.643 & & 3094.33 & .5722 & 520 & & & & & & & & \\
\hline 405 & 104 & 12:50:03 PM & 26.814 & 26.814 & 25.072 & 27.991 & & 48.656 & 53.146 & 16.761 & 32.397 & -1.222 & & 53.459 & 0.046 & 14.643 & & & & & $\frac{13.1}{13.1}$ & & 794 & 007 & .007 & 0.000 & & \\
\hline 406 & $6 / 16 / 2004$ & 12:51:03 PM & 26.79 & 26.8 & 25.028 & 27.917 & & 48.476 & 52.924 & 16.649 & 32.29 & & & 48.966 & 0.046 & 14.643 & & & & & $\frac{12.0}{12.0} \mathrm{C}$ & & & & & & & \\
\hline & 6/16/2004 & 12:52:03 PM & 26.779 & 26.804 & 25.022 & 27.931 & & 48.71 & 53.187 & 16.498 & 32.643 & -1.222 & & 52.291 & 0.046 & 14.643 & & & & & & & & & & & & \\
\hline 2408 & 6/16/2004 & 12:53:03 PM & 26.779 & 26.809 & 25.027 & 27.966 & 26.75 & $\begin{array}{lll}48.532 \\
\end{array}$ & 52.94 & 16.954 & 32.076 & \begin{tabular}{|l|l|} 
& -1.219 \\
\end{tabular} & & 57.797 & 0.045 & 14.643 & & 098.33 & 1.6389 & 51.5 & 14.2 & & & & & & & \\
\hline $240 S 5>>3$ & & 12:54:03 PM & 26.745 & 26.79 & 24.968 & 27.942 & & 48.764 & 53.28 & 16.693 & 32.54 & -1.222 & & 46.807 & 0.046 & 14.643 & & & & 51.8 & 11.5 & & 80 & 007 & 0.007 & & & 0.14 \\
\hline & & & 26.757 & & 24.985 & 27.989 & & & 53.343 & 16.647 & 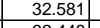 & $\begin{array}{l}-1.219 \\
-1202 \\
\end{array}$ & & 50.12 & 346 & 14.643 & & & & & & & & & & & & \\
\hline & $6 / 2004$ & 12:56:03 PM & 26.748 & 26.807 & 24.961 & & & 48.54? & 53.164 & 16.543 & 32.448 & -1.222 & & 51.711 & 0.046 & 14.643 & & & & & & & & & & & & \\
\hline 241 & & $12: 5: 03 \mathrm{PM}$ & 26.746 & & 24.954 & 28.0487 & & & 53.092 & $16.5 / 3$ & 32.474 & -1.222 & & & & 14.643] & & & 7056 & & 12.5 & & & & & & & \\
\hline$\frac{241}{241}$ & $\begin{array}{l}6 / 16 / 12004 \\
6 / 16 / 2004\end{array}$ & 12:58:03 PM & 26.715 & $\frac{26.815}{26.97}$ & $\frac{24.928}{249}$ & 28.052 & & $\frac{48.628}{49.115}$ & $\begin{array}{l}53.361 \\
53.29\end{array}$ & $\frac{16.593}{16.673}$ & 32.427 & $\begin{array}{l}-\frac{-1.222}{-1.222} \\
\end{array}$ & & $\frac{49.683}{49145}$ & 0.046 & $\frac{14.643}{14643}$ & & & & $\frac{1 .}{2}$ & 120 & & & & & & & \\
\hline 2415 & 6/16/2004 & $1: 00: 03 \mathrm{PM}$ & 20.695 & & $\frac{24.5}{24.888}$ & 28.007 & & 48.54 & 52.998 & $\begin{array}{c}16.61 \\
16.07 \\
\end{array}$ & $\begin{array}{l}32.058 \\
32.388\end{array}$ & $\frac{-1.22}{-1.202}$ & & $\begin{array}{l}52.445 \\
52.445\end{array}$ & $\begin{array}{l}0.040 \\
0.046\end{array}$ & 14.043 & & & & 51.5 & 29 & & & & & & & \\
\hline 2416 & $6 / 16 / 2004$ & 1:01:03 PM & 26.677 & $\frac{26.796}{20.796}$ & 24.849 & 27.929 & & & 53.624 & 16.531 & & \begin{tabular}{|l|l|}
-1.185 \\
\end{tabular} & & 47.52 & & 14.643 & & & & $\frac{52.2}{52.2}>$ & $\frac{11.6}{11.6}$ & & & & $\frac{0.00}{0.09}$ & & 0 & \\
\hline & 6/16/2004 & 1:02:03 PM & 26.681 & 26.816 & 24.849 & 27.918 & & 48.932 & 53.564 & 16.45 & 873 & -1.196 & & & 0.046 & 14.643 & & & & & 120 & & & & & & & \\
\hline & $6 / 1$ & & & & 24.84 & & & & & & & & & & & & & & & $=2$ & & & & & & & & \\
\hline 2419 & $6 / 1$ & 1:04:03 PM & 26.663 & 26.822 & 24.835 & 27.92 & & & 53.195 & & 32.47 & -1.159 & & 50.159 & & 14.643 & & & & & 12.3 & & & & & & & \\
\hline & $\mid 2004$ & & & & 24.834 & & & & 53.429 & & & & & & & 14.643 & & & & & & & & & & & & \\
\hline 421 & 6/16/2004 & 1:06:03 PM & & & 24.814 & 27.968 & & & \begin{tabular}{|l|}
53.429 \\
\end{tabular} & & 32.684 & -1.156 & & & & 14.643 & & & & 52.0 & 13.0 & & & & & & 69 & \\
\hline 2422 & 6/16/2004 & 3 PM & 26.6 & & 24.816 & 27.915 & & & 53.257 & 16.626 & & -1.1 & & 46.146 & 0.0 & 14.643 & & & & 51.8 & 111.3 & & & & .007 & & 0.166 & \\
\hline 242. & 6/16/2004 & & & & 24.798 & & & & & & & & & & & & & & & 52. & 12.7 & & & & & & 0.169 & \\
\hline & 6/16/2004 & 1:09:03 $\mathrm{P}$ & 26.601 & 26.7 & 24.803 & 27.883 & & 48.742 & 53.494 & 16.552 & 32.554 & -1.11 & & 49.22 & 0.046 & 14.643 & & & & & 12.1 & & & & & & & \\
\hline 2425 & $6 / 11$ & & & & 24.818 & & & & & & & & & & & & & & & 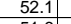 & 10.5 & & & & & & & \\
\hline & & & & & 24.793 & 27.863 & & & & & & & & & & & & & & & & & & & & & & \\
\hline$\frac{2427}{2428}$ & 6/16/2004 & $\begin{array}{l}1: 12: 03 \mathrm{PM} \\
1.13 .03 \mathrm{PM}\end{array}$ & 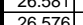 & $\frac{26.781}{26.771}$ & 24.783 & 27.873 & & & $\begin{array}{l}53.651 \\
55364\end{array}$ & $\frac{16.63}{16698}$ & $\begin{array}{ll}32.651 \\
32501\end{array}$ & $-\frac{-1.15}{-1116}$ & & & 0.046 & $\frac{14.643}{14643}$ & & & & 523 & 12. & & & & & & 66 & \\
\hline 2429 & 6/16/2004 & & & & 24.76 & 27.954 & & & 53.048 & & & & & & & $\frac{14.04}{14.64}$ & & & & 516 & 113 & & & & & & & \\
\hline 2430 & & & 26.562 & & 2473 & 27.934 & & & \begin{tabular}{|l|l|}
53.425 \\
\end{tabular} & & & & & & & & & & & & 139 & & & & & & & \\
\hline 2431 & 6/16/2004 & & 26.527 & & 24.699 & 27.904 & & & \begin{tabular}{|l|}
53.707 \\
\end{tabular} & & & & & & & 14.6 & & & & $\frac{52.2}{52.2}$ & $\frac{\frac{11.8}{11.8}}{4}$ & & & & & & (1.169 & \\
\hline & & & & & & & & & & & & & & & & & & & & & & & & & & & & \\
\hline 2433 & & & 26.56 & & 24.6 - 25 & 28.02 & & & $\begin{array}{l}53.266 \\
\end{array}$ & & & & & & & & & & & & $\frac{12.3}{12 .}$ & & & & & & & \\
\hline & & & & & 24.65 & 28.107 & & & & & & & & & & & & & & & & & & & & & & \\
\hline 2435 & $6 / 16 / 2$ & & & 26.7 > $>$ & 24.69 & 28.12 & & & $\mid 53.146$ & & & \begin{tabular}{|c|c|}
-1.098 \\
\end{tabular} & & & & & & & & 51.7 & 12.5 & & & & & & 71 & \\
\hline 243 & $6 / 16 / 2 C$ & 1:21: & 26.5 & & 24.657 & 28.117 & & & 53.16 & 16.6 & & -1.1 & & 52.4 & & 14.6. & & & & 51. & .9 & & & & & & 0.1 & \\
\hline 243 & & & & & $24.6-3$ & & & & & & & & & & & & & & & DI. & 2.6 & & & & & & 8 & \\
\hline 2438 & & 1:23: & 26.566 & & 24.6 & 28.221 & & & 53.286 & & & -1.1 & & & & 14.6 & & & & 51. & 12.2 & & & & & & 1 & \\
\hline 2435 & & & & & 24 & & & & & & & & & & & & & & & 51 & & & & & & & & \\
\hline 4 & & & 26.544 & 26.19 & 24.63 & 28.216 & & & 53.1434 & & & & & & & & & & & 51.1 & $\begin{array}{lll}0.1 \\
0.1\end{array}$ & & & & & & & \\
\hline$\frac{2444}{2417}$ & & 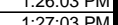 & $\frac{20.50}{2655}$ & 20.0 & $\begin{array}{ll}24.052 \\
22612\end{array}$ & 20.354 & & & 势. & 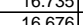 & $\frac{1}{6}$ & $\begin{array}{l}-1.049 \\
-1.75\end{array}$ & & & & & & & & 51 & 0 & & & & & & 年 & \\
\hline 2443 & $6 / 16 / 2004$ & $\frac{1.4703}{1 \cdot 28.03}$ & $\frac{2.030}{2652}$ & & 年.044 & $\frac{2.470}{28220}$ & & & $\frac{3.45}{53515}$ & 些16.05 & & \begin{tabular}{|c|c|}
-1.025 \\
\end{tabular} & & & & $\frac{11.0}{146}>0$ & & & & $\frac{5.3}{520}>0$ & $\frac{\frac{1.5}{116}}{116}+2$ & & & & & & 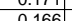 & \\
\hline 2444 & & & & & 2462 & 年 28194 & & & & & & & & & & 146 & & & & & 1 & & & & & & 1067 & \\
\hline 2445 & & & 26.54 & & $\frac{24.6}{206}$ & & & & 53.239 & & & & & & & 146 & & & & & 23 & & & & & & $\frac{68}{68}$ & \\
\hline 2446 & & & & & & & & & & & & & & & & & & & & & & & & & & & & \\
\hline & & & & & & & & & & & & & & & & & & & & & & & & & & & & \\
\hline 4448 & & $1: 33$ & 2654 & $26.8 \mathrm{r} \quad \mathrm{l}$ & 24.604 & 28.064 & & & 53.17 & 16.582 & & & & 46. & & 14.6 & & & & & & & & & & & 58 & \\
\hline 449 & & 1:34: & 26517 & & 24.569 & 234 & & & & $55 .>>>$ & & -0.9 & & & & $46.2 \div 25$ & & & & & & & & & & & & \\
\hline 2450 & 6/16/2004 & 1:35:03 PM & 26.518 & $\begin{array}{ll}26.832 \\
\end{array}$ & 24.57 & \begin{tabular}{|l|}
28.014 \\
\end{tabular} & $\begin{array}{l}426.805 \\
7\end{array}$ & \begin{tabular}{ll|l}
48.816 \\
\end{tabular} & 53.581 & 16.536 & 32.723 & \begin{tabular}{|l|l|}
-1.008 \\
\end{tabular} & 6.658 & 47.571 & 0.046 & 14.643 & & & 52.3389 & 52.1 & 11.7 & & 2.811 & 0.007 & 0.007 & 0.000 & 0.170 & \\
\hline
\end{tabular}


WSRC-TR-2005-00105, REVISION 0

SRNL-RPP-2005-00012, REVISION 0

RUN \# 3.03A AND B; FIRST AND SECOND HALF OF SLURRY DEWATERING - CONT.

\begin{tabular}{|c|c|c|c|c|c|c|c|c|c|c|c|c|c|c|c|c|c|c|c|c|c|c|c|c|c|c|c|c|}
\hline & A & B & D & E & $\mathrm{F}$ & $G$ & $\mathrm{H}$ & 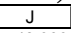 & K & $\begin{array}{ll}L \\
\end{array}$ & $\mathrm{M}$ & $\mathrm{N}$ & 0 & Q & \begin{tabular}{l|l|l|}
$R$ \\
\end{tabular} & & 1 & & & $x$ & & & $A A$ & & $A C$ & $A D$ & $A E$ & \\
\hline & 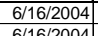 & 1:36:03 PM & & $\begin{array}{l}26.836 \\
36.82\end{array}$ & $\frac{24.58}{24.57}$ & \begin{tabular}{|l|l|}
28.059 \\
28.057
\end{tabular} & $\begin{array}{l}26.794 \\
26792 \\
\end{array}$ & $\begin{array}{l}48.909 \\
8733\end{array}$ & \begin{tabular}{|l|l|}
53.543 \\
55.573
\end{tabular} & \begin{tabular}{|l|}
16.524 \\
16329
\end{tabular} & $\begin{array}{l}32.891 \\
32795 \\
3\end{array}$ & $\begin{array}{l}-0.996 \\
-0994\end{array}$ & $\begin{array}{l}-6.655 \\
6649\end{array}$ & $\begin{array}{r}49.84 \\
51736\end{array}$ & $\begin{array}{l}0.046 \\
001\end{array}$ & $\begin{array}{l}14.643 \\
1.642\end{array}$ & 0.004 & & \begin{tabular}{|l|l|}
52.3556 \\
527020
\end{tabular} & 52. & 12.2 & & 2.82 & 0.007 & $\begin{array}{l}0.007 \\
007\end{array}$ & 0.000 & & \\
\hline$\frac{245}{245}$ & $\frac{6 / 116 / 2004}{661 / 2604}$ & $\begin{array}{l}1: 37: 03 \mathrm{PM} \\
1 \cdot 38: 03 \mathrm{PM}\end{array}$ & $\frac{26.50}{2650}$ & 26.83 & $\frac{24.57}{24.58}$ & 28.057 & $\frac{26.792}{26842}$ & $\begin{array}{r}48.733 \\
9078\end{array}$ & \begin{tabular}{|l|}
53.573 \\
537661 \\
\end{tabular} & $\begin{array}{l}16.349 \\
16.372\end{array}$ & $\begin{array}{r}32.795 \\
33187\end{array}$ & $\begin{array}{l}-0.994 \\
-0.965\end{array}$ & $\begin{array}{l}-6.649 \\
-6661\end{array}$ & $\frac{51.726}{5528}$ & $\begin{array}{l}0.046 \\
0.046\end{array}$ & $\frac{14.643}{11643}$ & 0.004 & $\begin{array}{l}3142.33 \\
3113232\end{array}$ & $\begin{array}{l}52.3722 \\
523889\end{array}$ & $\frac{52.1}{52.3}$ & $\frac{12.7}{13.5}$ & 40. & $\begin{array}{l}2.811 \\
2836 \\
\end{array}$ & $\begin{array}{l}0.007 \\
007\end{array}$ & 0.007 & & & \\
\hline 2454 & $6 / 16 / 2004$ & $\begin{array}{l}1.35: 03 \mathrm{PM} \\
13\end{array}$ & 26.49 & 26.836 & $\begin{aligned} 24.58 \\
24.58\end{aligned}$ & 28.048 & 20.842 & & & $\begin{array}{l}10.3512 \\
16.351\end{array}$ & 33.306 & $\begin{array}{l}-0.950 \\
-0.939 \\
-\frac{1}{2}\end{array}$ & $\begin{array}{l}-6.661 \\
-6.652\end{array}$ & $\begin{array}{r}55.28 \\
50.057\end{array}$ & $\begin{array}{l}0.046 \\
0.046\end{array}$ & $\begin{array}{l}14.643 \\
14.643 \\
\end{array}$ & 0.00 & $\begin{array}{l}31434.33 \\
3144.33 \\
\end{array}$ & $\begin{array}{l}55.38899 \\
52.4056\end{array}$ & 52.7 & $\begin{array}{l}\frac{13.5}{12.3} \\
\end{array}$ & 41. & \begin{tabular}{|l|l|}
2.836 \\
2.846
\end{tabular} & \begin{tabular}{|l|}
0.007 \\
0.007
\end{tabular} & $\frac{0.007}{0.007}$ & $\frac{0.000}{0.000}$ & & $\frac{0.14}{0.14}$ \\
\hline 2455 & $6 / 16 / 2004$ & 1:40:03 PM & 26.49 & 26.831 & 24.57 & 27.988 & 26.788 & 48.557 & \begin{tabular}{|r|}
53.26 \\
\end{tabular} & 16.24 & 32.749 & -0.936 & -6.649 & 50.055 & 0.045 & 14.643 & & 3145.33 & 2.4222 & 51.8 & 12.3 & & 2.803 & 0.007 & 0.007 & & & \\
\hline 2456 & $6 / 16 / 2004$ & 1:41:03 PM & 26.47 & & 24.56 & $\begin{array}{ll}27.953 \\
\end{array}$ & 26.768 & 47.738 & & $\begin{array}{l}17.229 \\
\end{array}$ & 30.98 & -0.941 & -6.658 & 53.148 & $\begin{array}{l}0.045 \\
\end{array}$ & 14.643 & & 146. & & & 13.0 & & & & & & & \\
\hline & $6 / 16 / 2004$ & & & 26.825 & 24.57 & 27.987 & 26.737 & $\begin{array}{l}48.372 \\
\end{array}$ & \begin{tabular}{|r|}
53.22 \\
\end{tabular} & 16.696 & 32.134 & -0.939 & -6.658 & & 0.044 & 14.643 & & 3147. & & 51.7 & 13.4 & 40 & & & 0.007 & & & \\
\hline 2458 & $6 / 16 / 2004$ & 1:43:03 PM & 26.46 & 26.81 & 24.56 & 27.972 & 26.757 & 48.387 & 53.201 & $\begin{array}{r}16.93 \\
\end{array}$ & 31.865 & -0.939 & -6.655 & 44.571 & $\begin{array}{l}0.045 \\
\end{array}$ & 14.643 & & & 52.4722 & 51.7 & 10.9 & & 2.767 & 0.007 & 0.007 & 0.000 & 0.16 & \\
\hline$\frac{2459}{3460}$ & 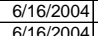 & $\begin{array}{ll}: 44: 03 \mathrm{PM} \\
1: 503 \mathrm{PM}\end{array}$ & $\begin{array}{l}26.48 \\
26.47 \\
2\end{array}$ & $\begin{array}{l}26.82 \\
2668\end{array}$ & $\begin{array}{l}24.56 \\
2456\end{array}$ & \begin{tabular}{|l|l|l|}
28.057 \\
28.027
\end{tabular} & $\begin{array}{l}26.762 \\
26772\end{array}$ & \begin{tabular}{|l|l|l|l}
48.377 \\
48119
\end{tabular} & \begin{tabular}{|l|l|}
53.189 \\
5.891 \\
\end{tabular} & $\begin{array}{l}16.792 \\
1661\end{array}$ & $\begin{array}{l}32.019 \\
301090\end{array}$ & $\begin{array}{r}-0.9399 \\
-0.933\end{array}$ & $\begin{array}{l}-6.652 \\
-6.652\end{array}$ & $\begin{array}{l}62.668 \\
4915\end{array}$ & \begin{tabular}{|l|l|}
0.044 \\
0.04
\end{tabular} & $\begin{array}{l}14.643 \\
1.642\end{array}$ & & $\frac{149.33}{11503}$ & $\begin{array}{l}52.4889 \\
5.5056\end{array}$ & 51.7 & $\begin{array}{l}15.4 \\
12.2\end{array}$ & & & \begin{tabular}{|l|l|}
0.007 \\
\end{tabular} & 0.007 & & & \\
\hline$\frac{|l| l 00}{2461}$ & $6 / 116 / 2604$ & $\begin{array}{ll}\text { 1:45:03 PM } \\
1: 46: 03 \mathrm{PM}\end{array}$ & $\frac{26.47}{26.46}$ & $\begin{array}{r}26.82 \\
26.793 \\
\end{array}$ & $\begin{array}{l}24.56 \\
24.52\end{array}$ & $\frac{28.027}{27.98}$ & $\frac{26.772}{26.701}$ & $\begin{array}{r}48.119 \\
48.348\end{array}$ & \begin{tabular}{|c|}
52.884 \\
53.061 \\
\end{tabular} & $\begin{array}{r}\mid 16.621 \\
16.63\end{array}$ & $\begin{array}{r}31.949 \\
32.167\end{array}$ & $\begin{array}{l}-0.933 \\
-0.941\end{array}$ & $\begin{array}{l}-6.652 \\
-6.652 \\
\end{array}$ & $\begin{array}{r}49.815 \\
44.575\end{array}$ & $\begin{array}{l}0.044 \\
0.045\end{array}$ & $\begin{array}{l}14.643 \\
14.643 \\
\end{array}$ & & $\begin{array}{l}150.3 \\
115.33\end{array}$ & $\begin{array}{l}52.5056 \\
52.5222\end{array}$ & $\frac{51.4}{51.6}$ & $\frac{12.2}{10.9}$ & $\frac{40 .}{40.3}$ & $\mid$\begin{tabular}{|c|}
2.770 \\
2776
\end{tabular} & \begin{tabular}{|l|l|}
0.007 \\
0.007
\end{tabular} & $\frac{0.007}{0.007}$ & $\begin{array}{l}0.000 \\
0.000\end{array}$ & $\frac{0.16}{0.16}$ & \\
\hline \begin{tabular}{|l|l|}
2462 \\
\end{tabular} & $6 / 16 / 2004$ & 1:47:03 PM & 26.46 & 26.801 & 24.52 & 27.934 & 26.679 & 48.24 & \begin{tabular}{|l|}
52.932 \\
\end{tabular} & 16.636 & 32.029 & -0.939 & & 51.408 & 0.044 & 14.643 & & 3152.3 & 52.5389 & 51.5 & 12.6 & & & & & & & \\
\hline & $6 / 16 / 2004$ & 1:48:03 PM & 26.45 & 26.791 & 24.52 & 27.998 & 26.713 & 48.188 & \begin{tabular}{|l|l|}
53.158 \\
\end{tabular} & $\begin{array}{l}16.666 \\
\end{array}$ & 31.91 & -0.901 & -6.646 & 49.784 & $\begin{array}{l}0.045 \\
\end{array}$ & 14.643 & & & 52.5556 & 51.7 & 12.2 & 40.0 & \begin{tabular}{|l|}
2.761 \\
\end{tabular} & 0.007 & $\begin{array}{l}0.007 \\
\end{array}$ & 0.000 & & \\
\hline & $6 / 16 / 2004$ & 1:49:03 PM & & & & & 26.746 & & & $\begin{array}{l}16.494 \\
\end{array}$ & & -0.936 & -6.643 & & & 14.643 & & 154. & 52.5722 & 51.9 & & 40.6 & & 0.007 & 0.007 & & & \\
\hline 2465 & $6 / 16 / 2004$ & 1:50:03 PM & 26.44 & 26.781 & 24.49 & 27.979 & 26.704 & 48.468 & 53.68 & 16.62 & 32.218 & $\begin{array}{r}-0.75 \\
\end{array}$ & & 52.28 & $\begin{array}{l}0.033 \\
\end{array}$ & 14.643 & & & 52.5889 & & 12.8 & & 2.782 & $\begin{array}{l}0.005 \\
\end{array}$ & & 0.000 & 0.12 & \\
\hline$\frac{2466}{2467}$ & $6 / 16 / 2004$ & 1:51:03 PM & $\begin{array}{l}26.46 \\
26.47\end{array}$ & $\begin{array}{l}26.771 \\
26792\end{array}$ & $\begin{array}{l}24.48 \\
24.51\end{array}$ & \begin{tabular}{|l|}
28.009 \\
28.019
\end{tabular} & $\begin{aligned} 26.704 \\
26.71\end{aligned}$ & $\begin{array}{l}48.576 \\
48.292 \\
\end{array}$ & \begin{tabular}{|l|}
53.902 \\
53519 \\
\end{tabular} & \begin{tabular}{|l|}
16.547 \\
16566
\end{tabular} & $\begin{array}{r}32.45 \\
33.148 \\
3\end{array}$ & & $\begin{array}{l}-6.175 \\
-6.166\end{array}$ & $\begin{array}{l}50.747 \\
550777\end{array}$ & $\begin{array}{l}0.044 \\
0044\end{array}$ & $\begin{array}{l}14.643 \\
16463\end{array}$ & & 56.3. & $\frac{6056}{6202}$ & 52.4 & $\begin{array}{l}12.4 \\
12.4\end{array}$ & 40.5 & \begin{tabular}{|l|l|}
2.793 \\
2773
\end{tabular} & \begin{tabular}{|l|l|}
0.007 \\
007
\end{tabular} & \begin{tabular}{|l|l|}
0.007 \\
\end{tabular} & $\begin{array}{l}0.000 \\
\end{array}$ & $\frac{0.16}{0.16}$ & \\
\hline$\frac{246 r}{2468}$ & 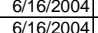 & $\begin{array}{l}1: 52: 03 \mathrm{PM} \\
115503 \mathrm{PM}\end{array}$ & $\frac{26.47}{26.48}$ & $\frac{26.792}{26.79}$ & 24.51 & $\begin{array}{l}28.019 \\
28.294\end{array}$ & $\begin{array}{l}26.71 \\
26.83\end{array}$ & $\begin{array}{r}48.292 \\
48.314 \\
\end{array}$ & \begin{tabular}{|l|}
53.519 \\
53.477 \\
\end{tabular} & \begin{tabular}{|l|}
16.536 \\
16.557
\end{tabular} & $\begin{array}{r}32.148 \\
32.212 \\
\end{array}$ & & $\begin{array}{l}-6.166 \\
-6.164\end{array}$ & 50.747 & $\begin{array}{r}0.044 \\
0.045\end{array}$ & $\begin{array}{l}14.643 \\
14.643 \\
\end{array}$ & $\frac{0.00}{0.00}$ & $\begin{array}{l}3157.33 \\
31158.33\end{array}$ & $\begin{array}{l}52.6222 \\
52.63899\end{array}$ & $\frac{52.0}{520}$ & $\frac{12.4}{120}$ & 40.2 & \begin{tabular}{|c|}
2.773 \\
2776 \\
\end{tabular} & 0.007 & 0.007 & 0.000 & & \\
\hline 2469 & $6 / 16 / 2004$ & 1:54:03 PM & $\frac{20.48}{26.45}$ & 26.77 & $\frac{24.05}{24.49}$ & 28.479 & $\begin{array}{l}2.00 \\
26.94\end{array}$ & $\frac{48.514}{48.323}$ & \begin{tabular}{|l|}
53.4206 \\
\end{tabular} & $\begin{array}{l}16.4251 \\
16.4\end{array}$ & $\frac{24.42}{32.351}$ & $\begin{array}{l}-0.0855 \\
-0.85\end{array}$ & -0.104 & $\begin{array}{l}49.014 \\
53.903\end{array}$ & $\begin{array}{l}0.045 \\
0.044\end{array}$ & $\frac{14.643}{14.643}$ & & $\frac{3158.5 .3}{3159.3}$ & $\begin{array}{l}52.6389 \\
52.6556\end{array}$ & $\frac{52.0}{51.7}$ & $\frac{12.0}{13.2}$ & 40.3 & \begin{tabular}{|l|}
2.78161 \\
\end{tabular} & \begin{tabular}{|l|}
0.007 \\
0.007
\end{tabular} & $\begin{array}{l}0.007 \\
0.007\end{array}$ & 0 & 0.16 & 0.14 \\
\hline & $6 / 16 / 2004$ & 1:55:03 PM & 26.45 & 26.77. & 24.47 & 28.587 & 27.008 & 48.449 & 53.606 & \begin{tabular}{|l|l|}
16.471 \\
\end{tabular} & 32.438 & $\begin{array}{l} \\
\end{array}$ & -6.227 & 54.21 & $\begin{array}{l}0.036 \\
\end{array}$ & 144.643 & & & 52.6722 & 52.1 & 13.3 & 40.4 & \begin{tabular}{|l|}
2.788 \\
\end{tabular} & 0.005 & 0.005 & 0.000 & & \\
\hline & $6 / 16 / 2004$ & 1:56:03 PM & & 26.78 & 24.46 & & 27.016 & 48.242 & \begin{tabular}{|l|l|}
53.388 \\
\end{tabular} & $\begin{array}{l}16.36 \\
\end{array}$ & 32.304 & $\begin{array}{l}-0.71 \\
\end{array}$ & & 50.968 & 0.045 & 14.643 & & 161. & 52.6889 & 51.9 & & 40.3 & & 0.007 & 0.007 & 0.000 & 0.16 & \\
\hline & $6 / 16 / 2004$ & 1:57:03 PM & 26.49 & 26.81 & 24.5 & 28.359 & 27.005 & 48.698 & \begin{tabular}{|l|l|}
53.714 \\
\end{tabular} & 16.428 & 32.688 & & -6.22 & 58.221 & 0.045 & 14.643 & & 62.3 & 52.7056 & 52.2 & 14.3 & & \begin{tabular}{|l|}
2.806 \\
\end{tabular} & 0.007 & 0.007 & & 0.16 & \\
\hline 247 & $6 / 16 / 2004$ & $\begin{array}{ll}1: 58: 03 \mathrm{PM} \\
1.5 \cdot 03\end{array}$ & $\frac{26.49}{26.48}$ & $\frac{26.83}{26.82}$ & 24.51 & 28.327 & $\frac{26.923}{26834}$ & $\begin{array}{l}48.553 \\
4864\end{array}$ & \begin{tabular}{|l|}
53.289 \\
53.324 \\
\end{tabular} & \begin{tabular}{|r|}
16.39 \\
\end{tabular} & $\begin{array}{l}32.706 \\
32.776\end{array}$ & $\begin{array}{r}-0.71 \\
1.104\end{array}$ & $\begin{array}{l}-6.233 \\
7152 \\
\end{array}$ & 52.549 & 0.045 & $\begin{array}{l}14.643 \\
1643\end{array}$ & & \begin{tabular}{|l|l|}
3163.3 \\
3643
\end{tabular} & $\begin{array}{l}52.7222 \\
527389\end{array}$ & $\frac{51.8}{51 .}$ & $\begin{array}{l}12.9 \\
12.2\end{array}$ & & \begin{tabular}{|l|}
2.801 \\
2814 \\
\end{tabular} & \begin{tabular}{|l|l|}
0.007 \\
0.007
\end{tabular} & 0.007 & 0.000 & 0.16 & 57 \\
\hline$\frac{2442}{2475}$ & $\frac{6 / 11 / 26004}{6 / 16 / 2004}$ & $\begin{array}{l}1.59: 003 \mathrm{PM} \\
2 \cdot 0003\end{array}$ & $\frac{26.48}{26.47}$ & $\frac{26.82}{26.82}$ & $\begin{array}{r}24 . \\
24.49\end{array}$ & $\begin{array}{l}28.184 \\
28.035\end{array}$ & & $\begin{array}{r}48.864 \\
49.134 \\
\end{array}$ & \begin{tabular}{|l|}
53.324 \\
53.714 \\
\end{tabular} & $\begin{array}{l}16.552 \\
16.582\end{array}$ & $\begin{array}{l}32.166 \\
32.928 \\
\end{array}$ & \begin{tabular}{|l}
-1.104 \\
-1.101
\end{tabular} & & $\begin{array}{l}49.834 \\
53.428 \\
\end{array}$ & & $\frac{14.643}{14.643}$ & & & & & $\frac{12.2}{13.1}$ & & & & & & & \\
\hline 2476 & $6 / 16 / 2004$ & 2:01:03 PM & 26.46 & $\frac{2.02}{26.81}$ & $\frac{24.45}{24.48}$ & 28.021 & $\frac{2.101}{26.776}$ & $\begin{array}{l}45.154 \\
48.741\end{array}$ & \begin{tabular}{|l|}
53.1494 \\
53.49
\end{tabular} & $\begin{array}{l}16.002 \\
16.459\end{array}$ & $\frac{25.920}{32.712}$ & $\frac{-1.11}{-1.159}$ & $\begin{array}{l}-7.103 \\
-7.248\end{array}$ & $\frac{03.460}{48.684}$ & \begin{tabular}{|l|}
0.046 \\
\end{tabular} & $\frac{14.045}{14.643}$ & & 3166.33 & 52.7722 & $\frac{52.4}{52.0}$ & $\frac{10.1}{11.9}$ & & 2.000 & \begin{tabular}{|l|}
0.007 \\
0.007
\end{tabular} & $\begin{array}{l}.007 \\
0.007\end{array}$ & 500 & $\begin{array}{l}0.17 \\
0.17\end{array}$ & \\
\hline \begin{tabular}{|l|}
2477 \\
\end{tabular} & $6 / 16 / 2004$ & 2:02:03 PM & 26.45 & 26.81 & 24.47 & 27.987 & 26.752 & 48.959 & 53.564 & 16.544 & 32.807 & -1.156 & -7.265 & 56.296 & 0.046 & 14.643 & & 3167.33 & 52.7889 & 52.1 & 13.8 & 40.9 & 2.819 & 0.007 & 0.007 & 0.000 & 0.17 & \\
\hline 2179 & $6 / 16 / 2004$ & 2:03:03 PM & 26.45 & & 24.47 & 27.969 & 26.719 & & & 16.509 & & & & & & 14.643 & & & 52.8056 & 52.2 & 11.1 & & & 0.008 & 0.008 & & 0.18 & \\
\hline 2479 & $6 / 16 / 2004$ & 2:04:03 PM & 26.43 & 26.80 & 24.46 & 27.914 & & & \begin{tabular}{|l|}
53.734 \\
\end{tabular} & 16.5 & & & & 50.245 & & 14.643 & & & 52.8222 & 52.3 & 12.3 & & 2.818 & 0.007 & & & 0.16 & \\
\hline$\frac{2486}{248}$ & 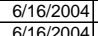 & 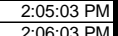 & $\frac{26.41}{26.40}$ & $\begin{array}{l}26.80 \\
26.80\end{array}$ & $\begin{array}{l}24.45 \\
24.44\end{array}$ & \begin{tabular}{|l|}
27.884 \\
27.884
\end{tabular} & $\begin{array}{l}26.674 \\
26.704\end{array}$ & $\begin{array}{l}48.744 \\
48.953 \\
\end{array}$ & \begin{tabular}{|l|l|}
53.456 \\
53.459 \\
\end{tabular} & \begin{tabular}{|c|}
16.378 \\
16.46
\end{tabular} & $\begin{array}{r}32.813 \\
32.94\end{array}$ & & $\begin{array}{l}-7.274 \\
-7.285\end{array}$ & $\begin{array}{l}48.624 \\
55011\end{array}$ & .043 & $\begin{array}{l}14.643 \\
1643\end{array}$ & & & $\begin{array}{l}52.8389 \\
5.8556\end{array}$ & $\begin{array}{l}52.0 \\
52.0\end{array}$ & $\begin{array}{l}11.9 \\
12.3\end{array}$ & & \begin{tabular}{|l|l|l|l|}
2.812 \\
2832
\end{tabular} & & $\begin{array}{l}0.007 \\
0.007 \\
\end{array}$ & & & \\
\hline$\frac{2481}{2482}$ & 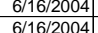 & $\begin{array}{l}2: 00: 03 \mathrm{PM} \\
2: 07: 03 \mathrm{PM}\end{array}$ & 26.40 & 26.80 & 24.44 & $\begin{array}{l}27.884 \\
27.915\end{array}$ & 26.704 & $\begin{array}{r}48.953 \\
48.853 \\
\end{array}$ & \begin{tabular}{|l|}
53.459 \\
53.504 \\
\end{tabular} & 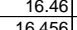 & $\begin{array}{r}32.94 \\
32.838 \\
\end{array}$ & & $\begin{array}{r}-7.285 \\
-7199 \\
-190\end{array}$ & $\begin{array}{r}50.011 \\
4989 \\
\end{array}$ & & $\frac{14.643}{14643}$ & & \begin{tabular}{|l|l|l}
3171.33 \\
3172.33
\end{tabular} & 52.8556 & & $\frac{12.3}{12.2}$ & 40.9 & $\frac{2.823}{2816}$ & $\begin{array}{l}0.007 \\
0.007\end{array}$ & 0.007 & 0.000 & & \\
\hline 248 & $6 / 16 / 2004$ & $\begin{array}{l}2: 0: 0003 \mathrm{PM} \\
2: 00: 03 \mathrm{P}\end{array}$ & $\frac{20.41}{26.40}$ & $\frac{20.80}{26.80}$ & 24.40 & $\frac{27.915}{27.989}$ & $\begin{array}{r}26.156 \\
26.84\end{array}$ & $\begin{array}{l}48.853 \\
49.065\end{array}$ & \begin{tabular}{|l|}
53.504 \\
53.651 \\
\end{tabular} & \begin{tabular}{|l|}
16.4564 \\
16.404
\end{tabular} & $\begin{array}{l}32.838 \\
33.158 \\
\end{array}$ & $\frac{-1.101}{-1.098}$ & $\begin{array}{l}-7.1999 \\
-7.219\end{array}$ & $\begin{array}{r}49.083 \\
51.083\end{array}$ & $\begin{array}{l}0.044 \\
0.045\end{array}$ & $\frac{14.643}{14.643}$ & & \begin{tabular}{|l|l|l|l|}
3173.3 \\
\end{tabular} & & $\frac{52.0}{52.2}$ & $\frac{12.2}{12.5}$ & 40.8 & $\frac{2.816}{2.834}$ & \begin{tabular}{|l|}
0.007 \\
0.007
\end{tabular} & & & & \\
\hline 248 & $6 / 16 / 2004$ & 2:09:03 PM & 26.38 & 26.79 & 24.42 & 28.024 & 26.894 & 49.343 & \begin{tabular}{|l|}
53.912 \\
\end{tabular} & 16.454 & 33.39 & & -7.225 & 48.645 & 0.046 & 14.643 & & & 056 & 52.4 & 11.9 & & 2.852 & 0.007 & 0.007 & 0.000 & 0.16 & \\
\hline & $6 / 16 / 2004$ & & & & 24.45 & & & & & & & & & & & & & & & 52.3 & 12.1 & & & & & & & \\
\hline 2486 & $6 / 16 / 2004$ & 2:11: & 26.39 & 26.80 & 24.44 & 28.13 & 26.97 & & \begin{tabular}{|l|l|}
53.971 \\
\end{tabular} & $\begin{array}{l}16.442 \\
\end{array}$ & 33.102 & & & 49.61 & & & & & 52.9389 & 52.5 & 12.2 & & \begin{tabular}{|l|l|}
$2.83 !$ \\
\end{tabular} & 0.007 & & & 0. & \\
\hline$\frac{2487}{2488}$ & $6 / 16 / 2004$ & $\begin{array}{l}\text { 2:12:03 PM } \\
20.1230\end{array}$ & $\frac{26.38}{26.40}$ & 26.80 & 24.43 & $\begin{array}{r}28.15 \\
282015\end{array}$ & $\begin{array}{l}26.995 \\
26.981\end{array}$ & $\begin{array}{l}48.986 \\
40011\end{array}$ & $\begin{array}{l}53.792 \\
55.583\end{array}$ & $\begin{array}{l}16.365 \\
11659\end{array}$ & $\begin{array}{l}33.137 \\
33091 \\
3\end{array}$ & $\begin{array}{r}-1.098 \\
-1.101\end{array}$ & $\begin{array}{r}-7.213 \\
7213\end{array}$ & $\begin{array}{l}54.191 \\
5504\end{array}$ & & $\begin{array}{l}14.643 \\
1643\end{array}$ & & 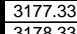 & $\begin{array}{l}52.9556 \\
5.9722\end{array}$ & $\frac{52.3}{52.1}$ & $\begin{array}{l}13.3 \\
1.5\end{array}$ & 41. & \begin{tabular}{|l|}
2.83 \\
2827
\end{tabular} & \begin{tabular}{|l|l|}
0.007 \\
\end{tabular} & \begin{tabular}{|l|l|}
007 \\
077
\end{tabular} & 0.000 & 0.1 & \\
\hline$\frac{2488}{2489}$ & 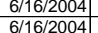 & $\begin{array}{l}2: 13: 03 \mathrm{PM} \\
2 \cdot 214: 03 \mathrm{PM}\end{array}$ & $\frac{26.40 .}{26.4}$ & $\frac{26.82}{26.83}$ & 24.44 & 28.245 & & $\begin{array}{l}49.011 \\
49277\end{array}$ & \begin{tabular}{|l|}
53.583 \\
53.975 \\
\end{tabular} & $\begin{array}{c}16.519 \\
16.554\end{array}$ & $\begin{array}{r}32.981 \\
33182\end{array}$ & $\begin{array}{l}-1.101 \\
-1.101\end{array}$ & $\begin{array}{l}-7.213 \\
-7.216\end{array}$ & $\begin{array}{l}55.048 \\
48888\end{array}$ & & $\frac{14.643}{14643}$ & & 3178.35 & \begin{tabular}{|l|l|}
52.9722 \\
5
\end{tabular} & $\frac{52.1}{52.5}$ & $\begin{array}{l}13.5 \\
11.9\end{array}$ & 41.6 & & & & & & \\
\hline 2490 & $6 / 16 / 2004$ & $2: 15: 03 \mathrm{PM}$ & $\frac{20.4}{26.41}$ & 26.83 & \begin{tabular}{|l|}
24.40 \\
24.48 \\
\end{tabular} & $\frac{28.201}{28.226}$ & & $\begin{array}{l}49.271 \\
49.077\end{array}$ & \begin{tabular}{|l|}
53.915 \\
53.776 \\
\end{tabular} & $\begin{array}{l}16.554 \\
16.463\end{array}$ & 33. & $\frac{-1.101}{-1.098}$ & $\frac{-.1216}{-7.21}$ & $\begin{array}{r}48.488 \\
52.94 \\
\end{array}$ & $\begin{array}{l}0.045 \\
0.046\end{array}$ & $\frac{14.643}{14.643}$ & & & & 52.3 & $\frac{11.9}{13.0}$ & 41 & & & & & $\begin{array}{l}0.16 \\
0.16\end{array}$ & \\
\hline 2491 & $6 / 16 / 2004$ & $2: 16: 03$ & 26.43 & 26.86 & 24.51 & 28.247 & 26.953 & & \begin{tabular}{|l|l|}
53.805 \\
\end{tabular} & 16.532 & 32.955 & & & 53 & & 14. & & & 53.0222 & 52.3 & 13.0 & & 2.826 & 0.007 & 0.007 & 0.000 & & \\
\hline 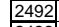 & $6 / 16 / 2$ & & 26.41 & 26.85. & 24.48 & & & & & & & & & & & & & & & & 111.9 & & & & & & & \\
\hline$\frac{2493}{2494}$ & & & 26.40 & & & & & & 53. & & & & & & & & & & & 51.9 & 12.0 & & \begin{tabular}{|l|}
2.803 \\
\end{tabular} & & & & & \\
\hline & $6 / 16 / 2004$ & 2:19: & 26.41 & $\frac{26.85}{26.86}$ & 24.4 & $28.144 \mid$ & & & \begin{tabular}{|l|l}
53.2 \\
5.3.
\end{tabular} & $\begin{array}{l}16.368 \\
16.407\end{array}$ & & & & & & 14.4 & & & & $\frac{51.8}{52.2}$ & $\begin{array}{l}13.0 \\
11.5\end{array}$ & & 2.807 & & & 00 & 0.15 & \\
\hline 249 & $6 / 16 / 6 / 2$ & & $\frac{26.42}{26.4}$ & $\frac{26.86}{26.87}$ & $24.4 \pi$ & & & & & \begin{tabular}{l|l|}
16.407 \\
165.515
\end{tabular} & & & & & & 14. & & & & & 11.5 & & & & & & & \\
\hline & $\frac{01016 / 2}{6 / 16 / 2}$ & & & & $\frac{24.41}{24.46}$ & & & & & & & & & & & & & & & & & & & & & & & \\
\hline & $6 / 16 / 2004$ & $2: 22:$ & 26.40 & 26.85 & $\frac{24.4}{24.4}$ & 28.065 & & & 53.343 & $\mid \begin{array}{c}10.44 \\
16.406\end{array}$ & 32.725 & & & & 0.04 & 14. & & & & 51.9 & 0.2 & & & & & & & \\
\hline & $6 / 16 / 2004$ & & & & & & & & & & & & & & & & & & & & & & & & & & & \\
\hline & & & & & 24.4 & & & & & & & & & & & & & & & & & & & & & & & \\
\hline & $6 / 16 /$ & & & $26 . \varepsilon$ & 24.4 & & & & & & & & & & & & & & & & & & & & & & & \\
\hline & $6 / 1$ & 2:26: & & & 24.4 & 28.0 & & & & 16.3 & & & & & & 14. & & & & 51 & & & & & & & & \\
\hline & $6 / 16 / 20$ & 2:27. & 26. & 26.85 & 24.42 & $27 .$. & & & & & & & & & & 14. & & & & 51 & .0 & & & & & & & \\
\hline & $6 / 16 / 2$ & 2:28: & & & 24.4 & 27.9 & & & & & & & & & & & & & & & 2.2 & & & & & & & \\
\hline & $6 / 16 / 2$ & & 26.36 & 26.83 & 24.4 & 27.9 & & & & & & & & & & & & & & 51 & 2.0 & & & & & & & \\
\hline & 6/16/20 & & & & 24. & & & & & & & & & & & & & & & & & & & & & & & \\
\hline & & & & & & 27. & & & & & & & & & & & & & & & & & & & & & & \\
\hline & 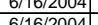 & & & & 24.42 & 28.06 & & & & & & & & & & & & & & & & & & & & & & \\
\hline & $\frac{1161}{6 / 6 / 2}$ & & & & 2.42 & $\frac{20.1}{280}$ & & & & & & & & & & & & & & & & & & & & & & \\
\hline & $6 / 16 / 2$ & & & & 24,44 & & & & & & & & & & & & & & & & & & & & & & & \\
\hline & $\frac{2616 /}{6 / 6 /}$ & & & $\frac{26.8}{26.8}$ & & & & & & & & & & & & & & & & & & & & & & & & \\
\hline & $\frac{0}{6 / 1616 / 20}$ & & & & & & & & & & & & & & & & & & & & & & & & & & & \\
\hline & & & & & & & & & & & & & & & & & & & & & & & & & & & & \\
\hline & $6 / 16 / 200$ & & & & 24.47 & & & 48 & & & & -0. & & & & 14. & & & & & & & & & & & & \\
\hline & $6 / 16 / 2$ & $2: 40: 46$ & & & 24.4 & 28.15 & & 48.2 & & 16.9 & 31. & -0.8 & & & 0.0 & 14.6 & & & & & & & & & & & & \\
\hline & $6 / 2$ & & & & 24.4 & & & & & & & & & & & 14. & & & & & & & & & & & & \\
\hline & 6/16/2 & & & 26.83 & 24.47 & 28.2 & & & & 17.047 & 31. & & & & & & & & & & & & & & & & & \\
\hline & & & 26.39 & 26.83 & 24.46 & 28.4 & & & 53.088 & 17.069 & 31.366 & & & & & & & & & 51.6 & 12.2 & & & & & & & \\
\hline & 6/16/2004 & $2: 44: 46 \mathrm{P}$ & & & 24.44 & \begin{tabular}{|l|}
28.218 \\
\end{tabular} & & $\begin{array}{l}48.447 \\
\end{array}$ & & \begin{tabular}{|l|}
17.125 \\
\end{tabular} & 31.723 & -0.889 & & 48.743 & $\mid 0.044$ & 14.643 & & & & & 11.9 & & & 0.007 & $\mid 0.007$ & 0.000 & 0.16 & 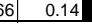 \\
\hline
\end{tabular}


WSRC-TR-2005-00105, REVISION 0

SRNL-RPP-2005-00012, REVISION 0

RUN \# 3.03A AND B; FIRST AND SECOND HALF OF SLURRY DEWATERING - CONT.

\begin{tabular}{|c|c|c|c|c|c|c|c|c|c|c|c|c|c|c|c|c|c|c|c|c|c|c|c|c|c|c|c|c|}
\hline & $\mathrm{A}$ & & D & $E$ & $\mathrm{~F}$ & G & $\mathrm{H}$ & $\mathrm{J}$ & K & $\begin{array}{ll}\text { L } \\
\end{array}$ & M & $\mathrm{N}$ & 0 & Q & $\mathrm{R}$ & $\mathrm{s}$ & $\begin{array}{ll}T \\
\end{array}$ & $\mathrm{v}$ & w & $x$ & \begin{tabular}{l|l} 
\\
\end{tabular} & 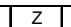 & $\mathrm{AA}$ & $A B$ & $A C$ & $A D$ & AE & AF \\
\hline 2521 & $6 / 16 / 2004$ & 2:45:46 PM & 26.409 & 26.833 & 24.476 & 28.2 & 26.855 & 48.248 & 53.239 & $\begin{array}{l}16.986 \\
\end{array}$ & 31.697 & -0.855 & -6.944 & $\begin{array}{ll}4.989 \\
\end{array}$ & 0.043 & 14.643 & 0.004 & 3211.05 & \begin{tabular}{|l|l|}
53.5175 \\
\end{tabular} & 51.8 & 12.0 & 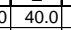 & 2.756 & 0.006 & 0.007 & 0.000 & 0.163 & \\
\hline & $6 / 11 / 2004$ & & 26.409 & 26.838 & 24.476 & 28.175 & 26.83 & 48.341 & 53.175 & \begin{tabular}{|l|l|}
17.017 \\
\end{tabular} & 31.805 & $\begin{array}{r}-0.8699 \\
-0.869\end{array}$ & $\begin{array}{l}-6.938 \\
\end{array}$ & 49.483 & \begin{tabular}{|l|l|}
0.045 \\
\end{tabular} & \begin{tabular}{|l|l|}
14.643 \\
\end{tabular} & 0.004 & & 53.5342 & 51.7 & 12.1 & & & & & & 0.170 & \\
\hline$\frac{2523}{253}$ & $6 / 16 / 2004$ & 2:47:46 PM & $\frac{26.421}{26.420}$ & 26.84 & 24.477 & 28.177 & 26.817 & $\begin{array}{l}48.298 \\
\end{array}$ & \begin{tabular}{|l|}
53.367 \\
5.39
\end{tabular} & \begin{tabular}{|l|}
16.917 \\
1.923
\end{tabular} & 31.746 & -0.834 & -6.941 & 51.258 & \begin{tabular}{|l|l|}
0.043 \\
\end{tabular} & \begin{tabular}{|l|l|}
14.643 \\
\end{tabular} & & | & & 51.9 & 12.6 & & 275 & & 0.007 & 0.000 & 0.163 & \\
\hline \begin{tabular}{|l|}
2524 \\
2525
\end{tabular} & $6 / 11 / 2004$ & 2:48:46 PM & 26.422 & 26.841 & 24.478 & $\begin{array}{l}28.227 \\
2.2727\end{array}$ & 26.868 & 48.287 & $\begin{array}{l}53.189 \\
53.0\end{array}$ & $\begin{array}{l}17.026 \\
\end{array}$ & 31.693 & -0.823 & $\begin{array}{l}-6.944 \\
\end{array}$ & 54.166 & $\begin{array}{l}0.043 \\
\end{array}$ & $\begin{array}{l}14.643 \\
\end{array}$ & 0.004 & 3214.05 & \begin{tabular}{|l|l}
53.5675 \\
\end{tabular} & 51.7 & \begin{tabular}{|l|l|}
13.3 \\
\end{tabular} & & 2.757 & 0.006 & 0.007 & 0.000 & 0.163 & \\
\hline 2526 & $\frac{6 / 16 / 2004}{66162004}$ & \begin{tabular}{l|}
$2: 49: 46 \mathrm{PM}$ \\
$2.50 \cdot 66 \mathrm{PM}$
\end{tabular} & $\frac{26.423}{26.429}$ & $\frac{26.836}{26.843}$ & $\begin{array}{l}24.484 \\
24.491\end{array}$ & \begin{tabular}{r|}
28.273 \\
28.3
\end{tabular} & $\frac{26.894}{26.92}$ & \begin{tabular}{|l|}
48.233 \\
48.194 \\
\end{tabular} & \begin{tabular}{|l|}
53.106 \\
52982 \\
\end{tabular} & \begin{tabular}{|c|}
16.842 \\
16934
\end{tabular} & $\begin{array}{l}31.826 \\
33.1727\end{array}$ & $\begin{array}{r}-0.86 \\
-0833\end{array}$ & $\begin{array}{l}-6.947 \\
6018\end{array}$ & $\begin{array}{r}52.31 \\
51728\end{array}$ & $\begin{array}{l}0.041 \\
0.045 \\
\end{array}$ & \begin{tabular}{|l|l|l|}
14.643 \\
14643
\end{tabular} & $\begin{array}{l}0.004 \\
0.003\end{array}$ & \begin{tabular}{|l|}
3215.05 \\
321605
\end{tabular} & \begin{tabular}{|l|l|}
53.5842 \\
5.536008
\end{tabular} & \begin{tabular}{|l|l|}
51.6 \\
\end{tabular} & \begin{tabular}{|l|l|}
12.8 \\
127
\end{tabular} & & & 0.006 & $\begin{array}{l}0.006 \\
0.027\end{array}$ & & $\frac{0.155}{0.170}$ & \\
\hline 2527 & $6 / 116 / 2004$ & $\begin{array}{l}2: 5: 5: 46 \mathrm{PM} \\
2: 51: 46 \mathrm{PM}\end{array}$ & $\begin{array}{r}26.429 \\
26.43\end{array}$ & $\frac{26.843}{26.839}$ & $\begin{array}{l}24.491 \\
24.537\end{array}$ & $\begin{array}{r}28.3 \\
28.36\end{array}$ & $\begin{array}{r}26.92 \\
26.896 \\
\end{array}$ & $\begin{array}{r}48.194 \\
48.032 \\
\end{array}$ & \begin{tabular}{|l|}
52.982 \\
52.222 \\
\end{tabular} & \begin{tabular}{|l|}
16.934 \\
16.8655 \\
\end{tabular} & $\begin{array}{l}31.127 \\
31.625 \\
\end{array}$ & $\begin{array}{l}-0.823 \\
-0.82\end{array}$ & $\begin{array}{l}-6.918 \\
-6.924 \\
\end{array}$ & $\begin{array}{l}51.728 \\
49.083 \\
\end{array}$ & $\begin{array}{l}0.045 \\
0.044\end{array}$ & \begin{tabular}{|l|}
14.643 \\
14.643 \\
\end{tabular} & $\begin{array}{l}0.003 \\
0.004\end{array}$ & $\begin{array}{l}3216.05 \\
3217.05\end{array}$ & \begin{tabular}{|c|c|}
53.6008 \\
55.6175
\end{tabular} & \begin{tabular}{|l}
51.5 \\
51.3
\end{tabular} & \begin{tabular}{|l|l|}
12.7 \\
12.0
\end{tabular} & \begin{tabular}{|c|c|} 
& 40.0 \\
& 39.8
\end{tabular} & $\mid$\begin{tabular}{|c|}
2.755 \\
2.746
\end{tabular} & \begin{tabular}{|l|l|l|l}
0.007 \\
0.007
\end{tabular} & $\begin{array}{l}0.007 \\
0.007\end{array}$ & $\begin{array}{l}0.000 \\
0.000\end{array}$ & 0.170 & 0.14 \\
\hline 2528 & $6 / 16 / 2004$ & & & 26.855 & 24.542 & & 26.872 & & 53.434 & & & & & 47.545 & 0.044 & \begin{tabular}{|l|l|}
14.643 \\
14.643
\end{tabular} & 0.004 & \begin{tabular}{|l|}
3218.05 \\
3218
\end{tabular} & 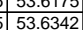 & \begin{tabular}{|l|l|l|}
52.0 \\
\end{tabular} & $\frac{12.0}{11.7}$ & $\begin{array}{|ll|}7 & 3.8 .1 \\
\end{array}$ & & & & 0.000 & & \\
\hline & 6/16/2004 & 2:53:46 PM & 26.447 & 26.856 & 24.553 & 28.242 & 26.838 & 48.08 & 52.93 & 16.84 & 31.725 & -0.82 & -6.927 & 50.437 & 0.043 & 14.643 & 0.004 & & 553.6508 & 51.5 & 12.4 & & 2.75 & & & & 0.163 & \\
\hline Luou & $6 / 16 / 2004$ & 2:54:46 PM & & 26.841 & 24.538 & \begin{tabular}{|l|}
28.207 \\
\end{tabular} & 26.843 & 48.408 & 53.556 & 16.799 & & -0.817 & -6.9 & & .045 & & & & & 52.1 & $\begin{array}{ll}11.6 \\
\end{array}$ & 40.2 & 2.773 & & & & & \\
\hline 2531 & 6/116/2004 & & & & 24.528 & & & & 53.502 & \begin{tabular}{|l|l|l|}
1634 \\
\end{tabular} & 32.165 & & & 53.59 & . & 14.643 & & & 3.6842 & & 13.1 & & & 0.007 & 0.007 & 0.000 & $\frac{172}{172}$ & \\
\hline & & $2: 56: 4$ & & 26.862 & 24.554 & \begin{tabular}{|l|l|}
28.213 \\
\end{tabular} & & & 53.374 & \begin{tabular}{|l|l|}
16.729 \\
\end{tabular} & & & & & & 14.643 & & & & 510 & & & & & & & 169 & \\
\hline & $6 / 16 / 2004$ & 2:57:46 PM & 26.473 & 26.852 & 24.524 & 28.263 & & & 53.164 & \begin{tabular}{|l|l|}
16.73 \\
\end{tabular} & & & & & & 146 & & & & & 11.8 & & & & & & & \\
\hline & 6/16/2004 & 2:58:46 PM & 26.483 & 26.852 & 24.52 & 28.284 & & 4 & 52.967 & 16.645 & 31.916 & & & 的.11 & 046 & $\begin{array}{l}14.643 \\
\end{array}$ & & 224. & & 51.5 & 12.2 & & 2.756 & 0.007 & 0.007 & & & \\
\hline & $6 / 16 / 2004$ & 2:59:46 PM & 26.485 & 26.849 & 24.512 & 28.231 & 26.896 & 48.443 & 53.544 & 16.87 & 31.927 & -0.817 & -6.924 & 51.238 & 0.044 & $\begin{array}{l}14.643 \\
\end{array}$ & & 3225.0 & 53.7508 & 52.1 & 12.6 & & & & & & & \\
\hline & & 3:00:46 PM & 26.482 & $\frac{26.846}{20.992}$ & 24.488 & 28.252 & 26.893 & & \begin{tabular}{|l|l|}
53.253 \\
5.203
\end{tabular} & $\begin{array}{l}16.844 \\
\end{array}$ & 31.787 & -0.779 & & 58.653 & $\begin{array}{l}0.045 \\
\end{array}$ & 14.643 & & & & & 14.4 & & & & & & & \\
\hline & 6/16//2004 & 3:01:46 PM & & & & & 26.975 & & & & & & & & 0.044 & 14.643 & & & 53.7842 & 51.6 & 12.0 & & 2.75 & 0.007 & 0.007 & & 0.167 & \\
\hline & 6/16/2004 & 3:02:46 PM & 26.494 & 26.873 & 24.505 & 28.284 & & 48.424 & 53.305 & & & $\begin{array}{r}-0.773 \\
\end{array}$ & & 53.515 & & 14.643 & & & 53.8008 & 51.8 & 13.1 & & & & & 0.000 & 0.165 & \\
\hline & $6 / 16 / 2004$ & 3:03:46 PM & 26.506 & $\begin{array}{r}26.88 \\
268666\end{array}$ & $\begin{array}{r}24.508 \\
24.489\end{array}$ & $\begin{array}{l}28.297 \\
282019\end{array}$ & & $\begin{array}{l}48.072 \\
4845 \\
\end{array}$ & $\begin{array}{l}53.166 \\
53.481\end{array}$ & $\begin{array}{l}16.644 \\
1678\end{array}$ & $\begin{array}{l}31.818 \\
32076\end{array}$ & $\begin{array}{l}-0.773 \\
-0773\end{array}$ & $\begin{array}{l}-6.904 \\
6007\end{array}$ & $\begin{array}{l}57.954 \\
55257\end{array}$ & $\begin{array}{l}0.044 \\
0044\end{array}$ & $\begin{array}{l}14.643 \\
11643\end{array}$ & & & & 51.7 & \begin{tabular}{|l|l|}
14.2 \\
\end{tabular} & $\frac{2}{2}$ & & 0.007 & 0.007 & 0.000 & D.167 & 0.14 \\
\hline$\frac{2540}{2541}$ & 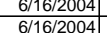 & $\begin{array}{l}3: 04: 46 \text { PM } \\
\text { 3:45:46 }\end{array}$ & $\frac{26.492}{26.498}$ & $\frac{26.866}{26.872}$ & $\begin{array}{r}24.489 \\
24.499\end{array}$ & $\frac{28.248}{28.243}$ & & & \begin{tabular}{|}
$\mid 53.481$ \\
53.498 \\
\end{tabular} & \begin{tabular}{|c|}
16.728 \\
16.664
\end{tabular} & $\begin{array}{r}32.076 \\
32.31 \\
\end{array}$ & & & $\begin{array}{l}52.527 \\
53.962 \\
\end{array}$ & $\begin{array}{l}044 \\
543\end{array}$ & $\begin{array}{l}\frac{14.643}{14.643} \\
\end{array}$ & & 3231.05 & 53.8342 & $\frac{52.0}{52.0}$ & $\begin{array}{l}12.9 \\
13.2\end{array}$ & & 2.776 . 2.76 & $\begin{array}{l}0.007 \\
0.006\end{array}$ & 0.007 & 0.000 & 1.165 & 0.14 \\
\hline & $6 / 16 / 2004$ & 3:06:46 PM & 26.487 & 26.866 & 24.479 & 28.208 & $\frac{20.874}{26.868}$ & 48.404 & \begin{tabular}{|l|}
53.32 \\
\end{tabular} & 16.585 & 32.236 & -0.773 & $\begin{array}{l}-0.904 \\
-6.915 \\
\end{array}$ & 55.307 & $\begin{array}{l}0.043 \\
0.044\end{array}$ & $\begin{array}{l}14.645 \\
14.643 \\
\end{array}$ & & $\frac{3231.0}{3232.0}$ & $\begin{array}{l}53.8006 \\
53.8675\end{array}$ & $\begin{array}{l}51.0 \\
51.8\end{array}$ & $\begin{array}{l}13.2 \\
13.6\end{array}$ & \begin{tabular}{|cc}
6 & 4.4 .3 \\
6 & 40.3
\end{tabular} & $\mid$\begin{tabular}{|l|}
2.780 \\
2.780 \\
\end{tabular} & \begin{tabular}{|l|}
0.000 \\
\end{tabular} & 0.007 & 0.000 & $\begin{array}{l}0.101 \\
0.165\end{array}$ & \\
\hline & $6 / 16 / 2004$ & 3:07:46 PM & 26.47 & 26.849 & 24.462 & 28.191 & 26.847 & 48.632 & 53.79 & 16.525 & 32.555 & \begin{tabular}{|c|c|c|}
-0.773 \\
\end{tabular} & -6.904 & 41.269 & 0.044 & 14.643 & & & 53.8842 & 52.3 & 10.1 & \begin{tabular}{|l|l|}
1 & 40.6 \\
\end{tabular} & 2.799 & 0.007 & 0.007 & 0.000 & 0.164 & \\
\hline 2544 & 6/116/2004 & 3:08:46 PM & & 26.85 & 24.45 & 28.179 & & & 53.26 & 16.545 & & & & & & 14.643 & & & & & & & & & & & & \\
\hline 2545 & & 3:09:46 PM & 26.474 & 26.87 & 24.475 & 28.169 & & 48.459 & & 16.544 & 32.446 & & & 5.668 & & 14.643 & & & .9175 & & & & & .006 & 0007 & & 161 & \\
\hline 2546 & & 3:10:46 PM & & 26.85 & 24.438 & \begin{tabular}{|c|}
28.108 \\
\end{tabular} & & & 53.394 & \begin{tabular}{|l}
16.53 \\
\end{tabular} & 32.288 & & & 50.11 & & 14.643 & & & & & & & & & & & & \\
\hline & 6/116/2004 & 3:11:46 PM & 26.457 & 26.86 & 24.463 & 28.128 & & $48.71 !$ & 53.722 & & 32.665 & & & & & 14.643 & & & 508 & & & & & & & & & \\
\hline & 6/16/2004 & 3:12:46 PM & 26.46 & 26.86 & 24.467 & 8.136 & & & 53.311 & 16.606 & 32.087 & & & 48.476 & 0.051 & 14.643 & & & 3.9675 & 51.8 & 11.9 & & & .008 & 1008 & & 0.192 & \\
\hline & 6/16/2004 & 3:13:46 PM & 26.46 & 26.87. & 24.467 & 28.161 & & 48.267 & 53.392 & 16.46 & 32.238 & -0.704 & & 51.676 & 0.043 & 14.643 & & & 53.98 & 51.9 & $\begin{array}{ll}12.7 \\
\end{array}$ & & $2.77 \mathrm{~S}$ & & $\overline{0.00}$ & & & \\
\hline & & 3:14: & $\frac{26.451}{2.962}$ & 26.85 & 24.437 & 1.15 & & & & 16.534 & 2.142 & & & & & 14.643 & & & & & & & & & & & & \\
\hline & $6 / 16 / 2004$ & & & 26.866 & 24.463 & & & & & & & & & & & 14.643 & & & & & & & & & & & & \\
\hline & & 3:10:46 & 26.469 & 26.888 & $24.4 / 5$ & 28.204 & & & 3.324 & 16.534 & 32.396 & & & & & 14.643 & & & & 51.5 & & & & & & & & \\
\hline & $\frac{6 / 116 / 2004}{6 / 16 / 2004}$ & $\begin{array}{l}3: 17: 46 \mathrm{PM} \\
3.18 .46 \mathrm{PM}\end{array}$ & $\frac{26.454}{26.44}$ & $\frac{26.87}{2686}$ & $\begin{array}{r}24.47 \\
24.446\end{array}$ & 28.204 & & & $\begin{array}{ll}3.475 \\
3.902 \\
9\end{array}$ & $\frac{16.568}{16.683}$ & 32.327 & & & 0.1 .1 & & $\frac{14.643}{14643}$ & & & & & & & & & & & & \\
\hline 2555 & 6/16/2004 & 3:19:46 PM & $\begin{array}{l}26.44 \\
26.44\end{array}$ & 26.87 & 24.4401 & 28.135 & & 48.27 & 53.336 & $\begin{array}{l}0.000 \\
16.559 \\
\end{array}$ & $\begin{array}{l}32.455 \\
32.169\end{array}$ & -0.681 & & & 0.044 & $\begin{array}{l}14.040 \\
14.643 \\
\end{array}$ & & & 540842 & 51.9 & 122 & & & & & & & \\
\hline & $6 / 16 / 2004$ & 3:20:46 PM & 26.429 & 26.86 & 24.45 & 28.13 & & & 53.635 & $\mid 26.677$ & 32.189 & -0. & & 57.46 & & 14.643 & & & & 2 & $\frac{14.1}{4.1}$ & & & & & & & \\
\hline & & 3:21:4 & 26.445 & 26 & 24.466 & 28.185 & & & 53.854 & \begin{tabular}{|c|}
16.61 \\
\end{tabular} & 32.538 & $\begin{array}{l}-0.669 \\
\end{array}$ & & & & 14. & & & & & & & & & & & & \\
\hline & $6 / 16 / 2004$ & & & 26.86 & 24.449 & 28.213 & & & & & & & & & & & & & & & & & & & & & & \\
\hline & & 3:23:46 & 26.448 & 26.87 & 24.459 & 28.278 & & & 53.521 & $\begin{array}{l}16.582 \\
\end{array}$ & 32.282 & & & & & 14.6 & & & 4.15 & & 11.4 & & $2.7 \quad$ & & & & 0.161 & \\
\hline & $6 / 16 /$ & & 26.46 & 26.88 & 24.474 & & & & 53.434 & & & & & & & & & & & & & & & & & & & \\
\hline & & & & & 24.454 & 28.248 & & & & \begin{tabular}{|l|l|}
16.599 \\
\end{tabular} & & & & & & & & & & & 21 & & & & & & & \\
\hline & 6/16/2004 & 3:26:4 & 26.463 & 26.88 & 24.459 & 28.253 & & & \begin{tabular}{|l|l|}
53.417 \\
\end{tabular} & \begin{tabular}{|l|l|l|}
16.638 \\
\end{tabular} & 32.195 & & & 54.3 & & 14.643 & & & & 51.9 & 13.3 & & & & & & 0.169 & \\
\hline & 6/116/2004 & & 26.453 & & 24.459 & 28.238 & & & & & & & & & & & & & & & 2.3 & & & & & & & \\
\hline & 6/16/2004 & & 26.459 & 26.89 & 24.465 & 28.194 & & 48. & 53.742 & 16.644 & & & & 48.4 & & & & & & & 11.9 & & & .007 & & & & \\
\hline & $6 / 16 / 2004$ & & 26.454 & & 24. & & & & & & & & & & & & & & & & 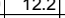 & & & & & & & \\
\hline & & & & & 24.51 & & & & $53.498 \mid$ & & & & & & & & & & & & & & & & & & & \\
\hline & $6 / 16 / 2 / 2$ & & 60.46. & 20.87 & $\frac{24.509}{24.493}$ & 28.149 & & $\begin{array}{ll}48.652 \\
48.318\end{array}$ & | & 16.593 & & & & & & $\frac{14.6}{146} \mathrm{r}-\mathrm{r}$ & & & & 520 & 120 & & & & & & & \\
\hline 2569 & $6 / 16 / 2004$ & & & & & & & & & & & & & & & & & & & 51.9 & \begin{tabular}{|l|}
11.4 \\
\end{tabular} & & & & & & 0.158 & \\
\hline & $6 / 161 / 20$ & & & & & & & & & & & & & & & & & & & 51.8 & 13.7 & & & & & & 0.173 & \\
\hline & $6 / 16 / 20$ & & 26.468 & 26.8 & 24.504 & 28.1 & & & & 16.4 & 31. & & & 49.1 & & & & & & 51.7 & 12.0 & & 2.7 & & & 0. & 163 & \\
\hline & 6/16/2004 & & 26.457 & & & & & & & 16. & & & & & & & & & & & \begin{tabular}{|l|}
12.7 \\
\end{tabular} & & & & & & & \\
\hline & 6/16/2004 & 3:37:4 & 26.446 & 26.8 & 24.488 & 28.1 & 26. & 48.213 & \begin{tabular}{|l|l|}
53.572 \\
\end{tabular} & 16.579 & 32.072 & & & 52. & & & & & & 52.1 & \begin{tabular}{|l|l}
12.8 \\
\end{tabular} & & & & & 0 & 166 & \\
\hline & 6/16/2004 & $\begin{array}{l}3: 38: 46 \mathrm{PM} \\
3: 30 \cdot 46 \mathrm{PM}\end{array}$ & 26.452 & $\frac{26.896}{26.883}$ & 24.493 & $\begin{array}{l}28.148 \\
28145 \\
2151\end{array}$ & & $\begin{array}{l}47.945 \\
4719\end{array}$ & \begin{tabular}{|l}
53.191 \\
53379
\end{tabular} & $\begin{array}{r}16.49 \\
16.453 \\
\end{array}$ & 31.873 & -0. & & $\begin{array}{r}48.48 \\
49608 \\
\end{array}$ & 0.6 & $\frac{14.643}{14.643}$ & & 326 & $\begin{array}{ll}54.46 \\
544\end{array}$ & 51.7 & \begin{tabular}{|l|l|}
11.9 \\
122
\end{tabular} & $\begin{array}{ll}9 & 39 \\
2 & 39 \\
\end{array}$ & $\begin{array}{l}2.752 \\
2.755 \\
\end{array}$ & 007 & 0.007 & 000 & 0.167 & 7 \\
\hline 2576 & | $6 / 1116 / 2004$ & $3: 49: 4$ & $\begin{array}{r}26.449 \\
26.45 \\
\end{array}$ & & $\begin{array}{l}24.466 \\
24.457\end{array}$ & $\frac{28.145}{28151}$ & & & & & & & & & & $\frac{14.6}{14.6}$ & & & & & 12.2 & & & & & & & \\
\hline 25 & $6 / 16 / 20$ & & & & 24.45 & & & & & & & & & & & & & & & & & & & & & & & \\
\hline & $6 / 16 / 2$ & & 26. & $\frac{26.8}{26.8}$ & 24.491 & 28.145 & & & (1914 & & & & & & & & & & & 52.4 & 13.0 & & & & & & & \\
\hline & 6/16/2004 & & 26.4 & & & & & & & & & & & & & & & & & & & & & & & & & \\
\hline & & & & & & 28.2 & & & & & & & & & & & & & & & & & & & & & & \\
\hline & $6 / 16 / 200$ & & & & 24.4 & 28.3 & & & & & & & & & & & & & & & & & & & & & & \\
\hline & $6 / 16 / 2$ & 3:46:4 & 26.47 & 26.8 & 24.47 & 28.38 & & 48.2 & 53.65 & 16.5 & & & & & & 14.6 & & & & & & & & & & & & \\
\hline & $6 / 16 / 200$ & 3:47: & $26.47 \quad$ & 260 & 24.472 & 28.42 & & 48.3 & 3.626 & 16.5 & 32.241 & & & 48.7 & & 14.6 & & & & & $110 \mathrm{c}$ & & & & & & 62 & \\
\hline & 6/16/2 & & & 26.5 & 24.4 & 28.34 & & & 883 & 16 & & & & & & $14.6 \mathrm{C}$ & & & & & & & & & & & & \\
\hline & $6 / 16 / 2$ & & 26.47: & 26.9 & 24.4 & 28. & & & & 16.5 & & & & & & & & & & 5 & 12.3 & & & & & & & \\
\hline & $6 / 16 / 2 / 2$ & & 26.44 & & 24.46 & & & & & & & & & & & & & & & & & & & & & & & \\
\hline & $6 / 16 / 2 / 2$ & & 20.41 & 26.9 & 24.43 & 28.3987 & & & & & & & & & & & & & & & & & & & & & & \\
\hline & 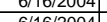 & $3.52 .40 \mathrm{~F}$ & 20.400 & 20.942 & 24.45 & 28.583 & & 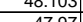 & \begin{tabular}{|l|l|}
53000 \\
5256
\end{tabular} & 10.509 & 1.935 & 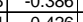 & & 40.206 & & $\begin{array}{l}14.045 \\
1.643\end{array}$ & & & & | & 10 & & & & & & & \\
\hline 2590 & \begin{tabular}{|l|}
$6 / 16 / 2004$ \\
6
\end{tabular} & 3:54:46 PM & $\frac{2.405}{26.49}$ & 26.924 & \begin{tabular}{|l|}
24.401 \\
24.451 \\
\end{tabular} & \begin{tabular}{|l|}
2.0731 \\
28.31 \\
\end{tabular} & 26.936 & 48.534 & \begin{tabular}{|l|}
54.014 \\
54.014 \\
\end{tabular} & \begin{tabular}{|c|}
10.500 \\
16.66
\end{tabular} & $\frac{01.104}{32.245}$ & \begin{tabular}{|l|l|l|l|l|l|}
5 & -0.388 \\
\end{tabular} & -0.400 & $\begin{array}{l}40.059 \\
48.991 \\
\end{array}$ & 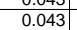 & \begin{tabular}{|c|c|}
14.643 \\
\end{tabular} & & 3280.05 & \begin{tabular}{|l|l|}
54.6675 \\
\end{tabular} & \begin{tabular}{|l|}
52.5 \\
\end{tabular} & \begin{tabular}{|l|l|}
12.0 \\
\end{tabular} & & \begin{tabular}{|l|l|}
2.785 \\
\end{tabular} & 0.006 & $\begin{array}{l}.007 \\
0.007\end{array}$ & 0.000 & \begin{tabular}{l|l}
0 & 0.161 \\
\end{tabular} & 0.1 \\
\hline
\end{tabular}


WSRC-TR-2005-00105, REVISION 0

SRNL-RPP-2005-00012, REVISION 0

RUN \# 3.03A AND B; FIRST AND SECOND HALF OF SLURRY DEWATERING - CONT.

\begin{tabular}{|c|c|c|c|c|c|c|c|c|c|c|c|c|c|c|c|c|c|c|c|c|c|c|c|c|c|c|c|c|}
\hline & $\mathrm{A}$ & & D & $E$ & $\mathrm{~F}$ & G & $\mathrm{H}$ & $\mathrm{J}$ & $\mathrm{K}$ & $\mathrm{L}$ & $\mathrm{M}$ & $\mathrm{N}$ & 0 & Q & R & $\mathrm{s}$ & $\begin{array}{ll}T \\
\end{array}$ & $\mathrm{~J} \quad \mathrm{v}$ & w & $x$ & \begin{tabular}{|r|} 
\\
\end{tabular} & $z$ & $A A$ & & & $A D$ & & AF $\mid A$ \\
\hline & $6 / 16 / 2004$ & 3:55:46 PM & 26.489 & 26.928 & 24.446 & 28.275 & 26.905 & 48.186 & 53.401 & 16.536 & 32.14 & -0.36 & -6.401 & 53.74 & 0.043 & 14.643 & 0.004 & 3281.05 & 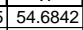 & 51.9 & 13.2 & 40.2 & 2.769 & 0.006 & 0.007 & 0.000 & 0.162 & \\
\hline & $6 / 11 / 2004$ & & 26.502 & 26.951 & 24.463 & 28.342 & 26.938 & 48.291 & 53.658 & & \begin{tabular}{|l|}
32.076 \\
\end{tabular} & \begin{tabular}{|r|}
-0.368 \\
\end{tabular} & -6.398 & 47.696 & 0.044 & $\begin{array}{r}14.643 \\
\end{array}$ & 0.004 & & 5.74 .7008 & 52.2 & $\begin{array}{l}11.7 \\
\end{array}$ & & & & & & 0.166 & \\
\hline (250) & $6 / 16 / 2004$ & 3:57:46 PM & 26.503 & 26.951 & 24.449 & \begin{tabular}{|l|}
28.343 \\
3030
\end{tabular} & 26.944 & $\begin{array}{l}48.466 \\
\end{array}$ & \begin{tabular}{|l|l|}
53.805 \\
5.85
\end{tabular} & $\begin{array}{l}16.614 \\
196\end{array}$ & \begin{tabular}{|c|}
32.298 \\
221
\end{tabular} & \begin{tabular}{|l|l|}
-0.362 \\
\end{tabular} & $\begin{array}{l}-6.395 \\
\end{array}$ & \begin{tabular}{|l|l|l|} 
\\
\end{tabular} & $\begin{array}{l}0.044 \\
\end{array}$ & $\begin{array}{l}14.643 \\
\end{array}$ & & 3283.05 & 54.7175 & 52.3 & 12.0 & & 2.784 & 0.007 & $\begin{array}{l}0.007 \\
\end{array}$ & 0.000 & 0.165 & \\
\hline \begin{tabular}{|l|}
2594 \\
2505
\end{tabular} & $6 / 16 / 2004$ & 3:58:46 PM & 26.509 & 26.958 & 24.466 & \begin{tabular}{|l|}
28.369 \\
\end{tabular} & 26.98 & 48.395 & \begin{tabular}{|l|l|}
53.854 \\
\end{tabular} & $\begin{array}{l}16.618 \\
\end{array}$ & $\begin{array}{r}32.13 \\
\end{array}$ & $\begin{array}{l}-0.36 \\
\end{array}$ & $\begin{array}{l}-6.406 \\
\end{array}$ & \begin{tabular}{|l|l|}
52.733 \\
\end{tabular} & $\begin{array}{l}0.043 \\
\end{array}$ & $\begin{array}{l}14.643 \\
\end{array}$ & 0.004 & 3284.05 & 554.7342 & \begin{tabular}{|l|} 
\\
\end{tabular} & \begin{tabular}{|l|}
12.9 \\
\end{tabular} & 40.3 & 2.776 & 0.006 & 0.007 & 0.000 & 0.162 & \\
\hline 2596 & $\frac{6 / 16 / 2004}{66162004}$ & 3:59:46 PM & $\frac{26.507}{26.508}$ & $\frac{26.966}{26.972}$ & & \begin{tabular}{|l|}
28.392 \\
28393 \\
\end{tabular} & & $\begin{array}{r}48.09 \\
48.123\end{array}$ & \begin{tabular}{|l|}
53.562 \\
53.444 \\
\end{tabular} & \begin{tabular}{|l|l|}
16.547 \\
16526
\end{tabular} & \begin{tabular}{|l|}
31.978 \\
32033
\end{tabular} & $\begin{array}{l}-0.36 \\
-0.354\end{array}$ & $\begin{array}{l}-6.409 \\
-63838\end{array}$ & $\begin{array}{l}48.428 \\
50241 \\
\end{array}$ & $\begin{array}{l}0.042 \\
0.046\end{array}$ & \begin{tabular}{|l|l|l|}
14.643 \\
14643
\end{tabular} & & 3285.05 & \begin{tabular}{|l|l|}
54.7508 \\
5.77566
\end{tabular} & \begin{tabular}{|l|} 
\\
\end{tabular} & \begin{tabular}{|l|l|}
11.9 \\
\end{tabular} & & & & $\begin{array}{l}0.006 \\
0.027\end{array}$ & 0.000 & $\begin{array}{l}0.159 \\
\end{array}$ & \\
\hline 2597 & $\begin{array}{l}66116621204 \\
6 / 16 / 2004\end{array}$ & $\begin{array}{l}4: 00: 03 \mathrm{PM} \\
4: 01: 03 \mathrm{PM}\end{array}$ & $\frac{26.508}{26.494}$ & $\frac{26.972}{26.968}$ & $\begin{array}{r}24.479 \\
24.466\end{array}$ & $\begin{array}{l}28.393 \\
28.275 \\
\end{array}$ & $\frac{26.92}{26.83}$ & $\begin{array}{r}48.123 \\
48.151\end{array}$ & $53.446 \mid$ & \begin{tabular}{|l|}
16.526 \\
1.612
\end{tabular} & $\begin{array}{l}32.033 \\
31.963\end{array}$ & $\begin{array}{r}-0.354 \\
-0.36\end{array}$ & $\begin{array}{l}-6.386 \\
-6.401\end{array}$ & $\begin{array}{l}50.241 \\
52.915 \\
\end{array}$ & $\mid \begin{array}{l}0.0464 \\
0.044\end{array}$ & $\begin{array}{l}\frac{14.643}{14.643} \\
\end{array}$ & 0.004 & \begin{tabular}{|}
3285.33 \\
3286.33
\end{tabular} & $\begin{array}{l}544.7556 \\
55.7722\end{array}$ & $\begin{array}{l}52.0 \\
52.1 \\
\end{array}$ & \begin{tabular}{|l|l|}
12.3 \\
\end{tabular} & $\begin{array}{l}\mid 40.1 \\
\quad 40.1 \\
\end{array}$ & \begin{tabular}{|l|}
2.763 \\
2.762 \\
\end{tabular} & 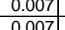 & \begin{tabular}{|c|}
0.007 \\
0.007
\end{tabular} & 0.000 & 0.174 & \\
\hline 2598 & $6 / 16 / 2004$ & & 26.484 & 26.967 & 24.46 & 28.264 & & 48.412 & & & 32.158 & & $\begin{array}{l}-6.4012 \\
-6.42\end{array}$ & 54.252 & 0.043 & $\begin{array}{l}14.043 \\
14.643\end{array}$ & $\begin{array}{l}0.004 \\
0.004\end{array}$ & $\begin{array}{l}3280.53 \\
3287.33 \\
\end{array}$ & \begin{tabular}{|l|l|}
54.7882 \\
\end{tabular} & \begin{tabular}{|l|l|}
52.1 \\
52.4
\end{tabular} & 13.3 & & & & & 0.000 & $\begin{array}{l}0.160 \\
0.162 \\
\end{array}$ & \\
\hline & $6 / 16 / 2004$ & 4:03:03 PM & 26.489 & 26.967 & 24.455 & 28.249 & & 47.777 & 53.226 & 16.472 & 31.719 & -0.36 & -6.383 & 47.61 & 0.044 & 14.643 & 0.004 & 3288.3 & \begin{tabular}{|l|l|}
54.8056 \\
\end{tabular} & \begin{tabular}{|l|}
51.8 \\
\end{tabular} & & & & \begin{tabular}{|l|l|}
0.007 \\
\end{tabular} & & & 0.168 & \\
\hline & $6 / 16 / 2004$ & 4:04:03 PM & & 26.977 & 24.479 & 28.308 & & 48.175 & \begin{tabular}{|l|l|}
53.593 \\
\end{tabular} & 16.581 & & -0.333 & -6.372 & 52.172 & 0.044 & 14.643 & & & $\begin{array}{l}54.8222 \\
\end{array}$ & 52.1 & 12.8 & 40.1 & 2.767 & & & 0.000 & 0.166 & \\
\hline 2601 & $6 / 16 / 2004$ & 4:05:03 PM & 26.493 & 26.972 & 24.464 & $\begin{array}{l}28.403 \\
2.2523\end{array}$ & & $\begin{array}{r}48.229 \\
\end{array}$ & $\begin{array}{l}53.796 \\
5.707\end{array}$ & $\begin{array}{l}16.524 \\
\end{array}$ & 32.113 & -0.357 & -6.372 & \begin{tabular}{|l|l|}
45.804 \\
\end{tabular} & $\begin{array}{l}0.044 \\
\end{array}$ & 14.643 & & 3290.33 & $\begin{array}{l}54.8389 \\
\end{array}$ & 52.3 & 11.2 & 40.2 & 2.770 & \begin{tabular}{|l|l|}
0.007 \\
\end{tabular} & 0.007 & 0.000 & 0.166 & \\
\hline 2603 & 6/16/2004 & $\begin{array}{ll}4: 06: 03 ~ P M \\
\end{array}$ & $\begin{array}{l}26.488 \\
26505\end{array}$ & $\begin{array}{l}26.972 \\
26.094\end{array}$ & $\begin{array}{l}24.444 \\
22.467\end{array}$ & \begin{tabular}{|l|}
28.353 \\
28361 \\
\end{tabular} & & $\begin{array}{l}48.072 \\
4804 \\
\end{array}$ & \begin{tabular}{|l|}
53.587 \\
53668 \\
\end{tabular} & 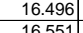 & \begin{tabular}{|c|}
31.963 \\
31.962
\end{tabular} & $\begin{array}{r}-0.348 \\
.362\end{array}$ & $\begin{array}{l}-6.404 \\
6.415\end{array}$ & \begin{tabular}{|r|r|}
52.13 \\
48.497
\end{tabular} & \begin{tabular}{|c|}
0.047 \\
\end{tabular} & $\begin{array}{l}14.643 \\
1.643\end{array}$ & & & 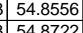 & 52.1 & \begin{tabular}{|l|l|} 
& 12.8 \\
\end{tabular} & & \begin{tabular}{|l|}
2.759 \\
\end{tabular} & 0.007 & $\begin{array}{l}0.007 \\
0.077\end{array}$ & $\begin{array}{l}0.000 \\
0.000\end{array}$ & 0.178 & \\
\hline$\frac{2603}{2604}$ & 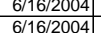 & $\begin{array}{l}4: 07: 03 \mathrm{PM} \\
40: 08: 03 \mathrm{PM}\end{array}$ & $\frac{26.505}{26.511}$ & $\begin{aligned} 26.994 \\
26.995\end{aligned}$ & $\begin{array}{r}24.467 \\
24.478\end{array}$ & $\begin{array}{r}28.361 \\
28.416\end{array}$ & $\begin{array}{l}27.04 \\
27.03 \\
\end{array}$ & & \begin{tabular}{|l|}
53.6648 \\
53.641 \\
\end{tabular} & $\begin{array}{l}16.551 \\
16.598\end{array}$ & \begin{tabular}{|l|}
31.926 \\
3.109
\end{tabular} & $\begin{array}{l}-0.362 \\
-0.36 \\
\end{array}$ & & $\begin{array}{l}48.497 \\
54.969 \\
\end{array}$ & 044 & $\begin{array}{l}14.643 \\
14.643 \\
\end{array}$ & $\begin{array}{l}0.003 \\
0.004\end{array}$ & & 54.8722 & 522 & \begin{tabular}{|l|l|}
13.9 \\
13.5
\end{tabular} & 402 & \begin{tabular}{|l|}
$\mid 2.759$ \\
2.771 \\
\end{tabular} & \begin{tabular}{|l|l|}
0.007 \\
0.006
\end{tabular} & $\begin{array}{l}0.007 \\
0.07\end{array}$ & 0.000 & 1.166 & 0.14 \\
\hline 2605 & $6 / 16 / 2004$ & $\begin{array}{l}4.000: 03 \mathrm{PM} \\
\text { 4:09 }\end{array}$ & $\frac{20.511}{26.513}$ & $\frac{26.995}{27.007}$ & $\begin{array}{l}24.418 \\
24.489\end{array}$ & \begin{tabular}{|l|}
28.408 \\
28.408
\end{tabular} & 27.034 & $\begin{array}{r}48.273 \\
48.526\end{array}$ & 53.041 & 16.721 & 32.206 & & & $\begin{array}{r}54.969 \\
44.54 \\
\end{array}$ & $\begin{array}{l}0.045 \\
0.045\end{array}$ & $\begin{array}{l}14.645 \\
14.643 \\
\end{array}$ & 0.004 & 2294.35 & \begin{tabular}{|l|l}
4.60009 \\
54.9056
\end{tabular} & \begin{tabular}{|l|l|}
52.4 \\
52.3
\end{tabular} & $\begin{array}{l}13.5 \\
10.9\end{array}$ & & $\frac{2.111}{2.783}$ & & & & & \\
\hline & $6 / 16 / 2004$ & 4:10:03 PM & 26.509 & 27.008 & 24.485 & 28.389 & & & 53.115 & 16.556 & 31.849 & & -6.43 & 49.195 & 0.044 & 14.643 & & & & & \begin{tabular}{|l|l|}
12.1 \\
\end{tabular} & & & 0.007 & & & 0.167 & $\frac{0.14}{0.14}$ \\
\hline & $6 / 16 / 2004$ & 4:11:03 PM & 26.509 & 27.003 & 24.48 & 28.354 & & 48.269 & & 16.512 & 32.255 & -0.36 & $\begin{array}{l}-6.444 \\
\end{array}$ & 50.96 & & \begin{tabular}{|l|l|}
14.643 \\
\end{tabular} & & & .9389 & 52.1 & & 40. & & & 0.007 & & & $\frac{0.14}{0.14}$ \\
\hline 2608 & & & & 27.023 & & & & & 53.763 & & 32.265 & & & & & 14.643 & & & 54.9556 & & & & .782 & \begin{tabular}{|l|l|}
0.007 \\
\end{tabular} & & 000 & $\frac{165}{165}$ & \\
\hline 2609 & $6 / 16 / 2$ & 4:13:03 PM & & & 24.54 & 28.373 & & & 53.711 & & 32.302 & -0.345 & & 499.634 & .042 & $\begin{array}{l}14.643 \\
\end{array}$ & & & & & 2.2 & & & & & & $\frac{157}{157}$ & \\
\hline & & 4:14: & & & 24.545 & 28.493 & & & 53.554 & & 31.955 & & & & & & & & & & & & & & & & & \\
\hline & & & & 27.047 & 24.58 & 28.5 & & & 53.842 & 16.544 & 32.124 & -0.339 & & 47.06 & & $\begin{array}{l}14.643 \\
\end{array}$ & & & 55.0056 & & 11.5 & & 771 & 0.006 & 0.006 & & & \\
\hline 26. & $6 / 16 / 2004$ & 4:16:03 PM & 26.534 & 27.048 & 24.571 & 28.469 & 27.04 & $48.10 \mathrm{~s}$ & 53.568 & 16.541 & 31.965 & -0.357 & -6.435 & 56.008 & $\begin{array}{l}0.043 \\
\end{array}$ & 14.643 & & 3301. & 55.0222 & 52.1 & 13.7 & & 2.760 & 0.006 & 0.006 & 0.000 & .162 & \\
\hline & $6 / 16 / 2004$ & 7:03 PM & 26.539 & 27.048 & 24.566 & 28.509 & & 48.206 & 53.523 & 16.505 & 32.195 & $\begin{array}{l}-0.357 \\
-0.07\end{array}$ & -6.464 & 47.137 & 0.045 & 14.643 & & & 55.0389 & & 11.6 & & 77: & 0.007 & 0.007 & 0.000 & . 169 & \\
\hline & $6 / 16 / 2004$ & 4:18:03 PM & 26.56 & 27.049 & 24.572 & 28.515 & & & 33.161 & 16.616 & 32.191 & $\begin{array}{l}-0.333 \\
\end{array}$ & -6.49 & 3.594 & & 14.643 & & & .0556 & 52. & & & & & & & & \\
\hline & & 4:19:03 PM & 26.561 & & 24.553 & 28.561 & & & 53.1995 & & 31.73 & & & & & 14.643 & & & & & & & & & & & & \\
\hline & $6 / 16 / 2004$ & 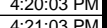 & $\frac{26.567}{26588}$ & 27.061 & $\frac{24.544}{24545}$ & $\begin{array}{l}28.592 \\
28652\end{array}$ & 27.148 & $\begin{array}{l}47.982 \\
48.024\end{array}$ & 53.415 & $\begin{array}{l}16.489 \\
16523\end{array}$ & $\begin{array}{r}31.99 \\
31.871\end{array}$ & -0.3 & & $\begin{array}{l}50.824 \\
4567\end{array}$ & & $\frac{14.643}{14643}$ & & & & & 112 & & & & & & & \\
\hline 2618 & $\frac{1 / 16 / 2004}{6}$ & 4:22: & 26.589 & 27.073 & 24.5336 & 20.006 & & $\begin{array}{l}40.024 \\
48.235\end{array}$ & 53.707 & $\begin{array}{l}10.020 \\
16.636\end{array}$ & 32.013 & $\begin{array}{r}-0.287 \\
-0.287\end{array}$ & & $\frac{43.044}{51.085}$ & $\begin{array}{l}0.041 \\
0.042\end{array}$ & $\begin{array}{l}14.045 \\
14.643\end{array}$ & & & & 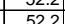 & 125 & & 2766 & 0.006 & 0.006 & & . & \\
\hline 261 & $6 / 16 / 2004$ & 4:23:03 PM & 26.596 & 27.084 & 24.547 & 28.655 & 27.2 & $\begin{array}{l}48.598 \\
\end{array}$ & 53.784 & $\mid 16.477$ & 32.513 & -0.684 & & 50.84 & 0.055 & 14.643 & & 3308.3 & 551389 & 52.3 & 12.5 & & & \begin{tabular}{|l|}
0.008 \\
\end{tabular} & 0.008 & & & \\
\hline & & & 26.596 & 27.08 & & & & & & & 32.786 & -1.014 & & & & 14.643 & & & & & & & & & & & & \\
\hline & 6/16/2004 & & & & & 28.613 & & 47.296 & 53.486 & 16.52 & 165 & & & $5.5 \pm$ & & 14.643 & & & & & & & & & & & & \\
\hline & 6/16/2004 & \begin{tabular}{|l|l|l}
$4: 26: 03$ \\
\end{tabular} & 26.605 & 27.089 & 24.517 & 28.57 & & & 53.718 & $\begin{array}{l}16.546 \\
\end{array}$ & 30.272 & & & 51.36 & & 14.643 & & & 1889 & $\frac{52.2}{52.2}$ & $\frac{12.6}{12.6}$ & & 646 & & & & & \\
\hline & $6 / 16 / 2$ & $4: 2$ & & & 24.543 & & & & $\begin{array}{l}53.56 \\
\end{array}$ & $\begin{array}{l}16.543 \\
\end{array}$ & $\begin{array}{l}32.228 \\
\end{array}$ & & & & & $\begin{array}{l}14.643 \\
\end{array}$ & & & & & & & & & & & & \\
\hline & 6/16/2004 & 4:28:03 PM & 26.62 & 27.124 & 24.537 & \begin{tabular}{|l|l|}
28.57 \\
\end{tabular} & & 48.766 & 53.488 & 16.611 & 32.626 & -1.011 & & & & 14.643 & & & .2222 & & 130 & & & & & & & \\
\hline & 6/16/2004 & & 26.636 & 27.1 & 24.538 & 28.531 & & & $\begin{array}{l}53.369 \\
\end{array}$ & $\begin{array}{ll}16.496 \\
\end{array}$ & 33.004 & \begin{tabular}{|l|l|} 
\\
\end{tabular} & & 46.71 & & 14.643 & & & & 51.9 & 11.4 & & & 0.006 & . .006 & & 0.140 & \\
\hline & $6 / 16 / 2$ & 4:30:03 PM & 26.631 & 27.1 & 24.538 & 28.506 & & 49.127 & \begin{tabular}{|l|l|} 
& \\
\end{tabular} & 16.537 & 33.024 & -1.222 & & 53 & & 14.643 & & & & 52.2 & 13.1 & & & 0.0 & & & & \\
\hline & & & 26.641 & 27.1 & 24.558 & 28.491 & & & \begin{tabular}{|l|l|l|l|} 
\\
383
\end{tabular} & 16.554 & 32.862 & -1.216 & & & & 14.6 & & & & 0.0 & . & & & & & & & \\
\hline & $6 / 16 / 2004$ & & & & & & & & & & & & & & & & & & & & & & & & & & & \\
\hline & & & 26.638 & 27.131 & 24.535 & 28.508 & & & 53.618 & 16.58 & 33.158 & -1.225 & & & & 14.6 & & & 55.3056 & 52.1 & 13.0 & & & & & & & \\
\hline & $\frac{61016,2}{6 / 16 / 2}$ & & 643 & 27.144 & $\begin{array}{l}2.35 \\
2454\end{array}$ & 28.415 & & & 5.8.824 & $\frac{10.05}{1660}$ & |3.3800 & $\begin{array}{l}-1.222 \\
-1.1219 \\
\end{array}$ & & & & . & & & & $\begin{array}{cc}5.4 \\
5.2\end{array}$ & 116 & & & & & & & \\
\hline & $6 / 16 / 2004$ & & 26.053 & & 24.555 & 28.450 & & & 3.979 & 1659 & $\mid$ & & & & & 14643 & & & & $\frac{2.25}{525}$ & 36 & & & & & & & \\
\hline 2633 & $6 / 16 / 2004$ & & 26.649 & 27.153 & 24546 & $\frac{2.5969}{28.579}$ & & & & 1.65 & & \begin{tabular}{|l|}
-1. \\
\end{tabular} & & & & 14643 & & & & $J$ & $\frac{6.0}{41}$ & & & & & & . & \\
\hline 263 & $6 / 16 / 2004$ & 4:38: & 26.64 & $\frac{27.149}{27.149}$ & 24.532 & 28.56 & & 49.2 & \begin{tabular}{|l|}
53.599 \\
\end{tabular} & 16.558 & 33.109 & & & 49. & & & & & & $\frac{52.1}{52.1}$ & 12.0 & & 8.839 & 0 & 0.007 & & & \\
\hline & $6 / 16 / 2004$ & & & & & & & & & & & & & & & & & & & & & & & & & & & \\
\hline & 6/116/2004 & & 26.661 & 27.1 & 24.548 & 28.491 & & & 53.886 & 16.5 & $\mid 33.338$ & -1.2 & & & & & & & & & 1.9 & & & & & & & \\
\hline & $6 / 16 / 2$ & & & & & 28.473 & & & & 16.62 & & & & & & & & & & & & & & & & & & \\
\hline & $6 / 16 / 2$ & & & 27.15 & 24.561 & 28.434 & & 48.78 & 53.096 & 17.554 & \begin{tabular}{|l|l|}
31.649 \\
\end{tabular} & \begin{tabular}{|l|}
-1.222 \\
\end{tabular} & & & & & & & & 516 & 122 & & & & & & & \\
\hline & 6/16/2004 & & 26.671 & 27.1 & 24.578 & 28.466 & & & \begin{tabular}{|l|l|}
52.088 \\
\end{tabular} & 17.22 & 31.346 & -1.2 & & & & 14.643 & & & & 50 & 13.1 & & & \begin{tabular}{|l|l|l|}
0.006 \\
\end{tabular} & .0 & 0.000 & 0.164 & \\
\hline & $6 / 16 / 2$ & & & & & 28.4 & & & & & & -1. & & & & & & & & & 3.4 & & & & & & & \\
\hline 26 & 6/116/2004 & & 26.672 & 27.16. & 24.58 & 28.4 & & & & 17.1. & & -1.22 & & & & 14.6 & & & & 51. & $2.7 \mathrm{~T}$ & & & & & & & \\
\hline$=0$ & $6 / 16 / 2004$ & & 26. & & & & & & & & & -1.2 & & & & & & & & & & & & & & & & \\
\hline & & & 606 & 27. & 24.5 & 28. & & & 5.9 & & & -1.222 & & & & & & & & & & & & & & & & \\
\hline & $\frac{6 / 10 / 2}{6 / 6 / 2}$ & & & 年17.15 & $\begin{array}{l}24.004 \\
24603\end{array}$ & 28.4 & & & 51848 & & $\begin{array}{r}31.37 \\
31.192\end{array}$ & $\frac{-1.2}{-12}$ & & & & 年 & & & & 504 & 10.0 & & & & & & & \\
\hline & $6 / 16 / 200$ & & 26686 & 2716 & 2466 & $\frac{20.4}{28.4}$ & & & $\mid$ & & 31.048 & & & & & & & & & & & & & & & & & \\
\hline & $6 / 16 / 200$ & & & & & & & & & & & $\frac{1.4}{-1.2}$ & & & & & & & & & 35 & & & & & & & \\
\hline & $\frac{26 / 16 / 2}{6.16 / 2}$ & & 26.677 & 27.14 & 24.579 & $\frac{28.36}{28.36}$ & & & 51.564 & & & $\frac{-1.222}{-1.22}$ & & & & & & & & & 114 & & & & & & & \\
\hline 264 & $6 / 16 / 2004$ & & & & & & & & & & & & & & & & & & & & & & & & & & & \\
\hline & & & & & & & & & & & & & & & & & & & & & & & & & & & & \\
\hline & $6 / 16 / 200$ & & & & 24.536 & 28.3 & & 48.2 & & & & -1.2 & & & & 14. & & & & & & & & & & & & \\
\hline & $6 / 16 / 2$ & 4:56: & & 27.141 & 24.509 & $\begin{array}{l}28.328 \\
\end{array}$ & & 48. & 52.61 & 16.42 & 31.97 & -1.2 & & & & 14.6 & & & & & & & & & & & & \\
\hline & $6 / 16 / 200$ & 4:57: & & 27. & 24.488 & 28.302 & & 48.6 & 2.478 & 16.433 & 32.062 & -1.2 .7 & & & & 14.64 & & & & & & & & & & & 166 & \\
\hline & $6 / 16 / 2$ & & & 27.1 & 24. & 28.2 & & 48.4 & 52. & 16.43 & & -1.219 & & & & $14.6 \mathrm{C}$ & & & & 51.3 & & & & & & & 65 & \\
\hline & $6 / 16 / 2$ & & 26. & 27. & 24.447 & 28.132 & & 47. & 52.368 & 16.373 & & -1.222 & & & & & & & & 5 & 12.5 & & & & & & 64 & \\
\hline & $6 / 16 / 2004$ & & & & 24. & & & & & & & -1.2 & & & & & & & & & & & & & & & & \\
\hline & $6 / 16 / 2 / 2$ & & & & 24.405 & 28.1144 & & 48.2 & 52.6 & & 32.358 & & & & & & & & & & & & & & & & & \\
\hline & 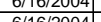 & 5.02 .03918 & . & 27.114 & 24.391 & 28.151 & & 40.111 & 52.006 & 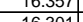 & $\begin{array}{l}32.063 \\
32515\end{array}$ & 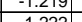 & & 5015 & & $\begin{array}{l}14.045 \\
1.643\end{array}$ & & & & S1.4 & & & & & 000 & 0.000 & 0.166 & \\
\hline 2660 & $6 / 116 / 2004$ & 5:04:03 PM & $\frac{0.005}{26.594}$ & 27.098 & \begin{tabular}{|l|}
24.350 \\
\end{tabular} & \begin{tabular}{|l|}
28.15 \\
\end{tabular} & 26.86 & 48.273 & \begin{tabular}{|l|}
52.723 \\
\end{tabular} & \begin{tabular}{|l|}
16.418 \\
\end{tabular} & 32.325 & 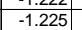 & $\begin{array}{l}-7.040 \\
-7.054\end{array}$ & $|44.7|$ & $\begin{array}{l}0.045 \\
0.042\end{array}$ & \begin{tabular}{|c|c|}
14.643 \\
\end{tabular} & & 3349.33 & $\begin{array}{l}355.8222 \\
\end{array}$ & \begin{tabular}{|l|l|} 
& 1.4 .3 \\
\end{tabular} & \begin{tabular}{|l|l|l|l}
11.0 \\
\end{tabular} & & \begin{tabular}{|l|}
2.778 \\
\end{tabular} & $\begin{array}{l}.006 \\
0.006\end{array}$ & \begin{tabular}{|c|}
0.006 \\
\end{tabular} & 0.000 & \begin{tabular}{|l|}
0.158 \\
0.158 \\
\end{tabular} & 0.1 \\
\hline
\end{tabular}


WSRC-TR-2005-00105, REVISION 0

SRNL-RPP-2005-00012, REVISION 0

RUN \# 3.03A AND B; FIRST AND SECOND HALF OF SLURRY DEWATERING - CONT.

\begin{tabular}{|c|c|c|c|c|c|c|c|c|c|c|c|c|c|c|c|c|c|c|c|c|c|c|c|c|c|c|c|c|}
\hline & A & $\mathrm{B}$ & D & $\mathrm{E}$ & $\mathrm{F}$ & $G^{-1}$ & $\mathrm{H}$ & $\mathrm{J}$ & $\mathrm{K}$ & L & $\mathrm{M}$ & $\mathrm{N}$ & $\mathrm{O}$ & Q & \begin{tabular}{l|l}
$R$ \\
\end{tabular} & $\mathrm{~s}$ & T & & & $x$ & & $L_{2}$ & $A A$ & & $A C$ & $A D$ & $\mathrm{AE}$ & \\
\hline 266 & 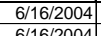 & 5:05:03 PM & $\begin{array}{l}26.582 \\
657\end{array}$ & 27.086 & 24.34 & $\begin{array}{l}28.153 \\
28.159\end{array}$ & 26.838 & $\begin{array}{l}48.242 \\
\end{array}$ & & $\begin{array}{ll}16.338 \\
1326\end{array}$ & $\begin{array}{l}32.321 \\
2050\end{array}$ & $\begin{array}{l}-1.222 \\
.220\end{array}$ & $\begin{array}{r}-7.045 \\
705\end{array}$ & 47.571 & $\begin{array}{l}0.043 \\
\end{array}$ & $\begin{array}{l}14.643 \\
1.62\end{array}$ & 0.004 & $\begin{array}{l}3350.33 \\
3251.22\end{array}$ & \begin{tabular}{|l|l|}
55.8389 \\
\end{tabular} & 51.3 & 11.7 & 40. & 2.77 & 0.006 & $\begin{array}{ll}0.007 \\
\end{array}$ & 0.000 & 0.162 & \\
\hline 2665 & $6 / 16 / 2004$ & 5:06:03 PM & $\begin{array}{r}26.57 \\
26.563\end{array}$ & 27.074 & $\frac{24.32}{24.3}$ & $\frac{28.156}{28129}$ & $\frac{26.842}{26.85}$ & $\begin{array}{r}48.011 \\
48.219 \\
\end{array}$ & \begin{tabular}{|l|}
52.368 \\
52652 \\
\end{tabular} & $\begin{array}{l}16.316 \\
16.242\end{array}$ & $\begin{array}{l}32.158 \\
33.442\end{array}$ & $\begin{aligned}-1.225 \\
-12219\end{aligned}$ & $\begin{array}{r}-7.045 \\
-7045\end{array}$ & $\begin{array}{l}46.732 \\
5.1690\end{array}$ & 0.044 & $\frac{14.643}{11643}$ & 0.004 & $\frac{3351.33}{325020}$ & \begin{tabular}{|l|l|}
55.8556 \\
5.87222
\end{tabular} & 50.9 & 11.5 & 40. & 2.764 & 0.007 & 0.007 & & 0.167 & \\
\hline 2664 & $6 / 16 / 2004$ & $\begin{array}{l}5.00 .03 \mathrm{PM} \\
\text { 5:08:03 PM }\end{array}$ & 20.530 & 27.055 & $\begin{array}{r}24.3 \\
24.28\end{array}$ & $\begin{array}{l}28.129 \\
28.057\end{array}$ & $\begin{array}{r}26.85 \\
26.797\end{array}$ & \begin{tabular}{|l|}
48.219 \\
48611
\end{tabular} & \begin{tabular}{|l|}
52.652 \\
53.104 \\
\end{tabular} & $\begin{array}{l}\frac{16.242}{16.343} \\
\end{array}$ & $\begin{array}{l}32.442 \\
32.698 \\
\end{array}$ & $\begin{array}{l}-1.2199 \\
-1.222\end{array}$ & $\begin{array}{l}-7.045 \\
-7.048\end{array}$ & $\begin{array}{r}51.469 \\
49.16 \\
\end{array}$ & $\begin{array}{l}0.043 \\
0.044 \\
\end{array}$ & $\begin{array}{l}14.643 \\
14.643 \\
\end{array}$ & $\frac{0.004}{0.004}$ & $\begin{array}{l}\frac{33252.33}{3353.33} \\
3\end{array}$ & $\begin{array}{l}55.8722 \\
55.8889\end{array}$ & $\frac{51.2}{51.6}$ & $\frac{12.6}{12.0}$ & & & $\begin{array}{l}0.006 \\
0.007\end{array}$ & $\begin{array}{l}0.0007 \\
0.007\end{array}$ & $\frac{0.000}{0.000}$ & $\frac{0.162}{0.165}$ & $\frac{0.14}{0.14}$ \\
\hline 2665 & $6 / 16 / 2004$ & 5:09:03 PM & 26.533 & 27.052 & 24.28 & 28.054 & 26.78 & 48.065 & \begin{tabular}{|l|}
52.598 \\
\end{tabular} & 16.248 & 32.251 & -1.225 & -7.048 & 46.793 & 0.043 & 14.643 & & 3354.33 & 55.9056 & 51.1 & 11.5 & & 2.769 & & & & & \\
\hline & $6 / 16 / 2004$ & 5:10:03 PM & 26.526 & 27.05 & 24.27 & 28.037 & 26.797 & 48.264 & \begin{tabular}{|l|l|}
52.65 \\
\end{tabular} & \begin{tabular}{|l|l|}
16.298 \\
\end{tabular} & 32.466 & -1.222 & -7.048 & 53.789 & $\begin{array}{l}0.043 \\
\end{array}$ & 14.643 & & & & & 13.2 & & & & & & & \\
\hline & $6 / 16 / 2004$ & 5:11:03 PM & & $27.04 \mathrm{C}$ & 24.26 & 28.046 & 26.757 & & & $\begin{array}{l}6.319 \\
\end{array}$ & & -1.225 & $\begin{array}{r}-7.048 \\
.705\end{array}$ & 48.918 & \begin{tabular}{|l|l|}
0.043 \\
\end{tabular} & 14.643 & & & 55.9389 & 51.4 & \begin{tabular}{|l|l|}
12.0 \\
\end{tabular} & & & & & & & \\
\hline 2668 & $6 / 16 / 2004$ & 5:12:03 PM & 26.499 & 27.028 & 24.2 & 27.95 & 26.711 & 48.457 & 52.843 & $\begin{array}{l}16.248 \\
16201\end{array}$ & 32.645 & -1.225 & -7.054 & 47.062 & 0.042 & 14.643 & & $3357.3 \mathrm{~s}$ & 55.9556 & 51.4 & 11.5 & 40.6 & 2.796 & 0.006 & 0.006 & 0.000 & 0.158 & \\
\hline 2669 & 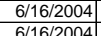 & $\begin{array}{l}5: 13: 03 \mathrm{PM} \\
5: 11: 03 \mathrm{P}\end{array}$ & $\begin{array}{l}26.483 \\
26.472\end{array}$ & $\begin{array}{l}27.027 \\
27016\end{array}$ & $\begin{array}{l}24.22 \\
24.22 \\
\end{array}$ & $\begin{array}{l}27.949 \\
27.924\end{array}$ & $\begin{array}{l}26.695 \\
26669\end{array}$ & $\begin{array}{r}48.563 \\
489 \\
\end{array}$ & $\begin{array}{l}53.108 \\
53257\end{array}$ & $\begin{array}{l}16.301 \\
16234\end{array}$ & $\begin{array}{l}32.762 \\
32.862 \\
\end{array}$ & $\begin{array}{c}-1.222 \\
-2222\end{array}$ & $\frac{-7.045}{-7.04}$ & $\begin{array}{l}49.306 \\
5089\end{array}$ & $\begin{array}{l}0.043 \\
0.43\end{array}$ & $\begin{array}{l}14.643 \\
1.642\end{array}$ & & & $\begin{array}{l}55.9722 \\
55.0889\end{array}$ & $\frac{51.6}{51.9}$ & $\begin{array}{l}12.1 \\
124\end{array}$ & & & & \begin{tabular}{|l|l|}
0.007 \\
\end{tabular} & & 0.161 & \\
\hline$\frac{2070}{2671}$ & $6 / 116 / 2604$ & $\begin{array}{l}\text { 5:14:03 PM } \\
\text { 5:03 }\end{array}$ & $\frac{26.472}{26.446}$ & $\frac{27.016}{26.994}$ & $\frac{24.22}{24.19}$ & $\begin{array}{l}27.924 \\
27.867\end{array}$ & $\frac{26.669}{26.628}$ & $\begin{array}{r}48.69 \\
48.426\end{array}$ & \begin{tabular}{|l|}
53.257 \\
52.876 \\
\end{tabular} & $\begin{array}{l}16.234 \\
16.252\end{array}$ & $\begin{array}{l}32.862 \\
32.655 \\
\end{array}$ & $\begin{array}{l}-1.222 \\
-1.222\end{array}$ & $\begin{array}{c}-7.04 \\
-7.042\end{array}$ & $\frac{50.789}{48.979}$ & $\begin{array}{l}0.043 \\
0.044\end{array}$ & $\begin{array}{l}14.643 \\
14.643 \\
\end{array}$ & 0.004 & $\begin{array}{l}3359.3 \\
336.03 \\
33\end{array}$ & $\begin{array}{l}55.9889 \\
56.0056\end{array}$ & $\frac{51.8}{55.4}$ & $\frac{12.4}{12.0}$ & \begin{tabular}{|l|l} 
\\
0.5
\end{tabular} & $\frac{2.811}{2.795}$ & $\frac{0.006}{0.007}$ & $\frac{0.007}{0.007}$ & $\begin{array}{l}0.000 \\
0.000\end{array}$ & 0.161 & \\
\hline 2672 & $6 / 16 / 2004$ & 5:16:03 PM & 26.438 & 26.987 & 24.19 & & 26.64 & 48.632 & \begin{tabular}{|l|}
53.063 \\
\end{tabular} & 16.163 & 32.883 & -1.222 & & 44.513 & 0.044 & 14.643 & & & \begin{tabular}{|l|}
56.02222 \\
\end{tabular} & 51.6 & 10.9 & & & & & & $\begin{array}{l}0.160 \\
0.165 \\
\end{array}$ & 0.14 \\
\hline 2673 & $6 / 16 / 2004$ & 5:17:03 PM & 26.411 & 26.97 & 24.17 & 27.868 & 26.648 & 48.352 & \begin{tabular}{|l|l|}
52.828 \\
\end{tabular} & 16.095 & 32.669 & -1.222 & $\begin{array}{r}-7.048 \\
\end{array}$ & 54.902 & 0.043 & 144.643 & & 3362.33 & 56.0389 & 51.4 & 13.5 & 40.5 & 2.793 & 0.006 & 0.007 & 0.000 & 0.162 & \\
\hline & $6 / 16 / 2004$ & 5:18:03 PM & & & & & & & & & & -1.216 & & 47.944 & 0.044 & 14.643 & & 3363.3 & 56.0556 & 51.6 & 11.7 & & 2.798 & 0.007 & 0.007 & & 0.165 & \\
\hline 2675 & $6 / 16 / 2004$ & 5:19:03 PM & 26.374 & 26.953 & 24.14 & 27.82 & 26.621 & 48.422 & \begin{tabular}{|l|l|}
52.944 \\
\end{tabular} & 16.14 & 32.756 & -1.222 & & 50.845 & 0.044 & 14.643 & & & 56.0722 & 51.5 & 12.5 & & 2.798 & \begin{tabular}{|l|l|l}
0.007 \\
\end{tabular} & & 0.000 & 0.166 & \\
\hline$\frac{2676}{2677}$ & 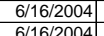 & $\begin{array}{l}5: 20: 03 \mathrm{PM} \\
5 \cdot 21: 03 \mathrm{PM}\end{array}$ & $\begin{array}{l}26.367 \\
26.341\end{array}$ & $\begin{array}{l}26.946 \\
22.935 \\
\end{array}$ & $\begin{array}{l}24.15 \\
24.12\end{array}$ & $\begin{array}{l}27.819 \\
27868\end{array}$ & $\begin{array}{l}26.589 \\
26.603\end{array}$ & \begin{tabular}{|l|l}
48.466 \\
48663
\end{tabular} & \begin{tabular}{|l|l|}
53.065 \\
53239
\end{tabular} & \begin{tabular}{|l|l|l|l|l|l|}
16.186 \\
16.186
\end{tabular} & $\begin{array}{l}32.791 \\
33.951 \\
3.951\end{array}$ & $\begin{array}{l}-1.222 \\
-1.222\end{array}$ & $\begin{array}{c}-7.04 \\
-7.04\end{array}$ & $\begin{array}{l}56.448 \\
5.1058\end{array}$ & \begin{tabular}{|c|c|}
0.043 \\
0043
\end{tabular} & $\begin{array}{l}14.643 \\
16463\end{array}$ & & & \begin{tabular}{|l|}
56.0889 \\
561056
\end{tabular} & 51.6 & \begin{tabular}{|l|l|}
13.8 \\
125
\end{tabular} & & \begin{tabular}{|l|l|}
2.801 \\
2813
\end{tabular} & \begin{tabular}{|l|l|}
0.006 \\
0006
\end{tabular} & \begin{tabular}{|l|l|}
0.007 \\
\end{tabular} & $\begin{array}{l}0.000 \\
\end{array}$ & $\begin{array}{l}0.162 \\
0.161\end{array}$ & \\
\hline$\frac{26 / 1}{2678}$ & 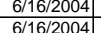 & $\begin{array}{l}5: 21: 03 \mathrm{PM} \\
\text { 5:02:03 }\end{array}$ & $\frac{26.341}{26.329}$ & $\frac{26.93}{26.90}$ & $\frac{24.12}{24.10}$ & $\begin{aligned} 27.868 \\
2782\end{aligned}$ & $\frac{26.603}{26.596}$ & \begin{tabular}{|}
48.663 \\
48.619
\end{tabular} & \begin{tabular}{|l|}
53.239 \\
53.099 \\
\end{tabular} & \begin{tabular}{|c|}
16.186 \\
16.123
\end{tabular} & $\begin{array}{r}32.951 \\
32.969 \\
\end{array}$ & $\begin{array}{l}-1.2222 \\
-1.222\end{array}$ & $\begin{array}{c}-7.04 \\
-7.037\end{array}$ & $\frac{51.058}{55.547}$ & $\begin{array}{l}0.043 \\
0.044\end{array}$ & $\frac{14.643}{14643}$ & 0.00 & \begin{tabular}{|l}
3366.33 \\
367.33
\end{tabular} & \begin{tabular}{|l|l|l|l}
56.1056 \\
5.1223
\end{tabular} & $\begin{array}{l}51.8 \\
51.5\end{array}$ & $\frac{12.5}{136}$ & \begin{tabular}{|l|}
40.8 \\
40.8
\end{tabular} & 2.813 & 0.006 & 0.007 & 0.000 & 0.161 & \\
\hline 2679 & $6 / 16 / 2004$ & 5:23:03 PM & $\frac{26.329}{26.332}$ & $\frac{20.90}{26.92}$ & $\frac{24.10}{24.11}$ & 27.828 & $\frac{26.596}{26.614}$ & \begin{tabular}{|l}
48.619 \\
48.036
\end{tabular} & \begin{tabular}{|r|}
5.009 \\
52.54 \\
\end{tabular} & $\begin{array}{l}10.125 \\
16.357\end{array}$ & $\begin{array}{r}32.969 \\
32.154 \\
\end{array}$ & $\frac{-1.242}{-1.222}$ & & $\begin{array}{l}55.54 ! \\
52.137\end{array}$ & \begin{tabular}{|l|l|l}
0.043 \\
\end{tabular} & $\frac{14.643}{14.643}$ & & \begin{tabular}{|l|}
33678.35 \\
3368.33
\end{tabular} & \begin{tabular}{|l|l|}
56.12424 \\
5689
\end{tabular} & $\frac{51.5}{51.1}$ & $\frac{13.6}{12.8}$ & \begin{tabular}{|l|}
40.8 \\
0.1
\end{tabular} & $\frac{2.813}{2.764}$ & \begin{tabular}{|l}
0.001 \\
0.006
\end{tabular} & $\begin{array}{l}0.007 \\
0.007\end{array}$ & 0 & $\begin{array}{l}0.165 \\
0.164 \\
\end{array}$ & 0. \\
\hline & $6 / 16 / 2004$ & 5::24:03 PM & 26.316 & 26.90 & 24.12 & 27.802 & 26.577 & 48.219 & 52.735 & 16.392 & 32.3 & -1.219 & -7.048 & 47.812 & 0.042 & 14.643 & & 3369.3 & 56.1556 & 51.3 & 11.7 & 40.3 & 2.776 & 0.006 & 0.006 & 0.000 & 0.159 & \\
\hline & 6/16/2004 & 5:25:03 PM & & 26.89 & 24.15 & \begin{tabular}{|l|l|}
27.797 \\
\end{tabular} & 26.582 & & \begin{tabular}{|l|}
52.907 \\
\end{tabular} & 16.551 & 32.376 & -1.225 & -7.04 & 51.292 & $\begin{array}{l}0.043 \\
\end{array}$ & 14.643 & & \begin{tabular}{|l|}
3370.3 \\
\end{tabular} & 56.1722 & 51.4 & 12.6 & 40.4 & 2.787 & 0.006 & $\begin{array}{l}0.007 \\
\end{array}$ & 0.000 & 0.162 & \\
\hline 2682 & $6 / 16 / 2004$ & 5:26:03 PM & 26.295 & 26.89 & 24.16 & 27.771 & 26.576 & 48.488 & $\begin{array}{l}52.955 \\
5.2006\end{array}$ & $\begin{array}{l}16.439 \\
1.47\end{array}$ & $\begin{array}{l}32.522 \\
\end{array}$ & $\begin{array}{l}-1.219 \\
\end{array}$ & & 54.12 & $\begin{array}{l}0.043 \\
\end{array}$ & 14.643 & & & 56.1889 & 51.5 & 13.3 & 40.5 & \begin{tabular}{|l|l|}
2.793 \\
\end{tabular} & 0.006 & $\begin{array}{l}0.007 \\
\end{array}$ & & 0.162 & \\
\hline$\frac{2004}{2685}$ & $6 / 11 / 2 / 2004$ & $\begin{array}{l}5: 28: 03 \mathrm{PM} \\
5: 2903 \mathrm{PM}\end{array}$ & $\frac{26.266}{26.254}$ & $\frac{26.8}{26.85}$ & $\frac{24.14}{24.12}$ & $\begin{aligned} 27.818 \\
27.86\end{aligned}$ & & \begin{tabular}{|}
$\mid 48.233$ \\
48.084 \\
\end{tabular} & & \begin{tabular}{l|l|}
16.349 \\
16.259
\end{tabular} & $\begin{array}{r}32.339 \\
32.29 \\
\end{array}$ & $\begin{array}{l}-1.2222 \\
-1.222\end{array}$ & & $\begin{array}{r}49.46 \\
47.539 \\
\end{array}$ & $\mid$\begin{tabular}{|c|c|c|}
0.041 \\
0.043
\end{tabular} & $\frac{14.643}{14.643}$ & & & \begin{tabular}{|l|l|}
56.22222 \\
56.2389
\end{tabular} & & & & & & & & & \\
\hline & $6 / 16 / 2004$ & 5:30:03 PM & 26.247 & $\frac{20.05}{26.83}$ & $\frac{24.12}{24.11}$ & 27.853 & 26.604 & $\frac{40.004}{48.2}$ & \begin{tabular}{|l|}
52.756 \\
\end{tabular} & $\frac{10.250}{16.22}$ & $\begin{array}{l}32.413 \\
32.413\end{array}$ & $\frac{-1.22}{-1.225}$ & & $\begin{array}{l}50.7059 \\
50.799\end{array}$ & $\begin{array}{l}0.045 \\
0.041\end{array}$ & $\frac{14.045}{14.643}$ & & & \begin{tabular}{|l|l|}
56.23589 \\
5.256
\end{tabular} & $\frac{51.1}{51.3}$ & $\frac{11.7}{12.4}$ & 40. & & $\frac{0.006}{0.006}$ & 0.007 & 00 & 1.156 & $\frac{0.14}{0.13}$ \\
\hline 2687 & 6/116/2004 & 5:31:03 PM & 26.236 & 26.8 & 24.10 & 27.827 & 26.593 & 48.677 & 53.195 & 16.265 & 32.819 & -1.161 & & 47.616 & 0.042 & 14.643 & & \begin{tabular}{|l|l|}
3376.33 \\
\end{tabular} & \begin{tabular}{|l|l|}
56.2722 \\
\end{tabular} & 51.7 & 11.7 & 40.7 & 2.809 & 0.006 & 0.006 & $\begin{array}{l}0.000 \\
0.000\end{array}$ & $\begin{array}{l}0.158 \\
0.158 \\
\end{array}$ & 0.13 \\
\hline & $6 / 16 / 2004$ & 5:32:03 PM & 26.24 & & 24.09 & 27.786 & 26.546 & 48.5 & \begin{tabular}{|l|l|l|}
53.104 \\
\end{tabular} & \begin{tabular}{|l|l|}
16.257 \\
\end{tabular} & 32.684 & -1.219 & & 54.08 & 0.042 & 14.643 & & & 56.2889 & 51.6 & 13.3 & 40.6 & 2.799 & 0.006 & 0.006 & & & \\
\hline 2689 & $6 / 16 / 2004$ & 5:33:03 PM & 26.239 & 26.82 & 24.14 & 27.74 & & & 52.996 & $\begin{array}{l}16.182 \\
\end{array}$ & & & & & \begin{tabular}{|l|l|l|}
0.043 \\
\end{tabular} & & & & & 51.5 & & & 2.784 & \begin{tabular}{|l|l|}
0.006 \\
\end{tabular} & & 0.000 & 0.163 & \\
\hline 2690 & $6 / 16 / 2$ & 5:34:03 PM & & & 24.20 & & & & & & 32.686 & -1.199 & & 446.617 & & 14.643 & & & & & & & & & & & & \\
\hline 2691 & 6/16/2004 & 5:35:03 PM & & & 24.24 & & 26 & 48.18 & 52.835 & & 32.534 & & & 47.149 & .042 & 14.643 & & & 5.3389 & & 1.6 & & & & & & & \\
\hline & 6/116/2004 & 5:36: & 26.211 & 26.80 & 24.26 & 27.688 & & & 52.787 & 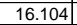 & 32.589 & -1.159 & & 53.071 & 0.04 & 14.643 & & & & 51.3 & 3.0 & & & 0.006 & 0.006 & & & \\
\hline & $6 / 16 / 2$ & 5:37:03 PM & & $26.80 !$ & 24.31 & 27.772 & 26.54 & & 52.884 & 16.02 & 32.827 & -1.115 & & 48.662 & $\begin{array}{l}0.044 \\
\end{array}$ & 14.643 & & & & 51.4 & 1.9 & & 2.79 & & & & & \\
\hline 26 & 6/16/2004 & 5:38:03 PM & 26.205 & 26.78 & 24.31 & 27.732 & & 48.009 & 52.615 & \begin{tabular}{|l|l|}
16.013 \\
\end{tabular} & 32.431 & -1.159 & & 47.195 & 0.043 & 14.643 & & & 56.3889 & 51.1 & 11.6 & 40 & 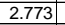 & 0.006 & 0.007 & 0.000 & 0.163 & \\
\hline & $6 / 2004$ & & & 26.78 & 24.37 & & & & 年 & ( & & & & & & & & & & & & & & & & & & \\
\hline & & & & 26.18 & 24.38 & 27.757 & & & 52.94 & 15.942 & 32.749 & -1.141 & & 52.1 & & & & & & 51.5 & 12.9 & & & & & & & \\
\hline & 6 & $\begin{array}{ll}5: 4.0394 \\
5\end{array}$ & $\frac{26.214}{26202}$ & 20.188 & 24.43 & $\begin{array}{l}21.145 \\
27.723\end{array}$ & $\frac{26.5}{26.41}$ & 48.217 & 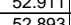 & $\begin{array}{l}16.016 \\
16027\end{array}$ & 32944 & $\begin{array}{r}-1.138 \\
1.06\end{array}$ & & 0278 & & $\frac{14.643}{14643}$ & & & & 514 & 123 & & & & & & 0160 & \\
\hline & $6 / 16 / 2$ & & & & $\frac{24.45}{24.2}$ & 27712 & & & & 年 & & -1.100 & & & & $\frac{14.6}{146}$ & & & & & 资, & & & & & & & \\
\hline & 6 & & 26205 & 26.14 & 24.47 & 27677 & & & 55063 & $\mid$ & & $-1,101$ & & & & 146 & & & & $\frac{5.1 .}{516}$ & 17 & & & & & & 1.160 & \\
\hline & $\frac{1 / 1612004}{6}$ & 5:45: & $\frac{20.225}{26.225}$ & $\frac{26.75}{26.75}$ & 24.49 & 27.791 & & 48.372 & $\mid$\begin{tabular}{|c|}
52.772 \\
\end{tabular} & 15.922 & 32.975 & -1.104 & & 49.788 & $\mid$ & 14.6 .6 & & & & 51.3 & 望. & & 2.804 & 0.006 & $\frac{0.007}{0.007}$ & 0.000 & 0160 & \\
\hline & & & 26.244 & & & & & & & & & & & & & & & & & & & & & & & & & \\
\hline & $6 / 1$ & & 26.237 & 26.7 & 24.55 & 27.9 & & & & 15.8 & & & & 50 & & & & & & th & $\frac{5.3}{2.3}$ & & & & & & & \\
\hline & & & & & 24.55 & & & & & & & & & & & & & & & & & & & & & & & \\
\hline & $6 / 16 / 2$ & & 26.262 & 26.76 & 24.55 & 27.9 & & & & 16.0 & & -1.1 & & & & & & & & & 1.3 & & & & & & 159 & \\
\hline & $6 / 16 / 2004$ & & & 26.77 & 24.59 & & & & & $\mid 16.118$ & & -1.1 & & 49.31 & & 14.6 & & & & 51 & 2.1 & & & & & & 0.163 & \\
\hline & $6 / 16 / 2$ & & & & 24.60 & & & & & & & & & & & & & & & 51.6 & 2.2 & & & & & & & \\
\hline & 6/16/2004 & 5:52: & 26.284 & 26.77 & 24.60 & 27.915 & & 48.791 & 53.452 & 15.971 & 33. & -1.0 & & & 0.044 & 14. & & & & & 11.5 & & & & & & & \\
\hline 10 & 6/116/2004 & & 26.294 & 26.77. & 24.6 & & & & & & & & & & & & & & & & tis & & & & & & & \\
\hline & & & & 26.7 & 24.6 & 27.8 & & & & & & & & & & & & & & 51.6 & 10 & & & & & & & \\
\hline & 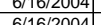 & & & & 24.6 & & & & & & & & & & & & & & & & & & & & & & & \\
\hline & $6 / 16 / 200$ & & & & $\frac{24.0}{246}$ & 27.67 & & & & & & & & & & & & & & & & & & & & & & \\
\hline 271 & $6 / 16 / 200$ & & & & $\frac{24.0}{246}$ & 27.6 & & $\begin{array}{l}40.40 \\
48.28\end{array}$ & & 161 & & -1.0 & & & & & & & & 554 & $\frac{0.30}{20}$ & & & & & & & \\
\hline & $6 / 16 / 2$ & 5:59: & 26.318 & 26.75 & $\frac{2.4 .7}{24.7}$ & $\frac{1.7 .6}{27.6}$ & & 48 & \begin{tabular}{|l|}
53.291 \\
\end{tabular} & 10.3 & & -1.025 & & & & & & & & $\frac{1.18}{51.8}$ & 12. & & & & & & & \\
\hline $2 / 1$ & $6 / 16 / 2004$ & & & & & & & & & & & & & & & & & & & & & & & & & & & \\
\hline & & & & & & & & & & & & & & & & & & & & & & & & & & & & \\
\hline & $6 / 16 / 2004$ & & & 26.7 & 24.78 & 27.5 & & & & & & & & & & 14. & & & & & & & & & & & & \\
\hline & $6 / 16 / 2$ & 6:03 & 26.34 & 26.7 & 24.79 & 27.612 & & 48.5 & 53.083 & 16. & 2.918 & $-0.9 \subseteq$ & & 45.4 & & 14.6 & & & & & 1. & & & & & & & \\
\hline & 6/16/2 & & 26.343 & & 24.7 & 27.57 & & 48.38 & & 16.076 & & -0.9 & & & & 14. & & & & & 2 . & & & & & & 159 & \\
\hline & $6 / 16 / 2$ & & & 26.69 & 24.7 & 27.5. & & & & & & & & & & & & & & & & & & & & & & \\
\hline & $6 / 16 / 2$ & & 26.3 & 26.69 & 24.8 & & & 48.2 & & & 32. & -0. & & & & & & & & 51 & 2.2 & & & & & & 0.163 & \\
\hline $2 \pi$ & 6/116/2004 & & & & & & & & & & & & & & & & & & & & & & & & & & & \\
\hline & $66116 / 2004$ & & (3) & 26.69 & 24.8 & 27.68 & & & & 10 & & & & & & & & & & & & & & & & & & \\
\hline & $\frac{61 / 60 / 2}{61 / 2 \pi}$ & & & & 2.82 & $\frac{27.160}{2701}$ & & 48.36 & $\frac{53.073}{5.525}$ & 10.0 & 32.823 & -0.959 & & & & & & & & & & & & & & & & \\
\hline & 01012004 & & & 20.00 & 24. & 27.04 & & 40.14 & 50.45 & & 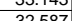 & -0.941 & & & & & & & & & & & & & & & & \\
\hline & 34 & 6.12 .03 & 26409 & 2670 & 24.13 & 27.91 & & 48.377 & 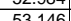 & & $\frac{3 x}{32}$ & -0.5 & & & & & & & & & & & & & & & & \\
\hline & & & & 26.7 & 2482 & 28.017 & & 48.3 & 53.106 & & 32715 & -0.939 & & & & 14. & & & & & 20 & & & & & & & \\
\hline 73 & 6/16/2004 & & $\frac{6.421}{6.42}$ & .71 & 24.80 & 28.022 & & \begin{tabular}{|l|l|}
48.694 \\
\end{tabular} & \begin{tabular}{|l|}
53.407 \\
\end{tabular} & $\mid$ & 33.094 & -0.939 & & & 1043 & $\frac{14.045}{14.643}$ & & & & 819 & 210 & & & 006 & 006 & 1000 & 158 & \\
\hline
\end{tabular}


WSRC-TR-2005-00105, REVISION 0

SRNL-RPP-2005-00012, REVISION 0

RUN \# 3.03A AND B; FIRST AND SECOND HALF OF SLURRY DEWATERING - CONT.

\begin{tabular}{|c|c|c|c|c|c|c|c|c|c|c|c|c|c|c|c|c|c|c|c|c|c|c|c|c|c|c|c|c|}
\hline & A & B & D & $E$ & $F$ & G & $\mathrm{H}$ & $\mathrm{J}$ & $\mathrm{K}$ & $\frac{L}{L}$ & $\mathrm{M}$ & $\mathrm{N}$ & 0 & $Q$ & $\mathrm{R}$ & $\mathrm{s}$ & T & 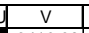 & & $x$ & & & $A A$ & & $A C$ & $A D$ & $\mathrm{AE}$ & \\
\hline 2731 & $6 / 16 / 2004$ & 6:14:42 PM & 26.432 & 26.72 & 24.79 & $\begin{array}{l}28.043 \\
27034\end{array}$ & $\begin{array}{l}26.873 \\
2.70\end{array}$ & $\begin{array}{l}48.644 \\
\end{array}$ & 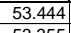 & $\begin{array}{l}16.055 \\
\end{array}$ & $\begin{array}{l}33.049 \\
22011\end{array}$ & -0.939 & -7.005 & \begin{tabular}{|l|l|}
51.567 \\
\end{tabular} & 0.043 & $\begin{array}{l}14.643 \\
1.620\end{array}$ & 0.004 & & 56.9997 & 52.0 & 12.6 & & 2.816 & 0.006 & 0.006 & 0.000 & 0.158 & \\
\hline$\frac{2733}{2733}$ & $6 / 16 / 2004$ & 6:15:42 PM & $\frac{26.442}{26.442}$ & $\frac{26.73}{26.73}$ & $\frac{24.82}{24.81}$ & \begin{tabular}{|r|r|}
27.994 \\
27.929
\end{tabular} & 26.794 & $\begin{array}{r}48.665 \\
4858\end{array}$ & \begin{tabular}{|l|}
53.355 \\
53.427 \\
\end{tabular} & \begin{tabular}{|l|}
16.037 \\
16162
\end{tabular} & $\begin{array}{r}33.111 \\
332844\end{array}$ & $\begin{aligned}-0.939 \\
-0.939\end{aligned}$ & $\begin{array}{l}-6.999 \\
-7005\end{array}$ & $\begin{array}{r}49.26 \\
48.684 \\
\end{array}$ & 0.044 & $\begin{array}{l}14.643 \\
14643\end{array}$ & 0.004 & $\begin{array}{l}3420.98 \\
342109\end{array}$ & \begin{tabular}{|l|l|}
57.0164 \\
5703231
\end{tabular} & 51.9 & 12.1 & 40. & $\frac{2.819}{207}$ & 0.007 & 0.007 & & $\frac{0.161}{0.162}$ & \\
\hline 2734 & $6 / 16 / 2004$ & & & & 24.81 & & 20.174 & $\begin{array}{r}4.536 \\
48.534 \\
\end{array}$ & \begin{tabular}{|l|}
53.4262 \\
53.26 \\
\end{tabular} & $\begin{array}{l}1.102 \\
16.093\end{array}$ & $\begin{array}{l}32.844 \\
32.873 \\
\end{array}$ & $\begin{array}{l}-.0 .959 \\
-0.9399\end{array}$ & $\begin{array}{l}-7.005 \\
-7.002\end{array}$ & $\begin{array}{l}48.684 \\
47.869\end{array}$ & \begin{tabular}{|l}
.044 \\
0.043
\end{tabular} & $\begin{array}{l}14.643 \\
14.643 \\
\end{array}$ & $\frac{0.004}{0.004}$ & & $\begin{array}{l}57.0331 \\
57.0497\end{array}$ & $\begin{array}{l}52.0 \\
51.8 \\
\end{array}$ & \begin{tabular}{|l|}
11.9 \\
11.7
\end{tabular} & & $\frac{2.807}{2.806}$ & \begin{tabular}{|l|}
0.0007 \\
0.006
\end{tabular} & $\begin{array}{l}0.007 \\
0.006\end{array}$ & $\frac{0.000}{0.000}$ & $\frac{0.162}{0.158}$ & 0.14 \\
\hline 2735 & $6 / 16 / 2004$ & 6:18:42 PM & 26.441 & 26.7 & 24.80 & 27.787 & 26.652 & $\begin{array}{l}8.192 \\
\end{array}$ & \begin{tabular}{|l|}
52.872 \\
\end{tabular} & 15.971 & 32.729 & -0.939 & -7.002 & 49.827 & 0.044 & 14.643 & & 3423.98 & \begin{tabular}{|l|l|}
57.0664 \\
\end{tabular} & 51.4 & \begin{tabular}{|l|l|}
12.2 \\
\end{tabular} & & 2.790 & \begin{tabular}{|l|}
0.007 \\
\end{tabular} & & & 0.163 & \\
\hline 2736 & $6 / 16 / 2004$ & 6:19:42 PM & 26.454 & 26.74 & 24.81 & 27.766 & 26.606 & 48.617 & \begin{tabular}{|l|l|}
53.618 \\
\end{tabular} & 16.111 & 32.836 & -0.939 & -7.011 & 49.677 & 0.043 & $\begin{array}{l}14.643 \\
\end{array}$ & & & 57.0831 & & & & & & & & & \\
\hline & $6 / 16 / 2004$ & & 26.448 & 26.73 & 24. & \begin{tabular}{|l|}
27.745 \\
\end{tabular} & 26.575 & 48.52 & \begin{tabular}{|l|l|}
53.224 \\
5.24
\end{tabular} & 16.031 & & -0.939 & & & 0.044 & $\begin{array}{l}14.643 \\
\end{array}$ & & & 57.0997 & 51.8 & & & & 0.007 & & & & \\
\hline 2738 & $6 / 16 / 2004$ & 6:21:42 PM & 26.463 & 26.75. & 24.8 & \begin{tabular}{l|l|}
27.745 \\
77
\end{tabular} & $\begin{array}{r}26.55 \\
6521\end{array}$ & $\begin{array}{r}48.352 \\
\end{array}$ & $\begin{array}{l}53.141 \\
5.252\end{array}$ & $\begin{array}{ll}16.016 \\
1.06\end{array}$ & $\begin{array}{l}32.809 \\
2508\end{array}$ & -0.912 & $\begin{array}{r}-7.005 \\
-7005\end{array}$ & 46.895 & 0.043 & 14.643 & & \begin{tabular}{|l|l|}
3426.98 \\
\end{tabular} & 57.1164 & 51.7 & 11.5 & & 2.798 & 0.006 & 0.006 & 0.000 & 0.159 & \\
\hline 2739 & 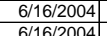 & $\begin{array}{ll}6: 22: 42 \mathrm{PM} \\
6 \cdot 62: 2: 2 \mathrm{P}\end{array}$ & $\begin{array}{l}26.454 \\
26.453\end{array}$ & $\begin{array}{l}26.73 \\
26.72 \\
267\end{array}$ & $\begin{array}{l}24.82 \\
24.81\end{array}$ & $\begin{array}{l}27.671 \\
27.625\end{array}$ & & \begin{tabular}{|l|l|}
48.279 \\
48.468
\end{tabular} & \begin{tabular}{|l|l|}
53.253 \\
5.37 \\
\end{tabular} & \begin{tabular}{|l|l|}
16.076 \\
1602
\end{tabular} & $\begin{array}{l}32.598 \\
32095\end{array}$ & \begin{tabular}{|r|}
-0.93 \\
\end{tabular} & $\begin{array}{l}-7.005 \\
.7002\end{array}$ & $\begin{array}{l}48.678 \\
5.575\end{array}$ & $\begin{array}{l}0.043 \\
0.043\end{array}$ & $\begin{array}{l}14.643 \\
1.643\end{array}$ & & \begin{tabular}{|l|}
3427.98 \\
32280.9
\end{tabular} & \begin{tabular}{|l|l|}
57.1331 \\
571907
\end{tabular} & 51.8 & \begin{tabular}{|l|l|}
11.9 \\
126
\end{tabular} & & & & 0.006 & & 0.159 & \\
\hline$\frac{2740}{2741}$ & $\frac{6 / 1 / 162004}{6 / 16 / 2004}$ & 6:23:42 PM & $\begin{aligned} 26.453 \\
26.447\end{aligned}$ & $\frac{26.72}{26.72}$ & 24.81 & $\begin{array}{l}27.625 \\
27.609 \\
\end{array}$ & $\frac{26.475}{26.41}$ & \begin{tabular}{|l}
48.468 \\
48.422
\end{tabular} & \begin{tabular}{|l|}
53.376 \\
53.307 \\
\end{tabular} & $\begin{array}{r}16.043 \\
15.976\end{array}$ & $\begin{array}{r}32.885 \\
32885 \\
\end{array}$ & $\begin{array}{r}-0.93 \\
-0.939\end{array}$ & $\begin{array}{l}-7.002 \\
-7.002\end{array}$ & $\begin{array}{r}51.575 \\
49.298\end{array}$ & $\begin{array}{l}0.043 \\
0.044\end{array}$ & $\begin{array}{l}14.643 \\
14.643 \\
\end{array}$ & $\begin{array}{l}0.004 \\
0.004\end{array}$ & \begin{tabular}{|c|}
3428.98 \\
3429.98 \\
\end{tabular} & \begin{tabular}{|l|l|}
57.1497 \\
57.1664
\end{tabular} & 51.9 & $\frac{12.6}{12.1}$ & $\frac{40.7}{40.7}$ & 2.805 & 0.006 & $\begin{array}{l}0.006 \\
0.007\end{array}$ & $\begin{array}{l}0.000 \\
0.000\end{array}$ & 0.158 & \\
\hline \begin{tabular}{|l|}
2742 \\
\end{tabular} & $6 / 16 / 2004$ & 6:25:42 PM & & 26.70 & 24.82 & 27.494 & 26.354 & 48.58 & \begin{tabular}{|l|}
53.357 \\
\end{tabular} & 16.068 & 32.928 & & & & $\begin{array}{l}0.044 \\
0.042\end{array}$ & $\begin{array}{l}14.045 \\
14.643\end{array}$ & & \begin{tabular}{|}
$\mid \begin{array}{l}3424.98 \\
3430.98\end{array}$ \\
\end{tabular} & \begin{tabular}{|l|l|}
57.16064 \\
57.1831
\end{tabular} & 51.8 & $\frac{12.1}{12.7}$ & & & & $\begin{array}{l}0.006 \\
0.006\end{array}$ & & $\begin{array}{l}0.162 \\
0.154 \\
\end{array}$ & $\begin{array}{l}0.14 \\
0.13\end{array}$ \\
\hline & $6 / 16 / 2004$ & 6:26:42 PM & 26.441 & 26.7. & 24.82 & \begin{tabular}{|l|l|}
27.513 \\
\end{tabular} & 26.348 & 48.547 & 53.309 & $\begin{array}{l}16.088 \\
\end{array}$ & 32.875 & -0.904 & -7.013 & 48.426 & 0.041 & $\begin{array}{l}14.643 \\
\end{array}$ & & 3431.98 & 57.1997 & 51.8 & 11.9 & 40.7 & 2.807 & 0.006 & 0.006 & 0.000 & 0.151 & \\
\hline & $6 / 16 / 2004$ & $6: 27: 42 \mathrm{PM}$ & 26.434 & & & & 26.396 & 48.511 & 53.376 & 16.106 & 32.844 & -0.884 & & 46.465 & 0.043 & 14.643 & & & 57.2164 & 51.9 & & & & 0.006 & 0.006 & & 0.158 & \\
\hline 2745 & $6 / 16 / 2004$ & $6: 28: 42 \mathrm{PM}$ & 26.423 & 26.66 & 24.8 & 27.515 & 26.436 & 48.671 & 53.562 & 16.105 & 33.027 & & -7.002 & 49.179 & 0.043 & $\begin{array}{l}14.643 \\
\end{array}$ & & \begin{tabular}{|l|l|}
3433.98 \\
\end{tabular} & 57.2331 & & 12.1 & & 2.816 & 0.006 & 0.006 & 0.000 & 0.158 & \\
\hline \begin{tabular}{|l|l|}
2746 \\
2747
\end{tabular} & $6 / 16 / 2004$ & $\begin{array}{ll}6: 29: 42 \mathrm{PM} \\
6 \cdot 63: 42 \mathrm{PM}\end{array}$ & $\begin{array}{l}26.418 \\
26.422\end{array}$ & $\frac{26.66}{26.64}$ & 24.83 & 27.594 & $\begin{array}{l}26.485 \\
26509\end{array}$ & \begin{tabular}{|l|l|}
48.715 \\
48.497
\end{tabular} & $\begin{array}{r}53.405 \\
53.456\end{array}$ & \begin{tabular}{|l|}
16.179 \\
16176
\end{tabular} & $\begin{array}{l}32.998 \\
32.715\end{array}$ & -0. & $\begin{array}{l}-7.0 \\
-7.0\end{array}$ & $\begin{array}{l}52.773 \\
6705\end{array}$ & 0.043 & $\begin{array}{l}14.643 \\
11.643\end{array}$ & & $\mid$ & \begin{tabular}{|l|}
57.2497 \\
572664
\end{tabular} & 51.9 & $\begin{array}{l}12.9 \\
11.4\end{array}$ & 40.9 & \begin{tabular}{|l|}
2.817 \\
2800
\end{tabular} & \begin{tabular}{|l|l|}
0.006 \\
0006
\end{tabular} & $\begin{array}{l}0.006 \\
0.06\end{array}$ & $\begin{array}{l}0.000 \\
\end{array}$ & $\begin{array}{l}0.158 \\
0.155\end{array}$ & 10 \\
\hline$\frac{2747}{2748}$ & $6 / 11 / 26 / 2044$ & $\begin{array}{l}6: 30: 42 \mathrm{PM} \\
6: 31: 42 \mathrm{PM}\end{array}$ & $\frac{26.422}{26.426}$ & $\frac{26.64}{26.6}$ & $\frac{24.81}{2481}$ & $\begin{array}{l}27.648 \\
27.673\end{array}$ & $\frac{26.509}{26.543}$ & $\begin{array}{l}48.497 \\
48.586\end{array}$ & & $\begin{array}{l}16.116 \\
16.276\end{array}$ & 32.715 & $\begin{array}{l}-0.8866 \\
-0.884\end{array}$ & & $\frac{46.105}{47.352}$ & $\begin{array}{l}0.042 \\
0.045\end{array}$ & $\frac{14.643}{14.643}$ & & \begin{tabular}{|c|}
3436.68 \\
\end{tabular} & \begin{tabular}{|l|l|}
57.2664 \\
57.2831
\end{tabular} & & & & & \begin{tabular}{|l|l|}
0.006 \\
0.007
\end{tabular} & & & & \\
\hline 2749 & $6 / 16 / 2004$ & 6:32:42 PM & 26.42 & 26.63 & $\frac{24.01}{24.83}$ & 27.717 & 26.577 & 48.559 & \begin{tabular}{|l|}
53.334 \\
\end{tabular} & 16.203 & 32.823 & -0.886 & -6.999 & 48.881 & $\begin{array}{l}0.044 \\
\end{array}$ & $\frac{14.045}{14.643}$ & & 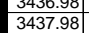 & \begin{tabular}{|l|l|}
57.283911 \\
5
\end{tabular} & $\frac{51.9}{51.9}$ & $\frac{11.6}{12.0}$ & \begin{tabular}{|l|}
40.7 \\
0.7
\end{tabular} & \begin{tabular}{|l|}
2.803 \\
2.806 \\
\end{tabular} & \begin{tabular}{|l|}
0.007 \\
0.007
\end{tabular} & $\begin{array}{l}0.007 \\
0.007\end{array}$ & 0 & $\begin{array}{l}0.166 \\
0.162 \\
\end{array}$ & 0. \\
\hline & $6 / 16 / 2004$ & 6:33:42 PM & 26.441 & 26.66 & 24.86 & 27.752 & & 48.569 & \begin{tabular}{|l|}
53.5355 \\
\end{tabular} & 16.214 & 32.749 & -0.884 & -6.999 & 47.153 & 0.044 & 14.643 & & 3438.98 & 57.3164 & 52.1 & 11.6 & 40.7 & \begin{tabular}{|l|}
2.803 \\
\end{tabular} & 0.007 & 0.007 & 0.000 & 0.162 & \\
\hline 2751 & $6 / 16 / 2004$ & 6:34:42 PM & 26.41 & 26.62 & 24.81 & 27.787 & 26.647 & 48.76 & \begin{tabular}{|l|l|}
53.618 \\
\end{tabular} & 16.355 & 32.817 & & & 48.13 & 0.043 & $\begin{array}{l}14.643 \\
\end{array}$ & & & 57.3331 & 52.1 & & 40.8 & \begin{tabular}{|l|l|}
2.812 \\
\end{tabular} & 0.006 & & 0.000 & 0.158 & \\
\hline 2752 & $6 / 16 / 2004$ & 6:35:42 PM & 26.437 & 26.65 & 24.86 & $\begin{array}{l}27.768 \\
27.705\end{array}$ & \begin{tabular}{|l|l|}
26.688 \\
6.72
\end{tabular} & 48.584 & \begin{tabular}{|l|l|}
53.465 \\
5.67 \\
\end{tabular} & $\begin{array}{l}16.206 \\
1.202\end{array}$ & $\begin{array}{l}32.844 \\
2756\end{array}$ & & \begin{tabular}{|l|l|}
-7.005 \\
701
\end{tabular} & 53.215 & 0.042 & 14.643 & & & \begin{tabular}{|l|l|}
57.3497 \\
\end{tabular} & 52.0 & 13.0 & & \begin{tabular}{|l|}
2.807 \\
\end{tabular} & 0.006 & 0.006 & 0.000 & 0.154 & \\
\hline & 6/16/2004 & $\begin{array}{l}6: 36: 42 \mathrm{PM} \\
6 \\
6 \cdot 673: 02\end{array}$ & $\frac{26.428}{26.444}$ & 26.64 & $\frac{24.8}{24.87}$ & 27.785 & $\begin{array}{r}26.73 \\
26.756\end{array}$ & \begin{tabular}{|l|l|l|}
487013 \\
48704
\end{tabular} & \begin{tabular}{|l|}
53.672 \\
53.492
\end{tabular} & \begin{tabular}{|l|}
16.239 \\
16255
\end{tabular} & $\begin{array}{r}32.756 \\
330955\end{array}$ & & & $\begin{array}{l}49.337 \\
48.097\end{array}$ & 0.041 & $\begin{array}{l}14.643 \\
14643\end{array}$ & 0.00 & \begin{tabular}{|l|}
3441.98 \\
34420.9
\end{tabular} & \begin{tabular}{|l|}
57.3664 \\
53831
\end{tabular} & 52.2 & $\begin{array}{l}12.1 \\
120\end{array}$ & & $\begin{array}{l}2.805 \\
2815\end{array}$ & $\begin{array}{l}0.006 \\
0.06\end{array}$ & $\begin{array}{l}0.006 \\
\end{array}$ & 0.000 & 0.151 & 12 \\
\hline & 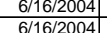 & $\begin{array}{l}6: 37: 42 \mathrm{PM} \\
6 \cdot 630.42 \mathrm{P}\end{array}$ & 26.444 & $\frac{26.66 .}{26.65}$ & 24.87 & 27.836 & 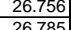 & $\begin{array}{r}48.704 \\
48.33 \\
\end{array}$ & \begin{tabular}{|l|}
53.492 \\
53.231 \\
\end{tabular} & $\begin{array}{l}16.265 \\
11.255 \\
\end{array}$ & $\begin{array}{r}32.955 \\
33.694\end{array}$ & & & $\begin{array}{l}48.997 \\
48.924\end{array}$ & 0.042 & $\begin{array}{l}14.643 \\
14643\end{array}$ & & \begin{tabular}{|c|}
3442.98 \\
344309 \\
343
\end{tabular} & \begin{tabular}{|l|}
57.3831 \\
573997
\end{tabular} & 52.0 & $\frac{12.0}{120}$ & \begin{tabular}{|l|l|}
40.8 \\
40.
\end{tabular} & 2.815 & 0.006 & 0.006 & 0.000 & 0.154 & \\
\hline \begin{tabular}{l|l}
2756 \\
\end{tabular} & $6 / 116 / 2004$ & $\begin{array}{l}6.83: 42 \mathrm{PM} \\
6: 39: 42 \mathrm{PM}\end{array}$ & $\frac{26.429}{26.434}$ & $\frac{26.65}{26.66}$ & $\begin{array}{l}24.85 \\
24.89\end{array}$ & $\frac{27.821}{27.905}$ & $\frac{26.185}{26.775}$ & $\begin{array}{r}48.43 \\
48.435 \\
\end{array}$ & \begin{tabular}{|r|}
53.231 \\
53.34 \\
\end{tabular} & \begin{tabular}{|l|l|l}
16.295 \\
169
\end{tabular} & $\begin{array}{r}32.694 \\
32.548 \\
\end{array}$ & $\frac{-0.845}{-0.878}$ & $\frac{-1.016}{-6.99}$ & $\frac{48.924}{50.309}$ & 0.042 & $\frac{14.643}{14.643}$ & & & \begin{tabular}{|l|l|}
57.499764 \\
57164
\end{tabular} & $\frac{51.8}{51.9}$ & $\frac{12.0}{12.3}$ & 40.6 & \begin{tabular}{|l|}
2.797 \\
2.792 \\
\end{tabular} & \begin{tabular}{|l|}
0.006 \\
0.007
\end{tabular} & $\begin{array}{l}0.006 \\
0.007\end{array}$ & & $\frac{0.155}{0.166}$ & 0.13 \\
\hline 2757 & $6 / 16 / 2004$ & 6:40:42 PM & 26.448 & 26.68 & 24.9 & 27.935 & 26.775 & 48.526 & 53.357 & 16.399 & 32.579 & -0.872 & -6.996 & 47.915 & 0.044 & 14.643 & & 3445.98 & 57.4331 & 51.9 & 11.7 & 40.6 & \begin{tabular}{|l|}
2.796 \\
\end{tabular} & 0.007 & $\begin{array}{l}0.007 \\
\end{array}$ & 0.000 & .162 & \\
\hline 2758 & $6 / 16 / 2004$ & 6:41:42 PM & 26.443 & 26.68 & 24.89 & 27.834 & 26.709 & 48.571 & & 16.336 & 32.729 & -0.878 & & 49.512 & 0.043 & $\begin{array}{l}14.643 \\
\end{array}$ & & \begin{tabular}{|l|l|}
3446.98 \\
\end{tabular} & 57.4497 & 51.8 & 12.1 & \begin{tabular}{|l|l|}
40.7 \\
\end{tabular} & & 0.006 & 0.006 & & & \\
\hline 2759 & & & 26.448 & 26.68 & 24.9 & 27.73 & & $\begin{array}{l}48.713 \\
\end{array}$ & & 16.303 & 32.827 & & & & & $\begin{array}{l}14.643 \\
\end{array}$ & & & \begin{tabular}{|l|l|}
57.4664 \\
\end{tabular} & 52.8 & & & 2.81. & 0.007 & & & & \\
\hline 2760 & 6/116/2004 & & 26.47 & 26.69 & 24.95 & & & & 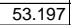 & & & & & & & \begin{tabular}{|l}
14.643 \\
\end{tabular} & & & & & & & & & & & & \\
\hline & 6/16/2004 & 6:44:42 PM & & 26.68 & 24.93 & & & 48.534 & & & & & & & & 14.6 & & & & & 1.9 & & 2798 & & & & & \\
\hline & 6/16/2004 & & 26.465 & 26.67 & 24.92 & 27.637 & & 48.43 & 53.241 & 16.327 & 32.555 & -0.817 & & 0.48 & & $\begin{array}{l}14.643 \\
\end{array}$ & & & & 51.8 & 2.4 & & & .006 & .006 & & & \\
\hline & & & & 26.67 & 24.94 & 27.598 & & 48.42 & 53.27 & $\begin{array}{l}16.276 \\
\end{array}$ & & & & & .038 & $\begin{array}{l}14.643 \\
\end{array}$ & & & & & 2.1 & & & & & & & \\
\hline 276 & 6/16/2004 & 6: & 26.466 & 26.67: & 24.93 & 27.568 & 26.438 & 48.52 & 53.411 & 16.29 & 32.665 & -0.817 & & 48.749 & 0.043 & 14.643 & & $3452.9 \varepsilon$ & & 51.9 & 11.9 & & & 0.006 & 0.006 & 0.000 & 0.158 & \\
\hline & 6/2004 & & & 26.64 & & 27.557 & & 47.93 & 52.814 & 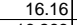 & & & & & & & & & & & & & & & & & & \\
\hline & & & & 26.64 & 24.91 & 27.492 & & 48.528 & 53.206 & 16.263 & 32.749 & -0. & & & & & & & & & 2.4 & & & & & & .158 & \\
\hline & 6 & 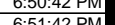 & $\begin{array}{l}26.449 \\
26457\end{array}$ & $\frac{26.63}{26.32}$ & 24.90 & 2 & 2.606 & $\begin{array}{r}48.6 \\
18480\end{array}$ & $\begin{array}{l}53.143 \\
5.473\end{array}$ & 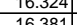 & $\frac{32.618}{32512}$ & & & & & $\frac{11.6}{14.6}$ & & & & 50 & . & & & 00 & مी & & 0.151 & \\
\hline & $6 / 16 / 2$ & & & 26.03 & 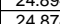 & 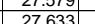 & & & & & & & & & & 146 & & & & & 1.9 & & & & & & & \\
\hline & $6 / 16 / 2004$ & & 20.401 & 20.00 & 24.90 & 27.066 & & 48.33 & 53287 & 16185 & & & & & & 14. & & & & & 25 & & & & & & 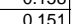 & \\
\hline 2771 & $6 / 16 / 2004$ & 6:54: & 26.463 & 26.62 & 24.89 & 27.719 & & 48.435 & \begin{tabular}{|l|l|}
53.305 \\
\end{tabular} & $\mid 16.168$ & 32.725 & & & & $\frac{.0442}{0.042}$ & 14.6 & & & & 51.8 & 1.7 & & 2798 & 0.006 & $\frac{0.006}{0.006}$ & 0.000 & D.1.155 & \\
\hline 2772 & & & & & & & & & & & & & & & & & & & & & & & & & & & & \\
\hline 2773 & $6 / 16 / 2004$ & & 26.464 & 26.62 & 24.89 & 27.7 & & & 53.206 & $\frac{1.6232}{11.232}$ & & & & & & & & & & & 6 & & & & & & & \\
\hline & $6 / 16 / 2$ & & 26.4 & 26.62 & 24.8 & & & 48.105 & 53.139 & $\begin{array}{l}16.18 \\
\end{array}$ & & & & & & & & & & & & & & & & & & \\
\hline & $6 / 16 / 2$ & & & 26.6 & 24.90 & 27.7 & & 48.44 & & 16.281 & & & & & & & & & & & 2. & & & & & & 0.155 & \\
\hline & $6 / 16 / 2004$ & 6:59: & 26.464 & 26.62 & 24.85 & & & $\begin{array}{l}48.484 \\
\end{array}$ & & & & & & & & 14.6 & & & & 52 & 1.7 & & & & & & 0.159 & \\
\hline & $6 / 16 / 2$ & $7: 00:$ & 26.479 & 26.63 & & 27.801 & & 48. & & & & & & & & & & & & & 2.0 & & & & & & & \\
\hline 27 & 6/16/2004 & 7:01: & 26.475 & 26.63 & 24.89 & 27.841 & & 48.221 & 53.208 & 16.2 & 32. & & & & & 14. & & & & & 11.9 & & & & & & & \\
\hline & $6 / 16 / 2004$ & & & 26.64 & & & & & & & & & & & & & & & & & & & & & & & & \\
\hline & & & & & & 2.7 .14 & & & & & & & & & & & & & & & & & & & & & & \\
\hline & 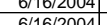 & & 20.49 & & 24.9 & & & & & & & & & & & & & & & & & & & & & & $\frac{55}{57}$ & \\
\hline & $6 / 16 / 200$ & $=0.036$ & $\frac{20.474}{26485}$ & $\frac{2.06}{26.39}$ & $\frac{24.6}{248}$ & 27.0 & & & & & & & & & & & & & & & & & & & & & & \\
\hline & $61 / 6120$ & & & & & 274 & & 年 & & & & & & & & & & & & & $\frac{1.1}{16}$ & & & & & & & \\
\hline & $\frac{1 / 1612004}{6}$ & $7: 08:$ & $\frac{20.464}{26.464}$ & $\frac{20.60}{26.60}$ & 24.8 & 27.5 & & 48 & $\begin{array}{l}53.452 \\
53.452\end{array}$ & 16. & & & & & & & & & & & 120 & & & & & & & \\
\hline & $6 / 116 / 2004$ & & & & & & & & & & & & & & & & & & & & & & & & & & & \\
\hline & $6 / 16 / 2004$ & & & & & & & & & & & & & & & & & & & & & & & & & & & \\
\hline & $6 / 16 / 2004$ & $7 \cdot 11$ & 26 & & 2487 & 27.47 & & & & & & & & & & 14. & & & & & & & & & & & & \\
\hline & $6 / 16 / 2$ & $7: 12: 42$ & 26.4 & 26.5 & 24.89 & 27.559 & & 48.277 & 53.315 & 16.2 & & & & & & 14.6 & & & & & & & & & & & & \\
\hline & $6 / 16 / 20$ & 7:13: & 26.445 & 26.56 & 24.8 & 27.58 & & 48.2 & & $16.19 !$ & & & & & & 14. & & & & & $1.2 \mathrm{Y}$ & & & & & & & \\
\hline & $6 / 16 / 2$ & 7:14: & & & 24. & & & 48. & & & & & & & & & & & & & .8 & & & & & & & \\
\hline & $6 / 16 / 2$ & & 26. & 26.5 & 24.9 & 27.639 & & 48.41 & 396 & 16.252 & & & & & & & & & & & 2.3 & & & & & & 0.1. & \\
\hline & 6/16/2004 & & & 26. & 24. & 27.6 & & & & & & & & & & & & & & & & & & & & & & \\
\hline & $66116 / 2004$ & 7:1: & tot & 26.55 & 24.92 & 27.71 & & 48.271 & 53.28 & 16. & & & & & & & & & & & & & & & & & & \\
\hline & 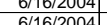 & $\frac{7.180 .42}{7.042}$ & 6150 & 85 & 24.92 & $\frac{2.1355}{27.725}$ & & 48.009 & $\frac{53.1233}{5.219}$ & 16. & & & & & & & & & & & & & & & & & & \\
\hline & 011012004 & $\frac{1.19 .44}{7.20 .1}$ & 20.4544 & $\frac{2.5}{26.5}$ & 24.94 & 27.1507 & & 40.4 & 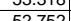 & & & & & & & & & & & & & & & & & & & \\
\hline & $6 / 16 t$ & $7 \cdot 21 \cdot 4$ & 26.45 & 2656 & $\frac{24.31}{2490}$ & 27776 & & 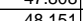 & 53137 & & & & & & & & & & & & & & & & & & & \\
\hline & & $7.22 \cdot 4$ & $466-2$ & 265 & 24.91 & 27.773 & & 482 & 53,419 & & & & & & & & & & & & 20 & & & & & & & \\
\hline & 6/16/2004 & & 5.471 & 5.58 & \begin{tabular}{|l|}
24.90 \\
\end{tabular} & 27.792 & & 48.07 & 53.038 & $\mid 16.274$ & 306 & -0.649 & & & 1042 & $\frac{14.045}{14.643}$ & & & & & $20-2$ & & & 006 & 006 & 1000 & 156 & \\
\hline
\end{tabular}


WSRC-TR-2005-00105, REVISION 0

SRNL-RPP-2005-00012, REVISION 0

RUN \# 3.03A AND B; FIRST AND SECOND HALF OF SLURRY DEWATERING - CONT.

\begin{tabular}{|c|c|c|c|c|c|c|c|c|c|c|c|c|c|c|c|c|c|c|c|c|c|c|c|c|c|c|c|c|}
\hline & A & $\mathrm{B}$ & D & $\mathrm{E}$ & 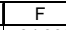 & $G$ & $\mathrm{H}$ & & $\mathrm{K}$ & $L^{-}$ & $\mathrm{M}$ & $\mathrm{N}$ & 0 & 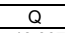 & $\mathrm{R}$ & & T & 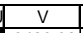 & & $x$ & & 2 & $A A$ & & $A C$ & $A D$ & $\mathrm{AE}$ & \\
\hline 2801 & 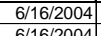 & 7:24:42 PM & $\begin{array}{l}26.461 \\
26.466\end{array}$ & 26. & 24.866 & $\begin{array}{l}27.763 \\
27663\end{array}$ & $\begin{array}{l}26.633 \\
2.563\end{array}$ & \begin{tabular}{|l|l|}
48.565 \\
4814
\end{tabular} & $\begin{array}{l}53.767 \\
53238\end{array}$ & \begin{tabular}{|l|l|}
16.304 \\
6269
\end{tabular} & $\begin{array}{l}32.682 \\
32255\end{array}$ & $\begin{array}{l}-0.652 \\
-0.65\end{array}$ & $\begin{array}{l}-6.785 \\
-6779\end{array}$ & $\begin{array}{l}46.607 \\
5.156\end{array}$ & $\begin{array}{l}0.041 \\
0041\end{array}$ & $\begin{array}{l}14.643 \\
1.643\end{array}$ & $\begin{array}{l}0.004 \\
0.004\end{array}$ & \begin{tabular}{|l|l|}
34899.98 \\
34909
\end{tabular} & \begin{tabular}{|l|l|}
58.1664 \\
581833 \\
\end{tabular} & 52.3 & 11.4 & 30 & 2.801 & 0.006 & $\begin{array}{l}0.006 \\
0\end{array}$ & 0.000 & $\begin{array}{l}0.151 \\
\end{array}$ & \\
\hline 2803 & $6 / 1 / 16 / 2004$ & $\begin{array}{ll}7: 25: 42 \mathrm{PM} \\
7726: 42 \mathrm{PM}\end{array}$ & 26.466 & 26. & 24.89 & $27.663 \mid$ & $\frac{26.563}{26518}$ & 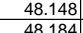 & \begin{tabular}{|l|}
53.228 \\
53309
\end{tabular} & \begin{tabular}{|l|}
16.269 \\
16209 \\
\end{tabular} & $\begin{array}{r}32.255 \\
33.333 \\
3\end{array}$ & $\begin{array}{l}-0.655 \\
-0.055 \\
-\frac{1}{2}\end{array}$ & $\begin{array}{l}-6.779 \\
-67774\end{array}$ & 51.536 & $\begin{array}{l}0.041 \\
0.043\end{array}$ & $\begin{array}{l}14.643 \\
11.643 \\
\end{array}$ & 0.004 & $\begin{array}{l}3490.98 \\
349198\end{array}$ & \begin{tabular}{|l|}
58.1831 \\
5.109077
\end{tabular} & 51.8 & 12.6 & 40.2 & 2.772 & 0.006 & 0.006 & & 0.153 & \\
\hline 2804 & $6 / 16 / 2000$ & $7: 27: 42 \mathrm{PM}$ & 20.401 & 26.5 & $\begin{array}{l}24.868 \\
24.868\end{array}$ & $\begin{array}{l}27.638 \\
27.588\end{array}$ & $\frac{26.518}{26.453}$ & \begin{tabular}{|}
$\mid 48.184$ \\
48.281
\end{tabular} & \begin{tabular}{|l|}
53.309 \\
53.224 \\
\end{tabular} & \begin{tabular}{|l|}
10.244 \\
16.216 \\
\end{tabular} & $\begin{array}{l}32.333 \\
32.534 \\
\end{array}$ & $\begin{array}{l}-.0 .055 \\
-0.652\end{array}$ & $\begin{array}{l}-6.714 \\
-6.779\end{array}$ & $\begin{array}{l}\frac{47.552}{52.013} \\
\end{array}$ & $\begin{array}{l}0.043 \\
0.042\end{array}$ & $\begin{array}{l}14.643 \\
14.643 \\
\end{array}$ & $\frac{0.003}{0.004}$ & $\begin{array}{l}3499.198 \\
3492.98\end{array}$ & $\begin{array}{l}58.1997 \\
58.2164\end{array}$ & $\frac{51.8}{51.8}$ & $\begin{array}{l}11.7 \\
12.7\end{array}$ & & \begin{tabular}{|l|}
2.776 \\
2.786
\end{tabular} & \begin{tabular}{|l}
0.006 \\
0.006
\end{tabular} & 0.0006 & $\frac{0.000}{0.000}$ & $\frac{0.160}{0.156}$ & 0.14 \\
\hline \begin{tabular}{|l|}
2805 \\
\end{tabular} & $6 / 16 / 2004$ & 7:28:42 PM & 26.461 & 26.5 & 24.878 & 27.533 & 26.373 & 48.109 & \begin{tabular}{|l|}
53.172 \\
\end{tabular} & $\begin{array}{l}116.302 \\
\end{array}$ & 32.236 & $\begin{array}{r}-0.652 \\
\end{array}$ & -6.774 & 47.735 & 0.043 & 14.643 & 0.004 & 3493.98 & 58.2331 & 51.7 & 11.7 & & & 0.006 & 0.006 & & & \\
\hline & $6 / 16 / 2004$ & 7:29:42 PM & 26.449 & & 24.87 & 27.496 & 26.337 & $\begin{array}{l}48.061 \\
\end{array}$ & \begin{tabular}{|l|l|}
53.139 \\
\end{tabular} & 16.16 & 32.37 & -0.652 & -6.771 & 51.375 & 0.037 & $\begin{array}{l}14.643 \\
\end{array}$ & & & 8.2497 & 51.7 & 12.6 & & & & & & & \\
\hline & $6 / 16 / 2004$ & & 26.455 & 26.5 & 24.86 & 27.462 & 26.308 & $\begin{array}{l}47.864 \\
\end{array}$ & & & & $\begin{array}{l}-0.652 \\
\end{array}$ & -6.779 & 46.006 & 0.041 & $\begin{array}{l}14.643 \\
\end{array}$ & & \begin{tabular}{|l|}
3495.98 \\
\end{tabular} & \begin{tabular}{|l|l|}
58.2664 \\
\end{tabular} & 51.4 & 11.3 & & & & & & & \\
\hline 2808 & $6 / 16 / 2004$ & $7: 31: 42 \mathrm{PM}$ & 26.444 & 26.5 & 24.845 & 27.421 & 26.256 & $\begin{array}{l}48.074 \\
\end{array}$ & \begin{tabular}{|l|}
53.3344 \\
\end{tabular} & $\begin{array}{l}16.183 \\
\end{array}$ & 32.337 & -0.649 & -6.762 & 47.529 & 0.043 & \begin{tabular}{|l|l|}
14.643 \\
\end{tabular} & & \begin{tabular}{|l|}
3496.98 \\
\end{tabular} & \begin{tabular}{|l|l|}
58.2831 \\
\end{tabular} & 51.9 & \begin{tabular}{|l|}
11.6 \\
\end{tabular} & 40.2 & 2.772 & 0.006 & 0.006 & 0.000 & 0.160 & \\
\hline 2810 & 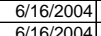 & $\begin{array}{ll}: 32: 42 \mathrm{PM} \\
7 \cdot 3: 3: 2 \mathrm{PM}\end{array}$ & $\begin{array}{l}26.443 \\
26.426\end{array}$ & $\frac{26.5}{26.5}$ & 24.899 & \begin{tabular}{|l|l}
27.395 \\
27.423
\end{tabular} & $\begin{array}{l}26.265 \\
26299\end{array}$ & $\begin{array}{r}48.306 \\
47.97 \\
\end{array}$ & $\begin{array}{l}53.376 \\
53034\end{array}$ & \begin{tabular}{|l|l|}
16.248 \\
1.122
\end{tabular} & $\begin{array}{r}32.435 \\
236\end{array}$ & $\begin{array}{l}-0.614 \\
-0582\end{array}$ & $\begin{array}{ll}-6.762 \\
-6748\end{array}$ & $\begin{array}{l}49.465 \\
56.15\end{array}$ & 0.041 & $\begin{array}{l}14.643 \\
1.643\end{array}$ & & & \begin{tabular}{|c|}
8.2997 \\
8.3145
\end{tabular} & 51.9 & $\begin{array}{l}12.1 \\
124\end{array}$ & 40.4 & & & 0.006 & & 0.152 & \\
\hline$\frac{\mid 2810}{2811}$ & $6 / 116 / 2604$ & $\begin{array}{l}\text { 7:33:42 PM } \\
7: 34: 42 \mathrm{PM}\end{array}$ & $\frac{26.426}{26.424}$ & 26. & $\frac{24.85}{22.856}$ & $\frac{27.423}{27.471}$ & $\frac{26.299}{26.362}$ & \begin{tabular}{|}
48.97 \\
48.59 \\
\end{tabular} & \begin{tabular}{|l|}
53.034 \\
53.293 \\
\end{tabular} & & $\begin{array}{r}32.36 \\
32.378 \\
\end{array}$ & $\begin{array}{l}-0.582 \\
-0.606\end{array}$ & $\begin{array}{l}-6.748 \\
-6.745\end{array}$ & $\frac{50.645}{49.49}$ & $\begin{array}{l}0.043 \\
0.044\end{array}$ & $\begin{array}{l}\frac{14.643}{14.643} \\
\end{array}$ & 0.004 & $\begin{array}{r}3498.98 \\
349998\end{array}$ & \begin{tabular}{|l|l|}
58.3164 \\
58.3331
\end{tabular} & $\frac{51.6}{51.8}$ & $\frac{12.4}{12.1}$ & & $2.769 \mid$ & \begin{tabular}{|l|l|}
0.006 \\
0.007
\end{tabular} & $\begin{array}{l}0.006 \\
0.007\end{array}$ & $\begin{array}{l}0.000 \\
0.000\end{array}$ & 0.160 & \\
\hline \begin{tabular}{|l|}
2812 \\
\end{tabular} & $6 / 16 / 2004$ & 7:35:42 PM & 26.419 & 26.5 & 24.85 & 27.451 & 26.361 & 48.399 & \begin{tabular}{|l|}
53.318 \\
\end{tabular} & \begin{tabular}{|l|}
15.849 \\
\end{tabular} & 33.041 & -0.585 & -6.75 & 50.783 & 0.044 & 14.643 & 0.004 & & \begin{tabular}{|l|}
58.35051 \\
5
\end{tabular} & 51.8 & $\frac{12.1}{12.4}$ & $\begin{array}{l}40.3 \\
\end{array}$ & $\frac{2.176}{2.808}$ & & & & $\begin{array}{l}0.164 \\
0.162 \\
\end{array}$ & 0.14 \\
\hline \begin{tabular}{|l|}
2813 \\
2
\end{tabular} & $6 / 16 / 2004$ & 7:36:42 PM & 26.407 & 26.5 & 24.85 & 27.519 & 26.379 & 48.518 & $\begin{array}{l}53.755 \\
\end{array}$ & 15.976 & 32.907 & -0.603 & -6.742 & 48.265 & 0.042 & 14.643 & 0.00 & \begin{tabular}{|l|l|}
3501.98 \\
\end{tabular} & 58.3664 & 52.3 & 11.8 & 40.7 & 2.807 & 0.006 & 0.006 & 0.000 & 0.154 & \\
\hline & $6 / 16 / 2004$ & 7:37:42 PM & & & 24.842 & & 26.438 & & $\begin{array}{l}53.738 \\
\end{array}$ & & & -0.603 & & 45.775 & 0.043 & $\begin{array}{l}14.643 \\
\end{array}$ & & \begin{tabular}{|l|}
3502.98 \\
\end{tabular} & 58.3831 & 52.3 & 11.2 & 40.9 & & 0.006 & 0.006 & 0.000 & 0.157 & \\
\hline 2815 & $6 / 16 / 2004$ & 7:38:42 PM & 26.405 & 26.5 & 24.822 & 27.542 & 26.448 & 48.333 & \begin{tabular}{|l|l|}
53.604 \\
\end{tabular} & 15.968 & 32.766 & -0.545 & -6.675 & 45.944 & 0.039 & 14.643 & & & \begin{tabular}{|l|l|l} 
\\
\end{tabular} & 52.1 & 111.3 & & \begin{tabular}{|l|l|}
2.796 \\
\end{tabular} & 0.006 & 0.006 & 0.000 & 0.144 & \\
\hline$\frac{2816}{2817}$ & 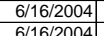 & 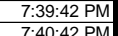 & $\begin{array}{l}26.404 \\
26.398\end{array}$ & 226.5 & 24.85. & $\begin{array}{l}27.606 \\
27.6\end{array}$ & $\begin{aligned} 26.456 \\
26.48\end{aligned}$ & \begin{tabular}{|l|l|l}
48.499 \\
48.576
\end{tabular} & \begin{tabular}{|l|}
53.714 \\
53709 \\
\end{tabular} & \begin{tabular}{|c|}
15.959 \\
16.06
\end{tabular} & $\begin{array}{l}32.934 \\
32.963 \\
3\end{array}$ & $\begin{array}{l}-0.542 \\
-0.542\end{array}$ & $\begin{array}{l}-6.664 \\
-6.658\end{array}$ & $\begin{array}{l}46.597 \\
46841\end{array}$ & $\begin{array}{l}0.042 \\
0.043\end{array}$ & $\begin{array}{l}14.643 \\
14.643\end{array}$ & & & \begin{tabular}{|l|l|}
58.4164 \\
5.3331
\end{tabular} & 52.2 & $\begin{array}{l}11.4 \\
1.5\end{array}$ & & $\frac{2.007}{2811}$ & $\begin{array}{l}0.006 \\
0.06\end{array}$ & $\begin{array}{l}0.006 \\
0.06\end{array}$ & $\begin{array}{l}0.000 \\
\end{array}$ & 0.154 & \\
\hline$\frac{\mid 017}{2818}$ & $\frac{6 / 11 / 26004}{6 / 16 / 2004}$ & $\begin{array}{l}\text { :4:4:424PM } \\
7: 44: 42 \mathrm{PM}\end{array}$ & $\begin{array}{l}26.398 \\
26.392\end{array}$ & $\frac{26.5}{26.5}$ & $\begin{array}{rl}24.84 & 24.82\end{array}$ & & $\frac{26.48}{26.504}$ & \begin{tabular}{|}
48.576 \\
48.374 \\
\end{tabular} & & \begin{tabular}{|r|}
16.06 \\
16.003
\end{tabular} & $\begin{array}{l}32.963 \\
32.823 \\
\end{array}$ & $\frac{-0.542}{-0.542}$ & & $6 \frac{6.841}{49.8}$ & $\frac{0.043}{0.041}$ & $\frac{14.643}{14.643}$ & & 3506.9 & $\begin{array}{l}58.4331 \\
58.4497\end{array}$ & & $\frac{11.5}{12.2}$ & & & 0.006 & & & & \\
\hline 2819 & $6 / 16 / 2004$ & 7:42:42 PM & 26.386 & 26.5 & 24.83 & 27.568 & $\frac{2.006}{26.508}$ & 48.671 & 53.948 & 16.025 & $\frac{32.020}{33.014}$ & -0.542 & -0.0667 & $\begin{array}{r}47.065 \\
47.45\end{array}$ & 0.043 & $\frac{14.045}{14.643}$ & & \begin{tabular}{|l|}
35007.98 \\
3507.98
\end{tabular} & \begin{tabular}{|l|l|}
58.44664 \\
5
\end{tabular} & $\frac{52.1}{52.5}$ & $\frac{12.4}{11.6}$ & 40.8 & \begin{tabular}{|l|}
2.199 \\
2816 \\
\end{tabular} & $\frac{0.006}{0.006}$ & $\begin{array}{l}0.006 \\
0.006\end{array}$ & 0 & $\begin{array}{l}0.151 \\
0.158\end{array}$ & 0.13 \\
\hline & $6 / 16 / 2004$ & 7:43:42 PM & 26.406 & 26. & 24.86 & 27.662 & & 48.65 & 53.817 & & 33.139 & -0.542 & -6.664 & 47.673 & 0.042 & 14.643 & & 3508.98 & 58.4831 & & 11.7 & 40.9 & & 0.006 & 0.006 & 0.000 & 0.154 & \\
\hline & $6 / 16 / 2004$ & 7:44:42 PM & 26.386 & 26. & 24.82 & 27.607 & 26 & 48.783 & \begin{tabular}{|l|l|}
54.099 \\
\end{tabular} & 16.131 & 32.988 & -0.542 & -6.661 & 49.254 & 0.044 & $\begin{array}{l}14.643 \\
\end{array}$ & & & 58.4997 & 52.6 & 12.1 & 40.9 & & 0.007 & $\begin{array}{l}0.007 \\
\end{array}$ & 0.000 & 0.161 & \\
\hline 2822 & $6 / 16 / 2004$ & 7:45:42 PM & 26.386 & 26. & 24.81 & 27.592 & 26.557 & $\begin{array}{l}48.432 \\
\end{array}$ & $\begin{array}{l}53.628 \\
5.620\end{array}$ & $\begin{array}{ll}16.097 \\
1907\end{array}$ & 32.768 & $\begin{array}{l}-0.542 \\
-0512\end{array}$ & -6.661 & 54.531 & 0.044 & 14.643 & & & 58.5164 & 52.2 & 13.4 & & \begin{tabular}{|l|}
2.799 \\
\end{tabular} & 0.007 & 0.007 & & 0.162 & \\
\hline$\frac{2823}{2824}$ & $6 / 16 / 2004$ & $\begin{array}{ll}7: 46: 42 \mathrm{PM} \\
7\end{array}$ & $\begin{aligned} 26.391 \\
26.386\end{aligned}$ & 26.5 & 24.842 & 27.602 & $\frac{26.552}{26.563}$ & \begin{tabular}{|l|l|}
48548 \\
48542
\end{tabular} & \begin{tabular}{|l|}
53.649 \\
53.798 \\
\end{tabular} & \begin{tabular}{|l|}
16.007 \\
161727
\end{tabular} & $\begin{array}{r}32.749 \\
33.854\end{array}$ & & & $\begin{array}{l}51.365 \\
5692\end{array}$ & 0.042 & $\begin{array}{l}14.643 \\
11.643\end{array}$ & 0.00 & & & \begin{tabular}{|l|l|}
52.2 \\
52.3
\end{tabular} & $\begin{array}{l}12.6 \\
1.2\end{array}$ & \begin{tabular}{|l|l|}
40.6 \\
407
\end{tabular} & \begin{tabular}{|l|}
2.796 \\
2806
\end{tabular} & $\begin{array}{l}0.006 \\
0.06\end{array}$ & $\begin{array}{l}0.006 \\
\end{array}$ & 0.000 & 0.155 & 0.10 \\
\hline & 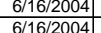 & $\begin{array}{ll}7: 47: 42 \mathrm{PM} \\
7\end{array}$ & 26.386 & 26.5 & $\begin{array}{l}24.848 \\
24.82\end{array}$ & 27.648 & $\frac{26.563}{26532}$ & $\begin{array}{r}48.542 \\
48688\end{array}$ & \begin{tabular}{|l|}
53.798 \\
53.819 \\
\end{tabular} & $\begin{array}{r}16.127 \\
1621\end{array}$ & $\begin{array}{r}32.854 \\
32.94\end{array}$ & $\begin{array}{l}-0.542 \\
-0.545\end{array}$ & & $\begin{array}{r}45.693 \\
4368 \\
\end{array}$ & 0.041 & $\begin{array}{l}14.643 \\
14643\end{array}$ & & \begin{tabular}{|l|}
3512.98 \\
3513.98 \\
\end{tabular} & \begin{tabular}{|l|l|}
58.5497 \\
555664
\end{tabular} & $\begin{array}{l}52.3 \\
5.2 .3\end{array}$ & $\frac{11.2}{10.7}$ & \begin{tabular}{|l|}
40.7 \\
40.8 \\
\end{tabular} & \begin{tabular}{|l|}
2.806 \\
2814
\end{tabular} & 0.006 & 0.006 & 0.000 & 0.151 & 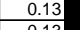 \\
\hline \begin{tabular}{|l|l|}
2826 \\
\end{tabular} & $6 / 116 / 2004$ & $\begin{array}{ll}.44: 494 \mathrm{PM} \\
7: 49: 22 \mathrm{M}\end{array}$ & $\frac{26.386}{26.385}$ & $\frac{26.5}{26.5}$ & $\frac{24.82}{24.85}$ & $2 \frac{27.014}{27.537}$ & $\frac{26.534}{26.447}$ & \begin{tabular}{|l|l|}
48.684 \\
4852
\end{tabular} & \begin{tabular}{|l|}
53.81 .877 \\
53.877 \\
\end{tabular} & \begin{tabular}{|c|}
16.21 \\
16.075
\end{tabular} & $\begin{array}{l}32.94 \\
32.893\end{array}$ & $\frac{-0.545}{-0.539}$ & & $\begin{array}{r}43.68 \\
45.136\end{array}$ & 0.044 & $\frac{14.643}{14.643}$ & & $\begin{array}{l}\frac{3513.98}{3514.98} \\
\end{array}$ & \begin{tabular}{|l|l|}
58.58604 \\
5831
\end{tabular} & $\frac{52.3}{52.4}$ & $\frac{10.7}{11.1}$ & \begin{tabular}{|l}
40.8 \\
40.7 \\
\end{tabular} & \begin{tabular}{|l|}
2.814 \\
2.807 \\
\end{tabular} & $\begin{array}{l}0.006 \\
0.006\end{array}$ & $\frac{0.006}{0.006}$ & $\frac{0.000}{0.000}$ & $\frac{0.154}{0.151}$ & $\frac{0.13}{0.13}$ \\
\hline \begin{tabular}{|l|}
2827 \\
\end{tabular} & $6 / 16 / 2004$ & 7:50:42 PM & 26.384 & 26.5 & 24.84 & 27.471 & 26.371 & 48.59 & \begin{tabular}{|l|}
53.898 \\
\end{tabular} & 16.197 & 32.747 & -0.545 & & 48.78 & 0.042 & 14.643 & & 3515.98 & 58.5997 & 52.4 & \begin{tabular}{|l|l|}
12.0 \\
\end{tabular} & 40.7 & \begin{tabular}{|l|}
2.804 \\
\end{tabular} & 0.006 & 0.006 & 0.000 & 0.155 & \\
\hline & $6 / 16 / 2004$ & 7:51:42 PM & 26.379 & & 24.82 & 27.421 & 26.311 & $\begin{array}{l}48.667 \\
\end{array}$ & \begin{tabular}{|l|}
53.904 \\
\end{tabular} & 16.217 & 32.883 & -0.519 & -6.66 & 51.06 & 0.041 & $\begin{array}{l}14.643 \\
\end{array}$ & & 3516.98 & 58.6164 & 52.4 & 12.5 & 40.8 & \begin{tabular}{|l|}
2.811 \\
\end{tabular} & 0.006 & 0.006 & & & \\
\hline \begin{tabular}{|l|}
2829 \\
\end{tabular} & & 7:52:42 PM & 26.377 & 26.5 & 24.83. & & & 48.576 & 53.79 & 16.2 & 32.797 & & & 47.504 & & $\begin{array}{l}14.643 \\
\end{array}$ & & & & & 11.6 & & & 0.006 & & 0.000 & 0.151 & \\
\hline 2830 & $6 / 16 / 2$ & & & 26 & 24.84 & & & & 53.74 & & & & & 45 & & \begin{tabular}{|l}
14.643 \\
\end{tabular} & & & & & & & & & & & & \\
\hline & $6 / 16 / 2004$ & 7:54:42 PM & 26.354 & 26.4 & 24.816 & & & 48.656 & & \begin{tabular}{|c|}
16.283 \\
\end{tabular} & 32.832 & & & & & $\begin{array}{l}14.643 \\
\end{array}$ & & & & & 1.6 & & & & & & & \\
\hline & 6/16/2004 & & 26.357 & 26.4 & $24.81:$ & 27.274 & & & & 16.239 & .715 & & & & & $\begin{array}{l}14.643 \\
\end{array}$ & & & & & 0.9 & & & .006 & 06 & & & \\
\hline & $6 / 16 / 2$ & 7:56:42 PM & & 26.4 & 24.84 & 27.297 & & & 53.676 & $\begin{array}{l}16.269 \\
\end{array}$ & & -0.519 & & 982 & 0.042 & $\begin{array}{l}14.643 \\
\end{array}$ & & & & & 11.5 & & & & & & & \\
\hline 2834 & $6 / 16 / 2004$ & 7:57:4 & 26.343 & 26.4 & 24.899 & 27.32 & & 48.333 & 53.401 & 16.243 & 32.596 & -0.498 & & 46.707 & 0.042 & 14.643 & & 3522. & 58.7164 & 51.9 & 11.4 & & 2.790 & 0.006 & 0.006 & 0.000 & 0.155 & \\
\hline & 0044 & & & 26.4 & 24.918 & 27.378 & & & 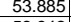 & 6.144 & & & & & & & & & & & 1.8 & & & & & & & \\
\hline & & & & 26.4 & 24.92 & 27.381 & & & 53.942 & 16.184 & 32.973 & & & & & & & & & & 12.4 & & & & & & & \\
\hline & $6 / 16 / 2004$ & $8000424 \mathrm{M}$ & $\frac{26.344}{26354}$ & 26.44 & $\frac{24.99}{24}$ & 2 & & $\begin{array}{l}48.404 \\
48642\end{array}$ & 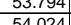 & $\begin{array}{l}16.068 \\
15921\end{array}$ & $\begin{array}{l}32.791 \\
33.119\end{array}$ & & & & & 14.6 & & & & 526 & $\frac{11.9}{11.9}$ & & & 00 & & & & \\
\hline & $\frac{0101}{6 / 6 / 2}$ & & & 26.4 & 24.07 & 27.005 & & & $\begin{array}{l}54.024 \\
53.941\end{array}$ & | & & & & & & $\frac{14 .}{14 .}$ & & & & & 11.8 & & & & & & & \\
\hline 2840 & 6 & & 26354 & $\frac{2.4}{264}$ & 24865 & 27.476 & & & $\mid$ & 告, & 33008 & & & & & 146 & & & & & 15 & & & & & & & \\
\hline 2841 & 6 & $8: 04: 3$ & $\frac{20.364}{26.364}$ & $\frac{2.4}{26.4}$ & 24.86 & 27.511 & & & $\begin{array}{l}54.124 \\
\end{array}$ & $\mid$\begin{tabular}{|c|c|c|}
15.683 \\
\end{tabular} & 33.355 & & & & 0.041 & 14.6 .6 & & & & & $\frac{1.1 .1}{12.1}$ & & 2.826 & 0.006 & 0.006 & 0.000 & 0 & \\
\hline & & & & 264 & & & & & & & & & & & & & & & & & 104 & & & & & & & \\
\hline & $6 / 1$ & & 26.356 & 26. & 24.808 & 27.5 & & & 54.0 & & $\frac{3.3 .451}{33.351}$ & & & & & & & & & & $\frac{5.2}{1.2}$ & & & & & & & \\
\hline 284 & $6 / 16 / 2$ & & 26.351 & & 24.8 & & & & & \begin{tabular}{|l|l|l|}
15.663 \\
\end{tabular} & & & & & & & & & & & 1.4 & & & & & & & \\
\hline 84 & 204 & & 26.34 & 26.4 & 24.797 & 27.562 & & & & 15.83 & & & & & & & & & & & 0.9 & & & & & & & \\
\hline & $6 / 16 / 2004$ & & & 26.4 & 24. & & & & 54.2 & \begin{tabular}{|c|}
15.743 \\
\end{tabular} & & & & & & \begin{tabular}{|l|l|}
14.643 \\
\end{tabular} & & & & 52. & $\mid 1.1$ & & & 0.000 & & & 0.157 & \\
\hline 284 & $6 / 16 / 2$ & & 26.35 & 26.4 & & 27.5 & & & & & & & & & & & & & & & 2.1 & & & & & & & \\
\hline 284 & $6 / 2004$ & & 26.357 & 26.4 & & 27.574 & & & \begin{tabular}{|l|l|l|} 
\\
4.026
\end{tabular} & 15.75 & 33.316 & & & & & 14. & & & & 52.6 & 11.2 & & & & & & D.150 & \\
\hline .04 & 6/116/2004 & & 20.00 & 26.4 & & & & & & & & & & & & & & & & & 1.0 & & & & & & & \\
\hline & & & & & & 27.5 & & & & & & & & & & & & & & & 1.2 & & & & & & & \\
\hline & 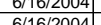 & & & $2.2^{2.4}$ & 24.172 & $\frac{2.5 .5}{275}$ & & & & & & & & & & & & & & & 107 & & & & & & 0.150 & \\
\hline & $6 / 16 / 200$ & & & $\frac{2.4}{26.4}$ & 24.1476 & & & & \begin{tabular}{|l|l|l|l|l|}
54035 \\
\end{tabular} & 15786 & & & & & & & & & & & $\frac{1.1}{12.3}$ & & & & & & & \\
\hline & $6 / 61 / 1200$ & & & & & 27.4 & & & & & & & & & & & & & & & 115 & & & & & & & \\
\hline & $\frac{21 / 6 / 2}{6 / 6 / 2}$ & & $\frac{2.3 .316}{26.316}$ & $\frac{2.4}{26.4}$ & $\frac{1.4 .74}{2.442}$ & 27.393 & & & \begin{tabular}{|c|}
5.4 .08 \\
\end{tabular} & $\frac{15.7}{115.7}$ & & & & & & & & & & & $\frac{11.6}{11.6}$ & & & & & & & \\
\hline & $6 / 116 / 2004$ & & & & & & & & & & & & & & & & & & & & & & & & & & & \\
\hline & & & & & & & & & & & & & & & & & & & & & & & & & & & & \\
\hline & $6 / 16 / 2004$ & & & & 247 & 27 & & & & 15 & & & & & & 14. & & & & & & & & & & & & \\
\hline & $6 / 16 / 2$ & $8: 22: 33$ & & 26.4 & 24.721 & 27.202 & & 48.6 & 54.167 & 15.742 & 33.32 & & & & & 14.6 & & & & & 11.7 & & & & & & & \\
\hline & $6 / 16 / 20$ & & & 26. & 24.7 & 27.196 & & 48.7 & 54.283 & 15.83 & 33.338 & 0.2 & & & & 14. & & & & & 11.7 & & & & & & 150 & \\
\hline & $6 / 16 / 2$ & & & 26.3 & 24.7 & & & & & & & & & & & & & & & & 1.9 & & & & & & & \\
\hline 286 & $6 / 16 / 2$ & 8:25: & 26. & & 24.712 & 27.2 & & & & 15 & 33.443 & & & & & & & & & 35 & 11.1 & & & & & & & \\
\hline & $6 / 16 / 2004$ & & & & & & & & & & & & & & & & & & & & 1.3 & & & & & & & \\
\hline & $6 / 16 / 2004$ & & 20 & 26.3 & 24.724 & 27.285 & & & & & 33. & & & & & & & & & & 12.0 & & & & & & & \\
\hline & $\frac{61 / 60 / 2}{61 / 2 \pi}$ & & & & $\frac{2.10}{219}$ & $\frac{2.2 .25}{2739}$ & & 48.15 & 53595 & 167 & $\frac{3.1 .7}{215}$ & & & & 0.06 & & & & & & & & & & & & & \\
\hline & 61012004 & & & & 24. & 27.4 & & 4 & 534 & & 21 & & & & & & & & & & & & & & & & & \\
\hline & 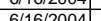 & 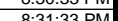 & & 263 & 24.65 & $\frac{27.021}{27345}$ & & 47.927 & 53.429 & 16639 & $\frac{31.74}{31.736}$ & & & & 804 & . & & & & & 126 & & & & & & & \\
\hline & & & & $\frac{2.63}{26.3}$ & 24768 & 27369 & & 48.059 & & & 31.955 & & & $45 / 15$ & & 14.6 & & & & 52 & 112 & & & & & & 54 & \\
\hline 87 & 6/16/2004 & & & 5.3 & 24.755 & 27.39 & & $\begin{array}{l}48.07 \\
\end{array}$ & 53.49 & $\mid 16.634$ & 31.88 & 189 & & & .042 & $\frac{14.045}{14.643}$ & & & & & 111 & & & 006 & 006 & 5001 & 158 & \\
\hline
\end{tabular}


WSRC-TR-2005-00105, REVISION 0

SRNL-RPP-2005-00012, REVISION 0

RUN \# 3.03A AND B; FIRST AND SECOND HALF OF SLURRY DEWATERING - CONT.

\begin{tabular}{|c|c|c|c|c|c|c|c|c|c|c|c|c|c|c|c|c|c|c|c|c|c|c|c|c|c|c|c|c|}
\hline & A & B & D & E & $F$ & $G_{G}$ & $\mathrm{H}$ & $\mathrm{J}$ & K & L & $\mathrm{M}$ & $\mathrm{N}$ & $\mathrm{O}$ & $Q$ & \begin{tabular}{l|l|l|l|} 
& \\
\end{tabular} & $\mathrm{s}$ & T & & & $x$ & & 2 & AA & $A B$ & $A C$ & $A D$ & $\mathrm{AE}$ & \\
\hline 2871 & $\begin{array}{l}6 / 116 / 2004 \\
661612004\end{array}$ & $\begin{array}{l}8: 34: 33 \mathrm{PM} \\
8: 53 \cdot 33 \mathrm{PM}\end{array}$ & $\frac{26.234}{26228}$ & $\frac{26.3}{26.3}$ & $\begin{array}{l}24.79 \\
24.77\end{array}$ & \begin{tabular}{|l|r|}
27.385 \\
27.395
\end{tabular} & $\begin{array}{l}26.291 \\
26.305\end{array}$ & $\begin{array}{l}47.763 \\
48.254\end{array}$ & \begin{tabular}{|l|}
53.363 \\
53.749 \\
\end{tabular} & \begin{tabular}{|c|}
16.399 \\
16482
\end{tabular} & $\begin{array}{l}31.769 \\
32.169 \\
3\end{array}$ & $\begin{array}{l}-0.218 \\
-0.197\end{array}$ & $\begin{array}{l}-6.276 \\
-6.265\end{array}$ & $\begin{array}{l}45.783 \\
48.486\end{array}$ & \begin{tabular}{|c|}
0.04 \\
0.041
\end{tabular} & $\begin{array}{l}14.643 \\
14.643 \\
\end{array}$ & $\begin{array}{l}0.004 \\
0.004\end{array}$ & $\begin{array}{l}3559.83 \\
3560.83\end{array}$ & \begin{tabular}{|l|l|}
59.3306 \\
59.3472
\end{tabular} & $\frac{51.9}{5.3 .3}$ & $\frac{11.2}{11.9}$ & 39. & 2.742 & 0.006 & $\begin{array}{l}0.006 \\
0.006\end{array}$ & $\begin{array}{l}0.000 \\
0.000\end{array}$ & 0.151 & $\begin{array}{l}0.13 \\
0.13\end{array}$ \\
\hline$\frac{28 / 2}{2873}$ & $\begin{array}{l}6 / 11 / 66 / 2044 \\
/ 12004\end{array}$ & $\begin{array}{l}8: 353: 33 \mathrm{PM} \\
8: 36 \mathrm{PM}\end{array}$ & $\frac{26.228}{26.239}$ & $\frac{26.3}{26.3}$ & $\frac{24.11}{24.7}$ & \begin{tabular}{|r|}
27.395 \\
27.43
\end{tabular} & $\frac{26.30}{26.33}$ & \begin{tabular}{|l|}
48.254 \\
48.157
\end{tabular} & \begin{tabular}{|l|}
53.794 \\
53.705 \\
\end{tabular} & $\begin{array}{l}16.3824 \\
16.335\end{array}$ & $\begin{array}{r}32.169 \\
32.234 \\
\end{array}$ & $\frac{-0.197}{-0.183}$ & $\frac{-6.265}{-6.262}$ & & \begin{tabular}{|c|}
.0 .44 \\
0.04 \\
\end{tabular} & $\frac{14.643}{14.643}$ & $\begin{array}{l}0.004 \\
0.004\end{array}$ & $\begin{array}{l}3560.83 \\
3561.83\end{array}$ & \begin{tabular}{|l|l|l|l|l|}
59.3639 \\
\end{tabular} & $\frac{52.3}{52.2}$ & $\frac{11.9}{11.6}$ & $\frac{40 .}{40 .}$ & & $\begin{array}{l}0.006 \\
0.006\end{array}$ & & $\begin{array}{l}0.000 \\
0.000\end{array}$ & $\frac{0.153}{0.149}$ & \\
\hline 2874 & $6 / 16 / 2004$ & & 26.239 & 26.3 & 24.81 & \begin{tabular}{l|l}
27.395 \\
\end{tabular} & & 47.815 & \begin{tabular}{|l|}
53.425 \\
\end{tabular} & 16.261 & 32.002 & -0.183 & -6.268 & 47.226 & $\begin{array}{l}0.04 \\
\end{array}$ & $\begin{array}{l}14.045 \\
14.643 \\
\end{array}$ & & 3562.83 & \begin{tabular}{|l|}
59.38006 \\
59.386
\end{tabular} & 52.0 & 11.6 & & 2.752 & 0.006 & 0.006 & 0.000 & 0.150 & \\
\hline 2875 & $6 / 16 / 2004$ & 8:38:33 PM & 26.232 & 26.3 & 24.80 & 27.379 & 26.32 & 47.92 & \begin{tabular}{|l|}
53.456 \\
\end{tabular} & 16.338 & 32.027 & -0.183 & -6.271 & 46.029 & 0.039 & 14.643 & & 3563.83 & 59.3972 & 52.0 & 11.3 & 40.0 & 2.756 & 0.006 & 0.006 & & 0.146 & \\
\hline 2876 & $6 / 16 / 2004$ & & 26.249 & 26.3 & 24.8 & 27.435 & 26.36 & 48.345 & \begin{tabular}{|l|}
53.745 \\
\end{tabular} & \begin{tabular}{|l|l|l|l}
16.447 \\
\end{tabular} & & $\begin{array}{l}-0.183 \\
\end{array}$ & -6.271 & 46.939 & 0.04 & 14.643 & & \begin{tabular}{|l|}
3564.83 \\
\end{tabular} & 59.4139 & 52.3 & & & & & & & 0.149 & \\
\hline Lort & $6 / 16 / 2004$ & 8:40:33 PM & 26.255 & 26.3 & 24.82 & 27.352 & & 47.904 & \begin{tabular}{|l|}
53.494 \\
\end{tabular} & 16.355 & 31.941 & -0.186 & -6.279 & 44.423 & 0.04 & 14.643 & & 3565.8 & 59.4306 & 52.0 & 10.9 & & & & 0.006 & & & \\
\hline \begin{tabular}{|l|l|}
2878 \\
270
\end{tabular} & $6 / 16 / 2004$ & 8:41:33 PM & 26.255 & 26.3 & 24.83 & 27.392 & 26.362 & 48.41 & 53.716 & $\begin{array}{l}16.422 \\
\end{array}$ & 32.477 & -0.183 & -6.259 & & \begin{tabular}{|l|l|}
0.041 \\
\end{tabular} & $\begin{array}{l}14.643 \\
\end{array}$ & & 3566.83 & 59.4472 & 52.2 & \begin{tabular}{|l|}
12.2 \\
\end{tabular} & 40.4 & 2.788 & 0.006 & 0.006 & 0.000 & 0.152 & \\
\hline $2 \frac{2879}{2880}$ & 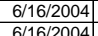 & 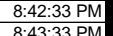 & $\begin{array}{l}26.254 \\
26249\end{array}$ & $\frac{26.3}{263}$ & & \begin{tabular}{|l|l|}
27.456 \\
27.44
\end{tabular} & $\begin{array}{l}26.346 \\
26.366\end{array}$ & $\begin{array}{l}48.294 \\
4892\end{array}$ & \begin{tabular}{|l|}
53.815 \\
53801 \\
\end{tabular} & \begin{tabular}{|c|}
16.398 \\
1647
\end{tabular} & \begin{tabular}{|l|l|}
32.333 \\
3205
\end{tabular} & $\begin{array}{l}-0.183 \\
.0189\end{array}$ & $\begin{array}{l}-6.288 \\
6.282\end{array}$ & $\begin{array}{l}46.786 \\
4704\end{array}$ & $\begin{array}{l}0.041 \\
0.041\end{array}$ & $\begin{array}{l}14.643 \\
1.643\end{array}$ & & \begin{tabular}{|l|}
3567.83 \\
2568923
\end{tabular} & \begin{tabular}{|l|}
59.4639 \\
50806
\end{tabular} & $\frac{52.3}{52.3}$ & \begin{tabular}{|l|l}
11.5 \\
11.7
\end{tabular} & 40.3 & \begin{tabular}{|l|l|}
2.779 \\
2766
\end{tabular} & & $\begin{array}{l}0.006 \\
0.066\end{array}$ & & 0.152 & \\
\hline$\frac{2880}{2881}$ & $\frac{6 / 1 / 162004}{6 / 16 / 2004}$ & $\begin{array}{l}8: 43: 33 \mathrm{PM} \\
8: 44: 33 \mathrm{PM}\end{array}$ & $\frac{26.249}{26.274}$ & $\frac{26.3}{26.3}$ & 24.81 & $\begin{array}{r}27.44 \\
27.466\end{array}$ & $\frac{26.366}{26.396}$ & \begin{tabular}{|l|}
48.192 \\
48.248 \\
\end{tabular} & \begin{tabular}{|l|}
53.801 \\
53.718 \\
\end{tabular} & \begin{tabular}{|l|l|}
16.487 \\
16.456
\end{tabular} & $\begin{array}{r}32.05 \\
32.22 \\
\end{array}$ & $\begin{array}{l}-0.186 \\
-0.18\end{array}$ & $\begin{array}{l}-6.282 \\
-6.285\end{array}$ & $\begin{array}{l}47.704 \\
46.863\end{array}$ & $\begin{array}{r}0.041 \\
0.04\end{array}$ & $\begin{array}{l}14.643 \\
14.643 \\
\end{array}$ & $\frac{0.004}{0.004}$ & \begin{tabular}{|l|}
3568.83 \\
3569.83
\end{tabular} & $\begin{array}{l}59.4806 \\
59.4972\end{array}$ & $\frac{52.3}{52 .}$ & \begin{tabular}{|l|}
11.7 \\
11.5 \\
\end{tabular} & 40.1 & $\begin{array}{l}2.766 \\
2774 \\
\end{array}$ & $\begin{array}{l}0.006 \\
0.006\end{array}$ & 0.006 & $\begin{array}{l}0.000 \\
\end{array}$ & 0.153 & 0.13 \\
\hline 2882 & & & & 26.3 & $\begin{aligned} 24.00 \\
24.82\end{aligned}$ & 27.446 & 26.37 & & & $\begin{array}{l}16.4503 \\
16.0\end{array}$ & & & & & $\begin{array}{l}0.049 \\
\end{array}$ & $\begin{array}{l}14.045 \\
4.643\end{array}$ & & & $\begin{array}{l}\mid 99.49 / 2 \\
59.5139\end{array}$ & 52.4 & $\begin{array}{l}11.5 \\
11.6\end{array}$ & \begin{tabular}{|l|}
40.2 \\
40.1
\end{tabular} & & & & $\begin{array}{l}0.000 \\
0.000\end{array}$ & $\begin{array}{l}0.144 \\
0.146\end{array}$ & $\frac{0.13}{0.12}$ \\
\hline & $6 / 16 / 2004$ & 8:46:33 PM & 26.259 & 26.3 & 24.93 & 27.491 & 26.39 & 47.806 & 53.429 & 16.337 & 31.906 & -0.177 & -6.276 & 52.036 & $\begin{array}{l}0.041 \\
\end{array}$ & 14.643 & 0.00 & 3571.8 & 59.5306 & 52.0 & 12.8 & & \begin{tabular}{|l|}
2.748 \\
\end{tabular} & 0.006 & 0.006 & 0.000 & 0.154 & \\
\hline & $6 / 16 / 2004$ & 8:47:33 PM & 26.254 & & & 27.386 & & & 53.272 & 16.439 & & -0.183 & -6.276 & 49.423 & \begin{tabular}{|l|l|} 
& 0.041 \\
\end{tabular} & $\begin{array}{l}14.643 \\
\end{array}$ & & 3572.83 & 59.5472 & 51.8 & & & 2.752 & 0.006 & 0.006 & & 0.154 & \\
\hline 2885 & $6 / 16 / 2004$ & 8:48:33 PM & 26.27 & 26.3 & 24.91 & 27.417 & & 48.024 & \begin{tabular}{|l|l|}
53.684 \\
\end{tabular} & \begin{tabular}{|l|l|}
16.419 \\
\end{tabular} & 31.966 & -0.186 & -6.273 & 49.225 & $\begin{array}{l}0.041 \\
\end{array}$ & $\begin{array}{l}14.643 \\
\end{array}$ & & \begin{tabular}{|l|l|}
3573.83 \\
30
\end{tabular} & 59.5639 & 52.2 & 12.1 & & & 0.006 & 0.006 & 0.000 & 0.153 & \\
\hline$\frac{2000}{2887}$ & 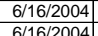 & 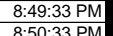 & $\begin{array}{r}26.28 \\
26.282 \\
\end{array}$ & $\frac{26.3}{26.3}$ & $\begin{array}{l}24.91 \\
24.89 \\
\end{array}$ & $\begin{array}{l}27.422 \\
27.424\end{array}$ & & $\begin{array}{r}47.8 \\
47.964\end{array}$ & $\begin{array}{l}53.258 \\
53.631\end{array}$ & \begin{tabular}{|c|}
16.311 \\
16576
\end{tabular} & $\begin{array}{l}31.883 \\
33.1805\end{array}$ & $\begin{array}{r}-0.16 \\
-0.142\end{array}$ & $\begin{array}{l}-6.285 \\
-6.268\end{array}$ & $\begin{array}{l}45.414 \\
55317\end{array}$ & $\begin{array}{l}0.04 \\
0.044\end{array}$ & $\begin{array}{l}14.643 \\
14643\end{array}$ & & \begin{tabular}{|l|}
3574.83 \\
3575.83
\end{tabular} & \begin{tabular}{|l|}
59.5806 \\
59972
\end{tabular} & $\frac{51.8}{52.2}$ & $\begin{array}{l}11.1 \\
13.1\end{array}$ & 39.8 & \begin{tabular}{|l|l|}
2.747 \\
2750
\end{tabular} & \begin{tabular}{|l|l|}
0.006 \\
0006
\end{tabular} & $\begin{array}{l}0.006 \\
0.006\end{array}$ & $\begin{array}{l}0.000 \\
\end{array}$ & $\begin{array}{l}0.150 \\
0.150\end{array}$ & (3) \\
\hline 2 & 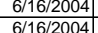 & 8:5:5:33 PM & $\frac{26.282}{26.281}$ & 26.3 & 24.89 & 27.424 & 374 & $\begin{array}{l}47.964 \\
47758\end{array}$ & \begin{tabular}{|l|}
53.631 \\
53.425 \\
\end{tabular} & \begin{tabular}{|c|}
16.576 \\
16.302
\end{tabular} & $\begin{array}{r}31.805 \\
31.873 \\
\end{array}$ & $\begin{array}{l}-0.142 \\
-0.148\end{array}$ & $\begin{array}{l}-6.268 \\
-6.271\end{array}$ & 53.317 & $\begin{array}{l}0.04 \\
0.04\end{array}$ & $\frac{14.643}{14643}$ & .00 & \begin{tabular}{|c|}
3575.83 \\
3576.83
\end{tabular} & \begin{tabular}{|l|l|}
59.5972 \\
596139
\end{tabular} & $\frac{52.2}{520}$ & 13.1 & 39.9 & $2.750 \mid$ & 0.006 & 0.006 & 0.000 & 0.150 & \\
\hline 2889 & $6 / 16 / 2004$ & 8:52:33 PM & $\frac{20.201}{26.29}$ & 26.3 & 24.05 & 27.307 & & 48.101 & \begin{tabular}{|l|}
53.4583 \\
53.53 \\
\end{tabular} & $\begin{array}{l}10.5063 \\
16.563\end{array}$ & $\frac{31.873}{32.013}$ & -0.113 & $\frac{-6.271}{-6.273}$ & $\begin{array}{r}46.83 \\
49.348 \\
\end{array}$ & $\begin{array}{l}0.04 \\
0.04\end{array}$ & $\frac{14.643}{14.643}$ & & \begin{tabular}{|c|}
$55 / .87$ \\
357.83
\end{tabular} & \begin{tabular}{|l|}
59.6139 \\
59.6306
\end{tabular} & $\frac{52.0}{52.1}$ & $\frac{11.5}{12.1}$ & 49.1 & \begin{tabular}{|l|}
2.762 \\
2.762 \\
\end{tabular} & & $\begin{array}{l}0.006 \\
0.006\end{array}$ & 0 & $\begin{array}{l}0.150 \\
0.149 \\
\end{array}$ & 0.13 \\
\hline & $6 / 16 / 2004$ & 8:53:33 PM & 26.273 & 26.3 & 24.86 & $\begin{array}{l}27.19 \\
\end{array}$ & 26.1 & 48.136 & 53.743 & 16.461 & 32.132 & -0.113 & -6.273 & 49.725 & 0.04 & $\begin{array}{l}14.643 \\
\end{array}$ & & 3578.83 & 59.6472 & 52.3 & & 40.1 & \begin{tabular}{|l|}
2.767 \\
\end{tabular} & 0.006 & 0.006 & 0.000 & 0.149 & \\
\hline & 6/16/2004 & 8:54:33 PM & & & 24.87 & 27.154 & & 48.038 & 53.573 & 16.405 & 32.074 & -0.113 & & 50.011 & 0.041 & 14.643 & & 3579.8 & 59.6639 & 52.1 & 12.3 & 40.1 & 2.762 & 0.006 & & 0.000 & 0.153 & \\
\hline 2892 & $6 / 16 / 2004$ & 8:55:33 PM & 26.286 & 26. & 24.88 & 27.168 & 26 & 48.018 & $\begin{array}{l}53.496 \\
\end{array}$ & $\begin{array}{l}16.424 \\
\end{array}$ & 32.101 & -0.113 & -6.262 & & \begin{tabular}{|l|l|} 
& 0.04 \\
\end{tabular} & 14.643 & & & 59.6806 & 52.0 & 11.3 & 40.1 & \begin{tabular}{|l|}
2.762 \\
\end{tabular} & 0.006 & 0.006 & 0.000 & 0.149 & \\
\hline 2894 & $6 / 16 / 2004$ & 8:56:33 PM & $\begin{array}{l}26.285 \\
26.272\end{array}$ & 26.3 & 24.87 & 27.142 & $\frac{26.05}{2604}$ & $\begin{array}{l}48.123 \\
4815\end{array}$ & $\begin{array}{r}53.591 \\
53.863\end{array}$ & \begin{tabular}{|l|}
16.442 \\
1.657 \\
\end{tabular} & $\begin{array}{l}32.197 \\
32357\end{array}$ & $\begin{array}{l}-0.111 \\
\end{array}$ & $\begin{array}{r}-6.25 \\
6.245\end{array}$ & $\begin{array}{l}48.482 \\
4783\end{array}$ & 0.041 & $\begin{array}{l}14.643 \\
14643\end{array}$ & & \begin{tabular}{|l|l|}
3581.83 \\
352832
\end{tabular} & \begin{tabular}{|l|}
59.6972 \\
57139
\end{tabular} & 52.1 & $\frac{11.9}{11.6}$ & 40.2 & \begin{tabular}{|l|}
2.769 \\
2778
\end{tabular} & $\begin{array}{l}0.006 \\
0.06\end{array}$ & $\begin{array}{l}0.006 \\
\end{array}$ & 0.000 & 0.153 & 10 \\
\hline 2094 & $6 / 11 / 2 / 2004$ & $8: 5: 5: 3 \mathrm{PM}$ & $\frac{26.272}{26.276}$ & $\frac{26.3}{26.2}$ & $\frac{24.83}{24.86}$ & $\frac{27.139}{27.139}$ & & $\begin{array}{l}48.215 \\
48.126\end{array}$ & & $\begin{array}{l}16.257 \\
16.174\end{array}$ & $\begin{array}{r}32.357 \\
32.38 \\
\end{array}$ & $\frac{-0.113}{-0.113}$ & & $\frac{47.18}{46.966}$ & $\mid$\begin{tabular}{|c|c|}
0.041 \\
0.041
\end{tabular} & $\frac{14.643}{14.643}$ & & & & & $\frac{11.6}{11.5}$ & & & & & & & \\
\hline & $6 / 16 / 2004$ & 8:59:33 PM & $\frac{20.210}{26.265}$ & 26.2 & $\frac{24.00}{24.85}$ & 27.145 & $\frac{20.0}{26.07}$ & $\frac{40.120}{48.25}$ & \begin{tabular}{|l|}
53.054 \\
53.854 \\
\end{tabular} & $\mid \frac{10.174}{16.136}$ & $\begin{array}{l}32.567 \\
32.567\end{array}$ & $\frac{-.110}{-0.113}$ & $\begin{array}{l}-0.240 \\
-6.245\end{array}$ & $\begin{array}{l}40.500 \\
49.886\end{array}$ & $\begin{array}{l}.0 .041 \\
0.0399\end{array}$ & $\frac{14.040}{14.643}$ & & & 59.7472 & $\frac{32.4}{52.4}$ & $\frac{11.5}{12.2}$ & & 2.78 & 0.006 & .006 & & 1.144 & $\frac{0.13}{0.12}$ \\
\hline & 6/116/2004 & 9:00:33 PM & 26.279 & 26.2 & 24.8 & 27.226 & 26.12 & 48.078 & \begin{tabular}{|r|}
53.57 \\
\end{tabular} & 16.235 & 32.284 & -0.096 & -6.239 & 47.078 & 0.04 & 14.643 & & $\frac{3584.83}{3585.83}$ & $\mid$ & 52.1 & \begin{tabular}{|l|l|}
11.5 \\
\end{tabular} & 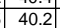 & 2.770 & $\begin{array}{l}0.006 \\
\end{array}$ & 0.006 & 0.000 & $\begin{array}{l}0.1449 \\
0.149\end{array}$ & $\frac{0.12}{0.13}$ \\
\hline & $6 / 16 / 2004$ & 9:01:33 PM & 26.262 & 26.2 & & 27.21 & 26.1 & \begin{tabular}{|l|l|l|l|}
48.134 \\
\end{tabular} & 53.842 & 16.185 & 32.36 & -0.111 & & 46.584 & $\begin{array}{l}0.041 \\
\end{array}$ & $\begin{array}{l}14.643 \\
\end{array}$ & & & 59.7806 & 52.4 & 11.4 & & & & & & & \\
\hline 28899 & & 9:02:33 PM & 26.256 & 26.2 & 24.84 & 27.223 & & & \begin{tabular}{|l|l|}
53.765 \\
\end{tabular} & & 32.438 & & & 46.3 & & $\begin{array}{l}14.643 \\
\end{array}$ & & & & 52.3 & 11.4 & & & 0.006 & .006 & & 153 & \\
\hline & $6 / 16 / 2$ & & & & & & & & & & & & & & 0.04 & \begin{tabular}{|l}
14.643 \\
\end{tabular} & & & & & & & & & & & & \\
\hline 2901 & 6/16/2004 & 9:04:33 PM & 26.251 & & 24.81 & 27.218 & & & 54.008 & & 32.747 & & & & $\begin{array}{l}0.042 \\
\end{array}$ & $\begin{array}{l}14.643 \\
\end{array}$ & & & & & & & & & & & & \\
\hline & $6 / 16 / 2004$ & & & 26.2 & 24.85 & 27.273 & & 48.128 & \begin{tabular}{l|l|l|}
53.657 \\
\end{tabular} & 16.108 & & -0.084 & & & 0.04 & $\begin{array}{l}14.643 \\
\end{array}$ & & & & $J_{4}$ & 1.6 & & & 0.006 & 06 & & & \\
\hline & $6 / 16 / 2$ & 9:06:33 PM & 26.25 & 26.2 & 24.83 & 27.252 & & & \begin{tabular}{|l|l}
53.73 \\
\end{tabular} & \begin{tabular}{|l|l|}
16.094 \\
\end{tabular} & & -0.09 & & & $\begin{array}{l}0.042 \\
\end{array}$ & $\begin{array}{l}14.643 \\
\end{array}$ & & & & & 11.0 & & & & & & & \\
\hline & 6/16/2004 & 9:07:33 PM & 26.251 & 26.2 & 24.82 & 27.268 & & 48.588 & \begin{tabular}{|l|l|}
54.429 \\
\end{tabular} & 16.226 & 32.678 & -0.08 & & 48.457 & 0.042 & $\begin{array}{l}14.643 \\
\end{array}$ & & 3592.83 & 59.8806 & & 11.9 & & 2.802 & 0.006 & 0.006 & 0.000 & 0.155 & \\
\hline & $6 / 2004$ & & & 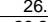 & 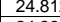 & & & & 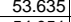 & ( & & & & & 041 & & & & & & & & & & & & & \\
\hline & & 9: & 26.24 & 26.2 & 24.83 & 27.257 & & & 54.051 & $16.1 / 1$ & & & & & 0.041 & & & & & & & & & & & & & \\
\hline & 6 & $\frac{910: 333}{0.1230}$ & 26234 & 26.2 & 24.81 & 2 & & 48.551 & $\begin{array}{l}54.323 \\
53263\end{array}$ & 16.147 & $\begin{array}{l}32.16 \\
32.776\end{array}$ & $\begin{array}{r}-0.09 \\
\end{array}$ & & 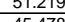 & 0.041 & 14.6 & & & & & $\frac{11.6}{1.1 .}$ & & & & & & 0.151 & \\
\hline & $6 / 16 / 2$ & & & $\frac{2.4}{262}$ & 24.18 & & & & 年 & 15.201 & & & & & & 14. & & & & & 14 & & & & & & & \\
\hline & 61/62004 & & $\frac{10.45}{26209}$ & & 24.81 & 27285 & & & \begin{tabular}{|l|l|l|l|l|}
54.055 \\
\end{tabular} & 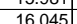 & 842 & & & & $|0.04|$ & 14. & & & & & $\frac{1.44}{23}$ & & & & & & & \\
\hline 291 & $\frac{1 / 1612004}{6}$ & $9: 14: 33$ PN & $\frac{2.254}{26.234}$ & $\frac{2.2}{26.2}$ & $\frac{24.8}{24.8}$ & 27.33 & & 48.281 & $\begin{array}{l}53.668 \\
53.668\end{array}$ & $\mid \begin{array}{l}\mid 1.04048 \\
1.048\end{array}$ & 32.749 & -0.0 & & & $\mid 0.039$ & 14.643 & & & & & 11.4 & & 2.793 & 0.006 & 0.006 & 0.000 & 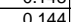 & \\
\hline & & & & & & & & & & & & & & & & & & & & & & & & & & & & \\
\hline & $6 / 1$ & & 26.219 & 26.2 & 24 & 27.261 & & & 54.068 & & 809 & & & & & & & & & & 11 & & & & & & & \\
\hline & $6 / 16 / 2$ & & & 26.2 & 24.80 & & & & & & 401 & & & & & & & & & & & & & & & & & \\
\hline & $6 / 16 / 2$ & $9: 18: 33 \mathrm{~F}$ & 26.2 & 26.2 & 24.77 & 27.307 & & & 54 & $\begin{array}{l}16.267 \\
\end{array}$ & & & & & & & & & & 52 & 1.6 & & & & & & & \\
\hline & $6 / 16 / 2004$ & & 26.199 & 26.2 & 2 & & & & 54.149 & 16.1 & & & & & 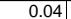 & $\begin{array}{l}14.643 \\
\end{array}$ & & & & 52 & 1. & & & & & & 0.147 & \\
\hline & $6 / 16 / 2$ & & & & 24.78 & & & & & & & & & & & & & & & & . & & & & & & & \\
\hline & 6/16/2004 & & 26.196 & 26. & 24.77 & 27.36 & & & \begin{tabular}{|l|}
53.979 \\
\end{tabular} & 16.2 & 32.676 & & & & & 14. & & & & 52. & 11.7 & & & & & & & \\
\hline (5) & $6 / 16 / 2004$ & & 26.2 & 26.2 & 24.18 & 27.2 & & & & & & & & & & & & & & & & & & & & & & \\
\hline & & & 20.208 & 26.2 & 24.9 & 27. & & & & & 32.791 & & & & & & & & & & & & & & & & & \\
\hline & 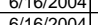 & & & & 24.81 & 27.26 & & & & & & & & & & & & & & & & & & & & & & \\
\hline & $6 / 6 / 200$ & $\frac{1.25 .5}{9 \cdot 2 \cdot 3}$ & $\frac{2.250}{26213}$ & $\frac{2.4}{26.2}$ & $\frac{24.05}{2487}$ & 27.275 & & & & 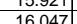 & & & & & & & & & & & 18 & & & & & & & \\
\hline & $6 / 16 / 200$ & & $\frac{20.45}{26219}$ & & 24.8 & & & & & & & & & & & & & & & & & & & & & & & \\
\hline 292 & $6 / 16 / 2$ & 9.28 & $\frac{2.252}{26.222}$ & $\frac{2.2}{26.2}$ & 24.83 & 27.294 & & 48.4 & $|54.194|$ & & 32.729 & & & & . & & & & & 52 & 捛1.5 & & & & & & & \\
\hline 292 & $6 / 16 / 2004$ & & & & & & & & & & & & & & & & & & & & & & & & & & & \\
\hline & & & & & 24 & & & & & & & & & & & & & & & & & & & & & & & \\
\hline & $6 / 16 / 2004$ & & 26.23 & & 248 & 27.3 & & & & & & & & & & & & & & & & & & & & & & \\
\hline & $6 / 16 / 2$ & $9: 32: 33$ & & 26.3 & 24.88 & 27.343 & & 48.636 & 54.263 & 17.4 & 31.5 & -0.0 & & & & 14.6 & & & 6 & & 22 & & & & & & 0.145 & \\
\hline & $6 / 16 / 20$ & & 224 & 26.3 & 24.90 & 27.3 & & 48.40 & 53.964 & 17.1 & 31.764 & & & & 0.6 & 14. & & & & & & & & & & & 149 & \\
\hline & $6 / 16 / 2$ & & & 26.3 & $24 . \mathrm{C}$ & & & 48.6 & & & & & & & & & & & & & & & & & & & & \\
\hline & $6 / 16 / 2$ & & 26.223 & & 24.5 & 27.3 & & 48.7 & & 17.0 & 32.044 & & & & & & & & & 53 & & & & & & & & \\
\hline & 6/16/2004 & & 26.21 & & 24. & & & & & & & & & & & & & & & & & & & & & & & \\
\hline & $6 / 16 / 2004$ & & 0.218 & 26.3 & 24.96 & 27.3 & & 48.60 & 54.503 & 16.721 & 32.24 & & & & & & & & & & & & & & & & & \\
\hline & $6 / 11 / 2004$ & & 2602 & 62 & 24.96 & $\frac{27.345}{27277}$ & & 48.8 & 54.41 & 10.1 & $\frac{32.41}{3250}$ & $80 ?$ & & & & & & & & & & & & & & & & \\
\hline & 011012004 & & 26 & 262 & $\frac{24.91}{2.07}$ & 年 & & 40.00 & $\frac{3.51}{5.57}$ & & 20701 & & & & & & & & & & & & & & & & & \\
\hline & 4 & & & 263 & $\frac{2.31}{2504}$ & 27302 & & 年 48.78 & 54646 & 16874 & $\begin{array}{l}32.145 \\
32253\end{array}$ & & & & 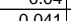 & & & & & & & & & & & & & \\
\hline & & & 2624 & $\frac{2.63}{26.3}$ & 2505 & $\frac{27.357}{2757}$ & & 48.85 & 54,652 & 116.6 & & & & 51886 & & 14.6 & & & & 53 & 27. & & & & & & 54 & \\
\hline 940 & 6/16/2004 & & 5.241 & 5.3 & $\frac{25.03}{25}$ & 27.372 & & 49.181 & 54.965 & 16.67 & .864 & & & 151 & & $\frac{14.045}{14.643}$ & & & & & & & & .006 & & & 145 & \\
\hline
\end{tabular}


WSRC-TR-2005-00105, REVISION 0

SRNL-RPP-2005-00012, REVISION 0

RUN \# 3.03A AND B; FIRST AND SECOND HALF OF SLURRY DEWATERING - CONT.

\begin{tabular}{|c|c|c|c|c|c|c|c|c|c|c|c|c|c|c|c|c|c|c|c|c|c|c|c|c|c|c|c|c|}
\hline & $\mathrm{A}$ & & D & $E$ & $\mathrm{~F}$ & G & $\mathrm{H}$ & $\mathrm{J}$ & $\mathrm{K}$ & $\mathrm{L}$ & M & $\mathrm{N}$ & o & $Q$ & $\mathrm{R}$ & $\mathrm{s}$ & $\begin{array}{ll}T \\
\end{array}$ & $\mathrm{~J} \quad \mathrm{~V}$ & w & $x$ & \begin{tabular}{l|l} 
\\
\end{tabular} & z & $\mathrm{AA}$ & $A B$ & $\mathrm{AC}$ & $A D$ & AE & \\
\hline & $6 / 16 / 2004$ & 9:44:33 PM & 26.241 & 26.295 & 25.053 & 27.313 & 26.308 & 49.175 & 55.054 & \begin{tabular}{|l|l|}
16.611 \\
\end{tabular} & $\begin{array}{l}32.977 \\
\end{array}$ & 0.086 & -6.031 & $\begin{array}{l}49.352 \\
\end{array}$ & $\begin{array}{l}0.042 \\
\end{array}$ & 14.643 & 0.004 & 3629.83 & 360.4972 & 53.6 & 12.1 & $\begin{array}{ll}1 & \\
1 & 41.1 \\
\end{array}$ & 2.832 & 0.006 & 0.006 & 0.000 & 0.152 & \\
\hline & $6 / 16 / 2004$ & & 26.271 & 26.325 & 25.088 & 27.393 & 26.328 & 49.071 & 54.992 & 16.59 & 32.836 & 0.089 & & & 0.041 & 14.643 & 0.004 & 3630.83 & & 53.5 & 13.1 & & & & & & 0.149 & \\
\hline 2943 & $6 / 16 / 2004$ & 9:46:33 PM & & 26.304 & 25.046 & 27.402 & 26.31 & 49.442 & 55.245 & $\begin{array}{l}16.382 \\
1632\end{array}$ & 33.431 & 0.089 & -6.0 & 52.193 & $\begin{array}{l}0.04 \\
\end{array}$ & \begin{tabular}{|l|}
14.643 \\
\end{tabular} & 0.004 & 3631.83 & 360.5306 & 53.8 & 12.8 & & & \begin{tabular}{|l|l|}
0.006 \\
\end{tabular} & 0.006 & 0.000 & 0.144 & \\
\hline 2944 & $6 / 16 / 2004$ & 9:47:33 PM & 26.265 & 26.309 & 25.041 & 27.422 & 26.33 & $\begin{array}{l}49.127 \\
\end{array}$ & 54.876 & $\begin{array}{l}16.312 \\
1.30\end{array}$ & $\begin{array}{l}33.223 \\
2207\end{array}$ & 0.121 & -6.0 & 47.671 & $\begin{array}{l}0.041 \\
\end{array}$ & 14.643 & 0.004 & 3632.83 & 360.5472 & 53.4 & 11.7 & $\begin{array}{ll}7 & 41.2 \\
\end{array}$ & 2.839 & \begin{tabular}{|l|l|} 
\\
\end{tabular} & 0.006 & 0.000 & 0.148 & \\
\hline $\mid \frac{2945}{2946}$ & $6 / 116 / 2004$ & 9:48:33 PM & $\frac{26.266}{26.27}$ & $\frac{26.294}{26.314}$ & $\begin{array}{l}25.022 \\
25061\end{array}$ & \begin{tabular}{|l|}
27.387 \\
2747
\end{tabular} & 26 & $\begin{array}{l}49.376 \\
9121\end{array}$ & \begin{tabular}{|l|}
55.083 \\
55.092 \\
\end{tabular} & $\begin{array}{r}16.493 \\
16.222\end{array}$ & $\begin{array}{l}33.287 \\
332.263 \\
\end{array}$ & $\begin{array}{l}0.109 \\
0139\end{array}$ & & $\begin{array}{l}46.638 \\
461777\end{array}$ & \begin{tabular}{|l|l|}
0.039 \\
0.043
\end{tabular} & \begin{tabular}{|l|l|l|}
14.643 \\
14643
\end{tabular} & 0.004 & 3633.83 & \begin{tabular}{|l|l|}
60.5639 \\
5.0 .506
\end{tabular} & 53.6 & \begin{tabular}{|l|l|} 
\\
\end{tabular} & & & \begin{tabular}{|l|}
0.006 \\
0.02
\end{tabular} & $\begin{array}{l}0.006 \\
\end{array}$ & & $\begin{array}{l}0.141 \\
0155\end{array}$ & 0.12 \\
\hline 2947 & $6 / 116 / 2004$ & 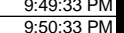 & $\frac{26.27}{26.281}$ & $\frac{26.314}{26.309}$ & $\begin{array}{l}25.061 \\
25.052\end{array}$ & $\begin{array}{l}27.4577 \\
27.467\end{array}$ & $\frac{26.32}{26.34 \varepsilon}$ & $\begin{array}{r}49.121 \\
49.218\end{array}$ & \begin{tabular}{|l|l}
55.092 \\
54.868
\end{tabular} & \begin{tabular}{|l|}
6.226 \\
16.151
\end{tabular} & $\begin{array}{r}33.263 \\
33.57 \\
\end{array}$ & $\begin{array}{l}0.138 \\
0.104\end{array}$ & $\begin{array}{r}-6.01 \\
-6.013\end{array}$ & $\begin{array}{l}46.177 \\
51.333\end{array}$ & $\begin{array}{c}0.043 \\
0.042\end{array}$ & \begin{tabular}{|l|}
14.643 \\
14643 \\
\end{tabular} & $\begin{array}{l}0.004 \\
0.004\end{array}$ & \begin{tabular}{|l|}
36345.83 \\
3635.83
\end{tabular} & $\begin{array}{l}360.5506 \\
36.5972\end{array}$ & 53.6 & \begin{tabular}{|l|}
11.3 \\
12.6
\end{tabular} & 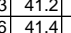 & $\begin{array}{l}2.840 \\
2.854 \\
\end{array}$ & \begin{tabular}{|l|l|} 
& 0.006 \\
\end{tabular} & $\begin{array}{l}0.006 \\
0.006\end{array}$ & 0.000 & $\begin{array}{l}0.155 \\
0.151\end{array}$ & 0.13 \\
\hline 2948 & & & 26.276 & 26.309 & 25.032 & 27.487 & 26.35 & 49.476 & 55.44 & \begin{tabular}{|l|l|}
16.351 \\
\end{tabular} & 33.501 & & $\begin{array}{r}-6.025 \\
\end{array}$ & 44.532 & 0.043 & $\begin{array}{l}14.643 \\
14.643\end{array}$ & 0.004 & & 360.6139 & \begin{tabular}{|l|l|}
5.4 \\
54.0
\end{tabular} & 10.9 & & & & & 0.000 & $\begin{array}{l}0.151 \\
0.154\end{array}$ & $\begin{array}{l}0.13 \\
0.13 \\
\end{array}$ \\
\hline 2949 & $6 / 16 / 2004$ & & & & 25.027 & 27.482 & 26.36 & 49.43 & $\begin{array}{ll}55.467 \\
\end{array}$ & 16.299 & 33.554 & 0.196 & -5.747 & 46.405 & 0.039 & 14.643 & 0.004 & \begin{tabular}{|l|}
3637.83 \\
\end{tabular} & 360.6306 & 54.0 & 11.4 & & 2.861 & & & & 0.140 & \\
\hline & $6 / 16 / 2004$ & 9:53:33 PM & & & 25.041 & 27.457 & 26.3 & 48.978 & \begin{tabular}{|l|l|}
54.961 \\
\end{tabular} & $\begin{array}{l}16.243 \\
\end{array}$ & & 0.199 & & & \begin{tabular}{|l|l|} 
\\
\end{tabular} & 14.643 & & & 360.6472 & 53.5 & 11.6 & & & 0.006 & & 0.000 & 0.145 & \\
\hline 2951 & $6 / 16 / 2004$ & 9:54:33 PM & $\begin{array}{ll}26.276 \\
20.276\end{array}$ & 26.309 & 25.042 & \begin{tabular}{ll|}
27.462 \\
27.27
\end{tabular} & & $\begin{array}{l}48.926 \\
10.050\end{array}$ & \begin{tabular}{|l|l|}
54.779 \\
5.12
\end{tabular} & $\begin{array}{ll}16.267 \\
1.2 \%\end{array}$ & 33.117 & 0.222 & & \begin{tabular}{|l|l|}
47.18 \\
\end{tabular} & \begin{tabular}{|l|l|}
0.041 \\
\end{tabular} & 14.643 & 0.00 & 3639.83 & 360.6639 & 53.3 & 11.6 & 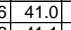 & 2.828 & \begin{tabular}{|l|l|} 
\\
\end{tabular} & 0.006 & 0.000 & 0.149 & \\
\hline$\frac{1504}{3953}$ & $6 / 16 / 2004$ & 9:55:33 PM & $\begin{array}{l}26.276 \\
26281\end{array}$ & $\begin{array}{l}26.314 \\
26.314\end{array}$ & $\begin{array}{l}25.032 \\
25027\end{array}$ & \begin{tabular}{|l|l|l|l}
27.427 \\
27.457
\end{tabular} & & $\begin{array}{l}49.059 \\
4088\end{array}$ & \begin{tabular}{|l|}
55.142 \\
550 \\
\end{tabular} & \begin{tabular}{r|r|}
16.265 \\
1619
\end{tabular} & 1.152 & 0.231 & $\begin{array}{l}-5.768 \\
5759\end{array}$ & $\begin{array}{l}50.272 \\
70.012\end{array}$ & \begin{tabular}{|l|l|}
0.042 \\
\end{tabular} & $\begin{array}{l}14.643 \\
1.643\end{array}$ & & \begin{tabular}{|l|}
3640.83 \\
36418
\end{tabular} & $\begin{array}{l}360.6806 \\
3606072\end{array}$ & $\begin{array}{l}53.7 \\
5.6\end{array}$ & \begin{tabular}{|l|l|}
12.3 \\
\end{tabular} & & $\begin{array}{l}2.834 \\
2842 \\
28\end{array}$ & \begin{tabular}{|l|l|}
0.006 \\
\end{tabular} & \begin{tabular}{|l|l|}
0.006 \\
0.006
\end{tabular} & $\begin{array}{l}0.000 \\
0.000\end{array}$ & $\frac{0.152}{0.152}$ & \\
\hline$\frac{2953}{2954}$ & 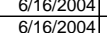 & $\begin{array}{l}9: 5: 5: 33 \mathrm{PM} \\
9: 53: 33 \mathrm{PM}\end{array}$ & $\frac{26.281}{26.296}$ & $\frac{26.314}{26.319}$ & $\begin{array}{r}25.027 \\
25.027\end{array}$ & $27.457 \mid$ & & $\begin{array}{r}49.088 \\
49.181 \\
\end{array}$ & $\begin{array}{r}55.09 \\
55.127\end{array}$ & $\begin{array}{r}\frac{16.19}{16.308} \\
\end{array}$ & $\begin{array}{l}33.343 \\
33.267\end{array}$ & & $\begin{array}{r}-5.759 \\
-5.77 \\
\end{array}$ & $\begin{array}{l}47.012 \\
47.879\end{array}$ & $\begin{array}{r}0.042 \\
0.04\end{array}$ & $\begin{array}{l}14.643 \\
14.643 \\
\end{array}$ & \begin{tabular}{|l|l|}
0.004 \\
0.004
\end{tabular} & \begin{tabular}{|l|}
3641.83 \\
36428
\end{tabular} & $\begin{array}{l}360.6972 \\
607139\end{array}$ & $\begin{array}{l}53.6 \\
53.7\end{array}$ & \begin{tabular}{|l|l|}
11.5 \\
11.7
\end{tabular} & $\frac{5}{7}$ & \begin{tabular}{l|l}
2.842 & \\
2842
\end{tabular} & \begin{tabular}{|l|l|} 
& .006 \\
& 006 \\
\end{tabular} & 0.006 & 0000 & $\begin{array}{l}.152 \\
2.145 \\
\end{array}$ & 0.13 \\
\hline 2955 & $6 / 16 / 2004$ & 9:58:33 PM & 26.278 & 26.312 & $\begin{array}{r}25.02 t \\
25.04\end{array}$ & 27.345 & $\frac{20.29}{26.22}$ & 49.021 & 54.947 & 16.252 & 33.148 & 0.231 & $\begin{array}{r}-5.11 \\
-5.753 \\
\end{array}$ & $\begin{array}{l}41.879 \\
46.503\end{array}$ & $\begin{array}{l}0.044 \\
0.042\end{array}$ & $\begin{array}{l}14.645 \\
14.643 \\
\end{array}$ & $\begin{array}{l}0.004 \\
0.004\end{array}$ & \begin{tabular}{|l|}
304.83 \\
3643.83
\end{tabular} & 360.7306 & \begin{tabular}{|l|l|}
53.5 \\
\end{tabular} & \begin{tabular}{|l|l|}
11.7 \\
11.4
\end{tabular} & & 2.844 & \begin{tabular}{|l|l|}
0.000 \\
\end{tabular} & $\begin{array}{l}0.000 \\
0.006\end{array}$ & & 0.145 & $\begin{array}{l}0.12 \\
0.13\end{array}$ \\
\hline & $6 / 16 / 2004$ & 9:59:33 PM & 26.281 & 26.30 & 25.032 & 27.313 & & 49.03 & 55.052 & 16.322 & 33.102 & 0.254 & & 47.11 & & 14.643 & & \begin{tabular}{|l|}
3644.83 \\
\end{tabular} & 360.7472 & & & & & & & & 0.149 & \\
\hline 2957 & $6 / 16 / 2004$ & 10:00:33 PM & & & 25.006 & & & 49.225 & 55.237 & 16.291 & & & & & .041 & 14.643 & & & 360.7639 & 53.8 & & & $2.846 \mid$ & & & & 0.148 & \\
\hline 2958 & $6 / 16 / 2004$ & $\begin{array}{ll}10: 01: 33 \mathrm{PM} \\
10: 02028\end{array}$ & 26.266 & 26.28 & 25.013 & 27.178 & & 49.528 & 55.633 & & & & & & & 14.643 & & & & 54.2 & 11.4 & & 2.878 & & 0.006 & 0.000 & 0.146 & \\
\hline 2955 & $\begin{array}{l}6 / 116 / 2004 \\
61612004\end{array}$ & $\begin{array}{l}10: 02: 33 \mathrm{PM} \\
10.0333 \mathrm{PM}\end{array}$ & $\frac{26.275}{26.273}$ & 26.289 & $\begin{array}{r}25.046 \\
25.05\end{array}$ & $\begin{array}{l}27.172 \\
27.165\end{array}$ & & $\begin{array}{l}49.426 \\
48.821\end{array}$ & $\begin{array}{l}55.436 \\
53817\end{array}$ & \begin{tabular}{|l|}
15.967 \\
17649
\end{tabular} & 1.878 & & $\begin{array}{l}-5.747 \\
5742\end{array}$ & $\begin{array}{l}46.444 \\
7.493\end{array}$ & .042 & $\begin{array}{r}14.643 \\
1.643\end{array}$ & & & $\begin{array}{l}360.7972 \\
608139\end{array}$ & $\begin{array}{l}54.0 \\
52.3\end{array}$ & \begin{tabular}{|l|l|}
11.4 \\
116
\end{tabular} & & $\begin{array}{l}2.872 \\
2700\end{array}$ & \begin{tabular}{|l|}
0.006 \\
0.006
\end{tabular} & $\begin{array}{l}0.006 \\
0.006\end{array}$ & 0.000 & 0.150 & \\
\hline 2961 & 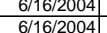 & $\begin{array}{l}10: 03: 33 \mathrm{PM} \\
1003: 33 \mathrm{PM}\end{array}$ & $\frac{26.273}{26.283}$ & $\frac{26.272}{26.276}$ & $\begin{array}{r}25.05 \\
25.074 \\
\end{array}$ & 27.165 & & $\begin{array}{l}47.821 \\
49.409\end{array}$ & \begin{tabular}{|l}
53.817 \\
55.455
\end{tabular} & $\begin{array}{l}17.609 \\
16.065\end{array}$ & $\begin{array}{r}30.508 \\
33.737 \\
\end{array}$ & & $\begin{array}{r}-5.742 \\
-5.75 \\
\end{array}$ & $\begin{array}{l}47.493 \\
48.322 \\
\end{array}$ & .042 & $\begin{array}{l}14.643 \\
14.643 \\
\end{array}$ & .004 & & $\begin{array}{l}360.8139 \\
36.8306\end{array}$ & 52.3 & \begin{tabular}{|l|l|}
11.6 \\
11.8
\end{tabular} & & $\begin{array}{l}2.700 \\
2.866\end{array}$ & \begin{tabular}{|l|}
0.006 \\
0.006
\end{tabular} & $\begin{array}{l}0.006 \\
0.006\end{array}$ & 0.000 & $\frac{160}{1147}$ & \\
\hline & $6 / 16 / 2004$ & $10: 05: 33$ PM & 26.27 & 26.259 & 25.037 & 27.132 & 26.118 & 49.631 & 55.683 & 15.565 & 34.488 & 0.295 & $\begin{array}{r}-5.15 \\
-5.736 \\
\end{array}$ & $\begin{array}{l}48.324 \\
46.682\end{array}$ & 0.042 & $\begin{array}{l}14.645 \\
14.643 \\
\end{array}$ & & \begin{tabular}{|}
304.83 \\
3650.83 \\
\end{tabular} & \begin{tabular}{|l|l|}
0.00300 \\
60.8472
\end{tabular} & \begin{tabular}{|l|l}
54.0 \\
54.2
\end{tabular} & 11.4 & & $\frac{2.800}{2.900}$ & \begin{tabular}{|l|}
0.000 \\
\end{tabular} & $\begin{array}{l}0.000 \\
0.006\end{array}$ & 0.000 & 0 & $\begin{array}{l}0.12 \\
0.13\end{array}$ \\
\hline 2963 & $6 / 16 / 2004$ & 10:05:57 PM & 26.279 & 26.263 & 25.06 & 27.161 & & 49.102 & 55.011 & 16.616 & 32.869 & 0.26 & & 52.41 & 0.041 & 14.643 & & 3651.23 & 360.8539 & 53.5 & 12.8 & & 2.826 & 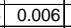 & 0.006 & 0.000 & 0.149 & \\
\hline 2964 & $6 / 16 / 2004$ & 10:06:57 PM & 26.267 & 26.251 & 25.069 & 27.114 & & 49.357 & 55.15 & 16.077 & & & $\begin{array}{r}-5.776 \\
\end{array}$ & & & 14.643 & & & 360.8706 & 53.7 & 11.8 & & 2.863 & \begin{tabular}{|l|l|} 
\\
\end{tabular} & 0.006 & 0.000 & 0.147 & \\
\hline 2965 & $6 / 16 / 2004$ & 10:07:57 PM & $\begin{array}{l}26.26 \\
36.269\end{array}$ & 26.244 & 25.056 & 27.172 & & \begin{tabular}{|l|l|} 
\\
\end{tabular} & \begin{tabular}{|l|}
55.948 \\
\end{tabular} & 15.565 & & & & & & 14.643 & & & 60.8872 & 54.5 & \begin{tabular}{|l|l|}
11.6 \\
\end{tabular} & & 2.907 & 0.006 & 0.006 & 0.000 & 0.152 & \\
\hline$\frac{2966}{2967}$ & $\begin{array}{l}6 / 116 / 2004 \\
661 / 2604\end{array}$ & $\begin{array}{l}\frac{10: 08: 57 \mathrm{PM}}{10.0957} \\
\end{array}$ & $\begin{array}{l}26.268 \\
26.265\end{array}$ & $\frac{26.257}{26.243}$ & $\begin{array}{l}25.035 \\
25.001\end{array}$ & $\begin{array}{r}27.22 \\
27.227\end{array}$ & $\frac{26.1}{26.1}$ & \begin{tabular}{|c|}
50.324 \\
50.633
\end{tabular} & \begin{tabular}{|c|}
56.236 \\
56.65 \\
\end{tabular} & \begin{tabular}{|l|}
15.014 \\
15.021
\end{tabular} & $\begin{array}{l}35.744 \\
36.031 \\
\end{array}$ & $\mid \begin{array}{l}0.321 \\
0.324\end{array}$ & $\begin{array}{l}-5.756 \\
-5.75\end{array}$ & $\begin{array}{l}43.487 \\
45.564\end{array}$ & \begin{tabular}{|c|c|c|}
0.044 \\
0.043
\end{tabular} & $\begin{array}{l}14.643 \\
14.643 \\
\end{array}$ & & \begin{tabular}{|c|}
3654.23 \\
3655.23 \\
3
\end{tabular} & $\begin{array}{l}360.9039 \\
6.020206\end{array}$ & \begin{tabular}{|l|l|}
54.8 \\
55.2
\end{tabular} & $\begin{array}{l}10.7 \\
11.2\end{array}$ & & $2.967 \mid$ & \begin{tabular}{|l|l|} 
& 0.007 \\
\end{tabular} & $\begin{array}{l}0.007 \\
0.006\end{array}$ & 0.000 & 0.152 & $\frac{0.13}{0.13}$ \\
\hline 2968 & $6 / 11 / 2004 / 2004$ & $\begin{array}{l}10: 095 / \mathrm{PM} \\
10: 057 \mathrm{P}\end{array}$ & $\begin{array}{l}26.265 \\
26.273\end{array}$ & $\frac{26.243}{26.257}$ & $\begin{array}{l}25.001 \\
25.014\end{array}$ & $\begin{array}{r}27.227 \\
27.19\end{array}$ & & $\begin{array}{l}50.633 \\
49.046\end{array}$ & \begin{tabular}{|l|}
56.652 \\
55.096 \\
\end{tabular} & $\begin{array}{l}\frac{15.021}{16.823} \\
\end{array}$ & $\begin{array}{l}36.031 \\
32.593\end{array}$ & $\begin{array}{l}.0 .344 \\
0.251\end{array}$ & & $\begin{array}{l}455.564 \\
46.071\end{array}$ & $\begin{array}{l}0.045 \\
0.04 \\
\end{array}$ & $\begin{array}{l}\frac{14.643}{14.643} \\
\end{array}$ & & & $\begin{array}{l}360.9206 \\
36.9372\end{array}$ & & $\frac{11.2}{11.3}$ & & |. 2.888 & \begin{tabular}{|l|}
0.006 \\
0.006 \\
\end{tabular} & $\frac{0.006}{0.006}$ & & & 0.13 \\
\hline & $6 / 16 / 2004$ & 10:11:57 PM & \begin{tabular}{|l|}
26.27 \\
\end{tabular} & 26.259 & 25.031 & 27.152 & 26. & 48.883 & \begin{tabular}{|l|l|}
54.893 \\
\end{tabular} & 16.817 & 32.47 & 0.251 & -5.7 & 51.075 & 0.041 & 14.643 & & & 60.9539 & \begin{tabular}{|l|} 
\\
\end{tabular} & \begin{tabular}{|l|l|} 
& 12.5 \\
\end{tabular} & & & \begin{tabular}{|l|l|}
0.000 \\
0.006
\end{tabular} & 0.006 & & 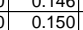 & $\frac{0.12}{0.13}$ \\
\hline 2970 & $6 / 16 / 2004$ & 10:12:57 PM & 26.274 & 26.247 & & & & & \begin{tabular}{|l|}
54.94 \\
\end{tabular} & 16.334 & 33.104 & 0.254 & & & 0.04 & 14.643 & & & & & \begin{tabular}{|l|l|}
11.5 \\
\end{tabular} & & & & & & & 0.13 \\
\hline 2971 & $6 / 16 / 2004$ & 10:13:57 PM & 26.271 & 26.26 & 25.038 & 27.218 & & 48.329 & \begin{tabular}{|l|l|l|}
54.298 \\
\end{tabular} & 17.13 & 31.623 & 0.257 & & 48.843 & $\begin{array}{l}0.04 \\
\end{array}$ & \begin{tabular}{|l|l|}
14.643 \\
\end{tabular} & & & 60.9872 & 52.8 & & & & & & & & \\
\hline 2972 & $6 / 16 / 2004$ & 10:14:5 & 26.274 & 26.268 & 25.045 & 27.261 & & & 54.797 & 16.686 & 32.604 & 0.257 & & & 0.04 & 14.643 & & & & 53.3 & 1.9 & & & 0.006 & 5.0 & & & \\
\hline 2973 & $6 / 16 / 2004$ & & & & 25.044 & & & 49.291 & 55.394 & 16.374 & 33.306 & & & 47.4. & $\begin{array}{ll}0.041 \\
\end{array}$ & 14.643 & & & & & & & & & & & & \\
\hline 2974 & $6 / 16 / 2004$ & $10: 16: 57 \mathrm{PM}$ & & & 25.033 & 27.219 & & 48.586 & & 16.771 & 32.269 & & & & 0.04 & $\begin{array}{l}14.643 \\
\end{array}$ & & & & & & & & & & & & \\
\hline & $6 / 16 / 2004$ & 10:17 & 26.258 & 26.25 & 25.045 & 27.22 & & & 54.142 & $\begin{array}{ll}17.063 \\
\end{array}$ & 31.764 & & & 53 & 0.038 & $\begin{array}{l}14.643 \\
\end{array}$ & & & & 52. & 3.1 & & & 0.006 & 1006 & & & \\
\hline & $6 / 16 / 2$ & $10: 18: 57 \mathrm{PN}$ & 26.27 & 26.27. & 25.061 & 27.227 & & & 54.762 & 16.992 & 32.132 & 0.306 & & 49.4 & 0.039 & 14.643 & & & & 53 & 2.1 & & & & & & & \\
\hline & & & & 26.27 & 25.098 & 27.249 & & 48.6 & 54.644 & 16.509 & & & & & .04 & 14. & & & & & & & & & .006 & & & \\
\hline & $6 / 16 / 2004$ & & & & 25.09 & & & & & 16.541 & & & & & & & & & & & & & & & & & & \\
\hline & $6 / 16 / 2$ & 10:221:57 PM & 26.264 & & 25.01 & & & 49.18 & 5.281 & & 33.31 & 0.32 & & 47.6 & & 14.6 & & & & 53.8 & 11.7 & & .84 & & & & & \\
\hline & $\frac{61016,2}{6 / 16 / 2}$ & & & $\frac{20.22}{26.27}$ & 250.083 & $\mid$ & & & 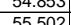 & & $\begin{array}{l}33.094 \\
33.144 \\
3\end{array}$ & & & & & 14. & & & & 5.40 & 11.5 & & & & & & & \\
\hline & $6 / 16 / 2004$ & & 年 & 26.17 & 25083 & & & & 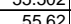 & & 706 & & & & & 14.643 & & & & & & & & & & & & \\
\hline 298 & $6 / 16 / 2004$ & & $\frac{2.275}{26.275}$ & 2626 & 25076 & & & & 54.988 & & & & & & 0.041 & $\frac{14.045}{14.643}$ & & & & $\frac{47}{53}$ & $\frac{\frac{1.1 .1}{1114}}{4}$ & & & & & & $\frac{0.474}{0147}$ & \\
\hline 29 & $6 / 16 / 2 C$ & $10: 26$ & 26.272 & $\frac{26.26}{26.26}$ & 25.059 & 27.269 & & 49.8 & 55.363 & 16.056 & 33.648 & & & $\frac{4.61}{44.61}$ & & 14. & & & & & $\frac{1.2}{10.2}$ & & & 0.006 & 0.006 & & & \\
\hline & $6 / 16 / 2004$ & & & & & & & & & & & & & & & & & & & & & & & & & & & \\
\hline & $6 / 16 / 2004$ & & 26.288 & 26.24 & 25.065 & 27.245 & & & 55.336 & 16.1 & & & & & & & & & & & 0.8 & & & & & & & \\
\hline & $6 / 16 / 2$ & & & 20.4 & 25.097 & 27.293 & & & 54.12 & 17.134 & & & & & & & & & & & 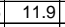 & & & & & & & \\
\hline & & & & 26.2. & 25.064 & 27.289 & & & 54.474 & 16.619 & & & & & & & & & & & & & & & & & & \\
\hline & 6/116/2004 & & 26.289| & & 25.065 & 27.216 & & 48. & 54.52 & 16.711 & 32.247 & & & 51. & & 14.6 & & & & 53 & 12 & & & 0.006 & 6 & & 0.144 & \\
\hline & $6 / 16 / 2$ & & 26.29 & & & & & & & & & & & & & & & & & & & & & & & & & \\
\hline & $6 / 16 / 2$ & & 26.286 & 26.21 & 25.0 & 27.223 & & & 54.487 & & & & & & & 14. & & & & 53.0 & 12.0 & & & & & & & \\
\hline & $6 / 116 / 2004$ & & & 26.24 & & & & & & & & & & & & & & & & & & & & & & & & \\
\hline & & & 294 & 26. & 25.0 & 2.72 .28 & & & 54. & & 32.645 & & & & & & & & & & & & & & & & & \\
\hline & $\frac{6 / 101}{6 / 16 \mid}$ & & 263 & 2621 & $\frac{25.11}{2511}$ & 27187 & & 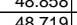 & $\begin{array}{l}54.926 \\
54868\end{array}$ & & $\begin{array}{l}32.649 \\
32516\end{array}$ & & & & $\begin{array}{r}0.035 \\
0.04\end{array}$ & . & & & & 534 & 110 & & & & & & & \\
\hline & $6 / 16 / 2004$ & & $\frac{26}{263}$ & & 251 & 271 & & & 5 & & & & & & & & & & & & & & & & & & & \\
\hline & & & & & & & & 48.5 & 54712 & & & & & & & & & & & & & & & & & & & \\
\hline & $6 / 16 / 2$ & & $\frac{26.309}{26.309}$ & $\frac{2.2 .202}{26.202}$ & $\frac{25.124}{25.12}$ & $\frac{27.191}{27.191}$ & & 49.3 & 55.5 & & & & & & & & & & & & 3 & & & & & & & \\
\hline & $6 / 16 / 2004$ & & & & & & & & & & & & & & & & & & & & & & & & & & & \\
\hline & & & & 26.1 & & & & & & & & & & & & & & & & & & & & & & & & \\
\hline & $6 / 16 / 2004$ & & & 26.2 & & 27.2 & & 48 & 55 & & & & & & & 14. & & & & & & & & & & & & \\
\hline & $6 / 16 / 2$ & 10:44: & & 6.20 & 25.12 & 27.164 & & 48.8 & 54.82 & $16.4 \mathrm{~s}$ & & 0.3 & & 48.4 & 0 & 14.6 & & & & & & & & & & & 45 & \\
\hline & $6 / 16 / 200$ & 10:45: & & 26.19 & 25.12 & 27.179 & & 49.0 & 55.18 & 16.4 & 32.95 & 0.3 & & & & 14.6 & & & & & & & & & & & 49 & \\
\hline & $6 / 16 / 2$ & & & 26.2 & 25.1. & 27.195 & & 48. & 54.907 & & & & & & & 14. & & & & & & & & & & & & \\
\hline & $6 / 16 / 2$ & & & 26 & & & & & 55.0 & & & & & & & & & & & 5 & 11 & & & & & & & \\
\hline & $6 / 16 / 2 / 2$ & & & & & & & & & & & & & & & & & & & & & & & & & & & \\
\hline & $6 / 11 / 2004$ & & 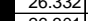 & 2.2011 & $\frac{2.1 .1}{2.5}$ & 27.234 & & 48. & & & & & & & & & & & & & & & & & & & & \\
\hline & 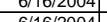 & & (3) & $\frac{2.1 .16}{26.16}$ & 20.1501 & 27.220 & & 4 & (55.391 & 10.510 & 35.1 & 0.468 & & 年 & & $\begin{array}{l}14.645 \\
1.637\end{array}$ & & & & & & & & & & & 148 & \\
\hline 3010 & $\begin{array}{l}6 / 16 / 2004 \\
6\end{array}$ & 10:52:57 PM & \begin{tabular}{|l|l|l|l}
26.298 \\
\end{tabular} & $\frac{20.100}{26.161}$ & \begin{tabular}{|l|}
25.051 \\
25.079 \\
\end{tabular} & \begin{tabular}{|l|}
27.165 \\
27.165 \\
\end{tabular} & 26.185 & 4.5905 & \begin{tabular}{|l|}
55.408 \\
55.208
\end{tabular} & \begin{tabular}{|l|l|}
16.568 \\
\end{tabular} & $\begin{array}{l}00.250 \\
33.111 \\
\end{array}$ & \begin{tabular}{|l|l|} 
& 0.440 \\
\end{tabular} & -5.666 & $\begin{array}{l}47.727 \\
4.727\end{array}$ & $\frac{0.053}{0.04}$ & \begin{tabular}{|l|}
14.645 \\
14.643 \\
\end{tabular} & & 3698.23 & $\begin{array}{l}361.6372 \\
\end{array}$ & \begin{tabular}{|l|l|}
53.7 \\
\end{tabular} & \begin{tabular}{|l|l|}
11.7 \\
\end{tabular} & & 2.838 & \begin{tabular}{|l|}
0.006 \\
\end{tabular} & 0.006 & 0.000 & 0.145 & \begin{tabular}{|ll}
5 & 0.1 \\
5 & 0.1
\end{tabular} \\
\hline
\end{tabular}


WSRC-TR-2005-00105, REVISION 0

SRNL-RPP-2005-00012, REVISION 0

RUN \# 3.03A AND B; FIRST AND SECOND HALF OF SLURRY DEWATERING - CONT.

\begin{tabular}{|c|c|c|c|c|c|c|c|c|c|c|c|c|c|c|c|c|c|c|c|c|c|c|c|c|c|c|c|c|}
\hline & $\mathrm{A}$ & & D & $E$ & $\mathrm{~F}$ & G & $\mathrm{H}$ & $\mathrm{J}$ & K & $\mathrm{L}$ & M & $\mathrm{N}$ & 0 & Q & $\mathrm{R}$ & $\mathrm{s}$ & $\begin{array}{ll}T \\
\end{array}$ & $\mathrm{v}$ & w & $x$ & $Y \mid$ & & $A A$ & $A B$ & $A C$ & $A D$ & AE & \\
\hline 3011 & 6/16/2004 & 10:53:57 PM & 26.291 & 26.1 & 25.098 & 27.134 & 26.159 & 48.895 & 55.162 & 16.475 & 32.85 & 0.463 & -5.658 & 50.975 & 0.042 & 14.643 & 0.004 & 3699.23 & 361.6539 & 53.7 & 12.5 & 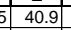 & 2.818 & 0.006 & 0.006 & 0.000 & 0.153 & \\
\hline & & 10:54:57 PM & 26.3 & 26.17 & 25.131 & & 26.157 & 48.999 & 55.316 & $\begin{array}{l}16.58 \\
\end{array}$ & 32.889 & 0.474 & -5.655 & & 0.04 & 14.643 & 0.004 & & 361.6706 & 53.8 & 11.8 & & & & & & 0.145 & \\
\hline 资 & 6/116/2004 & 10:55:57 PM & 26.306 & 26.18. & $\begin{array}{l}25.112 \\
2.10\end{array}$ & 27.183 & 26.178 & $\begin{array}{ll}49.028 \\
\end{array}$ & \begin{tabular}{|l|l|}
55.254 \\
5.571
\end{tabular} & $\begin{array}{ll}16.666 \\
19.76\end{array}$ & 32.704 & 0.457 & $\begin{array}{l}-5.646 \\
-5607\end{array}$ & 46.757 & $\begin{array}{l}0.041 \\
\end{array}$ & $\begin{array}{l}14.643 \\
\end{array}$ & & 3701.23 & Oravere & 53.8 & 11.5 & $\begin{array}{|ll|}5 & 40.9 \\
\end{array}$ & 2.010 & 0.006 & 0.006 & 0.000 & 0.149 & \\
\hline 3014 & 6/16/2004 & 10:56:57 PM & 26.311 & 26.1 & 25.118 & 27.193 & 26.184 & $\begin{array}{l}49.343 \\
\end{array}$ & \begin{tabular}{|l|l|}
55.571 \\
5.72
\end{tabular} & $\begin{array}{l}16.746 \\
\end{array}$ & 32.916 & 0.442 & $\begin{array}{l}-5.687 \\
\end{array}$ & $\begin{array}{l}47.629 \\
\end{array}$ & 0.041 & 14.643 & 0.004 & 02.23 & 361.7039 & 54.1 & 11.7 & $\begin{array}{lll}7 & 41.1 \\
\end{array}$ & 2.836 & \begin{tabular}{|l|l|} 
\\
\end{tabular} & 0.006 & 0.000 & 0.148 & \\
\hline$\frac{3015}{3016}$ & 6/16/2004 & $\frac{10: 57: 57 \text { PM }}{10.58 .57 \text { PM }}$ & $\frac{26.324}{26321}$ & $\frac{26.20}{26.2}$ & 25.145 & 27.136 & & $\begin{array}{l}49.287 \\
49227\end{array}$ & \begin{tabular}{|l|}
55.428 \\
55.411 \\
\end{tabular} & \begin{tabular}{|l|l|}
16.623 \\
16725 \\
\end{tabular} & $\begin{array}{l}33.104 \\
32.975 \\
\end{array}$ & 0.419 & & \begin{tabular}{|l|l|}
50.785 \\
46306
\end{tabular} & $\begin{array}{l}0.044 \\
0.039 \\
\end{array}$ & \begin{tabular}{|l|l|}
14.643 \\
14634 \\
\end{tabular} & & & $\begin{array}{l}361.7206 \\
6.7272 \\
6\end{array}$ & 54.0 & $\begin{array}{l}0.12 .4 \\
0 \\
0\end{array}$ & & \begin{tabular}{|l|}
2.840 \\
2820
\end{tabular} & & 0.007 & & 0.159 & \\
\hline$\frac{5010}{3017}$ & $\begin{array}{l}661166 / 2004 \\
6 / 16 / 2004\end{array}$ & $\begin{array}{l}\text { 10:58:5:57 PM } \\
0: 57 \text { PM }\end{array}$ & $\frac{26.321}{26.334}$ & $\frac{26.2}{26.22}$ & $\frac{25.157}{25.171}$ & \begin{tabular}{|l|l|}
27.183 \\
27.182
\end{tabular} & $\begin{array}{l}26.20 \\
26.207 \\
\end{array}$ & $\begin{array}{r}49.227 \\
49.241 \\
\end{array}$ & \begin{tabular}{|l}
55.3411 \\
55.372
\end{tabular} & \begin{tabular}{|l|}
16.725 \\
16.709 \\
\end{tabular} & 32.975 & $\begin{array}{ll}5 & 0.474 \\
3 & 0.477\end{array}$ & $\begin{array}{l}-5.571 \\
-5.536 \\
\end{array}$ & \begin{tabular}{|l|}
46.306 \\
46.065 \\
\end{tabular} & \begin{tabular}{|l|}
0.039 \\
0.041
\end{tabular} & \begin{tabular}{|l|}
14.643 \\
14.643 \\
\end{tabular} & $\begin{array}{l}0.004 \\
0.004\end{array}$ & $\begin{array}{l}3704.23 \\
3705.23\end{array}$ & $\begin{array}{l}361.7772 \\
36.7539 \\
3\end{array}$ & 53.9 & \begin{tabular}{l|l}
9 & 11.3 \\
9 & 11.3
\end{tabular} & $\begin{array}{l}3 \\
3\end{array}$ & \begin{tabular}{|l|}
2.834 \\
2.835 \\
\end{tabular} & \begin{tabular}{|l|}
0.006 \\
0.006 \\
\end{tabular} & $\begin{array}{l}0.006 \\
0.006\end{array}$ & $\begin{array}{l}0.000 \\
0.000\end{array}$ & \begin{tabular}{|l|l|l|}
0.141 \\
0.148
\end{tabular} & 0.12 \\
\hline 3018 & & & & 26.22 & 25.154 & & 26.2 & & & & 32.647 & & -5.559 & 45.868 & 0.039 & $\begin{array}{l}14.045 \\
14.643 \\
\end{array}$ & 0.004 & 3706.23 & 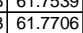 & \begin{tabular}{|l|l|}
5.9 \\
54.0
\end{tabular} & $\frac{11.5}{11.2}$ & 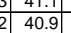 & 20.835 & & & 0.000 & $\begin{array}{l}0.148 \\
0.142 \\
\end{array}$ & $\frac{0.13}{0.12}$ \\
\hline 3019 & $6 / 16 / 2004$ & 11:01:57 PM & 26.343 & & 25.159 & 27.15 & & 49.469 & 55.757 & 16.689 & 33.164 & 0.474 & -5.548 & 46.371 & 0.041 & 14.643 & 0.004 & 3707.23 & 361.7872 & 54.3 & 11.4 & & 2.849 & & & & 0.147 & \\
\hline & 6/16/2004 & & & & & 27.187 & & 49.623 & & & & & -5.548 & 45.382 & & $\begin{array}{l}14.643 \\
\end{array}$ & & 08.23 & 361.8039 & 54.4 & 11.1 & & 2.855 & 0.006 & & 0.000 & D.143 & \\
\hline 3021 & 6/116/2004 & $\begin{array}{l}\text { 11:03:57 PM } \\
11: 015 \mathrm{PM}\end{array}$ & $\begin{array}{ll}26.347 \\
2.237 \\
\end{array}$ & 26.23 & 25.188 & 27.209 & & 49.295 & $\begin{array}{l}55.428 \\
5.515\end{array}$ & 16.813 & 32.957 & 0.474 & -5.548 & 47.742 & 0.04 & $\begin{array}{ll}14.643 \\
\end{array}$ & & & 361.8206 & 54.0 & 11.7 & $\begin{array}{ll}7 & 41.1 \\
\end{array}$ & 2.835 & \begin{tabular}{|l|l|} 
\\
\end{tabular} & 0.006 & 0.000 & 0.144 & \\
\hline 023 & $\begin{array}{l}6 / 116 / 2004 \\
61612004\end{array}$ & $\begin{array}{l}11: 04: 57 \mathrm{PM} \\
11.0557 \mathrm{PM}\end{array}$ & $\frac{26.337}{26.34}$ & $\frac{26.23}{26.23}$ & $\frac{25.178}{25.156}$ & $\frac{27.214}{27.237}$ & 26.18. & \begin{tabular}{|l|}
49.457 \\
49.316
\end{tabular} & \begin{tabular}{|c|}
55.515 \\
5.508
\end{tabular} & \begin{tabular}{|c|}
16.818 \\
16766
\end{tabular} & $\begin{array}{l}33.109 \\
33004\end{array}$ & $\begin{array}{l}0.544 \\
0.58\end{array}$ & & $\begin{array}{l}44.719 \\
32.247\end{array}$ & $\begin{array}{l}0.038 \\
00399\end{array}$ & \begin{tabular}{|l|l|l|}
14.643 \\
14643
\end{tabular} & 0.004 & \begin{tabular}{|c|}
3710.23 \\
371123
\end{tabular} & $\begin{array}{l}61.8372 \\
6159539\end{array}$ & 54.0 & 11.0 & 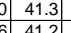 & \begin{tabular}{|l|}
2.846 \\
2838 \\
\end{tabular} & \begin{tabular}{|l|l|} 
\\
\end{tabular} & $\begin{array}{l}0.006 \\
0.006\end{array}$ & $\begin{array}{l}0.000 \\
\end{array}$ & 0.137 & 0.12 \\
\hline \begin{tabular}{|l|l|l|l|l|}
3024 \\
\end{tabular} & $6 / 116 / 2004$ & $\begin{array}{l}\frac{11005575 \mathrm{PM}}{11} \\
1105: 57\end{array}$ & $\begin{array}{r}26.34 \\
26.367 \\
\end{array}$ & $\frac{26.23}{26.25}$ & $\frac{25.156}{25.224}$ & 27.237 & & $\begin{array}{r}49.316 \\
49.44 \\
\end{array}$ & $\begin{array}{r}r 5.508 \\
55.62\end{array}$ & $\begin{array}{c}16.766 \\
16.826\end{array}$ & $\begin{array}{r}33.004 \\
33.02 \\
\end{array}$ & $\begin{array}{l}0.538 \\
0.544\end{array}$ & $\begin{array}{l}-5.55 \\
-5.54 \\
\end{array}$ & 47.422 & $\begin{array}{l}0.039 \\
0.04\end{array}$ & $\begin{array}{l}\frac{14.643}{14.643} \\
\end{array}$ & & $\begin{array}{l}3711.23 \\
37712.23\end{array}$ & $\begin{array}{l}361.8399 \\
36706\end{array}$ & 54.1 & $\frac{10.6}{11.6}$ & $\begin{array}{l}6 \quad 41.2 \\
6 \\
6\end{array}$ & $\begin{array}{l}2.838 \\
2.843\end{array}$ & \begin{tabular}{|l|}
0.006 \\
0.006
\end{tabular} & $\begin{array}{l}0.006 \\
0.006\end{array}$ & $\begin{array}{l}0.000 \\
0.000\end{array}$ & \begin{tabular}{|l|l|l|l|l}
0.144 \\
\end{tabular} & 0.12 \\
\hline $\begin{array}{ll}3025 \\
\end{array}$ & $6 / 16 / 2004$ & 11:07:57 PM & 26.349 & 26.23 & 25.216 & 27.221 & 26.226 & 49.519 & 55.83 & \begin{tabular}{|l|}
16.956 \\
\end{tabular} & 32.967 & 0.541 & & 51.062 & 0.041 & 14.643 & 0.004 & 3713.23 & 361.8872 & 54.4 & 12.5 & & $\frac{2.843}{2.844}$ & \begin{tabular}{|l|}
0.006 \\
\end{tabular} & 0 & & & 013 \\
\hline & $6 / 16 / 2004$ & 11:08:57 PM & 26.346 & 26.2 & 25.262 & 27.253 & & 49.658 & 56.064 & $\begin{array}{l}16.638 \\
\end{array}$ & 33.351 & 0.544 & & 45.372 & .038 & 14.643 & & 371423 & & 54.6 & & & & & & & & \\
\hline & $6 / 16 / 2004$ & & & 26.2 & 25.243 & & 26.194 & 49.59 & \begin{tabular}{|l|l|}
56.053 \\
\end{tabular} & 16.837 & 33.125 & 0.544 & & & & $\begin{array}{l}14.643 \\
\end{array}$ & & & 361.9206 & 54.6 & & & & & & & & \\
\hline 3028 & & 11:10:57 PM & 26.353 & & 25.255 & 27.235 & & & & & & & & & 038 & $\begin{array}{l}14.643 \\
\end{array}$ & & & $\begin{array}{l}361.9372 \\
\end{array}$ & 53.3 & 12.6 & & & & 0.006 & 0.000 & 0.142 & \\
\hline & $\begin{array}{l}6 / 116 / 2004 \\
61612004\end{array}$ & $\begin{array}{l}\frac{11: 11: 57 \mathrm{PM}}{11} \\
11.1257\end{array}$ & & $\frac{26.22}{26.22}$ & $\begin{array}{l}25.307 \\
25.309\end{array}$ & & & & \begin{tabular}{|r|r|r|}
55.21 \\
55.372
\end{tabular} & $\begin{array}{l}17.251 \\
16.992\end{array}$ & $\begin{array}{l}32.119 \\
32596\end{array}$ & & & $\begin{array}{l}48.371 \\
44536\end{array}$ & $\begin{array}{l}0.039 \\
\end{array}$ & $\begin{array}{l}14.643 \\
14643\end{array}$ & & & & 53.7 & 11.9 & & 2.793 & \begin{tabular}{|l|l|}
0.006 \\
0.06
\end{tabular} & 0.006 & 0.000 & 0.142 & 0.12 \\
\hline 3031 & $\frac{6 / 116 / 2004}{66112604}$ & $\begin{array}{l}\frac{11: 12: 57 \mathrm{PM}}{111.1557} \\
\end{array}$ & $\frac{26.368}{26.373}$ & $\frac{26.22}{26.22}$ & $\begin{array}{r}25.309 \\
25.3\end{array}$ & $\begin{array}{r}27.26 \\
27.225\end{array}$ & $\frac{26}{26 .}$ & & $\frac{55.372}{55.544}$ & $\begin{array}{c}\mid 16.992 \\
16.756\end{array}$ & 5.596 & & & $\begin{array}{l}44.536 \\
47.326\end{array}$ & $\begin{array}{l}0.04 \\
0.04\end{array}$ & $\frac{14.643}{14.643}$ & 0.004 & & & 54.1 & $\frac{1.9}{11.6}$ & & $2.819 \mid$ & \begin{tabular}{|l|}
0.006 \\
0.006
\end{tabular} & $\begin{array}{l}0.006 \\
0.006\end{array}$ & 0.000 & $\frac{145}{144}$ & $\frac{5}{4}$ \\
\hline 3032 & $6 / 16 / 2004$ & 11:14:57 PM & 26.381 & 26.2 & 25.282 & 27.303 & 26.2 & 49.283 & 55.384 & 16.82 & 32.877 & 0.55 & -5.533 & 45.583 & 0.039 & $\begin{array}{l}14.643 \\
14.043\end{array}$ & $\begin{array}{l}0.004 \\
0.004\end{array}$ & $\begin{array}{l}3719.25 \\
3720.23\end{array}$ & \begin{tabular}{|l|l|}
0.1 .5712 \\
62.0039
\end{tabular} & 53.9 & $\frac{11.6}{11.2}$ & & $\frac{2.031}{2.832}$ & \begin{tabular}{|l|}
0.000 \\
0.006
\end{tabular} & $\begin{array}{l}0.006 \\
0.006\end{array}$ & $\begin{array}{l}0.000 \\
0.000\end{array}$ & 0.144 & $\begin{array}{l}0.12 \\
0.12\end{array}$ \\
\hline & 6/16/2004 & 11:15:57 PM & 26.397 & 26.23 & 25.324 & 27.279 & & 49.111 & 55.558 & 16.743 & 32.864 & 0.596 & & & 0.04 & $\begin{array}{l}14.643 \\
\end{array}$ & & 3721.23 & 362.0206 & 54.1 & 11.3 & & 2.826 & \begin{tabular}{|l|l|} 
\\
\end{tabular} .006 & 0.006 & 0.000 & 0.144 & \\
\hline & $6 / 16 / 2004$ & 11:16:57 PM & 26.392 & 26.21 & 25.304 & 27.274 & & 49.366 & 55.705 & $\begin{array}{l}16.633 \\
\end{array}$ & 33.146 & 0.593 & & \begin{tabular}{|l|l|}
49.064 \\
\end{tabular} & 0.041 & \begin{tabular}{|l|l|}
14.643 \\
\end{tabular} & & |22.23 & & 54.2 & 12.0 & $\begin{array}{lll}041 \\
\end{array}$ & 2.844 & \begin{tabular}{|l|} 
\\
\end{tabular} & 0.006 & & & \\
\hline 3035 & & 11:17:57 PM & 26.389 & & & 27.306 & & & & 16.422 & & & & & & 14.643 & & & & & & & & & & & 0.144 & \\
\hline$\frac{3036}{3027}$ & & & & & & & & 48.806 & & & 31.896 & & & 48.13 & \begin{tabular}{|c|c|}
0.04 \\
\end{tabular} & 14.643 & & & & & & & & & & & & \\
\hline 3037 & $6 / 16 / 2004$ & 11:19:57 PM & & & 25.294 & 27.29 & & 48.47 & 54.748 & 17.255 & $\begin{array}{l}31.647 \\
\end{array}$ & 0.564 & & & & 14.643 & & & & & & & & & & & & \\
\hline 3038 & $6 / 16 / 2004$ & 11:20:57 PM & 26.408 & 26.21 & 25.305 & 27.2 & & 48.95 & 55.266 & \begin{tabular}{|l|l|l|l|l}
17.323 \\
\end{tabular} & 31.988 & 0.587 & & 48.3 & 0.039 & 14.643 & & & & 53.8 & 11.9 & & 8790 & 0.006 & 1006 & & 0.142 & \\
\hline$\frac{3039}{3004}$ & $6 / 16 / 2004$ & 11:21:57 PM & 26.42 & 26.21 & 25.322 & 27.212 & & 48.816 & 55.254 & 17.136 & 32.025 & 0.596 & & 52.137 & 0.04 & 14.643 & & & 62.1206 & 53.8 & 12.8 & & 2787 & 0.006 & 0.006 & & & \\
\hline$\frac{3040}{3041}$ & & $11: 22: 5$ & $\frac{26.426}{2.20}$ & & .298 & & & 48.86 & 55.046 & 10500 & 32.427 & 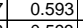 & & 10 & 0.04 & 14.643 & & & & & & & & & & & & \\
\hline & $6 / 16 / 2004$ & $11: 2$ & 26.423 & 26.21 & $\frac{25.28}{252.21}$ & & & 48.98 & & $\frac{16.588}{16720}$ & $32.8 / 3$ & & & & & $\frac{11.643}{14.642}$ & & & & & & & & & & & & \\
\hline & & 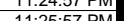 & & & $\frac{25.251}{2050}$ & & & & & & 32.944 & & & & & $\begin{array}{l}14.045 \\
1.612\end{array}$ & & & & & & & & 0.006 & & & & \\
\hline & $\begin{array}{l}6 / 1 / 16 / 2004 \\
6 / 1642004\end{array}$ & $\begin{array}{ll}11.255 \\
1126.57 \mathrm{PM}\end{array}$ & $\frac{26.431}{26.412}$ & $\frac{20.20}{26.19}$ & $\begin{array}{l}25.278 \\
25238\end{array}$ & $\begin{array}{l}27.195 \\
27.189\end{array}$ & & $\begin{array}{r}49.28 \\
49\end{array}$ & $\begin{array}{l}55.343 \\
55618\end{array}$ & \begin{tabular}{|l|}
10.4606 \\
16.546
\end{tabular} & $\begin{array}{r}3.1 \\
33.139 \\
\end{array}$ & $\begin{array}{l}0.5933 \\
0.593\end{array}$ & & 13 & $\frac{39}{04}$ & $\frac{14.643}{14.643}$ & & & & & & & & & & & & \\
\hline 04 & $6 / 16 / 2004$ & 11:27:57 PM & 26.429 & 26.20 & 25.256 & 27.211 & & 48.52 & 54.98 & \begin{tabular}{|l|l|}
17.425 \\
\end{tabular} & 31.487 & 0.593 & -5.5 & 50.91 & 0.038 & 14.643 & 0.00 & 3733.2 & \begin{tabular}{|l|l|}
62.2206 \\
\end{tabular} & 53.5 & 12.5 & & 758 & \begin{tabular}{|l|l|}
0.006 \\
\end{tabular} & 0.006 & 0.000 & 0.141 & \\
\hline & 6/116/2004 & $11: 28: 57 \mathrm{PP}$ & \begin{tabular}{l|l}
26.432 \\
\end{tabular} & 26.20 & 25.274 & 27.234 & & 49.11 & 55.355 & \begin{tabular}{|l|l|}
17.681 \\
\end{tabular} & 31.847 & $\begin{array}{l}0.593 \\
\end{array}$ & & 51.142 & & $\begin{array}{l}14.643 \\
\end{array}$ & & & & 53.9 & 12.5 & & & 0.006 & & 0.000 & 0.146 & 6 \\
\hline & $6 / 16 / 2$ & & 26.427 & 26.20 & 289 & & & 48.9 & 55.214 & 17.8 & 31.69 & 0.6 & & & & 14. & & & & 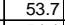 & & & & & 0.006 & & & \\
\hline 004 & $6 / 16 / 2004$ & & & & 25.304 & 27.164 & & & & 17.6 & - & & & & & & & & & & & & & & & & & \\
\hline & $6 / 166 / 2004$ & 11:31:57 PM & 26.423 & 26.199 & 25.325 & 27.21 & & & $55.4 / 3$ & 1.5 & 31.865 & 0.71 & & 45.5 & & 14.6 & & & & & $1.2 \mathrm{r}$ & & & & & & 0.110 & \\
\hline & $\frac{61016,2}{6 / 16 / 2}$ & 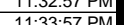 & $\frac{20.4<3}{26428}$ & $\frac{26.19}{26.9}$ & 252.34 & 20.1.106 & & 年 & S5.534 & & & & & & & 14. & & & & & & & & & & & & \\
\hline & $6 / 16 / 2004$ & & $\begin{array}{l}20.425 \\
26,35\end{array}$ & & $\frac{2.05}{2532}$ & & & 4886 & & & & & & & & 14643 & & & & & & & & & & & & \\
\hline 305 & $6 / 16 / 2004$ & 11:35:5 & $\frac{2.401}{26.451}$ & 26.19 & 25379 & & & & 55.486 & & & & & & & $\frac{14.045}{14.643}$ & & & & (5) & $\frac{1.6}{122}$ & & & & & & & \\
\hline 301. & $6 / 16 / 2004$ & $11: 36:$ & 26.427 & 26.17 & 25.354 & 27.129 & & 48.9 & 55.338 & 17.2 & 32.044 & & & & & & & & & & $2.1 \mathrm{Cl}$ & & 278 & 0.006 & 0.006 & & 149 & \\
\hline & $6 / 16 / 2004$ & & & & & & & & & & & & & & & & & & & & & & & & & & & \\
\hline & & & 26.454 & 26.17 & 25.361 & & & & 56.054 & $\begin{array}{l}17.122 \\
\end{array}$ & & 0.8 & & & & & & & & & 16 & & & & & & & \\
\hline & $6 / 16 / 200$ & & 20.40 & 26.19 & 25.362 & & & 48.6 & & & 31.309 & & & & & & & & & & & & & & & & & \\
\hline & $6 / 16 / 2004$ & 11:40:57 PM & 26.461 & 26.1 & 25.403 & 27.164 & & & & \begin{tabular}{|l|l|l|l|l}
17.373 \\
\end{tabular} & 32.002 & & & & & & & & & 50 & 15 & & & & & & 42 & \\
\hline & 6/16/2004 & & 26.467 & 26.19 & 25 & 27.204 & & 49.2 & 55.857 & 17.0 & 32.626 & 0.822 & & & .04 & 14.6 & & & & 54 & 12. & & & .006 & & & D.144 & \\
\hline & $6 / 16 / 2$ & 11:42: & & 26. & & & & & & & & & & & & & & & & & & & & & & & & \\
\hline (3) & 6/16/20 & & 26.463 & 26. & 25.381 & 27.221 & & 49.1 & 55.55 & 17.1. & 32.54 & & & & 0.6 & 14. & & & & & 11.4 & & & & & & & \\
\hline & $6 / 16 / 20$ & & 26.49 & 26.19 & & & & & & & & & & & & & & & & & & & & & & & & \\
\hline & & & & 26.16 & 25.376 & & & 49.1 & 53.608 & & 32.569 & & & & & & & & & & & & & & & & & \\
\hline & $\frac{6 / 116}{616}$ & & $\frac{26 .}{26}$ & $\frac{26.17}{2617}$ & $\begin{array}{l}\frac{2.5406}{25397} \\
20\end{array}$ & 20.1864 & & 494.297 & $\begin{array}{l}55.815 \\
55.991\end{array}$ & $\frac{16.887}{1687}$ & $\begin{array}{r}32.83 \\
33.051\end{array}$ & & & & & . & & & & 54 & se & & & & & & & \\
\hline 306 & $6 / 16 / 2004$ & & 26.475 & 26.16 & 25.41 & & & & 5514 & & & & & & & & & & & & & & & & & & & \\
\hline & & & & & & & & & & & & & & & & & & & & & & & & & & & & \\
\hline 306 & $6 / 16 / 2$ & & $\frac{26.493}{26.493}$ & 26.18 & $\frac{25.42}{25.42}$ & 27.285 & & 49.1 & 55.743 & $\begin{array}{l}17.67 \\
17\end{array}$ & 31.888 & 0.8 & & & & & & & & & 12.6 & & & & & & & \\
\hline & 6/16/2004 & & & & 25.4 & & & & & & & & & & & & & & & & & & & & & & & \\
\hline & & & & & & & & & & & & & & & & & & & & & & & & & & & & \\
\hline & $6 / 16 / 200$ & & & 26 & 25 & 27.2 & & 48. & 55.36 & & & & & & & & & & & & & & & & & & & \\
\hline & $6 / 16 / 2$ & 11:54:57 & & 26.18 & 25.398 & 27.213 & & 48.9 & 55.627 & 17.48 & $31.8 \mathrm{C}$ & 0. & & 45.94 & & 14.6 & & & & & & & & & & & & \\
\hline & $6 / 16 / 20$ & & & & 25.37: & 27.195 & & 48.8 & 55.469 & 17.35 & 31.99 & 0.87 & & 50. & & 14.6 & & & & & & & & & & & & \\
\hline & $6 / 16 /$ & & & 26.18 & 25 & 27.22 & & & & & 31.945 & 0.8 & & & & 14. & & & & & & & & & & & & \\
\hline & $6 / 16 / 2$ & & 26. & 26. & 25.35 & 27.1 & & 49. & 55.834 & 17. & 32.095 & & & & & & & & & 5 & 12.3 & & & & & & 42 & \\
\hline & $6 / 16 / 2 / 2$ & & 26.5 & & & & & & & & & & & & & & & & & & & & & & & & & \\
\hline & $6 / 110 / 2$ & & & 26.17 & $\frac{25.31}{25.30}$ & 27.184 & & $4 . .2$ & & 18.209 & 30.12 & & & & & & & & & & & & & & & & & \\
\hline & $0 / 1712004$ & & 20.051 & 2.17 & 20.354 & 27.1944 & & 48.09 & 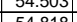 & 17.915 & 30.005 & 0.8 & & & & 14.045 & & & & & 0 & & & & & & & \\
\hline $\begin{array}{l}3079 \\
3080\end{array}$ & \begin{tabular}{|l|}
$6 / 177 / 2004$ \\
$6 / 172004$
\end{tabular} & $\begin{array}{l}\text { 12:01:19 A AM } \\
1202: 16 \mathrm{AM}\end{array}$ & $\frac{26.518}{26.518}$ & $\frac{26.18}{26.17}$ & \begin{tabular}{|l|}
25.411 \\
25.411 \\
\end{tabular} & \begin{tabular}{|l|}
27.2181 \\
27.181 \\
\end{tabular} & 26.196 & $\begin{array}{l}48.348 \\
48.515 \\
\end{array}$ & \begin{tabular}{|l|}
54.818 \\
55.044 \\
\end{tabular} & \begin{tabular}{|l|}
17.7377 \\
17.787 \\
\end{tabular} & $\begin{array}{l}31.089 \\
31.132 \\
\end{array}$ & 0.882 & & $\mid$\begin{tabular}{|c|}
5.2973 \\
4.638 \\
\end{tabular} & $\begin{array}{l}0.038 \\
0.037 \\
\end{array}$ & \begin{tabular}{|l|l|}
14.643 \\
14.643 \\
\end{tabular} & & 3767.55 & 562.7925 & \begin{tabular}{|l|}
53.6 \\
\end{tabular} & \begin{tabular}{l|l}
3 & 13.0 \\
6 & 12.2
\end{tabular} & & \begin{tabular}{|l|}
2.746 \\
\end{tabular} & \begin{tabular}{|l|}
0.006 \\
0.006 \\
\end{tabular} & \begin{tabular}{|l}
0.0065 \\
0.005
\end{tabular} & 0.000 & 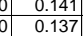 & 0.1 \\
\hline
\end{tabular}


WSRC-TR-2005-00105, REVISION 0

SRNL-RPP-2005-00012, REVISION 0

RUN \# 3.03A AND B; FIRST AND SECOND HALF OF SLURRY DEWATERING - CONT.

\begin{tabular}{|c|c|c|c|c|c|c|c|c|c|c|c|c|c|c|c|c|c|c|c|c|c|c|c|c|c|c|c|c|}
\hline & $A$ & & D & $\mathrm{E}$ & $\mathrm{F}$ & $\begin{array}{ll}G \\
\end{array}$ & $\begin{array}{lll}\mathrm{H} & \mathrm{I} \\
\end{array}$ & $\mathrm{J}$ & $\begin{array}{ll}\mathrm{K} & \mathrm{s} \\
\end{array}$ & \begin{tabular}{|l|} 
\\
\end{tabular} & M & \begin{tabular}{l|l}
$\mathrm{N}$ \\
\end{tabular} & 0 & Q & \begin{tabular}{l|l}
$R$ & \\
\end{tabular} & \begin{tabular}{l|l} 
\\
\end{tabular} & $T$ & $\mathrm{v}$ & \begin{tabular}{l|l} 
w & \\
\end{tabular} & $x \mid$ & $\begin{array}{llll} & & & \end{array}$ & & & & & & & $\overline{A F}$ \\
\hline 08 & 6/17/2004 & 12:03:16 AM & 26.518 & 26.178 & 25.406 & 27.206 & 26.176 & 48.76 & \begin{tabular}{|l|}
55.187 \\
\end{tabular} & \begin{tabular}{|l|l|}
17.701 \\
\end{tabular} & \begin{tabular}{l|l|}
31.592 \\
\end{tabular} & 0.891 & -5.056 & 49.759 & 0.038 & 14.643 & 0.004 & 3768.55 & 62.8092 & 53.7 & 12.2 & 40.2 & 2.770 & 0.006 & 0.006 & 0.000 & 0.139 & \\
\hline & & & & 26.162 & & & 26.185 & 49.314 & \begin{tabular}{|l|}
55.782 \\
\end{tabular} & \begin{tabular}{|l|l|}
16.888 \\
\end{tabular} & \begin{tabular}{|l|l|}
32.907 \\
\end{tabular} & \begin{tabular}{|l|l|}
0.932 \\
\end{tabular} & -5.048 & 50.372 & 0.038 & 14.643 & & & & & & & & & 0.006 & & & 0.12 \\
\hline 3083 & $\begin{array}{l}6 / 17 / 2004 \\
/ 17 / 204\end{array}$ & & 26.511 & 26.17 & $\frac{25.363}{25.34}$ & $\frac{27.194}{2794}$ & 26.184 & 48.694 & \begin{tabular}{|l|}
55.386 \\
5.63
\end{tabular} & \begin{tabular}{r|}
17.72 \\
\end{tabular} & $\begin{array}{l}31.257 \\
2.293\end{array}$ & \begin{tabular}{|l|}
0.935 \\
\end{tabular} & $\begin{array}{l}-5.03 \\
505\end{array}$ & 47.055 & 0.041 & 14.643 & & $\begin{array}{l}3770.55 \\
37755\end{array}$ & \begin{tabular}{|l|l|}
62.8425 \\
6.825
\end{tabular} & 53.9 & $\frac{11.5}{1.5}$ & 40.6 & & .006 & $\begin{array}{l}.006 \\
0.06\end{array}$ & 0.000 & 0.151 & \\
\hline 3084 & 6/17/2004 & 12:06:16 AM & 26.497 & 26.156 & 25.344 & 27.169 & 26.19 & 49.048 & 55.633 & 17.466 & $\begin{array}{l}31.943 \\
\end{array}$ & 0.929 & -5.056 & $\begin{array}{l}46.872 \\
\end{array}$ & $\begin{array}{l}0.038 \\
\end{array}$ & 14.643 & 0.004 & 3771.55 & \begin{tabular}{|l|l|}
62.8592 \\
\end{tabular} & 54.2 & 11.5 & 40.5 & 2.792 & 0.006 & 0.006 & 0.000 & 0.139 & \\
\hline 3086 & 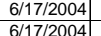 & $\begin{array}{l}12: 07: 16 \mathrm{AM} \\
12088.16 \mathrm{AM}\end{array}$ & \begin{tabular}{|l|l|}
26.511 \\
26.522
\end{tabular} & $\begin{array}{r}26.16 \\
26.171 \\
\end{array}$ & & & & 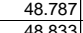 & \begin{tabular}{|l|}
55.539 \\
551 \\
\end{tabular} & 17.42 & & \begin{tabular}{|l|}
0.932 \\
0.935 \\
\end{tabular} & & \begin{tabular}{|l|l|}
51.208 \\
1.402
\end{tabular} & $\begin{array}{ll}0.038 \\
0039 \\
\end{array}$ & $\begin{array}{l}14.643 \\
14643 \\
1464\end{array}$ & & & & $\begin{array}{l}54.1 \\
5.12\end{array}$ & \begin{tabular}{|l|l|}
12.5 \\
\end{tabular} & & & \begin{tabular}{|l|l|l|l|l|}
0.006 \\
\end{tabular} & \begin{tabular}{|l|l|}
0.006 \\
\end{tabular} & & 0.139 & \\
\hline $\begin{array}{l}3000 \\
3087 \\
\end{array}$ & $6 / 1 / 172004$ & $\begin{array}{l}\text { 12:08:16 AM } \\
1209: 16 \mathrm{AM}\end{array}$ & $\begin{array}{l}26.522 \\
26.517 \\
\end{array}$ & $\frac{26.171}{26.176}$ & \begin{tabular}{|l|}
25.359 \\
25.349 \\
\end{tabular} & \begin{tabular}{|l|}
27.1944 \\
27.204 \\
\end{tabular} & $\begin{aligned} 26.185 \\
26.18\end{aligned}$ & $\begin{aligned} 48.833 \\
48.92 \\
\end{aligned}$ & $\begin{array}{l}55.51 \\
55.575 \\
\end{array}$ & $\begin{array}{l}17.473 \\
17.467\end{array}$ & $\begin{array}{r}31.74 \\
31.869 \\
\end{array}$ & \begin{tabular}{|l|}
0.935 \\
0.935 \\
\end{tabular} & $\begin{array}{l}-5.033 \\
-\frac{-539}{4}\end{array}$ & $\begin{array}{r}44.463 \\
48.034 \\
\end{array}$ & $\begin{array}{l}0.039 \\
0.039 \\
\end{array}$ & $\begin{array}{l}14.643 \\
14.643\end{array}$ & $\frac{0.004}{0.004}$ & $\begin{array}{l}3773.55 \\
3774.55 \\
\end{array}$ & \begin{tabular}{|c|}
562.8925 \\
562.9092
\end{tabular} & \begin{tabular}{|l}
54.0 \\
54.1
\end{tabular} & $\frac{10.9}{11.8}$ & \begin{tabular}{|l|}
40.3 \\
40.4
\end{tabular} & $\begin{array}{l}2.778 \\
2.785 \\
\end{array}$ & $|0.006|$ & $\begin{array}{l}0.006 \\
0.006\end{array}$ & $\begin{array}{l}0.000 \\
0.000\end{array}$ & $\begin{array}{l}0.143 \\
0.133\end{array}$ & 0.12 \\
\hline 3088 & $6 / 17 / 2004$ & & 26.502 & & & & & & 55.349 & & & & -5.033 & 53.935 & & 14.643 & 0.004 & 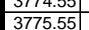 & \begin{tabular}{|l|}
62.9092 \\
62.9258 \\
\end{tabular} & 54.1. & $\begin{array}{ll}11.8 \\
13.2\end{array}$ & $\begin{array}{r}40.4 \\
40.1\end{array}$ & . & 0.000 & $\begin{array}{l}0.000 \\
0.006\end{array}$ & & & $\frac{0.12}{0.13}$ \\
\hline & 6/17/2004 & & 26.513 & 26.157 & & 27.2 & 26.18 & 49.017 & 55.751 & 17.77 & \begin{tabular}{|l|l}
31.643 \\
\end{tabular} & 0.932 & -5.045 & 50.228 & $\begin{array}{ll}0.038 \\
\end{array}$ & 14.643 & 0.004 & 3776.55 & 62.9425 & 54.3 & & & & & & & & $\frac{0.13}{0.12}$ \\
\hline 3090 & 6/17/2004 & 12:12:16 AM & 26.508 & 26.172 & 25.33 & 27.215 & & & 55.237 & 17.466 & & \begin{tabular}{|l|}
0.935 \\
\end{tabular} & & 51.25 & $\begin{array}{l}0.038 \\
\end{array}$ & 14.643 & & & & 53.8 & 12.6 & 40. & & & 0.006 & & & \\
\hline 3091 & 6/17/2004 & & 26.503 & & & 27.231 & & & \begin{tabular}{|l|l|} 
\\
\end{tabular} & 17.512 & 31.752 & & & & 0.04 & 14.643 & & & 9758 & & 11.6 & & & 006 & .006 & 0.000 & $\frac{0.147}{0.147}$ & \\
\hline & & 12:14:16 AM & & 26.173 & 25.301 & & & & 55.554 & 17.422 & & & & 45.616 & 0.04 & 14.643 & & & & 54.1 & 11.2 & & & & & & 146 & \\
\hline & & 12:15:16 AM & 26.497 & 26.166 & 25.279 & 27.184 & & & 55.747 & 17.428 & & & & & & 14.643 & & & & 54.3 & 112 & & & & & & 139 & \\
\hline & 6/177/2004 & 12:16:16 AM & 26.493 & 26.167 & 25.27 & & & 48 & 55.338 & 17.409 & 31.623 & & & & $\begin{array}{ll}0.039 \\
\end{array}$ & 14.643 & & & | & 53.9 & 11.7 & & 767 & . & 0.006 & & 0.144 & \\
\hline 309 & 6/17/2004 & 12:17:16 AM & 26.488 & 26.162 & 25.26 & 27.21 & 26 & 49.046 & $55.768 \mid$ & 17.614 & 31.803 & 0.932 & -5.042 & 49.15 & 0.04 & 14.643 & & 782.5 .5 & .0425 & 54.3 & 12.0 & 40. & .787 & 0.006 & 0.006 & & & \\
\hline & & 12:18:16 AM & $\begin{array}{ll}26.488 \\
2.970\end{array}$ & 26.163 & 25.266 & & & .545 & 55.307 & 17.324 & 31.619 & 0.932 & & 44.559 & 0.04 & 14.643 & & & & & & & & & & & 0.148 & \\
\hline (1) & & 12:19:16 AM & 26.49 & 26.163 & 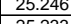 & $27.197 \mid$ & & 48.802 & 55.62 & 17.166 & 32.056 & 0.961 & & 46.815 & 0.039 & 14.643 & & & & 54.1. & & & & & & & & \\
\hline 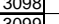 & $\frac{\pi}{7.21204}$ & & 20.416 & & & & & & $5.8 / 5$ & & 32.195 & 0.935 & & 46.615 & 0.04 & & & & & & & & & & & & & \\
\hline & & 12:2.1.10 AMM & 26.4777 & 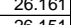 & 25.229 & 27.185 & & & (5.356! & $\frac{17.143}{17209}$ & 31.8889 & 0.958 & & 45.137 & 0.04 & 14.663 & & & & 5. 5.9 & 11.2 & & & & & . & 0.147 & \\
\hline & - $6 / 17 / 2004$ & 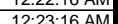 & $\frac{2.0 .47}{2644}$ & 20.151 26.153 & 25.199 & 27.1210 & & $\begin{array}{ll}49.040 \\
49.192\end{array}$ & $\begin{array}{l}5.0015 \\
55.855\end{array}$ & 17.156 & $\begin{array}{r}32.21 \\
32.493\end{array}$ & & & & & 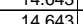 & & & & S4.ct & 121 & & & 0 & & & & \\
\hline 3102 & 6/17/2004 & 12:24:16 AM & 26.4.458 & 26.157 & 25.18 & 27.126 & $\frac{20.141}{26.146}$ & 48.953 & 55.71 & 17.034 & 32.292 & 1.001 & -5.013 & 50.299 & 0.04 & $\begin{array}{l}14.043 \\
14.643 \\
\end{array}$ & & 3789.55 & 63.1592 & 54.2 & $\frac{1.1}{12.3}$ & 40. & 801 & .006 & 0.006 & 0.000 & 0.146 & $\frac{0.12}{0.12}$ \\
\hline & $6 / 17 / 2004$ & $12: 25: 16 \mathrm{AM}$ & 26.449 & 26.153 & 25.166 & 27.141 & 26.137 & 48.988 & 55.45 & 17.007 & 32.444 & 0.955 & & 49.462 & 0.039 & 14.643 & & (3790.55 & $\frac{\mid c .1758}{63.1758}$ & 54.0 & $\frac{12.1}{12.1}$ & & 807 & .006 & 0.006 & 0.000 & $\frac{0.142}{0.142}$ & 0.12 \\
\hline & $6 / 1$ & 12:26:16 AM & 26.446 & 26.16 & & & & & 55.824 & & & & & & & & & & & 54.4 & & & & & & & & \\
\hline & $7 / 2004$ & $12: 27: 16 \mathrm{AM}$ & 26.431 & 26.14 & 25.138 & 27.138 & & & 55.776] & $16.917 \mid$ & 32.47 & 1.00 & & 43.393 & 0.04 & 14.643 & & & 3.2092 & 54.3 & $\frac{10.6}{10.6}$ & & 808 & 006 & .006 & 5.000 & 0.146 & \\
\hline & & 12:28:16 AM & 26.437 & & 25.139 & & & & & & 32.489 & & & & $\begin{array}{ll}0.04 \\
\end{array}$ & 14.643 & & & & 54.2 & $\begin{array}{l}11.8 \\
\end{array}$ & & & & & & & \\
\hline 107 & $7 / 2004$ & 12:29:16 AM & 26.428 & 26.147 & 25.13 & 27.115 & 26.14 & 49.152 & 55.823 & 17.022 & 32.546 & 1.001 & & & \begin{tabular}{|c|c|}
0.039 \\
\end{tabular} & 14.643 & & 7945 & .2425 & 54.4 & & & & & & & & \\
\hline 3108 & 6/17/2004 & 12:30:16 AM & 26.418 | & 26.142 & 25.11 & 27.095 & $26.136 \mathrm{r}-\mathrm{s}$ & 49.183 & \begin{tabular}{|l|l|} 
& 55.643 \\
\end{tabular} & 7.017 & 32.559 & 1.001 & & .798 & 0.039 & 14.643 & & & & 54.2. & 11.2 & & & 506 & .006 & & & \\
\hline 10 & $7 / 22$ & 12:31:16 AM & 26.406 & 26.12 & 25.088 & 27.099 & 26.1. & 49.623 & 56.352 & 16.804 & 33.24 & 0.998 & & 48.50 & 0.04 & 14.643 & & 7796.55 & 63.2758 & 54.9 & 11.9 & $\overline{41}$ & .857 & .006 & 0.006 & & 0.144 & \\
\hline & & & $\begin{array}{ll}26.406 \\
2.200\end{array}$ & 26.13 & & 27.129 & & & 55.817 & 889 & 20. & 1.001 & & & 0.041 & 14.643 & & & & 54.3. & & & & & & & & \\
\hline & . & 12:33:16 AM & 26.3814 & 26.12 & & & & & 56.054 & 16.863 & 32.803 & & & & & 14.643 & & & & 54.6. & & & & & & & & \\
\hline 3112 & .17200 & 12:34:16 AM & 20.391 & 26.13 & 505.013 & 27.114 & & & S6.134] & 16./99 & 32.8999 & 0.998 & & 48.922 & & 14.643] & & & 3.3.3258 & & 12.0 & & & & & & 0.138 & \\
\hline 势14 & $\frac{6112044}{6 / 17 / 2004}$ & $\begin{array}{l}12.35: 10 \mathrm{AMM} \\
12.36 \mathrm{AM}\end{array}$ & 资. & $\frac{26.135}{26.134}$ & (50.082 & 27.133 & & & $\begin{array}{l}56.265 \\
56227\end{array}$ & \begin{tabular}{ll|l}
16.758 \\
16.85
\end{tabular} & $\begin{array}{l}33.059 \\
32.920 \\
\end{array}$ & 1.001 & & & $\begin{array}{l}0.039 \\
0.04\end{array}$ & 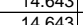 & & & & $\begin{array}{l}54.8 \\
55.7 \\
\end{array}$ & 11.8 & & & & & & 141 & \\
\hline 3115 & 6/17/72004 & 12:37:16 AM & $\frac{26.364}{26.64}$ & 26.104 & 25.031 & 27.106 & & & S5.4179 & & on & 0.995 & & & & 14643 & & & & 550 & 14 & & & & & & & \\
\hline & $6 / 17 / 20$ & $12: 38: 16$ AM & $\frac{2.004}{26.374}$ & $\frac{2.14}{26.123}$ & 25.041 & 27.146 & & & \begin{tabular}{|l|}
56.451 \\
561
\end{tabular} & & & 0.998 & & & 0.0 & $\begin{array}{l}\frac{1.045}{14.643} \\
\end{array}$ & & & & $\frac{5.50}{550}$ & $\frac{\frac{1.1 .5}{121}}{4}$ & $\frac{41}{41}+2$ & & & .006 & & $\frac{0.143}{0.143}>0$ & \\
\hline 3117 & 6/17/2004 & $12: 39: 16 \mathrm{~A}$ & 26.359 & & 25.021 & 27.126 & & & \begin{tabular}{|l|l|}
56.279 \\
\end{tabular} & 16.731 & & 0.99 & & & 0.04 & 14.643 & & 3804 & & 54.8 & 11.5 & & & & & & & \\
\hline & 7/200 & 12:40:16 A & 26.343 & & 25.019 & & & & 56.126 & & & & & & & & & & & 54.7 & 17 & $\frac{u_{41}}{41}-2$ & & & & & 45 & \\
\hline 3119 & & 12:41:16 AM & 26.348 & 26.116 & 25.009 & 27.1 & & & \begin{tabular}{|l|l|}
56.369 \\
\end{tabular} & 16.724 & 33.4 & 1.001 & & & & 14.643 & & & & 54.9 & 11.7 & & & & & & 0.144 & \\
\hline & $7 / 200$ & & & 26.111 & 25.003 & & & & 56.404 & 16.433 & & & & & 0.04 & 14.643 & & & & 54.9 & & & & & & & & \\
\hline 121 & $6 / 17 / 2$ & 12:43:16 AM & 26.347 & 26.121 & & 27.144 & & & & 16.687 & & & & & \begin{tabular}{|l|l|}
0.038 \\
\end{tabular} & 14.643 & & & & 54.9 & 11.4 & 41 & & & & & 0.136 & $\overline{01}+2$ \\
\hline & 6/17/2004 & 12:44:16 A & & 26.116 & 24.978 & 27.094 & & & 56.597 & 16.5 & 33.626 & & & & & 14.643 & & & & 55.1 & $11.8 \quad 2$ & 41 & 874 & & & 0.000 & 0.143 & \\
\hline $01 \mathrm{Se}_{0}$ & 1/1/2 & $12: 45: 16 \mathrm{~A}$ & & & 24.968 & & & & 56.464 & 16.697 & & & & & & & & & & & 1.4 & & & & & & 0.144 & \\
\hline 3124 & $7 / 2004$ & 12: & 26.332 & 26.116 & & 27.114 & & & 57.136 & 16.71 & 33.8977 & 1.024 & & & 0.039 & 14.643 & & & & 55.7 & 11.2 & & 0 & & & & 0.138 & \\
\hline 5 & 4 & & & & & 27.08 & & & 56.88 & & & & & & & & & & & 0.4 & 10.0 & & & & & & 0.140 & \\
\hline 3126 & & & 20.318 & & 24.999 & & & & & & & & & & & & & & & & 11.8 & & & & & & & \\
\hline 12 & 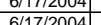 & 12:49:16 A & 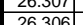 & 26.101 & & 27.1244 & & $\frac{48.87}{4900}-x$ & 55.508 & 18.107 & $\begin{array}{ll}31.153 \\
31.366\end{array}$ & 1.027 & & & & $\frac{14.643}{14643}$ & & & & (5.3. & 宸1.1. & & & & & & 0.138 & \\
\hline 3129 & 6/17/2004 & & 20.0.305 & & 25072 & 27108 & & & 55.73 & 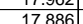 & & $\frac{1.021}{1016}$ & & & & $\frac{14.6}{14.6}$ & & & & & 10 & & & & & & & \\
\hline 3130 & & $12.52 .16 \mathrm{BA}$ & $\frac{2.005}{26.304}$ & & & 271006 & & & $\mid$\begin{tabular}{|l|l|}
55.504 \\
5
\end{tabular} & & & & & & & & & & & 0.4. & $\frac{10}{22}+3$ & & & & & & $\frac{0.104}{0141}$ & \\
\hline 3131 & 6/17/2004 & & $\frac{26.2}{26.2}-2$ & $\frac{26.086}{26.08}$ & $\frac{25.069}{25.069}$ & 27.079 & & & $|55.616|$ & $\frac{17.658}{17.658}$ & & & & & & $\frac{14.6}{14.6}-2$ & & & & 54.1 & 1.9 & & & & & & $\frac{0.141}{0.14}$ & \\
\hline & & & & & & 27.074 & & & & & & & & & & & & & & & & & & & & & & \\
\hline 3133 & & & 26.3 & & & 27.092 & & & 55.5 & & & & & & & & & & & & & & & & & & & \\
\hline & & & 26.3 & & & 27.117 & & & 55.915 & \begin{tabular}{ll|l}
17.58 \\
\end{tabular} & & & & & & & & & & 54.4] & 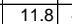 & & & & & & & \\
\hline 3135 & $6 / 17 / 2$ & $12: 57: 16$ & 26.305 & & & 27.117 & & $48.6 \mathrm{C}-\mathrm{s}$ & 55.548 & 17.553 & 31.574 & 1.05 & & & & & & & & 54.1 & 1.9 & & & & & & 0.137 & \\
\hline 3136 & 6/177/20 & 12:58:16 A & 26.309 & 26.09 & 25.0 & 27.061 & & & 55.76 & 17.38 & 31.86 & 1.047 & & & & 14.6 & & & & 54.3 & 1.6 & & & & & & 0.140 & \\
\hline 3137 & & & & & & 27.0 & & & & & & & & & & & & & & & 3 & & & & & & 6 & \\
\hline 3138 & & & 26.2 & 26.0 & 25.039 & 27.07 & & & 55.799 & 17.282 & & 1.0 & & & & & & & & 54. & 11.3 & & & & & & 0.137 & \\
\hline 1399 & & & & 26.0 & & 27.0 & & & & & & & & & & & & & & & & & & & & & & \\
\hline 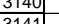 & & & & & & 27.088 & & & & 1.2 & & & & & & & & & & 54.6 & & & & & & & 99 & \\
\hline 势1 & (1) & 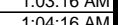 & 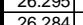 & & & 20.087 & & & & $\begin{array}{l}10.352 \\
11760\end{array}$ & & & & & & & & & & & 4 & & & & & & 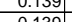 & \\
\hline (3) & $177 / 200-2>3$ & 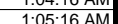 & $\frac{2.0 .04 \mid}{26294}$ & & & 年 & & & & 1..2031 & & 1.070 & & & & 望.60 & & & & & $\frac{4}{2}$ & & & & & & 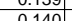 & \\
\hline 1344 & & 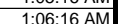 & $6284 \mathrm{G}$ & & & 27076 & & & & & & $\frac{1.06}{106} \Rightarrow$ & & & & & & & & 45 & 0 & & & & & & 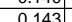 & \\
\hline 3145 & & & 26.28 & & & $\frac{27.075}{27}$ & & $\frac{49.2}{49.2}-2$ & & 17.279 & & & & & & & & & & & 129 & & & & & & 0 & \\
\hline 3146 & & & & & & & & & & & & & & & & & & & & & & & & & & & & \\
\hline 3147 & & & & & & 27.074 & & & & & & & & & & & & & & & & & & & & & & \\
\hline 1148 & & $1: 1 \mathrm{C}$ & 26.28 & & $24.9 \varepsilon$ & 27.08 & & 49.6 & 56.085 & \begin{tabular}{ll|l|}
17.07 \\
\end{tabular} & 32. & $1.07 \mathrm{r}-\mathrm{C}$ & & 463 & & 146 & & & & & T & & & & & & 43 & \\
\hline & & & 20 & & 974 & 27.095 & & & & 229 & & & & 46.4 & & $46.2 \div 25$ & & & & & & & & & & & 36 & \\
\hline 3150 & 6/17/2004 & 1:12:16 AM & 26.268 & $\begin{array}{l}26.067 \\
\end{array}$ & \begin{tabular}{|l|l|}
24.964 \\
\end{tabular} & \begin{tabular}{|l|l|}
27.095 \\
\end{tabular} & 26.036 & 49.129 & \begin{tabular}{|l|l|}
56.064 \\
\end{tabular} & 177.291 & 32.245 & 1.111 & -4.96 - & 49.379 & 0.038 & 14.643 & & 37.55 & $\mid$ & 54.6 & 12.1 & & $\mid 2.805$ & $\mid 0.006$ & $\begin{array}{l}0.006 \\
\end{array}$ & 0.000 & $\begin{array}{ll}0.139 \\
\end{array}$ & 0.1 \\
\hline
\end{tabular}


WSRC-TR-2005-00105, REVISION 0

SRNL-RPP-2005-00012, REVISION 0

RUN \# 3.03A AND B; FIRST AND SECOND HALF OF SLURRY DEWATERING - CONT.

\begin{tabular}{|c|c|c|c|c|c|c|c|c|c|c|c|c|c|c|c|c|c|c|c|c|c|c|c|c|c|c|c|c|}
\hline & A & & \begin{tabular}{l|l} 
D \\
\end{tabular} & $\mathrm{E}$ & $\mathrm{F}$ & \begin{tabular}{ll|}
$\mathrm{G}$ \\
\end{tabular} & $\mathrm{H}$ & $\mathrm{J}$ & $\begin{array}{ll}\mathrm{K} \\
\end{array}$ & \begin{tabular}{l|l|l|}
$\mathrm{L}$ \\
\end{tabular} & \begin{tabular}{l|l} 
\\
\end{tabular} & \begin{tabular}{l|l}
$\mathrm{N}$ \\
\end{tabular} & 0 & \begin{tabular}{l|l} 
Q \\
\end{tabular} & $R$ & \begin{tabular}{l|l} 
s \\
\end{tabular} & $\begin{array}{lll}T & \end{array}$ & $\mathrm{v}$ & w & \begin{tabular}{|c|}
$x \mid$ \\
\end{tabular} & $\begin{array}{lll} & \end{array}$ & $z$ & & $\mathrm{AB}$ & $\overline{A C}$ & & $\overline{\mathrm{AE}}$ & \\
\hline & $6 / 17 / 2004$ & $1: 13: 16 \mathrm{AM}$ & 26.278 & 26.067 & 24.969 & 27.065 & 26.036 & 48.907 & 55.817 & 17.172 & \begin{tabular}{|l|}
32.13 \\
\end{tabular} & 1.114 & -4.958 & 49.905 & 0.03 & 14.643 & 0.004 & 3838.55 & 63.9758 & 54.3 & 12.2 & 40.5 & 2.794 & 0.006 & 0.006 & 0.000 & 0.140 & \\
\hline & $6 / 17 / 2004$ & & 26.279 & 26.077 & 24.98 & 27.121 & 26.021 & 48.889 & \begin{tabular}{|l|}
55.933 \\
\end{tabular} & $\begin{array}{l}17.175 \\
\end{array}$ & $\begin{array}{l}32.029 \\
3\end{array}$ & 1.114 & -4.944 & $\begin{array}{l}50.195 \\
\end{array}$ & & \begin{tabular}{|l}
14.643 \\
\end{tabular} & & & & 54.5 & 12.3 & & & & 0.006 & & & \\
\hline & $6 / 17 / 2004$ & $1: 15: 16 \mathrm{AM}$ & $\frac{26.269}{26.252}$ & 26.072 & 24.965 & 27.081 & 26.021 & 48.899 & \begin{tabular}{|l|}
56.037 \\
\end{tabular} & \begin{tabular}{|l|}
17.108 \\
1.20
\end{tabular} & \begin{tabular}{|l|l|}
32.083 \\
\end{tabular} & 1.114 & $\begin{array}{l}-4.952 \\
\end{array}$ & \begin{tabular}{|l|l|}
49.339 \\
5032
\end{tabular} & & \begin{tabular}{|l}
14.643 \\
\end{tabular} & & \begin{tabular}{l|}
3840.55 \\
29155
\end{tabular} & 54.0092 & 54.6 & $\frac{12.1}{1.27}$ & 4.5 & $\begin{array}{l}.792 \\
72 \\
\end{array}$ & 8.006 & $\begin{array}{l}.006 \\
0.006\end{array}$ & 0.000 & 0.144 & \\
\hline 154 & 6/17/2004 & 1:16:16 AM & 26.253 & 26.062 & 24.949 & 27.045 & 26.026 & 48.785 & \begin{tabular}{|l|}
55.813 \\
\end{tabular} & $\begin{array}{l}17.229 \\
\end{array}$ & 31.9 & 1.111 & -4.949 & $\begin{array}{l}51.934 \\
\end{array}$ & $\begin{array}{l}0.04 \\
\end{array}$ & 14.643 & 0.004 & 3841.55 & \begin{tabular}{|l|l|}
64.0258 \\
\end{tabular} & 54.3 & 12.7 & 40.3 & 2.781 & . 0006 & 0.006 & 0.000 & 0.148 & \\
\hline 156 & 6/17//2004 & $\begin{array}{l}1: 17: 16 \mathrm{AM} \\
1 \cdot 18 \cdot 16 \mathrm{AM}\end{array}$ & \begin{tabular}{|l|}
26.253 \\
26.259
\end{tabular} & $\begin{array}{l}26.057 \\
26.072\end{array}$ & $\begin{array}{l}24.949 \\
25005\end{array}$ & 27.045 & 26.041 & $\begin{array}{l}48.997 \\
48746\end{array}$ & \begin{tabular}{|l|}
55.906 \\
556
\end{tabular} & \begin{tabular}{|l|l|}
17.119 \\
177171
\end{tabular} & & $\frac{1.114}{1.114}$ & $\begin{array}{l}941 \\
997\end{array}$ & 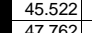 & $\begin{array}{l}0.04 \\
0.33\end{array}$ & $\frac{14.643}{14643}$ & & & & \begin{tabular}{|l|}
54.4 \\
5.4
\end{tabular} & 11.2 & 40.6 & \begin{tabular}{|l|l|}
2.799 \\
2795
\end{tabular} & & \begin{tabular}{|l|l|l|l|} 
& 0.006 \\
\end{tabular} & & 0.147 & \\
\hline 157 & $6 / 1 / 1 / 2004$ & $\begin{array}{l}\frac{1: 18: 16 \text { AM }}{1: 19: 16} \\
\end{array}$ & $\frac{26.259}{26.253}$ & $\begin{array}{l}26.072 \\
26.067\end{array}$ & \begin{tabular}{|l|}
25.005 \\
25.024 \\
\end{tabular} & \begin{tabular}{|l|}
27.036 \\
27.045 \\
\end{tabular} & $\frac{26.046}{26.016}$ & $\begin{array}{l}48.746 \\
49.175\end{array}$ & \begin{tabular}{|r}
55.6 \\
55.964
\end{tabular} \mid & \begin{tabular}{|l|}
17.171 \\
17.198 \\
\end{tabular} & $\begin{array}{l}32.0229 \\
32.362\end{array}$ & $\begin{array}{r}1.114 \\
1.14\end{array}$ & $\begin{array}{l}-4.947 \\
-4.438 \\
\end{array}$ & \begin{tabular}{r|r|r}
47.762 .59 \\
499
\end{tabular} & $\mid \begin{array}{l}0.038 \\
0.039\end{array}$ & $\begin{array}{l}\frac{14.643}{14.643} \\
\end{array}$ & $\frac{0.004}{0.004}$ & $\begin{array}{l}3884.55 \\
33844.55\end{array}$ & \begin{tabular}{|l|l|}
64.0592 \\
66.0758
\end{tabular} & 54.1 & $\frac{11.7}{12.2}$ & $\frac{40.4}{40.8}$ & 2.7855 & |.006 & \begin{tabular}{|l|l|l|}
0.006 \\
0.006
\end{tabular} & $\begin{array}{l}0.000 \\
0.000\end{array}$ & $\begin{array}{l}0.140 \\
0.143\end{array}$ & \\
\hline 158 & & & 26.242 & 26.051 & 25.009 & 27.044 & & & & 16.687 & & 1.123 & & 47.819 & 0.038 & 14.643 & $\begin{array}{l}0.004 \\
0.003\end{array}$ & 3845.55 & $\mid$ & $\begin{array}{l}54.5 \\
54.6 \\
\end{array}$ & $\begin{array}{ll}\frac{11.2}{11.7} \\
1\end{array}$ & \begin{tabular}{|}
40.8 \\
40.9 \\
\end{tabular} & $\frac{2.811}{2.822}$ & $\begin{array}{l}0.006 \\
0.006\end{array}$ & $\mid$ & 0.000 & $\begin{array}{l}0.143 \\
0.138 \\
\end{array}$ & \\
\hline & $6 / 17 / 2004$ & $1: 21: 16 \mathrm{AM}$ & 26.247 & 26.066 & & & & 48.683 & \begin{tabular}{|l|}
55.548 \\
\end{tabular} & 17.621 & 31.426 & 1.114 & -4.944 & 51.861 & 0.038 & 14.643 & 0.004 & 3846.55 & & 54.1 & & & & & & & & \\
\hline & $6 / 17 / 2004$ & $1: 22: 16 \mathrm{AM}$ & & $\begin{array}{r}26.05 \\
2.050\end{array}$ & & 27.044 & & & & 17.495 & & 1.111 & & & & 14.643 & & & 564.1258 & 54.2 & & & & & & & 0.140 & \\
\hline 1161 & $6 / 17 / 2004$ & 1:23:16 AM & 26.257 & 26.066 & 25.064 & 27.059 & & \begin{tabular}{|l|l|}
48.797 \\
\end{tabular} & \begin{tabular}{|l|}
55.566 \\
\end{tabular} & 17.394 & 31.883 & 1.129 & -4.932 & \begin{tabular}{|l|l|}
47.831 \\
\end{tabular} & 0.038 & 14.643 & 0.004 & 3848.55 & 64.1425 & 54.1 & 11.7 & 40. & 2.781 & 0.006 & 0.006 & 0.000 & 0.140 & \\
\hline & $6 / 17 / 2004$ & $\begin{array}{l}1: 24: 16 \mathrm{AM} \\
125 \cdot 6 \mathrm{~A}\end{array}$ & \begin{tabular}{|l|}
26.249 \\
26264
\end{tabular} & $\begin{array}{l}26.042 \\
26067\end{array}$ & 25.05 & \begin{tabular}{l|l|}
27.041 \\
27061
\end{tabular} & & & \begin{tabular}{|r|r|}
55.84 \\
55734
\end{tabular} & $\begin{array}{l}17.494 \\
17351\end{array}$ & \begin{tabular}{|l|}
31.795 \\
31932 \\
\end{tabular} & $\begin{array}{l}1.146 \\
1.129 \\
\end{array}$ & & \begin{tabular}{|l|l|}
48.939 \\
4796
\end{tabular} & 3 & $\begin{array}{l}14.643 \\
14643\end{array}$ & & \begin{tabular}{l|}
3849.55 \\
385055
\end{tabular} & $\begin{array}{l}34.1592 \\
4.1778 \\
4\end{array}$ & $\begin{array}{l}54.4 \\
5.3 \\
\end{array}$ & $\frac{12.0}{117}$ & & 赑 & 1.006 & $\begin{array}{l}0.006 \\
0006\end{array}$ & & $\begin{array}{l}0.140 \\
0.144\end{array}$ & \\
\hline$\frac{\frac{0.64}{164}}{4}$ & 6/17/72004 & $\begin{array}{l}\frac{1: 25: 16 \mathrm{AM}}{12616 \mathrm{AM}} \\
\end{array}$ & $\begin{array}{l}26.264 \\
26.248\end{array}$ & $\begin{array}{l}26.067 \\
26.047\end{array}$ & $\begin{array}{r}25.08 \\
25.054\end{array}$ & $\begin{array}{l}27.061 \\
27.055 \\
\end{array}$ & & $\begin{array}{r}48.88 \\
49.013\end{array}$ & \begin{tabular}{|l|}
$\mid 55.743$ \\
55.94
\end{tabular} & $\begin{array}{r}17.351 \\
17.39\end{array}$ & .933 & 129 & B87 & \begin{tabular}{|}
$\mid 47.796$ \\
49.93 \\
\end{tabular} & $|0.039|$ & $\begin{array}{l}14.643 \\
14.643\end{array}$ & $\frac{0.004}{0.004}$ & 3851.55 & \begin{tabular}{|l|l|l}
6.1758 \\
56.1925
\end{tabular} & $\begin{array}{l}54.3 \\
54.5 \\
\end{array}$ & $\frac{11.7}{12.1}$ & 40.5 & \begin{tabular}{|c|c|}
2.786 \\
.2792
\end{tabular} & 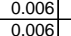 & \begin{tabular}{|l|l|}
0.006 \\
0.006
\end{tabular} & 0.000 & 0.144 & \\
\hline 165 & $6 / 17 / 2004$ & $1: 27: 16 \mathrm{AM}$ & 26.243 & 26.047 & $\begin{array}{l}25.034 \\
25.039\end{array}$ & 27.050 & 26.016 & 49.003 & $|56.06|$ & 17.429 & 31.847 & 1.166 & -4.894 & \begin{tabular}{|}
4.195 \\
47.904 \\
\end{tabular} & $\begin{array}{l}0.038 \\
0.037 \\
\end{array}$ & $\mid \begin{array}{l}14.6434 \\
14.643\end{array}$ & & $\begin{array}{l}3851.55 \\
3852.55\end{array}$ & \begin{tabular}{|l|}
64.1925 \\
564.2092
\end{tabular} & $\begin{array}{l}54.5 \\
54.6 \\
\end{array}$ & $\frac{1.1}{11.7}$ & \begin{tabular}{|l|}
40.5 \\
40.4
\end{tabular} & & $\begin{array}{l}\mid 0.006 \\
0.006\end{array}$ & \begin{tabular}{|l}
0.006 \\
0.006
\end{tabular} & & & $\frac{0.12}{0.12}$ \\
\hline & $6 / 17 / 2004$ & $1: 28: 16$ AM & 26.259 & 26.047 & 25.07 & 27.101 & & 48.684 & \begin{tabular}{|l|}
55.728 \\
\end{tabular} & 17.295 & 31.74 & 1.192 & & 46.586 & & 14.643 & & & & & 11.4 & & & & & & & $\frac{0.12}{0.12}$ \\
\hline & $6 / 17 / 2004$ & $1: 29: 16 \mathrm{AM}$ & 26.259 & 26.042 & 25.19 & 27.111 & & & \begin{tabular}{|l|}
55.906 \\
\end{tabular} & 17.365 & 31.738 & 1.207 & -4.877 & & & 14.643 & & 3854.55 & & 54.4 & 11.6 & 40. & & & & & & \\
\hline 168 & & & & & & 27.106 & & & & & & & & 50.482 & & & & & & & & & & 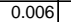 & .006 & 000 & & \\
\hline & & 1:31:16 AM & & & & & & 48.683 & 55.606 & 17.455 & 31.567 & $\begin{array}{l}1.198 \\
\end{array}$ & 366 & & & 14.643 & & & & Fis & $\frac{12.8}{12.8}$ & & & & & & & \\
\hline & & 1:32:16 AM & 26.258 & & 25.135 & & & & 55.906 & $\mid$\begin{tabular}{|l|l|}
17.46 \\
\end{tabular} & & & & 17069 & & & & & & & 2 & & & & & & & \\
\hline & 6/17/2004 & & & & 25.123 & 26.994 & & & 55.566 & 17.382 & 31.662 & 1.189 & & & & 14.643 & & & & 54.1 & & & & & & & & \\
\hline 172 & $7 / 2004$ & $1: 34: 16 \mathrm{AM}$ & 26.271 & 26.03 & 25.123 & 27.024 & & 48.615 & 55.357 & 17.374 & 31.651 & 1.221 & -4.866 & $\begin{array}{l}47.32 \\
\end{array}$ & 0.038 & 14.643 & & 3859.55 & 564.3258 & 53.9 & 11.6 & 40 & 2.767 & 0.006 & 0.006 & 0.000 & 0.141 & \\
\hline & 004 & $: 16 \mathrm{AM}$ & $\begin{array}{ll}26.27 \\
26.27\end{array}$ & 26.024 & 25.106 & 27.027 & & 48.831 & \begin{tabular}{|l|l|}
55.799 \\
\end{tabular} & 17.453 & \begin{tabular}{|l|l|}
31.779 \\
\end{tabular} & 1.236 & -4.863 & \begin{tabular}{|l|l|}
46.334 \\
\end{tabular} & $\begin{array}{l}0.038 \\
\end{array}$ & 14.643 & & \begin{tabular}{l|}
3860.55 \\
2015
\end{tabular} & 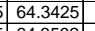 & 54.3 & 11.4 & & 2.779 & 0.006 & $\begin{array}{l}.006 \\
\end{array}$ & .000 & 0.140 & \\
\hline & 72004 & $16 \mathrm{AM}$ & 26.278 & 26.027 & 25.11 & 27.001 & & 48.88 & \begin{tabular}{|l|}
55.966 \\
\end{tabular} & 17.413 & 31.838 & 1.236 & & \begin{tabular}{|l|l|}
48.276 \\
\end{tabular} & & 14.643 & & 3861.55 & 564.3592 & 54.5 & 11.8 & & 2.783 & 0.006 & 0.006 & & 0.140 & \\
\hline 175 & 6/17/2004 & :16 AM & 26.277 & 26.025 & 25.098 & 27.009 & & 48.679 & \begin{tabular}{|l|}
55.608 \\
\end{tabular} & 17.282 & & 1.239 & & 52.493 & $\begin{array}{l}0.038 \\
\end{array}$ & 14.643 & & 3862.55 & \begin{tabular}{|l|l|}
6458 \\
\end{tabular} & 54.1 & 12.9 & & 2.773 & 0.006 & 0.006 & & 0.140 & \\
\hline 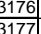 & 6/17/1/2004 & $\begin{array}{l}\frac{1: 38: 16 \mathrm{AM}}{1.39 \cdot 16 \mathrm{AM}} \\
\end{array}$ & $\begin{array}{l}26.266 \\
26.279\end{array}$ & $\begin{array}{r}26.01 \\
26.008\end{array}$ & $\frac{25.062}{25.071}$ & 27.013 & $\frac{26.004}{26.007}$ & \begin{tabular}{|l|l}
48.364 \\
48.677
\end{tabular} & \begin{tabular}{|l|}
55.262 \\
55.66 \\
\end{tabular} & \begin{tabular}{|l|l|}
17.195 \\
17315
\end{tabular} & $\begin{array}{l}31.619 \\
31777\end{array}$ & $\frac{1.236}{1.236}$ & $\begin{array}{l}-4.857 \\
-4.857 \\
\end{array}$ & \begin{tabular}{|l|}
48.038 \\
47337
\end{tabular} & \begin{tabular}{|l|l|}
0.037 \\
0.037
\end{tabular} & $\begin{array}{l}14.643 \\
14.63\end{array}$ & & $\begin{array}{l}3863.55 \\
3864.55 \\
3\end{array}$ & $\begin{array}{l}3925 \\
4092\end{array}$ & 53.8 & $\frac{11.8}{11.6}$ & 40.8 & 2.757 & $\begin{array}{l}0.006 \\
0.06\end{array}$ & $\mid$ & $\begin{array}{l}0.000 \\
0.000\end{array}$ & $\begin{array}{l}0.138 \\
0.137\end{array}$ & 0.12 \\
\hline 3178 & $6 / 1 / 1 / 2004$ & $\begin{array}{l}\frac{139.16 \mathrm{AM}}{1: 40: 16 \mathrm{AM}} \\
\end{array}$ & 20.269 & $\begin{array}{l}26.008 \\
26.033\end{array}$ & 25.081 & 27.027 & & $\begin{array}{r}48.671 \\
48.2 \\
\end{array}$ & \begin{tabular}{|}
$\mid 55.160$ \\
55.129 \\
\end{tabular} & $\frac{17.315}{17.104}$ & $\frac{31.717}{31.51}$ & $\begin{array}{l}\frac{1.236}{1.236} \\
\end{array}$ & & $\begin{array}{l}47.357 \\
48.171\end{array}$ & 38 & $\frac{14.645}{14.643}$ & & \begin{tabular}{|l|l}
386455.55 \\
3865.55
\end{tabular} & \begin{tabular}{|l|l|}
644.4092 \\
664.4258
\end{tabular} & $\begin{array}{l}54.2 \\
53.7\end{array}$ & $\frac{11.6}{11.8}$ & & 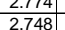 & & \begin{tabular}{|c|}
0.006 \\
0.006
\end{tabular} & & & \\
\hline 3179 & $6 / 17 / 2004$ & 1:41:16 AM & 26.289 & 26.032 & 25.08 & 27.031 & & 48.71 & 55.701 & 17.275 & \begin{tabular}{|l|}
31.808 \\
\end{tabular} & 1.236 & & 49.729 & 0.041 & $\begin{array}{l}14.043 \\
14.643\end{array}$ & & & & 54.2 & $\frac{1.0}{12.2}$ & & .776 & .006 & 0.006 & & 0.1451 & 0.12 \\
\hline & $6 / 177 / 2004$ & 1:42:16 AM & & & & 27.014 & & & 55.832 & 17.319 & 31.984 & & 834 & 47.308 & & 14.643 & & & & 54.4 & 11.6 & & & & & & & 0.13 \\
\hline & $6 / 17 / 2004$ & 1:43:16 AM & 26.284 & & 25.06 & 27.096 & & & 55.852 & 17.359 & .087 & 1.239 & & 48.05 & & 14.643 & & & & 54.4 & & & & & & & & \\
\hline 3182 & & 1:44: & 26.268 & & 25.054 & 27.03 & & 48.6 & 55.506 & 17.278 & 31.795 & 1.21 & & 46.18 & & 14.643 & & & & & $11.3 \mathrm{H}$ & & & & & & 141 & \\
\hline 183 & & & & 26.017 & 25.055 & & & & 55.678 & \begin{tabular}{|l|l|}
17.37 \\
\end{tabular} & 31.894 & 1.239 & & & & 14.643 & & & & & & & & & & & & \\
\hline & $6 / 17 / 2004$ & $16 \mathrm{AM}$ & & 25.997 & 55.029 & 26.98 & & & & 17.173 & 31.885 & 1.236 & & & & 14.643 & & & & & & & & & & & & \\
\hline 185 & 6/17/2004 & & 26.268 & & 25.034 & 26.98 & & & 56.083 & 421 & 31.961 & 1.239 & & & & 14.643 & & & & 54.6 & 1.5 & & & & & & & \\
\hline & $6 / 177 / 2$ & $1: 4$ & 26.272 & & & 27.009 & & & 55.568 & 17.289 & 31.787 & 1.247 & & 46 & & 14.643 & & & & 54. & 11.5 & & & & & & 0.141 & \\
\hline 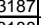 & 004 & & 26.263 & & 25.044 & 27.01 & & 48.76 & 55.709 & 17.121 & 32.0 & 1.279 & & 45.476 & 0.038 & 14.643 & & & & 54.2. & & & & & & & & \\
\hline & 04 & & & & 年 & 26.995 & & & 55.1866 & & & & & & & & & & & & & & & & & & & \\
\hline 189 & & 1:51:16 & 26.267 & & 20.544 & & & & 56.016 & 10.141 & 32.364 & 1.279 & & & & 14.643 & & & & & 11.9 & & & & & & 0.135 & \\
\hline 191 & $\frac{61 / 174}{61712}$ & $16 \mathrm{AM}$ & & $\frac{25 .}{259}$ & 20.5048 & $\mid$ & & & 年 & 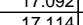 & 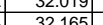 & $\frac{1.27}{127}$ & & & & 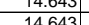 & & & & 54 & 24 & & & & & & 0140 & \\
\hline & $6 / 17 / 2004$ & & & & 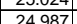 & 26.078 & & & $55^{5.9004}$ & & & & & & & 14643 & & & & & & & & & & & & \\
\hline & S1717 & & & & 25011 & 27037 & & & $\mid$ & 1173 & & 1.279 & & & & $\frac{14.043}{14643}$ & & & & 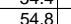 & 18 & & & & & & $\begin{array}{l}0.136 \\
0.142 \\
\end{array}$ & \\
\hline 319 & $6 / 17 / 2004$ & & $\frac{2.2636}{26.236}$ & 25.869 & 24.982 & $\frac{16.968}{26.96}$ & & & 56.093 & $\frac{17.119}{17.19}$ & 32.487 & $\frac{1.2}{1.2}$ & & $\frac{47.22}{44.2}$ & & 14.643 & & & & 54. & 1.6 & & & & & & 0 & \\
\hline & & & & & & & & & & & & & & & & & & & & & & & & & & & & \\
\hline 3196 & 7/2004 & & 26.251 & & 25.017 & 27.018 & & & 56.163 & 16.953 & & & & & & & & & & & 115 & & & & & & & \\
\hline & & & & & 24.986 & $26.997 \mid$ & & & & & 32.368 & 1.273 & & & & 14.6 & & & & & & & & & & & & \\
\hline & $6 / 17 / 2004$ & $2: 00: 16 \mathrm{~A}$ & & 25.823 & 25.006 & 27.027 & & & 56.172 & 17.308 & 32.037 & 1.279 & & & & & & & & 54 & 22 & & & & & & 40 & \\
\hline & $6 / 17 / 20$ & & & & 25.01 & 26.963 & & & \begin{tabular}{|l|}
55.946 \\
\end{tabular} & & & 1.282 & & & & 14.64 & & & & 54. & 1.1 & & & & & & 143 & \\
\hline & 6/17//2 & & & & & & & & & & & & & & & & & & & & .1 & & & & & & & \\
\hline 20 & 6/177/2 & & 26.228 & & 25.05 & 26.916 & & & \begin{tabular}{|l|}
55.728 \\
\end{tabular} & 16.906 & & 1.2 & & & & & & & & & 1.5 & & & & & & D.143 & \\
\hline & & & & & & & & & & & & & & & & & & & & & 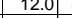 & & & & & & & \\
\hline & & & 26.228 & 25.1724 & 25.01 & 26.941 & & & 56.35 & & 32.6 & 1.2 & & & & & & & & & & & & & & & & \\
\hline & & 年 & $\frac{2.2 .2}{262}$ & 年. & $\begin{array}{l}25.5085 \\
25096\end{array}$ & $\frac{2.9}{269}$ & & & 5 & & & $\frac{1.343}{1343}$ & & & & $\frac{1.6}{146}$ & & & & 54. & 10 & & & & & & 40 & \\
\hline & 6 & 2 & $\frac{2.4}{262}$ & 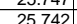 & 251 & 20.506 & & & $\mid$\begin{tabular}{|l|l|}
$\mid 5618$ \\
\end{tabular} & 1735) & 31.96 & 1.045 & & & & & & & & & & & & & & & & \\
\hline & & & & 25 & 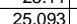 & & & & & & & & & & & & & & & & & & & & & & & \\
\hline 208 & & & & & $\frac{25.08}{25.08}$ & $\frac{26.964}{26.694}$ & & & & & & $\frac{1.3 .}{1.3}$ & & & & & & & & & 6 & & & & & & & \\
\hline & & & & & & & & & & & & & & & & & & & & & & & & & & & & \\
\hline 21 & & & & & & & & & & & & & & & & & & & & & & & & & & & & \\
\hline & & & & & 25.0 & & & & 55.9 & & 31 & & & & & & & & & & & & & & & & & \\
\hline 21 & $6 / 17 / 2$ & 2:13:26 & 26.241 & 25.69 & 25.05 & & & 49.1 & 56.34 & 17.03 & 32.48 & $1.39 !$ & & & & 14.6 & & & & 54 & & & & & & & & \\
\hline 21 & $117 / 20$ & 2:14:26 & 26.236 & 25.6 & 25.19 & 26.968 & & 48.692 & 55.84 & 17.20 & 31.8. & 1.36 & & & & 46. & & & & 54 & 8 & & & & & & & \\
\hline 221. & & & & 25.68 & 25.173 & 26.963 & & & & & 32.1 & 1.389 & & & & 14.6 & & & & & 4 & & & & & & & \\
\hline 21 & & & 26.246] & & & 26.94 & & 49.6 & & & & 1.395 & & & & & & & & & & & & & & & & \\
\hline 2216 & & & & & & 26.96 & & & & & & & & & & & & & & & & & & & & & & \\
\hline (2) & & & $\frac{26.25}{2025}$ & & 25.142] & 2 & & & 56.4 & 17.2. & & & & & & & & & & & & & & & & & & \\
\hline & o1/17212004 & & $\frac{2.201}{2625]}$ & 25.0899 & 20.124] & 20.945 & & 49.0 & $\mid$\begin{tabular}{|l|}
50.340 \\
5.925
\end{tabular} & $\mid$ & 年 & 1.395 & & & & $\begin{array}{ll}14.06 \\
11.6\end{array}$ & & & & (5).9. & & & & & & & al & \\
\hline$\frac{3229}{3220}$ & $\frac{6 / 17 / 1 / 2004}{6 / 17 / 2004}$ & 2:20:26 AM & $\frac{26.256}{26.25}$ & $\begin{array}{r}25.689 \\
25.6899\end{array}$ & \begin{tabular}{|l|}
25.117 \\
25.097
\end{tabular} & \begin{tabular}{|l|}
26.918 \\
26.923 \\
\end{tabular} & \begin{tabular}{|l|l|}
25.898 \\
\end{tabular} & $\begin{array}{l}48.791 \\
48.468\end{array}$ & \begin{tabular}{|l|}
55.92525 \\
55.707 \\
\end{tabular} & \begin{tabular}{|l|}
17.138 \\
17.105 \\
\end{tabular} & \begin{tabular}{|l|}
5 \\
5
\end{tabular} & \begin{tabular}{|l|}
1.395 \\
1.395 \\
\end{tabular} & -4.669 & |3.657| & 0.039 & \begin{tabular}{|l|}
14.643 \\
\end{tabular} & & (9006.72 & $\begin{array}{l}\frac{6}{65.0953} \\
65.1119\end{array}$ & \begin{tabular}{|l|}
54.5 \\
54.2 \\
\end{tabular} & \begin{tabular}{|l|}
12.0 \\
\end{tabular} & & \begin{tabular}{|l|l|}
2.763 \\
\end{tabular} & \begin{tabular}{|l|l|}
0.006 \\
\end{tabular} & $\mid$ & $\begin{array}{l}0.0000 \\
0.000\end{array}$ & $\begin{array}{l}0.140 \\
0.145\end{array}$ & 0.1 \\
\hline
\end{tabular}


WSRC-TR-2005-00105, REVISION 0

SRNL-RPP-2005-00012, REVISION 0

RUN \# 3.03A AND B; FIRST AND SECOND HALF OF SLURRY DEWATERING - CONT.

\begin{tabular}{|c|c|c|c|c|c|c|c|c|c|c|c|c|c|c|c|c|c|c|c|c|c|c|c|c|c|c|c|c|}
\hline & A & $\mathrm{B}$ & $\mathrm{L}$ & E & $r$ & 0 & $\mathrm{H}$ & 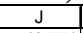 & K & 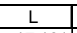 & $\mathrm{M}$ & $\mathrm{N}$ & 0 & $Q^{-}$ & $\begin{array}{ll}\mathrm{R} \\
\end{array}$ & 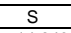 & T & $\sqrt{-5}$ & & $x$ & & & $\mathrm{AA}$ & & $A C$ & $A D$ & $\mathrm{AE}$ & \\
\hline & 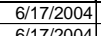 & 2:22:26 AM & $\begin{array}{l}26.254 \\
26263\end{array}$ & 25.6 & $\begin{array}{l}25.09 \\
25.11\end{array}$ & \begin{tabular}{|l|l|}
26.912 \\
26.965
\end{tabular} & $\begin{array}{l}25.907 \\
25.927\end{array}$ & \begin{tabular}{|l|l|}
48.771 \\
48922
\end{tabular} & $\begin{array}{l}56.058 \\
5.051\end{array}$ & $\begin{array}{l}17.181 \\
17.56\end{array}$ & \begin{tabular}{|l|l|}
32.009 \\
2.07
\end{tabular} & $\begin{array}{l}1.395 \\
1401\end{array}$ & $\begin{array}{l}-4.672 \\
4692\end{array}$ & 51.263 & \begin{tabular}{|l|l|}
0.037 \\
0.037
\end{tabular} & $\begin{array}{l}14.643 \\
1.62\end{array}$ & 0.004 & & 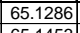 & 54.6 & 12.6 & & 2.785 & 0.006 & 0.006 & 0.000 & 0.136 & \\
\hline & $6 / 117712004$ & $\begin{array}{l}2: 23: 26 \mathrm{AM} \\
222: 26 \mathrm{AM}\end{array}$ & $\frac{26.263}{26.253}$ & 25.7 & $\frac{25.11}{2507}$ & $\begin{aligned} 26.965 \\
26.92\end{aligned}$ & $\begin{array}{l}25.921 \\
25926\end{array}$ & $\begin{array}{l}48.922 \\
48797\end{array}$ & \begin{tabular}{|l|}
56.051 \\
55.933
\end{tabular} & $\begin{array}{l}17.156 \\
17.108\end{array}$ & $\begin{array}{l}32.197 \\
32.142\end{array}$ & $\frac{1.401}{1.395}$ & -4.692 & $\begin{array}{r}46.38 \\
51.071\end{array}$ & 0.037 & $\begin{array}{l}14.643 \\
14643\end{array}$ & $\begin{array}{l}0.004 \\
0.004\end{array}$ & $\begin{array}{l}3908.72 \\
300797\end{array}$ & \begin{tabular}{|l|l|}
65.1453 \\
\end{tabular} & $\frac{54.6}{5.5}$ & $\frac{11.4}{125}$ & 40. & $\frac{2.796}{2709}$ & 0.006 & 0.005 & 0.000 & 0.136 & \\
\hline 322. & $6 / 17 / 2004$ & $\begin{array}{l}2.24 .20 \mathrm{AM} \\
2: 25: 26 \mathrm{Am}\end{array}$ & $\frac{20.200}{26.253}$ & 25.6 & 25.06 & $\begin{array}{l}26.94 \\
26.94\end{array}$ & $\begin{array}{l}25.920 \\
25.906\end{array}$ & $\begin{array}{r}\quad 88.971 \\
48.81\end{array}$ & \begin{tabular}{|l|}
56.933 \\
56.093 \\
\end{tabular} & $\begin{array}{l}17.108 \\
17.133\end{array}$ & $\begin{array}{l}32.142 \\
32.013\end{array}$ & 1.395 & $\begin{array}{l}-4.678 \\
-4.634\end{array}$ & $\begin{array}{l}\frac{51.0 / 1}{51.219} \\
\end{array}$ & $\mid \begin{array}{c}0.038 \\
0.038\end{array}$ & $\begin{array}{l}14.643 \\
14.643 \\
\end{array}$ & & $\begin{array}{l}3909.72 \\
3910.72\end{array}$ & $\begin{array}{l}5.1619 \\
65.1786\end{array}$ & $\frac{54.5}{54.6}$ & $\frac{12.5}{12.6}$ & & & $\begin{array}{l}0.006 \\
0.006\end{array}$ & $\begin{array}{c}0.006 \\
0.006\end{array}$ & $\frac{0.000}{0.000}$ & $\frac{0.147}{0.140}$ & $\frac{0.13}{0.12}$ \\
\hline 3225 & $6 / 17 / 2004$ & 2:26:26 AM & 26.253 & 25.7 & 25.07 & $\begin{array}{l}26.96 \\
\end{array}$ & 25.926 & $\begin{array}{l}48.939 \\
\end{array}$ & 55.96 & $\begin{array}{l}17.163 \\
\end{array}$ & 32.193 & 1.395 & -4.637 & 47.003 & 0.038 & $\begin{array}{l}14.643 \\
\end{array}$ & & 3911.72 & \begin{tabular}{|l|l|}
65.1953 \\
\end{tabular} & 54.5 & 11.5 & & & & 0.006 & & & \\
\hline & $6 / 17 / 2004$ & & & & & 26.961 & 25.911 & 48.978 & 56.234 & $\begin{array}{l}17.102 \\
\end{array}$ & 32.306 & & -4.626 & 46.184 & & $\begin{array}{l}14.643 \\
\end{array}$ & & & & 54.8 & & & & & & & & \\
\hline & $6 / 17 / 2004$ & $2: 28: 26 \mathrm{AM}$ & 26.244 & 25.7 & 25.0 & 26.936 & 25.906 & 48.667 & \begin{tabular}{|l|}
55.782 \\
\end{tabular} & $\begin{array}{l}17.09 \\
\end{array}$ & $\begin{array}{l}31.961 \\
3\end{array}$ & 1.395 & $\begin{array}{r}-4.652 \\
-6.652\end{array}$ & 51.133 & & 14.643 & & 3913.7. & 65.2286 & 54.3 & 12.5 & & & 0.006 & 0.006 & & 0.137 & \\
\hline 3228 & $6 / 17 / 2004$ & 2:29:26 AM & 26.234 & 25.6 & 25.03 & 26.911 & 25.906 & 48.677 & \begin{tabular}{|l|}
55.981 \\
\end{tabular} & $\begin{array}{ll}17.031 \\
\end{array}$ & 32.015 & 1.58 & -4.305 & 48.889 & \begin{tabular}{|l|l|}
0.038 \\
\end{tabular} & $\begin{array}{l}14.643 \\
\end{array}$ & & \begin{tabular}{|l|l|l}
3914.72 \\
\end{tabular} & 65.2453 & 54.5 & \begin{tabular}{|l|}
12.0 \\
\end{tabular} & 40.3 & 2.782 & 0.006 & 0.006 & 0.000 & 0.140 & \\
\hline 323 & 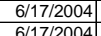 & $\begin{array}{ll}230: 26 \mathrm{AM} \\
2 \cdot 32 \cdot 26 \mathrm{~A}\end{array}$ & $\begin{array}{l}26.249 \\
26.238\end{array}$ & 25.7 & $\begin{array}{r}25.0 \\
25.03 \\
25\end{array}$ & \begin{tabular}{|l|}
26.911 \\
26.885
\end{tabular} & & \begin{tabular}{|l|l|}
48.549 \\
48837
\end{tabular} & \begin{tabular}{|r|r|}
55.78 \\
56.13 \\
\end{tabular} & $\begin{array}{l}16.864 \\
16999\end{array}$ & \begin{tabular}{|l|l|}
32.103 \\
32063
\end{tabular} & $\begin{array}{l}1.577 \\
1577\end{array}$ & \begin{tabular}{|c|}
-4.311 \\
.419
\end{tabular} & 50.017 & \begin{tabular}{|c|c|c|}
0.038 \\
0.39
\end{tabular} & $\begin{array}{l}14.643 \\
1.643\end{array}$ & & $\frac{15.72}{16.72}$ & \begin{tabular}{|l|l|}
65.2619 \\
652786
\end{tabular} & $\begin{array}{l}54.3 \\
5.6\end{array}$ & 12.3 & & \begin{tabular}{|l|l|}
2.780 \\
2706
\end{tabular} & & $\begin{array}{l}0.006 \\
0.006\end{array}$ & & 0.140 & \\
\hline $\begin{array}{l}3230 \\
3231 \\
3231\end{array}$ & $6 / 1 / 1712004$ & $\begin{array}{l}2: 31: 26 \mathrm{AM} \\
2232 \cdot 26 \mathrm{AM}\end{array}$ & $\frac{26.238}{26.249}$ & $\frac{25.7}{25.7}$ & $\frac{25.03}{25.05}$ & $\frac{26.885}{26.886}$ & 8.891 & \begin{tabular}{|l|}
48.837 \\
48.656
\end{tabular} & \begin{tabular}{|l|}
56.103 \\
55.981 \\
\end{tabular} & $\begin{array}{l}16.949 \\
16.936\end{array}$ & $\begin{array}{r}32.263 \\
32.16 \\
\end{array}$ & $\begin{array}{r}1.577 \\
1.58 \\
\end{array}$ & $\begin{array}{l}-4.319 \\
-4.302\end{array}$ & $\begin{array}{l}47.923 \\
45.633 \\
\end{array}$ & \begin{tabular}{|c|c|c|}
0.038 \\
0.038
\end{tabular} & $\begin{array}{l}\frac{14.643}{14.643} \\
\end{array}$ & $\frac{0.004}{0.004}$ & \begin{tabular}{|l|l|}
3916.72 \\
3917.72
\end{tabular} & $\begin{array}{l}65.2786 \\
65.2953\end{array}$ & $\begin{array}{l}54.6 \\
54.5 \\
\end{array}$ & \begin{tabular}{|l|}
11.7 \\
11.2
\end{tabular} & \begin{tabular}{|l|}
$\mid 40.6$ \\
40.4 \\
\end{tabular} & \begin{tabular}{|l|}
2.796 \\
2.786
\end{tabular} & $\begin{array}{l}0.006 \\
0.006\end{array}$ & $\begin{array}{l}0.006 \\
0.006\end{array}$ & $\begin{array}{l}0.000 \\
\end{array}$ & 0.140 & 0.12 \\
\hline 3232 & $6 / 17 / 2004$ & $2: 33: 26 \mathrm{AM}$ & & 25.7 & 25.02 & & 25.895 & & \begin{tabular}{|l|}
56.219 \\
\end{tabular} & 16.92 & 32.294 & 1.58 & & 48.242 & & 14.643 & & 3918.72 & & 54.7 & 11.8 & & & & & & $\begin{array}{l}0.140 \\
0.140\end{array}$ & $\frac{0.12}{0.12}$ \\
\hline & $6 / 17 / 2004$ & 2:34:26 AM & 26.236 & 25.7 & 25.03 & 26.889 & & 48.853 & 56.153 & 17.055 & 32.241 & 1.592 & -4.316 & 46.732 & \begin{tabular}{|l|}
0.037 \\
\end{tabular} & 14.643 & & 3919.72 & 65.3286 & 54.7 & 11.5 & 40.5 & \begin{tabular}{|l|}
2.796 \\
\end{tabular} & 0.006 & 0.006 & 0.000 & 0.136 & \\
\hline & $6 / 17 / 2004$ & 2:35:26 AM & & & 25.02 & & 25.896 & 48.8 & 56.284 & 16.974 & $\begin{array}{l}32.13 \\
\end{array}$ & 1.58 & & & & $\begin{array}{l}14.643 \\
\end{array}$ & & 3920.72 & 65.3453 & 54.8 & 11.4 & 40.5 & 2.790 & 0.006 & 0.006 & & 0.140 & \\
\hline 3235 & $6 / 17 / 2004$ & $2: 36: 26 \mathrm{AM}$ & 26.233 & 25.7 & 25.02 & 26.94 & 5.896 & 49.044 & 56.53 & $\begin{array}{l}16.937 \\
\end{array}$ & 32.466 & 1.589 & & 44.638 & \begin{tabular}{|l}
0.038 \\
\end{tabular} & $\begin{array}{l}14.643 \\
\end{array}$ & & \begin{tabular}{|l|l|}
3921.72 \\
\end{tabular} & 65.3619 & 55.1 & 10.9 & & & 0.006 & 0.006 & 0.000 & 0.139 & \\
\hline 年 & $6 / 177 / 2004$ & $\begin{array}{l}2: 37: 26 \mathrm{AM} \\
2 \cdot 33: 26 \mathrm{~A}\end{array}$ & $\begin{array}{l}26.233 \\
26.223\end{array}$ & $\begin{array}{l}25.7 \\
257\end{array}$ & $\frac{25.01}{2500}$ & \begin{tabular}{|l|}
26.945 \\
26.925
\end{tabular} & & $\begin{array}{r}48.81 \\
48.065\end{array}$ & $\begin{array}{l}56.321 \\
56306\end{array}$ & \begin{tabular}{|l|l|}
17.027 \\
1.6975
\end{tabular} & \begin{tabular}{|l|l|}
32.142 \\
32.429
\end{tabular} & $\begin{array}{l}1.58 \\
1.6\end{array}$ & \begin{tabular}{|c|}
-4.316 \\
.302
\end{tabular} & $\begin{array}{r}48.04 \\
47079 \\
\end{array}$ & \begin{tabular}{|c|c|c|}
0.037 \\
\end{tabular} & $\begin{array}{l}14.643 \\
14643\end{array}$ & & \begin{tabular}{|l|}
3922.72 \\
392372
\end{tabular} & $\begin{array}{l}65.3786 \\
653953\end{array}$ & 54.8 & $\begin{array}{ll}11.8 \\
1.8\end{array}$ & & \begin{tabular}{|l|}
2.791 \\
2806
\end{tabular} & $\begin{array}{l}0.006 \\
0.066\end{array}$ & $\begin{array}{l}0.006 \\
0.06\end{array}$ & $\begin{array}{l}0.000 \\
\end{array}$ & $\begin{array}{l}0.136 \\
0.139\end{array}$ & 0.14 \\
\hline$\frac{3<51}{3238}$ & $6 / 1 / 172004$ & $\begin{array}{l}2: 33: 86 \mathrm{AM} \\
2: 39 \cdot 26 \mathrm{AM}\end{array}$ & $\frac{26.223}{26.234}$ & & & 26.925 & & $\begin{array}{r}48.965 \\
49.028\end{array}$ & $56.306 \mid$ & $\begin{array}{l}16.957 \\
16.965\end{array}$ & $\begin{array}{r}32.429 \\
32.38\end{array}$ & $\frac{1.6}{1.635}$ & & $\begin{array}{l}47.979 \\
48.551 \\
\end{array}$ & .038 & $\frac{14.643}{14.643}$ & & \begin{tabular}{|l}
3923.72 \\
3924.72
\end{tabular} & $\frac{65.3953}{65.4119}$ & & & & $2.806 \mid$ & 0.006 & & & & 0.12 \\
\hline 3239 & $6 / 17 / 2004$ & $2: 40: 26 \mathrm{AM}$ & 26.229 & 25.7 & 25.01 & 26.937 & $\frac{2.000}{25.897}$ & $\begin{array}{l}45.020 \\
48.999\end{array}$ & $\frac{50.400}{56.261}$ & \begin{tabular}{|l|}
16.971 \\
\end{tabular} & $\begin{array}{l}32.45 \\
32\end{array}$ & 1.615 & $\begin{array}{l}-4.311 \\
-4.311 \\
-10\end{array}$ & $\frac{40.051}{46.688}$ & $\begin{array}{l}0.037 \\
0.037\end{array}$ & $\frac{14.045}{14.643}$ & & $\frac{3924.1 / 2}{3925.72}$ & \begin{tabular}{|l|}
65.41149 \\
65286
\end{tabular} & $\frac{55.0}{54.8}$ & $\frac{11.5}{11.4}$ & \begin{tabular}{|l|}
40.7 \\
\end{tabular} & \begin{tabular}{|l|}
2.8068 \\
2.808 \\
\end{tabular} & & $\begin{array}{l}0.006 \\
0.006\end{array}$ & 0 & $\begin{array}{l}0.139 \\
0.135 \\
\end{array}$ & $\frac{0.12}{0.12}$ \\
\hline & $6 / 17 / 2004$ & 2:41:26 AM & 26.213 & 25.7 & 24.99 & 26.93 & & 48.725 & 56.114 & 16.939 & 32.169 & 1.638 & & 47.035 & 0.039 & 14.643 & & & 65.4453 & 54.6 & 11.5 & 40.4 & \begin{tabular}{|l|}
2.789 \\
\end{tabular} & 0.006 & 0.006 & 0.000 & 0.144 & \\
\hline & $6 / 17 / 2004$ & 2:42:26 AM & 26.204 & & 24.96 & 26.851 & & 49.075 & \begin{tabular}{|l|l|}
56.294 \\
\end{tabular} & 16.985 & $\begin{array}{r}2.52 \\
\end{array}$ & & & 45.835 & & $\begin{array}{l}14.643 \\
\end{array}$ & & & 65.4619 & 54.8 & \begin{tabular}{|l|l}
11.2 \\
\end{tabular} & 40.8 & 2.813 & 0.006 & 0.006 & 0.000 & 0.139 & \\
\hline 3242 & $6 / 17 / 2004$ & 2:43:26 AM & 26.214 & 25.7 & 24.99 & 26.921 & 5.896 & 49.105 & 56.468 & \begin{tabular}{ll|}
17.001 \\
\end{tabular} & 32.583 & 1.638 & & 49.231 & $\begin{array}{l}0.038 \\
\end{array}$ & 14.643 & & 3928.72 & 65.4786 & 55.0 & \begin{tabular}{|l|l}
12.1 \\
\end{tabular} & & \begin{tabular}{|l|}
2.816 \\
\end{tabular} & 0.006 & 0.006 & 0.000 & 0.139 & \\
\hline 3244 & $6 / 17 / 2004$ & $\begin{array}{l}2: 44: 26 \mathrm{AM} \\
2045 \cdot 26 \mathrm{AM}\end{array}$ & $\begin{array}{l}26.203 \\
26.207\end{array}$ & 25.7 & 24.98 & $\begin{array}{r}26.92 \\
26.909\end{array}$ & $\frac{25.876}{2588}$ & \begin{tabular}{|l|l|}
49.198 \\
49215
\end{tabular} & \begin{tabular}{|l|l|}
56.52 \\
5.63
\end{tabular} & \begin{tabular}{|l|l|}
16.976 \\
1701
\end{tabular} & $\begin{array}{r}32.643 \\
32.674 \\
32.6\end{array}$ & $\begin{array}{l}1.635 \\
1635\end{array}$ & $\begin{array}{l}-4.308 \\
-4308 \\
-130\end{array}$ & $\begin{array}{l}48.274 \\
4337\end{array}$ & 0.037 & $\begin{array}{l}14.643 \\
11.643\end{array}$ & & \begin{tabular}{|l|}
3929.72 \\
392072
\end{tabular} & \begin{tabular}{|l|l|}
65.4953 \\
655119
\end{tabular} & 55.0 & $\begin{array}{ll}11.8 \\
11.4\end{array}$ & \begin{tabular}{|l|}
40.9 \\
410
\end{tabular} & \begin{tabular}{|l|}
2.821 \\
2821 \\
\end{tabular} & \begin{tabular}{|l|l|l|}
0.006 \\
\end{tabular} & $\begin{array}{l}0.006 \\
\end{array}$ & 0.000 & 0.135 & 0.11 \\
\hline$\frac{3<44}{3245}$ & $6 / 1 / 172004$ & $2: 2: 4: 26 \mathrm{AM}$ & & $\frac{25.1}{25.7}$ & $\frac{24.98}{24.98}$ & 26.909 & & & \begin{tabular}{r|r|}
56.63 \\
56.565
\end{tabular} & $\begin{array}{r}17.001 \\
17.103\end{array}$ & 32.674 & $\begin{array}{l}1.635 \\
1.635\end{array}$ & & & & $\frac{14.643}{14.643}$ & & & $\begin{array}{l}65.5119 \\
665586\end{array}$ & & & & & 0.005 & & & & 0.11 \\
\hline 3246 & $6 / 17 / 2004$ & $\begin{array}{l}2.40 .07 \mathrm{AM} \\
\end{array}$ & $\frac{20.214}{26.207}$ & 25.7 & $\frac{24.50}{24.98}$ & 26.059 & $\frac{2.00}{25.88}$ & $\begin{array}{l}45.000 \\
48.912\end{array}$ & \begin{tabular}{|l|}
56.331 \\
\end{tabular} & \begin{tabular}{|l|l|}
17.013 \\
\end{tabular} & $\begin{array}{l}32.000 \\
32.302\end{array}$ & $\begin{array}{l}.050 \\
1.638\end{array}$ & - -4.299 & $\frac{1.1004}{48.032}$ & $\begin{array}{l}0.051 \\
0.038\end{array}$ & $\frac{14.045}{14.643}$ & & \begin{tabular}{|l|l|}
3932.7 \\
3932
\end{tabular} & $\frac{65.5286}{65.5453}$ & $\frac{55.1}{54.9}$ & $\frac{12.5}{11.8}$ & & 2.800 & \begin{tabular}{|l|l}
0.0006 \\
0.006
\end{tabular} & 0.006 & & $\frac{1.156}{140}$ & $\frac{0.12}{0.12}$ \\
\hline 3247 & 6/177/2004 & $2: 48: 26 \mathrm{AM}$ & 26.197 & 25.7 & 24.97 & 26.894 & 25.875 & 49.254 & \begin{tabular}{|l|}
56.702 \\
\end{tabular} & 17.187 & $\begin{array}{l}32.52 \\
\end{array}$ & 1.638 & -4.293 & 45.428 & 0.038 & 14.643 & & 3933.72 & \begin{tabular}{|l|}
65.5619 \\
65619
\end{tabular} & 55.2 & 11.1 & 40.9 & \begin{tabular}{|l|}
2.819 \\
\end{tabular} & 0.006 & 0.006 & 0.000 & 0.139 & $\frac{0.12}{0.12}$ \\
\hline 3248 & $6 / 17 / 2004$ & 2:49:26 AM & & 25. & 24.97 & \begin{tabular}{|l|}
26.899 \\
\end{tabular} & & & \begin{tabular}{|r|}
56.57 \\
\end{tabular} & $\begin{array}{l}17.122 \\
\end{array}$ & 32.372 & 1.635 & & & & $\begin{array}{l}14.643 \\
\end{array}$ & & 3934.72 & 65.5786 & 55.1 & 10.7 & 40.8 & & 0.006 & 0.006 & & & \\
\hline 3249 & $6 / 17 / 2004$ & 2:50:26 AM & 26.201 & 25. & 25.00 & \begin{tabular}{|l|l|}
26.884 \\
\end{tabular} & & 49.347 & & & & & & & .038 & $\begin{array}{l}14.643 \\
\end{array}$ & & & & 55.2 & & & & 0.006 & & 0.000 & . & \\
\hline & $6 / 17 / 2004$ & 2:51:26 AM & & & 24.99 & 26.899 & & & 56.69 & 17.022 & 32.819 & & & & & \begin{tabular}{|l}
14.643 \\
\end{tabular} & & & & & & & & & & & & \\
\hline 3251 & 6/17//2004 & 2:52:26 AM & 26.191 & 25.7 & 25.00 & 26.913 & & 49 & 56.74 & 17.087 & & & & & 038 & 14.643 & & & & & & & & & & & & \\
\hline & 6/177/2004 & $2: 53$ & 26.19 & 25.7 & 25.01 & 26.9 & & & 56.715 & 177.049 & 32.684 & 1.641 & & & & $\begin{array}{l}14.643 \\
\end{array}$ & & & & 55.2 & 1.4 & & & 0.006 & & & & \\
\hline & $6 / 17 / 2004$ & 2:54:26 AM & 26.2 & 25.7 & 25.02 & 26.907 & & $\frac{49}{49}$ & 56.653 & $\mid 17.24$ & |. & 1.641 & & & $\begin{array}{l}0.037 \\
\end{array}$ & $\begin{array}{l}14.643 \\
\end{array}$ & & & & & 11.5 & & & & & & .136 & \\
\hline & 6/117/2004 & $2: 55: 26$ & 26.19 & 25.7 & 25.01 & 26.907 & & 49.015 & $\begin{array}{l}56.476 \\
\end{array}$ & $\begin{array}{l}17.335 \\
\end{array}$ & \begin{tabular}{|l|l|}
32.083 \\
\end{tabular} & 1.635 & & 49.348 & .037 & $\begin{array}{l}14.643 \\
\end{array}$ & & & 65.6786 & 55.0 & 12.1 & & 2.796 & 0.006 & 0.006 & 0.000 & 0.136 & \\
\hline & $6 / 17 / 2004$ & 56:26 AM & 26.189 & 25.7 & 25.08 & 36 & & & & & 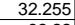 & & & & & & & & & & 2.8 & & & & & & & \\
\hline & & & 26.188 & & 25.0 & & & & & & & 1.635 & & & & & & & & & & & & & & & & \\
\hline & 601172004 & $\begin{array}{l}2.58 .20 \mathrm{AM} \\
2.50 .3 \mathrm{~A}\end{array}$ & $\frac{26.192}{26191}$ & 25.14 & $\frac{25.05}{2502}$ & $\mid 26.919$ & & $\begin{array}{c}48.862 \\
.9016\end{array}$ & 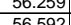 & $\mid \begin{array}{l}\mid 1.158 \\
1.698\end{array}$ & $\begin{array}{l}32.048 \\
32652\end{array}$ & $\frac{1.638}{1.653}$ & & & & $\frac{14.66}{14.6}$ & & & & & 111 & & & Dof & & & 年.1366 & \\
\hline & 6 & & & & 2501 & & & & & 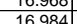 & 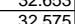 & & & & & & & & & & & & & & & & & \\
\hline & 6 & & $\frac{20.100}{26202}$ & 257 & 2502 & & & & $\frac{5.000}{56.3}$ & 17009 & 388 & & & & & $\frac{14 .}{14 .}$ & & & & $\frac{55 .}{55}$ & $\frac{11.5}{112}$ & & & & & & . & \\
\hline & $6 / 17 / 2004$ & 3:02:26 & $\frac{16.202}{26.202}$ & $\frac{25.7}{25.7}$ & 25.00 & 26.919 & & 49.109 & 56.642 & $\frac{1.5036}{10.936}$ & 32.579 & $\frac{1.705}{1.705}$ & & 49.719 & $\mid$ & 14.6 .6 & & & 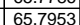 & 55.2 & 12.2. & & & $\frac{0.006}{0.006}$ & 0.006 & 0.000 & 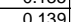 & \\
\hline & $6 / 177 / 2$ & & & & & & & & & & & & & & & & & & & & & & & & & & & \\
\hline & $6 / 1$ & & 26.18 & 25.7 & 24.96 & & & & & & 817 & & & & & & & & & & $\frac{1.6}{0.6}$ & & & & & & 131 & \\
\hline & $6 / 17 / 2004$ & & & & & & & & & & & & & & & & & & & 54.8 & & & & & & & & \\
\hline & 6/177/2004 & 3:06: & 26.195 & 25.7 & 24.98 & \begin{tabular}{|c|}
26.908 \\
\end{tabular} & & & & & 5.577 & & & & & & & & & & 1.0 & & & & & & 135 & \\
\hline & $6 / 17 / 2004$ & & 26.195 & 25.7 & 24.96 & & & & & & & & & & & 14.6 & & & & 55.3 & 11.7 & & & & & & 0.138 & \\
\hline & $6 / 117 / 2004$ & & 26.185 & & 24.96 & & & & & & & & & & & & & & & & $\frac{11.8}{11.8}$ & & & & & & & \\
\hline 326 & 6/177/2004 & 3:09: & 26.186 & & 24.97. & 26.8 & & & & 16.94 & 32.255 & & & & & 14. & & & & 54.9 & 11.9 & & & & & & 136 & \\
\hline & $6 / 17 / 2$ & & 20.170 & 25.7 & 24.9 & & & & & & & & & & & & & & & & & & & & & & & \\
\hline & & & 26.17 & & & & & & & & & & & & & & & & & & & & & & & & & \\
\hline & 年 & & 20.18 & & 24.95 & & & & & & & & & & & & & & & & & & & & & & & \\
\hline & 6 & & $\frac{2.11}{26.164}$ & & $\frac{24.54}{2493}$ & & & & & & & & & & & & & & & & 113 & & & & & & & \\
\hline & 6 & & $\frac{2.1044}{26.44}$ & & 24.95 & & & & & & & & & & & & & & & & 11.5 & & & & & & & \\
\hline 3275 & $6 / 17 / 20$ & & $\frac{20.148}{26.148}$ & $\frac{2.1 .}{25.7}$ & $\frac{1.49}{24.9}$ & 26 & & & & & & & & & & & & & & $\frac{25.0}{55.0}$ & $\frac{11.8}{11.8}$ & & & & & & & \\
\hline 3276 & $6 / 17 / 21$ & & 26.147 & & & & & & & & & & & & & & & & & & & & & & & & & \\
\hline & & & & & & & & & & & & & & & & & & & & & & & & & & & & \\
\hline & $6 / 17 / 2004$ & & 26.146 & & 2500 & 26. & & & & & & & & & & 14. & & & & & & & & & & & & \\
\hline & $6 / 177 / 2$ & 20: & & 25.7 & 24.99 & 26.9 & & 48.746 & 56.279 & 16.8 & 32.2 & 1.73 & & & & 14.6 & & & & & 1.9 & & & & & & & \\
\hline & $6 / 17 / 20$ & 21 & 145 & 25.7 & 25.00 & 26.96 & & & 56.812 & 16. & 32.942 & & & & & 14. & & & & & 11.4 & & & & & & 0.138 & \\
\hline & $6 / 17 / 2$ & & 26.157 & 25 & & & & & & & & & & & & & & & & & 1.6 & & & & & & & \\
\hline & $6 / 17 / 2$ & & 26.167 & 25 & 25.01 & 27.1 & & & & & & & & & & & & & & & 11.6 & & & & & & & \\
\hline & $6 / 171$ & & 26. & & & 27. & & & & & & & & & & & & & & & & & & & & & & \\
\hline & $6 / 17 / 2004$ & & 26.179 & 25.8 & 25.0 & 27.226 & & & 56.3 & 17 & $1 / 3$ & & & & & & & & & & & & & & & & & \\
\hline & 1 & $\frac{3.25 .2}{3 \cdot 2 \cdot 2.2}$ & $\frac{1175}{169}$ & & 25.02 & $\frac{27.197}{2712}$ & & 4.680 & $\frac{56.1}{5.6}$ & 17. & $\frac{32.15}{322^{2}}$ & $\frac{1.731}{1.720}$ & & & & & & & & & 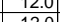 & & & & & & & \\
\hline & 011712004 & & 26.17 & 258 & 2.01 & $\frac{21.145}{27.01}$ & & 49.020 & 5677 & 171 & 220 & & & & & & & & & & 120 & & & & & & & \\
\hline & $6 / 17 / 2004$ & & $\frac{2.1170}{26168}$ & $\frac{2.0}{258}$ & 2500 & | & & & 57.044 & 1744 & 年 & 1734 & & & & & & & & & & & & & & & & \\
\hline & & & & $\frac{25.8}{25.8}$ & & & & & 56727 & $\frac{16.898}{16}$ & 32.384 & 1.734 & & 5120 & & & & & & & 26 & & & & & & $\frac{51}{32}$ & \\
\hline & $6 / 17 / 2004$ & & $\frac{6.191}{6.191}$ & 25. & $\frac{25.02}{25}$ & \begin{tabular}{|l|}
27.064 \\
\end{tabular} & & 49.034 & & 17.172 & 32.308 & 1.731 & & 46.373 & 037 & $\frac{14.045}{14.643}$ & & & $.2786 \mathrm{C}$ & & 11.4 & & & 0.006 & 006 & 5001 & 136 & \\
\hline
\end{tabular}


WSRC-TR-2005-00105, REVISION 0

SRNL-RPP-2005-00012, REVISION 0

RUN \# 3.03A AND B; FIRST AND SECOND HALF OF SLURRY DEWATERING - CONT.

\begin{tabular}{|c|c|c|c|c|c|c|c|c|c|c|c|c|c|c|c|c|c|c|c|c|c|c|c|c|c|c|c|c|}
\hline & \begin{tabular}{l|}
$A$ \\
$A$
\end{tabular} & \begin{tabular}{l|l}
$\mathrm{B}$ \\
\end{tabular} & $\mathrm{D}$ & $\mathrm{E}$ & $\mathrm{F}$ & \begin{tabular}{l|l|l|} 
& \\
\end{tabular} & $\mathrm{H}$ & $\mathrm{J}$ & $\mathrm{K}$ & \begin{tabular}{l|l|l|}
$\mathrm{L}$ & \\
\end{tabular} & $\begin{array}{ll}M \\
\end{array}$ & $\mathrm{~N}$ & 0 & \begin{tabular}{l|l|l|} 
& \\
\end{tabular} & \begin{tabular}{l|l|l|} 
& \\
\end{tabular} & \begin{tabular}{l|l|l|} 
& \\
\end{tabular} & \begin{tabular}{l|l} 
& 1
\end{tabular} & $\mathrm{v}$ & \begin{tabular}{l|l|} 
\\
\end{tabular} & $x$ & \begin{tabular}{r|r} 
&
\end{tabular} & $\mathrm{z}$ & $A A$ & $\begin{array}{ll}A B \\
\end{array}$ & AC & $\mathrm{AD}$ & $\mathrm{AE}$ & AF \\
\hline 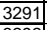 & $6 / 17 / 2004$ & 3:32:26 AM & 26.193 & 25.871 & 25.044 & 27.075 & 26.01 & 49.078 & 56.638 & 17.062 & 32.384 & 1.748 & -4.206 & $\frac{6.425}{46.425}$ & 0.036 & 14.643 & 0.004 & 3977.72 & $\begin{array}{ll}66.2953 \\
\end{array}$ & 55.2 & 11.4 & \begin{tabular}{|l|l|}
40.7 \\
\end{tabular} & 2.808 & 0.005 & 0.005 & 0.000 & 0.132 & 0.11 \\
\hline & $6 / 17 / 2004$ & $3: 33: 26$ AM & 26.172 & 25.846 & 25.003 & 27.004 & 25.98 & 48.963 & & 17.05 & & 1.731 & -4.198 & & & 14.643 & 0.004 & & & 50.0 & & & & & & & & 0.12 \\
\hline & 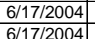 & $\begin{array}{l}3: 34: 26 \mathrm{AM} \\
3.35 \cdot 26 \mathrm{AM}\end{array}$ & $\begin{array}{l}26.183 \\
26.189\end{array}$ & $\begin{array}{l}25.856 \\
25.867\end{array}$ & $\frac{25.014}{2503}$ & $\begin{array}{l}26.995 \\
27011\end{array}$ & $\begin{array}{l}25.986 \\
25991\end{array}$ & \begin{tabular}{|l|l|l|l}
49.301 \\
94.472
\end{tabular} & \begin{tabular}{|l|}
56.849 \\
57063 \\
\end{tabular} & $\begin{array}{l}17.314 \\
17286\end{array}$ & $\begin{array}{l}32.321 \\
32528\end{array}$ & $\begin{array}{l}1.737 \\
1.742\end{array}$ & $\begin{array}{l}-4.209 \\
-4201\end{array}$ & \begin{tabular}{|l|l|}
46.767 \\
4679
\end{tabular} & $\begin{array}{l}0.036 \\
\end{array}$ & 14.643 & 0.004 & $\begin{array}{l}3979.72 \\
330072\end{array}$ & $\begin{array}{l}66.3286 \\
662155\end{array}$ & 55.4 & $\begin{array}{l}11.5 \\
11.5\end{array}$ & 01.00 & 814 & .005 & 0.005 & 0.000 & 0.131 & 0.11 \\
\hline 2294 & $6 / 17 / 2004$ & $\begin{array}{l}3: 35: 26 \mathrm{AM} \\
336.26 \mathrm{AM}\end{array}$ & $\frac{26.189}{26.184}$ & $25.867 \mid$ & $\begin{array}{l}25.03 \\
25055 \\
2055\end{array}$ & $27.011 \mid$ & 25.9911 & $\begin{array}{l}49.472 \\
49077\end{array}$ & \begin{tabular}{|r|}
57.063 \\
5663
\end{tabular} & $\begin{array}{r}17.286 \\
1712\end{array}$ & $\begin{array}{r}32.528 \\
32.39 \\
\end{array}$ & $\begin{array}{l}1.742 \\
1.734\end{array}$ & $\begin{array}{l}-4.201 \\
-41199\end{array}$ & \begin{tabular}{|c|}
46.784 \\
45562
\end{tabular} & 0.036 & $\frac{14.643}{14.63}$ & 0.004 & 3980.72 & 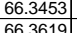 & 55.6 & 11.5 & \begin{tabular}{|l|}
$\mid 41.0$ \\
407
\end{tabular} & 2808 & $\begin{array}{l}0.005 \\
0.006\end{array}$ & \begin{tabular}{|c|c|}
0.005 \\
0.006
\end{tabular} & 0.000 & $\begin{array}{l}0.131 \\
0.125\end{array}$ & 0.11 \\
\hline & $6 / 17 / 2004$ & $\begin{array}{l}\frac{3.30 .20 \mathrm{AMI}}{3: 37: 26 \mathrm{Am}} \\
\end{array}$ & & $\frac{25.851}{25.858}$ & 25.053 & $\frac{27.050}{27.062}$ & $\frac{2.00}{25.937}$ & & \begin{tabular}{|l|}
56.657 \\
\end{tabular} & & & $\frac{1.154}{1.76}$ & $\begin{array}{l}-\frac{-1.189}{-4.195} \\
\end{array}$ & $\begin{array}{l}43.50641 \\
49.561\end{array}$ & 0.037 & $\frac{14.643}{14.643}$ & & & & & $\frac{11.2}{12.1}$ & & & & & & & $\frac{0.12}{0.11}$ \\
\hline 297 & $6 / 17 / 2004$ & $3: 38: 26 \mathrm{AM}$ & 26.195 & 25.863 & 25.091 & 27.007 & 25.967 & 48.982 & \begin{tabular}{|l|}
56.566 \\
\end{tabular} & 17.203 & 32.218 & 1.742 & -4.209 & 45.881 & 0.036 & 14.643 & 0.004 & 3983.72 & \begin{tabular}{|c|c|}
66.3953 \\
\end{tabular} & 55.1 & \begin{tabular}{l|l}
11.2 \\
11.2
\end{tabular} & 40.6 & 2.799 & 0.005 & 0.005 & 0.000 & 0.132 & \\
\hline 298 & $6 / 17 / 2004$ & $3: 39: 26$ AM & 26.21 & 25.868 & 25.096 & 27.007 & 25.937 & 48.719 & \begin{tabular}{|l|l|}
56.379 \\
\end{tabular} & \begin{tabular}{|l|l|}
17.078 \\
\end{tabular} & 31.988 & $\begin{array}{l}1.78 \\
\end{array}$ & -4.183 & 47.546 & 0.037 & 14.643 & 0.004 & 3984.72 & 66.4119 & 54.9 & 11.7 & 40.4 & 2.782 & 0.006 & 0.006 & 0.000 & 0.136 & 0.12 \\
\hline 3299 & $6 / 17 / 2004$ & $3: 40: 26 \mathrm{AM}$ & & 25.858 & 25.061 & 27.002 & 25.937 & 49.021 & \begin{tabular}{|l|}
56.721 \\
5.21 \\
\end{tabular} & $\begin{array}{l}17.165 \\
1760\end{array}$ & & 1.748 & -4.186 & \begin{tabular}{|l|l|}
46.004 \\
6.72
\end{tabular} & & 14.643 & 0.00 & 3985.72 & 66.4286 & 55.2 & 11.3 & 40.6 & 2.802 & 0.005 & 0.005 & 0.000 & 0.132 & 0.1 \\
\hline$\frac{3300}{3301}$ & $6 / 17 / 2004$ & $\begin{array}{l}3: 41: 26 \mathrm{AM} \\
30206\end{array}$ & 26.204 & 25.857 & $\begin{array}{l}25.06 \\
25.06\end{array}$ & $\begin{array}{l}26.981 \\
26091\end{array}$ & 25.941 & $\begin{array}{ll}48.789 \\
\end{array}$ & \begin{tabular}{|r|}
56.321 \\
5659 \\
\end{tabular} & $\begin{array}{l}17.103 \\
17101\end{array}$ & 32.181 & $\begin{array}{l}1.777 \\
1.790\end{array}$ & $\begin{array}{l}-4.1922 \\
\end{array}$ & $\begin{array}{l}47.264 \\
1955\end{array}$ & $\begin{array}{l}0.036 \\
\end{array}$ & 14.643 & 0.003 & $\begin{array}{l}3986.72 \\
300772\end{array}$ & $\begin{array}{l}66.4453 \\
66.615\end{array}$ & 54.8 & \begin{tabular}{ll|}
11.6 \\
1.1
\end{tabular} & 40.5 & $\begin{array}{l}2.791 \\
2.700 \\
\end{array}$ & $\begin{array}{l}0.005 \\
0.06\end{array}$ & 0.005 & 0.000 & 0.132 & 0.11 \\
\hline$\frac{3301}{3302}$ & $6 / 17 / 2004$ & $\begin{array}{l}3: 42: 26 \mathrm{AM} \\
3.43 .26 \mathrm{AM}\end{array}$ & $\frac{26.219}{26.213}$ & $\frac{25.862}{25.856}$ & $\frac{25.06}{250}$ & $\frac{26.991}{26.955}$ & 25.926 & 48.965 & \begin{tabular}{|r|}
56.59 \\
56.342
\end{tabular} & $\begin{array}{l}17.101 \\
17.166\end{array}$ & $\begin{array}{l}32.236 \\
32056\end{array}$ & $\begin{array}{r}1.789 \\
1786\end{array}$ & $\begin{array}{l}-4.175 \\
-41178\end{array}$ & $\begin{array}{r}\mid 49.558 \\
4974\end{array}$ & $0.037 \mid$ & $\frac{14.643}{14.633}$ & 0.004 & 3987.72 & 66.4619 & 55.1 & 12.1 & 40.6 & 2.799 & $\begin{array}{ll}0.006 \\
0.006\end{array}$ & $\begin{array}{l}0.006 \\
0006\end{array}$ & $\begin{array}{l}0.000 \\
0.000\end{array}$ & $\begin{array}{l}0.136 \\
0.201\end{array}$ & 0.12 \\
\hline & $6 / 17 / 2004$ & $\begin{array}{l}3: 43: 26 \mathrm{AM} \\
3: 44: 26 \mathrm{AM}\end{array}$ & $\frac{26.213}{26.214}$ & $\frac{25.856}{25.857}$ & 25.534 & $\frac{26.955}{26.946}$ & $\frac{25.921}{25.926}$ & $\frac{48.818}{49.034}$ & & $\begin{array}{l}\frac{17.166}{17.117} \\
\end{array}$ & $\begin{array}{l}32.056 \\
32.319\end{array}$ & $\begin{array}{l}\frac{1.186}{1.792} \\
\end{array}$ & $\begin{array}{l}-4.1 / 8 \\
-4.166\end{array}$ & $\begin{array}{r}49.754 \\
47.514\end{array}$ & $\begin{array}{l}0.037 \\
0.037 \\
\end{array}$ & $\begin{array}{l}14.643 \\
14.643\end{array}$ & $\frac{0.003}{0.004}$ & 3988.72 & $\begin{array}{l}66.4786 \\
66.493\end{array}$ & 54.9 & $\begin{array}{l}\frac{12.2}{11.6} \\
\end{array}$ & & 2.788 . 2805 & \begin{tabular}{|l|l|}
0.006 \\
0.006
\end{tabular} & \begin{tabular}{|l|l|}
0.006 \\
0.006
\end{tabular} & 0.000 & & 0.12 \\
\hline & $6 / 17 / 2004$ & 3:45:26 AM & 26.213 & 25.856 & 25.034 & 26.945 & 25.906 & 49.136 & \begin{tabular}{|l|}
56.874 \\
\end{tabular} & 17.101 & 32.392 & 1.792 & -4.169 & 47.518 & 0.037 & $\begin{array}{l}14.045 \\
14.643\end{array}$ & 0.004 & 3990.72 & $\frac{60.4953}{66.5119}$ & 55.4 & $\begin{array}{l}\frac{11.0}{11.6} \\
\end{array}$ & $\mid$\begin{tabular}{|l|}
40.8 \\
\end{tabular} & $\frac{2.805}{2.811}$ & $\begin{array}{l}0.006 \\
0.006\end{array}$ & $\begin{array}{l}0.006 \\
0.006\end{array}$ & & & $\frac{0.12}{0.12}$ \\
\hline & $6 / 17 / 2004$ & $3: 46: 26 \mathrm{AM}$ & 26.218 & 25.846 & 25.024 & 26.98 & 25.906 & 48.684 & \begin{tabular}{|l|}
56.284 \\
\end{tabular} & 17.043 & 32.105 & 1.792 & -4.163 & 48.468 & 0.037 & 14.643 & 0.004 & 3991.72 & 66.5286 & 54.8 & 11.9 & \begin{tabular}{|l|}
40.4 \\
\end{tabular} & 2.785 & 0.006 & 0.006 & 0.000 & 0.136 & \\
\hline 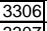 & $6 / 17 / 2004$ & $\begin{array}{l}3: 47: 26 \text { AM } \\
2: 0 ? 0\end{array}$ & 26.203 & 25.851 & 25.009 & $\begin{array}{r}26.95 \\
26.96 \\
\end{array}$ & 25.901 & 49.241 & \begin{tabular}{|l|l|}
56.852 \\
\end{tabular} & $\begin{array}{r}16.9 \\
\end{array}$ & 32.745 & $\begin{array}{l}1.789 \\
1.790\end{array}$ & -4.163 & \begin{tabular}{|l|l|}
47.498 \\
\end{tabular} & 0.037 & 14.643 & 0.004 & $\overline{3992.72}$ & 66.5453 & $\begin{array}{l}55.4 \\
\end{array}$ & \begin{tabular}{ll|}
11.6 \\
\end{tabular} & 41.0 & & 0.006 & $\begin{array}{l}0.006 \\
\end{array}$ & 0.000 & 0.135 & \\
\hline & $6 / 17 / 2004$ & $\begin{array}{l}3: 48: 26 \text { AM } \\
2: 00\end{array}$ & 26.209 & 25.847 & $\begin{array}{r}25.01 \\
25.01\end{array}$ & $\begin{array}{l}26.966 \\
26.025\end{array}$ & 25.911 & 49.111 & \begin{tabular}{|l|}
56.709 \\
56.827 \\
\end{tabular} & 17.113 & $\begin{array}{l}32.399 \\
22.502\end{array}$ & 1.789 & -4.154 & \begin{tabular}{|l|l|}
44.448 \\
4.95
\end{tabular} & $\begin{array}{l}0.038 \\
0.037\end{array}$ & 14.643 & 0.004 & $\begin{array}{l}3993.72 \\
390.72\end{array}$ & $\begin{array}{l}66.5619 \\
66579\end{array}$ & 55.2 & \begin{tabular}{l|l|}
10.9 \\
117
\end{tabular} & \begin{tabular}{|l|l|} 
\\
10.8 \\
\end{tabular} & 2.810 & 0.006 & $\begin{array}{l}0.006 \\
\end{array}$ & 0.000 & 0.139 & 0.12 \\
\hline$\frac{1309}{3309}$ & $6 / 17 / 72004$ & $\begin{array}{l}3: 49: 26 \mathrm{AM} \\
3350 \cdot 26 \mathrm{AM}\end{array}$ & $\frac{26.208}{26.213}$ & $\frac{25.841}{25846}$ & 25.004 & $\frac{26.935}{26.955}$ & $\frac{25.901}{25886}$ & $\begin{array}{ll}49.133 \\
48.849\end{array}$ & \begin{tabular}{|l|}
56.8233 \\
56514 \\
\end{tabular} & $\frac{16.909}{1685}$ & $\frac{32.593}{32.431}$ & $\frac{1.789}{1.789}$ & $\begin{array}{l}-4.154 \\
-4146\end{array}$ & 47.685 & $\mid \frac{0.037}{0.037}$ & $\frac{14.643}{14643}$ & & & & 55.4 & & & & & & & & 0.11 \\
\hline 3310 & $6 / 17 / 2004$ & $\begin{array}{l}\frac{3.50 .20 \mathrm{AMI}}{3: 51: 26 \mathrm{Am}} \\
\end{array}$ & $\frac{2.210}{26.203}$ & $\frac{25.840}{25.851}$ & $\frac{25.009}{24.999}$ & 26.950 26.94 & $\frac{25.800}{25.891}$ & 49.133 & \begin{tabular}{|l|}
56.592 \\
\end{tabular} & 10.076 & $\begin{array}{l}32.451 \\
32.651\end{array}$ & $\frac{1.169}{1.823}$ & $\begin{array}{l}-4.140 \\
-4.149\end{array}$ & $\begin{array}{l}44.352 \\
46.936\end{array}$ & $\frac{0.057}{0.037}$ & $\frac{14.645}{14.643}$ & & $\begin{array}{l}39956.72 \\
3996.72\end{array}$ & 66.5653 & 55.1 & \begin{tabular}{l|l}
111.5 \\
\end{tabular} & 40.9 & 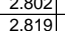 & $\mid$ & 0.006 & 0.000 & & 0.12 \\
\hline & $6 / 17 / 2004$ & 3:52:26 AM & 26.202 & 25.841 & 24.993 & 26.919 & 25.895 & 49.19 & \begin{tabular}{|l|l|}
56.754 \\
\end{tabular} & 16.796 & 32.842 & 1.809 & -4.146 & 45.856 & 0.037 & 14.643 & 0.004 & 3997.72 & 6 & 55.3 & $\begin{array}{ll}11 . .2 \\
11.2\end{array}$ & \begin{tabular}{|l|}
41.0 \\
\end{tabular} & 2.828 & 0.006 & $\mid$ & & 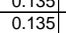 & 0. \\
\hline & $6 / 17 / 2004$ & 3:53:26 AM & 26.202 & 25.846 & 24.998 & 26.934 & 25.88 & 49.113 & \begin{tabular}{|l|l|}
56.711 \\
\end{tabular} & 16.903 & 32.612 & 1.841 & -4.14 & 45.061 & 0.037 & 14.643 & & 3998.72 & 66.6453 & 55.2 & 11.0 & \begin{tabular}{|l|}
40.9 \\
\end{tabular} & 2.817 & 0.006 & 0.006 & 0.000 & 0.135 & \\
\hline 3 & $6 / 17 / 2004$ & $3: 54: 26 \mathrm{AM}$ & 26.198 & $\frac{25.851}{2.811}$ & 24.994 & 26.955 & 25.896 & 49.055 & \begin{tabular}{|r|}
56.7 \\
\end{tabular} & 16.984 & 32.503 & 1.826 & -4.126 & \begin{tabular}{|l|l|}
48.576 \\
\end{tabular} & 0.037 & 14.643 & & 3999.72 & 66.6619 & 55.2 & \begin{tabular}{ll|}
11.9 \\
1.2
\end{tabular} & 40.8 & & 0.006 & 0.006 & 0.000 & 0.135 & \\
\hline 314 & $6 / 17 / 2004$ & $\begin{array}{l}3: 55: 26 \mathrm{AM} \\
356: 26 \mathrm{~A}\end{array}$ & $\frac{26.208}{26.107}$ & 25.841 & 25.004 & $\begin{array}{r}26.93 \\
36.950\end{array}$ & 25.886 & 48.903 & \begin{tabular}{|l|}
56.698 \\
566
\end{tabular} & $\begin{array}{l}16.936 \\
17053\end{array}$ & $\begin{array}{l}32.364 \\
23250\end{array}$ & 1.818 & & \begin{tabular}{|l|l|}
45.666 \\
\end{tabular} & 0.037 & 14.643 & & 4000.72 & $\begin{array}{ll}66.6786 \\
\end{array}$ & 55.2 & 11.2 & 40.6 & 2.802 & 0.006 & 0.006 & 0.000 & 0.136 & \\
\hline & $6 / 177 / 2004$ & $\begin{array}{l}3: 56: 26 \mathrm{AM} \\
3\end{array}$ & $\begin{array}{l}26.197 \\
26.199\end{array}$ & $\begin{array}{l}25.841 \\
25847\end{array}$ & $\begin{array}{r}24.983 \\
25\end{array}$ & 26.859 & $\begin{array}{r}25.9 \\
25866\end{array}$ & $\begin{array}{l}49.024 \\
48.576\end{array}$ & \begin{tabular}{|r|}
56.76 \\
56.242 \\
\end{tabular} & $\begin{array}{c}17.053 \\
175\end{array}$ & $\begin{array}{r}32.259 \\
31.446\end{array}$ & $\frac{1.812}{1.812}$ & $\begin{array}{r}-4.143 \\
-414 \\
\end{array}$ & \begin{tabular}{|c|}
48.568 \\
4974 \\
\end{tabular} & \begin{tabular}{|c|}
0.037 \\
0.036
\end{tabular} & $\begin{array}{l}14.643 \\
14.643\end{array}$ & 0.0 & $\begin{array}{l}4001.72 \\
4402.72\end{array}$ & \begin{tabular}{|c|c|}
66.6953 \\
667119
\end{tabular} & $\begin{array}{l}55.3 \\
54.8 \\
\end{array}$ & $\frac{11.9}{12 .}$ & & $\begin{array}{l}2.802 \\
2759\end{array}$ & $\begin{array}{l}0.006 \\
0.005\end{array}$ & 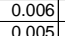 & 0.000 & \begin{tabular}{|l|l|l|l|l|l|}
0.136 \\
0.134
\end{tabular} & 0.12 \\
\hline$\frac{3310}{3317}$ & $6 / 1 / 1 / 2004$ & $\begin{array}{l}3: 57: 26 \mathrm{AM} \\
3 \text { 3:58:26 }\end{array}$ & $\frac{26.199}{26.195}$ & 25.8478 & $\begin{array}{r}25 \\
25.006\end{array}$ & $\frac{26.811}{26.927}$ & $\begin{array}{l}255.866 \\
25.787\end{array}$ & $\begin{array}{l}48.566 \\
48.362\end{array}$ & \begin{tabular}{|l|}
56.242 \\
55.991 \\
\end{tabular} & $\begin{array}{r}17.52 \\
17.494\end{array}$ & $\begin{array}{l}31.446 \\
31.309 \\
\end{array}$ & $\frac{1.812}{1.803}$ & $\begin{array}{r}-4.14 \\
-4.137 \\
\end{array}$ & $\begin{array}{r}49.74 \\
48.096\end{array}$ & \begin{tabular}{|l|l|}
0.036 \\
0.036
\end{tabular} & $\begin{array}{l}14.643 \\
14.643\end{array}$ & & $\begin{array}{l}4002.72 \\
4003.72\end{array}$ & \begin{tabular}{|c|}
66.7119 \\
66.7286
\end{tabular} & $\begin{array}{l}54.8 \\
54.5 \\
\end{array}$ & \begin{tabular}{|l|l|l|l|l|}
11.8 & \\
\end{tabular} & \begin{tabular}{|l|}
40.0 \\
33.8
\end{tabular} & 2.7 .799 & $\mid$\begin{tabular}{l|l}
0.005 \\
0.005
\end{tabular} & $\mid$ & 0.000 & 0.0 .134 & $\frac{0.11}{0.11}$ \\
\hline 2328 & 6/17/2004 & 3:59:26 AM & 26.185 & 25.818 & 25.001 & 26.922 & 25.737 & 48.092 & \begin{tabular}{|l|}
55.867 \\
\end{tabular} & 17.618 & 30.866 & 1.832 & -4.134 & 47.153 & 0.036 & 14.643 & & 4004.72 & 66.7453 & 54.4 & 11.6 & \begin{tabular}{|l|}
39.5 \\
\end{tabular} & 2.722 & 0.005 & 0.005 & & 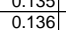 & $\frac{0.11}{0.12}$ \\
\hline & $6 / 17 / 2004$ & 4:00:26 AM & 26.18 & 25.818 & 25.011 & 26.982 & & 48.567 & 56.238 & 17.688 & 31.272 & 1.835 & -4.134 & 46.332 & 0.037 & 14.643 & & 4005.72 & 66.7619 & 54.8 & 11.4 & \begin{tabular}{|l|l}
39.9 \\
\end{tabular} & 2.752 & 0.006 & 0.006 & & 138 & $\frac{0.12}{0.12}$ \\
\hline 3320 & $6 / 17 / 2004$ & $4: 01: 26 \mathrm{AM}$ & 26.174 & 25.807 & 25.015 & 26.996 & 25.732 & 48.42 & 56 & 17.643 & 31.219 & 1.835 & -4.128 & 49.225 & 0.036 & 14.643 & & 4006.72 & & 54.5 & 12.1 & 39.8 & 2.745 & & & & & \\
\hline 3321 & $6 / 17 / 2004$ & 4:02:26 AM & 26.18 & 25.813 & 25.026 & 26.997 & 25.737 & & 56.59 & 17.721 & 31.635 & 1.87 & & \begin{tabular}{l|l}
48.983 \\
\end{tabular} & 0.036 & 14.643 & & & 66.7953 & 55.1. & & 40.3 & 2.778 | & & 0.005 & & 0.133 & \\
\hline$\frac{3322}{2022}$ & 6/17/2004 & 4:03:26 AM & 26.172 & 25.811 & 25.044 & 27.054 & 25.755 & \begin{tabular}{|l|l|}
48.704 \\
\end{tabular} & \begin{tabular}{|l|l|}
56.423 \\
\end{tabular} & 17.743 & 31.305 & 1.844 & -4.12 & 47.051 & 0.036 & 14.643 & & & 66.8119 & 53.0 & 1.5 & & & & & & 0.134 & \\
\hline 33233 & $6 / 17 / 2004$ & 4:04:26 AM & 26.178 & & 25.049 & 27.1 & & 48.289 & & 17.581 & & 1.844 & & & 0.036 & & & & & 54.4 & 2.4 & & 2700 & & & & & \\
\hline 3324 & 6/17/2004 & 4:05:26 AM & 26.162 & 25.786 & 25.034 & 27.129 & & 48.399 & \begin{tabular}{|l|l|}
56.143 \\
\end{tabular} & $\begin{array}{lll}17.52 & \\
\end{array}$ & 31.311 & 1.902 & & 47.934 & & 14.643 & & & & 54.7 & 11. & $39 . .5 \quad>\quad>0$ & 2.748 & 006 & 36] & & 0.138 & \\
\hline 332 & 6/17/2004 & 4:06:26 AM & 26.185 & 25.803 & 25.066 & 27.207 & $25.96 \mathrm{r}-2$ & 48.184 & 55.861 & 17.511 & 31.114 & 1.884 & -4.11 & 48.92 & 0.036 & 14.6 & & & & 54.4 & 12.0 & 99.6 & & & & & & \\
\hline (3) & 6/17/2004 & 4:07:26 AM & 26.185 & 25.804 & 25.072 & 27.237 & & 48.603 & 56.223 & 17.3 & 31.691 & 1.902 & -4. & 46.002 & 0.036 & 14.6 & & & & 54.8 & & 4. & & & & & & \\
\hline 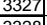 & 6/177/2004 & & 26.1911 & 25.809 & 25.087 & $27.288 \mathrm{H}$ & 26.118 & 48.5944 & 56.29 & 17.547 & 31.346 & 1.907 & & 46.914 & 0.035 & & & & & 54.8 & & & & & & & & \\
\hline 3328 & 61172004 & & 26.214 & & 25.126 & 2 & & & 56.304 & 1.1 .111 & 31.091 & & & 46.97 & 0.037 & & & & & & & & & & & & & \\
\hline 3330 & $6 / 17 / 12004$ & $\begin{array}{l}4: 10: 26 \mathrm{AM} \\
4 \cdot 11 \cdot 26\end{array}$ & $\frac{26.217}{26.206}$ & $\frac{25.841}{25.83}$ & 25.124 & 27.334 & $\begin{array}{r}26.12 \\
26.129\end{array}$ & \begin{tabular}{|l|l|}
48.258 \\
486699
\end{tabular} & 56.016 & 17.39 & $\begin{array}{l}31.247 \\
31.29\end{array}$ & 1.841 & $\frac{-4.19}{422}$ & 47.806 & 0.038 & $\frac{14.643}{14643}$ & & 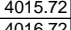 & ats & (54. & & 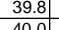 & 275 & (2) & & & 0.142 & 0.12 \\
\hline 3331 & $6 / 177 / 2004$ & $\begin{array}{l}\frac{1.11 .20 \mathrm{AM}}{4: 12: 26 \mathrm{Am}} \\
\end{array}$ & $\frac{20.200}{26.226}$ & \begin{tabular}{r|}
25.045 \\
25.845
\end{tabular} & $\frac{2.110}{25.123}$ & 27.318 & & $\begin{array}{l}-40.009 \\
-4.353\end{array}$ & $\frac{50.011}{2.883}$ & $\begin{array}{l}11.807 \\
.895\end{array}$ & $\begin{array}{l}3.29 \\
-7.733 \\
\end{array}$ & $\begin{array}{l}1.194 \\
1.392\end{array}$ & 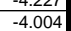 & $\mid-0.062$ & & $\begin{array}{l}\frac{14.045}{14.643} \\
\end{array}$ & & & & $\begin{array}{r}3.0 \\
1.4\end{array}$ & $\begin{array}{c}1 . .0 \\
0.0\end{array}$ & \begin{tabular}{|l|}
4.0 \\
-6.0 \\
\end{tabular} & & 0.000 & 0.000 & & \begin{tabular}{|c|c|}
-0.074 \\
-0.074
\end{tabular} & \\
\hline 3332 & $6 / 17 / 2004$ & 4:13:26 AM & 26.238 & 25.842 & 25.13 & 27.32 & 26.016 & -4.272 & 0.889 & 1.941 & $\begin{array}{r}-5.707 \\
\end{array}$ & -0.614 & -3.49 & $\begin{array}{l}-0.07 \\
\end{array}$ & & 14.643 & & 4018.72 & 66.9786 & -0.6 & $\begin{array}{ll}0.0 \\
\end{array}$ & -5.0 & 0.34 & & & & - & -0.8 \\
\hline 3333 & $6 / 17 / 2004$ & 4:14:26 AM & 26.258 & 25.861 & 25.139 & 27.13 & 25.99 & -3.224 & $\begin{array}{l}0.723 \\
\end{array}$ & 1.806 & -4.579 & -1.216 & -3.307 & -0.07 & $\begin{array}{l}.003 \\
\end{array}$ & 14.643 & & 4019.72 & 66.9953 & $\begin{array}{l}-0.7 \\
\end{array}$ & 0.0 & -3.9 & -0.269 & $\begin{array}{ll}0.000 \\
\end{array}$ & 0.000 & 0.000 & 0.114 & 0.10 \\
\hline 3334 & & & & & & & & & & & & & & & & & & & & & & & & & & & & \\
\hline t & & & & & & & & & & & & & & & & & & & & & & & & & & & & \\
\hline & & & & & & & & & & & & & & & & & & & & & & & & & & & & \\
\hline 3 & & Averages & 26.4 & 26.6 & 25.0 & 27.7 & & 46.9 & 51.5 & 14.1 & 33.3 & -0.5 & & 48.5 & 0.1 & 14.6 & & & & 50.1 & 11.9 & 401 & 2.763 & & & 0.000 & 0.3 & \\
\hline 3339 & & Maximum & 28.9 & 28.1 & $28.7 \mathrm{r}$ & 29.2 & & 52.0 & 57.1. & 18.5 & 41.5 & 74.0 & & 63.4 & 0.5 & & & & & 55.7] & 15.5 & 46.8 & & & & & 2.1 & \\
\hline 3340 & & Median & 26.3 & 26.6 & 24.9 & 27.7 & & 47.3. & 52.1 & 14.7 & 33.3 & -1.2 & & 48.5 & 0.1 & 14.6 & & & & 50.6 & 11.9 | & \begin{tabular}{|l|l|}
40.4 \\
\end{tabular} & 2.785 & & 99 & & .2 & \\
\hline 3411 & & Minim & 25.4 & 24.9 & 24.0 & 26.7 & & -7.3 & -0.8 & 1.3 & -18.5 & -1.2 & $=0.0$ & -0.1 & 0.0 & r & & & & -2.3 & & 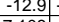 & 0.84 & & & 0.0000 & 0.11 & \\
\hline 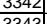 & & $2 \times$ Sta Dev & . & 1.086 & 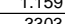 & 1.1655 & 0.919 & (8.097 & 6.803 & 50.046 & 1.019 & 6.216 & 10.515 & 8.072 & 0.106 & 0 & & & & 6.803 & (2) & $\frac{108}{102}$ & . & 0 & (2) & & 15 & \\
\hline 3433 & Number of & ff Points Used*| & 3303 & 3303 & 3303 & 3303 & 3303 & 3303 & 3303 & 3303 & 3303 & 3303 & 3303 & 3303 & 3303 & 3303 & & & & 3303 & 3303 & 3303 & 3303 & 3303 & 3303 & 3303 & 3303 & \\
\hline & & & & & & & & & & & & & & & & & & & & & & & & & & & & \\
\hline 3346 & & & & & & & & & & & & & & & & & & & & & & & & & & & & \\
\hline 3347 & & & & & & & & & & & & & & & & & & & & & & & & & $|0.018|$ & & & \\
\hline$\overline{3348}$ & & & & & & & & & & & & & & & & & & & & & Averag & gef flux & to $20 \%$ & UDS $=$ & $\mid 0.014$ & & & \\
\hline
\end{tabular}


RUN \# WASH1 AND WASH 2; CAMPAIGN III SLURRY WASHING

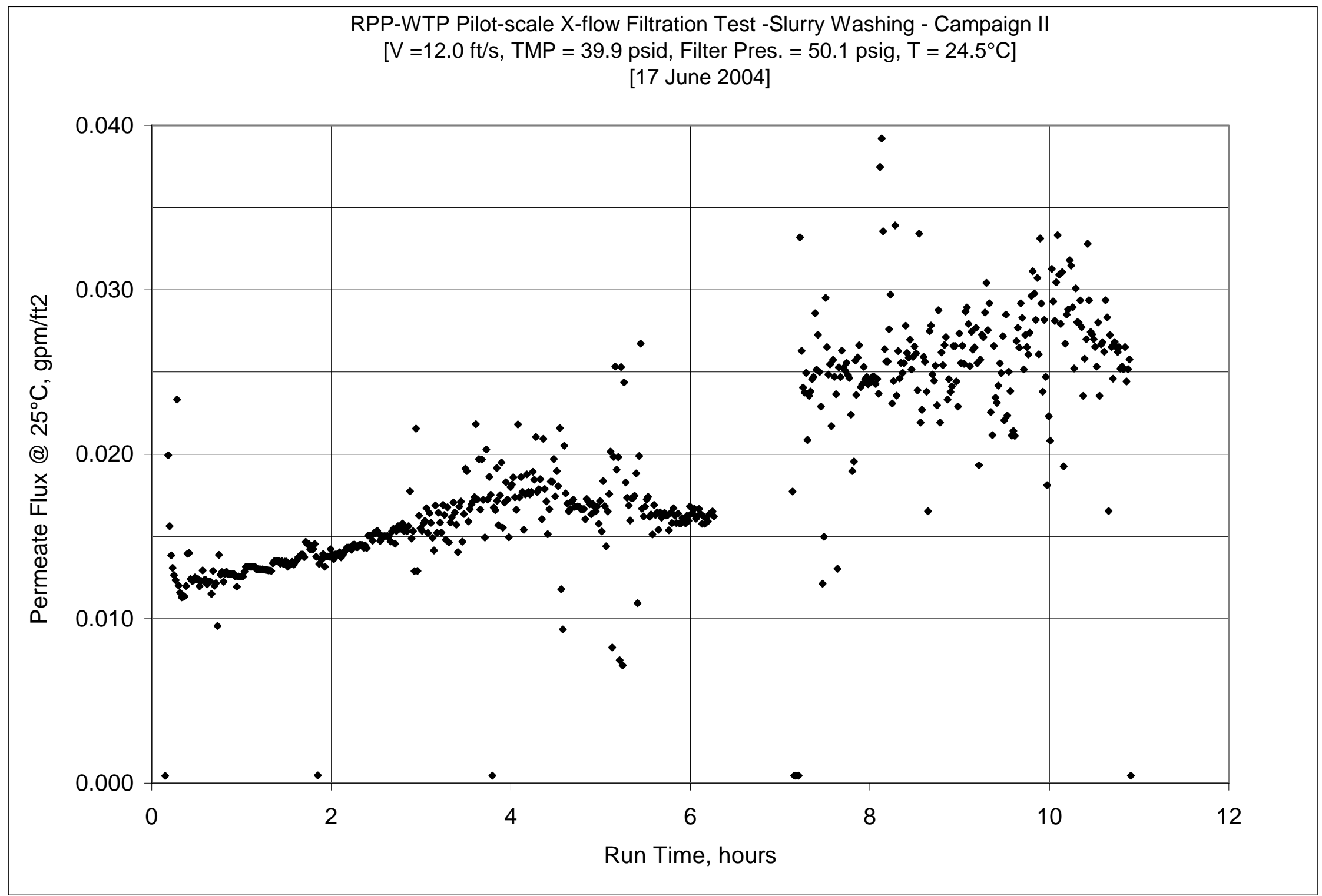


WSRC-TR-2005-00105, REVISION 0

SRNL-RPP-2005-00012, REVISION 0

RUN \# WASH1 AND WASH 2; CAMPAIGN III SLURRY WASHING - CONT.

\begin{tabular}{|c|c|c|c|c|c|c|c|c|c|c|c|c|c|c|c|c|c|c|c|c|c|c|c|c|c|c|c|c|}
\hline & A & B & $\frac{D}{D}$ & $\begin{array}{l}\mathrm{E} \quad \mathrm{P} \\
\ll \text { Kempe }\end{array}$ & $F$ & G G & $\mathrm{H}$ & $\mathrm{J}$ & K & P & $\frac{M}{M}$ & $\mathrm{~N}$ & 10 & $\frac{Q}{\ll \ll \ll<}$ & $\frac{\mathrm{R}}{\ll<\mathrm{F}}$ & \begin{tabular}{|c|}
$s$ \\
s
\end{tabular} & $T$ & d $v$ & $\frac{1 w}{s<\ll<\ll<}$ & $x$ & \begin{tabular}{|l|l|}
$\mathrm{Y}$ \\
\end{tabular} & $z$ & & $A B$ & 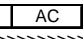 & \begin{tabular}{|l|l|}
$\mid$ & $A D$ \\
\end{tabular} & AE & AF $A$ \\
\hline & DATE & TIME & Filtrate & Cleaning | & \begin{tabular}{|l} 
Slaturry \\
\end{tabular} & Hi Amb. & \begin{tabular}{|l|} 
Lo Amb. \\
\end{tabular} & & & $\begin{array}{l}\mid \text { Filter dP } \\
\end{array}$ & $\begin{array}{l}\text { Nesosuremen } \\
\text { TopTMP }\end{array}$ & Filtrate & Pulsepot & & Filtrate & | & & & & & & & & & & & & \\
\hline 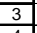 & & & $\operatorname{deg} C$ & & $\operatorname{deg} C$ & $\operatorname{deg} \mathrm{C}$ & & psid & psig & psid & & psig & nsia & $\mathrm{gpm}$ & $\mathrm{gpm}$ & & & & & & & & & & & & & \\
\hline & & & & T3 & & T4 & & $\mathrm{dP} 2$ & $\mathrm{P1}$ & dP1 & $\mathrm{dP3}$ & & & Q1 & $\mathrm{Q}^{2}$ & $\mathrm{Q}^{3}$ & bp & & & & & & & & & & & \\
\hline & & & & & & & & & & & & & & & & & & & & & & & & & & & & \\
\hline & $6 / 8 / 2004$ & 9:09:09 AM & 22.545 & 24.705 & \begin{tabular}{|l|}
27.987 \\
\end{tabular} & 26.054 & 24.911 & -0.004 & 0.128 & -0.01 & -0.003 & -0.129 & 0.111 & 45.741 & 0.293 & $\begin{array}{l}-0.013 \\
\end{array}$ & 0.003 & & & Filter Su & Surface $A$ & 6.707 & FT2 & & & & & \\
\hline 8 & $6 / 10 / 2004$ & 1:47:47 PM & 25.596 & $\begin{array}{l}26.216 \\
\end{array}$ & \begin{tabular}{|l|}
26.68 \\
\end{tabular} & 26.834 & 26 & -0.019 & 0.196 & -0.012 & $\begin{array}{ll}-0.02 \\
\end{array}$ & -0.106 & 0.073 & -0.071 & 0.003 & 14.643 & 0.003 & & & Convers & ersion & 851 & $1 \mathrm{~m} 3 / \mathrm{m} 2 /$ & 2/day/bar & $\arg / \mathrm{gpm} / \mathrm{ft}$ & /barg & & \\
\hline & $6 / 114212004$ & 7:41:41 AM & & & & 24.174 & & & 0.12 & & & & & & 0.003 & 14.643 & & & & & & & & & & & & \\
\hline & & & & & & & & & & & & & & & & & & & & Note: & ge & essur & & oxi & ely 1. & SIg & & \\
\hline & 6/177/2004 & 7:22:28 AM & 24.492 & 23.951 & & & 23.743 & -0.01 & 0.164 & $\begin{array}{l}-0.001 \\
-1\end{array}$ & -0.014 & -0.18 & & -0.075 & & 14.643 & 0.006 & & & & & & & & & & & \\
\hline & $6 / 17 / 2004$ & 4:57:57 PM & & 26.737 & & 27.805 & 26.45 & -0.002 & 0.197 & & -0.012 & -0.183 & -0.009 & 47.043 & 0.098 & 14.643 & & & & & P1is & orrec & $d$ for $4 \mathrm{C}$ & & & & & \\
\hline & $6 \frac{61 / 212004}{61203}$ & 6:52:52 AM & 23.109| & 23.008 & \begin{tabular}{|l|l|}
22.621 \\
\end{tabular} & 23.418 & 22.888 & -0.01 & 0.075 & & -0.01 & -0.197 & & & 0.003 & & & & & vwv & & & & & & & & \\
\hline & 6/22/2004 & 8:12:12 AM & 23.36 & 23.555 & 23.63 & 23.829 & & & 0.108 & -0.004 & -0.012 & -0.194 & & -0.083 & 0.003 & 14.643 & & & & & tyet & TMP & & & & & & \\
\hline$\frac{16}{16}$ & Data - Per M & & & & & & & & & & & & & & & & & Jime & Hour & plas. & 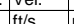 & si & $\mathrm{IMP}$ & $\mathrm{gpm}$ & & 20 & pinsi & av/bar \\
\hline & $6 / 17 / 2004$ & 10:09:44 AM & 24.904 & 24.615 & \begin{tabular}{|l|l|}
24.649 \\
\end{tabular} & 26.251 & 25.377 & 49.432 & 54.333 & 18.433 & 31.352 & -0.82 & -6.08 & 56.492 & 0.038 & 14.643 & 0.004 & & & 52.9 & $\frac{55}{13.8}$ & 40.4 & 2.785 & 0.006 & 0.006 & 0.000 & 0.142 & $\frac{d y D d u d}{0.12}$ \\
\hline & & & & & & & & & & & & & & 51.74 & & 14.643 & & & & & & & & & & & & \\
\hline 19 & 611721204 & 10:11:44 AM & 25.019 & 24.655 & 24.833 & 26.301 & 25.461. & 48.609 & 54.725 & 17.842 & 31.202 & $0.361 \mid$ & $-2.1930 \mathrm{Y} \quad \mathrm{l}$ & 48.935 & 0.003 & 14.643 & & & .0333 & 53.3 & 12.0 & & 2.75 & & & 0.000 & 011 & 0.01 \\
\hline & 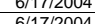 & $\frac{10.12 .44 \mathrm{AM}}{10.12 .44}$ & $\frac{2.0971}{25.12}$ & $24.678 \mathrm{j}$ & \begin{tabular}{|l|l|}
2.941 \\
2490
\end{tabular} & $\frac{20.31}{2635}$ & 25.4804 & 年55.311 & 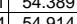 & $\frac{17.181}{17711}$ & 27.9686 & $\frac{3.360}{7107}$ & & 40.996 & 0.003 & $\frac{14.643}{1.643}$ & & & & 5.9 .9 & 些.0 & & 2 & & & & & 0.0 \\
\hline 22 & $6 / 1 / 17 / 2004$ & $\begin{array}{l}10.13 .44 \mathrm{AM} \\
0.14 .44 \mathrm{AM}\end{array}$ & $\frac{25.13}{25162}$ & $\frac{24.0090}{24709}$ & \begin{tabular}{|l|l|}
25.094 \\
\end{tabular} & $\frac{20.352}{2634}$ & $\frac{23.301}{25.525}$ & (41.045 36.725 & 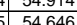 & 年 & $\begin{array}{ll}24.500 \\
19.431\end{array}$ & 12182 & 5.966 & 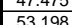 & 年 & 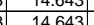 & 00 & $\frac{4.6}{5.6}-2$ & - & 55.44 & $\frac{1.60}{130}>>>$ & 23.4. & 2.924 & 0000 & 0.000 & 0.000 & 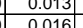 & \\
\hline & $6 / 17 / 2004$ & $10.15 .4 \mathrm{AM}$ & 25.203 & 24.739 & \begin{tabular}{|l|}
25.04128 \\
2.128
\end{tabular} & 26.379 & 25.545 & 30.987 & 54.943 & $\begin{array}{l}\frac{17.124}{17.59} \\
\end{array}$ & 13.994 & $\begin{array}{ll}12.106 \\
18.288\end{array}$ & $\frac{5.200}{69.221}$ & 50.059 & 0.003 & $\frac{14.045}{14.643}$ & 00 & $\frac{3.6}{66}$ & - & 53.5 & $\frac{1.03}{123}$ & & & & & & & $\overline{0.6}-(-3)$ \\
\hline & $\frac{0}{6 / 177 / 2004}$ & $\frac{10: 16: 44 \mathrm{AM}}{10}$ & & 24.743 & & $\frac{26.408}{26.408}$ & 25.58 & & 54.302 & 17.482 & $\frac{3.131}{7.131}$ & & & 45.099 & 0.003 & 14.643 & & & & 528 & & & & & & & & 0.6 \\
\hline 25 & $6 / 17 / 2004$ & $10: 17: 44 \mathrm{AM}$ & 25.362 & 24.773 & 25.242 & 26.449 & 25.624 & 9.171 & 53.401 & 12.141 & $\frac{-2.177}{-2.17 \mid}$ & 38.793 & 54.044 & 51.724 & 0.003 & 14.643 & & 8.6 & 0.13333 & 51.9 & 12.7 & 3.5 & 0.241 & $\frac{000}{0.000}$ & $\frac{0.000}{0.000}$ & $\frac{0.000}{0.000}$ & 0.127 & \\
\hline & & & & & & & & 9.142 & 53.541 & & & 38.863 & & 55.878 & 0.003 & 14.643 & & 9.0 & & 52.1 & 13.7 & 3.4 & & & & & & \\
\hline & $6 / 17 / 2004$ & 10:19:44 AM & 25.76 & 24.801 & 25.3 & 26.491 & 25.682 & 37.387 & 53.819 & 13.038 & 24.805 & $\begin{array}{ll}10.992 \\
\end{array}$ & $0.36-36$ & 49.875 & 0.366 & 14.643 & & $\frac{10.0}{10.0}-2$ & & 52.3 & 12.2 & 31.1 & $\mid \frac{2.144}{11}$ & & 0.054 & & $\frac{1.740}{1.740}$ & \\
\hline & 6/17/2004 & 10:20:44 AM & 25.643 & 24.814 & 25.343 & 26.51 & & 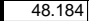 & 54.491 & 15.277 & 33.423 & 0.544 & & 60.061 & 0.135 & $\begin{array}{ll}14.643 \\
\end{array}$ & & 11.0 & 0.1833 & 53.0 & 14.7 & 40.8 & & & & & & \\
\hline & 6/177/2004 & 10:21:44 AM & 25.681 & 24.838 & 25.402 & 26.558 & 25.753 & 48.576 & 54.872 & 16.25 & 32.869 & 0.477 & 0.471 & 43.136 & 0.106 & 14.643 & 0.00 & 12.0 & $0.8>$ & 53.4 & 10.6 & 40.7 & 2.808 & 0.016 & 0.016 & 0.000 & $\overline{384}$ & \\
\hline & 6/177/2004 & 10:22:44 AM & 25.713 & 24.86 & 25.429 & 26.595 & 25.78 & 48.559 & 54.779 & 16.876 & 32.202 & 0.477 & 0.506 & 46.19 & 0.094 & 14.643 & & 13.0 & 0.0 .21667 & 53.3 & 11.3 & 40.4 & 2.784 & $\begin{array}{lll}4 & 0.011 \\
\end{array}$ & 0.014 & 0.000 & 0.343 & \\
\hline & $6 / 17 / 2004$ & 3:44 AM & 25.762 & 24.879 & \begin{tabular}{|l|}
25.478 \\
\end{tabular} & 26.609 & & 48.522 & 54.88 & 17.114 & 31.927 & 0.477 & & 49.867 & & 14.643 & & $14 . c \mathrm{C}$ & 23333 & 53.4 & $12.2 \mathrm{rt}$ & 40.2 & & & & & & \\
\hline$\frac{32}{32}$ & $6 / 17 / 2004$ & $10: 24: 44 \mathrm{AM}$ & 25.8212 & 24.903 & \begin{tabular}{|l|}
25.492 \\
2
\end{tabular} & 26.669 & 25.884 & 48.688 & 55.102 & 17.25 & $31.877 \mathrm{t}$ & 0.474 & & 51.684 & 0.086 & 14.643 & & 15.0 & & 53.6 & 12.7 & 40.3 & & & & 0.000 & & \\
\hline . & 0011722004 & $\begin{array}{l}10.250 .44 \\
10.26 .44\end{array}$ & 250.8006 & 24.039 & & & & 46.069) & 53.020 & $\begin{array}{l}1.232 \\
1.7205\end{array}$ & 31.9869 & & & 57.0007 & & 14.6 & & & 0.2007 & (53.0 & 14.2 & t. 4.5 & 2.181 & & 0.01 & & & \\
\hline$\frac{34}{35}$ & 0 & $10.20 .424 \mathrm{AM}$ & (25.0.045 & 24967 & \begin{tabular}{|l|}
25.511 \\
2511
\end{tabular} & $\frac{20.112}{26792}$ & 26007 & 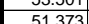 & $\frac{5.0044}{5243}$ & 20.545 & $\begin{array}{l}30.100 \\
31.701\end{array}$ & $\frac{1.45}{-1219}$ & 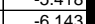 & 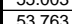 & 0.002 & $\begin{array}{l}14.045 \\
14643\end{array}$ & 000 & 180 & 0 & - & $\frac{15.5}{132}$ & $\frac{4.1 .1}{415}$ & $\frac{3.114}{284}$ & $\frac{1}{4}$ & 0.1 & 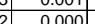 & 288 & 0.7 \\
\hline & & $\frac{16 \cdot 6 \cdot 44 \mathrm{AM}}{10 \cdot 08}$ & 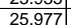 & 24.984 & \begin{tabular}{|l|}
25.627 \\
\end{tabular} & 26.809 & $\frac{2.0 .978}{25.978}$ & $\begin{array}{l}1.07 \\
48.87\end{array}$ & $\frac{2.43}{50.33}$ & 18.263 & 31.077 & -1.216 & & $\frac{53.96}{5296}$ & 0.079 & 14,643 & & $\frac{1.6}{19.6}$ & 0.31667 & $\frac{3.6}{48.6}$ & 13.0 & $\frac{4.0 .0}{40.0}$ & 2756 & & 1.1. & $00 c_{-1}$ & & \\
\hline & $6 / 171200$ & 10. & & 2498 & 25597 & & & 47.178 & 48280 & 17254 & 30469 & 1219 & & 43.731 & 0.077 & 146 & 010 & & s.ton & 468 & 107 & 40.6 & & & 0.01 & ond & & \\
\hline$\overline{38}$ & $6 / 17 / 2004$ & $10: 30: 44 \mathrm{~A}$ & 26.039 & 25.001 & 25.5 & 26.776 & 25.941 & 47.238 & 488.314 & 17.061 & 30.781 & -1.219 & & $54.5 \mathrm{c}$ & 0.07 & 14.643 & & 210 & 035 & 468 & 134 & 39.6 & 2.690 & 0001 & 0.01 & & & \\
\hline & & & 26.039 & 25006 & & 26.746 & & 47.572 & & 16.865 & & & & 53.901 & & 14.643 & & & & 471 & 13.2 & 39.4 & 2.718 & & & & & \\
\hline 40 & $6 / 17 / 2004$ & & 26.034 & 25.016 & \begin{tabular}{|l|}
25.255 \\
\end{tabular} & 26.731 & 25.896 & 47.085 & 48.03 & \begin{tabular}{|l|l|}
16.23 \\
\end{tabular} & 31.479 & -1.222 & -6 & 52.151 & 0.081 & $\begin{array}{l}14.643 \\
\end{array}$ & & 23.0 & 0.38333 & 46.6 & 12.8 & 39.3 & 2.708 & & & .000 & 0.305 & \\
\hline 41 & 6/177/2004 & $10: 33: 44 \mathrm{AM}$ & 26.067 & \begin{tabular}{|l|}
25.07 \\
\end{tabular} & \begin{tabular}{|l|}
25.158 \\
\end{tabular} & $\begin{array}{l}26.764 \\
\end{array}$ & 25.87 & \begin{tabular}{ll|l}
47.116 \\
\end{tabular} & 48.003 & $\begin{array}{ll}16.232 \\
\end{array}$ & 31.37 & \begin{tabular}{|l|l|}
-1.2222 \\
\end{tabular} & -7.0 & \begin{tabular}{l|l}
48.862 \\
\end{tabular} & 0.094 & \begin{tabular}{|c|}
14.643 \\
\end{tabular} & 0.00 & 24.0 & 0.4 & 46.5 & 12.0 & 39.2 & \begin{tabular}{|l|l|l|}
2.706 \\
\end{tabular} & & 0.014 & 0.000 & 0.356 & \\
\hline 42 & $6 / 17 / 2004$ & 10:34:44 & 26.064 & 25.101 & \begin{tabular}{|l|l|}
25.045 \\
\end{tabular} & 26.781 & 25.851 & \begin{tabular}{ll|l}
47.698 \\
\end{tabular} & 48.322 & 16.257 & 31.99 & -1.219 & & 51.185 & 0.094 & 14.643 & 0.0 & 25.0 & 0.41667 & 46.8 & 12.5 & 39.8 & 2.747 & .014 & 0.014 & 0.000 & 0.351 & \\
\hline 43 & 6/117/2004 & $10: 35: 44 \mathrm{f}$ & 20.035 & 25.117 & \begin{tabular}{|l}
24.9 \\
\end{tabular} & 26.777 & 25.8 & 48.038 & 48.513 & 16.229 & 32.382 & -1.222 & & 44.723 & 0.083 & & & 26.0 & & 47.0 & 11.0 & 40.2 & 2.772 & & & 0.000 & & \\
\hline & & & 25.9699 & & 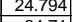 & 26.716 & & 48.018 & 48.507 & 16.232 & & & & 57.837 & & 14.643 & & 27.0 & 0.45 & 47.0 & 14.2 & 40.2 & 2.770 & $\begin{array}{lll}0 & 0 \\
0\end{array}$ & & & & \\
\hline$\frac{4}{4}$ & 611722004 & & $\frac{25.925}{25875}$ & 255.128 & 24.11 & 26.182 & 25.95 & $\begin{array}{l}48.051 \\
47809\end{array}$ & $\begin{array}{l}48.503 \\
18205\end{array}$ & $\frac{16.179}{16.99}$ & $\begin{array}{l}3.2 .44 \\
32.20\end{array}$ & -1.219 & & 48.419 & 0.082 & . & & 28.0 & 0.466067 & 4.0 & 11.9 & 40.2 & $2.7 / 5$ & & & 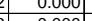 & 306 & \\
\hline & 0 & & $\begin{array}{l}23.015 \\
25825\end{array}$ & 年 & $\begin{array}{r}24.51 \\
253\end{array}$ & $\frac{20.037}{26852}$ & & 4 & $\begin{array}{l}46.305 \\
48602\end{array}$ & | & 32.24 & $\frac{-1.29}{-1219}$ & & (44.1097 & & & & 29.6 & & $\begin{array}{ll}46.9 \\
1171\end{array}$ & $\frac{11.0}{116}$ & 40.1 & 2.101 & & & & & \\
\hline 48 & 6 & 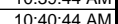 & 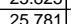 & 25.98 & \begin{tabular}{|l|}
24.545 \\
\end{tabular} & $\frac{20.054}{26.922}$ & 2610 & 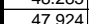 & 年 48206 & 16.163 & (32.345 & -1.222 & & 50.989 & 0.0 & 14. & & . & 051.07 & 46.1 & 1.0 & 40 & 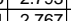 & & 0.014 & 0.0000 & 0 & \\
\hline & 6 & & & & & & & & 48735 & & & & & & & & & 320 & 年 & 473 & 128 & 404 & 278 & & & & & \\
\hline$\frac{10}{50}$ & $6 / 1772004$ & $10: 42: 44$ & 25.716 & 25.239 & 2436 & 27.013 & & 48.372 & 48.899 & 16.223 & 32.659 & $\frac{-1.219}{-1219}$ & & 50.278 & & & & 330 & 0.055 & 474 & 123 & 40.5 & 2.793 & & 0.012 & & 0303 & \\
\hline 5 & $6 / 177 / 2$ & & 25.673 & 25265 & 24282 & 27059 & & 48 & 48.824 & 16.241 & 32.645 & $-1,21$ & & 416 & & & & 34. & & 474 & 102 & & 2 & & & & & \\
\hline 5 & $6 / 17 / 200$ & & 25.619 & 25.276 & \begin{tabular}{|l|}
24.223 \\
\end{tabular} & 27.016 & & 48.215 & 48.635 & 16.145 & 32.594 & & & 48.40 & & 14.6 & & 35. & 0.58333 & 47.2 & 11.9 & $\overline{40.4}$ & 786 & & & & 06 & \\
\hline & $6 / 17 / 2$ & & 25.586 & 25.304 & \begin{tabular}{|l|}
24.17 \\
\end{tabular} & 27.083 & & & $\begin{array}{l}48.447 \\
\end{array}$ & & & & & & & & & 36.1 & & 47.0 & $\overline{12.7}$ & 40.3 & '82 & & & & & \\
\hline 5 & 6/17l2004 & $\begin{array}{l}10: 46: 44 \\
\end{array}$ & 25.553 & $\mid 25.321$ & \begin{tabular}{|l|}
24.087 \\
\end{tabular} & 27.075 & 26.2 & $\begin{array}{l}48.206 \\
\end{array}$ & 48.861 & 16.072 & 32.626 & -1.219 & & 54.448 & 0. & 14.6 & & 37. & 0.61667 & \begin{tabular}{|l|l|}
47.4 \\
\end{tabular} & 13.3 & 40.4 & \begin{tabular}{|l|}
2.787 \\
\end{tabular} & & & & 0.299 & \\
\hline & $6 / 17 / 2004$ & $\begin{array}{ll}10: 47: 44 \\
\end{array}$ & 25.517 & 25.34 & \begin{tabular}{|l|}
24.056 \\
\end{tabular} & $\begin{array}{l}27.094 \\
\end{array}$ & 26.2. & 48.387 & 48.932 & 16.191 & 32.68 & -1.219 & & $47.52 !$ & 0.08 & 14.643 & & 38. & 0.6333 & 47.5 & 11.6 & 40. & & & 0.6 & & & \\
\hline & & & 25.49 & & & & & 48.451 & 48.894 & & & & & & & & & 39.6 & & 47.4 & & 40.6 & & & & & & \\
\hline & & & 25.1777 & 25.384 & \begin{tabular}{|l|l|}
23.9955 \\
\end{tabular} & 27.143 & & 42.29 & 48.495 & 16.102 & 26.786 & 0.634 & & & & 14.643 & & & 0.66667 & 47.0 & 11.9 & 34. & & & & & & \\
\hline & & & 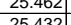 & 25.4 & \begin{tabular}{|l}
2.995 \\
23894
\end{tabular} & 27.144 & 2.2 & $\begin{array}{l}41.4355 \\
17495\end{array}$ & 48.131 & $\begin{array}{l}16.176 \\
1.6068\end{array}$ & $\mid \begin{array}{l}3.1 .13 \\
31.090\end{array}$ & $-\frac{1.219}{1.272}$ & & & 0.8 & 14. & & 41.6 & 0.68333 & 41.3 & 12.2 & $\begin{array}{l}3.6 .6 \\
30.7\end{array}$ & 25 & & & 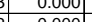 & 260 & \\
\hline & 6 & 10.5. & $\frac{2.453}{25372}$ & $\frac{25.419}{2544}$ & \begin{tabular}{|l|l|}
23.049 \\
23889
\end{tabular} & 270 & $\frac{20.1}{26.1}$ & 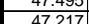 & $\begin{array}{l}48.456 \\
8.486\end{array}$ & $\frac{10.000}{1575}$ & $\frac{15.96}{3199}$ & -12 & & & & & & & 0.116 & 47,0 & $\frac{1.1}{125}$ & 年 & \begin{tabular}{|l|l|l|}
.500 \\
2731
\end{tabular} & & & & & \\
\hline & $6 / 171 / 200$ & $\frac{10.53 .44}{10.53 .44}$ & 25352 & $\frac{2.3444}{25.434}$ & \begin{tabular}{|l|}
23824 \\
\end{tabular} & 1270033 & & 47099 & 40.406 & $\frac{1.37}{15.747}$ & 31818 & & & & & & & 400 & & 470 & $\frac{1.26}{126}$ & (3.0 & 2.271 & & & & & \\
\hline & & & 25.401 & & \begin{tabular}{|l|}
23803 \\
\end{tabular} & & & 47.804 & 48488 & & & & & 48.82 & & & & 450 & 075 & 470 & 120 & 4023 & & & & & & \\
\hline & & & 25289 & 25462 & \begin{tabular}{|l|l|}
23717 \\
\end{tabular} & 26.981 & & 47715 & & 15725 & & -1216 & & & & & & & & 468 & 129 & 401 & & & & & & \\
\hline & & & 25.243 & 25.491 & & & & 47.852 & & & & & & & & & & & & & & & & & & & & \\
\hline & $6 / 17 / 200$ & 10:57:44 & 25.192 & 25.5 & 23.624 & 26.999 & 260 & 47.873 & 48.465 & 15.713 & 3269 & -1.216 & & 43. & (20) & 14.6 & & 48.0 & 0.8 & 470 & 108 & 40.3 & 2.777 & 70 & & & $\frac{304}{304}$ & \\
\hline & $6 / 17 / 2004$ & 10:58: & 25.157 & 25.52 & 23.614 & 27.004 & & 47.73 & 48.349 & 15.688 & & -1.2 & & & & 14.6 & & 49.0 & 0.81667 & 46.9 & 13.4 & 401 & 2.766 & & & & & \\
\hline & $6 / 17 / 2004$ & $10: 59: 44$ & 25.143 & 25.541 & 23.635 & 27.015 & 26.1 & 47.651 & 48.331 & 15.705 & 32.39 & -1.219 & & 47.6 & & 14.6 & & 50.6 & 0.83333 & 46.9 & 11.7 & 40.0 & 275 & & & & & \\
\hline 68 & $6 / 17 / 2004$ & $11: 00: 44$ & 25.119 & 25.546 & \begin{tabular}{|l|}
23.616 \\
\end{tabular} & 27.05 & 26. & 47.819 & 48.385 & 15.666 & 32.692 & -1.222 & -8.00 & $\begin{array}{l}44.323 \\
\end{array}$ & 0.082 & 14.643 & 0.0 & 51.0 & 0.85 & $\begin{array}{l}46.9 \\
\end{array}$ & 10.9 & 40.3 & \begin{tabular}{|l|l|}
3 & 2.775 \\
\end{tabular} & \begin{tabular}{l|l|l|}
5 & 0.012 \\
\end{tabular} & 0.013 & 0.000 & 0.316 & 0 \\
\hline & $\begin{array}{l}6 / 17 / 2004 \\
6 / 17 / 2004 \\
\end{array}$ & $\begin{array}{l}1: 01: 44 \mathrm{AM} \\
11: 02.44 \mathrm{AM}\end{array}$ & 25.119 & $\begin{array}{l}25.561 \\
25584 \\
\end{array}$ & \begin{tabular}{|l|}
23.621 \\
23623 \\
\end{tabular} & \begin{tabular}{|l|}
27.105 \\
32147
\end{tabular} & $\frac{26.26}{26.227}$ & $\begin{array}{ll}47.588 \\
47841\end{array}$ & $\begin{array}{l}8.403 \\
48565\end{array}$ & $\frac{15.722}{15769}$ & \begin{tabular}{|l|l|}
32.337 \\
32624
\end{tabular} & $\begin{array}{c}-1.216 \\
-1219 \\
\end{array}$ & -8. & $\begin{array}{l}53.519 \\
50.641\end{array}$ & $\begin{array}{l}0.082 \\
0.082 \\
\end{array}$ & \begin{tabular}{|l|l|}
14.643 \\
14643
\end{tabular} & 0.0 & & \begin{tabular}{|l|}
0.86667 \\
088333
\end{tabular} & $\begin{array}{l}46.9 \\
471\end{array}$ & $\begin{array}{l}13.1 \\
124\end{array}$ & & & & $\begin{array}{l}0.013 \\
0.13 \\
\end{array}$ & $\begin{array}{ll}0.000 \\
0.000\end{array}$ & 0.318 & $\frac{0}{0}$ \\
\hline
\end{tabular}


WSRC-TR-2005-00105, REVISION 0

SRNL-RPP-2005-00012, REVISION 0

RUN \# WASH1 AND WASH 2; CAMPAIGN III SLURRY WASHING - CONT.

\begin{tabular}{|c|c|c|c|c|c|c|c|c|c|c|c|c|c|c|c|c|c|c|c|c|c|c|c|c|c|c|c|c|}
\hline & A & \begin{tabular}{l|l}
$\mathrm{B}$ & $\mathrm{C}$ \\
\end{tabular} & $\begin{array}{ll} \\
\end{array}$ & $E$ & $\mathrm{~F}$ & G & $\mathrm{H}$ & $\mathrm{J}$ & $\mathrm{K}$ & \begin{tabular}{l|l}
$\mathrm{L}$ \\
\end{tabular} & $\mathrm{M}$ & $\mathrm{N}$ & 0 & Q & \begin{tabular}{l|l}
$R$ &
\end{tabular} & \begin{tabular}{l|l} 
\\
\end{tabular} & $\begin{array}{lll} & \mathrm{T} \\
\end{array}$ & $\begin{array}{ll}\mathrm{v} & \mathrm{g} \\
\mathrm{v}\end{array}$ & \begin{tabular}{ll|} 
\\
\end{tabular} & $x$ & $\mathrm{Y}$ & $z$ & $\mathrm{AA}$ & $A B$ & $\mathrm{AC}$ & $\mathrm{AD}$ & $\mathrm{AE}$ & AF $A$ \\
\hline 71 & $\begin{array}{l}6 / 17 / 2004 \\
6 / 17 / 2004\end{array}$ & \begin{tabular}{|l|}
$11: 03: 44$ AM \\
$11: 04: 44 \mathrm{MM}$
\end{tabular} & $\begin{array}{l}25.087 \\
2584 \\
25\end{array}$ & \begin{tabular}{l|l}
25.605 \\
$2567 \mid$
\end{tabular} & \begin{tabular}{|l|l|}
23.624 \\
23631
\end{tabular} & \begin{tabular}{l|l}
27.194 \\
27271
\end{tabular} & $\begin{array}{l}26.338 \\
26395 \\
\end{array}$ & $\begin{array}{l}47.742 \\
47524\end{array}$ & \begin{tabular}{|l|l|}
48.38 \\
48.202
\end{tabular} & \begin{tabular}{|l|l|}
15.748 \\
15739
\end{tabular} & \begin{tabular}{l|l}
32.524 \\
32333
\end{tabular} & \begin{tabular}{|l|l|}
-1.216 \\
-1216
\end{tabular} & $\begin{array}{l}-8.014 \\
-8011 \\
\end{array}$ & $\begin{array}{l}47.097 \\
4533\end{array}$ & $\begin{array}{l}0.082 \\
0.083 \\
\end{array}$ & \begin{tabular}{|l|l|}
14.643 \\
1643
\end{tabular} & 0.004 & $\begin{array}{l}54.0 \\
550\end{array}$ & \begin{tabular}{r|r}
0 & 0.9 \\
0 & 0.1667
\end{tabular} & 46.9 & $\begin{array}{l}11.5 \\
11.6\end{array}$ & 40.1 & $\begin{array}{l}2.767 \\
2.753 \\
\end{array}$ & $\begin{array}{l}0.012 \\
0012 \\
\end{array}$ & 0.013 & 0.000 & $\begin{array}{l}0.317 \\
0.318 \\
\end{array}$ & 0.27 \\
\hline & $\begin{array}{l}6 / 1 / 1 / 2004 \\
6 / 17 / 2004\end{array}$ & \begin{tabular}{|l|}
$11: 04: 44 \mathrm{AMI}$ \\
$11: 05: 44 \mathrm{AM}$
\end{tabular} & $\frac{25.004}{25.071}$ & 25.6234 & \begin{tabular}{|l|}
23.631 \\
23.603 \\
\end{tabular} & $\mid$\begin{tabular}{|l|}
27.271 \\
27.283 \\
\end{tabular} & $\frac{20.395}{26.412}$ & $\begin{array}{l}47.524 \\
47862\end{array}$ & \begin{tabular}{|l|}
48.2027 \\
48.772 \\
\end{tabular} & $\begin{array}{r}15.1399 \\
15805 \\
\end{array}$ & $\begin{array}{l}32.353 \\
32.481 \\
\end{array}$ & $\begin{array}{l}-1.216 \\
-1.129\end{array}$ & $\begin{array}{l}-8.011 \\
-7.999 \\
\end{array}$ & 47.523 & $\mid 0.081$ & $\begin{array}{l}14.643 \\
14643 \\
\end{array}$ & 0.004 & 55.0 & 0.91667 & 46.7 & 11.6 & 39.9 & 2.153 & $\begin{array}{l}0.012 \\
0.012 \\
\end{array}$ & 0.013 & 0.000 & $\begin{array}{l}0.318 \\
0.313 \\
\end{array}$ & \\
\hline & & 11:06:44 AM & & & & & $2 \frac{2.414}{26.429}$ & & 48.223 & $\begin{array}{l}15.000 \\
15.679\end{array}$ & $\begin{array}{l}32.401 \\
32.117\end{array}$ & $\begin{array}{l}-1.216 \\
-1.216 \\
\end{array}$ & -6.771 & 54.414 & $\begin{array}{l}0.017 \\
0.077\end{array}$ & $\begin{array}{l}14.045 \\
14.643\end{array}$ & 0.004 & 57.0 & 0.95 & 46.7 & 13.3 & $\frac{40.6}{39.7}$ & & on & 012 & 0.000 & 0.301 & \\
\hline 75 & $6 / 17 / 2004$ & & 25.116 & 25.659 & \begin{tabular}{|l|}
23.010 \\
23.593 \\
\end{tabular} & 27.253 & 26.4392 & & \begin{tabular}{|l|}
48.648 \\
48.648 \\
\end{tabular} & 15.807 & 32.435 & $\begin{array}{ll}-1.222 \\
-1.222 \\
\end{array}$ & & & 0.081 & $\begin{array}{l}14.045 \\
14.643\end{array}$ & & 58.0 & & 47.2 & $\begin{array}{l}13.5 \\
12.2 \\
\end{array}$ & & 2.766 & $\begin{array}{l}0.0112 \\
0.012\end{array}$ & & 0.000 & $\begin{array}{l}0.313 \\
0.313 \\
\end{array}$ & \\
\hline 76 & $6 / 17 / 2004$ & $11: 08: 44$ AM & 25.098 & 25.696 & 23.635 & 27.239 & 26.364 & 47.844 & \begin{tabular}{|l|}
48.72 \\
\end{tabular} & 15.832 & 32.472 & -1.219 & -8.008 & 46.592 & 0.081 & $\begin{array}{l}14.040 \\
14.643 \\
\end{array}$ & 0.004 & 59.0 & \begin{tabular}{|l|l|}
0 & 0.98333 \\
\end{tabular} & 47.2 & 11.4 & 40.2 & 2.769 & 0.012 & 0.013 & 0.000 & 0.313 & \\
\hline & $6 / 17 / 2004$ & 11:09:44 AM & 25.053 & 25.691 & & 27.184 & 26.284 & 47.827 & 48.652 & 15.812 & 32.518 & -1.219 & -8.008 & 49.706 & 0.081 & \begin{tabular}{l|l|l}
14.643 \\
\end{tabular} & 0.004 & 60.0 & & 47.2 & 12.2 & 40.2 & 2.770 & 0.012 & & & & \\
\hline ( & & $\begin{array}{l}11: 10: 44 \mathrm{AM} \\
11: 14 \mathrm{M}\end{array}$ & $\frac{25.049}{2009}$ & 25.697 & \begin{tabular}{|l|l|}
23.611 \\
3001
\end{tabular} & & 26.25 & 47.63 & $\begin{array}{ll}48.461 \\
7.777\end{array}$ & 15.764 & $\begin{array}{l}32.388 \\
32.60 \\
\end{array}$ & \begin{tabular}{|l|l|}
-1.219 \\
\end{tabular} & & 46.346 & $\begin{array}{ll}0.081 \\
.009 \\
\end{array}$ & \begin{tabular}{|l|l|l|}
14.643 \\
\end{tabular} & 0.004 & 61.0 & 1.01667 & 47.0 & 11.4 & 40.0 & 2.758 & 0.012 & & 0.000 & 0.314 & \\
\hline & 6/177/2004 & 11:11:44 AM & & 25.692 & 23.641 & 27.131 & 26.23 & 46.966 & \begin{tabular}{|l|l|}
47.777 \\
\end{tabular} & 15.33 & 32.158 & \begin{tabular}{|l|}
$\mid-1.222$ \\
\end{tabular} & -7.988 & 53.06 & 0.083 & \begin{tabular}{ll|}
14.643 \\
\end{tabular} & 0.004 & 62.0 & $\begin{array}{ll}0 & 1.03333 \\
\end{array}$ & 46.3 & & & & 0.012 & 0.013 & 0.000 & & \\
\hline 80 & 6/17/2004 & 11:12:44 AM & $\begin{array}{ll}25.05 \\
2505\end{array}$ & \begin{tabular}{l|l}
25.728 \\
25757
\end{tabular} & \begin{tabular}{|l|}
23.682 \\
2320
\end{tabular} & 27.156 & 26.221 & 47.35 & \begin{tabular}{|l|l|}
48.092 \\
\end{tabular} & 15.407 & 32.507 & -1.219 & $\begin{array}{l}-7.973 \\
7.907 \\
\end{array}$ & 49.999 & 0.085 & \begin{tabular}{ll|}
14.643 \\
\end{tabular} & 0.004 & $\begin{array}{ll}63.0 \\
6.0\end{array}$ & 1.05 & 46.6 & 12.3 & 39.9 & 2.753 & 0.013 & 0.013 & & 0.329 & \\
\hline 82 & $\begin{array}{l}6 / 17 / 2004 \\
6 / 17 / 2004\end{array}$ & $\begin{array}{l}11: 13: 44 \text { AM } \\
11: 14: 44 \text { AM }\end{array}$ & $\begin{array}{l}25.095 \\
25117\end{array}$ & $\frac{25.753}{2576}$ & $\begin{array}{l}23.702 \\
23679\end{array}$ & $\begin{array}{l}27.151 \\
27.094 \\
\end{array}$ & $\frac{26.181}{26.079}$ & $\begin{array}{ll}47.211 \\
47.167\end{array}$ & \begin{tabular}{|l|}
48.206 \\
48.067 \\
\end{tabular} & \begin{tabular}{|l|l|}
15.482 \\
15401 \\
\end{tabular} & \begin{tabular}{|l|l|}
32.193 \\
32.23 \\
\end{tabular} & \begin{tabular}{|c|}
-1.216 \\
-1.219 \\
\end{tabular} & $\begin{array}{l}-7.973 \\
-7.941 \\
\end{array}$ & \begin{tabular}{r|r|r|}
56.16 & \\
6.899
\end{tabular} & $\begin{array}{l}0.085 \\
0.085\end{array}$ & \begin{tabular}{|l|l|}
14.643 \\
14643
\end{tabular} & 0.004 & $\begin{array}{l}64.0 \\
65.0 \\
65\end{array}$ & \begin{tabular}{l|l|l|l|l}
0 & 1.06667 \\
0 & 1.08333 \\
\end{tabular} & \begin{tabular}{l|l}
46.7 \\
66
\end{tabular} & $\begin{array}{l}13.8 \\
11.5 \\
\end{array}$ & \begin{tabular}{l|l}
39.7 \\
39.7 \\
\end{tabular} & $\begin{array}{l}2.737 \\
2.737 \\
\end{array}$ & $\begin{array}{l}0.013 \\
0.013\end{array}$ & 0.013 & $\begin{array}{l}0.000 \\
0.000\end{array}$ & $\begin{array}{l}0.331 \\
0.31 \\
\end{array}$ & 0.28 \\
\hline 83 & $\frac{6 / 1 / 1 / 2004}{6 / 17 / 2004}$ & \begin{tabular}{|c|}
$11: 11: 44 \mathrm{AM}$ \\
$11: 15: 44 \mathrm{AM}$
\end{tabular} & $\frac{25.11 / 4}{25.153}$ & $\frac{20.100}{25.781}$ & \begin{tabular}{|l|}
23.671 \\
23.671 \\
\end{tabular} & $\frac{21.094}{27.07}$ & $\frac{2.079}{25.99}$ & 447.209 & \begin{tabular}{|l|l|}
48.132 \\
\end{tabular} & \begin{tabular}{|l|}
15.437 \\
15
\end{tabular} & $\begin{array}{ll}32.260 \\
32.263 \\
\end{array}$ & \begin{tabular}{|c|}
-1.219 \\
\end{tabular} & $\begin{array}{l}-7.941 \\
-7.962\end{array}$ & 40.094 & 年 & $\begin{array}{l}14.043 \\
14.643\end{array}$ & $\begin{array}{l}0.004 \\
0.004\end{array}$ & $\frac{65.0}{66.0}$ & \begin{tabular}{|c|c|} 
& 1.08333 \\
0 & 1.1 \\
\end{tabular} & $\begin{array}{l}46.6 \\
46.7 \\
\end{array}$ & $\begin{array}{l}11.5 \\
10.3 \\
\end{array}$ & $\begin{array}{ll}39.17 \\
39.7 \\
\end{array}$ & $\frac{2.151}{2.740}$ & & $\frac{0.013}{0.013}$ & $\begin{array}{l}0.000 \\
0.000\end{array}$ & $\frac{0.331}{0.331}$ & $\frac{0.28}{0.28}$ \\
\hline 84 & 6/17/2004 & $11: 16: 44$ AM & 25.175 & 25.788 & 23.653 & 27.087 & 26.017 & 47.325 & \begin{tabular}{|l|l|}
48.316 \\
\end{tabular} & 15.45 & 32.362 & -1.219 & -7.965 & 51.046 & 0.085 & 14.643 & 0.004 & 67.0 & \begin{tabular}{|l|l|}
0 & 1.11667 \\
\end{tabular} & 46.8 & 12.5 & 39.8 & \begin{tabular}{|l|}
2.747 \\
\end{tabular} & 0.013 & 0.013 & & 0.330 & 0.28 \\
\hline 85 & 6/17/2004 & 11:17:44 AM & 25.174 & & 23.651 & 27.096 & 26.076 & 47.184 & 48.13 & 15.51 & 32.134 & -1.219 & & 44.456 & & $\begin{array}{l}14.643 \\
\end{array}$ & 0.004 & 68.0 & \begin{tabular}{l|l|}
0 & 1.13333 \\
\end{tabular} & 46.7 & 10.9 & 39.7 & \begin{tabular}{|l|l|}
2.734 \\
\end{tabular} & $\mid 0.013$ & & 0.000 & & $\frac{0.28}{0.28}$ \\
\hline 00 & & & & & & & & & & & & & & & 0 & & & & & 46.8 & & & & & & & & \\
\hline 87 & $6 / 17 / 2004$ & 11:19:44 AM & 25.178 & 25.806 & 23.656 & 27.15 & 26.13 & 47.097 & 47.964 & 15.411 & 32.222 & $=\frac{1.222}{-1.22}$ & & 53.764 & $0.08-2>$ & 14.643 & & & .16667 & 46.5 & 13.2 & 39.7 & 2.734 & 然13 & 0.013 & & .328 & \\
\hline$=$ & $6 / 17 / 2004$ & 11:20:44 AM & 25.186 & 25.819 & 23.644 & 27.22 & 26.1 & 46.95 & $47.968 \mid$ & \begin{tabular}{|c|c|}
15.43 \\
\end{tabular} & 20 & 1.222 & & $54.53 .5>3$ & & 14.643 & & 71. & 1.18333 & & 13.4 & & & & & 500 & & \\
\hline & & & & & & & & & & 15.4 & & & & & & 14.643 & & & 1.2 & & & & & & & & & \\
\hline & $6 / 17 / 2004$ & $11: 22: 44 \mathrm{AM}$ & 25.197 & 25.845 & 23.659 & 27.328 & 26.268 & 47.051 & 47.983 & 15.477 & 32.083 & 1.219 & & 43.806 & 0.084 & 14.643 & & & 21667 & 46.5 & 10.7 & 39.6 & 2.728 & 013 & & & & \\
\hline & 6/17/2004 & 11:23:44 AM & 25.204 & 25.852 & 23.662 & 27.33 & 26.29 & 47.012 & 48.153 & 15.512 & 31.926 & 1.225 & & 51.262 & 0.084 & 14.643 & & 74.0 & 23333 & 46.7 & 12.6 & 39.5 & 2.721. & 0.013 & 013 & 500 & 30 & \\
\hline 93 & $\begin{array}{l}6 / 171 / 2004 \\
6 / 17 / 2004\end{array}$ & $\begin{array}{l}11: 24: 44 \text { AM } \\
112.25: 44 \text { AM }\end{array}$ & $\frac{25.209}{2526 \mid}$ & $\frac{25.872}{2584}$ & $\frac{23.717}{23.754}$ & $27.356 \mid$ & $\frac{26.296}{262282}$ & $\begin{array}{ll}46.916 \\
46.923\end{array}$ & 47.775 & $\frac{15.482}{15.543}$ & $31.984 \mid$ & $\begin{array}{l}-1.219 \\
-1.219\end{array}$ & -7.956 & $\begin{array}{l}48.324 \\
577755\end{array}$ & 0.084 & $\frac{14.643}{14643}$ & 0.004 & $\begin{array}{l}75.0 \\
76.0\end{array}$ & $\begin{array}{r}1.25 \\
22667 \\
\end{array}$ & 46.3 & $\frac{11.8}{1142}$ & 39.5 & 2.720 & 0.013 & $\frac{0.013}{0.013}$ & .000 & 0.329 & \\
\hline$y_{4}>2>$ & $6 / 17 / 2004$ & 11:26:44 AM & 25.246 & 25.899 & 23.789 & 27.338 & & 47.16 & 48.308 & 15.542 & 32.132 & -1.219 & & $\frac{5.11}{49.34}$ & & & & & 280073 & & & (39. & 2.1.74 & & 0.013 & & & \\
\hline 95 & 6/17/2004 & 11:27:44 AM & & 25.914 & 23.845 & 27.343 & & 46.93 & 47.999 & 15.532 & 31.924 & & & 44.93. & & 14.643 & & 78.0 & 1.3 & 46.5 & 11.0 & & & & & & & \\
\hline 96 & 6/17/2004 & 11:28:44 AM & 25.285 & 25.913 & 23.863 & 27.297 & 26.192 & 46.82 & 48.038 & 15.516 & 31.75 & -1.222 & -7.92 & 44.171 & 0.084 & 14.643 & & 79. & 31667 & 46.6 & $\overline{10.8} \quad>$ & 39. 39. & 2.709 & 0 & & & 29 & \\
\hline & 6/177/2004 & 11:29:44 AM & 25.293 & 25.926 & 23.957 & 27.23 & 26.155 & 46.224 & 47.294 & 15.049 & 31.701 & -1.219 & & 52.068 & 0.084 & 14.643 & 0.004 & 80.0 & 1.33333 & 45.8 & 12.8 & 39.0 & 2.686 & 0.013 & & & & \\
\hline$\frac{98}{8}>>2$ & 6/17/2004 & 11:30:44 AM & 25.295 & 25.928 & 23.939 & 27.177 & 26.102 & 46.728 & 48.117 & 15.216 & 31.892 & -1.222 & & & 0.087 & 14.643 & & & 1.35 & 46.6 & 11.9 & 39. & 710 & D13 & & & .340 & \\
\hline & $6 / 11 / 2004$ & 11:31:44 AM & 25.329 & 25.952 & 23.978 & 27.171 & & 46.292 & 47.392 & 15.182 & & & & 42.844 & 088 & & & & & 45.9 & & & 2.688 & & & & 0.346 & \\
\hline & $\frac{6 / 1 / 2004}{6 / 17 / 2004}$ & 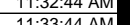 & 25.342 & $\begin{array}{ll}25.96 \\
25988\end{array}$ & $\begin{array}{l}23.981 \\
23092\end{array}$ & | & 26.054 & $\frac{46.554}{46.61}$ & $\begin{array}{l}47.935 \\
47.968\end{array}$ & 15.271 & \begin{tabular}{ll|l}
31.672 & \\
31.777 &
\end{tabular} & $\frac{1.219}{1.222}$ & & $\frac{51.959}{4883}$ & 0.088 & 14.643 & & 83.0 & 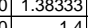 & $\frac{46.5}{46.5}-x$ & 年. & 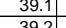 & . & & & & & \\
\hline & $\frac{17172044}{6 / 71 / 2004}$ & 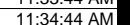 & $\begin{array}{ll}25.305 \\
25.352\end{array}$ & 25.95 & $\frac{23.075}{23.975}$ & $\frac{27.100}{27.083}$ & $\frac{20.021}{26.019}$ & $\begin{aligned} 40.01 \\
6.263\end{aligned}$ & $\begin{array}{l}47.900 \\
47.363 \\
\end{array}$ & 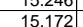 & 31.623 & $\frac{1.224}{1219}$ & & 53.594 & $\begin{array}{l}0.080 \\
0.088 \\
\end{array}$ & $\frac{14.045}{14.643}$ & & 85. & 1464 & $45 \mathrm{~s}>\mathrm{T}$ & $\frac{1}{131}$ & 389 & & & & & 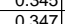 & \\
\hline & & 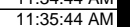 & & 25.963 & $\begin{array}{ll}20.378 \\
2098\end{array}$ & 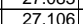 & & 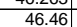 & 47.039 & $\begin{array}{l}15.172 \\
15.262\end{array}$ & $\begin{array}{l}31.020 \\
31.608\end{array}$ & $\frac{1.219}{1.219}$ & & (36) & 0.0 & 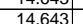 & & & & & & & & & & & & \\
\hline & $6 / 17 / 2004$ & 1:36:44 AM & $25.387 \mid$ & 25.96 & 24.006 & 27.154 & 26.0 & 46.634 & 48.161 & 15.246 & 31.791 & 1222 & & & 0.0 & 14.643 & & 87 & 1.45 & 46 & $2.4 .5-5$ & $39-7$ - & 704 & & & & 344 & \\
\hline & & 11:37: & 25.399 & 25.967 & 24.038 & 27.241 & & 46.64 & 48.08 & 15.261 & 31.808 & -1.219 & & 50.103 & & 14.64 & & & & 46 & 2.3 & & & & & & 340 & \\
\hline & $6 / 17 / 2004$ & & & & 24.056 & 27314 & & $46.29 \mathrm{r}-\mathrm{s}$ & & 15.186 & 31.571 & & & & & 14.643 & & & 8333 & & $\frac{113}{113}$ & & 2684 & & & & & \\
\hline 107 & $6 / 17 / 2004$ & 11:39:44 AM & 25.45 & 25.978 & \begin{tabular}{|l|l|}
24.069 \\
\end{tabular} & 27.366 & 26.2 & 46.348 & \begin{tabular}{|l|l|}
47.856 \\
\end{tabular} & $\mid 15.126$ & 31.67 & -1.222 & & 50.45 & 0.0 & 14.643 & & 90 . & 1.5 & 46 & 12.4 & 39.0 & 2.690 & & & & 0.345 & \\
\hline & $6 / 17 / 2004$ & 11:40:4 & 25.484 & 25.997 & 24.097 & 27.3 & & 46.30 & 47.574 & $\begin{array}{ll}15.243 \\
\end{array}$ & 31.5 & & & & & 14.6. & & & & & 12.0 & 38. & & & & & & \\
\hline & 6/17//2004 & & & 26.005 & 24.106 & 27.41 & & & 48.219 & & & & & & & & & & & & 12.5 & 39.2 & & & & & & \\
\hline & 6/177/2004 & 11:42:44 AM & 25.51 & 26.018 & 24.104 & 27.421 .19 & 26.3 & 46.186 & 47.572 & 15.244 & 31.426 & -1.222 & & 48.03 & 0.0 & 14.643 & & 93.0 & 1.55 & 46.1 & 11.8 & 38.8 & 2.676 & 0.013 & 0.013 & 0.000 & 0.343 & \\
\hline 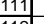 & 6/17/2004 & & $20.5<5$ & 26.036 & 24.122 & $27.43{ }^{\circ} \mathrm{Cl}$ & & 46.41 & 47.918 & 15.264 & 31.606 & -1.219 & & & & 14.643 & & & 66667 & 46. & 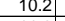 & 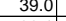 & 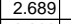 & & & & .345 & \\
\hline 113 & $6 / 1172004$ & & 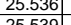 & & 24.145 & 2.7 .452 & & $\frac{46.466}{4.502}>2$ & 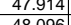 & 15360 & & & & & & 14.663 & & 06 & 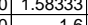 & & & ( & 2690 & & & & & \\
\hline & 0 & & $\frac{2.359}{2050}$ & 2.0051 & 24.153 & 27.45 & & 46.5024 & 4.090 & 15.3099 & 31.5 & & & & & 1.643. & & & & & 1.3 & 3.6 & 2.0964 & & & & & \\
\hline & $\frac{61172044}{6617 / 2004}$ & & 20.552 & 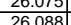 & (24.1919 & $\frac{2.45}{2078}-3$ & & $\frac{45}{45}$ & $\frac{47.4}{47 .}$ & $\frac{14.9}{150}$ & & $\frac{-1.22}{1.21}$ & & & & & & & & 46 & 12.6. & & & & & & 53 & \\
\hline 116 & 6 & & $\frac{25.50}{25.37}$ & 26096 & 24.1 & 27.42 & & 45.741 & 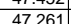 & 14.9 & (31.239 & & & & & $\frac{14.6}{146}$ & & & 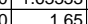 & & 100 & & & & & & & \\
\hline & $6 / 171 / 2004$ & & 25.581 & 26.094 & 24.14 & 27.36 & & 45.641 & & & & & & & & & & & & & 124 & & & & & & (2) & \\
\hline & 6/17/2004 & $\frac{11.50}{11.50}$ & 25.588 & 26.111 & 24.137 & $\frac{2736}{2736}$ & & 45.828 & & 15.019 & 31257 & -1.222 & & 53.92 & & 14.6 & & 101 & 68333 & $\frac{46}{46}>2$ & 132 & & & & & & 0.361 & \\
\hline & $6 / 17 / 2004$ & & 25.594 & & 24.149 & & & & 47.384 & & & -1.222 & & & & & & & & & 146 & & & & & & & \\
\hline & $6 / 17 / 2004$ & $11: 52: 44$ & 25.582 & 26.115 & 24.111 & 27.29 & & 47.55 & 49.56 & 13.621 & 34.278 & -1.219 & & 44.454 & & 14.643 & & 103 & 71667 & 48 & 10.9 & $\frac{40.9}{40.9}>0$ & 2007 & $\frac{14}{4}$ & & & 359 & \\
\hline & 6/17/2004 & & 25.589 & 26.12 & $4.1 .7-3$ & 27.2 & & 47.6. & 49.784 & & 34.3 & -1.219 & & & & 14.6 & & 10 & & & & & & & & & 54 & \\
\hline & 6/17/2004 & 1:54:4 & & 26.121 & 24.077 & & & 47.9 & 49. & 13. & & & & & & 14.6 & & & 1.75 & 48 & 12.4 & & & & & & & \\
\hline & 6/17/2004 & & 25.614] & 26.143 & & 27.24 & & 47.7 & 49.4 & & & $-1.2 .2>0$ & & & & & & & & & & & & & & & & \\
\hline & 6/177/2004 & & 25.601 & 26.144 & 24.06 & 27.23 & & 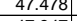 & 49.549 & $14.22>>$ & & -1.219 & & & & & & & & 48 & 10.8 & & & & & & & \\
\hline & & & & & 24.061 & $\frac{21}{271}+2-3$ & & & & & & & & & & & & & & & 11.3 & & & & & & & \\
\hline$\frac{21}{12}+2>$ & $\frac{6 / 1 / 1 / 2004}{6 / 17 / 2004}$ & & 告5.019 & & 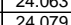 & 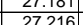 & & (48.3505] & & & & 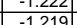 & & & & & & & $\mid$ & & 11.6. & 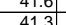 & & & & & 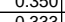 & \\
\hline 128 & $6 / 17 / 2004$ & & & & & & & 45.477 & 50.25 & & & -1. & & & & & & 111 & 1.85 & 48 & 9.5 & & & & & & $\begin{array}{l}0.050 \\
0.012 \\
\end{array}$ & \\
\hline 129 & $6 / 17 / 2$ & & 25.388 & & \begin{tabular}{|l|}
24.047 \\
\end{tabular} & & & 47. & & & & $\mid-1.216$ & & & & & & 112 & \begin{tabular}{l|l|l|}
0 & 1.86667 \\
\end{tabular} & 49 & 10.9 & 41.0 & & & & & $\frac{15}{25}$ & \\
\hline & 6/177/2 & 12:02:44 PM & 25.443 & 26.13 & 24.027 & & & 47.6 & & & & & & & & & & 113.0 & & 48 & 11.5 & 40. & & & & & 0.334 & \\
\hline & 6/17/2004 & $12: 03: 44$ PM & 25.503 & 26.156 & 24.082 & 27.489 & & 47.982 & 50.0 & 13.57 & 34 & 1.216 & & 40.7 & & 14.6 & 0.0 & 114 & 1.9 & 48 & 10.0 & & & & 0.014 & & & \\
\hline & & & 25.469 & & & & & & & & & \begin{tabular}{|c|} 
\\
\end{tabular} & & & & & & & & 48 & 8.8 & & & & & & 37 & \\
\hline & 6/17/2004 & $12: 05: 24 \mathrm{P}$ & 25.506 & 26.17 & 24.065 & 27.592 & & 47.236 & 49.57 & 14.452 & 33.066 & $1.219 \mid$ & & & & 14.6. & & 115.7 & $7 \mid \begin{array}{ll}7.92778 \\
\end{array}$ & 48 & 11.7 & 40.2 & 2.7 & 13 & & & 28 & \\
\hline & 6/17/2004 & 12:06:24 F & 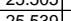 & & & & & 年77.543 & & & 33.587 & & & & & & & 116.74 & 1.94444 & & & & $\begin{array}{l}797 \\
750 \\
7\end{array}$ & 13 & & & 40 & 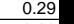 \\
\hline & $\frac{601 / 1724}{6 / 17 / 22}$ & & $\frac{25.5}{25.5}-3$ & & & $\frac{21.68}{27.68}-10$ & & & $\frac{49 .}{49.8}$ & & $\begin{array}{l}32.649 \\
33.847\end{array}$ & $-1.21>0$ & & & & & & & & & & & & & & & & \\
\hline & 6/17/2004 & & & 26204 & 24.111 & & & & & & & & & & & & & & & & 10 & & & & & & & \\
\hline $138->$ & & & & & & & & 47.4 & & & & $12255 \mathrm{C}$ & & & & & & & & & 107 & & & & & & & \\
\hline & & & & & & 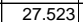 & & & & & & & & & & 14.643 & & & & ( & 10.3 & 20 & & & & & & \\
\hline 40[ & $6 / 17 / 2004$ & 12:12:24 PM & 25.619 & 26.247 & \begin{tabular}{|l|l|}
24.118 \\
\end{tabular} & 27.51 & 26.395 & $46.61 \mid$ & \begin{tabular}{|l|}
49.006 \\
\end{tabular} & 15.081 & 31.881 & $\mid-1.222$ & & 16211 & 0.091 & 14.643 & & & \begin{tabular}{l|l|}
72.04444 \\
\end{tabular} & 47.5 & $\frac{11.3}{11.3}$ & & 2.706 & $\overline{0.014} \mid$ & 0.014 & 000 & $\mid 354$ & \\
\hline
\end{tabular}


WSRC-TR-2005-00105, REVISION 0

SRNL-RPP-2005-00012, REVISION 0

RUN \# WASH1 AND WASH 2; CAMPAIGN III SLURRY WASHING - CONT.

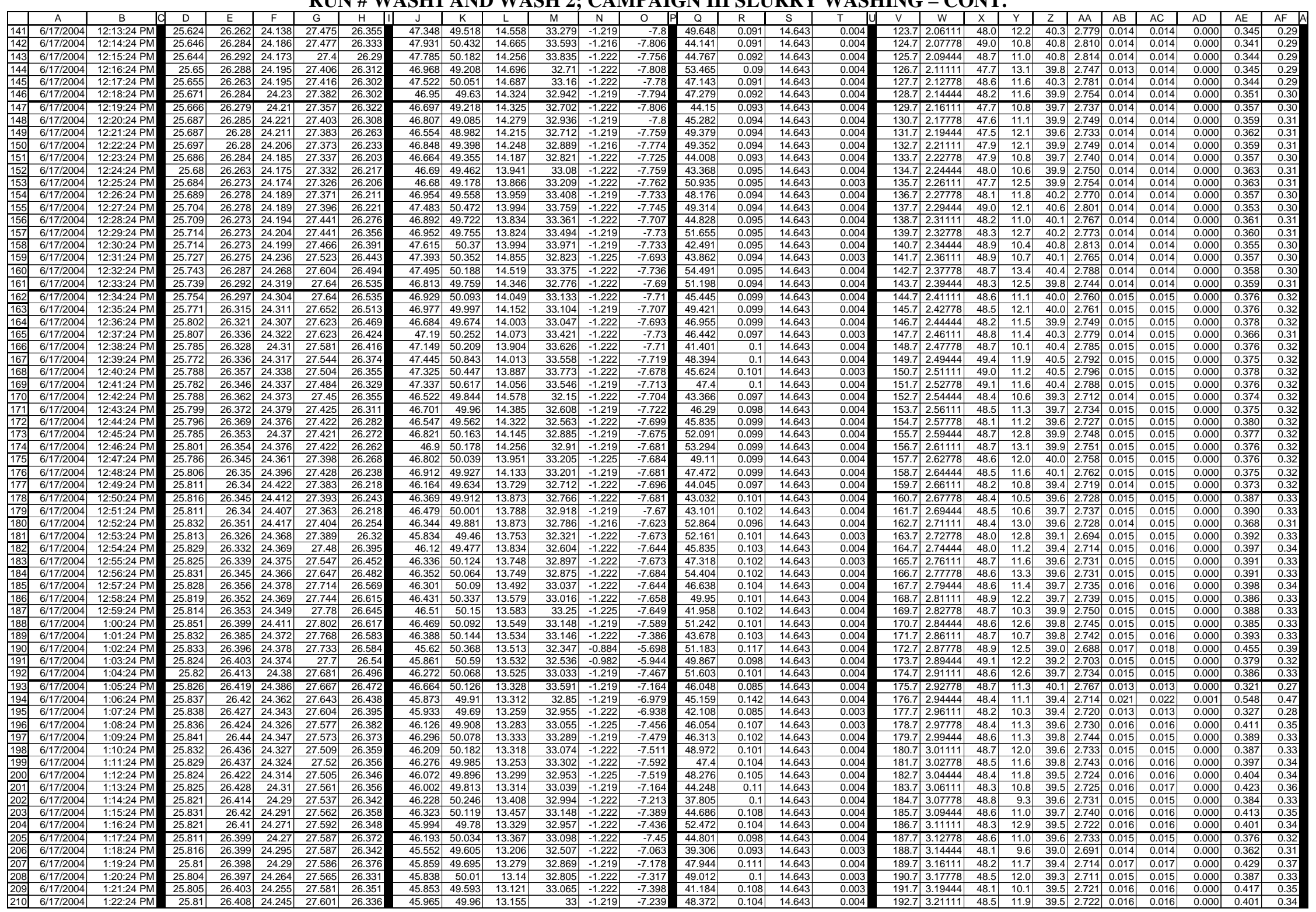


WSRC-TR-2005-00105, REVISION 0

SRNL-RPP-2005-00012, REVISION 0

RUN \# WASH1 AND WASH 2; CAMPAIGN III SLURRY WASHING - CONT.

\begin{tabular}{|c|c|c|c|c|c|c|c|c|c|c|c|c|c|c|c|c|c|c|c|c|c|c|c|c|c|c|c|c|}
\hline & A & \begin{tabular}{l|l}
$\mathrm{B}$ & $\mathrm{C}$ \\
\end{tabular} & & $E$ & & $\mathrm{G}$ & $\mathrm{H}$ & $\mathrm{J}$ & $\mathrm{K}$ & \begin{tabular}{l|l}
$\mathrm{L}$ \\
\end{tabular} & M & $\mathrm{N}$ & 0 & $Q$ & $\mathrm{R}$ & $\mathrm{s}$ & & $\mathrm{v}$ & w & $\mathrm{x}$ & $\mathrm{Y}$ & $z$ & AA & $A B$ & $\mathrm{AC}$ & $A D$ & $\mathrm{AE}$ & AF $A$ \\
\hline & $\begin{array}{l}6 / 117 / 2004 \\
6 / 172004\end{array}$ & \begin{tabular}{|l}
$1: 23: 24 \mathrm{PM}$ \\
$1.24: 24 \mathrm{PM}$
\end{tabular} & $\begin{array}{l}25.816 \\
25827\end{array}$ & $\begin{array}{l}26.399 \\
2641 \\
\end{array}$ & \begin{tabular}{|l|}
24.235 \\
24.27
\end{tabular} & \begin{tabular}{|l|l|}
27.647 \\
27.683
\end{tabular} & $\begin{array}{l}26.372 \\
26.394 \\
\end{array}$ & $\begin{array}{ll}45.448 \\
6.099 \\
\end{array}$ & \begin{tabular}{|l|}
49.174 \\
50.107 \\
\end{tabular} & \begin{tabular}{|l|l|}
13.088 \\
13.233
\end{tabular} & $\frac{32.682}{33.07}$ & \begin{tabular}{|c|}
-1.219 \\
-1.219 \\
\end{tabular} & $\begin{array}{r}-7.19 \\
-7.259 \\
\end{array}$ & $\begin{array}{l}44.763 \\
57.153\end{array}$ & $\begin{array}{r}0.1 \\
0.111 \\
\end{array}$ & $\begin{array}{l}14.643 \\
14.643 \\
\end{array}$ & $\begin{array}{l}0.004 \\
0.004\end{array}$ & \begin{tabular}{|l|}
193.7 \\
194.7
\end{tabular} & \begin{tabular}{|l|l|}
7 & 3.22778 \\
7 & 3.24444
\end{tabular} & $\begin{array}{l}47.7 \\
48.6 \\
\end{array}$ & $\frac{11.0}{140}$ & $\begin{array}{l}39.1 \\
3396\end{array}$ & \begin{tabular}{|l|}
2.693 \\
2.729
\end{tabular} & \begin{tabular}{|l|l|}
0.015 \\
0017 \\
\end{tabular} & 0.015 & 0.000 & 0.390 & 0.33 \\
\hline 51 & & & & & & & & & & & & & & & & $\frac{44.045}{14.643}$ & $\begin{array}{l}0.004 \\
0.004\end{array}$ & & & & & & & & & & & $\begin{array}{l}0.36 \\
0.35\end{array}$ \\
\hline & & & 25.829 & 26.412 & 24.198 & & 26.405 & 45.998 & \begin{tabular}{|l|}
49.949 \\
\end{tabular} & 13.167 & 33.094 & $\begin{array}{l}-1.222 \\
\end{array}$ & & 46.949 & 0.097 & $\frac{14.045}{14.643}$ & 0.004 & & \begin{tabular}{|l|l|}
7 & 3.27778 \\
\end{tabular} & 48.5 & 11.5 & & & & & & & \\
\hline & $6 / 117 / 2004$ & $1: 27: 24 \mathrm{PM}$ & 25.82 & 26.413 & 24.204 & 27.641 & 26.411 & 45.805 & \begin{tabular}{|l|l|}
49.769 \\
\end{tabular} & 13.185 & 32.795 & \begin{tabular}{|c|c|}
-1.222 \\
\end{tabular} & -7.268 & 45.941 & 0.11 & 14.643 & 0.004 & & 3.29444 & 48.3 & 11.3 & 39.3 & 2.710 & 0.016 & \begin{tabular}{|l|l|}
0.017 \\
\end{tabular} & 0.000 & 0.427 & \\
\hline & 6/17/2004 & 1:28:24 PM & 25.81 & 26.403 & 24.194 & 27.676 & 26.406 & 45.956 & 49.431 & 13.189 & 33.082 & $\begin{array}{r}-1.219 \\
\end{array}$ & -6.985 & 48.876 & 0.096 & 14.643 & 0.004 & 198.7 & \begin{tabular}{|l|l|}
7 & 3.31111 \\
\end{tabular} & 48.0 & 12.0 & 39.5 & & \begin{tabular}{|l|l|}
0.014 \\
\end{tabular} & 0.015 & 0.000 & 0.371 & \\
\hline$\frac{x_{1}}{21}$ & $\begin{array}{l}6 / 17 / 2004 \\
6 / 17204 \\
\end{array}$ & \begin{tabular}{|l|}
$1: 29: 24 \mathrm{PM}$ \\
$1: 30 \cdot 24 \mathrm{PM}$
\end{tabular} & $\begin{array}{l}25.816 \\
25808\end{array}$ & 26.405 & $\begin{array}{l}24.196 \\
241.8 \\
\end{array}$ & \begin{tabular}{|l|l|}
27.657 \\
27.654
\end{tabular} & $\begin{array}{ll}26.413 \\
26394 \\
6394\end{array}$ & $\begin{array}{l}46.504 \\
6008\end{array}$ & \begin{tabular}{|r|}
50.42 \\
49775 \\
\end{tabular} & $\begin{array}{l}13.304 \\
1317 \\
\end{array}$ & $\begin{array}{ll}33.406 \\
3.164\end{array}$ & $\begin{array}{l}-1.222 \\
-1.222 \\
\end{array}$ & $\begin{array}{l}-7.167 \\
-7.724 \\
\end{array}$ & $\begin{array}{ll}46.225 \\
47164\end{array}$ & $\begin{array}{l}0.104 \\
0.106\end{array}$ & $\begin{array}{l}14.643 \\
1.613\end{array}$ & 0.004 & $\begin{array}{l}199.7 \\
2007\end{array}$ & \begin{tabular}{l|l|}
73.32778 \\
\end{tabular} & 48.9 & $\begin{array}{l}11.3 \\
116\end{array}$ & $\begin{array}{l}40.0 \\
396 \\
\end{array}$ & \begin{tabular}{|l|l|}
2.755 \\
2.730
\end{tabular} & \begin{tabular}{|l|}
0.016 \\
0016
\end{tabular} & $\begin{array}{l}0.016 \\
0016\end{array}$ & 0.000 & 0.397 & 0.34 \\
\hline & $\frac{6}{6 / 17 / 72004}$ & $1.31: 24 \mathrm{PM}$ & & & & & & & & & & & & & & $\frac{14.643}{14643}$ & & & & & & & & & & & & \\
\hline & 6/17/2004 & $1: 32: 24 \mathrm{PM}$ & 25.789 & 26.4247 & \begin{tabular}{|l|}
24.203 \\
24.203
\end{tabular} & $\frac{27.050}{27.58}$ & 26.37 & 45.753 & $\begin{array}{l}49.429 \\
49.429\end{array}$ & 12.920 & 33.037 & \begin{tabular}{|l|l|}
-1.19 \\
-1.222 \\
\end{tabular} & & 44.631 & 0.108 & $\begin{array}{l}14.045 \\
14.643\end{array}$ & $\begin{array}{l}0.004 \\
0.004 \\
\end{array}$ & $\begin{array}{l}201.7 \\
2027 \\
\end{array}$ & \begin{tabular}{|l|l|}
7.301111 \\
7.37778
\end{tabular} & $\begin{array}{c}48.4 \\
48.0\end{array}$ & $\frac{10.0}{10.4}$ & $\begin{array}{r}39.4 \\
39.4 \\
\end{array}$ & & & $\frac{0.017}{0.016}$ & 0.000 & & 0.37 \\
\hline & $6 / 17 / 2004$ & 1:33:24 PM & $\frac{25.8}{25.8}$ & 26.419 & 24.19 & 27.551 & 26.372 & 46.018 & 49.912 & 12.724 & 33.499 & -1.222 & -6.953 & 44.882 & 0.103 & 14.643 & 0.004 & 203.7 & \begin{tabular}{|l|l|}
7 & 3.39444 \\
\end{tabular} & 48.4 & 11.0 & 39.8 & 2.741 & 0.015 & 0.016 & 0.000 & $\begin{array}{l}0.418 \\
0.395 \\
\end{array}$ & $\frac{0.36}{0.34}$ \\
\hline & $6 / 17 / 2004$ & 1:34:24 PM & 25.796 & 26.409 & & & 26.368 & 45.927 & 49.96 & 12.577 & & -1.222 & & & & 14.643 & 0.004 & 204.7 & \begin{tabular}{|l|l|}
7 & 3.41111 \\
\end{tabular} & 48.5 & & & & & & & & 0.34 \\
\hline & $6 / 17 / 2004$ & 1:35:24 PM & 25.771 & 26.394 & 24.116 & 27.537 & 26.32 & 45.958 & \begin{tabular}{|l|l|l|}
49.923 \\
\end{tabular} & 12.693 & 33.46 & -1.222 & -7.28 & 44.369 & 0.11 & $\begin{array}{l}14.643 \\
\end{array}$ & & \begin{tabular}{|l}
205.7 \\
\end{tabular} & $\begin{array}{l}3.42778 \\
\end{array}$ & 48.5 & 10.9 & 39.7 & & & & & & \\
\hline & & & \begin{tabular}{l|l|}
25.76 \\
\end{tabular} & & & & & & & 12.759 & 33.607 & -1.219 & & 48.119 & & & & & & & & & & & & & & \\
\hline & $6 / 17 / 2004$ & 1:37:24 PM & 25.755 & 26.394 & 24.095 & 27.576 & & $\begin{array}{l}46.404 \\
\end{array}$ & 50.484 & & 33.919 & & -7.057 & & 0.096 & 14.643 & & 2007.7 & $\begin{array}{l}773.46111 \\
\end{array}$ & 49.0 & & 10 & 2770 & & & 0.000 & $\begin{array}{l}0.426 \\
0.366\end{array}$ & \\
\hline & $6 / 17 / 2004$ & 1:38:24 PM & 25.75 & 26.398 & 24.089 & 27.616 & 26.326 & 45.933 & \begin{tabular}{|l|l}
49.821 \\
\end{tabular} & 12.467 & 33.755 & -1.225 & -6.748 & $\begin{array}{l}44.588 \\
\end{array}$ & 0.107 & $\begin{array}{l}14.643 \\
\end{array}$ & & 208.7 & 3.47778 & $\begin{array}{ll}48.3 \\
\end{array}$ & & 39.8 & 2.747 & & 0.016 & 0.000 & 0.411 & \\
\hline & $6 / 17 / 2004$ & 1:39:24 PM & 25.75 & 26.404 & 24.09 & 27.636 & 26.347 & 46.338 & 50.138 & 12.562 & 34.046 & -1.219 & -7.334 & 42.923 & 0.12 & 14.643 & & 209.7 & 3.49444 & \begin{tabular}{ll|}
48.7 \\
\end{tabular} & & 40 & & & 0.019 & & 0.476 & \\
\hline & $6 / 17 / 2004$ & 1:40:24 PM & $\begin{array}{l}25.741 \\
25.71\end{array}$ & 26.404 & 24.086 & 27.627 & 26.388 & 46.328 & 50.248 & 12.663 & 33.841 & -1.222 & -7.2. & 44.465 & 0.124 & 14.643 & & 210. & 5111 & \begin{tabular}{ll|}
48.8 \\
\end{tabular} & & 40 & 2.764 & & & 0.000 & 0.473 & \\
\hline & $6 / 17 / 2004$ & $\begin{array}{l}1: 41: 24 \mathrm{PM} \\
\end{array}$ & & & & & & 46.493 & 50.405 & & & & & 45.251 & 0.104 & 14.643 & & & & 48.9 & & & & & & & & \\
\hline & $6 / 1 / 172004$ & $1: 42: 24 \mathrm{PM}$ & 25.733 & 26.406 & \begin{tabular}{|l|}
24.107 \\
24128
\end{tabular} & 27.619 & $\frac{26.3}{26.37}$ & 46.166 & $\begin{array}{l}50.128 \\
50.289\end{array}$ & $\begin{array}{r}13.633 \\
13655\end{array}$ & $\begin{array}{l}32.727 \\
32097\end{array}$ & $\frac{-1.222}{1.222}$ & & 48.221 & 0.16 & $\frac{14.643}{14643}$ & & $\frac{212.7}{2137}$ & 54444 & 48.7 & & 39 & & & & 0.000 & 0.422 & \\
\hline & $6 / 1772004$ & $\begin{array}{l}1.434 .24 \mathrm{FM} \\
144: 24 \mathrm{PM}\end{array}$ & 25.729 & 26.397 & 24.123 & 27.653 & $\frac{20.315}{26.38}$ & $\frac{40.439}{46.077}$ & \begin{tabular}{|l|}
0.209 \\
49.782 \\
\end{tabular} & 213.556 & $\frac{32.96}{32.77}$ & $\frac{-1.222}{-1.222}$ & $\frac{-1.271}{-7.277}$ & $\frac{40.501}{52.241}$ & 0.11 & $\frac{14.045}{14.643}$ & $\frac{0.004}{0.004}$ & $\frac{13.11}{214.7}$ & $\begin{array}{ll}73.50111 \\
73.5778\end{array}$ & $\begin{array}{l}40.0 \\
48.3 \\
\end{array}$ & $\frac{1.4}{12.8}$ & $\frac{39.1}{39.4}$ & \begin{tabular}{|l|l|}
2.71818 \\
\end{tabular} & \begin{tabular}{|l|}
0.017 \\
0.017
\end{tabular} & \begin{tabular}{|l|l|}
0.017 \\
\end{tabular} & 0.000 & $\frac{0.427}{0.434}$ & 0.37 \\
\hline & & 1:45:24 PM & & 26.403 & 24.175 & & & 46.07 & 50.115 & \begin{tabular}{|l|l|}
13.324 \\
\end{tabular} & 32.953 & \begin{tabular}{|l|l|}
-1.219 \\
\end{tabular} & & & 0.11 & $\begin{array}{l}14.643 \\
\end{array}$ & & & 3.59444 & 48.6 & & & & & & & ( & \\
\hline 23 & $6 / 17 / 2004$ & 1:46:24 PM & 25.746 & 26.409 & 24.161 & \begin{tabular}{l|l|}
27.657 \\
\end{tabular} & 26.42 & 45.8 & 49.912 & 13.349 & 32.723 & & & 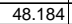 & 0.1 & $\begin{array}{l}14.643 \\
\end{array}$ & & 216.7 & & 48.4 & & $\sqrt{n}$ & & & & & & \\
\hline & $6 / 17 / 2004$ & 1:47:24PM & 25.731 & 26.394 & $\begin{array}{l}24.156 \\
\end{array}$ & 27.682 & & 45.983 & \begin{tabular}{|l|l|}
49.618 \\
\end{tabular} & \begin{tabular}{|l|l|l}
13.308 \\
\end{tabular} & $\overline{32.996}$ & -1.219 & -7.19 & $\begin{array}{l}48.857 \\
\end{array}$ & 0.113 & $\begin{array}{l}14.643 \\
\end{array}$ & & 217 & 3.62778 & 48.1 & & & & & $\overline{0.01}$ & 00 & 0.437 & \\
\hline & 6/117/2004 & 1:48:24 PM & 25.736 & 26.399| & 24.156 & 27.632 & & 45.958 & 49.925 & \begin{tabular}{ll|}
13.27 \\
\end{tabular} & 32.754 & -1.222 & $-7.21 \mathrm{Cl}$ & 54.195 & 0.12 & 14.643 & & 218 & 64444 & 48.5 & 10 & & & & 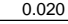 & & 0.500 & \\
\hline & & $1: 49: 24 \mathrm{PM}$ & & 26.4 & & & & & 49.6866 & & & & & & & 14.643 & & & & & & & & & & & 0.422 & \\
\hline & 6 & 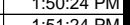 & 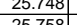 & $\frac{26.41}{2.42}$ & 20.187 & 27.619 & & 46. & $\begin{array}{l}49.993 \\
50252\end{array}$ & $\frac{1.3289}{13230}$ & $\frac{33.014}{23.15}$ & $\frac{-1.222}{-1210}$ & $-\frac{-7.15}{-772}$ & & 0.1 & 14.643 & & & $\frac{3.66778}{36944}$ & 48 & & & & & & & 0.497 & \\
\hline & $\frac{6 / 1 / 17204}{6 / 17 / 2004}$ & 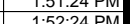 & 20.1506 & 20.424 & $\frac{2.240}{2.212}$ & 27.064 & 26. & $\frac{40.290}{4641}$ & $\begin{array}{l}50.204 \\
49.981\end{array}$ & $\begin{array}{r}13.350 \\
13.22\end{array}$ & $\frac{35.152}{33486}$ & $\frac{-1.219}{-1219}$ & $\frac{-1.2}{72}$ & $\begin{array}{l}46.305 \\
5019\end{array}$ & 0.1 & $\frac{14.643}{14.643}$ & & $\frac{21.91}{207}$ & $\begin{array}{l}5.03944 \\
77\end{array}$ & 年 & (25 & 年 & & & 0 & & 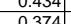 & \\
\hline & $\frac{111 / 2004}{61 / 2004}$ & $\begin{array}{l}1.5 .25 \\
1.5324 \mathrm{PM}\end{array}$ & 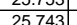 & 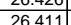 & 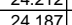 & 27.604 & $\begin{array}{l}\frac{20.304}{26384} \\
263\end{array}$ & $\frac{e_{0.44}}{46.12}$ & 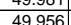 & $\mid \frac{13.26}{1348}$ & $\frac{3.406}{33 .}$ & $\frac{-1.21}{-1219}$ & 048 & 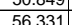 & 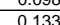 & $\frac{14.045}{14643}$ & & 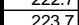 & 73.72778 & 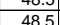 & 12. & (39. & & 装 & & 01 & 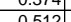 & \\
\hline & $\frac{61}{6 / 17 / 2004}$ & & 25.73 & & & 27.574 & $\frac{20.00}{26.40}$ & & & & & & & & & 14643 & & 232 & 37444 & 487 & & & & & & & 1434 & \\
\hline & 66 & 1.55 & $\frac{2.10}{2572}$ & $\frac{2.4115}{2640}$ & 24.177 & & & & (19.19074 & 13204 & 287 & 1.125 & & & 0.1 & 14.045 & & & & $\frac{40.4}{185}$ & 136 & & & & & & & \\
\hline & $6 / 17712004$ & $1.56 .24 \mathrm{PM}$ & 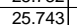 & 26.417 & $\frac{\mid}{24.203}$ & $\frac{27.659}{27.659}$ & 2.6 .4 & 46.207 & 50.175 & $\frac{13.336}{11.336}$ & $\frac{3.063}{33.063}$ & $\frac{-1.219}{-1.219}$ & -7.2 & 48192 & 01 & 14.643 & & 226 & $\frac{0.778}{7778}$ & 48 & 1118 & 396 & & & 0.018 & 0000 & 0.442 & $2 \pi$ \\
\hline & $6 / 17 / 2004$ & $1: 57: 2$ & & 26.429 & & 27.722 & & 42.709 & 50.034 & \begin{tabular}{|c|}
13.298 \\
\end{tabular} & 29.48 & 1.731 & & & & $\begin{array}{l}14.643 \\
\end{array}$ & & & 79444 & 48. & & 36 & & & & & & \\
\hline & $6 / 17 / 2004$ & 1:58:24 PM & 25.755 & 26.433 & 24.22 & 27.771 & & 45.645 & 50.453 & 13.428 & 32.312 & -0.82 & & 49.187 & 0.11 & $\begin{array}{l}14.643 \\
\end{array}$ & & & 3.81111 & 49 & & Fr & & & & & 0.430 & \\
\hline & 6/177/2004 & 1:59:24 PM & 25.775 & 26.438 & 24.25 & 27.856 & 26.5 & 45.409 & \begin{tabular}{|l|l|l|l|}
4978 \\
\end{tabular} & \begin{tabular}{|l|}
13.271 \\
\end{tabular} & 32.325 & \begin{tabular}{|c|c|c|}
-0.884 \\
\end{tabular} & -5.799 & 41.615 & 0.109 & $\begin{array}{l}14.643 \\
\end{array}$ & & 229 & 3.82778 & 48.5 & 10.2 & 38. & 268 & $\overline{1016}$ & & 0.000 & 0.427 & \\
\hline & $6 / 177 / 2004$ & & 25.786 & 26.459 & 24.311 & 27.926 & 26.5 & 45.56 & 49.562 & 13.027 & 32.557 & -1.222 & -7.1 & 47.56 & & 14.643 & & & 3.84444 & 48 & 11.7 & 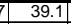 & & & $01 \mathrm{~s}$ & & & \\
\hline & $6 / 17 / 2004$ & & & $26.449 \mid$ & 24.321 & 27.921 & & 45.788 & \begin{tabular}{|l|l|l}
49.547 \\
\end{tabular} & & 32 & 1.222 & & & & 14. & & & & & & & & & & & & \\
\hline & 6611722004 & 2.01 .01710 & $\frac{25.955}{2577]}$ & 26.448 & $\begin{array}{l}24.26 \\
24.26\end{array}$ & 27.885 & 26.59 & 45.705 & 49.9433 & $\begin{array}{l}13.046 \\
3.096\end{array}$ & & -1.250 & 712 & 50 & 0.10 & 14.643 & & 2321. & \begin{tabular}{|l|l|}
3.86306 \\
\end{tabular} & 8 & 12.4 & (3. & & & then & 0.000 & 0.399 & \\
\hline & 0.17172004 & $2.02 .31 \mathrm{PM}$ & $\begin{array}{l}25.187 \\
5787\end{array}$ & 26.455 & 24.251 & 27.842 & & 46.099 & & 12.996 & & -1.222 & & 50.956 & 0.1 & 14.643 & & & & & & 39. & & 017 & & 0.000 & 0.441 & \\
\hline & $6 / 1 / 72004$ & 2:03.31 PM & 25.173 & 26.4566 & $\frac{2.438}{2.329}$ & 21.189 & & $\begin{array}{l}46.286 \\
45.206\end{array}$ & $\begin{array}{l}50.146 \\
5.1907\end{array}$ & $\frac{13.12}{1327}$ & $\begin{array}{l}3.3306 \\
3.3072\end{array}$ & 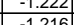 & & & 0.1 & 14 & & 233 & | & & $\frac{11.4}{122}$ & 39 & & & & 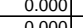 & 190 & \\
\hline & $\frac{01172004}{6 / 17 / 2004}$ & & 25 & $\frac{2.4016}{2646}$ & $\frac{24.45}{34219}$ & 27.140 & & & & $\mid$ & $\frac{3.0024}{3322}$ & & & $\frac{49.698}{45814}$ & & $\frac{14.6}{146}$ & & & & & & & & & & & & \\
\hline & $6 / 17 / 2004$ & $\frac{0.0631 \mathrm{PM}}{20.31}$ & $\frac{2.57}{25.78}$ & 资. & $\frac{24.43}{24.2}$ & $\frac{2.176}{27.736}$ & 26.45 & 46.168 & 50.148 & $\mid$ & 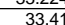 & -1.122 & & 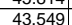 & & $\frac{14.6}{146}$ & & & 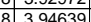 & 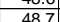 & & & & & & & 0 & \\
\hline & $6 / 17 / 2004$ & 1. PM & 25.785 & 26.473 & 24.19 & 2772 & & 46.458 & 50.416 & $\mid$ & $\begin{array}{l}33.445 \\
3375\end{array}$ & -1222 & & 45107 & 0.1 & 146 & & & 300306 & & 111 & & & & & & 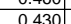 & \\
\hline & $6 / 17 / 2004$ & $2: 08: 31 \mathrm{PM}$ & 25.764 & 26.47 & 24.194 & 27.685 & & 46.78 & 50.503 & 12849 & 34.081 & -1.219 & & 50. & & 146 & & & & & & & & & & & . & \\
\hline & $6 / 6117 / 2004$ & $2 \cdot 09 \cdot 31$ & 25.759 & 26.472 & 24.179 & & & 46.045 & 49.979 & 12.649 & & $\frac{-1.222}{-1.222}$ & & 45.4 & & 14 & & & & 48 & 11 & & & & & & 0.452 & \\
\hline 25 & 6/117/2004 & 2:10:31 PM & 25.754 & 26.467 & 24.179 & 27.735 & & & 50.922 & 12.984 & 34.048 & \begin{tabular}{|l|} 
\\
\end{tabular} & & 52.5 & 0.1 & $\begin{array}{l}14.643 \\
\end{array}$ & & 24 & $\mid 4.01306$ & & 12.9 & & & 18 & & 0.000 & $\mid 0.448$ & \\
\hline 26 & $6 / 17 / 2004$ & 2:11:31 PM & $\mid 25.743$ & 26.471 & 24.208 & 27.854 & & 46.4 & & 12.732 & 33.893 & -1.225 & & 47 & & 14. & & & & & 11.6 & $4 c$ & & 18 & 0.0 & & 4 & \\
\hline 26 & 6/177/2004 & 2:12:31 PM & 25.746 & 26.484 & 24.206 & 27.9 & & 46.4 & 50.3 & 12.619 & 33.987 & -1.225 & & 51.6 & & 14.6 & & & & & 12.7 & 40 & & & & & & \\
\hline & 6/117/2004 & & 25.692 & & 24.201 & 27. & & & & 12.618 & & -1.222 & & & & & & & & & $11.7 \mathrm{r}$ & & & & & & & \\
\hline & & 2:14:31 PM & & & & & & & \begin{tabular}{|l|l|} 
\\
\end{tabular} & & & -1.228 & & & & & & & & & & & & & & & & \\
\hline & 661172004 & 2:15:31 PM & 25.636 & 26.509 & 24.196 & 27.98 & & & 50.457 & 12.733 & & -1.219 & & & & & & & & & & & & & & & & \\
\hline & 606172004 & $2.16 .31 P M$ & $\frac{25.62}{2561}$ & 20.51 & 2 & & & & \begin{tabular}{|l|l|l|l|l|}
5.54 \\
\end{tabular} & $\frac{\mid 12.651}{12717}$ & $\frac{33.856}{3.212}$ & \begin{tabular}{|c|}
$\mid-1.219$ \\
\end{tabular} & & & & & & 24678 & & & 1.0 & 10 & & & & & 0.464 & \\
\hline & $\frac{01172004}{6117004}$ & $\frac{2.17 .35}{2 \cdot 18.31 \mathrm{PM}}$ & $\mid \frac{23.044 \mid}{25637}$ & $\frac{2.5324}{26535}$ & $\frac{2.459}{34.91}$ & $\frac{17.96}{27.57}$ & & $\begin{array}{l}40.030 \\
46427\end{array}$ & \begin{tabular}{|c|}
5.3456 \\
5065
\end{tabular} & 12681 & & $-1.2 \angle 2$ & & 53.914 & & $\frac{14.6}{14.6}$ & & 247.8 & & & $\frac{13.8}{132}$ & & & & & & 0.436 & \\
\hline & 6 & & 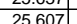 & & 22.191 & 2778 & & & 50.054 & $\begin{array}{l}12.081 \\
12592\end{array}$ & (34.248 & $-\frac{1.242}{1.202}$ & & | & & 14.6 & & & & $\frac{49}{48}$ & $\frac{1.4 .4}{122}$ & & & & & & | & \\
\hline & & & 25592 & & & & & & & & & & & & & & & & & & 124 & & & & & & & \\
\hline & $6 / 17 / 2004$ & & 2559 & & 24167 & 27858 & & & 50478 & 12715 & & 1225 & & & & 14 & & & & & 128 & & & & & & (39 & \\
\hline & 6 & 2.223 .31 & 25614 & 2653 & 24174 & 27 & & 467 & 50816 & 12736 & 3422 & -1225 & & & & 14 & & & 421 & 44 & 1 & & & & & & 3 & \\
\hline 272 & 6/17/2004 & $2: 23: 31$ & 25.61 & 26.528 & 24. & 27.865 & & 46 & & 12. & & -1.2 & & & & & & & & & & & & & & & & \\
\hline 27. & 6/177/2004 & & 25.604 & 26.5 & \begin{tabular}{l|l|}
24.164 \\
\end{tabular} & 27.88 & & 46.759 & 50.638 & 12.7 & & \begin{tabular}{l|l}
-1.222 \\
\end{tabular} & & & & & & 25 & & & 12.5 & & & & & & 4 & \\
\hline 27 & $6 / 177 /$ & & 25.6 & 26 . & 24 & 27.84 & & & & 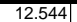 & $\overline{33}$ & \begin{tabular}{|c|}
-1.219 \\
\end{tabular} & & & & & & & & & $\overline{10.3}$ & & & & & & (61) & \\
\hline & 6/17//2004 & & 25.634 & 26.542 & 24.179 & 27.84 & & 46.021 & 50.063 & 12.3 & 33.802 & -1.219 & & & & & & 256 & & & 11.4 & & & & & & & \\
\hline 277 & $6 / 17 / 2004$ & 2:27:31 PM & 25.63 & 26.548 & 24.165 & 27.836 & 26.5 & 46.437 & 50.374 & 12.56 & 34.028 & -1.222 & & 50.1 & 0.1 & 14.6 & & 257.8 & 4.29639 & & 13.5 & 40 & & & & 0.000 & 0.440 & \\
\hline & & 2:28:311 & (2) & & 24.146 & 27.852 & & 46.659 & \begin{tabular}{|l|l|} 
\\
\end{tabular} & & & -1.219 & & & & & & & & & 11.0 & & & & & & 0.442 & \\
\hline & 6061772004 & $2: 29: 31 \mathrm{PM}$ & $2.5 .5 \mathrm{~kg}$ & 20.529 & 2 & 21.891 & & & 4.9.953 & (1) & & & & & & & & & & & & & & & & & & \\
\hline 280 & $\begin{array}{l}6 / 17 / 2004 \\
6 / 17 / 200\end{array}$ & $2.31 .33 \mathrm{PM}$ & 25607 & 2654 & 24126 & 28002 & & $\begin{array}{r}46.412 \\
16.616\end{array}$ & \begin{tabular}{|c|}
50.548 \\
50.814
\end{tabular} & 12469 & 34233 & 1219 & & 47521 & 0137 & 14.045 & & 2618 & 8436306 & 493 & 11.4 & & 278 & (200? & 8021 & 8001 & 0518 & 0 \\
\hline
\end{tabular}


WSRC-TR-2005-00105, REVISION 0

SRNL-RPP-2005-00012, REVISION 0

RUN \# WASH1 AND WASH 2; CAMPAIGN III SLURRY WASHING - CONT.

\begin{tabular}{|c|c|c|c|c|c|c|c|c|c|c|c|c|c|c|c|c|c|c|c|c|c|c|c|c|c|c|c|c|}
\hline & A & B & D & E & $\mathrm{F}$ & G & $\mathrm{H}$ & $\mathrm{J}$ & $\mathrm{K}$ & & $M$ & $\mathrm{~N}$ & 0 & Q & $R$ & $\mathrm{~s}$ & & $\mathrm{v}$ & w & $x$ & & $z$ & $\mathrm{AA}$ & $A D$ & $A C$ & $A D$ & $\mathrm{AE}$ & Af IA $^{-1}$ \\
\hline & $\frac{6 / 117 / 2004}{661 / 72004}$ & $2: 32: 31 \mathrm{PM}$ & $\begin{array}{l}\frac{25.583}{25.595} \\
25\end{array}$ & $\begin{array}{r}26.536 \\
26.558 \\
\end{array}$ & \begin{tabular}{|l|}
24.113 \\
24.104
\end{tabular} & $\begin{array}{l}27.969 \\
27.995\end{array}$ & 26.599 & $\begin{array}{l}46.193 \\
46.514 \\
\end{array}$ & \begin{tabular}{|l|}
50.109 \\
50.28 \\
\end{tabular} & \begin{tabular}{|l|l|}
12.447 \\
12.435
\end{tabular} & $\begin{array}{l}33.915 \\
33.233 \\
\end{array}$ & $\begin{array}{l}-1.222 \\
-1.222\end{array}$ & $\begin{array}{l}-6.973 \\
-7.057 \\
-\frac{1}{2}\end{array}$ & \begin{tabular}{|l|l|l}
51.196 \\
49.089
\end{tabular} & $\frac{0.117}{0.112}$ & $\begin{array}{l}14.643 \\
14.643 \\
\end{array}$ & $\begin{array}{l}0.004 \\
0.004\end{array}$ & $\begin{array}{l}262.8 \\
263.8 \\
\end{array}$ & \begin{tabular}{|l|}
4.37972 \\
4.39639 \\
\end{tabular} & \begin{tabular}{l|l|}
48.6 & 48.8 \\
\end{tabular} & $\frac{12.5}{12.0}$ & $\begin{array}{l}40.1 \\
40.4\end{array}$ & 2.762 & 0.017 & 0.018 & $\begin{array}{l}0.000 \\
0.000\end{array}$ & $\begin{array}{l}0.447 \\
0.44\end{array}$ & $\begin{array}{l}0.38 \\
0.36\end{array}$ \\
\hline & & & 25.593 & $\frac{20.050}{26.566}$ & $\frac{24.104}{24.107}$ & 28.043 & $\frac{20.500}{26.603}$ & & \begin{tabular}{|l|}
0.20 .536 \\
\end{tabular} & & $\frac{34.250}{34.213}$ & & & & 0.114 & $\frac{14.045}{14.643}$ & & & & & & & & & & & & \\
\hline 284 & $6 / 17 / 2004$ & 2:35:31 PM & 25.631 & 26.584 & 24.105 & 28.116 & 26.627 & 46.539 & \begin{tabular}{|l|l|}
51.164 \\
\end{tabular} & 12.401 & 34.143 & \begin{tabular}{|l|}
-1.222 \\
\end{tabular} & $\begin{array}{r}-6.019 \\
-6.04\end{array}$ & 44.661 & 0.109 & 14.643 & & 265.8 & 4.42972 & 49.7 & 10.9 & & 2.781 & \begin{tabular}{|l|}
0.016 \\
\end{tabular} & 0.017 & 0.000 & 0.413 & 0.35 \\
\hline & $6 / 17 / 2004$ & 2:36:31 PM & 25.643 & 26.596 & 24.132 & 28.167 & 26.688 & 46.278 & 50.323 & \begin{tabular}{|l|l|}
12.063 \\
\end{tabular} & 34.272 & $\begin{array}{l}1.222 \\
\end{array}$ & -6.771 & \begin{tabular}{|l|l|}
39.367 \\
\end{tabular} & 0.12 & $\begin{array}{l}14.643 \\
\end{array}$ & 0.004 & 266.8 & 4.44639 & 48.9 & 9.6 & 40.3 & & & 0.018 & & 0.455 & 0.39 \\
\hline 286 & $6 / 17 / 2004$ & & 25.661 & 26.619 & \begin{tabular}{r|}
24.15 \\
\end{tabular} & 28.171 & 26.711 & 46.348 & \begin{tabular}{|l|}
50.308 \\
\end{tabular} & \begin{tabular}{|l|l|}
12.178 \\
\end{tabular} & 34.235 & \begin{tabular}{|c|}
-1.222 \\
\end{tabular} & $\begin{array}{l}-.611 \\
-7.011 \\
\end{array}$ & 49.047 & 0.12 & 14.643 & 0.004 & 267.8 & 4.46306 & 48.8 & $\begin{array}{l}12.0 \\
12.0\end{array}$ & 40.3 & & 0.018 & 0.018 & 0.000 & 0.455 & \\
\hline & $6 / 117 / 2004$ & $\begin{array}{ll}2: 38: 31 \mathrm{PM} \\
2 \cdot 32 \cdot 31 \mathrm{P}\end{array}$ & $\begin{array}{l}25.651 \\
25666\end{array}$ & $\begin{array}{l}26.614 \\
26609\end{array}$ & $\begin{array}{l}24.125 \\
2.095\end{array}$ & $\begin{array}{l}28.111 \\
28031\end{array}$ & 26.71 & 46.678 & \begin{tabular}{|l|l|}
50.642 \\
5.20 \\
\end{tabular} & $\begin{array}{l}12.091 \\
205\end{array}$ & 34.666 & $\begin{array}{l}-1.219 \\
\end{array}$ & $\begin{array}{l}-6.892 \\
.630\end{array}$ & $\begin{array}{l}52.485 \\
5.707\end{array}$ & 0.129 & $\begin{array}{l}14.643 \\
14.62\end{array}$ & & 268.8 & 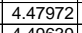 & 49.2 & 12.9 & & & & & 0.000 & 0.485 & \\
\hline$\frac{288}{289}$ & $6 / 17 / 2004$ & $\begin{array}{l}2: 39: 31 \mathrm{PM} \\
2 \cdot 20 \cdot 31 \mathrm{P}\end{array}$ & $\begin{array}{r}25.666 \\
25.65\end{array}$ & $\begin{array}{l}26.609 \\
26.608\end{array}$ & 24.095 & 28.031 & $\begin{array}{l}26.632 \\
26.646\end{array}$ & 46.081 & \begin{tabular}{|l|l|}
50.294 \\
50.23
\end{tabular} & $\begin{array}{l}12.095 \\
12076\end{array}$ & $\begin{array}{l}34.092 \\
2510\end{array}$ & \begin{tabular}{|c|}
-1.219 \\
-1220
\end{tabular} & $\begin{array}{l}-6.369 \\
6.641\end{array}$ & $\begin{array}{l}50.783 \\
\end{array}$ & 0.114 & $\begin{array}{l}14.643 \\
1643\end{array}$ & 0.004 & 269.8 & 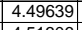 & 48.8 & $\begin{array}{c}12.4 \\
11.9\end{array}$ & 40.1 & $\begin{array}{l}2.764 \\
2.701 \\
\end{array}$ & \begin{tabular}{|c|c|c|}
0.017 \\
0018
\end{tabular} & \begin{tabular}{|l|l|}
0.017 \\
0.019
\end{tabular} & 0.000 & 0.435 & 0.37 \\
\hline 290 & $\begin{array}{l}6 / 1 / 172004 \\
6 / 172004\end{array}$ & 2:40:31 PM & $\begin{array}{r}25.65 \\
25.674 \\
\end{array}$ & $\frac{26.608}{26.632}$ & & 28.005 & $\frac{26.646}{26.615}$ & $\begin{array}{l}46.431 \\
46.805\end{array}$ & \begin{tabular}{|l|}
50.403 \\
50.832
\end{tabular} & \begin{tabular}{r|r}
12.076 \\
12.06
\end{tabular} & $\begin{array}{l}34.519 \\
34.904\end{array}$ & \begin{tabular}{l|l}
-1.222 \\
-1.222
\end{tabular} & $\begin{array}{l}-6.641 \\
-6.927\end{array}$ & $\begin{array}{r}48.002 \\
53.54\end{array}$ & $\begin{array}{l}0.124 \\
0.118\end{array}$ & $\begin{array}{l}14.643 \\
14.643 \\
\end{array}$ & & $\begin{array}{l}270.8 \\
271.8 \\
\end{array}$ & $\mid$\begin{tabular}{|l|}
4.55306 \\
4.52972
\end{tabular} & $\begin{array}{l}48.9 \\
49.4 \\
\end{array}$ & $\begin{array}{l}\mid 11.8 \\
13.1\end{array}$ & $\begin{array}{l}40.5 \\
40.9 \\
\end{array}$ & $2.791 \mid$ & \begin{tabular}{|c|}
0.018 \\
0.018
\end{tabular} & 0.019 & $\frac{0.000}{0.000}$ & $\begin{array}{l}0.469 \\
0.44\end{array}$ & $\begin{array}{l}0.40 \\
0.38\end{array}$ \\
\hline & $6 / 17 / 2004$ & 2:42:31 PM & & 26.633 & 24.054 & 28.065 & 26.66 & 48.163 & & 12.167 & & & & & 0.141 & 14.643 & 0.004 & & \begin{tabular}{|l|}
4.54639 \\
\end{tabular} & 49.4 & $\frac{13.1}{10.0}$ & & & & $\begin{array}{l}0.018 \\
0.022\end{array}$ & & & $\frac{0.38}{0.44}$ \\
\hline & $6 / 17 / 2004$ & 2:43:31 PM & 25.761 & 26.644 & 24.04 & 28.061 & 26.73 & 45.284 & 50.911 & 12.165 & 33.154 & \begin{tabular}{|l|l|} 
\\
\end{tabular} & -0.856 & 44.838 & 0.077 & 14.643 & & 273.8 & \begin{tabular}{|l|l|}
4.56306 \\
\end{tabular} & 49.4 & 11.0 & 39.2 & 2.704 & & 0.012 & 0.000 & 0.301 & 0.26 \\
\hline 293 & $6 / 17 / 2004$ & 2:44:31 PM & 25.707 & 26.65 & 24.026 & 28.022 & 26.687 & 47.57 & 50.497 & \begin{tabular}{|l|l|}
12.173 \\
\end{tabular} & 35.489 & \begin{tabular}{|r|}
-1.222 \\
\end{tabular} & $\begin{array}{l}-6.057 \\
-.073\end{array}$ & 48.128 & 0.061 & 14.643 & & 274.8 & 4.57972 & 49.0 & 111.8 & 41.5 & 2.863 & 0.009 & 0.009 & 0.000 & 0.225 & \\
\hline & $6 / 17 / 2004$ & $\begin{array}{l}2: 45: 31 \mathrm{PM} \\
2: 02: 12\end{array}$ & 25.737 & 26.665 & 24.056 & 28.042 & 26.65 & 47.323 & $\begin{array}{l}49.931 \\
0.952\end{array}$ & \begin{tabular}{l|l|}
12.785 \\
1270
\end{tabular} & 34.66 & \begin{tabular}{|l|l|} 
\\
\end{tabular} & $\begin{array}{l}-6.733 \\
\end{array}$ & $\begin{array}{l}45.566 \\
\end{array}$ & 0.134 & 14.643 & & 275.8 & \begin{tabular}{|l|l|} 
\\
\end{tabular} & 48.5 & 11.2 & 41.0 & 2.826 & & 0.021 & 0.001 & 0.501 & \\
\hline & $6 / 17 / 2004$ & 2:46:31 PM & $\begin{array}{l}25.724 \\
25751\end{array}$ & 26.657 & \begin{tabular}{|l|}
24.023 \\
2405
\end{tabular} & $\begin{array}{l}27.994 \\
28.076\end{array}$ & 26.62 & $\begin{array}{l}47.184 \\
4.881\end{array}$ & \begin{tabular}{|l|l|}
49.852 \\
40.86
\end{tabular} & 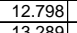 & \begin{tabular}{|l|l|}
34.523 \\
3565
\end{tabular} & \begin{tabular}{|l|l|}
-1.222 \\
-219
\end{tabular} & $\begin{array}{l}-6.956 \\
6.964\end{array}$ & $\begin{array}{l}44.744 \\
1712\end{array}$ & 0.115 & $\begin{array}{r}14.643 \\
194.3\end{array}$ & & $\begin{array}{l}276.8 \\
277\end{array}$ & \begin{tabular}{|l|l|}
4.61306 \\
\end{tabular} & $\begin{array}{l}48.4 \\
\end{array}$ & $\begin{array}{ll}11.0 \\
102\end{array}$ & 40.9 & & & 0.018 & .000 & $\begin{array}{l}0.431 \\
\end{array}$ & \\
\hline$\frac{297}{297}$ & $\frac{6}{661177 / 204204}$ & $2: 47: 31 \mathrm{PM}$ & $\begin{array}{l}25.751 \\
25.754\end{array}$ & $\frac{26.679}{26.682}$ & $\begin{array}{l}24.055 \\
2.069\end{array}$ & $\begin{array}{l}28.076 \\
28.134\end{array}$ & $\frac{26.70 .}{26.7}$ & $\begin{aligned} \frac{46.881}{46.06} \\
\end{aligned}$ & $\begin{array}{r}49.86 \\
49.355 \\
\end{array}$ & \begin{tabular}{|l|}
3.289 \\
13.185
\end{tabular} & $\begin{array}{r}33.56 \\
33.043 \\
\end{array}$ & $\begin{array}{l}-1.219 \\
-1.222\end{array}$ & $\begin{array}{l}-6.964 \\
-5.658 \\
\end{array}$ & \begin{tabular}{|c|}
41.713 \\
50.13
\end{tabular} & $\frac{0.111}{0.08}$ & $\begin{array}{l}14.643 \\
14.643 \\
\end{array}$ & & $\begin{array}{l}277.8 \\
278.8 \\
\end{array}$ & \begin{tabular}{|l|}
4.62972 \\
4.46639
\end{tabular} & $\begin{array}{l}48.4 \\
47.9 \\
\end{array}$ & & $\begin{array}{l}40.2 \\
39.6\end{array}$ & $\frac{2.773}{2.727}$ & $\frac{0.017}{0.016}$ & $\frac{0.017}{0.017}$ & 0.000 & $\begin{array}{l}0.423 \\
0.418 \\
\end{array}$ & 0.36 \\
\hline 298 & $6 / 17 / 2004$ & 2:49:31 PM & 25.771 & 26.709 & 24.105 & 28.126 & & 46.075 & 49.51 & 13.181 & 33.057 & \begin{tabular}{|l|}
-1.219 \\
\end{tabular} & $\begin{array}{r}-5.713 \\
\end{array}$ & 50.374 & 0.109 & 14.643 & & \begin{tabular}{|l|}
278.8 \\
279.8
\end{tabular} & \begin{tabular}{|l|}
4.640539 \\
4.66306 \\
\end{tabular} & 48.0 & 12.3 & 39.6 & 2.728 & 0.016 & 0.017 & $\begin{array}{l}0.000 \\
0.000\end{array}$ & $\begin{array}{l}0.416 \\
0.421\end{array}$ & $\frac{0.36}{0.36}$ \\
\hline & $6 / 17 / 2004$ & 2:50:31 PM & 25.753 & 26.691 & 24.093 & 28.009 & 26.674 & 46.307 & 49.328 & 13.301 & 33.057 & \begin{tabular}{|l|}
-1.225 \\
\end{tabular} & -6.99 & 51.788 & 0.112 & 14.643 & & 280.8 & \begin{tabular}{|l|l|}
4.67972 \\
\end{tabular} & 47.9 & 12.7 & 39.7 & 2.736 & 0.017 & 0.017 & 0.000 & 0.432 & 0.36 \\
\hline 300 & $6 / 17 / 2004$ & 2:51:31 PM & & 26.716 & 24.172 & 28.028 & 26.69 & & 48.872 & & & \begin{tabular}{|l|}
-1.222 \\
\end{tabular} & -6.996 & 50.295 & 0.113 & 144.643 & & 281.8 & & 47.4 & 12.3 & 39.4 & & & 0.017 & & & \\
\hline 301 & $6 / 17 / 2004$ & 2:52:31 PM & 25.76 & 26.708 & 24.14 & 28.005 & & 45.871 & \begin{tabular}{|l|l|l|l}
48.805 \\
\end{tabular} & $\begin{array}{l}12.96 \\
\end{array}$ & 33.08 & $\mid-1.225$ & & 47.723 & 0.11 & $\begin{array}{l}14.643 \\
\end{array}$ & & & & $\begin{array}{l}47.3 \\
\end{array}$ & 11.7 & & & .016 & 0.017 & 0.000 & $\frac{1.426}{0.426}$ & \\
\hline & $6 / 17 / 2004$ & 2:53:31 PM & 25.751 & 26.704 & 24.115 & 28.001 & 26.69 & 45.96 & $\begin{array}{l}48.936 \\
\end{array}$ & 13.144 & 32.975 & -1.222 & -1.0 & 51.918 & 0.11 & \begin{tabular}{l|l|}
14.643 \\
\end{tabular} & & 283.8 & 4.72972 & 47.5 & & 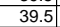 & & & & & & \\
\hline & $6 / 17 / 2004$ & 2:54:31 PM & & & & & 26.64 & 45.94 & 49.481 & 13.166 & & & & & 0.11 & $\begin{array}{l}14.643 \\
\end{array}$ & & 284.8 & 4.74639 & & & & & & & & 0.428 & \\
\hline & $6 / 17 / 2004$ & 2:55:31 PM & 25.752 & 26.705 & 24.101 & 28.022 & 26.628 & $\begin{array}{l}45.794 \\
\end{array}$ & 49.011 & \begin{tabular}{|c|}
13.057 \\
\end{tabular} & 32.848 & -1.222 & -7.005 & 52.431 & 0.11 & $\begin{array}{l}14.643 \\
\end{array}$ & & 285.8 & \begin{tabular}{|l|l|}
4.76306 \\
\end{tabular} & 47.5 & 12.8 & 39.3 & 2.711 & $\begin{array}{l}0.016 \\
\end{array}$ & 0.017 & 0.000 & 0.428 & 0.36 \\
\hline & $6 / 17 / 2004$ & 2:56:31 PM & 25.752 & 26.706 & 24.097 & 28.092 & 26. & 45.855 & $\begin{array}{l}49.191 \\
\end{array}$ & 13.071 & 32.836 & -1.222 & -6.996 & 40.564 & 0.109 & $\begin{array}{l}144.643 \\
\end{array}$ & & 286.8 & \begin{tabular}{|l|l|} 
& 4.77972 \\
\end{tabular} & 477.7 & 9.9 & 39.3 & $2.713 \mathrm{~s}$ & 0.016 & 0.017 & 0.000 & 0.424 & 0.36 \\
\hline & 6/17/72004 & $1 \mathrm{PM}$ & 25.763 & 26.72. & 24.108 & 28.158 & 26.6 & 45.728 & 48.787 & 13.003 & 32.881 & & -6.6. & 2.318 & $0.10 \mathrm{~S}$ & 14.643 & & 287.8 & \begin{tabular}{|l|}
4.79639 \\
\end{tabular} & 47.3 & & & & & & & & \\
\hline 30 & $6 / 17 / 2004$ & , & 25.757 & 26.71 & 24.091 & 28.166 & & 46.176 & 49.465 & 12.96 & 33.386 & -1.222 & & 46.786 & $0.10 \mathrm{~s}$ & 14.643 & & 88.8 & 81306 & 48.0 & & & & & & & $0.419 \mid$ & \\
\hline 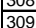 & $\frac{6 / 17 / 2004}{6}$ & 2:59:31 PM & 255755 & $\frac{26.713}{26721}$ & 20.4994 & $\begin{array}{r}28.17 \\
28087\end{array}$ & & 46.203 & 50.119 & \begin{tabular}{|c|}
12.913 \\
12832
\end{tabular} & 33.25 & \begin{tabular}{|c|}
-1.219 \\
\end{tabular} & & $\begin{array}{l}48.186 \\
45.341\end{array}$ & 0.10 & 14.643 & & 289.8 & 82972 & 48 & 11. & & 2.73 & & & & 0.404 & \\
\hline & $6 / 1 / 172004$ & $\begin{array}{l}3: 00.31 \text { PM } \\
3: 01: 31\end{array}$ & $\frac{23.152}{25.745}$ & $\frac{20.121}{26.723}$ & $\frac{24.102}{24.089}$ & $\frac{28.007}{28.02}$ & & $\begin{array}{l}46.155 \\
46.13 !\end{array}$ & $\frac{49.101}{49.641}$ & $\begin{array}{l}1.8285 \\
12882\end{array}$ & $\frac{33.384}{33326}$ & $\frac{-1.219}{-1219}$ & -6.5 & $\frac{45.3 .}{47.8}$ & $\frac{0.11}{0.11}$ & $\frac{14.045}{14643}$ & & $\frac{290.0}{2018}$ & (5) & & & 397 & 273 & & 0101 & & 0.435 & \\
\hline & $6 / 17 / 2004$ & 3:02:31 PM & $\begin{array}{ll}25.764 \\
\end{array}$ & 26.737 & 24.129 & 27.989 & 26.6 & $\frac{40.150}{45.345}$ & \begin{tabular}{|l|}
48.04442 \\
\end{tabular} & 12.511 & 32.926 & \begin{tabular}{|l|}
-1.21 .222 \\
-1.22
\end{tabular} & -0.90 & $\frac{47.029}{51.611}$ & $\frac{.111}{0.111}$ & $\frac{14.045}{14.643}$ & & \begin{tabular}{|l|l}
292.8 \\
\end{tabular} & \begin{tabular}{|l|}
4.000000 \\
4.87972
\end{tabular} & $\frac{40.4}{47.5}$ & & 39. & & & & 0.000 & $\begin{array}{l}.413 \\
0.433\end{array}$ & \\
\hline & $6 / 177 / 2004$ & 3:03:31 PM & 25.773 & 26.746 & 24.138 & 27.994 & & $\begin{array}{l}45.367 \\
\end{array}$ & \begin{tabular}{|l|l|}
49.017 \\
\end{tabular} & 12.57 & 32.934 & -1.216 & & 51.121 & 0.107 & $\begin{array}{l}14.643 \\
\end{array}$ & & & & 47.5 & 12.5 & 39. & & & 0.016 & & 0.418 & \\
\hline & $6 / 17 / 2004$ & $3: 04: 31 \mathrm{PM}$ & 25.752 & 26.73 & $\begin{array}{l}24.076 \\
\end{array}$ & 27.932 & 26.6 & 45.228 & 48.946 & 12.666 & 32.637 & -1.222 & -6.808 & 52.185 & 0.111 & $\begin{array}{l}14.643 \\
\end{array}$ & & \begin{tabular}{|l|l|}
294.8 \\
\end{tabular} & \begin{tabular}{|l|l|}
4.91306 \\
\end{tabular} & 47.5 & 12.8 & 38.9 & \begin{tabular}{|l|l|}
2.684 \\
\end{tabular} & & 0.017 & 0.000 & $\begin{array}{l}0.436 \\
\end{array}$ & \\
\hline & $6 / 17 / 2004$ & $1 \mathrm{PM}$ & $\frac{25.768}{25702}$ & 26 & 24.108 & 27.964 & & 45.544 & (250ल) & 12.68 & $\begin{array}{l}32.842 \\
2305\end{array}$ & .219 & & & 0.11 & 14.643 & & 29 & & 48.1 & & $\overline{3}$ & & & 101 & & 0.429 & \\
\hline & 601172004 & 资 & $\frac{25.1399}{25762}$ & $\frac{26.132}{26766}$ & 20.4609 & $\begin{array}{l}27.944 \\
27094\end{array}$ & & $\begin{array}{l}45.658 \\
45255\end{array}$ & $\begin{array}{l}49.562 \\
49187\end{array}$ & $\frac{12.621}{12.623}$ & 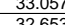 & $=\frac{-1.222}{-1216}$ & & 447 & 0.1 & $\frac{14.6}{11.6}$ & & & & & & 390 & 568 & 5016 & & (2) & & \\
\hline 31 & & 3.00 .53 PM & 25739 & & & & & (45.250 & 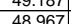 & $\begin{array}{r}12.633 \\
12582\end{array}$ & 势 & & & 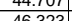 & $\frac{0.1}{0.12}$ & 14.6 & & & & & & & & & & & & \\
\hline $\begin{array}{ll}318 \\
318\end{array}$ & $6 / 17 / 2004$ & $3 \cdot 309 \cdot 31 \mathrm{PM}$ & 25.717 & 26.725 & $\mid 24041$ & 27.862 & 26. & 45.307 & 49226 & 1.2.58 & $\frac{3.264}{32692}$ & -1.225 & & 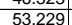 & 0.112 & 14.643 & & 20.0. & \begin{tabular}{|l|l|l|}
499639 \\
\end{tabular} & 478 & $\frac{1.40}{130}$ & 年 39.0 & 2.689 & 0.017 & 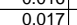 & 0.000 & 0 & \\
\hline & $6 / 17 / 2004$ & 3:10:31 PM & 25.734 & 26.737 & 224.074 & 27.86 & & 43.996 & 48.196 & 13.656 & 30.418 & -1.222 & & 50.484 & & 14.6 & & & & & & & & & & & & \\
\hline & $6 / 17 / 26$ & 3:11:31 PM & $\frac{5.708}{25.708}$ & 26.736 & 24.063 & $\frac{27.865}{27.86}$ & & 45.216 & 49.272 & $\mid$ & & $1+20$ & & $\frac{21.3}{51.3}$ & 0.12 & $\begin{array}{l}14.643 \\
\end{array}$ & & & & & & & & & & & $\frac{172}{772}$ & \\
\hline 321 & $6 / 17 / 2004$ & & & & & & & 45.6 & 49.549 & 12.544 & 33.18 & -1.219 & & & 0.1 & & & & & & & & & & & & 428 & \\
\hline & $6 / 117 / 2004$ & 3:13:31 PM & 25.688 & 26.731 & 24.033 & 27.83 & & & 50.115 & $\mid 12.239$ & 33.792 & $\frac{-1.219}{-1.219}$ & & ol & 0.09 & 14. & & & & & & & & & & & (n) & \\
\hline & $6 / 177 / 2004$ & 3:14:31 PM & 25.697 & $\overline{26.72}$ & 24.10 & 27.77 & & $45 !$ & & 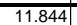 & 34.116 & -1.219 & & 48.3 & 0.10 & 14.643 & & & & & & & & & & & & \\
\hline & $6 / 17 / 2004$ & & 25.6 & & & & & & 49.7 & 11. & & & & & & & & & & & & & & & & & & \\
\hline 325 & 6/117/2004 & 3:16:31 PM & 25.702 & 26 & 24.137 & 27.818 & & & 50.155 & \begin{tabular}{|l|l|}
11.699 \\
\end{tabular} & 34.584 & -1.225 & & 36. & 0.1 & 14. & & & & & & 40 & & & & & & \\
\hline & $6 / 177 / 2004$ & & 25.598 & & 24.153 & 27.82 & & & & & & -1.222 & & & & & & & & & & & & & & & & \\
\hline & 6/17//2004 & & 25.661 & 26.734 & & & & & 50.227 & 11.378 & & & & 6.282 & 0.1 & 14.6 & & & & & & & & & & & 0.477 & \\
\hline & $6 / 17 / 2004$ & . & 25.677 & 26.126 & 24.182 & 27.91 & & 47. & S. & 11.247 & & 1.226 & & 44. & 0.16 & 14.6 & & & & & & & & & & & & \\
\hline 331 & 601172004 & 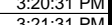 & 2573 & & $\frac{2.491}{2.192}$ & & & & S.6065 & & & & & & & & & & & & & 2 & & & & & & \\
\hline & $\frac{111 / 2004}{6}$ & 3.21 .31 & 20.15 & 20.151 & 24.953 & 21.014 & & & \begin{tabular}{|c|}
51.001 \\
51
\end{tabular} & 11.254 & & -1.252 & & & & & & & & & & & & & & & & \\
\hline & $\frac{1}{61 / 772004}$ & 13 PM & $\frac{25.1545}{25.665}$ & & $\begin{array}{l}24.2445 \\
24215\end{array}$ & & & & 50.901 & $\frac{1.15004}{11.04}$ & & -1.219 & & & 0.6 & & & & & & & $4 c$ & & & & & 0.1740 & \\
\hline & $6 / 17 / 26$ & 3:24:31 PM & 25.665 & & $\mid 24.215$ & & & & & & & & & & & & & & & & & & & & & & & \\
\hline & $6 / 17 / 22$ & & 25.7 & & 24.24 & 27.78 & & 48. & 51054 & 115 & 374 & -1222 & & 42 & & & & & & 4 & & & & & & & 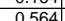 & \\
\hline & & & 25.104 & & & & & & & & & & & & & & & & & & & & & & & & & \\
\hline 336 & & & 25.673 & & 24.27 & 27.7 & & 46.9 & 48.6 & 12877 & 34. & & & 53. & & & & & & & & & & & & & & \\
\hline 337 & $6 / 17 / 2$ & & 25.6 & & 24.2 & 27.7 & & 46.8 & & 12.1 & & & & & & & & & & & & & & & & & & \\
\hline 338 & 6/117/2004 & 3:29:31 PM & 25.69 & 26.687 & 24.294 & 27.895 & & 47.0 & 48.9 & 11.7 & 35.389 & -1.222 & & & 0. & 14.6 & & 31 & & & & 41. & & & & & & \\
\hline 339 & $6 / 17 / 2$ & & 25.749 & 26.702 & 24.314 & 27.92 & & 47. & 49 & 11.6 & & & & & 0.1 & & & & & & & & & & & & & \\
\hline 340 & $6 / 17 / 24$ & 3:31:3 & 25.78 & 26.691 & 24.302 & 27.923 & & 47.3 & 49.39 & 11.8 & 35.4 & -1.222 & & 54. & 0.1 & 14.6 & & 32 & & & 13.4 & 41 & & & & & | & \\
\hline$\frac{341}{342}$ & $6 / 17722$ & & 25.18 & & & 27.8 & & & & & & & & & & & & & & & & & & & & & & \\
\hline & 6.1172 & & 25.192 & 26.6 & 24.327 & 2.8 & & 48.1 & 50.113 & 11. & & -1.2222 & & & & & & & & & 11.1. & 42.2 & & & & & & \\
\hline 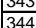 & $\frac{10172004}{6117004}$ & 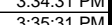 & 20.186 & $\begin{array}{l}\frac{20.085}{26679} \\
2.07\end{array}$ & 24.337 & 27.917 & & $\frac{48.186}{48100^{\circ}}$ & 50.1 & $\frac{11.9}{117}$ & 36.2717 & -1.22 & & 50.144 & 0.0 & 14. & & $\frac{32}{32}$ & & 48 & $\begin{array}{l}\frac{1.2 .3}{111} \\
\end{array}$ & & & & & & $\begin{array}{l}59 \\
\frac{51}{71}\end{array}$ & \\
\hline 345 & $6 / 1712004$ & & $\frac{2.021}{25.86}$ & $\frac{20.075}{26.668}$ & 24.34 & & & $\begin{array}{l}47.1 \\
47.9\end{array}$ & $\frac{50.101}{50.82}$ & $\frac{11.154}{11.422}$ & & & & 44. & $\frac{0.1}{0.1}$ & $\frac{14.6}{14.6}$ & & $\frac{0<0}{326}$ & & & $\frac{11.1}{11.0}$ & 42 & & 1026 & & & & \\
\hline 34 & & & & 26.672 & & & & & & & & & & 53. & & & & & & & 13.1 & & & 016 & & & & \\
\hline 347 & & & 25.778 & & & 27. & & 47.8 & \begin{tabular}{|l|l|}
49 \\
\end{tabular} & & 34.543 & -1.219 & & & 0. & & & 32 & & 47 & 12.8 & 40 & & & & & 997 & \\
\hline 348 & 6/177/2004 & & & & 24. & & & & & & & -1.219 & & & & & & & & & 11.2 & 40 & & & & & 0.415 & \\
\hline 350 & $\frac{6 / 17 / 24}{6.177212}$ & $\begin{array}{l}3: 40: 3 \\
2: 01: 0\end{array}$ & $\frac{20.044}{25876}$ & $\frac{26.681}{26.67}$ & \begin{tabular}{l|}
24.463 \\
24.52
\end{tabular} & $\begin{array}{l}27.843 \\
27.952\end{array}$ & & 46.637 & \begin{tabular}{|l|l|l|}
48.673 \\
\end{tabular} & $\begin{array}{r}12.399 \\
1223\end{array}$ & $\begin{array}{r}34.256 \\
3.621\end{array}$ & -1.222 & & 19.139 & 0.114 & $\begin{array}{l}14.643 \\
11643\end{array}$ & & 330.8 & 5.51306 & 17 & $\frac{11.1}{1.0}$ & 40.4 & & & 017 & 0.000 & 0.427 & 0.06 \\
\hline & & 3:41:3 & & & & & & 46.842 & 48.861 & 12.26 & 34.621 & & & $49.139]$ & & 14.643 & & & & & & & 2.808 & $0.017 \mid$ & 0.017 & 0.000 & 0.427 & 0.36 \\
\hline
\end{tabular}


WSRC-TR-2005-00105, REVISION 0

SRNL-RPP-2005-00012, REVISION 0

RUN \# WASH1 AND WASH 2; CAMPAIGN III SLURRY WASHING - CONT.

\begin{tabular}{|c|c|c|c|c|c|c|c|c|c|c|c|c|c|c|c|c|c|c|c|c|c|c|c|c|c|c|c|c|}
\hline & A & $\mathrm{B}$ & D & E & $\mathrm{F}$ & G & $\mathrm{H}$ & $\mathrm{J}$ & $\mathrm{K}$ & $\mathrm{L}$ & $\mathrm{M}$ & $\mathrm{N}$ & $\mathrm{O}$ & Q & $R$ & \begin{tabular}{l|l} 
\\
\end{tabular} & $\begin{array}{lll}T & 1\end{array}$ & $\mathrm{~V}$ & w & $x$ & $\mathrm{Y}$ & z & $\mathrm{AA}$ & $A B$ & $A C$ & $A D$ & $\mathrm{AE}$ & AF $|A|$ \\
\hline & $6 / 17 / 2004$ & $\begin{array}{l}3: 42: 31 \mathrm{PM} \\
3.43 .31 \mathrm{PM}\end{array}$ & \begin{tabular}{|l|}
25.916 \\
25886
\end{tabular} & $\begin{array}{l}26.66 \\
26.66\end{array}$ & \begin{tabular}{l|l}
24.472 \\
24487
\end{tabular} & $\begin{array}{l}27.847 \\
27832\end{array}$ & $\begin{array}{l}26.568 \\
25568\end{array}$ & $\frac{46.556}{46.22}$ & \begin{tabular}{|l|l|}
48.37 \\
8.65
\end{tabular} & \begin{tabular}{|l|l|}
13.216 \\
1283
\end{tabular} & \begin{tabular}{|l|l|}
33.39 \\
23.921
\end{tabular} & $\frac{1.222}{1.222}$ & $\begin{array}{l}-6.733 \\
\end{array}$ & 60.326 & \begin{tabular}{l|l|}
0.107 \\
\end{tabular} & $\begin{array}{l}14.643 \\
1.623\end{array}$ & $\begin{array}{l}0.004 \\
\end{array}$ & $\begin{array}{ll}332.8 \\
3238\end{array}$ & 8.54639 & 46.9 & 14.8 & 40.0 & $\frac{2.756}{2.772}$ & 0.016 & & 0.000 & 0.405 & 0.34 \\
\hline & $6 / 17 / 2004$ & 3:43:31 PM & 25.886 & 26.66 & \begin{tabular}{|l|}
24.487 \\
2.509 \\
\end{tabular} & $\begin{array}{l}27.832 \\
27.827\end{array}$ & 26.568 & $\begin{array}{l}46.62 \\
6.201\end{array}$ & \begin{tabular}{|l|l|}
48.654 \\
\end{tabular} & \begin{tabular}{|r|}
12.83 \\
\end{tabular} & $\begin{array}{l}33.821 \\
32652\end{array}$ & \begin{tabular}{|l|l|} 
\\
\end{tabular} & $\begin{array}{l}-6.756 \\
6.577 \\
\end{array}$ & 46.922 & $\begin{array}{l}0.108 \\
\end{array}$ & \begin{tabular}{|l|l|}
14.643 \\
\end{tabular} & $\begin{array}{l}0.003 \\
\end{array}$ & & 5.56306 & 47.2 & 11.5 & 40.2 & & \begin{tabular}{|l|l|}
0.016 \\
\end{tabular} & & 0.000 & 0.406 & 0.35 \\
\hline$\frac{354}{354}$ & $6 / 117 / 2004$ & $\begin{array}{l}3: 44: 31 \text { PM } \\
\text { 3:45:31 PM }\end{array}$ & $\begin{array}{l}\frac{25.892}{25.922} \\
2.5\end{array}$ & $\frac{26.671}{26.676}$ & \begin{tabular}{|l|}
24.508 \\
2.4528 \\
\end{tabular} & $\begin{array}{l}27.883 \\
27.953\end{array}$ & $\frac{26.573}{26.593}$ & $\begin{array}{l}46.381 \\
47.008\end{array}$ & \begin{tabular}{|c|}
48.536 \\
49.156 \\
\end{tabular} & \begin{tabular}{|l|}
12.8899 \\
12.653
\end{tabular} & \begin{tabular}{|l|}
33.652 \\
34.445
\end{tabular} & \begin{tabular}{|c|}
$\mid-1.212$ \\
-1.222 \\
\end{tabular} & $\begin{array}{l}-6.5777 \\
-6.669\end{array}$ & $\begin{array}{r}64.03 \\
46.196\end{array}$ & \begin{tabular}{r|}
0.1 \\
0.112
\end{tabular} & \begin{tabular}{|l|}
14.643 \\
14.643
\end{tabular} & $\begin{array}{l}0.004 \\
0.004\end{array}$ & $\begin{array}{l}334.8 \\
355.8\end{array}$ & \begin{tabular}{l|l|l|}
8 & 5.57972 \\
8 & 5.56939
\end{tabular} & $\begin{array}{l}47.1 \\
47.7 \\
\end{array}$ & $\begin{array}{l}15.7 \\
11.3 \\
\end{array}$ & $\begin{array}{l}40.0 \\
40.7\end{array}$ & \begin{tabular}{|l|}
2.759 \\
2.808
\end{tabular} & \begin{tabular}{|c|c|}
0.015 \\
0.017
\end{tabular} & $\begin{array}{l}0.015 \\
0.017\end{array}$ & $\begin{array}{l}0.000 \\
0.000\end{array}$ & $\begin{array}{l}0.378 \\
0.046\end{array}$ & \begin{tabular}{|l|}
0.32 \\
0.35
\end{tabular} \\
\hline 355 & $6 / 17 / 2004$ & 3:46:31 PM & 25.948 & 26.676 & & & & & \begin{tabular}{|l|l|}
48.559 \\
\end{tabular} & & & & $\begin{array}{l}-6.742 \\
\end{array}$ & 51.803 & & & 0.004 & 336.8 & \begin{tabular}{|l|l|}
8 & 5.61306 \\
\end{tabular} & 47.1 & & & & & 0.017 & 0 & $\frac{0.410}{0.410}$ & $\frac{0.35}{0.35}$ \\
\hline & & 3:47:31 PM & 25.939 & 26.672 & & & 26.605 & 46.502 & 48.789 & 12.825 & 33.691 & \begin{tabular}{|l|l|}
-1.219 \\
\end{tabular} & -6.768 & 46.037 & 0.109 & 14.643 & 0.004 & & \begin{tabular}{|l|l|}
8 & 5.62972 \\
\end{tabular} & 47.3 & 11.3 & & & 0.016 & 0.016 & & & $\begin{array}{l}0.35 \\
0.35\end{array}$ \\
\hline 357 & $6 / 17 / 2004$ & 3:48:31 PM & 25.933 & 26.666 & \begin{tabular}{|l|}
24.549 \\
\end{tabular} & 27.954 & 26.624 & 45.907 & $\begin{array}{l}48.942 \\
\end{array}$ & \begin{tabular}{|l|l|}
12.5699 \\
\end{tabular} & \begin{tabular}{|l|l|}
33.343 \\
\end{tabular} & \begin{tabular}{|l|l|}
-1.219 \\
\end{tabular} & -5.776 & $\begin{array}{l}56.734 \\
\end{array}$ & $\begin{array}{ll}0.102 \\
\end{array}$ & \begin{tabular}{|l|l|}
14.643 \\
\end{tabular} & 0.004 & & \begin{tabular}{|l|l|}
5.64639 \\
\end{tabular} & $\begin{array}{l}47.5 \\
\end{array}$ & 13.9 & & & & & & & \\
\hline 358 & $6 / 17 / 2004$ & 3:49:31 PM & 25.951 & 26.66 & 24.547 & 27.937 & 26.613 & 45.878 & 49.141 & \begin{tabular}{|l|l|}
12.58 \\
\end{tabular} & & -1.225 & & & & & & & & 47.7. & & & & & & & & \\
\hline (n) & & & & & & 27.934 & & & & & 33.384 & & & & & 14.643 & & & & 47.6 & & & & & & & & \\
\hline 360 & $6 / 17 / 2004$ & 3:51:31 PM & 25.986 & 26.674 & \begin{tabular}{|l|}
24.586 \\
\end{tabular} & 27.926 & 26.667 & 45.69 & 48.992 & 12.565 & .133 & -1.222 & & & 108 & 14.643 & & & 5.69639 & 47 & & & & & & & 0.413 & \\
\hline$\frac{361}{362}$ & $6 / 17 / 2004$ & 3:52:31 PM & 25.986 & 26.685 & 24.602 & 27.932 & 26.638 & 46.205 & 49.255 & 12.563 & 33.657 & -1.219 & -5.556 & 51.265 & \begin{tabular}{|l|l|l|} 
\\
\end{tabular} & 14.643 & 0.004 & 342.8 & $\begin{array}{l}85.71306 \\
\end{array}$ & 47.8 & 12.6 & 39.9 & 2.753 & 016 & & .000 & & \\
\hline 362 & $6 / 17 / 2004$ & 3:53:31 PM & 25.986 & $\frac{26.675}{2.67}$ & \begin{tabular}{|l|}
24.597 \\
2.50
\end{tabular} & 27.912 & 26.603 & 46.03 & 49.162 & 12.604 & \begin{tabular}{|c|c|}
33.423 \\
2.20
\end{tabular} & $\frac{1.222}{1.220}$ & $\begin{array}{l}-5.54 \\
5.58\end{array}$ & 44.744 & 0.108 & 14.643 & 0.004 & 343.8 & \begin{tabular}{l|l|l}
8 & 5.72972 \\
\end{tabular} & 47.7 & 11.0 & & & .016 & & & & \\
\hline & $6 / 1 / 72004$ & $35.54 .31 \mathrm{PM}$ & $\frac{2.5966}{2.920}$ & $\frac{26.67}{20.61}$ & $\frac{2.492}{2.962}$ & $\frac{27.907}{20.077}$ & & $\frac{45.851}{4.512}$ & 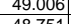 & $\frac{1.2 .371}{12309}$ & 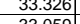 & $\frac{1.222}{1222}$ & & 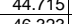 & 0.108 & $\frac{14.663}{103}$ & & 344 & .14039 & 4.7.5 & & & & & & & & \\
\hline & & $\begin{array}{l}3.553 .31 \mathrm{MM} \\
3.56 \mathrm{PM}\end{array}$ & & & 2 & & 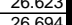 & $\begin{array}{ll}45.5517 \\
45690\end{array}$ & 48.151 & \begin{tabular}{|l|l}
1.2439 \\
1246
\end{tabular} & 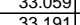 & 1.2224 & & 46.325 & 1024 & 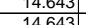 & & & & & & & & & & & 391 & \\
\hline & $6 / 17 / 2004$ & 30 & $\frac{20.000}{26.015}$ & $\frac{20.070}{26.698}$ & $\mid$\begin{tabular}{|}
$\mid 24.144$ \\
24.646
\end{tabular} & $\frac{20.150}{28.23}$ & $\begin{array}{l}20.094 \\
26.726\end{array}$ & $\frac{43.0 .65}{45.678}$ & 48.924 & $\begin{array}{c}12.401 \\
12.49\end{array}$ & $\begin{array}{l}3.191 \\
33.221\end{array}$ & $\begin{array}{l}-\frac{1.2<0}{-1.222} \\
-1\end{array}$ & & 49.437 & $\begin{array}{l}0.105 \\
0.105\end{array}$ & $\begin{array}{l}14.049 \\
14.643\end{array}$ & 0.004 & & 5.79639 & $\begin{array}{l}4.1 .0 \\
47.5\end{array}$ & & & .720 & $\begin{array}{l}0.016 \\
0.016\end{array}$ & & & $\frac{0.41 /}{0.401}$ & 0.35 \\
\hline 36 & 6/177/2004 & 3:58:31 PM & 26.04 & 26.699 & \begin{tabular}{|l|}
24.627 \\
\end{tabular} & 28.215 & 26.672 & 45.722 & $\begin{array}{l}49.066 \\
\end{array}$ & \begin{tabular}{|l|l|}
12.47 \\
\end{tabular} & 33.23 & -1.222 & -5.481 & -25.023 & 0.111 & 14.643 & 0.004 & 348.8 & \begin{tabular}{l|l|l|}
8 & 5.81306 \\
\end{tabular} & 47.6 & -6.1 & 39.5 & 2.722 & 0.017 & 0.017 & 0.000 & 0.424 & 36 \\
\hline & $6 / 17 / 2004$ & 3:59:31 PM & 26.07 & 26.704 & 24.647] & & 26.672 & 45.705 & 49.023 & 12.457 & 33.275 & -1.222 & & 52.107| & 0.108 & 14.643 & & 349.8 & $\begin{array}{l}8 \quad 5.82972 \\
\end{array}$ & 47.6 & & & & 016 & & 0.000 & 0.412 & 0.35 \\
\hline 3665 & 661172004 & 4:00:31 PM & 26.065 & 26.6944 & $24.647 \mid$ & 28.1111 & 26.687 & 45.900 & 49.612 & 12.519 & 33.295 & $\begin{array}{l}-1.222 \\
\end{array}$ & & & 0.105 & 14.643 & 0.004 & & 8.84639 & 48.1 & 12.3 & 39.6 & .730 & 016 & & & & \\
\hline & & $1.31 \mathrm{PM}$ & $\frac{2.091}{2602}$ & & & & & 45.556 & 49.1/16] & & & & & & & & & & & & & & & & & & & \\
\hline 37 & $6 / 1 / 1 / 12004$ & $31 \mathrm{PM}$ & $\frac{26.082}{26.076}$ & 26.206 & $\begin{array}{l}\frac{24.663}{24.667} \\
2\end{array}$ & $\frac{28.058}{28.027}$ & $\frac{26.663}{26.652}$ & $\begin{array}{r}45.49 \\
45.714\end{array}$ & 49.249 & $\frac{12.453}{12.553}$ & $\begin{array}{l}33.043 \\
33.172\end{array}$ & $\frac{1.225}{-1.222}$ & & $\begin{array}{l}46.74 \\
10.11\end{array}$ & 0 & $\begin{array}{l}\frac{14.643}{14643} \\
14\end{array}$ & & 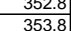 & 5.889639 & $\frac{47.6}{478}$ & L. & 9.4 & 10 & \begin{tabular}{|c|c|c|}
116 \\
16
\end{tabular} & & & $\frac{02}{01}$ & \\
\hline 373 & $6 / 177 / 2000$ & 4:04:31 PM & $\frac{26.091}{26.0}$ & 26.71 & 24.667 & 28.032 & 26.657 & 45.531 & 49.321 & 12.402 & 33.148 & $\frac{-1.222}{-1.22}$ & $\frac{-.450}{-5.51}$ & 54.535 & $\begin{array}{l}0.107 \\
0.10\end{array}$ & 14.643 & 0.003 & $\frac{354.8}{35.8}$ & 5.91306 & 47.8 & $\frac{13.4}{113.4}$ & $\frac{3.4}{39.3}$ & $\frac{2.112}{2.712}$ & 0.016 & $\frac{016}{.016}$ & 0.000 & $\begin{array}{l}0.409 \\
0.409\end{array}$ & 0.35 \\
\hline & & 4:05:31 PM & 26.082 & 26.706 & \begin{tabular}{|l|}
24.673 \\
\end{tabular} & 27.983 & 26.643 & $\begin{array}{l}45.483 \\
\end{array}$ & 49.332 & 12.193 & 33.217 & \begin{tabular}{|l|l|l|} 
\\
\end{tabular} & -5.571 & 53.108 & 0.107 & 14.643 & 0.004 & 355.8 & \begin{tabular}{|l|l|}
8.929272 \\
\end{tabular} & 47.9 & $\frac{13.0}{13.0}$ & 39.4 & & & .016 & 0.000 & $\frac{0.409}{0.409}$ & $\begin{array}{l}0.35 \\
0.35 \\
\end{array}$ \\
\hline 37 & $6 / 177 / 2004$ & 4:06:31 PM & 26.084 & 26.722 & 24.685 & 28.044 & & 45.546 & 49.315 & 12.224 & 33.302 & \begin{tabular}{l|l}
-1.222 \\
\end{tabular} & & 51.749 & 0.105 & 14.643 & 0.004 & 35 & 5.94639 & 47.8 & $12.7 \mathrm{C}$ & 39.4 & 2.718 & $\mid \overline{016}$ & & & 0.401 & \\
\hline & $6 / 17 / 2004$ & 4:07:31 PM & 26..093 & 26.701 & 24.666 & 28.108 & 26.664 & 45.369 & 49.118 & & 33.187] & -1.222 & & 49.575 & $\mid 0.108$ & 14.643 & & 357. & 5.96306 & 47.6 & 12.1 & 39.3 & 2.708 & 0 & & & & \\
\hline & $6 / 17 / 2004$ & 4:08:31 PM & & & & & & 45.641 & 49.465 & & 33.492 & & & & 0.106 & 14.643 & & & 5.97972 & & 11.4 & & & & & & 403 & \\
\hline & $6 / 17 / 2004$ & 4:09:31 PM & 26.129 & 26.742 & 24.71 & 28.30 & & & 49.693 & 12.274 & 33.519 & -1.219 & & 47.483 & & 14.643 & & & 5.99639 & & & 39. & & & & & & \\
\hline & $6 / 17 / 2004$ & 4:10:31 PM & 26.131 & 26.73 & 24.698 & 28.271 & 26.8 & 45.527 & 49.56 & 12.313 & 33.102 & -1.225 & & 43.728 & 0.109 & 14.643 & & 360.8 & $\begin{array}{l}86.01306 \\
\end{array}$ & 48.1 & 10.7 & 39.3 & 2.711 & 0.016 & .016 & 0.000 & 0.417 & \\
\hline & 6/17//2004 & 4:11:31 PM & 26.133 & $\frac{26.736}{20720}$ & 24.714 & 28.313 & 26.814 & & 49.301 & 12.207 & 33.068 & 1.222 & $\begin{array}{l}-5.31 \\
.50\end{array}$ & 54.32 & & 14.643 & & & 6.02972 & 47.8 & 13.3 & 392 & .700 & 16 & 016 & & 0.418 & \\
\hline & 72004 & & 26.135 & 26.739 & 24.707 & 28.29 & & & 49.154 & & (n) & & & ent & & & & & & & & & 694 & & & & & \\
\hline 382 & $6 / 177 / 2004$ & 4:13:31 PM & $\frac{26.143}{20.14}$ & $\frac{26.756}{62.751}$ & 24.724 & 28.288 & & $\begin{array}{l}45.909 \\
4.9097\end{array}$ & 49.877| & $\frac{12.431}{12}$ & 33.482 & 1.222 & & 50.681 & 0.107 & $\begin{array}{l}14.643 \\
1\end{array}$ & & 3663 & 6.06306 & 48.4 & 12.4 & 39.7 & .737 & & & & 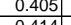 & \\
\hline 384 & 6 & $4.45 \cdot 31 \mathrm{PM}$ & $\frac{2.0 .45}{2616}$ & 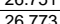 & 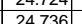 & $\frac{2.54}{2827}$ & $\frac{2.06}{26.8}$ & 年 45309 & 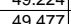 & 告. & | & -1.211 & & 年. & 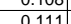 & $\mid$ & & & 609639 & 48 & 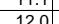 & 392 & 701 & & & & .444 & \\
\hline 385 & $6 / 17 / 2004$ & $4 \cdot 46 \cdot 31 \mathrm{PM}$ & $\frac{20.15}{26.155}$ & 20779 & 24722 & 28.256 & 26.83 & 45.544 & 4973 & 1236 & 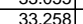 & - & & 5 & & 1464 & & 3668 & $\begin{array}{l}80.011306 \\
\end{array}$ & 483 & 125 & 39.4 & & & & & 0 & \\
\hline$\frac{386}{386}$ & $6 / 171 / 2004$ & 4:17:31 PM & $\frac{2.155}{2.655}$ & $\frac{2.779}{26.779}$ & $\frac{\mid c 4.737}{24.737}$ & 28.206 & $\frac{26.8}{26.8}$ & 45.303 & 49.518 & $\frac{12.231}{12.31}$ & 年3.1.104 & $\frac{1.222}{1.222}$ & & $\frac{19.442}{44.942}$ & 0.105 & 14.643 & & & 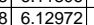 & $\frac{48}{48}$ & $\frac{\frac{12.1}{12.1}}{1.0}$ & $\frac{39.2}{39.2}$ & . & . & $\frac{016}{016}$ & 0.000 & 0 & \\
\hline & $6 / 177 / 2004$ & & & 26.796 & 24.744 & & & & 49.574 & & 32.969 & & & 4918 & & & & & & & & & & & & & & \\
\hline$\overline{38 \varepsilon}$ & $6 / 17 / 2004$ & $1 \mathrm{PM}$ & 26.179 & 26.797 & 24.74 & 28.184 & & & 49.692 & 12.21 & 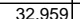 & 1098 & & 71 & & 14.643 & & & 6.16 & & 12.4 & $\overline{39.1}$ & 6995 & & & & 5.403 & \\
\hline & 6/17/2004 & & 26.175| & 26.803 & 24.746 & 28.175 & & $44.9 !$ & 49.147 & 12.19 & & 1.159 & & 8.9 & & 14.643 & & & & & 12.6 & & & & & & & \\
\hline & $6 / 17 / 2004$ & 1:31 PM & 26.176 & 26.799 & \begin{tabular}{|l|l|}
24.747 \\
\end{tabular} & 28.166 & 26.81 & 44.76 & 48.884 & 12.186 & 32.616 & -1.159 & & 546 & & 14.643 & & & & 47.4 & 13.6 & 38.7 & 2.667 & & & & & \\
\hline 391. & $6 / 17 / 2004$ & 1 PM & 26.181 & 26.804 & \begin{tabular}{|l|}
24.747 \\
\end{tabular} & 28.186 & & 45. & 49.535 & 12.084 & 33.078 & -0.936 & & 49.243 & & 14.643 & & & & 48 & 12.1 & 39.1 & 2.696 & & & & & \\
\hline & $6 / 17 / 2004$ & 4:23:3 & 26.181 & 26.805 & \begin{tabular}{|l|}
24.748 \\
\end{tabular} & 28.246 & 26.8 & & 49.792 & 12.314 & 33.022 & $\begin{array}{c}-1.08 \\
\end{array}$ & & 46.06 & 0.1 & 14.643 & & & & & 11.3 & 39. & & & & & 417 & \\
\hline 393 & $6 / 17 / 72004$ & 4:24: & & & 24.746 & & & & 49.388 & 12.246 & 32.645 & -0.884 & & & 0.14 & 14.643 & & & & 47. & 12.1 & 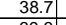 & 年 & 116 [1] & & & & \\
\hline & & 4:25:3 & 2 & 26.802 & 24.355 & & & & & & 33.084 & & & & & & & & & & & 39.3 & & & & & 0.413 & \\
\hline 染 & $6 / 17 / 2004$ & $5: 18: 0$ & $26.017 \mid$ & 26.7111 & 24.708 & 27.758 & 26.5 & 47.31 & 50.358 & 11.99 & 35.497] & 1.228 & & 46.18 & 0.118 & 14.643 & & & & 48. & 11.3 & & 2.855 & .018 & 0.018 & & & \\
\hline | & $6 / 17 / 2004$ & $5: 20: 07 \mathrm{PM}$ & $\frac{20.111}{25.549}$ & $\frac{26.12}{26.692}$ & \begin{tabular}{|l|}
24.705 \\
\end{tabular} & 27.755 & $\frac{20.513}{26.466}$ & 295.010 & \begin{tabular}{|l|}
50.198 \\
50.252
\end{tabular} & \begin{tabular}{rl|}
11.948 \\
11.92
\end{tabular} & 18.076 & \begin{tabular}{|l|}
14.99 \\
\end{tabular} & $\frac{19.244}{20.123}$ & 49.26 & $\begin{array}{l}0.003 \\
0.003 \\
\end{array}$ & $\frac{14.643}{14.643}$ & 0.0 & $\frac{429.4}{430.4}$ & \begin{tabular}{|l|l|}
47.17306 \\
\end{tabular} & $\begin{array}{l}48.7 \\
48.8 \\
\end{array}$ & $\begin{array}{l}11.0 \\
12.1 \\
\end{array}$ & $\frac{39.7}{24.0}$ & 1.653 & \begin{tabular}{|l|l|}
0.000 \\
0.000
\end{tabular} & $\begin{array}{l}0.000 \\
0.000 \\
\end{array}$ & 0.000 & $\begin{array}{l}0.011 \\
0.019\end{array}$ & \\
\hline 398 & $6 / 17 / 2004$ & & 25.696 & 26.704 & \begin{tabular}{|l|}
24.712 \\
\end{tabular} & 27.747 & & & 50.383 & 11.845 & \begin{tabular}{|c|}
-1.763 \\
\end{tabular} & 35.282 & & & & & & & & & 9.5 & $\begin{array}{lll}4.1 \\
\end{array}$ & 0.280 & & & & & \\
\hline & $6 / 17 / 2004$ & & 26.149 & 26.6 & 24.6 & & & 5.6 & 50.175 & & & \begin{tabular}{|l|}
39.4 \\
\end{tabular} & & & & 14.6. & & 43 & $\begin{array}{l}4.7 .20 \\
4\end{array}$ & & $11.0 \mathrm{C}$ & 1.8 & & & & & & \\
\hline 40 & $6 / 177 / 2004$ & 5:23:0 & 25.57 & & 24.741 & 27.74 & & & 50.0 & & & 0.576 & & & & & & 43. & & & 11.8 & 38.0 & & & & & 0.874 & \\
\hline 40 & $6 / 17 / 2004$ & & 25.56 & 26.673 & 24.726 & 27.761 & & 44.06 & 50.016 & & & 0.474 & & & & & & & & & 11.9 & 37.6 & & & & & & \\
\hline & $6 / 1 / 122$ & & 25.506] & 26.6 & \begin{tabular}{|l|}
24.707 \\
\end{tabular} & 27.757 & & & 50.3 & & & 0.54 & & & & & & & & & 11.1. & 政 & & & & & & \\
\hline 40 & $6 / 1772$ & & & & & 27.7 & & & & 13.75 & & & & & & & & & & & & & & & & & & \\
\hline & $\frac{101717004}{6117004}$ & & $\frac{25.12}{2.71}$ & & 24.127 & 2.84 & & & & 1.5217 & & -1.22 & & & & & & & & & $\frac{11.8}{11.8}$ & - & 2.100 & & & & & \\
\hline 然 & $6 / 1717200^{4}$ & & $\frac{2.119}{2571}$ & & 年, & 2.04 & & & (4.9.5 & 14.040) & & & & & & & & & & & 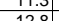 & & & & & & & \\
\hline$\frac{40}{40}$ & 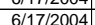 & 5:30.07 & $\frac{2.0154}{25.75}$ & & 24831 & $\frac{17.05}{27.555}$ & 26.6 & & 4 & 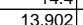 & $\frac{32.6}{32}$ & $\frac{-1.4}{-1.2}$ & & & & & & & & & $\frac{1}{13.0}$ & 398 & $\frac{2.657}{2743}$ & & & & 年 & \\
\hline 40 & $6 / 177 / 2002$ & $\frac{2: 31: 0}{513}$ & $\frac{\mid}{25.816}$ & 26.674 & $\frac{\mid 24.837}{24}$ & 27.971 & & & 49.514 & $\frac{13.751}{11.751}$ & 33.16 & & & & & $\frac{14.643}{14}$ & & & & & & & & & & & & \\
\hline & $6 / 1721200$ & & 2586 & & 24846 & 27945 & & & 49693 & & & -122 & & & & & & & & 48 & 113 & & & & & & & \\
\hline 41 & & & & & & & & & & & & & & & & & & & & & & & & & & & & \\
\hline$\overline{411}$ & $6 / 17 / 2004$ & & \begin{tabular}{|l|l|}
25.93 \\
\end{tabular} & 26.668 & 24.851 & 27.826 & & 47.4 & 49.7 & 13.245 & 34.43 & -1.2 & & & & 14.64 & & 444. & & & 11.7 & & & & & & & \\
\hline & & & 25.9 & 26.6 & 24.82 & 27.7 & & & & & & $-1.2 \quad$ & & & & & & 445 & & & & & & & & & & \\
\hline 41 & $6 / 17 / 2004$ & 5:36:C & 25.951 & 26.674 & $24.842 \mid$ & 27.7 & 26.8 & 47. & $49.8 \mathrm{r}$ & 13. & 34.0 & & & & & 14.64 & & 446.4 & $\begin{array}{l}4.7 .43 \\
4\end{array}$ & 48 & 13.9 & 40. & 2.809 & & & & 0.614 & \\
\hline 41. & $6 / 17 / 200$ & 5:37:C & 25.835 & 26.668 & 24.851 & 27.666 & 26.4 & 47.638 & 49.835 & 13.36 & 34.399 & $\begin{array}{l}-1.219 \\
\end{array}$ & & & 0.1 & $14.6 \mathrm{C}$ & & 447. & & 48 & 11.6 & 41 & & & & & 0.558 & \\
\hline & $6 / 177 / 2$ & & 25.758 & & 24.859 & 27. & & & 49.5 & & & & & & 0.08 & & & 448.4 & & & 10.9 & 39.6 & & & & & & \\
\hline 416 & $6 / 17 / 2004$ & & 25.1 & 26.644 & 24.842 & 27.582 & 26.3 & 48.1888 & 49.864 & 13.427 & 34.925 & 1.222 & & 50.309 & 0.1 & 14.643 & & 4449.4 & $\begin{array}{l}4 \mid 7.48 \\
\end{array}$ & 48.4 & 12.3 & 41.6 & 2.865 & 0.015 & 0.015 & 0.000 & 0.360 & \\
\hline$\frac{418}{418}$ & $\frac{6 / 1 / 12004}{6 / 172004}$ & $\begin{array}{l}5.40 .07 \mathrm{PM} \\
5.41 .07 \mathrm{PM}\end{array}$ & $\frac{25.1 .89}{25.818}$ & $\begin{array}{l}26.627 \\
26.632\end{array}$ & \begin{tabular}{|l|}
24.835 \\
24.834
\end{tabular} & 27.595 & $\frac{26.331}{26.385}$ & $\frac{48.455}{48.128}$ & $\frac{49.817}{49.773}$ & 13.594 & $\begin{array}{r}35.058 \\
34.804\end{array}$ & $\mid$\begin{tabular}{|c|}
$\mid-1.225$ \\
-1.222 \\
\end{tabular} & -5.1 & $\begin{array}{l}42.8247 \\
52.547\end{array}$ & $\begin{array}{l}0.197 \\
0.177 \\
\end{array}$ & $\frac{14.643}{14643}$ & $\frac{0.00}{0.00}$ & $\frac{450.4}{451.4}$ & \begin{tabular}{|l|l|}
4.50039 \\
4 & 7.52306
\end{tabular} & \begin{tabular}{|c|c|}
488.3 \\
48.3 \\
\end{tabular} & $\begin{array}{l}0.5 .5 \\
12.9\end{array}$ & $\frac{41.8}{41.5}$ & $\frac{2.879}{2.859}$ & $\begin{array}{l}0.029 \\
0.026\end{array}$ & $\begin{array}{l}0.030 \\
0.027\end{array}$ & $\begin{array}{l}0.001 \\
0.001\end{array}$ & \begin{tabular}{|c|}
0.079 \\
0.639 \\
\end{tabular} & \\
\hline 410 & $6 / 1772004$ & 5:42:07 PM & 25.828 & 26.617 & 24.859 & 27614 & D. & 49.011 & 49.86 & 13.239 & 250,07 & & & 4.270 & & 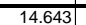 & 0.0 & & & 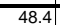 & & & 2098 & 0025 & & & \begin{tabular}{l|l}
0.585 \\
\end{tabular} & 0.5 \\
\hline 0 & $6 / 17 / 2004$ & & $\mid$ & & & 27.624 & & 49.017 & 49.912 & & 36.047 & 1.222 & & 992 & 0.17 & 14.643 & .004 & & 47.55639 & 48.4| & $\begin{array}{l}11.8 \\
\end{array}$ & & 2.932 & 0.025 & 0.025 & 0.001 & \begin{tabular}{|l|l|l|l|}
0.599 \\
\end{tabular} & \\
\hline
\end{tabular}


WSRC-TR-2005-00105, REVISION 0

SRNL-RPP-2005-00012, REVISION 0

RUN \# WASH1 AND WASH 2; CAMPAIGN III SLURRY WASHING - CONT.

\begin{tabular}{|c|c|c|c|c|c|c|c|c|c|c|c|c|c|c|c|c|c|c|c|c|c|c|c|c|c|c|c|c|}
\hline & A & & D & $E$ & $F$ & G & $\mathrm{H}$ & & $\begin{array}{ll}\mathrm{K} \\
\end{array}$ & $\begin{array}{ll}L \\
\end{array}$ & $\mathrm{M}$ & $\mathrm{N}$ & 0 & \begin{tabular}{l|l} 
\\
\end{tabular} & $R$ & \begin{tabular}{l|l} 
s \\
\end{tabular} & & $\mathrm{V}$ & w & $x$ & & & & & & & & AF $\| A$ \\
\hline 42 & $6 / 17 / 2004$ & 5:44:07 PM & 25.848 & 26.6 & 24.839 & 27.679 & 26.459 & 48.42 & 49.562 & 13.122 & 35.462 & -1.222 & -6.204 & 46.651 & 0.145 & 14.643 & 0.004 & 454. & 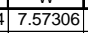 & 48.1 & 11.4 & 41.9 & 27 & ( & $\frac{\pi}{10}$ & 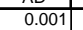 & $A E$ & 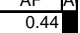 \\
\hline & 6/177/2004 & 5:45:07 PM & 25.86 & 26.613 & \begin{tabular}{|l|}
24.866 \\
\end{tabular} & 27.721 & 26.531 & 47.192 & 48.252 & 14.217 & 33.164 & 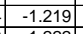 & -6.328 & 52.825 & 0.172 & \begin{tabular}{|l|l|}
14.643 \\
\end{tabular} & 0.004 & 455.4 & \begin{tabular}{|l|l|}
4 & 7.58972 \\
\end{tabular} & 46.8 & 12.9 & 40.2 & & & & & & \\
\hline (2) & $6 / 17 / 2004$ & $5: 46: 07 \mathrm{PM}$ & 25.889 & 26.602 & \begin{tabular}{|r|}
24.85 \\
24.85 \\
\end{tabular} & $\begin{array}{r}27.73 \\
\end{array}$ & 26.53 & 47.402 & \begin{tabular}{|l|l|}
48.698 \\
\end{tabular} & 14.185 & 33.289 & \begin{tabular}{|l|} 
\\
\end{tabular} & -6.47 & & 0.165 & \begin{tabular}{|l|l|}
14.643 \\
1.62
\end{tabular} & 0.004 & 456.4 & $\begin{array}{ll}47.60639 \\
\end{array}$ & 47.2 & 14.1 & 40.3 & 2.782 & 0.020 & & 0.001 & 0.612 & \\
\hline 424 & $6 / 17 / 2004$ & 5:47:07 PM & $\begin{array}{l}25.88 \\
25.8\end{array}$ & 26.588 & \begin{tabular}{|l|}
24.856 \\
\end{tabular} & 27.771 & 26.566 & $\begin{array}{l}47.393 \\
\end{array}$ & \begin{tabular}{|l|l|}
48.515 \\
\end{tabular} & 13.979 & 33.585 & -1.222 & -6.424 & \begin{tabular}{|l|l|}
53.653 \\
\end{tabular} & $\begin{array}{l}0.158 \\
\end{array}$ & $\begin{array}{l}14.643 \\
\end{array}$ & 0.004 & 457.4 & 62306 & 47.0 & 13.1 & 40.5 & \begin{tabular}{|l|}
2.792 \\
\end{tabular} & \begin{tabular}{l|l|}
0.024 \\
\end{tabular} & 0.024 & 0.001 & 0.584 & \\
\hline$\frac{425}{426}$ & $\frac{6 / 1 / 17 / 2004}{661 / 12004}$ & $\begin{array}{l}5: 48: 07 \text { Pि } \\
5\end{array}$ & $\frac{25.881}{25.962}$ & $\frac{26.579}{26.605}$ & \begin{tabular}{|l|}
24.842 \\
24.868 \\
\end{tabular} & $\begin{array}{l}27.772 \\
27.797\end{array}$ & $\frac{26.612}{26.608}$ & $\begin{array}{l}47.479 \\
47.972\end{array}$ & \begin{tabular}{|c|}
48.7771 \\
48716 \\
\end{tabular} & \begin{tabular}{|r|}
13.985 \\
13.81
\end{tabular} & $\begin{array}{l}33.622 \\
34.375 \\
\end{array}$ & $\begin{array}{l}-1.222 \\
-1.222\end{array}$ & $\begin{array}{l}-5.869 \\
-6.652 \\
\end{array}$ & \begin{tabular}{|l|l|}
49.039 \\
50.386
\end{tabular} & \begin{tabular}{|c|c|}
0.087 \\
0.199
\end{tabular} & \begin{tabular}{|l|l|l|l|l|l|}
14.643 \\
14.643
\end{tabular} & $\begin{array}{l}0.004 \\
0.004\end{array}$ & $\begin{array}{c}458.4 \\
459.4 \\
\end{array}$ & $\begin{array}{l}47.63972 \\
47 \\
4765639\end{array}$ & $\begin{array}{l}47.3 \\
47.2\end{array}$ & $\begin{array}{l}12.0 \\
12.3 \\
\end{array}$ & \begin{tabular}{l|l|l|l|l|}
40.6 & \\
41.2
\end{tabular} & \begin{tabular}{|l|}
$\mid 2.796$ \\
2.839 \\
\end{tabular} & $\begin{array}{l}0.013 \\
0.025\end{array}$ & $\begin{array}{l}0.013 \\
0.025 \\
\end{array}$ & $\begin{array}{l}0.000 \\
0.001\end{array}$ & $\begin{array}{l}0.321 \\
0614\end{array}$ & 0.27 \\
\hline 427 & $\frac{6 / 1 / 1 / 2004}{6 / 17 / 2004}$ & 5:49:07 PM & $\frac{25.962}{25.987}$ & $\frac{20.005}{26.586}$ & \begin{tabular}{|l|}
24.888 \\
24.869 \\
\end{tabular} & $\frac{27.191}{27.823}$ & $\frac{20.008}{26.648}$ & $\begin{array}{l}47.972 \\
47.147 \\
\end{array}$ & \begin{tabular}{|l|}
48.71629 \\
47.829 \\
\end{tabular} & $\begin{array}{r}\frac{13.81}{14.372} \\
\end{array}$ & $\frac{34.375}{32.969}$ & \begin{tabular}{|l|}
-1.222 \\
-1.225 \\
\end{tabular} & $\begin{array}{l}-6.652 \\
-6.606 \\
\end{array}$ & $\begin{array}{l}50.380 \\
51.142\end{array}$ & $\begin{array}{l}0.169 \\
0.165\end{array}$ & $\frac{14.643}{14.643}$ & $\frac{0.004}{0.003}$ & - 459.4 & $\begin{array}{l}47.67639 \\
47.67306\end{array}$ & $\begin{array}{l}47.2 \\
46.4 \\
\end{array}$ & $\frac{12.3}{12.5}$ & $\frac{41.24}{40.1}$ & \begin{tabular}{|l|}
2.839 \\
2.762 \\
\end{tabular} & $\begin{array}{l}0.025 \\
0.025\end{array}$ & $\frac{0.025}{0.025}$ & $\frac{0.001}{0.001}$ & $\mid \frac{0.614}{0.616}$ & \\
\hline 428 & & 5:51:07 PM & 26.017 & 26.611 & \begin{tabular}{|l|}
24.914 \\
\end{tabular} & 27.878 & 26.693 & 47.298 & \begin{tabular}{|l|}
47.976 \\
\end{tabular} & 14.063 & 33.396 & \begin{tabular}{|l|} 
\\
\end{tabular} & -6.609 & 51.778 & 0.176 & 14.643 & 0.003 & 461.4 & & 46.5 & 12.7 & 40.3 & & 0.026 & & & & 0.52 \\
\hline & $6 / 177 / 2004$ & 5:52:07 PM & $\frac{26.048}{26.048}$ & 26.617 & \begin{tabular}{|l|}
24.925 \\
\end{tabular} & 27.929 & 26.719 & 47.111 & 48.125 & \begin{tabular}{|l|l|}
13.753 \\
\end{tabular} & 33.494 & \begin{tabular}{|c|}
-1.2222 \\
\end{tabular} & $\begin{array}{r}-6.453 \\
\end{array}$ & 47.977 & 0.169 & \begin{tabular}{|c|}
14.643 \\
\end{tabular} & 0.004 & 462.4 & \begin{tabular}{|l|l}
47.70639 \\
\end{tabular} & 46.7 & 11.8 & 40.3 & \begin{tabular}{|l|l|}
2.779 \\
\end{tabular} & & & 0.001 & & \\
\hline 131 & $6 / 17 / 2004$ & 5:53:07 PM & \begin{tabular}{|l|l|}
26.08 \\
2609
\end{tabular} & & $\begin{array}{l}24.926 \\
2.015\end{array}$ & \begin{tabular}{r|r|}
27.98 \\
\end{tabular} & 26.746 & 47.651 & 48.416 & 13.927 & & \begin{tabular}{|l|}
$\mid$ \\
\end{tabular} & -6.672 & \begin{tabular}{|l|l|}
51.008 \\
\end{tabular} & $\begin{array}{l}0.168 \\
\end{array}$ & \begin{tabular}{|l|l|}
14.643 \\
\end{tabular} & 0.004 & 463.4 & \begin{tabular}{l|l|}
47.72306 \\
\end{tabular} & 46.9 & 12.5 & 40.8 & \begin{tabular}{|l|}
2.813 \\
\end{tabular} & & & & 0.615 & \\
\hline 431 & $6 / 17 / 2004$ & 5:54:07 PM & 26.084 & 26.617 & 24.915 & 27.979 & 26.765 & $\begin{array}{l}47.085 \\
7.29 \\
\end{array}$ & \begin{tabular}{|l|l}
48.08 \\
\end{tabular} & 13.857 & \begin{tabular}{|l|l}
33.34 \\
\end{tabular} & \begin{tabular}{|c|}
$\mid$ \\
\end{tabular} & -6.693 & 53.553 & $\begin{array}{l}0.171 \\
\end{array}$ & \begin{tabular}{|l|l|}
14.643 \\
\end{tabular} & 0.003 & $\begin{array}{l}464.4 \\
\end{array}$ & $\begin{array}{lll}47.73972 \\
\end{array}$ & 46.6 & 13.1 & 40.2 & \begin{tabular}{|l|l|}
2.773 \\
\end{tabular} & 0.025 & 026 & 0.001 & 0.636 & \\
\hline & $6 / 17 / 2004$ & 5:55:07 PM & \begin{tabular}{|l|}
26.059 \\
26115
\end{tabular} & 26.637 & 24.925 & $\begin{array}{l}27.975 \\
27.091\end{array}$ & $\begin{array}{l}26.765 \\
26717\end{array}$ & $\begin{array}{l}47.311 \\
47.389\end{array}$ & 48.438 & 13.876 & $\begin{array}{l}33.562 \\
33693 \\
3\end{array}$ & $\begin{aligned}-1.222 \\
1222\end{aligned}$ & & 48.891| & $\begin{array}{l}0.166 \\
\end{array}$ & \begin{tabular}{|l|l|}
14.643 \\
1.62
\end{tabular} & 0.003 & 465.4 & & 47.0 & 12.0 & 40.4 & & & & 0.001 & 0.613 & \\
\hline 4344 & $6 / 117 / 2004$ & $\begin{array}{l}5: 56: 07 \mathrm{PM} \\
5: 57: 07 \mathrm{PM}\end{array}$ & $\frac{26.115}{26.126}$ & $\frac{26.664}{26.655}$ & \begin{tabular}{|l|l|}
24.947 \\
2.438
\end{tabular} & $\begin{array}{l}27.991 \\
27.932\end{array}$ & 26.717 & $\begin{array}{l}47.389 \\
46.973 \\
\end{array}$ & $\begin{array}{l}48.582 \\
47.954\end{array}$ & $\begin{array}{l}13.845 \\
13.774\end{array}$ & $\begin{array}{l}33.693 \\
33.386\end{array}$ & $\begin{array}{l}-1.222 \\
-1.2219 \\
\end{array}$ & $\begin{aligned}-6.641 \\
-6.51\end{aligned}$ & 50.693 & $\frac{0.165}{0.15}$ & $\begin{array}{l}\frac{14.643}{14.643} \\
\end{array}$ & $\frac{0.004}{0.004}$ & $\frac{46}{46}$ & 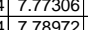 & $\begin{array}{l}47.1 \\
46.5 \\
\end{array}$ & $\begin{array}{l}12.4 \\
12.3 \\
\end{array}$ & $\begin{array}{l}40.5 \\
40.2\end{array}$ & 2.7770 & $\begin{array}{l}0.025 \\
0.022 \\
\end{array}$ & $\frac{0.025}{0.022}$ & 0.001 & \begin{tabular}{|c|c|}
0.608 \\
0.558
\end{tabular} & $\frac{52}{47}$ \\
\hline 435 & $6 / 17 / 2004$ & 5:58:07 PM & 26.096 & 26.665 & \begin{tabular}{|l|}
24.923 \\
\end{tabular} & 27.882 & 26.593 & 45.757 & 47.435 & 13.652 & 32.277 & $|-1.225|$ & -5.129 & 44.056 & \begin{tabular}{|c|}
0.15 \\
0.127
\end{tabular} & $\begin{array}{l}14.045 \\
14.643\end{array}$ & $\begin{array}{l}0.004 \\
0.004\end{array}$ & $\begin{array}{l}460.4 .4 \\
468.4\end{array}$ & 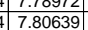 & $\begin{array}{l}46.0 \\
46.0\end{array}$ & $\frac{1.3}{10.8}$ & $\begin{array}{l}40.2 \\
39.0\end{array}$ & \begin{tabular}{|l|l|}
2.690 \\
2.690
\end{tabular} & $\begin{array}{l}\mid 0.022 \\
0.019 \\
\end{array}$ & $\frac{0.022}{0.019}$ & & & 0.041 \\
\hline 436 & $6 / 177 / 2004$ & 5:59:07 PM & 26.106 & 26.664 & 24.952 & 27.851 & 26.537 & 46.749 & 47.448 & 13.525 & 33.345 & -1.222 & -6.482 & 37.365 & 0.131 & \begin{tabular}{|c|c|}
14.643 \\
\end{tabular} & 0.004 & 469.4 & 47.82306 & 46.0 & 9.2 & 40.0 & 2.761 & & & & 0.488 & \\
\hline 437 & 6/177/2004 & 6:00:07 PM & 26.139 & 26.657 & \begin{tabular}{|l|}
24.92 \\
\end{tabular} & & 26.456 & 47.23 & 47.815 & 13.494 & 33.929 & \begin{tabular}{|l|} 
\\
\end{tabular} & -6.716 & 51.496 & & 14.643 & 0.004 & & & 46.3 & 12.6 & 40.6 & $2.798 \mid$ & \begin{tabular}{|c|}
0.026 \\
\end{tabular} & & & & \\
\hline 438 & $6 / 17 / 2004$ & 6:00:39 PM & 26.185 & & & 27.762 & & & & & & & & & \begin{tabular}{|l|l|l|}
0.158 \\
\end{tabular} & & & & & & 13.3 & & & & & & & \\
\hline$\frac{43}{414}$ & $6 / 17 / 2004$ & 6:01:39 PM & 26.18 & 26.643 & 24.881 & 27.686 & 26.421 & 47.08 & 48.115 & 13.552 & $\begin{array}{l}33.613 \\
3\end{array}$ & 1.224 & & 51.945 & 0.173 & 14.643 & & & & 46.6 & 12.7 & $0.4-4$ & & & & & & \\
\hline & & 6:02:39 PM & & & 24.88 & & 26.406 & & & $\mid 13.4$ & 33.776 & & & & & & & & & & & & & & & & & \\
\hline & 6/17/2004 & 6:03:39 PM & 26.114 & 26.647] & 24.875 & 27.66: & 26.40 & 46.6011 & 47.813 & 13.43 & 33.236 & -1.222 & & 49.921 & 0.161 & 14.643 & 0.004 & & 7.89861 & 46 & 12.2 & 39.9 & 2.752 & 0.024 & & & & \\
\hline & 6/17/2004 & 6:04:39 PM & 26.069 & 26.632 & 24.855 & 27.745 & 26.441 & 47.036 & 48.072 & 13.523 & 33.611 & -1.219 & & 47.281 & 0.162 & 14.643 & 0.004 & 47. & 7.9 & 46.6 & 11.6 & 40.3 & 78 & 024 & & & 6001 & \\
\hline & $6 / 17 / 2004$ & 6:05:39 PM & 26.085 & 26.628 & $24.841 \mid$ & 27.836 & 26.50. & 46.699 & 47.725 & 13.412 & 33.4377 & 1.222 & & 55.466 & 0.169 & 14.643 & 0.004 & & 7.93194 & 46.3 & 13.6 & 40.1 & 76 & & & & & \\
\hline & $\frac{61172004}{6117004}$ & $\begin{array}{l}6: 06: 39 \mathrm{PM} \\
6.07739 \mathrm{PM}\end{array}$ & $\frac{2.145}{26906}$ & $\frac{2.068}{2669}$ & | & $\frac{27.88}{27.93}$ & $\frac{26.58}{26.97}$ & $\frac{46.89}{46.51}$ & $\frac{47.922}{4762}$ & $\frac{13.483}{1336}$ & 3246 & $\begin{array}{c}-1.219 \\
1222\end{array}$ & & (972) & .164 & $\frac{14.643}{14643}$ & & & & & 32 & & & & & & & \\
\hline & 6 & 6:08:39 PM & $\frac{16.061}{26.61}$ & $\frac{2.054}{26625}$ & 势, & $\frac{2.30}{2700}$ & & 40 & 41.026 & 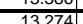 & 30.240 & & & & 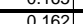 & $\frac{1.4045}{14643}$ & & & & & & & & & & & & \\
\hline & & 6.00.39: & & & & $\frac{21.35}{2802}$ & & 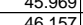 & $\mid \frac{4.29}{47.45}$ & 1 & 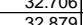 & $\frac{1.252}{1210}$ & & 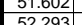 & 0.16 & 14.643 & & & & $\begin{array}{ll}45 \\
46\end{array}$ & 12 & & & & & & 617 & \\
\hline 448 & $6 / 17 / 2004$ & 6:10:39 PM & & 26.649 & \begin{tabular}{|l|}
24.842 \\
\end{tabular} & 28.03 & 26.7 & 45.77 & 4. 4.4587 & $\frac{10.34}{13.245}$ & $\begin{array}{l}32.079 \\
32.635\end{array}$ & $\frac{1.222}{1.222}$ & & $\frac{52.29}{52.33}$ & $\frac{1.16}{0.16}$ & 14.643 & & & & & $\begin{array}{ll}12.0 \\
12.8\end{array}$ & & & & & & & \\
\hline & 6/177/2004 & 6:11:39 PM & 26.092 & 26.646 & \begin{tabular}{|l|}
24.828 \\
\end{tabular} & 28.088 & 26.793 & 46.037 & 47.421 & 13.192 & 32.918 & -1.222 & -6.652 & 42.953 & 0.165 & 14.643 & 0.004 & 481.9 & \begin{tabular}{|l|l|}
8.03194 \\
\end{tabular} & 45.9 & 10.5 & 39.5 & 2.722 & 0.025 & 0.025 & 0.001 & 0.626 & \\
\hline & & 6:12:39 PM & 26.104 & 26.672 & 24.84 & 28.165 & 26.834 & 46.224 & 47.682 & 13.313 & 33.008 & -1.216 & & & \begin{tabular}{|c|c|}
0.165 \\
\end{tabular} & \begin{tabular}{|l|l|l|}
14.643 \\
\end{tabular} & & & 8.04861 & 46.2 & 13.4 & 39.6 & 2731 & & & & 0.624 & \\
\hline & $6 / 17 / 2004$ & 6:13:39 F & 26.082 & $26.681 \mid$ & $\begin{array}{l}24.823 \\
201915 \\
-19\end{array}$ & 28.19 & 26.8 & 45.9 & 47.415 & 13.206 & $\begin{array}{ll}32.784 \\
20727\end{array}$ & 20 & & 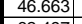 & & 14.643 & & & & 45 & 11.4. & ( & & & & & 17 & \\
\hline & $\frac{6117172004}{6}$ & $\frac{6.14 .39 \mathrm{PM}}{6.15 .29 \mathrm{M}}$ & & $\frac{2.6087}{2675}$ & $\frac{24.85}{2.8312}$ & $\frac{28.21}{2.21}$ & $\frac{26.88}{2.85}$ & 45.94 & 47.4 & & $\frac{32.137}{301}$ & & & & & $\frac{14.64}{1.64}$ & & & & & & & & & & & & \\
\hline & $011717200^{2}$ & 0.10 .5 & & 2.105 & 24.020 & & & & 4.288 & 13.14 & 31.451 & & & & & 14.0439 & & & & 45 & 1.3.2 & 38.0 & & & & & 623 & \\
\hline & 6 & 6.17.39 PM & 26039 & 26717 & 2287 & 28184 & & 44,41 & 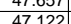 & 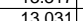 & 31.383 & . & & 57195 & 0.262 & 1464 & & & & $\frac{45}{45}$ & 然140 & 379 & 2614 & & & & 10301 & 0.80 \\
\hline 456 & $6 / 17 / 2004$ & $6: 18:$ & 26.06 & & $\frac{24.826}{24.826}$ & $\frac{28.10}{28,10}$ & & 45.554 & 47.458 & 13.057 & 32.518 & $\frac{1.219}{1.219}$ & & $\frac{1.918}{518}$ & $\frac{0.224}{0.224}$ & $\frac{21.643}{14.63}$ & $\frac{0.04}{0.004}$ & & 8.14861 & 46.8 & 127 & 39.0 & & & & & & 88 \\
\hline tut & $6 / 17 / 2004$ & & 26.165 & 26.724 & 24.802 & 28.06 & & 46.543 & 47.184 & 13.128 & 33.60 & & & & & 14.643 & & & & & & & & & & & & \\
\hline & 6/177/2004 & $6: 20: 39 \mathrm{~F}$ & & 26.726 & & 28.01 & & & & & & & & & & 14.643 & & & & & 12.8 & & & & & & & \\
\hline 45 & $6 / 177 / 2004$ & $\begin{array}{c}6: 21: 3 \\
\end{array}$ & 26.14 & 26.734 & 24.792 & 27.94 & & 46.3 & 47.4 & & 33.283 & -1.222 & & 48.1 & & 14.6 & & & & & $\overline{11.8}$ & 39. & & & & & & \\
\hline & $6 / 17 / 2004$ & & & 26.735 & 24.783 & & & & & & & & & & & 14.64 & & & & & 11.0 & & & & & & & \\
\hline & $6 / 17 / 2004$ & & & & & & & & & & & -1.220 & & & & & & & & & 14.0 & & & & & & & \\
\hline 462 & $6 / 177 / 2004$ & 6:24: & 26.117 & 26.741 & \begin{tabular}{|l|}
24.803 \\
\end{tabular} & $27.88 \varepsilon$ & & 46.22. & \begin{tabular}{|l|l|}
47.062 \\
\end{tabular} & 12.835 & 33.478 & $\mid-1.222$ & & 48.9 & & 14.643 & & & & & 12.0 & $\overline{39.9}$ & & & & & & \\
\hline 40 & 6/177/2004 & & 26.063 & 26.746 & \begin{tabular}{|l|}
24.784 \\
\end{tabular} & $27.95 \mathrm{c}$ & & & & & & -1.219 & & & & & & & & & 12.4 & & & & & & & \\
\hline & $6 / 17 / 2004$ & 6:26:39 F & 26.085 & 26.738 & 24.771 & 28.02 & 26. & 46.074 & 47.611 & 12.987 & 33.076 & \begin{tabular}{|c|c|} 
& -1.219 \\
\end{tabular} & & 47..466 & & 14.643 & & & & & 11.6 & 39.6 & $\mid 2.729$ & 0.034 & & 0.001 & 357 & \\
\hline 40 & 6/17//2004 & & 20.05 & 26.749 & 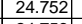 & 28.0 & & & & & 53.000 & 1.224 & & & & & & & & & 10.5 & & & & & & 590 & \\
\hline & & & & 26.156 & $\mid 24.53$ & & & & & & & & & & & & & & & & 12.2 & & & & & & & \\
\hline & $\frac{10172004}{61172004}$ & $\begin{array}{l}6: 29: 39 \mathrm{PM} \\
6.30 \cdot 39 \mathrm{PM}\end{array}$ & 26.204 & $\frac{26.772}{26754}$ & \begin{tabular}{|l|}
24.765 \\
24702
\end{tabular} & $\frac{28.12}{28.08}$ & $\frac{2.89}{2691}$ & & (17.0472) & $\frac{12.965}{1205}$ & $\begin{array}{l}33.363 \\
33.252\end{array}$ & $|-125|$ & & 514 & & 1464 & & & & 45 & 11.8 & \begin{tabular}{l|l|l|}
39.8 & \\
397 &
\end{tabular} & 2.074 & 0025 & & & 0.045 & \\
\hline & $\frac{6 / 17 / 2004}{6 / 17 / 204}$ & & & & & & & & & & & & & & & & & & & & & $\frac{39.1}{40.0}$ & & & & & & \\
\hline 47. & 6/117/2004 & & & & \begin{tabular}{|l|}
24.735 \\
\end{tabular} & 28.6 & & & & & & -1.219 & & & & & & & & & 13.3 & 39.8 & & & & & 42 & \\
\hline 47 & 6/177/2004 & & 26.134 & & 24.71 & & & & & & & & & & & & & & & & 11.8 & & & & & & & \\
\hline & $6 / 17 / 2004$ & & 26.1 & & 24.706 & & & & & & & -1.2 & & & & & & & & & & & & & & & & \\
\hline & & & & & & & & & & & & & & & & & & & & & & & & & & & & \\
\hline & $6 / 17 / 2004$ & & 26.05 & 26.789 & 24.62 & 27.8 & & 45.3 & 46.5 & & $32.8 \mathrm{C}$ & -1.2 & & & & 14.6. & & & & & & & & & & & & \\
\hline & $6 / 17 / 2004$ & & 26.1 & 26.8 & 24.62 & 27.9 & & & & & & & & & & & & & & & & & & & & & & \\
\hline 47. & 6/17/2004 & $6: 38: 33$ & 26.04 & 26.812 & 24.61 & 27.9 & & 45.2 & 46.43. & & & & & & & 14.64 & & & & & 10.6 & 39. & & & & & & \\
\hline 47 & $6 / 17 / 2004$ & & 26.027 & 26.791 & 24.563 & 27.9 & 26.8 & 46.3 & 47.6 & & & & & & & & & & & & $12.6 \mathrm{C}$ & 40 & & & & & & \\
\hline & $6 / 177 / 2$ & & 25.992 & 26.8 & 24.5 & 27.9 & & 46. & 47.748 & 12.196 & & -1.219 & & & & & & & & & 10.5 & 40.4 & & & & & 47 & \\
\hline $4 i$ & 661172004 & & 25.991 & 26.199 & 24.50 & 27.93 & & 45.965 & & 12.422 & 30.4 & 1.228 & & & & $14.6 \mathrm{C}$ & & & & & 11.0 & 5 & & & & & & \\
\hline & & & & & & & & & & & & & & & & & & & & & & & & & & & & \\
\hline & 6) & & $\frac{20.0<1}{26071}$ & $\frac{26.803}{26.814}$ & $24.486 \mid$ & 27.935 & & $\frac{46.234}{47566}$ & $\begin{array}{ll}48.623 \\
48.401\end{array}$ & $\begin{array}{l}12.077 \\
11999\end{array}$ & $\frac{34.129}{356}$ & $\begin{array}{l}-1.222 \\
-1219\end{array}$ & & & 0.145 & 14.643 & & & & $\frac{4 t}{46}$ & $\frac{1.1}{118}$ & $\begin{array}{l}40.2 \\
416\end{array}$ & . & & & & & \\
\hline 48 & $6 / 17 / 2004$ & & $\frac{20.6}{26}$ & & & & & 46. & & $\mid \frac{11.955}{13.148}$ & 018 & & & & & $\frac{14.0}{14.6}$ & & $51 !$ & & $\frac{44}{45}$ & $\begin{array}{l}11.0 \\
10.3 \\
\end{array}$ & $\begin{array}{l}41.0 \\
39.6\end{array}$ & & & & & 5 & \\
\hline$\overline{48}$ & 6/117/2004 & & & & & 27.891 & & & 48.2 & & & -1.2 & & & & & & 516 & & $\begin{array}{l}46.8 \\
\end{array}$ & \begin{tabular}{|c|}
11.6 \\
\end{tabular} & 41.2 & 2.840 & & & 0.0 & 0.622 & \\
\hline 48 & 6/177/2004 & & 25.94 & 26.8 & 24.39 & & & 46.8 & & $\begin{array}{l}12.546 \\
\end{array}$ & & $\mid-1.219$ & & & & 14. & & & & & 11.4 & 40.6 & \begin{tabular}{|l|l|}
2.802 \\
\end{tabular} & & & & 86 & \\
\hline & $6 / 17 / 2004$ & & 25.96 & & 24.3 & & & $46.8 \mathrm{~s}$ & & & & & & & & & & & & & 12.6 & & & & & & & \\
\hline 48 & $6 / 1 / 122$ & & 25.951 & 26.804 & 24.336 & 28.081 & & & & & 35.268 & & & & & & & & & & 11.3 & & & & & & & \\
\hline $\bar{n}$ & 0 & & 20.090 & 20.199 & 24.300 & 28.130 & 2.9 & 40.6 & 48.44 & 12.504 & 34.24 & -1.228 & & & & 14.6 & & & & & 12.5 & 40.4 & & & & 0.001 & 0.688 & 0.5 \\
\hline$\frac{409}{490}$ & $6 / 17 / 2004$ & 6:52:39 PMM & 25.945 & 26.0528 & \begin{tabular}{|l|}
24.425 \\
24.325 \\
\end{tabular} & 28.215 & & 47.058 & \begin{tabular}{|l|l|}
48.051 \\
\end{tabular} & \begin{tabular}{|l|}
12.200 \\
12.601
\end{tabular} & 34.479 & $\mid-1.222$ & -6.369 & 51.262 & 0.161 & 14.643 & 0.004 & 522.9 & \begin{tabular}{|l|}
8.71528 \\
\end{tabular} & 46.6 & \begin{tabular}{l|l|}
12.4 \\
12.6
\end{tabular} & 40.8 & 2.811 & 0.024 & 0.024 & 0.001 & \begin{tabular}{|l|}
0.6000 \\
\end{tabular} & 0.5 \\
\hline
\end{tabular}


WSRC-TR-2005-00105, REVISION 0

SRNL-RPP-2005-00012, REVISION 0

RUN \# WASH1 AND WASH 2; CAMPAIGN III SLURRY WASHING - CONT.

\begin{tabular}{|c|c|c|c|c|c|c|c|c|c|c|c|c|c|c|c|c|c|c|c|c|c|c|c|c|c|c|c|c|}
\hline & A & B & D & $\mathrm{E}$ & $\mathrm{F}$ & $\mathrm{G}$ & $\mathrm{H}$ & $\mathrm{J}$ & $\mathrm{K}$ & $\mathrm{L}$ & $\mathrm{M}$ & $\mathrm{N}$ & 0 & $\mathrm{Q}$ & $\mathrm{R}$ & \begin{tabular}{l|l}
$\mathrm{s}$ \\
\end{tabular} & $\mathrm{T}$ & $\mathrm{v}$ & w & $x$ & $\mathrm{Y}$ & $z$ & $\mathrm{AA}$ & $A B$ & $A C$ & $\mathrm{AD}$ & $\mathrm{AE}$ & AF $A$ \\
\hline & $6 / 17 / 2004$ & 6:53:39 PM & 25.873 & $\frac{26.846}{26.854}$ & $\begin{array}{r}24.313 \\
22.291\end{array}$ & $\begin{array}{l}28.237 \\
28.161\end{array}$ & $\begin{array}{l}26.978 \\
26.921\end{array}$ & $\begin{array}{l}46.431 \\
47163\end{array}$ & & \begin{tabular}{|l|l|}
12.705 \\
12332
\end{tabular} & \begin{tabular}{|l|}
33.673 \\
34867
\end{tabular} & $\frac{-1.222}{-1.222}$ & $\begin{array}{l}-6.276 \\
-207\end{array}$ & \begin{tabular}{|l|l|l|l}
47.028 \\
38587
\end{tabular} & $\begin{array}{l}0.167 \\
0.151\end{array}$ & \begin{tabular}{|c|c|}
14.643 \\
14634
\end{tabular} & $\begin{array}{l}0.004 \\
0.021\end{array}$ & 523.9 & \begin{tabular}{|l|l|}
8.73194 \\
87961
\end{tabular} & \begin{tabular}{|l|}
46.1 \\
6
\end{tabular} & 11.5 & 40.1 & \begin{tabular}{|l|l|}
2.761 \\
\end{tabular} & \begin{tabular}{|l|l|}
0.025 \\
0.23
\end{tabular} & $\begin{array}{l}0.025 \\
023\end{array}$ & 0.001 & 0.634 & 0.54 \\
\hline & 6/17/2004 & & 25.921 & 26.854 & & $\begin{array}{r}28.161 \\
2.0115\end{array}$ & 26.921 & 47.163 & \begin{tabular}{|l}
47.825 \\
\end{tabular} & 12.332 & 34.867 & & -6.207 & 38.587 & 0.151 & \begin{tabular}{|l|l|}
14.643 \\
\end{tabular} & & 524.9 & $\begin{array}{ll}98.74861 \\
\end{array}$ & $\begin{array}{l}46.4 \\
\end{array}$ & $\begin{array}{r}9.5 \\
\end{array}$ & \begin{tabular}{|l|}
41.0 \\
\end{tabular} & 2.828 & \begin{tabular}{|l|l|}
0.023 \\
\end{tabular} & \begin{tabular}{|l|l|} 
\\
\end{tabular} & \begin{tabular}{|l|}
0.001 \\
\end{tabular} & & \\
\hline 494 & 6/17/72004 & 6:55:39 PM & \begin{tabular}{r|r}
25.8899 \\
25.92
\end{tabular} & $\frac{26.863}{26.863}$ & $\begin{array}{l}24.275 \\
24.255\end{array}$ & $\begin{array}{r}28.115 \\
28.1\end{array}$ & 26.8851 & $\begin{array}{l}46.649 \\
46.085 \\
46\end{array}$ & \begin{tabular}{|l|}
47.539 \\
47.089 \\
\end{tabular} & \begin{tabular}{r|}
12.26 \\
12.86
\end{tabular} & \begin{tabular}{|l|}
34.441 \\
33.263 \\
\end{tabular} & \begin{tabular}{|c|}
-1.222 \\
-1.219 \\
\end{tabular} & $\begin{array}{l}-6.349 \\
-6.175 \\
\end{array}$ & $\begin{array}{r}53.912 \\
50.751\end{array}$ & $\begin{array}{l}0.189 \\
0.144\end{array}$ & \begin{tabular}{|l|}
14.663 \\
14.463
\end{tabular} & $\begin{array}{l}0.004 \\
0.004\end{array}$ & $\begin{array}{l}525.9 \\
526.9\end{array}$ & $\begin{array}{l}98.76528 \\
9 \\
\end{array}$ & \begin{tabular}{|l|}
46.1 \\
45.6
\end{tabular} & \begin{tabular}{|c|}
13.2 \\
12.4
\end{tabular} & \begin{tabular}{|l|}
40.5 \\
39.7 \\
\end{tabular} & \begin{tabular}{|l|l|} 
& 2.795 \\
7 & 2.735 \\
\end{tabular} & \begin{tabular}{|c|}
0.028 \\
0.021 \\
\end{tabular} & $\begin{array}{l}0.0229 \\
0.022\end{array}$ & \begin{tabular}{|l|}
0.001 \\
0.001 \\
\end{tabular} & $\begin{array}{l}0.709 \\
0.553\end{array}$ & \begin{tabular}{|l|l}
9 & 0.60 \\
3 & 0.4
\end{tabular} \\
\hline 495 & & 6:57:39 PM & 25.922 & 26.865 & & 28.067 & 26.838 & 46.471 & \begin{tabular}{|l|}
47.216 \\
\end{tabular} & & $\begin{array}{l}34.200 \\
\end{array}$ & -1.222 & $\begin{array}{l}-0.175 \\
-6.525 \\
\end{array}$ & 56.694 & $\begin{array}{l}0.144 \\
0.172\end{array}$ & $\begin{array}{l}14.045 \\
14.643 \\
\end{array}$ & $\begin{array}{l}.004 \\
0.004\end{array}$ & $\frac{526.9}{527.9}$ & \begin{tabular}{l|l|}
9 & 8.789194 \\
9 & 8.79861
\end{tabular} & $\begin{array}{l}45.6 \\
45.7 \\
\end{array}$ & $\frac{12.4}{13.9}$ & $\frac{39.1}{40.3}$ & $\begin{array}{l}2.135 \\
2.779\end{array}$ & \begin{tabular}{|l|}
0.0261 \\
\end{tabular} & 0.024 & $\frac{0.001}{0.001}$ & $\frac{0.553}{0.650}$ & 0.47 \\
\hline 496 & & & 25.937 & & \begin{tabular}{|l|}
24.267 \\
\end{tabular} & & 26.808 & 46.678 & 47.506 & 12.199 & 34.535 & & & 52.652 & 0.167 & 14.643 & & & 98.81528 & 46.0 & $\begin{array}{l}12.9 \\
\end{array}$ & & & & 0.025 & & & $\begin{array}{l}0.55 \\
0.53\end{array}$ \\
\hline 49 & 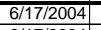 & 6:59:39 PM & 25.908 & 26.871 & \begin{tabular}{|l|l|}
24.263 \\
\end{tabular} & 28.078 & 26.789 & 46.473 & 47.473 & $\begin{array}{l}12.02 \\
\end{array}$ & 34.516 & -1.219 & -6.19 & 43.828 & 0.175 & 14.643 & 0.004 & 529.9 & \begin{tabular}{l|l}
98.83194 \\
\end{tabular} & 46.0 & $\begin{array}{l}10.7 \\
\end{array}$ & 40.5 & 2.792 & & $\begin{array}{l}0.027 \\
\end{array}$ & & & 0.56 \\
\hline & 6/17/2004 & & & & \begin{tabular}{|l|}
24.22 \\
\end{tabular} & & & & & & & & & & & & & & & & & & & & & & & \\
\hline 499 & $6 / 17 / 2004$ & 7:01:39 PM & 25.901 & 26.879 & \begin{tabular}{|l|}
24.216 \\
\end{tabular} & 28.057 & 26.762 & 46.917 & 48.005 & 11.751 & $\begin{array}{l}35.179 \\
\end{array}$ & -1.222 & -6.25 & 52.668 & & 14.6 & & & 6 & 46.5 & 12.9 & & & $\overline{023}$ & 023 & & 568 & \\
\hline & & & & & & & & & & & & & & & & & & & & 46.2 & & & & & & & & \\
\hline & $6 / 17 / 2004$ & 7:03:39 PM & 25.828 & 26.886 & \begin{tabular}{|l|l|l|l|}
248 \\
\end{tabular} & 27.948 & 26.719 & 46.61 & 47.823 & 11.943 & 34.631 & -1.222 & -6.036 & 51.077 & 0.156 & $\begin{array}{l}14.643 \\
\end{array}$ & & 533.9 & 8.89861 & 46.4 & 12.5 & 40.6 & 2.801 & 0.023 & 0.024 & & 0.586 & \\
\hline 50 & $6 / 17 / 2004$ & 7:04:39 PM & 25.828 & 26.876 & \begin{tabular}{|l|l|l|}
2433 \\
\end{tabular} & 27.983 & 26.714 & 47.541 & 48.449 & 12.127 & 35.454 & -1.222 & -6.262 & 45.91 & 0.158 & 14.643 & & 534.9 & 9 8.91528 & 47.0 & 11.3 & 41.5 & 2.861 & 0.024 & 0.024 & 0.001 & 0.581 & \\
\hline 50 & $6 / 17 / 2004$ & 7:05:39 PM & $\begin{array}{l}25.852 \\
25720\end{array}$ & 26.87 & 24.137 & 27.938 & 26.718 & 47.443 & 48.343 & 12.191 & 35.284 & & & 47.009 & 0.174 & 14.643 & & 535.9 & $\begin{array}{l}9 \\
8.93194\end{array}$ & 46.9 & 11.5 & 41. & 2.852 & 0.026 & 0.027 & 0.001 & 0.643 & 0.55 \\
\hline & $611 / 72$ & 7:06:39 PM & 2 & & & 2.91 & & 46.54 & 4.581 & 1855 & & & & 51.544 & & 14.66 & & & 8.94861 & 46.1 & & & & & & & & \\
\hline 50 & & & 25.996 & & & & & & 564 & 1655 & 344 & & & & & 14.6 & & & & 46.1 & 12.4 & & & 024 & 0.024 & & 604 & \\
\hline & $\frac{61172020}{61172004}$ & $7.00 .30 .39 \mathrm{PM}$ & $\frac{25.050}{2574}$ & $\begin{array}{l}\frac{20.003}{26867} \\
20\end{array}$ & $\mid \frac{2.1530}{24129}$ & 27884 & $\frac{2.051}{26625}$ & $\frac{40.025}{46699}$ & 年 47.4564 & $\mid \frac{1.200}{1204}$ & 年4.1.144 & -1.225 & & 53,403 & $=0.15$ & $\frac{14.6}{146}$ & & 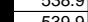 & $\begin{array}{l}8.980194 \\
0.0961\end{array}$ & 45.9 & $\frac{11.1}{1.1}$ & & 2805 & 2027 & 0 & & (502 & \\
\hline & 66 & $7 \cdot 710 \cdot 39 \mathrm{PM}$ & & 26849 & 24.101 & 27857 & 26.632 & 46.562 & 47286 & 12336 & $\begin{array}{r}34.056 \\
34.334 \\
\end{array}$ & 1.25 & & 55.763 & 年 & 146. & & 540.9 & 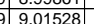 & 45.8 & & $\frac{40.1}{40.4}$ & 2700 & & $\frac{0.021}{0.026}$ & & $\frac{612}{621}$ & \\
\hline & $6 / 17 / 2004$ & 7:11:39 PM & 25.826 & 26.864 & 24.111 & 27.897 & 26.607 & 46.238 & 47.129 & 12.248 & $\begin{array}{l}34.034 \\
33.985 \\
\end{array}$ & $\begin{array}{l}-1.219 \\
-1.219\end{array}$ & & 44.162 & 0.174 & 14.643 & & 541.9 & $\begin{array}{l}9.03194 \\
9.3194\end{array}$ & 45.7 & 10.8 & $\frac{4.4}{40.1}$ & 2.766 & $\frac{0.026}{0.0}$ & $\frac{0.02 \mathrm{c}}{0.027}$ & $\frac{011}{001}$ & $\frac{0.031}{0.663}$ & $\begin{array}{l}0.54 \\
0.56\end{array}$ \\
\hline & $6 / 17 / 2004$ & 7:12:39 PM & 25.812 & 26.865 & \begin{tabular}{|l|}
24.157 \\
\end{tabular} & 27.803 & 26.583 & 46.083 & 47.172 & 12.181 & 33.862 & -1.228 & -6.181 & 52.199 & 0.167 & 14.643 & & 542.9 & $\begin{array}{l}99.04861 \\
\end{array}$ & 45.7 & 12.8 & 40.0 & 2.756 & $\frac{0.025}{-025}$ & 0.025 & .001 & 0.638 & \\
\hline & $6 / 17 / 2004$ & & & & & & & & & & & & & & & & & & & & & & & & & & & \\
\hline & 6/17/12004 & $7: 14: 39 \mathrm{PM}$ & $\frac{25.706}{20}$ & $\frac{26.844}{26}$ & \begin{tabular}{|l|}
24.251 \\
\end{tabular} & 27.797 & $\begin{array}{l}26.533 \\
\end{array}$ & 46.413 & 477.609 & $\frac{12.215}{12}$ & 34.268 & -1.222 & & 46.974 & 0.19 & 14.643 & & 544.9 & 9.08194 & 46.1 & 11.5 & & $2.781 \mathrm{C}$ & .028 & 0.029 & & 717 & 61 \\
\hline & $6 / 17 / 2004$ & 7:15:39 PM & 25.704 & 26.837 & \begin{tabular}{|l|}
24.359 \\
\end{tabular} & \begin{tabular}{|l|l|}
27.809 \\
\end{tabular} & 26.5 & 46.386 & 47.512 & 12.207 & 34.139 & & & 43.0 & 0.184 & 14.64 & & 545.9 & 9.09861 & 46.0 & & & & & & & 694 & \\
\hline & 6/17/2004| & 7:16:39 PM & $25.728 \mid$ & 26.821 & 24.353 & 27.804 & 26.514 & 46.103 & 47.129 & 11.987 & 34.075 & 1.22 & & 47.04 & 0.167 & 14.64 & & 54 & 9.11528 & 45.7 & 11.5 & & & & & & & \\
\hline & $6 / 17 / 2004$ & 7:17:39 PM & 25.85 & 26.833 & & & & 46.398 & 47.473 & 11.905 & 34.453 & & & 40.016 & 0.101 & $\begin{array}{l}14.643 \\
\end{array}$ & & 54 & 9.13194 & 46.0 & 11.8 & & & & 0.027 & & & \\
\hline 51 & $6 / 17 / 2004$ & 7:18:39 PM & 25.872 & 26.835 & 24.428 & 27.843 & & 46.34 & 47.477 & 11.919 & 34.401 & & & 45.875 & 0.174 & 14.643 & & 548 & 9.14861 & 46.0 & $11.2 \mathrm{C}$ & 40. & 2.783 & 026 & 0.026 & & 0.653 & \\
\hline & 6/17//2004 & 7:19:39 PM & 25.854 & 26.822 & 24.4499 & 27.864 & 26.685 & 46.421 & 47.404 & 11.923 & 34.451 & -1.222 & -6. & 50.612 & 0.175 & 14.643 & & 549.9 & 9.16528 & 45.9 & 12.4 & 40.4 & 2.788 & 0.026 & 0.026 & 0.001 & 0.655 & \\
\hline 51 & $6 / 17 / 72004$ & 7:20:39 PM & 25.166 & 26.824 & 24.4867 & 27.907 & 26.722 & 46.294 & 47.707 & 11.966 & 34.225 & -1. & & 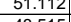 & 0.183 & 14.643 & & 550.9 & 9 9.18194 & 46.2 & 12.5 & 40.3 & 2.776 & & . & & 0.688 & \\
\hline & 011712004 & $=1.1 .39 \mathrm{PM}$ & $\frac{2.084}{25025}$ & $\frac{20.824}{2.820}$ & 24.54 & 2.909 & & 46.1871 & . & & 34.103 & & & & & & & & & & & & & & & & & \\
\hline & $\frac{617172004}{661 / 72004}$ & $\frac{1.223}{7.39} \mathrm{PM}$ & 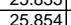 & $\frac{2.065}{26812}$ & $\frac{24.541}{24.64}$ & 28.029 & $\frac{26.81}{26869}$ & $\frac{46.021}{46.703}$ & $\begin{array}{r}47.873 \\
47.84\end{array}$ & $\frac{12.156}{12.198}$ & $\frac{33.843}{34.484}$ & $\frac{-1.219}{-1.222}$ & & 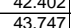 & $\frac{0.128}{0.171}$ & $\frac{14.643}{14.643}$ & & S55.9 & \begin{tabular}{l|l}
9 & 923194
\end{tabular} & 46.44 & $\frac{10.4}{107}$ & & & & $\frac{0.016}{0.026}$ & & 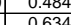 & \\
\hline 52 & $6 / 17 / 2004$ & 7:24:39 PM & 25.893 & 26.821 & \begin{tabular}{|l|}
24.609 \\
\end{tabular} & 28.088 & 26.873 & 46.274 & 47.24 & 11.851 & 34.443 & -1.225 & -6.3 & 44.944 & 0.181 & 14.643 & & 554.9 & \begin{tabular}{|l|l}
9 & 9.24861
\end{tabular} & 458 & $\frac{1.0}{110}$ & & & 0.027 & $\frac{0.027}{0.027}$ & & 0.676 & \\
\hline 52 & $6 / 17 / 2004$ & 7:25:39 PM & 25.914 & 26.822 & \begin{tabular}{|l|}
24615 \\
\end{tabular} & 28.064 & 26.869 & \begin{tabular}{|l|l|}
46.539 \\
\end{tabular} & 47.419 & 11.868 & 34.697 & \begin{tabular}{|l|l|} 
& -1.219 \\
\end{tabular} & -6.3 & 45.113 & \begin{tabular}{|l|l|}
0.18 \\
\end{tabular} & $\mid 14.643$ & & 555.9 & 9926528 & 45.9 & $\frac{71.1}{111}$ & 40.6 & 2800 & 0.027 & 0.027 & & & \\
\hline & $6 / 17 / 2004$ & 7:26:39 PM & 25.967 & 26.845 & \begin{tabular}{|l|}
24.633 \\
\end{tabular} & 28.067 & 26.843 & 46.375 & $\begin{array}{l}47.384 \\
\end{array}$ & 11.936 & 34.416 & & & & 0.19 & \begin{tabular}{|l|l|}
14.643 \\
\end{tabular} & & & & 45.9 & $\frac{2.9}{12.9}$ & & & & & & $\frac{0.709}{0.709}$ & \\
\hline & $6 / 17 / 2004$ & & & 26.843 & 24.636 & 28.04 & 26.801 & 46.278 & 47.545 & & 34.28 & -1.219 & & $\begin{array}{l}41.636 \\
\end{array}$ & 0.202 & 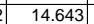 & & 55 & 9.29861 & 46.1 & 10.2 & 40 & 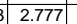 & .030 & & & & \\
\hline & & & 25.972 & 26.861 & 24.659 & 27.968 & & 46.717 & 47.663 & 11.854 & 34. & & & $50.87 \mathrm{~s}$ & 0.18 & \begin{tabular}{ll|l}
14.643 \\
\end{tabular} & & 55 & & 46.2 & 12.5 & & & & & & 0.675 & \\
\hline & & 7:29:39 PM & 25.949 & 26.852 & 24.68 & 27.925 & & 46.056 & 47.292 & 12.24 & 33. & -1.219 & & & 0.19 & 14.6 & & & & 45.8 & 12.5 & & & & & & & \\
\hline & $6 / 17 / 2004$ & $7: 30: 39 \mathrm{PM}$ & 26.003 & 26.841 & 24.689 & 27.984 & 26.6 & & & & & & & & 0.1 & 14. & & & & & 11.7 & & & & & & & \\
\hline 52 & 6/17/2004 & 7:31:39 PM & 25.966 & 26.824 & 24.767 & 27.921 & 26.65 & 45.863 & 47.066 & 12.073 & 33.73 & -1.225 & & 45.116 & 0.141 & 14.643 & & & & \begin{tabular}{l|l}
45.6 \\
\end{tabular} & 11.1 & 39.8 & 2.744 & & & 0.001 & 0.532 & \\
\hline$\frac{53}{52}$ & 6/17/2004 & 7:32:39 PM & 25.964 & 26.827 & 24.735 & 27.91 & 26.6 & 46.052 & 47.184 & 12.042 & & & & 56.08 & & 14.6 & & 56 & & 45.7 & 13.7 & & & & & & & \\
\hline & $6 / 17 / 2004$ & 7:33:39 PM & 25.967 & 26.825 & \begin{tabular}{|l}
24.733 \\
\end{tabular} & 27.868 & 26.6 & 45.674 & 47.162 & 12.025 & 33.616 & -1.219 & & 49.043 & 0.156 & 14.6 & & 56 & 9.39861 & 45.7 & 12.0 & 39.6 & 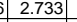 & & & & 0.591 & \\
\hline 53 & $6 / 17 / 72004$ & & 26.02 & 26.833 & $\mid \begin{array}{r}24.5151 \\
\end{array}$ & 27.895 & & 46. & (19.007 & 12.084] & 2000 & 1220 & & 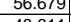 & 0.104 & & & 564.9 & 0 & 45.6 & 13.9 & & 2...151 & 0 & 802 & & 年 & \\
\hline & & & & & & & & & & & & & & & & & & & & & & & & & & & & \\
\hline & 6611722004 & $7.366 .39 \mathrm{PM}$ & 2 & 26.816 & 24.44 & 2.829 & 26.564 & 46.13 & 4.085 & 12.079 & 34.003 & & & 4.0 .046 & 0.116 & $14.6 \mathrm{C}$ & & 566.9 & & 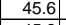 & 11.5 & & & & 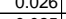 & & & \\
\hline & & & & & & & & & & & & & & & & & & & & 45.8 & & & & & & & & \\
\hline & 011712004 & 1.38 .39 & 23.9411 & 20.804 & 24.152 & 21. & & & & 12.225 & & -1.222 & & & & 14. & & & & & 12.5 & & & & & & & \\
\hline & $6 / 177 / 2$ & & 25.968 & 26.797 & \begin{tabular}{|l}
24.75 \\
\end{tabular} & 27.739 & & & & & & -1 & & & 0.1 & & & & & & 11.1 & & & & & & & \\
\hline & 6/17//2004 & 7:40:39 & 25.99 & 26.803 & \begin{tabular}{|l|}
24.801 \\
\end{tabular} & 27.756 & & $45 . .5$ & 46.84 & 11.897] & 34.112 & & & 51.6 & 0.19 & 14. & & 57 & & 45.4 & 12.5 & & & & & & & \\
\hline & $6 / 1 / 72004$ & $7: 14: 39 \mathrm{PM}$ & 2.957 & $\frac{26.191}{2.72}$ & 24.79 & $\frac{27.679}{2701}$ & 26. & 45.82 & 47.10 & $\frac{11.000}{11038}$ & $\begin{array}{l}30.010 \\
32005\end{array}$ & 1210 & & 41. & 0.149 & & & $\frac{5 / 1 .}{572}$ & & & $\frac{10.2}{115}$ & & & & & & & \\
\hline & & & & & & & & & & & & $\frac{1.219}{1.210}$ & & & & & & & & & & & & & & & & \\
\hline (3) & (61772 & & $\frac{25.994}{25096}$ & $\frac{2.106}{26770}$ & 24.014 & 21.140 & 20. & 4 & 41.170 & 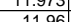 & & $\frac{1.219}{1.225}$ & & & 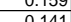 & & & $\frac{51}{57}$ & & & 114 & & & & & & & \\
\hline 54 & 6 & $7 \cdot 74: 39$ & 26.011 & 20.8 & $\mid$\begin{tabular}{|c|c|c|}
$\mid 24838$ \\
\end{tabular} & 27.867 & & & 47.493 & & & & & & & & & & & & 110 & & & & & & 0.535 & \\
\hline & $6 / 171 / 2004$ & $7: 46: 39$ & 25.986 & 26.789 & \begin{tabular}{|l|}
24.837 \\
\end{tabular} & 27.906 & & & & & & & & & & & & & & & 115 & & & & & & & \\
\hline 54 & 6/17/12004 & & 25.976 & 26.789 & & 27.931 & 26.7 & 46.027 & 47. & 12.017 & 33.987 & -1.222 & & 49. & 0.1 & 14. & & 577 & & 45.6 & 12.1 & & & & & & & \\
\hline 546 & & & & & & & & & & & & & & & & & & & & & & & & & & & & \\
\hline & $6 / 17 / 2004$ & & 25.992 & 26 & 24.8 & 28.032 & & 46.141 & & 11 & & & & & & 14.6 & & 579 & & 45.8 & 117 & & & & & & & \\
\hline 548 & $6 / 17 / 200$ & $7: 50$ & $\overline{25.987}$ & 26.801 & 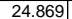 & 28.013 & & $45 . \varsigma$ & 47.10 & 11.90 & & -1.219 & & & 0.1 & 14. & & & & & 12 & & & & & & & \\
\hline 549 & $6 / 17 / 2004$ & 7:51:39 PI & 25.983 & 26.792 & 24.85 & 27.984 & & 46.13 & 47.236 & \begin{tabular}{|l|l|}
11.93 \\
\end{tabular} & & $-1.21 \mathrm{C}$ & & 53.3 & 0.189 & 14. & & 58 & & 45 & 13.1 & & & & & & & \\
\hline & $6 / 17 / 22$ & 7:52: & 26.072 & 26.7. & 24.8 & & & 45. & 46.8 & & & & & & & 14. & & & & & 12.2 & & & & & & & \\
\hline & $6 / 17 / 200$ & 7:53:39| & 26. & 26.788 & 24.846 & 27.8 & & 45. & 47.1 & 11.975 & 33.8 & -1.2 & & 49. & 0.1 & 14.6 & & 58 & & 3.7 & 12.2 & & & & & & 0.683 & 83 \\
\hline & $6 / 17 / 2004$ & 7:54:39 PI & 25.973 & 26.766 & 24.824 & 27.864 & & $46 . C$ & 47.299 & 12.074 & & & & $34 . c$ & 0.177 & 14.6 & & 584 & & 45 & 8.3 & & & & & & 663 & 63 \\
\hline & $6 / 17 / 72004$ & $7.553 .39 \mathrm{PM}$ & 2.952 & 26.17 & 24.834 & 27.828 & & 45.5 & & $\frac{12.284}{1.2001}$ & 33.181 & -1.2222 & & & 0.174 & 14.6 & & 58 & & 45.9 & 1.6 & & & & & & & \\
\hline & 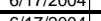 & & & & & & & & & & & & & & & & & & & & 1.4 .4 & & & & & & & \\
\hline & (1) & 7.5950 & 2507073 & $\frac{2.101}{20.791}$ & $\frac{2.0 .0}{2.8}$ & $\frac{2.1 .44}{27691}$ & & 46.6 & 4 & $\mid \frac{1.075}{11769}$ & $\begin{array}{l}34.067 \\
32178\end{array}$ & & & 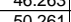 & 0.1900 & 14. & & 501. & & 45. & 1.1 .3 & & & & & & & \\
\hline 55 & $6 / 171 / 7004$ & $7 \cdot 59 \cdot 39 \mathrm{PM}$ & 25.982 & 26.801 & 24.874 & 27.713 & 26.5 & 46.979 & 48194 & 11.431 & 35504 & -1.216 & & 4136 & 0.079 & 14643 & & 5890 & & 467 & 101 & 41 & 2843 & & & & & \\
\hline 558 & $6 / 117 / 2004$ & $8: 00: 39 \mathrm{~F}$ & 25.975 & 26793 & 24831 & 27686 & & 470 & 48202 & 11587 & 35.423 & 1222 & & & 0188 & 1464 & & & & & & & & & & & & \\
\hline & $6 / 17 / 1 / 2004$ & $8 \cdot 01 \cdot 39 \mathrm{PM}$ & 25.97 & 26.798 & 24.816 & 27.746 & 26.5 & 46.931 & 47.868 & 1189 & & & & & 0.20 & 14.6 & & & & 46.4 & 103 & $4 \mathrm{II}$ & & & & & 750 & \\
\hline 5600 & 6 & 8:02:39 PM & 25.981 & 26.804 & 24.8 & 27.816 & & 46.86 & 48.196 & $\begin{array}{r}12.116 \\
\end{array}$ & 34.664 & -1.222 & & 39.244 & 0.174 & 14.643 & & 592.9 & $\begin{array}{l}9 \\
\end{array}$ & 46.7 & 9.6 & & 810 & 026 & 0.026 & 0.001 & 0.640 & 40 \\
\hline
\end{tabular}


WSRC-TR-2005-00105, REVISION 0

SRNL-RPP-2005-00012, REVISION 0

RUN \# WASH1 AND WASH 2; CAMPAIGN III SLURRY WASHING - CONT.

\begin{tabular}{|c|c|c|c|c|c|c|c|c|c|c|c|c|c|c|c|c|c|c|c|c|c|c|c|c|c|c|c|c|}
\hline & A & $\mathrm{B}$ & D & $E$ & $F$ & $G$ & $\mathrm{H}$ & $\mathrm{J}$ & $\mathrm{K}$ & \begin{tabular}{ll|}
$\mathrm{L}$ \\
\end{tabular} & M & $\mathrm{N}$ & 0 & Q & $R$ & $\mathrm{~s}$ & & $\mathrm{v}$ & w & $x$ & $\mathrm{Y}$ & $z$ & $\mathrm{AA}$ & $\mathrm{AB}$ & $A C$ & $A D$ & $\mathrm{AE}$ & AF $A$ \\
\hline & 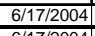 & 8:03:39 PM & & $\begin{array}{l}26.799 \\
.691\end{array}$ & \begin{tabular}{|l|}
24.812 \\
2.892
\end{tabular} & 27.8 & 26.657 & 47.8 & 48.907 & 12.236 & 35.547 & 1222 & -5.877 & & & 14.643 & 0.004 & & & 47.4 & & & & & & 0.00 & & \\
\hline & $6 / 17 / 2004$ & $8: 04: 19 \mathrm{PM}$ & \begin{tabular}{l|l|}
26.011 \\
25079
\end{tabular} & 26.81 & \begin{tabular}{|l|}
24.883 \\
2.845
\end{tabular} & 27.902 & $\begin{array}{l}26.662 \\
6673\end{array}$ & 46.2 & 48.439 & $\begin{array}{ll}11.624 \\
1.20\end{array}$ & 34.603 & -1.222 & $\begin{array}{l}-5.039 \\
-5.70\end{array}$ & 50.551 & 0.195 & $\begin{array}{l}14.643 \\
\end{array}$ & 0.004 & 594.6 & & 47.0 & 12.4 & 40.5 & & & 0.029 & 0.001 & 0.721 & \\
\hline 566 & $\begin{array}{l}6 / 17 / 2004 \\
6 \\
6 / 1 / 7204\end{array}$ & $\begin{array}{l}8: 05: 19 \text { PM } \\
8: 06 \cdot 19 P P\end{array}$ & $\begin{array}{l}25.979 \\
25.962\end{array}$ & $\begin{array}{l}26.802 \\
26.801\end{array}$ & \begin{tabular}{|l|}
24.845 \\
2.832
\end{tabular} & 27.93 & 26.73 & $\begin{array}{l}46.325 \\
46539\end{array}$ & 47.655 & $\begin{array}{l}12.395 \\
1170\end{array}$ & 33.86 & $\begin{array}{r}-1.219 \\
1.219\end{array}$ & $\begin{array}{l}-5.718 \\
6013\end{array}$ & $\begin{array}{l}45.635 \\
8.217\end{array}$ & 0.159 & 14.643 & 0.004 & $\begin{array}{l}595.6 \\
50 .\end{array}$ & 9.92639 & 46.2 & 11.2 & 40.1 & 2.764 & & 0.024 & 0.001 & 0.594 & \\
\hline 565 & $\begin{array}{ll}6 / 1 / 172004 \\
6 / 172004\end{array}$ & $\begin{array}{l}8.0619 \mathrm{PM} \\
807: 19 \mathrm{PM}\end{array}$ & $\frac{25.962}{26.002}$ & $\frac{26.801}{26.801}$ & \begin{tabular}{|l|}
24.833 \\
24.838
\end{tabular} & $\frac{27.953}{27.958}$ & & $\begin{array}{r}46.539 \\
46.35\end{array}$ & $\begin{array}{l}47.636 \\
477.998\end{array}$ & \begin{tabular}{|c|}
11.789 \\
12.321
\end{tabular} & $\begin{array}{l}34.697 \\
34.022\end{array}$ & $\begin{array}{l}-1.219 \\
-1.219\end{array}$ & $\begin{array}{r}-6.013 \\
-5.739 \\
\end{array}$ & $\begin{array}{r}48.317 \\
47.55\end{array}$ & $\begin{array}{l}0.188 \\
0.165\end{array}$ & $\begin{array}{l}14.643 \\
14.643\end{array}$ & & $\begin{array}{l}596.6 \\
597.6\end{array}$ & \begin{tabular}{|l|}
9.94306 \\
9.99572 \\
\end{tabular} & $\begin{array}{l}46.2 \\
46.3\end{array}$ & & $\begin{array}{l}40.6 \\
440.2\end{array}$ & & & 0.028 & $\begin{array}{l}0.001 \\
0.001\end{array}$ & $\begin{array}{l}0.693 \\
0.615\end{array}$ & \begin{tabular}{|l|}
0.59 \\
0.52 \\
\end{tabular} \\
\hline 566 & $6 / 17 / 2004$ & 8:08:19 PM & 26.013 & 26.816 & \begin{tabular}{|l|}
24.050 \\
\end{tabular} & 28.013 & 26.829 & 45.747 & 47.137 & 告. & 32.647 & -1.219 & -5.545 & 48.674 & 0.121 & $\begin{array}{l}14.045 \\
14.643\end{array}$ & 0.004 & 598.6 & \begin{tabular}{|l|l|}
9.97639 \\
\end{tabular} & $\begin{array}{l}40.5 \\
45.7\end{array}$ & $\frac{11.1}{11.9}$ & $\frac{40.2}{39.2}$ & $\frac{2.711}{2.702}$ & 0.025 & $\begin{array}{l}0.025 \\
0.018\end{array}$ & $\begin{array}{l}.001 \\
0.000\end{array}$ & \begin{tabular}{|l}
0.665 \\
0.462 \\
\end{tabular} & 0.52 \\
\hline 567 & $6 / 17 / 2004$ & & 26.001 & & \begin{tabular}{|l|}
24.843 \\
\end{tabular} & 27.967 & & 46.663 & \begin{tabular}{|l|l}
47.854 \\
\end{tabular} & 12.548 & 34.104 & -1.222 & -5.869 & 46.736 & 0.149 & $\begin{array}{l}14.643 \\
\end{array}$ & 0.004 & 599.6 & \begin{tabular}{|l|l|}
9.99306 \\
\end{tabular} & 46.4 & 11.5 & 40.4 & & & & & & \\
\hline 568 & $6 / 17 / 2004$ & $8: 10: 19$ PM & 25.991 & 26.794 & \begin{tabular}{|l|}
24.837 \\
\end{tabular} & & & 46.593 & 48.094 & \begin{tabular}{|l|l|}
12.308 \\
\end{tabular} & 34.204 & $\begin{array}{l}-1.225 \\
\end{array}$ & $\begin{array}{l}-6.097 \\
\end{array}$ & $\begin{array}{l}48.05 \\
\end{array}$ & 0.139 & \begin{tabular}{|l|l|}
14.643 \\
\end{tabular} & & 600.6 & \begin{tabular}{|l|}
10.0097 \\
\end{tabular} & 46.6 & 11.8 & 40.4 & 2.785 & 0.021 & 0.021 & 0.001 & 0.515 & \\
\hline 569 & 6/17/2004 & $8: 11: 19 \mathrm{PM}$ & 25.991 & & 24.873 & 27.952 & 26.732 & 46.945 & $\begin{array}{l}47.757 \\
\end{array}$ & 12.258 & 34.677 & $\begin{array}{r}-1.216 \\
\end{array}$ & -5.967 & 48.399 & 0.209 & 14.643 & 0.004 & 601.6 & & 46.3 & 11.9 & 40.8 & & & 0.031 & & & \\
\hline 570 & $6 / 117 / 2004$ & $8: 12: 19 \mathrm{PM}$ & \begin{tabular}{l|l|}
25.992 \\
25.024
\end{tabular} & $\begin{array}{l}26.796 \\
2670\end{array}$ & \begin{tabular}{|l|}
24.904 \\
\end{tabular} & 27.863 & 26.643 & $\begin{array}{l}46.477 \\
\end{array}$ & $\begin{array}{ll}47.419 \\
7691\end{array}$ & \begin{tabular}{|l|l|}
11.968 \\
\end{tabular} & 34.5 & $\begin{array}{l}-1.222 \\
.225\end{array}$ & $\begin{array}{l}-5.953 \\
5025\end{array}$ & \begin{tabular}{|l|l|}
58.73 \\
\end{tabular} & 0.196 & $\begin{array}{l}14.643 \\
1.642\end{array}$ & & $\begin{array}{l}602.6 \\
0.0\end{array}$ & \begin{tabular}{|l|}
10.0431 \\
\end{tabular} & 45.9 & 14.4 & & & 0.029 & 0.029 & 0.001 & 0.724 & \\
\hline 571 & $\frac{6 / 177 / 2004}{66 / 772004}$ & $\begin{array}{l}8: 13: 19 \mathrm{PM} \\
8: 14: 19 \mathrm{PM}\end{array}$ & $\begin{array}{l}25.996 \\
26.023\end{array}$ & $\begin{array}{r}26.79 \\
26.806 \\
\end{array}$ & \begin{tabular}{|l|}
24.903 \\
24.945 \\
\end{tabular} & \begin{tabular}{|l|}
27.837 \\
27.785
\end{tabular} & $\begin{array}{l}26.613 \\
26.529 \\
\end{array}$ & $\begin{array}{l}46.439 \\
44.118 \\
\end{array}$ & $\begin{array}{l}47.684 \\
47.541\end{array}$ & 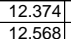 & $\begin{array}{l}33.989 \\
33.445 \\
\end{array}$ & \begin{tabular}{|c|c|}
-1.225 \\
-1.216
\end{tabular} & $\begin{array}{l}-6.005 \\
-6.06 \\
\end{array}$ & 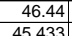 & \begin{tabular}{|l|l|l}
0.188 \\
0.204
\end{tabular} & $\begin{array}{l}14.643 \\
1.643\end{array}$ & $\begin{array}{l}0.004 \\
0.004\end{array}$ & $\begin{array}{l}603.6 \\
6046\end{array}$ & \begin{tabular}{|l|}
10.0597 \\
100764 \\
\end{tabular} & $\begin{array}{l}46.2 \\
46.1\end{array}$ & $\begin{array}{l}11.4 \\
11.1\end{array}$ & $\begin{array}{l}40.2 \\
39.8\end{array}$ & $\frac{2.175}{2743}$ & $\begin{array}{l}0.028 \\
0.030\end{array}$ & $\begin{array}{l}0.028 \\
0.030\end{array}$ & \begin{tabular}{|l|l|}
0.001 \\
0.001
\end{tabular} & \begin{tabular}{|l|l|}
0.699 \\
0776
\end{tabular} & $\begin{array}{l}0.59 \\
0.65\end{array}$ \\
\hline 573 & $6 / 17 / 2004$ & $\begin{array}{l}8.14 .19 \mathrm{PM} \\
8: 15: 19 \mathrm{PM}\end{array}$ & 26.018 & 26.781 & \begin{tabular}{|r|}
24.0492 \\
\end{tabular} & 27.684 & $\begin{array}{r}20.529 \\
26.47\end{array}$ & $\begin{array}{l}\frac{40.118}{46.269} \\
\end{array}$ & 47.28 & 12.429 & 33.831 & & & & $\frac{0.244}{0.223}$ & $\begin{array}{l}\frac{14.643}{14.643} \\
\end{array}$ & \begin{tabular}{|l|}
0.004 \\
0.003 \\
\end{tabular} & $\begin{array}{l}004.6 \\
605.6\end{array}$ & \begin{tabular}{|l|}
1.076464 \\
10.0931 \\
\end{tabular} & 40 & 11.0 & $\begin{array}{l}39.8 \\
40.1\end{array}$ & $\frac{2.143}{2.761}$ & & $\begin{array}{l}0.050 \\
0.033\end{array}$ & $\begin{array}{l}0.001 \\
0.001\end{array}$ & & \\
\hline 574 & $6 / 17 / 2004$ & $8: 16: 19 \mathrm{PM}$ & \begin{tabular}{|l|}
26.03 \\
\end{tabular} & 26.778 & 24.936 & 27.646 & 26.441 & 46.655 & 47.875 & 12.43 & 34.153 & -1.222 & $\begin{array}{r}-5.979 \\
\end{array}$ & 50.455 & 0.207 & 14.643 & 0.003 & 606.6 & \begin{tabular}{|l|l|}
10.1097 \\
\end{tabular} & 46.4 & 12.4 & 40.4 & 2.786 & 0.031 & 0.031 & 0.001 & $\begin{array}{l}0.032 \\
0.765\end{array}$ & $\begin{array}{l}0.71 \\
0.65\end{array}$ \\
\hline 575 & $6 / 17 / 2004$ & $8: 17: 19$ PM & 26.006 & & \begin{tabular}{|l|}
24.943 \\
\end{tabular} & & & 47.084 & 48.289 & 12.182 & & -1.222 & & 53.555 & 0.187 & 14.643 & & 607.6 & \begin{tabular}{|l|l|}
10.1264 \\
\end{tabular} & 46.8 & 13.1 & 41.0 & 2.825 & & 0.028 & 0.001 & 0.682 & \\
\hline 576 & $6 / 17 / 2004$ & $8: 18: 19$ PM & 26.001 & 26.75 & \begin{tabular}{|l|l|}
24.923 \\
\end{tabular} & 27.578 & 26.398 & 47.176 & 48.749 & 12.172 & 34.812 & -1.222 & & 48.399 & 0.208 & 14.643 & 0.003 & 608.6 & \begin{tabular}{|l|l|} 
& 10.1431 \\
\end{tabular} & 47.3 & 11.9 & 41.0 & 2.826 & 0.031 & $\begin{array}{l}0.031 \\
\end{array}$ & 0.001 & 0.758 & \\
\hline 577 & $6 / 17 / 2004$ & $8: 19: 19$ PM & 26.051 & & & & & & $\begin{array}{l}48.242 \\
\end{array}$ & 12.158 & 34.999 & $\begin{array}{l}-1.222 \\
\end{array}$ & & \begin{tabular}{|l|l|l|}
4.628 \\
\end{tabular} & 0.129 & 14.643 & & & & 46.8 & & & & & 0.019 & & 0.469 & 0.40 \\
\hline 578 & $6 / 17 / 2004$ & $8: 20: 19 \mathrm{PM}$ & 26.051 & $\frac{26.73}{26707}$ & $\begin{array}{r}24.943 \\
24.98 \\
\end{array}$ & & & 46.962 & 48.049 & 12.485 & 34.401 & $\frac{-1.222}{-1.219}$ & -6.019 & $\begin{array}{l}46.674 \\
5.0791\end{array}$ & 0.179 & 14.643 & 0.004 & 610.6 & \begin{tabular}{|l|l|} 
\\
\end{tabular} & 46.6 & 11.4 & & & & & 0.001 & 0.657 & 0.56 \\
\hline 579 & $\frac{66 / 1771204}{661 / 12004}$ & $\begin{array}{l}8: 21: 19 \mathrm{PM} \\
8: 22119 \mathrm{PM}\end{array}$ & $\frac{26.014}{26.034}$ & $\frac{26.707}{26.707}$ & \begin{tabular}{|l|}
24.98 \\
2.97 \\
\end{tabular} & $\frac{27.615}{27.64}$ & $\frac{26.446}{26.475}$ & $\begin{array}{l}46.276 \\
46.077\end{array}$ & $\begin{array}{r}47.39 \\
48.061\end{array}$ & \begin{tabular}{|r|}
12.24 \\
12.102
\end{tabular} & $\frac{34.046}{33.812}$ & \begin{tabular}{|c|}
-1.219 \\
-1.222 \\
\end{tabular} & $\begin{array}{r}-5.958 \\
-5.403 \\
\end{array}$ & $\begin{array}{l}56.974 \\
44.901\end{array}$ & $\begin{array}{l}0.191 \\
0.193 \\
\end{array}$ & $\begin{array}{l}14.643 \\
14.643 \\
\end{array}$ & 0.004 & $\frac{611.6}{6612.6}$ & \begin{tabular}{|l|}
10.1931 \\
10.2097 \\
\end{tabular} & $\begin{array}{l}45.9 \\
46.6\end{array}$ & $\begin{array}{l}14.0 \\
110\end{array}$ & $\begin{array}{l}40.2 \\
339.9\end{array}$ & \begin{tabular}{|l|l|}
2 & 2.769 \\
92.754
\end{tabular} & 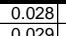 & $\begin{array}{l}0.028 \\
0.029\end{array}$ & $\begin{array}{l}0.001 \\
0001\end{array}$ & \begin{tabular}{|c|}
0.709 \\
0771
\end{tabular} & $\begin{array}{l}0.60 \\
0.661\end{array}$ \\
\hline & $6 / 17 / 2004$ & 8:23:19 PM & 26.035 & 26.688 & \begin{tabular}{|l|}
24.961 \\
\end{tabular} & 27.691 & 26.521 & 46.63 & $\begin{array}{l}40.011 \\
47.779\end{array}$ & 11.965 & 34.615 & \begin{tabular}{|l|l|}
-1.219 \\
\end{tabular} & $\begin{array}{l}-5.407 \\
-5.727\end{array}$ & 50.975 & 0.213 & $\begin{array}{l}\frac{14.643}{14.643} \\
\end{array}$ & $\begin{array}{l}.0004 \\
0.004\end{array}$ & \begin{tabular}{|l|}
612.6 \\
613.6
\end{tabular} & \begin{tabular}{|l|l|}
10.2099 \\
10.2264 \\
\end{tabular} & $\begin{array}{l}40.0 \\
46.3\end{array}$ & & $\begin{array}{l}30.5 \\
40.6\end{array}$ & $\frac{2.154}{2.801}$ & & & & & $\frac{0.61}{0.67}$ \\
\hline & $6 / 17 / 2004$ & $8: 24: 19 \mathrm{PM}$ & 26.057 & 26.701 & 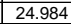 & 27.683 & & 46.939 & 48.275 & 11.872 & 34.99 & \begin{tabular}{|l|l|}
-1.219 \\
\end{tabular} & & $\begin{array}{l}43.445 \\
\end{array}$ & 0.211 & 14.643 & & $\begin{array}{l}014.6 \\
614.6\end{array}$ & $\mid 10.2431$ & 46. & 10.6 & & & & & & 0.0 .1868 & $\begin{array}{l}0.67 \\
0.65\end{array}$ \\
\hline & $6 / 17 / 2004$ & $8: 25: 19 \mathrm{PM}$ & 26.038 & 26.686 & \begin{tabular}{|l|}
24.975 \\
\end{tabular} & 27.659 & & 45.962 & 47.384 & 12.176 & 33.667 & \begin{tabular}{|l|l|}
-1.219 \\
\end{tabular} & -5.545 & \begin{tabular}{|l|l|l}
46.793 \\
\end{tabular} & 0.194 & \begin{tabular}{|l|l|l|}
14.643 \\
\end{tabular} & & & & 45.9 & 11.5 & 39. & & & 0.029 & & & \\
\hline & & & & & & & & & & & & & & & & 14.643 & & & & & & & & & & & 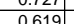 & \\
\hline & $6 / 17 / 12004$ & 8:27:19 PM & 26.04 & $\frac{16.673}{2673}$ & \begin{tabular}{|l|}
25.026 \\
\end{tabular} & 27.706 & & 46.45 & 488.032 & $\frac{12.211}{12.21}$ & 34.19 & $\frac{-1.222}{-1.22}$ & -5.143 & 45.781 & 0.20 & $\frac{14.045}{14.643}$ & & 617.6 & \begin{tabular}{|l|l|}
10.293 \\
\end{tabular} & 46.6 & 11.1 & 40 & 2.786 & & 0.036 & & 0.746 & 0.64 \\
\hline & $6 / 17 / 2004$ & $8: 28: 19 \mathrm{PM}$ & 26.073 & 26.66 & 25.005 & $27.74 \mathrm{G}$ & & 46.48 & \begin{tabular}{|l|l|l|}
47.68 \\
\end{tabular} & 12.316 & 34.077 & & & & 0.1 & 14.6 & & 618. & & 46.8 & & & & & $\overline{0.02}$ & & $\mid 0.696$ & \\
\hline 58 & $6 / 17 / 2004$ & 8:29:19 PM & 26.068 & 26.681 & 25.015 & & & & 47.526 & 12.204 & 33.948 & .219 & & 54.517 & 0.1 & & & & & 46.1 & 13.4 & & & & & & & \\
\hline & $6 / 117 / 2004$ & 8:30:19 PM & 26.063 & 26.686 & \begin{tabular}{|l|l|}
25.015 \\
\end{tabular} & 27.839 & & 46.116 & 47.79 & 11.929 & 34.114 & -1.219 & & |46.215 & 0.197 & $\begin{array}{l}144.643 \\
\end{array}$ & & 620.6 & \begin{tabular}{|l|l|} 
& 10.3431 \\
\end{tabular} & 46.3 & 11.3 & 44 & 2.766 & 0.029 & 0.029 & 0.001 & 0.732 & \\
\hline & $6 / 17 / 2004$ & 1:19 PM & 26.082 & 26 & 24.994 & 27.82 & & & 48.09 & 11.821 & 34.346 & -1.22 & & 53.294 & 0.18 & 14.643 & & & 10.3597 & 46.6 & 13.1 & & & & . & 0.00 & 0.688 & \\
\hline & & 8:32:19 PM & 26.102 & & & & & & & & & & & & & & & & & & & & & & & & & \\
\hline & 606172004 & $8.331 .14 \mathrm{PM}$ & $\frac{26.082}{26067}$ & $\frac{26.68}{26.89}$ & 24.969 & $\frac{2.158}{27718}$ & & 46.71. & 告8.006 & $\frac{12.118}{125}$ & $\frac{34.492}{23606}$ & $-\frac{1.225}{1.210}$ & & 39.3 & 0.1 & 14.6 & & $\frac{623.6}{6346}$ & 0.3931 & 46.6 & $\begin{array}{r}9.7 \\
107\end{array}$ & & & & & & 336 & \\
\hline 593 & $\frac{01172004}{61 / 7204}$ & 6.34.1.19 PM & $\frac{2.001}{26038}$ & $\frac{2.061}{26686}$ & $\begin{array}{r}24.9085 \\
25\end{array}$ & $\frac{2.1 .18}{2765}$ & $\frac{20.53}{2646}$ & $\frac{40.13}{45.676}$ & (4t.1.64) & $\mid$ & 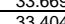 & $\frac{-1.219}{-1202}$ & -486 & $\begin{array}{ll}43.5064 \\
44354\end{array}$ & $\frac{0.181}{1027}$ & $\begin{array}{l}44.045 \\
11643\end{array}$ & & 624. & $\mid$ & 40.5 & 10.1 & 39.5 & 2726 & 0,033 & 0033 & & & 071 \\
\hline 594 & $6 / 17 / 2004$ & $8.53 .3 \cdot 19 \mathrm{PM}$ & 26.044 & 26.677 & 25005 & 27605 & & & \begin{tabular}{|l|}
47.0000 \\
7
\end{tabular} & $\frac{12.11}{12.14}$ & $\begin{array}{l}30.404 \\
33.661\end{array}$ & -1219 & & 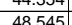 & 0.0197 & $\frac{1406}{146}$ & & 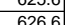 & $\mid$ & 4463 & 119 & 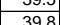 & & & & & 年 & \\
\hline & & 8:37.79 PM & 26.045 & 26.653 & 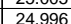 & 2753 & & & 48106 & $\frac{12.37}{12187}$ & $\frac{30.001}{33.89}$ & & & & $\frac{0.18}{0.18}$ & 14 & & & & 467 & . & & & & & & $\frac{105}{865}$ & \\
\hline & $6 / 17 / 26$ & $8: 38: 19$ & & $26.63 !$ & 24988 & 27.448 & & & 47.622 & 12.1699 & $\frac{53.30}{33.304}$ & & & 57.19 & & 14. & & & 0.4764 & 461 & 140 & & & & 0 & 0001 & 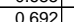 & \\
\hline 59 & $6 / 17 / 12004$ & & 26.039 & & \begin{tabular}{|l|l|}
2499 \\
\end{tabular} & 27.41 & & & 47.827 & 12.255 & $\frac{23.5}{33.5}$ & -1.122 & & $\frac{251}{52.1}$ & 0 & & & & & 46 & 12 & & & & 5.8 & & & \\
\hline & $6 / 17 / 72004$ & & & & & & & & 48239 & & & & & & & & & & & & & & & & & & & \\
\hline & 6/17/21 & 8:11:19 PM & & 26.631 & \begin{tabular}{|l|}
25.024 \\
\end{tabular} & 27.544 & & 45.8 & 48.014 & $\mid$\begin{tabular}{|l|l|}
12.113 \\
\end{tabular} & 33.605 & -1.22 & & & & 14.6 & & & & 46.5 & 10.6 & 39. & & & 0.0 & & & \\
\hline & 6/177/2004 & 8:42:19 PM & 26.036 & & \begin{tabular}{|l|}
25.018 \\
\end{tabular} & 27.608 & & 45.853 & \begin{tabular}{ll|l}
48.258 \\
\end{tabular} & & & & & 41.0 & & & & & & 46.8 & 10.1 & & & & & & & \\
\hline 60 & $6 / 1712044$ & 8:43:19 PM & 26.051 & 26.585 & 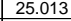 & 27.643 & & 45.264 & 47.873 & \begin{tabular}{|l|l|}
12.169 \\
\end{tabular} & 32.93 & -1.222 & & 50.568 & 0.158 & & & & & $\begin{array}{ll}46.4 \\
\end{array}$ & 12.4 & $3 !$ & & & & & & \\
\hline & 6/17/2004 & 8:44:19 F & 26.051 & 26.575 & 23.008 & 27.692 & & 46.143 & 48.563 & 12.211 & 33.015 & -1.24 & & 48. & 0.179 & 14. & & & & 47.1 & 11.0 & 40 & & & 0.0 & & & \\
\hline & & & 26.081 & & & 27.162 & & & & & & -1.219 & & & & & & & & & & & & & & & & \\
\hline & 6 & $8: 66.19 \mathrm{PM}$ & 26.149 & $\frac{26.5}{2.5}$ & 25.002 & $2.77 \pi$ & & 45.82. & $\begin{array}{r}48.25 \\
18.37\end{array}$ & $\frac{12.151}{12.155}$ & $\frac{33.542}{232020}$ & 120 & & 486.6 & 0.1 & 14. & & & & 46.8 & 10.0 & $\Delta$ & & & & & & \\
\hline & $\frac{0.1172004}{6}$ & $\frac{0.47 .19}{8: 4 \cdot 19}$ & $\frac{2.1449}{26.154}$ & & \begin{tabular}{|l|l|}
25000 \\
\end{tabular} & & & & 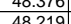 & $\frac{12.1}{12.1}$ & & & & $\frac{40.6}{49.3}$ & & & & & & & & & & & & & & \\
\hline & $\frac{111 / 72044}{6}$ & 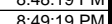 & 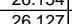 & $\frac{2.3111}{26496}$ & 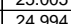 & 27.848 & & & (40.4219 & $\mid$ & & -1.22 & & & 0.1 & & & & & & 12.14 & & & & & & & \\
\hline & 6 & 8:50.5:19 PM & 20.14 & & 24.004 & & & & 48468 & & & & & & & & & & & & & & & & & & & \\
\hline |ct & $6 / 17 / 72004$ & $8.51 .19 \mathrm{PM}$ & 26.07 & 26.469 & \begin{tabular}{|l|l|}
25002 \\
\end{tabular} & 27896 & & 46.276 & 48.438 & 122 & 33962 & -1225 & & 44 & 01 & & & & & 470 & 100 & & & & 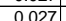 & & 51 & \\
\hline & $6 / 17 / 2004$ & $8: 52: 1 \mathrm{PM}$ & 26.064 & & 20.005 & 27.92 & & 46.26 & 48.211 & $\frac{12.251}{12.21}$ & $\frac{2.3845}{33.85}$ & -1.222 & & 46.7 & & & & & & & & & & & & & \begin{tabular}{ll|l}
144 \\
\end{tabular} & \\
\hline & $6 / 17 / 2004$ & 8:53:19 PM & 26.073 & 26.466 & 25.02 & 27.944 & & 46.26 & 48312 & 12.226 & 33.901 & -1.219 & & 48 & & 14.6 & & & & 46.8 & 118 & & 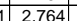 & & & & 0.669 & \\
\hline & $6 / 177 / 2$ & $8: 54: 1: 1$ & 26.06 & & 25 & 27. & & 46.2 & \begin{tabular}{|l|l|l|l|l|}
483 \\
\end{tabular} & $\overline{12.1}$ & 34. & -1.219 & & 48.6 & 0.1 & 14. & & & & $46 \quad-1$ & $\overline{11.9}$ & & & & & & (61) & \\
\hline & $6 / 17 / 2004$ & 8:55:19 PM & 26.068 & 26.4 & 25 & 27. & & 46.518 & 48.718 & \begin{tabular}{|l|l|}
12.099 \\
\end{tabular} & 34. & -1.219 & & & & & & 6 & & & 11 & & & & & & & \\
\hline & $6 / 17 / 2$ & 8:56:19 F & 26.04 & & 25.0 & 27. & & $45.91:$ & $\begin{array}{l}48.26 \\
\end{array}$ & 12.19 & $\overline{33.661}$ & -1.222 & & 49.8 & 0.1 & & & & & $\begin{array}{lll}46.8 \\
\end{array}$ & $\overline{12.2}$ & & & & & & | & \\
\hline & $6 / 177 / 2$ & & 26.03 & & & & & 46.2 & 48.666 & & & & & & & & & & & 47.2 & & & & & & & & \\
\hline 61 & $6 / 1772004$ & 8:58:19 PM & 26.0 & 26.435 & 25.0 & 27. & & 46 & 48.615 & 12.206 & 33.749 & -1.222 & & 50. & & & & & & 47.11 & 12.3 & & & & & & & \\
\hline & $\frac{6 / 17172004}{6}$ & $8: 59: 19 \mathrm{PM}$ & & & & & & 45.8 & 48.594 & & & & & & & & & & & & & & & & & & & \\
\hline & 0 & $9.000 .119 \mathrm{PM}$ & & & & & & & 4.004 & & & & & & & & & & & & & & & & & & & \\
\hline & $\frac{1}{61 / 72004}$ & $3.052 \cdot 9 \mathrm{PM}$ & 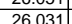 & 26.449 & \begin{tabular}{|l|l|}
25.035 \\
25.032
\end{tabular} & $\frac{2.046}{28046}$ & & 45718 & 14.452 & $\mid$ & 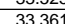 & -1216 & & 年 & 0.1166 & & & 65 & & 471 & 10.4 & 39 & & & & & & \\
\hline & $6 / 17 / 2004$ & & & & & & & 4584 & & & & $1.2+0$ & & & & & & & & & & & & & & & & \\
\hline & $6 / 17 / 2004$ & $9: 04: 19$ & 26.066 & 26.439 & 25012 & 28 & & -2.756 & 058 & 1265 & -4.068 & & & -0. & & & & & & -09 & 0 & & & & & & & \\
\hline & $6 / 17 / 2$ & & 26.1 & & 24.991 & 27.846 & & $-2.63 !$ & 0.128 & $\begin{array}{ll}1.025 \\
\end{array}$ & -3.71 & & & -0.0 & & 14 & & & & -1.5 & & & & & & & & \\
\hline & $6 / 17 / 2004$ & $\begin{array}{l}: 06: 19 \mathrm{P} \\
\end{array}$ & 26.2 & 26.42 & 250 & 27.626 & & -2.279 & $\begin{array}{l}-0.01 \\
\end{array}$ & 0.985 & -3.33 & -1216 & & -00 & & 14.6 & & 65 & & & n & & & & & & & \\
\hline & $6 / 177 / 2004$ & 9:07:19 PM & & & \begin{tabular}{|l|}
24.999 \\
\end{tabular} & & & -1.9 & -0.104 & & & -1219 & & -0.0 & & 14. & & 65 & & $1.6 \quad$ & $0.6 \quad-1$ & & & & & & 30 & \\
\hline 162 & 6/17/12004 & 9:08:19 PM & 26.241 & 26.369 & \begin{tabular}{|l|}
24.987 \\
\end{tabular} & 27.163 & & $\begin{array}{l}-1.692 \\
\end{array}$ & $\mid-0.18$ & $\mid 0.952$ & -2.75 & -1.225 & & -0.0 & 0.00 & & & & & -1.7 & 0.0 & & & & 0.000 & & & 0.1 \\
\hline & $6 / 17 / 7 /$ & 9:09:1 & 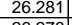 & & & & & -1.47 & -0.251 & 0.94 & -2.52 & & & & & & & & & & c. & & & & & & & \\
\hline 28 & & 9:10:1 & & & & & & & -0.325 & & 346 & & & & 0.003 & 14.643 & & & & $1.8 \mathrm{~S}$ & 0. & & & & 000 & 100 & 246 & \\
\hline
\end{tabular}


WSRC-TR-2005-00105, REVISION 0 SRNL-RPP-2005-00012, REVISION 0

RUN \# WASH1 AND WASH 2; CAMPAIGN III SLURRY WASHING - CONT.

\begin{tabular}{|c|c|c|c|c|c|c|c|c|c|c|c|c|c|c|c|c|c|c|c|c|c|c|c|c|c|c|c|c|}
\hline & A & B & D & $E$ & $F$ & G & $\mathrm{H}$ & $\mathrm{J}$ & K & L & M & $\mathrm{N}$ & $\mathrm{O}$ & $Q$ & $R$ & $\mathrm{~s}$ & $\begin{array}{lll}T & \mathrm{~L}\end{array}$ & |ul $v$ & w & $x$ & Y & $z$ & $\mathrm{AA}$ & $\mathrm{AB}$ & $\mathrm{AC}$ & $\mathrm{AD}$ & $\mathrm{AE}$ & AF $A$ \\
\hline $631 \mathrm{v}$ & Nash 1 and & & & & & & & & & & & & & & & & & & & & & & & & & & & \\
\hline \begin{tabular}{l|l}
632 \\
532
\end{tabular} & & \begin{tabular}{|l|l} 
Averages \\
\end{tabular} & $\frac{25.809}{2.029}$ & 26.430 & 24.456 & 27.693 & 26.470 & 45.657 & 48.431 & 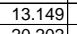 & 32.668 & 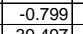 & $\begin{array}{l}-5.880 \\
0.081\end{array}$ & 47.728 & 0.125 & $\begin{array}{l}14.643 \\
\end{array}$ & 0.004 & & & 46.96 & 11.70 & 39.16 & 2.700 & 0.019 & 0.019 & 0.000 & 0.473 & \\
\hline$\left|\frac{633}{634}\right|$ & & \begin{tabular}{|l|} 
Maximum \\
Median
\end{tabular} & $26.281 \mid$ & \begin{tabular}{|l|}
26.886 \\
26.614
\end{tabular} & \begin{tabular}{|l|}
25.627 \\
24.369 \\
\end{tabular} & $28.328 \mid$ & $27.000 \mid$ & $\begin{array}{l}53.501 \\
46.411\end{array}$ & 55.102 & \begin{tabular}{|l|l|}
20.202 \\
12.918
\end{tabular} & \begin{tabular}{|c|}
37.956 \\
3.343
\end{tabular} & \begin{tabular}{|l|}
39.497 \\
-1.222 \\
\end{tabular} & $\begin{array}{l}93.981 \\
-6.579\end{array}$ & $\begin{array}{l}64.030 \mid \\
48.232\end{array}$ & $\begin{array}{l}0.366 \\
0.111\end{array}$ & \begin{tabular}{rl|l}
14.643 & 14.643 \\
\end{tabular} & $\begin{array}{l}0.005 \\
0.004\end{array}$ & & & $\begin{array}{r}53.63 \\
4.42 \\
\end{array}$ & $\frac{15.69}{11.82} \mid$ & $\begin{array}{l}45.13 \\
39.95\end{array}$ & $\begin{array}{l}3.112 \\
2.754 \\
\end{array}$ & 0.055 & $\begin{array}{l}0.054 \\
0.017\end{array}$ & 0.002 & $\begin{array}{l}1.740 \\
0.427 \\
\end{array}$ & $\begin{array}{l}1.481 \\
0.364\end{array}$ \\
\hline 635 & & Minimum & 25.024 & 24.739 & \begin{tabular}{|l|}
23.593 \\
\end{tabular} & 26.379 & 25.545 & $\begin{array}{ll}-2.411 \\
-2.756\end{array}$ & -0.325 & 0.928 & -4.068 & \begin{tabular}{|c|}
-1.228 \\
\end{tabular} & -8.048 & -25.023 & 0.003 & $\begin{array}{l}14.040 \\
14.643\end{array}$ & 0.003 & & & $\begin{array}{l}-1.44 \\
-1.80 \\
\end{array}$ & -6.13 & -3.41 & $\begin{array}{l}2.1235 \\
-0.235 \\
\end{array}$ & 0.000 & 0.000 & 0.000 & $\frac{-.476}{-0.246}$ & $\begin{array}{l}0.064 \\
-0.209 \\
\end{array}$ \\
\hline & & $2 \times$ Std Dev & 0.510 & 0.922 & 0.814 & 0.735 & 0.507 & 12.451 & 10.832 & 3.792 & 10.326 & 7.083 & 12.245 & 14.629 & 0.090 & 0.000 & 0.001 & & & 10.83 & & 11.30 & & & 0.013 & 0.000 & 0.351 & \\
\hline \begin{tabular}{|l|l|}
637 \\
\end{tabular} & Number o & of Points Used* & 606 & 606 & 606 & 606 & 606 & 606 & 606 & 606 & 606 & 606 & 606 & 606 & 606 & 606 & 606 & & & 606 & 606 & 606 & 606 & 606 & 606 & 606 & 606 & 606 \\
\hline \begin{tabular}{|c|}
638 \\
32
\end{tabular} & & ${ }^{\star}$ Backpulse poin & $s$ in boxa & re not incl & iluded & & & & & & & & & & & & & & & & & & & & & & & \\
\hline & & & & & & & & & & & & & & & & & & & & & & & & & & & & \\
\hline$\frac{64001}{641}$ & Wash 1 & Averages & & 26.252 & 24.264 & 27.587 & 26.406 & 46.332 & 49.626 & 13.745 & 32.842 & $\mid-0.799$ & -6.163 & 48.123 & 0.101 & 14.643 & 0.004 & & & 48.15 & 11.79 & 39.59 & 2.729 & 0.015 & 0.015 & 0.000 & 0.386 & 0.328 \\
\hline \begin{tabular}{|l|l|}
642 \\
\end{tabular} & & Maximum & 26.185 & 26.805 & 25.627 & 28.328 & 26.887 & 53.501 & 55.102 & 20.202 & 37.956 & 38.863 & 93.981 & 64.030 & 0.366 & 14.643 & 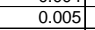 & & & 53.63 & 15.69 & 45.13 & 3.112 & & 0.054 & 0.002 & 1.740 & $\begin{array}{l}0.020 \\
1.481 \\
\end{array}$ \\
\hline 643 & & Median & 25.743 & 26.404 & 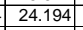 & 27.634 & 26.412 & 46.474 & 49.680 & 13.313 & 33.064 & \begin{tabular}{|l|}
-1.222 \\
\end{tabular} & -7.074 & 48.185 & 0.102 & 14.643 & 0.004 & & & 48.21 & 11.81 & 39.76 & 2.742 & 0.015 & 0.015 & 0.000 & 0.389 & 0.331 \\
\hline 644 & & Minimum & 25.024 & 24.739 & 23.593 & 26.379 & 25.545 & 9.142 & $\begin{array}{l}47.261 \\
\end{array}$ & 11.245 & -2.276 & -1.228 & -8.048 & -25.023 & 0.003 & 14.643 & 0.003 & & & 45.79 & -6.13 & 3.43 & 0.237 & 0.000 & 0.000 & 0.000 & 0.012 & 0.010 \\
\hline 645 & & $2 \times$ Std Dev & $\begin{array}{l}0.496 \\
077\end{array}$ & 1.009 & $\begin{array}{ll}0.742 \\
372\end{array}$ & $\begin{array}{l}0.823 \\
271\end{array}$ & \begin{tabular}{ll|l}
0.488 \\
\end{tabular} & 6.577 & 2.519 & 2.945 & 6.654 & $\begin{array}{ll}6.862 \\
\end{array}$ & 14.799 & 11.290 & 0.050 & 0.000 & 0.001 & & & 2.52 & 2.77 & 6.48 & $\begin{array}{ll}0.447 \\
377\end{array}$ & 0.007 & 0.007 & 0.000 & 0.204 & 0.174 \\
\hline \begin{tabular}{|c|}
646 \\
647
\end{tabular} & Number o & 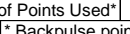 & $\begin{array}{r}372 \\
\sinh 2 x\end{array}$ & 372 & 372 & 372 & 372 & 372 & 372 & 372 & 372 & 372 & 372 & 372 & 372 & 372 & 372 & & & 372 & 372 & 372 & 372 & 372 & 372 & 372 & 372 & 372 \\
\hline $\mid \frac{647}{648}$ & & *Backpulse poin & sin box a & & & & & & & & & & & & & & & & & & & & & & & & & \\
\hline $\begin{array}{ll}0.049 \\
6649\end{array}$ & Nash 2 & & & & & & & & & & & & & & & & & & & & & & & & & & & \\
\hline & & Averages & 25.991 & 26.713 & 24.762 & 27.863 & 26.573 & 44.954 & 46.450 & 12.226 & 32.733 & -1.199 & -5.814 & 47.152 & \begin{tabular}{|c|c|}
0.167 \\
\end{tabular} & 14.643 & 0.004 & & & \begin{tabular}{l|l}
44.98 \\
\end{tabular} & 11.56 & 38.84 & 2.678 & 0.025 & 0.025 & 0.001 & 0.616 & 0.525 \\
\hline 651 & & Maximum & 26.281 & 26.886 & 25.039 & 28.237 & 27.000 & 49.017 & \begin{tabular}{|l|l|}
50.343 \\
\end{tabular} & 14.648 & 36.047 & $\begin{array}{ll}0.576 \\
\end{array}$ & 0.555 & 62.497 & 0.262 & 14.643 & 0.004 & & & 48.87 & 15.32 & & & & & 0.001 & $\begin{array}{ll}1.034 \\
\end{array}$ & 0.880 \\
\hline $6 \frac{652}{653}$ & & $\begin{array}{l}\text { Median } \\
\text { Minimum }\end{array}$ & 26.013 & $\frac{26.741}{26.342}$ & \begin{tabular}{|l|}
24.838 \\
24.101 \\
\end{tabular} & $27.892 \mid$ & $26.621 \mid$ & $\begin{array}{l}46.317 \\
-2.756\end{array}$ & $\begin{array}{l}47.725 \mid \\
-0.2325\end{array}$ & $\frac{12.240}{0.928}$ & $\begin{array}{ll}33.862 \\
-4.068\end{array}$ & \begin{tabular}{|l|l|}
-1.222 & \\
-1.228 &
\end{tabular} & $\begin{array}{l}-6.045 \\
-6.811 \\
\end{array}$ & $\begin{array}{l}48.317 \\
-0.075 \\
\end{array}$ & $\begin{array}{l}0.171 \\
0.003\end{array}$ & $\begin{array}{l}14.643 \\
14.643\end{array}$ & $\begin{array}{l}0.004 \\
0.003\end{array}$ & & & 46.25 & 11.84 & 40.0822 & $\begin{array}{l}2.764 \\
-0.255 \\
\end{array}$ & 0.025 & 0.026 & 0.001 & 0.639 & 0.544 \\
\hline$\left|\frac{653}{654}\right|$ & & $\begin{array}{l}\text { Minimum } \\
2 \times \text { Std Dev }\end{array}$ & $\begin{aligned} 25.506 \\
2.256\end{aligned}$ & $\begin{array}{r}26.342 \\
0.247 \\
\end{array}$ & \begin{tabular}{|l|}
24.1 .101 \\
0.491 \\
\end{tabular} & $\begin{array}{r}26.9611 \\
0.376\end{array}$ & $\frac{25.642}{0.474}$ & $\begin{array}{c}-2.756 \\
16.743\end{array}$ & \begin{tabular}{|}
$\mid-3.325$ \\
16.581 \\
\end{tabular} & \begin{tabular}{|c|}
0.228 \\
4.203
\end{tabular} & $\begin{array}{l}-4.068 \\
12.823 \\
\end{array}$ & 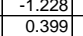 & , & $\begin{array}{r}-0.075 \\
18.846 \\
\end{array}$ & $\begin{array}{l}0.003 \\
0.074\end{array}$ & $\begin{aligned} 14.6033 \\
0.000\end{aligned}$ & $\begin{array}{l}0.003 \\
0.001 \\
\end{array}$ & & & \begin{tabular}{|c|}
-1.80 \\
16.58
\end{tabular} & $\begin{array}{c}-0.02 \\
.62 \\
\end{array}$ & $\begin{array}{l}-3.41-C \\
14.77 \\
\end{array}$ & \begin{tabular}{|c|}
-0.235 \\
1.018
\end{tabular} & \begin{tabular}{|l|}
0.000 \\
0.011 \\
\end{tabular} & $\begin{array}{l}0.000 \\
0.011\end{array}$ & $\begin{array}{l}0.000 \\
0.000\end{array}$ & $\begin{aligned}-0.246 \\
0.342\end{aligned}$ & $\begin{array}{l}-0.209 \\
0.291 \\
\end{array}$ \\
\hline 655 & Number o & of Points Used* & 229 & 229 & 229 & 229 & 229 & 229 & 229 & 229 & 229 & 229 & 229 & 229 & 229 & 229 & 229 & & & 229 & 229 & 229 & 229 & 229 & 229 & 229 & 231 & 231 \\
\hline 656 & & * Backpulse poin & 5 in box a & re not inc & iluded & & & & & & & & & & & & & & & & & & & & & & & \\
\hline
\end{tabular}


WSRC-TR-2005-00105, REVISION 0

SRNL-RPP-2005-00012, REVISION 0

RUN \# 3.18 AND 19; ACID CLEANING AND CAUSTIC RINSING

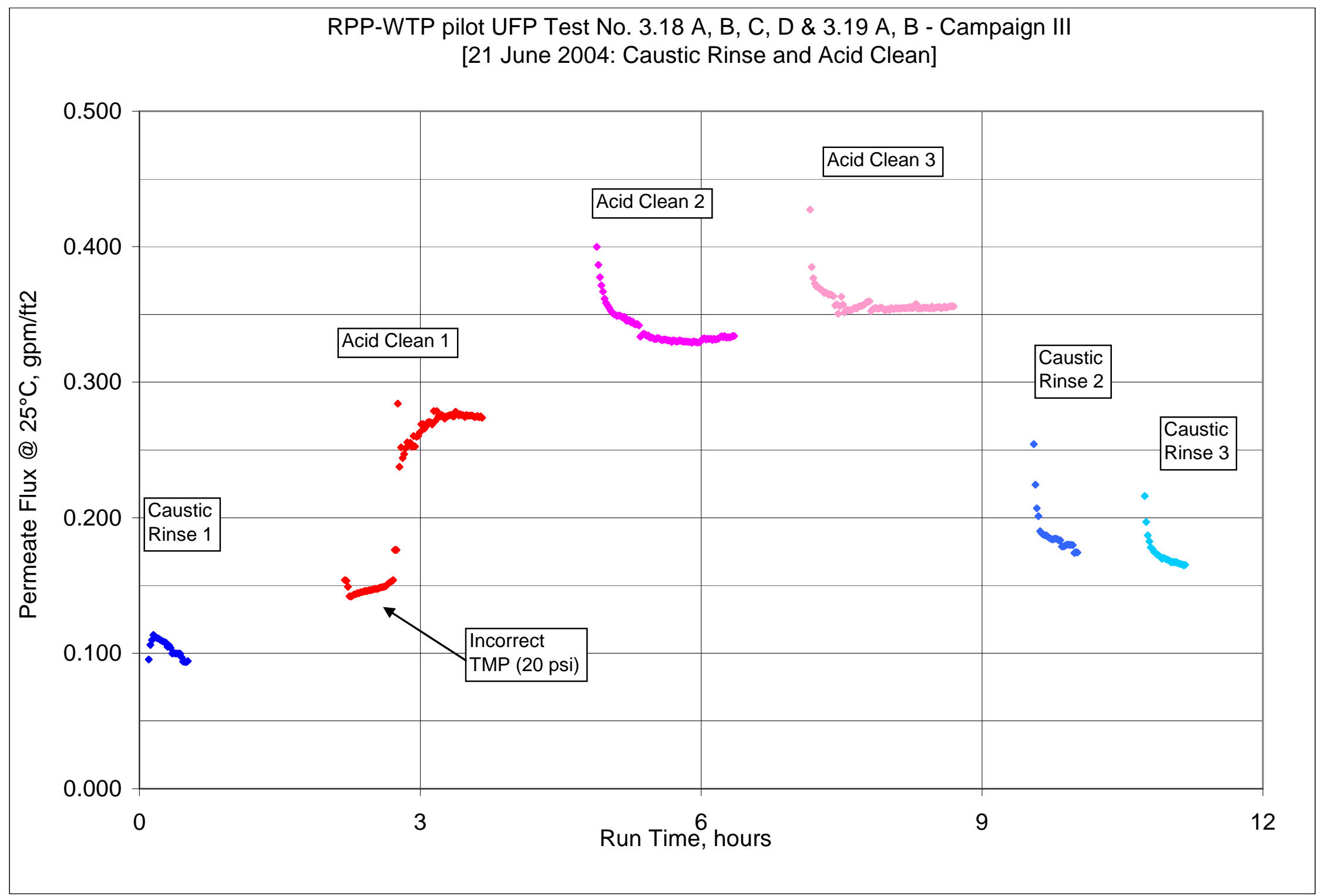


WSRC-TR-2005-00105, REVISION 0

SRNL-RPP-2005-00012, REVISION 0

RUN \# 3.18 AND 19; ACID CLEANING AND CAUSTIC RINSING - CONT.

\begin{tabular}{|c|c|c|c|c|c|c|c|c|c|c|c|c|c|c|c|c|c|c|c|c|c|c|c|c|c|c|c|c|}
\hline & A & B & $\frac{D}{R \ll \ll<}$ & $\begin{array}{l}\mathrm{E} \\
\mathrm{S} \text { Tempe }\end{array}$ & $F$ & $\frac{G}{G}$ & $\mathrm{H}$ & $\mathrm{J}$ & $\frac{K}{\ll \ll \ll<}$ & $L$ & $\mathrm{M}$ & $\left|\frac{N}{|l|}\right|$ & $\frac{0}{10}$ & $\frac{Q}{Q}$ & $\begin{array}{l}\mathrm{R} \\
\ll<\mathrm{Flow}\end{array}$ & $\frac{s}{s}$ & $\mathrm{~T}$ & $\mathrm{v}$ & w & $\frac{\mid x}{<\ll \ll<}$ & 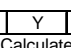 & \begin{tabular}{ll|}
$z$ \\
edFron
\end{tabular} & AA & $\mid$\begin{tabular}{|l|l|}
$A B$ \\
$D a t a \gg \gg \geqslant$
\end{tabular} & \begin{tabular}{|c|}
$A C$ \\
\end{tabular} & $A D$ & $\mathrm{AE}$ & $\begin{array}{lll}\mathrm{AF} & \mathrm{A} \\
\end{array}$ \\
\hline & DATE & TIME & Filtrate & Cleaning | & Slurry & Hi Amb. & Lo Amb. & BotTMP & Filter & Fiter dP & TopTMP & Filtrate & \begin{tabular}{|l|l|} 
\\
Pulsepot
\end{tabular} & Slurry & Filtrate & $\begin{array}{l}\text { Neasureme| } \\
\text { Hi Filtate| }\end{array}$ & Backpulse & & & & & & & & & & & \\
\hline & & & $\operatorname{deg} C$ & $\frac{1 \operatorname{deg} C}{\operatorname{deg}}$ & $\operatorname{deg} C$ & \begin{tabular}{|l|}
$\operatorname{deg} C$ \\
\end{tabular} & $\operatorname{deg} C$ & psid & psig & psid & psid & & & gpm & $\mathrm{gpm}$ & gpm & & & & & & & & & & & & \\
\hline & & & $\mathrm{T} 2$ & T3 & T1 & T4 & & $\mathrm{dP2}$ & $\mathrm{P1}$ & $\mathrm{dP1}$ & $\mathrm{dP3}$ & $\mathrm{P2}$ & & Q1 & $\mathrm{Q} 2$ & $\mathrm{P}_{3}$ & & & & & & & & & & & & \\
\hline & $04 / 16$ & & & & & & & & & & & & & & & & & & & & & & & & & & & \\
\hline & $88 / 2004$ & $\overline{~ 99: 09 ~ A M}$ & & 24.705 & & & .911 & & & & -0.003 & & & 45.741 & 0.29 & & & & & & & & & & & & & \\
\hline & & & & & 27.981 & & & $\frac{-0.01}{-0.01}$ & $\begin{array}{l}0.196 \\
0.196\end{array}$ & $\begin{array}{l}-0.012 \\
-0.012\end{array}$ & & $\begin{array}{l}-0.129 \\
-0.106\end{array}$ & & & & 年 14.643 & & & & & & & $\frac{12}{3 / \mathrm{m} 2}$ & aylba & $\mathrm{gpm} / /$ & barg & & \\
\hline & $6 / 114 / 2004$ & 7:41:41 AM & 23.83 & 23.885 & 66.603 & 24.174 & 24.204 & -0.012 & 0.12 & -0.01 & -0.016 & -0.163 & 0.107 & - -0.083 & 0.003 & 14.643 & & & & & & & & & & & & \\
\hline & $6 / 15 / 2004$ & 12:06:06 PM & 25.175 & 24.315 & 23.747 & 26.127 & \begin{tabular}{|l|l|}
23.997 \\
\end{tabular} & -0.012 & \begin{tabular}{|l}
0.139 \\
\end{tabular} & -0.002 & \begin{tabular}{|c|c|}
-0.012 \\
\end{tabular} & -0.177 & 0.04 & -0.073 & 0.003 & 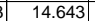 & & & & Note: & & & & oproxim & nately 1. & osig & & \\
\hline 11 & $6 / 177 / 2004$ & $7: 28: 28 \mathrm{AM}$ & 24.492 & 23.951 & 23.799 & 24.225 & \begin{tabular}{|l|l|l}
23.743 \\
\end{tabular} & -0.01 & 0.164 & $\mid-0.001$ & $\mid-0.014$ & $\mid-0.18$ & 0.032 & -0.075 & 0.003 & \begin{tabular}{|l|l|l|l|l}
14.643 \\
\end{tabular} & $5 x$ & & & & & & & & & & & \\
\hline & $6 / 17 / 2004$ & 4:57:57 PM & 26.259 & 26.737 & 24.765 & 27.805 & 26.456 & -0.002 & 0.197 & & \begin{tabular}{|c|c|c|}
-0.012 \\
\end{tabular} & -0.183 & -0.009 & 47.043 & 0.098 & $\begin{array}{l}14.643 \\
\end{array}$ & & & & & Dic & & ed for & & & tet & & \\
\hline & $6 / 21 / 2004$ & $6: 52: 52 \mathrm{AM}$ & & 23.008 & & 23.418 & & & 0.075 & & -0.01 & -0.197 & & & 0.003 & $\begin{array}{l}14.643 \\
\end{array}$ & & & & WWV & & & & $<$ < Filtrat & te Flux $=$ & $P E$ & $|A B| \mid$ & $\gg$ \\
\hline & 6/22/2004 & $8: 12: 12 \mathrm{AM}$ & 23.36 & 23.555 & 23.63 & 23.829 & 24.45 & -0.008 & 0.108 & -0.004 & -0.012 & -0.194 & 0.06 & -0.083 & 0.003 & 14.643 & & & & & & & & & & & & \\
\hline$\frac{15}{16}$ & Data - Per M & inute & & & & & & & & & & & & & & & & Time & $\begin{array}{l}\text { Time } \\
\text { Hour }\end{array}$ & $\begin{array}{l}\text { Press. } \\
\text { psii. }\end{array}$ & Vel. & $\begin{array}{ll}\text { TMP } \\
\text { ns }\end{array}$ & TMP & gpm $\frac{c}{d}$ & gpm & $\frac{g p m}{g t / p s i}$ & gpm & $\begin{array}{l}\text { meter } \\
\text { dav/lar }\end{array}$ \\
\hline 17 & $6 / 21 / 2004$ & 9:27:16 AM & & 24.021 & & 25.107 & \begin{tabular}{|l|}
24.404 \\
\end{tabular} & & \begin{tabular}{|l|l|}
26.986 \\
\end{tabular} & 8.631 & $\mid-1.057$ & 14.272 & $\begin{array}{l}67.403 \\
\end{array}$ & & 0.003 & \begin{tabular}{|l|l|l|}
14.643 \\
\end{tabular} & & & & 25.5 & & & & & & & 0.135 & 势 \\
\hline 18 & $6 / 21 / 2004$ & 9:28:16 AM & 24.782 & 24.031 & 25.423 & $\begin{array}{ll}25.058 \\
\end{array}$ & 24.41 & 7.311 & \begin{tabular}{|l|l}
27.019 \\
\end{tabular} & 8.587 & $\begin{array}{ll}-1.541 \\
\end{array}$ & \begin{tabular}{|l|l|}
14.382 \\
\end{tabular} & 66.935 & 53.375 & 0.003 & 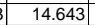 & & & 0.01667 & 25.5 & 13.1 & & 0.199 & 0.000 & 0.000 & 0.000 & 0.153 & 0.13 \\
\hline & 6/21/2004 & 9:29:16 AM & 24.368 & 24.043 & 25.514 & 25.129 & 24.451 & 4.488 & 26.242 & 5.474 & -1.026 & 16.637 & 30.712 & 52.333 & 0.003 & $\begin{array}{l}14.643 \\
\end{array}$ & & 2010 & 0.0333 & 24.8 & 12. & 1.7 & 0.119 & 0.000 & 0.000 & & & 0.22 \\
\hline & $6 / 21 / 2004$ & 9:30:16 AM & & & 25.61 & 25.165 & 24.532 & 4.26 & 26.168 & 5.422 & \begin{tabular}{|l|l|}
-1.24 \\
\end{tabular} & 16.782 & 21.3 & 52.176 & 0.003 & $\begin{array}{l}14.643 \\
\end{array}$ & & 3.0 & & 24.7 & 12. & 1.5 & 0.104 & 0.000 & & & & 0.25 \\
\hline & 6/21/12004 & $9: 31: 16$ AM & & & 25.675 & 25.16 & 24.572 & 4.381 & 26.298 & 5.367 & & 16.866 & & & 0.003 & 14.643 & & & & & & & & 0.000 & & & & 0.22 \\
\hline & $6 / 21 / 2004$ & 9:32:16 AM & 25.536 & 24.08 & 25.771 & 25.176 & 24.628 & 11.695 & 26.12 & 5.427 & 6.177 & 9.31 & & 49.496 & 0.397 & 14.643 & & & .08333 & 24.6 & & & & & & & & 152 \\
\hline 24 & $6 / 21 / 2004$ & $\begin{array}{l}9.3 .3: 16 \mathrm{AM} \\
0.346 \mathrm{M}\end{array}$ & 25.943 & 24.089 & & $\frac{25.204}{25236}$ & $\frac{24.62}{24.692}$ & $\frac{18.288}{12.152}$ & $\begin{array}{l}26.074 \\
28207\end{array}$ & 5.415 & $\frac{12.144}{1.5745}$ & 2.5622 & & 52.232 & 0.055 & $\begin{array}{l}14.643 \\
1464^{2}\end{array}$ & & 6.0 & & $\frac{24.6}{2.60}$ & $\frac{12 .}{11}$ & & $\frac{1.070}{1.238} \mathrm{r}$ & $\frac{0.098}{0.096}$ & & & & 0.4 \\
\hline$\frac{24}{25}$ & 0 & $9.5 .35 .16 \mathrm{AM}$ & $\begin{array}{l}20.095 \\
26.209\end{array}$ & $\begin{array}{l}24.095 \\
24103\end{array}$ & $\mid \begin{array}{l}25.349 \\
2549\end{array}$ & $\frac{25.250}{2526}$ & 24672 & 20.153 & 30.307 & $\begin{array}{l}4.312 \\
4.277 \\
\end{array}$ & $\begin{array}{l}15.145 \\
17.027 \\
\end{array}$ & 3.059 & $\frac{0.451}{0.803}$ & $4 \frac{45.210}{41.336}$ & 0.756 & $\frac{14.045}{14.643}$ & & 8.00 & \begin{tabular}{|l|l|}
.113013 \\
0.13333
\end{tabular} & $\frac{20.9}{28.8}$ & & $\frac{1.5}{192}$ & \begin{tabular}{|l|}
1.230 \\
1324 \\
\end{tabular} & $\begin{array}{l}0.1113 \\
0.113 \\
\end{array}$ & $\frac{0.100}{0.110}$ & 0.006 & & $\frac{5.03}{486}$ \\
\hline & $6 / 21 / 2004$ & $9: 36: 16 \mathrm{AM}$ & 26.335 & $\frac{24.105}{24.145}$ & \begin{tabular}{|l|}
26.051 \\
26.051
\end{tabular} & 25.306 & $\begin{array}{l}24.072 \\
24.698 \\
\end{array}$ & $\frac{21.004}{22.527}$ & $\begin{array}{l}30.2<4 \\
31.915 \\
\end{array}$ & 4.534 & $\begin{array}{ll}17.027 \\
17.899\end{array}$ & $\begin{array}{l}.0599 \\
4.299 \\
\end{array}$ & $\frac{0.03}{1.165}$ & $4 \frac{41.050}{48.649}$ & $\begin{array}{l}0.750 \\
0.784\end{array}$ & $\frac{14.045}{14.643}$ & & $\frac{0.00}{9.00}$ & \begin{tabular}{|l|l|}
.15030 \\
0.15 \\
\end{tabular} & $\frac{2.0}{30.4}$ & $\frac{10 .}{11 .}$ & 19.2. & $\frac{1.3244}{1394}$ & $\mid$ & $\frac{0.110}{0.113}$ & $\frac{0.000}{0.006}$ & $\frac{3.115}{5615}$ & $\frac{4.86}{478}$ \\
\hline & 6/21/2004 & $9: 37: 16 \mathrm{AM}$ & 26.42 & 24.155 & \begin{tabular}{|l|l|}
26.141 \\
\end{tabular} & 25.316 & 24.713 & 22.917 & 32.191 & 4.493 & 18.322 & 4.183 & 100 & 42126 & 0.779 & 14.643 & & 100 & 1666 & $\frac{30.7}{30.7}$ & & & 1.422 & 0116 & & 0.005 & & 4.78 \\
\hline & $6 / 21 / 2004$ & 9:38:16 AM & 26.503 & 24.179 & 26.239 & & 24.722 & 23.181 & 32.433 & \begin{tabular}{|l|l|l|l}
4.486 \\
\end{tabular} & 18.595 & & & 42.118 & 0.772 & 14.643 & & 11.00 & 018333 & 310 & 10 & 209 & 1.440 & 0 & $0.1-1$ & 0.005 & 5322 & $\frac{4.64}{4.53}$ \\
\hline & & & & 24.174 & 26.3 & 25.341 & & 23.291 & 32.363 & & 18.798 & 3.986 & & 41.549 & & 14.643 & & 12.00 & & 30.9 & 10. & 1. & & & & & 5.267 & $\frac{4.53}{4.48}$ \\
\hline & $6 / 21 / 2004$ & $9: 40: 16$ & 26.648 & 24.199 & 26.384 & 25.4 & 24.702 & 23.4 & 32.342 & \begin{tabular}{|l|l|}
4.436 \\
\end{tabular} & 18.856 & $\begin{array}{l}3.867 \\
\end{array}$ & & 45.149 & 0.768 & 14.643 & & $\frac{13.06}{13.06}$ & 0.21667 & 30.9 & $\frac{11 .}{11 .}$ & (1.1.1 & 1.457 & 0.115 & & 0.005 & 5.213 & $\frac{4.48}{4.44}$ \\
\hline & $6 / 21 / 2004$ & & & 24.198 & 26.428 & 25.434 & & 23.5 & 32.502 & 387 & $\begin{array}{ll}18.989 \\
\end{array}$ & $\begin{array}{l}3.772 \\
\end{array}$ & & & 0.763 & \begin{tabular}{|l|l|l|l|l|}
14.33 \\
\end{tabular} & & & & & & 21.2 & & & & & & . \\
\hline 32 & $6 / 21 / 2004$ & & 26.776 & 24.213 & 26.488 & 25.499 & 24 & 23.463 & \begin{tabular}{|l|}
32.367 \\
\end{tabular} & \begin{tabular}{|l|l|l|}
4.367 \\
\end{tabular} & \begin{tabular}{l|l|l|l|}
18.987 \\
\end{tabular} & 3.725 & & 45.987 & 0.76 & \begin{tabular}{|l|l|}
14.643 \\
\end{tabular} & & 15.00 & 0.25 & \begin{tabular}{l|l|}
30.9 \\
\end{tabular} & $\overline{11.3}$ & 21.2 & 1.463 & 0.113 & 0.109 & 0.005 & 5.121 & 4.36 \\
\hline & 6/21/2004 & 9:43:16 AM & 26.831 & 24.228 & 26.533 & 25.514 & 24.73 & 23.629 & 32.398 & \begin{tabular}{|l|l|l|}
4.347 \\
\end{tabular} & \begin{tabular}{l|l}
19.158 \\
\end{tabular} & 3.604 & & 42.491 & 0.757 & 14.643 & & 16.00 & \begin{tabular}{|l|l|l|}
0.26667 \\
\end{tabular} & 30.9 & 10.4 & 21.4 & \begin{tabular}{|l|l|}
1.475 \\
\end{tabular} & 0.113 & 0.108 & 0.005 & 5.054 & 4.30 \\
\hline 34 & $6 / 21 / 2004$ & 9:44:16 AM & 26.922 & 24.253 & $\begin{array}{l}26.613 \\
\end{array}$ & 25.505 & 24.746 & 23.645 & 32.386 & 4.33 & 19.201 & 3.549 & & $\begin{array}{l}46.461 \\
\end{array}$ & 0.755 & 14.643 & & 17.00 & 0.28333 & $\overline{30.9}$ & 11. & $2 . .4$ & 1.477 & .113 & .108 & & & \\
\hline 然 & 6/21/21/2004 & 9:45:1 & 26.967 & 24.274 & 26.679 & 25.535 & & 23.595 & 31.695 & 4.614 & 18.915 & 3.158 & & 50.416 & 0.736 & 14. & & 18.00 & 0.3 & 30.2 & $\mathrm{AL}$ & 21.0 & 1.465 & 0.110 & 0.105 & 0.005 & 4.926 & 4.19 \\
\hline & & & & & & 25.586 & & 23.672 & & 4.47 & 19.092 & 3.332 & & 2.056 & & & & & & & & & & & & & & 4.20 \\
\hline & $6 \frac{6 / 2122004}{6021204}$ & & 27.085 & 24.317 & 26.8 & 25.593 & & 23.649 & 31.832 & 4.58 & 18.946 & 3.0977 & & 14.258 & & 14.6 & & 0.00 & 0.33333 & 30.4 & 10. & 21.3 & 1.468 & 0.109 & 0.104 & 0.005 & 4.882 & 4.15 \\
\hline & $\begin{array}{l}6 / \angle 1 / 2004 \\
6 / 21 / 2004 \\
\end{array}$ & & 27.127 & $\frac{2.3 .34}{2.345}$ & \begin{tabular}{|l|l|}
2.0359 \\
20.600
\end{tabular} & 25.565 & & 23.243 & $\frac{30.694}{3251}$ & 4.878 & 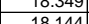 & 2.414 & & 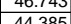 & 0.005 & & & & 0.35 & 29.2 & & 2.8 & & & & 0.005 & . & 4.08 \\
\hline 40 & $\begin{array}{l}-6 / \angle 1 / 2004 \\
6 / 21 / 2004 \\
\end{array}$ & (3.45.1. & 27.1619 & $\frac{24.354}{24351}$ & 26. & $\begin{array}{l}25.5000 \\
25.597\end{array}$ & & $\frac{23.004}{23.183}$ & & & 10.144 & $\frac{2.411}{2481}$ & & & & 热 & & 2.0 & $\begin{array}{l}0.30001 \\
0.38333\end{array}$ & $\frac{2.00}{294}$ & 10 & $z 0.0$ & $\frac{1.416}{1.436}$ & 0 & 0.100 & 0.005 & $\begin{array}{l}4.064 \\
4792 \\
\end{array}$ & $\begin{array}{l}4.14 \\
408\end{array}$ \\
\hline & $6 / 21 / 2004$ & 9.5:5.1.16 & 27.27 & 24.051 & 26.972 & 25.624 & 24.8 & 23.475 & 30.924 & 4.763 & $\begin{array}{l}1.400 \\
18.579\end{array}$ & 2417 & & $\begin{array}{l}01.400 \\
44.356\end{array}$ & 0.70 & & & 24.00 & (1) & 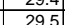 & & & & $\frac{0.105}{0.105}$ & 0 & 0.005 & 4744 & $\frac{4.08}{404}$ \\
\hline & & & & & & 25.66 & & $\frac{13.2}{23.2}$ & & & 18.48 & 2481 & & & & & & & & & & t.t. & & & & & & 4.04 \\
\hline & $6 / 61 / 2004$ & $9: 53: 16$ & 27.352 & 24.385 & 27.034 & 25.661 & & 23.421 & 30.931 & $\mid 4.63$ & 18.671 & 2,411 & & 5 & 0.70 & 14 & & 26.00 & \begin{tabular}{|l|l|l}
0.43333 \\
\end{tabular} & 29.5 & $\frac{11}{128}$ & . & , & 0.105 & 0.100 & 年 & 4.732 & $\frac{4.06}{403}$ \\
\hline 44 & $6 / 21 / 2004$ & & 27.4 & 24.4 & 27.1 & 25.713 & & 23.0 & 30.2 & & & 2.0 & & & & & & & & 28. & & 204 & & & & & & $407 \quad-2$ \\
\hline & $6 / 21 / 2004$ & & 27.42 & & 27.144 & & & 22.43 & & int & 17.709 & 1647 & & & & & & 28 & 0.46667 & 27.7 & 11. & 201 & & & 0.094 & & & 398 \\
\hline & $6 / 21 / 2004$ & & 27.45 & 24.44 & $\begin{array}{l}27 . \\
\end{array}$ & 25.75 & & 22.3 & 28.9 & 4.653 & $\begin{array}{ll}17.54 \\
\end{array}$ & $\begin{array}{l}1.647 \\
\end{array}$ & & & & & & 29.0 & \begin{tabular}{|l|}
0.48333 \\
\end{tabular} & 27.5 & 11.3 & $19.9]$ & 74 & 0.100 & 0.094 & & 4.702 & $\frac{3.0}{4.0}$ \\
\hline \begin{tabular}{|l|l|}
47 \\
\end{tabular} & $6 / 21 / 2004$ & & 27.469 & 24.468 & 27.186 & 25.759 & & & 29.1 & \begin{tabular}{|c|}
4.68 \\
\end{tabular} & & 1.58 & & & & & & & 0.5 & 27.7 & 11.4 & & & 099 & 0.093 & & & 3.98 \\
\hline \begin{tabular}{|l|l|}
48 \\
\end{tabular} & $6 / 21 / 2004$ & & 27.497 & \begin{tabular}{|l|l|l|l|}
2476 \\
\end{tabular} & & 25.787 & & 22.594 & 29.3 & \begin{tabular}{|l|l|}
4.542 \\
\end{tabular} & 17.951 & 1.716 & & 44.719 & & & & & 0.51667 & 27.9 & 11.0 & & & 0.100 & 0.094 & 0.005 & 4.641 & 3.95 \\
\hline 49 & $6 / 21$ & & 26.529 & & \begin{tabular}{l|l|}
26.345 \\
\end{tabular} & & & & 33.1 & 4.27 & $\begin{array}{ll}17.309 \\
\end{array}$ & 6.6 & & & & & & & & & 11 & 19 & & & 0.132 & & 6.805 & 5.79 \\
\hline & & & 26.65 & & & & & & $33.3 \mathrm{t}$ & & & 6.69 & & & 0 & & & & 2.00050 & 31. & 14 & & & 137 & & & & \\
\hline & $6 / 21 / 1 /$ & 11:31 & 26.654 & 24.796 & 26.585 & 25.736 & & 2.85 & 33.4. & 4.238 & -1.291 & 25.554 & & & & & & & 2.07722 & 32.0 & & 0.6 & & & & & & 0.47 \\
\hline & $601 / 2122$ & & 26.568 & 24.785 & & 25.741 & & $\begin{array}{l}2.869 \\
2072\end{array}$ & $\frac{33.2}{3.2}$ & 4.258 & -1.287 & 25.299 & & & & 14.6 & & & 2.09389 & 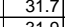 & $\frac{9.9}{120}$ & 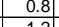 & & & & & 年 & 0.46 \\
\hline 54 & $\begin{array}{l}6 / 2 / 21 / 2004 \\
6 / 21204 \\
\end{array}$ & $\begin{array}{l}11.335 .54 \\
\end{array}$ & 2.547 & 24.8044 & 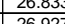 & 25.1790 & & $\begin{array}{l}3.273 \\
3.24\end{array}$ & $\begin{array}{l}33.383 \\
32320\end{array}$ & 4.286 & $=0.844$ & 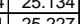 & & & 0.003 & & & & & | & & & & (2) & 0.000 & 0.000 & & \\
\hline & $\frac{0 / \angle 1 / 204}{6 / 21 / 2004}$ & & $\frac{2.5060}{26468}$ & $\frac{2.604}{2816}$ & & $\frac{23.199}{25816}$ & & & 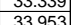 & $\begin{array}{l}4.200 \\
4.22\end{array}$ & & & & & & & & & & & 10.8 & & & & & & & 0.34 \\
\hline 56 & $6 / 21 / 2004$ & & 26. & & & 25874 & & 342 & 33 & 4.3 & & 25.0143 & & & 0.00 & & & & & 322 & $1 \pm .$. & & & & 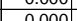 & & & $\begin{array}{l}-0.08 \\
-0.2\end{array}$ \\
\hline $3 T_{1}$ & & & & & & & & & & & & & & & & & & & & & & & & & & & & \\
\hline & & & 27.282 & & 27204 & & & & & & & & & & & & & & 219 & & & & & & & & & 68 \\
\hline & $6 / 21 / 2004$ & & 27.475 & 24.8 & & 25.965 & & & 38.2 & & 18.298 & & & & & & & & & 36.8 & & & & & & & & \\
\hline & $6 / 21 / 2$ & 11:40: & 27.619 & 24864 & 27 & 25.9 & & & 388 & & 17.98 & 10. & & & & & & 13 & & 374 & & & & & & & & \\
\hline $\begin{array}{ll}61 \\
\end{array}$ & $6 / 21 / 2004$ & 11:41: & 27.701 & 24.856 & 27.426 & 26.002 & & $\overline{21.92}$ & 36.6 & & & 9.45 & & & & 14. & & 13 & & 35. & & 108 & & 152 & & & 7.1 & 6.0 \\
\hline & $6 / 21 / 20$ & & 27.793 & 24.8 & 27.54 & 26.054 & & 21.8 & 36.5 & & & 9 & & & & & & & & 35. & & & & 152 & 0.142 & & & 6.1 \\
\hline 63 & $6 / 21 / 2$ & & 27.859 & 24.8 & 27. & 26.2 & & 21.745 & 36. & 4.346 & & & & & & & & & & 35.1 & 11.5 & 19 & & 153 & & & 7.240 & 6.16 \\
\hline 64 & & & $27.871 \mid$ & & & & & & & & & & & & & & & & & 35.4 & 11.4 & & & & & & & \\
\hline & $6 / 21 / 2$ & & $27.868 \mid$ & 24.934 & 27.416 & & & 21.499 & 36.5 & & & & & & 1 & & & & & 35.1 & & & & 154 & & & & \\
\hline 67 & 6 & 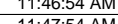 & 2.821 & 24.932 & 27.33 & 26.4 & & 21. & 37.2 & & & & & & & 14.6 & & 139 & & 35. & 11.1 & 26 & & 0.154 & 0.144 & & & 6.1 \\
\hline 68 & $\frac{612112004}{6 / 21204}$ & $\frac{11.446 .8}{11.0 .5}$ & 2.1.79 & 24.945 & 27.216 & 26.48 & 25.4 & $\frac{2.15}{216}$ & S0.509 & 4.129 & 17.586 & 9.817 & & 45.948 & 1002 & 19 & & 11 & 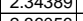 & 25 & 1. & & & 0.154 & & & & \\
\hline 69 & 0 & \begin{tabular}{|l|l}
11.46344 \\
1.49 .54
\end{tabular} & $\mid$ & $\begin{array}{r}24.980 \\
\end{array}$ & 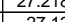 & $\begin{array}{l}2.0545 \\
26517\end{array}$ & $\frac{2.351}{2598}$ & $\frac{21.021}{21814}$ & $\begin{array}{l}30.089 \\
37057\end{array}$ & $\frac{4.2121}{4246}$ & 17.0021 & 9.040 & & 40.154 & 1033 & 1.645 & & $\begin{array}{l}14.103 \\
11262\end{array}$ & & 256 & 11.3 & 10.6 & 1.352 & 0.154 & 0 & 0.001 & & 6.2. \\
\hline$\frac{05}{70}$ & $6 / 21 / 2004$ & $\begin{array}{ll}11.44 .04 \mathrm{AN} \\
11: 50: 54 \mathrm{AM}\end{array}$ & 27.65 & 24.996 & 27.103 & \begin{tabular}{|l|}
20.011 \\
26.526 \\
\end{tabular} & \begin{tabular}{|l|}
25.4986 \\
25.486 \\
\end{tabular} & 21.814 & \begin{tabular}{|l|}
36.078 \\
36.678 \\
\end{tabular} & 4.224 & $\frac{17.121}{17.703}$ & \begin{tabular}{l|l} 
& 5.04 \\
3 & 9.834 \\
\end{tabular} & & $\begin{array}{ll}43.122 \\
44.504\end{array}$ & $\frac{1.035}{1.034}$ & $\begin{array}{l}14.643 \\
14.643 \\
\end{array}$ & & $\frac{14.63}{143.63}$ & $\frac{2.324}{2.39389}$ & $\begin{array}{l}35.6 \\
35.2\end{array}$ & $\begin{array}{l}10.9 \\
10.9\end{array}$ & \begin{tabular}{|l|}
19.8 \\
19.7
\end{tabular} & \begin{tabular}{|l|l|}
1.365 \\
1.357 \\
\end{tabular} & \begin{tabular}{|l|}
0.154 \\
0.154
\end{tabular} & \begin{tabular}{|l|}
0.145 \\
\end{tabular} & $\begin{array}{l}.0007 \\
0.007\end{array}$ & $\begin{array}{l}7.341 \\
7.383\end{array}$ & $\frac{6.2 .2}{6.28}$ \\
\hline
\end{tabular}


WSRC-TR-2005-00105, REVISION 0

SRNL-RPP-2005-00012, REVISION 0

RUN \# 3.18 AND 19; ACID CLEANING AND CAUSTIC RINSING - CONT.

\begin{tabular}{|c|c|c|c|c|c|c|c|c|c|c|c|c|c|c|c|c|c|c|c|c|c|c|c|c|c|c|c|c|}
\hline & & & & & & & & & & & & & & & & & & & & & & & & & & & & \\
\hline & A & $\mathrm{B}$ & $\mathrm{D}$ & $E$ & $F$ & $G$ & $\mathrm{H}$ & $\mathrm{J}$ & \begin{tabular}{l|l|}
$\mathrm{K}$ & \\
\end{tabular} & $\mathrm{L}$ & M & $\begin{array}{ll} \\
\end{array}$ & 0 & \begin{tabular}{l|l} 
\\
\end{tabular} & \begin{tabular}{l|l|} 
& \\
\end{tabular} & \begin{tabular}{l|l} 
\\
\end{tabular} & $\mathrm{T}$ & $\mathrm{V}$ & w & $x$ & $\begin{array}{r}Y \\
Y\end{array}$ & & AA & $A B$ & $\mathrm{AC}$ & $A D$ & $\mathrm{AE}$ & $\begin{array}{ll}\mathrm{AF} & \mathrm{A} \\
\end{array}$ \\
\hline & $6 / 21 / 2004$ & $1: 51: 54 \mathrm{AM}$ & 27.603 & 25.023 & \begin{tabular}{|l|}
27.031 \\
\end{tabular} & 26.498 & 25.429 & 21.393 & 36.498 & 421 & $\begin{array}{l}17.362 \\
\end{array}$ & 9.883 & 4.437 & \begin{tabular}{|c|}
39.267 \\
\end{tabular} & 1.036 & 14.643 & & 144.63 & 2.41056 & 35.0 & 9.6 & & & 0.154 & 0.146 & 0.008 & 7.531 & \\
\hline & & 11:52:54 AM & $\frac{27.500}{27007}$ & & $\begin{array}{r}27.01 \\
70.097\end{array}$ & 26.432 & & & \begin{tabular}{|l|}
37.124 \\
9.52
\end{tabular} & & & $\begin{array}{l}9.889 \\
\end{array}$ & 4.417 & & & 14.643 & & & & & & & . & & & & & 6.24 \\
\hline & $6 / 21 / 2004$ & 11:53:54 AM & 27.529 & 25.039 & \begin{tabular}{|l|}
26.987 \\
\end{tabular} & 26.419 & 25.335 & $\begin{array}{l}21.424 \\
\end{array}$ & \begin{tabular}{|l|}
36.583 \\
\end{tabular} & 4.239 & \begin{tabular}{|l|l|}
17.393 \\
\end{tabular} & $\begin{array}{l}9.921 \\
\end{array}$ & 4.46 & \begin{tabular}{|l|l}
43.028 \\
\end{tabular} & $\begin{array}{l}1.037 \\
\end{array}$ & \begin{tabular}{|l|l|}
14.643 \\
\end{tabular} & 0.0 & 146.63 & 2.44389 & 35.1 & & & 1.338 & 0.155 & 0.146 & 0.008 & 7.535 & 6.41 \\
\hline $\begin{array}{l}74 \\
75\end{array}$ & $6 / 21 / 2004$ & $11: 54: 54 \mathrm{AM}$ & 27.485 & $\begin{array}{l}25.045 \\
25042\end{array}$ & \begin{tabular}{|l|}
26.938 \\
26005 \\
\end{tabular} & \begin{tabular}{|l|l|}
26.37 \\
26.327
\end{tabular} & $\frac{25.301}{25.277}$ & \begin{tabular}{|l|l|}
21.779 \\
21719
\end{tabular} & $\begin{array}{r}37.122 \\
36.004\end{array}$ & $\begin{array}{l}4.342 \\
.200\end{array}$ & \begin{tabular}{|l|l|}
17.616 \\
17705
\end{tabular} & $\begin{array}{l}9.964 \\
9.961\end{array}$ & $\begin{array}{l}4.469 \\
4.79 \\
\end{array}$ & $\begin{array}{l}41.044 \\
177890\end{array}$ & \begin{tabular}{|l|l|}
1.037 \\
1037
\end{tabular} & \begin{tabular}{|l|l|}
14.643 \\
1.643
\end{tabular} & 0.00 & \begin{tabular}{|l|l|}
147.63 \\
148.63
\end{tabular} & \begin{tabular}{l|l|}
2.46056 \\
\end{tabular} & $\begin{array}{l}35.7 \\
35.4\end{array}$ & 117 & \begin{tabular}{|l|} 
\\
\end{tabular} & \begin{tabular}{|l|}
1.358 \\
1.359
\end{tabular} & \begin{tabular}{|l|l|}
0.155 \\
\end{tabular} & $\begin{array}{l}0.146 \\
\end{array}$ & 0.007 & 7.435 & 6.33 \\
\hline 76 & & $11: 155: 54 \mathrm{AM}$ & $\frac{27.452}{27.422}$ & $\begin{array}{l}25.042 \\
25.052\end{array}$ & \begin{tabular}{r|r}
26.9055 \\
2.699
\end{tabular} & $\frac{26.322}{26.337}$ & $\frac{25.277}{25.323}$ & \begin{tabular}{l|}
21.718 \\
21.449
\end{tabular} & $\begin{array}{l}36.904 \\
36.763\end{array}$ & $\frac{4.228}{4.24}$ & $\begin{array}{l}17.705 \\
17.405\end{array}$ & $\begin{array}{r}9.9611 \\
10.031\end{array}$ & $\begin{array}{l}4.478 \\
4512\end{array}$ & 47.879 & $\begin{array}{l}1.037 \\
1.039\end{array}$ & $\frac{14.643}{14.643}$ & & \begin{tabular}{|l|l|}
148.63 \\
149.63
\end{tabular} & \begin{tabular}{|c|}
2.47722 \\
3 \\
2.4938
\end{tabular} & $\begin{array}{l}35.4 \\
35.3 \\
\end{array}$ & & \begin{tabular}{|l|}
19.7 \\
19.4
\end{tabular} & $\begin{array}{l}1.359 \\
1.339\end{array}$ & & & 0.007 & $\begin{array}{l}7.436 \\
7563\end{array}$ & $\begin{array}{l}6.33 \\
6.44 \\
\end{array}$ \\
\hline 77 & $6 / 21 / 2004$ & $11: 57: 54 \mathrm{AM}$ & 27.378 & 25.053 & 26.846 & 26.328 & 25.344 & \begin{tabular}{|l|}
21.383 \\
\end{tabular} & 36.668 & 4.173 & 17.409 & \begin{tabular}{|l|l|}
10.074 \\
\end{tabular} & 4.559 & 41.887 & $\begin{array}{l}1.004 \\
1.042\end{array}$ & $\begin{array}{l}14.643 \\
14.643\end{array}$ & & \begin{tabular}{|l|}
1490.63 \\
150.63
\end{tabular} & \begin{tabular}{|l|} 
\\
3.2 .51056 \\
\end{tabular} & 35.2 & $\frac{9.1}{10.3}$ & $\frac{19.4}{19.4}$ & $\frac{1.339}{1.337}$ & \begin{tabular}{|l|}
0.155 \\
0.155 \\
\end{tabular} & $\frac{0.147}{0.148}$ & $\frac{0.008}{0.008}$ & $\frac{7.563}{7.606}$ & $\frac{6.44}{66.47}$ \\
\hline 78 & & 11:58:54 AM & 27.36 & 25.07 & 26.818 & 26.344 & & 21.903 & \begin{tabular}{l|l}
37.335 \\
\end{tabular} & 4.24 & 17.879 & 10.092 & 4.556 & 50.708 & 1.041 & 14.643 & 0.004 & 151.63 & 2.52722 & 35.9 & 12.4 & 19.9 & 1.371 & 0.155 & 0.148 & 0.007 & 7.416 & $\frac{0.41}{6.31}$ \\
\hline 19 & & & & 25.076 & & & & & 36.709 & & & & & & & & & & & & & & & & & & & 6.45 \\
\hline (1) & $6 / 21 / 2004$ & 12:00:54 PM & 27.278 & $\begin{array}{l}25.092 \\
2502\end{array}$ & $\begin{array}{l}26.686 \\
2.50 ?\end{array}$ & \begin{tabular}{ll|}
26.482 \\
2.89
\end{tabular} & 25.4 & $\begin{array}{l}21.519 \\
21500\end{array}$ & 36.966 & 4.259 & & & & 40.719 & 1.042 & \begin{tabular}{|l|l|}
14.643 \\
\end{tabular} & & $\begin{array}{l}153.63 \\
5.63\end{array}$ & 2.56056 & 35.5 & & & 342 & 0.155 & 0.148 & 0.008 & 7.614 & 6.48 \\
\hline 81 & $6 / 21 / 2004$ & $\begin{array}{ll}12: 01: 54 \mathrm{PM} \\
1000: 54 \mathrm{P}\end{array}$ & 27.214 & 25.084 & 26.592 & 26.494 & 25.489 & $\begin{array}{l}21.509 \\
21212\end{array}$ & & $\begin{array}{l}4.252 \\
\end{array}$ & 491 . & & & \begin{tabular}{|l|l}
43.778 \\
\end{tabular} & 1.042 & & . 0044 & 154.63 & 2.07024 & 35.3 & & 19.5 & 1.344 & 0.155 & 0.149 & 0.008 & 7.619 & 6.48 \\
\hline 83 & $\frac{6 / 21 / 2004}{66 / 212004}$ & $\frac{12: 02: 54}{12}$ & $27.178 \mid$ & $\frac{25.127}{25.124}$ & \begin{tabular}{|l|}
26.581 \\
26.528 \\
\end{tabular} & $26.567 \mid$ & $\begin{aligned} 25.533 \\
25.56\end{aligned}$ & $\begin{array}{l}21.212 \\
21.366\end{array}$ & $\begin{array}{l}36.446 \\
36.776\end{array}$ & $\begin{array}{l}4.259 \\
4.278\end{array}$ & . 1.48 & 129 & $\begin{array}{l}4.564 \\
4.605 \\
\end{array}$ & \begin{tabular}{|l|}
48.541 \\
50.553
\end{tabular} & $\begin{array}{l}1.042 \\
1.043\end{array}$ & \begin{tabular}{|c|}
14.643 \\
14.633
\end{tabular} & .004 & $\begin{array}{l}155.63 \\
156.63\end{array}$ & $2.59389 \mid$ & $\begin{array}{l}35.0 \\
35.3 \\
\end{array}$ & 11.9. & 19.3 & $\begin{array}{l}\frac{1.322}{1.331} \\
\end{array}$ & $\begin{array}{l}0.155 \\
0.156\end{array}$ & $\begin{array}{l}0.149 \\
0.149\end{array}$ & 0.008 & $\begin{array}{l}7.749 \\
77715\end{array}$ & 6.59 \\
\hline 84 & $6 / 21 / 2004$ & $12: 04: 54 \mathrm{PM}$ & 27.054 & 25.133 & \begin{tabular}{|l|}
26.456 \\
\end{tabular} & 26.593 & $\begin{array}{l}25.613 \\
25.613\end{array}$ & 21.443 & 36.991 & 4.282 & 17.389 & \begin{tabular}{|l|}
10.196 \\
10.19
\end{tabular} & $\begin{array}{l}4.0599 \\
4.599\end{array}$ & 43.735 & $\begin{array}{l}1.043 \\
1.044\end{array}$ & $\begin{array}{l}14.643 \\
14.643 \\
\end{array}$ & 0.004 & $\begin{array}{l}1566.63 \\
157.63\end{array}$ & & $\begin{array}{l}35.3 \\
35.5 \\
\end{array}$ & $\begin{array}{l}10.4 \\
10.7\end{array}$ & $\begin{array}{l}19.3 \\
19.4\end{array}$ & & & & & $\frac{1.15}{7.696}$ & $\frac{6.57}{6.55}$ \\
\hline & $6 / 21 / 2004$ & 12:05:54 PM & & $\frac{25.167}{25.167}$ & \begin{tabular}{|l|}
26.421 \\
2.272 \\
\end{tabular} & $\frac{26.677}{2.67}$ & 25.632 & 21.105 & 36.612 & $\begin{array}{r}4.23 \\
4.25\end{array}$ & & & & 47.437 & 1.048 & & & & & 35.1 & & & & & & & & 6.70 \\
\hline & & 12:06:54 PM & & & & & 25.665 & & 37.439 & & 627 & & 4.848 & 44.002 & & 14.643 & & & 2.66056 & 36.0 & & & 1.351 & & & & 7.740 & 6.59 \\
\hline & $6 / 21 / 2004$ & 12:07:54 PM & 26.915 & 25.183 & 26.242 & 26.833 & 25.694 & & 37.105 & 4.095 & & & 4.851 & 45.305 & 1.059 & & & & & 35.6 & & & & 0.158 & 0.152 & & 7.913 & 6.73 \\
\hline & $6 / 21 / 2004$ & & \begin{tabular}{l|l|}
26.818 \\
26711
\end{tabular} & $\begin{array}{l}25.197 \\
25205\end{array}$ & & $\begin{array}{ll}26.902 \\
26071\end{array}$ & & $\begin{array}{l}21.295 \\
21200\end{array}$ & & 4.112 & & & & \begin{tabular}{|l|}
40.007 \\
\end{tabular} & $\begin{array}{l}1.058 \\
\end{array}$ & & & 161.63 & & \begin{tabular}{|l|}
35.7 \\
357
\end{tabular} & & & & & $\begin{array}{ll}0.153 \\
\end{array}$ & & $\begin{array}{l}7.909 \\
7.950\end{array}$ & 6.73 \\
\hline 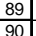 & $6 / 21 / 2004$ & 12:09:54 PM & $\frac{26.711}{26.615}$ & $\begin{array}{l}25.225 \\
25.233\end{array}$ & \begin{tabular}{|l|}
25.899 \\
25.69 \\
\end{tabular} & $26.974 \mid$ & $\frac{25}{256}$ & . & $\begin{array}{r}37.124 \\
51.9\end{array}$ & $\begin{array}{l}4.066 \\
1.825 \\
\end{array}$ & \begin{tabular}{ll|l}
421 & 427 \\
7
\end{tabular} & & & $\begin{array}{r}46.2 .2 \\
27398\end{array}$ & \begin{tabular}{|c|c|}
1.059 \\
1.204
\end{tabular} & \begin{tabular}{|c|}
14.643 \\
14.633
\end{tabular} & .004 & \begin{tabular}{|c|}
162.63 \\
163.63
\end{tabular} & $\begin{array}{l}32.71056 \\
32.272728\end{array}$ & $\begin{array}{l}35.7 \\
50.4 \\
\end{array}$ & $\frac{11.3}{6.7}$ & $\frac{19.3}{24.5}$ & $\begin{array}{l}1.334 \\
1.689\end{array}$ & $\begin{array}{l}0.158 \\
0.180\end{array}$ & 0.154 & $\begin{array}{l}0.008 \\
0.007\end{array}$ & $\begin{array}{l}7.959 \\
7.188\end{array}$ & 6.77 \\
\hline 91 & $6 / 21 / 2004$ & $12: 11: 54$ PM & 26.609 & 25.242 & \begin{tabular}{|l|}
25.651 \\
\end{tabular} & 26.917 & 25.652 & 28.123 & 57.971 & 2.031 & .355 & 24.639 & 11.1.33 & 30.696 & $\frac{1.204}{1.204}$ & $\begin{array}{l}14.6453 \\
14.643\end{array}$ & 004 & $\begin{array}{l}\mid 63.63 \\
164.63\end{array}$ & \begin{tabular}{|l|}
$\mid 2.747<2$ \\
2.7438
\end{tabular} & 56.5 & $\begin{array}{l}0.1 \\
7.5 \\
\end{array}$ & $\frac{24.5}{27.2}$ & $\begin{array}{l}1.689 \\
1.878\end{array}$ & \begin{tabular}{|l|}
0.100 \\
0.180 \\
\end{tabular} & $\begin{array}{l}0.176 \\
0.176\end{array}$ & $\frac{0.007}{0.006}$ & $\frac{7.188}{6.471}$ & $\frac{6.12}{551}$ \\
\hline & $6 / 21 / 2004$ & 12:12:54 PM & 26.597 & 25.266 & \begin{tabular}{|l|}
25.694 \\
\end{tabular} & 26.92 & 25. & 45.965 & 49.989 & 5.515 & .739 & -1.211 & -4.23 & 51.767 & 0.003 & 1.944 & & 165.63 & 2.76056 & 48.5 & 12.7 & 43.4 & 2.989 & 0.290 & 0.284 & & & $\begin{array}{l}5.51 \\
5.58\end{array}$ \\
\hline 93 & $6 / 21 / 2004$ & 12:13:54 PM & 26.445 & 25.263 & 25.702 & 26.828 & & 41.856 & \begin{tabular}{|l|l|}
44.393 \\
\end{tabular} & 3.833 & & & & 41.259 & 0.003 & 1.625 & & 166.63 & & 42.9 & & & 2.762 & 0.242 & & & & \\
\hline 94 & $6 / 21 / 2004$ & 12:14:54 PM & 26.363 & 25.271 & 25.639 & 26.81 & & \begin{tabular}{|l|l|}
42.723 \\
\end{tabular} & & 3.711 & & & & & $\begin{array}{l}0.003 \\
\end{array}$ & & & \begin{tabular}{|l|l|}
167.63 \\
\end{tabular} & \begin{tabular}{|l|l|}
2.79389 \\
\end{tabular} & 44.3 & 10.1 & 41. & 2.824 & $\begin{array}{l}0.256 \\
\end{array}$ & 0.252 & 0.006 & 6.150 & 5.23 \\
\hline & $6 / 21 / 2004$ & & 26.306 & 25.288 & $\begin{array}{l}25.602 \\
25.519\end{array}$ & \begin{tabular}{l|l|}
26.808 \\
6.700
\end{tabular} & & $\begin{array}{l}42.302 \\
2.252\end{array}$ & $\begin{array}{l}45.245 \\
\end{array}$ & $\begin{array}{l}3.942 \\
3.953\end{array}$ & & -1.214 & & & $\begin{array}{l}0.003 \\
\end{array}$ & $\begin{array}{l}1.665 \\
1682\end{array}$ & & & $\frac{2.81056}{2.20720}$ & 43.8 & 10.4 & & 2.789 & $\begin{array}{l}0.248 \\
\end{array}$ & 0.244 & & 6.034 & 5.14 \\
\hline 96 & $6 / 21 / 21 / 2004$ & 12:16:54 PM & $\frac{26.242}{26.201}$ & \begin{tabular}{r|}
25.29 \\
25.308
\end{tabular} & \begin{tabular}{|l|}
25.548 \\
25.50 \\
\end{tabular} & $\frac{26.799}{26.813}$ & $\begin{array}{l}25.635 \\
25.668\end{array}$ & $\begin{array}{l}42.252 \\
42.153\end{array}$ & \begin{tabular}{|r|r|}
45.039 \\
44.9
\end{tabular} & $\begin{array}{r}3.853 \\
3.929 \\
\end{array}$ & .6351 & $\begin{array}{l}-1.216 \\
-1.224\end{array}$ & $\begin{array}{l}-4.204 \\
-4.1166\end{array}$ & \begin{tabular}{|l|l|l|l|l|l|l|}
40.414 &
\end{tabular} & $\begin{array}{l}0.003 \\
0.03\end{array}$ & $\frac{1.682}{171}$ & & $\begin{array}{l}169.63 \\
170.63 \\
\end{array}$ & 2.82722 & \begin{tabular}{|l|l|l|l|}
43.6 & \\
43.4
\end{tabular} & 9.8 & 40.4 & 2.788 & $\begin{array}{l}0.251 \\
\end{array}$ & 0.247 & 0.006 & $\begin{array}{l}6.106 \\
6237 \\
237\end{array}$ & 5.20 \\
\hline 98 & $6 / 21 / 21 / 2004$ & $\begin{array}{l}12.1 .54 \\
12: 18: 54 \mathrm{PM}\end{array}$ & 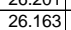 & $\frac{2.000}{25.316}$ & \begin{tabular}{|l|}
25.5069 \\
\end{tabular} & $\frac{20.010}{26.88}$ & 25.0001 & $\begin{array}{l}42 . .447 \\
42.45\end{array}$ & $\begin{array}{r}45.446 \\
45\end{array}$ & $\begin{array}{l}.929 \\
.767\end{array}$ & $\begin{array}{c}30.451 \\
38.91\end{array}$ & $-\frac{-1.214}{-1.219}$ & $\begin{array}{l}-2.100 \\
-4.146\end{array}$ & 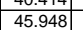 & $\begin{array}{l}0.003 \\
0.003\end{array}$ & $\begin{array}{l}1.11 \\
1.737 \\
\end{array}$ & & $\begin{array}{l}1 / 0.63 \\
171.63\end{array}$ & \begin{tabular}{|l|}
$\mid 2.04509$ \\
$\mid 2.86056$
\end{tabular} & $\begin{array}{l}43.4 \\
44.0\end{array}$ & \begin{tabular}{r|}
91.9 \\
11.3
\end{tabular} & $\frac{40.3}{40.7}$ & $\frac{2.179}{2.805}$ & | & $\begin{array}{l}0.251 \\
0.256\end{array}$ & $\begin{array}{l}0.006 \\
0.006\end{array}$ & $\begin{array}{l}6.283 \\
6.285 \\
\end{array}$ & $\begin{array}{l}5.31 \\
5.35\end{array}$ \\
\hline & $6 / 21 / 2004$ & 12:19:54 PM & 26.117 & 25.334 & \begin{tabular}{|l|}
25.433 \\
\end{tabular} & 26.904 & 25.7 & 42.132 & 45.039 & 3.955 & 38.364 & -1.214 & & & 0.003 & 1.72 & & & & 43.6 & 11.0 & & & 0.256 & & & & 5.36 \\
\hline & $6 / 21 / 2004$ & 12:20:54 PM & 26.074 & 25.346 & \begin{tabular}{|l|}
25.395 \\
\end{tabular} & 26.951 & 25. & & 45.528 & 3.995 & & & & \begin{tabular}{|l|}
45.599 \\
\end{tabular} & & 1.73 & & 173 & & 44.1 & & & 2.797 & $\mid 0.258$ & & & 6.295 & \\
\hline 101 & $6 / 21 / 2004$ & 12:21:5 & 26.042 & $\begin{array}{l}25.37 \\
25.37\end{array}$ & \begin{tabular}{|l|}
25.394 \\
25255 \\
\end{tabular} & \begin{tabular}{|l|}
26.994 \\
2.981
\end{tabular} & & 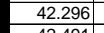 & \begin{tabular}{r|}
45.3 \\
\end{tabular} & & & & & $\begin{array}{l}44.559 \\
4\end{array}$ & $\begin{array}{l}0.003 \\
\end{array}$ & & & & \begin{tabular}{|l|l|}
2.91056 \\
\end{tabular} & 43.8 & 10.9 & & & 0.255 & 0.252 & 0.006 & 6.271 & 5.34 \\
\hline & $6 / 21 / 2004$ & $12: 22: 54 \mathrm{PM}$ & $\begin{array}{l}26.014 \\
25.992\end{array}$ & $\begin{array}{l}25.367 \\
25384\end{array}$ & \begin{tabular}{|l|}
25.365 \\
25.343 \\
\end{tabular} & $\begin{array}{l}26.981 \\
27054\end{array}$ & 25.8 & & $\begin{array}{l}45.562 \\
\end{array}$ & 304 & & $\begin{array}{l}-1.216 \\
\end{array}$ & & $\begin{array}{r}48.1 \\
615.5\end{array}$ & $\begin{array}{l}0.003 \\
0.023\end{array}$ & \begin{tabular}{|l|l|l|}
1.764 \\
171
\end{tabular} & & \begin{tabular}{|l|l|}
175.63 \\
$17 f 63$
\end{tabular} & 2.92722 & 44.1 & 11.8 & & 2.789 & $\begin{array}{l}0.263 \\
\end{array}$ & 0.260 & 0.006 & 6.436 & 5.48 \\
\hline & $6 / 21 / 2004$ & $\begin{array}{ll}12: 23: 54 \mathrm{PM} \\
12 \cdot 2454 \mathrm{PM}\end{array}$ & $\begin{aligned} 25.992 \\
25.99\end{aligned}$ & $\frac{25.384}{25412}$ & \begin{tabular}{|l|}
25.343 \\
25.346 \\
\end{tabular} & $\begin{array}{l}27.054 \\
27.112\end{array}$ & 25. & \begin{tabular}{|l|l|}
42.348 \\
42.534
\end{tabular} & $\begin{array}{l}45.342 \\
45.543\end{array}$ & 2944 & & \begin{tabular}{|c|}
-1.219 \\
\end{tabular} & & $\begin{array}{l}46.156 \\
45602\end{array}$ & 0.003 & $\frac{1.7}{175}$ & & \begin{tabular}{|l|l|}
176.63 \\
\end{tabular} & \begin{tabular}{|l|l|}
2.94389 \\
\end{tabular} & 43.9 & $\frac{11.3}{112}$ & 40.3 & 1.779 & 0.255 & 0.252 & 0.006 & $\begin{array}{l}6.265 \\
6.401 \\
6.401\end{array}$ & 5.33 \\
\hline & $6121 / 212004$ & $\frac{12: 24.54 \mathrm{PM}}{22: 25.54 \mathrm{PM}}$ & $\begin{array}{r}25.999 \\
25.983\end{array}$ & & \begin{tabular}{|l|}
25.343 \\
25334 \\
\end{tabular} & $\frac{27.112}{27.15}$ & $\frac{25}{25}$ & & $\mid$\begin{tabular}{|c|}
45.543 \\
46.316
\end{tabular} & $\begin{array}{l}174 \\
171 \\
\end{array}$ & \begin{tabular}{|}
38.013 \\
39.077 \\
\end{tabular} & 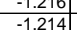 & & & 0 & & & & & & & & & & & & & 5.45 \\
\hline & $6 / 21 / 2004$ & $12: 26: 5$ & 25.961 & 25.449 & \begin{tabular}{|l|}
25.312 \\
\end{tabular} & 27.173 & 25. & & 4 & .39 & 38.744 & -1.219 & & | & & 1. & & & & $\begin{array}{l}44.0 \\
44.6 \\
\end{array}$ & & $4 c$ & 2.806 & & & & $\frac{6.344}{6.464}$ & $\begin{array}{l}5.40 \\
5.50\end{array}$ \\
\hline & $6 / 21 / 2004$ & 12:27:54 PM & 25.934 & 25.457 & \begin{tabular}{|l|}
25.285 \\
\end{tabular} & 27.146 & 25.8 & \begin{tabular}{|l|l|}
42.854 \\
\end{tabular} & 46.214 & 4.182 & 38.935 & -1.216 & & 42.473 & 0.003 & & & 180 & .01056 & 44.7 & & & & & & & 6.575 & \\
\hline & $6 / 21 / 2004$ & $\frac{12: 28}{12: 20}$ & \begin{tabular}{|l|}
25.901 \\
2500
\end{tabular} & $\begin{array}{l}25.459 \\
2.489\end{array}$ & \begin{tabular}{|l|}
25.242 \\
\end{tabular} & 27.098 & & & 46.225 & 4. & & \begin{tabular}{|l|}
-1.219 \\
\end{tabular} & & & & & & & 21200 & 44.8 & & & & & & & & \\
\hline & & & & & & & & & & & & -1.216 & & & & & & & & 44.7 & & & 793 & & & & & \\
\hline & & & & & & $\frac{27.0}{270}$ & & & & 4.146 & & & & & & & & & & $\frac{44.6}{44 .}$ & & & & & & & & 5.59 \\
\hline & $\frac{0.1 / 1 / 2}{6 / 21 / 2}$ & & $\frac{25.0}{25.8}$ & & & & & & 43.0 & 4.150 & & & & & & & & & & & & & & & & & & \\
\hline & & & 25.8 & 25.5 & & & & & & & & -1.2. & & 50.681 & & 1.8 & & & & & & & & & & & & \\
\hline & 6/21/2004 & $12: 34$ & 25.79 & 25.509 & \begin{tabular}{|l|}
25.138 \\
\end{tabular} & 26.954 & 25.7 & & 45.864 & 4.203 & & & & 38. & & & & 18 & 3.12 & 44.4 & & & & & 0.269 & & & \\
\hline & & & & & & & & & & & & & & & & & & & & & & & & & & & & \\
\hline & & & & & & & & & & & & & & & & & & & & & & & & & & & & \\
\hline & & & & & & & & & & & & & & & & & & & & & & & & & & & & \\
\hline & $\frac{0.1 / 1 / 2}{6 / 21 / 2}$ & & 25.74 & & & & & & & & & & & & & & & & & & & & & & & & & \\
\hline & & & & & & & & & & & & & & & & & & & & & & & & & & & & \\
\hline & $6 / 21 / 2004$ & & 25.73 & $\frac{25.5}{25.5}$ & & & & & & & & & & & & & & & & 44. & & & & & & & & \\
\hline & & & & & & & & & & & & & & & & & & & & & & & & & & & & \\
\hline & & & $\frac{25.713}{20.5}$ & 25.56 & & & & & & & & -1.2 & & & & & & & & & & & & & & & & \\
\hline & & & 25.71 & STr & & & & & & & & -1.216 & & & & & & & & & & & & & & & & \\
\hline & $6 / 21 / 2$ & & 25.7 & & & & & & & & & & & & & & & & & & & & & & & & & \\
\hline & $6 / 21 / 21$ & & 25.69 & 25.58 & & & & & & & & -1.2 & & & & & & & & & & & & & & & & \\
\hline & $6 / 21 / 2$ & & & & & & & 42. & & & & & & & & & & & & & & & & & & & & \\
\hline & $6 / 21 / 2$ & & 25.65 & 25.6 & 25.06 & 27.1. & & 42.173 & & & & -1.2 & & & & & & & & & & & & & & & & \\
\hline & & & & & & & & & & & & & & & & & & & & & & & & & & & & \\
\hline & & & & 25.6 & & 27.1 & & & & & & & & & & & & & & & & & & & & & & \\
\hline & & & & & & 2.314 & & & & & & & & & & & & & & & & & & & & & & \\
\hline & 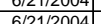 & 12.53 .5 & . & & & & & & & & & & & & & & & & & & & & & & & & & \\
\hline & (6) & & & & & & & 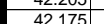 & & & & -1.2 & & & & & & & & & & & & & & & & \\
\hline & & & $\frac{1.067}{20.667}$ & & & $\frac{2.3}{27.2}$ & & $\frac{42.17}{42.4}$ & & & & & & & & & & & & & & & & & & & & \\
\hline & & & & & & & & & & & & & & & & & & & & & & & & & & & & \\
\hline & & & & & & & & & & & & & & & & & & & & & & & & & & & & \\
\hline & & & 25. & & & 271 & & & & & & -12 & & & & & & & & & & & & & & & & \\
\hline & & & & & & & & & & & & & & & & & & & & & & & & & & & & \\
\hline & $6 / 21 / 21$ & 1:00:54 & $5.639]$ & 25.712 & 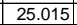 & 27.11 & & 42.574 & \begin{tabular}{|l|l|}
45.931 \\
\end{tabular} & 4.179 & 38.645 & $|-1.216|$ & & 5.116 & 003 & $1.847 \mid$ & & & & 44.5 & & & & 0.275 & $0.2 / 5$ & 0.007 & 6.778 & \\
\hline
\end{tabular}


WSRC-TR-2005-00105, REVISION 0

SRNL-RPP-2005-00012, REVISION 0

RUN \# 3.18 AND 19; ACID CLEANING AND CAUSTIC RINSING - CONT.

\begin{tabular}{|c|c|c|c|c|c|c|c|c|c|c|c|c|c|c|c|c|c|c|c|c|c|c|c|c|c|c|c|c|c|}
\hline & $\mathrm{A}$ & B & D & $\mathrm{E}$ & $\mathrm{F}$ & G & $\mathrm{H}$ & $\mathrm{J}$ & $\mathrm{K}$ & $\mathrm{L}$ & $\square$ & M & $\mathrm{N}$ & 0 & Q & \begin{tabular}{l|l|l|}
$\mathrm{R}$ & \\
\end{tabular} & $\mathrm{s}$ & $T$ & $\mathrm{v}$ & w & $x$ & Y & & AA & $A B$ & $\mathrm{AC}$ & $\mathrm{AD}$ & $\mathrm{AE}$ & AF $A$ \\
\hline & $6 / 21 / 2004$ & 1:01:54 PM & & 25.7 & 25.036 & 27.182 & 25.853 & 42.43 & 45.723 & 4.16 & 162 & 38.565 & -1.211 & -3.836 & 45.526 & \begin{tabular}{|c|}
0.003 \\
\end{tabular} & 1.842 & 0.005 & 214.63 & 3.57722 & 44.3 & 11.2 & $\frac{4}{40 .}$ & 2.792 & 0.275 & 0.274 & 0.007 & 6.774 & \\
\hline & 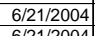 & 1:02:54 PM & & 25.73 & & $\begin{array}{l}27.148 \\
27.775\end{array}$ & $\begin{array}{l}25.914 \\
25.955\end{array}$ & & & 4.20 & & & -1.214 & & & & & & & & 44.4 & 10.8 & & & & & & & \\
\hline & $6 / 21 / 2004$ & 1:03:54 PM & 25.633 & $\begin{array}{l}25.726 \\
20726\end{array}$ & \begin{tabular}{l|l|l|}
6 & 25.024 \\
\end{tabular} & 27.175 & 25.955 & 42.331 & 45.864 & 4.16 & 168 & $\begin{array}{l}38.344 \\
2.395\end{array}$ & $\begin{array}{r}-1.216 \\
\end{array}$ & $\begin{array}{l}-3.834 \\
.2821\end{array}$ & 48.103 & \begin{tabular}{|l|}
0.003 \\
\end{tabular} & $\begin{array}{l}1.846 \\
\end{array}$ & 0.005 & 216.63 & 3.61056 & 44.4 & 11.8 & & 2.781 & . 275 & 0.275 & 0.007 & 6.818 & \\
\hline$\frac{144}{145}$ & $\begin{array}{l}6 / 211 / 2004 \\
6 / 21 / 204\end{array}$ & $\begin{array}{l}1: 04: 54 \mathrm{PM} \\
10554 \mathrm{PM}\end{array}$ & $\begin{array}{l}25.628 \\
25.636\end{array}$ & $\begin{array}{l}25.726 \\
25.749 \\
\end{array}$ & 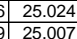 & $\begin{array}{r}27.21 \\
27.258 \\
\end{array}$ & $\begin{array}{r}25.99 \\
26.018\end{array}$ & \begin{tabular}{r|r|}
42.42 \\
42.343
\end{tabular} & $\begin{array}{l}45.881 \\
45.752\end{array}$ & $\frac{4.14}{4.15}$ & \begin{tabular}{|l|l|l|l|l|l|}
157 & \\
\end{tabular} & $\begin{aligned} 38.485 \\
38.371 \\
\end{aligned}$ & \begin{tabular}{|c|}
-1.214 \\
-1.222 \\
\end{tabular} & \begin{tabular}{|l|}
-3.831 \\
-3831
\end{tabular} & $\begin{array}{r}44.5 \\
5174 \\
\end{array}$ & \begin{tabular}{|c|}
0.003 \\
0.003
\end{tabular} & $\begin{array}{r}1.84 \\
1843\end{array}$ & 0.00 & \begin{tabular}{|c|}
217.63 \\
21863
\end{tabular} & 364389 & 44.4 & $\frac{10.9}{127}$ & $\begin{array}{l}40.5 \\
40.4\end{array}$ & $\begin{array}{l}2.789 \\
2.782\end{array}$ & $\begin{array}{l}0.274 \\
0.275\end{array}$ & $\begin{array}{l}0.274 \\
0.275\end{array}$ & \begin{tabular}{|l|l|}
0.007 \\
0.07
\end{tabular} & \begin{tabular}{|c|c|c|}
6.777 \\
6807
\end{tabular} & \begin{tabular}{|l|}
5.77 \\
5.79 \\
\end{tabular} \\
\hline$\frac{145}{146}$ & & & 25.632 & & & & $\frac{20.018}{26.044}$ & $\begin{array}{l}42.343 \\
42.499\end{array}$ & \begin{tabular}{|l|l|}
45.152 \\
45.924
\end{tabular} & & 175 & 38.508 & \begin{tabular}{|c|}
-1.221 \\
-1.214 \\
\end{tabular} & & 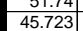 & \begin{tabular}{|c|c|c|}
0.003 \\
0.003
\end{tabular} & $\begin{array}{l}1.843 \\
1.837 \\
\end{array}$ & $\frac{0.005}{0.006}$ & $\begin{array}{r}218.63 \\
219.63\end{array}$ & \begin{tabular}{|l|l|}
3.64389 \\
3.66056
\end{tabular} & 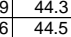 & & & & $\mid$ & $\mid 0.275$ & $\begin{array}{l}0.007 \\
0.007\end{array}$ & $6.807 \mid$ & $\begin{array}{l}5.79 \\
5.75\end{array}$ \\
\hline 147 & & & 24.64 & 25.293 & $\begin{array}{l}324.215 \\
\end{array}$ & 26.648 & 25.658 & & 48.561 & 4.06 & & 16.41 & 24.289 & 13.269 & & 0.697 & -0.013 & 0004 & & 4.80111 & & & & & & & & & \\
\hline 14 & & $2: 16: 20 \mathrm{PM}$ & & 25.326 & \begin{tabular}{|l|l|}
6 & 24.348 \\
\end{tabular} & 26.736 & 25.646 & 3.18 & 48.998 & 4.02 & & -0.69 & \begin{tabular}{|l|}
40.716 \\
\end{tabular} & 21.658 & 42.663 & 0.003 & \begin{tabular}{|c|}
-0.01 \\
\end{tabular} & (2) & 289.07 & \begin{tabular}{|l|l|}
4.81778 \\
\end{tabular} & 47.5 & 10.5 & & & -0.001 & -0.002 & & -1.218 & .04 \\
\hline 144 & $6 / 21 / 2004$ & $2: 17: 20 \mathrm{PM}$ & 24.741 & & 24.425 & $\begin{array}{r}26.738 \\
6771\end{array}$ & 25.608 & 2.891 & 48.476 & 4.03 & 039 & $\begin{array}{l}-1.002 \\
-1.0027\end{array}$ & \begin{tabular}{|l|l|}
40.7766 \\
\end{tabular} & 21.508 & 42.295 & 0.003 & \begin{tabular}{|c|c|c|}
-0.013 \\
\end{tabular} & 0.0 & 290.07 & 4.83444 & 47.0 & 10.4 & & 0.065 & -0.002 & $\begin{array}{l}-0.002 \\
\end{array}$ & & -2.086 & \\
\hline & & & & & & & & & 48.845 & 4.04 & & \begin{tabular}{|l|l|}
-0.677 \\
\end{tabular} & \begin{tabular}{|l|l|}
40.562 \\
\end{tabular} & & & 0.003 & $\begin{array}{l}0.834 \\
\end{array}$ & & 291.07 & 4.85111 & 47.4 & 10.5 & & & 0.124 & 0.126 & & & \\
\hline 15 & $6 / 21 / 2004$ & $\begin{array}{l}2: 19: 20 \mathrm{PM} \\
2: 020\end{array}$ & 25.026 & 25.323 & 24.601 & 26.653 & 25.46 & 4.607 & 48.383 & 4.02 & 026 & $\begin{array}{l}0.796 \\
\end{array}$ & \begin{tabular}{|l|}
38.984 \\
\end{tabular} & $\begin{array}{l}0.433 \\
\end{array}$ & $\begin{array}{l}45.441 \\
\end{array}$ & 0.476 & $\begin{array}{r}-0.007 \\
\end{array}$ & & 292.07 & 4.86778 & 46.9 & 11. & & 0.186 & 0.001 & 0.001 & & -0.391 & \\
\hline$\frac{152}{153}$ & 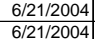 & $\begin{array}{l}2: 20: 20 \mathrm{PM} \\
2: 21: 20 \mathrm{MM}\end{array}$ & $\begin{array}{l}25.168 \\
25.275\end{array}$ & $\begin{array}{l}25.336 \\
25.338 \\
\end{array}$ & 24.688 & $\begin{array}{l}26.555 \\
26.482 \\
\end{array}$ & $\begin{array}{l}25.406 \\
25.338 \\
\end{array}$ & $\frac{40.585}{44.1311}$ & $\begin{array}{l}47.817 \\
47.893 \\
\end{array}$ & $\frac{4.09}{4.10}$ & 098 & \begin{tabular}{|l|}
37.098 \\
37.781
\end{tabular} & \begin{tabular}{|c|}
1.398 \\
0.932 \\
\end{tabular} & $\begin{array}{l}1.786 \\
1.723\end{array}$ & \begin{tabular}{r|}
42.88 \\
45.518 \\
\end{tabular} & \begin{tabular}{|l|l|}
0.004 \\
0.003
\end{tabular} & $\begin{array}{l}2.658 \\
2.575\end{array}$ & & \begin{tabular}{|l|}
293.07 \\
294.07
\end{tabular} & \begin{tabular}{|l|l|}
4.88444 \\
4.90111
\end{tabular} & $\frac{46.3}{46.3}$ & $\frac{10 .}{11 .}$ & $\begin{array}{l}38.8 \\
39.5\end{array}$ & $\frac{2.678}{2.727}$ & \begin{tabular}{|l|l|l|l|l}
0.396 \\
0.384
\end{tabular} & $\begin{array}{l}0.400 \\
0.386\end{array}$ & $\frac{0.010}{0.010}$ & $\frac{10.293}{9072}$ & \\
\hline 154 & $6 / 21 / 2004$ & $\begin{array}{l}\frac{2: 12: 20 \mathrm{PM}}{2: 22: 20 \mathrm{PM}} \\
\end{array}$ & $\frac{25.275}{25.378}$ & 25.350 & $\begin{array}{l}524.66 \\
5\end{array}$ & $\begin{array}{r}26.482 \\
26.569\end{array}$ & $\begin{array}{r}25.338 \\
25.295 \\
\end{array}$ & $\begin{array}{l}\frac{41.311}{41.779} \\
\end{array}$ & $\begin{array}{l}41.893 \\
48.101\end{array}$ & 4.10 & & \begin{tabular}{|l|}
3.1 .181 \\
38.196
\end{tabular} & $\begin{array}{l}0.932 \\
0.668 \\
\end{array}$ & $\begin{array}{l}1.723 \\
1.688\end{array}$ & $\begin{array}{l}45.518 \\
44.951 \\
\end{array}$ & $\begin{array}{l}0.003 \\
0.003\end{array}$ & $\begin{array}{l}2.575 \\
2.519\end{array}$ & & & 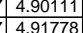 & $\begin{array}{l}46.4 \\
46.6\end{array}$ & $\frac{11.2}{11.0}$ & & $\begin{array}{l}2.727 \\
2.757 \\
\end{array}$ & $\begin{array}{l}0.384 \\
0.376\end{array}$ & $\begin{array}{l}0.386 \\
0.377 \\
\end{array}$ & $\frac{0.010}{0.009}$ & $\frac{9.772}{9.439}$ & 32 \\
\hline & $6 / 21 / 2004$ & 2:23:20 PM & 25.385 & 25.312 & 2) 24.785 & 26.472 & 25.298 & 42.08 & 48.409 & 4.14 & & 38.395 & 0.454 & 1.648 & 46.523 & 0.003 & 2.477 & & & 4.93444 & 46.9 & 11.4 & & 2.774 & & & & & $\frac{03}{86}$ \\
\hline & $6 / 21 / 2004$ & 2:24:20 PM & 25.422 & 25.314 & \begin{tabular}{|l|l}
44 & 24.837 \\
\end{tabular} & 26.449 & & 42.01 & 48.024 & & .09 & $\begin{array}{l}38.43 \\
\end{array}$ & 0.327 & 1.63 & 46.857 & 0.003 & 2.449 & 0.0 & 297.07 & 4.95111 & 46.6 & 11.5 & 40.2 & 2.773 & 0.365 & 0.367 & 0.009 & & 76 \\
\hline 157 & $6 / 21 / 2004$ & 2:25:20 PM & 25.444 & & 24.86 & 26.466 & & & & & & & & & 44.206 & $\begin{array}{l}0.003 \\
\end{array}$ & $\begin{array}{l}.416 \\
\end{array}$ & & 298.07 & & 46.8 & 10. & 40.5 & 2.790 & 0.360 & 0.362 & 0.009 & 8.935 & \\
\hline 158 & $6 / 21 / 2004$ & $\begin{array}{ll}2: 26: 20 \mathrm{PM} \\
2 \cdot 27 \cdot 20 \mathrm{P}\end{array}$ & $\begin{array}{l}25.477 \\
25.94\end{array}$ & $\begin{array}{l}25.299 \\
25206\end{array}$ & $\begin{array}{ll}924.877 \\
6\end{array}$ & $\begin{array}{l}26.493 \\
26546\end{array}$ & 25.409 & 42.371 & \begin{tabular}{|l|l|l|}
48.105 \\
\end{tabular} & & 087 & $\begin{array}{l}38.816 \\
28.027\end{array}$ & $\begin{array}{l}0.127 \\
.20\end{array}$ & 1.595 & $\begin{array}{l}4.06 \\
\end{array}$ & $\begin{array}{l}0.003 \\
002\end{array}$ & $\begin{array}{l}2.396 \\
2396\end{array}$ & & \begin{tabular}{|l|}
299.07 \\
30.07
\end{tabular} & \begin{tabular}{|l|l|}
4.98444 \\
\end{tabular} & 46.6 & 10.8 & 40.6 & 2.799 & 0.357 & 0.358 & 0.009 & 8.831 & \\
\hline & $\frac{6 / 21 / 2004}{6 / 21 / 204}$ & \begin{tabular}{|l|}
$2: 27: 20 \mathrm{PM}$ \\
$2 \cdot 28: 20 \mathrm{PM}$
\end{tabular} & $\begin{array}{l}25.494 \\
25515\end{array}$ & $\begin{array}{l}25.296 \\
25297\end{array}$ & \begin{tabular}{|l|l|}
24.904 \\
7
\end{tabular} & $\begin{array}{r}26.546 \\
25.57\end{array}$ & 25.426 & \begin{tabular}{|l|l|l|l|}
42.476 \\
2.69
\end{tabular} & \begin{tabular}{|l|l|}
48.281 \\
\end{tabular} & 4.04 & $\frac{543}{n g 0}$ & $\begin{array}{l}38.927 \\
39077\end{array}$ & $\begin{array}{l}-0.006 \\
0.21\end{array}$ & $\begin{array}{l}1.593 \\
\end{array}$ & \begin{tabular}{|l|l|l|}
44.696 \\
4079
\end{tabular} & \begin{tabular}{|l|l|}
0.003 \\
0.003
\end{tabular} & \begin{tabular}{|l}
2.386 \\
2371
\end{tabular} & & \begin{tabular}{|l|}
300.07 \\
30.07
\end{tabular} & & & $\frac{11.0}{100}$ & & & $\begin{array}{ll}0.356 \\
0.354\end{array}$ & 0.357 & 0.009 & 8.764 & \\
\hline 161 & $\begin{array}{l}66 / 21204 \\
6 / 21 / 2004\end{array}$ & $\begin{array}{l}2: 28: 20 \mathrm{PM} \\
22: 29 \cdot 20 \mathrm{PM}\end{array}$ & $\frac{25.515}{25.528}$ & $\begin{array}{l}25.297 \\
25.275 \\
\end{array}$ & $\begin{array}{l}7 \\
75 \\
5\end{array}$ & $\frac{26.567}{26.61}$ & $\frac{25 .}{25 .}$ & $\begin{array}{r}42.69 \\
42.7 \\
\end{array}$ & \begin{tabular}{|l|l|}
48.264 \\
48.457
\end{tabular} & $\frac{4.08}{4.10}$ & \begin{tabular}{|c|c|}
08 & \\
105 &
\end{tabular} & \begin{tabular}{|l|l|}
0777 \\
077
\end{tabular} & \begin{tabular}{|l|}
-0.021 \\
-0.076
\end{tabular} & $\begin{array}{l}\frac{1.50}{1.56} \\
1\end{array}$ & $\begin{array}{l}40.789 \\
43.587\end{array}$ & $\begin{array}{l}0.003 \\
0.003\end{array}$ & $\begin{array}{r}2.374 \\
2.36\end{array}$ & & \begin{tabular}{|c|}
301.07 \\
302.07
\end{tabular} & 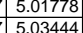 & $\begin{array}{ll}46.8 \\
47.0\end{array}$ & $\frac{10 .}{10 .}$ & & $\begin{array}{l}3.819 \\
2.819\end{array}$ & $\begin{array}{l}0.354 \\
0.352 \\
\end{array}$ & $\mid \begin{array}{l}0.355 \\
0.353\end{array}$ & 0.009 & 8.678 & \\
\hline & $6 / 21 / 2004$ & 2:30:20 PM & 25.566 & 25.308 & \begin{tabular}{|l|l|}
8 & 24.982 \\
\end{tabular} & 26.723 & 25.474 & 42.798 & 48.387 & 4.05 & & 39.235 & \begin{tabular}{|l|}
-0.079 \\
\end{tabular} & $\frac{1.001}{1.569}$ & 46.815 & 0.003 & $\begin{array}{l}2.350 \\
2.355\end{array}$ & & \begin{tabular}{|c|}
303.07 \\
\end{tabular} & 5.05111 & 46.9 & 11.5 & 41.0 & 2.82 & 0 & $\begin{array}{l}0.351 \\
0.351\end{array}$ & $\begin{array}{l}0.009 \\
0.009 \\
\end{array}$ & $\begin{array}{l}8.621 \\
8.565\end{array}$ & \\
\hline & $6 / 21 / 2004$ & $2: 31: 20 \mathrm{PM}$ & 25.58 & 25.302 & 24.975 & & 25.463 & 42.943 & 48.492 & 4.08 & 087 & 39.331 & \begin{tabular}{|l|l|} 
& -0.102 \\
\end{tabular} & & 45.403 & 0.003 & 2.347 & & 304.07 & & 47.0 & 11.1 & 411 & & & 0.350 & & & \\
\hline 164 & $6 / 21 / 2004$ & 2:32:20 PM & 25.597 & 25.284 & 24.983 & 26.539 & 25 & $\begin{array}{l}42.69 \\
\end{array}$ & & & 114 & \begin{tabular}{|l|l|}
39.038 \\
\end{tabular} & \begin{tabular}{|l|}
-0.108 \\
\end{tabular} & & 45.343 & & $\begin{array}{l}2.344 \\
\end{array}$ & & 305.07 & & 46.9 & 11. & 40. & & 0.349 & 0.350 & 0.009 & 8.556 & \\
\hline 165 & $6 / 21 / 2004$ & 2:33:20 F & $\begin{array}{l}25.614 \\
.561\end{array}$ & $\begin{array}{l}25.297 \\
2527\end{array}$ & 24.995 & 26.541 & & 42.876 & & 4.06 & 063 & \begin{tabular}{|r|}
39.3 \\
30.30
\end{tabular} & -0.102 & 1.474 & $\begin{array}{r}45.752 \\
\end{array}$ & \begin{tabular}{|l|l|}
0.003 \\
\end{tabular} & $\begin{array}{r}2.34 \\
\end{array}$ & & \begin{tabular}{|l|l|}
306.07 \\
0.7
\end{tabular} & 5.10111 & 47.0 & 11.2 & 41.1 & 2.833 & \begin{tabular}{l|l|}
0.349 \\
\end{tabular} & 0.349 & 0.008 & 8.492 & \\
\hline & & & $\begin{array}{r}25.61 \\
25.629\end{array}$ & $\begin{array}{l}25.267 \\
25.277\end{array}$ & $\begin{array}{r}24.976 \\
25.01\end{array}$ & 26.497 & & $\begin{array}{l}42.926 \\
42603\end{array}$ & $\begin{array}{l}48.298 \\
8.153\end{array}$ & & 108 & $\begin{array}{r}39.38 \\
38.925\end{array}$ & -0.111 & & 47. & & 2.3 & & (3) & $1 \frac{1778}{2444}$ & & & & & & & 0.008 & $\begin{array}{l}8.483 \\
8.563 \\
563\end{array}$ & \\
\hline & 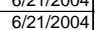 & $\begin{array}{l}2.53 .2 \mathrm{ZPM} M \\
2 \cdot 36 \cdot 20 \mathrm{PM}\end{array}$ & & & & & & & & & & & & & & & & & & & & & & & & & & & \\
\hline & $6 / 21 / 2004$ & $2: 37: 20$ & 25.649 & 25.266 & 25.034 & $\begin{array}{r}20.525 \\
26.53\end{array}$ & 25.4 & 42.947 & $\begin{array}{r}40.15 \\
4882\end{array}$ & & |lot & $\begin{array}{l}38.992 \\
39.251\end{array}$ & $\begin{array}{l}-0.1166 \\
-0.171\end{array}$ & & 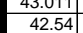 & 0.003 & $\begin{array}{l}2.5300 \\
2.337\end{array}$ & & 310.07 & & $\begin{array}{l}46.1 \\
47.1\end{array}$ & 10. & 41 & 2.834 & $\mid$ & & 0.008 & $\begin{array}{l}0.534 \\
8.470\end{array}$ & $\frac{26}{21}$ \\
\hline & $6 / 21 / 2004$ & & 25.675 & 25.287 & 725.056 & 26.587 & & & 48.167 & 4.08 & 082 & & \begin{tabular}{|l|}
-0.145 \\
\end{tabular} & & 45.725 & 0.003 & 2.338 & & 311.07 & 5.18444 & 46.7 & 11.2 & & .82 & 0.349 & 0.348 & 0.009 & 8.510 & \\
\hline & $6 / 21 / 2004$ & 2:39:20 & 25.678 & 25.29 & \begin{tabular}{l|l}
95.064 \\
9
\end{tabular} & 26.635 & & & 47.949 & & 226 & \begin{tabular}{|l|l|}
38.814 \\
\end{tabular} & & & 45.1 & & & & \begin{tabular}{|l|}
312.07 \\
\end{tabular} & & 46.5 & & & & 0.346 & & 0.008 & 8.486 & \\
\hline & 6/21/2004 & 2:40:20 PM & 25.692 & & 25.063 & 26.644 & & \begin{tabular}{|l|l|}
42.47 \\
\end{tabular} & 47.804 & & 194 & \begin{tabular}{|l|l|}
38.76 \\
20.7
\end{tabular} & -0.154 & 1.47 & 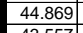 & $\begin{array}{l}0.003 \\
\end{array}$ & & & \begin{tabular}{|l|l|}
313.07 \\
21.07
\end{tabular} & 5.21778 & 46.3 & 11. & 40 & 2.800 & 0.346 & 0.346 & & 8.509 & \\
\hline & $\begin{array}{l}6 / 21 / 2004 \\
6 / 21 / 204\end{array}$ & $\begin{array}{l}2: 41: 20 \text { PM } \\
\\
\end{array}$ & $\begin{array}{l}25.716 \\
25.722\end{array}$ & & $\begin{array}{l}25.107 \\
25.108\end{array}$ & $\begin{array}{l}26.693 \\
26.64\end{array}$ & $\frac{25.54}{25.5}$ & $\begin{array}{r}42.893 \\
42.29\end{array}$ & $\begin{array}{l}48.476 \\
7734\end{array}$ & 4.17 & 179 & $\begin{array}{l}39.226 \\
38.728\end{array}$ & \begin{tabular}{|r|}
-0.189 \\
\end{tabular} & 1.47 & $\begin{array}{l}43.557 \\
5.745\end{array}$ & & 2.3. & & \begin{tabular}{|l|l|}
314.07 \\
325.07
\end{tabular} & & & 10. & 41 & & $\begin{array}{l}0.347 \\
0.345\end{array}$ & & & 8.424 & \\
\hline & $\frac{66 / 21 / 2004}{6 / 21 / 2004}$ & $2: 2: 2: 20 \mathrm{PM}$ & & & $\begin{array}{rl}4 & 25.108 \\
4 & 25.148\end{array}$ & $\frac{26.664}{26.699}$ & & & $\frac{41.734}{47.721}$ & & & $\frac{38.7}{38.6}$ & & & & & & & $\begin{array}{l}315.07 \\
316.07 \\
\end{array}$ & & & & & & & & & & \\
\hline & $6 / 21 / 2004$ & & 25.741 & 25.328 & 25.121 & 26.677 & & 42.46 & 47.85 & & & & \begin{tabular}{|l|} 
\\
\end{tabular} & & 48.8 & & & & 317 & & 4 & & & & & & & & \\
\hline & $6 / 21 / 2004$ & & 25.746 & 25.323 & \begin{tabular}{|l|l|}
3 & 25.117 \\
\end{tabular} & 26.688 & & 42.48 & 47.792 & & 276 & & \begin{tabular}{|l|}
-0.325 \\
\end{tabular} & & 48. & & 2.306 & & 318.07 & & 46.3 & 11.9 & $4 \mathrm{se}$ & & 0.344 & 0.3 & 008 & 8.450 & \\
\hline & $6 / 21 / 2004$ & & 25.743 & & 25.109 & 26.66 & & 42.234 & 47. & 4.25 & & \begin{tabular}{|l|l}
38.403 \\
\end{tabular} & \begin{tabular}{|l|}
-0.304 \\
\end{tabular} & & 44. & & 2.307 & & & & 46.1 & 19 & & & 0.344 & & & & \\
\hline & $6 / 21 / 2004$ & & 25.756 & 25.324 & 25.147 & 26.638 & & & & & & & -0.342 & & 41. & & 2.3 & & 320 & & 46.3 & & & & & & & 8.433 & \\
\hline & $6 / 21 / 2$ & $\begin{array}{l}2: 48: 20 \mathrm{PM} \\
: 0.020\end{array}$ & $\begin{array}{r}25.745 \\
2577\end{array}$ & $\begin{array}{l}25.317 \\
25227\end{array}$ & $\frac{25.126}{25.14}$ & $\begin{array}{l}26.602 \\
26.622\end{array}$ & 25. & & & & & & & & $\begin{array}{c}44.5 \\
5.5\end{array}$ & & 2.2 & & $\frac{321.0}{3220}$ & & & 10. & 20 & & 5 & & & 8.435 & \\
\hline & $\begin{array}{r}6 / 21 / 204 \\
66 / 212004\end{array}$ & & & & & $\frac{26.622}{26.601}$ & & & & & & & & & & & & & & & & & & & & & & & \\
\hline & $6 / 21 / 2004$ & & 25.783 & 25.335 & $\frac{25.109}{25.144}$ & 26.575 & 25. & $\frac{41 .}{42 .}$ & $\frac{47.021}{47.508}$ & & & & $\begin{array}{l}-0.404 \\
-0.522\end{array}$ & & $\frac{42}{45 .}$ & & & & $\mid \begin{array}{l}022.01 \\
324.07\end{array}$ & 5.40 & & 1.3 & $4 \mathrm{4c}$ & & & & & $\begin{array}{l}\frac{434}{334} \\
\end{array}$ & \\
\hline & $6 / 21 / 2004$ & & 25.792 & & 25.168 & 26.579 & & & & & & & -0.553 & & & & & & 325 & & & 11. & 40 & & & & & 340 & \\
\hline & $6 / 21 / 2004$ & & 25.791 & 25.339 & \begin{tabular}{|l|l|}
9 & 25.162 \\
\end{tabular} & 26.543 & & 42.001 & 47.093 & & & 38.061 & -0.536 & & 52.6 & & 2.254 & & 326. & \begin{tabular}{|l|l|} 
\\
\end{tabular} & & 12. & 4 & & 36 & & & & \\
\hline & $6 / 21 / 2$ & & 25.813 & 25.355 & 25.168 & 26.5 & & & & & & & -0.611 & & & & & & 327 & & & & & & 4 & & & & \\
\hline & $6 / 21 / 2004$ & & $\begin{array}{r}25.808 \\
25.81\end{array}$ & & \begin{tabular}{|l|l|}
6 & 25.164 \\
\end{tabular} & & & & & & & & & & & & 2.2 & & 328 & & & 11.1. & & & & & & & \\
\hline & 6/21/2004 & $\begin{array}{l}2: 56: 20 \mathrm{PM} \\
2 \cdot 5 \cdot 7 \cdot 20 \mathrm{PM}\end{array}$ & $\begin{array}{r}25.81 \\
25.811 \\
\end{array}$ & $\begin{array}{l}25.357 \\
25.369\end{array}$ & $\begin{array}{l}7 \quad 25.16 \\
9\end{array}$ & 26.552 & & & $\begin{array}{l}46.894 \\
46.998\end{array}$ & & & \begin{tabular}{|l|}
77.872 \\
3705
\end{tabular} & -0.556 & & & 0.0 & $\frac{2.2}{2.2}$ & & $\begin{array}{l}329 \\
330 \\
330\end{array}$ & 5.484 & 4 & $10.8 \mathrm{r}$ & 39.8 & & 34 & 0. & 08 & $8.361 \mid$ & \\
\hline & $\frac{60 / 21 / 2}{6 / 21 / 2}$ & & $\frac{25.81}{25.78}$ & $\frac{25.369}{25.343}$ & & & & & & & & & -0.62 & & & & & & & & & & & & & & & & \\
\hline & $6 / 21 / 2$ & & $\frac{2.176}{25.78}$ & & & & & & & & & & & & & & & & & & & & & & & & & & \\
\hline & & & 25.797 & $\frac{25.3}{253}$ & & 26. & & & & & & & -0.6 & & & & & & & & & & & & & & & & \\
\hline & & & & & & & & & & & & & & & & & & & & & & & & & & & & & \\
\hline & & & 25.79 & 25.326 & 25. & 26. & & & & & & & & & & & & & & & & & & & & & & & \\
\hline & & & 2578 & & & & & & & & & & & & & & & & & & & & & & & & & & \\
\hline & $6 / 21 /$ & & & 25. & 25.17 & 26.2 & & & & & & & & & & & & & & & & & & & & & & & \\
\hline & $6 / 21 / 2$ & & 25.78 & 25.28 & $\begin{array}{l}1 \\
\text { 1) } 25.1\end{array}$ & 26.3 & & & & & & & -0.6 & & & & & & & & & & & & & & & & \\
\hline & $6 / 21 / 2$ & & 25.79 & 25.277 & 25.1 & & & 41. & & & & & & & & & & & & & & & & & & & & & \\
\hline & & & 25.779 & 25.257 & 25.1 & & & & & & & & -0.64 & & & & 2.24 & & & & & & & & & & & & \\
\hline & & & 25. 169 & 25.241 & 25.164 & & & & 46. & & & & -0.6 & & & & & & & & & & & & & & & & \\
\hline & & & 25. & & & & & & & & & & & & & & & & & & & & & & & & & & \\
\hline & & & & $\frac{25.2}{25.2}$ & & & & & & & & & & & & & & & & & & & & & & & & & \\
\hline & $\frac{1 / 21 / 2}{6 / 212}$ & & $\frac{25.104}{25768}$ & 25 & & & & & & & & & & & & & & & & & & & & & & & & & \\
\hline & $6 / 21 / 2$ & & 257 & $\frac{25.6}{25.94}$ & $\frac{20.145}{25143}$ & 26.3 & & & & & & & & & & & & & & & & & & & & & & & \\
\hline & & & 25.744 & 25.176 & & & & 41.754 & & & & & -07 & & & & 222 & & & & & & & & & & & & \\
\hline & & & & 2518 & 25.144 & & & & & & & & & & & & & & & & & & & & & & & & \\
\hline & & & 25.756 & 25.178 & \begin{tabular}{l|l|l|}
8 & 25.142 \\
\end{tabular} & 26.3 & & 41.6 & & 4.26 & & & & & & & & & & & & & & & & & & & \\
\hline & & & & & & & & & & & & & & & & & & & & & & & & & & & & & \\
\hline 10 & $6 / 21 / 2004$ & 3:18:20 & 5.761 & 25.168 & \begin{tabular}{l|l|}
825.132 \\
\end{tabular} & 26.32 & & 41.829 & 46.743 & 4.31 & 317| & 37.888 & \begin{tabular}{|l|} 
\\
\end{tabular} & & | & 0.003 & & & & & 45.3 & & & & & 0.350 & 0.008 & 8.273 & \\
\hline
\end{tabular}


WSRC-TR-2005-00105, REVISION 0

SRNL-RPP-2005-00012, REVISION 0

RUN \# 3.18 AND 19; ACID CLEANING AND CAUSTIC RINSING - CONT.

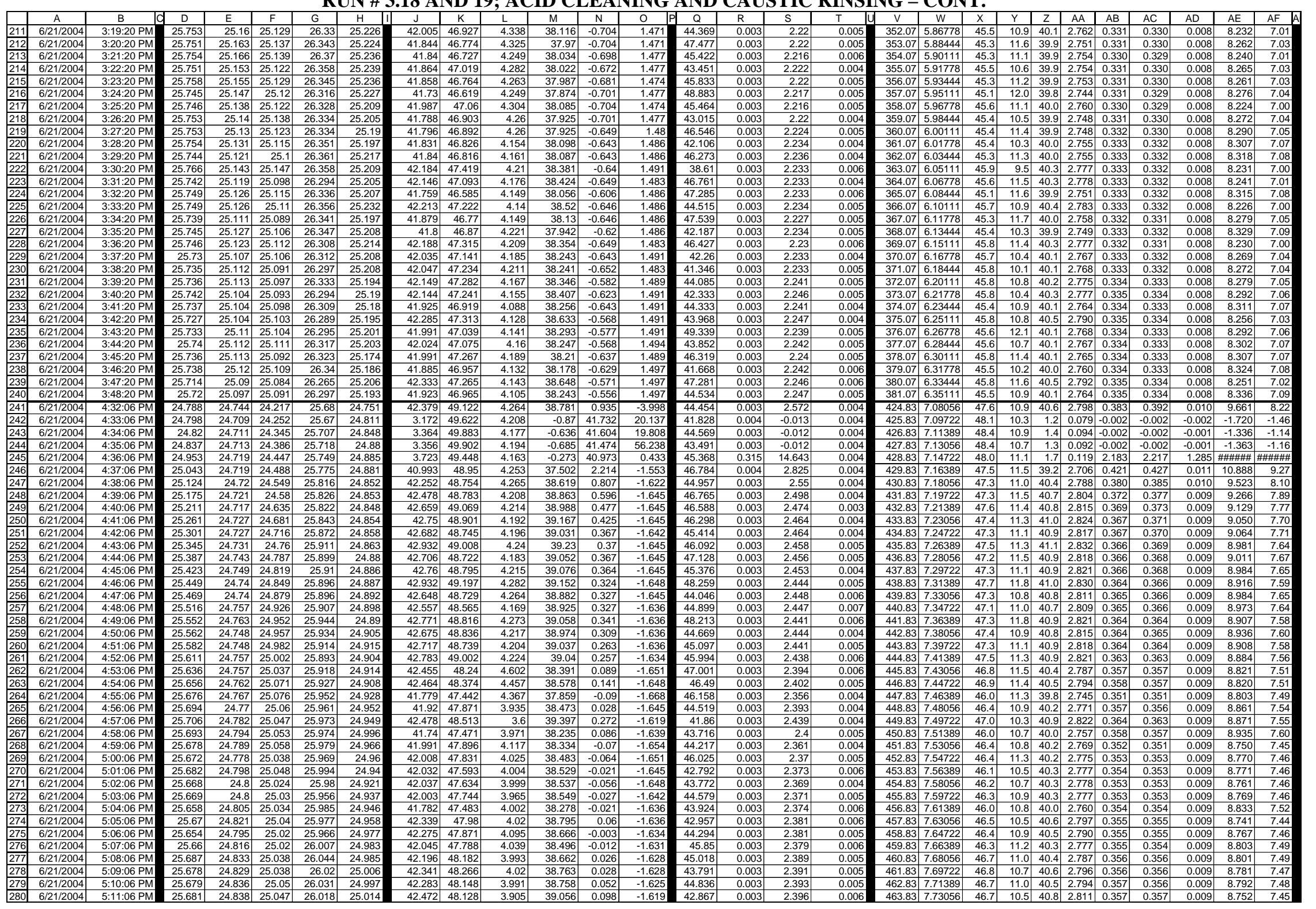


WSRC-TR-2005-00105, REVISION 0

SRNL-RPP-2005-00012, REVISION 0

RUN \# 3.18 AND 19; ACID CLEANING AND CAUSTIC RINSING - CONT.

\begin{tabular}{|c|c|c|c|c|c|c|c|c|c|c|c|c|c|c|c|c|c|c|c|c|c|c|c|c|c|c|c|c|}
\hline & & & & & & & & & & & & & & & & & & & & & & & & & & & & \\
\hline & A & B & D & $E$ & $F$ & $\begin{array}{ll} \\
\end{array}$ & $\mathrm{H}$ & $\mathrm{J}$ & $\mathrm{K}$ & $\mathrm{L}$ & \begin{tabular}{l|l|} 
\\
\end{tabular} & $\mathrm{N}$ & 0 & \begin{tabular}{l|l} 
\\
\end{tabular} & \begin{tabular}{l|l|l|l|l|l|} 
& \\
\end{tabular} & $\mathrm{s}$ & $\mathrm{T}$ & $\mathrm{v}$ & w & $x$ & Y & $z$ & $\mathrm{AA}$ & $A B$ & $\mathrm{AC}$ & $\mathrm{AD}$ & $\mathrm{AE}$ & $\begin{array}{ll}\mathrm{AF} & \mathrm{A} \\
\end{array}$ \\
\hline & $6 / 21 / 2004$ & 5:12:06 PM & 25.683 & 24.844 & 25.023 & $\begin{array}{l}26.01 \\
2.025\end{array}$ & 25.021 & 42.5 & 48.401 & 3.986 & 38.994 & 0.031 & -1.62 & 42.437 & 0.003 & 2.4 & 0.006 & 464.83 & 7.74722 & 46.9 & 10.4 & 40.8 & 2.810 & 0.358 & 0.358 & 0.009 & 8.773 & \\
\hline & & & & $\begin{array}{l}24.844 \\
2.84\end{array}$ & & & 25.006 & & & & \begin{tabular}{|l|}
39.403 \\
3231
\end{tabular} & 0.141 & & 42.139 & & 2.409 & & & & 47.5 & 10. & & & & & 0.009 & 8.735 & \\
\hline & $6 / 21 / 2004$ & 5:14:06 PM & 25.684 & 24.845 & \begin{tabular}{|l|l|}
25.009 \\
\end{tabular} & 26.01 & & 42.592 & 48.304 & 3.805 & 39.319 & $\begin{array}{l}0.185 \\
\end{array}$ & $\begin{array}{l}1.613 \\
\end{array}$ & 40.965 & $\begin{array}{l}0.003 \\
\end{array}$ & 2.411 & 11 & 466.83 & 7.78056 & 46.8 & 10.0 & & & 0.359 & 0.359 & 0.009 & 8.775 & \\
\hline & 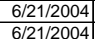 & $\begin{array}{l}5: 15: 06 \mathrm{PM} \\
5: 16: 06 \mathrm{PM}\end{array}$ & $\begin{array}{r}25.704 \\
25.69\end{array}$ & $\begin{array}{l}24.866 \\
24.851\end{array}$ & \begin{tabular}{|c|}
25.065 \\
25.046 \\
\end{tabular} & $\begin{array}{l}26.051 \\
26.027\end{array}$ & $\begin{array}{l}24.997 \\
25.023\end{array}$ & \begin{tabular}{|l|l|}
42.528 \\
42.08
\end{tabular} & $\begin{array}{l}48.358 \\
37.684\end{array}$ & $\begin{array}{l}3.827 \\
44058\end{array}$ & \begin{tabular}{|l|}
39.185 \\
38516
\end{tabular} & \begin{tabular}{|c|c|}
0.199 \\
-0.029
\end{tabular} & \begin{tabular}{|c|c|}
-1.605 \\
-1.631
\end{tabular} & \begin{tabular}{|l|l|}
42.262 \\
43699
\end{tabular} & \begin{tabular}{|l|l|}
0.003 \\
0.003
\end{tabular} & $\begin{array}{l}2.417 \\
2.368 \\
\end{array}$ & $\begin{array}{l}0.004 \\
0.005\end{array}$ & 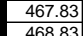 & 3781389 & $\begin{array}{l}46.9 \\
46.9\end{array}$ & $\begin{array}{ll}10.4 \\
10.7\end{array}$ & & \begin{tabular}{|l|l|} 
\\
\end{tabular} & $\begin{array}{l}7.360 \\
\end{array}$ & $\begin{array}{l}0.360 \\
0.353\end{array}$ & $\begin{array}{l}0.009 \\
\end{array}$ & $\begin{array}{l}8.804 \\
8755\end{array}$ & 7.49 \\
\hline & & $\begin{array}{l}5: 16: 06 \mathrm{PM} \\
5: 17: 06 \mathrm{PM}\end{array}$ & $\begin{array}{r}25.69 \\
25.675\end{array}$ & $\begin{array}{r}24.851 \\
24.856\end{array}$ & 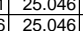 & $\frac{26.027}{26.022}$ & $\frac{25.023}{25.013}$ & $\begin{array}{r}42.08 \\
42.234 \\
\end{array}$ & $\begin{array}{l}47.684 \\
48.086 \\
\end{array}$ & $\begin{array}{l}4.058 \\
.052 \\
\end{array}$ & \begin{tabular}{|c|}
38.516 \\
38.641
\end{tabular} & $\begin{array}{l}-0.029 \\
-0.015\end{array}$ & $\begin{array}{c}-1.631 \\
-1.622 \\
\end{array}$ & $\begin{array}{l}43.6999 \\
457727\end{array}$ & \begin{tabular}{|c|}
0.003 \\
0.003
\end{tabular} & $\begin{array}{l}2.368 \\
2.377\end{array}$ & $\frac{0.005}{0.005}$ & $\begin{array}{l}468.83 \\
469.83 \\
\end{array}$ & 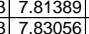 & $\begin{array}{l}46.2 \\
46.6 \\
\end{array}$ & $\frac{10.7}{112}$ & $\begin{array}{r}40.3 \\
40.4\end{array}$ & \begin{tabular}{|l|}
2.778 \\
2788
\end{tabular} & $\mid \begin{array}{l}0.353 \\
0.354\end{array}$ & $\begin{array}{l}0.353 \\
0.354 \\
\end{array}$ & $\begin{array}{l}0.009 \\
0.099\end{array}$ & $8.750 \mid$ & $\begin{array}{r}7.45 \\
7.45\end{array}$ \\
\hline 28 & $6 / 21 / 2004$ & 5:18:06 PM & 25.692 & 24.868 & 825.032 & 26.058 & 24.994 & $\begin{array}{l}42.204 \\
42.043\end{array}$ & 477.759 & 3.98 & 38.559 & \begin{tabular}{|l|}
-0.010 \\
\end{tabular} & -1.625 & $\begin{array}{l}45.127 \\
41.019\end{array}$ & $\begin{array}{l}0.003 \\
0.003\end{array}$ & $\frac{.3 .31}{2.379}$ & 0.005 & $\begin{array}{l}4699.83 \\
470.83\end{array}$ & $\begin{aligned} \\
3\end{aligned}$ & 年66.6 & $\frac{11.2}{10.1}$ & \begin{tabular}{|l|}
40.4 \\
0.3 \\
\end{tabular} & $\frac{2.788}{2.779}$ & $\begin{array}{l}0.354 \\
0.355\end{array}$ & $\begin{array}{l}.3544 \\
0.354 \\
\end{array}$ & $\begin{array}{l}0.0099 \\
0.009\end{array}$ & $\frac{8.753}{8.793}$ & $\begin{array}{l}7.45 \\
7.48\end{array}$ \\
\hline 288 & $6 / 21 / 2004$ & & 25.692 & 24.869 & 25.043 & 26.034 & 24.995 & 42.362 & 48.026 & 3.983 & 38.921 & 0.023 & & 44.337 & 0.003 & 2.382 & 0.005 & 471.83 & \begin{tabular}{|l|l}
37.86389 \\
\end{tabular} & 46.6 & 10.9 & 40.6 & 2.802 & 0.355 & 0.355 & 0.009 & & $\begin{array}{l}7.48 \\
7.43 \\
\end{array}$ \\
\hline & & & & & 25.054 & & & & & & 38.66 & & & & & & & & 88056 & 46.5 & & & & & & & & \\
\hline & $6 / 21 / 2004$ & 5:21:06 & 25.7 & 24.872 & 25.056 & \begin{tabular}{|l|}
26.047 \\
\end{tabular} & & 42.445 & 48.221 & & \begin{tabular}{|l|l|}
38.853 \\
\end{tabular} & 0.026 & $\begin{array}{l}-1.616 \\
\end{array}$ & $\begin{array}{l}43.236 \\
\end{array}$ & & 2.38 & & $\begin{array}{l}473.83 \\
\end{array}$ & 89722 & $\begin{array}{ll}46.7 \\
\end{array}$ & 10. & & & 0.355 & 354 & 0.009 & 8.716 & \\
\hline & $6 / 21 / 2004$ & 5:22:06 PM & \begin{tabular}{|l|l|}
25.709 \\
.5271
\end{tabular} & 24.88 & $\begin{array}{l}25.085 \\
25.071\end{array}$ & $\begin{array}{l}26.086 \\
20.087\end{array}$ & & & .215 & $\begin{array}{l}3.943 \\
3.056\end{array}$ & 39 & $\begin{array}{l}0.072 \\
0.321\end{array}$ & $\begin{array}{l}-1.608 \\
.160\end{array}$ & $\begin{array}{l}42.452 \\
\end{array}$ & . & 2.387 & 37 & $\begin{array}{l}474.83 \\
\end{array}$ & & 46.7 & 10.4 & & 年 & 0.356 & 0.030 & 0.009 & & \\
\hline & $\begin{array}{l}6 / 21 / 2004 \\
6 / 21 / 2004\end{array}$ & $\begin{array}{l}5: 23: 06 \mathrm{PM} \\
5: 24: 06 \mathrm{PM}\end{array}$ & $\begin{array}{r}25.71 \\
25.707\end{array}$ & $\frac{24.872}{24.878}$ & $\begin{array}{l}2 \quad 25.071 \\
3 \\
3\end{array}$ & $\frac{26.087}{26.104}$ & & $\begin{array}{r}42.393 \\
42.669\end{array}$ & \begin{tabular}{|l|l|}
48.324 \\
48.441
\end{tabular} & $\begin{array}{l}3.965 \\
3.974\end{array}$ & \begin{tabular}{|c|}
38.886 \\
39.218
\end{tabular} & $\begin{array}{l}0.031 \\
0.026\end{array}$ & $\begin{array}{r}-1.608 \\
-1.613 \\
\end{array}$ & \begin{tabular}{|l|}
40.858 \\
44.166
\end{tabular} & \begin{tabular}{|c|}
0.003 \\
0.003
\end{tabular} & $\frac{2.384}{2.379}$ & $\frac{0.0}{0.0}$ & $\begin{array}{r}475.83 \\
476.83\end{array}$ & $\begin{array}{l}37.93056 \\
3\end{array}$ & $\begin{array}{l}46.9 \\
47.0\end{array}$ & $\frac{10.6}{10.8}$ & $\frac{40.6}{40.9}$ & & \begin{tabular}{l|l}
0.355 \\
0.355
\end{tabular} & $\mid \begin{array}{l}0.355 \\
0.354 \\
\end{array}$ & 0.009 & $\begin{array}{l}8.729 \\
8648\end{array}$ & $\begin{array}{l}7.43 \\
736\end{array}$ \\
\hline 294 & $6 / 21 / 2004$ & 5:25:06 PM & 25.708 & 24.875 & $5 \quad 25.069$ & 26.12 & 25.0 & 42.25 & 47.974 & 4.087 & 38.643 & -0.021 & -1.616 & 43.428 & 0.003 & 2.372 & 0.004 & 477.83 & \begin{tabular}{|l|l}
3 & 7.96382 \\
\end{tabular} & 46.5 & 10.6 & 40.4 & 2.789 & 0.354 & $\begin{array}{l}0.353 \\
0.353\end{array}$ & $\begin{array}{l}0.009 \\
0.009 \\
\end{array}$ & & $\begin{array}{l}7.36 \\
7.43 \\
\end{array}$ \\
\hline & $\begin{array}{l}6 / 21 / 2004 \\
\end{array}$ & $\begin{array}{ll}5: 26: 06 \mathrm{PM} \\
\end{array}$ & $\begin{array}{l}25.71 \\
25710\end{array}$ & 24.866 & \begin{tabular}{|r|r|}
$6 \quad 25.07$ \\
\end{tabular} & $\begin{array}{l}26.077 \\
26.075\end{array}$ & & 42.144 & 47.846 & & 38.588 & $\begin{array}{l}0.02 \\
0.02\end{array}$ & -1.61 & & 0.003 & 2.377 & & 478.83 & & 46.4 & 10.7 & & & 0.354 & & & 8.762 & 46 \\
\hline & $6 / 21 / 2004$ & & 25.718 & 24.879 & & 26.125 & & 42.277 & 48.132 & 4.045 & 38.765 & 0.002 & & 43.881 & 0.003 & 2.379 & & 479.83 & $\begin{array}{l}37.99722 \\
\end{array}$ & 46.7 & 10. & & 2.794 & & 0.354 & 0.009 & 8.732 & \\
\hline & $6 / 21 / 2004$ & 5:28:06 PM & 25.725 & 24.881 & 25.101 & & & & 47.873 & 4.005 & & & & 43.305 & 0.003 & & 0.0 & & 8.01389 & 46.4 & 10. & & 2.790 & 0.354 & 0.353 & 0.009 & 8.734 & \\
\hline & \begin{tabular}{|c|}
$6 / 21 / 2004$ \\
$6 / 21 / 204$ \\
\end{tabular} & $\begin{array}{l}5: 29: 06 \mathrm{~F} \\
5 \cdot 3 \cdot 06 \mathrm{~F}\end{array}$ & $\frac{25.716}{25773}$ & $\begin{array}{l}24.873 \\
24.879 \\
\end{array}$ & \begin{tabular}{l|l}
25.072 \\
25079
\end{tabular} & & & & $\begin{array}{r}47.81 \\
48.368 \\
\end{array}$ & $\begin{array}{l}3.951 \\
3.975 \\
\end{array}$ & \begin{tabular}{|l|}
38.641 \\
38999 \\
\end{tabular} & $\begin{array}{ll}0.014 \\
0.026\end{array}$ & & \begin{tabular}{|l|l|}
43.551 \\
43323
\end{tabular} & & 2.384 & & & & & 10.7 & & & $\begin{array}{l}0.355 \\
0.255 \\
\end{array}$ & 0.355 & & & 47 \\
\hline & $\begin{array}{l}6 / 21 / 2004 \\
6 / 21 / 2004\end{array}$ & $\begin{array}{l}\text { 5:30:06 PM } \\
\text { 5:31:06 PM }\end{array}$ & $\frac{25.723}{25.719}$ & $\begin{aligned} 24.879 \\
24.88 \\
\end{aligned}$ & 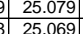 & $\begin{array}{r}26.11 \\
26.1 \\
\end{array}$ & 25.021 & $\begin{array}{l}42.472 \\
42.265\end{array}$ & $\begin{array}{l}48.368 \\
47.808\end{array}$ & & $\begin{array}{l}.959 \\
855\end{array}$ & $\begin{array}{l}0.026 \\
0.026\end{array}$ & $\begin{array}{l}-1.605 \\
-1.605 \\
\end{array}$ & \begin{tabular}{|l|l|}
43.032 & \\
43.685 &
\end{tabular} & $\begin{array}{l}0.003 \\
0.03\end{array}$ & $\begin{array}{r}2.38 \\
2.38 \\
\end{array}$ & 0.00 & $\begin{array}{l}482.83 \\
483.83 \\
\end{array}$ & \begin{tabular}{|l|l|l}
8.04722 \\
8.06389 \\
\end{tabular} & $\begin{array}{l}46.9 \\
46.3 \\
\end{array}$ & 10.5 & $\frac{40.7}{40.6}$ & 2.807 & 0.355 & $\begin{array}{l}0.354 \\
0.354\end{array}$ & 0.009 & $\begin{array}{l}8.696 \\
8.731 \\
\end{array}$ & $\begin{array}{l}7.40 \\
7.43 \\
74\end{array}$ \\
\hline 301 & $6 / 21 / 2004$ & $\begin{array}{l}\text { 5:31:06 PMI } \\
\text { 5:32:06 PM }\end{array}$ & 25.714 & 24.865 & 525.069 & 26.08 & $\frac{20.021}{25.027}$ & $\begin{array}{l}42.205 \\
42.331\end{array}$ & 48.121 & 3.969 & 38.855 & 0.008 & -1.599 & $\begin{array}{l}43.685 \\
44.433 \\
\end{array}$ & 0.003 & 2.384 & $\begin{array}{l}0.006 \\
0.004\end{array}$ & $\begin{array}{l}4854.85 \\
484.83\end{array}$ & \begin{tabular}{|c|c|}
3 & 8.06389 \\
3 & 8.08056 \\
\end{tabular} & $\begin{array}{l}46.3 \\
46.6 \\
\end{array}$ & $\frac{1.1 .}{10.9}$ & $\frac{40.6}{40.6}$ & $\frac{2.196}{2.799}$ & 0 & $\begin{array}{l}0.354 \\
0.355 \\
\end{array}$ & $\begin{array}{l}0.009 \\
0.009 \\
\end{array}$ & 8.731 & $\frac{7.43}{7.44}$ \\
\hline & $6 / 21 / 2004$ & 5:33:06 PM & 25.725 & 24.882 & 225.086 & 26.102 & & 42.225 & 47.922 & 3.981 & 38.664 & -0.006 & -1.602 & & 0.003 & 2.382 & & 485.83 & \begin{tabular}{|l|l|}
3 & 8.09722 \\
\end{tabular} & 46.4 & 10.7 & 40.4 & 2.789 & 0.355 & 0.354 & 0.009 & $\begin{array}{l}8.159 \\
8.760\end{array}$ & 7.44 \\
\hline & $6 / 21 / 2004$ & 5:34:06 PM & 25.721 & 24.882 & 25.087 & 26.093 & & 42.493 & 48.273 & 3.95 & 39.066 & -0.003 & -1.599 & & 0.003 & 2.384 & & 486.83 & \begin{tabular}{|l|l|}
3 & 8.11389 \\
\end{tabular} & 46.8 & 10.8 & & & & & 0.009 & & $\frac{45}{40}$ \\
\hline & $6 / 21 / 2004$ & & 25.732 & 24.884 & 25.088 & 26.109 & & 42.501 & & & & & -1.5 & & 0.003 & 2.382 & & & & & & & & & 354 & & 8.687 & \\
\hline & & & & & & 26.119 & & 42.182 & 7.808 & 3.94 & & & & & & & & & & & & & & & & & & \\
\hline & $6 / 21 / 2004$ & 5:37:C & 25.728 & 24.885 & 25.079 & 26.1 & & & 48.244 & 3.989 & 38 & .046 & & & 0 & 2.385 & & & & 46.8 & 11. & & & & & & & $\frac{40}{45}$ \\
\hline 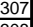 & & & & & & & & & & & & & & & & & & & & & & & & & & & 0.101 & \\
\hline $30 \varepsilon$ & $6 / 21 / 2004$ & 5:39:C & 25.725 & 24.886 & 25.085 & 26.146 & & $\begin{array}{l}42.59 \\
\end{array}$ & 48.152 & 3.963 & 39.167 & 0.026 & & 45.22 & $\begin{array}{l}0.003 \\
\end{array}$ & 2.386 & & & 8.19722 & 46 & 11. & & 2.818 & 0.356 & 0.355 & 0.009 & 8.681 & \\
\hline & $6 / 21 / 2004$ & 5:40:: & 25.74 & 24.912 & 25.121 & 26.247 & & 42.53 & 48.26 & & 38.996 & 0.02 & & 45. & & 387 & & & & & 11 & & & & & & & \\
\hline & $6 / 21 / 2004$ & 5 & 25.71 & & 25.055 & & & 42.315 & 48.126 & 3.94 & 38.879 & & & 44.849 & 0.003 & 2.389 & & & 8.23056 & 46.7 & & & & & .356 & & 760 & \\
\hline 2 & $\frac{6 / 21 / 2004}{66 / 21 / 2004}$ & $\begin{array}{l}5: 2: 2: 06 \mathrm{~F} \\
5 \cdot 33.06 \mathrm{~F}\end{array}$ & $\frac{25.735}{25.73}$ & 24.916 & $\begin{array}{r}25.08 \\
25.075 \\
\end{array}$ & $26.291 \mid$ & & $\begin{array}{r}42.24 \\
42.294 \\
\end{array}$ & \begin{tabular}{|l|}
48.173 \\
8.111 \\
\end{tabular} & $\frac{4}{3}$. & $\begin{array}{l}38.713 \\
38783 \\
\end{array}$ & 0.052 & $\frac{-1.58}{1.58}$ & $\begin{array}{r}42.89 \\
44.68\end{array}$ & 0.003 & $\frac{2.384}{2384}$ & & & 8.24722 & $\begin{array}{l}46.7 \\
46.6\end{array}$ & $\frac{10 .}{11}$ & & & 0.35 & 0.355 & 009 & 8.761 & $\frac{46}{45}$ \\
\hline & $\frac{1}{6} 6 / 212004$ & 5.:44: & 25.736 & 24.943 & 25.097 & 26.353 & & 42.746 & \begin{tabular}{|l|l}
48.468 \\
\end{tabular} & 3.384 & 39 & 0.13 & & 43.197 & 0.003 & 2402 & & 496.83 & 8.28056 & 47.0 & & & & D.358 & 0 & & 8.688 & \\
\hline & & & & & & \begin{tabular}{|l|l|l|l|}
26.348 \\
\end{tabular} & & \begin{tabular}{|l|}
42.426 \\
\end{tabular} & 48.208 & & & & & & & 2.4 & & & & & & & & & & & & \\
\hline & 6/21/2004 & & 25.747 & 24.978 & 25.093 & 26.354 & & \begin{tabular}{|l|l|l}
42.46 \\
\end{tabular} & & 3. & & 0.026 & & & & 2.38 & & & & & & & & & & & & \\
\hline & & & 25.743 & & 25.094 & & & 42.254 & & 887 & 38.762 & & & & & & & & & & & & & & & & & \\
\hline & 6/21/2004 & & 25.735 & 24.95 & 25.086 & 26.292 & & 42.493 & 48.144 & 3 & & 0.02 & & 44.442 & 0.003 & 2.388 & & & 8.34722 & 46 & & & & & 0.354 & & & \\
\hline & & & & & & & & & & & & & & & & & & & & & & & & & & & & \\
\hline & & & $\frac{25.724}{257212}$ & 25.00 & & & & & & & & & & & & & & & & & & & & & & & & \\
\hline & & & 25.142 & & 25.104 & & & & & & & & & & & & & & & & & & & & & & & \\
\hline & $\frac{0121 / 1 / 2}{6 / 21 / 2}$ & & $\frac{25.104}{25.734}$ & $\frac{20.01}{25.03}$ & 2.5 & $\frac{20.6}{262}$ & & & & & & 0.020 & & & & & & & & & & & & & & & & \\
\hline & & & 20.10 & & & & & & & & & & & & & & & & & & & & & & & & & \\
\hline & $6 / 21 / 2004$ & & 25.737 & 25.029 & 25.098 & 26.174 & & & 48.428 & 3.984 & & 0.028 & & & & & & & & & & & & & & & & \\
\hline & & & & & & & & & & & & & & & & & & & & & & & & & & & & \\
\hline & & & 25.745 & 25.04 & 25.11 & 26.20 & & & 48.29 & & & & & & & & & & & & & & & & & & & \\
\hline & & & 25.742 & 25.02 & 25.093 & 26.194 & & & & & & & & & & & & & & & & & & & & & & \\
\hline & $6 / 21$ & & & & 25.088 & 26.179 & & & & & & & & 41.5 & & & & & & & & & & & & & & \\
\hline & 6/21/2004 & & 25.726 & 25.01 & 25. & 26.138 & & & & & & & & & 0.00 & 2.3 & & & & & & & & & & & 8.793 & \\
\hline & $6 / 21 / 2$ & & 25.726 & & & 26.147 & & & & & & & & & & & & & & & & & & & & & & \\
\hline & 6/21/2004 & & 25.731 & 25.013 & 25.082 & 26.163 & & & & & & 0.043 & & & & & & & & & & & & & & & & \\
\hline & & & 25. & & & 26.194 & & & & & & & & & & & & & & & & & & & & & & \\
\hline 33 & & & & & & & & & & & & & & & & & & & & & & & & & & & & \\
\hline 235 & & & & & & & & & & & & & & & & & & & & & & & & & & & & \\
\hline & $\frac{121 / 21}{6212}$ & & $\frac{2.144}{25.729}$ & & & & & & & & & & & & & & & & & & & & & & & & & \\
\hline & & & 257 & & & & & & & & & & & & & & & & & & & & & & & & & \\
\hline & $\frac{15 / 1212}{66212}$ & & $\frac{2.5 .74}{25.74}$ & $\frac{25.012}{25.012}$ & 25.076 & $\frac{2.5 .16}{26.16}$ & & $\frac{42 .}{42 .}$ & & $\frac{3.395}{3.995}$ & & $\frac{0.0}{0.0}$ & & & & & & & & & & & & & & & & \\
\hline 33 & $6 / 21 / 2$ & & 24.409 & & 623.8 & 25.806 & & 22. & & 4.058 & & -1.214 & & & 0.003 & 1.188 & & 56 & & 22.2 & & 20. & & & & & & \\
\hline & $6 / 21$ & & 24.3 & 24.745 & 23.782 & 25.6 & & 2.962 & 23.95 & 3.961 & & 16.024 & & & & & & & & 2.5 & $10.7 \mathrm{rt}$ & 1. & & & & & & \\
\hline & & & 24.422 & 24.744 & $\begin{array}{l}423.745 \\
\end{array}$ & 25.629 & & 2.966 & 24.022 & & & 16.096 & & & & & & & & & 11.1 & & & & & & & \\
\hline & & & 24.454 & 24.736 & $\begin{array}{l}63.687 \\
\end{array}$ & 25.586 & & 2.854 & 24.105 & 3.864 & -1.03 & 16.194 & & & & & & & & .6 & 10.7 & & & & & & & \\
\hline & $6 / 21 / 2$ & & 24.785 & & & 25.5 & & & & 3.849 & -1 & & & & & & & & & $\sqrt{.7}$ & & & & & & & & \\
\hline & $6 / 21 / 2004$ & & 24.15 & & 23.6 & 25.6 & & 17.784 & 23.7 & 3.78 & 14.4 & & & & & 24 & & & & 28 & & & & & & & 23.620 & \\
\hline & $6 / 21 / 2$ & & 24.059 & & & 25.567 & & 21. & 24. & & & & & & & & & & & & & & & & & & & \\
\hline & $6 / 21 / 2$ & & 24.028 & 24. & & & & 22.169 & 24.165 & 3.706 & & & & & & 1.445 & & & & & & & & & & & & \\
\hline & $6 / 21 / 2$ & & 23.973 & 24 & 23.496 & 25.5 & & 22.193 & 23.95 & 3.882 & & -1. & & & & 1.00 & & & & & & & & & & & & \\
\hline & $6 / 21 / 2$ & & 23.965 & 24.717 & 23.568 & 25.42 & & 22.169 & \begin{tabular}{|l|l|}
23.828 \\
\end{tabular} & 3.842 & 18 & -1.214 & & & & 1.295 & & & & 22.4 & 11.0 & 20.5 & & & & & 9.892 & \\
\hline & $\frac{\mid 6 / 21 / 2004}{66 / 21 / 2004}$ & $\begin{array}{l}\text { 7:04:26 PM } \\
\text { 7:05:26 PM }\end{array}$ & $\frac{24.009}{24.053}$ & \begin{tabular}{|l|}
24.711 \\
24.705 \\
\end{tabular} & \begin{tabular}{|c|c|} 
& 23.628 \\
5 & 23.706 \\
\end{tabular} & \begin{tabular}{|l|}
25.396 \\
25.445 \\
\end{tabular} & \begin{tabular}{|l|}
24.618 \\
24.587 \\
\end{tabular} & $\begin{aligned} 22.139 \\
22.092 \\
\end{aligned}$ & \begin{tabular}{|r|}
23.705 \\
23.57
\end{tabular} & $\begin{array}{l}4.145 \\
4.148\end{array}$ & \begin{tabular}{|l|}
18.1487 \\
18.076
\end{tabular} & \begin{tabular}{|c|}
$\mid-1.2114$ \\
-1.216 \\
\end{tabular} & & $\begin{array}{l}45.961 \\
46.244 \\
\end{array}$ & $\begin{array}{l}0.003 \\
0.003\end{array}$ & $\begin{array}{l}1.226 \\
1.219\end{array}$ & & 578.17 & $\begin{array}{l}7 \\
7\end{array}$ & \begin{tabular}{|l|l|} 
& 22.2 \\
1 & 22.1
\end{tabular} & \begin{tabular}{|l|}
11.3 \\
11.3 \\
\end{tabular} & & \begin{tabular}{|l|l|} 
& 1.388 \\
1 & 1.385 \\
\end{tabular} & \begin{tabular}{|c|c|} 
\\
\end{tabular} & \begin{tabular}{|l|}
0.190 \\
0.189 \\
\end{tabular} & \begin{tabular}{|l|}
0.009 \\
0.009
\end{tabular} & \begin{tabular}{|c|}
9.433 \\
9
\end{tabular} & \begin{tabular}{|l}
8.03 \\
7.99 \\
\end{tabular} \\
\hline
\end{tabular}


WSRC-TR-2005-00105, REVISION 0

SRNL-RPP-2005-00012, REVISION 0

RUN \# 3.18 AND 19; ACID CLEANING AND CAUSTIC RINSING - CONT.

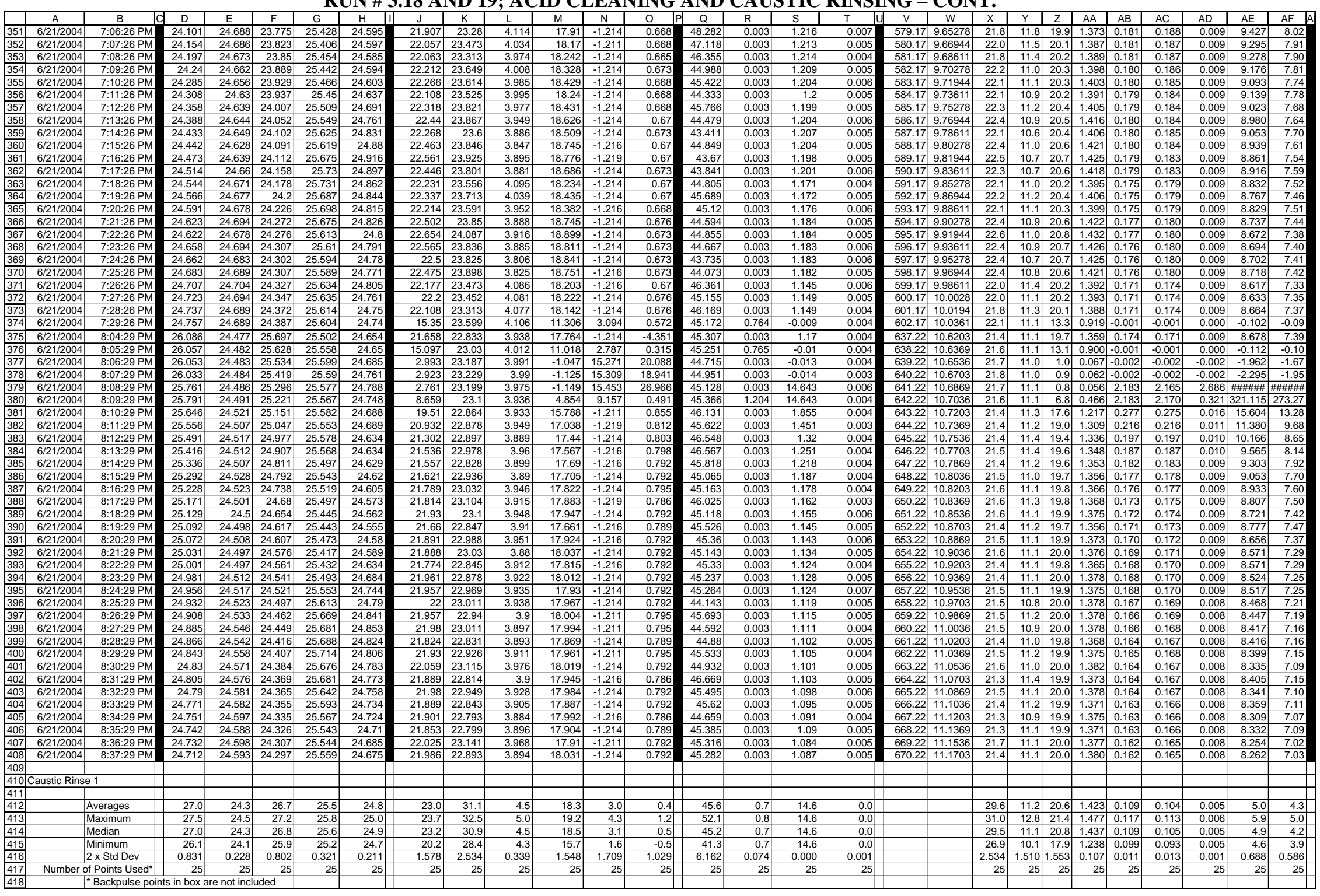


WSRC-TR-2005-00105, REVISION 0

SRNL-RPP-2005-00012, REVISION 0

RUN \# 3.18 AND 19; ACID CLEANING AND CAUSTIC RINSING - CONT.

\begin{tabular}{|c|c|c|c|c|c|c|c|c|c|c|c|c|c|c|c|c|c|c|c|c|c|c|c|c|c|c|c|c|}
\hline$\square$ & $\mathrm{A}$ & B & D & $E$ & $\mathrm{~F}$ & \begin{tabular}{l|l|l}
$G$ & \\
\end{tabular} & \begin{tabular}{|l|}
$\mathrm{H}$ \\
\end{tabular} & III $\mathrm{J}$ & $\mathrm{K}$ & $\mathrm{L}$ & \begin{tabular}{l|l}
$\mathrm{M}$ \\
\end{tabular} & \begin{tabular}{l|l|} 
\\
\end{tabular} & \begin{tabular}{|l|l}
$\mathrm{O}$ & $\mathrm{P}$ \\
\end{tabular} & \begin{tabular}{|l|l|l|}
$P$ & $Q$ & \\
\end{tabular} & \begin{tabular}{|l|l}
$\mathrm{R}$ & \\
\end{tabular} & \begin{tabular}{|l|}
$\mathrm{s}$ \\
\end{tabular} & \begin{tabular}{l|l|l} 
& $T$ \\
\end{tabular} & U $u$ V & \begin{tabular}{l|l} 
& $w$ \\
\end{tabular} & \begin{tabular}{l|l|} 
& $x$ \\
\end{tabular} & \begin{tabular}{|r|} 
\\
\end{tabular} & $\mathrm{z}$ & AA & $\mathrm{AB}$ & $\mathrm{AC}$ & $\mathrm{AD}$ & $\mathrm{AE}$ & $\begin{array}{ll}A F & A \\
A\end{array}$ \\
\hline 420 & Acid Clean 1 & & & & & & & & & & & & & & & & & & & & & & & & & & & \\
\hline & & & & & & & & & & & & & & & & & & & & & & & & & & & & \\
\hline & & Averages & 26.4 & 25.3 & 25.8 & 26.8 & $\begin{array}{l}25.7 \\
\end{array}$ & 34.6 & $\begin{array}{l}42.7 \\
\end{array}$ & 4.1 & 30.7 & 3.4 & -0.6 & $\begin{array}{l}44.3 \\
\end{array}$ & $\begin{array}{ll}0.4 \\
\end{array}$ & 6.7 & 0.0 & & & 41.3 & \begin{tabular}{|l|l|}
3 & 10.8 \\
\end{tabular} & 32.6 & 2.249 & 0.227 & 0.223 & 0.007 & 7.0 & \\
\hline 每23 & & Maximum & 27.9 & $\begin{array}{l}25.8 \\
25.44 \\
\end{array}$ & $\frac{27.5}{25.4}$ & 27.3 & $\begin{array}{l}26.0 \\
257 \\
\end{array}$ & $\begin{array}{l}46.0 \\
42.2\end{array}$ & \begin{tabular}{|l|}
58.0 \\
45.5 \\
\end{tabular} & $\frac{5.5}{42}$ & \begin{tabular}{|l|l|}
40.7 \\
383
\end{tabular} & 24.6 & 13.3 & \begin{tabular}{|l|}
56.1 \\
446
\end{tabular} & $\begin{array}{r}1.2 \\
0.0\end{array}$ & 14.6 & 0.0 & & & 56.5 & $\begin{array}{l}5 \quad 13.8 \\
5\end{array}$ & $\begin{array}{ll}43.4 \\
402\end{array}$ & \begin{tabular}{|l|}
2.989 \\
2771
\end{tabular} & 0.290 & 0.284 & 0.008 & 8.1 & \\
\hline 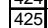 & & \begin{tabular}{|l} 
Median \\
Minimum
\end{tabular} & $\begin{array}{l}26.0 \\
25.6\end{array}$ & $\begin{aligned} 25.4 \\
24.8 \\
\end{aligned}$ & $\frac{25.4}{250}$ & $\begin{array}{r}22.0 \\
25.9 \\
\end{array}$ & 25.7 & $\frac{42.2}{21.1}$ & $\begin{array}{l}45.5 \\
33.0\end{array}$ & $\frac{4.2}{1.8}$ & $\begin{array}{l}38.3 \\
17.0\end{array}$ & \begin{tabular}{r|}
-1.2 \\
-1.2 \\
\end{tabular} & $\begin{array}{c}-3.9 \\
-4.3 \\
\end{array}$ & $\begin{array}{l}44.6 \\
27.4 \\
\end{array}$ & $\begin{array}{l}0.0 \\
0.0 \\
\end{array}$ & $\begin{array}{l}1.9 \\
1.6 \\
\end{array}$ & \begin{tabular}{l|l}
0.0 \\
0.0
\end{tabular} & & & \begin{tabular}{|l|l}
44.0 & \\
31.6 &
\end{tabular} & 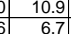 & $\begin{array}{l}40.2 \\
19.1 \\
\end{array}$ & & $\begin{array}{l}40.262 \\
0.152 \\
4\end{array}$ & & 0.007 & & $\begin{array}{c}5.8 \\
50\end{array}$ \\
\hline 426 & & $2 \times \operatorname{Std}$ Dev & 1.598 & 0.554 & 1.740 & 0.710 & 0.453 & $\begin{array}{ll}20.053 \\
\end{array}$ & 9.402 & 0.808 & 20.067 & $\begin{array}{lll}7 & 12.287 \\
\end{array}$ & 8.980 & 8.020 & 1.021 & $\begin{array}{ll}1.0 \\
12.468 \\
\end{array}$ & 0.002 & & & $\begin{array}{l}0.02 \\
9.402\end{array}$ & \begin{tabular}{|l|l|}
2 & 0.965 \\
\end{tabular} & $\frac{19.1}{4+\#+1}$ & \begin{tabular}{|l|}
1.315 \\
1.383 \\
\end{tabular} & \begin{tabular}{|l|l|}
0.152 \\
0.111
\end{tabular} & $\begin{array}{ll}.142 \\
0.118\end{array}$ & $\begin{array}{l}0.006 \\
0.001\end{array}$ & \begin{tabular}{r|}
5.994 \\
0.994
\end{tabular} & $\begin{array}{r}5.0 \\
0.846 \\
\end{array}$ \\
\hline \begin{tabular}{|l|l|}
427 \\
\end{tabular} & Number & of Points Used ${ }^{*}$ & 89 & 89 & $\begin{array}{ll}9 & 89 \\
\end{array}$ & 89 & 89 & 89 & 89 & 89 & 89 & $\begin{array}{ll}9 & 89 \\
\end{array}$ & 89 & 89 & 89 & 89 & 89 & & & 89 & \begin{tabular}{|l|l|}
9 & 89 \\
\end{tabular} & \begin{tabular}{|l|}
89 \\
\end{tabular} & \begin{tabular}{|l|} 
\\
\end{tabular} & 89 & 89 & 89 & 89 & 89 \\
\hline & & ${ }^{*}$ Backpulse poi & & are not incli & illuded & & & & & & & & & & & & & & & & & & & & & & & \\
\hline 429 & & & & & & & & & & & & & & & & & & & & & & & & & & & & \\
\hline & Acid Clean : & & & & & & & & & & & & & & & & & & & & & & & & & & & \\
\hline 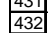 & & Averages & & & 25.1 & 26.4 & 25.3 & 42.1 & 47.3 & 4.2 & 38.3 & -0.4 & 1.5 & 45.3 & 0.0 & & 0.0 & & & & & & 2.769 & 0.340 & & 0.008 & & \\
\hline 433 & & Maximum & 25.8 & 25.4 & 25.2 & 26.7 & 25.6 & 42.9 & 48.6 & 4.5 & 39.4 & 1.4 & 1.8 & 52.7 & 0.0 & 2.7 & 0.0 & & & 47.1 & $\begin{array}{lll}1 & 1.1 .9 \\
\end{array}$ & 41.2 & \begin{tabular}{|l|}
2.837 \\
\end{tabular} & \begin{tabular}{|l|l|}
0.396 \\
\end{tabular} & 0.400 & 0.010 & $\begin{array}{r}0.4 \\
10.3 \\
\end{array}$ & $\begin{array}{l}7.2 \\
8.8 \\
\end{array}$ \\
\hline 434 & & Median & 25.7 & 25.3 & 25.1 & 26.4 & 25.2 & 41.9 & 47.1 & 4.2 & 38.1 & -0.6 & 1.5 & 45.3 & 0.0 & 2.2 & 0.0 & & & \begin{tabular}{l|l}
45.6 \\
\end{tabular} & \begin{tabular}{l|l|}
6 & 11.1 \\
\end{tabular} & 40.0 & 2.760 & 0.334 & 0.333 & 0.008 & 8.3 & 7.1 \\
\hline & & Minimum & 25.2 & 25.1 & 24.7 & 26.3 & 25.2 & 40.6 & 46.4 & 4.0 & 37.1 & $\begin{array}{l}-0.7 \\
\end{array}$ & 1.5 & 38.6 & 0.0 & 2.2 & 0.0 & & & 45.0 & $\begin{array}{ll}0.5 \\
\end{array}$ & \begin{tabular}{|l|}
38.8 \\
\end{tabular} & 2.678 & 0.330 & 0.329 & 0.008 & 8.2 & 7.0 \\
\hline \begin{tabular}{|l|}
436 \\
37
\end{tabular} & & $2 \times$ Std Dev & 0.244 & 0.180 & \begin{tabular}{l|l}
0 & 0.205 \\
\end{tabular} & 0.267 & 0.272 & 0.807 & 1.220 & 0.194 & 0.936 & 0.759 & 0.118 & 4.758 & 0.000 & $\begin{array}{ll}0.164 \\
\end{array}$ & 0.002 & & & 1.220 & & 0.865 & \begin{tabular}{|l|l|}
0.060 \\
\end{tabular} & 0.024 & 0.026 & 0.001 & 0.646 & 0.550 \\
\hline 4377 & Number & of Points Used ${ }^{*}$ & 89 & 89 & \begin{tabular}{l|r|}
9 & 89 \\
\end{tabular} & 89 & 89 & 89 & 89 & 89 & 89 & 89 & 89 & 89 & 89 & 89 & 89 & & & 89 & 89 & 89 & 89 & 89 & 89 & 89 & 89 & 89 \\
\hline |438 & & * Backpulse poi & ts in box & are not incli & icluded & & & & & & & & & & & & & & & & & & & & & & & \\
\hline $\begin{array}{l}439 \\
440 \\
\end{array}$ & Acid Clean 3 & & & & & & & & & & & & & & & & & & & & & & & & & & & \\
\hline 441 & 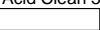 & & & & & & & & & & & & & & & & & & & & & & & & & & & \\
\hline 442 & & Averages & 25.7 & 24.9 & 25.0 & 26.1 & 25.0 & 42.4 & 48.2 & 4.0 & 38.8 & 0.1 & -1.6 & 44.2 & 0.0 & 2.4 & 0.0 & & & 46.8 & & 40.6 & \begin{tabular}{|l|}
2.799 \\
\end{tabular} & 0.358 & 0.358 & 0.009 & 8.8 & 7.5 \\
\hline 4443 & & Maximum & 25.7 & 25.0 & 25.1 & 26.4 & 25.3 & 42.9 & \begin{tabular}{|c|c|}
49.2 \\
\end{tabular} & 4.6 & 39.5 & 2.2 & -1.6 & 48.3 & 0.0 & 2.8 & 0.0 & & & 47.7 & 11.8 & 41.1 & \begin{tabular}{|l|l|}
2.834 \\
\end{tabular} & 0.421 & 0.427 & 0.011 & 10.9 & 9.3 \\
\hline 4444 & & Median & 25.7 & 24.9 & 25.1 & 26.1 & 25.0 & 42.4 & $\begin{array}{ll}48.2 \\
4\end{array}$ & 4.0 & 38.9 & 0.0 & -1.6 & 44.0 & 0.0 & 2.4 & 0.0 & & & 46.7 & $\begin{array}{l}7 \\
7\end{array}$ & $\begin{array}{ll}40.6 \\
\end{array}$ & 2.801 & 0.356 & 0.355 & 0.009 & 8.8 & 7.5 \\
\hline 4455 & & Minimum & 25.0 & 24.7 & 24.5 & 25.8 & 24.8 & 41.0 & 47.4 & 3.6 & 37.5 & -0.1 & \begin{tabular}{|c|}
-1.7 \\
\end{tabular} & 40.9 & 0.0 & 2.4 & 0.0 & & & & $\begin{array}{l}0 \\
0\end{array} 10.0$ & & 2.706 & 0.351 & 0.351 & 0.009 & 8.6 & 7.4 \\
\hline 4446] & & $2 \times \operatorname{Std}$ Dev & & & & & & & & & & & & & & 0.108 & & & & 0.760 & & 0.569 & & 0.016 & 0.019 & 0.001 & 0.503 & 0.428 \\
\hline 447 & Number & of Points Used ${ }^{*}$ & 93 & 93 & 93 & 93 & 93 & 93 & 93 & 93 & 93 & 93 & 93 & 93 & 93 & 93 & 93 & & & 93 & 93 & 93 & 93 & 93 & 93 & 93 & 93 & 93 \\
\hline & & |* Backpulse poi & & fare not incll & & & & & & & & & & & & & & & & & & & & & & & & \\
\hline - & aidcrar & & & & & & & & & & & & & & & & & & & & & & & & & & & \\
\hline 年50 & Acid Cleans & & & & & & & & & & & & & & & & & & & & & & & & & & & \\
\hline 452 & & Averages & 25.9 & 25.1 & 25.3 & 26.4 & 25.3 & 39.7 & 46.1 & 4.1 & 36.0 & 1.0 & -0.3 & 44.6 & 0.1 & 3.8 & 0.0 & & & 44.6 & 10.6 & 37.8 & 2.609 & 0.309 & 0.307 & 0.008 & 8.1 & 6.5 \\
\hline 453 & & Maximum & 27.9 & 25.8 & & & & 46.0 & & 5.5 & 40.7 & 24.6 & 13. & 56.1 & $1.2 \mathrm{C}>\mathrm{C}$ & 14.6 & & & & 56.5 & & \begin{tabular}{ll|l}
43.4 \\
\end{tabular} & $2.989 \mathrm{~S}$ & 0.421 & 0.427 & & 10.9 & \\
\hline 454] & & Median & 25.7 & 25.1 & 25.1 & 26.3 & 25.2 & 42.2 & 47.1 & 4.2 & 38.5 & -0.1 & -1.6 & 44.6 & 0.0 & 2.4 & 0.0 & & & 45.7 & 10.6 & $\begin{array}{l}40.3 \\
\end{array}$ & 2.78 & 0.334 & & 0.008 & 8.3 & 7.1. \\
\hline 4555 & & Minimum & 25.0 & 24.7 & 24.5 & 25.8 & 24.8 & 21.1 & 33.0 & 1.8 & 17.0 & -1.2 & $-4.3>3$ & 27.4 & 0.0 & 1.6 & 0.0 & & & 31.6 & 6.7 & $\begin{array}{lll}19.1 \\
\end{array}$ & 1.315 & 0.152 & 0.142 & 0.006 & 5.9 & 5.6 \\
\hline 456 & & $2 \times$ Std Dev & 1.178 & 0.537 & 1.249 & 0.775 & 0.630 & 13.558 & 7.260 & 0.525 & 13.709 & 7.807 & 5.760 & 5.722 & 0.696 & 8.243 & 0.002 & & & 7.260 & 1.402 & \#\#\#\# & 0.940 & 0.133 & 0.138 & 0.002 & 1.756 & 1.492 \\
\hline 4579 & Number & of Points Used ${ }^{*}$ & 271 & 1| $\quad 271$ & $\begin{array}{ll}1 & 271 \\
\end{array}$ & 271 & 271 & 271 & 271 & 271 & 271 & 271 & 271 & 271 & 271 & 271 & 271 & & & 271 & 271 & 271 & 27 & 271 & 271 & 271 & 271 & 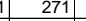 \\
\hline 458 & & ${ }^{*}$ Backpulse poi & ts in box & fare not incl & cluded & & & & & & & & & & & & & & & & & & & & & & & \\
\hline $\begin{array}{l}459 \\
460 \\
\end{array}$ & Caustic Rins & & & & & & & & & & & & & & & & & & & & & & & & & & & \\
\hline 461 . & & & & & & & & & & & & & & & & & & & & & & & & & & & & \\
\hline & & Averages & 24.4 & 24.7 & 24.0 & 25.6 & 24.7 & 21.9 & 23.7 & 4.0 & 18.1 & -1.0 & 0.7 & 45.1 & 0.0 & 1.2 & $\begin{array}{ll}0.0 \\
\end{array}$ & & & 22.2 & 11.1 & 20.0 & 1.377 & 0.183 & 0.188 & 0.009 & 9.4 & 8.4 \\
\hline 4663 & & Maximum & 24.8 & 24.8 & 24.4 & 25.7 & 24.9 & 22.7 & 24.2 & 4.1 & 18.9 & 3.1 & 0.8 & 48.3 & 0.8 & 2.5 & 0.0 & & & 22.7 & 11.8 & $20.8]$ & 1.432 & 0.366 & 0.381 & 0.024 & 23.6 & 20. \\
\hline 464 & & Media & 24.4 & 24.7 & 24.1 & 25.6 & $24.8 \mathrm{r}-\mathrm{l}$ & 22.2 & 23.7 & 4.0 & 18.4 & -1.2 & 0.7 & 44.9 & 0.0 & 1.2 & 0 & & & 22.2 & 11.0 & 20.3 & 1.40 & 0.179 & 0.184 & 0.009 & 9.0 & 7. \\
\hline 465 & & Minimum & 24.0 & 24.6 & 23.5 & 25.4 & 24.6 & 15.4 & 23.3 & 3.17 & 11.3 & -1.2 & 0.6 & 43.4 & 0.0 & 0.0 & 0.0 & & & 21.8 & 10.6 & 13.3 & 0.919 & -0.001 & -0.001 & 0.000 & -0.1 & -0.11 \\
\hline 4666 40 & & $2 \times$ Std Dev & 0.515 & 0.064 & 0.562 & 0.197 & 0.198 & 2.8999 & 0.445 & 0.234 & 2.891 & 1.594 & 0.051 & 2.109 & 0.269 & 0.654 & 0.002 & & & 0.445 & 0.517 & $2.891\}$ & 0.199 & 0.098 & 0.102 & 0.006 & 6.381 & 5.436 \\
\hline 467. & Number & of Points Used & 31 & 1) 31 & 1131 & 31 & 31 & 31 & 31 & 31 & 31 & 31 & 31 & 31 & 31 & 31 & 31 & & & 31 & 31 & 31 & 31 & 31 & 31 & 31 & 31 & 31 \\
\hline \begin{tabular}{|c|}
469 \\
\end{tabular} & & Danpuse pu & & & & & & & & & & & & & & & & & & & & & & & & & & \\
\hline 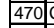 & Caustic Rins & & & & & & & & & & & & & & & & & & & & & & & & & & & \\
\hline & & & & & & & & & & & & & & & & & & & & & & & & & & & & \\
\hline & & Averages & 25.0 & 24.5 & 24.6 & 25.66 & 24.7 & 21.7 & 22.9 & 3.9 & 17.8 & -1.2 & 0.8 & 45.4 & 0.0 & 1.2 & 0.0 & & & 21.5 & 11.1 & $19.8 \mathrm{r}$ & 1.362 & 0.175 & $0.1 / 7$ & 0.009 & 9.0 & \\
\hline$\left|\frac{454}{474}\right|$ & & & 25.6 & $\frac{2.6}{2.5}$ & 23.2 & 25.14 & $\frac{24.9}{22.7}+2$ & 2.2 .1 & $\frac{23}{32}$ & $\begin{array}{l}4.0 \\
3.9\end{array}$ & 18.0 & -1.2 .2 & 0.9 & 46 & 0. & 1. & 0.0 & & & $\frac{21.1}{20.5}$ & 11.4 & 20.0 & 1.382 & 0.271 & 0.215 & 0.016 & $\frac{15.6}{.65}$ & 13.3 \\
\hline 475 & & Minimum & 24.7 & $\begin{array}{l}24.5 \\
24.5 \\
\end{array}$ & 24.3 & $\begin{array}{l}25.0 \\
25.4 \\
\end{array}$ & 24.6 & $\begin{array}{l}2.1 .5 \\
19.5\end{array}$ & 22.8 & $\begin{array}{l}3.9 \\
3.9\end{array}$ & $\begin{array}{l}1.9 \\
15.8\end{array}$ & $\frac{-1.4}{-1.2}$ & 0.0 & $\begin{array}{l}34.5 \\
44.1 \\
\end{array}$ & 0.0 & 1.1 & 0.0 & & & 21.5 & \begin{tabular}{|l|l|} 
& 11.1 \\
3 & 10.8
\end{tabular} & $\frac{19.9}{17.6}$ & \begin{tabular}{|l|}
1.070 \\
1217 \\
\end{tabular} & 0.162 & 0.165 & 0.008 & $\begin{array}{l}0.5 \\
8.3 \\
\end{array}$ & 7.3 \\
\hline 476 & & $2 \times \operatorname{Std}$ Dev & 0.526 & 0.069 & \begin{tabular}{l|l}
9 & 0.464 \\
9
\end{tabular} & 0.166 & 0.175 & 0.978 & 0.200 & 0.054 & 0.875 & 0.004 & 0.025 & 1.149 & 0.000 & 0.305 & 0.002 & & & 0.200 & 0.282 & 0.925 & \begin{tabular}{|l|l|} 
& 0.064 \\
\end{tabular} & 0.046 & 0.044 & \begin{tabular}{|l|} 
\\
\end{tabular} & 2.865 & 2.438 \\
\hline$\frac{47}{47}$ & Number & of Points Used & 28 & \begin{tabular}{|l|r|}
3 & 28 \\
\end{tabular} & $8 \quad 28$ & 28 & 28 & 28 & 28 & 28 & 28 & 28 & 28 & 28 & 28 & 28 & 28 & 8 & & 28 & \begin{tabular}{|l|}
8 \\
\end{tabular} & 28 & 28 & 3 & 28 & 28 & 28 & 28 \\
\hline & & $\square^{\star}$ Backpulse poi & ts in box & & & & & & & & & & & & & & & & & & & & & & & & & \\
\hline
\end{tabular}


WSRC-TR-2005-00105, REVISION 0 SRNL-RPP-2005-00012, REVISION 0

RUN \# 3.18 AND 19; ACID CLEANING AND CAUSTIC RINSING - CONT.

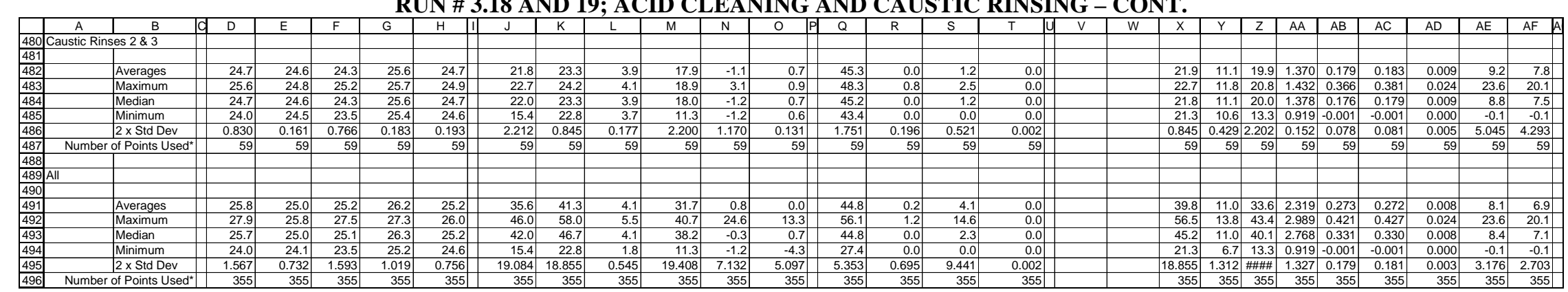


WSRC-TR-2005-00105, REVISION 0

SRNL-RPP-2005-00012, REVISION 0

RUN \# 3.21A, B, AND C; POST-TEST BASELINE, CAUSTIC RINSE 3 AND 0.1 M NAOH

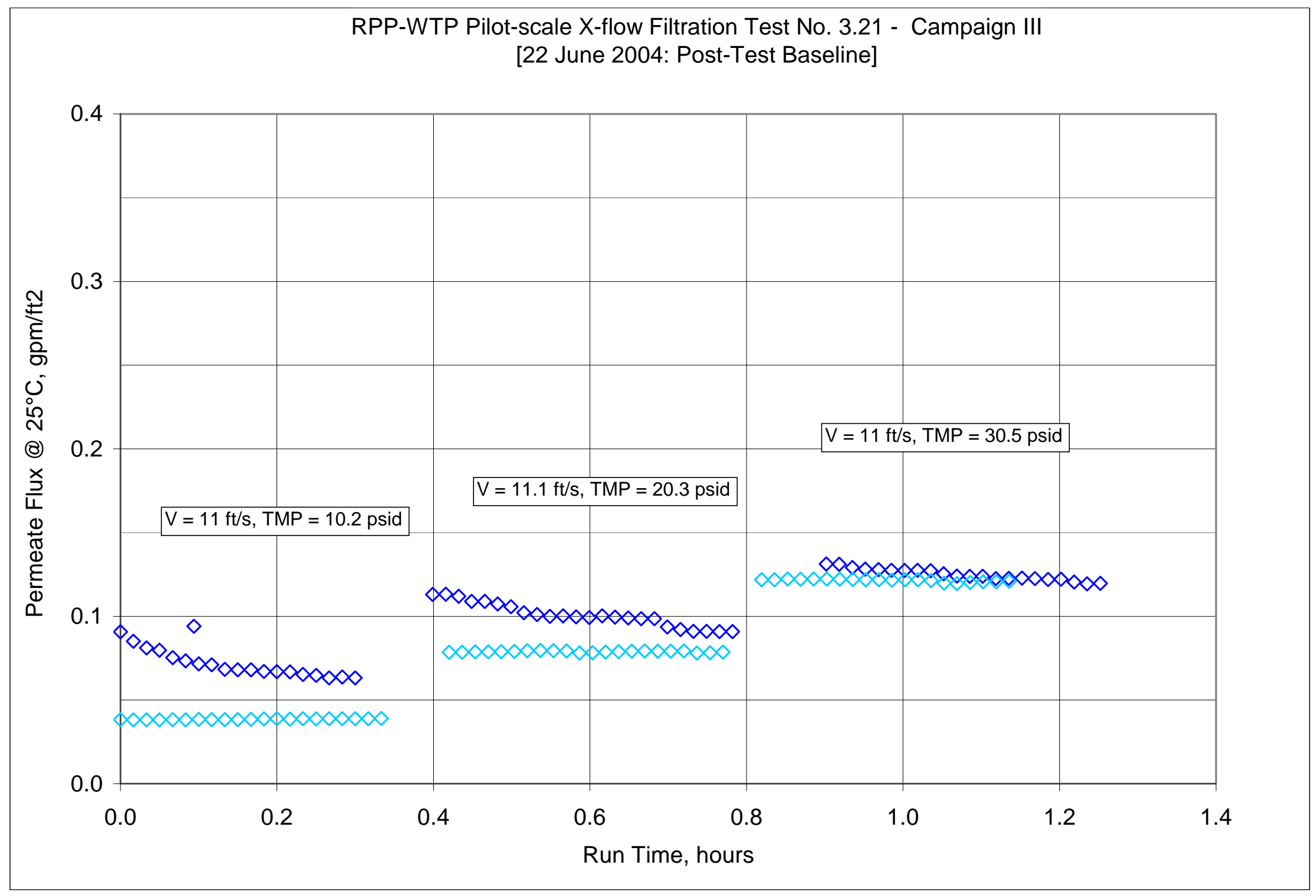


WSRC-TR-2005-00105, REVISION 0

SRNL-RPP-2005-00012, REVISION 0

RUN \# 3.21A, B, AND C; POST-TEST BASELINE WITH CAUSTIC RINSE 3 - CONT.

\begin{tabular}{|c|c|c|c|c|c|c|c|c|c|c|c|c|c|c|c|c|c|c|c|c|c|c|c|c|c|c|c|c|}
\hline & $\mathrm{A}$ & B & \begin{tabular}{l|l}
$\mathrm{D}$ & \\
\end{tabular} & $E$ & 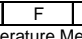 & $G$ & $\frac{H}{H+\lambda\rangle}$ & \begin{tabular}{l|l}
$\mathrm{J}$ & \\
\end{tabular} & $\frac{K}{K \ll<\ll}$ & \begin{tabular}{|l|l|l|} 
& $L$ \\
\end{tabular} & $M$ & I & $\frac{1}{10}$ & \begin{tabular}{l|l} 
\\
$<<<<<$
\end{tabular} & $\begin{array}{ll}\mathrm{R} \\
<<\mathrm{E}\end{array}$ & \begin{tabular}{|c|} 
is \\
\end{tabular} & $T^{2}$ & $\frac{v}{s e c<<}$ & W & \begin{tabular}{l|l}
$x$ \\
$x<x<$
\end{tabular} & \begin{tabular}{|l|} 
\\
\end{tabular} & \begin{tabular}{|l|}
$z$ \\
\end{tabular} & AA & $\begin{array}{ll}A B \\
\end{array}$ & $\begin{array}{ll}A C \\
y\end{array}$ & $A D$ & AE & $\mathrm{AF}$ \\
\hline & & & trate & Cleaning & $\begin{array}{l}\text { erature M- } \\
\text { Surry }\end{array}$ & Hi Amb & 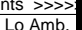 & & & & & & & & & & & & & & & & & & & & & \\
\hline & DATE & TIME & sale & dear & 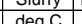 & $\operatorname{dec}$ & deg & osid & Fitier & Filier ar & TopTMP & Filifale & Isepc & Slurry & Filtrate & ii filtate & kpulse & & & & & & & & & & & \\
\hline 4 & & & $\mathrm{~T} 2$ & T3 & T1 & T4 & T5 & dP2 & $\frac{\text { pig }}{\text { P1 }}$ & dP1 & $\frac{\text { psid }}{\text { dP3 }}$ & $\frac{\mathrm{psg}}{\mathrm{P} 2}$ & $\frac{\mathrm{psig}}{\mathrm{P} 3}$ & $\frac{g p m}{Q 1}$ & $\frac{\mathrm{gpm}}{\mathrm{Q} 2}$ & $\frac{\mathrm{gpm}}{\mathrm{Q} 3}$ & & & & & & & & & & & & \\
\hline$\frac{5}{6}$ & & & & & & & & & & & & & & & & & & & & & & & & & & & & \\
\hline 7 & $6 / 8 / 2004$ & 9:09:09 AM & 22.545 & 24.705 & 27.987 & 26.054 & 24.911 & -0.004 & 0.128 & $\mid-0.01$ & -0.003 & -0.129 & 0.111 & 45.741 & 0.293 & $\mid-0.013$ & 0.003 & & & Filter Su & urface $A$ & 6.707 & FT2 & & & & & \\
\hline 8 & $6 / 10 / 2004$ & 1:47:47 PM & & 26.216 & \begin{tabular}{|l|}
26.68 \\
\end{tabular} & \begin{tabular}{|l|l|}
26.834 \\
\end{tabular} & 26 & -0.019 & 0.196 & & & & & & & & & & & Convers & & & & & /gpm & arg & & \\
\hline & & 7:41:41 AM & 23.83 & 23.885 & & 24.174 & & -0.012 & 0.12 & \begin{tabular}{|c|}
-0.01 \\
\end{tabular} & -0.016 & -0.163 & 0.107 & -0.083 & 0.003 & 14.643 & 0.005 & & & & & & & & & & & \\
\hline & 6/15/2004 & 12:06:06 PM & & 24.315 & 23.747 & 26.127 & 23.997 & \begin{tabular}{|c|}
-0.012 \\
\end{tabular} & 0.139 & & -0.012 & -0.177 & 0.043 & -0.073 & 0.003 & 14.643 & 0.006 & & & & & & & & & & & \\
\hline & $6 / 177 / 2004$ & $7: 28: 28 \mathrm{AM}$ & 24.492 & 23.951 & 23.799 & 24.225 & 23.743 & -0.01 & 0.164 & -0.001 & -0.014 & -0.18 & & -0.075 & 0.003 & 14.643 & & & & & & & & & & & & \\
\hline 12 & $6 / 17 / 2004$ & 4:57:57 PM & 26.259 & 26.737 & 24.765 & \begin{tabular}{|l|}
27.805 \\
\end{tabular} & 26.456 & - -0.002 & 0.197 & & -0.012 & -0.183 & -0.009 & 47.043 & 0.098 & 14.643 & 0.004 & & & & Plis & Eorrec & & & & r tubing & & \\
\hline & $6 / 21 / 2004$ & 6:52:52 AM & 23.109 & 23.008 & 22.621 & 23.418 & 22.888 & -0.01 & 0.075 & & -0.01 & $\mid-0.197$ & 0.055 & -0.079 & 0.003 & 14.643 & 0.006 & & & VWVV & & & & Filtrat & ate Flux $>$ & $<$ PERN & |ABILI & $\gg$ \\
\hline 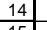 & $6 / 22 / 2004$ & $8: 12: 12 \mathrm{AM}$ & 23.36 & 23.555 & \begin{tabular}{|l|}
23.63 \\
\end{tabular} & 23.829 & 24.45 & -0.008 & 0.108 & -0.004 & -0.012 & -0.194 & 0.06 & -0.083 & 0.003 & 14.643 & 0.005 & & & & & & & & at $25 \mathrm{C}$ & & 1000 & \\
\hline \begin{tabular}{|l|}
16 \\
16
\end{tabular} & & & & & & & & & & & & & & & & & & Time & $\begin{array}{l}\text { Time } \\
\text { Thour }\end{array}$ & $\begin{array}{l}\text { Press. } \\
\text { psig. }\end{array}$ & $\begin{array}{l}\text { Vel. } \\
\text { ttls }\end{array}$ & $\begin{array}{l}\mathrm{MPP} \\
\mathrm{si}\end{array}$ & TMP & pm & & $\mathrm{jpm}$ & $\frac{p m}{2 / p s i}$ & neter \\
\hline & A & B & D & $\bar{E}$ & $\bar{F}$ & $\bar{G}$ & $\mathrm{H}$ & J & K & $\bar{L}$ & $\mathrm{M}$ & $\mathrm{N}$ & 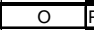 & Q & $R$ & s & T & $\mathrm{V}$ & $w$ & $x$ & $Y$ & $z$ & AA & $\overline{A B}$ & $\overline{A C}$ & AD & $\overline{\mathrm{AE}}$ & $\mathrm{AF}$ \\
\hline 107 & Baseline 2A & & & & & & & & & & & & & & & & & & & & & & & & & & & \\
\hline$\frac{108}{109}$ & $\frac{6}{6 / 22122004} 6$ & $\begin{array}{l}8: 26: 20 \mathrm{AM} \\
8: 27: 58 \mathrm{AM}\end{array}$ & $\begin{array}{l}24.052 \\
24.052\end{array}$ & $\begin{array}{l}23.606 \\
23.606\end{array}$ & \begin{tabular}{|l|}
23.721 \\
23.721 \\
\end{tabular} & $\begin{array}{l}\mid 24.367 \\
24.367\end{array}$ & $\begin{array}{l}23.675 \\
23.675 \\
\end{array}$ & $\begin{array}{l}21.104 \\
12.104\end{array}$ & $\frac{16.103}{16.103}$ & $\begin{array}{l}3.984 \\
3.984\end{array}$ & $\begin{array}{r}8.102 \\
8.102 \\
\end{array}$ & \begin{tabular}{|}
$\mid-1.211$ \\
-1.211
\end{tabular} & $\begin{array}{l}-2.197 \\
-2.197\end{array}$ & $\begin{array}{l}44.8466 \\
444.846\end{array}$ & $\frac{0.534}{0.534}$ & \begin{tabular}{|c|c|}
14.643 \\
11.443
\end{tabular} & $\frac{0.004}{0.004}$ & $\frac{0.00}{1.63}$ & & $\begin{array}{l}\frac{14.6}{14.6} \\
\end{array}$ & \begin{tabular}{|l|}
11.0 \\
11.0
\end{tabular} & 0.11 & $\mid \frac{0.697}{0.697}$ & $\begin{array}{l}0.080 \\
0.080 \\
\end{array}$ & $\begin{array}{l}0.083 \\
0.083 \\
\end{array}$ & 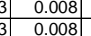 & $8.170 \mid$ & 6.955 \\
\hline & $6 / 22 / 2004$ & $8: 28: 58 \mathrm{AM}$ & 24.037 & 23.581 & 23.71 & 24.327 & 23.715 & $\begin{array}{r}2.829 \\
\end{array}$ & 16.347 & 3.914 & $\begin{array}{r}-1.08 \\
\end{array}$ & 8.566 & \begin{tabular}{|r|}
19.181 \\
\end{tabular} & 44.34 & 0.003 & 14.643 & 0.003 & 2.63 & \begin{tabular}{|l|l|}
0.04389 \\
\end{tabular} & \begin{tabular}{|l|}
14.9 \\
\end{tabular} & \begin{tabular}{|l|}
10.9 \\
\end{tabular} & \begin{tabular}{|l|}
0.9 \\
\end{tabular} & 0.060 & \begin{tabular}{|l|}
0.000 \\
\end{tabular} & $\begin{array}{l}0.000 \\
0.000 \\
0.0\end{array}$ & $\begin{array}{l}0.001 \\
0.001\end{array}$ & 0 & $\begin{array}{l}6.95 \\
0.45\end{array}$ \\
\hline & $6 / 22 / 2004$ & $8: 29: 58 \mathrm{AM}$ & $\begin{array}{l}24.037 \\
2.037\end{array}$ & 23.586 & $\begin{array}{l}23.715 \\
0.74\end{array}$ & \begin{tabular}{|l|l|}
24.357 \\
2.39
\end{tabular} & 23.74 & 2.906 & 16.467 & $\begin{array}{r}3.9 \\
3.0\end{array}$ & $\begin{array}{l}-1.04 \\
\end{array}$ & $\begin{array}{l}8.566 \\
1020\end{array}$ & $\begin{array}{l}19.516 \\
2150\end{array}$ & 41.745 & $\begin{array}{ll}0.003 \\
\end{array}$ & $\begin{array}{l}14.643 \\
\end{array}$ & 0.004 & 3.63 & 0.06056 & \begin{tabular}{ll|}
15.0 \\
\end{tabular} & $\begin{array}{ll}10.2 \\
\end{array}$ & \begin{tabular}{|l|}
0.9 \\
\end{tabular} & \begin{tabular}{|l|l|}
0.064 \\
\end{tabular} & $\begin{array}{l}0.000 \\
\end{array}$ & 0.000 & & 0.497 & 0.42 \\
\hline & $6 / 22 / 2004$ & $\begin{array}{l}8: 30: 58 \mathrm{AM} \\
8: 21: 58\end{array}$ & 24.046 & & & & & -6.215 & 16.679 & & -10.302 & & & & 0.003 & 14.643 & 1.619 & 4.63 & 0.07722 & 15.2. & 10.5 & & & & 0.000 & & 0.056 & -0.05 \\
\hline & 6/22/2004 & $8: 31: 58$ AM & \begin{tabular}{|r|r|}
24.07 \\
21.050
\end{tabular} & 23.604 & 23.759 & 24.405 & 23.753 & $\begin{array}{r}9.24 \\
11.270\end{array}$ & 16.322 & 3.994 & 5.149 & 1.939 & 0.485 & 44.703 & 0.609 & 14.643 & & 5.63 & 0.09389 & 14.8 & 1.00 & & 0.496 & 0.091 & 0.094 & & 3.071 & 11.12 \\
\hline$\frac{114}{115}$ & $\begin{array}{l}6 / 22 / 2004 \\
6 / 22 / 2004\end{array}$ & $\begin{array}{l}8: 32: 58 \text { AM } \\
8.33358\end{array}$ & 24.059 & $\frac{23.571}{23604}$ & $\frac{23.122}{32364}$ & $\frac{24.419}{24.455}$ & $\frac{23.167}{23833}$ & $\frac{11.218}{12199}$ & $\frac{16.242}{16.376}$ & | 3.0954 & $\frac{7.441}{8201}$ & \begin{tabular}{|c|c|c|}
-0.111 \\
-0.95
\end{tabular} & $\begin{array}{l}-1.394 \\
-2015 \\
\end{array}$ & $\begin{array}{r}44.3 \\
44.421\end{array}$ & 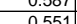 & $\begin{array}{c}\frac{14.643}{14643} \\
14\end{array}$ & 0.004 & 0.00 & 001667 & \begin{tabular}{ll|}
14.8 \\
14.9
\end{tabular} & 0.9 & & & & 0.091 & & $\begin{array}{l}9.695 \\
8332\end{array}$ & 8.25 \\
\hline & $6 / 22 / 2004$ & $8: 34: 58 \mathrm{AM}$ & 24.105 & 23.609 & 23.1844 & $\begin{array}{r}24.405 \\
24.43\end{array}$ & 23.908 & 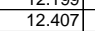 & $\frac{1.50}{16.302}$ & 3. 3.954 & $\frac{0.221}{8.264}$ & $\begin{array}{l}-0.214 \\
-1.214 \\
\end{array}$ & -2.203 & $\begin{array}{l}44.421 \\
44.202 \\
\end{array}$ & 0.526 & $\mid \frac{14.045}{14.643}$ & $\begin{array}{l}0.003 \\
0.003 \\
0.0\end{array}$ & 2.00 & \begin{tabular}{|l|l|}
0.03333 \\
\end{tabular} & \begin{tabular}{|l|}
14.8 \\
14.8
\end{tabular} & \begin{tabular}{|l|}
10.8 \\
\end{tabular} & \begin{tabular}{|l|l|}
10.3 \\
\end{tabular} & & $\begin{array}{l}078 \\
078 \\
\end{array}$ & 0.081 & $\mid 0.008$ & $\begin{array}{ll}0.0342 \\
7.842\end{array}$ & $\begin{array}{l}7.09 \\
6.67 \\
\end{array}$ \\
\hline & $6 / 22 / 2004$ & $8: 35: 58 \mathrm{AM}$ & 24.141 & 23.624 & 23.955 & \begin{tabular}{|l|l|}
24.416 \\
\end{tabular} & 23.959 & & 16.258 & & & -1.211 & -2.353 & 44.068 & 519 & & & 3.00 & \begin{tabular}{|l|} 
\\
\end{tabular} & $\begin{array}{l}14.8 \\
\end{array}$ & 10.8 & 10.5 & & & 0.080 & 0.008 & 7.578 & 6.45 \\
\hline 11 & $6 / 22 / 2004$ & $8: 36: 58 \mathrm{AM}$ & 24.228 & 23.636 & 24.027 & \begin{tabular}{|l|l|}
24.483 \\
\end{tabular} & 24.016 & 12.687 & 16.018 & $\begin{array}{l}4.069 \\
\end{array}$ & 8.469 & -1.214 & \begin{tabular}{|c|}
-2.64 \\
\end{tabular} & 46.181 & 491 & 14.643 & 0.004 & 4.00 & 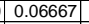 & 14.5 & \begin{tabular}{|l|l|}
11.3 \\
\end{tabular} & \begin{tabular}{|l|l|} 
& 10.6 \\
\end{tabular} & \begin{tabular}{|l|l|l|} 
\\
\end{tabular} & \begin{tabular}{|l|l|l|} 
\\
\end{tabular} & 0.075 & 0.007 & 7.113 & 6.05 \\
\hline & $6 / 22 / 2004$ & $8: 37: 58 \mathrm{AM}$ & 24.253 & 23.647 & 24.077 & 24.578 & 24.056 & 12.392 & 15.499 & & & -1.211 & \begin{tabular}{|r|} 
\\
\end{tabular} & $\begin{array}{l}45.02 \\
5\end{array}$ & 0.479 & 14.643 & 0.004 & 5.00 & \begin{tabular}{|l|}
0.08333 \\
\end{tabular} & $\begin{array}{l}14.0 \\
\end{array}$ & \begin{tabular}{|l|}
11.0 \\
\end{tabular} & \begin{tabular}{|l|}
10.3 \\
\end{tabular} & \begin{tabular}{|l|l|l|l|l|} 
& \\
\end{tabular} & 0.071 & 0.073 & 0.007 & 7.093 & 6.04 \\
\hline & 6/22/2004 & 8:38:58 AM & 24.315 & $\frac{23.668}{23691}$ & 24.174 & 24.584 & 24.117 & 12.346 & 15.329 & 3.931 & 8.256 & -1.211 & $\begin{array}{l}-2.836 \\
-2868 \\
\end{array}$ & $\begin{array}{r}45.41 \\
15\end{array}$ & 0.469 & 14.643 & 年, & 6.00 & $\begin{array}{r}0.1 \\
0.1097\end{array}$ & 13.9 & 11.1 & (10) & & & 0.072 & 0.007 & 6.948 & 5.91 \\
\hline & & $8: 39.58 \mathrm{AM}$ & 24.373 & $\frac{23.691}{23.71}$ & & $\frac{24.688}{24782}$ & $\frac{24.166}{2474}$ & $\frac{12.452}{12317}$ & $\frac{15.338}{15046}$ & \begin{tabular}{r|r|r|r|r|}
3995 \\
3995
\end{tabular} & & $\begin{array}{l}-1.216 \\
-1.214 \\
\end{array}$ & & & & $\frac{14.643}{14643}$ & & & $0.11667 \mid$ & & & & & & & & & 5.79 \\
\hline 123 & $6 / 22 / 2004$ & 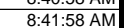 & $\begin{array}{r}24.41 \\
24.49\end{array}$ & 23.733 & 24.250 & 24.76 & 24.187 & 禁. & $\frac{1.040}{14.818}$ & & 8.4 & $\begin{array}{l}-1.214 \\
-1.211\end{array}$ & 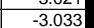 & 年4.500 & & $\begin{array}{l}14.0454 \\
14.643\end{array}$ & & 0.00 & 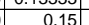 & 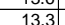 & $\frac{1.10}{110}$ & $\overline{0.1}$ & & & 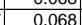 & & & 5.65 \\
\hline 124 & $6 / 22 / 2004$ & 8:42:58 AM & 24.542 & 23.746 & 24.397 & 24.752 & 24.175 & 12.222 & 14.9 & 3.919 & 8.125 & -1.214 & -3.041 & 44.515 & 0.448 & 14.643 & 0.00 & 10.00 & 0.16667 & 13.4 & 10.9 & & & & 0.068 & & 6.678 & 5.68 \\
\hline & $6 / 22 / 2004$ & 8:43:58 AM & 24.585 & 23.753 & 24.429 & 24.769 & 24.142 & 12.214 & & 3.95 & 8.049 & -1.214 & -3.091 & 45.226 & & & & 11.00 & 0.18333 & 13.4 & 11.1 & 10.1 & & & 0.067 & & & 50 \\
\hline & 6/22/2004 & 8:44:58 AM & 24.627 & 23.775 & 24.486 & 24.771 & 24.129 & 12.396 & 14.948 & 3.84 & 8.469 & -1.208 & $\begin{array}{l}3.088 \\
\end{array}$ & 44.663 & 0.4 & 14.643 & 0.004 & 12.00 & 0.2 & 13.5 & \begin{tabular}{|l|}
10.9 \\
\end{tabular} & & & 066 & 0.067 & 0.006 & 6.409 & 5.44 \\
\hline & & & 24.684 & & & 24.784 & & & & & & -1.214 & & & & & & & 0.21667 & 13.4 & 10.9 & & & & & 107 & 6.583 & \\
\hline & $6 / 22 / 2004$ & 8:46:58 A & 24.126 & 23.194 & 24.54 & 24.165 & 24.20 & 12..154] & 14.691 & $3.9 / 4$ & & $-1.214 \mid$ & -3.11 & & & & & 14.00 & & 10.2 & & & & & & & & .55 \\
\hline & $6 / 22 / 2004$ & $8: 47: 58$ & 24.756 & 23.815 & 24.586 & 24.761 & 24.22 & $12.537 \mid$ & $15.0 \mathrm{C}$ & & 8.402 & -1.214 ] & & & & $14.643 \mid$ & & 15.00 & & 13.6 & & & & & & & 6.181 & $5.26>-1$ \\
\hline & 6/22/2004 & $8: 48: 58 \mathrm{f}$ & 24.818 & 23.836 & 24.622 & 24.772 & 24.2 & 12.154 & 14.44 & 4.01 & 7.998 & -1.214 & -3.26 & 45.207 & 0.4 & 14.6 & & 16.00 & 0.26667 & 13.0 & & & & & 063 & 6 & & 5.3. \\
\hline & 6/22/2004 & 8:49:58 & 24.84 & 23.838 & 24.659 & 24.825 & & 12.376 & 14.751 & 3.98 & 8.231 & -1.214 & & 45.33 & 0.4 & 14.643 & & 7.00 & 0.28333 & 13.3 & & & & & 664 & & 180 & \\
\hline & 6/22/2004 & 8:50:58 A & & 23.849 & & 24.79 & 4.143 & 12.324 & 14.6 & 3.927 & 8.281 & -1.214 & & 45.464 & 0.42 & 14.643 & & 18.00 & & & & & & 0.063 & 0.063 & & & \\
\hline $\mid \frac{103}{134}$ & & & & & & & & & & & & & & & & & & & & & & & & & & & & \\
\hline & & & & & & & & & & & & & & & & & & & & & & & & & & & & \\
\hline & & & & & & & & & & & & & & & & & & & & & & & & & & & & \\
\hline 137] & & & 24.5 & 23.7 & 24.3 & 24.7 & & 12.3 & 15.3 & 9 & 8.2 & \begin{tabular}{ll|}
-1.1 \\
\end{tabular} & & 45 & 0.5 & & & & & 13.8 & & & 0.1 & 0.1 & 0.1. & & & \\
\hline 1388 & & Maximum & 24.9 & 23.8 & 24.7 & 24.8 & 22 & 12.7 & 16.4 & 4.1 & 8.5 & -0.1 & & 46.2 & 0.6 & 14.6 & & & & 14.9 & 11.3 & $\mid 10.6$ & 0.7 & 0.1 & 0.1 & .0 & 9.7 & \\
\hline 19 & & Median & 24.5 & 23.1 & $24.4]$ & 24.8 & & 12.3 & 15.0 & 4.0 & 8.3 & -1.2 & & & 0.4 & 14.6 & & & & 13.6 & 11.0 & 10.3 & 0.7 & 0.1 & J.1 & .0 & .7 & \\
\hline (1404) & & & 24.1 & $\begin{array}{l}23.6 \\
0.71\end{array}$ & 23.14 & 24.4 & 2.80 & $\frac{11.33}{055}$ & 14.4 & 3.7 & 1.4 & -1.2 & $\frac{-3.5}{2002}$ & 44.14 & 0.4 & 14.6 & & & & $\begin{array}{ll}13.04 \\
1257\end{array}$ & 10.8 & 9.4 & 0.6 & 0.1 & 0.1 & 0.0 & 6.14 & 5.2 \\
\hline$\frac{141}{142}$ & Number o & $2 \times$ Stad Dev & 0.532 & $\frac{0.1 / 1}{19}$ & $\frac{0.604}{19}$ & 0.292 & 0.260 & $\begin{array}{c}0.555 \\
19 \\
\end{array}$ & 1.258 & | & 0.4719 & 0.500 & 0.983 & $1.05 / 9$ & 0.092 & 0.000 & & & & 1.256 & 0.259 & 199 & . & 0.014 & 0.010 & 0.002 & 1.1 .16 & \\
\hline $\begin{array}{l}\frac{142}{143} \\
\end{array}$ & & $\begin{array}{l}\text { f Points Used } \\
\text { × Backpulse po }\end{array}$ & in box a & $\frac{19}{\text { re not inc }}$ & 19 & & & & & & & & & & & & & & & & & & & & & & & \\
\hline
\end{tabular}


WSRC-TR-2005-00105, REVISION 0

SRNL-RPP-2005-00012, REVISION 0

RUN \# 3.21A, B, AND C; POST-TEST BASELINE WITH CAUSTIC RINSE 3 - CONT.

\begin{tabular}{|c|c|c|c|c|c|c|c|c|c|c|c|c|c|c|c|c|c|c|c|c|c|c|c|c|c|c|c|c|}
\hline & A & B & $\mathrm{D}$ & $E$ & $\mathrm{~F}$ & $G$ & $\mathrm{H}$ & $\mathrm{J}$ & $\mathrm{K}$ & $\mathrm{L}$ & M & $\mathrm{N}$ & 0 & $Q$ & $R$ & $\mathrm{~s}$ & \begin{tabular}{l|l|l|l|} 
&
\end{tabular} & $\mathrm{V}$ & $w$ & $x$ & $Y$ & $z$ & AA & $A B$ & $\mathrm{AC}$ & $A D$ & $\mathrm{AE}$ & AF \\
\hline$\frac{148}{148}$ & Baseline 2B $\frac{1}{6 / 22 / 2004}$ & & & & & & 24207 & & 29.97 & & & & & & 0762 & & & & & & & & & & & & & \\
\hline $\begin{array}{ll}149 \\
149\end{array}$ & $6 / 22 / 2004$ & 8:57:54 AM & 25.519 & 23.933 & \begin{tabular}{|l|}
25.32 \\
\end{tabular} & 24.959 & 24.222 & 22.44 & 30.363 & 3.821 & 18.55 & $\frac{2.153}{2.822}$ & $\begin{array}{l}0.254 \\
0.277\end{array}$ & $\begin{array}{l}44.099 \\
44.579\end{array}$ & $\begin{array}{l}0.102 \\
0.766\end{array}$ & $\begin{array}{l}14.045 \\
14.643\end{array}$ & $\begin{array}{l}0.004 \\
0.004\end{array}$ & $\begin{array}{l}24.93 \\
24.93\end{array}$ & \begin{tabular}{|l|l|}
0.348889 \\
.41556
\end{tabular} & $\begin{array}{r}28.5 \\
28.9 \\
\end{array}$ & $\begin{array}{l}11.0 \\
10.9\end{array}$ & $\mid$\begin{tabular}{|l}
20.1 \\
20.5
\end{tabular} & \begin{tabular}{|l|}
1.388 \\
1.413 \\
\end{tabular} & $\mid$\begin{tabular}{|l|}
0.1114 \\
0.114
\end{tabular} & $\frac{0.113}{0.113}$ & $\begin{array}{l}0.006 \\
0.006\end{array}$ & $\begin{array}{l}5.611 \\
5.522\end{array}$ & $\begin{array}{l}4.78 \\
4.70\end{array}$ \\
\hline 150 & $6 / 22 / 2004$ & $8: 58: 54 \mathrm{AM}$ & 25.596 & 23.919 & \begin{tabular}{|l|l|}
25.391 \\
\end{tabular} & 24.981 & 24.208 & 22.444 & \begin{tabular}{|l|l|}
4 & 30.22 \\
\end{tabular} & 3.899 & 18.384 & 2.701 & 0.208 & 45.339 & 0.759 & 14.643 & 0.004 & 25.93 & \begin{tabular}{|l|l|} 
& 0.43222 \\
\end{tabular} & 28.7 & 11.1 & 20.4 & \begin{tabular}{|l|}
1.4107 \\
1.407 \\
\end{tabular} & \begin{tabular}{|l|}
0.1134 \\
\end{tabular} & 0.112 & 0.005 & 5.483 & 4.67 \\
\hline 151 & $6 / 22 / 2004$ & $8: 59: 54 \mathrm{AM}$ & 25.668 & 23.937 & 25.429 & 25.094 & 24.256 & 22.484 & \begin{tabular}{|l|}
429.759 \\
\end{tabular} & 4.04 & 18.396 & 2.307 & -0.029 & 46.288 & 0.739 & 14.643 & 0.004 & 26.93 & \begin{tabular}{|l|l|} 
& 0.44889 \\
\end{tabular} & 28.3 & & 20.4 & \begin{tabular}{|l|}
1.409 \\
\end{tabular} & \begin{tabular}{|l|}
0.110 \\
\end{tabular} & 0.109 & 0.005 & 5.326 & 4.53 \\
\hline$\frac{152}{152}$ & $6 / 22 / 2004$ & 9:00:54 AM & 25.67 & 23.944 & \begin{tabular}{|l|}
25.416 \\
\end{tabular} & 25.126 & 24.253 & 22.534 & \begin{tabular}{|l|l|} 
& 29.884 \\
\end{tabular} & 3.934 & 18.384 & 2.298 & $\begin{array}{l}-0.047 \\
-\end{array}$ & 45.624 & $\begin{array}{l}0.739 \\
\end{array}$ & 14.643 & 0.004 & 27.93 & \begin{tabular}{|l|l|} 
& 0.46556 \\
\end{tabular} & 28.4 & & \begin{tabular}{|l|}
20.5 \\
\end{tabular} & \begin{tabular}{|l|}
1.411 \\
\end{tabular} & \begin{tabular}{|l|l|}
0.110 \\
\end{tabular} & 0.109 & 0.005 & 5.323 & 4.53 \\
\hline 153 & $6 / 22 / 2004$ & 9:01:54 AM & 25.702 & 23.945 & \begin{tabular}{|l|}
25.392 \\
\end{tabular} & 25.147 & 24.284 & 22.716 & \begin{tabular}{|l|l|} 
& 29.903 \\
\end{tabular} & 4.066 & 18.503 & 2.075 & $\begin{array}{l}-0.171 \\
-10\end{array}$ & 45.683 & $\begin{array}{l}0.728 \\
\end{array}$ & $\begin{array}{l}14.643 \\
\end{array}$ & $\begin{array}{l}0.004 \\
\end{array}$ & 28.93 & \begin{tabular}{|l|l|} 
& 0.48222 \\
\end{tabular} & 28.4 & & \begin{tabular}{|l|}
20.6 \\
\end{tabular} & \begin{tabular}{|l|}
1.421 \\
\end{tabular} & \begin{tabular}{|l|l|}
0.109 \\
\end{tabular} & 0.107 & 0.005 & 5.209 & 4.43 \\
\hline$\frac{154}{155}$ & $\frac{6 / 22 / 2004}{6 / 22 / 2004}$ & $\begin{array}{l}9: 02: 54 \mathrm{AM} \\
90.54 \mathrm{~A}\end{array}$ & $\begin{array}{l}25.724 \\
25.732\end{array}$ & $\begin{array}{l}23.948 \\
23.971 \\
\end{array}$ & $\begin{array}{r}25.37 \\
25.393\end{array}$ & $\begin{array}{r}25.13 \\
25.158 \\
\end{array}$ & $\begin{array}{l}24.307 \\
24.345\end{array}$ & $\begin{array}{l}22.594 \\
22.351\end{array}$ & $\begin{array}{l}29.503 \\
128.893\end{array}$ & $\begin{array}{r}3.924 \\
4.12\end{array}$ & $\begin{array}{l}18.573 \\
18.095 \\
\end{array}$ & $\begin{array}{l}1.847 \\
1.433\end{array}$ & $\begin{array}{c}-0.31 \\
-0.55\end{array}$ & \begin{tabular}{|l|l|}
44.821 \\
4556
\end{tabular} & \begin{tabular}{|l|l|}
0.716 \\
0.693
\end{tabular} & \begin{tabular}{|l|l|l}
14.643 \\
14643
\end{tabular} & \begin{tabular}{|l|l|}
0.003 \\
0004
\end{tabular} & \begin{tabular}{|l|}
29.93 \\
3093
\end{tabular} & \begin{tabular}{|l|l|}
0.49889 \\
051556
\end{tabular} & \begin{tabular}{|l|}
28.0 \\
274
\end{tabular} & \begin{tabular}{|l|}
11.0 \\
112 \\
\end{tabular} & \begin{tabular}{|l|}
20.6 \\
202
\end{tabular} & \begin{tabular}{|l|}
1.419 \\
1394
\end{tabular} & \begin{tabular}{|l|l|}
0.107 \\
0.033
\end{tabular} & $\begin{array}{l}0.106 \\
0102 \\
\end{array}$ & $\begin{array}{l}0.005 \\
0005\end{array}$ & $\begin{array}{l}5.132 \\
5053\end{array}$ & $\begin{array}{l}4.37 \\
430\end{array}$ \\
\hline$\frac{105}{156}$ & $\begin{array}{l}6 / 221 / 2004 \\
6 / 21 / 2004\end{array}$ & $\begin{array}{l}9: 00554 \mathrm{AM} \\
9: 0454 \mathrm{Am}\end{array}$ & $\begin{array}{l}25.132 \\
25.733\end{array}$ & & \begin{tabular}{|l|}
25.350 \\
\end{tabular} & 25.173 & $\begin{array}{r}24.345 \\
24.34\end{array}$ & $\begin{array}{l}22.351 \\
22.195\end{array}$ & \begin{tabular}{|l|l|}
20.050 \\
28.549
\end{tabular} & $\begin{array}{r}4.12 \\
3.982 \\
\end{array}$ & $\begin{array}{l}18.005 \\
18.111\end{array}$ & $\begin{array}{l}1.433 \\
1.285 \\
\end{array}$ & $\begin{array}{r}-0.55 \\
-0.636\end{array}$ & \begin{tabular}{|l|}
46.556 \\
46.548
\end{tabular} & $\begin{array}{l}0.0585 \\
0.685\end{array}$ & \begin{tabular}{|l|}
14.643 \\
14.643
\end{tabular} & $\begin{array}{l}0.004 \\
0.004\end{array}$ & $\begin{array}{l}30.93 \\
31.93\end{array}$ & & & $\begin{array}{l}11.2 \\
11.4\end{array}$ & \begin{tabular}{|l|}
20.2 \\
20.2
\end{tabular} & \begin{tabular}{|l|}
1.094 \\
\end{tabular} & & $\begin{array}{l}0.102 \\
0.101\end{array}$ & $\begin{array}{l}0.005 \\
0.005\end{array}$ & $\begin{array}{l}5.053 \\
5.015\end{array}$ & $\begin{array}{l}4.30 \\
427\end{array}$ \\
\hline 157 & $6 / 22 / 2004$ & $9: 05: 54 \mathrm{AM}$ & 25.735 & 23.994 & $\begin{array}{l}25.355 \\
\end{array}$ & 25.205 & 24.352 & & \begin{tabular}{|l|l|}
728.304 \\
\end{tabular} & 3.994 & 17.906 & 1.12 & $\begin{array}{l}-0.009 \\
-0.709\end{array}$ & 45.633 & 0.677 & $\begin{array}{l}14.0443 \\
14643\end{array}$ & $\begin{array}{l}0.004 \\
0.004\end{array}$ & $\begin{array}{l}32.93 \\
32.03\end{array}$ & \begin{tabular}{|l|l|} 
\\
\end{tabular} & $\frac{1.1}{26.8}$ & 11.2 & \begin{tabular}{|l|}
20.0 \\
\end{tabular} & \begin{tabular}{|l|}
1.378 \\
\end{tabular} & \begin{tabular}{|l|}
0.104 \\
\end{tabular} & & & $\begin{array}{l}.010 \\
4.9999\end{array}$ & $\begin{array}{l}4.27 \\
4.25 \\
\end{array}$ \\
\hline 158 & $6 / 22 / 2004$ & 9:06:54 AM & 25.743 & 24.016 & \begin{tabular}{|l|}
25.363 \\
\end{tabular} & 25.208 & 24.35 & 22.482 & 28.582 & $\begin{array}{l}.983 \\
\end{array}$ & 18.378 & 1.12 & $\begin{array}{c}-0.709 \\
\end{array}$ & 46.747 & 0.679 & 14.643 & 0.004 & 33.93 & \begin{tabular}{|l|l|} 
& 0.56556 \\
\end{tabular} & 27.1 & 11.5 & 20.4 & \begin{tabular}{|l|}
1.409 \\
\end{tabular} & \begin{tabular}{|l|}
0.101 \\
\end{tabular} & 0.100 & 0.005 & $\begin{array}{l}4.955 \\
4.905\end{array}$ & 4.25 \\
\hline & $6 / 22 / 2004$ & 9:07:54 AM & 25.725 & 24.013 & \begin{tabular}{|l|}
25.315 \\
\end{tabular} & 25.2 & 24.352 & 22.251 & \begin{tabular}{|l|}
28.394 \\
\end{tabular} & 3.911 & & 1.053 & $\begin{array}{r}-0.743 \\
\end{array}$ & 44.554 & 0.674 & 14.643 & 0.00 & 34.93 & 0.58222 & 26.9 & 10.9 & 20.2 & & & & & & \\
\hline 160 & $6 / 22 / 2004$ & 9:08:54 AM & & & \begin{tabular}{|l|}
25.307 \\
\end{tabular} & & & & & & & 1.004 & & \begin{tabular}{|l|l|l|}
45.641 \\
\end{tabular} & & & & & & 27.2 & & 20.4 & .404 & \begin{tabular}{|l|l|l|l|l|l|} 
\\
\end{tabular} & 0.099 & 0.005 & 4869 & $\frac{4.19}{414}$ \\
\hline \begin{tabular}{|l|l|}
161 \\
\end{tabular} & $6 / 22 / 2004$ & 9:09:54 AM & 25.704 & 24.028 & \begin{tabular}{|l|l|}
25.275 \\
\end{tabular} & 25.175 & 24.372 & $\frac{22.641}{22.641}$ & 128.858 & 3.766 & 18.733 & 1.114 & 0.70 & 44.098 & 0.677 & 14.643 & & 36.93 & 61556 & $\frac{27.4}{22.4}$ & 10.8 & 20.7 & $\frac{1.426}{1.426}$ & $\frac{1.101}{0.101}$ & $\frac{0.100}{0.100}$ & $\frac{0.005}{0.005}$ & 4.842 & $\frac{4.14}{4.12}$ \\
\hline$\sqrt{102}$ & $6 / 22 / 2004$ & 9:10:54 AM & 25.707 & 24.045 & \begin{tabular}{|l|l|}
25.272 \\
\end{tabular} & 25.197 & 24.379 & 22.768 & \begin{tabular}{|l|l|}
329.046 \\
\end{tabular} & 3.815 & 18.788 & 1.056 & $\begin{array}{l}-0.749 \\
\end{array}$ & 42.844 & 0.673 & 14.643 & 0.00 & $37.9:$ & 63222 & 27.6 & 10 & 20.8 & $\begin{array}{l}1.433 \\
\end{array}$ & \begin{tabular}{|l|l|}
0.100 \\
\end{tabular} & 0.100 & 0.005 & 4.792 & \\
\hline $16^{3}$ & $6 / 22 / 2004$ & 9:11:54 AM & 25.694 & 24.043 & 25.254 & 25.179 & 24.361 & 22.758 & $\begin{array}{ll}328.852 \\
\end{array}$ & 3.84 & 18.823 & 0.998 & -0.81 & \begin{tabular}{|l|l|}
44.089 \\
\end{tabular} & 0.669 & 14.643 & & 38.93 & 0.64889 & 27.4 & 10. & $\mid 20.8$ & 1.433 & \begin{tabular}{|l|l|}
0.100 \\
\end{tabular} & 0.099 & 0.005 & 4.763 & 4.05 \\
\hline 164 & $6 / 22 / 2004$ & 9:12:54 AM & 25.696 & 24.065 & \begin{tabular}{|l|}
25.266 \\
\end{tabular} & 25.226 & 24.373 & 22.928 & 28.928 & 3.816 & 19.016 & 0.94 & -0.839 & 433.918 & 0.666 & $\begin{array}{l}14.643 \\
\end{array}$ & 0.00 & 39.93 & 66556 & 27.5 & 10.8 & 21.0 & 1.446 & \begin{tabular}{|l|l|}
0.099 \\
\end{tabular} & 0.099 & 0.005 & 4.699 & 4.00 \\
\hline 165 & $6 / 22 / 2004$ & 9:13:54 AM & 25.681 & 24.07 & \begin{tabular}{|l|}
25.247 \\
\end{tabular} & 25.242 & 24.409 & 22.903 & 28.961 & 3.836 & 18.95 & 0.932 & \begin{tabular}{|c|c|}
-0.847 \\
\end{tabular} & \begin{tabular}{|l|l|}
44.379 \\
\end{tabular} & 0.666 & 14.643 & & 40.9 & 68222 & 27.5 & 10 & 20.9 & 1.443 & 0.099 & 0.099 & 0.005 & 4.712 & 4.01 \\
\hline 166 & $6 / 22 / 2004$ & 9:14:54 AM & 25.678 & 24.087 & 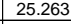 & 25.303 & 24.49 & 22.005 & 27.301 & 4.127 & 17.776 & 0.33 & -1.191 & 46.442 & 0.632 & 14.643 & & 41.9 & 69889 & 25.8 & & 19.9 & 1.371 & 0.094 & 0.094 & 0.005 & 4.702 & 4.00 \\
\hline & 6/22/2004 & $9: 15: 54 \mathrm{AM}$ & $\frac{25.675}{25.657}$ & 24.099 & 25.255 & 25.371 & 24.537 & $\frac{21.905}{21.971}$ & 27.092 & 4.089 & 17.702 & 0.141 & -1.313 & $\frac{47.226}{172}$ & 0.622 & 14.643 & & 42.93 & 1556 & 25.6 & 11 & $19.8 \mathrm{P}$ & 1.365 & 0.093 & & 0.005 & 4.649 & 3.96 \\
\hline$\frac{168}{169}$ & 0.124212004 & $\begin{array}{l}9: 16.54 \mathrm{AM} \\
0.75 .54\end{array}$ & $\frac{25.667}{25655}$ & 24.116 & 25.242 & 25.383 & 24.605 & $21.6 / 1$ & 26.144 & $\begin{array}{l}4.035 \\
.291\end{array}$ & 17.518 & 0.028 & $=\frac{-1.385}{1.391}$ & 455.543 & 0.614 & 14.643 & & 43.93 & 3222 & 25.3 & & 19.6 & & & & & & 3.95 \\
\hline$\frac{109}{170}$ & $6 / 2<2 / 2004$ & $\begin{array}{l}9: 17.54 \mathrm{AM} \\
9 \cdot 18: 54 \mathrm{AM}\end{array}$ & $\frac{25.655}{25.647}$ & $\begin{array}{l}\frac{24.139}{24.157} \\
\end{array}$ & \begin{tabular}{|l|}
25.24 \\
25.233 \\
\end{tabular} & $\begin{array}{r}25.426 \\
25.478\end{array}$ & $\begin{array}{r}24.051 \\
24.7\end{array}$ & $\frac{21.152}{21849}$ & \begin{tabular}{|l|l|l}
26.906 \\
26.868
\end{tabular} & 3.941 & $\frac{17.6 / 8}{17.746}$ & $\frac{-0.028}{-0.024}$ & $\frac{-1.394}{-1.408}$ & $\frac{45.249}{45.718}$ & $\begin{array}{l}0.614 \\
0.613\end{array}$ & $\frac{14.643}{14.643}$ & 0.0 & $\begin{array}{l}4.93 \\
45.93\end{array}$ & 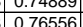 & $\frac{25.5}{25.4}$ & $\frac{11.1}{112}$ & $\frac{19.8}{19.8}$ & 1.5.395 & \begin{tabular}{|l|l|}
0.092 \\
0.091
\end{tabular} & 0.091 & 0.005 & 告.612 & $\begin{array}{l}3.92 \\
3.90 \\
\end{array}$ \\
\hline 171 & $6 / 22 / 2004$ & 9:19:54 AM & 25.64 & 24.18 & 25.231 & 25.526 & 24.688 & $\begin{array}{l}21.045 \\
21.847\end{array}$ & 27.011 & 3.958 & 17.725 & -0.024 & -1.408 & $\begin{array}{l}45.110 \\
45.839\end{array}$ & $\begin{array}{l}.010 \\
0.613\end{array}$ & $\begin{array}{l}14.040 \\
14.643\end{array}$ & 0.00 & $\begin{array}{l}4.50 \\
46.93\end{array}$ & $\mid 0.75222$ & 20.4 & $\frac{1.4}{112}$ & $\mid$ & | & 0.091 & 0.091 & 0.005 & 年.300 & $\begin{array}{l}3.90 \\
3.91\end{array}$ \\
\hline & & & & & & & & & & & & & & & & & & & & & & & & & & & & 3.91 \\
\hline 173 & & Averages & 25.7 & 24.0 & 25.3 & 25.2 & 24.4 & 22.3 & 28.6 & 4.0 & 18.3 & 1.2 & -0.7 & 45.3 & 0.7 & $\begin{array}{ll}14.6 \\
\end{array}$ & & & & 27.2 & 11.1 & 20.3 & 1.4 & 0.1 & 0.1 & 0.0 & 5.0 & 42 \\
\hline \begin{tabular}{|l|l|}
174 \\
\end{tabular} & & Maximum & 25.7 & 24.2 & 25.4 & 25.5 & 24.7 & 22.9 & 30.4 & 4.1 & 190 & 2.8 & 0.3 & 47.2 & 0.8 & 146 & & & & 28.9 & 11 & $\frac{210}{210}$ & 1.4 & 01 & 0.7 & 0.0 & 5.6 & \\
\hline $\begin{array}{ll}775 \\
\end{array}$ & & Median & 257 & 240 & 253 & 252 & 244 & 224 & 289 & 40 & 183 & & & 45.5 & 07 & 146 & & & & 274 & & & & & & & & $\frac{4.0}{42}$ \\
\hline 176 & & Minimum & 25.4 & 23.9 & 25.2 & 24.9 & 24. & 21.7 & 26.7 & 3.8 & 17.5 & 0.0 & -1.4 & 42.8 & 0.6 & 14.6 & & & & 25.3 & 10.5 & 19.6 & 1.4 & 0.1 & 0.1 & 0.0 & 4.6 & $\begin{array}{l}4.2 \\
3.9\end{array}$ \\
\hline 177 & & $2 \times \operatorname{Std} \mathrm{Dev}$ & 0.159 & 0.154 & 0.134 & 0.299 & 0.288 & 0.724 & 2.215 & 0.197 & 0.852 & 1.773 & 1.052 & 1.995 & 0.096 & 0.000 & & & & 2.215 & 0.489 & 0.783 & \begin{tabular}{|l|l|}
0.054 \\
\end{tabular} & \begin{tabular}{|l|}
0.014 \\
\end{tabular} & 0.014 & 0.001 & 0.615 & 0.523 \\
\hline 178 & Number & of Points Used ${ }^{*}$ & 24 & 24 & 24 & 24 & 24 & 24 & 24 & 24 & 24 & 24 & 24 & 24 & 24 & 24 & & & & 24 & 24 & 24 & 24 & 24 & 24 & 24 & 24 & \\
\hline tris & & & in box & re not inc & Iluded & & & & & & & & & & & & & & & & & & & & & & & \\
\hline & & & & & & & & & & & & & & & & & & & & & & & & & & & & \\
\hline$\frac{181}{182}$ & & & & & & & & & & & & & & & & & & & & & & & & & & & & \\
\hline & Baseline $2 \mathrm{C}$ & & & & & & & & & & & & & & & & & & & & & & & & & & & \\
\hline 184 & $6 / 22 / 2004$ & $9 \cdot 27: 05$ AM & 26.03 & 24.305 & 25.611 & 25.712 & 24.758 & 32.789 & 43.785 & 3.954 & 28.782 & 5781 & 2026 & 45,756 & 0.895 & 14.643 & & 541 & 90194 & 423 & 111 & 308 & 12 & 0.133 & 013 & 0.004 & 4261 & 36 \\
\hline 185 & $6 / 22 / 2004$ & 9:28:05 AM & 26.058 & 24.323 & \begin{tabular}{|l|}
25.011 \\
\end{tabular} & 25.84 & 24.766 & 32.652 & 43.634 & 3.9 & 28.688 & 5.694 & 2.006 & 44.559 & $\begin{array}{l}0.093 \\
\end{array}$ & $\frac{14.045}{14.643}$ & & 55.1 & 0.91861 & 422 & & & 2.11 & 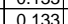 & 0.13 & $|0.04|$ & 4273 & 3.06 \\
\hline 186 & $6 / 22 / 2004$ & 9:29:05 AM & 26.057 & & & 25.878 & 24.815 & & & 3.976 & & & & & & & & & & & & & & & & & & \\
\hline & & & & 24.345 & 25.426 & & & 32.134 & 442.533 & 3.963 & 28.109 & 5.158 & & 44.823 & 0.87 & 14.643 & & 57.1 & 95194 & 41.1 & 11.6 & 30.1 & 2.077 & 0.130 & 0.128 & 0.004 & 4.255 & $\frac{3.06}{3.62} \Rightarrow$ \\
\hline & $6 / 22 / 2004$ & $9 \cdot 31 \cdot 1$ - & 25999 & 24,379 & 25.39 & 25.916 & 2486 & 32517 & $42647 \mathrm{r}$ & 386 & 28.624 & 5.147 & & 448 & & 14.643 & & 58 & & 412 & 116 & 306 & 2108 & 0.129 & 0.128 & 0.004 & 4.182 & 356 \\
\hline & 6/22/2004 & & 25.957 & 24.397 & \begin{tabular}{|l|l|}
25.338 \\
\end{tabular} & 25.918 & 24.8 & & & & & & & 44.2 & & 14.643 & & 59 & & 41.3 & 10. & & 2.11 & & & 0.004 & 4.154 & \\
\hline 190 & $6 / 22 / 2004$ & 9:33:05 AM & 25.914 & 24.404 & 25.305 & 25.936 & 24.87. & 32.719 & 42.656 & 3.893 & 28.86 & & 1.5 & 44.321 & & $\begin{array}{lll}14.643 \\
\end{array}$ & & 60.1 & 94 & 41.2 & 10.5 & 30.8 & $\mid$\begin{tabular}{|l|l|}
2.123 \\
\end{tabular} & 0.128 & 0.127 & 0.004 & $\begin{array}{l}4.134 \\
\end{array}$ & 3.52 \\
\hline 191 & $6 / 22 / 2004$ & 9:34:0 & 25.882 & 24.432 & 25.258 & 25.913 & 24.9 & 32.828 & & 3.836 & 28.98 & 4.967 & 1.5 & 44.903 & $0 . \varepsilon \quad \gamma$ & $\begin{array}{ll}14.643 \\
\end{array}$ & & 61. & & 41.4 & 11 & & & & 0.1 & 0.004 & \begin{tabular}{|l|l|l|l}
4.124 \\
\end{tabular} & 3.5 \\
\hline 192 & $6 / 22 / 2004$ & & 25.837 & 24.438 & 25.208 & 25.914 & 24.885 & 32.687 & 42.473 & 3.826 & 28.822 & & & 45.5 & & 14.6 & & 62. & & 41.0 & 11 & 30.8 & & 0.128 & 0.121 & 0.004 & 4.135 & \\
\hline & 6/22/2004 & & 25.799 & & 25.15 & 25.931 & & 32.783 & & 4.077 & 28.702 & & & & & & & & & & 11 & & & & & & & \\
\hline 年 & $6 / 22 / 2004$ & 9:37:05 & 25.757 & 24.472 & 25.122 & 25.928 & 24.8 & 32.401 & 42.0 & 4.015 & 28.347 & & & 45.3 & & & & 64.1 & & & & & & & & & 4.085 & \\
\hline$\frac{195}{196}$ & 6.122212004 & & $\frac{25.12}{25.96}$ & 24.485 & \begin{tabular}{|l|l|}
25.095 \\
2577
\end{tabular} & 25.906 & 24. & 32.441 3270 & & $\frac{3.89}{3762}>\mathrm{Cl}$ & & & & & & & & 65 & & & $\frac{11}{10}$ & & & 0.124 & & 004 & 4.061 & 3.46 \\
\hline 107 & 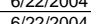 & 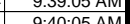 & $\frac{23.050}{25649}$ & $\frac{24.301}{2452}$ & 2.051 & 23.9008 & 24.009 & 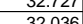 & $\mid \begin{array}{ll}424.424 \\
4091\end{array}$ & $\frac{1.103}{3086}$ & $\frac{20.912}{28074}$ & & & -44.5049 & & $\frac{14.045}{11643}$ & & 60. & & 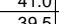 & 10. & & & 0.124 & & 0.004 & 4.021 & 0.442 \\
\hline 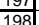 & 6 & $\frac{3.40 .05}{9 \cdot 105}$ & $\frac{25.045}{25612}$ & 24.517 & $\mid \begin{array}{ll}24.075 \\
24.977\end{array}$ & 25.930 & & 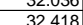 & (40.901 & $\frac{3.500}{4014}$ & $\frac{20.014}{23.58}$ & & & 45.0039 & & & & & & & & & & & & & & $3.4 t$ \\
\hline 199 & 6 & & 25.569 & $\frac{24.54}{24.54}$ & 24.935 & 25.896 & 24.85 & 32.621 & $\frac{41.00}{41.08}$ & $\begin{array}{r}4.014 \\
.93\end{array}$ & 28.631 & 4.001 & 0.93 & $\frac{4.5}{449}$ & & & & 69 & & 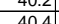 & & & & 0 & & & 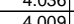 & 3.45 \\
\hline & $6 / 22 / 2004$ & & 25.542 & 24.54 & & 25.929 & 248 & 32578 & 431.64 & 4001 & 28.542 & & & & & & & & & & & & & & & & & \\
\hline 201 & & & 25.526 & 24561 & 24.916 & 25037 & & & & & & & & 460 & & & & & & & & & & & & & & \\
\hline 202 & $6 / 22 / 2004$ & & 25.488 & 24.563 & 24893 & 25.93 & 24 & 32584 & 41.497 & 3964 & 28.581 & 38 & & 45 & & 14 & & 7212 & & 40.0 & 112 & 3 & 21 & \begin{tabular}{|l|l|}
0.122 \\
\end{tabular} & 0.122 & D. & 3000 & 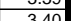 \\
\hline & $6 / 22 / 2004$ & & 25.466 & 24.581 & \begin{tabular}{|l|}
24.861 \\
\end{tabular} & 25.93 & 24. & 32.592 & $\begin{array}{ll}41.398 \\
\end{array}$ & 4.0 & 28.561 & 3.65 & & 45.3 & & 14.643 & & 73.12 & & 39.9 & 11. & 5 & 2.16 & 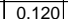 & 0.1 & 0.004 & 3.941 & \\
\hline 204 & $6 / 22 / 2004$ & 9:47:C & 25.444 & 24.589 & \begin{tabular}{|l|}
24.879 \\
\end{tabular} & 25.925 & 24.8 & 31.796 & \begin{tabular}{|l|l|}
40.233 \\
\end{tabular} & 3.901 & 27.869 & 3.52 & & 44. & 0.7 & $\begin{array}{l}14.643 \\
\end{array}$ & & 74.12 & \begin{tabular}{|l|l|}
1.23 \\
\end{tabular} & 38.8 & 10.9 & \begin{tabular}{|l|}
29.8 \\
\end{tabular} & 2.6 & \begin{tabular}{|l|l|}
0.119 \\
\end{tabular} & 0.1 & 0.004 & 4.002 & 3.41 \\
\hline 205 & $6 / 22 / 2004$ & 9:48:05 AM & 25.426 & 24.582 & \begin{tabular}{|l|}
24.871 \\
\end{tabular} & 25.868 & 24.874 & 32.022 & 240.508 & 3.827 & 28.212 & 3.523 & \begin{tabular}{|l|l|}
0.688 \\
\end{tabular} & \begin{tabular}{|l|l|}
44.498 \\
\end{tabular} & 0.799 & $\begin{array}{l}14.643 \\
\end{array}$ & 0.00 & 75.12 & \begin{tabular}{|l|l|}
1.25194 \\
\end{tabular} & 39.0 & \begin{tabular}{l|l}
10.9 \\
\end{tabular} & $\mid 30.1$ & \begin{tabular}{|l|}
2.076 \\
\end{tabular} & \begin{tabular}{|l|l|} 
\\
\end{tabular} & 0.120 & 0.004 & 3.970 & 3.38 \\
\hline 206 & & & & & & & & & & & & & & & & & & & & & & & & & & & & \\
\hline & & & & & & & & & & & & & & & & & & & & & & & & & & & & \\
\hline & & & & & & & & & & & & & & & & & & & & & & & & & & & & \\
\hline & & & & & & & & & & & & & & & & & & & & & & & & & & & & \\
\hline 211 & & & 25.7 & 24.5 & 25.2 & 25 & & 32.5 & 74 & 3.9 & 28 & 4.5 & & $4 !$ & \begin{tabular}{l|l}
0.8 \\
\end{tabular} & & & & & 40. & & & $\begin{array}{lll}2.1 & \end{array}$ & 0.1 & 0.1 & 0.0 & 4.1 & \\
\hline & & Maximun & 26.1 & 24.6 & 25.6 & 25.9 & 2 & 32.8 & 43.8 & 4.1 & 29.6 & 5.8 & & 4 & 0.9 & 14.6 & & & & 42.3 & 11.4 & 30.9 & 2.1 & & 0.1 & 0.0 & $4.3 \quad-3$ & 3.6 \\
\hline 21 & & & & 24.5 & 25.1 & 25.9 & & 32.6 & 42.2 & 3. & $2 \varepsilon$ & 4.3 & & & 0.8 & & & & & 40.7 & 11.0 & & 2.1 & 0.1 & 0.1 & 0.0 & & \\
\hline & & Minimum & 25.4 & 24.3 & 24.9 & 25.7 & 2 & 31.8 & 40.2 & 3.8 & 27.9 & 3.5 & & 43.6 & 0.8 & 14.6 & & & & 38.8 & 10.7 & 29.8 & 2.1 & 0.1 & 0.1 & 0.0 & & 3.4 \\
\hline & Number & $2 \times$ Std Dev & 427 & 0.178 & 0.474 & 0.097 & 0. & 0.539 & $1.75 \mathrm{t}$ & 0.153 & 0.564 & 341 & & & 0.057 & & & & & & & & & 20 & .007 & 20 & 20 & \\
\hline$\frac{216}{2177}$ & Number & I* Backpulse & nhox & not inc & & & & & & & & & & & & & & & & & & & & & & & & \\
\hline
\end{tabular}


WSRC-TR-2005-00105, REVISION 0

SRNL-RPP-2005-00012, REVISION 0

RUN \# 3.21A, B, AND C; POST-TEST BASELINE WITH 0.1 M NAOH

\begin{tabular}{|c|c|c|c|c|c|c|c|c|c|c|c|c|c|c|c|c|c|c|c|c|c|c|c|c|c|c|c|c|}
\hline & A & $B$ & $\mathrm{D}$ & $E$ & $F$ & $G$ & $\mathrm{H}$ & $\mathrm{J}$ & $\mathrm{K}$ & $\mathrm{L}$ & $M$ & $\mathrm{~N}$ & $\mathrm{O}$ & Q & $\mathrm{R}$ & $\mathrm{s}$ & \begin{tabular}{l|l} 
\\
\end{tabular} & $\mathrm{v}$ & W & $x$ & $\mathrm{Y}$ & $z$ & $\mathrm{AA}$ & $A B$ & $A C$ & $A D$ & $\mathrm{AE}$ & AF $\mathrm{KA}$ \\
\hline & $\begin{array}{c}\text { Baseline 3A } \\
6 / 22 / 2004 \\
\end{array}$ & & & & & & & & & & & & & & & & & & & & & & & & & & & \\
\hline$\frac{226}{227}>\mathrm{l}$ & $\mid 6 / 222 / 20004$ & $\begin{array}{l}\text { 12:12:33 3M } \\
\text { 12:13:33 PM }\end{array}$ & $\frac{24.623}{24.596}$ & $\begin{array}{l}24.268 \\
24.276\end{array}$ & \begin{tabular}{|r|}
24.126 \\
24.17 \\
\end{tabular} & $\frac{25.103}{25.117}$ & 24.516 & $\begin{array}{l}\frac{12.17}{12.249} \\
\end{array}$ & $\begin{aligned} 12.132 \\
12.84 \\
\end{aligned}$ & $\begin{array}{l}3.994 \\
4.038 \\
\end{array}$ & 8.018 & $\begin{array}{l}-1.216 \\
-1.216\end{array}$ & $\begin{array}{r}-4.49 \\
-4.49\end{array}$ & $\begin{aligned} 44.7 / 6 \\
44.83 \\
\end{aligned}$ & 0.25 & \begin{tabular}{|c|}
14.6634 \\
14.643
\end{tabular} & 0.004 & $\begin{array}{l}0.00 \\
1.00\end{array}$ & \begin{tabular}{|l|}
0 \\
0.01667 \\
\end{tabular} & \begin{tabular}{|l|}
11.3 \\
11.4 \\
\end{tabular} & $\begin{array}{l}11.0 \\
11.0 \\
\end{array}$ & & $\begin{array}{l}0.696 \\
0.699\end{array}$ & 0.037 & $\frac{0.038}{0.038}$ & 0.004 & $\begin{array}{l}3.785 \\
3.763 \\
\end{array}$ & $\begin{array}{l}3.22 \\
3.20\end{array}$ \\
\hline 228 & $6 / 22 / 2004$ & 12:14:33 PM & 24.594 & 24.274 & 24.178 & 25.225 & 24.647 & 12.106 & 12.654 & 3.926 & 7.987 & -1.214 & -4.487 & 46.088 & $\begin{array}{l}0.25 \\
0.25 \\
\end{array}$ & 14.643 & 0.004 & 2.00 & 0.03333 & 111.2 & 11.3 & $10.6 \mathrm{C}$ & 0.693 & 0.037 & 0.038 & 0.004 & 3.797 & $\begin{array}{l}3.20 \\
.23 \\
\end{array}$ \\
\hline 2290 & 6/22/2004 & 12:15:33 PM & 24.564 & & \begin{tabular}{|l|}
24.167 \\
2.109 \\
\end{tabular} & 25.38 & 24.707 & 12.193 & 12.747 & 3.969 & 8.037 & -1.214 & $\begin{array}{r}-4.49 \\
\end{array}$ & 45.43 & 0.25 & 14.643 & 0.004 & 3.00 & $\begin{array}{l}0.05 \\
0.07\end{array}$ & 11.3 & 11.1 & & & & 0.038 & 0.004 & 3.773 & 3.21 \\
\hline 230 & 6/22/2004 & 12:16:33 PM & 24.568 & $\begin{array}{r}24.303 \\
24317 \\
\end{array}$ & \begin{tabular}{|l|}
24.192 \\
241.69
\end{tabular} & $\begin{array}{r}25.494 \\
25.509\end{array}$ & 24.776 & $\begin{array}{ll}12.189 \\
12.207\end{array}$ & 12.791 & $\begin{array}{l}3.928 \\
3048\end{array}$ & $\begin{array}{r}8.102 \\
81 \\
\end{array}$ & -1.214 & $\begin{array}{r}-4.484 \\
-4.993 \\
\end{array}$ & 45.139 & 0.251 & 14.643 & 0.004 & 4.00 & $\mid 0.06667$ & 11.3 & 11.1 .1 & 10.1 & 0.699 & 0.037 & 0.038 & 0.004 & 3.774 & 3.21 \\
\hline 232 & $\begin{array}{l}0 / 222 / 2004 \\
6 / 2204 \\
\end{array}$ & $12: 18: 33 \mathrm{PM}$ & $\frac{24.52}{24.562}$ & $\begin{array}{l}24.337 \\
24.337\end{array}$ & \begin{tabular}{|l|}
24.1260 \\
24.226 \\
\end{tabular} & $\frac{25.506}{25.563}$ & $\begin{array}{l}24.85 \\
24.87\end{array}$ & $\frac{12.201}{12.089}$ & $\frac{12.601}{12.664}$ & $\frac{3.940}{3.926}$ & $\begin{array}{r}8.1 \\
7.987 \\
\end{array}$ & $\frac{-1.219}{-1.214}$ & $\begin{array}{l}-4.495 \\
-4.478 \\
\end{array}$ & $\frac{44.669}{44.709}$ & $\begin{array}{l}0.252 \\
0.252\end{array}$ & $\frac{14.643}{14.643}$ & $\begin{array}{l}0.003 \\
0.004 \\
\end{array}$ & $\frac{6.00}{6.00}$ & \begin{tabular}{|l|l|}
.00353 \\
\end{tabular} & $\frac{1.3}{11.2}$ & $\frac{1.9}{11.0}$ & $\frac{10.2}{10.0}$ & $\begin{array}{l}0.100 \\
0.692 \\
\end{array}$ & \begin{tabular}{|l|}
0.031 \\
038
\end{tabular} & 0.038 & $\begin{array}{l}0.004 \\
0.004 \\
\end{array}$ & $\frac{3.156}{3.826}$ & $\frac{3.20}{3.26}$ \\
\hline 233 & $6 / 22 / 2004$ & 12:19:33 PM & 24.551 & 24.361 & 24.21 & 25.622 & 24.909 & 12.237 & 12.826 & 3.995 & 8.032 & -1.214 & -4.493 & 44.846 & 0.251 & 14.643 & 0.004 & 7.00 & \begin{tabular}{|l|l|}
0.11667 \\
\end{tabular} & 11.4 & 11.0 & 10.1 & 0.699 & 0.037 & 0.038 & 0.004 & 3.776 & $\begin{array}{l}3.26 \\
3.21\end{array}$ \\
\hline 234 & 6/22/2004 & 12:20:33 PM & & 24.385 & 24.228 & & 24.897 & 12.197 & 12.751 & & & -1.214 & -4.49 & 44.947 & & 14.643 & 0.004 & & & & & & & & & & & $\frac{3.21}{3.22}$ \\
\hline 235 & $6 / 22 / 2004$ & 12:21:33 PM & 24.553 & 24.399 & 24.218 & 25.68 & 24.852 & $\begin{array}{ll}12.093 \\
\end{array}$ & 12.672 & 3.95 & 7.971 & & -4.49 & 46.015 & 0.251 & $\begin{array}{ll}14.643 \\
\end{array}$ & & & $\overline{0.15}$ & 11.2 & & & 692 & & 0.038 & 0.004 & 3.814 & $\frac{3.22}{3.25}$ \\
\hline 236 236 & & & & 24.423 & 24.226] & & 24.84 & & $12.857 \mathrm{r}$ & 4.001 & 8.049 & -1.214 & -4.49 & 44.638 & 0252 & $\begin{array}{ll}14.643 \\
\end{array}$ & & & & 11.4 & & & & & & & 3.783 & $\frac{3.25}{3.22}$ \\
\hline 237 & $6 / 22 / 2004$ & 12:23:33 PM & 24.55 & 24.436 & 24.235 & 25.717 & & 12.241 & 12.801 & 3.839 & 8.223 & -1.214 & -4.475 & 44.57: & 0.254 & $\begin{array}{ll}14.643 \\
\end{array}$ & 0.00 & 11.00 & 0.18333 & 11.3 & 10.9 & & . 705 & 038 & 0.039 & 0.004 & 3.782 & 3.22 \\
\hline & $6 / 22 / 2004$ & 12:24:33 PM & 24.553 & 24.439 & 24.207 & 25.72 & 24.826 & 12.33 & 12.907 & 3.902 & 8.273 & -1.216 & -4.472 & \begin{tabular}{ll|l}
44.644 \\
\end{tabular} & 0.255 & 14.643 & 0.0 & 12.00 & 0.2 & 11.4 & & & 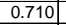 & & 0.03 & 0.004 & 3.774 & 3.21 \\
\hline & 6/22/2004 & 12:25:33 PM & 24.561 & 24.457 & 24.215 & 25.718 & 24.809 & 12.253 & 12.921 & 3.874 & 8.137 & -1.214 & -4.475 & 44.757 & 0.253 & 14.643 & 0.00 & 13.00 & \begin{tabular}{|l|l|l|l|l|l|} 
\\
\end{tabular} & 11.4 & 11.0 & & & & & 0.004 & 3.783 & 3.22 \\
\hline & $6 / 22 / 2004$ & 12:26:33 PM & 24.559 & 24.48 & 24.218 & 25.701 & 24.752 & 12.317 & 12.929 & 3.893 & 8.238 & -1.214 & -4.47 & 44.563 & 0.255 & 14.643 & 0.00 & 14.00 & 0.23333 & 11.5 & 10.9 & 10.3 & .709 & 0.038 & 0.039 & 0.004 & 3.782 & 3.22 \\
\hline 241 & 6/222/2004 & 12:27: 33 PM & 24.555 & 24.471 & 24.2444 & 25.702 & 24.728 & 12.131 & 12.772 & 3.898 & 8.024 & -1.208 & -4.47 & 44.775 & 0.254 & 14.643 & 0.00 & 15.00 & 0.25 & 11.3 & & & 695 & & 0.039 & 0.004 & 3.839 & 3.27 \\
\hline & $6 / 2$ & 12:28:33 PM & & & & 25.645 & & 12.048 & 12.635 & 3.861 & & & & 44.386 & & 14.643 & & & 0.26667 & & & & & & 0.039 & & & 3.30 \\
\hline & $6 \frac{6 / 22212004}{6232004}$ & 12:29:33 PM & 20.4655 & 24.491 & 24.2299 & 25.657 & 24.818 & 12.1044 & $\frac{12.12}{12.913}$ & 3.911 & 7.998 & -1.214 & -4.455 & 44.198 & 0.255 & 14.643 & 200 & 17.00 & | & 11.2 & 11.0 & & 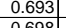 & . & 0.030 & 0.004 & 3.866 & 3.29 \\
\hline 245 & $6 / 22 / 2004$ & 12:31:33 PM & $\frac{24.00}{24.566}$ & 24.502 & 24.226 & 25.688 & $\begin{array}{l}24.041 \\
24.89\end{array}$ & 1..101 & $\begin{array}{l}\frac{12.010}{12.946} \\
\end{array}$ & $\begin{array}{l}0.004 \\
3.877 \\
\end{array}$ & 8.246 & - & $\frac{-4.47}{-4.47} \quad-1$ & 44.567 & 0.255 & 14643 & & 1900 & 0.31667 & $\frac{1.55}{11.5}$ & 109 & & 709 & - & - & 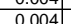 & $\begin{array}{l}3.856 \\
3780 \\
-10\end{array}$ & $\begin{array}{l}3.26 \\
322\end{array}$ \\
\hline 246 & $6 / 22 / 2004$ & 12:32:33 PM & 24.569 & 24.53 & 24.263 & 25.745 & 24.897 & 12.328 & 12915 & 3.923 & $\frac{6.24}{8.24}$ & -1.211 & -4.467 & 42.516 & 0.256 & 14.643 & 0.00 & 20.00 & $\mid 0.33333$ & 11.4 & 104 & 10.3 & 0.709 & $\mid 0.038$ & 0.039 & 0.004 & 3.789 & $\frac{3.22}{3.22}$ \\
\hline & & & & & & & & & & & & & & & & & & & & & & & & & & & & \\
\hline & & sos & & & & & & & & & & & & & & & & & & & & & & & & & & \\
\hline $24=$ & & Averages & 24.6 & 24.4 & 24.2 & 25.6 & & 12.2 & 12.8 & 3.9 & 8.1 & -1.2 & & 44.8 & 0.3 & 14.6 & & & & 11.3 & & & 0.695 & .038 & 0.039 & 0.004 & 3.8 & 3.2 \\
\hline |251 & & Mediann & $\frac{24.6}{246}>-1$ & $\frac{24.5}{24.4}$ & $\frac{24.3}{242}$ & 25.7 & 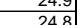 & $\frac{11.3}{122}$ & $\frac{12.5}{128}$ & $\frac{4.0}{39}$ & 然. & $\frac{-1.2}{-12}>\mathrm{C}$ & $-4.4-5>$ & 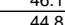 & 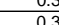 & $\frac{11.0}{14.6}$ & & & & $\frac{11.5}{113}$ & 110 & & 699 & 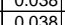 & 0.035 & 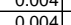 & & 3. \\
\hline 252 & & Minimum & 24.6 & $\begin{array}{l}24.4 \\
24.3 \\
\end{array}$ & 24.1 & 25.1 & 24.5 & 12.0 & 12.6 & 3.8 & 8.0 & -1.2 & -4.5 & 42.5 & 0.3 & 14.6 & & & & 11.2 & $\begin{array}{ll}10.4 \\
10.4\end{array}$ & & & & 0.038 & 0.004 & 3.8 & $\frac{3.4}{3.2}$ \\
\hline & & $2 \times$ Std Dev & 0.035 & 0.171 & 0.061 & 0.392 & 0.197 & 0.165 & 0.187 & 0.101 & 0.195 & 0.004 & 0.019 & 1.337 & 0.004 & 0.000 & 0. & & & 0.187 & 0.328 & & 0.012 & 0.001 & 0.001 & 0.000 & 0.064 & $\frac{3.2}{2.5}$ \\
\hline & Number $\mathrm{c}$ & of Points Used & 21 & & & 21 & & 21 & 21 & 21 & 21 & 21 & 21 & 21 & 21 & 21 & & & & 21 & 21 & 21 & 21 & 21 & 21 & 21 & $\frac{0.04}{21}$ & $\frac{.05}{2}$ \\
\hline & & “ Backpulse po & in box & re not incli & uded & & & & & & & & & & & & & & & & & & & & & & & \\
\hline 256 & & & & & & & & & & & & & & & & & & & & & & & & & & & & \\
\hline & & & & & & & & & & & & & & & & & & & & & & & & & & & & \\
\hline & & & & & & & & & & & & & & & & & & & & & & & & & & & & \\
\hline & aseline 3B & & & & & & & & & & & & & & & & & & & & & & & & & & & \\
\hline 260 & & 12:37:45 PM & 24.737 & 24.603 & 24.436 & 26.099 & $5.17 \mathrm{~s}$ & 22.156 & 25.805 & 3.854 & 18.177 & -1.214 & -2.388 & 44.477 & 0.518 & 14.643 & 0.002 & 25.20 & 0.42 & 24.3 & 10.9 & & 1.390 & . .077 & 0.078 & 0.004 & 3.891 & 3.3. \\
\hline$\frac{201}{20}$ & 6/2/22/2004 & 12:38:45 PM & 24.781 & 24.642 & 24.481 & 26.133 & 20.21 & 22.191| & 25.751 & 0.00 & 18.216 & -1.211 & -2.3711 & 44.5522 & 0.519 & 14.6. & & 20 & 0.43667 & 24.3 & & & & & $\sqrt{2}+2+3$ & 0.004 & 3.886 & (2) \\
\hline & 6/22/2004 & 12:39: & 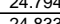 & 24.675 & $24.4 / 4$ & 26.146 & & 2.067 & 25.656 & 3.8549 & 18.051 & -1.214 & -2.374 & $44.838 \mathrm{r}$ & 0.52 & 14.6 & & & 0.45333 & 24.2 & 100 & & 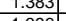 & & & & & 3.3. \\
\hline & & $\frac{12.40}{12 \cdot 41}$ & 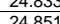 & 2 & 24.492 & 20.204 & 25.2 & $\frac{2.2 .35}{20505}>0$ & $\frac{23.900}{2.155}$ & & 18.273 & -1.1216 & & 44. & & 14.5 & & & 0.47 & 24.5 & & & & & & & 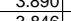 & 3.3 \\
\hline 265 & $0 / 26220004$ & $12: 42$ & $\begin{array}{l}\frac{2.001}{24.88} \\
24 .\end{array}$ & $\frac{24.056}{2476}$ & $\frac{24.300}{2452}$ & $\frac{20.420}{26202}$ & & 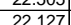 & 25736 & 年. 3000 & $\frac{10.354}{1823}$ & - & -233 & & & 年 & & & 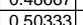 & 24.13 & . & & 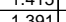 & & & & 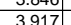 & 3.2 \\
\hline & $6 / 22 / 2004$ & $12: 43: 45$ PM & 24.003 & 24.73 & 24.533 & 26.175 & 25.141 & 22.193 & 25.938 & 3.706 & 18.369 & -1.214 & -2.31 & 42.857 & & 14.6 & 0.00 & 31 & 0.0052 & 24.5 & 10.5 & & 398 & & & (0.04 & 3.918 & $\frac{3.3}{3.3}$ \\
\hline 2017 & $6 / 22 / 2004$ & 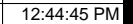 & 24.933 & 24.759 & 24.573 & $\begin{array}{ll}26.19 \\
\end{array}$ & 25.126 & 22.332 & 26.08 & 3.705 & 18.532 & -1.216 & -2.313 & & & 14.6 & & & \begin{tabular}{|l|}
0.53667 \\
\end{tabular} & 24.6 & 柆 & & & & & & & 沕 3 \\
\hline 200 & $6 / 22 / 2004$ & & 24.94 & 24.75 & & 26.132 & & 22.21 & 25.902 & 3.746 & 18.296 & -1.216 & & & & & & & & & & & & & & & & ה \\
\hline & $6 / 22 / 2004$ & $12: 46: 45 \mathrm{p}$ & 24.942 & 24.763 & 24.592 & 26.099 & 25. & 22.225 & 25.904 & 3.719 & 18.357 & -1.214 & -2.307 & 43.403 & 0.526 & 14.643 & & 34. & 0.57 & 24.4 & 106 & & 1399 & & 0.07 & 04 & 3.910 & 3.3 \\
\hline & $6 / 22 / 2004$ & 12:47:45 PM & 24.964 & 24.771 & $\mid$\begin{tabular}{|l|l|}
24.619 \\
\end{tabular} & 26.076 & 25.017 & 22.326 & 26.037 & 4.01 & 18.25 & -1.214 & -2.394 & 45.1 & 0.51 & 14.643 & & & & 24.6 & & & 1.399 & & & & 848 & $3.22>3$ \\
\hline & 6/22/2004 & $12: 48$ & 24.97.1. & 24.777 & 24.621 & 26.013 & & 22.112 & 25.651 & 3.848 & 18.109 & -1.216 & -2.385 & & & & & & 0.60333 & 24.2 & 100 & & 1387 & & & & 3.889 & 33 \\
\hline 272 & 6/22/2004 & 12:49:45 & 24.992 & 24.784 & \begin{tabular}{|l|l|}
24.632 \\
\end{tabular} & 25.989 & & 22.2 & 25.871 & 3.806 & 18.294 & -1.214 & -2.356 & $\begin{array}{lll}44.348 \\
\end{array}$ & 0.522 & 14.6 & 0.0 & 37 & 0.62 & 24.4 & 10.9 & 20. & 1.396 & & 0. & 0.004 & 3.884 & 3.3 \\
\hline 27. & $6 / 221 / 2 ; 0$ & 12:50:45 & 25.009 & 24. & 24.634 & 26.021 & & 22.424 & 26.066 & & & -1.214 & -2.342 & & 0.5 & & & & $\begin{array}{ll}0.63 \\
\end{array}$ & 24.6 & & & 12 & & & & & 3.2 \\
\hline 274 & 6/22/2004 & 12:51:45 & 25.02 & 24.796 & 24.635 & & & 22.289 & 26.076 & 3.782 & 18.413 & -1.211 & -2.33 & 44.4. & 0.525 & 14.643 & & & 0.653 & 24.6 & & & 1.403 & & & & 3.886 & 3.3 \\
\hline 270 & |l2004 & & 23.02 & 24.8 & 24. & 26.114 & 25.1 & 22.044 & & & & -1.216 & & & & & & & & 24.1 & & & & & & & 941 & \\
\hline$\frac{210}{277}$ & $6 / 22 / 2004$ & $\frac{12: 53: 4}{12.54 .4}$ & 20 & 24.809 & 24.643 & 26.149 & $\frac{25}{252}+2$ & 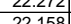 & 25.981 & 3.698 & 18.450 & -1.1.16 & -2. & 43.8 & 0.526 & & & & $\mid$ & 24.5 & 107 & 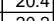 & & & & & (3.890 & . \\
\hline & (1) & 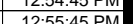 & $\frac{2.001}{25072}$ & $\frac{24.016}{24848}-1$ & 24680 & $\frac{20.194}{26179}$ & & 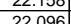 & 20.003 & & $\frac{10.250}{18144}$ & -1.124 & 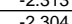 & & & & & & $\mid$ & 24.5 & & & & & & & . & 告, \\
\hline 279 & $\frac{01 / 21204}{6}$ & 12.5.45 & $\frac{2.5069}{25069}$ & $\frac{24.0401}{24851}$ & $\frac{24.064}{24704}$ & $\frac{20.175}{26206}$ & $\frac{25.4}{252}$ & 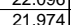 & 25.186 & $\frac{3.361}{3.845}$ & $\frac{10.147}{18107}$ & -1.146 & & & & 年 & & & \begin{tabular}{|l|l|} 
\\
\end{tabular} & $\frac{24.5}{220}$ & t & & & & & & & \\
\hline 280 & $6 / 2 / 2 / 2004$ & 12:57:45 & $\frac{25.086}{2506}$ & 24.862 & $\mid 2.716$ & 26.233 & & 22.108 & 25.662 & 3.892 & & -1.216 & & 44.4 & & & & & $\mid 07533$ & 24. & & & . & & & & 3889 & 它3 \\
\hline 281 & $6 / 22 / 2004$ & $12: 58: 45$ PM & 25.097 & 24.879 & 24.722 & 26.259 & 25.3 & 22.175 & 25.786 & 3.842 & 18.187 & -1.214 & -2.351 & 44.521 & 0.523 & 14.643 & 0.00 & 46.20 & 0.77 & 24.3 & 10.9 & 20.2 & 1.391 & 0.078 & 0.079 & 0.004 & 3.894 & 3.3 \\
\hline 28 & & nar & & & & & & & & & & & & & & & & & & & & & & & & & & \\
\hline & & Average & 23.6 & 24.8 & 24.00 & 20.1 & & 22.2 & 2.9 & 3.0 & 18.6 & -1.2 & & 44.5 & 0.5 & 14.00 & & & & 24.4 & 10.8 & 20.4 & 1.095 & 0.078 & 0.079 & 0.004 & 3.9 & \\
\hline & & & D.1 & 24.9 & 24.1 & 20 & & $2,2.5$ & 20 & 4.0 & & $\frac{-1.2}{1.2}$ & 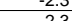 & 45 & 0.5 & 14.0 & & & & .1 & 1..5 & 20 & 1.4 & \begin{tabular}{|l|l} 
\\
\end{tabular} & & & (1) & \\
\hline |286 & & Minimum & 24.7 & 24.6 & 24.4 & 26.0 & 25. & 220 & 25.5 & 3.3 & 18.1 & -1.2 & 2.4 & 429 & 0.5 & 14.6 & & & & 24.0 & 10.5 & 20.0 & $\mid$ & 0.077 & 0.007 & $\mid 0.004$ & $\frac{3.8}{3.8}$ & 3. \\
\hline 287 & & & 0.207 & 0.146 & \begin{tabular}{|l|l|}
0.164 \\
\end{tabular} & 0.146 & 0.183 & 0.242 & 0.344 & 0.148 & 0.292 & 0.003 & 0.059 & 1.224 & 0.006 & 0.000 & 0.001 & & & 0.344 & 0.3000 & 0.258 & 0.018 & 0.001 & 0.001 & 0.000 & 0.050 & 0.043 \\
\hline \begin{tabular}{|c|}
288 \\
289 \\
\end{tabular} & Number c & $\begin{array}{l}\text { of Points Used } \\
I^{*} \text { Backpulse en }\end{array}$ & $\frac{22}{\sin \text { box }}$ & 22 & $\begin{array}{l}22 \\
\text { uded }\end{array}$ & & & & & & & 22 & & & & & & & & 22 & 22 & 22 & 22 & 22 & 22 & 22 & 22 & 2 \\
\hline
\end{tabular}


WSRC-TR-2005-00105, REVISION 0 SRNL-RPP-2005-00012, REVISION 0

RUN \# 3.21A, B, AND C; POST-TEST BASELINE WITH 0.1 M NAOH

\begin{tabular}{|c|c|c|c|c|c|c|c|c|c|c|c|c|c|c|c|c|c|c|c|c|c|c|c|c|c|c|c|c|}
\hline & A & $B$ & D & $E$ & $\mathrm{~F}$ & G & $\mathrm{H}$ & $\mathrm{J}$ & $\mathrm{K}$ & $\mathrm{L}$ & $\mathrm{M}$ & $\mathrm{N}$ & $\overline{0}$ & Q & $R$ & $\mathrm{~s}$ & $T$ & v & w & $x$ & $Y$ & $z$ & $\mathrm{AA}$ & $A B$ & $A C$ & $A D$ & AE & AF \\
\hline & Baseline $3 c$ & & & & & & & & & & & & & & & & & & & & & & & & & & & \\
\hline & $6 / 22 / 20004$ & $\begin{array}{l}\text { 1:01::2 PM } \\
\text { 1::202 PM }\end{array}$ & $\frac{25.311}{25.399}$ & $\frac{24.92}{24.94}$ & 24.992 & $\frac{26.548}{26.58}$ & $\begin{array}{l}25.394 \\
25.407\end{array}$ & $\begin{array}{ll}32.219 \\
32.194\end{array}$ & \begin{tabular}{|l|l|}
41.281 \\
4129
\end{tabular} & $\begin{array}{l}3.823 \\
3842 \\
\end{array}$ & $\begin{array}{r}28.343 \\
28325\end{array}$ & $\begin{array}{l}3.998 \\
3.995 \\
\end{array}$ & $\begin{array}{l}0.962 \\
0.957 \\
\end{array}$ & $\begin{array}{l}44.786 \\
44.652\end{array}$ & 0.817 & $\frac{14.643}{14.643}$ & 0.004 & $\begin{array}{l}49.1 \\
50.1\end{array}$ & $\mid 0.81917$ & 39.8 & $\frac{11.0}{109}$ & \begin{tabular}{|l|l|}
30.3 \\
30.3
\end{tabular} & \begin{tabular}{|l|}
2.088 \\
2086
\end{tabular} & & 0.122 & 0.004 & 4.023 & 3.42 \\
\hline & $6 / 22 / 2004$ & 1:00:42 PM & 25.477 & 24.96 & 25.118 & 26.602 & 25.35 & 32.327 & 41.344 & 3.819 & 28.483 & 4.096 & 1.023 & 44.264 & 0.823 & $\frac{14.045}{14.643}$ & & & 0.0525 & & 10.8 & & 096 & $\overline{0.123}$ & 0.12 & $\frac{0.004}{0.004}$ & & $\frac{3.44}{3.42}$ \\
\hline & & & & & 25.146 & $\overline{26.512}$ & & 32.036 & & 3.79 & & & & & & 146 & & & & $\frac{29.4}{39.4}$ & & & & & & & & 年.44 \\
\hline & $6 / 22 / 2004$ & 1:05:42 PM & 25.603 & 24.97 & 25.219 & 26.511 & 25.286 & 32.32 & 41.677 & 3.807 & 28.4 & & & 44 & 0.825 & & & & 0.88583 & & 10.8 & $\frac{30.4}{30.4}$ & 2.096 & & 0.122 & 0.004 & 4.021 & $\frac{3.45}{3.42}$ \\
\hline & $6 / 22 / 2004$ & 1:06:42 PM & 25.662 & 24.98 & 25.263 & 26.44 & 25.2 & 32.54 & 41.874 & 3.785 & 28.76 & & 1.06 & 43.351 & 0.826 & 14.643 & & 54.1 & 0.9025 & 40.4 & 10.6 & 30.7 & 2.113 & & 0.12 & 0.004 & 3.988 & 3.39 \\
\hline & $6 / 22 / 2004$ & 1:07:42 PM & 25.714 & 24.99 & 25.31 & 26.391 & 25.217 & 32.264 & \begin{tabular}{|l|l|}
41.584 \\
\end{tabular} & $\begin{array}{l}3.764 \\
\end{array}$ & 28.454 & & 1.049 & 44.348 & 0.826 & 14.643 & .004 & 55.1 & \begin{tabular}{|l|l|l|}
0.91917 \\
\end{tabular} & 40.1 & 10.9 & 30.4 & 2.09 & 0.123 & 0.122 & 0.004 & 4.021 & 3.42 \\
\hline & $6 / 22 / 2004$ & 1:08:42 PM & 25.746 & 24.99 & 25.332 & 26.297 & 25.189 & 32.573 & 41.997 & 3.835 & 28.702 & 4.134 & 1.055 & 44.048 & 0.826 & 14.643 & & 56.1 & 0.93583 & 40.5 & 10.8 & 30.6 & 2.112 & 0.123 & 0.122 & $\mid$ & 3.982 & 3.39 \\
\hline & $6 / 22 / 2004$ & 1:09:42 PM & 25.798 & 24.99 & 25.384 & 26.26 & 25.191 & 32.078 & 41.379 & 3.792 & 28.245 & 4.1 & .0 & 44.404 & 0.826 & 14.643 & & 57. & 0.9525 & 39. & & 30.2 & 2.080 & & & & & 3.44 \\
\hline & $6 / 22 / 2004$ & 1:10:42 PM & 25.845 & 24.99 & 25.436 & 26.32 & 25.258 & 32.179 & 41.354 & 3.736 & 28.473 & 4.12 & & 44.252 & 0.827 & 14.643 & & 58.1 & 0.96917 & & 10.8 & & 2.091 & & 0.12 & & & 3.42 \\
\hline & 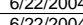 & $\frac{1.14: 42 \mathrm{PM}}{1.1 \% 20}$ & $\frac{25.881}{25019}$ & 25.00 & \begin{tabular}{|l}
25.478 \\
2520
\end{tabular} & $\frac{26.36}{2614}$ & 25 & $\frac{32.432}{32012}$ & $\frac{4.1 .692}{1192}$ & $\frac{3.803}{3.782}$ & $\frac{28.61}{28012}$ & $\frac{4.1}{4.1}$ & & 44.4 & & & & & 10027 & & 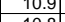 & & & & & 0.004 & & \\
\hline & 0.12421204 & 1:12:242 PM & & & & & & & & 3.185 & & & & 44.241 & & 14. & & & & & 10.8 & & & & & 0.004 & 4.018 & 3.42 \\
\hline & 6 & 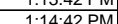 & $\frac{25.90}{2593}$ & $\frac{23.04}{2501}$ & \begin{tabular}{|l|l|}
25.345 \\
25.554
\end{tabular} & $\frac{2.425}{26505}$ & 25.405 & 32.026 & 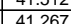 & o.t24 & $\frac{2.3}{28.3}$ & & $\frac{1.102}{1038}$ & 44.552 & 0.8 & 14. & & $\frac{10}{62}$ & 10353 & 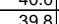 & 10.0 & 301 & 2.086 & $\mid \begin{array}{ll}0.122 \\
0.123\end{array}$ & & 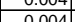 & & 3.43 \\
\hline & $6 / 22 / 2004$ & $\frac{1.1 .14: 2 \mathrm{PM}}{11}$ & 26.02 & $\frac{25.03}{25.03}$ & 25.586 & $\frac{2.056}{26.56}$ & 25.513 & $\frac{15.796}{31.796}$ & 40.637 & $\frac{3.059}{3.959}$ & 27.845 & $\frac{4.156}{3.96}$ & & 45.687 & $\frac{0.0217}{0.817}$ & 14.643 & & $\frac{23}{63}$ & $\frac{1.05025}{1.0525}$ & $\frac{3.62}{39.2}$ & $\frac{11.2}{112}$ & 298 & \begin{tabular}{l|l|}
2.056 \\
\end{tabular} & 0.122 & 0.12 & & $\frac{10.018}{4.018}$ & $\frac{3.43}{3.42}$ \\
\hline & $6 / 22 / 2000$ & 1:116:42 PM & 26.046 & 25.03 & 25.598 & 26.62 & 25.564 & 31.764 & 40.66 & & & & & 46.19 & & & & & & & & & & & & & & $\frac{3.4 \angle}{3.42}$ \\
\hline & 612012000 & $1: 17: 42 \mathrm{PM}$ & 26.084 & 25.05 & 25.63 & 26.67 & 25.601 & 31.893 & 40.931 & 3.998 & 27.886 & 3.998 & 0. & 45.32 & 0.82 & 14.643 & & 65.1 & 1.08583 & 39. & 111. & $\frac{2.9}{29.9}$ & 2.061 & 0.12 & 0.121 & 0.004 & 4.018 & $\frac{3.44}{3.42}$ \\
\hline & 6/22/2004 & 1:18:42 PM & 26.111 & 25.07 & 25.667 & 26.738 & 25.649 & 32.173 & 41.246 & 3.869 & 28.386 & & & 44.915 & 0.824 & & & 66.1 & & & & & & & & & & \\
\hline & $\frac{1 / 22 / 2000}{6}$ & 1:19:42 PM & $=26.15$ & $\frac{25.10}{25.10}$ & $\frac{25.701}{25.701}$ & $\frac{26.812}{26.812}$ & 25.687 & 31.92 & 41.335 & 3.904 & 27.953 & 4.116 & 1.035 & 44.915 & 0.024 & $\frac{14.043}{14.643}$ & & $\frac{00.1}{67.1}$ & $\begin{array}{l}1.10 \angle 5 \\
1.11917\end{array}$ & $\begin{array}{l}5.0 \\
39.9 \\
\end{array}$ & 11.0 & \begin{tabular}{|l|}
29.9 \\
29.9
\end{tabular} & 2.060 & \begin{tabular}{|l|l|l|l|} 
& .123 \\
\end{tabular} & 0.121 & 0.004 & $\begin{array}{l}3.002 \\
4.029\end{array}$ & 3.43 \\
\hline 316 & $6 / 22 / 2004$ & $1: 20: 42 \mathrm{PM}$ & 26.161 & 25.11 & 25.723 & 26.85 & 25.684 & 31.901 & 41.049 & 3.874 & 27.964 & 4.119 & 1.046 & 44.623 & 0.827 & 14.643 & 0.004 & 68.1 & 1.13583 & 39.6 & 10.9 & 29.9 & 2.064 & 0.123 & 0.121 & 0.004 & 4.036 & 3.44 \\
\hline & & & & & & & & & & & & & & & & & & & & & & & & & & & & \\
\hline & & & & & & & & & & & & & & & & & & & & & & & & & & & & \\
\hline 318 & & Averages & 25.8 & 25. & 25.4 & 26.5 & 25.4 & 32.1 & 41.3 & 3.8 & 28.3 & 4.1 & 1.0 & 44.6 & 0.8 & 14.6 & & & & 39.9 & 10.9 & 30.2 & 2.084 & \begin{tabular}{|l|l|l|}
0.123 \\
\end{tabular} & 0.121 & 0.004 & 4.0 & 34. \\
\hline & & Maximum & 26.2 & 25 & 25.7 & 26.6 & & 32.6 & \begin{tabular}{l|l|}
42.0 \\
\end{tabular} & 4.1 & 28.8 & 4.2 & & 46.2 & 80 & 14.6 & & & & 40.5 & 113 & 30.7 & 2.113 & 0.124 & 0.122 & 0.004 & 4.1 & \\
\hline & & Median & 25.9 & 25 . & 25.5 & 26.5 & 25 & 32.2 & $\begin{array}{l}41.3 \\
\end{array}$ & 3.8 & \begin{tabular}{l|l|}
28.4 \\
\end{tabular} & 4.1 & & 44.5 & 0.8 & 14.6 & & & & $\overline{39.9}$ & 10.9 & 30.3 & 2.087 & & .122 & 04 & 4.0 & \\
\hline & & Minimum & 25.3 & 24. & 25.0 & 26. & $2 !$ & 31.8 & 40.6 & 3.7 & 27.7 & 3.9 & & 43.4 & 0.8 & 14.6 & & & & 39.2 & 10.6 & 29.7 & 2.049 & 0.122 & 0.119 & 0.004 & 4.0 & 3.4 \\
\hline & & $2 \times$ Std Dev & 0.502 & 0.09 & 0.437 & 0.316 & 0.318 & 0.444 & 0.711 & 0.167 & 0.582 & 0.163 & 0.111 & 1.235 & 0.008 & 0.000 & & & & 0.711 & 0.303 & 0.510 & 0.035 & 0.001 & 0.002 & 0.000 & 0.039 & 0.033 \\
\hline & Number & ff Points Used* & & & & & & & & & & & & & & & & & & & 20 & 20 & 20 & 20 & 20 & 20 & 20 & 20 \\
\hline 324 & & * Backpulse po & in box & re not in & linded & & & & & & & & & & & & & & & & & & & & & & & \\
\hline
\end{tabular}


WSRC-TR-2005-00105, REVISION 0

SRNL-RPP-2005-00012, REVISION 0

CAMPAIGN IV NEW GKN BASELINE \#1 WITH DIF WATER

RPP-WTP Pilot-scale X-flow Filtration, Pre-Campaign IV Tests

[23-24 August 2004: New GKN Filter]

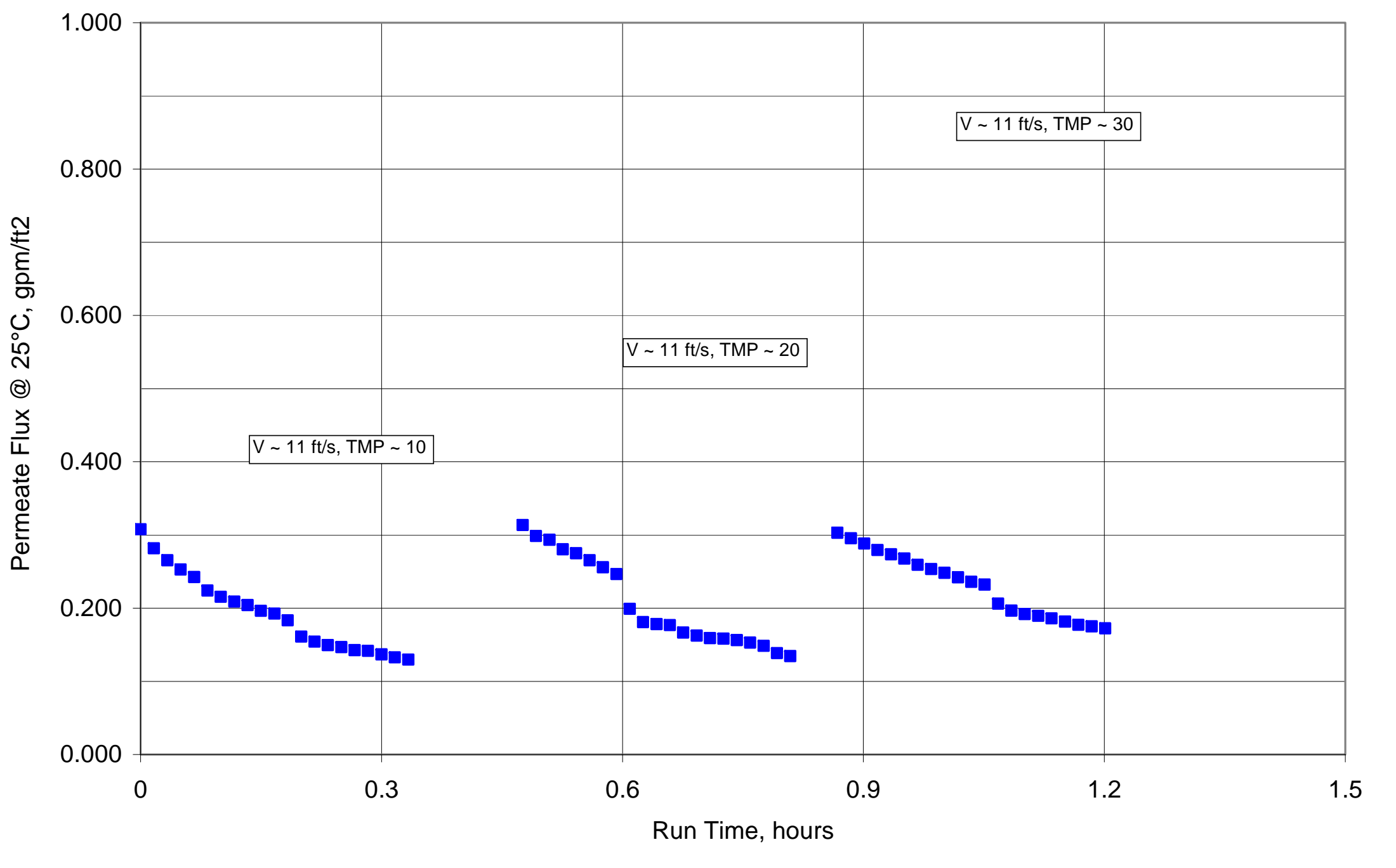


WSRC-TR-2005-00105, REVISION 0

SRNL-RPP-2005-00012, REVISION 0

CAMPAIGN IV NEW GKN BASELINE \#1 WITH DIF WATER

\begin{tabular}{|c|c|c|c|c|c|c|c|c|c|c|c|c|c|c|c|c|c|c|c|c|c|c|c|c|c|c|c|c|}
\hline & $\mathrm{A}$ & $B$ & $\frac{\mathrm{D}}{<<<<<}$ & $\begin{array}{l}\frac{T}{T} E \\
\ll<\text { Temn }\end{array}$ & I $F$ & $G$ & $\frac{H}{3}$ & 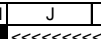 & $\frac{K}{\ll \ll \ll<\varepsilon}$ & $\frac{\mathrm{L}}{\mathrm{L}}$ & $\mathrm{M}$ & $N$ & $\frac{1}{110}$ & 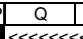 & $\begin{array}{ll}R \\
R<\text { Flow }\end{array}$ & $\frac{\mathrm{s}}{\mathrm{s}}$ & & $\mathrm{V}$ & $1 \mathrm{w}$ & $\mathrm{x}$ & $\frac{Y}{1 c u l a t}$ & $\frac{z}{2}$ & AA & $A B$ & 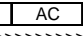 & $\frac{A D}{2}$ & $\mathrm{AE}$ & $\mathrm{AF}$ \\
\hline & DATE & TIME & Filtrate & $\begin{array}{l}\ll<\text { lemp } \\
\text { Cleaning }\end{array}$ & $\begin{array}{l}\text { elature Me } \\
\text { S Slurry }\end{array}$ & $\frac{\text { Neasureme }}{\text { Hi Amb. }}$ & 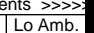 & BotTMP & Filter & $\begin{array}{l}\text { Pressure M } \\
\text { Filter dP }\end{array}$ & $\begin{array}{l}\text { Measuremer } \\
\text { TopTMP }\end{array}$ & 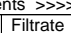 & Pulsepot & & $\begin{array}{l}\text { K< Hown } \\
\text { Filtrate }\end{array}$ & $\begin{array}{l}\text { Measureme } \\
\text { | Hi Filtate }\end{array}$ & 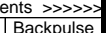 & & & & & & & & & & & \\
\hline & & & & $\operatorname{deg} C$ & \begin{tabular}{|l|}
$\operatorname{deg} C$ \\
\end{tabular} & & $\operatorname{deg} C$ & psid & psig & psid & psid & psig & psig & gpm & $\mathrm{gpm}$ & $\mathrm{gpm}$ & $\mathrm{gpm}$ & & & & & & & & & & & \\
\hline 4 & & & T2 & T3 & $\mathrm{T} 1$ & T4 & T5 & $\mathrm{dP2}$ & P1 & $\mathrm{dP1}$ & dP3 & P2 & P3 & Q1 & $\mathrm{Q} 2$ & Q3 & $\mathrm{Qbp}$ & & & & & & & & & & & \\
\hline & 012 & & & & & & & & & & & & & & & & & & & & & & & & & & & \\
\hline & $8 / 23 / 2004$ & 8:35:38 AM & 22.554 & 22.604 & 22.002 & 22.782 & 22.204 & -0.019 & 0.095 & -0.012 & 0.003 & -0.124 & 0.13 & -0.075 & 0.003 & $\begin{array}{ll}14.643 \\
\end{array}$ & 0.004 & & & Filter S & Surface & 6.985 & 5 5T2 & & & & & \\
\hline & $8 / 24 / 2004$ & 8:10:33 AM & & 22.93 & 23.679 & & 22.735 & & & -0.014 & -0.001 & -0.124 & 0.134 & 23.176 & 1.205 & $\begin{array}{ll}14.643 \\
\end{array}$ & 0.003 & & & Conver & ersion & 851 & & & & Ibarg & & \\
\hline & $8 / 26 / 2004$ & 9:14:11 AM & 25.819 & 23.352 & 24.932 & 25.316 & 23.242 & $\begin{array}{ll}-0.008 \\
-0.017\end{array}$ & 0.126 & -0.008 & 0.011 & $\begin{array}{l}-0.106 \\
\end{array}$ & 0.091 & 48.335 & 0.043 & $\begin{array}{l}14.643 \\
4.13\end{array}$ & 0.003 & & & & & & & & & & & \\
\hline$\frac{10}{11}$ & $8 / 27 / 2004 \mid$ & $\begin{array}{l}\text { 7:53:29 AM } \\
7: 28.55 \\
\text { AM }\end{array}$ & $\frac{22.649}{22511}$ & $\begin{array}{r}22.89 \\
22.848 \\
\end{array}$ & $\frac{22.298}{22329}$ & $\begin{array}{l}22.872 \\
22.69 \\
\end{array}$ & $\frac{22.871}{22.914}$ & $\begin{array}{l}-0.017 \\
-0.017 \\
-12\end{array}$ & $\begin{array}{r}0.14 \\
0.138 \\
\end{array}$ & $\begin{array}{l}-0.008 \\
-0.009 \\
\end{array}$ & 0.005 & $\begin{array}{l}-0.161 \\
-0.147\end{array}$ & 0.111 & \begin{tabular}{|l|}
-0.084 \\
-0.08
\end{tabular} & 0.003 & $\begin{array}{r}14.643 \\
14643 \\
\end{array}$ & 0.005 & & & Note: C & & ressur & & approxi & nately 1.4 & .4 psig & & \\
\hline & & & & & & & & & & & & & & & & & & & & Pressu & ure Plis & prrec & cted for 3 & & & vater tubin & & \\
\hline & & & & & & & & & & & & & & & & & & & & VVVVV & & & & $<$ Filtra & rate Flux $=$ & $\frac{3}{2}<\mathrm{PER}$ & |IABILI & $\gg$ \\
\hline & & & & & & & & & & & & & & & & & & & & & & & & & at $25 \mathrm{C}$ & & & \\
\hline$\frac{15}{16}$ & & & & & & & & & & & & & & & & & & Time & $\begin{array}{l}\text { Time } \\
\text { Hour }\end{array}$ & $\begin{array}{l}\text { Press. } \\
\text { psia }\end{array}$ & $\begin{array}{l}\text { Vel. } \\
\text { tit/s }\end{array}$ & MPP & $\frac{\text { TMP }}{\text { bar }}$ & $\frac{\mathrm{gpm}}{1 \mathrm{tat}}$ & $\mathrm{gpm}$ & $\mathrm{gpm}$ & $g \mathrm{gm}$ & neter \\
\hline & $\mathrm{A}$ & $\mathrm{B}$ & $\mathrm{D}$ & $E$ & $F$ & $G$ & $\mathrm{H}$ & $\mathrm{J}$ & $\mathrm{K}$ & $\mathrm{L}$ & $M$ & $\mathrm{~N}$ & 0 & $\mathrm{Q}$ & $\mathrm{R}$ & $\mathrm{s}$ & T & $\mathrm{v}$ & $w$ & $\mathrm{x}$ & $\mathrm{Y}$ & $z$ & $\mathrm{AA}$ & $A B$ & $\mathrm{AC}$ & $A D$ & $\mathrm{AE}$ & $\mathrm{AF}$ \\
\hline & $\begin{array}{l}\text { Monday, } 8 / 23 \\
\text { Baselelin XX }\end{array}$ & 304 & & & & & & & & & & & & & & & & & & & & & & & & & & \\
\hline$\frac{19}{19}$ & \begin{tabular}{|l|l|}
$8 / 23 / 2004$ \\
\end{tabular} & $8: 59: 50$ AM & & & & & & & & 3.951 & & -1.085 & & 44.545 & 0.003 & 2.1 & 0.004 & & & 13.8 & 10.6 & 8.9 & & 0.301 & 0.308 & & & 29.44 \\
\hline & 8/23/2004 & 9:00:50 AM & $\begin{array}{l}24.32 \\
\end{array}$ & 22.686 & 24.157 & & 22.616 & 11.129 & 14.78 & & 7.68 & -1.209 & 0.608 & 44.564 & & 1.921 & 0.003 & & $\begin{array}{l}500.0166 \\
0\end{array}$ & 13.7 & \begin{tabular}{l|l}
7 & 10.6 \\
\end{tabular} & & $\begin{array}{ll}4 & 0.648 \\
\end{array}$ & & 0.282 & 0.030 & 29.948 & \\
\hline & $8 / 23 / 2004$ & 9:01:50 AM & 24.317 & 22.698 & \begin{tabular}{|l|}
24.144 \\
\end{tabular} & 23.454 & 22.614 & 11.757 & 14.784 & 3.794 & 8.21 & -1.212 & 0.602 & $\begin{array}{l}44.583 \\
\end{array}$ & 0.003 & 1.81 & 0.003 & 2.00 & $\begin{array}{ll}0 & 0.0333 \\
\end{array}$ & 13.7 & \begin{tabular}{l|l}
7 & 10.6 \\
\end{tabular} & \begin{tabular}{l|l}
6 & 10.0 \\
\end{tabular} & $\begin{array}{ll}0 & 0.688 \\
\end{array}$ & $\begin{array}{l}8.259 \\
\end{array}$ & 0.265 & 0.027 & 26.591 & 22.6 \\
\hline & $8 / 23 / 2004$ & 9:02:50 AM & 24.294 & 22.7 & \begin{tabular}{|l|}
24.141 \\
\end{tabular} & 23.461 & 22.606 & 12.288 & 15.028 & & & -1.212 & & \begin{tabular}{|l|l|}
43.657 \\
\end{tabular} & & 1.722 & & 3.00 & \begin{tabular}{l|l}
0 & 0.0 \\
0
\end{tabular} & 13.9 & \begin{tabular}{l|l|}
9 & 10.4 \\
\end{tabular} & \begin{tabular}{l|l|}
4 & 10.3 \\
\end{tabular} & 30.711 & $\begin{array}{ll}1 & 0.247 \\
\end{array}$ & 0.253 & 0.024 & 24.493 & \\
\hline 年 & $8 / 23 / 2004$ & 9:03:50 AM & $\begin{array}{ll}24.282 \\
2.305\end{array}$ & $\begin{array}{l}22.717 \\
22.731\end{array}$ & 24.174 & $\begin{array}{l}23.559 \\
23.572\end{array}$ & $\begin{array}{l}22.734 \\
22.817 \\
\end{array}$ & $\frac{12.508}{12609}$ & 15.057 & & & $\begin{array}{l}-1.209 \\
.1200\end{array}$ & 0.599 & \begin{tabular}{|l|l|}
43.857 \\
3.039
\end{tabular} & & 1.655 & 0.003 & 4.00 & $\begin{array}{l}500.0666 \\
0.0022\end{array}$ & 13.9 & \begin{tabular}{l|l}
9 & 10.4 \\
7
\end{tabular} & 10.7 & & 0.237 & 0.243 & 0.023 & 22.676 & 19.30 \\
\hline & 8/23/2004 & $\begin{array}{l}9: 04: 50 \mathrm{AM} \\
0.50: 50 \mathrm{AM}\end{array}$ & 24.305 & $\begin{array}{l}22.731 \\
22.738\end{array}$ & $24.257 \mid$ & $\begin{array}{l}23.572 \\
23530\end{array}$ & 22.817 & \begin{tabular}{rl|l}
12.698 \\
1275
\end{tabular} & $\frac{14.858}{1472}$ & & & \begin{tabular}{|l|}
-1.209 \\
1209
\end{tabular} & 0.594 & \begin{tabular}{|l|}
45.488 \\
45.43 \\
\end{tabular} & & $\begin{array}{c}1.531 \\
1.477\end{array}$ & & 5.00 & 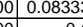 & 13.7 & $\begin{array}{l}7 \\
6\end{array}$ & 10.8 & & & 0.224 & & 20.637 & 17.56 \\
\hline 6 & $\begin{array}{l}8 / 2 / 23 / 2004 \\
8 / 2004\end{array}$ & $\begin{array}{l}\text { 9:05:50 AM } \\
\text { 9:06:50 AM }\end{array}$ & 24.357 & $\frac{22.138}{22.745}$ & 24.3255 & & $\frac{22.109}{22.596}$ & & & $\frac{4.152}{4.2}$ & $\begin{array}{r}8.67 \\
8.844 \\
\end{array}$ & & $\begin{array}{l}0.594 \\
0.591\end{array}$ & $\begin{array}{l}454.434 \\
44.712\end{array}$ & & & 0.004 & & & & & & & $\begin{array}{ll}8 & 0.211 \\
1 & 0.205\end{array}$ & & & $\frac{20.123}{19.181}$ & $\frac{17.13}{16.32}$ \\
\hline 27 & $8 / 23 / 2004$ & 9:07:50 AM & 24.442 & 22.753 & \begin{tabular}{|l|}
24.434 \\
\end{tabular} & 23.634 & 22.493 & 12.883 & $\frac{14.052}{14.504}$ & 4.152 & & -1.206 & 0.5 & 44.652 & 0.003 & $\begin{array}{l}.404 \\
1.403\end{array}$ & 0.8 & 8.6 & $\mid 0.1333$ & $\frac{1.4}{134}$ & $\frac{10}{10}$ & 109 & 0.749 & & 0.204 & & 18789 & 16.3 \\
\hline 28 & $8 / 23 / 2004$ & 9:08:50 AM & 24.497 & 22.763 & 24.5 & 23.615 & 22.418 & 13.232 & 14.738 & 4.235 & 9.031 & -1.209 & 0.58 & 44.896 & 0.003 & 1.351 & 0.003 & 9.00 & 0.1 & 13.6 & 10.7 & $\frac{11.1}{11.1}$ & & 0.193 & 0.196 & 0.018 & $\frac{0.622}{7.622}$ & \\
\hline & $8 / 23 / 2004$ & & 24.547 & 22.763 & 24.54 & 23.64 & & & & & & & & & & & & 10.00 & & 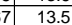 & & & & & & & & \\
\hline 30 & $8 / 23 / 2004$ & 9:10:50 & 24.572 & & 24.545 & $\begin{array}{l}23.629 \\
\end{array}$ & 22.347 & 13.041 & 14.361 & 4.2 & 8.782 & -1.22 & & 45.254 & & 1.264 & & 11.00 & \begin{tabular}{|l|l|l|l|l}
033 \\
\end{tabular} & 13.2 & & & & & & & 799 & \\
\hline & $8 / 23 / 2004$ & 9:11:50 AM & 24.627 & 22.788 & 24.625 & 23.614 & 22.377 & 12.701 & 13.53 & & 8.553 & -1.206 & & 44.122 & & 1.112 & & 12. & 0.8 & 12.4 & & & & & & & 5.140 & \\
\hline & $8 / 23 / 2004$ & 9:12:50 AM & 24.656 & 22.782 & 24.639 & \begin{tabular}{|l|l|l|}
23.623 \\
\end{tabular} & 22.291 & 12.522 & 13.262 & & 8.171 & -1.209 & 0.57 & 44.816 & 0.00 & 1.065 & & 13.00 & 0.2166 & 12.1 & 10.7 & 103 & & 0.152 & 0.154 & & 14.887 & $\frac{12.6}{12.6}$ \\
\hline & $8 / 23 / 2004$ & 9:13:50 AM & 24.659 & 22.77 & 24.617 & 23.641 & 22.294 & 12.356 & 13.101 & & 7.8 & -1.21 & & (44.846 & & & & 14.00 & \begin{tabular}{l|l}
0 & 0.23 \\
\end{tabular} & 12.0 & 10.7 & 0.1 & & $\begin{array}{l}7 \\
7.148\end{array}$ & & & .770 & \\
\hline 34 & 8/23/2004 & 9:14:5 & & 22.782 & 24.664] & 23.653 & 22.3 & 12.603 & 13.209 & & & -1.209 & & & & & & 15.0 & 0.2 & 12.1 & 10.7 & & & & & & .732 & \\
\hline 35 & 8/23/2004 & 9:15:5 & 24.723 & 22.779 & 24.696 & 23.67 & 22.348 & 12.369 & 12.987 & 4. & 8.128 & -1.209 & & 45.282 & 0.003 & 0.988 & & 16.00 & 0.2666 & 11.9 & 10.8 & & & & & & & \\
\hline 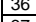 & 3/2004 & & 24.746 & & 24.689 & 23.698 & & 12.862 & 13.4 & & & -1.2 & & & & & & 17.6. & & $1<.0$ & & & & & & & & \\
\hline & 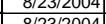 & & $\frac{24.188}{2.785}$ & $\frac{22.189}{20801}$ & 24.691 & 23.12 & & 12.4399 & 12.995 & & 8.4 & -1.206 & & 44. & & & & 18.6 & 0. & 11.9 & & & & & & & & \\
\hline & 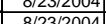 & 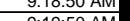 & $2 \frac{2.105}{2.787}$ & 22.001 & 20.0809 & & & & 13.110 & & 8.01 & -1.1212 & & & & & & 19.0 & 0.316 & 1.1 .1 & r & & & & & & & \\
\hline & $\frac{102512304}{8123204}$ & & 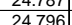 & $\frac{26.105}{22796}$ & $\frac{24.150}{24698}$ & 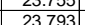 & & 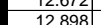 & $\frac{1.14}{13232}$ & & & $\frac{-1.21}{-121}$ & & $\frac{44514}{4474}$ & & & & & & $\frac{1.0 .6}{122}$ & & & & & & & & \\
\hline$\frac{\overline{4}^{41}}{4}$ & $8 / 2312004$ & 9.21 .5 & 24.814 & 22805 & 24,712 & 23.862 & & 12821 & $\frac{13.227}{13.227}$ & & $\frac{3.4}{8.1}$ & -1.2 & & 45092 & & & & & 036 & 121 & & & & & & & & \\
\hline 42 & $8 / 23 / 2004$ & & 24.828 & 22.809 & 24.721 & 23.901 & & $\frac{21.854}{12.85}$ & 13.128 & 3.98 & 8.936 & $\frac{-1.209}{-1.209}$ & & 45.275 & 0.003 & & & $\frac{23 .}{23 .}$ & 0.3833 & 120 & 10.8 & & & & & & & \\
\hline 45 & $8 / 23 / 2004$ & 9:23:50 AM & 24.833 & & 24.75 & 23.936 & 22.613 & 12.701 & 12.991 & 4.3 & 8.302 & $\frac{-1.209}{-1.209}$ & & $45.32 \mathrm{~s}$ & 0.005 & 0.861 & & & 0.4 & 11.9 & & & & 0.123 & & & & \\
\hline & & & & & & & & & & & & & & & & & & & & & & & & & & & & \\
\hline & & Averages & 24.6 & 22.8 & 24.5 & 23.6 & 22.5 & 12.5 & 14.0 & 4.1 & 8.4 & -1.2 & & 44.8 & 0.0 & 1.3 & 0.0 & & & 12.8 & \begin{tabular}{l|l|l|}
8 & 10.7 \\
\end{tabular} & $\begin{array}{ll}7 & 10.5 \\
\end{array}$ & 0.7 & 0.2 & 0.2 & 0.0 & 17.6 & \\
\hline 47 & & $\begin{array}{l}\text { IMaximum } \\
\text { Median }\end{array}$ & 24.8 & $\frac{22.8}{228}$ & 24.8 & 23.9 & & & & $\frac{4.5}{4.2}$ & 9.1 & & & & 0.0 & & & & & 13.9 & 107 & & & & & & & \\
\hline 48 & & Minimu & 24.3 & 22.7 & 24.1 & 23.4 & & & 13.0 & 4.2. & $\begin{array}{r}-8.4 \\
7.1 \\
\end{array}$ & -1.2 & & & 0.0 & $\begin{array}{l}1.1 \\
0.9\end{array}$ & t & & & 11.9 & \begin{tabular}{|l|l|}
9 & 10.4 \\
9
\end{tabular} & \begin{tabular}{lr|}
4 & 8.9 \\
4 & 8.9
\end{tabular} & 0.6 & 0.1. & 0.1 & 0.0 & 11.6 & 9.9 \\
\hline 49 & & & 0.399 & 0.080 & 0.429 & 0.278 & 0.294 & 1.156 & 1.618 & 0.405 & 0.930 & 0.049 & 0.042 & $\begin{array}{l}0.886 \\
\end{array}$ & 0.000 & 0.722 & 0.001 & & & 1.617 & \begin{tabular}{l|l|}
7 & 0.211 \\
\end{tabular} & 10.990 & $\begin{array}{ll}0 & 0.068 \\
\end{array}$ & \begin{tabular}{l|l}
8 & 0.103 \\
\end{tabular} & 0.107 & 0.012 & 11.899 & 10.12 \\
\hline & Number $\mathrm{C}$ & of Points Used ${ }^{*}$ & 25 & & & & & 25 & 25 & 25 & & 25 & & 25 & & & & & & 25 & & $\begin{array}{ll}5 & 25 \\
\end{array}$ & & $\begin{array}{l}5 \\
5\end{array}$ & 25 & 25 & & 25 \\
\hline 11 & & $\star$ * Backpulse p & ts in box & are noti & Eluded & & & & & & & & & & & & & & & & & & & & & & & \\
\hline
\end{tabular}


WSRC-TR-2005-00105, REVISION 0

SRNL-RPP-2005-00012, REVISION 0

CAMPAIGN IV NEW GKN BASELINE \#1 WITH DIF WATER - CONT.

\begin{tabular}{|c|c|c|c|c|c|c|c|c|c|c|c|c|c|c|c|c|c|c|c|c|c|c|c|c|c|c|c|c|}
\hline & & & & & & & & & & & & & & & & & & & & & & & & & & & & \\
\hline & $\mathrm{A}$ & $\mathrm{B}$ & $\mathrm{D}$ & $E$ & $\mathrm{~F}$ & $\mathrm{G}$ & $\mathrm{H}$ & $\mathrm{J}$ & $\mathrm{K}$ & $\mathrm{L}$ & M & $\mathrm{N}$ & \begin{tabular}{l|l}
0 \\
\end{tabular} & \begin{tabular}{l|l|}
$P$ & $Q$ \\
\end{tabular} & $\mathrm{R}$ & $\mathrm{s}$ & \begin{tabular}{l|l}
$T$ & $\mathrm{~L}$ \\
\end{tabular} & u $\quad v$ & w & $\mathrm{x}$ & \begin{tabular}{l|l} 
\\
\end{tabular} & $\mathrm{z}$ & AA & $\begin{array}{ll}A B \\
\end{array}$ & $\mathrm{AC}$ & $A D$ & $\mathrm{AE}$ & AF $A$ \\
\hline 5 & Monday, 8/2 & $32 / 04$ & & & & & & & & & & & & & & & & & & & & & & & & & & \\
\hline$\frac{75}{55}$ & & KB, water & 25003 & & & & & & & & & & & 45052 & & & & & & & & & & & & & & \\
\hline$\frac{55}{56}$ & $\frac{8 / 23 / 2004}{8 / 23 / 2004}$ & $\begin{array}{l}9.28: 23 \mathrm{AM} \\
9: 29: 23 \mathrm{AM}\end{array}$ & & $\begin{array}{r}22.848 \\
22.859 \\
\end{array}$ & $\begin{array}{r}24.81 \\
24.785 \\
\end{array}$ & $\frac{24.011}{24.081}$ & $\begin{array}{r}22.96 \\
23.005 \\
\end{array}$ & $\begin{array}{l}22.226 \\
22.423 \\
\end{array}$ & $\frac{26.805}{26.534}$ & $\begin{array}{l}3.938 \\
4.059 \\
\end{array}$ & $\begin{array}{l}18.6 .613 \\
18.613\end{array}$ & $\begin{array}{l}-0.807 \\
-1.209\end{array}$ & $\begin{array}{l}0.599 \\
0.594\end{array}$ & \begin{tabular}{|l|}
45.052 \\
44.841 \\
\end{tabular} & & $\frac{2.177}{2.073}$ & & & & $\frac{25.7}{25.4}$ & & & $\frac{1.41}{1.415}$ & $\begin{array}{l}0.312 \\
0.297 \\
\end{array}$ & 0.0 .299 & 0.015 & $\frac{5.321}{4.552}$ & $\frac{13.04}{12.38}$ \\
\hline$\frac{30}{57}+2$ & $8 / 23 / 2004$ & 9:30:23 AM & 25.013 & 22.874 & 24.756 & \begin{tabular}{|l|}
24.127 \\
\end{tabular} & $\frac{23.005}{23.02}$ & 22.904 & \begin{tabular}{|l|} 
\\
\end{tabular} & $\begin{array}{l}3.0593 \\
3.773 \\
\end{array}$ & $\begin{array}{l}19.2610 \\
19.261\end{array}$ & $\begin{array}{l}-1.209 \\
-1.212\end{array}$ & & \begin{tabular}{|l|l|}
44.084 \\
\end{tabular} & 0.003 & 2.035 & 0.005 & 30.55 & 50.50917 & $\begin{array}{l}25.4 \\
25.8\end{array}$ & 10.5 & 21.1 & 1.454 & \begin{tabular}{|l|l|}
0.291 \\
\end{tabular} & $\begin{array}{l}.299 \\
0.293 \\
\end{array}$ & $\begin{array}{l}0.014 \\
0.014\end{array}$ & $\begin{array}{ll}4 & 13.952 \\
4 & 13.914\end{array}$ & $\begin{array}{l}12.50 \\
11.84\end{array}$ \\
\hline & $8 / 23 / 2004$ & 9:31:23 AM & & 22.875 & & & 23.046 & & & 3.966 & & -1.209 & & \begin{tabular}{|l|}
43.0446 \\
4.76 \\
\end{tabular} & & & $\begin{array}{l}0.000 \\
0.004\end{array}$ & & & & & & & & & & & $\begin{array}{l}11.04 \\
11.06\end{array}$ \\
\hline 59 & $8 / 23 / 2004$ & 9:32:23 AM & 24.981 & 22.907 & 24.703 & \begin{tabular}{|l|l|}
24.155 \\
\end{tabular} & 23.073 & 23.711 & 27.22 & 3.815 & 19.959 & -1.212 & 0.582 & \begin{tabular}{|l|}
43.140 \\
4.555 \\
\end{tabular} & 0.003 & 1.942 & $\begin{array}{l}0.004 \\
0.004\end{array}$ & $\frac{1.50}{32.55}$ & 0.5425 & 26.1 & $\begin{array}{l}10.4 \\
10.4\end{array}$ & $\begin{array}{l}21.0 \\
21.8 \\
\end{array}$ & \begin{tabular}{|l|l|}
1.401 \\
1.505 \\
\end{tabular} & \begin{tabular}{|l|l|}
0.271 \\
\end{tabular} & 0.275 & 0.013 & $\frac{12.990}{12.582}$ & $\begin{array}{ll}\frac{11.00}{10.71} \\
\end{array}$ \\
\hline 60 & $8 / 23 / 2004$ & 9:33:23 AM & 24.967 & 22.918 & 24.69 & 24.161 & 23.099 & 23.939 & 26.809 & 3.748 & 20.552 & -1.212 & 0.582 & \begin{tabular}{|l|}
42.987 \\
\end{tabular} & 0.003 & 1.836 & 0.004 & & 50.55917 & 25.7 & & & 1.534 & \begin{tabular}{|l|l|}
4 & 0.263 \\
\end{tabular} & 0.265 & & 11.920 & \\
\hline$\frac{61}{61}$ & $8 / 23 / 2004$ & 9:34:23 AM & & & 24.68 & \begin{tabular}{|l|}
24.171 \\
\end{tabular} & 23.12 & 24.18 & \begin{tabular}{|l|l|}
27.1911 \\
\end{tabular} & 3.814 & 20.443 & -1.209 & 0.579 & 43.5 & 0.003 & 1.771 & 0.004 & 34.55 & 0.57583 & 26.1 & 10.4 & & 1.538 & $\begin{array}{|ll|}8 & 0.254 \\
\end{array}$ & 0.256 & 0.011 & 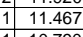 & 9.76 \\
\hline$\frac{62}{62}$ & $8 / 23 / 2004$ & $\begin{array}{l}9: 35: 23 \mathrm{AM} \\
90.323 \mathrm{AM}\end{array}$ & $\begin{array}{l}24.929 \\
24894\end{array}$ & $\begin{array}{l}22.956 \\
22.966 \\
\end{array}$ & $\begin{array}{l}24.652 \\
22.652 \\
\end{array}$ & $\begin{array}{ll}24.198 \\
24208 \\
\end{array}$ & $\begin{array}{l}23.127 \\
23217\end{array}$ & $\begin{array}{l}24.665 \\
2.231\end{array}$ & \begin{tabular}{|l|}
27.307 \\
23001
\end{tabular} & 3.855 & 21.028 & -1.212 & 0.579 & 43.185 & 0.003 & 1.705 & 0.005 & 35.55 & 0.5925 & 26.2 & 10.3 & 22.8 & 1.575 & 0.244 & 0.247 & 0.011 & 10.790 & 9.18 \\
\hline$\frac{63}{64}$ & $8 / 23 / 2004$ & 9:36:23 AM & 24.894 & 22.966 & 24.652 & \begin{tabular}{|l|}
24.208 \\
2.255
\end{tabular} & 23.147 & \begin{tabular}{l|l|}
22.317 \\
2207
\end{tabular} & 23.891 & $\begin{array}{ll}4.193 \\
\end{array}$ & 18.128 & -1.209 & 0.568 & 44.985 & 0.003 & 1.376 & 0.006 & 36.55 & 50.60917 & 22.8 & 10.7 & 20.2 & 1.394 & 0.197 & $\begin{array}{l}0.199 \\
\end{array}$ & 0.010 & 9.837 & 8.37 \\
\hline$\frac{64}{65}$ & $\begin{array}{l}8 / 23 / 2004 \\
88 / 23 / 2004\end{array}$ & & $\frac{24.861}{24827}$ & $\begin{array}{r}22.983 \\
22.999 \\
\end{array}$ & $\begin{array}{l}24.594 \\
24.555\end{array}$ & \begin{tabular}{|l|}
24.255 \\
24.267
\end{tabular} & $\frac{23.189}{23.175}$ & $\begin{array}{r}22.027 \\
22.469\end{array}$ & $\begin{array}{r}23.342 \\
23601\end{array}$ & $\begin{array}{r}4.105 \\
3.96\end{array}$ & $\frac{17.802}{18.536}$ & $\begin{array}{l}-1.212 \\
-1.209\end{array}$ & & $\begin{array}{r}45.108 \\
4.881\end{array}$ & $\begin{array}{l}0.003 \\
0.003\end{array}$ & $\begin{array}{r}1.25 \\
1.229\end{array}$ & $\begin{array}{l}0.004 \\
0.005\end{array}$ & $\begin{array}{r}37.55 \\
38.55\end{array}$ & $\begin{array}{l}5 \\
5\end{array}$ & $\frac{22.2}{22.5}$ & & \begin{tabular}{l|l}
19.9 \\
20.5
\end{tabular} & 1.373 & \begin{tabular}{l|l}
3 & 0.179 \\
& 0.176
\end{tabular} & 0.181 & $\begin{array}{l}0.009 \\
0.099\end{array}$ & 9.089 & $\begin{array}{l}7.74 \\
7739\end{array}$ \\
\hline 66 & $8 / 23 / 2004$ & $\begin{array}{l}\text { ::38:23 AM } \\
\text { 9:39:23 AM }\end{array}$ & 24.027 & $\begin{array}{l}22.999 \\
23.015\end{array}$ & $\frac{24.555}{24.521}$ & \begin{tabular}{|l|}
24.261 \\
24.278 \\
\end{tabular} & $\frac{2.115}{23.216}$ & $\frac{22.469}{22.402}$ & \begin{tabular}{|r|}
23.601 \\
23.58
\end{tabular} & $\begin{array}{r}3.96 \\
4.006\end{array}$ & $\begin{array}{l}18.53 \\
18.331 \\
\end{array}$ & $\begin{array}{l}-1.209 \\
-1.209\end{array}$ & $\begin{array}{l}0.502 \\
0.562\end{array}$ & \begin{tabular}{|l|}
44.881 \\
3.874
\end{tabular} & 0.003 & $\begin{array}{l}1.229 \\
1.217\end{array}$ & $\frac{0.005}{0.005}$ & $\begin{array}{l}3.55 \\
39.55\end{array}$ & \begin{tabular}{l|l}
5 & 0.6425 \\
5 & 0.65917
\end{tabular} & $\frac{2.5}{22.5}$ & $\begin{array}{l}1.1 . \\
10.5\end{array}$ & \begin{tabular}{|l|l|}
20.5 \\
520.4
\end{tabular} & \begin{tabular}{|l|l|} 
& 1.414 \\
& 1.404 \\
\end{tabular} & \begin{tabular}{ll|l}
4 & 0.176 \\
4 & 0.174
\end{tabular} & $\begin{array}{l}0.178 \\
0.177\end{array}$ & $\begin{array}{l}0.009 \\
0.009\end{array}$ & 8.690 & $\begin{array}{l}7.39 \\
7738\end{array}$ \\
\hline $\begin{array}{ll}67 \\
\end{array}$ & $8 / 23 / 2004$ & $9: 40: 23 \mathrm{AM}$ & 24.75 & 23.027 & 24.477 & 24.309 & 23.238 & 22.628 & \begin{tabular}{|l}
23.52 \\
\end{tabular} & 4.24 & 18.278 & -1.212 & 0.559 & \begin{tabular}{|l|}
45.599 \\
\end{tabular} & 0.003 & 1.147 & 0.005 & 40.55 & 0.67583 & 22.4 & 10.9 & 20.5 & 1.410 & $\begin{array}{lll}10 & 0.164\end{array}$ & 0.167 & 0.008 & $\frac{8.011}{8.148}$ & $\begin{array}{l}7.38 \\
6.93 \\
\end{array}$ \\
\hline 68 & $8 / 23 / 2004$ & 9:41:23 AM & & 23.043 & 24.448 & & 23.229 & 22.51 & & & & -1.212 & & & & & 0.004 & 41.55 & 0.6925 & 22.2 & 10.9 & 20.4 & & $\begin{array}{ll}7 & 0.160 \\
\end{array}$ & & & & 6.93 \\
\hline 69 & 8/23/2004 & 9:42:23 AM & 24.687 & 23.064 & 24.409 & 24.342 & 23.235 & $\begin{array}{l}22.365 \\
\end{array}$ & \begin{tabular}{|l|}
23.118 \\
\end{tabular} & 4.198 & 18.036 & -1.206 & 0.556 & \begin{tabular}{|l|l|}
45.484 \\
\end{tabular} & 0.003 & 1.091 & 0.004 & 42.55 & 50.70917 & 22.0 & $\begin{array}{l}10.8 \\
\end{array}$ & \begin{tabular}{|l|l|}
20.2 \\
\end{tabular} & 1.393 & \begin{tabular}{|l|l|}
3 & 0.156 \\
\end{tabular} & 0.159 & 0.008 & 7.862 & 6.179 \\
\hline 70 & & & & & & & & & & & & & & & & & & & & & & & & & & & & $\frac{6.69}{6.51}$ \\
\hline $11+2>$ & $8 / 23 / 2004$ & 9:44:23 AM & 24.64 & 23.102 & 24.342 & 24.364 & 23.283 & 22.796 & \begin{tabular}{|l|l|}
23.454 \\
\end{tabular} & 4.2 & 18.56 & -1.209 & & 444.937 & 0.003 & & & & & 22.3 & & $\frac{20.7}{20.7}$ & & & 0.156 & & 7.561 & $\frac{0.51}{6.43}$ \\
\hline 72 & $8 / 23 / 2004$ & 9:45:23 AM & & 23.108 & 24.298 & 24.366 & 23.289 & 22.684 & 23.34 & 4.253 & & -1.212 & & 45.058 & 0.003 & 1.047 & 0.004 & & \begin{tabular}{|l|l|l}
0.75917 \\
\end{tabular} & & & & & & & & & 6.45 \\
\hline 73 & $8 / 23 / 2004$ & 9:46:23 AM & 24.588 & 23.136 & 24.27 & 24.403 & 23.326 & 22.691 & \begin{tabular}{|l|l|}
23.141 \\
\end{tabular} & 4.11 & 18.65 & -1.212 & 0.553 & 45.476 & 0.003 & 1.015 & 0.004 & 46.55 & 0.77583 & 22.0 & 10.8 & 20.7 & 1.425 & $\begin{array}{lll}5 & 0.145 \\
\end{array}$ & 0.148 & 0.007 & 7.176 & 6.11 \\
\hline 74 & $8 / 23 / 2004$ & 9:47:23 AM & 24.554 & 23.142 & 24.236 & \begin{tabular}{|l|}
24.409 \\
\end{tabular} & 23.347 & 22.487 & \begin{tabular}{|l|l|}
23.033 \\
\end{tabular} & 4.281 & 18.132 & -1.209 & 0.553 & 45.077 & 0.003 & 0.947 & 0.005 & 47.55 & $\begin{array}{l} \\
\end{array}$ & 21.9 & 10.7 & 20.3 & 1.400 & \begin{tabular}{|l|l|}
0 & 0.136 \\
\end{tabular} & 0.139 & 0.007 & 6.821 & 5.80 \\
\hline 75 & $8 / 23 / 2004$ & 9:48:23 AM & 24.534 & 23.162 & 24.221 & 24.46 & 23.348 & 22.305 & \begin{tabular}{|l|}
22.751 \\
\end{tabular} & 4.217 & 17.962 & -1.209 & 0.55 & $\begin{array}{l}45.501 \\
\end{array}$ & 0.003 & 0.919 & 0.005 & 48.55 & $\begin{array}{ll}50.80917 \\
\end{array}$ & 21.6 & 10.8 & 20.1 & 1.388 & \begin{tabular}{|l|l|}
8 & 0.132 \\
\end{tabular} & 0.134 & 0.007 & 6.680 & 5.68 \\
\hline $\begin{array}{l}76 \\
77\end{array}$ & $8 / 23 / 2004$ & 9:49:23 AM & 24.501 & 23.169 & 24.168 & 24.446 & 23.365 & $\begin{array}{l}22.404 \\
2.02\end{array}$ & \begin{tabular}{|l|}
22.824 \\
2030
\end{tabular} & $\begin{array}{l}4.204 \\
\end{array}$ & 18.05 & -1.212 & 0.55 & 45.004 & 0.003 & 0.912 & 0.005 & 49.55 & 50.82583 & $\begin{array}{l}21.7 \\
\end{array}$ & 10.7 & \begin{tabular}{|l|l|}
20.2 \\
\end{tabular} & 1.395 & \begin{tabular}{|l|l|}
0.131 \\
\end{tabular} & $\begin{array}{l}0.134 \\
\end{array}$ & 0.007 & 6.608 & 5.62 \\
\hline & $8 / 23 / 2004$ & $\begin{array}{l}49: 50: 23 \mathrm{AM} \\
\end{array}$ & $\begin{array}{ll}24.482 \\
248\end{array}$ & 23.19 & $\begin{array}{l}24.164 \\
245\end{array}$ & $\begin{array}{l}24.468 \\
243 \\
\end{array}$ & 23.396 & \begin{tabular}{|l|l|}
22.431 \\
2.8
\end{tabular} & \begin{tabular}{|l|}
22.836 \\
246
\end{tabular} & 4.282 & $\begin{array}{l}18.005 \\
18.8\end{array}$ & -1.212 & 0.544 & \begin{tabular}{|l|l|}
44.428 \\
447
\end{tabular} & 0.003 & 0.883 & 0.005 & 50.55 & 0.8425 & 21.7 & 10.6 & 20.2 & 1.394 & $\begin{array}{l}4 \quad 0.126 \\
\end{array}$ & 0.129 & 0.006 & 6.402 & 5.45 \\
\hline$\frac{18}{79}$ & & \begin{tabular}{|l} 
Averages \\
Maximum
\end{tabular} & $\begin{array}{r}24.8 \\
25.0 \\
\end{array}$ & 23.0 & $\begin{array}{r}24.5 \\
22.8\end{array}$ & $\begin{array}{r}24.3 \\
24.5 \\
\end{array}$ & $\begin{array}{r}23.2 \\
23.4\end{array}$ & $\begin{array}{r}22.8 \\
24.7 \\
\end{array}$ & $\begin{array}{l}24.6 \\
27.3 \\
\end{array}$ & $\begin{array}{l}4.1 \\
4.3 \\
\end{array}$ & $\frac{18.8}{21 .}$ & $\begin{array}{l}-1.2 \\
-0.8\end{array}$ & $\begin{array}{l}0.6 \\
0.6\end{array}$ & $\begin{array}{l}44.7 \\
45.6\end{array}$ & $\begin{array}{l}0.0 \\
0.0\end{array}$ & $\begin{array}{l}1.4 \\
2.2 \\
\end{array}$ & 0.0 & & & $\begin{array}{ll}23.4 \\
26.2\end{array}$ & $\begin{array}{l}10.6 \\
10.9\end{array}$ & & 1.4 & 0.2 & $\frac{0.2}{0.3}$ & 0.0 & $\frac{9.6}{115.3}$ & 8.1 \\
\hline 80 & & Median & 24.8 & 23.0 & 24.5 & 24.3 & 23. & 22.5 & 23.5 & 4.1 & 18.5 & -1.2 & 0. & 45.0 & 0.0 & 1.2 & 0 & & & $\begin{array}{l}22.4 \\
22.4\end{array}$ & \begin{tabular}{|l|}
10.7 \\
\end{tabular} & \begin{tabular}{r|}
20.5 \\
20.5
\end{tabular} & 1.4 & 0.2 & & 0.0 & 87 & \\
\hline 81 & & Minimum & 24.5 & 22.8 & 24.2 & 24.0 & 23.0 & 22.0 & 22.8 & 3.7 & 17.8 & -1.2 & 0.5 & 43.0 & 0.0 & 0.9 & 0. & & & 21.6 & 10.2 & 19.9 & 1.4 & 0.1 & $\begin{array}{l}0.2 \\
0.1 \\
\end{array}$ & 0.0 & $\frac{8.1}{6.4}$ & 5.4 \\
\hline & & $2 \times \operatorname{Std}$ Dev & 0.359 & 0.212 & 0.412 & 0.249 & 0.243 & 1.348 & 3.570 & 0.352 & 1.801 & 0.165 & & 1.600 & 0.000 & 0.851 & 0.00 & & & 3.571 & 0.381 & 1.561 & $\begin{array}{ll} \\
\end{array}$ & 0.12 & .121 & .005 & 5.442 & $\frac{5.4}{631}$ \\
\hline 83 & Number & of Points Used* & 23 & 23 & 23 & 23 & 23 & 23 & 23 & 23 & 23 & 23 & 23 & 23 & 23 & 23 & 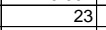 & & & 23 & 23 & 23 & 23 & $\frac{1.23}{23}$ & 23 & 23 & $\frac{.442}{23}$ & t.031 \\
\hline & & * ${ }^{\star}$ Backpulse poi & ints in box & are not inc & & & & & & & & & & & & & & & & & & & & & & & & \\
\hline & Oonday, $8 / 2$ & & & & & & & & & & & & & & & & & & & & & & & & & & & \\
\hline & Baseline $X X$ & KC, water & & & & & & & & & & & & & & & & & & & & & & & & & & \\
\hline & $8 / 23 / 2004$ & \begin{tabular}{|l|l|}
4 & $9: 51: 54 \mathrm{AM}$ \\
\end{tabular} & 24.53 & 23.213 & 24.317 & 24.505 & 23.398 & 31.312 & 35.413 & 3.92 & 27.60 & -1.212 & 0.6 & 44.881 & 0.003 & 2.076 & & 52. & & 34.3 & 10. & 29.5 & & 0.297 & 0.303 & 0.010 & 10.285 & \\
\hline & 8/23/2004 & 9:52:54 AM & 24.642 & 23.24 & 24.444 & 24.577 & 23.395 & 31.924 & 35.898 & 3.809 & 28.385 & -1.209 & 0.6 & 43.563 & 0.003 & 2.031 & 0.004 & & 0.88444 & 34.8 & 10. & & & & & & 9.795 & 8.34 \\
\hline$\frac{95}{90}$ & $8 / 23 / 2004$ & 9:53:54 AM & 24.739 & 23.252 & 24.542 & 24.584 & 23.432 & 32.131 & 35.989 & 3.8 & 28.36 & -1.212 & & 43.815 & 0.003 & $1.98 \mathrm{r}-\mathrm{r}$ & & 54 & & $34.99>>3$ & 10. & 30.2 & . & $\begin{array}{lll}5 & 0.284 \\
\end{array}$ & 0.200 & 0.010 & 0.021 & \\
\hline 90 & $8 / 23 / 2004$ & $9.54: 54 \mathrm{AM}$ & 24.82 & 23.268 & 24.613 & 24.591 & & 32.421 & 36.012 & & & & & 43.6 & & & & & & & & & & & & & & \\
\hline$\frac{91}{92}$ & $8 / 23 / 2004$ & & 24.943 & $\frac{23.291}{23202}$ & 24.136 & 24.685 & $\frac{23.466}{3250}$ & 32.538 & 35.961 & $\frac{3.15}{372}+2$ & 28.8 & -1.2122 & & 43.4. & 0.003 & 1.8 & & & 9344 & 34 & $\frac{1}{2}>0$ & 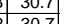 & 100 & 0.221 & & 然 & 8.906 & 50 \\
\hline 93 & $\begin{array}{l}8 / 23 / 2004 \\
8 / 23 / 2004\end{array}$ & $\begin{array}{l}\text { 9.506.54 AMM } \\
957: 54 \mathrm{AM}\end{array}$ & $\begin{array}{l}25.025 \\
25.092\end{array}$ & $\begin{array}{l}23.305 \\
23.315 \\
\end{array}$ & $\begin{aligned} 24.813 \\
24.88\end{aligned}$ & $\begin{aligned} 24.11 \\
24.712\end{aligned}$ & $\frac{23.503}{23.54}$ & $\begin{array}{l}32.5 / 9 \\
33.112\end{array}$ & \begin{tabular}{|l|}
35.886 \\
36.018 \\
\end{tabular} & 3.816 & $\frac{2.9}{29.3}$ & $\frac{-1.206}{-1.209}$ & 0. & $\begin{array}{l}43.033 \\
2.405 \\
\end{array}$ & 0.003 & $\begin{array}{l}1.86 \\
1.805 \\
\end{array}$ & 0.0 & & & $\begin{array}{l}34.8 \\
34.9 \\
\end{array}$ & 10 & 31.2 & & 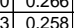 & 0.259 & $\begin{array}{l}.009 \\
0.008 \\
\end{array}$ & 8.07 & \\
\hline 9 & $8 / 23 / 2004$ & 9:58:54 AM & 25.184 & 23.342 & 24.952 & 24.749 & 23.577 & 33.336 & 36.313 & 3.84 & 29 & -1.212 & 0. & 43.517 & 0.003 & 1.767 & & & & 35.2 & 10 & 31.4 & 165 & & & 0.008 & 8.069 & \\
\hline 955 & 8/23/2004 & 9:59:54 & 25.24 & 23.349 & 24.994 & 24.776 & 23.61 & 33.328 & 36.292 & 3.74 & 29. & -1.212 & & 43.8 & 0.003 & 1.733 & & 60.4 & & 35. & 0. & 31.4 & & & & 0.008 & 7.896 & . \\
\hline & 8/23/2004 & $\begin{array}{l}4 \\
4\end{array}$ & & 23.356 & & 24.77 & & 33.583 & 36 & 3.78 & 29. & -1.22 & & 42 & & & & 01 & & > & 10 & & & & & & & \\
\hline 9 & 80.23212004 & & 25.37 & 23.388 & 25.108 & 24.84 & & 33.591 & 36 & 3.61 & & & & 42.6 & & & & & & 35.4 & & & & & & & & \\
\hline & $8 / 23 / 2004$ & & 25.416 & 23. & 25.159 & 24.8 & 23 & 33.975 & & & & -1. & & 42.11 & & & & & & 35 & & & & & & & & \\
\hline$\frac{3}{16}$ & $8 / 233 / 2004$ & & 25.474 2501 & $\frac{20.424}{2342}$ & (25.252 & $\frac{24}{240}-2>$ & & $\begin{array}{ll}32.0086 \\
32569\end{array}$ & & & & $\frac{-1.2}{-12}>0$ & & & & & & & & & & & & & & & & \\
\hline & $8 / 23 / 2004$ & \begin{tabular}{|l|l|l|}
4 & $10: 05: 54$ \\
4 & 10.04
\end{tabular} & 25.537 & 23.456 & 25295 & $\frac{25.054}{25.018}$ & 23.796 & 32.776 & 3. 35.441 & $\begin{array}{r}4.107 \\
4.37 \\
\end{array}$ & & $\frac{-1.212}{-1212}$ & & 45.5 & 0.0 & $\frac{1.5}{1.8}$ & & & & $\frac{2.0}{33 .}$ & & & & & & & & \\
\hline & $8 / 23 / 2004$ & \begin{tabular}{|l|l|}
4 & $10: 06: 5$ \\
\end{tabular} & 25.579 & 23.488 & 25.338 & 25.056 & 23.818 & 32.701 & \begin{tabular}{|l|}
34.181 \\
34.182 \\
\end{tabular} & 4.284 & 28.439 & $\frac{1.214}{-1.212}$ & & 45.14 & 0.003 & 1.336 & & $\begin{array}{ll}0.07 \\
67.07\end{array}$ & & 33.1 & 10.0 & & & & & 0.006 & 0.200 & \\
\hline & $8 / 23 / 2004$ & & 25.606 & 23.51 & 25.379 & 25.06 & & 32.589 & & 4.16 & & & & 45.3 & & & & & & & & & & & & & 6.104 & \\
\hline & & & 25.628 & & 25.367 & & & $32.3 / 8$ & 33.697 & 3. & & -1.2 & & 44.5 & & & & & & 32. & & & & & & & & \\
\hline & 8/23/2004 & $\begin{array}{l}4 \\
4\end{array}$ & 25.659 & 23.549 & 25.388 & 25.156 & 23.8 & 32.114 & 33 & 3.99 & & -1.2 & & 44. & & & & & & & & & & & & & & \\
\hline & 8/23/2004 & \begin{tabular}{l|l}
4 & $10: 10: 5$ \\
\end{tabular} & & & & 25.142 & & & & & & & & & & & & & & & & & & & & & & \\
\hline & 8/23/2004 & 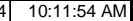 & 25.691 & 23.576 & 25.42 & 25.14 & & 32.828 & 33.79 & 4.0 & & -1.2 & & 44.5 & & & & & & & & & & & & & & \\
\hline & $8 / 23 / 2004$ & 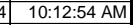 & 25.707 & 23.586 & 25.436 & 25.093 & 23.64 & 33.006 & 34 & 4.0 & & -1.21 & & 44.7 & & & & & & & & & & & & & & 4.7 \\
\hline & 8/23/2004 & $\begin{array}{l}4 \\
4\end{array}$ & 25.722 & 23.586 & 25.456 & 25.083 & 23.6 & 32.977 & 34.049 & 4.161 & 28.6 & -1.2 & & 44 & 0.0 & 1 & & & & 32 & & 30.8 & 3 & & & & 5.411 & \\
\hline & 8/23/2004 & & 25.736 & 23.596 & 25.455 & & & 32.992 & & & & -1.2 & & 45.1 & & & & & & 32.9 & & & & & & & & \\
\hline & $8 / 23 / 2004$ & & 25.751 & 23.60 & 25.48 & 25.0 & & 32.938 & 33.861 & 4.13 & & & & 45.0 & & & & & & 32. & & & & & & & & \\
\hline & 8/23/2004 & \begin{tabular}{l|l}
4 & $10: 1$ \\
\end{tabular} & 25.766 & 23.591 & 25.49 & 25.043 & $\frac{23.4}{23}$ & 32.961 & 33.95 & 4.008 & 28.839 & -1.212 & & 44.7 & .003 & 1.144 & & 77.0 & 1.28444 & 20.0 & & 30.9 & . & 0.164 & & 005 & . 228 & 4.45 \\
\hline & & & 25.4 & 23.4 & 25.1 & 24. & & 32.8 & 34. & 4.0 & 28 & -1 & & 44 & $=0$ & & & & & 33.8 & 110 & $\sqrt{3}+2$ & & & & 0.0 & & \\
\hline & & & 25.0 & 23 & 253 & 24 & & 328 & 34 & $\frac{4}{4}$ & $\frac{3}{28}$ & -1 & & $\frac{4}{4}$ & & & & & & $\frac{5.44}{332}$ & & & & & & 0.0 & $\frac{10.0}{6.6}$ & \\
\hline 116 & & & 24.5 & 23.2 & 243 & 245 & 23 & 313 & 33.1 & 36 & 27, & -12 & & 42 & 00 & 11 & & & & 320 & 10.0 & 295 & & $07-2$ & & 0 & 52 & \\
\hline & & & .138 & & 0.692 & 0.405 & & $\frac{1.132}{132}$ & $\frac{2.208}{2.208}$ & 0.376 & 1.208 & 0.004 & & 2.149 & 0.000 & 0.61 & & & & $\frac{2.208}{2.208}$ & 0.512 & $\mid \frac{1.144}{21.14}$ & & & 1093 & 003 & 081 & \\
\hline 11 & Number o & & 26 & & 26 & 26 & & 26 & 26 & 26 & 26 & 26 & & 26 & 26 & 2 & & & & 26 & 26 & 26 & & & & 26 & 26 & \\
\hline$\frac{11}{12}$ & & * Backpulse po & ints in box & are not inc & luded & & & & & & & & & & & & & & & & & & & Calc & & & & \\
\hline 12 & & & & & & & & & & & & & & & & & & & & & & & & & & & & \\
\hline \begin{tabular}{|l|l|}
122 \\
\end{tabular} & & & & & & & & & & & & & & & & & & & & & & & in & 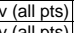 & \begin{tabular}{|l|l|}
0.121 \\
0.003
\end{tabular} & & & \\
\hline & & & & & & & & & & & & & & & & & & & & & & & in St Dv & $\mathrm{v}$ (all pts) & 0.003 & & & \\
\hline
\end{tabular}


WSRC-TR-2005-00105, REVISION 0

SRNL-RPP-2005-00012, REVISION 0

CAMPAIGN IV NEW GKN BASELINE \#2 WITH 0.1 M NAOH

RPP-WTP Pilot-scale X-flow Filtration, Pre-Campaign IV Tests

[23-24 August 2004: New GKN Filter]

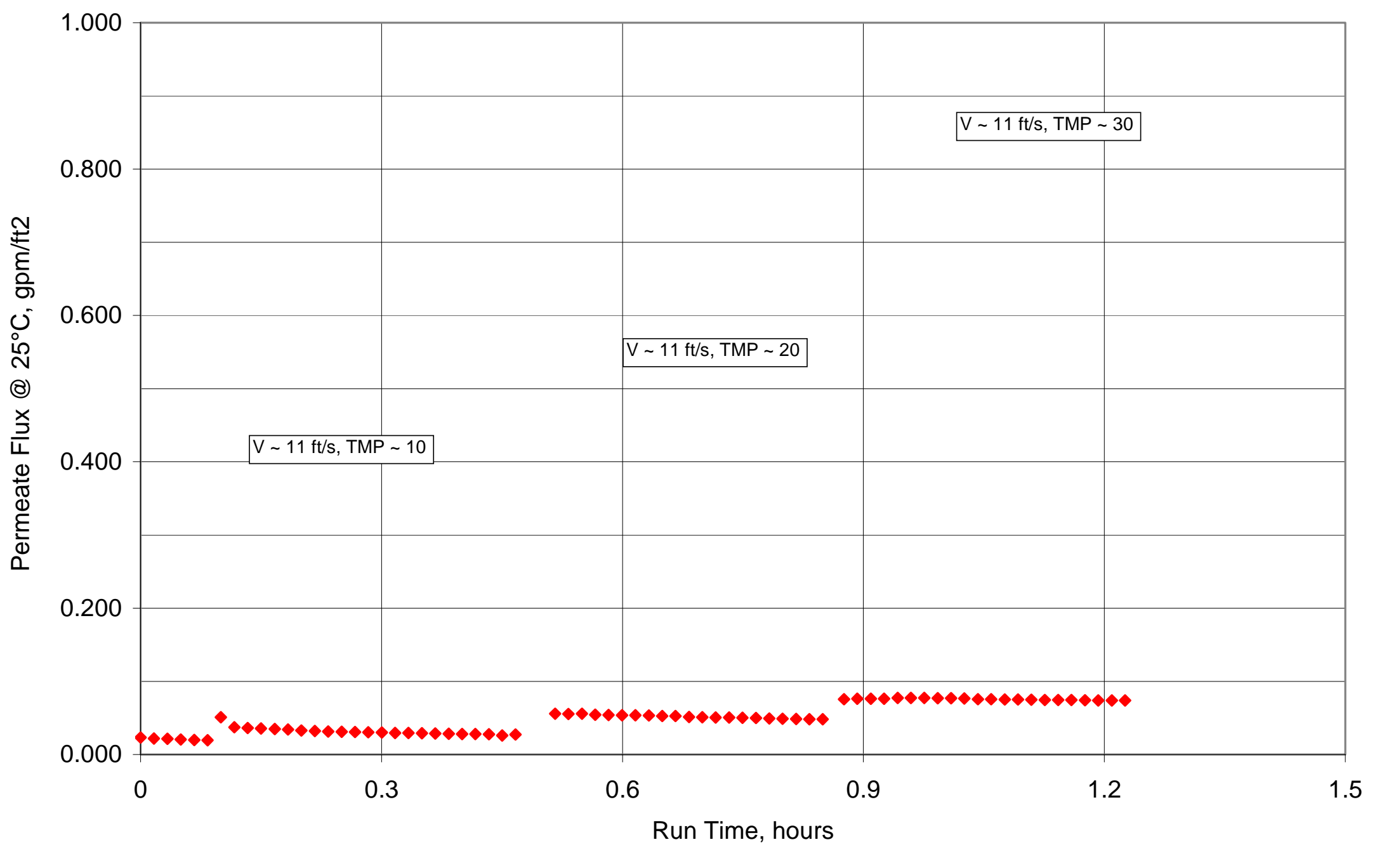


WSRC-TR-2005-00105, REVISION 0

SRNL-RPP-2005-00012, REVISION 0

CAMPAIGN IV NEW GKN BASELINE \#2 WITH 0.1 M NAOH

\begin{tabular}{|c|c|c|c|c|c|c|c|c|c|c|c|c|c|c|c|c|c|c|c|c|c|c|c|c|c|c|c|c|}
\hline & A & $B$ & D & $\mathrm{E}$ & $\mathrm{F}_{\mathrm{F}}$ & $G$ & $\mathrm{H}$ & \begin{tabular}{l|l}
$\mathrm{J}$ \\
\end{tabular} & $\mathrm{K}$ & $1 \mathrm{~L}$ & $1 \mathrm{M}$ & $\mathrm{N}$ & 10 & Q & R & $\mathrm{s}$ & $\mathrm{T}_{\mathrm{T}}$ & U] $\quad \mathrm{v}$ & $\begin{array}{l} \\
\end{array}$ & $\mathrm{x}$ & & & IAA & & $A C$ & & $\mathrm{AE}$ & $\mathrm{AF}$ \\
\hline$\frac{1}{2}$ & & & $\begin{array}{l}<<<<<< \\
\text { Filtrate }\end{array}$ & $\begin{array}{l}\mid<\ll \text { Temp } \\
\text { Cleaning }\end{array}$ & erature $M$ & $\begin{array}{l}\text { heasureme } \\
\text { HiAmb }\end{array}$ & ints >>>> & 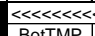 & 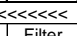 & $\begin{array}{l}\text { Pressure } \\
\text { FiterdP }\end{array}$ & Measureme & 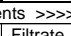 & , >>>>>>> & 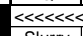 & $\ll<$ Flow & Measureme & & & & & & & rom Raw & & & & & \\
\hline & DAIE & TIME & & Cleaning & \begin{tabular}{|l|l|} 
Slurry \\
\end{tabular} & Hi Amb. & Lo Amb. & BotTMP & & Filter $d P$ & TopTMP & Filtrate & Pulsepot & & Filtrate & Hi Filtate & Backpulse & & & & & & & & & & & \\
\hline 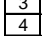 & & & $\frac{\operatorname{deg} C}{\mathrm{~T} 2}$ & $\operatorname{deg} C$ & \begin{tabular}{|l|}
$\operatorname{deg} C$ \\
1
\end{tabular} & deg C & \begin{tabular}{|l|}
$\operatorname{deg} C$ \\
5
\end{tabular} & $\begin{array}{l}\text { psid } \\
\text { dP2 }\end{array}$ & $\frac{\text { psig }}{\text { P1 }}$ & $\frac{\text { psid }}{d \text { dP1 }}$ & $\begin{array}{c}\text { psid } \\
\text { dP3 } \\
\end{array}$ & \begin{tabular}{|l|} 
Psig \\
P2
\end{tabular} & $\frac{\text { psig }}{\text { P3 }}$ & $\frac{\mathrm{gpm}}{\mathrm{O} 1}$ & $\mathrm{gpm}$ & gpm & gpm & & & & & & & & & & & \\
\hline & & & & & & & & & & & & & & & & & & & & & & & & & & & & \\
\hline & Eeros- 01/26 & & & & & & & & & & & & & & & & & & & & & & & & & & & \\
\hline & $8 / 23 / 2004$ & 8:35:38 AM & & & & & 22.204 & & & -0.012 & 0.003 & & & -0.075 & 0.00 & 14.643 & 0.004 & & & & urface $f$ & 46.98 & FT2 & & & & & \\
\hline & & 8:10:33 AM & & & 23.679 & 22.998 & 22.735 & -0.021 & 0.122 & -0.014 & -0.001 & -0.124 & 0.134 & 23.176 & 1.205 & 14.643 & & & & Convers & ersion & & $\mathrm{m} 3 / \mathrm{m} 2$ & ne/day/bar! & arg $/ \mathrm{gpm} / \mathrm{f}$ & 2/barg & & \\
\hline & $8 / 26 / 2004$ & 9:14:11 AM & & & 24.932 & 25.316 & 23.242 & -0.008 & 0.126 & -0.008 & 0.011 & -0.106 & 0.091 & 48.335 & 0.043 & 14.643 & & & & & & & & & & & & \\
\hline & $8 / 27 / 2004$ & 7:53:29 AM & 22.649 & 22.89 & 22.298 & 22.872 & 22.871 & -0.017 & & -0.008 & 0.005 & & & & 0.003 & 14.643 & 0.005 & & & Note: & & & & & & psig & & \\
\hline & 8/30/2004 & 7:28:55 AM & 22.511 & 22.848 & 22.397 & 22.669 & 22.914 & -0.017 & 0.138 & -0.009 & 0.003 & -0.147 & 0.12 & -0.082 & 0.003 & 14.643 & 0.006 & & & & & & & & & & & \\
\hline & & & & & & & & & & & & & & & & & & & & ressur & ure Plis & Eorrec & $d$ fo & $\begin{array}{l}1.25 \mathrm{inc} \\
<\text { Fitra }\end{array}$ & es of & if tub & & \\
\hline$\frac{13}{14}$ & & & & & & & & & & & & & & & & & & & & $w$ & & & & < Filtre & at $25 \mathrm{C}$ & & $\times 1000$ & \\
\hline & & & & & & & & & & & & & & & & & & Time & & Press. & $\begin{array}{l}\text { Vel. } \\
\end{array}$ & TMP & TMP & gam & $\mathrm{pm}$ & $\mathrm{gpm}$ & $\mathrm{gom}$ & \\
\hline 16 & & & & & & & & & & & & & & & & & & in. & Hour & psig & $t / s$ & si & & & & tit/psi & $2 / p s i$ & aylbar \\
\hline & $\mathrm{A}$ & B & $\mathrm{D}$ & E & $\mathrm{F}$ & $G$ & $\mathrm{H}$ & $\mathrm{J}$ & K & $\mathrm{L}$ & M & $\mathrm{N}$ & 0 & $Q$ & $R$ & $\mathrm{~s}$ & & $\mathrm{~V}$ & w & $x$ & $Y$ & Z & AA & $A B$ & $A C$ & $A D$ & $\mathrm{AE}$ & $\mathrm{AF}$ \\
\hline & $\begin{array}{l}\text { Monday, } 8 / 2 \\
\text { Baseline YY }\end{array}$ & $3 \frac{304}{A .01 M !}$ & & & & & & & & & & & & & & & & & & & & & & & & & & \\
\hline & & & 25.332 & & 25.1 & 25.208 & 23.726 & 7.97 & 13.329 & 4.216 & 3.56 & 0.259 & 0.339 & $\begin{array}{lll}44.698 \\
\end{array}$ & 0.163 & -0.013 & 0.00 & 0.6 & & 12.2 & 10.6 & 5.8 & 0.39 & & 0.023 & 0.004 & 4.036 & \\
\hline & $8 / 23 / 2004$ & $10: 39: 04 \mathrm{AM}$ & & 23.706 & 25.024 & 25.208 & 23.57 & 7.928 & 13.267 & 4.111 & 3.66 & 0.259 & 0.368 & 44.591 & 0.152 & -0.01 & 0.003 & & 0.01667 & & & & & & 0.04 & 0.004 & 3.753 & \\
\hline & $8 / 23 / 2004$ & 10:40:04 AM & 25.446 & 23.706 & 24.964 & 25.178 & 23.565 & 7.624 & 4) 13.035 & 4.17 & 3.186 & 0.262 & 0.406 & 44.654 & 0.151 & -0.0 & & 2.0 & 0.03333 & \begin{tabular}{|c|c|}
11.9 \\
\end{tabular} & 10.6 & 5.4 & & & & & 4.004 & \\
\hline & $8 / 23 / 2004$ & 10:41:04 AM & 25.511 & 23.701 & 24.914 & 25.138 & 23.509 & 7.833 & , 13.229 & 4.402 & 3.262 & 0.311 & 0.446 & 444.842 & 0.142 & - -0.013 & 0.003 & 3.00 & 0.05 & $\begin{array}{ll}12.1 \\
\end{array}$ & 10.7 & 5.5 & 0.382 & 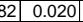 & 0.020 & 0.004 & 3.674 & \\
\hline & $8 / 23 / 2004$ & 10:42:04 AM & 25.561 & 23.706 & 24.889 & 25.103 & 23.489 & 7.928 & 13.316 & 4.28 & 3.693 & 0.331 & 0.484 & 44.662 & 0.139 & -0.0 & 0.0 & 4.0 & 0.06667 & 12.2 & 10.6 & $5.8 \mathrm{~s}->$ & 0.401 & & $0.02 C$ & & 3.436 & \\
\hline & 8/23/2004 & 10:43:04 AM & 25.516 & 23.681 & 24.814 & 25.077 & 23.504 & 7.817 & 13.204 & & 3.547 & 0.363 & (2) & 44.756 & 0.135 & & 0.6 & 5.00 & 0.08333 & 12.1 & 10.7 & 7 & 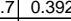 & .019 & 0.019 & 0.003 & 3.419 & \\
\hline & 80 & $10: 44: 04 \mathrm{AM}$ & 25.45 & 年3.685 & 24.173 & 25.0866 & 23.398 & 10.199? & & 4.392 & 5.755 & -1.212 & -2.595 & (44.844 & 0.354 & -0.013 & 0.003 & & 0.1 & 11.9 & 10.7 & & & 0.051 & & 0.006 & 6.395 & \\
\hline & $\frac{0.25 T 2004}{8 / 23204}$ & $\begin{array}{l}10.45 .04 \mathrm{AMM} \\
0.04 \mathrm{AM}\end{array}$ & 20.31 & 23.004 & 24.113 & 20.0000 & 23.410 & 12.0944 & 13.4081 & 4.247 & 8.3517 & -1.212 & -4.4717 & 44.9605 & 0.250 & $=0.0$ & & & 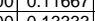 & $\frac{12.3}{1.9}$ & & & & & 然 0.031 & (2) & 告.500 & \\
\hline & $\mid$ & $\begin{array}{l}10.40 .04 \mathrm{AMM} \\
10.07 \mathrm{MA}\end{array}$ & $\frac{25.250}{2518}$ & 230.054 & 24.000 & 25.026 & 20.400 & $\frac{12.319}{1.390}$ & 13001 & 4.139 & 1.6161 & -1.142 & $-{ }^{-4.5515}$ & 44.894 & 0.25 & -0.6 & & 8.00 & 0.13333 & 11.9 & & & & 0 & & (2) & & \\
\hline 136 & $8 / 23 / 2004$ & 10:48:05 AM & 25.12 & 23.645 & 24.623 & 24.997 & 23.594 & 12.707 & 13.246 & $\begin{array}{l}4.3127 \\
4.327\end{array}$ & 8.132 & -1.209 & -4.567 & 44.687 & 0.241 & $\begin{array}{l}-0.013 \\
-0.013\end{array}$ & 0.003 & 10.0 & 0.16964 & 121 & 106 & $\frac{1.04}{10.4}-5$ & & 18 & $\frac{0.035}{0.035}$ & 0.003 & $\begin{array}{l}3.3 .347 \\
3.347\end{array}$ & $5.8-2 \times$ \\
\hline & $8 / 23 / 2004$ & $10: 49: 05 \mathrm{AM}$ & 25.071 & 23.641 & 24.584 & 25.027 & & 12.599 & 13.097 & & 8.126 & -1.212 & -4.56 & 44.756 & 0.237 & -0.013 & & & $\frac{1.18361}{0.1861}$ & & $\frac{\frac{10.7}{10.7}}{2}$ & $\frac{\frac{10.4}{10.4}}{2}$ & & $\begin{array}{l}140.034 \\
14\end{array}$ & $\frac{0.034}{0.034}$ & & & $2.8-8-8$ \\
\hline 138 & $8 / 23 / 2004$ & 10:50:05 AM & 25.032 & 23.636 & 24.549 & 25.078 & 23.69 & 12.506 & \begin{tabular}{|l|l|}
512.941 \\
\end{tabular} & & 8.001 & -1.212 & -4.6 & 44.581 & 0.227 & $-0.01:$ & & & & 11.8 & 10.6 & 10.3 & & & & & 3.210 & $=0$ \\
\hline & 8/23/2004 & 10:51:05 AM & 24.977 & 23.642 & 24.505 & 25.089 & 23.716 & 12.477 & 12.871 & 4.333 & 7.98 & -1.212 & -4.68 & 45.01 & 0.221 & -0.013 & 0.003 & 13.0 & 0.21694 & 11.7 & 10.7 & 10.2 & 0.705 & 0.032 & 0.032 & 0.003 & 3.137 & \\
\hline & 3/2004 & 10:52:05 AM & 24.944 & $\begin{array}{ll}23.648 \\
\end{array}$ & 24.481 & 25.11 & 23.7 & 12.421 & 12.823 & $\begin{array}{ll}4.208 \\
\end{array}$ & 8.009 & -1.212 & & 45.123 & 0.217 & -0.0 & & 14.0 & 0.23361 & $\begin{array}{ll}11.7 \\
\end{array}$ & 0.7 & 10.2 & 0.70 & & $0.0>-3$ & & 3.086 & \\
\hline & 8/23/2004 & 10:53:0 & 24.914 & 23.649 & 24.452 & 25.096 & $23.7 \mathrm{P}-\mathrm{C}$ & & 12.889 & & 8.269 & -1.212 & & & & & & & & & $107+2$ & & & & & & 2.998 & \\
\hline$\frac{142}{112}>>>$ & 8/23/2004 & 10:54:05 AM & 24.86 & 23.655 & 24.452 & 25.131 & 23.784 & 12.68 & $\begin{array}{l}32.902 \\
\end{array}$ & & 8.12 & -1.212 & & 45.0 & 0.211 & & & $16.0 \mathrm{r}>\mathrm{r}$ & & 11.8 & 10.7 & 10.4 & & 0.030 & & .003 & 2.950 & 2.5 \\
\hline & 8/23/2004 & $10: 55: 05 \mathrm{AM}$ & 24.84 & 23.66 & 24.428 & 25.157 & 23.8 & 12.466 & 12.887 & & 8.128 & -1.209 & & 44.977 & 0.208 & -0.013 & & & 0.28361 & 11.8 & 10.7 & 10.3 & $\begin{array}{ll}1.3 & 0.710 \\
\end{array}$ & $\begin{array}{l}10 \\
10\end{array}$ & & & 2.939 & 2 \\
\hline & $8 / 23 / 2004$ & 10:56 & 24.827 & 23.687 & 24.424 & 25.2099 & & 12.5955 & 12.831 & 4.198 4.198 & $8.368 \mathrm{~g}$ & -1.206 & $-4.7 .7-3$ & 44.825 & 0.206 & -0. & & 18. & & 11.7 & 0.1 & 10.5 & & & & & 860 & 2.4 \\
\hline & 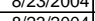 & $\begin{array}{l}1.57 .05 \mathrm{AMI} \\
\end{array}$ & 24.108 & 23.693 & 24.365 & 25.21 & & 12.522 & 12.908 & & & -1.212 & & $45.6 .0>-3$ & 0.201 & & & & 0.31694 & $11.8 \mathrm{r}$ & 0.7 & 0.2. & & & & & 2.869 & \\
\hline & 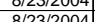 & & 24.159 & & 24.051 & & & & & 4.25 & & & & 450.012 & 0.202 & & & & & $\frac{12.1}{1.1}$ & & & & & & & & \\
\hline 148 & $\mid \frac{1212004 \mid}{8123204}$ & $\begin{array}{l}1.39 .05 \mathrm{Am} \\
11.00 .05 \mathrm{AM}\end{array}$ & $\frac{24.145}{24716}$ & $\frac{20.915}{23736}$ & 24.341 & 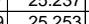 & $\frac{23.904}{23941}$ & 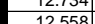 & 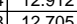 & (4.110 & 每.440 8171 & $\frac{-1.212}{-1209}$ & 年. & (44.909 & $\frac{0.2}{0.97}$ & 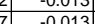 & & 220 & 0.36694 & 11.60 & 107 & 104 & & 0028 & & 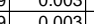 & $\frac{2.154}{2.772}$ & $\frac{2.3}{2.3}$ \\
\hline & $8 / 23 / 2004$ & $11: 01: 05$ & 24.687 & 23.732 & 24.319 & 25.274 & 23.916 & & & & 7.949 & -1.212 & & 45.0 & 0.195 & -0.6 & 0.003 & 23.02 & 0.38361 & 11.4 & \begin{tabular}{|l|l|}
4 & 10.7 \\
\end{tabular} & \begin{tabular}{l|l|l|}
7 & 10.1 \\
\end{tabular} & 0.698 & $\begin{array}{ll}98 & 0.028 \\
9\end{array}$ & 0.028 & 0.003 & 2.812 & 2.3 \\
\hline & $8 / 23 / 2004$ & 11:02: & 24.673 & $23.7 \mathrm{r}-\mathrm{C}$ & 24.3 & 25.325 & & 12.715 & \begin{tabular}{|l|l|}
512.978 \\
\end{tabular} & & 8.222 & -1.215 & & 45 & 0.193 & & & 24.0 & & 11.9 & 10.7 & 10.5 & 1.5 & & & & & 2. \\
\hline & 8/23/2004 & & 24.653 & 23.758 & 24.27 & 25.305 & & 12.657 & 12.877 & 4.368 & 8.062 & -1.212 & & 44.921 & 0.192 & -0. & & 25. & & 11.8 & 10.7 & & .4 & & & & 2.709 & 2. \\
\hline & | & & 24.623 & 23.758 & 24.265 & 25.245 & & 12.109 & 12.883 & 4.26 & 8.2 & -1.212 & & & 0.19 & & & 20 & & 11.8 & ont. & 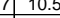 & & & & & 2.656 & \\
\hline & 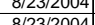 & $11: 05: 05 \mathrm{AM}$ & $\begin{array}{lll}24.603 \\
403\end{array}$ & 23.148 & 24.255 & 25.18 & & 12.487 & 12.661 & 4.19 & 8.163 & -1.215 & & 44.85 & 0.118 & -0.013 & & & & 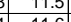 & 10. & 10.3. & & & & & 2.521 & \\
\hline & $8 / 23 / 2004$ & 11:066:05 AM & & 23.163 & 3. 24.265 & 25.164 & & 12.618 & | $12.7 / 1$ & 4.084 & 8.489 & -1.212 & & 44.9/9 & 0.187 & -0.013 & & & 0.46694 & 11.0 & 0. & $10.6-6$ & & 0.02 & & 0.000 & 2.590 & \\
\hline 156 & & erages & 24.9 & 23.7 & 24.4 & 25.2 & & 12.6 & 12.9 & 4.2 & 8.2 & -1.2 & & 44.9 & 0.2 & 0.0 & 0.0 & & & 11.8 & \begin{tabular}{l|l}
8 & 10.7 \\
\end{tabular} & 10.4 & & 0.030 & 0.0 & 0.003 & 3.0 & 2. \\
\hline & & & 25.3 & 23.8 & 24.7 & 25.3 & & 13.0 & 13.6 & 4.5 & 8.6 & -1.2 & & 45 & 0.3 & & & & & 12.5 & 10.7 & $0.8 \mathrm{O} \quad \mathrm{P}$ & $0.7 \quad>\quad>$ & & & & 3.6 & \\
\hline$\frac{1}{3}$ & & Median & 24.8 & 23.7 & 24.4 & 25.2 & & 12.6 & 12.9 & 4.2 & 8.1 & & & 45. & 0.2 & 0.0 & 0 & & & 11.8 & $10.7 \mathrm{r}$ & $10.4 \mathrm{Y}>\mathrm{C}$ & 0.110 & 0.030 & 0.030 & 0.003 & 2.9 & \\
\hline & & Minim & 24.6 & 23.6 & 24.3 & 25.0 & & 12.3 & 12.5 & 4.1 & 7.8 & -1.2 & -4.9 & 44.6. & 0.2 & 0.0 & 0.0 & & & 11.4 & 10. & to. & 0.005 & 205 & & 003 & 2.5 & 2 \\
\hline & & $\mid 2 \times \operatorname{sidd~Dev~}$ & 0.417 & 0.089 & 0.279 & 0.197 & 0.288 & 0.332 & 0.482 & 0.202 & 0.395 & & 0.2. & 0.264 & 0.044 & 0.000 & & & & & 0.06 & 0.03 & & & & 101 & 0.605 & \\
\hline & Number c & 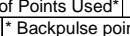 & th in box & are not inc & cluded 22 & & & & & & & & & & & & & & & 24 & & & & & & & $22-22>$ & \\
\hline
\end{tabular}


WSRC-TR-2005-00105, REVISION 0

SRNL-RPP-2005-00012, REVISION 0

CAMPAIGN IV NEW GKN BASELINE \#2 WITH 0.1 M NAOH - CONT.

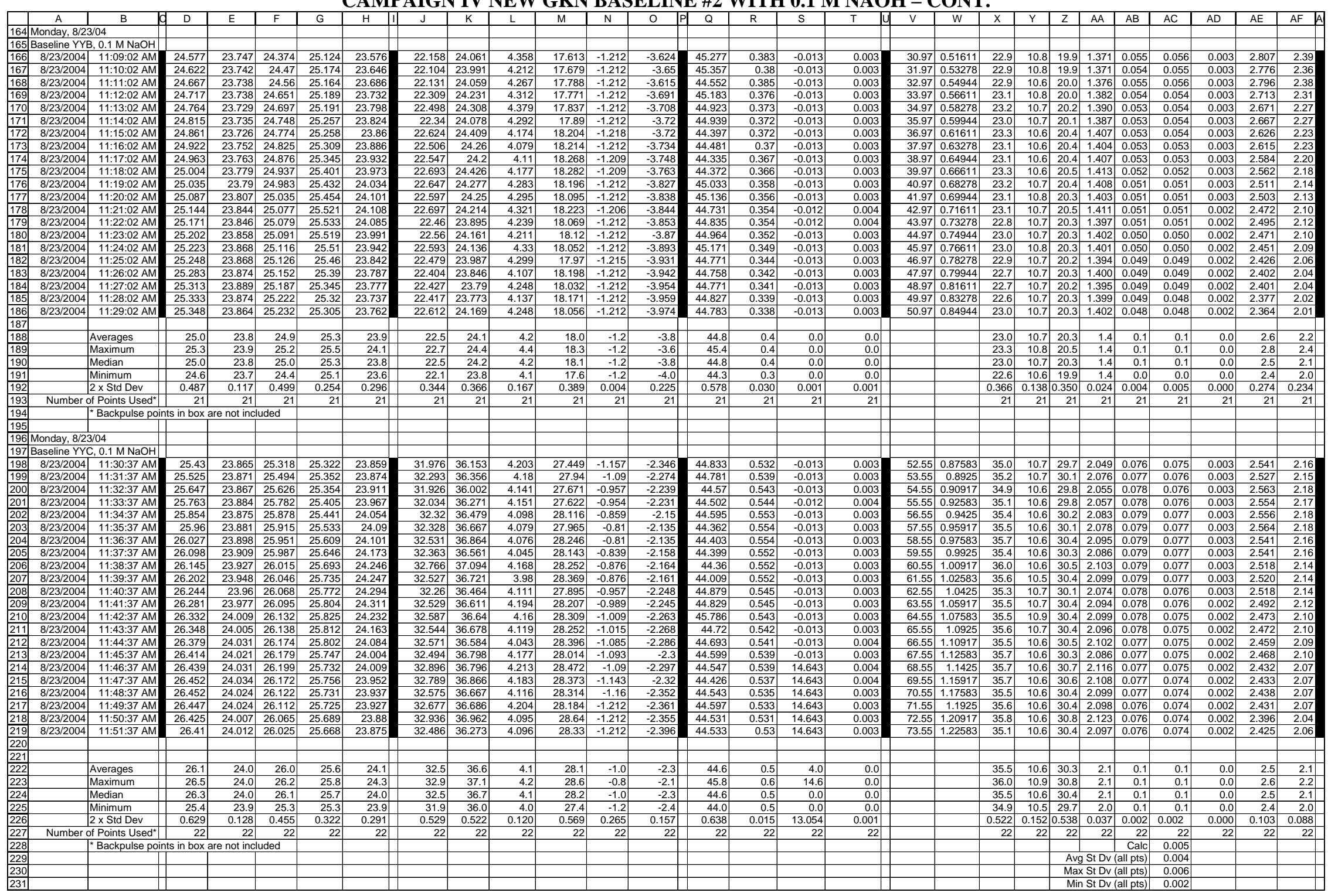


WSRC-TR-2005-00105, REVISION 0

SRNL-RPP-2005-00012, REVISION 0

CAMPAIGN IV NEW GKN BASELINE \#3 WITH 0.5 M HNO3

RPP-WTP Pilot-scale X-flow Filtration, Pre-Campaign IV Tests

[23-24 August 2004: New GKN Filter]

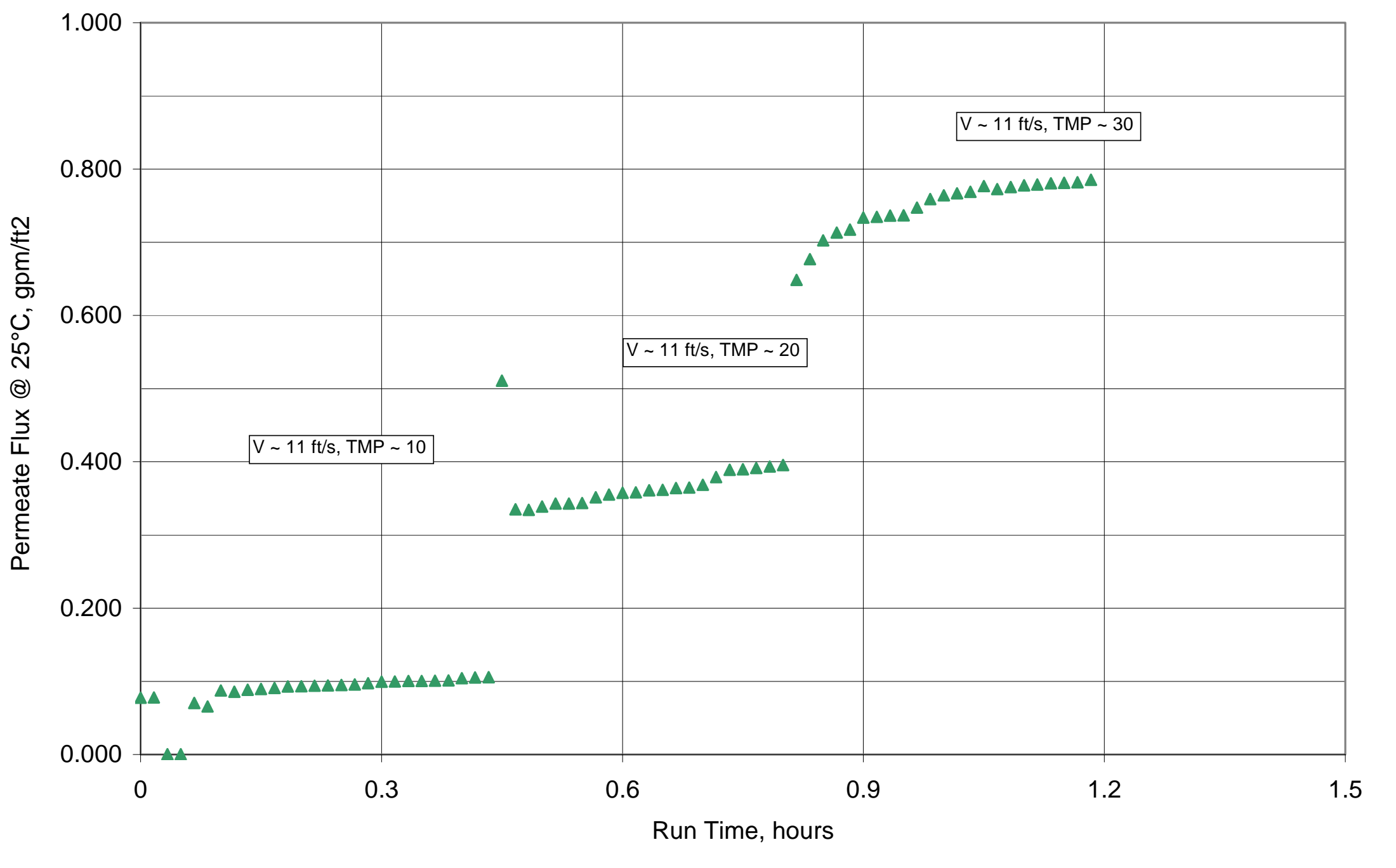


WSRC-TR-2005-00105, REVISION 0

SRNL-RPP-2005-00012, REVISION 0

CAMPAIGN IV NEW GKN BASELINE \#3 WITH 0.5 M HNO3

\begin{tabular}{|c|c|c|c|c|c|c|c|c|c|c|c|c|c|c|c|c|c|c|c|c|c|c|c|c|c|c|c|c|}
\hline & $\mathrm{A}$ & $B$ & $\frac{D}{\ll \ll \ll \ll}$ & $\begin{array}{l}\frac{1}{1} \\
\ll<\text { E }\end{array}$ & I F & $\begin{array}{ll}\text { e } G \\
\text { easurem }\end{array}$ & I & $\frac{\mathrm{J}}{\ll \ll \ll \ll<}$ & $\frac{1}{1<\ll<\ll<}$ & $\frac{1}{\text { L }}$ & $\mathrm{M}$ & $\frac{1 \mathrm{~N}}{\mathrm{~T} \text { ents } \gg \gg \geqslant}$ & $\begin{array}{ll}1 & 0 \\
y & 0\end{array}$ & $\frac{Q}{Q^{\prime}}$ & $\begin{array}{ll}R \\
R<\text { Flown }\end{array}$ & $\begin{array}{c}\mathrm{s} \\
\text { easurem }\end{array}$ & $\frac{1}{n e n}$ & $\frac{v}{v<s<<}$ & $\frac{w}{1}$ & $\frac{1}{1} \times$ & $\frac{\mathrm{C}}{\mathrm{C}}$ & $\mathrm{z}$ & 1 AA & $\mid A B$ & \begin{tabular}{|l|l|l|} 
I AC \\
\end{tabular} & $\frac{\mid A D}{\left.\prod \gg \gg \gg \gg \gg\right\rangle}$ & $\begin{array}{l}\mathrm{AE} \\
\gg \gg\end{array}$ & $\mathrm{AF}$ \\
\hline$\frac{1}{2}$ & DATE & TIME & $\begin{array}{l}\text { Filtrate } \\
\text { Fise }\end{array}$ & $\begin{array}{l}\text { elemp } \\
\text { Cleaning }\end{array}$ & $\begin{array}{l}\text { eprature M } \\
\text { If Slurry }\end{array}$ & $\begin{array}{l}\text { enasureme } \\
\text { Hi Amb }\end{array}$ & 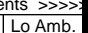 & $\begin{array}{l}\text { BotTMP } \\
\text { BotTMP }\end{array}$ & 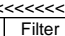 & $\begin{array}{l}\text { Pressure M } \\
\text { Filter } \mathrm{dP}\end{array}$ & $\begin{array}{l}\text { Measureme } \\
\text { TopTMP }\end{array}$ & $\begin{array}{l}\text { nents } \gg>>2 \\
\text { | Filtrate }\end{array}$ & Pulsep & & & Measureme & nents > > & & & & & & & & & & & \\
\hline & & & $\operatorname{deg} C$ & $\operatorname{deg} \mathrm{C}$ & & $\operatorname{deg} C$ & & psid & psig & psid & \begin{tabular}{|l} 
psid \\
\end{tabular} & psig & psig & $\mathrm{gpm}$ & gpm & $\mathrm{gpm}$ & & & & & & & & & & & & \\
\hline 4 & & & T2 & T3 & $\mathrm{T} 1$ & T4 & T5 & $\mathrm{dP2}$ & P1 & dP1 & dP3 & P2 & P3 & $\mathrm{Q1}$ & $\mathrm{Q} 2$ & $\mathrm{Q}^{3}$ & $\mathrm{Qbp}$ & & & & & & & & & & & \\
\hline 5 & & & & & & & & & & & & & & & & & & & & & & & & & & & & \\
\hline & Zeros- $01 / 26$ & 004 & & & & & & & & & & & & & & & & & & & & & & & & & & \\
\hline & 8/23/2004 & 8:35:38 AM & 22.554 & 22.604 & 22.002 & 22.782 & 22.204 & -0.015 & 0.099 & -0.012 & 0.005 & -0.124 & & -0.075 & 0.003 & 14.643 & 0.004 & & & Filter $\mathrm{S}$ & fface & & & & & & & \\
\hline & 8 & $8: 10: 33 \mathrm{AM}$ & 23.662 & & 23.679 & & 22.1350 & -0.0 & & -0.014 & & -0.124 & & 23.176 & 1.205 & 14.643 & 0.003 & & & Conver & ersion & & & & $\mathrm{g} / \mathrm{gpm}$ & barg & & \\
\hline & 8 & $\frac{14: 11 \mathrm{AM}}{5.2020 \mathrm{M}}$ & 25.819 & & $\begin{array}{l}24.932 \\
20200\end{array}$ & 25.316 & & & 0.121 & & 0.011 & $=0.1060$ & & 48.335 & 0.043 & 14.643 & & & & & & & & & & & & \\
\hline & $8 / 30 / 2004$ & $7: 28: 55 \mathrm{AM}$ & 22.511 & 22.848 & 22.397 & 22.669 & \begin{tabular}{|l|}
22.011 \\
\end{tabular} & -0.017 & $0.13 \varepsilon$ & $\begin{array}{l}-0.000 \\
-0.009\end{array}$ & $\begin{array}{l}0.003 \\
0.03\end{array}$ & $\begin{array}{l}-0.101 \\
-0.147\end{array}$ & 0.12 & $\mid$\begin{tabular}{|c|}
$\mid-0.084$ \\
-0.082
\end{tabular} & 0.003 & $\begin{array}{l}14.045 \\
14.643\end{array}$ & 0.006 & & & & & & & & allely & & & \\
\hline 12 & & & & & & & & & & & & & & & & & & & & Pressu & Sure P1is & & & & & vater tubir & & \\
\hline & & & & & & & & & & & & & & & & & & & & VWV & & & & F Filtra & rate Flux & SPE & $\mid A B \|$ & \\
\hline 12 & & & & & & & & & & & & & & & & & & & & & & & & & at 25C & & & \\
\hline$\frac{15}{16}$ & & & & & & & & & & & & & & & & & & $\frac{\text { Time }}{\text { Min }}$ & $\begin{array}{l}\text { Time } \\
\text { Hour }\end{array}$ & $\begin{array}{l}\text { Press. } \\
\text { psia }\end{array}$ & $\begin{array}{l}\text { Vel. } \\
\text { tit/s }\end{array}$ & TMP & $\frac{\mid \text { TMP }}{\text { bar }}$ & $\frac{\mathrm{gpm}}{\mathrm{dta} 2}$ & $\mathrm{gpm}$ & $\frac{\mathrm{gpm}}{\mathrm{ft} / \mathrm{psi}}$ & $g p m$ & neter \\
\hline & A & B & $\mathrm{D}$ & $E$ & $F$ & $G$ & $\mathrm{H}$ & $\mathrm{J}$ & $\mathrm{K}$ & $\mathrm{L}$ & $M$ & $\mathrm{~N}$ & $\mathrm{O}$ & $Q$ & $\mathrm{R}$ & $\mathrm{s}$ & $T$ & $\mathrm{v}$ & $\mathrm{w}$ & $\frac{x}{x}$ & $\mathrm{Y}$ & 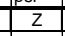 & AA & $A B$ & $A C$ & $A D$ & $A E$ & $\mathrm{AF}$ \\
\hline & 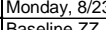 & & & & & & & & & & & & & & & & & & & & & & & & & & & \\
\hline & Datint/2004 & 3:50:11 PM & & & & & & & & & & & & 44.841 & 0.56 & 14.643 & & 0.0 & & 15.8 & & & & 0.080 & & & & \\
\hline & $\mid 8 / 23 / 2004$ & 3:51:11 PM & 26.696 & 24.25 & \begin{tabular}{|l|l|}
25.927 \\
\end{tabular} & 26.546 & 24.993 & $\begin{array}{ll}11.941 \\
\end{array}$ & $16.42 \mathrm{~S}$ & & 8.001 & -0.914 & -2.153 & \begin{tabular}{|l|l}
44.808 \\
\end{tabular} & 0.559 & 14.643 & 0.003 & & . 010166 & $\begin{array}{l}15.3 \\
15.3\end{array}$ & $\frac{1.1}{10.7}$ & $\frac{10.0}{10.0}$ & $\frac{1.17}{0.687}$ & & 0.078 & 0.008 & 7.820 & \\
\hline 36 & $8 / 23 / 2004$ & 3:52:11 PM & 26.673 & 24.276 & \begin{tabular}{|l|l|l|l|}
25.713 \\
\end{tabular} & 26.607 & \begin{tabular}{|l|l|}
24.929 \\
\end{tabular} & 2.345 & 16.87 & $\begin{array}{l}4.244 \\
\end{array}$ & \begin{tabular}{|c|c|}
-1.828 \\
\end{tabular} & $\begin{array}{l}9.338 \\
\end{array}$ & $\begin{array}{l}19.988 \\
\end{array}$ & 44.282 & $\begin{array}{l}0.003 \\
\end{array}$ & $\begin{array}{l}14.643 \\
\end{array}$ & 0.003 & 2.0 & 0.0333 & 15.8 & 10.5 & 0.3 & 0.018 & $\frac{0.000}{0.000}$ & 0.000 & 0.002 & 1.629 & \\
\hline & 8/23/2004 & 3:53:11 PM & 26.689 & 24.297 & 25.524 & & 25.02 & & 16.77 & & -1.912 & 9.34 & 21.847 & 44.501 & & 14.643 & & 3.0 & 0.0 & 15.6 & 10.6 & & 0.014 & 0.00 & 0.000 & & 2.159 & \\
\hline 238 & $8 / 23 / 2004$ & 3:54:11 PM & 26.616 & $24.32 \mathrm{~s}$ & 25.356 & 26.63 & 2 & 10.314 & 16.7 & & 6.234 & & & 44.347 & 0.498 & 14.643 & & & 0.06667 & 15.7 & 10.6 & & 0.570 & 0071 & 0.071 & 0.009 & 8.531 & \\
\hline & 8/23/2004 & 3:55:11 PM & 26.178 & & 25.193 & 26.742 & 25.134 & 10.127 & 16.68 & & 6.057 & 1.243 & & 44.908 & & $\begin{array}{l}14.643 \\
\end{array}$ & & & 0.0835 & 15.6 & & & & & 0.066 & & 8.130 & \\
\hline & 8/23/2004 & 3:56:11 PM & 26.011 & 24.384 & \begin{tabular}{|l|l|}
25.025 \\
\end{tabular} & 26.864 & 25.206 & \begin{tabular}{|l|l|}
11.45 \\
\end{tabular} & 16.82 & 4.22 & 7.325 & 0.12 & -1.45 & 44.405 & 0.611 & \begin{tabular}{|l|l|l|l|l|}
1433 \\
\end{tabular} & & $\begin{array}{ll}6.00 \\
\end{array}$ & 0.1 & 15.7 & 10.6 & 9.4 & 0.647 & 0.087 & $\mid 0.087$ & 0.009 & 9.312 & 79 \\
\hline & $8 / 23 / 2004$ & 3:57:11 PM & 25.769 & 24.422 & \begin{tabular}{|l|}
24.913 \\
\end{tabular} & 26.902 & 25.28 & 11.649 & 16.93 & & & -0.213 & & 44.47 & & $\begin{array}{l}14.643 \\
\end{array}$ & & & 0.11667 & 15.8 & 10.6 & $9.6 \mathrm{Cl}$ & 0.059 & 0.086 & & 0.009 & 8.991 & \\
\hline & 8/23/2004 & 3:58:11 PM & 25.576 & 24.443 & 24.779 & 26.973 & & 11.751 & 17.18 & & 7.377 & 0.027 & & 45.325 & & & & & 0.13333 & 16.1 & 10.8 & $9.6 \quad-\quad$ & 0.659 & & & & & \\
\hline 10 & $8 / 23 / 2004$ & 3:59:11 PM & 25.417 & 24.479 & 24.685 & 26.995 & 25.20 & 11.811 & 17.3 & & 7.535 & 0.125 & & 45.133 & 0.619 & 14.6 & & 9.00 & 0.15 & 16.2 & 10. & 9.7 & 0.667 & 0.089 & 0.089 & 0.009 & 9.243 & \\
\hline & $8 / 23 / 23 / 204$ & 4:00:11 PM & 25.284 & & 24.587 & 27.012 & & 11.892 & $\mid 17.54$ & & & 0.325 & & 45.2 & & & & 10 & 0.16667 & 16.4 & $\pi$ & & & & & & 5 & \\
\hline & & 4.01.11 P & $\frac{23.140}{25052}$ & & & & & & & & & (0.479 & & & & 14.6 & & & 0.18335 & 16.6 & & & & & & & & \\
\hline & 8 & $4.020 .11 \mathrm{PM}$ & 24058 & $\begin{array}{r}24.56 \\
2586\end{array}$ & $\begin{array}{l}24.435 \\
2.341\end{array}$ & $\frac{26.965}{26.921}$ & $\frac{25.14}{2507}$ & $\begin{array}{l}12.031 \\
11809\end{array}$ & 11.96 & & $\begin{array}{l}7.006 \\
7.529\end{array}$ & 0.51 & -1.24 & $\begin{array}{l}44.915 \\
44912\end{array}$ & 0.6 & 年 14.6 .6 & & $\frac{12}{13}$ & $\frac{.2}{0.6167}$ & 16.8 & 107 & 97 & 1006 & 0092 & | & 001 & 年 & \\
\hline & $8 / 23 / 2004$ & 4:04:11 PM & 24.859 & & & 26.842 & 25.0 & & \begin{tabular}{|l|l|}
177.42 \\
\end{tabular} & & & 6 & & & & & & & 0.2 & 16.3 & 10.7 & 9.4 & & & & & & \\
\hline & $8 / 23 / 2004$ & 4:05:11 PM & 24.779 & & 24.202 & & & 11.64 & 17.66 & & 7.531 & & & 44.8 & & 14. & & & 0.25 & 16.5 & 10.7 & 9.6 & & & & & & \\
\hline & 8/23/2004 & 4:06:11 PM & 24.674 & & 24.116 & 26.712 & 24. & 11.581 & \begin{tabular}{|l|l|} 
& 17.61 \\
\end{tabular} & & 7.549 & 0.664 & & 44.8 & & 14. & & & 0.26667 & 16.5 & 10. & 9.6 & & 093 & & & & \\
\hline & $\frac{8 / 23212004}{81200}$ & 4:07:1 & 24.6 & & 24.07 & 26.783 & & 11.87 & & & & 0.916 & & 45. & & & & & 0.2833 & 11. & & & & & & & & \\
\hline & $8 / 2325040$ & & 24.53 & & $\begin{array}{ll}24.027 \\
23072\end{array}$ & 26.819 & & $11.7 / 14$ & 18.2 & & 7.578 & 1.117 & & 44. & & 14. & & & 0.3 & 11. & & & & & & & & \\
\hline & $8 / 23 / 23 / 2004$ & 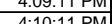 & 24.410 & $\frac{24.06}{246}$ & 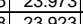 & 20.054 & & & \begin{tabular}{|l|l|l|l|} 
\\
\end{tabular} & & & $\mid$ & & & & & & & 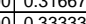 & & & & & & & & & \\
\hline & & 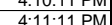 & $\frac{24.350}{24.373}$ & & $\frac{2.5305}{23995}$ & $\frac{21.044}{27.196}$ & & & 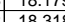 & & & $\mid$ & & & & & & & & & & & & & & & & \\
\hline & $8 / 23 / 2004$ & $4: 1: 1: 11$ & 24,363 & & & 27.222 & & 116 & 18.25 & & 7.303 & & & & & & & & 0.36 & 17.1 & & & & & & & & \\
\hline & $8 / 23 / 2004$ & $4: 1:$ & & & & & & & & & & & & 44.7 & & & & & 0.383 & 1 & 10.7 & & & & & & & \\
\hline & $8 / 23 / 2$ & & 24.3 & & & & & & & & & & & & & & & & & & & & & & & & & \\
\hline & $8 / 2312004$ & 4:15:IT & 24.31 & 24.716 & 24.135 & 27.265 & & 11.196 & 19. & & 7.545 & 1.0 & & & & & & & 0.41667 & & & & & & & & & \\
\hline & & 4.10. & & & & & & & & & & & & & & & & & & & & & & & 0.106 & & & \\
\hline & & & & & & & & & & & & & & & & & & & & & & & & & & & & \\
\hline & & & & & & & & & & & & & & & & & & & & & & & & & & & & \\
\hline & $8 / 23 / 2004$ & 4:19:11 P| & 24.635 & 24.739 & 24.428 & 27.083 & & 21.842 & 27.32 & 4.0 & 18.364 & -0.297 & & 45.0 & & 2.2 & & & 0.48 & 26.2 & 10 & & & & & & & \\
\hline & & 4:20:11 PM & 24.715 & 24.724 & 24.478 & 26.928 & 2 & 21.44 & 27.11 & 3.984 & 17.89 & $\begin{array}{l}-0.086 \\
-0.27\end{array}$ & & 44.389 & 0.003 & 2.333 & & & 0.5 & 26. & 10 & & & 0.334 & & & 17.237 & \\
\hline & $8 / 23 / 2004$ & 4:21:11 PM & 24.79 & \begin{tabular}{|l|l|}
$\mid$ & 24.729 \\
\end{tabular} & 24.553 & 6.898 & & 21.469 & 27.2 & 4.062 & 18.056 & \begin{tabular}{|c|}
$\mid 0.027$ \\
\end{tabular} & & 43.93 & 0.003 & 2.366 & 0.00 & 31.00 & 5166 & $\angle 6.1$ & 10.5 & & 1.36 & 0.336 & 0.343 & 0.017 & $7|17.357|$ & \begin{tabular}{l|l}
7 & 14 \\
7
\end{tabular} \\
\hline
\end{tabular}


WSRC-TR-2005-00105, REVISION 0

SRNL-RPP-2005-00012, REVISION 0

CAMPAIGN IV NEW GKN BASELINE \#3 WITH 0.5 M HNO3 - CONT.

\begin{tabular}{|c|c|c|c|c|c|c|c|c|c|c|c|c|c|c|c|c|c|c|c|c|c|c|c|c|c|c|c|c|}
\hline & & & & & & & & & & & & & & & & & & & & & & & & & & & & \\
\hline & \begin{tabular}{l|} 
\\
\end{tabular} & B & $\mathrm{D}$ & $E$ & $\mathrm{~F}$ & G & $\mathrm{H}$ & $\mathrm{J}$ & $\mathrm{K}$ & $\mathrm{L}$ & $M$ & $\mathrm{~N}$ & 0 & $\mathrm{Q}$ & $R$ & $\mathrm{~s}$ & $\mathrm{~T}$ & $\mathrm{v}$ & W & $x$ & $\mathrm{Y}$ & $z$ & $\mathrm{AA}$ & $A B$ & $\mathrm{AC}$ & $A D$ & $\mathrm{AE}$ & AF $A$ \\
\hline & $8 / 23 / 2004$ & 4:22:11 PM & 24.855 & 24.719 & 24.593 & 26.828 & 24.825 & 21.376 & \begin{tabular}{|l|l|}
6 & 27.166 \\
\end{tabular} & 3.999 & 17.962 & 0.027 & $\begin{array}{r}-4.474 \\
\end{array}$ & 44.466 & 0.003 & $\begin{array}{l}2.368 \\
\end{array}$ & 0.003 & 32.0 & \begin{tabular}{|l|l|}
0.53333 \\
\end{tabular} & 26.0 & 10.6 & & 1.356 & 0.339 & 0.343 & 0.017 & 17.435 & 14.84 \\
\hline & $8 / 23 / 2004$ & 4:23:11 PM & & 24.723 & 24.668 & 26.803 & 24.9 & 21.347 & $\begin{array}{ll}7 & 27.143 \\
\end{array}$ & & 18.081 & 0.027 & $\begin{array}{l}-4.468 \\
\end{array}$ & & & 2.378 & & 33.00 & 0.55 & 26.0 & & & & 0.340 & 0.344 & & . & 14.83 \\
\hline & 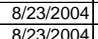 & $\begin{array}{l}4: 24: 11 \mathrm{PM} \\
4: 2 \cdot: 11 \mathrm{PM}\end{array}$ & $\begin{array}{l}24.984 \\
24.972 \\
\end{array}$ & $\frac{24.717}{24.65}$ & $\begin{array}{l}24.737 \\
24.761\end{array}$ & 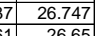 & \begin{tabular}{|l|}
24.874 \\
24697
\end{tabular} & $\begin{array}{l}21.538 \\
21.128\end{array}$ & $\begin{array}{l}827.659 \\
8\end{array}$ & $\begin{array}{r}4.13 \\
4.003 \\
\end{array}$ & \begin{tabular}{|c|}
18.009 \\
\end{tabular} & $\begin{array}{l}0.328 \\
0377\end{array}$ & $\begin{array}{l}-4.416 \\
405\end{array}$ & \begin{tabular}{|l|l|}
45.052 \\
45211 \\
\end{tabular} & $\begin{array}{l}0.003 \\
0.023\end{array}$ & \begin{tabular}{|l|l|}
2.437 \\
2.464
\end{tabular} & $\begin{array}{l}0.003 \\
0.03\end{array}$ & 34.00 & $\frac{0.56667}{0.5232}$ & 26.5 & $\begin{array}{l}10.7 \\
10\end{array}$ & \begin{tabular}{|l|l|}
19.8 \\
\end{tabular} & 1.363 & 0.349 & 0.351 & 0.018 & 17.776 & $\begin{array}{l}15.13 \\
\end{array}$ \\
\hline$\frac{250}{270}$ & $\begin{array}{l}8 / 23 / 2004 \\
8 / 23 / 204\end{array}$ & $\begin{array}{l}4: 25: 11 \mathrm{PM} \\
4 \cdot 26 \cdot 11 \mathrm{PM}\end{array}$ & $\begin{array}{l}24.972 \\
24.911\end{array}$ & $\begin{array}{r}24.65 \\
22574\end{array}$ & \begin{tabular}{|l|l|}
24.761 \\
1
\end{tabular} & \begin{tabular}{r|r}
1 & 26.65 \\
4
\end{tabular} & \begin{tabular}{|l|}
24.697 \\
24621 \\
\end{tabular} & 21.428 & $\begin{array}{l}8.27 .719 \\
8\end{array}$ & \begin{tabular}{|l|l}
4.003 \\
4.92
\end{tabular} & \begin{tabular}{|l|l|}
18.001 \\
17786
\end{tabular} & \begin{tabular}{|c|c|}
0.377 \\
0.50
\end{tabular} & $\begin{array}{l}-4.405 \\
\end{array}$ & \begin{tabular}{|l|}
45.211 \\
45233 \\
\end{tabular} & \begin{tabular}{|l}
0.003 \\
0.003
\end{tabular} & $\begin{array}{l}2.464 \\
.292\end{array}$ & 0.003 & 35.00 & 0.58333 & 26.6 & \begin{tabular}{|l|l|}
6 & 10.8 \\
7
\end{tabular} & \begin{tabular}{|l|l|}
19.7 \\
106
\end{tabular} & $\begin{array}{l}1.359 \\
1.252 \\
\end{array}$ & $\begin{array}{l}9 \\
9 \\
2\end{array}$ & 0.355 & $\begin{array}{l}0.018 \\
019\end{array}$ & 18.014 & \begin{tabular}{|l|l|}
15.33 \\
1551
\end{tabular} \\
\hline$\frac{270}{271}$ & $8 / 23 / 2004$ & & & $\begin{array}{ll}1 & 24.5 / 4 \\
3 & 24.596\end{array}$ & & & & $\frac{21.438}{21.461}$ & \begin{tabular}{|l|l|}
8 & 27.804 \\
1 & 27.788
\end{tabular} & $\begin{array}{l}4.192 \\
.135 \\
\end{array}$ & $\begin{array}{l}17.786 \\
18024\end{array}$ & 0.502 & $\begin{array}{r}-4.393 \\
-4.384 \\
\end{array}$ & \begin{tabular}{|l|}
45.236 \\
45.106
\end{tabular} & $\frac{0.003}{0.003}$ & \begin{tabular}{rl|l}
2.482 \\
2.494
\end{tabular} & $\frac{0.003}{0.003}$ & $\frac{36.00}{37.00}$ & $\begin{array}{r}0.6 \\
0.61667\end{array}$ & $\begin{array}{l}26.7 \\
267\end{array}$ & \begin{tabular}{|l|l|}
7 & 10.8 \\
7 & 107
\end{tabular} & \begin{tabular}{|l|l|}
19.6 \\
197
\end{tabular} & 1.352 & 0.355 & $\begin{array}{l}0.358 \\
0.358\end{array}$ & \begin{tabular}{|l|l|l|l|}
0.018 \\
0.018
\end{tabular} & $\frac{18.229}{18.151}$ & $\begin{array}{l}15.51 \\
1545\end{array}$ \\
\hline 272 & $8 / 23 / 2004$ & 4:28:11 PM & 25.064 & 24.632 & 24.947 & 26.717 & \begin{tabular}{|l|}
24.648 \\
\end{tabular} & $\frac{21.461}{21.15}$ & $\begin{array}{l}1 \\
5 \\
5\end{array}$ & 4.038 & 17.823 & 0.722 & $\begin{array}{l}-4.384 \\
-4.373 \\
\end{array}$ & \begin{tabular}{|c|}
45.088 \\
\end{tabular} & $\begin{array}{l}0.003 \\
0.003\end{array}$ & $\begin{array}{r}2.494 \\
2.519 \\
\end{array}$ & $\begin{array}{l}0.003 \\
0.003\end{array}$ & $\begin{array}{l}37.00 \\
38.00\end{array}$ & $\begin{array}{ll}0 & 0.61667 \\
& 0.63333\end{array}$ & $\begin{array}{l}26.7 \\
26.5\end{array}$ & \begin{tabular}{|l|}
10.7 \\
10.7
\end{tabular} & \begin{tabular}{|l|}
19.7 \\
19.5 \\
\end{tabular} & \begin{tabular}{|l|}
1.361 \\
1.344 \\
\end{tabular} & $\begin{array}{l}1 \\
\begin{array}{l}1 \\
4\end{array} \frac{0.357}{0.361}\end{array}$ & $\frac{0.358}{0.361}$ & $\mid \begin{array}{l}0.018 \\
0.019\end{array}$ & \begin{tabular}{|l|l|}
18.551 \\
18.534
\end{tabular} & $\begin{array}{l}15.45 \\
15.77\end{array}$ \\
\hline 273 & $8 / 23 / 2004$ & 4:29:11 PM & 25.144 & 24.677 & 25.027 & 26.777 & \begin{tabular}{|l|l|}
24.699 \\
\end{tabular} & 21.355 & \begin{tabular}{|l|l|}
5 & 27.844 \\
\end{tabular} & 4.061 & 18.079 & 0.742 & $\begin{array}{r}-4.364 \\
\end{array}$ & 44.754 & 0.003 & 2.529 & 0.003 & 39.00 & $\begin{array}{lll}0 & 0.65 \\
\end{array}$ & 26.7 & & & & & 0.362 & & \begin{tabular}{|l|l|}
18.349 \\
\end{tabular} & 15.62 \\
\hline 274 & $8 / 23 / 2004$ & 4:30:11 PM & & 24.722 & 25.082 & & 24.758 & 21.129 & \begin{tabular}{l|l|}
9 & 27.865 \\
\end{tabular} & 3.984 & 17.708 & 0.841 & -4.356 & & 0.003 & 2.549 & & & 0.66667 & 26.7 & 10.7 & \begin{tabular}{|l|l|}
19.4 \\
\end{tabular} & 1.339 & & 0.364 & & 18.749 & \\
\hline $\begin{array}{l}275 \\
276 \\
7\end{array}$ & $8 / 23 / 2004$ & $4: 31: 11 \mathrm{PM}$ & $\begin{array}{l}25.344 \\
25359\end{array}$ & $\begin{array}{l}24.797 \\
24792\end{array}$ & $\begin{array}{l}25.157 \\
25.177\end{array}$ & $\begin{array}{l}27.052 \\
27087\end{array}$ & $\begin{array}{l}24.888 \\
25009\end{array}$ & $\begin{array}{l}21.145 \\
21052\end{array}$ & \begin{tabular}{|l|l|}
5 & 27.877 \\
\end{tabular} & 3.966 & $\begin{array}{l}17.896 \\
1781\end{array}$ & $\begin{array}{l}0.89 \\
\end{array}$ & \begin{tabular}{|l|l|} 
& -4.344 \\
\end{tabular} & \begin{tabular}{|l|l|}
44.867 \\
\end{tabular} & 0.003 & $\begin{array}{l}2.559 \\
2596\end{array}$ & & 41.0 & 0.68333 & 26.8 & 10.7 & 19.5 & 1.346 & 0.366 & 0.365 & 019 & 18.685 & 15.90 \\
\hline & 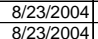 & $\begin{array}{l}4: 32: 11 \mathrm{PM} \\
4: 3: 311 \mathrm{PM}\end{array}$ & $\begin{array}{l}25.359 \\
25.402\end{array}$ & $\begin{array}{l}24.792 \\
24.806\end{array}$ & $\frac{25.177}{25.22}$ & $\begin{array}{l}27.087 \\
27.215\end{array}$ & $\begin{array}{l}25.009 \\
25082\end{array}$ & $\begin{array}{l}21.052 \\
21.426\end{array}$ & \begin{tabular}{|l|l|}
27.848 \\
6
\end{tabular} & $\begin{array}{l}3.957 \\
4119\end{array}$ & \begin{tabular}{|l|l|}
17.816 \\
179494 \\
\end{tabular} & \begin{tabular}{|l|l|}
1.003 \\
1399
\end{tabular} & -4. & \begin{tabular}{|l|l|}
44.887 \\
45231 \\
\end{tabular} & \begin{tabular}{|l}
0.003 \\
0.003
\end{tabular} & $\begin{array}{l}2.586 \\
2664\end{array}$ & & $\begin{array}{l}42.00 \\
4300\end{array}$ & $\begin{array}{r}0.7 \\
0716.67\end{array}$ & $\begin{array}{l}26.7 \\
275\end{array}$ & $\begin{array}{l}10.7 \\
10.9\end{array}$ & \begin{tabular}{|l|l|}
19.4 \\
10.7
\end{tabular} & $\begin{array}{l}1.340 \\
1.257\end{array}$ & 0.370 & $\begin{array}{l}0.368 \\
0370\end{array}$ & & $\begin{array}{l}18.956 \\
10.257\end{array}$ & 16.13 \\
\hline$\frac{271}{278}$ & $\begin{array}{l}88 / 232 / 20404 \\
8 / 232004\end{array}$ & $\begin{array}{l}\text { 4:3:3:11 PM } \\
\text { 4: }\end{array}$ & $\begin{array}{r}\frac{25.402}{25.43} \\
\end{array}$ & $\frac{24.806}{24.814}$ & $\begin{array}{r}25.22 \\
25.248\end{array}$ & $\begin{array}{r}27.215 \\
27.302 \\
\end{array}$ & $\begin{array}{l}25.082 \\
25.154 \\
\end{array}$ & $\frac{21.426}{21.753}$ & \begin{tabular}{|c|c|}
6 & 28.602 \\
& 29.201
\end{tabular} & $\begin{array}{l}4.119 \\
3.947\end{array}$ & \begin{tabular}{|l|}
17.941 \\
18.635
\end{tabular} & $\begin{array}{l}1.399 \\
1.735 \\
\end{array}$ & $\begin{array}{r}-4.286 \\
-4.26\end{array}$ & \begin{tabular}{|l|}
45.231 \\
44.59 \\
\end{tabular} & $\begin{array}{l}0.003 \\
0.003\end{array}$ & $\begin{array}{l}2.664 \\
2.736 \\
\end{array}$ & .003 & $\begin{array}{l}43.00 \\
44.00\end{array}$ & $\begin{array}{ll}0 & 0.71667 \\
0 & 0.73333\end{array}$ & $\frac{27.5}{281}$ & \begin{tabular}{|l|}
10.8 \\
10.6
\end{tabular} & \begin{tabular}{|l|}
19.7 \\
20.2
\end{tabular} & $\begin{array}{l}1.357 \\
1.392\end{array}$ & 0.381 & \begin{tabular}{|l|l|}
0.379 \\
0.389
\end{tabular} & $\begin{array}{l}0.019 \\
0.019\end{array}$ & $\begin{array}{l}19.257 \\
19.262\end{array}$ & $\begin{array}{l}16.39 \\
1.39\end{array}$ \\
\hline 279 & $8 / 23 / 2004$ & 4:35:11 PM & $\begin{array}{r}25.45 \\
25.484\end{array}$ & $\frac{24.014}{24.833}$ & \begin{tabular}{|l|l|l|l|l} 
& 25.340 \\
\end{tabular} & 27.317 & $\begin{array}{l}25.154 \\
25.234\end{array}$ & $\frac{21.153}{21.334}$ & $\begin{array}{ll}3 & 29.201 \\
4 & 28.988\end{array}$ & 3.857 & $\begin{array}{l}10.055 \\
18.225\end{array}$ & $\begin{array}{l}1.150 \\
1.758 \\
\end{array}$ & $\begin{array}{l}-4.20 \\
-4.26 \\
\end{array}$ & \begin{tabular}{|l|}
44.794 \\
\end{tabular} & 0.003 & $\begin{array}{l}2.150 \\
2.746\end{array}$ & $\begin{array}{l}0.003 \\
0.003\end{array}$ & & \begin{tabular}{|r|}
.1 .5355 \\
0.75
\end{tabular} & $\begin{array}{r}27.1 \\
27.9\end{array}$ & $\begin{array}{l}\frac{10.6}{10.7} \\
\end{array}$ & \begin{tabular}{|l|}
$\mid 20.2$ \\
19.8 \\
\end{tabular} & $\begin{array}{l}1.392 \\
1.364\end{array}$ & $\frac{0.392}{0.393}$ & $\begin{array}{l}0.389 \\
0.390\end{array}$ & $\frac{0.019}{0.020}$ & $\begin{array}{l}19.262 \\
19.696 \\
\end{array}$ & $\begin{array}{l}16.39 \\
16.76\end{array}$ \\
\hline 280 & $8 / 23 / 2004$ & 4:36:11 PM & 25.518 & 24.857 & 25.371 & 27.33 & 25.272 & 21.494 & \begin{tabular}{|l|l|}
4 & 29.205 \\
\end{tabular} & 3.956 & 18.391 & 1.854 & -4.243 & 44.787 & 0.003 & 2.763 & 0.003 & & 0.76667 & 28.1 & 10.7 & \begin{tabular}{|l|l|}
19.9 \\
\end{tabular} & 1.375 & 0.396 & 0.391 & 0.020 & 19.629 & 16.16 \\
\hline & $8 / 23 / 2004$ & 4:37:11 PM & 25.554 & 24.872 & 25.407 & 27.341 & 25.298 & 21.496 & & 3.996 & 18.268 & 1.915 & -4.23 & 44.568 & 0.003 & 2.78 & & 47.0 & 0.78333 & 28.2 & $\begin{array}{l}10.6 \\
\end{array}$ & \begin{tabular}{|l|l|l|}
19.9 \\
\end{tabular} & 1.371 & & 0.393 & .020 & & \\
\hline 282 & 8/23/2004 & 4:38:11 PM & 25.605 & & 25.448 & 27.363 & & & $\begin{array}{l}3 \quad 29.102 \\
\end{array}$ & 3.808 & & 1.961 & & 44.591 & 0.003 & 2.799 & & & 0.8 & 28.0 & & & & .401 & 0.396 & 020 & 0.181 & \\
\hline & & 4:39:11 PM & 25.717 & 24.896 & 25.525 & & 25.33 & 30.516 & $6 \quad 50.726$ & & 28.51 & 13.31 & -2.346 & 45.667 & & 4.598 & & & 18166 & & & & & & & & & \\
\hline & $8 / 23 / 2004$ & 4:40:11 PM & 26.031 & 24.92 & 25.83 & 27.404 & 25.26 & 30.202 & \begin{tabular}{l|l|l|}
252.299 \\
\end{tabular} & 3.745 & 28.642 & & & 44.729 & & 4.839 & & & 8333 & & & & & & & & & \\
\hline & $8 / 23 / 2004$ & 1:11 PM & 26.309 & $24.95 \varepsilon$ & 26.013 & & 25.27 & 30.542 & 254.366 & 3.66 & & 16.775 & & 44.923 & & 5.05 & & & 0.85 & & & & & & & & 3.466 & \\
\hline 2886 & 8/23/2004 & 4:42:11 PM & 26.447 & 24.922 & 26.121 & 27.48 & 25.227 & 30.737 & 7 75.305 & 3.756 & 29.393 & 17.557 & -1.635 & 45.081 & & 5.141 & & & 0.86667 & 54.2 & & 30. 30. & 2.073 & & 0.713 & & & \\
\hline 287 & $8 / 23 / 2004$ & 4:43:11 PM & 26.618 & 24.938 & 26.287 & 27.466 & 25.234 & 30.582 & \begin{tabular}{|l|l|}
2 & 55.738 \\
\end{tabular} & 3.602 & 29.413 & 17.979 & -1.56 & $\begin{array}{l}44.161 \\
\end{array}$ & 0.003 & 5.193 & 0.003 & 53.0 & 0.88333 & 54.6 & 10.5 & 30.0 & 2.068 & $\begin{array}{l}8 \\
8\end{array}$ & & 0.024 & 3.906 & 20.34 \\
\hline & 8/23/2004 & $\begin{array}{l}\text { 4:44:11 PM } \\
\end{array}$ & 26.782 & 24.987 & 26.351 & 27.625 & 25.243 & 31.15 & \begin{tabular}{|l|l|}
5 & 57.313 \\
& 57071
\end{tabular} & 3.65 & & 18.909 & -1.38 & 44.56 & 0.003 & 5.323 & & 54. & 0.6 & 56.2 & & 0.0 & 2.112 & 0.762 & & 0.024 & 3.957 & \\
\hline 289 & 8/23/2004 & 4:45:11 PM & 26.805 & 24.991 & 26.38 & & 25.19 & 30.797 & 7) 57.071 & 3.682 & 29.707 & 19.14 & & 44.668 & 0.003 & 5.333 & & & .91667 & 55.9 & $10.6 \mathrm{r}>\mathrm{C}$ & 30.3 & 2.086 & 0.763 & 0.735 & & 24.281 & 20.66 \\
\hline & 8 & 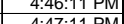 & 26.808 & 24.97. & 26.373 & 27.622 & $\frac{25.21}{25.20}$ & $\begin{array}{l}30.607 \\
21.026\end{array}$ & $\begin{array}{l}7 \quad 57.035 \\
657.792\end{array}$ & 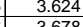 & 29.61 & $\frac{19.23}{10.24}$ & & 年4.585 & & 5.345 & & & (2) & & & & & & & & & 20.81 \\
\hline$\frac{\frac{291}{292}}{292}$ & $\begin{array}{l}88 \angle 3 / 20404 \\
8 / 23 / 2004\end{array}$ & $\begin{array}{l}\text { 4:4.111PM } \\
4: 48: 11 \mathrm{PM}\end{array}$ & $\begin{array}{l}26.855 \\
26.858\end{array}$ & 034 & $\frac{26.42}{26.363}$ & $\frac{27.069}{27.732}$ & 25.4 & $\frac{31.026}{30.532}$ & $\begin{array}{l}057.186 \\
2\end{array}$ & 3.678 & $\begin{array}{l}30.041 \\
29.789\end{array}$ & $\begin{array}{l}\frac{19.244}{19.893} \\
\end{array}$ & -1. & $\begin{array}{l}44.495 \\
44.666\end{array}$ & $\overline{003}$ & $\begin{array}{l}5.355 \\
5.423 \\
\end{array}$ & & & & $\begin{array}{l}56.1 \\
56.4\end{array}$ & 106 & & & & & & & 20.53 \\
\hline & $8 / 23 / 2004$ & 4:44.111 PM & 20.050 & 25.031 & 20.000 & 27.699 & 25.182 & 30.903 & \begin{tabular}{|l|l|}
3 & 58.959 \\
\end{tabular} & $\begin{array}{l}.044 \\
3.658\end{array}$ & 29.935 & \begin{tabular}{|}
$\mid 10.050$ \\
20.498 \\
\end{tabular} & -1.124 & \begin{tabular}{|l|}
44.052 \\
\end{tabular} & 003 & $\begin{array}{l}0.420 \\
5.496\end{array}$ & 0.003 & 59.00 & \begin{tabular}{|l|}
0.983013 \\
\end{tabular} & $\begin{array}{l}0.4 \\
57.8\end{array}$ & \begin{tabular}{|l|}
10.7 \\
\end{tabular} & \begin{tabular}{|l|}
30.4 \\
\end{tabular} & \begin{tabular}{|l|}
2.097 \\
2.097 \\
\end{tabular} & 0.787 & 0.759 & 0.025 & 24.955 & 21.24 \\
\hline 294 & $8 / 23 / 2004$ & 4:50:11 PM & 26.758 & 25.054 & 26.267 & 27.711 & 25.154 & 30.596 & \begin{tabular}{|l|l|}
6 & 58.654 \\
\end{tabular} & 3.575 & $\begin{array}{l}29.766 \\
\end{array}$ & \begin{tabular}{|l|}
20.756 \\
\end{tabular} & -1.077 & \begin{tabular}{|l|}
44.777 \\
\end{tabular} & 0.003 & 5.529 & & 60.00 & & 57.5 & 10.7 & 30.2 & 2.081 & & 0.764 & 0.025 & 25.312 & 21.54 \\
\hline r & $8 / 23 / 2004$ & 4:51:11 PM & 26.708 & 25.059 & 26.233 & 27.702 & 25.169 & 30.65 & \begin{tabular}{|l|l|}
5 & 58.897 \\
\end{tabular} & & & \begin{tabular}{|l|}
21.016 \\
\end{tabular} & & 44.574 & & 5.544 & & 61.00 & 1.01667 & 57.8 & 10.0 & 30.2 & & 0.794 & 0.767 & & 25.396 & \\
\hline 296 & 8/23/2004 & 4:52:11 PM & 26.685 & 25.07 & 26.194 & $\begin{array}{l}27.698 \\
27.717\end{array}$ & 25.155 & & \begin{tabular}{|l|l|} 
& 59.019 \\
\end{tabular} & $\begin{array}{l}3.605 \\
2.765\end{array}$ & & \begin{tabular}{|r|}
21.08 \\
21200 \\
\end{tabular} & & 44.272 & 0.003 & 5.553 & & 62.00 & 1.03333 & 57.9 & $\begin{array}{l}10.5 \\
\end{array}$ & \begin{tabular}{|l|l|}
30.2 \\
\end{tabular} & 2.083 & $\begin{array}{ll}3 & 0.795 \\
\end{array}$ & $\begin{array}{l}0.769 \\
\end{array}$ & 0.025 & \begin{tabular}{|l|l|}
25.447 \\
\end{tabular} & 21.66 \\
\hline & $8 / 23 / 2004$ & 4:53:11 PM & $\begin{array}{l}26.684 \\
26.995\end{array}$ & $\begin{array}{l}25.104 \\
25.126\end{array}$ & $\begin{array}{l}26.183 \\
\end{array}$ & $\begin{aligned} 27.717 \\
27.712\end{aligned}$ & 25.184 & $\begin{array}{l}30.627 \\
30502\end{array}$ & $\begin{array}{l}759.208 \\
25.001\end{array}$ & $\begin{array}{l}3.765 \\
3.762 \\
\end{array}$ & $\begin{array}{l}29.676 \\
\end{array}$ & \begin{tabular}{|r|}
21.358 \\
211 \\
\end{tabular} & -0.979 & \begin{tabular}{|l|l}
4.708 \\
6.141
\end{tabular} & 0.003 & \begin{tabular}{|l|l|}
5.609 \\
5571
\end{tabular} & & 63.00 & $\begin{array}{r}1.05 \\
10.667\end{array}$ & \begin{tabular}{|l|l|}
58.1 \\
579
\end{tabular} & $\begin{array}{l}10.6 \\
110\end{array}$ & \begin{tabular}{|l|}
30.2 \\
\end{tabular} & \begin{tabular}{|l|l|}
2.079 \\
\end{tabular} & $\begin{array}{l}9 \quad 0.803 \\
9\end{array}$ & $\begin{array}{l}0.777 \\
\end{array}$ & 0.026 & \begin{tabular}{|l|l|}
25.764 \\
\end{tabular} & 21.93 \\
\hline$\frac{298}{299}$ & $\begin{array}{l}8 / 2323204 \\
8 / 232004\end{array}$ & $\begin{array}{l}4: 5: 11 \mathrm{PM} \\
4: 55: 11 \mathrm{PM}\end{array}$ & $\begin{aligned} 26.685 \\
26.639 \\
\end{aligned}$ & $\frac{25.136}{25.115}$ & $\frac{26.159}{26.148}$ & $\frac{27.713}{27.737}$ & 25.246 & $\frac{30.4}{30.6}$ & \begin{tabular}{|c|c|}
3 & 58.901 \\
6 & 59.467
\end{tabular} & & $\begin{array}{r}29.582 \\
29.68 \\
\end{array}$ & $\begin{array}{r}21.1 \\
21366\end{array}$ & $\frac{-1 .}{-0 .}$ & \begin{tabular}{|l|}
46.141 \\
45.027
\end{tabular} & & & & & 1.06667 & $\begin{array}{l}57.8 \\
58.3\end{array}$ & \begin{tabular}{l|l|}
$\frac{11.0}{107}$ & \\
\end{tabular} & \begin{tabular}{|l|}
30.0 \\
30.2
\end{tabular} & 2.071 & & & & & $\frac{21.88}{2187}$ \\
\hline 300 & $8 / 23 / 2004$ & 4:56:11 PM & 26.612 & 25.117 & 26.121 & 27.73 & 25.397 & 30.715 & 559.516 & $\begin{array}{l}3.140 \\
3.643\end{array}$ & $\begin{array}{r}29.060 \\
29.865\end{array}$ & \begin{tabular}{|l|}
21.517 \\
\end{tabular} & -0.947 & \begin{tabular}{|l|}
44.585 \\
\end{tabular} & & 5.606 & & 66. & 1.1 & $\begin{array}{l}58.4 \\
58.4\end{array}$ & $\begin{array}{l}10.1 \\
10.6\end{array}$ & \begin{tabular}{|l|}
0.4 \\
30.3
\end{tabular} & 2.088 & & & & 25.677 & 21.87 \\
\hline 301 & $8 / 23 / 2004$ & 4:57:11 PM & 26.595 & 25.131 & 26.109 & 27.753 & 25.54 & 30.642 & \begin{tabular}{|l|l|}
25 & 59.784 \\
\end{tabular} & 3.604 & & \begin{tabular}{|l|}
21.589 \\
\end{tabular} & & \begin{tabular}{|l|l|}
44.529 \\
\end{tabular} & & 5.612 & & & 1.11667 & 58.7 & 10.6 & \begin{tabular}{|l|}
30.2 \\
\end{tabular} & 2.08 & & & & 25.802 & 21. \\
\hline 302 & 8/23/2004 & 4:58:11 PM & 26.594 & 25.17 & 26.119 & 27.847 & 25.6 & 30. & \begin{tabular}{|l|l|}
6 & 59.3 \\
\end{tabular} & & & & & 44.516 & & & & & \begin{tabular}{l|l|l|l|l|l|l|l}
0 & 1.13333 \\
\end{tabular} & 58.2 & 10.6 & 30.0 & 2.069 & & & & 26.014 & \\
\hline 303 & 8/23/2004 & 4:59:11 PM & 26.586 & 25.186 & 26.115 & 27.888 & 25.601 & 30.505 & $\begin{array}{l}559.566 \\
\end{array}$ & 3.639 & & \begin{tabular}{|l|l|}
21.618 \\
\end{tabular} & & \begin{tabular}{|l|l|}
4.545 \\
\end{tabular} & & 5.631 & & 69.00 & \begin{tabular}{l|l}
0 & 1.15 \\
\end{tabular} & 58.4 & $\begin{array}{l}10.6 \\
\end{array}$ & \begin{tabular}{|l|l|}
30.1 \\
\end{tabular} & 2.073 & 806 & 0.781 & 0.026 & 25.984 & 22 \\
\hline$\frac{304}{305}$ & $8 / 23 / 2004$ & 5:00:11 PM & 26.587 & 25.202 & 26.121 & 27.954 & 25.662 & 30.387 & $\begin{array}{l}759.651 \\
\end{array}$ & 3.605 & 29.684 & \begin{tabular}{|l|}
21.821 \\
\end{tabular} & -0.92. & 44.543 & & 5.636 & & 70.00 & 1.16667 & 58.5 & $\begin{array}{l}10.6 \\
\end{array}$ & 30.0 & 2.071 & 10.807 & 0.782 & 0.026 & 26.033 & 22.15 \\
\hline$\frac{305}{306}$ & 8/23/2004 & 5:01:11 PM & 26.579 & 25.225 & 26.113 & & 25.754 & 30.298 & \begin{tabular}{l|l|}
8 & 59.815 \\
\end{tabular} & 3.639 & 29.452 & \begin{tabular}{|l|}
21.922 \\
\end{tabular} & & 44.451 & 0.003 & 5.658 & & 71.00 & \begin{tabular}{l|l|l}
0 & 1.18333 \\
\end{tabular} & 58.7 & \begin{tabular}{ll|}
10.6 \\
\end{tabular} & \begin{tabular}{|l|}
29.9 \\
\end{tabular} & $2.060 \mid$ & 0.810 & 0.785 & 0.026 & 26.281 & 22.37 \\
\hline & & & & & & & & & & & & & & & & & & & & & & & & & & & & \\
\hline 308 & & Averages & 24.8 & 24.6 & 24.3 & 27.0 & 25.0 & 11.7 & 17.9 & 4.3 & & 0.9 & -1.6 & 44.9 & 0.7 & 14.6 & $0 . c^{2}-1$ & & & 16.8 & 10.7 & 9.6 & 0.7 & & 0.1 & 0.0 & & \\
\hline & & Maximum & & 24.7 & 25.0 & & & & & 4.5 & & 2.1 & & & 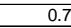 & 14.6 & & & & 18.0 & 10.8 & 9.8 & 0.7 & & 0.1 & 0.0 & 11.1 & \\
\hline 310 & & Median & 24.7 & 24.6 & 24.2 & & & 11.7 & 18 & 4.3 & 7 & & -1 & & 0.7 & 14 & & & & 16.8 & \begin{tabular}{ll|}
10.7 \\
\end{tabular} & 9.6 & 0.7 & 0.1 & 0.1 & 0.0 & 10.0 & 8.5 \\
\hline$\frac{311}{312}$ & & & 24.4 & 24.4 & 23.9 & 26.7 & & & & & 1.0 & -0.2 & & 44.4 & 5000 & 14.6 & & & & 15.7 & 10.6 & \begin{tabular}{|r|} 
\\
\end{tabular} & \begin{tabular}{|l|} 
\\
\end{tabular} & 0.1 & 0.1 & 0.0 & 9.0 & \\
\hline & Number c & $2 \times$ Std Dev & $\frac{1.000}{21}$ & $\frac{0.212}{21}$ & $\frac{0.638}{21}$ & $\frac{0.366}{21}$ & $\frac{0.254}{21}$ & 0.305 & $\begin{array}{r}1.241 \\
21\end{array}$ & 0.188 & $\frac{0.233}{21}$ & $\begin{array}{r}1.260 \\
21\end{array}$ & $\frac{0.79}{2}$ & $\begin{array}{r}0.487 \\
21 \\
\end{array}$ & 0.069 & 0.000 & $\frac{0.000}{21}$ & & & $\frac{1.241}{21}$ & $\begin{array}{ll}1 & 0.116] 0 \\
11 & 21\end{array}$ & $|0.248|$ & $|0.017|$ & 0.010 & 0.011 & 0.001 & $\begin{array}{r}1.229 \\
21\end{array}$ & 1.046 \\
\hline$\frac{313}{314}$ & Number c & 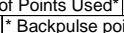 & in box & not in & & & & & & & & 21 & 2 & 21 & 21 & 21 & 21 & & & 21 & 21 & 21 & 21 & 21 & 21 & & & 21 \\
\hline & & & & & & & & & & & & & & & & & & & & & & & & & & & & \\
\hline & & & & & & & & & & & & & & & & & & & & & & & & & & & & \\
\hline 317 & & Averages & 25.1 & 24.7 & 24.9 & 27.0 & 24.9 & 21.4 & 28.0 & 4.0 & 18.1 & 0.8 & & 44.8 & 0.0 & 2.5 & $0.1 \quad-\quad>>$ & & & 26.9 & $10.7 \mathrm{C}-\mathrm{C}$ & 19.7 & 1.4 & 0.4 & & 0.0 & 18.4 & 15.6 \\
\hline & & & 25.6 & 24 & 25.4 & 27.4 & 25 & 21.9 & 29.3 & 4.2 & & 2. & & & & 2.8 & & & & 28.2 & $10.6 \mathrm{c}-\mathrm{s}$ & 20.2 & 1.4 & & & & & \\
\hline & & Mediar & & & 24.9 & & & & & & & 0 & & & & & & & & 26.7 - & & & & & & & & \\
\hline & & |Minimum & 24.6 & 24.6 & 24.4 & 26.6 & & 21.1 & 27.1 & 3.8 & $\begin{array}{ll}17.7 \\
\end{array}$ & -0. & & 43.9 & 0.0 & 2.3 & & & & 26.0 & 10.5 & 19.4 & 1.3 & & & 0.0 & $16.6 \mathrm{r}>\mathrm{l}$ & 14.1 \\
\hline & & $2 \times \mathrm{Std}$ Dev & 0.625 & 0.170 & 0.670 & 0.506 & 0.43 & 0.434 & 1.470 & 0.183 & 0.448 & 1.461 & 0.18 & 0.648 & 0.00 & 0.321 & 0.0 & & & 1.470 & 0.1540 & $\mid 0.409$ & \begin{tabular}{|l|l|}
0.028 \\
\end{tabular} & 0.046 & 0.039 & 0.002 & 2.015 & 1.715 \\
\hline & Number c & of Points Used $\mathrm{d}^{*}$ & 21 & 21 & 21 & 21 & 21 & 21 & 21 & 21 & 21 & 21 & 21 & 21 & 21 & 21 & 21 & & & 21 & 21 & 21 & 21 & 21 & 21 & 21 & 21 & 21 \\
\hline & & & ts in box & & & & & & & & & & & & & & & & & & & & & & & & & \\
\hline & & & & & & & & & & & & & & & & & & & & & & & & & & & & \\
\hline 26 & & Average & 26.6 & 25.1 & 26.2 & .2 & & 30.6 & & 3.7 & 29.6 & 19 & & 44 & 0 & 5.4 & (c) & & & 56. & & & & & & & 24.9 & \\
\hline & & Maximum & 26.9 & 25.2 & 26.4 & 28.0 & 25 & 31. & & 4 & 30 & 21. & & 46 & & 5.7 & & & & 58.7 & & & & & & & & \\
\hline & & Median & 26.6 & 25.1 & 26.2 & 27.7 & 25 & 30.6 & 58.9 & 3.7 & 29 & 20 & & 44 & 0. & 5 & & & & 57.8 & & & & & & 0.0 & 25.3 & \\
\hline & & Minimum & 25.7 & 24.9 & 25.5 & 27.4 & 25 & 30.2 & 50.7 & 3.5 & 28.5 & 13. & -2 . & 44.2 & 0.0 & 4.6 & & & & 49.6 & $10.5 \mathrm{P}-\mathrm{s}$ & 29.4 & 2.0 & $0.7 \quad-7$ & 0.6 & 0.0 & 22.0 & \\
\hline & & $2 \times \operatorname{Std} \mathrm{C}$ & & 0.189 & 0.381 & 0.311 & 0.35 & 0.416 & 4.827 & 0.182 & 0.730 & 4.464 & 0.76 & 0.840 & 0.000 & 0.544 & 0.001 & & & 4.827 & 0.200 & 1.524 & 0.036 & 0.078 & 0.072 & 0.002 & 2.222 & 1.891 \\
\hline 331 & Number c & of Points Used* & 23 & 23 & $\begin{array}{l}3 \\
\end{array}$ & 23 & 23 & 23 & 23 & 23 & 23 & 23 & 23 & 23 & 23 & 23 & 23 & & & 23 & 23 & 23 & 23 & 23 & 23 & 23 & 23 & 23 \\
\hline & & & sin box & $x$ are not inc & cluded & & & & & & & & & & & & & & & & & & $\frac{1}{S t D v e}$ & $\begin{array}{l}\mid \text { Calc } \\
\text { (all pts) }\end{array}$ & $\begin{array}{l}0.049 \\
0.041\end{array}$ & & & \\
\hline & & & & & & & & & & & & & & & & & & & & & & Max & x St Dv ( & (all pts) & 0.072 & & & \\
\hline 335 & & & & & & & & & & & & & & & & & & & & & & Min & $\overline{n S t D v(c}$ & (all pts) & \begin{tabular}{|l|}
0.011 \\
\end{tabular} & & & \\
\hline
\end{tabular}


WSRC-TR-2005-00105, REVISION 0

SRNL-RPP-2005-00012, REVISION 0

CAMPAIGN IV NEW GKN BASELINE \#4 WITH DIF WATER

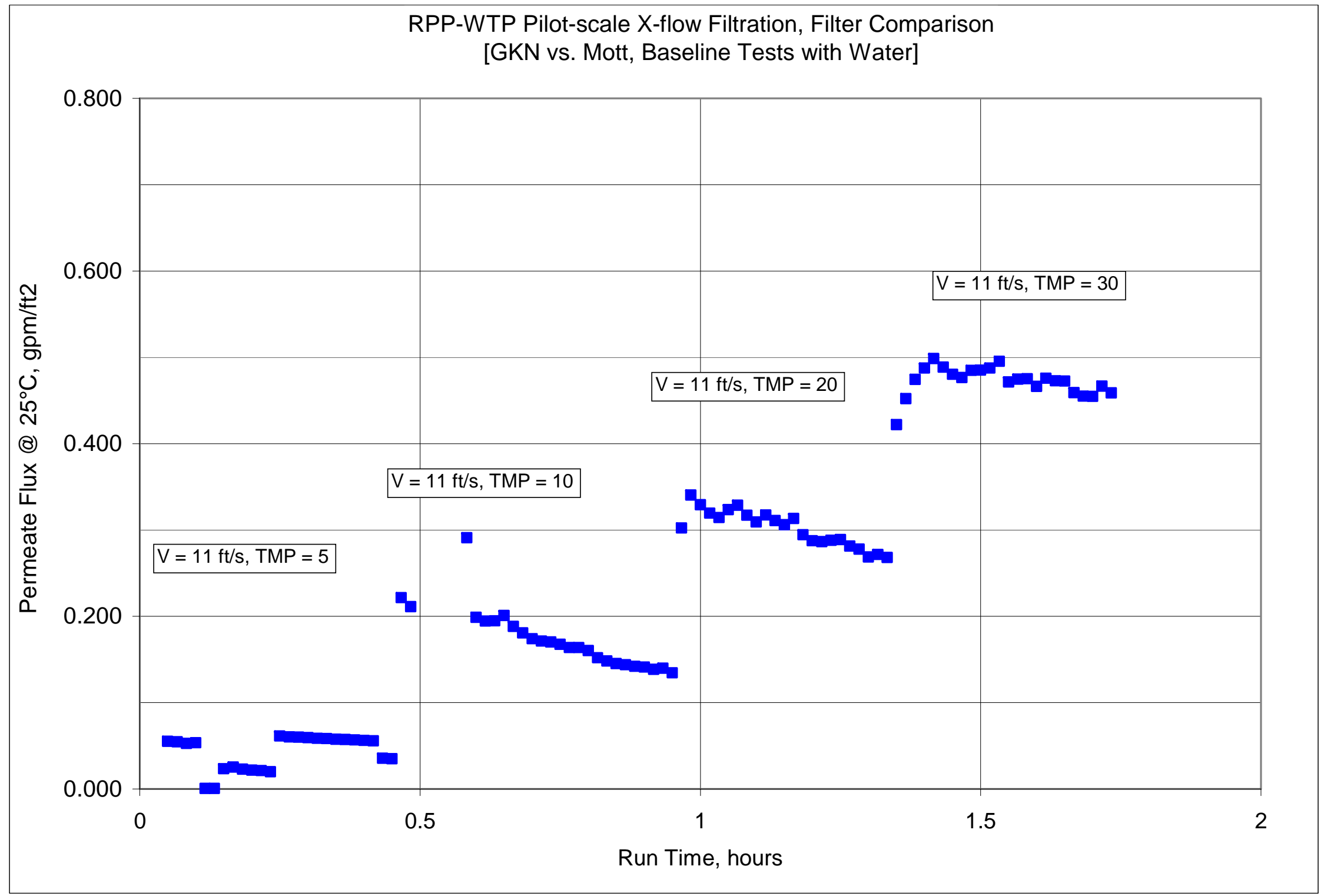


WSRC-TR-2005-00105, REVISION 0

SRNL-RPP-2005-00012, REVISION 0

CAMPAIGN IV NEW GKN BASELINE \#4 WITH DIF WATER

\begin{tabular}{|c|c|c|c|c|c|c|c|c|c|c|c|c|c|c|c|c|c|c|c|c|c|c|c|c|c|c|c|c|}
\hline & $\mathrm{A}$ & $B$ & $\mathrm{D}$ & $\begin{array}{l}\text { E } \\
<\text { Temp }\end{array}$ & 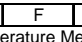 & Geasuremet & 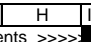 & $\mathrm{J}$ & $\frac{K}{K \ll \ll \ll}$ & $\frac{L}{\text { Pessure I }}$ & easureme & 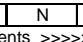 & $\frac{10}{110}$ & 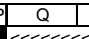 & $\frac{\mathrm{R}}{\mathrm{R}}$ & s 1 & $\frac{T}{T}$ & $\frac{v}{k \ll \ll<}$ & $\frac{w}{1 \leq \ll<}$ & $\frac{1}{x<<}$ & $\mathrm{Y}$ & $\frac{\mathrm{z}}{\mathrm{z}}$ & AA & \begin{tabular}{ll|}
$A B$ \\
$2 t a$
\end{tabular} & $\mid$ & $A D$ & $\mathrm{AE}$ & $\begin{array}{lll}\mathrm{AF} & \mathrm{A} \\
\end{array}$ \\
\hline & DATE & TIME & Filtrate & $\begin{array}{l}\text { Leaning } \\
\text { Cleani }\end{array}$ & $\begin{array}{l}\text { elature we } \\
\text { Slurry } \\
\end{array}$ & $\begin{array}{l}\text { eleasuremel| } \\
\text { Hi Amb. }\end{array}$ & Lo Amb. & BotTMP & $\begin{array}{l}\text { Filter } \\
\end{array}$ & Filter dP & \begin{tabular}{|l} 
Thastreme \\
TopTMP
\end{tabular} & | filtrate & Pulsepot & $\begin{array}{l}\text { Slurry } \\
\text { Sles }\end{array}$ & Fitrate & $\begin{array}{l}\text { Measuremer } \\
\text { Hi Filtate }\end{array}$ & Backpulse & & & & & & & & & & & \\
\hline & & & $\operatorname{deg} C$ & $\operatorname{deg} C$ & \begin{tabular}{|l|}
$\operatorname{deg} C$ \\
\end{tabular} & $\operatorname{deg} \mathrm{C}$ & $\operatorname{deg} C$ & psid & psig & psid & psid & \begin{tabular}{|l|} 
psig \\
\end{tabular} & psig & $\mathrm{gpm}$ & $\mathrm{gpm}$ & gpm & $\mathrm{gpm}$ & & & & & & & & & & & \\
\hline & & & & & & & & & & & & & & & & & & & & & & & & & & & & \\
\hline 6 & eros- $01 / 261$ & 504 & & & & & & & & & & & & & & & & & & & & & & & & & & \\
\hline 7 & $8 / 23 / 2004$ & 8:35:38 AM & 22.554 & 22.604 & 22.002 & \begin{tabular}{|l|l|}
22.782 \\
\end{tabular} & 22.204 & -0.019 & 0.095 & -0.012 & 0.003 & -0.124 & 0.13 & -0.075 & 0.003 & 14.643 & 0.004 & & & & urface & .985 & T2 & & & & & \\
\hline 9 & $\begin{array}{l}8 / 24 / 2004 \\
8 / 26 / 2004\end{array}$ & $\begin{array}{l}\text { 8:10:33 AM } \\
9: 14: 11 \mathrm{AM}\end{array}$ & $\frac{23.662}{25.819}$ & $\begin{array}{r}22.93 \\
23.352 \\
\end{array}$ & \begin{tabular}{|l|}
23.679 \\
24.932 \\
\end{tabular} & \begin{tabular}{|l|}
22.998 \\
25.316 \\
\end{tabular} & \begin{tabular}{|l|l|} 
& 22.735 \\
23.242
\end{tabular} & $\begin{array}{l}-0.021 \\
-0.008 \\
-1\end{array}$ & $\begin{array}{ll}0.122 \\
0.126\end{array}$ & $\begin{array}{l}-0.014 \\
-0.008 \\
-1\end{array}$ & $\frac{-0.001}{0.011}$ & $\begin{array}{l}-0.124 \\
-0.106 \\
\end{array}$ & 0.134 & \begin{tabular}{|l|l|}
23.176 \\
8.335
\end{tabular} & \begin{tabular}{|c|}
1.205 \\
0.043 \\
\end{tabular} & $\frac{14.643}{14.633}$ & 0.003 & & & Convers & & & & & & tz/barg & & \\
\hline & & & & & & & & & & & & & & -0.084 & & & & & & Note: & & essu & & & ely 1 & 4 psig & & \\
\hline & $8 / 30 / 2004$ & $7: 28: 55 \mathrm{AM}$ & 22.511 & 22.848 & 22.397 & 22.669 & 22.914 & -0.017 & 0.138 & -0.009 & 0.003 & -0.147 & & -0.082 & 0.003 & 14.643 & 0.006 & & & & & & & & & & & \\
\hline & & & & & & & & & & & & & & & & & & & & & P Plis c & correcte & ted for 31 & 31.25 inch & ches of wa & ater tubing & & \\
\hline$\frac{10}{14}$ & & & & & & & & & & & & & & & & & & & & VWVV & & & & $<$ Filtrat & te Flux & $\Leftrightarrow<$ PERN & $\begin{array}{l}\text { MIAABLIT } \\
\times 1000\end{array}$ & 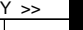 \\
\hline 15 & & & & & & & & & & & & & & & & & & Time & Time & Press. & Vel. & TMP & TMP & gpm & $\mathrm{pm}$ & gpm & gpm & meter \\
\hline 16 & & & & & & & & & & & & & & & & & & & Hour & sig & & & & & & $2 / p s i$ & $\mathrm{t} 2 / \mathrm{psi}$ & ay/bar \\
\hline & $\mathrm{A}$ & B & D & $E$ & $\mathrm{~F}$ & G & $\mathrm{H}$ & $\mathrm{J}$ & $\mathrm{K}$ & L & $\mathrm{M}$ & $\mathrm{N}$ & $\mathrm{O}$ & $Q$ & $\mathrm{R}$ & $\mathrm{s}$ & T & $\mathrm{V}$ & w & $\mathrm{x}$ & $\mathrm{Y}$ & $z$ & $\mathrm{AA}$ & $A B$ & $A C$ & $A D$ & $\mathrm{AE}$ & $\mathrm{AF} / \mathrm{A}$ \\
\hline & Tuesday, 8/2 & 104 & & & & & & & & & & & & & & & & & & & & & & & & & & \\
\hline 38 & Soselme XYL & $\begin{array}{l}\text { water } \\
\text { 8:42:31 AM }\end{array}$ & 24.822 & 23.079 & \begin{tabular}{|l|}
24.655 \\
\end{tabular} & 23.935 & 22.954 & 11.933 & 14.208 & 4.164 & 7875 & -1.209 & & 44.829 & & & 50 & 0.00 & & & & & 683 & 0.219 & & & & \\
\hline & $8 / 24 / 2004$ & 8:43:31 AM & 24.771 & $\begin{array}{l}23.083 \\
\end{array}$ & 24.599 & & 22.903 & 12.112 & \begin{tabular}{|l|l|}
14.206 \\
\end{tabular} & 4.137 & 8.331 & $\mid-1.209$ & -3.954 & 44.62 & 0.003 & 1.458 & 0.003 & & \begin{tabular}{|l|l|}
0.01667 \\
\end{tabular} & $\frac{1.1}{13.1}$ & & & & & & & & 19.03 \\
\hline & $8 / 24 / 2004$ & & 24.745 & 23.087 & 24.538 & 23.989 & & 7.563 & 14.295 & & 3.57 & $\mid$\begin{tabular}{|c|}
1.278 \\
\end{tabular} & & 44.349 & $\begin{array}{l}0.6 \\
\end{array}$ & & 0.003 & 2.00 & 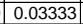 & 13.2 & $\frac{10.6}{10.6}$ & & & $\frac{-0.001}{-0.001}$ & 0.001 & & 0.182 & $\frac{7.58}{0.16}$ \\
\hline 341 & $8 / 24 / 2004$ & $8: 45: 31 \mathrm{AM}$ & 24.73 & 23.102 & 24.487| & 24.058 & 22.866 & 2.599 & 14.637 & 4.107 & -1.356 & 6.813 & 21.255 & 43.855 & 0.003 & -0.013 & 0.003 & 3.00 & 0.05 & 13.5 & 10.4 & & 0.043 & $\frac{-0.002}{-0.02}$ & 0.002 & -0.003 & 3.038 & $\frac{106}{59}$ \\
\hline & $8 / 24 / 2004$ & & & & & & & & & & & & & & & & & & & & & & & & & & & \\
\hline & $8 / 24 / 2004$ & & 24.453 & 23.116 & $24.421 \mid$ & 24.082 & & & & & & \begin{tabular}{|l|l|l|l} 
\\
\end{tabular} & & 44.08 & 0.65 & & & & 0.08333 & 13.5 & 10.5 & 5.1 & & 2.096 & 2.131 & $\overline{0.414}$ & & 2.46 \\
\hline & 8/24/2004 & & 24.608 & 23.125 & 24.39 & & 22.915 & 7.118 & & & & 2.088 & & 44.19 & 0.62 & & & 6.00 & & 13.4 & 10.5 & 5.1 & & 2.096 & & & & 58.94 \\
\hline & 8/24/2004 & & 24.577 & 23.125 & 24.354 & 24.106 & & 12.418 & & & & -1.206 & & 44.4 & 0.003 & & & & 0.11667 & & & & 221 & & & & & 23.70 \\
\hline & $8 / 24 / 2004$ & 8:50:3 & 24.542 & 23.13 & 24.324 & 24.081 & & 12.607 & 14.514 & & & -1.209 & & 44.531 & & $1.3 \mathrm{C}$ & & 8.00 & 0.13333 & 13.4 & & & & & & & & 16.03 \\
\hline & 8/24/2004 & & 24.501 & 23.109 & 24.273 & 24.07 & & 12.36 & & & & -1.212 & & 44.8 & 0.0 & & & 9.00 & 0.15 & 12.9 & 10 & 0. & & 0.191 & 95 & & & $158^{6}$ \\
\hline & $8 / 24 / 2004$ & 8:52:3 & 24.482 & $\frac{23.13}{23.13}$ & 24.294] & 24.076 & & 12.385| & 14.062 & 3.89 & 8.663 & -1.209 & & 44.4 & 0.003 & & & & 0.16667 & 12.9 & 10.6 & & & & & & 18.499 & 15.74 \\
\hline & $8 / 22 / 12004$ & 8:53: & $24.446]$ & 23.129 & & 24.145 & & 12.661 & & & & -1.209 & & & & & & 11.00 & 0.18333 & 13.1 & & & & & 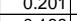 & & & \\
\hline & (1) & & $\frac{2.435}{24,45}$ & & 2.2434 & 24.1253 & & & & & & $-\frac{-1.212}{-120}$ & & & & & & & & 13.2 & & & & & & & & $4.8 \quad-8$ \\
\hline & $8 / 24 / 2004$ & 8:56:31 AM & $\begin{array}{l}24.4138 \\
24.38\end{array}$ & 23.133 & 24.178 & \begin{tabular}{r|r|}
24.115 \\
\end{tabular} & 22.5 & 12.553 & $\begin{array}{l}13.725 \\
13.725\end{array}$ & & $\frac{0.39}{8.538}$ & -1.212 & & 44.7 & & 8 & & 14.00 & \begin{tabular}{|l|}
0.2300 \\
\end{tabular} & \begin{tabular}{|l|}
12.8 \\
12.6
\end{tabular} & $\mid 10.7$ & & 727 & & $\begin{array}{l}0.181 \\
0.174\end{array}$ & & & $\frac{14.69}{1.05}$ \\
\hline & $8 / 24 / 2004$ & & 24.39 & 23.158 & 24.268 & 24.155 & & 12.3 & & & & \begin{tabular}{|c|}
-1.206 \\
\end{tabular} & & & & & & 15.00 & 0.25 & $\mid$\begin{tabular}{|l|l|}
12.5 \\
\end{tabular} & & 10.4 & & & 71 & & & 14.02 \\
\hline & $8 / 24 / 2 C$ & & 24.40 & 23.148 & & & & & & & & -1.212 & & & & & & & 0.266 & $12.5 \mathrm{r}$ & & & & & & & & 1407 \\
\hline & $8 / 24 / 2004$ & 8:59: & 24.435 & 23.153 & 24.348 & 23.984 & & 12. & & & 8.5 & -1.209 & & 44.3 & & & & 17.00 & \begin{tabular}{|l|l|} 
& 0.28333 \\
\end{tabular} & 12.8 & & 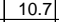 & 36 & & & & & 13.3 \\
\hline & $8 / 21 / 2004$ & & $\begin{array}{l}24.466 \\
2.51]\end{array}$ & 23.158 & 24.343 & 24.0499 & & $\begin{array}{l}12.313 \\
12587\end{array}$ & 13.3999 & 4.066 & 8.2 & -1.212 & & & 0.0 & & & 18.0 & 0.3 & 12.3 & & & & & & & 5.940 & \\
\hline & & & 24.511 & & & 24.09 & & & & 3.884 & & & & & & & & & & & & & & & & & & \\
\hline & 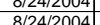 & & $\frac{24.500}{24591}$ & $\frac{23.1999}{23179}$ & $\frac{2.460}{24.78}$ & $\frac{24.055}{24.55}$ & & $\frac{12.414}{12317}$ & 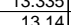 & & & -1.209 & & & & & & & \begin{tabular}{|l|l}
.33333 \\
\end{tabular} & $\frac{12.2}{120}$ & & & & & & & & \\
\hline & $8 / 24 / 2004$ & & 2461 & $\frac{2.1}{231}$ & 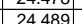 & & & 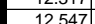 & $\mid \begin{array}{l}13.14 \\
1332\end{array}$ & & & -12 & & & & & & & & $\frac{120}{122}$ & & & & & & & & \\
\hline & $8 / 24 / 2004$ & & & 23.18 & & & & 12.53 & & & & -1.209 & & & & & & 230 & 0.38333 & 121 & & & & & & & & \\
\hline 362 & $8 / 24 / 2$ & 9:06: & 24.645 & 23.1 & $24.548 \mid$ & 24.1 & & & & & 8.298 & -1.21 & & 45.0 & 0.0 & 0.992 & & 24.00 & 0.4 .4 & 12.0 & & 0.5 & & & & & 13.903 & \\
\hline & $8 / 24 / 2004$ & & 24.681 & 23.199 & 24.553 & 24.2 & & 12.433 & & & & -1.212 & & & & & & 25.00 & 0.41667 & 12.0 & & & & & & & & \\
\hline & & & & & & & & & & & & & & & & & & & & 11.6 & & & & & & & & \\
\hline & & & 24.705 & & $24.557 \mid$ & 24.2 & & & & & & -1.21 & & & & & & & & 11.6 & & & & & & & & \\
\hline & $8 / 24 / 2004$ & $9: 10: 3$ & 24.72 & 23.20 & 24.6 & 24.2 & & 12.248 & 13.0 & & & $\begin{array}{l}-1.212 \\
\end{array}$ & & & & 0.9 & & 28 & 0.4 & 11.9 & & & & & & & & \\
\hline & $8 / 24 / 2004$ & 9:11: & 24.7 & 23.202 & 24.6 & 24.2 & & 12.404 & & & 8. & & & 45.0 & & & & 29.0 & & 11.9 & & & & & & & & \\
\hline & 8/24/26 & & 24.81 & & 24.6 & & & 20.733 & & & & & & & & & & & & 24.1 & & & & & & & & \\
\hline 36 & $8 / 24 / 2004$ & & 24.95 & 23.223 & \begin{tabular}{|l|}
24.803 \\
\end{tabular} & 24 & & 21.7 & & 4.0 & & -0.118 & & 45.826 & 0.003 & 2.364 & & 31.0 & 0.51667 & 26.1 & & 20.0 & $\mid$\begin{tabular}{|l|}
$\mid$ \\
$\mid 1.382$
\end{tabular} & 0.338 & 340 & $f$ & 16.976 & 14.45 \\
\hline & \begin{tabular}{|l|}
$8 / 24 / 2004$ \\
$8 / 242004$
\end{tabular} & $\begin{array}{l}\text { 9:14:31 AM } \\
9: 15: 31 \mathrm{AM}\end{array}$ & $25.015 \mid$ & $\begin{array}{l}23.228 \\
23.218\end{array}$ & \begin{tabular}{|l|}
24.803 \\
24588
\end{tabular} & \begin{tabular}{|r|}
24.475 \\
245 \\
\end{tabular} & & $\frac{21.774}{21.907}$ & $\begin{array}{l}27.079 \\
27.185\end{array}$ & $\begin{array}{l}3.924 \\
3.964 \\
\end{array}$ & $\begin{array}{l}18.485 \\
18.475\end{array}$ & $\begin{array}{l}-0.341 \\
-0.0419\end{array}$ & & $\begin{array}{l}44.985 \\
44.781\end{array}$ & $\begin{array}{l}0.003 \\
0.003\end{array}$ & $\begin{array}{l}2.286 \\
2.216\end{array}$ & & $23.00 \mid$ & \begin{tabular}{|l|l|}
0.5333 \\
0.53
\end{tabular} & 26.0 & $\frac{10.7}{10.7}$ & & \begin{tabular}{|l|}
1.388 \\
1392
\end{tabular} & $\mid \begin{array}{l}0.321 \\
0.317\end{array}$ & $\mid \begin{array}{c}0.329 \\
0.319\end{array}$ & $\frac{0.016}{0.016}$ & $\begin{array}{c}6 \\
6\end{array}$ & 13.46 \\
\hline
\end{tabular}


WSRC-TR-2005-00105, REVISION 0

SRNL-RPP-2005-00012, REVISION 0

CAMPAIGN IV NEW GKN BASELINE \#4 WITH DIF WATER - CONT.

\begin{tabular}{|c|c|c|c|c|c|c|c|c|c|c|c|c|c|c|c|c|c|c|c|c|c|c|c|c|c|c|c|c|}
\hline & A & B & D & $E$ & $F$ & G & $\begin{array}{ll}\mathrm{H} \\
\end{array}$ & $\mathrm{J}$ & $\mathrm{K}$ & $L$ & M & \begin{tabular}{l|l}
$N$ \\
\end{tabular} & 0 & 0 & $R$ & $\mathrm{~s}$ & $T$ & $\mathrm{v}$ & w & & 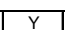 & $z$ & $\mathrm{AA}$ & $A B$ & $A C$ & & $\mathrm{AE}$ & AF \\
\hline & $8 / 24 / 2004$ & $9: 16: 31 \mathrm{AM}$ & 25.015 & 23.223 & 24.733 & 24.57 & 23.117 & 21.92 & 27.056 & 4.111 & 18.426 & -0.471 & $\begin{array}{l}-4.107 \\
\end{array}$ & 44.818 & 0.00 & 2.179 & 0.003 & 34.0 & 0.56667 & 25.9 & 10.7 & 20.2 & 1.391 & 0.312 & 0.314 & 0.016 & 15.578 & Ar 13.26 \\
\hline & $\begin{array}{l}8 / 24 / 2004 \\
\end{array}$ & $9: 17: 31 \mathrm{AM}$ & 25.001 & 23.234 & 24.714 & 24.616 & 23.179 & 22.211 & 27.483 & 3.913 & 18.725 & $\begin{array}{l}-0.456 \\
-0.451\end{array}$ & $\begin{array}{r}-4.101 \\
\end{array}$ & 43.874 & & 2.242 & 0.003 & 35.00 & $\begin{array}{l}0.58333 \\
\end{array}$ & 26.4 & 10.5 & & 1.411 & & 0.324 & & 15.809 & $\begin{array}{l}13.45 \\
\end{array}$ \\
\hline & $8 / 24 / 2004$ & $9: 18: 31 \mathrm{AM}$ & 24.992 & 23.245 & 24.684 & 24.642 & 23.219 & 22.326 & 27.406 & 3.926 & 18.91 & $\frac{-0.514}{-0.514}$ & -4.107 & 44.013 & & 2.275 & & 36.00 & \begin{tabular}{|r|r|}
0.6 \\
\end{tabular} & 26.3 & 10.5 & 20.6 & $1.4 \angle 24$ & 0.326 & 0.329 & 0.016 & 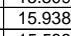 & \\
\hline 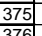 & $8 / 24 / 2004$ & 9:19:31 AM & 24.962 & 23.25 & 24.69 & 24.663 & \begin{tabular}{|l|l|}
23.13 \\
3.17
\end{tabular} & 22.16 & $\begin{array}{r}27 \\
\end{array}$ & $\begin{array}{l}4.078 \\
\end{array}$ & 18.545 & $\begin{array}{l}-0.76 \\
\end{array}$ & $\begin{array}{l}-4.13 \\
\end{array}$ & 455.234 & 0.003 & 2.196 & 0.003 & 37.00 & 61667 & 25.9 & 10.8 & 20.4 & 1.403 & 0.314 & 0.317 & 0.016 & 15.583 & 13.26 \\
\hline$\frac{376}{377}$ & 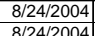 & $\begin{array}{l}9: 20: 31 \mathrm{AM} \\
\cdot 92 \cdot 131 \mathrm{AM}\end{array}$ & $\begin{array}{l}24.943 \\
24989\end{array}$ & $\begin{array}{l}23.256 \\
23266\end{array}$ & \begin{tabular}{|l|l|}
24.666 \\
24641
\end{tabular} & $\begin{array}{l}24.643 \\
21.694 \\
\end{array}$ & \begin{tabular}{|l|}
23.17 \\
23.191
\end{tabular} & \begin{tabular}{|l|l|}
22.425 \\
2489
\end{tabular} & $\frac{27.272}{27202}$ & \begin{tabular}{|l|l|}
3.931 \\
4.096
\end{tabular} & $\begin{array}{r}19 \\
18855\end{array}$ & \begin{tabular}{|c|c|c|}
-0.807 \\
-0.83
\end{tabular} & $\begin{array}{l}-4.133 \\
-4.139\end{array}$ & \begin{tabular}{|l|l|}
45.735 \\
45035
\end{tabular} & & $\begin{array}{l}2.139 \\
.195 \\
\end{array}$ & & 38.00 & & \begin{tabular}{|l|l|}
26.1 \\
36.
\end{tabular} & \begin{tabular}{|l|}
10.7 \\
107 \\
\end{tabular} & $\begin{array}{l}20.7 \\
20.7\end{array}$ & 1.428 & 0.306 & $\begin{array}{l}0.309 \\
0.317 \\
\end{array}$ & $\begin{array}{l}0.015 \\
\end{array}$ & 14.925 & 12.70 \\
\hline$\frac{371}{378}$ & $8 / 24 / 2004$ & $\begin{array}{c}9: 12: 31 \mathrm{AM} \\
\text { 9:22:31 }\end{array}$ & 2 & $\begin{array}{r}23.266 \\
23.282\end{array}$ & 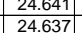 & $\begin{array}{l}24.694 \\
24.755\end{array}$ & $\begin{array}{l}\frac{23.191}{23.142} \\
\end{array}$ & $\begin{array}{r}22.489 \\
22.502\end{array}$ & $\frac{27.222}{27.035}$ & $\begin{array}{l}4.096 \\
3.941\end{array}$ & $\begin{array}{l}18.855 \\
19.021 \\
\end{array}$ & \begin{tabular}{|r|}
$\mid-0.83$ \\
-0.975
\end{tabular} & $\begin{array}{r}-4.139 \\
-4.142 \\
\end{array}$ & $\mid$\begin{tabular}{|}
$\mid 45.035$ \\
44799
\end{tabular} & $\begin{array}{l}0.003 \\
0.003\end{array}$ & $\begin{array}{r}2.195 \\
215\end{array}$ & 003 & $\begin{array}{l}39.00 \\
40.00\end{array}$ & \begin{tabular}{r|r}
0 \\
0
\end{tabular} & $\begin{array}{r}26.1 \\
25.9 \\
\end{array}$ & \begin{tabular}{|l|}
10.7 \\
107
\end{tabular} & 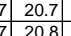 & \begin{tabular}{|l|l|}
1.425 \\
1.43
\end{tabular} & $\begin{array}{l}0.314 \\
0.308\end{array}$ & \begin{tabular}{|l|l|}
0.317 \\
0.311
\end{tabular} & $\begin{array}{l}0.015 \\
0.15\end{array}$ & $\begin{array}{l}15.356 \\
11078\end{array}$ & $\begin{array}{l}13.07 \\
1275\end{array}$ \\
\hline$\frac{1799}{379}$ & $8 / 24 / 2004$ & 9:23:31 AM & 24.895 & 23.273 & 24.598 & 24.751 & \begin{tabular}{|l|}
23.178 \\
\end{tabular} & 22.666 & 27.251 & 4.113 & 19.058 & \begin{tabular}{|c|}
-1.015 \\
\end{tabular} & -4.15 & \begin{tabular}{|r|}
44.67 \\
\end{tabular} & 0.003 & $\begin{array}{l}2.113 \\
\end{array}$ & 0.003 & & \begin{tabular}{|l|l|l}
0 & 0.68333 \\
\end{tabular} & 26.1 & & & $\begin{array}{l}1.431 \\
1.438\end{array}$ & 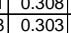 & $\begin{array}{l}0.311 \\
0.306\end{array}$ & \begin{tabular}{|l}
0.015 \\
0.015 \\
\end{tabular} & \begin{tabular}{|l|l|l|l|l|}
14.666 \\
\end{tabular} & $\begin{array}{l}12.75 \\
12.48\end{array}$ \\
\hline & $8 / 24 / 2004$ & & 24.881 & 23.289 & 24.599 & 24.792 & \begin{tabular}{|l|l|}
23.234 \\
\end{tabular} & 22.633 & 26.998 & 3.814 & 19.557 & -1.114 & $\frac{-4.15}{-4.15}$ & $\begin{array}{l}44.606 \\
\end{array}$ & & 2.164 & 0.004 & 42.00 & \begin{tabular}{|r|}
.00535 \\
\end{tabular} & & 10.6 & 21.1 & $\begin{array}{l}1.450 \\
1.454\end{array}$ & 0.310 & 0.313 & & 14.853 & 12.64 \\
\hline & $8 / 24 / 2004$ & & & 23.284 & 24.563 & 24.777 & 23.244 & 22.176 & 26.488 & 3.986 & 18.492 & & -4.179 & 44.614 & 0.003 & & & 43.00 & 0.71667 & 25.4 & 10.6 & 20.3 & 1.402 & & 0.294 & \begin{tabular}{|l|l|l|}
0.014 \\
\end{tabular} & 14.477 & 12.32 \\
\hline 382 & $8 / 24 / 2004$ & 9:26:31 AM & 24.852 & 23.295 & \begin{tabular}{|l|}
24.559 \\
\end{tabular} & $\begin{array}{l}24.787 \\
2.707\end{array}$ & 23.249 & 22.118 & 26.233 & $\begin{array}{l}4.109 \\
\end{array}$ & \begin{tabular}{|l|l|}
18.321 \\
19.727
\end{tabular} & -1.209 & $\begin{array}{l}-4.197 \\
\end{array}$ & \begin{tabular}{|l|l|}
44.958 \\
\end{tabular} & 0.003 & $\begin{array}{l}1.984 \\
\end{array}$ & 0.003 & 44.00 & 0.73333 & 25.1 & 10.7 & 20.2 & 1.394 & 0.284 & 0.288 & 0.014 & 14.224 & 12.10 \\
\hline 584 & 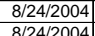 & $\begin{array}{l}9: 27: 31 \mathrm{AM} \\
9 \cdot 28.31 \mathrm{~A}\end{array}$ & \begin{tabular}{|l|}
24.827 \\
2812
\end{tabular} & $\begin{array}{l}23.295 \\
23.31\end{array}$ & \begin{tabular}{|r|}
24.549 \\
24.53
\end{tabular} & $\begin{array}{l}24.787 \\
24.818\end{array}$ & $\begin{array}{r}23.35 \\
23.315 \\
\end{array}$ & $\begin{array}{l}22.241 \\
22.344 \\
2\end{array}$ & $\frac{26.045}{26.26}$ & $\begin{array}{l}4.046 \\
2\end{array}$ & $\begin{array}{l}18.767 \\
18.452\end{array}$ & \begin{tabular}{|c|}
-1.212 \\
1.21 \\
\end{tabular} & \begin{tabular}{|l|l|} 
& -4.199 \\
\end{tabular} & \begin{tabular}{|l|l|}
45.198 \\
45063
\end{tabular} & & $\begin{array}{l}1.975 \\
\end{array}$ & & 45.00 & $\begin{array}{r}0.75 \\
\end{array}$ & 24.9 & 10.8 & 20.5 & 1.414 & 0.283 & 0.286 & $\begin{array}{l}0.014 \\
\end{array}$ & 13.967 & 11.89 \\
\hline$\frac{384}{385}$ & $\frac{8 / 24 / 2004}{8 / 24 / 204}$ & $\begin{array}{l}9: 28: 31 \mathrm{AM} \\
9 \cdot 29 \cdot 31 \mathrm{AM}\end{array}$ & \begin{tabular}{|l|l|}
24.812 \\
24792
\end{tabular} & $\begin{array}{r}23.31 \\
23.305\end{array}$ & \begin{tabular}{|l|}
24.53 \\
24.51
\end{tabular} & $\begin{array}{l}24.818 \\
24843\end{array}$ & $\begin{array}{l}23.315 \\
23.255 \\
\end{array}$ & \begin{tabular}{|l|l|}
22.344 \\
22.334
\end{tabular} & \begin{tabular}{|l|}
26.26 \\
26068
\end{tabular} & \begin{tabular}{r|}
4.2 \\
40.7
\end{tabular} & $\begin{array}{l}18.452 \\
18.773\end{array}$ & & $\begin{array}{r}-4.199 \\
-4.208 \\
\end{array}$ & \begin{tabular}{|l|}
45.063 \\
44.855 \\
\end{tabular} & & $\begin{array}{r}1.985 \\
1.99\end{array}$ & & $\begin{array}{l}46.00 \\
47.00\end{array}$ & 0.76667 & $\frac{25.1}{24.9}$ & $\begin{array}{l}10.7 \\
10.7\end{array}$ & $\begin{array}{l}20.4 \\
20.6 \\
\end{array}$ & $\begin{array}{l}1.406 \\
1.417\end{array}$ & $\begin{array}{l}0.284 \\
0285\end{array}$ & $\begin{array}{l}0.288 \\
0.289\end{array}$ & .014 & \begin{tabular}{|l|l|l|l|} 
\\
\end{tabular} & $\begin{array}{l}12.01 \\
11.06\end{array}$ \\
\hline 386 & $8 / 24 / 2004$ & $9: 30: 31 \mathrm{AM}$ & 24.788 & 23.322 & \begin{tabular}{|l|}
24.511 \\
24.511
\end{tabular} & $\begin{array}{r}24.045 \\
24.869\end{array}$ & 23.316 & $\frac{2 Z .354}{22.491}$ & \begin{tabular}{|l|}
26.2300 \\
26.239
\end{tabular} & \begin{tabular}{|l|l|}
3.984 \\
\end{tabular} & $\begin{array}{l}10.170 \\
19.003\end{array}$ & $\begin{array}{l}-1.212 \\
-1.212\end{array}$ & $\begin{array}{l}-4.200 \\
-2.208\end{array}$ & \begin{tabular}{|l|}
44.854 \\
44.973 \\
\end{tabular} & $\begin{array}{l}0.003 \\
0.003\end{array}$ & \begin{tabular}{|c|}
1.939 \\
1.938
\end{tabular} & \begin{tabular}{|l}
.003 \\
0.003
\end{tabular} & $\begin{array}{l}47.00 \\
48.00\end{array}$ & $\begin{array}{r}.88333 \\
0.8\end{array}$ & $\begin{array}{r}25.9 \\
25.1\end{array}$ & & \begin{tabular}{|l|}
20.6 \\
20.7
\end{tabular} & \begin{tabular}{|l|}
1.417 \\
1.430 \\
\end{tabular} & & $\begin{array}{l}0.289 \\
0.281 \\
\end{array}$ & & \begin{tabular}{|l|l|}
14.054 \\
13.559 \\
\end{tabular} & $\begin{array}{l}11.1 .56 \\
11.54\end{array}$ \\
\hline 387 & 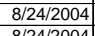 & $9: 31: 31 \mathrm{AM}$ & $\begin{array}{l}24.763 \\
2.7754\end{array}$ & $\begin{array}{l}23.327 \\
23232\end{array}$ & \begin{tabular}{|l|}
24.501 \\
2406
\end{tabular} & $\frac{24.885}{2.892}$ & 23.316 & 22.16 & 25.8 & 4.065 & $\begin{array}{l}18.489 \\
\end{array}$ & -1.209 & -4.22 & 45.384 & 0.003 & $\begin{array}{l}1.913 \\
\end{array}$ & & 49.0 & 0.81667 & 24.7 & & & \begin{tabular}{|l|}
1.401 \\
\end{tabular} & 10.274 & 0.278 & $\begin{array}{l}0.0144 \\
0.014\end{array}$ & 13.666 & $\begin{array}{l}11.64 \\
11.63\end{array}$ \\
\hline 300 & $8 / 24 / 2004$ & 9:32:31 AM & 24.754 & 23.332 & 24.496 & 24.92 & 23.292 & 22.102 & 25.603 & 4.013 & 18.372 & $\begin{array}{l}-1.209 \\
-1.209\end{array}$ & $\begin{array}{r}-4.225 \\
\end{array}$ & \begin{tabular}{|l|}
45.075 \\
\end{tabular} & 0.003 & $\begin{array}{r}1.85 \\
\end{array}$ & 0.004 & 50.00 & 0.83333 & 24.5 & & 20.2 & 1.395 & 0.265 & 0.269 & 0.013 & 13.275 & 11.30 \\
\hline$\frac{389}{390}$ & $8 / 24 / 2004$ & 9:33:31 AM & $\frac{24.735}{2.737}$ & 23.338 & 24.457 & 24.901 & $\begin{array}{r}23.298 \\
3.27\end{array}$ & & 25.591 & & & & & 44.927 & 0.003 & $\begin{array}{l}1.869 \\
\end{array}$ & & & 0.85 & 24.5 & 10. & & 1.404 & 0.268 & 0.272 & 0.013 & 13.344 & 11.36 \\
\hline$\frac{390}{391}$ & 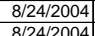 & $\begin{array}{l}9: 34: 31 \mathrm{AM} \\
\cdot 93 \cdot 31 \mathrm{~A}\end{array}$ & \begin{tabular}{|l|}
24.72 \\
247525
\end{tabular} & $\begin{array}{l}23.343 \\
23.354\end{array}$ & $\begin{array}{l}24.462 \\
24.48\end{array}$ & $\begin{array}{l}24.911 \\
24.947\end{array}$ & $\begin{array}{l}23.338 \\
23.398\end{array}$ & \begin{tabular}{|l|l|}
22.33 \\
30.632
\end{tabular} & $\begin{array}{l}25.858 \\
38.912 \\
\end{array}$ & \begin{tabular}{|l|l|}
4.061 \\
4385
\end{tabular} & $\begin{array}{l}18.615 \\
27047\end{array}$ & $\begin{array}{r}-1.209 \\
2384\end{array}$ & \begin{tabular}{|l|}
-4.231 \\
.793
\end{tabular} & \begin{tabular}{|l|l|}
44.91 \\
4790
\end{tabular} & $\begin{array}{l}0.003 \\
0.03\end{array}$ & $\begin{array}{l}1.845 \\
2002\end{array}$ & & 52.00 & $\begin{array}{l}0.86667 \\
0.8923\end{array}$ & $\begin{array}{ll}24.7 \\
279\end{array}$ & & 20.5 & \begin{tabular}{|c|c|}
1.412 \\
1009
\end{tabular} & 0.264 & $\begin{array}{l}0.268 \\
\end{array}$ & $\begin{array}{l}0.013 \\
0.15\end{array}$ & $\begin{array}{l}13.099 \\
1.632\end{array}$ & 11.15 \\
\hline$\frac{391}{392}$ & $\begin{array}{l}8 / 24 / 2004 \\
8824(204\end{array}$ & $\begin{array}{l}9: 35: 31 \mathrm{AM} \\
9 \cdot 36 \cdot 31 \mathrm{~A}\end{array}$ & $\begin{aligned} 24.725 \\
2455\end{aligned}$ & $\begin{array}{r}23.354 \\
23.359\end{array}$ & $\begin{array}{l}24.458 \\
24603\end{array}$ & $\frac{24.947}{24942}$ & $\begin{array}{r}23.398 \\
23.464\end{array}$ & \begin{tabular}{|c|c|}
30.632 \\
30.909
\end{tabular} & $\frac{38.912}{41.233}$ & $\begin{array}{l}4.385 \\
3.969\end{array}$ & $\begin{array}{r}27.047 \\
28.022\end{array}$ & $\begin{array}{r}2.384 \\
4337 \\
\end{array}$ & $\begin{array}{l}-3.7 \\
-3.5 \\
\end{array}$ & \begin{tabular}{|l|}
47.58 \\
4509
\end{tabular} & 003 & $\begin{array}{r}2.903 \\
3.122\end{array}$ & & $\frac{53.00}{54.00}$ & \begin{tabular}{cc|}
0 & 0.88333 \\
00 & 0.0
\end{tabular} & $\frac{37.8}{40.1}$ & 10. & $\frac{28.8}{29.5}$ & 1.988 & $\frac{0.416}{0.447}$ & $\begin{array}{l}0.422 \\
0.452\end{array}$ & & 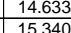 & $\begin{array}{l}12.45 \\
1305\end{array}$ \\
\hline 393 & $8 / 24 / 2004$ & 9:37:31 AM & 25.027 & 23.365 & 24.784 & 24.983 & 23.49 & 30.75 & 41.642 & 3.811 & 28.201 & 4.934 & -3.497 & 44.733 & 0.003 & 3.294 & & 55.00 & \begin{tabular}{|r|}
0.91667 \\
\end{tabular} & 40.5 & 10.7 & 29.5 & 2.032 & 0.472 & 0.474 & $\begin{array}{l}0.016 \\
0.016\end{array}$ & $\frac{15.340}{16.097}$ & $\begin{array}{l}13.05 \\
13.70\end{array}$ \\
\hline 394 & $8 / 24 / 2004$ & 9:38:31 AM & 25.157 & 23.381 & 24.865 & 25.039 & 23.44 & 30.594 & 41.664 & 3.799 & 27.919 & 5.059 & & 44.8 & 0.003 & & & 56.00 & 0.93333 & 40.5 & 10.7 & & 2.017 & 0.486 & 0.487 & & 16.662 & $\begin{array}{l}\frac{13.10}{14.18} \\
\end{array}$ \\
\hline & $8 / 24 / 2004$ & $9: 39: 31 \mathrm{AM}$ & 25.228 & 23.386 & 24.901 & 25.134 & 23.476 & 30.685 & 41.781 & 3.802 & 27.952 & 5.105 & -3.483 & \begin{tabular}{|l|l}
44.698 \\
\end{tabular} & & 3.472 & & 57.00 & $\begin{array}{cc}0 & 0.95 \\
\end{array}$ & 40.7 & 10. & 29.3 & 2.021 & 0.497 & 0.498 & & 17.001 & 14.47 \\
\hline 396 & $8 / 24 / 2004$ & 9:40:31 AM & $\begin{array}{l}25.284 \\
2.28\end{array}$ & $\begin{array}{l}23.402 \\
302\end{array}$ & 24.947 & $\begin{array}{r}25.225 \\
2.257\end{array}$ & 23.517 & 31.239 & 42.359 & 3.832 & 28.556 & \begin{tabular}{|l|l|}
5.053 \\
\end{tabular} & & \begin{tabular}{|l|}
44.26 \\
\end{tabular} & 0.003 & 3.408 & & 58.00 & \begin{tabular}{|l|l|}
0.96667 \\
\end{tabular} & 41.2 & 10.5 & 29.9 & 2.061 & 0.488 & 0.489 & & 16.344 & 13.91 \\
\hline$\frac{397}{398}$ & $\begin{array}{l}8 / 24 / 2004 \\
8 / 24 / 204\end{array}$ & $\begin{array}{l}9: 11: 31 \mathrm{AM} \\
9: 22: 31 \mathrm{AM}\end{array}$ & \begin{tabular}{|l|}
25.305 \\
25.315
\end{tabular} & $\begin{array}{l}23.408 \\
23.404 \\
\end{array}$ & \begin{tabular}{|l|l|}
24.928 \\
24.938
\end{tabular} & $\begin{array}{l}25.257 \\
25.277\end{array}$ & $\begin{array}{l}23.553 \\
23.549 \\
2\end{array}$ & \begin{tabular}{|l|l|}
31.316 \\
31.366
\end{tabular} & $\begin{array}{l}42.487 \\
42.252 \\
\end{array}$ & $\begin{array}{l}3.821 \\
3.795 \\
\end{array}$ & $\begin{array}{l}28.662 \\
28.786 \\
\end{array}$ & \begin{tabular}{|l|}
5.053 \\
4.972 \\
\end{tabular} & $\begin{array}{l}-3.485 \\
-3.491\end{array}$ & \begin{tabular}{|l|}
44.358 \\
44099 \\
\end{tabular} & $\begin{array}{l}0.003 \\
0.003\end{array}$ & \begin{tabular}{|l|l|}
3.348 \\
3.323
\end{tabular} & & $\begin{array}{l}59.00 \\
60.00\end{array}$ & 0.98333 & \begin{tabular}{|l|l|}
41.4 \\
413
\end{tabular} & $\frac{10.6}{10 .}$ & $\begin{array}{l}30.0 \\
301\end{array}$ & $\begin{array}{l}2.068 \\
2.074 \\
\end{array}$ & 0.479 & 0.480 & 0.016 & $\begin{array}{l}16.016 \\
15845\end{array}$ & $\begin{array}{l}13.63 \\
13.48\end{array}$ \\
\hline 399 & $8 / 24 / 2004$ & 9:43:31 AM & 25.341 & 23.43 & 24.989 & $\begin{array}{l}25.271 \\
25.343\end{array}$ & $\begin{array}{r}23.549 \\
23.554\end{array}$ & | & $\begin{array}{l}42.752 \\
42.701\end{array}$ & $\begin{array}{l}3.759 \\
3.911 \\
\end{array}$ & $\begin{array}{l}28.886 \\
29.015\end{array}$ & $\begin{array}{l}\mid 4.9 / 2 \\
5.105\end{array}$ & $\begin{array}{r}-3.3491 \\
-3.471\end{array}$ & \begin{tabular}{|l|}
44.049 \\
45.152 \\
\end{tabular} & & $\begin{array}{l}3.323 \\
3.386\end{array}$ & & $\frac{60.00}{6100}$ & \begin{tabular}{|r|}
101667 \\
\end{tabular} & $\begin{array}{l}41.3 \\
41.6 \\
\end{array}$ & $\frac{10.5}{10.8}$ & $\begin{array}{l}30.1 \\
30.3 \\
\end{array}$ & $\frac{2.074}{2092}$ & 0.476 & $\begin{array}{l}0.447 \\
0.485\end{array}$ & & $\begin{array}{l}15.845 \\
15.978\end{array}$ & 13.48 \\
\hline & 8/24/2004 & 4:31 AM & 25.357 & 23.436 & $\mid 25.005$ & 25.354 & 23.606 & 31.957 & 43.047 & 3.869 & 29.27 & 5.117 & & 45.286 & & & & 62.00 & 1.03333 & 41.9 & 10.8 & 30.6 & & & 0.485 & & $\frac{15.998}{15.842}$ & $\begin{array}{l}13.60 \\
1348\end{array}$ \\
\hline & $8 / 24 / 2004$ & 9:45:31 AM & 25.388 & 23.442 & \begin{tabular}{|l|}
25.041 \\
\end{tabular} & 25.39 & 23.6 & 31.679 & 42.746 & 3.876 & & & & 45.046 & & & & 63.00 & \begin{tabular}{c|c}
0 & 1.05 \\
\end{tabular} & 41.6 & & & & & 0.487 & & & $\frac{13.48}{13.69}$ \\
\hline & $8 / 24 / 2004$ & 9:46:31 AM & 25.414 & 23.448 & 25.062 & 25.421 & 23.6 & 31.722 & 42.755 & 3.798 & 28.991 & 4.986 & & 44.806 & & 3.465 & & 64.00 & \begin{tabular}{|l|l|}
1.06667 \\
\end{tabular} & 41.6 & 10.7 & 30.4 & & & & & & $\frac{13.69}{13.88}$ \\
\hline 403 & $8 / 241$ & & 25.445 & 23.469 & 25.089 & & 23.5 & 31.837 & 42.949 & & 28. & & & $\begin{array}{lll}44.8 & \end{array}$ & & & & & 1.08333 & 41.8 & & & 09 & & & & & \\
\hline 402 & $8 / 24 / 2004$ & & 25.487 & 23.491 & 25.115 & 25.444 & 23.6 & 31.946 & 42.1011 & 3.87 & 29.3 & & & & & 3.32 & & & 1.1 & & & & & & & & & \\
\hline & $8 / 24 / 2004$ & 9:31 AM & 25.492 & 23.476 & $\frac{25.096}{25}$ & 25.449 & & 32.407 & 43.418 & & & & & & & & & & . $11667 \mathrm{r}$ & & & & & & & & & \\
\hline & $8 / 24 / 2004$ & D:31 AM & 25.509 & 23.488 & 25.102 & 25.466 & & 32.098 & 42.981 & 3.84 & & $4.7 .7-3$ & & & & & & & .1333 & & & & & & & & & \\
\hline & $8 / 24 / 2$ & $1: 31 \mathrm{AM}$ & 25.54 & 23.505 & 25.133 & 25.502 & 23.7 & 32.28 & .078 & 3. & 29.47 & 4.7 & & & & & & 69 & $1.1 .1 \mathrm{C}$ & & & & $2.1 .12-3$ & & & & & \\
\hline & $8 / 24$ & 9:52: & 25.55 & 23.52 & 25.149 & 25.518 & 23.6 & 32.202 & & $3.8 \mathrm{r}>\mathrm{l}$ & & & & & & & & & & & & & & & & & & \\
\hline & & & 25.567 & & 25.165 & 25.539 & & 32.025 & & & & & & & & & & & & & & & & & & & & \\
\hline & & 9:54:31 & 25.583 & 23.537 & 25.176 & 25.57 & & 32.475 & & & $29.8 \mathrm{r}>\mathrm{r}$ & & & & & & & & & & & & & & & & & \\
\hline & $\frac{82424}{8021}>$ & $1 \mathrm{AM}$ & 25.594 & 2.3.59 & 望5.188 & 255671 & $\frac{23.8}{23}$ & $\begin{array}{ll}32.052 \\
32.169\end{array}$ & 42.328 & 3.9 & & & 35 & & & & & & & & & & & & & & & \\
\hline 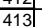 & $\begin{array}{l}8 / 2 / 24 / 2004 \\
8 / 2 / 204 \\
\end{array}$ & $\begin{array}{l}9.50 .31 \mathrm{AM} \\
9.57 \cdot 31 \mathrm{AM}\end{array}$ & $\frac{25.506}{25603}$ & 23.577 & $\frac{25.195}{25196}$ & 25.009 & & $\begin{array}{l}32.100 \\
32.258\end{array}$ & $\frac{41.975}{4227}$ & & & & & & & & & & & & & & & & & & & \\
\hline 41 & $8 / 24 / 2004$ & 9:58:31 AM & $\frac{25.599}{25}$ & 23.584 & 25.193 & $\mid 25.642$ & 23.793 & $\mid 31.957$ & 41.843 & 3.786 & 29.178 & 3.935 & $-3.581->$ & 44.533 & 0.003 & 3.222 & & & & & & 30.6 & 2.108 & & & & .144 & \\
\hline & & & & & & & & & & & & & & & & & & & & & & & & & & & & \\
\hline 416 & & Averages & 24.5 & 23.2 & 24.4 & 24.1 & & 12.4 & 13.6 & 4 & 8.5 & -1.2 & & $\begin{array}{ll}44.9 \\
\end{array}$ & 0.0 & $\begin{array}{ll}1.2 \\
\end{array}$ & & & & 12.5 & 10.7 & 10.4 & 0.7 & 0.2 & 0.2 & 0.0 & 16.2 & \\
\hline 417 & & & $\begin{array}{l}24.7 \\
\end{array}$ & 23.2 & 24 & 24.3 & & 12.8 & 1 & 4 & 90 & -1.2 & & & 80 & 2.0 & & & & 13.4 & \begin{tabular}{|l|}
10.9 \\
\end{tabular} & \begin{tabular}{l|l|}
9 & 10.8 \\
\end{tabular} & 0.7 & & & & 27.8 & \\
\hline 418 & & Media & $\begin{array}{l}24.5 \\
24 . \\
\end{array}$ & 23.2 & $\begin{array}{l}24.4 \\
2.42\end{array}$ & $\begin{array}{l}24.1 \\
2.1 \\
\end{array}$ & $\frac{22}{23}$ & $\begin{array}{l}12.4 \\
12.1\end{array}$ & 13.6 & 4.2 & 8.5 & -1.2 & -4. & 44.8 & 0.0 & 1.1 & 0.0 & & & $\begin{array}{ll}12.5 \\
11.6\end{array}$ & \begin{tabular}{|l|}
10.7 \\
10
\end{tabular} & $\begin{array}{ll}77 & 10.5 \\
6 & 10.0\end{array}$ & $\begin{array}{l}0.7 \\
0.7\end{array}$ & 70.2 & 0.2 & 0.0 & 15.7 & 13.4 \\
\hline & & $\frac{\text { Minimun }}{2 \times \operatorname{Std} D}$ & $\begin{array}{r}24.4 \\
0227 \\
\end{array}$ & $\begin{array}{r}23.1 \\
0.056 \\
\end{array}$ & \begin{tabular}{r|r|r|}
24.2 & \\
0267 &
\end{tabular} & \begin{tabular}{r|}
24.0 \\
0.16
\end{tabular} & $\begin{array}{r}22.9 \\
0.077 \\
\end{array}$ & \begin{tabular}{r|r}
12.1 \\
0.333
\end{tabular} & $\begin{array}{r}12.8 \\
1020 \\
\end{array}$ & $\begin{array}{r}3.9 \\
0.273 \\
\end{array}$ & \begin{tabular}{r|}
7.8 \\
0.68 \\
\end{tabular} & $\begin{array}{r}-1.2 \\
0.005 \\
\end{array}$ & \begin{tabular}{r|r}
-4.5 \\
0.043
\end{tabular} & $\begin{array}{r}44.3 \\
0.696\end{array}$ & $\begin{array}{r}0.0 \\
0.000 \\
\end{array}$ & $\begin{array}{r}0.9 \\
0.49 \\
\end{array}$ & $\begin{array}{r}0.0 \\
0.000\end{array}$ & & & $\begin{array}{r}11.6 \\
1019 \\
\end{array}$ & \begin{tabular}{|r|}
10.6 \\
0.166
\end{tabular} & $\begin{array}{ll}6 & 10.0 \\
6 & 0.450 \\
\end{array}$ & $\begin{array}{r}0.7 \\
0.031 \\
\end{array}$ & $\begin{array}{r}0.1 \\
0.064\end{array}$ & $\frac{0.1}{0.066}$ & $\begin{array}{r}0.0 \\
0.006\end{array}$ & $\begin{array}{r}12.9 \\
6.120 \\
-120\end{array}$ & $\begin{array}{r}11.0 \\
5208\end{array}$ \\
\hline & Number $\mathrm{C}$ & of Points Used* & 23 & & & 23 & 23 & 23 & 23 & 23 & 23 & 23 & 23 & 23 & $\frac{23}{23}$ & 23 & 23 & & & $\frac{1.019}{23}$ & $\begin{array}{r}.1 .00 \\
23 \\
\end{array}$ & 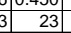 & $\frac{0.011}{23}$ & $\frac{0.004}{23}$ & $\frac{0.000}{23}$ & $\begin{array}{r}-.000 \\
23 \\
\end{array}$ & $\frac{0.120}{23}$ & 5.208 \\
\hline & & ${ }^{*}$ Backpulse poi & ts in box & re not inc & luded & & & & & & & & & & & & & & & & & & & & & & & \\
\hline & & & & & & & & & & & & & & & & & & & & & & & & & & & & \\
\hline 424 & & Averages & 24.9 & 23.3 & 24.6 & $\begin{array}{ll}24.7 \\
\end{array}$ & 23.2 & 22.2 & 26.7 & 4.0 & $\begin{array}{ll}18.7 \\
\end{array}$ & $\begin{array}{ll}-0.9 \\
\end{array}$ & -4.2. & 44.9 & 0.0 & 2.1 & 0.0 & & & 25.5 & 10.7 & 20.5 & 1.4 & 0.3 & 0.3 & 0.0 & 14.8 & \\
\hline & & Maximum & $\begin{array}{l}25.0 \\
249 \\
\end{array}$ & 23.3 & 24.8 & $\begin{array}{l}24.9 \\
21.9\end{array}$ & \begin{tabular}{|l|l|}
23.4 \\
323
\end{tabular} & $\begin{array}{l}22.7 \\
320 \\
\end{array}$ & 27.5 & 4.2 & 19.6 & $\begin{array}{ll} & -0.1 \\
-1 .\end{array}$ & -4 & 45 & 0.0 & $\begin{array}{l}2.4 \\
2 . \\
\end{array}$ & 0.0 & & & $\begin{array}{ll}26.4 \\
25.9\end{array}$ & $\begin{array}{ll}10.9 \\
107\end{array}$ & $\begin{array}{ll}9 & 21.1 \\
7 & 20.4\end{array}$ & 1.5 & 0.3 & 0.3 & 0.0 & 17.0 & 14.4 \\
\hline & & \begin{tabular}{|l} 
Median \\
Minimum
\end{tabular} & $\begin{array}{r}24.9 \\
247 \\
\end{array}$ & $\frac{23.3}{23.2}$ & \begin{tabular}{l|}
24.6 \\
24.5
\end{tabular} & $\begin{array}{r}24.8 \\
24.4 \\
\end{array}$ & $\frac{23.2}{23.1}$ & \begin{tabular}{r|r}
22.2 \\
21.8 \\
\end{tabular} & 27.0 & $\begin{array}{l}4.0 \\
3.8 \\
\end{array}$ & $\begin{array}{r}18.6 \\
18.3\end{array}$ & $\begin{array}{l}-1.1 \\
-1.2 \\
\end{array}$ & -4 & & & & & & & $\begin{array}{r}25.9 \\
2.5 \\
\end{array}$ & $\begin{array}{l}10.7 \\
10.5 \\
\end{array}$ & \begin{tabular}{|l|l|}
7 & 20.4 \\
5 & 200 \\
\end{tabular} & $\begin{array}{r}1.4 \\
1.4\end{array}$ & 0.3 & 0.3 & & & 12.6 \\
\hline & & $2 \times \operatorname{Std} \mathrm{Dev}$ & $\begin{array}{l}24.1 \\
0.193 \\
\end{array}$ & 0.079 & $\begin{array}{r}24.5 \\
0.211 \\
\end{array}$ & $\begin{array}{l}24.4 \\
0.289 \\
\end{array}$ & $\begin{array}{l}20.1 \\
0.170 \\
\end{array}$ & $\begin{array}{l}21.0 \\
0.485 \\
\end{array}$ & $\frac{25.6}{1.243}$ & \begin{tabular}{r|}
0.0 \\
0.179 \\
\end{tabular} & $\begin{array}{r}10.5 \\
0.605 \\
\end{array}$ & \begin{tabular}{r|}
-1.2 \\
0.707
\end{tabular} & $\begin{array}{l}-4.2 \\
0.099 \\
\end{array}$ & \begin{tabular}{r|}
43.9 \\
0.798
\end{tabular} & 0.000 & $\begin{array}{r}1.0 \\
0.301\end{array}$ & 0.001 & & & \begin{tabular}{r|}
24.5 \\
1.243
\end{tabular} & \begin{tabular}{|l|}
10.5 \\
0.190 \\
\end{tabular} & $\begin{array}{l}5 \\
5\end{array}$ & \begin{tabular}{|l|}
1.4 \\
0.036
\end{tabular} \mid & $\begin{array}{l}0.3 \\
0.043 \\
5\end{array}$ & \begin{tabular}{r|}
0.3 \\
0.042
\end{tabular} & $\begin{array}{r}0.002 \\
0.02\end{array}$ & $\frac{1.1}{2.104}$ & $\begin{array}{r}11.1 \\
1.791 \\
\end{array}$ \\
\hline 429 & Number $\mathrm{c}$ & of Points & 22 & 22 & 22 & 22 & 22 & 22 & 22 & 22 & 22 & 22 & 22 & 22 & 22 & 22 & 22 & & & 22 & 22 & 22 & 22 & 222 & 22 & 22 & 22 & $\frac{1.191}{22}$ \\
\hline & & * Backpulse & in boxa & re not inc & liuded & & & & & & & & & & & & & & & & & & & & & & & \\
\hline & & & & & & & & & & & & & & & & & & & & & & & & & & & & \\
\hline & & Averages & 25.4 & 23.5 & $\begin{array}{l}25.0 \\
252 \\
25\end{array}$ & 25.4 & 23.6 & $\begin{array}{l}31.7 \\
325\end{array}$ & 42.5 & 3 & 29 & $\frac{4.7}{5 .}$ & & & 0 & 3 & & & & 41.4 & & 30.4 & & & & & 15.6 & 13.3 \\
\hline & & Med & $\mid \begin{array}{l}25.6 \\
25.4\end{array}$ & 23.5 & 25.4 & $\frac{25.0}{25.4}$ & $\frac{23}{23}$ & $\frac{3.5}{31.9}$ & 42.6 & \begin{tabular}{ll|}
3.8 \\
\end{tabular} & 29.9 & $\begin{array}{l}5.1 \\
4.8 \\
\end{array}$ & & $\frac{45}{44}$ & 0.0 & 33 & & & & $\frac{4.3}{41.5}$ & 106 & 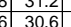 & 21 & 0.5 & 0.5 & $\frac{0.0}{0.0}$ & 1.0 & \\
\hline & & & 24.9 & 23.4 & 24.6 & 24.9 & & 30.6 & 4 & 3.7 & 27.9 & 3.9 & & & 0.0 & 3.1 & & & & 40.1 & 10.5 & \begin{tabular}{|l|l|}
5 & 29.3 \\
\end{tabular} & 2.0 & 0.4 & 0.5 & 0.0 & 14.7 & 12 \\
\hline & & & 0.386 & 0.139 & 0.297 & 0.397 & 0.235 & 1.108 & 1.103 & 0.120 & 1.073 & 0.764 & 0.072 & 0.558 & 0.000 & 0.172 & 0.000 & & & 1.103 & 0.133 & $\begin{array}{ll}3 & 1.085 \\
\end{array}$ & \begin{tabular}{|l|l|} 
& .075 \\
\end{tabular} & 5.025 & 0.025 & 0.001 & 1.184 & 1.008 \\
\hline 437 & Number c & of Points Used* & 23 & 23 & 23 & 23 & 23 & 23 & 23 & 23 & 23 & 23 & 23 & 23 & 23 & 23 & 23 & & & 23 & 23 & 23 & 23 & 23 & 23 & 23 & 23 & 23 \\
\hline 438 & & * Backpulse poi & ts in box a & are not inc & Eluded & & & & & & & & & & & & & & & & & & & Calc & & & & \\
\hline$\frac{439}{440}$ & & & & & & & & & & & & & & & & & & & & & & Avg & gSt Dv & ( (all pts) & 0.044 & & & \\
\hline$\frac{440}{441}$ & & & & & & & & & & & & & & & & & & & & & & Max & in StDv & 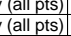 & 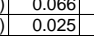 & & & \\
\hline
\end{tabular}


WSRC-TR-2005-00105, REVISION 0 SRNL-RPP-2005-00012, REVISION 0

CAMPAIGN IV NEW GKN BASELINE \#4 WITH DIF WATER - CONT.

\begin{tabular}{|c|c|c|c|c|c|c|c|c|c|c|c|c|c|c|c|c|c|c|c|c|c|c|c|c|c|c|c|c|}
\hline & $\mathrm{A}$ & B & D & $E$ & $\mathrm{~F}$ & G & $\mathrm{H}$ & $\mathrm{J}$ & $\mathrm{K}$ & $\mathrm{L}$ & $\mathrm{M}$ & $\mathrm{N}$ & O & Q & $\mathrm{R}$ & $\mathrm{s}$ & $T$ & $\mathrm{~V}$ & w & $x$ & $\mathrm{Y}$ & $Z$ & $\mathrm{AA}$ & $A B$ & $\mathrm{AC}$ & $A D$ & $\mathrm{AE}$ & $A F \quad A$ \\
\hline & $\begin{array}{l}\text { Tuesday, 8/2 } \\
\text { Baseline bas }\end{array}$ & & & & & & & & & & & & & & & & & & & & & & & & & & & \\
\hline & $8 / 24 / 2004$ & 10:43:11 AM & 25.725 & 23.986 & 25.499 & & 23.879 & & 9.814 & 4.357 & 3.301 & -1.212 & & 44.844 & 0.399 & $\begin{array}{ll}14.643 \\
\end{array}$ & 0.003 & $0 .(9>3$ & & 8.7 & $10.7 \mathrm{P}-\mathrm{s}$ & & 0.367 & & & & & \\
\hline 446 & $8 / 24 / 2004$ & $10: 44: 11 \mathrm{AM}$ & 25.683 & 23.984 & 25.397 & 25.3 & 23.702 & 7.35 & $\begin{array}{l}.014 \\
9.813 \\
\end{array}$ & 4.083 & $\begin{array}{l}3.001 \\
3.194 \\
\end{array}$ & -1.212 & $\begin{array}{l}-0.454 \\
-3.509 \\
\end{array}$ & 45.043 & 0.392 & $\begin{array}{l}14.045 \\
14.643 \\
\end{array}$ & 0.003 & 1.00 & 0.01667 & $\begin{array}{l}8.1 \\
8.7\end{array}$ & 10.7 & 5.3 & 0.363 & 0.056 & 0.055 & 0.011 & 10.527 & 8.96 \\
\hline \begin{tabular}{|l|l|}
447 \\
\end{tabular} & $8 / 24 / 2004$ & 10:45:11 AM & 25.625 & 23.981 & 25.304 & & 23.619 & 7.192 & 9.552 & 4.196 & 3.174 & \begin{tabular}{|l|}
-1.212 \\
\end{tabular} & -3.546 & 44.789 & & 14.643 & 0.003 & 2.00 & 0.03333 & 8.4 & 10.7 & 5.2 & 0.357 & 0.056 & 0.055 & 0.011 & 10.654 & \\
\hline 448 & $8 / 24 / 2004$ & $10: 46: 11 \mathrm{AM}$ & 25.572 & 23.983 & 25.2 & 25.399 & 23.54 & 7.273 & 9.586 & 4.085 & 3.282 & -1.209 & -3.569 & \begin{tabular}{|l|l|l|}
44.764 \\
\end{tabular} & 0.388 & \begin{tabular}{|l|l|}
14.643 \\
\end{tabular} & 0.004 & 3.00 & 0.05 & 8.5 & $\begin{array}{ll}10.7 \\
\end{array}$ & 5.3 & & & 0.055 & 0.010 & 10.466 & 8.91 \\
\hline 449 & $8 / 24 / 2004$ & 10:47:11 AM & 25.516 & 23.986 & 25.104 & 25.423 & 23.519 & 7.325 & 9.575 & 4.188 & 3.287 & -1.212 & -3.601 & 44.706 & 0.383 & 14.643 & 0.003 & 4.00 & 0.06667 & 8.4 & 10.6 & 5.3 & 0.366 & 0.055 & 0.055 & 0.010 & 10.304 & 8.77 \\
\hline & $8 / 24 / 2004$ & $10: 48: 11 \mathrm{AM}$ & 25.469 & & 25.058 & 25.446 & 23.543 & 7.474 & 9.722 & 4.111 & 3.574 & -1.206 & -3.561 & & 0.368 & \begin{tabular}{ll|}
14.643 \\
\end{tabular} & 0.004 & 5.00 & 0.08333 & 8.6 & 10.7 & 5.5 & 0.381 & & 0.053 & & 9.522 & 8.10 \\
\hline$\frac{451}{452}$ & $8 / 24 / 2004$ & 10:49:11 AM & 25.413 & 23.989 & 24.991 & 25.45 & 23.612 & 7.574 & 9.787 & 4.332 & 3.319 & $\begin{array}{r}-1.215 \\
-1.201\end{array}$ & -3.668 & $\begin{array}{l}44.61 \\
\end{array}$ & 0.373 & 14.643 & 0.003 & 6.00 & 0.1 & 8.7 & 10.6 & 5.4 & 0.376 & 0.053 & 0.053 & & 9.807 & 8.35 \\
\hline$\frac{452}{453}$ & 8/24/2004 & 10:50:11 AM & $\begin{array}{l}25.412 \\
25411 \\
\end{array}$ & 24.003 & 24.946 & $\begin{array}{l}25.494 \\
25.528\end{array}$ & $\begin{aligned} 23.671 \\
2374\end{aligned}$ & $\begin{array}{r}2.41 \\
2.458 \\
\end{array}$ & $\begin{array}{l}9.625 \\
9724\end{array}$ & $\begin{array}{l}4.106 \\
232\end{array}$ & & & $\begin{array}{l}19.948 \\
48.822 \\
\end{array}$ & \begin{tabular}{|l|}
44.685 \\
44.599
\end{tabular} & 0.003 & \begin{tabular}{|r|} 
\\
\end{tabular} & 0.003 & $\begin{array}{r}7.00 \\
8000\end{array}$ & 0.11667 & $\begin{array}{r}8.5 \\
8 .\end{array}$ & $\begin{array}{l}10.6 \\
106\end{array}$ & 0.5 & & 0.000 & 0.000 & 0.001 & 0.946 & 0.81 \\
\hline$\frac{45}{45}$ & $\begin{array}{l}8 / 24 / 2004 \\
8 / 242004\end{array}$ & $\begin{array}{l}10: 51: 11 \mathrm{AM} \\
10: 52: 11 \mathrm{AM}\end{array}$ & $\begin{array}{l}25.411 \\
25.262\end{array}$ & $\frac{24.012}{24.013}$ & $\begin{array}{l}24.909 \\
24.835 \\
\end{array}$ & 25.528 & $\begin{array}{r}23.74 \\
23.745\end{array}$ & 2.458 & $\begin{array}{l}9.724 \\
9697 \\
\end{array}$ & $\begin{array}{r}4.232 \\
4.19 \\
\end{array}$ & $\begin{array}{l}-1.741 \\
0.062 \\
\end{array}$ & $\begin{array}{r}1.984 \\
0.23\end{array}$ & $\begin{array}{r}48.822 \\
0.328 \\
\end{array}$ & \begin{tabular}{|l|}
44.599 \\
44.714 \\
\end{tabular} & $\begin{array}{l}0.003 \\
0.162 \\
\end{array}$ & \begin{tabular}{|l|}
$\mid 14.643$ \\
14.643 \\
\end{tabular} & 0.003 & 8.00 & 0.13333 & $\begin{array}{l}8.6 \\
8.6\end{array}$ & \begin{tabular}{l|l}
10.6 \\
10.7
\end{tabular} & $\frac{0.4}{2.1}$ & 0.025 & 0.000 & $\begin{array}{l}0.000 \\
0.03 \\
\end{array}$ & 0.001 & $\begin{array}{r}1.201 \\
11027\end{array}$ & 1.02 \\
\hline 455 & $\begin{array}{l}\mid 8 / 24 / 2004 \\
8 / 24 / 2004 \\
\end{array}$ & 10:53:11 AM & 25.247 & $\frac{24.013}{24.003}$ & $\begin{array}{l}24.035 \\
24.765\end{array}$ & 25.539 & 23.826 & $\begin{array}{l}-4.104 \\
4.378 \\
\end{array}$ & $\begin{array}{l}9.097 \\
9.961\end{array}$ & $\begin{array}{r}4.19 \\
4.402 \\
\end{array}$ & $\begin{array}{c}-0.0624 \\
-0.034\end{array}$ & $\begin{array}{l}0.259 \\
0.259 \\
\end{array}$ & $\begin{array}{l}0.328 \\
0.374\end{array}$ & $\begin{array}{l}44 . .144 \\
44.814\end{array}$ & 0.172 & $\begin{array}{l}\frac{14.043}{14.643} \\
\end{array}$ & $\begin{array}{l}0.004 \\
0.003 \\
\end{array}$ & $\begin{array}{r}9.00 \\
10.00\end{array}$ & $\begin{array}{l}0.15 \\
0.16667 \\
\end{array}$ & 8.6 & $\frac{1.1}{10.7}$ & $\frac{2.1}{2.2}$ & $\frac{0.146}{0.150}$ & & 0.025 & 0.011 & $\frac{11.027}{11.612}$ & $\begin{array}{l}9.38 \\
9.88 \\
\end{array}$ \\
\hline 456 & $8 / 24 / 2004$ & 10:54:11 AM & 25.247 & 24.008 & 24.77 & 25.549 & 23.936 & 4.137 & 9.718 & 4.029 & 0.368 & 0.331 & 0.429 & 44.579 & 0.159 & 14.643 & 0.003 & 11.00 & 0.18333 & 8.6 & 10.6 & 6 & 0.155 & 0.023 & 0.023 & .010 & $\begin{array}{l}\frac{1.1 .14}{10.171} \\
\end{array}$ & \\
\hline 457 & $8 / 24 / 2004$ & 10:55:11 AM & 25.213 & 24.019 & 24.826 & 25.515 & 23.977 & 4.195 & 9.834 & 4.135 & 0.179 & $\begin{array}{ll}0.328 \\
\end{array}$ & 0.464 & \begin{tabular}{|l|l}
44.7 \\
\end{tabular} & 0.151 & $\begin{array}{ll}14.643 \\
\end{array}$ & & 12.00 & & & & & & & & & & $\frac{8.66}{8.45}$ \\
\hline$\frac{458}{456}$ & 8/24/2004 & 10:56:11 AM & 25.214 & 24.04 & 24.857 & 25.561 & 23.953 & & & & & & 0.498 & 45.061 & 0.147 & & 0.003 & 13.00 & .21667 & 8.8 & 10.7 & & 14 & & $.02-28$ & & 0.159 & 8.45 \\
\hline & & 10:57:11 AM & & & & & & 3.944 & 9.664 & 4.104 & -0.063 & 0.369 & & 44.56 & & 14.643 & & & & 8.5 & & & & & & & & 8.05 \\
\hline & $8 / 24 / 2004$ & 10:58:11 AM & 25.105 & 24.046 & 24.868 & & 24.064 & 6.873 & 9.619 & $\begin{array}{lll}4.218 & \\
\end{array}$ & 2.802 & -1.209 & -3.2 & 45.179 & 0.426 & 14.643 & 0.003 & 15.00 & 0.25 & 8.5 & $10.8 \mathrm{Y} \quad \mathrm{s}$ & $\begin{array}{l}4.8 \\
\end{array}$ & 0.334 & & & & 2.654 & 10.77 \\
\hline 46 & 8/24/2004 & 10:59:11 AM & 25.081 & 24.052 & 24.879 & 25.644 & 23.985 & 7.033 & 9.834 & & 2.78 & -1.215 & -3.25. & $45.0 \angle 35$ & 0.419 & 14.043 & 0.0 & 16. & .26667 & 8.1 & 10. & 4. & & . & $=$ & & .268 & 10.44 \\
\hline & $8 / 24 / 2004$ & 11:00:11 AM & 25.072 & 24.068 & 24.875 & 25.734 & 24.051 & 7.037 & 9.629 & 4.218 & 3.034 & -1.206 & -3.312 & 45.134 & 0.418 & 14.643 & 0.00 & & 0.2833 & 8.5 & $10.8 \quad-\quad>$ & 5. & 0.347 & & 0.066 & .012 & $1.926 \mathrm{Fl}$ & 10.15 \\
\hline 463 & 8/24/2004 & 11:01:11 AM & 25.067] & 24.069 & 24.866 & 25.715 & 24.047 & 6.833 & 9.459 & 4.031 & 2.942 & -1.2122 & -3.35 & 44.8811 & 0.414 & 14.643 & 0.005 & 18.00 & 0.3 & $8.5>3$ & 10.7 & 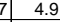 & 0.337 & 0.059 & 0.055 & 0.012 & 2.173 & 10.36 \\
\hline 4662 & 8/24/2004 & 11:02:11 AM & 25.054 & 24.075 & 24.857 & 25.706 & 24.0 & 7.138 & 9.7 & 4.2. & 2.973 & -1.218 & -3.39 & sol & 0.407 & 14. & & & 0.31667 & r & & & & & & & & \\
\hline$\frac{46}{46}$ & 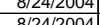 & $11: 03: 11 \mathrm{AM}$ & 25.044 & 24.015 & 24.882 & 25.166 & & $\frac{1.213}{2037}$ & 9.896 9.896 & 4.043 4.040 & 3.124 $\mathrm{rl}$ & -1.212 & 3.4. & 45.094 & $0.40 t$ & 14.643 & & & . 33333 & 8.8 & $10.7 \mathrm{r}-\mathrm{l}$ & & 0.356 & & & & $1 \frac{11.311}{11255}$ & 9.63 \\
\hline & 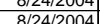 & 11.04.1.14 & $\frac{2.30444}{25099}$ & $\frac{24.000}{24096}$ & 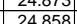 & $\frac{23.054}{25822}$ & 24.034 & 7233 & 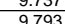 & (4.454 & $\frac{2.94}{2.856}$ & $\frac{-1.124}{-1.12}$ & & 年44.950 & 0.4 & 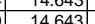 & & & 0.05 & 年. & 10 & & & & 0.0 & & . & 9.58 \\
\hline 468 & $\begin{array}{l}8 / 24 / 2004 \\
8 / 2004\end{array}$ & 11.06.1.1. & | & 24095 & 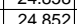 & 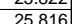 & $\frac{2.39}{239}>\mathrm{C}$ & 7.48 & 作 & $\frac{4.0}{42}+2$ & $\frac{2.05}{3.36}$ & -1.21 & -350 & (4) & & & & & & & & & (374 & & & & 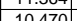 & \\
\hline$\frac{465}{465}$ & $8 / 24 / 2004$ & 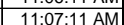 & $\frac{2.0 .044}{25}$ & 24.105 & 24.892 & 25.001 & & $\frac{1.406}{7746}$ & $\begin{array}{l}9.0789 \\
9789\end{array}$ & & $\frac{.032}{3.32}$ & -1.209 & & 45.211 & & 14.643 & & & 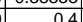 & & & $\frac{3.4}{5.4}-3$ & . & & & & 0.4125 & 年. \\
\hline 474 & $8 / 24 / 2004$ & $11: 08: 11 \mathrm{AM}$ & 25.038 & 24.1 & 24.871 & 25.746 & 23.897 & 7.261 & 9.569 & 4.269 & 3.12 & $\frac{-1.212}{-1.212}$ & -3.565 & 44.958 & 0.387 & 14.643 & 0.00 & 2500 & 0.41667 & $8.2-2$ & & $52-2+3$ & 358 & 055 & $0.05-2>$ & 0.011 & 10.707 & 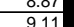 \\
\hline 4711 & $8 / 24 / 2004$ & 11:09:11 AM & & 24.118 & & 7570 & & & & & & & & & & & & & & & & & & & & & & \\
\hline & $8 / 24 / 2004$ & $11 \cdot 10 \cdot 11 \mathrm{AM}$ & 2516 & 241111 & 25.929 & 25.672 & & 8.544 & 9.635 & 424 & 43 & -1.21 & & 44.929 & & & & 2700 & 0.45 & & & & & & & & 405 & 4.0 \\
\hline 4 & $8 / 24 / 2004$ & $11: 11: 11 \mathrm{AM}$ & 25.249 & 24.126 & 25.688 & 25.707 & 23.668 & 8.696 & 9.801 & 4.326 & 4.542 & -1.212 & 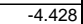 & 45.152 & 0.253 & 14.643 & & & 0.46667 & 8. & 10.8 & $6.6-6>2$ & 0.456 & 0.036 & 0.036 & 0.005 & 5.368 & $\frac{4.00}{4.57}$ \\
\hline 474 & & & & & & & & & & & & & & & & & & & & & & & & & & & & \\
\hline & & Averaces & 251 & 241 & & & & & & & & & & & & & & & & & & & & & & & & \\
\hline 477 & & Maximum & 25.2 & 24.1 & 25.9 & 25.8 & 24.1 & 8.7 & 9.9 & 4.5 & 4.6 & -1.2 & -3. & 45.3 & 0.4 & 14.6 & 00 & & & 8.8 & 10.8 & 6.6 & 0.5 & 0.1 & 0.1 & 0.0 & 12.7 & $\frac{8.6}{108}$ \\
\hline 478 & & Median & 25.1 & 24.1 & 24.9 & 25.1 & & 7.2 & 9.8 & 4.3 & 3.1 & -1.2 & S. & 45.1 & 0.4 & 14.6 & & & & 8.6 & 10.7 & 5.1 & 0.4 & 0.1 & 0.1 & 0.0 & 11.3 & 9.6 \\
\hline 4779 & & Minimum & 25.0 & 24.0 & 24.9 & 25.6 & & \begin{tabular}{ll|l}
6.8 & \\
\end{tabular} & 9.5 & 4.0 & 2.8 & -1.2 & 4.5 & 44.7 & 0.3 & $\begin{array}{ll}14.6 \\
\end{array}$ & & & & 8.3 & 10.7 & 4.8 .8 & 0.3 & & 0.0 & & & 4.6 \\
\hline 48 & & $2 \times \mathrm{Std}$ Dev & 0.114 & 0.047 & 0.709 & 0.138 & 0.262 & 1.236 & 0.247 & 0.218 & 1.254 & 0.006 & & 0.282 & 0.128 & 0.000 & & & & .247 & & & & .018 & 019 & 0.005 & 5.141 & 4.375 \\
\hline & Number o & of Point: & & & 14 & & 14 & 14 & 14 & 14 & 14 & 14 & 14 & 14 & 14 & 14 & 14 & & & 14 & 14 & 14 & 14 & 14 & 14 & 14 & 14 & 14 \\
\hline & & * Backpulse poi & ints in box a & are not inc & lluded & & & & & & & & & & & & & & & & & & & re & 00443 & & & \\
\hline
\end{tabular}


WSRC-TR-2005-00105, REVISION 0

SRNL-RPP-2005-00012, REVISION 0

CAMPAIGN IV NEW GKN BASELINE \#5 WITH 0.1 M NAOH

RPP-WTP Pilot-scale X-flow Filtration, Pre-Campaign IV Tests

[23-24 August 2004: New GKN Filter]

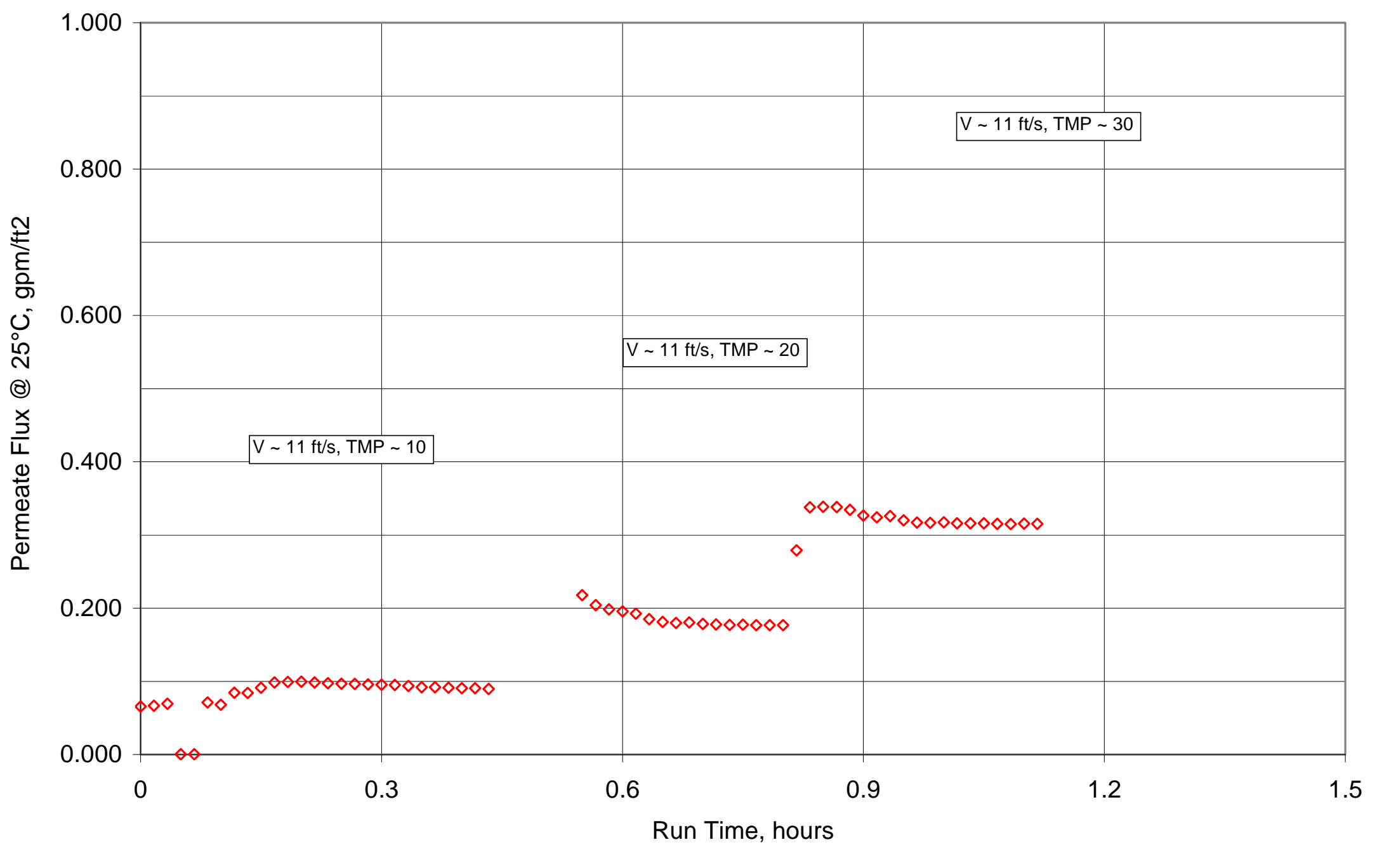


WSRC-TR-2005-00105, REVISION 0

SRNL-RPP-2005-00012, REVISION 0

CAMPAIGN IV NEW GKN BASELINE \#5 WITH 0.1 M NAOH

\begin{tabular}{|c|c|c|c|c|c|c|c|c|c|c|c|c|c|c|c|c|c|c|c|c|c|c|c|c|c|c|c|c|}
\hline & $\mathrm{A}$ & $B$ & $\frac{D}{1 \ll \ll \ll \ll}$ & E & $F$ & $\begin{array}{ll}G \\
\text { eazsureme }\end{array}$ & 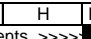 & $\frac{J}{1<<<<<}$ & $\frac{1}{1<\ll<\ll}$ & $\begin{array}{ll}\text { L } \\
\text { Pressure }\end{array}$ & $\mathrm{M}$ & $\frac{1 \mathrm{~N}}{\mathrm{I}}$ & $\begin{array}{ll}1 \\
1\end{array}$ & $\frac{Q}{k \ll \ll<<}$ & $\begin{array}{ll}R \\
R \text { R }\end{array}$ & $\mathrm{s}$ & $\mathrm{T}$ & $\mathrm{v}$ & $\mathrm{w}$ & $\perp x$ & Y I I I t & $z$ & AA & $\mid A B$ & 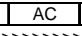 & $\frac{A D}{2}$ & $\mathrm{AE}$ & $\mathrm{AF}$ \\
\hline$\frac{1}{2}$ & DATE & TIME & Filtrate & $\begin{array}{l}\text { Cleaning } \\
\text { Cleaning }\end{array}$ & $\begin{array}{l}\text { elature } \mathrm{N} \\
\text { D Slurry }\end{array}$ & $\begin{array}{l}\text { enasureme } \\
\text { Hi Amb. }\end{array}$ & 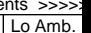 & BotTMP & $\begin{array}{l}k \ll<<< \\
\text { | Filter }\end{array}$ & $\begin{array}{l}\text { Pressuree } \\
\text { Filter dP } \\
\end{array}$ & $\begin{array}{l}\text { Measurem } \\
\text { TopTMP }\end{array}$ & $\begin{array}{l}\text { ents }>>>- \\
\text { | Filtrate }\end{array}$ & Pulsep & & $\begin{array}{l}\text { «< Hllow I } \\
\text { Filtrate }\end{array}$ & $\begin{array}{l}\text { Neasurumeme } \\
\text { Hi ivitate }\end{array}$ & Backpulse & & & & & & & & & & & \\
\hline & & & & & & & & psid & & psid & psid & psig & & gpm & $\mathrm{gpm}$ & gpm & & & & & & & & & & & & \\
\hline$\frac{3}{4}$ & & & T2 & $\frac{13}{\text { T3 }}$ & T1 & T4 & $\frac{\mathrm{Teg}}{\mathrm{T} 5}$ & $\frac{d P 2}{\mathrm{dP} 2}$ & $\frac{P S 19}{\mathrm{P} 1}$ & 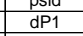 & $\mathrm{dP3}$ & P2 & $\frac{P_{019}}{\mathrm{P} 3}$ & $\frac{\mathrm{gpm}}{\mathrm{Q} 1}$ & gp2 & $\mathrm{Q}^{3}$ & $\begin{array}{l}\text { gpmp } \\
\text { Qbp }\end{array}$ & & & & & & & & & & & \\
\hline$\frac{5}{6}$ & $25-01 / 2$ & 204 & & & & & & & & & & & & & & & & & & & & & & & & & & \\
\hline & $\frac{|c| c \mid c-011 / 26}{8 / 23 / 2004}$ & 8:35:38 AM & 22.554 & 22.604 & 22.002 & 22.782 & 22.204 & -0.019 & 0.095 & -0.012 & 0.00 & -0.124 & 0.13 & -0.075 & 0.003 & \begin{tabular}{|c|c|c|}
14.643 \\
\end{tabular} & 0.004 & & & Filter Su & Surface & & FT2 & & & & & \\
\hline 8 & $8 / 24 / 2004$ & 8:10:33 AM & 23.662 & 22.93 & 23.679 & 22.998 & 22.735 & -0.021 & 0.122 & -0.014 & & -0.124 & & 23.176 & 1.205 & 14.643 & 0.003 & & & Convers & rsion & & $1 \mathrm{~m} 3 / \mathrm{m} 2 /$ & 2/day/bar & $\mathrm{arg} / \mathrm{gpm} / \mathrm{tt}$ & flt2/barg & & \\
\hline & $8 / 26 / 2004$ & & & & & & & -0.008 & 0.126 & -0.008 & 0.011 & -0.106 & & 48.335 & 0.043 & \begin{tabular}{|c|c|}
14.643 \\
\end{tabular} & & & & & & & & & & & & \\
\hline & $8 / 27 / 2004$ & 7:53:29 AM & 22.649 & 22.89 & 22.298 & 22.872 & 22.871 & $-0.017 \quad-\quad>$ & 0.14 & & & & & $\mid-0.084$ & & & & & & Note- & ge & & D1 is & iporo: & ately 1 & & & \\
\hline & $8 / 30 / 2004$ & 7:28:55 AM & 22.511 & 22.848 & 22.397 & 22.669 & 22.914 & -0.017 & 0.138 & -0.009 & 0.003 & -0.147 & & -0.082 & 0.003 & 14.643 & 0.006 & & & & & & & & & & & \\
\hline & & & & & & & & & & & & & & & & & & & & & re Plis & orrecte & ted for & & & ter tubi & & \\
\hline & & & & & & & & & & & & & & & & & & & & VWV & & & & < Filtra & ate Flux & $\ll$ PEF & IABIL & $\gg$ \\
\hline & & & & & & & & & & & & & & & & & & & & & & & & & at $25 \mathrm{C}$ & & 1000 & \\
\hline$\frac{15}{16}$ & & & & & & & & & & & & & & & & & & Time & $\begin{array}{l}\text { Time } \\
\text { Hour }\end{array}$ & \begin{tabular}{|l} 
Press. \\
psig
\end{tabular} & $\begin{array}{l}\text { Vel. } \\
\text { Vtls. } \\
\text { fls }\end{array}$ & TMP & TMP & $g \mathrm{pm}$ & $\frac{\mathrm{gpm}}{\mathrm{t} \mathrm{H}^{2}}$ & $g$ gpm & $\frac{\mathrm{gpm}}{\mathrm{g} / \mathrm{psi}}$ & $\begin{array}{l}\text { neter } \\
\text { alyybar }\end{array}$ \\
\hline & A & $\bar{B}$ & $\frac{D}{D}$ & $E$ & $F$ & $G$ & $\mathrm{H}$ & $\mathrm{J}$ & $\mathrm{K}$ & $\bar{L}$ & $M$ & $\bar{N}$ & 0 & $Q$ & $\bar{R}$ & $\mathrm{~s}$ & & $\mathrm{v}$ & w & $\frac{19}{x}$ & $\mathrm{Y}$ & $\frac{1}{z}$ & $\mathrm{AA}$ & $A B$ & $A C$ & $A D$ & $\mathrm{AE}$ & AF \\
\hline & Tuesday, 8/2 & $4 / 04$ & & & & & & & & & & & & & & & & & & & & & & & & & & \\
\hline & & & & & & & & & & & & & & & & & & & & & & & & & & & & \\
\hline & 8/24/2004 & 11:13:15 AM & 25.563 & 24.124 & 25.547 & 25.725 & 23.827 & 11.448 & 14.678 & 4.409 & 7.202 & -1.215 & -2.962 & 44.445 & 0.465 & 14.643 & 0.003 & 0.00 & & 13.6 & 10.6 & 9.3 & 0.643 & 067 & 0.066 & 0.007 & & \\
\hline & 8/2/24/2004 & $\begin{array}{l}\text { 11:14:15 AM } \\
1115 \cdot 15 \mathrm{AM}\end{array}$ & 25.617 & $\frac{24.134}{24.129}$ & $\begin{array}{l}25.496 \\
25.446\end{array}$ & $\frac{25.805}{25835}$ & $\begin{aligned} 23.841 \\
23.981\end{aligned}$ & $\frac{11.817}{12031}$ & \begin{tabular}{|l|l|}
15.076 \\
15.511 \\
\end{tabular} & 4.091 & 7.918 & $\begin{array}{l}-1.209 \\
-1212 \\
\end{array}$ & $\begin{array}{l}-2.896 \\
-2.768 \\
\end{array}$ & $\begin{array}{l}44.259 \\
4841 \\
\end{array}$ & 0.471 & $\begin{array}{l}14.643 \\
1.643 \\
\end{array}$ & 0.003 & $\frac{1.0}{20}$ & 0.0166 & $\begin{array}{l}14.0 \\
144\end{array}$ & 10.5 & 9.5 & \begin{tabular}{|l|l|}
0.680 \\
0686
\end{tabular} & 0.067 & 0.066 & 0.007 & 6.739 & $\frac{3.14}{5.92}$ \\
\hline & $8 / 24 / 2004$ & $11: 16: 15$ AM & 25.613 & 24.144 & 25.452 & 25.891 & 24.012 & 2.518 & \begin{tabular}{|l|l|l|}
15.408 \\
\end{tabular} & 4.268 & -1.629 & 7.774 & 19.896 & 44.72 & 0.003 & 14.643 & 0.003 & 3.0 & 0.05 & 14.3 & 10.7 & 0.4 & $\begin{array}{l}.000 \\
0.031 \\
\end{array}$ & .000 & 0.000 & $\frac{0.001}{0.001}$ & 0.954 & \\
\hline & & & 25.574 & & & & & & 15.594 & & & & & & & & & & & 14.5 & & & & & & & & \\
\hline & $8 / 24 / 2004$ & 11:18:15 AM & 25.484 & 24.156 & 25.443 & 25.917 & 24.026 & 8.916 & 15.501 & & & 1.249 & 0.3 & 44.996 & 503 & 14. & & 5.0 & 0.08333 & 14.4 & 10.7 & 6.8 & 0.472 & 0.072 & 0.071 & $\overline{010}$ & 10.383 & \\
\hline & 8/24/2004 & & 25.596 & 24.162 & 25.439 & 25.988 & & & & & & & & $\begin{array}{lll}44.8 & \end{array}$ & & & & & & \begin{tabular}{ll|l}
14.4 \\
\end{tabular} & $10.7 \mathrm{r}-\mathrm{C}$ & \begin{tabular}{ll|l}
6.8 \\
\end{tabular} & 0.471 & & & & 9.9 & \\
\hline & 8/24/2004 & 11:20 & 25.601 & & 25.4 & 25.999 & 24.126 & 10.235 & 15.397 & & & -0.257 & & 45.111 & & 14. & & & 0.11667 & 14.3 & 10.7 & 8.2 & & 0.085 & & 0.010 & $10.332 \mathrm{r}$ & \\
\hline & 8/24/2004 & 11:21:15 AM & 25.602 & 24.174 & 25.381 & 26.05 & 24.132 & 10.533 & 15.493 & 4.26 & 6.412 & -0.346 & -1 & 45.092 & 0.594 & 14.643 & & & 0.1333 & 14.4 & 10. & & & Wo & & & 9.90 & \\
\hline & 8/24/2004 & 11:22:15 AM & 25.594 & & & 26.067 & 24.144 & 11.373 & 17.101 & & & 0.484 & & 43.59 & 0.643 & & & & 0.1 & & 10.4 & 9.4 & & & & & & \\
\hline & $8 / 24 / 2004$ & 11:23:15 AM & 25.581 & 24.192 & 25.375 & 26.084 & 24.285 & 11.751 & 18.631 & & & 1.532 & & 45.388 & 0.696 & 14.643 & & & & 17.5 & 10. & 9.5 & & 0.100 & & & 10.343 & \\
\hline & $8 / 24 / 2004$ & $\begin{array}{l}11.24 .1 .1 \mathrm{AMM} \\
11.25 \mathrm{MM}\end{array}$ & 25.571 & 24.189 & 25.416 & 26.095 & $\frac{2.3}{2.3}$ & $\begin{array}{l}12.035 \\
1.217\end{array}$ & $\frac{18.929}{10901}$ & & 8.02 & $\begin{array}{r}1.59 \\
1645\end{array}$ & & (44. & $\begin{array}{r}0.7 \\
0703\end{array}$ & 14. & & 11. & 0.18333 & 17.8 & 10. & 10 & & 0.0101 & & & 8 & \\
\hline & 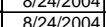 & & 25.0645 & & 告 25.425 & $\begin{array}{l}20.147 \\
26.173\end{array}$ & & & & & & & & & & & & & & & & & & & & & & \\
\hline & $8 / 24 / 2004$ & $11: 27: 15$ AM & 25.605 & & 25.429 & 26.178 & $\frac{1}{2}$ & & 18.832 & & & 1.356 & -0. & 44.7 & & 14 & & 14 & 0.23333 & 17.7 & t10 & 10.2 & & 0.099 & & $\frac{10}{10}$ & 9.572 & \\
\hline & $8 / 24 / 2$ & 11:28 & 25.64 & 24 & 25.449 & 26.138 & 24.1 & 12.414 & 18. & & 8. & 1.2 & & 45.5 & & & & & & 17.9 & $10.8 \mathrm{C}$ & 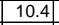 & & 0.098 & & & & \\
\hline & & 11:29 & 25.655 & 24.232 & 25.474 & 26.138 & & & & & & & & & & & & & & 17.9 & $10>>2>$ & (3.J & & & & & & \\
\hline & & 11 & 25.677 & & 25.514 & 26.088 & & & & & & 1.1 & & & & & & & 0.283 & 17.9 & & & & & & & & \\
\hline & $8 / 27 / 24$ & $\begin{array}{l}11.31 .1 \mathrm{AMM} \\
1.32 .15 \mathrm{M}\end{array}$ & 25.675 & 24.237 & 告5.534 & 26.093 & & 12.576 & $\mid \frac{19.161}{18010}$ & & & $\frac{1.121}{1050}$ & & $\frac{44}{44}>3$ & & & & & 0.3 & $\frac{18.0}{17.8}>-1$ & 107 & 10.4 & & & & & & \\
\hline & $8 / 2 / 24 / 2004$ & $\frac{11.32 .2}{1.23}$ & | & $\frac{24.240}{2426}$ & 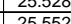 & $\frac{20.002}{26051}$ & & & & & & 1.052 & & & & & & & 0.31 & 11.0 & & 102 & & & & 0.009 & & \\
\hline & & $\frac{11.35}{11.34}$ & (25, & & $\frac{2.352}{25.53}$ & & & $\frac{12 .}{12 .}$ & & & & & & & & & & $211>->$ & & 些17. & & & & & & & & \\
\hline & $8 / 24 / 2004$ & & 25.726 & & 25.525 & 26.019 & 23. & 12.224 & & & & 0.6 & & 45.0 & & & & 22. & 0.3 & 17.0 & 10. & 10 & $0.7-7>$ & 0.0 & & 09 & & \\
\hline & & & 25.7 & & 25 . & & & & & & & & & & & & & & & $7.0 .6-2$. & & & & & & & & \\
\hline & $8 / 24 / 2$ & $11: 37$ & 25.747 & 24.284 & 25.591 & 26.07 & & & & & & 0.4 & & 45. & & & & & & & & & & & & & & \\
\hline & & 11:38:15 & 25.158 & 24.29 & 25.602 & 26.096 & & 12.659 & 18.542 & 4.31 & & 0.487 & & 45.038 & 0.643 & & & & $\begin{array}{ll}0.41667 \\
\end{array}$ & 17.4 & \begin{tabular}{|l|l|} 
& 10.7 \\
\end{tabular} & 10.5 & 0.1. & \begin{tabular}{|l|l|} 
\\
\end{tabular} & 0.091 & 0.00 & 8.611 & \\
\hline $51 \mathrm{ll}+2$ & $8 / 201200$ & 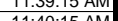 & 2.713 & 28207 & 23.002 & 20123 & & $\frac{13.482}{1832}$ & 19.35 & $\begin{array}{l}3.935 \\
20212\end{array}$ & 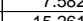 & 15.277 & & 2076 & 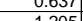 & 18 & & & 80 & & & & & & & (1) & 87007 & \\
\hline & & & & & & & & & & & & & & & & & & & & & & & & & & & & \\
\hline
\end{tabular}


WSRC-TR-2005-00105, REVISION 0

SRNL-RPP-2005-00012, REVISION 0

CAMPAIGN IV NEW GKN BASELINE \#5 WITH 0.1 M NAOH - CONT.

\begin{tabular}{|c|c|c|c|c|c|c|c|c|c|c|c|c|c|c|c|c|c|c|c|c|c|c|c|c|c|c|c|c|}
\hline & A & B $\quad k$ & $\mathrm{D}$ & $E$ & $\mathrm{~F}$ & $G$ & $\mathrm{H}$ & 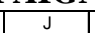 & $\mathrm{K}$ & $\mathrm{L}$ & $M$ & 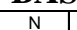 & 0 & 0 & $\mathrm{R}$ & $\mathrm{s}$ & $T^{T}$ & $\mathrm{v}$ & $w$ & $x$ & & $z$ & AA & $A B$ & $A C$ & & & AF \\
\hline & $8 / 24 / 2004$ & 11:41:15 AM & 26.03 & 24.302 & 25.939 & 26.198 & \begin{tabular}{|l|l|}
24.445 \\
\end{tabular} & 20.204 & 48.359 & 3.816 & 16.608 & 23.205 & 12.611 & 43.434 & 1.205 & 14.643 & 0.004 & 28.0 & 0.46667 & 47.2 & 10.3 & 18.4 & 1.269 & 0.173 & 0.168 & 0.009 & 9.129 & 7.77 \\
\hline & $8 / 24 / 2004$ & $11: 42: 15 \mathrm{AM}$ & 26.315 & 24.298 & 26.285 & 26.244 & \begin{tabular}{|l|}
24.586 \\
\end{tabular} & 29.377 & 50.948 & 3.537 & 26.475 & \begin{tabular}{|l|}
16.199 \\
16.04
\end{tabular} & 8.538 & $\begin{array}{l}43.457 \\
42.57 \\
\end{array}$ & 1.205 & $\begin{array}{r}\quad 4.044 \\
4.74 \\
\end{array}$ & 0.004 & 29.00 & $\begin{array}{ll}50 & 0.48333 \\
\end{array}$ & 49.8 & 10.1 & 27.99 & $\begin{array}{l}\frac{1.925}{1.925} \\
\end{array}$ & 0.679 & 0.655 & & 23.440 & 19.95 \\
\hline & $8 / 24 / 2004$ & $11: 43: 15 \mathrm{AM}$ & 26.746 & 24.294 & \begin{tabular}{|l|}
26.551 \\
\end{tabular} & 26.28 & 24.622 & 28.271 & 36.704 & 5.925 & 22.941 & \begin{tabular}{|l|}
2.528 \\
\end{tabular} & -4.014 & 52.851 & 0.003 & 2.929 & 0.003 & 30.00 & \begin{tabular}{|l|l}
0 & 0.5 \\
\end{tabular} & 35.6 & 12.6 & 25.6 & 1.765 & 0.419 & 0.402 & 0.016 & 15.680 & 13.34 \\
\hline 518 & $\begin{array}{l}8 / 24 / 2004 \\
8 / 24204 \\
\end{array}$ & \begin{tabular}{|l|}
$11: 44: 15 \mathrm{AM}$ \\
$11: 45: 15 \mathrm{AM}$
\end{tabular} & $\begin{array}{l}26.868 \\
26.908 \\
\end{array}$ & $\begin{array}{l}24.316 \\
22.322 \\
\end{array}$ & \begin{tabular}{|l|}
26.733 \\
2673 \\
\end{tabular} & 26.347 & 24.634 & 23.292 & 27.313 & $\begin{array}{l}4.281 \\
5011\end{array}$ & 19.479 & $\begin{array}{ll}-1.212 \\
.212\end{array}$ & $\begin{array}{l}-4.726 \\
-804\end{array}$ & \begin{tabular}{|l|l|}
47.397 \\
5034 \\
\end{tabular} & $\begin{array}{ll}0.003 \\
0.032\end{array}$ & $\begin{array}{ll}1.948 \\
17 n g\end{array}$ & 0.003 & 31.00 & 0.51667 & 26.2 & \begin{tabular}{|l|l|}
2 & 11.3 \\
\end{tabular} & 21.4 & 1.474 & 0.279 & 0.266 & 0.012 & 12.423 & 10.57 \\
\hline$\frac{1250}{520}$ & \begin{tabular}{|l|}
$8 / 24 / 2004$ \\
$8 / 24 / 204$
\end{tabular} & 11:45:15 AM & $\frac{26.908}{26.934}$ & $\frac{322}{333}$ & \begin{tabular}{|l|}
26.743 \\
267 \\
\end{tabular} & $\begin{array}{l}26.408 \\
26.424 \\
\end{array}$ & $\begin{array}{r}24.5 \\
24476\end{array}$ & $\begin{array}{l}22.854 \\
2173 \\
\end{array}$ & $\begin{array}{l}26.136 \\
24.49 \\
\end{array}$ & $\begin{array}{l}5.011 \\
4186 \\
\end{array}$ & $\begin{array}{l}18.132 \\
17907 \\
\end{array}$ & \begin{tabular}{|l|l|}
-1.212 \\
-1215 \\
\end{tabular} & $\begin{array}{l}-4.804 \\
-4882 \\
\end{array}$ & \begin{tabular}{|l|}
50.346 \\
46021 \\
\end{tabular} & $\begin{array}{l}0.003 \\
0.003 \\
\end{array}$ & $\begin{array}{ll}1.748 \\
1597\end{array}$ & & 32.00 & & 25.0 & $0 \quad 12.0$ & 20.5 & $\frac{1.413}{1.267}$ & 0.250 & 0.238 & $\begin{array}{ll}0.012 \\
001\end{array}$ & 111.630 & 9.90 \\
\hline$\frac{520}{521}$ & $8 / 24 / 2004$ & \begin{tabular}{|l|}
11.46 .15 AMM \\
\end{tabular} & $\begin{array}{l}20.934 \\
26.929\end{array}$ & 24.343 & \begin{tabular}{|l|}
26.16 \\
26.73 \\
\end{tabular} & $\frac{26.424}{26.449}$ & \begin{tabular}{|l|}
24.476 \\
24.366 \\
\end{tabular} & $\begin{array}{l}21.743 \\
21.919\end{array}$ & $\begin{aligned} 24.491 \\
24.41\end{aligned}$ & $\begin{array}{l}4.1 .06 \\
4.069 \\
\end{array}$ & & \begin{tabular}{|c|}
$\mid-1.215$ \\
-1.212 \\
\end{tabular} & $\begin{array}{l}-4.882 \\
-4.89 \\
\end{array}$ & $\mid$\begin{tabular}{|c|}
$\mid 46.012$ \\
44.914
\end{tabular} & $\begin{array}{l}0.003 \\
0.003 \\
\end{array}$ & $\begin{array}{l}1.597 \\
1.497 \\
\end{array}$ & $\begin{array}{l}0.003 \\
0.03\end{array}$ & $\begin{array}{l}33.00 \\
34.00\end{array}$ & \begin{tabular}{|l|r|}
0 & 0.55 \\
0 & 0.56667 \\
\end{tabular} & \begin{tabular}{|l|}
23.4 \\
23.3 \\
\end{tabular} & \begin{tabular}{|l|l|}
4 & 11.0 \\
3 & 10.7
\end{tabular} & \begin{tabular}{|l|l|}
0 & 19.8 \\
7 & 20.0
\end{tabular} & \begin{tabular}{|l|}
1.367 \\
1.381 \\
\end{tabular} & \begin{tabular}{|l|}
0.229 \\
0.214
\end{tabular} & $\begin{array}{ll}0.218 \\
0.204\end{array}$ & \begin{tabular}{|c|c|}
0.011 \\
0.010
\end{tabular} & \begin{tabular}{|l|}
10.978 \\
10199 \\
\end{tabular} & 9.34 \\
\hline & $8 / 24 / 2004$ & 11:48:15 AM & 26.914 & 24.354 & 26.69 & 26.419 & \begin{tabular}{|l|}
24.316 \\
\end{tabular} & $\frac{21.9}{21.9}$ & 24.165 & 4.149 & 18.02 & $\frac{1.212}{-1.212}$ & -4.908 & $\begin{array}{l}44.645 \\
\end{array}$ & 0.003 & 1.451 & 0.003 & & \begin{tabular}{|l|l|}
0 & 0.58333 \\
\end{tabular} & & & & & & & & \begin{tabular}{|r|}
10.1995 \\
9.927 \\
\end{tabular} & $\begin{array}{l}8.68 \\
8.45 \\
\end{array}$ \\
\hline & $8 / 24 / 2004$ & $11: 49: 15$ AM & 26.895 & 24.359 & 26.645 & 26.399 & 24.216 & 22.07 & 24.445 & 3.976 & 18.411 & -1.212 & -4.913 & 44.389 & 0.003 & 1.43 & & 36.00 & 0.6 & 23.3 & & & & & 0.196 & 0.010 & $\begin{array}{l}9.521 \\
9.659 \\
\end{array}$ & $\frac{8.25}{8.22}$ \\
\hline & $8 / 24 / 2004$ & $11: 50: 15 \mathrm{AM}$ & 26.879 & 24.363 & 26.624 & 26.364 & 24.125 & 22.207 & 24.451 & 3.952 & 18.483 & -1.212 & -4.925 & 44.211 & 0.003 & 1.407 & & 37.00 & \begin{tabular}{l|l|}
0 & 0.61667 \\
\end{tabular} & 23.3 & 10.5 & 20.3 & 1.403 & 0.201 & 0.192 & 0.009 & 9.461 & 8.22 \\
\hline & 8/24/2004 & 11:51:15 AM & 26.858 & 24.367 & 26.594 & 26.353 & 24.0 & 21.822 & 23.966 & 4.085 & 17.87 & 1.212 & -4.945 & 45.292 & 0.003 & 1.351 & & & & 22.8 & & & 1.368 & 0.193 & 0.185 & 0.009 & 9.320 & $\begin{array}{l}8.05 \\
7.93 \\
\end{array}$ \\
\hline 526 & 8/24/2004 & 11:52:15 AM & 26.843 & 24.377 & & & 24.069 & 21.873 & 23.773 & & & -1.212 & & 44.889 & & 1.323 & & & 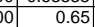 & 22.6 & & & 270 & & & & 9.068 & \\
\hline$\frac{527}{528}$ & $8 / 24 / 2004$ & $11: 53: 15$ AM & 26.816 & 24.36 & 26.527 & 26.296 & 24.077 & 21.678 & 23.605 & & 17.9 & -1.215 & -4.963 & 44.954 & & 1312 & & & & $\frac{25}{20}$ & & 1. & .364 & & & .009 & 9.094 & $\frac{7.22}{7.74}$ \\
\hline$\frac{528}{529}$ & $8 / 24 / 2004$ & 11:54:15 AM & 26.806 & 24.38 & 26.531 & 26.3 & 24.197 & 22.118 & 23.939 & 4.009 & 18.52 & -1.215 & -4.96 & 44.539 & 0.00 & 1.317 & & 41.0 & 0.68333 & $22.8 \mathrm{~S} \quad \mathrm{~s}$ & & 20.3 & 1.401 & $0.185^{0.9}$ & 0.181 & 0.009 & 8.890 & 7.57 \\
\hline & 8/24/2004 & 11:55:15 AM & 26.81 & 24.394 & 26.56 & 26.335 & 24.136 & 22.261 & 24.271 & 4.062 & 18.448 & -1.206 & -4.968 & 455.013 & 0.00 & 1.302 & 0.00 & $\begin{array}{lll}42 . \\
.0\end{array}$ & 0.7 & 23.1 & 10.7 & 20.4 & 1.403 & 0.186 & 0.178 & 0.009 & 8.766 & 7.46 \\
\hline & $\begin{array}{l}8 / 24 / 2004 \\
8 / 24 / 204\end{array}$ & 11:56:15 AM & 26.796 & 24.4 & 26.561 & 26.325 & 24.237 & 22.336 & 24.306 & 4.049 & 18.59 & -1.209 & -4.966 & 44.954 & 0.00 & 1.298 & & 43.0 & 0.71667 & & & & 1.41 & 0.186 & 0.178 & $\frac{0.099}{09}$ & 8.693 & 7.40 \\
\hline & $\begin{array}{r}8 / 24 / 2004 \\
8 / 24204\end{array}$ & 11:57:15 AM & 26.776 & 24.405 & $26.551 \mid$ & 26.35 & 24.292 & $\begin{array}{ll}22.149 \\
22097\end{array}$ & 23.955 & 4.107 & 18.331 & -1.209 & -4.971 & 44.892 & .003 & 1.292 & & 44.0 & 0.13335 & 22. & & & .395 & & & 0.009 & 8.750 & 7.45 \\
\hline & \begin{tabular}{|c|}
$8 / 24 / 24 / 2004$ \\
$8 / 2404$
\end{tabular} & $\begin{array}{l}11.58 .15 \mathrm{AM} \\
11.50 .15 \mathrm{M}\end{array}$ & $\frac{20.162}{26.710}$ & $\frac{24.41}{20.47}$ & $\frac{2.0484}{26363}$ & 26.461 & 24.268 & 22.097 & $\frac{23.928}{23.881}$ & 4.0989 & $\frac{18.319}{18.354}$ & \begin{tabular}{|c|c|c|}
-1.212 \\
1212
\end{tabular} & $\begin{array}{r}-4.96 \\
-4071 \\
\end{array}$ & (44.616 & 003 & 1.292 & & & 0.75 & & & & .39s & 0.185 & .1777 & & & \\
\hline & $\begin{array}{l}8 / \angle 2 / 2004 \\
8 / 24 / 2004\end{array}$ & $\begin{array}{l}11.59 .15 \mathrm{AM} \\
12: 00: 15 \mathrm{PM}\end{array}$ & $\frac{20.110}{26.654}$ & 24.401 & $\frac{26.363}{26.259}$ & $\frac{26.562}{26.643}$ & 24.299 & 22.106 & $\frac{23.081}{24.03}$ & $\begin{array}{r}4.08 \\
4.278 \\
\end{array}$ & $\frac{18.354}{1896} \mathrm{rat}$ & -1.212 & -4.977 & 44.904 & & $\frac{1.282}{1.270}$ & & & .76667 & & & & 0 & 0.184 & & & . .733 & 7.43 \\
\hline & $8 / 24 / 2004$ & 12:01:15 PM & 20.0566 & $\frac{24.424}{24.429}$ & 26.146 & $\begin{aligned} 20.645 \\
26.67 \\
\end{aligned}$ & $\frac{24.3}{24.4}$ & $\begin{array}{l}22.2 / 4 \\
22.075\end{array}$ & $\begin{array}{l}24.100 \\
23.81\end{array}$ & $\begin{array}{l}4.218 \\
4.002 \\
\end{array}$ & $\frac{1}{1}$ & $\begin{array}{l}-1.209 \\
-1.212\end{array}$ & -4.96 & $\left|\frac{44.827}{44814}\right|$ & 0 & & & & $\frac{78333}{0.8}$ & & & & 396 & & & & & 7.43 \\
\hline 536 & 8/24/2004 & 12:02:15 PM & 26.437 & 24.411 & 25.982 & 26.666 & 24.523 & 29.752 & 34.004 & 3.661 & 26.487 & $\mid-1.218$ & $\begin{array}{l}-4.928 \\
-4.728 \\
-10\end{array}$ & \begin{tabular}{|l|l|}
42.23 \\
\end{tabular} & 0.003 & 2.2004 & 0.004 & $\frac{48.0}{49.00}$ & $\begin{aligned} 0.81667 \\
0.9\end{aligned}$ & 32.9 & $\frac{10.1}{10.1}$ & $\frac{20.2}{28.1}$ & $\begin{array}{l}1.396 \\
1.939\end{array}$ & \begin{tabular}{|l|l|}
0.287 \\
\end{tabular} & $\begin{array}{l}0.1 / 7 \\
0.279 \\
\end{array}$ & $\frac{0.009}{0.010}$ & $\frac{8.31}{9.926}$ & $\begin{array}{l}7.43 \\
8.45 \\
\end{array}$ \\
\hline & 8/24/2004 & 12:03:15 PM & 26.42 & 24.433 & 26.055 & 26.739 & 24.596 & 31.679 & $\begin{array}{ll}37.747 \\
\end{array}$ & 3.971 & 28.313 & 0.262 & -4.486 & 44.95 & 0.003 & 2.43 & 0.004 & 50.00 & 0 & 36.6 & 10.7 & $\begin{array}{ll}\frac{20.1}{30.0} \\
-1\end{array}$ & 告.505 & \begin{tabular}{|l|l|}
0.2018 \\
\end{tabular} & 0.338 & 0.011 & & $\begin{array}{l}8.45 \\
9.58 \\
\end{array}$ \\
\hline & $\mid 8 / 24 / 2004$ & $12: 04: 15$ PM & 26.457 & 24.44 & 26.137 & 26.751 & 24.613 & 31.507 & 37.625 & 3.816 & 28.361 & 0.288 & -4.468 & 44.72 & 0.003 & 2.441 & & 51.00 & 0.85 & 36.5 & 10.7 & $\frac{2.9}{29.9}->$ & 2.064 & & & & & $\begin{array}{l}9.58 \\
9.62 \\
\end{array}$ \\
\hline & $8 / 24 / 2004$ & 12:05:15 PM & 26.503 & 24.457 & \begin{tabular}{|l|l|}
26.193 \\
\end{tabular} & 26.742 & 24.564 & 31.579 & 37.645 & 3.843 & 28.207 & 0.328 & & 44.62 & . 003 & 2.443 & & & & & & & 20.01 & & $0.3 \mathrm{0}-\mathrm{s}$ & & 1.315 & $\frac{9.62}{9.63}$ \\
\hline & & 12:06:15 PM & 26.539 & 24.452 & & & & & & 4.15 & 28.396 & 0.204 & -4.49 & $\begin{array}{ll}45.891 \\
\end{array}$ & & 2.417 & & & & & & & & & & & & $\frac{63}{41}$ \\
\hline & $8 / 24 / 2004$ & 12:07:15 PM & 26.579 & 24.453 & 26.259 & 26.694 & 24.495 & 31.463 & 37.154 & 4.007 & 28.063 & -0.028 & -4.53 & \begin{tabular}{|l|}
45.023 \\
4.02
\end{tabular} & 0 & 2.362 & & 54.00 & 0.9 & 36.0 & $\frac{10.9}{10.7}$ & $\begin{array}{ll}29.8 \\
29.8\end{array}$ & 2.052 & & 0.326 & 0.011 & $\frac{11.045}{10.968}$ & $\frac{41}{33}$ \\
\hline 542 & $8 / 24 / 2004$ & 12:08:15 PM & 26.63 & 24.479 & 26.335 & 26.719 & 24.36 & 31.77 & 37.449 & 3.944 & 28.3 & -0.031 & & \begin{tabular}{|l|}
44.837 \\
\end{tabular} & & 2.349 & & 55.00 & 0.91667 & 36.3 & 10.7 & 30.1 & 2.074 & 0.336 & 0.324 & 0.011 & 10.770 & 9.17 \\
\hline 543 & $8 / 24 / 2004$ & $12: 09: 15$ PM & 26.664 & 24.478 & 26.344 & 26.684 & 24.375 & 31.841 & 37.486 & 3.831 & 28.515 & \begin{tabular}{r|c|c|c|}
-0.022 \\
\end{tabular} & -4.526 & \begin{tabular}{|l|l|}
44.17 \\
\end{tabular} & & 2.362 & & 56.00 & 0.93333 & 36.4 & \begin{tabular}{|l|l|}
4 & 10.5 \\
\end{tabular} & 30.2 & 2.081 & 0.338 & 0.326 & 0.011 & 10.791 & 9.18 \\
\hline 544 & $8 / 24 / 2004$ & $12: 10: 15 \mathrm{PM}$ & 26.678 & 24.472 & 26.353 & 26.647 & 24.324 & 31.739 & 37.434 & 4.13 & & \begin{tabular}{r|r|}
-0.173 \\
-12
\end{tabular} & & 45.259 & & & & & 0.95 & 36.3 & \begin{tabular}{|l|l|}
3 & 10.8 \\
\end{tabular} & & & & & & 10.705 & 9.11 \\
\hline 545 & $8 / 24 / 2004$ & $12: 11: 15$ PM & 26.667 & 24.481 & 26.272 & 26.701 & & 31.814 & 37.283 & & 28.209 & & & 45.35 & & 2.295 & & 58.0 & 0.96667 & 36.2 & 10.8 & 30.0 & 2.069 & & 0.317 & 0.011 & 10.564 & 8.99 \\
\hline 546 & $8 / 24 / 2004$ & 12:12:15 PM & 26.616 & 24.49 & \begin{tabular}{|l|l|}
26.176 \\
\end{tabular} & 26.76 & & 31.907 & 37.478 & 4.099 & & & & \begin{tabular}{|l|}
45.215 \\
\end{tabular} & 003 & 2.286 & & 9.00 & 0.98333 & 36.4 & \begin{tabular}{|l|l|}
4 & 10.8 \\
\end{tabular} & 30.1 & 2.077 & 0.327 & 0.317 & 011 & 10.513 & 8.95 \\
\hline 547 & 8/24/2004 & 12:13:15 PM & 26.535 & 24.484 & 26.065 & 26.734 & 24.105 & $\begin{array}{l}32.025 \\
2.70 \\
\end{array}$ & 37.39 & 4.062 & $\begin{array}{l}28.447 \\
2.150\end{array}$ & \begin{tabular}{|l|l|}
-0.349 \\
\end{tabular} & -4.575 & \begin{tabular}{|l|l|}
45.021 \\
\end{tabular} & $\begin{array}{ll}0.003 \\
\end{array}$ & $\begin{array}{ll}2.284 \\
\end{array}$ & 0.005 & 60.00 & 1 & 36.3 & \begin{tabular}{|l|l|}
3 & 10.7 \\
\end{tabular} & \begin{tabular}{l|l|}
7 & 30.2 \\
\end{tabular} & 2.085 & 0.327 & 0.317 & 0.010 & $\begin{array}{l}10.496 \\
1069\end{array}$ & 8.93 \\
\hline$\frac{548}{549}$ & 8/24/2004 & 12:14:15 PM & 26.469 & 24.507 & $\mid$\begin{tabular}{|c|}
26.019 \\
\end{tabular} & 26.788 & 24.149 & 31.723 & \begin{tabular}{|l|}
37.032 \\
27.502
\end{tabular} & 4.042 & 28.158 & $\begin{array}{r}-0.422 \\
\end{array}$ & -4.575 & $\begin{array}{r}45.28 \\
\end{array}$ & 0.003 & $\begin{array}{r}2.27 \\
\end{array}$ & & & 1.01667 & 35.9. & \begin{tabular}{|l|l|}
9 & 10.8 \\
\end{tabular} & \begin{tabular}{|c|c|}
8 & 29.9 \\
& 30.9
\end{tabular} & 2.064 & 0.325 & 0.316 & 0.011 & 10.548 & 8.98 \\
\hline 549 & 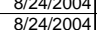 & $\frac{12: 11: 15 \mathrm{PM}}{12: 16: 15 \mathrm{PM}}$ & $\frac{26.388}{26.322}$ & $\frac{24.497}{24.495}$ & \begin{tabular}{|l|}
25.933 \\
2582 \\
\end{tabular} & $\frac{26 . / 211}{26.741}$ & \begin{tabular}{|l|}
24.198 \\
24.057 \\
\end{tabular} & \begin{tabular}{|l}
32.34 \\
31.996 \\
\end{tabular} & \begin{tabular}{|l|}
3.7592 \\
37.276 \\
\end{tabular} & $\begin{array}{l}4.0959 \\
3.961 \\
\end{array}$ & 28. & $\frac{-0.448}{-0.442}$ & & \begin{tabular}{|l|}
45.1537 \\
44.837 \\
\end{tabular} & 0.003 & 2.265 & & & $\begin{array}{l}0.0333 \\
0 \\
0.05\end{array}$ & $\begin{array}{l}36.5 \\
36.2 \\
\end{array}$ & 10.8 & 30.6 & 2.107 & & 0.316 & 0.010 & 0.0 .335 & $\begin{array}{l}8.80 \\
8.87 \\
\end{array}$ \\
\hline & $8 / 24 / 2004$ & $12: 17: 15$ PM & $\frac{20.024}{26.251}$ & 24.4504 & \begin{tabular}{|l|}
25.802 \\
\end{tabular} & $\begin{array}{l}26.1414 \\
26.714\end{array}$ & $\frac{24.0}{24.0}$ & 31.953 & 37.227 & 3.94 & $\frac{28 .}{28 .}$ & & & 44.91 & & & & & & & & & & & & & & $\begin{array}{l}8.87 \\
8.87\end{array}$ \\
\hline & 8/24/2004 & 12:18:1 & 26.19 & 24.508 & 25.74 & 26.689 & & 32.098 & 37.19 & & & -0.4 & & & & 2.2. & & & son & & & & & & & & & 8.87 \\
\hline 53 & $8 / 24 / 2$ & 12:19: & 26.129 & 24.502 & 25.673 & 26.657 & & 32.272 & 37.6 & 4.0 & 28. & $-0.5>>3$ & & 44.76 & & & & & & & & & & & & & 364 & 8.81 \\
\hline 554 & $8 / 24 / 2004$ & $12: 20: 15 \mathrm{~F}$ & 26.077 & 24.5 & 25.616 & 26.64 & 3.87 & 32.017 & 37.231 & 3.9 & 3.55 & -0.53 & & 44.716 & 0.004 & 2.23 & & & .1166 & 36. & & 30.3 & 2.088 & 32 & 0.31 & 0.010 & 0.403 & \\
\hline & & & & & & & & & & & & & & & & & & & & & & & & & & & & \\
\hline 500 & & Averages & 25.7 & 24.2 & 25.5 & 26.1 & & 12.4 & $18.8 \mathrm{\gamma}$ & 4 & 82 & 1.0 & & 45.6 & 0. & 14 & & & & 176 & & & & & & & & \\
\hline 558 & & Maximum & 25.8 & 24.3 & 25.7 & 26.2 & 24. & \begin{tabular}{|c|}
13.4 \\
\end{tabular} & $\begin{array}{l}1.0 \\
19.4\end{array}$ & 6.0 & $\frac{0.2}{8.7}$ & $\begin{array}{l}1.0 \\
1.6 \\
\end{array}$ & -0. & 55.1 & 0.7 & 14 & 0. & & & $\begin{array}{ll}18.2 \\
18.2\end{array}$ & 13.1 & \begin{tabular}{l|l|l|}
1 & 10.6 \\
\end{tabular} & 0.7 & 0.1 & 0.1 & 0.0 & $\begin{array}{l}3.9 \\
9.9 \\
\end{array}$ & $\frac{7.8}{8.4}$ \\
\hline 559 & & Median & 25.7 & 24.2 & 25.5 & 26.1 & 2 & 12.4 & & 4.2 & 8.4 & 1.1 & -0. & & 0. & 14 & te & & & $\begin{array}{lll}17.8 \\
\end{array}$ & 10.7 & 10.3 & 0.7 & 0.1 & 0.1 & 0.0 & 9.1 & 7.8 \\
\hline 560 & & Minimum & 25.6 & 24.2 & 25.4 & 26.0 & & 12.0 & 18.1 & & 7.6 & 0.4 & & 44.4 & 0.6 & 14 & & & & 17.0 & 10.6 & 10.0 & 0.7 & 0.1 & 0.1 & 0.0 & 8.5 & 7.2 \\
\hline$\frac{561}{562}$ & Numberc 0 & $\mid$ & $\begin{array}{ll}0.128 \\
16\end{array}$ & $\begin{array}{l}0.066 \\
16\end{array}$ & 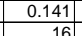 & $\begin{array}{l}0.097 \\
16\end{array}$ & $\begin{array}{r}0.296 \\
16\end{array}$ & $\begin{array}{l}0.633 \\
16\end{array}$ & $\begin{array}{r}0.799 \\
16\end{array}$ & $\begin{array}{ll}0.871 \\
16\end{array}$ & 0.471 & $\begin{array}{ll}0.813 \\
16\end{array}$ & 0.494 & \begin{tabular}{rl|l}
4.902 \\
16
\end{tabular} & $\begin{array}{ll}0.043 \\
16\end{array}$ & $\begin{array}{l}0.000 \\
16\end{array}$ & $\begin{array}{ll}0.001 \\
16\end{array}$ & & & $\begin{array}{l}0.799 \\
\end{array}$ & $\begin{array}{l}9.1 .168 \\
9\end{array}$ & 0.326 & 0.023 & .006 & 0.006 & 0.001 & 0.768 & 0.654 \\
\hline 563 & Number c & of Points Used* & 16 & 16 & 16 & 16 & 16 & & & & & 16 & & & & & & & & & & & & 16 & & & 16 & 16 \\
\hline & & * Backpulse poir & ts in box & & & & & & & & & & & & & & & & & & & & & & & & & \\
\hline & & & & & & & & & & & & & & & & & & & & & & & & & & & & \\
\hline$\overline{6}$ & & Averages & 26.8 & 24.4 & 26.5 & 26.4 & & & & 4 & & -1.2 & & & & 1. & & & & & & & $148+2$ & & & & & \\
\hline$\frac{\mid 567}{567}$ & & & 20.9 & & 26.8 & & & $\frac{22}{22}$ & $\frac{2}{2}$ & 5. & 18 & -1.2 & -4 & & 0 & 1 & & & & 233.3 & 0.8 & & 1.4 & & & & 9.9 & \\
\hline & & Median & 268 & & 266 & & & & & & & & & & & & & & & & 107 & & & & & & & \\
\hline & & Minimum & 26.6 & 24.3 & 26.1 & 26 & 24 & 217 & 23.6 & 4.0 & 17.9 & -1.2 & -5 & & 0. & 1.3 & 20 & & & 22.5 & $\begin{array}{ll}5 \\
5 & 10.5 \\
\end{array}$ & \begin{tabular}{l|l|l|}
5 & 19.8
\end{tabular} & $\mid 1.4$ & 0.2 & $\frac{0.2}{0.2}$ & 0.0 & $\begin{array}{l}0.7 \\
8.7 \\
\end{array}$ & $\begin{array}{r}7.5 \\
7.4 \\
\end{array}$ \\
\hline & & $2 \times S t d D$ & 0.195 & 0.061 & 0.321 & 0.220 & 0.260 & 0.531 & 1.092 & 0.466 & 0.448 & 0.005 & 0.088 & 2.687 & 0.000 & 0.255 & 0.002 & & & 0.493 & \begin{tabular}{|l|l|}
3 & 0.127 \\
\end{tabular} & 70.387 & \begin{tabular}{|l|}
0.027 \\
\end{tabular} & 0.016 & 0.014 & 0.001 & 0.771 & $\begin{array}{r}1.656 \\
0.656\end{array}$ \\
\hline 571 & Number $\mathrm{c}$ & of Points Used & 17 & & & 17 & 17 & 17 & 17 & 17 & 17 & 17 & 17 & 17 & 17 & 17 & 17 & & & 14 & 14 & 14 & 14 & 14 & 14 & 14 & 14 & 14 \\
\hline & & * Backpulse poir & ts in box & are not inc & dided & & & & & & & & & & & & & & & & & & & & & & & \\
\hline & & & & & & & & & & & & & & & & & & & & & & & & & & & & \\
\hline & & & & & & & & & & & & & & & & & & & & 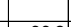 & & & & & & & & \\
\hline 75 & & & 26.5 & 24.5 & 26.1 & 26.7 & 24 & 31.9 & 37.5 & 4 & 28 & -0. & -4 & & 0 & 2.3 & 0 & & & 36.2 & 10. & 30.2 & 2.1 & & & & & 9.0 \\
\hline 77 & & Invaxim Media & 2.7 .5 & 24.5 & $\frac{2.4}{26.1}$ & $\frac{2}{2}$ & $\frac{24}{24}$ & $\begin{array}{l}32.3 \\
31.9 \\
\end{array}$ & 38.4 & 400 & $\frac{28}{28}$ & $\begin{array}{c}0.3 \\
-0.3 \\
\end{array}$ & $-46-3)$ & $\frac{43}{44}$ & 00 & $\frac{2.4}{2.3}$ & 0 & & & $\begin{array}{l}30.5 \\
36.2 \\
\end{array}$ & 10.07 & $\frac{30.6}{302}$ & $\frac{2.1}{2.1}$ & 0.3 & 03 & $\frac{0.0}{0.0}$ & $\frac{11.0}{10.5}$ & 9.3 \\
\hline & & & 26.1 & 2 & 25.6 & 26.6 & & 31.5 & 37.0 & 3.8 & 28.1 & -0.5 & -4. & 4 & 0.0 & 2.2 & & & & 35.9 & \begin{tabular}{|l|l|}
9 & 10.5 \\
\end{tabular} & \begin{tabular}{l|l|l|}
5 & 29.8 \\
\end{tabular} & \begin{tabular}{|l|}
2.1 \\
\end{tabular} & 0.3 & 0.3 & 0.0 & 10.3 & $\begin{array}{l}0.9 \\
8.8 \\
\end{array}$ \\
\hline & & & 0.368 & 0.046 & 0.461 & 0.079 & 0.471 & 0.476 & 0.537 & 0.200 & 0.421 & 0.593 & 0.084 & 0.710 & 0.000 & 0.141 & 0.001 & & & 0.339 & \begin{tabular}{|l|l|}
9 & 0.141 \\
\end{tabular} & 10.434 & \begin{tabular}{|l|} 
\\
\end{tabular} & 0.012 & 0.008 & 0.000 & 0.372 & 0.317 \\
\hline 580 & Number c & of Points Used* & 18 & 18 & 18 & 18 & 18 & 18 & 18 & 18 & 18 & 18 & 18 & 18 & 18 & 18 & 18 & & & 14 & \begin{tabular}{|l|l|}
4 & 14 \\
\end{tabular} & 14 & 14 & 14 & 14 & 14 & 14 & 14 \\
\hline 581 & & * Backpulse poir & ts in box & are not in & Cluded & & & & & & & & & & & & & & & & & & & Calc & & & & \\
\hline 582 & & & & & & & & & & & & & & & & & & & & & & Avg & g St Dv ( & (all pts) & 0.010 & & & \\
\hline 584 & & & & & & & & & & & & & & & & & & & & & & Max & $\overline{n S t D v(c}$ & |all pts) & $\left|\begin{array}{|c|}0.014 \\
0.006\end{array}\right|$ & & & \\
\hline
\end{tabular}


WSRC-TR-2005-00105, REVISION 0 SRNL-RPP-2005-00012, REVISION 0

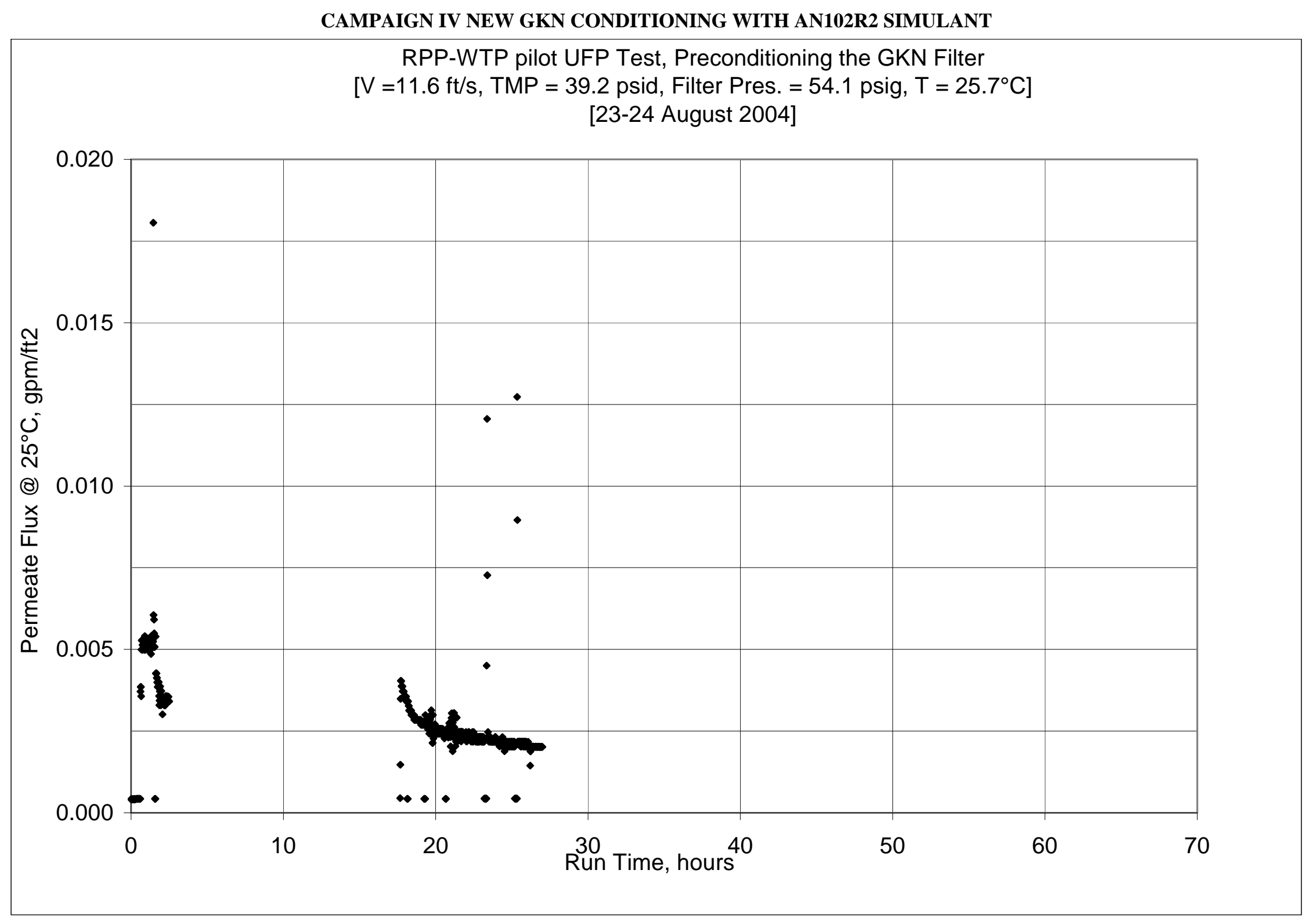


WSRC-TR-2005-00105, REVISION 0

SRNL-RPP-2005-00012, REVISION 0

CAMPAIGN IV NEW GKN CONDITIONING WITH AN102R2 SIMULANT

\begin{tabular}{|c|c|c|c|c|c|c|c|c|c|c|c|c|c|c|c|c|c|c|c|c|c|c|c|c|c|c|c|c|}
\hline & A & B & \begin{tabular}{l|l|l|} 
D & \\
\end{tabular} & $E$ & $\mathrm{~F}$ & G & $\mathrm{H}$ & $\mathrm{J}$ & $\mathrm{K}$ & $\mathrm{L}$ & \begin{tabular}{|l|} 
\\
\end{tabular} & \begin{tabular}{|l|}
$\mathrm{N}$ \\
\end{tabular} & 0 & \begin{tabular}{l|l} 
\\
\end{tabular} & \begin{tabular}{|l|l}
$\mathrm{R}$ \\
\end{tabular} & \begin{tabular}{|l|l|} 
& $s$ \\
\end{tabular} & $\mathrm{~T}$ & $\mathrm{~V}$ & W & $|x|$ & 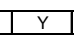 & $z$ & AA & $A B$ & AC & AD & \begin{tabular}{l|l}
$\mathrm{AE}$ \\
\end{tabular} & $\mathrm{AF}$ \\
\hline 1 & & TIME & Filtrate & $\begin{array}{l}\text { K< Tempe } \\
\text { Cleaning| }\end{array}$ & Derature 1 & teasureme & ents > >> & 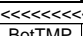 & 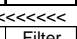 & $\begin{array}{l}\text { Pressure } \\
\text { Filter } d P\end{array}$ & $\begin{array}{l}\text { Measuremer| } \\
\text { TonTMP }\end{array}$ & & & 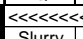 & 《< Flow M & Measuremer| & & & & & & & & & & & & \\
\hline & DAIL & TIME & $\begin{array}{l}\text { Filfitate } \\
\operatorname{deg} C\end{array}$ & $\frac{\text { Cleaning }}{\operatorname{deg} C}$ & \begin{tabular}{|l|} 
Slurry \\
$\operatorname{deg} C$ \\
\end{tabular} & \begin{tabular}{|l|} 
HiAmb. \\
deg C
\end{tabular} & \begin{tabular}{|c|} 
Lo Ammb. \\
$\operatorname{deg}$ C
\end{tabular} & $\frac{\text { BothMP }}{\text { psid }}$ & $\begin{array}{l}\text { Filter } \\
\text { psig }\end{array}$ & \begin{tabular}{|l|} 
Filter dP \\
psid
\end{tabular} & \begin{tabular}{|l|} 
TopTMP \\
psid
\end{tabular} & \begin{tabular}{|l|l|l|l} 
Filtrate \\
psig
\end{tabular} & \begin{tabular}{|l|} 
Pulsepot \\
psiq
\end{tabular} & Slurry & Filtrate & \begin{tabular}{|l|} 
Hi Filtate \\
\end{tabular} & Backpulse & & & & & & & & & & & \\
\hline 4 & & & $\mathrm{~T} 2$ & & & T4 & & $\mathrm{dP2}$ & $\frac{P 19}{\mathrm{P} 1}$ & dP1 & $\begin{array}{l}\text { psid } \\
\mathrm{dP3} 3\end{array}$ & $\begin{array}{l}\text { Psig } \\
\text { P2 }\end{array}$ & $\frac{\mathrm{psig}^{2}}{\mathrm{P} 3}$ & $\frac{\mathrm{gpm}}{\mathrm{Q1}}$ & $\frac{\mathrm{gpm}}{\mathrm{Q} 2}$ & \begin{tabular}{|l}
$\mathrm{gpm}$ \\
$\mathrm{Q} 3$
\end{tabular} & $\begin{array}{l}\text { gpm } \\
\text { obp }\end{array}$ & & & & & & & & & & & \\
\hline & & & & & & & & & & & & & & & & & & & & & & & & & & & & \\
\hline & s- $04 / 19$ & & & & & & & & & & & & & & & & & & & & & & & & & & & \\
\hline & $8 / 23 / 2004$ & $8: 35: 38 \mathrm{AM}$ & 22.554 & 22.604 & 22.002 & 22.782 & 22.204 & -0.019 & 0.095 & -0.012 & 0.003 & -0.124 & 0.137 & -0.075 & 0.003 & \begin{tabular}{ll|l}
14.643 \\
\end{tabular} & 0.004 & & & & Surface $A$ & 46.985 & FT2 & & & & & \\
\hline 8 & 8/24/2004 & 8:10:33 AM & & & 23.679 & 22.998 & 22.735 & -0.02 & 0.122 & $\begin{array}{l}-0.014 \\
\end{array}$ & -0.001 & -0.124 & 0.134 & 23.176 & 1.205 & 14.643 & & & & Convers & ersion & & $\mathrm{m} 3 / \mathrm{m} 21$ & e/day/barc & $\mathrm{arg} / \mathrm{gpm} / \mathrm{f}$ & t2/barg & & \\
\hline & 8/266/2004 & 9:14:11 AM & 25.819 & 23.352 & 24.932 & 25.316 & 23.242 & -0.00 & 0.126 & -0.008 & 0.011 & -0.106 & & 48.335 & 0.043 & 14.643 & 0.005 & & & & & & & & & & & \\
\hline$\frac{10}{11}$ & & & 22.6499 & 22.89 & 22.298 & & & & 0.14 & -0.008 & & & & & & & 0.6 & & & & & & (1) & & & & & \\
\hline$I 1$ & & & 22.511 & 22.848 & 22.397 & 22.669 & & -0.017 & 0.138 & -0.005 & 0.003 & -0.147 & & -0.082 & 0.003 & 14.643 & 0.00 & & & & & & & & & & & \\
\hline & & & & & & & & & & & & & & & & & & & & $\begin{array}{l}\text { Pressu } \\
\text { vavr }\end{array}$ & & - & & $\frac{1.88 \text { incl }}{<\text { K Fitrat }}$ & $\begin{array}{l}\text { ates of ow } \\
\text { ate Flux }\end{array}$ & $\begin{array}{l}\text { entubin } \\
K \text { KER }\end{array}$ & & \\
\hline 14 & & & & & & & & & & & & & & & & & & & & & & & & & at $25 \mathrm{C}$ & & $\times 1000$ & \\
\hline 15 & & & & & & & & & & & & & & & & & & Time & Time & Press. & Vel. & TMP & TMP & $\mathrm{gpm}$ & gpm & gpm & gpm & \\
\hline 16 & Data - Per M & hinute & & & & & & & & & & & & & & & & & Hour & psig t & $\mathrm{ft} / \mathrm{s}$ & psi & bar & $1+12$ & & ftr2/psi & $f$ tz/psi & \\
\hline 17 & 8/24/2004 & 1:44:56 PM & & 24.569 & 29.415 & 27.194 & & 45.531 & 48.544 & 9.93 & 33.448 & -0.08 & & $\begin{array}{ll}46.902 \\
\end{array}$ & 0.003 & $\begin{array}{l}14.643 \\
\end{array}$ & & & & 47.4 & \begin{tabular}{l|l}
4 & 11.2 \\
\end{tabular} & 39.5 & 2.723 & 0.000 & 0.000 & 0.000 & 0.011 & \\
\hline$\frac{18}{19}$ & $8 / 24 / 2004$ & 1:45:56 PMC & 26.104 & 24.577 & 29.374 & $\begin{aligned} 27.362 \\
27520\end{aligned}$ & 23.948 \# & $\begin{aligned} 45.51 \\
\end{aligned}$ & 48.593 & & & -0.077 & 0.105 & \begin{tabular}{|l|l|}
47.3999 \\
\end{tabular} & & $\begin{array}{l}14.643 \\
1.613\end{array}$ & 0.005 & 1.0 & 0.01667 & 47.5 & 11.3 & 39.5 & $\frac{2.722}{2.609}$ & 0.000 & 0.000 & 0.000 & 0.011 & \\
\hline$\frac{19}{20}$ & 8/24/2004 & $\begin{array}{l}\text { 1:46:56 PM } \mid 0 \\
\text { 1.47.56 PM0 }\end{array}$ & $26.107 \mid$ & $\begin{array}{l}24.565 \\
2454 \\
\end{array}$ & 29.207 & 27.529 & $\frac{24.046|\#|}{24.131 \#}$ & $\begin{array}{ll}45.062 \\
46.062\end{array}$ & $\begin{array}{ll}48.463 \\
49485\end{array}$ & $\begin{array}{l}10.224 \\
10546\end{array}$ & $\begin{array}{l}32.913 \\
33549\end{array}$ & $\begin{array}{r}-0.08 \\
\end{array}$ & & \begin{tabular}{|l|}
47.082 \\
48488 \\
\end{tabular} & .003 & \begin{tabular}{|r|r|}
14.643 \\
14.643
\end{tabular} & 0.005 & $\frac{2.00}{300}$ & 0.03333 & $\begin{array}{l}47.3 \\
484 \\
\end{array}$ & $\frac{11.2}{111.5}$ & $\begin{array}{ll}39.0 \\
39.8 \\
\end{array}$ & $\begin{array}{l}2.688 \\
2744 \\
\end{array}$ & $\begin{array}{ll}0.000 \\
0.000 \\
\end{array}$ & 0.000 & 0.000 & 0.011 & \\
\hline 21 & $\begin{array}{l}0 / 24 / 2004 \\
8 / 24 / 2004 \\
\end{array}$ & $\begin{array}{l}1: 4: 56 \mathrm{PM} / 0 \\
: 48: 56 \mathrm{PM}\end{array}$ & \begin{tabular}{|l|}
26.1316 \\
26.165 \\
\end{tabular} & $\begin{array}{l}24.564 \\
24563 \\
\end{array}$ & $\begin{array}{l}29.018 \\
28.768 \\
\end{array}$ & $\frac{27.113}{27.712}$ & 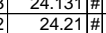 & $\begin{array}{l}46.062 \\
46.103 \\
\end{array}$ & $\begin{aligned} 49.485 \\
49.41\end{aligned}$ & $\begin{array}{l}10.546 \\
10.367 \\
\end{array}$ & $\begin{array}{l}33.5494 \\
34.064\end{array}$ & \begin{tabular}{|l|}
-0.083 \\
-0.097 \\
\end{tabular} & $\frac{0.108}{0.105}$ & $\begin{array}{l}48.486 \\
48.498\end{array}$ & 0.003 & \begin{tabular}{|l|}
14.643 \\
14.643 \\
\end{tabular} & $\begin{array}{l}0.005 \\
0.005\end{array}$ & & $\begin{aligned} 0.05 \\
0.06667\end{aligned}$ & $\begin{array}{l}48.4 \\
48.3 \\
\end{array}$ & 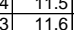 & & & 0.000 & $\frac{0.000}{0.000}$ & $\frac{0.000}{0.000}$ & & \\
\hline 22 & $8 / 24 / 2004$ & 1:49:56 PM 0 & 26.185 & 24.583 & 28.509 & 27.597 & $24.3 \#$ & 45.97 & 49.313 & 10.472 & 33.857 & \begin{tabular}{|l|}
-0.083 \\
\end{tabular} & 0.111 & \begin{tabular}{|l|l|}
48.932 \\
\end{tabular} & 0.003 & 14.643 & 0.005 & 5.00 & 0.08333 & 48.2 & \begin{tabular}{|l|l|}
2 & 11.7 \\
\end{tabular} & $\begin{array}{ll} \\
7 & 39.9 \\
\end{array}$ & 2.754 & 0.000 & 0.000 & & $\frac{0.010}{0.010}$ & \\
\hline & $8 / 24 / 2004$ & 1:50:56 PM 0 & 26.21 & 24.599 & & $\begin{array}{ll}27.483 \\
\end{array}$ & 24.395 \# & & 49.536 & 10.615 & 33.916 & -0.103 & & 49.345 & 0.003 & 14.643 & 0.005 & 6.00 & \begin{tabular}{|l|l}
0 & 0.1 \\
\end{tabular} & 48.4 & 11.8 & \begin{tabular}{|l|l|}
8 & 40.0 \\
\end{tabular} & \begin{tabular}{|l|} 
\\
\end{tabular} & 0.000 & .000 & & & 0 \\
\hline & $8 / 24 / 2004$ & 1:51:56 PM 0 & & 24.605 & \begin{tabular}{|l|l|}
527.943 \\
\end{tabular} & 27.4 & 24.442 \# & 45.705 & 49.321 & 10.612 & 33.672 & -0.083 & & \begin{tabular}{|l|l|}
49.063 \\
\end{tabular} & 0.003 & 14.643 & & & $\begin{array}{lll}0 & 0.11667 \\
\end{array}$ & & $\begin{array}{ll}11.7 \\
\end{array}$ & & & & & & & 0 \\
\hline 25 & $8 / 24 / 2004$ & 1:52:56 PM & 26.237 & & \begin{tabular}{|l|l|}
27.664 \\
\end{tabular} & 27.315 & $24.497 \#$ & & & & 34.208 & & & $\begin{array}{lll}48.342 \\
\end{array}$ & & & & 8.00 & \begin{tabular}{l|l}
00 & 0.13333
\end{tabular} & 48.5 & & 40.1 & 2.76: & .000 & .000 & 0.000 & 0 & \\
\hline 26 & & & & & & & & 45.914 & 49.648 & 10.46 & & -0.08 & & & & $\begin{array}{ll}14.643 \\
\end{array}$ & & & 0.15 & & & & & & & & & \\
\hline & $8 / 24 / 2004$ & $1: 54: 56 \mathrm{~F}$ & & 24.645 & \begin{tabular}{|l|l|}
5 & 27.089 \\
\end{tabular} & 27.344 & 24.486 \#\# & 45.408 & 49.481 & 10.362 & 33.797 & -0.103 & & 47.482 & & $\begin{array}{ll}14.643 \\
\end{array}$ & & $10.6>-1$ & & 48.4 & 11.3 & & & & & & & \\
\hline 28 & $8 / 24 / 2004$ & 1:55:56 & \begin{tabular}{|l|l|}
26.282 \\
\end{tabular} & 24.651 & 26.831 & 27.37 & $24.488 \| \#$ & 45.506 & & 10.598 & 33.77 & -0.08 & & 48.473 & 0.003 & 14.6 & & 11.0 & 0.1833 & 48.6 & 11.5 & 39.6 & & & & 500 & & \\
\hline 29 & $8 / 24 / 2004$ & 1:56:56 PM 0 & 26.279 & 24.647 & 26.538 & 27.277 & 24.479 \# & 45.817 & 49.924 & 10.61 & 34.257 & -0.109 & 0.10 & 48.488 & 0.003 & $\begin{array}{ll}14.643 \\
\end{array}$ & 0.00 & 12.0( & 0.2 & 48.8 & 11.5 & \begin{tabular}{l|l|}
5 & 40.0 \\
\end{tabular} & 2.76 & .000 & 0.000 & 0.000 & 0.010 & \\
\hline 30 & $8 / 24 / 2004$ & 7:56 PM 0 & 26.301 & 24.674 & 26.315 & 27.334 & 24.486 \# & 45.416 & 49.841 & 10.488 & 34.019 & -0.077 & & 49.142 & & $\begin{array}{ll}14.643 \\
\end{array}$ & & 13. & 0.21667 & 48.7 & 11.7 & & 2.73 & & & & & \\
\hline & 8/24/2004 & 1:58:56 PM & \begin{tabular}{|l|l|}
26.317 \\
\end{tabular} & 24.6 & 26.09 & 27.295 & & & M. & 10.595 & 33.969 & -0.103 & & & 0.0 & 14. & & 14. & 0.233 & 48.5 & 11.5 & 39. & & & & & & \\
\hline & (4)/2004 & 0.565 & 26.323 & & 25.955 & 27.267 & & & 49.526 & & 33.647 & & & 48.0 & & 14. & & & 0.2 & 48.4 & $\frac{11 .}{11}$ & 30 & & & & & & \\
\hline 34 & 80124212004 & & 26.336 & & 25.831 & 27.359 & 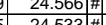 & 45.12 & 45.63 & & 34.1 & $=0.08$ & & $4 . .151$ & & & & 16.00 & 0.26606 & 48.5 & 11.4 & & & & & & & \\
\hline & 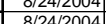 & & $\mid$\begin{tabular}{|l|l|}
20.342 \\
\end{tabular} & 24.1 .17 & 23.071 & 27.415 & 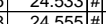 & 年53.441 & 50.09 & 10.3 & & & & & & & & & 0.28355 & 450. & & & & & & & & \\
\hline 36 & $\begin{array}{l}0 / \angle 1 / 2004 \\
8 / 24 / 2004 \\
\end{array}$ & $\frac{2.02 .50}{2 \cdot 0.56}$ & 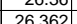 & $\frac{24.621}{2741}$ & $\frac{2.51}{25.491}$ & $\frac{21.450}{27425}$ & & $\frac{44.251}{4657}$ & S1.1380 & $\frac{1.11}{1.44^{2}}$ & $\frac{30.241}{34595}$ & $=0.0036$ & & $\frac{40.103}{50.694}$ & 0.003 & 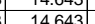 & & $\frac{10.0}{190}$ & 031667 & 50.3 & $\frac{11.6}{12.1}$ & 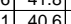 & & & & & & \\
\hline & $8 / 24 / 2004$ & & $\mid$\begin{tabular}{|l|l|}
$\mid 26.302$ \\
\end{tabular} & & $\frac{20.451}{25.443}$ & 27.316 & 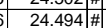 & & 51.537 & & & & & & & $\frac{14.6}{14.6}$ & & & 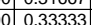 & 50.4 & 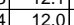 & 40.00 & & & & & & \\
\hline 38 & 8/24/2004 & $2: 05: 5$ & \begin{tabular}{|l|}
26.39 \\
\end{tabular} & 24.759 & 25.415 & 27.274 & & \begin{tabular}{|c|c|}
46.634 \\
\end{tabular} & 51.63 & & 35.131 & $\frac{-0.083}{-0.083}$ & & 50.2 & 0.003 & 14.643 & & 21.00 & 0.35 & 50.5 & 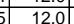 & 40.9 & & $\frac{000}{000}$ & .000 & 0.000 & $\frac{0.010}{0.010}$ & \\
\hline & $8 / 24 / 2004$ & & & & & 27.345 & & & 52.444 & & & & & & & 14.6 & & & 0.36667 & 51.3 & & & & & & & & \\
\hline & & & 26.408 & & 25.28 & 27.29 & & & 52.635 & & & & & 488 & & & & & 0.38333 & 51.5 & 11, & 110 & & & & & & \\
\hline & $8 / 24 / 2004$ & & 26.41 & 24.78 & 25.255 & 27.43 & $24.54 \#$ & & & & & & & & & & & & & & & & & & & & & \\
\hline & $8 / 24 / 2004$ & & 26.42. & & 25.171 & 27.424 & & & 52.496 & & 36.9 & -0.106 & & 48.711 & & 14. & & 25.0 & 0.41667 & 51.4 & 11.6 & 42. & & & & & & \\
\hline 43 & $8 / 24 / 2004$ & & \begin{tabular}{|l|}
26.428 \\
\end{tabular} & & 25.143 & 27.356 & & & 52.716 & & 36.937 & -0.106 & & 48.135 & & & & 26.00 & 0.43333 & 51.6 & 11.5 & 42.1 & 2.904 & 0.000 & 500 & .000 & 0.010 & \\
\hline 44 & $8 / 24 / 2004$ & & 26.454 & & 25.154 & 27.388 & & & 52.954 & & & -0.1 & & & & & & & & $\begin{array}{lll}51.8 & \end{array}$ & $11.4 \mathrm{r}>\mathrm{l}$ & & & & & & & \\
\hline 45 & 8/24/2004 & & \begin{tabular}{|l|l|}
26.461 \\
\end{tabular} & 24.836 & 25.09 & 27.4 & & & 52.838 & 10.563 & 37.159 & -0.089 & & 48.834 & & & & 28. & 0.4666 & 51.7 & $11.6 \mathrm{r}$ & & & & & & & \\
\hline 40 & $8 / 24 / 2004$ & 2:13:56 F & 26.474 & 24.859 & 25.109 & 27.477 & & 45.7. & $51.3 / 1$ & 11.55 & 34.78 & -0.089 & & 年 & & 14. & & 29 & 0.4833 & 50.2 & 12. & & 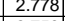 & & & & 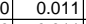 & \\
\hline & & & & & & & & & & & & & & & & & & & & & & & & & & & & \\
\hline$\frac{48}{109}$ & $\frac{821242004}{821204}$ & & 20.481 & 24. & 25.09 & 27.6 & & & 51.4 & 11.475 & & & & $50.5^{5}$ & & & & 31. & 0.51 & 50.4 & tat & 40 & & & & & . & \\
\hline & $\frac{0.21242044}{821204}$ & & & & & & & & $\frac{51.9}{51 .}$ & & & & & & & & & & & & & & & & & & & \\
\hline & 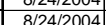 & & & & & 27.06 & & & (51.344 & & & $=0.0$ & & & & & & & & $\frac{5.0}{502}$ & & & & & & & & \\
\hline 52 & $801 / 242004$ & & $|26.53|$ & 24.4 & t) 25.094 & 27.75 & & & 51.976 & $\frac{1.4}{11.4}$ & & & & $\frac{51.6}{49.9}$ & & & & & \begin{tabular}{|l|l|}
0.5800133 \\
\end{tabular} & 50.9 & 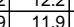 & & & & & & & \\
\hline & & & & & & & & & & & & & & & & & & & & & & & & & & & & \\
\hline 54 & $8 / 24 / 20$ & $2 \cdot 21: 5$ & 26.486 & & & 27.7 & 24.981 & & 51.414 & & & & & & & & & & $0.61667>$ & 50.3 & & & & & & & & \\
\hline & & & $26.3 \varepsilon$ & & & & & & & & & & & & & & & & & & & & & & & & & \\
\hline & $8 / 24 / 2$ & & \begin{tabular}{|l|}
26.302 \\
\end{tabular} & 25.0 & 25.121 & 27.635 & & 43.867 & 51.624 & 11.3 & 33.3 & 1.22 & & & & & & & & 50.5 & 12 & & & & & & & \\
\hline & $8 / 24 / 20$ & & 26.255 & 25.6 & 25.0 & 27.6 & & 4 & 51.7 & 11.4 & & 22 & & & & & & & 0.66 & 50 & & & & & & & & \\
\hline 58 & 8/24/2004 & & 26.21 & 25. & 25.12 & 27.5 & & \begin{tabular}{|l|l|l|}
42.447 \\
\end{tabular} & 51.895 & & 31.6 & 3. & & & & 14. & & & 0.68 & 50.8 & 8 & & & & & & & \\
\hline & 8/24/2004 & 2:26:56 & 26.172 & 25.0 & 25. & 27.59 & 24.8 & & 51.367 & 11.2 & 31.714 & 3.37 & & 51.1 & & 14. & & & 0 & 50.2 & & & & & & & & \\
\hline 60 & $8 / 24 / 2 / 2$ & & 26. & & & & & & & & & & & & & & & & & & 12.1 .187 & & & & & & & \\
\hline 6 & $8 / 24 / 4,2>$ & & 26.14 & 25. & t. 25 & 27. & & & 51.433 & & & & & & & & & 44 & & 50.3 & 2.4. & & & & & & & \\
\hline & $8 / 242120$ & & 26.106 & 25. & 25.14 & 27.50 & & 2.144 & 51.367 & & & & & & & & & & & & & & & & & & & \\
\hline & 80.12420 & & 20.14 & $\frac{25.0}{25 .}$ & 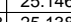 & 210 & & & $\frac{51.60}{5127}>2$ & & & & & & & & & & & & & & & & & & & \\
\hline 65 & $\frac{112421204}{824004}$ & & $\mid$ & (25.1153 & 25 & 27.45 & & & 517 & & & & & & & & & & & & & & & & & & & \\
\hline 66 & (1/24/2) & & & & 5 & 27.49 & & 年 42.004 & & & & & & & & & & & & & & & & & & & & \\
\hline 67 & $8 / 24 / 2004$ & & 26.101 & 2513 & 2517 & 27,444 & & 42.329 & 51.937 & 11388 & & 3.09 & & & & & & 50.0 & & 508 & 12 & & & & & & & \\
\hline & & & & & & & & & & & & & & & & & & & & & & & & & & & & \\
\hline & & & & & & & & & & & & & & & & & & & & & & & & & & & & \\
\hline 0 & 8/24/2004 & 2:37:56 PM & $\mid$ & 25.148 & \begin{tabular}{|l|l|}
3 & 25.178 \\
\end{tabular} & 27.491 & 24.704 \# \# & 42.285 & 51.68 & 11.448 & 31.655 & & & 50.256 & 0.037 & $\begin{array}{l}14.045 \\
14.643 \\
\end{array}$ & & $53.0-2=0$ & 8.88333 & 50.6 & & & & 0.005 & 0.005 & .000 & 0.143 & \\
\hline
\end{tabular}


WSRC-TR-2005-00105, REVISION 0

SRNL-RPP-2005-00012, REVISION 0

CAMPAIGN IV NEW GKN CONDITIONING WITH AN102R2 SIMULANT - CONT.

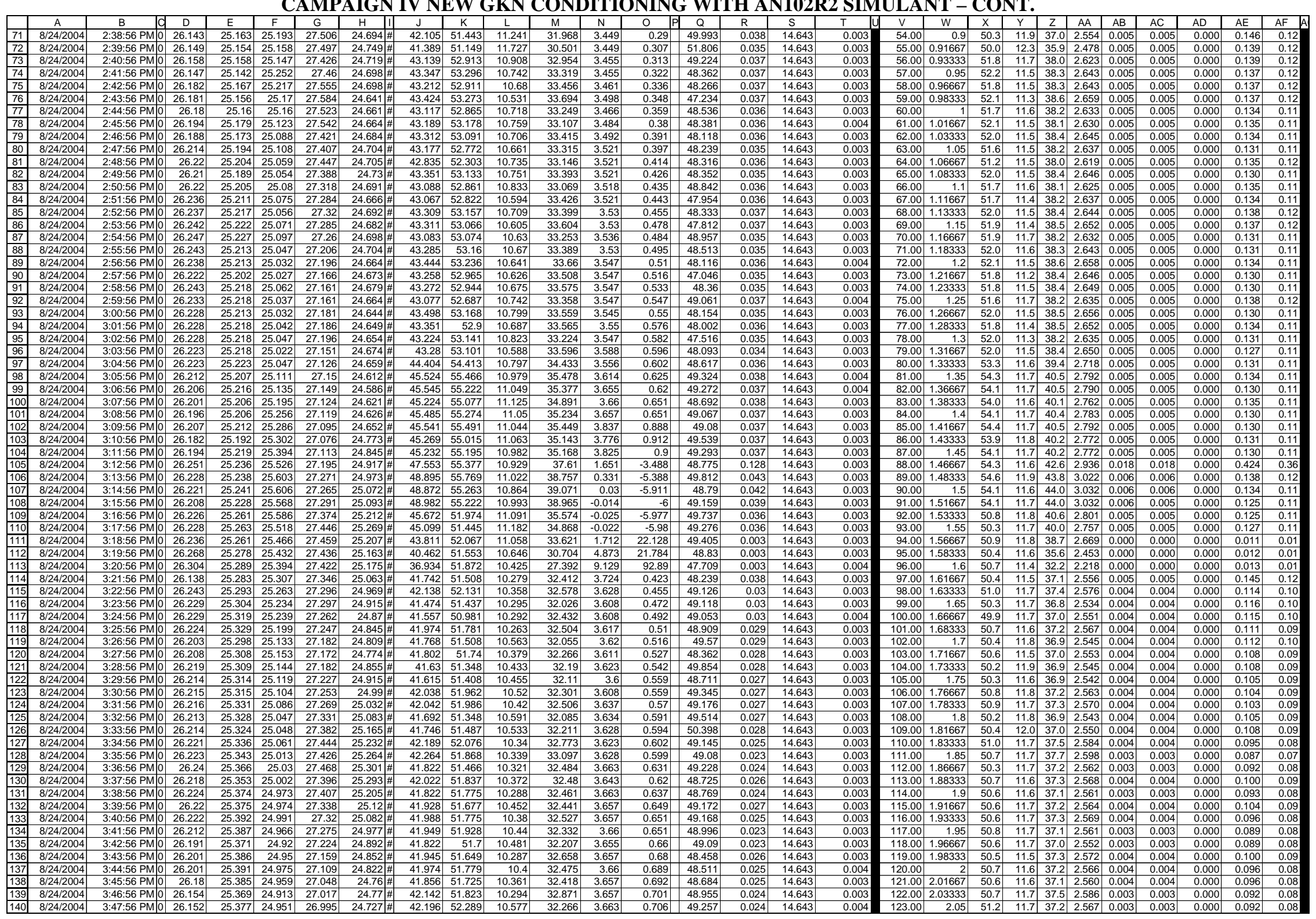


WSRC-TR-2005-00105, REVISION 0

SRNL-RPP-2005-00012, REVISION 0

CAMPAIGN IV NEW GKN CONDITIONING WITH AN102R2 SIMULANT - CONT.

\begin{tabular}{|c|c|c|c|c|c|c|c|c|c|c|c|c|c|c|c|c|c|c|c|c|c|c|c|c|c|c|c|c|}
\hline & A & \begin{tabular}{l|l}
$\mathrm{B}$ & $\mathrm{C}$ \\
\end{tabular} & D & $E$ & $\mathrm{~F}$ & $\mathrm{G}$ & \begin{tabular}{l|l|l}
$\mathrm{H}$ & $\mathrm{I}$ \\
\end{tabular} & $\mathrm{J}$ & $\mathrm{K}$ & $\mathrm{L}$ & $\begin{array}{ll} \\
\end{array}$ & $\mathrm{N}$ & $\mathrm{O}$ & Q & $\mathrm{R}$ & $\mathrm{s}$ & $\mathrm{T}$ & & $\mathrm{w}$ & $\mathrm{x} \mid$ & $\mathrm{Y}$ & $z$ & $\mathrm{AA}$ & $\mathrm{AB}$ & $\mathrm{AC}$ & $\mathrm{AD}$ & $\mathrm{AE}$ & \\
\hline & $\begin{array}{l}8 / 24 / 2004 \\
8 / 242004 \\
\end{array}$ & $\begin{array}{l}\text { 3:48:56 PM } 0 \\
3 \text { 3.4:56 PM } 0\end{array}$ & \begin{tabular}{|l|}
26.157 \\
26.146
\end{tabular} & $\begin{array}{l}25.377 \\
25.371\end{array}$ & 24.971 & $\begin{array}{r}26.96 \\
26.975 \\
\end{array}$ & $\begin{array}{l}24.737 \text { \# } \\
24.777 \#\end{array}$ & $\begin{array}{l}41.851 \\
44.727\end{array}$ & \begin{tabular}{|l|}
51.816 \\
51.650 \\
\end{tabular} & $\begin{array}{l}10.339 \\
10.35 \\
\end{array}$ & \begin{tabular}{|r|}
32.43 \\
32.363 \\
\end{tabular} & \begin{tabular}{|l|}
3.727 \\
3737 \\
\end{tabular} & \begin{tabular}{ll|l}
0.822 \\
0.894 \\
\end{tabular} & \begin{tabular}{|l|l|}
47.976 \\
49462
\end{tabular} & $\begin{array}{l}0.021 \\
0.024\end{array}$ & \begin{tabular}{|l|l|}
14.643 \\
14634 \\
\end{tabular} & $\begin{array}{ll}0.003 \\
0.004\end{array}$ & $\begin{array}{l}124.00 \\
12500 \\
\end{array}$ & \begin{tabular}{|l|l|}
2.06667 \\
20833 \\
\end{tabular} & \begin{tabular}{|l|l|}
50.7 \\
505 \\
\end{tabular} & $\begin{array}{l}11.4 \\
118\end{array}$ & \begin{tabular}{|l|l|}
37.1 \\
37.
\end{tabular} & $\begin{array}{l}2.561 \\
2554 \\
\end{array}$ & $\begin{array}{l}0.003 \\
0.003\end{array}$ & $\begin{array}{l}0.003 \\
0.003 \\
\end{array}$ & 0.000 & 0.081 & 0.07 \\
\hline$\overline{14}+2>$ & & & & & & & $24.871 \#$ & & 51.81 & 10.465 & 32.443 & & $\begin{array}{l}0.094 \\
0.894\end{array}$ & & & $\begin{array}{l}114.643 \\
14.643 \\
\end{array}$ & 0.004 & 125.00 & & & & & & & $\frac{0.003}{0.003}$ & 0.000 & & 0.08 \\
\hline$\overline{14}$ & $8 / 24 / 2004$ & 3:51:56 PM 0 & 26.15 & 25.371 & 24.995 & 27.054 & $24.916 \#$ & 41.905 & 52.071 & 10.503 & 32.143 & 3.773 & 0.897 & 48.802 & 0.024 & 14.643 & 0.003 & 127.00 & \begin{tabular}{|l|l|}
2.11667 \\
\end{tabular} & 50.9 & 11.6 & 37.0 & 2.553 & .003 & $\begin{array}{l}0.003 \\
0.003 \\
\end{array}$ & $\begin{array}{c}0.000 \\
0.000\end{array}$ & 0.092 & $\frac{0.08}{0.08}$ \\
\hline & & & 26.12 & 25.371 & 24.995 & 27.054 & $24.986 \#$ & 41.723 & 51.506 & 10.367 & 32.389 & 3.773 & 0.897 & 48.598 & 024 & $\begin{array}{ll}14.643 \\
\end{array}$ & & & 2.13333 & 50.4 & & & & & 0.003 & & & \\
\hline & $8 / 24 / 2004$ & 3:53:56 PM 0 & \begin{tabular}{|l|}
26.136 \\
\end{tabular} & 25.371 & 25.021 & 27.109 & 25.047 \# & 41.883 & 51.758 & 10.382 & $\begin{array}{l}32.408 \\
\end{array}$ & 3.773 & 0.897 & 48.961 & 0.024 & 14.643 & 0.003 & 129.00 & 2.15 & 50.6 & 11.7 & 37.1 & 2.561 & \begin{tabular}{l|l}
.003 \\
\end{tabular} & 0.003 & 0.000 & 0.092 & \\
\hline & 8/24/2004 & & & & & & $25.118 \#$ & & & & 32.375 & & & 48.69 & $\begin{array}{ll}0.024 \\
\end{array}$ & 14.643 & & & 2.16667 & & & & & & 0.000 & & & \\
\hline & 8/24/2004 & 3:55:56 PM 0 & 26.113 & 25.373 & 24.987 & 27.146 & $25.113 \#$ & 41.88 & 51.802 & 10.347 & 32.322 & $\begin{array}{ll}3.773 \\
377\end{array}$ & $\begin{array}{ll}0.894 \\
\end{array}$ & $\begin{array}{l}48.757 \\
\end{array}$ & $\begin{array}{l}0.023 \\
\end{array}$ & $\begin{array}{l}14.643 \\
\end{array}$ & 0.003 & 131.00 & 2.18333 & 50.7 & \begin{tabular}{|l|}
11.6 \\
\end{tabular} & 37.1 & 2.558 & 0.003 & 0.003 & & 0.089 & \\
\hline & $\begin{array}{l}8 / 24 / 2004 \\
8 / 242004 \\
\end{array}$ & $\begin{array}{l}\text { 3:56:56 PM } 0 \\
3.57 .56 \text { PM0 }\end{array}$ & \begin{tabular}{|l|}
26.115 \\
26116 \\
\end{tabular} & $\begin{array}{l}25.375 \\
25.381\end{array}$ & $\begin{array}{l}25.009 \\
25.026\end{array}$ & $\begin{array}{l}27.198 \\
27.209\end{array}$ & $\begin{array}{r}25.16 \text { \# } \\
25.232 \text {; }\end{array}$ & $\begin{array}{l}41.625 \\
41943 \\
\end{array}$ & $\begin{array}{l}51.506 \\
51.876\end{array}$ & $\begin{array}{l}10.357 \\
10.365 \\
\end{array}$ & \begin{tabular}{|l|}
32.225 \\
32.512 \\
\end{tabular} & \begin{tabular}{|r|}
3.77 \\
3.737 \\
\end{tabular} & \begin{tabular}{|l|l|}
0.894 \\
0.894
\end{tabular} & \begin{tabular}{|l|}
48.775 \\
48951
\end{tabular} & 0 & \begin{tabular}{|l|l|}
14.643 \\
14643 \\
\end{tabular} & \begin{tabular}{|l}
0.003 \\
0.003
\end{tabular} & \begin{tabular}{|l|}
132.00 \\
13300
\end{tabular} & \begin{tabular}{r|}
2.2 \\
221667 \\
\end{tabular} & \begin{tabular}{|l|}
50.4 \\
508 \\
\end{tabular} & \begin{tabular}{|l|}
11.6 \\
117
\end{tabular} & \begin{tabular}{|l|l|}
36.9 \\
372
\end{tabular} & $\begin{array}{l}2.546 \\
2.567\end{array}$ & $\begin{array}{l}0.003 \\
0.003\end{array}$ & $\frac{0.003}{0.003}$ & 0.000 & 0.089 & \\
\hline 15 & $\begin{array}{l}|l| \angle 4 / 2004 \\
8 / 24 / 2004 \\
\end{array}$ & $\begin{array}{l}3: 57: 56 \mathrm{PM} 0 \\
3: 58: 56 \mathrm{PM} 0\end{array}$ & \begin{tabular}{|l|}
26.116 \\
26.108
\end{tabular} & $\begin{array}{l}25.381 \\
25.388 \\
\end{array}$ & $\begin{array}{l}25.026 \\
24.992 \\
\end{array}$ & $\begin{array}{l}27.209 \\
27.251\end{array}$ & 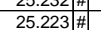 & & $\begin{array}{l}51.8 / 6 \\
52.181\end{array}$ & 10.348 & \begin{tabular}{|l|}
32.752 \\
32.736
\end{tabular} & 3.77 & $\begin{array}{l}.8944 \\
0.891 \\
\end{array}$ & $\begin{array}{l}48.951 \\
49.059\end{array}$ & \begin{tabular}{|l|l|}
0.023 \\
0.023 \\
\end{tabular} & $\frac{14.643}{14.643}$ & $\begin{array}{l}0.0003 \\
0.003 \\
\end{array}$ & $\begin{array}{l}133.00 \\
134.00\end{array}$ & 2.21661 & $\begin{array}{l}50.8 \\
51.1\end{array}$ & $\frac{11.1}{11.7}$ & & $2.567 \mid$ & \begin{tabular}{|c|c|}
0.003 \\
0.003
\end{tabular} & $\begin{array}{l}0.003 \\
0.003 \\
\end{array}$ & $\frac{0.000}{0.000}$ & 0.088 & \\
\hline & $8 / 24 / 2004$ & 3:59:56 PM 0 & 26.11 & 25.395 & \begin{tabular}{|l|}
24.999 \\
\end{tabular} & 27.278 & $25.295 \#$ & 41.719 & 51.702 & 10.306 & 32.33 & 3.77 & 0.891 & 48.729 & & 14.643 & 0.004 & & & 50.6 & 11.6 & & & & 0.003 & & & 0.07 \\
\hline & & 4:00:56 PM 0 & 26.1 & 25.401 & 25.05 & 27.313 & $25.351 \#$ & 44.633 & 54.561 & 10.449 & 35.09 & 3.822 & 0.903 & 49.199 & 0.025 & 14.643 & & & 2.26667 & 53.4 & 11.7 & & 2.748 & 0.004 & 0.004 & & 0.090 & \\
\hline & $8 / 24 / 2004$ & 4:01:56 PM 0 & 26.102 & 25.417 & & 27.31 & 25.332\# & 4.724 & 54.735 & 10.373 & 35.299 & & 0.9 & $\begin{array}{l}49.22 \\
\end{array}$ & & $\begin{array}{l}14.643 \\
\end{array}$ & & & 2.28333 & 53.6 & 11.7 & 40.0 & & 0.004 & & 0.000 & & $\frac{0.08}{0.08}$ \\
\hline & $8 / 24 / 2004$ & & 26.113 & & & & & & & & & & & & & & & & 2.3 & $\begin{array}{ll}53.3 \\
\end{array}$ & & & & 004 & & & .090 & \\
\hline & & $\mathrm{PM} 0$ & & 25.429 & & & $25.14 \| \#$ & & 5.29 & & & & & & & 14.643 & & & & & & & & & & & & \\
\hline & $8 / 24 / 2$ & 1:56 PM 0 & 26.1 & 25.43 & 25.139 & 27.193 & 25.065 \# & & 675 & .068 & 35.87 & 3.817 & & 498 & & & & & 33333 & & & & & & & & & \\
\hline & $8 / 24 / 2004$ & $56 \mathrm{PM} 0$ & 26.11 & & & & & .98 & & & & & & & & 14.643 & & & 2.35 & & & & & & & & & \\
\hline & $8 / 24 / 2004$ & 6:56 PM 0 & 26.11 & 25.445 & 25.229 & 27.123 & $24.92 \#$ & 45.203 & 55.224 & 10.012 & 36.104 & 3.811 & 0.9 & 48.183 & 0.025 & 14.643 & 0.003 & 142.0 & 2.36667 & 54.1 & 11.5 & 40. & 2.80 & 504 & 0.004 & & 0.087 & \\
\hline & $8 / 24 / 2$ & :56 PM0 & \begin{tabular}{|l|l|}
26.108 \\
\end{tabular} & 25.438 & 25.198 & 27.077 & 24.859\#\# & 45.441 & 55.649 & 10.077 & 36.089 & 3.825 & 0.9 & 47.78 & 025 & $\begin{array}{ll}14.643 \\
\end{array}$ & 0.00 & & 2.38333 & 54.5 & 11.4 & & 2.8 & .004 & 5.004 & .000 & 0.087 & \\
\hline & $8 / 24 / 2$ & $3: 56$ PM 0 & 26.108 & 25.438 & 25.227 & 27.026 & 24.833 \# & 45.304 & 8719 & 10.085 & 35.94 & 3.828 & 0.897 & & 025 & $\begin{array}{ll}14.643 \\
\end{array}$ & & 144 & 2.4 & 54.6 & & & & & & & & \\
\hline & & 56 PM] 00 & & 25.427 & 25.242 & 26.95 & 24.772|\#| & & 5.174 & 9.849 & 36.381 & 3.785 & 0.897 & & & 14.643 & & & & 54.0 & & & & & & & & \\
\hline & $\frac{821242004}{821204}$ & & 26.11 & 25.436 & 25.24 & 26.949 & 24.726 \# & $\frac{423}{223}$ & & & 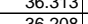 & $\begin{array}{l}3.825 \\
3779 \\
\end{array}$ & 0.9 & & & & & & .43333 & 54.4. & & & & & & & $\begin{array}{l}087 \\
087 \\
\end{array}$ & \\
\hline & $\frac{10242004 \mid}{8 / 24204}$ & (5.56 & $|26.995|$ & $\frac{25.409}{25.393}$ & (25.259 & 20.081 & 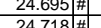 & .238 & .323 & 9.968 & $\begin{array}{l}36.208 \\
36.024\end{array}$ & 3.71 & & & & & & & & & & & & & & & & \\
\hline 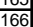 & $8 / 24 / 2004$ & 3:56 PM & \begin{tabular}{|l|}
26.000 \\
26.098 \\
\end{tabular} & 25.398 & $\mid$\begin{tabular}{|l|l|}
25.232 \\
25232
\end{tabular} & $\frac{20.020}{26.871}$ & 24.7903 \#\# & 45.352 & 55.097 & $\begin{array}{r}5.504 \\
10.203\end{array}$ & 30.0L4 & 3.005 & & 48.045 & 024 & $\begin{array}{l}\frac{14.045}{14.643} \\
\end{array}$ & 0.003 & 14900 & 2.43033 & 54.0 & 11.25 & & 280 & & & 0.000 & 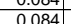 & 0.07 \\
\hline & & 4:56 PM 0 & \begin{tabular}{|l|}
26.087 \\
26
\end{tabular} & 25.387 & 25.221 & 26.86 & $24.867 \#$ & & 55.33 & 10.016 & \begin{tabular}{|l|l|}
36.446 \\
\end{tabular} & 3.788 & & & & $\begin{array}{l}14.045 \\
14.643 \\
\end{array}$ & & & $\frac{2.40505}{25}$ & 54.2 & tit. & & & & & & & $\frac{0.07}{0.07}$ \\
\hline & & 7:24:57 AM 0 & 22.515 & 22.726 & 23.475 & 22.492 & $22.185 \#$ & 48.807 & 55.906 & 9.731 & 39.935 & 0.794 & -0.349 & 6.673 & 003 & 14.643 & 0.003 & & $\frac{17.6669}{17.69}$ & 54.8 & 11. & 44. & 3.05 & .000 & 500 & & 0.010 & $\begin{array}{l}0.01 \\
0.01 \\
\end{array}$ \\
\hline & $8 / 25 / 2$ & & & 22.726 & & 22.819 & & 45.499 & 4.0 & & & & & 0.868 & & 14.643 & & & 17.6836 & 52.9 & & & & & & & & \\
\hline & & & 22.56 & & \begin{tabular}{|l|l|}
24.484 \\
\end{tabular} & 23.121 & & & & & & & & & & & & & 17.7003 & 530 & 11. & & 265 & & 003 & $n x^{2}$ & 0.089 & \\
\hline 171 & $8 / 25 / 2$ & & & & \begin{tabular}{|l|l|}
24.715 \\
\end{tabular} & 23.368 & $22.588|\#|$ & & 4.3 .3 & 10.706 & 34.372 & & & & & $\begin{array}{ll}14.643 \\
\end{array}$ & & & 17.716 & $53.2>>-2$ & & & & & & & & \\
\hline & & & & & & & 22.716 \# & & & & & 3.73 & & & & & & & & & & & & & & & & \\
\hline & $8 / 25 / 2004$ & AM 0 & \begin{tabular}{|l|l|}
23.019 \\
\end{tabular} & 22.743 & 24.911 & 23.695 & 22.819 \#\# & & & & & 3.7 & & & & & & & 17.7503 & 52.9 & 12. 2 & & & & & & .099 & \\
\hline & $8 / 25 / 2$ & 7:30:57 AM 0 & \begin{tabular}{|l|l|}
23.122 \\
\end{tabular} & 22.746 & 24.909 & 23.818 & $22.903|\#|$ & $\begin{array}{lll}44.363 \\
\end{array}$ & 54.015 & 10.657 & 34.601 & 3.73 & & & & 14.643 & & & 17.7669 & 52.9 & 11. & 39 & & & & & 0.098 & \\
\hline & & & \begin{tabular}{|l|l|}
23.228 \\
\end{tabular} & 22.762 & & 23.935 & 22.989|\# & & 54501 & 10.585 & 34.583 & 3.733 & & & & & & & & & & & & & & & & \\
\hline$\frac{11}{17}$ & & AMlo & 23.308 & 22.766 & 24.934 & & 23.118|\# & & & $10.7 \mathrm{r}$ & & 3.7 & & & & & & & & 53. & & & & & & & & \\
\hline & & & 23.394 & 22 & 24.97 & & $23.14 \mid \#$ & & & & & 3. & & & & & & & & 53 & & & & & & & & \\
\hline & & & & & 25033 & $24.14 t$ & & & & & & & & & & & & & & & & & & & & & & \\
\hline & & & $\mid \frac{2.5}{235}$ & $\frac{24.0}{208}$ & 250305 & 24.105 & & & & & & & & & & & & & & 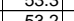 & & & & & & & & \\
\hline 18 & $801 / 25 / 2$ & & $\mid$\begin{tabular}{|l|l|l|l|l|}
23636 \\
\end{tabular} & 228 & 25.066 & $\frac{24.207}{24.227}$ & & & & & & 3.1. & & & & & & & & 532 & & & & & & & & \\
\hline $\bar{\varepsilon}->1$ & & & & & & 24.252 & & & & & & & & & & & & & & & & & & & & & & \\
\hline & $8 / 25 / 2$ & & $\mid 23.718$ & 22.825 & 25.118 & 24.304 & $22.941 \#$ & & & & & & & & & & & & & 53.2 & $11.8 \mathrm{~s}$ & & & & & & & \\
\hline & & & & & & & & & & & & & & & & & & & & & & & & & & & & \\
\hline & & & 23.819 & 22.852 & 25.139 & 24.3 & & & & & & & & & & & & & & & & & & & & & & \\
\hline & & & & & & & & & & & 35.164 & & & & & & & & & & & & & & & & & \\
\hline & $8 / 25 / 2$ & & $\mid$\begin{tabular}{|l|l|}
23.916 \\
\end{tabular} & 22.868 & 25.191 & 24.372 & $22.929 \mid \#$ & & & & 34 & 3.73 & & & & & & & & 53.0 & 11 & 39 & & & & & & \\
\hline & $8 / 25 / 2004$ & & \begin{tabular}{|l|l|}
23.956 \\
\end{tabular} & 22.868 & 25.196 & & & & & & 35.02 & & & & & & & & & 53 & & 44 & & & & & & \\
\hline & $8 / 25 / 2 / 2>19$ & & & 22.869 & 25.196 & 24.4 & & & & & & & & & & & & & & & & & & & & & & \\
\hline & & & 24.0 & 22.9 & & & 23.036"\# & & & & 35.56 & & & & & & & & & 53.6 & 11. & & & & & & & \\
\hline & 8/25/2 & & & 22.9 & 25.238 & & 22.976|\# & & & & & & & & & & & & & 53.4 & . & & & & & & & \\
\hline & & & & 22.881 & 25.228 & & & & & & & & & & & & & & & 53.4 & 11.6 & & & & & & & \\
\hline & & & $\mid 24.1555$ & 22.912 & 25.229 & & & & & & & & & & & & & & & & & & & & & & & \\
\hline & & & & & & & & & & & & & & & & & & & & & & & & & & & & \\
\hline & $8 / 25$ & & & & 25.251 & & & & & & & & & & & & & & & & & & & & & & & \\
\hline & $8 / 25 / 2$ & & & 22.949 & & 24.62 & 23.166 \# & & & & & & & & & & & & & & & & & & & & & \\
\hline & & & & & & & & & & & & & & & & & & & & & & & & & & & & \\
\hline & & & & & & & & & & & & & & & & & & & & & & & & & & & & \\
\hline & & & & & & & & & & & & & & & & & & & & & & & & & & & & \\
\hline & & & 24.56 & 23.003 & 25.21 & 24.7 & 23.2 & & 54.1 & & 35.26 & & & & & & & & & & & & & & & & & \\
\hline & $8 / 25 / 2$ & & 24.5 & 23.007 & 25.209 & 24.8 & 23.55 & 44.5 & 54. & & 34.98 & 3.5 & & & & 14 & & & & 5 & & & & & & & & \\
\hline & $8 / 25 / 2 / 2$ & & 24.6 & 23.0 & 25 . & 24.9 & 23.50 & & 54 & & & & & & & & & & & & & & & & & & & \\
\hline & 8/25/2 & & & & 25 . & & & & 53.. & & & & & & & & & & & $52-2>2$ & & & & & & & & \\
\hline & & & 24. & 23.04 & 25.195 & 25.0 & & & & & & & & & & & & & & & & & & & & & & \\
\hline & & & & & & & & & & & & & & & & & & & & & & & & & & & & \\
\hline & & & & 23.082 & 25.224 & 25.082 & & & 54.4344 & & & 3.5 & & & & & & & & & & & & & & & & \\
\hline & & & & & & & & 44 & & & & & & & & & & & & & & & & & & & 18 & \\
\hline 10 & $8 / 25 / 2$ & $8: 06: 57 \mathrm{AM} 0$ & \begin{tabular}{|l|l|} 
& 24.753 \\
\end{tabular} & 23.115 & 25.216 & 25.064 & $23.386 \#$ \# & 44.995 & \begin{tabular}{|l|l|}
54.482 \\
\end{tabular} & 9.915 & $\mid 35.9041$ & 3.524 & 0.51 & 48.173 & 0.022 & 14.643 & & & & 03.4 & & & & & .003 & .000 & 0.077 & \\
\hline
\end{tabular}


WSRC-TR-2005-00105, REVISION 0

SRNL-RPP-2005-00012, REVISION 0

CAMPAIGN IV NEW GKN CONDITIONING WITH AN102R2 SIMULANT - CONT.

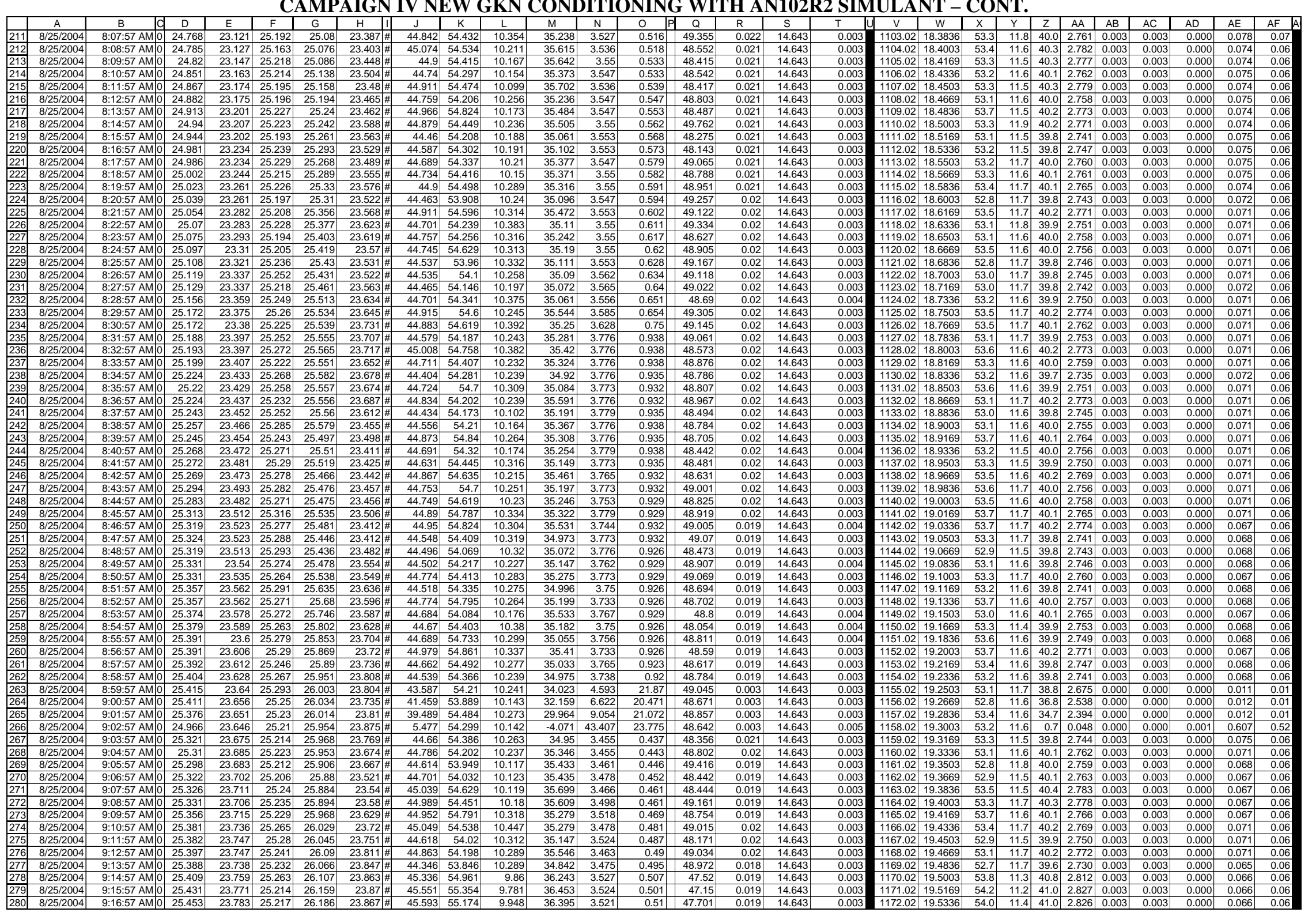


WSRC-TR-2005-00105, REVISION 0

SRNL-RPP-2005-00012, REVISION 0

CAMPAIGN IV NEW GKN CONDITIONING WITH AN102R2 SIMULANT - CONT.

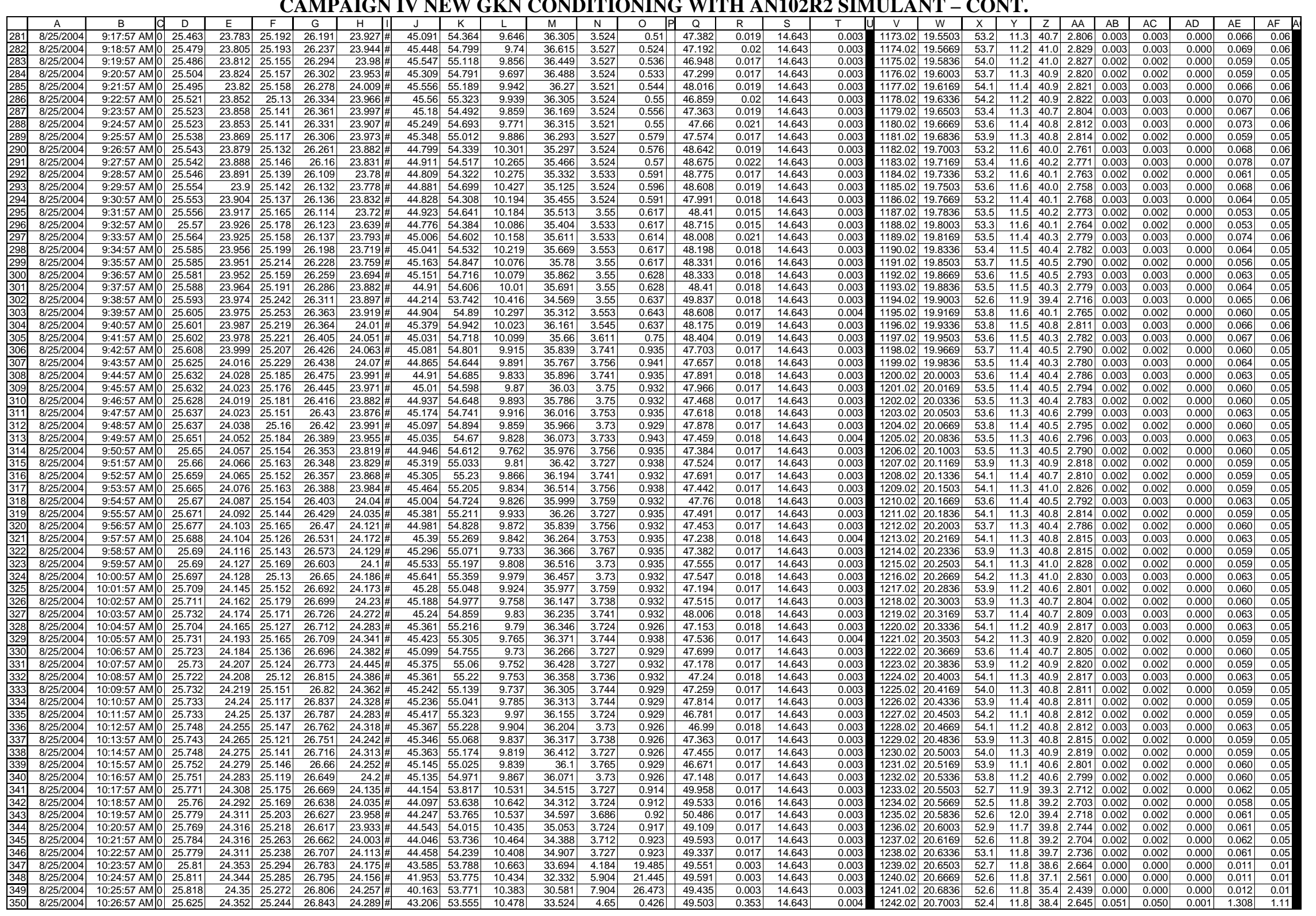


WSRC-TR-2005-00105, REVISION 0

SRNL-RPP-2005-00012, REVISION 0

CAMPAIGN IV NEW GKN CONDITIONING WITH AN102R2 SIMULANT - CONT.

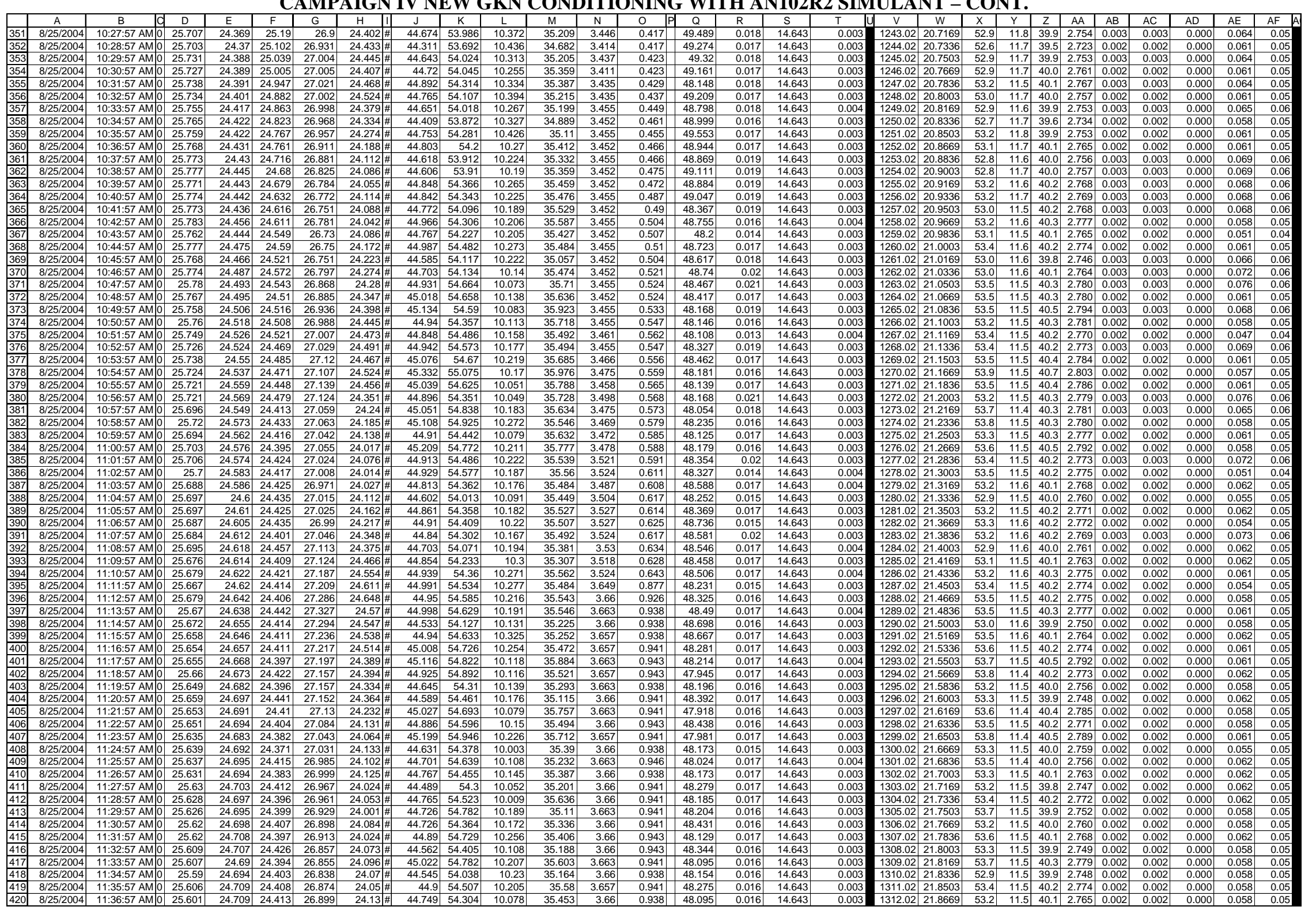


WSRC-TR-2005-00105, REVISION 0

SRNL-RPP-2005-00012, REVISION 0

CAMPAIGN IV NEW GKN CONDITIONING WITH AN102R2 SIMULANT - CONT.

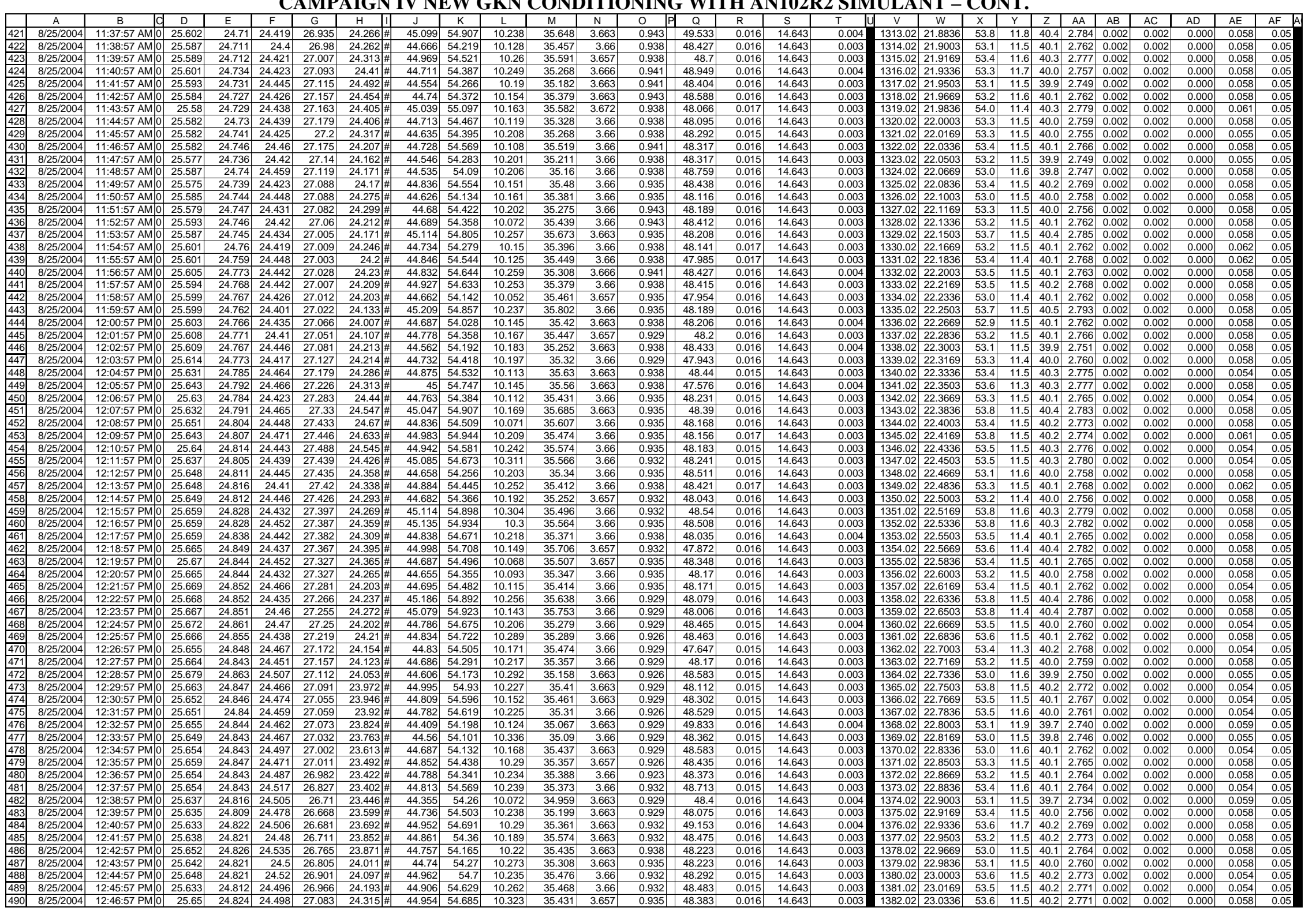


WSRC-TR-2005-00105, REVISION 0

SRNL-RPP-2005-00012, REVISION 0

CAMPAIGN IV NEW GKN CONDITIONING WITH AN102R2 SIMULANT - CONT.

\begin{tabular}{|c|c|c|c|c|c|c|c|c|c|c|c|c|c|c|c|c|c|c|c|c|c|c|c|c|c|c|c|c|}
\hline & & $\mathrm{B}$ & $\mathrm{D}$ & \begin{tabular}{|l|l|}
$\mathrm{E}$ & \\
\end{tabular} & $\mathrm{F}$ & $\mathrm{G}$ & $\begin{array}{lll}\mathrm{H} & \mathrm{H} \\
\end{array}$ & $\mathrm{J}$ & & & $\mathrm{M}$ & $\mathrm{N}$ & & & $\mathrm{R}$ & & $T$ & & & $x$ & & & $A A$ & & & & & \\
\hline & & 12:47:57 PM & 25.643 & $\begin{array}{l}24.816 \\
2.80\end{array}$ & 24.511 & & $24.458 \#$ & & & & & 3.66 & & & 0.01 & 14.643 & & & 8050 & 53.6 & & & & & & & & 0.05 \\
\hline & & & & & & & & & & & & 3.66 & & & & & & & & & & & & & & & & \\
\hline & $8 / 25 / 2004$ & 12:49:57 PM0 & 25.657 & \begin{tabular}{|l|l}
24.83 \\
\end{tabular} & 24.535 & 27.324 & $24.557 \#$ & 44.78 & 54.731 & $\begin{array}{ll}10.182 \\
\end{array}$ & 35.332 & 3.66 & 0.914 & 48.15 & & 14.643 & & & .0836 & 53.6 & & & & & 0.002 & 000 & 0.054 & \\
\hline & & & 25.66 & 24.823 & 24.512 & 27.307 & $24.599 \#$ & $\begin{array}{l}44.647 \\
4\end{array}$ & 54.31 & 10.154 & 35.318 & 3.655 & 0.912 & 48.642 & 0.016 & 14.643 & & & 3.1003 & 53.2 & & 40 & 2.757 & & & & & \\
\hline 495 & 8/25/2004 & 12:51:57 PM 0 & 25.665 & 24.839 & 24.528. & 27.338 & 24.445|\# & 44.539 & 54.252 & 10.17 & 35.121 & 3.66 & 0.917 & 48.298 & 0.015 & 14.643 & & 1387.02 & 23.1169 & 53.1 & 11.5 & $39.8 \mathrm{r} \quad \mathrm{C}$ & 2.746 & 0.002 & 0.002 & 0.000 & 0.055 & \\
\hline & 8/25/2004 & & 25.68 & 24.8444 & & 27.318 & 24.335|\#| & 44.575 & 54.14 & 10.135 & 35.301 & 3.666 & 0.917 & 48.185 & 0.016 & 14.643 & & & 3.1336 & & & & & & 0.002 & & & \\
\hline & 8/225/2004 & & 25.681 & 24.85 & 24.514 & 27.249 & 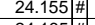 & 45.182 & 54.93 & 10.342 & 35.587 & 3.66 & 0.917 & 48.0411 & 0.015 & 14.643 & & & 1503 & 53.8 & & 40. & 2.784 & 1002 & 0.002 & 0.000 & 0.054 & \\
\hline & & & & & 24.543 & & & & 54.465 & & & 3.663 & & & & & & & & & & & & & & & & \\
\hline & & 12.55.57PM & t2.669 & 24.837 & $\begin{array}{ll}24.502 \\
20.57\end{array}$ & 2.151 & & 44.805 & S4.691 & 1.1.19 & 35.449 32096 & 3.06 & 0.912 & 48.5234 & & 14.6 & & & & 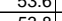 & & & 2.16 & & 0.002 & & & \\
\hline & 8 & $12.57 .57 \mathrm{PMO}$ & 25723 & 24.045 & 24516 & 27.1061 & 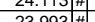 & 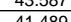 & 54207 & $\frac{1.204}{1009}$ & 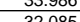 & 5.000 & 8754 & $\frac{14.4609}{48.629}$ & 0.0003 & 年 & & & 2321609 & 53.0 & 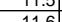 & & 2536 & 10000 & 0000 & 0.000 & 然 & \\
\hline & 88252004 & $12.51 .57 \mathrm{rMT}$ & 23.123 & 24.041 & 2.0925 & (1).001 & 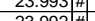 & 4.4095 & 5.257 & 10.08 & (32.065 & 0 & $\frac{0.154}{0.721}$ & (1) & 0.003 & 14.643 & & & 20.109 & (33.2 & 甚1.6. & (30.8) & \begin{tabular}{|l|l|} 
\\
\end{tabular} & 0.0000 & 0.000 & 0.000 & & \\
\hline & $8 / 25 / 2004$ & 12:59:57 PM 0 & 25.681 & 24.839 & 24.508 & 27.008 & $24.025 \#$ & $\begin{array}{l}39.903 \\
38.156 \\
\end{array}$ & 54.729 & $\frac{10.01}{10.253}$ & $\frac{30.514}{28.404}$ & 10.724 & $\begin{array}{l}0.151 \\
49.672\end{array}$ & 年0.044 & 0.003 & $\begin{array}{l}\frac{14.045}{14.643} \\
\end{array}$ & & $\frac{059404}{395.02}$ & $\begin{array}{l}\frac{20.2050}{23.2503} \\
\end{array}$ & 53.6 & $\frac{11.1}{11.5}$ & $\begin{array}{ll}30.4 \\
33.3\end{array}$ & \begin{tabular}{|l|}
2.400 \\
2.295 \\
\end{tabular} & 0.000 & 0.000 & 0.000 & $\frac{0.012}{0.013}$ & \\
\hline & $8 / 25 / 2004$ & 1:00:57 PM 0 & 25.69 & 24.844 & 24.498 & 27.048 & $24.064 \#$ & 35.872 & 54.753 & 10.118 & 26.278 & 13.066 & 91.502 & 48.031 & 0.003 & 14.643 & & 396.02 & 23.2669 & 53.6 & 11.4 & $\frac{0.0}{31.1}$ & 2.143 & 0.000 & 0.000 & 0.000 & 0.014 & $\begin{array}{l}0.01 \\
0.01 \\
\end{array}$ \\
\hline & 8/25/2004 & 1:01:57 PM 0 & 25.504 & 24.847 & 24.521 & 27.041 & $24.048 \#$ & & & 10.007 & & & & & & & & & & & & & & & & & & \\
\hline 506 & & & & & & & & 8.231 & 54.105 & 10.076 & & 40.743 & 64.198 & 48.041 & 0.003 & 14.643 & & & .3003 & 53. & & $-2+2>$ & 0.244 & 000 & 0.000 & 0.000 & 0.123 & \\
\hline & & 1:03:57 PM0 & & 24.865 & 24.524 & 27.014 & 23.936 \# & 8.35 & 54.416 & 10.041 & & 40.665 & & 48.081 & & 14.643 & & & & & & & & & & & 0.119 & \\
\hline & 8/25/2004 & 1:04:57 PM 0 & 25.645 & 24.854 & 24.488 & 26.978 & $23.959 \#$ & 8.312 & 54.399 & 10.057 & -1.142 & 40.74 & $\begin{array}{ll}0.357 \\
\end{array}$ & & & & & & 3.3336 & & & & & & 0.000 & 00 & & \\
\hline & $8 / 25 / 2004$ & 1:05:57 PM 0 & & & & & 23.904 \# & 10.326 & 54.074 & 9.996 & 1.054 & 38.67 & & 48.135 & & 14.64 & & & & & & & & & & & & \\
\hline 510 & 8/25/2004 & 1:06:57 PM 0 & 25.268 & 24.847 & 24.481 & 27.006 & $24.068 \mid \#$ & 36.072 & 54.498 & 10.064 & 26.725 & 12.823 & 0.377 & 48.11 & 0.083 & 14.643 & & 402.02 & 23.3669 & 53.4 & 11.5 & 31.4 & \begin{tabular}{|l|}
2.165 \\
\end{tabular} & 0.012 & 0.012 & 0.000 & 0.384 & \\
\hline & & 1:07:57 PM 00 & 25.593 & 24.851 & 24.46 & 27.045 & 24.027]\# & 44.74 & 54.181 & 10.021 & 35.488 & 3.547 & 0.406 & 48.294 & & 14.643 & & 3.0 & $23.3836 \mathrm{~F}$ & 53.11 & 11.5 .5 & 40.11 & 2.766 & 0.007 & 0.007 & 0.000 & 0.181 & \\
\hline & $8 / 25 / / 4$ & 1:08:57 PM 0 & 25.647 & 24.861 & 24.515 & 27.045 & 23.981|\# & 278 & 54.529 & 10.058 & 36.124 & 3.397 [ & 0.406 & 48.208 & 0. & 14.643 & & & 23.4003 & & & & 2.806 & & 0.002 & & 0.057 & \\
\hline & 8/25/2004 & $\begin{array}{l}\text { 1:09:57 PM } 10 \\
1.10 .57 \mathrm{P} M 0\end{array}$ & $\begin{array}{l}25.626 \\
256411\end{array}$ & 24.835 & $24.429 \mid$ & 26.989 & $\frac{24.026 \text { \# }}{2309}$ & 45.199 & 54.749 & $\begin{array}{r}9.977 \\
10025\end{array}$ & 35.927 & $\begin{array}{l}3.394 \\
3.391\end{array}$ & 0.409 & 48.619| & 0.016 & 14.643 & & & . 3.4169 & & & & 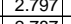 & 002 & 0.002 & 0.000 & 0.0577 & \\
\hline & 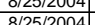 & 1.1.5.57 & & 2.04959 & 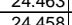 & 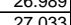 & 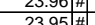 & (45.045 & (54.3501 & 10.025 & 35.194 & 年.391 & 0.049 & (46.0089 & & 14.645 & & & 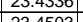 & & & & & & 0.002 & & 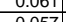 & \\
\hline & 8.252004 & 1.1.5. & 25664 & $\frac{24.044}{2483}$ & 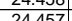 & 27017 & 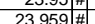 & & & $\frac{10.153}{10048}$ & & $\begin{array}{l}3.394 \\
3.397 \\
\end{array}$ & & 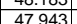 & & 14.643 & & & $\frac{20.406}{2346}$ & & & & & .002 & 0.002 & & - & \\
\hline 517 & $8 / 25 / 2004$ & 1:13:57 PM 0 & 25.0699 & 24.852 & 24.466 & 27051 & $24.113 \#$ & 45 & $\frac{34.586}{54,486}$ & 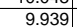 & 35.845 & 3.397 & 0.426 & 48.006 & & 14643 & & & $\frac{2.5436}{234836}$ & 53.4 & $\frac{\overline{11.4}}{1.4}>\mathrm{s}$ & 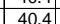 & 2788 & 002 & 0.002 & 0.000 & - & \\
\hline 518 & $8 / 25 / 2004$ & 1:14:57 PM 0 & 25.674 & 24.858 & $\frac{2.442}{24.42}$ & $\begin{array}{l}27.077 \\
27.077\end{array}$ & 24.1 & & 54.795 & 10.092 & 35.919 & 3.394 & 0.429 & 47.511 & & 14.643 & & & & & & 40.6 & $\frac{2.799}{2.799}$ & & $\frac{0.002}{0.002}$ & & 0.054 & \\
\hline 519 & $8 / 25 / 2004$ & 1:15:57 PM 0 & 25.674 & 24.853 & 24.472 & 27.067 & $24.119 \mid \#$ & 45.334 & 54.652 & 10.05 & 36.132 & 3.397 & 0.435 & 48.108 & 0.016 & 14.643 & & 1.06 & 23.5169 & 53.5 & 11.5 & 40. & \begin{tabular}{|c|c|}
2.808 \\
\end{tabular} & 0.002 & 0.002 & 0.000 & 0.057 & \\
\hline & 5/2004] & 1:16:57 PM 0 & 25.674 & & 24.456 & & & & 54.745 & 10.118 & & 3.397 & $\mid 0.44$ & & & & & & & & & & & & & & & \\
\hline & 8/25/2004 & 1:17:57 PM & 25.679 & 24.868 & 24.502 & 27.107 & $24.109 \#$ & 101 & 54.59 & 10.1 & 35.802 & 3.4 & & 48.093 & & 14.6 & & & & 53.5 & & & 2.789 & & 0.002 & 0.000 & 0.057 & \\
\hline & 8/25/2004 & 1:18:57 PM/0 & 25.674 & 24.848 & 24.452 & 27.072 & $24.154 \#$ & 44.956 & 54.592 & 10.019 & 35.706 & 3.397 & 0.446 & 48.342 & & 14.6 & & & & & 11.5 & & 2.781 & & & & & \\
\hline & 8/25/2004 & 1:19:57 PM 0 & 25.675 & 24.859 & 24.462 & 27.088 & & & & 10.046 & 35.839 & 3.403 & & 48.304 & & 14.6 & & & 583 & & & & 2.788 & & & & 554 & \\
\hline 524 & 8/25/2004 & 1:20:57 PM 0 & 25.68 & 24.854 & 24.473 & 27.063 & 24.004 \# & 45 & 54.443 & 10.058 & 36.048 & & 0.45 & 48.4. & & 14.6 & & & $23.6 \mathrm{c}-\mathrm{r}$ & 53.3 & 11.5 & & & 0.002 & & 500 & 0.054 & \\
\hline sce & 8/25/2004 & 1:21:57 PM 0 & 25.669 & $24.847 \mathrm{P}$ & 24.431 & 27.007 & 24.028 \# & 45.079 & 54.625 & 10.086 & 35.728 & 3.429 & & & & 14.6 & & & 23.6169 & & 11.4 & 40. & 2.786 & & & 00 & 0.054 & \\
\hline & $8 / 25 / 2004$ & 1:22:57 PM] & 25.667 & 24.846 & 24.465 & 26.96 & 23.911|\# & 45.2. & 54.527 & 10.032 & 36. & 3.397] & 0.46 & 48.18 & & 14 & & & 23.6 & & 1.5 & & 2.8 & & & & 554 & \\
\hline$\frac{521}{528}$ & 8/25/2004 & 1:23:57 & 25.672 & 24.8566 & 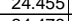 & 26.925 & & & 54.525 & 9.99 & & 3.423 & & 100 & & & & & & 50 & 11.4 & 40. & 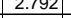 & & & & 0.054 & \\
\hline 528 & $8125 / 2004$ & 1.24 .51 & & & 20.413 & 20.883 & & & & 10.140 & & & & 48.0 & & & & & & & $\frac{1.4}{1.5}>2>$ & & & & & & 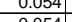 & \\
\hline & & 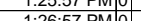 & 25.015 & 24.050 & 2.4 .424$. & 26.883 & & & 54.942 & & & & & 48 & & & & & & & 11 & & $278>$ & & & & (504) & \\
\hline & & & 20.070 & & 2.401 & & & & & & & $\frac{3.4201}{3.47}$ & & & & & & & & & & & & & & & & \\
\hline & $8 / 25 / 2004$ & 1:28:57 PM & $\frac{25.677}{25.677}$ & 24.846 & 22.46 & $\frac{26.05}{26.85}$ & $\frac{2.3 .9}{23.9}$ & & 53.989 & 10.011 & 35.427 & $\frac{1.46}{3.458}$ & & $68 \mathrm{6}-2$ & & & & & & 52 & 盐1.4 & & $2758 \mathrm{r}-2$ & 02 & & 00 & 555 & \\
\hline & $8 / 25 / 2004$ & 1:29:57 P & & & 24.474 & & & & 54.816 & & & & & & & & & & & & & & & & & & 554 & \\
\hline 534 & & 1:30:57 $\mathrm{P}$ & & 24.824 & 24.483 & 26.8 & & & 54.119 & & & 3.452 & & & & & & & & & 政 & & the & & & & 0.054 & \\
\hline & $8 / 25 / 2 / 2$ & $1: 31: 57 \mathrm{PM} 0$ & $\frac{15.676}{25}$ & & 24.493 & 26.934 & & & 54.7 & & & 3.443 & & & & & & & & & & & & & & & 54 & \\
\hline 536 & & & & 24.8 & 24.482 & & & & & & & & & 48. & & & & & & & 11 & & & & & & & \\
\hline & & & 25.6 & 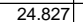 & 24.481 & $26.8 \mathrm{P} \quad \mathrm{P}$ & & & 54.681 & & & & & & & & & & & 53.6 & 11.4 & & & & & & $\mid$ & \\
\hline 538 & $\mid 8 / 25 / 2004$ & 1:34: & 25.667 & $24.8 \mathrm{C} 2>$ & 24.48 & $26.8 \mathrm{r}-\mathrm{x}$ & & & & & & & & & & & & & & & 11.4 & & & & & & 0.055 & \\
\hline 539 & 8/25/2004 & 1:35:57 F & 25.661 & 24 & 24.494 & 26.834 & 23.77 & & 54.37 & & 35.6 & 3.455 & & & & & & & & 53. & 11.4 & & & 0.002 & $0.6-6>$ & 500 & 0.054 & \\
\hline 540 & $8 / 25 / 2 / 2>0$ & 1:36:57 F & 25.6 & & 24.448| & 26. & & & 54.41 & & & & & & & & & & & & 11 & & & & & & & \\
\hline 541 & & & 25.6 & & 24.5 & & & & 54. & & & & & & & & & & & & 11. & & & & & & & \\
\hline 542 & $8 / 25 / 2,2>2$ & & & & 24.4 & & & & 54.872 & & & & & & & & & & & & 11. & & & & & & & \\
\hline 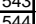 & & & 20.044 & 2.1.97! & 24.45 & & & & (5.0 & & & & & & & & & & & & & & & & & & & \\
\hline 545 & & & $\frac{2.0}{256}>9$ & & 24.8 & & & & S.3.0 & & & & & & & & & & & & & & & & & & & \\
\hline 546 & $\frac{6125 / 20}{8 / 520}$ & $\frac{1.44 .56}{1.425}$ & 25.6 & & 告, 24,496 & & & & 54.49 & & & & & & & & & & & & $\frac{\frac{1.4}{11.4}}{4}>0$ & & & & & & 54 & \\
\hline 547 & $8 / 25 / 2004$ & 1:43:57 $\mathrm{P}$ & 25.6 & & 24.491 & $\frac{26.9}{26.9}>0$ & & & 54.51 & & & & & & & & & & & & & & & & & & & \\
\hline 548 & $8 / 25 / 2004$ & & 25.6 & 24.8 & 24.51 & 26.9 & & & & & & & & & & & & & & & 11.5 & & 2.1 & & & & 0.054 & \\
\hline 549 & & & & & & & & & & & & & & & & & & & & & & & & & & & & \\
\hline & & & & & 24.531 & & & & & & & & & & & & & & & & & & & & & & & \\
\hline & & & 25.6 & 24 & & & & & & & & & & & & & & & & & & & & & & & & \\
\hline & $8 / 25 / 20$ & $1: 4 \varepsilon^{2}$ & 25.647 & 24.785 & 24.499 & 26.94 & 23.796 & & 54.72 & & & & & 48.5 & & 14. & & & & & 11.6 & & & & & & 54 & \\
\hline & $8 / 25 / 2$ & 1:49 & 25.645 & & 24.468 & 26.9 & 23.79 & & 55.0 & & & & & & & 14. & & & & & & & & & & & 54 & \\
\hline 55. & $8 / 25 / 2$ & 1:50:57 P & 25.656 & 24.78 & 24.483 & 26.9 & 23.92 & 44.99 & $54.5 \mathrm{C}$ & & & 3.458 & & 48.0 & & 14.6 & & & & 53 & 11.5 & & & & & & 0.054 & \\
\hline & & 1:51:57 P & 25.656 & 24.784 & 24.488| & 26.969 & & 45 & 54.644 & & & 3.481 & & & & 14. & & & & & & & & & & & 54 & \\
\hline & & 1.5 & & 24.78 & 24.455 & & & & & & & & & 47.1 & & & & & & & 11.4 & & & & & & 54 & \\
\hline 55 & {$[5 / 2004$} & & & & 2.5 .52 & 27.15 & 24.2. & & 54.3 & 10.1 & & & & & & 14.6 & & & & & & & & & & & 54 & \\
\hline & & 1.54 .57 T & $\frac{23.013}{25706}$ & 24.192 & 24.4000 & 27.2 & $2.4 .481+1+1]$ & 4. 44.001 & 34.37 & 10.09 & 35.511 & 3.018 & 0.075 & (4t.1793 & & & & & & 3.2 & 1.4 & & & & & & & \\
\hline 60 & $8 / 25 / 26$ & 1:56:57 PM 0 & 25.705 & $\begin{array}{r}4.02 \\
24.824 \\
\end{array}$ & \begin{tabular}{|l|}
24.513 \\
24.513
\end{tabular} & 27.493 & $\begin{array}{l}24.511+\# \\
24.575 \#\end{array}$ & $\begin{array}{l}44.950 \\
45.012\end{array}$ & \begin{tabular}{|l|}
54.492 \\
54.6 \\
\end{tabular} & \begin{tabular}{|c|}
10.091 \\
10.19
\end{tabular} & $\begin{array}{l}35.145 \\
35.562 \\
\end{array}$ & \begin{tabular}{|c|c|}
3.50 \\
3.608
\end{tabular} & 每. & \begin{tabular}{|l}
41.90 \\
48.216
\end{tabular} & 0.014 & $\begin{array}{l}14.643 \\
14.643 \\
\end{array}$ & & & & & & & & & & 0.000 & 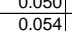 & \\
\hline
\end{tabular}


WSRC-TR-2005-00105, REVISION 0

SRNL-RPP-2005-00012, REVISION 0

CAMPAIGN IV NEW GKN CONDITIONING WITH AN102R2 SIMULANT - CONT.

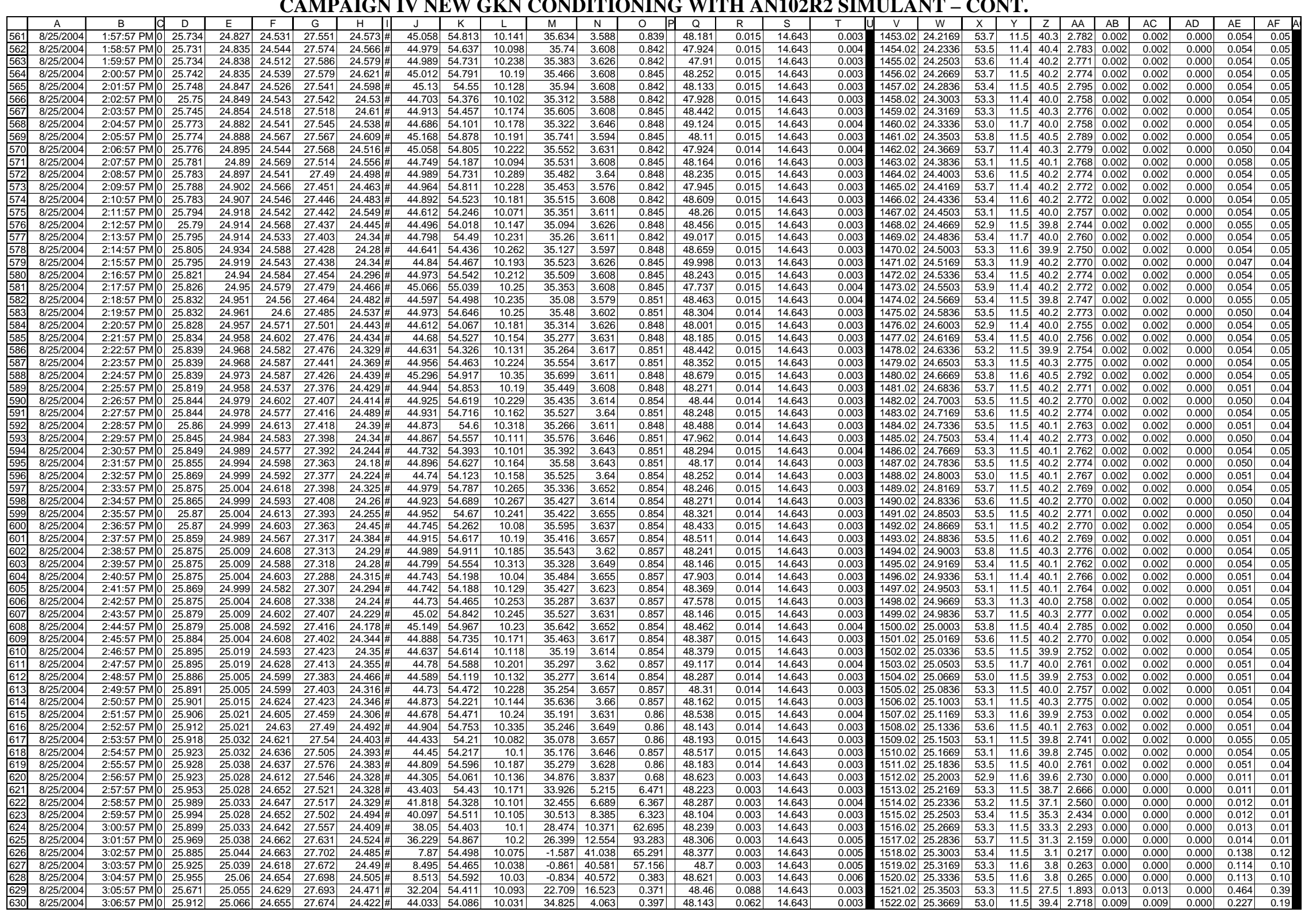


WSRC-TR-2005-00105, REVISION 0

SRNL-RPP-2005-00012, REVISION 0

CAMPAIGN IV NEW GKN CONDITIONING WITH AN102R2 SIMULANT - CONT.

\begin{tabular}{|c|c|c|c|c|c|c|c|c|c|c|c|c|c|c|c|c|c|c|c|c|c|c|c|c|c|c|c|c|}
\hline & & & $D$ & & & & & & $\mathrm{~K}$ & L & M & $\mathrm{N}$ & & & $R$ & & & & w & & & & & & & & & \\
\hline & $8 / 25 / 2004$ & 3:07:57 PM 0 & \begin{tabular}{|l|}
25.957 \\
\end{tabular} & \begin{tabular}{|l|}
25.066 \\
\end{tabular} & 24.62 & 27.704 & $24.392 \#$ & 44.861 & \begin{tabular}{|l|}
54.119 \\
\end{tabular} & 10.151 & 35.548 & 3.423 & 0.394 & \begin{tabular}{r|r}
48.437 \\
\end{tabular} & 0.015 & 14.643 & 0.003 & & No & 53.0 & & 40.2 & & & 0.002 & 0.000 & 0.054 & \\
\hline$\frac{632}{633}$ & 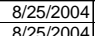 & \begin{tabular}{ll|}
$3: 08: 57$ PM 0 \\
$309.57 \mathrm{PM}$
\end{tabular} & \begin{tabular}{|l|}
25.963 \\
25.933 \\
\end{tabular} & \begin{tabular}{|l|}
25.077 \\
25077 \\
\end{tabular} & \begin{tabular}{|l|}
24.621 \\
24626
\end{tabular} & $\begin{array}{l}27.705 \\
27715 \\
\end{array}$ & 24.528 \# & 44.859 & \begin{tabular}{|l|l|}
54.192 \\
5.369 \\
\end{tabular} & 10.165 & \begin{tabular}{|l|l|}
35.488 \\
35607
\end{tabular} & 3.397 & 0.406 & 48.552 & 0.015 & \begin{tabular}{|l|l|}
14.643 \\
1.63
\end{tabular} & 0.004 & 1524.0 & \begin{tabular}{|l|l|}
25.4003 \\
2.5160
\end{tabular} & \begin{tabular}{|l|}
53.1 \\
5.3
\end{tabular} & \begin{tabular}{|l|}
11.6 \\
\end{tabular} & \begin{tabular}{|l|}
40.2 \\
\end{tabular} & 2.770 & 0.002 & 0.002 & 0.000 & 0.054 & \\
\hline 63 & $\frac{88 / 255 / 2004}{88 / 25 / 2004}$ & $\begin{array}{l}3: 09: 57 \mathrm{PMM}|0| \\
3: 10: 57 \mathrm{PM} \mid\end{array}$ & \begin{tabular}{|l|}
$\mid 25.963$ \\
25.984 \\
\end{tabular} & 25.077 & $\mid \begin{array}{l}24.626 \\
24.603\end{array}$ & $\begin{array}{l}27.715 \\
27687 \\
\end{array}$ & $\frac{24.4288^{*}}{24.454 \pi}$ & \begin{tabular}{|l|}
45.112 \\
44.87
\end{tabular} & \begin{tabular}{|l|}
$\mid 54.386$ \\
54.291 \\
\end{tabular} & $\begin{array}{r}\frac{10.221}{10.12} \\
\end{array}$ & \begin{tabular}{|l|}
35.697 \\
3567
\end{tabular} & $\begin{array}{r}3.42 \\
3.4 \\
\end{array}$ & $\begin{array}{l}0.406 \\
0.409\end{array}$ & \begin{tabular}{|c|}
48.214 \\
48629 \\
\end{tabular} & $\begin{array}{l}0.015 \\
0.015 \\
\end{array}$ & \begin{tabular}{|l|}
14.643 \\
14643
\end{tabular} & \begin{tabular}{|l}
0.004 \\
0.003
\end{tabular} & $\begin{array}{l}1525.02 \\
152002\end{array}$ & \begin{tabular}{|l|}
25.4169 \\
25.3236
\end{tabular} & \begin{tabular}{|l|}
53.3 \\
53.2
\end{tabular} & \begin{tabular}{|l|}
11.5 \\
11.6
\end{tabular} & \begin{tabular}{|l|}
40.4 \\
402 \\
\end{tabular} & \begin{tabular}{|l|}
2.786 \\
2774
\end{tabular} & 0.002 & 0.002 & 0.000 & 0.054 & \\
\hline & \begin{tabular}{|l}
$8 / 25 / 2004$ \\
$8 / 25 / 2004$ \\
\end{tabular} & $\begin{array}{l}3: 10: 57 \mathrm{PM}|0| \\
3: 11: 57 \mathrm{PM} 0\end{array}$ & \begin{tabular}{|l|}
25.984 \\
25.995 \\
\end{tabular} & $\begin{array}{r}25.089 \\
25.1 \\
\end{array}$ & \begin{tabular}{|l|l|}
$\mid 24.603$ \\
\end{tabular} & $\begin{array}{l}27.681 \\
27.662 \\
\end{array}$ & 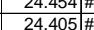 & \begin{tabular}{|l|}
45.018 \\
45
\end{tabular} & \begin{tabular}{|l|}
54.291 \\
54.652 \\
\end{tabular} & & $\begin{array}{l}355.601 \\
35.626\end{array}$ & $\begin{array}{r}3.4 \\
3.394 \\
\end{array}$ & $\begin{array}{l}0.409 \\
0.414\end{array}$ & $\begin{array}{l}8.629 \\
48.569 \\
\end{array}$ & \begin{tabular}{|l|}
0.015 \\
0.015
\end{tabular} & $\begin{array}{l}114.643 \\
14.643 \\
\end{array}$ & $\begin{array}{l}0.003 \\
0.003\end{array}$ & 1526.02 & $\mid \begin{array}{l}25.43356 \\
25.4502\end{array}$ & $\begin{array}{l}53.2 \\
53.5 \\
\end{array}$ & \begin{tabular}{|l|}
11.6 \\
116
\end{tabular} & 40.2 & $2.1 / 4$ & 0.002 & 0.002 & 0.0000 & 0.054 & 0.05 \\
\hline & & & 25.995 & 25.095 & 24.613 & 27.657 & & & 863 & & & 3.417 & & & & & & & & 53 & & & & & & & & \\
\hline & & 3:13:57 PM & \begin{tabular}{|l|}
25.986 \\
\end{tabular} & 25.1 & & & & & & & & 3.4 & & & & & & & & & & & & & & & & \\
\hline & $8 / 25 / 2004$ & 3:14:51 PM & \begin{tabular}{|l|}
26.001 \\
\end{tabular} & 25.095 & 24.604 & \begin{tabular}{|l|l|}
27.623 \\
\end{tabular} & 3311\# & & 54.32 & 10.104 & & 3.408 & & & & & & & & 53.2 & & & & & 0.00 & & & \\
\hline & 8/25/2004 & 3:15:57 PMC & \begin{tabular}{|l|l|}
26.006 \\
\end{tabular} & 25.105 & 24.589 & 27.608 & $24.371 \#$ & & 54.511 & & .701 & 3.449 & & 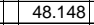 & & $\begin{array}{l}14.643 \\
\end{array}$ & & & 25.5169 & 53.4 & 11.5 & & & 002 & 0.002 & 0.000 & 0.054 & \\
\hline & & 3:16:57 PMC & 26.006 & 25.11 & 24.619 & 27.558 & $24.271 \#$ & & & 10.05 & 35.669 & 3.42 & & & & 14.643 & & & & 53.1 & $11.6 \mathrm{C}$ & & & & & & & \\
\hline 64 & 8/25/2004 & 3:17:57 PM0 & 26.006 & 25.11 & 24.594 & 27.528 & 24.306\#\# & 44.74 & 54.084 & 10.077 & 35.574 & 3.432 & 0.437 & 48.306 & 015 & 14.643 & & & 25.5503 & 53.0 & 11.5 & 40 & $.78 \mathrm{.}$ & 002 & 0.002 & 0.000 & 0.054 & \\
\hline & 8025322040 & 8:57 PM & 26.006 & & 24.619 & 27.453 & & 5.174 & & & & 3.452 & & & & & & & & & & & & & & & & \\
\hline & $8 / 25 / 2 / 2$ & 9:57 PM & 25.995 & 25.11 & 24.588 & 27.433 & & & 4992 & $\frac{10.149}{10.15}$ & & 3.452 & & & & & & & & & & & & & & & & \\
\hline & $\frac{01252004}{825204}$ & $3.20 .5157 \mathrm{PM}$ & 20.9999 & | & 24.5966 & 27.4019 & $\frac{24.174 \pi}{24.208}$ & & 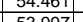 & & & 3.440 & & 19508 & & & & & 250160 & 559 & 然 & 10 & 276 & & & 0.0000 & 0.051 & \\
\hline 6 & $\begin{array}{l}0 / 25 / 2004 \\
8 / 25 / 204 \\
\end{array}$ & 3:22:57 PMC & $\mid$\begin{tabular}{|}
$\mid 25.960$ \\
25.97
\end{tabular} & 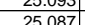 & 24.504 & $\mid \frac{2.415}{274}$ & $24.232+\theta$ & $\begin{array}{l}44.145 \\
45.145\end{array}$ & 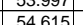 & $\frac{10.060}{1014}$ & . & $\begin{array}{l}3.442 \\
3.452\end{array}$ & & 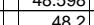 & 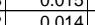 & 14.045 & & & $\frac{2.00035}{25636}$ & 年 & 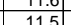 & & 2.10 & & & 0.000 & & \\
\hline 647 & $8 / 25 / 2004$ & 3:23:57 PMO & \begin{tabular}{|l|}
25.992 \\
\end{tabular} & 25.01 & \begin{tabular}{|l|}
24.605 \\
\end{tabular} & 27.404 & $24.287 \#$ & 4..1977 & 54.434 & 10.137 & 35.648 & $\begin{array}{l}3.452 \\
3.452 \\
\end{array}$ & 0.464 & $\begin{array}{l}48.314 \\
48.314\end{array}$ & 年 & $\begin{array}{l}14.0443 \\
14.643\end{array}$ & 003 & 539. & \begin{tabular}{|l}
25.6503 \\
2503
\end{tabular} & 53.3. & $\frac{11.5}{11.5}$ & 40.3 & 2.779 & \begin{tabular}{|l|}
0.002 \\
0.002
\end{tabular} & 0.002 & 0.000 & 0.054 & $\frac{0.04}{0.05}$ \\
\hline & $8 / 25 / 2004$ & 3:24:57 PM0 & \begin{tabular}{|l|}
25.991 \\
\end{tabular} & 25.106 & 24.574 & \begin{tabular}{|l|l|l|l}
27.459 \\
\end{tabular} & $24.301 \#$ & & 54.386 & 10.142 & \begin{tabular}{|l|}
35.656 \\
\end{tabular} & 3.455 & & 48.719 & 0.015 & 14.643 & & & 25.6669 & 53.3 & 11.6 & & 2.782 & \begin{tabular}{|l|l|}
0.002 \\
\end{tabular} & 0.002 & & 0.054 & \\
\hline & $8 / 25 / 2004$ & 3:25:57 PM0 & \begin{tabular}{|l|}
26.001 \\
\end{tabular} & 25.116 & 24.614 & & $24.311 \#$ & 4.801 & 54.256 & 10.21 & 35.459 & 3.458 & & 48.285 & & \begin{tabular}{|l|l|l|}
14.643 \\
\end{tabular} & 004 & & 55.6836 & & 11.5 & 40. & & & & & 0.054 & $\begin{array}{l}0.05 \\
0.05 \\
\end{array}$ \\
\hline & $8 / 25 / 2004$ & 3:26:57 PMI & $\mid$ & 25.095 & \begin{tabular}{|l|l|}
24.584 \\
\end{tabular} & 27.508 & & & & & & & & & & & & & & 53.5 & & & & & & & 054 & \\
\hline & & & & & & & & & t.254 & 10.096 & & 3.455 & & & & & & & & & & & & & & & & \\
\hline & $8 / 25 / 2004$ & $3: 28: 57 \mathrm{PM}$ & \begin{tabular}{|l|l|}
25.989 \\
\end{tabular} & 25.098 & 24.592 & 27.522 & $24.414 \#$ & .772 & 4.391 & 10.257 & 35.191 & 3.452 & & & & & & & $\overline{336}$ & 53. & & & & & & & & \\
\hline & $8 / 25 / 2004$ & $3: 29: 5$ & & 25.109 & & & $24.254 \#$ & & & & & 0.450 & & & & & & & & J5.1 & . & & & & & & & \\
\hline 65 & $8 / 25 / 2004$ & 3:30:57 PMC & 25.988 & 25.098 & 24.607 & 27.491 & 24.358 \# & 4.703 & 54.115 & 10.084 & 515 & 3.452 & & 48.465 & 014 & 14.6 & & & 25.7669 & 53.0 & 11.5 & 40.1 & 2.765 & .002 & 0.002 & 0.000 & 0.051 & \\
\hline & $8 / 25 / 2$ & 1:57 PM/0 & 25.994 & 25.109 & 24.597| & 27.527 & 24.409 \# & 44.892 & 54.513 & 10.188 & 35.426 & 3.452 & & 48.663 & 0.014 & 14.643 & & & 25.7836 & 53.4 & $11.6 \mathrm{C}$ & & .7 & .002 & 0.0 & & 0.050 & \\
\hline 6 & 8/25/2004 & 3:32:57 PM0 & 25.988 & 25.098 & 24.622 & 27.4966 & 24.378 \# & 44.873 & 54.358 & 10.224 & 35.449 & 3.452 & 0.498 & 48.454 & 014 & 14.643 & 0.003 & 54 & 25.8003 & 53.2 & 11. & 4 & 2.76 & 002 & 0.00 & & & \\
\hline & 8/25/2004 & 7 PM & 25.988 & 25.103 & $\frac{24.607}{21.07}$ & 27.566 & & & $\begin{array}{l}4.299 \\
1.202 \\
\end{array}$ & & & 3.455 & & & & & & & & 533 & & & & & & & 5154 & \\
\hline & 812522004 & & 25.994 & & & & & & & & & 3.452 & & & & & & & & & & & & & & & & \\
\hline & $\frac{182512}{8125 / 2}$ & 3.353 .50 & $\mid$ & 20.109 & & 27.091 & & $\frac{45.016}{44892}$ & & 10.2 & & 3.452 & & 48.287 & & & & & & $\frac{53}{52}$ & & & & & & & & \\
\hline & $8 / 25 / 2004$ & 3.35 .57 PMC & $\mid-26$ & $\frac{2.050}{25.1}$ & \begin{tabular}{|l|l|}
24.0569 \\
\end{tabular} & 27713 & 24.42 & $\begin{array}{l}44.054 \\
44.85 \\
\end{array}$ & 年 & & 35 & $\frac{1.455}{3.475}$ & & & & 14.643 & & & $\frac{250003}{25836}$ & 532 & & & & & & & & \\
\hline & $8 / 25 / 2004$ & 3:38:57 PMC & 26.011 & $\frac{25.116}{25.116}$ & \begin{tabular}{|l|l|l|l|l|}
24.619 \\
\end{tabular} & 27.798 & $24.341 \#$ & 44.95 & 54.523 & $\frac{1.220}{10.32}$ & & 3.452 & & $\frac{40.000}{48.59}$ & & 14. & & & & 53.4 & $\frac{11.0}{11.6}$ & & & & & & & \\
\hline 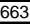 & $8 / 25 / 2004$ & 3:39:57 PM & 26.016 & 25.111 & 24.634 & 27.768 & $24.471 \#$ & 44.687 & 54.094 & 10.18 & 35.375 & 3.455 & 0.53 & 48.2 & & & & & 25.9169 & 53.0 & 11.5 & & 2.76 & & 0.00 & 0.000 & 0.054 & \\
\hline & $8 / 25 / 2004$ & & & & & & & & & & & 3.495 & & & & & & & & 53.6 & & & & & & & & \\
\hline & $5 / 2004$ & & \begin{tabular}{|l|}
26.032 \\
\end{tabular} & 25.137 & 24.636 & 675 & & & & & & & & & & & & & & 53.5 & 11. & & & & & $\frac{600}{.000}$ & 054 & \\
\hline & $8 / 25 / 2$ & & 26.0 & & $\begin{array}{l}24.645 \\
\end{array}$ & 27.574 & & & 54.5 & & & $\begin{array}{l}3.513 \\
\end{array}$ & & & & & & & & 53. & & & & & & & & \\
\hline & $8 / 25 / 2$ & & & & & & & & & & & & & & & & & & & & & & & & & & & \\
\hline & $8 / 25 / 2$ & & & & 24.646 & 27.505 & & & 54.268 & & & 3.481 & & & & & & & & 53. & 11. & & & & & & & \\
\hline & 8/25/2004 & $\mathrm{PM}[\mathrm{PO}$ & 26.012 & 25.132 & 24.621 & 27.48 & 24.402 \# & & 54.188 & 10.22 & & 3.507 & & & & & & & & 53.1 & 11. & & & & & & 051 & \\
\hline & $8 / 25 / 2$ & $\mathrm{PM}[\mathrm{PO}$ & \begin{tabular}{|l|}
26.017 \\
\end{tabular} & & 24.651 & 27.47 & & & & 10.2 & & 3.475 & & & & & & & & 53. & & & & & & & & \\
\hline & $8 / 25 / 2 / 2$ & & & & & 27.454 & $24.351 \| \#$ & & & & & & & & & & & & & 53.2 & & & & & & & & \\
\hline & & & & & & & & & & & & & & & & & & & & & & & & & & & & \\
\hline & 8 & & & & & (4899 & & & & & & & & & & & & & & $\frac{52.6}{5.2}$ & & & & & & & & \\
\hline & & & & & & & & & & & & & & & & & & & & & & & & & & & & \\
\hline$\frac{67}{67}$ & $8 / 25 / 2$ & $\frac{3.5}{3.5}$ & $\mid$ & $\frac{2.5136}{25.136}$ & | & 27.598 & & & & & & $\frac{3.52}{352}$ & & & & & & & & $\frac{2.35}{53.5}$ & & & & & & & & \\
\hline & $8 / 25 / 2$ & & & & & 27.698 & & & & & & & & & & & & & & & & & & & & & & \\
\hline & $8 / 25 / 2$ & & 26.032 & 25.141 & 24.655 & 724 & & & & & & & & & & & & & & 53.3 & & & & & & & & \\
\hline & & & & & & & & & & & & & & & & & & & & & & & & & & & & \\
\hline & $8 / 2$ & & 26.0 & 25.1 & & 27.6 & & & & & & & & & & & & & & & & & & & & & & \\
\hline & $8 / 2$ & & 26.0 & 25.146 & 24.65 & 27.634 & & & 54.15 & & & 3.611 & & & & & & & & 53.0 & & & & & & & & \\
\hline & $8 / 25 / 2$ & & 26. & 25.153 & 24.681 & 27.685 & & 44. & 54.515 & & & & & & & & & & & 53.4 & & & & & & & & \\
\hline & $8 / 25 / 2$ & & & & 24. & 27 & & & & & & & & & & & & & & 53. & & & & & & & & \\
\hline & $8 / 25 / 2$ & & & 25. & 24. & & & & & & & & & & & & & & & 53 & & & & & & & & \\
\hline & $8 / 25 / 2$ & & & & & & & & & & & & & & & & & & & & & & & & & & & \\
\hline & & & & & & & & & & & & & & & & & & & & & & & & & & & & \\
\hline & & & & & 24.683 & 2.687 & & 44 & & & & & & & & & & & & & & & & & & & & \\
\hline & $\frac{0152 / 2}{8 / 25 / 2}$ & & 20.079 & $\frac{2.153}{2516}$ & 20772 & 27097 & & & & $\frac{10.1}{10}$ & & & & & & & & & & & & & & & & & & \\
\hline & & & & & $\frac{2.103}{2467}$ & $\frac{21.08}{27637}$ & & & & & & & & & & & & & & & & & & & & & & \\
\hline & $8 / 25 / 2$ & $4:$ & & $\frac{25.1}{251}$ & 24.6 & & & & & & & & & & & & & & & & & & & & & & & \\
\hline & $8 / 25 / 2$ & & $\frac{26.0}{26.0}$ & 25.165 & 24. & & & & & & & & & & & & & & & & & & & & & & & \\
\hline & & & & & & & & & & & & & & & & & & & & & & & & & & & & \\
\hline & & & & & & & & & & & & & & & & & & & & & & & & & & & & \\
\hline & & & & & & & & & & & & & & & & & & & & & & & & & & & & \\
\hline & $8 / 25 / 21$ & 4. & 26.112 & 25.19 & 24.726 & 27.884 & 24.82 & 44.7 & 4.293 & 10.3 & 35.24 & 3.66 & & & & & & & & 53.2 & & & & & & & & \\
\hline & $8 / 25 / 2004$ & 13:57 PMI & 26.116 & 25.196 & 24.71 & 27.9 & & 44.8 & 54.6 & & 35.143 & 3.628 & & 47.9 & 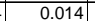 & 14. & & & & 53. &. .44 & & & & & & & \\
\hline & $8 / 25 / 2004$ & 4:14:57 & 26.124 & $25.209 \mid$ & 24.708 & 28.016 & 24.955|\# & 44.8 & 54.5 & 10.235 & 35.314 & 3.663 & & 48.4 & 0.014 & 14. & & & & 53.5 & & & & & & & 050 & \\
\hline & 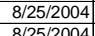 & \begin{tabular}{|l|} 
4:15:57 PM 0 \\
$4: 16: 57 \mathrm{PM}$
\end{tabular} & 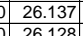 & \begin{tabular}{|l|}
25.212 \\
25203
\end{tabular} & $\begin{array}{l}24.736 \\
24702\end{array}$ & \begin{tabular}{|l|l|}
28.024 \\
2803
\end{tabular} & \begin{tabular}{|l|l|}
$24.917 \# \#$ \\
24799
\end{tabular} & \begin{tabular}{ll|}
$\#$ & 44.604 \\
$H$ & 44.639
\end{tabular} & $\begin{array}{l}454.413 \\
54.364 \\
5\end{array}$ & 10.298 & $\begin{array}{l}35.084 \\
35219 \\
\end{array}$ & $\begin{array}{ll}4 \quad 3.655 \\
4 & 3.623 \\
\end{array}$ & $\begin{array}{l}0.836 \\
0836\end{array}$ & 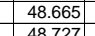 & \begin{tabular}{|c|}
0.014 \\
0014
\end{tabular} & \begin{tabular}{|l|}
14.643 \\
\end{tabular} & $\begin{array}{l}0.003 \\
0.003\end{array}$ & $\begin{array}{ll}1591.02 \\
159202\end{array}$ & \begin{tabular}{|l|l|}
26.5169 \\
265336
\end{tabular} & \begin{tabular}{|l|}
53.3 \\
532
\end{tabular} & 11.6 & & \begin{tabular}{|l|}
$\mid 2.747$ \\
2753
\end{tabular} & \begin{tabular}{|l|l|}
0.002 \\
0002
\end{tabular} & $\begin{array}{l}0.002 \\
002\end{array}$ & $\begin{array}{l}0.000 \\
0.000\end{array}$ & \begin{tabular}{|l}
0.051 \\
0.051
\end{tabular} & 0.0 \\
\hline
\end{tabular}


WSRC-TR-2005-00105, REVISION 0

SRNL-RPP-2005-00012, REVISION 0

CAMPAIGN IV NEW GKN CONDITIONING WITH AN102R2 SIMULANT - CONT.

\begin{tabular}{|c|c|c|c|c|c|c|c|c|c|c|c|c|c|c|c|c|c|c|c|c|c|c|c|c|c|c|c|c|}
\hline \\
\hline & \begin{tabular}{l|} 
\\
\end{tabular} & \begin{tabular}{l|l} 
B & C
\end{tabular} & 7 & \begin{tabular}{|l|}
$E$ \\
\end{tabular} & $\mathrm{~F}$ & $G$ & \begin{tabular}{l|l|} 
\\
\end{tabular} & $\mathrm{J}$ & \begin{tabular}{l|l} 
\\
\end{tabular} & $\mathrm{L}$ & \begin{tabular}{|l|} 
\\
25232
\end{tabular} & $\begin{array}{ll}\mathrm{N} \\
36\end{array}$ & 0 & \begin{tabular}{|l|}
$Q$ \\
\end{tabular} & $\begin{array}{ll}R \\
\end{array}$ & \begin{tabular}{|c|}
$\mathrm{s}$ \\
\end{tabular} & $T^{T}$ & $\mathrm{v}$ & W & \begin{tabular}{|c|}
$x$ \\
\end{tabular} & & & AA & $\mathrm{AB}$ & $A C$ & $\mathrm{AD}$ & $\mathrm{AE}$ & AF $A$ \\
\hline$\left|\frac{701}{702}\right|$ & $\begin{array}{r}88 / 252504 \\
8825 / 2004\end{array}$ & $\begin{array}{l}4: 17: 57 \mathrm{PM} \mid 0 \\
4\end{array}$ & \begin{tabular}{|c|c|}
56.13 \\
56.136
\end{tabular} & \begin{tabular}{|r|}
25.21 \\
25.211
\end{tabular} & $\begin{array}{l}24.714 \\
24.705\end{array}$ & $\begin{array}{l}28.017 \\
28.013 \\
\end{array}$ & $\begin{array}{l}24.776 \text { \# } \\
24.7211\end{array}$ & \begin{tabular}{|l|}
44.545 \\
44.562 \\
\end{tabular} & \begin{tabular}{|l|}
54.02 \\
54.067
\end{tabular} & $\begin{array}{l}10.087 \\
10.207\end{array}$ & \begin{tabular}{|l|}
35.353 \\
35.24
\end{tabular} & \begin{tabular}{|l|}
3.66 \\
3.66
\end{tabular} & $\mid \begin{array}{l}0.836 \\
0.834\end{array}$ & \begin{tabular}{|r|}
48.54 \\
48.275 \\
\end{tabular} & $\begin{array}{l}0.014 \\
0.014\end{array}$ & \begin{tabular}{ll|} 
& 14.643 \\
4 & 14.643
\end{tabular} & $\begin{array}{l}0.004 \\
0.003\end{array}$ & $\frac{1593.02}{159.402}$ & \begin{tabular}{ll|}
2 & 26.5503 \\
2 & 26.5669
\end{tabular} & \begin{tabular}{|c|}
52.9 \\
52.9
\end{tabular} & \begin{tabular}{|r|}
11.6 \\
11.5
\end{tabular} & \begin{tabular}{|c|}
39.9 \\
39.9 \\
\end{tabular} & $\begin{array}{l}2.754 \\
2.751 \\
\end{array}$ & \begin{tabular}{|l|}
0.002 \\
0.002 \\
\end{tabular} & $\begin{array}{l}0.002 \\
0.002\end{array}$ & $\begin{array}{l}0.000 \\
0.000\end{array}$ & 0.051 & 0.04 \\
\hline & $8 / 25 / 2004$ & & & & 24.701 & 28.029 & & & \begin{tabular}{|l|}
53.98 \\
\end{tabular} & 10.221 & 35.172 & 3.66 & 0.836 & 48.052 & & \begin{tabular}{|l|l|}
4 & 14.643 \\
\end{tabular} & & & & & & & & & & & 0.051 & $\frac{0.04}{0.04}$ \\
\hline 704 & $8 / 25 / 2004$ & & & 25.238 & 24.732 & 28.025 & 24.638 \# & 44.761 & \begin{tabular}{|l|l|l|}
54.488 \\
\end{tabular} & $\begin{array}{l}10.152 \\
\end{array}$ & \begin{tabular}{|l|l|}
35.373 \\
\end{tabular} & $\begin{array}{l}3.66 \\
\end{array}$ & $\begin{array}{l}0.839 \\
\end{array}$ & \begin{tabular}{|l|}
48.258 \\
4.036
\end{tabular} & 0.014 & \begin{tabular}{|l|}
14.643 \\
\end{tabular} & & 159602 & \begin{tabular}{|l|l|}
26.6003 \\
\end{tabular} & 53.4 & 11.5 & 40.1 & 2.762 & 0.002 & 0.002 & 0.000 & 0.050 & 0.04 \\
\hline 705 & $8 / 25 / 2004$ & 4:21:57 PM0 & 26.169 & 25.239 & 24.722 & 27.956 & 24.714 \# & 44.937 & 54.671 & 10.235 & $\begin{array}{l}35.466 \\
\end{array}$ & 3.66 & 0.836 & $\begin{array}{l}48.367 \\
\end{array}$ & 0.014 & \begin{tabular}{|l|}
14.643 \\
\end{tabular} & & 1597.02 & \begin{tabular}{l|l|l|l|l|l|}
2 & 26.6169 \\
\end{tabular} & $\begin{array}{l}53.5 \\
\end{array}$ & 11.5 & 40.2 & \begin{tabular}{|l|}
2.772 \\
\end{tabular} & 0.002 & 0.002 & 0.000 & 0.050 & 0.04 \\
\hline 706 & $8 / 25 / 2004$ & & 26.164 & 25.244 & & 27.961 & 24.704 \# & 44.861 & 54.656 & & 35.209 & $\begin{array}{l}3.66 \\
\end{array}$ & & 48.317 & & & & & & $\begin{array}{l}53.5 \\
5.5\end{array}$ & & & & & & & 0.050 & \\
\hline 707 & $8 / 25 / 2004$ & 4:23:57 PM 0 & 26.174 & 25.259 & 24.743 & $\begin{array}{l}27.971 \\
\end{array}$ & & & 54.557 & $\begin{array}{l}10.199 \\
\end{array}$ & 35.213 & 3.663 & & 48.148 & & & & & & 53.4 & & & & & & & 0.051 & 04 \\
\hline $\begin{array}{ll}708 \\
709\end{array}$ & $\frac{8 / 25 / 2004}{80 / 25004}$ & $\begin{array}{l}4: 24: 57 \mathrm{PM} \\
4\end{array}$ & $\begin{array}{l}0.26 .17 \\
0 \\
\end{array}$ & $\begin{array}{r}25.25 \\
25.249\end{array}$ & 24.709 & $\begin{array}{l}27.902 \\
27.882 \\
\end{array}$ & $\begin{array}{l}24.5966 \text { \# } \\
24.55 \text { 해 }\end{array}$ & $\begin{array}{l}44.776 \\
44.479\end{array}$ & \begin{tabular}{|l|}
54.451 \\
53.298 \\
\end{tabular} & $\begin{array}{l}10.115 \\
10.093\end{array}$ & \begin{tabular}{|l|}
35.541 \\
35.365 \\
\end{tabular} & \begin{tabular}{|l|l|}
3.657 \\
3.657
\end{tabular} & \begin{tabular}{|c|c|c|}
0.839 \\
0.833
\end{tabular} & \begin{tabular}{r|r|}
48.1 \\
48095
\end{tabular} & \begin{tabular}{l|l|l|l|}
0.014 \\
0.014
\end{tabular} & $\begin{array}{l}\frac{14.643}{14.643} \\
\end{array}$ & & & \begin{tabular}{l|l|}
2 & 26.6669 \\
2 & 26.6836
\end{tabular} & \begin{tabular}{|c|}
53.3 \\
52.8
\end{tabular} & \begin{tabular}{|l|l|}
11.5 \\
11.
\end{tabular} & (3). & | & 0.002 & 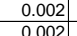 & $\begin{array}{l}0.000 \\
0.00\end{array}$ & 0.050 & 0.04 \\
\hline & $8 / 25 / 2004$ & 4:26:57 PM0 & D 26.159 & 25.244 & 24.683 & 27.002 & 24.5055 \# \# & $\begin{array}{l}44.479 \\
44.668\end{array}$ & | 50.920 & 10.095 & $\mid$ & $\begin{array}{l}3.057 \\
3.657\end{array}$ & \begin{tabular}{|c|} 
\\
0.8399 \\
\end{tabular} & \begin{tabular}{|}
48.095 \\
47.926 \\
\end{tabular} & \begin{tabular}{|l}
0.014 \\
0.014 \\
\end{tabular} & $\begin{array}{l}14.643 \\
14.643\end{array}$ & & $\begin{array}{l}01.02 \\
0.02 .02 \\
\end{array}$ & 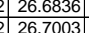 & \begin{tabular}{|l|}
52.8 \\
53.1
\end{tabular} & \begin{tabular}{|l|}
11.5 \\
\end{tabular} & & \begin{tabular}{|l|}
2.7562 \\
2762 \\
\end{tabular} & $\frac{0.002}{0.002}$ & $\begin{array}{l}0.002 \\
0.002\end{array}$ & $\begin{array}{l}0.000 \\
0.000\end{array}$ & $\frac{0.051}{0.050}$ & $\frac{0.04}{0.04}$ \\
\hline & $8 / 25 / 2004$ & 4:27:57 PM0 & 26.18 & 25.27 & 24.739 & 27.867 & $24.581 \#$ & 44.923 & \begin{tabular}{|l|}
54.451 \\
\end{tabular} & 10.157 & 35.582 & 3.66 & & 48.233 & 0.014 & 14.643 & & & 226.7169 & 53.3 & 11.5 & & 2.775 & 0.002 & & 0.000 & 0.050 & $\begin{array}{l}0.04 \\
0.04 \\
\end{array}$ \\
\hline & $8 / 25 / 2004$ & 4:28:57 PM0 & 26.175 & & 24.709 & 27.832 & 24.515 \# & & \begin{tabular}{|l|l|}
54.534 \\
\end{tabular} & & 35.213 & 3.657 & & 48.095 & 0.014 & \begin{tabular}{|l|l|}
4 & 14.643 \\
\end{tabular} & & & & 53.4 & 11.5 & & & & & 0.000 & 0.051 & \\
\hline & $8 / 25 / 2004$ & 4:29:57 PM 0 & $\begin{array}{ll}0 & 26.175 \\
\end{array}$ & $\begin{array}{l}25.27 \\
\end{array}$ & 24.699 & $\begin{array}{l}27.822 \\
27.770\end{array}$ & 24.525 \# & \begin{tabular}{|l|l|}
4.894 \\
\end{tabular} & \begin{tabular}{|l|}
54.668 \\
\end{tabular} & & & $\begin{array}{l}3.66 \\
\end{array}$ & & \begin{tabular}{|l|l|}
48.187 \\
\end{tabular} & 0.014 & & & & & 53.5 & 11.5 & & & & & 0.000 & & \\
\hline & $8 / 25 / 2004$ & $\begin{array}{l}4: 30: 57 \mathrm{PM} \\
4: 51: 57 \mathrm{PM}\end{array}$ & $\begin{array}{l}026.186 \\
0.2619\end{array}$ & \begin{tabular}{|l|}
25.271 \\
2529
\end{tabular} & $\begin{array}{l}24.725 \\
24719\end{array}$ & $\begin{array}{l}27.778 \\
27.797\end{array}$ & $\begin{array}{l}24.596 \text { \# } \\
24586\end{array}$ & \begin{tabular}{|l|l|l|l|}
44.724 \\
44657
\end{tabular} & \begin{tabular}{|l|}
54.447 \\
5.32
\end{tabular} & $\begin{array}{r}0.108 \\
\end{array}$ & \begin{tabular}{|l|l|}
35.431 \\
2539
\end{tabular} & $\begin{array}{r}.66 \\
366\end{array}$ & & $\begin{array}{l}48.277 \\
\end{array}$ & $\begin{array}{l}0.014 \\
\end{array}$ & & & & & \begin{tabular}{|l|}
53.3 \\
5.3
\end{tabular} & 11.5 & & \begin{tabular}{|l|}
2.763 \\
\end{tabular} & 0.002 & 0.002 & 0.000 & 0.050 & 04 \\
\hline $\begin{array}{l}715 \\
716 \\
\end{array}$ & $\frac{8 / 25 / 2004}{81 / 252004}$ & $\begin{array}{ll}4: 31: 57 \mathrm{PM} & 0 \\
4 & 0\end{array}$ & & \begin{tabular}{|l|}
25.29 \\
2.29
\end{tabular} & 24.719 & $\begin{array}{l}27.797 \\
27.772 \\
\end{array}$ & $\begin{array}{l}24.586 \text { \# } \\
24.535\end{array}$ & $\begin{array}{l}44.657 \\
44.767\end{array}$ & \begin{tabular}{|l|}
54.436 \\
54538 \\
\end{tabular} & \begin{tabular}{r|}
10.1 \\
10.072
\end{tabular} & \begin{tabular}{|r|}
35.361 \\
35.49 \\
\end{tabular} & \begin{tabular}{|l|l|}
3.663 \\
367
\end{tabular} & \begin{tabular}{|l}
0.845 \\
0.842
\end{tabular} & \begin{tabular}{|c|}
48.202 \\
48.15
\end{tabular} & $\begin{array}{l}0.014 \\
0.014\end{array}$ & & & & \begin{tabular}{l|l}
26.7836 \\
\end{tabular} & \begin{tabular}{|l|l|}
53.3 \\
53.4
\end{tabular} & \begin{tabular}{|r|}
11.5 \\
11.5
\end{tabular} & \begin{tabular}{|l|}
40.0 \\
\end{tabular} & \begin{tabular}{|l|}
2.758 \\
2767 \\
\end{tabular} & 0.002 & $\begin{array}{l}0.002 \\
0.002\end{array}$ & 0.000 & 0.050 & 0.04 \\
\hline & $8 / 25012004$ & 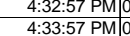 & & & $\frac{24.734}{24.707}$ & $\frac{27.772}{27.761}$ & & & & & & $\begin{array}{l}3.657 \\
3.66 \\
\end{array}$ & & & & & & & $\begin{array}{l}26.8003 \\
\end{array}$ & \begin{tabular}{|l|}
53.4 \\
52.9
\end{tabular} & $\mid 11.5$ & 40.1 & $2.767 \mid$ & \begin{tabular}{|l|l|}
0.002 \\
\end{tabular} & & & & 0.04 \\
\hline & $8 / 25 / 2004$ & $34: 57 \mathrm{PMO}$ & \begin{tabular}{|l|l|}
026.234 \\
\end{tabular} & 25.284 & 24.728 & 27.752 & 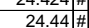 & & $\begin{array}{r}54.43 \\
54.461\end{array}$ & 10.6 & $\frac{35.410}{35.701}$ & $\begin{array}{l}.00 \\
3.66 \\
\end{array}$ & & & & & & & & 52.9 & 11 & & & & & & & 0.04 \\
\hline & $8 / 25 / 2004$ & 4:35:57 PM 0 & $\begin{array}{l}026.233 \\
\end{array}$ & 25.283 & 24.717 & 27.74 & & 101 & \begin{tabular}{|l|l|}
54.845 \\
\end{tabular} & 10. & & 3.657 & & & 114 & & & & & & \begin{tabular}{|l|l|}
1.5 \\
11.5
\end{tabular} & & & & & & 0.050 & $\frac{0.04}{0.04}$ \\
\hline & $8 / 25 / 2004$ & 4:36:57 PM0 & \begin{tabular}{|l|l|}
0 & 26.248 \\
\end{tabular} & 25.283 & 24.707 & 27.76 & & & & & & 3.657 & & 48. & & & & & & & & & & & & & & $\begin{array}{l}0.04 \\
0.04 \\
\end{array}$ \\
\hline & $8 / 25 / 2004$ & 4:37:57 PM0 & \begin{tabular}{l|l}
0 & 26.257 \\
\end{tabular} & 25.302 & 24.761 & 27.79 & & & $\begin{array}{l}54.755 \\
\end{array}$ & & 35.3 & & & & D14 & & & & & & & & & & & & & $\frac{.04}{0.04}$ \\
\hline & 55/2004 & & & 25.287 & & & & & & & & & & & & & & & & & & & & & & & & \\
\hline & $8 / 25 / 2004$ & 9:57 PM & 026.252 & 25.287 & 24.725 & 27.734 & & & 54.66 & & & & & & & & & & & & & & 276 & & & & 0.050 & \\
\hline & $8 / 25 / 2004$ & 4:40:57 PM 0 & 26.256 & 25.296 & 24.73 & 27.743 & & & & & & & & & & & & & & 52 & & & & & & & & \\
\hline & $8 / 25 / 2004$ & 4:41:57 PM0 & 26.236 & 25.286 & 24.69 & 27.703 & & & 54.3 & & & & & & & & & & & 53. & & & & & & & 0.050 & 0.04 \\
\hline & $8 / 25 / 2004$ & 4:42:57 PM0 & 26.242 & 25.282 & 24.72 & 27.714 & & & & & & & & & & & & & & & & & & & & & & 0.04 \\
\hline & $8 / 25 / 2004$ & 4:43:57 PM0 & $\begin{array}{l}0 \\
0\end{array}$ & 25.301 & 24.72 & 27.758 & & & & & & & & & & & & & & & & & & & & & & 0.04 \\
\hline & $8 / 25 / 2004$ & 4:44:57 PM0 & 26.25 & 25.3 & 24.734 & 27.738 & 24.506 \# & 44.579 & \begin{tabular}{|l|l|}
54.221 \\
\end{tabular} & 10.206 & 35.164 & 3.657 & 0.845 & 48.517 & $\begin{array}{ll}0.014 \\
\end{array}$ & \begin{tabular}{l|l|}
4 & 14.643 \\
\end{tabular} & & & \begin{tabular}{|l|l|}
27.0003 \\
\end{tabular} & 53.1 & 11.6 & & \begin{tabular}{|l|l|}
2.749 \\
\end{tabular} & 0.002 & 0.002 & \begin{tabular}{|c|}
0.000 \\
\end{tabular} & \begin{tabular}{|l|l|} 
& 0.051 \\
\end{tabular} & \\
\hline & & & & & & & & & & & & & & & & & & & & & & & & & & & & \\
\hline & & & & & & & & & & & & & & & & & & & & & & & & & & & & \\
\hline & & & & & & & & & & & & & & & & & & & & & & & & & & & 0.078 & \\
\hline & & & & & & 28 & 25 & & & & & 62 & & & & & & & & & & & & & & & & \\
\hline & & & & 24.8 & 24. & & & & & & 35.4 & 3. & & & & & & & & & & & & & & & & \\
\hline & & & & & 23.5 & 22.5 & & & & & & & & & & & & & & 40. & & & & & & & & \\
\hline & & $12 \times 3 x$ & 1.159 & 1.394 & 0.827 & 1.785 & & 9.077 & 2.238 & & 9.158 & 9.446 & & & & & & & & 2.238 & & & 228 & & & & & \\
\hline & & 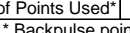 & intsin box & $\begin{array}{l}5] \\
\text { xare not inclu }\end{array}$ & 705 & & & & & & & & & & & & & & & & & & & & & & & \\
\hline
\end{tabular}


WSRC-TR-2005-00105, REVISION 0

SRNL-RPP-2005-00012, REVISION 0

CAMPAIGN IV NEW GKN ACID CLEAN AND CAUSTIC RINSE

RPP-WTP pilot UFP - Campaign IV, Pre-Test Activities

[26 August 2004: Caustic Rinse and Acid Clean]

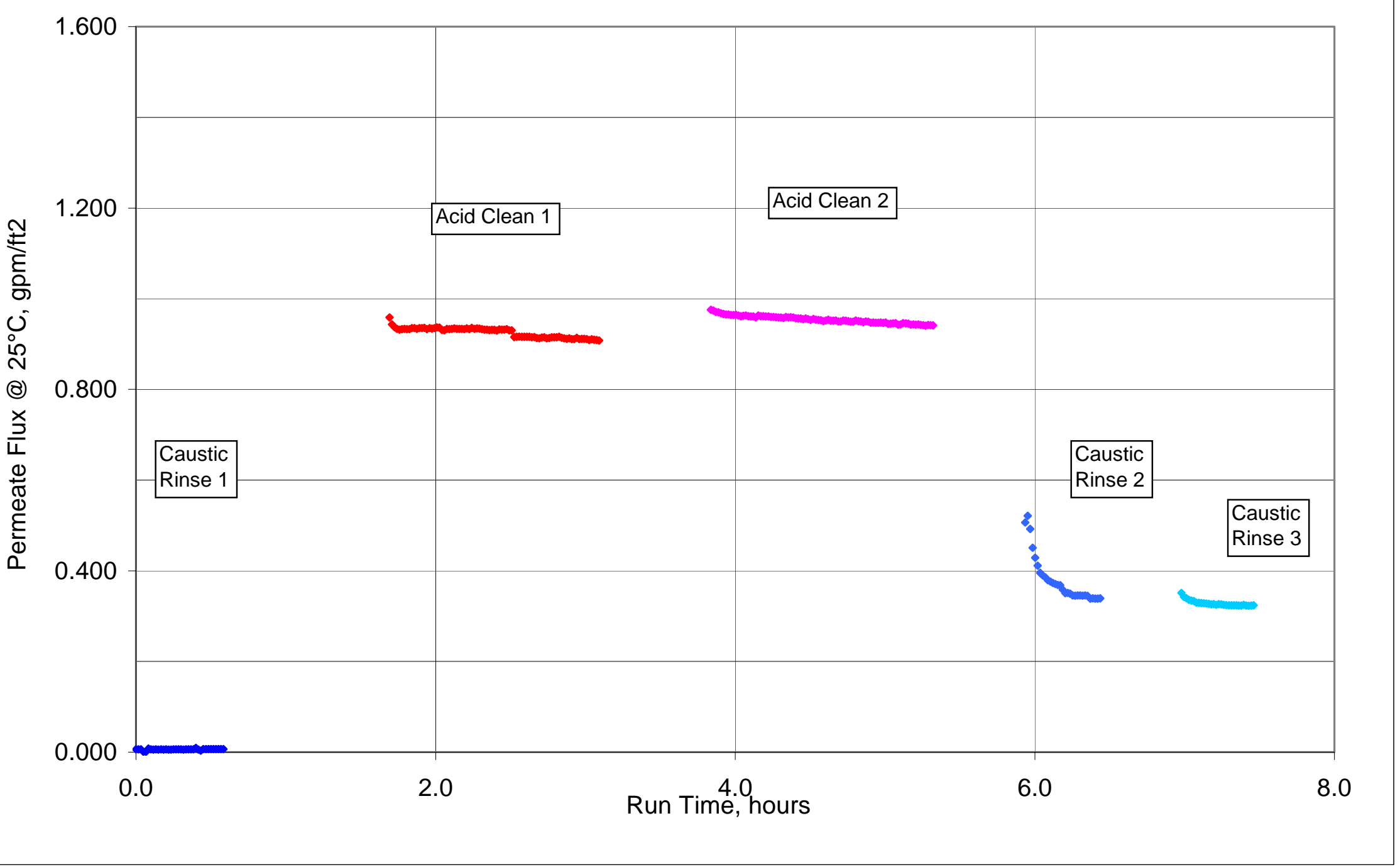


WSRC-TR-2005-00105, REVISION 0

SRNL-RPP-2005-00012, REVISION 0

CAMPAIGN IV NEW GKN ACID CLEAN AND CAUSTIC RINSE

\begin{tabular}{|c|c|c|c|c|c|c|c|c|c|c|c|c|c|c|c|c|c|c|c|c|c|c|c|c|c|c|c|c|}
\hline 1 & A & B & D & $\begin{array}{l}E \\
<\text { Temn }\end{array}$ & $\frac{F}{F}$ & e & $\frac{\mathrm{H}}{\mathrm{H} \text { ents } \gg \gg \geqslant}$ & \begin{tabular}{l|l|}
$\mathrm{J}$ \\
$1<<<<<$
\end{tabular} & I K & $\frac{\mathrm{L}}{\mathrm{p}}$ & \begin{tabular}{|c|} 
M \\
escures
\end{tabular} & $\mid \mathrm{N}$ & 10 & 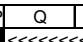 & $\frac{R}{<<<\text { R }}$ & s & 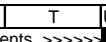 & $\mathrm{V}$ & $\frac{w}{1}$ & $\frac{|x| x \mid}{1<\ll<<c}$ & $\begin{array}{ll}\text { Calculat } \\
\text { Cal }\end{array}$ & $\frac{\mathrm{z}}{\mathrm{z}}$ & AA & $\mid$\begin{tabular}{|c|}
$\mid \mathrm{AB}$ \\
$D 2 \operatorname{ta} \gg \gg \geqslant$
\end{tabular} & $\frac{\mid A C}{|A \gg \gg \gg \gg \geqslant|}$ & 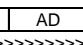 & $\frac{\mid A E}{\gg \gg \gg \gg}$ & AF \\
\hline$\frac{1}{2}$ & DATE & TIME & Filtrate & Cleaning & Slurry & Hi Amb. & Lo Amb. & BotTMP & Filter & & The & Filtrate & Pulsepot & & & & & & & & & & & & & & & \\
\hline & & & $\operatorname{deg} C$ & $\operatorname{deg} C$ & $\operatorname{deg} C$ & $\operatorname{deg} C$ & & psid & psig & psid & & psig & psig & & gpm & gpm & & & & & & & & & & & & \\
\hline 4 & & & & & [1] & T4 & & $\mathrm{dP2}$ & & $\mathrm{dP1}$ & $\mathrm{dP3}$ & P2 & & & $\mathrm{Q} 2$ & $\mathrm{Q}^{3}$ & $\mathrm{Qbp}$ & & & & & & & & & & & \\
\hline 5 & & & & & & & & & & & & & & & & & & & & & & & & & & & & \\
\hline & Zeros- 04/19 & & & & & & & & & & & & & & & & & & & & & & & & & & & \\
\hline & $\begin{array}{l}8 / 23 / 2004 \\
8 / 24 / 201\end{array}$ & $8: 35: 38 \mathrm{AM}$ & 22.554 & 22.604 & 22.002 & 22.782 & 22.204 & $\begin{array}{l}-0.019 \\
\end{array}$ & 0.095 & -0.012 & 0.003 & -0.124 & & -0.075 & 0.003 & \begin{tabular}{|l|l|}
14.643 \\
\end{tabular} & 0.004 & & & Filter Su & iufface & 6.985 & T2 & & & & & \\
\hline & $8 / 24 / 2004$ & & & & 23.679 & 22.950 & 22.7 & & 0.122 & -0.014 & & -0.124 & & 23.176 & 1.205 & 14.643 & & & & Convers & & & & & ginn & /lbarg & & \\
\hline & $8 / 27 / 27 / 2004$ & & $\frac{2.019}{22649}$ & $\frac{20.356}{3280}$ & 24.934 & & & 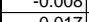 & & & & & & & & 14.045 & & & & & & & & & & & & \\
\hline & $\frac{1 / 21 / 2004}{8}$ & $\begin{array}{l}1.53 .29 \mathrm{Al} \\
7: 28: 55 \mathrm{AM}\end{array}$ & $\frac{22.049}{22.511}$ & $\frac{2.648}{22.848}$ & 322.397 & 22669 & 22914 & -0.017 & 0.138 & $\frac{-0.009}{-0.009}$ & 0.003 & - & & -0.082 & & 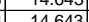 & & & & & & & & & & & & \\
\hline & & & & & & & & -0.017 & & & 0.003 & -0.147 & & $=-0.082$ & 0.003 & 14.043 & & & & & ire P1i & orrect & ted for 3 & 31.25 inch & ches of & ith & & \\
\hline 13 & & & & & & & & & & & & & & & & & & & & VWVV & & & & < Filtrat & ate Flux $=$ & $\mid x<$ PER & ABBIL & $\gg$ \\
\hline 14 & & & & & & & & & & & & & & & & & & & & & & & & & at $25 \mathrm{C}$ & & 1000 & \\
\hline & & & & & & & & & & & & & & & & & & Time & Time & Press. & el. & TMP & IMP & $\mathrm{gpm}$ & $\mathrm{pm}$ & $\mathrm{gpm}$ & gpm & \\
\hline & Data - Per M & & & & & & & & & & & & & & & & & & our & psig & & psi & ar & & & & $2 \mathrm{psi}$ & \\
\hline$\frac{11}{18}$ & $\begin{array}{l}8 / 26 / 2004 \\
8 / 26 / 200\end{array}$ & 9:15:47 AM & & $\begin{array}{r}23.377 \\
23.371\end{array}$ & 24.761 & $\begin{aligned} 25.305 \\
25265\end{aligned}$ & $\begin{array}{l}23.280 \\
23206\end{array}$ & $\frac{22.682}{20.761}$ & 27.732 & 5.027 & $\begin{array}{l}17.796 \\
17775 \\
\end{array}$ & $\begin{array}{r}-0.095 \\
-0.077\end{array}$ & & $\begin{array}{l}48.502 \\
48709\end{array}$ & 0.042 & $\frac{14.643}{14643}$ & 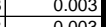 & 100 & \begin{tabular}{|l} 
\\
\end{tabular} & $\frac{26.6}{26.6}$ & 11.6 & $\frac{20.2}{303}$ & 1.395 & 0.006 & 0006 & 0.000 & 0.299 & \\
\hline 19 & $8 / 26 / 2004$ & $\begin{array}{l}9.10 .47 \mathrm{AM} \\
9.17: 47 \mathrm{AM}\end{array}$ & $\begin{array}{l}23.048 \\
25.599\end{array}$ & $\frac{23.741}{23.402}$ & $\begin{array}{r}24.04 \\
24.561\end{array}$ & $\begin{array}{r}25.200 \\
25.29\end{array}$ & $\frac{23.300}{23231}$ & 2 & 27.978 & & $\frac{17.155}{18.112}$ & $\begin{array}{l}-0.071 \\
-0.077\end{array}$ & $\begin{aligned}-4.00 \\
=40\end{aligned}$ & 年 & (.0.045) & $\begin{array}{l}14.045 \\
14643\end{array}$ & & & 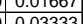 & & 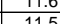 & & 1.59t & & & & & \\
\hline 20 & $8 / 26 / 2004$ & 9:18:47 AM & 25.593 & 23.406 & 24.455 & 25.265 & 23.376 & 20.274 & \begin{tabular}{|l|l|}
27.947 \\
\end{tabular} & 4.976 & 15.347 & 2.415 & 19.682 & 48.511 & 0.003 & 14.643 & 0.003 & 3.00 & 0.05 & 26.8 & 11.6 & \begin{tabular}{|l|}
17.8 \\
\end{tabular} & $\begin{array}{l}1.228 \\
\end{array}$ & \begin{tabular}{|l|}
0.000 \\
\end{tabular} & 0.000 & 0.000 & 0.024 & \\
\hline & $8 / 26 / 2004$ & 9:19:47 AM & 25.569 & 23.417 & 24.381 & 25.27 & 23.442 & 17.01 & 27.941 & 4.976 & \begin{tabular}{|l|l|}
11.996 \\
\end{tabular} & 5.788 & & 48.25 & & \begin{tabular}{|l|l}
14.643 \\
\end{tabular} & & 4.00 & 0.06667 & 26.8 & 11.5 & 14.5 & 1.000 & & & & 0.030 & \\
\hline & $8 / 26 / 2004$ & 9:20:47 AM & 25.219 & 23.417 & 24.31 & 25.295 & 23.372 & 19.714 & 28.093 & 4.972 & 14.847 & 3.162 & & 48.379 & 0.053 & 14.643 & 0.00 & 5.0 & \begin{tabular}{|l|l|}
0.08333 \\
\end{tabular} & 27.0 & $11 .:$ & 17.3 & 1.191 & 0.008 & 0.008 & 0.000 & 0.448 & \\
\hline 24 & $8 / 26 / 212004$ & 9:21:47 AM & 25.275 & 23.428 & 24.252 & 25.337 & 23.503 & 19.665 & 27.916 & 4.893 & 14.865 & 3.099 & & 47.747 & 0.04 & 14.643 & & 6.0 & 0.1 & 26.8 & 11. & $17.3 \mathrm{r}$ & 1.190 & & 0.006 & 0.000 & 0.339 & \\
\hline 24 & 8212622004 & 9:22:4/ AM & 25.201 & 23.435 & 24.173 & & & 19.683 & & & & 3.096 & & & 0.039 & 14.643 & & & 0.116067 & & & & & & & & & \\
\hline 26 & 8212012004 & $\begin{array}{l}\frac{9.3 .44}{0.4617} \\
\end{array}$ & 25.152 & 23.446 & 24.214 & 25.314 & 23.65 & 19.565 & 27.809 & 4.958 & & 3.096 & & 47.879 & & 14.643 & & 8.00 & 0.13333 & 26.9 & 11.4 & 17.1 & 1.182 & 0.000 & 0.000 & 0.000 & 0.350 & \\
\hline & $8 / 2 / 26 / 2004$ & $\begin{array}{l}9.24 .41 \\
0.25 .47\end{array}$ & & $\frac{20.46 \mathrm{C}}{2.470}$ & $\begin{array}{r}24.25 \\
24.257\end{array}$ & 2 & & & & & & S.0.096 & & (46.010 & & & & & \begin{tabular}{|l|l|} 
\\
\end{tabular} & 20.8 & & & & & & & & \\
\hline 28 & $8 / 26 / 2004$ & $\begin{array}{l}3.25 .47 \mathrm{AM} \\
9: 26: 47\end{array}$ & 25.032 & $\frac{2.045}{23.486}$ & \begin{tabular}{|l|l|l|l|l|}
24.268 \\
\end{tabular} & $\frac{30.016}{25.329}$ & 20.02 & $\begin{array}{l}\frac{19.004}{19.953} \\
\end{array}$ & \begin{tabular}{|l|}
20.101 \\
28.256 \\
\end{tabular} & $\frac{0.05}{5.05}$ & $\begin{array}{l}14.8206 \\
14.869\end{array}$ & $\begin{array}{l}3.050 \\
.151\end{array}$ & & $4 \frac{47.929}{47.922}$ & $\begin{array}{l}0.031 \\
0.039\end{array}$ & 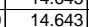 & 0.003 & $\frac{1.00}{11.00}$ & 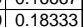 & 27.1 & $\frac{11 .}{11 .}$ & $\frac{17.0}{17.4}$ & $\frac{1.152}{1.200}$ & 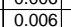 & $\frac{0.006}{0.006}$ & 0.000 & $\frac{0.041}{0.327}$ & \\
\hline & $8 / 26 / 2004$ & & 25.003 & 23.497 & 24.29 & \begin{tabular}{|l|l|}
25.33 \\
\end{tabular} & & & \begin{tabular}{|l|l|}
28.101 \\
\end{tabular} & & & & & & $\frac{0.04}{0.04}$ & & & 1200 & $\mid$ & 270 & 11: & 174 & 1.200 & 0.006 & & & & \\
\hline & 8/26/2004 & 9:28: & & & 24.296 & & 23.6 & $\begin{array}{ll}19.723 \\
\end{array}$ & 27.92 & 4.98 & 14.806 & 3.159 & 0.469 & 48.179 & 0.038 & $\begin{array}{l}14.643 \\
\end{array}$ & $\frac{0.003}{0.003}$ & $\frac{13.00}{13.00}$ & 0.21667 & $\frac{26.8}{26.8}$ & $\frac{\frac{c 1}{11 .}}{11 .}$ & $\frac{17.3}{17.3}$ & .1.190 & 0.005 & 5.006 & $\frac{.000}{.000}$ & $\frac{0.321}{0.321}$ & \\
\hline & 8/26/2004 & 9:29: & 24.945 & 23.51 & 24.307 & 25.393 & 23.6 & $\begin{array}{l}19.876 \\
\end{array}$ & 28.082 & & 14. & 3.162 & & \begin{tabular}{|l|l|l|l}
489 \\
\end{tabular} & 0.038 & \begin{tabular}{|l|l|}
14.643 \\
\end{tabular} & & 14.00 & 0.23333 & 27.0 & $\frac{11 .}{11 .}$ & 17.3 & 1.196 & & .006 & & & \\
\hline & $8 / 26 / 2004$ & & & 23.515 & 24.312 & & 23.5 & & 27.854 & & $14 . \varepsilon$ & & & 48.116 & 0.04 & $\begin{array}{l}14.643 \\
\end{array}$ & & & 0.25 & 26.7 & $\frac{\frac{11}{11 .}}{4}$ & 17.3 & $\frac{1.19}{1.19}$ & 006 & 0.006 & & $\frac{0.338}{0.338}$ & \\
\hline & $8 / 26 / 2$ & & 24.88 & 23.519 & & & & & & & $\begin{array}{ll}14 . \varepsilon \\
\end{array}$ & 3.159 & & & 0.04 & 14.6 & & & 0.26667 & 26.8 & 11.5 & 17.3 & & & .006 & & & \\
\hline 34 & $8 / 26 / 2004$ & & 24.859 & 23.523 & 24.311 & 25.331 & & \begin{tabular}{|l|l|}
19.827 \\
\end{tabular} & 28.088 & & 14.9 & 3.162 & & 47.768 & 0.042 & 14.6 & & & \begin{tabular}{|l|}
0.28333 \\
\end{tabular} & & 11. & & & & & & 0.353 & \\
\hline & 8/26/2004 & & 24.853 & 23.538 & \begin{tabular}{|l|l} 
& 24.331 \\
\end{tabular} & 25.3 & & \begin{tabular}{|l|l|l|}
19.603 \\
\end{tabular} & 27.782 & & 14.884 & 3.217 & & 48.058 & 041 & 14. & & 18.00 & 0.3 & 26.7 & $11.4 \mathrm{~S}$ & 17.2 & 1.189 & 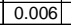 & 506 & 0.000 & 0.347 & \\
\hline & & & 24.847 & 23.552 & 24.349 & 25.304 & & 19.5 & 27.993 & & 14.5 & 3.197 & & & & & & & 0.31667 & 26.9 & 11.4 & 17. & & & & & & \\
\hline 37 & $8 / 26 / 2004$ & & 24.826 & 23.545 & 24.328 & 25.233 & 23.2 & 19.685 & 28.005 & 4.964 & 14.627 & 3.214 & & 48.235 & 0.041 & 14.643 & 0.00 & 20.00 & 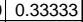 & 26.9 & 11.4 & 17.2 & 1.183 & 0.006 & 0.006 & 0.000 & 0.349 & \\
\hline & 012012004 & & & 23.564 & \begin{tabular}{|l|l|} 
& 24.342 \\
\end{tabular} & 25.242 & & & & 4.97 & & 3.217 & & & 0.04 & & & 21.00 & 0.05 & 20.9 & 11.4 & 17.0 & & & & & 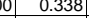 & \\
\hline 39 & & & 24.81 & & & & & & 27.91 & & & & & 48.133 & & & & & 0.36667 & 26.8 & 11. & 17.2 & & & & & & \\
\hline$\frac{40}{41}$ & 8212621004 & & $\begin{array}{r}24.81 \\
2406\end{array}$ & $\frac{23.579}{2595}$ & 24.362 & 25.242 & 23.3 & $\frac{19.735}{22042}$ & 212.985 & $\begin{array}{r}4.91 \\
4036\end{array}$ & $\begin{array}{l}14.892 \\
18090\end{array}$ & 3.217 & & $47 . .141$ & & & & 2000 & 0 & 2699 & $\frac{11.4}{11.5}$ & 1.3 & 1 & - 0.006 & 0000 & 000 & 0.345 & \\
\hline$\frac{41}{42}$ & $\frac{1}{8 / 26 / 2004}$ & & 24.000 & 20.0503 & 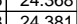 & & & & & & & -0.072 & & & & & & & 0.4 & & 些1.6 & & & & & & & \\
\hline 43 & $8 / 26 / 2004$ & & 24.777 & 2301 & \begin{tabular}{|l|l|}
24.381 \\
\end{tabular} & $\begin{array}{r}25.103 \\
25\end{array}$ & & 21.7 & \begin{tabular}{|l|}
27.001 \\
\end{tabular} & & 1669 & \begin{tabular}{|c|} 
\\
\end{tabular} & & (4) & & & & $\frac{25}{26}$ & $\mid$ & 26.5 & $\frac{1.6}{118}$ & 10.4 & $\frac{1.40}{132}$ & (1) & & & $\mid$ & \\
\hline 44 & $8 / 26 / 2004$ & & 24.74 & 23.61 & $\mid \begin{array}{l}24.393 \\
24,393\end{array}$ & 25.052 & 23.3 & 22.099 & 27.873 & 5.069 & 17.075| & $|.0 .57|$ & & 48. & & & & & 0.0 .45 & 26.7 & $\frac{1.1 .}{11 .}$ & 19.6 & & & & & & \\
\hline & $8 / 26 / 2004$ & & 24.724 & 23.609 & 24.386 & 24.965 & 23.2 & 22.015 & $\begin{array}{l}27.952 \\
\end{array}$ & & \begin{tabular}{|l|}
16.889 \\
\end{tabular} & \begin{tabular}{|c|}
0.629 \\
\end{tabular} & & 48.8 & & & & & 0.46667 & \begin{tabular}{l|l}
26.8 \\
\end{tabular} & T. & $\begin{array}{ll}19.5 \\
\end{array}$ & & & & & & \\
\hline 46 & $8 / 26 / 2004$ & $9: 44: 4$ & 24.711 & 23.621 & 24.378 & $\begin{array}{l}24.977 \\
\end{array}$ & 23.0 & \begin{tabular}{|l|l|}
21.903 \\
\end{tabular} & 27.748 & 4.995 & \begin{tabular}{|c|}
16.903 \\
\end{tabular} & \begin{tabular}{|c|c|c|}
0.664 \\
\end{tabular} & & $\begin{array}{l}48.156 \\
\end{array}$ & \begin{tabular}{|c|}
0.044 \\
\end{tabular} & 14.6 & & 29.00 & 0.40050 & 26.6 & 11.5 & 19.4 & & .006 & & & & \\
\hline & & & & & 24.368 & & & 22.16 & & & & & & & 0.045 & & & & 0.5 & 26.9 & 11. & 19.6 & & & & & & \\
\hline & $80<0 / 2004$ & & 24.115 & 23.020 & 24.368 & 25.012 & & 22.064 & 27.856 & & 16.989 & 0.66 & & 48.934 & & 14. & & & 0.51 & 26.7 & 11. & 19.5 & & 506 & 7 & 0.000 & 0.3 & \\
\hline & $8 / 26 / 20$ & & 24.118 & 23.633 & 24.316 & 25.07 & & & 27.9 & 5.002 & 17.03 & 0.6 & & 48. & & & & & \begin{tabular}{|l|l|} 
\\
\end{tabular} & 26.8 & 11. & $\begin{array}{l}19.6 \\
\end{array}$ & 348 & .006 & & & 0.328 & \\
\hline & 8/26/2004 & & 24.727 & 23.646 & 24.389 & 25.143 & & 21.8 & 27.6 & & 16.809 & \begin{tabular}{|c|c|} 
\\
\end{tabular} & & 48.3 & & & & & .55 & 26.5 & 11.5 & 19.3 & & & & & 0.339 & \\
\hline & $8 / 26 / 2004$ & & 24.735 & 23.645 & 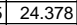 & 25.202 & 23. & 21.9 & 27.8 & 5.1 & \begin{tabular}{|l|}
16.747 \\
\end{tabular} & 0.7 & & 48.4 & & & & & & 26.7 & $11.5 \mathrm{~S}$ & \begin{tabular}{ll|}
19.4 \\
\end{tabular} & & & & & 0.331 & \\
\hline & $8 / 26 / 2$ & & 24.733 & 23.648 & 24.38 & 25.24 & & 22.4 & & 5.062 & 17 & \begin{tabular}{|l|l|} 
\\
\end{tabular} & & 48.3 & & 14. & & & & 26.8 & & 19.5 & & & & & & \\
\hline & & & 25.69 & & 25.0 & \begin{tabular}{|l|}
25.678 \\
\end{tabular} & & & & & & & & & & & & & & 62.2 & & 24 & & & & & & \\
\hline & & $10: 51$ & 25.785 & 24.312 & 25.183 & 25.682 & 24. & 24 & 63. & 3. & 24.439 & 31.1 & & 43.986 & 0.003 & & & & & 62.7 & 10.5 & 24.4 & & \begin{tabular}{|l|}
0.926 \\
\end{tabular} & & & & \\
\hline & $8 / 26 / 2004$ & $\begin{array}{l}10: 52: 18 \\
010.52 \cdot 18\end{array}$ & $\begin{array}{r}25.89 \\
25.034\end{array}$ & $\begin{array}{r}24.333 \\
2.307\end{array}$ & $\begin{aligned} 25.279 \\
25.313\end{aligned}$ & \begin{tabular}{|l|l|}
25.668 \\
25.622
\end{tabular} & 24. & $\begin{array}{r}24.478 \\
8983 \\
\end{array}$ & 64.1 & $\begin{array}{l}3.703 \\
3.567\end{array}$ & $\begin{array}{r}24.851 \\
56799\end{array}$ & \begin{tabular}{|l|}
31.357 \\
51.481 \\
\end{tabular} & & $\begin{array}{ll}43.7 \\
390\end{array}$ & & $\begin{array}{r}6.487 \\
\end{array}$ & & & $\frac{1.00}{162}$ & $\frac{63.04}{64.1}$ & $\frac{10.4}{9.4}$ & 24.1. & & 0.929 & & & 37.360 & \\
\hline & $\frac{1}{8 / 26 / 2004}$ & 10:54: & $\begin{array}{r}23.904 \\
25.959\end{array}$ & & \begin{tabular}{|l|}
25.310 \\
\end{tabular} & $\begin{array}{l}25.024 \\
25.631\end{array}$ & & & & & & \begin{tabular}{|l|}
51.401 \\
58.962 \\
\end{tabular} & & 37.7 & & & & $\begin{aligned} 97.02 \\
0.52\end{aligned}$ & $\frac{1.64}{164}$ & $\begin{array}{l}04.1 \\
65.0 \\
65\end{array}$ & & & & & & & & \\
\hline & 8/26/2004 & 10:55:1 & 25.944 & 24.326 & 25.438 & 25.626 & 24. & 2.072 & 65.882 & & -1.2 & \begin{tabular}{|l|l|} 
& 58.985 \\
\end{tabular} & & 37.5 & & & & & $\begin{array}{l}1.65 \\
\end{array}$ & 64 & 8. & 04 & & & & -0.004 & -3.993 & \\
\hline & $8 / 26 / 2004$ & 10:56: & 26.757 & 24.331 & \begin{tabular}{|l}
25.477 \\
\end{tabular} & 25.646 & & 2.23 & 65. & 3.4 & -1.077 & 59. & & & & 14.6 & & & & 64.8 & & 0.6 & & 96 & 68 & & \#\#\#\#" & \\
\hline & $8 / 26 / 2$ & 10:57:1 & 26.137 & 24.32 & 25.507 & 25.62 & & 20.8 & 63. & & 21. & 34.5 & & 43.8 & & & & & & \begin{tabular}{l|l|}
62.7 \\
\end{tabular} & $\overline{0 .}$ & 21.2 & & & & & & $\overline{38}$ \\
\hline & 8/26/2004 & & 26.192 & 24.319 & 25.566 & 25.629 & & 21.5 & 63.426 & 3.8 & 21.74 & & & 46.5 & & & & & 1.70 & 62.3 & & 21. & & 0.958 & & & & \\
\hline & $8 / 26 / 2$ & & 26.246 & 24.319 & \begin{tabular}{l|l}
95.61 \\
\end{tabular} & & & 21.946 & & & & & & & & & & & & & & & & & & & & \\
\hline 63 & 8126622004 & $11: 00$ & 26.255 & 24.302 & $25.5 / 4$ & 25.708 & & 22.143 & $\begin{array}{l}63.26 \\
\end{array}$ & 3.817 & 22.508 & \begin{tabular}{|l|}
32.648 \\
\end{tabular} & & 45.042 & & & & & $1.14 \mathrm{~s}$ & 62.1 & & 22. & & & & & & \\
\hline & 821262004 & & $\frac{26.2}{2003}$ & 24.322 & 25.499 & 25.143 & 24 & 22. & 63.697 & 3.83 & 23.082 & \begin{tabular}{|l|}
32.498 \\
\end{tabular} & & 44.6. & & & & & & $62.6]$ & & 20 & & & & & & \\
\hline & $\frac{01201204}{812604}$ & 11.02 & $\frac{2.09}{2601}$ & 24.301 & 年35.355 & $\frac{25.130}{25715}$ & & (2.014) & $\begin{array}{l}03.944 \\
0.707\end{array}$ & 3.80 & 23.201 & $\begin{array}{l}3<.428 \\
32373\end{array}$ & & 44.02 & & - & & & $\frac{1.17528}{17018}$ & 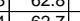 & & & & . & & & 40.382 & \\
\hline & $\frac{1.25004}{8262004}$ & $\frac{1.1 .04 .18}{11.04 .18}$ & 25.958 & $\frac{24.435}{24295}$ & $\frac{23.310}{25242}$ & $\frac{20.150}{25721}$ & & $\frac{2.35}{2296}$ & $\frac{03.151}{66^{2357}}$ & & 23.5 & $\frac{3.2 .3}{320}$ & & $\frac{44.45}{44.93}$ & & & & & & 然..1 & & & & (1) & & & & \\
\hline & 8 & 11.05. & 25.914 & 24.306 & $\begin{array}{l}25.472 \\
25192\end{array}$ & 25706 & 24 & & 636 & 382 & 23 & 320 & & & & & & & & & & & & & & & & \\
\hline & & & 25.87 & 24.317 & 25.164 & 25.783 & & $\frac{13.238}{23.23}$ & 63.568 & 3.742 & $\mid$\begin{tabular}{|c|}
23.608 \\
2.008
\end{tabular} & \begin{tabular}{|l|l|}
32.174 \\
\end{tabular} & & 43.847 & 0.003 & 6.56 & & $\frac{11.02}{110.52}$ & 18494 & 624 & 104 & 23. & & | & 35 & 40 & 39.923 & (3) \\
\hline & & 7.1 .25 & & $\frac{1.319}{319}$ & & 25.799 & & & 64.053 & 3.749 & 23.874 & \begin{tabular}{|l|}
31.959 \\
\end{tabular} & & 43.715 & & 6.655 & & & & & & & & & & & 39.4 & \\
\hline
\end{tabular}


WSRC-TR-2005-00105, REVISION 0

SRNL-RPP-2005-00012, REVISION 0

CAMPAIGN IV NEW GKN ACID CLEAN AND CAUSTIC RINSE - CONT.

\begin{tabular}{|c|c|c|c|c|c|c|c|c|c|c|c|c|c|c|c|c|c|c|c|c|c|c|c|c|c|c|c|c|}
\hline & A & $\mathrm{B}$ & $\mathrm{D}$ & $\mathrm{E}$ & $\mathrm{F}$ & $\mathrm{G}_{\mathrm{G}}$ & $\mathrm{H}$ & $\begin{array}{ll}\mathrm{J} \\
\mathrm{N}\end{array}$ & $\mathrm{K}$ & $\mathrm{L}$ & $\mathrm{M}$ & $\mathrm{N}$ & $\mathrm{O}$ & & $\mathrm{R}$ & $\mathrm{s}$ & 1 & & & $x$ & & & $A A$ & (1) & $A C$ & $A D$ & $\mathrm{AE}$ & \\
\hline & 8/26/2004 & 11:08:18 AM & $\begin{array}{l}25.783 \\
25725\end{array}$ & $\begin{array}{ll}24.341 \\
2.207 \\
\end{array}$ & $\begin{array}{ll}25.097 \\
25059\end{array}$ & $\begin{array}{ll}25.836 \\
25.307 \\
2\end{array}$ & 24.633 & & \begin{tabular}{|l}
63.548 \\
\end{tabular} & 3.718 & 23.716 & $\begin{array}{l}31.957 \\
2.955\end{array}$ & 3.565 & & 0.003 & 6.539 & 0.005 & 112.52 & 1.87528 & \begin{tabular}{ll|}
62.4 \\
\end{tabular} & 10.5 & & 1.61 & 0.936 & $\begin{array}{ll}0.934 \\
\end{array}$ & 0.040 & 39.749 & 33.83 \\
\hline & 8/26/2004 & 11:09:18 AM & 25.735 & $\begin{array}{l}24.347 \\
2439\end{array}$ & $\begin{array}{l}25.058 \\
25005 \\
\end{array}$ & $\begin{array}{l}25.887 \\
25.904 \\
25\end{array}$ & $\begin{array}{l}24.669 \\
24706\end{array}$ & $\begin{array}{l}23.616 \\
23524 \\
\end{array}$ & \begin{tabular}{|l|l|}
63.805 \\
63.26
\end{tabular} & $\begin{array}{l}3.794 \\
3715\end{array}$ & $\begin{aligned} 23.86 \\
23.911\end{aligned}$ & \begin{tabular}{|l|l|}
31.855 \\
31.777 \\
\end{tabular} & $\begin{array}{r}3.56 \\
3542\end{array}$ & \begin{tabular}{|l|l|l|l|l}
43.984 & \\
43832 &
\end{tabular} & $\begin{array}{l}0.003 \\
0.003\end{array}$ & $\begin{array}{l}6.543 \\
6.533 \\
\end{array}$ & 0.006 & 113.52 & 1.89194 & \begin{tabular}{l|l}
62.7 \\
625
\end{tabular} & 10.5 & 23 & 1.625 & 0.935 & $\begin{array}{l}0.935 \\
0.925 \\
\end{array}$ & 0.039 & \begin{tabular}{l|l|l|}
39.397 \\
39429
\end{tabular} & \\
\hline & $\mid 8 / 26 / 2004$ & $\begin{array}{c}11: 11: 18 \mathrm{AM} \\
11: 18 \mathrm{AM}\end{array}$ & $\frac{2.004}{25.649}$ & \begin{tabular}{|l|}
24.3496 \\
24.367
\end{tabular} & $\frac{25.005}{24.973}$ & $\begin{array}{l}25.904 \\
2592 \\
\end{array}$ & $\frac{24.706}{24.689}$ & $\begin{array}{l}23.524 \\
23.825 \\
\end{array}$ & \begin{tabular}{|l|l|}
63.626 \\
64.136
\end{tabular} & $\frac{3.115}{3.84}$ & $\frac{23.911}{24.014}$ & \begin{tabular}{|l|}
31.75 \\
31722 \\
\end{tabular} & & \begin{tabular}{ll|l}
43.832 \\
43.997
\end{tabular} & $\begin{array}{l}0.003 \\
0.003\end{array}$ & 6.533 & $\begin{array}{l}0.005 \\
0.005 \\
\end{array}$ & $\frac{114.5}{115.5}$ & 1.90861 & $\frac{62.5}{63.0}$ & $\frac{10.4}{10.5}$ & 23 & $\frac{1.635}{1640}$ & 0.935 & & 0.039 & $\begin{array}{ll}39.429 \\
391266\end{array}$ & 33.55 \\
\hline & $8 / 26 / 2004$ & $11: 12: 18 \mathrm{AM}$ & 25.636 & 24.394 & 24.944 & 26.024 & 24.696 & 23.73 & 63.579 & 3.773 & 23.994 & \begin{tabular}{|l|}
31.519 \\
\end{tabular} & 3.496 & 43.909 & 0.003 & $\begin{array}{l}6.532 \\
6.505 \\
\end{array}$ & $\begin{array}{l}0.005 \\
0.006\end{array}$ & & 941928 & $\begin{array}{l}63.0 \\
62.5 \\
\end{array}$ & $\frac{10.5}{10.5}$ & \begin{tabular}{|l|l|}
23.9 \\
23.9
\end{tabular} & 1.649 & $\begin{array}{l}0.9351 \\
0.931 \\
\end{array}$ & $\begin{array}{l}0.936 \\
0.933 \\
\end{array}$ & $\begin{array}{l}0.039 \\
0.039 \\
\end{array}$ & $\begin{array}{l}39.126 \\
39.090\end{array}$ & \\
\hline & & 11:13:18 AM & 25.617 & 24.409 & 24.935 & 26.03 & 24.642 & 23.908 & 64.248 & 3.809 & 24.16 & \begin{tabular}{|l|}
31.583 \\
\end{tabular} & 3.522 & 44.093 & & 6.519 & 0.005 & 117.52 & 95861 & 63.1 & 10.5 & 24.0 & 1.657 & & & & & \\
\hline & $8 / 26 / 2004$ & 11:14:18 AM & 25.582 & 24.41 & 24.886 & \begin{tabular}{|l|l|}
25.97 \\
\end{tabular} & 24.577 & 23.912 & 63.931 & 3.775 & 24.281 & 31.667 & 3.51 & 43.602 & 0.003 & 6.504 & 0.00 & 118.52 & 1.97528 & 62.8 & 10.4 & 24. & 1.661 & .931 & 0.934 & 0.039 & 38.767 & \\
\hline & $8 / 26 / 2004$ & 11:15:18 AM & & 24.416 & 24.867 & 25.907 & 24.579 & & & 3.764 & & & & 43.717 & & & & & & & & & & & & & 38.935 & \\
\hline $7 \mathrm{~g}$ & $8 / 26 / 2004$ & 11:16:18 AM & 25.539 & 24.432 & 24.863 & $\begin{array}{ll}25.922 \\
25.029 \\
\end{array}$ & 24.549 & 23.972 & 64.128 & 3.726 & $\begin{array}{l}24.346 \\
\end{array}$ & \begin{tabular}{|l|l|}
31.505 \\
3150 \\
\end{tabular} & 3.531 & 43.511 & 0.003 & 6.516 & 0.0 & 120.5 & 00861 & 63.0 & 10.4 & 24.2 & 1.66 & 0.933 & 0.936 & 0.039 & 38.763 & \\
\hline & 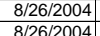 & 11:17:18 AM & $\begin{array}{ll}25.52 \\
25.905 \\
\end{array}$ & 24.438 & 24.843 & $\begin{array}{ll}25.928 \\
25.898 \\
\end{array}$ & 24.5 & 23.906 & \begin{tabular}{|l|l|}
63.794 \\
\end{tabular} & $\begin{array}{l}3.734 \\
3805 \\
\end{array}$ & 24.297 & \begin{tabular}{|l|}
31.569 \\
21302 \\
\end{tabular} & 3.516 & $\begin{array}{r}43.73 \\
4509\end{array}$ & 0.003 & 6.512 & & 121.5 & (1) & 62.7 & 10.4 & 24.1 & . & .932 & 0.936 & 0.039 & 38.853 & \\
\hline & $\begin{array}{l}8 / 26 / 2004 \\
8 / 26 / 2004\end{array}$ & $\begin{array}{l}\text { 11:11:18 AM } \\
1: 19: 18 \mathrm{AM}\end{array}$ & $\frac{25.485}{25.471}$ & $\begin{array}{r}24.443 \\
24.433 \\
\end{array}$ & 24.828 & $\begin{array}{l}25.888 \\
25.878 \\
\end{array}$ & 24.475 & 24.007 & & $\begin{array}{l}3.805 \\
3.777\end{array}$ & $\begin{array}{l}24.211 \\
24.088\end{array}$ & \begin{tabular}{|l|}
$\mid 31.392$ \\
31.904 \\
\end{tabular} & & 44.599 & & $\begin{array}{l}6.474 \\
6.463 \\
\end{array}$ & & & $\begin{array}{l}05194 \\
05861\end{array}$ & $\begin{array}{l}62.6 \\
62.3 \\
\end{array}$ & $\frac{10.6}{10.5}$ & $\frac{24 .}{24 .}$ & & & 0.931 & & $\begin{array}{l}38.631 \\
38.805\end{array}$ & \\
\hline & $8 / 26 / 2004$ & $11: 20: 18 \mathrm{AM}$ & 25.461 & 24.448 & 24.779 & 25.903 & 24.445 & 24.296 & \begin{tabular}{|c|} 
\\
\end{tabular} & 3.871 & 24.488 & \begin{tabular}{|l|l|}
31.236 \\
\end{tabular} & 3.484 & 44.222 & 0.003 & 6.481 & 0.005 & 124.52 & 2.07528 & 62.8 & 10.5 & 24.4 & 1.682 & \begin{tabular}{|c|c|} 
& 0.928 \\
\end{tabular} & 0.934 & 0.038 & 38.277 & \\
\hline & $8 / 26 / 2004$ & 11:21:18 AM & 25.446 & 24.448 & 24.754 & 25.903 & & 24.258 & 63.921 & 3.901 & 24.293 & 31.184 & 3.482 & 44.391 & & 6.472 & & 125.5 & 2.09194 & \begin{tabular}{|l|}
62.8 \\
\end{tabular} & & & 1.674 & & & & & \\
\hline & $8 / 26 / 2004$ & 11:22:18 AM & 25.434 & 24.442 & 24.732 & 25.952 & 24.39 & 24.119 & 63.49 & 3.831 & 24.248 & 31.224 & 3.467 & 43.734 & 0.003 & 6.471 & & 126. & 2.10861 & $\begin{array}{ll}62.4 \\
\end{array}$ & 10.4 & 24.2 & & 0.926 & & & & \\
\hline t & & 11:23:18 AM & 25.414 & & 24.707 & & & & & & & & & & & & & & & & 10.5 & 24. & & & & & & \\
\hline$\frac{87}{88}$ & $\begin{array}{l}8 / 26 / 2004 \\
8262004 \\
\end{array}$ & $\begin{array}{l}11: 24: 18 \mathrm{AM} \\
11.25 .18 \mathrm{AM}\end{array}$ & $\begin{array}{l}25.413 \\
25397 \\
\end{array}$ & \begin{tabular}{|l|}
24.43 \\
24.49 \\
\end{tabular} & $\begin{array}{l}24.72 \\
24.71 \\
\end{array}$ & \begin{tabular}{l|l}
25.98 \\
25.9055
\end{tabular} & & $\begin{array}{l}24.163 \\
24.122\end{array}$ & \begin{tabular}{|l|l|}
63.654 \\
63.654
\end{tabular} & $\begin{array}{l}3.812 \\
3.753 \\
\end{array}$ & $\begin{array}{l}24.287 \\
22.351 \\
\end{array}$ & \begin{tabular}{|l|l|}
31.311 \\
31.95 \\
\end{tabular} & $\begin{array}{r}3.49 \\
3.41\end{array}$ & $\begin{array}{ll}44.009 \\
3.817\end{array}$ & $\overline{003}$ & & & $\begin{array}{l}128.52 \\
1255 \\
\end{array}$ & $\begin{array}{l}2.14194 \\
215961\end{array}$ & $\begin{array}{l}62.5 \\
6.5 \\
6.5\end{array}$ & 10.5 & $\frac{24.2}{24.2}$ & & $\begin{array}{l}0.926 \\
0.256\end{array}$ & $\begin{array}{ll}0.933 \\
0.92\end{array}$ & $\begin{array}{l}0.039 \\
0.039 \\
\end{array}$ & 38.527 & \\
\hline $\begin{array}{ll}88 \\
89 \\
\end{array}$ & $\begin{array}{l}8 / 26 / 2004 \\
8 / 26 / 2004\end{array}$ & $\begin{array}{l}11: 25: 18 \mathrm{AM} \\
1126.18 \mathrm{AM}\end{array}$ & $\begin{array}{l}25.397 \\
25.392 \\
\end{array}$ & $\begin{array}{l}24.429 \\
24.434 \\
\end{array}$ & \begin{tabular}{|l|}
24.71 \\
2472
\end{tabular} & \begin{tabular}{rl|}
25.905 & \\
25.93 &
\end{tabular} & $\begin{array}{l}24.351 \\
24.321 \\
\end{array}$ & \begin{tabular}{l|l}
24.122 \\
24.296
\end{tabular} & \begin{tabular}{|l|l|}
63.654 \\
64.076
\end{tabular} & $\begin{array}{l}3.753 \\
3729 \\
\end{array}$ & 1.351 & \begin{tabular}{|l|}
31.195 \\
31.177 \\
\end{tabular} & $\begin{array}{l}3.461 \\
3.476 \\
\end{array}$ & $\begin{array}{ll}43.817 \\
43.632\end{array}$ & $\frac{0}{0}$ & & & $\frac{129.52}{130.52}$ & $\begin{array}{l}2.15861 \\
2.17528 \\
\end{array}$ & $\begin{array}{ll}62.5 \\
63.2\end{array}$ & 10.4 & 24.2 & 1.671 & $\begin{array}{l}0.926 \\
0.926 \\
\end{array}$ & 0.934 & $\begin{array}{l}0.039 \\
0.38 \\
\end{array}$ & \begin{tabular}{l|l|l}
38.520 \\
38186
\end{tabular} & \\
\hline 90 & 8/26/2004 & \begin{tabular}{|l}
11.27 .10 \\
18 AM
\end{tabular} & 25.3921 & $\frac{24.444}{24.424}$ & $\begin{array}{r}24 . / 72 \\
24.709\end{array}$ & \begin{tabular}{r|r|}
25.933 \\
25.919
\end{tabular} & $\begin{array}{l}24.32 \\
24.28\end{array}$ & $\begin{array}{l}24.296 \\
24.078\end{array}$ & \begin{tabular}{|l|l|}
$64.0 / 61$ \\
63.421
\end{tabular} & 3.729 & 24.5953 & \begin{tabular}{|l|}
31.133 \\
31.056 \\
\end{tabular} & $\frac{3.4 / 6}{3.47}$ & $\begin{array}{l}43.632 \\
43.847 \\
\end{array}$ & $\begin{array}{l}0.003 \\
0.003 \\
\end{array}$ & $\frac{6.649}{6.463}$ & 0006 & $\begin{array}{l}130.52 \\
131.52 \\
\end{array}$ & $\frac{2.1 / 528}{2.19194}$ & $\begin{array}{l}63.0 \\
62.3 \\
\end{array}$ & $\begin{array}{l}\frac{10.4}{10.4} \\
\end{array}$ & \begin{tabular}{|l|}
24.4 \\
24.2
\end{tabular} & $\frac{1.005}{1.670}$ & & $\begin{array}{l}0.935 \\
0.933 \\
\end{array}$ & $\begin{array}{l}0.038 \\
0.039\end{array}$ & $\begin{array}{l}38.186 \\
38.525\end{array}$ & \\
\hline & $8 / 26 / 2004$ & 11:28:18 AM & 25.351 & 24.408 & 24.668 & 25.908 & 24.3 & 24.371 & 63.983 & 3.765 & 24.554 & 31.215 & 3.473 & 43.717 & & 6.467 & & 132.5 & \begin{tabular}{|l|l|}
2.20861 \\
\end{tabular} & \begin{tabular}{|l|}
62.9 \\
\end{tabular} & 10.4 & \begin{tabular}{|l|l|}
24.5 \\
\end{tabular} & & 0.926 & 0.935 & & & \\
\hline & 8/26/2004 & \begin{tabular}{|l|l}
$11: 29: 18 ~ A M$ \\
\end{tabular} & 25.36 & 24.422 & 24.688 & 25.888 & 24.314 & 24.315 & 63.757 & 3.808 & 24.488 & 31.01 & 3.458 & 43.579 & & 6.459 & & & & \begin{tabular}{|l|}
62.6 \\
\end{tabular} & 10.4 & 24.4 & 1.68 & 0.925 & & & & \\
\hline (2) & 8/26/2004 & 11:30:18 AM & 25.349 & 24.402 & 24.657 & 25.887 & 24.384 & 24.445 & 63.981 & & & 31.221 & & 43.59 & & & & 134.5 & 2.24194 & 62.9 & 10.4 & 24.6 & & & 0.936 & & & \\
\hline 94 & 8/26/2004 & \begin{tabular}{|l|}
$11: 31: 18 \mathrm{AM}$ \\
\end{tabular} & 25.349 & 24.402 & 24.642 & 25.962 & 24.449 & 24.285 & 63.531 & 3.751 & & \begin{tabular}{|l|l|}
31.076 \\
\end{tabular} & & $\begin{array}{l}43.665 \\
42.805\end{array}$ & .003 & 6.455 & & 135.52 & \begin{tabular}{|l|l|}
2.25861 \\
\end{tabular} & \begin{tabular}{ll|l}
62.4 \\
6.9
\end{tabular} & 10.4 & & 1.684 & 0.924 & 0.934 & 0.038 & 38.226 & \\
\hline 95 & 8/26/2004 & 11:32:18 AM & $\begin{array}{l}25.341 \\
25.253 \\
\end{array}$ & 24.403 & 24.638 & \begin{tabular}{|l|l|}
26.028 \\
2608
\end{tabular} & & \begin{tabular}{|l|l|l|l}
24.449 \\
\end{tabular} & \begin{tabular}{|l|l|}
63.817 \\
6.803
\end{tabular} & $\begin{array}{l}3.677 \\
3752 \\
\end{array}$ & & \begin{tabular}{|r|}
30.88 \\
31.737 \\
\end{tabular} & $\begin{array}{l}3.45 \\
3.47 \\
\end{array}$ & $\begin{array}{l}43.805 \\
43.712\end{array}$ & & $\begin{array}{r}6.46 \\
6.41 \\
6\end{array}$ & & & & \begin{tabular}{l|l}
62.7 \\
6.7
\end{tabular} & 10.4 & \begin{tabular}{|l|l|}
24.6 \\
2.5
\end{tabular} & & & 0.934 & & $\begin{array}{ll}37.954 \\
39103\end{array}$ & \\
\hline$\frac{96}{97}$ & 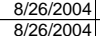 & 11:33:18 AM & 25.3534 & 24.436 & 24.666 & 26.081 & 24.663 & $\begin{array}{r}24.4 \\
24393\end{array}$ & 63.803 & $\begin{array}{l}3.752 \\
3.682 \\
\end{array}$ & $\frac{24.611}{24.695}$ & $\begin{array}{l}31.273 \\
3095 \\
\end{array}$ & 3.47 & $\begin{array}{l}43.713 \\
43.924 \\
\end{array}$ & 0.003 & $\begin{array}{l}6.461 \\
6.452 \\
\end{array}$ & & $\begin{array}{l}137.52 \\
13852 \\
\end{array}$ & $\begin{array}{l}2.29194 \\
230861 \\
\end{array}$ & 62.7 & $\frac{10.4}{105}$ & 24.5 & 1.696 & \begin{tabular}{ll|l}
0.925 \\
$n .021$
\end{tabular} & 0.934 & $\begin{array}{l}0.038 \\
0.038\end{array}$ & \begin{tabular}{ll|l}
38.103 \\
38009
\end{tabular} & 32.43 \\
\hline 98 & $8 / 26 / 2004$ & \begin{tabular}{|l}
$11.35: 18 \mathrm{AM}$ \\
\end{tabular} & 25.342 & $\frac{24.471}{24.45}$ & 24.68 & $\frac{26.141}{26.21}$ & 24.8 & 24.447 & \begin{tabular}{|l|l|}
63.813 \\
\end{tabular} & 3.002 & $\frac{4.055}{24.431}$ & \begin{tabular}{|l|}
31.053 \\
31.55
\end{tabular} & 3.453 & $\begin{array}{l}43.924 \\
43.577\end{array}$ & $\begin{array}{l}0.003 \\
0.003 \\
\end{array}$ & & 0.005 & $\frac{138.52}{139.52}$ & $\frac{2.30861}{2.32528}$ & $\begin{array}{l}0 . .0 \\
62.7 \\
\end{array}$ & $\frac{10.5}{10.4}$ & $\begin{array}{l}24.5 \\
24.4 \\
\end{array}$ & 1.05: & & $\begin{array}{l}0.5032 \\
0.932\end{array}$ & $\begin{array}{ll}0.038 \\
0.038 \\
\end{array}$ & $\begin{array}{ll}38.000 \\
38.133\end{array}$ & \\
\hline & & 11:36:18 AM & & 24.463 & 24.673 & & & 24.47 & & & & 31.198 & & & & & & 140. & & 62.4 & & 24.6 & & & & & & \\
\hline & $8 / 26 / 2004$ & 11:37:18 AM & 25.334 & 24.486 & 24.672 & 26.347 & 24. & 24.702 & 64.014 & 3.766 & 24.86 & \begin{tabular}{|l|l|}
31.068 \\
\end{tabular} & & 43.607 & 0 & 6.442 & & 141.5 & 2.35861 & 62.9 & 10.4 & \begin{tabular}{|l|}
24.8 \\
\end{tabular} & 1.70 & 0.922 & 0.931 & 0.038 & 37.562 & \\
\hline & $8 / 26 / 2004$ & 11:38: & 25.316 & 24.504 & 24.674 & 26.374 & & 24.57 & 63.707 & & & & & & & & & & & 62.6 & 10.5 & 24.7 & & & & & 37.651 & \\
\hline & 8/26/2004 & 11:39:18 AM & 25.323 & 24.531 & 24.676 & & & 24.485 & 63.577 & 3.713 & & \begin{tabular}{|l|}
30.784 \\
\end{tabular} & & 43.473 & & 444 & & 143.5 & & 62.5 & 10.4 & 24.7 & & 0.923 & 0.931 & 0.038 & 37.647 & \\
\hline & 8/26/2004 & \begin{tabular}{|l}
$11: 40: 18$ AM \\
$11 \cdot 11 \cdot 18 \mathrm{AM}$
\end{tabular} & 25.311 & 24.544 & 24.654 & 26.409 & 24.5 & 24.512 & 63.513 & 3.773 & 24.747 & \begin{tabular}{|l|}
30.874 \\
21.055 \\
\end{tabular} & & 43.256 & & 6.429 & & 144.52 & $\begin{array}{l}2.40861 \\
\end{array}$ & \begin{tabular}{|l|}
62.4 \\
\end{tabular} & $\frac{10.3}{101}$ & 24.6 & $1.6 \mathrm{~s}$ & 0.920 & 0.929 & 038 & 37.736 & \\
\hline & & & & $\begin{aligned} & 24.556 \\
& 24.57\end{aligned}$ & & & & & & & & \begin{tabular}{|l|}
31.0565 \\
30.969 \\
\end{tabular} & & & & & & & & & & & & & & & & \\
\hline & $8 / 26 / 2004$ & \begin{tabular}{|l|l}
$11: 43: 18 \mathrm{AM}$ \\
\end{tabular} & 25.305 & $\begin{array}{l}24.0583 \\
24.583\end{array}$ & 24.623 & 2.014 & 24.8 & 24.6043 & \begin{tabular}{|l|l|}
63.774 \\
63.774
\end{tabular} & 3.005 & 24.741 & \begin{tabular}{|l|}
30.917 \\
\end{tabular} & $\begin{array}{l}0.4 \\
3.43 \\
\end{array}$ & $\begin{array}{l}43.402 \\
43.607\end{array}$ & & & & 140 & $\frac{2.441}{2.458}$ & $\begin{array}{l}0.4 \\
62.6 \\
\end{array}$ & 10. & 24.7 & & & & & 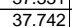 & \\
\hline & $8 / 26 / 2004$ & 11:44:18 AM & 25.316 & 24.604 & 24.628 & 26.199 & 24. & 348 & 63.987 & 3.782 & 25.1 & \begin{tabular}{|l|l|}
30.877 \\
\end{tabular} & & 43.678 & & 6.448 & & 14 & 2.47 & $\begin{array}{ll}62.9 \\
\end{array}$ & 10.4 & 25.6 & & & 0.6 & 0.037 & 37.350 & \\
\hline & & 11:45:18 & 25.307 & 24.605 & 24.63 & 26.145 & & 24.644 & 63.61 & 3.753 & & & & 43.5 & & 6.43 & & 14 & 2.4 & 62.5 & 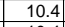 & & & & & & & \\
\hline & & 11:46:18 & & 24.621 & 24.645 & & & & & & & & & & & & & & & & & 24.9 & & & & & 37.278 & \\
\hline & $\begin{array}{l}8 / 26 / 2004 \\
8 / 26 / 204\end{array}$ & 11:47: & $\begin{array}{l}25.293 \\
25209 \\
2529\end{array}$ & 24.631 & $\begin{array}{l}24.666 \\
24.671\end{array}$ & 26.176 & & & 62.864 & & & 29.5 & & 45.968 & & & & 151 & 2.52528 & 61.7 & in & 24.8 & 1.11 & 0.907 & & & $\begin{array}{l}36.892 \\
27.275 \\
\end{array}$ & \\
\hline & 8/26/2004 & \begin{tabular}{|l|}
$11: 48: 1$ \\
$11 \cdot 49 \cdot 1$
\end{tabular} & $\begin{array}{ll}25.298 \\
252098\end{array}$ & 24.626 & \begin{tabular}{|l|}
24.671 \\
24.66
\end{tabular} & $\begin{array}{ll}26.086 \\
2607\end{array}$ & & 24.659 & \begin{tabular}{|l|}
62.681 \\
63.077
\end{tabular} & 4.092 & & 29.7 & & $\begin{array}{l}46.018 \\
4572 \\
\end{array}$ & & 6 & & $\begin{array}{l}152.52 \\
153.52\end{array}$ & \begin{tabular}{|l|l|}
2 & 2.54194 \\
2 & 255861
\end{tabular} & 61.6 & & 24.5 & & \begin{tabular}{|l|l|}
0.907 & \\
0.908 &
\end{tabular} & 0.916 & 0.037 & 37.375 & \\
\hline & $\begin{array}{l}8 / 26 / 2004 \\
8 / 26 / 2004\end{array}$ & $\begin{array}{l}11.49 \\
11: 50: \\
\end{array}$ & $\begin{array}{ll}25.298 \\
25.313\end{array}$ & \begin{tabular}{|l|}
24.636 \\
24.631
\end{tabular} & 24.6676 & $\begin{array}{ll}26.07 \\
26.105\end{array}$ & & & & & & & & $\frac{45}{456}$ & & & & & & & & 24 & & & & & $\begin{array}{l}37.053 \\
36767 \\
\end{array}$ & \\
\hline & $8 / 26 / 2004$ & & & 24.631 & 24.676 & & & & & & & & & & & & & & & & & & & & & & & \\
\hline & $8 / 26 / 2004$ & $11: 52: 18 \mathrm{AM}$ & 25.321 & 24.624 & 24.649 & 26.079 & & 24.945 & 62.992 & 4.053 & 24 & \begin{tabular}{|l|}
29.768 \\
\end{tabular} & & $45.7 .7-3$ & & & & 156 & 2.60861 & 61.9 & 1 & 24 & & 907 & & & & \\
\hline & $8 / \angle 6 / \angle 004$ & $11: 53$ & 25.346 & 24.634 & 24.67. & 26.143 & & 24.93 & & & & & & 45.4 & & & & & & & & & & & & & & \\
\hline & & & t5.335. & & & & & & & & & & & & & & & & & & & & & & & & & \\
\hline & & & & & & & & & & & & & & & & & & & & & & & & & & & & \\
\hline & & & & 24.044 & & & & & & & & & & & & & & & & & & & & & & & & \\
\hline & $8 / 262004$ & & 25.5 & 2463 & & & & & & & & & & & & & & & & & & & & & & & 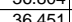 & \\
\hline & & & & & & & & & & & & & & & & & & & & & & & & & & & & \\
\hline & & & 25.349 & & & & & & & & & & & & & & & & & & & & & & & & & \\
\hline & & & & & & & & & & & & & & & & & & & & & & & & & & & & \\
\hline & & & 25.35 & 24.6 & 24.6 & & & & & & & & & & & & & & & & & & & & & & & \\
\hline & & & & 24. & & & & & & & & & & & & & & & & & & & & & & & & \\
\hline & $8 / 26 / 2$ & 12:04:18 P & & 24.627 & 24.69 & 26.267 & & 25 . 25 & & & & 29.7 & & & & & & & & & & & & & & & & \\
\hline & $8 / 26 / 20$ & & & 24. & 24. & & & & & & & & & & & & & & & & & & & & & & & \\
\hline & $8 / 26 / 2$ & $12: 0$ & & 24. & 24.7 & & & & & & & & & & & & & & & & & & & & & & & \\
\hline & 8/26/2 & & 25.354 & 24.612 & & & & & & & & & & & & & & & & & & & & & & & & \\
\hline & $8 / 26$ & & & & & & & & & & & & & & & & & & & & & & & & & & & \\
\hline & $8 / 26 / 2$ & & & & & & & & & & & & & & & & & & & & & & & & & & & \\
\hline & & 1.210.18 & & 24. & $\begin{array}{ll}20.691 \\
2097\end{array}$ & 2. & & 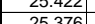 & & 4.0 & & & & & & & & & & & & & & & & & & \\
\hline & (1) & 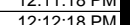 & & 24.01 & 24.7 & & & 年 & & $4.0>-3$ & & 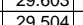 & & & & & & & & & & & & & & & & \\
\hline & 8 & 12.13.18 & & $\frac{24.6}{246}-8$ & 2469 & 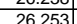 & & 25.098 & & 400 & & & & & & & & & & & & & & & & & & \\
\hline & $8 / 26 / 2004$ & 12:14:18 & & & & $\frac{2.62}{26.2}>0$ & & & & & & & & & & & & & & & & & & & & & & \\
\hline & 8/26/2004 & 12:15:18 $\mathrm{F}$ & & & & & & 25.42 & & 3.98 & & & & $45.2 .2>3$ & & 6.314 & & & & & & & & & & & & \\
\hline & & & & & & & & & & & & & & & & & & & & & & & & & & & & \\
\hline & & $12: 17: 18 \mathrm{P}$ & & & & .279 & & & 891 & 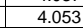 & & 29.476 & & t5.24 & & 6.298 & & & & & & & & & & $0 x$ & 5.955 & \\
\hline
\end{tabular}


WSRC-TR-2005-00105, REVISION 0

SRNL-RPP-2005-00012, REVISION 0

CAMPAIGN IV NEW GKN ACID CLEAN AND CAUSTIC RINSE - CONT.

\begin{tabular}{|c|c|c|c|c|c|c|c|c|c|c|c|c|c|c|c|c|c|c|c|c|c|c|c|c|c|c|c|c|}
\hline & & & & & & & & & & & & & & & & & & & & & & & & & & & & \\
\hline & A & \begin{tabular}{|l|l}
$\mathrm{B}$ \\
\end{tabular} & $\mathrm{D}$ & $E$ & $\mathrm{~F}$ & $G^{\prime}$ & $\mathrm{H}$ & $\mathrm{J}$ & $\mathrm{K}$ & $\mathrm{L}$ & $\mathrm{M}$ & $\mathrm{N}$ & $\mathrm{O}$ & $\mathrm{Q}$ & $\mathrm{R}$ & \begin{tabular}{l|l} 
\\
\end{tabular} & $\begin{array}{lll} \\
\end{array}$ & $\mathrm{v}$ & w & $x$ & $\mathrm{Y}$ & $\mathrm{z}$ & $\mathrm{AA}$ & $A B$ & $\mathrm{AC}$ & $A D$ & $\mathrm{AE}$ & AF \\
\hline 14 & $8 / 26 / 2004$ & \begin{tabular}{|l|l|}
$12: 18: 18 \mathrm{PM}$ \\
\end{tabular} & 25.361 & 24.619 & 24.719 & 26.264 & 24.636 & 25.515 & 63.206 & 3.943 & 25.397 & 29.403 & 3.282 & $\frac{6}{45.325}$ & 0.003 & 6.31 & 0.006 & 182.52 & \begin{tabular}{|l|}
3.04194 \\
\end{tabular} & \begin{tabular}{|l|}
62.1 \\
\end{tabular} & 10.8 & & & & 0.911 & 0.036 & 35.770 & \\
\hline & $8 / 26 / 2004$ & & & 24.615 & \begin{tabular}{|l|l|} 
& 24.72 \\
\end{tabular} & 26.295 & 24.697 & 25.339 & \begin{tabular}{|l|l|}
62.746 \\
\end{tabular} & 4.009 & 25.145 & \begin{tabular}{|l|}
29.496 \\
\end{tabular} & & & 0.003 & $\begin{array}{l}6.301 \\
\end{array}$ & 0.005 & & 3.05861 & \begin{tabular}{|l|}
61.6 \\
\end{tabular} & 10.8 & & & & 0.909 & 0.036 & 36.020 & \\
\hline$\frac{143}{144}$ & \begin{tabular}{|l|l|}
$8 / 26 / 2004$ \\
$8 / 26204$
\end{tabular} & \begin{tabular}{|l|}
$12: 20: 18 \mathrm{PM}$ \\
\end{tabular} & $\begin{array}{l}25.346 \\
25356\end{array}$ & 24.609 & \begin{tabular}{|l|}
24.699 \\
2.719
\end{tabular} & \begin{tabular}{|l|}
26.269 \\
2.224
\end{tabular} & 24.636 & \begin{tabular}{l|l|l|}
25.675 \\
\end{tabular} & $\begin{array}{ll}63.255 \\
6.2305\end{array}$ & 4.079 & $\begin{array}{l}25.401 \\
25305\end{array}$ & \begin{tabular}{|l|l|}
29.476 \\
29218
\end{tabular} & 3.25 & 45.121 & 0.003 & $\begin{array}{l}6.296 \\
.201\end{array}$ & 0.005 & 184.52 & \begin{tabular}{|l|l|}
3.07528 \\
\end{tabular} & 62.1 & 10.7 & | 20.5 & & 0.901 & 0.909 & 0.036 & \begin{tabular}{|l|l|}
35.596 \\
\end{tabular} & \\
\hline & $8 / 26 / 2004$ & 12:21:18 PM & & & & & 24.581 & & 63.285 & & & \begin{tabular}{|l|}
29.218 \\
32636 \\
\end{tabular} & & 45.309 & 0.003 & & 0.005 & & \begin{tabular}{|l|l|}
3.09194 \\
\end{tabular} & 62.2 & 10.8 & 25.5 & & 0.901 & 0.908 & 0.036 & 35.637 & \\
\hline & 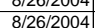 & $\begin{array}{l}\text { 1::00:04 PM } \\
1: 01: 04 \mathrm{PM}\end{array}$ & $\begin{array}{l}25.394 \\
25.368\end{array}$ & $\begin{array}{l}24.547 \\
24.546 \\
\end{array}$ & $\frac{24.717}{24701}$ & 26.147 & & $\frac{21.403}{2.138}$ & \begin{tabular}{rl|}
63.62 \\
63.249 \\
\end{tabular} & $\begin{array}{l}3.976 \\
3905 \\
\end{array}$ & & \begin{tabular}{|l|l|}
33.636 \\
22754 \\
\end{tabular} & & & & & & & & 62.5 & & & & $\begin{array}{ll}0.960 \\
0.950 \\
\end{array}$ & 0.968 & 0.045 & 44.980 & 38.28 \\
\hline$\frac{14}{147}$ & $8 / 26 / 2004$ & 1:02:04 PM & 25.353 & $\begin{array}{l}24.546 \\
24.536\end{array}$ & $\begin{array}{l}24.701 \\
24.651 \\
\end{array}$ & \begin{tabular}{|l|}
26.181 \\
26.231
\end{tabular} & 24.593 & $\begin{array}{r}21.38 \\
2435 \\
\end{array}$ & 63.249 & $\begin{array}{l}3.905 \\
3.719\end{array}$ & $\begin{array}{l}21.826 \\
-11115 \\
\end{array}$ & $\begin{array}{r}33.754 \\
58.05 \\
\end{array}$ & & & 0.003 & $\begin{array}{r}6.696 \\
-0.01 \\
\end{array}$ & & & $\begin{array}{l}3.75472 \\
377139 \\
\end{array}$ & $\frac{62.1}{642}$ & $\frac{10.7}{94} \times-3$ & & & $\begin{array}{l}959 \\
001 \\
\end{array}$ & $\begin{array}{r}0.967 \\
-0.001\end{array}$ & 0.045 & $\begin{array}{l}44.751 \\
-2.191\end{array}$ & 38.08 \\
\hline 148 & $8 / 26 / 2004$ & 1:03:04 PM & 25.353 & 24.536 & 24.626 & 26.276 & 24.763 & 2.231 & 65.469 & 3.74 & $\begin{array}{c}1.115 \\
-1.413\end{array}$ & \begin{tabular}{|l|}
58.449 \\
58.449
\end{tabular} & 20.549 & $\begin{array}{l}39.295 \\
39.162\end{array}$ & 0.003 & -0.011 & 0.003 & 227.28 & \begin{tabular}{|l|l|}
.778006 \\
.7880
\end{tabular} & $\begin{array}{l}04.2 \\
64.3 \\
\end{array}$ & 9.4 & 0.4 & 0.046 & & $\begin{array}{l}-0.002 \\
-0.002 \\
\end{array}$ & $\begin{array}{l}-0.002 \\
-0.004 \\
\end{array}$ & $\begin{array}{l}-2.191 \\
-3.891\end{array}$ & $\begin{array}{l}-1.86 \\
-3.31\end{array}$ \\
\hline$\overline{149}$ & & & & & 24.613 & 26.378 & 24.815 & & \begin{tabular}{|l|l|}
65.623 \\
\end{tabular} & & & \begin{tabular}{|l|}
58.438 \\
\end{tabular} & 105.615 & 38.874 & & \begin{tabular}{c|c|c|}
-0.012 \\
\end{tabular} & & & 3.80472 & \begin{tabular}{|l|}
64.5 \\
\end{tabular} & & & & & & & & $\begin{array}{l}-3.31 \\
-2.76\end{array}$ \\
\hline 150 & $8 / 26 / 2004$ & 1:05:04 PM & 25.341 & 24.569 & 24.574 & 26.399 & 24.906 & 7.563 & 64.64 & 3.73 & 4.311 & 52.199 & 30.251 & 40.636 & 1.205 & -0.012 & 0.003 & 229.2 & 3.82139 & \begin{tabular}{|l|}
63.5 \\
\end{tabular} & 9.7 & & 0.409 & & -0.002 & 0.000 & & $\begin{array}{l}-2.76 \\
-0.25 \\
\end{array}$ \\
\hline & & & & & & 26.456 & & & & & & & & & & & & & & & & & & & & & & \\
\hline 152 & & 1:07:04 PM & 25.279 & 24.597 & $\frac{24.617}{24}$ & 26.482 & & 20.7 & 63.351 & & & 34.009 & & 44.958 & & 6.73 & & & & 62.2 & & & & .964 & 974 & & $\frac{704}{404}>2$ & \\
\hline$\frac{15}{15}$ & & 1:08:04 PM & & 24.619 & 24.629 & 26.499 & & 21.173 & 63.539 & 3.949 & 21.516 & 754 & & & & & & & 3.87139 & 62.4 & & & & & & & & \\
\hline$\frac{15}{155}$ & $8 / 26 / 2004$ & 1:09:04 PM & 25.281 & & 24.624 & 26.439 & & 21.255 & 63.483 & 3.923 & & & & & & & & 2323 & 3.88806 & 62.1 & & & & & 0.970 & & & \\
\hline 155 & $8 / 26 / 2004$ & 1:10:04 PM & 25.298 & 24.646 & 24.646 & 26.491 & 24.963 & 21.145 & 63.05 & 3.859 & 21 & & 2.74 & 444.898 & 0.003 & 694 & & 234.2 & 3.90472 & 61.9 & 10 & 21.4 & 1.474 & .958 & 0.968 & & 45.261 & \\
\hline$\frac{156}{15}$ & $8 / 26 / 2004$ & 1:11:04 PM & 25.303 & 24.666 & 24.666 & 26.531 & 24.913 & 21.311 & 63.32 & 3.893 & 21.701 & 33.503 & & 44.975 & 0.003 & 6.686 & & 235.2 & 3.92139 & 62.2 & 10 & 21.5 & 1.48. & .957 & 0.966 & 0.045 & 44.930 & \\
\hline$\frac{15}{158}$ & 8/26/2004 & 1:12:04 PM & 25.304 & 24.672 & 24.657 & 26.462 & 24.839 & 21.419 & 63.343 & 3.874 & 21.896 & 33.491 & & 44.981 & & 6.678 & & 236.2 & 3.93806 & 62.2 & & & 1.49 & .956 & 0.965 & & 44.573 & \\
\hline$\frac{15}{15}$ & 8/26/2004 & 1:13:04 PM & 25.299 & & 24.667 & 26.437 & 24.844 & 21.508 & 63.515 & 3.916 & 21.952 & 33.523 & & 44.748 & & 6.677 & & 237. & & 62.4 & 10. & & & & & & & \\
\hline$\frac{15 s}{160}$ & $8 / 26 / 2004$ & 1:14:04 PM & 25.311 & $\begin{array}{r}24.704 \\
2.701\end{array}$ & 24.658 & 26.413 & 24.8 & 21.365 & 63.003 & $\begin{array}{l}3.921 \\
2.092\end{array}$ & & $\begin{array}{l}33.256 \\
2.27\end{array}$ & & 44.931 & & 6.67 & & & 97139 & 61.9 & & & & & 0.964 & 0.045 & 44.898 & \\
\hline & $8 / 26 / 20404$ & $\begin{array}{l}1: 15.04 \mathrm{PM} \\
1.0401\end{array}$ & $\frac{25.32}{25.325}$ & 24.704 & 24.6533 & 26.448 & 24.84 & $\frac{21.511}{21.72}$ & 63.2266 & 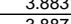 & 22.052 & $\frac{187}{205}$ & & 44.1899 & & & & $\frac{25.42}{2102}$ & . & 6.1 & & & & & & & 44.251 & \\
\hline & 8 & 1.17.04 PM & 25351 & 24.734 & 24684 & 26.4609 & 24.048 & 2170 & (63.578 & 年. & & & & (44.010 & & & & 240.45 & (1) & & & & & & & & & \\
\hline 100 & $8 / 26 / 2004$ & 1:18:04 PM & 25.34 & 24.724 & \begin{tabular}{|l|l|} 
& 24.673 \\
\end{tabular} & $|26.463|$ & 24.865 & $\frac{21.191}{212.861}$ & $\mid$\begin{tabular}{|c|}
63.707 \\
6370
\end{tabular} & 3.966 & 22.218 & \begin{tabular}{|l|}
33.335 \\
\end{tabular} & 2.698 & 44.931 & 0.003 & 6.052 & 0.006 & 2428 & 4.03806 & 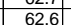 & 10.7 & $\frac{21}{22}$ & 1520 & 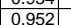 & 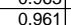 & $\frac{0.044}{0.04}$ & 43.610 & \\
\hline & $8 / 26 / 200$ & 1:19:04 PM & $\begin{array}{ll}25.34 \\
\end{array}$ & 24.728 & 24.673 & 26.463 & 24.86 & 21.716 & 63.459 & 3.899 & & \begin{tabular}{|l|l|}
33.366 \\
\end{tabular} & 2.707 & 44.821 & & & & 243.28 & \begin{tabular}{|l|l|l|l|}
4.05472 \\
\end{tabular} & \begin{tabular}{|l|l|}
62.3 \\
\end{tabular} & $10.7 \mathrm{r}>\mathrm{C}$ & & \begin{tabular}{|c|c|}
1.510 \\
\end{tabular} & \begin{tabular}{|c|c|}
0.954 \\
\end{tabular} & 0.963 & & $\begin{array}{ll}43.949 \\
\end{array}$ & \\
\hline & 8/26/2004 & 1:20:04 PM & 25.35 & 24.733 & 24.683 & 26.493 & 24.8 & 21.658 & 63.27 & 3.87 & 22.054 & \begin{tabular}{|l|}
33.303 \\
\end{tabular} & & 44.921 & 0.003 & 6.665 & & 244.28 & 4.07139 & 62.1 & 10. & 21.9 & 1.50 & 0.954 & 0.963 & 0.044 & & \\
\hline & 8/26/2004 & 1:21:04 PM & 25.345 & 24.743 & 24.688 & 26.513 & & 21.797 & \begin{tabular}{|l|l|}
63.396 \\
\end{tabular} & 3.968 & & 32.984 & & 44.768 & & 656 & & & 08806 & 62.3 & & & & & & & 43.986 & \\
\hline & 80126212004 & 1:22:04 PM & 25.345 & 24.754 & 24.6933 & 26.513 & & 22.058 & 63.977 & 3.987 & & 268 & & $44 . / 23$ & & 6.652 & & & 4.10472 & 62.9 & & & . 1.52 & 1.952 & & & 43.356 & \\
\hline & 8012622004 & $1.23 .04 \mathrm{~F}$ & 25.341 & 24.154 & 24.709 & 26.599 & $24.8 \mathrm{~s}$ & 22.021 & 63.678 & 3.916 & 2.497 & & & 44.852 & & & & 2 & 4.12139 & 62.6 & & & & & 05050 & & 43.156 & \\
\hline & & $\begin{array}{l}1.24 .04 \mathrm{PM} \\
1.2501\end{array}$ & 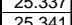 & 24.155 & $\frac{24.6969}{24.679}$ & 20.045 & & $\frac{21.851}{2072}$ & \begin{tabular}{|c|c|}
6.300 \\
6.137
\end{tabular} & & & & & & & & & & 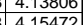 & & & & & & & & & \\
\hline$\frac{17}{171}$ & $\frac{1<1<1<04}{8 / 26 / 2004}$ & 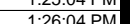 & (25.34t & 24773 & 24675 & 20.4549 & & 21.012 & $\mid$ & $\frac{3.091}{3.95}$ & & $\frac{5}{3}$ & & (44.950 & & & & $\frac{249}{250}>-1$ & & & & & & & 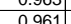 & & (43.910 & \\
\hline & $8 / 26 / 2$ & $\frac{1.20 .04 \mathrm{~F}}{1: 27: 04 \mathrm{~F}}$ & 25.347 & $\frac{24.16}{24.76}$ & 24.665 & $\frac{20.45}{26.48}$ & & 21.0544 & \begin{tabular}{|l|l|}
63.544 \\
\end{tabular} & 3.871 & 2.298 & & & 44.014 & & & & 251 & & & & & & & & & & \\
\hline & $8 / 26 / 2004$ & 1:28:04 PM & 25.357 & 24.76 & 24.66 & $\frac{26.465}{26}$ & & 21.658 & $\mid 63.056$ & 3.872 & 22.138 & \begin{tabular}{|l|l|}
33.039 \\
\end{tabular} & & 44.8 & 0.003 & 6.647 & & 252.28 & 4.20472 & $\frac{6.9}{61.9}$ & & & 1.5 & 952 & 0.9 & & 43.875 & \\
\hline & $8 / 26 / 2004$ & & & 24.776 & 24.696 & 26.495 & & & & & & $\mid$\begin{tabular}{|r|}
$\mid 33.19$ \\
\end{tabular} & & 44.614 & & & & & & & & & & & & & & \\
\hline & $8 / 26 / 2004$ & & 25.352 & 24.77 & 24.68 & 26.425 & & 21.7 & $|63.084|$ & & & & & 44.681 & & & & 254 & 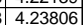 & $\frac{62.0}{62.0}$ & & & & & & & & \\
\hline & 8/26/2004 & 1:31:04 PM & & 24.764 & \begin{tabular}{|l|l|l|}
24.688 \\
\end{tabular} & & & 22.06 & 63.5 & 3.919 & 22.419 & & & 44.362 & & & & & 472 & 62.4 & & & & & & & 43134 & \\
\hline & $8 / 26 / 2004$ & 1:32:04 PM & 25.35 & 24.774 & 24.698 & 26.428 & 24.7 & 21.923 & \begin{tabular}{|l|}
63.378 \\
\end{tabular} & 3.874 & & 32.97 & & 44.75 & & & & 256. & .27139 & & & & & & & & & \\
\hline & $8 / 26 / 2$ & & 25.345 & 24.768 & \begin{tabular}{|l|}
24.698 \\
\end{tabular} & 26.438 & & \begin{tabular}{|l|l|}
22.062 \\
\end{tabular} & 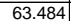 & 3. & 22.536 & & & 44.635 & & & & & & & & & 1.53 & & & & 42.975 & \\
\hline & $8 / 26 / 2$ & $1: 34: 04 \mathrm{PM}$ & 25.344 & 24.772 & 24.692 & $\begin{array}{l}26.422 \\
\end{array}$ & 24.8 & 22.085 & 63.55 & 3.867 & 22.502 & \begin{tabular}{|l|}
33.002 \\
\end{tabular} & & & & & & 258 & & & & & & & & & & \\
\hline & $8 / 26 / 2$ & 1:35:04 & 25.343 & 24.762 & 24.671 & 26.471 & 24. & 22.077 & \begin{tabular}{|l|l|}
63.527 \\
\end{tabular} & 3. & 22.257 & & & $\begin{array}{l}44.593 \\
\end{array}$ & & & & $25 \mathrm{c}$ & & 62.4 & & & & & & & 43.193 & \\
\hline & 8/26/2004 & 1:36:04 & 25.354 & 24.76 & 24.662 & 20.501 & & 22.209 & & & & & & 44.602 & & & & & & 02.1 & & & & & & & & \\
\hline & & & & & & & & & & & 22.499 & & & 44.499 & & & & & & & & & & & & & & \\
\hline & $8 / 26622004$ & & $\frac{25.359}{25364}$ & 24.782 & 24.679 & 26.6017 & & & & & & & & 44 & & & & & & & & 20.4 & & & & & & \\
\hline & & & $\frac{23.304}{25254}$ & & 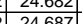 & & & & & & & & & & & & & & & & & & & & & & & \\
\hline & 8 & & $\frac{25.354}{25354}$ & 24.7 & 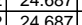 & $\begin{array}{l}2.0 .047 \\
26587\end{array}$ & & & & & & & & (44.4535 & & & & & & & & 20 & & & & & 42.900 & \\
\hline & $8 / 26 / 2004$ & & 25355 & 24.77 & 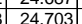 & & & & & & & & & & & & & & & & & & & & & & & \\
\hline 188 & $8 / 26 / 2004$ & 1:43:04 PM & 25.35 & & \begin{tabular}{|l|l|}
24.703 \\
\end{tabular} & 26.608 & & 22.0 & 63. & 3.8 & & & & 44.2 & & & & & & & 10.5 & & & & & & & \\
\hline & $8 / 26 / 2004$ & & & & & & & & & & & & & & & & & & & & & & & & & & & \\
\hline & & & 25.346 & 24.7 & 24.6 & 26.724 & & & \begin{tabular}{|l|l|l|l|} 
\\
\end{tabular} & 3.828 & & & & & & & & & & & & & & & & & & \\
\hline & & & & & 24.6 & & & & & & & & & 44. & & & & & & & & & & & & & & \\
\hline & $8 / 26 /$ & & 25.357 & 24.8 & 24.686 & & & 22. & & 3. & & & & 44.5 & & & & & & & & & & & & & & \\
\hline & $8 / 26 /$ & & 25.357 & 24.7 & \begin{tabular}{|l|l|}
24.67 \\
\end{tabular} & 26 & & & & & & & & & & & & & & & & & & & & & & \\
\hline & $8 / 26 / 2$ & 1:49:04 F & 25.369 & $\begin{array}{l}24.807 \\
\end{array}$ & 24.702 & 26.752 & & \begin{tabular}{l|l|l|}
22.776 \\
\end{tabular} & \begin{tabular}{|l|}
64.219 \\
\end{tabular} & & 22.9 & & & 44.391 & & & & & & & & 22 & & & & & & \\
\hline & $8 / 26$ & & 25.364 & 24.792 & 24.707 & 26.707 & & 22.429 & \begin{tabular}{|l|l|}
63.264 \\
\end{tabular} & 3.8 & 22.84 & & & 44. & & & & & 4.57139 & & & & & & & & & \\
\hline & & & & & 24. & & & 22.4 & & & & & & & & & & & & & & & & & & & & \\
\hline & & & & & 24. & & & 22. & & & & & & & & & & & & & & & & & & & & \\
\hline & & & 20.3 .3$. & & & & & & & & & & & & & & & & & & & & & & & & & \\
\hline & & & the & & 24.68 & & & & 63.5 & & & & & 44.2 & & & & & & & & & & & & & & \\
\hline & 0 & & 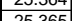 & 24.06 & 2.1000 & & & 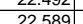 & & & & & & & & & & & & & & & & & & & & \\
\hline & 8 & & & 24.89 & & & & & & & & & & & & & & & & & & & & & & & & \\
\hline 203 & & & 25.36 & 24.80 & 24.4 & & & 22.6 & & & & & & 44.416 & & & & & & & & & & & & & & \\
\hline 204 & & & & & 24. & & & & & & & & & & & & & & & & & & & & & & & \\
\hline 2 & & & 25.366 & 24.814 & \begin{tabular}{|l|l|} 
& 24.709 \\
\end{tabular} & 26.649 & & 22.674 & & & & & & 44.5 & & & & & & & & & & & & & & \\
\hline & & & & & 24.708 & & & & 63. & & 23.049 & & & 44. & & & & & & & & & & & & & & \\
\hline & 04 & & 25.35 & 24.809 & \begin{tabular}{|l|l|}
24.688 \\
\end{tabular} & 26.643 & & 22.606 & & 3.8 & \begin{tabular}{|c|}
22.807 \\
\end{tabular} & 32.5 & & 44.3 & & & & & & & & & & & & & & \\
\hline & 8/26/2004 & 2:03 & 25.36 & 24.829 & 24.718 & 26.748 & 24.8 & 22.57 & 62.974 & 3.799 & 22.908 & 32 & & 44.387 & & 6.5 & & 287 & & 1.8 & & & & & & & & \\
\hline & $8 / 26 / 2$ & & 25.345 & 24.814 & 24.678 & 26.743 & 24.8 & $22.57 \mathrm{C}$ & 63.343 & 3.768 & 23.002 & 32.31 & & 44.437 & 0.00 & 6.58 & & $888-1$ & & 622 & & 28 & & 43 & 0.952 & 0.04 & 41.762 & \\
\hline 10 & & 2:05:C & 5.346 & 24.809 & 24.674 & & & 22.84 & \begin{tabular}{|l|}
63.564 \\
\end{tabular} & 3.887 & 23.054 & 32.342 & & 4.428 & 00 & 6.577 & & & & 2.4 & & & & & & 0.0 & 1.412 & \\
\hline
\end{tabular}


WSRC-TR-2005-00105, REVISION 0

SRNL-RPP-2005-00012, REVISION 0

CAMPAIGN IV NEW GKN ACID CLEAN AND CAUSTIC RINSE - CONT. 
WSRC-TR-2005-00105, REVISION 0

SRNL-RPP-2005-00012, REVISION 0

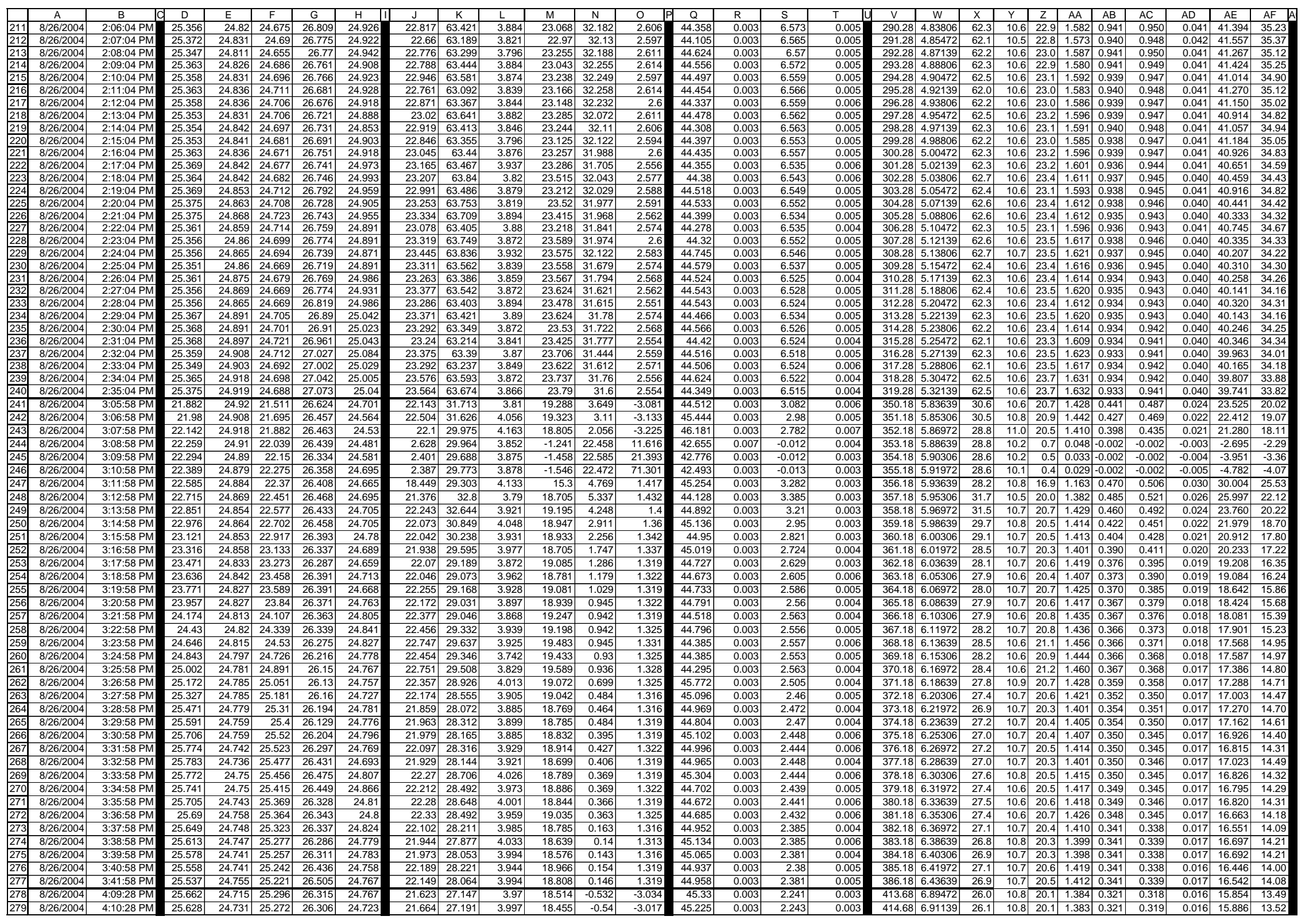


WSRC-TR-2005-00105, REVISION 0

SRNL-RPP-2005-00012, REVISION 0

CAMPAIGN IV NEW GKN ACID CLEAN AND CAUSTIC RINSE - CONT.

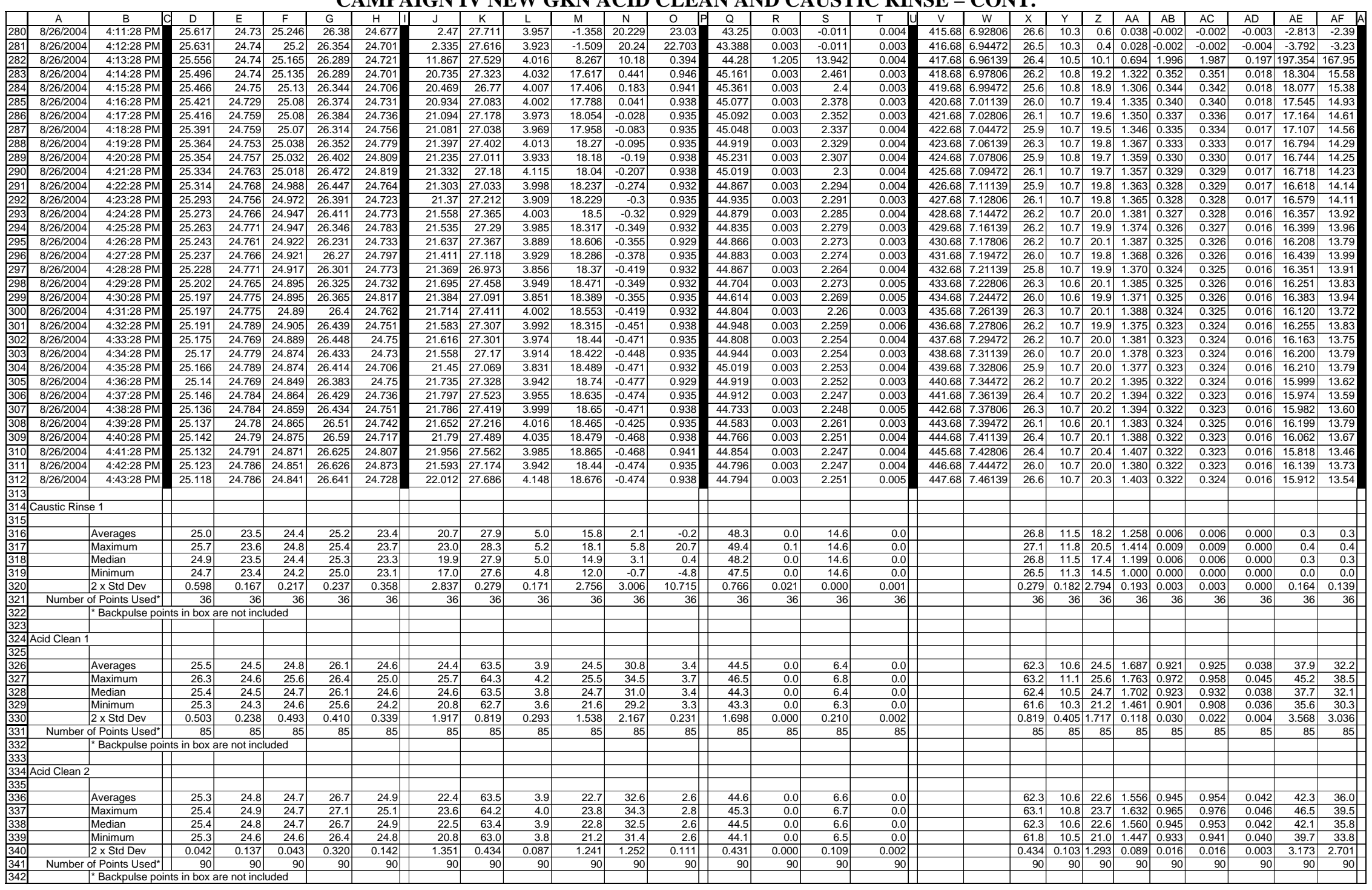


WSRC-TR-2005-00105, REVISION 0

SRNL-RPP-2005-00012, REVISION 0

CAMPAIGN IV NEW GKN ACID CLEAN AND CAUSTIC RINSE - CONT.

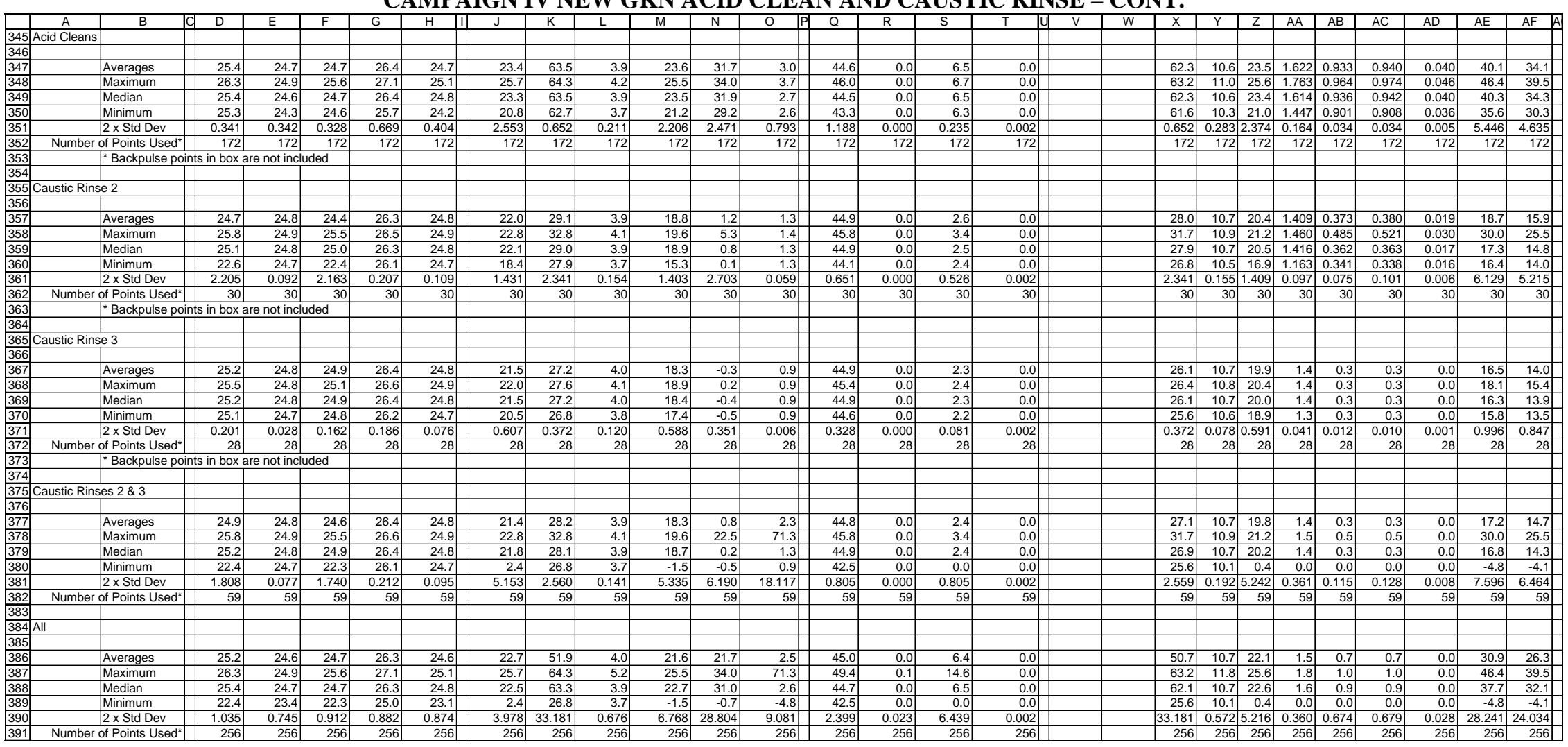


WSRC-TR-2005-00105, REVISION 0

SRNL-RPP-2005-00012, REVISION 0

RUN \# 4.00 A, B AND C; PRE-TEST BASELINE WITH 0.1 M NAOH

RPP-WTP Pilot-scale X-flow Filtration, Test No. 4.00

[27 August 2004: New GKN Filter]

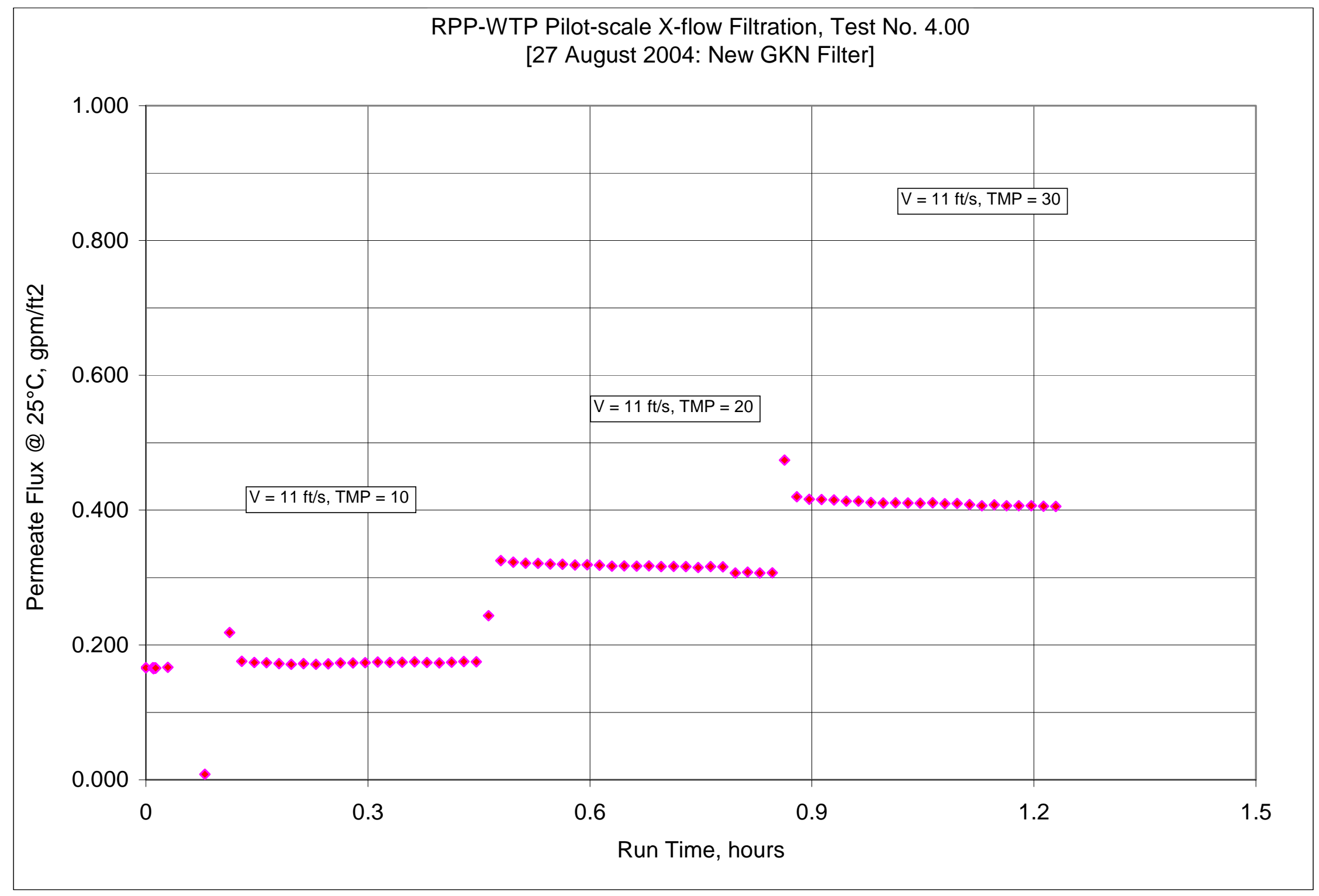


WSRC-TR-2005-00105, REVISION 0

SRNL-RPP-2005-00012, REVISION 0

RUN \# 4.00 A, B AND C; PRE-TEST BASELINE WITH 0.1 M NAOH - CONT.

\begin{tabular}{|c|c|c|c|c|c|c|c|c|c|c|c|c|c|c|c|c|c|c|c|c|c|c|c|c|c|c|c|c|}
\hline & A & $B$ & D & E & $\frac{F}{\text { nerature }}$ & G G & $\frac{\mathrm{H}}{\text { ents } \gg \gg>}$ & $\frac{\mathrm{J}}{1<<<<<}$ & $\frac{|c|}{K \ll \ll \ll<}$ & $\frac{\mathrm{L}}{\mathrm{L}}$ & $\mathrm{M}$ & $\frac{1 N}{\text { ents }}$ & 10 & $\frac{Q}{\ll \ll \ll \ll \ll}$ & $\begin{array}{l}\mathrm{R} \\
\ll<\text { Elown }\end{array}$ & \begin{tabular}{c|c}
$\mathrm{s}$ \\
$\mathrm{e}$ essuren
\end{tabular} & 1 & $\frac{\mathrm{V}}{<<<<}$ & $\frac{1}{1} \frac{w}{2<\ll \ll \ll<}$ & $\frac{1 x}{1 \leq \ll<c}$ & $\frac{1}{1} \quad \mathrm{Y}$ & $\frac{1}{2}$ & $\frac{1 \mathrm{AA}}{1 \mathrm{at}}$ & $|A B|$ & $\frac{\mid \mathrm{AC}}{\mid \mathrm{AC}}$ & $\frac{A D}{\gg \gg \gg \gg \geqslant}$ & $\frac{\mathrm{AE}}{\mathrm{D}}$ & $\mathrm{AF}$ \\
\hline 1 & DATE & TIME & Filtrate & & $\begin{array}{l}\text { perature } \\
\text { g) Slurry }\end{array}$ & $\begin{array}{l}\text { Neasurem } \\
\text { Hi Amb }\end{array}$ & 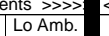 & BotTMP & Filter & $\begin{array}{l}\text { Pressure } \\
\mid \text { Fitter } \mathrm{dP}\end{array}$ & $\begin{array}{l}\text { Measuremer } \\
\text { TopTMP }\end{array}$ & $\begin{array}{l}\text { ents } \\
\text { |Filtrate }\end{array}$ & Sulsepot & & $\begin{array}{l}\text { 《< Hlitrot } \\
\text { Fittate }\end{array}$ & $\begin{array}{l}\text { Measuremer } \\
\text { |Hi Filtate }\end{array}$ & & & & & & & & & & & & \\
\hline 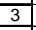 & & & $\operatorname{deg} C$ & $\operatorname{deg} C$ & $\operatorname{deg} C$ & $\operatorname{deg} C$ & $\operatorname{deg} C$ & psid & psig & psid & psid & psig & psig & $\mathrm{gpm}$ & $\mathrm{gpm}$ & \begin{tabular}{|l|l}
$\mathrm{gpm}$ \\
\end{tabular} & & & & & & & & & & & & \\
\hline 4 & & & & & & & & & & & & & & & Q2 & $\mathrm{Q}_{3}$ & & & & & & & & & & & & \\
\hline & s- 0 & & & & & & & & & & & & & & & & & & & & & & & & & & & \\
\hline & $8 / 23 / 2000$ & 8:35:38 AM & 22.554 & 22.604 & \begin{tabular}{|l|l|}
22.002 \\
\end{tabular} & 22.78 & 22.204 & -0.019 & \begin{tabular}{l|l}
9 & 0.095 \\
\end{tabular} & $\begin{array}{l}-0.012 \\
\end{array}$ & 0.003 & \begin{tabular}{|l|l|}
-0.124 \\
\end{tabular} & \begin{tabular}{|l|l|}
0.137 \\
\end{tabular} & $\begin{array}{l}-0.075 \\
\end{array}$ & 0.003 & \begin{tabular}{|l|l}
14.643 \\
\end{tabular} & 0.004 & & & Filter $S$ & Surface & & FT2 & & & & & \\
\hline 8 & $8 / 24 / 2004$ & $8: 10: 33 \mathrm{AM}$ & 23.662 & 22.93 & \begin{tabular}{l|l|l|}
3 & 23.679 \\
\end{tabular} & 22.998 & 22.735 & -0.021 & 0.122 & -0.014 & -0.001 & 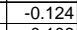 & 0.134 & \begin{tabular}{|l|l|}
23.176 \\
\end{tabular} & 1.205 & \begin{tabular}{|l|l|}
14.643 \\
\end{tabular} & 0.003 & & & Conver & ersion & 85 & & & & & & \\
\hline $\begin{array}{l}9 \\
10\end{array}$ & $8 / 26 / 2004$ & $\begin{array}{l}9: 14: 11 \mathrm{AM} \\
7 \cdot 5 \cdot 20\end{array}$ & 25.819 & $\begin{array}{r}23.352 \\
2380\end{array}$ & \begin{tabular}{l|l}
24.932 \\
92009
\end{tabular} & $\begin{array}{l}25.31 \\
2307 \\
\end{array}$ & \begin{tabular}{|l|}
23.242 \\
22871
\end{tabular} & $\begin{array}{l}-0.008 \\
0.017\end{array}$ & $\begin{array}{l}0.126 \\
.14\end{array}$ & \begin{tabular}{|c|c|}
-0.008 \\
0009
\end{tabular} & \begin{tabular}{|l}
0.011 \\
\end{tabular} & \begin{tabular}{|l|l|} 
& -0.106 \\
\end{tabular} & $\begin{array}{l}0.091 \\
0.111\end{array}$ & \begin{tabular}{|l|l|} 
& \\
\end{tabular} & $\begin{array}{l}0.043 \\
0.023\end{array}$ & \begin{tabular}{|l|l|}
14.643 \\
1.643
\end{tabular} & $\begin{array}{l}0.003 \\
0.025\end{array}$ & & & & & & & & & & & \\
\hline$\frac{10}{11}$ & $\frac{8 / 27 / 2004}{8 / 30 / 2004}$ & $\begin{array}{l}\text { 7:53:29 AM } \\
7: 28: 55\end{array}$ & $\frac{22.649}{22.511}$ & $\frac{22.89}{22.848}$ & $\begin{array}{l}9 \\
8 \\
8\end{array}$ & $\frac{22.87}{22.66 !}$ & $\begin{array}{l}22.82 .71 \\
2.214\end{array}$ & $\frac{-0.017}{-0.017}$ & \begin{tabular}{l|l|l|}
7 & 0.14 \\
7 & 0.138
\end{tabular} & $\begin{array}{c}-0.008 \\
-0.009\end{array}$ & $\mid \begin{array}{l}0.005 \\
0.003\end{array}$ & $\begin{array}{l}5-0.161 \\
3 \\
5\end{array}$ & $\begin{array}{l}0.1111 \\
0.122\end{array}$ & \begin{tabular}{|c|}
-0.0884 \\
-0.082
\end{tabular} & $\begin{array}{l}0.003 \\
0.003\end{array}$ & \begin{tabular}{|c|}
14.643 \\
14.643
\end{tabular} & 0.005 & & & & & & & & & & & \\
\hline 12 & & & & & & & & & & & & & & & & & & & & Pressu & ure Plis & & & 31.25 inch & ches of wa & vater tubin, & & \\
\hline$\frac{13}{14}$ & & & & & & & & & & & & & & & & & & & & vave & & & & $<$ Filtrat & ate Flux & 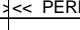 & IABILIT & $y \gg$ \\
\hline 15 & & & & & & & & & & & & & & & & & & Time & Time & Press. & $\begin{array}{l}\text { Vel. } \\
\end{array}$ & TMP & TMP & gpm & gpm & gpm & $\mathrm{pm}$ & meter \\
\hline 16 & & & & & & & & & & & & & & & & & & & & & & & & & & & $12 / \mathrm{psi}$ & day/bar \\
\hline & $A$ & B & D & $E$ & $\mathrm{~F}$ & $G$ & $\mathrm{H}$ & $\mathrm{J}$ & $K$ & $\mathrm{~L}$ & $M$ & $\mathrm{~N}$ & 0 & $Q$ & $R$ & $\mathrm{~s}$ & & $\mathrm{~V}$ & w & $\mathrm{x}$ & $Y$ & $z$ & $\mathrm{AA}$ & $A B$ & $A C$ & $A D$ & $\mathrm{AE}$ & $\mathrm{AF}$ \\
\hline & $\begin{array}{c}\text { Fridayl } 8 / 27 / \\
\text { Baseline }\end{array}$ & $0.1 \mathrm{M} \mathrm{NaOH}$ & & & & & & & & & & & & & & & & & & & & & & & & & & \\
\hline 587 & $8 / 27 / 2004$ & 7:59:56 AM & & & \begin{tabular}{|l|l|}
223.736 \\
\end{tabular} & 23.32 & $22.592 \#$ & 11.941 & 113.142 & 4.266 & 7.869 & & -3.711 & & 0.00 & 1.119 & & 0.00 & & 12.0 & 10.7 & & & \begin{tabular}{l|l|}
3 & 0.160
\end{tabular} & & & \begin{tabular}{|l|l|}
16.762 \\
\end{tabular} & \\
\hline & & $8: 00: 30 \mathrm{AM}$ & & & 23.736 & 23.32 & $22.592 \#$ & 11.941 & 133.142 & & & & & \begin{tabular}{|l|l|}
45.037 \\
\end{tabular} & & $\begin{array}{l}1.119 \\
\end{array}$ & & & 0.00944 & 12.0 & 10.7 & 9.9 & \begin{tabular}{|l|l|}
0.683 \\
\end{tabular} & & & & 16.762 & 14.26 \\
\hline & $7 / 2004$ & 8:00:31 AM & 23.879 & 22.901 & 23.761 & 23.35 & 22.611 \# & 11.898 & 13.124 & 4.177 & 7.943 & \begin{tabular}{|l|l|} 
\\
\end{tabular} & \begin{tabular}{|l|l|l|}
-3.714 \\
\end{tabular} & 45.061 & 0.00 & 1.118 & 0.003 & 0.58 & \begin{tabular}{|l|l|l|}
8 & 0.00972 \\
\end{tabular} & 12.0 & 10.7 & 9.9 & 0.684 & \begin{tabular}{|l|l|}
4 & 0.160 \\
\end{tabular} & 0.166 & 0.01 & $\begin{array}{l}16.709 \\
\end{array}$ & 14.2. \\
\hline & 7/2004 & 00:32 AM & & 22.906 & & 23.34 & 22.606 \# & & \begin{tabular}{|l|l|l|} 
& 13.076 \\
\end{tabular} & 4.148 & 7.97 & \begin{tabular}{l|l} 
& -1.209 \\
\end{tabular} & \begin{tabular}{|c|}
-3.714 \\
\end{tabular} & 44.914 & 0.00 & 1.114 & 0.003 & 0.60 & 0.01 & 11.9 & 10.7 & 9.9 & 旬 0.685 & $\begin{array}{ll}5 & 0.159 \\
\end{array}$ & 0.165 & & \begin{tabular}{|l|l|l|}
16.637 \\
\end{tabular} & 14.16 \\
\hline & $8 / 2$ & 8:00:33 AM & & & 23.755 & $\frac{23.3}{23.25}$ & & 11.796 & & & & & & 44.985 & & & & & & & & & & & & & 16.742 & 14.25 \\
\hline & 8 & $\begin{array}{l}8: 00: 34 \mathrm{AM} \\
8: 00: 35 \mathrm{AM}\end{array}$ & $\frac{23.878}{23.878}$ & $\begin{array}{r}22.906 \\
22.906\end{array}$ & $\frac{23.755}{23.75}$ & $\frac{23.35}{23.35}$ & $\frac{22.611}{22.616}$ & $\frac{11.873}{11811}$ & $\frac{13.037}{13007}$ & $\begin{array}{r}4.02 \\
4.173 \\
\end{array}$ & $\begin{array}{l}8.208 \\
7875\end{array}$ & $\begin{array}{l}-1.209 \\
-1.209 \\
\end{array}$ & $\begin{array}{l}-3.714 \\
-3.717 \\
\end{array}$ & $\frac{45.086}{44.944}$ & 0. & $\frac{1.11}{1.11}$ & & 0 & D.10 & 11.9 & 107 & & & & & & $\frac{1.6408}{10801}$ & $\begin{array}{l}14.01 \\
1.30\end{array}$ \\
\hline & $8 / 27 / 2004$ & 8:00:36 AM & 23.873 & 22.906 & \begin{tabular}{l|l}
6 & 23.755 \\
6
\end{tabular} & 23.3 & $22.616 \#$ & 11.807 & $\begin{array}{ll}7 & 12.937 \\
\end{array}$ & 4.18 & 7.914 & \begin{tabular}{|l|l|}
4 & -1.212 \\
\end{tabular} & $\begin{array}{r}-3.717 \\
-3.71\end{array}$ & $\begin{array}{l}44.944 \\
45.004\end{array}$ & 0.003 & $\begin{array}{l}1.110 \\
1.115\end{array}$ & 0.003 & $\begin{array}{l}0.05 \\
0.67\end{array}$ & \begin{tabular}{|l|}
0.011111 \\
0.00
\end{tabular} & $\begin{array}{l}11.9 \\
11.8\end{array}$ & 10.7 & $\begin{array}{l}9.0 \\
9.9 \\
\end{array}$ & \begin{tabular}{|l|l|l}
0.075 \\
0
\end{tabular} & \begin{tabular}{|l|l|} 
& 0.100 \\
\end{tabular} & 0 & 0.017 & \begin{tabular}{|l|}
16.00169 \\
\end{tabular} & $\frac{14.3}{14.2}$ \\
\hline & $8 / 27 / 2004$ & $8: 00: 37 \mathrm{AM}$ & 23.878 & & \begin{tabular}{l|l}
1 & 23.75 \\
\end{tabular} & 23.3 & $22.621 \#$ & 11.67 & & 4.176 & & -1.212 & & $\begin{array}{l}44.992 \\
\end{array}$ & & & & & & 11.7 & & 9.7 & & & & & \begin{tabular}{|l|l|l|}
17.144 \\
\end{tabular} & 14.5 \\
\hline & $8 / 27 / 2004$ & 8:00:38 AM & 23.883 & 22.906 & 23.755 & 23.3 & 22.621 \#1 & 11.852 & $\begin{array}{l}2 \\
\end{array}$ & 4.13 & 8.033 & -1.212 & -3.71 & 44.931] & 0.00 & 1.119 & & 0.7 & 0.0116 & 11.9 & 10. & 9.9 & 0.686 & 160 & .166 & & 16.690 & 14.22 \\
\hline & 年 & & & & & & 621 \#\# & & & 4.193 & & -1.21 & & & & & & & & 11 & & & & & & & 16.760 & \\
\hline & $8 / 27 / 2004$ & & 23.883 & 22.916 & 23.77 & 23.365 & $22.621 \mid \#$ & 11.782 & 12.95 & 4.2 & $\begin{array}{l}7.842 \\
0.025 \\
\end{array}$ & & & 44.946 & & & & & & 11 & & & & & & & & 14.3 \\
\hline & 8 & 8.004 & $\frac{23.883}{23872}$ & $\frac{22.906}{2291}$ & $\frac{23.165}{2276}$ & $\begin{array}{l}23.35 \\
23.35\end{array}$ & $\frac{22.621}{2.615} \#$ & $\frac{11.811}{11.923}$ & $\begin{array}{l}13.003 \\
13115\end{array}$ & $\frac{4.083}{4.15}$ & $\begin{array}{l}8.052 \\
7.727 \\
\end{array}$ & $-\frac{1.200}{-1.200}$ & $\frac{-3.111}{3.74}$ & $\frac{45.012}{44973}$ & & $\frac{1.1}{11}$ & & 0.75 & 0.0125 & $\frac{1.5}{1.0}$ & 10. & 98 & 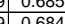 & & & & $\frac{16.704}{16.687}$ & $\frac{14.2}{1.2}$ \\
\hline & $8 / 27 / 2004$ & $\begin{array}{l}0.00 .42 \mathrm{Al} \\
8: 00: 43\end{array}$ & $\frac{25.075}{23878}$ & $\frac{2.91}{22.911}$ & $\frac{2.10}{23765}$ & 年 & 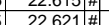 & 势1.950 & 8 & $\frac{4.15}{4.277}$ & 7836 & -1209 & $\begin{array}{l}-3.1144 \\
-3.714\end{array}$ & 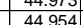 & & & & 0.78 & 0.0130 & 1119 & 107 & $\frac{5.8}{98}$ & 0.678 & \begin{tabular}{l|l|}
80 & 0.160
\end{tabular} & 0.166 & & 16019 & $\frac{14.20}{1421}$ \\
\hline & $8 / 27 / 2004$ & 8:01:43 AM & 23.901 & 22.914 & 22.783 & 23.26 & & $\begin{array}{ll}11.050 \\
11.944\end{array}$ & 413148 & & & -1.209 & -3.714 & & & & & & & & 10.1 & 9.9 & & & & & & $\frac{14.34}{1137}$ \\
\hline & $8 / 27 / 2004$ & 8:02: & 23.91 & 22.928 & 23.827 & 23.32 & $\frac{1}{22.658}$ & 2.507 & 7.13 .376 & & -1.36 & 5.571 & 20.564 & 44.187 & 0 & -0.012 & & $\frac{2.7}{2.7}$ & $\frac{0.04639}{0.0439}$ & $\frac{1.2}{12.2}$ & 10. & $\frac{1.6}{0.6}$ & 0.040 & $\mid-0.002$ & .002 & 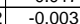 & -3097 & $\frac{14.3}{-2.6}$ \\
\hline & $8 / 27 / 2004$ & & & 22.91 & & 23.38 & & 2.439 & & & & & & 44.2 & & & & & 0.063 & & & & & & & & $\mid-3.367$ & \\
\hline & & & 23.841 & & 23.783 & 23.41 & & & & & -1.206 & & & & & & & & & & net & & & & & & & \\
\hline & & & 23.799 & 22.932 & \begin{tabular}{l|l|}
2 & 23.776 \\
\end{tabular} & 23.46 & & & \begin{tabular}{l|l}
6 & 13.3 \\
\end{tabular} & & -1.491 & 5.632 & & 44.1 & & & & & & 12.2 & 10.5 & 0.4 & & 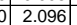 & . & 501 & \#\#\#n & N\#\#\# \\
\hline & & & 23.842 & & 23.8 & 23.4 & & 11.875 & & & & & & & & & & & & 12.2 & & & & & & & 21.913 & \\
\hline & $8 / 27 / 2004$ & & 23.906 & 22.924 & 23.803 & 23.54 & $22.624 \#$ & 11.63 & \begin{tabular}{l|l|}
3 & 12.941 \\
\end{tabular} & 4.23 & 7.652 & -1.209 & 0.5 & 44.7 & & & & & \begin{tabular}{|l|l|l|} 
\\
0.12972
\end{tabular} & 111.8 & 10.7 & 9.6 & & & 0.176 & & 18.233 & 15.5 \\
\hline & $8 / 27 / 2004$ & 8:08:43, & 23.941 & 22.944 & 23.803 & 23.59 & $22.628|\#|$ & 11.813 & & & & -1.209 & & 44.912 & & & & 0.1 & 0.14639 & 11.9 & 10.7 & 10.1. & 0.001 & 668 & .174 & & & 14.8 \\
\hline & & & 23.95 & 22.942 & 23.872 & 23.56 & 22.657|\# & 11.912 & 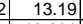 & & & 209 & & 44.866 & & & & & & 12.11 & $10.7 \mathrm{C}$ & 0.0 & & & & & & $14.6 \leqq$ \\
\hline & $8 / 27 / 2004$ & & 24.003 & 22.94 & 23.975 & 23.48. & & 11.95 & & & & & & 44.9 & & & & & & & 10.1 & & & & & & & 14.6 \\
\hline & & & 24.052 & 22.949 & 24.0240 & 23.424 & & & & & & -1.212 & & & & & & & . & 1.2 .0 & 10.8 & 101 & & 70.90 & 0.770 & & & \\
\hline & & & 2 & & & & & & & & & & & & & & & & & & 10.1 & & & & & & & \\
\hline & & & $\frac{24.170}{24235}$ & & $\frac{24.150}{24.192}$ & 2356 & $\frac{22.50}{22.56}$ & $\frac{12.04 t}{11.944}$ & $\frac{13 .}{413}$ & & & $\frac{1.21}{-121}$ & & & & & & & & $\frac{12.1}{122}$ & $\frac{1.6}{10.7}$ & & & & & & & $\frac{14.35}{14.77}$ \\
\hline & $8 / 27 / 2$ & & 24.299 & 22.966 & 24.221 & 23.64 & & 12.213 & $\begin{array}{ll}3 \\
3 & 13 \\
\end{array}$ & & & -1.21 & & & & & & & & 123 & $\begin{array}{l}1.16 \\
10.7\end{array}$ & & & & & & & \\
\hline & & & 24.3 & 22.9 & 24.316 & 23.67 & & & & & & & & 44 & & & & & & & 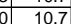 & & & & & & & \\
\hline & & & 24.388 & & 24.33 & 23.6 & & & & & & & & 45. & & & & & & & & & & & & & & \\
\hline & & & & & & & & & & & & & & & & & & & & & & & & & & & & \\
\hline & & & 24.478 & 22.985 & 24.435 & 23.75 & & 11 & \begin{tabular}{l|l}
11 & 13.1 \\
\end{tabular} & & & & & 44.9 & & & & & & 12.0 & 10.7 & 10.0 & & & 0.174 & & & \\
\hline & $8 / 27 / 200$ & & 24.5 & 22 & 24.45 & & & & & & & -1.2 & & & & 1.1 & & & & & 10.7 & & & & & & 17.790 & \\
\hline & $8 / 27 / 2$ & & 24.5 & 22. & 24.4 & & & & $\begin{array}{l}3813 . \\
3\end{array}$ & & & & & & & & & & & 12.0 & 10.7 & & & & & & & \\
\hline & $8 / 27 / 2$ & & 24.595 & 23.007 & 24.543 & 23.82 & 22.99 & 11.96 & $\begin{array}{l}6 \\
6\end{array}$ & & 7.81 & -1.209 & & 44.8 & & & & 22. & & 12.1 & 10.7 & $y$ & & & & & & \\
\hline & & & 24.6 & & 24.5 & 23.85 & & & & & & & & & & & & & & & & & & & & & & \\
\hline & $8 / 2$ & & 24.65 & 23 & 24.56 & 23.90 & & & & & & & & & & & & & & & & & & & & & & \\
\hline & & & 24.685 & 23.021 & 24.608 & (2.995 & 23.183 & $11.1 / 74$ & $\begin{array}{l}4.13 . \\
\end{array}$ & & & -1.212 & & 44.8 & & & & & & $\frac{11.9}{1.0}$ & 10.1 & 9.8 & & & & & & \\
\hline & $\frac{812 \pi / 2}{82720}$ & & $\frac{24.101}{24.73}$ & & 24.634 & 24.00 & & 11.904 & 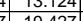 & & & -1.203 & & (45. & & & & & & & 1.6 & & & & & & & \\
\hline & 8 & & 248 & & & & & & & & & & & & & & & & & $\frac{1.0 .3}{258}$ & & & & & & & & \\
\hline & 8 & & 24.9 & 23.062 & 24.793 & 24.25 & & 21.587 & 726.8 & & & & & 45.054 & & & & & & 257 & 107 & 199 & & & & & & \\
\hline & & & 249 & & 24809 & & & & & & & & & & & & & & & & & & & & & & & \\
\hline & & & 250 & 23.07 & 24.82 & 24.43 & & 21.844 & & & & & & & & & & & & & & & & & & & & \\
\hline & & & 250 & 230 & 24.81 & 24,46 & 231 & & \begin{tabular}{|l|l|}
2 & 271
\end{tabular} & & 18.5 & & & 44. & & & & 32 & & & & & & & & & & \\
\hline & $8 / 27 / 20$ & & 25.07 & 23.095 & 24.836 & 24.508 & 23.11 & 21.986 & 27.0 & 3.922 & 18.797 & -0.56 & & 44.8 & & 2.223 & & 33.7 & 0.563 & 26.0 & 10.7 & 0.4 & 1406 & 18 & 0.320 & & & \\
\hline & $8 / 27 / 2004$ & $8: 34: 4$ & 25.084 & 23.106 & 24.841 & $24.51:$ & $23.141 \#$ & $21.81 \varepsilon$ & 26.9 & 3.974 & 18.4 & -0.6 & & 44.8 & & 2.21 & & 34 & & $5.8 \quad-8$ & 10.7 & 20.2 & \begin{tabular}{ll|}
1.3 \\
\end{tabular} & 17 & 0.319 & & & \\
\hline & $8 / 27 / 2004$ & $8: 35: 43 \mathrm{AM}$ & 25.089 & 23.116 & \begin{tabular}{|l|l|l|l|l|l} 
\\
\end{tabular} & 24.50 & $23.206 \#$ & 21.807 & 7 7. 26.973 & 3.935 & \begin{tabular}{|l|l|}
18.492 \\
\end{tabular} & -0.642 & 0.5 & $\begin{array}{l}44.70 \\
\end{array}$ & & & & 35.78 & \begin{tabular}{l|l|l|}
8 & 0.59 \\
\end{tabular} & 25.8 & 10.6 & 20.1 & 1.389 & $\begin{array}{l}9 \\
9\end{array}$ & $\begin{array}{l}7 \\
7\end{array}$ & 0.016 & $\begin{array}{l}6 \\
6\end{array}$ & \\
\hline & $8 / 27 / 2004$ & 8:36:43 AM & 25.109 & 23.121 & \begin{tabular}{l|l}
1 & 24.847 \\
\end{tabular} & 24.50 & $\mid 23.212$ \# & 21.847 & $7 \quad 26.785$ & 3.892 & 18.727 & \begin{tabular}{|l|l|} 
& -0.639 \\
\end{tabular} & 0.591 & \begin{tabular}{|l|l|} 
& 44.693 \\
\end{tabular} & 0.003 & \begin{tabular}{|l|}
2.213 \\
\end{tabular} & & 36.78 & \begin{tabular}{|l|l|l|}
8 & 0.61306 \\
\end{tabular} & \begin{tabular}{l|l}
6 & 25.7
\end{tabular} & 10.6 & \begin{tabular}{l|l|l|l|}
6 & 20.3 \\
\end{tabular} & $\begin{array}{ll}3 & 1.399 \\
\end{array}$ & $\begin{array}{l}9 \\
9\end{array}$ & 0.318 & 0.016 & \begin{tabular}{l|l}
6 & 15.685 \\
\end{tabular} & 13. \\
\hline
\end{tabular}


WSRC-TR-2005-00105, REVISION 0

SRNL-RPP-2005-00012, REVISION 0

RUN \# 4.00 A, B AND C; PRE-TEST BASELINE WITH 0.1 M NAOH - CONT.

\begin{tabular}{|c|c|c|c|c|c|c|c|c|c|c|c|c|c|c|c|c|c|c|c|c|c|c|c|c|c|c|c|c|}
\hline & & & & \\
\hline $\mathrm{T}$ & A & $\mathrm{B}$ & $\mathrm{D}$ & $E$ & $\mathrm{~F}$ & G & \begin{tabular}{l|l}
$\mathrm{H}$ & $\mathrm{I}$ \\
\end{tabular} & $\mathrm{J}$ & $\mathrm{K}$ & $\mathrm{L}$ & \begin{tabular}{l|l} 
\\
\end{tabular} & $\mathrm{N}$ & \begin{tabular}{l|l|} 
\\
\end{tabular} & Q & $R$ & \begin{tabular}{l|l} 
\\
\end{tabular} & $\mathrm{T}$ & $\mathrm{V}$ & W & $x$ & \begin{tabular}{l|l} 
\\
\end{tabular} & $\mathrm{z}$ & AA & $A B$ & $A C$ & $A D$ & $\mathrm{AE}$ & AF \\
\hline 638 & $8 / 27 / 2004$ & $8: 37: 43 \mathrm{AM}$ & 25.12 & 23.143 & 24.863 & 24.53 & 23.243 \# & 22.017 & 27.056 & 3.929 & \begin{tabular}{|l|l|}
18.738 \\
\end{tabular} & -0.659 & 0.591 & \begin{tabular}{|l|}
44.562 \\
\end{tabular} & 0.003 & 2.206 & 0.005 & 37.7 & 0.62972 & 25.9 & 10.6 & 20.4 & 1.405 & 0.316 & 0.317 & 0.01 & 15.559 & 13.24 \\
\hline senth & 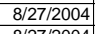 & $8: 38: 43 \mathrm{AM}$ & 25.12 & 23.143 & & 24.53 & 23.238 \# & & & 3.975 & 18.641 & -0.659 & & & 0.003 & 2.209 & & & 0.64639 & 26.0 & & & & & & & 5.617 & 13.29 \\
\hline (640) & $8 / 27 / 2004$ & $8: 39: 43$ AM & $\begin{array}{l}25.121 \\
25.32\end{array}$ & $\begin{array}{l}23.153 \\
3219\end{array}$ & \begin{tabular}{|l|l|}
24.874 \\
\end{tabular} & 24.596 & 23.304 \# & 22.108 & \begin{tabular}{|l|l|}
327.079 \\
\end{tabular} & 3.937 & $\begin{array}{l}18.84 \\
\end{array}$ & $\begin{array}{l}-0.682 \\
0.697\end{array}$ & \begin{tabular}{|l|l|l|}
0.588 \\
0501
\end{tabular} & 44.485 & $\begin{array}{l}0.003 \\
0.03\end{array}$ & $\begin{array}{l}2.206 \\
2.21\end{array}$ & 0.005 & 39.78 & 0.66306 & 26.0 & & & & 316 & 0.317 & & 5.480 & \begin{tabular}{|l|l|}
13.17 \\
1231
\end{tabular} \\
\hline | $\frac{641}{642}$ & $8 / 27 / 2004$ & $\begin{array}{l}8: 40: 43 \mathrm{AM} \\
8: 41: 34 \mathrm{AM}\end{array}$ & $\begin{array}{l}25.132 \\
25.133\end{array}$ & $\begin{array}{r}23.18 \\
23.65\end{array}$ & \begin{tabular}{|r|}
24.92 \\
24.906 \\
\end{tabular} & \begin{tabular}{|l|}
24.608 \\
24.57 \\
\end{tabular} & $\begin{array}{r}23.35 \text { \# } \\
23.391+\end{array}$ & $\begin{array}{l}21.959 \\
22019\end{array}$ & $\begin{array}{l}26.986 \\
2706\end{array}$ & $\begin{array}{l}3.964 \\
3.949\end{array}$ & \begin{tabular}{|c|}
18.584 \\
18643
\end{tabular} & \begin{tabular}{|c|c|c|} 
& -0.697 \\
\end{tabular} & \begin{tabular}{|c|}
0.591 \\
0.591
\end{tabular} & \begin{tabular}{|l|l|}
44.658 \\
44639
\end{tabular} & \begin{tabular}{|l|l|}
0.003 \\
0.003
\end{tabular} & \begin{tabular}{|l|}
2.21 \\
2204 \\
\end{tabular} & $\begin{array}{l}0.005 \\
0.005\end{array}$ & $\begin{array}{l}40.78 \\
41.78\end{array}$ & \begin{tabular}{|l|}
0.67972 \\
0.69639
\end{tabular} & $\begin{array}{l}25.9 \\
25.9\end{array}$ & $\begin{array}{l}10.6 \\
106\end{array}$ & 203 & 1.402 & $\begin{array}{l}0.316 \\
0.316\end{array}$ & $\begin{array}{l}0.317 \\
0.316\end{array}$ & $\begin{array}{l}0.016 \\
0.16\end{array}$ & $\begin{array}{l}15.643 \\
15.561\end{array}$ & \begin{tabular}{|l|l|}
13.31 \\
1324
\end{tabular} \\
\hline \begin{tabular}{|l|l|}
643 \\
643
\end{tabular} & $8 / 27 / 2004$ & $8: 42: 43 \mathrm{AM}$ & & & 24.901 & \begin{tabular}{|l|}
24.573 \\
24.6333
\end{tabular} & $\frac{23.3911 \#}{23.361 \#}$ & 21.958 & \begin{tabular}{|l|l|}
26.928 \\
\end{tabular} & $\begin{array}{l}3.949 \\
.962\end{array}$ & \begin{tabular}{|l|}
18.6433 \\
18.693
\end{tabular} & & & \begin{tabular}{|l|}
44.5659 \\
\end{tabular} & $\frac{0.003}{0.003}$ & $\frac{2.044}{2.204}$ & $\frac{0.005}{0.005}$ & $\frac{41.18}{42.78}$ & & $\begin{array}{l}25.9 \\
25.8\end{array}$ & & & $\begin{array}{l}1.402 \\
1.411 \\
\end{array}$ & $\mid \frac{0.316}{0.316}$ & & $\frac{0.016}{0.016}$ & $\frac{15.561}{15567}$ & $\begin{array}{l}13.24 \\
1325 \\
\end{array}$ \\
\hline 64 & $8 / 27 / 2004$ & 8:43:43 AM & 25.149 & 23.197 & 24.922 & 24.66 & 23.347 \# & 21.882 & 26.888 & 3.808 & 18.779 & 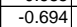 & 0.594 & 44.597 & 0.00 & 2.205 & 0.005 & 43.78 & \begin{tabular}{|l|l}
0.72972 \\
\end{tabular} & 25.8 & 10. & 20. & $\begin{array}{l}1.401 \\
1.402\end{array}$ & $\begin{array}{l}0.316 \\
20.316\end{array}$ & 0.316 & $\mid$ & $\frac{15.567}{15.562}$ & $\frac{13.25}{13.24}$ \\
\hline \begin{tabular}{|l|l|}
645 \\
\end{tabular} & $8 / 27 / 2004$ & $8: 44: 43 \mathrm{AM}$ & 25.16 & 23.217 & 24.948 & 24.71 & 23.353 \# & 22.052 & 27.027 & 4.02 & 18.557 & \begin{tabular}{|l|}
-0.694 \\
\end{tabular} & 0.591 & 44.527 & 0.003 & 2.197 & 0.006 & 44.78 & 0.74639 & 25.9 & 10.6 & 20.3 & & & & & 15.514 & $\begin{array}{l}15.24 \\
13.20\end{array}$ \\
\hline 646 & & $8: 45: 43 \mathrm{AM}$ & & 23.212 & 24.913 & $\begin{array}{r}24.7 \\
\end{array}$ & 23.343 \# & 21.919 & \begin{tabular}{|l|}
26.934 \\
\end{tabular} & 3.899 & $\begin{array}{l}18.635 \\
\end{array}$ & & 0.591 & 444.589 & 0.003 & 2.203 & & 45.78 & 0.76306 & 25.8 & & & 1.398 & 0.315 & 0.316 & & 5.592 & 13.27 \\
\hline 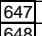 & $\frac{8 / 27 / 2004}{8 / 27 / 204}$ & $8: 46: 43 \mathrm{AM}$ & $\begin{array}{l}25.161 \\
25.82\end{array}$ & $\begin{array}{l}23.219 \\
23255\end{array}$ & $\begin{aligned} 24.914 \\
2494\end{aligned}$ & $\begin{array}{l}24.722 \\
24748\end{array}$ & $\begin{array}{l}23.354 \text { \# } \\
23.355 \pi\end{array}$ & $\begin{array}{l}21.992 \\
21.627\end{array}$ & 27.071 & 3.87 & $\begin{array}{l}18.695 \\
18110\end{array}$ & $\begin{array}{l}-0.697 \\
\end{array}$ & $\begin{array}{l}0.591 \\
0590\end{array}$ & $\begin{array}{l}44.527 \\
4.520\end{array}$ & $\begin{array}{l}0.003 \\
0.03\end{array}$ & $\begin{array}{l}2.202 \\
\end{array}$ & & 46.78 & $\begin{array}{l}0.77972 \\
\end{array}$ & 25.9 & $\begin{array}{l}10.6 \\
\end{array}$ & 20.3 & 1.403 & .315 & 0.316 & & 15.534 & 13.22 \\
\hline \begin{tabular}{|l|l|}
648 \\
649
\end{tabular} & $\frac{8 / 27 / 2004}{8 / 27 / 204}$ & $\begin{array}{l}8: 47: 43 \mathrm{AM} \\
884: 43 \mathrm{AM}\end{array}$ & $\begin{array}{l}25.182 \\
25.157\end{array}$ & $\frac{23.255}{23.23}$ & \begin{tabular}{l|l|}
24.94 \\
2.49
\end{tabular} & $\begin{array}{l}24.748 \\
24.718\end{array}$ & $\begin{array}{l}23.3555 \text { \# } \\
23.345 \text { \#\# }\end{array}$ & $\begin{array}{l}21.627 \\
21.533\end{array}$ & $\begin{array}{l}26.432 \\
3 \\
3\end{array}$ & $\begin{array}{l}4.037 \\
4.004\end{array}$ & \begin{tabular}{|c|}
18.141 \\
18.058
\end{tabular} & \begin{tabular}{|c|c|}
-0.925 \\
-0.928 \\
\end{tabular} & \begin{tabular}{|l|l|}
0.588 \\
0.582
\end{tabular} & $\begin{array}{l}45.029 \\
44762\end{array}$ & \begin{tabular}{|l|l|}
0.003 \\
0.003
\end{tabular} & $\begin{array}{l}2.139 \\
2154\end{array}$ & $\begin{array}{l}0.006 \\
0.005\end{array}$ & $\begin{array}{l}47.78 \\
48.78 \\
\end{array}$ & \begin{tabular}{|l|l|l|l|}
30.79639 \\
0.81306
\end{tabular} & $\frac{25.3}{25.3}$ & $\begin{array}{l}10.7 \\
107\end{array}$ & \begin{tabular}{|l|l|}
19.9 \\
198
\end{tabular} & \begin{tabular}{|l|}
1.371 \\
1365
\end{tabular} & $\begin{array}{l}0.306 \\
0.307\end{array}$ & $\begin{array}{l}0.307 \\
0.308\end{array}$ & $\begin{array}{l}0.015 \\
0.016\end{array}$ & $\begin{array}{l}15.427 \\
15.561\end{array}$ & $\begin{array}{l}13.13 \\
1224\end{array}$ \\
\hline 650 & $8 / 27 / 2004$ & 8:484:45 AM & 25.174 & $\begin{array}{r}23.251 \\
23.251\end{array}$ & $\begin{array}{r}24.989 \\
24.912\end{array}$ & 24.710 & 23.3405 \# & $\frac{11.533}{21.537}$ & 2 & $\begin{array}{l}4.004 \\
4.047\end{array}$ & 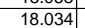 & \begin{tabular}{|c|}
-0.928 \\
-0.928 \\
\end{tabular} & $\begin{array}{l}0.582 \\
0.588\end{array}$ & $\frac{44.762}{44.823}$ & $\begin{array}{l}0.003 \\
0.003\end{array}$ & $\begin{array}{l}2.1 .15 \\
2.136\end{array}$ & $\frac{0.005}{0.005}$ & $\begin{array}{l}48.78 \\
49.78 \\
\end{array}$ & $\begin{array}{l}0.81306 \\
0.82972\end{array}$ & $\frac{25.2}{25.2}$ & \begin{tabular}{|c|}
10.7 \\
10.7
\end{tabular} & & \begin{tabular}{|c|}
$\mid 1.365$ \\
1.364
\end{tabular} & & $\frac{0.308}{0.307}$ & $\begin{array}{l}0.016 \\
0.015\end{array}$ & $\frac{15.561}{15.944}$ & $\begin{array}{l}13.24 \\
1319\end{array}$ \\
\hline 651 & $8 / 27 / 2004$ & 8:50:43 AM & 25.17 & 23.273 & 24.908 & 24.775 & 23.408 \# & 21.654 & $\begin{array}{r} \\
\end{array}$ & 4.07 & 18.212 & -0.923 & 0.588 & 44.923 & 0.00 & 2.138 & 0.006 & $\begin{array}{l}\frac{49.18}{50.78} \\
\end{array}$ & 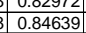 & 25.2 & $\begin{array}{l}10.7 \\
10.7\end{array}$ & \begin{tabular}{|l}
19.8 \\
19.9
\end{tabular} & $\mid$ & \begin{tabular}{|l|} 
\\
\end{tabular} & 0.307 & $\begin{array}{l}0.015 \\
0.015 \\
\end{array}$ & $\frac{15.494}{15.396}$ & $\begin{array}{l}\frac{13.19}{13.10} \\
\end{array}$ \\
\hline 652 & $8 / 27 / 2004$ & $8: 51: 43 \mathrm{AM}$ & 25.28 & 23.288 & 25.039 & 24.836 & 23.489 \# & 33.969 & \begin{tabular}{|l|l}
44.889 \\
\end{tabular} & 4.173 & 30.953 & 4.807 & 0.672 & 46.521 & 0.003 & 3.316 & 0.005 & 51.78 & \begin{tabular}{|l|l|}
0.86306 \\
\end{tabular} & 43.8 & 11.1 & 32.5 & \begin{tabular}{|l|}
2.238 \\
\end{tabular} & \begin{tabular}{|l|l|}
3 & 0.475 \\
\end{tabular} & 0.474 & 0.015 & 14.609 & $\frac{13.10}{12.43}$ \\
\hline 653 & $8 / 27 / 2004$ & 8:52:43 AM & 25.416 & 23.294 & 25.144 & $\begin{array}{l}24.872 \\
2.032\end{array}$ & 23.439 \# & 31.44 & \begin{tabular}{|l|}
40.093 \\
2.093 \\
\end{tabular} & 4.005 & \begin{tabular}{|l|l|}
28.398 \\
\end{tabular} & $\begin{array}{r}2.74 \\
2.77\end{array}$ & $\begin{array}{c}0.64 \\
\end{array}$ & $\begin{array}{l}44.998 \\
\end{array}$ & 0.003 & 2.944 & 0.004 & \begin{tabular}{|l|l|}
52.78 \\
5.70
\end{tabular} & 0.87972 & 39.0 & 10.7 & 29.9 & 2.063 & & 0.420 & 0.014 & 14.030 & 11.94 \\
\hline 654 & $8 / 27 / 2004$ & $8: 53: 43 \mathrm{AM}$ & 25.491 & 23.304 & \begin{tabular}{|l|l|}
25.189 \\
25205
\end{tabular} & $\begin{array}{l}24.932 \\
25019\end{array}$ & 23.414 \# & 31.366 & \begin{tabular}{|l|}
39.948 \\
\end{tabular} & 3.887 & 28.426 & \begin{tabular}{|r|}
2.679 \\
\end{tabular} & 0.64 & $\begin{array}{l}44.987 \\
4.909\end{array}$ & 0.003 & $\begin{array}{l}2.922 \\
.021\end{array}$ & 0.005 & \begin{tabular}{|l}
53.78 \\
5.78 \\
\end{tabular} & $\begin{array}{l}0.89639 \\
\end{array}$ & 38.8 & \begin{tabular}{|l|l}
10.7 \\
\end{tabular} & \begin{tabular}{|l|l|}
29.9 \\
\end{tabular} & 2.061 & 0.418 & 0.416 & 0.014 & 13.919 & 11.84 \\
\hline \begin{tabular}{|c|}
6555 \\
656
\end{tabular} & $\frac{8 / 27 / 2004}{8 / 27 / 204}$ & $8: 54: 43 \mathrm{AM}$ & $\frac{25.522}{25.547}$ & $\begin{array}{r}23.305 \\
232.20\end{array}$ & 25.205 & $\begin{array}{r}25.018 \\
25.079 \\
\end{array}$ & $\frac{23.415 \text { \# }}{23.425+4}$ & $\begin{array}{l}31.546 \\
31.588\end{array}$ & \begin{tabular}{|l|l|}
40.141 \\
340.15 \\
\end{tabular} & $\begin{array}{r}4.034 \\
384\end{array}$ & \begin{tabular}{|l|}
28.414 \\
2836
\end{tabular} & \begin{tabular}{|r|}
2.63 \\
2638 \\
\end{tabular} & $\frac{0.64}{0.64}$ & \begin{tabular}{|l|l|}
44.998 \\
4.593
\end{tabular} & $\begin{array}{l}0.003 \\
0.003\end{array}$ & \begin{tabular}{|l|}
2.921 \\
2917
\end{tabular} & & \begin{tabular}{|l|l|}
54.78 \\
55.78
\end{tabular} & \begin{tabular}{|l|l|} 
& 0.91306 \\
\end{tabular} & $\begin{array}{l}39.0 \\
390\end{array}$ & $\begin{array}{l}10.7 \\
106\end{array}$ & \begin{tabular}{|l|}
30.0 \\
302 \\
\end{tabular} & \begin{tabular}{|l|l|}
2.067 \\
2080
\end{tabular} & 0.418 & 0.416 & $\begin{array}{l}0.014 \\
\end{array}$ & $\begin{array}{l}13.869 \\
13762\end{array}$ & $\begin{array}{l}11.80 \\
1.71\end{array}$ \\
\hline 657 & $8 / 27 / 2004$ & $\begin{array}{l}8: 5: 5: 43 \mathrm{AM} \\
8: 534\end{array}$ & $\frac{25.547}{25.569}$ & $\begin{array}{r}23.32 \\
23.327 \\
\end{array}$ & $\begin{array}{l}25.2166 \\
25.222\end{array}$ & $\begin{array}{l}25.079 \\
25.105\end{array}$ & $\begin{array}{l}23.4252 \# \\
23.492]\end{array}$ & $\begin{array}{r}31.588 \\
31.59 \\
\end{array}$ & \begin{tabular}{|l|l|}
40.145 \\
39.961
\end{tabular} & $\begin{array}{r}3.84 \\
3.815 \\
\end{array}$ & $\begin{array}{l}28.736 \\
28.771\end{array}$ & $\begin{array}{l}2.638 \\
2.552\end{array}$ & $\begin{array}{r}0.644 \\
0.637\end{array}$ & $\frac{44.593}{44.533}$ & $\frac{0.003}{0.003}$ & $\frac{2.917}{2.905}$ & & $\frac{55.78}{56.78}$ & $\begin{array}{l}30.92972 \\
0.94639 \\
\end{array}$ & $\begin{array}{l}39.0 \\
38.8\end{array}$ & $\begin{array}{l}10.6 \\
10.6\end{array}$ & \begin{tabular}{|l|}
30.2 \\
30.2 \\
\end{tabular} & $\frac{2.080}{2.081}$ & \begin{tabular}{|c|}
$\mid 0.418$ \\
$\mid 10.46$ \\
\end{tabular} & $\begin{array}{l}0.415 \\
0.413\end{array}$ & $\begin{array}{l}0.014 \\
0.014\end{array}$ & $\frac{13.762}{13.694}$ & \begin{tabular}{|l|l|l|l|l|}
11.71 \\
11.65
\end{tabular} \\
\hline |658 & $8 / 27 / 2004$ & 8:57:43 AM & 25.589 & 23.342 & 25.243 & 25.156 & 23.508 & 31.525 & 39.888 & 3.84 & 28.712 & 2.552 & 0.643 & 44.562 & 0.003 & 2.906 & & 50.18 & $\begin{array}{l}0.94639 \\
0.96306\end{array}$ & $\begin{array}{l}38.8 \\
38.8\end{array}$ & $\begin{array}{l}10.6 \\
10.6\end{array}$ & \begin{tabular}{|l|}
30.2 \\
30.1 \\
\end{tabular} & \begin{tabular}{|}
$\mid 2.081$ \\
2.077 \\
\end{tabular} & \begin{tabular}{|c|}
$\mid$ \\
7 \\
7
\end{tabular} & $\begin{array}{l}0.413 \\
0.413\end{array}$ & $\mid \begin{array}{l}0.014 \\
0.014\end{array}$ & \begin{tabular}{|l|l|}
13.694 \\
13.719
\end{tabular} & \begin{tabular}{r|r|r|}
11.65 \\
11.68
\end{tabular} \\
\hline \begin{tabular}{|l|}
659 \\
\end{tabular} & $8 / 27 / 2004$ & 8:58:43 AM & 25.595 & 23.348 & 25.238 & 25.171 & 23.593 \# & 31.627 & 39.998 & 3.846 & 28.802 & 2.543 & 0.643 & 44.506 & 0.003 & 2.893 & 0.005 & 58.78 & \begin{tabular}{|l|l|l}
0.97972 \\
\end{tabular} & 38.9 & 10.6 & \begin{tabular}{|l|}
30.2 \\
\end{tabular} & \begin{tabular}{|l|}
2.083 \\
\end{tabular} & \begin{tabular}{|l|l|}
3 & 0.414 \\
\end{tabular} & 0.411 & 0.014 & \begin{tabular}{|l|}
13.1116 \\
13.616 \\
\end{tabular} & \begin{tabular}{|c|}
11.68 \\
11.59
\end{tabular} \\
\hline 660 & $8 / 27 / 2004$ & 8:59:43 AM & 25.621 & 23.375 & 25.275 & 25.233 & 23.565 \# & 31.637 & 40.151 & 3.759 & 28.816 & 2.531 & $\begin{array}{l}0.643 \\
\end{array}$ & 44.47 & 0.00 & 2.891 & & 59.78 & , 0.99639 & 39.0 & 10.6 & 30.2 & 2.084 & & & 0.014 & & \\
\hline & & & & 23.375 & 25.26 & 25.203 & & 32.087 & 40.614 & & & 2.482 & 0.64 & & 0.00 & 2.891 & & 60.78 & 1.01306 & 39.5 & & 306 & 2.110 & 0.414 & & & 13.425 & $\begin{array}{l}\frac{11.56}{11.43} \\
\end{array}$ \\
\hline & $8 / 27 / 2004$ & & 25.638 & 23.391 & 25.296 & 25.26 & 23.5911 \# & 31.919 & 40.423 & 3.818 & 29.028 & 2.48 & 0.64 & 44.416 & & & & & & & & & & & & & & \\
\hline (1) & $8 / 27 / 2004$ & $3 \mathrm{AM}$ & 25.644 & 23.397 & 25.302 & 25.245 & 23.647 \# & 31.924 & 40.508 & 3.884 & 28.781 & 2.43 & 0.64 & 44.36 & & 2.8 & & & $\overline{04639}$ & 39.4 & & & 509 & & & & 1025 & $\frac{11.41}{11.50}$ \\
\hline 6 & 8/27/2004 & $3 \mathrm{AM}$ & 25.655 & 23.408 & 25.318 & 25.267 & $23.598 \mid \#$ & 31.61 & 40.077 & 3.809 & 28.722 & 2.439 & 0.643 & 44.322 & 0.003 & $2.8 \mathrm{~s} \quad \mathrm{~s}$ & & & 1.06306 & 39.0 & 10.6 & & 2.080 & & & & 13.627 & 11.60 \\
\hline 665 & $8 / 27 / 2004$ & 9:04:43 AM & 25.666 & 23.425 & 25.325 & \begin{tabular}{|l|l|}
25.263 \\
\end{tabular} & 23.55 \# & 31.733 & \begin{tabular}{|l|l|} 
\\
\end{tabular} & 3.715 & \begin{tabular}{|l|}
28.89 \\
\end{tabular} & $\begin{array}{r}2.47 \\
\end{array}$ & 0.64 & 44.355 & 0 & 2.886 & & 64.78 & 1.07972 & 39.0 & $\begin{array}{l}10.6 \\
\end{array}$ & \begin{tabular}{|l|}
30.3 \\
\end{tabular} & \begin{tabular}{|l|l|}
2.090 \\
\end{tabular} & 0.413 & 0.409 & 0.014 & 13.507 & 11.49 \\
\hline & $8 / 27 / 2004$ & 9:05:43 AM & 25.656 & 23.43 & 25.315 & 25.268 & $23.63 \#$ & 31.81 & \begin{tabular}{|l|} 
\\
\end{tabular} & 3.842 & 28.913 & 2.462 & 0.643 & 44.257 & 0.003 & 2.889 & & 65.78 & 1.09639 & 39.1 & 10.5 & \begin{tabular}{|l|}
30.4 \\
\end{tabular} & \begin{tabular}{|l|}
2.093 \\
\end{tabular} & & & 0.014 & 13.503 & 10140 \\
\hline & 8/27/2004 & & 25.683 & 23.442 & 25.322 & 25.3 & 23.697 \# & 31.696 & & 3.819 & & & 0.64 & 44.892 & & & & & 1.11306 & 38.8 & $\mid 10.7$ & 30.2 & & & & 0.013 & 13.495 & 11.48 \\
\hline 000 & $8 / 27 / 2004$ & & 25.689 & 23.463 & \begin{tabular}{|l|l|} 
& 25.323 \\
\end{tabular} & 25.306 & 23.698 \# & 31.7 & 39.859 & 3.876 & & 2.375 & $\begin{array}{l}0.643 \\
\end{array}$ & 44.808 & & & & & \begin{tabular}{|l|l|} 
& 1.12972 \\
\end{tabular} & 38.7 & $\begin{array}{l}10.7 \\
\end{array}$ & 30.2 & & 410 & & \begin{tabular}{|l|l|}
0.013 \\
\end{tabular} & 13.440 & \begin{tabular}{|l|l|}
11.44 \\
\end{tabular} \\
\hline 69 & $8 / 27 / 2004$ & & 25.695 & 23.469 & 25.319 & 25.322 & 23.694 \# & 31.845 & & 3.926 & & 2.36 & & $\begin{array}{l}44.867 \\
\end{array}$ & 0.003 & 2.874 & & & \begin{tabular}{|l|l|} 
& 1.14639 \\
\end{tabular} & 39.3 & 10.7 & 30.3 & 2.086 & & & $\begin{array}{ll}0.013 \\
\end{array}$ & 13.475 & $\begin{array}{l}11.47 \\
\end{array}$ \\
\hline \begin{tabular}{|l|l|}
670 \\
671
\end{tabular} & $8 / 27 / 2004$ & & $\begin{array}{l}25.696 \\
25.717\end{array}$ & $\begin{array}{r}23.469 \\
23.491\end{array}$ & \begin{tabular}{|l|}
25.329 \\
25.25
\end{tabular} & $\begin{array}{l}25.328 \\
2.250\end{array}$ & $23.769 \#$ & $\begin{array}{r}31.735 \\
3108\end{array}$ & \begin{tabular}{|l|l|}
5 & 40.104 \\
\end{tabular} & 3.93 & & 2.366 & 0.64 & 44.892 & 0.003 & 2.866 & 0.004 & $\begin{array}{l}69.78 \\
-7.70\end{array}$ & \begin{tabular}{c|c}
8 & 1.16306 \\
8 & 1.1707
\end{tabular} & 39.0 & 10.7 & \begin{tabular}{|l|}
30.2 \\
3.2
\end{tabular} & 2.085 & \begin{tabular}{|l|l|} 
& 0.410 \\
\end{tabular} & 0.407 & 0.013 & 13.443 & $\begin{array}{ll}11.44 \\
1.27\end{array}$ \\
\hline$\frac{671}{672}$ & $\frac{88 / 27 / 2004}{8 / 27 / 2004}$ & $\begin{array}{l}9: 10: 43 \mathrm{AM} \\
9 \cdot 1 \cdot 13 \mathrm{AM}\end{array}$ & $\begin{array}{l}25.717 \\
25.723 \\
\end{array}$ & $\begin{array}{l}23.491 \\
23.507\end{array}$ & $\begin{array}{l}25.356 \\
25.372\end{array}$ & 25.359 & $\begin{array}{l}23.776 \text { \# } \\
23.792 \mid \#\end{array}$ & $\begin{array}{r}31.98 \\
31.652 \\
\end{array}$ & \begin{tabular}{|l|l|l|l|l|l|l|l|l} 
& 40.502 \\
2 & 39.921
\end{tabular} & $\frac{3.908}{3.928}$ & \begin{tabular}{|l|}
28.882 \\
22.876 \\
\end{tabular} & $\begin{array}{r}2.36 \\
2369 \\
\end{array}$ & \begin{tabular}{|c|}
0.643 \\
0.643 \\
\end{tabular} & $\frac{44.693}{44.793}$ & $\begin{array}{l}0.003 \\
0.003\end{array}$ & $\begin{array}{l}2.8868 \\
2.868\end{array}$ & $\begin{array}{l}0.006 \\
0.005 \\
\end{array}$ & 71.18 & \begin{tabular}{l|l}
8 & 1.17972 \\
8 & 119639
\end{tabular} & $\begin{array}{l}39.4 \\
388\end{array}$ & $\begin{array}{l}10.6 \\
10.7\end{array}$ & \begin{tabular}{|l|}
$\mid 30.4$ \\
30.1 \\
\end{tabular} & $\frac{2.098}{2.076}$ & $\mid \begin{array}{l}3 \\
\mid 0.4411 \\
5\end{array}$ & 0.407 & $\begin{array}{l}0.013 \\
0.013 \\
\end{array}$ & \begin{tabular}{|l|l|}
13.358 \\
13.493
\end{tabular} & \begin{tabular}{|l|l|}
11.37 \\
11.48
\end{tabular} \\
\hline 673 & $8 / 27 / 2004$ & $\begin{array}{l}9.1 .45 \mathrm{AVM} \\
9: 12: 43 \mathrm{AM}\end{array}$ & 25.734 & 23.529 & 25.403 & $\begin{array}{l}25.4<5 \\
25.447\end{array}$ & 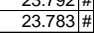 & $\frac{31.052}{31.554}$ & \begin{tabular}{|l|l|}
4 & 39.921 \\
\end{tabular} & $\frac{3.920}{3.812}$ & $\frac{20.570}{28.662}$ & 2.372 & $\begin{array}{l}0.045 \\
0.646 \\
\end{array}$ & $\begin{array}{l}44.195 \\
44.731 \\
\end{array}$ & 0.003 & 2.866 & 0.005 & & 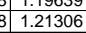 & $\begin{array}{l}38.0 \\
38.7 \\
\end{array}$ & $\frac{10.1}{10.7}$ & \begin{tabular}{|l|}
30.1 \\
30.1
\end{tabular} & $\frac{2.070}{2.076}$ & 0.411 & $\begin{array}{l}0.400 \\
0.406 \\
\end{array}$ & $\begin{array}{l}0.013 \\
0.013 \\
\end{array}$ & \begin{tabular}{|l|}
13.495 \\
13.474 \\
\end{tabular} & $\begin{array}{l}\mid 11.48 \\
11.47 \\
\end{array}$ \\
\hline$\frac{674}{675}$ & $8 / 27 / 2004$ & 9:13:43 AM & 25.746 & 23.53 & 25.409 & 25.448 & 23.77 \# & 31.714 & $\begin{array}{l}4 \quad 40.095 \\
\end{array}$ & 3.884 & 28.634 & 2.369 & 0.64 & 44.816 & 0.0 & 2.865 & 0.00 & & 1.22 & 39.0 & 10.7 & 30.2 & 2.080 & 0.410 & 0.405 & 0.013 & 13.438 & 11.44 \\
\hline & & & & & & & & & & & & & & & & & & & & & & & & & & & & \\
\hline & & Averages & & & 24.3 & & \begin{tabular}{ll|l}
22.8 & \\
\end{tabular} & 11.9 & 13.1 & 4.2 & 8.0 & -12 & 0.5 & & & 12 & & & & 120 & 10 & & & & & & & \\
\hline & & Maximum & 24.7 & 23.0 & 24.6 & 24.1 & 23.2 & 12.2 & 13.5 & 4.4 & 8.3 & -1.2 & 0.6 & 45.2 & 0.0 & $\frac{1.2}{1.2}$ & 0. & & & 12.3 & $\begin{array}{l}0.1 \\
10.8\end{array}$ & \begin{tabular}{|c|}
9.9 \\
10.2 \\
\end{tabular} & 0.7 & 0.2 & 0.2 & 0.0 & 18.5 & 15 \\
\hline 107 & & Median & 24.4 & 23.0 & 24.3 & 23.7 & 22.7 & 11.9 & 13.1 & 4.1 & 8.0 & -1.2 & 0.5 & 45.0 & 0.0 & 1.2 & 0. & & & 12.0 & \begin{tabular}{|l|l|}
10.7 \\
\end{tabular} & \begin{tabular}{|l|l|}
9.9 \\
\end{tabular} & 0.7 & 0.2 & 0.2 & 0.0 & 17.5 & \\
\hline & & Minimum & 23.9 & 22.9 & 23.8 & 23.5 & 22.6 & 11.6 & 12.9 & 4.0 & 7.7 & -1.2 & 0.5 & 44.8 & 0.0 & 1.2 & 0.6 & & & 11.8 & $\begin{array}{l}10.7 \\
\end{array}$ & \begin{tabular}{|l|l|}
9.6 \\
\end{tabular} & \begin{tabular}{|l|} 
\\
\end{tabular} & 0.2 & 0.2 & 0.0 & 16.9 & \\
\hline & & $2 \times \operatorname{Std}$ Dev & 0.528 & 0.060 & 0.531 & 0.328 & 0.437 & 0.256 & 0.266 & 0.182 & 0.325 & 0.004 & 0.005 & 0.236 & 0.000 & 0.029 & 0.001 & & & \begin{tabular}{|l|l|l|l|l}
0.266 \\
\end{tabular} & 0.0560 & 0.257 & \begin{tabular}{|l|}
0.018 \\
\end{tabular} & \begin{tabular}{|l|l|} 
& 0.004 \\
\end{tabular} & 0.002 & 0.001 & 0.626 & 0.5 \\
\hline & Number $\mathrm{c}$ & of Points Used ${ }^{*}$ & & 20 & 20 & 20 & 20 & 20 & 20 & 20 & 20 & 20 & 20 & 20 & 20 & 20 & 20 & & & 20 & 20 & 20 & 20 & 20 & 20 & 20 & 20 & 20 \\
\hline & & * Backpulse po & oints in box & are not ing & cliuded & & & & & & & & & & & & & & & & & & & & & & & \\
\hline & & & & & & & & & & & & & & & & & & & & & & & & & & & & \\
\hline 686 & & Averages & 25.1 & 23.2 & 24.9 & 24.6 & 23.3 & 21.9 & 26.9 & 4.0 & 18.5 & \begin{tabular}{|c|c|}
-0.7 \\
\end{tabular} & 0.6 & 44.7 & 0.0 & 2.2 & $\begin{array}{ll}0.0 \\
\end{array}$ & & & 25.8 & 10.7 & 20.2 & 1.4 & 0.3 & 0.3 & 0.0 & 15.7 & \\
\hline 687 & & & 25.2 & 23.3 & 24.9 & 24.8 & & 22.1 & 27.3 & 4.1 & 18.8 & -0.5 & 0.6 & 45.1 & 0.0 & 2.3 & $0.6 \mathrm{C}-\mathrm{s}$ & & & 26.2 & 10.7 & & 1.4 & 0.3 & 0.3 & 0.0 & 16.3 & \\
\hline 688 & & & 25.1 & 23.2 & 24.9 & 24.6 & & 21.9 & & & 18.6 & & 0.6 & 44.7 & 0.0 & 2.2 & & & & 25.8 & 10.6 & & 1.4 & $\begin{array}{ll}0.3 \\
\end{array}$ & 0.3 & 0.0 & 15.6 & \\
\hline & & & 24.8 & 23.0 & 24.7 & 24.2 & 23.1 & 21.5 & 26.3 & 3.8 & 18.0 & $\begin{array}{r}-0.9 \\
\end{array}$ & 0.0 & 44.5 & 0.0 & 2.1 & 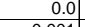 & & & 25.2 & 10.0 & 19.8 & 1.4 & $\begin{array}{ll}4 & 0.3 \\
\end{array}$ & 0.3 & 0.0 & 15.4 & \\
\hline 690 & & $2 \times$ Std Dev & 0.162 & 0.133 & 0.111 & 0.311 & $\begin{aligned} 0.192 \\
23\end{aligned}$ & $\frac{0.359}{23}$ & $\begin{array}{l}0.540 \\
23\end{array}$ & 0.132 & 0.477 & 0.256 & 0.006 & 0.345 & 000 & 0.064 & .001 & & & 0.540 & $0.082[0$ & 0.408 & 0.028 & 0.009 & 0.010 & 0.000 & 0.437 & 0.372 \\
\hline & Number c & 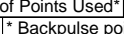 & $\frac{23}{\sin \text { box }}$ & $\frac{23}{2}$ & 23 & & 23 & & & 23 & 23 & 23 & 23 & & & 23 & 23 & & & 23 & 23 & 23 & 23 & 23 & 23 & 23 & 23 & \\
\hline & & & & & & & & & & & & & & & & & & & & & & & & & & & & \\
\hline & & & & & & & & & & & & & & & & & & & & & & & & & & & & \\
\hline & & Averag & 25.6 & 23.4 & 25.3 & 25.2 & 23.6 & 31.8 & 40.3 & 3.9 & 28.8 & 2.6 & 0.6 & 44.7 & 0.0 & 29 & 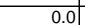 & & & 39.2 & $10.7 \mathrm{C}>\mathrm{C}$ & 30.3 & 2.1 & 0.4 & 0.4 & & 13.6 & \\
\hline & & & 25.7 & 23.5 & 25.4 & 25.4 & & 34.0 & & 4.2 & & & & 46. & & & & & & & & & & & & & & \\
\hline & & & 25.6 & 23.4 & 25.3 & 25.3 & 23.6 & 31.7 & & 3.9 & 28.8 & $2.5+2>$ & 0 & & & 2. & & & & 39.0 & 10.6 & & & & & & & \\
\hline & & Minimum & & & 25.0 & 24.8 & 23.4 & 31.4 & & & 28.4 & 2.4 & 0. & 44 & & & & & & & 10.5 & & 2.1 & .4. & 0.4 & & 13.4 & \\
\hline & & $2 \times$ Std Dev & 0.216 & 0.151 & 0.167 & 0.334 & 0.250 & 0.988 & 1.987 & 0.188 & 0.967 & 0.973 & 0.013 & 0.896 & 0.00 & 0.178 & 0.00 & & & 1.987 & 0.2120 & 0.971 & 0.067 & $\mid 0.026$ & 0.027 & 0.001 & 0.538 & 0.458 \\
\hline & Number & $\bar{f}^{f}$ Points Used & 23 & & 23 & & 23 & 23 & 23 & & 23 & 23 & 23 & 23 & 23 & 23 & & & & 23 & 23 & 23 & 23 & $3 \quad 23$ & 23 & & 23 & 23 \\
\hline & & & box & & ed & & & & & & & & & & & & & & & & & & & \begin{tabular}{|l|l|l|} 
Calc \\
\end{tabular} & & & & \\
\hline & & & & & & & & & & & & & & & & & & & & & & Avg & $\mathrm{gSt} \mathrm{Dv}(\mathrm{c}$ & (all pts) & 13 & & & \\
\hline & & & & & & & & & & & & & & & & & & & & & & $\begin{array}{l}\text { Max } \\
\text { Min }\end{array}$ & n StDv & (all pts & $\mid \begin{array}{c}0.027 \\
0.002 \\
\end{array}$ & & & \\
\hline
\end{tabular}


RUN \# 4.03A AND B; FIRST AND SECOND HALF OF SLURRY DEWATERING

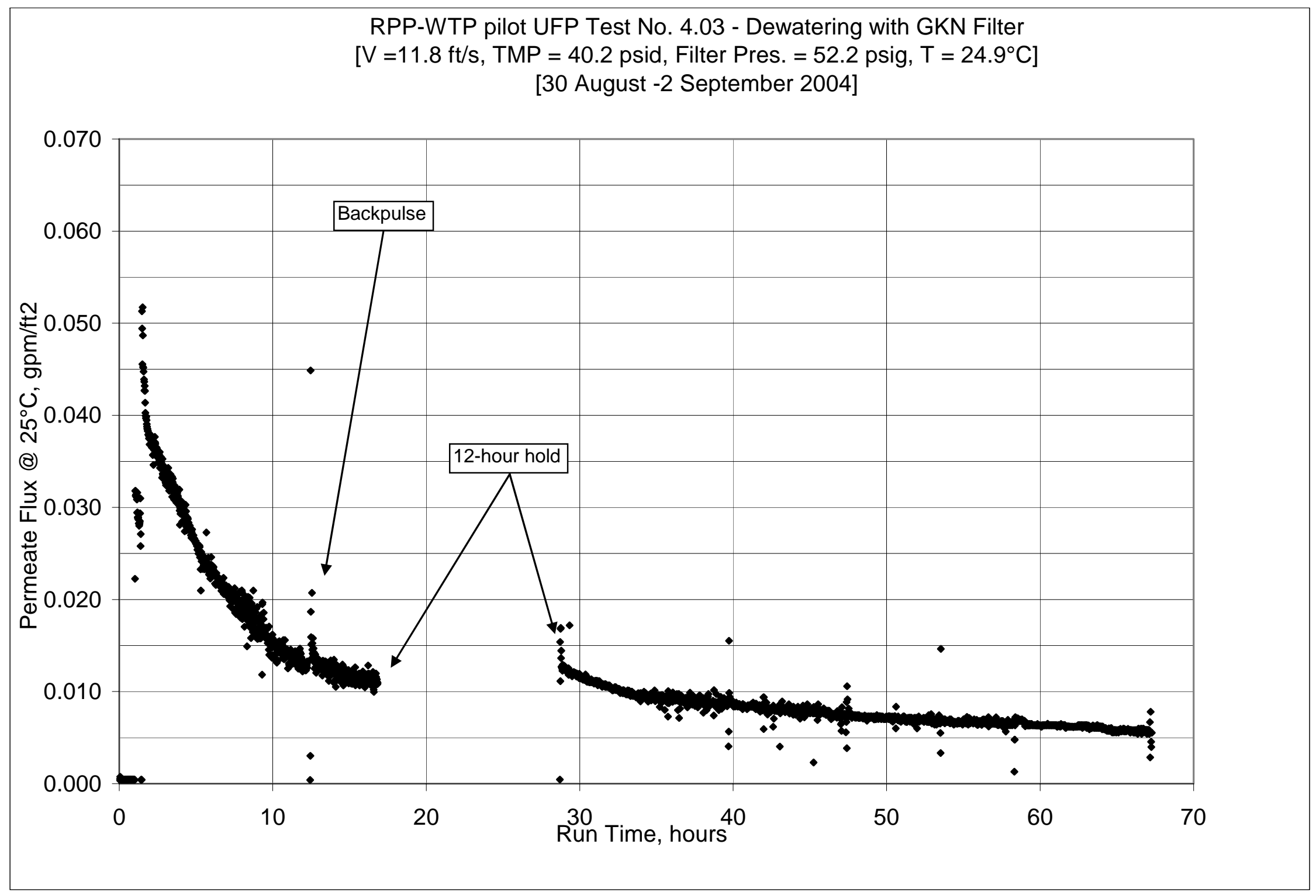


WSRC-TR-2005-00105, REVISION 0

SRNL-RPP-2005-00012, REVISION 0

RUN \# 4.03A AND B; FIRST AND SECOND HALF OF SLURRY DEWATERING - CONT.

\begin{tabular}{|c|c|c|c|c|c|c|c|c|c|c|c|c|c|c|c|c|c|c|c|c|c|c|c|c|c|c|c|c|}
\hline & $\mathrm{A}$ & $B$ & $\frac{D}{\ll \ll \ll \ll<}$ & $E$ & $\frac{F}{1}$ & $\frac{1}{1} \mathrm{G}$ & 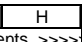 & 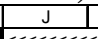 & $\frac{\mid k}{<\ll \ll \ll}$ & $\frac{L}{\text { Pressure }}$ & $\frac{M}{M}$ & $\frac{|N|}{\text { ents }}$ & $\frac{10}{10}$ & $\frac{Q}{Q}$ & $\mathrm{R}$ & $\mathrm{s}$ & 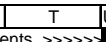 & 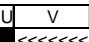 & $\frac{W}{1}$ & \begin{tabular}{|l|}
$|x|$ \\
\end{tabular} & \begin{tabular}{ll|}
$Y$ \\
\end{tabular} & $z$ & AA & \begin{tabular}{|l|l|}
$\mathrm{AB}$ \\
\end{tabular} & I $A C$ & $\begin{array}{ll}\mathrm{AD} \\
\end{array}$ & I $\mathrm{AE}$ & AF \\
\hline & DATE & TIME & $\begin{array}{l}\text { Filtrate } \\
\text { Fele }\end{array}$ & $\begin{array}{l}\text { k }<\text { Temp } \\
\text { Cleaning }\end{array}$ & $\begin{array}{l}\text { perature } \mathrm{N} \\
\text { D. Slury }\end{array}$ & $\begin{array}{l}\text { Measurume } \\
\text { DHi Amb. }\end{array}$ & $\begin{array}{l}\text { nents s > >> } \\
\text { Lo Amb. }\end{array}$ & $\begin{array}{l}\text { BotTMP } \\
\text { BotMe }\end{array}$ & $\begin{array}{l}\text { k } \\
\text { Filter }\end{array}$ & $\begin{array}{l}\text { Pressure } \\
\text { Filter dP } \\
\end{array}$ & $\begin{array}{l}\text { Measuremen } \\
\text { TopTMP }\end{array}$ & $\begin{array}{l}\text { ents }>>> \\
\text { in Filtrate }\end{array}$ & $\begin{array}{l}\text { p>>>>>>> } \\
\text { | Pulsepot }\end{array}$ & $\begin{array}{l}\text { Slurry } \\
\text { Sluse }\end{array}$ & $\begin{array}{l}\text { 《e Flow } \\
\text { Filtrate }\end{array}$ & $\begin{array}{l}\text { Measurem } \\
\text { Hi Filtate }\end{array}$ & $\begin{array}{l}\text { ents } \gg>\gg>7 \\
\text { Backpulse }\end{array}$ & & & & & & & & & & & \\
\hline & & & $\operatorname{deg} C$ & $\operatorname{deg} \mathrm{C}$ & $\operatorname{deg} C$ & $\operatorname{deg} C$ & $\operatorname{deg} C$ & psid & psig & psid & psid & psig & & $\mathrm{gpm}$ & gpm & $\mathrm{gpm}$ & & & & & & & & & & & & \\
\hline & & & $\mathrm{T} 2$ & & $\mathrm{~T} 1$ & & & & P1 & dP1 & dP3 & & P3 & Q1 & $\mathrm{Q} 2$ & & & & & & & & & & & & & \\
\hline & ros- $04 / 19$ & $1 / 2004$ & & & & & & & & & & & & & & & & & & & & & & & & & & \\
\hline 7 & $8 / 23 / 2004$ & $8: 35: 38 \mathrm{AM}$ & 22.554 & 22.604 & 22.002 & 22.782 & 22.204 & -0.019 & 0.095 & -0.012 & 0.003 & -0.124 & 0.137 & -0.075 & 0.003 & 14.643 & 0.004 & & & Filter St & Surface $A$ & $A 6.985$ & FT2 & & & & & \\
\hline 8 & $8 / 24 / 2004$ & 8:10:33 AM & 23.662 & 22.93 & 23.679 & 22.998 & 22.735 & -0.021 & 0.122 & -0.014 & -0.001 & -0.124 & $\begin{array}{l}0.134 \\
\end{array}$ & 23.176 & 1.205 & 144.643 & & & & Conver: & ersion & & $\mathrm{m} 3 / \mathrm{m} 21$ & Iday/barg & $\mathrm{arg} / \mathrm{gpm} / \mathrm{ft}$ & 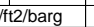 & & \\
\hline & $8 / 26 / 2004$ & 9:14:11 AM & 25.819 & & & 25.316 & & & & & & & & & 0.043 & 14.643 & & & & & & & & & & & & \\
\hline & 8/27/2004 & 7:53:29 AM & 22.649 & 22.89 & 22.298 & & & & 0.14 & -0.008 & & -0.161 & & & 0.003 & 14.643 & 0.005 & & & te: & anc & & & & & & & \\
\hline & 8/30/2004 & 7:28:55 AM & 22.511 & 22.848 & 22.397 & 22.669 & 22.914 & -0.017 & 0.138 & -0.009 & 0.003 & -0.147 & & -0.082 & 0.003 & 14.643 & 0.00 & & & & & & & & & & & \\
\hline & & & & & & & & & & & & & & & & & & & & $\begin{array}{l}\text { Pressur } \\
\text { vavv } \\
\end{array}$ & & & & $\frac{1}{1<\text { < Filtrat }}$ & 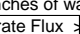 & $\begin{array}{l}\text { er tubin } \\
<\text { PER }\end{array}$ & & \\
\hline 14 & & & & & & & & & & & & & & & & & & & & & & & & & at $25 \mathrm{C}$ & & $\times 1000$ & \\
\hline 15 & & & & & & & & & & & & & & & & & & Time & Time & Press. & Vel. & TMP & TMP & $\mathrm{gpm}$ & gpm & gpm & gpm & \\
\hline 16 & Data - Per M & linute & & & & & & & & & & & & & & & & & Hour & psig & $\mathrm{ft} / \mathrm{s}$ & psi & bar & $1+2$ & & $f(t 2 / p s i$ & ft2/psi & \\
\hline 17 & 8/30/2004 & 7:53:22 AM & & & 24.506 & 23.324 & 22.77 & 35.192 & 34.464 & 4.366 & $\begin{array}{l}27.478 \\
\end{array}$ & \begin{tabular}{|l|l|}
8 & -1.209 \\
\end{tabular} & & 26.459 & 0.003 & 14.643 & 0.006 & & & 33.3 & \begin{tabular}{l|l}
3 & 6.3 \\
\end{tabular} & 31.3 & 2.160 & 0.000 & 0.000 & \begin{tabular}{c|l}
0 & 0.000 \\
\end{tabular} & 0.014 & \\
\hline \begin{tabular}{|c|}
18 \\
19
\end{tabular} & $8 / 30 / 2004$ & 7:54:37 AM & $\begin{array}{r}23.1 \\
23.13\end{array}$ & 23.025 & 24.506 & 23.324 & $\begin{aligned} 22.77 \\
22701\end{aligned}$ & $\begin{array}{l}35.192 \\
232159 \\
\end{array}$ & 34.464 & & $\begin{array}{l}27.478 \\
2570\end{array}$ & \begin{tabular}{|c|c|}
3 & -1.209 \\
\end{tabular} & & 26.459 & 0.003 & $\begin{array}{l}14.643 \\
1.62\end{array}$ & 0.006 & & $5 \quad 0.02083$ & 33.3 & 6.3 & & 2.160 & 0.000 & 0.000 & 0.000 & 0.014 & \\
\hline $\begin{array}{l}\frac{19}{20} \\
\end{array}$ & 8/30/2004 & 7:55:37 AM & $\begin{array}{r}23.13 \\
23.099\end{array}$ & $\begin{array}{l}23.036 \\
23.035\end{array}$ & $\frac{24.306}{24.44}$ & $\begin{aligned} 23.691 \\
2374 \\
\end{aligned}$ & $\begin{aligned} 22.791 \\
2288 \\
\end{aligned}$ & $\begin{array}{l}33.458 \\
47.613 \\
\end{array}$ & 34.642 & $\frac{4.363}{9602}$ & \begin{tabular}{|l|}
25.779 \\
34696
\end{tabular} & $\begin{array}{l}-0.309 \\
-0.17 \\
\end{array}$ & & 25.967 & $\begin{array}{ll}0.003 \\
0.005\end{array}$ & $\frac{14.643}{14643}$ & 0.005 & $\frac{2.25}{325}$ & \begin{tabular}{l|l}
5 & 0.0375 \\
5 & 0.05417
\end{tabular} & $\begin{array}{l}33.5 \\
479 \\
\end{array}$ & $\frac{6.2}{108}$ & \begin{tabular}{|l|}
29.6 \\
411
\end{tabular} & $\begin{array}{l}2.042 \\
2.837 \\
\end{array}$ & 0.000 & 0.000 & 0.000 & 0.015 & \\
\hline \begin{tabular}{|l|l|l|l|l|}
21 & \\
\end{tabular} & $\begin{array}{l}8 / 3 / 20 / 2004 \\
8 / 30 / 2004\end{array}$ & 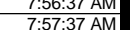 & 23.108 & 23.033 & $\begin{array}{l}0 \\
\begin{array}{l}3 \\
3\end{array} 24.669 \\
\end{array}$ & $\begin{array}{r}23.14 \\
23.829 \\
\end{array}$ & $\begin{array}{r}22.88 \\
22.938 \\
\end{array}$ & $\frac{47.613}{47.032}$ & $\begin{array}{l}49.027 \\
48.637 \\
\end{array}$ & $\begin{array}{r}9.002 \\
10.206 \\
\end{array}$ & $\begin{array}{r}34.609 \\
3.372 \\
\end{array}$ & 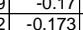 & & $\begin{array}{l}45.474 \\
47246\end{array}$ & 0.005 & $\frac{14.643}{14.643}$ & $\begin{array}{l}0.003 \\
0.005 \\
\end{array}$ & & $\frac{0.05411}{0.07083}$ & $\frac{4.9}{47.5}$ & & $\frac{41.1}{40.2}$ & 2.83t & 0.0000 & $\frac{0.001}{0.000}$ & $\frac{0.000}{0.000}$ & & \\
\hline 22 & & 7:58:37 AM & 23.103 & 23.034 & \begin{tabular}{l|l}
4 & 24.87 \\
\end{tabular} & 23.885 & 22.999 & 47.733 & 49.232 & 9.604 & 34.616 & $\begin{array}{|ll|}6 & -0.173 \\
\end{array}$ & 0.186 & 45.982 & $\begin{array}{l}0.0003 \\
0.003\end{array}$ & 14.643 & $\begin{array}{l}0.005 \\
0.00\end{array}$ & 5.2 & $\frac{0.0875}{0.085}$ & 48.1 & $\frac{11.0}{11.0}$ & $\frac{40.2}{41.2}$ & $\frac{2.174}{2.839}$ & 0.000 & 0.000 & & $\frac{0.011}{0.011}$ & \\
\hline 23 & $8 / 30 / 2004$ & 7:59:37 AM & 23.126 & 23.047 & 25.068 & 23.953 & 23.092 & 48.038 & 49.632 & 9.492 & 35.074 & 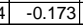 & 0.195 & 48.458 & 0.003 & 14.643 & 0.005 & 6.25 & $\begin{array}{ll}50.10417 \\
\end{array}$ & 48.5 & 11.5 & $\begin{array}{lll}5 & 41.6 \\
\end{array}$ & 2.865 & 0.000 & .000 & $\frac{0.000}{0.000}$ & 0.011 & \\
\hline 24 & $8 / 30 / 2004$ & 8:00:37 AM & & 23.045 & 25.262 & & 23.171 & \begin{tabular}{|l|l|}
46.634 \\
\end{tabular} & 48.079 & 10.372 & \begin{tabular}{|l|}
32.917 \\
\end{tabular} & $\begin{array}{l}-0.17 \\
\end{array}$ & & \begin{tabular}{|l|l|}
48.746 \\
\end{tabular} & 0.003 & 144.643 & 0.006 & 7.25 & $\begin{array}{lll}5 & 0.12083\end{array}$ & 47.0 & 11.6 & & & & & & & \\
\hline 25 & $8 / 30 / 2004$ & $8: 01: 37 \mathrm{AM}$ & \begin{tabular}{|l|l|l|}
23.173 \\
\end{tabular} & 23.069 & 25.43 & & 23.229 & & & & & & & & 0.003 & & & & $\begin{array}{ll}0.1375 \\
\end{array}$ & & 11.1 & 40.4 & 2.782 & .000 & 0.000 & 0.000 & $\frac{0.011}{0.011}$ & \\
\hline 26 & & & & & & 24.304 & & & & 10.597 & 32.824 & -0.173 & & & & 14.643 & & & & & & & & & & & & \\
\hline & $8 / 30 / 2004$ & 8:03:37 AM & 23.249 & 23.124 & 25.605 & 24.376 & & $\begin{array}{ll}46.947 \\
\end{array}$ & 48.604 & 10.719 & \begin{tabular}{|l|l|}
32.748 \\
\end{tabular} & -0.176 & & 488.477 & & 14.643 & & & 0.17083 & 47.5 & $\frac{11.5}{11.5}+2$ & $\frac{39.8}{39.8}$ & & & & & & \\
\hline 28 & $8 / 30 / 2004$ & & 23.287 & 23.147 & & 24.475 & 23.5 & 46.283 & 47.795 & 11.086 & 31.798 & -0.167 & & 46.448 & 0.003 & 14.643 & 0.005 & $11.2 \mathrm{r}$ & 0.1875 & 46.7 & 11.1 & 39.0 & & & & 000 & & \\
\hline 29 & $8 / 30 / 2004$ & 8:05:37 AM & 23.324 & 23.159 & $\begin{array}{l}9 \\
99\end{array}$ & 24.487 & 23.57 & $\begin{array}{l}46.607 \\
\end{array}$ & 48.096 & 11.005 & 32.289 & -0.17 & 0.186 & \begin{tabular}{|l|l|}
49.507 \\
\end{tabular} & 0.003 & 14.643 & 0.00 & 12.2 & 0.20417 & 47.0 & 11.8 & 39.4 & 2.720 & 0.000 & 0.000 & 0.000 & 0.011 & \\
\hline 30 & $8 / 30 / 2004$ & $37 \mathrm{AM}$ & 23.353 & 23.173 & $\begin{array}{ll}3 & 25.774\end{array}$ & 24.616 & 23.549 & 46.706 & 48.438 & 10.748 & 32.3 & -0.173 & & 45.636 & 0.003 & 14.643 & 0.0 & & 0.22083 & 477.3 & 10.9 & 39.5 & & .000 & .000 & & & \\
\hline & $8 / 30 / 2004$ & 8:07:37 AM & 23.392 & 23.198 & 25.759 & 24.7 & & 45.16 & 46.78 & 11.818 & 29.844 & -0.19 & & 48.636 & 0.0 & $14.6 \mathrm{C}$ & & & 0.237 & 45.7 & 11.6. & 37. & & & & & & \\
\hline & 8) 830120044 & & 23.421 & 23.212 & 25.687 & 24.72 & & 45.52 & .157 & 11.504 & 30.581 & -0.207 & & 51.205 & & 14. & & & & 46.0 & $\frac{0}{2}+2>$ & 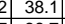 & & & & & & \\
\hline 象 & $8 / 30 / 2004$ & & 23.462 & 年32.223 & 25.564 & & & $\frac{45.893}{46.207}$ & 055 & & $\frac{31.50 r}{23.9}$ & $=0.1 / 3$ & & (53.112 & & . & & $\frac{16.2}{17}>2$ & 02075 & 46.2 & $\frac{1.8}{120}+2$ & 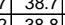 & & & & & & \\
\hline & $8 / 3 / 30 / 2004$ & & 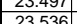 & 年3.24t & 年35.445 & 24.109 & & & 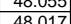 & 11.201 & & $=0.117$ & & 年1510 & & 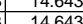 & & & & & & & & & & & & \\
\hline 36 & $8 / 30 / 2004$ & 8:12:37 AM & 23.582 & 23.293 & \begin{tabular}{|l|l}
325.218 \\
3
\end{tabular} & 24.815 & 23.588 & 4.5.57 & 48.229 & 11.475 & \begin{tabular}{|c|}
31.657 \\
\end{tabular} & \begin{tabular}{|ll|l|}
7 & -0.1179 \\
\end{tabular} & & 53.464 & 0.003 & 14.643 & 0.006 & 19.25 & 50.32083 & 47.1 & $\frac{12.7}{12.7}$ & 39.1 & 2.697 & 0.000 & 0.000 & 0.000 & 0.011 & \\
\hline & 8/30/2004 & & 23.605 & 23.3 & 25.12 & 24.863 & & 46.57 & & 11.233 & 32.4 & \begin{tabular}{|l|l|}
3 & -0.173 \\
\end{tabular} & & 48.825 & & 14. & & & & 47.0 & 11.6 & 39.3 & & & & & & \\
\hline 38 & 8/30/2004 & 1:37 AM & 23.632 & 23.312 & 25.037 & 24. & & 46.648 & 48.293 & 11.331 & 31.732 & -0.173 & & 50.273 & 0.06 & 14. & & 21. & 0.35 & 47.2 & 12.0 & 39.2 & & & 0.000 & & & \\
\hline S & 8/30/2004 & & 20.002 & 23.317 & 7] 24.942 & $24.7-7>$ & & 46.918 & & & & & & 51.2 & & 14. & & & & 47.4 & 4 & 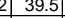 & & & & & & \\
\hline & & $8: 16: 37$ & & 23.349 & & & & & .9 & 11.262 & & & & & & & & & & 46.8 & & 39. & & & & & & \\
\hline 42 & 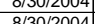 & $\begin{array}{l}8.1 .53 \mathrm{AMM} \\
0.1 .82 \mathrm{AM}\end{array}$ & 年3.154 & $\frac{2.37}{23.386}$ & 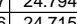 & $\frac{2.8}{2.8}$ & & & & 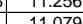 & $\frac{32.125}{32099}$ & - -0.173 & & (51.182 & & 年 & & 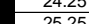 & & 47.4 & 110 & 395 & & & & & & \\
\hline 43 & $8 / 30 / 2004$ & & \begin{tabular}{|l|}
23.816 \\
23.00
\end{tabular} & $\frac{10.300}{23402}$ & 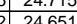 & $\frac{2.0}{247}$ & & & 年 & 11.0108 & | & & & (5.0040 & & 14. & & 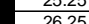 & & 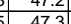 & $\frac{1.5}{124}>0$ & 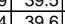 & & 0000 & & ( & & \\
\hline \begin{tabular}{|l|l|l|l|}
44 &
\end{tabular} & $8 / 30 / 2004$ & & 23.840 & $\frac{20.402}{23.42}$ & 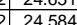 & 24848 & & & & & 年 324 & -0.213 & & & & 144 & & & & 年 47.3 & $1.4 .4>0$ & 396 & & & & & & \\
\hline 45 & $8 / 30 / 2004$ & $8: 21: 3$ & 23.866 & 23.432 & \begin{tabular}{|l|l|}
2 & 24.52 \\
\end{tabular} & 24.8 & & 47.0 & 48.763 & 10.973 & $\frac{15.74}{32.74}$ & & & $54.17-3$ & & & & & & 47.6 & $\frac{\frac{\pi}{129}}{129}>0$ & 39. & & & & & & \\
\hline 40 & & & & 23.454 & & & & & & & 32.303 & & & & & & & & & & & & & & & & & \\
\hline & 8/30/2004 & 8:23:37 & 23.931 & 23.477 & $\begin{array}{l}7724.405 \\
7\end{array}$ & 24.899 & & 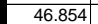 & 48.44 & 10.778 & \begin{tabular}{|r|}
32.64 \\
\end{tabular} & -0.173 & & 48.913 & & 14. & & & & 47.3 & $\frac{11 .}{11 .}$ & 39.7 & 2.74 & & & & 0.011 & \\
\hline 48 & $8 / 30 /$ & & 23.94 & 23.4 & 24.3 & 24. & & & 48.3 & $10 . \varepsilon$ & & -0.1 & & 51.2 & & 14. & & 31 & & 47.2 & & & & & & & 0.01 & \\
\hline \begin{tabular}{|l|l}
49 \\
\end{tabular} & $8 / 30 / 2004$ & & $\begin{array}{ll}23.978 \\
\end{array}$ & 23.489 & & $24 . \varepsilon$ & & & 48.38 & & & & & 52 & & & & & & 47.3 & 12.5 & 39 & 27 & & & & & \\
\hline & $8 / 30 / 2004$ & & 24.0 & 23 & 24.2 & 24. & & & \begin{tabular}{|l|l|}
48.206 \\
\end{tabular} & 10.8 & 32.64 & -0.1 & & 53.8 & & 14. & & 33 & & $\begin{array}{l}47.1 \\
\end{array}$ & 12.6 & & & & & & 0.011 & \\
\hline & $8 / 30 / 2004$ & & 24.042 & 23.5 & 24.2 & 24. & & & 48.5 & & 32.3 & & & & & & & & & 47.4 & 12. & & & & & & & \\
\hline 52 & $8 / 30 / 2004$ & & 24.069 & 23.5 & 24.141 & 24. & & & 48.66 & & & -0.1 & & 52.634 & & & & 35. & & 47.5 & 12.5 & 35 & & & & & 0.011 & \\
\hline 0 & 8/30/2004 & & 24.101 & 23.56 & $\begin{array}{l}724.1 \\
77\end{array}$ & & & & & & & & & 51.4 & & & & & & 47.2 & . & & & & & & & \\
\hline 54 & $8 / 30 / 2004$ & & 24.107 & 23.568 & $\begin{array}{l}824.0 \\
8\end{array}$ & 24 & & & & & & & & & & & & & & 47.6 & 1 & & & & & & & \\
\hline & 8013012004 & & 24.154 & 23.599 & $\begin{array}{l}94.041 \\
1\end{array}$ & 24.99 & & & & & 32. & -0.1 & & o. & & & & & & 47.1 & 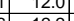 & 30 & & & & & & \\
\hline 5 & 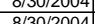 & & & $\frac{23.011}{23620}$ & 20.995 & & & & & & & & & & & & & & & & 11 & & & & & & & \\
\hline 58 & 8 & & 24207 & 23.024 & $\begin{array}{ll}5 \\
6\end{array}$ & & & 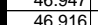 & & & & & & & & & & & & & & & & & & & & \\
\hline & 8 & & 24221 & 23.0532 & 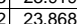 & & & & & & & & & & & & & & & 47.4 & 124 & & & & & & & \\
\hline 60 & & & $\frac{2.251}{24.251}$ & & & & & & & & & & & & & & & & & 454 & & & & & & & & \\
\hline 61 & $8 / 30 / 2004$ & & & 23.676 & \begin{tabular}{l|l|l}
6 & 23.958 \\
\end{tabular} & & & & & & & & & & & & & & & & & & & & & & & \\
\hline & & & 24.295 & 23.685 & & & & 44.7 & & 10 & & -0 & & & & & & & & 450 & 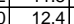 & & & & & & & \\
\hline 63 & $8 / 30 / 2004$ & & 24.319 & 23.7 & 24.161 & 25.6 & & & 49.373 & 11. & 33.2 & -0.1 & & 48. & & & & & 0.7 & 48.2 & & & & & & & & \\
\hline & $8 / 30 / 2004$ & & 24.3 & 23.71 & 24. & 25 . & & 47. & \begin{tabular}{l|l}
49.42 \\
\end{tabular} & & 33 & -0.1 & & & & 14 & & & & 48.3 & & & & & & & & \\
\hline 65 & $8 / 30 / 2004$ & & 24.345 & 23. & \begin{tabular}{l|l}
6 & 24.4
\end{tabular} & 25.047 & & 47.94 & 49 & & 33.5 & -0.17 & & 52.4 & & 14. & & 48. & & 48.4 & 1 & & & & & & & \\
\hline 66 & $8 / 30 / 20$ & & & & & & & 47.7 & & & & & & & & & & & & 48.3 & $12.2 \mathrm{r}$ & & & & & & & \\
\hline 67 & $8 / 30 / 2004$ & & 24.385[ & 23.756 & 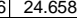 & 25.082 & & 47.982 | & \begin{tabular}{|l|l|}
49.586 \\
\end{tabular} & 10.991 & 33.608 & -0.176 & & 50.663 & & 14. & & 50. & & 48.5 & 12.1 & & & & & & 0.011 & \\
\hline & $8 / 30 / 2004$ & & 24.3 & & & & & 47.063 & \begin{tabular}{|l|l|} 
\\
\end{tabular} & & & & & & & & & & & 47.8 & & & & & & & & \\
\hline 70 & $8 / 30 / 2004$ & $8: 45: 37 \mathrm{AM}$ & $\frac{24.32}{24323}$ & $\begin{array}{r}23.81 \\
37994\end{array}$ & $\begin{array}{l}124.828 \\
2428\end{array}$ & 25.1625 & $\frac{23.83}{2389}$ & $\begin{array}{l}44.049 \\
614147\end{array}$ & $\begin{array}{l}4.936 \\
\end{array}$ & 10.901 & $\frac{3.352}{3.3967}$ & -0.167 & & $\begin{array}{ll}5.8069 \\
50275\end{array}$ & 0 & $\frac{14.643}{1.62}$ & & & & & & & & & & & & \\
\hline & & & 24.323 & & \begin{tabular}{l|l|l}
4 & 24.907
\end{tabular} & & & & 48.83 & 10.804 & $32.867 \mid$ & -0.173 & & 50.375 & 0.003 & 14.643 & & & & 77.7 & & & & .000 & 0.000 & .000 & 0.01 & \\
\hline
\end{tabular}


WSRC-TR-2005-00105, REVISION 0

SRNL-RPP-2005-00012, REVISION 0

RUN \# 4.03A AND B; FIRST AND SECOND HALF OF SLURRY DEWATERING - CONT.

\begin{tabular}{|c|c|c|c|c|c|c|c|c|c|c|c|c|c|c|c|c|c|c|c|c|c|c|c|c|c|c|c|c|}
\hline & A & B $\quad$ C & D & $E$ & $F$ & G & $\mathrm{H}$ & $\mathrm{J}$ & $\mathrm{K}$ & $\mathrm{L}$ & $\mathrm{M}$ & $\mathrm{N}$ & 0 & $\mathrm{Q}$ & $\mathrm{R}$ & 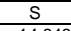 & & $\mathrm{v}$ & W & \begin{tabular}{l|l|}
$x$ \\
\end{tabular} & $\mathrm{Y}$ & $z$ & AA & & & & $\mathrm{AE}$ & \\
\hline & $\begin{array}{l}8 / 30 / 2004 \\
8 / 30 / 2004\end{array}$ & 8:47:37 AM & $\begin{array}{l}24.324 \\
24.326 \\
\end{array}$ & $\begin{array}{r}23.805 \\
23.812\end{array}$ & $\begin{array}{r}24.983 \\
25.045\end{array}$ & $\begin{array}{l}25.167 \\
25.163 \\
\end{array}$ & $\begin{array}{r}23.889 \\
23.931 \\
\end{array}$ & $\begin{array}{l}45.908 \\
45.423\end{array}$ & 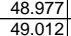 & $\frac{10.786}{10.847}$ & $\begin{array}{r}33.161 \\
32.99 \\
\end{array}$ & \begin{tabular}{|l}
-0.175 \\
-0.173 \\
\end{tabular} & $\begin{array}{l}0.195 \\
0.192\end{array}$ & $50.761 \mid$ & $\begin{array}{l}0.003 \\
0.003\end{array}$ & $\begin{array}{l}14.643 \\
11.643 \\
\end{array}$ & $\begin{array}{l}0.006 \\
0.006\end{array}$ & \begin{tabular}{|l}
54.25 \\
5.25
\end{tabular} & \begin{tabular}{|l|l|l|}
0.90417 \\
0.92083
\end{tabular} & \begin{tabular}{|r|}
47.9 \\
47.9
\end{tabular} & $\begin{array}{l}12.1 \\
12.1\end{array}$ & \begin{tabular}{|l|}
39.5 \\
39.2
\end{tabular} & \begin{tabular}{|l|}
2.726 \\
2.703
\end{tabular} & $\begin{array}{l}0.000 \\
0.000\end{array}$ & 0.000 & $\begin{array}{l}0.000 \\
0.000\end{array}$ & \begin{tabular}{|l|l|}
0.011 \\
0.011
\end{tabular} & $\begin{array}{l}0.01 \\
0.01\end{array}$ \\
\hline 73 & & & & & & & & & & 10.938 & & & & & 0.003 & 14.643 & 0.005 & & \begin{tabular}{|l|l|}
0.9375 \\
\end{tabular} & $\begin{array}{l}47.6 \\
\end{array}$ & & & & & 0.000 & 0.000 & 0.011 & $\begin{array}{l}0.01 \\
0.01\end{array}$ \\
\hline 74 & & $8: 50: 37 \mathrm{AM}$ & 24.357 & 23.853 & 25.201 & 25.234 & 23.917 & \begin{tabular}{|l|l|}
44.344 \\
\end{tabular} & \begin{tabular}{|l|l|}
48.84 \\
\end{tabular} & 10.797 & 32.943 & -0.173 & 0.189 & 50.747 & 0.003 & 14.643 & 0.006 & $\begin{array}{l}57.25 \\
\end{array}$ & \begin{tabular}{|l|} 
\\
\end{tabular} & 47.7 & 12.1 & 38.6 & 2.664 & 0.000 & 0.000 & 0.000 & & \\
\hline 75 & $8 / 30 / 2004$ & $8: 51: 37 \mathrm{AM}$ & $\begin{array}{l}24.369 \\
2.275\end{array}$ & $\begin{array}{r}23.86 \\
\end{array}$ & 25.233 & 25.286 & $\begin{array}{l}23.908 \\
.200\end{array}$ & 43.637 & \begin{tabular}{|l|l|l|l|}
48.624 \\
\end{tabular} & 10.818 & 32.672 & $\begin{array}{l}-0.173 \\
\end{array}$ & & $\begin{array}{l}48.004 \\
5.8023\end{array}$ & 0.003 & $\begin{array}{l}14.643 \\
1.642\end{array}$ & $\begin{array}{l}0.005 \\
0.0\end{array}$ & \begin{tabular}{|l|l|}
58.25 \\
5.25
\end{tabular} & \begin{tabular}{|l|l|}
0.97083 \\
\end{tabular} & 47.5 & 11.4 & \begin{tabular}{|l|l|}
38.2 \\
\end{tabular} & 2.631 & 0.000 & 0.000 & 0.000 & & \\
\hline $\begin{array}{l}76 \\
777\end{array}$ & $8 / 30 / 2004$ & $\begin{array}{l}8: 52: 37 \mathrm{AM} \\
8: 53: 37\end{array}$ & & 23.871 & $\begin{array}{l}25.199 \\
25.151\end{array}$ & $\begin{array}{l}25.403 \\
25.15\end{array}$ & $\begin{array}{r}23.96 \\
\end{array}$ & 43.461 & \begin{tabular}{|l|l|}
48.72 \\
50.919
\end{tabular} & 10.847 & 32.964 & $\begin{array}{l}-0.17 \\
\end{array}$ & & 50.000 & & $\begin{array}{l}14.643 \\
14.6\end{array}$ & 0.006 & & \begin{tabular}{|l|l|} 
\\
\end{tabular} & $\begin{array}{l}47.6 \\
\end{array}$ & 12.1 & & & | & 0.000 & 0.000 & 0.011 & \\
\hline $\begin{array}{ll}77 \\
78\end{array}$ & $8 / 30 / 2004$ & $\begin{array}{l}8: 53: 37 \mathrm{AM} \\
0: 05 \cdot 27\end{array}$ & 24.382 & $\frac{23.883}{23896}$ & 25.151 & $\begin{array}{l}25.415 \\
25.417\end{array}$ & & 45.078 & 50.819 & 10.806 & & -0.176 & & 52.649 & & $\begin{array}{l}14.643 \\
1.692\end{array}$ & $\begin{array}{l}0.004 \\
0.023\end{array}$ & & & $\begin{array}{l}49.7 \\
\end{array}$ & 12.5 & & 2.759 & $\begin{array}{ll}0.000 \\
\end{array}$ & 0.000 & 0.000 & 0.011 & \\
\hline $\begin{array}{l}788 \\
79\end{array}$ & $\frac{8 / 3012004}{88 / 3 / 2004}$ & $8: 54: 37 \mathrm{AM}$ & $\begin{array}{r}24.81 \\
25096\end{array}$ & $\begin{array}{l}23.896 \\
23.902\end{array}$ & $\begin{array}{r}25.149 \\
25.145\end{array}$ & $\begin{array}{l}25.417 \\
25.378\end{array}$ & 24.014 & $\begin{array}{l}45.116 \\
43386\end{array}$ & 51.352 & $\begin{array}{l}10.674 \\
10.605\end{array}$ & $\begin{array}{l}35.203 \\
33536\end{array}$ & 0.073 & $\begin{array}{l}0.186 \\
0319\end{array}$ & \begin{tabular}{rl|}
49.606 \\
47906
\end{tabular} & 0.156 & $\begin{array}{l}14.643 \\
14643\end{array}$ & 0.003 & \begin{tabular}{|l|l|}
61.25 \\
62.25
\end{tabular} & \begin{tabular}{|l|l|} 
\\
\end{tabular} & $\begin{array}{l}50.2 \\
50.1\end{array}$ & 11.8 & & & & 0.022 & 0.001 & 0.554 & \\
\hline \begin{tabular}{|l|}
80 \\
80
\end{tabular} & $\frac{88 / 30 / 2004}{8 / 30 / 2004}$ & $\begin{array}{l}8: 55: 37 \mathrm{AM} \\
85637 \mathrm{AM}\end{array}$ & $\begin{array}{r}25.096 \\
25.22\end{array}$ & $\begin{array}{l}23.902 \\
23.935\end{array}$ & $\begin{array}{l}25.145 \\
25.138\end{array}$ & $\begin{array}{l}25.378 \\
25.427\end{array}$ & $\begin{aligned} 24.026 \\
24.039\end{aligned}$ & $\begin{array}{l}43.386 \\
43.278\end{array}$ & $\begin{array}{l}51.249 \\
51.166\end{array}$ & $\frac{10.605}{10.631}$ & $\begin{array}{l}33.536 \\
33.444 \\
\end{array}$ & $\begin{array}{l}1.718 \\
1.71 \varepsilon\end{array}$ & $\begin{array}{l}0.319 \\
0.394\end{array}$ & $\begin{array}{r}47.96 \\
53.208\end{array}$ & \begin{tabular}{|l}
0.223 \\
0.223
\end{tabular} & $\frac{14.643}{14.643}$ & \begin{tabular}{|l}
0.003 \\
0.004
\end{tabular} & $\frac{62.25}{63.25}$ & \begin{tabular}{|l|}
1.0375 \\
1.05417 \\
\end{tabular} & \begin{tabular}{|c|}
50.1 \\
50.0
\end{tabular} & $\begin{array}{l}\frac{11.4}{12.7} \\
\end{array}$ & \begin{tabular}{|l|}
38.5 \\
38.4 \\
\end{tabular} & $\begin{array}{l}2.652 \\
2.645 \\
\end{array}$ & \begin{tabular}{|c|c|}
0.032 \\
0.032
\end{tabular} & $\frac{0.032}{0.032}$ & $\begin{array}{l}0.001 \\
0.001\end{array}$ & \begin{tabular}{|c|c|c|c|}
0.829 \\
0.829
\end{tabular} & 0.70 \\
\hline \begin{tabular}{|l|}
81 \\
\end{tabular} & $8 / 30 / 2004$ & 8:57:37 AM & 25.263 & 23.944 & 25.111 & 25.475 & 24.092 & $\begin{array}{r}43.32 \\
\end{array}$ & 51.178 & 10.643 & 33.436 & 1.74 & & \begin{tabular}{|l|}
49.099 \\
\end{tabular} & 0.219 & 14.643 & 0.003 & & \begin{tabular}{|l|l|}
1.07083 \\
\end{tabular} & 50.1 & 11.7 & & 2.645 & & 0.031 & 0.001 & \begin{tabular}{|l|l|l|l|l|l|}
0.814 \\
\end{tabular} & $\begin{array}{l}0.11 \\
0.69\end{array}$ \\
\hline & $8 / 30 / 2004$ & $8: 58: 37 \mathrm{AM}$ & & & 25.12 & & 24.085 & 43.506 & 51.431 & 10.49 & & $1.79 \mathrm{~S}$ & & 47.526 & $\begin{array}{l}0.22 \\
\end{array}$ & 144.643 & 0.004 & 65.25 & \begin{tabular}{|l|l|}
1.0875 \\
\end{tabular} & 50.3 & $\begin{array}{ll}11.3 \\
\end{array}$ & & & & & & & 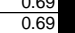 \\
\hline (1) & $8 / 30 / 2004$ & $8: 59: 37 \mathrm{AM}$ & 25.36 & 23.966 & 25.118 & 25.537 & 24.124 & 43.141 & 51.18 & 10.452 & 33.458 & 1.912 & & 51.216 & 0.219 & 144.643 & 0.003 & $\begin{array}{l}66.25 \\
\end{array}$ & \begin{tabular}{|l|l|}
1.10417 \\
\end{tabular} & $\begin{array}{l}50.1 \\
\end{array}$ & 12.2 & 38.3 & & & 0.031 & & & \\
\hline 84 & $8 / 30 / 2004$ & & & 23.99 & & & & & & & & 1.96 & & & 218 & & & & & $\begin{array}{l}50.3 \\
\end{array}$ & & & & & 0.031 & 0.001 & 0.806 & \\
\hline 85 & & & 25.39 & & & & & 43.479 & 51.375 & 10.399 & & 2.022 & & & & 14.643 & & & 1.1375 & 50.2 & & & 2.669 & & & & & \\
\hline 86 & $8 / 30 / 2004$ & $37 \mathrm{AM}$ & 24.852 & 24.003 & 25.06 & 25.574 & & 48.507 & 51.785 & 10.273 & 39.151 & -1.212 & & 48.533 & 206 & 14.643 & & 69.2 & 1.15417 & 50.7 & & & & & & & & \\
\hline & $8 / 30 / 2004$ & $37 \mathrm{AM}$ & 25.014 & 24.02 & 25.032 & 25.561 & & & 48.108 & 11.585 & 34.515 & $-1.20 \mathrm{~s}$ & & 55.454 & & 14.643 & & & 1.17 & 47.0 & 12 & & 2.72. & & & & & \\
\hline \begin{tabular}{|l|l|}
88 \\
\end{tabular} & $8 / 30 / 2004$ & $37 \mathrm{AM}$ & 25.125 & 24.052 & 25.029 & 25.603 & & 45.112 & 7.862 & 11.29 & 34.688 & -1.206 & & 51.693 & 0.206 & 14.643 & 004 & 71.25 & 1.1875 & \begin{tabular}{|l|l|}
46.7 \\
\end{tabular} & $12.3 \mathrm{C}$ & & & & 0.029 & 0.001 & 0.739 & \\
\hline & & $37 \mathrm{AM}$ & 25.146 & 24.057 & 24.984 & 25.643 & & 45.392 & 48.127 & 10.941 & 35.367 & $-1.20 \mathrm{~s}$ & & 52.23 & & 14.643 & & 72.2 & 1.20417 & 47.0 & 12.4 & 40. & & & 0.029 & 0.001 & 0.717 & \\
\hline 90 & 8/30/2004 & 9:06:37 AM & 25.177 & 24.068 & 24.955 & 25.659 & 24.206 & 44.966 & 47.644 & 10.863 & 34.885 & -1.212 & & 49.912 & 0.201 & 14.643 & 0.00 & 73.25 & 1.22083 & 46.5 & 11.9 & 39.5 & 2.75 & .029 & 0.029 & 0.001 & 0.722 & \\
\hline & 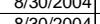 & & 25.198 & 24.094 & 24.971 & 25.6 .5 & & 45.46 & 48.148 & 10.761 & 35.472 & -1.205 & & דראח & 0.202 & 14.643 & & & & & & & & & & & & \\
\hline & 8 & & 25.199 & & 24.937 & 25.101 & & 45. & & 10.655 & 35.149 & -1.212 & & 0.638 & 0.2 & & & & & 47.1 & 12. & & & & & & 0.106 & \\
\hline 94 & 8 & $37 \mathrm{AM}$ & 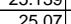 & $\frac{24.150}{24146}$ & $\begin{array}{l}24.950 \\
24888\end{array}$ & 25787 & $\frac{24.304}{22.254}$ & $\frac{4.145}{4505}$ & $\begin{array}{l}41.144 \\
47.555 \\
\end{array}$ & 10.001 & 35.055 & $\frac{-1.205}{-1200}$ & & 52.047 & 0.195 & $\begin{array}{l}14.045 \\
14643\end{array}$ & & 70 & 12075 & $\begin{array}{ll}46.6 \\
46.4\end{array}$ & & & & & & 0 & $\frac{155}{702}$ & \\
\hline 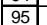 & $8 / 30 / 2004$ & $37 \mathrm{AM}$ & 25.086 & 24.172 & 24.874 & 25.818 & $\frac{24}{24}$ & 45.074 & 7.744 & 10.903 & $\frac{3.58}{35.08}$ & -1.20 & & 51.157 & 然.196 & 14.643 & & 78.25 & 1.204 & 46.4 & & & & & & & & \\
\hline \begin{tabular}{|l|}
96 \\
\end{tabular} & $8 / 30 / 2004$ & 9:12:37 AM & 25.075 & 24.166 & 24.828 & 25.762 & 24. & 45.371 & 47.988 & 10.652 & 35.527 & -1.215 & & 52.042 & 0.197 & 14.643 & 0.003 & 79.25 & 51.32083 & 46.9 & $\frac{12.4}{12.4}$ & 40. & & D28 & .02 & 001 & 0.701 & \\
\hline \begin{tabular}{|l|}
97 \\
\end{tabular} & & & & 24.182 & 24.803 & & & 45.591 & 47.982 & & 36.34 & & & & & & & & & 46.9 & & & & & & & & \\
\hline & $8 / 30 / 2004$ & & 25.11 & 24.218 & 24.809 & 25.769 & 24. & 45.63 & 48.208 & 10.46 & 36.13 & -1.212 & & 50.415 & 0.2 & 14.643 & & 81 & 1.35417 & 47.1 & & 40 & & & & & 718 & \\
\hline \begin{tabular}{|l|}
99 \\
9
\end{tabular} & $8 / 30 / 2004$ & & 25.137 & 24.229 & 24.771 & 25.795 & & 46.217 & \begin{tabular}{|l|l|}
48.633 \\
\end{tabular} & 10.514 & 36.422 & -1.212 & & & & 14.643 & & & 1.37083 & 47.5 & 11.7 & & 2.84 & & 031 & 001 & 0.750 & \\
\hline & & & & & & & & & & & & & & & & & & & & & & & & & & & & \\
\hline & $8 / 30 / 2004$ & & 25.169 & 24.256 & 24.747 & 25.83 & & 44.828 & 46.941 & 10.987 & $\begin{array}{l}34.63 \\
\end{array}$ & -1.21 & & 52.334 & & 14.643 & & 84.24 & 1.40 & $\mid$\begin{tabular}{l|l|}
45.8 \\
\end{tabular} & & & & & & & & \\
\hline & & & & & 24.734 & & & 9.6 & & 11.019 & -0.6 & & & & & & & & & & & & & & & & & \\
\hline & 8/301/2004 & & 25.212 & 24.294 & 24.725 & 25.925 & 24. & 7.035 & 46.873 & 10.997 & -3.145 & $34.29 \mathrm{~S}$ & & 52.467 & 0.003 & 14.643 & & 86.2 & 1.4375 & 45.7 & 12.5 & 1 & 0.13 & 0.000 & .000 & 0.000 & 0.223 & \\
\hline & & $37 \mathrm{AM}$ & $25.219 \mid$ & 24.311 & 24.697 & 25.937 & 24. & 6.247 & & 9.787 & & 35.188 & & & & 14. & & & 1.45 & 46.2 & te & & & & & & & \\
\hline & $8 / 3012004$ & 9:21:37 AM & 25.177] & 24.324 & 24.66 & 25.939 & & 38.846 & 46.666 & 9.935 & 29.812 & $\frac{1.718}{18}$ & & 48.187 & 0.355 & $14.6 \mathrm{C}$ & & 88 & 1.4708 & 45.5 & $\frac{11}{12}$ & 34 & 2.3 & & & & & \\
\hline & 8 & & 25.189 & 24.331 & 24.657 & 25010 & & 38.709 & 46.558 & 9.9 & 29.709 & & & 50051 & & 14. & & & 1. & 45.4 & . & & & & & & 1.445 & \\
\hline & 8 & & & & 24.050 & & & & & 10 & & & & & & & & & & & 11. & & & & & & & \\
\hline & 8 & & 2518 & 24387 & 24.000 & 25.95 & & & & & & & & & & & & & & & & & & & & & & \\
\hline & $8 / 30 / 2004$ & & 25.162 & 24.404 & 2466 & 25.984 & & & & 9.645 & & & & & & & & & & 448 & & & & & & & & \\
\hline & $8 / 30 / 2004$ & & $\frac{25.119}{25}$ & 24.417 & 24.607 & 25.942 & & & & & & & & & & 14.6 & & (1) & & 44.4 & 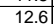 & 40 & & & & & 1126 & \\
\hline & $8 / 30 / 2004$ & & 25.096 & 24.439 & 24.599 & 25.954 & 24 & 44. & & 9.553 & 36.1 & & & 51.024 & & 14. & & 95. & & 44.9 & $\frac{12.0}{12.2}$ & & 2.7. & . & 0.045 & & 1.104 & \\
\hline & & & & & & & & & & & & & & & & & & & & \begin{tabular}{|c|}
44.6 \\
\end{tabular} & & & & & & & & \\
\hline 112 & $8 / 30 / 2004$ & & 25.082 & 24.45 & 24.55 & 25.9 & & & & $\begin{array}{l}9.458 \\
\end{array}$ & & & & & & & & & & 44.5 & 11.8 & & & & & & 1075 . & \\
\hline & & & & 24.448 & 24. & 25.9 & & & & & & & & 52.1 & & & & & & & & & & & & & & \\
\hline & & & 25.01 & 24.437 & $\begin{array}{l}24.442 \\
\end{array}$ & & & & & & & & & & & & & & & 44.9 & 11 & & & & & & & \\
\hline & 8/30/2004 & & & 24.44 & & & & & & & & & & & & & & & & 44.5 & & & & & & & & \\
\hline 11 & 8/30/2004 & & 25.0 & 24.433 & 24.378 & 25.7 & & & & 9.6 & & & & 51. & & & & & & 43.7 & 12 & & & & & & 1.034 & \\
\hline & 8/30/2 & & & 24.4 & 24. & 25.7 & & & & & & & & & & 14. & & & & 44.2 & 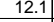 & & & & & & 1.004 & \\
\hline & & & 25. & 24.4 & & & & & & 9.8 & & & & & & & & & & 43.7 & 12 & & & & & & & \\
\hline & 8 & & 25.006 & 24.45 & 24.313 & 25.779 & & & & 9.772 & 35.6 & -1.214 & & 54.9 & & 14. & & & & 44.0 & ta & & & & & & 0.990 & \\
\hline & 8 & & & & 2.43 & & & & & & & & & & & & & & & & & & & & & & & \\
\hline & $8 / 30 / 2004$ & & 240 & 24.452 & 242 & 25.8 & & & & 96 & & & & & & & & & & & & & & & & & & \\
\hline & $8 / 30 / 2004$ & & 24.884 & & & $\frac{2.09}{25.9}$ & & & & & & & & & & & & & & 443 & & & & & & & & \\
\hline & & & & & & & & & & & & & & & & & & & & 44.1 & & & & & & & & \\
\hline 12 & $8 / 30 / 2$ & & \begin{tabular}{|l|l|}
24.84 \\
\end{tabular} & 24.452 & 24. & 25.95 & & & & & & & & & & & & & & \begin{tabular}{|c|}
44.6 \\
\end{tabular} & & & & & & & & \\
\hline & & & & & & & & & & & & & & & & & & & & & & & & & & & & \\
\hline & & & 24.782 & 24.4 & & 26.04 & & & & & & & & & & & & & & & & & & & & & & \\
\hline & & & & & & & & & & & & & & & & & & & & & & & & & & & & \\
\hline & 8/30/2004 & & 24.7 & 24.467 & 24.081 & 26.167 & & & & & 35.394 & -1.2 & & 52.4 & & & & 114 & & 44.2 & & & & & & & & \\
\hline & $8 / 30 / 2004$ & & 24.764 & 24.482 & 24.051 & 26.1 & & 44. & & 9.6 & 35.814 & & & 49. & & 14. & & 115 & $1.9 \mathrm{t}^{\mathrm{r}}$ & $\begin{array}{l}44.6 \\
\end{array}$ & & & & & & & & \\
\hline & $8 / 30 / 2$ & & 24.757 & 24.5 & 24.099 & 26.2 & 24. & & & 9.7 & & & & & & & & & & 44 & & & & & & & & \\
\hline & $8 / 30 / 2$ & & & 24.544 & 24.11 & 26.3 & & & & & 35.658 & & & 52. & & 14. & & & & 44.7 & & & & & & & & \\
\hline & & & & & & & & & & & & & & & & & & & & .6. & 11. & & & & & & & \\
\hline$\frac{13}{12}$ & $8 / 3012004$ & & 24.1774 & 24.590 & 24 & 2 & & 44 & & $\frac{9.023}{9072}$ & 35.14 & & & & & & & & & 45.0 & 12.5 & & & & & & & \\
\hline & $8 / 30 / 500$ & & 24767 & 2462 & 年 & $\frac{2.30}{26.32}$ & & & 45.56) & 年 & $\begin{array}{r}35.24 \\
35257\end{array}$ & $=\frac{-1.22}{-121}$ & & & & & & & & 44.5 & & & & & & & & \\
\hline & & & 年 & 2465 & 24.0 & & & & & 968 & 35.94 & & & & & & & & & 449 & & & & & & & & \\
\hline 140 & 8/30/2004 & 9:56:37 AM & 24.82 & 1.653 & 24.388 & 26.348 & & 44.614 & 45.998 & 9.488 & 35.874 & -1.212 & & 47.486 & 0.26 & 14.643 & & & 2.05417 & 44.9 & & & & & $03 \varepsilon^{03}$ & .001 & 0.941 & \\
\hline & & & & & & & & & & & & & & & & & & & & & & & & & & & & \\
\hline
\end{tabular}


WSRC-TR-2005-00105, REVISION 0

SRNL-RPP-2005-00012, REVISION 0

RUN \# 4.03A AND B; FIRST AND SECOND HALF OF SLURRY DEWATERING - CONT.

\begin{tabular}{|c|c|c|c|c|c|c|c|c|c|c|c|c|c|c|c|c|c|c|c|c|c|c|c|c|c|c|c|c|}
\hline & & & & & & & & & & & & & & & & & & & & & & & & & & & & \\
\hline & A & B & $\mathrm{D}$ & $E$ & $F$ & G & $\mathrm{H}$ & $\mathrm{J}$ & $\mathrm{K}$ & $\mathrm{L}$ & $\mathrm{M}$ & $\mathrm{N}$ & 0 & Q & \begin{tabular}{l|l|l|l|l} 
& \\
\end{tabular} & $\mathrm{s}$ & $T$ & $\mathrm{v}$ & w & $x$ & $\mathrm{Y}$ & $z$ & AA & $A B$ & $A C$ & $A D$ & $\mathrm{AE}$ & \\
\hline 14 & $8 / 30 / 2004$ & 9:57:37 AM & 24.859 & 24.663 & \begin{tabular}{|l|}
24.437 \\
\end{tabular} & 26.388 & 24.9 & 44.575 & 45.984 & 9.435 & 35.989 & \begin{tabular}{|c|} 
\\
\end{tabular} & -6.168 & 49.407 & 0.259 & 14.643 & 0.003 & 124.25 & 2.07083 & 44.9 & 11.8 & 40.3 & 2.777 & 0.037 & 0.038 & 0.001 & 0.935 & \\
\hline & & 9:58:37 AM & & 24.686 & \begin{tabular}{|l|l}
24.486 \\
\end{tabular} & 26.476 & 24.928 & & 46.344 & & 36.132 & & $\begin{array}{l}-6.168 \\
-.168\end{array}$ & & & 14.643 & 0.004 & & & & & & & & & & 0.925 & \\
\hline & $8 / 30 / 2004$ & 9:59:37 AM & 24.922 & 24.7 & 24.515 & 26.455 & $\begin{array}{l}24.907 \\
\end{array}$ & \begin{tabular}{|l|l|l|}
45.139 \\
\end{tabular} & 46.55 & 9.585 & 36.403 & & -6.183 & 48.869 & $\begin{array}{l}0.259 \\
\end{array}$ & 14.643 & 0.003 & 126.25 & $\begin{array}{l}2.10417 \\
.1070\end{array}$ & \begin{tabular}{|l|l|}
45.4 \\
\end{tabular} & 11.6 & & 2.81 & 0.037 & $\begin{array}{l}0.038 \\
\end{array}$ & 0.001 & $\begin{array}{l}0.922 \\
\end{array}$ & \\
\hline$\frac{144}{145}$ & $8 / 30 / 2004$ & $\begin{array}{ll}10: 00: 08 ~ A M \\
10.01000\end{array}$ & $\frac{24.948}{24.972}$ & $\begin{array}{l}24.707 \\
21.725\end{array}$ & \begin{tabular}{|l|}
24.542 \\
\end{tabular} & $\begin{aligned} 26.472 \\
26.47\end{aligned}$ & $\begin{array}{r}24.929 \\
2.947\end{array}$ & $\begin{array}{r}44.51 \\
44.736\end{array}$ & \begin{tabular}{|l|}
45.524 \\
\end{tabular} & $\begin{array}{r}9.312 \\
0809\end{array}$ & $\begin{array}{l}36.274 \\
25.572\end{array}$ & -1.212 & $\begin{array}{l}-6.165 \\
6223\end{array}$ & $\begin{array}{l}49.752 \\
500^{232}\end{array}$ & 0.259 & $\begin{array}{l}14.643 \\
11.643\end{array}$ & 0.003 & $\begin{array}{l}126.77 \\
127.77\end{array}$ & \begin{tabular}{ll|}
7 & 2.11278 \\
7
\end{tabular} & $\begin{array}{l}44.4 \\
15.3\end{array}$ & 11.9 & & 0.00 & \begin{tabular}{|l|l|}
0.037 \\
036
\end{tabular} & 0.038 & 0.001 & 0.930 & \\
\hline$\frac{\frac{145}{146}}{146}$ & $8 / 3 / 3 / 2004$ & $\begin{array}{l}10: 01: 08 \mathrm{AM} \\
10.0200 \mathrm{AM}\end{array}$ & $\frac{24.972}{24.997}$ & $\frac{24.725}{24.73}$ & \begin{tabular}{|l|}
24.585 \\
324.645 \\
\end{tabular} & $\frac{26.47}{26.44}$ & $\begin{array}{l}24.947 \\
24.942\end{array}$ & $\begin{array}{l}44.736 \\
44.487\end{array}$ & \begin{tabular}{|r|}
46.4 \\
45.897 \\
\end{tabular} & $\begin{array}{l}9.809 \\
9.529\end{array}$ & $\begin{array}{l}35.572 \\
35.798 \\
\end{array}$ & $\frac{-1.212}{-1.212}$ & $\begin{array}{l}-6.223 \\
-6.183\end{array}$ & $\begin{array}{r}50.033 \\
49.699 \\
\end{array}$ & $\begin{array}{l}0.252 \\
0.255\end{array}$ & $\begin{array}{l}14.643 \\
14.643 \\
\end{array}$ & $\begin{array}{l}0.003 \\
0.003\end{array}$ & $\begin{array}{l}127.77 \\
128.77\end{array}$ & \begin{tabular}{ll|} 
& 2.12944 \\
7 & 2.14611
\end{tabular} & $\begin{array}{l}45.3 \\
44.8 \\
\end{array}$ & & \begin{tabular}{|l|}
40.2 \\
30.1 \\
\end{tabular} & \begin{tabular}{|l|}
2.768 \\
2778 \\
\end{tabular} & $\begin{array}{l}0.036 \\
0.037\end{array}$ & $\begin{array}{l}0.037 \\
0.037\end{array}$ & $\begin{array}{l}0.001 \\
0.001\end{array}$ & $\begin{array}{l}0.909 \\
0.919\end{array}$ & 0.77 \\
\hline & $8 / 30 / 2004$ & 10:03:08 AM & 25.019 & 24.728 & \begin{tabular}{|l|l|}
24.640 \\
24.657
\end{tabular} & 26.372 & 24.869 & 44.614 & \begin{tabular}{|l|}
46.003 \\
\end{tabular} & 9.495 & & & & 49.806 & 0.254 & $\begin{array}{l}14.045 \\
14.643\end{array}$ & 0.003 & & \begin{tabular}{|l|l|}
7 & 2.140211
\end{tabular} & $\begin{array}{l}44.8 \\
44.9\end{array}$ & $\begin{array}{l}11.8 \\
11.9\end{array}$ & 年 $\frac{40.1}{40.3}$ & \begin{tabular}{|l|l|}
2.768 \\
2775
\end{tabular} & & & 0.001 & $\begin{array}{l}0.919 \\
0.912\end{array}$ & 0.78 \\
\hline 148 & & 10:04:08 AM & & 24.733 & \begin{tabular}{|l|l}
24.673 \\
\end{tabular} & 26.278 & 24.795 & 44.574 & \begin{tabular}{|l|}
46.117 \\
\end{tabular} & 9.556 & 35.892 & -1.218 & -6.133 & 48.077 & 0.247 & 14.643 & & 130.77 & & & & & & & & & 0.887 & 0.78 \\
\hline & $8 / 30 / 2004$ & 10:05:08 AM & 25.09 & 24.748 & 24.693 & 26.273 & 24.75 & 44.454 & \begin{tabular}{|l|}
45.617 \\
\end{tabular} & 9.423 & 36.054 & & -6.214 & 49.695 & 0.256 & 14.643 & & 131.77 & 2.19611 & 44.5 & & & & & 0.037 & & & $\frac{0.15}{0.78}$ \\
\hline 150 & & & & & 24.745 & & & 44.861 & 253 & & & & & & & & & & & & & & & & & & & $\begin{array}{l}0.88 \\
0.77\end{array}$ \\
\hline & & & & & & & & 44.701 & 6250 & 9.539 & & & & & & & & & & & & & & & & & & \\
\hline & $8 / 30 / 2004$ & 10:08:08 AM & 25.19 & 24.778 & 24.793 & 26.138 & 24.55 & \begin{tabular}{|c|c|}
44.676 \\
\end{tabular} & \begin{tabular}{|l|l|}
46.191 \\
\end{tabular} & 9.552 & & & & 504 & & 14.643 & & & 4611 & & & & & .036 & & & 0.905 & \\
\hline & & 10:09:08 AM & & 24.786 & \begin{tabular}{|l|l|}
24.846 \\
\end{tabular} & & & 44.546 & \begin{tabular}{|l|l|}
45.882 \\
\end{tabular} & 9.534 & 35.919 & & & & & $\begin{array}{l}14.643 \\
\end{array}$ & & $125=$ & & 44.8 & & & & & & & & \\
\hline & $8 / 30 / 2004$ & 10:10:08 AM & 25.226 & 24.765 & 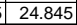 & 26.039 & 24.481 & 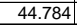 & \begin{tabular}{|l|l}
45.895 \\
\end{tabular} & 9.566 & 36.264 & -1.212 & & 48.734 & 0.253 & $\begin{array}{l}14.643 \\
\end{array}$ & & 136.7 & 2.27944 & \begin{tabular}{|l|l|}
44.8 \\
\end{tabular} & 11.6 & 40. & 2.794 & 0.036 & $\begin{array}{l}0.036 \\
\end{array}$ & 0.001 & 0.898 & \\
\hline & 8/3012004 & 10:11:08 AM & 25.24 & 24.753 & \begin{tabular}{|l|l|l|}
24.859 \\
\end{tabular} & 26.068 & 24.42 & 45.018 & 46.591 & 9.589 & 36.169 & -1.209 & -6.177 & 49.487 & 0.255 & 14.643 & & 137.7 & 2.29611 & 45.5 & 11 & & 2.799 & 0.037 & 0.037 & & 0.903 & \\
\hline & & $10: 12: 08 \mathrm{AM}$ & 25.266 & 24.74 & 24.86 & 26.074 & 24.50 & 44.371 & 45.93 & 9.553 & 35.632 & & & 50.749 & 0.262 & 14.643 & & 138.7 & . & 44.8 & & & & 0.038 & 0.038 & & 9411 & \\
\hline & & $10: 13: 08 \mathrm{AM}$ & & 24.741 & $\begin{array}{l}1 \quad 24.876 \\
\text { 1) }\end{array}$ & 26.131 & & 44.29 & 45.731 & 9.428 & & & & .99 & & 14.643 & & & & 44. & & & & & & & & \\
\hline & $8 / 30 / 2004$ & $10: 14: 08 \mathrm{AM}$ & 25.3 & 24.748 & $\begin{array}{l}84.898 \\
\end{array}$ & 26.233 & 24.60 & 44. & 46.411 & 9.491 & 36.13 & & & .115 & 0.257 & 14.643 & & & & 45 & & & & & .037 & & 0.912 & \\
\hline & 8 & $\begin{array}{ll}10.15080 \mathrm{AM} \\
10.16\end{array}$ & 255311 & 24.145 & $\begin{array}{l}0.94 .935 \\
7 \\
\end{array}$ & $\frac{20.259}{26311}$ & 24.62 & $\frac{44.124}{4.815}$ & $\begin{array}{l}46.141 \\
46.394\end{array}$ & $\begin{array}{r}9.48 \\
9539 \\
\end{array}$ & 36.143 & -1.24 & & & & $\begin{array}{l}14.043 \\
14643\end{array}$ & & 14127 & 2.30278 & & & & . & & & & & \\
\hline$\frac{16}{16}$ & 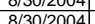 & 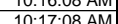 & $\frac{25.350}{25366}$ & 24.1411 & 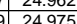 & $\frac{20.311}{26359}$ & $\frac{24.045}{24716}$ & $\frac{44.015}{4478}$ & $\begin{array}{l}40.394 \\
45.93\end{array}$ & 年. & & $\frac{-1.21}{-121}$ & & 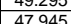 & 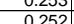 & 14.045 & & 1437 & 年 & 4 & 114 & & & 0.036 & . & & 0 & \\
\hline & $8 / 30 / 2004$ & $10: 18: 08 \mathrm{AM}$ & $\frac{20.000}{25.383}$ & 24.767 & $7 \quad 24.972$ & 26.376 & 24.7188 & $\begin{array}{l}4.10 \\
44.85\end{array}$ & $\begin{array}{r}4.50 .396 \\
46\end{array}$ & $\begin{array}{l}9.450 \\
9.434\end{array}$ & $\begin{array}{l}30.001 \\
36.198\end{array}$ & $\frac{-1.212}{-1.215}$ & $\frac{-0.140}{-6.107}$ & $\frac{47.345}{50.425}$ & $\begin{array}{l}0.204 \\
0.249\end{array}$ & $\frac{14.045}{14.643}$ & & $\begin{array}{l}14.1 .1 \\
144.7\end{array}$ & $\frac{2.0901}{2.4127}$ & $\begin{array}{r}44.0 \\
45.3\end{array}$ & $\frac{11}{12}$ & 40 & 2794 & 0 & 0.030 & $\begin{array}{l}0.001 \\
0.001\end{array}$ & $\frac{0.891}{0.880}$ & $\frac{0.76}{0.75}$ \\
\hline & $8 / 30 / 2004$ & 10:19:08 AM & 25.4 & 24.779 & \begin{tabular}{|l|l|}
9 & 24.912 \\
\end{tabular} & 26.358 & 24.83 & 44.726 & 46.313 & 9.591 & 35.917 & -1.212 & $\frac{.1 .11}{-6.11}$ & 48.909 & 0.255 & 14.643 & & 145.7 & 2.42944 & 45.2 & & & 2.780 & 0.037 & 0.037 & 0.001 & 0.905 & $\begin{array}{l}0.15 \\
0.77\end{array}$ \\
\hline & & $10: 20: 08 \mathrm{AM}$ & & & & 26.381 & & & & & 35.81 & & & & & 14.643 & & $\begin{array}{l}14.1 \\
146.7\end{array}$ & & 44.9 & & & & & 0.036 & & & \\
\hline & $8 / 30 / 2004$ & 10:21:08 AM & 25.452 & 24.816 & \begin{tabular}{|l|l|}
6 & 25.041 \\
\end{tabular} & 26.39 & 24.872 & 44.883 & \begin{tabular}{|l|l|}
46.425 \\
\end{tabular} & 9.546 & 36.256 & -1.212 & & 49.303 & 0.254 & 14.643 & & & & 45.3 & & & .797 & .036 & 0.036 & 0.001 & 0.895 & \\
\hline & & & & & & & & & $\begin{array}{l}46.432 \\
\end{array}$ & & 35.845 & & & 50.119 & 0.248 & 14.643 & & 148. & & & & & & & & & & \\
\hline & $\mid 04$ & $10: 23: 08$ AM & $\begin{array}{l}25.483 \\
25\end{array}$ & 24.826 & \begin{tabular}{|l|l|}
5625.097 \\
\end{tabular} & 26.406 & $24.89 \varepsilon$ & $\begin{array}{l}44.442 \\
\end{array}$ & 45.841 & 9.665 & & -1.212 & & 503 & 0.249 & 14.643 & & 149. & & 44 & & & & & & & 0.887 & \\
\hline & & 10:24:08 & 25.51 & 24.843 & $\begin{array}{ll}325.094 \\
\end{array}$ & 26.453 & 24.94 & 44.85 & \begin{tabular}{|l|l|}
46.318 \\
\end{tabular} & 9.822 & 35.784 & -1. & & 56 & 0.25 & 14.643 & & & & 45 & & & & .036 & 0.036 & & & \\
\hline & $8 / 30 / 2004$ & $10: 25: 08 \mathrm{AM}$ & 25.538 & 24.866 & \begin{tabular}{l|l|}
5 & 25.067 \\
\end{tabular} & 26.551 & 24.93 & 44.745 & 46.332 & 9.709 & 35.78 & -1.212 & & 47.192 & $\begin{array}{ll}0.244 \\
\end{array}$ & 14.643 & & $\begin{array}{l}151.7 \\
\end{array}$ & 2.52944 & \begin{tabular}{l|l|}
45.2 \\
\end{tabular} & 11.2 & & 277. & 0.035 & 0.035 & 0.001 & & \\
\hline & $8 / 30 / 2004$ & $10: 26: \mathrm{C}$ & 25.535 & 24.873 & $\begin{array}{ll}3 & 25.029 \\
\end{array}$ & 26.533 & 24.915 & 444.664 & \begin{tabular}{|l|}
46.044 \\
\end{tabular} & 9.603 & 36.015 & -1.215 & & 49.441 & 0.25 & 14.643 & & 152. & 2.54611 & 44 & 11 & & & 0.036 & 0.036 & & 0.887 & \\
\hline & $81 !$ & 27:08 AM & 25.533 & 24.881 & 25.016 & 26.55 & & 44.869 & 46.353 & 9.785 & & -1 & & 49.253 & 0.244 & 14 & & & & 45 & & & & & & & & \\
\hline & & & 25.508 & & 25.002 & 26.576 & $\frac{24.8}{2.7}$ & 44.56 & .843 & 9.447 & & & & & 0.252 & 14.643 & & & & & & & & & & & & \\
\hline & & $\begin{array}{l}10290.08 \\
0.020\end{array}$ & $\frac{25.513}{25518}$ & & 24.992 & 26.551 & 24.15 & -4.4.94 & 46.517 & $\begin{array}{l}9.62 \\
9.58\end{array}$ & & -1.20 & & 20.2 & & & & 155.1 & & & & & & & & & & \\
\hline & & 10.30 .00 & 23.518 & 24.097 & $\frac{24.902}{5.92}$ & $\frac{20.501}{26399}$ & $\frac{24.05}{2.58}$ & (44.1010 & (40.251 & 势 & & & & 49. & & & & & & & & & & & & & & \\
\hline & $8 / 30 / 2004$ & $\begin{array}{l}10.51 .0 \mathrm{AM} \\
10.32: 08 \mathrm{AM}\end{array}$ & $\begin{array}{r}25.400 \\
25.495\end{array}$ & 24.875 & $\begin{array}{l}24.92 \\
3 \quad 24.923 \\
\end{array}$ & $\mid$\begin{tabular}{|l|l|}
26.3898 \\
\end{tabular} & $\frac{24.50}{24.53}$ & $\frac{43.020}{44.444}$ & \begin{tabular}{|r|}
46.036 \\
\end{tabular} & 9.681 & 35.507 & $\frac{-1.212}{-1.212}$ & & \begin{tabular}{|l|}
5.004 \\
49847 \\
\end{tabular} & 0.231 & $\frac{14.045}{14.643}$ & & 158.77 & $\frac{2.02944}{2.64611}$ & $\frac{74.0}{44.9}$ & 11.1 & 4 & 2756 & 0.034 & $\begin{array}{l}.030 \\
0.034\end{array}$ & & 0.8 & \\
\hline & $8 / 30 / /$ & $10: 33: 08$ & 25.49 & 24.883 & $\begin{array}{ll}3 & 24.948 \\
\end{array}$ & 26.348 & 24.455 & & \begin{tabular}{|l|l|}
46.011 \\
\end{tabular} & & & -1. & & 52.711 & 0. & 14.643 & & 159.77 & & 44.9 & 12 & & & & 0.6 & & & \\
\hline & $8 / 30 / 2$ & 10:34:08 AM & 25.483 & & \begin{tabular}{|l|l}
24.927 \\
\end{tabular} & 26.301 & 24.443 & $\begin{array}{l}44.546 \\
\end{array}$ & \begin{tabular}{|l|l|}
45.988 \\
\end{tabular} & 9.661 & 35.792 & & & & & 14.643 & & 160.77 & \begin{tabular}{|l|l|}
2.67944 \\
\end{tabular} & \begin{tabular}{|l|l|}
44.9 \\
\end{tabular} & 12 & & 2.7 & & 0.6 & & & 0 \\
\hline & & 10:35: & 25.481 & & \begin{tabular}{l|l|l}
6 & 24.92 \\
\end{tabular} & 26.334 & 24.44 & & \begin{tabular}{|l|l} 
& 45.88 \\
\end{tabular} & & & -1 & & & & 14 & & 161. & 2.69611 & 44.8 & 11 & & & & & & & \\
\hline & & 10:36:08 AM & 25.49 & & & 26.318 & 24.4 & & 46.512 & 9.753 & & & & 48.465 & & & & 16 & & 45 & & & & & & & & \\
\hline & $8 / 30 / 2004 \mid$ & 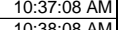 & $\begin{array}{r}25.493 \\
25.482\end{array}$ & 24.872 & $\begin{array}{l}2424.907 \\
1\end{array}$ & $\begin{array}{r}26.276 \\
26.25\end{array}$ & $\frac{24.42}{24.51}$ & 44.633 & $\begin{array}{l}46.071 \\
44417\end{array}$ & \begin{tabular}{|l|l|}
9.684 \\
9827
\end{tabular} & $\begin{array}{l}35.866 \\
35712 \\
5712\end{array}$ & -1.212 & & \begin{tabular}{|l|}
51.149 \\
50.584
\end{tabular} & 0.247 & $\frac{14.643}{14.643}$ & & $\begin{array}{l}163.77 \\
164.77\end{array}$ & \begin{tabular}{l|l}
7 & 2.72944 \\
7 & 274611
\end{tabular} & $\begin{array}{r}44.9 \\
45.3 \\
\end{array}$ & $\frac{12.2}{120}$ & 40 & 2776 & & & 0.00 & 0.881 & 75 \\
\hline$\frac{\frac{1}{18}}{2}$ & $\mid 8 / 3 / 30 / 2004$ & $\begin{array}{l}\frac{10: 38: 00 \mathrm{AM}}{1039 \cdot 08 \mathrm{AM}} \\
\end{array}$ & $\frac{25.482}{25.481}$ & & $\begin{array}{l}124.896 \\
524945\end{array}$ & $\begin{array}{r}26.25 \\
26.269\end{array}$ & $\frac{24.512}{24.576}$ & & \begin{tabular}{|l|}
46.417 \\
46.17 \\
\end{tabular} & & & $\frac{-1.215}{-1.212}$ & & \begin{tabular}{|l|}
50.584 \\
51.251
\end{tabular} & & & & 164.11 & & & $\frac{12.0}{12.2}$ & & & & & & & \\
\hline & & $10: 40:$ & 25.5 & & & $\mid \frac{20.203}{26.313}$ & & & $\begin{aligned} 46.114 \\
66.034\end{aligned}$ & & & & & & & & & & & & & & & & & & & \\
\hline & $8 / 30 / 20$ & 10:41: & 25.494 & 24.898 & & 26.342 & 24.68 & & 46.595 & 9.6 & & -1.2 & & & & & & $\frac{16}{16}$ & & & & & & & & & 0.847 & \\
\hline & $8 / 3$ & & & & & & & & & & & & & & & & & & & & & & & & & & & \\
\hline & & $10: 43: 08$ & 25.517 & 24.941 & 124.97 & 26.5 & 24.8 & 44.738 & 123 & 9.7 & & -1.20 & & 53.22 & 0.235 & & & 169. & & 45 & & & & & $n 0$ & & 0.835 & \\
\hline & & & 25.50 & & & 26.5 & & & & & & & & & & & & & & & & & & & & & & \\
\hline & & & 25.50 & & 24.9 & 26.59 & 24.9 & & 46 & & & & & & & & & & & & & & & & & & & \\
\hline & & & & & & 26. & 24. & & 46 & & & & & & & & & & & & & & & & & & & \\
\hline & & 10:47 & 25 & & & 26. & & & & & & & & & & & & & & & & & & & & & & \\
\hline & & & 25.51 & & & & 24.9 & & \begin{tabular}{|l|l|}
46.419 \\
\end{tabular} & & & -1.21 & & & & & & & & 45. & 10 & & & & & & & \\
\hline & & & 25.54 & 25.025 & & & & & 45.5 & 10.2 & & & & & & & & & & & & & & & & & & \\
\hline & & & 25.53 & 25.043 & & 26.7 & & & 45.285 & & & & & & & & & & & & & & & & & & & \\
\hline & & & 25554 & $\frac{25.056}{25082}$ & 2.011 & 26.805 & 25.1 & & 45. 408 & & & -1.22 & & & & & & & & 44 & & & & & & & & \\
\hline & & & & & $\frac{25.016}{2507}$ & $\frac{20.01}{26786}$ & & & & & & & & & & & & & & & & & & & & & & \\
\hline & & 10.5.5: & 年 25.54 & & 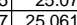 & $\frac{2.1}{26}$ & & & \begin{tabular}{|l|l|}
45.500 \\
46063
\end{tabular} & & & $\frac{-1.4}{-12}$ & & & & & & & & & & & & & & & & \\
\hline & & 10.5:5: & 25.567 & 25.112 & $\begin{array}{l}2 \\
2\end{array}$ & 267 & & & \begin{tabular}{|l|l|l|l|l|}
45762 \\
\end{tabular} & & & & & & & & & & & & & & & & & & & \\
\hline & & & & & & & & & & & & & & & & & & & & & & & & & & & & \\
\hline$\overline{2}$ & & & 25.5 & & & 26. & & 44. & \begin{tabular}{|l|l|}
45.656 \\
\end{tabular} & & & & & & & & & & & & & & & & & & & \\
\hline & & & & & \begin{tabular}{l|l}
4 & 24.
\end{tabular} & 26.6 & & & \begin{tabular}{|l|}
45.8 \\
\end{tabular} & & & & & & & & & & & & & & & & & & & \\
\hline & & $10: 59$ & 52 & 25.15 & 24.9 & 26.608 & 24 & 44.87: & 46.307 & & & -1 & & & & & & 18 & & 4 & & & & & & & & \\
\hline & & & & 25.165 & 24.8 & 26.5 & & 44. & $\overline{46.1}$ & & & -1. & & & & & & & & & & & & & & & & \\
\hline & & & & & 24 & 26.5 & & 44.7 & & & & & & & & & & 18 & & 44 & 12 & & & & & & & \\
\hline & & & & & & 26.579 & & & & & & & & & & & & & & & & & & & & & & \\
\hline & & 11:03 & & 25.18 & 24.8 & 26.52 & & 44.894 & 46.255 & 9.9 & & -1.212 & & & 0.2 & & & 18 & & 45. & 12 & & & & & & 0.837 & \\
\hline & & 11:04 & 25.4 & 25.18 & 24. & 26.4 & 24.7 & & \begin{tabular}{|l|}
46.344 \\
\end{tabular} & 10.0 & & -1.212 & & 49. & & & & 199 & & & 1 & & & & 0.034 & & & \\
\hline & $8 / 3 / 20 / 2004$ & $\begin{array}{l}110508 \mathrm{AM} \\
1106: 08 \mathrm{AM}\end{array}$ & \begin{tabular}{|l|}
25.462 \\
25.448 \\
\end{tabular} & \begin{tabular}{|l|}
25.201 \\
25.203 \\
\end{tabular} & 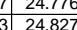 & $\begin{array}{r}26.46 \\
26.501\end{array}$ & $\begin{array}{l}24.832 \\
24.898 \\
\end{array}$ & $\begin{array}{l}45.025 \\
44.881\end{array}$ & \begin{tabular}{|l|}
46.759 \\
46.429 \\
\end{tabular} & $\begin{array}{l}9.945 \\
9.889 \\
\end{array}$ & $\begin{array}{l}35.833 \\
3.767 \\
\end{array}$ & \begin{tabular}{|l|l|l|} 
& -1.212 \\
-1.215 \\
\end{tabular} & $\begin{array}{l}-6.029 \\
-6.136\end{array}$ & $\begin{array}{r}49.191 \\
49.295 \\
\end{array}$ & 0.238 & $\frac{14.643}{14.643}$ & & $\begin{array}{l}191.77 \\
192.77\end{array}$ & $\begin{array}{ll}1 & 3.19611 \\
7 & 3.21278 \\
\end{array}$ & $\begin{array}{l}45.6 \\
45.3 \\
\end{array}$ & \begin{tabular}{|l|l|}
111. \\
11.7
\end{tabular} & 40.4 & $\mid$\begin{tabular}{|l|l|}
2.788 \\
2780
\end{tabular} & $\begin{array}{l}0.034 \\
0.032\end{array}$ & $\begin{array}{l}0.034 \\
0.033\end{array}$ & $\begin{array}{l}0.001 \\
0.001 \\
\end{array}$ & $\begin{array}{l}0.848 \\
0.810\end{array}$ & $\frac{0.76}{0.65}$ \\
\hline
\end{tabular}


WSRC-TR-2005-00105, REVISION 0

SRNL-RPP-2005-00012, REVISION 0

RUN \# 4.03A AND B; FIRST AND SECOND HALF OF SLURRY DEWATERING - CONT.

\begin{tabular}{|c|c|c|c|c|c|c|c|c|c|c|c|c|c|c|c|c|c|c|c|c|c|c|c|c|c|c|c|c|}
\hline & A & & D & 25210 & & 50 & & 5 & \begin{tabular}{|l|}
$\mathrm{K}$ \\
\end{tabular} & $\mathrm{L}$ & $\mathrm{M}$ & N & & 470 & & 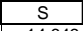 & & 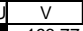 & 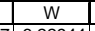 & $x$ & & 7 & & & & & $\mathrm{AE}$ & \\
\hline$\frac{21}{21}$ & $\begin{array}{l}88 / 30 / 2004 \\
8 / 30 / 2004\end{array}$ & $\begin{array}{l}\text { 11:07:08 AM } \\
11: 0808 \mathrm{AM}\end{array}$ & $\frac{25.455}{25.451}$ & $\frac{25.219}{25.211}$ & \begin{tabular}{|l|}
24.828 \\
24.82 \\
\end{tabular} & $\begin{array}{l}26.573 \\
26.659 \\
\end{array}$ & $\begin{array}{r}24.96 \\
24.981 \\
\end{array}$ & $\begin{array}{r}44.807 \\
45.222 \\
\end{array}$ & \begin{tabular}{|l|}
$\mid 46.587$ \\
4.619 \\
\end{tabular} & $\begin{array}{l}9.82 \\
9.99\end{array}$ & $\begin{array}{l}35.773 \\
36.061\end{array}$ & \begin{tabular}{|l|l|}
-1.212 \\
-1.212
\end{tabular} & $\begin{array}{l}-6.012 \\
-5.969 \\
\end{array}$ & $\begin{array}{l}47.828 \\
46.566\end{array}$ & 0.224 & $\begin{array}{l}14.643 \\
14.643 \\
\end{array}$ & 0.003 & \begin{tabular}{|l|}
193.77 \\
194.77
\end{tabular} & \begin{tabular}{l|l|l}
7 & 3.22944 \\
7 & 3.24611
\end{tabular} & $\begin{array}{l}45.5 \\
45.7 \\
\end{array}$ & $\begin{array}{l}11.4 \\
11.1 \\
\end{array}$ & \begin{tabular}{|l|}
4 \\
$\mid 40.3$ \\
140.6
\end{tabular} & \begin{tabular}{|l|}
2.778 \\
2.802 \\
\end{tabular} & \begin{tabular}{|l|}
0.032 \\
0.032 \\
\end{tabular} & 0.032 & & \begin{tabular}{|l|l}
0.800 \\
0.782 \\
\end{tabular} & $\begin{array}{l}0.68 \\
0.67\end{array}$ \\
\hline & & & 25.464 & & 24.833 & 26.682 & 25.024 & 45.022 & \begin{tabular}{|l|l|}
46.429 \\
\end{tabular} & 9.86 & 36.145 & & & 50.692 & 0.223 & 14.643 & & 195.77 & \begin{tabular}{|l|l|}
7 & 3.26278 \\
\end{tabular} & 45.3 & & & & & 0.032 & & $\begin{array}{l}0.182 \\
0.790\end{array}$ & 0.67 \\
\hline 214 & $8 / 30 / 2004$ & 11:10:08 AM & 25.452 & 25.242 & \begin{tabular}{|l|l|}
24.841 \\
\end{tabular} & 26.755 & 25.072 & 44.463 & \begin{tabular}{|l|l|}
46.038 \\
\end{tabular} & 9.76 & 35.5 & -1.212 & -6.029 & 49.199 & 0.233 & 14.643 & & 196.77 & 3.27944 & 44.9 & 11.7 & & 2.75 & & 0.034 & 0.001 & 0.838 & \\
\hline$\frac{215}{215}$ & $8 / 30 / 2004$ & 11:11:08 AM & 25.457 & 25.252 & \begin{tabular}{|l|l|}
24.831 \\
2.816
\end{tabular} & 26.79 & 25.152 & 44.859 & \begin{tabular}{|l|l|}
46.214 \\
\end{tabular} & 9.8 & $\begin{array}{l}35.925 \\
3.127\end{array}$ & -1.215 & -5.983 & 47.255 & 0.232 & 14.643 & 0.0 & 197.77 & 3.29611 & 45.1 & 11.3 & 40 & 2.78 & 0.033 & 0.033 & 0.001 & 0.826 & \\
\hline$\frac{21}{21}$ & 8/30/2004 & 11:12:08 AM & 25.462 & $\begin{array}{l}25.262 \\
2577 \\
\end{array}$ & \begin{tabular}{|l|l|}
24.846 \\
2.811
\end{tabular} & $\frac{26.815}{2683}$ & 25.167 & 45.11 & \begin{tabular}{|l|}
46.473 \\
\end{tabular} & 9.93 & $\begin{array}{l}36.137 \\
3507 \\
\end{array}$ & & & 010008 & & 14.643 & & $\begin{array}{l}198.77 \\
19077\end{array}$ & 2001 & 45.3 & 12.6 & & & & 0.023 & & 0.818 & \\
\hline$\frac{21}{21}$ & 8/30/2004 & 11:13:08 AM & 25.467 & 25.272 & 24.911 & 26.83 & 25.182 & 44.915 & \begin{tabular}{|l|}
46.577 \\
\end{tabular} & 9.84 & $\begin{array}{ll}35.929 \\
3589\end{array}$ & -1.212 & $\begin{array}{r}-5.96 \\
\end{array}$ & 49.998 & 0.231 & 14.643 & & $\begin{array}{l}199.77 \\
20.77\end{array}$ & & 45.5 & 11 & & & 0.033 & 0.033 & 0.001 & 0.820 & \\
\hline$\frac{21}{21}$ & 8/30/2004 & $\begin{array}{l}11: 14: 08 \mathrm{AM} \\
111.1550 \mathrm{AM}\end{array}$ & $\begin{array}{r}25.464 \\
25.474\end{array}$ & $\begin{array}{l}25.283 \\
25288\end{array}$ & \begin{tabular}{|l|}
24.902 \\
24.892
\end{tabular} & 26.877 & $\begin{aligned} 25.259 \\
25239\end{aligned}$ & $\begin{array}{l}45.213 \\
45.184\end{array}$ & \begin{tabular}{|l|}
46.861 \\
65.281 \\
\end{tabular} & $\frac{10.00}{0.01}$ & 5.886 & $\begin{array}{l}-1.215 \\
-1212 \\
\end{array}$ & -6 & $\begin{array}{r}50.3 \\
50021\end{array}$ & 0.234 & $\begin{array}{l}14.643 \\
14.643 \\
\end{array}$ & & 200.77 & .34611 & 45.7 & & & \begin{tabular}{|l|l|}
2.796 \\
\end{tabular} & 0.034 & 0.034 & 0.001 & 0.828 & \\
\hline$\frac{\frac{213}{220}}{220}$ & $\begin{array}{l}88 / 30 / 2004 \\
8 / 30 / 2004\end{array}$ & $\begin{array}{l}\frac{11: 15: 00 \mathrm{AM}}{11: 16: 08 \mathrm{AM}} \\
\text { 19. }\end{array}$ & $\begin{array}{l}25.474 \\
25.489\end{array}$ & $\frac{25.28 \varepsilon}{25.29 \varepsilon}$ & \begin{tabular}{|l|}
24.892 \\
24.887 \\
\end{tabular} & 26.917 & $\begin{array}{l}25.239 \\
25.199 \\
\end{array}$ & $\begin{array}{l}45.184 \\
45.195 \\
\end{array}$ & \begin{tabular}{|l|}
46.384 \\
46.912 \\
\end{tabular} & 9.84 & $\begin{array}{r}36.387 \\
36.21 \\
\end{array}$ & \begin{tabular}{|l|l|}
-1.212 \\
-1.212 \\
\end{tabular} & $\begin{array}{l}-6.021 \\
-5.972 \\
\end{array}$ & \begin{tabular}{|l|}
50.021 \\
48.702
\end{tabular} & $\begin{array}{l}0.225 \\
0.228\end{array}$ & $\begin{array}{l}\frac{14.643}{14.643} \\
\end{array}$ & 0.0 & $\frac{201.77}{202.77}$ & 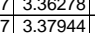 & $\frac{45.3}{45.8}$ & 11.6 & & \begin{tabular}{|l|}
2.812 \\
2.806 \\
\end{tabular} & \begin{tabular}{|l|}
0.032 \\
0.033
\end{tabular} & $\begin{array}{l}0.032 \\
0.033\end{array}$ & 0.001 & $\begin{array}{l}0.792 \\
0.805 \\
\end{array}$ & 0.67 \\
\hline 221 & $8 / 30 / 2004$ & 11:17:08 AM & 25.49 & 25.31 & 24.889 & 26.948 & 25.19 & 45.143 & \begin{tabular}{|l|l|}
46.734 \\
\end{tabular} & 9.95 & 36.003 & -1.212 & -5.948 & 49.545 & 0.227 & 14.643 & 0.003 & 203.77 & \begin{tabular}{|l|l|}
7 & 3.39611 \\
\end{tabular} & 45.6 & 11.8 & & \begin{tabular}{|l|}
2.0007 \\
2.797 \\
\end{tabular} & \begin{tabular}{|l|}
0.035 \\
0.032 \\
\end{tabular} & $\begin{array}{l}0.035 \\
0.033\end{array}$ & 0.001 & $\begin{array}{l}0.003 \\
0.803\end{array}$ & $\begin{array}{l}0.68 \\
0.68\end{array}$ \\
\hline & $8 / 30 / 2004$ & 11:18:08 AM & 25.481 & 25.315 & \begin{tabular}{|l|l|}
24.874 \\
\end{tabular} & 26.889 & 25.136 & 45.193 & \begin{tabular}{|l|}
46.628 \\
\end{tabular} & 9.93 & 36.104 & \begin{tabular}{|l|l|} 
\\
\end{tabular} & & 45.709 & 0.229 & 14.643 & & 204.77 & $\begin{array}{ll}77 & 3.41278 \\
\end{array}$ & 45.5 & & & 2.803 & & & & & \\
\hline 223 & $8 / 30 / 2004$ & 11:19:08 AM & $\begin{array}{l}25.476 \\
25.798\end{array}$ & 25.321 & 24.87 & 26.849 & 25.081 & 45.344 & \begin{tabular}{|l|}
46.885 \\
\end{tabular} & 9.98 & \begin{tabular}{|l|l|}
36.128 \\
6
\end{tabular} & & & 48.385 & 0.232 & 14.643 & & $\begin{array}{l}205.77 \\
23.77\end{array}$ & $\begin{array}{ll}73.42944 \\
\end{array}$ & 45.8 & 11 & & 2.809 & & 0.033 & & 0.818 & \\
\hline$\frac{224}{325}$ & $8 / 30 / 2004$ & $\begin{array}{ll}11: 20: 08 \mathrm{AM} \\
111: 21\end{array}$ & $\begin{array}{l}25.478 \\
25.474\end{array}$ & & \begin{tabular}{|l|l|}
24.906 \\
2490
\end{tabular} & $\begin{array}{l}26.826 \\
26.802\end{array}$ & $\begin{array}{l}25.073 \\
25.024\end{array}$ & 45.261 & \begin{tabular}{|l|}
46.817 \\
\end{tabular} & 9.85 & 36.204 & \begin{tabular}{|c|}
-1.215 \\
\end{tabular} & \begin{tabular}{|l|l|}
-5.948 \\
.006
\end{tabular} & \begin{tabular}{|l|l|}
49.072 \\
\end{tabular} & 0.217 & $\begin{array}{l}14.643 \\
1.642\end{array}$ & & 206.77 & 44611 & 45.7 & & & $\begin{array}{l}2.808 \\
2.012\end{array}$ & \begin{tabular}{|l|l|}
0.031 \\
032
\end{tabular} & 0.031 & 0.001 & 0.765 & \\
\hline$\frac{22}{22}$ & $8 / 30 / 2004$ & $\begin{array}{l}11: 21: 08 \mathrm{AM} \\
1112020\end{array}$ & $\begin{array}{l}25.474 \\
25.495\end{array}$ & $\begin{aligned} 25.339 \\
25.34\end{aligned}$ & \begin{tabular}{|l|}
24.918 \\
24.898
\end{tabular} & 26.802 & $\frac{25.024}{24.98}$ & $\begin{array}{l}45.263 \\
45.103\end{array}$ & \begin{tabular}{|r|}
46.631 \\
4678 \\
\end{tabular} & $\frac{9.81}{977}$ & 6.332 & $\begin{array}{l}-1.218 \\
-1.218\end{array}$ & & $\begin{array}{r}50.63 \\
47.211\end{array}$ & 0.228 & $\begin{array}{r}14.643 \\
114.643\end{array}$ & & $\begin{array}{l}207.77 \\
20.77\end{array}$ & & $\begin{array}{r}45.5 \\
\end{array}$ & & & 2.813 & 0.033 & 0.033 & & 0.802 & \\
\hline & $\begin{array}{l}88 / 30 / 2004 \\
8 / 30 / 2004\end{array}$ & $\frac{11: 22: 00 \mathrm{AM}}{11: 23: 08 \mathrm{AM}}$ & $\frac{25.495}{25.504}$ & $\begin{array}{r}25.34 \\
25.354\end{array}$ & \begin{tabular}{|l|}
24.898 \\
24.923 \\
\end{tabular} & $\frac{26.778}{26.747}$ & & $\frac{45.103}{45.302}$ & \begin{tabular}{|l|}
46.78 \\
46.782 \\
\end{tabular} & $\frac{9.774}{9.89}$ & $\begin{array}{r}36.223 \\
36.202 \\
\end{array}$ & $\frac{-1.218}{-1.212}$ & & $\frac{47.211}{49.808}$ & 0.228 & $\frac{14.643}{14.643}$ & & $\frac{208.77}{209.77}$ & $\frac{3.47944}{3.49611}$ & & $\frac{11.2}{110}$ & & $\frac{2.804}{2.810}$ & & $\frac{0.033}{0.033}$ & 0.001 & $\begin{array}{r}0.805 \\
0.813\end{array}$ & 0.69 \\
\hline & $8 / 30 / 2004$ & 11:24:08 AM & 25.499 & 25.353 & \begin{tabular}{|l|}
24.917 \\
\end{tabular} & 26.712 & 24.939 & 44.543 & \begin{tabular}{|c|}
45.926 \\
\end{tabular} & & 35.076 & -1.212 & & $\begin{array}{r}45.718 \\
45.718\end{array}$ & 222 & $\begin{array}{l}14.645 \\
14.643 \\
\end{array}$ & & 210.77 & $\begin{array}{l}3.49011 \\
3.51278\end{array}$ & $\begin{array}{l}45.1 \\
44.8 \\
\end{array}$ & 10.9 & & $\frac{2.810}{2.745}$ & 0.032 & 0.035 & 0.001 & $\begin{array}{l}.813 \\
0.800\end{array}$ & $\begin{array}{l}0.69 \\
0.68\end{array}$ \\
\hline & & 11:25:08 AM & 25.488 & & \begin{tabular}{|l|}
24.896 \\
\end{tabular} & 26.656 & & & \begin{tabular}{|l|l|}
45.835 \\
\end{tabular} & 10.18 & $\begin{array}{l}35.067 \\
\end{array}$ & & & & 0.22 & 14.643 & & 211.77 & \begin{tabular}{l|l}
7 & 3.52944 \\
\end{tabular} & \begin{tabular}{|l|l}
44.7 \\
\end{tabular} & 12.0 & & & \begin{tabular}{|l|}
0.031 \\
\end{tabular} & & & 0.796 & \\
\hline & $8 / 30 / 2004$ & 11:26:08 AM & 25.507 & 25.352 & 24.916 & 26.72 & 24.937 & 44.713 & \begin{tabular}{|l|}
46.378 \\
\end{tabular} & 10.33 & 35.141 & -1.212 & & \begin{tabular}{|l}
48.569 \\
\end{tabular} & 0.223 & 14.643 & & 212.77 & \begin{tabular}{c|c}
7 & 3.54611 \\
\end{tabular} & 45.3 & 11.6 & 39.9 & $\begin{array}{l}2.753 \\
\end{array}$ & \begin{tabular}{|l|}
0.032 \\
\end{tabular} & 0.032 & 0.001 & 0.801 & \\
\hline & & & & & 24.905 & 26.694 & & & & 10.33 & & & & & & 14.643 & & & & 45.2 & & & & & & & & \\
\hline$\frac{23}{3}$ & $8 / 30 / 2004$ & $\begin{array}{l}11: 28: 0 \\
111: 20: 0\end{array}$ & $\begin{array}{r}\frac{25.511}{25.2} \\
5\end{array}$ & $\begin{array}{l}25.36 \\
25.35\end{array}$ & \begin{tabular}{|l|}
24.999 \\
24979 \\
\end{tabular} & $\begin{array}{l}26.689 \\
26.678\end{array}$ & & & \begin{tabular}{|l|}
46.187 \\
46.47 \\
\end{tabular} & $\frac{10.15}{101}$ & $\begin{array}{r}35.42 \\
35.663\end{array}$ & $\begin{array}{r}-1.206 \\
-1215\end{array}$ & & \begin{tabular}{|l}
48.953 \\
51.600
\end{tabular} & 0.224 & $\begin{array}{l}14.643 \\
1.642\end{array}$ & & \begin{tabular}{|l|l|}
214.77 \\
215.77
\end{tabular} & 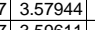 & 45.1 & 11.7 & & 2.762 & 0.032 & 0.032 & 0.001 & 0.800 & \\
\hline & $8 / 3012004$ & $\begin{array}{lll}11: 29: 08 \mathrm{AM} \\
111.3008 \mathrm{AM}\end{array}$ & $\begin{array}{r}25.52 \\
25.535 \\
\end{array}$ & $\begin{array}{r}25.35 \\
2536\end{array}$ & \begin{tabular}{|l|}
24.979 \\
24.984
\end{tabular} & 26.678 & $\frac{24.845}{2481}$ & $\begin{array}{l}44.977 \\
45045\end{array}$ & \begin{tabular}{|l|}
46.473 \\
46.531 \\
\end{tabular} & $\begin{array}{l}10.1 \\
10.1\end{array}$ & $\begin{array}{l}35.663 \\
35.683 \\
\end{array}$ & $\begin{array}{r}-1.215 \\
-1.212\end{array}$ & & \begin{tabular}{|l|}
51.068 \\
51.262
\end{tabular} & 0.219 & $\frac{14.643}{14.643}$ & & $\begin{array}{r}215.77 \\
21677\end{array}$ & $\begin{array}{l}7.59611 \\
7\end{array}$ & $\begin{array}{r}45.3 \\
45.4\end{array}$ & $\frac{12.2}{12 .}$ & $\frac{40 .}{40 .}$ & $2.780 \mid$ & \begin{tabular}{|l|}
0.031 \\
0.032
\end{tabular} & $\begin{array}{l}0.031 \\
0.032\end{array}$ & 0.001 & 0.778 & 0.66 \\
\hline 23 & $8 / 3012004$ & $\begin{array}{l}11: 31: 08 \mathrm{AM} \\
11: 3\end{array}$ & 25.535 & 25.354 & \begin{tabular}{|l|l|}
24.984 \\
24.973
\end{tabular} & $\begin{array}{l}20.023 \\
26.547\end{array}$ & & $\begin{array}{l}43.045 \\
45.105\end{array}$ & \begin{tabular}{|l|}
40.51543 \\
46.543
\end{tabular} & $\frac{1.1}{10.16}$ & $\begin{array}{l}35.003 \\
35.786\end{array}$ & $\frac{-1.212}{-1.215}$ & & & & $\begin{array}{l}\frac{14.043}{14.643} \\
\end{array}$ & & & & & $\frac{12.2}{11.6}$ & $\frac{40 .}{40 .}$ & & & & & & 0.68 \\
\hline & $8 / 30 / 2004$ & $11: 32: 08 \mathrm{AM}$ & 25.55 & 25.355 & \begin{tabular}{|l|}
24.969 \\
\end{tabular} & 26.573 & 24.9 & 44.854 & \begin{tabular}{|l|}
46.51 \\
\end{tabular} & 10.01 & $\begin{array}{l}35.78 \\
\end{array}$ & -1.212 & & \begin{tabular}{|l|l|}
49.495 \\
\end{tabular} & 0.216 & 14.643 & & 218.77 & $\begin{array}{l}73.024641 \\
3.6410\end{array}$ & 45.4 & 11.8 & & 2.780 & 0.031 & & & & 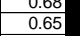 \\
\hline & & 11:33:08 AM & & 25.36 & \begin{tabular}{|l|l|}
24.979 \\
\end{tabular} & 26.643 & & & \begin{tabular}{|l|l|}
46.344 \\
\end{tabular} & 10.07 & & & & \begin{tabular}{|l|l|}
50.313 \\
5.721
\end{tabular} & & 14.643 & & & & 45.2 & & & & & & & & \\
\hline & $8 / 30 / 2004$ & 11:34:08 A & & 25.355 & \begin{tabular}{|l|}
24.979 \\
\end{tabular} & 26.683 & & & & 10.29 & 35.014 & & & \begin{tabular}{|l|}
50.724 \\
\end{tabular} & & 14.643 & & & & 44.9 & 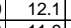 & & 2.740 & & & 0.001 & & \\
\hline$\frac{239}{240}$ & $8 / 30 / 2004$ & $\begin{array}{ll}11: 35: 08 \mathrm{AM} \\
1136\end{array}$ & $\begin{array}{l}25.554 \\
25.574\end{array}$ & $\begin{array}{l}25.344 \\
25354\end{array}$ & \begin{tabular}{|l|l|}
24.973 \\
24953
\end{tabular} & 26.717 & 25.0 & & \begin{tabular}{|l|l|}
45.916 \\
46.390 \\
\end{tabular} & 10.33 & \begin{tabular}{|l|}
34.957 \\
35.164
\end{tabular} & $\begin{array}{r}-1.215 \\
1218 \\
1218\end{array}$ & & \begin{tabular}{|l|l|}
49.977 \\
50.17 \\
\end{tabular} & 0.224 & 14.643 & & $\begin{array}{l}221.77 \\
202.77\end{array}$ & $\begin{array}{c}7 \\
7\end{array}$ & $\begin{array}{l}44.8 \\
4.5\end{array}$ & 11.9 & & 2.735 & \begin{tabular}{|l|l|}
0.032 \\
\end{tabular} & 0.032 & 0.001 & 0.809 & 0.69 \\
\hline & $8 / 30 / 2004$ & $\begin{array}{lll}11: 36: 08 \mathrm{AM} \\
1113700 \mathrm{AM}\end{array}$ & $\frac{25.574}{25.564}$ & $\begin{array}{r}25.354 \\
25354\end{array}$ & \begin{tabular}{|l|}
24.953 \\
25.073 \\
\end{tabular} & $\frac{26.772}{26847}$ & $\frac{25.12}{25.17}$ & & \begin{tabular}{|l|}
46.369 \\
46517
\end{tabular} & 10.40 & \begin{tabular}{|l|}
35.164 \\
35774
\end{tabular} & \begin{tabular}{|l|l|} 
& -1.218 \\
-1218
\end{tabular} & & \begin{tabular}{|l|}
50.417 \\
51.161
\end{tabular} & 0.213 & $\frac{14.643}{14643}$ & & & & 45.2 & 12.0 & & & & 0.031 & & 0.764 & \\
\hline$\frac{241}{242}$ & 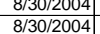 & $\begin{array}{ll}\frac{11: 37}{11: 088} \mathrm{AM}\end{array}$ & & & & & & & & & & & & $\begin{array}{l}51.164 \\
5.265\end{array}$ & & $\frac{14.643}{14.643}$ & & 223.77 & & & & 40.4 & & & & & & 0.67 \\
\hline & $8 / 30 / 2004$ & $11: 39: 08 \mathrm{AM}$ & 25.622 & 25.387 & \begin{tabular}{|l|}
25.096 \\
\end{tabular} & 26.935 & 25.31 & 44.763 & \begin{tabular}{|l|}
46.216 \\
\end{tabular} & 10.12 & \begin{tabular}{|c|}
35.663 \\
\end{tabular} & \begin{tabular}{|l|l|} 
& -1.212 \\
\end{tabular} & & \begin{tabular}{|l|}
47.747 \\
\end{tabular} & 0.216 & 14.643 & & 225.77 & & 45.1 & $\frac{12.0}{11.4}$ & 40 & 2.7 & 0.031 & 0.031 & 0.001 & $\begin{array}{l}0.105 \\
0.767\end{array}$ & \\
\hline & $8 / 30 / 2$ & 11:40: & 25.63 & 25.393 & & 26.956 & & & \begin{tabular}{|l|l|}
46.446 \\
\end{tabular} & 10.12 & 35. & & & 48.314 & 0.221 & 14.6 & & & & 45. & 11.5 & & & & & & & \\
\hline 245 & $8 / 30 / 2$ & 11:41:0 & 25.65. & 25.405 & \begin{tabular}{|l|l|}
25.084 \\
\end{tabular} & 27.023 & 25.3 & 44 & \begin{tabular}{|l|l|}
46.291 \\
\end{tabular} & 9.9 & & & & 49. & & 14.643 & & & & 45 & 11 & & & & & & & \\
\hline & $8 / 30 / 2$ & 11:42:08 & $\begin{array}{r}25.64 \\
25.648\end{array}$ & $\begin{array}{r}25.41 \\
25418\end{array}$ & \begin{tabular}{|l|}
25.074 \\
25087
\end{tabular} & $\begin{array}{l}27.043 \\
27.041\end{array}$ & $\frac{25}{252}$ & $\begin{array}{l}44.782 \\
4.995\end{array}$ & \begin{tabular}{|l|}
46.376 \\
\end{tabular} & 10.60 & & & & 49. & & 14.6 & & & & 45.3 & 12 & & & & & & & \\
\hline & & $\begin{array}{l}11: 43: 088 \\
11040.88\end{array}$ & & & & $\begin{aligned} 27.041 \\
26.988\end{aligned}$ & & & & & & -1 & & & & 14.6 & & & & 45.4 & 12. & & & & & & & \\
\hline & & $\frac{11.44}{11: 45}$ & $\frac{25.665}{25.643}$ & & & $\frac{26.9}{26.8}$ & & & & & & & & & & & & & & & & & & & & & & 0.65 \\
\hline & $8 / 30 / 2004$ & $11: 46$ & 25.655 & 25.44 & \begin{tabular}{|l|l|} 
& 25.164 \\
\end{tabular} & 26.833 & & & & 10.33 & & -1.21 & & & & 14.643 & & & & 45 & 11. & & & & & & & \\
\hline 25 & & & 25.67 & 25.435 & & 26.803 & & & & & & & & & & & & & & 45. & 11 & & & & & & & \\
\hline & $8 / 30 /$ & 11:48: & 25.679 & 25.434 & 25.153 & 26.852 & & 44.684 & & 10.21 & 35.385 & -1.212 & & & & 14.6 & & & & 45. & 12. & 4 & & & & & & \\
\hline & & & $\begin{array}{l}25.698 \\
25.707\end{array}$ & & & & & 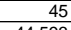 & & & & & & & & & & & & 45 & 11. & & & & & 0.0 & & \\
\hline & & & 25.70 & 25. & \begin{tabular}{|l|}
25.136 \\
25.12
\end{tabular} & & & & & & & & & & & & & & & 45 & & & & & & & & \\
\hline & $8 / 30 / 2$ & $\frac{11: 51: 0}{11.52: 0}$ & $\frac{25.713}{25.737}$ & $\begin{aligned} 25.452 \\
2546\end{aligned}$ & \begin{tabular}{|l|}
25.142 \\
25246
\end{tabular} & $\frac{26.7}{26}$ & & $\frac{45}{445}$ & \begin{tabular}{|l|}
46.566 \\
\end{tabular} & 10 & & $\begin{array}{r}-1.218 \\
-1209\end{array}$ & & \begin{tabular}{|l|}
51 \\
53
\end{tabular} & $\begin{array}{r}0.21 \\
0.11\end{array}$ & 14 & & & & 45.4 & 127 & & & & & 1001 & $\begin{array}{l}0.142 \\
0.710\end{array}$ & \\
\hline & $8 / 30 / 2$ & $\begin{array}{l}1115: 5: 0 \\
11: 53: 0\end{array}$ & $\begin{array}{l}25.737 \\
25.711\end{array}$ & $\begin{array}{r}25.467 \\
25.45\end{array}$ & \begin{tabular}{|l|}
25.246 \\
25.1
\end{tabular} & 26. & & $\begin{aligned} 44.921 \\
45\end{aligned}$ & \begin{tabular}{|r|}
46.32 \\
46833 \\
\end{tabular} & $\begin{array}{r}10.42 \\
10.3\end{array}$ & & $\begin{array}{l}-1.209 \\
-1.215\end{array}$ & & & 0.211 & & & & & $\begin{array}{l}45.2 \\
45.4 \\
\end{array}$ & \begin{tabular}{|l|}
12.7 \\
127
\end{tabular} & $\frac{40}{40}$ & & & & & $\frac{0 .}{0 .}$ & \\
\hline 25 & $8 / 30 /$ & & & & \begin{tabular}{|l|l|}
25.204 \\
\end{tabular} & 26.688 & & 44.844 & & & & \begin{tabular}{|l|} 
\\
-1.215 \\
\end{tabular} & & & & & & & & 45.1 & 11 & & & & & & & \\
\hline 25 & & & 25.749 & & & 26. & & & & & & & & & & 14. & & & & & 12 & & & & & & & \\
\hline 260 & & & 25.753 & 25 & & 26. & & & & & & & & 48. & & & & & & 45 & 11 & & & & & & & \\
\hline 26 & $8 / 30 / 2004$ & $\begin{array}{ll}11: 57: 0 \\
111.58 \cdot 0\end{array}$ & $\begin{array}{l}25.753 \\
25757\end{array}$ & $\begin{array}{l}25.453 \\
25.525\end{array}$ & \begin{tabular}{|l|}
25.177 \\
25.191
\end{tabular} & $\begin{array}{r}26.751 \\
26.81\end{array}$ & & $\begin{array}{r}44.981 \\
4.983\end{array}$ & \begin{tabular}{|l|}
46.452 \\
\end{tabular} & $\frac{10.22}{12.2}$ & $\begin{array}{r}35.626 \\
25.619\end{array}$ & & & {$[4$} & 0.215 & 14.6 & & & & 45. & 11.5 & & & & & & & \\
\hline & & & $\begin{array}{l}25.757 \\
25776\end{array}$ & & 25.181 & & & & \begin{tabular}{|l|l|}
46.6 \\
46.5
\end{tabular} & & & & & & 0.214 & & & & & 45. & 11. & & & & & & & \\
\hline$\frac{26}{26}$ & & & $\frac{23.1}{25.7}$ & $\frac{25.4}{25.4}$ & \begin{tabular}{|l|}
25.2 \\
\end{tabular} & & & & & & & & & & 0. & & & & & & 11. & & & & & & & \\
\hline & $8 / 30 / 2$ & & & 25.437 & 25.2 & & & 44. & 46 & & & & & & & & & & & \begin{tabular}{|l|l|}
45.4 \\
\end{tabular} & 12.2 & & & & & & & \\
\hline & & & & & & & & & & & & & & & & & & & & & & & & & & & & \\
\hline & & & & & & 26.7 & & & & & & & & & & & & & & & & & & & & & & \\
\hline & & & & & & & & & & & & & & & & & & & & & & & & & & & & \\
\hline & & & & & $\frac{25.239}{35241}$ & & & & & & & & & & & & & & & & & & & & & & & \\
\hline & 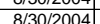 & & 80 & $\frac{25.41}{2542}$ & 25.206 & $\frac{2.00}{26.9}$ & & & & & 35.662 & & & & & 14. & & & & & & & & & & & & \\
\hline & $8 / 3$ & & & & 25.1 & 271 & & & & & & & & & & & & & & & & & & & & & & \\
\hline & & & 25.80 & 25.48 & \begin{tabular}{|l|}
25.1 \\
\end{tabular} & 27.129 & & & & & & & & & & & & & & & & & & & & & & \\
\hline & & & & & \begin{tabular}{|l|}
25.21 \\
\end{tabular} & 27.1 & & & & & & & & & & 14. & & & & & & & & & & & & \\
\hline & & & & & 20 & & & & & & & & & & & & & & & & & & & & & & & \\
\hline & 8/30/2004 & $12: 11$ & 25.802 & 25.532 & \begin{tabular}{|l|l|}
25.141 \\
\end{tabular} & 27.215 & 25.362 & & 46.228 & 10.48: & & -1.209 & & & 0.204 & 14 & & & & & & & & & & & & \\
\hline & & & & & & 27.743 & & & & & & & & & & & & & & & & & & & & & & \\
\hline & & & & & & 2.145 & & 45. & 46.647 & & & & & & & & & & & 45 & & & & & & & & \\
\hline & & & 704 & 25.549 & & .102 & & $\frac{45.412}{45.139}$ & $\mid$\begin{tabular}{|l|l|} 
& 46.958 \\
\end{tabular} & 34 & & -1.215 & & & 0.194 & & & 262.60 & \begin{tabular}{|l|l|} 
\\
\end{tabular} & $45.8]$ & 12.5 & & & & & 0.00 & 0.682 & \\
\hline & & & & & & & & & & & & & & & & & & & & & & & & & & & & \\
\hline
\end{tabular}


WSRC-TR-2005-00105, REVISION 0

SRNL-RPP-2005-00012, REVISION 0

RUN \# 4.03A AND B; FIRST AND SECOND HALF OF SLURRY DEWATERING - CONT.

\begin{tabular}{|c|c|c|c|c|c|c|c|c|c|c|c|c|c|c|c|c|c|c|c|c|c|c|c|c|c|c|c|c|}
\hline & A & & & & & & $\therefore$ & $\mathrm{J}$ & $\mathrm{K}$ & 2052 & $M$ & N & & 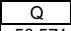 & & 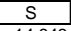 & & V & דרת & $x$ & & 7 & AA & & & & $\mathrm{AE}$ & \\
\hline & $\begin{array}{l}8 / 300 / 2004 \\
8 / 30 / 2004\end{array}$ & $\begin{array}{l}\text { 12:16:58 PM } \\
12: 17: 58 \mathrm{PM}\end{array}$ & $\frac{25.678}{25.684}$ & 25.548 & \begin{tabular}{|l|}
24.957 \\
24.963 \\
\end{tabular} & 27.081 & $\begin{array}{l}25.228 \\
25.264\end{array}$ & $\begin{array}{l}45.622 \\
44.929 \\
\end{array}$ & \begin{tabular}{|l|}
47.377 \\
46.374 \\
\end{tabular} & $\begin{array}{l}10.562 \\
10.292 \\
\end{array}$ & $\begin{array}{l}35.782 \\
3.503 \\
\end{array}$ & $\begin{array}{l}-1.215 \\
-1.209 \\
\end{array}$ & $\begin{array}{l}-5.575 \\
-5.833 \\
\end{array}$ & $\frac{50.571}{48.333}$ & $\frac{0.196}{0.202}$ & $\begin{array}{l}14.643 \\
14.643 \\
\end{array}$ & $\frac{0.003}{0.004}$ & $\begin{array}{l}263.60 \\
264.60 \\
\end{array}$ & $\begin{array}{r}4.39333 \\
4.41 \\
\end{array}$ & \begin{tabular}{r|r|}
46.3 & 45.2 \\
\end{tabular} & $\frac{12.0}{11.5}$ & $\begin{array}{l}40.7 \\
40.3 \\
\end{array}$ & \begin{tabular}{|l|}
2.806 \\
2.776 \\
\end{tabular} & \begin{tabular}{|l|}
0.028 \\
0.029
\end{tabular} & 0.028 & $\frac{0.00}{0.00}$ & $\frac{0.690}{0.719}$ & \\
\hline & $8 / 30 / 2004$ & & 25.663 & & \begin{tabular}{|l|}
24.937 \\
\end{tabular} & 27.026 & 25.208 & 45.522 & 47.424 & 10.429 & 35.882 & & & 47.186 & 0.197 & 14.643 & & & & & & & & & & & 0.119 & \\
\hline & & & 25.642 & 25.562 & 24.876 & 26.955 & 25.242 & 44.995 & 46.919 & 10.29 & 35.585 & -1.215 & -5.639 & 47.866 & 0.197 & 14.643 & & 266.60 & \begin{tabular}{|l|l|}
4.44333 \\
\end{tabular} & 45.8 & 11.4 & 40. & & & 0.028 & & 0.702 & \\
\hline 28 & 8/30/2004 & 12:20:58 PM & 25.667 & 25.577 & \begin{tabular}{|l|l|}
24.906 \\
2.801
\end{tabular} & 27.025 & 25.197 & 45.027 & 46.506 & 10.293 & 35.64 & -1.212 & -6.183 & 49.568 & 0.201 & 14.643 & & 267.60 & & \begin{tabular}{|c|}
45.4 \\
\end{tabular} & 11.8 & 40 & 2.781 & 0.029 & 0.029 & 0.001 & 0.715 & \\
\hline & 8/30/2004 & 12:21:58 PM & 25.642 & 25.582 & \begin{tabular}{|l|l}
24.891 \\
2.801
\end{tabular} & $\begin{array}{l}26.99 \\
2699\end{array}$ & 25.132 & 45.025 & \begin{tabular}{|l|l|}
46.398 \\
4.51
\end{tabular} & 10.234 & 35.927 & & -6.15 & 50.296 & 0.2 & 14.643 & & & 4.47667 & 45.3 & 12.0 & & & & 0.029 & & 0.710 & \\
\hline$\frac{28}{28}$ & $8 / 30 / 2004$ & $\begin{array}{l}12: 22: 58 \text { PM } \\
12 \cdot 23: 58 \mathrm{PM}\end{array}$ & 25.637 & 25.582 & \begin{tabular}{|l|l|}
24.901 \\
2.081
\end{tabular} & 26.98 & 25.062 & 44.879 & 46.51 & 10.242 & 35.642 & -1.212 & -6.214 & 48.761 & 0.197 & 14.643 & & & 49333 & 45.4 & 11.6 & & 2.7. & 0.028 & 0.028 & 0.001 & 0.702 & \\
\hline & 8/30/2004 & $\begin{array}{l}12: 23: 58 \mathrm{PM} \\
12 \cdot 24: 5 \mathrm{PM}\end{array}$ & $\frac{25.622}{25.627}$ & $\begin{array}{l}25.582 \\
25577\end{array}$ & \begin{tabular}{|l|}
24.981 \\
24.95
\end{tabular} & 26.92 & 25.0 & $\begin{array}{l}45.132 \\
45.159\end{array}$ & \begin{tabular}{|l|}
46.817 \\
46.948
\end{tabular} & 10.201 & 35.845 & -1.212 & -6.22 & $\begin{array}{l}49.339 \\
5.968\end{array}$ & 0.198 & 14.643 & & 270.60 & $\begin{array}{r}4.51 \\
53667\end{array}$ & 45.7 & 11. & & 2.79 & & 0.028 & 0.001 & 0.700 & \\
\hline & $\begin{array}{r}8 / 300 / 2004 \\
8 / 30 / 2004\end{array}$ & $\begin{array}{l}\frac{12: 24.58 \mathrm{PM}}{1222558 \mathrm{PM}} \\
\end{array}$ & $\frac{25.627}{25.632}$ & $\frac{25.577}{25.582}$ & \begin{tabular}{|l|}
24.95 \\
24.945 \\
\end{tabular} & $\begin{array}{r}26.91 \\
26.885 \\
\end{array}$ & $\frac{25.08}{25.04}$ & $\begin{array}{r}45.159 \\
45.363 \\
\end{array}$ & \begin{tabular}{|r|}
46.948 \\
47.24 \\
\end{tabular} & $\frac{10.321}{10.3}$ & $\begin{array}{r}35.673 \\
35.917 \\
\end{array}$ & $\frac{-1.212}{-1.212}$ & $\begin{array}{l}-6.194 \\
-6.185 \\
\end{array}$ & $\frac{50.868}{49.115}$ & $\begin{array}{l}0.194 \\
0.195 \\
\end{array}$ & $\begin{array}{l}14.643 \\
14.643 \\
\end{array}$ & & $\frac{271.60}{272.60}$ & $\frac{4.52667}{4.54333}$ & $\frac{45.8}{46.1}$ & 111.7 & & \begin{tabular}{|l|}
2.787 \\
2.802 \\
\end{tabular} & \begin{tabular}{|l|}
0.028 \\
0.028
\end{tabular} & $\begin{array}{l}0.028 \\
0.028 \\
\end{array}$ & $\frac{0.001}{0.001}$ & 0.688 & \\
\hline & $8 / 30 / 2004$ & 12:26:58 PM & 25.621 & 25.576 & 24.92 & 26.909 & 25.101 & 45.043 & 46.915 & 10.28 & 35.636 & -1.212 & -6.113 & 47.213 & 0.194 & 14.643 & 0.00 & 273.60 & 4.56 & 45.8 & 11.2 & & \begin{tabular}{|l|}
$2.00<1$ \\
2.781 \\
\end{tabular} & \begin{tabular}{|l|l|}
0.028 \\
\end{tabular} & $\begin{array}{l}0.020 \\
0.028 \\
\end{array}$ & 0.001 & $\begin{array}{l}0.000 \\
0.690\end{array}$ & $\begin{array}{l}0.59 \\
0.59\end{array}$ \\
\hline & 8/30/2004 & 12:27:58 PM & 25.607 & 25.577 & 24.881 & 26.9 & 25.047 & 44.786 & 46.595 & 10.219 & 35.517 & -1.215 & & 45.634 & 0.194 & $\begin{array}{l}14.643 \\
\end{array}$ & & 274.60 & \begin{tabular}{|l|l|} 
\\
\end{tabular} & 45.5 & & & \begin{tabular}{|l|}
2.768 \\
\end{tabular} & & & & 0.694 & \\
\hline & $8 / 30 / 2004$ & $\begin{array}{ll}12: 28: 58 \mathrm{PM} \\
12: 20: 58\end{array}$ & $\begin{array}{l}25.592 \\
25.577\end{array}$ & $\begin{array}{l}25.582 \\
2720\end{array}$ & \begin{tabular}{|l|l|}
24.861 \\
\end{tabular} & $\begin{array}{l}26.89 \\
\end{array}$ & $\begin{array}{l}25.047 \\
2.00\end{array}$ & $\begin{array}{l}44.821 \\
\end{array}$ & \begin{tabular}{|l|l|}
46.784 \\
\end{tabular} & 10.236 & $\begin{array}{l}35.449 \\
2.720\end{array}$ & & & 49.72 & 0.195 & 14.643 & & 275.60 & \begin{tabular}{|l|l|}
4.59333 \\
\end{tabular} & 45.7 & & & 2.767 & \begin{tabular}{|l|}
0.028 \\
\end{tabular} & 0.028 & 0.001 & 0.698 & \\
\hline 29 & $8 / 30 / 2004$ & & 25.577 & & \begin{tabular}{|r|}
24.85 \\
\end{tabular} & $\begin{array}{r}26.89 \\
26.895\end{array}$ & $\begin{array}{l}25.042 \\
25077\end{array}$ & 44.855 & \begin{tabular}{|l|l|}
46.964 \\
\end{tabular} & 10.357 & 35.275 & -1.212 & & \begin{tabular}{|l|l|}
46.581 \\
\end{tabular} & 0.193 & $\begin{array}{l}14.643 \\
1.642\end{array}$ & & & & 45.8 & 11. & & $\begin{array}{l}2.762 \\
\end{array}$ & \begin{tabular}{|l|l|}
0.028 \\
\end{tabular} & $\begin{array}{l}0.028 \\
\end{array}$ & 0.001 & 0.693 & \\
\hline & $8 / 30 / 2004$ & $\begin{array}{ll}122: 30: 58 \\
12.31\end{array}$ & $\begin{array}{l}25.577 \\
25.555\end{array}$ & $\begin{array}{r}25.577 \\
25.57\end{array}$ & \begin{tabular}{|l|}
24.855 \\
24.949 \\
\end{tabular} & $\begin{array}{l}26.865 \\
26.878\end{array}$ & $\begin{array}{l}25.077 \\
25035\end{array}$ & $\begin{array}{r}44.825 \\
45217\end{array}$ & \begin{tabular}{|l|}
46.881 \\
\end{tabular} & $\begin{array}{l}10.264 \\
10.275\end{array}$ & $\begin{array}{l}35.404 \\
35816\end{array}$ & -1.212 & & $\begin{array}{r}50.84 \\
\end{array}$ & & 14.643 & & & 4.62667 & 45.8 & 12. & & $\begin{array}{l}2.766 \\
2702\end{array}$ & \begin{tabular}{|l|}
0.028 \\
0027
\end{tabular} & 0.028 & & 0.692 & \\
\hline & $88 / 3012004$ & $\frac{12: 31: 58 \mathrm{PM}}{1223258 \mathrm{PM}}$ & $\frac{25.555}{25.56}$ & $\begin{array}{r}25.57 \\
25.565\end{array}$ & \begin{tabular}{|l|}
24.949 \\
24.909 \\
\end{tabular} & $\frac{26.878}{26.898}$ & $\frac{25.035}{24.985}$ & $\frac{45.217}{44.935}$ & \begin{tabular}{|l}
47.431 \\
47.086 \\
\end{tabular} & $\frac{10.275}{10.29}$ & $\begin{array}{l}35.816 \\
3513\end{array}$ & $\frac{-1.212}{-1.212}$ & -6.194 & \begin{tabular}{|r|}
48.792 \\
50.87
\end{tabular} & $\begin{array}{l}0.192 \\
0.188 \\
\end{array}$ & $\frac{14.643}{14.643}$ & & & $\begin{array}{r}4.64333 \\
4.66\end{array}$ & 46.3 & 11.6 & & $\frac{2.793}{2.773}$ & & 0.028 & 0.001 & $\frac{0.679}{0.671}$ & \\
\hline & $8 / 30 / 2004$ & $12: 33: 58$ PM & 25.57 & 25.57 & \begin{tabular}{|l|}
24.909 \\
24.903 \\
\end{tabular} & 26.898 & 24.98 & 44.371 & \begin{tabular}{|l|}
46.417 \\
\end{tabular} & $\begin{array}{l}10.29 \\
10.153 \\
\end{array}$ & $\begin{array}{l}35.1813 \\
35.182 \\
\end{array}$ & $\frac{-1.212}{-1.212}$ & & \begin{tabular}{|r|}
09.81 \\
\end{tabular} & $\begin{array}{l}0.180 \\
0.191\end{array}$ & $\begin{array}{l}14.645 \\
14.643 \\
\end{array}$ & & 280.60 & $\begin{array}{r}4.64667 \\
4.67667\end{array}$ & $\begin{array}{l}45.0 \\
45.3\end{array}$ & 11.7 & 39 & $\frac{2.113}{2.742}$ & \begin{tabular}{|l|}
0.027 \\
0.027
\end{tabular} & 0.027 & 0.001 & $\begin{array}{l}.011 \\
0.689\end{array}$ & \\
\hline & 8/30/2004 & 12:34:58 PM & 25.569 & 25.564 & \begin{tabular}{|l|l|}
24.893 \\
\end{tabular} & 26.872 & 24.974 & & \begin{tabular}{|l|l|}
46.763 \\
\end{tabular} & 10.241 & 35.308 & & & \begin{tabular}{|l}
47.97 \\
\end{tabular} & 0.186 & $\begin{array}{l}14.643 \\
\end{array}$ & & 281.60 & \begin{tabular}{|l|l|} 
\\
\end{tabular} & 45.6 & 11.4 & & 2.757 & \begin{tabular}{|l|l|}
0.027 \\
\end{tabular} & 0.027 & & 0.668 & \\
\hline & 8/30/2004 & 12:35:58 PM & 25.564 & 25.564 & \begin{tabular}{|l|l|}
24.858 \\
\end{tabular} & 26.812 & 25.019 & 44.512 & \begin{tabular}{|l|l|}
46.326 \\
\end{tabular} & 10.062 & 35.562 & -1.215 & -6.139 & 50.421 & 0.19 & 14.643 & & 282.60 & 4.71 & 45.2 & 12.0 & & \begin{tabular}{|l|}
2.760 \\
\end{tabular} & \begin{tabular}{|l|l|}
0.027 \\
\end{tabular} & 0.027 & 0.001 & 0.682 & \\
\hline & & & & 25.572 & 24.855 & 26.895 & & & & 10.306 & & & & & & 14.643 & & & & 45.9 & & & & & & & & \\
\hline & $8 / 30 / 2004$ & $\begin{array}{ll}12: 37: 58 \mathrm{PM} \\
2038.5 \mathrm{PM}\end{array}$ & $\begin{array}{l}25.546 \\
25.551\end{array}$ & $\begin{array}{l}25.561 \\
25566\end{array}$ & \begin{tabular}{|l|}
24.834 \\
2482
\end{tabular} & $\begin{array}{l}26.889 \\
26019\end{array}$ & & $\begin{array}{l}44.728 \\
44877\end{array}$ & \begin{tabular}{|l|}
47.057 \\
47348 \\
\end{tabular} & $\begin{array}{r}10.2 \\
10319\end{array}$ & $\begin{array}{l}35.418 \\
35360 \\
350\end{array}$ & -1.212 & & \begin{tabular}{|r|}
48.317 \\
5286
\end{tabular} & $\begin{array}{l}0.192 \\
0.188\end{array}$ & 14.643 & & $\begin{array}{l}284.60 \\
\end{array}$ & 4.74333 & 45.9 & & & \begin{tabular}{|l|}
2.763 \\
2760
\end{tabular} & \begin{tabular}{|l|}
0.027 \\
0.027
\end{tabular} & 0.028 & 0.001 & 0.689 & \\
\hline & 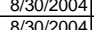 & $\begin{array}{l}12: 38: 58 \mathrm{PM} \\
122.395 \\
\end{array}$ & $\begin{array}{l}25.551 \\
25.557\end{array}$ & $\begin{array}{l}25.566 \\
25.57\end{array}$ & \begin{tabular}{|r}
24.82 \\
24.855
\end{tabular} & $\begin{array}{l}26.919 \\
26.935 \\
\end{array}$ & $\begin{array}{r}24.961 \\
24.982\end{array}$ & $\begin{array}{r}44.877 \\
44.799 \\
\end{array}$ & \begin{tabular}{|l|}
47.348 \\
47.296 \\
\end{tabular} & $\begin{array}{r}10.319 \\
10.305\end{array}$ & $\begin{array}{r}35.369 \\
35373 \\
\end{array}$ & $\begin{array}{l}-1.215 \\
-1.212\end{array}$ & $\begin{array}{r}-6.148 \\
-6099 \\
-6.09\end{array}$ & \begin{tabular}{|r|}
52.86 \\
50.233
\end{tabular} & $\begin{array}{l}0.188 \\
0.187\end{array}$ & $\begin{array}{l}14.643 \\
14.643 \\
\end{array}$ & & $\begin{array}{r}285.60 \\
28660\end{array}$ & $\begin{array}{r}4.76 \\
477666 \\
\end{array}$ & $\begin{array}{l}46.2 \\
46.2\end{array}$ & $\frac{12.6}{120}$ & 40 & 2.766 2. & \begin{tabular}{|l|}
0.027 \\
0.027
\end{tabular} & $\begin{array}{l}0.027 \\
0.027\end{array}$ & 0.001 & $\begin{array}{l}0.674 \\
0671\end{array}$ & 57 \\
\hline & $8 / 3012004$ & $\begin{array}{ll}12.39 \\
12: 40: 58 \mathrm{PM}\end{array}$ & $\frac{23.551}{25.542}$ & $\frac{25.587}{25.572}$ & \begin{tabular}{|l|}
24.855 \\
24.946 \\
\end{tabular} & 20.935 & $\begin{array}{l}24.986 \\
24.997\end{array}$ & $\begin{array}{r}44.199 \\
44.78\end{array}$ & \begin{tabular}{|l|l|}
46.296 \\
46999
\end{tabular} & & $\begin{array}{l}35.375 \\
35.474\end{array}$ & $\frac{-1.212}{-1.206}$ & & $\begin{array}{l}50.233 \\
51.185 \\
\end{array}$ & $\begin{array}{l}0.187 \\
0.186\end{array}$ & $\begin{array}{l}\frac{14.043}{14.643} \\
\end{array}$ & & & & & & 40 & & & & & & \\
\hline & $8 / 30 / 2004$ & 12:41:58 PM & 25.536 & 25.561 & \begin{tabular}{|l|} 
\\
\end{tabular} & 26.884 & 24.98 & 44.4 & \begin{tabular}{|l|}
46.645 \\
\end{tabular} & 10.145 & 35.211 & -1.209 & -6.125 & \begin{tabular}{|l|l|}
49.143 \\
\end{tabular} & 0.185 & 14.643 & & & 4.81 & 45.5 & 11.7 & & 2.744 & \begin{tabular}{|l|}
0.021 \\
0.026 \\
\end{tabular} & 0.027 & & 0.667 & \\
\hline & & $\begin{array}{ll}12: 42: 58 \text { PM } \\
12: 4250\end{array}$ & & & 24.915 & & & 44.156 & \begin{tabular}{|l|l|}
46.564 \\
\end{tabular} & & & & & & & 14.643 & & & & $\begin{array}{l}45.4 \\
51.5\end{array}$ & & & & & & & & \\
\hline & $8 / 30 / 2004$ & & & 25.561 & \begin{tabular}{|l|l|}
24.904 \\
\end{tabular} & 26.899 & & $\begin{array}{r}44.456 \\
.202\end{array}$ & \begin{tabular}{|l|l|}
46.906 \\
920
\end{tabular} & & & & & & & 14.643 & & & \begin{tabular}{|l|l|} 
\\
\end{tabular} & 45.8 & & & 2.741 & & & & & \\
\hline & $8 / 30 / 2004$ & $\begin{array}{ll}12: 44: 58 \mathrm{PM} \\
12.455 .5 \mathrm{PM}\end{array}$ & $\begin{array}{l}25.554 \\
25.552\end{array}$ & $\begin{array}{l}25.549 \\
25.547\end{array}$ & \begin{tabular}{|l|l|}
24.873 \\
\end{tabular} & $\begin{array}{l}26.892 \\
26.025\end{array}$ & 24.9 & & \begin{tabular}{|l|l|}
46.635 \\
46997
\end{tabular} & $\begin{array}{l}10.235 \\
10285\end{array}$ & $\begin{array}{l}35.059 \\
35143\end{array}$ & -1.215 & & 47.724 & 0.185 & 14.643 & & & $\begin{array}{r}4.86 \\
\end{array}$ & 45.5 & \begin{tabular}{|c|}
11.4 \\
\end{tabular} & & 2.734 & \begin{tabular}{|l|l|}
0.026 \\
020
\end{tabular} & 0.027 & 0.001 & 0.670 & \\
\hline & $8 / 30 / 2004$ & $\begin{array}{ll}12: 45: 58 \mathrm{PM} \\
12.46 .5 \mathrm{PM}\end{array}$ & $\frac{25.552}{25.55}$ & $\frac{25.547}{25545}$ & \begin{tabular}{|l|}
24.846 \\
24.834 \\
\end{tabular} & $\frac{26.925}{26.993}$ & & $\begin{array}{l}44.512 \\
44.626\end{array}$ & \begin{tabular}{|l|}
46.997 \\
4707
\end{tabular} & $\frac{10.285}{10447}$ & $\begin{array}{l}35.143 \\
35069\end{array}$ & -1.212 & & & 0.184 & $\frac{14.643}{11.642}$ & & & 4.87667 & $\begin{array}{r}45.9 \\
15.9\end{array}$ & $\begin{array}{l}11.5 \\
12\end{array}$ & & $2.746 \mid$ & & 0.026 & & 0.664 & \\
\hline & $\begin{array}{r}88 / 3012004 \\
8 / 30 / 2004\end{array}$ & & & & & & & $\begin{array}{l}44.626 \\
44.508 \\
\end{array}$ & \begin{tabular}{|r|}
47.07 \\
46.834 \\
\end{tabular} & & & & & & 0.185 & $\frac{14.643}{14.643}$ & & & 4.89333 & & $\mid 12.2$ & 39.8 & & & & 0.001 & & \\
\hline & $8 / 30 / 2004$ & $12: 48: 58 \mathrm{PM}$ & 25.549 & 25.564 & \begin{tabular}{|l|l|}
24.837 \\
\end{tabular} & 26.927 & 25.05 & 44.577 & \begin{tabular}{|l|l|}
47.093 \\
\end{tabular} & 10.495 & 35.061 & -1.215 & -6 & \begin{tabular}{|l|}
51.103 \\
\end{tabular} & 0.184 & 14.643 & & & 4.92667 & 46.0 & 12.2 & 39 & 2.745 & \begin{tabular}{|l|}
0.026 \\
\end{tabular} & 0.026 & 0.001 & 0.061 & \\
\hline & $8 / 30 /$ & 12:49: & 25.554 & 25.574 & \begin{tabular}{|l|l|}
24.848 \\
\end{tabular} & 26.912 & 24. & 44.11 & \begin{tabular}{|l|}
46.67 \\
\end{tabular} & 10.308 & 34.84 & -1. & & & 0. & 14.643 & & & \begin{tabular}{|l|l} 
\\
\end{tabular} .94333 & 45.5 & 11.8 & & & & & & 0.667 & \\
\hline & $8 / 30 /$ & 12:50: & 25.539 & 25.559 & \begin{tabular}{|l|l|}
24.812 \\
\end{tabular} & 26.937 & & 44 & \begin{tabular}{|l|l|}
47.379 \\
\end{tabular} & & 35.2 & & & \begin{tabular}{|l|l}
51.451 \\
\end{tabular} & 0.182 & 14.643 & & & \begin{tabular}{|l|}
4.96 \\
\end{tabular} & 46. & & & & & & & & \\
\hline & $8 / 30 / 2004$ & $\begin{array}{ll}12: 51: 58 \mathrm{PM} \\
12.52 \cdot 5 \mathrm{PM}\end{array}$ & $\begin{array}{r}25.529 \\
25.524\end{array}$ & $\begin{array}{r}25.554 \\
25540\end{array}$ & \begin{tabular}{|l|}
24.802 \\
24807
\end{tabular} & $\begin{array}{l}26.872 \\
26.867\end{array}$ & 25.0 & $\begin{array}{r}44.369 \\
44.402\end{array}$ & \begin{tabular}{|l|l|}
47.014 \\
\end{tabular} & 10.429 & $\begin{array}{r}34.95 \\
34.07\end{array}$ & & & $\begin{array}{l}48.066 \\
0.025\end{array}$ & 0.183 & 14.64 & & & 4.97667 & 45. & 11 & & & & & & & \\
\hline & & $\frac{12: 52: 58 \mathrm{PM}}{1205.58 \mathrm{PM}}$ & $\frac{25.524}{25.524}$ & & & 26.867 & & $\begin{array}{l}44.402 \\
44.427\end{array}$ & \begin{tabular}{|l|}
47.227 \\
10.005
\end{tabular} & $\begin{array}{r}10.44 \\
10.438 \\
\end{array}$ & & -1.2 & & & & 14.64 & & & & 46.1 & 11 & & & & & & & \\
\hline & & $\frac{12253}{12: 54:}$ & $\frac{25.524}{25.534}$ & $\frac{25.55}{25.55}$ & & $\frac{26.901}{26.997}$ & & & & & & & & & & & & & & & & & & & & & & \\
\hline & $8 / 30 / 2004$ & 12:55:58 PM & 25.56 & 25.565 & \begin{tabular}{|l|l|}
24.939 \\
\end{tabular} & 27.103 & & & & 10.442 & & -1. & & & & 14.643 & & & & & 11 & & & & & & & \\
\hline & & $12: 56:$ & 25.582 & 25.582 & \begin{tabular}{|l|}
24.941 \\
\end{tabular} & \begin{tabular}{|l|}
27.23 \\
\end{tabular} & & & & & & & & & & & & & & 46 & 1 & & & & & & & \\
\hline & $8 / 30 /$ & 12:57: & 25.596 & 25.601 & \begin{tabular}{|l|}
24.935 \\
\end{tabular} & 27.289 & 25.5 & & \begin{tabular}{|l|l|}
47.232 \\
\end{tabular} & 10.418 & & -1. & & & 0.18 & 14.6 & & & 5.0 & 46 & 12 & & & & & & & \\
\hline & & & 25.615 & 25.61 & 24 & & & 44. & & & & & & & & & & & & 45 & 11 & & & & & & 0.6 & \\
\hline & & & $\begin{array}{r}25.658 \\
25.557\end{array}$ & $\begin{array}{r}25.623 \\
2527\end{array}$ & & & & & & & & & & & & & & & 5. & & & & & & & & & \\
\hline & $8 / 30 / 2$ & 1:00:! & $\frac{25.657}{25.645}$ & $\begin{array}{r}25.637 \\
25.65 \\
\end{array}$ & & $\frac{27.509}{27.413}$ & & & & & & $\frac{-1.212}{-1212}$ & & & & 14 & & & & 45.8 & & & & & & & & \\
\hline & $\begin{array}{l}8 / 300 / 2 \\
8 / 30 / 2\end{array}$ & $\begin{array}{l}1.015 \\
1.02 \cdot 5\end{array}$ & $\begin{array}{l}25.645 \\
25.647\end{array}$ & $\begin{array}{r}25.65 \\
25.647\end{array}$ & \begin{tabular}{|l|l|}
24.96 \\
24.89
\end{tabular} & $\begin{array}{r}27.413 \\
27.37\end{array}$ & & & \begin{tabular}{|l|}
46.8 \\
47.2
\end{tabular} & & & $\frac{-1.212}{-1.212}$ & & & & $\frac{14 .}{14 .}$ & & & & 5.7 & & & & & & & & \\
\hline & $8 / 30 /$ & & 25.632 & & & & & & & & & & & & & & & & & & & & & & & & & \\
\hline & $8 / 30$ & & & & & 27. & & & & & & & & & & 14.6 & & & & & & & & & & & & \\
\hline 3 & & & 25.64 & & & & & & & & & & & & & & & & 5.21 & 45 & & & & & & & & \\
\hline & $8 / 30 / 2004$ & & $\frac{25.621}{25.61}$ & $\begin{array}{r}25.681 \\
25695\end{array}$ & 24.87 & $\begin{array}{r}27.168 \\
271.43\end{array}$ & & $\begin{array}{l}44.467 \\
44.465\end{array}$ & 47.3 & & $\frac{34.8}{35.0}$ & & -6. & & & 14.6 & & & 5.22667 & 46 & $\frac{12}{12}$ & & & & & & & \\
\hline & & & $\begin{array}{r}25.61 \\
25.605\end{array}$ & $\begin{array}{r}\frac{25.685}{25.68} \\
\end{array}$ & & 27.1 & & $\begin{array}{r}44.465 \\
441 \\
\end{array}$ & & & & & -6. & & & & & & & & & & & & & & & \\
\hline & & & & & & 27. & & & & & & & & & & & & & & 46. & & & & & & & & \\
\hline & $8 / 30 / 2004$ & 1:10: & 25.73 & 25. & & & & 43.8 & \begin{tabular}{|l|l|}
46.805 \\
\end{tabular} & & & -1. & & & & & & & & \begin{tabular}{|l|l|}
45.7 \\
4
\end{tabular} & 12 . & & & & & & & \\
\hline & & & & & & & & & & & & & & & & & & & & & & & & & & & & \\
\hline & & & & & & & & & & & & & & & & & & & & & & & & & & & & \\
\hline & & & & & & & & & & & & & & & & & & & & & & & & & & & & \\
\hline & & & & & & & & & & & & & & & & & & & & & & & & & & & & \\
\hline & $8 / 30 / 2$ & & & 2574 & & $\frac{27.10}{27.10}$ & & & & & & & & & & $\frac{14 .}{14}$ & & & & & & & & & & & & \\
\hline & $8 / 30 / 2004$ & & & & & 27.1 & & & & & & -1. & & & & & & & & & 1 & & & & & & & \\
\hline & $8 / 30 / 2004$ & & 25. & 25. & 25. & 27.1 & & 44 & 47. & & & -1 & & & & & & & & & & & & & & & & \\
\hline & & & & & 25. & 27.18 & & 44 & & & & & & & & & & & & & & & & & & & & \\
\hline & & & & & & 27.2 & & & & & & & & & & & & & & & & & & & & & & \\
\hline & 8035012004 & & & 25 & 2.1 .2 & 27.292 & & 44. & & & & $-\frac{1.125}{1.212}$ & & & & & & & & & & & & & & & & \\
\hline & 8 & 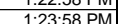 & 25.15 & 2576 & 24096 & 27285 & 25 & 年 44.5 & 47534 & & & -1.215 & & & & 146 & & & & & & & & & & & & \\
\hline & & 1.24 .58 & & 25.7 & 20 & 27.274 & 25.3 & 44.5 & 47.269 & & & 1215 & & & & 14.6 & & & & 46.1 & 11.9 & & & & & & 0.606 & \\
\hline & 2004 & $88 \mathrm{PM}$ & 0.11 & 25.76 & 24.873 & 27.232 & 25.35 & 444.151 & \begin{tabular}{|l}
47.099 \\
\end{tabular} & 10.335 & 34.764 & \begin{tabular}{|l|l|} 
& -1.212 \\
\end{tabular} & & 47.246 & 0.162 & 14.64 & & 332.60 & 5.54333 & 46.0 & 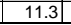 & 39.5 & & & & 0.001 & 0.590 & \\
\hline
\end{tabular}


WSRC-TR-2005-00105, REVISION 0

SRNL-RPP-2005-00012, REVISION 0

RUN \# 4.03A AND B; FIRST AND SECOND HALF OF SLURRY DEWATERING - CONT.

\begin{tabular}{|c|c|c|c|c|c|c|c|c|c|c|c|c|c|c|c|c|c|c|c|c|c|c|c|c|c|c|c|c|}
\hline 351 & A & $\begin{array}{c}B \\
\end{array}$ & D & $E$ & $F$ & G & $\mathrm{H}$ & $\mathrm{J}$ & $\mathrm{K}$ & $\mathrm{L}$ & \begin{tabular}{c|} 
\\
\end{tabular} & $\mathrm{N}$ & 0 & Q & $\mathrm{R}$ & 5 & & $\mathrm{v}$ & w & \begin{tabular}{l|l}
$x$ \\
\end{tabular} & $\mathrm{Y}$ & $\mathrm{Z}$ & AA & & & & $\mathrm{AE}$ & \\
\hline$\frac{351}{352}$ & $\begin{array}{l}8 / 301 / 2004 \\
8 / 3012004\end{array}$ & $\begin{array}{l}1: 26: 58 \mathrm{PM} \\
12: 57: 58 \mathrm{PM}\end{array}$ & $\begin{array}{l}25.705 \\
25.696\end{array}$ & $\begin{array}{l}25.776 \\
25.786\end{array}$ & 24.819 & $27.313 \mid$ & $\begin{array}{l}25.375 \\
25.431\end{array}$ & \begin{tabular}{|l|l}
44.042 \\
44.187
\end{tabular} & $\begin{array}{r}46.9 \\
46.765\end{array}$ & \begin{tabular}{|c|}
10.41 \\
10.45
\end{tabular} & $\begin{array}{r}34.573 \\
34.766\end{array}$ & \begin{tabular}{|l|}
-1.212 \\
-1.212 \\
\end{tabular} & $\begin{array}{r}-7.105 \\
-7.157 \\
\end{array}$ & \begin{tabular}{r|r|}
48.9055 \\
48.88
\end{tabular} & $\begin{array}{l}0.168 \\
0.169 \\
\end{array}$ & $\begin{array}{l}\frac{14.643}{14.643} \\
\end{array}$ & $\begin{array}{l}0.003 \\
0.003 \\
\end{array}$ & $\begin{array}{l}333.60 \\
334.60\end{array}$ & \begin{tabular}{|r|r}
5.56 \\
5.57667
\end{tabular} & \begin{tabular}{|l|}
45.8 \\
45.6
\end{tabular} & $\begin{array}{l}11.6 \\
11.6\end{array}$ & $\begin{array}{l}39.3 \\
39.5 \\
\end{array}$ & $\begin{array}{c}2.710 \\
2.722 \\
\end{array}$ & \begin{tabular}{|l|l|l|l|l|l|}
0.024 \\
0.024
\end{tabular} & $\mid$\begin{tabular}{l|l|}
0.024 \\
0.024
\end{tabular} & $\begin{array}{l}0.001 \\
0.001\end{array}$ & $\begin{array}{l}0.615 \\
0.617\end{array}$ & $\begin{array}{l}0.52 \\
0.52 \\
\end{array}$ \\
\hline & & & & & & & & & & 10.511 & & & & & & 14.643 & & & & & & & & & 0.024 & 0.001 & & \\
\hline 354 & & 1:29:58 PM & 25.652 & 25.812 & \begin{tabular}{|l|l|}
24.871 \\
\end{tabular} & 27.24 & 25.352 & 44.394 & 47.381 & 10.395 & $\begin{array}{r}34.95 \\
\end{array}$ & $\begin{array}{r}-1.209 \\
\end{array}$ & $\begin{array}{l}-7.171 \\
\end{array}$ & 49.003 & 0.164 & 14.643 & 0.003 & 336.60 & 5.61 & 46.3 & 11.7 & 39.7 & & & 0.024 & 0.001 & & \\
\hline 355 & $8 / 30 / 2004$ & $1: 30: 58 \mathrm{PM}$ & 25.631 & 25.806 & $\begin{aligned} 24.82 \\
4.755\end{aligned}$ & 27.214 & 25.376 & $\begin{array}{l}44.643 \\
\end{array}$ & 47.574 & 10.568 & \begin{tabular}{|l|l|}
34.993 \\
3520
\end{tabular} & \begin{tabular}{|l|l|}
-1.212 \\
\end{tabular} & $\begin{array}{l}-7.122 \\
.7061\end{array}$ & 50.617 & 0.168 & 14.643 & 0.003 & 337.60 & 5.62667 & 46.4 & 12.1 & 39.8 & 2.745 & .024 & 0.024 & 0.001 & 0.607 & \\
\hline 30 & $8 / 30 / 2004$ & 1:31:58 PM & & $\begin{array}{l}25.806 \\
25.032\end{array}$ & 24.755 & 27.279 & 25.406 & 44.931 & 48.027 & 10.616 & \begin{tabular}{|l|}
35.26 \\
35151
\end{tabular} & \begin{tabular}{|l|l|}
-1.212 \\
\end{tabular} & $\begin{array}{r}-7.064 \\
7.121\end{array}$ & 47.084 & 0.165 & 14.643 & $\begin{array}{l}0.004 \\
004\end{array}$ & 338.60 & \begin{tabular}{|l|l|}
5.64333 \\
\end{tabular} & 46.9 & $\frac{11.2}{11.2}$ & & & & 0.024 & 0.001 & $\begin{array}{l}0.593 \\
\end{array}$ & \\
\hline 357 & $8 / 30 / 2004$ & $\begin{array}{l}1: 32: 58 \mathrm{PM} \\
1 \cdot 23: 58 \mathrm{P}\end{array}$ & $\begin{array}{l}25.632 \\
25.607 \\
\end{array}$ & \begin{tabular}{|l|}
25.823 \\
25819
\end{tabular} & $\begin{array}{l}24.746 \\
2.701\end{array}$ & 27.335 & $\begin{array}{l}25.452 \\
2.272\end{array}$ & $\begin{array}{l}44.599 \\
1.782\end{array}$ & $\begin{array}{l}47.549 \\
77909\end{array}$ & $\begin{array}{l}10.45 \\
1.1\end{array}$ & $\begin{array}{l}35.151 \\
25121\end{array}$ & \begin{tabular}{|c|}
-1.209 \\
\end{tabular} & $\begin{array}{r}-7.134 \\
\end{array}$ & \begin{tabular}{|l|l|}
49.726 \\
5.250
\end{tabular} & 0.167 & $\begin{array}{l}14.643 \\
1.642\end{array}$ & $\begin{array}{l}0.004 \\
0.023\end{array}$ & 339.60 & $\begin{array}{r}5.66 \\
5.67677\end{array}$ & $\begin{array}{l}46.4 \\
19.7\end{array}$ & 11.8 & 39.5 & & 0.024 & $\begin{array}{l}0.024 \\
\end{array}$ & 0.001 & $\begin{array}{l}0.604 \\
\end{array}$ & \\
\hline$\frac{358}{359}$ & $\frac{8 / 3012004}{88 / 3 / 2004}$ & $\begin{array}{l}1: 33: 58 \mathrm{PM} \\
1.3458 \mathrm{P}\end{array}$ & \begin{tabular}{|l|}
25.607 \\
2554
\end{tabular} & $\begin{array}{l}25.818 \\
25.814\end{array}$ & $\begin{array}{l}24.701 \\
24.657\end{array}$ & \begin{tabular}{r|}
27.33 \\
27.321
\end{tabular} & $\begin{array}{l}25.372 \\
25.349\end{array}$ & $\begin{aligned} 44.782 \\
44.883\end{aligned}$ & $\begin{array}{l}47.789 \\
47.882\end{array}$ & $\begin{array}{r}10.61 \\
10.537\end{array}$ & \begin{tabular}{|l|}
35.131 \\
35367
\end{tabular} & $\begin{array}{c}-1.212 \\
-1.21\end{array}$ & $\begin{array}{c}-7.08 \\
-7.07\end{array}$ & 50.763 & $\begin{array}{l}0.189 \\
0.165\end{array}$ & $\begin{array}{l}14.643 \\
1.643 \\
\end{array}$ & 0.003 & $\begin{array}{l}340.60 \\
301\end{array}$ & \begin{tabular}{|l|l|}
5.67667 \\
560232
\end{tabular} & \begin{tabular}{|l|}
46.7 \\
\end{tabular} & 12.1 & & & & 0.027 & 0.001 & $\begin{array}{l}0.683 \\
0591\end{array}$ & \\
\hline 360 & $8 / 3012004$ & $\begin{array}{l}1: 3458 \mathrm{PM} \\
1: 35: 58 \mathrm{PM}\end{array}$ & $\begin{array}{l}25.594 \\
25.574\end{array}$ & & $\begin{array}{l}24.657 \\
24.653\end{array}$ & $\frac{27.321}{27.277}$ & & $\begin{array}{l}44.883 \\
44.641\end{array}$ & $\begin{array}{l}47.882 \\
47.702\end{array}$ & $\begin{array}{l}\frac{10.537}{10.559} \\
\end{array}$ & \begin{tabular}{|}
35.367 \\
34.938
\end{tabular} & $\begin{array}{c}\frac{-1.212}{-1.212} \\
\end{array}$ & $\begin{array}{l}-7.02 \\
-7.09 \\
\end{array}$ & $\begin{array}{l}58.712 \\
50.434\end{array}$ & $\begin{array}{l}0.165 \\
0.166\end{array}$ & $\begin{array}{l}14.643 \\
14.643 \\
\end{array}$ & $\begin{array}{l}0.0033 \\
0.004\end{array}$ & $\begin{array}{l}341.60 \\
342.60\end{array}$ & \begin{tabular}{|r|r|}
5.69333 \\
5.71
\end{tabular} & $\begin{array}{l}46.8 \\
46.6 \\
\end{array}$ & $\begin{array}{l}14.0 \\
12.0\end{array}$ & $\begin{array}{l}40.1 \\
39.8 \\
4\end{array}$ & \begin{tabular}{|l|}
2.766 \\
2.743
\end{tabular} & $\begin{array}{l}0.024 \\
0.024\end{array}$ & $\frac{0.024}{0.024}$ & $\frac{0.001}{0.001}$ & \begin{tabular}{|c|}
0.594 \\
0.603 \\
\end{tabular} & \\
\hline 361 & $8 / 30 / 2004$ & 1:36:58 PM & 25.565 & 25.825 & \begin{tabular}{|l|l|}
24.623 \\
\end{tabular} & 27.248 & 25.335 & & 47.648 & 10.558 & 35.121 & \begin{tabular}{|r|}
-1.212 \\
\end{tabular} & $\begin{array}{l}-7.096 \\
\end{array}$ & 49.587 & 0.163 & 14.643 & 0.004 & 343.60 & 5.72667 & 46.5 & \begin{tabular}{l|l|}
11.8 \\
\end{tabular} & & & & $\begin{array}{l}.024 \\
0.024\end{array}$ & 0.001 & $\begin{array}{l}.0090 \\
0.591\end{array}$ & $\begin{array}{l}0.51 \\
0.50 \\
\end{array}$ \\
\hline & $8 / 30 / 2004$ & $1: 37: 58 \mathrm{PM}$ & 25.534 & 25.82 & 24.583 & 27.202 & 25.294 & $\begin{array}{l}44.993 \\
\end{array}$ & 47.845 & 10.679 & & & & 51.331 & & 14.643 & & & 5.74333 & $\begin{array}{l}46.7 \\
\end{array}$ & 12.2 & & & & & & & 0 \\
\hline 363 & $8 / 30 / 2004$ & $1: 38: 58$ PM & 25.529 & 25.834 & \begin{tabular}{|l|l|}
24.582 \\
\end{tabular} & 27.251 & 25.309 & $\begin{array}{l}4.904 \\
\end{array}$ & 47.926 & 10.692 & 35.262 & $\begin{array}{l}-1.206 \\
\end{array}$ & $\begin{array}{r}7.038 \\
\end{array}$ & \begin{tabular}{|l|l}
48.946 \\
\end{tabular} & & $\begin{array}{ll}14.643 \\
\end{array}$ & 0.004 & 345.60 & 5.76 & 46.8 & $\begin{array}{ll}11.7 \\
\end{array}$ & 40 & & & & & 0.589 & \\
\hline 364 & $8 / 30 / 2004$ & 1:39:58 PM & 25.508 & 25.833 & \begin{tabular}{|l|l|}
24.701 \\
\end{tabular} & 27.301 & 25.343 & 44.95 & $\begin{array}{ll}47.862 \\
\end{array}$ & 10.691 & & & $\begin{array}{r}.087 \\
\end{array}$ & 47.374 & 166 & & & & & & & & & & 0.024 & & 0.597 & \\
\hline & & & & & & & & & & & \begin{tabular}{|l|l|}
35.349 \\
\end{tabular} & -1.215 & & & & $\begin{array}{l}14.643 \\
\end{array}$ & & & & $\begin{array}{ll}46.7 \\
\end{array}$ & $\overline{11.4}$ & & & & & & 0.613 & \\
\hline 366 & 8/301/2004 & 1:41:58 PM & 25.529 & 25.839 & 24.657 & 27.256 & & 44.612 & 477.692 & 10.351 & 35.236 & -1.212 & & 47.958 & 165 & $\begin{array}{l}14.643 \\
\end{array}$ & & 348.60 & 5.81 & 46.6 & 114 & & & & & & 0.597 & \\
\hline 367 & & 1:42:58 PM & 25.516 & 25.836 & 24.634 & 27.204 & & & 48.309 & 10.455 & 35.543 & -1.212 & & & & $\begin{array}{l}14.643 \\
\end{array}$ & & & & 47.2 & & & & & & & 0.585 & \\
\hline$\frac{368}{360}$ & 8/30/2004 & $1: 43: 58 \mathrm{PM}$ & 25.504 & 25.844 & 24.607] & 27.201 & & 44.185 & 47.261 & 10.913 & 34.242 & -1.212 & & 49.303 & 57 & 14.643 & & 350.60 & 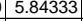 & 46.1 & $11.7 \mathrm{r}$ & 36 & & & & 0.001 & 0.580 & \\
\hline & & $8 \mathrm{PM}$ & 25.482 & 25.838 & 24.571 & & & 44.095 & 47.232 & 10.757 & 34.331 & -1.212 & & 49.743 & & 14.643 & & 351.60 & 5.86 & 46.1 & 111.8 & 36 & $\pi$ & & & & 0.584 & \\
\hline$\frac{3 \pi}{37}$ & 801302004 & $\begin{array}{l}1.455 .58 \mathrm{PM} \\
\end{array}$ & 25.466 & $\begin{array}{r}25.831 \\
25.92\end{array}$ & 24.5699 & 27.129 & & 44.533 & 47.965 & 10.888 & $\begin{array}{r}34.55 \\
20.912\end{array}$ & $\begin{array}{r}-1.215 \\
\end{array}$ & & 48.627 & 0.159 & 14.643 & 0.003 & 352.60 & 5.87667 & 46.8. & 11.6. & 39 & 2.72 & 023 & 0.023 & 0.001 & 0.583 & \\
\hline | & nitorent & $\begin{array}{l}1.465 .58 \mathrm{PM} \\
\end{array}$ & $\frac{25.45}{25.42}$ & 25.82 & 24.518 & 27.093 & & 44.44 & $47.6 / 1$ & 1.11 & $\frac{34.618}{32.018}$ & $\frac{-1.218}{-2.218}$ & & 4 & 0.156 & $\frac{14.643}{1.632}$ & & 353.60 & & 46.5 & 110 & & & & & & 年 & \\
\hline & 8 & $\begin{array}{l}1.47 .58 \mathrm{PM} \\
1.580\end{array}$ & $\begin{array}{l}25.443 \\
25419\end{array}$ & $\begin{array}{l}25.833 \\
25839\end{array}$ & 24.530 & 27.056 & & 44.92 & & 1.01 & 34.926 & $\frac{-1.212}{-1.215}$ & & $\begin{array}{l}46.206 \\
5.217\end{array}$ & 0.16 & 14.643 & & 354.60 & 5.91 & & $\frac{11.6}{120}$ & & & & & & 0 & \\
\hline 374 & $8 / 30 / 2004$ & $\frac{1.46 .59}{1.58}$ & 25.427 & 25.037 & $\frac{24.021}{2461}$ & 27079 & $\frac{2}{2}$ & $\frac{44.954}{4462}$ & 年18.945 & 10899 & 34.0413 & $\frac{1.45}{-1218}$ & & 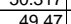 & & 14.645 & & 3566 & . & 44.0 & 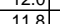 & & & & & & 0.073 & \\
\hline 375 & $8 / 30 / 2004$ & 1:50:58 PM & 25.445 & 25.83 & 24.608 & 27.123 & 25 & & 48.374 & 10.644 & 35.295 & $\frac{-1.212}{-1.212}$ & & 50.477 & 0.164 & 14.643 & & 357.60 & $\frac{5.96}{5.96}$ & 47.2 & $\frac{1.50}{120}$ & & 876 & & & & & \\
\hline & $8 / 30 / 2004$ & 1:51:58 PM & 25.444 & 25.829 & 24.577 & 27.221 & 25.29 & 44.975 & 48.112 & 10.698 & 35.156 & -1.215 & & 50.421 & 0.163 & 14.643 & 0.00 & 358.60 & 5.97667 & 47.0 & 12.0 & 4 & & & 0.024 & 001 & 0.589 & \\
\hline & & & 25.459 & & & & & & \begin{tabular}{|l|l}
49.014 \\
\end{tabular} & 10.834 & & & & & & & & & & & 121 & & & & & & & \\
\hline & $8 / 30 / 2004$ & 1:53:58 PM & 25.45. & 25.834 & 24.577 & 27.316 & & & 48.156 & 10.999 & 35.307 & -1.218 & & 50.125 & 0.1 & 14.643 & & 360.60 & 6.01 & 47.0 & 1.9 & & & & & & & \\
\hline $3 / 5$ & 8/30/2004 & 1:54:5 & 25.463 & 25.833 & 24.581 & 27.331 & & 45.464 & 48.898 & 11.069 & 35.266 & -1.215 & & \begin{tabular}{|l|l|}
52.93 \\
\end{tabular} & & 14.643 & & 361.60 & 5.02667 & \begin{tabular}{ll|}
47.8 \\
\end{tabular} & 12.6 & & & & 0.023 & 0.001 & 0.571 & \\
\hline & & & & & & & & & & & & & & & & & & & & & & & & & & & & \\
\hline 88 & $8 / 30 / 2004$ & $1: 56: 58 \mathrm{PM}$ & 25.471 & 25.836 & \begin{tabular}{|l|l|l|l|}
2414 \\
\end{tabular} & 27.378 & & 45.52 & 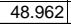 & 10.933 & \begin{tabular}{|l|l|l}
35.589 \\
\end{tabular} & $\mid-1.215$ & & 52.106 & & 14.6 & & 363.60 & 6.06 & 47.8 & 12.4 & & & & & & 569 & \\
\hline & $8 / 30 / 2004$ & & & & 24.744 & & & & & & & -1.212 & & & & & & & 6.07 & 47.7 & 12.7 & & & & & & & \\
\hline 383 & 8/30/2004 & 1:58:58 PM & 25.547 & 25.872 & 24.75 & 27.469 & 25. & 45.454 & 48.575 & 10.978 & 35.541 & -1.209 & & 50.934 & 0.161 & 14.643 & & 365.60 & 6.09333 & 47.4 & 12.1 & 40 & & & 0.023 & 0.001 & 0.573 & \\
\hline & & & & 25.868 & 24.721 & 27.46 & & & 49.099 & 11.011 & 35. & -1.215 & & 52.4 & & 14. & & 366.6 & 6.11 & 48.0 & . & & & & & & 0.575 & \\
\hline 38 & 8/30/2004 & 2:00:58 PM & 25.569 & 25.874 & 24.722 & 27.511 & & $\begin{array}{r}45.69 \\
5.252\end{array}$ & 49.112 & 10.994 & 35.621 & -1.215 & & 50 & 0. & 14. & & 367.60 & 6.12667 & 48.0 & 12.1 & & & & & & & \\
\hline$\sqrt{28}$ & 803012004 & 2:01:58 PM & 25577 & 25.881 & 24.109 & 27474 & & $\begin{array}{r}45.352 \\
5711\end{array}$ & 49354 & $\frac{11.068}{11130}$ & $\begin{array}{l}35.211 \\
35.367\end{array}$ & -1.215 & & & 0.163 & & & & & 47.8 & 11.1 & & & & & & 0.584 & \\
\hline & 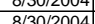 & & & & & & & & & & & & & & & & & | & & & 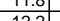 & & & & & & & \\
\hline & & & 25.579 & 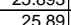 & 24.873 & $\frac{27.34}{27.462}$ & & & & 11 & & & & & & & & & & & & & & & & & & \\
\hline & $8 / 30 / 2004$ & & 25.617 & 25.902 & 24.875 & 27.484 & 25. & & & 11.033 & & -1.215 & & & & & & & & & & & & & & & 0569 & \\
\hline & & & 25.6 & 25.901 & 24.835 & 27.383 & & & & & & $\frac{-1.212}{-1.212}$ & & & & 14.6 & & 272 & & 47.5 & $\frac{12.6}{12.6}$ & & & & & & 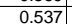 & \\
\hline$\sqrt{39}$ & $8 / 30 / 2004$ & & 25.631 & 25.906 & 24.85 & 27.388 & & 45.315 & 488.629 & 11.014 & 35.33 & -1.212 & & 49.197 & 0.151 & 14. & & 374.60 & 6.24333 & 47.5 & 11.7 & & & & & & 0.563 & \\
\hline & & & & & & & & & & & & & & & & & & & & & 110 & & & & & & & \\
\hline \begin{tabular}{|l|l|}
394 \\
\end{tabular} & $8 / 30 / 2004$ & & 25.6 & 25. & 24.835 & 27.40 & & & 48.378 & 10.9 & & & & & & & & & & 47.3 & 12. & & & & & & 0.570 & \\
\hline & & & & 25.9 & 24.8 & 27.4 & & & & 11.03 & & -1.2 & & & & & & & & & & & & & & & & \\
\hline & & & & & 24.82 & & & & & & & -1.21 & & & & & & & & \begin{tabular}{ll|}
47.6 \\
\end{tabular} & 11. & & & & & & & \\
\hline & 8/30/2004 & & & & 24. & 27.47 & & 45.556 & & 11.2 & & & & & & & & & & & 12 & & & & & & & \\
\hline 398 & 8/30/2004 & & 25. & & 24.819 & 27.48 & & & & 11.176 & & -1.2 & & & & & & & & $\begin{array}{ll}47.4 \\
\end{array}$ & 11. & & & & & & & \\
\hline & & & 25.6 & & 24. & 27.5 & & & & 11.127 & & & & & & 14. & & & 6.35 & 47 & \pm & & & & & & & \\
\hline 400 & $8 / 30 / 2$ & & & 25.9 & & 27. & & & & & & & & & & & & & & & $\begin{array}{lll}1.4 \\
1.7\end{array}$ & & & & & & & \\
\hline$\frac{40}{10}$ & 8013012004 & & $\frac{25.4}{25}$ & 25.935 & 24.93 & 27.52 & & 44.964 & 40.2 & 10.9 & & -1.212 & & & 0.1 & 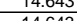 & & & & & $\frac{11 .}{11 .}$ & & & & & & & \\
\hline & & & & & & & & & & & & & & & & & & & & & $\frac{11 .}{11 .}$ & & & & & & & \\
\hline & & & & 258 & & 272 & & & & & & & & & & & & & & & $\frac{1 x .6}{116}$ & & & & & & & \\
\hline & $8 / 30 / 200$ & & & 25.8 & & & & & 48.747 & 11.0 & & & & & & & & & & & $\frac{17.5}{12.5}$ & & & & & & & \\
\hline & & & & & & & & & & & & & & & & & & & & & & & & & & & & \\
\hline 4 & $8 / 30 / 2$ & & 25.689 & 25.8 & 24.868 & 27.32. & & 44 & 48.765 & & & & & & & & & & & & & & & & & & 0.544 & \\
\hline & & & & & & & & & & & & & & & & & & & & & & & & & & & & \\
\hline & & & $\overline{25.7}$ & 25.8 & 24.95 & 27.29 & & & & & & & & & & & & & 6.5 & & & & & & & & & \\
\hline & & & & & & & & & & 11.387 & & & & & & & & & & & 11.8 & & & & & & & \\
\hline & $8 / 30 / 2$ & & 25.72 & 25.821 & & 27.289 & & 44. & & 11.405 & 34.448 & -1.215 & & & 0.1 & & & 393 & & 47 & 11.6 & & & & & & & \\
\hline 41 & $8 / 30 / 2004$ & & 25. & 25.8 & 25.006 & 27.24 & & & 48.26 & 11.3 & & -1.21 & & & 0.1 & & & & 6.5 & & 11.4 & & & & & & & \\
\hline I41 & 8/30/2 & 2:28:2 & 25. & 25.8 & 24.992 & 27.2 & & 44. & 48.0 & & & -1.2 & & & & & & & & & 12.4 & & & & & & & \\
\hline 414 & $8 / 30 / 2$ & & & & & 27.1 & & & & 11.0 & & & & & & & & & & & & & & & & & & \\
\hline 415 & & & & & & & & & & & & & & & & & & & & 48 & 11.9 & & & & & & & \\
\hline 41 & $8 / 30 / 2004$ & & 25.153 & 25.759 & 24.942 & 27.01 & & 45. & & 11.0 & 34. & -1.215 & & & 0.1 & & & & & 47.6 & 11.4 & & & & & & & \\
\hline & 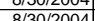 & & 2577 & 25727 & 24.941 & 26.34 & & 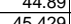 & 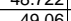 & 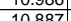 & |34.850 & $\begin{array}{l}-1.215 \\
-1215\end{array}$ & & (48.606 & $\begin{array}{l}0.140 \\
0.154\end{array}$ & . & & 年 & & 4 & $\frac{11.6}{119}$ & & & & & & 5 & \\
\hline & & & & & & 20.5 & & 45250 & 40.00 & & & 1.1212 & & 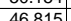 & 0 & & & 400.1 & & 47.8 & $\frac{1.5}{112}$ & & & & & & 37 & \\
\hline 420 & $8 / 30 / 2004$ & 2:35:28 PM & $25.728 \mid$ & 25.683 & 25.067] & 26.901 & & 45.387 & 49.153 & 10.854 & 35.468 & -1.212 & & 50.106 & 0.15 & 14.643 & & 402.10 & 6.70167 & 48.0 & 11.9 & & & & & 0.001 & 0.530 & \\
\hline & & L. $35.20 \mathrm{PHV}$ & -0.120 & 20.002 & 20.007 & 20.901 & & 45.001 & 4.150 & 10.034 & 30.400| & -1.212 & & 50.100 & 0.10 & 14.045 & & 40Z.1. & 0.10107 & & & & & & & & & \\
\hline
\end{tabular}


WSRC-TR-2005-00105, REVISION 0

SRNL-RPP-2005-00012, REVISION 0

RUN \# 4.03A AND B; FIRST AND SECOND HALF OF SLURRY DEWATERING - CONT.

\begin{tabular}{|c|c|c|c|c|c|c|c|c|c|c|c|c|c|c|c|c|c|c|c|c|c|c|c|c|c|c|c|c|}
\hline & A & B & D & $E$ & $F$ & \begin{tabular}{l|l|l} 
G \\
\end{tabular} & $\mathrm{H}$ & $\mathrm{J}$ & \begin{tabular}{ll|}
$\mathrm{K}$ \\
\end{tabular} & $\mathrm{L}$ & M & $\mathrm{N}$ & $\mathrm{O}$ & $\mathrm{Q}$ & $\begin{array}{ll}R \\
\end{array}$ & $\mathrm{~s}$ & $\mathrm{~T}$ & $\mathrm{v}$ & w & $x$ & $Y$ & $z$ & AA & $A B$ & $A C$ & AD & $\mathrm{AE}$ & \\
\hline & $8 / 30 / 2004$ & $2: 36: 28$ PM & & 25.672 & 25.201 & 26.9 & 24.967 & 45.32 & \begin{tabular}{|l|l}
48.886 \\
\end{tabular} & 10.82 & 35.541 & & $\begin{array}{l}-7.096 \\
\end{array}$ & 48.533 & 0.153 & 14.643 & 0.0 & 403.10 & 6.71833 & 47.8 & 11,6 & & 2.788 & & 0.022 & 0.00 & 0.539 & \\
\hline & $8 / 30 / 2004$ & $2: 37: 28 \mathrm{PM}$ & $\frac{20.100}{25015}$ & $\begin{array}{l}25.645 \\
5.645\end{array}$ & 23.254 & & & 44.95 & & $\begin{array}{r}11.29 \\
1.272\end{array}$ & & & $\begin{array}{r}-7.09 \\
\end{array}$ & & & 14.643 & & & & 47.6 & & & & & 4002 & & & \\
\hline & $8 / 30 / 2004$ & $\begin{array}{l}2: 38: 28 \mathrm{PM} \\
2 \cdot 39 \cdot 28 \mathrm{P}\end{array}$ & $\begin{array}{l}25.815 \\
25.862\end{array}$ & $\begin{array}{l}25.645 \\
25637\end{array}$ & 25.409 & \begin{tabular}{|l|l|}
26.968 \\
227
\end{tabular} & $\begin{array}{r}25.055 \\
24097\end{array}$ & \begin{tabular}{|l|l|l}
4427 \\
4.52
\end{tabular} & \begin{tabular}{|l|l|}
48.662 \\
4.00
\end{tabular} & $\begin{array}{l}11.273 \\
11259\end{array}$ & \begin{tabular}{|l|l|}
34.443 \\
\end{tabular} & \begin{tabular}{|c|}
-1.209 \\
\end{tabular} & $\begin{array}{r}-7.09 \\
\end{array}$ & $\begin{array}{l}48.775 \\
5725\end{array}$ & 0.151 & $\begin{array}{l}14.643 \\
1.62\end{array}$ & $\begin{array}{l}0.004 \\
.002\end{array}$ & & \begin{tabular}{l|l}
0.75167 \\
0.7
\end{tabular} & 47.5 & & & 2733 & 0.022 & 0.021 & 0.001 & 0.539 & \\
\hline 42 & $8 / 30 / 2004$ & 2:39:28 PM & $\begin{aligned} 25.862 \\
25.927\end{aligned}$ & $\begin{array}{r}25.637 \\
25642\end{array}$ & \begin{tabular}{|l|l|}
25.516 \\
25637
\end{tabular} & \begin{tabular}{r|}
27 \\
26995
\end{tabular} & $\begin{array}{r}24.947 \\
24.973\end{array}$ & $\begin{array}{l}44.535 \\
44813\end{array}$ & \begin{tabular}{|l|}
48.048 \\
48.39 \\
\end{tabular} & $\frac{11.258}{11.171}$ & $\begin{array}{r}34.318 \\
34.682\end{array}$ & $\begin{array}{r}-1.215 \\
\end{array}$ & $\begin{array}{r}-7.053 \\
-7.116 \\
\end{array}$ & $\begin{array}{r}51.235 \\
5.186\end{array}$ & $\begin{array}{l}0.151 \\
0.159\end{array}$ & $\frac{14.643}{14.643}$ & 0002 & $\begin{array}{l}406.10 \\
\end{array}$ & 6.16833 & $\begin{array}{r}46.9 \\
7.3\end{array}$ & 12.2 & $\frac{3.4}{307}$ & 270 & 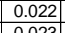 & 0.021 & $\begin{array}{l}0.001 \\
0.01\end{array}$ & $\begin{array}{l}0.540 \\
0.563\end{array}$ & $\begin{array}{l}0.46 \\
0.9\end{array}$ \\
\hline $4 \frac{426}{426}$ & $8 / 30 / 2004$ & $\begin{array}{l}2: 40: 28 \mathrm{PM} \\
2: 41: 28 \mathrm{PM}\end{array}$ & $\frac{25.927}{26.002}$ & & $\begin{array}{l}25.63 t \\
25.787\end{array}$ & $\begin{array}{r}26.995 \\
27.02\end{array}$ & $\begin{array}{r}24.993 \\
24.973 \\
\end{array}$ & $\begin{array}{l}44.813 \\
44.535\end{array}$ & \begin{tabular}{|l|}
48.3494 \\
4.287 \\
\end{tabular} & $\begin{array}{l}\frac{11.1 / 1}{11.167} \\
\end{array}$ & \begin{tabular}{|l|l|}
34.682 \\
34.392
\end{tabular} & $\begin{array}{r}-1.215 \\
-1.1212\end{array}$ & $\begin{array}{l}-7.116 \\
-7.116\end{array}$ & $\begin{array}{l}52.186 \\
50.031\end{array}$ & $\begin{array}{l}0.159 \\
0.147\end{array}$ & $\begin{array}{l}14.643 \\
14.643 \\
\end{array}$ & & $\begin{array}{l}407.10 \\
408.10\end{array}$ & \begin{tabular}{l|l} 
& 6.785 \\
0 & 6.80167
\end{tabular} & \begin{tabular}{|l|l|l}
477.3 & \\
7.2
\end{tabular} & & & & \begin{tabular}{|l|}
0.023 \\
0.021
\end{tabular} & $\begin{array}{l}0.022 \\
0.021\end{array}$ & \begin{tabular}{|l}
0.001 \\
0.001
\end{tabular} & $\begin{array}{l}0.563 \\
0.522\end{array}$ & $\begin{array}{l}0.48 \\
0.44\end{array}$ \\
\hline 427 & $8 / 30 / 2004$ & 2:42:28 PM & 26.058 & 25.643 & 25.883 & 27.016 & 24.968 & 44.827 & \begin{tabular}{|l|l|}
48.262 \\
\end{tabular} & 11.298 & 34.725 & -1.212 & -7.07 & 50.006 & 0.15 & 14.643 & 0.003 & 409.10 & \begin{tabular}{|c|c|c|}
0 & 6.81833 \\
\end{tabular} & $\begin{array}{l}47.2 \\
47.1\end{array}$ & & & $\frac{.1 .71}{2.742}$ & & 0.021 & 0.001 & $\begin{array}{l}0.522 \\
0.527\end{array}$ & 0.44 \\
\hline & & & 26.13 & 25.655 & & 27.048 & 25.02 & 45.219 & \begin{tabular}{|l|}
49.196 \\
\end{tabular} & 11.315 & 34.778 & -1.212 & $\begin{array}{l}-7.174 \\
\end{array}$ & 51.387 & 0.152 & 14.643 & & 410.10 & 6.835 & 48.1 & & & & & & & & \\
\hline 429 & $8 / 30 / 2004$ & $2: 44: 28 \mathrm{PM}$ & & 25.634 & 26.063 & & 24.984 & 45.01 & $\begin{array}{l}48.753 \\
\end{array}$ & & $\begin{array}{l}34.731 \\
\end{array}$ & -1.218 & & 48.068 & 0.152 & 14.643 & & & \begin{tabular}{l|l}
0 & 6.85167 \\
\end{tabular} & 47.6 & & & 2.749 & 0.022 & 0.021 & 0.001 & 0.530 & \\
\hline 430 & $8 / 30 / 2004$ & & 26.25 & 25.645 & 26.18 & 27.038 & 24.94 & 44.745 & $\begin{array}{l}48.62 \\
\end{array}$ & 11.199 & 34.511 & -1.215 & $\begin{array}{r}-7.015 \\
\end{array}$ & 50.188 & 0.151 & 14.643 & 0.0 & & 6.86833 & 47.5 & 12.0 & & & \begin{tabular}{|l|l|}
0.022 \\
\end{tabular} & 0.021 & 0.001 & 0.528 & \\
\hline 43 & $8 / 30 / 2004$ & \begin{tabular}{|l|l|l}
$2: 46: 28 \mathrm{PM}$ \\
$2: 24: 28 \mathrm{PM}$
\end{tabular} & \begin{tabular}{|l|l|}
26.331 \\
263838
\end{tabular} & $\begin{array}{l}25.652 \\
25654\end{array}$ & \begin{tabular}{|l|l|}
26.311 \\
26408
\end{tabular} & $\begin{array}{l}26.989 \\
27.002\end{array}$ & 24.932 & \begin{tabular}{|l|l|}
44.989 \\
4.809
\end{tabular} & \begin{tabular}{|r|}
48.73 \\
\end{tabular} & $\begin{array}{l}10.921 \\
1007\end{array}$ & $\begin{array}{l}35.166 \\
3500\end{array}$ & \begin{tabular}{|l|l|} 
& -1.212 \\
\end{tabular} & $\begin{array}{l}-6.995 \\
\end{array}$ & \begin{tabular}{|r|}
50.39 \\
\end{tabular} & 0.153 & 14.643 & & 413.10 & $\begin{array}{r}6.885 \\
600167\end{array}$ & 47.6 & 12.0 & & $\begin{array}{l}2.763 \\
\end{array}$ & & 0.021 & 0.001 & & \\
\hline & $\frac{8 / 30 / 2004}{88 / 3 / 2004}$ & $\begin{array}{l}2: 47: 28 \mathrm{PM} \\
2 \cdot 28 \cdot 28 \mathrm{PM}\end{array}$ & $\frac{26.388}{26.451}$ & $\frac{25.654}{25.652}$ & 26.408 & $\begin{array}{l}27.002 \\
27.005\end{array}$ & $\begin{array}{l}24.924 \\
22.008\end{array}$ & $\begin{aligned} 44.898 \\
4502 \\
\end{aligned}$ & \begin{tabular}{|l|}
48.394 \\
48.902 \\
\end{tabular} & $\begin{array}{r}10.97 \\
10.977\end{array}$ & \begin{tabular}{|l|}
35.008 \\
34.932
\end{tabular} & \begin{tabular}{|l|}
-1.209 \\
-1212
\end{tabular} & $\begin{array}{r}-6.94 \\
6.983\end{array}$ & \begin{tabular}{|l|}
48.364 \\
50402
\end{tabular} & $\begin{array}{l}0.154 \\
0.154\end{array}$ & $\begin{array}{r}14.643 \\
14643\end{array}$ & 0.003 & & $\frac{6.90167}{6.91833}$ & \begin{tabular}{|l|l|}
47.3 \\
47.
\end{tabular} & & 40.0 & 2.755 & 0.022 & $\begin{array}{l}0.021 \\
0.021\end{array}$ & $\begin{array}{l}0.001 \\
0.0101\end{array}$ & $\begin{array}{l}0.530 \\
0.529\end{array}$ & 0.45 \\
\hline 434 & $\begin{array}{l}8 / 30 / 2004 \\
\end{array}$ & $\begin{array}{l}2: 48.28 \mathrm{PM} \\
2: 49: 28 \mathrm{PM}\end{array}$ & 20.451 & $\frac{25.652}{25.632}$ & \begin{tabular}{|l}
26.492 \\
26.521 \\
\end{tabular} & & $\begin{array}{l}24.988 \\
24.892 \\
\end{array}$ & $\begin{array}{r}45.092 \\
45.093\end{array}$ & \begin{tabular}{|l|}
48.092 \\
49.083 \\
\end{tabular} & $\frac{1.971}{10.921}$ & $\begin{array}{l}3.9432 \\
35.176\end{array}$ & $\begin{array}{l}-1.212 \\
-1.212 \\
\end{array}$ & $\begin{array}{l}-6.983 \\
-7.021 \\
\end{array}$ & $\begin{array}{l}50.402 \\
51.474\end{array}$ & $\begin{array}{l}0.154 \\
0.157\end{array}$ & $\begin{array}{l}14.643 \\
14.643\end{array}$ & 0.003 & $\begin{array}{l}4115.10 \\
416.10\end{array}$ & $\begin{array}{r}6.91833 \\
6.935 \\
\end{array}$ & $\begin{array}{l}478.8 \\
48.0\end{array}$ & 12.3 & $\begin{array}{l}40.0 \\
40.1\end{array}$ & \begin{tabular}{|l|}
$\mid 2.756$ \\
2.767
\end{tabular} & \begin{tabular}{|l|}
0.022 \\
0.022 \\
\end{tabular} & $\frac{0.021}{0.022}$ & $\frac{0.001}{0.001}$ & $\begin{array}{l}0.529 \\
0.537\end{array}$ & $\frac{0.45}{0.46}$ \\
\hline & $8 / 30 / 2004$ & 2:50:28 PM & 26.556 & 25.637 & & 27.03 & 24.858 & 44.846 & \begin{tabular}{|l|}
48.631 \\
\end{tabular} & 10.903 & 35.059 & -1.215 & -7.102 & & 0.151 & 14.643 & & 417.10 & \begin{tabular}{l|l}
0 & 6.95167 \\
\end{tabular} & 47.5 & 12.8 & & 2.755 & & & & 0.518 & \\
\hline 436 & $8 / 30 / 2004$ & 2:51:28 PM & 26.598 & 25.639 & 26.503 & 26.972 & 24.829 & 44.753 & \begin{tabular}{|l|}
48.709 \\
\end{tabular} & 10.974 & 34.655 & -1.218 & $\begin{array}{l}-7.082 \\
-1\end{array}$ & 46.479 & 0.149 & 14.643 & & & \begin{tabular}{l|l}
0 & 6.96833 \\
\end{tabular} & 47.6 & & 39. & 2.737 & \begin{tabular}{|l|l|}
0.021 \\
\end{tabular} & & & & \\
\hline & $8 / 30 / 2004$ & $\begin{array}{ll}252: 28 \mathrm{PM} \\
2: 202 \mathrm{P}\end{array}$ & & & 26.489 & & & & \begin{tabular}{|l|l|}
48.933 \\
\end{tabular} & $\begin{array}{l}1.103 \\
1.002\end{array}$ & & & & 45.985 & & 14.643 & & & & 47.8 & & & 2.745 & & 0.021 & & & \\
\hline & $8 / 30 / 2004$ & $\begin{array}{l}2: 53: 28 \mathrm{PM} \\
2.55: 28\end{array}$ & $\begin{array}{l}26.674 \\
26.705\end{array}$ & $\begin{array}{l}25.625 \\
25632\end{array}$ & 26.429 & $\begin{array}{r}26.943 \\
2602\end{array}$ & & $\begin{array}{r}44.863 \\
1.640\end{array}$ & $\begin{array}{l}18.446 \\
182021\end{array}$ & $\begin{array}{l}11.005 \\
10.019\end{array}$ & $\begin{array}{r}34.932 \\
3.811\end{array}$ & $\begin{array}{r}-1.215 \\
.215\end{array}$ & & \begin{tabular}{|l|l|}
50.801 \\
\end{tabular} & 0.15 & 14.643 & & & 7.00167 & 47.3 & & 39.9 & 2.751 & \begin{tabular}{|l|l|}
0.021 \\
\end{tabular} & 0.021 & 0.001 & 0.517 & \\
\hline 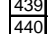 & $\begin{array}{l}8 / 30 / 2004 \\
8 / 301204\end{array}$ & $2: 54: 28 \mathrm{PM}$ & $\frac{26.705}{26.71}$ & $\begin{array}{l}25.632 \\
25.627\end{array}$ & 26.376 & $\begin{array}{r}26.92 \\
26.915 \\
\end{array}$ & $\frac{24.847}{24.872}$ & \begin{tabular}{|r|r|}
44.649 \\
45.19
\end{tabular} & \begin{tabular}{|r|}
48.231 \\
4934
\end{tabular} & $\begin{array}{l}\frac{10.918}{11107} \\
107\end{array}$ & $\begin{array}{r}34.844 \\
3487\end{array}$ & & & \begin{tabular}{|l|}
50.067 \\
49.964
\end{tabular} & $\begin{array}{l}0.149 \\
0.154\end{array}$ & $\begin{array}{l}14.643 \\
14.643 \\
\end{array}$ & & & $\frac{7.01833}{7035}$ & & & & $\begin{array}{l}2.740 \\
2.760\end{array}$ & \begin{tabular}{|l|}
0.021 \\
0.022 \\
\end{tabular} & 0.021 & 0.001 & $\begin{array}{l}0.516 \\
0.531\end{array}$ & 0.44 \\
\hline 441 & $8 / 30 / 2004$ & $\begin{array}{l}. .5 .50 \mathrm{PM} \\
2: 56: 28 \mathrm{PM}\end{array}$ & $\begin{array}{l}20.11 \\
26.73\end{array}$ & $\begin{array}{l}25.627 \\
25.637\end{array}$ & $\begin{array}{l}20.011 \\
26.266\end{array}$ & $\begin{array}{r}20.910 \\
26.87\end{array}$ & $\begin{array}{l}24.8 / 2 \\
24.902\end{array}$ & $\begin{array}{r}45.19 \\
45.253 \\
\end{array}$ & \begin{tabular}{|}
$\mid 45.04$ \\
49.06
\end{tabular} & 11.009 & $\begin{array}{r}34.21 \\
35.273 \\
\end{array}$ & $\frac{-1.212}{-1.212}$ & & \begin{tabular}{|l|}
49.964 \\
50.938
\end{tabular} & $\begin{array}{l}0.154 \\
0.152\end{array}$ & $\begin{array}{l}\frac{14.643}{14.643} \\
\end{array}$ & & & $\begin{array}{r}1.035 \\
7.05167\end{array}$ & $\begin{array}{l}48.2 \\
47.9 \\
\end{array}$ & 12.1 & 40.3 & $\frac{2.600}{2.776}$ & \begin{tabular}{|l|}
0.022 \\
0.022 \\
\end{tabular} & & 0.001 & $\begin{array}{l}0.531 \\
0.522\end{array}$ & \\
\hline & & 2:57:28 PM & 26.732 & 25.643 & 26.267 & 26.881 & 24.873 & $\begin{array}{l}45.259 \\
\end{array}$ & \begin{tabular}{|l|}
49.136 \\
\end{tabular} & 11.077 & 35.373 & -1.209 & -6.98 & \begin{tabular}{|l|}
50.868 \\
\end{tabular} & $\begin{array}{l}0.104 \\
\end{array}$ & $\begin{array}{l}\frac{14.045}{14.643} \\
\end{array}$ & & $\begin{array}{l}424.10 \\
424.10\end{array}$ & \begin{tabular}{|l|l|l|}
0 & 7.06833 \\
\end{tabular} & 48.0 & $\frac{1.1}{12.1}$ & 40. & 2.780 & \begin{tabular}{|l|l|}
0.021 \\
\end{tabular} & 0.021 & 0.001 & 0.514 & \\
\hline 443 & 8/30/2004 & 2:58:28 PM & 26.733 & 25.64 & 26.204 & 26.887 & 24.875 & \begin{tabular}{|l|l}
45.085 \\
\end{tabular} & \begin{tabular}{|l|l|}
49.033 \\
\end{tabular} & 11.04 & $\begin{array}{l}35.123 \\
2.172\end{array}$ & & & 47.092 & 0.152 & 14.643 & & & \begin{tabular}{|l|r}
0 & 7.085 \\
\end{tabular} & $\begin{array}{l}47.9 \\
\end{array}$ & 11. & 40. & 2.765 & \begin{tabular}{|l|l|}
0.022 \\
\end{tabular} & 0.021 & 0.001 & 0.525 & \\
\hline 444 & $8 / 30 / 2004$ & 2:59:28 PM & 26.725 & 25.641 & \begin{tabular}{|l|} 
\\
\end{tabular} & 26.899 & & 45.193 & & 11.12 & 35.172 & & -6.969 & 53.206 & & 14.643 & & & \begin{tabular}{l|l}
0 & 7.10167
\end{tabular} & 48.0 & 12. & & 2.770 & \begin{tabular}{|l|l|}
0.022 \\
\end{tabular} & 0.021 & 0.001 & 0.524 & \\
\hline $\begin{array}{l}445 \\
446 \\
\end{array}$ & $8 / 30 / 2004$ & 3:00:28 PM & 26.706 & 25.632 & 26.102 & $\begin{array}{l}26.93 \\
\end{array}$ & 24.857 & $\begin{array}{l}45.417 \\
5.212\end{array}$ & \begin{tabular}{|l|}
49.358 \\
\end{tabular} & 11.333 & $\begin{array}{r}35 \\
\end{array}$ & $\begin{array}{l}-1.218 \\
\end{array}$ & $\begin{array}{r}-7.018 \\
-70 \\
\end{array}$ & \begin{tabular}{|l|l} 
\\
\end{tabular} & 0.154 & 14.643 & & & \begin{tabular}{l|l}
0 & 7.11833 \\
\end{tabular} & 48.2 & 11.3 & & 2.772 & \begin{tabular}{|l|l|}
0.022 \\
\end{tabular} & 0.021 & 0.001 & 0.532 & \\
\hline & $8 / 30 / 2004$ & $\begin{array}{ll}3: 01: 28 \mathrm{PM} \\
3 \cdot 202 \cdot 28 \mathrm{PM}\end{array}$ & 26.688 & $\begin{array}{r}25.634 \\
25636\end{array}$ & 26.058 & 26.932 & $\begin{array}{r}24.949 \\
25036\end{array}$ & $\begin{array}{r}45.313 \\
4.244\end{array}$ & \begin{tabular}{|l|}
49.145 \\
48.957 \\
\end{tabular} & $\begin{array}{l}11.064 \\
10.071\end{array}$ & \begin{tabular}{|l|}
35.223 \\
35.451
\end{tabular} & \begin{tabular}{|c|}
-1.215 \\
-1212
\end{tabular} & $\begin{array}{r}-7.073 \\
-7015\end{array}$ & \begin{tabular}{|l|}
51.059 \\
46302
\end{tabular} & 0.148 & $\frac{14.643}{11643}$ & & $\begin{array}{r}428.10 \\
\end{array}$ & $\begin{array}{r}7.135 \\
7.97\end{array}$ & & 12.2 & & \begin{tabular}{|l|l|}
2.776 \\
\end{tabular} & \begin{tabular}{|l|l|}
0.021 \\
\end{tabular} & & 0.001 & 0.511 & 0.43 \\
\hline 448 & $\begin{array}{r}8 / 3 / 2004 \\
8 / 30 / 2004\end{array}$ & $\begin{array}{l}\text { 3:02:28 PM } \\
\text { 3:03:28 PM }\end{array}$ & 26.675 & $\frac{25.636}{25.634}$ & 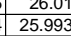 & $\begin{array}{l}26.949 \\
27.012\end{array}$ & $\begin{array}{l}25.036 \\
25.089\end{array}$ & \begin{tabular}{|l|}
45.2448 \\
45.188
\end{tabular} & \begin{tabular}{|l|}
48.995 \\
49.132 \\
\end{tabular} & $\begin{array}{l}10.911 \\
10.968 \\
\end{array}$ & $\begin{array}{l}3.35 .4151 \\
35.371 \\
\end{array}$ & $\begin{array}{l}-1.212 \\
-1.215 \\
\end{array}$ & $\begin{array}{l}-7.015 \\
-6.986 \\
\end{array}$ & \begin{tabular}{|l|}
46.383 \\
43.185 \\
\end{tabular} & $\frac{0.146}{0.152}$ & $\begin{array}{l}\frac{14.643}{14.643} \\
\end{array}$ & & $\frac{42}{43}$ & $\begin{array}{l}7.15167 \\
7.16833\end{array}$ & \begin{tabular}{|l|}
47.8 \\
48.0
\end{tabular} & $\begin{array}{l}11.0 \\
10.3\end{array}$ & 40 & & & & $\frac{0.001}{0.001}$ & $\begin{array}{l}0.504 \\
0.525\end{array}$ & $\frac{0.43}{0.45}$ \\
\hline & $8 / 30 / 2004$ & $3: 04: 28$ & 26.643 & 25.649 & 25.953 & 27.097 & 25.17 & 45.487 & \begin{tabular}{|l|}
49.53 \\
\end{tabular} & 11.185 & 35.215 & -1.212 & & \begin{tabular}{|l|}
48.652 \\
\end{tabular} & 0.143 & 14.643 & & & 7.185 & 48.4 & 11.6 & & 2.782 & \begin{tabular}{|l|l|}
0.020 \\
\end{tabular} & 0.020 & 0.000 & 0.494 & \\
\hline 450 & $8 / 30 / 2004$ & 3:05: & 26.621 & 25.653 & \begin{tabular}{|l|l|}
25.917 \\
\end{tabular} & 27.22 & 25.2 & 45.292 & \begin{tabular}{|l|}
49.298 \\
\end{tabular} & 11.06 & & -1.212 & & 50.246 & 0.15 & 14.643 & & & \begin{tabular}{l|l}
0 & 7.20167 \\
\end{tabular} & 48.2 & 12. & & 2.776 & & & 0. & & \\
\hline 451 & & 3:06:28 PM & 26.6 & 25.651 & $\begin{array}{r}25.9 \\
\end{array}$ & \begin{tabular}{|l|l|}
27.214 \\
\end{tabular} & 25.2 & 45.3 & \begin{tabular}{|l|}
49.327 \\
\end{tabular} & $\begin{array}{l}11.067 \\
1.07\end{array}$ & $\begin{array}{l}35.117 \\
2.127\end{array}$ & -1.212 & & \begin{tabular}{|l|l}
51.896 \\
\end{tabular} & 0.138 & & & & \begin{tabular}{l|l}
0 & 7.21833 \\
\end{tabular} & 48.2 & & & 2.7 & \begin{tabular}{|l|l|}
0.020 \\
\end{tabular} & & & 0.479 & \\
\hline$\frac{45}{45}$ & $8 / 30 / 2004$ & 3:07: & \begin{tabular}{|l|l|}
26.58 \\
26564
\end{tabular} & $\begin{array}{l}25.661 \\
25666\end{array}$ & \begin{tabular}{|l|l|}
25.945 \\
25895
\end{tabular} & \begin{tabular}{|l|l|}
27.294 \\
27.343
\end{tabular} & & $\begin{array}{l}45.207 \\
5257\end{array}$ & \begin{tabular}{|l|l|}
49.045 \\
49199 \\
\end{tabular} & $\begin{array}{r}11.11 \\
11.25\end{array}$ & & $\frac{-1.2}{1.2}$ & & \begin{tabular}{|l|l|l|}
50.183 \\
\end{tabular} & 0.144 & 14 & & 43 & & 47.9 & & & 2.7 & \begin{tabular}{|l|l|}
0.021 \\
\end{tabular} & 0.020 & 0.000 & 0.499 & \\
\hline & $\begin{array}{r}88 / 30 / 2004 \\
8 / 30 / 2004\end{array}$ & & $26.564 \mid$ & $\begin{array}{r}25.666 \\
25.676\end{array}$ & 25.895 & & & $\begin{array}{l}45.2 \\
45.1 \\
\end{array}$ & & $\frac{11.125}{11028}$ & & & & & & & & & & & & & & & & & & \\
\hline & $8 / 30 / 2004$ & $\begin{array}{l}3.0 .20 .28 \mathrm{PM} \\
3: 10: 28\end{array}$ & 26.54 & 25.676 & \begin{tabular}{|l|l|l|l}
25.80 \\
\end{tabular} & $\mid$ & 25. & $\begin{array}{l}45.1 \\
45.3\end{array}$ & \begin{tabular}{|l|}
48.942 \\
49.462 \\
\end{tabular} & $\frac{11.028}{11.105}$ & $\begin{array}{r}35.334 \\
35.191 \\
\end{array}$ & $\frac{-1.212}{-1.215}$ & & $\frac{5.1}{48 .}$ & $\begin{array}{l}0.147 \\
0.147\end{array}$ & $\frac{14.643}{14.643}$ & & & $\frac{7.206}{7.2}$ & 48 & $\frac{11}{11}$ & & & & & & & \\
\hline & $8 / 30 / 2004$ & $3: 11: 2$ & 26.53 & 25.696 & 25.815 & 27.389 & 25. & 45.09 & \begin{tabular}{|l|}
49.362 \\
\end{tabular} & 11.081 & & -1.215 & & \begin{tabular}{|l|}
49.726 \\
\end{tabular} & 0.148 & 14. & & & & 48.2 & 11.8 & & 2.7 & & & & & \\
\hline & & & & 25.687 & 25.78 & 27.364 & & 45. & \begin{tabular}{|l|}
49.3 \\
\end{tabular} & 11.164 & & -1. & & \begin{tabular}{|l|}
47.664 \\
\end{tabular} & & 14. & & & & 48. & 11 & & 2.7 & & & & & \\
\hline & $8 / 301$ & 3:13:1 & 26.473 & 25.684 & 25.75 & 27.286 & & & \begin{tabular}{|l|}
49.076 \\
\end{tabular} & 10.988 & & & & 51.293 & 144 & 14. & & & & 48.0 & & & 2.7 & & & & & \\
\hline 45 & $8 / 30$ & & 26.464 & 25.6 & & & & & & 11.142 & & -1 & & & 0. & 14 & & & & & 14 & & & & & & & \\
\hline & $8 / 30 / 2004$ & $3: 15: 1$ & 26.465 & $\begin{array}{l}25.721 \\
25711\end{array}$ & \begin{tabular}{|l|}
25.725 \\
25720
\end{tabular} & $\begin{array}{l}27.199 \\
27.220\end{array}$ & & & & & & -1. & -7 & & 0.144 & & & & & & & & & & & & & \\
\hline & $\begin{array}{l}8 / 30 / 2004 \\
8 / 3 / 2004\end{array}$ & $\begin{array}{l}3: 16: 12 \mathrm{PM} \\
3 \cdot 11: 12 \mathrm{PM}\end{array}$ & \begin{tabular}{l|l|}
26.468 \\
26.443
\end{tabular} & $\frac{25.744}{25.744}$ & $\begin{array}{l}25.728 \\
125683\end{array}$ & $\begin{array}{l}27.232 \\
27.202\end{array}$ & $\begin{array}{l}25.41 \\
25.42\end{array}$ & $\frac{44.8}{45.0}$ & \begin{tabular}{|c|}
48.877 \\
49153 \\
\end{tabular} & $\begin{array}{l}11.134 \\
11.202\end{array}$ & $\begin{array}{l}34.708 \\
34.768 \\
\end{array}$ & $\begin{array}{r}-1.209 \\
.215\end{array}$ & & \begin{tabular}{|l|}
49.14 \\
5171 \\
\end{tabular} & $\begin{array}{l}0.145 \\
0.143\end{array}$ & $\frac{14 .}{14 .}$ & & 44 & $\begin{array}{ll}3 & 7.38056 \\
\end{array}$ & $\begin{array}{l}47.8 \\
48.0\end{array}$ & $\begin{array}{l}11.7 \\
123\end{array}$ & 39 & $\begin{array}{l}2.742 \\
27750 \\
\end{array}$ & & 0.020 & 1.001 & $\begin{array}{l}0.511 \\
0.504\end{array}$ & 44 \\
\hline 46 & 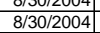 & & 20.4436 & $\begin{array}{l}25.144 \\
25.757\end{array}$ & \begin{tabular}{|l|l|}
25.683 \\
25.771
\end{tabular} & $\begin{array}{l}27.204 \\
27.185\end{array}$ & 25. & & \begin{tabular}{|r|}
49.1533 \\
49.47 \\
\end{tabular} & $\begin{array}{ll}11.204 \\
11.221\end{array}$ & & $\frac{-1.215}{-1.212}$ & & \begin{tabular}{|l|}
50.11 \\
50.819
\end{tabular} & $\begin{array}{l}.143 \\
0.141\end{array}$ & & & & & $\begin{array}{l}48.0 \\
48.3 \\
\end{array}$ & $\begin{array}{l}\frac{12.3}{12.1} \\
\end{array}$ & & & & & & & \\
\hline 46 & & & 26.42 & 25.75 & 25.735 & 27.188 & & & 49.6 & & & -1. & & \begin{tabular}{|l|}
49.929 \\
\end{tabular} & & & & & & & & & & & & & & \\
\hline & & & 26.415 & & 25.71 & 27.154 & 25. & & 49. & $\begin{array}{l}11.074 \\
\end{array}$ & & -1. & & 47.564 & & & & & & 48.0 & 1 & & 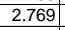 & & & & & \\
\hline & $8 / 30 / 2$ & & 26.41 & 25.761 & 25.7 & 27.199 & 25.34 & & & 111.114 & & -1. & & \begin{tabular}{|l|l}
49.03 \\
\end{tabular} & 0.144 & & & & & 48. & 11 & & 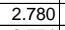 & & & & 0.501 & \\
\hline & & & $\begin{array}{r}26.39 \\
26.207\end{array}$ & & \begin{tabular}{|l}
25.66 \\
\end{tabular} & 27.219 & 25.2 & & 49 & & & & & 48 & & & & & & & & & & & & & & \\
\hline$\frac{44}{44}$ & $8 / 30 / 2004$ & $3: 23: 12 \mathrm{PM}$ & $26.397 \mid$ & $\begin{array}{l}25.773 \\
25770\end{array}$ & 25.677 & $\begin{array}{r}27.25 \\
27.312\end{array}$ & 25.2 & 45.0 & \begin{tabular}{|l|}
49.346 \\
48.565
\end{tabular} & $\begin{array}{l}11.109 \\
1109\end{array}$ & 35 & $\begin{array}{r}-1.209 \\
.206\end{array}$ & & 49.79 & $\begin{array}{l}0.148 \\
0.151\end{array}$ & 14 & & & 22 & 48.2 & 11. & & & & 0.021 & 0.001 & 0.519 & 0 \\
\hline & & & & & & & & & & & & & & & & & & & & & & & & & & & & \\
\hline & & & & & & 27.2 & & & & 1 & & & & & & & & & & & & & & & & & & \\
\hline & & & 26 & & 25.6 & & & & & $\frac{11.25}{11.25}$ & & & & & & & & & & & & & & & & & & \\
\hline & & & 20.54 & & & & & & & & & & & & & & & & & & & & & & & & & \\
\hline & & & 263 & & 25.6 & 27.1 & & & 49.28 & & & & & & & & & & & & & & & & & & & \\
\hline & & & & & & & & & & & & & & & & & & & & & & & & & & & & \\
\hline & & & 32 & 25.7 & 25 & 27.233 & & 45.8 & 49.246 & 11.1 & & & & & & & & & & & & & & & & & & \\
\hline & & & & & 25.4 & 27.3 & & & & & & & & & & & & & & & & & & & & & & \\
\hline & & & & & 25. & 27.25 & & & \begin{tabular}{|l|l|} 
& 49.279 \\
\end{tabular} & & & & & & & & & & & & & & & & & & & \\
\hline & & & & & $25.58 \mathrm{C}$ & 27.2 & & 44.8 & 48.77 & & & & & & 0. & & & & & & & & & & & & & \\
\hline & & & & & & 27.1 & & & & & & & & & & & & & & & & & & & & & & \\
\hline & & & & & & 27.231 & & 44.751 & & & & & & & & & & & & & & & & & & & & \\
\hline & & & & & & 27.22 & & 44.65 & 49.008 & & & & & & & & & & & 4 & & & & & & & & \\
\hline & & & & & & $\frac{21}{272}$ & & $\frac{44}{445}$ & & & & & & & & & & & & & & & & & & & & \\
\hline & & & & & $\frac{2.35}{256}$ & 27.26 & & & & & & & & & & & & & & & & & & & & & & \\
\hline & & & & & & 27.2 & & & & & & & & & & & & & & & & & & & & & & \\
\hline & & & & & 25.5 & 27.15 & & 44.6 & & 11.006 & & $\frac{-1.212}{-1.212}$ & & & & & & & & 47 & & & & & & & & \\
\hline & & & & & & 271 & & & & & & & & & & & & & & & & & & & & & & \\
\hline & & & & & & & & & & & & & & & & & & & & & & & & & & & & \\
\hline & $8 / 30 / 2004$ & & & & 25.597 & 27.166 & & & & & & & & 8.682 & 0.141 & 14.643 & & & & & & & & & & 0.00 & 0.498 & \\
\hline
\end{tabular}


WSRC-TR-2005-00105, REVISION 0

SRNL-RPP-2005-00012, REVISION 0

RUN \# 4.03A AND B; FIRST AND SECOND HALF OF SLURRY DEWATERING - CONT.

\begin{tabular}{|c|c|c|c|c|c|c|c|c|c|c|c|c|c|c|c|c|c|c|c|c|c|c|c|c|c|c|c|c|}
\hline & A & B & \begin{tabular}{l|l|}
$\mathrm{D}$ \\
\end{tabular} & $E$ & $\mathrm{~F}$ & G & $\mathrm{H}$ & $\mathrm{J}$ & \begin{tabular}{ll|}
$\mathrm{K}$ \\
\end{tabular} & $\mathrm{L}$ & M & $\mathrm{N}$ & 0 & Q & $\begin{array}{ll}R \\
\end{array}$ & \begin{tabular}{l|l|} 
\\
\end{tabular} & $\mathrm{T}$ & $\mathrm{v}$ & w & $x$ & $\mathrm{Y}$ & $z$ & $\mathrm{AA}$ & $A B$ & $\mathrm{AC}$ & $A D$ & $\mathrm{AE}$ & \\
\hline 49 & $8 / 30 / 2004$ & $3: 46: 12 \mathrm{PM}$ & 26.322 & 25.768 & \begin{tabular}{|l|l|}
85.562 \\
\end{tabular} & 27.276 & 25.173 & 44.91 & \begin{tabular}{|l|}
49.398 \\
\end{tabular} & 11.171 & $\begin{array}{l}34.626 \\
\end{array}$ & & -6.908 & 49.549 & 0.135 & 14.643 & 0.003 & 472.83 & 7.88056 & 48.3 & 111.8 & 39.8 & \begin{tabular}{|l|}
2.742 \\
\end{tabular} & & 0.019 & 0.000 & 0.478 & \\
\hline & & & & \begin{tabular}{|l|l|}
25.768 \\
55757
\end{tabular} & & & \begin{tabular}{|l|}
25.188 \\
25117 \\
\end{tabular} & & \begin{tabular}{|l|}
49.217 \\
\end{tabular} & $\begin{array}{l}11.149 \\
1.282\end{array}$ & \begin{tabular}{|l|}
34.657 \\
3.
\end{tabular} & & $\begin{array}{r}-7.018 \\
.702 \\
\end{array}$ & & & 14.643 & & & & 48.1 & & & & & & & 0.454 & \\
\hline & $8 / 30 / 2004$ & $\begin{array}{ll}3: 48: 12 \mathrm{PM} \\
3 \cdot 4 \cdot 12 \mathrm{P}\end{array}$ & \begin{tabular}{|l|l|}
26.306 \\
263255
\end{tabular} & \begin{tabular}{|l}
25.757 \\
25.77
\end{tabular} & \begin{tabular}{l|l}
7 & 25.556 \\
7 & 2555
\end{tabular} & 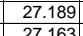 & \begin{tabular}{|l|l|}
25.147 \\
\end{tabular} & $\begin{array}{l}44.726 \\
.619\end{array}$ & \begin{tabular}{|l|l|}
49.091 \\
\end{tabular} & $\begin{array}{l}11.282 \\
1.220\end{array}$ & \begin{tabular}{|l|l}
34.443 \\
\end{tabular} & $\begin{array}{r}-1.215 \\
\end{array}$ & \begin{tabular}{|l|l|}
-7.024 \\
.009 \\
\end{tabular} & $\begin{array}{l}52.374 \\
552070\end{array}$ & 0.131 & \begin{tabular}{|l|l|}
14.643 \\
\end{tabular} & $\begin{array}{l}0.003 \\
\end{array}$ & 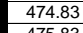 & 7.91389 & $\begin{array}{l}48.0 \\
\end{array}$ & & & 2720 & 0.019 & 0.018 & 0.000 & 0.466 & \\
\hline $\begin{array}{ll}494 \\
495 \\
\end{array}$ & $8 / 30 / 2004$ & $\begin{array}{l}3: 49: 12 \mathrm{PM} \\
3.50 \cdot 12 \mathrm{P}\end{array}$ & $\frac{26.325}{26.31}$ & $\begin{array}{r}25.77 \\
25766\end{array}$ & $\begin{array}{l}7 \\
6 \\
6\end{array}$ & 27.163 & $\begin{array}{r}25.115 \\
25.106\end{array}$ & $\begin{array}{l}44.618 \\
44.508\end{array}$ & \begin{tabular}{|l|}
48.948 \\
48.95 \\
\end{tabular} & $\frac{11.028}{11.144}$ & \begin{tabular}{|l|}
34.618 \\
34.347 \\
\end{tabular} & $\begin{array}{r}-1.209 \\
-1.212\end{array}$ & $\begin{array}{l}-6.989 \\
-6.983\end{array}$ & $\begin{array}{l}50.379 \\
48.404\end{array}$ & 0.139 & $\begin{array}{r}14.643 \\
14.643 \\
\end{array}$ & \begin{tabular}{|l|l|}
0.003 \\
0.003
\end{tabular} & $\begin{array}{r}475.83 \\
476.83\end{array}$ & $\begin{array}{ll}3 & 7.93056 \\
\end{array}$ & \begin{tabular}{|l|l}
47.8 \\
44.8
\end{tabular} & $\begin{array}{l}12.0 \\
11.5\end{array}$ & $\begin{array}{l}39.6 \\
394\end{array}$ & 270 & (1) & $\begin{array}{l}0.020 \\
0019\end{array}$ & $\begin{array}{l}0.000 \\
0000\end{array}$ & 0.495 & \\
\hline & $8 / 30 / 2004$ & $\begin{array}{l}3.0 .12 \mathrm{PM} \\
3: 11: 12 \mathrm{PM}\end{array}$ & $\frac{20.31}{26.295}$ & & \begin{tabular}{|l|l|}
5 & 25.545 \\
5 & 25.522
\end{tabular} & $\frac{27.184}{27.188}$ & \begin{tabular}{|l|}
255.106 \\
25.105 \\
\end{tabular} & $\begin{array}{l}44.506 \\
44.386\end{array}$ & \begin{tabular}{|l|}
$\mid 48.952$ \\
4889 \\
\end{tabular} & $\begin{array}{l}11.144 \\
11.124\end{array}$ & $\begin{array}{l}34.34 t \\
34.411\end{array}$ & $\frac{-1.212}{-1.215}$ & $\begin{array}{l}-6.983 \\
-6.868 \\
-6.8\end{array}$ & $\begin{array}{l}48.404 \\
50.511 \\
\end{array}$ & 0.137 & $\frac{14.643}{14.643}$ & & $\begin{array}{r}476.83 \\
477.83 \\
\end{array}$ & \begin{tabular}{l|l|} 
\\
3 & 7.9479622 \\
\end{tabular} & $\begin{array}{l}47.8 \\
47.5 \\
\end{array}$ & & $\begin{array}{l}39.4 \\
39.4 \\
\end{array}$ & \begin{tabular}{|l|}
2.718 \\
2716 \\
\end{tabular} & \begin{tabular}{|l|l|}
0.020 \\
0.019
\end{tabular} & $\begin{array}{l}0.019 \\
0.019\end{array}$ & $\begin{array}{l}0.000 \\
0.000\end{array}$ & $\begin{array}{l}0.490 \\
0.476\end{array}$ & 0.42 \\
\hline & $8 / 30 / 2004$ & 3:52:12 PM & 26.31 & 25.766 & \begin{tabular}{l|l}
5 & 25.555 \\
\end{tabular} & 27.204 & 25.391 & \begin{tabular}{|l|l|l|}
44.417 \\
\end{tabular} & \begin{tabular}{|l|l|}
48.691 \\
\end{tabular} & \begin{tabular}{|l|l|}
11.11 \\
\end{tabular} & 34.427 & -1.212 & $\mid-6.928$ & 51.725 & 0.149 & 14.643 & 0.003 & 478.83 & & 47.6 & & & & & & $\begin{array}{l}0.000 \\
0.001\end{array}$ & $\begin{array}{l}0.476 \\
0.533\end{array}$ & $\begin{array}{l}0.41 \\
0.45\end{array}$ \\
\hline & & & & & \begin{tabular}{|l|l|}
625.599 \\
\end{tabular} & 27.237 & & 44.846 & \begin{tabular}{|l|}
49.416 \\
\end{tabular} & 11.172 & 34.558 & -1.212 & $\begin{array}{l}-7.024 \\
\end{array}$ & 50.82 & 0.148 & 14.643 & 0.00 & 479.83 & $\begin{array}{ll}3 & 7.99722 \\
\end{array}$ & & & & & & & & 0.525 & $\begin{array}{l}0.45 \\
0.45\end{array}$ \\
\hline & $8 / 30 / 2004$ & 3:54:12 PM & 26.329 & 25.775 & \begin{tabular}{|l|l|}
5 & 25.63 \\
\end{tabular} & & 25.709 & 45.051 & \begin{tabular}{|l|l|}
49.752 \\
\end{tabular} & 11.212 & 34.731 & -1.212 & -6.995 & 49.614 & 0.142 & 14.643 & & 480.83 & 8.01389 & $\begin{array}{l}48.6 \\
\end{array}$ & & 39.9 & 2.750 & & 0.020 & 0.001 & 0.501 & \\
\hline 500 & $8 / 30 / 2004$ & 3:55:12 PM & \begin{tabular}{|l|l|}
26.34 \\
26.252
\end{tabular} & 25.791 & 25.64 & \begin{tabular}{|l|l|}
27.204 \\
\end{tabular} & 25.72 & 45.118 & \begin{tabular}{|l|l|}
49.549 \\
\end{tabular} & 11.284 & 34.821 & -1.212 & -6.966 & \begin{tabular}{|l}
49.679 \\
\end{tabular} & & $\begin{array}{l}14.643 \\
\end{array}$ & 0.004 & 481.83 & 3.03056 & \begin{tabular}{|l|l|}
48.4 \\
\end{tabular} & 11.8 & & \begin{tabular}{|l|l|}
2.756 \\
\end{tabular} & 0.018 & 0.018 & 0.000 & $\begin{array}{l}0.447 \\
\end{array}$ & \\
\hline 501 & $8 / 30 / 2004$ & $\begin{array}{l}3: 56: 12 \mathrm{PM} \\
3.57 \cdot 12 \mathrm{P}\end{array}$ & $\begin{array}{l}26.352 \\
26369\end{array}$ & $\begin{array}{l}25.798 \\
25815\end{array}$ & \begin{tabular}{l|l|l|}
8 & 25.611 \\
5 & 25616
\end{tabular} & \begin{tabular}{|c|}
27.27 \\
27.288
\end{tabular} & $\begin{array}{l}25.842 \\
25819\end{array}$ & \begin{tabular}{|l|l|l|l|l}
45.045 \\
44.902
\end{tabular} & \begin{tabular}{|l|}
49.424 \\
49271 \\
\end{tabular} & $\begin{array}{r}11.32 \\
11.283 \\
\end{array}$ & $\begin{array}{r}34.7 \\
\end{array}$ & $\begin{array}{l}-1.212 \\
.212\end{array}$ & \begin{tabular}{|l|l|}
-6.983 \\
7061
\end{tabular} & \begin{tabular}{|l|l}
45.703 \\
\end{tabular} & 0.134 & $\begin{array}{l}14.643 \\
1.643\end{array}$ & 0.003 & $\begin{array}{l}482.83 \\
\end{array}$ & 3.04722 & \begin{tabular}{|l|l|}
48.3 \\
19.1
\end{tabular} & 10.9 & $\begin{array}{l}39.9 \\
\end{array}$ & \begin{tabular}{|l|l|}
2.749 \\
\end{tabular} & 0.019 & \begin{tabular}{|l|l|l|}
0.019 \\
\end{tabular} & 0.000 & 0.473 & \\
\hline$\frac{50}{50}$ & $\frac{8 / 30 / 2004}{88 / 3 / 204}$ & $\begin{array}{l}3: 57: 12 \mathrm{PM} \\
3 \cdot 5 \cdot 12 \mathrm{PM}\end{array}$ & $\frac{26.369}{26.372}$ & $\frac{25.815}{25828}$ & & $\frac{27.288}{27265}$ & $\frac{25.819}{25872}$ & $\begin{array}{l}44.902 \\
44.577\end{array}$ & \begin{tabular}{|l|}
49.271 \\
48.933 \\
\end{tabular} & $\frac{11.283}{11094}$ & $\begin{array}{r}34.575 \\
34.536 \\
\end{array}$ & $\frac{-1.212}{-1.1212}$ & $\begin{array}{r}-7.061 \\
-7.015\end{array}$ & $\begin{array}{r}48.371 \\
49485\end{array}$ & $\begin{array}{l}0.136 \\
0.132\end{array}$ & $\frac{14.643}{14.643}$ & $\frac{0.003}{0.003}$ & $\begin{array}{r}483.83 \\
484.83 \\
\end{array}$ & 8.06389 & & & & $\frac{2.740}{2727}$ & 0.019 & 0.019 & 0.000 & 0.482 & $\frac{41}{40}$ \\
\hline 504 & $8 / 30 / 2004$ & 3.50.12 & 26.391 & 25.852 & \begin{tabular}{|l|l}
2 & 25.616 \\
2
\end{tabular} & 27.354 & 25.881 & 44.728 & \begin{tabular}{|l|}
49.205 \\
\end{tabular} & $\begin{array}{l}11.094 \\
11.169\end{array}$ & 34.56 & $\frac{-1.212}{-1.212}$ & $\begin{array}{l}-7.015 \\
-7.056\end{array}$ & $\begin{array}{r}\frac{49.485}{50.78} \\
\end{array}$ & 0.134 & $\begin{array}{l}\frac{14.643}{14.643} \\
\end{array}$ & $\begin{array}{l}0.003 \\
0.004\end{array}$ & $\begin{array}{r}484.83 \\
485.83\end{array}$ & \begin{tabular}{l|l|l|} 
& 8.08056 \\
3 & 8.09722
\end{tabular} & $\begin{array}{l}44.8 \\
48.1 \\
\end{array}$ & 12.1 & 39.6. & \begin{tabular}{|l|l|}
2.727 \\
2.733 \\
\end{tabular} & $\frac{0.019}{0.021}$ & $\begin{array}{l}0.019 \\
0.021\end{array}$ & 0.000 & $\begin{array}{l}0.470 \\
0.518\end{array}$ & 0.40 \\
\hline & $8 / 30 / 2004$ & 4:00:12 PM & 26.404 & 25.865 & \begin{tabular}{|l|l|}
5 & 25.611 \\
\end{tabular} & 27.402 & 25.874 & 44.433 & \begin{tabular}{|c|}
49.058 \\
\end{tabular} & 11.158 & 34.322 & -1.212 & -7.05 & \begin{tabular}{|l|l|}
49.113 \\
\end{tabular} & 0.141 & 14.643 & & 486.83 & \begin{tabular}{c|c|c|}
3 & 8.11389
\end{tabular} & 47.9 & $\begin{array}{l}11.7 \\
\end{array}$ & & & & & & 0.504 & $\frac{0.44}{0.43}$ \\
\hline 506 & $8 / 30 / 2004$ & 4:01:12 PM & 26.402 & 25.878 & \begin{tabular}{|l|l|}
825.582 \\
\end{tabular} & 27.435 & 25.937 & 44.548 & \begin{tabular}{|l|}
49.066 \\
\end{tabular} & 11.162 & 34.431 & -1.212 & -6.989 & 49.629 & 0.136 & 14.643 & & 487.83 & $\begin{array}{ll}3 & 8.13056 \\
\end{array}$ & 47.9 & & 39.5 & 2.72 & 0.019 & & 0.000 & 0.485 & $\frac{0.43}{0.41}$ \\
\hline & $8 / 30 / 2004$ & 4:02:12 PM & & 25.895 & \begin{tabular}{|l|l|}
5525.614 \\
\end{tabular} & 27.402 & 25.884 & 44.321 & \begin{tabular}{|r|}
48.84 \\
\end{tabular} & $\begin{array}{l}11.163 \\
\end{array}$ & 34.22 & & & & & $\begin{array}{l}14.643 \\
\end{array}$ & & & & \begin{tabular}{|l|l|}
47.7 \\
\end{tabular} & & & & & 0.019 & & & \\
\hline 508 & $8 / 30 / 2004$ & 4:03:12 PM & \begin{tabular}{|l|l|}
26.41 \\
\end{tabular} & 25.896 & 25.58 & 27.398 & 25.85 & & 48.774 & 11.145 & & & & $\begin{array}{l}48.613 \\
\end{array}$ & & & & & & & & & & & & & 0.435 & \\
\hline 50 & & & & & & & & 44.35 & |48.687 & 11.148 & .308 & & & 48.782 & 0.132 & 14.643 & & & & & & & & & & & & \\
\hline 510 & $8 / 30 / 2004$ & 5:12 PM & 26.429 & 25.935 & \begin{tabular}{|l|l|}
5 & 25.594 \\
\end{tabular} & 27.257 & 25.794 & 43.894 & 8.204 & 11.067 & 33.908 & -1.212 & & & 0.132 & 14.643 & & & & 47 & 11 & & & & & & 0.478 & \\
\hline 511 & $8 / 30 / 2004$ & 4:06:12 PM & 26.419 & 25.935 & \begin{tabular}{|l|l|}
5 & 25.636 \\
\end{tabular} & 27.222 & 25.8 & 44.081 & 48.626 & 11.033 & 34.101 & -1.22 & & 49.434 & 0.128 & 14.643 & & & 8.21389 & 47.5 & 11 & & & & 0.018 & & & \\
\hline $5 \frac{512}{512}$ & $8 / 30 / 2004$ & $4: 07: 12$ PM & 26.434 & 25.961 & 125.695 & 27.263 & 25.77 & 44.78 & $\begin{array}{l}49.36 \\
\end{array}$ & 11.136 & 34.661 & -1.21 & & 48.17 & 0.13 & 14.643 & & & 8.230 & 48.2 & 11 & 39. & 2.739 & \begin{tabular}{|l|l|l|l|}
0.019 \\
\end{tabular} & 0.018 & 0.000 & $\begin{array}{l}0.460 \\
\end{array}$ & \\
\hline$\frac{51}{51}$ & $8 / 30 / 2004$ & & 26.434 & 25.966 & $\begin{array}{c}625.665 \\
\end{array}$ & 27.278 & 25.64 & 44.649 & 49.172 & 11.096 & 34.718 & & & 48.488 & 0.13 & 14.643 & & 494.83 & & 48.0 & & & 736 & .019 & .018 & 0.000 & 0.460 & \\
\hline & $8 / 30 / 2004$ & 4:09:12 PM & 26.445 & 25.976 & 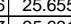 & 27.269 & 25.646 & 44.747 & 9.412 & 11.223 & 34.45 & & & & 0.142 & 14.643 & & & & 48.3 & & & & & & .001 & & \\
\hline & $8 / 30 / 2004$ & 4:10:12 PM & 20.4500 & & 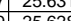 & 27.329 & 25.819 & & 9.505 & 11.317 & 34.934 & $\frac{-1.212}{1.212}$ & & 30.910 & 0.128 & 14.643 & & & & & & & & .018 & 0.018 & 0.000 & & \\
\hline & $\frac{1530404}{8 / 20204}$ & & & & & & $\frac{25.68}{2567}$ & & $\begin{array}{r}49 \\
48927\end{array}$ & 11.19 & & & & $\frac{48.433}{5524}$ & & $\frac{14.643}{14642}$ & & & & & & & & & & & & \\
\hline & $8 / 30 / 2004$ & 4:13:12 PM & 26.438 & 25.999 & 925.598 & 27.192 & 25.51 & 44.713 & 49.41 & 11.207 & 34.565 & -1.212 & -6.949 & 48.25 & 0.106 & 14.643 & & $\begin{array}{r}498.05 \\
499.83\end{array}$ & \begin{tabular}{c|c|}
38.31509 \\
38.3056
\end{tabular} & 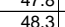 & 11.5 & 59.6 & $\frac{2.112}{2.733}$ & $\frac{0.019}{0.015}$ & $\begin{array}{l}0.019 \\
0.015\end{array}$ & 0.000 & 0.470 & \\
\hline & $8 / 30 / 2004$ & 4:14:12 PM & 26.433 & 26.009 & \begin{tabular}{|l|l|}
925.613 \\
\end{tabular} & 27.197 & 25.6 & 44.263 & 48.809 & 11.274 & 34.062 & -1.212 & & 50.77 & 0.126 & 14.643 & & & & \begin{tabular}{|l|l|}
47.7 \\
\end{tabular} & 12.1 & 39. & 2700 & 0.018 & 0.018 & 0.000 & $\begin{array}{l}0.453 \\
\end{array}$ & 0.39 \\
\hline & $8 / 30 / 2004$ & & & 26.004 & $\begin{array}{ll}425.593 \\
4\end{array}$ & 27.206 & 25.6 & 444.429 & \begin{tabular}{|l}
49.253 \\
\end{tabular} & 11.138 & 34.265 & -1 & & 50.734 & 0.135 & 14.643 & & 501.8 & & 48 & & & & $\overline{019}$ & & & 0.483 & \\
\hline & $8 / 3$ & 4:16:12 PM & & & 25.567 & 27.166 & & & 48.345 & 11..151 & & & & & 0.144 & & & & & 47 & & & & & & & & \\
\hline $5 \frac{5}{5}$ & & & & & & 27.225 & & & .624 & $\frac{11.286}{11.270}$ & & & & 49.86 & 0.122 & 14.643 & & & & & & & & & & & & \\
\hline & $8 / 30 / 2004$ & & & & & & & & . & & & & & 40422 & 0.13 & & & & & & & & & & & & & \\
\hline & $8 / 30 / 2004$ & 4:20:12 PM & 26.437 & 26.048 & \begin{tabular}{|l|l}
3 & 25.617 \\
\end{tabular} & 27.4 & 25.8 & 44.877 & \begin{tabular}{|c|}
49514 \\
\end{tabular} & 11.385 & 34.536 & -1.215 & & \begin{tabular}{|l|}
4.424 .752 \\
\end{tabular} & 0.122 & 14643 & & 506.83 & $\begin{array}{l}384 \\
\end{array}$ & 48.4 & 11. & & & & $\begin{array}{l}.017 \\
0.017 \\
\end{array}$ & & $\frac{0.484}{0.432}$ & \\
\hline & $8 / 30 / 2004$ & & 26.436 & 26.048 & \begin{tabular}{|l|l|}
825.647 \\
\end{tabular} & 27.405 & 25.9 & 44.788 & \begin{tabular}{|l|}
49.52 \\
\end{tabular} & 11.364 & & -1.215 & & 51.232 & 0.138 & 14. & & & & 48.4 & 12 & & & & & & & \\
\hline & & & 26.46 & & & 27.44 & & 45.304 & 50.034 & 11.306 & & & & & & 14. & & & & & & & & & & & 0.2 & \\
\hline & 8/30/2004 & B:12 PM & 26.462 & 26.06 & 25.742 & 27.49 & & 45.41 & 50.562 & 11.444 & & & & 48.863 & 0.144 & 14.643 & & & & & & & & & & & & \\
\hline & & & & $20.0 \%$ & 25.77 & 27.3 & & 45.6 & & 11.478 & & & & & 0.13 & & & & & & & & & & & & & \\
\hline & & & & & & & & & 50.498 & & & & & & & & & & & & & & & & & & & \\
\hline & & & & $\frac{26.095}{26111}$ & 25.776 & 27.462 & & $\frac{45.51}{15142}$ & 年 & $\begin{array}{ll}1.4 .46 \\
1125\end{array}$ & $\begin{array}{l}35.022 \\
34864 \\
3486\end{array}$ & $\frac{-1.21}{121}$ & & 5.1.1089 & 0 & $\frac{14.6}{1146}$ & & & & $\frac{49}{48}$ & & & & & & & & \\
\hline 533 & $8 / 301<004$ & & & & 25.8 & 27.517 & & $\frac{45 .}{44.8}$ & & $\frac{11.276}{11.276}$ & & $\frac{-1.21 \mathrm{c}}{-1.218}$ & & 51. & $\begin{array}{l}0.150 \\
0.113\end{array}$ & & & & & 48 & $\frac{14}{12}$ & & & & & & & \\
\hline & $8 / 30 / 2004$ & & 26.575 & & \begin{tabular}{|l|l|l|}
525845 \\
\end{tabular} & 27.463 & & & & 11.424 & & $\frac{-1.215}{-1.215}$ & & & & & & & & & & & & & & & & \\
\hline & $8 / 30 / 2004$ & & 26.595 & 26. & \begin{tabular}{|l|l|}
25.844 \\
\end{tabular} & 27.503 & 26.06 & 44.745 & \begin{tabular}{|l|l|}
49.228 \\
\end{tabular} & 11.276 & 34.645 & -1.212 & & \begin{tabular}{|l|l|}
47.507 \\
\end{tabular} & 0.131 & 14 & & 51 & & & 11 & & & & & & & \\
\hline & $8 / 30 / 26$ & & 26.609 & 26.181 & 25.86 & 27.448 & & 45.0 & & 11.356 & & -1.21 & & & 0.14 & & & & & & & & & & & & & \\
\hline & & & & & & & & & & & & & & & & & & & & & & & & & & & 0.424 & \\
\hline & & & & 26.17 & 25.88 & 27.31 & & & & 11.359 & & & & & & & & & & & & & & & & & & \\
\hline & & & & & & & & & & & & & & & & & & & & & & & & & & & & \\
\hline $\begin{array}{l}541 \\
541 \\
\end{array}$ & $\frac{0 / 301 / 2}{8 / 30 / 2}$ & & & & & 2730 & & & & & & & & & & & & & & & & & & & & & & \\
\hline$\sqrt{542}$ & $8 / 30 / 2$ & & 26.63 & & 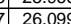 & 27.382 & & 46. & & 11.631 & & -1.24 & & & & & & & & & & & & & & & & \\
\hline 54 & & & & & & & & & & & & & & & & & & & & & & & & & & & & \\
\hline & & & 26.65 & 26.169 & 926.1 & 27.381 & 25.8 & 45.4 & 50.115 & 11.456 & & & & & & & & & & & & & & & & & & \\
\hline & & & & & & & & & & & & & & & & & & & & & & & & & & & & \\
\hline & $8 / 30 / 2004$ & & 26.69 & 26.16 & 26.103 & 27.297 & 25. & 45.4 & 50.324 & 11.389 & & -1.2 & & & 0.121 & & & & & 49 & 12 & & & & & & & \\
\hline & $8 / 30 / 2$ & & & & 26.1 & 27.40 & & & 50.214 & 11.364 & & -1. & & & 0.1 & & & & & 4 & & & & & & & & \\
\hline & $8 / 30 / 2004$ & & & & & 27.3 & & & \begin{tabular}{|l|}
50.104 \\
\end{tabular} & & & & & & & & & & & & 11 & & & & & & & \\
\hline & $8 / 30 / 2004$ & & & & & 27.313 & 25 & & & 11.34 & 34.314 & -1.212 & & & 0.1 & & & & & 48.4 & 11 & & & & & & & \\
\hline & & & & & & 27.328 & & & & & & & & & & & & & & & & & & & & & & \\
\hline $5=$ & & & & & & 27.348 & & 44. & & 11.337 & & -1.209 & & & 0.1 & & & & & 48.4 & & & & & & & & \\
\hline & 8313012004 & & & 26.19 & 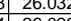 & 27.34 & & 43.1. & & I1.1199 & & & & & 0.1 & & & & & $4 i$ & & & & & & & & \\
\hline & & & & & & 27.4 & $\frac{25 .}{26 .}$ & 44.14 & $\frac{49.275}{51519}$ & & & & & & & & & & & & & & & & & & & \\
\hline & & & & & & 27.454 & & 4.456 & & & & & & & & & & & & & $110^{8}$ & & & & & & & \\
\hline & & & & & & 27.53 & $\frac{26}{26}$ & & $\mid$ & & & & & & & & & & & & & & & & & & & \\
\hline & $\frac{1 / 30 / 2004}{8}$ & & & $\frac{26.23}{26.23}$ & & 27.445 & 25.9 & 45.59 & 50.819 & $\frac{11.509}{11.509}$ & & -1.157 & & & 0.138 & & & & & 49 & 11. & & & & & & & \\
\hline & & & & & & & & & & & & & & & & & & & & & & & & & & & & \\
\hline & & & & & & & & & & & & & & & 0.1 & & & & & & & & & & & & & \\
\hline 560 & $8 / 30 / 2004$ & & 6.759 & 26.236 & \begin{tabular}{|l|l|}
6 & 25.966 \\
\end{tabular} & 27.368 & & & \begin{tabular}{|l|l|l|}
49.178 \\
\end{tabular} & 1.19 & 34.091 & $\frac{-1.215}{-1.215}$ & & 49.364 & 0.12 & 14.643 & & 541.83 & & & 11.8 & & & .017 & 0.017 & 0.000 & 0.42 & \\
\hline
\end{tabular}


WSRC-TR-2005-00105, REVISION 0

SRNL-RPP-2005-00012, REVISION 0

RUN \# 4.03A AND B; FIRST AND SECOND HALF OF SLURRY DEWATERING - CONT.

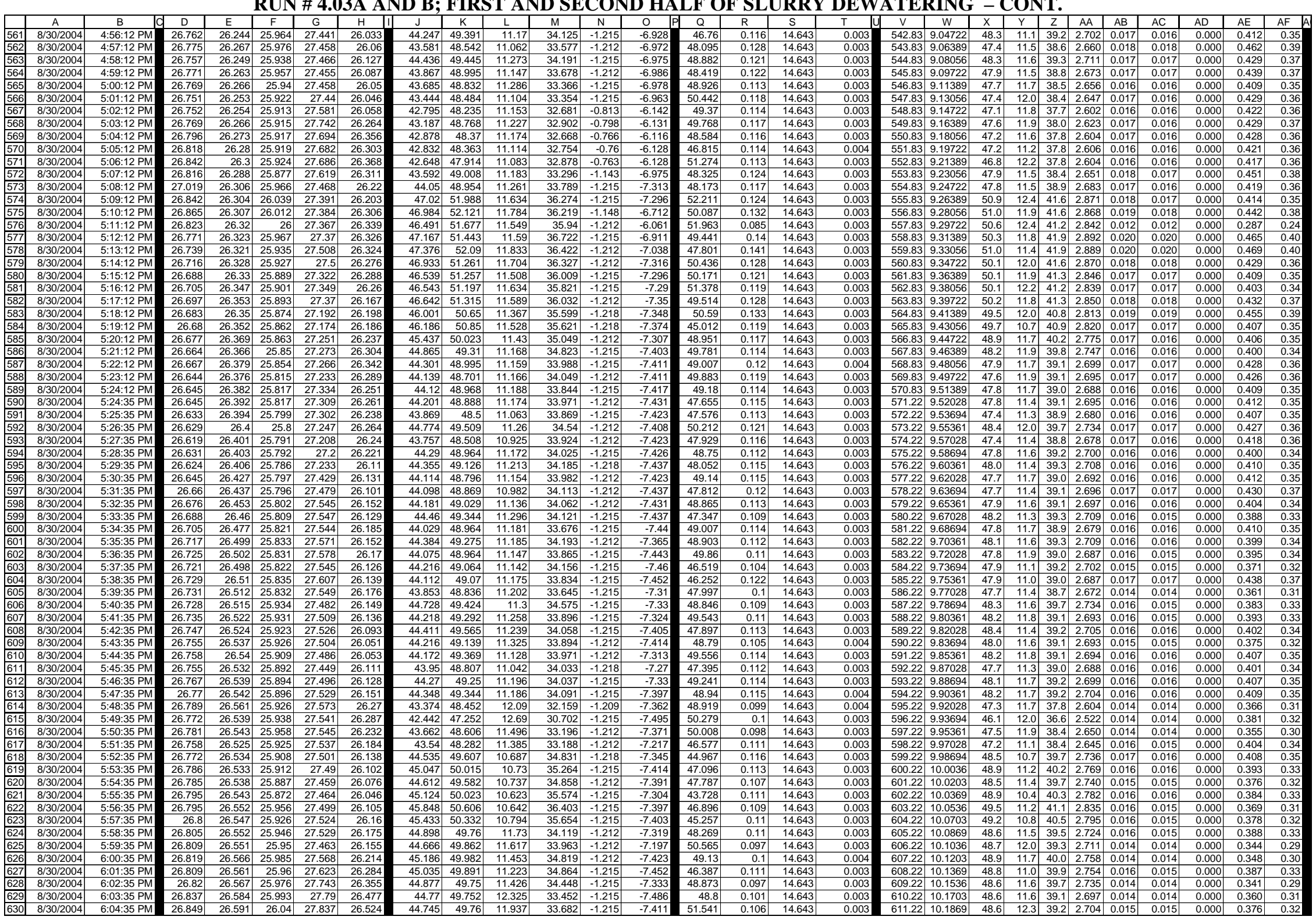


WSRC-TR-2005-00105, REVISION 0

SRNL-RPP-2005-00012, REVISION 0

RUN \# 4.03A AND B; FIRST AND SECOND HALF OF SLURRY DEWATERING - CONT.

\begin{tabular}{|c|c|c|c|c|c|c|c|c|c|c|c|c|c|c|c|c|c|c|c|c|c|c|c|c|c|c|c|c|}
\hline & & & & & & & & & & & & & & & & & & & & & & & & & & & & \\
\hline & A & $\begin{array}{ll} \\
\end{array}$ & D & $E$ & $F$ & G & $\mathrm{H}$ & $\mathrm{J}$ & \begin{tabular}{ll|}
$\mathrm{K}$ \\
\end{tabular} & $\mathrm{L}$ & \begin{tabular}{l|l|}
$\mathrm{M}$ \\
\end{tabular} & $\mathrm{N}$ & 0 & $\mathrm{Q}$ & $\begin{array}{ll}R \\
\end{array}$ & $\mathrm{~s}$ & \begin{tabular}{ll|} 
\\
\end{tabular} & $\mathrm{V}$ & w & $x$ & $\mathrm{Y}$ & $z$ & $\mathrm{AA}$ & $A B$ & $A C$ & $A D$ & $\mathrm{AE}$ & \\
\hline 63 & $8 / 30 / 2004$ & 6:05:35 PM & 26.861 & 26.603 & \begin{tabular}{|l|l|}
26.072 \\
\end{tabular} & \begin{tabular}{|l|}
27.914 \\
\end{tabular} & 26.525 & 44.801 & \begin{tabular}{l|l|}
49.526 \\
\end{tabular} & 12.162 & $\begin{array}{l}33.789 \\
\end{array}$ & \begin{tabular}{|l|}
-1.218 \\
\end{tabular} & \begin{tabular}{|l|l|}
-7.348 \\
\end{tabular} & 50.406 & 0.102 & 14.643 & 0.003 & 612.22 & 10.2036 & 48.4 & & 39.3 & 2.709 & & 0.014 & 0.000 & 0.361 & \\
\hline & & & & 26.609 & & & & & & \begin{tabular}{|l|l|}
12.079 \\
\end{tabular} & & & $\begin{array}{r}-7.368 \\
.768\end{array}$ & 48.074 & & \begin{tabular}{|l|l|}
14.643 \\
\end{tabular} & & & & & & & & & & & & \\
\hline 633 & $8 / 30 / 2004$ & 6:07:35 PM & \begin{tabular}{|l|l|}
26.893 \\
2.019
\end{tabular} & 26.616 & 26.13 & \begin{tabular}{l|l|}
27.862 \\
770
\end{tabular} & \begin{tabular}{|l|l|}
26.498 \\
2.20
\end{tabular} & \begin{tabular}{|l|l|l|l|}
4.017 \\
\end{tabular} & \begin{tabular}{|l|l|}
49.252 \\
\end{tabular} & $\begin{array}{l}2.177 \\
\end{array}$ & \begin{tabular}{|l|l|}
33.153 \\
\end{tabular} & \begin{tabular}{|l|l|} 
& -1.218 \\
\end{tabular} & $\begin{array}{r}-7.446 \\
\end{array}$ & 47.716 & 0.105 & \begin{tabular}{|l|}
14.643 \\
\end{tabular} & 0.003 & $\begin{array}{ll}614.22 \\
1.20\end{array}$ & 10.2369 & $\begin{array}{l}48.1 \\
\end{array}$ & 111.4 & & 2.674 & 0.015 & $\begin{array}{l}0.015 \\
\end{array}$ & $\begin{array}{l}0.000 \\
\end{array}$ & 0.376 & \\
\hline $6 \frac{634}{635}$ & $8 / 30 / 2004$ & 6:08:35 PM & $26.911 \mid$ & 26.628 & \begin{tabular}{|l|}
26.142 \\
26217
\end{tabular} & \begin{tabular}{|r|}
27.799 \\
27684
\end{tabular} & $\begin{array}{l}26.246 \\
26266\end{array}$ & \begin{tabular}{|l|l|}
44.817 \\
4095
\end{tabular} & $\mid$ & $\begin{array}{l}11.845 \\
11.820\end{array}$ & \begin{tabular}{|l|l|l|}
34.121 \\
3141
\end{tabular} & $\begin{array}{r}-1.212 \\
-1215\end{array}$ & $\begin{array}{r}-7.466 \\
7275 \\
\end{array}$ & \begin{tabular}{|l|l|}
49.451 \\
50215
\end{tabular} & 0.106 & $\begin{array}{r}14.643 \\
14643\end{array}$ & $\begin{array}{l}0.003 \\
\end{array}$ & $\begin{array}{l}615.22 \\
619.22 \\
\end{array}$ & \begin{tabular}{l|l|}
2 & 10.2536 \\
\end{tabular} & \begin{tabular}{|l|l|}
48.5 \\
497
\end{tabular} & & & , & 0.015 & $\begin{array}{l}0.015 \\
0.0\end{array}$ & $\begin{array}{l}0.000 \\
\end{array}$ & 0.372 & \\
\hline & $8 / 3 / 3 / 2004$ & & $\begin{aligned} 26.906 \\
26.936\end{aligned}$ & $\frac{26.618}{26.643}$ & \begin{tabular}{|l|}
26.217 \\
26.297 \\
\end{tabular} & \begin{tabular}{r|r|}
27.684 \\
27.684
\end{tabular} & $\begin{array}{l}26.266 \\
26.351\end{array}$ & $\begin{array}{r}44.985 \\
45.3 \\
\end{array}$ & \begin{tabular}{|l|}
49.8514 \\
50.18 \\
\end{tabular} & $\begin{array}{l}11.828 \\
11.524 \\
\end{array}$ & $\begin{array}{r}34.14 \\
34.784\end{array}$ & $\begin{array}{r}-1.215 \\
-1.209 \\
\end{array}$ & $\begin{array}{l}-7.275 \\
-7.255 \\
\end{array}$ & \begin{tabular}{|r|}
50.215 \\
47.63
\end{tabular} & 0.095 & \begin{tabular}{|c|}
14.643 \\
14.643
\end{tabular} & $\begin{array}{l}0.003 \\
0.004\end{array}$ & $\frac{616.22}{6617.22}$ & \begin{tabular}{ll|} 
& 10.2703 \\
2 & 10.02863
\end{tabular} & $\begin{array}{l}48.7 \\
49 . \\
\end{array}$ & $\begin{array}{l}12.0 \\
11.3 \\
\end{array}$ & \begin{tabular}{|l|}
$\mid 39.6$ \\
30.0
\end{tabular} & $\begin{array}{l}2.728 \\
2761\end{array}$ & & $\begin{array}{l}0.013 \\
0.015\end{array}$ & 0.000 & $\begin{array}{l}0.332 \\
0.365\end{array}$ & $\begin{array}{l}0.28 \\
0.331\end{array}$ \\
\hline 637 & $8 / 30 / 2004$ & 6:11:35 PM & 26.946 & & \begin{tabular}{|l|}
20.231 \\
26.262 \\
\end{tabular} & & \begin{tabular}{|l|}
20.051 \\
2.356
\end{tabular} & $\begin{array}{r}45.728 \\
4.728\end{array}$ & \begin{tabular}{|l|}
50.140 \\
50.724
\end{tabular} & & & \begin{tabular}{|l|}
-1.215 \\
-1.215 \\
\end{tabular} & & \begin{tabular}{|l|}
48.060 \\
\end{tabular} & 0.111 & $\begin{array}{l}11.643 \\
14.643 \\
\end{array}$ & 0.004 & & \begin{tabular}{|l|l}
10.2809 \\
2 & 10.3036
\end{tabular} & $\begin{array}{l}49.0 \\
49.6\end{array}$ & $\frac{11.3}{11.5}$ & $\begin{array}{l}40.0 \\
40.5 \\
4\end{array}$ & $\begin{array}{l}2.761 \\
2.790\end{array}$ & $\frac{0.015}{0.016}$ & 0.015 & $\begin{array}{l}0.000 \\
0.000\end{array}$ & $\begin{array}{l}0.365 \\
0.379\end{array}$ & $\begin{array}{l}0.31 \\
0.32\end{array}$ \\
\hline & & & & 26.634 & & 27.665 & & 45.278 & \begin{tabular}{|l|}
50.158 \\
\end{tabular} & 11.587 & 34.634 & \begin{tabular}{|l|}
-1.218 \\
\end{tabular} & -7.298 & 50.01 & 0.103 & 14.643 & & & \begin{tabular}{|l|l}
2 & 10.3203 \\
\end{tabular} & & & & & & & & & $\frac{0.32}{0.30}$ \\
\hline 639 & $8 / 30 / 2004$ & 6:13:35 PM & 26.986 & 26.659 & \begin{tabular}{|l|l|}
26.278 \\
\end{tabular} & 27.72 & 26.357 & 45.379 & \begin{tabular}{|l|}
50.137 \\
\end{tabular} & 11.554 & & -1.212 & $\begin{array}{l}-7.376 \\
\end{array}$ & \begin{tabular}{|l|l|}
47.803 \\
\end{tabular} & 0.097 & $\begin{array}{l}14.643 \\
\end{array}$ & 0.003 & 620.22 & \begin{tabular}{|l|l|l|l|l|}
2 & 1036
\end{tabular} & 49.0 & & 40.2 & 2.768 & 0.014 & & & & 0.30 \\
\hline 640 & & & 27.001 & & & & & & \begin{tabular}{|l|}
50.778 \\
\end{tabular} & $\begin{array}{ll}11.663 \\
\end{array}$ & 35.063 & & & & & \begin{tabular}{l|l}
14.643 \\
\end{tabular} & & & & & & & & & & 0.000 & 0.379 & $\frac{0.28}{0.32}$ \\
\hline 641 & & & & 26.6 & \begin{tabular}{|l|}
26.283 \\
\end{tabular} & 27.73 & & & 50.198 & & & & & & & & & & & & & & & & & & & \\
\hline & $8 / 30 / 2004$ & 6:35 PM & 27 & 26.657 & \begin{tabular}{|l|l|}
26.261 \\
\end{tabular} & 27.674 & 26.38 & 45.732 & \begin{tabular}{|l|}
50.786 \\
\end{tabular} & 11.547 & $\begin{array}{l}35.067 \\
\end{array}$ & & & 46.258 & & 14.643 & & & & & & & 2.785 & .015 & & & 0.359 & \\
\hline 64 & & $6: 17: 35 \mathrm{PM}$ & & $\begin{array}{l}26.677 \\
\end{array}$ & & & & & 50207 & 11.554 & & & & & 0.105 & $\begin{array}{l}14.643 \\
\end{array}$ & & & 10.40 & 49.2 & & & & & & & & \\
\hline & $8 / 30 / 2004$ & 6:18:35 PM & 27.04 & 26.692 & \begin{tabular}{|l|}
26.286 \\
\end{tabular} & 27.649 & 26.35 & 45.622 & \begin{tabular}{|l|}
50.436 \\
\end{tabular} & 11.384 & 35.24 & -1.212 & $\begin{array}{ll}-7.374 \\
\end{array}$ & \begin{tabular}{|l|l|} 
\\
\end{tabular} 9.424 & 0.112 & $\begin{array}{l}14.643 \\
\end{array}$ & & 625.2 & 10.4203 & 49.3 & 11.8 & 40.4 & 2.788 & 0.016 & 0.015 & 0.000 & $\begin{array}{ll}0.383 \\
\end{array}$ & \\
\hline 64 & 8/3012004 & 6:19:35 PM & 27.05 & 26.697 & 26.296 & 27.684 & 26.335 & 45.508 & 50.347 & 11.292 & 35.201 & & -7.463 & 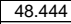 & 0.112 & 14.643 & & 626.2 & 10.4369 & 49.2 & 11 & & 2.78 & 0.016 & & 0.000 & 0.383 & \\
\hline & 8/3012004 & 6:20:35 PM & 27.05 & 26.697 & 26.281 & 27.649 & 26.32 & 45.622 & 50.365 & 11.424 & 35.197 & -1.218 & & 48.544 & 0.103 & 14.643 & & & 0.4536 & 49.2 & & & & & & & & \\
\hline 64 & 8/30/2004 & & & 26.701 & 26.285 & 27.592 & & 45.914 & 50.821 & 11.226 & 35.64 & & & 46.475 & & 14.643 & & & .47 & 49. & & & & & & & & \\
\hline & 883012004 & $6: 22: 35 \mathrm{PM}$ & 27.058 & 26.706 & $\mid 26.295$ & 27.597 & 26. & 45.234 & 49.837 & 11.186 & & & & 46.925 & 0.109 & 14.643 & & & & 48.1 & & & & .016 & & & $0.3 / 4$ & \\
\hline & 8 & $\begin{array}{l}0.3 .35 \mathrm{PM} \\
6.2025 \mathrm{PM}\end{array}$ & 20.060 & $\frac{20.119}{26708}$ & \begin{tabular}{|l|l|}
2.35 \\
2628
\end{tabular} & 27.000 & & 年 & 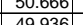 & 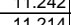 & $\begin{array}{l}35.5817 \\
35312\end{array}$ & 1.121 & & $\begin{array}{r}46.6 \\
47205\end{array}$ & & $\begin{array}{l}14.043 \\
14643\end{array}$ & & & $\frac{0.5030}{0.532}$ & (3) & & & 2.86 & & & & & \\
\hline 651 & $8 / 30 / 2004$ & $6.42 .535 \mathrm{PM}$ & 27059 & $\frac{20.100}{26712}$ & \begin{tabular}{|l|l|}
2.205 \\
26296
\end{tabular} & 27.554 & $\frac{20.24}{26.2}$ & 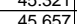 & | & $\frac{11.214}{11308}$ & 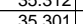 & $\frac{1.410}{-1212}$ & & 41.2032 & 0.1308 & 14.045 & & & 0.0535 & & & & 2.10 & 015 & 015 & & 0 & \\
\hline & $8 / 30 / 2004$ & $6: 26: 35 \mathrm{PM}$ & 27.067 & 26.71 & \begin{tabular}{|l|}
20.250 \\
26.294 \\
\end{tabular} & $\mid 27.556$ & $\frac{20.2}{26.23}$ & 444.971 & 50.071 & $\begin{array}{l}11.000 \\
11.083\end{array}$ & 34.862 & $\frac{-1.214}{-1.212}$ & $\frac{-7.575}{-7.301}$ & $\begin{array}{r}-47.502 \\
46.99 \\
\end{array}$ & $\begin{array}{l}0.100 \\
0.103\end{array}$ & $\frac{14.045}{14.643}$ & & & \begin{tabular}{|l|l|}
10.55336 \\
\end{tabular} & $\begin{array}{r}43.0 \\
48.9\end{array}$ & $\frac{11.2}{11.2}$ & & 2.752 & 0.015 & 0.014 & 0.0000 & 0.356 & \\
\hline & $8 / 30 / 2004$ & $6: 27: 35 \mathrm{PM}$ & 27.066 & 26.719 & \begin{tabular}{|l|l|}
26.373 \\
\end{tabular} & 27.536 & 26.24 & 45.759 & \begin{tabular}{|l|}
50.826 \\
\end{tabular} & 11.203 & 35.371 & -1.215 & -7.486 & \begin{tabular}{|l|l|}
44.704 \\
\end{tabular} & 0.107 & 14.643 & & 634.2 & \begin{tabular}{|l|l|}
10.5703 \\
\end{tabular} & 49.7 & & & 2797 & $\frac{0.015}{0.015}$ & & & 0.0363 & \\
\hline & $8 / 30 / 2004$ & $: 35 \mathrm{PM}$ & & \begin{tabular}{l|l|l|}
26.707 \\
\end{tabular} & \begin{tabular}{|l|}
26.381 \\
\end{tabular} & 27.519 & & & & \begin{tabular}{|l|l|}
11.08 \\
\end{tabular} & \begin{tabular}{|c|c|c|c|}
35.443 \\
\end{tabular} & & $\begin{array}{l}-7.466 \\
\end{array}$ & & & 14.643 & & & & 49.4 & & & & & & & & \\
\hline 65 & $8 / 30 / 2004$ & 6:29:35 PM & 27.08 & & \begin{tabular}{|l|l|}
26.386 \\
\end{tabular} & 27.509 & & 45.763 & 50.608 & $\begin{array}{ll}11.334 \\
\end{array}$ & $\begin{array}{l}35.426 \\
\end{array}$ & -1.215 & $\begin{array}{l}-7.293 \\
\end{array}$ & 46.748 & 0.104 & 14.643 & & & & 49.5 & & & 2.799 & 0.015 & .014 & 0.000 & 0.353 & \\
\hline & & & & & & & & & & & & & & & 0.11 & 14.643 & & & & 49.4 & & & & & & & & \\
\hline & $8 / 30 / 2004$ & $6: 31: 35 \mathrm{PM}$ & 27.071 & $\begin{array}{l}26.714 \\
\end{array}$ & \begin{tabular}{|l|l|}
26.353 \\
\end{tabular} & 27.456 & 26.1 & 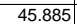 & & $\begin{array}{ll}11.156 \\
\end{array}$ & $\begin{array}{l}35.78 \\
\end{array}$ & -1.212 & & \begin{tabular}{|l|}
45.701 \\
\end{tabular} & 0.099 & 14.643 & & 638.2 & & 49.5 & & & & & & & 0.334 & \\
\hline & $8 / 30 / 2004$ & & 27.09 & 26.728 & 26.372 & 27.46 & & 46.005 & \begin{tabular}{|l|}
50.668 \\
\end{tabular} & 11.083 & 36.038 & -1.209 & & \begin{tabular}{|l|}
46.975 \\
\end{tabular} & & 14.643 & & & & & 11. & & & & & & 0.359 & \\
\hline & $8 / 30 / 2004$ & 6:33:35 PM & 27.073 & 26.706 & 26.34 & 27.418 & 26.14 & 572 & \begin{tabular}{|l|}
50.602 \\
\end{tabular} & 11.096 & \begin{tabular}{|l|l|l|}
35.344 \\
\end{tabular} & -1.215 & $\begin{array}{ll}-7.405 \\
\end{array}$ & 46.669 & 0.105 & 14.643 & & & 10.6703 & 49.5 & 11 & & 2.789 & 0.015 & \begin{tabular}{|l|l|l|}
0.014 \\
\end{tabular} & 0.000 & 0.358 & \\
\hline 66 & 8/30/2004 & 6:34:35 PM & 27.066 & 26.704 & 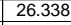 & 27.381 & 26.1 & 45.848 & \begin{tabular}{|l|} 
\\
\end{tabular} & 11.141 & & -1.21 & & \begin{tabular}{|l|l}
48.608 \\
\end{tabular} & 0.113 & 14.643 & & & & & 11 & & 2.809 & 0.016 & & & 0.383 & \\
\hline 66 & $8 / 30 / 2004$ & $6: 35$ & 27.059 & 26.6 & 26.3 & 27.379 & & & \begin{tabular}{|l|}
50.386 \\
\end{tabular} & 11.037 & 35.26 & -1.218 & & 6.514 & 0.105 & 14 & & & & 49 & & & $2.18 \mathrm{x}$ & & & & & \\
\hline & & & 27.0677 & & $\frac{20.334}{20372}$ & 27.377 & & 45.663 & 50.266 & 11.066 & 54 & & & 777.497 & 0.106 & 14.643 & & & & 49 & & & & & {$[\pi$} & & & \\
\hline$\frac{10}{66}$ & 年 & & 27.05 & 26.103 & 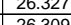 & $\begin{aligned} 27.4 \\
2730 \mathrm{~g}\end{aligned}$ & & 年 45.4817519 & $\frac{5.30 \%}{5.323}$ & 10.961 & & $-\frac{1.1212}{-1215}$ & & $49.5 / 2$ & & 14.64 & & & & & 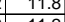 & & & & & & & \\
\hline & $8 / 301<004$ & & 27.040 & $\frac{20.6}{26.6}$ & \begin{tabular}{|l|l|}
2.303 \\
2634
\end{tabular} & 年, & & & $\mid$\begin{tabular}{|c|}
$\mid 50.30$ \\
5058
\end{tabular} & $\frac{10.945}{11.171}$ & & - & & & & & & & & & $\frac{11}{10}$ & & & & 然 0.013 & & 0.303 & \\
\hline & $8 / 30 / 2004$ & & 27.056 & $\frac{20.005}{26.668}$ & \begin{tabular}{|l|}
20.314 \\
26.317 \\
\end{tabular} & $\frac{27.506}{2731}$ & & & \begin{tabular}{|l|}
30.49 .464 \\
49.464 \\
\end{tabular} & 11.174 & 34.501 & $\frac{-1.210}{-1.218}$ & & \begin{tabular}{|l|}
43.511 \\
48.969 \\
\end{tabular} & 0.095 & $\frac{14.045}{11643}$ & & & & $\frac{4.4}{483}$ & 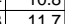 & & & & & & 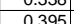 & \\
\hline 66 & $8 / 30 / 2004$ & $6: 41: 3$ & 27.034 & 26.676 & 26.36 & $27.298 \mid$ & 25. & & \begin{tabular}{|l|}
50.231 \\
\end{tabular} & 11.547 & \begin{tabular}{|c|}
34.788 \\
\end{tabular} & \begin{tabular}{|l|l|}
-1.212 \\
\end{tabular} & -7.481 & 48.256 & & 14.6 & & & & 49. & & & & & 0.014 & & 0.341 & \\
\hline & $8 / 30 / 2$ & & 27.016 & 26.654 & \begin{tabular}{|l|}
26.413 \\
\end{tabular} & 27.246 & 25.9 & 45.611 & \begin{tabular}{|l|}
50.372 \\
\end{tabular} & 11.642 & & -1.215 & & \begin{tabular}{|l|l|}
50.09 \\
\end{tabular} & 0.107 & $\begin{array}{l}14.643 \\
\end{array}$ & & & & 49 & & & & & & & & \\
\hline & & & & & 26.43 & 27.25 & & & .384 & 11.664 & & -1.21 & & 47.042 & & & & & & & & & & & & & & \\
\hline & & & .026 & & & & & & & & & -1. & & & & & & & & & & & & & & & & \\
\hline & & & 27.037 & 26.655 & 26.443 & 27.347 & & & 49.872 & 11.517 & 34.587 & -1.212 & & 50.367 & 0.102 & $14.6 \mathrm{r}$ & & & & & & & 2.746 & & & 0.000 & 0.352 & \\
\hline & 8 & & 27.054 & 20.040 & \begin{tabular}{|l|l|}
20.452 \\
6
\end{tabular} & 27.353 & & & 年 & 11.400 & & 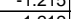 & & & & & & 65 & & 49.7 & 10.9 & & & & & & & \\
\hline $\begin{array}{ll}674 \\
674\end{array}$ & 8 & & 2706 & & 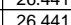 & 20.4744 & & & $\mid$ & 11.46 & & -1.215 & & & & & & & $\begin{array}{l}\frac{10.9036}{10.9203} \\
\end{array}$ & $\begin{array}{l}49.8 \\
49.6 \\
\end{array}$ & $\frac{11.3}{11.4}$ & & & & & & & 30 \\
\hline 675 & 8 & 6.49 & 27.081 & 26.659 & \begin{tabular}{|l|l|l|l|}
2653 \\
\end{tabular} & 22.476 & & 45.823 & \begin{tabular}{|l|l|}
50.65 \\
\end{tabular} & 11.478 & $\mid$ & \begin{tabular}{|l|l|}
-1.215 \\
\end{tabular} & & $\frac{41}{46.7}$ & 0 & & & & & & & & & & & & $\frac{0.040}{0.359}$ & \\
\hline 67 & & $6: 50: 35 \mathrm{~F}$ & 27.077 & & \begin{tabular}{|l|l|}
26.448 \\
\end{tabular} & & & & & & & & & & & & & & & & & & & & & & & \\
\hline & $8 / 30 / 2004$ & 6:51:35 PM & 27.093 & 26.666 & \begin{tabular}{|l|l|}
26.455 \\
\end{tabular} & 27.558 & 26. & 45.528 & $\mid$ & $\frac{11.541}{11.541}$ & & -1.215 & & 494 & 0.101 & 14.64 & & & & 49 & 11 & & & .014 & 0.014 & 0.000 & 0.345 & \\
\hline & & & 27.09 & 26.6 & 26.4 & 276 & & & 50.2 & 11.4 & & & & & & & & & & & & & & & & & & \\
\hline & $8 / 30 / 2004$ & & 27.088 & 26.655 & $26.44 \mathrm{~s}$ & 27.577 & & & \begin{tabular}{|l|l|}
50.165 \\
\end{tabular} & 11.4 & & & & & & & & & & & & & & & & & 0.348 & \\
\hline & $8 / 30 / 2$ & & 27.0 & & 26. & 27.5 & & & \begin{tabular}{|l|} 
\\
\end{tabular} 0.0199 & & & -1. & & & & & & & & & 11. & & & & & & & \\
\hline 66 & $8 / 30 / 2$ & & 27.09 & 26.6 & 26. & 27.5 & & & 50.5 & & & & & & & & & & & & 11. & & & & & & & \\
\hline 68 & & & 27.093 & 26. & 26.45 & 27.4 & & & 50.1 & $11.4 \mathrm{C}$ & & -1.215 & & & & & & & & 49 & 11. & & & & & & 0.3 & \\
\hline & & & 27.088 & & \begin{tabular}{|l}
26.529 \\
\end{tabular} & 27.4 & & & 50.3 & & & -1.2 & & 48.2 & & & & & & & 11 & & & & & & & \\
\hline & & & 27.098 & & 26.534 & 27. & & & 50.75 & 11.3 & & -1.2 & & & & & & & & & 12 & & & & & & & \\
\hline & & & 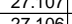 & 26. & \begin{tabular}{|l|l|} 
\\
\end{tabular} & 27.401 & & & 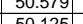 & & & -1.22 & & & & & & & & 49. & $\frac{11}{11}>>$ & & & & & & & \\
\hline & 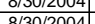 & & t.1.100 & & $\mid 2.515$ & 27.320 & & & $\frac{5.125}{51315}$ & & & & & & & & & & & & & & & & & & & \\
\hline$\frac{6}{66}$ & 8 & & 2710 & & 26.45 & & & & & & & $\frac{1.4}{-12}$ & & & & & & & & & & & & & & & nat & \\
\hline & 8 & & 27.09 & $\frac{26}{266}$ & \begin{tabular}{|l|l|l|l|l|}
26488 \\
\end{tabular} & 27.146 & & & | $50.094 \mid$ & 11271 & & & & & & & & & & & & & & & & & & \\
\hline & & & & & & & & & & & & & & & & & & & & & & & & & & & & \\
\hline 6. & & & 27.083 & & & & & 45. & & & & & & & & & & & & & & & & & & & & \\
\hline & & & & & 26. & 27.127 & & & $50.1 \mathrm{c}$ & & & -1218 & & & 0.1 & & & & & & & & & & & & & \\
\hline & $8 / 30 / 2004$ & $7: 07$ & .084 & 26 & 26.485 & 27.134 & 25. & 45.058 & \begin{tabular}{|l|}
50.123 \\
\end{tabular} & 11.36 & & -1.2 & & & 0.0 & 14 & & & & 4 & & & & & & & & \\
\hline & & & & & \begin{tabular}{|l|l|}
26.478 \\
\end{tabular} & 27.062 & 25. & 45.1 & & & & -1.2 & & & & 14 & & & & 4 & & & & & & & & \\
\hline & | & & 7.06 & & 26 & 27.068 & & 45.1 & 49 & & & & & & $0 . c_{1}$ & & & & & & 112 & & & & & & & \\
\hline & & & $7.0-2$ & & \begin{tabular}{|l|}
26.467 \\
\end{tabular} & 27.091 & & & 50.39 & & & & & & 0 & & & & & & & & & & & & & \\
\hline 699 & $8 / 30 / 2004$ & & 27.054 & & \begin{tabular}{|l|l|} 
& 26.476 \\
\end{tabular} & 27.059 & & 45.002 & 49.874 & 11.293 & 34. & -1.212 & & & 0.099 & 14. & & & & 48 & & & & & 0.014 & & 0.341 & \\
\hline & & & & & 26. & 27. & & & & & & & & 48 & & & & & & & & & & & & & & \\
\hline | & $8 / 30 / 2004$ & $\begin{array}{l}\text { 7:13:23 PM } \\
77 \cdot 1 \cdot 423 \mathrm{PM}\end{array}$ & 27033 & 2655 & \begin{tabular}{|l|l|l|} 
\\
\end{tabular} & $\begin{array}{l}26.985 \\
2702\end{array}$ & & & \begin{tabular}{|l|l|l|l|} 
\\
\end{tabular} & 罢1.1155 & $\begin{array}{l}34.536 \\
34.864\end{array}$ & \begin{tabular}{|c|}
-1.212 \\
-1215
\end{tabular} & & 180087 & 0.099 & 14.643 & & & & 48 & & & & & & & 0.344 & \\
\hline & & & 27.033 & 26.551 & \begin{tabular}{|l|l|}
26.474 \\
\end{tabular} & $\begin{array}{l}4|27.043| \\
\end{array}$ & & 45.02 & 49.75 & 11.326 & $34.864 \mid$ & 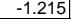 & & 46.087 & 0.097 & 14.643 & & & & & & & & 0.014 & 0.013 & 0.000 & 0.334 & \\
\hline
\end{tabular}


WSRC-TR-2005-00105, REVISION 0

SRNL-RPP-2005-00012, REVISION 0

RUN \# 4.03A AND B; FIRST AND SECOND HALF OF SLURRY DEWATERING - CONT.

\begin{tabular}{|c|c|c|c|c|c|c|c|c|c|c|c|c|c|c|c|c|c|c|c|c|c|c|c|c|c|c|c|c|}
\hline & & & & & & & & & & & & & & & & & & & & & & & & & & & & \\
\hline & A & B & D & $E$ & $\mathrm{~F}$ & \begin{tabular}{l|l|} 
\\
\end{tabular} & $\mathrm{H}$ & $\mathrm{J}$ & \begin{tabular}{ll|}
$\mathrm{K}$ \\
\end{tabular} & $\mathrm{L}$ & $\mathrm{M}$ & $\mathrm{N}$ & 0 & $\mathrm{Q}$ & $\begin{array}{ll}R \\
\end{array}$ & $\mathrm{~s}$ & $T$ & $\mathrm{~V}$ & w & $x$ & $\mathrm{Y}$ & $z$ & AA & $A B$ & $A C$ & $A D$ & $\mathrm{AE}$ & \\
\hline & $8 / 30 / 2004$ & 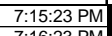 & 27.037 & 26.539 & 26.468 & $\begin{array}{l}27.122 \\
7727\end{array}$ & 25.923 & 45.066 & \begin{tabular}{|l|}
50.003 \\
\end{tabular} & 11.361 & 34.667 & \begin{tabular}{|l|l|} 
& -1.215 \\
\end{tabular} & \begin{tabular}{|c|c|}
-7.394 \\
\end{tabular} & 47.632 & 0.098 & 14.643 & 0.003 & & 11.3669 & $\begin{array}{l}\lambda .98 \\
48.9\end{array}$ & $\frac{1}{11 .}$ & 39.9 & 2.749 & $\frac{\mathrm{AD}}{0.014}$ & 0.013 & 0.000 & $\begin{array}{l}A L \\
0.338 \\
\end{array}$ & \\
\hline & & & & 26.534 & & $\begin{array}{l}27.147 \\
\end{array}$ & & & & $\begin{array}{l}11.374 \\
1.322\end{array}$ & $\begin{array}{l}35.258 \\
2.290\end{array}$ & & \begin{tabular}{|r|}
-7.408 \\
\end{tabular} & & & 14.643 & & & & 49.4 & & & & & & & 0.326 & \\
\hline & $8 / 30 / 2004$ & 7:17:23 PM & 27.036 & 26.529 & 26.562 & $\begin{array}{l}27.216 \\
\end{array}$ & 25.967 & $\begin{array}{l}45.526 \\
\end{array}$ & \begin{tabular}{|l|l|}
50.272 \\
\end{tabular} & $\begin{array}{l}11.322 \\
\end{array}$ & 35.289 & -1.215 & $\begin{array}{l}-7.379 \\
\end{array}$ & 48.216 & 0.104 & \begin{tabular}{|l|l}
14.643 \\
\end{tabular} & 0.003 & $\begin{array}{l}684.02 \\
\end{array}$ & 1.4003 & \begin{tabular}{|l|l|}
49.1 \\
\end{tabular} & 11.5 & & 2.786 & 0.015 & \begin{tabular}{|l|l|}
0.014 \\
\end{tabular} & $\begin{array}{l}0.000 \\
\end{array}$ & 0.353 & \\
\hline$\frac{704}{7705}$ & $8 / 30 / 2004$ & $\begin{array}{l}7: 18: 23 \mathrm{PM} \\
7 \cdot 19: 23 \mathrm{PM}\end{array}$ & $27.057 \mid$ & $\begin{array}{r}26.539 \\
20551\end{array}$ & 26.573 & \begin{tabular}{|l|}
27.236 \\
27273
\end{tabular} & \begin{tabular}{|l|}
26.023 \\
260069
\end{tabular} & \begin{tabular}{|l|l|}
45.717 \\
45.173
\end{tabular} & \begin{tabular}{|l|}
50.471 \\
5023 \\
\end{tabular} & $\begin{array}{l}11.406 \\
11.286\end{array}$ & $\begin{array}{l}35.463 \\
35283 \\
352\end{array}$ & \begin{tabular}{|l|l|} 
\\
\end{tabular} & \begin{tabular}{|l|}
-7.504 \\
-7055
\end{tabular} & \begin{tabular}{|l|l|}
45.668 \\
46591
\end{tabular} & 0.101 & \begin{tabular}{|r|}
14.643 \\
1.643
\end{tabular} & 0.0023 & \begin{tabular}{|l|l|}
685.02 \\
68022
\end{tabular} & \begin{tabular}{|l|}
11.4169 \\
1.1236
\end{tabular} & \begin{tabular}{|l|l|}
49.3 \\
49.1
\end{tabular} & $\begin{array}{l}10.9 \\
111\end{array}$ & & $\begin{array}{l}2.799 \\
2794\end{array}$ & 0.014 & $\begin{array}{l}0.014 \\
0.1\end{array}$ & $\begin{array}{l}0.000 \\
\end{array}$ & 0.341 & \\
\hline & $\begin{array}{r}8 / 30 / 2004 \\
8 / 30 / 2004\end{array}$ & & 27.068 & $\frac{26.541}{26.546}$ & \begin{tabular}{|l|}
26.569 \\
26.565
\end{tabular} & $\begin{array}{l}27.273 \\
27.293\end{array}$ & $\begin{array}{r}26.069 \\
26.14\end{array}$ & $\begin{array}{l}45.473 \\
45.568\end{array}$ & \begin{tabular}{|l|}
50.243 \\
50.361 \\
\end{tabular} & & $\begin{array}{r}35.283 \\
35.34 \\
\end{array}$ & $\begin{array}{r}-1.215 \\
-1.215\end{array}$ & $\begin{array}{r}-7.455 \\
-7.186 \\
\end{array}$ & \begin{tabular}{|l|}
46.594 \\
46.535 \\
\end{tabular} & $\begin{array}{l}0.101 \\
0.105\end{array}$ & $\begin{array}{l}14.643 \\
14.643\end{array}$ & $\frac{0.003}{0.004}$ & \begin{tabular}{|l|}
686.02 \\
68.702
\end{tabular} & \begin{tabular}{|l|l|l}
$\mid 11.4336$ \\
11.4503
\end{tabular} & $\begin{array}{l}49.1 \\
49.2 \\
\end{array}$ & $\begin{array}{l}11.1 \\
11.1 \\
\end{array}$ & \begin{tabular}{|l|l|} 
& 40.4 \\
& 40.5 \\
\end{tabular} & $\begin{array}{l}2.784 \\
27899\end{array}$ & 0.014 & $\begin{array}{l}0.014 \\
0.014\end{array}$ & $\begin{array}{l}0.000 \\
0.000\end{array}$ & $\begin{array}{l}0.343 \\
0.356\end{array}$ & $\begin{array}{l}0.29 \\
0.33\end{array}$ \\
\hline & $8 / 30 / 2004$ & 7:21:23 PM & 27.074 & & & 27.358 & 26.12 & 45.628 & \begin{tabular}{|r|}
50.39 \\
\end{tabular} & 11.384 & 35.307 & & & & 0.102 & $\begin{array}{l}14.643 \\
14.643 \\
\end{array}$ & 0.004 & $\begin{array}{l}688.02 \\
688.02 \\
\end{array}$ & & & & & $\begin{array}{l}2.789 \\
2.790\end{array}$ & $\frac{0.015}{0.015}$ & & 0 & $\begin{array}{l}0.356 \\
0.346\end{array}$ & $\begin{array}{l}0.30 \\
0.29\end{array}$ \\
\hline & & & & 26.537 & 26.561 & 27.424 & & 46.026 & \begin{tabular}{|l|}
50.792 \\
\end{tabular} & 11.536 & 35.576 & -1.212 & -7.394 & \begin{tabular}{|l|}
48.358 \\
\end{tabular} & 0.096 & 14.643 & 0.004 & $\begin{array}{l}689.02 \\
\end{array}$ & 11.4836 & & & & & & & & & $\frac{0.29}{0.27}$ \\
\hline & $8 / 30 / 2004$ & 7:23:23 PM & 27.096 & 26.534 & 26.552 & 27.43 & 26.192 & 45.39 & \begin{tabular}{|l|}
50.252 \\
\end{tabular} & 11.24 & & & -7.246 & \begin{tabular}{|l|l|}
46.779 \\
\end{tabular} & 0.1 & 14.643 & 0.003 & 690.02 & 11.5003 & 49.1 & & & & & & 0.000 & 0.340 & $\begin{array}{l}0.27 \\
0.29\end{array}$ \\
\hline 710 & & & & & 26.549 & & & 45.246 & \begin{tabular}{|l|}
50.125 \\
\end{tabular} & & & & & \begin{tabular}{|l|l}
46.986 \\
\end{tabular} & & $\begin{array}{l}14.643 \\
\end{array}$ & & & & & & & & & & & 0.322 & \\
\hline 711 & & & & & 26.544 & 27.433 & & & 50.363 & 11.363 & & & & & & & & & & & & & & & & & & \\
\hline & $8 / 30 / 2004$ & 7:26:23 PM & 27.103 & 26.536 & \begin{tabular}{|l|l|}
826.554 \\
\end{tabular} & 27.408 & & 44.817 & 49.781 & 11.233 & & & & 45.546 & 998 & 14.643 & & & 1.5503 & & & & & & & & 0.339 & \\
\hline & & 7:27:23 PM & & 26.52 & 26.534 & 27.342 & & \begin{tabular}{|l|l|l|}
44.844 \\
\end{tabular} & \begin{tabular}{|l|l|}
49.69 \\
\end{tabular} & 11.306 & 34.517 & -1.218 & & & & $\begin{array}{l}14.643 \\
\end{array}$ & & & 15669 & 48.6 & & & & & & & & \\
\hline & $8 / 30 / 2004$ & 7:28:23 PM & 27.102 & 26.53 & \begin{tabular}{l|l}
326.539 \\
\end{tabular} & 27.277 & 26.009 & $\begin{array}{l}45.634 \\
\end{array}$ & 50.409 & 11.132 & 35.687 & -1.215 & $\begin{array}{ll}-7.533 \\
\end{array}$ & 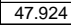 & 0.102 & $\begin{array}{l}14.643 \\
\end{array}$ & & 695.02 & 1.5836 & \begin{tabular}{|l|l|}
49.3 \\
\end{tabular} & 11.4 & 40.7 & 2.803 & 0.015 & 0.014 & 0.000 & $\begin{array}{ll}0.344 \\
\end{array}$ & \\
\hline & $8 / 30 / 2004$ & 7:29:23 PM & 27.096 & 26.539 & 26.527 & 27.196 & 25.937 & 45.614 & 50.511 & 11.24 & 35.361 & -1.212 & -7.45 & \begin{tabular}{|l|l|}
47.908 \\
\end{tabular} & 0.097 & 14.643 & & $\begin{array}{l}6966.0 \\
\end{array}$ & 1.6003 & \begin{tabular}{|l|l|}
49.4 \\
\end{tabular} & & & 2.791 & 0.014 & 0.01 & 0.000 & & \\
\hline & $8 / 30 / 2004$ & 7:30:23 PM & 27.104 & 26.532 & 26.546 & 27.194 & 25.886 & 44.877 & 49.6866 & & 34.95 & & & \begin{tabular}{|l|l}
47.299 \\
\end{tabular} & 0.097 & 14.643 & & 697.0 & 1.6169 & 48.6 & & & & & & & .333 & \\
\hline & 8/30/2004 & & & 26.516 & \begin{tabular}{|l|l|} 
& 26.509 \\
\end{tabular} & 27.143 & & & 50.376 & 11.147 & .373 & -1.212 & & 5.866 & 0.099 & 14.643 & & & & 49. & & & & 014 & & & & \\
\hline$\frac{11}{71}$ & $8 / 30 / 2004$ & $\begin{array}{l}7: 32: 23 \mathrm{PM} \\
7 \\
7 \cdot 220232\end{array}$ & 27.083 & 26.521 & 26.519 & 27.133 & 25.83 & 45.808 & 50.112 & 11.146 & & -1.212 & & 46.339 & 0.096 & 14.643 & & & & 49.6 & & & 2.806 & & & & 0.324 & \\
\hline & 813012004 & $\frac{1.35 .2 \mathrm{PM}}{7.252 \mathrm{PM}}$ & (2.7.024 & $\frac{20.524}{20.927}$ & 20.518 & 2.072 & 25.1 & 40.259 & S1.26 & $\begin{array}{r}11.35 \\
11139\end{array}$ & 35.829 & 1.42 & & $47^{35}$ & & $\frac{14.643}{14.643}$ & & & 1.6669 & 40 & & & & & & & & \\
\hline & $8 / 30 / 2004$ & 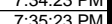 & (27.054 & 20.496 & $\begin{array}{l}20.400 \\
25503\end{array}$ & $\frac{20.944}{27}$ & 25.120 & 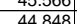 & 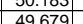 & $=1.1158$ & $\frac{35.075}{3.821}$ & $\frac{-1.120}{-1212}$ & $\frac{-1.300}{-7408}$ & $\begin{array}{l}41.536 \\
4564\end{array}$ & 0.0095 & 14.045 & & & 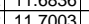 & | & & & & 014 & . & & 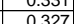 & \\
\hline & $8 / 30 / 2004$ & $7: 36: 23 \mathrm{PM}$ & $\frac{27.044}{27}$ & 26.482 & 26.501 & 26.95 & 25.686 & 45.051 & \begin{tabular}{|l|}
50.231 \\
\end{tabular} & $\begin{array}{l}11.193 \\
1.193\end{array}$ & 334.677 & $\frac{-1.212}{-1.212}$ & -7.518 & 466.354 & 0.1 & 14.643 & & \begin{tabular}{|c|}
703.02 \\
\end{tabular} & 11.7169 & $\mid \begin{array}{l}49.1 \\
\end{array}$ & 11.6 & 39 & 2.748 & 0.014 & 0.014 & 0.000 & 0.344 & \\
\hline 72 & $8 / 30 / 2004$ & $\begin{array}{l}7: 37: 23 \mathrm{PM} \\
\end{array}$ & 27.047 & 26.48 & $\begin{array}{ll}326.559 \\
3\end{array}$ & 26.927 & 25.669 & \begin{tabular}{|l|l|l|l|}
46.619 \\
\end{tabular} & \begin{tabular}{|l|}
51.524 \\
\end{tabular} & $\begin{array}{ll}11.446 \\
\end{array}$ & 36.116 & & $\begin{array}{l}-7.463 \\
\end{array}$ & \begin{tabular}{|l|}
46.239 \\
\end{tabular} & 0.106 & 14.643 & & \begin{tabular}{|c|}
704.02 \\
\end{tabular} & 11.7336 & 50.4 & & & $\frac{2.140}{2.852}$ & 0.015 & 0.015 & 0.000 & 0.351 & \\
\hline & $8 / 30 / 2004$ & 7:38:23 PM & 27.05 & 26.473 & 26.581 & 26.965 & 25.682 & 46.209 & \begin{tabular}{|l|}
51.043 \\
\end{tabular} & $\begin{array}{l}11.316 \\
\end{array}$ & & & -7.492 & & & $\begin{array}{l}14.643 \\
\end{array}$ & & & & & & & & & & & & \\
\hline 725 & $8 / 30 / 2004$ & 7:39:23 PM & 27.039 & 26.467 & 26.561 & 27.019 & 25.686 & \begin{tabular}{|l|l}
45.738 \\
\end{tabular} & & & 35.519 & -1.212 & $\begin{array}{l}-7.446 \\
\end{array}$ & 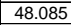 & 0.107 & $\begin{array}{l}14.643 \\
\end{array}$ & & & .7669 & 49.4 & & & 2.801 & 0.015 & 0.015 & 0.000 & 0.361 & \\
\hline & & & & & & & & & 50.826 & & & & & 44.405 & 0.104 & 14.643 & & & & & & & & & & & & \\
\hline & $8 / 30 / 2004$ & 7:41:23 PM & 27.033 & 26.446 & $\begin{array}{l}26.549 \\
5\end{array}$ & 26.983 & 25.6 & 46 & 51.331 & 11.369 & & -1.212 & & 46817 & 0.1 & 14.643 & & & 1.8003 & 50.2 & & & & & & & 0.331 & \\
\hline & $8 / 30 / 2004$ & & 27.044 & 26.452 & 26.546 & 26.984 & 25. & & 51.155 & 11.357 & 35.964 & -1.215 & & 45.378 & & 14.643 & & & & & & & & 014 & $\overline{014}$ & & & \\
\hline & $8 / 30 / 2004$ & 7:43:23 PM & 27.028 & 26.421 & 26.534 & 27.008 & 25.8 & 46.171 & 50.942 & 11.298 & 35.882 & -1.218 & -7.426 & \begin{tabular}{l|l}
47.067 \\
\end{tabular} & 0.103 & 14.643 & & 710.0 & $\begin{array}{ll}11.8336 \\
\end{array}$ & \begin{tabular}{|l|l|}
49.8 \\
\end{tabular} & 11.2 & & 2.829 & 0.015 & 0.014 & & 0.344 & \\
\hline & $8 / 30 / 2004$ & 7:44:23 PM & 27.034 & 26.421 & 26.55 & 27.094 & 25. & & 51.232 & 11.254 & 35.652 & -1.2. & & \begin{tabular}{|l}
49.163 \\
\end{tabular} & 0.096 & 14.643 & & 71 & & 50.1 & 11 & & 281 & 0.014 & & & & \\
\hline & $8 / 30 / 2$ & 7:45:23 PM & & 26.4 & 26.556 & 27.124 & 25. & 46.281 & 51.249 & 11 & 35.929 & & & & 0.1 & 14. & & 7 & 11.8 & & & & & & & & & \\
\hline & & & 27.054 & & 26.576 & 27.174 & & & 51.049 & 388 & & & & .391 & 0.099 & 14.643 & & & & 49 & & & & 014 & & & & \\
\hline & . & & 2 & & $\begin{array}{l}26.581 \\
26597\end{array}$ & 27.226 & & 告5.663 & 5. 50.598 & 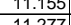 & & -1.212 & & 4 & & & & & & 49.5 & & & & & & & 年 & \\
\hline & $\frac{13012004}{81201304}$ & & 年. & $\frac{20 .}{26}$ & $\begin{array}{l}20.301 \\
26.589\end{array}$ & $\frac{21.220}{27207}$ & & & 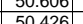 & $\frac{11.471}{11108}$ & & & & 45.31 & & & & & & & & & & & & & & \\
\hline & $8 / 30 / 2004$ & & 27.083 & $\frac{20.420}{26.431}$ & $\begin{array}{l}20.305 \\
26584\end{array}$ & 27.303 & & & $\mid$ & $\frac{11.190}{11.199}$ & $\begin{array}{l}35.400 \\
34.967\end{array}$ & $\frac{-1.210}{-1.215}$ & & & 0.095 & $\frac{14.045}{14643}$ & & & & $\frac{4.5}{492}$ & $\frac{11}{10}$ & & & & & & $\frac{0.301}{0.334}$ & \\
\hline & $8 / 30 / 2$ & & 27.079 & & 26.606 & 27.294 & & & $\mid 5.0388$ & 势1.169 & & & & $\frac{15.065}{44646}$ & 0.093 & $\frac{14.043}{14.643}$ & & & & & & & & & & & & \\
\hline & $8 / 3$ & 7:5:22:23 PM & 27.08 & 26.428 & \begin{tabular}{r|r|}
3 & 26.581 \\
\end{tabular} & 27.25 & 25. & 44. & \begin{tabular}{|c|}
49.822 \\
\end{tabular} & 11.118 & 34.398 & -1.212 & & & & 14.643 & & & & & & & & & & & & \\
\hline & & & & & \begin{tabular}{|l|}
26.58 \\
\end{tabular} & 27.209 & & & & & & & & 45.465 & 0.094 & & & & & & & & & & & & & \\
\hline & & & 27.068 & 26 & & 27.118 & & & 51.416 & $\frac{11.445}{11.45}$ & & -1 & & & & & & & & & & & & & & & & \\
\hline & & & 27.073 & & 26.614 & 27.098 & & & 50.734 & $\frac{11.285}{11.285}$ & & & & & 0.097 & & & & & & & & & & & & & \\
\hline & $8 / 30 / 2004$ & & & 26.414 & & 27.042 & 25.74 & & 51.027 & 11.316 & & -1.21 & & & 0.092 & 14.6 & & & & 49.9 & 11.8 & & & & & & & \\
\hline 78 & $8 / 30 / 2004$ & & 27.06 & & \begin{tabular}{l|l|}
326.596 \\
\end{tabular} & 27.025 & & & $|3|$ & . 379 & & -1.215 & & 46.986 & 0.094 & & & & & $\begin{array}{ll}49.9 \\
\end{array}$ & 11 & & 2.829 & .013 & 0.013 & & 0.314 & \\
\hline 74 & $8 / 30 / 2004$ & & 27.068 & 26.401 & 26.604 & 26.933 & & & 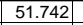 & 904 & & & & & & & & & & & & & & & & & & \\
\hline & $8 / 30 / 26$ & & 27.071 & 26.40 & 26.63 & 26.941 & 25.6 & & & 11.499 & & & & \begin{tabular}{|l|l|}
44.8 \\
\end{tabular} & 0.093 & & & & & & 10 & & & & & & 0.318 & \\
\hline & 8/30/2004 & $8: 00: 23 \mathrm{PM}$ & 27.054 & 26.387 & 26.616 & 26.97 & 25. & & 49.001 & 11.546 & & -1.215 & & 47.6 & 0.09 & 14. & & & & & & & & & 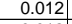 & & 0.001 & \\
\hline & & & & & & & & & & & & & & & & & & & & & & & & & & & & \\
\hline & 813012004 & & 27.064 & 26.38 & 26.6 & 26.839 & 25. & & 50.04] & 11.5000 & & -1.212 & & & & & & & & & $\frac{11}{11}$ & & & & & & 02 & \\
\hline & 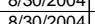 & & $\frac{2.044}{27052}$ & & & $\frac{20.159}{26.783}$ & & & $50 . / 95]$ & & & & & & & & & & & & & & & & & & & \\
\hline 751 & $\frac{103012}{83 / 212}$ & & 27036 & & $\mid \begin{array}{l}26.0 \\
26.5\end{array}$ & & & & & & & & & & & & & & & & & & & & & & & \\
\hline & $8 / 30 / 2$ & & 27.034 & & & $\frac{2.6}{26.7}$ & & & & & & & & & & & & & & & 11 & & & & & & 0.323 & \\
\hline & & & & & & & & & & & & & & & & & & & & & 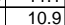 & & & & & & & \\
\hline 78 & & & 27.043 & 26.3 & \begin{tabular}{|l|l|}
26.584 \\
\end{tabular} & 26.7 & & & 316 & 11.3 & & & & 47 & & & & & & & & & & & & & 0.3 & \\
\hline & & & & & & & & & & & & & & & & & & & & & & & & & & & & \\
\hline & $8 / 30 / 2$ & $8: 19: 13$ & 26.854 & 26.181 & 26.519 & 26.763 & 25 & \begin{tabular}{|l|}
6.427 \\
\end{tabular} & 50.332 & 9.804 & & 38. & & 47 & & & & & & 49 & 11.4 & & & & & & 0.223 & \\
\hline & $8 / 30 / 2$ & & 26.88 & & 26.5 & 26.84 & 25. & 7.14 & \begin{tabular}{|l|l|}
49.134 \\
\end{tabular} & & & 36.7 & & & & & & & & 48 & 11. & & & & & & & \\
\hline & $8 / 30 / 26$ & & 26.978 & & & 26.798 & 25 & 36.457 & \begin{tabular}{|l|l|}
49.87 \\
\end{tabular} & & & 7.5 & & & & & & & & \begin{tabular}{|l|l|}
48.7 \\
\end{tabular} & 11. & & & & & & & \\
\hline & $8 / 30 / 2$ & & 26.968 & & 26. & 26.778 & 25 & 40.317 & \begin{tabular}{|l|l|}
49.429 \\
\end{tabular} & & & 3.157 & & & 0. & 14. & & & & & & & & & & & & \\
\hline & & & & & & & & & & & & & & & & & & & & & & & & & & & & \\
\hline & $8 / 3$ & & 26 & & 26. & 26. & & 39.9 & & & & & & & 0. & & & & & 47.8 & & & & & & & & \\
\hline & & & 1 & 26.129 & 26.48 & 26.58 & & $\begin{array}{l}39.52 \\
\end{array}$ & 48.6 & & & 3.0 & & 45. & & & & & & 47 & & & & & & & & \\
\hline & & & 260 & & & 26.5 & & 45.64 & St.3. & & & $=0.286$ & & & 0. & & & & & 50 & & & & & & & & \\
\hline & & & 260 & & & 2.49 & & $\begin{array}{l}435.949 \\
46163\end{array}$ & 51 & & 34 & & & & & & & & & $\frac{45}{55}$ & 110 & & & & & & & \\
\hline & $8 / 3$ & & & & & 26.487 & 25.1 & & & & & & & & & & & & & & & & & & & & & \\
\hline & $\frac{1 / 30 / 2004}{8}$ & & 26.96 & $\frac{20.091}{26.091}$ & 26.509 & 26.478 & 25 & 46.082 & 50.952 & $\frac{12.903}{12.903}$ & & $\frac{-1.212}{-1.212}$ & & & 0.1 & 146 & & & & 498 & 11. & & & & & & 0.393 & \\
\hline & & & & & & & & & & & & & & & & & & & & & & & & & & & & \\
\hline & & & & & & & & 45.98 & & & & & & & 0. & & & & & & & & & & & & & \\
\hline & $8 / 30 / 2004$ & 8:33:13 PM & 26.972 & 26.065 & \begin{tabular}{|l|l|}
5 & 26.473 \\
\end{tabular} & 26.517 & 25.254 & 43.11 & \begin{tabular}{|l|}
47.733 \\
\end{tabular} & $\frac{14.042}{14.042}$ & 30.208 & \begin{tabular}{|l|l|}
3 & -1.212
\end{tabular} & & 49.608 & 0.107 & 14.643 & & 759.85 & 5 & 46.6 & 11.8 & & & \begin{tabular}{|l|l|}
0.015 \\
\end{tabular} & 0.015 & 0.000 & 0.401 & \\
\hline
\end{tabular}


WSRC-TR-2005-00105, REVISION 0

SRNL-RPP-2005-00012, REVISION 0

RUN \# 4.03A AND B; FIRST AND SECOND HALF OF SLURRY DEWATERING - CONT.

\begin{tabular}{|c|c|c|c|c|c|c|c|c|c|c|c|c|c|c|c|c|c|c|c|c|c|c|c|c|c|c|c|c|}
\hline & & & & & & & & & & & & & & & & & & & & & & & & & & & & \\
\hline & A & B & \begin{tabular}{l|l|l|} 
& \\
\end{tabular} & $E$ & $\mathrm{~F}$ & $G$ & $\mathrm{H}$ & $\mathrm{J}$ & \begin{tabular}{l|l}
$\mathrm{K}$ \\
\end{tabular} & $\mathrm{L}$ & $\mathrm{M}$ & $\mathrm{N}$ & 0 & Q & \begin{tabular}{l|l|l|l|l} 
& \\
\end{tabular} & $\mathrm{s}$ & $T$ & $\mathrm{v}$ & w & $x$ & $\mathrm{Y}$ & $\mathrm{z}$ & AA & $A B$ & $A C$ & $A D$ & $\mathrm{AE}$ & \\
\hline 77 & $8 / 30 / 2004$ & $8: 34: 13$ PM & 26.946 & 26.058 & 26.352 & 26.471 & 25.352 & 43.013 & \begin{tabular}{l|l}
47.885 \\
\end{tabular} & $\begin{array}{r}3.22 \\
\end{array}$ & 30.795 & -1.215 & $\begin{array}{l}-7.348 \\
\end{array}$ & 48.281 & 0.096 & 14.643 & 0.003 & 760.85 & 12.6808 & 46.8 & 11.5 & 36.9 & \begin{tabular}{|l|l|}
2.544 \\
\end{tabular} & 0.014 & 0.013 & 0.000 & 0.359 & \\
\hline & & & 26.929 & 26.042 & & 26.409 & 25.321 & & & & 34.14 & & & & & 14.643 & & & & 49.4 & & & & & & & & \\
\hline 773 & $8 / 30 / 2004$ & $\begin{array}{l}8: 36: 13 \mathrm{PM} \\
0: 27: 200\end{array}$ & \begin{tabular}{l|l|}
26.885 \\
\end{tabular} & 26.022 & 26.145 & 26.474 & 25.336 & $\begin{array}{l}44.877 \\
\end{array}$ & \begin{tabular}{|l|}
49.76 \\
\end{tabular} & $\begin{array}{l}2.022 \\
\end{array}$ & 33.826 & \begin{tabular}{|c|} 
\\
\end{tabular} & \begin{tabular}{|l|l|}
-7.267 \\
-10 \\
\end{tabular} & \begin{tabular}{|r|}
44.77 \\
\end{tabular} & 0.1 & \begin{tabular}{|l|l}
14.643 \\
\end{tabular} & 0.003 & 762.85 & \begin{tabular}{|l|l|}
12.7142 \\
\end{tabular} & \begin{tabular}{l|l}
48.6 \\
\end{tabular} & \begin{tabular}{|l|}
10.7 \\
\end{tabular} & & 2.71 & 0.014 & \begin{tabular}{|l|l|}
0.014 \\
\end{tabular} & $\begin{array}{l}0.000 \\
\end{array}$ & 0.352 & \\
\hline$\frac{774}{775}$ & \begin{tabular}{|l|l|}
$8 / 30 / 2004$ \\
$8 / 312004$
\end{tabular} & $\begin{array}{ll}8: 37: 13 \mathrm{PM} \\
8 \cdot 38 \cdot 13 \mathrm{PM}\end{array}$ & \begin{tabular}{l|l}
26.874 \\
26.855
\end{tabular} & 26.021 & \begin{tabular}{|l|l|l|l|} 
\\
26.101
\end{tabular} & $\begin{array}{l}26.524 \\
26.625\end{array}$ & $\begin{array}{l}25.315 \\
25367\end{array}$ & \begin{tabular}{|l|l}
45.083 \\
45008
\end{tabular} & \begin{tabular}{|r|}
50.334 \\
51066
\end{tabular} & $\begin{array}{r}12.821 \\
12.284\end{array}$ & $\frac{33.122}{32.31}$ & -1.215 & \begin{tabular}{|l|}
-7.035 \\
-7301
\end{tabular} & \begin{tabular}{|l|l}
48.039 \\
46143
\end{tabular} & $\begin{array}{l}0.09 \\
0095\end{array}$ & \begin{tabular}{|r|}
14.643 \\
1.643
\end{tabular} & $\begin{array}{l}0.003 \\
\end{array}$ & $\begin{array}{l}763.85 \\
764.55\end{array}$ & \begin{tabular}{|l|}
12.7308 \\
12.775
\end{tabular} & 49.2 & \begin{tabular}{|l|l|}
11.4 \\
110
\end{tabular} & & & 0.013 & $\begin{array}{l}0.012 \\
0.032\end{array}$ & $\begin{array}{l}0.000 \\
\end{array}$ & 0.319 & \\
\hline & $8 / 3 / 3 / 2004$ & 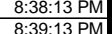 & \begin{tabular}{l|l}
26.855 \\
26.815
\end{tabular} & $\frac{26.017}{26.002}$ & \begin{tabular}{|l|}
26.101 \\
26.066 \\
\end{tabular} & $\frac{26.625}{26.61}$ & $\begin{array}{l}25.367 \\
25.327\end{array}$ & $\begin{array}{l}45.908 \\
47.128\end{array}$ & 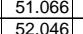 & $\begin{array}{r}12.284 \\
11.56\end{array}$ & $\begin{array}{r}34.31 \\
36.582 \\
\end{array}$ & $\frac{-1.212}{-1.215}$ & $\begin{array}{r}-7.391 \\
-7.437 \\
\end{array}$ & \begin{tabular}{|l|}
46.143 \\
43.874
\end{tabular} & 0.095 & $\begin{array}{l}14.643 \\
14.643 \\
\end{array}$ & $\begin{array}{l}0.003 \\
0.003\end{array}$ & $\begin{array}{l}764.85 \\
765.85\end{array}$ & \begin{tabular}{|l|}
12.7475 \\
127642
\end{tabular} & $\begin{array}{l}49.9 \\
50.9\end{array}$ & $\begin{array}{l}11.0 \\
10.5\end{array}$ & \begin{tabular}{|l|}
50.1 \\
51.9 \\
\end{tabular} & \begin{tabular}{|c|}
2.765 \\
2868
\end{tabular} & 0.014 & $\begin{array}{l}0.013 \\
\end{array}$ & 0.000 & $\begin{array}{l}0.329 \\
0.335\end{array}$ & $\begin{array}{l}0.28 \\
0.99\end{array}$ \\
\hline \begin{tabular}{|l|l|}
777 \\
\end{tabular} & $8 / 30 / 2004$ & 8:40:13 PM & 26.801 & & \begin{tabular}{|l|l|}
26.117 \\
\end{tabular} & 26.596 & 25.412 & 4t.1.796 & \begin{tabular}{|l|}
59.512 \\
\end{tabular} & $\begin{array}{r}1 . .500 \\
12.709\end{array}$ & 33.257 & & & \begin{tabular}{|l|}
45.899 \\
\end{tabular} & $\begin{array}{l}0.101 \\
0.091\end{array}$ & $\begin{array}{l}14.643 \\
14.643 \\
\end{array}$ & 0.003 & & & $\begin{array}{l}50.9 \\
48.4\end{array}$ & $\begin{array}{l}10.5 \\
10.9\end{array}$ & & \begin{tabular}{|l|}
2.886 \\
2.691 \\
\end{tabular} & $\frac{0.014}{0.013}$ & $\frac{0.014}{0.013}$ & $\begin{array}{l}0.000 \\
0.000\end{array}$ & $\begin{array}{l}0.335 \\
0.324\end{array}$ & \\
\hline & & & \begin{tabular}{|l|}
26.791 \\
\end{tabular} & 26.003 & \begin{tabular}{|l|}
26.132 \\
\end{tabular} & & 25.432 & 45.672 & \begin{tabular}{|l|}
50.465 \\
\end{tabular} & 12.829 & 33.834 & \begin{tabular}{|l|} 
\\
\end{tabular} & -7.455 & \begin{tabular}{|l|}
47.002 \\
\end{tabular} & 0.094 & 14.643 & & 767.85 & & & & & & & & & & $\frac{0.28}{0.28}$ \\
\hline & $8 / 30 / 2004$ & 8:42:13 PM & 26.751 & 25.992 & \begin{tabular}{|l|}
26.206 \\
\end{tabular} & 26.675 & 25.487 & 46.252 & \begin{tabular}{|l|}
51.224 \\
\end{tabular} & 13.48 & & -1.218 & $\begin{array}{l}-7.463 \\
\end{array}$ & 48.644 & 0.098 & 14.643 & & 768.85 & \begin{tabular}{|l|l|} 
& 12.8142 \\
\end{tabular} & 50.1 & 11.6 & 39.9 & 2.753 & 0.014 & & & & \\
\hline 780 & & & & & & & & & & & & & & & & $\begin{array}{l}14.643 \\
\end{array}$ & & & 2.8308 & & & & & & & 0.000 & 0,300 & 0.29 \\
\hline 781 & & 13 PM & & & 20207 & 26.751 & & 46.814 & 51.694 & 12.473 & .324 & & & & & & & & & & & & & $\frac{014}{.014}$ & & & & \\
\hline & $8 / 30 / 2004$ & $13 \mathrm{PM}$ & 26.752 & 25.994 & 26.307 & 26.731 & & & \begin{tabular}{|l|l|}
51.027 \\
\end{tabular} & 13.312 & & -1.209 & & 46.375 & 095 & 14.643 & & & 28642 & 49.9 & & & & 014 & & & & \\
\hline & & 5:13 PM & 26.747 & 25.979 & \begin{tabular}{|l|l|}
26.352 \\
\end{tabular} & 26.706 & 25.458 & & 51.624 & $\begin{array}{ll}3.157 \\
\end{array}$ & & -1.212 & & $\begin{array}{l}47.847 \\
\end{array}$ & 0.094 & $\begin{array}{l}14.643 \\
\end{array}$ & & 772.85 & & & & & & & & & & \\
\hline 7884 & $8 / 30 / 2004$ & 8:47:13 PM & 26.768 & 25.994 & \begin{tabular}{|l|}
26.388 \\
\end{tabular} & 26.767 & 25.369 & \begin{tabular}{|l|l|l|l}
45.999 \\
\end{tabular} & \begin{tabular}{|l|}
51.037 \\
\end{tabular} & 13.078 & 33.818 & -1.212 & -7.359 & \begin{tabular}{|l|l|}
47.171 \\
\end{tabular} & 0.095 & $\begin{array}{l}14.643 \\
\end{array}$ & & 773.85 & 12.8975 & \begin{tabular}{|l|l|}
49.9 \\
\end{tabular} & 11.2 & 39.9 & 2.752 & 0.014 & 0.013 & 0.000 & $\begin{array}{ll}0.328 \\
\end{array}$ & \\
\hline & $8 / 30 / 2004$ & 8:13 PM & 26.759 & 26.001 & \begin{tabular}{|l|l}
26.439 \\
\end{tabular} & 26.673 & 25.22 & 46.565 & 51.644 & 12.916 & 34.591 & -1.212 & -7.356 & \begin{tabular}{|l|l|}
46.579 \\
\end{tabular} & 0.099 & 14.643 & & 774.85 & 12.9142 & 50.5 & 11. & & 2.798 & 0.014 & 0.014 & 0.000 & & \\
\hline 78 & 8/301/2004 & 8:13 PM & 26.71 & 25.987 & 26.461 & 26.57 & 25.187 & 45.197 & 50.303 & 12.963 & 33.118 & -1.212 & -7.382 & 45.555 & 0.094 & 14.643 & & 775.8. & 12.9308 & 49.2 & & & & .013 & & & & \\
\hline & & : 13 PM & & 25.962 & 26.461 & 26.485 & 25.11 & & 51.28 & 13.171 & 34.234 & -1.215 & & 8.181 & & 14.643 & & & .94 & & & & -1 & & & & & \\
\hline 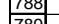 & 8313012004 & $13 \mathrm{PM}$ & 26.621 & 25.947 & 26.481 & 26.44 & 25. & 45.123 & 50.834 & 12.937 & 33. & -1.215 & & 47.165 & 0.097 & 14.643 & & $777.8 \mathrm{~s}$ & 2.9642 & 49.1 & & & 2.738 & .014 & & & 0.335 & \\
\hline & 813012004 & 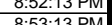 & $\mid$ & 25.932 & 20.496 & $\begin{array}{l}2.355 \\
26268\end{array}$ & & $\begin{array}{l}45.9716 \\
15.47\end{array}$ & S & 12.998 & $\begin{array}{r}34.015 \\
33.43\end{array}$ & - & & & 0.091 & $\begin{array}{l}14.043 \\
14643\end{array}$ & & $\frac{778.8}{779.8}$ & 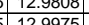 & 494 & & & & & & & & \\
\hline 791 & 8 & 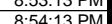 & $\frac{2.5044}{2654 ?}$ & $\frac{25.911}{25899}$ & \begin{tabular}{|l|l|}
26.452 \\
\end{tabular} & $\frac{20.200}{26246}$ & & 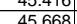 & 50.484t & $\frac{1.910}{12936}$ & $\frac{3.45}{33.64}$ & $\frac{-1.40}{-1.1212}$ & & 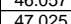 & 0.091 & 14.045 & & 780.05 & 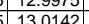 & 4 & & & & 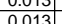 & . & 0.000 & 0 & \\
\hline 792 & $8 / 30 / 2004$ & $8.55: 13 \mathrm{PM}$ & 20.0436 & 25.0832 & \begin{tabular}{|l|l|l|l|l|}
26.601 \\
\end{tabular} & $\frac{20.240}{26.2}$ & 24.054 & $\begin{array}{l}45.000 \\
47.258\end{array}$ & 52.075 & $\frac{1.500}{13.253}$ & $\frac{30.004}{35.016}$ & $\frac{-1.212}{-1.212}$ & $\begin{array}{l}-1.25 \\
-7.296\end{array}$ & \begin{tabular}{|l|}
47.025 \\
47.255 \\
\end{tabular} & & $\frac{14.643}{14.643}$ & & 781.85 & \begin{tabular}{|l|l|}
13.0301428 \\
\end{tabular} & $\begin{array}{l}45.1 \\
50.9\end{array}$ & $\frac{11.2}{11.3}$ & 41 & $\frac{2.956}{2836}$ & $\frac{0.014}{0.014}$ & 0.013 & 0.0000 & $\frac{0.515}{0.326}$ & \\
\hline 793 & $8 / 30 / 2004$ & 8:56:13 PM & 26.519 & 25.86 & 26.619 & 26.183 & 24.585 & 46.794 & 52.125 & 13.155 & 34.351 & -1.151 & & 47.029 & 0.091 & 14.643 & & 782.85 & 13.0475 & 51.0 & & & $\frac{2.097}{2.797}$ & & & 0.000 & $\frac{0.0507}{0.307}$ & \\
\hline & & & & & & 26.156 & & & 51.694 & & & & & & & 14.643 & & & $\frac{10.047}{13.0642}$ & & & & & 0.014 & & & & \\
\hline 795 & $8 / 30 / 2004$ & :13 PM & 26.493 & 25.81 & \begin{tabular}{|l|l|}
26.603 \\
\end{tabular} & 26.112 & & 46.621 & 51.518 & 12.968 & 34.712 & -1.212 & & 47.42 & 0.097 & 14.643 & & 784.85 & 13.0808 & 50.4 & & & 2.804 & 0.014 & 0.013 & 0.000 & 0.326 & \\
\hline 7966 & & & & & & & & & & & 34.314 & & & & & 14.643 & & & & & & & & & & & & \\
\hline & $8 / 3$ & 9:00:13 PM & 26.488 & 25.754 & \begin{tabular}{|l|l|}
26.638 \\
\end{tabular} & 26.082 & & 46.781 & & 13.166 & & -1.212 & & 477.211 & 0.091 & 14.643 & & 786.8 & & 50.8 & & & & & & & & \\
\hline & $0 / 2004$ & & 26.482 & 25.738 & 26.641 & 26.09 & & & .665 & 13.101 & 34.357 & -1.212 & & & 0.094 & 14.643 & & 787.85 & 1308 & 50.5 & & & 2.790 & & & & & \\
\hline & $8 / 30 / 2004$ & 9:02:13 PM & 26.484 & 25.715 & \begin{tabular}{|l|}
26.644 \\
\end{tabular} & 26.118 & 24.6 & & 51.174 & 13.059 & 33.838 & -1.212 & & 46.168 & 0.0 & 14.643 & & 78 & 13.1475 & 50.0 & 11.0 & & 2.75 & 0.014 & 0.013 & & 0.325 & \\
\hline & $8 / 30 / 2004$ & & 4.488 & 25.694 & 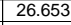 & 26.147 & 24.61 & 45.974 & 50.915 & 13.046 & 33.959 & -1.212 & & 47.68 & 0.096 & 14.643 & & 789.8 & 13.1642 & 49.8 & 11 & & 2.75 & 0.014 & .013 & & & \\
\hline & 0/2004 & 9:04:13 PM & 4882 & 25.668 & 26.646 & 26.141 & 24.6 & & 51.503 & 13.091 & 34.22 & -1.21 & & 47.843 & 093 & 14. & & & $13.1 \mathrm{C}$ & & & & 2.7. & & & & & \\
\hline & & & 4.455 & & 26.67 & 26.16 & 24. & $\begin{array}{l}45.385 \\
\end{array}$ & 50.49 & 2.95 & $\begin{array}{l}3.284 \\
2.215 \\
\end{array}$ & & & $\begin{array}{l}47.144 \\
1017\end{array}$ & .095 & 14.643 & & & & & & & & & & & & \\
\hline & 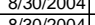 & & |. & & 26.691 & $\frac{26.175}{26.172}$ & & & . & $\frac{12.635}{12796}$ & $\begin{array}{l}33.315 \\
32694\end{array}$ & $-\frac{1.1215}{-1212}$ & & 4 & 0.091 & 14.64 & & 92.85 & & 48.8 & & & & & 50 & & 0.014 & \\
\hline & $\frac{13012004}{81201304}$ & & | & & \begin{tabular}{|l|l|}
2.075 \\
26908
\end{tabular} & $\frac{20.17}{26197}$ & 24. & & | & $\frac{12.100}{12725}$ & & & & & & & & & & 49 & & & & & & & & \\
\hline 806 & $8 / 30 / 2004$ & $\begin{array}{l}9.00 .13 \mathrm{PM} \\
9.09: 13 \mathrm{PM}\end{array}$ & $\frac{\mid 20.510}{26.533}$ & 25 & \begin{tabular}{|l|l|l|l}
2670 \\
\end{tabular} & $\frac{20.191}{26.222}$ & 24. & & | & $\frac{12.153}{12846}$ & $\begin{array}{l}34.035 \\
3.418\end{array}$ & $\frac{-1.12}{-1.206}$ & & 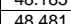 & 0.098 & $\frac{14.045}{11643}$ & & & & $\frac{4.50}{502}$ & $\frac{1}{11}$ & & & 014 & 013 & & 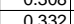 & \\
\hline & $8 / 30 / 2$ & & & & \begin{tabular}{|l|l|l|l|}
26.687 \\
\end{tabular} & 20.216 & 24. & 45.8 & & $\frac{1}{12.0408}$ & 34.0 & -1.154 & & 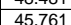 & 0 & $\frac{14.043}{14.643}$ & & & & & & & & & & & & \\
\hline & $8 / 3$ & & 521 & 25.572 & \begin{tabular}{|l|l|}
26.696 \\
\end{tabular} & 26.2 & & 45.4 & 50.347 & 12.616 & 33.641 & -1.215 & & 455.167 & 0.089 & 14.643 & & & 13. & 49 & 10. & & & & & & 0.307 & \\
\hline & & & & & \begin{tabular}{|l|}
26.709 \\
\end{tabular} & & & & & & & $-\frac{1.212}{-1.212}$ & & & 0.09 & & & & & & & & & & & & & \\
\hline & & 9:13:13 PM & 26.534 & & 26.739 & 26.23 & & & $\mid$ & 12.97 & & $\frac{-1.2}{-1.2}$ & & 45.9 & & & & & & & & & & & & & & \\
\hline & & & 26.549 & & 26.749 & \begin{tabular}{|l|l|}
26.248 \\
\end{tabular} & & & & & & & & & $0.0 !$ & & & & & & & & & & & & & \\
\hline & $8 / 30 / 2004$ & & 574 & 25.55 & 26.794 & 26.273 & 24.8 & & 162 & 13.32 & 32.744 & -1.205 & & 47.87 & 0.093 & 14.643 & & & 13.36 & 49 & 11. & & & & & & & \\
\hline & $8 / 30 / 2004$ & & 26.584 & & \begin{tabular}{|l|}
26.824 \\
\end{tabular} & 26.243 & 24 & & . & 13.403 & 33.4 & -1.215 & & 49.013 & 0.09 & & & 802.8 & & & 11 & & & 013 & 0.012 & 0. & 0.309 & \\
\hline & 8/30/2004 & & 26.594 & & 26.879 & 26.27 & & & & & & -1.209 & & & & & & & & & & & & & & & & \\
\hline & 8/3012004 & 9:18: & 26.609 & & \begin{tabular}{|l}
26.929 \\
\end{tabular} & 26.303 & 24. & & 52.376 & 13.598 & 34.766 & -1.212 & & $4 \mathrm{c}$ & 0.098 & & & 80 & & & 11 & & & 014 & & & 0.324 & \\
\hline & $8 / 30 / 20$ & 9:19:13 PM & 26.62 & 25.526 & 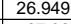 & 26.279 & & 46.532 & $0.1 / 27$ & 13.3 & 34.6 & -1.2 & & 4.506 & 0.09 & 14.64 & & & & 0. & +4 & & & & 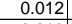 & 0.00 & 0.000 & \\
\hline & & & & & & & & & & & & & & & & & & & & & & & & & & & & \\
\hline & & & & 25.51 & 27.0 & 20.4 & 24. & 41.04 & (163) & & & -1.212 & & 46. & & & & & & 51 & & & & & & & $=0$ & \\
\hline & & & & & & & & & & & & & & & & & & & & & & & & & & & & \\
\hline & $\frac{103012}{83 / 212}$ & & & & & & & & & & & & & & & & & & & & & & & & & & & \\
\hline 822 & $8 / 30 / 2$ & & 26.72 & & 27.138 & 20.3 & & & & & & -1.1212 & & & & & & & & & $\frac{11}{11}$ & & & & & & & \\
\hline & & & 26.14 & & & & & & & & & & & & & & & & & & $=$ & & & & & & & \\
\hline 82 & & & 26.76 & 25.515 & 27208 & 26.3 & & 46. & 51 & & & -1.145 & & & & & & & & & & & & & & & & \\
\hline & & & & & & & & & & & & & & & & & & & & & & & & & & & & \\
\hline & $8 / 30 / 20$ & $9: 29: 1$ & & 25.4 & 27.226 & 26.2 & 24. & & .648 & & & -1.1 & & 47. & & & & & & & 11 & & & & & & & \\
\hline & $8 / 30 / /$ & & & & 27.246 & & & & 5 & & & -1.2 & & 46. & & & & & & 49 & & & & & & & & \\
\hline & $8 / 30 / 20$ & & 26.8 & 25.4 & 27.276 & 26.2 & 24. & & 51.508 & & 33.8 & -1.2 & & & & & & & & & 11 & & & & & & & \\
\hline & $8 / 3012004$ & & & 25.45 & \begin{tabular}{|l}
27.273 \\
\end{tabular} & 26.113 & & 45.9 & & & & -1.09 & & 48. & & 14. & & & & & & & & & & & & \\
\hline & & & & & 27. & 26.0 & & & & & & & & & & & & & & & & & & & & & & \\
\hline $8=$ & 8/30/2004 & & & & 27.31 & 26.02 & 24. & & 331 & & & & & & & & & & & & & & & & & & 0.306 & \\
\hline & 8313012004 & & & 2 & 27.32 & 26.02 & 24. & & 51.696 & & 3 & & & $4 r$ & & & & & & & & & & & & & & \\
\hline & & & & $\frac{25}{25}$ & \begin{tabular}{|l|l|}
27.36 \\
\end{tabular} & 25.95 & & 45. & $\frac{50.06}{51.76}$ & & & $-\frac{-1.1}{1}$ & & & & & & & & $\frac{45}{55}$ & & & & & & & & \\
\hline & 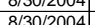 & 势 & & & 2.0 & 26.045 & & 40.3 & & & & & & & & & & & & & 11 & & & & & & & \\
\hline & & & & & $\frac{27}{27}$ & $\frac{2.00}{260}$ & & & & 132 & & & & & & & & & & & & & & & & & & \\
\hline 83 & $8 / 30 / 2004$ & & 26.81 & 25.39 & \begin{tabular}{|l|}
27.424 \\
\end{tabular} & 26.089 & 24.6 & 46.406 & & 13.209 & 34.181 & -1.143 & & 47.338 & 0.1 & 14.6 & & & & 50 & 11 & & & & 0.013 & & 0.332 & \\
\hline & & & & & \begin{tabular}{|l|l|}
27.409 \\
\end{tabular} & & & & & & & & & & & & & & & & & & & & & & & \\
\hline & $8 / 30 / 2004$ & 9:42:13 PM & \begin{tabular}{l|l|}
26.814 \\
0.9
\end{tabular} & 25.366 & \begin{tabular}{|l|l}
27.433 \\
\end{tabular} & 26.079 & 24.686 & & 1.2.200 & 13.158 & 34.119 & -1.212 & & 47.009 & 0.089 & 14.643 & & & & & & & & & 0.012 & & 0.296 & \\
\hline [840| & 8/30/2004 & 9:43:13 PM & 26.824 & & 27.428 & $26.079 \mid$ & & $\begin{array}{l}46.076 \\
\end{array}$ & $|51.265|$ & 13.141 & 33.791 & -1.151 & & 47.954 & 0.092 & 14.643 & & 829.85 & $\begin{array}{ll}5 & 13.8308\end{array}$ & 50.1 & & & & 0.013 & 0.012 & 0.000 & 0.308 & \\
\hline
\end{tabular}


WSRC-TR-2005-00105, REVISION 0

SRNL-RPP-2005-00012, REVISION 0

RUN \# 4.03A AND B; FIRST AND SECOND HALF OF SLURRY DEWATERING - CONT.

\begin{tabular}{|c|c|c|c|c|c|c|c|c|c|c|c|c|c|c|c|c|c|c|c|c|c|c|c|c|c|c|c|c|}
\hline & A & B & $\mathrm{D}$ & $E$ & \begin{tabular}{|l}
$F$ \\
\end{tabular} & $G$ & $\mathrm{H}$ & $\mathrm{J}$ & \begin{tabular}{|l|}
$K$ \\
51227 \\
\end{tabular} & $\mathrm{~L}$ & \begin{tabular}{c|}
$M$ \\
2303
\end{tabular} & $\mathrm{~N}$ & 0 & $\mathrm{Q}$ & & s & $\mathrm{T}$ & $\mathrm{v}$ & $\mathrm{w}$ & $\mathrm{x}$ & $\mathrm{Y}$ & \begin{tabular}{l|l}
$\mathrm{z}$ \\
\end{tabular} & AA & $\mathrm{AB}$ & $A C$ & $\mathrm{AD}$ & & AF $A$ \\
\hline$\frac{841}{842}$ & $\begin{array}{l}8 / 30 / 2004 \\
8 / 30 / 2004\end{array}$ & $\begin{array}{l}\text { 9:44:13 PM } \\
\text { 9:45:13 PM }\end{array}$ & 26.8394 & $\frac{25.356}{25.355}$ & \begin{tabular}{|l|}
27.463 \\
27.477 \\
\end{tabular} & 26.109 & $\begin{array}{r}24.736 \\
24.73 \\
\end{array}$ & $\begin{array}{l}46.043 \\
47.086\end{array}$ & $\left|\begin{array}{|l}\mid 51.277 \\
52.223\end{array}\right|$ & $\begin{array}{l}13.04 \\
13.30 \\
\end{array}$ & \begin{tabular}{|l|}
33.863 \\
3.708 \\
\end{tabular} & $\begin{array}{r}-1.209 \\
-1.1015 \\
\end{array}$ & $\begin{array}{r}-7.223 \\
-6.94 \\
\end{array}$ & $\begin{array}{l}47.443 \\
49.143 \\
\end{array}$ & \begin{tabular}{|c|}
0.095 \\
0.094
\end{tabular} & $\begin{array}{l}14.643 \\
14.643 \\
\end{array}$ & $\begin{array}{l}0.003 \\
0.004\end{array}$ & \begin{tabular}{|l|}
830.85 \\
831.85 \\
\end{tabular} & \begin{tabular}{|l|l|l|l|l} 
& 13.8475 \\
5 & 13.8642 \\
\end{tabular} & \begin{tabular}{|l|}
50.2 \\
51.1 \\
\end{tabular} & \begin{tabular}{|l|}
11.3 \\
11.7
\end{tabular} & $\mid$\begin{tabular}{|l|}
3 \\
$\mid 70.0$ \\
70.9
\end{tabular} & & \begin{tabular}{|l|l|}
0.014 \\
0.013
\end{tabular} & \begin{tabular}{|l}
0.013 \\
0.013 \\
\end{tabular} & $\begin{array}{l}0.000 \\
0.000\end{array}$ & $\begin{array}{l}0.318 \\
0.307 \\
\end{array}$ & 0.27 \\
\hline & & & & & & 26.133 & & & & 13.1 & & & & & 0.095 & 14.643 & & & & 50.0 & & & & & & 0.000 & & $\begin{array}{l}0.20 \\
0.27\end{array}$ \\
\hline 844 & $8 / 30 / 2004$ & 9:47:13 PM & 26.849 & 25.336 & 27.478 & 26.114 & 24.766 & 47.074 & \begin{tabular}{|l|}
52.407 \\
\end{tabular} & 13.28 & 34.602 & -1.154 & -7.174 & 47.484 & 0.095 & 14.643 & & 833.85 & 13.8975 & 51.3 & 11.3 & & \begin{tabular}{|l|}
2.816 \\
\end{tabular} & 0.014 & 0.013 & 0.000 & 0.311 & \\
\hline 845 & 8/30/2004 & 9:48:13 PM & 26.864 & 25.335 & 27.497 & 26.138 & 24.8 & 46.435 & \begin{tabular}{|l|l|}
51.423 \\
\end{tabular} & 13.36 & 33.974 & \begin{tabular}{|c|}
-1.148 \\
\end{tabular} & -7.203 & 47.741 & 0.095 & 14.643 & 0.003 & 834.85 & 513.9142 & 50.3 & 11.4 & 40.2 & \begin{tabular}{|l|}
2.772 \\
\end{tabular} & 0.014 & 0.013 & 0.000 & 0.316 & \\
\hline 846 & $8 / 30 / 2004$ & 9:49:13 PM & & & & 26.178 & 24.775 & 46.787 & \begin{tabular}{|l|l|}
52.015 \\
5015
\end{tabular} & $\begin{array}{ll}13.26 \\
1201\end{array}$ & \begin{tabular}{|l|l|}
34.417 \\
32281
\end{tabular} & -1.088 & -7.05 & & 0.1 & 14.643 & & 835.85 & 513.9308 & 50.9 & 11.6 & & 2.799 & & 0.013 & 0.000 & 0.329 & \\
\hline 847 & $8 / 30 / 2004$ & 9:50:13 PM & 26.888 & 25.33 & \begin{tabular}{|l|l|}
27.517 \\
27569
\end{tabular} & 26.158 & 24.8 & 45.305 & \begin{tabular}{|l|}
50.465 \\
5.057 \\
\end{tabular} & $\begin{array}{l}12.91 \\
1282\end{array}$ & 33.282 & -1.148 & -7.217 & 48.415 & 0.09 & $\begin{array}{l}14.643 \\
1.642\end{array}$ & 0.003 & $\begin{array}{l}836.85 \\
83795\end{array}$ & 13.9475 & 49.3 & 11.5 & 39.3 & \begin{tabular}{|l|}
2.709 \\
268
\end{tabular} & 0.013 & 0.012 & 0.000 & 0.306 & \\
\hline 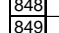 & 8/30/2004 & $\begin{array}{l}9: 51: 13 \mathrm{PM} \\
9.5 \cdot 13 \mathrm{PM}\end{array}$ & $\frac{26.925}{26.92}$ & 25.351 & \begin{tabular}{|l|}
27.568 \\
27.544
\end{tabular} & $\frac{26.225}{26.21}$ & 24.812 & $\begin{array}{r}45.035 \\
4668 \\
\end{array}$ & \begin{tabular}{|l|}
50.057 \\
52077
\end{tabular} & 12.83 & .231 & \begin{tabular}{r|r|r|r|r|} 
& -1.143 \\
-109 &
\end{tabular} & $\begin{array}{l}-6.998 \\
-7085 \\
\end{array}$ & 48.437 & 0.09 & 14.643 & & 837.85 & 13.9642 & 48.9 & 11.5 & & \begin{tabular}{|l|}
2.698 \\
2780
\end{tabular} & 0.013 & 0.012 & 0.000 & 0.306 & \\
\hline 849 & $\begin{array}{l}88 / 30 / 2004 \\
8 / 30 / 2004\end{array}$ & $\begin{array}{l}\text { 9:52:13 PM } \\
953: 13 \mathrm{PM}\end{array}$ & $\begin{array}{l}26.92 \\
26.94\end{array}$ & $\frac{25.336}{25.331}$ & \begin{tabular}{|l|}
27.544 \\
27.563 \\
\end{tabular} & 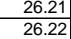 & 24.812 & $\begin{array}{r}46.68 \\
45.815 \\
\end{array}$ & \begin{tabular}{|r|}
52.076 \\
51.02
\end{tabular} \mid & $\frac{13.23}{13.04}$ & $\begin{array}{r}34.22 \\
33.719\end{array}$ & $\begin{array}{r}-1.09 \\
-1.148\end{array}$ & $\begin{array}{r}-7.085 \\
-7.053 \\
\end{array}$ & $\begin{array}{r}48.986 \\
49.512 \\
\end{array}$ & $\begin{array}{l}0.101 \\
0.082 \\
\end{array}$ & $\begin{array}{l}14.643 \\
14.643 \\
\end{array}$ & $\begin{array}{l}0.003 \\
0.004\end{array}$ & \begin{tabular}{|l|}
838.85 \\
839.85 \\
\end{tabular} & \begin{tabular}{|l|l|}
5 & 13.9808 \\
55 & 13.9975 \\
\end{tabular} & $\begin{array}{l}51.0 \\
49.9\end{array}$ & $\frac{11.7}{11.8}$ & 39.8 & \begin{tabular}{|l|}
$\mid 2.789$ \\
2.742 \\
\end{tabular} & $|0.014|$ & $\begin{array}{l}0.013 \\
0.011 \\
\end{array}$ & 0.000 & 0.333 & 0.28 \\
\hline 851 & $8 / 30 / 2004$ & 9:54:13 PM & 26.949 & 25.336 & \begin{tabular}{|l|l|}
27.578 \\
\end{tabular} & 26.229 & 24.866 & 45.087 & \begin{tabular}{|l|l|}
49.937 \\
\end{tabular} & 12.74 & 33.549 & \begin{tabular}{|c|}
-1.143 \\
\end{tabular} & & 45.968 & 0.091 & 14.643 & 0.003 & 840.85 & \begin{tabular}{|l|l|}
514.0142 \\
\end{tabular} & 48.8 & 10.9 & & & \begin{tabular}{|l|l|}
0.013 \\
\end{tabular} & $\begin{array}{l}0.011 \\
0.012\end{array}$ & 0.000 & $\begin{array}{l}0.270 \\
0.308\end{array}$ & $\begin{array}{l}0.23 \\
0.26 \\
\end{array}$ \\
\hline & $8 / 30 / 2004$ & 9:55:13 PM & 26.953 & 25.33 & \begin{tabular}{|l|}
27.572 \\
\end{tabular} & 26.223 & 24.84 & 45.977 & \begin{tabular}{|l|}
51.186 \\
\end{tabular} & 13.03 & \begin{tabular}{|l|l|}
33.887 \\
\end{tabular} & \begin{tabular}{|c|}
-1.148 \\
\end{tabular} & -7.215 & \begin{tabular}{|l|l|}
46.713 \\
\end{tabular} & 0.09 & $\begin{array}{l}14.643 \\
\end{array}$ & & 841.85 & & 50.1 & $\begin{array}{l}11.1 \\
\end{array}$ & & \begin{tabular}{|l|}
2.753 \\
\end{tabular} & & & & & \\
\hline 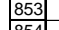 & $8 / 30 / 2004$ & 9:56:13 PM & 26.958 & 25.324 & \begin{tabular}{|l|l|}
27.586 \\
\end{tabular} & 26.218 & 24.835 & 46.541 & \begin{tabular}{|l|}
51.613 \\
\end{tabular} & 13.12 & 34.333 & -1.145 & $\begin{array}{r}-7.2 \\
\end{array}$ & \begin{tabular}{|l}
47.336 \\
\end{tabular} & 0.092 & 14.643 & & 842.85 & 14.0475 & 50.5 & 11.3 & 40.4 & \begin{tabular}{|l|}
2.788 \\
\end{tabular} & 0.013 & 0.012 & 0.000 & 0.303 & \\
\hline 854 & $8 / 30 / 2004$ & 9:57:13 PM & $\begin{array}{l}26.968 \\
\end{array}$ & 25.33 & \begin{tabular}{|l|l|}
27.602 \\
\end{tabular} & \begin{tabular}{|l}
26.223 \\
.627
\end{tabular} & 24.9 & 45.273 & \begin{tabular}{|l|}
50.183 \\
5120 \\
\end{tabular} & $\begin{array}{l}12.84 \\
1202\end{array}$ & $\begin{array}{l}33.454 \\
\end{array}$ & \begin{tabular}{|l|l|}
-1.148 \\
.1205
\end{tabular} & \begin{tabular}{|l|l|}
-7.076 \\
7.702
\end{tabular} & 46.786 & 0.093 & 14.643 & 0.00 & 843.85 & 14.0642 & 49.1 & 11.1 & & \begin{tabular}{|l|}
2.714 \\
\end{tabular} & 0.013 & 0.012 & 0.000 & 0.315 & \\
\hline 85 & $8 / 30 / 2004$ & $\begin{array}{l}9: 58: 13 \mathrm{PM} \\
9 \cdot 5 \cdot 13 \mathrm{PM}\end{array}$ & \begin{tabular}{r|}
26.98 \\
27.109
\end{tabular} & $\begin{array}{l}25.346 \\
25.326\end{array}$ & \begin{tabular}{|l|}
27.643 \\
27.623
\end{tabular} & $\begin{array}{r}26.27 \\
26.225\end{array}$ & & $\begin{array}{r}46.296 \\
57713\end{array}$ & \begin{tabular}{|l|}
51.309 \\
50.948 \\
\end{tabular} & 13.02 & $\begin{array}{l}34.339 \\
33699\end{array}$ & $\begin{array}{r}-1.145 \\
\end{array}$ & & $\begin{array}{r}48.36 \\
46.324 \\
\end{array}$ & $\begin{array}{l}0.092 \\
0.085\end{array}$ & & & & \begin{tabular}{|c|c|c|} 
\\
\end{tabular} & 50.2 & 11.5 & $\begin{array}{ll}40.3 \\
20.7\end{array}$ & \begin{tabular}{|l|}
2.780 \\
2.737 \\
\end{tabular} & \begin{tabular}{|l|l|}
0.013 \\
0.012
\end{tabular} & 0.012 & & 0.303 & \\
\hline & $8 / 3012004$ & $\begin{array}{l}9: 59: 13 \mathrm{PM} \\
\cdot 95 \cdot 20 \mathrm{PM}\end{array}$ & & $\frac{25.326}{25341}$ & \begin{tabular}{|l|}
27.623 \\
27.628 \\
\end{tabular} & $\frac{26.225}{26.23}$ & $\frac{24.887}{24.882}$ & $\frac{45.713}{45.195}$ & \begin{tabular}{|l|}
50.948 \\
50285 \\
\end{tabular} & $\begin{array}{r}12.9 \\
1277\end{array}$ & $\begin{array}{l}33.696 \\
33.469\end{array}$ & $\frac{-1.145}{-1.099}$ & $\begin{array}{l}-6.905 \\
-6.6752 \\
-6.07\end{array}$ & $\begin{array}{r}46.324 \\
46.99\end{array}$ & $\begin{array}{l}0.085 \\
0.079\end{array}$ & $\frac{14.643}{14.643}$ & & & \begin{tabular}{|c|}
14.0975 \\
714.094
\end{tabular} & $\begin{array}{l}49.8 \\
49.9 \\
\end{array}$ & $\frac{11.0}{11.2}$ & & $|2.737|$ & & 0.011 & 0.000 & 0.285 & 24 \\
\hline 858 & $8 / 3012004$ & $\begin{array}{c}1.59 .20 \mathrm{PM} \\
10: 00: 20 \mathrm{PM}\end{array}$ & 27.035 & $\frac{23.341}{25.317}$ & \begin{tabular}{|l|l|}
27.028 \\
27.619
\end{tabular} & $\begin{array}{l}20.23 \\
26.21\end{array}$ & 24.8862 & $\begin{array}{l}45.195 \\
45.553\end{array}$ & \begin{tabular}{|l|}
50.285 \\
50.504 \\
\end{tabular} & $\begin{array}{l}12.17 \\
12.84\end{array}$ & $\begin{array}{r}3.439 \\
33.75 \\
\end{array}$ & \begin{tabular}{|}
-1.209 \\
-1.212
\end{tabular} & $\begin{array}{l}-0.152 \\
-7.249\end{array}$ & $\begin{array}{l}40.99 \\
45.878\end{array}$ & $\begin{array}{l}0.094 \\
0.094\end{array}$ & $\begin{array}{l}14.045 \\
14.643\end{array}$ & & & .1161 & $\frac{49.2}{49.4}$ & & & & $\frac{0.011}{0.013}$ & $\frac{0.011}{0.013}$ & $\begin{array}{l}0.000 \\
0.000\end{array}$ & $\frac{0.267}{0.315}$ & $\frac{0.23}{0.27}$ \\
\hline & $8 / 30 / 2004$ & 10:01:20 PM & 27.041 & 25.318 & \begin{tabular}{|l|}
27.61 \\
\end{tabular} & 26.216 & 24.843 & & \begin{tabular}{|l|l|}
51.794 \\
\end{tabular} & 13.12 & \begin{tabular}{|l|l|}
34.638 \\
\end{tabular} & \begin{tabular}{|c|}
-1.198 \\
\end{tabular} & $\begin{array}{l}-7.267 \\
\end{array}$ & 44.368 & 0.088 & 14.643 & & & & 50.7 & 10.6 & & & 年 & & & 0.287 & $\begin{array}{l}0.27 \\
0.24\end{array}$ \\
\hline 86 & 8/30/2004 & 10:02:20 PM & 27.071 & 25.328 & 27.645 & 26.246 & 24.873 & 46.285 & \begin{tabular}{|l|l|}
51.315 \\
\end{tabular} & 13.03 & 34.181 & -1.145 & \begin{tabular}{|l|l|} 
& -7.082 \\
\end{tabular} & 47.87 & 0.094 & 14.643 & & & 14.1494 & 50.2 & $\begin{array}{l}11.4 \\
\end{array}$ & 40.2 & 2.774 & \begin{tabular}{|l|l|}
0.013 \\
\end{tabular} & 0.013 & 0.000 & 0.311 & \\
\hline & & & & 25.317 & & 26.23 & & & & & 34.425 & -1.148 & & & & 14.643 & & & & & & & & & & & & \\
\hline & $8 / 30 / 2004$ & 10:04:20 PM & 27.066 & 25.328 & 27.65 & 26.236 & & 46.348 & 1.418 & 12.97 & 34.296 & -1.145 & & 48.12 & 0.09 & 14.643 & & & & 50.3 & & & & .013 & .012 & & 0.297 & \\
\hline & 8313012004 & $\begin{array}{ll}10.02020 \mathrm{PM} \\
10.0602\end{array}$ & 27.062 & 25.324 & 27.666 & 26.242 & 24.864 & 46.171 & 51.924 & 13.162 & 34.597 & -1.148 & $\begin{array}{l}-1.20 \\
-720\end{array}$ & 48.25 & 0.093 & 14.643 & & & $\begin{array}{l}4.1994 \\
1.201\end{array}$ & 50. & & & & & & & & \\
\hline & & & & & & & & & & & & & & & & & & & 4.2161 & & & & & & .012 & & & \\
\hline & $8 / 30 / 2004$ & & 27.053 & 25.32 & 27.682 & 26.248 & 24. & & $51.537 /$ & 13.08 & 34.2 & -1.143 & & & 0.087 & 14.643 & & & & 50.4 & 11 & & & & & & & \\
\hline & 8/30/2004 & 10:08:20 PM & 27.059 & 25.321 & 27.698 & 26.249 & 24.88 & 45.636 & 50.817 & 12.99 & 33.612 & -1.09 & -7.105 & 45.947 & 0.095 & 14.643 & & & 14.2494 & 49.7 & & & 2.732 & 0.014 & 0.013 & & & \\
\hline & & & & & & & & & 51.454 & & $\begin{array}{r}34.123 \\
22.256\end{array}$ & $\begin{array}{r}-1.09 \\
1.19\end{array}$ & & 45.734 & & 14.643 & & & & & & & & & & & & \\
\hline & 8313012004 & & 27.05 & 25.312 & \begin{tabular}{|l|l|}
27.714 \\
\end{tabular} & 26.251 & & 45.267 & 5. & $\frac{12.199}{109}$ & & $\begin{array}{l}-1.148 \\
-148\end{array}$ & & 46.128 & & 14.643 & & & & (4) & 111 & & & & & & & \\
\hline & $8 / 3012004$ & $10: 11: 20 \mathrm{PM}$ & 27.076 & 25.304 & \begin{tabular}{|l|l|}
27.699 \\
776
\end{tabular} & 26.236 & 24.8 & 46.089 & - & $\begin{array}{ll}13.09 \\
13070\end{array}$ & $\begin{array}{l}33.898 \\
3.89\end{array}$ & -0.931 & & 46.11 & 094 & 14.643 & & & & & 11.1 & & 757 & 0.013 & 0.012 & .000 & & \\
\hline & $\frac{0}{8 / 2012004}$ & 10.12 .2007 & 2700 & & $\begin{array}{l}21.124 \\
\end{array}$ & 20.210 & 24.05 & $\begin{array}{l}46.45 \\
46.294\end{array}$ & (. & 年 & $\begin{array}{l}33.000 \\
31207\end{array}$ & & & $\begin{array}{l}44.51 \\
477\end{array}$ & . & $\frac{14.045}{1.612}$ & & & & & & & & & & & & \\
\hline 然 & $\frac{15302004}{8}$ & $10.10 .420 \mathrm{PM}$ & 27.05 & & \begin{tabular}{|l|l|l|l|}
27.18 \\
\end{tabular} & $\frac{20.28}{26.27}$ & & & 218 & & $\begin{array}{l}34.2017 \\
3.2775\end{array}$ & -1.212 & & & & $\frac{14.643}{14643}$ & & & & & 11 & & & & & & & \\
\hline 873 & $8 / 30 / 2004$ & $10: 15: 2$ & 27.119 & 25.316 & \begin{tabular}{|l|l|}
27.753 \\
\end{tabular} & 26.439 & 24.89 & 43.817 & \begin{tabular}{|l|}
49.182 \\
\end{tabular} & $\frac{13.06}{13.06}$ & 31.544 & -1.148 & & $\begin{array}{r}40.10 \\
48.511\end{array}$ & 0.088 & 14.643 & & & & 48.1 & $\frac{71.6}{11.6}$ & & 2.598 & \begin{tabular}{|l|l|}
0.013 \\
\end{tabular} & 0.012 & 0.000 & 0.310 & \\
\hline 874 & $8 / 30 / 2004$ & 10:16: & & & 27.746 & 26.508 & & & & & & \begin{tabular}{|c|c|}
-1.157 \\
-157
\end{tabular} & & & 0.084 & 14.643 & & & & & 11. & & & & & & & \\
\hline 875 & $8 / 30 / 2004$ & 10:17:20 PM & 27.311 & 25.328 & 27.79 & 26.442 & 24.8 & 45 & 50.368 & 13.33 & 32.66 & -1.148 & -7.165 & 47.897 & 0.09 & 14.643 & & & 14.3 & 49 & 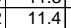 & & & & & & 306 & \\
\hline & & & & & & & & & & & & & & & & & & & & & & & & & & & & \\
\hline & $8 / 30 / 2004$ & 10:19:20 PM & 27.417 & 25.345 & 27.806 & 26.07 & & $\begin{array}{l}45.504 \\
\end{array}$ & 50.508 & 13.25 & 3.265 & -1.105 & & & 0.086 & 14.64 & & & & 49. & & & & & & & 028 & \\
\hline & & & 27.434 & 25.3 & \begin{tabular}{|l|l|}
27.798 \\
\end{tabular} & 25.99 & & & . 254 & & & & & & & 14. & & & & & & & & & & & & \\
\hline & & 10:21 & 27.462 & 25.345 & & 25.92 & & & & & & -1.093 & & & & & & & & & & & & & & & & \\
\hline & $8 / 30 / 2004$ & & 27.4911 & 25.354 & 27.83 & 25.932 & & & & & & -1.09 & & & & 14.645 & & & & 49.4 & 11 & & & & & & & \\
\hline & 8/30/2004 & 10:23: & 27.501 & 25.354 & 27.836 & & & & & & & -1.09 & & & & & & & & & & & & & & & & \\
\hline 88 & 0/2004 & & 27.528 & 25.367 & 27.858 & 25.975 & 25.10 & & \begin{tabular}{|l|l|}
50.608 \\
\end{tabular} & 13.04 & & $\frac{-1.03}{-1}$ & & \begin{tabular}{|l|l|} 
\\
\end{tabular} & 0.083 & 14.64 & & & & 49.5 & 11 & & & & & 0.000 & & \\
\hline & & & 27.549 & 25.373 & $\begin{array}{l}27.899 \\
\end{array}$ & 26.001 & & & & & & & & & & & & & & 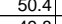 & & & & & & & 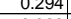 & \\
\hline & & & 2.513 & & & & & & & & & & & & & & & & & & & & & & & & & \\
\hline & & & 27.021 & 25.370 & \begin{tabular}{|l}
2.919 \\
\end{tabular} & & & & & $\frac{13.1}{121}$ & $\frac{3.1}{321}$ & -1.0 & & & & & & & & & & & & & & & & \\
\hline & & & 27.65 & 25.48 & 27.978 & & & & & & & & & & & & & & & & & & & & & & & \\
\hline 88 & $8 / 30 / 200$ & & 27.691 & & \begin{tabular}{|l|l|}
27.991 \\
\end{tabular} & 26.143 & & & & & & & & & & & & & & & & & & & & & & \\
\hline & $8 / 30 / 200$ & & 27.703 & & & 26.165 & & & & & & & & & & & & & & & & & & & & & & \\
\hline & $8 / 30 / 4$ & & \begin{tabular}{|l|l|}
27.723 \\
\end{tabular} & & & 26. & & & & & & & & & & & & & & & & & & & & & & \\
\hline & $8 / 30 / 2004$ & & 27.751 & 25.455 & \begin{tabular}{|l|}
28.006 \\
\end{tabular} & 26.163 & 25. & & 51.114 & 13.2 & & & & & & & & & & & & & & & & & & \\
\hline & & & & 25.469 & 28.044 & & & & & & & & & & & & & & & & & & & & & & & \\
\hline & $8 / 30 / 200$ & $10: 35$ & 27.188 & 25.45 & 28.023 & 26.1 & 25. & & $51.36 \mathrm{C}$ & & & & & & & & & & & & 11 & & & & & & & \\
\hline & & & 27.80 & 25.4 & & 26.1 & & & 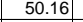 & & & -1.6 & & & & & & & & & 11 & & & & & & & \\
\hline & $8 / 30 / 2$ & & 27.83 & 25.49 & 28.07 & 26.1 & & & 3] & 13. & & & & & & & & & & & 11.3 & & & & & & & \\
\hline & & & & & & & & & & & & & & & & & & & & & & & & & & & & \\
\hline 89 & 8/30/2 & & 27.845 & 25.495 & 28.07 & 26.1 & & & & & & & & & & & & & & & & & & & & & & \\
\hline & & & & & 20. & & & & & & & & & & & & & & & & & & & & & & & \\
\hline & & & $27.06 / 4$ & & 28.094 & 20.4 & & & & & & & & & & & & & & & & & & & & & & \\
\hline & $8 / 30 / 2$ & & 788 & 25.52 & 28112 & $\frac{26.18}{2619}$ & $\frac{25.4}{252}$ & & 623 & $\frac{13.28}{130}$ & $\begin{array}{r}33.214 \\
33.561\end{array}$ & $\begin{array}{r}-1.004 \\
-1.09\end{array}$ & & & & $\frac{14 .}{14}$ & & & & & $\frac{1.4}{114}$ & & & & & & & \\
\hline 90 & $8 / 30 / 200$ & & 7.84 & 25 & 28.116 & 26.22 & & & 51. & $\frac{13.1}{131}$ & & & & & & & & & & & $113^{2}$ & & & & & & & \\
\hline & $8 / 30 / 2$ & & 27.90 & 25.5 & $\frac{28.091}{28.01}$ & $\frac{20.19}{26.19}$ & & & 51.5 & & & & & & & & & & & & & & & & & & & \\
\hline & & & $6.94 \mathrm{c}$ & & 28.101 & 26.20 & & & & & & -0.9 & & & & 14.6 & & & & & & & & & & & & \\
\hline & & & & & & & & & & & & & & & & & & & & & & & & & & & & \\
\hline & $8 / 30 / 2004$ & & 27.93 & 25.572 & \begin{tabular}{|l|}
28.121 \\
\end{tabular} & 26.219 & 25. & & & 13.14 & 33.348 & & & & & & & & & & & & & & & & & \\
\hline & & & & & 28.127 & 26.185 & & & & & & & & & & & & & & & $\overline{11.4}$ & & & & & & & \\
\hline & 8/30// & & 952 & 25.58 & 28.147 & $26.17 \mathrm{~s}$ & 25.2 & 45. & 51.20 & & & -0.928 & & 47.9 & 0. & & & & & & & & & & & & & \\
\hline & & & & & & & & & & & & & & & & & & & & & & & & & & & & \\
\hline 910 [1] & 8/30/2004 & 10:52:20 & 7.962 & 25.597 & \begin{tabular}{|l|l|}
28.147 \\
\end{tabular} & 26.22 & 25.30 & $\begin{array}{l}46.433 \\
\end{array}$ & | 51.731 & 13.3 & $33.943 \mid$ & -1.114 & & 45.131 & .086 & 14.643 & & 898.97 & 14.9828 & 50.6 & 10.7 & & & 0.012 & .01 & 0.000 & 0.281 & \\
\hline
\end{tabular}


WSRC-TR-2005-00105, REVISION 0

SRNL-RPP-2005-00012, REVISION 0

RUN \# 4.03A AND B; FIRST AND SECOND HALF OF SLURRY DEWATERING - CONT.

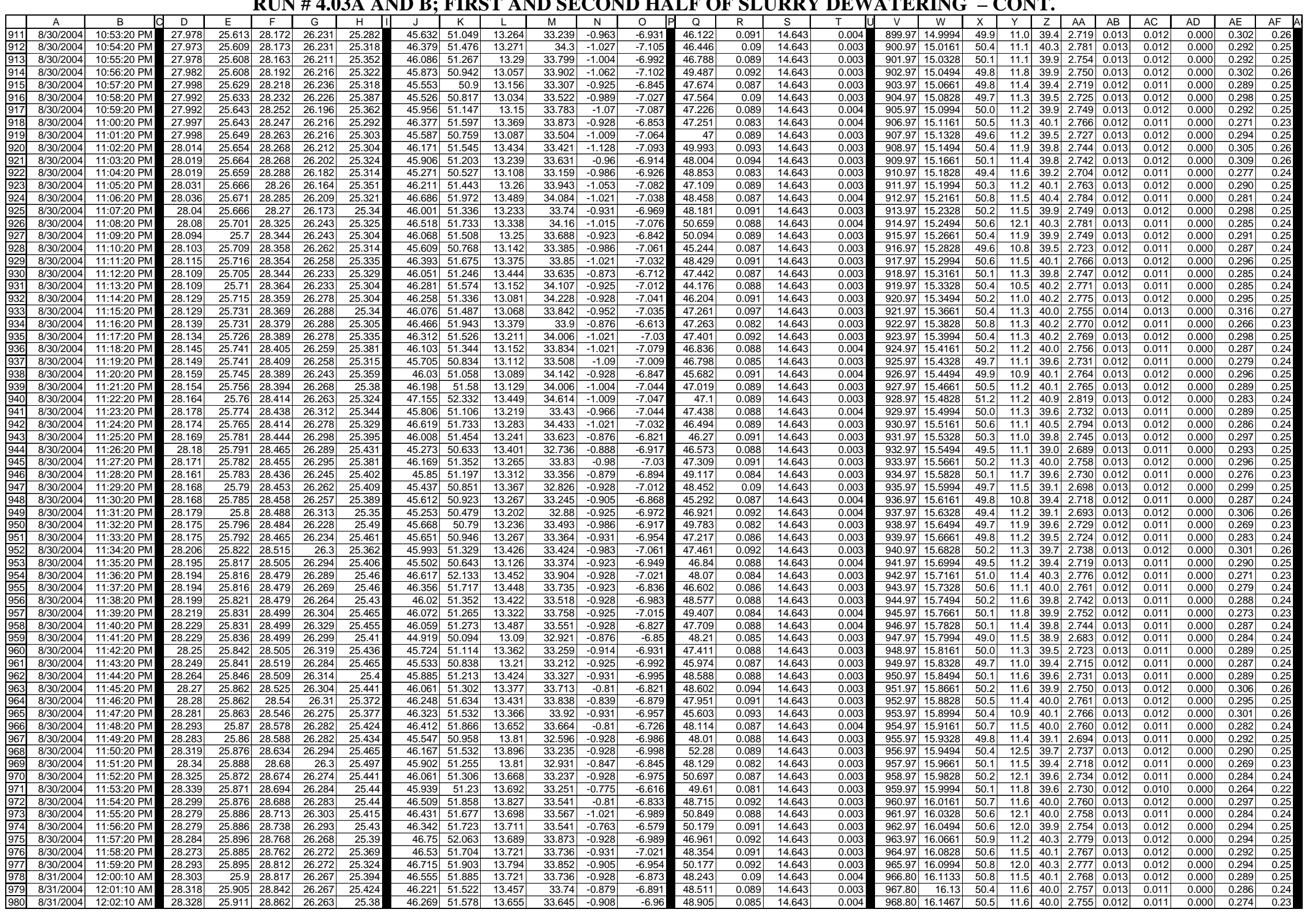


WSRC-TR-2005-00105, REVISION 0

SRNL-RPP-2005-00012, REVISION 0

RUN \# 4.03A AND B; FIRST AND SECOND HALF OF SLURRY DEWATERING - CONT.

\begin{tabular}{|c|c|c|c|c|c|c|c|c|c|c|c|c|c|c|c|c|c|c|c|c|c|c|c|c|c|c|c|c|}
\hline & A & & D & $E$ & $F$ & $G$ & $\mathrm{H}$ & $\mathrm{J}$ & K & $\mathrm{L}$ & $M$ & $\mathrm{~N}$ & 0 & 0 & R & \begin{tabular}{|c|} 
\\
\end{tabular} & $\begin{array}{ll}T \\
\end{array}$ & $\mathrm{~V}$ & w & $x$ & $\begin{array}{ll} \\
\end{array}$ & $z$ & & & & & & \\
\hline 981 & $8 / 31 / 2004$ & $12: 03: 10 \mathrm{AM}$ & 28.343 & \begin{tabular}{|l|l|}
25.911 \\
\end{tabular} & 28.872 & 26.278 & 25.33 & 45.692 & 51.244 & 13.464 & 32.993 & -0.859 & -6.885 & 47.403 & 0.09 & 14.643 & 0.003 & 969.80 & 16.1633 & 50.1 & 11.3 & 39.3 & 2.713 & 0.013 & 0.012 & 0.000 & 0.294 & 0.25 \\
\hline & & & 28.353 & 25.91 & 28.896 & \begin{tabular}{|l|}
26.267 \\
\end{tabular} & $\begin{array}{r}25.309 \\
\end{array}$ & $\begin{array}{r}46.655 \\
\end{array}$ & \begin{tabular}{|l|}
51.686 \\
\end{tabular} & \begin{tabular}{|l|l}
13.707 \\
\end{tabular} & $\begin{array}{r}34.07 \\
\end{array}$ & -0.989 & & 45.982 & $\begin{array}{l}0.092 \\
\end{array}$ & $\begin{array}{l}14.643 \\
\end{array}$ & 0.003 & 970.80 & 16.18 & \begin{tabular}{|l|}
50.6 \\
\end{tabular} & \begin{tabular}{|l|l}
11.0 \\
\end{tabular} & & & & & & & \\
\hline & $8 / 31 / 2004$ & $12: 05: 10 \mathrm{AM}$ & 28.358 & 25.91 & 28.896 & \begin{tabular}{|l|l|}
26.242 \\
2.27
\end{tabular} & \begin{tabular}{|l|}
25.364 \\
5.29
\end{tabular} & \begin{tabular}{|l|l|}
45.815 \\
\end{tabular} & \begin{tabular}{|l|}
51.306 \\
5.320
\end{tabular} & 13.606 & $\begin{array}{l}33.263 \\
3.13\end{array}$ & $\begin{array}{l}-0.723 \\
-0.97\end{array}$ & & $\begin{array}{l}50.058 \\
0.78\end{array}$ & 0.093 & 14.643 & 0.003 & 971.80 & 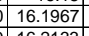 & \begin{tabular}{|c|}
50.2 \\
\end{tabular} & \begin{tabular}{|l|l|}
11.9 \\
11.9
\end{tabular} & 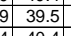 & 2.726 & 0.013 & 0.012 & 0.000 & 0.302 & \\
\hline \begin{tabular}{|l|l|}
984 \\
985
\end{tabular} & $8 / 31 / 2004$ & $\begin{array}{l}12: 06: 10 \mathrm{AM} \\
1200710\end{array}$ & $\begin{array}{l}28.378 \\
28.287\end{array}$ & 25.915 & 28.916 & \begin{tabular}{|l|l|}
26.297 \\
\end{tabular} & $\begin{array}{l}25.389 \\
32.3\end{array}$ & $\begin{array}{l}46.752 \\
\end{array}$ & \begin{tabular}{|l|l|}
52.134 \\
\end{tabular} & \begin{tabular}{|l|l|}
13.636 \\
\end{tabular} & 34.015 & -0.876 & & $\begin{array}{l}47.991 \\
9\end{array}$ & $\begin{array}{r}0.1 \\
\end{array}$ & $\begin{array}{l}14.643 \\
\end{array}$ & 0.003 & 972.80 & 16.2133 & \begin{tabular}{|l|}
51.0 \\
\end{tabular} & $\begin{array}{l}11.4 \\
\end{array}$ & $\begin{array}{ll}4 & 40.4 \\
\end{array}$ & 2.784 & 0.014 & 0.013 & 0.000 & $\begin{array}{l}0.318 \\
\end{array}$ & \\
\hline \begin{tabular}{|l|l|}
985 \\
886 \\
\end{tabular} & $8 / 31 / 2004$ & $\frac{12: 07: 10 \mathrm{AM}}{12200 \cdot 10 \mathrm{AM}}$ & $\frac{28.387}{28.413}$ & $\frac{25.915}{25.921}$ & $\begin{array}{r}28.941 \\
28.947\end{array}$ & \begin{tabular}{|l|}
26.317 \\
26.333 \\
6
\end{tabular} & $\begin{array}{l}25.364 \\
25425 \\
\end{array}$ & \begin{tabular}{|l|l|}
46.095 \\
46698
\end{tabular} & \begin{tabular}{|l|}
51.19 \\
51905 \\
\end{tabular} & & \begin{tabular}{|l|}
33.711 \\
33.912
\end{tabular} & \begin{tabular}{|c|}
-0.957 \\
\end{tabular} & $\begin{array}{l}-6.986 \\
6775\end{array}$ & $\begin{array}{r}46.763 \\
4914\end{array}$ & & \begin{tabular}{|l|l|l|}
14.643 \\
14634 \\
\end{tabular} & & $\begin{array}{l}973.80 \\
974.80\end{array}$ & \begin{tabular}{|r|r|} 
\\
\end{tabular} & \begin{tabular}{|l|}
50.1 \\
550 \\
\end{tabular} & \begin{tabular}{|l|}
11.1 \\
1.1 .1 \\
\end{tabular} & & \begin{tabular}{|l|}
2.751 \\
\end{tabular} & \begin{tabular}{|l|}
0.013 \\
0.12 \\
\end{tabular} & & & $\begin{array}{l}0.296 \\
\end{array}$ & \\
\hline \begin{tabular}{|l|}
987 \\
\end{tabular} & $8 / 3 / 31 / 2004$ & $\begin{array}{l}12: 08: 0: 10 \mathrm{AM} \\
12209: 10 \mathrm{~A}\end{array}$ & $\begin{array}{l}28.413 \\
28.428\end{array}$ & $\frac{25.921}{25.931}$ & $\begin{array}{l}28.947 \\
28.937\end{array}$ & \begin{tabular}{|l|}
26.333 \\
26.348
\end{tabular} & $\begin{array}{r}25.425 \\
25.39\end{array}$ & \begin{tabular}{|l|}
46.6988 \\
46.903
\end{tabular} & \begin{tabular}{|r|}
51.995 \\
5.185 \\
\end{tabular} & $\begin{array}{r}3.694 \\
13.584 \\
\end{array}$ & $\begin{array}{l}33.912 \\
34.253 \\
\end{array}$ & $\begin{array}{l}-0.8399 \\
-0.0873\end{array}$ & $\begin{array}{l}-6.775 \\
-6.937 \\
\end{array}$ & $\begin{array}{r}49.14 \\
47.926\end{array}$ & $\mid 0.0899$ & \begin{tabular}{|l|}
14.6634 \\
14.643
\end{tabular} & $\frac{0.004}{0.004}$ & $\begin{array}{l}974.80 \\
975.80\end{array}$ & $\begin{array}{l}\mid 16.2467 \\
0 \\
16.2633\end{array}$ & \begin{tabular}{|}
50.9 \\
51.1 \\
\end{tabular} & \begin{tabular}{|l|}
11.7 \\
11.4 \\
\end{tabular} & \begin{tabular}{|l|l|l|l|l|l|l|}
4 & 40.3 & 40.6
\end{tabular} & \begin{tabular}{|l|}
2.779 \\
2.798 \\
\end{tabular} & $\frac{0.013}{0.013}$ & $\begin{array}{l}0.011 \\
0.012\end{array}$ & \begin{tabular}{|c|}
0.000 \\
0.000
\end{tabular} & $\begin{array}{l}0.283 \\
0.0294\end{array}$ & $\begin{array}{l}0.24 \\
0.25\end{array}$ \\
\hline \begin{tabular}{|l|}
988 \\
\end{tabular} & $8 / 31 / 2004$ & 12:10:10 AM & 28.423 & 25.921 & & \begin{tabular}{|l|}
20.040 \\
\end{tabular} & & & & & & & & & 0.093 & 14.643 & $\begin{array}{l}0.004 \\
0.004\end{array}$ & & \begin{tabular}{|l|}
10.2033 \\
5
\end{tabular} & \begin{tabular}{|l|}
51.1 \\
50.9 \\
\end{tabular} & $\frac{11.4}{11.5}$ & & & & & $\begin{array}{l}0.000 \\
0.000\end{array}$ & & $\frac{0.25}{0.25}$ \\
\hline & $8 / 31 / 2004$ & $12: 11: 10 \mathrm{AM}$ & 28.449 & 25.936 & 28.977 & \begin{tabular}{|l|}
26.339 \\
\end{tabular} & 25.4 & 46.146 & \begin{tabular}{|l|l|}
51.464 \\
\end{tabular} & 13.938 & 33.14 & -0.873 & & 49.482 & 0.085 & 14.643 & & & 16.2967 & 50.3 & & & & & & & & $\begin{array}{l}0.25 \\
0.23\end{array}$ \\
\hline 990 & $8 / 31 / 2004$ & $12: 12: 10 \mathrm{AM}$ & 28.45 & 25.933 & 28.979 & \begin{tabular}{|l|}
26.306 \\
\end{tabular} & 25.382 & 46.088 & \begin{tabular}{|l|}
51.483 \\
\end{tabular} & 13.877 & & -0.807 & & 49.101 & \begin{tabular}{|c|}
0.089 \\
\end{tabular} & \begin{tabular}{|l|l|}
14.643 \\
\end{tabular} & & 978.80 & 16.3133 & 50.4 & $\begin{array}{ll}11.7 \\
\end{array}$ & 39.6 & 2.729 & .0.013 & & 0.000 & & \\
\hline 991 & & 12:13:10 AM & 28.445 & & 28.999 & 26.301 & 25.382 & & & & 33.147 & & & 48.154 & & $\begin{array}{l}14.643 \\
\end{array}$ & & 979.80 & \begin{tabular}{|l|l|} 
& 16.33 \\
\end{tabular} & 50.1 & & & & 0.013 & 0.011 & & 0.288 & \\
\hline 992 & & & & & & & & & 51.016 & 13.71 & 32.761 & -0.804 & & & 0.09 & \begin{tabular}{ll|l}
14.643 \\
\end{tabular} & & 0.80 & & 49.9 & 11.9 & & & & & & 0.294 & \\
\hline (1) & 004 & 12:15:10 AM & 28.472 & 25.94 & 29.03 & & 25.364 & 45.869 & 51.34 & $\begin{array}{ll}13.624 \\
\end{array}$ & 33.2 & -0.876 & & & .084 & \begin{tabular}{|l|l|l|l|}
14.643 \\
\end{tabular} & & 31.80 & 16.3633 & 50.2 & & 20 & & $n_{1}$ & & & 0.272 & \\
\hline & $11 / 2004$ & $12: 16: 10 \mathrm{AM}$ & 28.47 & 25.923 & 29.049 & & 25.442 & 45.732 & 51.132 & 13.712 & 32.976 & -0.81 & & 52.46 & 0.084 & \begin{tabular}{l|l}
14.643 \\
\end{tabular} & 0.003 & 982.80 & \begin{tabular}{|l|l|} 
& 16.38 \\
\end{tabular} & 50.0 & 12.5 & 39.4 & $\pi \pm$ & & 0.011 & 0.000 & 0.273 & \\
\hline & $8 / 31 / 2004$ & $12: 17: 10 \mathrm{AM}$ & 28.479 & 25.932 & 29.053 & \begin{tabular}{|l}
26.269 \\
\end{tabular} & 25.41 & 45.85 & 51.257 & 13.683 & 33.085 & -0.827 & & 46.921 & 0.088 & \begin{tabular}{|l|l|}
14.643 \\
\end{tabular} & 0.003 & 983.80 & \begin{tabular}{|l|l|}
16.3967 \\
\end{tabular} & 50.1 & 11.2 & & & 0.013 & 0.011 & 0.000 & & \\
\hline & & 12:18:10 AM & 28.495 & 25.938 & 29.069 & 26.306 & 25.372 & 46.064 & 51.445 & 13.756 & $\begin{array}{r}33.142 \\
\end{array}$ & $\begin{array}{l}-0.902 \\
-0.702 \\
-10\end{array}$ & & 50.34 & 0.087 & 14.643 & 0.00 & 984.80 & 16.4133 & 50.3 & & & & & & & & \\
\hline & 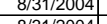 & & & & & 26.271 & 25.422 & & 51.396] & 13.741 & 33.11 & - -0.195 & & 48.986 & & 14.643 & & 985.80 & \begin{tabular}{|l}
16.43 \\
\end{tabular} & 50.3 & 11.2 & 列.6 & & & & & & \\
\hline & $8 / 31 / 2004$ & $12: 20: 10 \mathrm{AM}$ & 28.515 & & & 26.261 & 25.412 & 45.85 & & & 32.964 & -0.81 & & 50.694 & 088 & 14.643 & & 86.80 & \begin{tabular}{|l|l|} 
& 16.4467 \\
\end{tabular} & 50.3 & 12.1 & & & & & & 0.285 & \\
\hline & $8 / 31 / 2$ & 12:22:10 AM & 28.535 & & 29.114 & 26.266 & 25.36 & 46.599 & 51.968 & $\begin{array}{l}13.904 \\
13.714\end{array}$ & 33.616 & -0.876 & & 49.68 & |.091 & 14.643 & & & 16.4633 & 50.8 & & & & 01 & & & 0.290 & \\
\hline 1001 & $31 / 2004$ & $\begin{array}{ll}12.2 .23 \\
12.20 \mathrm{AM}\end{array}$ & $\frac{2.0535}{28555}$ & $\begin{array}{l}25.350 \\
25.53\end{array}$ & 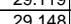 & $\frac{20}{26}$ & $\frac{23.051}{25.402}$ & $\begin{array}{r}45.154 \\
45.844\end{array}$ & 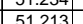 & 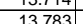 & 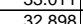 & $\begin{array}{l}-0.149 \\
-0.876\end{array}$ & & & & 14643 & & & & 501 & & 394 & & & & & & \\
\hline & $8 / 31 / 2004$ & $12: 24: 10 \mathrm{AM}$ & 28.57 & 25.948 & 29.143 & \begin{tabular}{|l|}
20.286 \\
\end{tabular} & 25.39 & 46.0418 & 51.611 & 13.762 & 33.567 & -0.81 & & 48.621 & 0.084 & $\begin{array}{l}14.0453 \\
14.643\end{array}$ & 0.003 & 990.80 & 16.5133 & 50.5 & $\begin{array}{ll}11.2 \\
11.6\end{array}$ & 40.0 & 6757 & 0.012 & 0.01 & 0.000 & 0.268 & \\
\hline & $8 / 31 / 200$ & $12: 25: 10 \mathrm{AM}$ & 28.575 & 25.953 & 29.099 & 26.251 & 25.43 & 46.539 & 52.032 & 13.822 & $\begin{array}{l}33.547 \\
\end{array}$ & -0.807 & & 49.115 & 0.095 & 14.643 & 0.004 & 991.80 & $\begin{array}{l}16.53 \\
\end{array}$ & $\frac{50.9}{50.9}$ & 11.7 & 40.0 & 761 & 0.014 & & & & \\
\hline & 8 & 12:26:10 AM & & & 29.104 & & & & & & & & & 48.018 & & 14.643 & 0.003 & 992.80 & 16.5467 & 50.7 & & & & & & & & \\
\hline & $8 / 31 / 2004$ & 12:27:10 AM & 28.768 & 25.952 & 29.112 & 26.289 & & \begin{tabular}{|l|l|}
46.13 \\
\end{tabular} & 51.638 & 13.747 & 33.354 & -0.81 & & 50.868 & 587 & 14.643 & & 993.80 & \begin{tabular}{|l|l|}
16.5633 \\
6
\end{tabular} & 50.5 & $\frac{12.1}{12.1}$ & 39. & & $\frac{012}{012}$ & & & 0 & \\
\hline & & & & & & 26.324 & 25.43 & & & & & & & $\overline{46.4}$ & 0.08 & & & & 16.58 & & & & & & & & & \\
\hline & $8 / 31 / 2004$ & 12:29:10 AM & 28.698 & 25.967 & 29.142 & \begin{tabular}{|l|}
26.324 \\
\end{tabular} & 25.42. & 46.082 & 51.765 & 13.701 & 33.186 & -0.708 & & & & 14.643 & & 995.80 & 16.5967 & 50.6 & & & & & & & & \\
\hline & $8 / 31 / 2004$ & $12: 30: 10$ & 28.672 & 25.951 & 29.132 & \begin{tabular}{|l|l|} 
& 26.269 \\
\end{tabular} & $25.43 !$ & \begin{tabular}{|l|l|l|l|l|}
4676 \\
\end{tabular} & 51.394 & \begin{tabular}{|c|c|}
13.594 \\
\end{tabular} & 33.415 & -0.853 & & 47.205 & \begin{tabular}{|l|l|l|} 
\\
\end{tabular} & \begin{tabular}{l|l}
14.643 \\
\end{tabular} & & 996.80 & \begin{tabular}{|l|l|l|l|l|l|}
1613 \\
\end{tabular} & 50.3 & 11.2 & 39 . & $.740 \mathrm{Cl}$ & & & & & \\
\hline & $8 / 31 / 2004$ & $12: 31: 10 \mathrm{AM}$ & 28.658 & 25.967 & 29.152 & \begin{tabular}{|l}
26.279 \\
\end{tabular} & 25.426 & 46.339 & 51.64 & 13.694 & 33.557 & -0.876 & & 48.285 & & $\begin{array}{l}14.643 \\
\end{array}$ & & 997.80 & 5.63 & 50.5 & 11.5 & 39.9 & 2.754 & .012 & 0.01 & & & 0.23 \\
\hline & & & 28.643 & $\begin{array}{l}25.971 \\
2.097\end{array}$ & & \begin{tabular}{|l|l|}
26.334 \\
\end{tabular} & & 46.028 & \begin{tabular}{|l|l|l|}
51.42 \\
50.7
\end{tabular} & & & $\begin{array}{l}-0.821 \\
-0.050\end{array}$ & & 48.6 & 089 & 14.643 & & & 467 & 50.3 & & & & & & & & \\
\hline & $8 / 31 / 2$ & & & 25.967 & 29.172 & & 25.41 & 45.686 & & & 33.163 & -0.859 & & 48.442 & & 14.643 & & & 16.6 & 49.9 & 20 & & & & & & 0.278 & \\
\hline & $8 / 31 / 2 / 2$ & $12: 34: 10 \mathrm{AM}$ & 28.633 & 25.972 & 29.202 & $26.3 / 4$ & & & 51.1924 & 13.646 & 33.04 & -0.801 & & $47.5 \mathrm{c}$ & & & & 0.80 & & & 11.3 & 39 . & & & & & & \\
\hline & $8 / 31 / 2004$ & $\begin{array}{ll}12: 35: 10 \mathrm{AM} \\
123.36\end{array}$ & $\frac{28.628}{28633}$ & 25.972 & $\frac{29.212}{29217}$ & $\frac{26.344}{26.339}$ & $\begin{array}{l}\frac{25.35}{2539} \\
25\end{array}$ & $\begin{array}{l}46.182 \\
46.171\end{array}$ & 51.524 & $\begin{array}{r}13.659 \\
1377\end{array}$ & $\begin{array}{l}33.397 \\
33.173\end{array}$ & $\begin{aligned}-0.813 \\
-0.813\end{aligned}$ & & $\frac{48.0}{493}$ & .084 & 14.643 & & & $\begin{array}{l}16.0601 \\
167132\end{array}$ & 50.4 & 118 & 39 & & & & & & \\
\hline 1015 & $8 / 31 / 2004$ & $12.37 .10 \mathrm{AM}$ & 28.050 & 25.976 & 2925 & \begin{tabular}{|l|l|}
26358 \\
\end{tabular} & & 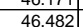 & 51.762 & 13.683 & 33764 & $\begin{array}{l}-.0 .019 \\
-0891\end{array}$ & & $\begin{array}{l}4.012 \\
47.451\end{array}$ & 0.094 & 14643 & & 0380 & & 506 & $\frac{1.4}{113}$ & & & & & & & \\
\hline & $8 / 31 / 2004$ & $12: 38: 10 \mathrm{AM}$ & $\frac{20.011}{28.613}$ & 25.992 & \begin{tabular}{|l|}
29.257 \\
\end{tabular} & \begin{tabular}{|l|}
20.000 \\
26.339
\end{tabular} & 25.41 & 4 & 51.271 & 13.465 & 33.167 & -0.694 & & $\frac{4.41}{51.22}$ & 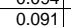 & $\begin{array}{l}14.045 \\
14.643\end{array}$ & & & 16.467 & $\frac{3.01}{501}$ & $\frac{11.0}{12.2}$ & & & & & & 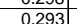 & \\
\hline & $8 / 31 / 2$ & & 28.619 & 25.983 & & 26.38 & & & 51.605 & 13.769 & 33.095 & -0.538 & & & 0.086 & & & & 16.7 & $\frac{0.5}{50.5}$ & 11.4 & & & & & & & \\
\hline & 8 & 12:40:11 & 28.613 & 25.977 & \begin{tabular}{|l|}
29.247 \\
\end{tabular} & & 25 & & 51.105 & & 32.7 & -0.645 & & 45.92 & & 14.6 & & & & 50.0 & 100 & & & & & & 0.289 & \\
\hline & & $12: 41: 10 \mathrm{AM}$ & 28.619 & 25.988 & \begin{tabular}{|l|}
29.267 \\
\end{tabular} & 26.36 & & 45.8 & 51.483 & 13.726 & 33.073 & -0.535 & & 48.325 & 0.087 & & & & 16.7967 & 50.4 & $\frac{11.5}{11.5}$ & & & 01 & 0.0 & & 0.280 & \\
\hline & $8 / 31 / 2004$ & & & & & & & & & & 33.288 & & & & & & & & & 51.0 & & & & & & & & \\
\hline & $8 / 31 / 2$ & 12:43:10 AM & 28.6 & 25.983 & 29.273 & \begin{tabular}{|l|}
26.396 \\
\end{tabular} & 25.51 & & 51.414 & \begin{tabular}{|l|l|}
13.677 \\
\end{tabular} & 32.993 & -0.471 & & 48.556 & & & & & & 50.3 & 11.6 & 39.4 & & & & & \begin{tabular}{|c|}
0.278 \\
\end{tabular} & 024 \\
\hline & $8 / 31 / 2004$ & $12: 35: 24$ & 23.992 & 23.689 & \begin{tabular}{|l|l|}
23.066 \\
\end{tabular} & 24.649 & 23.8 & -0.129 & 2.051 & 0.641 & & $\mid-1.209$ & & -0. & 0.003 & \begin{tabular}{l|l}
14.643 \\
\end{tabular} & & & 28.7 & 0.9 & 0.0 & -0. & & .000 & 0.000 & & $\mid-8.249$ & \\
\hline & $8 / 31 / 2004$ & $12: 36: 24$ & 24.007 & & \begin{tabular}{|l|l|}
25.821 \\
\end{tabular} & 24.598 & & & 65.289 & & & -1.212 & & & & & & & & 64.2 & 12. & & & & & & & \\
\hline & $8 / 31 / 2004$ & 12:37: & 23.967 & 23.694 & \begin{tabular}{|l|}
26.462 \\
\end{tabular} & \begin{tabular}{|l|} 
\\
\end{tabular} 4.82 & $23.89=$ & 60.363 & & 18.083 & 43.153 & -0.532 & & 54.8 & & & & & & 65.0 & 13.1 & 51 & & & & 0.000 & 0.215 & \\
\hline & & & 24.389 & & $26.95 \%$ & & & & & & & & & & & & & & & - & $=0$ & & & & & & 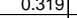 & \\
\hline & & & & & 27.308 & & & & & & & & & & & & & & & & & & & & & & & \\
\hline & 8 & $\begin{array}{l}\frac{1}{12.24} \\
12.4\end{array}$ & & $\frac{2.3124}{2373}$ & 27.4451 & & & $\begin{array}{r}50.73 \\
481616\end{array}$ & 年6.030 & $\frac{15.615}{15.65}$ & $\begin{array}{l}36.083 \\
33.48\end{array}$ & $\begin{array}{l}-1.088 \\
-1.1212\end{array}$ & & & & $\frac{14 .}{14 .}$ & & & & $\frac{54.9}{51 .}$ & 126 & & & & 01 & & $\mid \begin{array}{l}0.314 \\
0.354\end{array}$ & \\
\hline & $8 / 31 / 200$ & & & 23762 & 27,393 & & & & 52009 & 15517 & 33.475 & -121 & & 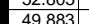 & & & & & & & 10 & & & & & & & \\
\hline 1030 & $8 / 31 / 200$ & & & $\frac{23.572}{23772}$ & 27.323 & & & & $\mid$ & 15.443 & & & & $\frac{4.00}{53.9}$ & & & & & & 0.0 & 129 & & & & & & & \\
\hline & $8 / 31 / 2$ & & 25.607 & $\frac{23.802}{23.80}$ & \begin{tabular}{|l|l|}
27.259 \\
\end{tabular} & 25.4 & & & 52.502 & 15.528 & 33.247 & $\frac{-1.2 .1}{-1.2}$ & & 50. & & & & & & $\frac{51.4}{51.4}$ & 12.0 & & & & & & $\frac{0.308}{0.308}$ & \\
\hline & & & & & & & & & & & & & & & & & & & & & & & & & & & & \\
\hline & & & & 23.8 & 26.86 & 25. & & & 52.40 & $\frac{14.7}{14.7}$ & & -1.1. & & & & & & & & & & & & & & & & \\
\hline & & & & & 26.502 & & & \begin{tabular}{|l|l|l|}
48.069 \\
\end{tabular} & \begin{tabular}{|c|}
52.38 \\
\end{tabular} & & 33.99 & & & & & & & & & & & & & & & & & \\
\hline & $8 / 31 / /$ & & & 23 & 26.208 & 25.522 & & \begin{tabular}{|l|l|}
47.24 \\
\end{tabular} & \begin{tabular}{|l|l|l|}
51.688 \\
\end{tabular} & 15.803 & 32.387 & $\begin{array}{l}-1.209 \\
\end{array}$ & & & & 14.6 & & & & 50.6 & 11.9 & & $2.7 \quad$ & & & & & \\
\hline & $8 / 31 / 206$ & & & 23.906 & \begin{tabular}{|l|}
25.944 \\
\end{tabular} & 25. & & $\begin{array}{l}47.381 \\
\end{array}$ & 51.69 & 15.3 & 32.995 & -1.2 & & & & 14.6 & & & & & 11 & & & & & & 0.309 & \\
\hline & $8 / 31 / /$ & & & & $25.7 \mathrm{r}-\mathrm{x}$ & & & & & 15.467 & & & & & & 14. & & & & 50 & 11.9 & & & & & & & \\
\hline & $8 / 31 / 2$ & & 25.986 & 23.973 & 25.54 & 25. & & 47. & 52.229 & 15.211 & $33.6 \mathrm{~F}$ & -1.212 & & 49.3 & & & & & & 51.1 & 11.8 & & & & $0.0>-1$ & & 0.315 & \\
\hline & & & & & & & & & & & & & & & & & & & & & & & & & & & & \\
\hline & $\begin{array}{lll}8 / 31120 \\
0.12120\end{array}$ & & & & 25.151 & & & 41. & 51.4 & 15.4 & & & & 50. & & & & & & 50.3 & $\frac{1}{1}$ & & & & & & & \\
\hline & 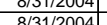 & & & 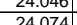 & 24.918 & 25.090 & & & 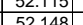 & 15.4 & 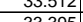 & - & & $\frac{41.5}{49}>>2$ & & & & & & & 望1.3. & & & & & & 年 & \\
\hline & $8 / 31 / 200$ & & & 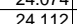 & $\frac{24.01}{24.710}$ & \begin{tabular}{|c|c|c|}
26.548 \\
\end{tabular} & & (4t.7505t & $\frac{3.14}{5182}$ & 15.321 & 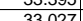 & $-\frac{1.14}{1212}$ & & $\frac{43.6}{516}-3$ & & $\begin{array}{l}1.0 \\
146\end{array}$ & & & & & $12^{2}+3$ & & & & & & 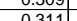 & \\
\hline & $8 / 31 / 2$ & & & 24.124 & 24.555 & & & & 5196 & & & -12 & & 501 & & 146 & & & & $508-2>$ & . & & & & & & & \\
\hline & & & & & 24.402 & & & 47 & \begin{tabular}{|l|r|}
52.08 \\
52
\end{tabular} & 15.493 & 33.089 & $\frac{-1212}{-1212}$ & & & & $14.6 \mathrm{C}-\mathrm{C}$ & & & & 510 & 124 & & & & & & & \\
\hline & & & & 24.148 & 24.264 & & & & & & & & & & & & & & & & & & & & & & & \\
\hline & & & & & & & & & & & & & & & & & & & & & & & & & & & & \\
\hline & $8 / 31 / 200$ & & & 24.176 & 24.001 & 26.118 & 24.6 & 47.4. & 51.71 & 15.675 & 32.734 & -1.209 & & 50. & & 14.4 & & & & 50.6 & $\frac{12.1}{12}>$ & & & & & & 308 & \\
\hline & 311 & & & & 856 & & & 47.4 & 1646 & 15.614 & & -1.212 & & $50.4 \varepsilon$ & & 14.643 & & & $\overline{06}$ & 50.5 & 120 & & & & & & $306 \mathrm{G}$ & $\overline{02}+2-3$ \\
\hline 1050 & 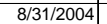 & 1:03:24 PM & 25.611 & 24.182 & \begin{tabular}{|l|l|}
23.731 \\
\end{tabular} & \begin{tabular}{|l|l|}
25.993 \\
\end{tabular} & & 47.745 & $\mid$ & 15.662 & 33.134 & \begin{tabular}{|l|}
$\mid-1.212$ \\
\end{tabular} & & 51.18 & 0.083 & $\begin{array}{ll}14.643 \\
\end{array}$ & & 50.03 & 329.1672 & 50.5 & 12.2 & & 2.788 & \begin{tabular}{|l|l|}
0.012 \\
\end{tabular} & 0.012 & 0.000 & 0.305 & 0.2 \\
\hline
\end{tabular}


WSRC-TR-2005-00105, REVISION 0

SRNL-RPP-2005-00012, REVISION 0

RUN \# 4.03A AND B; FIRST AND SECOND HALF OF SLURRY DEWATERING - CONT.

\begin{tabular}{|c|c|c|c|c|c|c|c|c|c|c|c|c|c|c|c|c|c|c|c|c|c|c|c|c|c|c|c|c|}
\hline & A & $\mathrm{B}$ & $\mathrm{D}$ & $E$ & $F$ & $G$ & $\mathrm{H}$ & $\mathrm{J}$ & K & $\mathrm{L}$ & $M$ & $\mathrm{~N}$ & 0 & Q & $\mathrm{R}$ & \begin{tabular}{l|l} 
\\
\end{tabular} & \begin{tabular}{l|l}
$T$ \\
\end{tabular} & $\mathrm{~V}$ & w & $x$ & $Y$ & $z$ & $\mathrm{AA}$ & $A B$ & $A C$ & AD & AE & \\
\hline & $8 / 31 / 2004$ & 1:04:24 PM & 25.561 & 24.198 & 23.606 & 25.949 & 24.445 & $\begin{array}{l}47.857 \\
\end{array}$ & 52.173 & $\begin{array}{l}15.451 \\
\end{array}$ & \begin{tabular}{|l|l|}
33.393 \\
\end{tabular} & -1.218 & $\begin{array}{l}-7.692 \\
\end{array}$ & 50.473 & 0.082 & 14.643 & 0.003 & 1751.03 & 29.1839 & 51.0 & 12.6 & 40.6 & 2.801 & 0.012 & 0.012 & 0.000 & 0.301 & \\
\hline & $8 / 31 / 2004$ & 1:05:24 PM & 25.51 & 24.201 & 23.494 & \begin{tabular}{|l|}
25.932 \\
\end{tabular} & 24.389 & 47.994 & \begin{tabular}{|l|l|}
452.243 \\
\end{tabular} & 15.352 & 33.612 & $\begin{array}{l}-1.215 \\
\end{array}$ & & 50.254 & 0.082 & $\begin{array}{l}14.643 \\
\end{array}$ & 0.003 & & & \begin{tabular}{|l|}
51.1 \\
\end{tabular} & & & & & & & & \\
\hline & $8 / 31 / 2004$ & 1:06:24 PM & 25.459 & 24.21 & 23.393 & 25.911 & 24.493 & 47.42 & \begin{tabular}{|l|l|}
51.387 \\
\end{tabular} & 15.626 & \begin{tabular}{|l|}
32.826 \\
2.82
\end{tabular} & \begin{tabular}{|c|}
-1.212 \\
-1.212
\end{tabular} & $\begin{array}{l}-7.69 \\
.709\end{array}$ & 49.643 & $\begin{array}{l}0.082 \\
\end{array}$ & 14.643 & 0.003 & $\begin{array}{l}1753.03 \\
77.03\end{array}$ & 29.2172 & \begin{tabular}{|l|}
50.3 \\
5.3
\end{tabular} & 11.8 & 40.1 & $\frac{766}{7.76}$ & 0.012 & 0.012 & 0.000 & $\begin{array}{l}0.306 \\
\end{array}$ & \\
\hline 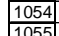 & $8 / 31 / 2004$ & 1:07:24 PM & 25.403 & 24.219 & \begin{tabular}{|l|}
23.281 \\
\end{tabular} & 25.915 & 24.447 & $\begin{array}{l}47.264 \\
\end{array}$ & $\begin{array}{l}451.313 \\
\end{array}$ & 15.482 & $\begin{array}{l}32.793 \\
\end{array}$ & -1.212 & $\begin{array}{l}-7.70 \\
-7.71\end{array}$ & 49.324 & $\begin{array}{l}0.082 \\
\end{array}$ & 14.643 & 0.003 & 1754.03 & \begin{tabular}{|l|l|}
29.2339 \\
\end{tabular} & 50.2 & 11.7 & 40.0 & $\begin{array}{l}760 \\
7775\end{array}$ & 0.012 & 0.012 & 0.000 & 0.308 & \\
\hline & $8 / 31 / 2004$ & $\begin{array}{ll}1: 08: 24 \mathrm{PM} \\
1 \cdot 09 \cdot 24 \mathrm{PM}\end{array}$ & $\frac{25.351}{2531}$ & $\frac{24.228}{24.247}$ & & \begin{tabular}{|l|}
25.914 \\
\end{tabular} & $\begin{array}{l}24.446 \\
24.43 \\
\end{array}$ & $\begin{array}{l}47.492 \\
47663\end{array}$ & & $\begin{array}{l}15.502 \\
15535\end{array}$ & \begin{tabular}{|l|}
33.003 \\
3301
\end{tabular} & \begin{tabular}{|c|}
-1.209 \\
1212
\end{tabular} & $\begin{array}{r}-7.71 \\
77\end{array}$ & $\begin{array}{l}53.429 \\
49.58\end{array}$ & \begin{tabular}{|l|l|}
0.081 \\
0083
\end{tabular} & \begin{tabular}{|l|l|l|}
14.643 \\
14634 \\
\end{tabular} & & $\begin{array}{l}1755.03 \\
175502\end{array}$ & 329.2506 & \begin{tabular}{|l|}
50.4 \\
\end{tabular} & \begin{tabular}{|l|}
12.7 \\
1.0 \\
\end{tabular} & 740.2 & & 0.012 & 0.012 & & & \\
\hline 1057 & $8 / 31 / 2004$ & $\begin{array}{l}1: 0.924 \mathrm{PM} \\
1: 10: 24 \mathrm{PM}\end{array}$ & $\begin{array}{r}25.31 \\
25.253\end{array}$ & $24.247 \mid$ & $\frac{23.089}{22.996}$ & \begin{tabular}{|l|}
25.928 \\
25.906 \\
\end{tabular} & $\begin{array}{r}24.43 \\
24.453 \\
\end{array}$ & $\begin{array}{r}47.663 \\
47.206\end{array}$ & \begin{tabular}{|l|l|}
51.7295 \\
51.294
\end{tabular} & $\begin{array}{l}\frac{15.535}{16.111} \\
\end{array}$ & \begin{tabular}{|l|}
33.001 \\
32.026
\end{tabular} & 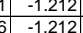 & $\begin{array}{r}-7.72 \\
-7.741 \\
\end{array}$ & $\begin{array}{l}4.5 .58 \\
51.598\end{array}$ & $\begin{array}{l}0.083 \\
0.078\end{array}$ & $\begin{array}{r}\mid 14.643 \\
14.643\end{array}$ & $\begin{array}{l}0.003 \\
0.003 \\
\end{array}$ & $\frac{1756.03}{1757.03}$ & \begin{tabular}{|l|}
29.2672 \\
329.2832
\end{tabular} & \begin{tabular}{|l|}
50.7 \\
50.2
\end{tabular} & $\begin{array}{l}11.8 \\
12.3\end{array}$ & $\begin{array}{l}40.4 \\
39.6 \\
\end{array}$ & 2.7731 & .012 & \begin{tabular}{|c|}
0.013 \\
0.012
\end{tabular} & $\begin{array}{c}0.000 \\
0.000\end{array}$ & 0.311 & 0.26 \\
\hline 1058 & $8 / 31 / 2004$ & 1:11:24 PM & 25.201 & 24.258 & 22.909 & \begin{tabular}{|l|} 
\\
\end{tabular} & 24.516 & & 5 & 16.011 & 32.055 & \begin{tabular}{|l|l|}
5 & -1.209 \\
\end{tabular} & $\begin{array}{l}-7.697 \\
\end{array}$ & 51.009 & 0.079 & 14.643 & $\begin{array}{l}0.003 \\
0.003\end{array}$ & 1758.03 & $\begin{array}{l}29.2859 \\
29.3006\end{array}$ & $\begin{array}{l}30.2 \\
50.0\end{array}$ & $\begin{array}{l}12.3 \\
12.1\end{array}$ & $\begin{array}{l}39.6 \\
39.6 \\
\end{array}$ & $\frac{2.151}{2.727}$ & 0.011 & 0.012 & $\begin{array}{l}0.000 \\
0.000\end{array}$ & $\begin{array}{l}.290 \\
0.303\end{array}$ & $\begin{array}{l}0.25 \\
0.26\end{array}$ \\
\hline & $8 / 31 / 2004$ & 1:12:24 PM & & 24.256 & \begin{tabular}{|l|}
22.817 \\
\end{tabular} & \begin{tabular}{|l|}
25.947 \\
\end{tabular} & 24.544 & 47.289 & 51.512 & 16.053 & 32.182 & \begin{tabular}{|l|l|}
2 & -1.212 \\
\end{tabular} & $\begin{array}{r}-7.715 \\
\end{array}$ & 51.134 & 0.079 & \begin{tabular}{|l|l|}
14.643 \\
\end{tabular} & 0.003 & & 29.3172 & 50.4 & & & & & & & & \\
\hline 1060 & $8 / 31 / 2004$ & 1:13:24 PM & & & \begin{tabular}{|l|l|}
22.846 \\
\end{tabular} & & & 47.03 & & 15.96 & & $\begin{array}{l}-1.212 \\
\end{array}$ & & & 1113 & \begin{tabular}{|l|l|}
14.643 \\
\end{tabular} & & & 29.3339 & 50.2 & & & & & & 0.000 & 0.435 & \\
\hline 1061 & $8 / 31 / 2004$ & 1:14:24 PM & 25.057 & \begin{tabular}{l|l|}
24.284 \\
2.202
\end{tabular} & \begin{tabular}{|l|}
22.774 \\
2.692
\end{tabular} & $\begin{array}{l}26.04 \\
\end{array}$ & $\begin{array}{l}24.687 \\
2711\end{array}$ & $\begin{array}{l}46.823 \\
\end{array}$ & $\begin{array}{l}50.929 \\
\end{array}$ & 15.829 & \begin{tabular}{|l|}
32.01 \\
\end{tabular} & -1.212 & $\begin{array}{r}-7.692 \\
\end{array}$ & 51.253 & & $\begin{array}{l}14.643 \\
\end{array}$ & 0.003 & 1761.03 & 29.3506 & 49.8 & 12.2 & 39.4 & 2.718 & 0.011 & 0.012 & 0.000 & 0.302 & \\
\hline 106 & $8 / 31 / 2004$ & $\begin{array}{ll}1: 15: 24 \mathrm{PM} \\
1 \cdot 16.24 \mathrm{PM}\end{array}$ & $\begin{array}{l}25.001 \\
24064\end{array}$ & $\begin{array}{l}24.293 \\
24.306\end{array}$ & \begin{tabular}{|r|}
22.682 \\
2259
\end{tabular} & \begin{tabular}{|l|l|} 
& 26.049 \\
\end{tabular} & $\begin{array}{r}24.741 \\
22.769\end{array}$ & $\begin{array}{r}47.02 \\
47285 \\
\end{array}$ & $\begin{array}{l}51.296 \\
51.45\end{array}$ & $\begin{array}{r}15.808 \\
158\end{array}$ & \begin{tabular}{|l|}
32.147 \\
32376
\end{tabular} & & $\begin{array}{r}-7.69 \\
-768\end{array}$ & $\begin{array}{r}50.98 \\
50.82\end{array}$ & 078 & $\begin{array}{l}14.643 \\
14643 \\
\end{array}$ & & $\begin{array}{l}1762.03 \\
170203\end{array}$ & $\begin{array}{l}29.3672 \\
29.3839\end{array}$ & $\begin{array}{l}50.2 \\
50.3\end{array}$ & 12.1 & $\begin{array}{l}39.6 \\
39.8\end{array}$ & .729 & & & 0.000 & 0.301 & 0.26 \\
\hline 1064 & 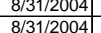 & $\begin{array}{l}\text { 1:16:24 PM } \\
1: 17: 24 \mathrm{P}\end{array}$ & $\begin{array}{l}24.964 \\
24.918\end{array}$ & $\begin{array}{r}24.306 \\
24.32\end{array}$ & $\begin{array}{r}22.59 \\
22.504\end{array}$ & \begin{tabular}{|l|}
26.102 \\
26.171 \\
\end{tabular} & $\begin{array}{r}24.769 \\
24.833 \\
\end{array}$ & $\begin{array}{r}47.285 \\
47.515 \\
\end{array}$ & \begin{tabular}{|}
$\mid 51.435$ \\
51.746
\end{tabular} & $\begin{array}{r}15.86 \\
15.788\end{array}$ & \begin{tabular}{|c|}
32.367 \\
32.752
\end{tabular} & & $\begin{array}{r}-7.689 \\
-7.694 \\
\end{array}$ & $\frac{50.826}{49.418}$ & $\begin{array}{l}079 \\
078 \\
\end{array}$ & $\begin{array}{r}14.643 \\
14643\end{array}$ & $\begin{array}{r}0.003 \\
0.003\end{array}$ & & $\begin{array}{r}29.3839 \\
29.4006\end{array}$ & $\begin{array}{l}50.3 \\
50.6\end{array}$ & 118 & 401 & 2.746 & .011 & $\begin{array}{l}0.012 \\
0.012\end{array}$ & 0.000 & $\begin{array}{l}0.304 \\
0.2299\end{array}$ & 0.26 \\
\hline 1065 & $8 / 31 / 2004$ & 1:18:24 PM & 24.883 & 24.345 & $\frac{22.504}{22.428}$ & $\begin{array}{l}20.111 \\
26.216 \\
\end{array}$ & $\begin{aligned} 24.033 \\
24.923\end{aligned}$ & $\begin{array}{r}47.515 \\
47.275\end{array}$ & \begin{tabular}{|l|l|}
51.440 \\
51.416
\end{tabular} & $\begin{array}{l}15.160 \\
15.823\end{array}$ & $\begin{array}{l}32.152 \\
32.379\end{array}$ & \begin{tabular}{|l|l|}
9 & -1.212 \\
\end{tabular} & & $\begin{array}{r}4.418 \\
51.86\end{array}$ & $\begin{array}{l}0.077 \\
0.077\end{array}$ & \begin{tabular}{|l|l}
14.645 \\
14.643
\end{tabular} & $\begin{array}{l}.0003 \\
0.003\end{array}$ & 1764.0 & $\begin{array}{l}29.4000 \\
29.4172\end{array}$ & $\begin{array}{l}50.0 \\
50.3\end{array}$ & $\begin{array}{l}11.8 \\
12.4\end{array}$ & $\begin{array}{l}40.1 \\
39.8 \\
\end{array}$ & $\frac{2.161}{2.746}$ & & 0.012 & & $\begin{array}{l}0.299 \\
0.298\end{array}$ & $\begin{array}{l}0.25 \\
0.25\end{array}$ \\
\hline & $8 / 31 / 2004$ & 1:19:24 PM & 24.858 & 24.361 & 22.358 & 26.256 & & 47.573 & \begin{tabular}{|l|l|}
351.619 \\
\end{tabular} & 15.712 & 32.894 & \begin{tabular}{|l|l|}
4 & -1.212 \\
\end{tabular} & & $\begin{array}{l}49.017 \\
497\end{array}$ & & \begin{tabular}{|l|}
14.643 \\
\end{tabular} & & & 29.4339 & 50.5 & & & & & & & & \\
\hline 1067 & $8 / 31 / 2004$ & 1:20:24 PM & 24.797 & 24.354 & \begin{tabular}{|l|}
22.251 \\
\end{tabular} & 26.22 & 24.957 & 47.463 & 51.696 & \begin{tabular}{|l|l|}
15.81 \\
\end{tabular} & & & & 49.67 & & $\begin{array}{l}14.643 \\
\end{array}$ & & & 29.4506 & 50.6 & 11. & 40.1 & & 0.011 & & & 0.301 & \\
\hline 1068 & $8 / 31 / 2004$ & 1:21:24 PM & 24.786 & 24.394 & & 26.269 & & & & & & & & & & & 0.004 & & & & 11. & & 2.764 & & & & 0.298 & \\
\hline & $8 / 31 / 200$ & $\begin{array}{ll}1: 22: 24 \mathrm{PM} \\
1 \cdot 23: 24 \mathrm{PM}\end{array}$ & $\begin{array}{l}24.735 \\
247044\end{array}$ & $\begin{array}{l}24.388 \\
24.401\end{array}$ & $\begin{array}{l}22.124 \\
22062\end{array}$ & $\begin{array}{l}26.223 \\
26.217\end{array}$ & & & $\begin{array}{r}51.7 \\
51901\end{array}$ & $\begin{array}{r}15.86 \\
15.791\end{array}$ & \begin{tabular}{|l|l|}
32.633 \\
32835 \\
\end{tabular} & & & $\begin{array}{r}49.48 \\
50315\end{array}$ & & $\begin{array}{l}14.643 \\
14643\end{array}$ & & & $\begin{array}{r}29.4839 \\
295006\end{array}$ & $\begin{array}{l}50.6 \\
50.8\end{array}$ & 11. & 40.1 & 764 & & & & 0.298 & 0.25 \\
\hline 1071 & $88 / 31 / 2004$ & $\begin{array}{l}1: 23: 24 \mathrm{PM} \\
1: 24: 24 \mathrm{PM}\end{array}$ & $\frac{24.704}{24.663}$ & $\begin{array}{r}24.401 \\
24.415\end{array}$ & $\frac{22.062}{22}$ & $\frac{26.217}{26.2}$ & & $\begin{array}{r}47.538 \\
47.575 \\
\end{array}$ & $\begin{array}{r}351.901 \\
5 \\
5\end{array}$ & $\frac{15.791}{15.831}$ & \begin{tabular}{|l|}
32.2835 \\
32.718
\end{tabular} & 2.215 & -7.7 & $\frac{50.315}{50.16}$ & & $\begin{array}{l}\frac{14.643}{14.643} \\
\end{array}$ & $\begin{array}{r}0.003 \\
0.003\end{array}$ & & 9.5006 & $\begin{array}{l}50.8 \\
50.6\end{array}$ & $\frac{12.0}{119}$ & $\frac{4.2}{40.1}$ & 1.774 & .011 & $\frac{0.012}{0.012}$ & 0.000 & $\begin{array}{l}0.290 \\
0.299\end{array}$ & 0.25 \\
\hline & $8 / 31 / 2004$ & 1:25:24 PM & 24.605 & 24.418 & 21.908 & 26.139 & 24.896 & 47.206 & \begin{tabular}{|c|}
51.441 \\
5
\end{tabular} & 15.608 & 32.566 & \begin{tabular}{|l|l|}
6 & -1.215 \\
\end{tabular} & -7.718 & 51.447 & 0.076 & $\begin{array}{l}14.045 \\
14.643\end{array}$ & $\begin{array}{l}0.003 \\
0.003\end{array}$ & $\frac{171.0}{1772.0}$ & \begin{tabular}{|l|}
29.5172 \\
29339
\end{tabular} & $\begin{array}{l}50.0 \\
50.3\end{array}$ & $\begin{array}{l}11.9 \\
12.3\end{array}$ & $\frac{40.1}{39.9}$ & $\frac{2.108}{2.750}$ & 0.011 & & 0.000 & $\begin{array}{l}0.299 \\
0.298\end{array}$ & \\
\hline & $8 / 31 / 2004$ & 1:26:24 PM & 24.584 & 24.442 & 21.861 & 26.152 & 24.904 & 47.716 & 51.733 & 15.761 & 32.978 & \begin{tabular}{|l|l|}
8 & -1.212 \\
\end{tabular} & & 51.064 & 0.077 & 14.643 & & & 29.5506 & 50.6 & 12.2 & 40.3 & 2.782 & 0.011 & & 0.000 & 0.299 & \\
\hline & $8 / 31 / 2004$ & 1:27:24 PM & 24.535 & 24.453 & 21.792 & 26.143 & & 47.389 & 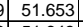 & 15.714 & & \begin{tabular}{|l|l|}
3 & -1.212 \\
\end{tabular} & & 49.184 & & \begin{tabular}{|l|l|}
14.643 \\
\end{tabular} & & & 29.5672 & 50.5 & 11.7 & 40.0 & 2.758 & & & 0.000 & 0.298 & \\
\hline 075 & $8 / 31 / 2004$ & 1:28:24 PM & 24.507 & 24.47 & & 26.161 & 24.798 & & 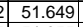 & $\begin{array}{l}15.676 \\
\end{array}$ & \begin{tabular}{|l|l|}
32.734 \\
\end{tabular} & & $\begin{array}{c}-7.65 \\
\end{array}$ & 48.888 & & \begin{tabular}{|l|l|}
14.643 \\
\end{tabular} & & & 29.5839 & 50.5 & 11.6 & & 2.765 & & & 0.000 & 0.298 & \\
\hline$\frac{1076}{1077}$ & $\begin{array}{l}8 / 31 / 2004 \\
8 / 31 / 204\end{array}$ & $\begin{array}{l}1: 29: 24 \mathrm{PM} \\
1: 20: 24 \mathrm{PM}\end{array}$ & $\begin{array}{l}24.459 \\
24.421\end{array}$ & $\begin{array}{l}24.462 \\
24.479\end{array}$ & \begin{tabular}{|l|}
21.676 \\
21.617 \\
\end{tabular} & $\begin{array}{r}\frac{26.138}{26.07} \\
\end{array}$ & $\frac{24.845}{22.822}$ & $\begin{array}{r}47.775 \\
4.569\end{array}$ & \begin{tabular}{|l|l|}
51.955 \\
9
\end{tabular} & \begin{tabular}{|l|}
15.778 \\
15.711
\end{tabular} & \begin{tabular}{|l|}
32.978 \\
32.789
\end{tabular} & \begin{tabular}{|c|c|}
8 & -1.212 \\
9 & -1.215 \\
\end{tabular} & $\begin{array}{r}-7.70 \\
-7 \\
\end{array}$ & $\begin{array}{r}50.43 \\
49.604\end{array}$ & $\begin{array}{l}0.076 \\
0.077\end{array}$ & $\begin{array}{l}\frac{14.643}{14.643} \\
\end{array}$ & $\begin{array}{l}0.003 \\
0.003\end{array}$ & $\frac{1776.0}{1777.0}$ & $\begin{array}{l}29.6006 \\
29.6172\end{array}$ & $\frac{50.8}{50.6}$ & $\frac{12.0}{11 .}$ & & . & 0.011 & 0.012 & 0.000 & $\begin{array}{l}0.296 \\
0.302 \\
\end{array}$ & 0.25 \\
\hline 1078 & $8 / 3 / 31 / 2004$ & $\begin{array}{l}1.03: 24 \mathrm{PM} \\
1: 31: 24 \mathrm{PM}\end{array}$ & $\frac{24.421}{24.382}$ & $\begin{array}{l}24.479 \\
24.486\end{array}$ & \begin{tabular}{|l|}
21.611 \\
21.574 \\
\end{tabular} & 26.046 & $\frac{24.8}{24.8}$ & $\begin{array}{r}4.569 \\
47.648\end{array}$ & \begin{tabular}{|l|l|}
51.877 \\
\end{tabular} & 15.751 & \begin{tabular}{|l}
32.289 \\
32.806
\end{tabular} & $\begin{array}{l} \\
6 \\
6\end{array}$ & & & & $\begin{array}{l}\frac{14.643}{14.643} \\
\end{array}$ & & & $\frac{29.61 / 2}{29.6339}$ & & & & 77 & & & & & $\begin{array}{ll}0.26 \\
0.25 \\
\end{array}$ \\
\hline 1079 & $8 / 31 / 2004$ & 1:32:24 PM & 24.334 & 24.487 & \begin{tabular}{|l|}
21.504 \\
\end{tabular} & \begin{tabular}{|l|} 
\\
\end{tabular} & 24 & 47.634 & \begin{tabular}{l|l}
47 & 51.85
\end{tabular} & 15.688 & $\begin{array}{l}32.8002 \\
32.03\end{array}$ & \begin{tabular}{|l|l|}
2 & -1.212 \\
\end{tabular} & -7. & 48.408 & & 14.643 & 0.003 & & 29.6506 & 50.7 & 11.5 & 40. & 2.776 & 0.01 & & & 0.296 & $\begin{array}{l}0.25 \\
0.25 \\
\end{array}$ \\
\hline & $8 / 31 / 2$ & 1:33:24 PM & 24.295 & 24.504 & \begin{tabular}{|l|l|}
21.526 \\
\end{tabular} & & 24. & & \begin{tabular}{|l|l|}
151.595 \\
\end{tabular} & $\begin{array}{l}15.7656 \\
\end{array}$ & & $\begin{array}{l}-1.209 \\
-1.209\end{array}$ & & & & \begin{tabular}{|l|}
14.643 \\
\end{tabular} & & & 29.6672 & 50.5 & 11.5 & & & & & & & \\
\hline & $8 / 31 / 2004$ & 1:34:24 PM & & 24.505 & \begin{tabular}{|l|l|}
21.598 \\
\end{tabular} & & 24.752 & 47.412 & \begin{tabular}{|l|l|} 
& 51.713 \\
\end{tabular} & $\begin{array}{l}5.699 \\
\end{array}$ & \begin{tabular}{|l|l|}
32.679 \\
27
\end{tabular} & -1.209 & & 48.406 & & \begin{tabular}{|l|l|}
14.643 \\
\end{tabular} & & & & 50.6 & 11.5 & & 2.761 & & & & 0.295 & \\
\hline & $8 / 31 / 2004$ & 1:35:24 PM & \begin{tabular}{|l|l|}
24.213 \\
\end{tabular} & 24.516 & \begin{tabular}{|l|}
21.534 \\
\end{tabular} & & $\begin{array}{l}24.774 \\
\end{array}$ & & \begin{tabular}{|l|}
51.829 \\
\end{tabular} & $\begin{array}{l}15.745 \\
\end{array}$ & $\begin{array}{l}32.876 \\
\end{array}$ & & & $\begin{array}{l}48.542 \\
532\end{array}$ & & $\begin{array}{l}14.643 \\
\end{array}$ & 203 & & \begin{tabular}{|l|l|}
29.7006 \\
\end{tabular} & 50.7 & 11.6 & 40.3 & 2.775 & & 0.012 & 00 & 0.298 & \\
\hline$\frac{108}{108}$ & $\begin{array}{l}8 / 31 / 2004 \\
8 / 31 / 204\end{array}$ & $\begin{array}{l}1: 36: 24 \mathrm{PM} \\
1 \cdot 17 \cdot 24 \mathrm{PM}\end{array}$ & \begin{tabular}{|l|l|}
24.169 \\
24.125
\end{tabular} & $\begin{array}{l}24.508 \\
24.523\end{array}$ & \begin{tabular}{|l|}
21.455 \\
21.395 \\
\end{tabular} & \begin{tabular}{|l|}
26.018 \\
25.999 \\
\end{tabular} & $\begin{array}{r}24.82 \\
24.866 \\
\end{array}$ & $\begin{array}{r}47.586 \\
47.694 \\
\end{array}$ & \begin{tabular}{|l|l|}
51.939 \\
4 & 51.945 \\
\end{tabular} & $\begin{array}{l}15.696 \\
15.804\end{array}$ & $\begin{array}{r}32.8 \\
32.802\end{array}$ & \begin{tabular}{|l|l|} 
& -1.212 \\
2 & -1.215 \\
\end{tabular} & $\begin{array}{l}-7.70 \\
-7.71 \\
\end{array}$ & $\begin{array}{l}52.386 \\
48.219\end{array}$ & 0.076 & \begin{tabular}{l|l|}
14.643 \\
14.643
\end{tabular} & 0.6 & $\begin{array}{l}1783.0 \\
1784.0\end{array}$ & \begin{tabular}{|l|}
29.7172 \\
29.7339 \\
\end{tabular} & $\begin{array}{l}50.8 \\
50.8\end{array}$ & $\begin{array}{l}12.5 \\
11.5\end{array}$ & $\frac{40.2}{40.2}$ & 2.775 & 0.011 & 0.012 & 500 & $\mid 0.299$ & 0.25 \\
\hline$\frac{1085}{1085}$ & $8 / 3 / 31 / 2004$ & $\begin{array}{l}1.3: 24 \mathrm{PM} \\
1: 38: 24 \mathrm{PM}\end{array}$ & $\begin{array}{l}24.1255 \\
24.091\end{array}$ & $\begin{array}{r}24.523 \\
24.52\end{array}$ & \begin{tabular}{|l|}
21.395 \\
21.336 \\
\end{tabular} & \begin{tabular}{|r|}
25.994 \\
\end{tabular} & $\begin{array}{l}24.8606 \\
24.802\end{array}$ & $\begin{array}{r}47.6944 \\
47.634\end{array}$ & $\begin{array}{l}51.945 \\
\mid 51.698 \\
\end{array}$ & $\begin{array}{r}15.804 \\
15.71\end{array}$ & $\begin{array}{l}33.802 \\
32.859\end{array}$ & \begin{tabular}{|l|l|}
9 & -1.215 \\
\end{tabular} & & $\begin{array}{l}48.219 \\
50.502\end{array}$ & & $\begin{array}{l}\frac{14.643}{14.643} \\
\end{array}$ & & & & & & & & & & & & $\frac{0.25}{0.25}$ \\
\hline & $8 / 31 / 2004$ & 1:39:24 PM & 24.066 & 24.535 & \begin{tabular}{|l|}
21.281 \\
\end{tabular} & 25. & 24. & 47.383 & \begin{tabular}{|l|l|}
3 & 51.53 \\
\end{tabular} & 15.643 & 32.674 & -1.212 & & 52.223 & & $\frac{14.045}{14.643}$ & & & & 50 & 12. & & & & & & $\frac{0.296}{0.294}$ & $\frac{0.25}{0.25}$ \\
\hline 108 & $8 / 31 / 2$ & & 24.032 & 24.536 & \begin{tabular}{|l|}
21.236 \\
\end{tabular} & 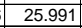 & 24. & & \begin{tabular}{|l|l|}
51.949 \\
\end{tabular} & 15.705 & & & & 51.61 & & & & & & & & & & & & & & $\frac{0.25}{0.25}$ \\
\hline & $8 / 31 / 2004$ & 1:41:24 PM & 24.004 & & \begin{tabular}{|l|l|}
21.188 \\
\end{tabular} & & & & & 15.605 & & -1.212 & -7 & & & 14.6 & & & & & & & & & & & & $\frac{0.25}{0.25}$ \\
\hline & & & & 24.564 & \begin{tabular}{|l|}
21.199 \\
\end{tabular} & 26.019 & & 47.9 & 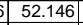 & 15.635 & 33.2 & 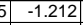 & & 50. & & & & & & 51. & 12 & & & & & & 0.295 & \\
\hline & & & & 24.581 & 21.276 & & & 47.7 & 51 & & & & & & & & & & & & & & & & & & & \\
\hline & $8 / 31 / 2$ & & & 24.599 & 21.35 & & & 47.9 & 52.26 & & 33.282 & \begin{tabular}{|l|l|l|l|}
2 & -1.212 \\
\end{tabular} & & & & & & & & 51.1 & 12. & & & & & & & 0.25 \\
\hline & $8 / 31 / 2004$ & & 23. & 24.602 & 21.408 & & & & 51.588 & 15.988 & 32.414 & -1.2 & & & & 14.6 & & & & 50 & & & & & & & 0.298 & \\
\hline & $8 / 31 / 2$ & & & & 21.487 & & & & & & & & & & & & & & & & & & & & & & & \\
\hline & & & 23.864 & 24.644 & \begin{tabular}{|l|}
21.566 \\
\end{tabular} & \begin{tabular}{|l|l|} 
& 26.314 \\
\end{tabular} & & 47.578 & $\begin{array}{l}3.51 .742 \\
3\end{array}$ & 15.586 & 32.968 & -1.209 & & & & & & & & 50. & & & & & & & 0.290 & \\
\hline & & & & 24.641 & 21.619 & & & & & & & & & & & & & & & & & & & & & & & \\
\hline & & & 23.819 & & $21.6 / 6$ & & & & 51.66 & & & & & & & & & & & & & & & & & & & \\
\hline & $8 / 31 / 4$ & $\begin{array}{l}1: 50: 24 \mathrm{PN} \\
1: 51: 24\end{array}$ & 23.786 & $\frac{24.655}{24.666}$ & $\begin{array}{l}21.718 \\
21.755\end{array}$ & \begin{tabular}{|l}
26 \\
26
\end{tabular} & & & 51.5374 & $\frac{15.89}{15.91}$ & $\begin{array}{l}32.53 \\
32336\end{array}$ & -1.21 & & & & 14. & & & & 50 & & & & & & & 300 & \\
\hline & $8 / 31 / 200$ & & & & \begin{tabular}{|l|l|}
2181 \\
2181
\end{tabular} & & & & & & & & & & & & & & & & & & & & & & & \\
\hline & $8 / 31 / 200$ & & & 24695 & \begin{tabular}{|l|l|}
21.84 \\
\end{tabular} & & & & & & & & & & & & & & & & & & & & & & & \\
\hline & $8 / 31 / 2$ & & 23.765 & $\mid 24.69$ & \begin{tabular}{|l|}
21.914 \\
\end{tabular} & 26. & & 47.4 & 51.678 & 15.736 & & & & & & & & & & & $\frac{12.2}{12.2}$ & & & & & & 年 & \\
\hline & & & & & & & & & & & & & & & & & & & & & & & & & & & & \\
\hline & & & 23.77 & 24.71 & $\begin{array}{l}21.993 \\
\end{array}$ & & & & 51.63 & 15.7 & 32.7 & & & & & & & & & & & & & & & & & \\
\hline & & & & 24.73 & 22.275 & & & 47.256 & & & & & & & & & & & & & & & & & & & & \\
\hline & $8 / 31 / 2$ & $1: 58$ & & 24.726 & 22.272 & 26. & & & 51.412 & & 32.689 & -1.212 & & & & 14. & & & & & 12.3 & & & & & & & \\
\hline & $8 / 31 / 206$ & & 23 & $24.7 \mathrm{r}$ & 22.268 & & & & 50.98 & 15. & 32.62 & -1.2 & & & & $14.6 \mathrm{C}$ & & & & & 11. & & & & & & & \\
\hline & & & & & & & & & 51.4 & & & & & & & 14. & & & & & & & & & & & & \\
\hline & $8 / 31 / 2$ & & $23.80 \varepsilon$ & 24.758 & \begin{tabular}{|l|}
22.294 \\
\end{tabular} & 26. & & 47.5 & B 51.80 & 15.755 & 32.8 & -1.209 & & & & & & & & & 11.8 & & & & & & & \\
\hline & & & & & & & & & & & & & & & & & & & & & & & & & & & & \\
\hline & $8 / 312 / 2$ & & & 24.763 & 2.2 .24 & & & 4.1 .2 & 51.2 & & 32 & & & & & & & & & & & & & & & & & \\
\hline & & & & $\frac{24.16}{2478}$ & 资.3044 & & & & 5187 & 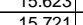 & $\begin{array}{l}32.765 \\
32.751\end{array}$ & $\frac{-1.2}{-12}$ & & & & & & & & & & & & & & & & \\
\hline & 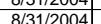 & & & 2479 & $\frac{251}{2231}$ & & & 471 & $\frac{3.0130}{51.30}$ & 15738 & $\frac{52.154}{32348}$ & -1.26 & & 515 & & 146 & & & & & & & & & & & & \\
\hline & & & & 24799 & & & & & 51.24 & & 32.516 & & & & & & & & & & & & & & & & & \\
\hline & & & & 24.80 & & & & & & & & & & & & 14.6 & & & & & & & & & & & & \\
\hline & & & & 24.86 & & & & & & & & & & & & & & & & & & & & & & & & \\
\hline & & & & & 22355 & & & & & & & & & & & & & & & & & & & & & & & \\
\hline & & & & 24.835 & 22.357 & & & 47.4 & 951.69 & & 32.5 & -1.218 & & & & & & & & & & & & & & & & \\
\hline & & & $946>2$ & & 36 & & & 47.. & & & 327 & & & & & & & & & & & & & & & & & \\
\hline $120 \mathrm{P}$ & $8 / 31 / 2004$ & 2:13:24 P & 23.972 & 24.847 & $\begin{array}{l}22.353 \\
\end{array}$ & 26.356 & & 47.391 & $\begin{array}{l}151.561 \\
\end{array}$ & \begin{tabular}{|c|}
15.509 \\
\end{tabular} & $\mid$ & \begin{tabular}{|c|c|}
8 & -1.215 \\
\end{tabular} & & 53.934 & | & 14.643 & & & 3339 & 50.4 & & & 2.767 & .011 & 0.011 & 0.000 & 0.285 & 0.2 \\
\hline
\end{tabular}


WSRC-TR-2005-00105, REVISION 0

SRNL-RPP-2005-00012, REVISION 0

RUN \# 4.03A AND B; FIRST AND SECOND HALF OF SLURRY DEWATERING - CONT.

\begin{tabular}{|c|c|c|c|c|c|c|c|c|c|c|c|c|c|c|c|c|c|c|c|c|c|c|c|c|c|c|c|c|}
\hline & A & B & D & $E$ & $F$ & $G$ & $\mathrm{H}$ & $\mathrm{J}$ & K & $\mathrm{L}$ & $M$ & $\mathrm{~N}$ & 0 & 0 & R & $\mathrm{s}$ & $\begin{array}{ll}T \\
\end{array}$ & $\mathrm{v}$ & w & $x$ & $\begin{array}{ll} \\
\end{array}$ & $z$ & AA & & & AD & & \\
\hline & $8 / 31 / 2004$ & 2:14:24 PM & $\begin{array}{l}23.977 \\
\end{array}$ & 24.852 & 22.374 & 26.322 & 25.029 & 47.424 & \begin{tabular}{|l|}
51.667 \\
\end{tabular} & 15.586 & 32.835 & -1.212 & -7.715 & 50.648 & 0.074 & 14.643 & 0.003 & 1821.03 & 30.3506 & 50.5 & 12.1 & 40.1 & 2.767 & 0.011 & 0.011 & 0.000 & $\begin{array}{ll}A E \\
0.284\end{array}$ & \\
\hline & $8 / 31 / 2004$ & 2:15:24 PM & 24.009 & 24.874 & 22.416 & 26.308 & 25.06 & $\begin{array}{l}47.605 \\
\end{array}$ & \begin{tabular}{|l|}
51.895 \\
\end{tabular} & 15.595 & 32.888 & \begin{tabular}{|r|}
-1.215 \\
\end{tabular} & & 51.009 & 0.074 & \begin{tabular}{|l|}
14.643 \\
\end{tabular} & 0.003 & & \begin{tabular}{|l|l|l}
30.3672 \\
\end{tabular} & \begin{tabular}{|l|}
50.8 \\
\end{tabular} & 12.1 & & & & & & & \\
\hline & $8 / 31 / 2004$ & $2: 16: 24 \mathrm{PM}$ & 24.004 & 24.874 & \begin{tabular}{|l|}
22.391 \\
2.29 \\
\end{tabular} & 26.293 & 24.99 & \begin{tabular}{|l|}
47.418 \\
7.78
\end{tabular} & \begin{tabular}{|l|l|}
51.597 \\
5.1737
\end{tabular} & $\begin{array}{l}5.653 \\
\end{array}$ & \begin{tabular}{|l|l|}
32.767 \\
32727
\end{tabular} & \begin{tabular}{|l|}
-1.212 \\
\end{tabular} & & \begin{tabular}{|l|l|}
49.916 \\
5.980
\end{tabular} & $\begin{array}{l}0.077 \\
\end{array}$ & \begin{tabular}{|l|}
14.643 \\
\end{tabular} & $\begin{array}{l}0.003 \\
.003\end{array}$ & 20.03 & 30.3839 & \begin{tabular}{|l|}
50.5 \\
\end{tabular} & 11.9 & 40.1 & $\begin{array}{l}2.764 \\
.779 \\
\end{array}$ & 0.011 & 0.012 & 0.000 & 0.296 & \\
\hline$\frac{1124}{1125}$ & $8 / 31 / 2004$ & 2:17:24 PM & \begin{tabular}{l|l|}
24.014 \\
2402
\end{tabular} & $\begin{array}{r}24.874 \\
2489 \\
\end{array}$ & \begin{tabular}{|l|}
22.406 \\
2.207
\end{tabular} & 26.238 & $\begin{array}{l}24.95 \\
\end{array}$ & $\begin{array}{l}47.631 \\
\end{array}$ & \begin{tabular}{|l|}
51.731 \\
51921 \\
\end{tabular} & $\begin{array}{l}15.466 \\
15.51\end{array}$ & $\begin{array}{l}33.227 \\
32011\end{array}$ & $\begin{array}{l}-1.212 \\
.202\end{array}$ & & $\begin{array}{l}51.885 \\
5.251\end{array}$ & $\begin{array}{l}0.074 \\
0075\end{array}$ & $\begin{array}{l}14.643 \\
\end{array}$ & 0.003 & $\begin{array}{l}824.03 \\
20.03\end{array}$ & 30.4006 & \begin{tabular}{|l|}
50.6 \\
\end{tabular} & 12.4 & \begin{tabular}{|l|l|}
40.4 \\
\end{tabular} & 2.787 & 0.011 & 0.011 & 0.000 & 0.282 & \\
\hline$\frac{1125}{1126}$ & $8 / 31 / 2004$ & $\begin{array}{l}2: 18: 24 \mathrm{PM} \\
2 \cdot 19224 \mathrm{PM}\end{array}$ & \begin{tabular}{r|}
24.02 \\
24026
\end{tabular} & $\begin{array}{r}24.89 \\
24.91\end{array}$ & & & $\begin{array}{l}25.046 \\
25.477\end{array}$ & & & \begin{tabular}{|c|}
15.614 \\
15.572
\end{tabular} & \begin{tabular}{|l|l|}
33.011 \\
33301
\end{tabular} & \begin{tabular}{|r|}
-1.209 \\
\end{tabular} & & \begin{tabular}{|l}
56.251 \\
49407
\end{tabular} & & \begin{tabular}{|l|l|l|}
14.643 \\
14634 \\
\end{tabular} & & & 30.4172 & \begin{tabular}{|l|}
50.8 \\
\end{tabular} & \begin{tabular}{|l|}
13.4 \\
1.1
\end{tabular} & & & & & & 0.290 & \\
\hline 1127 & $8 / 3 / 31 / 2004$ & $\begin{array}{l}2: 19: 24 \mathrm{PM} \\
2: 20: 24 \mathrm{PM}\end{array}$ & $\frac{24.026}{24.042}$ & $\frac{24.901}{24.912}$ & $\frac{22.423}{22.45}$ & \begin{tabular}{|l|}
26.255 \\
26.282 \\
\end{tabular} & $\begin{array}{l}25.147 \\
25.224\end{array}$ & $\begin{array}{r}47.725 \\
46.98 \\
\end{array}$ & \begin{tabular}{|r|}
51.9144 \\
51.34
\end{tabular} & $\begin{array}{l}15.752 \\
16.195\end{array}$ & \begin{tabular}{|}
33.307 \\
31.712 \\
\end{tabular} & \begin{tabular}{|c|}
$\mid-1.212$ \\
-1.212 \\
\end{tabular} & $\begin{array}{r}-7.715 \\
-7.726 \\
\end{array}$ & \begin{tabular}{|l|}
49.4077 \\
50.578 \\
\end{tabular} & $\begin{array}{c}0.074 \\
0.072\end{array}$ & $\begin{array}{r}\mid 14.643 \\
14.643\end{array}$ & $\begin{array}{l}0.003 \\
0.003\end{array}$ & $\frac{1826.03}{1827.03}$ & \begin{tabular}{|}
$\mathbf{3} 30.4339$ \\
$\mathbf{3} 30.4506$
\end{tabular} & \begin{tabular}{|l|}
50.8 \\
50.2 \\
\end{tabular} & \begin{tabular}{|l|}
$\mid 11.8$ \\
12.0 \\
\end{tabular} & \begin{tabular}{|c|c|}
8 & 40.5 \\
0 & 39.3 \\
\end{tabular} & 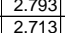 & 0.011 & \begin{tabular}{|c|}
0.011 \\
0.011
\end{tabular} & $\begin{array}{l}0.000 \\
0.000\end{array}$ & 0.281 & 0.24 \\
\hline \begin{tabular}{|l|}
1128 \\
\end{tabular} & $8 / 31 / 2004$ & 2:21:24 PM & 24.037 & 24.912 & 22.45 & 26.262 & 25.189 & & & 16.113 & & & $\begin{array}{l}-7.729 \\
\end{array}$ & & & 14.643 & $\begin{array}{l}0.003 \\
0.003\end{array}$ & $\begin{array}{l}1827.03 \\
1828.03\end{array}$ & $\begin{array}{l}30.4500 \\
30.4672\end{array}$ & $\begin{array}{l}30.2 \\
49.8\end{array}$ & $\begin{array}{ll}12.0 \\
12.3\end{array}$ & \begin{tabular}{|l|l|}
3 & 39.9 \\
\end{tabular} & $\frac{2.113}{2.700}$ & 0.010 & 0.011 & $\begin{array}{l}0.000 \\
0.000\end{array}$ & & $\begin{array}{l}0.24 \\
0.24\end{array}$ \\
\hline & $8 / 31 / 2004$ & $2: 22: 24 \mathrm{PM}$ & 24.032 & 24.912 & 22.459 & 26.201 & 25.143 & $\begin{array}{l}47.437 \\
\end{array}$ & \begin{tabular}{|r|}
51.76 \\
\end{tabular} & 16.221 & 32.143 & -1.215 & & 52.394 & & $\begin{array}{l}14.643 \\
\end{array}$ & & & 30.4839 & 50.6 & & & & & & & & \\
\hline 1130 & $8 / 31 / 2004$ & 2:23:24 PM & & 24.922 & $\begin{array}{r}22.45 \\
22.95\end{array}$ & & & $\begin{array}{r}46.92 \\
\end{array}$ & & & & $\begin{array}{l}-1.212 \\
\end{array}$ & & $\begin{array}{r}50.1 \\
\end{array}$ & 072 & \begin{tabular}{|l|l|}
14.643 \\
\end{tabular} & & & 30.5006 & 49.9 & 11.9 & 39.4 & & & & 0.000 & 0.281 & \\
\hline 1131 & $8 / 31 / 2004$ & $\begin{array}{l}2: 24: 24 \mathrm{PM} \\
2: 25: 24 \mathrm{P}\end{array}$ & 24.053 & 24.933 & $\begin{array}{l}22.485 \\
22511\end{array}$ & 26.237 & $\begin{array}{l}25.055 \\
2500\end{array}$ & $\begin{array}{l}47.405 \\
\end{array}$ & \begin{tabular}{|l|}
51.812 \\
51819 \\
\end{tabular} & 15.911 & \begin{tabular}{|l|l}
32.383 \\
325
\end{tabular} & $\begin{array}{l}-1.212 \\
\end{array}$ & & $\begin{array}{l}48.189 \\
\end{array}$ & .072 & 14.643 & $\begin{array}{l}0.003 \\
\end{array}$ & $\begin{array}{l}831.03 \\
8203\end{array}$ & \begin{tabular}{|l|}
30.5172 \\
\end{tabular} & 50.7 & 11.5 & 39.9 & 2.751 & 0.010 & 0.011 & 0.000 & 0.278 & \\
\hline$\frac{\frac{1132}{1133}}{1133}$ & $8 / 31 / 2004$ & $\begin{array}{l}2: 25: 24 \mathrm{PM} \\
2 \cdot 22: 24 \mathrm{PM}\end{array}$ & $\begin{array}{l}24.044 \\
24065\end{array}$ & $\begin{array}{r}24.939 \\
24.95 \\
\end{array}$ & $\frac{22.511}{22.613}$ & & $\begin{array}{r}25.08 \\
25.162 \\
\end{array}$ & \begin{tabular}{|c|}
47.569 \\
47179 \\
\end{tabular} & \begin{tabular}{|c|}
51.818 \\
51.29 \\
\end{tabular} & \begin{tabular}{|c|}
16.006 \\
15593
\end{tabular} & \begin{tabular}{|r|}
32.51 \\
32.166 \\
\end{tabular} & \begin{tabular}{l|l|l|}
-1.212 \\
-1.209
\end{tabular} & & \begin{tabular}{|l|}
53.279 \\
52.916
\end{tabular} & & \begin{tabular}{|l|l|}
14.643 \\
14634 \\
\end{tabular} & & & $\begin{array}{l}3 \quad 30.5339 \\
\end{array}$ & $\begin{array}{l}50.7 \\
50.3\end{array}$ & $\begin{array}{l}12.7 \\
126\end{array}$ & $\begin{array}{l}440.0 \\
3327\end{array}$ & $\begin{array}{l}2.761 \\
2.736\end{array}$ & 0.010 & & 0.000 & 0.280 & 0.24 \\
\hline$\frac{1133}{1134}$ & $8 / 3 / 1 / 2004 \mid$ & $\begin{array}{l}2: 22: 24 \mathrm{PM} \\
2: 27: 24 \mathrm{PM}\end{array}$ & $\begin{aligned} 24.065 \\
24.065\end{aligned}$ & $\begin{array}{r}24.95 \\
24.946\end{array}$ & $\frac{22.613}{22.609}$ & $\begin{array}{r}26.22 \\
26.225 \\
\end{array}$ & $\begin{array}{l}25.162 \\
25.098 \\
\end{array}$ & \begin{tabular}{|c|}
47.179 \\
47.308
\end{tabular} & \begin{tabular}{|l|}
51.429 \\
55.597
\end{tabular} & $\begin{array}{l}15.893 \\
15.969\end{array}$ & $\begin{aligned} 32.186 \\
32.26\end{aligned}$ & \begin{tabular}{|c|}
-1.209 \\
-1.218 \\
\end{tabular} & $\begin{array}{r}-7.718 \\
-7.726\end{array}$ & $\begin{array}{l}52.916 \\
51.178\end{array}$ & $\begin{array}{l}0.074 \\
0.073\end{array}$ & $\begin{array}{l}\mid 14.643 \\
14.643\end{array}$ & $\begin{array}{r}0.003 \\
0.003\end{array}$ & & \begin{tabular}{|l}
30.5506 \\
30.5672
\end{tabular} & $\begin{array}{l}50.3 \\
55.5 \\
\end{array}$ & 122 & 398 & $\frac{2.743}{2.743}$ & 0.011 & 0.011 & 0.000 & 0.286 & 0.24 \\
\hline 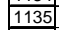 & $8 / 31 / 2004$ & $2: 28: 24 \mathrm{PM}$ & 24.073 & $\begin{array}{l}24.946 \\
24.968\end{array}$ & \begin{tabular}{|l|}
22.009 \\
22.636 \\
\end{tabular} & $\mid$\begin{tabular}{|l|l|}
20.225 \\
26.262
\end{tabular} & 25.089 & 47.132 & \begin{tabular}{|l|}
51.367 \\
\end{tabular} & $\begin{array}{l}15.969 \\
15.831\end{array}$ & 32.311 & \begin{tabular}{|l|}
-1.215 \\
\end{tabular} & -7.1718 & S1.1161 & $\begin{array}{l}0.074 \\
0.074\end{array}$ & \begin{tabular}{l|l}
$\frac{14.043}{14.643}$ &
\end{tabular} & $\begin{array}{l}.0003 \\
0.00\end{array}$ & & $\frac{30.50 / 2}{30.5839}$ & 50.2 & $\frac{12.2}{12.2}$ & $\frac{39.8}{39.7}$ & & & $\frac{0.011}{0.011}$ & $\begin{array}{l}0.000 \\
0.000\end{array}$ & & $\frac{0.24}{0.24}$ \\
\hline & $8 / 31 / 2004$ & 2:29:24 PM & \begin{tabular}{|l|l|}
24.08 \\
\end{tabular} & 24.98 & & 26.354 & 25.256 & 47.202 & \begin{tabular}{|r|}
51.24 \\
\end{tabular} & 15.818 & 32.399 & \begin{tabular}{|l|}
-1.212 \\
\end{tabular} & & 50.148 & 0.072 & 14.643 & & & & 50.1 & & & & & & & & \\
\hline & $8 / 31 / 2004$ & 2:30:24 PM & 24.083 & 24.983 & 22.601 & \begin{tabular}{|l|}
26.393 \\
\end{tabular} & 25.39 & 47.33 & 51.464 & 16.029 & 32.276 & -1.212 & -7.72 & 50.686 & & \begin{tabular}{|l|l|}
14.643 \\
\end{tabular} & & & & & & & & & & & & $\frac{0.24}{0.24}$ \\
\hline 1138 & $8 / 31 / 2004$ & $2: 31: 24 \mathrm{PM}$ & & & & & & & & & & & & & & & & & 6339 & 50.2 & & 39 . & & & & & 0.281 & \\
\hline$\frac{1139}{1190}$ & 81 & $2: 32: 24 \mathrm{PM}$ & & & & 26.549 & & $\begin{array}{l}47.295 \\
\end{array}$ & & \begin{tabular}{|l|l|}
15.871 \\
\end{tabular} & 32.383 & -1.215 & & 52.092 & & $\begin{array}{l}14.643 \\
\end{array}$ & & & & 50.4 & & & & nt & & & & \\
\hline 1140 & & 2:33:24 PM & 24.144 & 25.039 & 22.616 & & & 47.528 & \begin{tabular}{|l|l|}
51.825 \\
\end{tabular} & 15.896 & & & & 51.725 & & & & & & 577 & & & 76 & & & & & \\
\hline & 1:/2004 & & & 25.047 & \begin{tabular}{|l|}
22.629 \\
\end{tabular} & 26.736 & & 47.181 & \begin{tabular}{|l|}
51.634 \\
\end{tabular} & \begin{tabular}{|l|l|l|}
16.009 \\
\end{tabular} & 31.995 & -1 & & & & \begin{tabular}{l|l}
14.643 \\
\end{tabular} & & & & 50.5 & 12.4 & & & & & & & \\
\hline & $31 / 2004$ & 2:35:24 PM & 24.159 & 25.054 & \begin{tabular}{|l|}
22.627 \\
\end{tabular} & 26.693 & 25.645 & 47.225 & \begin{tabular}{|l|}
51.593 \\
\end{tabular} & 15.91 & 32.254 & -1.218 & -7.7 & 50.765 & 0.072 & 14.643 & 0.003 & 1842.0 & 30.7006 & 50.5 & 12.1 & 139.7 & 2.740 & 0.010 & 0.011 & 0.000 & 0.277 & \\
\hline & $8 / 31 / 2004$ & 2:36:24 PM & 24.176 & 25.066 & \begin{tabular}{|l|}
22.629 \\
\end{tabular} & $\begin{array}{r}26.75 \\
6.107\end{array}$ & 25.552 & 47.509 & \begin{tabular}{|l|}
51.819 \\
5.052
\end{tabular} & 16.037 & 32.516 & -1.212 & & 50.277 & 0.072 & 14.643 & 0.00 & & 30.7172 & 50.7 & $12.6 \mathrm{C}$ & 40.0 & 759 & .010 & .011 & & 0.276 & \\
\hline & $8 / 31 / 2004$ & $2: 37: 24 \mathrm{PM}$ & 24.178 & 25.068 & 22.6 & $26.74 t$ & & & & 15.967 & 32.664 & -1.21 & & 50.204 & & 14.643 & & & .7339 & 50.9 & & & & & & & 0.278 & \\
\hline & $8 / 31 / 2004$ & $2,38: 24 \mathrm{PM}$ & 24.189 & 25.089 & 2.617 & 26.118 & & $47.1 / 79$ & & 15.854 & 32.297 & & & $50.8 / 6$ & & & & & & & & & & & & & & \\
\hline 步1474 & $8 / 31 / 2004$ & $\frac{2: 39: 24 \mathrm{PM}}{2 \cdot 40 \cdot 24 \mathrm{P}}$ & $\begin{aligned} 24.189 \\
2421\end{aligned}$ & $\frac{25.094}{25.12}$ & $\frac{22.637}{22643}$ & $\frac{26.638}{26679}$ & $\frac{25.325}{25.401}$ & $\begin{array}{l}47.043 \\
471196\end{array}$ & \begin{tabular}{|l|}
51.601 \\
51.688 \\
\end{tabular} & $\frac{16.032}{15.871}$ & $\begin{array}{l}31.808 \\
32083\end{array}$ & $\begin{array}{r}-1.215 \\
-1.209\end{array}$ & & $\frac{50.121}{46.79}$ & & $\frac{14.643}{14643}$ & & & 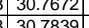 & $\frac{50.5}{50.6}$ & & & & & & & & \\
\hline$\frac{1148}{1148}$ & $8 / 31 / 2004$ & $2: 41: 24 \mathrm{PM}$ & 24.211 & $\frac{25.116}{25}$ & 22,643 & 26.61 & 25.35 & 47.268 & \begin{tabular}{|l|}
51.553 \\
\end{tabular} & 15.071 & $\begin{array}{l}32.005 \\
32.432\end{array}$ & $\frac{-1.25}{-1.212}$ & & 53.031 & .072 & 10 & & & . & 50.4 & 126 & & 748 & & & & & \\
\hline & $8 / 31 / 2004$ & $2: 42: 24 \mathrm{PM}$ & & 25.116 & \begin{tabular}{|l|}
22.639 \\
22.639 \\
\end{tabular} & 20.63 & 25.402 & 47.192 & \begin{tabular}{|r|}
51.42 \\
\end{tabular} & 15.072 & 32.204 & -1.21 & $-7.72 \quad$ & 50.97 & & $\begin{array}{l}14.045 \\
14.643\end{array}$ & & & 0.0172 & 50.3 & 121 & 39. & & 0.010 & & & 0.278 & 0.24 \\
\hline & & & 24.238 & & & $\frac{26.662}{26 .}$ & & $\mid 47.186$ & \begin{tabular}{|r|}
51.28 \\
\end{tabular} & $\begin{array}{l}15.86 \\
\end{array}$ & 32.358 & -1.2 & & 52.497 & & & & & & & $x$ & & & & & & & \\
\hline & $8 / 31 / 2$ & 2:44:24 PM & 24.244 & 25.139 & 22.753 & & 25.4 & \begin{tabular}{|l|l|}
47.213 \\
\end{tabular} & & 15.847 & 32.282 & -1.21 & & 48.358 & & 14.643 & & & & 50.4 & & & & & & & & \\
\hline & $8 / 31 / 2$ & 2:44:41 PM & 24.249 & 25.144 & 22.763 & 26.648 & & 47.493 & \begin{tabular}{|r|} 
\\
\end{tabular} & 15.844 & 32.625 & -1.215 & & & & 14.643 & & & .8553 & 50.6 & 12.4 & $40 \quad-1$ & 76 & & & & .278 & \\
\hline & $8 / 31 / 2$ & 2:45:41 PM & & $\begin{array}{l}25.167 \\
\end{array}$ & 22.766 & & & 47.221 & 51.497| & 15.831 & & -1.212 & & & & 14.643 & & & & 50.4 & & & & & & & & \\
\hline & $8 / 31 / 2004$ & 2:46:41 PM & 24.294 & 25.169 & 22.757 & 26.593 & 25.42 & 46.999 & \begin{tabular}{|l|l|}
51.354 \\
\end{tabular} & 15.771 & 32.221 & -1.215 & & & & & & & 8886 & 50.2 & 11. & 39 & & & & & & 0.24 \\
\hline & $8 / 31 / 2004$ & $2: 47$ & 24.305 & 25.17 & \begin{tabular}{|l|l|}
22.728 \\
\end{tabular} & 26.623 & & & \begin{tabular}{|l|l|l|}
51.704 \\
\end{tabular} & 15.766 & 32.584 & -1.2 & & & & $\begin{array}{l}14.643 \\
\end{array}$ & & & .9053 & 50.6 & 12 & & & & & & & \\
\hline & $8 / 31 / 2$ & 2:48:41 PM & 24.315 & 25.185 & 22.728 & 26.634 & 25.3 & 47.2 & \begin{tabular}{|l|l|}
51.466 \\
\end{tabular} & 15.679 & 32.594 & -1.21 & & 50.747 & & 14. & & & & 50.3 & 12.1 & & & & & & 0.275 & \\
\hline & $8 / 3$ & 2:49: & 24.326 & 25.191 & 22.699 & 26.69 & & 47.262 & \begin{tabular}{|l|}
51.663 \\
\end{tabular} & 15.803 & 32.43 & -1.2 & & 52.125 & & & & & & 50.5 & 12. & & & & & & & \\
\hline & 004 & & & & & & & 47.67 & & & & & & & & & & & & 51.0 & & & & & & & 0.274 & \\
\hline & $8 / 31 / 2 / 2$ & $2: 51: 41 \mathrm{PM}$ & 24.351 & 25.221 & 22.713 & 26.62 & & 41.2 & 51.59 & 15.606 & & -1.21 & & & & & & & & 50.5 & 11. & & & & & & & \\
\hline & 8 & $2.5 .42 .41 \mathrm{PM}$ & $\begin{array}{ll}24.3586 \\
2.348\end{array}$ & $\begin{array}{l}25.286 \\
25213\end{array}$ & 22.100 & & & (4).254 & $|51.068|$ & 15.647 & (32. & $\frac{-1.212}{-1212}$ & & & & 14. & & & & 50.5 & 120 & 40 & & & & & 876 & \\
\hline & $8 / 31 / 2004$ & $2: 54:$ & 24.353 & & 22686 & & & & & 15.532 & & & & & & 146 & & & & 508 & & & & & & & & \\
\hline & $8 / 31 / 2$ & 2:55: & 24.363 & 25.239 & 22.691 & & & & & & & & & & & & & & & & & & & & & & & \\
\hline & $8 / 31 / 2004$ & $2: 56$ & 24.37 & 25.245 & \begin{tabular}{|l|}
22.673 \\
\end{tabular} & 26. & & 47.605 & \begin{tabular}{|l|}
51.962 \\
\end{tabular} & 15.549 & 33.068 & -1.215 & & & & 14.6 & & & \begin{tabular}{|l|}
31.0553 \\
\end{tabular} & 50.8 & 12. & & & & 0.0 & & & \\
\hline & & & & & & & & & & & & & & & & & & & & & $11 .$. & & & & & & & \\
\hline & & & 24.365 & 25.265 & $\begin{array}{l}22.668 \\
\end{array}$ & 26.4 & & & \begin{tabular}{|l|}
51.926 \\
\end{tabular} & 15.5 & & -1.21 & & & & & & & & 50.8 & & & & & & & 0.273 & \\
\hline & $8 / 31 / 2$ & 2:59:41 PM & 24.364 & 25.259 & \begin{tabular}{|l|}
22.677 \\
\end{tabular} & 26.518 & 25. & \begin{tabular}{|l|l|}
47.714 \\
\end{tabular} & \begin{tabular}{|l|l|}
51.843 \\
\end{tabular} & 15.606 & 33.171 & -1.212 & & & & $\begin{array}{l}14.643 \\
\end{array}$ & & & 01.1050 & 50.7 & & & & & & & 0.216 & \\
\hline & $8 / 31 / 2$ & 3:00:41 PM & 24.364 & $\begin{aligned} 25.249 \\
2.275\end{aligned}$ & 22.622 & 26.573 & 25.4 & & & & & -1.218 & & & & 14.6 & & & 31.1219 & 50.8 & 12. & & & & & & 0.274 & \\
\hline & $8 / 31 / 2004$ & $\begin{array}{l}3: 01: 41 \text { PM } \\
3 \cdot 30.04\end{array}$ & $\frac{24.380}{24.389}$ & $\frac{25.275}{25.279}$ & \begin{tabular}{|l|}
22.663 \\
22682 \\
\end{tabular} & \begin{tabular}{|l|}
26.539 \\
\end{tabular} & $\frac{25.39}{253}$ & \begin{tabular}{|l|}
47.974 \\
7.736
\end{tabular} & \begin{tabular}{|l|}
52.484 \\
51765
\end{tabular} & $\begin{array}{r}15.545 \\
15.528 \\
\end{array}$ & $\begin{array}{r}33.296 \\
33.126\end{array}$ & \begin{tabular}{|r|}
-1.215 \\
1201
\end{tabular} & $-1 . .11$ & 54.486 & 0.073 & $\frac{1.4 .643}{4}$ & 0.00 & $\frac{1868.32}{100922}$ & \begin{tabular}{|l|l|}
31.1386 \\
\end{tabular} & \begin{tabular}{|l|}
51.4 \\
5
\end{tabular} & 13.0 & \begin{tabular}{|l|l|} 
& 40.6 \\
\end{tabular} & 2.802 & \begin{tabular}{|l|}
0.010 \\
0010
\end{tabular} & 0.011 & 0.000 & 0.275 & 0.23 \\
\hline & $8 / 31 / 2004$ & & 369 & 25.269 & \begin{tabular}{|l|}
22.002 \\
22.697 \\
\end{tabular} & & & & \begin{tabular}{|l|}
52.080 \\
52.086
\end{tabular} & 15.020 & 33.1.337 & -1.215 & & & & $\frac{14.6}{14.6}$ & & & & 51.0 & $\begin{array}{ll}\frac{11.4}{11.5} \\
11.5\end{array}$ & & & & & & 0.264 & $\frac{0.23}{0.22}$ \\
\hline & & & & & & & & & & & & & & & & & & & & & & & & & & & & \\
\hline & & & & $\frac{25.302}{25.302}$ & 22.76 & & & & & 15.4 & 33.159 & -1. & & & & & & & 31.2 & $\frac{21.1}{51 .}$ & & & & & & & & \\
\hline & & & & 25.303 & 22.746 & & & & |52.14 & & & & & & & & & & & & & & & & & & & \\
\hline & $8 / 31 / /$ & & & 25.3 & 22.733 & 26. & & & \begin{tabular}{|l|l|}
52.293 \\
\end{tabular} & 15.432 & 33.216 & -1.215 & & & & 14. & & & & 512 & 12. & & & & & & 268 & \\
\hline & $8 / 31 / 206$ & 3:08 & 24. & 25.31 & 22.722 & & & 47.636 & 51.825 & 15.37 & 33.362 & -1.212 & & & & 14.6 & & & & 50.7 & 11.9 & & & & & & & \\
\hline & $8 / 31 / 2$ & & & 25.3 & 22.7 & & & & \begin{tabular}{|l|} 
\\
\end{tabular} & & & & & & & 14. & & & & 51.1 & & & & & & & & \\
\hline & $8 / 31 / 2$ & & & 25.342 & 22.725 & 26. & & & \begin{tabular}{|l|}
52.053 \\
\end{tabular} & 15.476 & 33.3 & -1.212 & & & & & & & & 50.9 & 11.8 & & & & & & 0.275 & \\
\hline & & & & & & & & & & & & & & & & & & & & & & & & & & & & \\
\hline & $8 / 3121 / 2$ & & & 25.3 & 22.749 & & & & 51. & & & & & & & & & & & & 14 & & & & & & & \\
\hline & & 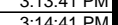 & & 5362 & 2.144 & 26. & & (47.202 & S1.30 & 13.04 & $\begin{array}{l}32.555 \\
32.50\end{array}$ & $\frac{-1.21}{-1.21}$ & & & & & & & & & 82 & & & & & & 12 & \\
\hline & $8 / 31 / 200$ & $\frac{3.14 .417}{3 \cdot 15: 12}$ & & $\frac{25.548}{25348}$ & $\frac{2.715}{20781}$ & $\frac{260}{2625}$ & & $\frac{41.400}{4731}$ & $\frac{1.047}{51663}$ & 15724 & 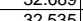 & -1209 & & 505 & & 146 & & & & & & & & & & & & \\
\hline & $8 / 31 / 2$ & 3.16:41 & & 25.3 & 22781 & & & & 51.45 & 15617 & 32767 & -1209 & & & & 146 & & & & 504 & & & & & & & & \\
\hline & $8 / 31 / 2$ & & & 25.34 & $\frac{22.752}{22.75}$ & & & 47 & & 15.827 & & -1.212 & & & & 14.4 & & & & 506 & & & & & & & & \\
\hline & & & & 25.3 & & & & & & & & & & & & & & & & & & & & & & & & \\
\hline & & & & & 22789 & & & & & & & & & & & & & & & & & & & & & & & \\
\hline & $8 / 31 / 2$ & & & 25.37 & 22.793 & & & 47.6 & 5211 & 15.8 & 32.681 & -1.215 & & & & & & & & 51.0 & 120 & & & & & & & \\
\hline & & & & 25.3 & & & & & & 5.724 & & 215 & & 512 & & 14.643 & & & 314719 & 50.7 & & & & & & & & 02 \\
\hline 190 & $8 / 31 / 2004$ & 3:22:41 PM & 24.408 & \begin{tabular}{l|l|}
25.363 \\
\end{tabular} & \begin{tabular}{|l|l|}
22.821 \\
\end{tabular} & \begin{tabular}{|l|}
26.237 \\
\end{tabular} & 24.80 & \begin{tabular}{|l|l|}
47.441 \\
\end{tabular} & \begin{tabular}{|l|l|}
51.74 \\
\end{tabular} & \begin{tabular}{|l|l|}
15.753 \\
\end{tabular} & 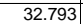 & $|-1.212|$ & & 51.347 & 0.071 & 14.643 & & & 1.4886 & 50.6 & & & 2.766 & \begin{tabular}{|l|l|}
0.010 \\
0.0
\end{tabular} & 0.011 & 0.000 & 0.270 & 0.2 \\
\hline
\end{tabular}


WSRC-TR-2005-00105, REVISION 0

SRNL-RPP-2005-00012, REVISION 0

RUN \# 4.03A AND B; FIRST AND SECOND HALF OF SLURRY DEWATERING - CONT.

\begin{tabular}{|c|c|c|c|c|c|c|c|c|c|c|c|c|c|c|c|c|c|c|c|c|c|c|c|c|c|c|c|c|}
\hline & A & B & D & $E$ & $\mathrm{~F}$ & G & $\mathrm{H}$ & $\mathrm{J}$ & $\mathrm{k}$ & $\mathrm{L}$ & $M$ & $\mathrm{~N}$ & 0 & $Q$ & R & \begin{tabular}{l|l} 
\\
\end{tabular} & \begin{tabular}{l|l}
$T$ \\
\end{tabular} & $\mathrm{~V}$ & w & $x$ & Y & $z$ & $\mathrm{AA}$ & & $A C$ & AD & & \\
\hline & $8 / 31 / 2004$ & 3:23:41 PM & 24.423 & 25.373 & 22.836 & 26.302 & 24.929 & 47.474 & \begin{tabular}{|l|l|}
51.686 \\
\end{tabular} & 15.745 & 32.754 & -1.212 & $\begin{array}{l}-7.72 \\
\end{array}$ & 49.729 & 0.07 & \begin{tabular}{|l|l|}
14.643 \\
\end{tabular} & 0.004 & 1890.32 & 31.5053 & 50.6 & 11.8 & 40.1 & 2.766 & 0.010 & 0.011 & 0.000 & 0.266 & \\
\hline & $8 / 31 / 2004$ & $\begin{array}{l}3: 24: 41 \mathrm{PM} \\
\end{array}$ & 24.413 & 25.363 & & 26.222 & 24.969 & 47.474 & \begin{tabular}{|l|}
51.682 \\
\end{tabular} & 15.703 & 32.904 & $\begin{array}{l}-1.212 \\
-1.212\end{array}$ & & 51.489 & $\begin{array}{l}.07 \\
\end{array}$ & $\begin{array}{l}14.643 \\
\end{array}$ & 0.003 & 1891.32 & \begin{tabular}{|l|l|l}
31.5219 \\
\end{tabular} & 50.6 & 12.3 & & & & 0.011 & & & \\
\hline & $8 / 31 / 2004$ & $3: 25: 41 \mathrm{PM}$ & 24.41 & 25.35 & 22.973 & \begin{tabular}{|l|l|}
26.208 \\
9.20
\end{tabular} & 24.96 & & \begin{tabular}{|l|}
51.603 \\
5.06 \\
\end{tabular} & \begin{tabular}{|l|l|}
15.793 \\
1.714
\end{tabular} & $\begin{array}{l}32.26 \\
32.507\end{array}$ & \begin{tabular}{|c|}
-1.218 \\
-212
\end{tabular} & \begin{tabular}{|c|c|}
-7.718 \\
7725
\end{tabular} & 50 & $\begin{array}{l}0.07 \\
\end{array}$ & 14.643 & 0.003 & $\frac{1892.32}{198232}$ & 31.5386 & 50.5 & 111.9 & 39.7 & 736 & 0.010 & 0.011 & 0.000 & 0.268 & \\
\hline & $8 / 31 / 2004$ & 3:26:41 PM & 24.393 & $\begin{array}{l}25.323 \\
\end{array}$ & 22.982 & \begin{tabular}{|l|l|}
26.162 \\
\end{tabular} & 24.939 & $\begin{array}{l}47.291 \\
\end{array}$ & 51.661 & 15.714 & 32.597 & -1.212 & \begin{tabular}{|r|r|}
-7.715 \\
-7.71
\end{tabular} & 51.003 & $\begin{array}{l}0.07 \\
\end{array}$ & $\begin{array}{l}14.643 \\
\end{array}$ & 0.004 & 1893.32 & $\begin{array}{l}31.5553 \\
2.50\end{array}$ & 50.5 & 12.1 & 39.9 & .754 & 0.010 & 0.011 & 0.000 & 0.266 & \\
\hline & $8 / 31 / 2004$ & 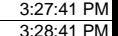 & $\begin{array}{l}24.387 \\
24387\end{array}$ & $\frac{25.313}{25.308}$ & \begin{tabular}{|l|}
22.936 \\
22.926 \\
\end{tabular} & \begin{tabular}{|l|l|}
26.156 \\
26.161
\end{tabular} & $\begin{aligned} 24.988 \\
25083\end{aligned}$ & & & $\begin{array}{l}15.588 \\
15651\end{array}$ & \begin{tabular}{|l|}
32.638 \\
32732
\end{tabular} & \begin{tabular}{|c|}
-1.215 \\
1215
\end{tabular} & & $\begin{array}{r}48.73 \\
51351\end{array}$ & $\begin{array}{l}0.071 \\
\end{array}$ & \begin{tabular}{|l|}
14.643 \\
1.4633 \\
\end{tabular} & & $\begin{array}{l}894.32 \\
805.22 \\
8\end{array}$ & \begin{tabular}{|l|}
31.5719 \\
33.588
\end{tabular} & \begin{tabular}{|l|}
50.4 \\
55.6 \\
\end{tabular} & \begin{tabular}{|l|l|}
11.6 \\
122
\end{tabular} & 39.9 & & & $\begin{array}{l}0.011 \\
0.11\end{array}$ & & & \\
\hline 1197 & $8 / 3 / 31 / 2004$ & $\begin{array}{l}3: 28: 41 \mathrm{PM} \\
3: 29: 41 \mathrm{P}\end{array}$ & $\begin{array}{l}24.381 \\
24.382\end{array}$ & $\begin{array}{l}25.308 \\
25.298\end{array}$ & \begin{tabular}{|l|}
22.9296 \\
22.896 \\
\end{tabular} & \begin{tabular}{|l|}
26.161 \\
26.271 \\
\end{tabular} & $\begin{array}{l}25.083 \\
25.118\end{array}$ & \begin{tabular}{|l|}
47.3867 \\
47.563 \\
\end{tabular} & \begin{tabular}{|l|}
51.775 \\
51.976 \\
\end{tabular} & \begin{tabular}{|l|}
5.651 \\
15.614 \\
\end{tabular} & \begin{tabular}{|l|}
32.732 \\
33.023
\end{tabular} & \begin{tabular}{|c|}
$\mid-1.215$ \\
-1.212 \\
\end{tabular} & $\begin{array}{l}-7.7712 \\
-7.712\end{array}$ & $\begin{array}{l}\frac{51.351}{50.484} \\
\end{array}$ & $\begin{array}{l}0.07 \\
0.07\end{array}$ & $\begin{array}{r}\mid 14.643 \\
14.643\end{array}$ & $\begin{array}{l}0.003 \\
0.003\end{array}$ & $\begin{array}{l}885.32 \\
896.32\end{array}$ & \begin{tabular}{|c|}
31.5886 \\
31.6053
\end{tabular} & \begin{tabular}{|l|}
50.6 \\
50.8 \\
\end{tabular} & \begin{tabular}{|l|l}
12.2 \\
12.0
\end{tabular} & $\begin{array}{l}40.1 \\
0.3 \\
\end{array}$ & \begin{tabular}{|l|}
2.762 \\
2.778 \\
\end{tabular} & $\frac{0.010}{0.010}$ & $\begin{array}{l}0.011 \\
0.011\end{array}$ & $\begin{array}{l}0.000 \\
0.000\end{array}$ & 0.265 & 0.23 \\
\hline 1198 & $8 / 31 / 2004$ & 3:30:41 PM & 24.388 & 25.308 & \begin{tabular}{|l|}
22.892 \\
\end{tabular} & \begin{tabular}{|l|}
26.272 \\
\end{tabular} & 25.209 & & \begin{tabular}{|r|}
51.69 \\
\end{tabular} & 15.753 & 32.828 & & $\begin{array}{l}-7.706 \\
\end{array}$ & 50.244 & 0.071 & 14.643 & $\begin{array}{l}0.003 \\
0.003\end{array}$ & $\begin{array}{l}\frac{1}{1890.32} .32 \\
1897.32\end{array}$ & \begin{tabular}{|l|}
31.6053 \\
31.6219 \\
\end{tabular} & \begin{tabular}{|l|}
50.8 \\
50.6
\end{tabular} & $\begin{array}{l}12.0 \\
12.0\end{array}$ & $\begin{array}{l}40.5 \\
40.2 \\
\end{array}$ & 2.770 & \begin{tabular}{|l|l|}
0.010 \\
\end{tabular} & $\frac{0.011}{0.011}$ & 0.000 & & $\begin{array}{l}0.22 \\
0.23\end{array}$ \\
\hline & $8 / 31 / 2004$ & 3:31:41 PM & & & & 26.362 & & 47.445 & \begin{tabular}{|l|}
51.707 \\
\end{tabular} & 15.802 & 32.613 & \begin{tabular}{|l|}
-1.212 \\
\end{tabular} & & 50.607 & 0.071 & \begin{tabular}{|l|l|}
14.643 \\
\end{tabular} & 0.003 & $\frac{1898.32}{11998.32}$ & 31.6386 & 50.6 & & & & & & & & \\
\hline & $8 / 31 / 2004$ & 3:32:41 PM & & 25.308 & \begin{tabular}{|l|l|}
22.846 \\
\end{tabular} & 26.412 & & 47.354 & & & & -1.212 & & 51.47 & $\begin{array}{l}0.069 \\
\end{array}$ & \begin{tabular}{|l|l|}
14.643 \\
\end{tabular} & & 1899.32 & 31.6553 & 50.7 & 12.3 & 40.1 & & & & 0.000 & 0.262 & \\
\hline 1201 & $8 / 31 / 2004$ & 3:33:41 PM & 24.4 & 25.315 & \begin{tabular}{|l|l|}
22.848 \\
2.801
\end{tabular} & $\begin{array}{l}26.498 \\
36.519\end{array}$ & $\begin{array}{r}25.38 \\
\end{array}$ & $\begin{array}{l}47.434 \\
\end{array}$ & \begin{tabular}{|r|}
51.91 \\
5206
\end{tabular} & $\begin{array}{l}15.62 \\
15.567 \\
19\end{array}$ & \begin{tabular}{|l|}
32.793 \\
32325 \\
\end{tabular} & $\begin{array}{l}-1.212 \\
\end{array}$ & & 50.279 & $\begin{array}{l}0.071 \\
\end{array}$ & $\begin{array}{l}14.643 \\
\end{array}$ & 0.003 & 1900.32 & 31.6719 & 50.8 & 12.0 & 40.1 & 2.766 & 0.010 & 0.011 & 0.000 & $\begin{array}{l}0.269 \\
\end{array}$ & \\
\hline & $\begin{array}{l}8 / 31 / 2004 \\
8 / 312004\end{array}$ & 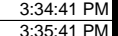 & 24.397 & $\begin{array}{l}25.318 \\
25.348\end{array}$ & \begin{tabular}{|l|}
22.801 \\
22.851 \\
\end{tabular} & $\frac{26.581}{26.642}$ & $\begin{array}{l}25.418 \\
25.409\end{array}$ & \begin{tabular}{|l|}
47.756 \\
47.45 \\
\end{tabular} & \begin{tabular}{|l|}
52.067 \\
51.73 \\
\end{tabular} & \begin{tabular}{|c|}
15.567 \\
1567
\end{tabular} & & \begin{tabular}{l|l|l|}
-1.218 \\
-1.212
\end{tabular} & & $\begin{array}{l}50.738 \\
49856\end{array}$ & \begin{tabular}{|c|c|}
0.071 \\
\end{tabular} & $\begin{array}{l}14.643 \\
14643 \\
\end{array}$ & & $\begin{array}{l}1901.32 \\
1902.32\end{array}$ & $\begin{array}{l}31.6886 \\
31.705\end{array}$ & $\begin{array}{l}50.9 \\
506\end{array}$ & 12.1 & 40.5 & 2.792 & 0.010 & & 0.000 & $\begin{array}{l}0.267 \\
0.256\end{array}$ & \\
\hline & 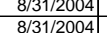 & $\begin{array}{l}3: 5: 4: 41 \mathrm{PM} \\
3\end{array}$ & $\begin{array}{l}24.408 \\
24.408\end{array}$ & $\begin{array}{l}25.348 \\
25.343 \\
\end{array}$ & \begin{tabular}{|l|}
22.8511 \\
22.806 \\
\end{tabular} & $\frac{26.642}{26.667}$ & $\begin{array}{l}25.409 \\
25.284 \\
\end{array}$ & \begin{tabular}{|}
47.445 \\
47.625 \\
\end{tabular} & \begin{tabular}{|l|}
51.731 \\
52.125 \\
\end{tabular} & $\begin{array}{r}15.67 \\
15.638\end{array}$ & \begin{tabular}{|l|}
32.876 \\
33.085 \\
\end{tabular} & $\begin{array}{l}-1.212 \\
-1.215\end{array}$ & $\begin{array}{r}-7.703 \\
-7.703 \\
\end{array}$ & $\begin{array}{l}45.086 \\
51.028\end{array}$ & $\begin{array}{r}0.07 \\
0.069\end{array} \mid$ & $\begin{array}{r}\mid 14.643 \\
14.643\end{array}$ & $\begin{array}{r}0.003 \\
0.003\end{array}$ & & 31.7053 & $\begin{array}{l}50.6 \\
510\end{array}$ & $\frac{11.9}{12.2}$ & 40.4 & $\begin{array}{l}2.769 \\
2.782\end{array}$ & 0.010 & 0.011 & 0.000 & 0.265 & 0.23 \\
\hline & $8 / 31 / 2004$ & 3:37:41:41 PM & 24.427 & 25.378 & \begin{tabular}{|l|}
22.800 \\
22.846 \\
\end{tabular} & \begin{tabular}{|l|}
20.601 \\
26.661 \\
\end{tabular} & 25.243 & 47.339 & \begin{tabular}{|l|}
51.901 \\
\end{tabular} & 15.661 & 32.664 & \begin{tabular}{|l|}
-1.212 \\
\end{tabular} & & $\begin{array}{l}51.028 \\
50.511\end{array}$ & 0.07 & \begin{tabular}{|l|l}
14.645 \\
14.643
\end{tabular} & $\begin{array}{l}0.003 \\
0.003\end{array}$ & $\begin{array}{l}1903.32 \\
1904.32\end{array}$ & $\begin{array}{l}31.7219 \\
31.7386\end{array}$ & $\begin{array}{l}51.0 \\
50.8\end{array}$ & 12.0 & & & & & & & $\begin{array}{l}0.22 \\
0.23 \\
\end{array}$ \\
\hline & & 3:38:41 PM & 24.449 & 25.394 & 22.862 & 26.647 & 25.195 & 47.428 & \begin{tabular}{|l|l|}
51.847 \\
\end{tabular} & 15.557 & 32.951 & \begin{tabular}{|c|}
-1.212 \\
\end{tabular} & & 47.566 & 0.07 & $\begin{array}{l}14.643 \\
\end{array}$ & & & & 50.7 & & & & & & & & \\
\hline & $8 / 31 / 2004$ & 3:39:41 PM & 24.441 & & 22.854 & & & & \begin{tabular}{|l|l|}
51.798 \\
\end{tabular} & & 32.949 & -1.215 & & 51.176 & 0.07 & 14.643 & & 1906.32 & 31.7719 & 50.7 & 12.2 & 40.3 & 2.776 & & 0.011 & & 0.265 & \\
\hline & $8 / 31 / 2004$ & 3:40:41 PM & 24.442 & & 22.841 & 26.576 & 25.083 & & & & & & & 50.991 & & 14.643 & 0.003 & & $\begin{array}{l}31.7886 \\
2.78-7\end{array}$ & 50.6 & 12.1 & & & & & & & \\
\hline & \begin{tabular}{|l|}
$8 / 31 / 2004$ \\
$8 / 21204$
\end{tabular} & $\begin{array}{l}3: 41: 41 \mathrm{PM} \\
3.2: 11 \mathrm{PM}\end{array}$ & $\begin{array}{l}24.432 \\
24.407 \\
\end{array}$ & $\begin{array}{l}25.407 \\
25.383 \\
\end{array}$ & \begin{tabular}{|l|l|}
22.845 \\
2.836
\end{tabular} & 26.515 & $\begin{array}{l}25.017 \\
24893 \\
2489\end{array}$ & & \begin{tabular}{|l|}
51.684 \\
5.094
\end{tabular} & & $\begin{array}{ll}32.796 \\
33095\end{array}$ & $\begin{array}{l}-1.209 \\
.212 \\
\end{array}$ & $\begin{array}{r}-7.697 \\
77\end{array}$ & $\begin{array}{l}51.339 \\
50763\end{array}$ & $\begin{array}{l}0.07 \\
0.07 \\
\end{array}$ & \begin{tabular}{|l|l|}
14.643 \\
14633
\end{tabular} & & & & \begin{tabular}{|l|l|}
50.6 \\
510
\end{tabular} & $\frac{12.2}{12 .}$ & 40.0 & $\frac{761}{783}$ & .010 & & & $\begin{array}{ll}0.266 \\
0246\end{array}$ & 0.032 \\
\hline & 8/3/13/2004 & $\begin{array}{l}\text { 3:24:41 PM } \\
\text { 3:43:41 PM }\end{array}$ & 24.407 & $\begin{array}{l}25.383 \\
25.383\end{array}$ & \begin{tabular}{|l|}
22.836 \\
22.811 \\
\end{tabular} & \begin{tabular}{|l|l|}
26.431 \\
26.377 \\
\end{tabular} & $\begin{array}{l}24.893 \\
24.869 \\
\end{array}$ & \begin{tabular}{|l|}
47.652 \\
47316 \\
\end{tabular} & \begin{tabular}{|l|}
52.094 \\
51.899 \\
\end{tabular} & $\begin{array}{l}15.637 \\
15.702\end{array}$ & $\begin{array}{l}33.085 \\
32.603\end{array}$ & $\begin{array}{l}-1.212 \\
-1.215\end{array}$ & $\begin{array}{c}-7.7 \\
-703 \\
\end{array}$ & $\begin{array}{l}50.753 \\
49.512\end{array}$ & $\begin{array}{r}0.07 \\
0.069\end{array}$ & $\frac{14.643}{14.643}$ & & & 13.8219 & $\begin{array}{l}51.0 \\
50.8\end{array}$ & $\frac{12.1}{11.8}$ & $\frac{40.4}{40.0}$ & $\begin{array}{l}.783 \\
7755\end{array}$ & .010 010 & $\frac{0.011}{0.011}$ & 0.000 & 0.264 & $\frac{22}{22}$ \\
\hline & $8 / 31 / 2004$ & 3:44:41 PM & 24.407 & 25.388 & \begin{tabular}{|l|}
22.811 \\
22.831 \\
\end{tabular} & \begin{tabular}{|l|l|}
26.2811 \\
26.286
\end{tabular} & $\begin{array}{l}24.009 \\
24.768\end{array}$ & 47.505 & \begin{tabular}{|l|}
51.8987 \\
51.887 \\
\end{tabular} & 15.667 & $\begin{array}{l}32.869 \\
32.06\end{array}$ & -1.212 & & $\frac{49.512}{51.76}$ & $\begin{array}{l}0.009 \\
0.07 \\
\end{array}$ & $\begin{array}{l}14.643 \\
14.643 \\
\end{array}$ & $\begin{array}{l}0.003 \\
0.003 \\
\end{array}$ & 1911.32 & $\mid \begin{array}{l}11.05000 \\
31.8553\end{array}$ & $\begin{array}{l}50.8 \\
50.8\end{array}$ & $\begin{array}{ll}11.8 \\
12.3 \\
\end{array}$ & $\begin{array}{l}40.0 \\
40.2\end{array}$ & $\frac{2.755}{2.771}$ & $\frac{0.010}{0.010}$ & $\frac{0.011}{0.011}$ & $\begin{array}{l}0.000 \\
0.000\end{array}$ & $\begin{array}{l}0.205 \\
0.265 \\
\end{array}$ & $\begin{array}{l}0.22 \\
0.23 \\
\end{array}$ \\
\hline & $8 / 31 / 2004$ & 3:45:41 PM & 24.397 & 25.382 & 22.82 & 26.23 & 24.717 & \begin{tabular}{|l|l}
47.831 \\
\end{tabular} & 52.067 & 15.783 & 33.11 & -1.212 & -7.703 & 50.075 & 0.07 & 14.643 & & & 31.8719 & 50.9 & 11.9 & 40.5 & & 0.010 & 0.011 & & 0.263 & \\
\hline & $8 / 31 / 2004$ & 3:46:41 PM & 24.401 & 25.386 & 22.849 & & 24. & 47.436 & 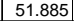 & 15.761 & 32.599 & $\begin{array}{l}-1.212 \\
\end{array}$ & & 49.877 & & \begin{tabular}{l|l|l}
14.643 \\
\end{tabular} & & & & 50.8 & & & & & & & & 0.22 \\
\hline & $8 / 31 / 2004$ & 3:47:41 PM & 24.363 & 25.353 & 22.781 & 26.142 & & $\begin{array}{l}47.364 \\
\end{array}$ & & & 32.923 & & & 49.898 & & \begin{tabular}{|l|l|l|l}
14.643 \\
\end{tabular} & & & .0053 & 50.7 & & & & & & & & \\
\hline & & 3:48:41 PM & & & & 26.145 & & & & 15.691 & 32.997 & -1.218 & & & 0.07 & 14.643 & & & & & & & & & & & & \\
\hline & $8 / 31 / 2$ & 3:49:41 PM & 24.382 & 25.373 & 22.851 & 26.131 & $24 . \varepsilon$ & 47.341 & $\begin{array}{l}51.669 \\
\end{array}$ & & 32.822 & $\begin{array}{l}-1.209 \\
\end{array}$ & & 49.1 & (n) & 14.643 & & & & 50 & & & & & & & 0.266 & \\
\hline & $8 / 31 / 2004$ & 3:50:41 PM & 24.382 & 25.367 & 22.83 & 26.135 & & 47.67: & 52.02 & 15.767 & 32.954 & -1.21 & & & 0.068 & 14.643 & & & 1.9553 & 50.9 & 12 & & & & & & & \\
\hline & $8 / 31 / 2$ & 3:51:41 PM & 24.366 & 25.351 & 22.829 & 26.089 & 24. & 47.484 & 51.804 & 15.661 & 32.904 & -1.21 & -7.7 & 52.075 & & 14.643 & & प18. & 19 & 50.7 & 12.4 & & & & & & & \\
\hline & & 3:52:41 PM & 24.377 & 2 & 22.905 & 26.12 & & 47.9 & 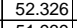 & 15.75 & 33.2 & & & 49.551 & 0.068 & 14.643 & & & & 51.2 & & & & & & & & \\
\hline & $8 / 31 / 2$ & $441 \mathrm{PM}$ & & 25.348 & & & & & 51.93 & 15.706 & 32.83 & -1.212 & & 51.1 & . 069 & 14.643 & & & & & & & & & & & & \\
\hline & $8 / 31 / 2$ & 3:54:41 PM & 24.36 & 25.341 & 2.949 & 26.289 & & 4.1 .8 & 52.333 & 15.50 & 33.282 & -1.212 & & & 060 & & & & & 51.24 & & & & & & & & \\
\hline & $8 / 31 / 2004$ & $\begin{array}{l}\text { 3:5:5:14 PM } \\
\text { 3:56:41 PM }\end{array}$ & $\begin{aligned} 24.385 \\
24.4\end{aligned}$ & $\frac{25.355}{25.371}$ & 22.979 & $\frac{26.344}{26.414}$ & & $\begin{array}{ll}47.25 \\
47.38\end{array}$ & $\frac{51.908}{51.847}$ & $\frac{15.583}{15.688}$ & 32.703 & 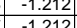 & & & 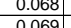 & $\frac{14.643}{14643}$ & & & & 507 & & & & & & & & \\
\hline & $8 / 31 / 2004$ & 3:55: & 24.412 & 25.383 & 22.986 & 26.486 & & 47.16 & 51.582 & 15.547 & 32.7 & & & & 0.069 & 14643 & & & & & 118 & & & & & & & \\
\hline & $8 / 31 / 2004$ & 3:58:41 PM & 24.448 & 25.428 & 23.001 & \begin{tabular}{|l|}
26.521 \\
\end{tabular} & 25.211 & 47 & \begin{tabular}{|l|l|}
51.632 \\
\end{tabular} & 15.521 & 32.906 & \begin{tabular}{|c|}
$\mid-1.212$ \\
\end{tabular} & & & & 14.6 & & & & 50 & $\frac{11}{11}$ & & & & & & & \\
\hline & & & 24.468 & 25.449 & 23.002 & & & 47.801 & \begin{tabular}{|l|l|} 
& 52.304 \\
\end{tabular} & 15.864 & & -1.212 & & 50.7 & 0.06 & & & & & 51.2 & & & & & & & & \\
\hline & & & & 25.461 & & 26.5 & & & & & & & & & & & & & & & & & & & & & & \\
\hline & & & & 25.459 & 22.992 & 26.6 & 25.3 & 47.5 & 51.95 & $15.5 \mathrm{\varepsilon}$ & 33.017 & -1.21 & & 50.4 & & 14. & & & 32.1386 & 50.8 & & & & & & & 256 & \\
\hline & & & 24.467 & 25.468 & 23.011 & & & & & & & & & & & & & & & & & & & & & & & \\
\hline & $8 / 31 / 2$ & & & 25.46 & \begin{tabular}{|l|l|}
22.979 \\
\end{tabular} & & & & 52.08 & & & & & & & & & & & 510 & $110 \mathrm{C}$ & & & & & & & \\
\hline & $8 / 31 / 2004$ & & 24.433 & 25.448 & \begin{tabular}{|l|}
22.941 \\
\end{tabular} & & & & 52.16 & 15.642 & 33.144 & -1.2. & & & 0.068 & 14.6 & & & & 51.8 & 12 & & & & & & 0.255 & \\
\hline & $8 / 31 / 2$ & & 24. & 25.45 & & 26.5 & & & 51.94 & & & & & & 0.00 & 14. & & & & 0.0 & & & & & & & & \\
\hline & 8/31/2004 & & & 25.452 & 22.97 & & & 47.3 & \begin{tabular}{|l|}
51.76 \\
\end{tabular} & 15.624 & 32.674 & -1.205 & & & 0.068 & & & & & 50.6 & & & & & & & 0.258 & \\
\hline & & & & & & & & & & & & & & & & & & & & , & & & & & & & & \\
\hline & & & & & & & & & & & 32.163 & & & & & & & & & & & & & & & & & \\
\hline & $8 / 31 / 2$ & & $\frac{24.387}{24.392}$ & $\frac{25.41}{25.41}$ & 22.925 & $26.255^{2}$ & & & $\begin{array}{r}51.843 \\
52.09 \\
\end{array}$ & $\frac{15.641}{15.686}$ & 33.027 & -1.21 & & & & $\frac{14 .}{14 .}$ & & & & 510 & & & & & & & 258 & \\
\hline & $8 / 31 / 2004$ & & 24 & & & & & & & 15.66 & & & & & & & & & & 51.0 & 12.1 & & & & & & 0.257 & \\
\hline & $8 / 31 / 2$ & & & & & & & & & & & & & & & & & & & 50.9 & 12 & & & & & & & \\
\hline & $8 / 31 / 2004$ & & 24.381 & 25.412 & \begin{tabular}{|l|}
22.95 \\
\end{tabular} & 26.145 & & & \begin{tabular}{|l|l|}
52.218 \\
\end{tabular} & 15.71 & 32. & -1.209 & & 51.3 & 0 & & & & 32. & 51.1 & 12.2 & & & & 0.4 & 0. & 0.252 & \\
\hline & $8 / 31 / /$ & & & & & & & & & & & & & & & & & & & & & & & & & & & \\
\hline & $8 / 31 / 2004$ & & 24.355 & 25.39 & 22.923 & & 24. & 47.6 & \begin{tabular}{|l|}
52.059 \\
\end{tabular} & 15.744 & 33.013 & -1.212 & & 50.9 & $\begin{array}{ll}0.067 \\
\end{array}$ & 14. & & & \begin{tabular}{|l|}
32.3719 \\
\end{tabular} & 50.9 & 12.1 & & & & & & 0.252 & \\
\hline & $8 / 31 / 200$ & $\begin{array}{l}\text { 4:16:41 PM } \\
147: 41 \mathrm{PM}\end{array}$ & $\frac{24.378}{24384}$ & 25.403 & $\frac{22.947}{22.953}$ & $\frac{26.062}{26053}$ & 24.6 & $\begin{array}{l}47.779 \\
47.453\end{array}$ & \begin{tabular}{|l|}
52.279 \\
51707 \\
\end{tabular} & $\frac{15.668}{15699}$ & $\begin{array}{r}33.243 \\
33.941 \\
\end{array}$ & $\begin{array}{l}-1.212 \\
-1.212\end{array}$ & 770 & $\frac{50.4}{542}$ & $\begin{array}{ll}0.068 \\
0.067\end{array}$ & $\begin{array}{l}14.643 \\
1.643 \\
\end{array}$ & & & 32.3 & $\frac{51.2}{50.6}$ & 12.0 & $\frac{40}{40}$ & 277 & & & $\begin{array}{l}00 \\
00\end{array}$ & 0.255 & \\
\hline & $8 / 31 / 200$ & & 24.384 & $\frac{25.415}{25.396}$ & $\frac{22.953}{22929}$ & & & & \begin{tabular}{|l|}
51.00 \\
52051 \\
\end{tabular} & $\frac{15.649}{15719}$ & & $\frac{-1.212}{-1.215}$ & & & & & & & & & & & & & & & & \\
\hline & & & & & & & & & & & & & & & & & & & & & & & & & & & & \\
\hline & $8 / 31 / 2$ & & $\frac{24}{2}$ & 25.385 & $\frac{25.963}{22.963}$ & & & & $\mid 51.818$ & 15.565 & & $\frac{-1.21}{-1.21}$ & & & & 14. & & & & 50.7 & $\mid$ & & & & & & & \\
\hline & & & & & & & & & & & & & & & & & & & & & & & & & & & & \\
\hline & & & & & & & & & & & & & & & & & & & & & & & & & & & & \\
\hline & $8 / 31 / 20$ & & & & 22.966 & & & & 52.3 & & & & & & & & & & & & & & & & & & & \\
\hline & $8 / 31 / 2$ & 4:24: & $24.36 \mathrm{~K}$ & 25.3 & 958 & 26.2 & & 47. & 52.544 & 15.8 & 32.91 & -1.21 & & & & 14. & & & & & 1 & & & & & & & \\
\hline & $8 / 31 / 200$ & $25:$ & & 25.37 & 955 & 26. & & 47.4 & 52.0 & 15.6 & 32.8 & -1.21 & & & & 14.6 & & & & & & & & & & & & \\
\hline & $8 / 31 / 2$ & $4: 26: 4$ & & 25.38 & 22.987 & & & & & 15.7 & & -1.21 & & & & 14. & & & & & & & & & & & & \\
\hline & $8 / 31 / 2$ & & & 25.3 & 23. & & & & & 15.637 & & -1.212 & & & & & & & & .6 & & & & & & & & \\
\hline & & & & & & & & & & & & & & & & & & & & & & & & & & & & \\
\hline & & & 24 & 25.391 & 22.99 & 26 & & $4 \pi$ & 51.8 & & & -1.215 & & & & & & & & & & & & & & & & \\
\hline & $8 / 121 / 200^{4}$ & 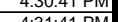 & $\begin{array}{l}2.4 .40 \\
20.23\end{array}$ & 20.411 & $\frac{23.024}{23027}$ & 20.4 & 25.5 & 417.0 & 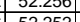 & 13.1.1998 & $\begin{array}{l}32.051 \\
32702\end{array}$ & - & & (51. & 年 & $\begin{array}{l}1.6435 \\
1.643\end{array}$ & & & & S1.1. & $\frac{12.2}{1.21}$ & & & & & & . & \\
\hline 1260 & $\mid$ & $4: 32: 41 \mathrm{PM}$ & \begin{tabular}{|l|}
24.420 \\
24.417 \\
\end{tabular} & 25.418 & \begin{tabular}{|l|}
23.011 \\
23.011 \\
\end{tabular} & $\begin{array}{l}1 \\
1126.526 \\
\end{array}$ & 25.283 & 4 & \begin{tabular}{|l|}
52.223 \\
\end{tabular} & \begin{tabular}{|l|l|}
15.741 \\
\end{tabular} & 32.871 & \begin{tabular}{|l|l|} 
& -1.21 \\
\end{tabular} & & 50.709 & 年 & \begin{tabular}{|l|}
14.04043 \\
14.643
\end{tabular} & & 1959.32 & \begin{tabular}{|l|}
32.6553 \\
\end{tabular} & \begin{tabular}{|l|}
51.1 \\
51.1 \\
\end{tabular} & \begin{tabular}{|l|}
12.1 \\
12.1 \\
\end{tabular} & & \begin{tabular}{|l|l|l|}
2.773 \\
\end{tabular} & \begin{tabular}{|l|}
0.009 \\
\end{tabular} & \begin{tabular}{|c|}
0.010 \\
\end{tabular} & 0.000 & \begin{tabular}{|l|}
0.249 \\
0.249
\end{tabular} & \\
\hline
\end{tabular}


WSRC-TR-2005-00105, REVISION 0

SRNL-RPP-2005-00012, REVISION 0

RUN \# 4.03A AND B; FIRST AND SECOND HALF OF SLURRY DEWATERING - CONT.

\begin{tabular}{|c|c|c|c|c|c|c|c|c|c|c|c|c|c|c|c|c|c|c|c|c|c|c|c|c|c|c|c|c|}
\hline & A & $B$ & D & $E$ & $\mathrm{~F}$ & G & $\mathrm{H}$ & $\mathrm{J}$ & K & $\mathrm{L}$ & $\mathrm{M}$ & $\mathrm{N}$ & 0 & Q & $\mathrm{R}$ & $\mathrm{s}$ & $\begin{array}{lll}T \\
\end{array}$ & $\mathrm{u} \quad \mathrm{v}$ & w & $x$ & $\mathrm{Y}$ & $z$ & & & & & & \\
\hline & $8 / 31 / 2004$ & 4:33:41 PM & 24.418 & 25.423 & 23.097 & 26.472 & 25.229 & 47.291 & 51.858 & 15.68 & 32.629 & -1.215 & \begin{tabular}{|c|}
-7.689 \\
\end{tabular} & 48.732 & 0.066 & \begin{tabular}{|l|l|}
14.643 \\
\end{tabular} & $\begin{array}{l}0.003 \\
\end{array}$ & 1960.32 & \begin{tabular}{|l|}
32.6719 \\
\end{tabular} & \begin{tabular}{|l|}
50.7 \\
\end{tabular} & 11.6 & 40.0 & 2.755 & 0.009 & 0.010 & 0.000 & 0.250 & \\
\hline & $8 / 31 / 2004$ & 4:34:41 PM & 24.417 & 25.417 & 23.206 & & 25.137 & $\begin{array}{l}47.322 \\
\end{array}$ & 51.756 & \begin{tabular}{|l|l|}
15.653 \\
\end{tabular} & 32.679 & $\begin{array}{l}-1.212 \\
\end{array}$ & & 50.738 & 0.066 & \begin{tabular}{|l|l|}
14.643 \\
\end{tabular} & 0.003 & 1961.32 & $\begin{array}{ll}32.6886 \\
\end{array}$ & \begin{tabular}{|l|l|}
50.6 \\
\end{tabular} & & & & & & & & \\
\hline & $8 / 31 / 2004$ & 4:35:02 PM & 24.397 & 25.398 & \begin{tabular}{|l|}
23.202 \\
3.20 \\
\end{tabular} & $\frac{26.361}{2.321}$ & 25.133 & $\begin{array}{l}47.528 \\
\end{array}$ & \begin{tabular}{|l|l|}
52.015 \\
5.190
\end{tabular} & \begin{tabular}{|l|l|}
15.698 \\
1562
\end{tabular} & \begin{tabular}{|l|l|}
32.913 \\
2.53
\end{tabular} & $\begin{array}{l}-1.215 \\
-1.215\end{array}$ & $\begin{array}{r}-7.692 \\
.782 \\
\end{array}$ & \begin{tabular}{|l}
48.296 \\
5.237
\end{tabular} & \begin{tabular}{|l|}
0.067 \\
\end{tabular} & \begin{tabular}{|l|}
14.643 \\
\end{tabular} & 0.003 & 1961.67 & 32.6944 & \begin{tabular}{|l|}
50.9 \\
\end{tabular} & 11.5 & 40.2 & 2.773 & 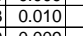 & 0.010 & 0.000 & 0.251 & \\
\hline$\frac{1264}{1265}$ & $8 / 31 / 2004$ & 4:36:02 PM & 24.41 & 25.4 & 23.169 & 26.338 & $\begin{array}{l}25.07 \\
\end{array}$ & $\begin{array}{l}47.262 \\
\end{array}$ & \begin{tabular}{|l|l|}
51.889 \\
\end{tabular} & \begin{tabular}{|l|}
15.713 \\
\end{tabular} & 32.518 & $\begin{array}{l}-1.215 \\
\end{array}$ & $\begin{array}{r}-7.689 \\
\end{array}$ & 51.37 & $\begin{array}{l}0.066 \\
\end{array}$ & $\begin{array}{l}14.643 \\
\end{array}$ & 0.003 & 1962.67 & 32.7111 & \begin{tabular}{|l|}
50.8 \\
\end{tabular} & 12.2 & 39.9 & 2.750 & 0.009 & 0.010 & 0.000 & $\begin{array}{l}0.249 \\
\end{array}$ & \\
\hline & $\frac{8 / 31 / 2004}{88 / 312004}$ & $\begin{array}{l}4: 37: 02 \mathrm{PM} \\
4.38 .02 \mathrm{PM}\end{array}$ & $\frac{24.406}{24.47}$ & $\begin{array}{l}25.386 \\
25.377\end{array}$ & & & $\begin{array}{r}25.012 \\
20957 \\
\end{array}$ & & \begin{tabular}{|l|}
52.571 \\
511441 \\
\end{tabular} & \begin{tabular}{|l|}
15.742 \\
15716
\end{tabular} & & & $\begin{array}{r}-7.689 \\
\end{array}$ & $\begin{array}{l}53.033 \\
51.207\end{array}$ & & \begin{tabular}{|l|l|l|}
14.643 \\
14634 \\
\end{tabular} & & 1963.67 & & \begin{tabular}{|l|}
51.4 \\
50.8 \\
\end{tabular} & \begin{tabular}{|l|l|} 
& 12.6 \\
122 & \\
\end{tabular} & & \begin{tabular}{|l|}
2.786 \\
.7552
\end{tabular} & \begin{tabular}{|l|l|}
0.0099 \\
\end{tabular} & $\begin{array}{l}0.010 \\
\end{array}$ & & $\begin{array}{l}0.247 \\
\end{array}$ & \\
\hline 1267 & & $\begin{array}{l}4: 38: 02 \text { PM } \\
4: 3902 \mathrm{P}\end{array}$ & $\begin{array}{l}24.407 \\
24.407\end{array}$ & $\begin{array}{l}25.377 \\
25.373\end{array}$ & \begin{tabular}{|l|}
23.101 \\
23.062 \\
\end{tabular} & $\begin{array}{l}26.205 \\
26.191\end{array}$ & $\begin{array}{r}24.957 \\
24.903 \\
\end{array}$ & \begin{tabular}{|l|}
47.254 \\
47.242
\end{tabular} & \begin{tabular}{|}
$\mid 51.941$ \\
51.837
\end{tabular} & $\begin{array}{l}\frac{15.716}{15.651} \\
\end{array}$ & $32.568 \mid$ & \begin{tabular}{|c|}
$\mid-1.212$ \\
-1.212 \\
\end{tabular} & $\begin{array}{c}-7.694 \\
-7.689 \\
\end{array}$ & $\begin{array}{l}51.207 \\
51.698\end{array}$ & $\mid \begin{array}{l}0.066 \\
0.066\end{array}$ & \begin{tabular}{|l|}
14.6634 \\
14.643
\end{tabular} & $\begin{array}{l}0.003 \\
0.003\end{array}$ & 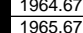 & \begin{tabular}{|c|}
32.72744 \\
32.7611
\end{tabular} & \begin{tabular}{|l|}
50.8 \\
50.7 \\
\end{tabular} & $\begin{array}{l}32.2 \\
12.3 \\
3\end{array}$ & 39.9 & \begin{tabular}{|l|}
2.752 \\
2.756 \\
\end{tabular} & \begin{tabular}{|c|}
0.009 \\
0.009
\end{tabular} & \begin{tabular}{|c|c|}
0.010 \\
0.010
\end{tabular} & \begin{tabular}{|c|}
0.000 \\
0.000
\end{tabular} & 0.250 & \\
\hline 1268 & $8 / 31 / 2004$ & 4:40:02 PM & 24.413 & 25.373 & & & & & \begin{tabular}{|l|}
52.044 \\
\end{tabular} & 15.55 & & & $\begin{array}{r}-7.689 \\
\end{array}$ & 52.855 & 0.067 & 14.643 & $\begin{array}{l}0.003 \\
0.003\end{array}$ & $\begin{array}{l}1963.07 \\
1966.67\end{array}$ & 32.1711 & \begin{tabular}{|l|}
50.9 \\
5
\end{tabular} & $\begin{array}{l}12.3 \\
12.6\end{array}$ & 40.0 & & $\begin{array}{l}0.009 \\
0.010\end{array}$ & $\frac{0.010}{0.010}$ & $\begin{array}{l}0.000 \\
0.000\end{array}$ & & $\begin{array}{l}0.21 \\
0.21\end{array}$ \\
\hline & $8 / 31 / 2004$ & & 24.443 & 25.388 & 23.072 & & 24.729 & 47.453 & \begin{tabular}{|l|}
52.015 \\
\end{tabular} & 15.655 & & \begin{tabular}{|l|}
-1.212 \\
\end{tabular} & -7.694 & 49.679 & & 14.643 & & 1967.67 & 32.7944 & \begin{tabular}{|r|}
50.9 \\
\end{tabular} & & & & & & & & \\
\hline & $8 / 31 / 2004$ & 4:42:02 PM & 24.437 & 25.388 & 23.047 & 26.216 & $\begin{array}{l}24.723 \\
\end{array}$ & 47.225 & \begin{tabular}{|l|l|l|}
51.713 \\
\end{tabular} & 15.65 & 32.646 & -1.212 & \begin{tabular}{|l|}
-7.694 \\
\end{tabular} & 49.735 & .066 & \begin{tabular}{|l|l|}
14.643 \\
\end{tabular} & & 1968.67 & 22.8111 & 50.6 & 11.8 & 39.9 & 2.753 & 0.009 & 0.010 & 0.000 & & \\
\hline 1271 & & & 24.447 & & 23.036 & 26.25 & & & 51.951 & & 32.646 & & & 51.053 & & \begin{tabular}{l|l}
14.643 \\
\end{tabular} & & & & & & & & .009 & & & 0.246 & \\
\hline 1272 & & & 24.456 & 25.391 & & & & 47.447 & & 15.722 & 32.685 & & & & & 14.643 & & 70.67 & .8444 & 51.0 & 12.6 & & & & & & 0.253 & \\
\hline 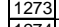 & & $02 \mathrm{PM}$ & 24.461 & 25.401 & 23.01 & 26.37 & & 47.405 & 52.144 & 15.756 & & & & & & & & & & & & & & & & & 0.254 & \\
\hline & 8/31/2004 & 16:02 PM & 24.471 & 25.421 & 23.015 & 26.41 & & 477.611 & 52.303 & $\begin{array}{l}15.816 \\
\end{array}$ & 32.75 & -1.215 & -7.68 & 49.883 & 066 & \begin{tabular}{|l|l|l|l}
14.643 \\
\end{tabular} & 0.00 & 2.67 & & 51.2 & & 40.2 & 2.770 & 0.009 & & 0.000 & 0.249 & \\
\hline & $8 / 31 / 2004$ & 4:47:02 PM & 24.482 & 25.428 & 23.036 & 26.481 & 25.113 & 47.745 & 52.589 & 15.805 & 32.855 & -1.215 & -7.68 & 50.653 & 0.065 & $\begin{array}{l}14.643 \\
\end{array}$ & 0.003 & 973.67 & 32.8944 & 51.5 & 12.1 & 40.3 & 2.779 & 0.009 & 0.010 & 0.000 & 0.244 & \\
\hline & & 3:02 PM & 24.489 & 25.434 & 23.023 & 26.577 & 25.17 & 47.464 & \begin{tabular}{|l|}
52.076 \\
5.077 \\
\end{tabular} & 15.749 & $\begin{array}{l}32.845 \\
2.720\end{array}$ & $-\frac{1.215}{-2.12}$ & & 52.044 & 666 & 14.643 & & 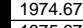 & 2.9111 & 50.9 & & & & & & & $\begin{array}{ll}0.249 \\
\end{array}$ & \\
\hline & |8/31/12004 & 4:49:02 PM & & & 23.03 & 615 & & 47.082 & 51.677 & 15.511 & 32.738 & -1.212 & & 52.048 & & 14.643 & & 975.6 & & & & & & & & & 0.247 & \\
\hline & $8 / 31 / 2004$ & $0.02 \mathrm{PM}$ & & & 23.015 & 26.635 & & & & & 33.099 & & & & & & & & & & & & & & & & & \\
\hline & $8 / 31 / 2$ & $1: 02 \mathrm{PM}$ & & $\frac{25.448}{25.44}$ & 23.002 & 26.612 & & 47.565 & $\begin{array}{c}52.374 \\
5309\end{array}$ & $\frac{15.769}{15.923}$ & $\begin{array}{l}32.773 \\
32.486\end{array}$ & $-\frac{1.212}{1.212}$ & & 50.569 & & $\frac{14.643}{14643}$ & & 77.6 & 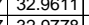 & 51.2 & & & & 00 & & & 0.249 & \\
\hline$\overline{1281}$ & $8 / 3131 / 2004$ & & 24506 & $\frac{25.44}{25.456}$ & $\frac{2.300}{23015}$ & & & & & & $\begin{array}{r}32.4866 \\
32.57\end{array}$ & & & $\frac{43.040}{4878}$ & 2066 & $\begin{array}{l}1.0 .045 \\
114643\end{array}$ & & & $\frac{32.978}{32.9944}$ & 505 & & & 749 & & & & & \\
\hline & $8 / 31 / 2004$ & 4:54:02 PM & 24.52 & $\frac{2.400}{25.47}$ & \begin{tabular}{|l|}
23.014 \\
23.014
\end{tabular} & 26.568 & 25.14 & $\begin{array}{l}47.11 \\
47.424\end{array}$ & \begin{tabular}{|l|}
51.833 \\
\end{tabular} & 15.616 & \begin{tabular}{|c|}
33.095 \\
\end{tabular} & \begin{tabular}{|l|}
-1.212 \\
\end{tabular} & -7.67 & 50.16 & & $\begin{array}{l}14.045 \\
14.643\end{array}$ & 0.004 & 1980.67 & 33.0111 & 50.7 & $\frac{11.0}{11.9}$ & 40.3 & 2.776 & 0.009 & 0.010 & 0.000 & 0.245 & \\
\hline & $8 / 31 / 2004$ & 4:55:02 PM & 24.511 & 25.466 & \begin{tabular}{|l|l|}
23.005 \\
\end{tabular} & 26.485 & 25.092 & \begin{tabular}{|l|}
47.37 \\
\end{tabular} & \begin{tabular}{|l|}
52.036 \\
\end{tabular} & 15.73 & 32.613 & \begin{tabular}{|l|}
-1.212 \\
\end{tabular} & -7.677 & 50.306 & 0.064 & 14.643 & 0.003 & 1981.67 & 33.0278 & $\frac{50.9}{50.9}$ & & & $\frac{2.757}{2.757}$ & 0.009 & $\frac{0.010}{0.010}$ & & & \\
\hline & $8 / 31 / 2004$ & & & 25.474 & & 26.447 & & & \begin{tabular}{|l|}
51.879 \\
\end{tabular} & & & & -7.67 & 48.631 & & 14.643 & 0.00 & 1982.67 & 3.0444 & & & & & 0.009 & & & 0.246 & \\
\hline & $8 / 31 / 2004$ & 4:57:02 PM & 24.51 & 25.476 & 23.014 & 26.469 & & & 52.393 & 15.819 & 32.681 & -1.212 & & 45.09 & & 14.643 & & & .0611 & $\frac{21.3}{51.3}$ & & & 766 & & 0.01 & & 0 & \\
\hline & $8 / 31 / 2$ & & & & & 26.482 & & & & & & & & 51.9 & & $\begin{array}{ll}14.643 \\
\end{array}$ & & & & & & & & & & & & \\
\hline & $8 / 31 / 2004$ & $02 \mathrm{PM}$ & & 25.482 & 23.02 & 26.435 & 24.982 & & \begin{tabular}{|c|}
51.843 \\
\end{tabular} & 15.626 & 32.656 & -1.212 & & & & $\begin{array}{l}14.643 \\
\end{array}$ & & & .0944 & & & & & & & & 0.247 & \\
\hline & $8 / 31 / 2004$ & 5:00:02 PM & 24.513 & 25.479 & 23.027 & 26.422 & 24.9 & \begin{tabular}{|l|l|}
47.47 \\
\end{tabular} & \begin{tabular}{|l|}
52.165 \\
\end{tabular} & 15.736 & 32.874 & -1.212 & & 49.011 & 1.067| & $\begin{array}{l}14.643 \\
\end{array}$ & 0.004 & $186-2$ & 33.1111 & 51.0 & 11.7 & 40 & & & & & & \\
\hline & $8 / 31 / 2004$ & 5:01:02 PM & 24.512 & 25.487 & \begin{tabular}{|l|}
23.006 \\
\end{tabular} & 26.436 & 24. & 47.349 & 52.181 & 15.67 & 32.623 & -1.212 & -7.68 & 49.708 & 064 & 14.643 & & & & 51.1 & 11.8 & & 1.757 & & 0.01 & & 0.242 & \\
\hline & & & & 25.491 & 23.024 & 26.389 & & & \begin{tabular}{|l|}
51.988 \\
\end{tabular} & 15.678 & 32.83 & -1.2 & & 51.013 & & 14.643 & & & 33.1444 & & & & & & & & 0.249 & \\
\hline & $8 / 31 / 2$ & & & 25.484 & & 26.392 & & 47.466 & 22.084 & 15.742 & 32.83 & -1.215 & & & & 14.643 & & & 3.1611 & 51.0 & & & & & & & 0.245 & \\
\hline & $8 / 31 / 2$ & $02 \mathrm{PM}$ & 24.514 & 25.495 & 23.073 & 26.403 & & & 52.078 & 15.835 & 32.726 & -1.209 & & 49 & & & & & 3.1778 & & 11. & 40 & & .005 & & & .249 & \\
\hline & $8 / 31 / 2004$ & 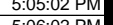 & 24.512 & $\frac{25.492}{2592}$ & 23.161 & 26.4611 & 25.118 & 47.186 & S2.02024 & 15.765 & 32.375 & -1.212 & & 48.1. & & 14.643 & & & & 50.9 & & 10 & & & & & 46 & \\
\hline & & & & & & & & & & & & & & & & & & & & & & & & & & & & \\
\hline & $8 / 31 / 2004$ & & 24.521 & 25.496 & 23.13 & 26.479 & 24. & 46.98 & 51.702 & 15.779 & 32.258 & -1.2 & & 51.577 & & 14.643 & & 202 & & 50.6 & 12. & & & & & & & \\
\hline & $8 / 31 / 2$ & $5: 08$ & & 25.504 & \begin{tabular}{|l|l|}
23.148 \\
\end{tabular} & 26.482 & & 47.314 & \begin{tabular}{|l|}
51.854 \\
\end{tabular} & 15.725 & 32.736 & -1.209 & & 50.044 & & 14.6 & & & $\begin{array}{c}3.2444 \\
\end{array}$ & 50.7 & 11.9 & & & & & & 0.245 & \\
\hline & $8 / 3$ & & 527 & 25.492 & \begin{tabular}{|l|}
23.096 \\
\end{tabular} & 26. & & 47 & \begin{tabular}{|l|} 
\\
\end{tabular} & 15.873 & 32 & -1.215 & & & 1064 & & & & & 51.0 & 11. & & & & & & 241 & \\
\hline & 8 & & & 25.484 & & & & & & & & & & & & & & & & & 11. & & & & & & & \\
\hline & $8 / 31 / 2$ & 5:11:02 PM & 24.533 & 25.493 & 23.077 & 26.4 & & 47.18 & 51.893 & 15.628 & 32.611 & -1.209 & & 48.9 & & & & & 33.2944 & 50.8 & 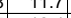 & & & & & & 0.239 & 0.2 \\
\hline & 8 & $02 \mathrm{PM}$ & $\begin{array}{l}24.523 \\
24518\end{array}$ & $\begin{array}{l}25.474 \\
25468\end{array}$ & \begin{tabular}{|l|l|}
23.010 \\
2303
\end{tabular} & $\frac{20.424}{26466}$ & 24. & $\begin{array}{l}47.163 \\
47509\end{array}$ & $\begin{array}{l}51.843 \\
5.071\end{array}$ & $\begin{array}{l}15.121 \\
15715\end{array}$ & $\begin{array}{l}32.471 \\
32898\end{array}$ & $\begin{array}{r}-1.215 \\
-1.215\end{array}$ & & & & . & & & 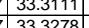 & $\frac{50.7}{509}$ & $\frac{12.4}{12.2}$ & 40 & & & & & 437 & \\
\hline & $8 / 31 / 2004$ & & 24526 & 25.471 & 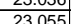 & 26485 & & & 5 & 15714 & $\mid$ & -1212 & & & & 14643 & & & 3.3444 & 512 & 12 & & & & & & & \\
\hline & $8 / 31 / 2004$ & 5:15:02 PM & 24.53 & $\frac{25.475}{25.475}$ & \begin{tabular}{|l|l|}
23.054 \\
\end{tabular} & & & & 55044 & 1573 & & $\frac{-1.212}{1212}$ & & & & 14643 & & & 733611 & 50.9 & 12 & & & & & & & \\
\hline & $8 / 31 / 2004$ & & 24.54 & 25.481 & \begin{tabular}{|l|}
23.049 \\
\end{tabular} & 26.479 & & 47.414 & \begin{tabular}{|l|}
51.899 \\
\end{tabular} & 15.652 & $\frac{22.886}{33.286}$ & -1.212 & & $\frac{50.8}{50.8}$ & & 14.643 & & & & 50.8 & $\frac{12.1}{12.1}$ & & & 0.009 & $\frac{0.0}{0.0}$ & 500 & $\mid$ & \\
\hline & & & & & & & & & & & & & & & & & & & & & 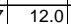 & & & & & & & \\
\hline & & & 24.546 & 25.4 & \begin{tabular}{|l|}
23.039 \\
\end{tabular} & 26. & & & 52.2 & 15.7 & & -1.1. & & & & & & & & 51.2 & 12 & & & & & & $\frac{0.250}{0.250}$ & \\
\hline & & & 24.541 & 25.486 & 23.055 & & & & 51.881 & 15.593 & 32.482 & -1.212 & & & & & & & & & & & & & & & & \\
\hline & $8 / 31 / 2$ & & 24.543 & 25.489 & \begin{tabular}{|l|}
23.037 \\
\end{tabular} & 26. & & 47.443 & 52.227 & 15.693 & 32.8 & \begin{tabular}{|l|l|} 
& -1.215 \\
\end{tabular} & & & & & & & 3.4444 & $\overline{51.1}$ & 11.4 & & & & & & 0.245 & \\
\hline & $8 / 31 / 200$ & 5:21:02 PM & 24.551 & 25.507 & 23.06 & 26.48 & & 47.704 & \begin{tabular}{|l|l|} 
& 52.54 \\
\end{tabular} & 15.756 & $\mid 32.898$ & $\begin{array}{l}-1.212 \\
\end{array}$ & & & & 14.64 & & & & 51.4 & 12. & & & 0.00 & & & 0.236 & 0.20 \\
\hline & $8 / 31 / 2$ & & & 25.515 & 23.044 & & & & & & & & & & & 14. & & & & 51. & & & & & & & & \\
\hline & 8/31/2004 & & 24. & 25.532 & \begin{tabular}{|l|}
23.076 \\
\end{tabular} & 26. & & & 52.1 & 15.687 & 32.767 & -1.2. & & & & & & & & 51.0 & $11 . .5$ & & & & & & 0.238 & \\
\hline & & & $24.5 / 4$ & & & & & & & & & & & & & & & & & & & & & & & & & \\
\hline & & & & 25.542 & 23.075 & & & 47 & \begin{tabular}{|l|}
1.982 \\
\end{tabular} & 15.17 & 32.785 & & & & & & & & & & 111. & & & & & & & \\
\hline & 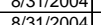 & $\begin{array}{l}5.60 .06 \\
5.570\end{array}$ & & $\begin{array}{l}25.534 \\
25568\end{array}$ & 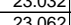 & 26. & & & $\begin{aligned} 52.21 \\
5471\end{aligned}$ & $\begin{array}{l}15.1 \\
157\end{array}$ & $\begin{array}{r}32.72 \\
32882\end{array}$ & $\begin{array}{l}-1.218 \\
-1.212\end{array}$ & & & & & & & & 51.1 & 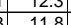 & & & & & & 0.242 & \\
\hline & $8 / 31 / 20$ & & & 25.56 & $\mid 23074$ & 2688 & & 474 & $\frac{52.4}{52 .}$ & 15587 & 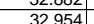 & -1209 & & 503 & & 146 & & & & & & & & & & & & \\
\hline & $8 / 31 / 20$ & & & & & & & & & & & & & & & 14.6 & & & & 51.0 & 12 & & & & & & & \\
\hline & $8 / 31 / 2$ & 5:30:02 PM & 24.587 & 25.567 & 23.151 & 26.8 & & & 51. & 15.685 & 32.662 & \begin{tabular}{|l|l|} 
\\
\end{tabular} & & 51.3 & 0.0 & 14.6 & & & & 50.8 & 12.2 & & & & & & 0.242 & \\
\hline & & & & & & & & & & & & & & & & & & & & & & & & & & & & \\
\hline & & & & & 23.123 & 26.7 & & & & 15. & & & & & & & & & & & & & & & & & & \\
\hline & & & & & 20.140 & & & & & & & & & & & & & & & & & & & & & 000 & & 0 \\
\hline & $8 / 31 / 200$ & 年. & & $\frac{2.05}{25}$ & 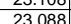 & $\frac{2.05}{265}$ & & 4 & 5205 & $\frac{15.0064}{1574}$ & 年 & -1210 & & $\frac{5.1}{109}$ & & $\frac{1.40}{146}$ & & 020.67 & 33.6 & $\begin{array}{r}50.8 \\
\end{array}$ & 12.2 & $\begin{array}{l}2 \\
2\end{array}$ & 55] & \begin{tabular}{|c|}
0.009 \\
\end{tabular} & & & 0.234 & \\
\hline & $8 / 31 / 2$ & & & 25.61 & 23.078 & & & & & 15679 & 32742 & -1.212 & & & & 146 & & & & & $11.7 \mathrm{C}$ & & & & & & & \\
\hline & & & & $\frac{25.61}{25.61}$ & $=0$ & & & & 51.787 & 15 & 32. & $\frac{-1.215}{-1.215}$ & & & & 14.6 & & & & 507 & 11.9 & & & & & & 0.239 & \\
\hline & & & & 25.5 & & & & & & & & $\frac{-1.2}{-1.2}$ & & & & & & & & & & & & & & & & \\
\hline & & & & & & & & & & & & & & & & & & & & & & & & & & & & \\
\hline & $8 / 31 / 2$ & & & 25.625 & 23.064 & 26.418 & & 47.3 & & 15.84 & 32.3 & -1.209 & & & & 144 & & & & 51.1 & 12.0 & & & & & & 239 & \\
\hline & 311 & & & 624 & (52) & & & 47.4 & & & 22617 & 212 & & 492 & & 4.643 & & & & 51.1 & & & & & & & 2021 & $\overline{02}$ \\
\hline 33. & $8 / 31 / 2004$ & 5:42:02 PM & 24.577 & \begin{tabular}{|l|l|}
25.638 \\
\end{tabular} & \begin{tabular}{|l|}
23.041 \\
\end{tabular} & 26.391 & 24.978 & \begin{tabular}{|l|l}
47.453 \\
\end{tabular} & \begin{tabular}{|l|l|}
52.461 \\
\end{tabular} & \begin{tabular}{|c|}
15.676 \\
\end{tabular} & \begin{tabular}{|l|l|}
32.726 \\
\end{tabular} & \begin{tabular}{|l|}
$\mid-1.212$ \\
\end{tabular} & & 50.444 & 0.065 & \begin{tabular}{|l|l|l|l|l|}
1433 \\
\end{tabular} & & 28.67 & 33.8111 & 51.3 & 12.0 & & $\mid 2.764$ & 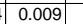 & 0.010 & 0.000 & 0.245 & 0.2 \\
\hline
\end{tabular}


WSRC-TR-2005-00105, REVISION 0

SRNL-RPP-2005-00012, REVISION 0

RUN \# 4.03A AND B; FIRST AND SECOND HALF OF SLURRY DEWATERING - CONT.

\begin{tabular}{|c|c|c|c|c|c|c|c|c|c|c|c|c|c|c|c|c|c|c|c|c|c|c|c|c|c|c|c|c|}
\hline & A & & D & $E$ & $\mathrm{~F}$ & G & $\mathrm{H}$ & $\mathrm{J}$ & $\mathrm{K}$ & $\mathrm{L}$ & $\mathrm{M}$ & $\mathrm{N}$ & 0 & Q & $\mathrm{R}$ & $\mathrm{s}$ & $T$ & $\mathrm{v}$ & w & $x$ & Y & $z$ & $\mathrm{AA}$ & $\mathrm{AB}$ & & & & \\
\hline & $8 / 31 / 2004$ & 5:43:02 PM & 24.575 & 25.64 & 23.054 & 26.333 & 24.931 & $47.46 \varepsilon$ & 52.403 & 15.662 & 32.923 & -1.212 & -7.533 & 50.78 & 0.064 & \begin{tabular}{|l|}
14.643 \\
\end{tabular} & 0.003 & 2029.67 & 33.8278 & \begin{tabular}{|l|}
51.3 \\
\end{tabular} & 12.1 & 40.2 & 2.771 & 0.009 & 0.010 & 0.000 & 0.241 & 0.20 \\
\hline & & & 24.575 & 25.645 & \begin{tabular}{|l|l|}
23.064 \\
\end{tabular} & 26.358 & 24.911 & 47.09 & \begin{tabular}{|l|l|}
52.076 \\
\end{tabular} & $\begin{array}{l}15.612 \\
\end{array}$ & 32.48 & \begin{tabular}{|c|c|}
3 & -1.212 \\
\end{tabular} & $\begin{array}{l}-7.527 \\
-7.527 \\
\end{array}$ & $\begin{array}{r}51.798 \\
\end{array}$ & 0.063 & $\begin{array}{l}14.643 \\
\end{array}$ & & & 73.8444 & 50.9 & 12.3 & & & & 0.010 & & & \\
\hline & $8 / 31 / 2004$ & 5:45:02 PM & 24.568 & 25.644 & \begin{tabular}{|l|}
23.047 \\
\end{tabular} & 26.327 & 24.909 & \begin{tabular}{|l|}
47.497 \\
\end{tabular} & \begin{tabular}{|l|}
52.247 \\
5.78 \\
\end{tabular} & \begin{tabular}{|l|}
15.738 \\
1.58
\end{tabular} & 32.787 & 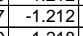 & $\begin{array}{l}-7.527 \\
-7523\end{array}$ & \begin{tabular}{|l|l}
49.145 \\
5.185
\end{tabular} & $\begin{array}{l}0.064 \\
\end{array}$ & \begin{tabular}{|l|}
14.643 \\
\end{tabular} & & $\frac{2031.67}{2032.67}$ & & 51.1 & 11.7 & & 2.768 & .0.009 & 0.010 & 0.000 & 0.241 & \\
\hline 1334 & $8 / 31 / 2004$ & $\begin{array}{l}5: 46: 02 \mathrm{PM} \\
5.57: 02 \mathrm{P}\end{array}$ & $\begin{array}{l}24.551 \\
24534\end{array}$ & $\begin{array}{l}25.617 \\
25.605\end{array}$ & $\begin{array}{r}23.03 \\
2300\end{array}$ & $\begin{aligned} 26.23 \\
\end{aligned}$ & $\begin{array}{l}24.952 \\
25.025\end{array}$ & $\begin{array}{l}47.074 \\
14727\end{array}$ & \begin{tabular}{|l|l|}
51.7655 \\
5216
\end{tabular} & $\begin{array}{l}15.642 \\
1559\end{array}$ & $\begin{array}{r}32.459 \\
2.720\end{array}$ & $\begin{array}{l}-1.218 \\
121\end{array}$ & $\begin{array}{r}-7.533 \\
7.533\end{array}$ & 51.925 & \begin{tabular}{|c|}
0.063 \\
\end{tabular} & \begin{tabular}{|l|l|}
14.643 \\
\end{tabular} & & 2032.67 & $\begin{array}{l}33.8778 \\
20.9\end{array}$ & 50.6 & 12.4 & 39.8 & $\begin{array}{l}2.742 \\
.772\end{array}$ & 0.009 & 0.010 & 0.000 & 0.240 & \\
\hline$\frac{1335}{1336}$ & $\frac{8 / 31 / 2004}{8 / 31204}$ & $\begin{array}{l}5: 47: 02 \mathrm{PM} \\
5.0802 \mathrm{PM}\end{array}$ & $\frac{24.534}{24.529}$ & $\frac{25.605}{25.605}$ & \begin{tabular}{|l|}
23.018 \\
23033
\end{tabular} & $\frac{26.208}{26.197}$ & & $\begin{array}{l}47.279 \\
477277\end{array}$ & \begin{tabular}{|r|}
52.16 \\
52.683 \\
\end{tabular} & $\begin{array}{l}15.592 \\
1563\end{array}$ & $\begin{array}{l}32.769 \\
33032\end{array}$ & \begin{tabular}{|c|}
-1.212 \\
-1213
\end{tabular} & \begin{tabular}{|l|}
-7.533 \\
7.5255
\end{tabular} & $\begin{array}{l}44.422 \\
51.122\end{array}$ & \begin{tabular}{|c|}
0.064 \\
0.064
\end{tabular} & \begin{tabular}{|l|l|}
14.643 \\
14643 \\
\end{tabular} & & & $\begin{array}{l}33.8944 \\
230111\end{array}$ & 51.0 & \begin{tabular}{|l|} 
\\
\end{tabular} & & & & 0.010 & & 0.242 & \\
\hline $\begin{array}{l}1337 \\
1337\end{array}$ & $\begin{array}{l}8 / 31 / 204 \\
8 / 31 / 2004\end{array}$ & $\begin{array}{l}\text { 5:48:02 PM } \\
\text { 5:49:02 PM }\end{array}$ & $\frac{24.529}{24.512}$ & $\frac{25.605}{25.597}$ & \begin{tabular}{|l|}
23.033 \\
23.011 \\
\end{tabular} & $\begin{array}{r}26.197 \\
26.27\end{array}$ & $\begin{array}{r}25.14 \\
25.122 \\
\end{array}$ & $\begin{array}{l}47.727 \\
477.192\end{array}$ & \begin{tabular}{|l|}
52.683 \\
52.075 \\
\end{tabular} & \begin{tabular}{|l|}
15.673 \\
15.748 \\
\end{tabular} & $\begin{array}{r}33.032 \\
32.469 \\
\end{array}$ & \begin{tabular}{|c|c|}
-1.218 \\
-1.212
\end{tabular} & $\begin{array}{r}-7.535 \\
-7.53 \\
\end{array}$ & $\begin{array}{l}51.1222 \\
50.861\end{array}$ & 0.0664 & $\begin{array}{r}14.643 \\
14.643 \\
\end{array}$ & .003 003 & $\begin{array}{l}2034.67 \\
2035.67\end{array}$ & 33.9111 & $\begin{array}{l}51.6 \\
50.9\end{array}$ & $\begin{array}{l}\frac{12.2}{12.1} \\
\end{array}$ & $\begin{array}{l}40.4 \\
39.8 \\
\end{array}$ & \begin{tabular}{|l|}
$\mid 2.784$ \\
\\
\end{tabular} & $\mid .009$ & $\frac{0.010}{0.0010}$ & $\begin{array}{l}0.000 \\
0.000\end{array}$ & $\frac{0.240}{0.240}$ & 0.20 \\
\hline \begin{tabular}{|l|}
1338 \\
\end{tabular} & $8 / 31 / 2004$ & 5:50:02 PM & 24.503 & 25.588 & & 26.291 & 25.143 & 47.198 & \begin{tabular}{|l|}
52.026 \\
\end{tabular} & 15.479 & 32.732 & & & 50.066 & 0.065 & $\begin{array}{l}14.643 \\
\end{array}$ & 0.003 & 20356.67 & 7. 33.9444 & 50.9 & 11.9 & & $\frac{2.146}{2.755}$ & \begin{tabular}{|l|}
0.0099 \\
\end{tabular} & 0.010 & & & $\begin{array}{l}0.20 \\
0.21\end{array}$ \\
\hline & $8 / 31 / 2004$ & 5:51:02 PM & 24.513 & 25.594 & & 26.417 & 25.119 & 47.244 & 52.094 & \begin{tabular}{|l|}
15.531 \\
\end{tabular} & 32.8 & -1.212 & -7.431 & 50.843 & 0.059 & 14.643 & & & 733.9611 & 51.0 & & & & 0.008 & & & & 19 \\
\hline 1340 & 8/31/2004 & 5:52:02 PM & $\begin{array}{l}24.523 \\
2.505\end{array}$ & & & 26.426 & & \begin{tabular}{|l|l|}
47.169 \\
\end{tabular} & & \begin{tabular}{|l|}
15.611 \\
15
\end{tabular} & $\begin{array}{l}32.636 \\
200\end{array}$ & $\begin{array}{l}-1.212 \\
\end{array}$ & $\begin{array}{l}-7.507 \\
-7501 \\
\end{array}$ & $\begin{array}{l}49.697 \\
5.717\end{array}$ & & 14.643 & & & 33.9778 & & 11.8 & & 2.751 & 0.009 & 0.010 & 0.000 & 0.239 & \\
\hline 1341 & $8 / 31 / 2004$ & 5:53:02 PM & 24.505 & $\begin{array}{l}25.585 \\
25590\end{array}$ & \begin{tabular}{|l|}
23.019 \\
23032
\end{tabular} & $\begin{array}{l}26.423 \\
2632\end{array}$ & $\begin{array}{r}25.24 \\
\end{array}$ & $\begin{array}{l}47.422 \\
1759\end{array}$ & \begin{tabular}{|l|}
52.131 \\
5.2901 \\
\end{tabular} & 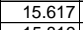 & $\begin{array}{l}33.009 \\
3200\end{array}$ & $\begin{array}{l}-1.215 \\
\end{array}$ & $\begin{array}{r}-7.501 \\
\end{array}$ & $\begin{array}{l}50.747 \\
\end{array}$ & $\begin{array}{l}0.062 \\
\end{array}$ & \begin{tabular}{|l|l|}
14.643 \\
\end{tabular} & & & 33.9944 & 51.0 & 12.1 & & & 0.009 & 0.009 & 0.000 & 0.233 & \\
\hline$\frac{1342}{1342}$ & $8 / 31 / 2004$ & $\begin{array}{ll}\text { 5:54:02 PM } \\
5: 5: 02 \mathrm{P}\end{array}$ & $\begin{array}{l}24.503 \\
2.507\end{array}$ & $\begin{array}{l}25.589 \\
25.592\end{array}$ & \begin{tabular}{|l|}
23.032 \\
23096
\end{tabular} & $\frac{26.422}{26.42}$ & $\begin{array}{l}25.179 \\
25117\end{array}$ & \begin{tabular}{|l|l|}
47.546 \\
17488 \\
\end{tabular} & \begin{tabular}{|l|}
52.691 \\
52323 \\
\end{tabular} & \begin{tabular}{|l|}
15.819 \\
15693
\end{tabular} & $\begin{array}{l}32.722 \\
32685\end{array}$ & \begin{tabular}{|c|}
-1.215 \\
.215
\end{tabular} & & \begin{tabular}{|l|}
50.027 \\
50632
\end{tabular} & $\begin{array}{l}0.065 \\
0.63\end{array}$ & \begin{tabular}{|l|l|}
14.643 \\
1464
\end{tabular} & & 2040.67 & $\begin{array}{l}34.0111 \\
34078\end{array}$ & 51.6 & $\begin{array}{l}11.9 \\
121\end{array}$ & & $\begin{array}{l}2.767 \\
27672\end{array}$ & 0.009 & 0.010 & 0.000 & 0.245 & 21 \\
\hline$\frac{1343}{1344}$ & $\frac{8 / 31 / 2004}{88 / 312004}$ & $\begin{array}{l}\text { 5:55:02 PM } \\
\text { 5:56:02 PM }\end{array}$ & $\frac{24.507}{24.503}$ & $\begin{array}{l}25.592 \\
25.589 \\
\end{array}$ & \begin{tabular}{|l|}
23.096 \\
23.173 \\
\end{tabular} & $\begin{array}{r}26.42 \\
26.332 \\
\end{array}$ & $\begin{array}{l}25.117 \\
25.029\end{array}$ & \begin{tabular}{|l|}
47.428 \\
47.457
\end{tabular} & \begin{tabular}{|l|}
52.332 \\
52.202 \\
\end{tabular} & \begin{tabular}{|c|}
15.693 \\
15.5688
\end{tabular} & $\begin{array}{r}32.685 \\
32.832 \\
\end{array}$ & $\begin{array}{l}-1.215 \\
-1.212\end{array}$ & $\begin{array}{l}-7.501 \\
-7.501\end{array}$ & $\begin{array}{r}50.632 \\
49.639 \\
\end{array}$ & 0.063 & $\begin{array}{l}14.643 \\
14.643 \\
\end{array}$ & 0.003 & 2042.67 & $\begin{array}{l}34.0278 \\
34.0444\end{array}$ & $\frac{51.1}{51 .}$ & 11.8 & 40.1 & 2.762 & | 0.009 & $\frac{0.010}{0.010}$ & $\frac{0.000}{0.000}$ & 0.238 & $\frac{0.20}{0.20}$ \\
\hline 1345 & $8 / 31 / 2004$ & 5:57:02 PM & 24.517 & 25.597 & \begin{tabular}{|l|}
23.173 \\
\end{tabular} & 26.34 & $\frac{23.029}{24.952}$ & 47.105 & \begin{tabular}{|l|}
51.827 \\
\end{tabular} & $\mid$ & 32.518 & \begin{tabular}{|l|l|}
-1.212 \\
\end{tabular} & $\begin{array}{l}-7.501 \\
-7.504\end{array}$ & $\begin{array}{r}49.639 \\
49.66 \\
\end{array}$ & $\begin{array}{l}0.064 \\
0.062\end{array}$ & $\begin{array}{l}4.643 \\
14.643 \\
\end{array}$ & & $\frac{2424.61}{2043.67}$ & 34.0611 & 50.7 & $\begin{array}{ll}11.8 \\
11.8\end{array}$ & $\frac{40.1}{39.8}$ & $\begin{array}{l}2.768 \\
2.745\end{array}$ & \begin{tabular}{|l|}
0.0099 \\
0.009 \\
\end{tabular} & $\frac{0.010}{0.009}$ & & & $\frac{0.20}{0.20}$ \\
\hline & $8 / 31 / 2004$ & 5:58:02 PM & 24.516 & 25.587 & 23.12 & 26.265 & 24.9 & 47.242 & & 15.64 & 32.642 & \begin{tabular}{|l|l|}
2 & -1.215 \\
\end{tabular} & & 50.434 & & 14.643 & & & & & & & 2.754 & & & & 0.234 & 0.20 \\
\hline 1347 & $8 / 31 / 2004$ & 5:59:02 PM & 24.504 & 25.57 & \begin{tabular}{|l|l|}
23.088 \\
\end{tabular} & & 24.8 & 47.335 & 52.428 & & 32.5 & -1.212 & & & 0.064 & & & & 34.0944 & & & & & & & & & \\
\hline 1348 & & & & & & & & & & & & & & 49.629 & & 14.6 & & & & 51.0 & & & $.74 \varepsilon$ & & & & & \\
\hline & & & 24.53 & 25.601 & 23.079 & 26.194 & & $\begin{array}{l}46.893 \\
\end{array}$ & 51.939 & \begin{tabular}{l|l|l|l}
15.506 \\
\end{tabular} & 32.473 & -1.215 & & 49.599 & . & 14.6 & & & 3412 & 50 & & & & & & & .244 & \\
\hline & $8 / 31 /$ & & 24.526 & 25.591 & \begin{tabular}{|l|}
23.065 \\
\end{tabular} & 26.139 & & & & & & 1215 & & & & & & & & & & & & & & & & \\
\hline & 2004 & & & 25.593 & 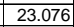 & 26.136 & 24. & & 52.212 & $\begin{array}{l}15.583 \\
\end{array}$ & 32.582 & -1.212 & & 50.605 & 0.062 & 14.643 & & 049.67 & & & & & & & & & & \\
\hline & $8 / 31 / 2004$ & 6:04:02 PM & 24.496 & 25.571 & 23.045 & 26.084 & 25. & \begin{tabular}{|l|l|}
47.264 \\
\end{tabular} & \begin{tabular}{|l|}
52.301 \\
\end{tabular} & 15.799 & 32.404 & -1.212 & -7.498 & 50.676 & 0.064 & 14.643 & 0.00 & 2050.67 & 34.1778 & 51.2 & 12.1 & 39. & 2.746 & \begin{tabular}{|l|l|}
0.009 \\
\end{tabular} & 0.010 & 0.000 & 0.243 & \\
\hline & $8 / 31 / 2$ & & 24.484 & 25.57 & \begin{tabular}{|l|l|}
23.013 \\
\end{tabular} & 26.088 & 24.95 & $\begin{array}{l}47.183 \\
\end{array}$ & \begin{tabular}{|l|}
52.218 \\
\end{tabular} & 15.593 & 32.623 & \begin{tabular}{|l|l|}
3 & -1.212 \\
\end{tabular} & & 52.104 & 0.066 & 14.643 & & 2051.67 & 734.1944 & 51.1 & 12.4 & & 2.751 & \begin{tabular}{|l|l|}
0.009 \\
\end{tabular} & 0.010 & 0.000 & 0.251 & \\
\hline & 31/2004 & & 24.484 & & & & 24.9 & & \begin{tabular}{|l|}
52.252 \\
\end{tabular} & 15.63 & & -1.215 & & 49.474 & 0.063 & & & & 734.2111 & 51.1 & 11.8 & 40 & 2.757 & \begin{tabular}{|l|l|}
0.009 \\
\end{tabular} & 0.010 & 0.000 & & \\
\hline & $8 / 31 / 2$ & 6:07:02 PM & 24.469 & 25.564 & \begin{tabular}{|l|}
23.017 \\
\end{tabular} & 26.002 & 24.90 & 47.347 & \begin{tabular}{|l|l|}
52.044 \\
\end{tabular} & \begin{tabular}{|l|l|}
15.644 \\
1.74
\end{tabular} & 32.82 & -1.215 & & 49.053 & .062 & $\begin{array}{l}4.643 \\
4.19\end{array}$ & & & 34.2278 & 50.9 & 11.7 & 40.1 & & 0.009 & & & 0.234 & \\
\hline & $\begin{array}{l}8 / 31 / 2004 \\
8 / 31 / 204\end{array}$ & 6:08:02 PM & $\begin{array}{r}24.475 \\
24.464\end{array}$ & $\begin{array}{r}25.576 \\
25.564\end{array}$ & \begin{tabular}{|l|}
23.009 \\
23.002
\end{tabular} & \begin{tabular}{l|l}
26.028 \\
2 \\
22.007
\end{tabular} & $\begin{array}{l}24.88 \\
24.81 \\
\end{array}$ & $\begin{array}{r}47.252 \\
47.26 \\
\end{array}$ & \begin{tabular}{|l|}
52.333 \\
52.326 \\
\end{tabular} & \begin{tabular}{|c|}
15.743 \\
15.73
\end{tabular} & $\begin{array}{r}32.467 \\
32.5\end{array}$ & $\frac{-1.212}{-1.212}$ & $\begin{array}{l}-7.472 \\
-7.469 \\
\end{array}$ & \begin{tabular}{|l|}
50.044 \\
50.905
\end{tabular} & $\begin{array}{l}0.063 \\
0.064\end{array}$ & $\begin{array}{l}14.643 \\
14.643 \\
\end{array}$ & & 20 & $\begin{array}{l}74.2444 \\
742611\end{array}$ & $\frac{51.2}{51.2}$ & $\frac{11.9}{12.1}$ & \begin{tabular}{|l|l|}
39 \\
39
\end{tabular} & $\frac{2.748}{2.750}$ & 0.009 & 0.010 & 0.000 & 0.239 & 0.20 \\
\hline$\frac{\mid 007}{1358}$ & $8 / 31 / 2004$ & $6: 10: 02 \mathrm{PM}$ & 24.4043 & 25.559 & \begin{tabular}{|l|l|}
22.982 \\
\end{tabular} & \begin{tabular}{|l}
25.9016 \\
25.916
\end{tabular} & $\frac{2.019}{24.784}$ & \begin{tabular}{|l|}
47.094 \\
47.094
\end{tabular} & \begin{tabular}{|l|}
52.1111 \\
\end{tabular} & \begin{tabular}{|c|}
15.596 \\
150
\end{tabular} & $\begin{array}{r}32.609 \\
32.69\end{array}$ & \begin{tabular}{|l|l|l|l|}
9 & -1.215 \\
\end{tabular} & & $\begin{array}{l}50.905 \\
50.394\end{array}$ & $\begin{array}{l}0.064 \\
0.065\end{array}$ & $\begin{array}{l}14.643 \\
14.643 \\
\end{array}$ & & $\frac{2055.61}{2056.67}$ & & $\begin{array}{l}51.2 \\
51.0\end{array}$ & 1.t. & $\mid 39$ & $\frac{2.1500}{2.748}$ & 然.009 & & & & 0.21 \\
\hline 1359 & $8 / 31 / 2004$ & 6:11:02 PM & 24.452 & 25.562 & & $\begin{array}{ll}3 & 25.875 \\
\end{array}$ & & 47.314 & \begin{tabular}{|l|}
52.088 \\
\end{tabular} & 15.608 & 32.833 & \begin{tabular}{|l|l|}
3 & -1.212 \\
\end{tabular} & & 51.047 & 0.063 & 14.643 & & 67 & 34.2944 & 51.0 & 12.2 & 40.1 & 2.763 & & 0.010 & & & $\frac{0.21}{0.20}$ \\
\hline & $8 / 31 / 2004$ & & 24.44 & & 22.979 & \begin{tabular}{|l|l|}
925.888 \\
\end{tabular} & 24.7 & 47.02 & \begin{tabular}{|l|}
51.839 \\
\end{tabular} & 15.531 & & \begin{tabular}{|l|l|}
2 & -1.212 \\
\end{tabular} & & 49.774 & 0.063 & & & & 74.3111 & & & & & & & & & 0.20 \\
\hline 1361 & $8 / 31 / 2004$ & 6:13:02 PM & 24.434 & 25.564 & \begin{tabular}{|l|}
22.987 \\
\end{tabular} & 25.902 & 24.8 & & \begin{tabular}{|l|}
52.26 \\
\end{tabular} & 15.655 & 32.543 & -1.212 & -7.4 & & 0.061 & $\begin{array}{l}14.643 \\
\end{array}$ & & 0.059 .67 & & 51.1 & 12.2 & 3 & & & & & & $\begin{array}{l}0.20 \\
0.20\end{array}$ \\
\hline 1362 & & & & 25.563 & 22.991 & 25.941 & & & 52.185 & 15.533 & 32.64 & -1.215 & & & 2663 & & & & 734.34 & 51.1 & 1.8 & & & & & & & \\
\hline 1363 & $8 / 31 / 2004$ & & 24.42 & 25.562 & 23.015 & 25.9 & & 47.103 & & 15.395 & & -1.215 & & & .061 & 14.6 & & & & & & & & & & & & \\
\hline & $8 / 31 / 2004$ & & 24.425 & 25.555 & 23.044 & 26.03 & & 47.32 & 2.225 & 15.613 & 32.828 & -1.212 & & & .063 & 14.6 & & & & & & & & & & & & \\
\hline & $8 / 31 / 2004$ & & 24.42. & 25.568 & 323.036 & 26.05 & & 47.194 & \begin{tabular}{|l|} 
\\
\end{tabular} & 15.466 & 32.884 & $\begin{array}{ll}4 & -1.21 \\
\end{array}$ & & & | & 14.6 & & 263.6 & & 50 & 11.4 & & & & & & & \\
\hline & $8 / 31 / 2$ & & 24.44 & 25.582 & 23.025 & 26.1 & & & 52.04 & 15.549 & 32.8 & \begin{tabular}{|l|l|}
5 & -1.215 \\
\end{tabular} & & 49. & 0.063 & 14.6 & & & 34. & 50 & 11.8 & & & & & & & \\
\hline & $8 / 31 /$ & & 24.44 & 25.591 & 23.029 & 26.16 & & 47. & 52.121 & 15.622 & & -1.2 & & & 0.062 & 14. & & & & & & & & & & & & \\
\hline & 004 & & & & & & & & & 15.654 & & -1.22 & & & & & & & & 51.1 & & & & & & & & \\
\hline & & 6:21:02 & & 25.60 & 23.153 & 26.1 & & 47.546 & 52.453 & 15.619 & 32.935 & -1.21 & & & & & & 067 & 34.4611 & 51.3 & 12. & & $2 \pi$ & & & & & \\
\hline 37 & - $8 / 131 / 2$ & & 24.48 & $\begin{array}{l}25.64 \\
25.625\end{array}$ & $\begin{array}{l}23.264 \\
23285\end{array}$ & $\frac{20.26}{2627}$ & & & $\frac{52.268}{52.735}$ & $\frac{15.4}{15.5}$ & & $\begin{array}{r}-1.209 \\
-1.218\end{array}$ & & & 64 & & & & & $\frac{51.1}{51 .}$ & & & & & & & & \\
\hline & & & 24.489 & & 23.374 & & & & & $\begin{array}{l}15.020 \\
15.544\end{array}$ & & \begin{tabular}{|l|l}
9 & -1.210 \\
\end{tabular} & & & & $\frac{14.6}{14.6}$ & & & & 516 & & & & & & & & \\
\hline & $8 / 31 / 2004$ & & & 25.6 & 23.419 & & & & \begin{tabular}{|l|}
52.911 \\
\end{tabular} & & & & & & & & & & & & 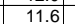 & & & & & & & \\
\hline 374 & $8 / 31 / 2004$ & & 24.518 & 25.643 & \begin{tabular}{|l|l|}
3 & 23.448 \\
\end{tabular} & 26.406 & & $\begin{array}{l}477.474 \\
\end{array}$ & 52.42 & $\begin{array}{l}15.4799 \\
\end{array}$ & 33.081 & -1.215 & & & 0.063 & 14. & & & & 51.3 & $\begin{array}{ll}11.9 \\
\end{array}$ & & & 009 & & 0 & 0.234 & \\
\hline & & & & & & & & & & & & & & & & & & & & & & & & & & & & \\
\hline & & & & 25.657 & 23.557 & 26. & & & 52.685 & 15.645 & & -1.2 & & & & & & & & 51.6 & 11.9 & & & & & & & \\
\hline & $8 / 3$ & & 24.54 & 25.63 & \begin{tabular}{|l|l|}
3.575 \\
\end{tabular} & 26.21 & 2 & & $\begin{array}{l}52.407 \\
\end{array}$ & $\begin{array}{l}15.537 \\
\end{array}$ & 33.167 & -1.215 & & & .000 & 14. & & & & 51.3 & 12.0 & & & 0 & & & & \\
\hline & $8 / 31 / 2004$ & & & 25.643 & 23.638 & 26.15 & & & \begin{tabular}{|r|}
52.89 \\
\end{tabular} & & & -1.21 & & & & & & & & & 12.0 & & & & & & & \\
\hline $13 \pi$ & $8 / 31 / 20$ & & $\frac{24.588}{24611}$ & $\begin{array}{r}25.634 \\
25637\end{array}$ & $\begin{array}{l}23.669 \\
723.607\end{array}$ & $\begin{array}{l}926 \\
72\end{array}$ & 24.83 & 47. & \begin{tabular}{|l|}
52.558 \\
52602
\end{tabular} & $\frac{15.448}{15399}$ & & -1.2 & & 50.6 & & 14. & & & & 51.4 & $\frac{12.1}{120}$ & & & \begin{tabular}{|l|l|} 
\\
\end{tabular} & 0.0 & 0.0 & 0.239 & \\
\hline & $\begin{array}{r}8 / 31 / 2004 \\
8 / 31 / 204\end{array}$ & $\begin{array}{l}\text { 6:32:02 PM } \\
\text { 6:33:02 }\end{array}$ & $\begin{array}{r}24.611 \\
24.64\end{array}$ & $\begin{array}{l}25.637 \\
25.645\end{array}$ & \begin{tabular}{|l|l|}
23.697 \\
23.756
\end{tabular} & \begin{tabular}{l|l|}
6 & 26.14 \\
6 & 26.143
\end{tabular} & $\frac{24.6}{24.7}$ & $\frac{47.5}{47 .}$ & \begin{tabular}{|l|}
52.693 \\
52.119 \\
\end{tabular} & $\begin{array}{r}\frac{15.3999}{15.33} \\
\end{array}$ & & \begin{tabular}{|c|c|} 
& -1.209 \\
1 & -1.206 \\
\end{tabular} & & $\begin{array}{l}50.45 \\
47.25\end{array}$ & \begin{tabular}{|c|}
0.066 \\
0.061
\end{tabular} & $\frac{14.6}{14.6}$ & & 37 & & \begin{tabular}{|l|l|}
51.6 \\
510
\end{tabular} & $\frac{12.0}{11.3}$ & & $\frac{2.785}{2.775}$ & $\mid \begin{array}{l}0.0099 \\
0009\end{array}$ & $\begin{array}{l}0.010 \\
0.009\end{array}$ & $\frac{0.000}{0.000}$ & $\begin{array}{l}0.243 \\
0.225\end{array}$ & 0 \\
\hline & | & & & & & & & & & & & & & & & & & & & & & & & & & & & \\
\hline & & & & & 23.817 & & & & $\begin{array}{l}52.498 \\
\end{array}$ & $15.4 !$ & 32.8 & $\frac{-1.2}{-1.2}$ & & & & & & & & & & & & & & & & \\
\hline & & & 24.6 & & & & & & & & & & & & & & & & & & & & & & & & & \\
\hline & $8 / 31 / 2$ & & 24.7 & & & 26. & & 47.3 & \begin{tabular}{|l|}
52.084 \\
\end{tabular} & $15.1:$ & 33.4 & -1.2 & & & & & & & & 510 & & & & & & & & \\
\hline & $8 / 31 / 206$ & & 24.7 & & 23.966 & & & 47 & & 15. & & -1.2 & & & & 14.6 & & & & & & & & & & & & \\
\hline & $8 / 31 / 2$ & & 24.7 & & & & & & & & & -1.2 & & & & & & & & & & & & & & & & \\
\hline & & & 24.7 & & & & & & & & & -1.2 & & & & & & & & 51.3 & 12.0 & & & & & & & \\
\hline & & & & & & & & & & & & & & & & & & & & & & & & & & & & \\
\hline & $8 / 31 / 2$ & & 24.8 & & & & & & & & & -1. & & & & & & & & & 2.4 & & & & & & & \\
\hline & 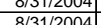 & & $\frac{2.0}{2.8}$ & $\frac{25.044}{2568}$ & & & & & & 15312 & & $=1.2$ & & & & & & & & & & & & & & & & \\
\hline & 8 & & 2488 & $\frac{2.04}{25.64}$ & 2408 & & & & 52476 & $\frac{1.51}{1535}$ & & $\frac{-1.2}{92}$ & & & & & & & & & & & & & & & & \\
\hline & $8 / 31 / 20$ & & 24 & & 24.1 & & & & & 15.41 & & \begin{tabular}{l|l}
4 & -1.2 \\
4
\end{tabular} & & & & & & & & & $1.7 \mathrm{Y}$ & & & & & & & \\
\hline & & & 24.90 & 25.644 & & & & & & & & \begin{tabular}{|c|c|}
9 & -1.1. \\
\end{tabular} & & & & & & & & & & & & & & & & \\
\hline & & & & & & & & & & & & & & & & & & & & & & & & & & & & \\
\hline & & & & & & & & & & & & & & & & & & & & & & & & & & & & \\
\hline & $8 / 31 / 2$ & & 24.94 & & 24.111 & 26.7 & & 47.2 & & & & -1.2 & & & & & & & & & & & & & & & 38 & \\
\hline & & & 24.944 & & & & & & 69 & 44 & & 122 & & & & & & & & & & & & & & & & \\
\hline $40 \mathrm{C}$ & $8 / 31 / 2004$ & $\begin{array}{ll}6: 52: 02 \\
\end{array}$ & 24.97 & 25.64 & 24.147 & \begin{tabular}{l|l|}
7 & 26.163
\end{tabular} & & 47.609 & \begin{tabular}{|l|}
52.652 \\
\end{tabular} & 15.488 & 33.253 & \begin{tabular}{|c|}
$|3|-1.215$ \\
\end{tabular} & & $\overline{49.434}$ & 0.065 & 14.643 & & & 9778 & & 1.8 & & $2.788 \mid$ & 0.009 & 0.01 & 0.000 & 0.236 & \\
\hline
\end{tabular}


WSRC-TR-2005-00105, REVISION 0

SRNL-RPP-2005-00012, REVISION 0

RUN \# 4.03A AND B; FIRST AND SECOND HALF OF SLURRY DEWATERING - CONT.

\begin{tabular}{|c|c|c|c|c|c|c|c|c|c|c|c|c|c|c|c|c|c|c|c|c|c|c|c|c|c|c|c|c|}
\hline & A & $\mathrm{B}$ & $\mathrm{D}$ & $E$ & $F$ & G & $\mathrm{H}$ & $\mathrm{J}$ & $\mathrm{k}$ & $\mathrm{L}$ & M & $\mathrm{N}$ & 0 & Q & $\mathrm{R}$ & $\mathrm{s}$ & $\begin{array}{ll}T \\
\end{array}$ & $\mathrm{~V}$ & w & $x$ & Y & $z$ & & & $\mathrm{AC}$ & & & $\overline{A F}$ \\
\hline & $8 / 31 / 2004$ & $6: 53: 02$ PM & 24.963 & 25.628 & 24.155 & 26.086 & 24.823 & 47.399 & 52.643 & \begin{tabular}{ll|}
15.357 \\
\end{tabular} & 33.036 & -1.212 & $\begin{array}{l}-7.215 \\
\end{array}$ & 47.32 & 0.064 & 14.643 & 0.003 & 2099.67 & 34.9944 & 51.5 & 11.3 & 40.2 & 2.773 & 0.009 & 0.009 & 0.000 & 0.233 & 0.20 \\
\hline & $\frac{131 / 2004}{31 / 200}$ & 6:54:02 PM & 24.982 & 25.632 & 24.164 & 26.1 & & 47.772 & 52.716 & 15.355 & 33.516 & -1.215 & & 46.733 & 0.064 & $\begin{array}{l}14.643 \\
\end{array}$ & 0.003 & 2100.67 & & 51.6 & & & & & & & & \\
\hline$\frac{1403}{1001}$ & $8 / 31 / 2004$ & $6: 55: 02 \mathrm{PM}$ & 24.99 & 25.63 & \begin{tabular}{|l|}
24.162 \\
2172 \\
\end{tabular} & 26.068 & 24.84 & 47.729 & S2.051 & 15.444 & \begin{tabular}{|l|}
33.229 \\
3229
\end{tabular} & \begin{tabular}{|c|}
-1.215 \\
-212
\end{tabular} & $\frac{-7.238}{-7238}$ & 48.235 & 0.065 & 14.643 & 0.003 & 2101.67 & 35.0278 & 51.7 & 11.5 & 40.5 & .791 & 0.009 & 0.010 & 0.000 & 0.235 & \\
\hline $\begin{array}{l}1404 \\
1105\end{array}$ & $8 / 31 / 2004$ & 6:56:02 PM & 24.995 & \begin{tabular}{|l|l|}
25.63 \\
\end{tabular} & \begin{tabular}{|l|l|}
24.172 \\
\end{tabular} & 26.078 & 24.97 & 47.555 & \begin{tabular}{|l|l|}
52.648 \\
\end{tabular} & 15.306 & \begin{tabular}{|l|l|}
33.263 \\
\end{tabular} & -1.212 & $\begin{array}{l}-7.20 \\
\end{array}$ & 49.08 & 0.065 & $\begin{array}{l}14.643 \\
\end{array}$ & 0.004 & 2102.67 & 35.0444 & 51.5 & 11.7 & 40.4 & 2.786 & 0.009 & 0.010 & 0.000 & 0.236 & \\
\hline & $\frac{8 / 31 / 2004}{88 / 312004}$ & $6: 57: 02 \mathrm{PM}$ & $\begin{array}{l}24.994 \\
25.024\end{array}$ & $\frac{25.614}{25639}$ & \begin{tabular}{|l|}
24.176 \\
24266 \\
\end{tabular} & $\begin{array}{l}26.057 \\
26.087\end{array}$ & $\begin{array}{l}25.014 \\
25.089 \\
\end{array}$ & $\begin{array}{l}477.642 \\
47.408\end{array}$ & \begin{tabular}{|l|}
52.786 \\
52223 \\
\end{tabular} & $\frac{15.422}{15363}$ & \begin{tabular}{|l|}
33.233 \\
33062
\end{tabular} & \begin{tabular}{|c|}
-1.212 \\
1215
\end{tabular} & & $\begin{array}{l}49.437 \\
47.98\end{array}$ & $\begin{array}{l}0.064 \\
0064\end{array}$ & \begin{tabular}{|l|l|l|}
14.643 \\
14634 \\
\end{tabular} & & 2103.67 & 35.0611 & \begin{tabular}{|l|}
51.7 \\
\end{tabular} & \begin{tabular}{|l|}
11.8 \\
\end{tabular} & 40.4 & 2.788 & 0.009 & & & 0.232 & \\
\hline 1407 & & $\begin{array}{l}6: 5: 02 \mathrm{PM} \\
6: 59: 02 \mathrm{PM}\end{array}$ & $\frac{25.024}{25.034}$ & $\begin{array}{l}25.639 \\
25.639\end{array}$ & \begin{tabular}{|l|}
24.266 \\
24.276 \\
\end{tabular} & \begin{tabular}{|l|}
$\mid 26.087$ \\
26.142 \\
\end{tabular} & $\begin{array}{l}25.089 \\
25.074\end{array}$ & $\begin{array}{r}\quad 47.408 \\
47.54 \\
\end{array}$ & \begin{tabular}{|l|}
$\mid 52.223$ \\
52.461 \\
\end{tabular} & $\begin{array}{l}\frac{15.363}{15.376} \\
\end{array}$ & $\begin{array}{l}33.062 \\
33.382\end{array}$ & \begin{tabular}{|c|}
-1.215 \\
-1.215 \\
\end{tabular} & $\begin{array}{l}-7.281 \\
-7.272 \\
\end{array}$ & $\begin{array}{l}44.908 \\
49.366\end{array}$ & $\begin{array}{c}0.064 \\
0.064\end{array}$ & $\begin{array}{r}\mid 14.643 \\
14.643\end{array}$ & $\begin{array}{l}0.003 \\
0.003 \\
\end{array}$ & $\begin{array}{l}\frac{1}{104.667} \\
105.67\end{array}$ & \begin{tabular}{|l|}
35.0778 \\
7350948
\end{tabular} & \begin{tabular}{|l|}
51.1 \\
51.3 \\
\end{tabular} & $\begin{array}{l}11.4 \\
11.8\end{array}$ & $\begin{array}{l}40.2 \\
40.5 \\
4\end{array}$ & 2.7774 & $\begin{array}{l}0.009 \\
0.009 \\
\end{array}$ & 0.009 & $\begin{array}{l}0.000 \\
0.000\end{array}$ & $\begin{array}{l}0.232 \\
0.231\end{array}$ & 0.20 \\
\hline 1408 & $8 / 31 / 2004$ & 7:00:02 PM & 25.031 & 25.636 & \begin{tabular}{|l|}
24.263 \\
\end{tabular} & \begin{tabular}{|l|l|}
26.184 \\
\end{tabular} & & & & 15.453 & & & & & 0.064 & 14.643 & $\begin{array}{l}0.003 \\
0.003\end{array}$ & 2106.67 & \begin{tabular}{|l|}
35.0944 \\
35.1111
\end{tabular} & $\begin{array}{l}1.5 \\
51.5 \\
\end{array}$ & & 40.5 & $\frac{2.190}{2.791}$ & $\begin{array}{l}0.009 \\
0.009\end{array}$ & 0.009 & $\begin{array}{l}0.000 \\
0.000\end{array}$ & 0.231 & $\frac{0.20}{0.20}$ \\
\hline & $8 / 31 / 2004$ & & 25.044 & 25.639 & 24.261 & \begin{tabular}{|l|}
26.217 \\
\end{tabular} & 25.214 & 47.652 & \begin{tabular}{|l|}
52.768 \\
\end{tabular} & 15.392 & 33.272 & \begin{tabular}{|r|}
-1.215 \\
\end{tabular} & & 50.008 & 0.063 & \begin{tabular}{|l|l|}
14.643 \\
\end{tabular} & & 20767 & & 51.6 & & & & & & & & \\
\hline 1410 & $8 / 31 / 2004$ & 7:02:02 PM & & 25.63 & \begin{tabular}{|l|}
24.262 \\
\end{tabular} & & 25.25 & 47.561 & 52.691 & 15.378 & & -1.212 & -7.26 & 49.167 & & \begin{tabular}{|l|l|}
14.643 \\
\end{tabular} & & 2108.67 & 35.1444 & 51.6 & 11.7 & & 2.781 & 0.009 & 0.010 & 0.000 & $\begin{array}{l}0.239 \\
\end{array}$ & \\
\hline 1411 & $8 / 31 / 2004$ & 7:03:02 PM & $\begin{array}{l}25.061 \\
25071\end{array}$ & $\begin{array}{l}25.636 \\
25617\end{array}$ & \begin{tabular}{|l|}
24.273 \\
2.281
\end{tabular} & 26.289 & 25.201 & $\begin{array}{l}47.656 \\
7022\end{array}$ & 52.936 & $\begin{array}{l}15.392 \\
15251\end{array}$ & $\begin{array}{l}33.114 \\
32957\end{array}$ & $\begin{array}{l}-1.212 \\
\end{array}$ & & 49.243 & $\begin{array}{l}0.064 \\
\end{array}$ & \begin{tabular}{|l|l|}
14.643 \\
\end{tabular} & $\begin{array}{l}0.003 \\
\end{array}$ & 2109.67 & 35.1611 & 51.8 & 11.7 & 40.4 & 2.784 & 0.009 & 0.009 & 0.000 & 0.232 & \\
\hline$\frac{1412}{1413}$ & $\begin{array}{l}8 / 31 / 2004 \\
8 / 312004\end{array}$ & $\begin{array}{l}7: 03: 18 \mathrm{PM} \\
7 \cdot 04 \cdot 18 \mathrm{PM}\end{array}$ & \begin{tabular}{|l|l|}
25.071 \\
25.098
\end{tabular} & $\begin{array}{l}25.647 \\
25.663\end{array}$ & \begin{tabular}{|r|}
24.284 \\
2432 \\
\end{tabular} & \begin{tabular}{|l|}
26.309 \\
26.326 \\
\end{tabular} & $\begin{array}{l}25.186 \\
25308\end{array}$ & $\begin{array}{l}47.022 \\
431616\end{array}$ & \begin{tabular}{|c|}
52.059 \\
5.254
\end{tabular} & \begin{tabular}{l|l|l|}
15.254 \\
15.378
\end{tabular} & \begin{tabular}{|l|}
32.857 \\
32872
\end{tabular} & \begin{tabular}{|c|c|}
-1.192 \\
1.172
\end{tabular} & $\begin{array}{l}-7.16 \\
.1 .1 \\
\end{array}$ & $\begin{array}{l}49.662 \\
50.23\end{array}$ & & \begin{tabular}{|l|l|l|}
14.643 \\
14643
\end{tabular} & & 2109.93 & $\begin{array}{l}35.1656 \\
3251820\end{array}$ & \begin{tabular}{|l|}
50.9 \\
51.5
\end{tabular} & 11.8 & 39.9 & $\begin{array}{l}2.754 \\
2764 \\
2764\end{array}$ & 0 & & 0.000 & 0.230 & \\
\hline$\frac{1413}{1414}$ & 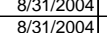 & $\begin{array}{l}7: 40: 18 \text { PM } \\
7: 05: 18\end{array}$ & $\begin{array}{l}25.098 \\
25.093\end{array}$ & $\begin{array}{l}25.663 \\
25.648\end{array}$ & \begin{tabular}{|r|}
24.32 \\
24.225 \\
\end{tabular} & \begin{tabular}{|l|}
26.326 \\
26.356
\end{tabular} & $\begin{array}{r}25.308 \\
25.278 \\
\end{array}$ & $\begin{array}{l}47.316 \\
47.486\end{array}$ & \begin{tabular}{|l|}
52.5944 \\
52.645 \\
\end{tabular} & $\begin{array}{l}15.378 \\
15.334\end{array}$ & \begin{tabular}{|l|}
32.872 \\
3.3136
\end{tabular} & $\begin{array}{l}-1.172 \\
-1.212\end{array}$ & -7.258 & $\begin{array}{l}50.423 \\
51.794\end{array}$ & $\begin{array}{ll}0664 \\
064\end{array}$ & $\begin{array}{l}14.643 \\
14.643 \\
\end{array}$ & 0.003 & 11193 & $\begin{array}{l}35.1822 \\
35.1989\end{array}$ & $\begin{array}{r}51.5 \\
51.5\end{array}$ & 123 & 403 & 2.764 & 0.009 & 0.009 & 0.000 & 0.233 & 0.20 \\
\hline 1415 & $8 / 31 / 2004$ & 7:06:18 PM & 25.103 & $\begin{array}{l}25.648 \\
25.659 \\
\end{array}$ & 24.291 & \begin{tabular}{|l|}
20.350 \\
\end{tabular} & $\begin{array}{l}25.278 \\
25.333 \\
\end{array}$ & & \begin{tabular}{|l|}
52.24467 \\
\end{tabular} & $\begin{array}{l}15.354 \\
15.456 \\
\end{array}$ & \begin{tabular}{|}
3.150 \\
33.171 \\
\end{tabular} & \begin{tabular}{|l|l|}
-1.212 \\
-1.212 \\
\end{tabular} & & $\begin{array}{l}31.194 \\
49.489 \\
\end{array}$ & $\begin{array}{l}0.044 \\
0.057\end{array}$ & \begin{tabular}{|l|l}
14.645 \\
14.643
\end{tabular} & \begin{tabular}{|l}
0.004 \\
0.003 \\
\end{tabular} & $\begin{array}{l}21111.93 \\
2112.93\end{array}$ & $\begin{array}{l}35.1989 \\
35.2156\end{array}$ & $\begin{array}{l}51.5 \\
51.3\end{array}$ & $\begin{array}{l}11.3 \\
11.8\end{array}$ & $\frac{40.3}{40.3}$ & & & $\frac{0.009}{0.008}$ & & $\mid$ & $\frac{0.20}{0.18}$ \\
\hline & & 7:07:18 PM & & 25.669 & 24.302 & \begin{tabular}{|l|}
26.397 \\
\end{tabular} & 25.409 & 47.445 & \begin{tabular}{|l|}
52.5833 \\
\end{tabular} & 15.345 & \begin{tabular}{|l|l|}
33.12 \\
3.12
\end{tabular} & \begin{tabular}{|r|}
-1.212 \\
\end{tabular} & & $\begin{array}{l}49.092 \\
990\end{array}$ & 0.064 & \begin{tabular}{|l|l|}
14.643 \\
\end{tabular} & & & & $\begin{array}{l}51.5 \\
51.5\end{array}$ & & & & & & & & \\
\hline & $8 / 31 / 2004$ & 7:08:18 PM & 25.13 & & 24.312 & 26.433 & & 47.636 & \begin{tabular}{|l|}
52.67 \\
\end{tabular} & $\begin{array}{l}15.47 \\
\end{array}$ & & \begin{tabular}{|l|l|} 
& -1.212 \\
\end{tabular} & & 50.054 & 0.064 & \begin{tabular}{|l|l|}
14.643 \\
\end{tabular} & & 114.93 & 35.2489 & 51.5 & & 40.4 & & 0.009 & 0.009 & 0.000 & 0.231 & \\
\hline & $8 / 31 / 2004$ & 7:09:18 PM & 25.125 & $\begin{array}{r}25.66 \\
25.661\end{array}$ & 24.302 & 26.393 & 25.195 & 47.576 & \begin{tabular}{|r|}
52.72 \\
\end{tabular} & 15.341 & $\begin{array}{l}33.288 \\
32.0\end{array}$ & & & & & & & & 35.2656 & 51.6 & & & 2.788 & & & & 0.235 & \\
\hline 1419 & $\frac{8 / 31 / 2004}{8 / 312004}$ & $\begin{array}{l}7: 10: 18 \mathrm{PM} \\
7 \cdot 711.18 \mathrm{PM}\end{array}$ & $\begin{array}{l}25.136 \\
25.141\end{array}$ & $\begin{array}{l}25.661 \\
25.661\end{array}$ & $\begin{array}{l}24.298 \\
24.293\end{array}$ & $\begin{array}{l}26.389 \\
26.334\end{array}$ & $\begin{array}{r}\frac{25.185}{25.03} \\
\end{array}$ & $\begin{array}{l}47.617 \\
47.408\end{array}$ & \begin{tabular}{|r|}
$\mid 52.921$ \\
52.38
\end{tabular} & $\begin{array}{l}15.453 \\
15.374\end{array}$ & \begin{tabular}{|l|}
33.161 \\
33301
\end{tabular} & \begin{tabular}{|c|}
-1.212 \\
1212
\end{tabular} & -7.24 & $\begin{array}{l}49.626 \\
49099\end{array}$ & & $\begin{array}{l}14.643 \\
14643\end{array}$ & & & $\frac{2822}{2989}$ & $\begin{array}{l}51.8 \\
51.3\end{array}$ & 11. & & & & & & 0.228 & 0.19 \\
\hline 1421 & $88 / 31 / 2004$ & $\begin{array}{l}7: 11: 18 \mathrm{PM} \\
7: 1218 \mathrm{PM}\end{array}$ & $\begin{array}{r}25.141 \\
25.14\end{array}$ & $\begin{array}{l}25.661 \\
25.665\end{array}$ & $\begin{array}{l}24.293 \\
24.302\end{array}$ & $\begin{array}{l}26.334 \\
26.283 \\
\end{array}$ & $\begin{array}{r}25.03 \\
24.98\end{array}$ & $\begin{array}{l}47.408 \\
47.011 \\
\end{array}$ & \begin{tabular}{|r|}
52.38 \\
52.038 \\
\end{tabular} & $\frac{15.374}{15.195}$ & \begin{tabular}{|l|l|}
.091 \\
.972
\end{tabular} & \begin{tabular}{|c|}
$\mid-1.212$ \\
-1.148
\end{tabular} & & $\begin{array}{l}49.929 \\
49.606\end{array}$ & & $\begin{array}{l}\frac{14.643}{14.643} \\
\end{array}$ & & & $\begin{array}{r}35.2989 \\
35.3156\end{array}$ & $\begin{array}{l}51.3 \\
50.9\end{array}$ & $\frac{11.5}{11.8}$ & $\frac{40.2}{40.0}$ & \begin{tabular}{|c|c|}
2.775 \\
2.757
\end{tabular} & \begin{tabular}{|l|l|l}
0.009 \\
0.0009
\end{tabular} & 0.010 & 0.000 & 0.239 & 0.20 \\
\hline & $8 / 31 / 2004$ & 7:13:18 PM & 25.124 & 25.634 & \begin{tabular}{|l|}
24.086 \\
\end{tabular} & \begin{tabular}{|l|}
20.202 \\
\end{tabular} & 24.909 & 47.217 & \begin{tabular}{|l|}
52.368 \\
\end{tabular} & 15.266 & 32.962 & \begin{tabular}{|l|}
-1.215 \\
\end{tabular} & $\begin{array}{l}-0.952 \\
-7.157 \\
\end{array}$ & 49.414 & $\begin{array}{l}0.060 \\
0.064\end{array}$ & $\begin{array}{l}14.045 \\
14.643\end{array}$ & $\begin{array}{l}0.003 \\
0.003\end{array}$ & 2119.93 & \begin{tabular}{|l|}
55.3150 \\
35.3322
\end{tabular} & $\begin{array}{l}30.9 \\
51.2\end{array}$ & $\begin{array}{l}11.8 \\
11.8\end{array}$ & 40.1 & $\frac{2.151}{2.764}$ & $\begin{array}{l}0.009 \\
0.009\end{array}$ & $\frac{0.009}{0.009}$ & $\begin{array}{l}0.000 \\
0.000\end{array}$ & 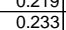 & $\frac{0.19}{0.20}$ \\
\hline & $8 / 31 / 2004$ & 7:14:18 PM & 25.143 & 25.639 & 24.301 & 26.146 & 24.863 & 47.358 & \begin{tabular}{|l|}
52.189 \\
\end{tabular} & 15.386 & 33.091 & \begin{tabular}{|l|}
-1.215 \\
\end{tabular} & & 49.733 & 0.064 & $\begin{array}{l}14.643 \\
\end{array}$ & 0.003 & & 35.3489 & 51.1 & 11.8 & 40.2 & 2.773 & 0.009 & & 0.000 & & 0.20 \\
\hline 124 & $8 / 31 / 2004$ & 7:15:18 PM & 25.142 & 25.637 & 24.279 & 26.13 & 24.802 & 47.169 & \begin{tabular}{|l|l|}
52.105 \\
\end{tabular} & $\begin{array}{l}15.216 \\
\end{array}$ & \begin{tabular}{|l|}
33.087 \\
\end{tabular} & -1.215 & -7 & 50.017 & 064 & \begin{tabular}{|l|l|}
14.643 \\
\end{tabular} & & 121.93 & 35.3656 & 51.0 & 11.9 & 40.1 & 2.767 & 0.009 & 0.009 & 0.000 & & $\frac{0.20}{0.20}$ \\
\hline & $8 / 31 / 2004$ & 7:16:18 PM & & 25.641 & 24.293 & & & & & & 33.186 & & & & & & & & 35.3822 & & & & & & & & & \\
\hline$\frac{26}{27}$ & $8 / 31 / 2$ & $7: 17: 18 \mathrm{PM}$ & 5.143 & & & 26.071 & & $\begin{array}{l}47.47 \\
\end{array}$ & $\begin{array}{l}52.413 \\
\end{array}$ & & 33.321 & & & 48.5 & & 14.643 & & & & 51. & & & & & & & & \\
\hline & $8 / 31 / 200$ & 7:18:18 PM & 25.15 & 25.635 & 24.373 & 26.038 & 24.9 & & & 15.395 & 33.259 & -1.212 & & & & & & & 5.4156 & & & 40. & & & & & & \\
\hline$\frac{42}{27}$ & $8 / 31 / 2004$ & 7:19: & & 25.634 & \begin{tabular}{|l|}
24.356 \\
\end{tabular} & 26.102 & 4. & 46.868 & \begin{tabular}{|l|l|l|l|} 
\\
\end{tabular} & 15.204 & 32.775 & -1.212 & & 48.404 & .064 & \begin{tabular}{l|l}
14.643 \\
\end{tabular} & & & & 50.7 & 11.5 & & .746 & & & & & \\
\hline & $8 / 31 / 2$ & 7:20:18 PM & 25.168 & 25.643 & 24.345 & 26.136 & 25. & 47.464 & 52.505 & 15.296 & 33.366 & -1.212 & & 50.97 & & 14.643 & & 126.9 & & 51.4 & 12.1 & 40.4 & 2.786 & 0.009 & & & 0.227 & \\
\hline & & & & 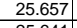 & 24.369 & 2.100 & & 47.358 & 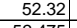 & $\begin{array}{l}15.376 \\
15.207\end{array}$ & 33.245 & -1.212 & & 48.656 & & 14.643 & & & & & & & & & & & & \\
\hline & $8 / 31 / 2$ & $8 \mathrm{PM}$ & & $25.641 \mid$ & \begin{tabular}{|}
24.359 \\
\end{tabular} & 26.164 & & 47.524 & 52.475 & & 33.274 & -1.212 & & 52.106 & & 14.643 & & & & & & 40. & & & & & & \\
\hline & $8 / 31 / 2$ & 1:23:18 PM & 25.196 & 25.661 & $24.3 / 8$ & 26.214 & & & 52.523 & 15.335 & 33.122 & -1.212 & & & & & & & & & & & & 0.00 & & & 228 & \\
\hline 1434 & $8 / 131 / 200$ & $\begin{array}{l}\text { 7:24:18 PM } \\
7 \cdot 75 \cdot 18\end{array}$ & $\frac{25.192}{25.198}$ & $\begin{array}{l}25.652 \\
25.653\end{array}$ & $\frac{24.354}{2436}$ & $\frac{26.245}{26.331}$ & 25. & $\frac{47.202}{47.712}$ & $\frac{52.227}{52.633}$ & $\begin{array}{r}15.38 \\
15.448 \\
\end{array}$ & $\begin{array}{r}32.89 \\
33.411 \\
\end{array}$ & $\begin{array}{l}-1.212 \\
-1.209\end{array}$ & & 51.668 & & $\frac{14.643}{14643}$ & & 231 & & $\frac{5.1}{515}$ & & & 79 & & & & & \\
\hline & $8 / 31 / 2004$ & $7: 26$ & & 25.658 & \begin{tabular}{|l|}
24.356 \\
2.356 \\
\end{tabular} & $\frac{20.001}{26.331}$ & & & 52.6008 & 15.334 & $\mid$ & $\begin{array}{l}-1.20 \\
-1.148 \\
\end{array}$ & & 49.6 & & $\begin{array}{l}14.045 \\
14.643\end{array}$ & & & & 515 & & & & & & & & \\
\hline & $8 / 31 / 200$ & 7:27:18 PM & 25.205 & 25.655 & \begin{tabular}{|l|}
24.367 \\
\end{tabular} & \begin{tabular}{|l|l|}
26.318 \\
\end{tabular} & 25 & 47.113 & \begin{tabular}{|l|}
52.25 \\
\end{tabular} & 15.316 & 32.966 & $|-1.212|$ & & 49.6 & & 14.6 & & & & 51.1 & $\frac{11}{11}$ & & 76 & & & & 0.233 & \\
\hline & & & & 25.66 & \begin{tabular}{|l|}
24.363 \\
\end{tabular} & & & 47.6 & \begin{tabular}{|l|l|} 
\\
\end{tabular} & 15.385 & \begin{tabular}{|l|l|}
33.251 \\
\end{tabular} & & & 49.9 & & & & & & & & & & & & & & \\
\hline & 8 & & & & & & & 47.4 & & & & -1.1 & & & & 14. & & & & 51.5 & & & & & & & & \\
\hline & & 7:30:18 PM & 25.212 & 25.667 & 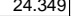 & 26.3 & & 47.6 & 52.8 & 15.515 & 33.103 & -1.212 & & 49.6 & & 14.6 & & 136 & & 51.8 & & & & & & & 0.235 & \\
\hline & & & & 25.68 & & & & & & & & & & & & & & & & & & & & & & & & \\
\hline & $8 / 31 / 2$ & & 25.214 & 25.665 & $\begin{array}{l}24.352 \\
\end{array}$ & & & & & & 33.278 & \begin{tabular}{|c|}
-1.212 \\
\end{tabular} & & & & & & & & 51.7 & 11.6 & 40 & & & & & 0.230 & \\
\hline & $8 / 31 / 2004$ & & & 25.68 & 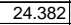 & \begin{tabular}{|l|}
26.382 \\
\end{tabular} & & & \begin{tabular}{|l|l|l|}
52.768 \\
\end{tabular} & 15. & 33.278 & -1.206 & & & & 14.6 & & & & 51.6 & & & & 00 & & & 0.234 & \\
\hline & $8 / 31 / 2$ & & & & & & & & & & & & & & & & & & & & & & & & & & & \\
\hline & & & & 25.668 & \begin{tabular}{|l|}
24.361 \\
\end{tabular} & \begin{tabular}{|l}
26.261 \\
\end{tabular} & & 47.484 & \begin{tabular}{|l|}
52.762 \\
\end{tabular} & 15.492 & 32.949 & -1.145 & & & & & & & & 51.6 & 1 & & & & 0.0 & & 0.232 & \\
\hline & & & & 25.657 & 24.349 & & & & & & & -1.172 & & & & & & & & 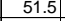 & & & & & & & 0 & \\
\hline & & & & & & & & & & & & & & & & & & & & & & & & & & & & \\
\hline & $8 / 31 / 2$ & $7: 38$ & 213 & $\frac{25.654}{25658}$ & $\frac{24.346}{24356}$ & 26.067 & & $\begin{array}{l}47.194 \\
7.53\end{array}$ & $\frac{51.961}{52.687}$ & $\frac{15.248}{15.355}$ & $\begin{array}{l}33.235 \\
33235\end{array}$ & $\begin{array}{l}-1.215 \\
-1.1148\end{array}$ & & & & 14. & & & & 516 & & & & & & & 0249 & \\
\hline$\overline{1044}$ & $8 / 31 / 2$ & & & 25.662 & & & & & & 15312 & & -1.1215 & & & & & & & & & & & & & & & & \\
\hline & $8 / 31 / 2$ & & & & & & & & & & & -1.215 & & & & & & & & & & & & & & & $\frac{0.526}{0226}$ & \\
\hline & $8 / 31 / 2004$ & & & 25.666 & & 26. & & & & 15.263 & & & & & & & & & & $\frac{51.4}{51.4}$ & $\frac{12.0}{12.0}$ & & & & & & 217 & \\
\hline & & & & & & & & & & & & & & & & & & & & & & & & & & & & \\
\hline & & & & & \begin{tabular}{|l|}
24.436 \\
\end{tabular} & & & & & $\frac{15.2}{15.2}$ & 33.151 & $\frac{-1.2}{-1.2}$ & & & & & & & & & & & & & & & & \\
\hline & & & & & & & & & & & 33.294 & & & & & & & & & & & & & & & & & \\
\hline & $8 / 31 / /$ & & & 25.669 & \begin{tabular}{|l|l|}
24.422 \\
\end{tabular} & 26. & & & 52.473 & 15.471 & 33.001 & $\begin{array}{l}-1.212 \\
\end{array}$ & & & & 14. & & & & 51 & 2.1 .12 & & & & & & & \\
\hline & $8 / 31 / 206$ & & & 25.664 & 24.382 & 26. & & 47.4 & 52.46 & 15.31 & 33.2 & -1.27 & & & & 14.6 & & & & & & & & & & & 0.234 & \\
\hline & $8 / 31 / 2$ & & & & 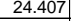 & & & & & 15.1 & 33.483 & & & & & 14. & & & & 01.4 & & & & & & & & \\
\hline & $8 / 31 / 2$ & & 25.251 & 25.676 & \begin{tabular}{|l|l|}
24.408 \\
\end{tabular} & 26.2 & & & \begin{tabular}{|l|}
52.5966 \\
\end{tabular} & 15.53 & 32.5 & -1.212 & & & & & & & & 51.5 & 10.9 & & & & & & 0.231 & \\
\hline & & & & & & & & & & & & & & & & & & & & & & & & & & & & \\
\hline & $\frac{8 / 3121 / 20}{8 / 2120}$ & & & & 24.405 & & & & & & & & & & & & & & & & & & & & & & 23 & \\
\hline & & & & 25696 & 24.510 & 20. & & & 5247 & $\begin{array}{l}15.405 \\
1.5365\end{array}$ & 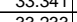 & -1.21 & & & & & & & & & & & & & & & & \\
\hline & 8 & & & & $\frac{2.4 .410}{2.404}$ & & & & $\frac{3<.41}{5.92}$ & 15.0053 & $\frac{3.256}{33.36}$ & -120 & & & & 146 & & & & & & & & & & & 0230 & \\
\hline & $8 / 31 / 2$ & & & & 20.44 & & & & 52.2 & 15397 & $\begin{array}{r}35.50 \\
33.085 \\
\end{array}$ & -121 & & & & 146 & & & & & & & & & & & & \\
\hline & $8 / 31 / 2$ & & & $\frac{25.703}{25.703}$ & 24.43 & $\frac{26.3}{26.3}$ & & & & 15.506 & & $\frac{-1.148}{-1.148}$ & & & & 14.6 & & 216 & & & 123 & & & & & & & \\
\hline & & & & 25.6 & 24.35 & & & & & & & & & & & & & & & & & & & & & & & \\
\hline & & & & & \begin{tabular}{|l|l|}
24,404 \\
\end{tabular} & & & & & & & & & & & & & & & & & & & & & & & \\
\hline & $8 / 31 / 2$ & & & 25.686 & \begin{tabular}{|l|l|}
24.388 \\
\end{tabular} & 26.128 & 24 & 47.3 & $52.3 \varepsilon$ & & \begin{tabular}{|l|l|l|}
33.229 \\
\end{tabular} & -1.215 & & & & & & & & 51.3 & 5.3 & & & & & & 31 & \\
\hline & & & & 686 & 393 & & & & & 366 & & -1.212 & & & & 13 & & 166 & & 1.4 & & & & & & & & 11 \\
\hline 470 & $8 / 31 / 2004$ & $8: 01: 18 \mathrm{PM}$ & $\mid 25.244$ & 25.669 & \begin{tabular}{|l|l|}
24.382 \\
\end{tabular} & \begin{tabular}{|l|}
26.022 \\
\end{tabular} & & $\begin{array}{l}48.065 \\
\end{array}$ & \begin{tabular}{|l|}
53.508 \\
\end{tabular} & 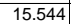 & \begin{tabular}{|l|}
33.397 \\
\end{tabular} & \begin{tabular}{|c|}
-1.215 \\
\end{tabular} & & 50.905 & 0.064 & 14.643 & & 67.6 & 5.1322 & 52.4 & 12.1 & & 2.808 & 0.009 & 0.009 & 0.000 & \begin{tabular}{|l|l|}
0.229 \\
\end{tabular} & 0.1 \\
\hline
\end{tabular}


WSRC-TR-2005-00105, REVISION 0

SRNL-RPP-2005-00012, REVISION 0

RUN \# 4.03A AND B; FIRST AND SECOND HALF OF SLURRY DEWATERING - CONT.

\begin{tabular}{|c|c|c|c|c|c|c|c|c|c|c|c|c|c|c|c|c|c|c|c|c|c|c|c|c|c|c|c|c|}
\hline & A & & D & $E$ & $\mathrm{~F}$ & G & $\mathrm{H}$ & $\mathrm{J}$ & $\mathrm{K}$ & $\begin{array}{ll} \\
\end{array}$ & $\mathrm{M}$ & $\mathrm{N}$ & 0 & $\mathrm{Q}$ & $\mathrm{R}$ & $\mathrm{s}$ & $T$ & $\mathrm{v}$ & \begin{tabular}{l|l} 
w \\
\end{tabular} & $x$ & $\mathrm{Y}$ & $z$ & $\mathrm{AA}$ & $\mathrm{AB}$ & $A C$ & & & \\
\hline & $8 / 31 / 2004$ & 8:02:18 PM & 25.237 & 25.662 & \begin{tabular}{l|l|}
2 & 24.374 \\
\end{tabular} & 26.03 & 24.927 & 47.403 & 52.471 & \begin{tabular}{|l|l|}
15.308 \\
\end{tabular} & 33.268 & -1.198 & -7.232 & 48.337 & 0.063 & \begin{tabular}{|l|}
14.643 \\
\end{tabular} & 0.003 & 168.93 & 36.1489 & 51.3 & 11.5 & 40.3 & 2.781 & 0.009 & 0.009 & 0.000 & 0.228 & \\
\hline & & & 25.23 & 25.665 & 524.392 & 26.028 & 24.925 & 47.582 & \begin{tabular}{|l|l|}
52.625 \\
\end{tabular} & $\begin{array}{l}15.449 \\
\end{array}$ & 33.311 & \begin{tabular}{|c|c|c|}
1 & -1.212 \\
\end{tabular} & -7.22 & 48.754 & 0.064 & $\begin{array}{l}4.643 \\
\end{array}$ & & & $\begin{array}{l}36.1656 \\
\end{array}$ & 51.5 & 11.6 & & & & & & & \\
\hline 1473 & $8 / 31 / 2004$ & 8:04:18 PM & 25.244 & 25.669 & 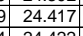 & 26.042 & 25.029 & & & \begin{tabular}{|l|l|}
15.364 \\
154
\end{tabular} & 33.284 & \begin{tabular}{|l|l}
44 & -1.143 \\
\end{tabular} & $\begin{array}{l}-7.073 \\
.7232\end{array}$ & 51.942 & 0.062 & $\begin{array}{l}14.643 \\
\end{array}$ & 0.004 & & 36.1822 & 51.5 & 12.4 & & 2.788 & . 009 & 0.009 & 0.000 & & \\
\hline $\begin{array}{l}1474 \\
1475\end{array}$ & \begin{tabular}{|l|l|}
$8 / 31 / 2004$ \\
$8 / 21 / 204$
\end{tabular} & $8: 05: 18 \mathrm{PM}$ & $\begin{array}{l}25.249 \\
25237\end{array}$ & $\begin{array}{l}25.674 \\
25.662\end{array}$ & \begin{tabular}{ll|l|}
4 & 24.422 \\
2 & 2.4205
\end{tabular} & $\begin{array}{ll}26.062 \\
2607\end{array}$ & $\begin{array}{l}25.054 \\
2517\end{array}$ & $\begin{array}{l}47.561 \\
\end{array}$ & \begin{tabular}{r|r|}
52.82 \\
52.827
\end{tabular} & $\begin{array}{l}15.444 \\
15.2302\end{array}$ & 33.058 & $\begin{array}{r}-1.206 \\
1.105\end{array}$ & $\begin{array}{l}-7.223 \\
7.223 \\
\end{array}$ & 50.74 & 0.062 & \begin{tabular}{|l|l|}
14.643 \\
\end{tabular} & 0.003 & & \begin{tabular}{|l|l|}
36.1989 \\
\end{tabular} & 51.7 & 12.1 & & 2.779 & 0.009 & 0.009 & 0.000 & \begin{tabular}{|l|l|}
0.224 \\
\end{tabular} & \\
\hline$\frac{1475}{1476}$ & $\frac{8 / 31 / 2004}{8 / 31 / 204}$ & $\begin{array}{l}8: 06: 18 \text { PM } \\
8: 07: 18\end{array}$ & $\frac{25.237}{25241}$ & $\frac{25.662}{25.671}$ & $\begin{array}{l}224.425 \\
\end{array}$ & & \begin{tabular}{|l|}
25.117 \\
25106
\end{tabular} & & & \begin{tabular}{|l|}
15.382 \\
15.217
\end{tabular} & $\begin{array}{r}33.149 \\
33.181\end{array}$ & \begin{tabular}{|c|}
-1.195 \\
1200
\end{tabular} & $\begin{array}{r}-7.223 \\
7226 \\
\end{array}$ & $\begin{array}{l}50.336 \\
49614\end{array}$ & $\begin{array}{l}0.064 \\
\end{array}$ & \begin{tabular}{|c|}
14.643 \\
14643 \\
\end{tabular} & & & $\begin{array}{l}36.2156 \\
2.2020\end{array}$ & 51.5 & \begin{tabular}{|l|}
12.0 \\
\end{tabular} & & \begin{tabular}{|l|l|}
2.779 \\
\end{tabular} & & 0.009 & & & \\
\hline \begin{tabular}{|l|l|}
1477 \\
147
\end{tabular} & $\begin{array}{l}8 / 31 / 204 \\
8 / 31 / 2004\end{array}$ & $\begin{array}{l}8: 70: 18 \mathrm{PM} \\
8: 08: 18 \mathrm{PM}\end{array}$ & $\begin{array}{r}\frac{25.241}{25.25} \\
\end{array}$ & $\frac{25.671}{25.665}$ & \begin{tabular}{|l|}
24.489 \\
24.482 \\
\end{tabular} & $\frac{26.139}{26.117}$ & $\begin{array}{l}255.106 \\
25.154 \\
\end{array}$ & $\begin{array}{r}47.223 \\
7.632 \\
\end{array}$ & \begin{tabular}{|l|}
52.355 \\
52.645
\end{tabular} & \begin{tabular}{|l|}
15.217 \\
15.475
\end{tabular} & $\begin{array}{l}33.181 \\
33.284 \\
\end{array}$ & \begin{tabular}{|c|}
-1.209 \\
-1.183
\end{tabular} & $\begin{array}{l}-7.226 \\
-7.217 \\
\end{array}$ & $\begin{array}{l}49.614 \\
50.099\end{array}$ & \begin{tabular}{|c|c|}
0.062 \\
0.063
\end{tabular} & $\begin{array}{l}44.643 \\
14.643 \\
\end{array}$ & $\frac{0.003}{0.003}$ & 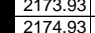 & \begin{tabular}{|}
836.2322 \\
336.2429
\end{tabular} & $\begin{array}{l}51.2 \\
51.5 \\
\end{array}$ & $\begin{array}{ll}\frac{11.8}{12.0} \\
\end{array}$ & \begin{tabular}{|l|l|}
40.2 \\
40.
\end{tabular} & $\begin{array}{l}\frac{2.772}{2.789} \\
2.78\end{array}$ & .0.009 & $\begin{array}{l}0.009 \\
0.009\end{array}$ & $\frac{0.000}{0.000}$ & $\begin{array}{l}0.224 \\
0.026\end{array}$ & $\begin{array}{l}0.19 \\
0.19\end{array}$ \\
\hline \begin{tabular}{|l|}
1478 \\
\end{tabular} & $8 / 31 / 2004$ & 8:09:18 PM & 25.259 & 25.679 & $\begin{array}{ll}9 & 24.482 \\
\end{array}$ & 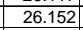 & 25.139 & & 52.691 & 15.378 & & & -7.082 & 50.044 & 0.064 & 14.643 & 0.004 & 2175.93 & \begin{tabular}{|l|}
30.2469 \\
36.2656
\end{tabular} & $\begin{array}{l}51.5 \\
51.6\end{array}$ & $\begin{array}{l}11.0 \\
11.9\end{array}$ & \begin{tabular}{|l|}
40.3 \\
0.3
\end{tabular} & $\frac{2.189}{2.776}$ & \begin{tabular}{|l}
0.009 \\
0.099
\end{tabular} & $\begin{array}{l}0.009 \\
0.009 \\
\end{array}$ & $\begin{array}{l}0.000 \\
0.000\end{array}$ & & $\frac{0.19}{0.20}$ \\
\hline 1479 & $8 / 31 / 2004$ & 8:10:18 PM & & 25.674 & \begin{tabular}{|l|l|}
4 & 24.457 \\
\end{tabular} & 26.172 & 25.164 & 47.159 & 52.502 & 15.386 & 32.718 & -1.189 & & $\begin{array}{l}49.143 \\
\end{array}$ & 0.064 & \begin{tabular}{|l|l|}
14.643 \\
\end{tabular} & & & $3 \quad 36.2822$ & 51.4 & & & & & & & & \\
\hline & $8 / 31 / 2004$ & 8:11:18 PM & & & & & 25.14 & 47.534 & \begin{tabular}{|l|}
52.805 \\
\end{tabular} & $\begin{array}{l}15.41 \\
\end{array}$ & & -1.148 & & 48.01 & & 14.643 & & & \begin{tabular}{|l|l|}
36.2989 \\
\end{tabular} & & $\begin{array}{ll}11.4 \\
\end{array}$ & & 2.780 & .009 & 0.009 & 0.000 & 0.227 & \\
\hline 1481 & $8 / 31 / 2004$ & 8:12:18 PM & $\begin{array}{l}25.265 \\
25275\end{array}$ & $\begin{array}{r}25.68 \\
\end{array}$ & 824.448 & $\begin{array}{l}26.208 \\
26262\end{array}$ & $\begin{array}{l}25.16 \\
2519\end{array}$ & 47.451 & \begin{tabular}{|l|}
52.722 \\
5.877 \\
\end{tabular} & $\begin{array}{l}15.304 \\
15.36\end{array}$ & $\begin{array}{l}3.222 \\
3278\end{array}$ & $\begin{array}{l}-1.148 \\
1.123\end{array}$ & & 49.51 & $\begin{array}{l}0.063 \\
\end{array}$ & \begin{tabular}{|l|l|}
14.643 \\
\end{tabular} & 0.003 & & $\begin{array}{l}36.3156 \\
\end{array}$ & 51.6 & 11.8 & & 2.781 & 0.009 & 0.009 & 0.000 & 0.227 & \\
\hline$\frac{1482}{1823}$ & 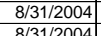 & $8: 13: 18 \mathrm{PM}$ & 25.275 & 25.685 & \begin{tabular}{|l|l|}
5 & 24.473 \\
2 & 24.439
\end{tabular} & $\begin{array}{l}26.263 \\
26235\end{array}$ & $\begin{array}{r}25.18 \\
25.206\end{array}$ & $\begin{array}{l}47.656 \\
47619\end{array}$ & \begin{tabular}{|l|}
52.871 \\
5253 \\
\end{tabular} & \begin{tabular}{r|r|}
15.36 \\
15559
\end{tabular} & $\begin{array}{l}33.378 \\
33185\end{array}$ & $\begin{array}{r}-1.143 \\
.1155\end{array}$ & & \begin{tabular}{|l|l|}
49.028 \\
52077
\end{tabular} & $\begin{array}{l}0.067 \\
0.046\end{array}$ & \begin{tabular}{|l|l|}
14.643 \\
14634
\end{tabular} & & $\begin{array}{l}1779.93 \\
18002\end{array}$ & & 51.7 & \begin{tabular}{|c|c|}
11.7 \\
12.
\end{tabular} & & $\begin{array}{l}2.794 \\
2786\end{array}$ & 4.010 & .010 & 0.000 & 0.240 & 0.20 \\
\hline$\frac{1483}{1484}$ & $\frac{8 / 31 / 2004}{88 / 312004}$ & $\begin{array}{l}8: 14: 118 \mathrm{PM} \\
\text { 8:15:18 }\end{array}$ & $\begin{array}{l}25.272 \\
25.265\end{array}$ & $\begin{array}{l}25.682 \\
25.675 \\
\end{array}$ & \begin{tabular}{|l}
2 \\
5 \\
5
\end{tabular} & $\frac{26.235}{26.278}$ & $\begin{array}{l}25.226 \\
25.275 \\
\end{array}$ & \begin{tabular}{|}
47.619 \\
47.509
\end{tabular} & \begin{tabular}{|l|}
52.536 \\
52.511 \\
\end{tabular} & \begin{tabular}{|l|}
15.559 \\
15.329
\end{tabular} & $\begin{array}{l}3.185 \\
33.28 \\
\end{array}$ & $\begin{array}{l}-1.151 \\
-1.093 \\
\end{array}$ & $\begin{array}{r}-7.108 \\
-7.128 \\
\end{array}$ & $\begin{array}{l}52.077 \\
48.777\end{array}$ & $\begin{array}{l}0.064 \\
0.062\end{array}$ & \begin{tabular}{|c|}
14.643 \\
11.4633
\end{tabular} & $\frac{0.003}{0.003}$ & 年1.93 & \begin{tabular}{|c|c|}
36.3489 \\
36.3656
\end{tabular} & 51.4 & $\frac{12.4}{11.6}$ & 40.4 & $\begin{array}{l}2.786 \\
2.785 \\
\end{array}$ & | 0.009 & $\begin{array}{l}0.009 \\
0.009\end{array}$ & $\frac{0.000}{0.000}$ & $\frac{0.230}{0.223}$ & $\frac{0.20}{0.19}$ \\
\hline 1485 & $8 / 31 / 2004$ & 8:16:18 PM & 25.27 & 25.685 & $5[24.453$ & \begin{tabular}{|l|l|} 
& 0.210 \\
\end{tabular} & 25.26 & 47.569 & \begin{tabular}{|l|}
52.511 \\
52.907 \\
\end{tabular} & 15.467 & 33.118 & \begin{tabular}{|l|l|}
8 & -1.088 \\
\end{tabular} & & 49.847 & $\begin{array}{l}0.062 \\
0.064\end{array}$ & $\begin{array}{l}14.643 \\
14.643 \\
\end{array}$ & & 2182.93 & \begin{tabular}{|l|}
30.3050 \\
36.3822
\end{tabular} & $\begin{array}{l}51.4 \\
51.8\end{array}$ & $\begin{array}{ll}11.6 \\
11.9\end{array}$ & & & \begin{tabular}{|c|}
0.009 \\
0.009 \\
\end{tabular} & $\begin{array}{l}0.009 \\
0.009\end{array}$ & & & $\begin{array}{l}0.19 \\
0.20\end{array}$ \\
\hline & $8 / 31 / 2004$ & & 25.277 & 25.692 & 2. 24.464 & 26.32 & & & 53.356 & 15.442 & 33.458 & \begin{tabular}{|l|l|}
8 & -1.143 \\
\end{tabular} & & 49.468 & 0.055 & 14.643 & & & & & & & & 0.008 & & & & $\begin{array}{l}0.20 \\
0.17\end{array}$ \\
\hline 1487 & $8 / 31 / 2004$ & 8:18:18 PM & 25.267 & 25.687 & $\begin{array}{l}7 \\
24.434 \\
\end{array}$ & 26.265 & 25.361 & 47.472 & 52.813 & $\begin{array}{l}15.537 \\
\end{array}$ & 33.05 & -1.09 & -7.05 & 49.677 & 0.066 & & & 34.99 & 36.4156 & 51.7 & \begin{tabular}{|c|}
11.8 \\
\end{tabular} & & & & & & & $\frac{17}{20}$ \\
\hline 1488 & & & & & & & & & & 15.444 & & & & 51.37 & & 14.6 & & & & 51.5 & & & & & & & 234 & \\
\hline & & & & 25.699 & $\begin{array}{l}924.437 \\
\end{array}$ & 26.312 & & $\begin{array}{l}47.613 \\
\end{array}$ & 52.836 & 15.426 & 33.282 & $\mid-1.145$ & & 51.093 & .067 & 14.6 & & & 89 & 51.7 & 122 & & & & & & & \\
\hline & $8 / 31 /$ & & 25.264 & 25.694 & \begin{tabular}{|l|}
24.432 \\
\end{tabular} & 26.297 & & 47.42 & & 15.382 & & -1.215 & & & & & & & & & & & & & & & & \\
\hline & 2004 & & 25.265 & 25.69 & $\begin{array}{l}9 \\
\end{array}$ & 26.243 & & & 52.44 & 15.291 & 3.155 & -0.87 & & 48.798 & | & 14.6 & & & & & \begin{tabular}{|l|l|}
11.6 \\
\end{tabular} & & & & & & & \\
\hline & $8 / 31 / 2004$ & 8:23:18 PM & 25.251 & 25.681 & 124.423 & 26.164 & 25.04 & 47.588 & \begin{tabular}{|l|}
52.867 \\
\end{tabular} & 15.484 & 33.128 & \begin{tabular}{|l|l|}
8 & -1.212 \\
\end{tabular} & & 49.535 & 0.061 & 14.643 & & 189.93 & \begin{tabular}{|l|}
36.4989 \\
\end{tabular} & 51.7 & 11.8 & 40. & 2.783 & 0.009 & 0.009 & 0.000 & 0.220 & 19 \\
\hline & $8 / 31 / 2$ & & 25.251 & 25.676 & \begin{tabular}{l|l|}
624.403 \\
\end{tabular} & 26.104 & 24.901 & 47.385 & \begin{tabular}{|l|}
52.722 \\
\end{tabular} & \begin{tabular}{|l|l|}
15.38 \\
15.35
\end{tabular} & 32.96 & $\begin{array}{l}-1.195 \\
\end{array}$ & & 48.181 & 0.063 & 14.643 & & 190.93 & \begin{tabular}{|l|}
36.5156 \\
\end{tabular} & 51.6 & 11.5 & & 2.770 & \begin{tabular}{|l|l|}
0.009 \\
\end{tabular} & 0.009 & 0.000 & 0.228 & \\
\hline & 31/2004 & & & 25.666 & $\begin{array}{ll}624.388 \\
\end{array}$ & & & & & 15.452 & & & & & 0.056 & & & 191.93 & & 51.8 & 11.7 & 40.4 & $2.785 \mid$ & \begin{tabular}{|l|}
0.008 \\
\end{tabular} & 0.008 & 0.000 & 0.202 & \\
\hline & $11 / 2004$ & $\begin{array}{l}8: 26: 1 \\
8: 27-1\end{array}$ & $\begin{array}{r}25.24 \\
\end{array}$ & 25.67 & $\begin{array}{l}7.24 .407 \\
7.412\end{array}$ & $\begin{array}{l}25.968 \\
25.092\end{array}$ & 24.8. & & \begin{tabular}{|l|}
52.339 \\
5.2019 \\
\end{tabular} & \begin{tabular}{|r|}
15.47 \\
15.59
\end{tabular} & 2.982 & -1.154 & & & .063 & & & & & 51.2 & 12.0 & & \begin{tabular}{|l|l|}
2.769 \\
\end{tabular} & 0.009 & & & $\begin{array}{l}0.228 \\
\end{array}$ & \\
\hline & $\begin{array}{l}8 / 31 / 2004 \\
8 / 31 / 204\end{array}$ & $\begin{array}{l}8: 27: 18 \mathrm{PM} \\
8: 28: 18 \mathrm{PM}\end{array}$ & $\begin{array}{l}25.239 \\
25.238\end{array}$ & $\begin{array}{l}25.664 \\
25.663\end{array}$ & $\begin{array}{l}4 \quad 24.412 \\
3 \\
3 \\
24.426\end{array}$ & $\frac{25.942}{25.921}$ & $\frac{24.83}{24.82}$ & $\begin{array}{l}47.764 \\
47.773\end{array}$ & \begin{tabular}{|l|}
52.948 \\
53.058 \\
\end{tabular} & \begin{tabular}{|c|}
15.558 \\
15.481
\end{tabular} & $\begin{array}{l}33.198 \\
33.337 \\
\end{array}$ & $\begin{array}{ll}8 & -1.148 \\
7 & -1.206 \\
\end{array}$ & $\begin{array}{l}-7.154 \\
-7.194 \\
\end{array}$ & $\begin{array}{l}49.799 \\
49.833 \\
\end{array}$ & $\begin{array}{l}0.063 \\
0.064\end{array}$ & $\begin{array}{l}14.643 \\
14.463 \\
\end{array}$ & & $\mid \begin{array}{c}1.93 .93 \\
1199.93 \\
\end{array}$ & $\begin{array}{l}836.5656 \\
3 \\
3\end{array}$ & $\begin{array}{l}51.8 \\
51.9 \\
\end{array}$ & \begin{tabular}{ll|l}
11.9 \\
11.9
\end{tabular} & $\frac{40}{40}$ & $\frac{2.791}{2.796}$ & 0.009 & 0.009 & 0.000 & 0.227 & 0.19 \\
\hline 1498 & $\begin{array}{l}8311 / 204 \\
8 / 31 / 2004\end{array}$ & $\begin{array}{l}8.28 .18 \mathrm{PM} \\
8: 29: 18 \mathrm{PM}\end{array}$ & 25.223 & $\frac{25.663}{25.653}$ & $\begin{array}{l}3 \\
3 \\
3\end{array}$ & $\frac{25.921}{25.931}$ & $\begin{array}{l}24.823 \\
24.843\end{array}$ & $\begin{array}{l}47.173 \\
47.835\end{array}$ & \begin{tabular}{|l|}
53.0085 \\
52.691 \\
\end{tabular} & \begin{tabular}{|l}
15.501 \\
15.501
\end{tabular} & $\begin{array}{l}33.351 \\
33.499\end{array}$ & \begin{tabular}{|l|l|}
9 & -1.209 \\
\end{tabular} & & $\begin{array}{l}49.833 \\
52.572\end{array}$ & $\mid \begin{array}{l}0.064 \\
0.066\end{array}$ & $\begin{array}{l}14.643 \\
14.643 \\
\end{array}$ & & $\mid \begin{array}{c}194.93 \\
1195.93 \\
\end{array}$ & $\begin{array}{l}836.5822 \\
336.5989\end{array}$ & $\begin{array}{l}51.9 \\
51.6\end{array}$ & $\begin{array}{l}11.9 \\
12.5\end{array}$ & & $\frac{2.196}{2.804}$ & & & & & 0.20 \\
\hline & $8 / 31 / 2004$ & 8:30:18 PM & 25.233 & 25.658 & B 24.415 & 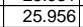 & 24.8 & & \begin{tabular}{|l|}
52.19 \\
\end{tabular} & 15.336 & 32.988 & \begin{tabular}{c|c|c|}
8 & -1.145 \\
\end{tabular} & & 48.848 & 0.065 & 14.643 & & 196 & 56 & 51.1 & 11.6 & 40 & 2.764 & \begin{tabular}{|l|}
0.009 \\
\end{tabular} & & & & $\frac{0.20}{0.20}$ \\
\hline & $8 / 31 / 2004$ & & 25.243 & 25.668 & 824.486 & & & & & 15.346 & & \begin{tabular}{|l|l|}
7 & -1.183 \\
\end{tabular} & & 50.117 & & & & & & & 110 & & & & & & & $\frac{0.20}{0.20}$ \\
\hline & $8 / 31 / 2004$ & $8: 32: 18$ F & & 25.657 & $7 \quad 24.474$ & 25.994 & 24.9 & 47.354 & \begin{tabular}{|l|}
52.575 \\
\end{tabular} & 15.519 & 32.82 & -1.212 & & 50.34 & 0.061 & $\begin{array}{l}14.643 \\
\end{array}$ & & & & 51.4 & 12.0 & 40 & 2.764 & & & & & 0.20 \\
\hline 1502 & & & 25.236 & 25.661 & 124.454 & & 25. & & 52.776 & 15.409 & 33.056 & -1.166 & & 48. & 0.062 & & & & & & \begin{tabular}{|c|}
11.6 \\
\end{tabular} & & & & & & & \\
\hline & $8 / 31 / 2004$ & & 25.25 & 25.67 & 24.468 & 26.06. & & & 52.714 & 15.354 & 33.056 & & & & 0.064 & 14.6 & & & & & & & & & & & & \\
\hline & $8 / 31 / 2004$ & & 25.245 & 25.65 & 24.447 & 26.06 & & & 52.397 & $\mid$\begin{tabular}{|c|c|}
15.23 \\
\end{tabular} & 33.337 & \begin{tabular}{|c|c|}
-1.169 \\
\end{tabular} & & & . & 14.6 & & & & 513 & 1118 & & & & & & & \\
\hline & $8 / 31 / 2004$ & & 25.25 & 25.655 & $5 \quad 24.457$ & 26.082 & & 47. & 52.48 & 15.397 & 32.974 & -1.16 & & 50.30 & | & 14.6 & & & & 51.4 & 12.0 & & & & & & & \\
\hline & $8 / 31 / 2$ & & 25.25 & 25.65 & $\begin{array}{l}5 \\
\end{array}$ & 26.09 & & & 52.614 & 15.339 & 33.1 & -1.148 & & 51.15 & & 14.6 & & & & 51.5 & 12.2 & & & & & & & \\
\hline & & & 25.245 & 25.65 & $\begin{array}{l}5 \\
5\end{array}$ & 26.12 & & & 53.048 & 15.4 & 202 & $-\frac{1.143}{1.10}$ & & & 664 & 14. & & & & & 11.8 & & & & & & & \\
\hline & 004 & & & 25.6 & & & & & & & & & & & & & & & & 51.4 & 11.9 & & & & & & & \\
\hline & & & 25.251 & 25.6 & 24.449 & 26. & & 41.25 & 52.832 & 15.518 & 33.27 & -1.1 & & & 0.062 & & & & & 51.7 & 11.8 & & & & & & & \\
\hline & 004 & & 25.241 & 250.646 & 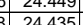 & 26.18 & & & $\frac{53.016}{5256}$ & $\begin{array}{r}15.19 \\
15175\end{array}$ & $\begin{array}{l}33.352 \\
33445\end{array}$ & $\begin{array}{r}-1.18 \\
-1.09\end{array}$ & & & 0.063 & & & & & (51.94 & 116 & & & & & & & \\
\hline & $8 / 31 / 2004$ & & 25.272 & 25.670 & \begin{tabular}{|l|l|}
24.450 \\
2
\end{tabular} & & & & & & & -1.145 & & & & $\frac{14.6}{14.6}$ & & & & & 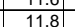 & & & & & & & \\
\hline & $8 / 31 / 2004$ & & $\frac{2.2617}{25.262}$ & $\frac{25.6}{256}$ & 24,434 & & & & 52799 & & & \begin{tabular}{|l|l|}
1 & -1.151 \\
\end{tabular} & & & & & & & & & $\frac{1.10}{119}$ & & & & & & $0 . \angle 20$ & \\
\hline & $8 / 31 / 2004$ & & 25.268 & 25.668 & $\begin{array}{ll}8 & 24.445 \\
\end{array}$ & 26.22 & & 47. & \begin{tabular}{|l|}
53.039 \\
\end{tabular} & $\mid 15.312$ & & \begin{tabular}{|l|l|}
4 & -1.148 \\
\end{tabular} & & & 0.063 & & & & & 51.9 & $\frac{\frac{x 1.5}{11.5}}{4}$ & & & 009 & 0.00 & 0 & $\frac{2.225}{0.225}$ & \\
\hline & & & & & & & & & & & & & & & & & & & & & & & & & & & & \\
\hline & & & 25.268 & & & 26.2 & & & 52.759 & 15.16 & & -1.1. & & & & & & & & & 11.9 & & & & & & & \\
\hline & $8 / 31 / 2004$ & & 20.27 & $25.67 \mathrm{~s}$ & 24.442 & 26.253 & & & 2.703 & 15.181 & 33.627 & -1.148 & & & 064 & & & 14.9 .9 & & 51.6 & 11.8 & 40. & & 0.009 & & & 0.229 & 1 \\
\hline & $8 / 31 / 2004$ & & & & 24.482 & 26.278 & & & & 15.204 & & $\begin{array}{l}-1.09 \\
\end{array}$ & & & & & & & & & 11.9 & 40.6 & & 8009 & & & 0.222 & \\
\hline & $8 / 31 / 20$ & & 25.27. & 25.6 & & & & & 52.963 & \begin{tabular}{|l|l|}
15.198 \\
\end{tabular} & & -1.108 & & & & & 3 & & & & $\begin{array}{l}11.8 \\
120\end{array}$ & 40.6 & & \begin{tabular}{|l|l|} 
\\
\end{tabular} & & 然 & 0.226 & 19 \\
\hline & $8 / 31 / 2004$ & & $\frac{25.277}{25.262}$ & $\frac{25.682}{25.662}$ & $\begin{array}{l}2 \quad 24.489 \\
2\end{array}$ & $\frac{26.315}{26.27}$ & $\frac{25.4}{25.3}$ & $\begin{array}{r}47 \\
476 \\
\end{array}$ & \begin{tabular}{|c|}
52.627 \\
52627 \\
\end{tabular} & \begin{tabular}{|r|r|}
15.178 \\
15.304
\end{tabular} & $\begin{array}{r}33.43 \\
33.397 \\
\end{array}$ & $\begin{array}{r}-1.09 \\
-1.093\end{array}$ & & $\begin{array}{l}50.248 \\
49.30\end{array}$ & $\begin{array}{l}0.064 \\
0.063\end{array}$ & $\frac{14.6}{14.6}$ & & & & $\begin{array}{l}51.5 \\
51.5 \\
\end{array}$ & $\begin{array}{l}12.0 \\
11.7\end{array}$ & $\frac{40}{40}$ & & $\mid \begin{array}{l}0.0099 \\
0009\end{array}$ & 0.009 & $\begin{array}{l}0.000 \\
0.000\end{array}$ & $\frac{0.230}{0.226}$ & $\frac{20}{19}$ \\
\hline & $015112<04$ & 8.5:53:18 & 20.202 & $\frac{25.004}{25.683}$ & \begin{tabular}{|l|l|}
3 & 24.439 \\
3
\end{tabular} & $\begin{array}{l}20.271 \\
26.271\end{array}$ & 25.28 & 47. & \begin{tabular}{|l|}
52.0287 \\
52.878 \\
\end{tabular} & $\begin{array}{r}35.304 \\
15.18\end{array}$ & \begin{tabular}{|l|}
3.091 \\
33.208
\end{tabular} & \begin{tabular}{|l|l|}
8 & -1.090 \\
8
\end{tabular} & $\frac{-1.1}{-7.11}$ & & 0.000 & & & & & S1.0 & $\begin{array}{ll}1.11 \\
11.7\end{array}$ & & 2.190 & & & .000 & $\begin{array}{l}0.220 \\
0.223 \\
\end{array}$ & \\
\hline & & & 25.27 & & & & & & 52.803 & 15.2 & & & & & & & & & & & 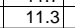 & & & & & & & \\
\hline & & & 25 & & & & & & & & & & & & & & & & & & 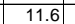 & & & & & & & \\
\hline & $8 / 31 / 2$ & & 5.2 & 25.67 & \begin{tabular}{|l|}
24.446 \\
\end{tabular} & 26.0 & & 47 & 53.12 & & & \begin{tabular}{|l|l|}
-1.09 \\
\end{tabular} & & & & & & & & & 11.5 & & & & & & & \\
\hline & $8 / 31 / 206$ & & 25.2 & 25.66 & 24.42 & & & 47.5 & 52.73 & 15.203 & & -1.1 .1 & & & & 14.6 & & & & & 111.6 & & & & & & .234 & \\
\hline & & & & & & & & & & & & -1 & & & & & & & & & 11.8 & & & & & & & \\
\hline & $8 / 31 / 20$ & & 25.25 & 25.6 & & 25 & & & 52.894 & 15.245 & & -1.09 & & & & & & & & & 12.1 & & & & & & & \\
\hline & & & 25 & & & & & & & & & & & & & & & & & & & & & & & & & \\
\hline & & & $\frac{25.2}{252}$ & & 24.40 & 25.4 & & & & & & & & & & & & & & & $\frac{11.1}{11.0}$ & & & & & & & \\
\hline & 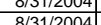 & & 5524 & & 24.4 & 25.94 & & & & & 33.16 & $=\frac{-1.05}{-1.03}$ & & & & & & & & & & & & & & & & \\
\hline & $8 / 31 / 20$ & & 23 & & $\frac{24.44}{24,44}$ & & & 47.056 & 526 & 1531 & 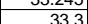 & -111 & & & & & & & & & $=0$ & & & & & & & \\
\hline & & & 25.246 & & & 25.993 & & 47.347 & 52.903 & & & $\begin{array}{l}-.1 .925 \\
-0.925\end{array}$ & & & & & & & & & & & & & & & & \\
\hline & & & 25.2. & $\frac{25.646}{25.646}$ & & $\frac{26.0}{26.0}$ & & 47.8 & & & & $\begin{array}{l}-1.09 \\
-1.09\end{array}$ & & & & & & & & & & & & & & & & \\
\hline & & & & & 24.41 & & & & & & & & & & & & & & & & & & & & & & & \\
\hline & & & & & & & & & & & & & & & & & & & & & & & & & & & & \\
\hline & $8 / 31 / /$ & & 2523 & 25. & 24.42 & 26.6 & & & & & & -1.0 & & & & & & & & & & & & & & & & \\
\hline & & & & & 2444 & & & & & 19 & & -100 & & & & & & & & & & & & & & & & \\
\hline & $8 / 31 / 2004$ & $9: 11: 18$ & 25.236 & 25.636 & \begin{tabular}{l|l|}
624.413 \\
\end{tabular} & \begin{tabular}{|l|}
26.088 \\
\end{tabular} & & $\begin{array}{ll}47.642 \\
\end{array}$ & \begin{tabular}{|l|l|}
52.865 \\
\end{tabular} & \begin{tabular}{|l|l|l|l|l}
15.199 \\
\end{tabular} & 33.452 & \begin{tabular}{|l|l|}
2 & -1.082 \\
\end{tabular} & & 49.595 & 0.061 & 14.643 & & & 2989 & & & & 2. 196 & 0.009 & 0.005 & 0.000 & 0.219 & \\
\hline
\end{tabular}


WSRC-TR-2005-00105, REVISION 0

SRNL-RPP-2005-00012, REVISION 0

RUN \# 4.03A AND B; FIRST AND SECOND HALF OF SLURRY DEWATERING - CONT.

\begin{tabular}{|c|c|c|c|c|c|c|c|c|c|c|c|c|c|c|c|c|c|c|c|c|c|c|c|c|c|c|c|c|}
\hline & A & & $\mathrm{D}$ & $E$ & $\mathrm{~F}$ & G & $\mathrm{H}$ & $\mathrm{J}$ & $\mathrm{K}$ & $\begin{array}{ll}\mathrm{L} \\
\end{array}$ & M & $\mathrm{N}$ & 0 & $Q$ & $\mathrm{R}$ & $\mathrm{s}$ & $T$ & $\mathrm{v}$ & w & $x$ & \begin{tabular}{l|l} 
\\
\end{tabular} & $z$ & AA & & & & & AF \\
\hline & $8 / 31 / 2004$ & 9:11:26 PM & 25.246 & 25.641 & \begin{tabular}{|l|}
24.433 \\
\end{tabular} & 26.118 & 25.13 & 47.439 & \begin{tabular}{|l|}
52.726 \\
\end{tabular} & 15.187 & 33.278 & -1.067 & -7.056 & 46.116 & 0.062 & \begin{tabular}{|l|}
14.643 \\
\end{tabular} & 0.003 & 238.07 & 37.3011 & 51.6 & 11.0 & \begin{tabular}{|l|} 
\\
\end{tabular} & 2.783 & 0.009 & 0.009 & 0.000 & 0.223 & \\
\hline & & & & 25.629 & \begin{tabular}{|l|}
24.407 \\
\end{tabular} & \begin{tabular}{|l|l|}
26.082 \\
\end{tabular} & $\begin{array}{l}25.119 \\
2.119\end{array}$ & 47.824 & \begin{tabular}{|l|l|}
52.888 \\
\end{tabular} & $\begin{array}{l}15.264 \\
\end{array}$ & 33.809 & \begin{tabular}{|r|}
-1.093 \\
\end{tabular} & & 49.518 & 0.065 & $\begin{array}{l}14.643 \\
\end{array}$ & & & 737.3178 & 51.8 & 11.8 & & & & 0.009 & & & \\
\hline 1543 & $8 / 31 / 2004$ & 9:13:27 PM & $\frac{25.229}{2522}$ & 25.634 & \begin{tabular}{|l|l|}
24.426 \\
\end{tabular} & \begin{tabular}{|l|l|}
26.106 \\
\end{tabular} & 25.133 & $\begin{array}{l}47.884 \\
\end{array}$ & \begin{tabular}{|l|}
52.994 \\
5.942 \\
\end{tabular} & \begin{tabular}{|l|}
15.343 \\
1.233
\end{tabular} & 33.639 & \begin{tabular}{|c|c|} 
& -1.09 \\
\end{tabular} & $\begin{array}{r}-7.07 \\
\end{array}$ & 47.876 & 0.059 & $\begin{array}{l}14.643 \\
\end{array}$ & & $\frac{10.08}{10.08}$ & $\frac{7.3347}{7.3270}$ & 51.9 & 11.4 & & 2.810 & \begin{tabular}{|l|l|}
0.008 \\
\end{tabular} & 0.009 & 0.000 & 0.211 & \\
\hline 1544 & $8 / 31 / 2004$ & 9:14:27 PM & $\begin{array}{r}25.23 \\
\end{array}$ & 25.635 & \begin{tabular}{|l|l|}
24.427 \\
\end{tabular} & 26.123 & $\begin{array}{l}25.115 \\
25.110\end{array}$ & $\begin{array}{l}47.685 \\
\end{array}$ & \begin{tabular}{|l|}
52.942 \\
5.125 \\
\end{tabular} & $\begin{array}{l}15.249 \\
\end{array}$ & 33.483 & -1.09 & \begin{tabular}{|l|l|}
-7.061 \\
.700
\end{tabular} & 48.435 & $\begin{array}{l}0.066 \\
\end{array}$ & \begin{tabular}{|l|l|}
14.643 \\
\end{tabular} & & & 3514 & 51.8 & 11.5 & & 8.798 & 0.009 & 0.010 & 0.000 & 0.237 & \\
\hline$\frac{1545}{1546}$ & $\frac{8 / 31 / 2004}{8 / 31204}$ & $\begin{array}{l}\text { 9:15:27 PM } \\
9 \cdot 16 \cdot 27\end{array}$ & $\frac{25.239}{25.241}$ & $\frac{25.649}{25.641}$ & \begin{tabular}{|l|l|}
24.432 \\
24.423
\end{tabular} & \begin{tabular}{|l|l|}
26.142 \\
26.138 \\
\end{tabular} & $\begin{array}{l}25.119 \\
25111 \\
\end{array}$ & \begin{tabular}{|l|l|}
47.569 \\
47746
\end{tabular} & \begin{tabular}{|l|}
52.645 \\
52662 \\
\end{tabular} & $\begin{array}{l}15.205 \\
15281 \\
\end{array}$ & $\begin{array}{r}33.495 \\
33.696 \\
\end{array}$ & $\frac{-1.07}{-1.021}$ & & \begin{tabular}{|l|}
50.098 \\
48.323 \\
\end{tabular} & \begin{tabular}{|l|l|}
0.063 \\
0.623
\end{tabular} & \begin{tabular}{|l|}
14.643 \\
14643 \\
\end{tabular} & & & & 51.5 & 11.9 & & & 0.009 & 0.009 & & 0.226 & 0.19 \\
\hline $\begin{array}{l}1547 \\
1547\end{array}$ & & $\begin{array}{l}\text { 9:16:27 PM } \\
\text { 9:17:27 PM }\end{array}$ & $\frac{25.241}{25.23}$ & $\frac{25.641}{25.63}$ & 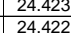 & \begin{tabular}{|l|}
26.138 \\
26.123
\end{tabular} & $\begin{array}{l}25.115 \\
25.195 \\
\end{array}$ & \begin{tabular}{|l|}
47.7646 \\
47.617
\end{tabular} & \begin{tabular}{|l|}
52.662 \\
52.911 \\
\end{tabular} & $\begin{array}{l}15.281 \\
15.186 \\
\end{array}$ & $\begin{array}{l}33.696 \\
33.436 \\
\end{array}$ & \begin{tabular}{|c|}
-1.021 \\
\end{tabular} & $\begin{array}{l}-7.061 \\
-7.053 \\
\end{array}$ & \begin{tabular}{|l|}
48.323 \\
49.987 \\
\end{tabular} & $\begin{array}{l}0.062 \\
0.063\end{array}$ & $\begin{array}{l}44.643 \\
14.643 \\
\end{array}$ & $\begin{array}{l}0.003 \\
0.003\end{array}$ & $\begin{array}{l}4.43 .08 \\
44.08\end{array}$ & 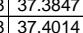 & $\begin{array}{l}51.5 \\
51.8\end{array}$ & $\begin{array}{l}\frac{11.5}{11.9} \\
\end{array}$ & \begin{tabular}{|l|l|}
40.7 \\
40.5
\end{tabular} & \begin{tabular}{|l|l|}
2.808 \\
2.794
\end{tabular} & 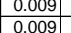 & $\mid \begin{array}{l}0.009 \\
0.009\end{array}$ & $\begin{array}{l}0.000 \\
0.000\end{array}$ & \begin{tabular}{|l}
0.222 \\
0.226
\end{tabular} & $\begin{array}{l}0.19 \\
0.19\end{array}$ \\
\hline \begin{tabular}{|l|}
1548 \\
\end{tabular} & $8 / 31 / 2004$ & 9:18:27 PM & 25.24 & 25.635 & & & & 47.638 & \begin{tabular}{|l|}
52.832 \\
\end{tabular} & 15.243 & & \begin{tabular}{|l|l|} 
& -.983 \\
\end{tabular} & & 49.184 & & & $\begin{array}{l}0.0003 \\
0.004\end{array}$ & & \begin{tabular}{|l|l|l|}
37.41814 \\
3
\end{tabular} & $\begin{array}{l}51.8 \\
51.7\end{array}$ & 11.7 & & $\frac{2.994}{2.801}$ & 0.009 & $\begin{array}{l}.0099 \\
0.009\end{array}$ & & & $\frac{0.19}{0.20}$ \\
\hline 1549 & $8 / 31 / 2004$ & 9:19:27 PM & & 25.636 & \begin{tabular}{|l|}
24.428 \\
\end{tabular} & \begin{tabular}{|l|}
26.128 \\
\end{tabular} & 25.26 & 47.673 & \begin{tabular}{|l|}
52.915 \\
\end{tabular} & 15.4 & 33.309 & \begin{tabular}{|l|l|} 
& -1.009 \\
\end{tabular} & & 48.212 & 0.06 & \begin{tabular}{|l|l|}
14.643 \\
\end{tabular} & & & 337.4347 & 51.8 & 11.5 & & & 0.009 & & & & 0.18 \\
\hline & $8 / 31 / 2004$ & 9:20:27 PM & 25.236 & 25.636 & \begin{tabular}{|l|l|}
24.408 \\
\end{tabular} & 26.148 & & $\begin{array}{l}47.343 \\
\end{array}$ & \begin{tabular}{|r|}
52.5 \\
\end{tabular} & $\begin{array}{l}15.157 \\
\end{array}$ & & -1.038 & $\begin{array}{l}-7.061 \\
\end{array}$ & 49.307 & & 14.643 & & & 37.4514 & 51.4 & $\begin{array}{lll}11.7 \\
\end{array}$ & & 2.782 & 0.009 & 0.009 & 0.000 & 0.220 & \\
\hline 1551 & $8 / 31 / 2004$ & 9:21:27 PM & 25.231 & 25.631 & 24.433 & 26.153 & 25.24 & \begin{tabular}{|l|l|}
47.739 \\
\end{tabular} & \begin{tabular}{|l|l|}
53.058 \\
5320
\end{tabular} & $\begin{array}{l}15.364 \\
15.252\end{array}$ & $\begin{array}{l}33.335 \\
22.52\end{array}$ & $\begin{array}{l}-1.085 \\
1015\end{array}$ & & 48.125 & $\begin{array}{l}0.062 \\
\end{array}$ & \begin{tabular}{|l|l|}
14.643 \\
\end{tabular} & 0.003 & & 37.4681 & 51.9 & 11.5 & & 795 & 0.009 & 0.009 & 0.000 & & \\
\hline$\frac{1552}{1553}$ & $\frac{8 / 31 / 2004}{8 / 31204}$ & $\begin{array}{l}\text { 9:22:27 PM } \\
9 \cdot 23: 27\end{array}$ & $\begin{array}{r}25.24 \\
25.245 \\
\end{array}$ & $\frac{25.645}{25.64}$ & \begin{tabular}{|l|l|}
24.452 \\
24.457
\end{tabular} & $\begin{array}{l}26.168 \\
26203\end{array}$ & $\begin{array}{l}25.245 \\
25.295\end{array}$ & 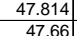 & \begin{tabular}{|l|}
53.139 \\
52623 \\
\end{tabular} & $\begin{array}{l}15.352 \\
15158\end{array}$ & \begin{tabular}{|l|}
33.452 \\
33625
\end{tabular} & $\begin{array}{r}-1.015 \\
-1079\end{array}$ & & $\begin{array}{r}50.69 \\
\end{array}$ & $\begin{array}{l}0.062 \\
0063\end{array}$ & \begin{tabular}{|l|l|}
14.643 \\
14643
\end{tabular} & & $\begin{array}{l}449.08 \\
5008\end{array}$ & $\begin{array}{l}37.4847 \\
375514\end{array}$ & $\begin{array}{l}52.0 \\
55.5\end{array}$ & $\begin{array}{l}12.1 \\
115\end{array}$ & & & \begin{tabular}{|l|}
0.009 \\
\end{tabular} & 0.009 & 0.000 & & 0.19 \\
\hline$\frac{1553}{1554}$ & $\begin{array}{r}88 / 31 / 2004 \\
88312004\end{array}$ & 9:23:27 PM & $\frac{25.245}{25.241}$ & $\begin{array}{r}25.64 \\
25.646 \\
\end{array}$ & \begin{tabular}{|l|}
24.457 \\
24.438
\end{tabular} & $\begin{array}{l}26.203 \\
26.204\end{array}$ & $\begin{aligned} 25.295 \\
22.3\end{aligned}$ & \begin{tabular}{|r|}
$\mid 47.66$ \\
47.781
\end{tabular} & \begin{tabular}{|l|}
52.623 \\
53.267 \\
\end{tabular} & \begin{tabular}{|c|}
15.182 \\
15.318
\end{tabular} & \begin{tabular}{|}
33.625 \\
33.514 \\
\end{tabular} & $\begin{array}{l}-1.079 \\
-0.989 \\
\end{array}$ & -6.94 & $\begin{array}{l}48.421 \\
49.666\end{array}$ & $\begin{array}{c}0.063 \\
0.058\end{array}$ & \begin{tabular}{|l|}
14.643 \\
14.643 \\
\end{tabular} & $\frac{0.004}{0.003}$ & 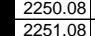 & $\begin{array}{l}37.5014 \\
3775181 \\
\end{array}$ & $\frac{51.5}{52.1}$ & \begin{tabular}{ll|}
11.5 \\
11.8
\end{tabular} & & & | 0.009 & $\frac{0.009}{0.008}$ & 0.000 & $\begin{array}{l}0.225 \\
0.208\end{array}$ & $\begin{array}{l}0.19 \\
0.18\end{array}$ \\
\hline 1555 & $8 / 31 / 2004$ & $9: 25: 27 \mathrm{PM}$ & 25.241 & 25.646 & & \begin{tabular}{|l|l|}
20.189 \\
\end{tabular} & 25.335 & 47.731 & \begin{tabular}{|l|}
52.82 \\
\end{tabular} & $\mid 15.278$ & 33.497 & & & 49.507 & $\begin{array}{l}.0586 \\
0.062\end{array}$ & \begin{tabular}{|l|}
14.043 \\
14.643 \\
\end{tabular} & & 2252.08 & \begin{tabular}{|l|l|}
37.5341 \\
3
\end{tabular} & $\begin{array}{l}32.1 \\
51.7 \\
\end{array}$ & \begin{tabular}{ll|l}
11.8 \\
11.8
\end{tabular} & & & \begin{tabular}{|c|}
0.008 \\
0.009 \\
\end{tabular} & $\frac{0.008}{0.009}$ & & & $\begin{array}{l}0.18 \\
0.19\end{array}$ \\
\hline & $8 / 31 / 2004$ & 9:26:27 PM & & 25.653 & 24.46 & 26.191 & 25.30 & 47.484 & 52.944 & 15.349 & 33.204 & 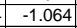 & & 49.41 & 0.064 & 14.643 & & & & 51.8 & & & & 0.009 & & & & $\begin{array}{l}0.19 \\
0.20\end{array}$ \\
\hline 1557 & $8 / 31 / 2004$ & 9:27:27 PM & 25.248 & 25.648 & 24.51 & 26.176 & 25.3 & 47.851 & 53.157 & 15.395 & 33.534 & & & & & 14.643 & & & 37.5681 & & \begin{tabular}{|c|c|}
11.8 \\
\end{tabular} & 40 & & 0.009 & & & & 0.20 \\
\hline 1 & & & & & & & 25.35 & & 53.232 & & & -1.03 & & 48.819 & & & & & & 52.1 & & & & & & & & \\
\hline 155 & & 9:29:27 PM & & 25.638 & 24.48 & 26.181 & & 47.557 & & 15.264 & 33.288 & -1.093 & & 49.005 & & 14.6 & & & 77.6014 & 51.9 & 11.7 & & & & & & & \\
\hline & $8 / 31 / 2004$ & & 25.268 & & & 26.211 & & 47.955 & \begin{tabular}{|l|l}
53.24 \\
\end{tabular} & 15.409 & & $\begin{array}{l}-1.09 \\
\end{array}$ & & & & 10 & & & & & 11.3 & & & & & & & \\
\hline & $8 / 31 / 2004$ & 9 & 25.263 & 25.653 & 24.49 & 26.176 & & & 53.446 & 15.407 & .746 & -1.09 & & 49.16 & 0.062 & 14.643 & & & & 52.3 & 11.7 & & & 0.009 & & & & \\
\hline & $8 / 31 / 2004$ & 9:32:27 PM & 25.263 & 25.663 & 24.501 & 26.176 & 25.26 & 48.138 & \begin{tabular}{|l|l|} 
& \\
\end{tabular} & 15.535 & 33.397 & -1.085 & & 50.302 & 0.063 & 14.643 & & 59.08 & 37.6514 & 52.4 & 12.0 & & 8.811 & 0.009 & .009 & .000 & .224 & 0.19 \\
\hline & $8 / 31 / 2004$ & & 25.249 & 25.644 & 24.441 & 26.167 & 25.2 & 47.513 & 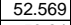 & 15.245 & $\begin{array}{l}33.403 \\
3.200\end{array}$ & -1.056 & & 49.789 & 0.063 & 14.643 & & 30.08 & & 51.4 & 1.9 & & & 009 & 0.009 & & & \\
\hline & $8 / 31 / 2004$ & 34:22: PM & 25.248 & 25.643 & 24.436 & 26.151 & & 47.797 & 53.24 & $15.3 / 1$ & & -1.093 & & 49.647 & .064 & 14.64 & & & & 52.1 & 11.8 & & & & & & & \\
\hline & $80131 / 2004$ & 9:35:2 PM & 25.264 & 25.654 & 24.467 & 26.167 & & & 52.59 & 15.049 & & & & & & & & & & 51.5 & 12.0 & & & & & & & \\
\hline 156 & $88 / 31 / 2004$ & 9:3:3:27 PM & $\frac{25.275}{25.244}$ & $\begin{array}{r}25.67 \\
25.644\end{array}$ & \begin{tabular}{|l|}
24.483 \\
24.436
\end{tabular} & $\frac{26.173}{26.092}$ & $\frac{25 .}{25.1}$ & $\frac{47}{478}$ & $\begin{array}{l}53.091 \\
53.311\end{array}$ & $\frac{15.237}{15.196}$ & $\begin{array}{l}33.674 \\
33.588 \\
\end{array}$ & $\begin{array}{l}-1.004 \\
-0.992 \\
-0.04\end{array}$ & & $\frac{48.859}{49147}$ & 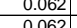 & $\frac{14.643}{14643}$ & & & & 523 & & & & & & & & \\
\hline & $8 / 31 / 2004$ & $8: 27 \mathrm{PM}$ & 25.254 & 25.649 & \begin{tabular}{|l|l|}
24.450 \\
24.431
\end{tabular} & $\frac{20.054}{26.022}$ & 24.9 & 477.001 & 52.795 & 10.15 .465 & 33.44 & $\begin{array}{l}-0.969 \\
-0.069 \\
-10\end{array}$ & & & 0.061 & $\begin{array}{l}14.045 \\
14.643\end{array}$ & & & & $\begin{array}{l}55.7 \\
51.7\end{array}$ & 11.6 & & & & 0.006 & & 0.218 & \\
\hline & $8 / 31 / 2004$ & 9:39:27 PM & 25.253 & 25.648 & \begin{tabular}{|l|l|l} 
& 44.451 \\
\end{tabular} & 25.981 & 24.9 & 47.787 & \begin{tabular}{|l|}
53.13 \\
\end{tabular} & 15.324 & 33.491 & -1.018 & & 47.831 & $\mid 0.06$ & 14.643 & & & & & $\frac{11.4}{11.4}$ & & & & & & & \\
\hline & & & & & \begin{tabular}{|l|l|}
24.439 \\
\end{tabular} & \begin{tabular}{|r|}
25.92 \\
\end{tabular} & & 47.808 & & 15.339 & & & & 49.8 & 0.062 & & & & & & 117 & & & & & & & \\
\hline & 31/2004 & 9 & 25 & 25.641 & 24.463 & 25.898 & & & & & 33.674 & -1.018 & & 254 & & 14.643 & & & & & & & & & & & 224 & \\
\hline & $8 / 31 / 2$ & & 25.241 & 25.631 & 24.433 & 25.863 & & & 52.929 & 15.359 & 33.699 & \begin{tabular}{|c|c|}
-1.093 \\
\end{tabular} & & 48.6 & 0.066 & 14.6 & & & & 51.8 & 11.6 & & & & & & & \\
\hline & $8 / 31 / 2004$ & & 25.243 & 25.628 & \begin{tabular}{|l|}
24.486 \\
\end{tabular} & 25.891 & 24.9 & & \begin{tabular}{|l|}
53.242 \\
\end{tabular} & \begin{tabular}{|l|l|}
15.293 \\
\end{tabular} & 33.432 & -0.925 & & & .064 & $\begin{array}{l}14.643 \\
\end{array}$ & & & & 52.1 & 11.6 & & & 0.005 & & & 0.229 & 0.19 \\
\hline & $8 / 31 / 2004$ & 4:27 PM & 25.237 & 25.617 & \begin{tabular}{|l|l|}
24.499 \\
\end{tabular} & \begin{tabular}{|l|l|}
25.889 \\
\end{tabular} & 24.98 & 47.65 & 52.975 & \begin{tabular}{|l|l|}
15.278 \\
\end{tabular} & $\begin{array}{r}33.421 \\
\end{array}$ & -1.085 & & & & 14.643 & & & & 51.8 & 11.8 & 40 & & 0.009 & 0.009 & 0.000 & & 0.19 \\
\hline & $8 / 31 / 2004$ & 9:45:27 PM & $\frac{25.241}{25.26}$ & $\frac{25.626}{25.63}$ & \begin{tabular}{|l|}
24.519 \\
24513
\end{tabular} & \begin{tabular}{|l|}
25.934 \\
25.953 \\
\end{tabular} & 24.991 & $\begin{array}{l}47.551 \\
48067\end{array}$ & \begin{tabular}{|l|}
52.693 \\
5.251 \\
\end{tabular} & \begin{tabular}{|l|}
15.177 \\
15325 \\
\end{tabular} & $\begin{array}{r}33.53 \\
33.777 \\
\end{array}$ & \begin{tabular}{|l|l|} 
& -1.067 \\
& -1088 \\
\end{tabular} & & 49.3 & $\begin{array}{l}0.062 \\
\end{array}$ & $\begin{array}{r}14.643 \\
14633 \\
\end{array}$ & 0.0 & 年 & & 51.6 & 11.8 . & 40 & 2.795 & \begin{tabular}{|l|l|} 
\\
\end{tabular} & 0.009 & 0.000 & 0.222 & 0.19 \\
\hline & $8 / 31 / 2004$ & $9: 47: 27 \mathrm{PM}$ & $\begin{array}{r}25.250 \\
25.255\end{array}$ & 25.63 & \begin{tabular}{|l|}
24.410 \\
\end{tabular} & \begin{tabular}{|l|l|}
25.977 \\
\end{tabular} & & & \begin{tabular}{|l|}
52.054 \\
52.973 \\
\end{tabular} & 15.273 & 33.307 & \begin{tabular}{|l|}
-1.000 \\
-1.07
\end{tabular} & & $\begin{array}{r}50.738 \\
49.088 \\
\end{array}$ & $\begin{array}{l}0.062 \\
0.062\end{array}$ & 14.643 & & & & 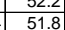 & 最.1. & & & & & & & $\frac{0.19}{0.19}$ \\
\hline & $8 / 31 / 2004$ & & 2525 & & \begin{tabular}{|l|l|}
24.492 \\
\end{tabular} & 25.99 & & & \begin{tabular}{|l|l|}
52.449 \\
\end{tabular} & 15.905 & & & & 48. & & & & & & S.: & 1.5 & & & & & & & $\frac{19}{19}$ \\
\hline & 31/2004 & 9:49:27 PM & 25.254 & 25.624 & \begin{tabular}{|l|}
24.497 \\
\end{tabular} & 26.02 & & & \begin{tabular}{|l|}
53.288 \\
\end{tabular} & 15.374 & 33.3 & -1.088 & & & 0.063 & $\frac{14.6}{14.6}$ & & & & 52.2 & $\frac{71.5}{11.5}$ & & & & & & & \\
\hline & & & 25.254 & & & 26.017 & & & & 15.154 & & & & & & & & & & & & & & & & & & \\
\hline & $8 / 31 / 2004$ & 1:27 PM & 25.239 & 25.604 & 24.461 & 26.00 & & & \begin{tabular}{|l|}
52.558 \\
\end{tabular} & 15.3 & 33.276 & & & & & 14.6 & & & & 51.4 & $\frac{11.9}{11.9}$ & & & & & & & 019 \\
\hline & $8 / 31 / 2004$ & 9:52: & 25.249 & 25.614 & 24.471 & 26.02 & & & \begin{tabular}{|l|l|}
53.045 \\
\end{tabular} & 15.369 & & -1.082 & & & & 14.6 & & & & 51.9 & \begin{tabular}{ll|l}
11.5 \\
\end{tabular} & & & & & & & \\
\hline & $8 / 31 / 2004$ & & 25.243 & & 24.465 & & & & & & & -1.0 & & & & & & & & & 11.7. & & & & & & 0.225 & \\
\hline & 8/31/2004 & 9:54:2 & 25.243 & 25.603 & 24.46 & 26.0 & & 47.5699 & 53.157 & 15.218 & 33.294 & \begin{tabular}{|l|l|} 
& -1.001 \\
\end{tabular} & & & 0.06 & & & & & 52.0 & 12.2. & & & 0.009 & 0.009 & 0.000 & 0.216 & \\
\hline & 87 & & & & 24.51 & 26.0 & & & 52.809 & 10.219 & & -1.0 & & & & & & & & & 11.9 & & & & & & & \\
\hline & & & 25.253 & & & & & & & 15.316 & & & & & & & & & & & & & & & & & & \\
\hline & $8 / 31 / 2004$ & 9: & 25 & $\frac{25.62}{2562}$ & $\frac{24.493}{24.488}$ & 26.113 & & $\frac{47.86}{48.05}$ & 53.178 & $\frac{15.269}{15.497}$ & $\begin{array}{l}33.653 \\
33.477 \\
3\end{array}$ & $\begin{array}{l}-0.923 \\
-0.086 \\
\end{array}$ & & & 0.064 & $\frac{14.6}{146}$ & & & & 524 & 120 & & & (50) & & & 214 & \\
\hline & $8 / 31 / 2004$ & & 25.25 & & & & & & $\frac{5306}{5296}$ & & & 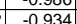 & & & & & & & & & $\mid$ & & & & & & & \\
\hline & $8 / 31 / 200$ & & & & & & & & & & & & & & & & & & & & 118 & & & & & & & \\
\hline & $8 / 131 / 2004$ & 10:01: & 25.261 & & & 26.083 & & & \begin{tabular}{|l|l|}
52.813 \\
\end{tabular} & $\begin{array}{l}15.279 \\
\end{array}$ & & $\mid-0.923$ & & & & & & & & & 11.5 & & & & & & $\frac{0.220}{0.220}$ & \\
\hline & & & & & & & & & & & & & & & & & & & & & & & & & & & & \\
\hline & $81 /$ & & 25.25 & 25.62 & 24.55 & & & & 53.445 & & & & & & & & & & & & $\frac{\frac{11.7}{11.7}}{4}$ & & & & & & & \\
\hline & & & 25.245 & & & & & & & & & & & & & & & & & & 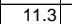 & & & & & & & \\
\hline & $8 / 31 / 2$ & & 25.245 & 25.6 & \begin{tabular}{|l|}
24.508 \\
\end{tabular} & 26.058 & & 47.75 & \begin{tabular}{|l|l|}
52.975 \\
\end{tabular} & 15.425 & 33.358 & -0.928 & & & & 14.6 & & & & & $\overline{11.0}$ & & & & & & & \\
\hline & $8 / 31 / 2004$ & 10:06:2 & 25.26 & 25 & 24.5 & 26. & & 47.862 & 53.336 & 15.38 & & & & & & 14.6 & & & & & $\mid 11.8$ & & & & & & 0.221 & \\
\hline & $8 / 31 / 200$ & & & & 24.4 & & & & & & & -0.9 & & & & & & & & & 11.6 & & & & & & & \\
\hline & $8 / 31 / 200$ & & 25.252 & 25.622 & 24.5 & 26. & & & 53.135 & 15.474 & & -0.92 & & & & & & & & 52.0 & 11.5 & & & & & & 0.218 & \\
\hline & & & & & & & & & & & & & & & & & & & & & & & & & & & & \\
\hline & $8 / 13 / 200$ & & $\frac{25.25}{2525}$ & & & & & & & & & & & & & & & & & & 11.6 & & & & & & & \\
\hline & 年 81312120 & & & $\frac{23.02}{25.32}$ & 24.46 & $\frac{20.1}{26.1}$ & & & \begin{tabular}{|l|l|}
52.049 \\
53957
\end{tabular} & & & -0.6 & & & & & & & & & $\begin{array}{ll}1.144 \\
11.6\end{array}$ & & & & & & & \\
\hline & $8 / 31 / 1200$ & $0.13 \cdot 27$ & 525 & & 24.45 & & & (4t. & \begin{tabular}{|l|}
52.031 \\
5
\end{tabular} & 15.48 & $\frac{35.3}{32}$ & 0.0 & & & & 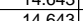 & & & & & 10 & & & & & & & \\
\hline & $8 / 31 / 200$ & 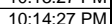 & 2524 & & & & & & & 153 & 33.694 & -0.031 & & & & & & & & & 18 & & & & & & & \\
\hline & $8 / 3 / 1 /$ & & 25.243 & & 24 & & & & & & & -0.928 & & & & & & & & & 116 & & & & & & 0.205 & \\
\hline & & & & & 24.4 & & & & & & & & & & & & & & & & & & & & & & & \\
\hline & & & & & & & & & & & & & & & & & & & & & & & & & & & & \\
\hline & $8 / 31 / 2004$ & & 25.25 & & 24.47 & 260 & & 47.3 & 52.7 & 15.29 & 33.1 & -0.8 & & & & 146 & & & & & 12.0 & & & & & & & \\
\hline & & & 23 & & 21 & & & & & 396 & & & & 2667 & & 146. & & & & & & & & & 0009 & & 222 & 0 \\
\hline & $8 / 31 / 2004$ & 10:20:27 & 25.233 & 25.608 & 24.45 & \begin{tabular}{|l|}
25.906 \\
\end{tabular} & & 47.849 & \begin{tabular}{|l|l|}
53.203 \\
\end{tabular} & \begin{tabular}{|l|l|l|l|}
15.34 \\
\end{tabular} & 33.586 & \begin{tabular}{|c|c|}
$5-0.928$ \\
\end{tabular} & & 48.869 & 0.061 & 14.643 & & 7.08| & $\begin{array}{l}338.4514 \\
\end{array}$ & 52.1 & 11.6 & & 2.807 & \begin{tabular}{|l|l|} 
\\
\end{tabular} & 0.009 & 0.000 & 0.218 & 0.1 \\
\hline
\end{tabular}


WSRC-TR-2005-00105, REVISION 0

SRNL-RPP-2005-00012, REVISION 0

RUN \# 4.03A AND B; FIRST AND SECOND HALF OF SLURRY DEWATERING - CONT.

\begin{tabular}{|c|c|c|c|c|c|c|c|c|c|c|c|c|c|c|c|c|c|c|c|c|c|c|c|c|c|c|c|c|}
\hline & A & & D & $E$ & $\mathrm{~F}$ & G & $\mathrm{H}$ & $\mathrm{J}$ & $\mathrm{K}$ & $\mathrm{L}$ & $\mathrm{M}$ & $\begin{array}{ll}N \\
\end{array}$ & 0 & \begin{tabular}{l|l} 
\\
\end{tabular} & $\mathrm{R}$ & $\mathrm{s}$ & $T$ & $\mathrm{~V}$ & w & $x$ & Y & $z$ & AA & & $\mathrm{AC}$ & & & \\
\hline & $8 / 31 / 2004$ & 10:21:27 PM & 25.247 & 25.617 & 24.474 & 25.869 & 24.901 & 47.857 & 53.197 & 15.436 & 33.454 & -0.928 & -6.905 & 47.63 & 0.061 & \begin{tabular}{|l|l|}
14.643 \\
\end{tabular} & 0.004 & 2308.08 & 38.4681 & 52.1 & 11.3 & 40.7 & 2.803 & 0.009 & 0.009 & 0.000 & 0.218 & \\
\hline & & & 25.24 & 25.615 & \begin{tabular}{|l|l|}
24.478 \\
\end{tabular} & 25.883 & 24.895 & 47.719 & 53.066 & $\begin{array}{l}15.415 \\
\end{array}$ & 33.309 & \begin{tabular}{|c|c|}
-0.894 \\
\end{tabular} & $\begin{array}{l}-6.859 \\
\end{array}$ & 49.437 & 0.061 & $\begin{array}{l}14.643 \\
\end{array}$ & & & 38.4847 & 51.9 & 11.8 & & & & 0.009 & & & 0.19 \\
\hline & $8 / 31 / 2004$ & $\begin{array}{l}10: 23: 27 \mathrm{PM} \\
102: 20\end{array}$ & $\frac{25.238}{2.232}$ & 25.603 & 24.466 & 25.896 & 24.958 & $\begin{array}{l}47.441 \\
\end{array}$ & \begin{tabular}{|l|}
52.681 \\
\end{tabular} & \begin{tabular}{|l|}
15.461 \\
1.231
\end{tabular} & 33.144 & \begin{tabular}{|c|c|}
4 & -0.911 \\
\end{tabular} & $\begin{array}{l}-6.871 \\
-891\end{array}$ & 47.311 & \begin{tabular}{l|}
0.06 \\
\end{tabular} & \begin{tabular}{|l|}
14.643 \\
\end{tabular} & & & & 51.6 & 11.3 & (1) & 2.778 & . 009 & 0.009 & 0.000 & 0.216 & \\
\hline 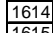 & $8 / 31 / 2004$ & $\begin{array}{ll}10: 24: 27 \mathrm{PM} \\
10: 25: 27\end{array}$ & $\begin{array}{l}25.243 \\
25227\end{array}$ & $\begin{array}{l}25.608 \\
2507\end{array}$ & \begin{tabular}{|r}
24.46 \\
\end{tabular} & $\begin{array}{l}25.905 \\
25.805\end{array}$ & 24.992 & \begin{tabular}{|l|l|}
47.571 \\
1751
\end{tabular} & \begin{tabular}{|r|}
52.94 \\
52.007
\end{tabular} & $\begin{array}{l}15.391 \\
\end{array}$ & 33.157 & -0.876 & $\begin{array}{l}-6.81 \\
\end{array}$ & 49.699 & $\begin{array}{l}0.061 \\
\end{array}$ & \begin{tabular}{|l|l|}
14.643 \\
\end{tabular} & 0.0 & 311.08 & 38.5181 & 51.8 & 11.8 & 40 & \begin{tabular}{|l|l|}
2.783 \\
\end{tabular} & 0.009 & $\begin{array}{l}0.009 \\
\end{array}$ & 0.000 & 0.220 & \\
\hline$\frac{1615}{1616}$ & $\frac{8 / 31 / 2004}{8 / 31204}$ & $\begin{array}{l}10: 25: 27 \mathrm{PM} \\
10.26 .2 \mathrm{PM}\end{array}$ & $\frac{25.237}{25.232}$ & $\begin{array}{l}25.607 \\
25.602\end{array}$ & \begin{tabular}{|r|}
24.515 \\
24.53 \\
\end{tabular} & & $\frac{25.002}{25017}$ & \begin{tabular}{|l|l|}
47.511 \\
47746
\end{tabular} & \begin{tabular}{|l|}
52.907 \\
52.989 \\
\end{tabular} & \begin{tabular}{|c|}
15.291 \\
15258
\end{tabular} & .309 & $\begin{array}{l}-0.876 \\
-0876\end{array}$ & & $\begin{array}{r}50.486 \\
4772\end{array}$ & $\begin{array}{l}0.061 \\
0.061\end{array}$ & \begin{tabular}{|l|l|}
14.643 \\
14643 \\
\end{tabular} & & & & 51.8 & \begin{tabular}{|l|}
12.0 \\
\end{tabular} & & & . & 0.009 & & & 0.19 \\
\hline$\frac{1017}{1617}$ & $\begin{array}{l}8 / 31 / 204 \\
8 / 31 / 2004\end{array}$ & $\begin{array}{l}10: 26: 227 \mathrm{PM} \\
10227: 27 \mathrm{PM}\end{array}$ & $\frac{25.232}{25.243}$ & $\frac{25.602}{25.608}$ & \begin{tabular}{|r|}
24.53 \\
24.525 \\
\end{tabular} & \begin{tabular}{|l|}
25.875 \\
25.885 \\
\end{tabular} & $\begin{array}{l}25.01 \\
24.99 \\
\end{array}$ & \begin{tabular}{|l|}
47.746 \\
47.889
\end{tabular} & \begin{tabular}{|l|}
52.989 \\
53.557 \\
\end{tabular} & \begin{tabular}{|l|}
15.258 \\
15.451 \\
\end{tabular} & $\begin{array}{l}33.565 \\
33.292 \\
\end{array}$ & $\begin{array}{l}-0.876 \\
-0.876 \\
\end{array}$ & $\begin{array}{r}-6.855 \\
-6.856\end{array}$ & $\begin{array}{r}47.72 \\
551.193\end{array}$ & $\begin{array}{r}0.061 \\
0.06\end{array}$ & $\begin{array}{l}14.643 \\
14.643 \\
\end{array}$ & $\begin{array}{l}.0003 \\
.0003\end{array}$ & $\begin{array}{l}13.08 \\
13.08\end{array}$ & $\begin{array}{l}38.5514 \\
88.5681\end{array}$ & $\frac{51.9}{52.4}$ & $\frac{11.4}{12.2}$ & $\frac{40.7}{40.6}$ & \begin{tabular}{|c|}
2.803 \\
2.799 \\
\end{tabular} & 吕9 & $\begin{array}{l}0.009 \\
0.009\end{array}$ & $\frac{0.000}{0.000}$ & & 0.19 \\
\hline 1618 & $8 / 31 / 2004$ & 10:28:27 PM & 25.238 & 25.603 & 24.511 & \begin{tabular}{|l|}
25.931 \\
\end{tabular} & & 47.445 & \begin{tabular}{|l|l|}
52.413 \\
\end{tabular} & 15.457 & 33.313 & & -6.859 & 49.968 & 0.06 & 14.643 & 0.003 & $\frac{2314.08}{2315.08}$ & \begin{tabular}{|l|l|}
38.5081 \\
3
\end{tabular} & 51.3 & $\frac{1.2}{11.9}$ & \begin{tabular}{|l|l|}
40.4 \\
0.4
\end{tabular} & 2.784 & \begin{tabular}{|l|}
0.009 \\
\end{tabular} & $\begin{array}{l}0.009 \\
0.009 \\
\end{array}$ & $\begin{array}{l}0.000 \\
0.000\end{array}$ & & $\frac{0.18}{0.18}$ \\
\hline 1619 & $8 / 31 / 2004$ & & & 25.604 & & \begin{tabular}{|l|}
25.957 \\
\end{tabular} & 25.04 & 48.023 & 53.514 & 15.52 & 33.399 & -0.876 & & 49.82 & 0.061 & \begin{tabular}{|l|l|}
14.643 \\
\end{tabular} & & & 88.6014 & 52.4 & & & & 0.009 & & & & 19 \\
\hline & $8 / 31 / 2004$ & 10:30:27 PM & 25.233 & 25.593 & & 25.965 & & 47.795 & \begin{tabular}{|l|l|}
53.584 \\
\end{tabular} & \begin{tabular}{|l|l|}
15.343 \\
\end{tabular} & 33.331 & -0.876 & & 48.371 & & 14.643 & & & 38.6181 & & 11.5 & & & \begin{tabular}{|l|l|}
0.009 \\
\end{tabular} & 0.009 & 0.000 & & \\
\hline 1621 & $8 / 31 / 2004$ & $\begin{array}{ll}0: 31: 27 \\
10: 22: 27\end{array}$ & 25.223 & 25.583 & \begin{tabular}{|l|}
24.485 \\
\end{tabular} & $\begin{array}{r}25.935 \\
2506\end{array}$ & 25.0 & $\begin{array}{l}47.592 \\
\end{array}$ & $\begin{array}{l}52.927 \\
5.2021\end{array}$ & \begin{tabular}{l|l|}
15.373 \\
15177
\end{tabular} & 33.276 & $\begin{array}{l}-0.876 \\
\end{array}$ & & 47.128 & $\begin{array}{l}0.06 \\
\end{array}$ & \begin{tabular}{|l|l|}
14.643 \\
\end{tabular} & 0.003 & & 38.6347 & 51.8 & 11.2 & & 2.788 & 0.009 & 0.009 & 0.000 & 0.216 & 0.18 \\
\hline & $\frac{8 / 31 / 2004}{8 / 31204}$ & $\begin{array}{l}10: 32: 27 \text { PM } \\
10.33 \cdot 27 \mathrm{PM}\end{array}$ & $\begin{array}{l}25.228 \\
25.239\end{array}$ & $\begin{array}{l}25.593 \\
25.604\end{array}$ & \begin{tabular}{|l|}
24.495 \\
24.492 \\
\end{tabular} & $\begin{array}{r}25.96 \\
26.032 \\
\end{array}$ & $\begin{array}{l}25.08 \\
25.10\end{array}$ & $\begin{array}{r}47.7 \\
47.364 \\
\end{array}$ & \begin{tabular}{|l|}
53.031 \\
5271 \\
\end{tabular} & \begin{tabular}{|l|l|}
15.427 \\
1527
\end{tabular} & \begin{tabular}{|l|}
33.266 \\
33171
\end{tabular} & $\begin{array}{l}-0.876 \\
\end{array}$ & & $\begin{array}{l}49.276 \\
47639\end{array}$ & $\begin{array}{l}0.06 \\
0.66\end{array}$ & \begin{tabular}{|l|l|}
14.643 \\
1463 \\
\end{tabular} & & |319.08 & $\begin{array}{l}3.6514 \\
36681\end{array}$ & $\frac{51.9}{51 .}$ & $\begin{array}{l}11.7 \\
11.3\end{array}$ & & $\begin{array}{l}2.791 \\
2776\end{array}$ & \begin{tabular}{|l|}
0.009 \\
\end{tabular} & 0.009 & 0.000 & & 0.18 \\
\hline$\frac{10<4}{1624}$ & $\begin{array}{l}8 \pi / 31 / 2004 \\
8831204\end{array}$ & $\begin{array}{l}\text { 10:33:277 } 7 \text { M } \\
10: 34: 27\end{array}$ & $\frac{25.239}{25.224}$ & $\begin{array}{l}25.604 \\
25.579\end{array}$ & \begin{tabular}{|l|}
24.492 \\
24.466 \\
\end{tabular} & $\begin{array}{l}26.032 \\
25.987\end{array}$ & $\begin{array}{l}25.10 \\
25.12 \\
\end{array}$ & $\begin{array}{l}47.764 \\
47.719\end{array}$ & $\begin{array}{r}52.71 \\
53.093 \\
\end{array}$ & \begin{tabular}{|c|}
15.267 \\
15.353
\end{tabular} & $\begin{array}{l}33.171 \\
33.229\end{array}$ & $\begin{array}{l}-0.876 \\
-0.879\end{array}$ & & $\begin{array}{l}47.6399 \\
49.871\end{array}$ & $\begin{array}{r}0.06 \\
0.062\end{array}$ & $\begin{array}{l}14.643 \\
14.643 \\
\end{array}$ & 0.003 & $2 \frac{2.08}{21.08}$ & & $\frac{51.6}{52.0}$ & 位.3. & $\frac{40.3}{40.5}$ & . 2.776 & 0.009 & 0.009 & $\frac{0.000}{0.000}$ & & 0.18 \\
\hline 1625 & $8 / 31 / 2004$ & $10: 35: 27 \mathrm{PM}$ & 25.229 & 25.594 & & \begin{tabular}{|l|l|} 
& 25.992 \\
\end{tabular} & 25.144 & 47.65 & 53.193 & \begin{tabular}{|l|l|}
15.309 \\
\end{tabular} & 33.372 & 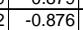 & & 50.223 & \begin{tabular}{|}
0.002 \\
0.06 \\
\end{tabular} & \begin{tabular}{|l|}
14.643 \\
14.643 \\
\end{tabular} & & $\frac{2311.08}{2322.08}$ & \begin{tabular}{|l|l|}
30.0647 \\
38.7014
\end{tabular} & 52.1 & $\frac{11.9}{12.0}$ & & $\frac{2.91}{2.793}$ & & $\begin{array}{l}0.009 \\
0.009\end{array}$ & & & $\begin{array}{l}0.19 \\
0.18\end{array}$ \\
\hline & & 10:36:27 PM & & & \begin{tabular}{|l|}
24.503 \\
\end{tabular} & 25.978 & & & \begin{tabular}{|l|}
52.675 \\
\end{tabular} & $\begin{array}{l}15.391 \\
\end{array}$ & & -0.815 & & 50.075 & 0.061 & $\begin{array}{l}14.643 \\
\end{array}$ & & & & & & & & & & & & $\frac{0.18}{0.19}$ \\
\hline & $8 / 31 / 2004$ & 10:37:27 PM & 25.226 & 25.596 & \begin{tabular}{|l|l|}
24.474 \\
\end{tabular} & 25.979 & & 47.748 & \begin{tabular}{|l|}
52.977 \\
\end{tabular} & $\begin{array}{l}15.313 \\
\end{array}$ & 33.45 & -1.128 & & 48.848 & 0.051 & \begin{tabular}{|l|}
14.643 \\
\end{tabular} & & & $\begin{array}{l}38.7347 \\
\end{array}$ & 51.9 & 11.6 & 40 & & 0.007 & & & & $\frac{1.19}{16}$ \\
\hline 1628 & & & & & & & 25.14 & & & & & & & 47.303 & 0.07 & & & & & & & & & & & & & \\
\hline & & 10:39:27 PM & & 25.593 & \begin{tabular}{|l|}
24.491 \\
\end{tabular} & 26.016 & & 47.905 & & 15.425 & 33.491 & - & & 49.649 & $\begin{array}{l}0.061 \\
\end{array}$ & 14.6 & & & & 52. & 111. & & & & & & & \\
\hline & $8 / 31 /$ & & 25.224 & 25.594 & 24.526 & 26.026 & & & 53.122 & 15.228 & 33461 & $|-0.81|$ & & & $\mid 0.06$ & & & & & & & & & & & & & \\
\hline & & & 25.224 & 25.599 & 24.536 & 26.056 & & & & 15.28 & & -0.873 & & & 0.061 & 14.6 & & & & & 11.3 & & & & & & & \\
\hline & $8 / 31 / 2004$ & 10:42:27 PM & 25.219 & 25.594 & \begin{tabular}{|l|}
24.506 \\
\end{tabular} & 26.056 & & $\begin{array}{l}47.646 \\
\end{array}$ & \begin{tabular}{|l|}
52.84 \\
\end{tabular} & 15.375 & 33.319 & -0.879 & & 46.416 & 0.058 & 14.643 & & 229.08 & 38.8181 & 51.7 & 11.1 & 40 & 2.791 & 0.008 & 0.008 & 0.000 & 0.208 & 18 \\
\hline & 004 & 10:43:27 PM & 25.239 & 25.604 & 24.536 & 26.056 & 25.2 & \begin{tabular}{|l|l|}
47.594 \\
\end{tabular} & \begin{tabular}{|l|}
53.274 \\
5.257
\end{tabular} & $\begin{array}{l}15.431 \\
1530\end{array}$ & 33.03 & \begin{tabular}{|c|c|}
-0.873 \\
\end{tabular} & & 49.701 & $\begin{array}{l}0.061 \\
\end{array}$ & \begin{tabular}{|l|l|}
14.643 \\
\end{tabular} & & 30.08 & 38.8347 & 52.1 & \begin{tabular}{ll|l}
11.8 \\
\end{tabular} & 40 & 2.779 & \begin{tabular}{|l|l|}
0.009 \\
\end{tabular} & 0.009 & 0.000 & 0.219 & \\
\hline & $11 / 2004$ & 10:44:27 PM & & 25.597 & & 26.045 & & & & 15.308 & 33.206 & & & 47.997 & 0.061 & & & 31.08 & & 51.7 & 11.4 & 40 & & \begin{tabular}{|l|l|}
0.009 \\
\end{tabular} & 0.009 & & & \\
\hline & $8 / 31 / 200$ & 10:45:27 PM & 25.232 & 25.602 & $\begin{array}{r}24.51 \\
\end{array}$ & 26.055 & 25.1 & & \begin{tabular}{|l|}
52.708 \\
\end{tabular} & 15.416 & 33.196 & -0.876 & & 48.456 & $\begin{array}{l}0.061 \\
\end{array}$ & & & 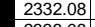 & & 51.6 & 11.5 & & & 0.009 & & & 0.219 & \\
\hline & $8 / 3131 / 2004$ & $\begin{array}{l}\frac{10: 46: 27}{10.4727 P M} \\
\end{array}$ & $\frac{25.253}{25.232}$ & $\begin{array}{l}25.608 \\
25.602\end{array}$ & \begin{tabular}{|l|}
24.516 \\
24.504
\end{tabular} & \begin{tabular}{|l|}
26.081 \\
26.069 \\
\end{tabular} & $\frac{25.20}{25.21}$ & \begin{tabular}{|c|}
47.437 \\
47229
\end{tabular} & \begin{tabular}{|l|}
52.896 \\
52.515 \\
\end{tabular} & \begin{tabular}{|c|}
15.365 \\
15.158
\end{tabular} & $\begin{array}{r}33.069 \\
33.153\end{array}$ & \begin{tabular}{|c|c|c|c|}
-0.876 \\
-0.911
\end{tabular} & & $\begin{array}{l}48.362 \\
50.357\end{array}$ & \begin{tabular}{|r|r|}
0.066 & \\
0.067
\end{tabular} & \begin{tabular}{|l|}
14.643 \\
14.463
\end{tabular} & 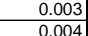 & $\begin{array}{l}33.08 \\
44.08\end{array}$ & $\begin{array}{l}38.8847 \\
38.9014\end{array}$ & 51.8 & $\frac{11.5}{120}$ & $\begin{array}{l}\frac{40}{40} \\
40\end{array}$ & $\frac{2.775}{2.771}$ & 0.009 & 0.009 & 0.000 & 0.216 & 0.18 \\
\hline$\frac{\mid 007}{1638}$ & $8 / 31 / 2004$ & $\begin{array}{l}10.48: 27 \mathrm{MM} \\
10: 48: 27 \mathrm{PM}\end{array}$ & $\frac{25.232}{25.251}$ & $\frac{25.602}{25.616}$ & \begin{tabular}{|l|}
24.504 \\
24.534 \\
\end{tabular} & \begin{tabular}{|l|}
20.009 \\
\end{tabular} & 25.2 & $\frac{44.229}{47.619}$ & \begin{tabular}{|l|}
52.515 \\
53.0777 \\
\end{tabular} & \begin{tabular}{|l|}
15.458 \\
15.461
\end{tabular} & $\begin{array}{l}33.153 \\
33.165\end{array}$ & $\begin{array}{l}-0.911 \\
-0.894\end{array}$ & & $\begin{array}{r}50.35 / \\
49.27 \\
\end{array}$ & \begin{tabular}{|c|c|c|}
0.067 \\
0.06
\end{tabular} & $\begin{array}{l}14.044 \\
14.643\end{array}$ & & & & $\frac{51.4}{52.0}$ & $\begin{array}{l}\frac{12.0}{11.7} \\
\end{array}$ & & 2.771 & & & & & 0.21 \\
\hline 1639 & $8 / 31 / 2004$ & 10:49:27 PM & 25.241 & 25.611 & \begin{tabular}{|l}
24.514 \\
\end{tabular} & 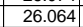 & 25. & 47.638 & \begin{tabular}{|l|l|}
53.074 \\
\end{tabular} & 15.409 & 33.208 & \begin{tabular}{|c|c|}
3 & -0.928 \\
\end{tabular} & & 48.654 & 0.06 & 14.643 & & $336.0 \varepsilon$ & & 51.9 & 11.6 & 40 & 2.787 & & & & & $\frac{0.18}{0.18}$ \\
\hline 1640 & & 10:50:27 PM & 25.247 & 25.612 & \begin{tabular}{|l|}
24.505 \\
\end{tabular} & & & 47.76 & \begin{tabular}{|l|}
53.002 \\
\end{tabular} & & & & & 49.795 & 0.06 & 14.643 & & & & & & & & 0.009 & & & & $\frac{0.18}{0.18}$ \\
\hline 1641 & $8 / 31 / 2004$ & 10:51:27 PM & 25.243 & 25.608 & \begin{tabular}{|l|}
24.496 \\
\end{tabular} & \begin{tabular}{|l|}
26.046 \\
\end{tabular} & & 47.681 & 53.157 & \begin{tabular}{|l|l|}
15.44 \\
\end{tabular} & 33.181 & -0.891 & & 46.758 & 0.06 & 14.6 & & & & 52.0 & \begin{tabular}{|l|l|}
11.1 \\
\end{tabular} & 40 & & & & & & $\frac{0.18}{0.18}$ \\
\hline 1642 & & 10:52:27 PM & 25.249 & & 24.501 & \begin{tabular}{|l|}
26.046 \\
\end{tabular} & 25.25 & 47.551 & 53.139 & 15.367 & 33.202 & & & & 0.06 & 14.6 & & & & 52.0 & $\begin{array}{ll}11.7 \\
\end{array}$ & & & & & & & 0.18 \\
\hline 1643 & $8 / 31 / 2004$ & 10:53:27 PM & 25.244 & 25.604 & 24.502 & 26.037 & 25.2 & 47.47 & 52.704 & 15.374 & 33.222 & 0.894 & & 48.317 & 0.061 & 14.6 & & & & & & & & & & & & \\
\hline & $8 / 31 / 2004$ & 10:54:27 & 25.244 & 25.614 & 24.521 & & & 48.1 & 53.456 & 15.575 & 33.465 & -0.905 & & & $\mid 0.06$ & 14.6 & & & & & 115 & & & & & & & \\
\hline & $8 / 31 / 2004$ & 10:55:27 P & 25.234 & 25.59 & 24.481 & 26.061 & & 47.681 & 53.039 & 15.257 & 33.3 & -0.876 & & 48.7 & 0.061 & $\begin{array}{l}14.643 \\
\end{array}$ & & & & 51. & 11.6 & & & & & & & \\
\hline & $8 / 31 / \mid$ & 10:56:27 & 25.244 & 25.61 & 24.492 & 26.08 & & 47.7 & 52.973 & 15.426 & 33.325 & -0.925 & & 49.685 & 0.061 & 14.6 & & & & 51. & 11.8 & & & & & & & \\
\hline & $8 / 3$ & 10:57 & 25.244 & 25.60 & 24.512 & 26.08 & & 47.84 & 53.39 & 15.408 & & -0.8 & & & 0.055 & 14. & & & & & & & & & & & 0.196 & \\
\hline & $1 / 2 / 2$ & & D. & & 24.523 & & & & 886 & 15.455 & & & & & 0.062 & & & & & & (1. & & & & & & & \\
\hline & & $10: 59:$ & 25.245 & 25.66 & 24.548 & & & & & $15.4 / 4$ & 33.5 & -0.86 & & & & & & & & 52.4 & 11.5 & & & & & & & \\
\hline & 然 & $\begin{array}{l}11.00 \\
11.01\end{array}$ & $\frac{25.245}{2525}$ & 25. & $\begin{array}{l}24.563 \\
24.558\end{array}$ & 26.09 & & & $\begin{aligned} 53.068 \\
53.05\end{aligned}$ & $\begin{array}{r}15.4 \\
15368\end{array}$ & & $\begin{array}{l}-0.88 \\
-0.9-9\end{array}$ & & & 0 & & & & & $\frac{51.9}{51.9}$ & $\frac{11.7}{11.7}$ & & & & & & & \\
\hline & $8 / 31 / 2004$ & 11.02 .04 & $\frac{25.20}{25255}$ & & 24.568 & & & & & 15319 & & & & & 然.050 & & & & & & $\frac{\pi .16}{118}$ & & & & & & & \\
\hline & $8 / 31 / 2004$ & 11:03:04 & 25.26 & & 22,543 & & & & 53066 & 15.522 & & & & & & & & & & & $\frac{1.6}{11.6}$ & & & & & & 0.21 & \\
\hline & $8 / 31 / 2004$ & 11:04:0 & 25.266 & 25.621 & $\begin{array}{ll}14.533 \\
14.53\end{array}$ & 26.098 & & 47.76 & 53.203 & $\frac{15.47}{1.57}$ & & $\begin{array}{r}-0.763 \\
-0.763\end{array}$ & & 48. & o. & 14. & & & & 52. & $\frac{\frac{0}{11.5}}{11.5}$ & & & 009 & 0.00 & 5000 & 0.233 & \\
\hline & & & & & & & & & & & & & & & & & & & & & & & & & & & & \\
\hline & & & 25.27 & 25.6 & 24.5 & 26. & & & 53.271 & 15.415 & & & & & \begin{tabular}{|c|}
0.06 \\
\end{tabular} & & & & & & 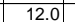 & & & & & & & \\
\hline & 81 & & 25.266 & 25.61 & 24.524 & 26.104 & 25.2 & & . & \begin{tabular}{ll|}
15.382 \\
\end{tabular} & 33.621 & 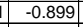 & & & 0.06 & & & & & 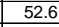 & 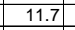 & & & & & & & \\
\hline & 81 & & 25.256 & & 24.504 & 26.139 & 25.1 & & & 15.295 & 33.346 & & & & & & & & & 51.9 & 11.6 & & & & & & & \\
\hline & $8 / 31 / 200$ & & 25.267 & 25.6 & 24.535 & 26 & & & 53.045 & 15.444 & & -0.914 & & & 0.06 & & & & & 51 & 11.6 & & & & & & 0.215 & \\
\hline & $8 / 31 / 2$ & & 25.263 & & 24 & & & & & 15.4 & & & & & 0.058 & & & & & & 12.2 & & & & & & & \\
\hline & 8/31/2004 & & 25.269 & & 24.5 & & & & 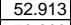 & 15.383 & & -0.9 & & & 0.061 & & & & & & 11.9 & & & & & & & \\
\hline & & & & & & & & & & & & & & & & & & & & & & & & & & & & \\
\hline & & & 25 & 25.62 & & & & & 53.607 & 15. & & & & & & & & & & & 型 & & & & & & & \\
\hline & - $8 / 131 / 2$ & $\frac{11.14}{11.15}$ & $\frac{24}{38}$ & 25.61 & \begin{tabular}{|l}
24.534 \\
2669
\end{tabular} & 26. & & & $\begin{array}{l}53.019 \\
53.137\end{array}$ & $\begin{array}{l}15.387 \\
15437\end{array}$ & $\begin{array}{ll}33.2 \\
332\end{array}$ & -0.9 & & & . & 14. & & & & 51. & $\frac{1.0}{118}$ & & & & & & & \\
\hline 166 & $8 / 31 / 200$ & 11.16 & & & 24.55 & & & & 52961 & t..45 & & & & & & & & & & & $\frac{1.06}{117}$ & & & & & & & \\
\hline 166 & & & & & & & & & & & & -0.9 & & & & & & & & & & & & & & & & \\
\hline & $8 / 31 / 20$ & & $\frac{25.25}{25.25}$ & 25.606 & 24.5 & & & & $\begin{array}{l}52.797 \\
\end{array}$ & & & -0.86 & & & & & & & & & $\frac{12.0}{12.0}$ & & & & & & & \\
\hline & & & & & & & & & & & & & & & & & & & & & & & & & & & & \\
\hline & & & & & & & & & & & & & & & & & & & & & & & & & & & & \\
\hline & $8 / 31 / 2$ & & 25 & 25.628 & 324.545 & & & & & & & -0.5 & & & & & & & & & 118 & & & & & & & \\
\hline & $8 / 31 / 2$ & $11: 22: 0:$ & & 25.60 & 24.5 & $25.89 !$ & & & 53.294 & 15.442 & 33.3 & -0.9 & & & & 14.6 & & & & & $\overline{1 . \varepsilon}>$ & & & & & & & \\
\hline & $8 / 31 / 20$ & 11:23 & & 25. & 24.54 & & & 47. & 52.602 & 15.33 & 33. & -0.6 & & & & 14.6 & & & & & 1.8 & & & & & & & \\
\hline & $8 / 31 / 2$ & & 25. & 25. & 24.5 & & & & 53.504 & & 33.329 & $-0.7 \quad$ & & & 0.061 & 14. & & & & & 11.5 & & & & & & & \\
\hline & & & 25.26 & 25.609 & 24 & & & & 52 & & & -0.6 & & & & & & & & & 11.5 & & & & & & & \\
\hline & & & & & 24. & & & & & & & & & & & & & & & & & & & & & & & \\
\hline & $8 / 31 / 200$ & & 20.20 & & 24.5 & & & & 213 & & & & & & & & & & & & 11.9 & & & & & & & \\
\hline 679 & $8151 / 2004$ & & 年3.25 & 25.59 & 24.53 & $\begin{array}{l}25.914 \\
5021\end{array}$ & & 4.4 .4 & 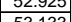 & 15.4524 & 3.04 & $\begin{array}{l}-0.598 \\
0.509\end{array}$ & & & (2.039) & & & & & 罗 & $\frac{11.4}{11.4}$ & & & & & & 213 & 装 \\
\hline 1680 & $\begin{array}{l}88 / 31 / 2004 \\
8 / 31 / 2004\end{array}$ & $\begin{array}{l}11: 29: 04 \mathrm{PM} M \\
11: 30: 04 \mathrm{PM}\end{array}$ & $\frac{25.251}{25.252}$ & $\begin{array}{l}25.586 \\
25.587\end{array}$ & $\begin{array}{l}24.534 \\
24.515 \\
\end{array}$ & \begin{tabular}{|l|}
25.934 \\
25.905 \\
\end{tabular} & \begin{tabular}{|l}
25.166 \\
25.187
\end{tabular} & $\begin{aligned} 47.4055 \\
46.81\end{aligned}$ & \begin{tabular}{|l|}
532.133 \\
52.357 \\
\end{tabular} & \begin{tabular}{|l|}
15.494 \\
15.151 \\
\end{tabular} & $\begin{array}{l}32.949 \\
32.785\end{array}$ & \begin{tabular}{|c|c|} 
& -0.598 \\
5 & -0.575 \\
\end{tabular} & & $\begin{array}{l}47.007 \\
\end{array}$ & $\mid 0.059$ & 14.643 & & $\mid 376.70$ & \begin{tabular}{|l|l|}
39.595 \\
\end{tabular} & 51.2 & \begin{tabular}{|l|l|}
11.2 \\
\end{tabular} & & \begin{tabular}{|l|l|l|l|}
2.744 \\
\end{tabular} & \begin{tabular}{|l|}
0.008 \\
\end{tabular} & $|0.009|$ & $\mid$ & 0.212 & \begin{tabular}{l|l}
12 & 0.1 \\
\end{tabular} \\
\hline
\end{tabular}


WSRC-TR-2005-00105, REVISION 0

SRNL-RPP-2005-00012, REVISION 0

RUN \# 4.03A AND B; FIRST AND SECOND HALF OF SLURRY DEWATERING - CONT.

\begin{tabular}{|c|c|c|c|c|c|c|c|c|c|c|c|c|c|c|c|c|c|c|c|c|c|c|c|c|c|c|c|c|}
\hline & A & $B$ & $\mathrm{D}$ & $E$ & $F$ & G & $\mathrm{H}$ & $\mathrm{J}$ & K & $\mathrm{L}$ & $M$ & $\mathrm{~N}$ & 0 & $Q$ & R & \begin{tabular}{l|l} 
\\
\end{tabular} & \begin{tabular}{l|l}
$T$ \\
\end{tabular} & $\mathrm{v}$ & w & $x$ & Y & $z$ & $\mathrm{AA}$ & & & & & $\overline{A F}$ \\
\hline & $8 / 31 / 2004$ & 11:31:04 PM & 25.253 & 25.593 & 24.521 & 25.891 & 25.178 & 47.464 & 52.894 & 15.529 & 32.853 & -0.584 & $\begin{array}{l}-6.295 \\
\end{array}$ & 47.862 & 0.06 & \begin{tabular}{|l|l|}
14.643 \\
\end{tabular} & 0.003 & 2377.70 & \begin{tabular}{|l|l|}
39.6283 \\
\end{tabular} & 51.8 & 11.4 & 40.2 & 2.769 & 0.009 & 0.009 & 0.000 & 0.217 & \\
\hline & & $\begin{array}{l}11: 32: 04 \text { PM } \\
\end{array}$ & 25.248 & 25.593 & 24.536 & \begin{tabular}{|l|}
25.881 \\
\end{tabular} & 25.198 & 47.725 & 53.458 & $\begin{array}{l}15.447 \\
\end{array}$ & 33.325 & \begin{tabular}{|l|l|}
-0.642 \\
\end{tabular} & & 48.045 & $\begin{array}{l}0.059 \\
\end{array}$ & $\begin{array}{l}14.643 \\
\end{array}$ & & 2378.70 & \begin{tabular}{|l|}
39.645 \\
\end{tabular} & 52.3 & 11.4 & & & & 0.009 & & & \\
\hline & $8 / 31 / 2004$ & $11: 33: 17 \mathrm{PM}$ & 25.243 & 25.588 & \begin{tabular}{|l|l|}
24.536 \\
2523
\end{tabular} & \begin{tabular}{|l|}
25.8411 \\
2582 \\
\end{tabular} & 25.158 & $\begin{array}{l}47.787 \\
\end{array}$ & $\begin{array}{l}53.332 \\
5323\end{array}$ & 15.377 & 33.549 & & $\begin{array}{l}-6.30 \\
-6.32\end{array}$ & 48.05 & & 14.643 & 0.003 & 2379.92 & \begin{tabular}{|l|l|}
39.66533 \\
2.653
\end{tabular} & 52.2 & 11.4 & \begin{tabular}{|l|l|}
4 & 40.7 \\
\end{tabular} & $\begin{array}{l}2.804 \\
.777\end{array}$ & 0.008 & 0.009 & 0.000 & 0.210 & \\
\hline$\frac{1684}{1685}$ & $8 / 31 / 2004$ & $\begin{array}{l}11: 34: 17 \mathrm{PM} \\
11.35 \cdot 17 \mathrm{PM}\end{array}$ & \begin{tabular}{r|r|}
25.24 \\
25.571
\end{tabular} & $\begin{array}{r}25.6 \\
25601\end{array}$ & \begin{tabular}{|l|}
24.533 \\
\end{tabular} & \begin{tabular}{|l|}
25.828 \\
2586 \\
\end{tabular} & $\begin{array}{r}25.15 \\
\end{array}$ & \begin{tabular}{|l|l|}
47.493 \\
\end{tabular} & \begin{tabular}{|l|l|}
53.392 \\
5005
\end{tabular} & $\begin{array}{l}15.537 \\
15201\end{array}$ & $\begin{array}{l}32.902 \\
3.02\end{array}$ & \begin{tabular}{|l|l|} 
& -0.564 \\
\end{tabular} & $\begin{array}{l}-6.327 \\
5\end{array}$ & \begin{tabular}{|l|l|}
48.692 \\
5150
\end{tabular} & $\begin{array}{l}0.062 \\
\end{array}$ & $\begin{array}{l}14.643 \\
\end{array}$ & 0.003 & 2380.92 & \begin{tabular}{|l|l|}
39.6819 \\
\end{tabular} & \begin{tabular}{|l|}
52.3 \\
\end{tabular} & 11.6 & 40.2 & 2.771 & 0.009 & $\begin{array}{l}0.009 \\
\end{array}$ & 0.000 & 0.224 & \\
\hline 1686 & $\frac{8 / 31 / 2004}{8831 / 2004}$ & $\begin{array}{ll}11: 35: 17 \mathrm{PM} \\
1113.6 \cdot 17 \mathrm{PM}\end{array}$ & $\frac{25.571}{25.336}$ & $\begin{array}{l}25.601 \\
25.596 \\
\end{array}$ & \begin{tabular}{|l|}
24.524 \\
24529 \\
\end{tabular} & \begin{tabular}{|l|}
25.869 \\
25.854 \\
\end{tabular} & $\begin{array}{l}25.151 \\
25136\end{array}$ & \begin{tabular}{|l|l|}
48.397 \\
46473
\end{tabular} & & 15.391 & $\begin{array}{l}34.062 \\
31044 \\
3\end{array}$ & \begin{tabular}{|c|}
-1.212 \\
0589
\end{tabular} & $\begin{array}{r}-5.139 \\
\end{array}$ & 51.502 & & \begin{tabular}{|l|l|l|}
14.643 \\
14634 \\
\end{tabular} & & $\frac{2381.92}{20202}$ & \begin{tabular}{|l|l|}
39.6986 \\
\end{tabular} & \begin{tabular}{|l|}
51.8 \\
\end{tabular} & \begin{tabular}{|l|}
12.3 \\
1.0 \\
\end{tabular} & & 2.843 & \begin{tabular}{|l|}
0.004 \\
0.004 \\
\end{tabular} & \begin{tabular}{|l|l|}
0.004 \\
\end{tabular} & & 0.099 & \\
\hline 1687 & & $\frac{11: 3: 17 \mathrm{PM}}{112717 \mathrm{PM}}$ & $\begin{array}{l}25.3360 \\
25.395\end{array}$ & $\begin{array}{r}25.596 \\
25.59\end{array}$ & 24.529 & \begin{tabular}{|l|}
25.8594 \\
25.893 \\
\end{tabular} & $\begin{array}{l}25.136 \\
25.135 \\
\end{array}$ & \begin{tabular}{|l|}
46.4373 \\
4807
\end{tabular} & \begin{tabular}{|l|}
53.237 \\
52.965
\end{tabular} & $\begin{array}{r}15.6 \\
15.512\end{array}$ & $\begin{array}{l}31.944 \\
33.428 \\
\end{array}$ & \begin{tabular}{|c|c|}
4 & 0.589 \\
8 & -1.212
\end{tabular} & $\begin{array}{l}-1.913 \\
-6.139 \\
\end{array}$ & $\begin{array}{l}49.993 \\
49.603 \\
\end{array}$ & $\begin{array}{l}0.0399 \\
0.107\end{array}$ & $\begin{array}{l}\mid 14.643 \\
14.643\end{array}$ & 0.0004 & $\begin{array}{l}2382.92 \\
32838.92 \\
\end{array}$ & \begin{tabular}{|l|}
39.7153 \\
239.735 \\
\end{tabular} & \begin{tabular}{|l|}
52.0 \\
51.8 \\
\end{tabular} & \begin{tabular}{|l|}
11.9 \\
11.8 \\
\end{tabular} & $\begin{array}{l}939.2 \\
8 \\
80.7 \\
\end{array}$ & 2.7.802 & \begin{tabular}{|l|}
0.006 \\
0.015
\end{tabular} & $\begin{array}{l}0.006 \\
0.016\end{array}$ & 0.000 & $\begin{array}{l}0.144 \\
0.381\end{array}$ & 0.12 \\
\hline 1688 & $8 / 31 / 2004$ & 11:38:17 PM & 25.355 & 25.6 & 24.553 & \begin{tabular}{|l|}
25.938 \\
\end{tabular} & 25.145 & & & 15.472 & 34.482 & \begin{tabular}{|l|l|}
2 & -1.21 \\
\end{tabular} & $\begin{array}{l}-7.533 \\
\end{array}$ & 49.451 & 0.068 & 14.643 & $\begin{array}{l}0.004 \\
0.004\end{array}$ & $\frac{2383.92}{2384.92}$ & $\mid$ & \begin{tabular}{|l|}
1.1 .6 \\
52.1 \\
\end{tabular} & \begin{tabular}{|l|}
11.8 \\
11.8
\end{tabular} & & $\frac{2.001}{2.874}$ & & $\begin{array}{l}0.010 \\
0.010\end{array}$ & 0.000 & & $\begin{array}{l}0.32 \\
0.20\end{array}$ \\
\hline & $8 / 31 / 2004$ & & 25.26 & & \begin{tabular}{|l|}
24.523 \\
\end{tabular} & \begin{tabular}{|l|}
25.948 \\
\end{tabular} & 25.175 & & \begin{tabular}{|l|l|}
53.062 \\
\end{tabular} & 15.624 & 34.302 & \begin{tabular}{|l|l|}
2 & -1.212 \\
\end{tabular} & & 50.505 & 0.065 & \begin{tabular}{|l|l|}
14.643 \\
\end{tabular} & & & & \begin{tabular}{|l|}
51.9 \\
\end{tabular} & & & & & & & & \\
\hline 1690 & $8 / 31 / 2004$ & 11:40:17 PM & & & & & 25.215 & & & & 34.708 & -1.212 & & & 063 & \begin{tabular}{|l|l|}
14.643 \\
\end{tabular} & & 2386.92 & & & 11.4 & & & & 0.009 & 0.000 & & \\
\hline 1691 & $8 / 31 / 2004$ & $\begin{array}{l}11: 41: 17 \text { PM } \\
11: 1017\end{array}$ & $\begin{array}{l}25.176 \\
25165\end{array}$ & \begin{tabular}{|l|}
25.591 \\
25595
\end{tabular} & \begin{tabular}{|l|}
24.519 \\
2.539 \\
\end{tabular} & $\begin{array}{l}25.969 \\
25079\end{array}$ & $\begin{array}{l}25.156 \\
25.105\end{array}$ & 49.193 & \begin{tabular}{|l|l|}
53.688 \\
\end{tabular} & 15.562 & 34.532 & $\begin{array}{l}-1.212 \\
\end{array}$ & $\begin{array}{l}-7.608 \\
-7.0\end{array}$ & 49.253 & $\begin{array}{l}0.063 \\
\end{array}$ & $\begin{array}{l}14.643 \\
\end{array}$ & $\begin{array}{l}0.003 \\
\end{array}$ & 2387.92 & 39.7986 & 52.6 & 11.7 & 41.9 & 2.886 & \begin{tabular}{|l|l|} 
& 0.009 \\
\end{tabular} & 0.009 & 0.000 & 0.218 & \\
\hline$\frac{1692}{162}$ & $8 / 31 / 2004$ & $\begin{array}{ll}11: 42: 17 \mathrm{PM} \\
110: 4 \cdot 17\end{array}$ & $\begin{array}{l}25.165 \\
25.187\end{array}$ & $\begin{array}{l}25.585 \\
25602\end{array}$ & $\begin{array}{l}24.528 \\
24539\end{array}$ & \begin{tabular}{|l|}
25.978 \\
25.994 \\
\end{tabular} & $\begin{array}{l}25.195 \\
25.196\end{array}$ & $\begin{array}{l}48.996 \\
4912\end{array}$ & \begin{tabular}{|l|}
52.929 \\
53072 \\
\end{tabular} & $\begin{array}{l}15.628 \\
15565\end{array}$ & \begin{tabular}{|l|}
34.454 \\
3471
\end{tabular} & \begin{tabular}{|l|l|}
212 \\
209
\end{tabular} & $\begin{array}{l}-7.666 \\
7688\end{array}$ & $\begin{array}{l}48.888 \\
49676\end{array}$ & & \begin{tabular}{|l|l|}
14.643 \\
14634 \\
\end{tabular} & & & \begin{tabular}{|l|l|}
39.8153 \\
\end{tabular} & \begin{tabular}{|l|}
51.8 \\
519
\end{tabular} & 11.6 & & 年 & $\mid$ & & 0.000 & 0.219 & \\
\hline 1694 & 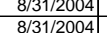 & 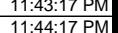 & $\begin{array}{r}25.1877 \\
25.16\end{array}$ & \begin{tabular}{r|r}
25.602 \\
25.57
\end{tabular} & $\begin{aligned} 24.539 \\
24.498\end{aligned}$ & \begin{tabular}{|l|}
25.9944 \\
25.968 \\
\end{tabular} & $\begin{array}{l}25.196 \\
25.245\end{array}$ & 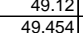 & \begin{tabular}{|l|}
53.042 \\
5.377 \\
\end{tabular} & $\begin{array}{l}15.556 \\
15.565 \\
\end{array}$ & .716 & \begin{tabular}{|l|l|}
-1.201 \\
-1.212
\end{tabular} & $\begin{array}{l}-7.668 \\
-7.668 \\
\end{array}$ & $\begin{aligned} \frac{49.676}{48.84} \\
\end{aligned}$ & $\begin{array}{l}0.063 \\
0.063 \\
\end{array}$ & $\begin{array}{l}\frac{14.643}{14.643} \\
\end{array}$ & $\begin{array}{l}0.004 \\
0.003 \\
\end{array}$ & & 39.8319 & $\begin{array}{r}51.9 \\
524 \\
\end{array}$ & $\frac{11.8}{11.6}$ & & 2.890 & 0009 & 0.009 & 0.000 & & 0.19 \\
\hline 1695 & $8 / 31 / 2004$ & $11: 45: 17 \mathrm{PM}$ & 25.165 & 25.58 & 24.512 & \begin{tabular}{|l|}
26.007 \\
\end{tabular} & 25.209 & \begin{tabular}{|l|}
49.685 \\
\end{tabular} & \begin{tabular}{|l|}
53.75 \\
\end{tabular} & $\begin{array}{r}15.66 \\
\end{array}$ & 35.016 & \begin{tabular}{|l|l|}
6 & -1.212 \\
\end{tabular} & & 49.391 & 0.063 & $\begin{array}{l}14.045 \\
14.643 \\
\end{array}$ & $\begin{array}{l}0.003 \\
0.003\end{array}$ & $\frac{2930.94}{2391.92}$ & \begin{tabular}{|l|}
39.84800 \\
39.8653 \\
\end{tabular} & $\begin{array}{l}52.4 \\
52.6 \\
\end{array}$ & \begin{tabular}{|l|}
11.6 \\
11.8
\end{tabular} & & & \begin{tabular}{|l|}
0.0099 \\
\end{tabular} & & & & $\frac{0.18}{0.18}$ \\
\hline & & $\begin{array}{l}11: 46: 17 \\
1: 46\end{array}$ & & 25.583 & 24.541 & \begin{tabular}{|l|}
25.991 \\
2.991 \\
\end{tabular} & 25.198 & $\begin{array}{l}48.998 \\
\end{array}$ & \begin{tabular}{|l|}
53.259 \\
\end{tabular} & \begin{tabular}{|l|l|}
15.547 \\
527
\end{tabular} & 34.552 & \begin{tabular}{|c|c|}
2 & -1.209 \\
\end{tabular} & & 51.03 & $\begin{array}{l}0.062 \\
\end{array}$ & \begin{tabular}{|l|}
14.643 \\
\end{tabular} & & & 39.8819 & & & & & & & & & \\
\hline & $8 / 31 / 2004$ & 11:47:17 PM & & 25.59 & & & 25.199 & 49.121 & \begin{tabular}{|l|l|}
53.023 \\
\end{tabular} & $\begin{array}{l}15.939 \\
\end{array}$ & & $\begin{array}{l}-1.212 \\
\end{array}$ & $\begin{array}{l}-7.68 \\
\end{array}$ & 48.181 & 061 & 14.643 & 0.004 & 2393.9 & 39.8986 & 51.9 & & & & 0.009 & 0.009 & 0.000 & 0.212 & \\
\hline$\frac{16}{16}$ & $8 / 31 / 2004$ & 11:48:17 PM & 25.183 & 25.578 & & 26.006 & 25.183 & & & & 33.688 & & & & & 14.643 & 0.004 & & 39.9153 & & 11.4 & & & & 0.009 & & 0.218 & \\
\hline & $\frac{8 / 31 / 2004}{8 / 312004}$ & $\begin{array}{ll}11: 49: 17 \mathrm{PM} \\
11050\end{array}$ & $\begin{array}{l}25.178 \\
25109\end{array}$ & $\begin{aligned} 25.578 \\
25.589\end{aligned}$ & \begin{tabular}{|l|}
24.531 \\
24567
\end{tabular} & $\begin{aligned} 26.021 \\
25097\end{aligned}$ & $\begin{array}{r}25.158 \\
25204\end{array}$ & \begin{tabular}{|l|}
48.461 \\
48.214 \\
\end{tabular} & \begin{tabular}{|l|}
52.299 \\
52336
\end{tabular} & $\begin{array}{l}16.128 \\
16.44 \\
\end{array}$ & \begin{tabular}{r|r|}
33.522 \\
33.886
\end{tabular} & $\begin{array}{l}-1.212 \\
1215\end{array}$ & $\begin{array}{r}-7.718 \\
7666\end{array}$ & $\begin{array}{l}49.019 \\
4934\end{array}$ & 061 & $\begin{array}{l}14.643 \\
14643 \\
\end{array}$ & & & 39.9319 & $\begin{array}{l}51.2 \\
512\end{array}$ & 11.7 & & & & & & 0.216 & \\
\hline & $88 / 31 / 2004$ & $\begin{array}{ll}11: 50: 17 \mathrm{PM} \\
1115 \cdot 17\end{array}$ & $\begin{array}{r}25.199 \\
25.2\end{array}$ & $\begin{array}{r}25.589 \\
2559\end{array}$ & \begin{tabular}{|l|}
24.567 \\
24.592
\end{tabular} & $\begin{array}{l}.997 \\
997\end{array}$ & $\begin{array}{r}25.204 \\
25.164\end{array}$ & & \begin{tabular}{|c|}
$\mid 52.366$ \\
5.2706
\end{tabular} & $\frac{16.434}{16.544}$ & & $\frac{215}{212}$ & $\begin{array}{r}-7.666 \\
-7.66\end{array}$ & $\begin{array}{l}49.364 \\
49.977 \\
4\end{array}$ & 0599 & $\frac{14.643}{14.643}$ & $\begin{array}{l}003 \\
003 \\
\end{array}$ & & $\begin{array}{l}9.9486 \\
9.9653\end{array}$ & $\begin{array}{r}51.2 \\
516\end{array}$ & 11.8 & $\begin{array}{l}4.06 \\
407\end{array}$ & $\begin{array}{ll}796 \\
8844\end{array}$ & $\begin{array}{l}0.008 \\
0.08\end{array}$ & 0.009 & 0.000 & & 0.18 \\
\hline & $8 / 31 / 2004$ & $11: 52: 17 \mathrm{PM}$ & 25.2 & $\begin{array}{r}25.595 \\
25.585\end{array}$ & \begin{tabular}{|l|}
24.663 \\
\end{tabular} & \begin{tabular}{|l|}
25.991 \\
26.002 \\
\end{tabular} & 25.104 & $\begin{array}{l}4.4742 \\
48.442\end{array}$ & \begin{tabular}{|l|}
52.902 \\
\end{tabular} & $\begin{array}{l}\frac{10.544}{16.504} \\
\end{array}$ & $\begin{array}{l}32.808 \\
32.917 \\
\end{array}$ & $\begin{array}{l}-1.212 \\
-1.209 \\
\end{array}$ & $\begin{array}{r}-1.00 \\
-7.66\end{array}$ & $\begin{array}{l}\frac{49.911}{50.227} \\
\end{array}$ & $\begin{array}{l}0.059 \\
0.0599\end{array}$ & \begin{tabular}{l|l}
$\frac{14.043}{14.643}$ &
\end{tabular} & $\begin{array}{l}0.003 \\
0.003\end{array}$ & 2398.92 & \begin{tabular}{|l|}
39.9053 \\
39.9819 \\
\end{tabular} & $\begin{array}{l}51.0 \\
51.8\end{array}$ & \begin{tabular}{|l|}
11.9 \\
12.0
\end{tabular} & $\begin{array}{ll}0 & 40.7 \\
0 & 40.7\end{array}$ & \begin{tabular}{|l|}
2.804 \\
2.805 \\
\end{tabular} & \begin{tabular}{|l|l|}
0.000 \\
\end{tabular} & 0.009 & 0.000 & \begin{tabular}{|c|c|}
0.210 \\
0.210
\end{tabular} & $\frac{0.18}{0.18}$ \\
\hline & $8 / 31 / 2004$ & 11:53:17 PM & 25.2 & 25.58 & \begin{tabular}{|l|}
24.642 \\
\end{tabular} & \begin{tabular}{|l|}
26.012 \\
\end{tabular} & 25.199 & 48.44 & \begin{tabular}{|l|}
52.426 \\
\end{tabular} & 16.401 & 33.11 & -1.212 & -7.671 & 50.163 & 0.059 & 14.643 & 0.003 & 23399.92 & 39.9986 & 51.3 & 11.9 & 40.8 & 2.811 & \begin{tabular}{|l|l|} 
& 0.008 \\
\end{tabular} & & 0.000 & 0.209 & \\
\hline & $8 / 31 / 2004$ & 11:54:17 PM & 25.205 & 25.58 & 24.637 & 26.032 & 25.129 & 328 & \begin{tabular}{|l|}
52.484 \\
\end{tabular} & 16.335 & 33.052 & -1.215 & -7.668 & 50.803 & & 14.643 & & & & 51.4 & 12.1 & $\begin{array}{ll}40.7 \\
\end{array}$ & & & 0.008 & & & \\
\hline & $8 / 31 / 2004$ & 11:55:17 PM & 25.219 & 25.589 & \begin{tabular}{|l|l|}
24.652 \\
\end{tabular} & 26.037 & & & & & 33.079 & .212 & & $\begin{array}{lll}47.947 \\
\end{array}$ & & $\begin{array}{l}14.643 \\
\end{array}$ & & & & & & & & $\overline{0.008}$ & & & & \\
\hline & $8 / 31 / 2004$ & 11:56:17 PM & 25.235 & $\begin{array}{l}25.585 \\
\end{array}$ & 24.663 & 26.032 & & & & \begin{tabular}{|l|l|}
16.264 \\
\end{tabular} & 33.068 & & & 51.627 & & $\begin{array}{l}14.643 \\
\end{array}$ & & & & & & & & & & & & \\
\hline & $8 / 31 / 2004$ & 11:57:17 PM & 25.245 & 25.595 & 24.653 & 26.033 & 25.1 & 48 & 52.403 & \begin{tabular}{|c|c|c|}
16.378 \\
\end{tabular} & 32.853 & -1.212 & 7.67 & 47.33 & & 14.643 & & & & 51.3 & 11.3 & & & & & & & \\
\hline & $8 / 31 / 2004$ & 11:58:17 PM & & 25.59 & 24.658 & 26.082 & 25.109 & 48.424 & 52.567 & \begin{tabular}{|l|l|l|}
16.43 \\
\end{tabular} & 33.077 & $\mid-1.209$ & & 49.568 & 0.059 & 14.643 & & & .0819 & 51.4 & 11.8 & & 8 & 0.008 & & & & \\
\hline & $8 / 31 / 2002$ & 11:59:17 PM & 25.26 & 25.585 & 24.648 & 26.062 & 25.109 & 48.523 & 52.905 & 16.393 & 33.138 & -1.212 & & 49.551 & .059 & 14.643 & & 2405.9 & & 51.8 & 11.8 & & 2.815 & & & & 0.209 & \\
\hline & & & & $\frac{25.585}{2.250}$ & 24.663 & 2000 & & 48.142 & & 16.272 & & 215 & & 52.82 & 0.06 & 14.643 & & & & & & & & & & & & \\
\hline & & 12:01:17/ AM & .27 & 25.59 & 24.668 & 26.042 & 5.1 & 48.548 & & 16.296 & 33.257 & -1.209 & & 49.572 & nom & 14.643 & & & & & & & & & & & & \\
\hline & & 2:1/ AM & & 25.591 & 24.659 & 26.049 & & & 5.619 & 16.214 & 33.225 & -1.212 & & & & & & & & & & & & & & & 0.213 & \\
\hline & $\begin{array}{l}9 / 1 / 22004 \\
9 / / 12004\end{array}$ & $\begin{array}{ll}12: 03: 17 \mathrm{AM} \\
12004 \cdot 17\end{array}$ & $\frac{25.261}{25227}$ & $\frac{25.571}{25.582}$ & 24.624 & $\frac{26.029}{26.08}$ & 25. & $\frac{074}{774}$ & $52.457 \mid$ & $\frac{16.298}{16293}$ & $\begin{array}{r}33.03 \\
33.637 \\
\end{array}$ & $\begin{array}{l}-1.215 \\
-1.212 \\
\end{array}$ & & 55.659 & . 0.06 & $\frac{14.643}{14643}$ & & & 1819 & 519 & 22 & & 8 & & & & & \\
\hline 171 & 9 & & & 25.579 & $\begin{array}{l}24.07 \\
24.667\end{array}$ & 26.05 & 25.1 & & & $\begin{array}{l}10.290 \\
16.114\end{array}$ & 3.05/ & 4 & & 50.611 & 0.061 & 14643 & & & & & 12 & & & & & & & \\
\hline & $9 / 1 / 2004$ & $12: 06: 17 \mathrm{AM}$ & 25.294 & 25.594 & \begin{tabular}{|l|l|l|}
24682 \\
\end{tabular} & 26.07 & 25. & & 52.098 & & & -1.209 & & & & 146 & & & & $\frac{1.6}{510}$ & 12 & & & & & & $\frac{1.251}{0.211}$ & \\
\hline & & 12:07:17 AM & & 25.584 & 24.667 & $\frac{26.07}{26.07}$ & & & 52.328 & 16.291 & 32.984 & -1.206 & & 49.8 & 0.059 & & & & & & & & & & & & & \\
\hline & & & & & & & & & & & & & & & & & & & & 51.9 & & & & & & & & \\
\hline & & 12:09:17 AM & 25.291 & 25.586 & 24.674 & & & & 52.399 & 16.2 & 33. & -1.212 & & 49.7 & & & & & 40.26 & $\frac{21.3}{51.3}$ & 11. & & & & & & & \\
\hline & & & & & & & & & & & & & & & & & & & & & & & & & & & & \\
\hline & & 12:11:17 AM & 25.299 & 25.594 & \begin{tabular}{|l|}
24.697 \\
\end{tabular} & \begin{tabular}{|l|}
26.081 \\
\end{tabular} & & & \begin{tabular}{|l|}
52.486 \\
\end{tabular} & 16.12 & 33.284 & \begin{tabular}{|c|}
-1.209 \\
\end{tabular} & & & & & & & & 51.4 & $\overline{11.5}$ & & & & & & 09 & 018 \\
\hline & $9 / 1 / 2004$ & 12:12:17 AM & 25.309 & 25.589 & & & & & 52.815 & 16.018 & 33.304 & -1.212 & & & 0.058 & 14.6 & & & & 51.7 & 11.6 & & & & & & 0.205 & \\
\hline & & 12:13:17 AM & & 25.604 & & & & & & & & & & & & & & & & 51.5 & 11.5 & & & & & & & \\
\hline & & $12: 14: 17 \mathrm{AM}$ & 25.309 & 25.584 & \begin{tabular}{|l|}
24.687 \\
\end{tabular} & 26.052 & & & \begin{tabular}{|l|l|} 
& 52.708 \\
\end{tabular} & 16.151 & 33.368 & -1.2. & & & 0.059 & & & & .3486 & 51.6 & $11.8 \mathrm{~S}$ & & & & & & 0.208 & \\
\hline & & & & 20.0 & & & & & & & & & & & & & & & & 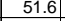 & & & & & & & 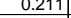 & \\
\hline & & & & & & & & & & & & & & & & & & & & & & & & & & & & \\
\hline & $9 / 1 / 2$ & $\begin{array}{l}12: 17: 17 \mathrm{AM} \\
12018\end{array}$ & 25.3254 & $\frac{25.585}{25.584}$ & $\begin{array}{l}24.753 \\
247757\end{array}$ & $\frac{26.043}{26037}$ & 2524 & & $\frac{52.822}{53255}$ & $\frac{15.997}{16059}$ & 33.565 & -1.1212 & & & & $\frac{14 .}{14 .}$ & & & & $\frac{5.1}{513}$ & 12 & & & & & & (n) & \\
\hline & $9 / 1 / 12004$ & & & 25.584 & & & & & & & & & & & & & & & & & 125 & & & & & & & \\
\hline & & $\frac{12.2017}{12.2017}$ & & 2559 & & & & & & & & & & & & & & & & 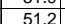 & 114 & & & & & & & \\
\hline & $9 / 1 / 2004$ & $\frac{12: 21: 17 \mathrm{AM}}{12}$ & $\frac{25.346}{25}$ & 25.601 & 24 & & & & $\mid$\begin{tabular}{|l|}
52.788 \\
\end{tabular} & 16.138 & & & & & & 14.6 & & & & $\frac{31.7}{51.7}$ & $\frac{12.1}{12.1}$ & & & & & & $\frac{0.204}{0.204}$ & \\
\hline & & & & & & & & & & & & & & & & & & & & & & & & & & & & \\
\hline & & & 25.341 & 25.591 & & & & & & & 33. & & & & & & & & & & & & & & & & & \\
\hline & & & 25.341 & 25.591 & & & & & & & & & & & & & & & & 51.4 & 11.9 & & & & & & & \\
\hline & & & & 25.596 & 24.694 & 26. & & & \begin{tabular}{|l|l|}
52.735 \\
\end{tabular} & 16 & 33.3 & -1.21 & & & & & & & & 516 & 11.4 & & & & & & & \\
\hline & & & 25 & 25.596 & 684 & 26.0 & & 48.4 & 52. & 16.1 & 33.35 & -1.2 & & & & 14.6 & & & & & 12. & & & & & & & \\
\hline & & & & & & & & & & & & & & & & & & & & 1.4 .4 & 1 & & & & & & & \\
\hline & & & 25.352 & 25.602 & & 26.05 & & & & 16.008 & & -1.212 & & & & & & & & 51.2 & 11.9 & & & & & & & \\
\hline & & & & & & & & & & & & & & & & & & & & & & & & & & & & \\
\hline & & 1.2:30: & 年 & 25. & & & & & & & & -1.2 & & & & & & & & & 12 & & & & & & & \\
\hline & & 0.33. & 343 & $\frac{12.003}{2563}$ & $2 \frac{2.006}{2.66}$ & $\frac{2.0030}{26041}$ & & & \begin{tabular}{|l|l|}
5.2017 \\
5276
\end{tabular} & & $\begin{array}{l}33.512 \\
32107\end{array}$ & $\frac{-1.20}{121}$ & & & & & & & & & 12.3 & & & & & & & \\
\hline & & & & 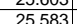 & $\frac{2.0400}{2462}$ & $\frac{2.0445}{26005}$ & & 48704 & $\begin{array}{l}0.3<41 \\
15300\end{array}$ & 16.035 & $\frac{35.101}{23711}$ & -1.4 & & 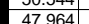 & & 146 & & & & & & & & & & & & \\
\hline & & & & 25.598 & 2464 & & & & & & & & & & & & & & & & 11 & & & & & & & \\
\hline & & & & $\frac{25.593}{25.593}$ & & & & & & & & $\frac{-1.215}{-1.215}$ & & & & 14.6 & & & & 514 & 120 & & & & & & & \\
\hline & & & & & & & & & & & & & & & & & & & & & & & & & & & & \\
\hline & & & & & & & & & & & & & & & & & & & & & & & & & & & & \\
\hline & & & & $25588^{8}$ & 24.621 & 26.6 & & 48.3 & 52.5 & 16.0 & 33.43 & 12 & & 49.5 & & & & & & & 110 & & & & & & & \\
\hline & & & & 591 & 620 & & & & & 160 & 2351 & & & & & & & & & & & & & & & & & 11 \\
\hline & $9 / 1 / 2$ & $12: 40: 17 \mathrm{AM}$ & \begin{tabular}{|l|}
25.342 \\
\end{tabular} & 25.617 & 24.66 & \begin{tabular}{|c|}
26.06 \\
\end{tabular} & 25.16 & 48.426 & \begin{tabular}{|l|l|}
52.561 \\
\end{tabular} & \begin{tabular}{|l|l|l|l|}
15.89 \\
\end{tabular} & 33.655 & 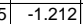 & & 499.791 & 0.058 & $\begin{array}{l}14.643 \\
\end{array}$ & & 446.9 & 0.7819 & 51.4 & 11.9 & & 2.830 & \begin{tabular}{|c|}
$\mid 0.008$ \\
\end{tabular} & 0.008 & 0.000 & \begin{tabular}{|c|}
0.204 \\
\end{tabular} & 0.1 \\
\hline
\end{tabular}


WSRC-TR-2005-00105, REVISION 0

SRNL-RPP-2005-00012, REVISION 0

RUN \# 4.03A AND B; FIRST AND SECOND HALF OF SLURRY DEWATERING - CONT.

\begin{tabular}{|c|c|c|c|c|c|c|c|c|c|c|c|c|c|c|c|c|c|c|c|c|c|c|c|c|c|c|c|c|}
\hline & A & $\mathrm{B}$ & $\mathrm{D}$ & $E$ & $\mathrm{~F}$ & $G$ & $\mathrm{H}$ & $\mathrm{J}$ & K & $\mathrm{L}$ & $M$ & $\mathrm{~N}$ & 0 & Q & $R$ & $\mathrm{~s}$ & \begin{tabular}{l|l}
$T$ \\
\end{tabular} & $\mathrm{v}$ & w & $x$ & $Y$ & $z$ & $\mathrm{AA}$ & & $\mathrm{AC}$ & AD & & \\
\hline & 9/1/2004 & 12:41:17 AM & 25.337 & 25.607 & 24.66 & 26.015 & 25.232 & 48.488 & 52.832 & 15.876 & 33.733 & -1.212 & -7.842 & 48.423 & 0.05 & 14.643 & 0.003 & 2447.92 & 40.7986 & 51.7 & 11.5 & 41.1 & $\frac{2.834}{2.83}$ & 0.008 & 0.008 & 0.000 & 0.204 & \\
\hline & & 12:42:17 AM & 25.327 & 25.597 & 24.64 & 26.015 & 25.222 & 48.519 & \begin{tabular}{|l|}
52.892 \\
\end{tabular} & 16.092 & 33.46 & -1.209 & $\begin{array}{l}-7.856 \\
-7.856 \\
\end{array}$ & 51.139 & 0.058 & 14.643 & 0.003 & & & 51.8 & 12.2 & & & & 0.008 & & & \\
\hline & $\begin{array}{l}9 / 1 / 2004 \\
0 / 1204\end{array}$ & 12:43:17 AM & $\frac{25.328}{25232}$ & 25.608 & \begin{tabular}{|l|}
24.641 \\
\end{tabular} & \begin{tabular}{|l|}
26.025 \\
2.025 \\
\end{tabular} & 25.247 & 488.322 & \begin{tabular}{|l|l|}
52.646 \\
5562
\end{tabular} & 15.898 & 33.518 & $\begin{array}{l}-1.215 \\
-1.212\end{array}$ & $\begin{array}{l}-7.856 \\
-7.851\end{array}$ & 53.03 & & 14.643 & 0.003 & 2449.92 & 40.8319 & 51.5 & 12.6 & 40 & & 0.008 & 0.008 & 0.000 & 0.205 & \\
\hline & $\begin{array}{l}\text { 9/1/2004 } \\
0 / 12004\end{array}$ & 12:44:17 AM & 25.322 & 25.597 & \begin{tabular}{|l|l|}
24.645 \\
\end{tabular} & \begin{tabular}{|l|}
26.0355 \\
\end{tabular} & 25.282 & $\begin{array}{l}48.409 \\
\end{array}$ & \begin{tabular}{|l|l|}
52.453 \\
\end{tabular} & \begin{tabular}{ll|l}
16.003 \\
\end{tabular} & 33.524 & -1.212 & $\begin{array}{r}-7.851 \\
-705\end{array}$ & 49.449 & 0.058 & 14.643 & 0.003 & 2450.92 & 40.8486 & \begin{tabular}{|l|l|}
51.3 \\
\end{tabular} & 11.8 & 41.0 & 2.824 & 0.008 & 0.008 & 0.000 & 0.205 & \\
\hline & 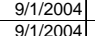 & $\begin{array}{l}12: 45: 17 \mathrm{AM} \\
12244 \cdot 17 \mathrm{AM}\end{array}$ & $\begin{array}{l}25.337 \\
25.333 \\
\end{array}$ & $\frac{25.612}{25.613}$ & \begin{tabular}{|r|}
24.67 \\
24.66 \\
\end{tabular} & $\begin{array}{r}26.05 \\
26055\end{array}$ & $\begin{array}{l}25.212 \\
25227 \\
527\end{array}$ & $\begin{array}{l}48.364 \\
48579 \\
\end{array}$ & & 15.995 & $\begin{array}{l}33.606 \\
33592 \\
\end{array}$ & \begin{tabular}{|c|}
-1.209 \\
1215
\end{tabular} & & $\begin{array}{l}50.916 \\
18.996\end{array}$ & & \begin{tabular}{|l|l|}
14.643 \\
14634 \\
\end{tabular} & & \begin{tabular}{|l|l|}
2451.92 \\
21523
\end{tabular} & .8653 & \begin{tabular}{|l|}
51.4 \\
\end{tabular} & 12.1 & & & & & & 0.204 & \\
\hline 1757 & $\begin{array}{l}9 / 1 / 2004 \\
9 / 1 / 2004\end{array}$ & $\begin{array}{l}\text { 12:44:4717 AM } \\
\text { 12:47: }\end{array}$ & $\frac{25.333}{25.332}$ & $\begin{array}{l}25.613 \\
25.607\end{array}$ & \begin{tabular}{r|}
24.661 \\
24.67 \\
\end{tabular} & \begin{tabular}{|l|}
26.055 \\
26.055 \\
\end{tabular} & $\begin{array}{l}25.227 \\
25.192 \\
\end{array}$ & $\begin{array}{r}48.579 \\
48.7 \\
\end{array}$ & \begin{tabular}{|l|}
53.081 \\
52.824 \\
\end{tabular} & $\begin{array}{r}16 \\
16.035\end{array}$ & \begin{tabular}{|l|}
33.592 \\
33.879 \\
\end{tabular} & \begin{tabular}{|c|}
-1.211 \\
-1.212 \\
\end{tabular} & $\begin{array}{l}-7.859 \\
-7.871 \\
\end{array}$ & $\begin{array}{l}48.966 \\
48.608\end{array}$ & $\frac{0.058}{0.056}$ & $\begin{array}{l}14.643 \\
14.643 \\
\end{array}$ & $\begin{array}{l}0.003 \\
0.003\end{array}$ & 2452.322 & $\begin{array}{l}40.88198 \\
40.896\end{array}$ & \begin{tabular}{|l|}
52.0 \\
51.7 \\
\end{tabular} & $\frac{11.7}{11.6}$ & $\begin{array}{l}41.1 \\
41.3 \\
\end{array}$ & .8.833 & 0.008 & \begin{tabular}{l|l}
0.000 \\
0.008
\end{tabular} & 0.000 & 0.204 & 0.17 \\
\hline 1758 & $9 / 1 / 2004$ & & 25.332 & 25.612 & 24.715 & 26.04 & 25.172 & 48.785 & \begin{tabular}{|l|l|}
53.137 \\
\end{tabular} & 16.071 & 33.836 & & & 51.201 & 0.058 & $\begin{array}{l}14.643 \\
14.643\end{array}$ & 0.003 & 2455.92 & \begin{tabular}{|l|}
40.0900 \\
40.9153 \\
\end{tabular} & \begin{tabular}{|l|}
52.0 \\
52.0 \\
\end{tabular} & $\frac{11.0}{12.2}$ & $\begin{array}{ll}41.35 \\
41.3 \\
\end{array}$ & 2.847 & $\mid \begin{array}{l}0.008 \\
0.008\end{array}$ & $\begin{array}{l}.008 \\
0.008 \\
\end{array}$ & 0.000 & & \\
\hline & & & & 25.612 & & 26.075 & & 48.364 & 52.836 & 16.021 & 33.407 & \begin{tabular}{|l|l|}
-1.212 \\
\end{tabular} & & 48.757 & & 14.643 & 0.004 & 2455.92 & & 51.7 & & & & & & & & \\
\hline & 9/1/2004 & & & & 24.72 & 26.08 & & $\begin{array}{l}48.249 \\
\end{array}$ & & 16.06 & 33.343 & -1.212 & -7.859 & 48.485 & & $\begin{array}{l}14.643 \\
\end{array}$ & & 2456.92 & \begin{tabular}{|l|l|}
40.9486 \\
\end{tabular} & 51.3 & 11 & & & & 0.009 & 0.000 & 0.209 & \\
\hline$\frac{1761}{1762}$ & 1/2004 & 12:51:17 AM & 25.326 & 25.601 & \begin{tabular}{|l|l|}
24.669 \\
2.69 \\
\end{tabular} & 26.048 & 25.155 & 48.824 & \begin{tabular}{|l|l|}
53.298 \\
5.290
\end{tabular} & 16.117 & 33.688 & -1.218 & -7.868 & 48.6 & 0.058 & 14.643 & 0.003 & & 0.9653 & 52.2 & 11.6 & & 2.844 & 0.008 & 0.008 & 0.000 & $\begin{array}{ll}0.203 \\
\end{array}$ & \\
\hline & $\begin{array}{l}9 / 1 / 2004 \\
9 / 1 / 2004\end{array}$ & $\begin{array}{ll}12: 52: 17 \mathrm{AM} \\
122.5 \cdot 17 \mathrm{AM}\end{array}$ & $\begin{array}{l}25.345 \\
25.344\end{array}$ & $\begin{array}{l}25.605 \\
25.609\end{array}$ & \begin{tabular}{|l|}
24.683 \\
24687 \\
\end{tabular} & $\begin{array}{l}26.063 \\
26.072\end{array}$ & $\begin{array}{l}25.145 \\
25.169\end{array}$ & \begin{tabular}{|c|}
48.084 \\
48488 \\
\end{tabular} & \begin{tabular}{|c|}
52.436 \\
52811 \\
\end{tabular} & \begin{tabular}{r|r|}
15.939 \\
16.14
\end{tabular} & \begin{tabular}{|l|}
33.294 \\
33.41
\end{tabular} & $\begin{array}{l}-1.212 \\
-1.212\end{array}$ & \begin{tabular}{|l|}
-7.862 \\
-7868 \\
\end{tabular} & 50.3 & & \begin{tabular}{|l|l|}
14.643 \\
14634 \\
\end{tabular} & & \begin{tabular}{l|}
2458.92 \\
245092
\end{tabular} & \begin{tabular}{|l|l|}
40.9819 \\
5
\end{tabular} & \begin{tabular}{|l|l|}
51.3 \\
51.7
\end{tabular} & $\frac{12.0}{117}$ & 40.7 & 283 & \begin{tabular}{|l|l|} 
& 0.008 \\
& 0.008 \\
\end{tabular} & 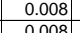 & 0.000 & $\begin{array}{l}0.206 \\
0201\end{array}$ & \\
\hline 64 & $\begin{array}{l}9 / 1 / 2004 \\
99 / 1 / 2004\end{array}$ & $\begin{array}{l}\text { 12:53: } 1717 \mathrm{AM} \\
\text { 12:55:17 }\end{array}$ & $\begin{array}{l}25.344 \\
25.344 \\
\end{array}$ & $\begin{array}{l}25.609 \\
25.609\end{array}$ & \begin{tabular}{|l|}
24.687 \\
24.687
\end{tabular} & $\frac{26.072}{26.052}$ & $\begin{array}{r}25.169 \\
25.169 \\
\end{array}$ & \begin{tabular}{|l|}
48.488 \\
47.684
\end{tabular} & \begin{tabular}{|r|}
52.8111 \\
52.25 \\
\end{tabular} & $\begin{array}{r}16.14 \\
15.8\end{array}$ & $\begin{aligned} \frac{33.401}{33.13} \\
\end{aligned}$ & \begin{tabular}{|l|}
-1.212 \\
-1.212 \\
\end{tabular} & $\begin{array}{r}-7.868 \\
-7.853 \\
\end{array}$ & $\begin{array}{l}499.207 \\
48.771\end{array}$ & $\begin{array}{l}0.057 \\
0.059\end{array}$ & \begin{tabular}{|c|}
14.643 \\
14.643
\end{tabular} & $\begin{array}{l}0.003 \\
0.004\end{array}$ & \begin{tabular}{|l|l}
2459.92 \\
260.92
\end{tabular} & \begin{tabular}{|l|l|}
40.9986 \\
41.0153 \\
\end{tabular} & $\begin{array}{l}51.7 \\
55.1\end{array}$ & $\frac{11.7}{11.5}$ & $\begin{array}{l}40.9 \\
40.5 \\
\end{array}$ & 2.823 & 0.008 & $\begin{array}{l}0.008 \\
0.009\end{array}$ & 0.000 & 0.201 & 0.17 \\
\hline & 9/1/2004 & $12: 55: 17 \mathrm{AM}$ & 25.349 & 25.604 & \begin{tabular}{|l|}
24.667 \\
\end{tabular} & \begin{tabular}{|l|}
26.047 \\
\end{tabular} & 25.149 & \begin{tabular}{|}
48.504 \\
8.59
\end{tabular} & \begin{tabular}{|r|}
52.89 \\
\end{tabular} & 16.072 & 33.709 & \begin{tabular}{|l|}
-1.212 \\
\end{tabular} & $\begin{array}{l}-1.853 \\
-7.865 \\
\end{array}$ & $\begin{array}{r}8.4811 \\
48.988\end{array}$ & $\begin{array}{l}0.059 \\
0.058\end{array}$ & $\begin{array}{l}14.045 \\
14.643 \\
\end{array}$ & $\begin{array}{l}0.004 \\
0.003\end{array}$ & $\frac{2440.92}{2461.92}$ & \begin{tabular}{|l|}
41.0153 \\
41.0319 \\
\end{tabular} & \begin{tabular}{|l|}
51.1 .8 \\
\end{tabular} & $\begin{array}{l}11.5 \\
11.7\end{array}$ & & 2..1947 & \begin{tabular}{|l|l|} 
\\
\end{tabular} & $\begin{array}{l}.009 \\
0.008\end{array}$ & $\begin{array}{l}0.000 \\
0.000\end{array}$ & & $\begin{array}{l}0.18 \\
0.17\end{array}$ \\
\hline & & 12:56:17 AM & 25.349 & 25.614 & \begin{tabular}{|l|}
24.672 \\
\end{tabular} & 26.076 & 25.183 & 48.5 & 52.961 & 15.94 & 33.643 & 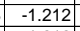 & & 49.215 & & 14.643 & & & 41.0486 & 51.8 & & & & & & & & \\
\hline & 9/1/2004 & & & & & 26.076 & & $\begin{array}{l}48.314 \\
\end{array}$ & & \begin{tabular}{|l|l}
16.08 \\
\end{tabular} & 33.307 & -1.212 & & 50.083 & & $\begin{array}{l}14.643 \\
\end{array}$ & & 2463.9 & .0653 & 51.6 & & & & & 0.008 & 0.000 & 0.205 & \\
\hline & & $\begin{array}{l}12: 58: 17 \mathrm{AM} \\
12: 50\end{array}$ & 25.363 & $\begin{array}{l}25.628 \\
25627\end{array}$ & $\begin{array}{l}24.696 \\
2.750\end{array}$ & 26.076 & $\begin{array}{l}25.158 \\
25.172\end{array}$ & & \begin{tabular}{|l|}
52.538 \\
\end{tabular} & 15.92 & 33.442 & & & $\begin{array}{l}48.807 \\
\end{array}$ & & $\begin{array}{l}14.643 \\
\end{array}$ & 0.003 & & .0819 & 51.4 & 11.6 & & & 0.008 & $\begin{array}{l}0.009 \\
\end{array}$ & 0.000 & 0.209 & \\
\hline & $\begin{array}{l}9 / 1 / 1 / 2004 \\
99 / 2004\end{array}$ & $\begin{array}{c}12: 59: 17 \mathrm{AM} \\
1: 00: 17\end{array}$ & $\begin{array}{l}25.362 \\
25.353\end{array}$ & $\frac{25.627}{25.618}$ & \begin{tabular}{|l|}
$\mid 24.705$ \\
24.671 \\
\end{tabular} & $\begin{array}{r}26.06 \\
26.056\end{array}$ & $\begin{array}{r}25.172 \\
25098 \\
\end{array}$ & & \begin{tabular}{|l|}
53.056 \\
52.411 \\
\end{tabular} & $\begin{array}{l}16.112 \\
15.962\end{array}$ & . 321 & $\begin{array}{l}-1.209 \\
-1.212\end{array}$ & $\begin{array}{c}-7.859 \\
-7.862\end{array}$ & $\begin{array}{r}49.117 \\
48.8\end{array}$ & & \begin{tabular}{|c|}
14.643 \\
14643
\end{tabular} & $\begin{array}{l}0.003 \\
0.04\end{array}$ & & $\mid \begin{array}{l}.0986 \\
1153 \\
\end{array}$ & $\begin{array}{l}51.9 \\
51.3\end{array}$ & $\frac{11.7}{11.7}$ & & & 0.008 & $\begin{array}{l}0.009 \\
0.09\end{array}$ & 000 & $\begin{array}{l}0.208 \\
020\end{array}$ & $\begin{array}{l}0.18 \\
018\end{array}$ \\
\hline 1771 & $\begin{array}{r}9 / 1 / 2004 \\
9 / 1 / 2004\end{array}$ & $\begin{array}{l}\text { 1:00:017 AM } \\
1: 01: 17\end{array}$ & $\begin{array}{l}25.353 \\
25.339 \\
\end{array}$ & $\begin{array}{l}25.618 \\
25.609\end{array}$ & \begin{tabular}{|l|}
24.671 \\
24.672 \\
\end{tabular} & $\frac{26.056}{26.026}$ & $\begin{array}{r}25.098 \\
25.123 \\
\end{array}$ & & \begin{tabular}{|l|}
52.4111 \\
52.635 \\
\end{tabular} & $\begin{array}{l}15.962 \\
15.954 \\
\end{array}$ & & $\frac{-1.212}{-1.212}$ & $\begin{array}{l}-7.862 \\
-7.862 \\
\end{array}$ & & & $\begin{array}{r}\mid 14.643 \\
14.643\end{array}$ & $\begin{array}{l}0.004 \\
0.003\end{array}$ & & .1153 & $\begin{array}{r}51.3 \\
51.5\end{array}$ & $\frac{11.6}{12.3}$ & $\frac{40.8}{40.8}$ & 810 & 0.008 & 0.009 & 0.000 & 0.209 & 0.18 \\
\hline & $9 / 1 / 2004$ & 1:02:17 AM & 25.349 & 25.614 & \begin{tabular}{|l|}
24.692 \\
\end{tabular} & 26.041 & 25.113 & \begin{tabular}{|l|l|}
48.223 \\
\end{tabular} & \begin{tabular}{|l|}
52.677 \\
\end{tabular} & 16.086 & 33.212 & \begin{tabular}{|l|l|} 
& -1.212 \\
\end{tabular} & $\begin{array}{l}-1.004 \\
-7.868 \\
\end{array}$ & $\begin{array}{l}31.817 \\
49.334 \\
\end{array}$ & 0.058 & $\begin{array}{l}14.045 \\
14.643\end{array}$ & $\begin{array}{l}0.003 \\
0.003\end{array}$ & $\frac{2407.92}{2468.92}$ & \begin{tabular}{|l|}
41.131486 \\
41.146
\end{tabular} & $\begin{array}{l}51.5 \\
51.6 \\
\end{array}$ & $\begin{array}{l}11.3 \\
11.8\end{array}$ & 40.8 & $\frac{2.810}{2.807}$ & \begin{tabular}{|l|l|} 
& 0.008 \\
\end{tabular} & $\begin{array}{l}.008 \\
0.008\end{array}$ & 0.000 & $\begin{array}{l}0.206 \\
0.206\end{array}$ & $\begin{array}{l}0.18 \\
0.18\end{array}$ \\
\hline & 9/1/2004 & 1:03:17 AM & 25.344 & 25.599 & \begin{tabular}{|l|}
24.682 \\
\end{tabular} & 26.051 & & 48.388 & \begin{tabular}{|l|}
53.045 \\
\end{tabular} & 15.873 & $\begin{array}{l}33.534 \\
3.939\end{array}$ & \begin{tabular}{|l|} 
\\
\end{tabular} & & 48.836 & 0.058 & 14.643 & & & 41.1653 & 51.9 & 11.6 & 41.0 & 2.824 & 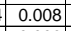 & 0.008 & 0.000 & 0.205 & \\
\hline & & 1:04:17 AM & & 25.614 & \begin{tabular}{|l|}
24.687 \\
\end{tabular} & & & $\begin{array}{l}48.164 \\
\end{array}$ & \begin{tabular}{|l|}
52.517 \\
\end{tabular} & 15.99 & & -1.212 & & & 0.06 & \begin{tabular}{|l|l|}
14.643 \\
\end{tabular} & & & 41.1819 & 51.4 & 11.0 & & & 0.009 & 0.009 & & & \\
\hline 1775 & $9 / 1 / 2004$ & 1:05:17 AM & 25.344 & $\begin{array}{l}25.599 \\
25500\end{array}$ & \begin{tabular}{|l|}
24.667 \\
2.720 \\
\end{tabular} & & 25.1 & & \begin{tabular}{|l|}
53.253 \\
5250 \\
\end{tabular} & $\begin{array}{l}15.846 \\
\end{array}$ & & & & $\begin{array}{l}49.241 \\
\end{array}$ & & $\begin{array}{l}14.643 \\
\end{array}$ & & & 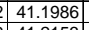 & \begin{tabular}{|r|}
52.1 \\
\end{tabular} & 11.7 & & 2.835 & 0.009 & $\begin{array}{l}0.009 \\
\end{array}$ & 0.000 & 0.211 & \\
\hline $\begin{array}{l}1776 \\
1777\end{array}$ & $\begin{array}{l}9 / 1 / 2004 \\
9 / 1 / 2004\end{array}$ & $\begin{array}{l}1: 06: 17 \mathrm{AM} \\
107: 17 \mathrm{AM}\end{array}$ & $\begin{array}{r}25.349 \\
25.35\end{array}$ & $\begin{array}{r}25.599 \\
25.6\end{array}$ & \begin{tabular}{|l|}
24.722 \\
24773 \\
\end{tabular} & \begin{tabular}{|l|}
26.042 \\
26.038 \\
\end{tabular} & $\begin{array}{r}25.184 \\
25.145\end{array}$ & \begin{tabular}{|r|r|}
48.376 \\
48.42
\end{tabular} & \begin{tabular}{|c|}
52.579 \\
53228 \\
\end{tabular} & $\begin{array}{l}15.952 \\
16.118\end{array}$ & \begin{tabular}{|l|}
33.493 \\
33.1599
\end{tabular} & \begin{tabular}{|l|} 
\\
\\
-1.21215 \\
\end{tabular} & $\begin{array}{l}-7.871 \\
-7.865 \\
\end{array}$ & $\begin{array}{l}50.079 \\
49631\end{array}$ & \begin{tabular}{|c|}
0.058 \\
0.06
\end{tabular} & $\begin{array}{l}14.643 \\
14.643\end{array}$ & $\begin{array}{l}0.003 \\
0.003\end{array}$ & 2472.92 & $\begin{array}{l}41.2153 \\
41.2319\end{array}$ & $\begin{array}{l}51.5 \\
52.1\end{array}$ & $\frac{11.9}{11.8}$ & $\frac{40.8}{40.8}$ & & 0.008 & $\begin{array}{l}0.008 \\
0.009\end{array}$ & 0.000 & 0.204 & $\frac{0.17}{0.18}$ \\
\hline & 9/1/1/2004 & 1:08:17 AM & 25.349 & 25.594 & \begin{tabular}{|l|}
24.712 \\
\end{tabular} & \begin{tabular}{|l|}
26.050 \\
26.012
\end{tabular} & 25.119 & $\begin{array}{l}40.465 \\
48.465\end{array}$ & \begin{tabular}{|l|}
52.733 \\
\end{tabular} & 15.929 & $\begin{array}{c}33.74 \\
3\end{array}$ & \begin{tabular}{|l|}
-1.212 \\
-1.212 \\
\end{tabular} & $\begin{array}{l}-\frac{-1.865}{-7.865} \\
\end{array}$ & $\begin{array}{l}\frac{49.631}{49.222} \\
\end{array}$ & \begin{tabular}{|l|}
0.058 \\
0.058
\end{tabular} & $\begin{array}{l}\frac{14.645}{14.643} \\
\end{array}$ & & & \begin{tabular}{|l|l|} 
\\
21.2319 \\
2
\end{tabular} & \begin{tabular}{|l|}
52.1 \\
51.6
\end{tabular} & $\begin{array}{l}11.1 .7 \\
11.7\end{array}$ & & .834 & $\begin{array}{l}0.009 \\
0.008\end{array}$ & 0.0 & & & $\begin{array}{l}0.18 \\
0.17\end{array}$ \\
\hline & 9/1/2004 & 1:09:17 AM & 25.365 & 25.615 & \begin{tabular}{|l|}
24.713 \\
\end{tabular} & 26.058 & & 48.455 & \begin{tabular}{|l|}
52.998 \\
\end{tabular} & 15.933 & 33.522 & -1.212 & & & & 14.643 & & 475.9 & \begin{tabular}{|l|l|}
41.2453 \\
4
\end{tabular} & 51.9 & 11.5 & & 2.8 & & 0.0 & & $\begin{array}{l}0.204 \\
0.204\end{array}$ & 0.17 \\
\hline & & 1:10:17 AM & 25.366 & 25.616 & \begin{tabular}{|l|}
24.729 \\
\end{tabular} & & & & & & & & & & & 14.643 & & & & 51.8 & & & & & & & & $\frac{0.17}{0.18}$ \\
\hline & 9/1/2004 & 1:11:17 AM & & 25.621 & 24.734 & 26.063 & 25 & 48.0 & 52.38 & $\begin{array}{ll}15.876 \\
\end{array}$ & 33.327 & -1.209 & -7.8 & 49.3 & 0.061 & $\begin{array}{l}14.643 \\
\end{array}$ & & & 41.2986 & 51.3 & 1.8 & & 304 & 009 & 0.009 & & & $\begin{array}{l}0.18 \\
0.18\end{array}$ \\
\hline 782 & & 1:11:39 AM & 25.361 & 25.611 & & & & & 52.72 & $\begin{array}{l}16.076 \\
\end{array}$ & & -1.212 & & & & & & & & 51.6 & & & & & & & & \\
\hline & & & 1.361 & 25.611 & 24.709] & 26.053 & & & & 16.057 & 33.426 & & & & & 14.643 & & & & & & & & & & & & \\
\hline & & 1:13:39 AM & 25.36 & 25.6 & 24.698 & 26.063 & 5.1 .1 & & 2.498 & 15.938 & 33.508 & -1.215 & & & & 14.643 & & & & 51.4 & 11.9 & & & & & & & \\
\hline & $9 / 1 / 2004$ & & & 25.61 & 24.683 & 26.078 & & & 53.189 & 15.975 & 33.313 & -1.2 & & 49.1 & .058 & $\begin{array}{l}14.643 \\
\end{array}$ & & & & & & & & 0.008 & & & & \\
\hline & & $1: 15$ & & 25.6 & 24.668 & & & & 53.052 & 15.843 & 33.764 & -1.212 & & & & 14. & & & & 51.9 & & & 8 & & & & & \\
\hline & & & 356 & 25.611 & 24.689 & 26 & & 48.594 & 53.331 & 15.97 & 33.407 & & & & 105 & 14. & & & & 0.2 & & & & & & & & \\
\hline & & & & 20.00 & 2 & & & & & & & & & & & & & & & & & & & & & & & \\
\hline & & 1:18:39 AM & 25.359 & & & & & & 53.429 & 15.856 & 33.534 & -1.212 & & 49.378 & & & & & & 52.3 & 11.8 & & & & & & 208 & \\
\hline & & & $\begin{array}{l}25.36 \\
2534\end{array}$ & $\begin{array}{l}\frac{25.015}{25605} \\
250\end{array}$ & $\begin{array}{l}24.068 \\
24653\end{array}$ & & 25 & & 52.979 & $\frac{15.826}{15801}$ & $\begin{array}{l}33.709 \\
33.902\end{array}$ & $\frac{-1.21}{-1.21}$ & & & & 14. & & & & $\frac{51.9}{520}$ & 114 & & & & & & & \\
\hline & $9 / 1 / 12004$ & & & & & & & & & & 3.564 & & & & & 14643 & & & & & $\frac{1.4 .4}{116}$ & & & & & & & \\
\hline & & & 5.344 & $\frac{2.000}{25.609}$ & 24 & & & & D.s. & 15.832 & & & & & & & & & & , & $\frac{1.5}{117}$ & & & & & & & \\
\hline & & & 25.339 & 25.609 & \begin{tabular}{|l|l|}
24.647 \\
\end{tabular} & $\frac{26.05}{26.5}$ & & & 52.865 & 15.893 & 33.764 & $\frac{-1.212}{-1.212}$ & & & 0.0 & 14.6 & & & & $\frac{51.7}{51.7}$ & $\frac{1}{1}$ & & 183 & & 0.0 & & 11 & \\
\hline & & & & & & & & & & & & & & & & & & & & & & & & & & & & \\
\hline & & & 5.348 & 25.618 & 24.686 & & & & 52.981 & & & $\frac{-1.2}{-1.2}$ & & & & & & & & 51.9 & & & & & & & & \\
\hline & & & & & & & & & & & & & & & & & & & & & & & & & & & & \\
\hline & & $1: 27: 39 \mathrm{AM}$ & & 25.62 & 24.661 & 26.09 & & 48.488 & 52.996 & 15.792 & 33.633 & $|-1.212|$ & -7.7 & & & $\frac{14.6}{14.6}$ & & $\begin{array}{l}2494.28 \\
24\end{array}$ & 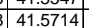 & 51.9 & 12.0 & 41 & & (2008 & & 000 & 0.204 & 0 \\
\hline & $9 / 1 / 2$ & & 25.353 & 25.61 & & & & & \begin{tabular}{|l|l|} 
& 53.267 \\
\end{tabular} & 15.804 & & -1.2 & & & & 14.6 & & & & 52.1 & 11.1 & & & 8 & & & 202 & \\
\hline & & & & & & & & & & & & & & & & & & & & & & & & & & & & \\
\hline & & & 25.348 & 25.6 & & & & & \begin{tabular}{|l|l|} 
\\
\end{tabular} & 15.759 & & -1.215 & & & & & & & & 51.8 & 11.6 & & & & & & 0.200 & \\
\hline & & & & & & & & & & & & & & & & & & & & & & & & & & & & \\
\hline & & & & 25.6 & 24.113 & & & & 52.675 & 15.7 & 33.621 & & & & & & & & & & & & & & & & & \\
\hline & & & & 25.62 & $\begin{array}{l}24.129 \\
24704\end{array}$ & & & & $\overline{34}$ & $\begin{array}{l}15.8044 \\
1.5914\end{array}$ & $\begin{array}{ll}33.859 \\
3374\end{array}$ & -1.22 & & & & (14.6 & & & & 520 & $\frac{1.4}{115}$ & & & & & & & \\
\hline & & & & 2560 & 24.664 & & & & 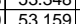 & 15879 & 33.766 & -1.2 & & & & & & & & & & & & & & & & \\
\hline & & & & & & & & & & & & & & & & & & & & & & & & & & & & \\
\hline & & & & $\frac{25.625}{25.625}$ & & & & & $\mid$\begin{tabular}{|l|l|}
52.925 \\
\end{tabular} & $\frac{15.6}{15.6}$ & & -1.212 & & & & & & & & & $\frac{11.4}{11.4}$ & & & & & & & \\
\hline & & & & & & & & & & & & & & & & & & & & & & & & & & & & \\
\hline & & & & & & & & & & & & & & & & & & & & & & & & & & & & \\
\hline & & & & & 24 & & & 48. & 53.26 & & & & & & & & & & & & & & & & & & & \\
\hline & & $1: 41: 39$ & & 25.611 & 24.669 & & & 48. & 53.54 & 15.7 & 33.807 & -1.21 & & 47. & & 14. & & & & & 1.2 & & & & & & & \\
\hline & & & & & 24.68 & & & 48.1 & 52.8 & 15.6 & 33.707 & -1.2 & & 50.4 & & 14.6 & & & & & & & & & & & & \\
\hline & & & & 25.616 & 24.664 & & & & 53.288 & 15.99 & 33.647 & -1.2 & & & & 14. & & & & & & & & & & & & \\
\hline & & & & 25.6 & 24. & & & 48 & & & & -1.2 & & & & & & & & & 1 & & & & & & & \\
\hline & & & & 25.6 & 24.6 & & & & & & & & & & & & & & & & & & & & & & & \\
\hline & & & & 25.61 & & & & & & 15.886 & 33.6 & -1.212 & & & & & & & & & & & & & & & & \\
\hline & & & 25.36 & 25.024 & 2 & 20.074 & 25.14 & 48.01 & | 53.4524 & $\begin{array}{l}13.1 .144 \\
15067\end{array}$ & $\begin{array}{r}3.93 \\
32.93\end{array}$ & $\begin{array}{l}-1.209 \\
-1212\end{array}$ & & 4 & & $\begin{array}{l}1.6435 \\
1.643\end{array}$ & & & & 告, & $\begin{array}{l}11.6 \\
11.6\end{array}$ & & 28 & & & & 0 & -2 \\
\hline 826 & 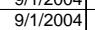 & $1: 49: 39 \mathrm{AM}$ & \begin{tabular}{|l|}
25.341 \\
25.31 \\
\end{tabular} & 25.616 & \begin{tabular}{|l|}
24.054 \\
24.649 \\
\end{tabular} & $\mid$\begin{tabular}{|l|}
$\mid 2.05038$ \\
26.038
\end{tabular} & 25.165 & $\begin{array}{ll}40.000 \\
48.22\end{array}$ & \begin{tabular}{|l|l|}
53.072 \\
\end{tabular} & 15.723 & $\begin{array}{l}0.44 \mathrm{c} \\
33.621 \\
\end{array}$ & \begin{tabular}{|l|l|}
$\mid-1.212$ \\
1.215 \\
\end{tabular} & \begin{tabular}{|l|}
-7.634 \\
\end{tabular} & $\begin{array}{l}49.837 \\
49.837\end{array}$ & $\begin{array}{l}0.055 \\
0.055\end{array}$ & \begin{tabular}{|l|}
14.04043 \\
14.643
\end{tabular} & 0.003 & | 2510.28 & \begin{tabular}{|l|}
41.5214 \\
41.9381
\end{tabular} & \begin{tabular}{|l|}
51.9 \\
51.0
\end{tabular} & \begin{tabular}{|l|}
11.9 \\
11.9 \\
\end{tabular} & & \begin{tabular}{|l|}
2.021 \\
2.821 \\
\end{tabular} & \begin{tabular}{|l|}
$\mid 0.008$ \\
\end{tabular} & \begin{tabular}{|l|}
0.008 \\
\end{tabular} & 0.000 & \begin{tabular}{|l|}
0.194 \\
0.194 \\
\end{tabular} & 0.1 \\
\hline
\end{tabular}


WSRC-TR-2005-00105, REVISION 0

SRNL-RPP-2005-00012, REVISION 0

RUN \# 4.03A AND B; FIRST AND SECOND HALF OF SLURRY DEWATERING - CONT.

\begin{tabular}{|c|c|c|c|c|c|c|c|c|c|c|c|c|c|c|c|c|c|c|c|c|c|c|c|c|c|c|c|c|}
\hline & \begin{tabular}{l|l} 
\\
\end{tabular} & B & D & $E$ & $\mathrm{~F}$ & G & $\mathrm{H}$ & $\mathrm{J}$ & $\mathrm{K}$ & $\begin{array}{ll}\mathrm{L} \\
\end{array}$ & $\mathrm{M}$ & $\mathrm{N}$ & 0 & \begin{tabular}{l|l|} 
& \\
\end{tabular} & \begin{tabular}{|l|}
$\mathrm{R}$ \\
\end{tabular} & $\mathrm{s}$ & $\mathrm{T}$ & $\mathrm{v}$ & $\mathrm{w}$ & $x$ & 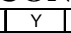 & $\mathrm{z}$ & AA & AB & $\begin{array}{ll}A C \\
\end{array}$ & $\mathrm{AD}$ & $\mathrm{AE}$ & \\
\hline & 9/1/2004 & 1:50:39 AM & 25.346 & 25.611 & 24.654 & 26.023 & 25.14 & 48.513 & 53.331 & \begin{tabular}{ll|}
15.958 \\
\end{tabular} & 33.495 & -1.212 & -7.553 & 49.022 & 0.06 & $\begin{array}{r}14.643 \\
\end{array}$ & 0.003 & & 41.9547 & 52.2 & 11.7 & & 2.827 & & 0.009 & 0.000 & 0.2. & \\
\hline & $9 / 1 / 12004$ & & & 25.615 & & 26.048 & $\begin{array}{r}25.1855 \\
\end{array}$ & 48.741 & & $\begin{array}{l}15.868 \\
\end{array}$ & 33.891 & -1.212 & & 49.472 & & $\begin{array}{l}14.643 \\
\end{array}$ & & & 341.9714 & 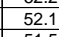 & & & 2.849 & & & & & \\
\hline & 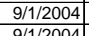 & $\begin{array}{l}1: 52: 39 \mathrm{AM} \\
1.5: 39\end{array}$ & \begin{tabular}{|l|l|}
25.349 \\
25324
\end{tabular} & $\begin{array}{l}25.614 \\
25590\end{array}$ & \begin{tabular}{|l|l|}
24.662 \\
24657 \\
\end{tabular} & $\begin{array}{l}26.022 \\
20007\end{array}$ & \begin{tabular}{|l|}
25.244 \\
25201 \\
\end{tabular} & \begin{tabular}{|l|l|l}
47.416 \\
17951
\end{tabular} & \begin{tabular}{|l|}
52.654 \\
5.418 \\
\end{tabular} & \begin{tabular}{|l|}
16.671 \\
16595
\end{tabular} & $\begin{array}{l}31.642 \\
3519\end{array}$ & \begin{tabular}{|l|l|}
-1.215 \\
\end{tabular} & \begin{tabular}{|l|l|}
-6.524 \\
7194
\end{tabular} & \begin{tabular}{|l|l|}
49.111 \\
5058
\end{tabular} & \begin{tabular}{|l}
0.041 \\
0.065
\end{tabular} & \begin{tabular}{|l|l|}
14.643 \\
1463
\end{tabular} & & & \begin{tabular}{|l|l|}
8 & 41.9881 \\
\end{tabular} & $\begin{array}{l}51.5 \\
51 .\end{array}$ & 1.1 & & \begin{tabular}{|l|l|} 
\\
\end{tabular} & \begin{tabular}{|l|l|}
0.006 \\
\end{tabular} & 0.006 & 0.000 & 0.150 & \\
\hline$\frac{1025}{1825}$ & $\begin{array}{l}9 / 1 / 12004 \\
9 / 1 / 2004\end{array}$ & $\begin{array}{l}1: 53: 39 \mathrm{AM} \\
1: 54: 39 \mathrm{AM}\end{array}$ & $\frac{25.324}{25.341}$ & $\begin{array}{l}25.599 \\
25611\end{array}$ & \begin{tabular}{|l|}
24.657 \\
24714
\end{tabular} & \begin{tabular}{|l|}
26.007 \\
\end{tabular} & $\begin{array}{l}25.219 \\
25216 \\
\end{array}$ & $\begin{array}{l}47.945 \\
48905\end{array}$ & \begin{tabular}{|l|}
52.418 \\
52832
\end{tabular} & \begin{tabular}{|l|}
16.585 \\
\end{tabular} & $\begin{array}{l}32.519 \\
33.309\end{array}$ & \begin{tabular}{|l|l|}
-1.212 \\
-1215
\end{tabular} & $\begin{array}{l}-7.194 \\
-7322\end{array}$ & $\begin{array}{l}50.586 \\
51.531\end{array}$ & $\begin{array}{r}0.065 \\
0.056\end{array}$ & $\begin{array}{r}14.643 \\
14643\end{array}$ & & 5120 & \begin{tabular}{|l|l|}
8 & 42.0047 \\
\end{tabular} & $\begin{array}{l}51.3 \\
51.7\end{array}$ & & 40.2 & \begin{tabular}{|l|l|}
2.774 \\
2765
\end{tabular} & \begin{tabular}{|l|l|}
0.009 \\
\end{tabular} & $\begin{array}{l}0.009 \\
0.00\end{array}$ & $\begin{array}{l}0.000 \\
0.000\end{array}$ & 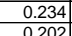 & 017 \\
\hline & 9/1/1/2004 & & & & & & & & & & & & & & & \begin{tabular}{|l|}
14.643 \\
14.643 \\
\end{tabular} & & 521.28 & & & & & & & & $\frac{0.000}{0.000}$ & 0.202 & $\begin{array}{l}0.17 \\
0.17\end{array}$ \\
\hline 1827 & 9/1/2004 & 1:56:39 AM & 25.348 & 25.608 & \begin{tabular}{|l|l|}
24.751 \\
\end{tabular} & 26.045 & 25.117 & 47.746 & \begin{tabular}{|l|}
52.252 \\
\end{tabular} & 16.336 & 32.58 & -1.209 & -7.379 & 50.774 & 0.057 & 14.643 & & $\frac{2.2}{23.2}$ & 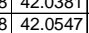 & 51.1 & $\frac{12.3}{12.1}$ & $\begin{array}{l}40 . \\
40 .\end{array}$ & $\frac{2.163}{2.769}$ & 0.000 & $\frac{0.008}{0.008}$ & $\frac{0.000}{0.000}$ & 0.202 & $\begin{array}{l}0.17 \\
0.17\end{array}$ \\
\hline \begin{tabular}{|l|}
1828 \\
\end{tabular} & 9/1/2004 & & 25.332 & 25.597 & \begin{tabular}{|l|l|}
24.705 \\
\end{tabular} & 25.995 & 25.152 & 48.146 & \begin{tabular}{|l|}
52.911 \\
\end{tabular} & 16.321 & 32.773 & \begin{tabular}{|l|}
-1.218 \\
\end{tabular} & -7.397 & 50.333 & 0.056 & 14.643 & & & \begin{tabular}{|l|l|}
42.0714 \\
\end{tabular} & 51.8 & & & 2.790 & \begin{tabular}{|l|}
0.008 \\
\end{tabular} & 0.008 & 0.000 & & \\
\hline 1829 & $9 / 1 / 2004$ & & & 25.618 & \begin{tabular}{|l|l}
24.726 \\
\end{tabular} & & 25.1 & 47.97 & 52.712 & $\begin{array}{l}16.586 \\
\end{array}$ & 32.381 & -1.215 & -7.35 & 50.805 & & 14.643 & & & & 51.6 & & & 2.770 & \begin{tabular}{|l|}
0.008 \\
\end{tabular} & 0.008 & 0.000 & & \\
\hline$\frac{1830}{1831}$ & 9/1/2004 & 1:59:39 AM & 25.363 & 25.618 & 24.736 & 26.025 & 25.22 & 47.982 & \begin{tabular}{|l|}
52.942 \\
\end{tabular} & \begin{tabular}{|l|l|}
16.299 \\
16.9
\end{tabular} & $\begin{array}{l}32.644 \\
2000\end{array}$ & -1.212 & & 49.597 & 0.055 & 14.6 & & 26.2 & \begin{tabular}{|l|l|}
42.1047 \\
\end{tabular} & 51.8 & 11.8 & & .779 & 0.008 & 0.008 & 0.000 & 0.197 & \\
\hline$\frac{1831}{1832}$ & $\begin{array}{l}9 / 1 / 2004 \\
9 / 1 / 204\end{array}$ & $\begin{array}{l}2: 00: 39 \text { AM } \\
2 \cdot 0.199\end{array}$ & $\begin{array}{l}25.368 \\
25.266\end{array}$ & 25.618 & 24.741 & 26.025 & 25.217 & 47.82 & $\begin{array}{l}52.183 \\
5.5757\end{array}$ & $\begin{array}{ll}16.406 \\
1633\end{array}$ & 2.609 & \begin{tabular}{|l|l|}
.212 \\
\end{tabular} & $\begin{array}{r}-7.25 \\
.720\end{array}$ & 48.415 & $\begin{array}{l}0.056 \\
\end{array}$ & 14. & & 27.28 & \begin{tabular}{|l|l|}
8 & 42.1214 \\
\end{tabular} & 51.1 & 11.5 & 40. & \begin{tabular}{|l|l}
2.773 \\
2.791
\end{tabular} & 0.008 & $\begin{array}{l}0.008 \\
\end{array}$ & 0.000 & 0.201 & \\
\hline$\frac{1832}{1833}$ & $\begin{array}{l}91 / 1 / 2004 \\
9 / 1 / 2004\end{array}$ & $\begin{array}{l}2: 01: 39 \mathrm{AM} \\
2 \cdot 202 \cdot 3 \mathrm{AM}\end{array}$ & $\begin{array}{l}25.366 \\
25.371\end{array}$ & $\frac{25.616}{25.616}$ & \begin{tabular}{|l|l|}
24.719 \\
24.724
\end{tabular} & $\frac{26.054}{26.049}$ & $\frac{25.16}{25.19}$ & $\begin{array}{r}47.955 \\
48.059\end{array}$ & $\begin{array}{r}52.577 \\
52.95 \\
\end{array}$ & \begin{tabular}{|c|}
16.336 \\
16478
\end{tabular} & $\begin{array}{l}32.711 \\
32588 \\
3\end{array}$ & $\frac{-1.212}{-1.212}$ & & $\begin{array}{r}49.403 \\
49.641\end{array}$ & $\begin{array}{l}0.057 \\
0.055\end{array}$ & $\frac{14.643}{14.643}$ & & 28.28 & $\begin{array}{l}42.1381 \\
42.154\end{array}$ & & & 40 & 2.781 & 0.008 & 0.008 & 0.000 & 0.204 & $\begin{array}{l}0.17 \\
\end{array}$ \\
\hline \begin{tabular}{|l|l|}
1834 \\
\end{tabular} & 9/1/2004 & 2:03:39 AM & & $\frac{25.010}{25.626}$ & & \begin{tabular}{|l|l} 
& 0.049 \\
& 26.074
\end{tabular} & 25.16 & $\begin{array}{l}40.059 \\
48.233\end{array}$ & $\begin{array}{l}52.95 \\
52.759\end{array}$ & \begin{tabular}{|l|}
16.476 \\
16.446
\end{tabular} & $\begin{array}{r}32.500 \\
32.814 \\
\end{array}$ & \begin{tabular}{|l|}
-1.212 \\
\end{tabular} & $\begin{array}{l}-7.34 \\
-7.44\end{array}$ & $\begin{array}{l}59.041 \\
52.641\end{array}$ & $\begin{array}{l}0.055 \\
0.061\end{array}$ & $\begin{array}{c}14.643 \\
14.643 \\
\end{array}$ & & 532.28 & \begin{tabular}{|l|}
42.15417 \\
42.1714 \\
\end{tabular} & $\begin{array}{l}51.8 \\
51.6\end{array}$ & $\begin{array}{l}\frac{11.8}{12.5} \\
\end{array}$ & $\begin{array}{l}50 . \\
5\end{array}$ & $\frac{2.880}{2.794}$ & $\mid$\begin{tabular}{|}
$\mid .008$ \\
0.009 \\
\end{tabular} & $\begin{array}{l}0.008 \\
0.009\end{array}$ & & & $\begin{array}{l}0.17 \\
0.18\end{array}$ \\
\hline & 9/1/2004 & 2:04:39 AM & 25.376 & 25.626 & \begin{tabular}{|l|l|}
24.749 \\
\end{tabular} & 26.034 & 25.14 & 47.546 & \begin{tabular}{|l|}
52.397 \\
\end{tabular} & 16.474 & 32.196 & \begin{tabular}{|l|} 
\\
\end{tabular} & -7.37 & 49.95 & 0.056 & $\begin{array}{l}14.643 \\
\end{array}$ & & 2531.28 & \begin{tabular}{|l|l|l}
8 & 42.1881 \\
\end{tabular} & 51.3 & 11.9 & & 2.749 & 0.008 & 0.008 & 0.000 & 0.203 & \\
\hline & 9/1/2004 & $2: 05: 39 \mathrm{AM}$ & \begin{tabular}{l|l|}
25.372 \\
2.372
\end{tabular} & 25.627 & 24.735 & 26.025 & & \begin{tabular}{|l|l|}
47.557 \\
\end{tabular} & \begin{tabular}{|l|l|}
52.415 \\
\end{tabular} & \begin{tabular}{|l|l|}
16.773 \\
\end{tabular} & 31.704 & & & 50.69 & $\begin{array}{l}0.055 \\
\end{array}$ & $\begin{array}{l}14.643 \\
\end{array}$ & & & \begin{tabular}{l|l|}
8 & 42.2047 \\
\end{tabular} & 51.3 & 12.1 & & $\begin{array}{ll}2.732 \\
\end{array}$ & \begin{tabular}{|l|l|}
0.008 \\
\end{tabular} & 0.008 & 0.000 & 0.200 & \\
\hline 337 & 9/1/2004 & 2:06:39 AM & 25.376 & & 24.729 & & 25.24 & 47.534 & \begin{tabular}{|l|}
52.102 \\
\end{tabular} & \begin{tabular}{ll|}
16.601 \\
165
\end{tabular} & 32.03 & & -7.39 & 52.348 & 0.056 & 14.643 & & & \begin{tabular}{l|l|}
8 & 42.2214 \\
\end{tabular} & 51.0 & 12.5 & & & \begin{tabular}{|l|}
0.008 \\
\end{tabular} & 0.008 & 0.000 & 0.203 & \\
\hline 838 & $\begin{array}{l}9 / 1 / 2004 \\
0 / 1 / 2001\end{array}$ & 2:07:39 AM & $\frac{25.371}{25.367}$ & $\begin{array}{l}25.626 \\
2522\end{array}$ & \begin{tabular}{|l|l|}
24.739 \\
\end{tabular} & 26.014 & 25.22 & 47.551 & \begin{tabular}{|l|}
52.129 \\
52677 \\
\end{tabular} & $\begin{array}{l}16.548 \\
1670\end{array}$ & $\begin{array}{l}32.127 \\
22212\end{array}$ & \begin{tabular}{|r|}
-1.215 \\
\end{tabular} & $\begin{array}{r}-7.50 \\
.75 .0\end{array}$ & $\begin{array}{r}49.42 \\
5.201\end{array}$ & $\begin{array}{l}0.052 \\
0.055\end{array}$ & $\begin{array}{r}14.643 \\
\end{array}$ & & 534.28 & \begin{tabular}{c|c|c|}
8 & 42.2381 \\
8
\end{tabular} & 51.0 & 11.8 & & & \begin{tabular}{|l|l|}
0.007 \\
\end{tabular} & 0.007 & 0.000 & 0.188 & \\
\hline$\frac{1839}{1840}$ & \begin{tabular}{c|}
$9 / 1 / 2004$ \\
$9 / 1 / 2004$
\end{tabular} & $\begin{array}{l}2: 08: 399 \mathrm{AM} \\
2 \cdot 00 \cdot 39 \mathrm{AM}\end{array}$ & $\frac{25.367}{25378}$ & $\frac{25.622}{25.628}$ & \begin{tabular}{|r}
24.75 \\
24.756
\end{tabular} & & & $\begin{array}{l}47.914 \\
47.822 \\
\end{array}$ & \begin{tabular}{|l|}
52.677 \\
52.563 \\
\end{tabular} & $\begin{array}{r}16.704 \\
16644\end{array}$ & 2.243 & $\begin{array}{l}-1.212 \\
-1.215\end{array}$ & & $\begin{array}{l}51.241 \\
51.157\end{array}$ & $\begin{array}{l}0.055 \\
0.056\end{array}$ & $\begin{array}{l}14.643 \\
14.643 \\
\end{array}$ & & & $\begin{array}{l}42.2547 \\
42.2714\end{array}$ & $\frac{51.6}{51.4}$ & & & 2.763 & $\begin{array}{ll}0.008 \\
0.008\end{array}$ & 0.008 & $\frac{0.000}{0.000}$ & & \\
\hline & 9/1/2004 & 2:10:39 AM & 25.379 & 25.624 & \begin{tabular}{|l|} 
\\
\end{tabular} & 26.037 & 25.18 & \begin{tabular}{|l|}
48.0192 \\
4801
\end{tabular} & \begin{tabular}{|l|}
52.500 \\
52.641 \\
\end{tabular} & 16.69 & 32.416 & \begin{tabular}{|l|}
-1.212 \\
\end{tabular} & & 48.21 & 0.056 & 14.643 & & 37.28 & \begin{tabular}{|l|}
42.27114 \\
42.2881
\end{tabular} & 51.4 & 11.5 & 40.2 & 2.773 & $\begin{array}{l}.000 \\
0.008\end{array}$ & $\frac{0.008}{0.008}$ & $\frac{0.000}{0.000}$ & & 0.17 \\
\hline 84 & $9 / 1 / 2004$ & 2:11:39 AM & 25.386 & 25.631 & \begin{tabular}{|l|}
24.759 \\
\end{tabular} & 26.039 & 25.20 & $|47.679|$ & \begin{tabular}{|l|l|}
52.395 \\
\end{tabular} & 16.536 & 32.266 & \begin{tabular}{|l|l|} 
\\
\end{tabular} & -7.54 & 49.747 & \begin{tabular}{|l|l|} 
\\
\end{tabular} & 14.643 & & 538.28 & \begin{tabular}{|l|l|}
42.3047 \\
\end{tabular} & 51.3 & 11.8 & & \begin{tabular}{|l|}
2.756 \\
\end{tabular} & \begin{tabular}{|l|}
0.008 \\
\end{tabular} & 0.008 & 0.000 & $\frac{201}{206}$ & 0.17 \\
\hline 1843 & $9 / 1 / 2004$ & 2:12:39 AM & 25.387 & 25.627 & 24.775 & 26.03 & 25.19 & 47.797 & \begin{tabular}{|l|}
52.297 \\
\end{tabular} & \begin{tabular}{|c|}
16.463 \\
\end{tabular} & 32.424 & 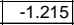 & & 50.924 & \begin{tabular}{|c|c|}
0.055 \\
\end{tabular} & 14.643 & & & 42.3214 & 51.2 & & 40. & 2.765 & \begin{tabular}{|l|}
0.008 \\
\end{tabular} & $\begin{array}{l}0.008 \\
\end{array}$ & 0.000 & & 0.17 \\
\hline & $9 / 1 / 2004$ & 2:13:39 AM & 25.373 & & 24.746 & & & 47.839 & 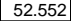 & 16.561 & & & & & 0.056 & & & & \begin{tabular}{|l|l|}
42.3381 \\
\end{tabular} & & & & & .008 & 0.008 & 0.000 & 0.202 & \\
\hline & & & 25.394 & & & & & & & & & -1.206 & & 50.215 & 0.056 & & & & & 51.4 & & & & & & & & \\
\hline & & $2: 15: 3$ & \begin{tabular}{|l|l|}
25.39 \\
\end{tabular} & & \begin{tabular}{|l|l|}
24.768 \\
\end{tabular} & 26.0 & & $\begin{array}{l}47.903 \\
\end{array}$ & 52.513 & 16.494 & & -1.212 & & 50.87 & 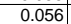 & & & & & 51. & & & & & & & & \\
\hline 1847 & & & 25.38 & & \begin{tabular}{|l|}
24.768 \\
\end{tabular} & 26.023 & & 47.607 & 52.364 & \begin{tabular}{l|l|l|l|}
16.468 \\
\end{tabular} & & -1.215 & & 50.982 & $\mid 0.057$ & & & & 42.3881 & & & & 2.75 & & & 000 & & \\
\hline & $\mid$ & 2:17:39 AN & 25.384 & 25.624 & 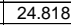 & 26.022 & & 47.972 & 52.596 & 16.424 & 32.621 & -1.212 & & 50.137 & 0.057 & 14.643 & & & \begin{tabular}{|l|l|}
42.4047 \\
\end{tabular} & 51.5 & 11.9 & 4 & & 08 & & & 0.204 & \\
\hline & $9 / 1 / 2004$ & 2:18:3 & 25.384 & 25.619 & 24.822 & 26.041 & & 47.721 & 52.316 & 16.587 & 32.278 & -1.212 & & 49.76 & 0.057 & 14.643 & & 45.28 & 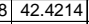 & 51.2 & 1. & & .758 & 0.008 & .008 & 0.000 & & \\
\hline & 004 & $2: 19: 3$ & 25.394 & 25.624 & 24.808 & & & 47.743 & \begin{tabular}{|l|} 
\\
\end{tabular} & 16.453 & 32. & -1.26 & & 50.792 & 0.054 & 14. & & & 42.4381 & 51.4 & 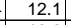 & & 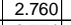 & & ( & 0.000 & 0.194 & \\
\hline & $91 / 12004$ & & & & & & & 47.928 & & & & & & 51.049 & & & & & & & & & & & & & & \\
\hline & & $2: 21:$ & 25.405 & & \begin{tabular}{|l|l|}
24.813 \\
2077
\end{tabular} & 26.04 & & & & 16.246 & & & & 49.12 & 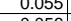 & & & & & 51. & $\frac{11}{12}$ & & & & & & 198 & \\
\hline 355 & $\mid \frac{11 / 2044}{11 / 2004}$ & & $\begin{array}{r}25.4 \\
25.401\end{array}$ & $\frac{25.625}{25.631}$ & 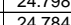 & $\frac{26.028}{26.043}$ & & $\begin{array}{l}47 ., 492 \\
47.845\end{array}$ & $\begin{array}{l}52.152 \\
55658\end{array}$ & $\frac{1.24}{16.441}$ & & $\frac{-1.215}{-1212}$ & & 51. & 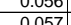 & & & & . & & & & & & & & 0205 & \\
\hline & $9 / 1 / 2$ & $2: 24: 39 \mathrm{~A}$ & $\frac{2.401}{25.401}$ & 25.631 & \begin{tabular}{|l|l|}
24.104 \\
\end{tabular} & $\frac{20.040}{26.063}$ & & $\begin{array}{r}41.040 \\
47.94\end{array}$ & \begin{tabular}{|l|}
02.050 \\
52.627 \\
\end{tabular} & $\frac{10.444}{16.36}$ & $\begin{array}{l}32.050 \\
32.796\end{array}$ & \begin{tabular}{|l|l|l|l|l} 
& -1.212 \\
\end{tabular} & & 50.60 & 0.057 & 14. & & & 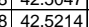 & S1.5 & 2.1 & & & & & & & \\
\hline & 9/1/2004 & & 25.392 & 25.622 & \begin{tabular}{|l|}
24.174 \\
24.76
\end{tabular} & & & 47.8 & & 16.521 & 32.412 & \begin{tabular}{|l|l|} 
& -1.212 \\
\end{tabular} & & 51.875 & 0.056 & 14. & & & & $\frac{51.4}{51.4}$ & & & & & & & & \\
\hline & 9/1/2004 & $2: 26: 39$ & & & & 26.0 & & 47.5 & & & & & & & & & & & & & & & & & & & & \\
\hline & & & 25.401 & 25.626 & 24.775 & & & \begin{tabular}{|c|}
47.938 \\
\end{tabular} & \begin{tabular}{|l|l|}
52.733 \\
\end{tabular} & $\mid 16.614$ & & -1.2 & & 50.28 & 0.056 & & & & $\begin{array}{ll}8 & 42.5 \\
\end{array}$ & 51.6 & 12. & & & & & 0.000 & 0.201 & \\
\hline & & & 25.411 & 25.636 & \begin{tabular}{|l|}
24.78 \\
\end{tabular} & 26.0 & & & \begin{tabular}{|l|}
52.84 \\
\end{tabular} & 16.6 & 32. & -1.2 & & 48.4 & & & & & & 51.7 & & & & & & & 0.201 & \\
\hline & & & 25.422 & & & & & & & 16.529 & & & & & & & & & & & & & & & & & & \\
\hline 36 & & & 25.41 & & \begin{tabular}{|l|}
24.796 \\
\end{tabular} & & & & & \begin{tabular}{|l|l|}
16.3599 \\
\end{tabular} & & -1.2. & & 51.0 & & & & & & & & & & & & & & \\
\hline & & 2:31:3 & 25.418 & 25.643 & 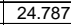 & 26 & & & \begin{tabular}{|l|} 
\\
\end{tabular} & 16.61 & 32.0 & -1.212 & & 50.632 & $\mid 0.058$ & & & & & 51.2 & 124 & & & & & & & \\
\hline & $9 / 1 / 2004$ & & 25.41 & 25.648 & 24.796 & & & 47.7 & 52.231 & 16.38 & & $-1.21:$ & & 50.279 & \begin{tabular}{|c|}
0.049 \\
\end{tabular} & & & & & 51. & & & & & & & & \\
\hline & & & 25.403 & & & & & & & 16.4 & & & & & & & & & & & & & & & & & & \\
\hline & & & 25.408 & & & & & & 52.517 & 16.4 & & -1.2. & & & & & & & & 51.4 & & & & & & & & \\
\hline & & & 25.4 & 25.6 & & & & & & & & & & & & & & & & 51. & & & & & & & & \\
\hline & & & $\begin{array}{l}25.4 .4 \\
25.4\end{array}$ & & $\mid 2.14$ & & & & & & & $\mid$ & & & & & & & & & & & & & & & & \\
\hline & & & $\frac{20.44}{254}$ & & 24.176 & & & & 5210 & 1632 & & -1215 & & & & & & & & & & & & & & & & \\
\hline 1870 & & & 25.41 & & 224795 & & & & & \begin{tabular}{|c|}
16.3747 \\
\end{tabular} & & $\mid-1.212$ & & & & & & & & & & & & & & & & \\
\hline & $9 / 1 / 2$ & & 25.4 & 25.64 & & & & & & $\mid \frac{16.407}{10.07}$ & & $\frac{-1.2}{-1.2}$ & & & & & & & & & & & & & & & & \\
\hline & & & 25.423 & & & & & & & 16.3 & & -1.26 & & & & & & & & & & & & & & & & \\
\hline & & & 25.41 & 25.63 & 24 & & & & \begin{tabular}{|l|}
52.708 \\
\end{tabular} & 16 & 32.679 & -1.215 & & & & & & & & 51.6 & & & & & & & & \\
\hline & & & 25.41 & 25.64 & 24.8 & & & & & & & -1.212 & & & & & & & & 5 & & & & & & & & \\
\hline & 9/1/20 & & 25.4 & 25.64 & 24.8 & & & & \begin{tabular}{|l|}
52.691 \\
\end{tabular} & \begin{tabular}{|l|l|}
16.164 \\
\end{tabular} & & -1. & & & & & & & & & & & & & & & 99 & \\
\hline 187 & $9 / 1 / 20$ & & 25.42 & 25.64 & & 26 & & 48.6 & \begin{tabular}{|l|l|} 
& 52.824 \\
\end{tabular} & 16.20 & & -1.215 & & 49.6 & & & & & & 51. & & & & & & & 0.195 & \\
\hline 87 & & & 25.42 & 25.637 & 24.8 & 26 & & & 53.0 & 16.093 & & -1.2 & & & & & & & & & & & & & & & & \\
\hline & & & 25.41 & & & & & & & & & & & & & & & & & & & & & & & & & \\
\hline & & & 25.429 & 25.64 & & 26.01 & & & \begin{tabular}{|l} 
\\
\end{tabular} & 16.1 & & -1.2 & & & & & & & & & & & & & & & & \\
\hline & $\frac{91 / 2004}{91 / 2004}$ & & $\frac{2.5 .424}{25.10}$ & $\frac{25.649}{25624}$ & 24.1 & & & & 52.349 & 16.165 & & $\begin{array}{l}-1.215 \\
-1.215\end{array}$ & & & & & & & & & & & & & & & & \\
\hline & 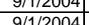 & & 254 & $\frac{23.03}{2564}$ & 24.17 & & & & \begin{tabular}{|l|l|}
52.109 \\
5
\end{tabular} & $\frac{10.1}{162}$ & & -12 & & & & & & & & & & & & & & & & \\
\hline 188 & $9 / 1 / 1 / 2$ & $\frac{2 \cdot 1 \cdot 36}{2 \cdot 5 \cdot 36}$ & 25.42 & 25.045 & & $\frac{20}{25}$ & & & & 163 & & -1218 & & $\frac{45.8}{494}$ & & & & & & & & & & & & & 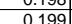 & \\
\hline 1884 & $9 / 1 / 200$ & & 25.41 & & & & & & \begin{tabular}{|l|}
53.131 \\
\end{tabular} & $\mid$ & & 212 & & & 0.057 & & & & & 52.0 & & & & & & & 0.202 & \\
\hline & & & 25.406 & & & & & & & & & \begin{tabular}{|l|l|} 
& -1.215 \\
\end{tabular} & & & 0.059 & & & & & & & & & & & & & \\
\hline 886 & & & 41 & 25.641 & & 26. & & & 53.135 & 16.1 & & -1.212 & & & 0.6 & & & & & & & & & & & & & \\
\hline & & & 25.411 & 25.641 & 24.7 & & & 47 & & $\begin{array}{l}16.266 \\
\end{array}$ & & -1.09 & & & & & & & & 51.9 & & & & & & & 16 & \\
\hline & $9 / 1 / 2004$ & 2:57:39 & 25.41 & 25.63 & 24.763 & 26013 & & $\begin{array}{lll}48.2 \\
\end{array}$ & 52975 & 16305 & 32.984 & -1215 & & & & 14 & & & & 518 & & & & & & & 0.188 & \\
\hline & & & 510 & & & & & 47.9 & 697 & 6.086 & & 1212 & & & 0.05 & & & & & & & & & & 08 & & & \\
\hline 890 & $9 / 1 / 2004$ & $2: 59: 39$ & 25.409 & 25.639 & \begin{tabular}{|l|l|} 
& 24.787 \\
\end{tabular} & 25.981 & & |48.05 & 522.78 & \begin{tabular}{|l|l|}
16.224 \\
\end{tabular} & 32.91 & -1.215 & & 49.387 & $\mid 0.056$ & 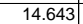 & & & \begin{tabular}{|c|c|}
8 & 43.1007
\end{tabular} & 51.7 & & & 2.791 & \begin{tabular}{|l|} 
\\
\end{tabular} & 0.008 & 0.000 & 0.199 & 0.1 \\
\hline
\end{tabular}


WSRC-TR-2005-00105, REVISION 0

SRNL-RPP-2005-00012, REVISION 0

RUN \# 4.03A AND B; FIRST AND SECOND HALF OF SLURRY DEWATERING - CONT.

\begin{tabular}{|c|c|c|c|c|c|c|c|c|c|c|c|c|c|c|c|c|c|c|c|c|c|c|c|c|c|c|c|c|}
\hline & $\mathrm{A}_{\mathrm{A}}$ & B & D & $E$ & $F$ & $G$ & $\mathrm{H}$ & $\mathrm{J}$ & $\mathrm{K}$ & $\mathrm{L}$ & $\begin{array}{ll}M \\
\end{array}$ & $N$ & 0 & \begin{tabular}{l|l} 
Q \\
\end{tabular} & $R$ & $\mathrm{~s}$ & $T$ & $\mathrm{~V}$ & w & $x$ & $\mathrm{Y}$ & $z$ & AA & $A B$ & $A C$ & $A D$ & $\mathrm{AE}$ & \\
\hline $18 \mathrm{CS}$ & $9 / 1 / 2004$ & 3:00:02 AM & 25.403 & 25.633 & 24.771 & 25.991 & 25.243 & 48.202 & 53.135 & 16.242 & \begin{tabular}{|l|l|}
32.984 \\
\end{tabular} & -1.215 & -7.33 & 49.812 & 0.054 & $\begin{array}{ll}14.643 \\
\end{array}$ & & 2586.67 & $7 \longdiv { 4 3 . 1 1 1 1 }$ & 52.0 & 11.9 & 40.6 & 2.799 & 0.008 & 0.008 & 0.000 & 0.192 & \\
\hline & & & 25.423 & 25.653 & & & 25.253 & 48.204 & & 16.366 & 32.839 & -1.209 & $\begin{array}{r}-7.29 \\
-7.29 \\
\end{array}$ & & & 14.643 & & & & 51.9 & & & & & & & & \\
\hline & 9/1/2004 & 3:02:02 AM & $\begin{array}{l}25.407 \\
25408\end{array}$ & $\begin{array}{l}25.637 \\
25643\end{array}$ & \begin{tabular}{|l|}
24.786 \\
24781 \\
\end{tabular} & \begin{tabular}{|l|}
25.975 \\
25091 \\
\end{tabular} & \begin{tabular}{|l|}
25.257 \\
25218
\end{tabular} & & 52.762 & 16.23 & \begin{tabular}{|l|l|}
33.087 \\
32076
\end{tabular} & -1.212 & \begin{tabular}{|l|l|}
-7.322 \\
7320
\end{tabular} & \begin{tabular}{|l|l|}
48.571 \\
5.30
\end{tabular} & $\begin{array}{l}0.057 \\
\end{array}$ & $\begin{array}{l}14.643 \\
1.63\end{array}$ & & $\begin{array}{l}2588.67 \\
258.67\end{array}$ & $\begin{array}{ll}743.1444 \\
\end{array}$ & 51.6 & 11.6 & & \begin{tabular}{|l|}
2.799 \\
\end{tabular} & 0.008 & $\begin{array}{l}0.008 \\
\end{array}$ & 0.000 & 0.202 & \\
\hline$\frac{1894}{1895}$ & $\begin{array}{c}9 / 1 / 2004 \\
9 / 1 / 204\end{array}$ & $\begin{array}{l}3: 03: 02 \mathrm{AM} \\
\text { 3.04:02 }\end{array}$ & $\begin{array}{l}25.408 \\
25.407\end{array}$ & $\begin{array}{l}25.643 \\
25.647\end{array}$ & \begin{tabular}{|r|}
24.781 \\
2478
\end{tabular} & \begin{tabular}{|l|}
25.991 \\
25994
\end{tabular} & $\begin{array}{r}25.218 \\
25246\end{array}$ & \begin{tabular}{|l|l|}
48.351 \\
48183
\end{tabular} & $\begin{aligned} 53.103 \\
5.691\end{aligned}$ & $\begin{array}{l}16.274 \\
16.207\end{array}$ & $\begin{array}{r}33.126 \\
33081\end{array}$ & $\begin{array}{l}-1.215 \\
-1.212\end{array}$ & $\begin{array}{r}-7.368 \\
-7.457\end{array}$ & $\begin{array}{l}50.369 \\
51681\end{array}$ & .059 & \begin{tabular}{|l|l|l|l|l|l|}
14643 \\
14643
\end{tabular} & & $\begin{array}{l}2589.67 \\
250967\end{array}$ & $\begin{array}{l}74 \\
7\end{array}$ & $\begin{array}{l}52.0 \\
516\end{array}$ & 12.0 & 40.7 & \begin{tabular}{|l|}
2.809 \\
\end{tabular} & 0.008 & 0.008 & $\begin{array}{l}0.000 \\
0.000\end{array}$ & 0.209 & $\begin{array}{l}0.18 \\
\end{array}$ \\
\hline 1896 & & $\begin{array}{l}3: 04: 02 \mathrm{AM} \\
\text { 3:05:02 }\end{array}$ & & & $\begin{array}{r}24.78 \\
24.785 \\
\end{array}$ & & & & & & & & $\begin{array}{l}-7.457 \\
-7.483 \\
\end{array}$ & & $\frac{.056}{062}$ & $\frac{14.643}{14.643}$ & & $\frac{2590.67}{259.67}$ & & $\begin{array}{l}51.6 \\
51.8\end{array}$ & & 40.6 & \begin{tabular}{|l|}
2.801 \\
2812 \\
\end{tabular} & 0.008 & & 0.000 & $\begin{array}{l}0.199 \\
0.219\end{array}$ & 0.17 \\
\hline \begin{tabular}{|l|l|}
1897 \\
\end{tabular} & 9/1/2004 & 3:06:02 AM & 25.4 & 25.64 & \begin{tabular}{|l|}
24.794 \\
\end{tabular} & \begin{tabular}{|l|}
25.968 \\
\end{tabular} & 25.27 & 48.167 & 53.031 & 16.255 & 32.773 & \begin{tabular}{|l|l} 
& -1.210 \\
\end{tabular} & $\begin{array}{l}-1.405 \\
-7.576\end{array}$ & 49.069 & 0.056 & 14.643 & & $\begin{array}{l}2591.61 \\
2592.67\end{array}$ & \begin{tabular}{|l|l|}
7 & 43.1944 \\
\end{tabular} & $\begin{array}{l}51.8 \\
51.9\end{array}$ & $\frac{12.0}{11.7}$ & $\frac{40.8}{40.5}$ & $\frac{2.812}{2.790}$ & .009 & $\frac{0.009}{0.008}$ & $\frac{0.000}{0.000}$ & 0.219 & 0.19 \\
\hline 1898 & & 3:07:02 AM & 25.406 & 25.641 & \begin{tabular}{|l|l|}
24.789 \\
\end{tabular} & 25.999 & & \begin{tabular}{|l|l|}
48.301 \\
\end{tabular} & 52.923 & 16.278 & 33.11 & -1.212 & -7.533 & 48.832 & 0.056 & \begin{tabular}{|l|l|l|l|l|}
14.343 \\
\end{tabular} & & 2593.67 & \begin{tabular}{|l|l|}
7 & 43.2278 \\
\end{tabular} & 51.8 & 11.6 & 40.7 & & 0.008 & 0.008 & 0.000 & 0.198 & \\
\hline & $9 / 1 / 2004$ & 3:08:02 AM & 25.401 & 25.646 & \begin{tabular}{|l|l|}
24.789 \\
\end{tabular} & 26.009 & 25. & 48.065 & 52.662 & 16.238 & 32.945 & \begin{tabular}{|l|} 
\\
\end{tabular} & & 50.659 & & 14.643 & & 2594.67 & \begin{tabular}{l|l}
77 & 43.2444 \\
\end{tabular} & 51.5 & 12.1 & 40.5 & 2.793 & & & & 0.196 & \\
\hline & 9/1/2004 & 3:09:02 AM & 25.407 & 25.652 & 24.805 & \begin{tabular}{|l|}
26.014 \\
\end{tabular} & 25.201 & 48.16 & 52.969 & 16.229 & $\begin{array}{l}32.988 \\
\end{array}$ & \begin{tabular}{|c|} 
\\
\end{tabular} & $\begin{array}{r}-7.53 \\
\end{array}$ & 50.872 & 0.056 & \begin{tabular}{|l|l|}
14.643 \\
\end{tabular} & & & \begin{tabular}{l|l|}
7 & 43.2611 \\
\end{tabular} & \begin{tabular}{|r}
51.8 \\
\end{tabular} & 12.1 & 40.6 & \begin{tabular}{|l|l|}
2.797 \\
\end{tabular} & 0.008 & 0.008 & 0.000 & 0.199 & \\
\hline & $\begin{array}{c}9 / 1 / 2004 \\
9 / 1 / 204\end{array}$ & $\begin{array}{l}3: 10: 02 \mathrm{AM} \\
311.02 \mathrm{AM}\end{array}$ & $\begin{aligned} 25.401 \\
25.006\end{aligned}$ & $\begin{array}{l}25.641 \\
25.651\end{array}$ & 24.819 & \begin{tabular}{|l|}
26.009 \\
\end{tabular} & $\begin{array}{l}25.226 \\
25.206\end{array}$ & 48.608 & $\begin{array}{l}53.207 \\
533\end{array}$ & $\begin{array}{l}16.405 \\
1.410\end{array}$ & $\begin{array}{l}33.196 \\
23.069\end{array}$ & $\begin{array}{l}-1.212 \\
.215\end{array}$ & $\begin{array}{r}-7.524 \\
\end{array}$ & $\begin{array}{r}49.145 \\
502\end{array}$ & (50 & $\begin{array}{r}14.643 \\
11.643\end{array}$ & & $\begin{array}{l}2596.67 \\
250767\end{array}$ & $\begin{array}{l}7 \\
\end{array}$ & $\begin{array}{l}52.1 \\
5.2\end{array}$ & 11.7 & & 2.820 & 0.008 & 0.008 & 0.000 & 0.193 & \\
\hline \begin{tabular}{|l|l|l|l}
1903 \\
\end{tabular} & $\begin{array}{r}991 / 2004 \\
9 / 1 / 2004\end{array}$ & $\begin{array}{l}\text { 3:11:02 AM } \\
\text { 3:12:02 }\end{array}$ & $\begin{array}{r}25.406 \\
25.39\end{array}$ & $\frac{25.651}{25.635}$ & $\begin{array}{r}24.844 \\
24848\end{array}$ & $\begin{array}{r}26.004 \\
25.987\end{array}$ & $\frac{25.246}{25254}$ & $\begin{array}{l}48.486 \\
48.025\end{array}$ & $\begin{array}{r}53.3 \\
52.751 \\
\end{array}$ & $\frac{16.419}{16.27}$ & \begin{tabular}{|l|}
33.068 \\
32.73 \\
\end{tabular} & & & $\begin{array}{r}50.2 \\
49.71 \\
\end{array}$ & .056 & $\frac{14.643}{14.643}$ & & & & $\frac{52.2}{51.6}$ & $\frac{12.0}{11 .}$ & $\begin{array}{l}40.8 \\
40.4\end{array}$ & \begin{tabular}{|l|l|}
2.811 \\
2785
\end{tabular} & & & 0.000 & 0.197 & 7 \\
\hline 1904 & 9/1/2004 & 3:13:02 AM & 25.395 & 25.635 & \begin{tabular}{|l|}
24.839 \\
\end{tabular} & 25.978 & 25.255 & 48.318 & 52.925 & 16.246 & 33.05 & \begin{tabular}{|l|l|}
-1.218 \\
\end{tabular} & $\begin{array}{l}-1.52 \\
-7.52\end{array}$ & $\begin{array}{l}5.9 .11 \\
50.342\end{array}$ & 0.050 & $\begin{array}{l}\frac{14.045}{14.643} \\
\end{array}$ & & $\begin{array}{l}2598.67 \\
2599.67\end{array}$ & \begin{tabular}{|l|}
43.3111 \\
43.3278 \\
\end{tabular} & $\begin{array}{l}51.6 \\
51.8\end{array}$ & $\frac{11.8}{12.0}$ & $\begin{array}{l}40.4 \\
40.7\end{array}$ & \begin{tabular}{|l|l|} 
\\
2.805 \\
\end{tabular} & & & & $\begin{array}{l}0.199 \\
0.194\end{array}$ & 0.17 \\
\hline 1905 & $9 / 1 / 2004$ & $3: 14: 02 \mathrm{AM}$ & 25.39 & 25.63 & \begin{tabular}{|l|l|}
24.833 \\
\end{tabular} & 25.962 & 25.294 & 48.028 & 52.795 & 16.147 & 32.982 & -1.218 & -7.518 & 49.797 & 0.056 & 14.643 & & 2600.67 & \begin{tabular}{|l|l|}
7 & 43.3444 \\
\end{tabular} & 51.7 & 11.9 & 40.5 & \begin{tabular}{|l|}
2.793 \\
\end{tabular} & 0.008 & 0.008 & 0.000 & 0.199 & \\
\hline & 9/1/2004 & 3:15:02 AM & 25.41 & 25.645 & \begin{tabular}{|l|}
24.858 \\
\end{tabular} & 25.987 & 25.284 & & 53.468 & 16.389 & & -1.212 & & 49.998 & 057 & \begin{tabular}{l|l}
14.643 \\
\end{tabular} & & \begin{tabular}{|l|}
2601.67 \\
\end{tabular} & 743.3611 & 52.3 & 11.9 & 40.9 & 2.820 & & & 0.000 & 0.200 & \\
\hline 1907 & 9/1/2004 & 3:16:02 AM & 25.411 & 25.646 & 24.829 & 25.969 & 25.251 & & 53.195 & 16.302 & 33.37 & -1.212 & -7.535 & 48.892 & .054 & $\begin{array}{l}4.643 \\
\end{array}$ & & \begin{tabular}{|l|}
2602.67 \\
6
\end{tabular} & \begin{tabular}{l|l|l}
7 & 43.3778 \\
\end{tabular} & 52.1 & 11.6 & 41.0 & 2.825 & 0.008 & 0.008 & 0.000 & 0.190 & \\
\hline 108 & $9 / 1 / 2004$ & 3:17:02 AM & $\frac{25.417}{25.512}$ & $\frac{25.652}{25.65}$ & 24.835 & 25.979 & $\frac{25.22}{25.22}$ & & \begin{tabular}{|c|}
53.259 \\
52320
\end{tabular} & $\begin{array}{l}16.106 \\
\end{array}$ & $\begin{array}{r}33.153 \\
2.2020\end{array}$ & -1.212 & & \begin{tabular}{|l|l|}
50.208 \\
51258
\end{tabular} & & $\begin{array}{r}14.643 \\
1.613\end{array}$ & & 2603.67 & $\begin{array}{l}7 \\
\end{array}$ & 52.1 & 12.0 & 40.7 & \begin{tabular}{|l|}
2.806 \\
\end{tabular} & 0.008 & 0.008 & 0.000 & 0.198 & \\
\hline$\frac{1903}{1910}$ & \begin{tabular}{c|}
$9 / 1 / 2004$ \\
\end{tabular} & $\begin{array}{l}3: 18: 02 \mathrm{AM} \\
3 \cdot 19 \cdot 02 \mathrm{AM}\end{array}$ & $\frac{25.412}{25.413}$ & $\frac{25.647}{25.643}$ & $\begin{array}{r}24.836 \\
24.831\end{array}$ & $\begin{array}{r}25.95 \\
25.976\end{array}$ & & & & & $\frac{33.032}{33.112}$ & $\frac{-1.212}{-1.212}$ & & $\frac{51.258}{51.598}$ & & & & 6056 & & & & & & & & & & \\
\hline & 9/1/2004 & 3:20:02 AM & 25.418 & 25.643 & $\begin{array}{l}24.051 \\
24.826\end{array}$ & 26.001 & 25.243 & $\begin{array}{r}40.015 \\
48.391\end{array}$ & 52.156 & 16.202 & $\frac{53.112}{32.99}$ & \begin{tabular}{|l|l|} 
& -1.212 \\
\end{tabular} & $\frac{-1.54}{-7.585}$ & $\begin{array}{l}41.590 \\
49.226\end{array}$ & 0.057 & $\begin{array}{l}\frac{14.045}{14.643} \\
\end{array}$ & & 2606.07 & \begin{tabular}{|l|l}
7 & 43.430 \\
\end{tabular} & $\begin{array}{l}51.0 \\
52.3\end{array}$ & $\begin{array}{l}11.5 \\
11.7\end{array}$ & $\frac{40.6}{40.7}$ & 2.805 & 0.008 & & 0.000 & $\begin{array}{l}0.202 \\
0.202\end{array}$ & 0 \\
\hline & 9/1/2004 & 3:21:02 AM & 25.408 & 25.648 & \begin{tabular}{|l|}
24.816 \\
\end{tabular} & 25.991 & 25.228 & 48.25 & 53.045 & 16.443 & 32.755 & 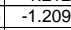 & -7.582 & 49.806 & 0.056 & 14.643 & & 2607.67 & \begin{tabular}{|l|l}
7 & 43.4611 \\
\end{tabular} & 51.9 & 11.9 & 40.5 & \begin{tabular}{|l|}
2.793 \\
\end{tabular} & 0.008 & 0.008 & 0.000 & 0.199 & \\
\hline & 9/1/2004 & 3:22:02 AM & 25.413 & 25.643 & \begin{tabular}{|l|}
24.821 \\
\end{tabular} & 25.976 & 25.243 & & \begin{tabular}{|l|l|l|l|}
52.867 \\
\end{tabular} & & $\begin{array}{l}32.81 \\
\end{array}$ & -1.212 & & 48.949 & & 14.643 & & 2608.67 & \begin{tabular}{|l|l|}
7 & 43.4778 \\
\end{tabular} & 51.7 & 11.7 & & \begin{tabular}{|l|}
2.783 \\
\end{tabular} & & & & & \\
\hline & 9/1/2004 & 3:23:02 AM & 25.397 & 25.637 & 24.816 & 25.955 & 25.237 & & 53.118 & 16.273 & 33.011 & \begin{tabular}{|c|} 
\\
\end{tabular} & $\begin{array}{r}-7.544 \\
\end{array}$ & 49.259 & & & & 2609.67 & 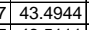 & 52.0 & 11.7 & 40.7 & \begin{tabular}{|l|}
2.803 \\
\end{tabular} & 0.008 & 0.008 & 0.000 & 0.195 & \\
\hline & 9/1/2004 & 3:24:02 AM & $\frac{25.397}{25.501}$ & $\begin{array}{r}25.637 \\
25.61\end{array}$ & \begin{tabular}{|l|}
24.805 \\
2.780
\end{tabular} & \begin{tabular}{|l|}
25.939 \\
\end{tabular} & $\begin{array}{r}25.231 \\
25.256\end{array}$ & 48.295 & $\begin{array}{l}52.952 \\
52.10\end{array}$ & & $\begin{array}{l}33.122 \\
32200\end{array}$ & \begin{tabular}{|l|l|} 
\\
\end{tabular} & -7.55 & \begin{tabular}{|l}
49.222 \\
10.621
\end{tabular} & & $\begin{array}{r}14.643 \\
1.643\end{array}$ & & 2610.67 & $\begin{array}{l}7 \\
7\end{array}$ & \begin{tabular}{|l|}
51.8 \\
\end{tabular} & 11.7 & & \begin{tabular}{|l|}
2.807 \\
\end{tabular} & 0.008 & 0.008 & 0.000 & 0.202 & 0 \\
\hline \begin{tabular}{|l|l|l|l|l|}
1917 \\
1917
\end{tabular} & $\begin{array}{c}9 / 1 / 2004 \\
/ 1 / 2004\end{array}$ & $\begin{array}{l}3: 25: 202 \mathrm{AM} \\
3: 2602 \mathrm{AM}\end{array}$ & $\begin{array}{l}25.401 \\
25.399\end{array}$ & $\frac{25.641}{25.629}$ & \begin{tabular}{|l|}
24.789 \\
24.802 \\
\end{tabular} & .959 & & & \begin{tabular}{|}
53.149 \\
53.081
\end{tabular} & $\begin{array}{l}16.343 \\
16.274 \\
\end{array}$ & $\begin{array}{l}33.229 \\
32.787 \\
\end{array}$ & \begin{tabular}{|l|l|}
-1.218 \\
\end{tabular} & & $\begin{array}{r}49.631 \\
49.789 \\
\end{array}$ & & & & & & & $\frac{11.8}{11.9}$ & & & & & & & \\
\hline & 9/1/2004 & 3:27:02 AM & 25.403 & 25.628 & \begin{tabular}{|l|}
24.817 \\
\end{tabular} & 25.961 & 25.27 & 48.05 & 52.629 & 16.225 & 32.945 & \begin{tabular}{|l|}
-1.212 \\
\end{tabular} & -7.56 & 49.931 & 0.058 & 14.6 & & 2613.67 & \begin{tabular}{|l|}
43.54644 \\
43.5611
\end{tabular} & 51.5 & $\frac{11.9}{11.9}$ & & 2792 & & & & 206 & 0.17 \\
\hline & 9/1/2004 & 3:28:02 AM & 25.408 & 25.638 & \begin{tabular}{|l|l|}
24.816 \\
\end{tabular} & 25.96 & 25.2 & 48.391 & 53.373 & 16.185 & 33.22 & \begin{tabular}{|l|l|} 
\\
\end{tabular} & -7.56 & 48.827 & $\begin{array}{ll}0.058 \\
\end{array}$ & $\begin{array}{l}14.045 \\
14.643\end{array}$ & & 261467 & \begin{tabular}{|l|l}
7 & 43.5778
\end{tabular} & 52.2 & 11.6 & & & 0.008 & 0.00 & & 200 & $\frac{0.18}{0.17}$ \\
\hline & $9 / 1 / 2004$ & 3:29:02 AM & 25.401 & 25.626 & \begin{tabular}{|l|l|}
24.794 \\
\end{tabular} & 25.968 & 25 & 48.20 & 52.942 & 16.334 & & \begin{tabular}{|l|}
-1.212 \\
\end{tabular} & -7.5 & & & 14.6 & & & & $\frac{21.8}{51 .}$ & & & & & & & & \\
\hline & \begin{tabular}{|c|}
$9 / 1 / 2004$ \\
\end{tabular} & & & & \begin{tabular}{|l|l|}
24.812 \\
\end{tabular} & 25.971 & & & \begin{tabular}{|l|l|}
52.813 \\
\end{tabular} & & & \begin{tabular}{|l|l|} 
& -1.212 \\
\end{tabular} & & 50.135 & & & & & & 5.17 & 11.9 & & 2.788 & & & 0.000 & 0.199 & \\
\hline & 9/1/2004 & & 25.408 & & & & & & & & & & & & & & & & & & & & & & & & & \\
\hline & 9/1/2004 & & & & & & & & & & & \begin{tabular}{|l|l|l|} 
& -1.218 \\
\end{tabular} & & 49.4 & & & & & & & 11.8 & & & & & & & \\
\hline & 9/1/2004 & & 25.408 & & \begin{tabular}{|l|l|}
24.856 \\
\end{tabular} & & & & 2.581 & & & \begin{tabular}{|l|l|} 
& -1.212 \\
\end{tabular} & & 49.021 & & & & & & & 11.7 & & & & & & & \\
\hline & 9/1/2004 & 3:34: & 25.412 & 25.637 & \begin{tabular}{|l|}
24.861 \\
\end{tabular} & 25.97 & 25.2 & 48.1 & 52.996 & 16.258 & 32.839 & -1.215 & & 50.636 & & & & & & 51.9 & 12.1 & & & & & & 88 & \\
\hline & 9/1/2004 & & 25.41 & & 24.861 & 5.96 & & & 52.838 & & 32.898 & & & 49.848 & & 14. & & 262 & 43.6944 & 51.7 & 11.9 & & & & & & 98 & \\
\hline & 9/1/2004 & & & 25.6 & 24.855 & & & & . & & & & & & & & & & & & 12.0 & & & & & & & \\
\hline & 9/1/2004 & & 25.417 & & 24.83 & 25.96 & & & & & & & & & & & & & & 51.7 & & & & & & & & \\
\hline & $\frac{91 / 2004}{0.1 / 204}$ & & 25.412 & 25.6 & 24.836 & & & & 52.9 & & & & & & & & & & & & 11.2 & & & & & & & \\
\hline 年 & $\frac{11 / 2044}{91 / 2004}$ & & & & $\frac{14.64}{348}$ & & & & & & & & & & & & & & & & 118 & & & & & & & \\
\hline 1932 & $9 / 1 / 2004$ & $3: 41: 02 \mathrm{AM}$ & 25.407 & 25.622 & 24.04 & 25.945 & 25. & & 5 & 10.015 & & $\mid-1215$ & & 53.683 & & & & & & 51.4 & 11.0 & & & & & & 205 & \\
\hline & 9/1/2004 & 3:42:02 AM & 25.392 & & \begin{tabular}{r|}
24.785 \\
24.0
\end{tabular} & 25.934 & & 47 & \begin{tabular}{|l|l|l|c|}
52.596 \\
\end{tabular} & & & & & 49.633 & & & & & & 51.5 & 11.8 & & & & & & 0.196 & \\
\hline & $9 / 1 / 200$ & & & & & 25.9 & & & & & & & & & & & & & & & & & & & & & & \\
\hline & $9 / 1 / 200$ & & 25.396 & & & & & & 52.67 & & & & & & & & & & & 51.5 & 11.8 & & & & & & & \\
\hline & & & & & & & & & & & & & & & & & & & & & & & & & & & & \\
\hline$\frac{153}{193}$ & $\frac{11 / 2044}{91 / 2004}$ & & & & & & & & & & & & & & & & & & & & 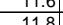 & & & & & & & \\
\hline & $9 / 1 / 1 / 2004$ & & & & 24.816 & & & & 5 & & & & & & & & & & & & . & & & & & & & \\
\hline 1940 & 9/1/1/2004 & & & & & & & & & & & & & 45.4 & & & & & & & & & & & & & & \\
\hline & $9 / 1 / 200$ & & $\frac{25.396}{25}$ & 25.6 & & & & & & & & & & & & & & & & & & & & & & & & \\
\hline & & & 25.401 & & & & & & & & & & & & & & & & & & & & & & & & & \\
\hline & $9 / 1 / 2004$ & & & & 24 & & & & & & & & & & & & & & & & & & & & & & & \\
\hline & & & & & 24.8 & & & & & & & & & & & & & & & & & & & & & & & \\
\hline & $9 / 1 / 200$ & 3:54:C & 25. & & 24.7 & & & & 53.3 & & & & & & & & & & & & & & & & & & & \\
\hline & $9 / 1 / 200$ & & 25. & 25. & 24. & 25. & & & 53.34 & & & & & 49.324 & & & & & & & 11 & & & & & & & \\
\hline & 9/1/200 & & 25. & & 24.8 & & & & 53.70 & & & & & 49.3 & & & & & & & 11. & & & & & & 86 & \\
\hline & $9 / 1 / 200$ & & & & & & & & & & & & & & & & & & & & 11.5 & & & & & & & \\
\hline & & & 25.388 & 25.608 & & & & & & & & & & & & & & & & & 11.6 & & & & & & & \\
\hline & $\frac{91 / 2004}{91 / 12004}$ & & $\frac{25.318}{25382}$ & $\frac{25.59}{25.0}$ & $\frac{2.00}{22.00}$ & 25.92 & & & 52.191 & & & -1. & & & & & & & & & 12. & & & & & & & \\
\hline & 年 & & 望.3.3 & & & & & & 52 & & & & & & & & & & & & & & & & & & & \\
\hline 195 & $\frac{11 / 200}{91 / 1200}$ & & & & & & & & 53 & & & & & & & & & & & & 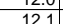 & & & & & & & \\
\hline 195 & $9 / 1 / 2004$ & & $\frac{2.004}{25.394}$ & 25.6 & & $25 . \varsigma$ & 25. & & 52.741 & & & -1.212 & & 49.5 & & & & & & 51.6 & 11.9 & & 1.7 & & .0 & & & \\
\hline & $9 / 1 / 200$ & & 25.37 & $\frac{25.5}{25.5}$ & & & 25. & & & & 32.949 & & & & & & & & & 52.2 & & & & & & & & \\
\hline 195 & 9/1/12004 & & 25.39 & 25. & 24 & & & & 53.315 & & 33.4 & & & & & & & & & & 11.8 & & & & & & & \\
\hline & & & & $25.59 !$ & & & & & & & & & & & & & & & & 52.1 & 11.6 & & & & & & & \\
\hline & 9/1/2004 & $4: 07: 02 \mathrm{~A}$ & 25.379 & 25.599 & 24.777 & 25.917 & 25.10 & 47.6 & 52.453 & 16.08 & 32.656 & -1.212 & & 48.43 & & 14.6 & & 653 & & 513 & 11.5 & & & & & & 97 & \\
\hline & 9/1/2004 & 4:08:02 AM & $\begin{array}{l}25.38 \\
\end{array}$ & 25.6 & 24.773 & \begin{tabular}{|l}
25.928 \\
\end{tabular} & 25.09 & 48.1933 & 53.085[ & 16.208 & 32.98 & 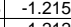 & $.72 \mathrm{~s}$ & 49.931 & 0.057 & 14.643 & & 2654.67 & 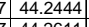 & 52.0 & 11.9 & & \begin{tabular}{|l|}
2.798 \\
2.70 \\
\end{tabular} & 0.008 & 0.008 & 0.000 & 0.202 & 0 \\
\hline & 9/1/2004| & 4:09:02 AM & 25.38 & & 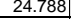 & 25.937 & & 48.119| & |53..006 & & 33.073 & -1.212 & & 49.58 & & 14.643 & & & 7) 44.2611 & & & & 2.799 & 0.008 & & & 0.195 & \\
\hline
\end{tabular}


WSRC-TR-2005-00105, REVISION 0

SRNL-RPP-2005-00012, REVISION 0

RUN \# 4.03A AND B; FIRST AND SECOND HALF OF SLURRY DEWATERING - CONT.

\begin{tabular}{|c|c|c|c|c|c|c|c|c|c|c|c|c|c|c|c|c|c|c|c|c|c|c|c|c|c|c|c|c|}
\hline & $\mathrm{A}$ & & D & $E$ & $F$ & G & $\mathrm{H}$ & $\mathrm{J}$ & $\mathrm{K}$ & $\mathrm{L}$ & M & $\mathrm{N}$ & 0 & Q & $R$ & $\mathrm{~s}$ & $T$ & $\mathrm{v}$ & w & $x$ & $\mathrm{Y}$ & $z$ & $\mathrm{AA}$ & $A B$ & & $\mathrm{AD}$ & $\mathrm{AE}$ & AF $\mathrm{A}$ \\
\hline 1961 & 9/1/2004 & 4:10:02 AM & 25.378 & 25.593 & 24.771 & 25.916 & 25.093 & 48.391 & 53.367 & 15.995 & \begin{tabular}{|l|}
33.446 \\
\end{tabular} & -1.215 & $\begin{array}{l}-7.405 \\
\end{array}$ & 49.925 & 0.056 & 14.643 & 0. & 2656.67 & \begin{tabular}{|l|l|}
44.2778 \\
\end{tabular} & \begin{tabular}{|l|}
52.2 \\
\end{tabular} & 11.9 & 40.9 & \begin{tabular}{|l|}
2.821 \\
\end{tabular} & 0.008 & 0.008 & 0.000 & 0.197 & 0.17 \\
\hline & $\begin{array}{l}9 / 1 / 12004 \\
\end{array}$ & & 25.378 & 25.593 & 24.766 & \begin{tabular}{|l|}
25.926 \\
\end{tabular} & & 48.32 & 52.909 & 16.244 & 33.142 & -1.215 & & 48.811 & 0.056 & 14.643 & & 2657.67 & 44.2944 & \begin{tabular}{|l|}
51.8 \\
\end{tabular} & 11.6 & & & & & & & \\
\hline 19034 & 9/1/2004 & 4:12:02 AM & $\begin{array}{l}25.378 \\
25.257\end{array}$ & $\begin{array}{l}25.603 \\
5677\end{array}$ & $\begin{array}{ll}3 & 24.786 \\
\end{array}$ & \begin{tabular}{|l|l|}
25.961 \\
25005
\end{tabular} & $\begin{array}{l}25.103 \\
250102\end{array}$ & \begin{tabular}{|l|l|}
48.27 \\
\end{tabular} & $\mid$ & 16.188 & 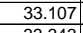 & $\begin{array}{l}-1.209 \\
.209\end{array}$ & \begin{tabular}{|c|c|c|}
7.403 \\
\end{tabular} & \begin{tabular}{|l|l|l|}
4928 \\
\end{tabular} & 0.055 & $\begin{array}{l}14.643 \\
\end{array}$ & & 2658.67 & 74.3111 & \begin{tabular}{|r|}
52.1 \\
\end{tabular} & \begin{tabular}{|l|l|}
11.8 \\
\end{tabular} & \begin{tabular}{|l|l|}
8 & 40.7 \\
\end{tabular} & & 0.008 & $\begin{array}{l}0.008 \\
\end{array}$ & 0.000 & 0.195 & \\
\hline 1964 & 9/1/2004 & 4:13:02 AM & $\begin{array}{l}25.357 \\
25.367\end{array}$ & $\begin{array}{l}25.577 \\
25502\end{array}$ & $\begin{array}{l}7.24 .755 \\
\end{array}$ & \begin{tabular}{|l|}
25.905 \\
\end{tabular} & 25.072 & $\begin{array}{l}48.229 \\
\end{array}$ & \begin{tabular}{|l|l|}
52.917 \\
\end{tabular} & 16.098 & 33.343 & -1.215 & \begin{tabular}{|l|l|}
-7.411 \\
-7.0
\end{tabular} & 50.73 & 0.055 & 14.643 & & 2659.67 & 44.3278 & \begin{tabular}{|r|}
51.8 \\
\end{tabular} & 12.1 & 40.8 & \begin{tabular}{|l|}
2.812 \\
\end{tabular} & \begin{tabular}{|l|l|}
0.008 \\
\end{tabular} & $\begin{array}{l}0.008 \\
\end{array}$ & 0.000 & 0.194 & \\
\hline$\frac{1965}{1966}$ & 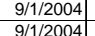 & $\begin{array}{ll}4: 02 \mathrm{AM} \\
5: 02 \mathrm{AM}\end{array}$ & $\begin{array}{l}25.367 \\
25.372\end{array}$ & $\frac{25.592}{25.597}$ & & \begin{tabular}{|r|}
25.93 \\
\end{tabular} & 25.112 & & \begin{tabular}{|l|}
53.576 \\
53234 \\
\end{tabular} & $\begin{array}{l}16.152 \\
16.119 \\
\end{array}$ & \begin{tabular}{|l|}
32.949 \\
332599
\end{tabular} & \begin{tabular}{|l|}
-1.212 \\
-1212 \\
\end{tabular} & $\begin{array}{r}-7.475 \\
\end{array}$ & $\begin{array}{l}49.971 \\
4964\end{array}$ & .056 & $\begin{array}{l}14.643 \\
1643\end{array}$ & & 2660.67 & $\begin{array}{l}4.3444 \\
261 .\end{array}$ & \begin{tabular}{|r|}
52.5 \\
517
\end{tabular} & 11.9 & & & & & 0.000 & 0.199 & 0.17 \\
\hline 1967 & 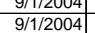 & $\begin{array}{l}\text { 4:15:02 AM } \\
\text { 4:16:02 }\end{array}$ & $\begin{array}{l}25.372 \\
25.376\end{array}$ & $\frac{25.597}{25.596}$ & $\begin{array}{l}724.765 \\
6 \\
6 \\
24.774\end{array}$ & \begin{tabular}{|l|}
25.945 \\
25.929
\end{tabular} & $\frac{25.122}{25.121}$ & \begin{tabular}{|l|}
48.1576 \\
48.573
\end{tabular} & $\mid$ & $\begin{array}{l}16.119 \\
16.262\end{array}$ & $\begin{array}{r}33.259 \\
3.185 \\
\end{array}$ & $\frac{-1.212}{-1.212}$ & $\begin{array}{r}-7.475 \\
-7.252 \\
\end{array}$ & $\begin{array}{l}\frac{49.664}{48.592} \\
48\end{array}$ & $\frac{049}{054}$ & $\begin{array}{l}\frac{14.643}{14.643} \\
\end{array}$ & & $\frac{261667.67}{2662.67}$ & \begin{tabular}{|l|l|}
44.3611 \\
7
\end{tabular} & \begin{tabular}{|l|}
51.7 \\
5.7
\end{tabular} & $\frac{11.8}{11.6}$ & $\begin{array}{l}40.7 \\
440.9\end{array}$ & \begin{tabular}{|l|}
$\mid 2.807$ \\
2818
\end{tabular} & \begin{tabular}{|l|}
0.007 \\
0.008 \\
\end{tabular} & \begin{tabular}{|c|}
0.007 \\
0.008
\end{tabular} & $\begin{array}{c}0.000 \\
0.000\end{array}$ & 0.173 & 0.15 \\
\hline 1968 & $9 / 1 / 2004$ & 4:17:02 AM & 25.376 & & & 25.918 & & & & & & \begin{tabular}{|l|l|} 
& -1.212 \\
\end{tabular} & $\begin{array}{l}-7.42 \\
\end{array}$ & 48.974 & 0.056 & 14.643 & & \begin{tabular}{|l|l|}
26063.01 \\
266367
\end{tabular} & \begin{tabular}{|l|}
44.3517 \\
44.3944
\end{tabular} & $\begin{array}{l}52.1 \\
52.3\end{array}$ & $\begin{array}{l}11.6 \\
11.7\end{array}$ & $\begin{array}{r}40.9 \\
40.8\end{array}$ & & \begin{tabular}{|l|}
0.0008 \\
\end{tabular} & $\begin{array}{l}0.0008 \\
0.008\end{array}$ & 0.000 & & $\begin{array}{l}0.16 \\
0.17\end{array}$ \\
\hline & $9 / 1 / 2004$ & $4: 18: 02 \mathrm{AM}$ & 25.369 & & \begin{tabular}{l|l}
9 & 24.782 \\
\end{tabular} & \begin{tabular}{|l|}
25.906 \\
\end{tabular} & 25.133 & 48.042 & 52.973 & 16.124 & 32.974 & -1.218 & $\begin{array}{r}-7.414 \\
\end{array}$ & 50.359 & 0.056 & 14.643 & & \begin{tabular}{|l|}
2664.67 \\
\end{tabular} & 44.4111 & 51.8 & & & & & & & & \\
\hline & 9/1/2004 & $4: 19: 02 \mathrm{AM}$ & 25.382 & 25.597 & & & 25.172 & 47.959 & & & & .206 & $\begin{array}{r}-7.414 \\
\end{array}$ & & .055 & 14.643 & & & 44.4278 & & 11.9 & 40.6 & & & & & & \\
\hline 1971 & 9/1/2004 & 4:20:02 AM & $\begin{array}{l}25.387 \\
25.277\end{array}$ & $\begin{array}{l}25.597 \\
\end{array}$ & $\begin{array}{l}7 \\
7\end{array}$ & $\begin{array}{l}25.96 \\
2505\end{array}$ & $\begin{array}{l}25.167 \\
25.102\end{array}$ & $\begin{array}{l}47.928 \\
19.2026\end{array}$ & 52.876 & 16.135 & $\begin{array}{l}32.849 \\
\end{array}$ & -1.212 & $\begin{array}{r}-7.423 \\
\end{array}$ & 50.763 & 0.055 & 14.643 & & 2666.67 & 44.4444 & 51.8 & 12.1 & 40.4 & \begin{tabular}{|l|}
2.785 \\
\end{tabular} & 0.008 & 0.008 & 0.000 & 0.196 & \\
\hline$\frac{1972}{1972}$ & $9 / 1 / 2004$ & $\begin{array}{l}21: 02 \mathrm{AM} \\
20.02 \mathrm{MM}\end{array}$ & $\begin{array}{l}25.377 \\
25383\end{array}$ & 25.587 & 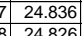 & $\begin{array}{r}25.95 \\
25.001\end{array}$ & $\begin{array}{l}25.192 \\
25.128\end{array}$ & $\begin{array}{l}48.026 \\
\end{array}$ & $\begin{array}{l}52.826 \\
53172\end{array}$ & $\begin{array}{l}16.147 \\
16121 \\
\end{array}$ & \begin{tabular}{|r|}
32.96 \\
32102 \\
\end{tabular} & $\begin{array}{r}-1.215 \\
.215\end{array}$ & $\begin{array}{r}-7.446 \\
7.433\end{array}$ & \begin{tabular}{|l|l|}
50.315 \\
47095
\end{tabular} & & $\begin{array}{l}14.643 \\
1643\end{array}$ & & \begin{tabular}{|l|l|}
2667.67 \\
268.67
\end{tabular} & \begin{tabular}{|l|l|}
44.4611 \\
4.4778
\end{tabular} & \begin{tabular}{|l|}
51.7 \\
50
\end{tabular} & $\begin{array}{l}12.0 \\
114\end{array}$ & & \begin{tabular}{|l|}
2.792 \\
3803
\end{tabular} & \begin{tabular}{|l|l|}
0.007 \\
0008
\end{tabular} & & $\begin{array}{l}0.000 \\
0.002\end{array}$ & $\begin{array}{l}0.185 \\
0.98\end{array}$ & 0.16 \\
\hline$\frac{1973}{1974}$ & $\begin{array}{r}9 / 1 / 2004 \\
9 / 1 / 2004\end{array}$ & $\begin{array}{l}\text { 4:22:02 AM } \\
\text { 4:23:02 AM }\end{array}$ & $\begin{array}{l}25.383 \\
25.378\end{array}$ & $\frac{25.588}{25.588}$ & $\begin{array}{l}8 \\
8 \\
8\end{array}$ & $\begin{array}{l}25.901 \\
25.931\end{array}$ & $\begin{array}{r}25.128 \\
25.108\end{array}$ & $\begin{array}{r}48.2 \\
48.422 \\
\end{array}$ & \begin{tabular}{|l|}
53.172 \\
5.395
\end{tabular} & $\frac{16.121}{16.332}$ & \begin{tabular}{|l|}
33.122 \\
33.188
\end{tabular} & $\frac{215}{212}$ & $\begin{array}{l}-7.443 \\
-7.359 \\
\end{array}$ & \begin{tabular}{|l|}
47.995 \\
47.887
\end{tabular} & & $\frac{14.643}{14.643}$ & & & $\begin{array}{l}4.4778 \\
.4944\end{array}$ & \begin{tabular}{|l|}
52.0 \\
52.1 \\
\end{tabular} & $\frac{11.4}{11.4}$ & 40.8 & \begin{tabular}{|l|}
2.803 \\
2813
\end{tabular} & \begin{tabular}{|l|l}
0.008 \\
0.008
\end{tabular} & $\begin{array}{l}0.008 \\
0.008\end{array}$ & $\begin{array}{l}0.000 \\
0.000\end{array}$ & .198 & 0.17 \\
\hline 1975 & 9/1/2004 & 4:24:02 AM & 25.378 & 25.583 & \begin{tabular}{|l|l|}
3 & 24.790 \\
\end{tabular} & \begin{tabular}{|l|}
25.916 \\
\end{tabular} & 25.113 & & 53.186 & $\begin{array}{l}16.2332 \\
16.232\end{array}$ & $\begin{array}{l}35.100 \\
33.202 \\
\end{array}$ & \begin{tabular}{|l|l|l|l|l|} 
& -1.218 \\
\end{tabular} & $\begin{array}{r}-1.559 \\
-7.426\end{array}$ & $\begin{array}{l}41.081 \\
50.784\end{array}$ & $\begin{array}{l}0.054 \\
0.054\end{array}$ & $\begin{array}{l}\frac{14.043}{14.643} \\
\end{array}$ & & $\begin{array}{l}2059.01 \\
2670.67\end{array}$ & \begin{tabular}{|l|}
44.49444 \\
44.5111
\end{tabular} & $\begin{array}{l}52.1 \\
52.1\end{array}$ & $\frac{11.4}{12.1}$ & & & & & 0.000 & & $\frac{0.16}{0.16}$ \\
\hline 1976 & 9/1/2004 & 4:25:02 AM & 25.383 & & & 25.931 & & 47.976 & & 16.195 & 32.734 & & & 48.782 & & 14.643 & & & & 51.9 & 11.6 & & \begin{tabular}{|l|}
2.782 \\
\end{tabular} & & & 0.000 & 0.196 & $\begin{array}{l}0.16 \\
0.17\end{array}$ \\
\hline & 9/1/2004 & 4:26:02 AM & 25.389 & 25.584 & \begin{tabular}{l|l|l}
4 & 24.817 \\
\end{tabular} & 25.916 & 25.143 & & 52.66 & & & & $\begin{array}{l}-7.345 \\
\end{array}$ & & .059 & 14.643 & & & 44.5444 & & & 40.3 & 2.780 & & & & & $\begin{array}{l}0.17 \\
0.18 \\
\end{array}$ \\
\hline 1978 & & & & & & & & & & & & & & 50.926 & & & & & 44.5611 & 51.8 & 12.1 & & & .008 & & & 189 & \\
\hline 1979 & 9/1/12004 & 4:28:02 AM & & & $\begin{array}{l}9 \\
\end{array}$ & 25.922 & 25. & 48. & 53.017 & 16.277 & \begin{tabular}{l|l|}
32.952 \\
\end{tabular} & -1.212 & & 50.843 & & $\begin{array}{l}14.643 \\
\end{array}$ & & 674.67 & & 51.9 & & & & & & & & \\
\hline 1980 & & 4:29:02 AM & 25.394 & 25.579 & $\begin{array}{l}9 \\
9\end{array}$ & & & & \begin{tabular}{|l|l|}
53.46 \\
\end{tabular} & & & & & & & & & & & $52=$ & & & & & & & & \\
\hline 1981 & 9/1/2004 & 6:02 AM & & & & 928 & & & 52.751 & 16.2 & .984 & 212 & & & & 14.643 & & & & 51.6 & 11.8 & & 2.794 & & & & & \\
\hline & 9/1/2004 & 4:31:02 AM & 25.39 & 25.585 & $\begin{array}{l}5 \\
5\end{array}$ & 25.923 & 25.095 & 48.366 & 53.452 & 16.269 & 32.894 & -1.215 & -7.28 & 48.341 & 0.05 & 14.643 & & 2677.67 & 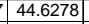 & 52.3 & 11.5 & 40. & 2.801 & 0.007 & 0.007 & & 177 & 0.15 \\
\hline & 9/1/2004 & 2:02 AM & 25.39 & 25.57 & $7 \quad 24.778$ & 25.898 & & 47.926 & 52.811 & 16.21 & 32.894 & -1.218 & & 49.447 & 0.058 & 14.643 & & 2678.67 & (44.6444 & 51.7 & 11.8 & & 2.786 & 008 & 0.008 & .000 & 207 & \\
\hline & 9/1/2004 & 3:02 AM & 25.39 & $25.5 / 5$ & 24.788 & 25.918 & & 48.264 & 53.166 & 16.199 & .068 & -1.215 & & 49.276 & & 14.643 & & & & & 11.8 & & 2.804 & & & & & \\
\hline & |2004| & 44:02 AM & 25.39 & 25.575 & $5 \mid 24.798$ & 25.903 & & & 52.61 & 15.844 & & & & & & & & & & 51.5 & & & & & & & & \\
\hline & $9 / 1 / 2004$ & $\begin{array}{l}5.02 \mathrm{AM} \\
3.62 \mathrm{AM}\end{array}$ & $\frac{25.389}{25.394}$ & $\frac{25.574}{25.569}$ & 24.807 & $\frac{25.907}{25881}$ & $\frac{25 .}{25 .}$ & & 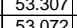 & $\frac{16.156}{16.118}$ & $\begin{array}{l}32.802 \\
32833\end{array}$ & $\frac{1.215}{1.215}$ & & $\begin{array}{l}47.457 \\
48661\end{array}$ & & $\frac{14.643}{14.643}$ & & & & 52.2 & 111.3 & & & & & & 9.99 & \\
\hline 1988 & 甚1/1/1/2004 & $4.37: 02 \mathrm{AM}$ & 25.397 & 2558 & $2 \quad 24.05$ & 25885 & & & 5 & 16165 & $\begin{array}{l}32.050 \\
32939\end{array}$ & -1.215 & & $\frac{40.00}{49.96}$ & & 14.643 & & 268367 & & 519 & 11.0 & & 2794 & 0,008 & & & 0.192 & \\
\hline & 9/1/2004 & 8:02 AM & 25.391 & 25.571 & 124804 & 25869 & 25 & 47.957 & 52.716 & 16.19 & 32.945 & -1.212 & -7. & 50.26 & & $\frac{14.045}{14.643}$ & & $\frac{20307}{26467}$ & 4 & 51.6 & $\frac{11.0}{12.0}$ & & 2786 & & & & 0.196 & $\frac{0.16}{0.17}$ \\
\hline & & & 25.381 & & 24.794 & & & 48.19 & 53.414 & & $\begin{array}{ll}32.962 \\
32.962\end{array}$ & -1.218 & & 50.649 & & 14.643 & & & & & & & & & & & & \\
\hline & $9 / 1 / 2004$ & 22 AM & 25.401 & 25.576 & 24.824 & 25.884 & & & 2.886 & 16.195 & & & & & & 14.643 & & & & 51.8 & 12.6 & & & & & & & \\
\hline & | & $02 \mathrm{AM}$ & 25.396 & 25.581 & $\begin{array}{lll}11 & 24.804\end{array}$ & 25.914 & & & 53.383 & & & 515 & & 49.524 & & 14.643 & & & & 52.3 & 11.8 & & 2.804 & 008 & & & 188 & \\
\hline & & & 25.396 & & & 25.879 & & & & & & & & 50.3 & & 14.643 & & & & & & & & & & & & \\
\hline & 9/1/2004 & 3:02 AM & 25.396 & 25.571 & $\begin{array}{lll}11 & 24.814\end{array}$ & 25.879 & & & \begin{tabular}{|l|l|}
53.57 \\
\end{tabular} & 16.19 & & -1.215 & & & & 14.643 & & & & 52.4 & 12.1 & & & & & & & 0.18 \\
\hline & 9/1/2004 & 4:44:02 AM & 25.391 & 25.57 & $\begin{array}{lll}11 & 24.824\end{array}$ & 25.879 & & 48.16 & 53.278 & 16.082 & 33.0 & $-1 \quad-1$ & & 51.545 & & 14.643 & & & 44.8444 & 52.2 & 12.3 & & 800 & 0.008 & 0.0 & 0.000 & 0.191 & \\
\hline & 9/1/2004 & $2 \mathrm{AM}$ & 25.407 & 25.587 & 24.85 & 25.914 & & & 53.475 & 16 & 33.239 & -1.212 & & 52.3 & & 14.643 & & & & 52.3 & 12.5 & & & & & & & 0.1 \\
\hline & 9/1/2004 & 4 & 25.392 & 25.57 & 24.815 & 9005 & & 48. & 53.381 & 16.334 & & & & 49.6 & & 14. & & & & 52. & $11.8 \mathrm{P}$ & & & & & & 87 & \\
\hline & & & & & & & & & & & & & & & & & & & & & $12.0 \mathrm{r}$ & & & & & & & \\
\hline & $9 / 1 / 200$ & 4:4 & 25.387 & 25.567 & 24.881 & 25.89 & & & 53.008 & 16.1 & 32.7 & -1.212 & & & & 14.6 & & & 44.91 & 51.9 & 11.6 & & 2.18 & & & & 196 & 6 \\
\hline & $\begin{array}{l}91 / 1 / 2044 \\
91 / 12004\end{array}$ & $02 \mathrm{AM}$ & $\begin{array}{l}25.381 \\
25392\end{array}$ & $\begin{array}{l}25.501 \\
25562\end{array}$ & & $\begin{array}{l}25.9005 \\
25005\end{array}$ & & & $\begin{array}{l}53.443 \\
5.9203\end{array}$ & & 32.9 & & & & & & & & & 517 & $\frac{11.0}{117}$ & & & & & & & $\frac{1}{7}$ \\
\hline & $9 / 1 / 1 / 2004$ & 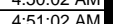 & & & & 25.91 & & & $\mid$ & & & & & & & 146 & & & & & 115 & & & & & & & \\
\hline & $\frac{1 / 1 / 2004}{91 / 2004}$ & & & & & & & & $\mid$ & & & & & & & & & & & 5 & 115 & & & & & & & \\
\hline & 9/1/1/2004 & $4: 5$ & 25.398 & 25.558 & \begin{tabular}{l|l|l|}
8 & 24.846
\end{tabular} & 25.871 & & 48.096 & 52.925 & 16.212 & & & & 50 & & & & & & 51.8 & $\frac{12.0}{12.0}$ & & 2.799 & \begin{tabular}{|l|l|}
0.008 \\
\end{tabular} & $\frac{0.00}{0.00}$ & 0.00 & , & \\
\hline & & & & & & & & & & & & & & & & & & & & & & & & & & & & \\
\hline & & & 25.387 & 25.547 & 24.81 & & & & 52.654 & & & & & 48.4 & & & & & & 51.5 & $\frac{x_{11}}{11}$ & & & & & & 99 & \\
\hline & 9/1/2004 & 4: & 25.402 & 25.552 & 24.83 & 864 & & & 53.611 & & & -1.209 & & & & & & & & $2<.0$ & 11.8 & & & & & & 0.199 & \\
\hline & 9/1/2004 & $4: 5$ & 25.387 & 25.542 & 24.81 & & & & & & & -1.218 & & & & & & & & 52.4 & 11.7 & & & & & & 0.194 & \\
\hline & $9 / 1 / 2004$ & & 25.3 & & \begin{tabular}{|l|}
24.814 \\
\end{tabular} & 25.8 & & & 53.052 & & & & & 49.3 & & 14.6 & & & & 51. & 11.7 & & & 0.008 & & & 0.192 & \\
\hline & $9 / 1 / 2004$ & $4: 59$ & & & & & & & & & & & & & & & & & & & 11.7 & & & & & & & \\
\hline & 9/1/2004 & & 25.402 & 25.5 & 24 & 25.859 & & & 53.048 & & & & & 48. & & & & & & $51 .$. & $11.6 \mathrm{C}$ & & & & & & 96 & \\
\hline & & & & & & & & & & & & & & & & & & & & & & & & & & & & \\
\hline & 9/1 & & 25.3 & & & & & & 53.3 & & & & & & & & & & & 52.2 & 12.0 & & & & & & & \\
\hline & 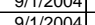 & & & 25.55 & \begin{tabular}{|l|l|}
24.804 \\
\end{tabular} & 25.879 & & & 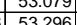 & & & -1.20 & & & & & & & & 522 & $\frac{11.8}{116}$ & & & & & & & \\
\hline & 年 $91 / 1 / 200$ & & & & & & & & & & & & & & & & & & & & 11.0 & & & & & & & \\
\hline & & & & & & & & & & & & & & & & & & & & 523 & 11.9 & & & & & & & \\
\hline & $9 / 1 / 200$ & & 25.385 & 25. & 24.813 & & & & & & & & & & & & & & & 519 & 117 & & & & & & 88 & \\
\hline & & & & & & & & & & & & & & & & & & & & & & & & & & & & \\
\hline & & & & & & & & & & & & & & & & & & & & & & & & & & & & \\
\hline & $9 / 1 / 200$ & & & & & 25. & & & 53.1 & & & & & & & & & & & & 11.8 & & & & & & & \\
\hline & $9 / 1 / 200$ & $5: 10: 32 \mathrm{~A}$ & 25.3 & 25.542 & 24.81 & 25.8 & & 47.969 & 52.944 & 16.9 & & -1.2 .1 & & & & 14. & & & 45.2 & 51.8 & 12.0 & & & & & & & \\
\hline & $9 / 1 / 200$ & 5:11:32 & 25.386 & 25.5 & 24.8 & & & 48.2 & 53.309 & 16.2 & 32.814 & -1.215 & & 21 & & 14.6 & & & $45.36 \mathrm{C}$ & 5 & 12.4 & & & & & & $\overline{999}$ & \\
\hline & 9/1/20 & & & & & & & & & & & & & & & 14. & & & & 51.5 & 11.7 & & & & & & 197 & \\
\hline & 9/1/20 & & 25.3 & 25.54 & 24.85 & & & & 53.358 & & & -1.2 & & & & & & & & 52 & 1 & & & & & & 99 & 9 \\
\hline & & & & & & & & & & & & & & & & & & & & & 11 & & & & & & & \\
\hline & $9 / 1 / 1200$ & & 25.396 & & & & & & 52.94 & & & & & & & & & & & 51.8 & $\frac{12.1}{12.1}$ & & & & & & & \\
\hline & & s.10. & 20.390 & $\frac{23.306}{25512}$ & 1) 124.053 & 250.009 & . & (4.3. & 53.290 & $\begin{array}{l}10.209 \\
1.077\end{array}$ & $\begin{array}{l}33.6 \\
327\end{array}$ & $\frac{-1.212}{1.12}$ & 7 & 30.051 & & $\begin{array}{l}14.043 \\
11.642\end{array}$ & & & & $\frac{52.2}{5.2}$ & $\frac{12.1}{12.1}$ & & 2.806 & 0 & 0.0 & 0.000 & 191 & \\
\hline 2030 & 9/1/1/2004 & $5: 18: 32 \mathrm{AM}$ & \begin{tabular}{|l|l|}
25.391 \\
\end{tabular} & 25.531 & 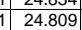 & \begin{tabular}{|l|}
25.0048 \\
\end{tabular} & 25.105 & \begin{tabular}{|l|}
4.58515 \\
48.5
\end{tabular} & \begin{tabular}{|l|}
53.551 \\
53.51 \\
\end{tabular} & 16.324 & 33.255 & \begin{tabular}{|l|}
-1.218 \\
\end{tabular} & \begin{tabular}{|r|r|} 
& -7.113 \\
\end{tabular} & \begin{tabular}{|l|l|}
49.049 \\
\end{tabular} & 0.054 & $\frac{14.045}{14.643}$ & & $\begin{array}{l}725.17 \\
\end{array}$ & 45.4194 & \begin{tabular}{|l|}
51.4 \\
52.4 \\
\end{tabular} & \begin{tabular}{|l|}
11.7 \\
11.7 \\
\end{tabular} & & \begin{tabular}{|l|}
2.819 \\
\end{tabular} & \begin{tabular}{|l|}
0.008 \\
\end{tabular} & \begin{tabular}{|c|}
0.008 \\
\end{tabular} & 0.000 & |.1954 & \begin{tabular}{|l|l|}
4 & 0.16 \\
\end{tabular} \\
\hline
\end{tabular}


WSRC-TR-2005-00105, REVISION 0

SRNL-RPP-2005-00012, REVISION 0

RUN \# 4.03A AND B; FIRST AND SECOND HALF OF SLURRY DEWATERING - CONT.

\begin{tabular}{|c|c|c|c|c|c|c|c|c|c|c|c|c|c|c|c|c|c|c|c|c|c|c|c|c|c|c|c|c|}
\hline & $\mathrm{A}$ & $\mathrm{B}$ & D & $E$ & $\mathrm{~F}$ & G & $\mathrm{H}$ & $\mathrm{J}$ & $\begin{array}{ll}\mathrm{K} \\
\end{array}$ & \begin{tabular}{l|l|l|}
$\mathrm{L}$ \\
\end{tabular} & M & $\mathrm{N}$ & 0 & \begin{tabular}{l|l} 
\\
\end{tabular} & $R$ & $\mathrm{~s}$ & T & u $\quad v$ & w & $x$ & Y & $\mathrm{z}$ & & & & & & AF \\
\hline & 9/1/2004 & 5:19:32 AM & 25.395 & 25.54 & \begin{tabular}{|l|}
24.823 \\
\end{tabular} & 25.873 & 25.09 & 48.086 & \begin{tabular}{|l|}
53.383 \\
\end{tabular} & 16.268 & 32.804 & -1.212 & $\begin{array}{l}-7.122 \\
\end{array}$ & 51.27 & 0.053 & \begin{tabular}{|l|}
14.643 \\
\end{tabular} & $\begin{array}{l}0.003 \\
\end{array}$ & 2726.17 & \begin{tabular}{|l|l|}
45.4361 \\
\end{tabular} & \begin{tabular}{|r|}
52.3 \\
\end{tabular} & 12.2 & 40.4 & 2.789 & 0.008 & 0.008 & 0.000 & 0.189 & \\
\hline & & & 25.396 & 25.541 & \begin{tabular}{|l|l|}
24.834 \\
\end{tabular} & \begin{tabular}{|l|}
25.888 \\
2.88 \\
\end{tabular} & & $\begin{array}{l}4.079 \\
\end{array}$ & \begin{tabular}{|l|}
53.126 \\
\end{tabular} & 16.213 & 32.876 & \begin{tabular}{|c|}
-1.212 \\
\end{tabular} & & & 0.053 & \begin{tabular}{|l|}
14.643 \\
\end{tabular} & 0.003 & & & \begin{tabular}{|l|}
52.0 \\
\end{tabular} & & & & & 0.008 & & & \\
\hline ( & $9 / 1 / 2004$ & 5:21:32 AM & $\begin{array}{l}25.39 \\
25.39 \\
\end{array}$ & 25.54 & \begin{tabular}{|l|}
24.833 \\
2.81 \\
\end{tabular} & \begin{tabular}{|l|l|}
25.883 \\
25870 \\
\end{tabular} & & \begin{tabular}{|l|}
47.949 \\
\end{tabular} & \begin{tabular}{|l|}
53.087 \\
5.283 \\
\end{tabular} & $\begin{array}{l}16.244 \\
\end{array}$ & 32.697 & \begin{tabular}{|l|}
-1.212 \\
\end{tabular} & & $\begin{array}{l}49.399 \\
9.356\end{array}$ & & \begin{tabular}{|l|}
14.643 \\
\end{tabular} & 0.003 & $\frac{2728.17}{272.17}$ & 45.4694 & \begin{tabular}{|l|}
52.0 \\
\end{tabular} & & 40.3 & 望. & 0.008 & 0.008 & 0.000 & $\begin{array}{l}0.196 \\
\end{array}$ & \\
\hline 0.034 & 9/1/2004 & 5:22:32 AM & 25.389 & $\begin{array}{l}25.539 \\
25554\end{array}$ & \begin{tabular}{|l|l|}
24.812 \\
\end{tabular} & \begin{tabular}{|l|l|}
25.872 \\
\end{tabular} & 25.079 & $\begin{array}{l}48.418 \\
\end{array}$ & \begin{tabular}{|l|l|}
53.823 \\
\end{tabular} & \begin{tabular}{|l|}
16.33 \\
\end{tabular} & 32.96 & $\begin{array}{l}-1.212 \\
\end{array}$ & $\begin{array}{r}-7.165 \\
7.71\end{array}$ & 48.456 & $\begin{array}{l}0.048 \\
\end{array}$ & 14.643 & 0.003 & 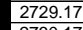 & 45.4861 & \begin{tabular}{|l|}
52.7 \\
\end{tabular} & 11.5 & 40.7 & 2.805 & \begin{tabular}{|l|}
0.007 \\
\end{tabular} & $\begin{array}{l}0.007 \\
\end{array}$ & 0.000 & $\begin{array}{l}0.170 \\
\end{array}$ & \\
\hline 2036 & 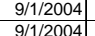 & $\begin{array}{l}5: 23: 32 \mathrm{AM} \\
5 \cdot 32 \cdot 32 \mathrm{AM}\end{array}$ & $\begin{array}{l}25.399 \\
25.379\end{array}$ & $\frac{25.554}{25.534}$ & \begin{tabular}{|l|}
24.847 \\
24822 \\
\end{tabular} & \begin{tabular}{|l|l|}
25.912 \\
25.856
\end{tabular} & $\begin{array}{l}25.124 \\
25088 \\
\end{array}$ & \begin{tabular}{|l|}
48.075 \\
48262 \\
\end{tabular} & & \begin{tabular}{|l|}
16.064 \\
16.154 \\
\end{tabular} & & \begin{tabular}{|l|}
-1.212 \\
\end{tabular} & & $\begin{array}{l}49.263 \\
507767\end{array}$ & \begin{tabular}{|c|}
0.054 \\
0.057 \\
\end{tabular} & \begin{tabular}{|l|l|l|}
14.643 \\
14634 \\
\end{tabular} & & 2730.17 & & \begin{tabular}{|l|}
51.8 \\
5.5 \\
\end{tabular} & \begin{tabular}{|l|l|} 
& 11.7 \\
& 121 \\
\end{tabular} & & \begin{tabular}{|l|}
2.797 \\
2.07 \\
\end{tabular} & & & & $\begin{array}{l}0.191 \\
0202\end{array}$ & \\
\hline & & $\begin{array}{l}5: 24: 32 \mathrm{AM} \\
5: 25: 32 \mathrm{AM}\end{array}$ & $\frac{25.3 / 9}{25.379}$ & & \begin{tabular}{|l|}
24.822 \\
24.812 \\
\end{tabular} & \begin{tabular}{|l|}
25.856 \\
25.856 \\
\end{tabular} & $\begin{array}{r}25.088 \\
25.088 \\
\end{array}$ & $\begin{array}{r}\mid 48.262 \\
48.42 \\
\end{array}$ & \begin{tabular}{|l|}
53.58 \\
53.458 \\
\end{tabular} & $\begin{array}{c}16.154 \\
16.244\end{array}$ & $\begin{array}{l}33.023 \\
33.185 \\
\end{array}$ & \begin{tabular}{|c|}
$\mid-1.2121$ \\
-1.218 \\
\end{tabular} & $\begin{array}{l}-7.267 \\
\end{array}$ & $\frac{50.767}{49.503}$ & $\begin{array}{l}0.057 \\
0.06\end{array}$ & \begin{tabular}{|l|}
14.6634 \\
14.643
\end{tabular} & $\begin{array}{l}0.003 \\
0.003\end{array}$ & $\frac{2731.17}{2732.17}$ & $\begin{array}{l}445.5194 \\
745331\end{array}$ & \begin{tabular}{|l|}
52.5 \\
52.3 \\
\end{tabular} & \begin{tabular}{|l|l}
32.1 \\
31.8
\end{tabular} & $\begin{array}{l}4^{40.6} \\
40.8 \\
\end{array}$ & $\begin{array}{l}2.802 \\
2.813 \\
\end{array}$ & \begin{tabular}{|l|l|}
0.008 \\
0.009
\end{tabular} & \begin{tabular}{|l|}
0.008 \\
0.009
\end{tabular} & \begin{tabular}{|c|}
0.000 \\
0.000
\end{tabular} & $\begin{array}{l}0.202 \\
0.212\end{array}$ & 0.17 \\
\hline 2038 & $9 / 1 / 2004$ & 5:26:32 AM & 25.383 & 25.538 & \begin{tabular}{|l|}
24.811 \\
\end{tabular} & \begin{tabular}{|l|}
25.861 \\
\end{tabular} & 25.113 & 48.471 & & & & & $\begin{array}{l}-7.405 \\
-1.40\end{array}$ & 48.548 & 0.054 & 14.643 & $\begin{array}{l}0.003 \\
0.003\end{array}$ & 2733.17 & $\mid$\begin{tabular}{|c|}
$\mid 35.53511$ \\
45.5528
\end{tabular} & \begin{tabular}{|l|}
52.2 \\
\end{tabular} & $\begin{array}{l}11.8 \\
11.6\end{array}$ & $\begin{array}{l}40.8 \\
40.9 \\
\end{array}$ & \begin{tabular}{|l|}
2.813 \\
2.817 \\
\end{tabular} & \begin{tabular}{|l|l|}
0.009 \\
0.008
\end{tabular} & $\begin{array}{l}0.009 \\
0.008\end{array}$ & $\begin{array}{l}0.000 \\
0.000\end{array}$ & $\frac{0.212}{0.190}$ & $\frac{0.18}{0.16}$ \\
\hline & & $5: 27: 32 \mathrm{AM}$ & & 25.544 & \begin{tabular}{|l|}
24.827 \\
\end{tabular} & & & 48.171 & \begin{tabular}{|l|l|}
53.168 \\
\end{tabular} & 16.258 & $\begin{array}{l}32.937 \\
\end{array}$ & 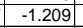 & -7.394 & 48.675 & & $\begin{array}{l}14.643 \\
\end{array}$ & 0.004 & & 45.5694 & \begin{tabular}{|r|}
52.0 \\
\end{tabular} & & & & & & & & $\begin{array}{l}0.16 \\
0.17\end{array}$ \\
\hline 2040 & 9/1/2004 & 5:28:32 AM & 25.384 & 25.544 & & & 25.044 & 48.04 & \begin{tabular}{|l|}
52.824 \\
\end{tabular} & $\begin{array}{l}16.062 \\
\end{array}$ & & \begin{tabular}{|l|} 
\\
\end{tabular} & -7.374 & 49.174 & 052 & \begin{tabular}{|l|l|}
14.643 \\
\end{tabular} & & & & \begin{tabular}{|r|}
51.7 \\
\end{tabular} & 11.7 & 40.5 & & & 0.007 & 0.000 & & \\
\hline 2041 & & & & & \begin{tabular}{|l|}
24.817 \\
\end{tabular} & & & & & & 32.847 & & & 50.853 & & \begin{tabular}{l|l}
14.643 \\
\end{tabular} & & & & 52.1 & & & & .008 & 0.008 & & 0.199 & \\
\hline 2042 & & & & & \begin{tabular}{|l|}
24.832 \\
\end{tabular} & & & 48.129 & 52067 & & & & & 48.821 & & 14.643 & & & & 51.8 & 11.6 & & & & & & & \\
\hline 2043 & & 5:31:32 AM & 25.384 & & 24.812 & 25.886 & & 48.32 & 52.989 & 16.263 & 33.304 & -1.212 & & $\begin{array}{ll}48.227 \\
\end{array}$ & & \begin{tabular}{|l|l|l|l|}
14.643 \\
\end{tabular} & & 738. & & & & & .814 & 007 & & & 0.183 & \\
\hline 2044 & & 5:32:32 AM & & 25.544 & \begin{tabular}{|l|}
24.837 \\
\end{tabular} & 25.886 & & \begin{tabular}{|l|l|}
48.37 \\
\end{tabular} & \begin{tabular}{|l|}
53.53 \\
\end{tabular} & 16.208 & 33.185 & -1.212 & & 49.989 & 0.056 & \begin{tabular}{|l|l|l|l|}
14.643 \\
\end{tabular} & 0.004 & & & 52.4 & & & 2.811 & 0.008 & 0.008 & 0.000 & & \\
\hline 2045 & 9/1/2004 & 5:33:32 AM & 25.394 & 25.534 & \begin{tabular}{|l|}
24.837 \\
\end{tabular} & 25.871 & $25.10 €$ & 48.102 & \begin{tabular}{|l|l|} 
\\
\end{tabular} & 16.163 & 33.114 & -1.212 & -7.36 & 49.391 & 0.053 & $\begin{array}{l}14.643 \\
\end{array}$ & 0.003 & 2740.17 & \begin{tabular}{|l|l|}
45.6694 \\
\end{tabular} & 51.7 & 11.8 & 40.6 & 2.800 & 0.008 & 0.008 & 0.000 & 0.188 & 0.16 \\
\hline & & $5: 34: 32 \mathrm{AM}$ & 25.393 & 25.538 & 24.851 & $\frac{25.886}{2.596}$ & & 48.177 & \begin{tabular}{|l|l|}
53.323 \\
\end{tabular} & 16.298 & 32.806 & -1.212 & & 48.615 & & 14.643 & & & 45.6861 & & & & & & & & 188 & \\
\hline & & 5:35:32 AM & & & & 8.856 & & 48.446 & 53.396 & 16.295 & 33.155 & -1.218 & & 48.992 & & 14.643 & & & & & & & & .008 & & & 194 & \\
\hline & & $5: 36: 32$ AM & 25.394 & & & 25.876 & & & & & 32.687 & -1.212 & & & & & & & . .71794 & & & & & & & & 0.189 & \\
\hline & & $\begin{array}{l}5: 37: 32 \text { AM } \\
5 \cdot 38 \cdot 32\end{array}$ & 25.004 & $\frac{25.528}{25.534}$ & 24.901 & 25.866 & & 48.349 & $\frac{53.504}{53201}$ & $\frac{16.292}{16.359}$ & $\begin{array}{l}33.048 \\
32.243 \\
3\end{array}$ & -1.212 & & 49.2766 & & 14.643 & & 44.1 & 7361 & 52.4 & & & 812 & 000 & & & 190 & \\
\hline & |1/2004 & & $\frac{23.094}{25.399}$ & $\frac{25.534}{25.539}$ & $\begin{array}{l}24.907 \\
24.898\end{array}$ & $\frac{2.010}{25.897}$ & & $\begin{array}{l}40.302 \\
47.982\end{array}$ & $\mid$ & $\begin{array}{l}10.059 \\
16.104\end{array}$ & $\begin{array}{l}53.245 \\
32.968\end{array}$ & $\begin{array}{l}-1.210 \\
-1.215\end{array}$ & & $\begin{array}{l}50.070 \\
46.859\end{array}$ & 0.055 & 14643 & & & & & & & & 0.008 & 0008 & & & 0.16 \\
\hline & 9/1/2004 & 5:40:32 AM & 25.394 & 25.534 & 24.872 & \begin{tabular}{|l|l|}
25.896 \\
\end{tabular} & 25.078 & $\mid 48.558$ & \begin{tabular}{|l|}
53.526 \\
\end{tabular} & 16.25 & 33.165 & $\mid$\begin{tabular}{|c|}
$\mid$ \\
$\mid-1.215$ \\
\end{tabular} & -7.319 & 499.649 & & \begin{tabular}{|c|}
14.643 \\
\end{tabular} & 0.003 & 2747.17 & 45.7861 & 52.4 & $\begin{array}{l}11.8 \\
11.8\end{array}$ & 40.9 & 2.817 & 0.008 & 0.008 & 0.000 & 0.193 & 0.16 \\
\hline & $9 / 1 / 2004$ & 5:41:32 AM & 25.393 & 25.523 & 24.866 & 25.876 & 25.073 & 48.135 & \begin{tabular}{|l|l|}
53.153 \\
\end{tabular} & 16.17 & \begin{tabular}{|l|}
33.021 \\
\end{tabular} & \begin{tabular}{|c|} 
\\
\end{tabular} & -7.316 & $\begin{array}{l}47.887 \\
\end{array}$ & 0.054 & $\begin{array}{l}14.643 \\
\end{array}$ & 0.003 & 2748.17 & & 52.0 & 11.4 & 40.6 & .798 & 0.008 & 0.008 & & & \\
\hline & & & & & & & & & & & & & & & & 14.643 & & 2749.1 & 8194 & & & & & & & & & \\
\hline & & 5:43:32 AM & 25.419 & 25.549 & 24.897 & 25.896 & & 47.943 & 52.936 & 16.238 & 32.839 & -1.209 & & 47.611 & & 14.643 & & & 5.8361 & 51.8 & & & & 0.008 & 0.0 & & 0 & \\
\hline & & & & & & & & & & & & & & & & $\begin{array}{l}14.643 \\
\end{array}$ & & & & & & & & & & & & \\
\hline & $9 / 1 / 2004$ & $5: 45: 32 \mathrm{AM}$ & 25.397 & 25.527 & 24.861 & 25.87 & & 48.471 & 53.172 & 16.393 & 33.194 & -1.212 & $-7.26 \mathrm{Cl}$ & & & $\begin{array}{l}14.643 \\
\end{array}$ & & 752.17 & 45.8694 & & & & $81 !$ & & & & & 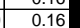 \\
\hline & |/2004 & 5:46: & 391 & 25.526 & 24.859 & 25.834 & 25.05 & 48.125 & \begin{tabular}{|l|}
53.197 \\
\end{tabular} & 16.231 & 32.892 & $\mid-1.215$ & & 49.326 & D.053 & $\begin{array}{ll}14.643 \\
\end{array}$ & & 53.17 & 45.8861 & 52.1 & 11.7 & & .793 & 0.008 & 0.008 & & & \\
\hline & $9 / 1 / 2004$ & 5:47:32 AM & 25.391] & 25.526 & 24.864 & \begin{tabular}{|l|}
25.829 \\
\end{tabular} & & 48.393 & \begin{tabular}{|r} 
\\
\end{tabular} & 16.421 & 33.017 & $\begin{array}{l}-1.212 \\
\end{array}$ & -7.262 & 49.059 & .057 & $\begin{array}{l}14.643 \\
\end{array}$ & & 2754.17 & $\begin{array}{l}45.9028 \\
\end{array}$ & 52.3 & 11.7 & 40.7 & 2.806 & & 0.0 & & & \\
\hline & & & 25.407 & $\begin{array}{l}25.552 \\
2.527\end{array}$ & 24.875 & & & 48.2 & & 16.232 & 33.009 & -1.209 & & 48.25 & & 14.643 & & & & & & & & & & & & \\
\hline & & $2 \mathrm{AM}$ & 25.402 & 25.527 & 24.861 & & & & & 16.164 & 33.186 & -1.212 & & 49.8 & & 14.643 & & & & 52.1 & 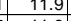 & & & & & & & \\
\hline & & 5:50:32 AM & 25.382 & 25.511 & 24.851 & 25.82 & & & 53.605 & 16.352 & 33.124 & -1.212 & & & & & & $5 \%$. & & & 11.6 & & & 0.008 & & & 0.187 & \\
\hline & $9 / 1 / 2004$ & $\begin{array}{l}5: 51: 32 \mathrm{AM} \\
5 \cdot 52.32\end{array}$ & $\frac{25.391}{25.36}$ & $\frac{25.521}{25.511}$ & $\begin{array}{l}24.859 \\
24829\end{array}$ & $\frac{25.844}{25524}$ & $\frac{25.096}{2510}$ & 48.067 & \begin{tabular}{|r|}
52.9655 \\
53754
\end{tabular} & $\begin{aligned} \frac{16.118}{1624} \\
1624\end{aligned}$ & $\begin{array}{l}33.062 \\
33075\end{array}$ & $\begin{array}{l}-1.212 \\
-1.218\end{array}$ & & & & 14.643 & & 5917 & $\begin{array}{l}45.9694 \\
45.9861\end{array}$ & $\frac{51.8}{52.6}$ & 116 & & $580 c^{2}$ & & & & 194 & \\
\hline & 9 & 5.53 & 25.50 & $\frac{2.015}{25.52}$ & $\begin{array}{l}24.053 \\
24838\end{array}$ & 25828 & 251. & & \begin{tabular}{|c|}
$\mid 52.706$ \\
52.706
\end{tabular} & \begin{tabular}{|l|l|l|l|l|}
16.227 &
\end{tabular} & 32607 & -1212 & & & 0.055 & 14643 & & & & 516 & & & & 0,008 & & & & \\
\hline 2066 & $9 / 1 / 2004$ & 5:54:32 AM & 25.39 & 25.52 & 24.858 & $\frac{2.020}{25.842}$ & 25.0 & 48.339 & \begin{tabular}{|l|}
53.445 \\
\end{tabular} & $\frac{10.241}{16.267}$ & 33.167 & \begin{tabular}{|l|l|} 
& -1.212 \\
\end{tabular} & & 49.399 & & $\begin{array}{l}14.045 \\
14.643\end{array}$ & & $\frac{761.17}{67617}$ & 746 & $\frac{51.0}{52.3}$ & $\frac{11 .}{11.8}$ & & 810 & 0.008 & & & 0.190 & \\
\hline & & & 25.387 & 25.512 & 24.855 & & & & \begin{tabular}{|l|}
53.201 \\
\end{tabular} & 16.317 & & -1.212 & & & & & & & & & $\frac{11 .}{11 .}$ & & & & & & 85 & \\
\hline & & 5:56: & & 25.499 & & & & & & & & -1.212 & & & & 14. & & & & 52.4 & 11.5 & & & & & & & \\
\hline & & & 25.366 & 25.475 & 24.829 & 25.813 & & 48.774 & \begin{tabular}{|l|}
53.744 \\
\end{tabular} & 16.212 & 33.643 & \begin{tabular}{|l|} 
\\
\end{tabular} & & 49.2 & & 14.6 & & & & 52.6 & $\frac{11.7}{11.7}$ & & 2.84 & & & & 182 & \\
\hline & & & & & & & & & \begin{tabular}{|r|} 
\\
\end{tabular} & \begin{tabular}{|l|l|}
16.279 \\
\end{tabular} & & & & & & & & & & & 11.4 & & & & & & & \\
\hline & & & & 25.471 & 24.844 & 25.804 & & & 53.412 & 16.235 & 33.083 & $\mid-1.212$ & & & & 14. & & & & 52.3 & 11.8 & & 180 & & & & 187 & 0.0 \\
\hline & $9 / 1 / 2004$ & & 25.352 & 25.457 & 24.84 & 25.779 & 25. & & \begin{tabular}{|l|}
53.609 \\
\end{tabular} & \begin{tabular}{l|l|}
16.292 \\
\end{tabular} & 32.7 & \begin{tabular}{|c|} 
\\
\end{tabular} & & & 0.055 & 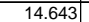 & & & & 52.5 & 11. & & & 0.008 & 0.0 & & 0.195 & \\
\hline & & & & 25.462 & \begin{tabular}{|l|l}
24.836 \\
\end{tabular} & & & & & & & & & & & & & & & & 11. & & & & & & & \\
\hline & & & 25.359 & 25.459 & \begin{tabular}{|l|}
24.842 \\
\end{tabular} & & & 48.446 & 53.559 & 16.478 & 32.939 & -1.192 & & 50.4 & .054 & & & & & 52.4 & 12.0 & & $.80 \mathrm{SP}$ & 0.008 & 0.0 & & 0.191 & \\
\hline & & & & 25.449 & & & & & & & & & & & & & & & & 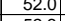 & 11 & & & & & & 1.194 & \\
\hline & & & & & & & & & & & & & & & & & & & & & & & & & & & & \\
\hline & & 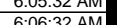 & & $\frac{25.425}{25.434}$ & \begin{tabular}{|l|l|}
2.834 \\
2484
\end{tabular} & & & & 53.075 & $\begin{array}{l}16.293 \\
1.637\end{array}$ & $\begin{array}{l}32.958 \\
32.923\end{array}$ & $\begin{array}{l}-1.163 \\
-1212\end{array}$ & & & & . & & & & 523 & 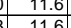 & & & & & & 0.1884 & \\
\hline 2079 & & & & 25,435 & & & & & | 53.046 & & & -1212 & & & & & & & & & 124 & & & & & & & \\
\hline & & & & 25.449 & & & & & & & & & & & & & & & & & 117 & & & & & & & \\
\hline 0 & & 6:09: & 25.384 & 25.453 & 24. & & & & & & & -1.212 & & & & & & & & 52.0 & 11.9 & & & & & & & \\
\hline & & & & & & & & & & & & & & & & & & & & & & & & & & & & \\
\hline & & & \begin{tabular}{|l|l|}
25.38 \\
\end{tabular} & & $\begin{array}{l}24.909 \\
\end{array}$ & & & & \begin{tabular}{|l|}
52.788 \\
\end{tabular} & & 31.88 & & & & & & & & & & & & & & & & & \\
\hline & & & & 25.45 & 24.934 & & & & & \begin{tabular}{|l|l|}
16.719 \\
\end{tabular} & & -1.212 & & & & & & & & & 11.8 & & & & & & 186 & \\
\hline & & & & 25.459 & \begin{tabular}{|l|l|}
24.952 \\
\end{tabular} & & & & \begin{tabular}{|l|}
52.703 \\
\end{tabular} & \begin{tabular}{ll|l}
16.806 \\
\end{tabular} & 31.925 & \begin{tabular}{|l|l|} 
& -1.209 \\
\end{tabular} & & & & 14. & & & & 51.6 & 11.8 & & & & & & 184 & \\
\hline & $9 / 1 / 200$ & & & 25.45 & 24.92 & & & 47.9 & \begin{tabular}{|l|}
53.07 \\
\end{tabular} & 16.9 & 32.157 & -1.215 & & & & 14.6 & & & & 51. & 1. & & & & & & D.183 & \\
\hline & & & & & & & & & & & & & & & & 14. & & & & 52.1 & 11.5 & & & & & & & \\
\hline & & & & & \begin{tabular}{|l|l|}
25.043 \\
\end{tabular} & \begin{tabular}{|l|l|}
25.8 \\
\end{tabular} & & & \begin{tabular}{|l|}
53.143 \\
\end{tabular} & 16.968 & 31.884 & -1.209 & & & & & & & & 52.0 & 11.9 & & & & & & 0.176 & \\
\hline & & & & & & & & & & & & & & & & 14. & & & & & & & & & & & & \\
\hline & & & & $25.4 / 3$ & 25 & & & & 52.3 & & & -1.212 & & & & & & & & & 11.8 & & & & & & & \\
\hline & & & & 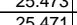 & $\frac{2.0060}{25020}$ & 23.091 & & | & & $\begin{array}{l}16.1 \\
1.67\end{array}$ & S2.320 & -1.145 & & & & & & & & & 11.9 & & & & & & 烈 & \\
\hline & & & & $\frac{2.4417}{25468}$ & $\frac{2.044}{25016}$ & $\frac{20.004}{25846}$ & & 47524 & 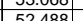 & 10.6 & 31807 & -1.140 & & 50.18 & & $\begin{array}{ll}1.40 \\
1146\end{array}$ & & & & 5..5 & $\frac{1.4 .2}{120}$ & & & & & & & \\
\hline & & & & 25 & \begin{tabular}{|l|l|}
24.996 \\
\end{tabular} & 25816 & & & $\mid$\begin{tabular}{|l|l|l|l|}
$\mid 53188$ \\
\end{tabular} & 16747 & & -1.145 & & 49774 & & 146 & & & & $\frac{5.4}{521}$ & $110^{\circ}$ & & & & & & & \\
\hline & & & & 25.463 & & & & 48 & 53.38 & & & $\mid-1.143$ & & & & $\frac{14.6}{14.6}$ & & & & 523 & 12 & & & & & & & \\
\hline & & & & & & & & & & & & -1.148 & & & & & & & & & & & & & & & & \\
\hline & & & & & & & & & & & & & & & & & & & & & 120 & & & & & & & \\
\hline & & & & 25.466 & 24.989 & & & 47.5 & & 16.686 & 31.91 & -1.145 & & 52.6 & & 14.4 & & 502 & & 51.5 & 12.4 & & & & & & 87 & $f(x)$ \\
\hline & & & 25.435 & 25.455 & & & & 47.8 & 52.942 & 702 & 202 & -1145 & & 507 & & 14.643 & & & & 51.8 & 120 & & & & & & & 016 \\
\hline 2100 & 9/1/2004 & 6:28:32 AM & 25.435 & \begin{tabular}{|l|l|l|l}
25.46 \\
\end{tabular} & \begin{tabular}{|l|l|}
24.973 \\
\end{tabular} & \begin{tabular}{|l|l|}
25.827 \\
\end{tabular} & 25.05 & 47.849 & \begin{tabular}{|l|}
52.801 \\
\end{tabular} & \begin{tabular}{|c|}
16.611 \\
\end{tabular} & 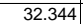 & $\mid-1.145$ & & 50.39 & $\overline{0.051}$ & $\begin{array}{l}14.643 \\
\end{array}$ & 00 & 795.17 & 46.5861 & 51.7 & 12.0 & & \begin{tabular}{|l|l|}
2.765 \\
\end{tabular} & \begin{tabular}{|l|}
0.007 \\
\end{tabular} & 0.007 & 0.000 & $\begin{array}{ll}0.182 \\
\end{array}$ & 0.16 \\
\hline
\end{tabular}


WSRC-TR-2005-00105, REVISION 0

SRNL-RPP-2005-00012, REVISION 0

RUN \# 4.03A AND B; FIRST AND SECOND HALF OF SLURRY DEWATERING - CONT.

\begin{tabular}{|c|c|c|c|c|c|c|c|c|c|c|c|c|c|c|c|c|c|c|c|c|c|c|c|c|c|c|c|c|}
\hline & $\mathrm{A}$ & $B$ & D & $E$ & $\mathrm{~F}$ & G & $\mathrm{H}$ & $\mathrm{J}$ & $\mathrm{K}$ & $\mathrm{L}$ & $\mathrm{M}$ & $\mathrm{N}$ & 0 & \begin{tabular}{l|l} 
\\
\end{tabular} & $\mathrm{R}$ & $\mathrm{s}$ & $T^{T}$ & $\mathrm{~V}$ & \begin{tabular}{l|l} 
w \\
\end{tabular} & $x$ & $\mathrm{Y}$ & $z$ & AA & $\mathrm{AB}$ & & & $\mathrm{AE}$ & AF \\
\hline & 9/1/2004 & $6: 29: 32 \mathrm{AM}$ & 25.43 & 25.45 & 24.979 & \begin{tabular}{|l|}
25.843 \\
\end{tabular} & 25.045 & 47.967 & 53.213 & 16.725 & 32.2 & -1.145 & -7.142 & 50.628 & 0.052 & \begin{tabular}{|l|}
14.643 \\
\end{tabular} & 0.004 & 2796.1 & \begin{tabular}{|l|l|}
46.6028 \\
\end{tabular} & 52.1 & 12.1 & 40.1 & 2.764 & 0.007 & 0.007 & 0.000 & 0.186 & 0.16 \\
\hline & $9 / 1 / 12004$ & & 25.431 & 25.451 & 1024.964 & \begin{tabular}{|l|}
25.809 \\
\end{tabular} & 25.066 & $\begin{array}{l}47.557 \\
\end{array}$ & \begin{tabular}{|l|l|}
52.496 \\
\end{tabular} & \begin{tabular}{|l|}
16.509 \\
\end{tabular} & 32.186 & $\begin{array}{l}-1.09 \\
\end{array}$ & & 50.496 & 0.051 & $\begin{array}{l}14.643 \\
\end{array}$ & & & $\begin{array}{ll}7 \\
7 & 46.6194 \\
\end{array}$ & 51.4 & 12.0 & & 2.749 & & 0.007 & & & 0.16 \\
\hline & $9 / 1 / 12004$ & $6: 31: 32 \mathrm{AM}$ & 25.431 & 25.456 & \begin{tabular}{|l|l}
624.949 \\
\end{tabular} & \begin{tabular}{|l|}
25.799 \\
\end{tabular} & 25.061 & \begin{tabular}{|l|}
47.793 \\
\end{tabular} & & \begin{tabular}{|l|}
16.623 \\
\end{tabular} & 32.127 & $\begin{array}{l}-1.125 \\
\end{array}$ & $\begin{array}{l}-7.096 \\
-7.097\end{array}$ & $\begin{array}{r}48.8 \\
\end{array}$ & 0.051 & \begin{tabular}{|l|}
14.643 \\
\end{tabular} & & $\frac{2798.17}{270.17}$ & $\begin{array}{ll}7466.6361 \\
7\end{array}$ & 51.9 & 11.6 & & 2.755 & $\begin{array}{l}.007 \\
007\end{array}$ & 0.007 & 0.000 & 0.183 & \\
\hline \begin{tabular}{|l|l|l|l|}
2104 \\
2105
\end{tabular} & 9/1/2004 & $6: 32: 32 \mathrm{AM}$ & 25.42 & 25.45 & \begin{tabular}{|l|l|}
5 & 24.969 \\
\end{tabular} & $\begin{array}{l}25.813 \\
2.70\end{array}$ & 25.07 & $\begin{array}{l}47.97 \\
\end{array}$ & 52.95 & 16.5 & 32.551 & -1.096 & \begin{tabular}{|l|l|}
-7.087 \\
.7 .0
\end{tabular} & 49.385 & $\begin{array}{l}0.052 \\
\end{array}$ & \begin{tabular}{|l|l|}
14.643 \\
\end{tabular} & 0.004 & 2799.17 & \begin{tabular}{|l|l|}
46.6528 \\
1.60
\end{tabular} & 51.8 & 11.8 & 40 & 2.776 & 0.007 & 0.007 & 0.000 & 0.185 & \\
\hline$\frac{2105}{2106}$ & $\begin{array}{l}9 / 1 / 2004 \\
9 / 1 / 2004\end{array}$ & $\begin{array}{ll}6: 33: 32 \mathrm{AM} \\
6: 33: 32 \mathrm{~A}\end{array}$ & $\frac{25.405}{25.402}$ & $\frac{25.425}{25.417}$ & & \begin{tabular}{|l|l|}
25.798 \\
\end{tabular} & $\begin{array}{r}25.14 \\
25.172\end{array}$ & \begin{tabular}{|l|}
48.082 \\
47943 \\
\end{tabular} & \begin{tabular}{|r|}
53.16 \\
53.238 \\
\end{tabular} & \begin{tabular}{|l|}
16.611 \\
16385 \\
\end{tabular} & $\begin{array}{r}32.58 \\
32.576\end{array}$ & \begin{tabular}{|c|c|}
-1.09 \\
-1.093
\end{tabular} & & $\begin{array}{l}50.354 \\
49827\end{array}$ & $\begin{array}{l}0.051 \\
0.053 \\
\end{array}$ & \begin{tabular}{|l|l|}
14.643 \\
14643 \\
\end{tabular} & & $\frac{2800.17}{28017}$ & 6694 & $\frac{52.0}{5.1}$ & \begin{tabular}{|l|}
12.0 \\
119
\end{tabular} & 40. & \begin{tabular}{|l|}
2.781 \\
2776 \\
\end{tabular} & & 0.007 & & 0.181 & 0.15 \\
\hline$\frac{2107}{2107}$ & $\begin{array}{l}91 / 1 / 12004 \\
91 / 2004\end{array}$ & 6:3:3:32 AM & $\begin{array}{l}\frac{25.402}{25.405} \\
25\end{array}$ & $\begin{array}{r}\frac{25.417}{25.42} \\
\end{array}$ & $\begin{array}{l}724.956 \\
224.943\end{array}$ & \begin{tabular}{|l|}
25.775 \\
3.778 \\
\end{tabular} & $\begin{array}{r}25.172 \\
25.22 \\
\end{array}$ & \begin{tabular}{|l|}
47.9434 \\
47.775 \\
\end{tabular} & \begin{tabular}{|l|}
53.238 \\
52.875 \\
\end{tabular} & $\begin{array}{r}16.385 \\
16.52\end{array}$ & $\begin{array}{l}32.576 \\
32.307 \\
\end{array}$ & 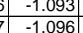 & & $\begin{array}{l}49.827 \\
49.165\end{array}$ & $\begin{array}{l}0.053 \\
0.051\end{array}$ & $\begin{array}{l}44.643 \\
14.643 \\
\end{array}$ & $\begin{array}{l}0.003 \\
0.003 \\
\end{array}$ & $\frac{2800.17}{2802.17}$ & \begin{tabular}{|l|}
46.68611 \\
46.7028 \\
\end{tabular} & $\frac{52.1}{51.7}$ & $\begin{array}{l}\frac{11.9}{11.7} \\
\end{array}$ & $\frac{40.3}{40.0}$ & $\frac{2.776}{2761}$ & |.008 & $\begin{array}{l}0.008 \\
0.007\end{array}$ & $\frac{0.000}{0.000}$ & $\begin{array}{l}0.189 \\
0.133 \\
\end{array}$ & $\begin{array}{l}0.16 \\
0.16\end{array}$ \\
\hline 2108 & $9 / 1 / 2004$ & $6: 36: 32 \mathrm{AM}$ & 25.426 & 25.44 & \begin{tabular}{|l|l|}
4 & 24.964 \\
\end{tabular} & \begin{tabular}{|l|l|} 
& 25.803 \\
\end{tabular} & 25.185 & $\begin{array}{l}48.137 \\
\end{array}$ & \begin{tabular}{|l|}
53.292 \\
\end{tabular} & & 32.625 & \begin{tabular}{|c|c|}
5 & -1.09 \\
\end{tabular} & -7.018 & 49.706 & $\begin{array}{l}0.051 \\
0.053 \\
\end{array}$ & \begin{tabular}{|l|}
14.043 \\
14.643 \\
\end{tabular} & $\begin{array}{l}.0004 \\
0.004\end{array}$ & $\frac{2802.11}{2803.17}$ & \begin{tabular}{|l|}
46.1020 \\
46.7194 \\
\end{tabular} & 52.2 & $\begin{array}{l}\frac{11 . t}{11.8} \\
\end{array}$ & \begin{tabular}{|l|l|}
40.0 \\
0.4
\end{tabular} & $\frac{2.161}{2.784}$ & $\begin{array}{l}0.007 \\
0.008\end{array}$ & $\begin{array}{l}.007 \\
0.008\end{array}$ & $\begin{array}{l}0.000 \\
0.000\end{array}$ & & $\begin{array}{l}0.16 \\
0.16\end{array}$ \\
\hline & 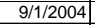 & & 25.433 & 25.453 & \begin{tabular}{|l|}
324.976 \\
\end{tabular} & \begin{tabular}{|l|l|}
25.806 \\
\end{tabular} & 25.148 & 48.009 & \begin{tabular}{|l|}
53.296 \\
\end{tabular} & 16.472 & 32.578 & -1.09 & & 49.464 & 0.053 & 14.643 & & & 46.7361 & & & & & & & & & $\frac{0.16}{0.16}$ \\
\hline & 9/1/2004 & $6: 38: 32 \mathrm{AM}$ & 25.442 & 25.457 & $\begin{array}{l}7 \\
724.985 \\
\end{array}$ & 25.789 & 25.136 & \begin{tabular}{|l|l|}
47.974 \\
\end{tabular} & 53.056 & $\begin{array}{l}16.395 \\
\end{array}$ & 32.521 & $\begin{array}{c}-1.09 \\
\end{array}$ & & 50.569 & 0.05 & 14.6 & & & \begin{tabular}{|l|l|}
46.7528 \\
\end{tabular} & 51.9 & 12.0 & 40.2 & 2.775 & & $\begin{array}{l}0.007 \\
\end{array}$ & & & $\begin{array}{l}0.16 \\
0.15\end{array}$ \\
\hline 2111 & & & & & \begin{tabular}{|l|l|}
5 & 24.974 \\
\end{tabular} & 25.768 & 25.1 & $\begin{array}{lll}47.8 \\
\end{array}$ & 53 & \begin{tabular}{ll|l}
16.576 \\
\end{tabular} & 32.34 & -1.09 & & & 0.051 & 14.6 & & & & 51.9 & & & 2.765 & & & & 0.182 & $\frac{.15}{16}$ \\
\hline & & & 25.434 & 25.449 & \begin{tabular}{l|l}
9 & 24.977 \\
\end{tabular} & 25.807 & & & & 16.551 & 32.471 & $\mid-1.088$ & & 50.676 & & 14.6 & & & & 52.0 & & & & & & & & \\
\hline & $1 / 2004$ & & 25.447 & 25.462 & \begin{tabular}{|l|l|}
2 & 24.986 \\
\end{tabular} & 25.8 & & & 53.63 & 16.625 & 32.666 & $\begin{array}{l}-1.09 \\
\end{array}$ & & 4987 & (5) & 14.6 & & & & 3 & 1 & & .796 & & & & 184 & \\
\hline & |1/2004 & $32 \mathrm{AM}$ & 25.454 & 25.474 & $\begin{array}{ll}44 & 25.018\end{array}$ & 25.797 & 25.0 & & 53.338 & 16.824 & 32.256 & $\mid-0.998$ & & 49.916 & 0.052 & 14.643 & & 2809.1 & \begin{tabular}{|l|l|}
46.8194 \\
\end{tabular} & & & & 2.767 & & & & & \\
\hline & 9/1/2004 & 6:43:32 AM & 25.442 & 25.462 & \begin{tabular}{|l|l|}
2 & 25.03 \\
\end{tabular} & \begin{tabular}{|l|l|}
25.789 \\
\end{tabular} & 25. & 48.198 & \begin{tabular}{|l|}
53.522 \\
\end{tabular} & \begin{tabular}{|l|l|}
16.871 \\
\end{tabular} & 32.303 & & & 50.669 & 0.052 & 14.643 & 0.0 & 2810.1 & 46.8361 & 52.4 & 12.1 & & & & & & & 16 \\
\hline & 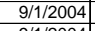 & & 25.425 & 25.445 & \begin{tabular}{|l|l|}
5525.043 \\
\end{tabular} & 25.767 & 25.0 & & \begin{tabular}{|l|l|}
53.448 \\
\end{tabular} & 16.76 & 32.27 & $\begin{array}{l}-1.056 \\
\end{array}$ & & 50.169 & 0.052 & $\begin{array}{l}14.643 \\
\end{array}$ & & & & & 11.9 & & & 0.007 & & & & 16 \\
\hline & 9/1/2004 & $6: 45: 32 \mathrm{AM}$ & 25.411 & 25.426 & & 25.794 & & 47.468 & & & 31.611 & \begin{tabular}{|c|}
-1.09 \\
\end{tabular} & & 51.27 & & 14.643 & & & \begin{tabular}{|l|l|}
46.8694 \\
\end{tabular} & 51.5 & & & 2.726 & & 0.007 & & 0.188 & .16 \\
\hline 2118 & & & 25.405 & & \begin{tabular}{|l|l|}
5 & 25.018 \\
\end{tabular} & 25.757 & 25.0 & & & $\begin{array}{l}16.88 \\
\end{array}$ & & & & 49.322 & & & & & & & 11.7 & & & $\begin{array}{l}0.008 \\
\end{array}$ & 0.008 & & & 0.16 \\
\hline 2119 & 9/1/2004 & 7:32 AM & 25.419 & 25.418 & \begin{tabular}{ll|l}
8 & 25.027 \\
\end{tabular} & $\begin{array}{l}7.75 .771 \\
9\end{array}$ & 25.10 & $\begin{array}{l}47.484 \\
\end{array}$ & 52.587 & 16.526 & 32.116 & -1.073 & & 50.625 & 0.053 & 14.643 & & 814.1 & .9028 & 51.5 & 12.1 & & & 0.008 & 0.008 & 0.000 & 0.191 & 0.16 \\
\hline & 9/1/2004 & $\begin{array}{l}8: 32 \mathrm{AM} \\
0.320 \mathrm{M}\end{array}$ & $\begin{aligned} 25.428 \\
25.422\end{aligned}$ & $\begin{array}{r}25.423 \\
25.406\end{array}$ & $\begin{array}{l}3 \quad 25.016 \\
6\end{array}$ & $\begin{array}{l}6 \quad 25.771 \\
5\end{array}$ & $\begin{array}{r}25.08 \\
25.06\end{array}$ & $\begin{array}{l}47.928 \\
47.921\end{array}$ & \begin{tabular}{|l|}
52.975 \\
\end{tabular} & $\begin{array}{r}16.85 \\
16.577\end{array}$ & . 2.149 & -1.018 & & $\begin{array}{r}51.103 \\
0.895\end{array}$ & $\begin{array}{r}0.05 \\
0.052\end{array}$ & & & 815.1 & & 51.8 & $\frac{11.2}{1.0}$ & & 2.761 & 0.007 & 0.007 & 0.000 & & 0.15 \\
\hline$\frac{2122}{2122}$ & \begin{tabular}{|}
$91 / 1 / 2004$ \\
$9 / 1 / 2004$
\end{tabular} & $\begin{array}{l}6.49 .52 \mathrm{AN} \\
6: 50: 32 \mathrm{AM}\end{array}$ & $\frac{25.42 L}{25.427}$ & $\frac{23.400}{25.416}$ & \begin{tabular}{c|c} 
& 24.905 \\
6 & 24.99
\end{tabular} & \begin{tabular}{|l|l|} 
& 25.154 \\
& 25.744
\end{tabular} & 25.06 & $\begin{array}{l}47.922 \\
47.795\end{array}$ & \begin{tabular}{|l|}
53.209 \\
52.983 \\
\end{tabular} & 16.571 & $\begin{array}{l}32.541 \\
32.092\end{array}$ & $\begin{array}{l}-1.0<1 \\
-1.012\end{array}$ & $\begin{array}{l}-\frac{-.03}{-7.04} \\
\end{array}$ & $\begin{array}{l}49.695 \\
50.997\end{array}$ & $\begin{array}{l}0.032 \\
0.05\end{array}$ & \begin{tabular}{|l|}
14.643 \\
14.643 \\
\end{tabular} & $\begin{array}{l}.0003 \\
0.003\end{array}$ & $\frac{2816.1}{2817.1}$ & \begin{tabular}{|l|}
46.9361 \\
46.9528 \\
\end{tabular} & $\begin{array}{l}52.1 \\
51.9\end{array}$ & $\begin{array}{l}\frac{11.9}{12.1} \\
\end{array}$ & $\frac{40 .}{39}$ & $\begin{array}{l}2.714 \\
2.754\end{array}$ & \begin{tabular}{|l|}
0.007 \\
0.007 \\
\end{tabular} & $\begin{array}{l}.007 \\
0.007\end{array}$ & $\begin{array}{l}0.000 \\
0.000\end{array}$ & $\begin{array}{l}0.185 \\
0.179\end{array}$ & $\begin{array}{l}0.16 \\
0.15\end{array}$ \\
\hline & 9/1/2004 & 6:51:32 AM & 25.442 & 25.421 & $\begin{array}{l}1 \quad 25.01 \\
\end{array}$ & 25.749 & 25.08 & 48.05 & \begin{tabular}{|l|}
53.388 \\
\end{tabular} & 16.668 & 32.383 & -1.015 & & 49.276 & 0.054 & 14.643 & & 2818.1 & 46.9694 & 52.3 & 11.7 & 40. & 2.773 & 0.008 & 0.008 & 0.000 & 0.192 & $\begin{array}{l}0.15 \\
0.16\end{array}$ \\
\hline 2124 & 9/1/2004 & 6:52:32 AM & 25.446 & 25.426 & 25.009 & 25.759 & 25.08 & \begin{tabular}{|l|l|}
48.063 \\
\end{tabular} & \begin{tabular}{|l|l|} 
& 53.388 \\
\end{tabular} & \begin{tabular}{|l|l|}
16.896 \\
\end{tabular} & 32.038 & -1.018 & & 49.403 & 0.052 & 14.643 & & & & & \begin{tabular}{l|l|l|}
11.8 \\
\end{tabular} & 40. & & & & & & $\begin{array}{l}0.16 \\
0.16\end{array}$ \\
\hline & & & 25.457 & 25.431 & $\begin{array}{l}11 \\
11\end{array}$ & 25.784 & & & & 16.686 & & $\begin{array}{l}-1.079 \\
\end{array}$ & & 49.952 & 0.05 & 14.6 & & & & 18 & & & & & & & & 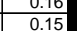 \\
\hline & & & 25.437 & 25.416 & & 25.779 & & & & & 32.297 & & & & 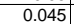 & 14.643 & & & & & & & & & & & & \\
\hline & |11/2004 & & 25.43 & 25.4 & $\begin{array}{ll}4424.994 \\
\end{array}$ & 25.763 & & $\frac{1016}{716}$ & & 16.648 & 32.05 & -0.986 & & 49.6 & & 14.643 & & & & 52.0 & 11. & & & & & & & $\frac{14}{16}$ \\
\hline & $9 / 1 / 2004$ & 6: & 25.417 & 25.392 & \begin{tabular}{l|l}
2 & 24.99 \\
\end{tabular} & \begin{tabular}{l|l}
9 & 25.765 \\
\end{tabular} & & 48.106 & 52.662 & $\begin{array}{l}16.732 \\
\end{array}$ & 32.484 & -1.212 & & 48.348 & 0.04 & 14.643 & & $23.1 \mathrm{~T}$ & & 51.5 & 11.5 & & .778 & & & & & \\
\hline & & & 25.44 & 25.404 & $\begin{array}{lll}4 & 24.983 \\
\end{array}$ & $\begin{array}{l}3 \quad 25.76 \\
3\end{array}$ & & 46.97 & 53.062 & 16.646 & 31.324 & -0.173 & & 51.049 & 0.046 & 14.643 & & 224.1 & \begin{tabular}{|l|l|l|l|}
47.0694 \\
\end{tabular} & It & 2.2. & & 6.69 & & & & & \\
\hline & & & 25.41 & 25.4 & $\begin{array}{ll}44 & 24.983 \\
\end{array}$ & $\begin{array}{l}25.743 \\
5720\end{array}$ & & 47.882 & 53.137 & 16.625 & .194 & -0.992 & & & .058 & 14.643 & & & & & & & & & & & & \\
\hline & 9/1/2004 & & 25.436 & 25.406 & 25 & 25.73 & & 47.88 & 52.846 & 16.743 & & -1.09 & & 11.2. & & 14.66 & & & & & & & & & & & & \\
\hline & & & 25.438 & 25.418 & 25.006 & 25.78 & & 47.797 & 52.82 & 16.665 & 32.141 & -1.108 & & & & 14.6 & & & & & 11. & & & & & & & \\
\hline 134 & $\begin{array}{l}9 / 1 / 22004 \\
9 / / 2004\end{array}$ & & $\frac{25.435}{25.435}$ & $\frac{25.41}{25.41}$ & $\begin{array}{l}124.983 \\
11 \\
1124.989\end{array}$ & $\begin{array}{l}25.747 \\
25.783\end{array}$ & & & $\frac{53.628}{53.081}$ & $\frac{16.773}{16.25}$ & $\begin{array}{l}32.453 \\
32.256\end{array}$ & $\frac{-1.131}{-109}$ & & & 0 & $\frac{14.6}{146}$ & & & & 520 & 些110.0 & & 2764 & & & & $180^{\circ}$ & 0.16 \\
\hline & 9/1/2004 & 7:03:32 AN & 25.427 & 25.406 & \begin{tabular}{|l|l}
6 & 24.98 \\
\end{tabular} & 25.734 & 24.98 & 47.92 & \begin{tabular}{|l|}
53.151 \\
\end{tabular} & 16.651 & 32.395 & -1.096 & & 50.519 & 0.051 & 14.643 & & & \begin{tabular}{r|r|}
7 & 4694 \\
\end{tabular} & 52.0 & 12. & & & & 0.0 & & 0.182 & \\
\hline & $9 / 1 / 2004$ & & 25.433 & 25.413 & \begin{tabular}{l|l|}
3 & 25.011 \\
\end{tabular} & 125.766 & & & \begin{tabular}{|l|l|}
53.398 \\
\end{tabular} & 16.737 & & $\begin{array}{ll}1 & -1.088 \\
\end{array}$ & & 49. & 0.051 & 14.643 & & & & 52.3 & 11.9 & & 2.77 & & & & 0.182 & \\
\hline & $9 / 1 / 2$ & & 25.434 & 25.403 & \begin{tabular}{|l|l}
3 & 25.002 \\
\end{tabular} & \begin{tabular}{|l|l|}
2 & 25.766 \\
\end{tabular} & & & \begin{tabular}{|r|}
52.82 \\
\end{tabular} & 16.775 & & & & 49.5 & 0.051 & 14.6 & & & & 51.7 & 11.8 & 4 & 2.760 & & 0. & & 0.182 & \\
\hline & 9/1/2004 & & 25.43 & 25.405 & \begin{tabular}{|l|l|}
5 & 24.983 \\
\end{tabular} & & & & \begin{tabular}{|l|}
52.784 \\
\end{tabular} & & & & & & & & & & & & & & & & & & 0.191 & \\
\hline 2139 & & & 25.435 & 25.41 & 25.018 & $\begin{array}{l}825.7 \\
\end{array}$ & 24.9 & 48.20 & \begin{tabular}{|l|l|}
53.416 \\
\end{tabular} & $\begin{array}{l}16.804 \\
\end{array}$ & 32.519 & \begin{tabular}{|c|}
-1.05 \\
\end{tabular} & & & 0.052 & & & & & 52.3 & 11.9 & & $\begin{array}{l}2.783 \\
\end{array}$ & & & & 0.184 & 0.16 \\
\hline$\frac{2140}{2141}$ & $\begin{array}{l}9 / 1 / 12004 \\
9 / 1 / 2004\end{array}$ & $\begin{array}{l}7: 08: 27 \mathrm{AM} \\
7700272 \mathrm{AM}\end{array}$ & $\begin{array}{l}25.431 \\
25.421\end{array}$ & $\begin{array}{r}25.411 \\
25.401\end{array}$ & $\begin{array}{l}1 \quad 25.015 \\
1\end{array}$ & \begin{tabular}{|l|l|} 
& 25.774 \\
1 & 25.733
\end{tabular} & & $\begin{array}{r}48.1 \\
47.98 \\
\end{array}$ & \begin{tabular}{|l|}
53.622 \\
53.147 \\
\end{tabular} & $\frac{16.77}{16.691}$ & $\begin{array}{r}32.28 \\
32.299 \\
\end{array}$ & $\begin{array}{r}-1.079 \\
-1.024\end{array}$ & & 47. & 0.051 & \begin{tabular}{|l|}
14.643 \\
14.643 \\
\end{tabular} & & & & $\begin{array}{l}52.5 \\
52.0 \\
\end{array}$ & \begin{tabular}{|l|l|}
11.4 \\
11.9
\end{tabular} & 40. & $\frac{2.771}{2.767}$ & \begin{tabular}{|l|}
0.007 \\
0.077 \\
\end{tabular} & 0.007 & 0.000 & 0.182 & 0.15 \\
\hline$\frac{2141}{2142}$ & $\begin{array}{l}9,1 / 1 / 1004 \\
9 / 1 / 200\end{array}$ & & $\frac{25.421}{25.422}$ & $\frac{25.401}{25.402}$ & $\begin{array}{l}1 \\
2 \\
25.091 \\
24.995\end{array}$ & & & & & & & & & & $\begin{array}{l}0.056 \\
0.053\end{array}$ & $\frac{14.6}{14.6}$ & & & & & $\frac{11.9}{12.6}$ & & & & & & & 0.16 \\
\hline 2143 & 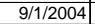 & & 25.428 & 25.407 & $7 \quad 25.011$ & 25.78 & & & \begin{tabular}{|l|}
52.876 \\
\end{tabular} & & & $\frac{1.1119}{-1.119}$ & & & & & & & & & 2.0 & & & & & & $\frac{0.190}{0.182}$ & $\frac{0.16}{0.15}$ \\
\hline 2144 & 9/1/2004 & & 25.423 & 25.403 & \begin{tabular}{l|l|l}
3 & 25.047
\end{tabular} & \begin{tabular}{|l|l|}
7 & 25.731 \\
\end{tabular} & 25.0 & 47.802 & 53.075 & 16.742 & 32.007 & -1.137 & & & 0.052 & 14.6 & & & & 51.9 & 11.8 & & & & & & $\frac{104}{186}$ & $\frac{0.15}{0.16}$ \\
\hline & $9 / 1 / 2$ & & 25.444 & & $\begin{array}{l}9 \\
9\end{array}$ & & & & 342 & & & & & & & & & & & & & & & & & & & \\
\hline & & & 25.421 & 25.396 & 25.069 & \begin{tabular}{l|l}
9 & 25.7 \\
\end{tabular} & & & 53.056 & 16.776 & & -0.9 & & & & & & & & & 18 & & & & & & & \\
\hline 2147 & $9 / 1 / 2004$ & & 25.411 & 25.391 & 25.064 & 25.733 & & & 618 & 16.753 & 31.985 & -1.212 & & & & 14. & & & & (1..0 & 12. & & & & & & & \\
\hline & & & & & 25.054 & $\begin{array}{l}4 \\
4\end{array}$ & & & & & & -1.212 & & & & & & & & & 11.7 & & & & & & & \\
\hline & 9/1/2004 & & 25.84 & & 25.064 & 25. & & & \begin{tabular}{|l|l|} 
\\
\end{tabular} & 16.7 & & -1.2 & & & & 14.6 & & & & & $1+$. & & & & & & 0.096 & \\
\hline & & & 25.58 & & & & & & & & & & & & & & & & & & & & & & & & & \\
\hline & & & 25.574 & & & 25.77 & & & \begin{tabular}{|l|}
53.197 \\
\end{tabular} & & & -1.11 & & & & & & & & & & & & & & & 0.178 & \\
\hline & & & 25.54 & & & & & & & & & & & & & & & & & & & & & & & & & \\
\hline & & & 25.48 & 25.388 & 25.026 & 25 & & & 53.162 & & 32.925 & -1.2 & & & & & & & & & & & & & & & & \\
\hline & & & 554 & 25.40 & 250.035 & $\begin{array}{l}25.164 \\
25797\end{array}$ & & 40.4 & $\frac{1124}{075}$ & & $\begin{array}{l}32.8 \\
32.8\end{array}$ & $\frac{-1.212}{-1209}$ & & & & . & & & & & & & & & & & & \\
\hline & $11 / 200$ & & .4 & 25.39 & 25037 & & & & $\mid 53274$ & & & -12 & & & & 146 & & & & & 1.9 & & & & & & & \\
\hline & & & & & & & & & & & & & & & & & & & & & & & & & & & & \\
\hline & $9 / 1 / 2004$ & & 25.413 & 25.403 & \begin{tabular}{l|l}
3 & 25.021 \\
\end{tabular} & \begin{tabular}{l|l}
1 & 25.7 \\
\end{tabular} & & & 53.242 & $\mid 16.716$ & & 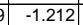 & & & & & & & & & 11.8 & & & & & & & \\
\hline & & & 25. & & & & & & & & & & & & & & & & & & & & & & & & & \\
\hline & & & & & & & & & & & & & & & & & & & & & the & & & & & & & \\
\hline & & & & & & & & & & & & & & & & & & & & & & & & & & & & \\
\hline & & & .3 & 25.38 & 25.02 & 25.7 & & 48. & & 17.04 & 32.1 & $-1.2 \mathrm{C}$ & & & & 14. & & & & & 1.9 & & & & & & & \\
\hline & $11 / 20$ & & 36 & $2538 \mathrm{C}$ & 25.016 & 25 & & 48.3 & 52.952 & 17.149 & 32.112 & -1.2 .2 & & & & 14.6 & & & & & & & & & & & & \\
\hline & & & .3 & 25.38 & 25 & 25.754 & & & 52.996 & & 32.246 & -1.209 & & & & 14. & & & & & & & & & & & & \\
\hline & $9 / 1 / 2$ & & 25.3 & 25.3 & 25 & $\begin{array}{l}4 \quad 25 \\
4\end{array}$ & & & 52 & & & -1.2 & & & & & & & & & & & & & & & & \\
\hline & & & 25.2 & & 25.054 & & & & & & & -1.2 & & & & & & & & & & & & & & & & \\
\hline & & & 25.32 & & & & & & 52.84 & $\frac{1.12}{1.12}$ & & -1.2. & & & & & & & & & 2.4 .4 & & & & & & & \\
\hline & 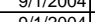 & & $\begin{array}{l}23.34 \\
2536\end{array}$ & 250.394 & 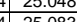 & $\begin{array}{l}0 \\
3\end{array}$ & & 40. & 53014 & 17.1186 & $\begin{array}{l}32.25 \\
32171\end{array}$ & - & & & 0 & & & & & 51.9 & 123 & & & & & & 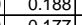 & \\
\hline $217 \mathrm{C}$ & $\begin{array}{r}9 / 1 / 2004 \\
9 / 1 / 2004\end{array}$ & $\begin{array}{l}7: 37: 27 \mathrm{AM} \\
7: 38: 27 \mathrm{AM}\end{array}$ & $\begin{array}{r}25.36 \\
25.388 \\
\end{array}$ & $\begin{array}{r}25.394 \\
25.393\end{array}$ & $\begin{array}{l}4 \\
\begin{array}{l}4 \\
3\end{array} 25.083 \\
3.142\end{array}$ & $\begin{array}{ll} & 25.742 \\
2 & 25.766\end{array}$ & 24.938 & $\begin{array}{l}48.4033 \\
48.359\end{array}$ & \begin{tabular}{|l|}
532.066 \\
52.952 \\
\end{tabular} & \begin{tabular}{|l|}
17.23 \\
17.038 \\
\end{tabular} & $\begin{array}{l}32.174 \\
32.379\end{array}$ & 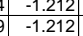 & -7.108 & $\begin{array}{r}51.6 \\
50.392\end{array}$ & $\begin{array}{l}0.05 \\
0.051\end{array}$ & 1 & & 2865.08 & \begin{tabular}{|l|l|}
8 & 47.77347 \\
8 & 47.7514
\end{tabular} & \begin{tabular}{|l|}
51.9 \\
51.8 \\
\end{tabular} & \begin{tabular}{ll|}
12.0 \\
\end{tabular} & & 2.783 & \begin{tabular}{|l|}
0.007 \\
\end{tabular} & \begin{tabular}{|l|}
0.007 \\
0.007 \\
\end{tabular} & \begin{tabular}{|c|}
0.000 \\
\end{tabular} & \begin{tabular}{|l|}
0.177 \\
0.180 \\
\end{tabular} & 0.1 \\
\hline
\end{tabular}


WSRC-TR-2005-00105, REVISION 0

SRNL-RPP-2005-00012, REVISION 0

RUN \# 4.03A AND B; FIRST AND SECOND HALF OF SLURRY DEWATERING - CONT.

\begin{tabular}{|c|c|c|c|c|c|c|c|c|c|c|c|c|c|c|c|c|c|c|c|c|c|c|c|c|c|c|c|c|}
\hline & $\mathrm{A}$ & $B$ & D & $E$ & $\mathrm{~F}$ & G & $\mathrm{H}$ & $\mathrm{J}$ & $\mathrm{k}$ & $\mathrm{L}$ & $M$ & $\mathrm{~N}$ & 0 & $Q$ & $\mathrm{R}$ & $\mathrm{s}$ & $T^{T}$ & $\mathrm{v}$ & w & $x$ & \begin{tabular}{l|l} 
\\
\end{tabular} & & $\mathrm{AA}$ & $\mathrm{AB}$ & & & & AF \\
\hline & 9/1/2004 & $7: 39: 27 \mathrm{AM}$ & 25.392 & 25.381 & 25.13 & 25.744 & 24.996 & 47.909 & 52.422 & 16.898 & 32.116 & -1.215 & -7.1 & 51.992 & 0.052 & 14.643 & 0.003 & 8866.08 & 347.7681 & 51.3 & 12.4 & $\frac{L}{40.0}$ & 2.759 & 0.007 & 0.007 & 0.000 & 0.185 & 0.16 \\
\hline & $9 / 1 / 2004$ & & 25.394 & 25.379 & \begin{tabular}{l|l}
95.097 \\
\end{tabular} & \begin{tabular}{|l|l|} 
\\
\end{tabular} & 25.044 & 48.314 & \begin{tabular}{|l|}
52.983 \\
\end{tabular} & $\begin{array}{l}17.288 \\
\end{array}$ & 32.118 & \begin{tabular}{|c|c|}
-1.212 \\
\end{tabular} & & $\begin{array}{l}49.422 \\
\end{array}$ & 0.052 & $\begin{array}{l}14.643 \\
\end{array}$ & & & 347.7847 & 51.9 & 11.8 & & & & 0.007 & & & 0.16 \\
\hline & $9 / / 1 / 2004$ & $7: 41: 27 \mathrm{AM}$ & 25.397 & 25.357 & \begin{tabular}{|l|l|}
7 & 25.076 \\
\end{tabular} & 25.695 & 25.017 & 47.878 & \begin{tabular}{|l|}
52.5922 \\
5.290 \\
\end{tabular} & \begin{tabular}{|l|}
17.317 \\
1.27 \\
\end{tabular} & $\begin{array}{l}31.589 \\
2.1706\end{array}$ & \begin{tabular}{|r|r|} 
& -1.215 \\
\end{tabular} & $\begin{array}{r}-7.105 \\
-7.105\end{array}$ & 51.134 & & \begin{tabular}{|l|}
14.643 \\
\end{tabular} & & 68.08 & 877.8014 & 51.5 & 12.2 & & 2.739 & $\begin{array}{l}.007 \\
007\end{array}$ & 0.007 & 0.000 & 0.187 & \\
\hline \begin{tabular}{|l|l|l|l|}
2174 \\
2175
\end{tabular} & \begin{tabular}{ll|}
$9 / 1 / 2004$ \\
$0 / 1 / 2001$
\end{tabular} & 7:42:27 AM & 25.406 & 25.356 & \begin{tabular}{ll|l}
6 & 25.095 \\
\end{tabular} & 25.683 & 25.036 & 48.142 & \begin{tabular}{|l|}
52.909 \\
\end{tabular} & $\begin{array}{l}17.347 \\
\end{array}$ & $\begin{array}{l}31.786 \\
2.77\end{array}$ & -1.212 & $\begin{array}{r}-7.105 \\
7.720\end{array}$ & 52.2 & 0.051 & $\begin{array}{l}14.643 \\
\end{array}$ & 0.003 & 669.08 & \begin{tabular}{|l|l|}
47.8181 \\
\end{tabular} & 51.8 & 12.4 & & 2.755 & 0.007 & 0.007 & 0.000 & 0.182 & \\
\hline$\frac{2175}{2176}$ & $\frac{9 / 1 / 2004}{9 / 1 / 2004}$ & $\begin{array}{l}7: 43: 27 \mathrm{AM} \\
7 \cdot 74: 27 \mathrm{AM}\end{array}$ & $\begin{array}{r}25.41 \\
25.408\end{array}$ & $\begin{array}{l}25.344 \\
25.342\end{array}$ & & $\begin{array}{r}25.657 \\
25.55\end{array}$ & $\frac{25.009}{25032}$ & $\begin{array}{r}48.098 \\
46.634\end{array}$ & \begin{tabular}{|l|}
52.884 \\
5.225 \\
\end{tabular} & \begin{tabular}{|c|}
17.24 \\
17.194
\end{tabular} & $\begin{array}{l}31.757 \\
31556\end{array}$ & $\begin{array}{l}-1.212 \\
-12121\end{array}$ & & $\begin{array}{l}51.879 \\
50684\end{array}$ & $\begin{array}{l}0.052 \\
0.051\end{array}$ & \begin{tabular}{|l|l|}
14.643 \\
14643 \\
\end{tabular} & & & & $\begin{array}{l}51.8 \\
51.1\end{array}$ & $\begin{array}{l}12.4 \\
12.1\end{array}$ & & & & $\begin{array}{l}0.007 \\
\end{array}$ & & 0.186 & \\
\hline$\frac{1177}{2177}$ & $\begin{array}{l}9 / 1 / 12004 \\
9 / 1 / 2004\end{array}$ & $\begin{array}{l}\text { 7:44:27 AM } \\
7: 45: 27\end{array}$ & $\begin{array}{l}25.408 \\
25.399\end{array}$ & $\frac{25.342}{25.314}$ & $\begin{array}{l}25.501 \\
4\end{array}$ & $\begin{array}{r}25.65 \\
25.622 \\
\end{array}$ & $\begin{array}{l}25.03 \\
25.06 \\
\end{array}$ & $\begin{array}{l}47.634 \\
47.974 \\
\end{array}$ & \begin{tabular}{|l|}
52.2225 \\
52.801 \\
\end{tabular} & $\begin{array}{l}17.194 \\
17.193 \\
\end{array}$ & $\begin{array}{l}31.556 \\
31.712 \\
\end{array}$ & $\begin{array}{l}-\frac{1.212}{1.212} \\
-12\end{array}$ & $\begin{array}{l}-7.105 \\
-7.105 \\
\end{array}$ & $\begin{array}{l}50.684 \\
50.684\end{array}$ & $\frac{0.051}{0.051}$ & $\begin{array}{l}14.643 \\
14.643 \\
\end{array}$ & $\begin{array}{l}0.003 \\
0.003 \\
\end{array}$ & $\begin{array}{l}71.08 \\
7708\end{array}$ & $\begin{array}{l}847.8514 \\
3\end{array}$ & $\begin{array}{l}51.1 \\
51.7\end{array}$ & 年12.1 & $\begin{array}{l}39.6 \\
39.8 \\
\end{array}$ & $\begin{array}{l}2.730 \\
2747 \\
\end{array}$ & $\begin{array}{l}0.007 \\
0.07\end{array}$ & $\begin{array}{l}0.007 \\
0.07\end{array}$ & 0.000 & $\begin{array}{l}0.184 \\
0.133\end{array}$ & 0.16 \\
\hline 2178 & & 7:46:27 AM & 25.411 & 25.311 & \begin{tabular}{|c|c|}
1 & 25.05 \\
\end{tabular} & 25.623 & 25.01 & 47.926 & \begin{tabular}{|l|}
52.807 \\
\end{tabular} & \begin{tabular}{|l|}
17.127 \\
\end{tabular} & $\frac{31.114}{31.782}$ & $\mid \begin{array}{l}-1.212 \\
-1.212\end{array}$ & -7.099 & 51.956 & $\begin{array}{l}0.051 \\
0.052\end{array}$ & \begin{tabular}{|l|}
14.043 \\
14.643 \\
\end{tabular} & 0.003 & $\frac{2872.08}{2873.08}$ & \begin{tabular}{|l|l|}
3.7 .8081 \\
\end{tabular} & $\begin{array}{l}51.1 \\
51.7 \\
\end{array}$ & $\begin{array}{l}\frac{12.1}{12.4} \\
\end{array}$ & $\begin{array}{l}39.6 \\
39.9 \\
\end{array}$ & $\frac{2.474}{2.748}$ & \begin{tabular}{|l|}
0.007 \\
\end{tabular} & $\begin{array}{l}0.007 \\
0.007\end{array}$ & $\begin{array}{l}0.000 \\
0.000\end{array}$ & & $\begin{array}{l}0.16 \\
0.16\end{array}$ \\
\hline 2179 & 9/1/2004 & $7: 47: 27 \mathrm{AM}$ & 25.419 & & \begin{tabular}{|l|l|}
3 & 25.042 \\
\end{tabular} & 25.596 & 25.00 & 47.816 & \begin{tabular}{|l|l|}
52.548 \\
\end{tabular} & 17.296 & 31.447 & \begin{tabular}{|l|l|} 
& -1.212 \\
\end{tabular} & $\frac{-7.105}{-7.105}$ & 50.26 & 0.051 & \begin{tabular}{|l|l|}
14.643 \\
\end{tabular} & & & \begin{tabular}{|l|l|}
87.9014 \\
\end{tabular} & 51.4 & & & & \begin{tabular}{|l|}
0.007 \\
\end{tabular} & & & 0.184 & 16 \\
\hline & 9/1/2004 & 7:48:27 AM & $\begin{array}{l}25.419 \\
25.255\end{array}$ & 25.288 & & 25.606 & & $\begin{array}{l}48.299 \\
\end{array}$ & \begin{tabular}{|l|}
52.886 \\
\end{tabular} & & & \begin{tabular}{|l|l|} 
& -1.212 \\
\end{tabular} & & & & 14.643 & & & \begin{tabular}{|l|l|}
47.9181 \\
\end{tabular} & & 12.2 & & 2.770 & \begin{tabular}{|l|l|}
0.007 \\
\end{tabular} & 0.007 & 0.000 & 0.182 & \\
\hline 2181 & \begin{tabular}{ll|}
$9 / 1 / 2004$ \\
$0 / 1 / 2001$
\end{tabular} & 7:49:27 AM & 25.425 & $\begin{array}{r}25.3 \\
\end{array}$ & $\begin{array}{l}325.039 \\
5\end{array}$ & $\begin{array}{l}25.622 \\
25509\end{array}$ & 25.01 & $\begin{array}{l}47.814 \\
\end{array}$ & \begin{tabular}{|r|}
52.49 \\
\end{tabular} & $\begin{array}{l}17.239 \\
1730\end{array}$ & $\begin{array}{l}31.654 \\
21914\end{array}$ & $\begin{array}{l}-1.206 \\
.212\end{array}$ & & 51.268 & $\begin{array}{l}0.052 \\
\end{array}$ & \begin{tabular}{|l|l|}
14.643 \\
\end{tabular} & 0.004 & & 47.9347 & 51.4 & 12.2 & & 2.740 & \begin{tabular}{|l|l|}
0.007 \\
\end{tabular} & 0.007 & 0.000 & 0.187 & 0.16 \\
\hline$\frac{104}{102}$ & $\begin{array}{l}9 / 1 / 2004 \\
9 / 1 / 2004\end{array}$ & $\begin{array}{ll}7: 50: 27 \mathrm{AM} \\
7 \cdot 75 \cdot 27 \mathrm{AM}\end{array}$ & $\begin{array}{l}25.421 \\
25.431\end{array}$ & $\begin{array}{l}25.285 \\
25.286\end{array}$ & \begin{tabular}{|l|l|}
5 & $25.02 c$ \\
6 & 25.04
\end{tabular} & $\begin{array}{l}25.588 \\
25.608\end{array}$ & $\frac{25.016}{25066}$ & \begin{tabular}{|l|}
47.967 \\
48.021 \\
\end{tabular} & \begin{tabular}{|r|}
52.71 \\
52.73 \\
\end{tabular} & \begin{tabular}{|l|}
17.286 \\
17177
\end{tabular} & $\begin{array}{l}31.644 \\
33704\end{array}$ & \begin{tabular}{|l|l|} 
& -1.212 \\
\end{tabular} & & \begin{tabular}{|l|}
50.465 \\
51305
\end{tabular} & $\begin{array}{r}0.05 \\
0052\end{array}$ & \begin{tabular}{|l|l|}
14.643 \\
14643
\end{tabular} & & & \begin{tabular}{|l|l|}
47.9514 \\
3
\end{tabular} & $\begin{array}{l}51.6 \\
51.6\end{array}$ & $\begin{array}{l}12.0 \\
12.2\end{array}$ & 39 & \begin{tabular}{|l|}
2.744 \\
2784 \\
\end{tabular} & 0.007 & $\begin{array}{l}0.007 \\
\end{array}$ & 0.000 & 0.180 & 0.15 \\
\hline$\frac{1284}{2184}$ & $\begin{array}{l}9 / 1 / 2004 \\
9 / 1 / 2004\end{array}$ & $\begin{array}{c}7: 51: 27 \mathrm{AM} \\
7: 52: 27\end{array}$ & $\begin{array}{l}25.431 \\
25.435\end{array}$ & $\begin{array}{r}\frac{25.286}{25.28} \\
\end{array}$ & \begin{tabular}{l|l}
6 & 25.04 \\
8 & 25.03.
\end{tabular} & $\begin{array}{l}25.608 \\
25.588\end{array}$ & $\frac{25.06}{25.0}$ & $\begin{array}{l}48 \\
47 \\
\end{array}$ & \begin{tabular}{|c|}
52.733 \\
5.236
\end{tabular} & \begin{tabular}{|c|}
17.177 \\
17.223
\end{tabular} & $\begin{array}{r}31.704 \\
31.901 \\
\end{array}$ & . 1.212 & & \begin{tabular}{|l|}
51.305 \\
50.832
\end{tabular} & $\begin{array}{l}0.052 \\
0.051\end{array}$ & \begin{tabular}{|l|}
14.643 \\
14.643 \\
\end{tabular} & 0.003 & \begin{tabular}{ll|l}
78.08 & 78.08 \\
7
\end{tabular} & $\begin{array}{l}47.9681 \\
47.9847\end{array}$ & $\begin{array}{l}51.6 \\
51.3 \\
\end{array}$ & $\frac{12.2}{12.1}$ & $\frac{39.9}{39.9}$ & $\begin{array}{l}2.748 \\
2799\end{array}$ & |0.007 & 0.007 & 0.000 & 0.187 & 0.16 \\
\hline 2185 & $9 / 1 / 2004$ & $\begin{array}{l}7.52 .27 \mathrm{Am} \\
7: 53: 27 \mathrm{MM}\end{array}$ & $\frac{25.435}{25.434}$ & 25.268 & & \begin{tabular}{|l|l|} 
& 3.5068 \\
25.576
\end{tabular} & 24.99 & $\begin{array}{l}41.853 \\
47.955 \\
\end{array}$ & \begin{tabular}{|l|}
52.3108 \\
52.708 \\
\end{tabular} & $\begin{array}{l}17.225 \\
17.05 \\
\end{array}$ & $\frac{31.901}{31.966}$ & \begin{tabular}{|l|l|} 
& -1.212 \\
\end{tabular} & & $\begin{array}{r}30.802 \\
51.42\end{array}$ & $\begin{array}{l}.051 \\
0.051\end{array}$ & \begin{tabular}{|c|}
14.643 \\
14.643
\end{tabular} & & $\frac{288900}{2880.08}$ & $\begin{array}{l}3 \\
38.984 t \\
3\end{array}$ & $\begin{array}{l}51.3 \\
51.6\end{array}$ & $\begin{array}{l}\frac{12.1}{12.2} \\
\end{array}$ & $\begin{array}{l}39.9 \\
40.0\end{array}$ & \begin{tabular}{|l|}
2.749 \\
2.755 \\
\end{tabular} & & $\begin{array}{l}.007 \\
0.007\end{array}$ & & & $\begin{array}{l}0.16 \\
0.16\end{array}$ \\
\hline & $9 / 1 / 2004$ & 7:54:27 AM & & 25.257 & \begin{tabular}{l|l|}
7 & 25.016 \\
\end{tabular} & 25.554 & 25.04 & 47.748 & \begin{tabular}{|l|}
52.523 \\
\end{tabular} & 17.106 & 31.698 & \begin{tabular}{|l|l|} 
& -1.212 \\
\end{tabular} & & 51.923 & 0.05 & 14.643 & & & & 51.4 & & & & 0.007 & 0.007 & & & $\begin{array}{l}0.16 \\
0.15\end{array}$ \\
\hline & 9/1/2004 & 7:55:27 AM & 25.441 & 25.256 & & 25.559 & & 48.198 & \begin{tabular}{|l|}
52.824 \\
\end{tabular} & \begin{tabular}{ll|}
17.266 \\
\end{tabular} & 31.936 & \begin{tabular}{|l|l|}
5 & -1.215 \\
\end{tabular} & & & 0.051 & & & & & 51.7 & $\begin{array}{ll}12.1 \\
\end{array}$ & 40 & 2.762 & & & & & $\begin{array}{l}0.15 \\
0.15\end{array}$ \\
\hline 2188 & & & & & 25.048 & 25.591 & & & & \begin{tabular}{|l|l|l|}
17.194 \\
\end{tabular} & & & & 52.876 & & 14.6 & & & & & & & & & & & 182 & \\
\hline 2189 & & & & & 25.036 & 25.595 & & 47.424 & 52.009 & \begin{tabular}{ll|l}
17.155 \\
\end{tabular} & 31.359 & -1.212 & & 51.182 & & 14.6 & & & 48.06 & 50.9 & & & 710 & & & & & \\
\hline & & & 25.46 & 25.25 & & 25.568 & & & 52.849 & \begin{tabular}{|c|c|}
17.048 \\
\end{tabular} & 31.993 & 129 & & 590 & & 196 & & & & & & & & & & & & \\
\hline & & & 25.468 & 25.253 & \begin{tabular}{l|l|l|}
3 & 25.062 \\
\end{tabular} & 25.576 & & & & \begin{tabular}{|l|l|l|l}
17.229 \\
\end{tabular} & 31.811 & -1.209 & & & 0.051 & 14.643 & & & & & & & & & & & & \\
\hline & 9/1/2004 & $8: 00: 27 \mathrm{~A}$ & 25.465 & 25.245 & \begin{tabular}{|l|l|}
5 & 25.054 \\
\end{tabular} & 25.548 & 24. & $\begin{array}{l}47.997 \\
\end{array}$ & \begin{tabular}{|l|l|}
52.7499 \\
\end{tabular} & \begin{tabular}{|l|l|}
17.297 \\
\end{tabular} & 31.702 & -1.212 & & 49.14 & 0.051 & 14.643 & & 87.08 & 8 48.1181 & 51.6 & 11.7 & & 2.747 & 0.007 & 0.007 & & 0.183 & 16 \\
\hline & 9/1/2004 & $8: 01: 27 \mathrm{AM}$ & 25.467 & 25.242 & \begin{tabular}{|l|l|}
2 & 25.051 \\
\end{tabular} & 25.56 & 25.6 & & \begin{tabular}{|l|l|}
52.5899 \\
502
\end{tabular} & \begin{tabular}{|l|}
17.158 \\
17.07
\end{tabular} & 31.572 & $\begin{array}{r}-1.206 \\
-1.261\end{array}$ & & 50.4 & 0.052 & $\begin{array}{l}14.643 \\
\end{array}$ & & & & 51.5 & \begin{tabular}{ll|}
12.0 \\
\end{tabular} & & 2.734 & \begin{tabular}{|l|l|}
0.007 \\
\end{tabular} & $\begin{array}{l}0.007 \\
\end{array}$ & 0.000 & 0.187 & \\
\hline & 9/1/2004 & 8:02:27 AM & 25.46 & 25.234 & $\begin{array}{ll}4 & 25.048 \\
\end{array}$ & 25.557 & & & \begin{tabular}{|l|}
52.498 \\
\end{tabular} & \begin{tabular}{ll|}
17.072 \\
\end{tabular} & 31.603 & -1.212 & & 50.479 & 0.05 & & & & & 51.4 & 12.0 & & 2.732 & \begin{tabular}{|l|l|}
0.007 \\
\end{tabular} & 0.007 & & 0.180 & \\
\hline 2195 & 9/1/2004 & 8:03:27 AM & 25.477 & 25.232 & $\begin{array}{ll}225.066 \\
20.06\end{array}$ & 25.56 & 24.9 & & \begin{tabular}{|l|}
53.245 \\
\end{tabular} & $\begin{array}{l}17.118 \\
\end{array}$ & 32.135 & -1.212 & & 50.769 & 0.05 & & & & & 52.1 & 12.1 & & 2.773 & 0.007 & 0.007 & & 0.178 & \\
\hline \begin{tabular}{|l|l|l|l|l|}
2196 \\
2197
\end{tabular} & $9 / 1 / 2004$ & $\begin{array}{l}8: 04: 27 \mathrm{AM} \\
880.527 \mathrm{Am}\end{array}$ & $\begin{array}{r}\frac{25.479}{25.48} \\
\end{array}$ & $\frac{25.233}{25.23}$ & \begin{tabular}{l|l|} 
& 25.078 \\
3 & 25.594
\end{tabular} & \begin{tabular}{|l|l|} 
& 25.571 \\
& 25.618 \\
\end{tabular} & $\frac{24.90}{24.9}$ & \begin{tabular}{|l|}
48.142 \\
47.839 \\
\end{tabular} & \begin{tabular}{|r|}
52.809 \\
5.256
\end{tabular} & \begin{tabular}{|c|}
17.232 \\
17.03
\end{tabular} & $\begin{array}{l}31.929 \\
31.722 \\
\end{array}$ & $\begin{array}{l}0 .-1.209 \\
2-1.212 \\
\end{array}$ & $-\frac{1.108}{1000}$ & $\begin{array}{r}48.805 \\
51.96\end{array}$ & $\begin{array}{l}0.051 \\
0.052\end{array}$ & $\begin{array}{l}14.643 \\
14.643 \\
\end{array}$ & & $\begin{array}{l}891.08 \\
892.08\end{array}$ & & $\begin{array}{l}51.7 \\
51.5 \\
\end{array}$ & \begin{tabular}{l|l|l|}
11.6 \\
12.4
\end{tabular} & 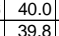 & $\frac{2.760}{2.743}$ & $\begin{array}{l}0.007 \\
0.007\end{array}$ & 0.007 & 0.000 & 0.182 & 0.15 \\
\hline 2198 & $9 / 1 / 12004$ & $8: 06: 27 \mathrm{AM}$ & $\begin{array}{r}25.402 \\
25.502\end{array}$ & 25.242 & $\begin{array}{ll}2 & 25.136\end{array}$ & \begin{tabular}{|l|} 
\\
\end{tabular} & 24.9 & $\begin{array}{l}4.055 \\
48.198\end{array}$ & \begin{tabular}{|l|}
53.027 \\
\end{tabular} & \begin{tabular}{|l|l|l|}
17.139 \\
\end{tabular} & 32.147 & 7. -1.212 & & $\begin{array}{l}51.96 \\
50.721\end{array}$ & $\begin{array}{l}.052 \\
0.052\end{array}$ & $\begin{array}{l}14.643 \\
14.643 \\
\end{array}$ & & & & $\begin{array}{l}51.5 \\
51.9\end{array}$ & $\begin{array}{l}12.4 \\
12.1\end{array}$ & & & $\begin{array}{l}0.007 \\
.007\end{array}$ & & & & 0.16 \\
\hline 2199 & \begin{tabular}{|c|}
$9 / 1 / 2004$ \\
\end{tabular} & $8: 07: 2$ & 25.504 & 25.244 & \begin{tabular}{l|l|l}
4 & 25.154
\end{tabular} & 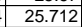 & 24 & 47.743 & \begin{tabular}{|l|}
52.631 \\
\end{tabular} & 17.211 & 31.4 & $\begin{array}{ll}4 & -1.215 \\
\end{array}$ & & 53.21 & 0.05 & 14.643 & & & & 51.5 & 12.7 & 3 & 2.728 & & 0.007 & & & $\begin{array}{l}0.16 \\
0.15\end{array}$ \\
\hline & & 8:08: & 25.507 & & \begin{tabular}{|l|l|}
6 & 25.146 \\
\end{tabular} & \begin{tabular}{|l|l|}
5.764 \\
\end{tabular} & & 47.864 & \begin{tabular}{|l|}
52.428 \\
\end{tabular} & $\begin{array}{l}17.248 \\
\end{array}$ & 31.654 & \begin{tabular}{|l|l|}
4 & -1.215 \\
\end{tabular} & & & 0.05 & 14.643 & & & & & 12.4 & & & 0.007 & & & & $\frac{0.15}{0.15}$ \\
\hline & 9/1/2004 & $8: 09: 2$ & 25.53 & 25.26 & \begin{tabular}{l|l|}
6 & 25.144 \\
\end{tabular} & 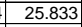 & & 47.853 & \begin{tabular}{|l|}
52.791 \\
\end{tabular} & $\begin{array}{l}17.336 \\
\end{array}$ & 31.533 & \begin{tabular}{|l|l|}
3 & -1.2099 \\
\end{tabular} & & 50.915 & 0.051 & & & & & 51.7 & 12.1 & & 2.737 & & & & & $\begin{array}{l}0.15 \\
0.16\end{array}$ \\
\hline 2202 & & & 25.529 & 25.238 & \begin{tabular}{l|l|l|}
825.083 \\
\end{tabular} & $\begin{array}{l}325.821 \\
\end{array}$ & & & 52.571 & 17.157 & 31.64 & $\mid-1.218$ & & 50.4 & 0.051 & 14.6 & & & & 51.4 & & & & & & & 184 & 0.16 \\
\hline 203 & $9 / 1 / 2004$ & & 25.552 & 25.261 & & 25.869 & & & & \begin{tabular}{|l|l|}
17.312 \\
\end{tabular} & 31.308 & -1.212 & & 51.57 & & 14.6 & & & & & & & & & & & & 16 \\
\hline & 9/1/2004 & & 25.549 & 25.259 & \begin{tabular}{l|l}
9 & 24.978 \\
\end{tabular} & 25.862 & & & 52.148 & 17.151 & 31.263 & -1.212 & & & 205 & 14.6 & & & & 51.0 & & & 71 & & & & & $\frac{10}{16}$ \\
\hline & 9/1/2004 & & 25.547 & 25.256 & \begin{tabular}{l|l}
6 & 24.92 \\
\end{tabular} & 25.819 & & 48.15 & \begin{tabular}{|l|} 
\\
\end{tabular} & 17.309 & 31.761 & $\begin{array}{l}11 \\
\end{array}$ & & 51.228 & 0.051 & 14.643 & & & & 51.7 & & & & & & & & \\
\hline & & $8: 14: 27 \mathrm{~A}$ & 25.544 & 25.258 & $\begin{array}{ll}824.88 \\
\end{array}$ & 25.801 & & & 52.878 & 17.364 & 31 & $\begin{array}{l}8 \\
\end{array}$ & & 50.536 & 0.05 & 14. & & & & 51.8 & 12. & & 8.74 & & & & & \\
\hline & & & 25.526 & 25.245 & \begin{tabular}{l|l|l}
5 & 24.76 \\
5
\end{tabular} & 25.7 & & 47.901 & 52.741 & 17.064 & 31.884 & $\begin{array}{ll}4 & -1.2 \\
\end{array}$ & & 51.931. & 0.05 & 14. & & & & 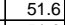 & & & & & & & & \\
\hline & & & & & & & & & 52.689 & & & & & & & & & & & 51.6 & & & & & & & & \\
\hline & & & 25.54 & 25.275 & 24.154 & 25.8 & & & 52.635 & $17.24 t$ & 31. & -1.206 & & & 0.05 & & & & & 51.5 & 2. & & & & & & 0.181 & 31 \\
\hline & & & 25522 & 25.271 & 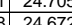 & 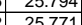 & & & $\frac{52.453}{5265}$ & $\begin{array}{l}16.955 \\
17182\end{array}$ & & $\begin{array}{l}-1.212 \\
-1.212\end{array}$ & & & 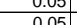 & & & & & (51.35 & & & & & & & & \\
\hline & $9 / 1 / 1 / 2004$ & & 25.495 & & $\mid 2462$ & 25.773 & & & 52.548 & $\begin{array}{l}17.102 \\
16.949\end{array}$ & & \begin{tabular}{|l|l|}
3 & -1.2 .2 \\
\end{tabular} & & 50.64 & 0.049 & 14.6 & & & & 51.4 & $12.1 \mathrm{C}$ & & & & & & & \\
\hline & $9 / 1 / 2004$ & & 25.498 & & $\begin{array}{l}724.626 \\
\end{array}$ & \begin{tabular}{|l|r|}
525.79 \\
\end{tabular} & & & 52.507 & & & \begin{tabular}{|l|l|}
3 & -1.215 \\
\end{tabular} & & & & & & & & $\begin{array}{l}51.4 \\
51.4\end{array}$ & $12.2 \mathrm{C}$ & & & & & & $\frac{1.178}{0.178}$ & \\
\hline & & & 25.478 & 25.273 & \begin{tabular}{l|l}
3 & 24.572 \\
\end{tabular} & 25.766 & & & \begin{tabular}{|l|}
52.733 \\
\end{tabular} & 17.252 & 31.696 & $\mid$\begin{tabular}{|c|}
$\mid 5.215$ \\
5
\end{tabular} & & & 0.05 & 14. & & & & 51.6 & $\frac{12.1}{12.1}$ & & & & & 2000 & 182 & \\
\hline & & & 25.4 & & & & & & & & & & & & & & & & & & & & & & & & & \\
\hline & & & 25.45 & & & & & & 52.407 & \begin{tabular}{l|l|}
17.02 \\
\end{tabular} & & $\begin{array}{l}4 \\
4\end{array}$ & & $50 . \varepsilon$ & & & & & & 1.3. & 2 & & & & & & & \\
\hline & & & 25.42 & 20.201 & 24.475 & 25.774 & & & 52.732 & 17.155 & & -1.212 & & & 0.049 & & & & & 51.6 & $12.2 \mathrm{CH}$ & & & 0.007 & & & 0.179 & \\
\hline & & & 25.42. & 25.272 & & 25.745 & & & \begin{tabular}{|l|}
52.538 \\
\end{tabular} & $\begin{array}{l}17.015 \\
\end{array}$ & & -1.215 & & & & & & & & 51.4 & 11.6 & & & 0.007 & & & 0.182 & \\
\hline & $9 / 1 / 2$ & & 25.408 & & & 25.7 & & & 52.25 & 17.0 & & \begin{tabular}{|l|l|}
5 & -1.209 \\
\end{tabular} & & & & & & & & 51.1 & 11.9 & & & & & & 0.187 & \\
\hline & & & 25.383 & & & & & & & & & -1.2 & & & & & & & & & & & & & & & & \\
\hline & & & 25.374 & & & 25 & & & 52.689 & 17.1 & & $\begin{array}{l}4 \\
\end{array}$ & & & 0.05 & & & & & 51.6 & 12.2 & & & & & & & \\
\hline & & & 25. & & & & & & & & & & & & & & & & & & & & & & & & & \\
\hline & & & 25.34 & 25 & & 25. & & & 53.008 & 11.2. & 31.8 & -1.2 & & & & & & & & & 2. & & & & & & & \\
\hline & & & 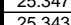 & 25.271 & & $\frac{25}{257}$ & & & \begin{tabular}{|l|}
52.737 \\
52604 \\
\end{tabular} & 1.2 .2 & & 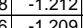 & & & & . & & & & 515 & 124 & & & & & & & \\
\hline & $9 / 1 / 1200$ & & 2532 & & & & & & \begin{tabular}{|l|l|}
5281 \\
5
\end{tabular} & & & \begin{tabular}{l|l}
4 & -1.2 \\
\end{tabular} & & & & & & & & & 16 & & & & & & & \\
\hline & & & & & & & & & & & & & & & & & & & & & 1. & & & & & & & \\
\hline & & & $\frac{25.29}{25.29}$ & & & & & & \begin{tabular}{|l|}
52.699 \\
\end{tabular} & & & \begin{tabular}{l|l}
4 & -1.212 \\
\end{tabular} & & & & & & & & & $12.1 \mathrm{Y}$ & & 2.8 & & & & & \\
\hline & & & & & & & & & & & & & & & & & & & & & & & & & & & & \\
\hline & & & 25.2 & & & & & & & & & & & & & & & & & & & & & & & & & \\
\hline & & & 25.28 & & $2 a$ & & & & & & 31.2 & -1. & & & & 14. & & & & & 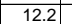 & & & & & & & \\
\hline & & & 25.27 & 25.267 & 24. & 25.745 & & 47.8 & 52.691 & 17.3 & 31.5 & -1.2 & & & & 14. & & & & & & & & & & & & \\
\hline & $11 / 20$ & & 5.26 & 252 & 24.3. & & & 47. & 52.73 & 17.1 & 31.7 & \begin{tabular}{|l|l|l|}
8 & -1.2 \\
\end{tabular} & & & 0.6 & 14.6 & & & & & & & & & & & & \\
\hline & & & 25.2 & 25.2 & 24.306 & & & 48. & 938 & & 31.7 & -1.2 & & & 0.05 & & & & & & & & & & & & & \\
\hline & & & 25.2 & 25.2 & $\begin{array}{llll}8 & 24 \\
\end{array}$ & 25 & & & & & & $\begin{array}{l}3 \\
3\end{array}$ & & & 0.049 & & & & & & & & & & & & & \\
\hline & & & 25.2 & & & & & & & & & -1. & & & & & & & & & & & & & & & & \\
\hline & & & $\frac{25.23}{2.21}$ & 25.269 & & 25. & & 4.5 & 52.608 & 1.0 & & -1.22 & & & & & & & & & 1.9 & & & & & & & \\
\hline 23 & 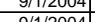 & & 23.214 & 25.259 & $\begin{array}{ll}74.272 \\
7\end{array}$ & $\mid \begin{array}{l}1 \\
25.1 .747\end{array}$ & & 4.1. & 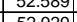 & $\begin{array}{l}17.295 \\
17201\end{array}$ & S1.49t & -1.215 & & & 0.049 & 14.6 & & & & $\begin{array}{ll}51.5 \\
519\end{array}$ & $\begin{array}{ll}11.1 .9 \\
11.1\end{array}$ & & 27 & & 0.007 & 0.000 & 0.181 & 81 \\
\hline 2240 & $9 / 1 / 2004$ & $8: 48: 27 \mathrm{AN}$ & 25.212 & $\begin{array}{r}25.271 \\
251\end{array}$ & \begin{tabular}{|l|l|}
1124.25 \\
1
\end{tabular} & \begin{tabular}{|l|l|} 
& 25.774 \\
\end{tabular} & 25.062 & $\begin{array}{l}40.14 \\
47.692 \\
\end{array}$ & \begin{tabular}{|l|}
52.542 \\
\end{tabular} & \begin{tabular}{|l|l|l|l}
17.154 \\
\end{tabular} & $\begin{array}{l}0.54 \\
31.54\end{array}$ & \begin{tabular}{|l|l|}
4 & -1.212 \\
\end{tabular} & & 49.508 & 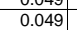 & 14.643 & & 2935.08 & \begin{tabular}{|l|l|}
4.0 .914 \\
48.9181
\end{tabular} & $\begin{array}{l}51.4 \\
51.4\end{array}$ & \begin{tabular}{|l|}
11.8 \\
\end{tabular} & & \begin{tabular}{|l|l|}
2.731 \\
\end{tabular} & \begin{tabular}{|l|}
0.007 \\
\end{tabular} & \begin{tabular}{|l|}
0.007 \\
\end{tabular} & \begin{tabular}{|c|}
0.000 \\
\end{tabular} & \begin{tabular}{|l|}
0.181 \\
\end{tabular} & 81 \\
\hline
\end{tabular}


WSRC-TR-2005-00105, REVISION 0

SRNL-RPP-2005-00012, REVISION 0

RUN \# 4.03A AND B; FIRST AND SECOND HALF OF SLURRY DEWATERING - CONT.

\begin{tabular}{|c|c|c|c|c|c|c|c|c|c|c|c|c|c|c|c|c|c|c|c|c|c|c|c|c|c|c|c|c|}
\hline & $\mathrm{A}_{\mathrm{A}}$ & B & D & $E$ & $F$ & $G$ & $\mathrm{H}$ & $\mathrm{J}$ & \begin{tabular}{l|l}
$\mathrm{K}$ & \\
\end{tabular} & $\mathrm{L}$ & $M$ & $\mathrm{~N}$ & 0 & O & $R$ & $\mathrm{~s}$ & $\begin{array}{ll}T \\
\end{array}$ & $\mathrm{v}$ & w & $x$ & Y & $z_{2}$ & & $A B$ & & & & \\
\hline & 9/1/2004 & 8:49:27 AM & 25.202 & 25.262 & 24.25 & 25.755 & 25.117 & 47.887 & 52.772 & 17.449 & 31.48 & -1.215 & $\begin{array}{l}-7.082 \\
\end{array}$ & 50.25 & 0.05 & 14.643 & 0.003 & 2936.08 & \begin{tabular}{|l|l|}
48.9347 \\
\end{tabular} & 51.6 & 12.0 & 39.7 & 2.736 & 0.007 & 0.007 & 0.000 & 0.184 & 0.16 \\
\hline & $\begin{array}{l}9 / 1 / 12004 \\
\end{array}$ & $8: 50: 27 \mathrm{AM}$ & 25.188 & 25.262 & 24.241 & 25.715 & 25.118 & $\begin{array}{r}47.758 \\
\end{array}$ & 52.589 & 17.174 & 31.554 & \begin{tabular}{|c|}
-1.215 \\
\end{tabular} & $\begin{array}{r}-7.099 \\
\end{array}$ & 51.433 & \begin{tabular}{|c|}
0.049 \\
\end{tabular} & $\begin{array}{l}14.643 \\
\end{array}$ & 0.003 & & \begin{tabular}{|l}
8.9514 \\
\end{tabular} & \begin{tabular}{|l|}
51.5 \\
\end{tabular} & 12.3 & & 2.734 & & 0.007 & & & \\
\hline & $9 / 1 / 2004$ & $8: 51: 27 \mathrm{AM}$ & 25.199 & 25.273 & \begin{tabular}{|l|l|}
24.246 \\
2251
\end{tabular} & \begin{tabular}{|l|l|}
25.756 \\
\end{tabular} & 25.179 & $\begin{array}{l}47.727 \\
\end{array}$ & \begin{tabular}{|l|}
52.558 \\
5.304
\end{tabular} & $\begin{array}{l}17.212 \\
1721\end{array}$ & 31.634 & \begin{tabular}{|c|}
-1.212 \\
\end{tabular} & $\begin{array}{l}-7.087 \\
-709 \\
\end{array}$ & 50.753 & \begin{tabular}{|l|l|}
0.049 \\
\end{tabular} & 14.643 & 0.003 & c50.000 & & \begin{tabular}{|l|l|}
51.4 \\
\end{tabular} & 12.1 & 39.1 & .736 & 0.007 & 0.007 & 0.000 & 0.181 & \\
\hline & $9 / 1 / 2004$ & $\begin{array}{l}8: 52: 27 \mathrm{AM} \\
885: 27 \mathrm{Am}\end{array}$ & $\begin{array}{l}25.194 \\
25.84\end{array}$ & $\begin{array}{l}25.273 \\
25274\end{array}$ & \begin{tabular}{|l|}
24.251 \\
24242 \\
\end{tabular} & \begin{tabular}{|l|l|}
25.746 \\
25.737 \\
\end{tabular} & $\begin{array}{l}25.214 \\
.25 .23\end{array}$ & $\begin{array}{l}48.044 \\
48.17\end{array}$ & \begin{tabular}{|l|l|}
53.064 \\
5219 \\
\end{tabular} & \begin{tabular}{|l|l|}
17.149 \\
17.32
\end{tabular} & $\begin{array}{l}31.749 \\
21.702\end{array}$ & $\begin{array}{l}-1.212 \\
.2125\end{array}$ & $\begin{array}{r}-7.085 \\
7095\end{array}$ & $\begin{array}{l}49.224 \\
5.0202\end{array}$ & $\begin{array}{l}0.05 \\
\end{array}$ & $\begin{array}{l}14.643 \\
1.43\end{array}$ & 0.003 & 2939.08 & \begin{tabular}{|l|l|l|}
8 & 48.9847 \\
\end{tabular} & \begin{tabular}{|l|l|}
51.9 \\
5.1 \\
\end{tabular} & 11.7 & 39.9 & 7.751 & 0.007 & $\begin{array}{l}0.007 \\
\end{array}$ & 0.000 & $\begin{array}{l}0.183 \\
\end{array}$ & \\
\hline & $\begin{array}{l}9 / 1 / 2004 \\
9 / 1 / 2004\end{array}$ & $\begin{array}{l}8: 53: 27 \mathrm{AM} \\
85.27 \mathrm{AM}\end{array}$ & $\begin{array}{l}25.184 \\
25.179 \\
\end{array}$ & $\frac{25.274}{25274}$ & & \begin{tabular}{|l|l|}
25.737 \\
25742
\end{tabular} & & \begin{tabular}{|l|l|}
48.167 \\
47.994
\end{tabular} & \begin{tabular}{|l|}
53.184 \\
53329 \\
\end{tabular} & \begin{tabular}{|c|c|}
17.33 \\
17.277
\end{tabular} & $\begin{array}{l}31.743 \\
31.704 \\
31\end{array}$ & $\begin{array}{l}-1.215 \\
-1.212\end{array}$ & & $\begin{array}{l}50.292 \\
50667\end{array}$ & $\begin{array}{l}0.05 \\
0.05 \\
\end{array}$ & \begin{tabular}{|l|l|l|}
14.643 \\
14634 \\
\end{tabular} & & $\begin{array}{l}2940.08 \\
201\end{array}$ & & \begin{tabular}{|l|}
52.1 \\
510 \\
\end{tabular} & \begin{tabular}{|l|}
12.0 \\
121 \\
\end{tabular} & & & & & & 0.183 & \\
\hline 247 & 9/1/1/2004 & $\begin{array}{l}8: 55: 27 \mathrm{AM} \\
\text { 8:55:27 }\end{array}$ & $\frac{25.1 / 9}{25.175}$ & $\begin{array}{l}25.274 \\
25.275\end{array}$ & \begin{tabular}{|l|}
24.247 \\
24.223 \\
\end{tabular} & \begin{tabular}{|l|}
25.742 \\
25.768 \\
\end{tabular} & $\begin{array}{l}25.185 \\
25.175\end{array}$ & \begin{tabular}{|l|}
47.994 \\
4.838 \\
\end{tabular} & \begin{tabular}{|r|}
53.029 \\
52.95 \\
\end{tabular} & \begin{tabular}{|l|}
17.7277 \\
17.163
\end{tabular} & $\begin{array}{l}31.704 \\
31.876\end{array}$ & $\mid$\begin{tabular}{|c|}
-1.2121 \\
-1.212 \\
\end{tabular} & $\begin{array}{r}-7.09 \\
-7.085 \\
\end{array}$ & $\begin{array}{l}50.667 \\
51.366\end{array}$ & $\begin{array}{l}0.05 \\
0.05\end{array}$ & \begin{tabular}{|l|}
14.6433 \\
14643 \\
\end{tabular} & $\begin{array}{l}0.0033 \\
0.003\end{array}$ & $\begin{array}{l}2944.08 \\
294.08\end{array}$ & $\begin{array}{l}99.01811 \\
99.0347\end{array}$ & \begin{tabular}{|l|}
$\mid 51.9$ \\
51.8 \\
\end{tabular} & \begin{tabular}{|l|}
12.1 \\
12.2 \\
\end{tabular} & $\begin{array}{l}39.8 \\
40.0\end{array}$ & 2.7475 & 0.007 & \begin{tabular}{|l|}
0.007 \\
0.007
\end{tabular} & $\begin{array}{l}0.000 \\
0.000\end{array}$ & \begin{tabular}{|c|c|}
0.183 \\
0.133
\end{tabular} & 0.16 \\
\hline 248 & $9 / 1 / 2004$ & $8: 56: 27 \mathrm{AM}$ & 25.165 & & & 25.753 & 25.265 & & & 17.078 & 32.149 & & $\begin{array}{l}-7.093 \\
\end{array}$ & 50.294 & & 14.643 & $\begin{array}{l}0.003 \\
0.003\end{array}$ & $\frac{2944.00}{2943.08}$ & \begin{tabular}{|l|l|}
49.0341 \\
49.0514
\end{tabular} & \begin{tabular}{|l|}
51.1. \\
51.7 \\
\end{tabular} & \begin{tabular}{|l|}
12.2 \\
\end{tabular} & 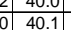 & $\frac{2.150}{2.763}$ & $\begin{array}{l}0.007 \\
0.007\end{array}$ & $\begin{array}{l}0.007 \\
0.007\end{array}$ & $\begin{array}{l}0.000 \\
0.000\end{array}$ & & $\begin{array}{l}0.16 \\
0.15\end{array}$ \\
\hline & & $8: 57: 27 \mathrm{AM}$ & & & \begin{tabular}{|l|}
24.262 \\
\end{tabular} & \begin{tabular}{|l|}
25.767 \\
\end{tabular} & & $\begin{array}{l}47.748 \\
\end{array}$ & & 17.166 & 31.558 & \begin{tabular}{|c|} 
\\
\end{tabular} & $\begin{array}{l}-7.087 \\
\end{array}$ & 49.729 & 0.049 & 14.643 & & & 349.0681 & \begin{tabular}{|l|}
51.6 \\
\end{tabular} & & & & & & & & $\frac{0.15}{0.15}$ \\
\hline & 9/1/2004 & 8:58:27 AM & & 25.275 & \begin{tabular}{|l|}
24.228 \\
\end{tabular} & & & 47.696 & 52.631 & \begin{tabular}{ll|}
17.272 \\
\end{tabular} & & -1.209 & $\begin{array}{l}-7.09 \\
\end{array}$ & 49.009 & \begin{tabular}{|c|c|}
0.049 \\
\end{tabular} & \begin{tabular}{|l|l|}
14.643 \\
\end{tabular} & 0.004 & & & 51.5 & 11.7 & 39.6 & 2.730 & & 0.007 & 0.000 & & \\
\hline & & & 25.154 & & & 752 & & 47.862 & & & 31.767 & & & 51.028 & & \begin{tabular}{l|l}
14.643 \\
\end{tabular} & & & & & & & & .007 & 0.007 & & 0.180 & \\
\hline & & & & & \begin{tabular}{|l|l|}
24.217 \\
\end{tabular} & & & 47.739 & \begin{tabular}{|l|}
52.687 \\
\end{tabular} & 17.196 & 31.406 & & & & & 14.643 & & & & 51.6 & & & & & & & . 181 & \\
\hline & & 9:01:27 AM & 25.16 & 25.28 & 24.243 & 8.758 & & & 50201 & 17.102 & .556 & & & & 049 & 14.643 & & & & 51.3 & & & & 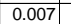 & & & .181 & \\
\hline & & 9:02:27 AM & & 25.281 & 24.224 & 25.804 & & \begin{tabular}{|l|l|}
47.961 \\
\end{tabular} & \begin{tabular}{|l|}
52.886 \\
\end{tabular} & 17.125 & 31.909 & $\mid-1.215$ & -7.087 & 50.632 & $\mid 0.05$ & \begin{tabular}{|l|l|l|l}
14.643 \\
\end{tabular} & & & \begin{tabular}{|l|l|l|}
491414 \\
\end{tabular} & & & 39.9 & 2.753 & 0.007 & 0.007 & 0.000 & & \\
\hline & 9/1/2004 & 9:03:27 AM & 25.142 & 25.276 & \begin{tabular}{|l|}
24.239 \\
\end{tabular} & \begin{tabular}{|l|l|}
25.759 \\
\end{tabular} & 25.282 & & \begin{tabular}{|l|}
52.743 \\
\end{tabular} & \begin{tabular}{|l|l|}
17.173 \\
\end{tabular} & 31.654 & -1.218 & $\begin{array}{l}-7.096 \\
\end{array}$ & 50.636 & 0.048 & & 0.003 & 2950.08 & \begin{tabular}{|l|l}
39.1681 \\
\end{tabular} & 51.6 & 12.1 & & & & & & & \\
\hline & & 9:04:27 AM & & $\frac{25.313}{25.313}$ & \begin{tabular}{|l|}
24.371 \\
\end{tabular} & \begin{tabular}{|l|l|}
25.806 \\
2.78 \\
\end{tabular} & 25.243 & $\begin{array}{l}47.646 \\
\end{array}$ & \begin{tabular}{|r|}
52.61 \\
\end{tabular} & $\begin{array}{l}17.19 \\
\end{array}$ & $\begin{array}{l}31.577 \\
377\end{array}$ & $\begin{array}{l}-1.212 \\
-1.212 \\
\end{array}$ & \begin{tabular}{|c|c|}
-7.087 \\
\end{tabular} & 51.141 & $\begin{array}{l}0.048 \\
\end{array}$ & 14.643 & & & & $\begin{array}{l}51.5 \\
5.5\end{array}$ & & & & & & & 0.177 & \\
\hline & 9/1/2004 & 9:05:27 AM & & & 24.376 & & & & \begin{tabular}{|l|}
52.507 \\
\end{tabular} & & 31.574 & $\begin{array}{l}-1.212 \\
\end{array}$ & & & 0.05 & $\begin{array}{l}4.643 \\
\end{array}$ & & & 49.2014 & 51.4 & & & & 0.007 & & & $\begin{array}{l}0.184 \\
\end{array}$ & \\
\hline & & 9:06:27 AM & 25.154 & \begin{tabular}{|l|}
25.283 \\
25203
\end{tabular} & $\begin{array}{l}24.327 \\
2.232\end{array}$ & \begin{tabular}{|l|l|}
25.801 \\
\end{tabular} & $\begin{array}{l}25.199 \\
25271\end{array}$ & $\begin{array}{r}47.658 \\
47917\end{array}$ & & & & & $\begin{array}{r}-7.087 \\
\end{array}$ & 53.003 & & \begin{tabular}{|l|l|}
14.643 \\
\end{tabular} & 0.003 & & 499.2181 & 51.5 & 12. & & & 0.007 & & & $\begin{array}{l}0.181 \\
\end{array}$ & \\
\hline & $\begin{array}{l}9 / 1 / 2004 \\
9 / 1 / 2004\end{array}$ & $\begin{array}{l}9: 07: 27 \mathrm{AM} \\
0902 \cdot 27 \mathrm{AM}\end{array}$ & $\begin{array}{r}25.159 \\
25.18\end{array}$ & $\begin{array}{l}25.293 \\
25.305\end{array}$ & $\begin{aligned} 24.322 \\
24.308\end{aligned}$ & \begin{tabular}{|l|}
25.801 \\
25.843
\end{tabular} & $\begin{array}{l}25.274 \\
25345\end{array}$ & \begin{tabular}{|l|l|l|}
4777 \\
\end{tabular} & \begin{tabular}{|l|l|}
52.844 \\
52556 \\
\end{tabular} & $\begin{array}{l}17.346 \\
17226\end{array}$ & \begin{tabular}{|l|l|}
31.513 \\
31324
\end{tabular} & \begin{tabular}{|c|}
-1.212 \\
.212
\end{tabular} & $\begin{array}{l}-7.09 \\
7.085\end{array}$ & \begin{tabular}{|l|}
51.084 \\
1973
\end{tabular} & \begin{tabular}{|c|c|}
0.048 \\
0019
\end{tabular} & 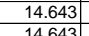 & & & $\begin{array}{l}49.2347 \\
492514\end{array}$ & \begin{tabular}{|l|l|}
51.7 \\
51.4
\end{tabular} & & 39.7 & & 0.007 & 0.007 & & 0.177 & 0.15 \\
\hline 261 & 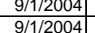 & $\begin{array}{l}9: 08: 27 \mathrm{AM} \\
9: 09: 18 \mathrm{~A}\end{array}$ & $\begin{array}{r}25.18 \\
25.166\end{array}$ & $\begin{array}{l}25.3055 \\
25.296\end{array}$ & \begin{tabular}{|l|}
24.308 \\
24.294 \\
\end{tabular} & \begin{tabular}{|l|}
25.843 \\
25.814 \\
\end{tabular} & $\begin{array}{l}25.345 \\
25.296 \\
\end{array}$ & \begin{tabular}{r|}
47.6 \\
47.02
\end{tabular} & \begin{tabular}{|r|}
52.5464 \\
55.484
\end{tabular} & $\begin{array}{r}17.226 \\
17.325 \\
\end{array}$ & $\begin{array}{l}31.334 \\
31.273\end{array}$ & $\begin{array}{l}-1.2121 \\
-1.215\end{array}$ & $\begin{array}{r}-7.085 \\
-7.09 \\
\end{array}$ & $\begin{array}{l}\frac{49.743}{51.151} \\
5\end{array}$ & $\begin{array}{l}0.049 \\
0.048\end{array}$ & $\begin{array}{l}\frac{14.643}{14.643} \\
\end{array}$ & $\begin{array}{l}0.003 \\
0.003\end{array}$ & & $\frac{2514}{2656}$ & $\frac{55.4}{51.4}$ & $\frac{11.8}{12.2}$ & $\begin{array}{l}33.5 \\
3.4 .4\end{array}$ & . & 0.007 & $\begin{array}{l}0.007 \\
0.007\end{array}$ & 0.000 & $\begin{array}{l}0.181 \\
0.178\end{array}$ & 0.15 \\
\hline & $9 / 1 / 2004$ & 9:10:18 AM & 25.16 & 25.295 & \begin{tabular}{|l|}
24.298 \\
\end{tabular} & \begin{tabular}{|l|}
25.814 \\
\end{tabular} & 25.245 & 47.368 & \begin{tabular}{|l|}
52.219 \\
\end{tabular} & 17.243 & 31.053 & $\begin{array}{l}-1.212 \\
\end{array}$ & $\begin{array}{l}-7.079 \\
-7.079\end{array}$ & $\begin{array}{l}51.151 \\
50.732\end{array}$ & 0.05 & $\begin{array}{l}14.045 \\
14.643\end{array}$ & $\begin{array}{l}0.003 \\
0.003\end{array}$ & $\begin{array}{l}2950.95 \\
2956.93\end{array}$ & \begin{tabular}{|l|}
49.26520 \\
49.2822
\end{tabular} & $\begin{array}{l}51.4 \\
51.1\end{array}$ & $\frac{1<.2}{12.1}$ & $\begin{array}{l}39.4 \\
39.2 \\
\end{array}$ & $\frac{2.119}{2.703}$ & 0.007 & $\begin{array}{l}0.007 \\
0.007\end{array}$ & 0.000 & $\begin{array}{l}0.186 \\
0.186 \\
\end{array}$ & $\begin{array}{l}0.15 \\
0.16\end{array}$ \\
\hline & 9/1/2004 & 9:11:18 AM & 25.156 & 25.285 & 24.249 & \begin{tabular}{|l|l}
25.789 \\
\end{tabular} & 25.296 & $\begin{array}{l}47.752 \\
\end{array}$ & \begin{tabular}{|r|}
52.49 \\
52
\end{tabular} & 17.307 & 31.587 & \begin{tabular}{|c|}
-1.218 \\
\end{tabular} & $\begin{array}{r}-7.09 \\
\end{array}$ & 50.642 & 0.048 & 14.643 & & & \begin{tabular}{|l|l|}
49.2989 \\
\end{tabular} & 51.4 & 12.1 & 39.7 & 2.735 & 0.007 & 0.007 & 0.000 & 0.177 & \\
\hline & & 9:12:18 AM & 25.166 & 25.296 & 24.264 & & & $\begin{array}{l}47.582 \\
\end{array}$ & \begin{tabular}{|l|l|l|}
52.498 \\
\end{tabular} & 17.115 & $\begin{array}{l}31.548 \\
\end{array}$ & -1.212 & & 50.212 & $\begin{array}{l}0.049 \\
\end{array}$ & $\begin{array}{l}14.643 \\
\end{array}$ & & 2958.93 & 49.3156 & 51.4 & 12.0 & 39.6 & 2.728 & 0.007 & 0.007 & 0.000 & 0.181 & \\
\hline & $9 / 1 / 2004$ & 9:13:18 AM & $\begin{array}{l}25.168 \\
25.69\end{array}$ & \begin{tabular}{|l|l|}
25.307 \\
25.302
\end{tabular} & $\begin{array}{l}24.26 \\
20.265\end{array}$ & \begin{tabular}{|l|}
25.835 \\
\end{tabular} & $\begin{array}{l}25.328 \\
25.3212\end{array}$ & & \begin{tabular}{|l|l|}
52.287 \\
5.675 \\
\end{tabular} & $\begin{array}{l}17.162 \\
17.223\end{array}$ & \begin{tabular}{|l|l|}
31.244 \\
3149
\end{tabular} & $\begin{array}{r}-1.215 \\
.215\end{array}$ & -7.079 & $\begin{array}{l}49.497 \\
4039\end{array}$ & 0.05 & \begin{tabular}{|l|l|l|}
1.643 \\
1613
\end{tabular} & $\begin{array}{l}0.003 \\
\end{array}$ & & 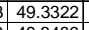 & 51.2 & 11.8 & $\begin{array}{l}39.3 \\
2.3 \\
\end{array}$ & 2.709 & 0.007 & $\begin{array}{l}0.007 \\
\end{array}$ & 0.000 & 0.186 & \\
\hline & $\begin{array}{l}9 / 1 / 2004 \\
9 / 1 / 2004\end{array}$ & $\begin{array}{l}9: 14: 18 \mathrm{AM} \\
9 \cdot 15: 18 \mathrm{AM}\end{array}$ & $\begin{array}{l}25.168 \\
25.163\end{array}$ & $\begin{array}{l}25.302 \\
25.302\end{array}$ & $\begin{array}{l}24.265 \\
24.245\end{array}$ & \begin{tabular}{|l|}
25.805 \\
25.86 \\
\end{tabular} & $\begin{array}{r}25.343 \\
25.348\end{array}$ & \begin{tabular}{|c|}
47.679 \\
47742
\end{tabular} & \begin{tabular}{|l|}
52.675 \\
52312 \\
\end{tabular} & $\begin{array}{r}17.223 \\
17.153\end{array}$ & \begin{tabular}{|l|}
31.419 \\
31.355
\end{tabular} & \begin{tabular}{r|r|}
-1.215 \\
-1.212
\end{tabular} & -7.067 & $\begin{array}{l}49.038 \\
51844\end{array}$ & $\begin{array}{l}0.049 \\
0.051\end{array}$ & \begin{tabular}{|l|l|}
14.643 \\
14.633
\end{tabular} & $\begin{array}{l}0.003 \\
0.03\end{array}$ & $\begin{array}{l}2960.93 \\
2961.93\end{array}$ & \begin{tabular}{|l|l|}
49.3489 \\
9.3656
\end{tabular} & $\frac{51.5}{51.2}$ & $\frac{11.7}{12.3}$ & $\frac{39.5}{39.4}$ & & 0.007 & 0.007 & 0.000 & $\begin{array}{l}0.181 \\
0.189\end{array}$ & 0.15 \\
\hline & 9/1/1/2004 & $\begin{array}{l}\text { :15:18 } \mathrm{AM} \\
9: 16: 18 \mathrm{~A}\end{array}$ & $\begin{array}{l}25.163 \\
25.163\end{array}$ & 25.308 & \begin{tabular}{|l|}
24.24 .246 \\
24.26 \\
\end{tabular} & \begin{tabular}{|l|}
25.86 \\
\end{tabular} & $\begin{array}{l}25.348 \\
25.298\end{array}$ & $\begin{array}{l}4.47 .62 \\
47.621\end{array}$ & \begin{tabular}{|c|}
52.212 \\
52.66
\end{tabular} & 17.097 & $\begin{array}{r}31.355 \\
31.47 \\
\end{array}$ & $\frac{-1.212}{-1.218}$ & $\begin{array}{l}-7.06 \\
-7.08 \\
-10\end{array}$ & $\begin{array}{l}51.644 \\
51.097\end{array}$ & $\begin{array}{l}0.051 \\
0.049\end{array}$ & $\begin{array}{l}\frac{14.645}{14.643} \\
\end{array}$ & 0.00 & & & $\begin{array}{l}51.2 \\
51.5\end{array}$ & $\frac{12.3}{12.2}$ & $\begin{array}{l}39.4 \\
39.5\end{array}$ & & $\begin{array}{l}0.007 \\
0.007\end{array}$ & 0.007 & & & $\frac{0.16}{0.15}$ \\
\hline & $9 / 1 / 2004$ & $9: 17: 18 \mathrm{AM}$ & 25.174 & 25.324 & \begin{tabular}{|l|}
24.257 \\
\end{tabular} & \begin{tabular}{|l|}
25.807 \\
\end{tabular} & 25. & 47.706 & \begin{tabular}{|l|}
52.867 \\
\end{tabular} & 17.02 & 31.572 & $\mid-1.212$ & -7.076 & 51.103 & 0.049 & 14.643 & & & & 51.7 & 12.2 & 39. & 2.73 & 0.007 & 0.007 & & & 0.15 \\
\hline & & $9: 18: 18 \mathrm{AM}$ & 25.164 & 25.313 & \begin{tabular}{|l|}
24.252 \\
\end{tabular} & \begin{tabular}{|l|}
25.782 \\
\end{tabular} & & 47.884 & \begin{tabular}{|l|l|}
52.873 \\
\end{tabular} & 17.084 & 31.95 & \begin{tabular}{|l|}
-1.212 \\
\end{tabular} & & 50.736 & 0.05 & 14.643 & & & & 51.7 & 121 & & & & & & & $\frac{0.15}{0.16}$ \\
\hline & 9/1/2004 & 9:19:18 AM & 25.159 & & 24.217 & \begin{tabular}{|l|l|}
25.782 \\
\end{tabular} & 25.1 & $\begin{array}{l}47.824 \\
\end{array}$ & \begin{tabular}{|l|l|}
52.776 \\
\end{tabular} & \begin{tabular}{|l|l|}
17.243 \\
\end{tabular} & 31.638 & $\begin{array}{l}-1.212 \\
\end{array}$ & -7.0 & 49.8 & $\begin{array}{l}0.048 \\
\end{array}$ & 14.643 & & & & & 11.9 & 39.7 & & 0.007 & & & & $\frac{0.16}{0.15}$ \\
\hline & & 9:20:18 AM & 25.15 & 25.309 & \begin{tabular}{|l|l|}
24.232 \\
\end{tabular} & \begin{tabular}{|l|l|}
25.772 \\
\end{tabular} & 25.28 & & 52.834 & & 31.85 & $\begin{array}{l}-1.212 \\
\end{array}$ & & 50.696 & 0.049 & & & & & 51.7 & & & & 0.007 & 0.6 & & 180 & \\
\hline & & 9:21:18 AM & 25. & & 24.213 & \begin{tabular}{|l|l|}
25.783 \\
\end{tabular} & 25.2 & 47.507 & \begin{tabular}{|l|}
52.42 \\
\end{tabular} & \begin{tabular}{|l|l|}
16.894 \\
\end{tabular} & 31.907 & -1.212 & & 51. & 0.048 & \begin{tabular}{|l|l|}
14.643 \\
\end{tabular} & & & & & & & & & & & & \\
\hline & & 9:22:18 AM & & 25.316 & 24.204 & \begin{tabular}{|l|l|}
25.739 \\
\end{tabular} & & 47.88 & & 17.222 & 31.663 & -1.212 & & & 0.049 & 14.643 & & & & 51.9 & & 39 & 2.742 & & & & 180 & \\
\hline & 9/1/2004 & & 138 & 25.327 & 24.23 & 25.735 & & 47.7 & \begin{tabular}{|l|l|l|} 
\\
\end{tabular} & 16.998 & 31.726 & -1.212 & & 49.88 & 0.049 & \begin{tabular}{l|l}
14.643 \\
\end{tabular} & & & & 51.8 & & & & & & & & \\
\hline & & 9:24:18 AN & 25.127 & 25.321 & 24.2 & 25.735 & & $47 . .9$ & \begin{tabular}{|l|l|}
52.743 \\
\end{tabular} & 17.144 & 31.845 & -1.212 & & 51.5 & 0.05 & 14.6 & & & & 51.6 & & & & & & & 184 & 0.16 \\
\hline & & & 25.118 & 25.332 & 24.205 & 25.775 & & 47.646 & \begin{tabular}{|r|}
52.59 \\
\end{tabular} & $\begin{array}{l}17.116 \\
.1700\end{array}$ & 31.503 & -1.218 & & 51.107 & 0.049 & 14. & & & & 51.5 & & & & & & & & \\
\hline & & $9: 4$ & & 25.342 & & & & & - 52.6/4 & 17.06 & & & & & 0.00 & & & & & 51.5 & & & & & & & & \\
\hline & & 9:27: & 25.113 & 25.332 & 24.195 & 25.7 & & 47.702 & 52.803 & 17.13 & 31.552 & -1.215 & & & 049 & & & & 49.5656 & 51.7 & & & & & & & 0.181 & \\
\hline & & (9.28. & 25118 & $\begin{array}{l}25.34 ! \\
25358\end{array}$ & $\frac{24.21}{24.231}$ & $\begin{array}{r}25.78 \\
25.726\end{array}$ & 254 & $\begin{aligned} 47.843 \\
75.52\end{aligned}$ & $\frac{52.5}{5.24}$ & $\begin{array}{l}17.134 \\
1.9076\end{array}$ & 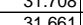 & $\begin{array}{l}-1.206 \\
-1.212\end{array}$ & & & - 0.05 & . & & & & $\begin{aligned} 51.5 \\
554\end{aligned}$ & & & & & & & 181 & \\
\hline & 9 & & & 25352 & $\frac{24.251}{242}$ & 25755 & & 47.9 & & 17046 & | & -1212 & & 49 & . & 14643 & & & & & & & & & & & & \\
\hline & & & $\frac{2.110}{25.118}$ & $\frac{2.054}{25.352}$ & 24.215 & 25. & & & \begin{tabular}{|l}
52.714 \\
\end{tabular} & 17.025 & 32.118 & -1.212 & & & 0.040 & & & & & $\frac{2.6}{516}$ & & & & & & & & \\
\hline & & 9:32:18 AM & 25.118 & 25.352 & 24.21 & & & 47.801 & \begin{tabular}{|l|}
52.979 \\
\end{tabular} & 17.188 & 31.556 & -1.215 & & $\frac{29.3}{49.3}$ & 0.05 & 14.6 & & & & $\frac{51.9}{51.9}$ & & & & 007 & 0.00 & & 184 & \\
\hline & & & & & & & & & & & & & & & & & & & & & & & & & & & & \\
\hline & & & 25.118 & 25.362 & 24.205 & & & & 52.2 & 16.9 & 31.552 & $\begin{array}{l}-1.209 \\
\end{array}$ & & & 048 & & & & & 51.2 & & & & & & & & \\
\hline & & & & & 24.271 & & & & 3.004 & 17.254 & 31.564 & -1.210 & & & & & & & & 51. & & & & & & & & \\
\hline & & 9:36:18 An & 25.113 & 25.362 & 24.291 & & & & 52.795 & & 31.462 & -1.212 & & & 049 & & & & & 517 & 12 & & & & & & 181 & 0.15 \\
\hline & $9 / 1 / 2$ & & 25. & 25.37 & & & & & & & 31.5 & $\begin{array}{l}-1.212 \\
\end{array}$ & & & 0.05 & 14.6 & & & & 51. & 11 & & & & & & 0.184 & \\
\hline & & & & & & & & & & & & & & & & & & & & & & & & & & & & \\
\hline & & & 131 & 25.39 & 24. & & & $47.6 \mathrm{C}$ & \begin{tabular}{|l|} 
\\
\end{tabular} & 17.159 & 31. & -1.2. & & 50.1 & 0.049 & & & & & 51.3 & 11.5 & & & & & & 80 & \\
\hline & & & & & & & & & & & & & & & & & & & & & & & & & & & & \\
\hline & & & 25.132 & 25.38 & 24.245 & & & & & 1.1999 & 31.5 & -1.2 & & & & & & & & & & & & & & & & \\
\hline & & & & $\begin{array}{l}25.398 \\
25309\end{array}$ & \begin{tabular}{|l|l|l}
2.250 \\
274
\end{tabular} & & & & & & 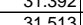 & $\begin{array}{l}-1.215 \\
-1.1212\end{array}$ & & & & & & & & S1.6 & 12. & & & & & & 81 & \\
\hline & & & & 25.00 & & & & & 528 & & & -1.21 & & & & & & & & & $\frac{14}{11}$ & & & & & & & \\
\hline & & & & & & & & & & & & -1.1 .14 & & & & & & & & & 11 & & & & & & & \\
\hline & & & & 25.41 & 24.193 & & & 47.41 & & & 31.47 & -1.16 & & & 0.048 & & & & & 51 & $\frac{12.1}{12.1}$ & & & & & & & \\
\hline & & & & & & & & & & & & & & & & & & & & & & & & & & & & \\
\hline & & & & & & & & & & & & & & & & & & & & & & & & & & & & \\
\hline & & & & & 214 & & & & & & 31. & -1.1 & & & & & & & & & 11 & & & & & & & \\
\hline & $9 / 1 / 2$ & & & 25.448 & 24.221 & 26. & & 47.789 & \begin{tabular}{|l|}
53.014 \\
\end{tabular} & 17.1 & 31.679 & -1.143 & & & & 14. & & & & & 12.2 & & & & & & & \\
\hline & & & & 25.444 & 24.192 & & & 4 & \begin{tabular}{|l|l|}
52.86 \\
\end{tabular} & 16. & 31.954 & -1.151 & & 49.6 & & $14.6 \mathrm{C}$ & & & & & 11.8 & & & & & & & \\
\hline & & & & 25.444 & 24. & & & 47. & & & 31.5 & -1.148 & & & & 14. & & & & & 12. & & & & & & & \\
\hline & & & & 25.445 & & & & 47. & & & & -1.1 .1 & & & & & & & & & 1 & & & & & & & \\
\hline & & & & & & & & & & & & -1.1 .1 & & & & & & & & & & & & & & & & \\
\hline & & & & & 24.18 & & & $4 !$ & & 16.826 & & -1.148 & & & & & & & & 51.1 & 12.4 & & & & & & & \\
\hline & & & $\frac{23.148}{25.50}$ & 20.4060 & 24.1000 & 20.001 & 2.4 & 47,506 & \begin{tabular}{|l|l|}
5.027 \\
5.72
\end{tabular} & 1.0489 & 31.441 & - & & 50.00 & 0.049 & $\begin{array}{l}14.643 \\
1.643\end{array}$ & & & & 年 & 12.14 & & & & ( & 0.000 & 年.182 & -2 \\
\hline 2310 & 9/1/1/2004 & $9: 58: 18 \mathrm{AM}$ & \begin{tabular}{|l|}
25.166 \\
2.160
\end{tabular} & $\frac{25.49}{25.49}$ & \begin{tabular}{|l|}
24.152 \\
24.203 \\
\end{tabular} & \begin{tabular}{|l|l|}
20.012 \\
26.028
\end{tabular} & 25.381 & $\begin{array}{l}47.000 \\
4.407\end{array}$ & \begin{tabular}{|l|}
52.442 \\
52.120
\end{tabular} & \begin{tabular}{|l|}
1.004 \\
16.767
\end{tabular} & $\begin{array}{l}1.010 \\
31.726 \\
\end{array}$ & \begin{tabular}{|l|}
$\mid-1.145$ \\
-1.119 \\
\end{tabular} & \begin{tabular}{|l|}
-7.050 \\
-7.053 \\
\end{tabular} & $\begin{array}{l}49.741 \\
49.74\end{array}$ & 0 & \begin{tabular}{|l|}
14.04043 \\
14.643
\end{tabular} & 0.003 & 3004.93 & \begin{tabular}{|l|l|}
50.0822 \\
\end{tabular} & \begin{tabular}{|l|}
51.3 \\
51.3
\end{tabular} & \begin{tabular}{|l|}
11.8 \\
11.8 \\
\end{tabular} & \begin{tabular}{|l|l|}
8 & 39.6 \\
8
\end{tabular} & \begin{tabular}{|l|l|}
2.728 \\
\end{tabular} & \begin{tabular}{|l|}
0.007 \\
\end{tabular} & \begin{tabular}{|l|}
0.007 \\
\end{tabular} & 0.000 & $\begin{array}{l}0.101 \\
0.181 \\
\end{array}$ & 0.1 \\
\hline
\end{tabular}


WSRC-TR-2005-00105, REVISION 0

SRNL-RPP-2005-00012, REVISION 0

RUN \# 4.03A AND B; FIRST AND SECOND HALF OF SLURRY DEWATERING - CONT.

\begin{tabular}{|c|c|c|c|c|c|c|c|c|c|c|c|c|c|c|c|c|c|c|c|c|c|c|c|c|c|c|c|c|}
\hline & $\mathrm{A}$ & $\mathrm{B}$ & D & $E$ & $F$ & G & $\mathrm{H}$ & $\mathrm{J}$ & $\mathrm{K}$ & $\mathrm{L}$ & $\mathrm{M}$ & $\mathrm{N}$ & 0 & $\mathrm{Q}$ & $R$ & $\mathrm{~s}$ & $T^{T}$ & $\mathrm{~V}$ & w & $x$ & $\mathrm{Y}$ & & $\mathrm{AA}$ & $A B$ & & & $\mathrm{AE}$ & \\
\hline & 9/1/12004 & $9: 59: 18 \mathrm{AM}$ & 25.166 & 25.496 & \begin{tabular}{|l|}
24.199 \\
\end{tabular} & 26.064 & 25.391 & 47.901 & 53.124 & $\begin{array}{l}17.037 \\
\end{array}$ & 31.936 & -1.111 & -7.056 & 49.439 & 0.048 & 14.643 & 0.003 & 3005.93 & 50.0989 & 52.0 & 11.8 & 39.9 & 2.752 & 0.007 & 0.007 & 0.000 & 0.176 & 0.15 \\
\hline & $9 / 1 / 2004$ & & 25.157 & 25.492 & 24.195 & 26.025 & 25.317 & 47.785 & 53 & $\begin{array}{l}16.921 \\
\end{array}$ & 31.808 & \begin{tabular}{|l|l|l|}
8 & -1.143 \\
\end{tabular} & $\frac{-3.047}{-7.040}$ & 51.729 & 0.05 & \begin{tabular}{|l|}
14.643 \\
\end{tabular} & & & 50.1156 & 51.9 & 12.3 & & & & 0.007 & & & 0.16 \\
\hline & $9 / 1 / 12004$ & 10:01:18 8M & 25.163 & 25.498 & \begin{tabular}{|l|l|}
34.236 \\
\end{tabular} & 26.071 & $\begin{array}{r}25.383 \\
2.23\end{array}$ & $\begin{array}{l}47.524 \\
771\end{array}$ & \begin{tabular}{|c|}
52.73 \\
52.93
\end{tabular} & \begin{tabular}{|l|l|}
16.856 \\
16.02
\end{tabular} & 31.622 & \begin{tabular}{|l|l|l|}
22 & -1.128 \\
\end{tabular} & $\begin{array}{l}-7.056 \\
.7050\end{array}$ & 50.432 & \begin{tabular}{|c|}
0.048 \\
\end{tabular} & \begin{tabular}{|l|}
14.643 \\
\end{tabular} & & \begin{tabular}{|l|}
3007.93 \\
208.92
\end{tabular} & 350.1322 & 51.6 & 12.0 & & 2.728 & 007 & 0.007 & 0.000 & 0.177 & \\
\hline $\begin{array}{l}2314 \\
2315\end{array}$ & 9/1/2004 & 10:02:18 AM & 25.149 & 25.493 & \begin{tabular}{|l|l|}
24.191 \\
\end{tabular} & 26.016 & 25.249 & $\begin{array}{l}47.714 \\
\end{array}$ & \begin{tabular}{|l|}
52.884 \\
\end{tabular} & \begin{tabular}{|l|l|}
16.993 \\
19.8
\end{tabular} & 31.796 & -1.108 & \begin{tabular}{|l|l|}
-7.058 \\
.705
\end{tabular} & 48.208 & \begin{tabular}{|c|}
0.048 \\
\end{tabular} & \begin{tabular}{|l|l|}
14.643 \\
\end{tabular} & 0.00 & 3008.93 & 50.1489 & 51.8 & 11.5 & & 2.741 & 0.007 & 0.007 & 0.000 & \begin{tabular}{|l|l|}
0.177 \\
\end{tabular} & \\
\hline & $\frac{9 / 1 / 2004}{9 / 1 / 2004}$ & $\begin{array}{ll}10: 03: 18 \mathrm{AM} \\
10.04 \cdot 1 \mathrm{AM}\end{array}$ & $\begin{array}{l}25.149 \\
25.149\end{array}$ & $\begin{array}{l}25.488 \\
25.493\end{array}$ & $\begin{array}{l}324.186 \\
32.186\end{array}$ & $\begin{aligned} 25.981 \\
25.911\end{aligned}$ & $\begin{array}{l}25.254 \\
25.354\end{array}$ & \begin{tabular}{|l|}
47.752 \\
47.984
\end{tabular} & \begin{tabular}{|l|}
53.126 \\
53040 \\
\end{tabular} & \begin{tabular}{|c|}
17.024 \\
17.033
\end{tabular} & $\begin{array}{r}31.755 \\
32094 \\
\end{array}$ & $\begin{array}{l}-1.096 \\
1.122\end{array}$ & & $\begin{array}{l}49.672 \\
51854\end{array}$ & \begin{tabular}{|c|}
0.049 \\
0.049
\end{tabular} & \begin{tabular}{|l|l|}
14.643 \\
14643 \\
\end{tabular} & & 3009.93 & $\begin{array}{l}50.1656 \\
50.1020\end{array}$ & 52.0 & 11.8 & & $\begin{array}{l}2.741 \\
.761\end{array}$ & & 0.007 & & $\frac{0.181}{0.170}$ & 0.15 \\
\hline 2317 & $\begin{array}{l}9 / 1 / 12004 \\
9 / 1 / 2004\end{array}$ & $\begin{array}{l}10: 04: 18 \mathrm{AM} \\
10: 05: 11 \mathrm{AM}\end{array}$ & $\begin{array}{l}25.149 \\
25.132\end{array}$ & $\begin{array}{l}25.493 \\
25.477 \\
\end{array}$ & $\begin{array}{r}24.186 \\
24.18\end{array}$ & $\frac{25.911}{25.815}$ & $\begin{array}{l}25.354 \\
25.322 \\
\end{array}$ & $\begin{array}{l}47.984 \\
47.963 \\
\end{array}$ & \begin{tabular}{|l|}
53.046 \\
53.201 \\
\end{tabular} & \begin{tabular}{|r|}
17.033 \\
17.113
\end{tabular} & $\begin{array}{r}32.094 \\
31.864 \\
\end{array}$ & $\begin{array}{l}-1.122 \\
-1.102 \\
\end{array}$ & $\begin{array}{l}-7.053 \\
-7.056\end{array}$ & $\begin{array}{l}51.854 \\
50.079\end{array}$ & $\begin{array}{l}0.049 \\
0.05\end{array}$ & \begin{tabular}{|c|}
14.643 \\
14.643
\end{tabular} & .003 003 & $\begin{array}{l}3010.93 \\
301.93 \\
\end{array}$ & $\begin{array}{l}350.1822 \\
3 \\
3 \\
5.0 .1989\end{array}$ & $\begin{array}{l}51.9 \\
52.1 \\
\end{array}$ & $\begin{array}{l}12.4 \\
11.9\end{array}$ & 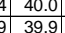 & $\begin{array}{l}2.761 \\
2.752 \\
\end{array}$ & 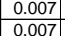 & $\begin{array}{l}0.007 \\
0.007\end{array}$ & $\frac{0.000}{0.000}$ & & 0.15 \\
\hline 2318 & 9/1/2004 & 10:06:18 AM & 25.134 & 25.479 & 24.172 & $\begin{array}{l} \\
25.802 \\
\end{array}$ & 25.159 & 47.646 & \begin{tabular}{|l|}
52.674 \\
\end{tabular} & 16.96 & 31.852 & -1.117 & $\begin{array}{l}-7.079 \\
-7.079\end{array}$ & 50.225 & $\mid \begin{array}{l}0.058 \\
0.048\end{array}$ & 14.643 & $\begin{array}{l}0.0003 \\
0.003\end{array}$ & $\begin{array}{l}301.95 \\
3012.93\end{array}$ & 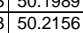 & $\begin{array}{l}32.1 \\
51.5 \\
\end{array}$ & 12.0 & \begin{tabular}{|l|l|}
0 & 39.7 \\
\end{tabular} & $\frac{2.154}{2.741}$ & 0.007 & $\begin{array}{l}0.007 \\
0.007\end{array}$ & $\begin{array}{l}0.000 \\
0.000\end{array}$ & & $\begin{array}{l}0.16 \\
0.15 \\
\end{array}$ \\
\hline & & & 25.148 & 25.492 & 24.205 & 25.79 & 25.048 & 47.799 & & $\begin{array}{l}17.024 \\
\end{array}$ & 31.761 & -1.122 & & $\begin{array}{l}49.608 \\
\end{array}$ & 0.048 & \begin{tabular}{|l|l|}
14.643 \\
\end{tabular} & & 30193 & & & & & & & & & & 15 \\
\hline & 9/1/2004 & 10:08:18 AM & & 25.465 & \begin{tabular}{|l|l|}
24.208 \\
\end{tabular} & 25.708 & 25.081 & 47.685 & \begin{tabular}{|l|l|}
52.8655 \\
\end{tabular} & $\begin{array}{l}16.967 \\
\end{array}$ & 31.747 & -1.096 & & 49.996 & & 14.643 & & 3014.93 & 50.2489 & 51.7 & 11.9 & & 2.738 & 0.007 & 0.007 & 0.000 & 0.181 & \\
\hline 2321 & \begin{tabular}{ll|}
$9 / 1 / 2004$ \\
$0 / 1 / 2001$
\end{tabular} & & $\begin{array}{r}25.13 \\
\end{array}$ & 25.474 & $\begin{array}{l}24.288 \\
\end{array}$ & $\begin{array}{l}25.727 \\
25766\end{array}$ & 25.175 & $\begin{array}{r}47.773 \\
\end{array}$ & \begin{tabular}{|l|}
52.782 \\
5.720 \\
\end{tabular} & \begin{tabular}{|c|}
17.058 \\
\end{tabular} & $\begin{array}{r}31.73 \\
3171\end{array}$ & \begin{tabular}{|l|l|}
-1.093 \\
\end{tabular} & & 52.046 & $\begin{array}{l}0.048 \\
\end{array}$ & \begin{tabular}{|l|l|}
14.643 \\
\end{tabular} & 0.003 & 3015.93 & 50.2656 & 51.7 & 12.4 & & & 0.007 & 0.007 & 0.000 & 0.176 & \\
\hline & $\begin{array}{l}9 / 1 / 2004 \\
9 / 1 / 2004\end{array}$ & $\begin{array}{l}10: 10: 18 \mathrm{AM} \\
10.111 .1 \mathrm{AM}\end{array}$ & $\begin{array}{l}25.129 \\
25.153\end{array}$ & $\begin{array}{r}25.469 \\
25.498\end{array}$ & $\begin{array}{l}24.302 \\
3 \\
24.321\end{array}$ & $\begin{array}{l}25.766 \\
25821\end{array}$ & $\frac{25.139}{2503}$ & \begin{tabular}{|l|}
47.646 \\
47.588 \\
\end{tabular} & \begin{tabular}{|l|}
52.722 \\
52.952 \\
\end{tabular} & \begin{tabular}{|c|}
16.971 \\
17042
\end{tabular} & $\begin{array}{l}31.761 \\
31.535 \\
\end{array}$ & $\begin{array}{r}-1.09 \\
1088\end{array}$ & & $\begin{array}{l}99.268 \\
51.068\end{array}$ & \begin{tabular}{|l|l|}
0.049 \\
0047
\end{tabular} & \begin{tabular}{|l|l|}
14.643 \\
14643
\end{tabular} & & $\begin{array}{l}3016.93 \\
3017\end{array}$ & & $\begin{array}{l}51.6 \\
51.8\end{array}$ & \begin{tabular}{l|l|l|}
11.7 \\
122
\end{tabular} & & & .007 & 0.007 & 0.000 & 0.180 & 0.15 \\
\hline 2324 & $\begin{array}{l}9 / 1 / 2004 \\
9 / 1 / 2004\end{array}$ & $\begin{array}{l}10: 11: 18 \mathrm{AM} \\
10.12218 \mathrm{AM}\end{array}$ & $\begin{array}{l}25.153 \\
25.137\end{array}$ & $\begin{array}{r}25.498 \\
25.477\end{array}$ & $\begin{array}{l}34.321 \\
724.295\end{array}$ & $\frac{25.821}{25.76}$ & $\frac{25.203}{25.228}$ & \begin{tabular}{|l|}
47.588 \\
47.532
\end{tabular} & \begin{tabular}{|l|}
52.952 \\
52.786 \\
\end{tabular} & \begin{tabular}{|c|c|}
17.042 \\
17.154
\end{tabular} & $\frac{535}{419}$ & $\begin{array}{r}-1.088 \\
-1.09\end{array}$ & & $\begin{array}{r}51.068 \\
49.906\end{array}$ & $\begin{array}{l}0.047 \\
0.048\end{array}$ & $\begin{array}{l}14.643 \\
14.643 \\
\end{array}$ & 0.003 & $\begin{array}{l}3017.93 \\
3018.93 \\
3\end{array}$ & $\begin{array}{l}50.2989 \\
50.136\end{array}$ & $\frac{51.8}{51.7}$ & \begin{tabular}{|l|l|}
12.2 \\
11.9
\end{tabular} & $\begin{array}{l}2 \\
\begin{array}{l}2 \\
\end{array} \frac{39.6}{39.5}\end{array}$ & 2.728 & 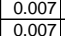 & 0.007 & $\frac{0.000}{0.000}$ & 0.173 & 0.15 \\
\hline 2325 & $9 / 1 / 2004$ & 10:13:18 AM & 25.143 & 25.483 & \begin{tabular}{|l|l}
24.260 \\
\end{tabular} & 25.806 & 25.253 & $\begin{array}{l}4.036 \\
4.936\end{array}$ & \begin{tabular}{|l|}
53.255 \\
\end{tabular} & $\mid \begin{array}{l}17.213 \\
17.213\end{array}$ & 31.683 & -1.09 & & 50.655 & $\begin{array}{l}0.048 \\
0.049\end{array}$ & $\begin{array}{l}14.043 \\
14.643 \\
\end{array}$ & & $\begin{array}{l}3018.95 \\
3019.93\end{array}$ & 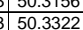 & $\begin{array}{l}51.1 \\
52.1 \\
\end{array}$ & $\begin{array}{ll}11.9 \\
12.1\end{array}$ & \begin{tabular}{l|l}
9 & 39.5 \\
1 & 39.8
\end{tabular} & $\frac{2.722}{2.745}$ & & $\begin{array}{l}.007 \\
0.007\end{array}$ & & & $\begin{array}{l}0.15 \\
0.15\end{array}$ \\
\hline & & 10:14:18 AM & 25.142 & 25.477 & 24.26 & \begin{tabular}{|l|}
25.825 \\
\end{tabular} & 25.27 & & \begin{tabular}{|l|}
52.987 \\
\end{tabular} & 17.149 & & -1.093 & & 50.513 & 0.05 & 14.643 & & & & & & & & 0.007 & & & & $\begin{array}{l}0.15 \\
0.16\end{array}$ \\
\hline & 9/1/2004 & & & 25.493 & \begin{tabular}{|l|l|}
24.251 \\
\end{tabular} & 25.876 & & 47.638 & & \begin{tabular}{ll|}
17.146 \\
\end{tabular} & 31.466 & -1.082 & & 50.183 & 0.048 & & & & 50.3656 & 51.7 & 12.0 & & & & $\begin{array}{l}0.007 \\
\end{array}$ & & & $\frac{1.16}{.15}$ \\
\hline 2328 & & & & & & 25.922 & 25.3 & & 53.085 & & & & & 50.129 & & 14.6 & & & & & & & & & & & & \\
\hline & & & & & & 25.953 & & 47.443 & 52.662 & \begin{tabular}{|l|l|}
16.964 \\
\end{tabular} & $\begin{array}{l}31.443 \\
\end{array}$ & -1.093 & & 50.49 & 0.046 & 14.6 & & & 50.3989 & 51 & & & & & & & & \\
\hline & & 10:18 & 25.156 & 25.496 & \begin{tabular}{|l|l|l|} 
f 24.239 \\
\end{tabular} & 25.909 & & 47.75 & 52.99 & & 31.804 & 107 & & & & & & & & & & & & & & & & 15 \\
\hline & & & 25.157 & 25.502 & $\begin{array}{ll}24.215 \\
\end{array}$ & 25.96 & & 47.916 & & 16.9 & 32.03 & -1.062 & & & 0.048 & 14.6 & & 25.9 & & & & & & & & & & \\
\hline & $9 / 1 / 200$ & $10: 20: 18 \mathrm{AM}$ & 25.168 & 25.518 & \begin{tabular}{|l|l|}
3 & 24.241 \\
\end{tabular} & 26.001 & 25.2 & 47.358 & \begin{tabular}{|l|}
52.417 \\
\end{tabular} & 16.914 & $\begin{array}{l}31.603 \\
\end{array}$ & -1.079 & & 49.749 & 0.048 & 14.643 & 0.004 & 3026.93 & 30.4489 & 51.3 & 11.8 & & 2.722 & 0.007 & 0.007 & 0.000 & 0.178 & \\
\hline & & 10:21:1 & 25.159 & 25.504 & $\begin{array}{l}424.227 \\
\end{array}$ & $\begin{array}{ll}7 & 25.967 \\
\end{array}$ & 25.25 & 47.648 & \begin{tabular}{|l|}
52.747 \\
50.70
\end{tabular} & 17.083 & 31.724 & -1.024 & & 51.093 & 0.048 & 14.643 & & & & 51.6 & 12.2 & & 2.736 & $\begin{array}{l}0.007 \\
\end{array}$ & 0.007 & 0.000 & 0.177 & \\
\hline & $11 / 200$ & & 25.166 & 25.51 & \begin{tabular}{|l|}
24.228 \\
\end{tabular} & 25.953 & 25.3 & & \begin{tabular}{|l|}
52.762 \\
\end{tabular} & \begin{tabular}{l|l|}
17.024 \\
\end{tabular} & & -1.07 & & 50.286 & $\begin{array}{l}0.049 \\
\end{array}$ & 14.643 & & & & 51.6 & 12.0 & & 2.725 & 0.007 & 0.007 & 0.000 & 0.181 & \\
\hline 2335 & 9/1/200 & 10:23: & 25.161 & 25.511 & 24.199 & \begin{tabular}{|l|l|}
9 & 25.969 \\
\end{tabular} & & & \begin{tabular}{|l|}
52.666 \\
\end{tabular} & \begin{tabular}{|l|l|}
16.937 \\
\end{tabular} & 31.646 & -1.021 & & 49.726 & 0.05 & & & & & 51.5 & \begin{tabular}{|l|l|}
11.8 \\
\end{tabular} & & 2.730 & .007 & 0.007 & & 0.185 & 0.16 \\
\hline$\frac{2336}{2337}$ & $9 / 1 / 2004$ & $\begin{array}{l}10: 24: 18 \mathrm{AM} \\
10.251 .18 \mathrm{~A}\end{array}$ & $\begin{array}{l}25.157 \\
25.157\end{array}$ & $\begin{array}{r}25.512 \\
25.517\end{array}$ & 24.215 & $\begin{array}{l}25.92 \\
25.92\end{array}$ & $\frac{25.3}{25.26}$ & $\begin{array}{l}47.677 \\
47.495 \\
\end{array}$ & \begin{tabular}{|l|}
52.946 \\
52.612 \\
\end{tabular} & $\mid$\begin{tabular}{|c|}
$\mid 17.047$ \\
16.929
\end{tabular} & $\begin{array}{l}31.593 \\
31.577 \\
3\end{array}$ & $\begin{array}{l}-1.021 \\
-1.024 \\
\end{array}$ & & $\begin{array}{l}49.973 \\
50.227\end{array}$ & \begin{tabular}{|c|c|}
0.048 \\
0.05
\end{tabular} & $\begin{array}{l}14.643 \\
14.643 \\
\end{array}$ & .003 & \begin{tabular}{|l|l|l}
3030.93 \\
3031.93
\end{tabular} & \begin{tabular}{|l|}
50.5156 \\
5.03522
\end{tabular} & $\begin{array}{l}51.8 \\
51.5 \\
\end{array}$ & $\frac{11.9}{12.0}$ & $\begin{array}{ll}8 & 39 \\
139 & 39\end{array}$ & $\frac{2.733}{2726}$ & $\begin{array}{l}0.007 \\
0.007 \\
\end{array}$ & 0.007 & 0.000 & 0.177 & 0.15 \\
\hline$\frac{23018}{2338}$ & $\begin{array}{l}991 / 1 / 2004 \\
91 / 2004\end{array}$ & $\begin{array}{l}10: 25: 18 \mathrm{AM} \\
10: 26: 18 \mathrm{AM}\end{array}$ & $\begin{array}{l}25.151 \\
25.157\end{array}$ & $\frac{25.511}{25.522}$ & \begin{tabular}{|l}
24.225 \\
24.205
\end{tabular} & $\begin{array}{l}25.92 \\
25.96 \\
\end{array}$ & $\frac{25.2}{25.3}$ & $\begin{array}{l}47.495 \\
47.619\end{array}$ & \begin{tabular}{|r|}
52.612 \\
52.95 \\
\end{tabular} & \begin{tabular}{|l|}
10.929 \\
17.099
\end{tabular} & 31.505 & $\begin{array}{c}-1.024 \\
-1.056\end{array}$ & & $\begin{array}{l}50.221 \\
49.983\end{array}$ & $\begin{array}{l}0.05 \\
0.05\end{array}$ & \begin{tabular}{|l|}
14.643 \\
14.643 \\
\end{tabular} & & $\begin{array}{l}3031.93 \\
3032.93\end{array}$ & \begin{tabular}{|l|l|}
50.52524 \\
50.5489
\end{tabular} & $\begin{array}{l}51.5 \\
51.8\end{array}$ & $\begin{array}{ll}12.0 \\
11.9\end{array}$ & & & & & & & 0.16 \\
\hline 2339 & 9/1/12004 & $10: 27: 18 \mathrm{AM}$ & 25.152 & 25.517 & 24.195 & \begin{tabular}{|l|l}
5 & 25.985 \\
\end{tabular} & & 47.528 & \begin{tabular}{|l|}
52.706 \\
\end{tabular} & 17 & 31.577 & $\begin{array}{ll}77 & -1.073\end{array}$ & & 50.765 & 0.049 & 14.643 & & 3033.93 & 50.5656 & 51.6 & 12.1 & 20 & 2.72 & & & & $\begin{array}{l}0.185 \\
0.181\end{array}$ & $\frac{0.16}{0.15}$ \\
\hline & & & 25.157 & & 24.195 & & & 47.455 & \begin{tabular}{|l|}
52.672 \\
\end{tabular} & 16.9 & & \begin{tabular}{|l|l|l|}
3 & -1.018 \\
\end{tabular} & & 49.674 & 0.049 & 14.643 & & & & & 11.8 & & & & & & & $\frac{0.15}{0.15}$ \\
\hline 2341 & 9/1/2004 & $10: 29$ & 25.163 & 25.528 & 24.211 & 26.041 & & $\begin{array}{l}47.748 \\
\end{array}$ & \begin{tabular}{|l|}
53.1499 \\
\end{tabular} & $\begin{array}{l}17.056 \\
\end{array}$ & 683 & -0.873 & & 51.314 & 0.041 & 14.643 & & & & 52.0 & \begin{tabular}{ll|}
12.2 \\
\end{tabular} & & & & & & & $\begin{array}{l}0.15 \\
0.13\end{array}$ \\
\hline 2342 & & & 25.154 & 25.533 & 24.181 & 26.026 & & 47.92 & \begin{tabular}{|l|}
53.448 \\
\end{tabular} & 16.871 & & -0.954 & & & & & & & & 52.3 & & & & & & & 209 & 0.18 \\
\hline 2343 & 9/1/2004 & & 25.149 & 25.528 & 24.181 & 26.016 & & 47.725 & & 16.99 & 31.765 & & & & 0.048 & 14.6 & & & & & & & & & & & & \\
\hline & $11 / 2004$ & & 25.174 & 25.549 & 24.212 & 26.087 & & 48.09 & 53.396 & 17.071 & 31.938 & -0.983 & & & 0.048 & & & & & 523 & $\overline{12}$ & & & & & & & \\
\hline & 9/1/2004 & $10: 33$ & 25.164 & 25.546 & 24.21 & 26.067 & & 47.4 & \begin{tabular}{|l|}
52.857 \\
\end{tabular} & 16.948 & 3 & -0.969 & & 50.14 & \begin{tabular}{|c|}
0.048 \\
\end{tabular} & 14.64 & & & & 51. & 111.9 & & & & & & & \\
\hline & & 10:34 & 25.16 & 25.8 & 24.178 & & & & \begin{tabular}{|l|l|}
53.006 \\
\end{tabular} & 17.088 & 31.614 & -0.989 & & 50.6 & $\mid 0.048$ & 14.6 & & & & 51. & 12.1 & & & & & & 0.177 & \\
\hline & & & 25.164 & 5.534 & 24.206 & 25 & & 47.6 & \begin{tabular}{|l|l|} 
& \\
& .9009 \\
\end{tabular} & 16.901 & & -0.9 & & 52.2 & 0.049 & 14. & & & & & 12.4 & & & & & & & \\
\hline & & & & & & & & & 52.662 & & & & & & 0.048 & & & & & & & & & & & & & \\
\hline & & & 25.17 & 25.546 & 24.214 & 25.91 & & & 53.504 & 17.108 & $\begin{array}{r}32.057 \\
\end{array}$ & -0.9 & & & 0.047 & & & & & 52. & 11.8 & & & & & & & 0.15 \\
\hline & & & $\begin{array}{l}25.160 \\
25153\end{array}$ & 255 & $\frac{24.223}{2206}$ & $\begin{array}{l}25.856 \\
25826\end{array}$ & & & $\begin{array}{l}52.853 \\
53033\end{array}$ & $\frac{1.18}{16.961}$ & & & & & 0.049 & & & & & (51.9 & 118 & & & & & & & 0.16 \\
\hline & $9 / 1 / 2004$ & & 25.158 & & 24.276 & \begin{tabular}{|l|l}
6 & 2.020 \\
\end{tabular} & & & \begin{tabular}{|l|}
52.768 \\
52.768 \\
\end{tabular} & & & -0.928 & & & | & 14.6 & & & & 51.6 & $\frac{11.0}{11.9}$ & & & & & & 0.174 & \\
\hline & 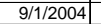 & & 25.145 & & $\begin{array}{l}324.283 \\
\end{array}$ & & & & & & & & & & $\begin{array}{l}0.049 \\
\end{array}$ & & & & & 51.9 & $\overline{12.0}$ & & & & & & 0.180 & \\
\hline & 9/1/2004 & & 25.149 & 25.524 & $\begin{array}{l}424.307 \\
\end{array}$ & 25.731 & & & \begin{tabular}{|l|} 
\\
\end{tabular} & \begin{tabular}{|l|l|l|l|}
17.067 \\
\end{tabular} & & $\begin{array}{l}-0.952 \\
\end{array}$ & & 50. & \begin{tabular}{|l|l|l|}
0.047 \\
\end{tabular} & & & 304 & & 52.1 & \begin{tabular}{|l|l|}
12.0 \\
\end{tabular} & & 2.745 & \begin{tabular}{|l|l|}
0.007 \\
\end{tabular} & 0.6 & 0.0 & $\begin{array}{l}0.172 \\
\end{array}$ & \\
\hline & & & 25.153 & 25.528 & \begin{tabular}{|l|l|}
24.321 \\
\end{tabular} & 25.7 & & & & & & & & & $\begin{array}{l}0.047 \\
\end{array}$ & & & & & 51.8 & \begin{tabular}{ll|}
12.3 \\
\end{tabular} & & & & & & \begin{tabular}{|l|l|l|}
0.174 \\
\end{tabular} & \\
\hline & & & 25.147 & 25.517 & 24.285 & 25. & & & \begin{tabular}{|l|}
52.973 \\
\end{tabular} & \begin{tabular}{ll|}
17.034 \\
\end{tabular} & & & & & & & & & & & & & & & & & & \\
\hline 357 & $\begin{array}{l}9 / 1 / 12004 \\
9 / 1 / 2004\end{array}$ & $\begin{array}{l}10: 45: 18 \mathrm{AM} \\
10.46 .18 \mathrm{~A}\end{array}$ & $\begin{array}{l}25.178 \\
25.162\end{array}$ & $\begin{array}{r}25.543 \\
25.522\end{array}$ & $\begin{array}{l}324.311 \\
24.275\end{array}$ & \begin{tabular}{l|l|}
1 & 25.856 \\
5 & 25.825
\end{tabular} & $\begin{array}{l}25.04 \\
25.05\end{array}$ & \begin{tabular}{|l|}
47.4555 \\
47.755 \\
\end{tabular} & \begin{tabular}{|l|}
52.745 \\
53.055 \\
\end{tabular} & \begin{tabular}{|l|}
17.251 \\
17.121
\end{tabular} & $\begin{array}{l}31.199 \\
31.745 \\
\end{array}$ & $\begin{array}{l}-0.928 \\
-0.925 \\
\end{array}$ & $\frac{-6}{-6.5}$ & $\begin{array}{r}50.452 \\
50.1\end{array}$ & \begin{tabular}{l|l|}
0.048 \\
0.048
\end{tabular} & $\begin{array}{l}14.6 \\
114.6\end{array}$ & & \begin{tabular}{|c|}
3051.93 \\
305.93
\end{tabular} & $\begin{array}{l}350.8656 \\
3508822 \\
3\end{array}$ & $\begin{array}{l}51.6 \\
51.9\end{array}$ & \begin{tabular}{l|l|l|}
12.0 \\
11.9
\end{tabular} & $\begin{array}{l}3 \mathrm{~s} \\
3 \mathrm{~s}\end{array}$ & $\frac{2.711}{2.742}$ & \begin{tabular}{|l|}
0.007 \\
0.007
\end{tabular} & 0.007 & $\frac{0.000}{0.000}$ & $\begin{array}{l}0.178 \\
0.176\end{array}$ & 0.15 \\
\hline & & & & & & & & & & & & & & & $\mid$\begin{tabular}{|c|} 
\\
0.0488 \\
\end{tabular} & & & & & & 11.9 & & & & & & & \\
\hline & & & & & & & & & & & & & & & & & & & & & \pm 2.0 & & & & & & & \\
\hline & 9/1/2 & & 25.163 & 25. & $\begin{array}{ll}3 & 24.276 \\
\end{array}$ & \begin{tabular}{l|l|l}
5 & 25.886
\end{tabular} & & & & 17.132 & & \begin{tabular}{l|l}
9 & -0.876 \\
\end{tabular} & & & 0.047 & & & & & 52.0 & 11. & & & & & & 174 & \\
\hline & & & 25.1 & & & & & & & & & & & & & & & & & & & & & & & & & \\
\hline & & & 25.165 & 25.524 & 24.2 & & & & 52.789 & 17.079 & & -0.9 & & & & & & & & & & & & & & & & \\
\hline & & & 25.165 & 25.525 & \begin{tabular}{|l|l|}
524.233 \\
\end{tabular} & 25.9 & & & & 16.708 & & & & & & & & & & & 12. & & & & & & & \\
\hline & & & & 25.546 & \begin{tabular}{|l|l|} 
& 24.279 \\
\end{tabular} & & & & & 17.1 & & -0 . & & & & & & & & & 11. & & & & & & & \\
\hline & |11/200 & & 25.1 & 25.542 & 24.2 & & & & & & & & & & & 14.6 & & & & & 12. & & & & & & 0.181 & \\
\hline & & & & & 24 & & & & & & & & & & & & & & & & & & & & & & & \\
\hline & & & 25.17 & & 24.24 & & & & 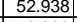 & & & -0.8 & & & & & & & & & $12.2 \mathrm{r}$ & & & & & & 0.180 & \\
\hline & & & 25.1 & & 24.2 & & & & & & & & & & & & & & & & & & & & & & & \\
\hline & & & $\frac{25.17}{2516}$ & & $\mid 24.222$ & & & & & & & & & & & & & & & & $\begin{array}{lll}11.5 \\
1.2\end{array}$ & & & & & & & \\
\hline & & & 2517 & $\begin{array}{l}23.350 \\
25549\end{array}$ & 年4.24 & & & & 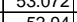 & & & & & & & & & & & & & & & & & & & \\
\hline & $\frac{3+17}{91 / 1}$ & & 年. & 25.354 & $\frac{24.25}{2425}$ & & & (4) & 53.329 & 1706 & & & & & & & & & & & $18 \mathrm{~s}$ & & & & & & & \\
\hline & & & 25.1 & & & & & & 53 & 17.17 & & -8.8 & & & & & & & & & & & & & & & & \\
\hline & & & $25.1 \mathrm{~T}$ & $\frac{25.5}{25.5}$ & $\frac{2.264}{24.264}$ & & & & & & & $\frac{-0.8}{-0.8}$ & & & & & & & & & & & & & & & & \\
\hline & & & & & $24.2 \quad$ & & & & & & & & & & & & & & & & & & & & & & & \\
\hline & & & 25.1 & & & & & & & & & & & & & & & & & & & & & & & & & \\
\hline & $9 / 1 / 2$ & & 25.1 & 25. & 24.23 & 26.0 & & & 53.1 & 16.96 & & -0.8 & & & & & & & & & & & & & & & & \\
\hline & & & 16 & & & & & & & & & & & & & & & & & & & & & & & & & \\
\hline & 9/1/2004 & 11:07:2 & 25.183 & 25.568 & \begin{tabular}{l|l}
3 & 24.26 \\
\end{tabular} & \begin{tabular}{l|l|l|}
6 & 25.955 \\
\end{tabular} & & 47.586 & \begin{tabular}{|l|}
52.956 \\
\end{tabular} & \begin{tabular}{|l|l|}
16.992 \\
\end{tabular} & 31.776 & \begin{tabular}{|l|l|}
76 & -0.813
\end{tabular} & & 49.985 & 0.046 & 14.643 & & & & 1.8 & & & 2.736 | & 0.007 & 0.00 & 0.000 & 0.169 & \\
\hline
\end{tabular}


WSRC-TR-2005-00105, REVISION 0

SRNL-RPP-2005-00012, REVISION 0

RUN \# 4.03A AND B; FIRST AND SECOND HALF OF SLURRY DEWATERING - CONT.

\begin{tabular}{|c|c|c|c|c|c|c|c|c|c|c|c|c|c|c|c|c|c|c|c|c|c|c|c|c|c|c|c|c|}
\hline & $\mathrm{A}$ & & D & $E$ & $F$ & G & $\mathrm{H}$ & $\mathrm{J}$ & $\mathrm{K}$ & $\begin{array}{ll}\mathrm{L} \\
\end{array}$ & $\mathrm{M}$ & $\mathrm{N}$ & 0 & $Q$ & $\mathrm{R}$ & $\mathrm{s}$ & $T$ & $\mathrm{~V}$ & w & $x$ & \begin{tabular}{l|l} 
\\
\end{tabular} & & & & & & $\mathrm{AE}$ & AF $\mathrm{AA}$ \\
\hline 2381 & 9/1/2004 & 11:08:21 AM & 25.171 & 25.556 & \begin{tabular}{|l|}
24.239 \\
\end{tabular} & 25.924 & 25.056 & 47.517 & 53.199 & 16.738 & 31.854 & -0.876 & -6.871 & 50.321 & $\begin{array}{l}0.049 \\
\end{array}$ & \begin{tabular}{|l|}
14.643 \\
\end{tabular} & 0.003 & 0744.98 & 51.2497 & 52.1 & 12.0 & 39.7 & 2.736 & 0.007 & 0.007 & 0.000 & 0.181 & 0.15 \\
\hline & & 11:09:21 AM & 25.174 & 25.554 & & 25.892 & 25.019 & 47.64 & 53.3 & $\begin{array}{l}16.981 \\
\end{array}$ & 31.501 & $\begin{aligned}-0.87 \\
\end{aligned}$ & -6.882 & 48.054 & $\begin{array}{l}0.047 \\
\end{array}$ & $\begin{array}{l}14.643 \\
\end{array}$ & & & 51.2664 & 52.2 & 11.4 & & & & 0.007 & & & 0.15 \\
\hline & $9 / 1 / 12004$ & & 25.182 & $\frac{25.562}{25565}$ & 24.25 & 25.85 & $\begin{array}{l}25.023 \\
\end{array}$ & 47.905 & \begin{tabular}{|l|}
53.4855 \\
5.82 \\
\end{tabular} & \begin{tabular}{|l|}
16.993 \\
\end{tabular} & 31.837 & \begin{tabular}{|c|c|} 
& -0.87 \\
\end{tabular} & & 51.109 & \begin{tabular}{|l|l|}
0.048 \\
\end{tabular} & $\begin{array}{r}4.643 \\
\end{array}$ & & $\begin{array}{l}776.98 \\
778\end{array}$ & 1.2831 & 52.4 & $\begin{array}{ll}12.2 \\
1.2\end{array}$ & & 2.749 & $\begin{array}{l}.007 \\
007\end{array}$ & \begin{tabular}{|l|l|}
0.007 \\
\end{tabular} & 0.000 & 0.176 & \\
\hline \begin{tabular}{|l|l|}
2384 \\
2385
\end{tabular} & 9/1/2004 & 11:11:21 AM & 25.165 & 25.545 & \begin{tabular}{|l|l|}
24.303 \\
\end{tabular} & 25.748 & $\begin{array}{r}24.96 \\
\end{array}$ & $\begin{array}{l}47.464 \\
\end{array}$ & \begin{tabular}{|l|l|}
52.824 \\
\end{tabular} & $\begin{array}{l}17.035 \\
\end{array}$ & 31.54 & -0.818 & $\begin{array}{l}-6.897 \\
-80\end{array}$ & 49.941 & $\begin{array}{l}0.046 \\
\end{array}$ & \begin{tabular}{|l|l|}
14.643 \\
\end{tabular} & & 77.98 & 2997 & 51.7 & $\begin{array}{l}11.9 \\
\end{array}$ & & 2.724 & 0.007 & $\begin{array}{l}0.007 \\
\end{array}$ & 0.000 & 0.170 & \\
\hline$\frac{2385}{2386}$ & $\frac{9 / 1 / 2004}{9 / 1 / 2004}$ & $\begin{array}{ll}11: 12: 21 \mathrm{AM} \\
111.132 .2 \mathrm{AM}\end{array}$ & $\begin{array}{l}25.174 \\
25.173\end{array}$ & $\frac{25.544}{25.548}$ & & & & & \begin{tabular}{|r|}
52.766 \\
5317
\end{tabular} & & $\begin{array}{l}31.451 \\
31821\end{array}$ & $\begin{array}{r}-0.81 \\
0.807\end{array}$ & & 49.996 & $\begin{array}{l}0.046 \\
0074\end{array}$ & \begin{tabular}{|l|}
14.643 \\
14643 \\
\end{tabular} & & & 1.3164 & 51.6 & \begin{tabular}{|l|}
11.9 \\
\end{tabular} & & & & $\begin{array}{l}0.007 \\
\end{array}$ & & $\begin{array}{l}0.170 \\
\end{array}$ & 0.14 \\
\hline$\frac{2380}{2387}$ & $\begin{array}{l}91 / 1 / 12004 \\
91 / 2004\end{array}$ & $\begin{array}{l}11: 13: 21 \text { AM } \\
111: 14: 21 \mathrm{AM}\end{array}$ & $\frac{25.173}{25.173}$ & $\frac{25.548}{25.543}$ & \begin{tabular}{|l|}
24.341 \\
24.326 \\
\end{tabular} & $\begin{array}{l}25.745 \\
25.761\end{array}$ & $\begin{array}{l}25.083 \\
25.048 \\
\end{array}$ & $\begin{array}{l}47.889 \\
47.378\end{array}$ & \begin{tabular}{|l|}
53.17 \\
5.78
\end{tabular} & \begin{tabular}{|l|}
17.078 \\
17.001 \\
\end{tabular} & $\begin{array}{r}31.1 .521 \\
31.533\end{array}$ & $\begin{array}{l}-0.807 \\
-0.81 \\
\end{array}$ & & $\begin{array}{l}49.6744 \\
50.081\end{array}$ & $|0.047|$ & $\begin{array}{l}14.643 \\
14.643 \\
\end{array}$ & $\begin{array}{l}0.003 \\
0.003 \\
\end{array}$ & $\begin{array}{l}78.98 \\
80.98\end{array}$ & $\begin{array}{l}851.331 \\
3 \\
3.13497\end{array}$ & \begin{tabular}{|l}
52.0 \\
51.7
\end{tabular} & \begin{tabular}{|c|}
11.8 \\
11.9
\end{tabular} & \begin{tabular}{|l|}
39.9 \\
39.5 \\
\end{tabular} & \begin{tabular}{|l|}
2.748 \\
2720 \\
\end{tabular} & $\begin{array}{l}0.007 \\
0.07\end{array}$ & $\begin{array}{l}0.007 \\
0.007\end{array}$ & $\frac{0.000}{0.000}$ & & $\begin{array}{l}0.15 \\
0.15\end{array}$ \\
\hline 2388 & $9 / 1 / 2004$ & & 25.189 & & \begin{tabular}{|l|}
24.320 \\
2432 \\
\end{tabular} & 25.806 & 25.109 & 47.495 & \begin{tabular}{|l|}
53.066 \\
\end{tabular} & & $\begin{array}{l}31.222 \\
31.220\end{array}$ & $\begin{array}{r}-0.81 \\
\end{array}$ & -6.827 & 50.058 & $\begin{array}{l}0.048 \\
0.047\end{array}$ & $\begin{array}{l}14.643 \\
14.643 \\
\end{array}$ & $\begin{array}{l}0.003 \\
0.03\end{array}$ & 3081.98 & 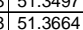 & $\begin{array}{l}51.1 .9 \\
51.9\end{array}$ & $\begin{array}{ll}11.9 \\
11.9\end{array}$ & \begin{tabular}{|l|}
39.5 \\
39.4 \\
\end{tabular} & \begin{tabular}{|l|}
2.720 \\
2.714 \\
\end{tabular} & \begin{tabular}{|l}
0.007 \\
0.007
\end{tabular} & $\begin{array}{l}.007 \\
0.007\end{array}$ & & & $\begin{array}{l}0.15 \\
0.15\end{array}$ \\
\hline & & 11:16:21 AM & & & \begin{tabular}{|l|}
24.286 \\
\end{tabular} & \begin{tabular}{|l|l|} 
& 25.851 \\
\end{tabular} & 25.173 & 47.627 & \begin{tabular}{|l|}
53.081 \\
\end{tabular} & 17.044 & 31.548 & -0.81 & & 51.587 & 0.048 & 14.643 & & & $\begin{array}{l}351.3831 \\
\end{array}$ & & & & & 0.007 & & & & $\begin{array}{l}0.15 \\
0.15\end{array}$ \\
\hline 23390 & 9/1/2004 & 11:17:21 AM & & 25.554 & & \begin{tabular}{|l|l|}
25.867 \\
\end{tabular} & 25. & \begin{tabular}{|l|l|}
47.943 \\
\end{tabular} & \begin{tabular}{|l|l|}
53.464 \\
\end{tabular} & \begin{tabular}{|l|}
17.25 \\
\end{tabular} & $\begin{array}{l}31.587 \\
\end{array}$ & -0.81 & & 48.759 & & 14.6 & & & & 52.3 & 11.6 & & 2.742 & & 0.007 & & & \\
\hline 2391 & & & 25.184 & & & 25.892 & & 47.513 & \begin{tabular}{|l|}
53.054 \\
\end{tabular} & & & & & 50.281 & $\mid$ & 14.6 & & & & & & & & & & & 0.177 & \\
\hline 2302 & & & & 25.545 & \begin{tabular}{|l|l|}
24.303 \\
\end{tabular} & \begin{tabular}{|l|l|l|}
25.908 \\
\end{tabular} & & & & \begin{tabular}{|l|l|l|}
17.019 \\
\end{tabular} & & -0.807 & & 51.894 & & 14.6 & & & & 521 & 12 & & & & & & & \\
\hline & $1 / 2004$ & 11:20:21 AM & 25.19 & 2554 & 24.278 & 25.968 & & & 53.363 & & & & & & & 146 & & & & 57 & & & $1717+3$ & & & & & \\
\hline & $\sqrt{12004}$ & $11: 21: 21 \mathrm{AM}$ & 25.2 & 25.545 & 24.288 & 25.968 & & 47.53 & 52.975 & \begin{tabular}{l|l|l|l|}
17.049 \\
\end{tabular} & 31.599 & -0.81 & & 50.066 & | & 14.643 & & B7.98 & $\begin{array}{l}351.4664 \\
\end{array}$ & & & & & |0.007 & 0.007 & & & \\
\hline & $9 / 1 / 2004$ & $11: 22: 21 \mathrm{AM}$ & 25.197 & 25.552 & 24.259 & 25.994 & 25.24 & 47.542 & \begin{tabular}{|l|l|}
53.238 \\
\end{tabular} & \begin{tabular}{|l|l|}
16.893 \\
\end{tabular} & 31.616 & -0.769 & & 51.414 & 0.048 & 14.643 & & 88.98 & 31.4831 & 52.1 & 12.2 & 396 & & & 0.007 & & & 0.15 \\
\hline & 12000 & & $\frac{25.191}{20.201}$ & 25.551 & \begin{tabular}{|l|}
24.264 \\
\end{tabular} & 25.999 & 25.2 & $\begin{array}{l}47.667 \\
\end{array}$ & 53.29 & \begin{tabular}{|l|l|}
17.087 \\
1.007
\end{tabular} & 31.425 & $\begin{array}{l}-0.763 \\
-0.70\end{array}$ & & 50.515 & 0.049 & 14.643 & & & & & & & & & & & & \\
\hline 2398 & $\frac{112006}{11203}$ & $11: 24: 21$ & & $\frac{25.563}{25.55}$ & \begin{tabular}{|l|}
24.276 \\
$2.26 ?$
\end{tabular} & 26.021 & & 47.899 & 53.419 & 16.959 & $\begin{array}{l}31.911 \\
21507\end{array}$ & -0.76 & & 9.593 & & 14.643 & & & .5164 & & & & & & & & & \\
\hline$\frac{2050}{2399}$ & & & & & 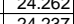 & $\frac{26.04 i}{20050}$ & & (47.669) & & & $\frac{31.501}{21.91}$ & $\frac{0.163}{0.834}$ & & $\begin{array}{l}49.1933 \\
1.927\end{array}$ & & $\frac{14.64}{1.67}$ & & & & & & & & & & & & \\
\hline$\frac{2399}{2400}$ & (1) & $\frac{11: 26: 2}{11 \cdot 27 \cdot 2}$ & $\frac{25.199}{252}$ & $\frac{25.554}{25.56}$ & \begin{tabular}{|l|}
24.237 \\
24243
\end{tabular} & $\frac{26.052}{26073}$ & & $\begin{array}{l}47.204 \\
47665\end{array}$ & $\begin{array}{r}52.66 \\
53079\end{array}$ & $\frac{16.766}{16.916}$ & $\begin{array}{l}31.484 \\
31.81\end{array}$ & 0.804 & & 年9.827 & .049 & 14.64 & & & & & & & .11 & & & & 182 & 0.16 \\
\hline & $9 / 1 / 12004$ & $\begin{array}{l}11.27 .21 \mathrm{AM} M \\
11: 28: 21 \mathrm{AM}\end{array}$ & 25.211 & 25.571 & \begin{tabular}{|l|}
24.245 \\
24.229 \\
\end{tabular} & $\frac{20.070}{26.094}$ & & $\begin{array}{l}47.005 \\
47.351\end{array}$ & 52.612 & $\begin{array}{l}\frac{10.910}{16.832} \\
\end{array}$ & $\begin{array}{l}31.01 \\
31.673\end{array}$ & $\begin{array}{l}-0.109 \\
-0.76\end{array}$ & & & 5.049 & 14.643 & & & 51.58 & & 11.8 & & & & & & & 0.15 \\
\hline 40 & 9/1/2004 & $11: 29: 21 \mathrm{AM}$ & 25.185 & 25.56 & $\begin{array}{l}24.213 \\
\end{array}$ & $\begin{array}{ll}3 & 26.038 \\
\end{array}$ & 25.3 & 47.395 & 52.88 & 16.989 & 31.455 & -0.778 & & 50.334 & 0.048 & 14.643 & 0.003 & 3095.98 & 51.5997 & 51.8 & 12.0 & 39. & 2.718 & \begin{tabular}{|l|}
0.007 \\
\end{tabular} & 0.007 & 0.000 & 0.178 & 0.15 \\
\hline & & & 25.189 & 25.559 & \begin{tabular}{|l|l|}
24.242 \\
\end{tabular} & $\begin{array}{l}25.957 \\
\end{array}$ & 25.29 & 47.52 & 53.103 & \begin{tabular}{ll|}
17.042 \\
1.02
\end{tabular} & 31.544 & -0.766 & & 48.905 & $\begin{array}{l}0.049 \\
\end{array}$ & 14.643 & & 3096.98 & 351.6164 & 52.0 & 11.6 & & & 0.007 & 0.007 & & 0.181 & \\
\hline & 1112004 & $11: 31$ & 182 & 25.552 & 24.205 & 25.92 & & 47.605 & 53.317 & 16.989 & & 0.763 & & $0.5 / 3$ & 047 & 14.64 & & & & 52.2 & & & & & & & & \\
\hline & & & & 25.552 & \begin{tabular}{|l}
24.219 \\
\end{tabular} & 25.864 & & 41.642 & 53.207 & 17.065 & & & & & & & & & & 52. & & & & & & & & \\
\hline$\frac{2406}{2407}$ & 告1/12004 & $\begin{array}{l}11: 33: 21 \mathrm{AN} \\
1134: 21\end{array}$ & $\frac{25.175}{25.172}$ & $\begin{array}{r}25.54 \\
25.542\end{array}$ & \begin{tabular}{|l|}
24.218 \\
24.22
\end{tabular} & $\frac{25.798}{25.775}$ & $\frac{25.06}{25.04}$ & $\frac{47.963}{47.576}$ & $\frac{53.371}{53.085}$ & $\begin{array}{l}\frac{16.875}{16.901} \\
16.9\end{array}$ & 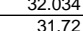 & $\begin{array}{l}-0.763 \\
-0.76\end{array}$ & & & . & $\frac{14.643}{14.643}$ & & & & 520 & $\frac{1.8}{1.8}$ & & & & & & & 0.15 \\
\hline 2408 & $9 / 1 / 12004$ & $11: 35: 21 \mathrm{AA}$ & 25.176 & 25.551 & \begin{tabular}{|l|}
24.24 \\
\end{tabular} & \begin{tabular}{|l|l|l|l|}
4 & 25.759 \\
4
\end{tabular} & 25. & 47.804 & 53.005 & $\mid$\begin{tabular}{|c|}
16.987 \\
\end{tabular} & $\begin{array}{l}0.12 \\
31.704\end{array}$ & -0.763 & & 48.961 & . & $\begin{array}{l}14.045 \\
14.643\end{array}$ & & 1.98 & & 52.2 & 11.7 & & & & & & & \\
\hline & & $11: 36: 21 \mathrm{AM}$ & 25.175 & 25.54 & \begin{tabular}{|l|}
24.218 \\
24.218 \\
\end{tabular} & \begin{tabular}{|l|l|}
8 & 25.707 \\
\end{tabular} & & 47.48 & \begin{tabular}{|l|}
53.050 \\
\end{tabular} & 16.885 & 31.55 & $\mid \begin{array}{l}5 \\
5\end{array}$ & & 49.927 & 0.047 & 14.643 & & 02 98] & 7164 & & 1.t.9 & \begin{tabular}{|l|l|}
35 \\
\end{tabular} & 2.724 & & 0.06 & & 174 & 015 \\
\hline & & 11:37:21 AM & 25.169 & & 24.242 & & & 47.588 & \begin{tabular}{|r|}
52.89 \\
\end{tabular} & & & & & 51.385 & .048 & & & & & & & & & & & & & \\
\hline & $9 / 1 / 2004$ & & 25.169 & 25.529 & 24.217 & 25.787 & 25 & 47.381 & & 16.617 & 31.739 & -0.76 & & 50.651 & .047 & 14.643 & & & & & 2 & & & & & & & .15 \\
\hline$\frac{412}{412}$ & & & 25.174 & 25.544 & \begin{tabular}{|l|}
24.247 \\
\end{tabular} & 25.832 & & & \begin{tabular}{|l|l|}
53.23 \\
\end{tabular} & 17.133 & 31.552 & & & & 046 & 14.6 & & & & 52.1 & 12.2 & & & & & & & \\
\hline & & & & & 24.302 & 25.862 & & & 53.421 & \begin{tabular}{|l|l|}
17.07 \\
\end{tabular} & 31.552 & -0.763 & & & & & & & & & & & & & & & & \\
\hline & $9 / 1 / 2004$ & 11:41:21 AM & 25.164 & 25.534 & 24.342 & $\begin{array}{l}25.882 \\
25\end{array}$ & 25 & 47.949 & 53.545 & 17.169 & $\begin{array}{l}31.673 \\
\end{array}$ & -0.697 & & & 0.049 & 14.6 & & & & 52.4 & 20 & & 2.745 & & 0.00 & & & 0.15 \\
\hline & $9 / 1 / 2004$ & 11:42:21 AM & 25.18 & 25.54 & 24.333 & \begin{tabular}{l|l}
3 & 25.928 \\
\end{tabular} & & & \begin{tabular}{|l|l|}
53.215 \\
\end{tabular} & $\begin{array}{l}17.142 \\
\end{array}$ & 31.683 & -0.694 & & & 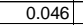 & 14.6 & & & & 52. & 12.5 & & & & & & & \\
\hline & & $11: 43: 21 \mathrm{~A}$ & 25.176 & 25.536 & $\begin{array}{l}24.329 \\
\end{array}$ & $\begin{array}{l}9 \\
\begin{array}{l}9 \\
\end{array} 25.918\end{array}$ & & 477.67 & \begin{tabular}{|l|l|}
53.238 \\
\end{tabular} & 17.217 & 31.533 & -0.7 & & 49.676 & $\mid 0.048$ & 14. & & & & 52. & 11.8 & & & & & & 0.177 & 0.15 \\
\hline & & & 25.181 & 25.546 & $\begin{array}{l}524.299 \\
5\end{array}$ & 25.946 & & 47.5 & \begin{tabular}{|l|l|l|} 
\\
\end{tabular} & 17.182 & & -0.697 & & & 0.045 & 14.6. & & & & -1 & 1.5 & & & & & & 166 & \\
\hline & 2004 & & 25.19 & & 24.326 & & & & $52.8 / 6$ & 16.989 & 31.1 & & & & & & & & & & 2.0 & & & & & & & \\
\hline 2419 & & $11: 46: 21 \mathrm{Al}$ & 25.17 & 25.531 & 24.255 & 25.9 & & 4.64 & 53.46 & 17.2 & 31.2 & - -0.703 & & & .048 & & & 124 & & 52.3 & 11.8 & & & & & & & \\
\hline & $\mid \frac{11 / 2004}{11 / 2004}$ & $\frac{11.4}{11.4}$ & $\begin{array}{l}25.182 \\
25198\end{array}$ & $\begin{array}{l}25.537 \\
25548\end{array}$ & $\begin{aligned} 24.26 \\
3251\end{aligned}$ & $\frac{25.915}{20.021}$ & & $\begin{array}{l}4.45 \\
4.445\end{array}$ & 5.94017 & $\frac{1.037}{1689}$ & $\begin{array}{l}31.361 \\
31652\end{array}$ & $\begin{array}{l}-0.0103 \\
-0.697\end{array}$ & & & | & . & & & & $\begin{array}{ll}51.80 \\
559\end{array}$ & 117 & & 727 & & & & 177 & \\
\hline 2422 & $11 / 2004$ & & 25.199 & 25.544 & $\begin{array}{l}24.251 \\
24.267\end{array}$ & \begin{tabular}{|l|l|}
77 & 2.021 \\
7 & 027
\end{tabular} & & & 53.446 & & & $\begin{array}{l}-.051 \\
-0.697\end{array}$ & & & 0.047 & $\frac{14.0}{14.6}$ & & & & & 11..8 & & & & & & & \\
\hline & 9/1/2004 & & 25.199 & 25.549 & $\frac{12424242}{24242}$ & 226.0 & & & \begin{tabular}{|l|l|l|l|l|} 
\\
\end{tabular} & & & -0.7 & & & 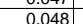 & & & & & & 118 & & 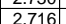 & & & & & \\
\hline & $9 / 1 / 2004$ & 11:51: & 25.2 & 25.56 & \begin{tabular}{|l|l|l}
5 & 24.263 \\
\end{tabular} & 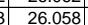 & & & \begin{tabular}{|l|}
53.087 \\
\end{tabular} & $\begin{array}{l}17.057 \\
\end{array}$ & & -0.7 & & & D.047 & $\frac{14.6}{14.6}$ & & & & 52.0 & $\frac{1.7 .7}{11.7}$ & & & & 0.6 & 0.000 & $\frac{174}{174}$ & \\
\hline & & & & & & 26.6 & & & & & & -0.6 & & & & & & & & & . & & & & & & & \\
\hline & & & 25.202 & 25.557 & 24.234 & 26. & & & 53.052 & 16.86 & & & & & & & & & & & $\mid 12.4$ & & & & & & 174 & \\
\hline & & & 25.207 & 25.557 & 24.235 & 26.15 & 25.487 & & 53.489 & $\begin{array}{ll}16.875 \\
\end{array}$ & 31.536 & -0.645 & & & 0.046 & & & & & 52.4 & 11.8 & 39. & $2.1<4$ & 0.007 & & & 0.170 & 14 \\
\hline & & & $25.20 \subseteq$ & 25.574 & 24.247 & $\begin{array}{l}726.157 \\
\end{array}$ & & & & & & -0.645 & & & & & & & & & 12.2 & 39 . & 2.728 & 0.007 & & & 0.177 & \\
\hline & 9/1/2004 & & $\frac{25.21}{25.20}$ & 25.5 & & 26.1 & 25.48 & & $\begin{array}{l}53.245 \\
5.221\end{array}$ & $\begin{array}{r}17.134 \\
16.955\end{array}$ & & -0.6. & & & 0.047 & & $\frac{3}{3}$ & & & & \begin{tabular}{|l|}
11.6 \\
119
\end{tabular} & \begin{tabular}{|l|}
39.6 \\
30.5 \\
\end{tabular} & \begin{tabular}{|l|}
2.729 \\
.7723 \\
\end{tabular} & 0.007 & & 0.000 & 0.174 & \\
\hline & $9 / 1 / 2004$ & & $\begin{array}{l}25.204 \\
25208\end{array}$ & $\frac{25.57}{25.56}$ & & $\begin{array}{l}7 \\
7\end{array}$ & $\begin{array}{r}25.45 \\
25.42\end{array}$ & & \begin{tabular}{|l|}
53.224 \\
53559
\end{tabular} & $\begin{array}{r}16.955 \\
17156\end{array}$ & & $\begin{array}{l}-0.648 \\
0.525\end{array}$ & & & 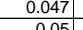 & $\begin{array}{r}14.643 \\
14643\end{array}$ & & & & 52.1 & $\frac{11.9}{120}$ & $\frac{39.5}{397}$ & $2.723 \mid$ & \begin{tabular}{|l|}
0.007 \\
0.007
\end{tabular} & & 0.000 & $\begin{array}{l}0.174 \\
0.184\end{array}$ & \\
\hline & & & $\begin{array}{l}25.200 \\
25.208\end{array}$ & $\frac{25.568}{25578}$ & $\begin{array}{l}24.236 \\
24.226\end{array}$ & 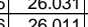 & $\frac{25.42}{25.39}$ & & \begin{tabular}{|l|}
53.559 \\
53.584
\end{tabular} & \begin{tabular}{|l|l|}
17.156 \\
17288
\end{tabular} & & -0.535 & & & $\begin{array}{r}0.05 \\
0048\end{array}$ & & & & & 52.4 & 12.0 & 39.1 & 2.137 & 0.007 & & 0.000 & 0.184 & \\
\hline$\frac{2432}{2433}$ & & $\begin{array}{l}\frac{11: 59: 21 \mathrm{AM}}{12200: 21 \mathrm{PM}} \\
\end{array}$ & $\frac{0.200}{25.191}$ & 25.561 & \begin{tabular}{|l|l|l|l|l|l|}
24.204 \\
\end{tabular} & \begin{tabular}{|l|l|}
4 & 25.944 \\
\end{tabular} & 25. & & & \begin{tabular}{|c|}
16.9420 \\
16.92
\end{tabular} & & $\begin{array}{l}-0.65 \\
-0.645 \\
\end{array}$ & & & $\frac{0.06}{0.02}$ & 14.6 & & & & & $\frac{11.0}{12.1}$ & 30 & & & & 0.0 & 0.171 & \\
\hline 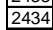 & 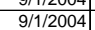 & 12:01:21 PM & 25. & 25.575 & 24.228 & \begin{tabular}{|l|l|}
8 & 25.928 \\
\end{tabular} & & & \begin{tabular}{|l|}
53.267 \\
\end{tabular} & 17.008 & & \begin{tabular}{|l|l|} 
& -0.639 \\
\end{tabular} & & & 0.046 & 14. & & & & 52.1 & 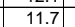 & $\frac{396}{39.6}$ & 2.730 & & & & 0.170 & \\
\hline & 9/1/2004 & & 25.17 & 25.547 & 24.21 & 25.8 & & & \begin{tabular}{|l|l|} 
& 53.11 \\
\end{tabular} & & 31.6 & -0.6 & & & & & & & & 520 & \begin{tabular}{|c|c|}
11.8 \\
\end{tabular} & & 2721 & & & & 177 & 0.15 \\
\hline & $9 / 1 / 2004$ & & 25.18 & 25.556 & 24.19 & 25.8 & & & \begin{tabular}{|l|l|} 
\\
\end{tabular} & & & -0.6 & & 50.03 & & & & & & & 1.9 & & & & & & 0.177 & \\
\hline & & & & & & & & & 3.17 & & & & & & & & & & & & 1.9 & & & & & & 78 & \\
\hline & & & 25.18 & 25 & \begin{tabular}{|l|l|l|} 
& 24.239 \\
\end{tabular} & 25. & & & 53.539 & & & & & & & & & & & & 11.8 & & 62 & & & & 0.175 & \\
\hline 24 & & & & & & & & & & & & & & & & & & & & & & & & & & & & \\
\hline & & & $\frac{25.18}{2517}$ & & 24.25 & 25. & & & & 16 & & -0 & & & & & & & & & $1 . .2$ & & & & & & 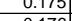 & \\
\hline 244 & & & 25.17 & 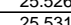 & 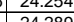 & $\begin{array}{l}4-2.12 \\
\text { g. } 257\end{array}$ & & & 53.01 & & & 0.5 & & & & & & & & & & & & & & & 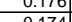 & \\
\hline & $9 / 1 / 20$ & & 20.1 & & 24.20 & & & & 5.582 & 17.12 & & 0 & & & & & & & & & 18 & & & & & & 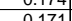 & \\
\hline & & & 25.16 & & & & & & & & & -0.5 & & & & & & & & & & & & & & & & \\
\hline & $9 / 1 /$ & & 25.18 & 25 & 24 & & & 47. & & & & -0.5 & & & & & & & & & & & & & & & 0.17 & \\
\hline & & & & & 24.46 & & & & & & & & & & & & & & & & & & & & & & & \\
\hline & & & & & & & & & & & & & & & & & & & & & & & & & & & & \\
\hline & & & 25.20 & 25.53 & \begin{tabular}{|l|l|}
24.381 \\
\end{tabular} & 25. & & 47.6 & & $\begin{array}{ll}17.228 \\
\end{array}$ & & -0. & & 50 & & & & & & 52.4 & & & & & & & 172 & \\
\hline & & & & & & 250 & & & 3.74 & & & & & & & & & & & & & & & & & & $\mid 776$ & n \\
\hline 450 & $9 / 1 / 2004$ & 12:17:21 & 25.203 & 25.528 & $3 \longdiv { 2 4 . 3 4 1 }$ & \begin{tabular}{l|l}
1 & 25.956
\end{tabular} & & 47.652 & 53.34 & \begin{tabular}{|l|l|}
17.122 \\
\end{tabular} & 31.443 & \begin{tabular}{|c|c|} 
& -0.538 \\
\end{tabular} & & 50.357 & 0.049 & $14.643 \mid$ & & & 52.3997 & 52.2 & & & 2.727 & 0.007 & 0.007 & 0.000 & 0.181 & \\
\hline
\end{tabular}


WSRC-TR-2005-00105, REVISION 0

SRNL-RPP-2005-00012, REVISION 0

RUN \# 4.03A AND B; FIRST AND SECOND HALF OF SLURRY DEWATERING - CONT.

\begin{tabular}{|c|c|c|c|c|c|c|c|c|c|c|c|c|c|c|c|c|c|c|c|c|c|c|c|c|c|c|c|c|}
\hline & $\mathrm{A}$ & $\mathrm{B}$ & $\mathrm{D}$ & $E$ & $F$ & G & $\mathrm{H}$ & $\mathrm{J}$ & K & $\mathrm{L}$ & $M$ & $\mathrm{~N}$ & 0 & Q & $\begin{array}{ll}R \\
\end{array}$ & \begin{tabular}{l|l} 
\\
\end{tabular} & $\mathrm{T}$ & $\mathrm{V}$ & w & $x$ & Y & $z$ & & & & AD & & AF \\
\hline 2451 & 9/1/2004 & 12:18:21 PM & 25.213 & 25.538 & 24.361 & 25.951 & 25.498 & 47.702 & \begin{tabular}{|l|}
53.559 \\
\end{tabular} & 17.106 & 31.552 & -0.561 & -6.582 & 50.089 & 0.048 & 14.643 & 0.003 & 3144.98 & 52.4164 & \begin{tabular}{|l|}
52.4 \\
\end{tabular} & 11.9 & 39.6 & 2.732 & 0.007 & 0.007 & 0.000 & 0.177 & \\
\hline & & & 25.222 & 25.532 & 24.34 & \begin{tabular}{|l|}
25.985 \\
\end{tabular} & 25.407 & 47.764 & \begin{tabular}{|l|}
53.518 \\
\end{tabular} & 17.058 & 31.677 & \begin{tabular}{|l|l|}
-0.552 \\
\end{tabular} & -6.596 & 51.36 & 0.046 & $\begin{array}{l}14.643 \\
\end{array}$ & 0.003 & 3145.98 & & \begin{tabular}{|l|}
52.4 \\
\end{tabular} & 12.2 & & & & 0.007 & & & \\
\hline$\frac{2435}{205}$ & $9 / 1 / 2004$ & & 25.227 & 25.552 & \begin{tabular}{|l|l}
24.365 \\
\end{tabular} & \begin{tabular}{|l}
26.04 \\
\end{tabular} & $\begin{array}{l}25.407 \\
3.09\end{array}$ & 48.05 & \begin{tabular}{|l|}
53.713 \\
5.256
\end{tabular} & $\begin{array}{l}17.163 \\
\end{array}$ & 31.862 & $\begin{array}{r}-0.535 \\
\end{array}$ & $\begin{array}{r}-6.57 \\
6.591\end{array}$ & 50.578 & \begin{tabular}{|l|l|}
0.049 \\
\end{tabular} & 14.643 & 0.003 & 8146.98 & 52.4497 & \begin{tabular}{|l|l|}
52.6 \\
\end{tabular} & & $\frac{40.0}{30.0}$ & 7.755 & 0.007 & 0.007 & 0.000 & 0.179 & \\
\hline 2454 & 9/1/2004 & 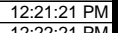 & $\begin{array}{l}25.243 \\
25.243\end{array}$ & $\begin{array}{l}25.552 \\
25559\end{array}$ & \begin{tabular}{|l|l|}
24.356 \\
2.31
\end{tabular} & $\begin{array}{r}26.11 \\
\end{array}$ & $\begin{array}{l}25.488 \\
25.9\end{array}$ & $\begin{array}{l}47.839 \\
\end{array}$ & \begin{tabular}{|l|l|}
53.566 \\
5306
\end{tabular} & \begin{tabular}{|l|l|}
17.173 \\
17217
\end{tabular} & $\begin{array}{l}31.683 \\
21.571\end{array}$ & \begin{tabular}{|l|l|} 
& -0.54 \\
& 525 \\
\end{tabular} & $\begin{array}{l}-6.561 \\
6561\end{array}$ & 49.989 & $\begin{array}{l}0.047 \\
\end{array}$ & 14.643 & 0.003 & $\begin{array}{l}3147.98 \\
20.9\end{array}$ & 2.4.4664 & \begin{tabular}{|l|l|}
52.4 \\
\end{tabular} & \begin{tabular}{ll|}
11.9 \\
\end{tabular} & 39.8 & 2.741 & 0.007 & $\begin{array}{l}0.007 \\
\end{array}$ & 0.000 & 0.172 & \\
\hline$\frac{2455}{2456}$ & $\frac{9 / 1 / 2004}{99 / 1 / 2004}$ & $\begin{array}{ll}12: 22: 21 \mathrm{PM} \\
122 \cdot 2 \cdot 21 \mathrm{PM}\end{array}$ & $\begin{array}{l}25.243 \\
25.251\end{array}$ & $\frac{25.558}{25.576}$ & \begin{tabular}{|l|}
24.361 \\
24359 \\
\end{tabular} & & $\begin{array}{l}25.493 \\
25546\end{array}$ & & & \begin{tabular}{|c|}
17.317 \\
1708
\end{tabular} & \begin{tabular}{|l|}
31.574 \\
31.761
\end{tabular} & $\begin{array}{l}-0.535 \\
-0.535\end{array}$ & $\begin{array}{l}-6.564 \\
6567\end{array}$ & $\begin{array}{l}49.998 \\
50475\end{array}$ & $\begin{array}{l}0.048 \\
0047\end{array}$ & \begin{tabular}{|l|l|l|}
14.643 \\
14634 \\
\end{tabular} & & 3148.98 & 4831 & \begin{tabular}{|l|}
52.7 \\
\end{tabular} & \begin{tabular}{|l|}
11.9 \\
10.0 \\
\end{tabular} & & $\begin{array}{l}2.744 \\
\end{array}$ & & & & $\begin{array}{l}0.176 \\
\end{array}$ & \\
\hline 2457 & & $\frac{122: 23: 21 \mathrm{PM}}{12: 22: 21 \mathrm{PM}}$ & $\frac{25.251}{25.258}$ & $\begin{array}{l}25.576 \\
25.578\end{array}$ & \begin{tabular}{|l|}
24.359 \\
24.371 \\
\end{tabular} & \begin{tabular}{|l|}
26.134 \\
26.181 \\
\end{tabular} & $\begin{array}{l}25.546 \\
25.483 \\
\end{array}$ & $\begin{array}{r}47.122 \\
48.125\end{array}$ & \begin{tabular}{|l|}
53.323 \\
53.835
\end{tabular} & $\begin{array}{r}17.08 \\
17.3\end{array}$ & \begin{tabular}{|l|}
31.761 \\
31.858 \\
\end{tabular} & $\begin{array}{r}-0.535 \\
-0.52 \\
\end{array}$ & $\begin{array}{l}-6.567 \\
-6.477\end{array}$ & $\begin{array}{l}50.455 \\
50.584\end{array}$ & \begin{tabular}{|l|}
0.047 \\
0.048
\end{tabular} & \begin{tabular}{|c|}
14.643 \\
14643 \\
\end{tabular} & $\begin{array}{l}0.003 \\
0.003\end{array}$ & $\begin{array}{l}3349.98 \\
3150.98 \\
\end{array}$ & \begin{tabular}{|c|}
52.4997 \\
$\mathbf{3}$
\end{tabular} & \begin{tabular}{|l|}
52.2 \\
52.7 \\
\end{tabular} & $\frac{12.0}{12.0}$ & $\begin{array}{l}39.8 \\
40.0\end{array}$ & $\begin{array}{l}2.743 \\
2.757 \\
\end{array}$ & $\begin{array}{l}0.007 \\
0.007\end{array}$ & $\begin{array}{l}0.007 \\
0.007\end{array}$ & $\begin{array}{l}0.000 \\
0.000\end{array}$ & $\begin{array}{l}0.172 \\
0.075\end{array}$ & $\begin{array}{l}0.15 \\
0.15\end{array}$ \\
\hline 2458 & $9 / 1 / 2004$ & & 25.255 & 25.585 & & \begin{tabular}{|l|}
26.208 \\
\end{tabular} & & & & & & & & & 0.047 & 14.643 & $\begin{array}{l}0.003 \\
0.003\end{array}$ & 3151.98 & \begin{tabular}{|l|}
52.5164 \\
52.5331
\end{tabular} & \begin{tabular}{|l|}
25.1 \\
52.3 \\
\end{tabular} & $\begin{array}{ll}12.8 \\
11.8\end{array}$ & \begin{tabular}{|l|l|}
8 & 40.0 \\
8
\end{tabular} & $\frac{2.757}{2.735}$ & & 0.007 & $\begin{array}{l}0.000 \\
0.000\end{array}$ & & $\frac{0.15}{0.15}$ \\
\hline & & & & 25.587 & 24.35 & 26.23 & & 47.953 & & 17.209 & 31.679 & & -6.547 & 49.122 & 0.049 & 14.643 & & & 2.5497 & \begin{tabular}{|r|}
52.6 \\
\end{tabular} & & & & & & & & $\begin{array}{l}0.15 \\
0.15\end{array}$ \\
\hline 2460 & 9/1/2004 & 12:27:21 PM & 25.254 & 25.589 & \begin{tabular}{|l|}
24.357 \\
\end{tabular} & & 25.684 & $\begin{array}{l}47.797 \\
\end{array}$ & 53.696 & \begin{tabular}{|l|l|}
17.063 \\
\end{tabular} & 31.669 & & & 50.626 & 049 & \begin{tabular}{|l|l|}
14.643 \\
\end{tabular} & & 53.98 & 5664 & \begin{tabular}{|c|}
52.6 \\
\end{tabular} & 12.1 & 39.7 & 2.739 & & 0.007 & 0.000 & & \\
\hline 2461 & & & 25.266 & & 24.349 & 26.209 & & & & & & & & & & $\begin{array}{l}14.643 \\
\end{array}$ & & & & 52.3 & & & & .007 & 0.007 & 0.000 & 0.173 & \\
\hline 2462 & & 12:29:21 PM & & 25.591 & 24.359 & 26.159 & & 47.858 & 53.479 & 16.816 & 32.051 & & & 49.462 & & & & & & 52.4 & $\frac{11 . \varepsilon}{11.8}$ & & & & & & & \\
\hline & & 12:30:21 PM & 25.257 & 25.592 & 24.365 & 26.105 & & 47.739 & 533 & & & 506 & & & & $\begin{array}{l}14.643 \\
\end{array}$ & & & 6164 & & & & & 007 & & & & \\
\hline & & 12:31:21 PM & & 25.587 & 24.35 & 26.08 & 25.442 & 47.754 & \begin{tabular}{|l|l|}
53.336 \\
\end{tabular} & 16.945 & 31.907 & -0.471 & & 50.528 & 0.046 & \begin{tabular}{|l|l|l|l|}
14.643 \\
\end{tabular} & & & & 52.2 & 12.0 & 39.8 & 2.746 & 0.007 & 0.007 & 0.000 & & \\
\hline & 9/1/2004 & 12:32:21 PM & 25.256 & 25.591 & 24.349 & 26.059 & 25.366 & 48.04 & \begin{tabular}{|l|}
53.843 \\
\end{tabular} & 17.454 & 31.503 & -0.491 & -6.45 & 50.824 & 0.048 & 14.643 & 0.003 & 158.98 & 52.6497 & 52.7 & 12.1 & & & & 0.007 & 0.000 & & 0.15 \\
\hline & & 12:33:21 PM & 25.254 & 25.594 & $\begin{array}{l}24.357 \\
2.301\end{array}$ & $\begin{array}{l}25.987 \\
25.070\end{array}$ & 25.28 .5 & $\begin{array}{l}47.52 \\
7.001\end{array}$ & \begin{tabular}{|l|l|}
53.203 \\
5203
\end{tabular} & 17.127 & 31.367 & -0.488 & & 49.9 & 0.047 & \begin{tabular}{|l|l|}
14.643 \\
1.63
\end{tabular} & & & 52.6664 & 52.1 & & & & 0.007 & & & $\begin{array}{l}0.174 \\
\end{array}$ & \\
\hline & & 12:34:21 PM & & & 24.341 & & & 47.801 & & & 31.515 & 503 & & 49.823 & & 14.643 & & & 52.6831 & & & & & .007 & & & & \\
\hline & & $12: 35: 21 \mathrm{PM}$ & 25.262 & & 24.365 & 25.93 & & 47.901 & & & 31.969 & .509 & & 50.219 & & 14.643 & & & & 52.4 & & & & .007 & & & & \\
\hline & & 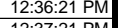 & 年 & $\begin{aligned} 25.591 \\
25.579\end{aligned}$ & 24.339 & 25.918 & & 48.069 & \begin{tabular}{|c|}
54.129 \\
5201
\end{tabular} & $\begin{array}{ll}1.7062 \\
1701\end{array}$ & $\begin{array}{l}31.852 \\
21.927\end{array}$ & -0.477 & -6 & $\begin{array}{l}49.401 \\
5.512\end{array}$ & & 14.643 & & 62.98 & $\begin{array}{l}52.7164 \\
527201\end{array}$ & & 11.8 & $\frac{40.0}{300}$ & & 00 & & & 0.168 & 0.14 \\
\hline 2471 & & $\begin{array}{l}12: 38: 21 \mathrm{PM} \\
13\end{array}$ & $\frac{25.249}{25269}$ & 25.389 & $\frac{24.344}{24.402}$ & $\frac{25.004}{25846}$ & & (4t.940 & $\mid \begin{array}{l}3.931 \\
5374\end{array}$ & $\frac{17.101}{17.149}$ & $\frac{31.827}{31.743}$ & $\begin{array}{l}-0.4 / 4 \\
-0.471\end{array}$ & & & & 14643 & & & 它.7497 & & $\frac{12.6}{11.7}$ & & $\mid 2.747$ & 0007 & 0007 & & & 0.16 \\
\hline & 9/1/2004 & $12: 39: 21$ PM & 25.263 & 25.588 & \begin{tabular}{|l|}
24.404 \\
\end{tabular} & 25.885 & 25.163 & 47.746 & \begin{tabular}{|l|}
53.578 \\
\end{tabular} & 17.102 & 31.63 & $\begin{array}{l}-.411 \\
-0.416\end{array}$ & -6.33 & \begin{tabular}{|l|}
50.609 \\
\end{tabular} & 0.049 & $\begin{array}{l}14.643 \\
14.643\end{array}$ & 0.004 & 3165.98 & 52.7664 & \begin{tabular}{|l|}
52.5 \\
\end{tabular} & \begin{tabular}{|l|}
11.1 \\
12.1 \\
\end{tabular} & \begin{tabular}{ll|}
1 & 39.7 \\
\end{tabular} & & 0.007 & $\begin{array}{l}0.007 \\
\end{array}$ & 0.000 & 0.180 & \\
\hline & & 12:40:21 PM & 25.268 & 25.578 & \begin{tabular}{|l|}
24.391 \\
\end{tabular} & 25.92 & & 47.88 & \begin{tabular}{|l|l|}
53.678 \\
\end{tabular} & 17.292 & 31.609 & -0.465 & & 50.102 & 0.047 & $\begin{array}{l}14.643 \\
\end{array}$ & & 3166.98 & \begin{tabular}{|l|l}
3 & 52.7831 \\
\end{tabular} & 52.6 & 11.9 & 39.7 & 2.740 & \begin{tabular}{|l|}
0.007 \\
\end{tabular} & 0.007 & 0.000 & 0.172 & \\
\hline & & 12:41:21 PM & 25.269 & 25.589 & 24.412 & 25.972 & & & \begin{tabular}{|l|}
53.487 \\
\end{tabular} & 17.389 & & & & 50.131 & & 14.643 & & & 52.7997 & 52.4 & 11.9 & & 2.724 & 0.007 & 0.007 & & 0.177 & \\
\hline 2475 & & 12:42:21 PM & 25.28 & 25.59 & 24.463 & 26.033 & 25.38 & 47.864 & \begin{tabular}{|l|l|}
53.74 \\
\end{tabular} & 17.282 & & & & 50.469 & & 14.643 & & & 52.8164 & 52.6 & & 39.7 & 2.735 & 0.007 & 0.007 & 0.000 & 0.172 & \\
\hline$\frac{2476}{2477}$ & $\begin{array}{l}9 / 1 / 2004 \\
9 / 1 / 2004\end{array}$ & $\begin{array}{l}\text { 12:43:21 PM } \\
\text { 1224:21 PM }\end{array}$ & 25.282 & 25.597 & $\frac{24.516}{24.461}$ & $\begin{array}{r}26.02 \\
26.061 \\
\end{array}$ & $\begin{array}{l}25.417 \\
25.393 \\
\end{array}$ & \begin{tabular}{|l|}
47.955 \\
47.71 \\
\end{tabular} & \begin{tabular}{|l|}
53.881 \\
55.362 \\
\end{tabular} & $\begin{array}{r}17.282 \\
17.21\end{array}$ & $\begin{array}{l}31.726 \\
31.515 \\
\end{array}$ & $\begin{array}{l}-0.471 \\
-0.47 \\
\end{array}$ & $\begin{array}{l}-6.475 \\
-6.457\end{array}$ & $\begin{array}{l}50.075 \\
5.076\end{array}$ & .048 & $\begin{array}{l}\frac{14.643}{14.643} \\
\end{array}$ & $\begin{array}{l}0.003 \\
0.003 \\
\end{array}$ & $\begin{array}{l}3169.98 \\
3170.98\end{array}$ & 52.8331 & $\begin{array}{l}52.8 \\
52.7\end{array}$ & $\begin{array}{ll}\frac{11.9}{12 .} \\
\end{array}$ & $\frac{39.8}{39.6}-x$ & 2.747 & 0.007 & 0.007 & & $\begin{array}{l}0.175 \\
0.169\end{array}$ & 0.15 \\
\hline 2478 & 9/1/2004 & $\begin{array}{l}\frac{1224: 421 \mathrm{PM}}{12: 45: 21 \mathrm{PM}} \\
\end{array}$ & $\begin{array}{r}25.273 \\
25.29\end{array}$ & 25.59 & $\begin{array}{l}24.461 \\
24.494 \\
\end{array}$ & \begin{tabular}{|l|}
26.061 \\
26.098 \\
\end{tabular} & $\begin{array}{l}25.393 \\
25.335 \\
\end{array}$ & $\begin{array}{r}44.81 \\
47.64 \\
\end{array}$ & \begin{tabular}{|l|}
53.421 \\
53.421
\end{tabular} & $\begin{array}{r}1.21 \\
16.906\end{array}$ & $\begin{array}{l}31.515 \\
31.747 \\
\end{array}$ & $\begin{array}{l}-0.4 / 4 \\
-0.459 \\
\end{array}$ & & $\begin{array}{l}50.516 \\
46.466\end{array}$ & $\begin{array}{l}0.046 \\
0.047 \\
\end{array}$ & $\begin{array}{l}\frac{14.643}{14.643} \\
\end{array}$ & & & 22.84967 & $\begin{array}{l}52.1 \\
52.3\end{array}$ & $\begin{array}{l}\frac{12.0}{11.1} \\
\end{array}$ & & 2.734 & $\begin{array}{l}0.007 \\
0.007 \\
\end{array}$ & $\frac{0.007}{0.007}$ & & & 0.14 \\
\hline 2479 & 9/1/2004 & 12:46:21 PM & 25.301 & 25.591 & 24.464 & 26.164 & 25.2 & 47.868 & \begin{tabular}{|l|}
53.86 \\
\end{tabular} & 17.21 & 31.48 & -0.454 & & 48.4 & & 14.643 & & 3172.9 & 331 & $\frac{52.3}{52.7}$ & $\frac{11.5}{11.5}$ & $\frac{39.7}{39.7}$ & 2.735 & 0.007 & & & 0.176 & $\begin{array}{l}0.15 \\
0.15 \\
\end{array}$ \\
\hline & & 12:47:21 PM & & 25.618 & 24.502 & 26.191 & & & 53.591 & & 31.638 & -0.462 & & 50.402 & & 14.643 & & & & & & & & & & & & $\frac{0.15}{0.16}$ \\
\hline 2481 & 9/1/2004 & 12:48:21 PM & 25.3 & 25.59 & 24.438 & 26.173 & 25.545 & $\begin{array}{l}47.847 \\
\end{array}$ & \begin{tabular}{|l|l|}
53.688 \\
\end{tabular} & 17.135 & 31.728 & -0.419 & & & 047 & 14.643 & & & & & & & & & $\overline{0.0}$ & & & $\begin{array}{l}0.16 \\
0.15\end{array}$ \\
\hline 482 & & 12:49:21 PM & 25.327 & 25.612 & & 26.225 & & & \begin{tabular}{|l|l|l|}
53.518 \\
\end{tabular} & 17.148 & 31.776 & -0.41 & & 50.448 & & & & & & 52.4 & & 39. & & 007 & 0.0 & & $\frac{168}{1168}$ & \\
\hline 2483 & & 12:50:21 PM & 25.328 & 25.618 & 24.446 & 26.241 & 25.6 & 47.849 & \begin{tabular}{|l|l|}
53.684 \\
\end{tabular} & & \begin{tabular}{|l}
31.63 \\
\end{tabular} & -0.419 & & & & 14.643 & & & & & & & & & & & & \\
\hline & $9 / 1 / 2$ & 12:51:21 PM & & 25.613 & 24.407 & 26.271 & 25.6 & 47.432 & |53.13 & 16.959 & 31.663 & -0.422 & & & & $\begin{array}{l}14.643 \\
\end{array}$ & & 778 & 2.9664 & 52.0 & $\overline{11.8}$ & $\overline{39}$ & & & & & & \\
\hline & 9/1/2004 & $12: 52: 21 \mathrm{PM}$ & 25.335 & 25.625 & 24.428 & 26.243 & & (47.667 & \begin{tabular}{|l|}
53.622 \\
\end{tabular} & 17.092 & 31.527 & -0.419 & & 49.0 & 2.048 & \begin{tabular}{l|l}
14.643 \\
\end{tabular} & & & & 52.5 & 11 & & & & 0.0 & & & \\
\hline & & 12:53:21 PM & 25.345 & 25.635 & 24.398 & 26.248 & & 47.8 & \begin{tabular}{|l|}
53.632 \\
\end{tabular} & 17.312 & 31.439 & -0.419 & & 51.4 & & 14. & & & & & 12 & & & & & & & 0.15 \\
\hline & & 12:54: & 25.335 & 25.625 & 24.408 & 26.183 & & 47.777 & 53.69 & 17.139 & 31.521 & -0.419 & & 51.2. & 047 & & & & & 52.6 & & & & & & & & \\
\hline & & & 25.346 & & 24.404 & $26.1 / 4$ & & & & & & & & & & & & & & & & & & & & & & \\
\hline & & 12:56:21 PM & 25.334 & 25.624 & 24.397 & 26.097 & & 47.735 & 53.68 & 17.102 & 31.626 & -0.41 & & & & & & & & 52.6 & 11. & 39 & & & & & 172 & \\
\hline & & 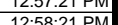 & 25342 & $\begin{array}{l}\frac{2.0628}{25672} \\
20\end{array}$ & $\begin{aligned} 24.391 \\
24385\end{aligned}$ & $\begin{array}{l}26.080 \\
26035\end{array}$ & & 年17.814 & $\begin{array}{ll}53.60 \\
5530\end{array}$ & $\frac{11 .}{17}$ & $\begin{array}{ll}31.675 \\
31.496\end{array}$ & & & & & & & & & 521 & 111 & 39 & & & & & 173 & \\
\hline & 9/1/2004 & $125.5 \cdot 21 \mathrm{PM}$ & & 25615 & 24368 & 26007 & & 年 478 & $\mid$ & 16937 & 32061 & & & & & 146 & & & & 525 & 11 & & & & & & & \\
\hline 2493 & & 12:59:24 PM & 25.335 & $\frac{2.015}{25.62}$ & 24.373 & \begin{tabular}{|l|}
20.0017 \\
26.017
\end{tabular} & & & \begin{tabular}{|l|}
53.868 \\
53
\end{tabular} & & & & & & & & & & & 527 & 11 & & & & & & & \\
\hline & $9 / 1 / 2$ & 1:00:24 PM & 25.342 & 25.632 & 24.391 & 25.995 & & 48.0 & \begin{tabular}{|l|l|}
53.945 \\
\end{tabular} & 17.179 & 31.835 & $\frac{-0.349}{-0.349}$ & & & $\overline{044}$ & 14.6 & & & & $\frac{52.8}{52.8}$ & $\frac{11}{11}$ & & 2.754 & 0.006 & $\frac{.006}{0.006}$ & & 0 & \\
\hline & & & & & & & & & & & & & & & & & & & & & & & & & & & & \\
\hline & & & 5.314 & 25.599 & 24.367 & 25.876 & & & 53.86 & \begin{tabular}{|l|l|l|}
17.167 \\
\end{tabular} & & & & 50.8 & & & & & & 52.7 & & & & & & & 172 & \\
\hline & & 1:03:24 PM & & 25.602 & 24.365 & 25.91 & & 47.969 & 53.901 & 17.322 & 31.667 & -0.41 & & & & 14. & & & & 52.8 & 12. & & 2.14 & & & & 100 & \\
\hline & & & & 25.606 & 24.39 & 25.864 & & & 53.354 & 16.849 & 31.718 & -0.40 & & & & & & & & 52.2 & & & 2.732 & & & & & \\
\hline & $9 / 1 / 2$ & $1: 05$ & & 25.606 & \begin{tabular}{|l|}
24.374 \\
\end{tabular} & \begin{tabular}{|l|}
25.873 \\
\end{tabular} & & $47 . .9$ & 53.752 & 17.107 & 31.872 & -0.39 & & & & 14.6 & & & & 52.6 & 12.0 & & & & & & . 168 & \\
\hline & & 1:06: & & & & & & & & & & & & & & & & & & 02.0 & & & & & & & & \\
\hline & & 1:07: & 319 & 25.594 & 24.387 & 25.882 & & & 53.744 & 17.23 & & & & & & & & & & 52.6 & 11 & & & & & & 169 & \\
\hline & & & & & & & & & & & & & & & & & & & & & & & & & & & & \\
\hline & & & & 25.595 & 24.388 & $26 . C$ & & & 53.8 & 17.07 & & -0.3 & & & & & & & & & & & & & & & & \\
\hline & & $\begin{array}{ll}1.1 .20 .24 \mathrm{~N} \\
1.11 .2 \mathrm{PN}\end{array}$ & & 2 & 24.409 & $\begin{array}{l}26.139 \\
26188\end{array}$ & & & $\begin{array}{l}53.18101 \\
55098\end{array}$ & $\begin{array}{l}16.941 \\
17195\end{array}$ & $\begin{array}{ll}31.815 \\
31.907\end{array}$ & $\begin{array}{l}-0.35 \\
-0.34\end{array}$ & & & & . & & & & $\frac{52 .}{529}$ & $\frac{11 .}{11}$ & & & & & & 717 & \\
\hline & & & & 2560 & 24 & & & & & & & & & & & & & & & & & & & & & & & \\
\hline & & & & & & 26.26 & & & & & & & & & & & & & & & & & & & & & & \\
\hline 25 & & & & 25.609 & \begin{tabular}{|l|l|}
24.397 \\
\end{tabular} & $\frac{26.292}{26.292}$ & & & 53.7 & $\frac{16.9}{16.9}$ & 31.847 & & & & & & & & & 52.6 & $\frac{1.1}{12.1}$ & & & & & & & \\
\hline & & & & & & & & & & & & & & & & & & & & & & & & & & & & \\
\hline & & & & & & & & & & & & & & & & & & & & & & & & & & & & \\
\hline & & & & & 24. & & & & 54.18 & & 32.1 & & & & & & & & & & 1. & & & & & & & \\
\hline & & $1: 18: 2$ & & 25.656 & 24.409 & 26.459 & & & 53.7 & 17.0 & 31.942 & -0.3 & & & & 14. & & & 53.2 & & 1 & & & & & & & \\
\hline & & & & 25.66 & 24.441 & 26.476 & & 48.0 & 54.05 & 16.987 & 32.12 & -0.3 & & & & $14.6 \mathrm{C}$ & & & & & & & & & & & 67 & \\
\hline & & & & 25.66 & & 26.4 & & & & & 31.915 & & & & & 14.6 & & & & & 11 & & & & & & 178 & \\
\hline & & 1:21: & & 25.666 & & 26. & & & 54.244 & & & & & & & & & & & & 11 & & & & & & & \\
\hline & & & & & 24.461 & & & & & & & & & & & & & & & & & & & & & & & \\
\hline & & & & 25.10 & 24.527 & 26.4 & & & $53.2 \%$ & 1.210 & 32.22 & & & & & & & & & 52.1 & 12.0 & & & & & & & \\
\hline & & $\begin{array}{l}1.24 .24 \mathrm{PM} \\
.25 .24\end{array}$ & 20.421 & 25.111 & 24.539 & \begin{tabular}{|l|l|l|}
20.409 \\
6
\end{tabular} & 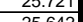 & 46.989 & 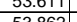 & $\begin{array}{l}17.504 \\
17271\end{array}$ & $\begin{array}{l}30.595 \\
32.457\end{array}$ & 0.484 & & 1022 & & $\begin{array}{l}14.643 \\
1.613\end{array}$ & & 212 & & $\begin{array}{ll}5,5,5 \\
5,7\end{array}$ & 11.9 & & 27 & & & 0.000 & 0.086 & 6] \\
\hline 2520 & 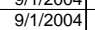 & $1: 26: 24 \mathrm{PM}$ & \begin{tabular}{|l|}
25.447 \\
25.44 \\
\end{tabular} & 25.719 & \begin{tabular}{|l|}
24.050 \\
24.542 \\
\end{tabular} & \begin{tabular}{|l|}
20.440 \\
26.417 \\
\end{tabular} & \begin{tabular}{|l|}
25.0414 \\
2514
\end{tabular} & $\begin{array}{l}48.1032 \\
48.032\end{array}$ & \begin{tabular}{|l|}
53.734 \\
53.734
\end{tabular} & $\mid 17.229$ & \begin{tabular}{|l|l|l|l|l|}
31.788 \\
\end{tabular} & \begin{tabular}{|l|}
-0.529 \\
-0.529
\end{tabular} & - -6.081 & $\begin{array}{l}50.2045 \\
50.275\end{array}$ & $\begin{array}{l}0.1016 \\
0.046\end{array}$ & \begin{tabular}{|l|}
14.04043 \\
14.643
\end{tabular} & 0.004 & 3213.03 & 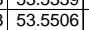 & \begin{tabular}{|l|}
52.6 \\
52.6 \\
\end{tabular} & \begin{tabular}{|l|}
12.0 \\
\end{tabular} & | & \begin{tabular}{|l|l|}
2.752 \\
\end{tabular} & \begin{tabular}{|l|}
0.014 \\
0.007 \\
\end{tabular} & $\begin{array}{l}.0107 \\
0.007\end{array}$ & 0.000 & 0.167 & \begin{tabular}{|l|l|}
7 & 0.1 \\
\end{tabular} \\
\hline
\end{tabular}


WSRC-TR-2005-00105, REVISION 0

SRNL-RPP-2005-00012, REVISION 0

RUN \# 4.03A AND B; FIRST AND SECOND HALF OF SLURRY DEWATERING - CONT.

\begin{tabular}{|c|c|c|c|c|c|c|c|c|c|c|c|c|c|c|c|c|c|c|c|c|c|c|c|c|c|c|c|c|}
\hline & $\mathrm{A}$ & $B$ & D & $E$ & $\mathrm{~F}$ & G & $\mathrm{H}$ & $\mathrm{J}$ & $\mathrm{K}$ & $\begin{array}{ll}\mathrm{L} \\
\end{array}$ & $\mathrm{M}$ & $\mathrm{N}$ & 0 & $\mathrm{Q}$ & $R$ & $\mathrm{~s}$ & $T$ & $\mathrm{~V}$ & w & $x$ & Y & $z$ & $\mathrm{AA}$ & $A B$ & & & $\mathrm{AE}$ & \\
\hline & 9/1/1/2004 & $1: 27: 24 \mathrm{PM}$ & 25.459 & 25.729 & \begin{tabular}{l|l}
9 & 24.522 \\
\end{tabular} & 26.382 & 25.704 & 48.202 & 53.908 & 17.287 & 31.888 & -0.642 & $\begin{array}{l}-6.388 \\
\end{array}$ & 49.434 & 0.048 & 14.643 & 0.0 & 214.03 & 53.5672 & 52.8 & 11.8 & 40.0 & 2.761 & 0.007 & 0.007 & 0.000 & 0.174 & 0.15 \\
\hline & $9 / 1 / 12004$ & $\begin{array}{l}1: 28: 24 \mathrm{PM} \\
\end{array}$ & 25.454 & 25.724 & $\begin{array}{ll}4 \quad 24.502 \\
\end{array}$ & 26.287 & 25.574 & & \begin{tabular}{|l|l|}
53.752 \\
\end{tabular} & 17.391 & 31.679 & \begin{tabular}{|c|c|} 
& -0.682 \\
\end{tabular} & $\begin{array}{l}-6.399 \\
\end{array}$ & 49.664 & 0.046 & \begin{tabular}{|l|l|}
14.643 \\
\end{tabular} & & & 353.5839 & & 11.8 & & & & 0.007 & & & 0.14 \\
\hline & $9 / 1 / 12004$ & $1: 29: 24 \mathrm{PM}$ & 25.474 & $\frac{25.734}{25717}$ & \begin{tabular}{l|l}
4 & 24.487 \\
\end{tabular} & 26.312 & $\frac{25.634}{25.512}$ & $\begin{array}{l}48.154 \\
\end{array}$ & \begin{tabular}{|l|}
53.912 \\
5.920 \\
\end{tabular} & \begin{tabular}{|l|}
17.302 \\
1.225
\end{tabular} & 31.65 & \begin{tabular}{|c|c|}
5 & -0.671 \\
\end{tabular} & $\begin{array}{l}-6.394 \\
-6391\end{array}$ & & $\begin{array}{l}0.047 \\
\end{array}$ & $\begin{array}{l}14.643 \\
\end{array}$ & & & & 520 & 12.1 & & & 007 & 0.007 & 0.000 & 0.171 & \\
\hline 2524 & 9/1/2004 & 1:30:24 PM & 25.457 & 25.717 & \begin{tabular}{l|l}
7 & 24.461 \\
\end{tabular} & 26.245 & 25.542 & 48.125 & \begin{tabular}{|l|}
53.827 \\
\end{tabular} & $\begin{array}{l}17.226 \\
\end{array}$ & 31.802 & -0.645 & $\begin{array}{l}-6.391 \\
\end{array}$ & 49.288 & $\begin{array}{l}0.048 \\
\end{array}$ & \begin{tabular}{|l|l|}
14.643 \\
\end{tabular} & 0.0 & & & 52.7 & 11.7 & 7 & 2.755 & 0.007 & 0.007 & 0.000 & 0.175 & \\
\hline & $\frac{9 / 1 / 12004}{9 / 1 / 2004}$ & \begin{tabular}{l|l}
$1: 31: 24 \mathrm{PM}$ \\
$1: 23: 24 \mathrm{PM}$
\end{tabular} & $\begin{array}{l}25.466 \\
25.464\end{array}$ & $\frac{25.721}{25.724}$ & $\begin{array}{l}1 \\
4\end{array}$ & \begin{tabular}{|l|l|}
26.204 \\
36.242
\end{tabular} & $\begin{array}{l}25.491 \\
25.474\end{array}$ & $\begin{array}{l}48.216 \\
48.579\end{array}$ & \begin{tabular}{|l|}
53.976 \\
5409 \\
\end{tabular} & \begin{tabular}{|c|}
17.534 \\
171699
\end{tabular} & $\begin{array}{r}31.542 \\
32.438 \\
\end{array}$ & \begin{tabular}{|c|c|c|c|}
-0.645 \\
-0.639
\end{tabular} & $\begin{array}{l}-6.405 \\
-6.391\end{array}$ & \begin{tabular}{|l|}
49.774 \\
49.843 \\
\end{tabular} & \begin{tabular}{|c|}
0.046 \\
0.048
\end{tabular} & \begin{tabular}{|l|l|}
14.643 \\
14643 \\
\end{tabular} & & $\frac{3218.03}{32100^{2}}$ & 6339 & $\frac{52.9}{53.0}$ & \begin{tabular}{|l|}
11.9 \\
119
\end{tabular} & & & & $\begin{array}{l}0.007 \\
\end{array}$ & & $\frac{0.168}{0.172}$ & 0.14 \\
\hline 2527 & 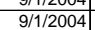 & $\begin{array}{l}1: 33: 24 \mathrm{PM} \\
1: 33: 24 \mathrm{PM}\end{array}$ & $\frac{25.464}{25.458}$ & $\frac{25.124}{25.723}$ & $\begin{array}{l}4 \\
\begin{array}{l}3 \\
3\end{array} 24.4443 \\
\end{array}$ & \begin{tabular}{|l|}
26.242 \\
\end{tabular} & $\begin{array}{l}25.474 \\
25.473 \\
\end{array}$ & $\begin{array}{r}48.579 \\
48.104 \\
\end{array}$ & \begin{tabular}{|l|}
53.89 \\
53.856 \\
\end{tabular} & \begin{tabular}{|}
1.1 .169 \\
17.312 \\
\end{tabular} & $\begin{array}{r}32.438 \\
31.72 \\
\end{array}$ & \begin{tabular}{|c|c|}
3 & -0.639 \\
2 & -0.6399
\end{tabular} & $\frac{391}{394}$ & $\begin{array}{r}49.8433 \\
48.02\end{array}$ & $\begin{array}{l}0.048 \\
0.048\end{array}$ & $\begin{array}{l}14.643 \\
14.643 \\
\end{array}$ & .003 & 220.03 & $\begin{array}{l}\frac{53.6506}{5.66672} \\
\frac{5}{5}\end{array}$ & $\begin{array}{l}53.0 \\
55.7 \\
\end{array}$ & $\frac{11.9}{11.4}$ & $\begin{array}{l}40.5 \\
39.9\end{array}$ & \begin{tabular}{|l|l|} 
& 2.793 \\
9 & 2755 \\
\end{tabular} & |.007 & $\begin{array}{l}0.007 \\
0.007\end{array}$ & $\frac{0.000}{0.000}$ & $\begin{array}{l}0.172 \\
0.175\end{array}$ & $\begin{array}{l}0.15 \\
0.15\end{array}$ \\
\hline 2528 & & 1:34:24 PM & 25.456 & 25.721 & \begin{tabular}{|l|l|}
1 & 24.42 \\
\end{tabular} & 26.184 & 25.436 & 48.148 & \begin{tabular}{|l|}
53.775 \\
\end{tabular} & \begin{tabular}{|l|l|}
17.1699 \\
\end{tabular} & 31.903 & $\begin{array}{l}-0.642 \\
-0.03\end{array}$ & -6.405 & $\begin{array}{l}51.328 \\
\end{array}$ & $\begin{array}{l}0.048 \\
0.046\end{array}$ & $\begin{array}{l}14.643 \\
14.643 \\
\end{array}$ & 0.003 & 3221.03 & \begin{tabular}{|l|}
53.60172 \\
53.6839
\end{tabular} & $\begin{array}{l}52.6 \\
52.6 \\
\end{array}$ & $\begin{array}{l}\frac{11.4}{12.2} \\
\end{array}$ & 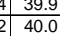 & $\frac{2.252}{2.760}$ & $\begin{array}{l}0.007 \\
0.007\end{array}$ & $\begin{array}{l}.007 \\
0.007\end{array}$ & & & $\frac{0.15}{0.14}$ \\
\hline & $9 / 1 / 2004$ & 1:35:24 PM & 25.461 & 25.721 & $\begin{array}{ll}124.439 \\
\end{array}$ & 26.144 & 25.561 & 48.16 & \begin{tabular}{|l|}
53.748 \\
\end{tabular} & \begin{tabular}{ll|}
17.407 \\
\end{tabular} & 31.771 & -0.645 & -6.405 & 50.617 & 0.047 & 14.643 & & & 53.7006 & & 12.1 & & & 0.007 & & & 0.171 & 0.145 \\
\hline & 9/1/2004 & 1:36:24 PM & & 25.715 & \begin{tabular}{|l|l|}
5 & 24.418 \\
\end{tabular} & 26.193 & 25.68 & 47.959 & \begin{tabular}{|l|}
53.497 \\
\end{tabular} & \begin{tabular}{|l|l|}
17.279 \\
\end{tabular} & 31.691 & & -6.402 & 50.313 & \begin{tabular}{|c|}
0.047 \\
\end{tabular} & 14.6 & & & 53.7172 & 52.4 & & 39 & 2.746 & & $\begin{array}{l}0.007 \\
\end{array}$ & & & .15 \\
\hline 2531 & & & 25.444 & & $\begin{array}{ll}4 & 24.427\end{array}$ & 26.177 & & $\begin{array}{ll}47.938 \\
\end{array}$ & \begin{tabular}{|l|}
53.512 \\
\end{tabular} & \begin{tabular}{|l|l|l|l|}
17.028 \\
\end{tabular} & & - 0.639 & & & & 14.6 & & & & & 11.8 & & & & & & 171 & \\
\hline & & & 25.45 & 25.715 & \begin{tabular}{l|l|l|l|l}
54431 \\
\end{tabular} & 26.243 & & & 527 & \begin{tabular}{|l|l|}
17.277 \\
\end{tabular} & .866 & 0.645 & & 64 & 048 & 14.6 & & & & & & & & & & & & \\
\hline & $1 / 2004$ & 1:39:24 PM & 25.456 & 25.721 & \begin{tabular}{l|l}
1 & 24.424
\end{tabular} & 26.319 & & & 53.904 & 17.212 & & & & & & & & & & & & & 76 & & & & & \\
\hline & |1/2004 & 1:40:24 PM & 25.455 & 25.72 & \begin{tabular}{l|l|l|}
2 & 24.418 \\
\end{tabular} & 26.318 & & & 53.615 & \begin{tabular}{|l|l|}
17.241 \\
\end{tabular} & $\begin{array}{l}31.677 \\
\end{array}$ & -0.624 & & 49.789 & 0.045 & 14.643 & & & 53.7839 & & & & & & & & & \\
\hline & 9/1/2004 & 1:41:24 PM & 25.444 & 25.719 & \begin{tabular}{l|l}
9 & 24.382 \\
\end{tabular} & 26.337 & 25.8 & 48.121 & \begin{tabular}{|l|}
53.713 \\
\end{tabular} & \begin{tabular}{|l|l|}
17.147 \\
\end{tabular} & $\begin{array}{l}32.022 \\
\end{array}$ & -0.593 & -6.396 & 51.577 & 0.048 & 14.643 & 0.00 & 28.0 & 53.8006 & 52.6 & 12.3 & 40. & 2.763 & & 0.007 & & & \\
\hline & & 1:42:24 PM & 25.461 & 25.736 & $\begin{array}{ll}624.425 \\
\end{array}$ & 26.414 & & 48.283 & \begin{tabular}{|l|l|}
53.957 \\
\end{tabular} & \begin{tabular}{ll|}
17.237 \\
1.379
\end{tabular} & & -0.593 & & 50.277 & 0.047 & 14.643 & & & & & & & & & & & & \\
\hline & $11 / 2004$ & $24 \mathrm{PM}$ & 25.468 & & $\begin{array}{l}8 \mid 24.406 \\
\end{array}$ & 26.516 & & 47.965 & 53.642 & 17.286 & 31.735 & -0.645 & & 51.671 & & & & & & & & & & & & & 0.172 & \\
\hline & & & 25.469 & & & 26.537 & & & 53.719| & 16.982 & 32.258 & -0.611 & & 99.716 & & 14.6 & & & 85 & & 11. & & & & & & 0.170 & \\
\hline & 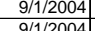 & $40 M$ & 25.474 & $\begin{array}{r}25.749 \\
25770\end{array}$ & $\begin{array}{l}9 \\
9\end{array}$ & 26.53 & & & 54.086 & 17.247 & 1.188 & 0.624 & & 49.8 & 046 & 14.6 & & & & & 11. & & .77 & & & & 0.166 & 0.14 \\
\hline 2541 & $11 / 1 / 2004$ & $\begin{array}{l}1.40 .24 \mathrm{PM} \\
1.47: 24 \mathrm{PM}\end{array}$ & 25.464 & $\frac{25.739}{25.739}$ & $\begin{array}{l} \\
9\end{array}$ & $\begin{array}{l}26.49 \\
26.42\end{array}$ & & & \begin{tabular}{|l|}
54.041 \\
54.032 \\
\end{tabular} & $\begin{array}{l}\frac{11.236}{17.216} \\
\end{array}$ & 096 & $\begin{array}{l}-0.598 \\
-0.578 \\
\end{array}$ & & & . & $\frac{14.6}{14.6}$ & & & & & 11.8 & & & & & & & $\frac{1.14}{14}$ \\
\hline & 9/1/12004 & 1:48:24 PM & 25.474 & 25.754 & $\begin{array}{lll}4 & 24.397 \\
\end{array}$ & 26.452 & 25.81 & 48.256 & \begin{tabular}{|l|}
53.939 \\
\end{tabular} & 17.175 & 32.129 & -0.572 & -6.41 & 50.417 & 0.046 & 14.643 & 0.00 & & 53.9172 & 52.8 & $\begin{array}{ll}11.0 \\
12.0\end{array}$ & 40. & 2.771 & 0.007 & 0.007 & 1.000 & 0.167 & $\frac{1.14}{14}$ \\
\hline & 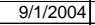 & 1:49:24 PM & 25.462 & 25.737 & \begin{tabular}{l|l}
7 & 24.39 \\
\end{tabular} & 26.385 & 25.7 & $\begin{array}{l}48.496 \\
\end{array}$ & \begin{tabular}{|l|}
54.231 \\
\end{tabular} & 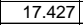 & $\begin{array}{ll}32.063 \\
\end{array}$ & $\begin{array}{l}-0.575 \\
\end{array}$ & & 50.196 & 0.047 & 14.643 & & 36. & 53.9339 & 53.1 & 12.0 & 40. & & 0.007 & .007 & & & \\
\hline 25544 & 9/1/2004 & 1:50:24 PM & 25.446 & 25.736 & 24.4 & & & & & 17.268 & 31.839 & & & & & & & & & & 1.9 & & & & & & & \\
\hline & & & 25.461 & 25.751 & $\begin{array}{ll}1224.409 \\
\end{array}$ & 26.364 & & 48.37 & 54.096 & 17.283 & 0.012 & 0.546 & & 48.362 & & 14.6 & & & & & 115 & & & & & & .167 & \\
\hline & & & & & & & & & & & & & & & & & & & & & & & & & & & & $\overline{14}$ \\
\hline & 9/1/2004 & 1:53:24 PM & 25.468 & 25.753 & 24.537 & 26.381 & & 48.291 & \begin{tabular}{|l|}
53.989 \\
\end{tabular} & 17.514 & 31.763 & -0.558 & & 47.929 & 0.047 & 14.643 & & & 54.00 & 52.9 & $\begin{array}{l}11.5 \\
11.4\end{array}$ & 440 & .760 & & 0.007 & & $\frac{100}{170}$ & $\frac{.14}{14}$ \\
\hline & $9 / 1 / 2004$ & 1:54:24 PM & 25.467 & 25.752 & \begin{tabular}{l|l|l|}
2 & 24.515 \\
\end{tabular} & \begin{tabular}{|l|l|}
5 & 26.325 \\
\end{tabular} & & & \begin{tabular}{|l|}
53.597 \\
\end{tabular} & $\mid 17.311$ & 31.546 & -0.561 & & & $\mid 0.046$ & \begin{tabular}{|l|l|}
14.643 \\
\end{tabular} & & & 54.01 & 52.5 & $\mid 11.7$ & & 2.740 & 0.007 & & 0.0 & $\begin{array}{ll}0.168 \\
\end{array}$ & \\
\hline & $9 / 1 / 2$ & 1:55:24 PM & 25.471 & 25.746 & \begin{tabular}{|l|l|}
6 & 24.51 \\
\end{tabular} & \begin{tabular}{|l|l|}
1 & 26.294 \\
\end{tabular} & 25. & 47.953 & \begin{tabular}{|l|}
53.736 \\
\end{tabular} & 17.251 & 31.735 & \begin{tabular}{|l|l|}
5 & -0.538 \\
\end{tabular} & & 49.662 & 0.047 & \begin{tabular}{|l}
14.643 \\
\end{tabular} & & & 54.0 & 52.6 & 11.8 & 39.8 & 2.747 & 007 & & & 0.171 & \\
\hline & 9/1/2004 & 1:56:24 PM & 25.476 & & \begin{tabular}{|l|l|}
6 & 24.485 \\
\end{tabular} & & & & \begin{tabular}{|l|}
53.908 \\
5
\end{tabular} & $\begin{array}{r}17.3 \\
\end{array}$ & & \begin{tabular}{|l|l|}
2 & -0.535 \\
\end{tabular} & & 49.904 & 0.047 & 14.643 & & & & & 11.9 & & 2.754 & & & & & \\
\hline & 9/1/2004 & 1:57:24 PM & 25.487 & 25.752 & \begin{tabular}{l|l}
2 & 24.5 \\
\end{tabular} & & & & \begin{tabular}{|l|}
53.605 \\
\end{tabular} & & 31.8 & & & & $\begin{array}{l}0.046 \\
\end{array}$ & 14.643 & & & 54.0672 & 52.5 & 12.1 & & & & & & 0.167 & \\
\hline 255 & & $1: 58: 24 \mathrm{PM}$ & 25.482 & 25.747 & $\begin{array}{l}7 \\
7\end{array}$ & 26.34 & & & \begin{tabular}{|l|}
53.918 \\
\end{tabular} & \begin{tabular}{ll|}
17.243 \\
\end{tabular} & & -0.535 & & & & & & & & 52.8 & 11.9 & & $\begin{array}{l}2.767 \\
.775\end{array}$ & 007 & 07 & & 0.170 & \\
\hline & $\begin{array}{l}9 / 1 / 12004 \\
9 / 1 / 2004\end{array}$ & $\begin{array}{l}1: 59: 24 \mathrm{PM} \\
2 \cdot 00 \cdot 24 \mathrm{PM}\end{array}$ & $\begin{array}{l}25.483 \\
25.462\end{array}$ & $\begin{array}{l}25.748 \\
25.737\end{array}$ & \begin{tabular}{l|l}
8 & 24.471 \\
7 & 24.435
\end{tabular} & \begin{tabular}{l|l|} 
& 26.271 \\
5 & 26.265
\end{tabular} & $\begin{array}{l}25.56 \\
25.56\end{array}$ & \begin{tabular}{|l|}
48.148 \\
48.318
\end{tabular} & \begin{tabular}{|l|}
53.887 \\
54.127 \\
\end{tabular} & \begin{tabular}{|c|}
17.428 \\
17382
\end{tabular} & $\begin{array}{l}31.667 \\
31.718 \\
\end{array}$ & $\begin{array}{l}-0.538 \\
-0.535 \\
-10\end{array}$ & & $\begin{array}{r}49.75 \\
51.087 \\
\end{array}$ & 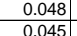 & \begin{tabular}{|l|}
14.643 \\
14.643 \\
\end{tabular} & & 17. & $\begin{array}{l}54.1006 \\
54.1172\end{array}$ & $\begin{array}{l}52.8 \\
53.0\end{array}$ & $\begin{array}{ll}\frac{11.8}{12.2} \\
\end{array}$ & $\frac{39}{40}$ & $\frac{2.751}{2.759}$ & $0.007 \mid$ & 0.007 & 0.000 & $\frac{175}{1764}$ & $\begin{array}{l}0.15 \\
0.14\end{array}$ \\
\hline 2555 & $\begin{array}{l}9,1 / 1 / 1004 \\
9 / 1 / 200\end{array}$ & $\begin{array}{l}2: 00: 24 \mathrm{PM} \\
2: 01: 24 \mathrm{PM}\end{array}$ & $\begin{array}{l}\frac{25.462}{25.476} \\
\end{array}$ & $\begin{array}{l}25.741 \\
25.741\end{array}$ & \begin{tabular}{l|l}
1 & 24.435 \\
1 & 24.449 \\
\end{tabular} & $\begin{array}{l}26.265 \\
26.269\end{array}$ & 25.5 & $\begin{array}{l}48.318 \\
48.115\end{array}$ & \begin{tabular}{|l|}
54.1212 \\
53.816 \\
\end{tabular} & $\begin{array}{l}17.8382 \\
17.224\end{array}$ & $\begin{array}{l}31.718 \\
31.864\end{array}$ & $\begin{array}{l}-0.535 \\
-0.532\end{array}$ & & $\begin{array}{l}1.081 \\
49.716\end{array}$ & 0.0045 & $\frac{14.6}{14.6}$ & & & & $\begin{array}{l}53.0 \\
52.7\end{array}$ & $\begin{array}{l}\frac{12.2}{11.8} \\
\end{array}$ & & & & $\frac{0.007}{0.007}$ & & & 0.14 \\
\hline 2556 & 9/1/2004 & $2: 02: 2$ & 25.465 & 25.7 & \begin{tabular}{|l|l}
5 & 24.418 \\
\end{tabular} & \begin{tabular}{|l|l}
8 & 26.218 \\
\end{tabular} & & 48.146 & \begin{tabular}{|l|}
53.968 \\
\end{tabular} & 17.294 & 31.714 & $\begin{array}{|ll|}4 & -0.535 \\
\end{array}$ & & 50.058 & 0.045 & 14.6 & & & 54.1 . & 52.8 & 11.9 & & 27 & & & & & $\frac{0.14}{0.14}$ \\
\hline & $9 / 1 / 2$ & & 25.46 & 25.7 & \begin{tabular}{l|l|}
5 & 24.398 \\
\end{tabular} & \begin{tabular}{|l|l}
8 & 26.163 \\
\end{tabular} & & 48.025 & \begin{tabular}{|l|}
53.495 \\
\end{tabular} & 17.26 & & \begin{tabular}{|l|l|}
6 & -0.538 \\
\end{tabular} & & & 0.046 & 14. & & & 54.1 & 52.4 & 11.8 & & & & & & & $\frac{0.14}{0.14}$ \\
\hline & 9/1/2004 & & 25.479 & 25.749 & \begin{tabular}{l|l}
9 & 24.447 \\
\end{tabular} & 726. & & 47.959 & \begin{tabular}{|l|}
53.651 \\
\end{tabular} & & & & & 50.6 & \begin{tabular}{|c|c|}
0.046 \\
\end{tabular} & & & & & & 121 & & & & & & & $\frac{14}{14}$ \\
\hline 255 & & & $25.45 \mathrm{C}$ & 25.734 & $\begin{array}{ll}4 & 24.392 \\
\end{array}$ & \begin{tabular}{|l|l|}
2 & 26.2 \\
\end{tabular} & & \begin{tabular}{|l|l} 
\\
\end{tabular} & \begin{tabular}{|l|}
53.993 \\
\end{tabular} & 17.248 & & $\begin{array}{l}-0.54 \\
\end{array}$ & & & & & & & 54.20 & & 11.7 & & & & & & & \\
\hline & & & 25.4 & & & & & & 53.518 & \begin{tabular}{|c|c|c|}
17.144 \\
\end{tabular} & & & & & .046 & & & & & & & & & & & & & \\
\hline & & & 25.46 & 25.74 & 24.414 & $\begin{array}{ll}4 & 26.40\end{array}$ & & & & & & & & & & & & & & & 120 & & & & & & & 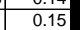 \\
\hline & $9 / 1 / 2004$ & & 25.463 & & \begin{tabular}{l|l|l|}
8 & 24.431
\end{tabular} & 126. & & & \begin{tabular}{|l|}
53.839 \\
\end{tabular} & 17.187 & & -0.538 & & & 0.046 & 14.6 & & & & & 12.0 & & & & & & 0.166 & \\
\hline & 9/1/2004 & & 25.46 & & \begin{tabular}{l|l|l|}
6 & 24.418 \\
\end{tabular} & & & & & & & & & & .046 & & & & & & 11.9 & & & & & & 0.167 & \\
\hline & & & 25.461 & & $\begin{array}{ll}1124.414 \\
\end{array}$ & 26.459 & & & \begin{tabular}{|l|l|} 
\\
\end{tabular} & 17.134 & & -0.538 & & & .0.047 & & & & & & 11.9 & & & & & & & \\
\hline & & & 25.46 & & & & & & & & & 0.000 & & & & & & & & & 地. & & & & & & & \\
\hline & & & $\frac{25.46}{25.48}$ & & & & & & 53.601 & 17.1 & & & & & & & & & & & & & & & & & & \\
\hline & $\begin{array}{r}9 / 1 / 2004 \\
99 / 1 / 2004\end{array}$ & & $\frac{25.472}{25.478}$ & $\frac{25.78}{2578}$ & $\frac{24.42}{24.41}$ & $\frac{26.66}{26.68}$ & & & $\begin{array}{r}53.75 \\
53895\end{array}$ & $\begin{array}{l}17.209 \\
17167\end{array}$ & $\frac{31}{31}$ & $\begin{array}{r}-0.5 \\
-0.488 \\
\end{array}$ & & & 0.046 & & & & & & & & 875 & & & & & \\
\hline & 9/1/2004 & & & & & & & & & & & & & & 0.044 & & & & & 52.8 & 12.0 & & & & & & 0.160 & \\
\hline & & & 25.48 & & & & & & & & & & & & & & & & & & 11.9 & & & & & & 0.167 & \\
\hline & 9/1/2004 & & 25.483 & & & 26.5 & & & \begin{tabular}{|l|}
53.653 \\
\end{tabular} & \begin{tabular}{|l|}
17.211 \\
\end{tabular} & & \begin{tabular}{|l|l|}
5 & -0.2 \\
\end{tabular} & & & 0.0 & & & & & 2.5 & 11.4 & & 49 & & & & 0.168 & \\
\hline & & & & & & & & & & & & & & & & & & & & & 12.0 & & & & & & & \\
\hline & 9/1/2004 & $2: 19: 2$ & 25.492 & 25.787 & \begin{tabular}{l|l}
7 & 24.42 \\
\end{tabular} & 26.45 & & 48. & \begin{tabular}{|l|l|}
54.084 \\
\end{tabular} & $\begin{array}{l}17.042 \\
\end{array}$ & & -0.4 & & 49.2 & 0.046 & & & & 54.4 & 53.0 & $\begin{array}{ll}11.7 \\
\end{array}$ & & 2.767 & & & & 0.167 & \\
\hline & $\begin{array}{l}9 / 1 / 1 / 2004 \\
9 / 1 / 2004\end{array}$ & $\frac{2: 20: 24 \mathrm{PM}}{2: 21 \cdot 24 \mathrm{PM}}$ & $\begin{array}{l}25.486 \\
25.497\end{array}$ & $\frac{25.796}{25.808}$ & 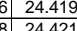 & \begin{tabular}{l|r|}
9 & 26.399 \\
1 & 26.37
\end{tabular} & $\frac{25}{25}$ & $\begin{array}{r}48.21 \\
48.542\end{array}$ & \begin{tabular}{|l|}
54.055 \\
54413
\end{tabular} & $\begin{array}{l}17.156 \\
17074\end{array}$ & $\begin{array}{r}32.104 \\
32.498 \\
\end{array}$ & $\begin{array}{l}4 \\
3\end{array}$ & & $\frac{50.1}{510}$ & 0.046 & 14.6 & & & 54.4 & \begin{tabular}{|l|l|}
52.9 \\
53.3
\end{tabular} & \begin{tabular}{l|}
11.9 \\
12.2
\end{tabular} & \begin{tabular}{|l|l|} 
\\
\end{tabular} & $\begin{array}{l}2.769 \\
27 \\
2794\end{array}$ & & 0. & 0.000 & $\begin{array}{l}0.167 \\
0.165\end{array}$ & 14 \\
\hline 2576 & $\begin{array}{l}9 / 1 / 1 / 2004 \\
9 / 1 / 2004\end{array}$ & & $\begin{array}{l}25.497 \\
25.481\end{array}$ & & \begin{tabular}{c|c|c}
1 & 24.4241 \\
1
\end{tabular} & & & $\begin{array}{l}\frac{48.542}{48.409} \\
\end{array}$ & \begin{tabular}{|l|}
54.418 \\
54.185 \\
\end{tabular} & $\begin{array}{l}17.074 \\
17.128\end{array}$ & & $=0.4 / 1$ & & & & $\frac{14.6}{146}$ & & & & & $\begin{array}{l}\frac{1.2 .}{11.7} \\
\end{array}$ & & & & & & & \\
\hline 2577 & & & & & & & & & & & & -0.4 & & & & & & & & & 势1 & & & & & & & \\
\hline & & & 25.4 & & & & & & & 17.1 & & -0 & & & & & & & & & $\mid$ & & & & & & 1.64 & \\
\hline & & & & & & & & & & & & & & & & & & & & & & & & & & & & \\
\hline & & & & & & & & & & & & & & & & & & & & & & & & & & & & \\
\hline & & & 25.4 & & 24.51 & 26.4 & & & & & & $-0.2 \quad$ & & & & & & & & & 11.9 & & & & & & & \\
\hline & & & 25.49 & 25.7 & 24.5 & 26.402 & & & 54.1 & & 31.7 & -0.47 & & & & & & & & & 116 & & & & & & & \\
\hline & $11 / 20$ & & 55.49 & & 24.5 & 26.4 & & 48. & 53.906 & 17.3. & & -0.4 & & 50.9 & & 14.6 & & & & & & & & & & & 64 & \\
\hline & & & 25.5 & & 24. & 26.446 & & 48. & 53.928 & $17.3^{-12}$ & & -0.4 & & & & & & & & & & & & & & & & \\
\hline & & & 25.4 & & 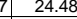 & & & & & & & -0.4 & & & & & & & & & 12. & & & & & & & \\
\hline & & & & & 24.4 & 266 & & & & & & & & & & & & & & & & & & & & & & \\
\hline & & & 25.49 & 25.17 & $24.4 t$ & 26.485 & & & 53.14 & 17.26 & & & & & & & & & & & 11.9 & & & & & & 108 & \\
\hline & 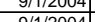 & & $\frac{25.49}{25.19}$ & 23.11 & 24.44 & $\begin{array}{l}4 \\
7\end{array}$ & & (47.994 & 53.5037 & 17.350 & $\frac{31}{315}$ & - -0.047 & & & 0.04 & & & & & 22.4 & 117 & & 27 & & 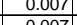 & & 年 0.168 & \\
\hline 259 & 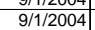 & $2: 36: 24 \mathrm{PM}$ & 25.514 & 25.789 & $\begin{array}{ll}9 & 24.4441 \\
\end{array}$ & \begin{tabular}{|l|l|l|}
2 & 26.462
\end{tabular} & 25.594 & 47.884 & \begin{tabular}{|l|}
53.763 \\
\end{tabular} & \begin{tabular}{|l|}
17.335 \\
\end{tabular} & $\begin{array}{l}31.054 \\
31.505\end{array}$ & \begin{tabular}{|c|c|}
5 & -0.43 \\
\end{tabular} & & 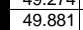 & 0 & \begin{tabular}{|c|}
54.643 \\
\end{tabular} & & 283.03 & \begin{tabular}{|l|l|}
354.7172 \\
\end{tabular} & \begin{tabular}{|l|l|}
52.6 \\
5
\end{tabular} & \begin{tabular}{|l|l|}
11.9 \\
\end{tabular} & & 8.737 & \begin{tabular}{|l|l|} 
\\
\end{tabular} & \begin{tabular}{|l|}
0.007 \\
\end{tabular} & \begin{tabular}{|c|}
0.000 \\
\end{tabular} & \begin{tabular}{|l|l|} 
& .11265 \\
\end{tabular} & 0.1 \\
\hline
\end{tabular}


WSRC-TR-2005-00105, REVISION 0

SRNL-RPP-2005-00012, REVISION 0

RUN \# 4.03A AND B; FIRST AND SECOND HALF OF SLURRY DEWATERING - CONT.

\begin{tabular}{|c|c|c|c|c|c|c|c|c|c|c|c|c|c|c|c|c|c|c|c|c|c|c|c|c|c|c|c|c|}
\hline & A & $B$ & D & $E$ & $F$ & $G$ & $\mathrm{H}$ & $\mathrm{J}$ & K & $\mathrm{L}$ & $M$ & $\mathrm{~N}$ & 0 & \begin{tabular}{l|l} 
\\
\end{tabular} & R & \begin{tabular}{|c|} 
\\
\end{tabular} & $\begin{array}{ll}T \\
\end{array}$ & $\mathrm{~V}$ & w & $x$ & $Y_{1}$ & $z$ & & & & & & \\
\hline 2591 & 9/1/2004 & 2:37:24 PM & 25.504 & 25.779 & 24.437 & 26.417 & 25.619 & 47.984 & 53.895 & 17.381 & 31.515 & -0.416 & -6.411 & 47.83 & 0.046 & $\begin{array}{l}14.643 \\
\end{array}$ & 0.004 & 3284.03 & \begin{tabular}{|l|l|}
54.7339 \\
\end{tabular} & 52.8 & 11.4 & 39.7 & 2.741 & 0.007 & 0.007 & 0.000 & 0.168 & 0.14 \\
\hline & $\begin{array}{l}9 / 1 / 1 / 2004 \\
\end{array}$ & & 25.495 & $\begin{array}{l}25.77 \\
\end{array}$ & 24.408 & \begin{tabular}{|l|}
26.363 \\
\end{tabular} & & 48.119 & $\begin{array}{lr}9 & 53.96 \\
\end{array}$ & $\begin{array}{l}17.281 \\
17.28\end{array}$ & $\begin{array}{l}31.895 \\
\end{array}$ & \begin{tabular}{|c|c|c|}
5 & -0.454 \\
\end{tabular} & & 50.575 & 0.045 & \begin{tabular}{|l|}
14.643 \\
\end{tabular} & 0.003 & 3285.03 & 054.7506 & \begin{tabular}{|l|l|}
52.8 \\
\end{tabular} & & & & & 0.007 & & & \\
\hline 2593 & $9 / 1 / 2004$ & $2: 39: 24 \mathrm{PM}$ & 25.506 & 25.791 & \begin{tabular}{|l|}
24.429 \\
\end{tabular} & \begin{tabular}{|l|}
26.419 \\
2.42
\end{tabular} & & 48.048 & \begin{tabular}{|l|l|}
853.986 \\
\end{tabular} & \begin{tabular}{|l|l|}
17.287 \\
17.252
\end{tabular} & & \begin{tabular}{|c|c|}
5 & -0.419 \\
\end{tabular} & -6.414 & 51.714 & \begin{tabular}{|l|}
0.046 \\
\end{tabular} & \begin{tabular}{|l|}
14.643 \\
\end{tabular} & 0.003 & 286.03 & \begin{tabular}{|l|l|}
54.7672 \\
59.792 \\
\end{tabular} & \begin{tabular}{|l|}
52.9 \\
\end{tabular} & & & 2.748 & 0.007 & 0.007 & 0.000 & 0.168 & \\
\hline \begin{tabular}{|l|}
2594 \\
2595
\end{tabular} & 9/1/2004 & 2:40:24 PM & 25.501 & 25.792 & \begin{tabular}{|r|}
24.43 \\
\end{tabular} & \begin{tabular}{|l|l|}
26.429 \\
\end{tabular} & 25.596 & 47.754 & \begin{tabular}{l|l|}
4 & 53.576 \\
\end{tabular} & \begin{tabular}{|l|l|}
17.153 \\
\end{tabular} & $\begin{array}{l}31.544 \\
2.14\end{array}$ & -0.419 & -6.414 & 49.397 & $\begin{array}{l}0.046 \\
\end{array}$ & $\begin{array}{l}14.643 \\
\end{array}$ & 0.003 & \begin{tabular}{|c|}
3287.03 \\
20.03
\end{tabular} & \begin{tabular}{|l|l|}
54.7839 \\
\end{tabular} & \begin{tabular}{|l|}
52.5 \\
\end{tabular} & \begin{tabular}{ll|l}
11.8 \\
\end{tabular} & \begin{tabular}{|l|l|}
8 & 39.6 \\
\end{tabular} & 2.734 & 0.007 & 0.007 & 0.000 & $\begin{array}{l}0.169 \\
\end{array}$ & \\
\hline$\frac{2595}{2596}$ & $\frac{9 / 1 / 2004}{99 / 1 / 2004}$ & $\begin{array}{l}2: 41: 24 \mathrm{PM} \\
2: 22: 24\end{array}$ & $\frac{25.519}{25.515}$ & $\begin{array}{r}\frac{25.804}{25.8} \\
25\end{array}$ & & \begin{tabular}{|l|}
26.562 \\
26.613 \\
\end{tabular} & $\begin{array}{r}25.649 \\
25805\end{array}$ & $\begin{array}{l}47.876 \\
48.121\end{array}$ & \begin{tabular}{l|l|l|}
1 & 53.678 \\
1 & 53.902
\end{tabular} & \begin{tabular}{|r|r|r|r|}
17.284 \\
17.284
\end{tabular} & \begin{tabular}{|l|}
31.671 \\
31.566 \\
\end{tabular} & \begin{tabular}{|c|c|c|}
-0.419 \\
-0.419
\end{tabular} & $\begin{array}{l}-6.414 \\
-6.411 \\
-x^{2}\end{array}$ & $\begin{array}{r}48.65 \\
50.628\end{array}$ & \begin{tabular}{|c|c|}
0.046 \\
0.046
\end{tabular} & \begin{tabular}{|l|l|l|}
14.643 \\
14634 \\
\end{tabular} & & \begin{tabular}{|l|}
3288.03 \\
328903
\end{tabular} & \begin{tabular}{|l|l|}
54.8006 \\
5817172
\end{tabular} & \begin{tabular}{|l|}
52.6 \\
55.8 \\
\end{tabular} & \begin{tabular}{|l|l|}
11.6 \\
121
\end{tabular} & & \begin{tabular}{|l|}
2.742 \\
27577 \\
\end{tabular} & & & & 0.168 & \\
\hline & & $\begin{array}{l}2: 42: 24 \mathrm{PM} \\
2: 43: 24 \mathrm{P}\end{array}$ & $\frac{25.515}{25.518}$ & $\begin{array}{r}25.8 \\
25.808\end{array}$ & \begin{tabular}{|l|}
24.438 \\
24.421 \\
\end{tabular} & \begin{tabular}{|l|}
26.613 \\
26.661
\end{tabular} & $\begin{array}{r}25.805 \\
25.888 \\
\end{array}$ & $\begin{array}{r}48.121 \\
48.249\end{array}$ & \begin{tabular}{l|l|} 
& 53.902 \\
9 & 54.127
\end{tabular} & $\begin{array}{ll}17.284 & \\
17.3\end{array}$ & \begin{tabular}{|c|}
31.256 \\
32.018
\end{tabular} & $\begin{array}{c}-0.419 \\
-0.419 \\
\end{array}$ & $\frac{-6.411}{-6.411}$ & $\begin{array}{l}50.628 \\
50.079\end{array}$ & $\mid \begin{array}{l}0.046 \\
0.046\end{array}$ & \begin{tabular}{|l|}
14.6634 \\
14.643
\end{tabular} & $\begin{array}{l}0.003 \\
0.003\end{array}$ & \begin{tabular}{|l|}
3289.03 \\
3290.03
\end{tabular} & \begin{tabular}{|l|l|}
54.8172 \\
558339
\end{tabular} & \begin{tabular}{|l|}
52.8 \\
530 \\
\end{tabular} & 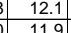 & $\begin{array}{l}1 \\
9 \\
9\end{array}$ & $2.757 \mid$ & 0.007 & 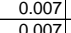 & $\begin{array}{r}0.000 \\
\end{array}$ & $\begin{array}{l}0.167 \\
0.167\end{array}$ & 0.14 \\
\hline 2598 & $9 / 1 / 2004$ & 2:44:24 PM & 25.515 & 25.816 & \begin{tabular}{|l|}
24.423 \\
24.439 \\
\end{tabular} & \begin{tabular}{|l|}
20.001 \\
26.703 \\
\end{tabular} & 25.905 & & & 17.214 & & \begin{tabular}{|cc|c|}
5 & -0.419 \\
\end{tabular} & & & 0.046 & \begin{tabular}{|l|l}
14.645 \\
14.643
\end{tabular} & $\begin{array}{l}0.003 \\
0.003\end{array}$ & S290.03 & \begin{tabular}{|l|}
54.85359 \\
54.850
\end{tabular} & \begin{tabular}{|}
53.0 \\
53.0 \\
\end{tabular} & $\begin{array}{l}111.9 \\
11.5\end{array}$ & $\begin{array}{l}40.1 \\
40.2\end{array}$ & $\begin{array}{l}2.67 \\
2.773\end{array}$ & & $\begin{array}{l}0.007 \\
0.007\end{array}$ & $\begin{array}{l}0.000 \\
0.000\end{array}$ & & $\frac{0.14}{0.14}$ \\
\hline & & 2:45:24 PM & & & \begin{tabular}{|l|}
24.426 \\
\end{tabular} & 26.726 & & & \begin{tabular}{|l|l|}
6 & 54.049 \\
\end{tabular} & 17.034 & 32.001 & -0.416 & & 50.611 & $\begin{array}{l}0.046 \\
\end{array}$ & 14.643 & & & 54.8672 & 52.9 & & & & & & & & $\frac{0.14}{0.14}$ \\
\hline 2600 & 9/1/2004 & 2:46:24 PM & & 25.829 & \begin{tabular}{|l|}
24.407 \\
\end{tabular} & & & $\begin{array}{l}48.334 \\
\end{array}$ & & 17.154 & 32.149 & -0.399 & & 47.999 & $\begin{array}{l}0.046 \\
0\end{array}$ & \begin{tabular}{|l|l|}
14.643 \\
\end{tabular} & & & \begin{tabular}{|l|l|}
54.8839 \\
\end{tabular} & $\begin{array}{l}53.1 \\
\end{array}$ & 111.4 & 40.2 & 2.775 & & $\begin{array}{l}0.007 \\
\end{array}$ & 0.000 & & $\begin{array}{l}0.14 \\
0.14\end{array}$ \\
\hline 2601 & & & 25.536 & & \begin{tabular}{|l|l|}
24.449 \\
\end{tabular} & & & & & & 31.946 & & & 49.314 & & \begin{tabular}{l|l}
14.643 \\
\end{tabular} & & & & 52.9 & & & & .007 & 0.007 & & 0.167 & \\
\hline 2602 & & 2:48:24 PM & & 25.837 & 21305 & & & 48.194 & \begin{tabular}{|l|l|}
4 & 54.123 \\
\end{tabular} & 17.266 & 31.934 & 419 & & & & 14.643 & & & 54.9172 & 53.0 & 12. & 40.1 & & & & & 0.167 & \\
\hline 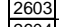 & & $2: 49: 24 \mathrm{PM}$ & 25.522 & 25.837 & 24.411 & 26.725 & & & 53.808 & & .813 & & & & & $\begin{array}{l}14.643 \\
\end{array}$ & & & & 52.7 & & 20 & 746 & 006 & & & 0.164 & \\
\hline & & 2:50:24 PM & 25.533 & 25.843 & 24.401 & 26.706 & 25.91 & $\begin{array}{l}48.415 \\
\end{array}$ & 54.188 & 17.309 & 32.192 & $\mid-0.361$ & & 50.924 & 0.044 & \begin{tabular}{|l|l|l|l|}
14.643 \\
\end{tabular} & & & 54.9506 & 53.1 & 12.1 & 40.3 & 2.779 & 0.006 & 0.006 & 0.000 & 0.159 & \\
\hline & $9 / 1 / 2004$ & 2:51:24 PM & 25.533 & 25.853 & 24.421 & 26.651 & 25.88 & 48.152 & \begin{tabular}{ll|l|}
2 & 54.092 \\
\end{tabular} & 17.178 & 31.96 & -0.37 & -6.41 & 49.985 & 0.046 & $\begin{array}{l}14.643 \\
\end{array}$ & 0.003 & & 54.9672 & 53.0 & 11.9 & 40.1 & 2.762 & & 0.007 & 0.000 & & 0.14 \\
\hline & & $\begin{array}{l}2: 52: 24 \mathrm{PM} \\
2: 2020\end{array}$ & & 25.848 & 24.411 & 26.656 & 25.7 & 47.882 & 53.651 & $\begin{array}{l}16.74 \\
17.04\end{array}$ & 32.055 & $\begin{array}{l}-0.358 \\
\end{array}$ & & 48.11 & 0.046 & 14.643 & & & 54.9839 & 52.5 & & & & & & & & \\
\hline & & $2: 53: 24 \mathrm{PM}$ & & & 24.406 & 26.611 & & 48.225 & 5 5 54.115 | & & 32.042 & - 0.349 & & 49.706 & 2048 & 14.643 & & & .0006 & 53.0 & & & & .007 & 0.007 & & 0.174 & \\
\hline & & $2: 54: 24 \mathrm{PM}$ & & & 24.402 & 26.592 & & 47.909 & & & 31.927 & -0.349 & & 45.1866 & 046 & & & & .0172 & & & & & & 0.00 & & 0.168 & \\
\hline & & $2: 55: 24 \mathrm{PM}$ & & & 24.392 & 26.747 & & 48.446 & \begin{tabular}{|c|c|}
54.422 \\
54034
\end{tabular} & $\frac{11.22}{16701}$ & $\begin{array}{l}32.098 \\
32.114\end{array}$ & -0.416 & & 49.994 & & 14.643 & & & . & 53.3 & & & & . & & & 174 & \\
\hline 2611 & & $2.05 .24 \mathrm{PM}$ & 25.534 & 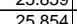 & $\frac{24.424}{24392}$ & 26782 & & $\begin{array}{l}47.955 \\
48.158\end{array}$ & $\begin{array}{l}54.004 \\
54.086\end{array}$ & 17187 & 32.1144 & 4.419 & & & 046 & 14643 & & & 5 & 530 & & & 76 & . & 0.007 & & & 0.14 \\
\hline 2612 & 9/1/2004 & 2:58:24 PM & 25.54 & $\frac{2.074}{25.85}$ & 24.403 & 26.738 & 25.774 & 48.278 & 54.287 & 17.091 & 32.202 & $\begin{array}{l}-.412 \\
-0.352\end{array}$ & -6.336 & 48.713 & 0.046 & $\begin{array}{l}14.045 \\
14.643\end{array}$ & 0.003 & 305.0 & 55.0839 & 53.2 & 11.6 & $\frac{40.1}{40.2}$ & 2.774 & 0.007 & 0.007 & 0.000 & 0.166 & $\begin{array}{l}0.14 \\
0.14\end{array}$ \\
\hline & 9/1/2004 & 2:59:24 PM & 25.555 & 25.86 & 24.418 & 26.783 & 25.804 & 48.337 & \begin{tabular}{|l|l|}
7 & 54.382 \\
\end{tabular} & 17.232 & 32.114 & -0.349 & -6.362 & 49.977 & 0.046 & 14.643 & 0.004 & & 55.1006 & & 11.9 & 40.2 & & 0.007 & 0.007 & & $\frac{0.166}{0.166}$ & \\
\hline & & 3:00:24 PM & & 25.87 & \begin{tabular}{|l|l|}
24.434 \\
\end{tabular} & 26.748 & & & & & & & & 48.344 & 0.046 & \begin{tabular}{ll|l}
14.643 \\
\end{tabular} & & & & 53.4 & & & & & & & & \\
\hline & & 3:01:24 PM & 25.57 & 25.875 & \begin{tabular}{|l|}
24.594 \\
\end{tabular} & 26.798 & & 48.108 & & 17.304 & 31.87 & -0.419 & & 50.371 & 546 & 14.643 & & & .1.1339 & 52.8 & & & & 5.007 & 0.007 & & & \\
\hline & & & & & $\begin{array}{l}24.59 \\
\end{array}$ & 26.834 & & & 53.678 & & & -0.413 & & & & $\begin{array}{l}14.643 \\
\end{array}$ & & & & & & & & & & & & \\
\hline & $9 / 1 / 2$ & 3:02:53 PM & 25.573 & 25.878 & \begin{tabular}{|l|}
24.571 \\
\end{tabular} & 26.741 & 25.7 & \begin{tabular}{|l|} 
\\
\end{tabular} & 53.373 & 17.239 & 31.529 & -0.419 & & 49.145 & & $\begin{array}{l}14.643 \\
\end{array}$ & & & 5.1586 & 52.2 & & & & & & & & \\
\hline & 1/2004 & 3:03: & 25.588 & 25.878 & \begin{tabular}{|l|}
24.541 \\
\end{tabular} & 26.736 & 25.70 & $\begin{array}{lll}48.247 \\
\end{array}$ & $\begin{array}{l}754.426 \\
\end{array}$ & 17.285 & 32.016 & -0.349 & & & 0.045 & \begin{tabular}{l|l}
14.643 \\
\end{tabular} & & & 55.1753 & 53.3 & 11.9 & 40.1 & 76 & .006 & 0.007 & & & \\
\hline & $1 / 2004$ & 3:04:53 PM & 25.588 & 25.874 & \begin{tabular}{|l|}
24.517 \\
\end{tabular} & 26.746 & 25. & 48.086 & \begin{tabular}{l|l|l|}
6 & 54.187 \\
\end{tabular} & 17.235 & 31.654 & -0.349 & & 49.795 & 0.046 & $\begin{array}{l}14.643 \\
\end{array}$ & & & 55.1919 & 53.1 & 11.9 & 39.5 & 2.749 & 0.007 & 0.0 & & 0.167 & 0.14 \\
\hline & & & & 25.879 & 24.502 & 26.701 & & & \begin{tabular}{l|l|}
5 & 53.875 \\
\end{tabular} & $\begin{array}{r}17.39 \\
17.025\end{array}$ & $\begin{array}{l}31.501 \\
2.092\end{array}$ & -0.37 & & 48.075 & 047 & 14.643 & & & & & & & & & & & & \\
\hline & & $3 \mathrm{PM}$ & & 25.868 & 24.471 & 26.731 & & 47.997 & 53.736 & & 31.962 & -0.407 & & & 0.05 & 14.643 & & & & 52.6 & & & & & & & & \\
\hline & & 3:0 I:53 PM & 5.598 & $25.8 / 3$ & 24.486 & 26.696 & & & 153.729 & 16.994 & 31.839 & -0.277 & & & & & & & & 52.6 & & & & & & & 179 & \\
\hline & $9 / 1 / 2004$ & 3:08:53 PM & 25.597 & 25.862 & 24.455 & $\begin{array}{r}26.72 \\
26.734 \\
\end{array}$ & $\frac{25.612}{2561}$ & $\begin{array}{l}47.936 \\
48.077 \\
\end{array}$ & $\begin{array}{r}54.1 \\
53877\end{array}$ & $\frac{16.914}{17249} \mid$ & $\begin{array}{c}31.966 \\
31799\end{array}$ & 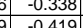 & & $\frac{50.7}{497}$ & (048 & $\frac{14.643}{14643}$ & & & 5753 & $\frac{53.0}{52.8}$ & 118 & & & & & & 178 & \\
\hline 2625 & $9 / 1 / 12004$ & 3:10:53 PM & 25.585 & 25.86 & \begin{tabular}{|l|l|}
24.434 \\
\end{tabular} & 26.773 & & & 54.1 & $\begin{array}{l}17.245 \\
17.216\end{array}$ & 32.104 & $\begin{array}{l}-.419 \\
-0.419\end{array}$ & & & 0.045 & 14643 & & & 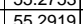 & 530 & & & & 006 & 0007 & & & \\
\hline & 9/1/2004 & 3:11:53 PM & 25.597 & 25.867 & 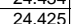 & $\frac{117}{26.8}$ & & & 53.844 & 10 & 31.589 & $\frac{.419}{-0.419}$ & & 49.624 & & $\begin{array}{l}14.045 \\
14.643\end{array}$ & & & & $\frac{5.0}{52.7}$ & $\frac{12.8}{11.8}$ & & & 000 & & & 0.161 & $\frac{1.14}{14}$ \\
\hline & & 3:12:53 PM & 25.602 & 25.872 & 24.445 & 26.765 & 25. & & 53.806 & 16.963 & & -0.349 & & & 047 & & & & & $\frac{2.7}{52.7}$ & & & & & & & & \\
\hline & & 3:13:! & & 25.867 & \begin{tabular}{|l|}
24.41 \\
\end{tabular} & & & & 54.393 & & & & & & & & & & & & & & & & & & & \\
\hline & & 3:14:53 PM & 25.602 & 25.872 & \begin{tabular}{|l|}
24.425 \\
\end{tabular} & 26 & & & 53.727 & $\begin{array}{l}17.227 \\
\end{array}$ & & & & 50.2 & & 14.6 & & & 55.3586 & 52.6 & 12 & & & & 0.0 & & 0 & \\
\hline & & & & & 24.401 & & & & \begin{tabular}{|c|}
54.04 \\
\end{tabular} & & & & & & & & & & & & & & & & & & & \\
\hline & & 3:16:53 PM & & 25.868 & \begin{tabular}{|l|}
24.411 \\
\end{tabular} & 26.646 & & & 53.904 & 17.314 & 31.696 & \begin{tabular}{|c|c|c|}
-0.419 \\
\end{tabular} & & & & 14. & & & & 52.8 & 11.8 & $\overline{39}$ & & & & & 168 & 0.14 \\
\hline & $9 / 1 / 2004$ & 3:17: & 25.603 & 25.874 & \begin{tabular}{|l|}
24.407 \\
\end{tabular} & 26.636 & & & 954.071 & 17.185 & & $\begin{array}{l}-0.419 \\
\end{array}$ & & 49.3 & | & 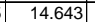 & & & & 52.9 & 11.8 & $40 \quad-$ & & 0.006 & 0.0 & & 0.164 & \\
\hline & & & & & & & & & 253.165 & 17.071 & & & & & & & & & & 52.6 & 11.7 & & & & & & & \\
\hline & & 3:19:53 PM & 25.599 & 25.874 & \begin{tabular}{|l|}
24.402 \\
\end{tabular} & 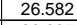 & & 48.048 & $\begin{array}{ll}8 & 54.061\end{array}$ & 17.176 & 31.893 & -0.323 & & & .0.045 & & & & & 52.9 & 11.4 & & 275 & 0.006 & 0.00 & & 0.164 & \\
\hline & & & & 25.864 & & & & & & & & & & & & & & & & 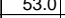 & 11 & & & & & & & \\
\hline & & & & & & & & & & & & & & & & & & & & & & & & & & & & \\
\hline & $\frac{9 / 1 / 2}{91 / 1 / 2}$ & $\begin{array}{l}3: 22: 53 \mathrm{PM} \\
3 \cdot 23.53 \mathrm{P}\end{array}$ & 572 & $\frac{25.869}{25.857}$ & $\frac{24.377}{2438}$ & $\frac{26.591}{265}$ & & & \begin{tabular}{|l|l|}
54.049 \\
5.26
\end{tabular} & $\begin{array}{l}17.247 \\
17058\end{array}$ & $\begin{array}{l}31.804 \\
32.383\end{array}$ & $\begin{array}{l}-0.41 \\
-0.35\end{array}$ & & & (1) & $\frac{14.6}{146}$ & & & & 531 & 120 & & 278 & & & & 170 & $\frac{14}{1}$ \\
\hline 2639 & & $3.24 \cdot 53$ & 2558 & 年. & \begin{tabular}{|l|l|}
24.309 \\
\end{tabular} & & & & $\frac{1}{6} 53580$ & 17133 & 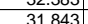 & - & & 50.945 & & & & & & 527 & $\frac{12}{12}$ & & & & & & & \\
\hline & & & 25.57 & 25.005 & \begin{tabular}{|l|l|}
24383 \\
\end{tabular} & & & & 0.000 & & & & & & & & & & & $J$ & & & & & & & 0 & .14 \\
\hline 644 & & & 25.57 & 25.865 & \begin{tabular}{|l|}
24.393 \\
\end{tabular} & & & & \begin{tabular}{l|l|l|}
8 & 54.552
\end{tabular} & 17.903 & 31.523 & $\frac{-0.422}{-0.422}$ & & & & & & & & 53.4 & $\frac{12.3}{12.3}$ & & & & $\frac{0.0}{0.0}$ & & & \\
\hline & & & & & & & & & & & & & & & & & & & & & & & & & & & & \\
\hline & & & & 25.867 & \begin{tabular}{|l|}
24.426 \\
\end{tabular} & 26.56 & & & & & & & & & & & & & & $\frac{03.4}{53.4}$ & $\frac{21:}{11:}$ & & & & & & & \\
\hline & & & & 25.857 & \begin{tabular}{|l|l|l|}
2425 \\
\end{tabular} & & & & & & & & & & & & & & & 0.11 & 11.7 & & & & & & & \\
\hline & & 3:30:53 PM & 25.566 & 25.866 & \begin{tabular}{|l|}
24.459 \\
\end{tabular} & 26.519 & & 48. & \begin{tabular}{l|l}
44 & 54.5
\end{tabular} & 17.4 & 31.93 & -0.349 & & & & 14. & & & & 535 & 11.8 & & & & & & 0.166 & \\
\hline & $9 / 1 / 2$ & $3: 31$ & 25.56 & 25.855 & \begin{tabular}{|l|l|}
24.448 \\
\end{tabular} & 26.5 & & & $\begin{array}{ll}44 & 54.52\end{array}$ & 17.528 & 32.0 & -0.34 & & & & 14.6 & & & & 53.4 & 11.8 & & 1.78 & & & & .162 & \\
\hline & & & & 25.8 & 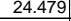 & & & & & & & & & & & 14. & & & & 53.2 & 12.1 & & & & & & 171 & \\
\hline 648 & & & & 25.85 & \begin{tabular}{|l|l|}
24.468 \\
\end{tabular} & \begin{tabular}{|l|l|} 
& 26.648 \\
\end{tabular} & & & \begin{tabular}{l|l|}
4 & 54.523 \\
\end{tabular} & 17.397 & 31.989 & -0.349 & & & & & & & & 53.4 & 11.9 & & & & & & 0.170 & \\
\hline 64 & & & & & 24.494 & & & & & & & & & & & & & & & & & & & & & & & \\
\hline & & & & 25.8 & 24.5 & 26 & & & 91 54. & 17.3 & & & & & & & & & & 53.4 & 11. & & & & & & & \\
\hline & & $\begin{array}{l}3.30 .531 \\
3.37 .53 \mathrm{P}\end{array}$ & & $\mid$ & \begin{tabular}{|l|l|}
2.541 \\
\end{tabular} & $\begin{array}{ll}20.06 \\
2656\end{array}$ & & & $\begin{array}{r}54.55 \\
54.554\end{array}$ & $\begin{array}{l}17.5 \\
176\end{array}$ & 31.06 & -0.3. & & & & & & & & 3 & $\frac{11.9}{120}$ & & & & & & 等 & \\
\hline & & 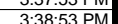 & & $\frac{2.0454}{25858}$ & $\frac{2.401}{24551}$ & & & & 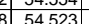 & $\frac{10.0406}{176616}$ & $\frac{31.050}{21891}$ & -0.54 & & & & 116 & & & & o. & & & & & & & 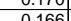 & \\
\hline & & & & $258 \mathrm{~g}>\mathrm{s}$ & 24612 & & & & & & & -0.35 & & & & 146 & & & & $\frac{5.4}{53.3}$ & 10 & & & & & & 166 & \\
\hline & & & & 25.847 & 24.676 & & & 48 & $\begin{array}{l}6 \\
6\end{array}$ & $17.6 \mathrm{Y}>\mathrm{l}$ & & $\frac{-0.344}{-0.344}$ & & & & $14.6 \mathrm{C}-\mathrm{s}$ & & & & 53. & 11.5 & & & & & & & \\
\hline & & & & 25.848 & 24.667 & & & & & & & & & & & & & & & & & & & & & & & \\
\hline & & & & & \begin{tabular}{|l|l|}
24.647 \\
\end{tabular} & & & & & & & & & & & & & & & & 120 & & & & & & & \\
\hline & & & & 25.837 & 24.641 & & & 48.2 & \begin{tabular}{|c|c|}
54.268 \\
\end{tabular} & 7.796 & 31.353 & -0.32 & & & & & & & & 53.1 & 11.9 & & & & & & 67 & in \\
\hline & & 6.44.53P & & 85 & 24.636 & & & & 196 & & 31.32 & -0.294 & & 50.094 & & 14.643 & & & & 53.1 & & & 2.7. & & & & & 11 \\
\hline 2660 & $9 / 1 / 2004$ & $3: 45: 53 \mathrm{PM}$ & 25.641 & 25.847 & 24.62 & \begin{tabular}{|l|l|}
26.625 \\
\end{tabular} & 25.512 & 48.368 & \begin{tabular}{l|l|}
8 & 54.505 \\
\end{tabular} & $\mid 17.774$ & 31.501 & \begin{tabular}{ll|}
11 & -0.297 \\
\end{tabular} & 281 & 49.234 & 0.046 & $\begin{array}{ll}14.643 \\
\end{array}$ & & 352. & 55.8753 & 53.4 & 11.7 & & 2.753 & \begin{tabular}{|l|}
0.007 \\
\end{tabular} & 0.007 & 0.000 & $\begin{array}{ll}0.167 \\
\end{array}$ & 0.1. \\
\hline
\end{tabular}


WSRC-TR-2005-00105, REVISION 0

SRNL-RPP-2005-00012, REVISION 0

RUN \# 4.03A AND B; FIRST AND SECOND HALF OF SLURRY DEWATERING - CONT.

\begin{tabular}{|c|c|c|c|c|c|c|c|c|c|c|c|c|c|c|c|c|c|c|c|c|c|c|c|c|c|c|c|c|}
\hline & $\mathrm{A}_{\mathrm{A}}$ & B & D & $E$ & $F$ & $G$ & $\mathrm{H}$ & $\mathrm{J}$ & \begin{tabular}{l|l}
$\mathrm{K}$ & \\
\end{tabular} & $\mathrm{L}$ & $M$ & $\mathrm{~N}$ & 0 & 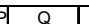 & $R$ & $\mathrm{~s}$ & $\begin{array}{ll}T \\
\end{array}$ & $\mathrm{v}$ & w & $x$ & Y & $z$ & & & & & & \\
\hline 2661 & 9/1/2004 & 3:46:53 PM & 25.626 & 25.836 & 24.609 & 26.614 & 25.546 & 48.63 & 54.542 & 17.953 & 31.626 & -0.303 & -6.336 & 51.326 & 0.045 & 14.643 & 0.003 & 3353.52 & 55.8919 & 53.4 & 12.2 & 40.1 & 2.767 & 0.006 & 0.007 & 0.000 & $\frac{A E}{0.162}$ & \\
\hline & $\begin{array}{l}9 / 1 / 12004 \\
\end{array}$ & & 25.624 & 25.829 & \begin{tabular}{|l|}
24.588 \\
\end{tabular} & \begin{tabular}{|l|}
26.647 \\
\end{tabular} & 25.474 & 47.522 & 53.493 & 17.931 & 30.624 & $\begin{array}{r}-0.323 \\
\end{array}$ & -6.333 & 52.88 & 0.044 & $\begin{array}{l}14.643 \\
\end{array}$ & 0.003 & 3354.52 & 55.9086 & \begin{tabular}{|l|}
52.4 \\
\end{tabular} & & & & 0.006 & & & & \\
\hline 2663 & $9 / 1 / 2004$ & 3:48:53 PM & 25.648 & 25.839 & \begin{tabular}{|l|}
24.632 \\
\end{tabular} & \begin{tabular}{|l|l|}
26.646 \\
2.60
\end{tabular} & 25.528 & 47.746 & \begin{tabular}{|r|}
53.47 \\
5.97
\end{tabular} & $\begin{array}{l}18.009 \\
18005\end{array}$ & & $\begin{array}{r}-0.303 \\
-0.303\end{array}$ & $\begin{array}{l}-6.324 \\
-.320\end{array}$ & 51.683 & 0.046 & 14.643 & $\begin{array}{l}0.003 \\
\end{array}$ & $\frac{3355.52}{325.52}$ & 55.9253 & \begin{tabular}{|l|}
52.3 \\
5.7 \\
\end{tabular} & 12.3 & 39.3 & 1710 & 0.007 & 0.007 & 0.000 & 0.169 & \\
\hline $\begin{array}{l}26644 \\
2665\end{array}$ & $9 / 1 / 2004$ & $\begin{array}{ll}3: 49: 53 \mathrm{PM} \\
3.55 \cdot 53 \mathrm{P}\end{array}$ & $\begin{array}{l}25.643 \\
25653\end{array}$ & $\begin{array}{l}25.833 \\
25.839 \\
\end{array}$ & \begin{tabular}{|l|}
24.601 \\
24607 \\
\end{tabular} & \begin{tabular}{|l|l|}
26.626 \\
26.621 \\
\end{tabular} & $\begin{array}{l}25.573 \\
25.558\end{array}$ & 47.866 & \begin{tabular}{|l|l|}
53.787 \\
53.91 \\
\end{tabular} & \begin{tabular}{l|l|}
18.005 \\
17051
\end{tabular} & $\begin{array}{l}30.832 \\
21021\end{array}$ & $\begin{array}{r}-0.3 \\
\end{array}$ & $\begin{array}{l}-6.339 \\
6333\end{array}$ & 50.738 & $\begin{array}{l}0.044 \\
0.155\end{array}$ & $\begin{array}{l}14.643 \\
1.43\end{array}$ & $\begin{array}{l}0.003 \\
0003\end{array}$ & $\begin{array}{l}3356.52 \\
325752\end{array}$ & \begin{tabular}{|l|l|}
55.9419 \\
55.0599 \\
\end{tabular} & \begin{tabular}{|l|l|}
52.7 \\
5.7 \\
\end{tabular} & 12.1 & 39.3 & 1.713 & 0.006 & 0.006 & 0.000 & 0.162 & \\
\hline$\frac{2665}{2666}$ & $\begin{array}{l}9 / 1 / 2004 \\
9 / 1 / 2004\end{array}$ & $\begin{array}{ll}3: 50: 53 \mathrm{PM} \\
3.5153\end{array}$ & $\frac{25.653}{25.653}$ & $\begin{array}{l}25.839 \\
25.839\end{array}$ & & & $\begin{array}{l}25.558 \\
25603\end{array}$ & $\begin{array}{r}47.876 \\
18.16 \\
\end{array}$ & & \begin{tabular}{|l|}
17.951 \\
180202
\end{tabular} & $\begin{array}{l}31.031 \\
31283 \\
3\end{array}$ & $\begin{array}{r}-0.3 \\
\end{array}$ & & $\begin{array}{l}53.594 \\
51318\end{array}$ & $\begin{array}{l}0.045 \\
0.047\end{array}$ & \begin{tabular}{|l|l|l|}
14.643 \\
14634 \\
\end{tabular} & & $\begin{array}{l}3357.52 \\
220852\end{array}$ & \begin{tabular}{|l|}
55.9586 \\
\end{tabular} & \begin{tabular}{|l|}
52.7 \\
\end{tabular} & $\begin{array}{l}12.8 \\
\end{array}$ & & & \begin{tabular}{|l|}
0.006 \\
0.007 \\
\end{tabular} & & & $\begin{array}{l}0.165 \\
\end{array}$ & \\
\hline 2667 & & $\begin{array}{l}3: 5: 53 \mathrm{PM} \\
3: 52: 53 \mathrm{PM}\end{array}$ & $\frac{25.653}{25.648}$ & $\begin{array}{l}25.839 \\
25.828\end{array}$ & \begin{tabular}{|l|}
24.622 \\
2.581 \\
\end{tabular} & \begin{tabular}{|l|}
26.651 \\
26.631 \\
\end{tabular} & $\begin{array}{l}25.603 \\
25.583 \\
\end{array}$ & $\begin{array}{r}48.16 \\
48.044 \\
\end{array}$ & \begin{tabular}{|l|}
54.094 \\
53.955 \\
\end{tabular} & $\begin{array}{l}18.042 \\
17.869 \\
\end{array}$ & \begin{tabular}{ll|l}
31.283 & 31.267 \\
3
\end{tabular} & $\begin{array}{r}-0.3 \\
-0.234\end{array}$ & $\begin{array}{l}-6.316 \\
-6.243\end{array}$ & $\begin{array}{l}51.318 \\
51.898\end{array}$ & $\begin{array}{l}0.047 \\
0.045\end{array}$ & $\begin{array}{l}\frac{14.643}{14.643} \\
\end{array}$ & $\begin{array}{l}0.004 \\
0.003\end{array}$ & $\begin{array}{l}3338.52 \\
3359.52 \\
\end{array}$ & \begin{tabular}{|l|}
55.9753 \\
255.9959
\end{tabular} & \begin{tabular}{|l|}
53.0 \\
52.8 \\
\end{tabular} & $\frac{12.2}{12.4}$ & $\begin{array}{l}239.7 \\
4 \\
4 \\
4\end{array}$ & 2.739 & 0.007 & $\begin{array}{l}0.007 \\
0.07\end{array}$ & $\begin{array}{r}0.000 \\
\end{array}$ & $\begin{array}{l}0.171 \\
0.164\end{array}$ & 0.15 \\
\hline 2668 & $9 / 1 / 2004$ & 3:53:53 PM & 25.653 & 25.823 & & \begin{tabular}{|l|}
20.0581 \\
\end{tabular} & & 48.042 & & & 31.004 & -0.173 & -6.197 & 51.654 & 0.045 & 14.643 & 0.003 & $\begin{array}{l}3359.52 \\
3360.52\end{array}$ & $\mid$ & \begin{tabular}{|l|}
52.9 \\
52.9
\end{tabular} & $\begin{array}{c}12.4 \\
12.3\end{array}$ & $\begin{array}{l}4 \\
\begin{array}{l}4 \\
3\end{array} 39.9 .5 \\
\end{array}$ & & \begin{tabular}{|l|l|}
0.000 \\
\end{tabular} & $\begin{array}{l}0.007 \\
0.007\end{array}$ & 0.000 & & $\begin{array}{l}0.14 \\
0.14\end{array}$ \\
\hline & & 3:54:53 PM & 25.643 & 25.813 & 24.571 & & 25.518 & 48.125 & \begin{tabular}{|l|l|}
54.103 \\
\end{tabular} & 17.841 & 31.283 & -0.286 & -6.278 & 51.537 & 0.045 & 14.643 & 0.003 & & & 53.0 & & & & & & & 0.164 & \\
\hline & 9/1/2004 & 3:55:53 PM & & 25.828 & & \begin{tabular}{|l|l|}
26.551 \\
\end{tabular} & 25.558 & 48.374 & & 17.987 & 31.314 & -0.236 & & 51.183 & 0.045 & $\begin{array}{l}14.643 \\
\end{array}$ & & 3362.52 & 56.0419 & 53.5 & 12.2 & & & & & 0.000 & 0.164 & \\
\hline 2671 & 9/1/2004 & 3:56:53 PM & 25.662 & 25.822 & \begin{tabular}{|l|}
24.601 \\
2.57
\end{tabular} & 26.595 & 25.5 & 47.714 & \begin{tabular}{|l|}
53.624 \\
54623 \\
\end{tabular} & 17.853 & \begin{tabular}{|r|}
30.895 \\
312
\end{tabular} & -0.234 & & $\begin{array}{l}49.531 \\
5.32\end{array}$ & $\begin{array}{l}0.047 \\
\end{array}$ & 14.643 & $\begin{array}{l}0.003 \\
\end{array}$ & $\begin{array}{l}3363.52 \\
20.52\end{array}$ & 56.0586 & 52.5 & 11.8 & 39.3 & 710 & 0.007 & $\begin{array}{l}0.007 \\
\end{array}$ & 0.000 & 0.173 & \\
\hline \begin{tabular}{|l|l|}
2672 \\
2673
\end{tabular} & $9 / 1 / 2004$ & 3:57:53 PM & $\begin{array}{l}25.656 \\
25.656\end{array}$ & $\begin{array}{l}25.816 \\
25816\end{array}$ & \begin{tabular}{|l|}
24.575 \\
2455
\end{tabular} & \begin{tabular}{|l|l|}
26.559 \\
26559 \\
\end{tabular} & $\begin{array}{l}25.511 \\
25506\end{array}$ & $\begin{array}{l}48.467 \\
8582\end{array}$ & \begin{tabular}{|l|}
54.633 \\
54431 \\
\end{tabular} & \begin{tabular}{l|}
18.074 \\
17171
\end{tabular} & \begin{tabular}{|r|}
31.26 \\
3205
\end{tabular} & $\begin{array}{l}-0.236 \\
-0193\end{array}$ & $\begin{array}{l}-6.295 \\
-6301\end{array}$ & $\begin{array}{l}51.289 \\
19822\end{array}$ & $\begin{array}{ll}046 \\
046\end{array}$ & \begin{tabular}{|l|l|}
14.643 \\
14634 \\
\end{tabular} & & & \begin{tabular}{|l|l|}
56.0753 \\
56.0919
\end{tabular} & \begin{tabular}{|l|}
53.5 \\
53.3
\end{tabular} & 12.2 & 39.9 & $\begin{array}{l}2.748 \\
5781\end{array}$ & 0.007 & 0.007 & 0.000 & 0.167 & \\
\hline$\frac{2673}{2674}$ & $\begin{array}{l}9 / 1 / 2004 \\
99 / 1 / 2004\end{array}$ & $\begin{array}{l}3: 58: 53 \mathrm{PM} \\
3\end{array}$ & $\frac{25.656}{25.656}$ & $\begin{array}{l}25.816 \\
25.816\end{array}$ & $\begin{array}{r}24.5 .55 \\
24.57\end{array}$ & \begin{tabular}{|l|}
26 \\
26
\end{tabular} & $\begin{array}{l}25.506 \\
25.561\end{array}$ & $\begin{array}{r}48.583 \\
48.53 \\
\end{array}$ & \begin{tabular}{|l|}
54.443 \\
54.486
\end{tabular} & $\begin{array}{l}17.421 \\
17.007\end{array}$ & $\begin{array}{l}32.085 \\
32.557 \\
\end{array}$ & \begin{tabular}{|c|c|c|c|c|}
-0.1999 \\
-0.199
\end{tabular} & $\begin{array}{l}-6.301 \\
-6.29 \\
\end{array}$ & $\begin{array}{l}\frac{49.8222}{49.399} \\
\end{array}$ & $\begin{array}{r}0.046 \\
0.046\end{array}$ & $\begin{array}{r}14.643 \\
14643\end{array}$ & $\begin{array}{r}0.003 \\
0.003\end{array}$ & 365.5 & $\frac{56.0919}{56.1086}$ & $\begin{array}{r}53.3 \\
5.34 \\
\end{array}$ & 118 & 40,5 & 2.781 & 0.007 & 0.007 & 0.000 & 0.165 & 0.14 \\
\hline 2675 & 9/1/2004 & 4:00:53 PM & 25.6561 & $\frac{25.810}{25.816}$ & \begin{tabular}{|r|}
24.585 \\
\end{tabular} & \begin{tabular}{|l|}
20.539 \\
26.559 \\
\end{tabular} & 25.501 & $\begin{array}{r}48.53 \\
48.102\end{array}$ & \begin{tabular}{|l|}
54.2140 \\
\end{tabular} & 17.345 & 31.737 & $\begin{array}{l}-0.179 \\
\end{array}$ & & 49.3916 & $\begin{array}{l}0.046 \\
0.047\end{array}$ & \begin{tabular}{|l|l}
14.645 \\
14.643
\end{tabular} & $\begin{array}{l}0.003 \\
0.003\end{array}$ & $\begin{array}{l}\frac{3500.52}{3367.52} \\
\end{array}$ & 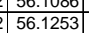 & $\begin{array}{l}53.4 \\
53.1\end{array}$ & $\begin{array}{l}11.8 \\
11.9\end{array}$ & & & & & & & $\begin{array}{l}0.14 \\
0.15\end{array}$ \\
\hline 2676 & & $4: 01: 53 \mathrm{PM}$ & 25.645 & 25.811 & \begin{tabular}{|l|}
24.564 \\
\end{tabular} & \begin{tabular}{|l|l|}
26.588 \\
\end{tabular} & $\begin{array}{r}25.58 \\
2.00\end{array}$ & 48.559 & \begin{tabular}{|l|}
54.532 \\
532
\end{tabular} & 17.454 & 32.274 & $\begin{array}{l}-0.176 \\
-0.197\end{array}$ & -6.174 & 51.062 & 0.045 & $\begin{array}{l}14.643 \\
\end{array}$ & & & 56.1419 & 53.4 & & & & & & & 0.161 & \\
\hline 2677 & 9/1/2004 & 4:02:53 PM & 25.665 & & 24.584 & & & 48.403 & & \begin{tabular}{|l|l|}
17.684 \\
\end{tabular} & & & & 51.164 & & \begin{tabular}{|l|l|}
14.643 \\
\end{tabular} & & 3369.5 & 56.1586 & 53.3 & 12.2 & 40.1 & 2.763 & & & & 0.170 & \\
\hline 2678 & & 4:03:53 PM & 25.649 & & & \begin{tabular}{|l|l|}
26.642 \\
\end{tabular} & 25.504 & 48.15 & & & & & & 51.328 & & $\begin{array}{l}14.643 \\
\end{array}$ & 0.003 & & 56.1753 & 53.2 & 12.2 & 39.8 & & 0.006 & & 0.000 & 0.164 & \\
\hline 2679 & $\begin{array}{l}9 / 1 / 2004 \\
0 / 1 / 2004\end{array}$ & $\begin{array}{l}4: 04: 53 \mathrm{PM} \\
05.53 \mathrm{P}\end{array}$ & $\begin{array}{l}25.648 \\
25.672\end{array}$ & $\begin{array}{l}25.798 \\
25.812\end{array}$ & \begin{tabular}{|l|}
24.537 \\
259
\end{tabular} & \begin{tabular}{|l|l|}
26.711 \\
26705 \\
\end{tabular} & 25.5 & $\begin{array}{l}48.084 \\
8314\end{array}$ & \begin{tabular}{|l|l|}
54.173 \\
5439 \\
\end{tabular} & \begin{tabular}{|c|}
17.877 \\
17739
\end{tabular} & 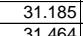 & $\begin{array}{r}-0.187 \\
-0173\end{array}$ & & $\begin{array}{l}52.421 \\
5.071\end{array}$ & & \begin{tabular}{|l|l|}
14.643 \\
14633
\end{tabular} & & & \begin{tabular}{|l|}
56.1919 \\
56208
\end{tabular} & $\begin{array}{l}53.0 \\
532 .\end{array}$ & & 39.6 & 747 & 0.006 & $\begin{array}{l}0.006 \\
0.007\end{array}$ & 500 & $\frac{0.161}{0.67}$ & 0.14 \\
\hline$\frac{2681}{2681}$ & | & $\begin{array}{l}4: 05: 53 \mathrm{PM} \\
4: 06: 53 \mathrm{PM}\end{array}$ & $\begin{array}{l}25.672 \\
25.666\end{array}$ & $\frac{25.812}{25.806}$ & \begin{tabular}{|r|}
24.59 \\
24.574
\end{tabular} & \begin{tabular}{|l|}
26.705 \\
26.639 \\
\end{tabular} & $\frac{25.51}{25.50}$ & & \begin{tabular}{|c|}
54.349 \\
55.204 \\
\end{tabular} & $\frac{17.739}{17.649}$ & $\begin{aligned} 31.464 \\
31.56\end{aligned}$ & $\begin{array}{l}-0.173 \\
-0.196 \\
\end{array}$ & & $\frac{50.071}{49.19}$ & \begin{tabular}{ll|l}
0464 & 047
\end{tabular} & $\frac{14.643}{14.643}$ & $\begin{array}{l}0.004 \\
0.003\end{array}$ & & $\begin{array}{l}56.2086 \\
56.2253\end{array}$ & $\begin{array}{l}53.2 \\
5.1 \\
\end{array}$ & $\begin{array}{ll}\frac{11.5}{11.7} \\
\end{array}$ & $\begin{array}{l}39.0 \\
39.9 \\
3.9\end{array}$ & \begin{tabular}{|l}
2.747 \\
.279
\end{tabular} & 0.007 & 0.007 & 0.000 & & 0.14 \\
\hline 2682 & 9/1/2004 & 4:07:53 PM & $\begin{array}{l}25.000 \\
25.676\end{array}$ & 25.811 & $\begin{array}{r}24.534 \\
24.59\end{array}$ & \begin{tabular}{|l|}
20.0039 \\
\end{tabular} & $\begin{array}{l}25.501 \\
25.471\end{array}$ & $\begin{array}{l}\frac{48.181}{48.592} \\
\end{array}$ & \begin{tabular}{|l|}
54.7764 \\
\end{tabular} & 17.544 & 31.944 & -0.213 & $\begin{array}{l}-\frac{-2.255}{-6.266} \\
\end{array}$ & 50.567 & $\begin{array}{l}0.0476 \\
0.046\end{array}$ & \begin{tabular}{l|l}
$\frac{14.043}{14.643}$ &
\end{tabular} & $\begin{array}{l}0.003 \\
0.003\end{array}$ & 3374.5 & $\begin{array}{l}50.2243 \\
56.2419\end{array}$ & $\begin{array}{l}53.1 \\
53.7\end{array}$ & $\frac{11.7}{12.0}$ & $\frac{39.9}{40.3}$ & $\frac{2.149}{2.776}$ & 0.007 & 0.007 & 0.000 & 0.165 & $\frac{0.15}{0.14}$ \\
\hline 2683 & 9/1/2004 & $4: 08: 53 \mathrm{PM}$ & 25.664 & 25.805 & 24.573 & \begin{tabular}{|l|}
26.517 \\
\end{tabular} & 25.549 & 48.615 & \begin{tabular}{|l|l|l|l|}
5408 \\
\end{tabular} & \begin{tabular}{|l|l|l|l}
17.447 \\
\end{tabular} & 31.954 & -0.179 & -6.258 & 49.376 & 0.048 & $\begin{array}{l}14.643 \\
\end{array}$ & 0.003 & 3375.5 & 56.2586 & 53.6 & 11.8 & 40.3 & 2.777 & 0.007 & 0.007 & & & \\
\hline 26 & & 4:09:53 PM & 25.675 & 25.815 & 24.579 & 26.503 & 25.605 & & \begin{tabular}{|c|}
54.888 \\
\end{tabular} & 17.393 & 32.258 & & & 49.639 & & 14.643 & & & & & & & & & & & & \\
\hline 2685 & & $4: 10: 53 \mathrm{PM}$ & & & 24.593 & & & & & & 32.397 & & & 51.506 & & 14.643 & & & 2919 & 53.4 & & & & & 0.00 & & 165 & \\
\hline 2686 & & $50 P M$ & 5.664 & 25.804 & & 26.467 & & 48.401 & 54.639 & & & & & & & 14.643 & & & & & & & & & & & & \\
\hline 2687 & $9 / 1 / 2$ & 53 PM & 25.647 & 25.782 & 24.551 & 26.4 & & & & 17.002 & 32.512 & -0.179 & & 49.08 & & 14.643 & & & & 53.4 & & & & & & & & \\
\hline & 1/2004 & & 25.668 & 25.803 & 24.571 & 26.466 & 25.5 & 48.436 & \begin{tabular}{|l|l|l|l|}
5486 \\
\end{tabular} & 17.162 & 32.194 & -0.152 & & 49.499 & 0.048 & \begin{tabular}{l|l}
14.643 \\
\end{tabular} & & & 6.3419 & 53.4 & 11.8 & & 780 & .007 & 0.007 & & & \\
\hline & 1/2004 & 4:14:53 PM & 25. & 25.797 & 24.581 & 26.415 & & 48.561 & \begin{tabular}{|l}
54.515 \\
\end{tabular} & 17.237 & 32.319 & -0.181 & & 51.558 & 1046 & 14.643 & & 3381.5 & & 53.4 & 12.3 & 40.2 & 278 & & & & 0.165 & \\
\hline & & & & 25.802 & 24.575 & 26.429 & & & \begin{tabular}{|l|l|}
54.8655 \\
5.150
\end{tabular} & 17.373 & 32.211 & -0.103 & & 49.608 & & 14.643 & & & 6.3753 & 53.7 & & & & & & & & \\
\hline & & $3 \mathrm{PM}$ & & 25.795 & 24.583 & $26.4 / 3$ & & & 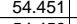 & 17.167| & 32.381 & -0.109 & & 50.7 & 04 & 14.643 & & & & & & & & & & & & \\
\hline & & 4:17:53 PM & 5.649 & 25.189 & $24.5 / 3$ & & & 48.3 & $\begin{array}{r}54.459 \\
\end{array}$ & 17.14 & 32.344 & -0.106 & & & & & & & 0.4086 & 53.3 & & & & 00 & & & & \\
\hline & (1) & $53 \mathrm{PM}$ & 5648 & $\frac{25.804}{25.794}$ & \begin{tabular}{|l|}
24.553 \\
24597 \\
\end{tabular} & $\frac{26.497}{26516}$ & & $\frac{48.695}{48619}$ & $\begin{array}{r}54.78 \\
54.67\end{array}$ & $\frac{17.048}{17101}$ & $\begin{array}{l}32.668 \\
32.358\end{array}$ & $\begin{array}{l}-0.164 \\
-0.109\end{array}$ & & $\frac{49.745}{50.46}$ & 2 & $\frac{14.643}{14643}$ & & & 56.449 & 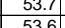 & 12 & & 79 & & & & 68 & \\
\hline & 9 & & 25648 & 25799 & \begin{tabular}{|l|l|}
24.582 \\
\end{tabular} & 26.546 & & 年 & $\mid$ & 17355 & 31.977 & -0.161 & & & 2047 & 14643 & & & & 532 & & & & & & & & \\
\hline$\frac{269}{269}$ & $9 / 1 / 2004$ & 4:21:53 PM & 25.647 & 25.787 & 24.6 & 26.45 & & 48.4 & \begin{tabular}{|l|}
54.4854 \\
\end{tabular} & 17.463 & 31.944 & -0.106 & & $\frac{7.5 .5}{48.5}$ & & $\begin{array}{ll}14.045 \\
14.643\end{array}$ & & & 66 & 53.4 & 11.6 & & & & & & $\frac{0.179}{0169}$ & $\frac{14}{14}$ \\
\hline & & & 25.647 & 25.792 & 24.6 & 26.565 & & 48.5 & \begin{tabular}{|l|}
54.729 \\
\end{tabular} & 17.38 & 32.03 & -0.106 & & & 0.04 & & & & & $\frac{13.6}{53.6}$ & & & & & & & & \\
\hline & & PM & & & 24.611 & & & & \begin{tabular}{|l|}
54.77 \\
\end{tabular} & & & -0.106 & & & & & & & & 53.6 & & & & & & & & \\
\hline & & 4:24:53 PM & 25.653 & 25.798 & \begin{tabular}{|l|}
24.611 \\
\end{tabular} & & & & \begin{tabular}{|l|}
54.387 \\
\end{tabular} & 16.752 & 32.596 & -0.121 & & 50.751 & & 14. & & & & 53.3 & 12. & & & & & & 0.168 & \\
\hline & & & & 25.794 & & & & & 54.842 & & & -0.106 & & & & & & & & & & & & & & & & \\
\hline & & 4:26:53 PM & 668 & 25.799 & 24.667 & \begin{tabular}{|l|l|}
26.676 \\
\end{tabular} & & & 54.633 & & 32.194 & 106 & & & & & & & & 53.5 & 11.2 & 404 & & & & & 68 & 014 \\
\hline & $9 / 1 / 2004$ & & 25.663 & 25.803 & 24.681 & 26. & & & 54.101 & 17.308 & 31.835 & -0.103 & & & & 14.6 & & & & 53.0 & 11. & & & 006 & 0.00 & & 0.163 & \\
\hline & & & 25.674 & & 24.703 & & & & 54.484 & & & & & & & & & & & 53.4 & 11. & & & & & & & \\
\hline & & & 25.673 & 25.804 & \begin{tabular}{|l|l|} 
& 4.672 \\
\end{tabular} & 26. & & 48.604 & \begin{tabular}{|l|}
54.726 \\
\end{tabular} & 17.401 & 32.18 & -0.106 & & & 0.047 & & & & 66.6086 & 53.6 & 12.1 & & & & 0.00 & & 0.168 & \\
\hline & & & & 25.798 & 24.666 & & & & 54.8 & & 32.127 & -0.109 & & & & & & & & , & $1 \pm$ & & & & & & 175 & \\
\hline & & & & & & & & & & & & & & & & & & & & & & & & & & & & \\
\hline & & & & $\frac{25.799}{25803}$ & $\frac{24.622}{24656}$ & $\frac{26.531}{26556}$ & & & $\begin{array}{r}54.49 \\
54.329\end{array}$ & $\begin{array}{l}17.295 \\
117261\end{array}$ & $\begin{array}{r}31.93 \\
32083\end{array}$ & $\begin{array}{l}-0.083 \\
-0.0106\end{array}$ & & & & $\begin{array}{l}14.6 \\
1.6\end{array}$ & & & & 5.4 & 12 & & & & & & 160 & \\
\hline & & & & 25809 & 24647 & & & & 34.5 & & & -0.086 & & & & & & & & & & & & & & & & \\
\hline & & & & 25.598 & \begin{tabular}{|l|l|l|l|}
24636 \\
\end{tabular} & & & & 54.57 & & & & & & & & & & & 0.0 & & & & & & & & \\
\hline & & & 25.688 & 25.803 & 24 & & & & \begin{tabular}{|l|}
54.482 \\
\end{tabular} & 17.107 & & $\frac{-0.092}{-0.092}$ & & & & & & & & 53.4 & $\frac{12.0}{12.0}$ & & & & & & 165 & \\
\hline & & & & & & & & & & & & & & & & & & & & & & & & & & & & \\
\hline & & & & 25.8 & 24.651 & & & & 54.49 & 17.3 & 31.987 & $\frac{-0.6}{-0.6}$ & & & & & & & & & & & & & & & & \\
\hline & & & & 25.7 & 24.626 & & & & 54.569 & & 32.231 & & & & & & & & & & & & & & & & & \\
\hline & & & & 25.79 & 24.641 & 26. & & & \begin{tabular}{|l|l|}
54.561 \\
\end{tabular} & & 31.798 & & & & & & & & & 530 & 12.0 & & & & & & & \\
\hline & $9 / 1 / 2$ & & & 25.80 & 24.626 & & & 48. & \begin{tabular}{|l|l|}
54.598 \\
\end{tabular} & 16.9 & 32.4 & & & & & 14.6 & & & & 53.5 & 11. & & & & & & . 168 & \\
\hline & & & & & & & & & 54.73 & & & & & & & 14. & & & & & & & & & & & & \\
\hline & & & & 25.818 & 24.666 & 26.5 & & 48. & 54. & 17.385 & & $\begin{array}{r}-0.08 \\
-\end{array}$ & & & & & & & & 53.8 & 12.3 & & & & & & 0.161 & \\
\hline & & & & & 24.64 & & & & & & & & & & & & & & & & & & & & & & & \\
\hline & & & & 25.8 & 24.665 & & & & 54.87 & & 32. & & & & & & & & & & & & & & & & & \\
\hline & & 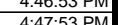 & & 20. & 2.05 & 20.504 & & & | & & $\frac{32.424}{3236}$ & & & & & & & & & & 11.3 & & & & & & & \\
\hline & & & & $\frac{2.01}{2580}$ & 24.6011 & $\frac{2.5}{265}$ & & 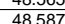 & \begin{tabular}{|l|}
54.9697 \\
5
\end{tabular} & 16954 & 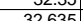 & & & & & 146 & & & & & $110^{\circ}$ & & & & & & & \\
\hline & & & & 25802 & & 26 & & & $\mid$ & & 3 & & & & & 146 & & & & & 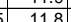 & & & & & & & \\
\hline & & & & 25.80 & 24646 & & & 48. & 54. & & 32402 & & & & & 146 & & & & 53 & 116 & & & & & & & \\
\hline & & & & & 24.631 & & & & 54.6 & & & & & & & & & & & & & & & & & & & \\
\hline & & & & & & $26.7 \mathrm{C}$ & & & & & & & & & & & & & & & & & & & & & & \\
\hline & & & & 25.80 & 24.646 & 26 & & 48.3 & \begin{tabular}{|l|l|}
54.329 \\
\end{tabular} & 17.1 & 32.196 & & & & & & & & & & 12.3 & & & & & & & \\
\hline & & & & 812 & 647 & & & & 5478 & & 3241 & R & & & & 4.6 & & & & & 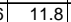 & & & & & & & 11 \\
\hline 2730 & $9 / 1 / 2$ & 4:55:53 PM & 5.699 & 25.819 & \begin{tabular}{|l|l|}
24.8533 \\
\end{tabular} & \begin{tabular}{|l|l|}
26.672 \\
\end{tabular} & $25.56 \mathrm{~s}$ & 48.241 & 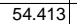 & 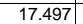 & \begin{tabular}{|l|l|l|}
3139 \\
\end{tabular} & -0.048 & & 51.097 & $\mid 0.046$ & $\begin{array}{l}14.643 \\
\end{array}$ & & 422.5 & 57.0419 & 53.3 & $12.2 \mathrm{r}$ & & 2.757 & \begin{tabular}{|l|}
0.007 \\
\end{tabular} & 0.007 & 0.000 & 0.165 & 0.1 \\
\hline
\end{tabular}


WSRC-TR-2005-00105, REVISION 0

SRNL-RPP-2005-00012, REVISION 0

RUN \# 4.03A AND B; FIRST AND SECOND HALF OF SLURRY DEWATERING - CONT.

\begin{tabular}{|c|c|c|c|c|c|c|c|c|c|c|c|c|c|c|c|c|c|c|c|c|c|c|c|c|c|c|c|c|}
\hline & $\mathrm{A}$ & B & D & $E$ & $\mathrm{~F}$ & \begin{tabular}{l|l|l|} 
G & \\
\end{tabular} & $\mathrm{H}$ & $\mathrm{J}$ & \begin{tabular}{l|l|}
$\mathrm{K}$ & \\
\end{tabular} & 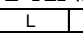 & M & $\mathrm{N}$ & 0 & \begin{tabular}{l|l} 
\\
\end{tabular} & \begin{tabular}{l|l|l|}
$\mathrm{R}$ & \\
\end{tabular} & \begin{tabular}{l|l|l|} 
& \\
\end{tabular} & $T$ & $\mathrm{v}$ & w & $x$ & $\mathrm{Y}$ & $z$ & $\mathrm{AA}$ & $\mathrm{AB}$ & $\mathrm{AC}$ & AD & AE & \\
\hline & 9/1/2004 & 4:56:53 PM & 25.691 & 25.821 & 24.795 & 26.689 & 25.571 & 48.859 & 54.934 & 17.633 & 32.321 & -0.066 & & 49.535 & 0.046 & 14.643 & & & 77.0586 & 53.8 & 11.8 & 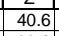 & 2.799 & & & 0.000 & 0.163 & \\
\hline & & & 25.696 & & 24.195 & 26.634 & & & & & & & & 48.404 & 0.047 & 14.643 & & & & & $1+$ & & & & & & & \\
\hline$\frac{2733}{2734}$ & 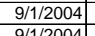 & $4: 58: 53 \mathrm{PM}$ & $\begin{array}{l}25.716 \\
25711\end{array}$ & $\begin{array}{l}25.821 \\
25812\end{array}$ & $\begin{array}{l}24.775 \\
24755\end{array}$ & $\begin{array}{l}26.639 \\
26699\end{array}$ & 25.641 & \begin{tabular}{|l|l|}
48.602 \\
4839
\end{tabular} & \begin{tabular}{|l|}
54.768 \\
5469
\end{tabular} & $\begin{array}{l}17.461 \\
1739\end{array}$ & $\begin{array}{l}32.143 \\
31.936\end{array}$ & $\begin{array}{l}-0.077 \\
-0.077\end{array}$ & $\begin{array}{l}-6.078 \\
5992\end{array}$ & $\begin{array}{l}49.076 \\
5223\end{array}$ & $\begin{array}{l}0.047 \\
0045\end{array}$ & $\begin{array}{l}14.643 \\
14643\end{array}$ & & & \begin{tabular}{|l|}
57.0919 \\
570694
\end{tabular} & 53.6 & r & & & 007 & 0.007 & 0.000 & 0.168 & \\
\hline$\frac{2734}{2735}$ & $\begin{array}{l}9 / 1 / 2004 \\
99 / 2004\end{array}$ & 4:59:09 PM & $\frac{25.711}{25706}$ & $\frac{25.812}{25.811}$ & $\frac{24.755}{24714}$ & 26.629 & 25611 & $\begin{array}{l}48.349 \\
48.351\end{array}$ & \begin{tabular}{|l}
54.699 \\
547766
\end{tabular} & $\begin{array}{l}17.396 \\
17.579\end{array}$ & $\begin{array}{l}31.936 \\
31767 \\
3\end{array}$ & $\begin{array}{l}-0.077 \\
0.027\end{array}$ & & 52.223 & 0.045 & $\begin{array}{l}14.643 \\
14643\end{array}$ & & 2678 & .0964 & 536 & 119 & & & & 0.006 & 0.000 & 0.162 & 0.14 \\
\hline & $9 / 1 / 2004$ & 5:01:09 PM & & & 24.724 & 26.579 & & & & & 31.88 & $\frac{-0.025}{-0.025}$ & & 51.378 & & 14.643 & & & & & $\frac{11.9}{1.2}$ & & & & & & & $\frac{0.14}{0.15}$ \\
\hline & 9/1/2004 & 5:02:09 PM & 25.721 & 25.811 & 24.694 & 26.539 & 25.626 & 48.422 & 54.548 & 17.56 & 31.866 & -0.022 & & 50.448 & 0.047 & 14.643 & & 3428.78 & \begin{tabular}{|l|l|}
57.1464 \\
\end{tabular} & 53.4 & 12.0 & 40.1 & 2.768 & .007 & 0.007 & & 0.169 & \\
\hline & & 5:03:09 PM & 25.725 & 25.815 & 24.699 & 26.473 & 25.525 & 48.364 & 54.482 & 17.601 & 31.778 & -0.025 & & 47.791 & 0.046 & 14.643 & & & 57.1631 & 53.4 & 11.4 & & 2.763 & 007 & 0.007 & 0.000 & & \\
\hline & & 5:04:09 PM & & 25.814 & & 26.497 & & & 54.293 & 17.278 & 31.564 & -0.022 & & 49.616 & 0.047 & 14.643 & & & & & 11.8 & & & & & & & \\
\hline 2740 & $9 / 1 / 2004$ & 5:05:09 PM & 25.713 & 25.799 & 24.647 & 26.491 & 25 & \begin{tabular}{|l|}
48.538 \\
\end{tabular} & \begin{tabular}{|r|}
54.66 \\
54.63
\end{tabular} & 17.481 & 32.155 & $\begin{array}{l}-0.025 \\
-.023\end{array}$ & & 49.954 & 0.045 & 14.643 & & & 57.1964 & 53.5 & 11.9 & 40.3 & 2.782 & $\begin{array}{l}0.66 \\
02\end{array}$ & 0.007 & 0.000 & 0.161 & \\
\hline & $\begin{array}{l}9 / 1 / 2004 \\
91 / 2004\end{array}$ & 5:06:09 PM & $\begin{array}{r}25.719 \\
25712\end{array}$ & $\begin{array}{l}25.794 \\
25.801\end{array}$ & $\begin{array}{l}24.663 \\
2.652\end{array}$ & $\begin{array}{l}26.467 \\
26531\end{array}$ & 25 & $\begin{array}{r}48.092 \\
\end{array}$ & $\begin{array}{l}54.403 \\
5.030\end{array}$ & \begin{tabular}{|l|l|}
17.411 \\
\end{tabular} & $\begin{array}{l}31.652 \\
321.007\end{array}$ & $\begin{array}{l}-0.022 \\
-0.222\end{array}$ & & $\begin{array}{l}48.208 \\
5.214\end{array}$ & 0.046 & 14.643 & & & 57.2131 & 53.3 & 11.5 & & $\begin{array}{l}2.749 \\
.2775\end{array}$ & 007 & 0.007 & 0.000 & 0.167 & \\
\hline 2743 & $\begin{array}{l}9 / 1 / 2004 \\
9 / 1 / 2004\end{array}$ & $\begin{array}{l}5: 07: 09 \mathrm{PM} \\
\text { 5.08:09 PM }\end{array}$ & 25.713 & $\begin{array}{l}25.804 \\
25809\end{array}$ & $\frac{24.652}{24648}$ & $\begin{array}{l}26.531 \\
26.467\end{array}$ & 25.47 & \begin{tabular}{|c|}
48.594 \\
48542
\end{tabular} & \begin{tabular}{|r|}
55.039 \\
54.77
\end{tabular} & 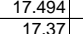 & $\begin{array}{l}31.907 \\
32.211\end{array}$ & $\begin{array}{l}-0.022 \\
-0.025\end{array}$ & & $\frac{52.144}{50.231}$ & $\begin{array}{l}0.044 \\
0.045\end{array}$ & $\frac{14.643}{14.643}$ & & $\begin{array}{r}3433.78 \\
3434.78\end{array}$ & $\frac{57.2297}{57246}$ & & $\frac{12.4}{120}$ & $\begin{array}{l}40.3 \\
40.4 \\
\end{array}$ & 2.775 & \begin{tabular}{|l|l|l|}
.006 \\
006
\end{tabular} & & $\begin{array}{l}0.000 \\
0.000\end{array}$ & 0.158 & \\
\hline \begin{tabular}{|l|l|}
2744 \\
\end{tabular} & $9 / 1 / 2004$ & 5:09:09 PM & 25.708 & 25.804 & 24.637 & 26.481 & 25.513 & $\begin{array}{r}-40.542 \\
48.21\end{array}$ & 54.329 & 17.411 & 31.854 & $\begin{array}{l}-0.022 \\
-\end{array}$ & & 49.28 & 0.046 & 14.643 & & $\begin{array}{l}3434.18 \\
3435.78\end{array}$ & 57.2631 & $\begin{array}{l}53.0 \\
53.2\end{array}$ & $\frac{12.0}{11.7}$ & & & & $\frac{0.001}{0.007}$ & & $\mid$ & 1.14 \\
\hline & 9/1/2004 & 5:10:09 PM & 25.703 & 25.793 & 24.611 & 26.456 & 25.473 & 48.376 & 54.602 & \begin{tabular}{|l|}
17.34 \\
\end{tabular} & 31.942 & -0.016 & & 49.334 & 0.045 & \begin{tabular}{|l|l|}
14.643 \\
\end{tabular} & & 3436.78 & \begin{tabular}{|l|l|}
57.2797 \\
\end{tabular} & 53.5 & 11.8 & 40.2 & 2.769 & 0.006 & 0.007 & 0.000 & 0.162 & \\
\hline 2746 & 9/1/2004 & 5:11:09 PM & 25.708 & & 24.621 & 26.436 & & 48.287 & 54.42 & $\begin{array}{l}17.351 \\
\end{array}$ & 32.018 & 0.03 & & 51.873 & .044 & & & & \begin{tabular}{|l|l|}
57.2964 \\
\end{tabular} & 53.3 & 12.4 & 40.2 & 2768 & 0.006 & 0.006 & & 0.159 & \\
\hline 2747 & 9/1/2004 & 5:12:09 PM & 25.703 & 25.798 & 24.611 & \begin{tabular}{|l|}
26.436 \\
\end{tabular} & 25.408 & \begin{tabular}{|l|l|}
48.276 \\
\end{tabular} & $\begin{array}{r}54.42 \\
5.97 \\
\end{array}$ & \begin{tabular}{|l|l|}
17.421 \\
17.26
\end{tabular} & 32.005 & $\begin{array}{l}0.03 \\
\end{array}$ & & 49.518 & 0.046 & \begin{tabular}{|l|l|}
14.643 \\
1.643
\end{tabular} & & & \begin{tabular}{|l|}
57.3131 \\
\end{tabular} & 53.3 & & & 2.768 & 0.007 & 0.007 & 0.000 & 0.166 & \\
\hline 274 & 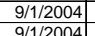 & 5:13:09 PM & 25.703 & $\begin{array}{l}25.793 \\
25.776\end{array}$ & 24.611 & \begin{tabular}{|l|}
26.416 \\
26309
\end{tabular} & 25.48 & \begin{tabular}{|l|l|}
4857 \\
\end{tabular} & 54.677 & $\begin{array}{l}17.436 \\
1736\end{array}$ & \begin{tabular}{r|}
31.884 \\
22.109
\end{tabular} & $\begin{array}{l}0.03 \\
0.07\end{array}$ & & 49.823 & 0.046 & $\begin{array}{r}14.643 \\
1.643\end{array}$ & & & \begin{tabular}{|l|}
57.3297 \\
7327 \\
\end{tabular} & $\begin{array}{l}53.6 \\
5.77\end{array}$ & 11.9 & & & & 0.007 & 0.000 & 0.166 & \\
\hline 2745 & $\frac{9 / 1 / 200}{9 / 1 / 200}$ & $\begin{array}{l}\text { 5:14:09 PM } \\
\text { 5.515.09 PM }\end{array}$ & & $\begin{array}{l}25.776 \\
25.776\end{array}$ & 24.605 & $\frac{26.389}{26.363}$ & & \begin{tabular}{|l|}
48.523 \\
48.436
\end{tabular} & \begin{tabular}{|c|}
54.791 \\
54338
\end{tabular} & \begin{tabular}{|r|r}
17.36 \\
17.124
\end{tabular} & \begin{tabular}{r|r|}
32.149 \\
3228
\end{tabular} & $\begin{array}{l}0.007 \\
0.024\end{array}$ & & $\frac{49.864}{49.047}$ & \begin{tabular}{ll|l|l|}
046 \\
206
\end{tabular} & & & & 57.3464 & & $\frac{11.9}{11.7}$ & & & & & & $\begin{array}{l}0.165 \\
0.165\end{array}$ & \\
\hline & $9 / 1 / 2004$ & 5:16:09 PM & 25.675 & 25.77 & 24.588 & $\frac{20.505}{26.288}$ & 25.405 & \begin{tabular}{|l|}
4.0 .432 \\
48.324
\end{tabular} & $\begin{array}{l}54.500 \\
54.507\end{array}$ & $\begin{array}{l}17.124 \\
17.373\end{array}$ & 31.925 & 0.013 & & 5. $\frac{49.041}{51.039}$ & 年 0.045 & $\begin{array}{l}14.045 \\
14.643\end{array}$ & & 3442.78 & \begin{tabular}{|l|l|}
57.3631 \\
\end{tabular} & $\begin{array}{l}53.1 \\
53.4 \\
\end{array}$ & $\frac{11.1}{12.2}$ & $\begin{array}{l}40.4 \\
40.1\end{array}$ & 2.76 & 0.006 & 0.007 & $\begin{array}{l}0.000 \\
0.000\end{array}$ & $\begin{array}{l}0.165 \\
0.162\end{array}$ & \\
\hline & 9/1/2004 & 5:17:09 PM & 25.673 & 25.768 & 24.572 & \begin{tabular}{|l|}
26.321 \\
\end{tabular} & & 48.614 & 54.84 & \begin{tabular}{|l|l|}
17.344 \\
\end{tabular} & 32.246 & 0.015 & & 49.311 & 0.046 & \begin{tabular}{|l|l|}
14.643 \\
\end{tabular} & & & 57.3964 & 53.7 & 11.7 & 40.4 & 2.788 & $\begin{array}{l}0.007 \\
\end{array}$ & 0.007 & 0.000 & 0.165 & \\
\hline & 9/1/2004 & 5:18:09 PM & 25.678 & & 24.612 & & & & & $\begin{array}{l}17.464 \\
1\end{array}$ & \begin{tabular}{|l|l|}
32.137 \\
\end{tabular} & 0.021 & & 48.723 & & & & & \begin{tabular}{|l|}
57.4131 \\
\end{tabular} & 53.9 & 11.6 & 40.4 & 2.784 & 0.006 & 0.007 & & & \\
\hline & 9/1/2004 & 5:19:09 PM & 25.652 & $\begin{array}{l}25.763 \\
25756\end{array}$ & 24.561 & 26.315 & & & 54.847 & \begin{tabular}{|l|l|l|}
17.322 \\
17017
\end{tabular} & $\begin{array}{l}32.221 \\
20.202\end{array}$ & $\begin{array}{l}0.027 \\
\end{array}$ & & 50.279 & & 14.643 & & & \begin{tabular}{|l|}
57.4297 \\
\end{tabular} & 53.7 & 12.0 & & & 0.006 & 0.007 & 0.000 & 0.162 & \\
\hline & $9 / 1 / 2004$ & 5:20:09 PM & 25.651 & $\begin{array}{r}25.756 \\
2576\end{array}$ & 24.564 & 26.274 & & $\begin{array}{l}48.546 \\
\end{array}$ & \begin{tabular}{|l|}
54.971 \\
5.7707
\end{tabular} & $\begin{array}{l}17.317 \\
17.277\end{array}$ & $\begin{array}{l}32.192 \\
21.920 \\
2\end{array}$ & $\begin{array}{l}0.03 \\
\end{array}$ & & 49.405 & 0.045 & \begin{tabular}{|l|l|}
14.643 \\
1.62
\end{tabular} & & & \begin{tabular}{|l|}
57.4464 \\
7.72 \\
\end{tabular} & 53.8 & 11.8 & & & & 0.007 & 0.000 & 0.162 & \\
\hline$\frac{2750}{2757}$ & $\begin{array}{l}9 / 1 / 1 / 2004 \\
91 / 2004\end{array}$ & $\begin{array}{l}5: 21: 09 \mathrm{PM} \\
5: 22: 09 \mathrm{PM}\end{array}$ & $\begin{array}{l}25.655 \\
25.629\end{array}$ & $\begin{array}{r}25.76 \\
25.729\end{array}$ & 24.594 & $\frac{26.248}{26.207}$ & & $\begin{array}{r}48.5 \\
48.782\end{array}$ & $\mid$\begin{tabular}{|}
$|5.47 .17|$ \\
55.131
\end{tabular} & $\begin{array}{l}17.377 \\
17.357\end{array}$ & $\begin{array}{l}31.829 \\
32.334\end{array}$ & $\begin{array}{r}0.03 \\
0.024\end{array}$ & & $\begin{array}{l}\frac{49.629}{51.101} \\
5\end{array}$ & $\begin{array}{l}.047 \\
.046\end{array}$ & $\frac{14 .}{14 .}$ & & & \begin{tabular}{|l|}
57.4631 \\
7.4797
\end{tabular} & $\begin{array}{l}53.7 \\
54.0\end{array}$ & $\frac{11.8}{12.2}$ & & $\frac{2.10}{2.79}$ & . $007 \mid$ & & Doo 00 & \begin{tabular}{|c|c|}
0.169 \\
0.165
\end{tabular} & \\
\hline 2758 & 9/1/2004 & 5:23:09 PM & 25.628 & 25.733 & 24.566 & \begin{tabular}{|l|}
26.201 \\
\end{tabular} & 25.3 & $\begin{array}{l}48.442 \\
\end{array}$ & 54.681 & 17.206 & 32.367 & 0.033 & & & 0.047 & 14.643 & & & \begin{tabular}{|l|l|}
57.4964 \\
\end{tabular} & 53.6 & 11.6 & 40.4 & 2.78 & & & & 0.169 & \\
\hline & & & 25.622 & 25.727 & 24.551 & \begin{tabular}{|l|}
26.225 \\
\end{tabular} & & 48.5 & 54.778 & 17.259 & 32.231 & $\begin{array}{l}0.03 \\
\end{array}$ & & 49.385 & 0.046 & 14.643 & & & 57.5131 & 53.7 & 11.8 & 40.4 & 2.784 & 0.007 & 0.007 & 0.000 & 0.165 & \\
\hline$\frac{2760}{2701}$ & & 5:25:09 PM & & 25.726 & 24.564 & 26.244 & & $\begin{array}{l}48.463 \\
\end{array}$ & 54.925 & \begin{tabular}{|l|l|}
17.134 \\
\end{tabular} & & 0.065 & & & 44 & & & & & 53.8 & 11.8 & 40.2 & & $\begin{array}{l}0.006 \\
\end{array}$ & & & $\begin{array}{ll}0.158 \\
\end{array}$ & \\
\hline 2761 & 9/1/2004 & 5:26:09 PM & 25.616 & 25.721 & 24.559 & 26.304 & & & 54.652 & \begin{tabular}{|l|l|l|}
17.119 \\
\end{tabular} & $\begin{array}{l}32.363 \\
32.230\end{array}$ & \begin{tabular}{|l|l|} 
\\
\end{tabular} & & & & & & & \begin{tabular}{|l|l|}
57.5464 \\
\end{tabular} & 53.5 & 11.5 & 40. & 2.78 & 0.007 & & 0.000 & 0.169 & \\
\hline$\frac{2762}{2763}$ & $9 / 1 / 2004$ & 5:27:09 PM & 25.615 & \begin{tabular}{|l|l|}
25.725 \\
25732
\end{tabular} & $\begin{array}{r}24.548 \\
20.57 \\
\end{array}$ & 26.353 & 25.4. & 48.6 & $\begin{array}{r}54.88 \\
5.53 \\
\end{array}$ & $\begin{array}{l}17.235 \\
16.096\end{array}$ & \begin{tabular}{|l|l|l|}
32.332 \\
32244
\end{tabular} & \begin{tabular}{|c|c|}
0.105 \\
033
\end{tabular} & & 49.278 & 847 & \begin{tabular}{|l|l|}
14.643 \\
1.63
\end{tabular} & & & \begin{tabular}{|l|}
57.5631 \\
55707
\end{tabular} & 53.8 & \begin{tabular}{ll|}
11.7 \\
11.6
\end{tabular} & & 2.79 & $\begin{array}{l}007 \\
007\end{array}$ & $0.6 \gamma$ & 0.000 & 0.168 & \\
\hline$\frac{2763}{2764}$ & $\begin{array}{l}9 / 1 / 22004 \\
9 / / 2004\end{array}$ & $\begin{array}{l}5: 22: 09 \mathrm{PM} \\
5.2909 \mathrm{P}\end{array}$ & $\frac{25.621}{25.627}$ & \begin{tabular}{l|}
25.732 \\
25.727
\end{tabular} & $\begin{array}{r}24.57 \\
24.556\end{array}$ & $\frac{26.414}{26.535}$ & & & \begin{tabular}{|c|}
54.538 \\
55.63
\end{tabular} & $\begin{array}{r}16.986 \\
17.072\end{array}$ & $\begin{array}{l}32.244 \\
33.083\end{array}$ & \begin{tabular}{|l}
0.033 \\
0.027
\end{tabular} & & & $\begin{array}{ll}046 \\
046\end{array}$ & & & & & & & & & & & & & \\
\hline & 9/1/2004 & 5:30:09 PM & 25.624 & 25.739 & 24.597 & \begin{tabular}{|l|}
26.567 \\
\end{tabular} & & 48.0 & $\begin{array}{r}54.3 \\
5\end{array}$ & 17.753 & 31.349 & 0.027 & & 50.075 & 0 & $\begin{array}{l}14.045 \\
14.643\end{array}$ & & & $\left|\begin{array}{|l}57.5964 \\
57.6131\end{array}\right|$ & 53.2 & $\begin{array}{l}11.0 \\
11.9 \\
\end{array}$ & 39. & & & & & 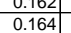 & \\
\hline & & & 25.616 & 25.736 & 24.569 & \begin{tabular}{|l|}
26.619 \\
\end{tabular} & & & 54.357 & $\begin{array}{l}17.468 \\
\end{array}$ & & 0.088 & & & & & & & & 53.2 & 10 & 40 & & & & & 0.153 & \\
\hline 2767 & & 5:32:0 & & 25.748 & 24.602 & & & & 54.409 & $\begin{array}{l}17.724 \\
\end{array}$ & 31.345 & & & 47. & & & & & & 53.3 & 11.3 & 39.7 & 2.73 & & & & 0.164 & \\
\hline 2768 & & $5: 33: 09$ & 25.625 & 25.755 & 24.619 & 26.7 & & & 54.482 & \begin{tabular}{|l|l|l|l|}
1733 \\
\end{tabular} & $\begin{array}{l}31.23 \\
3.91\end{array}$ & & & & & & & & \begin{tabular}{|l|l|}
57.6631 \\
\end{tabular} & 53.4 & 12.0 & 39.7 & 2.736 & 0.006 & & 00 & 0.160 & \\
\hline 276. & $9 / 1 / 2004$ & 5:34:09 PM & $\begin{array}{l}25.633 \\
25625\end{array}$ & $\begin{array}{l}25.763 \\
25755\end{array}$ & 24.606 & \begin{tabular}{|l|l|}
26.821 \\
26.709
\end{tabular} & & 48. & 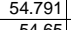 & \begin{tabular}{|l|l|l|l|}
17.677 \\
17556
\end{tabular} & $\begin{array}{l}31.616 \\
21.767\end{array}$ & 0.0 & & 51. & & & & & \begin{tabular}{|l|l|}
57.6797 \\
\end{tabular} & 53.7 & $\frac{12.2}{1.0}$ & 40.0 & 2.75 & 0.006 & & 500 & 0.163 & 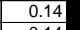 \\
\hline & 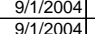 & $\begin{array}{l}5: 35: 09 \mathrm{PM} \\
\text { 5:36:09 PM }\end{array}$ & $\begin{array}{l}25.625 \\
25.637\end{array}$ & $\begin{array}{l}25.755 \\
25.772\end{array}$ & $\begin{aligned} 24.603 \\
24601\end{aligned}$ & $26.798 \mid$ & & & $\begin{array}{r}54.65 \\
55606\end{array}$ & $\begin{array}{r}17.556 \\
17.566\end{array}$ & & 0.0 & & & & & & & & $\begin{array}{l}53.5 \\
53.5\end{array}$ & $\frac{12.0}{116}$ & \begin{tabular}{|l|}
40.0 \\
39.9
\end{tabular} & & \begin{tabular}{|l|l}
0.007 \\
0.007
\end{tabular} & & & \begin{tabular}{l|l}
0.166 \\
0.167
\end{tabular} & \\
\hline 2772 & $\begin{array}{l}/ 1 / 1 / 2004 \\
\end{array}$ & 5:37:09 PM & 25.648 & 25.778 & 24.627 & \begin{tabular}{|l|}
26.800 \\
\end{tabular} & & & $\begin{array}{l}54.746 \\
547\end{array}$ & \begin{tabular}{|c|}
1.566 \\
17.244
\end{tabular} & $\begin{array}{l}31.517 \\
31.997\end{array}$ & $\begin{array}{r}0.03 \\
0.036\end{array}$ & & $\begin{array}{l}48.125 \\
50.819\end{array}$ & $\begin{array}{l}0.046 \\
0.046\end{array}$ & & & & & & $\frac{11 .}{12 .}$ & & & & & & 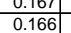 & \\
\hline & & & 25.654 & 25.784 & 24.627 & 26. & & & 55.174 & 17.366 & & & & & & & & & & 54.0 & 11. & & & & & & 0.158 & \\
\hline & $9 / 1 / 20$ & & 25.649 & 25.789 & & & & & & & & & & & & & & & & & 11. & & & & & & & \\
\hline & 9/1/2004 & & 25.664 & 25.8 & 24.648 & 26.772 & & & 54.527 & \begin{tabular}{|l|l|}
17.412 \\
\end{tabular} & & & & & & & & & & 53.4 & 11.9 & $4 \mathrm{C}$ & & & & & 6 & \\
\hline & $9 / 1 / 2004$ & & & $\frac{25.785}{25.811}$ & 24.614 & & & & 54.699 & & $\frac{32.1}{230}$ & & & & & & & & & & 11.4 & & & & & & 61 & \\
\hline & & & & & $\begin{array}{r}24.64 \\
24645\end{array}$ & & & & & & & & & & & & & & & & & & & & & & & \\
\hline & $9 / 1 / 200$ & & 25.676 & $\frac{20.001}{25.806}$ & 24.635 & & & & 54.899 & $\begin{array}{l}17.475 \\
17.538\end{array}$ & 31. & & & 50.6 & & & & & & \begin{tabular}{|l|l}
53.8 \\
5
\end{tabular} & 11. & & & & & & $\begin{array}{l}0.101 \\
0.162\end{array}$ & \\
\hline & & & 25.676 & 25.81 & 24.67 & & & & 55.035 & & & & & & & & & & & 53. & $\overline{11}$ & & & & & & & \\
\hline & $9 / 1 / 20$ & & & & & & & & & & & & & & & & & & & & & & & & & & & \\
\hline & & & & & 24.735 & & & & & & & & & & & & & & & & & & & & & & & \\
\hline & & & & & 24.135 & & & & 54.7. & 17. & & & & & & & & & & & & & & & & & & \\
\hline & & & & $\frac{25.8}{2582}$ & $\frac{24.11}{24.72}$ & & & & & & & & & & & & & & & & & & & & & & & \\
\hline & $9 / 1 / 26$ & 5.51 .1 & & 2581 & 24.68 & & & & 5581 & 1.13 & & & & & & & & & & & & & & & & & & \\
\hline & & & & & 24685 & & & & & & & & & & & & & & & & & & & & & & & \\
\hline & & & & & & & & & & & & & & & & & & & & & & & & & & & & \\
\hline & & & & 25.826 & 24.665 & & & & & & $32.1 \mathrm{C}$ & 0.1 & & & & & & & & & & & & & & & & \\
\hline & & & & & 24.68 & & & & & & & & & & & & & & & & & & & & & & & \\
\hline & & 5:56: & & 25.8 & 24.665 & 26.6 & & & 55.042 & 17.3 & & & & & & & & & & & & & & & & & & \\
\hline & $9 / 1 / 20$ & & & 25.8 & 24.64 & & & 47 & 54.15 & & & 0. & & & & & & & & & & & & & & & & \\
\hline & & 5:58: & & 25.82 & 24.66 & 26. & & 48.4 & 54. & & & 0.1 & & & & & & & & & & & & & & & & \\
\hline & 9/1/20 & & & 25.8 & 24.651 & & & & 54.959 & & & 0.1 & & & & & & & & & & & & & & & & \\
\hline & & & & & & & & & & & & & & & & & & & & & & & & & & & & \\
\hline & & & & 25.839 & 24.648 & 26.607 & & & 54.865 & 17.2 & & & & & & & & & & & & & & & & & & \\
\hline & $\begin{array}{lll}91120 \\
01200\end{array}$ & $6: 02:$ & 570 & 25.835 & 24.638 & 26.593 & & 48.6. & 55.1314 & $1.37 \mathrm{~s}$ & $\frac{32.01}{3020}$ & 0.1 & & & & 14.6 & & & & & . & & & & & & & \\
\hline & & & & & & & & & & & & 01 & & & & & & & & & & & & & & & & \\
\hline & & & & 2583 & 24.64 & & & & 54799 & 17135 & 266 & & & 8035 & $0.044 \mathrm{~T}$ & 14643 & & & & & & & & & & & 158 & \\
\hline & & & i.7: & & & & & 48.322| & & & & & & & & & & & & & & & & & & & & \\
\hline
\end{tabular}


WSRC-TR-2005-00105, REVISION 0

SRNL-RPP-2005-00012, REVISION 0

RUN \# 4.03A AND B; FIRST AND SECOND HALF OF SLURRY DEWATERING - CONT.

\begin{tabular}{|c|c|c|c|c|c|c|c|c|c|c|c|c|c|c|c|c|c|c|c|c|c|c|c|c|c|c|c|c|}
\hline & A & B & D & $E$ & $F$ & G & $\mathrm{H}$ & $\mathrm{J}$ & $\mathrm{K}$ & $\mathrm{L}$ & $\mathrm{M}$ & $\mathrm{N}$ & 0 & 0 & $R$ & $\mathrm{~s}$ & T & $\mathrm{V}$ & w & $x$ & $Y_{1}$ & $z_{2}$ & & & & & & \\
\hline & 9/1/2004 & 6:06:09 PM & 25.725 & 25.825 & 24.633 & 26.593 & 25.66 & 48.693 & 55.11 & 17.256 & 32.385 & 0.14 & -5.867 & 48.98 & 0.046 & 14.643 & 0.003 & 3492.78 & 58.2131 & 54.0 & 11.7 & 2 & 2.795 & 0.007 & 0.007 & 0.000 & $\begin{array}{l}\frac{A E}{0.164} \\
\end{array}$ & \\
\hline & $\begin{array}{l}9 / 1 / 12004 \\
\end{array}$ & 6:07:09 PM & $\begin{array}{r}25.72 \\
\end{array}$ & 25.825 & 24.613 & 26.568 & 25.719 & 48.726 & \begin{tabular}{|l|l|}
55.348 \\
\end{tabular} & $\begin{array}{l}16.828 \\
\end{array}$ & 32.777 & 0.146 & $\begin{array}{l}-5.87 \\
\end{array}$ & 49.03 & 0.045 & $\begin{array}{l}14.643 \\
\end{array}$ & 0.003 & 3493.78 & & 54.2 & 11.7 & & & .006 & & & & \\
\hline & $9 / 1 / 2004$ & 6:08:09 PM & $\frac{25.724}{25.724}$ & 25.824 & \begin{tabular}{|l|}
24.643 \\
261 \\
\end{tabular} & 26.557 & 25.649 & \begin{tabular}{|l|}
48.689 \\
8.969
\end{tabular} & \begin{tabular}{|l|}
54.961 \\
5.525
\end{tabular} & 17.094 & 32.654 & 0.204 & $\begin{array}{l}-5.873 \\
-5.893\end{array}$ & 49.261 & 0.045 & 14.643 & 0.003 & 3494.78 & 58.2464 & 53.8 & 11.7 & & 8.804 & 0.006 & 0.007 & 0.000 & 0.160 & \\
\hline 804 & 9/1/2004 & 6:09:09 PM & 25.724 & 25.829 & \begin{tabular}{|l|l|}
24.618 \\
\end{tabular} & 26.562 & 25.694 & $\begin{array}{l}48.758 \\
\end{array}$ & \begin{tabular}{|l|}
55.236 \\
\end{tabular} & $\begin{array}{r}16.9 \\
\end{array}$ & 32.716 & $\begin{array}{l}0.149 \\
\end{array}$ & $\begin{array}{l}-5.867 \\
\end{array}$ & 48.963 & $\begin{array}{l}0.045 \\
\end{array}$ & 14.643 & 0.003 & 495.78 & \begin{tabular}{|l|l|}
58.2631 \\
\end{tabular} & \begin{tabular}{|l|l|}
54.1 \\
\end{tabular} & 11.7 & 40.7 & 809 & 0.006 & $\begin{array}{l}0.007 \\
\end{array}$ & 0.000 & 0.160 & \\
\hline & 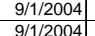 & 6:10:09 PM & $\frac{25.713}{25719}$ & $\frac{25.828}{25.829}$ & & \begin{tabular}{|l|l|}
26.516 \\
26542
\end{tabular} & $\begin{array}{l}25.678 \\
25694\end{array}$ & \begin{tabular}{|l|l|}
48.886 \\
48811
\end{tabular} & & & \begin{tabular}{|l|}
32.638 \\
32732
\end{tabular} & $\begin{array}{l}0.16 \\
0201\end{array}$ & $\begin{array}{r}-5.859 \\
5.839\end{array}$ & $\begin{array}{l}50.035 \\
47989\end{array}$ & \begin{tabular}{|c|c|}
0.045 \\
0048
\end{tabular} & \begin{tabular}{|l|}
14.643 \\
1.4632 \\
\end{tabular} & & 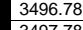 & & \begin{tabular}{|l|}
54.2 \\
\end{tabular} & \begin{tabular}{|l|l|}
11.9 \\
\end{tabular} & & 2.810 & & & & & \\
\hline 2807 & $\begin{array}{l}9 / 1 / 2004 \\
9 / 1 / 2004\end{array}$ & 6:11:09 PM & $\frac{25 . / 19}{25.668}$ & $\begin{array}{l}25.829 \\
25.823\end{array}$ & $\frac{24.623}{24.612}$ & \begin{tabular}{|l|}
26.542 \\
26.576
\end{tabular} & $\begin{array}{l}25.694 \\
25.673 \\
\end{array}$ & \begin{tabular}{|l|l|}
48.811 \\
5.033 \\
\end{tabular} & \begin{tabular}{|l|l|}
55.151 \\
55.185
\end{tabular} & \begin{tabular}{|c|}
17.156 \\
17.193
\end{tabular} & $\begin{array}{r}32.732 \\
34.934 \\
\end{array}$ & \begin{tabular}{|r|}
0.204 \\
-1.212 \\
\end{tabular} & $\begin{array}{l}-5.839 \\
-4.948\end{array}$ & $\begin{array}{r}47.989 \\
49.58\end{array}$ & $\begin{array}{l}0.048 \\
0.009\end{array}$ & $\begin{array}{r}\mid 14.643 \\
14.643\end{array}$ & $\begin{array}{l}0.003 \\
0.003\end{array}$ & \begin{tabular}{|l}
34979.78 \\
3498.78
\end{tabular} & \begin{tabular}{|c|}
58.2964 \\
3
\end{tabular} & \begin{tabular}{|l|}
54.0 \\
54.1
\end{tabular} & $\frac{11.4}{11.8}$ & $\begin{array}{l}40.8 \\
43.0 \\
\end{array}$ & 2.8111 & 0.007 & $\begin{array}{l}0.007 \\
0.001\end{array}$ & $\begin{array}{l}0.000 \\
0.000\end{array}$ & \begin{tabular}{l|l|l|l|l|}
0.170 \\
0.030
\end{tabular} & 0.14 \\
\hline \begin{tabular}{|l|l|}
2808 \\
\end{tabular} & $9 / 1 / 2004$ & 6:13:09 PM & 25.703 & 25.823 & $\frac{24.012}{24.627}$ & \begin{tabular}{|l|}
20.510 \\
26.516 \\
\end{tabular} & 25.673 & & \begin{tabular}{|l|l|}
55.543 \\
\end{tabular} & & 32.88 & \begin{tabular}{|c|} 
\\
\end{tabular} & -3.297 & $\begin{array}{r}48.928 \\
48.921\end{array}$ & 0.033 & \begin{tabular}{|l|l}
14.645 \\
14.643
\end{tabular} & $\begin{array}{l}0.003 \\
0.003\end{array}$ & $\frac{5498.18}{3499.78}$ & \begin{tabular}{|l|}
58.3131 \\
58.3297
\end{tabular} & \begin{tabular}{|l|}
54.1 \\
54.4 \\
\end{tabular} & $\begin{array}{l}11.8 \\
11.7\end{array}$ & & $\frac{2.964}{2.836}$ & $\frac{0.001}{0.005}$ & $\frac{0.001}{0.005}$ & $\begin{array}{l}0.000 \\
0.000\end{array}$ & & $\frac{0.03}{0.10}$ \\
\hline & & 6:14:09 PM & 25.723 & 25.823 & 24.612 & & 25.663 & 48.278 & & 17.546 & 31.642 & 1.269 & -0.69 & 49.253 & & 14.643 & & & 358.3464 & 54.6 & 11.7 & & & & & & & 0 \\
\hline & 9/1/2004 & 6:15:09 PM & & 25.828 & & & & 50.093 & 55.276 & \begin{tabular}{|l|l|}
17.943 \\
\end{tabular} & & -1.209 & -5.414 & 49.841 & $\begin{array}{l}0.044 \\
\end{array}$ & \begin{tabular}{|l|l|}
14.643 \\
\end{tabular} & 0.004 & & & 54.2 & & & 2.864 & & 0.006 & 0.000 & & \\
\hline 2811 & & & 25.717 & & 24.741 & 26.53 & & & & & 33.467 & & & & & $\begin{array}{l}14.643 \\
\end{array}$ & & & & & & & & .007 & & & & \\
\hline 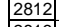 & & & & 25.812 & & & & 50.413 & 55.286 & 17.124 & & & & & & 14.643 & & & & 54.2 & & & & & & & & \\
\hline & & $: 09 \mathrm{PM}$ & & 25.811 & 24.7 & 26.574 & & & & 17519 & . 407 & -1.2 & & & & 14.643 & & & & 53.5 & & & & & & & 166 & \\
\hline & & 6:19:09 PM & & 25.805 & 24.664 & \begin{tabular}{|l|}
26.508 \\
\end{tabular} & & 50.475 & 55.054 & \begin{tabular}{|l|l|l|l|l}
17.603 \\
\end{tabular} & 33.885 & -1.212 & -6.399 & & 0.048 & \begin{tabular}{|l|l|l|l|}
14.643 \\
\end{tabular} & & & & & & & 2.908 & 0.007 & 0.007 & 0.000 & 0.164 & \\
\hline & $1 / 2004$ & 6:20:09 PM & 25.73 & 25.805 & 24.674 & \begin{tabular}{|l|}
26.448 \\
\end{tabular} & 25.615 & 50.33 & 55.315 & \begin{tabular}{|l|l}
17.378 \\
\end{tabular} & \begin{tabular}{|l|l|}
33.904 \\
\end{tabular} & -1.212 & -6.394 & 49.084 & 0.048 & 14.643 & 0.003 & 3506.78 & 38.4464 & \begin{tabular}{|r|}
54.2 \\
\end{tabular} & 11.7 & & & & & & & \\
\hline & & 6:21:09 PM & 25.734 & & 24.658 & \begin{tabular}{|l|l|}
26.462 \\
05
\end{tabular} & 25.619 & \begin{tabular}{|l|l|}
50.431 \\
577
\end{tabular} & $\begin{array}{l}55.222 \\
50202\end{array}$ & $\begin{array}{l}17.531 \\
7700\end{array}$ & & & & 49.43 & \begin{tabular}{|c|}
0.049 \\
\end{tabular} & \begin{tabular}{ll|}
14.643 \\
\end{tabular} & 0.004 & & 58.4631 & $\begin{array}{l}54.1 \\
5.1\end{array}$ & & & & & & & & \\
\hline & 1/2004 & 6:22:09 PM & 25.733 & & & & & & & & & & -6.408 & 49.345 & & $\begin{array}{l}14.643 \\
\end{array}$ & & 3508.78 & 358.4797 & 54.1 & & & & 0.007 & & & 0.164 & \\
\hline & & 6:23:09 PM & $\begin{array}{l}25.737 \\
25731\end{array}$ & $\begin{array}{l}25.797 \\
25707\end{array}$ & $\begin{array}{r}24.626 \\
216\end{array}$ & 26.445 & $\begin{array}{l}25.617 \\
2506\end{array}$ & & & & $\begin{array}{l}33.793 \\
2.102\end{array}$ & & & 49.203 & & $\begin{array}{l}14.643 \\
\end{array}$ & 0.003 & & 58.4964 & 54.0 & 11.7 & & 897 & 0.007 & & 0.000 & & \\
\hline & $\begin{array}{l}9 / 1 / 2004 \\
9 / 1 / 2004\end{array}$ & $\begin{array}{ll}6: 24: 09 \mathrm{PM} \\
6.25 .09 \mathrm{PM}\end{array}$ & $\begin{array}{l}25.731 \\
25.751\end{array}$ & $\begin{array}{l}25.797 \\
25811\end{array}$ & $\begin{array}{r}24.6 \\
24619\end{array}$ & \begin{tabular}{|l|}
26.499 \\
26.494 \\
\end{tabular} & $\begin{array}{r}25.596 \\
25.65\end{array}$ & & 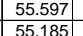 & $\begin{array}{l}17.421 \\
17.395\end{array}$ & $\begin{array}{r}34.103 \\
34.109\end{array}$ & \begin{tabular}{l|l|l|}
-1.212 \\
-1.209
\end{tabular} & $\begin{array}{l}-6.414 \\
-6.396\end{array}$ & $\begin{array}{l}49.716 \\
47883\end{array}$ & 048 & $\begin{array}{l}14.643 \\
14643 \\
\end{array}$ & & & 58.5131 & $\begin{array}{l}54.5 \\
541\end{array}$ & 11.8 & & & 0.007 & 0.007 & 00 & 0.164 & 0.14 \\
\hline & $\begin{array}{l}9 / 1 / 12004 \\
9 / 1 / 2004\end{array}$ & 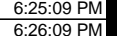 & $\frac{25.751}{25.728}$ & $\begin{array}{l}25.811 \\
25.784\end{array}$ & $\begin{array}{l}24.619 \\
24.577\end{array}$ & \begin{tabular}{|l|}
26.494 \\
26.476
\end{tabular} & $\begin{array}{r}25.65 \\
25.623 \\
\end{array}$ & $\begin{array}{r}50.475 \\
50.33 \\
\end{array}$ & \begin{tabular}{|r|}
55.185 \\
55.224 \\
\end{tabular} & $\begin{array}{l}17.395 \\
16.888\end{array}$ & $\begin{array}{l}34.109 \\
34.505 \\
\end{array}$ & $\begin{array}{l}-1.209 \\
-1.212\end{array}$ & $\begin{array}{l}-6.396 \\
-6.405 \\
\end{array}$ & $\begin{array}{l}47.883 \\
47.493 \\
\end{array}$ & $\begin{array}{l}049 \\
049 \\
\end{array}$ & $\begin{array}{l}\frac{14.643}{14.643} \\
\end{array}$ & $\begin{array}{l}0.003 \\
0.003\end{array}$ & & 8.5297| & $\begin{array}{l}54.1 \\
54.1\end{array}$ & $\frac{11.4}{11.3}$ & & |. 2.916 & 0.007 & 0.007 & 0.000 & & 0.14 \\
\hline 282 & 9/1/2004 & 6:27:09 PM & 25.733 & 25.794 & \begin{tabular}{|l|}
24.5717 \\
24.562 \\
\end{tabular} & \begin{tabular}{|l|}
20.410 \\
26.536 \\
\end{tabular} & 25.0258 & \begin{tabular}{|l}
50.493 \\
\end{tabular} & \begin{tabular}{|l|}
55.429 \\
\end{tabular} & 17.285 & 34.216 & -1.212 & $\begin{array}{l}-6.405 \\
-6.408\end{array}$ & $\begin{array}{l}4.495 \\
48.754\end{array}$ & $\begin{array}{l}0.049 \\
0.048\end{array}$ & $\begin{array}{l}14.045 \\
14.643\end{array}$ & $\begin{array}{l}0.004 \\
0.004\end{array}$ & $\frac{3512.16}{3513.78}$ & \begin{tabular}{|l|}
58.5404 \\
3
\end{tabular} & $\begin{array}{l}54.1 \\
54.3\end{array}$ & $\begin{array}{l}11.3 \\
11.6\end{array}$ & $\begin{array}{l}42.4 \\
42.4 \\
\end{array}$ & $\frac{2.920}{2.920}$ & 0.007 & 0.007 & 0.000 & $\begin{array}{l}0.167 \\
0.164\end{array}$ & \\
\hline & 9/1/2004 & 6:28:09 PM & 25.728 & 25.784 & 24.567 & 26.566 & & 50.659 & \begin{tabular}{|l|}
55.578 \\
\end{tabular} & 17.379 & 34.249 & -1.212 & & 49.618 & 0.048 & 14.643 & & 3514.78 & \begin{tabular}{|l|l|}
3 & 58.5797 \\
\end{tabular} & 54.5 & 11.8 & 42.5 & 2.927 & 0.007 & 0.007 & 0.000 & 0.164 & \\
\hline & & 6:29:09 PM & 25.739 & 25.799 & 24.578 & & & & \begin{tabular}{|l|l|}
55.396 \\
\end{tabular} & \begin{tabular}{|l|l|}
17.247 \\
\end{tabular} & 34.304 & & & & 0.048 & \begin{tabular}{|l|l|}
14.643 \\
\end{tabular} & & & 58.5964 & 54.3 & $11 .$. & 42. & & 0.007 & & 0.000 & 0.164 & \\
\hline & & 6:30:09 PM & 25.746 & 25.806 & & \begin{tabular}{|l|l|}
26.704 \\
\end{tabular} & & & 55.524 & 17.496 & 33.904 & -1.209 & & & & $\begin{array}{l}14.643 \\
\end{array}$ & & & 58.6131 & 54.4 & & & 2.911 & 0.007 & 0.007 & & & \\
\hline$\frac{2826}{2827}$ & $\begin{array}{l}9 / 1 / 2004 \\
9 / 1 / 2004\end{array}$ & 6:31:09 PM & $\begin{array}{l}25.728 \\
25.735\end{array}$ & \begin{tabular}{r|r}
25.803 \\
25.82
\end{tabular} & $\begin{array}{r}24.551 \\
24.544\end{array}$ & \begin{tabular}{|l|}
26.771 \\
26.828 \\
\end{tabular} & $\begin{array}{r}25.798 \\
25.825 \\
\end{array}$ & \begin{tabular}{|l|}
50.298 \\
50.091
\end{tabular} & \begin{tabular}{|l|}
55.224 \\
5.471
\end{tabular} & $\begin{array}{r}17.272 \\
16.96\end{array}$ & $\begin{array}{l}34.084 \\
34.166\end{array}$ & $\begin{array}{r}-1.215 \\
-1.215\end{array}$ & $\begin{array}{l}-6.405 \\
-6.396 \\
-\end{array}$ & $\begin{array}{r}48.88 \\
47.776 \\
\end{array}$ & $\begin{array}{l}.048 \\
0.047\end{array}$ & $\begin{array}{l}14.643 \\
14.643\end{array}$ & $\begin{array}{l}0.003 \\
0.003\end{array}$ & $\begin{array}{l}3517.78 \\
3318.78\end{array}$ & $\begin{array}{l}358.6297 \\
3 \\
3\end{array}$ & $\begin{array}{l}54.1 \\
5.6\end{array}$ & $\frac{11.6}{11.4}$ & & $\begin{array}{l}.909 \\
.905 \\
905\end{array}$ & 0.007 & 0.007 & 0.000 & 0.165 & 0.14 \\
\hline 2828 & $\begin{array}{l}91 / 1 / 2004 \\
9 / 12004\end{array}$ & $\begin{array}{l}6.35 .09 \mathrm{P} \\
6: 33: 09 \mathrm{PM}\end{array}$ & $\begin{array}{l}25.135 \\
25.737\end{array}$ & $\begin{array}{l}25.828 \\
25.828\end{array}$ & $\begin{array}{l}24.544 \\
24.556\end{array}$ & \begin{tabular}{|r|}
20.828 \\
\end{tabular} & & $\mid$\begin{tabular}{|c|c|c|}
50.446 \\
\end{tabular} & \begin{tabular}{|l|}
$\mid 54.4141$ \\
55.348
\end{tabular} & $\begin{array}{l}17.336 \\
17\end{array}$ & \begin{tabular}{|l|}
34.106 \\
34.302 \\
\end{tabular} & $\frac{-1.215}{-1.212}$ & & $\begin{array}{l}47.1 / 67 \\
47.407\end{array}$ & $\begin{array}{l}0.047 \\
0.047 \\
\end{array}$ & $\begin{array}{l}\frac{14.645}{14.643} \\
\end{array}$ & & & & $\begin{array}{l}53.6 \\
54.2\end{array}$ & & & & $\begin{array}{l}0.007 \\
0.007\end{array}$ & $\frac{0.007}{0.007}$ & & & 0.14 \\
\hline 2829 & $9 / 1 / 2004$ & 6:34:09 PM & 25.745 & 25.835 & \begin{tabular}{|l}
24.563 \\
\end{tabular} & \begin{tabular}{|l|}
26.933 \\
\end{tabular} & 25.999 & 50.334 & \begin{tabular}{|l|}
55.18 \\
\end{tabular} & 17.361 & $\begin{array}{l}4.025 \\
\end{array}$ & -1.212 & -6.405 & 48.974 & & 14.643 & 0.003 & 3520.78 & 58.6797 & \begin{tabular}{|l|}
54.1 \\
\end{tabular} & \begin{tabular}{|l|l|}
11.7 \\
\end{tabular} & & & & & & $\begin{array}{l}0.161 \\
0.162 \\
\end{array}$ & $\frac{0.14}{0.14}$ \\
\hline & & & & 25.837 & \begin{tabular}{|l|}
24.525 \\
\end{tabular} & \begin{tabular}{|r|}
26.96 \\
\end{tabular} & & \begin{tabular}{|l|l|}
50.411 \\
\end{tabular} & \begin{tabular}{|l|}
55.242 \\
\end{tabular} & $\begin{array}{l}17.076 \\
\end{array}$ & 34.308 & & & & & \begin{tabular}{|l|}
14.643 \\
\end{tabular} & & & & 54.1 & 11.6 & & & & & & 0.164 & \\
\hline & & 6:36:09 PM & 25.733 & 25.843 & & & 26.01 & & $\begin{array}{r}5.5 \\
\end{array}$ & \begin{tabular}{|l|l|}
17.103 \\
\end{tabular} & $\begin{array}{l}34.563 \\
2.1\end{array}$ & & & & & $\begin{array}{l}14.643 \\
\end{array}$ & & & & 54.4 & 11.7 & & & & & & 0.160 & \\
\hline & & 6:37:09 PM & 25.718 & $\begin{array}{l}25.829 \\
25.919\end{array}$ & \begin{tabular}{|l|}
24.492 \\
2.523 \\
\end{tabular} & \begin{tabular}{|l|l|}
26.861 \\
\end{tabular} & $\begin{array}{l}25.928 \\
5.500\end{array}$ & & \begin{tabular}{|l|l|}
55.628 \\
55122 \\
\end{tabular} & $\begin{array}{l}17.232 \\
17005\end{array}$ & & \begin{tabular}{|l|l|}
-1.218 \\
\end{tabular} & & & & & & & & 54.5 & \begin{tabular}{|l|l|}
11.6 \\
\end{tabular} & & 933 & 0.007 & 0.007 & & 0.160 & \\
\hline$\frac{2833}{2834}$ & $\frac{9 / 1 / 2004}{9 / 1 / 2004}$ & $\begin{array}{ll}6: 38: 09 \mathrm{PM} \\
6: 39009 \mathrm{PM}\end{array}$ & $\begin{array}{r}25.729 \\
25.72\end{array}$ & $\begin{array}{l}25.849 \\
25.85 \\
\end{array}$ & \begin{tabular}{|l|}
24.523 \\
24.58 \\
\end{tabular} & \begin{tabular}{|l|}
26.857 \\
26.778
\end{tabular} & $\begin{array}{l}25.959 \\
25.895 \\
\end{array}$ & \begin{tabular}{|l|}
50.394 \\
50.055
\end{tabular} & \begin{tabular}{|l|}
55.122 \\
55611 \\
\end{tabular} & $\begin{array}{r}17.095 \\
16.971\end{array}$ & $\begin{array}{r}34.22 \\
34.599 \\
\end{array}$ & \begin{tabular}{|l|}
-1.212 \\
-1.212 \\
\end{tabular} & $\begin{array}{l}-6.405 \\
-6.408\end{array}$ & $\begin{array}{l}49.038 \\
50.073 \\
\end{array}$ & $\begin{array}{l}0.047 \\
0.047\end{array}$ & $\frac{14.643}{14.633}$ & $\begin{array}{l}0.003 \\
0.004\end{array}$ & .78 & $\begin{array}{l}358.7 \\
358.7\end{array}$ & $\begin{array}{l}54.0 \\
55.5 \\
\end{array}$ & $\begin{array}{l}11.7 \\
11.9\end{array}$ & & 2.917 & 0.007 & 0.007 & 000 & $\frac{0.161}{0.160}$ & 0.14 \\
\hline 2835 & 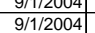 & $\begin{array}{l}6.5909 \mathrm{PM} \\
6: 40: 09 \mathrm{PM}\end{array}$ & $\begin{array}{l}25 . / 24 \\
25.71\end{array}$ & $\begin{array}{l}25.855 \\
25.845\end{array}$ & \begin{tabular}{|l|}
24.508 \\
24.478 \\
\end{tabular} & \begin{tabular}{|l|}
26.1788 \\
26.768
\end{tabular} & 25.885 & \begin{tabular}{|l|}
51.012 \\
5100
\end{tabular} & \begin{tabular}{|c|}
55.9611 \\
5566
\end{tabular} & 17.08 & $\begin{array}{l}34.599 \\
34.998\end{array}$ & $\frac{-1.212}{-1.215}$ & & $\frac{50.0 / 3}{49.001}$ & & $\begin{array}{l}\frac{14.643}{14.643} \\
\end{array}$ & & & & $\begin{array}{l}54.5 \\
54.8\end{array}$ & & & 2.965 & & & & & 0.14 \\
\hline 2836 & & 6:41:09 PM & 25.731 & 25.876 & \begin{tabular}{|l|}
24.519 \\
\end{tabular} & \begin{tabular}{|l|}
26.744 \\
\end{tabular} & 25.86 & & \begin{tabular}{|l|}
55.398 \\
\end{tabular} & 17.046 & 34.565 & -1.21 & $\frac{-.40}{-6.40}$ & 48.5 & & $\begin{array}{l}14.045 \\
14.643\end{array}$ & & & & $\begin{array}{l}54.8 \\
54.3\end{array}$ & $\frac{11.7}{11.6}$ & & & & & & $\frac{159}{164}$ & $\frac{0.14}{0.14}$ \\
\hline & & & 25.715 & 25.865 & 24.4 & & & 50.632 & \begin{tabular}{|l|l|}
55.498 \\
\end{tabular} & 16.909 & 34.61 & -1.212 & & 48. & 0.048 & 14.6 & & & & 54.4 & 11.5 & & & & & & & $\begin{array}{l}0.14 \\
0.14\end{array}$ \\
\hline & & 6:43:0 & 25.704 & 25.854 & & & 25. & & \begin{tabular}{|l|}
55.589 \\
\end{tabular} & & & -1.219 & & & & 14. & & & & 54.5 & 11.3 & & & & & & & $\frac{0.14}{0.14}$ \\
\hline & & & 25.703 & 25.864 & 24.4 & & & & \begin{tabular}{|l|}
55.97 \\
\end{tabular} & 17.015 & 34. & -1.21 & & & & & & & & 54.8 & 11.6 & & & & & & 0.159 & \\
\hline & & & & 25.87 & & & & & & & & & & & & & & & & 54.6 & & & & & & & & \\
\hline & & & & 25.861 & 24.495 & & & & $\mid 55.79$ & 17.018 & 34.893 & -1.21 & & & & & & & & 54.7 & 11. & & & & & & 59 & 014 \\
\hline & 9/1/2004 & & 694 & 25.85 & 24.472 & \begin{tabular}{|l|}
26.547 \\
\end{tabular} & & & 55.96 & 17.089 & 34. & -1.2 & & & & \begin{tabular}{|l|l|}
14.643 \\
\end{tabular} & & & & 54.8 & 11. & & & & 0.0 & & 0.159 & \\
\hline & & & & & 24.4 & & & & \begin{tabular}{|l|}
56.248 \\
\end{tabular} & 17.317 & & & & & & 14. & & & & 55.1 & 11. & & & & & & & \\
\hline & & & 25.694 & 25.864 & \begin{tabular}{|l|}
24.482 \\
\end{tabular} & & & & \begin{tabular}{|l|}
55.272 \\
\end{tabular} & 16.748 & 34.716 & -1.2. & & & & & & & & 54.1 & $11.4 \mathrm{r}$ & & & & 0.007 & & & \\
\hline & & & & 25.85 & & & & & & & & & & & & & & & & Th & & & & & & & & \\
\hline & & & & & & & & & & & & & & & & & & & & & & & & & & & & \\
\hline & & & 687 & $\frac{25.85}{25.85}$ & $\frac{24.476}{24.48}$ & 26.475 & & $\frac{51.201}{50803}$ & $\frac{56.001}{55665}$ & $\begin{array}{r}17.17 \\
16.993\end{array}$ & $\begin{array}{l}35.059 \\
34809\end{array}$ & $\frac{-1.21}{-1.21}$ & & & & $\frac{14 .}{14 .}$ & & & & 势, & 11 & & & & & & 56 & \\
\hline & & & & 2583 & 24.509 & & & & 5391 & & 31.154 & -12 & & & & & & & & & & & & & & & & \\
\hline & & & & & 24.504 & & & & & & & -121 & & & & & & & & $J 2.0$ & & & & & & & & \\
\hline & & & 25.659 & & 24.538 & & & & $\mid$\begin{tabular}{|l|l}
54.799 \\
\end{tabular} & 18.979 & 31. & $\frac{-1.21}{-1.21}$ & & & & & & & & 53.7 & 12.4 & & & & & & 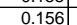 & \\
\hline & & & & & & & & & & & & & & & & & & & & & & & & & & & & \\
\hline & & & & & 24.589 & & & & & 18.9 & & -1.2 & & 52.3 & & & & & & & & & & & & & & \\
\hline & & & & & & & & & & & & & & & & & & & & & & & & & & & & \\
\hline & & & 25.664 & 25.80 & 24.597 & 26.452 & & & \begin{tabular}{|l|l|}
54.484 \\
\end{tabular} & & & -1.21 & & & & & & & & & 11.6 & & & & & & & \\
\hline & & & & 25. & 24.62 & 26.4 & & & 54.27. & 18. & 31.868 & -1.2 & & 48. & & 14.6 & & & & & & & & & & & & \\
\hline & & & & & & 26. & & & & & & & & & & 14. & & & & & & & & & & & & \\
\hline & & & & 25.79 & 24.639 & 26.3 & & & \begin{tabular}{|l|l|} 
& 54.722 \\
\end{tabular} & 18.462 & 32.356 & -1.2. & & & & & & & & 53.6 & 12.1 & & & & & & 158 & \\
\hline & & & & & & & & & & & & -1. & & & & & & & & & & & & & & & & \\
\hline & & & & & & 26. & & & & & & & & & & & & & & & & & & & & & & \\
\hline & & & & $\frac{23.18}{2579}$ & 24.104 & 2 & & & 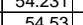 & 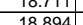 & 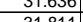 & -1.2 & & & & & & & & & & & & & & & & \\
\hline & & & & 20.578 & 24736 & $\frac{20.406}{26,416}$ & & & 543 & 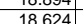 & $\frac{31.0}{310}$ & $\frac{-1.4}{-12}$ & & 4974 & & 146 & & & & & & & & & & & & \\
\hline & & & & 2578 & & & & & & & 31.85 & -12 & & 512 & & 146 & & & & & & & & & & & & \\
\hline & & & & 25.78 & 24.733 & 264 & & & & & & $\frac{-1.2}{-1.2}$ & & & & 14.6 & & & & & & & & & & & & \\
\hline & & & & & 24.7 & & & & & & & & & & & & & & & & & & & & & & & \\
\hline & & & & & 247 & & & & & & & & & & & & & & & & & & & & & & & \\
\hline & & & & 258 & 24.755 & 26.6 & & 49. & & 18.389 & 32.159 & -1.212 & & 51.9 & & & & & & 53.4 & 1 & & & & & & & \\
\hline & & & & & 2474 & & & 49 & & & & & & & & & & & & & & & & & & & & \\
\hline 870 & $9 / 1 / 2$ & 7:15:09 PM & 25.714 & 25.815 & \begin{tabular}{|l|l|l}
24.733 \\
\end{tabular} & \begin{tabular}{|l|l|}
26.667 \\
\end{tabular} & & 49.492 & \begin{tabular}{|l|l|}
54.469 \\
\end{tabular} & $\mid$\begin{tabular}{|c|}
18.298 \\
\end{tabular} & 32.192 & \begin{tabular}{|l|l|} 
& -1.212 \\
\end{tabular} & & 50.467 & 0.044 & 14.643 & & & 59.3631 & 53.3 & $12.0 \mathrm{C}$ & & 2.816 & 0.006 & 0.006 & 0.000 & o.155 & 0.1 \\
\hline
\end{tabular}


WSRC-TR-2005-00105, REVISION 0

SRNL-RPP-2005-00012, REVISION 0

RUN \# 4.03A AND B; FIRST AND SECOND HALF OF SLURRY DEWATERING - CONT.

\begin{tabular}{|c|c|c|c|c|c|c|c|c|c|c|c|c|c|c|c|c|c|c|c|c|c|c|c|c|c|c|c|c|}
\hline & $\mathrm{A}$ & B & $\mathrm{D}$ & $E$ & $\mathrm{~F}$ & G & $\mathrm{H}$ & $\mathrm{J}$ & \begin{tabular}{|l|}
$\mathrm{K}$ \\
\end{tabular} & $\mathrm{L}$ & \begin{tabular}{l|l|}
$\mathrm{M}$ \\
\end{tabular} & $\mathrm{N}$ & 0 & \begin{tabular}{l|l|}
$Q^{2}$ & \\
\end{tabular} & $\mathrm{R}$ & $\mathrm{s}$ & $\mathrm{T}$ & $\mathrm{V}$ & w & $x$ & $\mathrm{Y}$ & Z & $\mathrm{AA}$ & $\mathrm{AB}$ & $\mathrm{AC}$ & $A D$ & $\mathrm{AE}$ & \\
\hline & 9/1/2004 & $\begin{array}{lll}7: 16: 09 \\
71: 0 M\end{array}$ & $\frac{25.726}{2.73}$ & 25.826 & 24.74 & 26.699 & 25.671 & 49.268 & \begin{tabular}{|l|}
54.302 \\
\end{tabular} & 18.444 & \begin{tabular}{|l|}
31.743 \\
197
\end{tabular} & -1.215 & -6.405 & 50.53 & 0.044 & 14.643 & 0.003 & 3562.7 & 59.3797 & 53.2 & 12. & 40.5 & 2.793 & 0.006 & 0.006 & 0.000 & 0.157 & \\
\hline & & & & 25.843 & & & & $\begin{array}{l}49.407 \\
\end{array}$ & & & 31.687 & \begin{tabular}{|l|}
-1.212 \\
\end{tabular} & & & & 14.643 & & & & & & & & & & 0.000 & & \\
\hline & 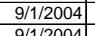 & 7:18:09 PM & \begin{tabular}{r|}
25.724 \\
2571
\end{tabular} & \begin{tabular}{|l|l|}
25.829 \\
25846
\end{tabular} & $\begin{array}{l}24.713 \\
24.734\end{array}$ & \begin{tabular}{|l|l|}
26.742 \\
26728
\end{tabular} & 25.799 & \begin{tabular}{|l|}
49.481 \\
49112 \\
\end{tabular} & 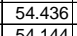 & \begin{tabular}{|l|l|}
18.511 \\
1817
\end{tabular} & \begin{tabular}{|l|}
32.012 \\
3281
\end{tabular} & \begin{tabular}{|l|l|}
-1.215 \\
\end{tabular} & \begin{tabular}{|l|l|}
-6.405 \\
6399
\end{tabular} & $\begin{array}{l}54.357 \\
5.30206\end{array}$ & 0.044 & $\begin{array}{l}14.643 \\
1.63\end{array}$ & $\begin{array}{l}0.003 \\
0.023\end{array}$ & 3564.78 & \begin{tabular}{|l|}
59.4131 \\
50207
\end{tabular} & \begin{tabular}{|l|l|}
53.3 \\
\end{tabular} & 12.9 & 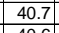 & & 0.006 & 0.006 & 0.000 & 0.156 & \\
\hline 875 & $\begin{array}{c}9 / 1 / 2004 \\
9 / 1 / 2004\end{array}$ & $\begin{array}{l}7: 19: 09 \mathrm{PM} \\
7 \cdot 20.09 \mathrm{PM}\end{array}$ & $\begin{array}{r}25.74 \\
25.725\end{array}$ & $\begin{array}{l}25.846 \\
25841\end{array}$ & $\begin{array}{l}24.734 \\
24719\end{array}$ & \begin{tabular}{|l|}
26.728 \\
26683
\end{tabular} & 25655 & $\begin{array}{l}49.152 \\
49597\end{array}$ & $\begin{array}{l}54.144 \\
54633\end{array}$ & $\begin{array}{r}18.17 \\
18.593\end{array}$ & $\begin{array}{l}32.081 \\
31.911\end{array}$ & \begin{tabular}{|l|}
-1.215 \\
-1.215
\end{tabular} & $\begin{array}{l}-6.399 \\
-6.396\end{array}$ & $\begin{array}{l}50.286 \\
50744\end{array}$ & 0.045 & \begin{tabular}{|r|}
14.643 \\
14643
\end{tabular} & 年 & 506678 & $\mid$ & \begin{tabular}{|r|}
53.0 \\
536
\end{tabular} & 121 & 40.6 & 2810 & 0.006 & 0.006 & 0.000 & 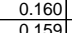 & \\
\hline & $2 / 1 / 1 / 2004$ & $7: 21: 09$ PM & 25.735 & 25.846 & & & & & & & & & & 51.312 & 0.045 & & $\frac{0.003}{0.003}$ & 3560.178 & & & & & & & & & & 0.14 \\
\hline & 9/1/2004 & 7:22:09 PM & 25.73 & $\begin{array}{l}25.84 \\
\end{array}$ & 24.714 & \begin{tabular}{|l|}
20.578 \\
\end{tabular} & & 49.174 & \begin{tabular}{|l|}
54.044 \\
\end{tabular} & $\begin{array}{c}18.44 \\
\end{array}$ & 31.646 & \begin{tabular}{|l|}
-1.212 \\
\end{tabular} & -6.402 & 50.965 & 0.044 & \begin{tabular}{|l|l|}
14.643 \\
\end{tabular} & 0.003 & 3568.78 & \begin{tabular}{|l|l|}
59.4797 \\
\end{tabular} & \begin{tabular}{|l|}
52.9 \\
\end{tabular} & 12.1 & 40.4 & 2.786 & 0.006 & 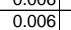 & $\begin{array}{l}0.000 \\
0.000\end{array}$ & $\begin{array}{l}0.155 \\
0.157\end{array}$ & $\frac{0.13}{0.13}$ \\
\hline 878 & & & 25.724 & 25.839 & 24.693 & \begin{tabular}{|l|}
26.497 \\
\end{tabular} & & 49.139 & \begin{tabular}{|l|}
53.931 \\
\end{tabular} & 18.482 & 31.72 & \begin{tabular}{|l|}
-1.215 \\
\end{tabular} & -6.402 & 49.307 & 0.044 & 14.643 & 0.003 & & \begin{tabular}{|l|l|}
59.4964 \\
\end{tabular} & 52.8 & & 40.4 & 2.787 & 0.006 & 0.006 & 0.000 & & \\
\hline 879 & & 7:24:09 PM & & 25.844 & \begin{tabular}{|l|l|}
24.718 \\
\end{tabular} & & 25.454 & & \begin{tabular}{|l|}
54.579 \\
\end{tabular} & 18.514 & & \begin{tabular}{|l|}
-1.212 \\
\end{tabular} & & 52.184 & 0.044 & 14.643 & & 3570.78 & \begin{tabular}{|l|l|}
59.5131 \\
\end{tabular} & 53.5 & & & & & 0.006 & & 0.155 & \\
\hline & 9/1/2004 & 7:25:09 PM & $\begin{array}{l}25.723 \\
25722\end{array}$ & $\begin{array}{l}25.843 \\
25848 \\
25\end{array}$ & \begin{tabular}{|l|}
24.702 \\
2.721 \\
\end{tabular} & \begin{tabular}{|l|}
26.441 \\
\end{tabular} & $\begin{array}{l}25.478 \\
25.393\end{array}$ & $\begin{array}{l}49.649 \\
\end{array}$ & \begin{tabular}{|l|}
54.648 \\
5.217 \\
\end{tabular} & $\begin{array}{l}18.322 \\
1.025\end{array}$ & \begin{tabular}{|l|}
32.404 \\
2.21
\end{tabular} & $\begin{array}{l}-1.215 \\
\end{array}$ & \begin{tabular}{|l|l|}
-6.399 \\
\end{tabular} & 50.125 & 0.044 & \begin{tabular}{|l|l|}
14.643 \\
1.63
\end{tabular} & 0.003 & $\begin{array}{l}3571.78 \\
257270\end{array}$ & \begin{tabular}{|l|}
59.5297 \\
50561 \\
\end{tabular} & $\begin{array}{l}53.5 \\
5.2\end{array}$ & 11.9 & & 2.829 & 0.006 & 0.006 & 0.000 & 0.155 & \\
\hline & $\begin{array}{l}9 / 1 / 2004 \\
9 / 1 / 2004\end{array}$ & $\begin{array}{l}7: 26: 09 \mathrm{PM} \\
77 \cdot 0709 \mathrm{PM}\end{array}$ & $\begin{array}{l}25.722 \\
25717 \\
\end{array}$ & $\begin{array}{l}25.848 \\
25833\end{array}$ & $\begin{aligned} 24.721 \\
247711\end{aligned}$ & \begin{tabular}{|l|l|}
26.48 \\
26.42
\end{tabular} & $\begin{array}{l}25.393 \\
\end{array}$ & 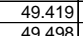 & \begin{tabular}{|l|}
54.347 \\
5.40 \\
\end{tabular} & $\begin{array}{l}18.235 \\
18552\end{array}$ & \begin{tabular}{|l|}
32.196 \\
31856
\end{tabular} & \begin{tabular}{|l|}
-1.215 \\
\end{tabular} & $\begin{array}{l}-6.396 \\
\end{array}$ & 50.169 & 0.044 & \begin{tabular}{|l|l|l|l|l|l|}
14.643 \\
14.643
\end{tabular} & 0.003 & \begin{tabular}{|l|l|}
3572.78 \\
257278
\end{tabular} & \begin{tabular}{|l|l|}
59.5464625 \\
55021
\end{tabular} & $\begin{array}{l}53.2 \\
52.2\end{array}$ & & & $\begin{array}{l}2.814 \\
2.805 \\
\end{array}$ & 0.006 & $\begin{array}{l}0.006 \\
\end{array}$ & 0.000 & 0.156 & \\
\hline & $\begin{array}{l}9 / 1 / 2004 \\
9 / 1 / 2004\end{array}$ & $\begin{array}{l}7: 27: 09 \text { PM } \\
7: 28: 09\end{array}$ & & $\begin{array}{l}25.833 \\
25.832\end{array}$ & $\begin{array}{l}24.711 \\
24.695\end{array}$ & \begin{tabular}{|r|}
26.42 \\
26.375
\end{tabular} & 25 & \begin{tabular}{|r|r|r|}
49.498 \\
49.44
\end{tabular} & \begin{tabular}{|l|}
54.442 \\
54.447
\end{tabular} & $\frac{18.552}{17.994}$ & \begin{tabular}{|l|}
31.856 \\
32313
\end{tabular} & \begin{tabular}{|l|}
-1.212 \\
-1.215 \\
\end{tabular} & $\begin{array}{l}-6.396 \\
-6.396\end{array}$ & $\frac{51.339}{50.442}$ & $\frac{0.044}{0.044}$ & $\begin{array}{l}14.643 \\
14.643\end{array}$ & 0.003 & \begin{tabular}{|c|}
3573.78 \\
357478
\end{tabular} & $\begin{array}{l}59.5631 \\
5.9797\end{array}$ & & $\frac{12.2}{120}$ & $\begin{array}{l}40.7 \\
40.9\end{array}$ & 2.805 & $\begin{array}{l}0.006 \\
0.006\end{array}$ & 0.006 & 0.000 & 0.156 & 0.13 \\
\hline 884 & 9/1/2004 & 7:29:09 PM & 25.721 & 25.836 & 24.745 & \begin{tabular}{|l|}
26.369 \\
\end{tabular} & 25.336 & 49.608 & \begin{tabular}{|l|}
54.4456 \\
\end{tabular} & 18.389 & 32.147 & \begin{tabular}{|l|}
-1.212 \\
\end{tabular} & $\begin{array}{l}-6.396 \\
-6.391\end{array}$ & tu.4828 & 0.044 & 14.643 & $\begin{array}{l}0.003 \\
0.003\end{array}$ & $\begin{array}{l}35 / 4.8 \\
3575.78\end{array}$ & \begin{tabular}{|l|}
59.979797 \\
99.5964
\end{tabular} & $\begin{array}{l}53.3 \\
53.4\end{array}$ & $\frac{12.0}{11.6}$ & $\begin{array}{r}40.9 \\
40.9 \\
\end{array}$ & $\frac{2.818}{2.818}$ & \begin{tabular}{|c|c|}
0.006 \\
0.006
\end{tabular} & 0.006 & $\begin{array}{l}0.000 \\
0.000\end{array}$ & $\begin{array}{l}0.155 \\
0.155\end{array}$ & 0.13 \\
\hline & 9/1/2004 & 7:30:09 PM & 25.699 & 25.81 & 24.698 & \begin{tabular}{|l|}
26.312 \\
\end{tabular} & & 49.419 & \begin{tabular}{|l|}
54.413 \\
\end{tabular} & 18.354 & 31.944 & \begin{tabular}{|l|}
-1.212 \\
\end{tabular} & -6.394 & 51.504 & 0.044 & 14.643 & 0.003 & 3576.78 & \begin{tabular}{|l|l|}
59.6131 \\
\end{tabular} & 53.3 & 12.3 & 40.7 & 2.805 & \begin{tabular}{|l|}
0.006 \\
\end{tabular} & 0.006 & 0.000 & 0.156 & \\
\hline & $9 / 1 / 2004$ & 7:31:09 PM & & 25.808 & \begin{tabular}{|l|}
24.717 \\
\end{tabular} & & 25.2 & & 54.204 & 18.387 & 31.891 & $\mid-1.212$ & & 49.47 & 0.044 & 14.643 & & & & 53.1 & 11.8 & 40.6 & & \begin{tabular}{|l|}
0.006 \\
\end{tabular} & $\begin{array}{l}0.006 \\
\end{array}$ & 0.000 & & \\
\hline & & 7:32:09 PM & 25.691 & & \begin{tabular}{|l|}
24.71 \\
\end{tabular} & 26.249 & & & \begin{tabular}{|l|}
53.93 \\
\end{tabular} & \begin{tabular}{|l|l|l|l|l|}
18.193 \\
\end{tabular} & 31.839 & & & 48.485 & & & & & & 52.8 & & & & 0.006 & 0.006 & & 0.157 & \\
\hline & & 7:33:09 PM & & & & & & 49.496 & & $\begin{array}{ll}18.349 \\
\end{array}$ & & $\frac{-1.212}{-1212}$ & & 50.676 & & $\begin{array}{ll}14.643 \\
\end{array}$ & & & & & & & $81 \mathrm{~s}$ & 0.006 & & & & \\
\hline & & 7:34:09 PM & $\begin{array}{l}25.686 \\
\end{array}$ & 25.796 & $\begin{array}{l}24.719 \\
\end{array}$ & $\begin{array}{l}26.244 \\
2\end{array}$ & & \begin{tabular}{|l|l|l|}
49.367 \\
\end{tabular} & $\mid 54.48$ & $\begin{array}{l}18.415 \\
\end{array}$ & 31.983 & -1.21 & & 50.261 & & $\begin{array}{l}14.643 \\
\end{array}$ & & & & $\overline{53.4}$ & & & .80. & & & & & \\
\hline & 200 & (2) & & 25.797 & 24.715 & 26.309 & & & \begin{tabular}{|l|}
54.273 \\
\end{tabular} & 17.983 & & -1.215 & & 50.24 & 044 & 14.6 & & & & 53.1 & & & .811 & 0.006 & 0.006 & & & \\
\hline & $9 / 1 / 200$ & 7:36:09 PM & 25.696 & 25.806 & 24.755 & \begin{tabular}{|l|}
26.319 \\
\end{tabular} & & 49.094 & \begin{tabular}{|l|}
54.129 \\
\end{tabular} & 18.218 & 31.694 & -1.212 & -6. & 52.015 & .044 & \begin{tabular}{|l|l|l|l|}
14.643 \\
\end{tabular} & 0.003 & 3582.78 & 59.7131 & 53.0 & 12.4 & 40.4 & & $\begin{array}{l}0.006 \\
\end{array}$ & 0.006 & 0.000 & 0.157 & \\
\hline & $9 / 1 / 2004$ & 7:37:09 PM & 25.691 & 25.802 & 24.74 & 26.369 & & 49.745 & \begin{tabular}{|l|l|}
54.648 \\
\end{tabular} & 18.195 & 32.519 & -1.212 & -6.388 & 50.686 & 0.044 & $\begin{array}{l}14.643 \\
\end{array}$ & 0.003 & 3583.78 & 59.7297 & 53.5 & 12.1 & 41. & 2.836 & 0.006 & 0.006 & 0.000 & 0.154 & \\
\hline & $9 / 1 / 2004$ & 7:38:09 PM & 25.671 & 25.786 & 24.729 & 26.364 & & 49.5699 & 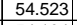 & 18.392 & 32.176 & -1.218 & & 50.663 & 044 & 14.643 & & & 59.7464 & 53.4 & 12.1 & & & 0.006 & .006 & & 0.155 & \\
\hline & 9 & 7:39:09 PM & 25.681 & & & & & & & & & & & 50.502 & & 14.643 & & & & & & & & 0.006 & & & & \\
\hline & & $7: 40.09 \mathrm{PM}$ & $\frac{2.6501}{25691}$ & $\frac{25.196}{25917}$ & 24.129 & $\frac{26.434}{2.44}$ & & $\begin{array}{r}49.4 \\
\end{array}$ & \begin{tabular}{|l|}
54.254 \\
5.7272 \\
\end{tabular} & 18.087 & $\frac{32.360}{32307}$ & -1.2122 & & $\frac{50.421}{5.012}$ & & $\frac{14.643}{11643}$ & & 507 & 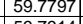 & & & & & & & & & \\
\hline$\frac{897}{897}$ & $\begin{array}{l}9 / / 1 / 2004 \\
9 / 1 / 2004\end{array}$ & & $\frac{25.691}{25.692}$ & $\frac{25.817}{25.812}$ & 24.776 & $\frac{26.444}{26.445}$ & & & \begin{tabular}{|l|}
$54 . / 242$ \\
5.523 \\
\end{tabular} & $\frac{18.368}{18.339}$ & $\begin{array}{l}32.307 \\
32.008\end{array}$ & $\frac{-1.212}{-1.215}$ & & $\frac{50.042}{49.904}$ & 0.044 & $\begin{array}{l}14.6 \\
1.6\end{array}$ & & & & (53.60 & $\frac{11.9}{119}$ & & . & | & 0 & & . & \\
\hline & $9 / 1 / 2004$ & 7:412:51 PM & 25.693 & $\frac{25.014}{25.828}$ & 24.757 & 26.411 & & $\begin{array}{l}45.036 \\
49.486\end{array}$ & \begin{tabular}{|r|}
54.054 \\
54.37 \\
\end{tabular} & 10.059 & $\begin{array}{l}32.000 \\
32.393\end{array}$ & $\begin{array}{l}-1.210 \\
-1.212\end{array}$ & & $\begin{array}{l}45 . .904 \\
51.137\end{array}$ & .045 & $\begin{array}{l}\frac{14.045}{14.643} \\
\end{array}$ & 0.00 & 5 & & 53.2 & $\frac{11.5}{12.2}$ & 40 & & 0.006 & 0.006 & 0.000 & o.158 & \\
\hline & 9/1/2004 & 7:43:51 PM & 25.689 & 25.819 & 24.773 & \begin{tabular}{|l|}
26.482 \\
\end{tabular} & & $\mid 49.289$ & 54.185 & 18.171 & 32.149 & $\mid$\begin{tabular}{r|}
$\mid$ \\
$\mid-1.221$
\end{tabular} & & 50.596 & 0.044 & 14.643 & 0.003 & 3590.48 & 59.8414 & 53.1 & 12. & & 2807 & | 0.006 | & 0.006 & 0.000 & 0.156 & \\
\hline & & & 25.69 & & 24.774 & & & & \begin{tabular}{|l|l|}
54.673 \\
\end{tabular} & 18.128 & 32.465 & -1.212 & & 49.843 & .044 & 14.6 & & & & & & & & & & & & \\
\hline & $9 / 1 / 2004$ & $51 \mathrm{PM}$ & 25.686 & 25.826 & 24.785 & 26.514 & & & & & 32.047 & -1.215 & & 50.354 & & & & & & 52.9 & 12. & & & 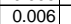 & 0.006 & 0.000 & 0.156 & \\
\hline & & & & & & 26.5 & & & 54.393 & $\begin{array}{l}18.499 \\
\end{array}$ & & & & 49.109 & & & & & & & & & & & & & & \\
\hline & & 11 PM & \begin{tabular}{|l|l|}
25.698 \\
\end{tabular} & & \begin{tabular}{|l|}
24.772 \\
\end{tabular} & 26.461 & & & \begin{tabular}{|l|l|}
54.484 \\
\end{tabular} & 18.206 & 32.428 & -1.21 & & 50.951 & & & & & & 53.4 & & & & & & & 0.155 & \\
\hline & $9 / 1 / 2004$ & 1 PM & & 25.843 & \begin{tabular}{|l|l|}
24.797 \\
\end{tabular} & 26.406 & & 49.842 & \begin{tabular}{|l|}
54.637 \\
\end{tabular} & 18.419 & 32.467 & -1.212 & & 50.594 & & 14.643 & & & & 53.5 & 12.1 & $\overline{41}$ & & & 0.006 & & 0.154 & \\
\hline & $9 / 1 / 20$ & 7:49:51 PM & 25.703 & 25.848 & \begin{tabular}{|l|}
24.802 \\
\end{tabular} & \begin{tabular}{|l|} 
\\
\end{tabular} & & & \begin{tabular}{|l|l|l} 
\\
\end{tabular} & 17.961 & 32.57 & -1.212 & & 51.71 & & $\begin{array}{ll}14.643 \\
\end{array}$ & & 596.48 & & 53.5 & 12 & & & & & & 0.154 & \\
\hline & & & 25.697 & 25.843 & 24.806 & 26. & & 49.4 & 54.42 & 18.151 & 32.346 & -1.212 & & 51.366 & & 14. & & & & 53.3 & & & & & & & 155 & \\
\hline & 9/1/2004 & 51 PM & & 25.827 & 24.801 & 26.25 & & & \begin{tabular}{|l|} 
\\
\end{tabular} & 17.732 & 32.693 & -1.215 & & & & 14. & & & & 52.8 & 12.4 & & & & & & 155 & \\
\hline & & 51 PM & 25.685 & 25.83 & & & & & & & & & & & & & & & & & & & & & & & 0.156 & \\
\hline & & 7.5 .5 & 年 & 25. & 24.934 & 26.193 & & & \begin{tabular}{|l|}
54.134 \\
54.19 \\
\end{tabular} & 18.468 & 31.529 & -1.21 & & 50.025 & & 14.6 & & & & 53.0 & 11.0 & 40 & 70 & & & & 0.157 & \\
\hline & $99 / 1 / 12004$ & & & & & & & & \begin{tabular}{|l|}
5.194 \\
5.03
\end{tabular} & 18.5325 & & - & & & & & & & & & & & & & & & & \\
\hline & $9 / 1 / 200$ & 7.55.5. PM & 682 & & 24861 & \begin{tabular}{|l|l|}
26045 \\
\end{tabular} & & (45.250 & $\mid$ & 18517 & 31786 & $\mid \begin{array}{l}-1.215 \\
1215\end{array}$ & & $\begin{array}{l}45320 \\
53229\end{array}$ & & 14 & & & & 520 & & & & & & & 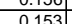 & \\
\hline & $9 / 1 / 200$ & & & 25801 & 24825 & & & & \begin{tabular}{|l|}
54.366 \\
\end{tabular} & 18.62 & 31.62 & \begin{tabular}{|c|c|} 
& -1209 \\
\end{tabular} & & 50.369 & & & & & & & & & & & & & & \\
\hline & $9 / 1 /$ & 7:58:51 PM & 年 & $\frac{25.789}{25.789}$ & 24.818 & & & & 54.436 & 18.541 & 31.691 & $\frac{-1.218}{-1.218}$ & & 50.767 & & 14.6 & & & & 53.3 & & & & & & & 年 & \\
\hline & & $7: 59 \cdot 51 \mathrm{PM}$ & 25.678 & 25.793 & \begin{tabular}{|l|}
24.797 \\
\end{tabular} & & & & 54.2 & 18.3 & 31.845 & -1.21 & & & & & & & & 53.1 & 118 & & & & & & 0.156 & \\
\hline & & & & 25.78 & 24.78 & & & & \begin{tabular}{|l|}
54.4 \\
\end{tabular} & 18.4 & 31.626 & -1.21 & & & & 14. & & & & & & & & & & & & \\
\hline & & & & & 24.78 & & & & 54 & & & -1.2 & & & & & & & & & & & & & & & 55 & \\
\hline & $9 / 1 / 2004$ & & & & 24.755 & & & & 54.52 & 18. & & -1.20 & & & & & & & & 53.4 & 12 & & & & & & 0.156 & \\
\hline & $9 / 1 / 200$ & 51 PM & & 25. & 24.764 & & & & \begin{tabular}{|l|l|}
54.48 \\
\end{tabular} & 18.2 & & -1.212 & & 51.716 & & 14. & & & & 53.4 & 12 & & & & & & $\begin{array}{l}0.156 \\
\end{array}$ & \\
\hline & & & & 25.7 & 24.754 & & & & 54.227 & 18.15 & 32.114 & -1.205 & & 52.407 & & 14.6 & & & & 53.1 & & & & & & & & \\
\hline & 9/1/2004 & & & 25.18 & & & & & 54.2 & & 32.218 & -1.212 & & & & & & & & & & & & & & & & \\
\hline & & & & & 24.732 & & & & 54.7 & 18. & 32.244 & -1.215 & & & & & & & & & & & & & & & & \\
\hline & & & & & 24.08 & & & & $54 .$. & 18.244 & 32.5 & -1.218 & & & & & & & & 30.6 & & & & & & & 55 & \\
\hline & & & & & & & & & \begin{tabular}{|l|l|}
54.3 \\
\end{tabular} & & & & & & & & & & & & & & & & & & & \\
\hline & $9 / 1 / 26$ & $8.030 .51 \mathrm{PM}$ & 25634 & 2578 & $\frac{24.11}{2469}$ & & & & 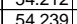 & 181 & & -121 & & & & & & & & & & & & & & & 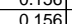 & \\
\hline & $9 / 1 / 200$ & & & 2577 & 24708 & & & & \begin{tabular}{|l|l|} 
& 54.6 \\
\end{tabular} & 1812 & 322 & -1215 & & & & & & & & $\frac{5.1}{536}$ & & & & & & & & \\
\hline & & & & & 24.718 & & & & 54.4 & & & $\frac{-1.212}{-1.212}$ & & 50.41 & & & & & & & & & & & & & 5 & \\
\hline & & & 25.63 & & 24.703 & & & & 54.4 & 18.239 & & -1.218 & & & & & & & & & & & & & & & & \\
\hline & & & & & & & & & & & 32.204 & $\frac{-1.212}{-1.212}$ & & & & & & & & & & & & & & & & \\
\hline & $9 / 1 / 200$ & & & 25.811 & 24.68 & & & & 543 & & 31.921 & -1.215 & & & & 14.6 & & & & & & & & & & & 157 & \\
\hline & $9 / 1 / 200$ & 8:16:51 & & & 24.73 & 26. & & & 54.2 & 18.0 & & $\begin{array}{l}-1.212 \\
\end{array}$ & & 50.97 & & 14. & & & & & & & & & & & 0.156 & \\
\hline & $9 / 1 / 2$ & $8: 17: 51 \mathrm{P}$ & 25. & 25.8 & 24.68 & & & 49. & 54.1 & 18. & & $\begin{array}{l}-1.212 \\
\end{array}$ & & 51.26 & & 14. & & & & $3.1 \quad \mathrm{C}$ & & & & & & & $\begin{array}{l}0.157 \\
\end{array}$ & \\
\hline & 9/1/2004 & & & 25.814 & 24.697 & & & 49. & \begin{tabular}{|l|}
54.625 \\
\end{tabular} & 18.047 & 32.406 & -1.215 & & 51.468 & & 14. & & & & 53.5 & & & & & & & 155 & \\
\hline & & & & & 24.706 & & & & & & & -1.2 & & & & & & & & & & & & & & & & \\
\hline & & & 25.608 & 25.998 & 4.656 & 26. & & 49.3 & 54.5 & $17.8 \mathrm{r}$ & 32.41 & -1.154 & & & & & & & & 53.4 & & & & & & & & \\
\hline & $\begin{array}{ll}911 / 2004 \\
0.1 / 204\end{array}$ & $8: 21: 51 \mathrm{PM}$ & 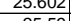 & 25.792 & 24.676 & 26.01 & & 49.556 & \begin{tabular}{|l|}
54.749 \\
\end{tabular} & 18.091 & 32.381 & $-1.1 / 4$ & & 51.103 & & 14.6 & & & 60.4 & (5.6. & 12.2 & & 2.0 & & & & 0.155 & \\
\hline & 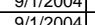 & $8.22 .51 P M$ & 20.59 & 20.1006 & th. 2.044 & \begin{tabular}{|l|l|}
20.003 \\
6030
\end{tabular} & & 49.1. & 5192 & 10 & 年 & -1.16 & & 50100 & & $\begin{array}{l}1.043 \\
\end{array}$ & & & & 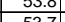 & & & & | & & 0.000 & 年 & \\
\hline 2940 & 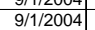 & 8:24:51 PM & \begin{tabular}{|l|}
25.582 \\
\end{tabular} & 25.768 & \begin{tabular}{|l|}
24.051 \\
24.651 \\
\end{tabular} & \begin{tabular}{|l|}
20.004 \\
25.955 \\
\end{tabular} & 24.888 & $\frac{4.1100}{49.45}$ & \begin{tabular}{|l|}
54.573 \\
5
\end{tabular} & $\begin{array}{l}18.076 \\
18.076\end{array}$ & $\mid$ & $\begin{array}{l}-1.105 \\
\end{array}$ & $\begin{array}{l}-0.044 \\
-6.347\end{array}$ & 50.867 & 0.044 & \begin{tabular}{|l|}
14.0453 \\
14.643
\end{tabular} & 0.003 & 3631.48 & 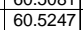 & \begin{tabular}{|l|}
53.4 \\
53.4
\end{tabular} & \begin{tabular}{|l|}
12.1 \\
12.1 \\
\end{tabular} & & \begin{tabular}{|c|}
2.818 \\
\end{tabular} & \begin{tabular}{|l|}
0.0006 \\
\end{tabular} & 年0 & 0.000 & $\mid 0.156$ & 0.1 \\
\hline
\end{tabular}


WSRC-TR-2005-00105, REVISION 0

SRNL-RPP-2005-00012, REVISION 0

RUN \# 4.03A AND B; FIRST AND SECOND HALF OF SLURRY DEWATERING - CONT.

\begin{tabular}{|c|c|c|c|c|c|c|c|c|c|c|c|c|c|c|c|c|c|c|c|c|c|c|c|c|c|c|c|c|}
\hline & $\mathrm{A}$ & B & D & $E$ & $\mathrm{~F}$ & G & $\mathrm{H}$ & $\mathrm{J}$ & \begin{tabular}{|l|}
$\mathrm{K}$ \\
\end{tabular} & $\mathrm{L}$ & $\begin{array}{ll} \\
\end{array}$ & $\mathrm{N}$ & 0 & \begin{tabular}{l|l} 
\\
\end{tabular} & \begin{tabular}{l|l|l}
$R$ &
\end{tabular} & $\mathrm{~s}$ & $\mathrm{~T}$ & $\mathrm{v}$ & w & $x$ & $Y$ & Z & $\mathrm{AA}$ & \begin{tabular}{ll|}
$\mathrm{AB}$ & \\
\end{tabular} & $\mathrm{AC}$ & $A D$ & $\mathrm{AE}$ & \\
\hline 2941 & 9/1/2004 & $8: 25: 51 \mathrm{PM}$ & 25.57 & 25.76 & 24.654 & 25.953 & 24.97 & 49.456 & \begin{tabular}{|l|}
54.449 \\
\end{tabular} & 18.069 & 32.387 & $\frac{1.148}{-1.148}$ & -6.34 & 50.269 & 0.044 & 14.643 & 0.003 & 3632.48 & 60.5414 & \begin{tabular}{|l|}
53.3 \\
\end{tabular} & 12. & & 2.821 & 0.006 & 0.006 & 0.000 & 0.155 & \\
\hline & & & & & & & & $\begin{array}{l}49.442 \\
\end{array}$ & & 17.761 & 32.779 & & & 51.22 & \begin{tabular}{|l|}
0.044 \\
\end{tabular} & 14.643 & & & & \begin{tabular}{|l|}
53.4 \\
\end{tabular} & & & & & & 0.000 & 0.150 & \\
\hline$\frac{2943}{2944}$ & 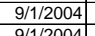 & $8: 27: 51 \mathrm{PM}$ & $\begin{array}{l}25.563 \\
25562\end{array}$ & \begin{tabular}{l|l}
25.753 \\
25757
\end{tabular} & $\begin{array}{l}24.632 \\
24661\end{array}$ & \begin{tabular}{|l|}
25.996 \\
\end{tabular} & $\begin{array}{l}25.053 \\
25127\end{array}$ & \begin{tabular}{|l|}
49.477 \\
4907 \\
\end{tabular} & \begin{tabular}{|l|l|}
54.617 \\
5.189 \\
\end{tabular} & \begin{tabular}{|l|l|}
18.084 \\
17822
\end{tabular} & \begin{tabular}{|l|l|}
32.377 \\
32166
\end{tabular} & \begin{tabular}{|r|}
-1.148 \\
\end{tabular} & \begin{tabular}{|l|l|}
-6.344 \\
-6347
\end{tabular} & \begin{tabular}{|l|}
50.772 \\
50546
\end{tabular} & $\begin{array}{l}0.044 \\
\end{array}$ & \begin{tabular}{ll|}
14.643 \\
14
\end{tabular} & $\begin{array}{l}0.003 \\
.0037\end{array}$ & 305.450 & \begin{tabular}{|l|}
60.5747 \\
\end{tabular} & \begin{tabular}{|l|}
53.5 \\
\end{tabular} & 12.1 & & & $\begin{array}{l}0.006 \\
\end{array}$ & 0.006 & 0.000 & $\begin{array}{l}0.156 \\
\end{array}$ & \\
\hline$\frac{2944}{2945}$ & $\begin{array}{l}9 / 1 / 2004 \\
9 / 1 / 2004\end{array}$ & 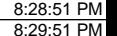 & $\begin{array}{l}25.562 \\
25.561\end{array}$ & $\begin{array}{l}25.757 \\
25.751\end{array}$ & $\begin{aligned} 24.661 \\
24.654\end{aligned}$ & $\begin{array}{r}26 \\
260^{24}\end{array}$ & $\begin{array}{l}25.127 \\
25.06\end{array}$ & \begin{tabular}{|l|}
49.071 \\
49.99 \\
\end{tabular} & \begin{tabular}{|l|}
54.188 \\
54.99 \\
\end{tabular} & $\begin{array}{l}17.822 \\
18091\end{array}$ & $\begin{array}{r}32.166 \\
32.715\end{array}$ & \begin{tabular}{|r|}
-1.151 \\
-11188 \\
\end{tabular} & $\begin{array}{l}-6.347 \\
-6347\end{array}$ & $\begin{array}{l}50.546 \\
50.525\end{array}$ & $\begin{array}{l}0.044 \\
0044\end{array}$ & \begin{tabular}{|r|}
14.643 \\
14643
\end{tabular} & $\begin{array}{l}0.003 \\
0.023\end{array}$ & $\begin{array}{l}3635.48 \\
363648\end{array}$ & \begin{tabular}{|l|}
60.5914 \\
60081
\end{tabular} & \begin{tabular}{|l|l|}
53.1 \\
53.9
\end{tabular} & \begin{tabular}{|l|l|}
12.0 \\
120
\end{tabular} & 40.6 & 2849 & $\begin{array}{l}0.006 \\
0.006\end{array}$ & $\begin{array}{l}0.006 \\
0.066\end{array}$ & $\begin{array}{l}0.000 \\
0.000\end{array}$ & \begin{tabular}{|c|c|}
0.157 \\
0
\end{tabular} & \\
\hline 2946 & $\frac{\mid 9 / 1 / 1 / 2004}{4}$ & & & & $\frac{24.654}{24.669}$ & & & & & & & \begin{tabular}{|c|}
-1.148 \\
-1.151 \\
\end{tabular} & & & & $\begin{array}{l}\frac{14.643}{14.643} \\
\end{array}$ & $\begin{array}{l}0.003 \\
0.003\end{array}$ & & & & & & & & & & $\begin{array}{l}0.154 \\
0.156\end{array}$ & $\begin{array}{l}0.13 \\
0.13\end{array}$ \\
\hline 2947 & 9/1/2004 & 8:31:51 PM & 25.556 & 25.757 & 24.675 & \begin{tabular}{|l|}
26.074 \\
\end{tabular} & 25.227 & 49.257 & \begin{tabular}{|l|}
54.339 \\
\end{tabular} & $\begin{array}{c}17.99 \\
\end{array}$ & 32.182 & \begin{tabular}{|l|}
-1.145 \\
\end{tabular} & -6.347 & 51.124 & 0.044 & \begin{tabular}{|l|l|}
14.643 \\
\end{tabular} & $\begin{array}{l}0.004 \\
0.004\end{array}$ & $\begin{array}{l}303.400 \\
3638.48\end{array}$ & \begin{tabular}{|l|l|}
60.6414 \\
\end{tabular} & 53.2 & 12.3 & $\begin{array}{l}40.8 \\
40.7\end{array}$ & 2.807 & 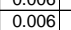 & 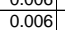 & $\begin{array}{l}0.000 \\
0.000\end{array}$ & $\begin{array}{l}0.156 \\
0.156\end{array}$ & $\frac{0.13}{0.13}$ \\
\hline 2948 & & & 25.541 & 25.747 & 24.65 & \begin{tabular}{|l|}
26.099 \\
\end{tabular} & & 49.705 & \begin{tabular}{|l|}
54.799 \\
\end{tabular} & 17.936 & 32.754 & \begin{tabular}{|l|}
-1.145 \\
\end{tabular} & -6.35 & 49.631 & 0.044 & 14.643 & 0.003 & 3639.48 & & 53.7 & & & 2.843 & 0.006 & 0.006 & 0.000 & & \\
\hline 2949 & & & & 25.752 & 24.691 & 26.08 & & 49.612 & \begin{tabular}{|l|l|}
54.563 \\
\end{tabular} & 18.064 & 32.484 & \begin{tabular}{|l|}
-1.148 \\
\end{tabular} & -6.344 & 52.77 & \begin{tabular}{l|}
0.044 \\
\end{tabular} & 14.643 & & 3640.48 & 0.6747 & 53.4 & & & & & 0.006 & & 0.155 & \\
\hline$\frac{2950}{2951}$ & 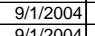 & $\begin{array}{l}8: 34: 51 \mathrm{PM} \\
8.55 .51 \mathrm{P}\end{array}$ & $\begin{array}{l}25.547 \\
25542\end{array}$ & \begin{tabular}{|l|l|}
25.747 \\
25742
\end{tabular} & $\begin{array}{l}24.666 \\
24.681\end{array}$ & $\frac{26.115}{2613}$ & $\begin{array}{l}25.207 \\
25207\end{array}$ & \begin{tabular}{|l|l|}
49.363 \\
\end{tabular} & \begin{tabular}{|l|}
54.459 \\
5.755 \\
\end{tabular} & 17.966 & \begin{tabular}{|l|l|}
32.463 \\
2.72
\end{tabular} & $\begin{array}{r}-1.151 \\
\end{array}$ & -6.34 & $\begin{array}{l}49.458 \\
5.923\end{array}$ & \begin{tabular}{|l|l|}
0.044 \\
\end{tabular} & $\begin{array}{l}14.643 \\
1.63\end{array}$ & 0.003 & & $\begin{array}{l}60.6914 \\
607021\end{array}$ & \begin{tabular}{|l|}
53.3 \\
5.3
\end{tabular} & 11.8 & & 2.821 & $\begin{array}{l}0.006 \\
\end{array}$ & 0.006 & 0.000 & 0.155 & \\
\hline$\frac{2951}{2952}$ & $\begin{array}{l}9 / 1 / 2004 \\
91 / 2004\end{array}$ & $8: 35: 51 \mathrm{PM}$ & 25.542 & $\begin{array}{l}25.742 \\
25763\end{array}$ & $\begin{array}{r}24.681 \\
21707\end{array}$ & $\begin{array}{r}26.13 \\
26.101\end{array}$ & $\begin{array}{l}25.237 \\
25.23\end{array}$ & 49.5 & \begin{tabular}{|l|}
54.758 \\
5.145 \\
\end{tabular} & 17.895 & $\begin{array}{l}32.72 \\
2.25 \\
\end{array}$ & $\begin{array}{l}-1.148 \\
\end{array}$ & $\begin{array}{l}-6.347 \\
-6.20 \\
\end{array}$ & 50.853 & $\begin{array}{l}0.044 \\
\end{array}$ & $\begin{array}{l}14.643 \\
\end{array}$ & 0.003 & $\begin{array}{l}3642.48 \\
3642.9\end{array}$ & \begin{tabular}{|l|}
60.7081 \\
607217
\end{tabular} & \begin{tabular}{|l|l|}
53.6 \\
532
\end{tabular} & 12.1 & & 2.836 & 0.006 & $\begin{array}{l}0.006 \\
\end{array}$ & 0.000 & 0.155 & \\
\hline 2953 & $\begin{array}{l}9 / 1 / 2004 \\
9 / 1 / 2004\end{array}$ & $8: 36: 51 \mathrm{PM}$ & $\begin{array}{l}25.563 \\
25.548\end{array}$ & $\begin{array}{l}25.763 \\
25.748\end{array}$ & $\frac{24.707}{24.677}$ & \begin{tabular}{|l|}
26.191 \\
26201
\end{tabular} & & \begin{tabular}{|l|}
49.206 \\
49736
\end{tabular} & \begin{tabular}{|c|}
54.453 \\
55.008 \\
\end{tabular} & $\begin{array}{l}17.905 \\
17.953 \\
\end{array}$ & $\begin{array}{r}32.25 \\
32.603 \\
\end{array}$ & \begin{tabular}{rl|l}
-1.143 \\
-1.145
\end{tabular} & & $\frac{51.839}{50.747}$ & $\mid \begin{array}{c}0.044 \\
0.044\end{array}$ & $\begin{array}{l}14.643 \\
14.643\end{array}$ & & $\frac{3643.48}{3644.48}$ & 60.7247 & & & 4 & $\frac{2.808}{2.838}$ & 0.006 & 0.006 & 0.000 & 0.156 & \\
\hline 2954 & 9/1/2004 & 8:38:51 PM & & 25.749 & \begin{tabular}{|l|}
24.071 \\
24.697 \\
\end{tabular} & 26.222 & & $\begin{array}{l}49.237 \\
49.237\end{array}$ & \begin{tabular}{|l|}
54.459 \\
\end{tabular} & 17.892 & \begin{tabular}{|l|}
32.000 \\
32.213 \\
\end{tabular} & \begin{tabular}{|l|}
-1.140 \\
-1.145 \\
\end{tabular} & $\begin{array}{l}-0.344 \\
-6.342 \\
\end{array}$ & 50.352 & $\begin{array}{l}0.0444 \\
0.044\end{array}$ & \begin{tabular}{|l|}
1.045 \\
14.643 \\
\end{tabular} & $\begin{array}{l}0.003 \\
0.004\end{array}$ & $\begin{array}{l}3644.48 \\
3645.48\end{array}$ & \begin{tabular}{|l|l|}
60.751414 \\
60151
\end{tabular} & $\begin{array}{l}53.9 \\
53.3\end{array}$ & $\frac{12.1}{12.0}$ & $\begin{array}{l}41.2 \\
40.7 \\
\end{array}$ & $\frac{2.838}{2.808}$ & $\mid$ & $\begin{array}{l} \\
0.006 \\
0.006\end{array}$ & $\begin{array}{l}0.000 \\
0.000\end{array}$ & & $\begin{array}{l}0.13 \\
0.13\end{array}$ \\
\hline & 9/1/2004 & $8: 39: 51 \mathrm{PM}$ & 25.549 & 25.755 & \begin{tabular}{|l|l|}
24.678 \\
\end{tabular} & & 25.345 & $\begin{array}{l}49.714 \\
\end{array}$ & \begin{tabular}{|l|l|}
54.787 \\
\end{tabular} & 18 & 32.662 & \begin{tabular}{|l|}
-1.128 \\
\end{tabular} & -6.342 & 49.161 & 0.044 & $\begin{array}{l}4.643 \\
\end{array}$ & 0.003 & 3646.48 & \begin{tabular}{|l|l|}
60.7747 \\
\end{tabular} & 53.7 & & & 2.840 & \begin{tabular}{|l|}
0.006 \\
\end{tabular} & 0.006 & 0.000 & 0.154 & \\
\hline & 9/1/2004 & $8: 40: 51 \mathrm{PM}$ & & 25.755 & 24.709 & 26.213 & 25.29 & & \begin{tabular}{|l|l|}
54.791 \\
\end{tabular} & 18.012 & $\begin{array}{l}32.636 \\
2.70 \\
\end{array}$ & \begin{tabular}{|l|l|}
-1.151 \\
\end{tabular} & & 49.956 & $\begin{array}{l}0.044 \\
\end{array}$ & $\begin{array}{l}14.643 \\
\end{array}$ & & & \begin{tabular}{|l|}
60.7914 \\
\end{tabular} & 53.7 & 11.9 & & 2.835 & \begin{tabular}{l|l}
0.006 \\
\end{tabular} & $\begin{array}{l}0.006 \\
\end{array}$ & & 0.154 & \\
\hline$\frac{2957}{2958}$ & 9/1/2004 & $8: 41: 51 \mathrm{PM}$ & 25.561 & 25.771 & $\begin{array}{r}24.725 \\
\end{array}$ & 26.274 & $\begin{array}{l}25.356 \\
25.227\end{array}$ & $\begin{array}{l}49.662 \\
\end{array}$ & \begin{tabular}{|l|l|}
54.728 \\
5.68 \\
\end{tabular} & & \begin{tabular}{|l|l|}
32.703 \\
2.077
\end{tabular} & $\begin{array}{l}-1.143 \\
\end{array}$ & -6.336 & 49.045 & .044 & 14.643 & 0.003 & 3648.48 & \begin{tabular}{|l|}
60.8081 \\
\end{tabular} & 53.6 & 11.7 & & 2.839 & $\begin{array}{ll}0.006 \\
\end{array}$ & 0.006 & 0.000 & 0.154 & \\
\hline & 9/1/2004 & 8:42:51 PM & $\frac{25.552}{25.557}$ & 25.752 & \begin{tabular}{|r|}
24.69 \\
21.71
\end{tabular} & $\begin{array}{r}26.26 \\
26.255\end{array}$ & 25.33 & & & 17.854 & $\begin{array}{l}32.677 \\
220267\end{array}$ & \begin{tabular}{|l|l|} 
\\
\end{tabular} & & 51.168 & $\begin{array}{l}0.044 \\
\end{array}$ & $\begin{array}{r}14.643 \\
1.613\end{array}$ & 0.003 & 3649.48 & \begin{tabular}{|l|}
60.8247 \\
\end{tabular} & 53.6 & & & 2.833 & \begin{tabular}{|l|l|}
0.006 \\
\end{tabular} & 0.006 & 0.000 & 0.155 & \\
\hline$\frac{2959}{2960}$ & $9 / 1 / 1 / 2004$ & $\begin{array}{l}8: 34: 51 \mathrm{PM} \\
8.4451 \mathrm{PM}\end{array}$ & & $\begin{array}{l}25.757 \\
25768\end{array}$ & \begin{tabular}{|r|}
24.71 \\
24717
\end{tabular} & $\frac{26.255}{26296}$ & & $\begin{array}{r}49.38 \\
49.483\end{array}$ & \begin{tabular}{|l|}
54.635 \\
54.671
\end{tabular} & $\begin{array}{l}\frac{18.058}{17.686} \\
\end{array}$ & $\begin{array}{l}32.266 \\
32.732 \\
\end{array}$ & $\begin{array}{l}-1.096 \\
-1.096\end{array}$ & & $\begin{array}{l}50.069 \\
51.022\end{array}$ & $\begin{array}{l}0.044 \\
0.044\end{array}$ & $\begin{array}{l}14.643 \\
14.643\end{array}$ & $\frac{03}{04}$ & & & & & & & $\begin{array}{l}0.006 \\
0.006\end{array}$ & 0.006 & & & \\
\hline & $9 / 1 / 2004$ & $8: 45: 51 \mathrm{PM}$ & 25.549 & 25.759 & \begin{tabular}{|l|}
24.711 \\
24.718 \\
\end{tabular} & \begin{tabular}{|l|}
20.290 \\
26.242
\end{tabular} & 25.219 & \begin{tabular}{|l|}
49.407 \\
49.407
\end{tabular} & \begin{tabular}{|l|}
54.0413 \\
\end{tabular} & 17.989 & 32..332 & $\begin{array}{l}-1.050 \\
-1.143\end{array}$ & $\begin{array}{l}-0.05 \\
-6.33\end{array}$ & $\frac{31.024}{49.82}$ & 年 & \begin{tabular}{|l|l|}
14.643 \\
\end{tabular} & & $\begin{array}{l}0001.40 \\
3652.48\end{array}$ & 60.8747 & 53.5 & $\frac{12.4}{11.9}$ & 40 & 2.818 & \begin{tabular}{|c|}
0.006 \\
0.006
\end{tabular} & $\begin{array}{l}0.000 \\
0.006\end{array}$ & $\begin{array}{l}0.000 \\
0.000\end{array}$ & $\frac{0.155}{0.155}$ & 013 \\
\hline & $9 / 1 / 2004$ & 8:46:51 PM & 25.554 & 25.764 & \begin{tabular}{|l|}
24.723 \\
\end{tabular} & 26.222 & 25.059 & 49.096 & \begin{tabular}{|l|l|}
54.391 \\
\end{tabular} & 17.76 & 32.336 & \begin{tabular}{|r|} 
\\
\end{tabular} & -6.336 & 51.11 & $\mid 0.044$ & 14.643 & 0.003 & 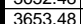 & \begin{tabular}{|l|l|}
600914 \\
\end{tabular} & 53.3 & $\frac{11.5}{12.2}$ & & 2.807 & $\frac{0.000}{0.006}$ & 0.006 & 0.000 & $\frac{0.155}{0.156}$ & \\
\hline & & 8:47:51 PM & 25.544 & 25.744 & & 26.112 & 25.11 & 49.174 & 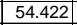 & 17.99 & 32.155 & $\mid-1.105$ & & 50.738 & \begin{tabular}{|c|c|}
0.044 \\
\end{tabular} & \begin{tabular}{|l|l|l|}
14.643 \\
\end{tabular} & & $\frac{3654.48}{3054}$ & & & & & & & & & & \\
\hline & 9/1/2004| & 8:48:51 PM & 25.549 & & 24.733 & & & & 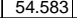 & & 32.332 & -1.128 & & 50.199 & & & & $55^{\circ}$ & 9247 & 53.5 & 12.1 & & 816 & 0.006 & .006 & 0.000 & 0.155 & \\
\hline & & & & & & 26.08 & & & & 18.192 & $\begin{array}{l}32.443 \\
\end{array}$ & & & & & & & & & & & & & & & & & \\
\hline & & $8: 50:$ & 25.536 & 25.736 & \begin{tabular}{|l|l|}
24.715 \\
\end{tabular} & & & 49.4 & & $\begin{array}{l}17.973 \\
\end{array}$ & 32.319 & $\begin{array}{c}-1.096 \\
\end{array}$ & & 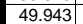 & & $\begin{array}{ll}14.643 \\
\end{array}$ & & 657.48 & & $\overline{53.4}$ & & & & & & & & \\
\hline & 2004 & & 25.544 & 25.744 & \begin{tabular}{|l|l|}
24.732 \\
\end{tabular} & & & & 54.793 & 18.071 & 32.455 & \begin{tabular}{|c|c|}
-1.088 \\
\end{tabular} & & 50.444 & . & & & & & 53.7 & 12.0 & & 827 & $\mid 0.006$ & $\mid .006$ & & & \\
\hline & $9 / 1 / 2004$ & 8:52:51 PM & 25.543 & 25.738 & \begin{tabular}{|l|}
24.752 \\
\end{tabular} & 25.936 & 24.95 & 49.334 & \begin{tabular}{|l|}
54.625 \\
\end{tabular} & 18.014 & 32.272 & -1.09 & & 48.763 & 0.044 & \begin{tabular}{l|l}
14.643 \\
\end{tabular} & 0,00 & 659.48 & 60.9914 & 53.5 & 11.6 & & 2.813 & & 0.0 & & 0.155 & \\
\hline & |2004 & 8:53:51 PM & 25.542 & 25.737 & \begin{tabular}{|l|l}
24.746 \\
\end{tabular} & 25.91 & & & 54.726 & 18.072 & 32.319 & -1.09 & & 51.718 & 0.044 & $\begin{array}{l}14.643 \\
\end{array}$ & 0.00 & 3660.48 & $\begin{array}{ll}61.0081 \\
\end{array}$ & 53.6 & & & $2.821 \mathrm{C}$ & 0.006 & 0.006 & 0.000 & 5.155 & \\
\hline & & & & & 24.73 & & & & \begin{tabular}{|l|}
54.855 \\
\end{tabular} & 17.937 & 32.66 & -1.093 & & 50.769 & & 14. & & & & (1) & 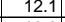 & & & & & & 0.154 & \\
\hline & $901 / 1200$ & $8.555 .51 \mathrm{P}$ & 25.536 & $25 . / 314$ & & & & & & & & -1.088 & & 51.839 & & & & & & & & & 841 & & & & & \\
\hline & & & $\frac{25.541}{25520}$ & & 24.139 & & & & & $\frac{17.671}{19016}$ & $\frac{32.859}{32537}$ & -1.093 & & 50.325 & & 14. & & & & 53.2 & & & 5 & & & & 0.154 & \\
\hline$\frac{2904}{2974}$ & $\begin{array}{l}9 / / 1 / 2004 \\
9 / 1 / 2004\end{array}$ & $8: 5.551 \mathrm{PM}$ & 25.528 & $\frac{25.144}{25724}$ & 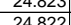 & 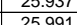 & & & $\begin{array}{l}5.4 .567 \\
5.795\end{array}$ & $\frac{18.016}{18.185}$ & $\begin{array}{l}32.5317 \\
32486\end{array}$ & $\frac{-1.09}{-1088}$ & & (5.452 & 044 & $\begin{array}{l}14.645 \\
1.643\end{array}$ & & & & $\begin{array}{ll}5.4 .4 \\
5.7\end{array}$ & 121 & & & & & & 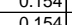 & \\
\hline & $9 / 1 / 1 / 2004$ & 8:59:51 PM & & 25.719 & \begin{tabular}{|l|l|l|}
24802 \\
4
\end{tabular} & 26.011 & 25.01 & & \begin{tabular}{|l|}
54.718 \\
\end{tabular} & $\begin{array}{l}10.105 \\
18.056\end{array}$ & $\begin{array}{l}32.400 \\
32.283\end{array}$ & $\begin{array}{l}-1.000 \\
-1.093\end{array}$ & & 49.918 & 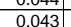 & $\begin{array}{l}\frac{14.045}{14.643} \\
\end{array}$ & & & & 53.6 & $\frac{12}{11}$ & & 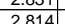 & & & & 0.152 & \\
\hline & & & & & 24808 & & & & \begin{tabular}{|l|}
54.252 \\
\end{tabular} & 18.229 & 31.85 & \begin{tabular}{|c|}
$\mid-1.085$ \\
\end{tabular} & & 51.329 & & 14.6 & & & & 531 & & & & & & & & \\
\hline & & & & & 24.764 & & & & \begin{tabular}{|l|}
54.401 \\
\end{tabular} & & & -1.073 & & & & & & & & & & & & & & & & \\
\hline & & 9:02: & 25.539 & 25.719 & \begin{tabular}{|l|l|}
24.793 \\
\end{tabular} & & & & & 17.921 & $\begin{array}{l}32.044 \\
32\end{array}$ & 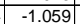 & & & & & & & 581 & 52.9 & 123 & & 791 & & & & 0.157 & \\
\hline & & & 25.546 & 25.721 & 24.78 & & & & 54.598 & & 32.237 & -1.085 & & & & 14.6 & & & & & & & & & & & & \\
\hline & & & & & 24.744 & & & & & & & & & & & & & & & 53.3 & & & 1007 & & & & 56 & \\
\hline 881 & $9 / 1 / 2004$ & & & & 24.765 & & & & 54.65 & & & -1.07 & & 51.061 & & & & & & 53.5 & & & .813 & & & & .155 & \\
\hline & 9/1/20 & 9:06:09 PM & 25. & 25.732 & 24.765 & 26.154 & & & 54.6 & 17.8 & 32.576 & \begin{tabular}{|c|c|} 
\\
\end{tabular} & & & & 14.8 & & & & 53.5 & 12 & & .82 & & & & & \\
\hline & $9 / 1$ & 9:07:C & 25.541 & 25.722 & 24.73 & & & & 54.76 & 17.969 & 32.424 & -1.053 & & 49.528 & & 14. & & & & 53.6 & & & & & & & & \\
\hline & & & & 25.732 & 24.77 & & & & & & & & & & & & & & & 53.4 & & & & & & & & \\
\hline & & & 25.547 & 25.12 & 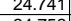 & & & & 54.5 & 17.8 & 32.43 & $-1.0]$ & & & & & & & & 53.4 & & & & & & & & \\
\hline & & & & 25.14 & & & & & & & 32 & & & & & & & & & & & & & & & & . & \\
\hline & & & & & \begin{tabular}{|l|l|l|l|}
24697 \\
\end{tabular} & & & & $\mid \begin{array}{l}5.4523 \\
5443\end{array}$ & 18. & & - & & & & & & & & $\frac{53.4}{5.2}$ & & & & & & & & \\
\hline & $9 / 1 / 1200$ & & 25.549 & 2578 & 24.732 & & & & \begin{tabular}{|l|}
54.463 \\
5
\end{tabular} & & 32354 & \begin{tabular}{|c|c|c|}
$\mid-1001$ \\
\end{tabular} & & & & & & & & 536 & & & & & & & & \\
\hline & & & $2.04+4$ & 2574 & \begin{tabular}{|l|l|}
24715 \\
\end{tabular} & & & & \begin{tabular}{|l|}
55116 \\
5
\end{tabular} & 18.012 & 32711 & \begin{tabular}{|c|c|c|} 
\\
-1015
\end{tabular} & & & & & & & & & & & & & & & & \\
\hline & & & & 25.746 & \begin{tabular}{|l|}
24.715 \\
\end{tabular} & & & & 55.135 & & $\frac{32.812}{32.812}$ & $\begin{array}{r}-0.983 \\
-0.983\end{array}$ & & & & & & & & 54.0 & & & & & & & & \\
\hline & & & 5.55 & 25.7 & 24.695 & & & & 55.2 & 17.5 & 33.183 & -0.9 & & & & & & & & & & & & & & & & \\
\hline & $9 / 1 / 200$ & & & 25.7 & $\begin{array}{l}24.67 \\
\end{array}$ & $\frac{26.24}{20.24}$ & & & 54 & 17.384 & 33.206 & $|-0.989|$ & & & & 14.6 & & & & & & & & & & & & \\
\hline & & & & 25.73 & 24.69 & & & & 54.629 & & 33.36 & -0.957 & & & & & & & & & & & & & & & 53 & \\
\hline & $9 / 1 / 20$ & & & & 24.66 & & & & 54.94 & 17.2 & 33.536 & -0.9 & & & & 14. & & & & & & & & & & & 53 & \\
\hline & $9 / 1 / 20$ & 9:20:09 F & & 25.7. & 24.66 & & & & \begin{tabular}{|l|l|l|}
55.12 \\
\end{tabular} & 17.6 & 33 & -0.9 & & & & 14. & & & & & & & & & & & 0.153 & \\
\hline & $9 / 1 / 200$ & & & 25.7. & 24.683 & & & & 55.185 & 17.537 & 33.27 & -0.946 & & & & & & & & 54.1 & & & & & & & & \\
\hline & 9/1/2004 & 9:22: & & 25.713 & 24.622 & & & & 54.905 & & 33.27 & & & & & & & & & & & & & & & & & \\
\hline & & & & 25.697 & 24.646 & 25.945 & & & 55.0 & & $33.1 / 9$ & -0.96 & & & & & & & & 53.9 & & & & & & & & \\
\hline & $\begin{array}{ll}91 / 1 / 20 \\
0.1 / 20\end{array}$ & & & $\frac{25.68}{2567}$ & $\frac{2.6019}{2.621}$ & & & & $\begin{array}{r}55.12 \\
55.754\end{array}$ & $\frac{1.474}{17.498}$ & $\begin{array}{l}33.233 \\
22.090 \\
2\end{array}$ & -0.975 & & & & 14. & & & & (5.6. & & & & & & & 0.154 & \\
\hline & 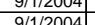 & & & 25.067 & 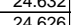 & & & & 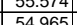 & $\frac{11.4}{177}$ & 3.4599 & $\begin{array}{c}-0.90 \\
0.01\end{array}$ & & & & & & & & & & & & & & & 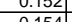 & \\
\hline & & & & $\frac{2.067}{2564}$ & $\frac{24.02}{2460}$ & 25. & & & $\frac{34.5}{549}$ & 172 & 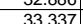 & $=0.031$ & & & & & & & & & & & & & & & 0.154 & \\
\hline & 9/1/1200 & & & 25.639 & 24623 & & & & \begin{tabular}{|l|l|}
54811 \\
\end{tabular} & 11.202 & 33.579 & -0.931 & & & & & & & & & & & & & & & & \\
\hline & & & & & & & & & 55.423 & & 33481 & -0.93 & & & & & & & & 54.3 & & & & & & & & \\
\hline & & & & 25.605 & 24.634 & 25. & & & $\mid$\begin{tabular}{|c|}
55.394 \\
\end{tabular} & 17.4 & 33.54 & & & & & & & & & & & & & & & & & \\
\hline & & & & & & & & & & 18.419 & & -0.986 & & & & & & & & & & & & & & & & \\
\hline & $9 / 1 / 2004$ & IIPM & 466 & 25.571 & 24.639 & 25698 & 247 & 48.7 & 5407 & 17877 & 31.87 & -0.957 & & 51354 & & 146 & & & & 52.9 & 122 & & & & & & 0.151 & \\
\hline & $9 / 1 / 2004$ & & 475 & 55 & & & & & $456:$ & 18.454 & 31.55 & -0.92 & & 51.011 & & 14.643 & & & & 53.4 & & & & & & & & \\
\hline 01 & $9 / 1 / 2$ & $09 \mathrm{PM}$ & 25.47 & 25.575 & \begin{tabular}{|l|l|}
24.679 \\
\end{tabular} & \begin{tabular}{|l|l|}
25.763 \\
\end{tabular} & & 49.067 & \begin{tabular}{|l|l|}
54.225 \\
\end{tabular} & 18.59 & 31.589 & \begin{tabular}{|l|}
$\mid-0.983$ \\
\end{tabular} & & 50.74 & 0.042 & 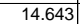 & & & & 53.1 & & & 2.780 & \begin{tabular}{|l|}
0.006 \\
\end{tabular} & 0.006 & 0.000 & $\mid 0.150$ & 0.1 \\
\hline
\end{tabular}


WSRC-TR-2005-00105, REVISION 0

SRNL-RPP-2005-00012, REVISION 0

RUN \# 4.03A AND B; FIRST AND SECOND HALF OF SLURRY DEWATERING - CONT.

\begin{tabular}{|c|c|c|c|c|c|c|c|c|c|c|c|c|c|c|c|c|c|c|c|c|c|c|c|c|c|c|c|c|}
\hline & A & B & D & $E$ & $F$ & G & $\begin{array}{ll}\mathrm{H} \\
\end{array}$ & $\mathrm{J}$ & & & \begin{tabular}{l|l} 
\\
\end{tabular} & $\mathrm{N}$ & 0 & Q & $\mathrm{R}$ & $\mathrm{s}$ & $\begin{array}{c}\mathrm{T} \\
\mathrm{H}\end{array}$ & & w & $x$ & 1 & $z$ & $\mathrm{AA}$ & $\mathrm{AB}$ & $\mathrm{AC}$ & AD & $\mathrm{AE}$ & AF $k$ \\
\hline \begin{tabular}{|c|}
3011 \\
3012
\end{tabular} & 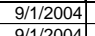 & $\begin{array}{ll}: 35: 09 \mathrm{PM} \\
03: 09 \mathrm{P}\end{array}$ & $\begin{array}{l}25.444 \\
25.454\end{array}$ & $\begin{array}{l}25.555 \\
25.54\end{array}$ & 24.699 & $\begin{array}{l}25.777 \\
25782\end{array}$ & $\begin{array}{l}24.87 \\
2.89\end{array}$ & & $\begin{array}{l}54.25 \\
54.374\end{array}$ & $\begin{array}{l}18.156 \\
18.023\end{array}$ & \begin{tabular}{|l|l|}
31.767 \\
31837
\end{tabular} & \begin{tabular}{|c|}
-0.923 \\
\end{tabular} & $\begin{array}{l}-6.295 \\
-6209\end{array}$ & $\begin{array}{l}50.857 \\
51.883\end{array}$ & $\begin{array}{l}0.043 \\
\end{array}$ & \begin{tabular}{|l|l|l|}
14.643 \\
\end{tabular} & 0.004 & 370178 & 61.6964 & \begin{tabular}{|l|}
53.1 \\
\end{tabular} & 12.1 & 40.3 & $\frac{2.781}{2796}$ & 0.006 & 0.006 & 0.000 & 0.154 & \\
\hline & & $\begin{array}{l}9: 3.3: 09 \mathrm{PM} \\
3: 09 \mathrm{PM}\end{array}$ & $\frac{25.454}{25.445}$ & $\frac{25.54}{25.52:}$ & $\frac{24.71}{24.68}$ & \begin{tabular}{|l|}
25.782 \\
25.808 \\
\end{tabular} & 24.875 & $\begin{array}{r}48.967 \\
49011\end{array}$ & $54.347 \mid$ & $\frac{18.023}{18.255}$ & $\begin{array}{l}31.837 \\
31.862\end{array}$ & -0.928 & & $\frac{51.883}{51.084}$ & & $\begin{array}{l}14.643 \\
14643\end{array}$ & & & & $\frac{53.2}{53.1}$ & & & 1.786 & & & & 0.154 & \\
\hline 3014 & 9/1/2004 & 9:38:09 PM & 25.459 & 25.52. & 24.728 & \begin{tabular}{|l|l|}
25.800 \\
\end{tabular} & $\begin{array}{r}24.875 \\
24.84\end{array}$ & 49.264 & \begin{tabular}{|l|}
54.72615 \\
\end{tabular} & 18.319 & $\begin{array}{l}1.006 \\
31.788 \\
\end{array}$ & - & $\begin{array}{l}-\frac{-2.295}{-6.292} \\
\end{array}$ & 50.93 & $\begin{array}{l}0.043 \\
0.043 \\
\end{array}$ & \begin{tabular}{l|l}
$\frac{14.043}{14.643}$ \\
\end{tabular} & \begin{tabular}{|c|}
0.003 \\
0.003 \\
\end{tabular} & $\begin{array}{l}703.18 \\
704.78\end{array}$ & \begin{tabular}{|l|l|}
61.742964 \\
61.746
\end{tabular} & \begin{tabular}{|}
$\mid 53.1$ \\
53.6
\end{tabular} & \begin{tabular}{|l|l|}
12.2 \\
12.1
\end{tabular} & $\begin{array}{l}40.4 \\
40.5 \\
\end{array}$ & & & $\mid$ & $\frac{0.000}{0.000}$ & & 0.13 \\
\hline 3015 & 9/1/2004 & 9:39:09 PM & $\begin{array}{l}25.47 \\
\end{array}$ & 25.525 & 24.72. & 25.863 & 24.905 & 49.322 & \begin{tabular}{|l|}
54.65 \\
\end{tabular} & 18.146 & 32.239 & -0.928 & -6.295 & 51.593 & 0.043 & \begin{tabular}{|l|l|}
14.643 \\
\end{tabular} & & 3705.78 & \begin{tabular}{|l|l|}
61.7631 \\
\end{tabular} & 53.5 & \begin{tabular}{|l}
12.3 \\
\end{tabular} & & & & 0.006 & 0.000 & & \\
\hline & 9/1/2004 & & & $25.51 \mathrm{~s}$ & & & & 49.444 & \begin{tabular}{|l|l|}
54.666 \\
\end{tabular} & 18.261 & \begin{tabular}{l|l|}
32.262 \\
\end{tabular} & -0.928 & -6.292 & 50.911 & 0.043 & 14.643 & & & \begin{tabular}{|l|l|}
61.7797 \\
\end{tabular} & 53.5 & & & & & & & & \\
\hline & 9/1/2004 & 9:41:09 PM & 25.464 & 25.51 & 24.74 & & & $\begin{array}{l}49.307 \\
\end{array}$ & \begin{tabular}{|l|l|}
54.824 \\
522
\end{tabular} & 18.041 & $\begin{array}{l}32.049 \\
2.19\end{array}$ & -0.931 & $\begin{array}{l}-6.29 \\
\end{array}$ & 49.968 & 0.043 & $\begin{array}{l}14.643 \\
\end{array}$ & & & 61.7964 & 53.7 & & & & & 0.006 & 0.000 & 0.152 & \\
\hline $\begin{array}{l}3018 \\
3019\end{array}$ & 9/1/2004 & 9:42:09 PM & 25.474 & 25.51. & 24.758 & \begin{tabular}{|l|l|}
25.937 \\
\end{tabular} & 25.065 & & & 18.264 & $\begin{array}{l}32.174 \\
\end{array}$ & -0.925 & & 51.349 & 0.043 & \begin{tabular}{|l|l|}
14.643 \\
\end{tabular} & 0.003 & & 1.8131 & 53.5 & \begin{tabular}{|l|l}
12.2 \\
\end{tabular} & 40.8 & 2.816 & 0.006 & 0.006 & 0.000 & 0.152 & \\
\hline 3019 & $\begin{array}{l}9 / 1 / 2004 \\
9 / 1 / 2004\end{array}$ & 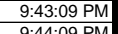 & $\begin{array}{l}25.475 \\
25.472\end{array}$ & 25.5. & 24.77 & \begin{tabular}{|l|}
25.943 \\
25050 \\
\end{tabular} & \begin{tabular}{|l|}
25.07 \\
25.117
\end{tabular} & $\begin{array}{l}49.583 \\
49525\end{array}$ & \begin{tabular}{|l|l|}
54.901 \\
5.920 \\
\end{tabular} & $\begin{array}{l}18.272 \\
18201\end{array}$ & \begin{tabular}{|l|}
32.231 \\
32072
\end{tabular} & $\begin{array}{l}-0.925 \\
-0.98\end{array}$ & $\begin{array}{l}-6.287 \\
.6287\end{array}$ & 50.532 & 0.043 & \begin{tabular}{|l|l|l|l|l|}
14643 \\
\end{tabular} & $\begin{array}{l}0.003 \\
0.023\end{array}$ & $\begin{array}{l}3709.78 \\
277070\end{array}$ & & \begin{tabular}{|l|l|}
53.8 \\
5.3 \\
\end{tabular} & \begin{tabular}{|l|l|}
12.0 \\
121
\end{tabular} & 40.9 & & & 0.0000 & & 0.151 & \\
\hline$\frac{3020}{3021}$ & $\begin{array}{l}9 / 1 / 2004 \\
9 / 1 / 2004\end{array}$ & $\begin{array}{l}9: 44: 09 \mathrm{PM} \\
9: 0509 \mathrm{PM}\end{array}$ & $\begin{array}{l}25.472 \\
25.472\end{array}$ & $\frac{25.496}{25.50}$ & $\frac{24.76}{24.7}$ & $\frac{25.959}{25.974}$ & & $\frac{49.535}{44949}$ & \begin{tabular}{|l|}
54.921 \\
54.465
\end{tabular} & $\frac{18.201}{18.148}$ & \begin{tabular}{|c|}
32.227 \\
32036
\end{tabular} & $\begin{array}{c}-0.928 \\
-0.931\end{array}$ & $\begin{array}{l}-6.287 \\
-6.284\end{array}$ & $\begin{array}{l}50.605 \\
51.187\end{array}$ & 0.043 & $\frac{14.643}{14.643}$ & $\begin{array}{l}0.003 \\
0.003\end{array}$ & 3710.78 & \begin{tabular}{|l|l|}
61.8464 .8631 \\
61.863
\end{tabular} & \begin{tabular}{|l|}
53.8 \\
53.3 \\
\end{tabular} & \begin{tabular}{|l|l|}
12.1 \\
12.2 \\
\end{tabular} & $\begin{array}{l}40.9 \\
40.6 \\
\end{array}$ & 2.819 & 0.006 & $\begin{array}{l}0.006 \\
0.006\end{array}$ & 0.000 & 0.152 & \\
\hline 3022 & 9/1/2004 & 9:46:09 PM & 25.482 & 25.496 & $24.7 \mathrm{~s}$ & & 25.152 & 49.083 & \begin{tabular}{|l|l|}
54.287 \\
\end{tabular} & 18.335 & 31.646 & -0.928 & & 50.131 & $\begin{array}{l}0.045 \\
0.043\end{array}$ & 14.643 & $\begin{array}{l}0.003 \\
0.003\end{array}$ & 3712.78 & 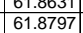 & \begin{tabular}{|l|}
53.2 \\
53.2
\end{tabular} & $\begin{array}{l}1.2 \\
11.9\end{array}$ & \begin{tabular}{|c|c|}
40.6 \\
40.4
\end{tabular} & \begin{tabular}{|l|}
2.802 \\
2.783 \\
\end{tabular} & \begin{tabular}{|c|c|}
0.006 \\
0.006
\end{tabular} & $\begin{array}{l}0.006 \\
0.006\end{array}$ & 0.0000 & $\frac{0.152}{0.153}$ & $\begin{array}{l}0.13 \\
0.13\end{array}$ \\
\hline & 9/1/2004 & 9:47:09 PM & 25.487 & 25.49 & 24.77 & 26.015 & 25.148 & 49.324 & \begin{tabular}{|l|l|}
54.459 \\
\end{tabular} & 18.246 & 32.083 & -0.928 & -6.284 & 50.588 & 0.043 & 14.643 & 0.003 & & \begin{tabular}{|l|l|}
61.8964 \\
\end{tabular} & 53.3 & 12.0 & 40.7 & 2.806 & 0.006 & 0.006 & 0.000 & & \\
\hline & 9/1/2004 & 9:48:09 PM & & & 24.80 & & & 49.226 & \begin{tabular}{|l|l|}
54.513 \\
\end{tabular} & 18.147 & \begin{tabular}{|l|}
32.067 \\
\end{tabular} & & & 50.707 & $\begin{array}{l}0.043 \\
\end{array}$ & \begin{tabular}{|l|l|}
14.643 \\
\end{tabular} & & 3714.78 & \begin{tabular}{|l|l|}
61.9131 \\
\end{tabular} & 53.4 & 12.1 & \begin{tabular}{l|l|}
40.6 \\
\end{tabular} & & & & 0.000 & & \\
\hline 3025 & & 9:49:09 PM & 25.484 & & 24.78 & & & & & & & .931 & & & & 14.643 & & & & & & & & 006 & & & 0.151 & \\
\hline & & & & & & 26.052 & & & & 18.181 & 31.897 & & & & & \begin{tabular}{|l|l|l|l|l|}
1433 \\
\end{tabular} & & & & 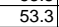 & & & 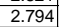 & & & & & \\
\hline & & 9:51:09 PM & 25.5 & 25.48 & 24.82 & 26.008 & & & & 18.057 & 32.502 & -0.928 & & & & $\begin{array}{l}14.643 \\
\end{array}$ & & & 9631 & 53.6 & & 41. & 87 & & & & & \\
\hline & & 9:52:09 PM & & 25.48. & 24.8 & 25.989 & 25.11 & & \begin{tabular}{|l|}
54.679 \\
\end{tabular} & 18.207 & 32.051 & -0.931 & & 52.749 & 0.043 & 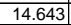 & & 18.78 & \begin{tabular}{|l|l|}
61.9797 \\
\end{tabular} & 53.6 & & & 2.803 & $\mid$ & 0.006 & & & \\
\hline & & 9:53:09 PM & 25.492 & 25.45 & 24.81. & 25.895 & 25.107 & 49. & \begin{tabular}{|l|l|}
54.677 \\
\end{tabular} & 18.401 & 31.85 & -0.931 & & 49.092 & 0.043 & $\begin{array}{l}14.643 \\
\end{array}$ & 0.003 & B719.78 & \begin{tabular}{|l|l|}
61.9964 \\
\end{tabular} & 53.6 & 11.7 & 40.6 & 2.796 & 0.006 & $\begin{array}{l}0.006 \\
\end{array}$ & 0.000 & 0.153 & 0.13 \\
\hline & $9 / 1 / 2004$ & 9:54:09 PM & 25.496 & 25.46. & 24.81. & 25.869 & & 49.436 & \begin{tabular}{|l|}
54.981 \\
\end{tabular} & 18.227 & 32.217 & -0.931 & & 50.012 & 0.043 & 14.643 & 0.003 & & 62.0131 & 53.9 & 11.9 & 40.8 & 2.815 & 0.006 & 0.006 & 0.000 & & \\
\hline & & 9:55:09 PM & & & & & & & & & 32.26 & & & & & 14.643 & & & & & & & .809 & & & & & \\
\hline & & 9:56:09 PM & 25.505 & 25.45 & $24.82 \xi$ & & & 49.108 & \begin{tabular}{|l|}
54.509 \\
\end{tabular} & 18.115 & 32.1 & 0.925 & & 52.071 & & 14.643 & & & & 53.4 & & & & .006 & & & & \\
\hline & & 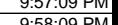 & 25.491 & 25.432 & $\begin{array}{ll}24.80 \\
2.820\end{array}$ & $\frac{25.16}{25723}$ & 24.895 & 49.011 & $\mid$\begin{tabular}{|l|l|}
54.451 \\
5.437
\end{tabular} & $\frac{18.129}{18.07}$ & $\begin{array}{l}31.946 \\
32020\end{array}$ & 0.928 & & $\begin{array}{l}50.348 \\
17486\end{array}$ & & 告14.643 & & 2478 & 年 & (5.3. & 113 & & 796 & (.) & 000 & 然 & 0 & \\
\hline & $11 / 2004$ & $9: 0.0009$ & 25.4909 & $\frac{25.42}{25.41}$ & 24.045 & $\frac{23.150}{25722}$ & & & \begin{tabular}{|l|l|}
54.675 \\
546
\end{tabular} & & $\begin{array}{l}32.024 \\
33026\end{array}$ & & & $\begin{array}{l}4.4070 \\
490707\end{array}$ & 0043 & 14.045 & & & (1) & $\frac{3.2}{5.2}$ & & & & . & & & & \\
\hline 3 & $\frac{1 / 204}{1 / 2004}$ & 10:00:09 PM & $\frac{2.450}{25.502}$ & $\frac{2.41}{25.412}$ & $\frac{24.015}{2485}$ & \begin{tabular}{|l|l|l|l|l|}
25.635 \\
\end{tabular} & & & \begin{tabular}{|l|}
54.986 \\
\end{tabular} & $\frac{10.200}{17.84}$ & $\begin{array}{l}32.020 \\
32.615\end{array}$ & 0 & & 50.571 & 0.040 & $\frac{14.045}{14.643}$ & & 2678 & \begin{tabular}{|l|l|}
62.05131 \\
\end{tabular} & 539 & $\frac{12 .}{12 .}$ & 40. & 2.80 & 006 & 0.006 & & 0151 & \\
\hline & 9/1/2004 & 10:01:09 PM & 25.489 & 25.395 & 24.92 & 25.602 & & 49.164 & \begin{tabular}{|l|}
54.577 \\
\end{tabular} & 18.332 & 31.769 & - 0.928 & & 51.233 & 0.043 & 14.643 & 0.003 & & \begin{tabular}{|l|l|}
62.1131 \\
62.1297
\end{tabular} & 53.5 & $\frac{1.2}{12.2}$ & 40.5 & 2.790 & 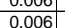 & $\mid$ & .000 & $\frac{0.152}{0.152}$ & $\frac{13}{13}$ \\
\hline & & 10:02:09 PM & 25.497 & & 24.991 & & & & \begin{tabular}{|l|l|} 
& 54.015 \\
\end{tabular} & 18.223 & & 0.891 & & 49.789 & & 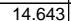 & & & & & & & & & & & 0.154 & \\
\hline & & $9 \mathrm{PM}$ & 25.495 & $25.37 !$ & 24.944 & 25.647 & & & & 18.285 & 31.691 & 0.925 & & 51.343 & & 14.643 & & & & 53.1 & 12.2 & & 78 & | & 0.006 & & 0.153 & \\
\hline & & 10:04:09 PM & 25.514 & $25.38 \mathrm{G}$ & 24.93 & 25.682 & 24.849 & & & 18.358 & 31.56 & 0.891 & & 51.731 & & $\begin{array}{l}14.643 \\
\end{array}$ & & & & 53.2 & 12.3 & & 2.775 & & & & & \\
\hline & $9 / 1 / 2004$ & 10:05:09 PM & 25.519 & $25.37 \mathrm{G}$ & 24.91 & 25.647 & & 48.874 & & 18.25 & 31.519 & -0.92 & & & & 14.643 & & & \begin{tabular}{|l|l|}
62.1964 \\
\end{tabular} & & & & & .006 & & & 0.154 & \\
\hline & $9 / 1 / 2004$ & 10:06: & & 25.37 & 24.88 & \begin{tabular}{|l|}
25.672 \\
\end{tabular} & & 49.148 & \begin{tabular}{|l|}
54.501 \\
\end{tabular} & 18.367 & 31.942 & -0.899 & & 50.815 & . .043 & 14.643 & & & $\mid 2131$ & 53.4 & & & 1.795 & 006 & & & & \\
\hline & & 10:07:09 PM & 25.513 & 25.36: & 24.88 & 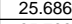 & & & \begin{tabular}{|l|} 
\\
\end{tabular} & 18.36 & 31.946 & -0.908 & & 48.802 & & 14.643 & & & & 53.8 & 11.6 & & & & & & 0.152 & 1 \\
\hline & & 10:08:09 PM & 25.524 & 25.366 & 24.86: & 25.762 & & 49.166 & 54.596 & 18.142 & 31.948 & -0.902 & & 52.34 & 0.043 & 14.643 & 0.003 & 734.78 & 62.2464 & 53.5 & 12.5 & 40.6 & 2.796 & 0.006 & 0.006 & 0.000 & 0.152 & \\
\hline & & & & & & & & & & & & & & & & & & & & 53.4 & & & & & & & & \\
\hline & & & & & & & & & & & & 0.914 & & & & & & & & 53.3 & & & & & & & & \\
\hline & 91/1/2004 & $10.1 .1050 \mathrm{PM}$ & $\begin{array}{r}2.533 \\
25515\end{array}$ & $\begin{array}{l}25.306 \\
2525\end{array}$ & 24.835 & $\begin{array}{l}25.848 \\
25832\end{array}$ & 24 & $\begin{array}{l}48.944 \\
49.34\end{array}$ & \begin{tabular}{|l|}
54.3441 \\
54542
\end{tabular} & $\frac{18.23}{18.289}$ & 31.589 & - & & 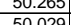 & 0043 & $\begin{array}{l}14.643 \\
1.643\end{array}$ & & & $\begin{array}{l}62.2964 \\
6623131\end{array}$ & 53.2 & & & 2804 & 0006 & م0006 & . & 0 & 011 \\
\hline & $9 / 1 / 2004$ & & 25.526 & 25.37 & $2481:$ & 25.064 & 25.117 & 493 & \begin{tabular}{|l|}
54.683 \\
\end{tabular} & 18.189 & 32088 & $\frac{-0.025}{-0.925}$ & & 50.43 & 0.043 & 14.643 & & & & 53.6 & 12. & & & & & & & \\
\hline & & 10:14 & & & 24.796 & \begin{tabular}{|l|}
25.905 \\
\end{tabular} & & & \begin{tabular}{|l|}
54.776 \\
\end{tabular} & 18.28 & 32.069 & -0.92 & & & & 14.643 & & & & 53.7 & 11.9 & & & & & & & 0.1 \\
\hline 3051 & & 10:15: & 25.524 & 25.368 & 24.79 & \begin{tabular}{|l|}
25.891 \\
\end{tabular} & & & \begin{tabular}{|l|l|} 
\\
\end{tabular} & 18.23 & 31.946 & -0.894 & & 48.9 & 0.043 & 14.643 & & & 62.3631 & 53.3 & 11.7 & & 2.796 & 0.006 & 0.006 & 0.000 & 0.153 & \\
\hline & & & & & & & & & 54.500 & & & & & & & & & & & & & & & & & & & \\
\hline & & & & & & 25.88 & & & & & & & & & & & & & & 53.2 & & & & & & & & \\
\hline & & & $\begin{array}{l}25.503 \\
25497\end{array}$ & & 24.152 & & & & 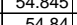 & 18.318 & 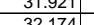 & -0.8855 & & & & & & & & (5.1 & 11 & & & & & & 0 & \\
\hline & $\frac{31 / 2}{91 / 2}$ & & $\frac{25.491}{25475}$ & $\frac{23.30}{2532}$ & $\frac{24.12}{2470}$ & 25.14 & & & \begin{tabular}{|r|r|}
54.654 \\
\end{tabular} & $\frac{10.2}{1.9 .1}$ & & - & & & & & & & & & & & & & & & & \\
\hline & & & $\frac{20.4196}{25469}$ & & 2469 & 25.0502 & & & \begin{tabular}{|l|}
54350 \\
5435
\end{tabular} & 17869 & 321 & - & & & & $\frac{14.0}{146}$ & & & & 532 & $\frac{11}{12}$ & & & 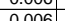 & & & 0.100 & 0.1. \\
\hline 305 & 9/1/2004 & 10:22: & 25.452 & $\frac{25.31}{25.31}$ & 24.6 & 25.604 & & & $\mid$\begin{tabular}{|c|c|c|}
54.666 \\
\end{tabular} & 17.82 & 32.256 & $\mid \begin{array}{l}-0.079 \\
-0.879\end{array}$ & & 49.199 & $\frac{0.044}{0.044}$ & 14.6 & & & & 53.5 & 11.7 & & & & & & . & \\
\hline & & & & & & & & & & & & & & & & & & & & & & & & & & & & \\
\hline & & & & & 24. & & & & & & & & & & & & & & & & & & & & & & & \\
\hline & & & & & 24. & & & & 54.641 & 17.8 & 32.486 & & & & & & & & & & & & & & & & & \\
\hline & & 10:26:09 PM & 25.448 & 25.3 & 24.6 & \begin{tabular}{|l|l|}
25.7 \\
\end{tabular} & & & \begin{tabular}{|l|l|}
54.393 \\
\end{tabular} & 17.4 & 32.6 & & & & & & & & & 53.3 & 12.0 & & 2.8 & 6 & & & 0.152 & \\
\hline & & & 25.448 & 25. & 24.6 & 25. & & & $\begin{array}{l}55.1 \\
\end{array}$ & 17.97 & 32 & -0.876 & & & 0.04 & 14.6 & & & & 54.0 & $\overline{11}$ & & $2.8 \mathrm{r}$ & D006 & & & 0.155 & \\
\hline & 9/1/2 & $10: 28$ & 25.443 & 25.31 & 24.6 & 25.7 & & & 54.8 & & & & & & & 14. & & & & & 11. & & & & & & & \\
\hline & & & 25.427 & 25.306 & & 25. & & & 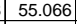 & 18.012 & & -0.8 & & & 0 & & & & & 53.9 & 11.4 & & & & & & 0.155 & \\
\hline & & & & & & 25 & & & & & & & & & & & & & & & & & & & & & & \\
\hline & & & & 25.312 & 24.66. & 25 & & & 54.492 & $17.9 \mathrm{~s}$ & 31.9 & & & & & & & & & & & & & & & & & \\
\hline & & & & 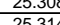 & 24.682 & 25.16 & & & & 1.1 & & & & & & & & & & & & & 20 & & & & 50 & \\
\hline & & & & $\frac{2.301}{2530}$ & $\frac{2.000}{2467}$ & $\frac{2.0}{258}$ & & & & 17.073 & & $=0.076$ & & & & & & & & & & & & & & & & \\
\hline & & & & 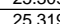 & 24678 & & & & & 173 & & & & & & & & & & & & & & & & & & \\
\hline 301 & & & & 25 & 24.66 & 25.884 & & & $\mid$\begin{tabular}{|l|}
$\mid 54.744$ \\
\end{tabular} & 17.72 & & -0.815 & & & 0.044 & & & & & 53.6 & 12 & & & & & & & \\
\hline & & & & & & & & & & & & & & & & & & & & & & & & & & & & \\
\hline & & & & & & & & & & & & & & & & & & & & & & & & & & & & \\
\hline & & & & 25.326 & 24.6 & $25.8 \varepsilon$ & & 49 & \begin{tabular}{|l|}
547 \\
\end{tabular} & 17.833 & & -0.847 & & & & 11 & & & & & & & & & & & 48 & \\
\hline 107 & $9 / 1 / 2$ & 10:40: & & 5344 & 24.676 & 25.8 & & 49.7 & \begin{tabular}{|l|l|}
55.174 \\
\end{tabular} & 17.8 & 32.8 & -0.81 & & $\overline{50.3}$ & & 14.6 & & & & 5 & & & 28 & & & & & \\
\hline & $\mid$ & & & & $24.66 \mathrm{t}>\mathrm{s}$ & & & 49. & & 17.463 & $32.9->$ & & & 50.0 & & 14.6 & & & & 53 & 110 & & & & & & & \\
\hline & & & & 25.3 & 24.70 & 25.876 & 25.0 & 49 & & 17.681 & 32.488 & -0.813 & & & 0.044 & 14.6 & & & & 53.5 & 12 & & & & & & 155 & \\
\hline sorg & & 10:43:09 PM & 25.408 & 25.33 & 24.66. & 25.85 & & 49.403 & 54.826 & 17.706 & 32.646 & -0.818 & & & 0.043 & 14.643 & & & & 53.7 & 12.0 & & & 006 & 0.0 & 0.000 & 0.151 & \\
\hline 3080 & 9/1/2004 & 10:44:09 PM & 25.403 & 25.34 & 24.69 & 25.881 & & 49.591 & 55.06 & 17.515 & \begin{tabular}{|l|}
33.114 \\
\end{tabular} & -0.81 & & 47.732 & 0.043 & 14.643 & & 770.78 & 62.8464 & 53.9 & 11.4 & $\mid 41.4$ & 2.851 & 0.006 & 0.006 & 0.000 & 0.150 & 0.1 \\
\hline
\end{tabular}


WSRC-TR-2005-00105, REVISION 0

SRNL-RPP-2005-00012, REVISION 0

RUN \# 4.03A AND B; FIRST AND SECOND HALF OF SLURRY DEWATERING - CONT.

\begin{tabular}{|c|c|c|c|c|c|c|c|c|c|c|c|c|c|c|c|c|c|c|c|c|c|c|c|c|c|c|c|c|}
\hline & $\mathrm{A}_{\mathrm{A}}$ & $B$ & D & $E$ & $F$ & $G$ & $\mathrm{H}$ & $\mathrm{J}$ & \begin{tabular}{l|l|}
$\mathrm{K}$ & \\
\end{tabular} & $\mathrm{L}$ & $M$ & $\mathrm{~N}$ & 0 & 0 & $R$ & $\mathrm{~s}$ & $\begin{array}{ll}T \\
\end{array}$ & $\mathrm{v}$ & w & $x$ & $Y_{Y}$ & $z_{2}$ & AA & & & & & \\
\hline & 9/1/2004 & 10:45:09 PM & 25.41 & 25.354 & 24.683 & 25.893 & 25.195 & 49.564 & 55.267 & 17.622 & 32.818 & -0.833 & -6.255 & 49.464 & 0.043 & 14.643 & 0.003 & 3771.78 & \begin{tabular}{|l|}
62.8631 \\
\end{tabular} & 54.1 & 11.8 & 41.2 & 2.840 & $\frac{A B}{0.006}$ & 0.006 & 0.000 & $\begin{array}{l}\frac{A E}{0.151} \\
\end{array}$ & \\
\hline & $\begin{array}{l}9 / 1 / 1 / 2004 \\
\end{array}$ & & 25.415 & 25.359 & 24.678 & \begin{tabular}{|l|}
25.918 \\
\end{tabular} & & $\begin{array}{r}49.882 \\
\end{array}$ & 55.371 & 17.842 & 32.976 & $\begin{array}{l}-0.81 \\
-0.81\end{array}$ & & 51.429 & 0.044 & \begin{tabular}{|l|l|}
14.643 \\
\end{tabular} & 0.003 & & \begin{tabular}{|l|l|}
62.8797 \\
\end{tabular} & 54.2 & 12.2 & & & 0.006 & 0.006 & & & \\
\hline & $9 / 1 / 2004$ & 10:47:09 PM & 25.431 & 25.375 & \begin{tabular}{|l|}
24.7144 \\
2.50 \\
\end{tabular} & \begin{tabular}{|l|}
25.913 \\
2503 \\
\end{tabular} & 25.061 & $\begin{array}{r}49.469 \\
\end{array}$ & 54.969 & \begin{tabular}{|l|}
17.65 \\
17.04
\end{tabular} & 32.644 & $\begin{array}{l}-0.81 \\
-0.89 \\
\end{array}$ & & 50.175 & 0.043 & 14.643 & 0.003 & \begin{tabular}{|l|l|}
3773.78 \\
277.78
\end{tabular} & 62.8964 & \begin{tabular}{|l|}
53.8 \\
\end{tabular} & $\frac{12.0}{12.0}$ & & - & 0.006 & 0.006 & 0.000 & 0.151 & \\
\hline 3084 & 9/1/2004 & 10:48:09 PM & 25.426 & 25.371 & \begin{tabular}{|l|l|}
24.695 \\
\end{tabular} & \begin{tabular}{|l|}
25.929 \\
\end{tabular} & 25.072 & $\begin{array}{l}49.569 \\
\end{array}$ & $\begin{array}{l}55.046 \\
\end{array}$ & $\begin{array}{l}17.794 \\
\end{array}$ & \begin{tabular}{|l|l|}
32.81 \\
\end{tabular} & -0.81 & -6.252 & 49.672 & $\begin{array}{l}0.043 \\
\end{array}$ & $\begin{array}{l}14.643 \\
\end{array}$ & 0.003 & 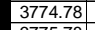 & 2.9131 & \begin{tabular}{|l|l|}
53.9 \\
\end{tabular} & 11.8 & $\begin{array}{l}41.2 \\
\end{array}$ & 2.840 & 0.006 & $\begin{array}{l}0.006 \\
\end{array}$ & 0.000 & 0.151 & \\
\hline & $\frac{9 / 1 / 2004}{99 / 1 / 2004}$ & $\begin{array}{l}10: 49: 09 \mathrm{PM} \\
100509 \mathrm{PM}\end{array}$ & $\frac{25.421}{25.422}$ & $\frac{25.371}{25.377}$ & \begin{tabular}{|l|}
24.705 \\
24.701 \\
\end{tabular} & \begin{tabular}{|l|}
25.899 \\
25.925 \\
\end{tabular} & $\begin{array}{r}25.027 \\
2507\end{array}$ & \begin{tabular}{|l|l|}
49.645 \\
491818
\end{tabular} & & \begin{tabular}{|r|}
17.601 \\
1757
\end{tabular} & & $\begin{array}{r}-0.81 \\
081 \\
\end{array}$ & & $\begin{array}{l}51.452 \\
50.548\end{array}$ & \begin{tabular}{|l|l|}
0.043 \\
0.044
\end{tabular} & \begin{tabular}{|l|l|l|}
14.643 \\
14634 \\
\end{tabular} & & \begin{tabular}{|l|}
3775.78 \\
377.
\end{tabular} & \begin{tabular}{|l|}
62.9297 \\
60961
\end{tabular} & \begin{tabular}{|l|}
53.9 \\
\end{tabular} & \begin{tabular}{|l|}
12.3 \\
10. \\
\end{tabular} & & \begin{tabular}{|l|}
2.849 \\
\end{tabular} & & & & 0.150 & \\
\hline $\begin{array}{l}3000 \\
3087 \\
\end{array}$ & & $\begin{array}{l}\frac{1050: 09 P M}{10: 51: 09 ~ P M} \\
\end{array}$ & $\frac{25.422}{25.429}$ & $\begin{array}{l}25.377 \\
25.383\end{array}$ & $\begin{array}{l}24.701 \\
24.707\end{array}$ & \begin{tabular}{|l|}
25.925 \\
25.951 \\
\end{tabular} & $\begin{array}{l}25.087 \\
25.144\end{array}$ & $\mid$\begin{tabular}{|}
$\mid 49.118$ \\
49.064
\end{tabular} & \begin{tabular}{|}
$\mid 5.4362$ \\
54.239
\end{tabular} & \begin{tabular}{|}
$\mid 17.57$ \\
17.668
\end{tabular} & $\begin{array}{l}32.438 \\
32.531 \\
\end{array}$ & $\begin{array}{r}-0.811 \\
-0.807\end{array}$ & $\begin{array}{l}-6.246 \\
-6.237\end{array}$ & $\begin{array}{l}50.548 \\
51.881\end{array}$ & $\begin{array}{l}0.044 \\
0.044\end{array}$ & \begin{tabular}{|c|}
14.643 \\
11.4633
\end{tabular} & $\begin{array}{l}0.0033 \\
0.003 \\
\end{array}$ & \begin{tabular}{|l|}
3776.78 \\
3777.78
\end{tabular} & \begin{tabular}{|l|}
62.9464 \\
62.9631 \\
\end{tabular} & \begin{tabular}{|l|}
53.2 \\
53.1 \\
\end{tabular} & \begin{tabular}{|l|}
12.0 \\
12.4 \\
\end{tabular} & 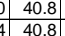 & \begin{tabular}{|l|}
2.812 \\
2.813 \\
\end{tabular} & \begin{tabular}{|c|c|}
0.006 \\
0.006
\end{tabular} & \begin{tabular}{|c|}
0.006 \\
0.006
\end{tabular} & $\begin{array}{l}0.000 \\
0.000\end{array}$ & 0.156 & 0.13 \\
\hline 3088 & $9 / 1 / 2004$ & 10:52:09 PM & 25.429 & 25.384 & \begin{tabular}{|l|}
24.708 \\
\end{tabular} & \begin{tabular}{|l|}
25.932 \\
\end{tabular} & 25.129 & & & & 32.898 & & -6.258 & 50.751 & 0.043 & 14.643 & $\begin{array}{l}0.003 \\
0.003\end{array}$ & \begin{tabular}{|c|}
3777.18 \\
378.78
\end{tabular} & 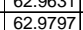 & \begin{tabular}{|l|}
53.6 \\
\end{tabular} & $\begin{array}{l}\frac{12.4}{12.1} \\
\end{array}$ & & $\frac{2.813}{2.835}$ & & $\begin{array}{l}0.006 \\
0.006\end{array}$ & $\begin{array}{l}0.000 \\
0.000\end{array}$ & & $\begin{array}{l}0.13 \\
0.13 \\
\end{array}$ \\
\hline & & 10:53:09 PM & & & 24.709 & 25.973 & & 49.649 & 55.184 & 18.042 & 32.504 & -0.772 & -6.246 & 50.206 & $\begin{array}{l}0.043 \\
\end{array}$ & 14.643 & & & \begin{tabular}{|l|l|}
62.9964 \\
\end{tabular} & \begin{tabular}{|l|}
54.1 \\
\end{tabular} & & & & & & & & \\
\hline & 9/1/2004 & 10:54:09 PM & 25.446 & 25.4 & \begin{tabular}{|l|l|}
24.734 \\
\end{tabular} & \begin{tabular}{|l|}
25.939 \\
\end{tabular} & 25.141 & $\begin{array}{l}49.608 \\
\end{array}$ & & \begin{tabular}{|l|l|}
17.863 \\
\end{tabular} & \begin{tabular}{|l|l|}
32.744 \\
\end{tabular} & $\begin{array}{r}-0.81 \\
\end{array}$ & -6.24 & 49.368 & .044 & \begin{tabular}{|l|l|}
14.643 \\
\end{tabular} & & 3780.78 & & \begin{tabular}{|l|l|} 
& 53.9 \\
\end{tabular} & 11.8 & & 2.839 & & \begin{tabular}{|c|}
0.006 \\
\end{tabular} & 0.000 & 0.154 & \\
\hline 3091 & & 10:55:09 PM & 25.442 & & & & & & & & & $\mid-0.81$ & & & & $\begin{array}{l}14.643 \\
\end{array}$ & & & & 53.8 & & & & . & . & 0.000 & 0.147 & \\
\hline & & 10:56:09 PM & & 25.403 & & & & 49.44 & & 17.568 & & -0.815 & & 49.902 & & \begin{tabular}{ll|l}
14.643 \\
\end{tabular} & & & & 539 & 11.9 & & & & & & .151 & \\
\hline & & 10:57:09 PM & 25.444 & 25.408 & 24.732 & 25.961 & & & 54.598 & 17.821 & 2.225 & -0.813 & & & & 14.643 & & & & 53.5 & 1 & & .806 & & & & & \\
\hline & & 10:58:09 PM & 25.449 & 25.408 & 24.717 & 25.966 & & 49.535 & \begin{tabular}{|l|l|}
55.07 \\
\end{tabular} & 17.868 & 32.34 & \begin{tabular}{|c|c|}
-0.772 \\
\end{tabular} & & 51.149 & 0.042 & \begin{tabular}{|l|l|l|l|}
14.643 \\
\end{tabular} & 0.003 & 7884.78| & \begin{tabular}{|l|l|}
63.0797 \\
\end{tabular} & & & & 2.822 & \begin{tabular}{|l|l|l|}
0.006 \\
\end{tabular} & $\begin{array}{l}0.006 \\
\end{array}$ & 0.000 & 0.148 & \\
\hline & $1 / 2004$ & 10:59:09 PM & 25.454 & 25.413 & \begin{tabular}{|l|}
24.757 \\
\end{tabular} & \begin{tabular}{|l|}
25.921 \\
\end{tabular} & 25.159 & & 55.442 & \begin{tabular}{|l|l|}
17.817 \\
\end{tabular} & 32.845 & -0.781 & & 49.512 & $\begin{array}{l}0.043 \\
\end{array}$ & \begin{tabular}{|l|l|}
14.643 \\
\end{tabular} & 0.003 & 3785.78 & \begin{tabular}{|l|l|}
63.0964 \\
\end{tabular} & $\begin{array}{l}54.3 \\
\end{array}$ & 11.8 & & 2.848 & & & & & \\
\hline & & 11:00:09 PM & & & \begin{tabular}{|l|l|}
24.748 \\
\end{tabular} & \begin{tabular}{|l|l|}
25.967 \\
\end{tabular} & 25.135 & $\begin{array}{l}49.649 \\
\end{array}$ & \begin{tabular}{|l|l|}
55.193 \\
5
\end{tabular} & 17.932 & $\begin{array}{l}32.58 \\
2.700\end{array}$ & -0.795 & & 50.778 & $\begin{array}{l}0.043 \\
\end{array}$ & $\begin{array}{l}14.643 \\
\end{array}$ & 0.003 & & \begin{tabular}{|l|l|}
63.1131 \\
\end{tabular} & $\begin{array}{l}54.1 \\
5.0\end{array}$ & 12.1 & & & & & & 0.151 & \\
\hline & 1/2004 & 11:00:47 PM & 25.449 & 25.414 & \begin{tabular}{|l|l|}
24.733 \\
\end{tabular} & \begin{tabular}{|l|}
25.972 \\
\end{tabular} & & & 55.149 & 17.726 & & & -6.246 & 49.952 & & $\begin{array}{l}14.643 \\
\end{array}$ & & 3787.42 & 3.1236 & 54.0 & 11.9 & & & & 0.006 & & 0.147 & \\
\hline & & 11:01:47 PM & $\begin{array}{l}25.465 \\
25.171\end{array}$ & 25.435 & & \begin{tabular}{|l|}
25.948 \\
\end{tabular} & 25.155 & & & 17.851 & 32.539 & & & & & $\begin{array}{l}14.643 \\
\end{array}$ & 0.003 & & 63.1403 & 53.9 & 11.5 & & 2.827 & 0.006 & 0.006 & 0.000 & 0.151 & \\
\hline & 1/12004 & $\begin{array}{ll}1: 02: 47 \text { PM } \\
11: 0: 07 \text { P }\end{array}$ & $\begin{array}{l}25.471 \\
25.471\end{array}$ & $\begin{array}{l}25.436 \\
25.366\end{array}$ & $\begin{array}{l}24.765 \\
24775\end{array}$ & \begin{tabular}{|l|l|}
25.999 \\
25.974
\end{tabular} & $\begin{array}{l}25.191 \\
25101\end{array}$ & & $\begin{array}{l}55.114 \\
55119\end{array}$ & \begin{tabular}{ll|l}
17.778 \\
17596
\end{tabular} & \begin{tabular}{|l|}
32.662 \\
3288
\end{tabular} & \begin{tabular}{|c|c|}
-0.763 \\
.0763
\end{tabular} & & \begin{tabular}{|l|l|}
49.109 \\
50118
\end{tabular} & $\begin{array}{l}0.044 \\
\end{array}$ & \begin{tabular}{|l|l|}
14.643 \\
14643
\end{tabular} & & & $\begin{array}{l}33.1569 \\
3.1732\end{array}$ & \begin{tabular}{|l|l|}
54.0 \\
540
\end{tabular} & $\begin{array}{ll}11.7 \\
119\end{array}$ & & 2.835 & $\begin{array}{l}0.006 \\
0006\end{array}$ & $\begin{array}{l}0.006 \\
006\end{array}$ & 0.000 & 0.154 & 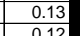 \\
\hline & 2004 & $\begin{array}{l}\frac{11003: 47 \text { PM }}{11: 4477} \\
\end{array}$ & $\frac{25.471}{25.482}$ & $\begin{array}{l}25.436 \\
25.442\end{array}$ & $\begin{array}{l}24.775 \\
24.781\end{array}$ & \begin{tabular}{|l|}
25.974 \\
25.915 \\
\end{tabular} & $\begin{array}{l}25.101 \\
25.068\end{array}$ & & $\mid$\begin{tabular}{|}
55.149 \\
5.4917
\end{tabular} & $\begin{array}{r}17.596 \\
17.245 \\
\end{array}$ & \begin{tabular}{|l|}
32.828 \\
33.214 \\
\end{tabular} & $\begin{array}{c}-0.763 \\
-0.76\end{array}$ & -6.24 & $\begin{array}{l}50.158 \\
49.226\end{array}$ & $\frac{1042}{0.42}$ & \begin{tabular}{|c|}
14.643 \\
14.643
\end{tabular} & $\begin{array}{l}0.003 \\
0.003\end{array}$ & 0.42 & 3..1736 & $\begin{array}{l}54.0 \\
53.8 \\
\end{array}$ & $\frac{11.9}{11.7}$ & & $2.841 \mid$ & \begin{tabular}{|l|l|}
0.006 \\
0.006
\end{tabular} & $\begin{array}{l}0.006 \\
0.006\end{array}$ & 0.000 & 0.147 & 0.12 \\
\hline 3102 & \begin{tabular}{|l|}
$91 / 2004$ \\
$9 / 1 / 2004$
\end{tabular} & $\begin{array}{l}11.04 .44 \mathrm{FM} \\
11: 05: 47 \mathrm{PM}\end{array}$ & $\frac{25.482}{25.472}$ & $\frac{25.444}{25.432}$ & 24.781 & \begin{tabular}{|l|}
25.910 \\
2545 \\
\end{tabular} & 25.008 & \begin{tabular}{|l|}
49.931 \\
\end{tabular} & 55.498 & 17.677 & $\begin{array}{l}3.2414 \\
3.144\end{array}$ & $\begin{array}{r}-0.176 \\
-0.763 \\
\end{array}$ & -6.249 & 51.076 & $\begin{array}{l}0.044 \\
0.041\end{array}$ & \begin{tabular}{l|l}
$\frac{14.043}{14.643}$ &
\end{tabular} & $\begin{array}{l}.0003 \\
0.00\end{array}$ & $\begin{array}{l}3791.42 \\
3792.42\end{array}$ & \begin{tabular}{|l|l|}
63.20695 \\
63.2069
\end{tabular} & $\begin{array}{l}33.8 \\
54.4 \\
\end{array}$ & \begin{tabular}{|l|}
11.1 \\
12.2 \\
\end{tabular} & $\frac{41.3}{2} 41.5$ & $\frac{2.849}{2.864}$ & \begin{tabular}{|l|l|}
0.006 \\
0.006
\end{tabular} & $\begin{array}{l}0.006 \\
0.006\end{array}$ & $\begin{array}{l}0.000 \\
0.000\end{array}$ & $\frac{0.140}{0.142}$ & $\begin{array}{l}0.12 \\
0.12\end{array}$ \\
\hline 3103 & 9/1/2004 & 11:06:47 PM & 25.487 & 25.442 & 24.801 & 25.9 & 25.048 & 50.066 & 55.632 & 17.916 & 32.925 & -0.76 & & $\begin{array}{l}49.883 \\
\end{array}$ & 0.043 & 14.643 & 0.003 & 3793.42 & $\begin{array}{l}63.2236 \\
\end{array}$ & 54.5 & 11.9 & & 2.861 & 0.006 & 0.006 & 0.000 & 0.149 & \\
\hline & & 11:07:47 PM & 25.477 & 25.426 & 24.775 & & 24.90 & & & 17.305 & & $\mid-0.763$ & & 49.092 & & \begin{tabular}{l|l|l}
14.643 \\
\end{tabular} & & & & & & & & & & & & \\
\hline & & 11:08:47 PM & & & & 25.778 & & & 55.187 & 17.782 & 32.818 & & & 49.568 & & \begin{tabular}{|l|l|l|l}
14.643 \\
\end{tabular} & & & 2569 & 54.1 & 11.8 & & & 0.006 & 5.006 & & 0.150 & \\
\hline & & 11:09:47 PM & & & & & & & & 18.152 & 32.64 & $\mid-0.76$ & & & & 14.643 & & & & & & & & & & & & \\
\hline & & 11:10:47 PM & 25.473 & 25.428 & 24.852 & \begin{tabular}{|l|l|}
25.73 \\
\end{tabular} & 24. & 49.442 & & & 31.995 & -0.763 & & & & 14.643 & & 3797.42 & & 53.9 & 1.8 & & 80 & & & & 0.148 & \\
\hline & 1004 & 11:11:47 & 25.482 & 25.426 & \begin{tabular}{|l|}
24.87 \\
\end{tabular} & \begin{tabular}{|l|}
25.689 \\
\end{tabular} & 24.80 & 49.349 & 54.923 & 17.981 & 32.352 & -0.763 & & 50.713 & 0.043 & 14.643 & & 3798.42 & 3.3069 & 53.8 & 12.1 & & 816 & .006 & 0.006 & & & \\
\hline & 1/2004 & 11:12:47 PM & 25.475 & 25.425 & \begin{tabular}{|l|}
24.844 \\
\end{tabular} & \begin{tabular}{|l|l}
25.713 \\
\end{tabular} & 24. & 49.892 & 55.388 & 18.387 & 32.492 & $\begin{array}{l}-0.763 \\
\end{array}$ & & 50.83 & .043 & 14.643 & & 3799.42 & & 54.3 & 12.1 & & 8.840 & & & & 0.150 & \\
\hline & & & 25.49 & 25.435 & 24.859 & \begin{tabular}{|l|l|}
25.753 \\
\end{tabular} & & 49. & 55.002 & 18.010 & $\begin{array}{l}32.143 \\
2.211\end{array}$ & $\begin{array}{l}-0.76 \\
\end{array}$ & & 49.583 & 043 & 14.643 & & & & & & & & & & & & \\
\hline & & $11: 14: 4 / \mathrm{PM}$ & 25.49 & 25.43 & 24.849 & 25.783 & & 49.1 & 54.78 & 17.79 & 32.311 & -0.758 & & & & 14.643 & & & & 53.7 & & & & & & & 148 & \\
\hline & & II:15: & 25.49 & 25.43 & 24.814 & \begin{tabular}{|l|}
25.813 \\
\end{tabular} & & & 55.052 & 1.1 .944 & 32.586 & $\begin{array}{l}-0.743 \\
\end{array}$ & & & & & & & $3 / 36$ & 53.9 & 11.8 & & & & & & 151 & \\
\hline & & $\begin{array}{ll}11: 16: 47 \text { PM } \\
11117: 77 \text { M }\end{array}$ & $\begin{array}{r}25.5 \\
55.11 \\
\end{array}$ & $\frac{25.435}{25.435}$ & $\begin{aligned} 24.844 \\
24825\end{aligned}$ & \begin{tabular}{|l|}
25.813 \\
25813
\end{tabular} & $\frac{24 .}{24}$ & $\frac{49.606}{4933}$ & 55.153 & $\frac{17.675}{17.771}$ & $\begin{array}{l}32.935 \\
32545\end{array}$ & $\begin{array}{l}-0.731 \\
-0.703\end{array}$ & & & & $\frac{14.643}{14643}$ & & & 84069 & $\frac{54.0}{53.8}$ & 116 & & & & & & & \\
\hline & $9 / 1 / 2004$ & 11:18: & 25.502 & 25.441 & 24.825 & \begin{tabular}{|l|}
25.804 \\
\end{tabular} & 24.8 & 49.3 & 54.814 & 17.788 & 32.588 & $\begin{array}{l}0.072 \\
-0.72\end{array}$ & & 48.098 & 0.043 & $\begin{array}{l}14.045 \\
14.643\end{array}$ & & & t236 & 537 & 11.5 & & & 年 & & & & \\
\hline & & 11:19:47 PM & $\frac{2.002}{25.502}$ & 25,431 & $\frac{24.020}{24.79}$ & \begin{tabular}{|l|}
25.784 \\
\end{tabular} & & 49.4 & 55.025 & 17.675 & 32.806 & $\mid-0.703$ & & 51.028 & & $\frac{14.045}{14.643}$ & & & & 539 & $\frac{11.2}{12.2}$ & & 836 & & & & $\frac{0.154}{0.154}$ & \\
\hline & & 11:20:47 & & 25.436 & 24.795 & 25.834 & 24. & & 55.087 & 17.653 & 32.941 & \begin{tabular}{|c|}
-0.697 \\
\end{tabular} & & 52.5 & .043 & & & & & 54.0 & & & 843 & & & & & \\
\hline & & 11:21:47 PM & 25.482 & 25.416 & & & & & & & & -0.7 & & & & & & & & 54.0 & 127 & & & & & & & \\
\hline & & :22:4 & 25.502 & 25.432 & 24.781 & 25.88 & & & 55.164 & 17.8 & 32.576 & -0.697 & & & & & & & 3 & 54.0 & 12.3 & & & & & & & \\
\hline & & 11:23: & 25.487 & 25.427 & & & & & & & \begin{tabular}{|l|l|l|l|}
32.984 \\
\end{tabular} & $\begin{array}{l}-0.7 \\
\end{array}$ & & & & & & & & 54.1 & & & & & & & & \\
\hline & & 11:24:47 PM & & 25.438 & \begin{tabular}{|l|l|}
24.772 \\
\end{tabular} & \begin{tabular}{|l|}
25.871 \\
\end{tabular} & & & 55.124 & 17.874 & 32.537 & -0.7 & & & & & & & & 54.0 & 11.7 & & & & & & & \\
\hline & $9 / 1 / 2004$ & 11:25: & 25.498 & 25.438 & & 25. & & & 55.404 & \begin{tabular}{l|l|l|l|}
17.658 \\
\end{tabular} & & \begin{tabular}{|l|l|} 
& -0.7 \\
\end{tabular} & & & 0.043 & 14.6 & & & 3.5403 & 54.3 & 11.7 & & .863 & (006 & & & 0.149 & \\
\hline & & 11:26:47 & 25.499 & 25.443 & & & 24.9 & & & & & -0.694 & & & & & & & & & 11.6 & & & & & & & \\
\hline & & 11:27: & 25.494 & 25.444 & \begin{tabular}{|l|}
24.748 \\
\end{tabular} & \begin{tabular}{|l|}
25.857 \\
\end{tabular} & & 49.481 & 55.025 & 17.672 & 32.847 & -0.7 & & & .043 & & & & 3.5736 & 53.9 & 11. & & & & & & 151 & \\
\hline & & & & 25.44 & & & & & 55.34 & & & -0. & & & & & & & & 54.2 & $I \pm$ & & & & & & & \\
\hline & & & & & & & & & & & 32.865 & -0 . & & & & & & & & & & & & & & & & \\
\hline & & $\begin{array}{ll}11: 30: 47 \text { PM } \\
1113 \cdot 3 \cdot 77 \text { M }\end{array}$ & $\frac{2.405}{25495}$ & $\frac{25.454}{25.455}$ & $\frac{24.763}{24764}$ & \begin{tabular}{|l|}
25.937 \\
25918
\end{tabular} & & $\frac{49.316}{49.83}$ & 5549 & $\frac{17.432}{17683}$ & $\begin{array}{l}33.023 \\
33069\end{array}$ & $\begin{array}{l}-0.694 \\
-0.697 \\
\end{array}$ & & & & $\frac{14.6}{146}$ & & & & 544 & 10 & 41 & & & & & 0.0150 & \\
\hline & & $11: 32: 47$ & 25.496 & 25.456 & 24.75 & 25.92 & & 49.6 & $\mid 55.174$ & & & $\begin{array}{l}-0.097 \\
-0.697\end{array}$ & & & & & & & & 540 & $\frac{1.3}{118}$ & & & & & & & \\
\hline & & $\frac{11: 33: 47}{11}$ & & 25.466 & \begin{tabular}{|l|}
24.75 \\
\end{tabular} & & & & & & & -0.694 & & & & & & & & & 116 & & & & & & & \\
\hline & & & 25.486 & 25.456 & \begin{tabular}{|l|}
24.745 \\
\end{tabular} & \begin{tabular}{|l|}
25.909 \\
\end{tabular} & & & 55.618 & 17.548 & 33.126 & $\begin{array}{c}-0.7 \\
\end{array}$ & & & & & & & & 54.5 & $\frac{11.5}{11.5}$ & & & & & & 49 & \\
\hline & & & & & & & & & & & & & & & & & & & & & & & & & & & & \\
\hline & & & & & 24.76 & & & & 55.05 & 17.56 & 33.093 & -0.648 & & & & & & & & 53 & & & & & & & & \\
\hline & & & & 25.4 & 24.725 & 25.914 & & & & & 33.112 & -0.653 & & & & & & & & $54 . \mathrm{C}^{2}$ & 12.0 & & & & & & & \\
\hline & & 11:38 & & 25.46 & 24.731 & 25.96 & & & & & 33.147 & -0.648 & & & & & & & 69 & 54.0 & 13.1 & & & & & & 147 & \\
\hline & & & & 25.46 & & 25.9 & & & 55.13 & 17.50 & 32.871 & -0.645 & & & & 14. & & & & 54. & 11.9 & & & & & & 0.151 & \\
\hline & & & & 25.48 & & & & & & & & -0.6 & & & & 14. & & & & . & 11.8 & & & & & & & \\
\hline & & & 25.505 & 25.494 & \begin{tabular}{|l|l|}
24.763 \\
\end{tabular} & $25 .$. & & & 55.004 & 17.433 & 33.03 & -0.674 & & & & 14. & & & & 53.9 & 11.5 & & & & & & & \\
\hline & & & & & & & & & & & & & & & & & & & & & & & & & & & & \\
\hline & & $\frac{11.43}{11.44}$ & & 25. & 24.728 & 25. & & & 55.1086 & 17. & 33.005 & -0.6 & & & & & & & & 54.0 & 11.6 & & & & & & & \\
\hline & & $\frac{11.44 .4}{11 \cdot 5 \cdot}$ & & $\begin{array}{l}23.489 \\
25495\end{array}$ & 2.1446 & \begin{tabular}{|l|l|l|} 
\\
\end{tabular} & & $\frac{49.12}{49.676}$ & 55114 & 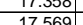 & $\begin{array}{l}30.424 \\
33239\end{array}$ & 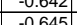 & & & & & & & & S.4. & $\begin{array}{ll}12.1 \\
119,8\end{array}$ & & & & & & (40 & \\
\hline & & $\frac{\frac{1.145}{11.46}}{11.0}$ & & $\frac{2.459}{25497}$ & $\frac{2.1544}{24736}$ & 250 & & $\frac{4.6}{49}$ & 55.2 & 17707 & 年 & -0.66 & & & & 146 & & & & 4 & $\frac{1.5}{11.5}$ & & & & & & 150 & \\
\hline & & & & 25.502 & 24746 & 255 & & & & & 33081 & $\frac{-0.642}{-0.642}$ & & & & 146 & & & & 540 & 118 & & & & & & & \\
\hline & $\frac{9}{9}$ & & & $\frac{25.481}{20.481}$ & & 25.93 & & & & & 33.128 & -0.64 & & & & 14.6 & & & & 542 & 11 & & & & & & & \\
\hline & & & & 25.505 & 24.764 & & & & & & & & & & & & & & & & & & & & & & & \\
\hline & & & & & & & & & & & & & & & & & & & & & & & & & & & & \\
\hline & & 11:51: & & 25.498 & 24.752 & 25.746 & & 49 & 5504 & 17.137 & 33.504 & -0.6 & & & & 114 & & & & 53.9 & 110 & & & & & & 49 & \\
\hline & & $17 \mathrm{PN}$ & 5477 & 5.492 & & & & 49.7 & 55.454 & 17.44 & & -0.64 & & & & 4625 & & & & 543 & 178 & & & & & & & 11 \\
\hline 3150 & $9 / 1 / 2$ & $11: 53: 47 \mathrm{PM}$ & 25.477 & 25.491 & \begin{tabular}{|l|l|}
24.745 \\
\end{tabular} & \begin{tabular}{|l|l|}
25.754 \\
\end{tabular} & 24.8 & 49.685 & $\mid 55.34$ & $\mid$\begin{tabular}{|c|}
17.448 \\
\end{tabular} & $\begin{array}{l}33.177 \\
\end{array}$ & \begin{tabular}{|l|l|} 
& -0.639 \\
\end{tabular} & & $\begin{array}{l}49.074 \\
\end{array}$ & 0.042 & 14.643 & & 8440.42 & \begin{tabular}{|l|l|}
64.0069 \\
\end{tabular} & 54.2 & 11.7 & & 2.857 & $\begin{array}{l}0.006 \\
\end{array}$ & 0.006 & 0.000 & 0.146 & 0.1 \\
\hline
\end{tabular}


WSRC-TR-2005-00105, REVISION 0

SRNL-RPP-2005-00012, REVISION 0

RUN \# 4.03A AND B; FIRST AND SECOND HALF OF SLURRY DEWATERING - CONT.

\begin{tabular}{|c|c|c|c|c|c|c|c|c|c|c|c|c|c|c|c|c|c|c|c|c|c|c|c|c|c|c|c|c|}
\hline & $\mathrm{A}$ & $\mathrm{B}$ & D & $E$ & $\mathrm{~F}$ & G & $\mathrm{H}$ & $\mathrm{J}$ & K & $\mathrm{L}$ & $M$ & $\mathrm{~N}$ & 0 & $\mathrm{Q}$ & $\mathrm{R}$ & \begin{tabular}{l|l} 
\\
\end{tabular} & \begin{tabular}{|l|l|}
$T$ \\
\end{tabular} & $\mathrm{v}$ & w & $x$ & $Y$ & $z$ & $\mathrm{AA}$ & & $\mathrm{AC}$ & AD & & \\
\hline & 9/1/2004 & 11:54:47 PM & 25.47 & 25.48 & \begin{tabular}{|l|l|}
24.739 \\
\end{tabular} & 25.723 & 24.835 & 49.844 & 55.709 & 17.53 & 33.093 & -0.645 & -6.214 & 50.258 & 0.043 & 14.643 & 0.003 & 3841.42 & 64.0236 & 54.6 & 12.0 & 41.5 & 2.859 & 0.006 & 0.006 & 0.000 & 0.150 & \\
\hline & & $\begin{array}{l}11: 55: 47 \mathrm{PM} \\
\end{array}$ & $\begin{array}{r}25.47 \\
\end{array}$ & 25.48 & \begin{tabular}{|l|l|}
24.7344 \\
\end{tabular} & \begin{tabular}{|l|}
25.763 \\
\end{tabular} & 24.75 & 49.848 & \begin{tabular}{|l|l|}
55.576 \\
\end{tabular} & 17.654 & 33.161 & -0.645 & -6.22 & 47.503 & 0.041 & 14.643 & 0.003 & $\begin{array}{ll}3842.42 \\
842.42\end{array}$ & \begin{tabular}{|l|l|}
64.0403 \\
\end{tabular} & \begin{tabular}{|l|l|}
54.5 \\
\end{tabular} & & & & 0.006 & 0.006 & & & \\
\hline & $\begin{array}{l}9 / 1 / 2004 \\
0 / 1204\end{array}$ & 11:56:47 PM & $\frac{25.475}{25.450}$ & \begin{tabular}{|l|}
25.48 \\
25.44
\end{tabular} & \begin{tabular}{|l|}
24.764 \\
2702
\end{tabular} & 25.753 & 24.78 & \begin{tabular}{|l|l|}
49.776 \\
\end{tabular} & \begin{tabular}{|l|}
55.456 \\
5.252 \\
\end{tabular} & 17.619 & 33.101 & -0.642 & -6.205 & 48.219 & 0.042 & 14.643 & 0.004 & $\frac{3843.42}{38.42}$ & 64.0569 & \begin{tabular}{|l|l|}
54.3 \\
\end{tabular} & 11.5 & & 2.857 & 0.006 & 0.006 & 0.000 & 0.146 & \\
\hline & $\begin{array}{l}\text { 9/1/2004 } \\
0 / 12004\end{array}$ & 11:57:47 PM & 25.459 & 25.464 & \begin{tabular}{|l|l|}
24.728 \\
\end{tabular} & \begin{tabular}{|l|}
25.737 \\
\end{tabular} & 24.85 & $\begin{array}{l}49.792 \\
\end{array}$ & \begin{tabular}{|l|l|}
55.352 \\
\end{tabular} & 17.671 & 33.083 & -0.645 & -6.211 & 49.013 & $\begin{array}{l}0.042 \\
\end{array}$ & 14.643 & 0.003 & 3844.42 & 64.0736 & \begin{tabular}{|l|l|}
54.2 \\
\end{tabular} & 11.7 & 41.4 & 2.857 & 0.006 & 0.006 & 0.000 & 0.146 & \\
\hline & $\begin{array}{c}9 / 1 / 2004 \\
9 / 1 / 2004 \\
\end{array}$ & $\begin{array}{l}11: 58: 47 \text { PM } \\
1115 \cdot 5 \cdot 77 \text { PM }\end{array}$ & 25.479 & \begin{tabular}{|l|l|}
25.474 \\
25.468
\end{tabular} & \begin{tabular}{|l|}
24.753 \\
24.762 \\
\end{tabular} & \begin{tabular}{|l|}
25.792 \\
25781 \\
\end{tabular} & $\begin{array}{r}24.86 \\
24.034 \\
\end{array}$ & \begin{tabular}{|r|}
49.631 \\
49.79 \\
\end{tabular} & \begin{tabular}{|l|}
55.33 \\
55.51 \\
\end{tabular} & \begin{tabular}{|c|}
17.45 \\
17.619 \\
\end{tabular} & $\begin{array}{l}33.155 \\
33.147 \\
\end{array}$ & $\begin{array}{l}-0.642 \\
-0651 \\
-0.515\end{array}$ & $\begin{array}{l}-6.214 \\
6206\end{array}$ & $\begin{array}{l}48.888 \\
49799 \\
\end{array}$ & $\begin{array}{l}0.043 \\
0.042\end{array}$ & \begin{tabular}{|l|l|}
14.643 \\
14634 \\
\end{tabular} & $\begin{array}{l}0.003 \\
0003\end{array}$ & 3845.42 & & \begin{tabular}{|l|}
54.2 \\
5.4 \\
\end{tabular} & \begin{tabular}{|l|l|}
11.6 \\
119
\end{tabular} & 41.4 & $\frac{2.854}{2.859}$ & & & & & \\
\hline & $\begin{array}{l}9 / 1 / 2004 \\
9 / 2 / 2004\end{array}$ & $\begin{array}{l}\text { 11:59:977 PM } \\
\text { 12:00:47 AM }\end{array}$ & $\frac{25.469}{25.469}$ & 25.468 & \begin{tabular}{|l|}
24.762 \\
24.757 \\
\end{tabular} & \begin{tabular}{|l|}
25.781 \\
25.861 \\
\end{tabular} & $\begin{aligned} 24.934 \\
24.884\end{aligned}$ & $\begin{array}{l}49.79 \\
50.07 \\
\end{array}$ & \begin{tabular}{|l|}
$\mid 55.71$ \\
55.736 \\
\end{tabular} & $\begin{array}{l}11.619 \\
17.904\end{array}$ & $\begin{array}{l}33.147 \\
33.048\end{array}$ & $\begin{array}{l}-0.645 \\
-0.642\end{array}$ & $\begin{array}{l}-6.206 \\
-6.211 \\
\end{array}$ & $\begin{array}{l}49.799 \\
4.321\end{array}$ & $\begin{array}{l}0.042 \\
0.042\end{array}$ & $\begin{array}{l}14.643 \\
14.643 \\
\end{array}$ & $\begin{array}{l}0.003 \\
0.003\end{array}$ & $\begin{array}{l}38466.42 \\
3847.42\end{array}$ & $\begin{array}{l}64.1069 \\
64.1236\end{array}$ & \begin{tabular}{|l|}
$\mid 54.4$ \\
54.6 \\
\end{tabular} & \begin{tabular}{|l|}
11.9 \\
1.5
\end{tabular} & \begin{tabular}{|l|l|}
41.6 \\
\end{tabular} & 2.859 & $\begin{array}{l}0.006 \\
0.006 \\
\end{array}$ & \begin{tabular}{|c|}
0.006 \\
0.006 \\
\end{tabular} & $\frac{0.000}{0.000}$ & $\begin{array}{l}\mid 0.146 \\
0.146\end{array}$ & 0.12 \\
\hline & & 12:01:47 AM & 25.463 & 25.463 & 24.772 & 25.841 & & & 55.585 & 17.523 & 33.364 & -0.645 & & & & 14.643 & 0.003 & $\frac{36874.42}{3848.42}$ & \begin{tabular}{|l|l|} 
& 64.1230 \\
64.1403
\end{tabular} & \begin{tabular}{|l|}
54.5 \\
54.5 \\
\end{tabular} & & & & & $\begin{array}{l}0.006 \\
0.006 \\
\end{array}$ & 0.000 & & $\frac{0.12}{0.12}$ \\
\hline & & 12:02:47 AM & & 25.473 & 24.777 & 25.886 & 24.994 & 49.759 & \begin{tabular}{|l|l|}
55.595 \\
\end{tabular} & 17.813 & 32.869 & -0.642 & -6.21 & 48.056 & 0.041 & 14.643 & 0.003 & 3849.42 & & \begin{tabular}{|l|}
54.5 \\
\end{tabular} & 11.4 & & & & & & 0.143 & \\
\hline & & & 25.469 & 25.473 & 24.782 & 25.896 & 25.004 & 49.71 & \begin{tabular}{|l|l|l|}
55.187 \\
\end{tabular} & & & & & 50.388 & 0.042 & $\begin{array}{ll}14.643 \\
\end{array}$ & & 3850.42 & 64.1736 & 54.1 & & & & & 0.006 & 0.000 & 0.146 & \\
\hline 61 & $2 / 2004$ & 12:04:47 AM & 25.474 & 25.474 & \begin{tabular}{|l|l|}
24.788 \\
2.793
\end{tabular} & \begin{tabular}{|l|l|}
25.907 \\
25012
\end{tabular} & $\begin{array}{l}24.97 \\
2.95\end{array}$ & 49.681 & 55.203 & \begin{tabular}{ll|l}
17.638 \\
\end{tabular} & 33.007 & -0.598 & & 49.176 & $\begin{array}{l}0.042 \\
\end{array}$ & \begin{tabular}{|l|l|}
14.643 \\
\end{tabular} & $\begin{array}{l}0.003 \\
\end{array}$ & 3851.42 & \begin{tabular}{|l|l|}
64.1903 \\
\end{tabular} & 54.1 & 11.7 & 41.3 & 2.851 & 0.006 & $\begin{array}{l}0.006 \\
\end{array}$ & 0.000 & $\begin{array}{l}0.146 \\
\end{array}$ & \\
\hline & 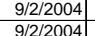 & $\begin{array}{l}12: 05: 47 \mathrm{AM} \\
12200: 47 \mathrm{AM}\end{array}$ & $\begin{array}{r}25.479 \\
25.48 \\
\end{array}$ & $\begin{array}{l}25.474 \\
25.475\end{array}$ & $\begin{array}{r}24.783 \\
24.809 \\
\end{array}$ & \begin{tabular}{|l|}
25.942 \\
25893 \\
\end{tabular} & $\begin{array}{r}24.95 \\
25.005\end{array}$ & 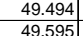 & \begin{tabular}{|c|}
55.299 \\
55253
\end{tabular} & \begin{tabular}{r|}
17.69 \\
18.446
\end{tabular} & \begin{tabular}{|l|}
32.835 \\
32073
\end{tabular} & $\begin{array}{l}-0.604 \\
-0.642\end{array}$ & & $\begin{array}{l}49.443 \\
51205\end{array}$ & $\begin{array}{l}0.041 \\
0041\end{array}$ & $\begin{array}{l}14.643 \\
14643\end{array}$ & & $\begin{array}{l}8852.42 \\
853.42\end{array}$ & \begin{tabular}{|l|l|}
64.2069 \\
6.22323
\end{tabular} & \begin{tabular}{|l|}
54.2 \\
54.
\end{tabular} & $\frac{11.8}{122}$ & & $\begin{array}{l}2.838 \\
28155\end{array}$ & 0.006 & 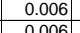 & 0.000 & $\begin{array}{l}0.143 \\
0.1515\end{array}$ & \\
\hline & $\begin{array}{c}9 / 2 / 2004 \\
9 / 2 / 2004\end{array}$ & 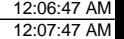 & $\begin{array}{r}25.48 \\
25.491\end{array}$ & $\begin{array}{l}25.475 \\
25.481\end{array}$ & 24.809 & \begin{tabular}{|l|}
25.893 \\
25.919 \\
\end{tabular} & $\begin{array}{l}25.005 \\
24.986\end{array}$ & \begin{tabular}{|}
$\mid 49.595$ \\
49.712
\end{tabular} & \begin{tabular}{|l|}
55.253 \\
55.41 \\
\end{tabular} & \begin{tabular}{|c|}
18.446 \\
18085
\end{tabular} & $\begin{array}{l}32.073 \\
32.642\end{array}$ & $\begin{array}{l}-0.642 \\
-0.619\end{array}$ & $\begin{array}{l}-6.211 \\
-6.206\end{array}$ & $\begin{array}{l}51.205 \\
50.845\end{array}$ & \begin{tabular}{|c|}
0.041 \\
0.042
\end{tabular} & $\frac{14.643}{14.643}$ & $\begin{array}{l}0.003 \\
0.003\end{array}$ & 3853.42 & $\begin{array}{ll}64.2236 \\
64.2403\end{array}$ & $\begin{array}{l}54.1 \\
54.3\end{array}$ & $\frac{12.2}{12.1}$ & \begin{tabular}{|l|}
40.8 \\
41.2 \\
\end{tabular} & $2.815 \mid$ & \begin{tabular}{|c|}
0.006 \\
0.006
\end{tabular} & $\begin{array}{l}0.006 \\
0.006\end{array}$ & 0.000 & $\mid \begin{array}{l}0.145 \\
0.147\end{array}$ & 0 \\
\hline & $2 / 2004$ & $12: 08: 47 \mathrm{AM}$ & 25.476 & 25.471 & $\begin{array}{l}24.815 \\
24.815\end{array}$ & \begin{tabular}{|l|}
25.919 \\
25.914 \\
\end{tabular} & 24.951 & 49.71 & \begin{tabular}{|l|}
55.431 \\
\end{tabular} & 18.152 & 32.539 & -0.616 & $\begin{array}{l}-.214 \\
-6.214 \\
\end{array}$ & 50.375 & 0.041 & 14.643 & $\begin{array}{l}0.0003 \\
0.003\end{array}$ & \begin{tabular}{|l}
38534.42 \\
3855.42
\end{tabular} & \begin{tabular}{|l|} 
\\
\\
264.25409 \\
\end{tabular} & \begin{tabular}{|l|}
54.3 \\
54.3
\end{tabular} & $\begin{array}{l}12.0 \\
\end{array}$ & & & \begin{tabular}{|l|}
0.006 \\
0.006 \\
\end{tabular} & $\begin{array}{l}0.000 \\
0.006\end{array}$ & & & 0.12 \\
\hline & & 12:09:47 AM & 25.492 & $\begin{array}{l}25.477 \\
25.923\end{array}$ & 24.831 & $\begin{array}{r}25.91 \\
25.906\end{array}$ & 24.953 & \begin{tabular}{|c|}
49.683 \\
\end{tabular} & \begin{tabular}{|l|l|}
55.5393 \\
\end{tabular} & 17.994 & 32.716 & \begin{tabular}{|c|c|c|}
-0.624 \\
\end{tabular} & & 50.213 & $\begin{array}{l}0.042 \\
\end{array}$ & \begin{tabular}{|l|}
14.643 \\
\end{tabular} & & 3856.42 & $\begin{array}{l}264.2736 \\
\end{array}$ & $\begin{array}{l}34.4 \\
5.4 \\
\end{array}$ & \begin{tabular}{|l|}
12.0 \\
\end{tabular} & & & & & & 0.147 & \\
\hline & & 12:10:47 AM & & 25.483 & 24.832 & & 24.918 & $\begin{array}{l}49.654 \\
\end{array}$ & \begin{tabular}{|l|l|}
55.015 \\
\end{tabular} & 18.252 & 32.551 & & & 49.432 & $\begin{array}{l}0.041 \\
\end{array}$ & \begin{tabular}{|l|l|}
14.643 \\
\end{tabular} & & 3857.42 & 64.2903 & 53.9 & & & 2.834 & & & & $\begin{array}{l}0.143 \\
\end{array}$ & \\
\hline & & $\begin{array}{l}12: 11: 47 \mathrm{AM} \\
121: 271 \mathrm{~A}\end{array}$ & 25.488 & \begin{tabular}{|l|l|}
25.473 \\
25.91
\end{tabular} & & \begin{tabular}{|l|}
25.861 \\
25027 \\
\end{tabular} & 24.973 & & & & $\begin{array}{l}32.451 \\
3.527\end{array}$ & -0.566 & & $\begin{array}{l}49.795 \\
9\end{array}$ & & \begin{tabular}{|l|l|}
14.643 \\
\end{tabular} & 0.003 & & $\begin{array}{l}64.3069 \\
\end{array}$ & 54.2 & & & 2.831 & 0.006 & 0.006 & 0.000 & 0.144 & \\
\hline & & $\begin{array}{l}12: 12: 47 \mathrm{AM} \\
12213: 47 \mathrm{AM}\end{array}$ & $\begin{aligned} 25.504 \\
25.51\end{aligned}$ & $\begin{array}{l}25.494 \\
25.489\end{array}$ & \begin{tabular}{|l|}
24.8773 \\
24.874 \\
\end{tabular} & \begin{tabular}{|l|}
25.937 \\
25.922 \\
\end{tabular} & $\begin{array}{r}25.004 \\
24.97\end{array}$ & & \begin{tabular}{|l|}
55.091 \\
55.081 \\
\end{tabular} & $\begin{array}{l}18.335 \\
18.238 \\
\end{array}$ & $\begin{array}{l}32.527 \\
32.229\end{array}$ & $\begin{array}{l}-0.558 \\
-0.569\end{array}$ & $\begin{array}{l}-6.206 \\
-6.209\end{array}$ & $\begin{array}{l}49.931 \\
47.922\end{array}$ & \begin{tabular}{|c|}
0.04 \\
0.041
\end{tabular} & $\begin{array}{l}14.643 \\
14.643 \\
\end{array}$ & & & $\begin{array}{l}64.3236 \\
64.3403\end{array}$ & $\begin{array}{l}54.0 \\
540\end{array}$ & 11.9 & & $\frac{2.851}{2.810}$ & $\begin{array}{ll}0.006 \\
0006\end{array}$ & 0.006 & 000 & .140 & \\
\hline & 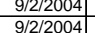 & $\begin{array}{l}\frac{12: 13: 47 \mathrm{AM}}{12: 41: 47} \\
\end{array}$ & $\begin{array}{r}25.51 \\
25.521\end{array}$ & $\begin{array}{l}25.489 \\
25.501\end{array}$ & $\begin{array}{r}24.874 \\
24.895 \\
\end{array}$ & \begin{tabular}{|l|}
25.922 \\
25.909
\end{tabular} & $\begin{array}{r}24.97 \\
24.936 \\
\end{array}$ & & \begin{tabular}{|l|}
55.081 \\
54.961 \\
\end{tabular} & $\begin{array}{l}18.238 \\
17.855 \\
\end{array}$ & .229 & $\begin{array}{c}-0.569 \\
-0.558\end{array}$ & & $\begin{array}{l}47.922 \\
48.972 \\
\end{array}$ & $\mid \begin{array}{l}0.041 \\
0.041\end{array}$ & \begin{tabular}{|c|}
14.643 \\
14643 \\
\end{tabular} & $\begin{array}{l}0.003 \\
0.003\end{array}$ & $\begin{array}{l}3860.42 \\
3361.42\end{array}$ & $\begin{array}{ll}64.3403 \\
64.3569\end{array}$ & $\begin{array}{r}54.0 \\
53.8\end{array}$ & $\frac{11.4}{11.7}$ & $\begin{array}{l}40.9 \\
40.9\end{array}$ & \begin{tabular}{|l|}
2.819 \\
2.818
\end{tabular} & \begin{tabular}{|c|}
0.006 \\
0.006
\end{tabular} & $\begin{array}{l}0.006 \\
0.006\end{array}$ & 0.000 & 0.144 & 0.12 \\
\hline & $2 / 2004$ & $12: 15: 47 \mathrm{AM}$ & 25.516 & 25.486 & 24.865 & \begin{tabular}{|l|}
25.919 \\
\end{tabular} & 24.951 & $\begin{array}{l}4.606 \\
49.606\end{array}$ & \begin{tabular}{|l|}
55.311 \\
\end{tabular} & 17.693 & 32.787 & -0.569 & -6.21 & 49.351 & 0.04 & $\begin{array}{l}14.643 \\
14.643\end{array}$ & $\begin{array}{l}0.003 \\
0.003\end{array}$ & \begin{tabular}{|l|l|l|l|}
3862.42 \\
\end{tabular} & \begin{tabular}{|l|l|}
64.359 \\
64.3736 \\
\end{tabular} & \begin{tabular}{|l|}
54.2 \\
\end{tabular} & 111.8 & $\begin{array}{l}40.9 \\
41.2\end{array}$ & $\mid \frac{2.880}{2.840}$ & \begin{tabular}{|l|}
0.006 \\
0.006 \\
\end{tabular} & $\begin{array}{l}0.000 \\
0.006 \\
\end{array}$ & 0.000 & $\begin{array}{l}0.144 \\
0.140\end{array}$ & \\
\hline & & 12:16:47 AM & 25.516 & 25.481 & 24.885 & \begin{tabular}{|l|}
25.894 \\
\end{tabular} & 24.941 & $\begin{array}{l}49.751 \\
\end{array}$ & \begin{tabular}{|l|}
55.427 \\
\end{tabular} & 17.934 & 32.742 & $\begin{array}{l}-0.569 \\
\end{array}$ & & 48.898 & 0.041 & \begin{tabular}{|l|l|}
14.643 \\
\end{tabular} & & & 64.3903 & 54.3 & 11.6 & 41.2 & 2.844 & 0.006 & 0.006 & 0.000 & 0.143 & \\
\hline & & 12:17:47 AM & 25.516 & 25.496 & & & & & \begin{tabular}{|l|}
55.151 \\
\end{tabular} & 18.049 & $\begin{array}{l}32.492 \\
\end{array}$ & & & 48.969 & $\begin{array}{l}0.04 \\
\end{array}$ & $\begin{array}{l}4.643 \\
\end{array}$ & & 3864.42 & 64.4069 & 54.0 & 11.7 & & & $\begin{array}{ll}0.006 \\
\end{array}$ & & & 0.140 & \\
\hline & & $\begin{array}{l}12: 18: 47 \mathrm{AM} \\
1210: 17 \mathrm{~A}\end{array}$ & $\begin{array}{l}25.531 \\
25.525\end{array}$ & 25.496 & \begin{tabular}{|r|}
24.92 \\
25.009
\end{tabular} & & & & \begin{tabular}{|l|l|}
55.694 \\
5500 \\
\end{tabular} & $\begin{array}{l}18.198 \\
1.190\end{array}$ & \begin{tabular}{|l|}
32.754 \\
3.26
\end{tabular} & .555 & & $\begin{array}{l}50.202 \\
5000\end{array}$ & & \begin{tabular}{|l|l|}
14.643 \\
1.643
\end{tabular} & & $\begin{array}{l}3865.42 \\
396642\end{array}$ & \begin{tabular}{|l|l|l}
64.4236 \\
\end{tabular} & \begin{tabular}{|l|}
54.6 \\
\end{tabular} & 12.0 & & 2.853 & 0.006 & 0.006 & & $\begin{array}{l}0.142 \\
\end{array}$ & \\
\hline $\begin{array}{l}76 \\
77\end{array}$ & |l|l|2004 & $\begin{array}{l}12: 19: 47 \mathrm{AM} \\
1220: 47 \mathrm{AM}\end{array}$ & $\begin{array}{r}25.525 \\
25.53 \\
\end{array}$ & $\begin{array}{r}25.489 \\
25.5\end{array}$ & \begin{tabular}{|l|}
25.009 \\
25.009 \\
\end{tabular} & \begin{tabular}{|l|}
25.897 \\
25.928 \\
\end{tabular} & $\begin{array}{r}25.045 \\
25.056\end{array}$ & \begin{tabular}{|l|}
49.581 \\
49.434
\end{tabular} & \begin{tabular}{|l|}
55.0899 \\
55.135 \\
\end{tabular} & \begin{tabular}{r|}
18.189 \\
18.255
\end{tabular} & \begin{tabular}{|l|}
32.463 \\
32003
\end{tabular} & 5.535 & $\begin{array}{l}-6.209 \\
-6.694\end{array}$ & $\begin{array}{r}50.909 \\
49.604\end{array}$ & \begin{tabular}{|c|}
0.04 \\
0.041
\end{tabular} & $\begin{array}{l}\frac{14.643}{14.643} \\
\end{array}$ & $\begin{array}{l}0.003 \\
0.03\end{array}$ & $\begin{array}{l}3866.42 \\
3867.42\end{array}$ & \begin{tabular}{|l|l|}
64.4403 \\
6
\end{tabular} & $\begin{array}{l}54.0 \\
54.0 \\
\end{array}$ & $\frac{12.1}{11.8}$ & $\frac{41.0}{40.7}$ & $\frac{2.828}{2809}$ & $\begin{array}{l}0.006 \\
0.006\end{array}$ & $\begin{array}{l}0.006 \\
0.006\end{array}$ & & $\begin{array}{l}0.140 \\
0.144\end{array}$ & $\frac{0.14}{0.12}$ \\
\hline & 12004 & $\begin{array}{l}12: 2: 247 \mathrm{AM} \\
12: 21: 47 \mathrm{AM}\end{array}$ & 25.542 & 25.507 & \begin{tabular}{|l|}
25.046 \\
\end{tabular} & \begin{tabular}{|l|}
25.820 \\
\end{tabular} & 25.147 & \begin{tabular}{|l|}
49.4545 \\
49.695
\end{tabular} & \begin{tabular}{|l|}
55.392 \\
\end{tabular} & 18.268 & \begin{tabular}{|l|}
32.301 \\
32.00
\end{tabular} & $\begin{array}{l}-0.535 \\
-0.535\end{array}$ & 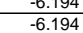 & $\begin{array}{l}\frac{49.604}{49.998} \\
\end{array}$ & \begin{tabular}{|l|}
0.041 \\
0.042
\end{tabular} & $\begin{array}{l}\frac{14.645}{14.643} \\
\end{array}$ & 0.00 & $\frac{3867.42}{3868.42}$ & \begin{tabular}{|l|l|}
64.4569 \\
64.4736
\end{tabular} & $\begin{array}{l}54.0 \\
54.3\end{array}$ & $\begin{array}{ll}11.8 \\
11.9\end{array}$ & \begin{tabular}{|l|}
40.7 \\
41.0
\end{tabular} & & $\begin{array}{l}0.006 \\
0.006\end{array}$ & $\begin{array}{l}.0060 \\
0.006\end{array}$ & & & \\
\hline & & 12:22:47 AM & 25.548 & 25.507 & \begin{tabular}{|l|l|}
25.022 \\
\end{tabular} & 25.945 & 25.133 & 49.415 & \begin{tabular}{|l|l|}
55.263 \\
\end{tabular} & 18.243 & 32.227 & -0.549 & & 51.043 & 0.041 & 14.643 & 0.00 & \begin{tabular}{|l|l|}
3869.42 \\
\end{tabular} & & 54.1 & 12.2 & 40 & 2.814 & 0.006 & & & $\begin{array}{c}146 \\
144 \\
\end{array}$ & $\frac{0.12}{0.12}$ \\
\hline & & 12:23:47 AM & & 25.513 & & & & & \begin{tabular}{|l|l|}
55.413 \\
\end{tabular} & 17.703 & & -0.532 & & & 0.04 & 14.643 & & & & 54.3 & & & & & & & & 0.12 \\
\hline & 2/2/2004 & 12:24:47 AM & 25.571 & & 24.995 & \begin{tabular}{|l|}
25.928 \\
\end{tabular} & 25.166 & 49. & \begin{tabular}{|l|l|}
55.384 \\
\end{tabular} & 17.776 & 32.677 & -0.532 & & 48.569 & 0.041 & 14.643 & & & & & & & & & & & 43 & 0.17 \\
\hline & & 12:25:47 AM & & 25.511 & 24.965 & \begin{tabular}{|l|}
25.939 \\
\end{tabular} & & & \begin{tabular}{|l|l|l|}
55.88 \\
\end{tabular} & 17.941 & 32.539 & -0.535 & & & 0.04 & & & & & 54.0 & & & & & & & 40 & \\
\hline 83 & & 12:26:47 AM & 5.567 & 25.502 & 24.966 & 25.925 & & 49.877 & 55.514 & 17.961 & 32.675 & -0.532 & & & & 14.643 & & & & & & & & & & & & \\
\hline & & 12:27:47 AM & 5.573 & 25.508 & 24.948 & 25.936 & & 50.046 & 55.711 & 17.98 & 33.046 & $-0.53 !$ & & & $\overline{04}$ & 14.643 & & 38744 & .5736 & 54.6 & & & 864 & & & & & \\
\hline & $2 / 2004$ & 12:28: & & 25.499 & 24.933 & \begin{tabular}{|l|}
25.907 \\
\end{tabular} & 25. & & 55.46 & 17.823 & 33.048 & -0.535 & & 49.4 & 0.041 & \begin{tabular}{l|l}
14.643 \\
\end{tabular} & & & & 54.3 & 11.8 & & .858 & 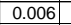 & & & & \\
\hline & & 12:29:47 AN & 25.591 & 25.51 & 24.945 & 25.888 & 25. & & \begin{tabular}{|l|l|}
55.226 \\
\end{tabular} & 17.767 & 32.931 & $\begin{array}{r}-0.52 \\
\end{array}$ & & 49.0 & & 14. & & & & 54.1 & 11 & & & & & & 146 & 0.1 \\
\hline & & & 20.592 & $\frac{25.507}{25.7}$ & 24.916 & & & & \begin{tabular}{|l|}
55.077 \\
5.42 \\
\end{tabular} & 17.669 & 33.06 & -0.526 & & & 0.041 & & & & & 54.0 & & & & & & & & \\
\hline & & 1: & & & & & & & & & & -0.535 & & & & & & & & & & & & & & & & \\
\hline & & 12:32: & 25.598 & 25.503 & 24.917 & 25.881 & & & 55.51 & .41 & 32.913 & - & & 48.8 & & & & & & 54.4 & & & & & & & & \\
\hline & & $\begin{array}{l}12.3534 \mathrm{AN} \\
2.34 .47\end{array}$ & & $\begin{array}{l}25.501 \\
25507\end{array}$ & 24.911 & $\frac{25.895}{2585}$ & & & 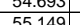 & & $\frac{30.513}{31.23}$ & $\begin{array}{cc}-0 \\
-c\end{array}$ & & & 0 & $\begin{array}{l}14.6 \\
1.6\end{array}$ & & & & 540 & 122 & & & & & & & \\
\hline & $1 / 2004$ & & & & 24.946 & & & & & 19.42 & & - 0.538 & & & & 146 & & & & & & & & & & & & \\
\hline & & 12:36:47 & & 25.505 & 24985 & & & & 54.74 & & & -0.535 & & & & & & & & 536 & & & & & & & & \\
\hline & 2004 & $\begin{array}{l}12: 37: 47 \\
\end{array}$ & 25.596 & 25.516 & 25.001 & \begin{tabular}{|l|}
25.909 \\
\end{tabular} & & 49.181 & \begin{tabular}{|l|}
54.925 \\
\end{tabular} & 19.318 & 30.963 & $\frac{-0.532}{-0.532}$ & & & 0.04 & $\frac{14.6}{14.6}$ & & & & 53.8 & 12.2 & & & & & & 43 & \\
\hline & & & & & & & & & & & & & & & & & & & & & & & & & & & & \\
\hline & & & 25.606 & 25.52 & 25.045 & 25.878 & & & & 18 & & & & & & & & & & 53 & & & & & & & & \\
\hline & & & & 25.5 & & & & & & & & -0.51 & & & & & & & & 04.0 & & & & & & & & \\
\hline & & & & 25.526 & 25.085 & \begin{tabular}{|l|}
25.813 \\
\end{tabular} & & & \begin{tabular}{|l|l|}
55.147 \\
\end{tabular} & & 30.983 & -0.477 & & & 004 & & & & 4.8069 & 54.0 & 12 & & & & & & 42 & \\
\hline & & & & 25.5 & & & & & 55.122 & & 31. & -0.4 & & & 0.0 & 14.6 & & & & 54.0 & 12.3 & & & & & & 0.139 & \\
\hline & & & & 25.51 & & & & & & & & & & & & & & & & & & & & & & & & \\
\hline & & & 25. & 25.502 & 25. & 25. & & & 54.959 & 19.1 & & & & & 0.04 & & & & & 53.8 & 1 & & & & & & & \\
\hline & & & & & & & & & & & & & & & & & & & & & & & & & & & & \\
\hline & & & $\frac{25}{2-2}$ & 25.8 & 25.129 & & & & & & & & & & & & & & & & & & & & & & & \\
\hline & & & & 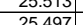 & 25.158 & & & & $\begin{array}{l}55.44 \\
5501\end{array}$ & & & & & & & (14.6 & & & & 54 & 12. & & & & & & 4 & \\
\hline & & & & $\mid$ & 25.15 & & & & 54.89 & & & & & & & & & & & & & & & & & & & \\
\hline & & & & & 25.15 & & & & & & & & & & & & & & & & & & & & & & & \\
\hline & & & 25. & $\frac{25.495}{25.495}$ & 25. & & & & 55.2 & & & & & & & 14. & & & & $\frac{5.1}{54.1}$ & $\frac{1}{1}$ & & & & & & & \\
\hline & & & & & & & & & & & & & & & & & & & & & & & & & & & & \\
\hline & & & & & & & & & & & & & & & & & & & & & & & & & & & & \\
\hline & & & & & 25.202 & & & & 55 & & & -0.2 & & & & & & & & & & & & & & & & \\
\hline & & & 642 & 25.491 & 25.201 & 25.6 & & & 55. & 18.8 & 31.747 & -0.4 & & 50 & & 14. & & & & & & & & & & & & \\
\hline & & & & 25.496 & 25. & & & & 55.38 & & 31.5 & 0.4 & & 50.35 & & 14.6 & & 90 & & & & & & & & & 40 & \\
\hline & & & & 25.486 & 25. & & & & & & 31.4 & & & & & 14. & & & & & 1 & & & & & & 44 & \\
\hline & & & & 25.49 & 25.2 & & & & 55. & & 32.6 & -0. & & & 0.0 & & & & & 5 & & & & & & & & \\
\hline & & & & & & & & & & & & & & & & & & & & & & & & & & & & \\
\hline & & & & 25.501 & 25.251 & & & & \begin{tabular}{|c|}
55.81 \\
\end{tabular} & & 31.1 & & & & & & & & & & & & & & & & & \\
\hline & & 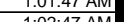 & 25.006 & 20.450 & $\frac{25.31}{25225}$ & $\begin{array}{l}2.1180 \\
\end{array}$ & 24. & 49.4 & $\mid$ & 10 & 31.44 & - 0.419 & & S. 50.124 & 0.041 & 1.6453 & & & $\frac{6.1403}{6.51560}$ & 54.1 & $\begin{array}{l}12.1 \\
12.1\end{array}$ & & 28 & & & 0.000 & 0 & \\
\hline 3220 & 9/2/2004 & $1: 03: 47 \mathrm{AM}$ & \begin{tabular}{|l|}
25.701 \\
2.701
\end{tabular} & $\begin{array}{l}25.545 \\
25.506 \\
\end{array}$ & \begin{tabular}{|l|}
25.325 \\
25.325 \\
\end{tabular} & \begin{tabular}{|l|l|}
25.794 \\
\end{tabular} & 25.001 & $\mid$ & \begin{tabular}{|l|}
55.39 \\
55.39
\end{tabular} & \begin{tabular}{|c|}
18.739 \\
\end{tabular} & $\begin{array}{l}1.145 \\
31.866 \\
\end{array}$ & \begin{tabular}{|l|}
-0.4119 \\
-0.419
\end{tabular} & & 53.095 & $\mid \frac{0.041}{0.04}$ & \begin{tabular}{|l|}
14.04043 \\
14.643
\end{tabular} & 0.003 & 3910.42 & \begin{tabular}{|l|l|}
65.1736 \\
\end{tabular} & \begin{tabular}{|l|}
54.3 \\
54.3
\end{tabular} & \begin{tabular}{|l|}
12.6 \\
\end{tabular} & & \begin{tabular}{|l|l|}
2.807 \\
\end{tabular} & \begin{tabular}{|l|}
0.006 \\
\end{tabular} & \begin{tabular}{|l|}
0.006 \\
\end{tabular} & 0.000 & $\begin{array}{l}0.1459 \\
0.139\end{array}$ & 0.1 \\
\hline
\end{tabular}


WSRC-TR-2005-00105, REVISION 0

SRNL-RPP-2005-00012, REVISION 0

RUN \# 4.03A AND B; FIRST AND SECOND HALF OF SLURRY DEWATERING - CONT.

\begin{tabular}{|c|c|c|c|c|c|c|c|c|c|c|c|c|c|c|c|c|c|c|c|c|c|c|c|c|c|c|c|c|}
\hline & $\mathrm{A}$ & $\mathrm{B}$ & D & $E$ & $\mathrm{~F}$ & G & $\mathrm{H}$ & \begin{tabular}{l|l}
$\mathrm{J}$ \\
\end{tabular} & K & $\mathrm{L}$ & $M$ & $\mathrm{~N}$ & 0 & Q & $\begin{array}{ll}R \\
\end{array}$ & \begin{tabular}{l|l} 
\\
\end{tabular} & $\begin{array}{ll}\mathrm{T} & \mathrm{T}\end{array}$ & $\mathrm{u} \quad \mathrm{v}$ & \begin{tabular}{l|} 
w \\
\end{tabular} & $x$ & Y & & & & & & & \\
\hline & 9/2/2004 & 1:04:47 AM & 25.706 & 25.501 & 25.29 & 25.804 & 24.921 & 49.608 & 55.458 & 18.556 & 32.003 & -0.399 & -6.122 & 47.833 & 0.04 & 14.643 & 0.003 & 3911.42 & 65.1903 & \begin{tabular}{|l|}
54.3 \\
\end{tabular} & 11.4 & 40.8 & 2.813 & 0.006 & 0.006 & 0.000 & 0.139 & \\
\hline & $9 / 2 / 2004$ & 1:05:47 AM & 25.712 & 25.507 & 25.312 & 25.79 & $\begin{array}{l}24.953 \\
\end{array}$ & $\begin{array}{r}49.51 \\
\end{array}$ & 55.437 & $\begin{array}{l}18.48 \\
\end{array}$ & 31.815 & $\begin{array}{l}-0.393 \\
\end{array}$ & -6.119 & 50.989 & 0.041 & \begin{tabular}{|l|l|}
14.643 \\
\end{tabular} & 0.003 & 3912.42 & \begin{tabular}{|l|l|l}
65.2069 \\
\end{tabular} & \begin{tabular}{|l|l|}
54.3 \\
\end{tabular} & & & & & 0.006 & & 0.143 & \\
\hline & $9 / 2 / 2 / 2004$ & 1:06:47 AM & $\frac{25.717}{25.712}$ & 25.497 & \begin{tabular}{|l|}
25.292 \\
2.296 \\
\end{tabular} & 25.81 & \begin{tabular}{|l|}
24.928 \\
2023
\end{tabular} & $\begin{array}{r}49.508 \\
\end{array}$ & 55.224 & \begin{tabular}{|l|l|}
18.433 \\
18.93
\end{tabular} & 32.166 & $\begin{array}{l}-0.381 \\
-0.3298 \\
\end{array}$ & -6.105 & $\begin{array}{l}49.833 \\
\end{array}$ & $\begin{array}{l}0.042 \\
\end{array}$ & \begin{tabular}{|l|}
14.643 \\
\end{tabular} & 0.003 & $\frac{391.42}{3013.42}$ & 65.2236 & \begin{tabular}{|l|}
54.1 \\
\end{tabular} & $\begin{array}{l}11.9 \\
1.9\end{array}$ & & 816 & 0.006 & 0.006 & 0.000 & 0.146 & \\
\hline & 9/2/2004 & $1: 07: 47 \mathrm{AM}$ & 25.712 & 25.492 & \begin{tabular}{|l|}
25.267 \\
\end{tabular} & $\begin{array}{l}25.79 \\
25.755\end{array}$ & 24.933 & $\begin{array}{l}49.316 \\
\end{array}$ & \begin{tabular}{|l|l|}
55.122 \\
\end{tabular} & $\begin{array}{l}18.436 \\
\end{array}$ & 31.735 & -0.387 & -6.116 & 50.77 & $\begin{array}{l}0.041 \\
\end{array}$ & $\begin{array}{l}14.643 \\
\end{array}$ & 0.003 & $\begin{array}{l}3914.42 \\
30.42\end{array}$ & 65.2403 & 54.0 & 12.1 & 40.5 & 2.794 & 0.006 & $\begin{array}{l}0.006 \\
\end{array}$ & 0.000 & 0.144 & \\
\hline & $\frac{9 / 2 / 2004}{9 / 2 / 2004}$ & $\begin{array}{ll}1: 08: 47 \mathrm{AM} \\
109 \cdot 47 \mathrm{AM}\end{array}$ & $\frac{25.717}{25.728}$ & $\frac{25.502}{25.508}$ & \begin{tabular}{|l|}
25.282 \\
252558 \\
\end{tabular} & $\frac{25.755}{25801}$ & $\begin{array}{r}24.973 \\
2.978\end{array}$ & & & $\frac{18.553}{184}$ & $\begin{array}{r}32.04 \\
32.051\end{array}$ & $\begin{array}{l}-0.352 \\
-0352\end{array}$ & \begin{tabular}{|c|c|} 
\\
6.119 \\
\end{tabular} & $\begin{array}{l}\frac{49.403}{50} \\
5\end{array}$ & $\begin{array}{l}0.041 \\
\end{array}$ & \begin{tabular}{|l|l|l|}
14.643 \\
14634 \\
\end{tabular} & & $\begin{array}{l}3915.42 \\
30.42\end{array}$ & & \begin{tabular}{|l|}
54.5 \\
5.8 \\
\end{tabular} & \begin{tabular}{|l|}
11.8 \\
119
\end{tabular} & \begin{tabular}{|l|l|}
40.9 \\
\end{tabular} & $\frac{2.817}{2.80}$ & & $\begin{array}{l}0.006 \\
0.066\end{array}$ & & 0.143 & \\
\hline & $\begin{array}{l}9 / 2 / 2004 \\
9 / 2 / 2004\end{array}$ & $\begin{array}{l}1: 90: 47 \mathrm{AM} \\
1: 10: 47 \mathrm{AM}\end{array}$ & $\frac{2.120}{25.749}$ & & $\begin{array}{l}25.258 \\
25.298\end{array}$ & $\frac{25.801}{25.852}$ & $\begin{array}{r}24.978 \\
24.939\end{array}$ & \begin{tabular}{|}
$\mid 49.944$ \\
49.919 \\
\end{tabular} & \begin{tabular}{|l|}
$\mid 55.887$ \\
55.672 \\
\end{tabular} & $\begin{array}{r}18.4 \\
18.552\end{array}$ & 32.4519 & $\begin{array}{l}-0.352 \\
-0.349\end{array}$ & -6.119 & $\begin{array}{r}50 \\
49.574\end{array}$ & $\begin{array}{r}0.04 \\
0.04\end{array}$ & $\begin{array}{r}\mid 14.643 \\
14.643\end{array}$ & $\begin{array}{l}0.003 \\
0.003\end{array}$ & $\begin{array}{l}3916.42 \\
3917.42\end{array}$ & \begin{tabular}{|l|}
$\mid 65.2736$ \\
265.2903
\end{tabular} & \begin{tabular}{|l|}
54.8 \\
54.5 \\
\end{tabular} & \begin{tabular}{|l|}
$\mid 11.9$ \\
1.8
\end{tabular} & \begin{tabular}{|l|}
41.2 .2 \\
14.2
\end{tabular} & $\begin{array}{l}2.840 \\
2.842\end{array}$ & $\mid$ & \begin{tabular}{|c|}
0.006 \\
0.006
\end{tabular} & $\frac{0.000}{0.000}$ & 0.138 & \\
\hline & $9 / 2 / 2004$ & $1: 11: 47 \mathrm{AM}$ & 25.739 & 25.509 & \begin{tabular}{|l|}
25.273 \\
\end{tabular} & \begin{tabular}{|l|}
25.791 \\
\end{tabular} & & & \begin{tabular}{|l|l|}
55.614 \\
\end{tabular} & 18.453 & & & & 51.005 & 0.041 & 14.643 & $\begin{array}{l}0.003 \\
0.003\end{array}$ & $\begin{array}{l}3917.4 \mathrm{~s} \\
3918.42\end{array}$ & 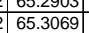 & \begin{tabular}{|l|}
54.5 \\
\end{tabular} & \begin{tabular}{|l|}
11.6 \\
12.1 \\
\end{tabular} & $\begin{array}{l}41.1 \\
40.9 \\
\end{array}$ & & \begin{tabular}{|l|}
0.006 \\
\end{tabular} & $\begin{array}{l}0.006 \\
0.006\end{array}$ & $\begin{array}{l}0.000 \\
0.000\end{array}$ & & $\begin{array}{l}0.12 \\
0.12\end{array}$ \\
\hline & $9 / 2 / 2004$ & 1:12:47 AM & 25.739 & 25.514 & & & 24.904 & 49.627 & & 18.47 & 31.979 & -0.349 & -6.11 & & 0.04 & \begin{tabular}{|l|l|}
14.643 \\
\end{tabular} & & 3919.42 & & \begin{tabular}{|l|l|}
54.4 \\
\end{tabular} & & & & & & & & \\
\hline & & 1:13:47 AM & & & \begin{tabular}{|l|}
25.273 \\
\end{tabular} & & 24.944 & $\begin{array}{l}49.653 \\
\end{array}$ & \begin{tabular}{|r|}
55.45 \\
\end{tabular} & 18.264 & & & & \begin{tabular}{|l}
51.404 \\
\end{tabular} & $\begin{array}{l}0.041 \\
\end{array}$ & \begin{tabular}{|l|l|}
14.643 \\
\end{tabular} & & 20.42 & \begin{tabular}{|l|l|}
65.3403 \\
\end{tabular} & \begin{tabular}{|l|l|}
54.3 \\
\end{tabular} & & & & & 0.006 & 0.000 & 0.142 & \\
\hline 3231 & |l/2004 & $1: 14: 47 \mathrm{AM}$ & 25.754 & 25.519 & $\begin{array}{l}25.284 \\
25.21\end{array}$ & 25.812 & $\begin{array}{r}24.87 \\
\end{array}$ & 50.014 & $\begin{array}{l}55.943 \\
5.52\end{array}$ & $\begin{array}{l}18.703 \\
1.971\end{array}$ & $\begin{array}{l}32.227 \\
\end{array}$ & -0.346 & $\begin{array}{l}-6.107 \\
\end{array}$ & 51.13 & $\begin{array}{l}0.041 \\
\end{array}$ & $\begin{array}{l}14.643 \\
\end{array}$ & 0.004 & 3921.42 & 65.3569 & 54.8 & 12.2 & & 2.835 & $\begin{array}{l}0.006 \\
\end{array}$ & $\begin{array}{l}0.006 \\
\end{array}$ & 0.000 & 0.142 & \\
\hline & 2004 & \begin{tabular}{ll|}
$1: 15: 47 \mathrm{AM}$ \\
$116: 47 \mathrm{AM}$
\end{tabular} & $\begin{array}{l}25.745 \\
25.745\end{array}$ & $\begin{array}{l}25.505 \\
25.515\end{array}$ & \begin{tabular}{|l|}
25.24 \\
25.27
\end{tabular} & \begin{tabular}{|l|}
25.823 \\
25.798 \\
\end{tabular} & $\begin{array}{l}24.896 \\
24.996\end{array}$ & & \begin{tabular}{|l|}
55.622 \\
554433 \\
\end{tabular} & \begin{tabular}{|l|}
18.474 \\
18.504
\end{tabular} & \begin{tabular}{|l|}
32.221 \\
32.299
\end{tabular} & $\begin{array}{l}-0.358 \\
-0.355 \\
-x^{2}\end{array}$ & $\begin{array}{l}-6.157 \\
-6.159\end{array}$ & $\begin{array}{l}49.449 \\
50217\end{array}$ & $\begin{array}{l}0.041 \\
0041\end{array}$ & $\begin{array}{l}14.643 \\
14643 \\
\end{array}$ & & $\begin{array}{r}3922.42 \\
3323.42\end{array}$ & \begin{tabular}{|l|l|}
65.3736 \\
56.3903
\end{tabular} & \begin{tabular}{|l|l|}
54.5 \\
543
\end{tabular} & \begin{tabular}{ll|}
11.8 \\
120
\end{tabular} & $\mid 4100$ & 2830 & \begin{tabular}{|l|}
0.006 \\
0.06
\end{tabular} & 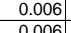 & 0.000 & 0.142 & \\
\hline 234 & 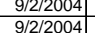 & $\begin{array}{l}1: 11: 474 \mathrm{AM} \\
1: 17: 47\end{array}$ & $\frac{25.745}{25.745}$ & $\begin{array}{r}25.515 \\
25.514\end{array}$ & $\begin{array}{r}25.27 \\
25.259\end{array}$ & \begin{tabular}{|l|}
25.798 \\
25.827 \\
\end{tabular} & $\begin{aligned} 24.996 \\
24.93\end{aligned}$ & \begin{tabular}{|}
49.809 \\
44.624 \\
\end{tabular} & \begin{tabular}{|l|}
55.413 \\
55.506
\end{tabular} & \begin{tabular}{|r|}
18.504 \\
17.814
\end{tabular} & $\begin{array}{l}32.289 \\
32.874 \\
\end{array}$ & $\begin{array}{l}-0.355 \\
-0.346\end{array}$ & $\begin{array}{l}-6.159 \\
-6.154\end{array}$ & $\begin{array}{l}50.217 \\
49.111\end{array}$ & \begin{tabular}{|c|}
0.041 \\
0.041
\end{tabular} & \begin{tabular}{|c|}
14.643 \\
14.643
\end{tabular} & $\begin{array}{r}0.0033 \\
0.004\end{array}$ & $\begin{array}{r}3923.42 \\
3924.42\end{array}$ & $\mid \begin{array}{l}65.3903 \\
655.4069\end{array}$ & $\begin{array}{r}54.3 \\
54.4 \\
\end{array}$ & $\frac{12.0}{11.7}$ & $\begin{array}{l}41.0 \\
41.2 \\
\end{array}$ & 2.830 & \begin{tabular}{|l|}
0.006 \\
0.006
\end{tabular} & $\begin{array}{l}0.006 \\
0.006\end{array}$ & $\frac{0.000}{0.000}$ & 0.142 & 0.12 \\
\hline & $2 / 2004$ & 1:18:47 AM & 25.75 & 25.514 & \begin{tabular}{|l|}
25.234 \\
\end{tabular} & \begin{tabular}{|l|}
25.837 \\
\end{tabular} & 24.895 & 49.763 & \begin{tabular}{|l|l|}
55.659 \\
\end{tabular} & 18.317 & 32.418 & \begin{tabular}{|c|}
$\mid-0.349$ \\
\end{tabular} & -6.157 & 52.117 & 0.042 & $\begin{array}{l}14.045 \\
14.643 \\
\end{array}$ & $\begin{array}{l}0.004 \\
0.003\end{array}$ & $\begin{array}{l}3924.42 \\
3925.42\end{array}$ & \begin{tabular}{|l|l|}
65.4069 \\
265.4236
\end{tabular} & \begin{tabular}{|l|}
54.4 \\
\end{tabular} & \begin{tabular}{|l|}
1.1 .1 \\
12.4
\end{tabular} & & $\frac{2.844}{2.833}$ & \begin{tabular}{|l|}
0.006 \\
0.006
\end{tabular} & 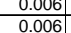 & $\begin{array}{l}0.000 \\
0.000\end{array}$ & $\frac{0.141}{0.145}$ & $\frac{0.12}{0.12}$ \\
\hline & & 1:19:47 AM & 25.754 & 25.519 & & \begin{tabular}{|l|}
25.797 \\
\end{tabular} & & & \begin{tabular}{|l|l|}
55.676 \\
\end{tabular} & 18.415 & 32.35 & -0.349 & & 50.986 & 0.041 & 14.643 & & & 65.4403 & 54.6 & & & & & & & 0.142 & \\
\hline & |2004 & 1:20:47 AM & 25.761 & 25.531 & 25.276 & & 24.92. & 499.732 & 55.748 & $\begin{array}{ll}18.342 \\
\end{array}$ & \begin{tabular}{|l|l|}
32.367 \\
\end{tabular} & -0.349 & -6.159 & 49.412 & $\begin{array}{l}0.04 \\
\end{array}$ & \begin{tabular}{|l|l|}
14.643 \\
\end{tabular} & & 3927.42 & \begin{tabular}{|l|l|}
65.4569 \\
\end{tabular} & 54.6 & 11.8 & 41.0 & & & & & & \\
\hline & & 1:21:47 AM & 25.756 & & & & & & & 18.52 & & & & & & & & & 65.4736 & 54.8 & & & & 0.006 & .006 & & 141 & \\
\hline & & $1: 22: 47 \mathrm{AM}$ & & & & 25.774 & & 49.7 & 55.603 & $\begin{array}{ll}18.626 \\
\end{array}$ & 32.348 & -0.349 & & 51.074 & & $\begin{array}{l}14.643 \\
\end{array}$ & & 201 & T. & 54.5 & & & & & & & 145 & \\
\hline & & 1:23:47 AM & 25.763 & 25.533 & 25.258 & 25.816 & 24.899 & & & & 32.285 & -0.352 & & 50.146 & & & & & & & & & & & & & & \\
\hline & & 1:24: & & & 25.258 & 25.841 & 24. & & 55.885 & 18.427 & & -0.352 & & & \begin{tabular}{|c|c|} 
\\
\end{tabular} & \begin{tabular}{l|l}
14.643 \\
\end{tabular} & & & 5.5236 & 54.8 & & & 843 & & 0.006 & & 0.138 & \\
\hline & & 1:25:47 AM & 25.763 & 25.533 & 25.328 & 25.821 & 24.969 & 49.533 & 55.439 & 18.721 & 31.761 & -0.346 & & 48.266 & 0.041 & 14.643 & 0.004 & 3932.42 & 65.5403 & 54.3 & 11.5 & 40. & 802 & 0.006 & 0.006 & 0.000 & 0.143 & 0.12 \\
\hline & & $1: 26: 47 \mathrm{AM}$ & 25.774 & 25.534 & 25.303 & 25.857 & 24.934 & 49.676 & \begin{tabular}{|l|}
55.551 \\
\end{tabular} & 18.53 & 32.024 & -0.349 & & 51.789 & 0.041 & 14.643 & 0.0 & 3933.42 & 65.5569 & 54.4 & & & .816 & . 006 & .006 & & 142 & \\
\hline & & $1: 27: 47 \mathrm{AM}$ & 5.764 & $25.53 \%$ & & 25.847 & 25.006 & & \begin{tabular}{|l|l|}
55.614 \\
\end{tabular} & & 32.315 & -0.346 & & 47.776 & 0.041 & 14.643 & & & & 54.5 & & & & & & & 142 & \\
\hline & & 1::28:45 AM & & & 25.304 & 25.802 & & & $55.4 \pi \mathrm{s}$ & 1.861 & & -0.349 & & & 0.04 & 14.643 & & & & & & & & .006 & & & 138 & \\
\hline 47 & & $\begin{array}{l}1: 29: 54 \mathrm{AM} \\
1: 30 \cdot 45 \mathrm{AM}\end{array}$ & 5765 & $\frac{25.535}{25.525}$ & $\begin{array}{r}25.28 \\
2526\end{array}$ & $\frac{25.813}{25.793}$ & 24.93 & 9.958 & \begin{tabular}{|c|c|}
55.168 \\
5548
\end{tabular} & $\frac{18.476}{18.518}$ & $\begin{array}{r}31.87 \\
32.395 \\
\end{array}$ & 染45 & & 52.855 & $\begin{array}{r}0.04 \\
0.041\end{array}$ & $\frac{14.643}{14643}$ & & 30373 & & $\begin{array}{l}54.0 \\
547 \\
\end{array}$ & & & & & & & $\frac{40}{42}$ & \\
\hline & 9/2/2004 & $1: 31: 45 \mathrm{f}$ & 25.781 & 25.536 & 25.281 & 25.804 & 25.0 & 499.726 & 55.711 & $\begin{array}{l}10.010 \\
18.643\end{array}$ & 31.966 & $\frac{-.052}{-0.306}$ & & 52.336 & 0.041 & $\begin{array}{l}14.045 \\
14.643\end{array}$ & & 93838 & 6397 & 546 & 12.5 & & 816 & 0006 & 0006 & & & \\
\hline & & $1: 32: 45 \mathrm{AM}$ & 25.782 & 25.542 & \begin{tabular}{|l|}
25.276 \\
\end{tabular} & 25.84 & 24.9 & 49. & 55.415 & 18.245 & 32.317 & -0.3 & & 50.248 & 0.041 & 14.643 & & 393938 & & 54.3 & & & 2.821 & & & & 142 & 0.12 \\
\hline & & & & & 25.266 & & 24. & & \begin{tabular}{|l|l|}
55.394 \\
\end{tabular} & \begin{tabular}{|l}
18.381 \\
\end{tabular} & $\begin{array}{l}31.946 \\
\end{array}$ & -0.32 & & 52.63 & 0.041 & 14.6 & & & & 54.3 & & & & & & & & \\
\hline & & $1: 34: 45$ AM & 782 & 25. & 25.261 & 25.845 & & & 55.278 & & & -0.303 & & 50.44 & & 14.643 & & & & 54.2 & & & & & & & 43 & \\
\hline & & 45 AM & 25.778 & 25.538 & 25.252 & 25.86 & & & 55.589 & 18.562 & 31.952 & $\begin{array}{ll} \\
\end{array}$ & & 47.743 & 0.041 & \begin{tabular}{|l|l|l|}
14.643 \\
\end{tabular} & & & & 54.5 & 11. & & & 006 & .006 & & 43 & \\
\hline & & 1:36:45 AM & 25.773 & 25.543 & 25.273 & 25.886 & 25.029 & 49.63 & \begin{tabular}{|l|l|}
55.634 \\
\end{tabular} & $\begin{array}{l}18.676 \\
\end{array}$ & 31.784 & $\begin{array}{l}-0.3 \\
\end{array}$ & & 50.795 & 0.042 & \begin{tabular}{|l|l|}
14.643 \\
\end{tabular} & 0.003 & & 365.7231 & 54.5 & 12 & 40.7 & 2.00 & 0.006 & 0.0 & 000 & 0.147 & 0.12 \\
\hline & & $\begin{array}{l}1: 37: 45 \text { AM } \\
1.20 .50\end{array}$ & & 25.543 & \begin{tabular}{|l|}
25.263 \\
\end{tabular} & 25.871 & 25.049 & & \begin{tabular}{|}
$\mid 55.535$ \\
5707
\end{tabular} & $\begin{array}{l}18.595 \\
\end{array}$ & 31.831 & -0.3 & & 50.175 & 0 & $\begin{array}{l}14.643 \\
1.643\end{array}$ & & 3944.38 & $\begin{array}{l}6.5 .7397 \\
3\end{array}$ & 54.4 & & & 2.806 & \begin{tabular}{|l|l|l|} 
& 0.006 \\
\end{tabular} & & & & 0.12 \\
\hline & $2 / 2004$ & $\begin{array}{l}1.50 .395 \mathrm{AM} \\
1: 39: 45 \mathrm{AM}\end{array}$ & $\frac{2.109}{25.779}$ & 25.534 & $\frac{23.254}{25.269}$ & \begin{tabular}{|l|}
25.8017 \\
25.817 \\
\end{tabular} & $\frac{2.562}{3.520}$ & & \begin{tabular}{|l|l|}
5.101 \\
55.869
\end{tabular} & $\begin{array}{l}18.1044 \\
18.477\end{array}$ & $\begin{array}{l}32.124 \\
32.438\end{array}$ & $\begin{array}{l}-0.3 \\
-0.3\end{array}$ & & $\frac{49.49}{50.88}$ & 0.041 & 14643 & & $\begin{array}{l}3945.38 \\
39130\end{array}$ & & $\begin{array}{l}54.0 \\
54.7\end{array}$ & $\frac{11 .}{12}$ & & . & & & & $\frac{0.142}{0.142}$ & $\frac{0.12}{0.12}$ \\
\hline & & & 25.778 & 25.538 & 25.258 & 25.816 & 24.9 & 49.803 & \begin{tabular}{|l|}
55.8939 \\
\end{tabular} & 18.724 & 31.964 & $\begin{array}{r}-0.3 \\
\end{array}$ & & 49.8 & 0.041 & & & & & 54.8 & & & & & & & & 0.12 \\
\hline & & & & & 25.244 & & & & & & 32.42 & & & & & & & & & 54.8 & 10 & & & & & & & \\
\hline & & $1: 42$ & 25.791 & 25.551 & 25.275 & 25.808 & 24. & & 55.49 & 18.583 & 31.971 & -0.283 & & 49.795 & & 14. & & & & 54.4 & 11 & & & & & & 143 & \\
\hline & & & & & & & & & & & & & & & & & & & & & & & & & & & & \\
\hline & & 1:44:45 AN & & 25.541 & 25.285 & \begin{tabular}{|l|}
25.778 \\
\end{tabular} & 24. & 49.542 & 55.524 & 18.611 & 31.962 & $\begin{array}{l}-0.268 \\
-0.268\end{array}$ & & 49.581 & 0.041 & $\begin{array}{l}14.045 \\
14.643\end{array}$ & & 3951.38 & \begin{tabular}{|l|l|l}
65.8564 \\
\end{tabular} & $\begin{array}{l}04.4 \\
54.4\end{array}$ & 111.8 & 40.8 & 2810 & 0.006 & 0.0 & 500 & 0.143 & \\
\hline & $\mid 1 / 2004$ & & & 25.534 & 25.269 & 25.672 & 24. & & 55.638 & 18.45 & 31.989 & -0.248 & & 50.559 & & \begin{tabular}{l|l}
14.643 \\
\end{tabular} & & & & 54.5 & 12.0 & & 6.8 & 006 & & & 0.136 & \\
\hline & & & & & 25.262 & & & & 55.81 & & & & & & & & & & & 54.7 & & & & & & & & \\
\hline & & & & 25.532 & 25.342 & 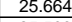 & & & 55.433 & 18.527 & 32 & -0.245 & & 50.0 & 0.04 & & & & 65.9064 & 54.3 & $11.6 \mathrm{~s}$ & & & & & & 139 & \\
\hline & & & & & & 25.593 & & & 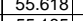 & & & & & & & & & & & 54.5 & & & & & & & & \\
\hline & & & & 25.519 & & & & & & & & & & & & & & & & & & & & & & & & \\
\hline & & & & $\frac{25.52}{25 .}$ & $\frac{5.318}{253}$ & $\frac{25.556}{25548}$ & & $\frac{49.54}{49.42}$ & 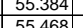 & $\frac{18.46}{1852}$ & $\begin{array}{l}31.934 \\
31.811\end{array}$ & 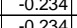 & & & & $\frac{14.6}{146}$ & & $\begin{array}{l}3955.3 \\
395.3\end{array}$ & & $\frac{54.5}{543}$ & 110 & & & & & & 年 & 0.1 \\
\hline & & & 25.76 & 25.51 & $\begin{array}{r}25.288 \\
25.0\end{array}$ & \begin{tabular}{|l|l|}
25.640 \\
\end{tabular} & & & \begin{tabular}{|c|}
55.460 \\
556
\end{tabular} & & 31.011 32.373 & $\begin{array}{l}-0.204 \\
-0.196\end{array}$ & & & 0.04 & & & & & $\frac{5.35}{543}$ & & & & & & & & \\
\hline & & & & & & & & & & & & & & & & & & & & & & & & & & & & \\
\hline & & & 25.783 & 25.5. & 25.323 & 25.706 & & & \begin{tabular}{|l|}
55.707 \\
\end{tabular} & 18. & 31. & & & & 0.04 & & & & & 54.6 & $\frac{12}{12}$ & & & & & & & \\
\hline & & & & & & & & & & & & & & & & & & & & & & & & & & & & \\
\hline & & & & 25.50 & 25.302 & 25.645 & & & 55.835 & & & & & & & & & & & & & & & & & & & \\
\hline & & & & & 25.332 & 25.67 & & & \begin{tabular}{|l|l|}
55.67 \\
\end{tabular} & & & -0.23 & & & 0. & & & & & 54.5 & & & & & & & & \\
\hline & & & & 25.51 & & 25.705 & & 49.6 & 55.529 & & 31.6 & & & & & & & & & 54.4 & & & & & & & & \\
\hline & & & & 25.51 & 25.346 & 25.684 & & 49 & 55.29 & 18.6 & 31.552 & -0.1 & & & & 14.6 & & & & 54.2 & & & & & & & 0.140 & \\
\hline & & & & & & & & 49.1 & & & & & & & & 14. & & & & 53.9 & 12 & & & & & & & \\
\hline & & & & 25.50 & 25.334 & 25.6 & & 49 & 55.547 & 18.5 & & & & & & & & & & 54.4 & 11.5 & & & & & & & \\
\hline & & & & & & & & & & & & & & & & & & & & & & & & & & & & \\
\hline & & & & 25. & 25.313 & 25.6 & & & 55. & & 31. & & & & & & & & & 54.0 & & & & & & & & \\
\hline & & & & 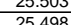 & $\frac{2.3524}{25207}$ & 25 & & & 年 & & 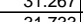 & $=0.17$ & & & & & & & & 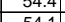 & $\frac{1}{1}$ & & & & & & & \\
\hline & & & & $\frac{2.456}{25498}$ & $\frac{2.0517}{25287}$ & $\frac{2.36}{257}$ & & 告, & 55.53 & 187.04 & $\frac{31.150}{31.449}$ & -0.176 & & & & & & & & 5.4.14 & & & & & & & 414 & \\
\hline & & & & 25,498 & 20.512 & 25 & & & 54.81 & & 31.841 & -0.176 & & & 0.04 & & & & & 537 & 119 & & & & & & & \\
\hline & & & & $\frac{25.5}{25.5}$ & 25308 & 25.746 & & & & & 31.86 & -0.158 & & & 0.039 & 14. & & & & 542 & 124 & & & & & & & \\
\hline & & & & & & & & & & & & & & & & & & & & & & & & & & & & \\
\hline & & & & & & & & & & & & & & & & & & & & & & & & & & & & \\
\hline & & & & 25.505 & 25.305 & 25.818 & & 49.8 & 56.13 & & 31.925 & -0.179 & & & & & & & & 55.0 & 20 & & & & & & 39 & \\
\hline & & & & 55.49 & & & & & 92 & & & -017 & & 521 & & & & & & 548 & & & & & & & & 11 \\
\hline 3290] & & 2:13:45 AM & 25.782 & 25.511 & 25.316 & 25.819 & 24.8 & 49.857 & $\mid$ & $\mid 18.712$ & 32.34 & $\mid$\begin{tabular}{|l|l|} 
& -0.164 \\
\end{tabular} & -6. & 54.199 & 0.041 & 14.643 & & 3980.38 & 366.3397 & 54.8 & $12.9 \quad>\quad \mathrm{s}$ & & 2.834 & \begin{tabular}{|l|l|}
0.006 \\
\end{tabular} & 0.006 & 0.000 & 0.142 & \\
\hline
\end{tabular}


WSRC-TR-2005-00105, REVISION 0

SRNL-RPP-2005-00012, REVISION 0

RUN \# 4.03A AND B; FIRST AND SECOND HALF OF SLURRY DEWATERING - CONT.

\begin{tabular}{|c|c|c|c|c|c|c|c|c|c|c|c|c|c|c|c|c|c|c|c|c|c|c|c|c|c|c|c|c|}
\hline & $\mathrm{A}$ & $B$ & D & $E$ & $F$ & G & \begin{tabular}{l|l}
$\mathrm{H}$ \\
\end{tabular} & $\mathrm{J}$ & $\mathrm{K}$ & \begin{tabular}{l|l|l|}
$\mathrm{L}$ & \\
\end{tabular} & $\mathrm{M}$ & $\mathrm{N}$ & 0 & Q & $\begin{array}{ll}R \\
\end{array}$ & $\mathrm{~s}$ & $T^{T}$ & u $\vee$ & w & $x$ & Y & $z$ & $\mathrm{AA}$ & $A B$ & AC & & & AF $A$ \\
\hline & 9/2/2004 & 2:14:45 AM & 25.773 & 25.493 & 25.283 & 25.811 & 24.898 & 49.568 & 55.504 & 18.436 & $\begin{array}{l}32.083 \\
\end{array}$ & -0.112 & -6.119 & 50.229 & 0.038 & 14.643 & 0.003 & 3981.38 & 866.3564 & 54.4 & 12.0 & 40.8 & 2.815 & 0.005 & 0.005 & 0.000 & 0.132 & 0.11 \\
\hline & 9/2/2004 & 2:15:45 AM & 25.772 & 25.492 & 25.312 & 25.775 & 24.858 & 49.49 & \begin{tabular}{|l|}
55.245 \\
\end{tabular} & \begin{tabular}{|l|}
18.338 \\
\end{tabular} & 32.001 & \begin{tabular}{|l|}
-0.132 \\
\end{tabular} & -6.11 & 49.841 & 0.041 & 14.643 & & 3982.38 & 66.3731 & 54.1 & 11.9 & & & & 0.006 & & & \\
\hline , & $9 / 2 / 2004$ & 2:16:45 AM & $\frac{25.767}{25.771}$ & 25.491 & $\frac{25.286}{2528}$ & \begin{tabular}{|l|l|}
25.699 \\
\end{tabular} & 24.757 & 49.788 & \begin{tabular}{|l|}
55.738 \\
5.738 \\
\end{tabular} & \begin{tabular}{|l|}
18.429 \\
\end{tabular} & \begin{tabular}{|l|l|}
32.328 \\
32.08
\end{tabular} & \begin{tabular}{|r|}
-0.158 \\
\end{tabular} & $\begin{array}{l}-6.11 \\
\end{array}$ & 48.604 & 0.042 & 14.643 & & \begin{tabular}{|l|}
3983.38 \\
382.38
\end{tabular} & 66.3897 & 54.6 & 11.6 & & & 0 & 0.006 & 0.000 & 0.145 & \\
\hline 3294 & $1 / 2004$ & $2: 17: 45 \mathrm{AM}$ & \begin{tabular}{|l|l|}
25.771 \\
\end{tabular} & 25.481 & 25.28 & 25.678 & $\begin{array}{l}24.691 \\
\end{array}$ & 49.734 & \begin{tabular}{|l|l|}
55.848 \\
\end{tabular} & 18.571 & \begin{tabular}{|l|l|}
31.909 \\
3.20
\end{tabular} & \begin{tabular}{|l|l|}
-0.135 \\
\end{tabular} & $\begin{array}{l}-6.113 \\
\end{array}$ & 50.415 & $\begin{array}{l}0.039 \\
\end{array}$ & \begin{tabular}{|l|l|}
14.643 \\
\end{tabular} & & 3984.38 & 66.4064 & 54.7 & 12.0 & 40.8 & 2.814 & 0.006 & $\begin{array}{l}0.006 \\
\end{array}$ & 0.000 & $\begin{array}{ll}0.136 \\
\end{array}$ & 0. \\
\hline & $9 / 2 / 2004$ & 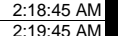 & $\begin{array}{l}25.77 \\
25.77\end{array}$ & & $\begin{array}{r}25.31 \\
25295\end{array}$ & $\begin{array}{l}25.638 \\
25.628\end{array}$ & $\begin{array}{r}24.67 \\
24.65 \\
\end{array}$ & $\begin{array}{l}49.678 \\
49627\end{array}$ & & $\begin{array}{l}18.392 \\
1845\end{array}$ & \begin{tabular}{|l|l|}
32.322 \\
32088
\end{tabular} & & $\begin{array}{l}-6.102 \\
-6096\end{array}$ & $\begin{array}{l}49.272 \\
43005\end{array}$ & $\begin{array}{l}0.042 \\
0.042\end{array}$ & \begin{tabular}{|l|l|}
14.643 \\
14634
\end{tabular} & & 3985.38 & $\begin{array}{l}66.4231 \\
66.207\end{array}$ & 54.7 & 11.7 & \begin{tabular}{|l|}
41.0 \\
\end{tabular} & & & 0.006 & & 0.145 & 0.12 \\
\hline$\frac{340}{3297}$ & $\begin{array}{l}9 / 2 / 2004 \\
9 / 2 / 2004\end{array}$ & $\begin{array}{l}2: 19: 45 \mathrm{AM} \\
2: 20: 45 \mathrm{MM}\end{array}$ & $\begin{array}{r}25.77 \\
25.759\end{array}$ & $\begin{array}{r}25.48 \\
25.464\end{array}$ & \begin{tabular}{|l|}
25.295 \\
25.264
\end{tabular} & \begin{tabular}{|l|} 
\\
$\quad 25.628$ \\
\end{tabular} & $\begin{array}{l}24.665 \\
24.55\end{array}$ & $\begin{array}{l}49.627 \\
49.803\end{array}$ & \begin{tabular}{|l|}
55.825 \\
55.993 \\
\end{tabular} & \begin{tabular}{|l|}
18.435 \\
18.579 \\
\end{tabular} & $\begin{array}{r}32.088 \\
31.79\end{array}$ & $\left|\begin{array}{|c|}-0.1229 \\
-0.026\end{array}\right|$ & $\begin{array}{l}-6.096 \\
-6.102 \\
\end{array}$ & $\begin{array}{l}43.905 \\
52.657\end{array}$ & $\mid \begin{array}{l}0.042 \\
0.041\end{array}$ & $\frac{14.643}{14.643}$ & $\begin{array}{l}0.003 \\
0.004\end{array}$ & $\begin{array}{c}3986.38 \\
3987.38\end{array}$ & $\begin{array}{l}366.4997 \\
36.4564\end{array}$ & $54.7 \mid$ & $\begin{array}{l}\frac{10.5}{12.5} \\
\end{array}$ & $\begin{array}{l}40.9 \\
40.8 \\
\end{array}$ & $\frac{2.817}{2.813}$ & \begin{tabular}{|c|c|}
0.006 \\
0.006
\end{tabular} & $\mid \begin{array}{l}0.006 \\
0.006\end{array}$ & $\frac{0.000}{0.000}$ & \begin{tabular}{|l|l|l|l|l|}
0.143 & \\
\end{tabular} & 0.12 \\
\hline 3298 & 9/2/2004 & 2:21:45 AM & 25.759 & 25.468 & \begin{tabular}{|l|l|}
25.293 \\
\end{tabular} & \begin{tabular}{|l|}
35.506 \\
\end{tabular} & 24.524 & & \begin{tabular}{|l|}
55.937 \\
\end{tabular} & 18.395 & 32.227 & & & 51.57 & 0.041 & 14.643 & $\begin{array}{l}0.004 \\
0.003\end{array}$ & $\begin{array}{l}3988.58 \\
3988.38\end{array}$ & & $\begin{array}{ll}54.9 \\
54.8\end{array}$ & $\begin{array}{l}12.5 \\
12.3 \\
\end{array}$ & & $\frac{2.813}{2.823}$ & $\begin{array}{l}0.006 \\
0.006\end{array}$ & $\begin{array}{l}0.000 \\
0.006\end{array}$ & $\begin{array}{l}0.000 \\
0.000\end{array}$ & & $\frac{0.12}{0.12}$ \\
\hline & $9 / 2 / 2004$ & 2:22:45 AM & & & & 25.49 & 24.518 & 49.444 & \begin{tabular}{|l|}
55.429 \\
\end{tabular} & & 31.685 & -0.109 & & 50.665 & 0.041 & 14.643 & & & 866.4897 & 54.3 & & & & & & & 0.144 & $\begin{array}{l}0.12 \\
0.12\end{array}$ \\
\hline & 9/2/2004 & 2:23:45 AM & 25.741 & 25.441 & 25.296 & 25.474 & 24.447 & & \begin{tabular}{|l|l|}
55.941 \\
\end{tabular} & \begin{tabular}{|l|l|l|} 
& \\
\end{tabular} & \begin{tabular}{|l|l|}
32.19 \\
\end{tabular} & \begin{tabular}{|l|}
-0.132 \\
\end{tabular} & & 53.07 & 0.041 & \begin{tabular}{|l|l|}
14.643 \\
\end{tabular} & & & & 54.8 & 12.6 & 41 & 2.833 & \begin{tabular}{|l|}
0.006 \\
\end{tabular} & 006 & & & \\
\hline 3301 & & & & & & & & 49.388 & & 18.266 & & & & 51.404 & & 14.643 & & & & & & & & $\begin{array}{l}0.006 \\
\end{array}$ & 0.006 & 0.000 & 1.139 & $\frac{0.12}{0.12}$ \\
\hline & & & & 25.421 & & 25.448 & 24.346 & 49.77 & 55.864 & & & $\begin{array}{l}-0.106 \\
\end{array}$ & & 48.423 & 0.04 & & & & & & & & & & & & & \\
\hline & 2004 & 2:26:45 & 25.735 & & 25.289 & 25.402 & 24.375 & & 55.622 & 18.738 & 751 & -0.106 & & 49.044 & $\begin{array}{l}0.04 \\
\end{array}$ & & & & & & & & & & & & & \\
\hline & & 2:27:45 & & 25.434 & 25.289 & 25.431 & 24.469 & 49.481 & 55.595 & 18.341 & & -0.103 & & 49.677 & 0.042 & 14.643 & & & 366.5731 & 54.5 & & & 2.81 & \begin{tabular}{|c|c|}
0.006 \\
\end{tabular} & 0.006 & & & \\
\hline & 12004 & $2: 28: 45 \mathrm{AM}$ & 25.733 & 25.417 & \begin{tabular}{|l|}
25.257 \\
\end{tabular} & 25.45 & 24.458 & 49.828 & 56.157 & 18.45 & \begin{tabular}{|l|l|}
32.112 \\
\end{tabular} & \begin{tabular}{|l|}
-0.103 \\
\end{tabular} & -6.084 & 51.529 & 0.042 & 14.643 & 0.003 & 3995.38 & 366.5897 & 55.0 & $\begin{array}{ll}12.3 \\
\end{array}$ & 41.0 & 2.825 & 0.006 & 0.006 & 0.000 & 0.146 & 0. \\
\hline & & : 45 AM & & $\frac{25.422}{25.27}$ & $\begin{array}{l}25.296 \\
2.251\end{array}$ & 25.429 & 24.452 & $\begin{array}{l}49.863 \\
\end{array}$ & \begin{tabular}{|l|}
56.287 \\
\end{tabular} & 18.425 & 32.363 & \begin{tabular}{r|r|}
-0.1 \\
\end{tabular} & $\begin{array}{l}-6.096 \\
\end{array}$ & 51.451 & 0.038 & 14.643 & & 3996.38 & & 55.2 & & & & & & & & 0. \\
\hline & 9/2/2004 & 2:30:45 AM & & 25.407 & & 25.404 & 24.467 & & & 18.368 & $\begin{array}{l}31.921 \\
22025\end{array}$ & & & 50.661 & 0.041 & 14.643 & & & 66.6231 & 54.3 & 12.1 & 40.7 & & & 0.006 & & 0.143 & \\
\hline & 9/2/2004 & 2:31:45 AM & 25.722 & 25.407 & 25.266 & 25.444 & 24.532 & 49.8077 & \begin{tabular}{|l|l|}
55.808 \\
\end{tabular} & & & -0.109 & & 50.984 & & 14.643 & & & & 54.7 & & & 2.842 & 0.006 & 0.006 & 0.000 & 0.138 & \\
\hline & $9 / 2 / 2004$ & $\begin{array}{l}2: 32: 45 \mathrm{AM} \\
22 \cdot 33 \cdot 45 \mathrm{AM}\end{array}$ & & & $\begin{array}{l}25.281 \\
25291\end{array}$ & & & \begin{tabular}{|l|}
49.72 \\
9.525
\end{tabular} & & $\begin{array}{l}18.497 \\
18313\end{array}$ & $\begin{array}{l}32.147 \\
332112 \\
\end{array}$ & $\begin{array}{r}-0.1 \\
\end{array}$ & & $\begin{array}{r}46.94 \\
48681\end{array}$ & & & & & & $\begin{array}{l}54.8 \\
54.5 \\
\end{array}$ & 11.2 & 40.9 & & & 0.006 & & & 0.12 \\
\hline & 9/2/20004 & $\begin{array}{l}\frac{2: 33: 45 \mathrm{AM}}{2: 34: 45 \mathrm{AM}} \\
\end{array}$ & $\begin{array}{r}\frac{25.732}{25.72} \\
25\end{array}$ & $\begin{array}{r}25.417 \\
25.4\end{array}$ & $\frac{25.291}{25.285}$ & $\begin{array}{l}25.479 \\
25.413\end{array}$ & $\frac{24.652}{24.561}$ & $\begin{array}{l}49.525 \\
49.552\end{array}$ & \begin{tabular}{|l|}
55.665 \\
55.713 \\
\end{tabular} & \begin{tabular}{|c|}
18.313 \\
18.312
\end{tabular} & $\frac{32.112}{32.1}$ & $\begin{array}{l}-0.092 \\
-0.006\end{array}$ & & $\begin{array}{l}48.681 \\
50.169\end{array}$ & & $\begin{array}{l}14.643 \\
14.643 \\
\end{array}$ & 0.003 & $\begin{array}{c}4000.38 \\
4001.38\end{array}$ & & $\frac{54.5}{54.6}$ & \begin{tabular}{l|l|l|}
11.6 \\
11.9
\end{tabular} & $\begin{array}{l}40.8 \\
40.8\end{array}$ & 2.814 & 0.006 & 0.006 & 0.000 & $\begin{array}{l}0.146 \\
0.143\end{array}$ & 0.12 \\
\hline 3312 & 9/2/2/2004 & $\begin{array}{l}\frac{2.544 .45 \mathrm{AMI}}{2: 35: 45} \\
\end{array}$ & $\frac{25.12}{25.711}$ & 25.386 & $\frac{25.285}{25.251}$ & $\frac{23.413}{25.418}$ & $\begin{array}{l}24.561 \\
24.636\end{array}$ & $\begin{array}{l}\frac{49.552}{49.421} \\
\end{array}$ & \begin{tabular}{|l|}
55.113 \\
55.593 \\
\end{tabular} & $\begin{array}{l}18.1612 \\
18.161\end{array}$ & $\begin{array}{r}32.243 \\
32.24\end{array}$ & $\begin{array}{l}-.0060 \\
-0.092 \\
-10\end{array}$ & $\begin{array}{l}-0.078 \\
-6.078\end{array}$ & $\begin{array}{l}0.109 \\
48.888\end{array}$ & $\begin{array}{l}0.041 \\
0.041\end{array}$ & $\frac{14.045}{14.643}$ & & $\begin{array}{l}401.50 \\
4002.38\end{array}$ & \begin{tabular}{|l|l|}
0.06097 \\
66.7064
\end{tabular} & $\begin{array}{l}54.0 \\
54.5 \\
\end{array}$ & $\frac{11.9}{11.6}$ & \begin{tabular}{|l|}
40.8 \\
40.8 \\
\end{tabular} & $\frac{2.815}{2.815}$ & \begin{tabular}{|l|}
0.000 \\
0.006 \\
\end{tabular} & $\frac{0.006}{0.006}$ & & \begin{tabular}{|c|}
0.143 \\
0.143
\end{tabular} & $\frac{0.12}{0.12}$ \\
\hline 3313 & 9/2/2004 & 2:36:45 AM & & 25.386 & & & & & & 18.191 & & -0.092 & & 48.183 & & & & & & 54.6 & 11.5 & 40.9 & & 0.006 & & & & \\
\hline 3314 & 9/2/2004 & $2: 37: 45$ AM & 25.697 & 25.382 & 25.241 & 25.474 & 24.662 & \begin{tabular}{|l|l|}
49.873 \\
\end{tabular} & \begin{tabular}{|l|l|}
56.049 \\
\end{tabular} & 18.833 & & \begin{tabular}{|c|c|} 
& -0.083 \\
\end{tabular} & -6.073 & 49.483 & 0.042 & 14.643 & & $\mid 4004.38$ & & 54.9 & \begin{tabular}{l|l|l|}
11.8 \\
\end{tabular} & 40.9 & 2.823 & \begin{tabular}{|c|}
0.006 \\
\end{tabular} & \begin{tabular}{|c|}
0.006 \\
\end{tabular} & 0.000 & & $\begin{array}{l}0.12 \\
0.12\end{array}$ \\
\hline 3315 & $\mid$ & & 25.713 & 25.392 & 25.257 & 25.53 & 24.5 & 49.848 & \begin{tabular}{|l|}
56.101 \\
\end{tabular} & \begin{tabular}{|l|l|}
18.65 \\
\end{tabular} & 31.942 & & & 52.165 & & & & & & & & & & \begin{tabular}{l|l|l|}
0.006 \\
\end{tabular} & 0.006 & 0.000 & & \\
\hline 3316 & & & & 25.387 & 25.237 & 25.545 & & & \begin{tabular}{|l|}
55.495 \\
\end{tabular} & 18.272 & 32.243 & 0.086 & & 48536 & 0.04 & 14.643 & & & & 54.4 & 11.6 & & & & & & & \\
\hline & & & 25.693 & 25.382 & 25.242 & 25.495 & & & & & & & & 50.36 & & & & & & & & & & & & & & \\
\hline & & & & & & 25.54 & & & \begin{tabular}{|l|}
56.059 \\
\end{tabular} & 18.435 & & & & & & & & & & 54.9 & $\overline{11.5}$ & & & & & & & \\
\hline & & $2: 42: 45 \mathrm{~A}$ & 25.697 & 25.377 & 25.226 & 25.554 & 24.7 & 49.622 & \begin{tabular}{|l|}
55.701 \\
\end{tabular} & \begin{tabular}{|l|l|}
18.13 \\
\end{tabular} & 32.521 & -0.08 & & 47.046 & 0.0 & 14.643 & & 09.38 & $\begin{array}{l}3 \mid 66.82 \\
3\end{array}$ & 54.6 & 11.2 & & .832 & .006 & 0.006 & 0.000 & \begin{tabular}{|l|l|}
0.139 \\
\end{tabular} & \\
\hline & & & 25.696 & 25.381 & $\frac{25.241}{25.20}$ & 25.559 & 24.7 & 49.726 & 55.982 & 18.296 & & -0.08 & & 52.768 & & 14.643 & & & & 54.9 & (2) & & & 0.006 & .006 & 000 & 0.142 & \\
\hline & & & & & & 25.604 & & & 55.912 & & & -0.0 & & 52.196 & & & & & & & 12.4 & & & & & & & \\
\hline & 9/2/2004 & & 25.696 & 25.386 & 25.236 & 25.604 & 24.65 & 49.902 & 55.997 & $18.081 \mid$ & 32.763 & -0.08 & & 49.151 & 0.041 & 14.643 & & & & 54.9 & & & 2.85 & 0.006 & 0.006 & .000 & 0.141 & 0. \\
\hline & & & $\begin{aligned} 25.706 \\
257\end{aligned}$ & $\frac{25.396}{25.384}$ & $\frac{25.246}{25.224}$ & $\frac{25.599}{25627}$ & & 49.541 & 55672 & $\frac{18.192}{18445}$ & & & & & & & & & & & 12 & & 8832 & & & & & 0.12 \\
\hline & & & $\frac{25.1}{52694}$ & & $\mid 25243$ & & & & 56.113 & $\begin{array}{l}18.458 \\
1858\end{array}$ & & & & & & & & & & & & & & & & & & 0.12 \\
\hline 332 & & & 25.705 & $\frac{2.004}{25.389}$ & \begin{tabular}{|l|}
25.240 \\
25.239 \\
\end{tabular} & 25.592 & & 49.981 & \begin{tabular}{|l|}
56.268 \\
\end{tabular} & 18.411 & 32.391 & -0.083 & & 49.87 & & & & & & $\overline{55.1}$ & 11 & & 840 & & & & 0 & \\
\hline & & & & 25.395 & 25.23 & $\frac{25.638}{25.638}$ & & & $\mid 56.24$ & 18.437 & 32.43 & & & 47.874 & & & & & & & & & & & & & & \\
\hline & & & & $\begin{array}{l}25.405 \\
505\end{array}$ & 25.275 & 25.618 & & & 56.24 & 18.576 & 32.01 & & & 51.952 & 0.04 & 14643 & & & & & & & & 0.006 & 年 & & & \\
\hline & $\mid$ & & 25.706 & 25.396 & 25.246 & 25.654 & 24.8 & 49.94 & 56.092 & 18.21 & 32.654 & -0.02 & & 50. & 0.041 & 14.643 & & & & 55.0 & $\frac{11 . !}{11 .}$ & & 2.84 & 06 & & 0.000 & & \\
\hline & & & & & & & & & & 18.584 & & & & & & & & & & & & & & & & & & \\
\hline & & $2: 54: 4$ & 25.713 & 25.398 & 25.273 & 25.646 & 24.82 & 50.079 & \begin{tabular}{|l|l|}
56.009 \\
\end{tabular} & 18.682 & 32.397 & & & 51.85 & 0.04 & 14.6 & & & & 54.9 & $\frac{12.3}{12.3}$ & $\begin{array}{ll}41.2 \\
1.2\end{array}$ & 2.843 & 06 & & & 0 & \\
\hline & & & & 25.3 & 25.8 & 25.6 & & & & 18.8 & & & & & & & & & & & & & & & & & $\begin{array}{l}.143 \\
\end{array}$ & \\
\hline & & & & 25.3 & & & 24.8 & & 55.7 & & & & & & & & & & & 54.6 & 11.5 & & & & & & & \\
\hline & & & 25.71 & 25.39 & 25.264 & 25.6 & 24 & 49.76 & \begin{tabular}{|l|l|}
55.8 \\
\end{tabular} & $18.6 \mathrm{C}$ & & & & & & & & & & 54.8 & $\begin{array}{ll}12.3 \\
\end{array}$ & & & & & & 39 & \\
\hline & & & & 25.394 & & & & & & & & & & & & & & & & 54.8 & 11.9 & & & & & & & \\
\hline & & & 25.708 & 25.3.388 & $\frac{25.268}{5320}$ & 25.6 & & & $\frac{56.1}{5.2}$ & 18.34 & & & & & & & & & & & 11.9 & & & & & & & \\
\hline 338 & & & 25774 & 20594 & $\frac{25.283}{20502}$ & 257 & $\frac{2.0}{2.0}$ & (5) & 5.8 .84 & $\frac{18.10}{10}$ & 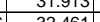 & & & & & & & & & & 120 & & & & & & & \\
\hline & & & $\frac{2.144}{25709}$ & 25.394 & 25269 & $\frac{2.1}{257}$ & & & 560 & 1870 & & 0. & & & & & & & & & & & & & & & & \\
\hline & & & & 25.00 & & & & 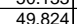 & $\frac{5.56}{56}$ & $\frac{1.1}{18.3}$ & & & & & & & & & & & 123 & & & & & & (9) & \\
\hline & & & $=25.72$ & 25.4 & $\frac{25.259}{25.259}$ & $\frac{25.707}{20.707}$ & & & & $\frac{19.042}{19.04}$ & & & & & & & & & & 552 & 117 & & & & & & & \\
\hline & & & & & & & & & & & & & & & & & & & & & & & & & & & & \\
\hline & & & & & & & & & & & & & & & & & & & & & & & & & & & & \\
\hline & & & 6.128 & 25.413 & 25.298 & 25.681 & & 49.627 & 55.8 & & & & & & & & & & & 84.8 & 11.5 & & & & & & 88 & \\
\hline & & & & & 25308 & & & 50089 & 56.4 & 1873 & 2 & 0247 & & 1797 & & & & & & 55.4 & & & & & & & & \\
\hline 46 & 9/2/2004| & 3:09:45 & & 25.409 & \begin{tabular}{|l|l|}
25.284 \\
\end{tabular} & \begin{tabular}{|l|l|} 
& 25.712 \\
\end{tabular} & 24.909 & 49.506 & 55.947 & \begin{tabular}{|l|l|}
18.7499 \\
\end{tabular} & $\overline{31.447}$ & 0.253 & -5.208 & 49.297 & 0.039 & 14.643 & & & $\begin{array}{l}367.2731 \\
3\end{array}$ & 54.8 & 11.7 & & 79 & 0.006 & 0.006 & 0.000 & |.137| & \\
\hline
\end{tabular}


WSRC-TR-2005-00105, REVISION 0 SRNL-RPP-2005-00012, REVISION 0

RUN \# 4.03A AND B; FIRST AND SECOND HALF OF SLURRY DEWATERING - CONT.

\begin{tabular}{|c|c|c|c|c|c|c|c|c|c|c|c|c|c|c|c|c|c|c|c|c|c|c|c|c|c|c|c|c|}
\hline & A & $\mathrm{B}$ & D & $E$ & $\mathrm{~F}$ & G & $\mathrm{H}$ & $\mathrm{J}$ & $\mathrm{K}$ & $\mathrm{L}$ & M & $\mathrm{N}$ & 0 & $Q$ & $\mathrm{R}$ & $\mathrm{s}$ & $T$ & u $v$ & W & $x$ & $\mathrm{Y}$ & $z$ & $\mathrm{AA}$ & $A B$ & $\mathrm{AC}$ & $A D$ & $\mathrm{AE}$ & AF $A$ \\
\hline 3349 & Dewater 1 $1 \mathrm{a}$ & and 2 & & & & & & & & & & & & & & & & & & & & & & & & & & \\
\hline 3350 & & Averages & 25.6 & 25.6 & 24.9 & 26.3 & 25.2 & 47.4 & 52.2 & 15.3 & 33.1 & -1.0 & -6.8 & 49.6 & 0.1 & 14.6 & 0.0 & & & 51.1 & 111.8 & & & 0.011 & 0.011 & 0.000 & & 0.2 \\
\hline 3351 & & Maximum & 28.9 & 26.7 & 29.3 & 27.9 & 26.6 & 61.4 & $\begin{array}{l}67.2 \\
5.7 \\
\end{array}$ & 19.4 & 43.9 & 38.5 & $\begin{array}{ll}0.8 \\
.8\end{array}$ & 58.7 & 0.4 & 14.6 & 0.0 & & & 666.1 & 14.0 & 52.7 & 3.630 & $\begin{array}{l}0.051 \\
5\end{array}$ & 0.052 & 0.001 & 1.5 & 1.3 \\
\hline $\begin{array}{l}3352 \\
3353 \\
\end{array}$ & & \begin{tabular}{|l|} 
Median \\
Minimum
\end{tabular} & \begin{tabular}{l|l}
25.5 \\
23.3 \\
\end{tabular} & 25.6 & 24.7 & $\frac{26.2}{24.6}$ & \begin{tabular}{l|l}
25.2 \\
23.9 \\
\end{tabular} & $\begin{array}{l}47.7 \\
-0.1 \\
\end{array}$ & $\frac{52.7}{21}$ & $\begin{array}{ll}16.0 \\
0.6\end{array}$ & $\begin{array}{l}33.0 \\
-2 . \\
\end{array}$ & -1.2 & $\begin{array}{l}-7.0 \\
-79\end{array}$ & $\begin{array}{l}49.7 \\
-0.1\end{array}$ & 0.1 & $\begin{array}{l}14.6 \\
146 \\
\end{array}$ & $\begin{array}{l}0.0 \\
0.0\end{array}$ & & & $\begin{array}{r}51.6 \\
0.9\end{array}$ & $\begin{array}{ll}11.8 \\
0.0\end{array}$ & $\begin{array}{l}40.3 \\
-0.1\end{array}$ & \begin{tabular}{|c|}
2.775 \\
-0.004 \\
\end{tabular} & \begin{tabular}{|l|l|l|}
5 & 0.008 \\
4 & 0.000 \\
\end{tabular} & $\begin{array}{l}0.009 \\
0.000\end{array}$ & $\begin{array}{l}0.000 \\
-0.008 \\
\end{array}$ & 0.2 & $\begin{array}{l}0.2 \\
-70 \\
\end{array}$ \\
\hline 33534 & & $2 \times \operatorname{Std} \mathrm{Dev}$ & $\begin{array}{r}23.8 \\
1.685\end{array}$ & $\frac{23.1}{0.803}$ & $\frac{21.2}{2.475}$ & $\frac{24.6}{1.010}$ & \begin{tabular}{|c|}
23.9 \\
0.746
\end{tabular} & \begin{tabular}{|c|}
-0.1 \\
4.297
\end{tabular} & $\frac{2.1}{5.234}$ & $\frac{0.6}{5.262}$ & $\frac{-2.7}{3.382}$ & $\frac{-1.2}{2093}$ & $\begin{array}{r}-7.9 \\
1.444 \\
\end{array}$ & $\begin{array}{r}-0.1 \\
3.657\end{array}$ & $\begin{array}{r}0.0 \\
0.103 \\
\end{array}$ & $\frac{14.6}{0.000}$ & $\begin{array}{r}0.0 \\
0.001\end{array}$ & & & $\frac{0.9}{5.34}$ & \begin{tabular}{l|l|}
9 & 0.0 \\
4 & 0.8712 \\
\end{tabular} & \begin{tabular}{|r|}
-0.1 \\
2.847
\end{tabular} & $\begin{array}{l}-0.004 \\
0.169\end{array}$ & 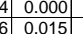 & 0.000 & $\begin{array}{c}-0.008 \\
0.000 \\
\end{array}$ & $\begin{array}{r}-8.2 \\
0.475 \\
\end{array}$ & \begin{tabular}{r|}
-7.0 \\
0.05 \\
\end{tabular} \\
\hline 3355 & Number & of Points Used & 3242 & 3242 & 3242 & 3242 & 3242 & 3242 & 3242 & 3242 & 3242 & 3242 & 3242 & 3242 & 3242 & 3242 & $\begin{array}{l}3 . .012 \\
3242\end{array}$ & & & 3242 & 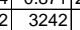 & 3242 & $\begin{array}{l}.196 \\
3242 \\
\end{array}$ & $\begin{array}{ll}6 & 0.015 \\
2 & 3242 \\
\end{array}$ & $\frac{0.015}{3242}$ & $\frac{0.000}{3242}$ & $\frac{0.475}{3242}$ & $\mid 0.405$ \\
\hline 3356 & & * Backpulse po & ints in box a & not inc & ded & & & & & & & & & & & & & & & & & & & & & & & \\
\hline 3357 & & & & & & & & & & & & & & & & & & & & & & & & & & & & \\
\hline \begin{tabular}{l|l}
3358 \\
3359 \\
\end{tabular} & Dewater 1 & Averages & 26.5 & 25.8 & 26.2 & 26.8 & 25.4 & 45,1 & 49.1 & 11.5 & 346 & -10 & -68 & 487 & 01 & 146 & 00 & & & & & & 2746 & & & & & \\
\hline 3360 & & \begin{tabular}{|l} 
Maximum \\
\end{tabular} & $\begin{aligned} 28.5 \\
28.9\end{aligned}$ & $\frac{25.8}{26.7}$ & 29.3 & $\frac{26.8}{27.9}$ & 20.6 & $\begin{array}{ll}45.14 \\
47.4 \\
\end{array}$ & $\begin{array}{ll}45.1 \\
52.6\end{array}$ & $\begin{array}{ll}14.0 \\
14.0\end{array}$ & $\begin{array}{l}34.0 \\
36.8 \\
\end{array}$ & 38.5 & $\begin{array}{l}-0.0 \\
0.8 \\
\end{array}$ & $\begin{array}{l}40.1 \\
58.7 \\
\end{array}$ & 0.1 & $\frac{14.6}{14.6}$ & 0.0 & & & $\frac{48.0}{51.4}$ & $\frac{11.6}{14.0}$ & $\begin{array}{l}39.8 \\
41.9 \\
\end{array}$ & \begin{tabular}{|l|}
2.746 \\
2.892 \\
\end{tabular} & 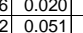 & $\frac{0.020}{0.052}$ & $\frac{0.001}{0.001}$ & $\frac{0.502}{1.495}$ & $\frac{0.4}{1.3}$ \\
\hline 361 & & Median & 26.6 & 25.8 & 25.9 & 26.9 & 25.3 & 45.1 & 49.4 & 11.2 & 34.8 & -1.2 & -7.0 & 48.7 & 0.1 & 14.6 & 0.0 & & & 48.2 & 11.6 & 40.0 & 2.758 & \begin{tabular}{|l|l|}
8 & 0.017 \\
\end{tabular} & 0.017 & 0.000 & 0.432 & \\
\hline 362 & & Minimum & 24.8 & 24.3 & 24.1 & 25.7 & 24.1 & 6.4 & 44.8 & 9.3 & -2.7 & -1.2 & -7.5 & $\begin{array}{l}43.1 \\
4.2 \\
\end{array}$ & 0.0 & $\begin{array}{l}14.0 \\
14.6 \\
\end{array}$ & 0.0 & & & 43.7 & $\begin{array}{l}1.0 \\
10.3 \\
\end{array}$ & 1.8 & \begin{tabular}{|l|l|}
2.127 \\
\end{tabular} & \begin{tabular}{l|l|}
7 & 0.000 \\
\end{tabular} & 0.000 & 0.000 & 0.223 & 0.2 \\
\hline 3363 & & $2 \times$ Std Dev & 1.898 & 1.078 & 2.618 & 1.031 & 1.011 & 4.041 & 3.897 & 2.520 & 4.056 & 3.718 & 1.809 & 3.960 & 0.116 & 0.000 & 0.001 & & & 3.897 & 0.943 & 3.849 & 0.265 & \begin{tabular}{|l|l|}
5 & 0.017 \\
\end{tabular} & 0.017 & 0.000 & 0.437 & 0.372 \\
\hline 3364 & Number & of Points Used & \begin{tabular}{|r|r|} 
& 917 \\
\end{tabular} & 917 & 917 & 917 & $\begin{array}{l}917 \\
\end{array}$ & $\begin{array}{l}917 \\
\end{array}$ & 917 & 917 & 917 & 917 & 917 & 917 & 917 & 917 & 917 & & & 917 & \begin{tabular}{|l|l|}
7 & 917 \\
\end{tabular} & 917 & \begin{tabular}{|l|l|}
917 \\
\end{tabular} & \begin{tabular}{|l|l|}
7 & 917 \\
\end{tabular} & 917 & 917 & 917 & 917 \\
\hline 3365 & & ${ }^{*}$ Backpulse po & ints in box a & enot inc & ided & & & & & & & & & & & & & & & & & & & & & & & \\
\hline$\frac{3366}{3367}$ & Delater? & & & & & & & & & & & & & & & & & & & & & & & & & & & \\
\hline |3367| & Dewater 2 & \begin{tabular}{|l|} 
Averages \\
\end{tabular} & 25.3 & 25.5 & 24.4 & 26.1 & 25.2 & $\begin{array}{l}48.3 \\
\end{array}$ & 53.5 & 16.7 & 32.5 & -0.9 & -6.9 & 50.0 & 0.1 & $\mid 14.6$ & 0.0 & & & 52.3 & 11.9 & \begin{tabular}{|l|l|}
40.4 \\
\end{tabular} & \begin{tabular}{|l|l|}
2.786 \\
\end{tabular} & \begin{tabular}{|l|l|}
6 & 0.008 \\
\end{tabular} & 0.008 & 0.000 & 0.190 & \begin{tabular}{l|l}
0.2 \\
\end{tabular} \\
\hline 3369 & & Maximum & 26.3 & 25.9 & 27.4 & 27.0 & 26.1 & 61.4 & 67.2 & 19.4 & 43.9 & 1.3 & -0.7 & 56.3 & 0.1 & 14.6 & 0.0 & & & 66.1 & 13.4 & & 3.630 & \begin{tabular}{ll|}
0 & 0.018 \\
\end{tabular} & 0.017 & 0.000 & 0.435 & $\begin{array}{r}0.4 \\
0.4 \\
\end{array}$ \\
\hline 3370 & & Median & 25.4 & 25.6 & 24.6 & 26.0 & 25.2 & 48.0 & 53.2 & 16.9 & 32.5 & -1.1 & -7.0 & 50.0 & 0.1 & 14.6 & 0.0 & & & 52.0 & 11.9 & 40.4 & 2.783 & \begin{tabular}{|l|l|}
3 & 0.007 \\
\end{tabular} & 0.007 & 0.000 & 0.183 & 0.2 \\
\hline 3371 & & Minimum & 23.8 & 23.7 & 21.2 & 24.6 & 23.9 & -0.1 & 2.1 & 0.6 & 0.0 & -1.2 & $\begin{array}{r}-7.9 \\
\end{array}$ & -0.1 & 0.0 & 14.6 & 0.0 & & & 0.9 & 0.0 & -0.1 & -0.004 & \begin{tabular}{|l|l|}
4 & 0.000 \\
\end{tabular} & 0.000 & -0.008 & -8.249 & $\begin{array}{r}-7.0 \\
-0.0\end{array}$ \\
\hline 3372 & & $2 \times$ Std Dev & $\begin{array}{ll}0.847 \\
2325\end{array}$ & $\begin{array}{ll}0.609 \\
2320 \\
\end{array}$ & 1.522 & $\begin{array}{l}0.615 \\
2252 \\
\end{array}$ & $\begin{array}{l}0.584 \\
2325 \\
\end{array}$ & 2.810 & 3.337 & $\frac{2.171}{2205}$ & 2.188 & 0.797 & 1.266 & 3.283 & 0.022 & & 0.001 & & & 3.337 & 0.7822 & & 0.156 & \begin{tabular}{l|l|}
6 & 0.003 \\
\end{tabular} & 0.003 & 0.000 & 0.360 & 0.307 \\
\hline \begin{tabular}{|c|}
3373 \\
3274
\end{tabular} & Number & of Points Used ${ }^{*}$ & 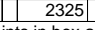 & 2325 & 2325 & 2325 & 2325 & 2325 & 2325 & 2325 & 2325 & 2325 & 2325 & 2325 & 2325 & 2325 & 2325 & & & 2325 & 2325 & 2325 & 2325 & \begin{tabular}{|l|l|}
5 & 2325 \\
\end{tabular} & 2325 & 2325 & 2325 & 2325 \\
\hline 33745 & & ${ }^{*}$ Backpulse po & ints in box a & & & & & & & & & & & & & & & & & & & & & & & & & \\
\hline 3376 & & & & & & & & & & & & & & & & & & & & & & & & & & & & \\
\hline & & & & & & & & & & & & & & & & & & & & & & & & & & & & \\
\hline (3) & & & & & & & & & & & & & & & & & & & & & $\begin{array}{l}\text { Averag } \\
\text { Averac }\end{array}$ & age flux & to $17 \%$ & $\%$ UDS $=$ & $\begin{array}{l}0.016 \\
0.013\end{array}$ & & & \\
\hline
\end{tabular}


WSRC-TR-2005-00105, REVISION 0 SRNL-RPP-2005-00012, REVISION 0

CAMPAIGN IV BACKPULSE DURING FIRST HALF OF SLURRY DEWATERING, RUN \# 4.03A

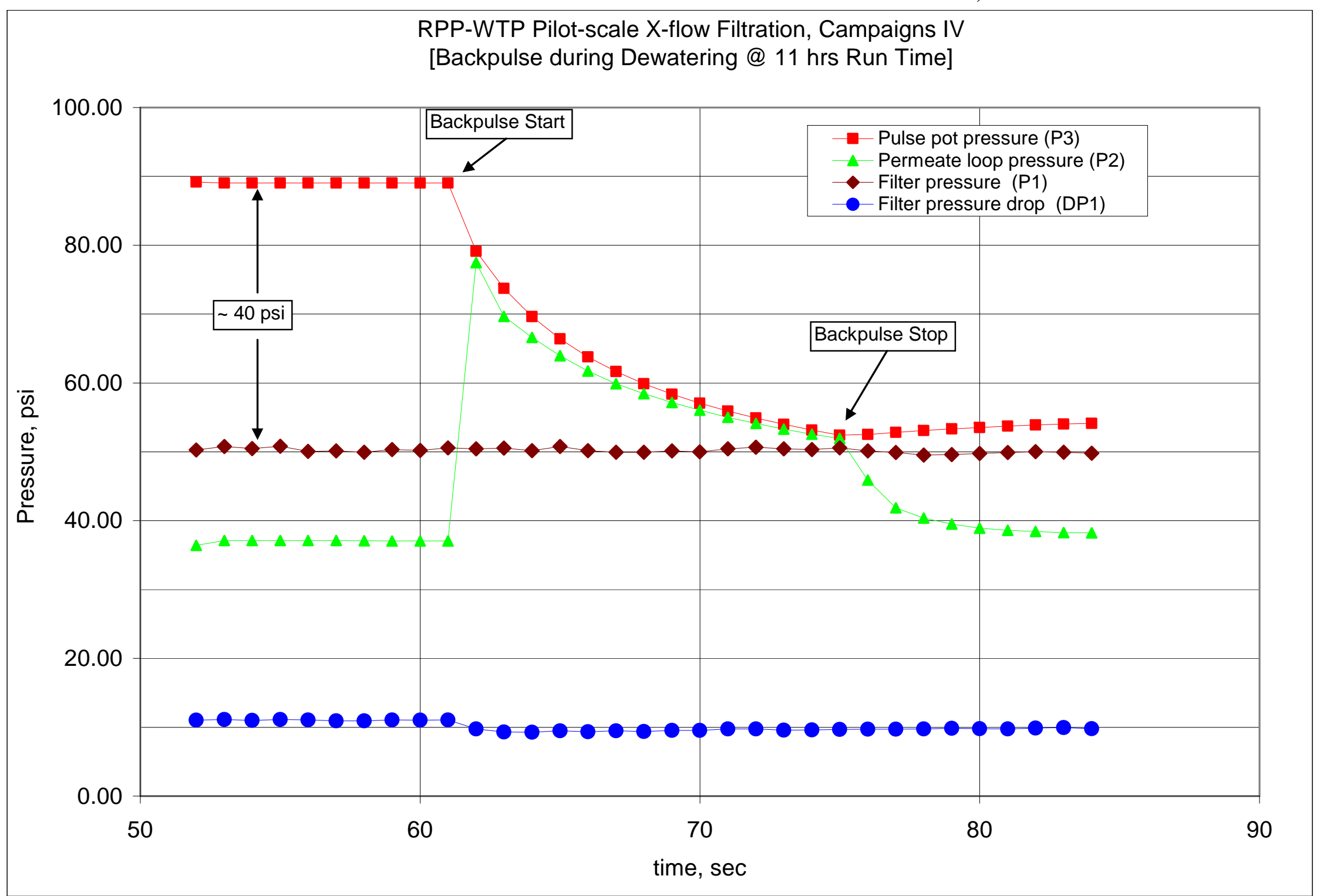


WSRC-TR-2005-00105, REVISION 0

SRNL-RPP-2005-00012, REVISION 0

CAMPAIGN IV BACKPULSE DURING FIRST HALF OF SLURRY DEWATERING, RUN \# 4.03A - CONT.

\begin{tabular}{|c|c|c|c|c|c|c|c|c|c|c|c|c|c|c|c|c|c|c|c|c|c|c|c|c|c|c|c|c|c|}
\hline & $\mathrm{A}$ & $\mathrm{B}$ & $\frac{\mathrm{D}}{\ll \ll \ll<}$ & \begin{tabular}{|c|}
$E<\mathrm{Tem}$ \\
$\ll<$
\end{tabular} & $\frac{F}{1 . \quad F}$ & & $\begin{array}{l}G \\
\text { easurem }\end{array}$ & $\frac{\mathrm{H}}{\mathrm{e}}$ & $\frac{1}{\ll \ll<\ll<<}$ & $\frac{K}{k \ll \ll<<}$ & $\frac{\mathrm{L}}{\mathrm{L}}$ & $\frac{M}{\text { Teasureme }}$ & $\frac{N}{e n t s ~}$ & $\frac{0}{1}$ & \begin{tabular}{|c|} 
Q \\
$\ll<\ll<<<$
\end{tabular} & $\begin{array}{l}\mathrm{R} \\
\ll<\text { Flow }\end{array}$ & \begin{tabular}{|c|}
$S$ \\
Measurer
\end{tabular} & \begin{tabular}{l|} 
\\
ents $\gg \gg \gg>$
\end{tabular} & $\frac{v}{v \ll<}$ & $\frac{w}{1}$ & 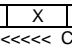 & \begin{tabular}{|c|}
$\mathrm{Y}$ \\
Calculatec
\end{tabular} & $\frac{z}{|c|}$ & \begin{tabular}{|l|} 
AA \\
$n$ Raw $D a$
\end{tabular} & \begin{tabular}{|l|}
$A B$ \\
ata $\gg \gg$ \\
\end{tabular} & $\frac{\mid A C}{|l|}$ & 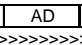 & 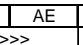 & AF \\
\hline \begin{tabular}{|l}
2 \\
\end{tabular} & DATE & TIME & Filtrate & Cleaning & 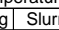 & & Hi Amb. & J Lo Amb. & BotTMP & Filter & Filter dP & TopTMP & Filtrate & Pulsepot & & Filtrate & & & & & & & & & & & & & \\
\hline & & & $\operatorname{deg} C$ & $\operatorname{deg} C$ & deg & & $\operatorname{deg} C$ & $\operatorname{deg} C$ & psid & psig & psid & psid & psig & psig & $\mathrm{gpm}$ & $\mathrm{gpm}$ & gpm & & & & & & & & & & & & \\
\hline 4 & & & $\mathrm{~T} 2$ & T3 & $\begin{array}{ll}\mathrm{T} 1 \\
\end{array}$ & & & & $\mathrm{dP} 2$ & P1 & dP1 & $\mathrm{dP3}$ & $\mathrm{P} 2$ & P3 & Q1 & Q2 & Q3 & Qbp & & & & & & & & & & & \\
\hline & & & & & & & & & & & & & & & & & & & & & & & & & & & & & \\
\hline & Zeros- 01/26 & $6 / 2004$ & & & & & & & & & & & & & & & & & & & & & & & & & & & \\
\hline$\frac{7}{8}$ & $8 / 23 / 2004$ & $\begin{array}{l}\quad 8: 35: 38 \mathrm{AM} \\
\end{array}$ & \begin{tabular}{|l|l|}
22.554 \\
23662
\end{tabular} & \begin{tabular}{|l|}
22.60 \\
209
\end{tabular} & \begin{tabular}{l|l}
4 & 22.0 \\
236
\end{tabular} & & $\begin{array}{ll}22.782 \\
22009\end{array}$ & $\begin{array}{l}22.204 \\
22725 \\
\end{array}$ & \begin{tabular}{|c|c|}
-0.019 \\
\end{tabular} & $\begin{array}{l}0.095 \\
\end{array}$ & $\begin{array}{c}-0.012 \\
0.014\end{array}$ & 0.003 & -0.124 & 0.137 & $\begin{array}{l}-0.075 \\
-2.172 \\
\end{array}$ & 0.003 & \begin{tabular}{|l|l|} 
& 14.643 \\
\end{tabular} & 0.004 & & & Filter Su & Surface $\mathrm{A}$ & $\begin{array}{l}\text { A A } 6.985 \text {. } \\
\end{array}$ & FT2 & & & & & \\
\hline \begin{tabular}{|l|}
8 \\
9 \\
\end{tabular} & $\frac{8 / 24 / 2004}{88 / 2604}$ & $\begin{array}{l}\text { 8:1::3:3 AM } \\
911: 11 \mathrm{AM}\end{array}$ & $\frac{23.662}{25.819}$ & \begin{tabular}{|r}
22.39 \\
23.35 \\
\end{tabular} & $\begin{array}{ll}1 & 23.6 \\
2 & 24.9 \\
\end{array}$ & & $\frac{22.998}{25.316}$ & $\frac{22.735}{23.242}$ & $\begin{array}{l}-0.021 \\
-0.008 \\
\end{array}$ & $\mid \begin{array}{l}0.122 \\
0.126\end{array}$ & $\frac{-0.014}{-0.008}$ & $\begin{array}{c}-0.001 \\
0.011 \\
\end{array}$ & \begin{tabular}{|l|l|l|l|l|l|}
-0.106 \\
\end{tabular} & $\begin{array}{l}0.134 \\
0.091 \\
\end{array}$ & \begin{tabular}{|l|}
23.176 \\
4.335 \\
\end{tabular} & $\frac{1.205}{0.043}$ & \begin{tabular}{|l}
14.643 \\
14.643 \\
\end{tabular} & \begin{tabular}{|l}
0.003 \\
0.003 \\
\end{tabular} & & & Convers & & 851 & & & $\mathrm{~g} / \mathrm{gpm} / \mathrm{t}$ & t2/barg & & \\
\hline$\frac{10}{10}$ & $8 / 27 / 2004$ & 7:53:29 AM & & 22.8 & & & 22.872 & 22.871 & & 0.14 & & & -0.161 & & & & $\begin{array}{l}14.045 \\
14.643 \\
\end{array}$ & 0.005 & & & Note: $\mathrm{C}$ & uge $F$ & ssur & & proxi & ately $1.4 \mathrm{p}$ & & & \\
\hline 11 & $8 / 30 / 2004$ & 7:28:55 AM & 22.511 & $22.84 \varepsilon$ & 22.3 & & 22.669 & 22.914 & -0.017 & 0.138 & -0.009 & $\begin{array}{l}0.003 \\
\end{array}$ & -0.147 & 0.122 & -0.082 & 0.003 & 14.643 & 0.006 & & & & & & & & & & & \\
\hline$\frac{12}{13}$ & & & & & & & & & & & & & & & & & & & & & Pressur & ire Plis cc & corrected & ed for 31 . & $\begin{array}{l}1.25 \text { inch } \\
1<\text { Fiturat }\end{array}$ & & $\begin{array}{l}\text { ter tubinc } \\
\text { tes PPEF }\end{array}$ & & \\
\hline & & & & & & & & & & & & & & & & & & & & & vor & & & & Filltal & at $25 \mathrm{C}$ & $<<P E F$ & ALEIII & $\gg$ \\
\hline 15 & & & & & & & & & & & & & & & & & & & Time & Time & Press. & Vel. & TMP & TMP & gpm & $\mathrm{gpm}$ & gpm & $\mathrm{pm}$ & \\
\hline$\frac{16}{17}$ & Backpulse & & & & & & & & & & & & & & & & & & & Hour & psig & & & & & & & & \\
\hline \begin{tabular}{|l}
18 \\
18 \\
\end{tabular} & $8 / 30 / 2004$ & 8:15:39 PM & 27.017 & 26.226 & & & 26.772 & 25.629 & 8.043 & & & -2.396 & & & & & & & & & & & 2.8 & & & 0.00 & 0.00 & & 0.1 \\
\hline 10 & $8 / 30 / 2004$ & 8:16:31 PM & 27.017 & 26.225 & 26.5 & & 26.772 & 25.629 & 8.043 & & & -2.396 & 36.432 & 89.169 & 45.434 & 0.003 & 14.643 & 0.006 & & 0.01444 & 49.1 & & & & & & & & \\
\hline 20 & $\begin{array}{l}8 / 30 / 2004 \\
8 / 30 / 2004\end{array}$ & 8:16:32 PM & 27.008 & 26.225 & 26.5 & & 26.823 & 25.629 & 8.238 & 50.759 & 11.119 & -2.213 & 37.11 & 89.051 & 46.089 & 0.003 & 14.643 & 0.00 & 0.88 & 0.01472 & 49.6 & 11.6 & 3.0 & 0.208 & 0.000 & 0.000 & 0.000 & 0.136 & 0.12 \\
\hline 21 & $\begin{array}{l}88 / 301 / 2004 \\
8 / 30 / 2004\end{array}$ & 8:16:33 PM & 27.013 & 26.22 & 26.5 & & 26.828 & 25.629 & 7.883 & -50.475 & 10.991 & $\begin{array}{l}-2.353 \\
\end{array}$ & $\begin{array}{l}37.11 \\
27.11\end{array}$ & 89.045 & 48.275 & 0.003 & 14.643 & 0.005 & 0.90 & 0.015 & 49.3 & 11.5 & $2.8 \mathrm{~s}$ & 0.191 & 0.000 & 0.000 & 0.000 & 0.149 & \\
\hline 22 & $\begin{array}{l}8 / 30 / 2004 \\
8 / 30 / 2004\end{array}$ & $8: 16: 34 \mathrm{PM}$ & 27.008 & $\frac{26.22}{2023}$ & 26.5 & & 26.833 & 25.629 & 8.841 & 5. 5. & 11.1233 & -2.546 & 37.107 & 89.048 & 47.105 & 0.003 & 14.643 & 0.006 & 0.9 & 0 & 49.7 & 111 & $\frac{2.1}{23}$ & 0.189 & 0.000 & 0.000 & 0.006 & 0.150 & 0.1 \\
\hline & $8 / 301 / 2004$ & $8: 10.35 \mathrm{PM}$ & 27.008 & $\frac{20.24}{26.216}$ & 20.5 & & $\frac{20.833}{26838}$ & 25.634 & 7.526 & & & $\frac{-2.8124}{-2809}$ & $\frac{3.11}{3711}$ & $\frac{89.048}{80.045}$ & $\begin{array}{l}47.458 \\
4.918\end{array}$ & 0 & $\begin{array}{l}14.643 \\
14643\end{array}$ & & & $\frac{0.0156}{0.0158}$ & 49.0 & & & & & & & & \\
\hline & $8 / 30 / 2004$ & 8.0 .560 & 27013 & $\frac{2.21}{2623}$ & $\frac{2.5}{265}$ & & $\frac{20.030}{26848}$ & 23.034 & $\mid$ & 30.144 & 10.945 & $\frac{-2.495}{-2973}$ & 37.11 & $\frac{63.045}{8042}$ & $\frac{4.01}{46.229}$ & 年 & $\frac{14.045}{14643}$ & & 09 & 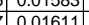 & $\frac{4.0}{488}$ & 11 & & & & & 0.00 & 0.100 & 0.1 \\
\hline 25 & $8 / 30 / 2004$ & 8:16:38 PM & 27.013 & $\frac{26.225}{26.22}$ & 26.5 & & 26.838 & 25.634 & 7.775 & $\begin{array}{l}5.354 \\
50.334\end{array}$ & $=11.06$ & $\begin{array}{l}-2.510 \\
-2.616\end{array}$ & 3706 & & 45622 & 0.003 & 14643 & & & & $\begin{array}{ll}40.6 \\
492\end{array}$ & & & & & & & & \\
\hline 26 & $8 / 30 / 2004$ & 8:16:39 PM & 27.008 & 26.22 & 26.5 & & 26.843 & 25.634 & 7.566 & 50.193 & \begin{tabular}{|l|}
11.036 \\
\end{tabular} & -2.895 & 37.055 & 89.042 & 46.817 & 0.003 & \begin{tabular}{|l|l|}
3 & 14.643 \\
\end{tabular} & 0.005 & 1.00 & 0.01667 & 49.1 & 11.2 & 2.3 & 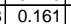 & \begin{tabular}{|l|} 
\\
\end{tabular} & 0.000 & 0.000 & \begin{tabular}{|c|}
0.176 \\
\end{tabular} & 0.14 \\
\hline & $8 / 30 / 2004$ & $8: 16: 4$ & 27.013 & 26.226 & 26.5 & & 26.858 & 25.64 & $\begin{array}{l}7.725 \\
\end{array}$ & 50.565 & 11.062 & -2.802 & 37.055 & & 46.466 & & 14.643 & 0.0 & 1.02 & 0.01694 & 49.4 & 11.1. & 2.5 & 0.170 & 0.000 & & 0.000 & 0.167 & 0.14 \\
\hline$\frac{28}{20}$ & 8/30/2004 & 8:16:41 PM & 27.023 & 26.225 & 26.5 & & 26.848 & 25.63 & -7.261 & 50.44 & 9.747 & -18.541 & 77.47 & 79.13 & 46.529 & 0.003 & 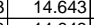 & & $1 . \mathrm{C}^{-1}$ & 0.01 & 49.3 & 11 & 2.9 & 889 & & & & & \\
\hline$\frac{25}{30}$ & $\begin{array}{l}8 / 30 / 2004 \\
8 / 30 / 2004\end{array}$ & 8:16:42 PM & 26.869 & 26.226 & 26.5 & & 26.858 & 25.6 & $\begin{array}{l}-7.267 \\
-2.267\end{array}$ & -50.544 & 9.31 & -18.543 & $\mid \begin{array}{l}69.668 \\
\end{array}$ & 73.744 & 46.569 & 0.003 & 14.643 & & 1.0 & 0.0175 & 49.4 & 11 & & & 0.000 & .00 & & & \\
\hline$\frac{3}{31}$ & $8 / 30130004$ & & & $\frac{20.24}{2620}$ & $\frac{2.5}{265}$ & & 20.000 & $\frac{23.034}{2564}$ & & & & & & & & & & & & & & & & & 2000 & & 000 & & \\
\hline 34 & $8 / 30 / 2004$ & $\begin{array}{l}0.1 .0 .44 \mathrm{PMM} \\
8: 165 \mathrm{PM}\end{array}$ & 26.618 & $\frac{20.24}{26.22}$ & 26.5 & & 20.050 & 25.63 & $\begin{array}{r}-7.205 \\
-7.269 \\
\end{array}$ & 50.173 & 9.34 & \begin{tabular}{|c|c|}
-10.045 \\
-18.543 \\
\end{tabular} & \begin{tabular}{|l|l|}
61.72 \\
\end{tabular} & $\frac{00 .}{63 .}$ & (6.577 & & \begin{tabular}{|l|l|l}
14.643 \\
14
\end{tabular} & & 1. & & 495.0 & $\frac{10.4}{11.1}$ & 12.9. & & & & 0.000 & - & $\frac{1}{2}$ \\
\hline 33 & $8 / 30 / 2004$ & $8: 16: 4$ & & 26.226 & 26.5 & & 26.873 & & \begin{tabular}{|c|}
-7.269 \\
\end{tabular} & & & \begin{tabular}{|c|}
-18.543 \\
\end{tabular} & & & 45.448 & & & & 111 & & \begin{tabular}{|l|l|}
48.8 \\
\end{tabular} & 10. & 2.9 & & & & & & -0.0 \\
\hline 34 & 8/30/2004 & & & 26.23. & & & & & -7.269 & & & & 58.446 & & & & & & & & 48.8 & 11. & & & & & 0 & & \\
\hline 35 & 8/30/2004 & 8:16: & 26.734 & 26.22 & 26.5 & & 26.868 & 25.65 & -7.269 & 50.144 & 9.548 & -18.545 & 57.158 & 58.376 & 44.551 & 0.003 & 14.6 & & 1. & 0.01917 & 49.0 & 10. & 12.9 & & & & 0.000 & & \\
\hline 37 & $8 / 30 / 2004$ & & 26.169 & & 26.5 & & $26.8 / 3$ & 25. & $\mid-1.269$ & & & -18.543 & 56.0 & & (1) & & 14 & & & & 48.9 & 1 & 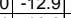 & & & & & & \\
\hline$\frac{31}{38}$ & $8 / 30 / 2004$ & $8: 16: 50 \mathrm{PM}$ & 26.793 & 26.214 & 26.5 & & & 25.66 & $\begin{array}{c}-1.269 \\
7.720\end{array}$ & 50.43 & $\frac{9.146}{9.752}$ & $\frac{-18.544}{1794}$ & & & 46.4 & & & & & 0.01 & 49.3 & & 12.9 & & & & & & \\
\hline $\begin{array}{l}30 \\
39 \\
\end{array}$ & $8 / 30 / 2004$ & $\begin{array}{l}0.10 .5 \\
8.16 .5\end{array}$ & $\begin{array}{l}2.064 \\
26833\end{array}$ & $\frac{26.21}{26.205}$ & 年.5.5 & & 26.000 & $\begin{array}{r}25.64 \\
25.649\end{array}$ & \begin{tabular}{|c|}
-7.269 \\
-7257
\end{tabular} & $\frac{5.606}{50.433}$ & 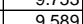 & -1723 & 353.12 & 54005 & 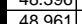 & & 14 & & $\frac{1.2}{1.2}$ & 028 & $\frac{49.5}{49.3}$ & 117 & 122 & -0.84 & & & & & \\
\hline 40 & $8 / 30 / 2004$ & 8:16:5 & & 26.21 & 26.5 & & 26.848 & 25.65 & \begin{tabular}{|c|c|}
-7.223 \\
\end{tabular} & \begin{tabular}{|l|}
50.334 \\
5
\end{tabular} & & $|-1.63|$ & 352.54 & & 49.814 & & & & 123 & & 492 & 11.1. & 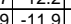 & & 0.000 & & 0.000 & & \\
\hline 41 & $8 / 30 / 2004$ & $8: 16: 5$ & & & 20.5 & & 26.858 & $25.6 !$ & -6.8 & \begin{tabular}{|c|}
50.56 \\
\end{tabular} & & -15.826 & 51.96 & 52. & 47.468 & & & & & & $\frac{0.4}{9.4}$ & 11.3 & 11.3 & 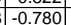 & & & & & \\
\hline 42 & $8 / 30 / 2004$ & $8: 16: 55$ & 26.888 & 26.20 & 26.5 & & $\begin{array}{l}26.863 \\
\end{array}$ & 25.64 & \begin{tabular}{|l|l|}
0.168 \\
\end{tabular} & 50.127 & & \begin{tabular}{|c|}
-8.957 \\
\end{tabular} & & & $\begin{array}{l}47.334 \\
\end{array}$ & & & & & & 49.0 & 11 & -4.4 & & & & 0.00 & & \\
\hline 43 & & & & & & & & & & & & & & & & & & & & & & & & & & & & & \\
\hline & $8 / 30 / 2004$ & 8:16:57 & 26.894 & 26.22. & 26.5 & & 26.873 & 25 & 4.233 & 49.528 & 9.7 & \begin{tabular}{|c|c|}
-4.658 \\
\end{tabular} & & 53. & 48. & & & & 1.3 & & 48.4 & 11.6 & -0.2 & & 0.001 & & -0.005 & -4.515 & \\
\hline 45 & & & $26.8 \mathrm{c}$ & & 26.5 & & & 25 & & & & & & & & & & & & & 48. & & 0.4 & & & & & & \\
\hline 46 & $8 / 30 / 2004$ & 8:16: & 26.8 & 26. & 26.5 & & 26.8 & & & 49.76 & & & & & & & & & 1.3 & & & 11 & \begin{tabular}{ll|}
0.7 \\
\end{tabular} & & & & & & \\
\hline 47 & 8/30i2004 & $8: 17: 00$ & & 26. & 26.5 & & & 25. & 5.819 & 49 & 9 & & & & & & & & 1.3 & & & & 18 & & & & & & \\
\hline 48 & $8 / 30 / 2004$ & & & 26.2 & 26.5 & & & & 6.05 & 50.6 & & -3.1 & & & & & & & & & & & & & & & & & \\
\hline 49 & 8/30/2004 & $8: 17: c$ & 26.878 & 26.215 & 26.5 & & 26. & & 6.317 & 49. & 9.9 & -2.948 & & & 47.324 & & 14 & & 1.38 & & 48.8 & 11.5 & 1.7 & 0.11 & 0.000 & & 0.000 & . 244 & 4 \\
\hline & 8/30/2004 & 8:17:03 PM & $26.8 / 3$ & 26.2. & 26.5 & & 26.873 & 25.634 & 6.12 & 49.791 & 9.795 & -2.928 & 38.245 & & 48.152 & 0.003 & 14.643 & & 1.40 & & 48.7 & 11. & 1.6 & 0.114 & 0.000 & 0.000 & 0.00 & 0.258 & $0.2^{2}$ \\
\hline$\frac{5}{5}$ & & & & & & & & & & & & & & & & & & & & & & & & & & & & & \\
\hline & & & & & & & & & & & & & & & & & & & & & & & & & & & & & \\
\hline 5 & & & 26.9 & 26.2 & 26 & 6.6 & 26.9 & & 0.8 & 5 & 10 & & & & & & & & & & & & -4.3 & & & & & 0.0 & \\
\hline & & Maxim & 27.0 & 26.2 & 26 & & 26.9 & & 8.2 & 50.8 & 11.1 & & & & & & 14 & & & & 49.7 & 1 & 3.0 & & & & & 2.8 & \\
\hline 5 & & Mediar & 26.9 & 26.2 & 26 & 6.6 & 26.9 & & 4.6 & 50.2 & 9.8 & -4.4 & 39.8 & & 46 & 0.0 & 14.6 & & & & 49.0 & 11.1 & 0.1 & & 0.0 & & 0. & 0.1 & \\
\hline 5 & & & 26.5 & 26. & 26 & & 26.8 & & -7.3 & & 9.3 & -18.5 & 3.4 & & 44.6 & 0.0 & 14.6 & & & & 8.4 & 10.6 & -12.9 & & 0.0 & & 0.0 & 4.5 & \\
\hline 58 & & & 0.274 & 0.013 & 0.0 & 115 & 0.052 & 0.6 & $\begin{array}{r}13.764 \\
34\end{array}$ & 0.652 & 1.343 & 14.650 & 23.316 & & 2.299 & .002 & 0.000 & & & & 0.652 & 0.548 & \#\#+ & 0.978 & 0000 & 0.000 & .002 & 1.8566 & \\
\hline & & & 34 & $\frac{3}{3 \cos ^{2}}$ & 4 & & & & & 34 & & 34 & & & & & & & & & 34 & & 34 & & 34 & 34 & & 34 & \\
\hline
\end{tabular}


WSRC-TR-2005-00105, REVISION 0 SRNL-RPP-2005-00012, REVISION 0

RUN \# 4.07; STEADY STATE TEST AT 25 WT\% UDS

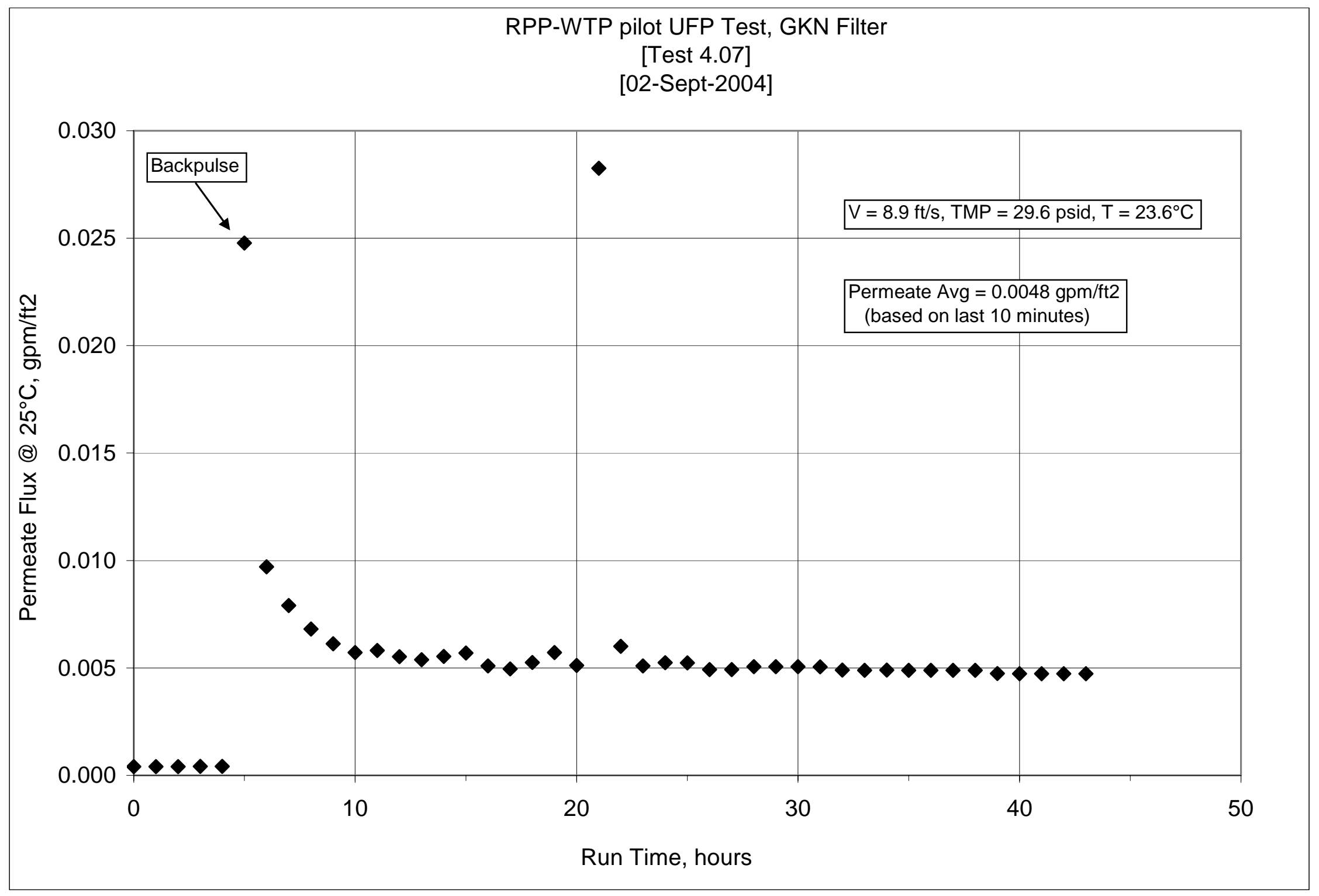


WSRC-TR-2005-00105, REVISION 0

SRNL-RPP-2005-00012, REVISION 0

RUN \# 4.07; STEADY STATE TEST AT 25 WT\% UDS

\begin{tabular}{|c|c|c|c|c|c|c|c|c|c|c|c|c|c|c|c|c|c|c|c|c|c|c|c|c|c|c|c|c|}
\hline & A & B & \begin{tabular}{l|l}
$\frac{D}{k}$ \\
$k \ll<<<$
\end{tabular} & $\frac{E}{\text { 《 Temp }}$ & erature $\mathrm{M}$ & eleasureme & :nts & $\mathrm{J}$ & $\frac{K}{\ll \ll \ll \ll}$ & 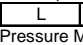 & \begin{tabular}{|c|} 
\\
Measuren
\end{tabular} & \begin{tabular}{l|l} 
\\
\end{tabular} & 0 & \begin{tabular}{l|l|}
$\mathrm{Q}$ \\
$\ll<<<<$
\end{tabular} & $\begin{array}{l}\mathrm{R} \\
<\text { Flow! }\end{array}$ & $\frac{\mathrm{s}}{\text { Measureme }}$ & $T$ & $\frac{v}{\ll \ll \ll<}$ & 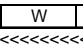 & \begin{tabular}{|l|l|}
$x<\ll<c$ & $x$ \\
$x$
\end{tabular} & $\begin{array}{l}\mathrm{Y} \\
\text { Calculatt }\end{array}$ & $\frac{\mathrm{z}}{\text { ted Fron }}$ & $\frac{\mid \mathrm{AA} A}{\mathrm{~m} \operatorname{Raw} \mathrm{D}}$ & \begin{tabular}{|l|}
$\mid \mathrm{AB}$ \\
Data $\gg \gg \gg$ \\
\end{tabular} & 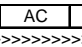 & \begin{tabular}{ll|}
$\mid A D$ \\
$\Rightarrow \gg \gg \gg \gg \gg \gg$
\end{tabular} & \begin{tabular}{|l|l|}
$A E$ \\
$D>$
\end{tabular} & AF $A$ \\
\hline & DATE & TIME & Filtrate & Cleaning & Slurry & Hi Amb. & Lo Amb. & & Filter & Filter dP & TopTMP & Filtrate & Pulsepot & & Filtrate & Hi Fittate & Backpulse & & & & & & & & & & & \\
\hline & & & $\frac{\operatorname{deg} C}{T 2}$ & $\frac{\operatorname{deg} C}{T 3}$ & $\operatorname{deg} C$ & \begin{tabular}{|l|l|}
$\operatorname{deg} C$ \\
\end{tabular} & $\operatorname{deg} C$ & $\begin{array}{l}\text { psid } \\
\text { P2 }\end{array}$ & psig & psid & \begin{tabular}{|l|} 
psid \\
\end{tabular} & \begin{tabular}{l|l} 
psig \\
p2
\end{tabular} & \begin{tabular}{|l|} 
psig \\
p2
\end{tabular} & $\mathrm{gpm}$ & $\mathrm{gpm}$ & gpm & gpm & & & & & & & & & & & \\
\hline & & & & & & & & & & & & & & & & & & & & & & & & & & & & \\
\hline & $0 s-04 / 1$ & & & & & & & & & & & & & & & & & & & & & & & & & & & \\
\hline & & $8: 35: 38 \mathrm{AM}$ & 22.554 & 22.604 & 22.002 & 22.782 & 22.204 & -0.019 & 0.095 & \begin{tabular}{|c|c|}
-0.012 \\
\end{tabular} & \begin{tabular}{|l|l|}
0.003 \\
\end{tabular} & -0.124 & $\begin{array}{l}0.137 \\
\end{array}$ & -0.075 & 0.003 & $\begin{array}{l}14.643 \\
\end{array}$ & 0.004 & & & Filter Su & Surface $A$ & & $\mathrm{FT2}$ & & & & & \\
\hline & 8/24/2004 & $8: 10: 33 \mathrm{AM}$ & & 22.93 & 23.679 & 22.998 & 22.735 & -0.021 & 0.122 & -0.014 & & -0.124 & & 23.176 & 1.205 & 14.643 & & & & & rsion & 85 & $\mathrm{~m} 3 / \mathrm{m}$ & lay/barg & $\mathrm{gpm} / \mathrm{tt2} 2$ & arg & & \\
\hline 9 & 8/26/2004 & 9:14:11 AM & 25.819 & 23.352 & 24.932 & 25.316 & 23.242 & -0.008 & 0.126 & -0.008 & 0.011 & -0.106 & 0.091 & 48.335 & 0.043 & 14.643 & & & & & & & & & & & & \\
\hline & 8/27/2004 & & 22.649| & 22.89 & 22.298 & 22.872 & 22.871 & -0.017 & 0.14 & -0.008 & 0.005 & -0.161 & 0.11 & -0.084 & 0.003 & 14.643 & 0.00 & & & Note: & get & & & proxil & $\operatorname{ly~} 1$ & $\lg$ & & \\
\hline & 8/30/2004 & $7: 28$ & & 22.848 & 22.397 & 22.669 & 22.914 & -0.017 & 0.138 & -0.009 & 0.003 & -0.147 & 0.122 & -0.082 & 0.003 & 14.643 & & & & & & & & & & & & \\
\hline & & & & & & & & & & & & & & & & & & & & $\frac{\text { Pressur }}{\text { VwVy }}$ & & & & < Filtrate & e Flux $><<$ & $\frac{1}{<<P E R I}$ & ABILII & \\
\hline & & & & & & & & & & & & & & & & & & & & t & & & & & \begin{tabular}{|l|l|} 
at $25 \mathrm{C}$ \\
\end{tabular} & & $\times 1000$ & \\
\hline & & & & & & & & & & & & & & & & & & Time & Time & Press. & el. & TMP & TMP & gpm & gpm & pm & ppm & eter \\
\hline & ata - Per Mi & $\pi$ & & & & & & & & & & & & & & & & & Hour & & & & atr & & & & & 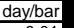 \\
\hline$\frac{11}{18}$ & $9 / 2 / 20044$ & $\frac{4: 10: 15 \mathrm{AM}}{4 \cdot 11 \cdot 15 \mathrm{AM}}$ & 年.658 & $\frac{25.404}{25304}$ & \begin{tabular}{|l|}
24.271 \\
24.182
\end{tabular} & 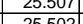 & 24.87 & $\begin{array}{l}30.175 \\
37618\end{array}$ & \begin{tabular}{|l|l}
4.452 \\
\end{tabular} & 14.278 & 17.158 & 5.678 & 21.055 & 38.298 & 0.003 & 14.643 & & & & 41.3 & & & 1.652 & 0.000 & & & & \\
\hline & & & & & & & 2501 & & $\mid 42649$ & & & & & | & & & & & 100 & & & & & & & & & \\
\hline & $\begin{array}{l}9 / 2 / 2004 \\
9 / 2 / 2004\end{array}$ & $\begin{array}{l}4: 12: 15 \mathrm{AM} \\
4: 13: 15 \mathrm{AM}\end{array}$ & $\frac{20.529}{26.211}$ & $\frac{25.415}{25.406}$ & $\frac{24.145}{24.083}$ & 25.009 & $\frac{25.090}{25.196}$ & $\begin{array}{r}24.404 \\
6.877\end{array}$ & $\begin{array}{r}42.049 \\
41.43\end{array}$ & $\begin{array}{l}\frac{14.042}{10.462} \\
\end{array}$ & $\frac{11.0 / 9}{-2.895}$ & 28.938 & $\begin{array}{l}\frac{0.1 .029}{49.192} \\
4\end{array}$ & 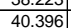 & 0.003 & $\frac{14.643}{14.643}$ & & & 00 & $\begin{array}{l}41.5 \\
40.3\end{array}$ & & 2.6 & 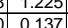 & 000 & 100 & & & \\
\hline 21 & 9/2/2004 & 4:14:15 AM & 26.221 & 25.411 & \begin{tabular}{|l|}
24.014 \\
\end{tabular} & 25.514 & 25.257 & 7.211 & 41.509 & 10.551 & -2.759 & 28.688 & 0.296 & 40.348 & 0.003 & 14.643 & 0.005 & 4.00 & 0.06667 & 40.4 & 9.6 & 2.2 & 0.153 & 0.000 & 0.000 & 0.000 & 0.186 & 0.16 \\
\hline & 9/2/2004 & 4:15:15 АM & 26.201 & $\begin{array}{r}25.416 \\
25.01\end{array}$ & \begin{tabular}{|l|}
23.944 \\
20.959
\end{tabular} & $\begin{array}{l}25.529 \\
25.172\end{array}$ & $\frac{25.167}{20501}$ & $\begin{array}{r}29.999 \\
2201\end{array}$ & \begin{tabular}{|l|l}
1.766 \\
\end{tabular} & $\begin{array}{l}11.684 \\
\end{array}$ & 18.992 & 5.785 & 0.374 & $\begin{array}{r}40.23 \\
20.201\end{array}$ & 0.179 & 14.643 & & & 0.08333 & 40.6 & & $\frac{24.5}{2.2}$ & \begin{tabular}{|l|l|}
1.689 \\
\end{tabular} & & & & & 0.86 \\
\hline & 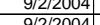 & 4:16:15 AM & 26.156 & $\begin{array}{l}25.401 \\
25.405\end{array}$ & 23.858 & 25.473 & 25.061 & $\begin{array}{l}3.21 \\
32.546\end{array}$ & \begin{tabular}{|l|}
41.986 \\
\end{tabular} & $\begin{array}{r}12.579 \\
13269\end{array}$ & 20.359 & 3.524 & 0.406 & 39.206 & $\begin{array}{r}0.07 \\
0.057\end{array}$ & 14.643 & & 6.0 & 0.1 & 40.9 & 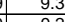 & 26.3 & 1.812 & 0.010 & 0.010 & & - & 0.31 \\
\hline & & 4.17.15 AMI & $\frac{2.115}{250}$ & & $\frac{23.004}{22717}$ & 20.4839 & 24.951 & & & 13.2600 & 2.084 & & & $\frac{38.600}{3205}$ & 0 & 1.6437 & & & 0.11001 & 40.9 & g. & 20.3 & 1..814 & 0.0006 & 0.008 & 0.000 & . & \\
\hline 登 & 9/2/2004 & 4.40 .90 & 20.001 & $\frac{25.415}{25.415}$ & $\frac{23.64 t}{23691}$ & $\begin{array}{l}2.4,401 \\
25483\end{array}$ & $\frac{24.500}{2486}$ & $\begin{array}{l}\frac{52.051}{33234} \\
3.25\end{array}$ & \begin{tabular}{|l|l|}
42.513 \\
403
\end{tabular} & $\frac{14.08}{14.451}$ & 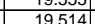 & 3.399 & $\frac{0.464}{0.469}$ & 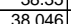 & - & $\frac{14.643}{14643}$ & 20 & . & 0.13333 & $\begin{array}{l}41.2 \\
41.6\end{array}$ & 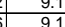 & $\frac{2.4}{264}$ & $\frac{1.807}{1818}$ & 0.0006 & 006 & & 然 & . \\
\hline \begin{tabular}{|l|}
27 \\
\end{tabular} & $9 / 2 / 2004$ & 4:20:15 AM & $\frac{25.943}{25.93}$ & 25.408 & \begin{tabular}{|l|}
23.609 \\
\end{tabular} & 25.455 & 24.833 & 32.985 & \begin{tabular}{|l|}
42.408 \\
\end{tabular} & \begin{tabular}{|l|}
14.401 \\
14.796 \\
\end{tabular} & 18.939 & 3.333 & 0.487 & 37.948 & 0.041 & 14.643 & 0.003 & 10.00 & \begin{tabular}{|l|}
0.16667 \\
\end{tabular} & 41.3 & 9.0 & $\frac{2.4}{26.0}$ & t..790 & 0.006 & 0.006 & 0.000 & 0.220 & $\frac{.20}{.19}$ \\
\hline & $9 / 2 / 2004$ & 4:21:15 AM & 25.903 & 25.413 & \begin{tabular}{|l}
23.569 \\
\end{tabular} & 25.455 & & 33.554 & .862 & 15.09 & 19.282 & 3.33 & 0.527 & 37.635 & 0.039 & 14.643 & 0.003 & 11.00 & 0.18333 & \begin{tabular}{|l|l|l}
41.7 \\
\end{tabular} & 9.0 & 26.4 & 1.821 & 0.006 & 0.006 & & & $\frac{.19}{.19}$ \\
\hline & 9/2/2004 & 4:22:15 AM & 25.841 & 25.406 & \begin{tabular}{|l|}
23.507 \\
\end{tabular} & 25.469 & 24.767 & $\begin{array}{r}33.759 \\
\end{array}$ & 43.136 & 15.237 & 19.251 & 3.324 & 0.516 & 37.478 & 0.037 & 14.643 & 0.00 & 12.00 & 0.2 & 42.0] & 5 & 26.5 & 1.827 & 0.005 & .006 & & & $\frac{1.19}{18}$ \\
\hline & 9/2/212004 & & 25.787 & 25.401 & 23.462 & 25.454 & 24.792 & 33.695 & 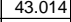 & 15.431 & 19.017 & 3.307 & 0.52 & 37.324 & & 14.643 & 0.0 & 13.00 & $0.21667 \mid$ & 41.9 & & 26.4 & 1.817 & . & . & 0.000 & 204 & \\
\hline & & & & & & & & & \begin{tabular}{|l|l|} 
& 2.734 \\
\end{tabular} & & & & & 37.241 & & 14.643 & & 14.0 & & $\begin{array}{l}41.6 \\
\end{array}$ & & & & & & & & 0.18 \\
\hline 2 & $9 / 2 / 2004$ & 4:25:15 AM & 25.687 & 25.412 & 23.387 & 25.45 & 24.813 & 33.935 & 43.36 & 15.834 & 18.853 & 3.275 & 0.56 & 37.228 & 0.038 & 14.643 & 0.0 & 15.00 & 0.25 & 42.2. & 8.9 & 26.4 & 1.820 & 0.005 & & 000 & & .18 \\
\hline 33 & 9/2/2004 & 4:26:15 & 25.653 & 25.423 & \begin{tabular}{|l|l|}
23.378 \\
\end{tabular} & 25.48 & 24.813 & 33.614 & 42.939 & 15.996 & 18.457 & 3.281 & 0.57 & 37.12 & 0.034 & 14.643 & 0.00 & 16.00 & 0.26667 & 41.8 & 8.8 & 26.0 & 1.795 & 0.005 & 0.005 & 0.000 & 0.196 & 0.17 \\
\hline $4^{4}$ & 9/2/2004 & 4:27:15 AM & 25.594 & 25.413 & \begin{tabular}{|l|}
23.324 \\
\end{tabular} & 25.461 & 24.809 & 33.672 & 42.995 & 16.133 & 18.307 & 3.243 & 0.57 & 37.038 & & 14.643 & 0.00 & 17.6 & 0.2833 & 41.9 & to & & 1.792 & 0.005 & 0.005 & 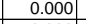 & 1911 & 0.16 \\
\hline & 9/2/212004 & $4: 28: 15 \mathrm{AM}$ & 25.554 & 25.413 & \begin{tabular}{|l|l|}
23.289 \\
\end{tabular} & $25.471 \mid$ & 24.784| & 33.651 & 43.028 & 16.039 & 18.5 & $3.238 \mid$ & 0.614 & 36.932 & & 14.6 & & 18.00 & 0.3 & 41.9 & 8.8 & 26.1 & 1.798 & 0.005 & 0.005 & $t$ & 202 & 0,17 \\
\hline 37 & 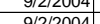 & $\begin{array}{l}4.29 .15 \\
4 \cdot 30 \cdot 15\end{array}$ & $\frac{25.503}{2548}$ & $\frac{25.408}{25408}$ & 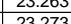 & 25.48 & $\frac{24.16}{2483}$ & $\begin{array}{l}30.312 \\
36872\end{array}$ & 45.60 & 1.071 & 21.6 & & 067 & $\frac{36.4}{36.5}$ & & 14.6 & & 19. & 0 & 44.5. & & & 1.979 & . & . & & & \\
\hline & 2004 & & $\frac{20.440}{25408}$ & 25.400 & 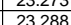 & 25.5 & & $\begin{array}{l}30.072 \\
38683\end{array}$ & $\frac{40.510}{46.8}$ & $\frac{10.44}{16539}$ & $\frac{2.5}{229}$ & & & & & 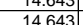 & & $\frac{20.1}{21 .}$ & 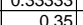 & 456 & 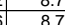 & & 年 & & & & & .15 \\
\hline (5) & $9 / 2 / 2004$ & & 25.412 & 25.402 & 23.322 & 25.47 & & $\begin{array}{l}39.015 \\
39.815\end{array}$ & 45.899 & 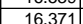 & 24,48 & & & 36.17 & & 14.64 & 0.0 & $\frac{250}{220}$ & 0.3667 & 44.8 & & & & | & & & & 0.16 \\
\hline \begin{tabular}{|l|}
40 \\
\end{tabular} & 9/2/2004 & 4:33:15 AM & 25.363 & 25.413 & \begin{tabular}{|l|}
23.418 \\
\end{tabular} & 25.49 & 24.87 & 39.496 & \begin{tabular}{|l|}
45.333 \\
\end{tabular} & 17.258 & 23.331 & -0.413 & & 37.802 & 0.0 & 14.643 & & 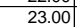 & 0.38333 & 44.2 & r & $\frac{31.4}{31.4}$ & 2.166 & 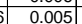 & .005 & 0.000 & 1.162 & $\frac{0.16}{0.14}$ \\
\hline & $9 / 2 / 2004$ & 4:34:15 AM & 25.287 & 25.392 & \begin{tabular}{|l|}
23.418 \\
\end{tabular} & 25.439 & 24.82 & 40.014 & 45.944 & 16.591 & 24.34 & - -0.387 & & 36.436 & & 14.643 & & 24.0 & \begin{tabular}{|l|}
0.4 \\
\end{tabular} & 44.8 & & 202 & 2.218 & 0.005 & .005 & 000 & 163 & 0.14 \\
\hline & 9//2/2004 & & & 25.411 & \begin{tabular}{|l|}
23.487 \\
\end{tabular} & & & & & & & & & & & & & & & 44.5 & & & & & & & & .1. \\
\hline & & 4:36:15 AM & 25.197| & 25.391 & 23.507 & 25.439 & & 38.965 & 44.782 & 17.604 & 22.342 & & & 38.065 & & 14.6 & & 26. & 0.43 & \begin{tabular}{|l|l|}
43.7 \\
\end{tabular} & 9.1 & & 2.113 & & & & & 11 \\
\hline & 2004 & & 25.162 & 25.3 & $23.563 \mid$ & 25.455 & & 39. & & & 22.9 & & & 37. & & & & & 0.45 & 43.9 & 9.0 & & & & & & & \\
\hline & 9/2/2004 & & 25.122 & & \begin{tabular}{|l|l|} 
& 23.578 \\
\end{tabular} & 25.439 & & & \begin{tabular}{|l|l|}
45.588 \\
\end{tabular} & & 23.5 & & & & & $14.643 \mid$ & & 28. & 0.46667 & 44.5 & $8.9 \mathrm{Y}$ & & & 0.005 & . 0005 & & 0.160 & 0.14 \\
\hline 46 & $9 / 2 / 2004$ & 4:39:15 AM & 25.102 & 25.397 & 23.633 & 25.46 & & 39.504 & \begin{tabular}{|l|}
45.352 \\
\end{tabular} & 17.181 & & & & 37. & & 14.6 & & & 0.48333 & 44.2. & $8.8 \quad$ & 31.3 & $2.161 \mid$ & 0.005 & 0.005 & 0.000 & 0.161 & .14 \\
\hline 47 & $9 / 2 / 2004$ & & 25.068 & 25.393 & \begin{tabular}{|l}
23.629 \\
\end{tabular} & 25.4 & 24.78 & 39.649 & \begin{tabular}{|l}
45.459 \\
\end{tabular} & 16.954 & 23.548 & & & 36.8 & & & & 30.00 & 0.5 & 44.3 & 0.0 & & & & & & 160 & \\
\hline 40 & (1) & $\begin{array}{l}4.4 .115 \mathrm{AM} \\
0.4015 \mathrm{~A}\end{array}$ & 250.020 & $\frac{25.383}{25277}$ & 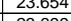 & 25.405 & & $\begin{array}{l}39.693 \\
30202\end{array}$ & 45.5074 & $\begin{array}{l}16.888 \\
17255\end{array}$ & $\frac{23.71}{23.17}$ & & & $\begin{array}{l}36.611 \\
37570\end{array}$ & & 14.6 & & 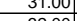 & 0.516672 & 44.4. & & & 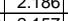 & & & & & 0.1. \\
\hline & & & & & & 20.4199 & & & & & & & & & & & & & & 4.4.2 $>3.3$ & & & & & & & & \\
\hline & 年 & & 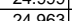 & 2537 & \begin{tabular}{|l|l|l|}
23734 \\
\end{tabular} & 2541 & 24.6 & & 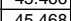 & 17.1 & $\frac{20.110}{23226}$ & & & 年 & & 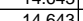 & & 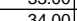 & 0.056 & 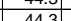 & 89 & 31.4 & 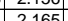 & 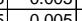 & & & 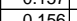 & $\overline{01}$ \\
\hline 52 & & $4: 45: 15 \mathrm{AM}$ & 24.953 & 25.378 & $\begin{array}{l}23.754 \\
23.754\end{array}$ & 25.445 & & & 45.459 & & & & & & & $\frac{14.643}{14}$ & & & 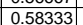 & $\frac{74.3}{44.3}$ & 8.8 & & & $\frac{5}{5}$ & & & & $\frac{0.13}{0.13}$ \\
\hline 53 & $9 / 2 / 2004$ & 4:46:15 AM & 24.938 & 25.373 & \begin{tabular}{|l|}
23.78 \\
\end{tabular} & 25.435 & 24.77 & 39.69 & \begin{tabular}{|l|}
45.418 \\
\end{tabular} & 16.992 & 23.737 & & & 36.7 & & 14.6 & 0.0 & $\frac{36 .}{36 .}>>>$ & 0.6 & $\begin{array}{l}44.3 \\
\end{array}$ & 5 & & 2.187 & 0.005 & 105 & & 0.154 & 0.13 \\
\hline 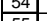 & 9/2/2004 & & 24.917 & 25.351 & \begin{tabular}{|l|l|l}
23.768 \\
\end{tabular} & 25.404 & & & & $\begin{array}{ll}17.002 \\
\end{array}$ & & -0.42 & & & & 14.6 & (n) & . & 0.61667 & 43.0 & & & 2.204 & - & & & & 0.13 \\
\hline & & & 24.911 & & 23.797 & & & 39.873 & 45 & & & & & & & & & & 0.63 & 44.7 & & & 2.194 & & & & 154 & 013 \\
\hline (1) & 91/212004 & 4:49:1 & 24.902 & 25.361 & 23.818 & 25.424 & 24.90 & 39.046 & 44.875 & 17.625 & 22.475 & -0.4 & & 38.135 & & 14.6 & & & 0.65 & 43.7 & 7 & 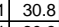 & 2.121 & & & & 154 & \\
\hline & & & 24.91 & 25.375 & 23.867 & 25.4277 & $24.8 \mathrm{~s}>\mathrm{l}$ & 39.172 & 44.989 & 17.606 & 22.565 & -0.4 & & & & 14.6 & & & 0.66667 & 43.9] & & & 2.128 & .005 & & & & \\
\hline & 9/2/2/2 30 & 4:51:1 & $24.88 \mathrm{r}$ & 25.359 & 23.8 & 25.412 & & 39. & $45 .{ }^{2}$ & & & & & & & 14.6 & & 41. & 0.68333 & 44.11 & & & 2.136 & & & & & .1 \\
\hline 59 & 9/2/2004 & & 24.889 & 25.359 & 23.881 & 25.412 & 24 & 39.23 & & 17.398 & 22.805 & & & 37.948 | & & 14.6 & & 42.00 & & 43.99 & & 31.0 & 139 & 0.005 & 05 & & 52 & \\
\hline 60 & 9/2/2004 & 4:53:15 AM & 24.884 & 25.359 & & 25.3911 & 24.68 & & 45.084 & 17.175 & & -0.419 & & 37.133 & & 14.643 & & & 0.71667 & & & & 2.153 & & & & . & \\
\hline & & & & & & & & & & & & & & & & & & & & & & & & & & & & \\
\hline & & Maximum & 26.1 & $\frac{25.4}{25.4}$ & \begin{tabular}{|l|}
23.9 \\
\end{tabular} & 25.5 & 25.6 & $\begin{array}{l}30.4 \\
40.1\end{array}$ & $\begin{array}{l}46.0 \\
46.7\end{array}$ & \begin{tabular}{|l|}
10.4 \\
17.6 \\
\end{tabular} & $\begin{array}{l}21.0 \\
24.5 \\
\end{array}$ & $\frac{1.1}{25}$ & 然. & $\begin{array}{l}38.7 \\
38.7\end{array}$ & 0.0 & $\begin{array}{l}14.0 \\
14.6 \\
\end{array}$ & & & & 年 45.5 & $\frac{0.9}{9.2}$ & $\begin{array}{l}29.0 \\
2232.2\end{array}$ & 2.218 & $\begin{array}{l}0.000 \\
0.027\end{array}$ & o. 0 & & $\begin{array}{l}0.4 \\
0.9 \\
\end{array}$ & \\
\hline & & Median & 25.3 & 25.4 & 23.6 & 25.5 & & 39.2 & 45.2 & 16.9 & 22. & & & 37. & 0.0 & 14 & & & & (44.1. & 8.9 & 1.0 & 2.1 & & & & 0.2 & \\
\hline 65 & & Minimum & 24.9 & 25.4 & 23.3 & 25.4 & 24. & 32.5 & 42.0 & 13.3 & $18.3]$ & -0.4 & \# & 36.2 & 0.0 & 14.6 & & & & 40.9 & & $\begin{array}{lll}6 & 26.0 \\
\end{array}$ & 1.790 & 0.005 & & & 0.2 & 0.1 \\
\hline $0 x+2$ & & $2 \times$ Sta Dev & 0.179 & 0.040 & 0.390 & 0.058 & 0.11 & 5.663 & 2.667 & 2.114 & 4.084 & 3.5966 & 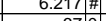 & 1.232 & 0.050 & 0.000 & & & & 2.6667 & $t$ & & 0.333 & $.00 \%$ & 008 & (2) & 248 & \\
\hline 67 & Number o & f Points Used ${ }^{\star} \mid$ & 37 & 37 & 37 & 37 & $37[0]$ & 37 & 37 & 37 & 37 & 37 & 3700 & 37 & 37 & 37 & $3 \pi 0$ & & & 37 & 37 & 37 & & 37 & $37 \mid$ & 37 & 37 & \\
\hline \begin{tabular}{|l|}
69 \\
69 \\
\end{tabular} & & & & & & & & & & & & & & & & & & & & rage for & & st 20 & & 0.0047 & $0.0049 / g$ & $\mathrm{~m} / \mathrm{tt} 2$ & & \\
\hline \begin{tabular}{|l|}
70 \\
70
\end{tabular} & & & & & & & & & & & & & & & & & & & & & XXStanda & ard Dev & viation = & $\mid 0.0003$ & \begin{tabular}{|l|l|l|l|}
$0.0003 \mid 9$ \\
\end{tabular} & $\mathrm{gpm} / \mathrm{tt} 2$ & & \\
\hline
\end{tabular}


WSRC-TR-2005-00105, REVISION 0

SRNL-RPP-2005-00012, REVISION 0

RUN \# 4.08; STEADY STATE TEST AT 25 WT\% UDS

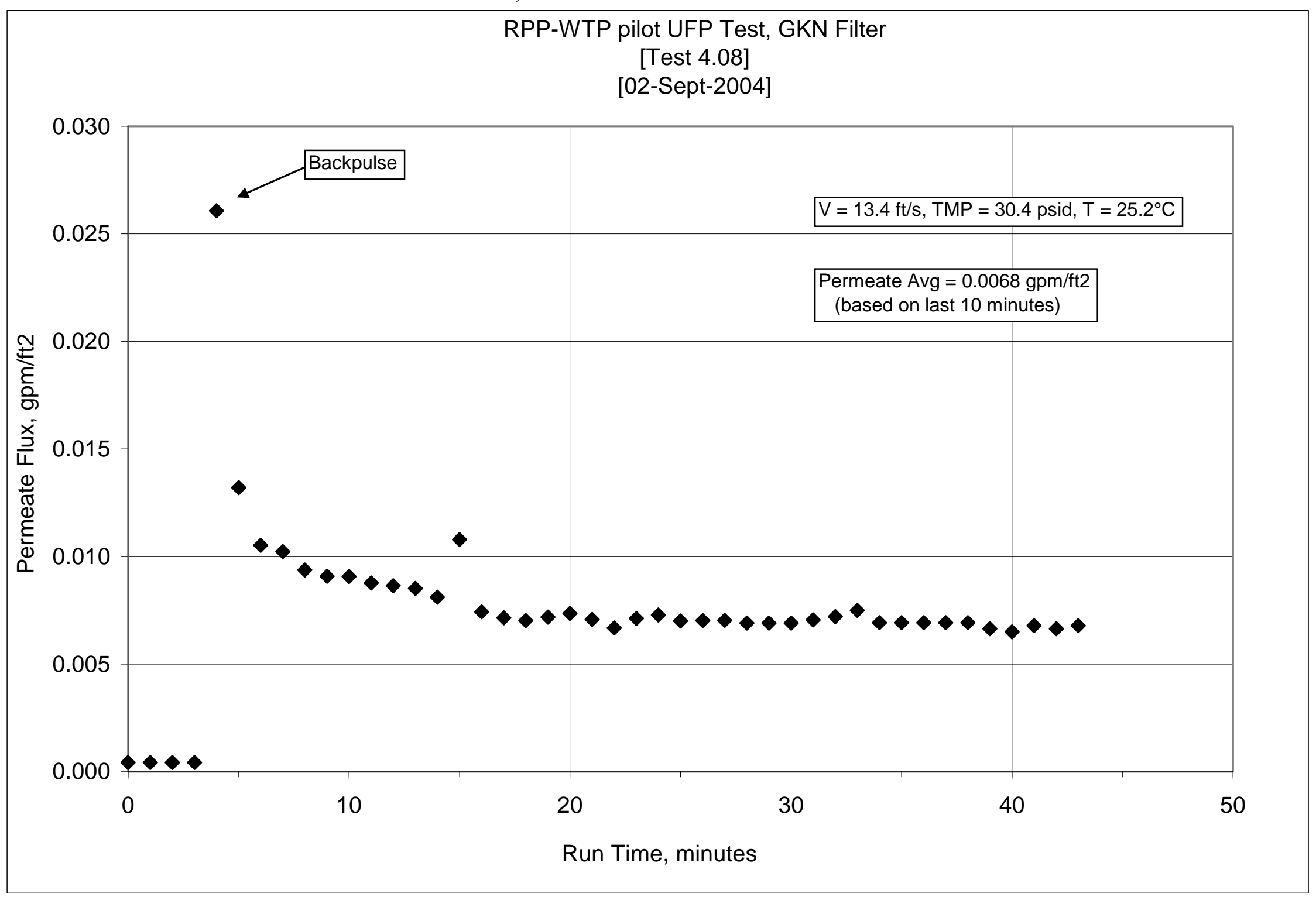


WSRC-TR-2005-00105, REVISION 0

SRNL-RPP-2005-00012, REVISION 0

RUN \# 4.08; STEADY STATE TEST AT 25 WT\% UDS

\begin{tabular}{|c|c|c|c|c|c|c|c|c|c|c|c|c|c|c|c|c|c|c|c|c|c|c|c|c|c|c|c|c|}
\hline & A & $B$ & $\mathrm{D}$ & $\mathrm{E}$ & $\mathrm{F}$ & $G$ & $\mathrm{H}$ & $\mathrm{J}$ & $\mathrm{K}$ & \begin{tabular}{l|l|l|l|l} 
&
\end{tabular} & \begin{tabular}{|l|} 
\\
\end{tabular} & $\mathrm{N}$ & $\mathrm{O}$ & Q & $\begin{array}{ll} \\
\end{array}$ & $\mathrm{s}$ & $\mathrm{T}$ & $\mathrm{V}$ & W & $|x|$ & \begin{tabular}{ll|} 
& $\mathrm{Y}$ \\
\end{tabular} & $\mathrm{z}$ & AA & $A B$ & \begin{tabular}{|l|l|} 
& \\
\end{tabular} & AD & $\mathrm{AE}$ & AF \\
\hline \begin{tabular}{|l}
1 \\
2
\end{tabular} & DATE & TIME & \begin{tabular}{|l|l} 
\\
Eilitrate
\end{tabular} & 《e Temp & 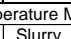 & teasureme & ents $\gg>$ & 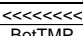 & 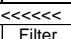 & $\begin{array}{l}\text { Pressure } \mathrm{M} \\
\end{array}$ & Measureme & & | & 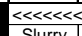 & << Flow & Measurem & tents $\gg \gg \gg \gg>$ & & & & & & & & & & & \\
\hline & DATE & & & & \begin{tabular}{|l|} 
Slurry \\
deg C \\
\end{tabular} & \begin{tabular}{|l|l|} 
AI Amb. \\
$\operatorname{deg} C$.
\end{tabular} & $\frac{\mid c o \text { Amb. }}{\text { deg } \mathrm{C}}$ & $\frac{\text { BottMP }}{\text { psid }}$ & $\begin{array}{l}\text { Filter } \\
\text { psiq }\end{array}$ & Filter dP & \begin{tabular}{|l} 
TopTMP \\
\end{tabular} & \begin{tabular}{|l|} 
Filtrate \\
nsigh
\end{tabular} & & \begin{tabular}{|l|l|l|l|} 
\\
\end{tabular} & Filtrate & Hi Filtate & Backpulse & & & & & & & & & & & \\
\hline & & & $\frac{\operatorname{deg} \mathrm{C}}{\mathrm{T} 2}$ & \begin{tabular}{|l}
$\operatorname{deg} \mathrm{C}$ \\
$\mathrm{T} 3$
\end{tabular} & $\frac{\operatorname{deg} \mathrm{C}}{\mathrm{T} 1}$ & $\frac{\mathrm{deg}}{\mathrm{T} 4}$ & $\frac{\mathrm{deg} C}{\mathrm{~T} 5}$ & $\frac{\text { PIId }}{\mathrm{dP2}}$ & $\frac{p_{p s i g}}{\mathrm{P} 1}$ & $\frac{\text { Psid }}{\mathrm{dP} 1}$ & $\begin{array}{l}\text { pSSI } \\
d P 3\end{array}$ & $\begin{array}{c}\text { PSilg } \\
\text { P2 }\end{array}$ & $\begin{array}{c}\text { pSSI } \\
\text { P3 }\end{array}$ & $\frac{\mathrm{gpm}}{\mathrm{Q} 1}$ & $\frac{\mathrm{gpm}}{\mathrm{Q} 2}$ & $\begin{array}{l}\mathrm{gpm} \\
\mathrm{Q} 3\end{array}$ & $\begin{array}{l}\text { gpm } \\
\text { Qbp }\end{array}$ & & & & & & & & & & & \\
\hline & & & & & & & & & & & & & & & & & & & & & & & & & & & & \\
\hline 7 & eros- 04/19 & & & & & & & & & & & & & & & & & & & & & & & & & & & \\
\hline 8 & $\frac{8 / 23 / 2004}{8 / 24204}$ & 8:35:38 AM & 22.554 & 22.604 & 22.002 & 22.782 & 2.204 & -0.019 & 0.095 & -0.012 & 0.003 & -0.124 & 0.13 & -0.075 & 0.003 & 14.643 & 0.004 & & & Filter S $\mathrm{S}$ & Surface & & & & & & & \\
\hline & $81246 / 2004$ & 8:10:33 AM & 23.662 & 22.93 & \begin{tabular}{|l}
23.679 \\
\end{tabular} & 22.998 & 22.735 & -0.021 & 0.122 & -0.014 & -0.001 & -0.124 & 0.134 & 23.176 & 1.205 & 14.643 & 0.003 & & & Conver: & ersion & 85 & $\mathrm{~m} 3 / \mathrm{m} 21$ & day/barg & ggm/ft2t2 & barg & & \\
\hline & 年12004 & $\begin{array}{l}9: 14: 11 \mathrm{AM} \\
7 \cdot 52 \cdot 29 \mathrm{~A}\end{array}$ & & 23.352 & \begin{tabular}{|l|l|}
4.932 \\
\end{tabular} & 25.316 & 23.242 & -0.008 & 0.126 & & 0.011 & -0.106 & & 48.335 & 0.043 & 14.643 & & & & & & & & & & & & \\
\hline & & $\begin{array}{l}\text { 7:33:29 AM } \\
\text { 7:28:55 AM }\end{array}$ & $\frac{22.649}{22.511}$ & $\begin{array}{r}22.89 \\
20.848\end{array}$ & $\frac{22.298}{22397}$ & 22.872 & 22.871 & $\begin{array}{r}-0.017 \\
\end{array}$ & 0.14 & -0.008 & 0.005 & -0.161 & & -0.084 & 0.003 & 14.643 & & & & & & & & pproxi & ely 1.1 & & & \\
\hline & & & & & & & & & 0.138 & -0.009 & 0.003 & -0.147 & & & 0.003 & 14.643 & & & & & re P1is & rrec & ted for 31 & 31.25 inche & hes of wate & ter tubing & & \\
\hline & & & & & & & & & & & & & & & & & & & & WVWV & & & & 1 F Filtrate & & 《 $\ll$ PERN & ABILI & $\gg$ \\
\hline 14 & & & & & & & & & & & & & & & & & & & & & & & & & at $25 \mathrm{C}$ & & א & \\
\hline 15 & & & & & & & & & & & & & & & & & & Time & Time & Press. & Vel. & TMP & TMP & $\mathrm{gpm}$ & gpm & gpm & pm & \\
\hline$\frac{10}{17}$ & Data - Per M & ute & 25338 & 25322 & 25727 & 2539 & 21603 & 25103 & 51598 & 36002 & 4790 & 10493 & 18051 & 54841 & 8003 & 14643 & & & Hour & psig & $\frac{\mathrm{ft} / \mathrm{s}}{\mathrm{F}}$ & psi & \begin{tabular}{|l|l|} 
bar \\
1,20
\end{tabular} & 112 & $\frac{12}{12000}$ & $2 / p s i$ & $2 / \mathrm{psi}$ & ay/bar \\
\hline 11 & $\begin{array}{l}9 / 2 / 2004 \\
\end{array}$ & 5:04:11 AM & & 25.316 & & & & & 51.149 & 26.093 & & $\begin{array}{l}14.450 \\
14.777\end{array}$ & $\begin{array}{l}\frac{18.951}{55.187} \\
\end{array}$ & $\begin{array}{r}54.841 \\
57.43\end{array}$ & 0.003 & $\begin{array}{l}\frac{14.643}{14.643} \\
\end{array}$ & 0.00 & 1.00 & \begin{tabular}{|l|}
0.01667 \\
0
\end{tabular} & $\begin{array}{l}50.5 \\
50.0\end{array}$ & \begin{tabular}{l|l} 
& 14.4 \\
0 & 14.1 \\
\end{tabular} & \begin{tabular}{|l|}
20.0 \\
15.5
\end{tabular} & \begin{tabular}{|l|l|}
1.376 \\
1.069 \\
\end{tabular} & 0.000 & & $\begin{array}{l}0.000 \\
0.000\end{array}$ & $\begin{array}{l}.021 \\
0.027 \\
\end{array}$ & $\begin{array}{l}0.02 \\
0.02\end{array}$ \\
\hline 10 & $9 / 2 / 2004$ & 5:05:11 AM & 25.252 & 25.316 & \begin{tabular}{|l|}
25.817 \\
\end{tabular} & 25.399 & 24.762 & $\begin{array}{l}-6.448 \\
\end{array}$ & $\begin{array}{l}44.811 \\
\end{array}$ & 17.59 & -18.564 & 45.922 & & $\begin{array}{l}62.223 \\
\end{array}$ & 0.003 & & 0.784 & 2.00 & \begin{tabular}{|l|}
0.03333 \\
\end{tabular} & \begin{tabular}{|l|l|}
43.7 \\
\end{tabular} & 15.2 & -12.5 & & & & & & \\
\hline & 9/2/2004 & 5:06:11 AM & 25.251 & 25.311 & 25.881 & $\begin{array}{l}25.408 \\
\end{array}$ & 24.876 & $\begin{array}{l}13.279 \\
\end{array}$ & $\begin{array}{l}45.186 \\
\end{array}$ & 18.862 & \begin{tabular}{|l|l|}
-4.982 \\
\end{tabular} & 26.366 & D.16 & 64.094 & 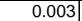 & & 0.00 & 3.00 & 0.05 & 44.1 & 15.7 & 4. & 0.286 & 0.000 & & & & \\
\hline 21 & & 5:07:11 AM & 25.941 & 25.316 & \begin{tabular}{|l|}
25.941 \\
\end{tabular} & $\begin{array}{l}25.448 \\
\end{array}$ & 25.001 & \begin{tabular}{|l|l|}
29.437 \\
\end{tabular} & 45.636 & 20.005 & 10.215 & 10.472 & 0.307 & 65.902 & 0.187 & $\begin{array}{l}14.643 \\
\end{array}$ & 0.003 & 4.00 & 0.06667 & 44.5 & 16.2 & 19.8 & 1.367 & & & 0.001 & 1.315 & \\
\hline & 9/2/2004 & 5:08:11 AM & 25.676 & 25.301 & 25.966 & 25.428 & 24.921 & 36.766 & 46.62 & 21.72 & 15.848 & 3.77 & 0.354 & 65.389 & 0.094 & 14.643 & 0.00 & 5.00 & \begin{tabular}{|l|l|} 
\\
\end{tabular} .0833333 & 45.5 & 16.0 & 26.3 & 1.814 & 0.013 & 0.013 & 0.001 & 0.502 & 0.43 \\
\hline 2 & 9/2/2004 & 5:09:11 AM & 25.726 & 25.311 & 26.026 & 25.443 & 24.856 & 37.413 & 47.163 & 22.622 & & 3.66 & 0.394 & 63.823 & 0.075 & 14.643 & 0.00 & 6.00 & 0.1 & 46.0 & 15.6 & 26.5 & 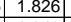 & 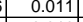 & 0.011 & 0.000 & 0.397 & \\
\hline 24 & $9 / 2 / 2004$ & 5:10:11 AM & 25.766 & & $26.006 \mid$ & 25.418 & 24.906 & 38.763 & 48.43 & 21.928 & & 3.64 & & 58.938 & & 14.643 & & & 0.11667 & & & & 1.943 & & & & & \\
\hline 25 & $\frac{9 / 212004}{21201}$ & 5:11:111 AM & 25.81 & 25.289 & \begin{tabular}{|l|}
26.034 \\
\end{tabular} & $25.42 t$ & 24.8 .8 & 39.093 & 48.741 & 22.614 & 17.252 & 3.608 & & 59.981 & 0.061 & 14.643 & & 8.00 & 0.1333 & 47.6 & 14. & 28.2 & 1.942 & 0.010 & 0.009 & & 0.333 & 0.28 \\
\hline \begin{tabular}{|l}
26 \\
27 \\
\end{tabular} & $\begin{array}{l}9 / 2 / 2004 \\
9 / 2 / 2004\end{array}$ & 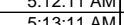 & 25.8606 & 25.300 & $\mid$\begin{tabular}{|l|l|}
20.041 \\
6025
\end{tabular} & $\begin{array}{l}25.458 \\
25.923\end{array}$ & 24.106 & & 年 & & 17.026 & $\begin{array}{l}3.565 \\
591\end{array}$ & & 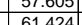 & $\begin{array}{l}0.065 \\
0.065\end{array}$ & & & 1.00 & 0.15 & 41.6 & 14. & & & 0.009 & & & & \\
\hline 28 & $9 / 2 / 2004$ & $\begin{array}{l}5.15 .11 \mathrm{AV} \\
5: 14: 11 \mathrm{AM}\end{array}$ & 25.095 & 25.290 & \begin{tabular}{|l|}
20.025 \\
25.984 \\
\end{tabular} & $\begin{array}{l}25.517 \\
25.517\end{array}$ & $\begin{array}{l}24.010 \\
24.915\end{array}$ & 40.918 & $\begin{array}{l}\frac{49.044}{50.608} \\
\end{array}$ & $\frac{22.450}{22.043}$ & $\begin{array}{l}\frac{10.001}{19.386} \\
\end{array}$ & $\begin{array}{l}3.591 \\
3.608\end{array}$ & 0.51 & $\begin{array}{l}01.424 \\
56.024\end{array}$ & 0.063 & $\frac{14.043}{14.643}$ & & $\frac{10.00}{11.00}$ & \begin{tabular}{|l|l|}
0.10001 \\
\end{tabular} & $\begin{array}{l}40.4 \\
49.5 \\
\end{array}$ & 13. & 302 & $\frac{1.990}{2.079}$ & $\begin{array}{l}0.009 \\
0.009 \\
\end{array}$ & $\begin{array}{l}0.009 \\
0.009 \\
\end{array}$ & 0.000 & 0.0 .291 & 0.25 \\
\hline 29 & 9/2/2004 & 5:15:11 A & $\begin{array}{r}25.94 \\
\end{array}$ & 25.3 & \begin{tabular}{|l|}
25.94 \\
\end{tabular} & 25.528 & 24841 & 41.146 & 50.824 & 22.24 & 19.592 & 3.585 & 0.53 & 54.286 & 0.062 & 14643 & 0.003 & 1200 & 0.10 & 497 & 13. & $\frac{30.4}{304}$ & 2094 & 0.009 & 0.009 & 0.000 & 0 & $\frac{0.25}{0.24}$ \\
\hline 30 & 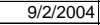 & 5:16:11 AM & 25.961 & 25.301 & & 25.528 & & 41.754 & 51.462 & 22.256 & 20.154 & $\begin{array}{l}3.547 \\
\end{array}$ & & & & 14.643 & & 13.00 & 0.21667 & 50.3 & 13.8 & 31.0 & 2.134 & & & & & \\
\hline \begin{tabular}{|l|l}
31 \\
\end{tabular} & 9/2/2004 & 5:17:11 AM & 25.976 & 25.301 & 25.826 & \begin{tabular}{|l|}
25.554 \\
\end{tabular} & 24.801 & 41.559 & \begin{tabular}{|l|}
51.027 \\
\end{tabular} & 22.359 & $\begin{array}{l}19.949 \\
\end{array}$ & 3.547 & & 53.844 & 0.058 & 14 & & 14.00 & $\begin{array}{l}0.23333 \\
0.2333\end{array}$ & 49.9 & 128 & 30.8 & 2120 & 0.008 & 0.008 & 0.000 & 0.264 & 0.22 \\
\hline & 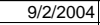 & 5:18:11 AM & 25.966 & & & & & 41.4 & \begin{tabular}{|l|l|}
51.203 \\
\end{tabular} & 22.309 & $\begin{array}{l}19.94 \\
\end{array}$ & 3.579 & & 56.351 & 0.077 & & & 15.00 & 0.25 & 50.1 & 13.4 & 30.7 & 2.115 & 0.011 & 0.011 & & 0.352 & \\
\hline 33 & $\mid 2004$ & 5:19:11 AM & \begin{tabular}{|l|l|}
25.927 \\
\end{tabular} & 25.306 & & & 24.792 & $\begin{array}{l}43.382 \\
\end{array}$ & $\begin{array}{l}49.456 \\
\end{array}$ & 23.558 & $\begin{array}{l}20.665 \\
\end{array}$ & -0.019 & & 54.691 & 0.053 & & & 16.00 & 0.26667 & $\begin{array}{l}48.3 \\
\end{array}$ & 13.6 & 32.0 & 2.208 & & & & 0.232 & \\
\hline 34 & 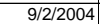 & 5:20:11 AM & 25.957 & 25.312 & \begin{tabular}{|l|}
25.717 \\
\end{tabular} & 25.635 & & & 47.876 & 22.469 & 20.178 & -0.147 & & 56.712 & 0.051 & & & 17.00 & 0.28333 & \begin{tabular}{ll|l}
46.8 \\
\end{tabular} & 13.5 & 31.0 & 2.134 & 0.007 & & & & \\
\hline 35 & 9/2/2004 & $5: 21: 11 \mathrm{AM}$ & 25.967| & 25.307 & 25.652 & 25.61 & & 40.665 & 46.631 & $\begin{array}{l}21.707 \\
\end{array}$ & $\begin{array}{ll}19.918 \\
\end{array}$ & -0.173 & & 58.076 & 0.05 & & 0.0 & 18.00 & 0.3 & 45.5 & 13.8 & 30.3 & 2.088 & $\begin{array}{l}0.007 \\
\end{array}$ & 0.6 & 0.000 & 0.232 & 0.20 \\
\hline 36 & 9/2/2004 & 5:22:11 AM & 25.952 & 25.302 & 25.542 & 25.55 & 24.658 & 40.833 & $\begin{array}{l}46.807 \\
\end{array}$ & 21.806 & 19.867 & -0.3 & & 57.43 & 0.051 & & & 19.00 & 0.31667 & 45.7 & 13.7 & 30.4 & 2.093 & 0.007 & & & & \\
\hline 20 & 9/2/20004 & 5:23:11 AM & 25.927 & 25.271 & 25.426 & 25.489 & & 41.151 & 47.109 & 21.826 & 20. & -0.335 & & 02.004 & 0.052 & & & 20.00 & .03333 & 46.0 & $12.6 \mathrm{C}$ & 30.7 & 2.117 & 0.007 & & 0.000 & 240 & \\
\hline 38 & $9 / 2 / 2004$ & 5:24:11 AM & 25.936 & & & & & & & & & & & & & & & & & & & & & & & & & \\
\hline & 2004 & 5:25:111 AM & 25.904 & 25.268 & 25.263 & 25.441 & & 40.7 & 46.56 & 21.639 & 20.024 & -0.349 & & 55.148 & $0.04 t$ & & & 22.00 & 0.36667 & 45.4 & 13.1 & 30.4 & 2.096 & $0.6-6>$ & & 0.000 & 0.220 & 0.19 \\
\hline$\frac{40}{41}$ & $\begin{array}{l}9 / 2 / 2004 \\
9 / 2 / 2004\end{array}$ & & 25.8064 & & & 253.4 & & & & 21.8 & & $\begin{aligned}-0.3 \\
-0.349\end{aligned}$ & & & & & & & & 456 & & 20 & & & & & & \\
\hline 42 & 9/2/2004 & $5: 28: 11 \mathrm{AM}$ & 25.835 & 25264 & \begin{tabular}{|l|l|}
25.034 \\
\end{tabular} & \begin{tabular}{|l|}
25.300 \\
\end{tabular} & & & \begin{tabular}{|l|}
40.051 \\
47.051 \\
\end{tabular} & $\begin{array}{l}21.400 \\
21.737\end{array}$ & 20.417 & $\begin{array}{l}-0.3459 \\
-0.349\end{array}$ & & 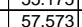 & 0.041 & & & 2500 & 0.4667 & 年 & 137 & 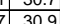 & 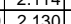 & 0.007 & & 0.000 & 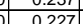 & 0.19 \\
\hline 43 & 9/2/2004 & 5:29:11 AM & 25.819 & 25253 & 24.948 & & & & & 21.376 & & -0352 & & $\mid 53.644$ & 0.049 & & & & 0.43333 & & & & & & & & & 0.15 \\
\hline 44 & & 5:30:11 AM & 25.794 & 25.258 & & 25.366 & & & \begin{tabular}{|l|l|}
47.339 \\
\end{tabular} & 21.644 & & -0.349 & & 53.4 & 0.049 & & & 27.00 & 0.45 & 46.2 & 12.7 & & 2.127 & & & & & \\
\hline 45 & 9/2/2004 & $5: 31: 11 /$ & 25.772 & 25.257 & \begin{tabular}{|l|}
24.811 \\
\end{tabular} & 25.359 & 24.5 & 41.117 & 47. & 21.7 & & & & 55.3 & 0.0 & & & 28.00 & 0.46667 & 45.9 & 13.2 & 30.7 & 2.115 & & & & 0.225 & $0.1 \mathrm{c}$ \\
\hline 46 & & $5: 32$ & 25.7 & & 24.7 & & & 41 & & & 20. & & & 53. & & & & & \begin{tabular}{|l|l|l|}
0.48333 \\
\end{tabular} & $\begin{array}{ll}45.8 \\
\end{array}$ & $12.8 \mathrm{Y}$ & & 2.122 & & & & 0.225 & \\
\hline 47 & & 5:33:11 & 25.732 & & 24.8 & & & & & 21. & & & & & & & & 30.00 & & 45.9 & & $\overline{30.9}>>$ & 2.131 & & & & 0.224 & \\
\hline 4 & 9/2/2004 & $5: 34: 11 \mathrm{~A}$ & 25.697 & 25.256 & 24 & & & 41 & & 21.84 & & -0.349 & & $54.8 \quad-3$ & 0.049 & & & 31. & 0.51667 & $46.2 \mathrm{~s}-2$ & 13.1 & 31.0 & 2.135 & & & & 0.228 & \\
\hline & $9 / 2 / 2004$ & 5:35:11 AM & 25.682 & 25.257 & 24.766 & 25.405 & & 41.211 & 47.095 & 21.521 & 20.585 & -0.3 & & 55. & 0.05 & & & 32.00 & 0.53333 & 46.0 & $13.2 \mathrm{r}$ & $30.6 \mathrm{r}$ & 2.130 & & & 0.000 & 0.233 & 0.20 \\
\hline & $\frac{9 / 2 / 2004}{90 / 20004}$ & 5:36:11 A & 25.647 & 25.247 & 24. & 25.3 & & & 47.559 & 21.567 & 20. & -0.346 & & 54. & 0.052 & & & 33.00 & & 46.4 & 13.1 & 31. & 2.156 & & & & & \\
\hline$\sqrt{5}+3$ & . & 5:3:1114 & 25.622 & 25.24 & & 25.395 & & & & & & & & & & & & & 0.56667 & 45.9 & & & 2.100 & & & & & \\
\hline & & & & & & & & & & & & & & & & & & & & 45.8 & 12. & & 2.156 & & & & & \\
\hline & & S & 23.579 & $\frac{23.24}{252}$ & 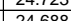 & 20.441 & & & 47.161 & 21.3 & & $=-0.34$ & & & 0.6 & & & 30.04 & & 46.0 & 13. & 31.1. & 2.145 & & & & 0.24 & 0. \\
\hline 55 & 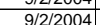 & 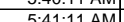 & 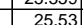 & $\frac{2.24}{2523}$ & 246 & & & & & $\frac{2.30}{214}$ & & & & 514 & & & & & 0.63 & 46.1 & 12 & 312 & & & & & & \\
\hline 56 & & $5: 42: 11 \mathrm{~A}$ & 25.515 & 252 & 24.6 & 25.483 & & 40.966 & 46.798 & 21.6 & & $-0.3 !$ & & $\frac{21.5}{51.5}$ & & & & 39. & & 457 & 123 & 306 & 2108 & & & & 0217 & 019 \\
\hline & $9 / / 2 / 2004$ & $5 \cdot 43 \cdot 11 \mathrm{~A}$ & 25.49 & 2524 & & & & & & 21.896 & & -0.33 & & 54.5 & & & & & & & & & & & & & 0214 & \\
\hline & & & 25.47 & & & & & & & & & & & & & & & 41.00 & t & & 14 & & & & & & & \\
\hline & & & 25.45 & 25.235 & & & & & 46 & 21 & & -0.3 & & $58.1-3$ & 0.0 & & & 42.00 & & 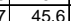 & 13 & & & & & & & \\
\hline & 9/2/2004 & $5: 46: 11 \mathrm{AM}$ & 25.435 & & 24.664 & 25.453 & & $\begin{array}{l}40.897 \\
\end{array}$ & \begin{tabular}{l|l}
46.707 \\
\end{tabular} & 21.948 & 19.871 & -0.349 & & 53.147 & 0.047 & 14.643 & & & 0.71667 & 45. & & & & & & & 0.224 & \\
\hline & & & & & & & & & & & & & & & & & & & & 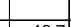 & & & & & & & & \\
\hline \begin{tabular}{|l|}
63 \\
63
\end{tabular} & & Iave & $\frac{2.8}{26.0}$ & $\frac{25.3}{25.3}$ & $\frac{25.2}{260}$ & 25.5 & & $\frac{4.9}{43.4}$ & $\frac{4 / 8}{51.5}$ & $\frac{21.9}{23.6}$ & & & & 63 & & & & & & 503 & & 30 & $\frac{96}{86}$ & & & & $\frac{0.3}{0.4}$ & \\
\hline 64 & & Median & 25.8 & 25.3 & $\begin{array}{l}25.1 \\
25.1\end{array}$ & 25.4 & & 41.1 & 47.1 & 21.8 & 20.2 & & & $\begin{array}{l}55.4 \\
5\end{array}$ & 01 & 14. & & & & 46.0 & \begin{tabular}{l|l}
0 & 13.3 \\
0
\end{tabular} & 30.7 & $\frac{2.200}{2.116}$ & 07 & 0.007 & & 0.8 & \\
\hline & & & 25.4 & 25.2 & & 25.3 & & 37.4 & & 21.3 & 15.6 & & \begin{tabular}{|c|c|}
-6.4 \\
\end{tabular} & 51.5 & & & & & & 45.4 & \begin{tabular}{|l|l|}
4 & 12.3 \\
\end{tabular} & & & & & & 0 & \\
\hline 66 & & $2 \times \operatorname{Std} \mathrm{Dev}$ & 0.340 & 0.052 & 1.052 & 0.156 & $0.217 \#$ & 1.945 & 2.882 & 0.925 & 2.358 & 3.447 & 6.002 & 5.137 & $\begin{array}{l}0.017 \\
\end{array}$ & 0.000 & & & & 2.882 & 21.435 & 2.097 & \begin{tabular}{|l|l|} 
\\
\end{tabular} & 002 & 02 & 0.000 & $\overline{092}$ & \\
\hline & Number & of Points Used ${ }^{*}$ & & & 38 & & & & & & & & 38 & 38 & & & & & & & 3 & 38 & & & & & & \\
\hline 68 & & ${ }^{\circ}$ Backpulse poi & ints in box & are not inc & Eluded & & & & & & & & & & & & & & & & & & & & & & & \\
\hline & & & & & & & & & & & & & & & & & & & Aver & erage for & in the last & st $10 \mathrm{mi}$ & inutes $=$ & $=0.0069$ & 0.0069 & $9 \mathrm{gpm} / \mathrm{ft} 2$ & & \\
\hline 70 & & & & & & & & & & & & & & & & & & & & & 2xStandar & ard Dev & viation $=1$ & $=|0.0005|$ & | 50.0004 & 14/gpm/ft2 & & \\
\hline
\end{tabular}


WSRC-TR-2005-00105, REVISION 0

SRNL-RPP-2005-00012, REVISION 0

RUN \# 4.09; STEADY STATE TEST AT 25 WT\% UDS

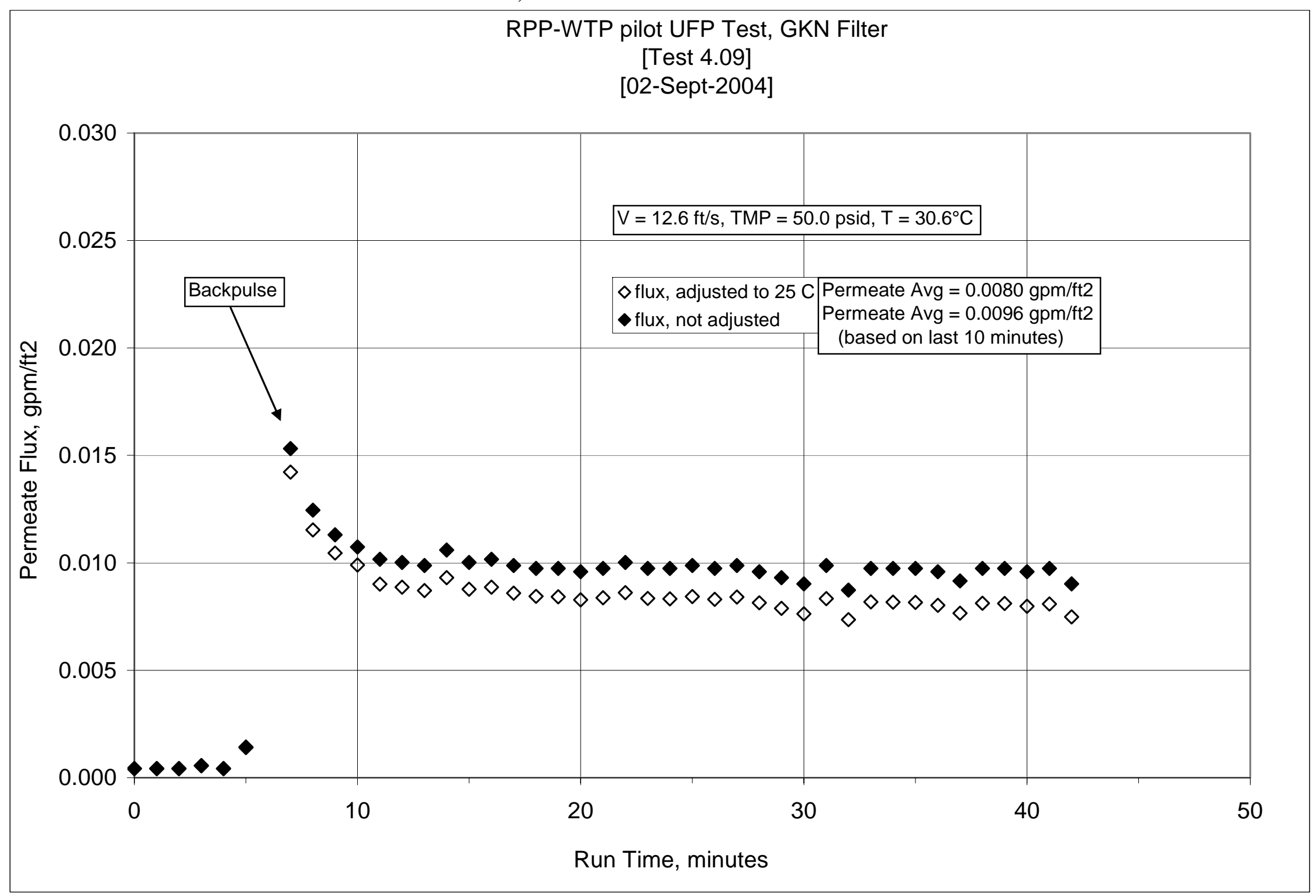


WSRC-TR-2005-00105, REVISION 0

SRNL-RPP-2005-00012, REVISION 0

RUN \# 4.09; STEADY STATE TEST AT 25 WT\% UDS

\begin{tabular}{|c|c|c|c|c|c|c|c|c|c|c|c|c|c|c|c|c|c|c|c|c|c|c|c|c|c|c|c|c|}
\hline & A & B & $\mathrm{D}$ & $\mathrm{E}$ & $\mathrm{F}$ & $\mathrm{G}$ & $\mathrm{H}$ & $\mathrm{J}$ & $\mathrm{K}$ & \begin{tabular}{|l|}
$\mathrm{L}$ \\
\end{tabular} & M & $\mathrm{N}$ & 0 & $Q$ & $R$ & \begin{tabular}{l|l}
$\mathrm{s}$ & \\
$\mathrm{s}$
\end{tabular} & $\mathrm{T}$ & $\mathrm{v}$ & w & $x$ & & & $\mathrm{AA}$ & & $A C$ & & $\mathrm{AE}$ & \\
\hline $\begin{array}{ll}1 \\
2\end{array}$ & & & $\begin{array}{l}\text { Fer<<< } \\
\text { Filtrate }\end{array}$ & $\begin{array}{l}<<\text { Temp } \\
\text { Cleaning }\end{array}$ & erature & teasureme & & BotTMP & 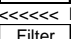 & $\begin{array}{l}\text { Pressure N } \\
\text { Fiter }\end{array}$ & Measureme & 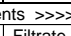 & & & $\ll<$ Flow & & & & & & & & om Raw & Data $>$ & & & & \\
\hline 2 & DAIE & TIME & & $\begin{array}{l}\text { Cleaning } \\
d e q\end{array}$ & \begin{tabular}{|l} 
Silury \\
\end{tabular} & Hi Amb. & Lo Amb. & BotrimP & & Filter dP & TopTMP & & \begin{tabular}{|l|l|} 
Pulsepot \\
\end{tabular} & & $\begin{array}{l}\text { Filtrate } \\
\text { a }\end{array}$ & \begin{tabular}{|l|} 
Hi Filtate \\
\end{tabular} & Backpulse & & & & & & & & & & & \\
\hline $\begin{array}{lll}4 & \end{array}$ & & & $\frac{\text { degC }}{\mathrm{T} 2}$ & $\frac{\operatorname{deg} C}{\text { T3 }}$ & $\frac{\operatorname{deg} C}{T 1}$ & $\frac{\operatorname{deg} C}{\text { T4 }}$ & $\frac{\operatorname{deg} C}{\text { T5 }}$ & $\frac{\text { psid }}{d d^{2}}$ & $\frac{p s i g}{p 1}$ & $\frac{p_{\text {sidd }}}{d \mathrm{~d} 1}$ & $\begin{array}{l}\text { psid } \\
\text { did3 }\end{array}$ & $\frac{p s i g}{P 2}$ & $\begin{array}{l}\text { Psig } \\
\text { P3 }\end{array}$ & $\frac{g p m}{01}$ & $\frac{g p m}{02}$ & $\frac{g p m}{03}$ & gpm & & & & & & & & & & & \\
\hline & & & & & & & & & & & & & & & & & & & & & & & & & & & & \\
\hline & Zeros- $04 / 19$ & & & & & & & & & & & & & & & & & & & & & & & & & & & \\
\hline & 8/23/2004 & 8:35:38 AM & 22.554 & & & & \begin{tabular}{|c|}
22.204 \\
\end{tabular} & & & & & & & -0.075 & & & 0.004 & & & & & & & & & & & \\
\hline 8 & 8/24/2004 & 8:10:33 AM & 23.662 & 22.93 & \begin{tabular}{|l|l|}
23.679 \\
\end{tabular} & 22.998 & 22.735 & -0.021 & 0.122 & -0.014 & $\begin{array}{l}-0.001 \\
\end{array}$ & -0.124 & 0.134 & 23.176 & 1.205 & 14.643 & 0.003 & & & Conver: & & & $1 \mathrm{~m} 3 / \mathrm{m} 2$ & 2/day//barg & /gpm/tt2 & Jbarg & & \\
\hline 9 & $8 / 26 / 2004$ & 9:14:11 AM & 25.819 & 23.352 & 24.932 & 25.316 & 23.242 & -0.008 & 0.126 & -0.008 & 0.011 & -0.106 & 0.091 & 48.33 & 0.043 & 14.643 & & & & & & & & & & & & \\
\hline 10 & $8 / 27 / 2004$ & 7:53:29 AM & 22.649 & 22.89 & \begin{tabular}{|l|l|}
22.298 \\
\end{tabular} & 22.872 & 22.871 & -0.017 & 0.14 & -0.008 & & -0.161 & & & 0.003 & 14.643 & & & & Note: & & & & pprox & ely 1 & sig & & \\
\hline & 8/30/2004 & 7:28:55 АM & 22.511 & 22.848 & 22.397 & 22.669 & 22.914 & -0.017 & 0.138 & -0.009 & 0.003 & -0.147 & 0.12 & -0.082 & 0.003 & 14.643 & & & & & & & & & & & & \\
\hline$\frac{12}{13}$ & & & & & & & & & & & & & & & & & & & & 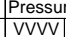 & re Plis & orrec & ted for 5 & $\frac{31.25 \text { inch }}{<<\text { Fitrate }}$ & $\begin{array}{l}\text { ens of } \mathrm{w}_{\mathrm{t}} \\
\text { Flux }\end{array}$ & $\begin{array}{l}\text { er tubing } \\
\ll<P E R \Lambda\end{array}$ & & \\
\hline 14 & & & & & & & & & & & & & & & & & & & & & & & & & at $25 \mathrm{C}$ & & $\times 1000$ & \\
\hline 15 & & & & & & & & & & & & & & & & & & Time & Time & Press. & Vel. & TMP & TMP & gpm & gpm & gpm & gpm & meter \\
\hline & ata-Per M & linute & & & & & & & & & & & & & & & & & Hour & psig & & psi & bar & & & & $2 / \mathrm{psi}$ & tay/ba \\
\hline$\frac{17}{10}$ & 9//2/2004 & 6:00:42 AM & 26.161 & & 27.718 & 25.609 & 24.901 & 19.868 & 67.511 & 22.41 & -1.904 & 42.202 & 1118 & 54.353 & 0.003 & 14.643 & 00 & & & 66.4 & 13. & & 0.619 & 0.000 & & & & \\
\hline & 9/2/2004 & 6:01:42 AM & 26.132 & 25.261 & 27.854 & & 24.942 & & 61.834 & & $\frac{-2.215}{-2.21}$ & & & 56.389 & 0.003 & 14.643 & & 1.0 & 0.0166 & & & & & & & & & \\
\hline$\frac{19}{20}$ & 9/2/2004 & 6:02:42 AM & 26.337 & 25.262 & 28.029 & 25.595 & 25.098 & $\frac{-7.253}{-1.238}$ & 69.695 & 19.891 & -18.564 & 87.848 & 94.792 & 54.739 & 0.003 & 14.643 & 0.841 & . & 1.0333 & 68.6 & 13.4 & -12.9 & & & & & & -0.03 \\
\hline$\frac{21}{21}$ & $\begin{array}{l}9 / 2 / 2004 \\
9 / 2 / 2004\end{array}$ & $\begin{array}{l}0.03 .42 \mathrm{AM} \\
6: 04: 42 \mathrm{AM}\end{array}$ & 25739 & 25.279 & $\frac{20.208}{28.424}$ & 25.612 & 25.189 & $\frac{11.18}{12.41}$ & $\frac{0.415}{66.172}$ & $\frac{15.318}{15823}$ & $\begin{array}{l}-3.354 \\
-2973\end{array}$ & $\begin{array}{l}40.004 \\
4834\end{array}$ & $\begin{array}{l}63.34 \\
29.97 \\
\end{array}$ & 50.009 & 0.003 & $\begin{array}{l}14.045 \\
14.643\end{array}$ & 0.004 & $\frac{3.0}{40}$ & \begin{tabular}{|l|}
0.05 \\
0.06667
\end{tabular} & 64.4 & 140 & $\frac{3.8}{4.7}$ & $\frac{0.263}{0.325}$ & 0.001 & & & & 0.13 \\
\hline 22 & $9 / 2 / 2004$ & 6:05:42 AM & 25.761 & 25.291 & 28.59 & 25.664 & 25.276 & 12.379 & 65.643 & 15.77 & -2.911 & \begin{tabular}{|l|}
47.937 \\
\end{tabular} & 0.287 & 56.262 & 0.01 & 14.643 & 0.003 & 5.00 & \begin{tabular}{|l|l|}
0.08333 \\
\end{tabular} & $\begin{array}{l}0.5 \\
\end{array}$ & 13.8 & \begin{tabular}{|l|l|}
8 & 4.7 \\
\end{tabular} & 70.326 & 0.001 & 0.001 & 0.000 & 0.296 & 0.00 \\
\hline 23 & 9/2/2004 & 6:06:42 AM & 28.534 & & 28.686 & 25.624 & 25.307 & 48.5 & 67.015 & 18.221 & 30.789 & 12.803 & 0.354 & 56.395 & 0.415 & 14.643 & & 6.00 & & 65.9 & 13.8 & \begin{tabular}{|l|l|}
8 & 39.6 \\
\end{tabular} & & 0.059 & 0.054 & 0.001 & 1.358 & 1.16 \\
\hline & 9/2/2004 & 6:07:42 AM & & & & & & & & & $\begin{aligned} 37.031 \\
20.069\end{aligned}$ & 3.657 & & 54.716 & 0.107 & 14.643 & & 7.0 & 0.1166 & 66.1 & 13.4 & 47.22 & 3.251 & & & & & \\
\hline 25 & 9/2/2004 & $6: 08: 42 \mathrm{AM}$ & 27.757 & & & & & & \begin{tabular}{|l}
67.5522 \\
\end{tabular} & & 36.069 & 3.53 & & 53.754 & 0.087 & 14.643 & & & 0.13333 & 66.4 & 13.2 & & 3.244 & & & & $\frac{0.245}{.02}$ & \\
\hline$\frac{26}{27}$ & 9/2/2004 & 6:00:42 AM & 27.826 & $\begin{array}{r}25.27 \\
\end{array}$ & 29.067 & 25.573 & 24.821 & 58.652 & \begin{tabular}{|l|}
68.321 \\
6.021
\end{tabular} & 24.019 & $\begin{array}{r}35.205 \\
25.202 \\
\end{array}$ & 3.521 & 0.48. & 55.079 & 0.079 & 14.643 & & 9.0 & & 67.2 & 13.5 & 46.9 & 3.236 & 0.011 & & & & 0.19 \\
\hline$\frac{21}{28}$ & $\begin{array}{l}9 / 2 / 2004 \\
9 / 2 / 2004\end{array}$ & 6.0 & 27.911 & 25.215 & 20.1019 & 25.511 & 24.090 & 258.97 & $\mid 0.0514$ & $\frac{24.210}{2466}$ & $\begin{array}{l}35.345 \\
35149\end{array}$ & $\begin{array}{l}3.0510 \\
3.495\end{array}$ & 0.53 & 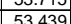 & . 071 & $\begin{array}{l}14.045 \\
14.643\end{array}$ & 0.000 & $\frac{10.0}{110}$ & 0 & 66.9 & $\frac{13.2}{12.7}$ & 4.0 & 3.241 & & & & & 0.18 \\
\hline 29 & $9 / 2 / 2004$ & 6:12:42 AM & 28.066 & 25.251 & 29.407 & 25.519 & 24.762 & 58.974 & \begin{tabular}{|l|}
68.419 \\
\end{tabular} & 25.072 & 34.677 & 3.478 & 0.55 & 53.498 & 0.07 & 14.643 & 0.003 & 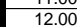 & 0.2 & 67.3 & 12.8 & 468 & 3.228 & 0.010 & 0.009 & 0.000 & 0.189 & $\frac{0.16}{0.16}$ \\
\hline 30 & $9 / 2 / 2004$ & 6:13:42 AM & 28.178 & & 29.548 & 25.566 & 24.809 & 59.306 & & 25.089 & 34.934 & 3.521 & & 53.483 & 0.069 & 14.643 & & 120 & 0.21667 & 67.5 & 12.7 & & $\frac{5.249}{3.249}$ & & & & & $\frac{0.16}{0.16}$ \\
\hline 31 & 9/2/2004 & $6: 14: 42 \mathrm{AM}$ & 27.949 & 25.299 & 29.678 & 25.622 & 24.835 & 62.313 & \begin{tabular}{|l|l|}
68.574 \\
\end{tabular} & 24.862 & 38.293 & 0.157 & -5.489 & 53.742 & 0.074 & 14.643 & 0.003 & 14.0 & 0.2333 & 67.4 & $\frac{12.8}{12.8}$ & 50 & 3.468 & 0.011 & 0.009 & 000 & 185 & 0.16 \\
\hline & 9/2/2004 & $6: 15: 42 \mathrm{AM}$ & & & & 25.666 & & 63.339 & & & & -0.083 & & 52.999 & 0.07 & $\begin{array}{l}14.643 \\
\end{array}$ & 0.0 & & 0.25 & 68.3 & 12.6 & 1.6 .6 & 8.516 & & & & & \\
\hline 33 & 9/2/2004 & $6: 16: 4$ & 28.437 & & 29.921 & 25.741 & 24.94 & 62.841 & \begin{tabular}{|l|l}
68.79 \\
\end{tabular} & & 38.53 & -0.179 & -6.19 & 53.986 & 0.071 & $\begin{array}{l}14.643 \\
\end{array}$ & & 16.0 & 0.26667 & 67.7 & 12. & & 3.495 & $\overline{0.010}$ & & & & .15 \\
\hline 34 & $9 / 2 / 2004$ & 6:17:42 AM & 28.591 & 25.368 & 30.05 & 25.796 & 24.93 & 63.178 & 69.146 & 25.294 & $\begin{array}{l}38.413 \\
\end{array}$ & -0.219 & -6.2. & 51.274 & 0.069 & $\begin{array}{l}14.643 \\
\end{array}$ & 0.003 & 17.00 & \begin{tabular}{|l|l|}
0.28333 \\
\end{tabular} & 68.0 & 12.2 & 50.8 & 3.502 & 0.010 & 0.009 & 0.000 & & \\
\hline 35 & 9//2/2004 & $6: 18: 42 \mathrm{AM}$ & 28.72 & 25.383 & 30.139 & 25.806 & & & \begin{tabular}{|l|l|} 
& 68.549 \\
\end{tabular} & 25.313 & 38.353 & -0.239 & & 48.256 & 0.0 & $\begin{array}{l}14.643 \\
\end{array}$ & & 18.00 & & 67.4 & 11.5 & & & & & & & 0.14 \\
\hline 36 & 9/2/2004 & $6: 19: 42 \mathrm{AM}$ & 28.824 & 25.383 & 30.238 & 25.876 & 25.058 & 62.842 & \begin{tabular}{|l|l|}
68.688 \\
\end{tabular} & 25.331 & 38.48 & -0.176 & -6.23 & 53.443 & 0.068 & 14.643 & 0.003 & 19.0 & 0.31667 & 67.6 & 12.7 & 50.7 & 3.493 & 0.010 & 0.008 & .000 & 0.166 & 0.14 \\
\hline 37 & 9/2/2004 & $6: 20: 42 \mathrm{AM}$ & 28.916 & 25.4 & 30.329 & 25.923 & 25. & 62.931 & \begin{tabular}{|l}
68.891 \\
\end{tabular} & 25.253 & 38.337 & -0.222 & -6.27 & 54.731 & 0.067 & 14.643 & 0.00 & 20.06 & 0.3333 & 67.8 & $13.0 \mathrm{C}$ & 50.6 & 3.491 & .010 & & & 163 & $\overline{0.14}$ \\
\hline 300 & 9/2/2004 & 6:21:42 AM & 29.003 & 25.403 & 30.427 & 25.906 & 25.11 & 63.374 & \begin{tabular}{|l|l|l|l|} 
\\
\end{tabular} & 25.67 & 38.638 & -0.176 & 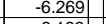 & & & 14.643 & & 21.00 & 0.35 & 68.2 & 12. & 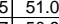 & (1) & & & & 164 & 4 \\
\hline & 9/2/2004 & $6: 22: 42 \mathrm{AM}$ & 29.067 & & 30.5 & 25.91 & 25.11 & 63 & | 68.987 & 25.398 & 38.659 & -0.205 & & 53.126 & 0.07 & 14.643 & & & 0.366067 & 67.9 & 12. & & & & & & & 0.14 \\
\hline$\frac{40}{41}$ & $9 / 2 / 2004$ & 6:23:42 AM & 29.168 & 25.424 & 30.601 & $\begin{array}{l}25.9246 \\
25926\end{array}$ & $\frac{25.07}{2511}$ & $\begin{array}{l}62.945 \\
63082\end{array}$ & $\begin{array}{l}68.74 \\
6892\end{array}$ & $\frac{25.51}{2500}$ & $\begin{array}{l}38.363 \\
38917\end{array}$ & $\begin{array}{l}-0.173 \\
-0.219\end{array}$ & -6.1 & $\begin{array}{l}49.282 \\
5.251\end{array}$ & 0.060 & $\begin{array}{l}14.643 \\
1.613\end{array}$ & & 23.00 & $\begin{array}{l}0.38333 \\
0.0 .0\end{array}$ & 6.6 & $\frac{11 .}{11 .}$ & 500 & $\frac{3.492}{3.52}$ & 然10 & & 年 & & 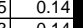 \\
\hline \begin{tabular}{|l|}
42 \\
2
\end{tabular} & (1/2/2004 & $\begin{array}{l}0.42 .4 \\
6.25 .4\end{array}$ & 20.253 & 25.415 & 20.049 & 25.913 & & & & & & & & & & & & & 0.4 & & 120 & & & & & & & \\
\hline \begin{tabular}{|l|l|}
43 \\
\end{tabular} & $9 / 2 / 2004$ & $6.26 .42 \mathrm{AM}$ & 29.324 & 25.401 & 30.787 & 25.904 & 25.1 & & 68781 & 25. & 38.626 & -0.234 & & 52.561 & 0 & 146 & & 2600 & \begin{tabular}{|l}
0.47333 \\
0.433
\end{tabular} & 677 & 125 & 507 & 3497 & & 00 & 0.000 & 164 & $\frac{0.14}{0.14}$ \\
\hline 44 & 9/2/2004 & 6:27:42 AM & 29.387 & 25.394 & 30.855 & 25.922 & $\frac{25.0}{25.0}$ & & 949 & & 38.792 & -0.3 & -6.281 & 53.633 & & 14.643 & & 27.00 & & 67.8 & 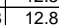 & & & & & & (165) & $\overline{0.12}$ \\
\hline 45 & 9/2/2004 & $6: 28: 42 \mathrm{~A}$ & 29.446 & 25.403 & 30.943 & 25.961 & 25.13 & 62.358 & \begin{tabular}{|l|}
68.321 \\
\end{tabular} & 26.093 & 37.023 & -0.216 & 50 & 55.984 & 0.067 & $\begin{array}{l}14.643 \\
\end{array}$ & & 28.00 & 0.46667 & 67.2 & 13.3 & & 8.426 & & & & 164 & $0.1-$ \\
\hline 46 & 9/2/2004 & 6:29:42 AM & & \begin{tabular}{|l|l|}
25.395 \\
\end{tabular} & & \begin{tabular}{|l|l|}
25.998 \\
\end{tabular} & 25.1 & $\begin{array}{ll}62.418 \\
\end{array}$ & & & 37.173 & & -6.28 & \begin{tabular}{|l|}
54.97 \\
\end{tabular} & & 14. & & 20 & & 67. & 12 & & & & & & & \\
\hline 47 & $\begin{array}{l}9 / 2 / 2004 \\
\end{array}$ & $6: 30: 4$ & 29.563 & 25.396 & 31.1 & 26.004 & 25.081 & 62.53 & 68.425 & 25.912 & 37.505 & -0.193 & -6.22 & 54.635 & & 14.643 & & 30.0 & 0.5 & 67. & 13.6 & & 3.449 & & & & & \\
\hline 48 & $9 / 2 / 2004$ & $6: 31: 4$ & 29.624 & 25.403 & 31.165 & 25.986 & 25.1 & 62.813 & .779 & 25 & 37.9 & -0.176 & & 56 & & 14.6 & & 31.00 & 0.51667 & 67.7 & 13.5 & 50.4 & 43.474 & & 08 & 0.000 & 6 & 5 \\
\hline \begin{tabular}{|l|l|}
49 \\
\end{tabular} & 9//2/2004 & & 29.685 & 25.404 & 31.216 & 26.002 & 25.1 & & \begin{tabular}{|l|l|}
68.763 \\
\end{tabular} & & 38.2 & -0.297 & & 53.3 & & 14. & & 32.00 & 0.53333 & 67.6 & 12.7 & & 3.493 & & & 0.000 & 145 & \\
\hline 50 & $9 / / 2 / 2004$ & $6: 33: 42 \mathrm{AM}$ & 29.757 & 25.417 & 31.303 & 25.96 & 25. & & \begin{tabular}{|l|l|}
68.723 \\
\end{tabular} & 25.74 & 37.887 & -0.17 & & 46.43 & 0.0 & 14. & & 33.00 & 0.55 & 67.6 & 11.1 & & & & & & 0.162 & 1 \\
\hline 51 & 9//2/2004 & $6: 34: 42 \mathrm{AM}$ & 29.818 & 25.408 & 31.349 & 25.986 & 25.11 & & & & 901 & -0.234 & & 50.657 & 0.068 & $14.6 \mathrm{C}$ & & 34. & 0.56667 & 67.4 & 12.1 & & 60 & & & 00 & 163 & \\
\hline 52 & 9/2/2004 & 6:35:4 & 29.873 & 25.404 & 31.404 & 25.992 & 25.1 & 62.792 & 68.564 & 25 & 38.423 & -0.3 & & & & 14 & & 35.0 & \begin{tabular}{|l}
0.58333 \\
\end{tabular} & 67.4 & 12.6 & & 3.489 & & & & 0.161 & \\
\hline 5 & $\frac{91212004}{0121004}$ & $6: 30: 42$ AM & $\begin{array}{l}29.955 \\
32027\end{array}$ & $\frac{25.421}{25.43}$ & 3.1452 & $\frac{26.049}{26066}$ & 25514 & $\frac{62.616}{6.2076}$ & 68.47 & 25.122 & 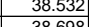 & -0.216 & 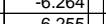 & 54.441 & 0.061 & 14.643 & & 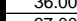 & 0.6 & 0 & 13.6 & 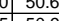 & & & & & & \\
\hline 55 & $\begin{aligned} 91212004 \\
9 / 2004\end{aligned}$ & $0.31 .42 \mathrm{~A}$ & S0.031 & 20.443 & S1.535 & 20.000 & & & & & 30.098 & $\begin{array}{l}-0.193 \\
0.172\end{array}$ & & 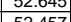 & & 14. & & 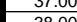 & 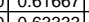 & & 年 & & & & & & 100 & \\
\hline 56 & $9 / 2 / 2004$ & & 30.005 & 20.414 & $\frac{31.01}{3.41}$ & 2.053 & & 62064 & \begin{tabular}{|l|l|l} 
\\
685056
\end{tabular} & & 3.04 & 0.173 & & & & & & 00.04 & 0.03030 & & 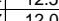 & & & & & & 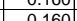 & \\
\hline \begin{tabular}{|l|l|}
57 \\
\end{tabular} & $9 / 2 / 2004$ & & 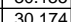 & 25.467 & 33,695 & & & & & & 38562 & 0.017 & & & & & & & & & 年 & & & & & & t57 & \\
\hline \begin{tabular}{|l|l|}
58 \\
\end{tabular} & 9/2/2004 & & 30.22 & 25.463 & 31726 & 26.081 & & & $\mid 68.584$ & $\frac{25.082}{25.082}$ & 38.669 & $\frac{-0.213}{-0.213}$ & & 53.281 & & 146 & & 41.00 & 0.68333 & & 127 & & & & & 000 & 159 & \\
\hline 59 & 9/2/2004 & 6:42:42 AM & 30.241 & 25.434 & 31.742 & 26.117 & $25.18 \mathrm{C}$ & 63.032 & 69.015 & 25.201 & 38.718 & -0.135 & -6.168 & 51.919 & 0.063 & $\begin{array}{l}14.643 \\
\end{array}$ & . & 42.0 & 0.1 & 67.9 & 12.4 & 50.9 & 3.508 & 0.009 & 0.007 & 0.000 & 0.147 & 0.13 \\
\hline 600 & & & & & & & & & & & & & & & & & & & & & & & & & & & & \\
\hline & & Averages & 29.1 & 25.4 & 30.6 & 25.9 & $25.0 \mid=$ & 62.2 & 68.7 & 25.2 & 37.8 & 0.4 & -5.1 & 52.9 & 0.1 & 14.6 & & & & 67.6 & 12.6 & 50.0 & 3.447 & 0.010 & 0.0 & 0.000 & 0.2 & \\
\hline & & |Maximum & 30.2 & & 31.7 & & & 63.4 & & & 38.8 & & & 56 & & & & & & & 13.5 & & & & & & 0.2 & \\
\hline 50 & & |Medial & 20.5 & 25 & & & & & & & & & & & & & & & & & 12.7 & & & & & & 0.2 & \\
\hline & & |Minimum & 27.8 & 25.3 & 28.9 & 25.5 & 24 & 58.0 & 67.6 & 22.7 & 34.7 & -0.3 & -6. & 46.4 & 0.1 & 14.6 & & & & 66.4 & 11.1 & $\begin{array}{l}1 \\
1\end{array}$ & 3.228 & 0.009 & 0.007 & 0.000 & 0.1 & \\
\hline & & & 1.555 & 0.122 & 1.677 & 0.369 & $0.277]$ & 3.157 & 0.712 & 1.195 & 2.506 & 2.796 & $5.077 \not \mid \#$ & 4.085 & 0.009 & 0.000 & 0.001 & & & 0.712 & 1.033 & 32.790 & 0.192 & 0.001 & 0.002 & 0.000 & 0.041 & \\
\hline 666 & Number & ff Points Used| & 35 & 35 & 35 & 35 & $35[\mathrm{c}$ & 35 & 35 & 35 & 35 & 35 & $35 \sqrt{0} \mathrm{C}-\mathrm{s}$ & 35 & 35 & 35 & 35 & & & 35 & 35 & 35 & 35 & 35 & 35 & 35 & 35 & 35 \\
\hline \begin{tabular}{|l|l|}
67 \\
68
\end{tabular} & & * Backpulse poir & ts in box & are not incl & & & & & & & & & & & & & & & & gige for & the las & $\frac{d}{s t} 10 \mathrm{~m}$ & inutes $=$ & $=0.0096$ & 0.0081 & $\mathrm{~m} / \mathrm{ft} 2$ & & \\
\hline \begin{tabular}{|l|}
69 \\
\end{tabular} & & & & & & & & & & & & & & & & & & & & & & & & $\begin{array}{ll}0.0007 \\
\end{array}$ & 0.0006 & $\mathrm{gpm} / \mathrm{tt} 2$ & & \\
\hline
\end{tabular}


WSRC-TR-2005-00105, REVISION 0 SRNL-RPP-2005-00012, REVISION 0

RUN \# 4.11; STEADY STATE TEST AT 25 WT\% UDS

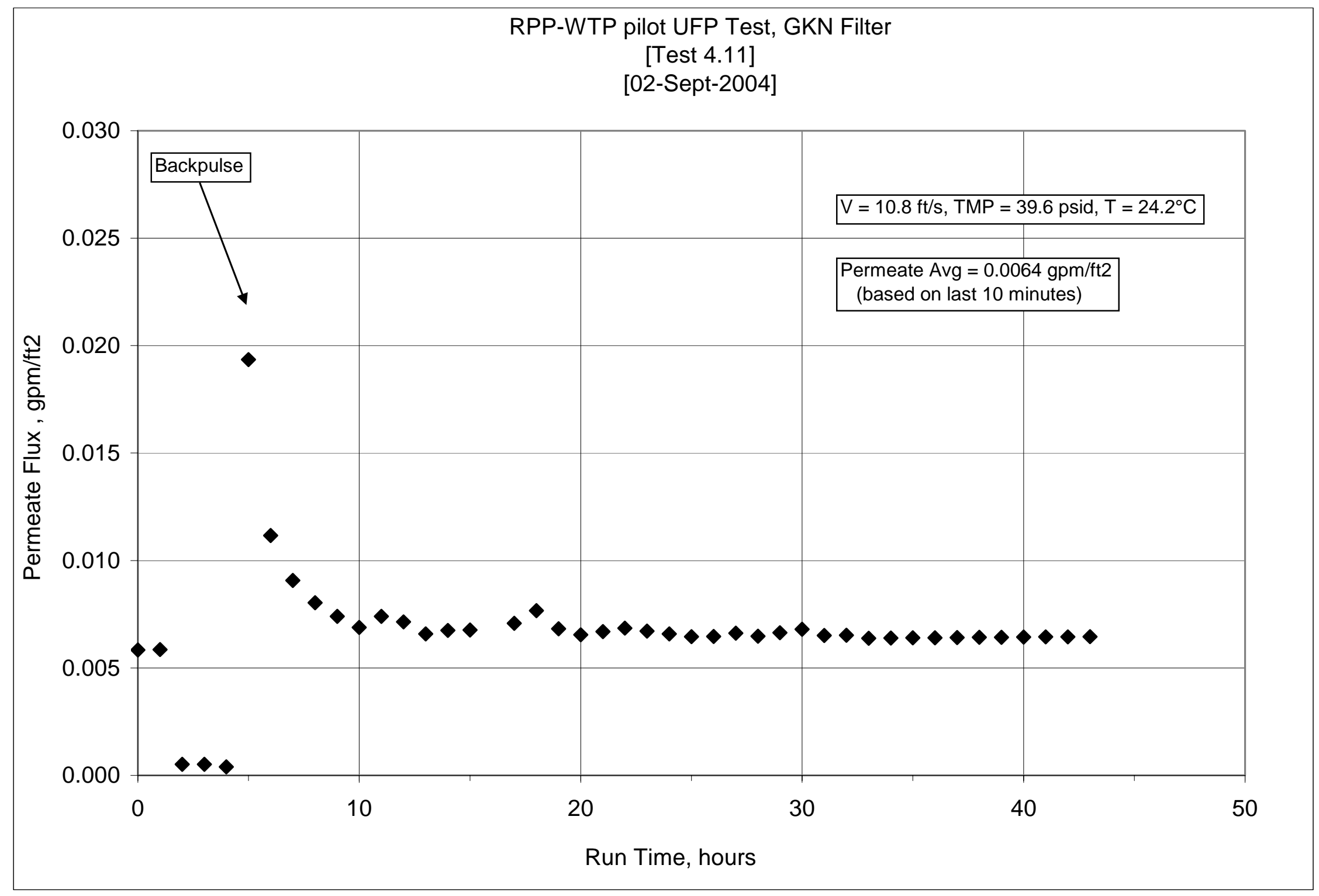


WSRC-TR-2005-00105, REVISION 0

SRNL-RPP-2005-00012, REVISION 0

RUN \# 4.11; STEADY STATE TEST AT 25 WT\% UDS

\begin{tabular}{|c|c|c|c|c|c|c|c|c|c|c|c|c|c|c|c|c|c|c|c|c|c|c|c|c|c|c|c|c|}
\hline & A & $B$ & 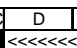 & 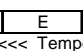 & \begin{tabular}{|l|}
$F$ \\
erature N
\end{tabular} & $\frac{G}{\text { easure }}$ & $\frac{H}{\text { ents } \gg \geqslant}$ & $\mathrm{J}$ & $\frac{K}{k \ll \ll<}$ & $\begin{array}{l}\text { L } \\
\text { Pressure }\end{array}$ & \begin{tabular}{c|}
$\mathrm{M}$ \\
easurement
\end{tabular} & \begin{tabular}{|l|}
$\mathrm{N}$ \\
$t \mathrm{ts} \gg \gg \gg{ }^{\prime}$
\end{tabular} & $\frac{O}{\partial>>>>}$ & \begin{tabular}{l|l} 
\\
$<\ll<<<<$
\end{tabular} & \begin{tabular}{l|}
$\mathrm{R} \mid$ \\
$\ll<$ Flow $\mathrm{M}$
\end{tabular} & $\frac{\mathrm{s}}{\text { Teasureme }}$ & $\frac{T}{1+15 \gg \gg}$ & $\frac{V}{\ll \ll<}$ & $\frac{w}{k \ll<\ll<s}$ & $\frac{1}{1<\ll<<c}$ & \begin{tabular}{|c|}
$\mathrm{Y}$ \\
alculate
\end{tabular} & \begin{tabular}{|l|}
$z$ \\
ed From
\end{tabular} & \begin{tabular}{|l|} 
AA \\
m Raw D
\end{tabular} & \begin{tabular}{|l|}
$A B$ \\
Data $\gg \gg$
\end{tabular} & $\frac{\mathrm{AC}}{\mid \mathrm{AC}_{1>\gg \gg \gg \gg}}$ & $A D$ & $\mathrm{AE}$ & AF \\
\hline & DATE & TIME & Filtrate & Cleaning & Slury & Hi Amb. & Lo Amb. & BotTMP & Filter & Filter dP & TOpTMP & Filtrate & Pulsepot & & Filtrate & Hi Filtate & Backpulse & & & & & & & & & & & \\
\hline & & & $\operatorname{deg} C$ & $\operatorname{deg} \mathrm{C}$ & $\operatorname{deg} C$ & $\operatorname{deg} \mathrm{C}$ & $\operatorname{deg} \mathrm{C}$ & psid & psig & psid & psid & psig & psig & $\mathrm{gpm}$ & $\mathrm{gpm}$ & gpm & $\mathrm{gpm}$ & & & & & & & & & & & \\
\hline & & & & & & & & $\mathrm{dP2}$ & & dP1 & $\mathrm{dP3}$ & & P3 & & & & & & & & & & & & & & & \\
\hline & eros- $04 / 19$ & & & & & & & & & & & & & & & & & & & & & 851 & & ay/lbarg & & & & \\
\hline & $8 / 23 / 2004$ & $8: 35: 38 \mathrm{AM}$ & 22.554 & & 22.002 & 22.782 & 22.204 & -0.019 & 0.095 & -0.012 & 0.003 & -0.124 & 0.137 & -0.075 & & $|14.643|$ & 0.004 & & & Vote: & & Sur & 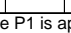 & oximatt & a & & & \\
\hline & $8 / 24 / 2004$ & 8:10:33 AM & 23.662 & 22.93 & \begin{tabular}{|l|}
23.679 \\
\end{tabular} & 22.998 & 22.735 & -0.021 & & -0.014 & & -0.124 & 0.134 & 23.176 & 1.205 & 14.643 & 0.003 & & & & & & & & & & & \\
\hline & $8 / 26 / 2004$ & 9:14:11 AM & & 23.352 & 24.932 & 25.316 & 23.242 & & & -0.008 & 0.011 & -0.106 & 0.091 & 48.335 & 0.043 & 14.643 & 0.003 & & & Pressur & re Plis c & correcte & ted for 3 & .25 inche & of water & & & \\
\hline & $8 / 27 / 2004$ & 7:53:29 AM & 22.649 & 22.89 & 22.298 & 22.872 & 22.871 & -0.017 & 0.14 & -0.008 & 0.005 & -0.161 & & -0.084 & 0.003 & & 0.005 & & & $\begin{array}{lll}\text { VVVV } \\
\end{array}$ & & & & $<$ Filtrate & & « PERN & ABILIT) & $\gg$ \\
\hline & $8 / 30 / 2004$ & 7:28:55 AM & 22.511 & 22.848 & 22.397 & 22.669 & 22.914 & -0.017 & 0.138 & -0.009 & 0.003 & -0.147 & 0.122 & -0.082 & 0.003 & 14.643 & 0.006 & & & & & & & & at $25 \mathrm{C}$ & & & \\
\hline 13 & & & & & & & & & & & & & & & & & & Time & \begin{tabular}{|l} 
Time \\
Hour
\end{tabular} & $\mid$\begin{tabular}{|l|} 
Press. \\
psig
\end{tabular} & Vel. & TMP & $\frac{\text { TMP }}{\text { bar }}$ & $\frac{g p m}{/ 1+2}$ & gpm & $\frac{\mathrm{gpm}}{\mathrm{ft} / \mathrm{si}}$ & pm & \begin{tabular}{|l|} 
meter \\
daybar
\end{tabular} \\
\hline 14 & $9 / 2 / 2004$ & $6: 55: 11 \mathrm{AM}$ & 29.37 & 25.452 & & & 24.832 & & & & & $\mid-0.349$ & & 45.707 & 0.046 & \begin{tabular}{|l|l|}
14.643 \\
\end{tabular} & 0.003 & & & & & & & & & & & \\
\hline & 9/2/2004 & 6:56:11 AM & 29.212 & 25.433 & 26.102 & 25.821 & 24.828 & 50.145 & 56.101 & 20.49 & 30.429 & -0.416 & & \begin{tabular}{|l|l|}
45.019 \\
\end{tabular} & 0.046 & \begin{tabular}{ll|}
14.643 \\
\end{tabular} & 0.003 & & 0.01667 & 55.0 & 10.7 & 40.3 & 2.778 & 0.007 & 0.006 & 0.000 & 0.145 & 0.12 \\
\hline & 9/2/2004 & 6:57:11 AM & 29.061 & 25.436 & \begin{tabular}{|l|l|}
26.03 \\
\end{tabular} & 25.879 & 24.871 & 47.171 & 55.933 & 20.351 & 27.587 & 2.702 & 21.39 & $\begin{array}{l}44.9 \\
\end{array}$ & 0.004 & 14.643 & 0.003 & 2.00 & 0.03333 & 54.8 & 10.7 & 37.4 & 2.577 & 0.001 & 0.001 & 0.000 & 0.014 & 0.01 \\
\hline & 9/2/2004 & 6:58:11 AM & 28.921 & $\begin{aligned} 25.44 \\
25.41\end{aligned}$ & \begin{tabular}{|l|l|}
25.919 \\
2591
\end{tabular} & 25.898 & $\begin{array}{l}24.92 \\
24.901\end{array}$ & 42.54 & $\begin{array}{l}55.939 \\
5.527\end{array}$ & 19.698 & 23.704 & 7.545 & 21.385 & 44.896 & 0.004 & $\begin{array}{ll}14.643 \\
1.612\end{array}$ & 0.003 & 3.00 & 0.05 & 54.8 & 10.7 & 33.1 & 2.284 & 0.001 & 0.001 & 0.000 & 0.016 & \\
\hline & $9 / 2 / 2004$ & 6:59:11 AM & 27.663 & $\begin{array}{r}25.441 \\
25.446\end{array}$ & & & $\frac{24.98}{24.94}$ & $\begin{array}{r}6.549 \\
45.688 \\
\end{array}$ & & $\begin{array}{r}10.89 \\
13.933\end{array}$ & & 41.771 & & $\frac{49.837}{47.83}$ & & \begin{tabular}{ll|l}
14.643 & 14.643 \\
\end{tabular} & & & $\begin{array}{l}.06667 \\
08232\end{array}$ & & & & & & & & & 0.24 \\
\hline 20 & $\begin{array}{l}9 / 2 / 2004 \\
9 / 2004\end{array}$ & $\begin{array}{l}7.0 .111 \mathrm{AM} \\
7: 01: 11 \mathrm{AM}\end{array}$ & $\frac{28.011}{28.103}$ & $\frac{25.440}{25.448}$ & \begin{tabular}{|l|l|}
25.643 \\
25
\end{tabular} & $\frac{23.924}{25.951}$ & $\begin{array}{l}24.941 \\
24.888\end{array}$ & $\frac{45.000}{46.76}$ & $\begin{array}{l}54.017 \\
55.498\end{array}$ & \begin{tabular}{|c|}
1.935 \\
16.37
\end{tabular} & $\frac{32.54 !}{31.217}$ & $\frac{3.5061}{2.751}$ & 0.30 & $\begin{array}{l}45.293 \\
45.292\end{array}$ & $\begin{array}{l}0.144 \\
0.085\end{array}$ & \begin{tabular}{|l|l|}
14.643 \\
14.643
\end{tabular} & 0.003 & 6.00 & $\frac{0.1}{0.1}$ & \begin{tabular}{|l|}
53.5 \\
5.4
\end{tabular} & 10.8 & \begin{tabular}{|l|}
39.1 \\
39.0 \\
\end{tabular} & \begin{tabular}{|l|l|} 
\\
\end{tabular} & $\frac{0.012}{0.012}$ & 0.011 & 0.000 & $\begin{array}{l}0.286 \\
0.286\end{array}$ & $\frac{0.42}{0.24}$ \\
\hline & 9/2/2004 & 7:02:11 AM & 28.061 & 25.431 & 25.535 & \begin{tabular}{|l|l|}
25.974 \\
\end{tabular} & 25.001 & 46.509 & 55.156 & \begin{tabular}{|l|l}
17.483 \\
\end{tabular} & \begin{tabular}{|l|}
29.812 \\
\end{tabular} & 2.638 & 0.45 & 44.46 & 0.069 & 14.643 & 0.003 & 7.00 & 0.11667 & 54.0 & 10.6 & 38.2 & 2.631 & 0.010 & 0.009 & 0.000 & 0.238 & 0.20 \\
\hline & $9 / 2 / 2004$ & 7:03:11 AM & 27.982 & 25.432 & 25.446 & 25.96 & 25.057 & 46.648 & 55.541 & 18.222 & 29.139 & 2.821 & 0.484 & 45.757 & 0.061 & 14.643 & 0.003 & 8.00 & 0.13333 & 54.4 & 10.9 & \begin{tabular}{|l|}
37.9 \\
\end{tabular} & 2.613 & 0.009 & 0.008 & 0.000 & 0.212 & 0.18 \\
\hline & $9 / 2 / 2004$ & 7:04:11 AM & $\begin{array}{l}27.871 \\
2789\end{array}$ & $\begin{array}{l}25.406 \\
2.522\end{array}$ & \begin{tabular}{|l|}
25.315 \\
25262
\end{tabular} & 25.929 & 25.056 & \begin{tabular}{|l|l|}
47.071 \\
7530
\end{tabular} & \begin{tabular}{|l|l|}
56.011 \\
5.20
\end{tabular} & $\begin{array}{l}19.016 \\
1.071\end{array}$ & \begin{tabular}{l|l|}
28.861 \\
28.817
\end{tabular} & \begin{tabular}{|l|l|}
2.751 \\
\end{tabular} & & \begin{tabular}{|l|l|l|}
4578 \\
\end{tabular} & $\begin{array}{l}0.056 \\
.0052\end{array}$ & \begin{tabular}{|l|l|}
14.643 \\
\end{tabular} & 0.003 & 9.00 & 0.15 & $\begin{array}{l}54.9 \\
5.2\end{array}$ & 10.8 & 38.0 & 2.618 & 0.008 & $\begin{array}{l}0.007 \\
\end{array}$ & 0.000 & 0.195 & 0.17 \\
\hline & $9 / 2 / 2004$ & 7:05:11 AM & & $\frac{25.433}{25.461}$ & \begin{tabular}{|l|l|}
25.262 \\
25.195
\end{tabular} & $\frac{25.946}{25.029}$ & $\frac{25.048}{25046}$ & $\begin{array}{l}47.538 \\
7561\end{array}$ & \begin{tabular}{|l|}
56.304 \\
56.287 \\
\end{tabular} & $\frac{19.871}{20119}$ & 28.402 & $\frac{2.702}{2770}$ & & 44.777 & 0.052 & $\frac{14.643}{11.643}$ & & $\frac{1.6}{110}$ & $\frac{0.16661}{0.1022}$ & 55.2 & & & & & & & & 0.15 \\
\hline $\begin{array}{l}26 \\
26\end{array}$ & 9/2/2/2004 & $\begin{array}{l}\text { 7:06:11 AM } \\
\text { 7:07:11 }\end{array}$ & 27.647 & $\begin{array}{l}25.461 \\
25.473\end{array}$ & \begin{tabular}{|l|}
25.185 \\
25.097 \\
\end{tabular} & $\begin{array}{l}25.939 \\
25.951\end{array}$ & $\begin{array}{l}25.046 \\
25.013\end{array}$ & \begin{tabular}{|c|c|}
47.661 \\
47.36
\end{tabular} & & 20.119 & 28.1669 & $\frac{2.702}{2.702}$ & & $\begin{array}{r}44.8 \\
43675\end{array}$ & & & & & 0.18333 & & & & & & & & & 0.17 \\
\hline & 9/2/2004 & 7:08:11 AM & 27.555 & 25.479 & \begin{tabular}{|l|l|}
25.012 \\
\end{tabular} & 25.977 & 25.004 & 47.459 & 50.140 & $\frac{20.074}{20.524}$ & 27.634 & 2.702 & 0.5 & 44.508 & 0.006 & \begin{tabular}{|l|l|}
14.6453 \\
14.643
\end{tabular} & 0.003 & 13.00 & $\mid 0.21667$ & 55.1 & \begin{tabular}{|l|}
10.6 \\
10.6
\end{tabular} & \begin{tabular}{|l|}
37.5 \\
\end{tabular} & $\frac{2.000}{2.589}$ & 0.007 & $\begin{array}{l}0.007 \\
0.00\end{array}$ & o.000 & 0 & $\frac{0.16}{0.15}$ \\
\hline 28 & $9 / 2 / 2004$ & 7:09:11 AM & 27.447 & 25.465 & 24.908 & 25.953 & 24.98 & 47.669 & \begin{tabular}{|l|l|}
56.581 \\
\end{tabular} & 20.637 & 27.768 & 2.702 & 0.58 & 44.579 & 0.047 & 14.643 & 0.003 & 14.00 & 0.23333 & 55.5 & 10.6 & \begin{tabular}{|l|} 
\\
\end{tabular} & 2.601 & 0.007 & 0.007 & 0.000 & 0.179 & 0.15 \\
\hline 29 & 9/2/2004 & $7: 10: 11 \mathrm{AM}$ & 27.338 & 25.461 & \begin{tabular}{|l|l|}
24.814 \\
\end{tabular} & 25.959 & 24.966 & $\begin{array}{l}47.486 \\
\end{array}$ & \begin{tabular}{|r|}
56.25 \\
\end{tabular} & $\begin{array}{l}20.765 \\
203\end{array}$ & \begin{tabular}{|l|l|}
27.564 \\
2707
\end{tabular} & \begin{tabular}{|l|}
2.699 \\
\end{tabular} & 0.6 & \begin{tabular}{|l|l|l|}
4.27 \\
\end{tabular} & $\begin{array}{l}0.047 \\
\end{array}$ & \begin{tabular}{|l|l|}
14.643 \\
\end{tabular} & 0.003 & 15.00 & 0.25 & 55.1 & 10.5 & 37.5 & 2.587 & 0.007 & 0.007 & 0.000 & 0.180 & 0.15 \\
\hline & $\frac{9 / 2 / 2004}{9 / 2 / 2004}$ & $7: 11: 11 \mathrm{AM}$ & 27.244 & 25.457 & \begin{tabular}{|l|}
24.735 \\
20.1641
\end{tabular} & $\frac{25.979}{25.092}$ & $\frac{24.987}{24.961}$ & & \begin{tabular}{|l|}
56.032 \\
56.023 \\
\end{tabular} & & & & & $\begin{array}{l}44.627 \\
15.5234\end{array}$ & & & & & \begin{tabular}{|l|l|} 
\\
\end{tabular} & & & \begin{tabular}{|l|}
39.3 \\
\end{tabular} & & & & 0.001 & $\begin{array}{l}0.770 \\
01709\end{array}$ & 0.66 \\
\hline & $9 / 2 / 2004$ & 7:12:11 AM & 27.123 & $\begin{array}{r}25.44 \\
25.451\end{array}$ & \begin{tabular}{|l|}
24.664 \\
22.609
\end{tabular} & \begin{tabular}{l|l}
25.993 \\
260009
\end{tabular} & 24.961 & $\begin{array}{r}50.27 \\
55218\end{array}$ & \begin{tabular}{|r|}
56.003 \\
5555
\end{tabular} & $\begin{array}{r}21.692 \\
2157\end{array}$ & \begin{tabular}{l|l|}
29.372 & \\
20.436
\end{tabular} & $\begin{array}{r}-0.645 \\
\end{array}$ & & 45.534 & 0.049 & $\begin{array}{r}14.643 \\
16443\end{array}$ & & 17.00 & 0.28333 & 54.9 & 10.8 & \begin{tabular}{|l|}
39.8 \\
39.8
\end{tabular} & 2.746 & 0.007 & 0.007 & 0.000 & 0.178 & 0.15 \\
\hline 33 & $\begin{array}{l}9 / 2 / 2004 \\
9 / 2 / 2004\end{array}$ & $\begin{array}{l}7: 13: 11 \mathrm{~A} \\
7: 14: 11 \mathrm{AM}\end{array}$ & 27.059 & $\begin{array}{l}25.451 \\
25.423\end{array}$ & \begin{tabular}{|l|}
24.5001 \\
24.501
\end{tabular} & $\begin{array}{l}26.009 \\
25.951\end{array}$ & $\frac{24.991}{24.948}$ & $\begin{array}{r}50.218 \\
50.44\end{array}$ & \begin{tabular}{|l|}
55.552 \\
55.92 \\
\end{tabular} & $\begin{aligned} 21.57 \\
21.293\end{aligned}$ & 29.436 & $\begin{array}{l}-0.841 \\
-0.879 \\
\end{array}$ & & $\begin{array}{l}45.494 \\
45.384\end{array}$ & $\mid \begin{array}{l}0.053 \\
0.047\end{array}$ & $\frac{14.643}{14.643}$ & & $\frac{18.00}{1900}$ & $\begin{array}{r}0.3 \\
0.31667\end{array}$ & $\begin{array}{l}54.4 \\
54.8 \\
\end{array}$ & $\frac{10.8}{10.8}$ & $\begin{array}{l}39.8 \\
40.1\end{array}$ & 2.746 & & & & 193 & $\frac{0.16}{0.14}$ \\
\hline & 9/2/2004 & 7:15:11 AM & 26.888 & 25.425 & 24.457 & 25.978 & 24.95 & 50.425 & 55.798 & 21.372 & 30.035 & -0.928 & & 46.204 & 0.045 & 14.643 & 200 & 20.00 & 0.3333 & 54.7 & $11.6 \quad \mathrm{C}$ & 40 & 774 & .006 & & 0.000 & D.163 & $\frac{0.14}{0.14}$ \\
\hline & $9 / 2 / 2004$ & $7: 16: 11 \mathrm{AM}$ & & 25.436 & & 25.954 & & & & & & & & 47.701 & & & & & & & & & & & & & & \\
\hline 36 & & 7:17:11 AM & 26.731 & 25.417 & & 25.865 & 24.90 & 50.757 & 56.132 & 21.4 & 29.937 & -0.943 & & & 0 & 14.6 & & & $\frac{1.66}{3667}$ & 55.0 & & $\frac{50}{40}$ & 70 & 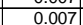 & & 2000 & 1770 & \\
\hline & 9/2/2004 & $1 \mathrm{AM}$ & 26.659 & 25.425 & \begin{tabular}{|l|l|}
24.292 \\
\end{tabular} & 25.853 & 24.8 & 50.57 & \begin{tabular}{|c|}
55.74 \\
\end{tabular} & 21.321 & 30.136 & $\mid-0.957$ & & 45.926 & $\begin{array}{ll}0.046 \\
\end{array}$ & 14 & & 23. & 0.38333 & 54.6 & 10. & 40. & 2.78 & & & & 166 & 0. \\
\hline & 9/2/2004 & 7:19:11 AM & 26.576 & 25.422 & & 25.785 & & & \begin{tabular}{|l|l|}
55.806 \\
\end{tabular} & 21.41 & 29.863 & & & 455.204 & & 14 & & 24. & & 54.7 & & & & & & & & \\
\hline 39 & 9/2/2004 & 7:20:11 AM & 26.499 & 25.415 & 24.147 & 25.757 & 24.805 & 50.516 & \begin{tabular}{|l|l|}
55.757 \\
\end{tabular} & 21.614 & 29.727 & \begin{tabular}{|c|}
-1.009 \\
\end{tabular} & & 44.998 & \begin{tabular}{|c|}
0.044 \\
\end{tabular} & & & 250 & 0.41667 & 54.6 & 10 & 40. & 765 & & & & & 0.14 \\
\hline 40 & 9/2/2004 & 7:21:11 AM & 26.42 & 25.396 & 24.093 & 25.774 & & 50.583 & 55.902 & 21.527 & 30 & -0.986 & & & 0.0 & & & 26. & 0.43333 & 54.8 & & & & & & & & \\
\hline & 9/2/2004 & $1 \mathrm{AM}$ & 26.357] & 25 & & 25.795| & & & 55.653 & 21.391. & & -0.98 & & & & & & 27. & 0.45 & 54 & & & & & & & & \\
\hline & $9 / 2 / 2004$ & 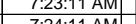 & 26.294 & & 23. & 25.777 & & & & & & & & & & & & & 0.46667 & & 10 & & & & & & & \\
\hline & & $\frac{T .24 .11 \mathrm{AM}}{2.5 .11}$ & 2 & & & 25.1.899 & & & 53.6 & 2 & & & & & & & & & & & & & & & & & & \\
\hline (4) & $\begin{array}{l}9 / 2 / 2004 \\
9 / 2 / 2004\end{array}$ & $\frac{1.25 .111 \mathrm{AN}}{7.26}$ & $\frac{2.11 / 1}{26.77}$ & $\begin{array}{l}\frac{25.350}{25397} \\
2.07\end{array}$ & 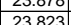 & | & $\frac{24.15}{2478}$ & 50. & 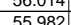 & $\frac{21.009}{20.622}$ & 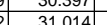 & $\begin{array}{l}=-.9060 \\
-0.06\end{array}$ & & 年 45.254 & $\mid$ & & & . & 0,51667 & $\begin{array}{ll}54.9 \\
54.9\end{array}$ & 10 & & T & & & & & \\
\hline & $9 / 2 / 2004$ & $7.5 .7 .11 \mathrm{AM}$ & 26.052 & 25397 & & 25734 & & 50.454 & & & & & & & & & & & & & & & & & & & & \\
\hline+0 & $9 / 2 / 2004$ & $7 \cdot 728.11 \mathrm{AM}$ & 26.012 & 25402 & 2378 & 25769 & 2470 & 409 & 55054 & 21128 & & & & & 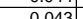 & & & & 0 & . & & & & & & & & \\
\hline & & $77^{7} 2 \cdot 1 \cdot \mathrm{AM}$ & 250 & & & & & 50.317 & & & & & & & & & & & & & & & & & & & & \\
\hline & $9 / 2 / 2004$ & $7 \cdot 30 \cdot 11 \mathrm{AM}$ & 25.885 & 25 & 23.621 & 25.683 & & & 55.5 & 21.5 & & & & 45.4 & & & & 35 & 0.58333 & & & & & & & & & \\
\hline & $9 / 2 / 2004$ & $7: 31:$ & 25.85 & 25.3 & 23 & 25.743 & 24 & 50. & 55.4 & 21.3 & 29.7 & & & & & & & 36 & & & & & & & & & & \\
\hline & $9 / 2 / 2004$ & $7: 32: 1$ & 25.788 & 25.383 & 23. & 25.726 & 24.91 & 49.987 & 55.1 & 21.405 & 29.481 & -1.021 & & 444.191 & & & & 37 & 0.61667 & 4.0 & & & & & & & & \\
\hline & 9/2/2004 & $1 \mathrm{AM}$ & 25.736 & $25.3 / 6$ & 3.516 & 25.703 & $24.8 \mathrm{r}$ & 50.35 & 55.68 & 21.289 & & & & 46.226 & & & & & & 54.6 & & & & & & & & \\
\hline & & 7:34: & 25.694| & & & 25.696 & & & & & & & & & & & & & & & & & & & & & & \\
\hline & 9//2/2004 & 7:35:1 & 25.651 & 25.381 & 23.436 & 25.638 & & & & & & & & & & & & & & & & & & & & & & \\
\hline & $\frac{9 / 212004}{9 / 2004}$ & & 25.592 & 255361 & \begin{tabular}{|l|l|l}
23.382 \\
\end{tabular} & 25.56 & & & & & & & & & & & & & & & & & & & & & & \\
\hline & $\begin{array}{l}91212004 \\
9 / 2 / 2004\end{array}$ & & $\begin{array}{l}2.5340 \\
2549\end{array}$ & $\frac{25.31}{2536}$ & & & & & & & & & & & & & & & & & & & & & & & & \\
\hline & $9 / 2 / 2004$ & & $25.449]$ & & & & & & & & & & & & & & & & & & & & & & & & & \\
\hline & 9/2/2004 & $7: 40: 11 \mathrm{~A}$ & 25.414 & 25.359 & 23.244 & 25.472 & 24.44 & 50.477 & 55.155 & 21.489 & 29.908 & & & 45.342 & .042 & 14.643 & & & & 54.0 & & & & & & & 0.157 & \\
\hline & & & & & & & & & & & & & & & & & & & & & & & & & & & & \\
\hline & & Averages & 26.6 & 25.4 & 24.2 & 25.8 & 24.8 & 49.6 & 55.8 & 20.8 & 29.6 & 0.0 & -4.5 & 45.3 & 0.1 & 14.6 & 0.0 & & & 54.7 & 10.8 & 39.6 & \begin{tabular}{|l|l|} 
& 2.7 \\
\end{tabular} & 0.0 & 0.0 & 0.0 & 0.2 & \\
\hline 63 & & Maximu & 28.1 & $\begin{array}{r}25.5 \\
254 \\
54\end{array}$ & $\frac{2}{2}$ & $\begin{array}{r}26.0 \\
258 \\
\end{array}$ & & $\frac{50.9}{50.4}$ & & & 31 & & & & & & & & & 547 & & & & & & & $\frac{0.8}{0.2}$ & \\
\hline 64 & & & 25.4 & $\begin{array}{l}25.4 \\
25.4\end{array}$ & $\frac{2.4 .1}{23.2}$ & 25.5 & $\frac{2.34}{24.4 \#}$ & 46.5 & $\begin{array}{l}35.0 \\
54.6\end{array}$ & \begin{tabular}{c|c|c|c|}
16.4 \\
\end{tabular} & 27.6 & -1.0 & -6. & $\begin{array}{l}45.4 \\
43.7 \\
\end{array}$ & 0.0 & $\begin{array}{l}14.6 \\
14.6 \\
\end{array}$ & & & & $\frac{54.1}{53.5}$ & \begin{tabular}{|l|}
10.8 \\
10.4
\end{tabular} & \begin{tabular}{|l|l|}
40.1 \\
37.5 \\
\end{tabular} & $\begin{array}{l}2.8 \\
2.6\end{array}$ & $\frac{0.0}{0.0}$ & 0.0 & $\frac{0.0}{0.0}$ & $\frac{0.2}{0.2}$ & \\
\hline 65 & & $2 \times \operatorname{Std}$ Dev & 1.648 & & 1.402 & 0.302 & 0.319 & 2.853 & 0.764 & 2.290 & 1.789 & 3.184 & 5.96 & 1.397 & 0.053 & 0.000 & 0.00 & & & 0.764 & \begin{tabular}{|l|} 
\\
\end{tabular} & 3.097 & 0.145 & 0.008 & 0.008 & 0.000 & 0.193 & \\
\hline 66 & Number c & of Points Used ${ }^{\star}$ & 40 & 40 & 40 & 40 & $40 \mathrm{dc}$ & 40 & 40 & 40 & 40 & 40 & $40-2$ & 40 & 40 & 40 & 40 & & & 40 & 40 & 40 & 40 & 40 & 40 & 40 & 40 & \\
\hline & & ${ }^{*}$ Backpulse poin & ts in box a & not inc & & & & & & & & & & & & & & & & & & & & & & & & \\
\hline$\frac{68}{69}$ & & & & & & & & & & & & & & & & & & & & & $\times$ XStandar & ard Devi & viation $=$ & $\mid 0.0003$ & \begin{tabular}{|l|l|}
3 & 0.0002 \\
\end{tabular} & $\frac{9 p m / t / 2}{g p m / t i 2}$ & & \\
\hline
\end{tabular}


WSRC-TR-2005-00105, REVISION 0

SRNL-RPP-2005-00012, REVISION 0

RUN \# STEADY5; STEADY STATE TEST AT 25 WT\% UDS

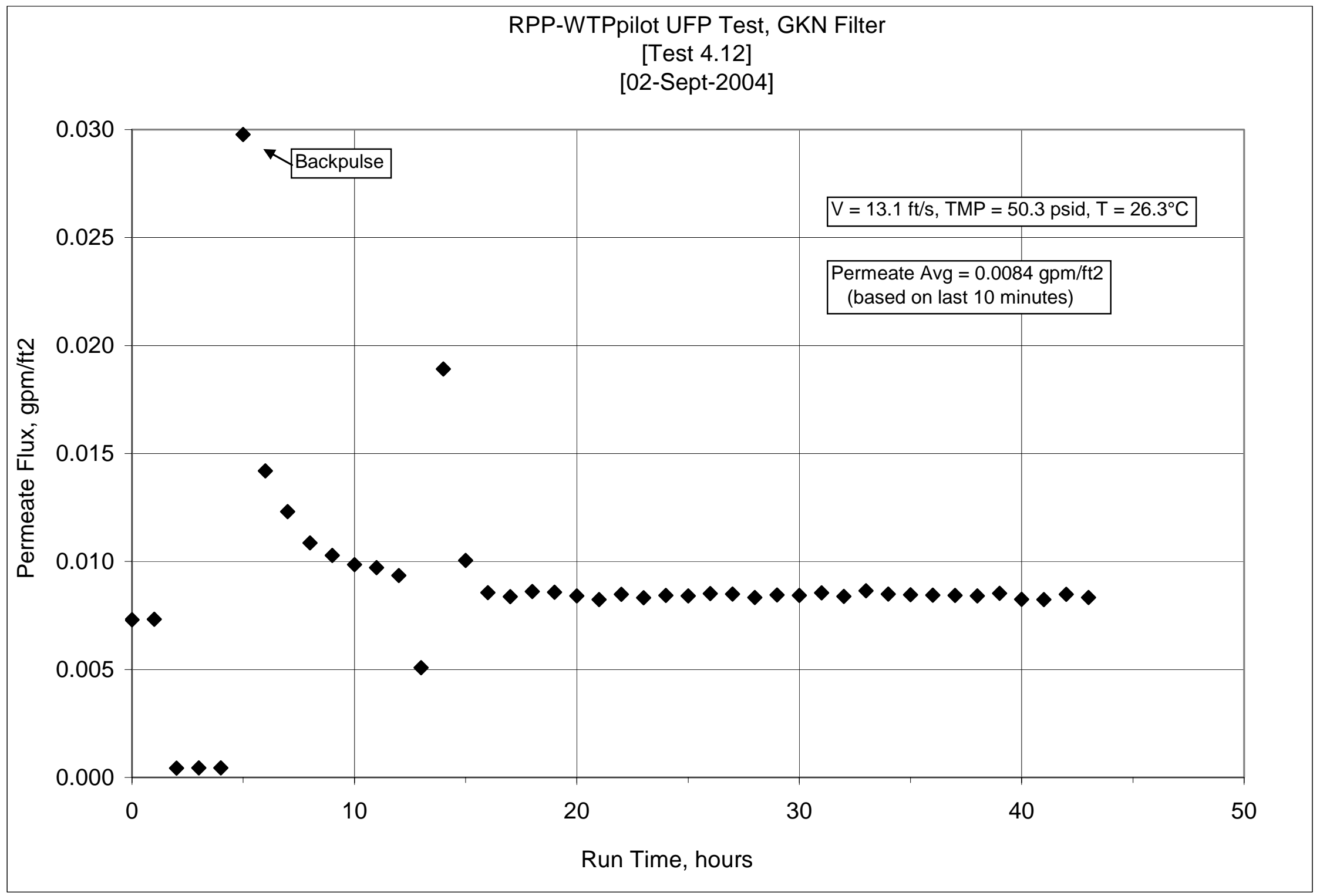


WSRC-TR-2005-00105, REVISION 0

SRNL-RPP-2005-00012, REVISION 0

RUN \# STEADY5; STEADY STATE TEST AT 25 WT\% UDS

\begin{tabular}{|c|c|c|c|c|c|c|c|c|c|c|c|c|c|c|c|c|c|c|c|c|c|c|c|c|c|c|c|c|}
\hline & A & B & D & $\frac{\mathrm{E}}{\mathrm{T} \text { Ter }}$ & & & & $\mathrm{J}$ & $\frac{K}{\ll \ll<F}$ & $\frac{L}{\text { ssur }}$ & Isuren & $\frac{\mathrm{N}}{323}$ & 0 & $\frac{Q}{2<\ll<<}$ & $\frac{\mathrm{R}}{\ll \text { Flon }}$ & \begin{tabular}{c|}
$\mathrm{s}$ \\
$\mathrm{s}$
\end{tabular} & T & $\mathrm{v}$ & $\frac{W}{W}$ & $x$ & $\mathrm{Y}$ & & AA & $\frac{A B}{\text { ta } \gg \geq}$ & $A C$ & $\frac{A D}{2>27}$ & $\mathrm{AE}$ & AF \\
\hline & DATE & TIME & rate IC & Cleaning & \begin{tabular}{|l|l|} 
Slurry \\
\end{tabular} & Hi Amb. L & Lo Amb. & BotTMP & Filter & Filter dP & \begin{tabular}{|l|} 
TopTMP \\
\end{tabular} & \begin{tabular}{|l|} 
Filtrate \\
\end{tabular} & ilsepot & & Filtrate & Hi Filtate & Backpulse & & & & & & & & & & & \\
\hline & & & $\frac{\operatorname{deg} C}{\operatorname{den}}$ & $\operatorname{deg} C$ & \begin{tabular}{|l|}
$\operatorname{deg} C$ \\
\end{tabular} & \begin{tabular}{|l|l|}
$\operatorname{deg} \mathrm{C}$ \\
\end{tabular} & $\operatorname{deg} C$ & psid & psig & psid & \begin{tabular}{|c|} 
psid \\
\end{tabular} & \begin{tabular}{|l|} 
psig \\
\end{tabular} & psig & gpm & gpm & \begin{tabular}{l|l}
$\mathrm{gpm}$ \\
\end{tabular} & & & & & & & & & & & & \\
\hline & & & T2 & & & & & & & & & & & & Q2 & & & & & & & & & & & & & \\
\hline & & & & & & & & & & & & & & & & & & & & & & & & ylbarg & & $\mathrm{rg}$ & & \\
\hline & 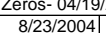 & & & & & & & & & & & & & & & & & & & & & & & & & & & \\
\hline & $8 / 24 / 2004$ & $\begin{array}{l}8: 355: 38 \mathrm{AM} \\
8: 10: 33 \mathrm{AM}\end{array}$ & $\frac{22.564}{23.662}$ & $\frac{22.064}{22.93}$ & & \begin{tabular}{|c|c|}
2.92 \\
2.998
\end{tabular} & $\frac{204}{735}$ & $\frac{-0.01}{-0.02}$ & $\frac{0.095}{0.122}$ & $\begin{array}{l}-0.012 \\
-0.014 \\
-0.0\end{array}$ & & & & 3.176 & 1.205 & $\begin{array}{l}14.643 \\
14.643 \\
\end{array}$ & $\frac{0.004}{0.003}$ & & & & & & & & $\mid y 1.1$ & & & \\
\hline & $8 / 26 / 2004$ & $9: 14: 11 \mathrm{AM}$ & $\frac{2.006}{25.819}$ & $\frac{23.352}{23.352}$ & \begin{tabular}{|l|}
24.932 \\
\end{tabular} & \begin{tabular}{|l|}
25.316 \\
\end{tabular} & 23.242 & -0.008 & o.126 & - & $\begin{array}{l}-0.001 \\
0.011\end{array}$ & $\frac{-.124}{-0.106}$ & & $\frac{2.116}{48.335}$ & $\begin{array}{ll}0.043 \\
\end{array}$ & $\begin{array}{l}14.043 \\
14.643\end{array}$ & $\frac{0.003}{0.003}$ & & & & & & for 3 & $1.25 \mathrm{inc}$ & ff wate & & & \\
\hline & $8 / 27 / 2004$ & 7:53:29 AM & 22.649 & \begin{tabular}{|l|l|}
22.89 \\
\end{tabular} & \begin{tabular}{|l|}
22.298 \\
\end{tabular} & 22.872 & 22.871 & $\mid-0.017$ & $\begin{array}{l}0.14 \\
\end{array}$ & $\mid-0.008$ & \begin{tabular}{|c|c|}
0.005 \\
\end{tabular} & -0.161 & 0.111 & -0.084 & \begin{tabular}{|l|l|l|l|} 
\\
\end{tabular} & 14.643 & 0.005 & & & VWVV & & & & < Filtrate & & 《 PERM & & $>>$ \\
\hline & $8 / 30 / 2004$ & 7:28:55 AM & 22.511 & 22.848 & \begin{tabular}{|l|l|}
22.397 \\
\end{tabular} & 22.669 & 22.914 & -0.017 & $\begin{array}{l}0.138 \\
\end{array}$ & \begin{tabular}{|c|}
-0.009 \\
\end{tabular} & $\begin{array}{ll}0.003 \\
\end{array}$ & -0.147 & 0.122 & -0.082 & 0.003 & 14.643 & 0.006 & & & & & & & & \begin{tabular}{|l|l|} 
at 25c \\
\end{tabular} & & $\begin{array}{l}1000 \\
1000\end{array}$ & \\
\hline & & & & & & & & & & & & & & & & & & ime & me & ress. & el. & IMP & MP & $\mathrm{pm}$ & & & pm & \\
\hline & Data - Per Mi & & & & & & & & & & & & & & & & & & & $\frac{s i g}{69.0}$ & & $\frac{s i}{50.4}$ & $\begin{array}{l}\frac{a r}{3.477} \\
30\end{array}$ & & & $\frac{\mathrm{t} / \mathrm{psi}}{0.000}$ & t2/psi & ay/bar \\
\hline & 9/2/2004 & 7:51:38 AM & 24.919 & 25.323 & \begin{tabular}{|l|}
21.192 \\
\end{tabular} & 25.481 & 24.619 & 64.947 & 70.129 & & 535 & -0.928 & & 53.081 & 0.051 & 14.643 & & 1.00 & 0.01667 & 69.0 & & 502 & 3.464 & 0.007 & & 0 & $\begin{array}{l}0.145 \\
0.146 \\
\end{array}$ & 0.12 \\
\hline & 9/2/2004 & $7: 52: 38$ AM & & 25.313 & \begin{tabular}{|l|}
21.574 \\
\end{tabular} & 25.456 & & \begin{tabular}{|c|}
61.914 \\
\end{tabular} & $\begin{array}{l}70.75 \\
\end{array}$ & & 32.465 & \begin{tabular}{|l|}
2.818 \\
\end{tabular} & & $\begin{array}{l}50.89 \\
\end{array}$ & & & & & & 69.6 & & & & & & & & \\
\hline & 9/2/2004 & 7:53:38 AM & 24.045 & 25.33 & \begin{tabular}{|l|l|}
21.974 \\
\end{tabular} & 25.503 & 24.616 & $\begin{array}{l}54.988 \\
\end{array}$ & 70.929 & 26.093 & 26.048 & \begin{tabular}{|l|l|}
10.139 \\
\end{tabular} & 20.301 & 53.256 & $\begin{array}{ll}0.003 \\
\end{array}$ & $\begin{array}{l}14.643 \\
\end{array}$ & 0.003 & 3.00 & 0.05 & \begin{tabular}{ll|}
69.8 \\
\end{tabular} & $12.7 \mathrm{r}$ & 40.5 & 2.794 & 0.000 & 0.000 & 0.000 & 0.011 & 0.01 \\
\hline & 9/2/2004 & 7:54:38 AM & \begin{tabular}{|l|}
24.341 \\
\end{tabular} & 25.316 & \begin{tabular}{|l|}
22.261 \\
\end{tabular} & 25.509 & 24.677 & \begin{tabular}{|l|l|l|}
43.148 \\
\end{tabular} & 70.568 & 26.093 & 14.892 & \begin{tabular}{|l|}
21.708 \\
\end{tabular} & 114.028 & 56.399 & 0.003 & \begin{tabular}{ll|}
14.643 \\
\end{tabular} & & 4.00 & 0.06667 & 69.4 & 13.4 & & 2.001 & & & & & \\
\hline & $9 / 2 / 2004$ & 7:55:38 AM & \begin{tabular}{|l|}
24.663 \\
2.575
\end{tabular} & $\begin{array}{l}25.318 \\
25320\end{array}$ & \begin{tabular}{|r|}
22.56 \\
2202
\end{tabular} & \begin{tabular}{|l|l|}
25.531 \\
25517
\end{tabular} & $\begin{array}{l}24.759 \\
2.625\end{array}$ & & $\begin{array}{ll}67.938 \\
\end{array}$ & \begin{tabular}{|l|l|}
18.171 \\
21300
\end{tabular} & \begin{tabular}{|l|l|}
41.269 \\
\end{tabular} & \begin{tabular}{|r|}
3.238 \\
\end{tabular} & 0.351 & 56.637 & $\begin{array}{ll}0.206 \\
\end{array}$ & \begin{tabular}{|l|l|}
14.643 \\
\end{tabular} & 0.0 & 5.00 & 0.08333 & $\begin{array}{ll}66.8 \\
6.8\end{array}$ & & 50.0 & $\begin{array}{l}3.444 \\
.42\end{array}$ & 0.029 & 0.030 & 0.001 & & \\
\hline & $\begin{array}{l}9 / 2 / 2004 \\
90 / 2 / 2004\end{array}$ & 7:56:38 AM & $\begin{array}{r}24.575 \\
24601\end{array}$ & $\begin{array}{l}25.305 \\
25335\end{array}$ & \begin{tabular}{|l|}
22.803 \\
23085 \\
\end{tabular} & $\begin{array}{l}25.517 \\
25.518\end{array}$ & \begin{tabular}{|l|}
24.635 \\
24666
\end{tabular} & \begin{tabular}{|c|}
61.413 \\
61.654 \\
\end{tabular} & \begin{tabular}{|r|}
68.64 \\
68.893
\end{tabular} & \begin{tabular}{|l|l|}
21.329 \\
23.336
\end{tabular} & \begin{tabular}{|l|}
40.851 \\
39.055
\end{tabular} & $\begin{array}{l}1.006 \\
1.246\end{array}$ & 0.391 & \begin{tabular}{|l|l|l|}
57.136 \\
56.05
\end{tabular} & \begin{tabular}{|c|c|}
0.098 \\
0.055
\end{tabular} & & 0.003 & $\begin{array}{ll}6.00 \\
770\end{array}$ & $\begin{array}{r}0.1 \\
\end{array}$ & $\begin{array}{l}67.5 \\
67.8\end{array}$ & & 51.1 & \begin{tabular}{|l|l}
3.525 \\
3 \\
3.471
\end{tabular} & 0.014 & $\begin{array}{l}0.014 \\
0.012\end{array}$ & 0.000 & $\begin{array}{l}0.278 \\
0244\end{array}$ & 0.24 \\
\hline & $\frac{9 / 2 / 2004}{9 / 2 / 2004}$ & 7:57:38 AM & \begin{tabular}{|l|}
24.601 \\
2587
\end{tabular} & $\frac{25.315}{25337}$ & & $\frac{25.518}{25.51}$ & & \begin{tabular}{|c|}
61.645 \\
62402
\end{tabular} & \begin{tabular}{|c|c|}
68.893 \\
6978
\end{tabular} & \begin{tabular}{|l|l|}
23.336 \\
24299
\end{tabular} & & $\begin{array}{l}1.246 \\
1.776\end{array}$ & & & & $\frac{14.643}{14643}$ & & 7.00 & 0.11667 & $\begin{array}{l}67.8 \\
6.77\end{array}$ & \begin{tabular}{|l|l}
13.3 \\
129
\end{tabular} & 50.4 & $\begin{array}{l}3.471 \\
3.491 \\
\end{array}$ & 0.012 & 0.012 & & $\frac{0.244}{0.215}$ & 0.21 \\
\hline & \begin{tabular}{|l|l|l|l|l|}
$9 / 212004$ \\
\end{tabular} & $\begin{array}{l}7.58: 38 \mathrm{AM} \\
7759: 38 \mathrm{Am}\end{array}$ & $\frac{24.587}{24.574}$ & $\begin{array}{l}25.307 \\
25.309\end{array}$ & $\mid$\begin{tabular}{|l|}
2.33232 \\
23.565
\end{tabular} & $\begin{array}{r}\frac{25.51}{25.527} \\
\end{array}$ & $\begin{array}{l}24.708 \\
24.685\end{array}$ & $\begin{array}{l}62.402 \\
62.481\end{array}$ & \begin{tabular}{|}
69.18 \\
69.627
\end{tabular} & \begin{tabular}{|}
24.299 \\
24.933
\end{tabular} & $\begin{array}{l}38.86 \\
38.423 \\
\end{array}$ & \begin{tabular}{|l|l|}
1.176 \\
1.118
\end{tabular} & & $\begin{array}{r}52.22 \\
52.972\end{array}$ & $\begin{array}{l}0.075 \\
0.071\end{array}$ & $\frac{14.643}{14.643}$ & & 8.000 & $\begin{array}{r}0.13333 \\
0.15\end{array}$ & 68.7 & & $\begin{array}{l}50.6 \\
505\end{array}$ & 3.491 & 0.011 & & 0.000 & $\frac{0.215}{0.204}$ & 0.18 \\
\hline 24 & 9/2/2004 & $8: 00: 38 \mathrm{AM}$ & 24.574 & 25.314 & \begin{tabular}{|l|}
23.796 \\
\end{tabular} & 25.562 & 24.645 & $\mid$\begin{tabular}{|l|}
62.86108 \\
6
\end{tabular} & $\begin{array}{l}79.021 \\
70.015\end{array}$ & 25.301 & $\begin{array}{l}30.45 \\
38.412 \\
\end{array}$ & $\begin{array}{l}1.115 \\
\end{array}$ & 0.52 & 53.464 & $\begin{array}{l}0.068 \\
0.068\end{array}$ & $\begin{array}{l}14.045 \\
14.643\end{array}$ & 0.003 & $\begin{array}{l}1.000 \\
10.00\end{array}$ & \begin{tabular}{|l|}
0.16667 \\
\end{tabular} & $\begin{array}{l}0.0 .5 \\
68.9 \\
\end{array}$ & 12.7 & 50.6 & 3.491 & 0.010 & 0.010 & 0.000 & 0.1954 & 0 \\
\hline & 9/2/2004 & $8: 01: 38$ AM & 24.587 & 25.302 & \begin{tabular}{|l|l|}
24.029 \\
\end{tabular} & 25.59 & \begin{tabular}{|l|l|}
24.767 \\
\end{tabular} & & 70.216 & 25.473 & 38.177 & 1.159 & 0.55 & 57.288 & 0.066 & \begin{tabular}{ll|}
14.643 \\
\end{tabular} & & 11.00 & \begin{tabular}{|l|l|l} 
\\
\end{tabular} & 69.1 & 13.6 & 50.6 & 3.489 & 0.009 & 0.010 & & & 0.16 \\
\hline & 9/2/2004 & \begin{tabular}{ll|}
$: 02: 38 \mathrm{AM}$ \\
$0.02: 38$
\end{tabular} & \begin{tabular}{|l|}
24.618 \\
2.69
\end{tabular} & $\begin{array}{l}25.303 \\
25230\end{array}$ & \begin{tabular}{|l|}
24.266 \\
2.196 \\
\end{tabular} & 25.681 & 24.849 & 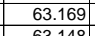 & \begin{tabular}{|l|l|}
70.384 \\
7079
\end{tabular} & \begin{tabular}{|l|}
25.935 \\
57232
\end{tabular} & 38 & $\begin{array}{l}1.162 \\
\end{array}$ & 0.58 & $\begin{array}{l}53.425 \\
5.039\end{array}$ & $\begin{array}{l}0.064 \\
\end{array}$ & \begin{tabular}{|l|}
14.643 \\
\end{tabular} & & 12.00 & 0.2 & $\begin{array}{ll}69.3 \\
69.7\end{array}$ & & 506 & $\begin{array}{l}3.488 \\
3.409\end{array}$ & $\begin{array}{l}0.009 \\
\end{array}$ & & & & \\
\hline & $\begin{array}{l}9 / 2 / 2004 \\
90 / 2 / 2004\end{array}$ & : $: 38 \mathrm{AM}$ & $\begin{array}{l}24.688 \\
24.769\end{array}$ & $\begin{array}{l}25.328 \\
25344\end{array}$ & \begin{tabular}{|l|}
24.496 \\
24707
\end{tabular} & $\begin{array}{l}25.801 \\
25887\end{array}$ & $\begin{array}{l}24.913 \\
24.944 \\
\end{array}$ & \begin{tabular}{|c|}
63.148 \\
6536
\end{tabular} & \begin{tabular}{|c|}
70.798 \\
7004919
\end{tabular} & \begin{tabular}{|l|}
25.733 \\
25.833
\end{tabular} & $\begin{array}{l}38.082 \\
11183\end{array}$ & $\begin{array}{l}1.405 \\
1212\end{array}$ & & & $\begin{array}{l}035 \\
131 \\
13\end{array}$ & $\frac{14.6}{146}$ & 0.00 & 1400 & \begin{tabular}{|l|}
0.21667 \\
\end{tabular} & $\begin{array}{ll}69.7 \\
69.3\end{array}$ & & 50.6 & $\begin{array}{l}3.490 \\
3689 \\
\end{array}$ & 0.005 & $\begin{array}{l}0.005 \\
0.019\end{array}$ & 0.000 & 0.100 & \\
\hline & 9/2/2004| & $\begin{array}{l}8: 04: 38 \\
80.05 .38\end{array}$ & $24.769 \mid$ & & \begin{tabular}{|l|}
24.707 \\
24.915 \\
\end{tabular} & $\begin{array}{l}25.847 \\
25889\end{array}$ & & & & & \begin{tabular}{|l|l|l|}
577 \\
573
\end{tabular} & $\begin{array}{l}-1.212 \\
-1215\end{array}$ & & & & & & $\begin{array}{ll}14.00 \\
15.00\end{array}$ & 0.23333 & $\begin{array}{l}69.3 \\
69.9\end{array}$ & $\begin{array}{ll}11.8 \\
1.8\end{array}$ & 53.5 & \begin{tabular}{|l|l|}
3.689 \\
3709
\end{tabular} & 0.019 & \begin{tabular}{ll|l|}
0.019 \\
0.010
\end{tabular} & & & 0.30 \\
\hline & $\begin{array}{l}9 / 2 / 212004 \\
9 / 212004\end{array}$ & $\begin{array}{l}8: 05: 38 \text { AM } \\
806: 38\end{array}$ & $\begin{array}{r}24.836 \\
24.9\end{array}$ & & \begin{tabular}{|l|}
$\mid 24.915$ \\
25.144 \\
$\mid$
\end{tabular} & & & & & & & & & & & & & & & & & & 3. & 0.010 & 0.010 & & & 0.16 \\
\hline & 9/2/2004 & $8: 0$ & \begin{tabular}{|l|l|}
24.994 \\
\end{tabular} & 25.393 & \begin{tabular}{|l|}
25.328 \\
\end{tabular} & 25.926 & 24.999 & 63.464 & $\begin{array}{l}67.693 \\
67.69\end{array}$ & 26.093 & 37.235 & -1.215 & & 53.98 & 0.0 & & & 17.00 & 0.28333 & $\frac{00.1}{66.6}$ & 12.9 & 50.3 & $\begin{array}{l}0.4741 \\
3.471\end{array}$ & $\mid$ & 0.008 & 00 & 0.166 & 0.14 \\
\hline & 9/2/2004 & $8: 08: 38$ & $\begin{array}{l}25.096 \\
\end{array}$ & 25.4 & 25.49 & 25.953 & \begin{tabular}{|l|l|}
25.011 \\
\end{tabular} & & 67.627 & \begin{tabular}{ll|}
26.093 \\
\end{tabular} & 37.641 & -1.212 & & 56.326 & & 14.6 & & 18.00 & 0.3 & 66.5 & \begin{tabular}{|l|}
13.4 \\
\end{tabular} & 50.6 & 3.486 & 0.009 & 0.009 & & 0.170 & 0.14 \\
\hline & $\frac{9 / 2 / 2004}{902004}$ & & \begin{tabular}{|l|}
25.202 \\
25206
\end{tabular} & $\frac{25.402}{25.40}$ & \begin{tabular}{|l|}
25.641 \\
25791 \\
\end{tabular} & $\begin{array}{l}25.95 \\
25.990 \\
\end{array}$ & & & & 25.9 & & & & & & & & & 0.31667 & $\begin{array}{ll}66.3 \\
6.9\end{array}$ & 12 & 501 & $\begin{array}{l}3.515 \\
2.72 \\
\end{array}$ & & 0 & & & \\
\hline \begin{tabular}{l|l}
34 \\
35
\end{tabular} & 9/2/2004 & $\begin{array}{l}8: 10: 33 \\
8 \cdot 1 \cdot 38\end{array}$ & $\begin{array}{l}25.286 \\
25.376\end{array}$ & $\begin{array}{l}25.406 \\
25401\end{array}$ & \begin{tabular}{|l|}
25.791 \\
25915 \\
\end{tabular} & \begin{tabular}{|l|l|}
25.969 \\
25.984
\end{tabular} & & & \begin{tabular}{|l|l|}
67.494 \\
66.13 \\
\end{tabular} & & & & & & & & & 20.00 & $\begin{array}{l}0.33333 \\
0.35\end{array}$ & $\begin{array}{ll}66.4 \\
65.4\end{array}$ & $\begin{array}{l}13.5 \\
13 . \\
\end{array}$ & 50.4 & $\begin{array}{l}3.473 \\
3.405 \\
3\end{array}$ & 0.009 & 0.008 & 10 & & 0.14 \\
\hline & $\frac{9 / 2 / 2004}{9 / 2 / 204}$ & & \begin{tabular}{r|r|r|}
25.376 \\
25.463
\end{tabular} & $\frac{25.401}{25398}$ & & \begin{tabular}{|l|}
25.984 \\
25.951
\end{tabular} & & & \begin{tabular}{|r|}
66.13 \\
66.199
\end{tabular} & \begin{tabular}{|l|}
25.988 \\
26048
\end{tabular} & & & & & & & & 21.00 & $\begin{array}{r}0.35 \\
036667\end{array}$ & 65.0 & \begin{tabular}{|l|}
13.0 \\
12.
\end{tabular} & \begin{tabular}{|l|}
49.4 \\
495.
\end{tabular} & \begin{tabular}{|l|}
3.405 \\
3.412
\end{tabular} & 0.008 & \begin{tabular}{ll|l}
0.008 \\
0.008
\end{tabular} & & .167 & 0.14 \\
\hline & $\frac{9 / 2 / 2004}{9 / 2 / 2004}$ & $\begin{array}{l}8: 12: 38 \mathrm{~A} \\
8 \cdot 13 \cdot 38 \mathrm{~A}\end{array}$ & \begin{tabular}{|l|}
25.463 \\
25.536
\end{tabular} & $\begin{array}{l}25.3988 \\
25.361\end{array}$ & $\mid$\begin{tabular}{|c|}
26.027 \\
26135
\end{tabular} & \begin{tabular}{|c|}
25.951 \\
25999
\end{tabular} & & & $\begin{array}{r}66.149 \\
66.19\end{array}$ & \begin{tabular}{|l|l|}
26.048 \\
25.831
\end{tabular} & $\begin{array}{l}36.957 \\
37009\end{array}$ & \begin{tabular}{|l|l|}
-1.212 \\
\end{tabular} & & $\frac{54.1}{56.4}$ & 0.0 & & & 22.00 & $\mid \begin{array}{l}0.36667 \\
0.038333\end{array}$ & $\begin{array}{l}65.0 \\
65.1 \\
65.1\end{array}$ & 12.9 & \begin{tabular}{|l|l|} 
& 49.5 \\
\end{tabular} & $\frac{3.412}{3.414}$ & 0.009 & \begin{tabular}{|c|c|}
0.008 \\
0.08
\end{tabular} & & & 0.15 \\
\hline 38 & 9//2/2004 & $8: 14: 38 \mathrm{~A}$ & \begin{tabular}{|l|l|}
25.625 \\
2.625
\end{tabular} & $\begin{array}{l}25.301 \\
25.38 \\
\end{array}$ & \begin{tabular}{|l|}
26.264 \\
\end{tabular} & \begin{tabular}{|l|}
26.008 \\
\end{tabular} & $\begin{array}{r}25.021 \\
25.02 \\
\end{array}$ & & \begin{tabular}{|c|}
66.389 \\
6.13
\end{tabular} & 25.864 & 37.271 & \begin{tabular}{|l|}
-1.209 \\
\end{tabular} & & $\begin{array}{l}56.44 \\
57.76\end{array}$ & $\begin{array}{l}0.061 \\
0.061\end{array}$ & $\frac{14.6}{14.6}$ & & $\frac{23.00}{24.00}$ & \begin{tabular}{|c|} 
\\
\end{tabular} & 65.1 & $\mid$ & $\begin{array}{l}\frac{49.5}{49.7} \\
4\end{array}$ & $\begin{array}{l}3.414 \\
3.427 \\
\end{array}$ & $\begin{array}{l}0.009 \\
0.009 \\
\end{array}$ & $\frac{0.008}{0.008}$ & 0.00 & 0.0 .170 & 0.14 \\
\hline & 9/2/2004 & 8:15: & 25.708 & 25.362 & & & & & $\begin{array}{l}66.36 \\
\end{array}$ & 25.5 & 789 & 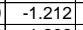 & & 54.5 & & & & & \begin{tabular}{|l|l|}
0.41667 \\
\end{tabular} & 65.2 & t. & 00. & 3.445 & & \begin{tabular}{|l|}
0.008 \\
\end{tabular} & & 0.168 & \\
\hline & $9 / 2 / 2004$ & $8: 16: 3$ & \begin{tabular}{|l|}
25.811 \\
2.09
\end{tabular} & 25.396 & 26.4 & & & & & & & & & & & & & & & 65.2 & 10.4 & ( & & & & & & \\
\hline & 9/2/2004 & & \begin{tabular}{|l|l|}
25.919 \\
25.973
\end{tabular} & $\begin{array}{r}25.409 \\
25383\end{array}$ & 26.5 & 26. & & & & & & -1. & & & & & & 27.4 & 0.45 & 65.1 & 125 & 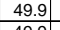 & & & . 008 & & & 0.1 \\
\hline & $\frac{9 / 2 / 2004}{9 / 2 / 204}$ & & & & & & & & & & & -1. & & & & & & & 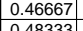 & $\begin{array}{l}65.3 \\
65.5 \\
5.5\end{array}$ & 127 & 502 & & & & & & 0.1 \\
\hline 44 & 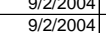 & & & & & \begin{tabular}{|l|}
25.8 \\
25.8 \\
\end{tabular} & & & & & & & & 53.4 & & & & 29.00 & \begin{tabular}{|c|}
0.48333 \\
0.5
\end{tabular} & $\frac{65.5}{65.7}$ & 12.7 & & & & & & & 0.1 \\
\hline & 9/2/2004 & & 26.232 & 25.407 & & & & & 66.4 & & & \begin{tabular}{|l|} 
\\
\end{tabular} & & 55.9 & & & & & \begin{tabular}{|l|}
0.51667 \\
\end{tabular} & 65.3 & & 50. & $\begin{array}{l}0.450 \\
3.452 \\
\end{array}$ & & & & & \\
\hline & 9/2/2004 & & 26.305 & 25.4 & & & & & & 25.2 & & -1.2 & & & & & & 32. & \begin{tabular}{|l|l|}
0.53333 \\
\end{tabular} & 65.3 & 13 & & 3.443 & & 0.008 & & & \\
\hline & & & \begin{tabular}{|l|l|}
26.37 \\
\end{tabular} & 25.375 & & & & & & & & & & & & & & & & & t.e & & & & & & & \\
\hline $\begin{array}{ll}48 \\
9\end{array}$ & $9 / 2 / 2004$ & & \begin{tabular}{r|r|}
26.44 \\
26.545
\end{tabular} & $\begin{array}{r}25.37 \\
25.405\end{array}$ & \begin{tabular}{|l|l|}
27.1 \\
272
\end{tabular} & & & & & & & -1.2 & & & & & & & \begin{tabular}{|l|l|}
0.56667 \\
058323 \\
\end{tabular} & 65.6 & 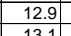 & 50 & & & & & & \\
\hline & $\frac{9 / 2 / 2004}{9 / 2 / 204}$ & & \begin{tabular}{|l|r|}
26.545 \\
26609
\end{tabular} & $\begin{array}{r}25.405 \\
254 \\
\end{array}$ & & & & & & & & & & & & & & & 0.58333 & 65 & 121 & & & & & & & \\
\hline & 9. & & & & & & & & & & & & & & & & & & & & & & & & & & & \\
\hline & 9/2/2004 & & & & 27.5 & & & & & 25. & & & & & & & & 38. & \begin{tabular}{|l|l|}
0.63333 \\
\end{tabular} & & & 50 & & & & & & \\
\hline & & & \begin{tabular}{|l|}
26.791 \\
\end{tabular} & & & & & & & & & & & & & & & & & 65.4 & & 00 & 3.448 & & & & & \\
\hline & & & $\begin{array}{l}26.852 \\
2.01\end{array}$ & & & & & & & & & & & & & & & & & 65. & t5. & & & & & & & \\
\hline & 9/2/2004 & & 26.921 & $\begin{aligned} 25.388 \\
-25.201\end{aligned}$ & \begin{tabular}{|l|}
27.6 \\
27.7
\end{tabular} & 25.9 & & & & & & -1.2 & & & & & & & & 65.3 & 13.1 & 50 & & & & & & \\
\hline & 9/2/2004| & & \begin{tabular}{l|l}
26.989 \\
27067
\end{tabular} & 25.3914 & \begin{tabular}{|l|}
27.767 \\
27355 \\
\end{tabular} & 26.0 & & & & & & -1.2 & & & & & & & & 65.6 & $\begin{array}{l}12.6 \\
130\end{array}$ & & & & & & & 0.1 \\
\hline & 9. & & & $\frac{25.40}{25.41}$ & & & & & & & & & & & & & & & & & & & & & & & & \\
\hline & 9/2/2004 & & 27.191 & 25. & \begin{tabular}{|l|}
27. \\
\end{tabular} & & & & & 24.9 & & \begin{tabular}{|l|l|} 
& -1.2 \\
\end{tabular} & & & & & & & & 65.2 & 13. & 50 & & & & & & \\
\hline & 9/2/20004 & & \begin{tabular}{|l|}
27.265 \\
\end{tabular} & & & & & 62.2 & 66.6 & 25.14 & & & & 56.63 & & & & & & 65.5 & 13 & 50.1 & 3.454 & & & & & \\
\hline & 9/2/2004 & 8:37:38 AM & 27.327 & 25.439 & 28.085 & 26.132 & 25.174 & 62.493 & 66.951 & 25.296 & 38.107 & -1.215 & & 53.97. & 0.063 & 14.643 & & & & 5 5. & & & 468 & & & 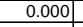 & 1.65 & \\
\hline & & & & & & & & & & & & & & & & & & & & & & & & & & & & \\
\hline & & & 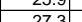 & 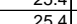 & & & & & & & & & & & & & & & & & & & & & & & & \\
\hline & & & 25.9 & 25 & 26 & 26 & & & $\frac{\pi}{66}$ & & & $\begin{array}{r}-1.2 \\
-1.2\end{array}$ & & 54 & & \begin{tabular}{|c|}
14.6 \\
\end{tabular} & & & & $\begin{array}{ll}65.6 \\
\end{array}$ & 13.0 & \begin{tabular}{|l|l|}
50. \\
\end{tabular} & 3.5 & .0 & & 0.6 & 0.4 & \\
\hline & & Minimum & $\begin{array}{l}24.6 \\
\end{array}$ & 25.3 & 23.3 & 25.5 & 24.6 & $\begin{array}{l}61.9 \\
\end{array}$ & $\begin{array}{l}66.1 \\
\end{array}$ & 24.3 & 36.8 & -1.2 & -6. & \begin{tabular}{|l|l|}
49.5 \\
\end{tabular} & & $\begin{array}{l}14.6 \\
\end{array}$ & & & & $\begin{array}{ll}65.0 \\
\end{array}$ & \begin{tabular}{|l|}
11.8 \\
\end{tabular} & \begin{tabular}{|l|}
49.4 \\
\end{tabular} & 3.4 & 0.0 & 0. & 0.0 & $\begin{array}{l}0.1 \\
\end{array}$ & \\
\hline & & & 1.789 & 0.070 & 2.699 & 0.299 & 0.237 \# & 1.762 & 2.932 & 0.791 & 1.776 & 1.721 & 4.869 \# & 3.335 & 0.024 & 0.000 & & & & 2.932 & \begin{tabular}{|l|l|l|}
0.794 & 1 \\
\end{tabular} & \begin{tabular}{|l|l|}
1.659 \\
\end{tabular} & 0.114 & 0.003 & 0.004 & 0.000 & 0.065 & \\
\hline & & of Points Used ${ }^{*}$ & 40 & 40 & 40 & 40 & 40 & 40 & 40 & 40 & 40 & 40 & 40 & 40 & 40 & 40 & 40 & & & 40 & 40 & \begin{tabular}{|l|}
40 \\
\end{tabular} & 40 & 40 & 40 & 40 & 40 & \\
\hline & & & sin box ar & re not incll & & & & & & & & & & & & & & & & ge for & $\begin{array}{l}\text { the last } 1 \\
\text { SStandarc }\end{array}$ & $\frac{10 \mathrm{~min}}{\mathrm{rd} \mathrm{Devic}}$ & $\begin{array}{l}\text { nutes }= \\
\text { uation }= \\
\text { and }\end{array}$ & \begin{tabular}{|l|}
0.0090 \\
0.0002 \\
\end{tabular} & $\frac{0.00842 \mathrm{~g}}{0.00022 \mathrm{~g}}$ & $\frac{\mathrm{gpm} / \mathrm{th} 2}{\mathrm{gpm} / \mathrm{tt2}}$ & & \\
\hline
\end{tabular}


WSRC-TR-2005-00105, REVISION 0

SRNL-RPP-2005-00012, REVISION 0

RUN \# STEADY6; STEADY STATE TEST AT 25 WT\% UDS

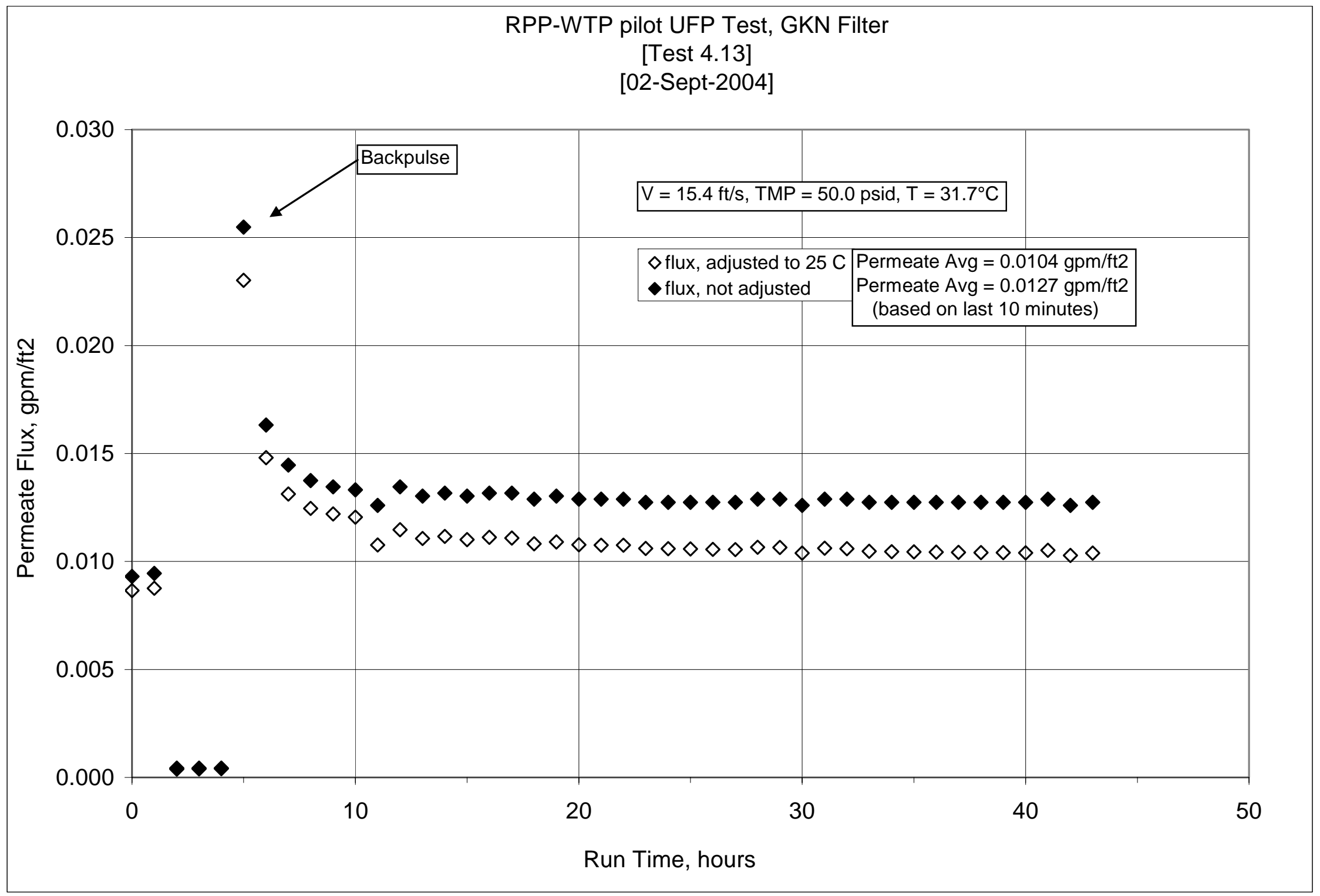


WSRC-TR-2005-00105, REVISION 0

SRNL-RPP-2005-00012, REVISION 0

RUN \# STEADY6; STEADY STATE TEST AT 25 WT\% UDS

\begin{tabular}{|c|c|c|c|c|c|c|c|c|c|c|c|c|c|c|c|c|c|c|c|c|c|c|c|c|c|c|c|c|}
\hline & A & B & $\mathrm{D}$ & $\mathrm{E}$ & \begin{tabular}{|l|l|} 
\\
\end{tabular} & $G$ & $\mathrm{H}$ & \begin{tabular}{l|l|l|}
$\mathrm{J}$ & $\mathrm{s}$
\end{tabular} & \begin{tabular}{l|l}
$\mathrm{K}$ & 1 \\
\end{tabular} & $\begin{array}{ll}\mathrm{L} \\
\end{array}$ & $M$ & \begin{tabular}{|l|}
$\mathrm{N}$ \\
\end{tabular} & 0 & \begin{tabular}{l|l}
$\mathrm{Q}$ & \\
\end{tabular} & $\mathrm{R}$ & \begin{tabular}{|l|}
$\mathrm{s}$ \\
\end{tabular} & \begin{tabular}{|l|} 
\\
\end{tabular} & $\begin{array}{lll}\mathrm{v} & \mathrm{I}\end{array}$ & w & $\mathrm{x}$ & $\mathrm{Y}$ & \begin{tabular}{|l|}
$z$ \\
\end{tabular} & AA & $\mathrm{AB}$ & $\mathrm{AC}$ & AD & $\mathrm{AE}$ & AF $\quad A$ \\
\hline & DATF & TIME & & ke Temp & perature M & $\frac{1}{\text { Measurem }}$ & ents $\gg \gg>$ & 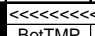 & 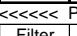 & $\begin{array}{l}\text { Sressure } M \\
\text { FititedPI }\end{array}$ & $\begin{array}{l}\text { leasuremen } \\
\text { ToTMP }\end{array}$ & & & 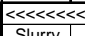 & 《< Flow $\mathrm{M}$ & Measureme & ents $\gg \gg \gg>$ & & & & & & & & & & & \\
\hline & DAIE & & Flitrate & $\begin{array}{l}\text { Cleaning } \\
\text { deq }\end{array}$ & 9 of slurry & & & BotlMP & $\begin{array}{l}\text { Filter } \\
\text { nesigh }\end{array}$ & Filfer ap & IopIMP & |- Hiltrate & Uusepot & Slurry & Filfrate & Hil Intate & Backpulse & & & & & & & & & & & \\
\hline 4 & & & $\frac{\mathrm{deg}}{\mathrm{T} 2}$ & $\frac{\text { aegC }}{\text { T3 }}$ & $\frac{\operatorname{aeg}}{\mathrm{d} 1}$ & \begin{tabular}{|l|l|} 
dege \\
T4
\end{tabular} & $\frac{\text { aegC }}{\mathrm{T} 5}$ & $\begin{array}{l}\text { psid } \\
\text { dP2 }\end{array}$ & $\frac{\text { PIg }}{\mathrm{P} 1 \mathrm{~g}}$ & $\begin{array}{l}\text { psid } \\
\text { dP1 }\end{array}$ & $\begin{array}{l}\text { Psila } \\
\text { dP3 }\end{array}$ & $\frac{\mathrm{PS} \text { PIg }}{\mathrm{P} 2}$ & $\begin{array}{l}\text { Psig } \\
\text { P3 }\end{array}$ & $\mathrm{gpm}$ & $\frac{g p m}{Q 2}$ & $\mathrm{gpm}$ & $\frac{g p m}{\mathrm{Obp}}$ & & & & & & & & & & & \\
\hline 定 & 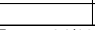 & & & & & & & & & & & & & & & & & & & Convers & ission & $\mid 851$ & & I/daylbarg & 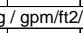 & $\frac{2 / \mathrm{barg}}{2 / 3}$ & & \\
\hline & eros- 04/19 & //2004 & & & & & & & & & & 0,120 & & 0.075 & & & & & & & & & P1 is & annroxima & tately & & & \\
\hline & $8 / 23 / 2004$ & & & & & & & & & & & & & & & & 0.004 & & & & & & & & ately $1.1 \mathrm{p}$ & & & \\
\hline & & & & & & & & & & & & -0.124 & & & 1.205 & 14.643 & & & & & & & & & & & & \\
\hline & 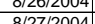 & & 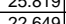 & $\frac{23.354}{2089}$ & $\begin{array}{l}2.4 .9324 \\
2.20\end{array}$ & $\frac{2.5316}{20272}$ & $\frac{23.242}{20271}$ & -0.008 & 0.1261 & $\begin{array}{l}-0.008 \\
-008\end{array}$ & 0 & 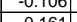 & 0.051 & 48.335| & 0.043 & $\begin{array}{l}14.663 \\
1263\end{array}$ & 0.0033 & & & & & & & Fittre & & DFP & & \\
\hline & $8 / 313020004$ & $\frac{1.53 .25 \mathrm{AM}}{7}$ & $\frac{2.20 .451}{2251}$ & $\frac{2.2098}{22848}$ & 822390 & $\frac{26.072}{22669}$ & 22914 & $\frac{-0.011}{-0.17}$ & 0.14 & 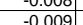 & 0.003 & 年. & 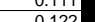 & - & & & 0.005 & & & & & & & & & & & \\
\hline & & & & & & & & -0.017 & & & & -0.147 & & -0.082 & 0.003 & 14.043 & & Time & Time & $\begin{array}{l}\text { Press. } \\
\text { Pon }\end{array}$ & ele & TMP & TMP & $\mathrm{pm}$ & $\mathrm{pm}$ & gpm & pm & neter \\
\hline 13 & ta - Per M & nute & & & & & & & & & & & & & & & & & Hour & psig & & $\mathrm{psi}$ & aar & & & $2 / \mathrm{psi}$ & $2 / \mathrm{psi}$ & laylbar \\
\hline & 9/2/2004 & 8:41:25 AM & & 25.442 & 28.988 & 26.18 & & 65.448 & \begin{tabular}{|l|l|}
69.853 \\
\end{tabular} & 26.093 & 35.117 & & & 60.948 & 0.065 & 14.643 & 0.00 & & & & & & & & & & & \\
\hline 5 & $9 / 21 / 2$ & 8:42:25 AM & 27.709 & 25.443 & 29.169 & 26.171 & 25.238 & 65.759 & 70.299 & 26.093 & 35.989 & -1.212 & -6.295 & 59.434 & 0.066 & 14.643 & & 1.00 & 0.01667 & 69.2 & 14.2 & & .508 & & & 0.000 & 172 & \\
\hline 17 & $\frac{9 / 2 / 22004}{9 / 2 / 2004}$ & $\begin{array}{l}8: 43: 25 \mathrm{AM} \\
8.44 \cdot 25 \mathrm{AM}\end{array}$ & $\frac{28.698}{28315}$ & $\frac{25.441}{25.452}$ & $\frac{2.350}{29466}$ & $\frac{26.209}{26.22}$ & $\frac{23.27}{25266}$ & $\frac{59.306}{46006}$ & \begin{tabular}{|l|}
6.9697 \\
6939
\end{tabular} & $\frac{26.0993}{26903}$ & $\frac{2.0015}{16525}$ & - & $\frac{21.139}{21081}$ & 60.548 & 年 & $\frac{14.643}{14642}$ & $\frac{0.003}{0.003}$ & $\frac{2.04}{30 x}$ & 005 & (68.6] & 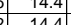 & 313 & 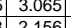 & & & & & , \\
\hline 18 & 9/2/2004 & $8.45 .25 \mathrm{AM}$ & $\frac{20.010}{26.468}$ & 25.454 & $\begin{array}{l}299.400 \\
9 \\
29.632\end{array}$ & $\begin{array}{l}26.247 \\
26.247\end{array}$ & $\begin{array}{l}25.200 \\
25314\end{array}$ & $\begin{array}{l}40.000 \\
11.141\end{array}$ & $\mid$\begin{tabular}{|l|}
$\mid 0.5949$ \\
67.254 \\
\end{tabular} & (18.525 & $\begin{array}{l}-1.0250 \\
-6.845\end{array}$ & \begin{tabular}{|l|}
1.19 \\
51.313 \\
\end{tabular} & $\begin{array}{l}2.0101 \\
58.466\end{array}$ & $\begin{array}{l}0.549 \\
64.192\end{array}$ & 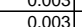 & $\begin{array}{l}14.045 \\
14.643 \\
\end{array}$ & 0.028 & $\frac{3.00}{4.00}$ & $\begin{array}{l}0.05 \\
0.06667 \\
\end{array}$ & $\begin{array}{l}0.2 \\
66.1 \\
\end{array}$ & $\begin{array}{l}14.4 \\
15.3\end{array}$ & $\frac{3.5}{2.1}$ & . & 0.000 & 0.00 & 0.000 & & 0.01 \\
\hline 19 & 9/2/2004 & 8:46:25 AM & 28.656 & 25.459 & \begin{tabular}{l|l|}
9929.791 \\
9
\end{tabular} & $\begin{array}{ll}11 & 26.262 \\
\end{array}$ & 25.334 & 59.919 & \begin{tabular}{|l|l|}
67.245 \\
\end{tabular} & 24.366 & 36.11 & \begin{tabular}{|l|}
1.295 \\
\end{tabular} & $\begin{array}{l}0.404 \\
0.394 \\
\end{array}$ & 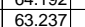 & 0.178 & $\begin{array}{l}14.045 \\
14.643 \\
\end{array}$ & $\begin{array}{l}0.020 \\
0.004\end{array}$ & $\frac{4.00}{5.00}$ & $\begin{array}{l}0.0000 \\
0.08333 \\
\end{array}$ & $\begin{array}{l}0.1 \\
6.1 \\
\end{array}$ & & & & & & & 0 & \\
\hline & & $8: 47: 25 \mathrm{AM}$ & & & & & & & & & & & & 67.037 & & 14.643 & & $\frac{6.00}{6.00}$ & & $\frac{0.1}{67.2}$ & & & & & & & & \\
\hline 21 & $9 / 2 / 2004$ & $8: 48: 25$ AM & 28.471 & 25.478 & \begin{tabular}{c|c|}
8 & 30.118 \\
\end{tabular} & 26.266 & 25.358 & $\begin{array}{l}61.572 \\
\end{array}$ & \begin{tabular}{|l|l|}
68.669 \\
\end{tabular} & 26.093 & 34.782 & 0.794] & $\mid$ & 63.089 & $\mid$ & 14.643 & 0.003 & 7.00 & .11667 & $\frac{0.5}{67.5}$ & 15.0 & & 322 & .014 & $\overline{013}$ & $\frac{0.000}{0.000}$ & 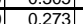 & \\
\hline & & & & & & 26.299 & & & & 26.093 & 35.211 & 0.812 & 0.498 & 65.813 & 0.096 & 14.643 & & 8.0 & & 67.9 & 15. & & & & & & & \\
\hline & 9/2/2004 & 8:50:25 AM & 28.515 & 25.463 & 30.421 & 26.236 & 25.217 & 61.975 & \begin{tabular}{|l|l|}
69.167 \\
\end{tabular} & 26.093 & 34.85 & $\mid 0.826$ & 0.539 & 65.836 & \begin{tabular}{|c|}
0.094 \\
\end{tabular} & 14.643 & & 9.00 & 0.15 & 68.0 & 15.7 & 48.4 & .338 & & & & & 0.21 \\
\hline 24 & $9 / 2 / 2004$ & 8:51:25 AM & 28.567 & 25.45 & 30.542 & 26.188 & 25.19 & 61.549 & \begin{tabular}{|l|}
68.576 \\
\end{tabular} & 26.093 & 34.526 & 0.881 & 0.582 & 66.985 & $\mid 0.093$ & 14.643 & 0.004 & 10.00 & 0.16667 & 67.5 & 16.0 & |48.0 & \begin{tabular}{|l|}
$\mid 3.312$ \\
\end{tabular} & 0.013 & 0.012 & 0.000 & & \\
\hline & & 8:52:25 AM & 28.463 & 25.45 & 30.677 & 26.173 & 25.12 & 64.847 & \begin{tabular}{|l|l|}
68.943 \\
\end{tabular} & 26.093 & 37.737 & $\mid-1.209$ & -5.168 & 60.072 & \begin{tabular}{|c|}
0.088 \\
\end{tabular} & & 0.003 & 11.00 & 0.18333 & 67.8 & $\overline{14.3}$ & 51.3 & 3.536 & 013 & & $\overline{0.000}$ & $\overline{0.210}$ & 0.18 \\
\hline 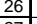 & & 8:53:25 AM] & 28.939 & 25.479 & 30.789| & 26.096 & 25.088 & 64.057 & \begin{tabular}{|l|l|}
67.956 \\
\end{tabular} & 26.093 & 36.884 & -1.218 & -6.099 & 69.822 & 0.094 & 14.643 & & 12.00 & 0.2 & 66.8 & 16.6 & 5 & 3.480 & & & 000 & & \\
\hline & & & 29.24 & 25.506 & & & & 63.968 & 67.37 & 26.093 & $37.368 \mathrm{P}$ & -1.212 & & 67.752 & 0.091 & 14.643 & & & & 66.2 & 16.1 & . & 3.493 & & & & 218 & \\
\hline 28 & $9 / 2 / 2004$ & 8:55:25 AM & 29.368 & $\begin{array}{r}25.5 \\
25.469\end{array}$ & 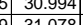 & $\frac{25.978}{25017}$ & 25.966 & 64.011 & $\frac{67.927}{6.9219}$ & 2. & $\begin{array}{l}36.832 \\
37302\end{array}$ & $-\frac{1.209}{-121}$ & -6.1199 & T.3.399 & 年 0.092 & $\begin{array}{l}14.643 \\
1.643\end{array}$ & & $\begin{array}{ll}14.00 \\
4\end{array}$ & 23333 & 66.8 & $\frac{16.8}{157}$ & 然 & 3.476 & . & & & & \\
\hline & $\begin{array}{l}9 / 2 / 2004 \\
9 / 2 / 2004\end{array}$ & $\frac{6.50 .25 \mathrm{AM}}{8.57 .25}$ & 29.4684 & $\frac{2.3409}{25.56}$ & 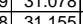 & 203.947 & $\frac{23.029}{25098}$ & 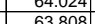 & 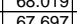 & | & 年 & $\frac{-1.251}{1.21}$ & 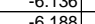 & 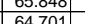 & $\mid$ & & & 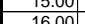 & 0.25 & 然. & & & & & & & & \\
\hline 31 & 9.1/2/2004 & 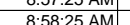 & $\frac{29.5074}{2971}$ & 25.4804 & 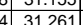 & $\frac{25.510}{26032}$ & 25.129 & $\begin{array}{l}0.000 \\
63.551\end{array}$ & \begin{tabular}{|l|}
67195 \\
67105
\end{tabular} & 26.093 & $\begin{array}{l}0.024 \\
36.918\end{array}$ & - & $\begin{array}{l}-0.100 \\
-6.185 \\
\end{array}$ & 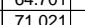 & $\mid$ & $\begin{array}{l}14.0453 \\
14643\end{array}$ & & 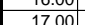 & $\begin{array}{l}0.2003 \\
02833\end{array}$ & 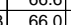 & $\frac{1.4}{16.9}$ & 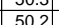 & 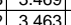 & 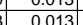 & & 然 & & \\
\hline 32 & 9/2/2004 & 8.59:25 AM & 29.809 & 25.504 & 31.35 & & 25.144 & 63.105 & \begin{tabular}{|l|l|}
66.837 \\
\end{tabular} & 26.093 & 36.724 & -1.215 & $\frac{1.203}{-6.203}$ & 60.944 & $\mid 0.09$ & 14. & 200 & 18.00 & 0.20 & 65.7 & $\frac{1.95}{14.5}$ & 49.9 & 3.441 & (13) & & 00 & & \\
\hline 33 & 9/2/2004 & $9: 00: 25 \mathrm{~A}$ & 29.904 & 25.525 & 31.44 & 26.108 & 25.155 & $\begin{array}{l}63.337 \\
637\end{array}$ & \begin{tabular}{|l|}
67.094 \\
\end{tabular} & & 36.873 & $\frac{-1.212}{-1.212}$ & & 71.593 & 0.091 & 14.643 & & 19.00 & 31667 & 660 & 171 & 501 & 3455 & 0.013 & $\overline{011}$ & 000 & 0218 & \\
\hline 34 & & & 29.939 & 25.515 & \begin{tabular}{|l|l|}
51.475 \\
\end{tabular} & & & & \begin{tabular}{|l|l|}
66.425 \\
\end{tabular} & & & -1.215 & & 67.953 & $\mid 0.09$ & & & $\frac{\frac{10.6}{20.6}}{2}$ & & 6.3 & $\frac{1.2}{16.2}$ & 49. & 3.428 & & & & & \\
\hline 35 & $9 / 2 / 2004$ & 9:02:25 AM & 29.994 & 25.53 & \begin{tabular}{l|l|l|}
3 & 31.569 \\
\end{tabular} & 26.108 & 25.225 & 62.939 & $\mid$ & 26.093 & 36.672 & -1.212 & & 63.975 & 0.6 & 14.6 & & $\frac{21.5}{21.5}$ & 0.35 & \begin{tabular}{ll|l}
65.7 \\
5
\end{tabular} & 15.2 & \begin{tabular}{cl|l}
49.8 \\
\end{tabular} & 3.434 & 0.013 & .011 & 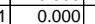 & 0.216 & 018 \\
\hline 36 & 9/2/2004 & & 30.031 & & 31.577 & 26.066 & 25.206 & 62.802 & \begin{tabular}{|l|l|}
66.578 \\
\end{tabular} & & 36.471 & -1.215 & & 70.074 & & 14.643 & & 22.00 & 0.36667 & 7. & 16.1 & 49.6 & 8.422 & & & 8.000 & & \\
\hline 37 & 9/2/2004 & 9:04:25 AM & 30.136 & 25.543 & 31.681 & 26.131 & 25.193 & 63.279 & \begin{tabular}{|l|}
67.109 \\
\end{tabular} & 26.093 & 36.879 & -1.209 & -6.194 & 68.806 & \begin{tabular}{|c|}
0.089 \\
\end{tabular} & & & 23.00 & 0.38333 & 66.0 & 16.4 & & 3.453 & & & 5.000 & & 0.18 \\
\hline 38 & $9 / 2 / 2004$ & 9:05:25 & 30.196 & 25.534 & 31.707 & 26.156 & 25.198 & 62.676 & \begin{tabular}{|l|}
66.584 \\
\end{tabular} & 26.093 & 36.621 & -1.212 & & $66.664]$ & \begin{tabular}{|c|}
0.089 \\
\end{tabular} & 14. & 0.003 & 24.00 & 0.4 & 65.5 & 15.9 & 49.6 & 3.423 & 0.013 & & .000 & 13 & 0.1 \\
\hline 年 & (2/2004 & & 30.239 & 25.538 & 31.76 & 26.165 & & & \begin{tabular}{|l|l|} 
& 66.7777 \\
\end{tabular} & 26.09 & 36.59 & & -6 & 68.2911 & 0.08 & & & 25.6 & & - & 16.3 & & 431 & & & & & \\
\hline 40 & 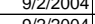 & & 30.304 & 25.553 & 31.82 & 26.176 & 25.2 & 62 & & & & -1.212 & & & & & & 26. & 0.43333 & 65.5 & 15.0 & $\frac{49}{90}$ & $\begin{array}{l}3.430 \\
\end{array}$ & 0.015 & & 0.000 & 0.214 & \\
\hline & & & 30.374 & & & & & & & & & & & & 0.089 & & & & & 60.0 & 16.1 & & 3.463 & & & & & \\
\hline$\frac{72}{43}$ & $\begin{array}{l}9 / 20004 \\
9 / 2 / 2004\end{array}$ & 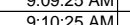 & 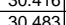 & 25.353 & 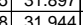 & $\frac{20.250}{26266}$ & $\frac{25.25}{25343}$ & 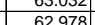 & $\mid$\begin{tabular}{|c|}
$\mid 0.125$ \\
67122
\end{tabular} & 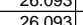 & 年, 3 & $|-1.12|$ & $\frac{-0.19}{-6}$ & | & 0 & 年 & & 2900 & 0.46867 & (5).0. & $\frac{1.1}{137}$ & 498 & $\begin{array}{l}3.456 \\
3.436\end{array}$ & 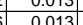 & & & 14 & \\
\hline 44 & 9/2/2004 & $9.91 .125 \mathrm{AM}$ & 30.568 & 25.564 & \begin{tabular}{|l|l|l|}
432024 \\
\end{tabular} & 26.327 & 2532 & 6292 & $\mid$\begin{tabular}{|c|c|}
$|66.64|$ \\
\end{tabular} & & & - & & & $\mid$ & & & & & & 标. & & 3.443 & & & & & \\
\hline & 9/2/2004 & $\frac{9: 12: 25}{9: 25}$ & 30.634 & 25.58 & 年. & $\frac{26.378}{26.378}$ & 25.36 & $\frac{163.3}{63.3}$ & \begin{tabular}{|l|l|}
67.351 \\
\end{tabular} & & & $\frac{1.212}{-1.212}$ & & 每4.096 & $\frac{0.09}{0.09}$ & & & $\frac{51.0}{31.0}$ & 051667 & 年 & 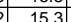 & & 3456 & & & & & 0. \\
\hline 46 & $9 / 2$ & & 30.69 & 25.591 & 32.106 & 26.454 & & 63.346 & & & & -1.212 & & & & & & & & & 16.4 & & & & & & & \\
\hline 47 & $9 / 2 / 2004$ & & 30.741 & 25598 & 832132 & 26.486 & & 63561 & & & 37.475 & -1218 & & 59182 & 0.08 & 146 & & 3300 & 0.55 & $\mid$ & 141 & 0 & 3.483 & & 10 & 000 & 0207 & \\
\hline 48 & $9 / 21 /$ & & 30.798 & & 32.184 & \begin{tabular}{l|l}
4 & 26.514 \\
\end{tabular} & & & & & $\begin{array}{l}36.9 \\
\end{array}$ & $\frac{-1.212}{-1.21}$ & & & & & & & & it & 14.6 & & & & & & & \\
\hline $4 \div$ & $9 / 2 / 2004$ & $9: 16: 25 \mathrm{~A}$ & 30.845 & 25.628 & \begin{tabular}{l|l|l|}
82.221 \\
821
\end{tabular} & 26.501 & 25.36 & 63.246 & \begin{tabular}{|l|l|}
67.239 \\
\end{tabular} & 26.0 & 37.161 & -1.212 & & 66.927 & $0.0 \varepsilon$ & 14.64 & & 35.00 & 0.58333 & 66.1 & 15.9 & 50.2 & 3.461 & 0.013 & 0.010 & 0.000 & 0.208 & 0. \\
\hline 50 & $9 / 2 / 2004$ & 9:17:25 AM & 30.876 & 25.644 & 32.262 & 26.392 & $25.38 \mathrm{~S}$ & 63.383 & \begin{tabular}{|l|l|}
67.378 \\
\end{tabular} & 26.093 & 37.116 & $\begin{array}{l}-1.209 \\
\end{array}$ & -6.20 & 63.428 & \begin{tabular}{|c|c|}
0.089 \\
\end{tabular} & 14.643 & & 36.00 & 0.6 & 66.3 & 10. & & 465 & 0.013 & 0.010 & .000 & 08 & 0.1 \\
\hline & & & & & & & & & & & & & & & & & & & & & 15.2 & & & & & & 9 & \\
\hline$\frac{52}{52}$ & & & 30.897 & 25.67 & 32.337 & 26.328 & & & 67.241 & 26.093 & 37.005 & -1.212 & & 55.162 & 0.0 & & & $38 . \mathrm{C}$ & 3333 & 66.1 & 13.1 & & & & & & & \\
\hline & & & 年0.912 & & 32.362 & & & & & & & -1.209 & & $\frac{65.64}{6.67}$ & & & & & & 6.1 & 13.00 & & & & & & & \\
\hline 5 & & & 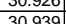 & 25.020 & S2.581 & $\frac{20.213}{26176}$ & & 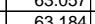 & | 0.0909 & & S3.9.944 & -1.212 & & of.8.89 & 0 & & & 4.00 & 0.06007 & 然.0.0 & 15.4 & $\mid$\begin{tabular}{|c|}
$\mid 50.0$ \\
\end{tabular} & | & & & . & 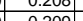 & \\
\hline 56 & $\frac{1 / 2 / 200}{902}$ & $\frac{1.25}{9 \cdot 25 \mathrm{AM}}$ & | & & 532.306 & 26.158 & $\frac{2.0 .56}{25154}$ & $\frac{6.5047}{63217}$ & $4,67.045$ & 26093 & 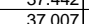 & $\mid$ & $\frac{-2.628}{-6214}$ & |c.1.104| & | & $\mid \frac{140453}{14643}$ & & 年 & & & & & & & & & & \\
\hline & & & 2013 & 2564 & 32.42 & 20.1504 & 2510 & 63072 & 07.017 & & 37031 & 1.215 & & 65.52 & 年 & & & $\frac{4.00}{4300}$ & & 整. & & & & & & & & \\
\hline 58 & 2004 & & $\frac{31.019}{319}$ & 25.639 & 32.44 & 26.056 & 25.10 & 63.041 & & & & -1.1215 & & 62484 & & & & 440 & & & & & & & & & & \\
\hline & & & nt & 25.622 & 32.458 & 26.039 & & & & & 37.579 & & & & & & & 4500 & 075 & & & & & & & & & \\
\hline 6 & 9//2/2004 & 9:27:25 AM & 31.041 & 25.64 & \begin{tabular}{l|l|l|}
4 & 32.496
\end{tabular} & 26.073 & 25.15 & 63.327 & 67.39 & 26.093 & 37.395 & -1.212 & & 66.121 & 0.089 & 14.643 & & 46.00 & 0.76667 & 66.3 & 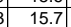 & & 3.472 & 013 & & 000 & 0.206 & \\
\hline & & & & & & & & & & & & & & & & & & & & & & & & & & & & \\
\hline 62 & & & 30.1 & & 31.7 & 26 & & 63 & 67.4 & 26.1 & & 1.0 & & 6 & & & & & & 66.2 & $\overline{15.4}$ & & $|4|$ & & & & .2 & \\
\hline & & & 31.00 & 25.7 & 32.5 & 26 & & 64.8 & & & & & & & & & & & & 68.0 & $17.1 \mathrm{~T}$ & & & & & & 0.3 & \\
\hline 64 & & & 30.3 & 25.5 & 31.8 & 26 & 25 & 63.2 & 67.1 & 26.1 & 37.0 & -1.2 & -6.2 & 65.6] & 0. & & & & & 66.0 & 15.6 & 50.1 & 3.5 & 0.0 & & 0.0 & 0.2 & \\
\hline & & $\frac{\text { Minimum }}{2 \times \text { Std Dey }}$ & $\frac{28.5}{1643}$ & $\begin{array}{r}25.5 \\
0139\end{array}$ & $\frac{30.1}{1341}$ & $\frac{25.9}{0295}$ & $\frac{25.07}{0.224 t}$ & \begin{tabular}{r|r}
61.5 \\
1228
\end{tabular} & $\begin{array}{r}66.4 \\
1355 \\
\end{array}$ & $\begin{array}{r}26.1 \\
0000\end{array}$ & $\begin{array}{r}34.5 \\
1444\end{array}$ & $\begin{array}{r}-1.2 \\
1.25\end{array}$ & \begin{tabular}{r|}
-6.2 \\
4022
\end{tabular} & 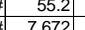 & $\begin{array}{r}0.1 \\
0.005\end{array}$ & \begin{tabular}{r|r}
14.6 \\
0000
\end{tabular} & 0.001 & & & $\begin{array}{r}65.3 \\
1355 \\
\end{array}$ & $\begin{array}{l}3 \\
5 \\
5\end{array}$ & \begin{tabular}{|r|}
48.0 \\
1271
\end{tabular} & \begin{tabular}{|r|}
3.3 \\
\end{tabular} & $\begin{array}{r}0.0 \\
0.001\end{array}$ & $\begin{array}{r}0.0 \\
0.001\end{array}$ & $\begin{aligned} 0.0 \\
0.000\end{aligned}$ & $\begin{array}{r}0.2 \\
0.030\end{array}$ & 025 \\
\hline$\frac{76}{67}$ & Number c & f Points Used ${ }^{*}$ & 40 & 40 & $\frac{1.5+1}{40}$ & 40 & 40 & $\frac{1.220}{40}$ & 40 & 40 & 1.40 & 40 & 40 & 40 & 40 & 40 & $40 d$ & & & 40 & 40 & 40 & 40 & 40 & 40 & 40 & 40 & 40 \\
\hline$\frac{68}{60}$ & & Backpulse poin & sin box & are not inc & icluded & & & & & & & & & & & & & & & & & tin & & & & & & \\
\hline$\frac{69}{70}$ & & & & & & & & & & & & & & & & & & & $\overline{A v E}$ & rage for & $\begin{array}{l}r \text { the last } \\
\text { rxStandar }\end{array}$ & $\begin{array}{l}t 10 \mathrm{~min} \\
\text { rdd Devi }\end{array}$ & $\begin{array}{l}\text { vinutes }= \\
\text { viation }=\end{array}$ & $\begin{array}{l}=0.0128 \\
=0.0022 \\
=0.00\end{array}$ & \begin{tabular}{|l|l|} 
& 0.0105 \\
0.0002
\end{tabular} & $\frac{5 \mathrm{gpm} / \mathrm{tt2}}{2 \mathrm{gam} / \mathrm{t} 2}$ & & \\
\hline
\end{tabular}


WSRC-TR-2005-00105, REVISION 0

SRNL-RPP-2005-00012, REVISION 0

RUN \# TEMP1; CAMPAIGN IV HIGH TEMPERATURE TEST

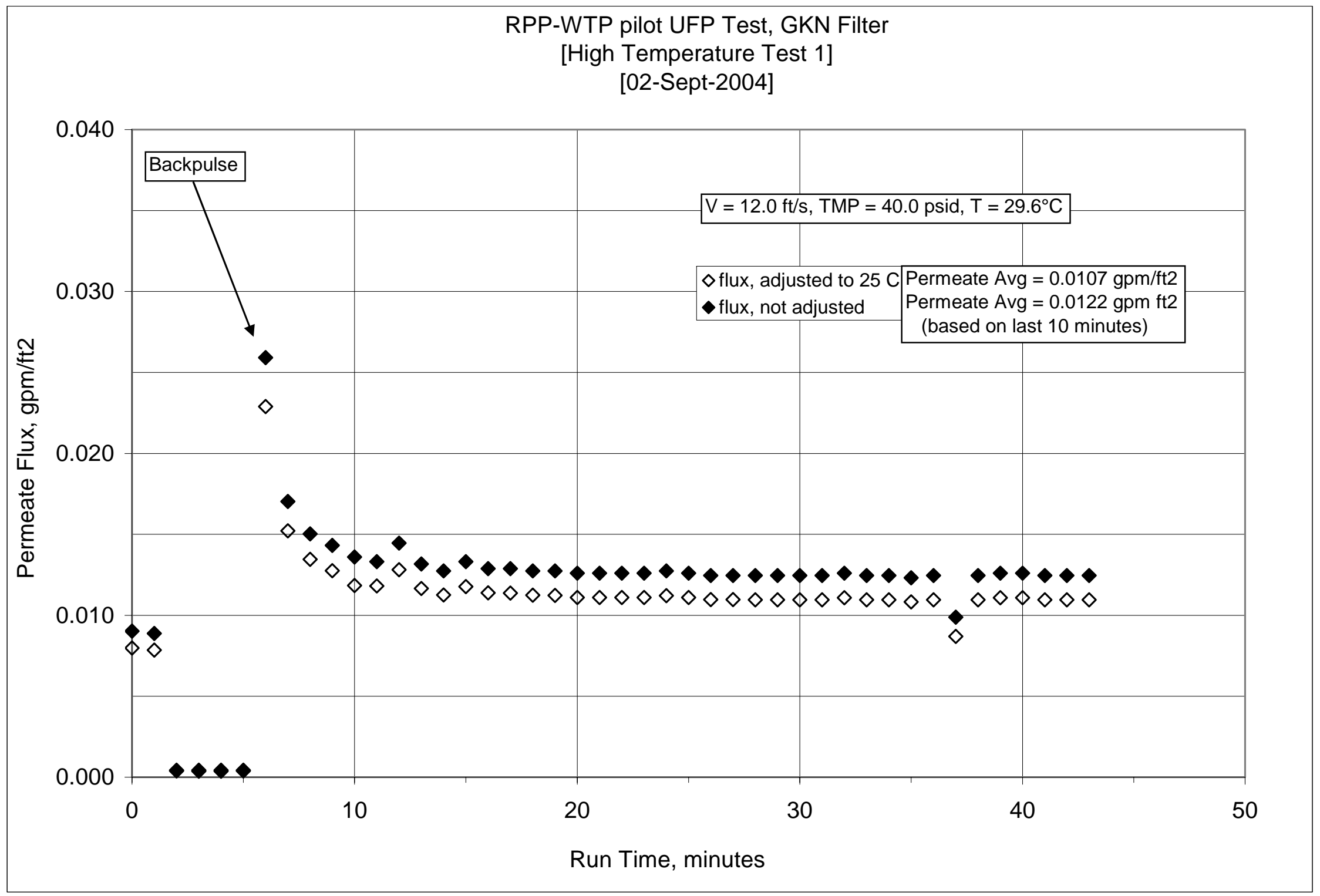


WSRC-TR-2005-00105, REVISION 0

SRNL-RPP-2005-00012, REVISION 0

RUN \# TEMP1; CAMPAIGN IV HIGH TEMPERATURE TEST

\begin{tabular}{|c|c|c|c|c|c|c|c|c|c|c|c|c|c|c|c|c|c|c|c|c|c|c|c|c|c|c|c|c|}
\hline & A & B & $\mathrm{D}$ & $E$ & $\mathrm{~F}$ & \begin{tabular}{|l|l|} 
\\
\end{tabular} & $\mathrm{H}$ & $\mathrm{J}$ & $\mathrm{K}$ & \begin{tabular}{l|l}
$\mathrm{L}$ & \\
\end{tabular} & \begin{tabular}{l|l} 
\\
\end{tabular} & $\mathrm{N}$ & $\mathrm{O}$ & \begin{tabular}{l|l}
$Q$ & 1 \\
\end{tabular} & $\mathrm{R}$ & \begin{tabular}{|l|l} 
& $s$ \\
\end{tabular} & $\mathrm{~T}$ & $\mathrm{~V}$ & w & $\mathrm{x} \mid$ & & $\mathrm{z}$ & $\mathrm{AA}$ & $\mathrm{AB}$ & $\mathrm{AC}$ & $\mathrm{AD}$ & $\mathrm{AE}$ & $\overline{A F}$ \\
\hline & DATF & TIME & Itrate & $\begin{array}{l}\text { Kempe } \\
\text { leanina }\end{array}$ & $\begin{array}{l}\text { erature } \mathrm{M} \\
\text { Slump }\end{array}$ & teasureme & ntsts >>>> & OTTPP & Filter & $\begin{array}{l}\text { ressure M } \\
\text { Fition }\end{array}$ & $\begin{array}{l}\text { Measuremen } \\
\text { TonTMP }\end{array}$ & 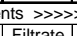 & senot & 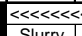 & 《< Flown & 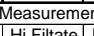 & & & & & & & Raw & & & & & \\
\hline & DAIL & & $\frac{\text { reg } \mathrm{C}}{\text { deg }}$ & deg $C$ & \begin{tabular}{|l|} 
Surry \\
deg C \\
\end{tabular} & 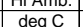 & deg $C$ & $\begin{array}{l}\text { oftMP } \\
\text { psid }\end{array}$ & $\begin{array}{l}\text { Fifiter } \\
\text { psig }\end{array}$ & $\begin{array}{l}\text { Hiter dP } \\
\text { psid }\end{array}$ & \begin{tabular}{|l|} 
TopIMP \\
psid
\end{tabular} & \begin{tabular}{|l|l|} 
Hiftrate \\
psig
\end{tabular} & unsepot & $\frac{\text { Slurry }}{\text { gam }}$ & $\begin{array}{c}\text { Filfrate } \\
\text { gom }\end{array}$ & \begin{tabular}{|c|} 
Hil intate \\
gpm
\end{tabular} & \begin{tabular}{|l|} 
Backpulse \\
gpm
\end{tabular} & & & & & 6.985 & FT2 & & & & & \\
\hline & & & $\mathrm{T} 2$ & T3 & $\frac{T}{T 1}$ & $\mathrm{~T} 4$ & & dP2 & Poly & dP1 & dP3 & P2 & P3 & 01 & $\mathrm{Q}^{2}$ & $\mathrm{Q}^{2}$ & Qbp & & & Convers & ision & 851 & $\mathrm{~m} 3 / \mathrm{m}$ & yy/barg & $1 \mathrm{pm} / \mathrm{tt} 2$ & $\arg$ & & \\
\hline & 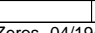 & 04 & & & & & & & & & & & & & & & & & & & & & 1 P1 is? & 背 & 年 & & & \\
\hline & elos- & 8:2004 & 22.554 & 22.604 & 22.002 & 22.782 & 22.204 & -0.019 & 0.095 & -0.012 & 0.003 & -0.124 & 0.137 & -0.075 & 0.003 & 14.643 & 0.004 & & & Note: $G$ & Gauge Pr & Pressure & $\mathrm{e} P 1$ is a & approximat & ately $1.1 \mathrm{p}$ & & & \\
\hline & $8 / 24 / 2004$ & & & & 23.679 & 22.998 & 22.735 & -0.021 & 0.122 & -0.014 & -0.001 & -0.124 & 0.134 & 23.176 & 1.205 & 14.643 & 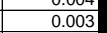 & & & & lis & & d for: & 25 inch & & r tubing & & \\
\hline & $8 / 26 / 2004$ & 9:14:11 AM & 25.819 & 23.352 & 24.932 & 25.316 & 23.242 & -0.008 & 0.126 & -0.008 & 0.011 & -0.106 & 0.091 & 48.335 & 0.043 & $\begin{array}{l}14.643 \\
\end{array}$ & 0.003 & & & & & & & 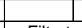 & & 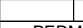 & & \\
\hline & $8 / 27 / 2004$ & 7:53:29 AM & $\frac{22.649}{22511}$ & $\begin{array}{r}22.89 \\
22848 \\
\end{array}$ & 22.298 & 22.872 & $\frac{22.871}{22091}$ & $\begin{array}{l}-0.017 \\
-0.017\end{array}$ & 0.14 & $\begin{array}{l}-0.008 \\
-0.08 \\
\end{array}$ & 0.005 & 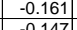 & 0.111 & $\begin{array}{l}-0.084 \\
-0.02\end{array}$ & & & & & & $\mathrm{VWV}$ & & & & Filtrate & & & ABILIT & \\
\hline$\frac{12}{12}$ & & 7:28:55 AM & 22.511 & 22.846 & 22.397 & 22.669 & 22.914 & $=0.017$ & 0.138 & -0.009 & 0.003 & -0.147 & 0.12 & -0.082 & 0.003 & 14.643 & & ime & Time & Press. $\mid$ & & TMP & TMP & $\mathrm{pm}$ & at 25C & $\mathrm{pm}$ & $\frac{1000}{\mathrm{pm}}$ & $-5 \mathrm{~T}$ \\
\hline & ata - Per M & nute & & & & & & & & & & & & & & & & in. & Hour & psig f f & & si & bar & tit2 & & $2 / 2 / p s i$ & $2 / \mathrm{psi}$ & ay/bar \\
\hline & 9/2/2004 & 11:34:18 AM & 29.397 & 25.599 & 30.309 & 25.707 & 25.319 & 49.21 & 53.638 & & 32.104 & -1.215 & -6.313 & 49.831 & $\begin{array}{ll}0.063 \\
\end{array}$ & 14.643 & & & & & 11.9 & & & & & & & 0.17 \\
\hline & 9/2/2004 & 11:35:18 AM & 29.417 & $\begin{array}{l}25.605 \\
25611\end{array}$ & 30.046 & 25.727 & 25.345 & 49.351 & $\begin{array}{l}53.877 \\
5.232\end{array}$ & \begin{tabular}{ll|}
18.072 \\
18902
\end{tabular} & 32.114 & $\begin{array}{ll}-1.212 \\
\end{array}$ & $\begin{array}{ll}-6.318 \\
-2.503\end{array}$ & 46.452 & 0.062 & 14.643 & 0.0 & 1.00 & 0.01667 & 52.8 & 11.1 & 40.7 & \begin{tabular}{|l|}
2.808 \\
\end{tabular} & 0.009 & 0.008 & 0.000 & 0.193 & 0.16 \\
\hline 17 & $\frac{9 / 2 / 2004}{9 / 2 / 2004}$ & 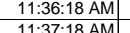 & 29.414 & $\begin{array}{l}25.611 \\
25.618 \\
\end{array}$ & \begin{tabular}{|l|}
29.794 \\
29.582 \\
\end{tabular} & $\begin{aligned} 25.724 \\
2574 \\
\end{aligned}$ & \begin{tabular}{|l|}
25.336 \\
25.368
\end{tabular} & $\begin{array}{l}47.889 \\
41.387\end{array}$ & \begin{tabular}{|l|}
53.323 \\
53.124
\end{tabular} & $\begin{array}{l}18.885 \\
18.546 \\
\end{array}$ & $\begin{array}{r}29.82 \\
23.515 \\
\end{array}$ & \begin{tabular}{|c|}
-0.703 \\
5724 \\
\end{tabular} & $\frac{20.503}{20.318}$ & \begin{tabular}{|l|}
49.478 \\
51.06 \\
\end{tabular} & $\begin{array}{l}0.003 \\
0.03 \\
\end{array}$ & \begin{tabular}{|l|l|}
14.643 \\
1.643
\end{tabular} & 0.003 & 2.00 & $\begin{array}{l}0.03333 \\
0.05 \\
\end{array}$ & $\frac{52.2}{52 .}$ & 11.8 & $\begin{array}{l}38.9 \\
325 \\
3.5\end{array}$ & \begin{tabular}{|l|}
2.679 \\
2.237
\end{tabular} & $\begin{array}{l}0.000 \\
000\end{array}$ & $\begin{array}{ll}0.000 \\
0.000\end{array}$ & $\begin{array}{l}0.000 \\
0.000 \\
\end{array}$ & $\frac{0.010}{0.011}$ & 0.01 \\
\hline 18 & 9/2/2004 & 11:38:18 AM & 30.206 & 25.619 & \begin{tabular}{|l|}
29.454 \\
\end{tabular} & 25.692 & \begin{tabular}{|l|}
25.4609 \\
\end{tabular} & 30.899 & \begin{tabular}{|l|}
53.095 \\
\end{tabular} & 18.588 & 12.833 & 16.627 & & 48.615 & $\begin{array}{l}0.003 \\
0.003\end{array}$ & $\begin{array}{l}14.045 \\
14.643\end{array}$ & $\begin{array}{l}0.003 \\
0.004\end{array}$ & $\frac{3.00}{4.00}$ & & $\begin{array}{l}52.0 \\
52.0\end{array}$ & \begin{tabular}{|l|}
12.3 \\
11.6 \\
\end{tabular} & 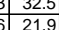 & \begin{tabular}{|l|}
2.231 \\
1.508 \\
\end{tabular} & $\begin{array}{l}0.000 \\
0.000\end{array}$ & $\frac{0.000}{0.000}$ & & & 0.01 \\
\hline 19 & 9/2/2004 & 11:39:18 AM & 28.438 & 25.611 & 29.346 & 25.638 & 25.56 & 8.843 & \begin{tabular}{|l|}
51.267 \\
\end{tabular} & 12.494 & $\begin{array}{ll}-2.969 \\
-2.969\end{array}$ & 36.919 & 0.278 & 53.297 & 0.003 & 14.643 & 0.004 & 5.00 & 0.08333 & 50.1 & \begin{tabular}{|l|} 
\\
\end{tabular} & \begin{tabular}{|l|l|}
7 & 2.9 \\
\end{tabular} & \begin{tabular}{|l|l|}
0.202 \\
\end{tabular} & 0.000 & 0.000 & & $\frac{0.017}{0.133}$ & $\frac{0.011}{0.11}$ \\
\hline 20 & 9/2/2004 & 11:40:18 AM & 29.464 & & \begin{tabular}{|l|}
29.268 \\
\end{tabular} & 25.624 & & 44.595 & 51.928 & 14.599 & 30.78 & 1.405 & & 50.759 & 0.181 & \begin{tabular}{ll|l}
14.643 \\
\end{tabular} & & 6.00 & & 50.8 & 12.1 & 37.7 & 2.598 & 0.026 & & 0.001 & & \\
\hline 21 & & 11:41:18 AM & 29.077 & 25.607 & & 25.594 & 25.332 & 45.135 & 52.757 & 15.586 & 30.253 & & & 53.264 & 0.119 & $\begin{array}{ll}14.643 \\
\end{array}$ & & & 0.11667 & 51.6 & 12.7 & 37.7 & 2.599 & 0.017 & 0.015 & & 0.404 & \\
\hline 22 & $\begin{array}{l}9 / 2 / 2004 \\
9 / 2 / 2004\end{array}$ & 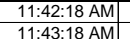 & $\begin{array}{l}29.003 \\
29.148 \\
\end{array}$ & $\begin{array}{l}25.614 \\
25.608 \\
\end{array}$ & \begin{tabular}{|r|}
29.21 \\
29.234
\end{tabular} & $\begin{array}{l}25.581 \\
25.521 \\
\end{array}$ & \begin{tabular}{|l|}
25.294 \\
25.294 \\
\end{tabular} & $\begin{array}{l}45.437 \\
45.723\end{array}$ & \begin{tabular}{|l|l|}
52.826 \\
53.508 \\
\end{tabular} & \begin{tabular}{|c|}
16.169 \\
1.6436
\end{tabular} & $\begin{array}{l}30.052 \\
30.06 \\
306\end{array}$ & $\begin{array}{l}1.367 \\
1.455 \\
\end{array}$ & $\begin{array}{l}0.472 \\
0.518\end{array}$ & \begin{tabular}{r|r|}
50.008 \\
50
\end{tabular} & 0.105 & \begin{tabular}{|l|l|}
14.643 \\
14643
\end{tabular} & & 8.00 & $\begin{array}{r}0.13333 \\
0.15\end{array}$ & $\begin{array}{l}51.7 \\
52.4 \\
\end{array}$ & \begin{tabular}{|l|}
11.9 \\
119 \\
\end{tabular} & $\begin{array}{l}937.7 \\
9 \\
9\end{array}$ & \begin{tabular}{|l|l|}
2.602 \\
2613
\end{tabular} & $\begin{array}{l}0.015 \\
0.014\end{array}$ & $\begin{array}{l}0.013 \\
0.013 \\
\end{array}$ & $\frac{0.000}{0.000}$ & & 0.30 \\
\hline 24 & 9/2/2004 & $11: 44: 18$ AM & 30.009 & 25.625 & \begin{tabular}{|l|}
29.286 \\
\end{tabular} & 25.557 & \begin{tabular}{|l|}
25.294 \\
\end{tabular} & 45.379 & \begin{tabular}{|l|}
52.726 \\
\end{tabular} & 16.375 & 29.769 & $\begin{array}{l}1.402 \\
1.402\end{array}$ & 0.556 & 50.413 & $\begin{array}{r}0.1 \\
0.095\end{array}$ & $\begin{array}{l}14.643 \\
14.643\end{array}$ & 0.003 & & 0.16667 & 51.6 & $\frac{11.0}{12.0}$ & \begin{tabular}{|l|l|}
5 & 37.9 \\
\end{tabular} & \begin{tabular}{|l|}
2.013 \\
2.591 \\
\end{tabular} & o. 0.014 & & & & 0.29 \\
\hline 25 & 9/2/2004 & 11:45:18 AM & 29.955 & 25.621 & 29.326 & 25.563 & 25.411 & 45.726 & 53.108 & 16.54 & 29.931 & 1.411 & 0.588 & 50.657 & 0.093 & 14.643 & & $\frac{11.00}{11.00}$ & 0.18333 & 52.0 & 12.1 & 37.8 & \begin{tabular}{|l|l|}
2.608 \\
\end{tabular} & 0.013 & 0.012 & 0.000 & & 0.27 \\
\hline 26 & $9 / 2 / 2004$ & $11: 46: 18 \mathrm{AM}$ & 28.896 & 25.606 & \begin{tabular}{|l|}
29.341 \\
\end{tabular} & 25.568 & & 48.712 & 53.35 & 16.667 & 32.826 & -1.218 & -5.208 & 48.681 & 0.101 & 14.643 & 0.0 & 12.00 & 0.2 & 52.2 & 11.6 & 40.8 & 2.811 & 0.014 & 0.013 & 0.000 & & \\
\hline 27 & $\begin{array}{ll}9 / 2 / 2004 \\
\end{array}$ & 11:47:18 AM & 28.852 & 25.616 & 29.421 & \begin{tabular}{|l|l|}
25.634 \\
\end{tabular} & 25.426 & 48.196 & 52.212 & 17.389 & & -1.209 & -6.09 & 49.042 & 0.092 & \begin{tabular}{|c|c|}
14.643 \\
\end{tabular} & & & 0.21667 & 51.1. & 11.7 & 40.0 & 2.757 & 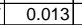 & .012 & & & \\
\hline 28 & & 11:48:18 AM & & & & 25.64 & & 47.758 & \begin{tabular}{|l|l|l} 
& \\
\end{tabular} & & 31.714 & & -6.15 & 51.18 & & 14.643 & & 14.0 & & & & & 2.740 & & & & & \\
\hline & 9/2/2004 & 11:49:18 AM & 28.774 & 25.613 & \begin{tabular}{|l|l|}
29.453 \\
\end{tabular} & 25.625 & & 48.121 & 52.237 & 16.516 & 32.477 & -1.218 & -6.14 & $50.67 \mathrm{5}-2$ & & & & 15.00 & 0.25 & E1.1. & $\frac{2.1}{2.1}-2$ & 40.3 & 2.778 & 0.013 & & & & 0. \\
\hline & 9/2/2004 & 11:50:18 & 28.739 & 25.613 & \begin{tabular}{|l|}
29.488 \\
\end{tabular} & 25.64 & 25.408 & 48.156 & 52.146 & 16.744 & 32.246 & -1.212 & -6.194 & 51.055 & & 14.643 & & & & 51.0 & & & & & & & & \\
\hline & 9/2/2004 & $11: 51: 18 \mathrm{AM}$ & 28.705 & 25.609 & \begin{tabular}{|l|}
29.508 \\
\end{tabular} & 25.651 & 25.384 & 48.034 & \begin{tabular}{|l|l|}
52.179 \\
\end{tabular} & 16.771 & 32.108 & -1.212 & -6.197 & 51.927 & 0.09 & 14.643 & 0.0 & 17.00 & 0.28333 & 51.1 & 12.4 & \begin{tabular}{|l|l|}
4 & 40.1 \\
\end{tabular} & 2.763 & $0 . c_{-1}$ & & & 0.284 & 0.8 \\
\hline & 9/2/2004 & 11:52:18 AM & 28.675 & 25.609 & \begin{tabular}{|l|}
29.538 \\
\end{tabular} & 25.651 & 25.374 & & \begin{tabular}{|l|l|}
51.939 \\
\end{tabular} & 16.784 & 32.182 & -1.212 & -6.2 & 52.951 & 0.089 & 14.643 & & 18.00 & 0.3 & 50.8 & 12.6 & 40.1 & 2.765 & 0.013 & & & 0.280 & \\
\hline & 9/2/2004 & 11:53:18 & 28.651 & 25.604 & 29.544 & 25.622 & & 48.098 & 52.088 & 16.694 & & & & $\begin{array}{l}50.348 \\
\end{array}$ & & $\begin{array}{l}14.643 \\
\end{array}$ & & 19.00 & 0.31667 & 51.0 & 12.0 & 40.2 & 2.771 & 0.013 & & & & \\
\hline & $9 / 2 / 2004$ & $\begin{array}{l}11: 54: 18 \mathrm{AM} \\
11: 55: 19 \mathrm{AM}\end{array}$ & 28.636 & 25.614 & \begin{tabular}{|l|l|}
29.574 \\
\end{tabular} & $\begin{array}{l}25.652 \\
25.502 \\
\end{array}$ & $\frac{25.379}{.5 .32}$ & 48.272 & \begin{tabular}{|l|l|}
52.548 \\
5.167
\end{tabular} & $\begin{array}{l}16.724 \\
1.567\end{array}$ & 32.268 & -1.212 & -6. & \begin{tabular}{|l|l|}
52.038 \\
5
\end{tabular} & 0.088 & \begin{tabular}{|l|l|l|l|}
14.643 \\
1.612
\end{tabular} & .0 & 20.00 & 0.33333 & 51.4 & 12.4 & 40.3 & 2.776 & 0.013 & & & .276 & 0.23 \\
\hline & 9/2/2004 & 11:55:18 AM & & & & & & & & $\begin{array}{l}16.567 \\
16.45\end{array}$ & 32.229 & $\begin{array}{l}-1.212 \\
1215\end{array}$ & & 51.985 & & & & & \begin{tabular}{|r|} 
\\
\end{tabular} & & & & \begin{tabular}{|l|}
2.763 \\
2.700
\end{tabular} & 0.013 & & & & 0.24 \\
\hline$\frac{57}{37}$ & 9/2/2004 & $\frac{11: 56: 18 \mathrm{AM}}{11: 57: 18 \mathrm{AM}}$ & $\frac{28.602}{28599}$ & $\frac{25.595}{25.602}$ & \begin{tabular}{|l|}
29.569 \\
29.586
\end{tabular} & 255.599 & $\frac{22.27}{25.347}$ & $\begin{aligned} 48.125 \\
4.93\end{aligned}$ & $\frac{52.347}{51881}$ & $\begin{array}{l}\frac{16.452}{16.392} \\
\end{array}$ & $\frac{32.523}{32.473}$ & $\begin{array}{l}-1.215 \\
-1.212 \\
\end{array}$ & 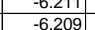 & $\begin{array}{l}5.0599 \\
56.103\end{array}$ & & $\frac{14.6}{146}$ & & $\frac{22.4}{23 .}$ & $\begin{array}{l}0.36667 \\
0.38333 \\
\end{array}$ & $\frac{51.2}{508}$ & $\frac{2.1}{34}$ & $\frac{40.3}{40.2}-19$ & $\frac{2.180}{2772}$ & 政 & & & & $\frac{0.22}{0.23}$ \\
\hline 38 & 9/2/2004 & 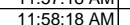 & 28.599 & 25.597 & \begin{tabular}{|l|l|}
29.601 \\
29.601
\end{tabular} & 25.625 & & 47.872 & 5193 & 16.452 & 32.313 & $\frac{x_{2}}{-12}$ & Sa & 51.98 & & & & $\frac{2.0}{24.0}-4$ & $\frac{0.005}{0.4}$ & 50.8 & & & & & & & & \\
\hline 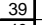 & 9/2/2004 & & & & & 25.645 & & 48.399 & & & 32.5 & $\frac{-1.2}{1.2}$ & & $49.88 \quad-1$ & & 14.6 & & & 41667 & & & 40 & & & & & & \\
\hline & 12004 & & 28.585 & 25 & & 25.651 & & & 52,403 & 16.497 & & -1.2 & & & & & & & 0.43 & 51.3 & & & & & & & & \\
\hline & $\frac{12004}{2004}$ & 12:01: & 28.58 & 25.588 & 29.592 & 25.636 & & & \begin{tabular}{|l|l|}
52.467 \\
\end{tabular} & & 32.4 & & & 46.287 & & & & & 0.45 & 51 & {$[1.0 \mathrm{C}-\mathrm{C}$} & $\frac{\pi}{40}-2>0$ & 2.788 & & & & & $\pi=$ \\
\hline & 9/2/2004 & & 28.59 & 25.588 & \begin{tabular}{|l|l|}
29.612 \\
\end{tabular} & 25.621 & & & 52.15 & 16.461 & 32.5 & -1.2 & & & & & & & 0.46667 & & & & & & & & & \\
\hline & 9/2/2004 & 12:03:18 & 28.585 & 25.583 & 29.612 & 25.621 & 25.39 & 47.758 & 51.69 & 16.204 & 32.512 & -1.21 & -6.21 & 50.749 & & & & 29. & 0.48333 & 506 & $2.1 .1>3$ & & 2.767 & & & & & \\
\hline $44-4 \div$ & 9/2/2004 & 12:04:1 & 28.596 & 25.589 & 29.623 & 25.641 & $25.40+2$ & 48.121 & \begin{tabular}{|l|l|} 
& \\
2.287 \\
\end{tabular} & 16.498 & 32.3 & -1.212 & -6.24 & 53.27 & & 14.6 & & 30. & & 玻 & & & & & & & & 0.2 \\
\hline & | $2 / 2004$ & & 28.602 & 25.584 & 29.613 & 25.647 & & & & & & -1.2 & & & & & & 31 & & & 1.8 & & & & & & & \\
\hline & 9 $9 / 2 / 20004$ & & 28.607 & 25.59 & 29.623 & 25.6577 & 25 & & - & & 32.52 & & & & & & & 32. & 0.53333 & 51.1 & . & ( & 2.773 & & & & & \\
\hline & $\begin{array}{ll}9 / 2120044 \\
0 / 21024\end{array}$ & & 28.5606 & & & & & & 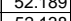 & & & & & 48.928 & & & & & & & & & & & & & & \\
\hline & $\begin{array}{l}9 / 21 / 2004 \\
9 / 2 / 2004\end{array}$ & $\frac{12.06 .18}{1209 \cdot 18}$ & $\frac{2.5917}{28617}$ & $\begin{array}{l}25.364 \\
25605\end{array}$ & 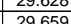 & (25.597 & $\frac{2.3}{25.33}$ & 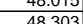 & \begin{tabular}{|l|l|l|l|}
52643 \\
5264
\end{tabular} & $\mid$ & 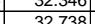 & 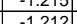 & & 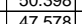 & & & & 年. & $\mid$ & $\begin{array}{l}51.0 \\
51.5 \\
\end{array}$ & 13 & & . & & & & 267 & \\
\hline & 9/2/2004 & 12:10.118 PM & 28608 & 25.596 & $\mid$ & 25 & 20.00 & & \begin{tabular}{|l|l|l|l|l|}
52059 \\
\end{tabular} & $\mid$ & (3255 & - -1.215 & & 50 & & & & & & & & & & & & & & \\
\hline & $9 / 2 / 2004$ & 12.11.1.18 PMA & 28701 & 2550 & 20.024 & 250.034 & 2537 & 48276 & 5231 & 1610 & 32056 & 1.212 & & (19.51) & & 146 & & 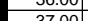 & 0.01607 & 512 & & to. & 年 & & & & & \\
\hline & & & 28.518 & $\frac{25.5}{25}$ & & 25.668 & & & & & & & & & & & & & & & & & & & & & & \\
\hline & $9 / 2 / 2004$ & & 28548 & & 29.61 & 25.664 & & $48.47 \mathrm{r}$ & 5254 & & & & & & & $146,>>2$ & & & 0.65 & 51.4 & & & & & & & & \\
\hline & 9/2/2004 & 12:14: & $\frac{28.572}{28}$ & 25.9 & 296 & 25.67 & & & & & & -1.2 & & 48.22 & & & & & 67 & & & & & & & & & \\
\hline & 9/2/2004 & 12:15:18 & 28.573 & 25.591 & 29.635 & 25.674 & & 48.1 & 52.447 & 16.2. & 32.7 & $-1.2 .1>0$ & & 46. & & 14.6 & & 41. & $\mid 0.68333$ & 51.3 & 1.0 & & & & & & & \\
\hline 56 & $9 / 2 / 2004$ & 12:16:18 PM & 28.559 & 25.592 & 29.631 & 25.664 & 25.46 & 48.21 & \begin{tabular}{|l|}
52.527 \\
\end{tabular} & 16.271 & 32.787 & -1.215 & & $\begin{array}{lll}47.28 \\
\end{array}$ & 0.087 & 14.643 & & 42 & & 51.4 & & & .19 & & & & & \\
\hline & 9/2/2004 & & & & & 25.6 & & & & & & & & & & & & & & & & & & & & & & \\
\hline & & 12:18:1 & 28.56 & 25.597 & 29.646 & 25.685 & & 48.494 & 52.579 & $16.298 \mathrm{Z}$ & 33.146 & 1.212 & & 48.8 & & & & 44. 4.4 & 0.73333 & 5 & & & & & & & & \\
\hline & & 12:19:18 & 20.856 & 25.593 & (4) & 25.666 & 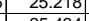 & 48.156 & 20.216 & 16.141 & 年 & & & 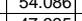 & & & & & & & & & & & & & & \\
\hline & & 12:20:18 PM & 28.576 & 25.599 & 29.822 & 25.531 & 25.434 & 48.351 & 52.536 & 16.136 & 32.949 | & $|-1.212|$ & & 47..995 & 0.086 & 14.643 & & & & & & & & & & & & \\
\hline & & & 28.7 & 25.6 & 29.6 & & & 47.9 & 52.4 & & & & & 50. & & & & & & 51.3 & $\overline{12.0}$ & 40.1 & & & & & 0.3 .3 & \\
\hline & & & 30.0 & 25.6 & 29.8 & & & 48.7 & 53.5 & 17.4 & & & & 56. & & & & & & 52.4 & 13.4 & & & & & & 0.4 & \\
\hline & & Medi & 28.6 & 25.6 & 29.6 & 25.6 & & 48.1 & 52.3 & 16.4 & & -1. & & 50 & & & & & & 1.2 & 12.0 & $\begin{array}{ll}0 & 40.3 \\
\end{array}$ & 2.776 & 0.013 & & & 0.3 & \\
\hline & & Minimum & $\begin{array}{r}28.5 \\
0.641\end{array}$ & $\begin{array}{r}25.6 \\
0.025 \\
\end{array}$ & \begin{tabular}{|l|}
29.2 \\
\end{tabular} & \begin{tabular}{r|r}
25.5 \\
0
\end{tabular} & \begin{tabular}{r|r}
25.2 \\
0.139
\end{tabular} & $\begin{array}{r}45.4 \\
1616\end{array}$ & $\begin{array}{r}51.7 \\
0.755 \\
\end{array}$ & \begin{tabular}{r|}
16.1 \\
0.493
\end{tabular} & \begin{tabular}{r|r}
29.8 \\
1695 \\
\end{tabular} & $\frac{-1.2}{1.583}$ & 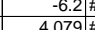 & $\begin{array}{r}46.3 \\
4044\end{array}$ & $\frac{0.1}{0.1}$ & \begin{tabular}{r|r}
14.6 \\
0000
\end{tabular} & 0.0 & & & \begin{tabular}{|l|r}
50.6 & \\
755 &
\end{tabular} & \begin{tabular}{|l|}
11.0 \\
50.963 \\
\end{tabular} & $\begin{array}{l}0.37 .6 \\
31.636 \\
3\end{array}$ & \begin{tabular}{|l|}
2.591 \\
0.113
\end{tabular} & \begin{tabular}{|c|c|} 
& 0.010 \\
0.002 &
\end{tabular} & 0.009 & \begin{tabular}{|c|c|}
0.000 \\
0.000
\end{tabular} & $\frac{0.2}{0.044}$ & \\
\hline & Number $\mathrm{c}$ & f Points Used $d^{*}$ & $\begin{array}{r}3.4+1 \\
39\end{array}$ & 39 & & 39 & 39 & 39 & 39 & 39 & 39 & 39 & 390 & 39 & \begin{tabular}{r|r|}
.011 \\
9
\end{tabular} & 39 & 39 & & & 39 & 39 & \begin{tabular}{|l|l|} 
& 30 \\
\end{tabular} & \begin{tabular}{|r|r|}
$\mid 39$ \\
$\mid$
\end{tabular} & $|39|$ & 39 & 39 & 39 & $39]$ \\
\hline & & Backpulse poin & in boxa & re not incll & Iuded & & & & & & & & & & & & & & & & & & tos - & | & & & & \\
\hline$\frac{69}{70}$ & & & & & & & & & & & & & & & & & & & Avere & rage for & $\begin{array}{l}r \text { the last } \\
\text { postandar }\end{array}$ & $\begin{array}{ll}\text { st } 10 \mathrm{mil} \\
\text { ard Dev }\end{array}$ & $\begin{array}{l}\text { inutes }= \\
\text { vitaion }= \\
\text { val }\end{array}$ & \begin{tabular}{|l|}
0.0123 \\
0.0021 \\
\end{tabular} & \begin{tabular}{|l|l|}
0.0108 \\
0.001
\end{tabular} & $\mathrm{gpm} / \mathrm{ft} 2$ & & \\
\hline & & & & & & & & & & & & & & & & & & & & & & & & & & & & \\
\hline
\end{tabular}


WSRC-TR-2005-00105, REVISION 0

SRNL-RPP-2005-00012, REVISION 0

RUN \# TEMP2; CAMPAIGN IV HIGH TEMPERATURE TEST

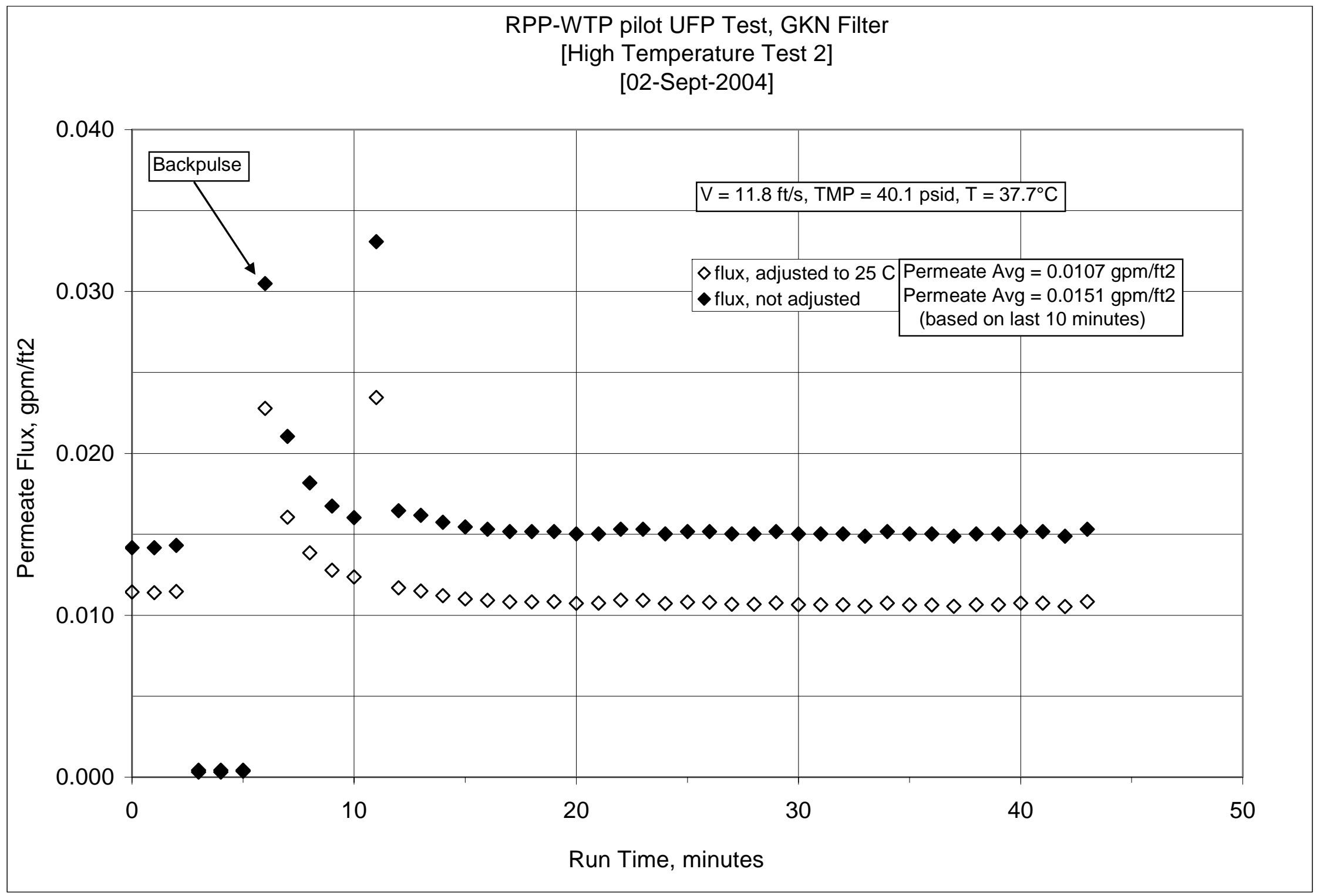


WSRC-TR-2005-00105, REVISION 0

SRNL-RPP-2005-00012, REVISION 0

RUN \# TEMP2; CAMPAIGN IV HIGH TEMPERATURE TEST

\begin{tabular}{|c|c|c|c|c|c|c|c|c|c|c|c|c|c|c|c|c|c|c|c|c|c|c|c|c|c|c|c|c|}
\hline & & & & & & \\
\hline & A & B & $\mathrm{D} /$ & \begin{tabular}{l|l|l}
$E$ & \\
\end{tabular} & \begin{tabular}{|l|l|}
$\mathrm{F}$ \\
\end{tabular} & \begin{tabular}{|l|l}
$G$ & \\
\end{tabular} & $\mathrm{H}$ & \begin{tabular}{l|l}
$\mathrm{J}$ & 1 \\
\end{tabular} & \begin{tabular}{l|l}
$\mathrm{K}$ \\
\end{tabular} & \begin{tabular}{l|l|l|l|l|} 
& $\mathrm{L}$ \\
\end{tabular} & $\mathrm{M}$ & \begin{tabular}{|l|} 
\\
$N$
\end{tabular} & 0 & \begin{tabular}{l|l} 
& \\
\end{tabular} & \begin{tabular}{l|l}
$\mathrm{R}$ & \\
\end{tabular} & $s$ & $\begin{array}{lll}T & U \\
\end{array}$ & $\mathrm{v}$ & $\mathrm{I} \mid \mathrm{w}$ & $|x|$ & $\begin{aligned} 1 \\
\end{aligned}$ & $|z|$ & $\mid \mathrm{AA}$ & $\mathrm{AB}$ & \begin{tabular}{ll|}
$\mathrm{AC}$ & \\
\end{tabular} & \begin{tabular}{|l|l|} 
AD \\
\end{tabular} & $\mathrm{AE}$ & $\mathrm{AF} A$ \\
\hline 1 & & & & 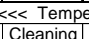 & $\begin{array}{l}\text { erature } \mathrm{M} \\
\text { Sury }\end{array}$ & leasuremen & ints >>> & 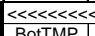 & 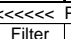 & $\begin{array}{l}\text { Pressure } M \\
\text { Fiter }\end{array}$ & Measuremer & & 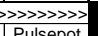 & 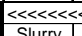 & 《< Flow M & $\begin{array}{l}\text { Measurem } \\
\text { Hij Fititate }\end{array}$ & ents $\gg>>>=$ & & & & & & & & & & & \\
\hline & DAIE & & & & & & & & & & & & & & & & \begin{tabular}{|l|l|} 
Backpulse \\
\end{tabular} & & & & & & & & & & & \\
\hline$\frac{3}{4}$ & & & $\frac{\operatorname{deg} \mathrm{C}}{\mathrm{T} 2}$ & $\frac{\mathrm{deg} C}{\mathrm{~T} 3}$ & $\frac{\mathrm{deg} \mathrm{C}}{\mathrm{T} 1}$ & $\frac{\mathrm{deg} C}{\mathrm{~T} 4}$ & $\begin{array}{l}\mathrm{deg} C \\
\mathrm{~T} 5\end{array}$ & $\frac{\mathrm{ps} \text { SId }}{\mathrm{dP2}}$ & $\begin{array}{l}\text { Pslg } \\
\text { P1 }\end{array}$ & $\begin{array}{l}\text { psid } \\
\text { dP1 }\end{array}$ & $\begin{array}{l}\frac{p s i d}{d P 3} \\
d P\end{array}$ & $\begin{array}{l}\text { psig } \\
\text { P2 }\end{array}$ & psig & gpm & $\mathrm{gpm}$ & $\frac{g p m}{\mathrm{O} 3}$ & $\frac{g p m}{O b p}$ & & & & & & & & & & & \\
\hline 5 & & & & & & & & & & & & & & & & & & & & Convers & & 851 & $\mathrm{~m} 3 / \mathrm{m} 2 / \mathrm{c}$ & $2 /$ day/barg & $\mid \mathrm{gpm} / \mathrm{tt} 2 / \mathrm{t}$ & barg & & \\
\hline 6 & eros- $04 / 19 /$ & & & & & & & & & & & & & & & & & & & & & & & & & & & \\
\hline 7 & $8 / 23 / 2004$ & 8:35:38 AM & 22.554 & 22.604 & \begin{tabular}{|l|}
22.002 \\
\end{tabular} & 22.782 & 22.204 & \begin{tabular}{|c|c|}
-0.019 \\
\end{tabular} & 0.095 & \begin{tabular}{|c|c|c|}
-0.012 \\
\end{tabular} & 0.003 & - -0.124 & 0.137 & - -0.075 & 0.003 & 14.643 & 0.004 & & & Note: $\mathrm{G}$ & Gauge PI & Pressure & $\mathrm{eP} 1$ is al & approxin & ely $1.1 \mathrm{ps}$ & sig & & \\
\hline$\frac{8}{9}$ & 8/24/2004 & 8:10:33 AM & 23.662 & & 23.679 & 22.998 & 22.735 & -0.021 & 0.122 & -0.014 & -0.001 & -0.124 & 0.134 & 23.176 & 1.205 & 14.643 & 0.003 & & & & & & & & & & & \\
\hline$\frac{9}{10}, 2>$ & 8/26/2004 & 9:14:11 AM & & 23.352 & 24.932 & 25.316 & 23.242 & -0.00 & 0.126 & -0.008 & 0.011 & -0.106 & 0.091 & & 0.043 & 14.643 & & & & & Plis C & & for 3 & & & & & \\
\hline 11 & 8/27/2004 & 7:53:29 AM & 22.649 & 22.89 & 22.298 & 22.8124 & 20.017 & -0.017 & 0.14 & -0.008 & 0.005 & -0.161 & & -0.084 & 0.003 & 14.643 & & & & vwo & & & & & & & & \\
\hline$\frac{11}{12}$ & 8/30/2004 & 7:28:55 AM & & $22.848 \mathrm{G}$ & 397 & 22.669] & 22.914 & -0.017 & 0.138 & -0.009 & 0.003 & -0.147 & 0.122 & -0.082 & 0.003 & 14.643 & & & & & & & & & & & & \\
\hline & ata - Per Mi & & & & & & & & & & & & & & & & & Ime & Ime & ress. & & TMP & MPP & m & & $\frac{m}{\operatorname{lnsi}}$ & & terer \\
\hline 14 & & $12: 35: 53 \mathrm{PM}$ & 32.815 & 25.497 & \begin{tabular}{|l|}
37.641 \\
\end{tabular} & 24.281 & 25.592 & 47.306 & 51.972 & 15.969 & 32.086 & -1.215 & -6.162 & 53.179 & 0.099 & 14.643 & 0.003 & & & 50.9 & $\begin{array}{ll}12.7 \\
\end{array}$ & 39.7 & 2.737 & $\mid 0.014$ & 0.011 & 0.000 & 0.288 & 0.25 \\
\hline & 9//2/2004 & 12:36:53 PM & 32.952 & 25.495 & \begin{tabular}{|l|l|}
37.708 \\
\end{tabular} & & 25.351 & 47.331 & 52.051 & 15.814 & 32.358 & $\mid-1.218$ & -6.162 & 54.91 & 0.099 & 14.643 & 0.003 & 1.00 & 0.01667 & 50.9 & 13.1 & 39.8 & 2.747 & 0.014 & & 0.000 & 0.286 & \\
\hline $10 \mid$ & 9 & 12:37:53 PM & 33.079| & 25.504 & 37.781 & 24.534 & 25.404 & & 52.113 & 15.27 & 32.553 & -1.209 & -6.151 & 51.016 & 0.1 & 14.643 & & 2.00 & 0.03333 & 11.0 & 12.2 & 39.9 & 2.749 & 0.014 & ( & & 0.288 & \\
\hline 11 & (9/2/2004] & 12:38:53 PM & 36.636] & 25.482 & $\mid \begin{array}{l}37.699 \\
\mid 7.69\end{array}$ & 20.633 & 25.398 & 39.014 & 52.1633 & $15.0 / 1$ & 24.4966 & 1.184 & & $4 . .422$ & 0.003 & 14.643 & & 3.00 & & & & & & & & & & .01 \\
\hline 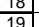 & 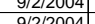 & 12:39:53 PM & 35.9077 & $25.487]$ & \begin{tabular}{|c|c|}
30.93 \\
3793
\end{tabular} & 24.732 & 25.112 & 17.323 & 52.084] & 15.026 & 2.853 & 29.085 & 21.07 & 47.515 & 0.003 & 14.643 & & 4.00 & 0.066672 & 5 & 11.3 & 10.1 & & 0.000 & & & & 0.03 \\
\hline 20 & (5) & $\frac{1.2 .453 \mathrm{FM}}{12.153 \mathrm{PM}}$ & 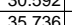 & 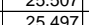 & 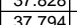 & (2.4306 & 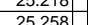 & $\frac{-7.277}{43137}$ & 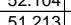 & $|0.213|$ & -18.557 & $\frac{66.13}{2138}$ & & 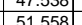 & $\begin{array}{l}0.003 \\
0.213 \\
\end{array}$ & 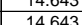 & 1.807 & 5.00 & $\frac{0.08333}{01}$ & 51.0 & 11.3 & & & & & & & -0.02 \\
\hline 21 & 多/2/2004 & \begin{tabular}{|l|}
$12: 42: 53 \mathrm{PM}$ \\
\end{tabular} & $\begin{array}{l}3.100 \\
34.919\end{array}$ & 25.508 & $\mid$\begin{tabular}{|l|l|}
37.795 \\
\end{tabular} & 25.05 & 25.448 & 44.1328 & 5 & $\begin{array}{l}\frac{12.041}{14.005} \\
\end{array}$ & $\begin{array}{l}31.408 \\
31.088\end{array}$ & $\mid$\begin{tabular}{|l|l|}
.1 .50 \\
1.466
\end{tabular} & 0.423 & 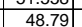 & 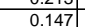 & $\begin{array}{l}14.045 \\
14.643 \\
\end{array}$ & 0.004 & 7.00 & 0.11667 & 50.8 & $\frac{11.6}{11.6}-5$ & 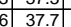 & 2.600 & 0.021 & 0.016 & & . & 0.52 \\
\hline & & \begin{tabular}{|l|}
$12: 43: 53$ PM \\
\end{tabular} & 34.955 & 25.51 & 37.791 & 25.111 & 25.33 & 44.846 & $\mid 52.247$ & 14.784 & 30.727 & 1.347 & 0.495 & 50.849 & 0.127 & 14.643 & 0.00 & 8.00 & 0.13333 & 51.1 & 1 & \begin{tabular}{|l|l|}
1 & 37.8 \\
\end{tabular} & 2.605 & 0.018 & & & & $\begin{array}{ll}0.36 \\
0.31\end{array}$ \\
\hline 20 & 9/2/2004 & 12:44:53 PM & 34.91 & 25.52 & 37.801 & 25.151 & 25.115 & 44.836 & & & 30.645 & 1.35 & & 49.507 & 0.117 & 14.643 & 0.002 & 9.00 & 0.15 & 51.0 & 11.8 & 37.7 & & 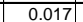 & & & 0.339 & \\
\hline 24 & 9/2/2004 & 12:45:53 PM & 34.493 & 25.526 & 37.663 & & & & & 15.389 & 30.253 & & & 48.221 & 0.112 & & & 10.00 & & $\begin{array}{lll}51.3 \\
\end{array}$ & 11.5 & & 2.595 & 0.016 & 0.012 & 0.000 & 0.329 & \\
\hline & & 12:46:53 PM & & 25.515[ & & & & & & & 32.921 & & & & 0.231 & 14.643 & & & 0.18333 & & 11.3 & & & 0.033 & & & & \\
\hline 20 & 9/2/20004 & |12:47:53 PM & $34.309 \mid$ & & $37.664 \mid$ & 25.272 & & 48.146] & & $15.458 \mathrm{i}$ & 33.861 & -1.215 & & $48.458 \mid$ & & 14.643 & & 12.00 & & 50.8 & & & & 0.016 & & & & \\
\hline & 9/2/20004 & \begin{tabular}{|c|}
$12: 48: 53 \mathrm{PM}$ \\
\end{tabular} & $34.364 \mid$ & 25.535 & 37.65 & & 25.361 & & & 15.692 & & -1.209 & & 47.05 & 0.113 & 14.643 & & 13.00 & 0.21667 & 51.4. & 11.2 & 年 & 2.811 & 0.010 & & & & 0.24 \\
\hline 28 & 9/2/2004 & 12:49:53 PM & 34.289 & 25.53 & 37.585 & 25.327 . & 25.215 & 48.345 & 52.453 & 15.75 & 33.467 & $|-1.212|$ & & 47.228 & 0.11 & 14.643 & 0.003 & $14.00 \mid$ & 0.23333 & 51.3 & 11.2 & 40.9 & 2.820 & 0.016 & 0.011 & 500 & & \\
\hline & 9/2/20004 & 12:50:53 PM & 34.191| & 25.54 & 37.541 & 25.347 & 25.311 & 47.872 & 51.876 & 16.075 & 32.668 & -1.215 & -6.136 & 52.265 & 0.108 & 14.643 & 0.003 & 15.00 & 0.25 & 50.8 & 12.4 & 40.3 & 2.776 & 0.015 & 0.011 & 0.000 & 0.274 & 0.23 \\
\hline & 9//2/2004 & 12:51:53 PM & 34.118| & 25.5514 & 37.513 & $25.348]$ & 25.416 & $47.951 \mid$ & & 16.139 & 32.785 & -1.215 & & 49.32 & 0.107 & 14.643 & & 16.00 & $\mid 0.26667$ & 50.6 & 10 & (7.-7 & 2.783 & & & & - & \\
\hline$\frac{31}{32}$ & (9/2/2004] & 12:52:53 PM & 34.029 & 25.551 & | $3 ., 498$ & 25.333 & 25.421 & 4.631 & & 16.122 & 32.317 & -1.215 & & 51.6/5 & 0.106 & & & & 0.28333 & & & 40.0 & & 0.015 & & & & \\
\hline & 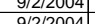 & \begin{tabular}{|l|}
2.53 .53 \\
\end{tabular} & 33.947] & & & & & 47.279 | & & & 32.4611 & -1.209 & & 4 & 0.106 & 14.643 & & 18.00| & 0.3 & 5 & 11.8 & 39.9 & 2.749 & (0.015 & 整1 & & .272 & 0.4 \\
\hline$\frac{35}{34}$ & 年 & 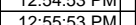 & (3.064) & 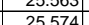 & $|37.41|$ & $\frac{25.35}{2536}$ & & 年, & 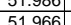 & \begin{tabular}{|c|}
15.987 \\
15.953
\end{tabular} & 32.6817 & $\begin{array}{l}-1.215 \\
-1.209 \\
\end{array}$ & & $\begin{array}{l}48.183 \\
4882\end{array}$ & $\begin{array}{l}0.106 \\
0.105 \\
-10\end{array}$ & 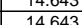 & & $\frac{19.00}{2000}$ & 0.31667 & 50.9 & $\frac{11.5}{1116}$ & 40.3 & & 0.015 & & & & \\
\hline 35 & 9/2/2004 & \begin{tabular}{|l|}
$12.56 .533 \mathrm{PM}$ \\
12.56
\end{tabular} & 33.785 & 25.569 & $\mid$\begin{tabular}{|c|}
$\mid 37.442$ \\
3742
\end{tabular} & 25351 & 25.409 & 年, & 52005 & 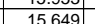 & 33025 & -1.1212 & & 4 & 0.105 & 14643 & $0.003 \quad 25$ & 2100 & 0 & 50.9 & 11. & 40.4 & $\mid 2785$ & 0.015 & 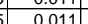 & & 0 & $\frac{0.4}{0.2}$ \\
\hline 36 & & \begin{tabular}{|l|}
$12.57 .53 \mathrm{PMM}$ \\
12.53
\end{tabular} & $\frac{23.785}{33.785}$ & & & & & 48.187 & 52.37 & 15.992 & & & & & & 14.643 & & & & & & (1) & & & & & & 0.2 \\
\hline & 9/2/2004 & 12:58:53 PM & 33.785 & 25.589 & 37.486 & 25.296 & 25.299 & 48.31 & 52.54 & $\frac{16.105}{16}$ & 32.933 & $\frac{-1.206}{-1.206}$ & & $\frac{06.468}{46.45}$ & 0.107 & 14.643 & & 23.00 & .383333 & $\frac{51.4}{51.4}$ & 11.1 & $\frac{20.6}{40.6}-2$ & 2.801 & 0.015 & & & 269 & 3 \\
\hline 38 & 9/2/2004 & \begin{tabular}{|l|} 
12:59:53 PM \\
\end{tabular} & 33.8 & 25.584 & \begin{tabular}{|l|l|}
37.511 \\
\end{tabular} & 25.301 & 25.384 & 47.951 & 51.937 & 16.19 & 32.728 & -1.212 & & 50.976 & 0.105 & 14.6 & & 24.00 & 0.4 & 50.8 & 12.1 & 40.3 & 2.781 & 0.015 & 011 & $5000+2$ & 0.266 & \\
\hline 39 & 9/2/2004 & 7:53 PM & 33.781 & 25.59 & 37. & & & 47.996 & 52.107 & & 32.796 & -1.215 & & 49.979 & 0.106 & $\begin{array}{ll}14.643 \\
\end{array}$ & & 25.00 & j.41667 & $\frac{51.0}{51.0}$ & $\frac{\frac{11.9}{11.9}}{2}$ & $\frac{40.4}{40.4}$ & & 0.015 & D11 & & & \\
\hline 40 & & 1:01:53 PM & 33.816 & & & 25.313 & & & & & & & & & & & & 26.00 & $\mid 0.43333$ & & 119 & 40.5 & 2.795 & & & & & \\
\hline 41 & 9/2/2004 & & 33.817 & 25.596 & & & & 47.924 & & 16.144 & & $\mid-1.212$ & & & & 14. & & $27.00 \mid$ & 0.45 & & $11.8 \mathrm{P}$ & & & & & & & $0.2-2>3$ \\
\hline 42 & 9/2/2004 & 1:03:53 PM & 33.842 & 25.607 & \begin{tabular}{|l|l|}
37.671 \\
\end{tabular} & 25.304 & 25.332 & 47.748 & 52.136 & 16.042 & 32.418 & $\mid-1.215$ & & 52.663 & 0.105 & 14.643 & 0.003 & 28.00 & 0.46667 & 51.0 & 12.5 & 40.1 & 2.764 & 15 & 5.011 & & 0.266 & 0.23 \\
\hline 4 & 9//2/2004] & 1:04:53 PM & 33.856 & 25.601 & 37.709 & 25.313 & 25.346 & 48.032 & 52.345 & 16.0 & 32.789 & -1.212 & & 49.357 & 0.106 & 14.6. & & 29.00 & 0.48333 & 51.2 & 11.8 & 40.4 & 2.786 & & & & 66 & $0.4-2+3$ \\
\hline & 9/2/2004] & 1:05:53 PM & & $25.618 \mathrm{G}$ & 37.74 & & & & & 16.115 & & & & & & & & 30.00 & & 51.1 & 11.9 & 40.1 & .768 & & & & & \\
\hline 45 & 91/212004 & 1:06:53 PM & 33.916] & 25.612 & 37.559 & 25.364 & 25.482 & 47.646] & 51.808 & 16.088 & 32.332 & -1.209 & & 46.535 & 0.105 & $14.6 \mathrm{r}$ & & 31.00 & 0.51667 & 50.7 & II.1. & & 2.157 & & & & & \\
\hline 17 & (1) & (535MM & 33.951 & 25.624 & $\mid$\begin{tabular}{|c|}
$\mid 37.74$ \\
37.70
\end{tabular} & |ch.539 & & $41.9 \mathrm{c}$ & & & & -1.2124 & & & 0.1 & 14. & & 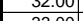 & 0.53335 & (5.4 & $\frac{\pi}{120}$ & ton & 2,7004 & & & & & \\
\hline$\frac{41}{48}$ & 管 & & 年3.5065 & $\frac{2.06}{256}>0$ & & 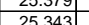 & & & & & & 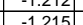 & & & & & & & 0.53 & & $\frac{1.5}{115}$ & & & & & & & \\
\hline 49 & 9/2/2004 & \begin{tabular}{|c|c|c|}
$1.0533 \mathrm{PM}$ \\
\end{tabular} & 34 & 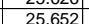 & \begin{tabular}{|c|}
37784 \\
37
\end{tabular} & 25.594 & 25392 & & & 16.113 & & 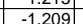 & & 50.146 & 0.105 & 146 & & 3500 & - & 510 & 11.9 & ( & 23 & & & & 0 & 0.2. \\
\hline 50 & 9/2/2004 & $1: 11: 53 \mathrm{PM}$ & 33.995 & $\frac{2.046}{25.646}$ & & $\frac{25.403}{25.43}$ & & 47.878 & & 16.201 & 32.586 & -1.206 & & 50.901 & & 14.6 & & 0 & & & & & 2.100 & & & & & \\
\hline & 9/2/2004 & 1.1253 PM & 33.98 & 25.646 & $\mid$\begin{tabular}{|l|l|}
37.768 \\
\end{tabular} & 25.378 & 25.306 & 47.943 & 25.361 & $\frac{16.12}{112.0}$ & & -1.212 & & 51.787 & 0.104 & 14.643 & & $\frac{37.00}{37.00}$ & D.61667 & 51.2 & & & . & & & & & \\
\hline 52 & 9/2/2004 & 1:13:53 & 33.985 & 25.662 & & 25.399 & $25.38 \mathrm{r}>\mathrm{C}$ & & 52.196 & $16.0 \mathrm{C}-\mathrm{s}$ & & -1.2 & & & & & & & $\mid 0.63333$ & 51.1 & & & 2.781 & & & & & \\
\hline & 9/2/2004 & $1: 14: 53 \mathrm{~F}$ & 33.976 & & \begin{tabular}{|l|l|}
37.765 \\
\end{tabular} & . & & & & & & & & & & & & & & - & & & & & & & & \\
\hline 54 & 9/2/2004 & 1:15:53 PM & 33.987 & 25.6 & \begin{tabular}{|l|l|}
37.756 \\
\end{tabular} & 25.401 & & & & 16.01 & & -1.212 & & & & 14. & & & & 510 & 12.3 & 10.4 | & 2.787 & & & & & \\
\hline & 9/2/2004 & $1: 16$ & 34.008 & 25.675 & \begin{tabular}{|l|}
37.767 \\
\end{tabular} & 25.40 & & & 51. & 15.9 & & -1.215 & & 52.44 & 0.1 & 14. $\mathrm{Y}$ & & & 0.68333 & 50. & 12 & 39.9 & 2.754 & & & & & \\
\hline 56 & 9/2/2004 & 1:17:53 PM & 34.029 & 25.681 & $\begin{array}{lll}37.782 \\
\end{array}$ & 25.413 & 25.55 & 47.957 & 52.4 & 16.0 & 32.808 & -1.209 & & $\begin{array}{l}44.72 \\
\end{array}$ & 0.10 & 14.6 & & 42.00 & | & 51. & 10.7 & 10.4[ & 2. & & & & 0.261 | & 0.22 \\
\hline 57 & 9/2/2004] & 1:18:53 PM & 34.05 & 25.697 & 37.808 & 25.449 & 25.492 & $47.38 \mathrm{~g}-\mathrm{s}$ & 51.767 & 16.016 & & -1.212 & -6.17 & 47.0 & 0.107 & 14.6. & & 43.00 & $\mid$\begin{tabular}{|l|l|}
0.71667 \\
\end{tabular} & 50.6 & 11.2 & $39.8[->3$ & 2.747 & & & & & $0.2+2+3$ \\
\hline & 9/2/2004] & 1:19:53 & 34.04] & 25.672 & 37.774 & 25.404 & & 47.9477 & & & & -1.215 & & & 0.104 & & & & 0.733 & 51.3 & 11.1. & & & & & & & \\
\hline & 91/212004] & 1:20:53 & 34.004 & 20.687 & 37.664 & 25.439 & & & 52.046 & & & $-1.2 .14>0$ & & & 0.105 & 14.6 & & 45.00 & & & 2.3 & & & & & & & \\
\hline & | & 1.21.55 & | & 20.0104 & $\mid 3.146$ & 20.5.46 & 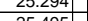 & 4.7480 & & $\frac{15.9}{15.1}$ & & -1.2124 & & & 0.104 & & & 4.000 & $\mid$ & & +4 & 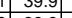 & & & & & & \\
\hline & & Averaces & | & $\frac{2.0 .1}{256}$ & $\mid$ & 20.2454 & $\frac{2.45}{25}$ & $\frac{4.1032\}}{477}$ & 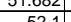 & $\frac{1.01}{16}$ & 32.342 & $\mid-1.213$ & & 年9.69 & 0.003 & 14.64 & & & & & & & & & & & & \\
\hline 63] & & & 34.9 & 257 & 378 & $\frac{25.45}{25.5}$ & & 483 & & & 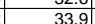 & $\frac{1.1}{14}$ & & & $\frac{3.1}{0.2}$ & & & & & 514 & (1) & & & & & & ( & \\
\hline 64 & & & 34.0 & 25.6 & 37.7 & 25.4 & & 47.9 & 52.1 & $16.6>>$ & 32.6 & -1.2 & & & $\frac{0.1}{0.1}-1$ & 14 & & & & 510 & 1118 & & & & & & 03 & \\
\hline & & & 33.8 & & & 25.2 & & 44.8 & 51.4 & & & & & & & & & & & & & & & & & & $22+2$ & \\
\hline 66 & & & 0.437 & 0.114 & 0.245 & 0.135 & & 1.379 & 0.537 & 0.524 & 1.190 & 1.116 & & 3.860 & 0.042 & 0.000 & & & & 0.537 & $\begin{array}{l}7 \\
7\end{array}$ & & \begin{tabular}{|l|}
0.087 \\
\end{tabular} & 0.006 & 0.004 & (200 & 1110 & \\
\hline 67 & Number o & of Points Us & & & 39 & & & 39 & & 39 & 39 & 39 & & 39 & 39 & & & & & 39 & & & 39 & & 39 & 39 & 39 & \\
\hline 68 & & & $n$ boxa & not incli & Iuded & & & & & & & & & & & & & & & & & & & & & & & \\
\hline 69 [ & & & & & & & & & & & & & & & & & & & & & & & & & $0.0002[\mathrm{~s}$ & gpm/tit2 & & \\
\hline
\end{tabular}


WSRC-TR-2005-00105, REVISION 0

SRNL-RPP-2005-00012, REVISION 0

RUN \# TEMP3; CAMPAIGN IV HIGH TEMPERATURE TEST

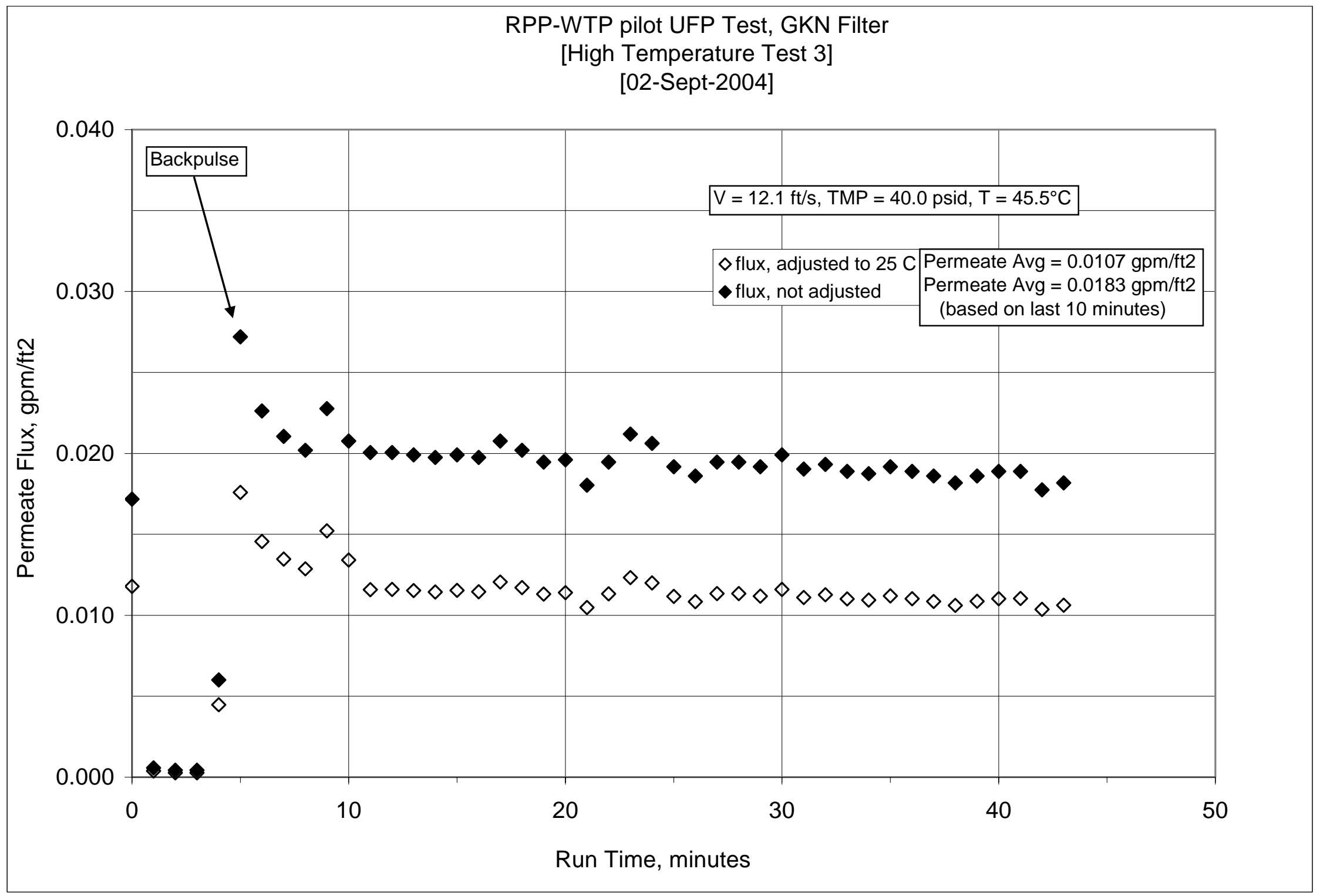


WSRC-TR-2005-00105, REVISION 0

SRNL-RPP-2005-00012, REVISION 0

RUN \# TEMP3; CAMPAIGN IV HIGH TEMPERATURE TEST

\begin{tabular}{|c|c|c|c|c|c|c|c|c|c|c|c|c|c|c|c|c|c|c|c|c|c|c|c|c|c|c|c|c|}
\hline & A & B & D & $\mathrm{E}$ & \begin{tabular}{|l|l|} 
\\
\end{tabular} & $\mathrm{G}^{\mathrm{G}}$ & $\mathrm{H}$ & $\mathrm{J}$ & $\mathrm{K}$ & \begin{tabular}{ll|} 
& $L$ \\
\end{tabular} & \begin{tabular}{|l|} 
\\
\end{tabular} & $\mathrm{N}$ & $\mathrm{O}$ & \begin{tabular}{|l|l|} 
& \\
\end{tabular} & $\mathrm{R}$ & \begin{tabular}{|l|} 
\\
\end{tabular} & & $\mathrm{v}$ & I w & $x$ & & & & $A B$ & $A C$ & & $\mathrm{AE}$ & $\mathrm{AF}$ \\
\hline$\frac{1}{2}$ & & TIME & 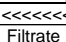 & \begin{tabular}{l|}
$\ll \ll<T e m p$ \\
$\mid$ Cleaning
\end{tabular} & $\begin{array}{l}\text { perature } \mathrm{M} \\
\text { pelary }\end{array}$ & Measureme & & & & $\begin{array}{l}\text { Pressure } \mathrm{M} \\
\text { FilterdPI }\end{array}$ & Measureme & & & 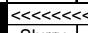 & « Flow $\mathrm{M}$ & Measureme & & & & & & & Tm Raw D & Data > & & & & \\
\hline & DAIE & & $\frac{\text { Filtrate }}{\text { deg } C}$ & \begin{tabular}{|c|} 
Cleaning \\
deg C
\end{tabular} & \begin{tabular}{|l|} 
Slury \\
deg C \\
\end{tabular} & \begin{tabular}{|c|} 
HiAmb. \\
deg C
\end{tabular} & $\begin{array}{l}\text { Lo Amb. } \\
\operatorname{deg} C\end{array}$ & $\frac{\text { BottMP }}{\text { psid }}$ & \begin{tabular}{|l|} 
Filster \\
psig
\end{tabular} & \begin{tabular}{|l|} 
Filter d \\
psid
\end{tabular} & \begin{tabular}{|l|} 
TopTMP \\
psid
\end{tabular} & \begin{tabular}{|l} 
Filtrate \\
ssig
\end{tabular} & \begin{tabular}{|l|} 
Pulsepot \\
psia
\end{tabular} & \begin{tabular}{|l|l|l|l|l} 
Slury \\
gpm
\end{tabular} & $\begin{array}{l}\text { Filtrate } \\
\text { grmm }\end{array}$ & Hi Filtate & Backpulse & & & & & & & & & & & \\
\hline 4 & & & $\frac{\mathrm{T}}{\mathrm{T} 2}$ & & & & T5 & dP2 & pro & $\frac{d P 1}{d P 1}$ & & & poly & $9 p 1$ & 92 & $\frac{\mathrm{gpm}}{\mathrm{O} 3}$ & $\frac{g p m}{O b p}$ & & & & & & & & & & & \\
\hline & & & & & & & & & & & & & & & & & & & & & & & & & $\mathrm{pm} / \mathrm{tt}$ & & & \\
\hline 6 & Zeros- 04/19 & $9 / 2 C$ & & & & & & & & & & & & & & & & & & & & & & & & & & \\
\hline & $8 / 23 / 2004$ & 8:35:38 AM & & & & 22.782 & 22.204 & -0.019 & 0.095 & -0.012 & 0.003 & -0.124 & & -0.075 & 0.003 & 14.643 & & & & & & & $P 1$ is & oprox & ely 1.1 & & & \\
\hline 8 & $8 / 24 / 2004$ & 8:10:33 AM & 23.662 & \begin{tabular}{|l|l|}
22.93 \\
\end{tabular} & \begin{tabular}{|l|l|l|}
3 & 23.679 \\
\end{tabular} & 22.998 & 22.735 & -0.021 & $\begin{array}{ll}0.122 \\
\end{array}$ & -0.014 & -0.001 & -0.124 & 0.134 & 23.176 & | 1.205 & 14.643 & 0.00 & & & & & & & & & & & \\
\hline & $8 / 26 / 2004$ & 9:14:11 AM & & 23.352 & \begin{tabular}{|l|}
24.932 \\
\end{tabular} & 25.316 & 23.242 & -0.008 & 0.126 & -0.008 & 0.011 & -0.106 & 0.091 & 48.335 & 0.043 & 14.643 & & & & & ePlis & correcte & cted for: & $1.25 \mathrm{incl}$ & es of wa & tubing & & \\
\hline 10 & 8/27//2004 & 7:53:29 AM & 22.649 & 22.89 & $22.298 \mid$ & 22.872 & 22.871 & -0.017 & 0.14 & -0.008 & 0.005 & -0.161 & 0.111 & -0.084 & 0.003 & 14.643 & 0.00 & & & WWV & & & & $<$ < Filtrate & & $<$ PERI & IABBILI & \\
\hline 11 & 8/30/2004 & 7:28:55 АM & 22.511 & 22.848 & 22.397] & 22.669 & 22.914 & -0.017 & 0.138 & -0.009 & 0.003 & -0.147 & 0.122 & -0.082 & 0.003 & 14.643 & & & & & & & & & at $25 \mathrm{C}$ & & & \\
\hline$\frac{12}{12}$ & & & & & & & & & & & & & & & & & & Time & Time & ress & Vel. & TMP & TMP & jpm & $\mathrm{pm}$ & $\mathrm{mm}$ & $\mathrm{pm}$ & \\
\hline 14 & \begin{tabular}{|c|} 
|ata - Per M M \\
$9 / 2 / 2004$
\end{tabular} & 1:42:02 PM & & & & & 25.848 & & & 15,391 & 32.477 & -1.015 & -6.145 & & & & & & & $\frac{51.4}{51 .}$ & & & & & & & & \\
\hline 15 & 9//2/2004 & 1:43:02 PM & 39.084 & 25.658 & \begin{tabular}{l|l|l|}
8 & 44.339
\end{tabular} & 24.302 & 25.937 & 46.31 & 52.598 & $\mid 15.561$ & 31.523 & 0.262 & 16.363 & $\begin{array}{r}45.000 \\
47.43 \\
\end{array}$ & 0.004 & $\frac{14.045}{14.643}$ & 0.0 & 1.00 & \begin{tabular}{|l|l|}
0.01667 \\
\end{tabular} & 51.5 & 11.3 & $\begin{array}{l}3.9 \\
38.9 \\
0\end{array}$ & $\frac{2.140}{2.683}$ & 0.001 & 0.000 & 0.000 & & \\
\hline & 9/2/2004 & 1:44:02 PM & 43.111 & 25.65 & \begin{tabular}{|l|l|l}
5 & 44.473 \\
\end{tabular} & 24.295 & 26.039 & 26.733 & 52.254 & 15.207 & 12.009 & 19.78 & 20.046 & 50.288 & 0.003 & 14.643 & & 2.00 & $\begin{array}{ll}0.03333 \\
\end{array}$ & 51.1 & 12.0 & 19.4 & 1.336 & 0.000 & & & & \\
\hline & 9/2/2004 & 1:45:02 PM| & 41.663 & 25.64 & 44.671 & 24.329 & 26.193 & 10.513 & 52.388 & 14.926 & -3.997 & 36.149 & 19.315 & 51.839 & 0.003 & 14.643 & 0.0 & 3.00 & \begin{tabular}{l|l}
0 & 0.05 \\
\end{tabular} & 51.3 & 12.3 & 3.3 & 0.225 & 0.000 & & 0.000 & 0.085 & \\
\hline 10 & & & & & & & & & & & & & & & & 14.643 & & & & & & & & & & & & \\
\hline 19 & 9/2/2004 & 1:47:02 PM & 41.33 & 25.633 & 3] 45.253 & 24.348 & 26.142 & 44.722 & \begin{tabular}{|l|l|}
51.895 \\
\end{tabular} & 13.695 & 31.792 & 1.168 & 0.409 & 51.583 & 0.19 & 14.643 & & 5.00 & 0.08333 & 50.8 & 12.3 & 38.3 & 2.638 & 0.027 & 0.018 & 0.000 & 0.460 & \\
\hline & & & & & & 24.371 & & \begin{tabular}{|c|c|c|}
45.298 \\
\end{tabular} & 52.558 & \begin{tabular}{ll|l|l|}
14.548 \\
\end{tabular} & 31.544 & & & 47.271 & $\begin{array}{l}0.158 \\
\end{array}$ & 14.643 & & 6.00 & & 51.4 & 11.3 & & & & & & & \\
\hline & $9 / 2 / 2004$ & 1:49:02 PM & $\begin{array}{l}41.756 \\
\end{array}$ & 25.61 & 145.741 & \begin{tabular}{|l|l|}
24.54 \\
\end{tabular} & 25.6 & 45.558 & \begin{tabular}{|l|}
52.619 \\
\end{tabular} & $\mid$\begin{tabular}{|l|l|l|}
15.18 \\
\end{tabular} & \begin{tabular}{|l|l|}
31.08 \\
\end{tabular} & 1.0 & & $\begin{array}{l}49.837 \\
\end{array}$ & 0.147 & $\begin{array}{l}14.643 \\
\end{array}$ & & & 0.11667 & 51. & 11.9 & $\begin{array}{l}38.3 \\
\end{array}$ & 32.642 & & 0.013 & 0.000 & 0.351 & \\
\hline & $9 / 2 / 2004$ & & $\begin{array}{l}41.902 \\
\end{array}$ & 25.625 & \begin{tabular}{|l|l|}
45.886 \\
\end{tabular} & 24.71 & & 45.348 & 52.25 & 15.168 & 31.045 & & & 51.364 & 0.141 & $\begin{array}{l}14.643 \\
\end{array}$ & & 8.0 & $\overline{0.13333}$ & 51. & 12.8 & & 2.634 & & & & & \\
\hline 23 & $9 / 2 / 2004$ & 1:51:02 PM & 40.016 & 25.624 & $\begin{array}{ll}4 & 45.953 \\
\end{array}$ & 24.795 & 25.589 & 48.368 & 52.37 & $\begin{array}{ll}15.287 \\
1\end{array}$ & 33.951 & -1.2. & -5.61 & 50.252 & 0.159 & $\begin{array}{l}14.643 \\
\end{array}$ & & 9.0 & 0.15 & 51. & 12.8 & 41.2 & 2.838 & & .015 & 0.000 & 370 & \\
\hline & 9/2/2004 & :02 PM & 41.359 & 25.623 & 45.87 & $24.899 \mid$ & 25.653 & 47.561 & 51.52 & 16.653 & 31.724 & & & 54.28 & 0.145 & 14.643 & & & 0.16667 & 50.4 & 12.9 & 39.6 & $\begin{array}{l}3.733 \\
\end{array}$ & & 0.013 & 0.000 & 0.338 & \\
\hline-1 & 9/2/2004 & 1:53:02 PM| & 41.687 & 25.634 & 4] 45.832 & 24.99 & 25.409 & 47.192 & 51.114 & 16 & 32.046 & -1.215 & -6.064 & 53.373 & 0.14 & 14.643 & & 11.00 & 0.1833 & 50. & 12. & 39.6 & 2.732 & & 012 & 0.000 & 292 & \\
\hline & $9 / 2 / 2004$ & & & & & & & & & 15.9 & 32.352 & & & & 0.14 & 14.643 & & 12.0 & & & & 39.9 & 2.153 & & & & & \\
\hline $2 t$ & $\frac{91212004}{912004}$ & & 41.908 & 25.65 & 45.76 & 25.152 & 25.6 & 47.41 & 51.412 & 15.853 & 32.432 & -1.215 & & 55.335 & 0.139 & 14. & & 13.00 & 0.21667 & 50. & 13.2. & 39 & 黑.753 & & & & & \\
\hline & 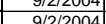 & $\frac{1.50 .02}{1.50}$ & $\begin{array}{l}42.040 \\
42071\end{array}$ & 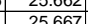 & $\mid$ & 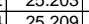 & $\frac{23.09}{2569}$ & $\frac{4.101}{4773}$ & 年 & $\begin{array}{l}13.035 \\
1584\end{array}$ & $\frac{32.21}{32519}$ & $\frac{-1.200}{-1215}$ & & 年 & 0.1380 & $\frac{14.045}{14643}$ & & $\begin{array}{l}14.00 \\
1500\end{array}$ & 0.20335 & 50. & $\frac{1.5}{116}$ & & 先 & & & & & \\
\hline 30 & 9/2/2004 & $1.58 .02 \mathrm{PM}$ & 4 & 25.078 & \begin{tabular}{|c|c|}
8.356 \\
8
\end{tabular} & 20 & $25.555^{2}$ & 47.509 & $\mid$ & $\frac{15.04}{15634}$ & 3272 & $\frac{-1.250}{-1209}$ & & $\mid$ & $\mid$ & 14643 & & 1600 & 0.2665 & 50.4 & $\frac{1.50}{121}$ & $\frac{40.1}{401}$ & $\mid \begin{array}{l}\mid 2.106 \\
\end{array}$ & $\frac{0.020}{0.020}$ & 0.011 & 0.000 & 0286 & \\
\hline & 9/2/2004 & & 42.101 & 25.669 & 45.662 & 25.251 & 25.6 & & \begin{tabular}{|l|l|}
51.903 \\
\end{tabular} & $|15.637|$ & 32.674 & -1.215 & & 5.2 .242 & 0.145 & 14.643 & & 17.0 & 0.28333 & - & 124 & & 2,768 & & & & & \\
\hline 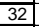 & $9 / 2 / 2004$ & 2: $200: 02$ PM & \begin{tabular}{|c|c|c|}
42.079 \\
\end{tabular} & 25.696 & \begin{tabular}{l|l|l|}
655.659 \\
6
\end{tabular} & $\frac{25.313}{25.313}$ & 25.76 & & \begin{tabular}{|l|}
51.597 \\
\end{tabular} & $\frac{\mid 15.599}{15.59}$ & & & & $\frac{21.764}{551.64}$ & 0.141 & & & 18.9 & 0.3 & $\frac{20.5}{50.5}$ & $\frac{12.3}{12.3}$ & 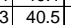 & $\frac{793}{793}$ & & & & & \\
\hline & 9/2/2004 & & 42.076 & & 45.642 & & & & 51.479 & & & & & & & 14. & & 190 & 0.31667 & 50.4 & 110 & 40 & 769 & & & & 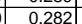 & \\
\hline 34 & & & 42.086 & 25.698 & 5.617 & 25.335 & 25.86 & & \begin{tabular}{|l|l|}
51.789 \\
\end{tabular} & \begin{tabular}{l|l|l|}
15.544 \\
\end{tabular} & 32.964 & -1.21 & & 50. & $\begin{array}{l}0.137 \\
\end{array}$ & & & 20.0 & 0.33333 & 50. & 12.0 & 40. & 2.781 & & & & & \\
\hline & & & 42.145 & & 5.613 & & & & & & & & & & & & & 21.0 & & 50 & 11.8 & & & & & & & \\
\hline 36 & & & 42.119 & 25.712 & & 25.254 & & & \begin{tabular}{|l|l|}
51.819 \\
\end{tabular} & & 33.366 & -1.2 & & 47.47 & & & & 22.4 & \begin{tabular}{|l|}
0.36667 \\
\end{tabular} & 50. & 11.3 & 40. & 2.803 & & & .000 & 0.279 & \\
\hline & $9 / 2 / 2004$ & & & 25.12 & .571 & 25.232 & 25. & & \begin{tabular}{|l|l|}
51.918 \\
\end{tabular} & & & & & & & & & 23. & 0.38333 & 50 & 11.6 & & 2.7999 & & & & & \\
\hline & & & 42.11 & 25.728 & .549 & 25.175 & 25.8 & & \begin{tabular}{|l|l|}
51.877 \\
\end{tabular} & 15.475 & 33.276 & -1.212 & & 46.249 & 0.144 & & & 24.0 & 0.4 & 50. & 11.0 & & 800 & & & & & \\
\hline 399 & 9/2/2004 & & 42.122 & 25 & 532 & 25.127 & 25.7 & & \begin{tabular}{|l|l|} 
& 51.765 \\
\end{tabular} & & & -1. & & & 0.134 & & & 25 . & 0.41667 & 50.6 & 12.2 & & 2.805 & 0.01 & & & 0.275 & \\
\hline & 9/2/2004 & & 42.1999 & 25. & (1) 45.000 & & & & & & & & & & & & & 26. & 0.43333 & 51. & 11.5 & & & & & & 0.2 & \\
\hline$\frac{41}{12}-2>$ & & & 42.1888 & & 45.506 & 25.135 & & & 51.964 & & & -1.215 & & & 0.136 & & & & 0.45 & & 12.0 & & 2.801 & & & & & \\
\hline$\frac{42}{42}>>>$ & 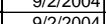 & & $\frac{42.24}{12.176}$ & & 15193 & 25.081 & & & $\begin{array}{ll}51.688 \\
5.061\end{array}$ & 15.4 & $\begin{array}{ll}33.239 \\
32231\end{array}$ & -1.2 & & & 0.136 & & & 2000 & 010923 & . & $\frac{1.50}{116}$ & 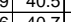 & 2802 & & & & & \\
\hline 44 & & & 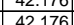 & & & & & & & & & & & & & & & & & & & & & & & & & \\
\hline 45 & 9/2/2004 & & 年 42.156 & & 5 & 25162 & & & $\mid$\begin{tabular}{|l|l|}
$\mid 51.412$ \\
51
\end{tabular} & & 32 & -1.2 & - & 50. & 0.1 & & & & 051.0 & 50 & 120 & & & & & & 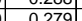 & \\
\hline $46-2>3$ & $9 / 2 / 2004$ & & 42.156 & $\frac{25.7}{25.7}$ & \begin{tabular}{|l|l|}
45.502 \\
\end{tabular} & 25.191 & & & $\mid$\begin{tabular}{|l|l|}
51.155 \\
5
\end{tabular} & & $\frac{2.192}{32.192}$ & $\frac{-1.209}{-1.209}$ & & $\frac{52.6}{5.6}$ & 0.135 & & & & & & 1200 & & & & & & & \\
\hline & & & 42.156 & & & & & & & & & & & & & & & ( & & & 134 & & & & & & & \\
\hline 48 & & & 42.133 & & 45.485 & & & & \begin{tabular}{|l|l|}
51.566 \\
\end{tabular} & & & & & & & & & & 0.56 & & 11.4 & & 59 & & & & 73 & \\
\hline & & & 42.119 & & & & & & & & & -1.215 & & & & & & & 0.58333 & & & & & & & & & \\
\hline & & & 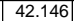 & 25. & 459 & 25.5 & 25 & & & & & -1.212 & & & 0.132 & 14 & & & 0.6 & $49.8 \mathrm{r}$ & 11.9 & & & & & & 79 & \\
\hline & & & 42.203 & & $5.47-27$ & 25.6 & 25. & & & & 32. & -1. & & 50. & & & & & & & 12.0 & & & & & & $\overline{73}$ & \\
\hline & & & 42.217 & & & & & & & & 32. & -1. & & 50. & & & & $38.8-2=$ & 0.63 & & 12.0 & 0 & & & & & 66 & \\
\hline & & & 42.163 & & & & & & & & & & & & & & & & & & 12.2 & & & & & & 74 & \\
\hline 54 & 9/2/2004 & & 42.1699 & & 4] 45.447] & 25.7 & & & 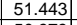 & 15.5 & & -1.2 & & & 0.132 & & & & 0.66667 & & 11.7 & & & & & & 0.276 & \\
\hline & & & & & & & & & & & & & & & & & & & & & 16 & & & & & & & \\
\hline & & & 年2.115 & & 45.3884 & 25.88 & & & $51.1 / 74$ & 15. & 32.4514 & -1.212 & & & & & & & 0.1 & & 11.6 & & & & & & & \\
\hline & & 2.26 .0 & 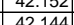 & & 40.5023 & (23.099 & 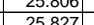 & & S5.90 & & 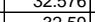 & -1.24 & & & & & & & 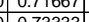 & & 11.0 & & & & & & (1) & \\
\hline & 992120 & & (42.144 & 250 & 45.304 & 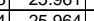 & & & $\mid$ & & & & & & & & & & 075 & & $\frac{1.5}{116}>0$ & & & & & & 0.2040 & \\
\hline & & & 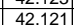 & & & & & & & & & & & & 0.125 & & & & 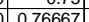 & & $\frac{1.0}{119}>0$ & & & & & & & \\
\hline & & & 42.114 & & & & & & $\mid$\begin{tabular}{|l|l|}
51.106 \\
5
\end{tabular} & 15.746 & & $\frac{-1.209}{-1.209}$ & & 49.449 & & & & & 333 & $500-2-3$ & & & & & & & 0269 & \\
\hline & & & 42069 & & & & & & & & & & & & & & & & & & 124 & & & & & & & \\
\hline & & & 41.278 & 26.046 & 15.235 & 25.993 & & 46.781 & .184 & 15.631 & 31.927 & -1.212 & & & & & & & & & & & & & 0.011 & 0.000 & 0.277 & \\
\hline & 9/2/2004 & $\mid$ 2:32:02 PM & \begin{tabular}{|l|l|}
40.979 \\
\end{tabular} & 26.06 & $\begin{array}{ll}6 & 45.191\end{array}$ & $1 \quad 25.942$ & $|25.744|$ & 47.051 & \begin{tabular}{|l|}
51.402 \\
\end{tabular} & $\mid 15.637$ & 32.184 & 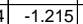 & -6.044 & \begin{tabular}{|l|}
52.117 \\
\end{tabular} & 0.132 & 14.643 & & & & & & & & & & & & \\
\hline
\end{tabular}


WSRC-TR-2005-00105, REVISION 0 SRNL-RPP-2005-00012, REVISION 0

RUN \# TEMP3; CAMPAIGN IV HIGH TEMPERATURE TEST - CONT.

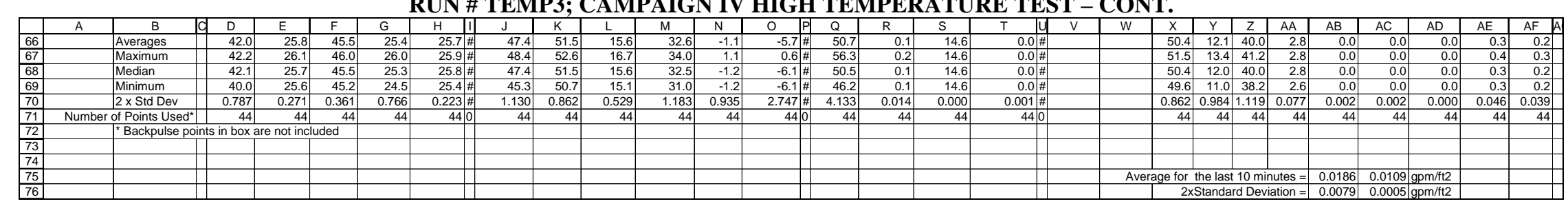


WSRC-TR-2005-00105, REVISION 0

SRNL-RPP-2005-00012, REVISION 0

RUN \# TEMP4; CAMPAIGN IV HIGH TEMPERATURE TEST

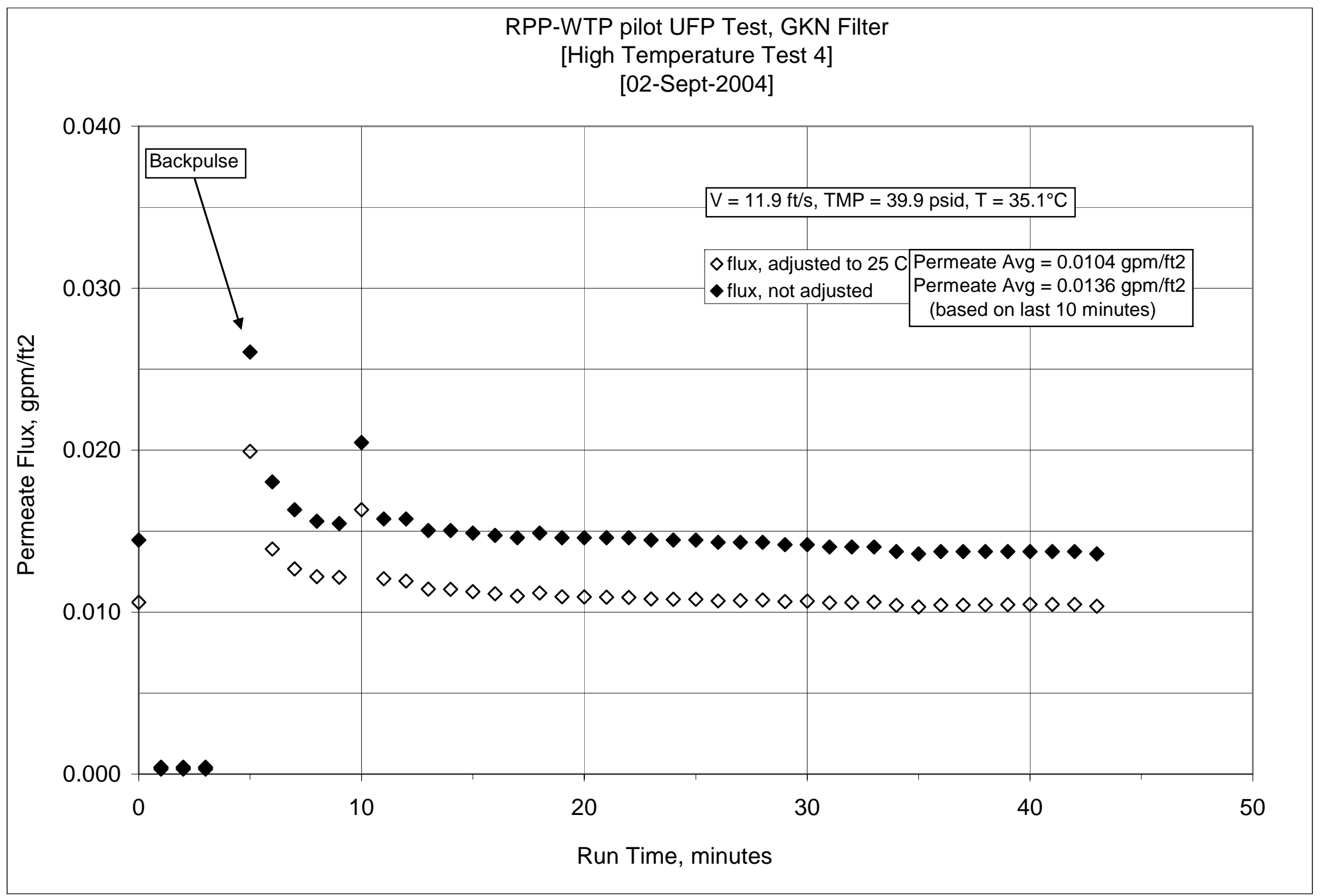


WSRC-TR-2005-00105, REVISION 0

SRNL-RPP-2005-00012, REVISION 0

RUN \# TEMP4; CAMPAIGN IV HIGH TEMPERATURE TEST

\begin{tabular}{|c|c|c|c|c|c|c|c|c|c|c|c|c|c|c|c|c|c|c|c|c|c|c|c|c|c|c|c|c|}
\hline & A & $B$ & $\mathrm{D}$ & $\frac{1}{E<E_{1}}$ & $F$ & $G$ & $\frac{H}{1-1}$ & $\mathrm{~J}$ & $\begin{array}{ll}l & K \\
<\ll<\ll<< & \end{array}$ & $\frac{L}{\mathrm{~L}}$ & $\begin{array}{l}\mathrm{M} \\
\text { sureme }\end{array}$ & $\frac{\mathrm{N}}{\mathrm{I}} \mathrm{N}$ & $\frac{1}{1} 0$ & \begin{tabular}{l|}
$Q$ \\
$<<<<<<$
\end{tabular} & $\begin{array}{l}\mathrm{R} \\
\ll<\mathrm{Flow}\end{array}$ & $\begin{array}{c}\mathrm{s} \\
\text { easurem }\end{array}$ & $1 \mathrm{~T}$ & $\mathrm{~V}$ & $\frac{w}{1}$ & $\frac{|x|}{<\ll \ll c c}$ & $\frac{1 \mathrm{Y}}{\mathrm{C} \text { Y }}$ & $\mathrm{z}$ & IAA & $A B$ & AC & AD & $\mathrm{AE}$ & AF $A$ \\
\hline 2 & DATE & TIME & Filtrate & Cleaning & S Slurry & $\begin{array}{l}\text { Hi Amb. } \\
\text { Hiteme }\end{array}$ & Lo Amb. & BotTMP & Filter & $\begin{array}{l}\text { Pressuren } \\
\text { Filter dP }\end{array}$ & TopTMP & Filtrate & Pulsepot & & Filtrate & $\begin{array}{l}\text { Hivedsureme } \\
\text { Hi Filtate }\end{array}$ & & & & & & & & & & & & \\
\hline & & & $\operatorname{deg} C$ & $\mid \operatorname{deg} C$ & $\operatorname{deg} C$ & \begin{tabular}{|l|}
$\operatorname{deg} \mathrm{C}$ \\
\end{tabular} & $\operatorname{deg} \mathrm{C}$ & psid & psig & psid & psid & psig & psig & $\mathrm{gpm}$ & $\mathrm{gpm}$ & $\mathrm{gpm}$ & $\mathrm{gpm}$ & & & & & & & & & & & \\
\hline 4 & & & & & & & & & & $\mathrm{dP1}$ & $\mathrm{dP3}$ & & & & & $\mathrm{Q}_{3}$ & Qbp & & & & & & & & & & & \\
\hline 5 & 7eros- 04/19 & & & & & & & & & & & & & & & & & & & & & & & & & & & \\
\hline & & $8: 35: 38 \mathrm{AM}$ & & & & & 22.204 & -0.019 & 0.095 & -0.012 & 0.003 & -0.124 & 0.13 & -0.075 & 0.003 & $\begin{array}{l}14.643 \\
\end{array}$ & & & & Filter \$ & & & & & & & & \\
\hline & $8 / 24 / 2004$ & 8:10:33 AM & & & 23.679 & 22.998 & 22.735 & & 0.122 & & -0.001 & $\mid-0.124$ & & 23.176 & 1.205 & 14.643 & & & & $\begin{array}{l}\text { Convers } \\
\end{array}$ & & 851 & $1 / \mathrm{m} 3 / \mathrm{m} 2 / \mathrm{I}$ & 2/day/barg & $\mathrm{gpm} / \mathrm{ttzl} / \mathrm{s}$ & $2 / \mathrm{barg}$ & & \\
\hline 9 & $8 / 26 / 2004$ & 9:14:11 AM & 25.819 & 23.352 & 24.932 & 25.316 & 23.242 & -0.008 & $\begin{array}{ll}0.126 \\
\end{array}$ & \begin{tabular}{|c|c|}
-0.008 \\
\end{tabular} & 0.011 & -0.106 & 0.09 & 48.335 & 0.043 & $\mid 14.643$ & & & & & & & & & & & & \\
\hline 10 & $8 / 27 / 2004$ & 7:53:29 AM & 22.649 & 22.89 & & 22.872 & 22.871 & -0.017 & 0.14 & \begin{tabular}{|c|c|}
-0.008 \\
\end{tabular} & 0.005 & -0.161 & 0.11 & \begin{tabular}{|c|c|}
-0.084 \\
\end{tabular} & 0.003 & $\begin{array}{l}14.643 \\
\end{array}$ & & & & Note: & nuge & & 1 is & proxim & $\mid \frac{1}{1 y} 1.1$ & & & \\
\hline & 8/30/2004 & 7:28:55 AM & 22.511 & 22.848 & 22.397 & 22.669 & 22.914 & $\begin{array}{l}-0.017 \\
\end{array}$ & 0.138 & 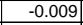 & $\begin{array}{l}0.003 \\
\end{array}$ & -0.147 & 0.122 & -0.082 & 0.003 & $\begin{array}{l}14.643 \\
\end{array}$ & & & & & & & & & & & & \\
\hline & & & & & & & & & & & & & & & & & & & & & & & & & & & & \\
\hline $\mid \frac{13}{14}$ & & & & & & & & & & & & & & & & & & & & WVV & & & & Filtrat & & & & \\
\hline & & & & & & & & & & & & & & & & & & & & & & & & & & & & \\
\hline \begin{tabular}{|l|l}
16 \\
\end{tabular} & Jata - Per M! & inute & & & & & & & & & & & & & & & & Mine & $\begin{array}{l}y_{1} \text { Hour } \\
\text { Hour }\end{array}$ & $\begin{array}{l}\text { Press. } \\
\text { psig. }\end{array}$ & $\begin{array}{l}\text { Welis } \\
\text { fits }\end{array}$ & psi & thar & 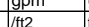 & & onsi & pim & ay/bar \\
\hline \begin{tabular}{l|l}
17 \\
\end{tabular} & 9/2/2004 & $2: 50: 23 \mathrm{PM}$ & 36.412 & 26.085 & 34.216 & $\begin{array}{l}26.413 \\
\end{array}$ & 25.635 & 47.858 & 52.138 & $\begin{array}{l}17.347 \\
\end{array}$ & 31.349 & -1.212 & $-6.24 \mathrm{~K}$ & 53.5 & 0.101 & $\begin{array}{ll}14.643 \\
\end{array}$ & & & & 51.0 & 12. & 39.6 & \begin{tabular}{|l|l|}
52.731 \\
\end{tabular} & $\mid$ & 0.011 & 0.000 & 0.268 & $\frac{0.23}{0.03}$ \\
\hline & & & & & & & & 43.971 & \begin{tabular}{|l|l|l} 
\\
\end{tabular} & 17.513 & 27.174 & & & & & & & & & & & 35.6 & & & & & & \\
\hline 19 & 9/2/2004 & 2:52:53 PM & 36.975 & 26.078 & 33.665 & 26.3 & & 27.106 & 51.785 & 17.467 & 10.104 & \begin{tabular}{|c|c|}
4 & 19.019 \\
\end{tabular} & 20.7 & 52.592 & 0.003 & 14.643 & & & 1.0333 & 50.7 & 12 & 18. & 1.283 & 0.000 & & & 0.017 & \\
\hline & $\frac{9 / 2 / 2004}{9 / 2 / 2004}$ & $2.53: 23 \mathrm{PM}$ & & $\frac{26.062}{26053}$ & & 26.294 & $25 . / 16$ & $\frac{12.943}{24.8}$ & & $\frac{11.1 / 5}{12721}$ & $\frac{-3.642}{1230}$ & $\begin{array}{l}233.343 \\
8\end{array}$ & $\frac{91.44}{9.35}$ & $\begin{array}{l}49.4344 \\
5.4764\end{array}$ & 0.003 & $\begin{array}{l}14.643 \\
14643\end{array}$ & & & & & & $\frac{4.1}{184}$ & & & & & & \\
\hline 22 & $\begin{array}{l}9 / 21 / 2004 \\
9 / 2004\end{array}$ & $\begin{array}{l}2.54 .23 \mathrm{PM} \\
2: 55: 23 \mathrm{PM}\end{array}$ & $\begin{array}{c}34.044 \\
34.844 \\
\end{array}$ & 26.053 & $\begin{array}{l}35.100 \\
32.923 \\
\end{array}$ & $\begin{aligned} 26.4 \\
26.45\end{aligned}$ & 25.807 & $\begin{array}{r}24.48 \\
44.203 \\
\end{array}$ & \begin{tabular}{|l|}
50.278 \\
50.625 \\
\end{tabular} & $\frac{12.131}{15.092}$ & 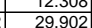 & $\frac{21.474}{0.484}$ & 0.354 & $\begin{array}{l}54.710 \\
50.744\end{array}$ & $\begin{array}{l}0.152 \\
0.182 \\
\end{array}$ & $\begin{array}{l}\frac{14.045}{14.643} \\
\end{array}$ & & $\begin{array}{l}4.00 \\
5.00\end{array}$ & 0.0833 & $\frac{49.2}{49.5}$ & $\frac{13}{12}$ & $\frac{18.4}{37.1}$ & $\frac{1.260}{2.555}$ & $\frac{0.108}{0.026}$ & & $\frac{0.004}{0.001}$ & $\frac{4.353}{0.538}$ & 3.69 \\
\hline & 9/2/2004 & 2:56:23 PM & 34.565 & 26.085 & $\begin{array}{l}32.798 \\
32.798\end{array}$ & 26.353 & 25.774 & 44.525 & 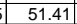 & \begin{tabular}{|l|l|}
15.897 \\
\end{tabular} & 29.321 & \begin{tabular}{|c|c|}
0.664 \\
\end{tabular} & & 51.975 & 0.126 & 14.643 & & 6.00 & & & & & 2546 & & & & & 0.46 \\
\hline & $9 / 2 / 2004$ & & 34.296 & & & & & $\begin{array}{l}44.608 \\
\end{array}$ & & & & & & & 0.114 & $\begin{array}{l}14.643 \\
\end{array}$ & & & & & & & & & & & & \\
\hline 25 & $9 / 2 / 2004$ & $2: 58: 23 \mathrm{PM}$ & 34.04 & \begin{tabular}{|l|l|}
26.088 \\
\end{tabular} & 33.062 & 26.27 & \begin{tabular}{l|l}
25.762 \\
\end{tabular} & & \begin{tabular}{|l|l|}
51.319 \\
\end{tabular} & 16.491 & 28.718 & \begin{tabular}{|c|c|}
0.603 \\
\end{tabular} & 0.5 & 51.222 & 0.109 & 14.643 & & 8.0 & 0.13333 & 50.2 & $\frac{12}{12} \mathrm{X}$ & 36. & 2524 & 0.016 & & & 0.333 & \\
\hline & & & & & & & & 44.94 & & 16.7 & 29.056 & \begin{tabular}{l|l|}
6 & 0.658 \\
\end{tabular} & & 53.852 & 0.108 & 14.643 & & 9.00 & & 50.6 & & 37.0 & & 0.015 & & 0.000 & & \\
\hline 227 & $9 / 2 / 2004$ & 3:00:23 PM & 33.273 & 26.096 & 34.103 & $\begin{array}{l}26.013 \\
\end{array}$ & 25.675 & 47.551 & & $\begin{array}{l}16.605 \\
\end{array}$ & 31.81 & \begin{tabular}{l|l|}
-1.212 \\
\end{tabular} & & 53.335 & 0.143 & \begin{tabular}{|l|l|l|l|l|}
1433 \\
\end{tabular} & & & & & & 39. & & & & & & \\
\hline $\begin{array}{ll}286 \\
20\end{array}$ & 9/2/2004 & 3:01:23 PM & 33.243 & 26.07 & 34.774 & 25.848 & 25.694 & $\begin{array}{l}477.437 \\
\end{array}$ & \begin{tabular}{|l|l|} 
\\
\end{tabular} & 16.365 & 32.03 & -1.212 & & 50.031 & 0.11 & $\mid 14.643$ & & 11.00 & 0.18333 & $\begin{array}{ll}49.8 \\
\end{array}$ & 11. & 39.7 & 2.739 & 0.016 & & & 0.304 & \\
\hline & 9/2/2004 & 3:02:23 PM & 33.435 & 26.06 & \begin{tabular}{|l|}
35.223 \\
\end{tabular} & 25.667 & 25.754 & 47.689 & 51.062 & 16.323 & 32.243 & -1.212 & -6.174 & 55.811 & 0.11 & 14.643 & & 12.00 & 0.2 & 49.9 & $13.3 \mathrm{r}$ & 40.0 & D. 2.756 & 0.016 & 0.012 & 0.000 & 0.298 & \\
\hline$\frac{3}{31}$ & 9/2/2004 & 3:03:23 PM| & 33.523 & 26.049 & \begin{tabular}{|l|}
35.083 \\
\end{tabular} & 25.441 & 25.603 & 46.897 & 50.374 & $\begin{array}{r}15.9 \\
\end{array}$ & 31.732 & -1.212 & & 50.976 & 0.105 & 14.643 & & 10.0 & 0.21667 & 49.3 & 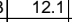 & 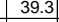 & 2.111 & 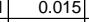 & & 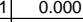 & 年 & \\
\hline & & & & & & 25.369 & & & & & 32.399 & & & & & & & & & & & & & & & & & \\
\hline & $\begin{array}{l}9 / 2 / 2004 \\
9 / 2 / 2004\end{array}$ & $3.050 .2 \mathrm{~s}$ & $\begin{array}{l}33.574 \\
33574\end{array}$ & $\frac{2.0449}{26046}$ & $\mid$\begin{tabular}{|c|}
3.532454 \\
3534
\end{tabular} & \begin{tabular}{|c|}
25.353 \\
\end{tabular} & $\frac{25.196}{25055}$ & $\begin{array}{l}471.41 \\
475\end{array}$ & 5 & $\mid 15.047$ & $\frac{32.599}{32.18}$ & \begin{tabular}{|c|c|c|}
8212 \\
8
\end{tabular} & $\frac{-0.21}{-622}$ & 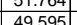 & $\begin{array}{l}0.144 \\
0.103\end{array}$ & $\begin{array}{l}14.045 \\
14643\end{array}$ & & $\frac{15.00}{1600}$ & $\begin{array}{l}0.25 \\
0.26667\end{array}$ & $\begin{array}{ll}49.6 \\
49.6\end{array}$ & $\frac{12.3}{111.8}$ & $\begin{array}{l}40.0 \\
39.7 \\
\end{array}$ & $\begin{array}{l}2.758 \\
72.736 \\
\end{array}$ & $\begin{array}{l}0.015 \\
0.015 \\
\end{array}$ & $\frac{0.011}{0.011}$ & $\frac{0.000}{0.000}$ & 0.282 & \\
\hline 34 & 9/2/2004 & 3:07:23 PM & 33.576 & 26.042 & $|35.422|$ & \begin{tabular}{|l|l|}
25.349 \\
\end{tabular} & 25.067 & 47.302 & $\mid 50.691$ & \begin{tabular}{|l|}
15.797 \\
\end{tabular} & 32.385 & \begin{tabular}{|l|l|}
5 & -1.215 \\
\end{tabular} & & 52.034 & 0.102 & 14.643 & & 17.00 & 0.28333 & 49.6 & 12. & 年 & \begin{tabular}{|l|l|}
8.747 \\
\end{tabular} & & & & 0.276 & $\frac{1}{6}$ \\
\hline - 35 & $9 / 2 / 2004$ & 3:08:23 PM & 33.572 & 26.033 & 35.516 & 25.385 & 25.033 & 477.743 & $\begin{array}{l}550.965 \\
\end{array}$ & $\begin{array}{l}15.834 \\
\end{array}$ & 32.705 & -1.212 & -6.214 & 50.225 & 0.104 & 14.643 & & 18.00 & 0.3 & \begin{tabular}{l|l}
49.8 \\
\end{tabular} & $\overline{12.0}$ & $\begin{array}{ll}40.2 \\
\end{array}$ & 2.773 & \begin{tabular}{|c|}
0.015 \\
\end{tabular} & $\overline{0.011}$ & 0.000 & 0.278 & \\
\hline 36 & 9/2/2004 & 3:09:23 PM & 33.602 & 26.034 & 35.591 & 25.456 & 24.979 & 47.399 & \begin{tabular}{|l|l|} 
\\
\end{tabular} & 15.697 & 32.648 & -1.212 & & 49.797 & 0.102 & 14.643 & & 19.00 & 0.31667 & 49.1 & 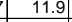 & 40.0 & 2.159 & 0.010 & 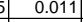 & 0.000 & 0.274 & \\
\hline & & 3:10:23 PM & & & & & & & & & & & & & & & & & & & & & & & & & & \\
\hline$\frac{38}{8}$ & 9/2/2004 & 3:11:23 PM & 33.693 & 26.017 & 35.687 & 25.569 & 24.91 & 47.702 & 50.969 & 15.707 & 32.943 & -1.212 & & 49.382 & 0.102 & 14.643 & & 21.0 & 0.35 & 49.8 & & 40.5 & 2.780 & & .01 & & & \\
\hline 年 & & 3:12:23 PMI & & & & & & & & & & & & & & & & & & & & & & & & & & \\
\hline & 9/2/2004 & 3:13:23 PM & 33.764 & 26.008 & 35.728 & 25.49 & 24.928 & 47.244 & 50.631 & 15.664 & 32.531 & $\begin{array}{l}1 \\
\text { 1) }\end{array}$ & & 49.034 & 0.101 & 14.6 & & 23.00 & 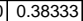 & 49.5 & 11. & & 2.750 & & & & 271 & \\
\hline & $9 / 2 / 2004$ & 3:14:23 PM & 33.766 & 25.991 & 35.745 & 25.482 & $24.97 \mathrm{~s}$ & 47.7 & 51.197 & 15.63 & 32.902 & -1.212 & & 50.283 & 0.101 & 14.643 & & 24.00 & 0.4 & 50 & 12 & 40.3 & $\begin{array}{l}32.779 \\
\end{array}$ & | & .011 & & 0.268 & \\
\hline 42 & 9/2/2004 & 3:15:23 PM & 33.809 & 25.994] & 35.787 & 25.56 & 25.03 & 47.88 & 51.331 & 15.774 & 32.888 & -1.212 & -6.22 .2 & 49.322 & 0.101 & 14.643 & & 25.00 & 0.41667 & 50.4 & 11. & 40.4 & 2.784 & 0.014 & .01 & 0.000 & 0.261 & \\
\hline & $9 / 21212004$ & & 33.861 & & & & & & $\begin{array}{l}80.846 \\
8\end{array}$ & & & & & & 0.1 & & & 26.00 & 0.43333 & 49.7 & & 40.2 & & & & & & \\
\hline 告4 & $9 / 2 / 20044$ & 3:17:23 PM & $\begin{array}{l}33.893 \\
32803\end{array}$ & 25.999 & \begin{tabular}{|l}
35.65 \\
35.65
\end{tabular} & 25.8911 & $\frac{25.28}{2525}$ & 47.729 & 50.9 & $\frac{15.526}{15.577}$ & $\begin{array}{l}33.208 \\
32.208\end{array}$ & $\begin{array}{l}8 \\
8\end{array}-1.212 .25$ & -6.23 & 48.692 & 0.1 & 14.643 & & 27.06 & 0.45 & 年. & 些1.4 & 40.5 & $\frac{2.790}{2.700}$ & 年14 & & & 265 & \\
\hline 46 & $\begin{array}{l}9 / 2 / 20044 \\
9 / 2 / 2004 \mid\end{array}$ & $3.19 .23 \mathrm{PM}$ & 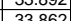 & 26007 & $\mid$ & 25.955 & & & 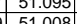 & $\frac{15.611}{15701}$ & 33.048 & $\begin{array}{l}8.1 .215 \\
6.1212\end{array}$ & & & & 14.6 & & 28.00 & 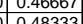 & & & 40.4 & & & & & 606 & \\
\hline 47 _ & $9 / 2 / 2004$ & $3.20 .23 \mathrm{PM}$ & 33852 & $\begin{array}{l}26.007 \\
26.007\end{array}$ & 35.417 & 26.06 & & & 50703 & 15.537 & 32.611 & 1.1212 & & & 0.099 & 146 & & 3000 & 0.40 & 496 & 111 & & 2757 & & & & 0267 & \\
\hline 48 & 9/2/2004 & $3: 21: 23 \mathrm{PN}$ & 33.847 & 26.002 & & 26.1 & & & & & & & & & & & & & & 50.2 & & & & & & & 262 & \\
\hline 49 & 9/2/2004 & 3:22:23 PM & 33.818 & 26.022 & 35.308 & 26.14 & 25.51 & 47.947 & 51.545 & 15.531 & 33.259 & \begin{tabular}{|c|c|}
9 & -1.209 \\
\end{tabular} & & & & & & $\begin{array}{l}32.00 \\
32.00\end{array}$ & 0.53333 & 500 & 114 & 406 & 2799 & & & & 261 & \\
\hline 56 & $9 / 2 / 2004$ & 3:23:23 PM & 33.794 & 26.023 & 35.245 & 26.176 & 25.562 & 47.679 & 51.06 & 15.495 & 33.179 & \begin{tabular}{|l|l|}
9 & -1.212 \\
\end{tabular} & & 48.097 & 0.098 & \begin{tabular}{|l|l|l|l|}
14.643 \\
\end{tabular} & & 33.00 & 0.55 & 49 & 1t. & 40.4 & 42.787 & 0.014 & & & & \\
\hline & 9/2/2004 & 3:24:23 PM & 33.759 & 26.013 & 35.176 & 26.196 & 25.56 & 46.872 & 50.318 & 15.76 & 31.891 & -1.212 & & & & $\begin{array}{l}14.643 \\
\end{array}$ & & 34.0 & \begin{tabular}{l|l}
0.56667 \\
\end{tabular} & 49 & & 39.4 & $\begin{array}{ll}42.715 \\
\end{array}$ & & & & 0.265 & \\
\hline & $9 / 2 / 2004$ & 3:25:23 PM & 33.706 & 26.035 & 35.157 & 26.192 & 25.5 & 47. & \begin{tabular}{|l|l|}
50.334 \\
\end{tabular} & \begin{tabular}{|l|l|l|}
16.019 \\
\end{tabular} & 32.075 & $\begin{array}{l}-1.212 \\
\end{array}$ & & & 0.095 & $\begin{array}{lll}14.6 \\
\end{array}$ & & 35. & $\begin{array}{l}0.58333 \\
\end{array}$ & 49. & 12.4 & 39.6 & 62.730 & & & & .261 & \\
\hline & $9 / 2 / 2004$ & 3:26:23 PM & 33.668 & 26.041 & 35.134 & 26.163 & & & 51.141 & $\begin{array}{ll}16.109 \\
\end{array}$ & 32.346 & $\begin{array}{l}6 \\
6\end{array}$ & & & & 14.6 & & 36 & 0.6 & 50 & 11.5 & 4 & 2.756 & & & & .261 & \\
\hline$\frac{54}{55}$ & $9 / 2 / 2004$ & 3:27:23 PN & 33.61 & 26.042 & 35.101 & 26.12 & & 47.723 & 51.286 & & 32.315 & -1.212 & & & & & & 37. & 0.61667 & 50. & $11.7 \mathrm{r}$ & & \begin{tabular}{|l|l|} 
& 2.759 \\
\end{tabular} & & & & 261 & \\
\hline & 9/2/2004 & & 33.565 & 26.032 & 35.042 & 26.085 & & 47.48 & 51.265 & $15 . .6$ & 32.402 & -1.212 & & & & & & 38.00 & 0.63333 & 50.1 & 12.3 & 39.9 & 9. 2.754 & & & & 0.262 & \\
\hline 告地 & $9 / 2 / 20044$ & 3:29:23 PM & 33.535 & 26.032 & 34.9977 & 26.039 & 25.35 & 47.768 & \begin{tabular}{|c|c|c|}
51.168 \\
\end{tabular} & 16.051 & 32.566 & -1.212 & & 52.138 & 0.096 & 14.643 & & $39 . c$ & 0.65 & 50 & 12.4 & 40.2 & 2.769 & 0.014 & 4 & & 0.261 & \\
\hline 58 & $\frac{9 / 2 / 2004}{9 / 2 / 2004 \mid}$ & & & & & & & & & & 2.416 & & & & & $\frac{14.6}{14.6}$ & & 24 & \begin{tabular}{|l|l|}
0.666673 \\
0.63333
\end{tabular} & 50.0 & 11.5 & $\frac{40.0}{40.4}$ & $\frac{2 .}{12 .}$ & & & & $\frac{0.262}{0259}$ & \\
\hline 59 & $\mid 9 / 2 / 2004$ & 3:32:23F & & & & 26.0 & & & & & 32.796 & \begin{tabular}{|l|l|}
6 & -1. \\
\end{tabular} & & & & 14.6 & & 42.00 & 0.7 & 50.1 & \begin{tabular}{|l|}
$\mid 11.4$ \\
$\mid 1$
\end{tabular} & 40.2 & \begin{tabular}{|l|}
2.2 .769 \\
2
\end{tabular} & 0.0 & \begin{tabular}{|l|l|}
4 & 0.010 \\
\end{tabular} & 0.0 & 0.261 & \\
\hline 60 & $9 / 2 / 2004$ & $3: 33: 23$ & 33.382 & & $\begin{array}{l}34.957 \\
\end{array}$ & \begin{tabular}{|l|l|}
25.949 \\
\end{tabular} & & 47.6 & \begin{tabular}{|l|l|}
551.193 \\
\end{tabular} & & 32.835 & \begin{tabular}{|l|l|}
5 & -1 \\
\end{tabular} & & & 0 & & & & \begin{tabular}{|l|l|}
0.71667 \\
\end{tabular} & $\begin{array}{l}50.1 \\
\end{array}$ & 112.0 & 40.3 & \begin{tabular}{|l|l|}
2.776 \\
\end{tabular} & 0.0 & 0.010 & 0.0 & 0.258 & \\
\hline & $9 / 2 / 2004$ & & 33.357 & 26.047 & \begin{tabular}{|l|l|}
34.977 \\
\end{tabular} & 25.904 & & & 51.163 & & |32.484 & & & & & & & 44. & 0.73 & 50.0 & 12.3 & & & & & & & \\
\hline 62 & 9/2/2004 & 3:35:23 PM & 33.311 & 26.04 & 34.975 & 25.842 & 25.294 & 47.797 & 51.224 & \begin{tabular}{|l|l|l|l|}
15.974 \\
\end{tabular} & $\mid 32.64$ & \begin{tabular}{ll|l}
4 & -1.212 \\
\end{tabular} & & $\begin{array}{lll}49.677 \\
\end{array}$ & 0.096 & & & & 0.75 & 1 & \begin{tabular}{l|l}
1111.8 \\
\end{tabular} & 40.2 & 2.773 & 14 & .010 & & 260 & \\
\hline 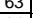 & 9/2/2004 & 3:36:23 PM & & & & & & & & & 32.5 & $\begin{array}{l}3 \mid \\
\mid-1.209 \\
\end{array}$ & & & & 14.6 & & & & & & 401 & & & & & 261 & \\
\hline & $9 / 2 / 2004$ & $3: 3$ & 33.263 & 26.036 & & 25.92 & & 47.36 & 50.8 & 16.0 & 32.38 & -1.212 & & & & 14.6 & & 470 & 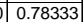 & & & & & & & & 257 & \\
\hline 65 & $9 / 2 / 2004$ & 3:38:23 PN & 33.248 & 26.036 & 35. & 25.968 & 25.2 & 47.522 & 50.95 & \begin{tabular}{ll|l|}
16.034 \\
\end{tabular} & 32.512 & \begin{tabular}{|l|l|}
2 & -1.212 \\
\end{tabular} & & 47.3 & 0.0 & 14.6 & & 48 & 0.8 & & & 40 & & & & & 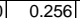 & \\
\hline 66 & 9/2/2004 & 3:39:23 PM & 33.259 & \begin{tabular}{|l|l|}
26.027 \\
\end{tabular} & 35.061 & 25.929 & 25.276 & 47.87 & 51.518 & $\begin{array}{ll}16.207 \\
\end{array}$ & 32.434 & \begin{tabular}{l|l}
4 & -1.212 \\
\end{tabular} & -6.255 & $\begin{array}{l}49.399 \\
\end{array}$ & 0.095 & 14.6 & & 49.0 & 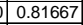 & 50.4 & 11.8 & 40.2 & 2.768 & 0.014 & 0.010 & 0. & 0.258 & 8 \\
\hline & $\begin{array}{r}9 / 2 / 2004 \\
9 / 2 / 204 \\
\end{array}$ & 3:40:23 PM & $\begin{array}{ll}33.234 \\
33075\end{array}$ & $\frac{26.037}{26.036}$ & $\begin{array}{l}35.085 \\
35.104 \\
\end{array}$ & $\frac{25.949}{25.943}$ & \begin{tabular}{|l|}
25.381 \\
\end{tabular} & $\begin{array}{r}47.2 \\
49203 \\
\end{array}$ & \begin{tabular}{|l|}
50.739 \\
51.468
\end{tabular} & \begin{tabular}{|l|}
15.821 \\
16004
\end{tabular} & $32.317 \mid$ & 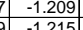 & -6.194 & $\begin{array}{r}49.61 \\
48246 \\
\end{array}$ & 0.095 & $\frac{14.643}{14643}$ & & & $\begin{array}{r}0.83333 \\
0.85 \\
\end{array}$ & 49.6] & 11.8 & & \begin{tabular}{|l|}
2.741 \\
2872 \\
\end{tabular} & 0.014 & 0.010 & 0.000 & 0.260 & 0. \\
\hline
\end{tabular}


WSRC-TR-2005-00105, REVISION 0 SRNL-RPP-2005-00012, REVISION 0

RUN \# TEMP4; CAMPAIGN IV HIGH TEMPERATURE TEST - CONT.

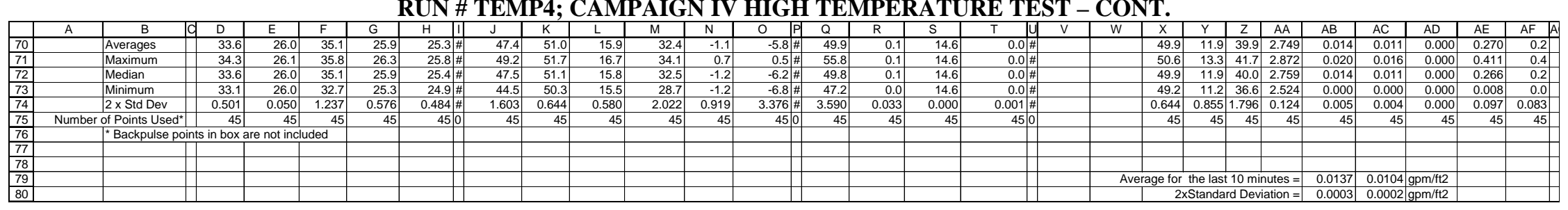


WSRC-TR-2005-00105, REVISION 0

SRNL-RPP-2005-00012, REVISION 0

RUN \# TEMP5; CAMPAIGN IV HIGH TEMPERATURE TEST

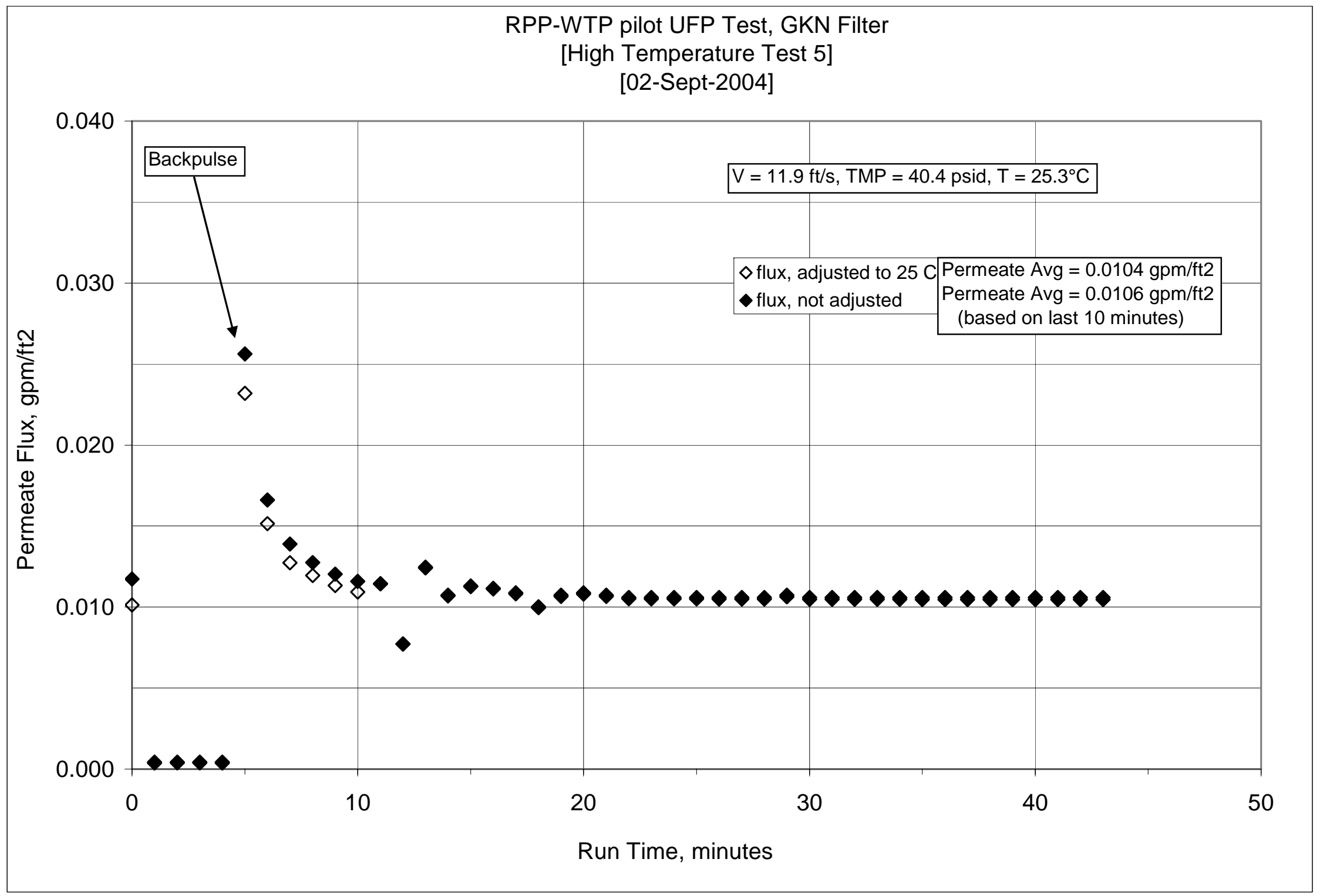


WSRC-TR-2005-00105, REVISION 0

SRNL-RPP-2005-00012, REVISION 0

RUN \# TEMP5; CAMPAIGN IV HIGH TEMPERATURE TEST

\begin{tabular}{|c|c|c|c|c|c|c|c|c|c|c|c|c|c|c|c|c|c|c|c|c|c|c|c|c|c|c|c|c|}
\hline & A & B & $\mathrm{D}$ & \begin{tabular}{|l|l} 
\\
\end{tabular} & \begin{tabular}{|l|l}
$F$ \\
\end{tabular} & \begin{tabular}{|l|l}
$G$ \\
\end{tabular} & $\mathrm{H}$ & \begin{tabular}{l|l}
$\mathrm{J}$ & 1 \\
\end{tabular} & \begin{tabular}{|l|l}
$\mathrm{K}$ & \\
\end{tabular} & \begin{tabular}{|l|l|}
$\mathrm{L}$ \\
\end{tabular} & \begin{tabular}{|l|} 
\\
\end{tabular} & $\mathrm{N}$ & 0 & \begin{tabular}{l|l} 
\\
\end{tabular} & \begin{tabular}{l|l}
$\mathrm{R}$ & \\
\end{tabular} & $\mathrm{s} /$ & \begin{tabular}{|l|l|}
$\mathrm{T}$ & $\mathrm{U}$ \\
\end{tabular} & \begin{tabular}{l|l} 
\\
\end{tabular} & W & $x$ & & & & $A B$ & $\begin{array}{ll}\mathrm{AC} \\
\end{array}$ & $A D$ & $\mathrm{AE}$ & AF $A$ \\
\hline 1 & & & & $\begin{array}{l}\text { 《e Tempe } \\
\text { Cleaninal }\end{array}$ & $\begin{array}{l}\text { erature Me } \\
\text { Sulury }\end{array}$ & |easureme & & BotTMP & 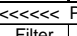 & $\begin{array}{l}\text { Pressure } \mathrm{N} \\
\text { Filtard }\end{array}$ & $\begin{array}{l}\text { Measuremer| } \\
\text { TonTMP }\end{array}$ & ents $\gg \gg>$ & |>>>>>>>| & 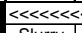 & $\ll<$ Flow $M$ & Measuremen & & & - & & & & & & & & & \\
\hline & DATE & TIME & Filtrate & Cleaning & \begin{tabular}{|l|l|} 
\\
$\mid$
\end{tabular} & Hi Amb. & Lo Amb. & & & & TopTMP & Filtrate & & & \begin{tabular}{l|l|} 
Filtrate & $\mathrm{B}$ \\
\end{tabular} & Hi Filtate & Backpulse & & & & & & & & & & & \\
\hline 4 & & & $\frac{\mathrm{deg} C}{\mathrm{~T} 2}$ & $\frac{\mathrm{deg} C}{\mathrm{~T} 3}$ & \begin{tabular}{|l|}
$\operatorname{deg} C$ \\
$T 1$
\end{tabular} & \begin{tabular}{|l|}
$\operatorname{deg} C$ \\
$T 4$
\end{tabular} & \begin{tabular}{|l|}
$\operatorname{deg} C$ \\
$T 5$
\end{tabular} & $\begin{array}{l}\frac{p s i d}{d P 2} \\
d P 2\end{array}$ & $\frac{\mathrm{psig}}{\mathrm{P} 1}$ & psid & $\begin{array}{ll}\text { psid } \\
d{ }^{2} 3\end{array}$ & \begin{tabular}{|l|} 
psig \\
P2
\end{tabular} & psig & gpm & gpm & gpm & gpm & & & & & & & & & & & \\
\hline & & & & & & & & & & & & & & & & & & & & & & & & & & & & \\
\hline & eros- $04 / 19 /$ & & & & & & & & & & & & & & & & & & & & & & & & & & & \\
\hline 7 & $8 / 23 / 2004$ & $8: 35: 38 \mathrm{Am}$ & & & \begin{tabular}{|l|}
22.002 \\
\end{tabular} & 22.782 & 22.204 & \begin{tabular}{|c|}
-0.019 \\
\end{tabular} & $\mid$\begin{tabular}{|c|c|} 
\\
\end{tabular} & \begin{tabular}{|c|c|c|}
-0.012 \\
\end{tabular} & \begin{tabular}{|l|l|}
0.003 \\
\end{tabular} & $\mid-0.124$ & & \begin{tabular}{|c|c|}
-0.075 \\
\end{tabular} & \begin{tabular}{|c|}
0.003 \\
\end{tabular} & \begin{tabular}{|l|l|}
14.643 \\
\end{tabular} & & & & Jote: G & & & 1 is & & |y 1.1 & & & \\
\hline 8 & 8/24/2004 & $8: 10: 33 \mathrm{AM}$ & 23.662 & 22.93 & \begin{tabular}{|l|}
23.679 \\
\end{tabular} & 22.998 & 22.735 & $\begin{array}{l}-0.021 \\
\end{array}$ & 0.122 & -0.014 & -0.001 & -0.124 & 0.134 & 23.176 & 1.205 & \begin{tabular}{|l|l|}
14.643 \\
\end{tabular} & .0 & & & & & & & & & & & \\
\hline & 8/26/2004 & 9:14:11 AM & 25.819 & 23.352 & 24.932 & 25.316 & 23.242 & -0.008 & 0.126 & -0.008 & 0.011 & -0.106 & 0.091 & 48.335 & 0.043 & 14.643 & & & & SS & $\mathrm{P}$ Plis ch & correcte & ted for 3 & 25 in & of $W_{c}$ & tubing & & \\
\hline & $8 / 27 / 2004$ & 7:53:29 AM & & 22.89 & \begin{tabular}{|l|}
22.298 \\
\end{tabular} & 22.872 & & -0.017 & 0.14 & -0.008 & 0.005 & -0.161 & 0.111 & -0.084 & 0.003 & 14.643 & & & & VWVV & & & & Filtrate I & e Flux $>$ & < PERI & ABBIL & $\gg>$ \\
\hline & 8/30/2004 & 7:28:55 АM & 22.511 & 22.848 & 22.397 & 22.669 & 22.914 & -0.017 & 0.138 & -0.009 & 0.003 & -0.147 & 0.122 & -0.082 & 0.003 & 14.643 & & & & & & & & & at $25 \mathrm{C}$ & & & \\
\hline & ata - Per Mi & & & & & & & & & & & & & & & & & Time & \begin{tabular}{|l} 
Time \\
Hour
\end{tabular} & $\begin{array}{l}\text { Press. V V } \\
\text { psig ff }\end{array}$ & \begin{tabular}{|l}
$\mid \begin{array}{l}\mid \text { Vel. } \\
\text { ftls }\end{array}$ \\
\end{tabular} & $\begin{array}{l}\text { TMP } \\
\text { psi }\end{array}$ & IMP & gtit & pm & $\frac{\mathrm{gmm}}{\mathrm{ft2} / \mathrm{psi}}$ & $\frac{\mathrm{pm}}{2 / \mathrm{psi}}$ & $\begin{array}{l}\text { neter } \\
\text { daylbar }\end{array}$ \\
\hline & 9/2/2004 & 4:00:30 PM & 30.3 & 26.004 & 25.022 & 26.491 & 25.558 & 48.934 & \begin{tabular}{|l|}
53.008 \\
\end{tabular} & 17.485 & 32.389 & 1.212 & -6.255 & 50.09 & 0.082 & \begin{tabular}{|l|l|}
14.643 \\
\end{tabular} & 0.003 & & & 51.9 & 11.9 & 40.7 & 2.803 & 0.012 & $\mid 0.010$ & 0.000 & 0.249 & $\frac{0.2}{0.2}$ \\
\hline & 9/2/2004 & 4:01:30 PM & & 25.987 & 25021 & 26.38 & & 45.686 & \begin{tabular}{|l|l|}
53.041 \\
\end{tabular} & 17.423 & & \begin{tabular}{|l|} 
\\
\end{tabular} & & & & & & & .01000 & & & 37.4 & & & & & & \\
\hline & 9/2/2004 & 4:02:30 PM & 29.409 & 25.997 & \begin{tabular}{|l|}
25.041 \\
\end{tabular} & 26.385 & 25.397 & 9994 & \begin{tabular}{|l|}
52.921 \\
\end{tabular} & 17.361 & 16.06 & \begin{tabular}{|l|l|l|l|l|} 
\\
\end{tabular} & 21.818 & 48.871 & 0.003 & 14.643 & & 2.00 & $\begin{array}{l}0.0 .03333 \\
0\end{array}$ & 51.8 & 11.6 & 24.5 & 1.691 & 0.000 & 0.000 & 0.000 & & 0.01 \\
\hline & 9/2/2004 & 3:30 PM & 29.512 & 25.996 & 25.064 & 26.309 & 25.265 & 16.365 & \begin{tabular}{|l|l|}
52.735 \\
5.192
\end{tabular} & 17.238 & $\begin{array}{l}-0.342 \\
-205\end{array}$ & 30.851 & 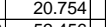 & $\begin{array}{ll}47.906 \\
5.9277\end{array}$ & 0.003 & 14.643 & & 3.00 & 0.05 & 51.6 & 11.4 . & & 0.552 & 0.000 & & & 0.047 & 0.04 \\
\hline 18 & 9/2/2004 & 4:30 PM & 30.309| & 25.988 & 25.006 & 26.335 & 25.382 & 7.69 & 51.462 & 12.08 & -3.855 & 38.43 & 52.456 & 51.827] & 0.003 & 14.643 & & 4.00 & 0.06667 & 50.3 & 12.3 & 1.99 & 0.132 & 0.000 & 0.000 & 0.000 & 0.193 & 0.16 \\
\hline & 9/2/2004 & & 28.577 & 25.991| & 24.989 & 26.258 & $\frac{25.3 \mathrm{~s}}{25.37}$ & 年4.153 & \begin{tabular}{|l|l|}
5.52 \\
5.52
\end{tabular} & 13.738 & & $1.564 \mid$ & & 48.967 & 0.179 & 14.643 & & 5.00 & 08333 & D.6.6 & & 年 & 2.594 & 0 & & & & \\
\hline & $\begin{array}{l}9 / 2 / 2004 \\
9 / 20004\end{array}$ & & 28.31 & 25.994 & 24.95 & 26.254 & $\frac{25.321}{2.520}$ & 45.1717 & $\mid$ & 10.035 & & 0.10 & & 4.0.095 & 0 & $\begin{array}{l}14.643 \\
1.637\end{array}$ & & & & $5.44 \mathrm{~s}$ & & & 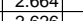 & & 0.015 & 0.000 & & \\
\hline & 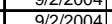 & & $\frac{28.126}{2731}$ & 25.998 & 20.4976 & $\frac{26.21}{26.19}$ & & 年5.549 & \begin{tabular}{|c|}
52.38 \\
52.47
\end{tabular} & $\frac{15.16}{10377}$ & & 0.122 & & $\begin{array}{l}4.8960 \\
4.0209\end{array}$ & on 0.097 & $\begin{array}{l}14.643 \\
1.163\end{array}$ & & & $\frac{0.1606}{0.1232}$ & . & $1 \frac{1.9}{11.7}$ & 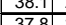 & 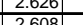 & 0.014 & & & . & 年 \\
\hline & 9/2/2004 & & 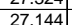 & 25.587 & 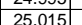 & 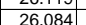 & & & 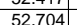 & 16.669 & & & & 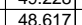 & | & & & 年 & 0.10003 & 16 & & & 年 & & & & & 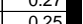 \\
\hline 24 & 9/2/2004 & $30 \mathrm{PM}$ & 27.112 & 25.975 & 25.038 & 26.047 & 25.08 & & \begin{tabular}{|l|}
52.817 \\
\end{tabular} & 16.69 & & 0.748 & & $\frac{4.017}{48.2}$ & 0.081 & 14.6 & & 10.0 & 0.16667 & 51.7 & 11.5 & 38.1 & 2.624 & 0.012 & & & 0.287 & 0.24 \\
\hline & 9/2/2004 & $30 \mathrm{PM}$ & 26.948 & 25.98 & 25.068 & 26.027 & 25.09 & 46.259 & 53.124 & 16.902 & 30.068 & 0.794 & & 47.824 & \begin{tabular}{|l}
0.08 \\
\end{tabular} & 14.643 & & 11.00 & \begin{tabular}{|l|l|}
0.18333 \\
\end{tabular} & E2.0 & $\frac{11.4}{11.4}$ & $\frac{38.2}{38.2}$ & .631 & 0.011 & & & & 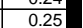 \\
\hline & 9/2/2004 & 2:30 PM & 27.111 & 25.969 & 25.072 & 26.032 & 25.134 & 49.641 & \begin{tabular}{|l|l|}
53.056 \\
\end{tabular} & 16.865 & \begin{tabular}{|l|l|}
33.83 \\
\end{tabular} & -1.215 & -5.448 & 46.343 & 0.054 & 14.643 & & 12.00 & 0.2 & \begin{tabular}{c|c|}
51.9 \\
\end{tabular} & 11.0 & 41.7 & 2.878 & 0.008 & $\overline{008}$ & 000 & 285 & $\overline{0.1}$ \\
\hline & 9/2/2004 & & & & & & & & & & & & & & & & & & 21667 & & & & & & & & & \\
\hline 28 & 9/2/2004 & 4:30 PM] & 27.03 & 25.972 & 25.135 & 26.055 & 25.04 & & 52.903 & & 323 & -1.212 & & 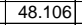 & | & 14. & & 14.00 & $\overline{23333}$ & (1.8 & 111 & $\begin{array}{ll}41 \\
4\end{array}$ & 2.860 & & & & & \\
\hline & 9/2/2004 & & 26.983 & & & & & & \begin{tabular}{|l|}
52.94 \\
\end{tabular} & 16.987 & & & & 47.463 & 0.079 & & & 15.00 & & $11.8 \mathrm{~B}$ & & & & & & & & \\
\hline & 9/2/2004 & 5:30 PM & & 25.961 & 25.164 & 25.968 & 24.9 & & \begin{tabular}{|l|}
53.006 \\
\end{tabular} & 17.023 & & -1.215 & & 50.202 & 0.078 & 14.6 & & 16.6 & 0.26667 & 51.9 & & 41.9| & .891 & & & & & 0.2 \\
\hline & 9/2/2004 & & 26.894 & 25.9 & 25.204 & 25.963 & & & \begin{tabular}{|r|}
52.243 \\
\end{tabular} & 17.929| & 32.141 & -1.215 & & 50.227 & 0.076 & $14.6 \mathrm{C}$ & & 17.00 & 0.28333 & 51.1 & 12.0 & 40.6] & $2.798 \mid$ & & 0.011 & 0.000 & 267 & 0.23 \\
\hline & 9/2/2004 & :30 PM & 26.844 & 25.956 & 25.214 & 25.928 & 24.94 & & \begin{tabular}{|l|l|}
52.561 \\
\end{tabular} & 17.632 & 32.432 & -1.212 & & 47.399] & 0.07 & 14. & & & & 51.4 & 11.3 & & 813 & & & & .244 & 0.21 \\
\hline & $\begin{array}{l}9 / 2 / 20004 \\
9 / 2 / 2004\end{array}$ & & $\frac{26.782}{27.2}$ & 25.939 & $\frac{2.2333}{20.233}$ & $\frac{25.927}{2.527}$ & $\frac{24.95}{20.9}$ & & \begin{tabular}{|l|}
5.2647 \\
\end{tabular} & $\frac{11.548}{\mid 1.545}$ & $\frac{32.504}{32.50}$ & $\frac{-1.212}{-\frac{1212}{2}}$ & & 50.859 & $\frac{0.075}{0.075}$ & $14.6 \mathrm{C}$ & & & 0.31667 & 51. & & 40.8 & 2.816 & & & 000 & & \\
\hline & 9/2/2004 & & 2 & & (3) & 2 & & & $\frac{417}{107}$ & 1.4 & & - 1.215 & & - 49.301 & & & & 20. & 0.33333 & 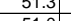 & 11.8 & (n) & ( & & & & & \\
\hline & $\begin{array}{l}9 / 2 / 20044 \\
9 / 2 / 2004\end{array}$ & & $\frac{2.1034}{26654}$ & $\frac{25.93}{25021}$ & $\frac{249}{264}$ & 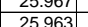 & & & 597 & & & 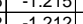 & & 年 & & & & 21. & 0.35 & S5.1.0 & $\frac{11.6}{110}$ & & 2.814 & & & & & \\
\hline & 9/2/2004 & $4: 23: 30 \mathrm{PM}$ & 26.614 & 25.916 & \begin{tabular}{|l|}
25.284 \\
25.8
\end{tabular} & 25.938 & 24.9 & & $\mid$\begin{tabular}{|}
$\mid 52.339$ \\
\end{tabular} & 17.892 & 31.87 & 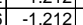 & & 51.153 & 0.074 & \begin{tabular}{|l|}
14.643 \\
\end{tabular} & & 23.00 & \begin{tabular}{|l|l|l|}
0.38333 \\
\end{tabular} & 51.2 & 12.2 & 40.4 & \begin{tabular}{|l|l|}
2.788 \\
\end{tabular} & 0.011 & 0.011 & 0.000 & 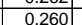 & + \\
\hline & & & 26.573 & & & $25.907 \mid$ & & & & & & & & 49.1 & & & & & & 51.0 & 11.7. & & & & & & & 0.2 \\
\hline & 9/2/2004 & & 26.537 & 25.904 & 25.317 & 25.911 & 24.94 & & \begin{tabular}{|l|}
52.036 \\
\end{tabular} & 17.805 & & -1.212 & & 51.135 & 0.074 & 14.6 & & 25 . & 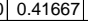 & 50.9 & 12.2 & & 2.789 & & 0.011 & 0.000 & & 0.22 \\
\hline & 9/2/2004 & 1 & 26.496 & 2.097 & 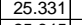 & 25.89 & & & 52.127 & 17.717 & 32.124 & 1.214 & & o.tor & $0.07-1$ & & & 20.4 & 0.43333 & 0 & & & 1.100 & & & & & \\
\hline & & & 26.46 & 25.886 & 20.345 & 2.588 & 24.94 & & 1924 & & & -1.215 & & 49.839] & 0.074 & & & $27.6 \mathrm{Cl}$ & & & & 0.4 & 186 & & & & & \\
\hline & $\begin{array}{ll}91 / 2120 \\
012120\end{array}$ & & 26.43 & & & & & & 52.14 & & & & & & & & & & & 5 & 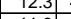 & & & & & & & \\
\hline 告4 & & & 20.412 & & 20.371 & & & & & & & - & & & & & & & 0.48335 & & & & 2.19 & & & & & \\
\hline & & & $\frac{2.050}{26.36}$ & & . & & & & & & & & & & & & & & & 5 & $\frac{1.8}{12.2}$ & & & & & & & + \\
\hline & 9/2/2004 & & 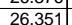 & & $\mid$ & & & & & & & & & & & & & & & 50 & & & & & & & & \\
\hline 47 & 9/2/2004 & & 26.341 & & $25.426 \mid$ & & & & & & & -1.2 & & 50.58 & & & & 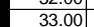 & & & 年 & & & & & & & \\
\hline & 9/2/2004 & & 26.321 & & & & & & & & & & & 52.4 & & & & & 0.56667 & & & & & & & & & \\
\hline & & & & & & & & & & & & & & & & & & & & & & & & & & & & \\
\hline & $9 / 2 / 200$ & & 26.293 & & & & & & & & & & & & & & & & & & 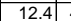 & & & & & & & \\
\hline & & & 26.269 & & & & & & & & & -1.2 & & & & & & & & & & & & & & & & \\
\hline & 9/2/2004 & & 26.261 & 25.9 & 25.455 & 26.3 & & & & 17.5 & & & & 51.5 & & & & & \begin{tabular}{|l|l|}
0.63 \\
\end{tabular} & & & & & & & & & \\
\hline & & & 26.246 & & 5 & & & & & & & & & & & & & & & & & & & & & & & \\
\hline & 9/2/2004 & & 26.221 & 25.8 & $25.451 \mid$ & & & & & 17.4 & 32.3 & -1.2 .7 & & & & & & & 0.66 & & 2. & & & & & & & $\mathrm{~T}$ \\
\hline & & & 26.208 & & & 26.1 & & & & & & & & & & & & & 0.68333 & & & & & & & & & \\
\hline & 9/2/200 & & 26.182 & & & 26.15 & & & 51.939 & & & & & & & & & & 0.7 & & & & & & & & 8 & t \\
\hline & & & & & & & & & & & & & & & & & & & & & & & & & & & & \\
\hline & & & $\frac{2.145}{2.515}$ & & (1) & 20.4 & & & & & & -1.213 & & & & & & & & & & & & & & & & \\
\hline & 9/2/20044 & & $\frac{2.1544}{2613}$ & & 2547 & 26071 & & & & 1017214 & & & & 47758 & & & & & & & & & & & & & & \\
\hline & MILILOU4 & 4.40.50 $\mathrm{FIII}^{\prime}$ & 20.104 & 20.090 & \begin{tabular}{|l|l|} 
\\
\end{tabular} & 20.011 & 20.100 & 40.004 & 01.901 & 17.600 & 32.211 & $\mid-1 .<4$ & & 47.100 & & $\mid 14.043$ & & 40.00 & & 50.8 & & & 2.100 & 0.010 & 0.004 & & 0.255 & \\
\hline & & & 26.6 & & 5.3 & 26 & & & & & & & & & 0.1 & & & 27.0 & 0.5 & 51.1 & 11.9 & 4 & 2.8 & & & & 6 & \\
\hline & & Maximum & 27.3 & 26 & 25.5 & 26.3 & $25.3:$ & & 53.1 & 18.1 & 33.9 & 0.8 & & 52.4 & 0.1 & 14. & 0.0 & 46.0 & 0.8 & 2.0 & 12.5 & & 2.9 & & & & 0.315 & \\
\hline & & & 26.5 & & 25. & & & & & & & & & & & & & & 0.5 & & 11.9 & & & & & & & \\
\hline & & & 20.14 & 24 & 25.04 & 25.9 & 24 & 45.7 & S1.5 & 10.4 & 30.0 & -1.24 & $=0$ & 40.3 & 0.1 & 1.4.6 & & 80 & 0.1 & 0.3 & 11.0 & 301.8 & 2.00 & & & & (849 & \\
\hline & & Pointst & 0.682 & & 0.239 & 0.241 & & 1.956 & $\frac{0.801}{39}$ & $\frac{21}{30}$ & $1 ., 944$ & 1.182 & 4.1060 & & 0.010 & & & & 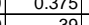 & & & 告3 & 27 & 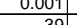 & 1 & , to & 年 & \\
\hline & Number $\mathrm{c}$ & Points 4 & 39 & & 39 & & 390 & 39 & 39 & 39 & 39 & 39 & 390 & 39 & 39 & 39 & 39 & 39 & 39 & 39 & 39 & 39 & 39 & 39 & 39 & 39 & 39 & \\
\hline & & & & & & & & & & & & & & & & & & & & & & & & & & $\mathrm{gpm} / \mathrm{ft} 2$ & & \\
\hline & & & & & & & & & & & & & & & & & & & & $2 \times S$ & Standard & rd Deviat & jiation $=\square$ & 0.0001 & $0.0001 \mathrm{~g}$ & $\mathrm{gpm} / \mathrm{tt} 2$ & & \\
\hline
\end{tabular}


WSRC-TR-2005-00105, REVISION 0

SRNL-RPP-2005-00012, REVISION 0

RUN \# TEMP6; CAMPAIGN IV HIGH TEMPERATURE TEST

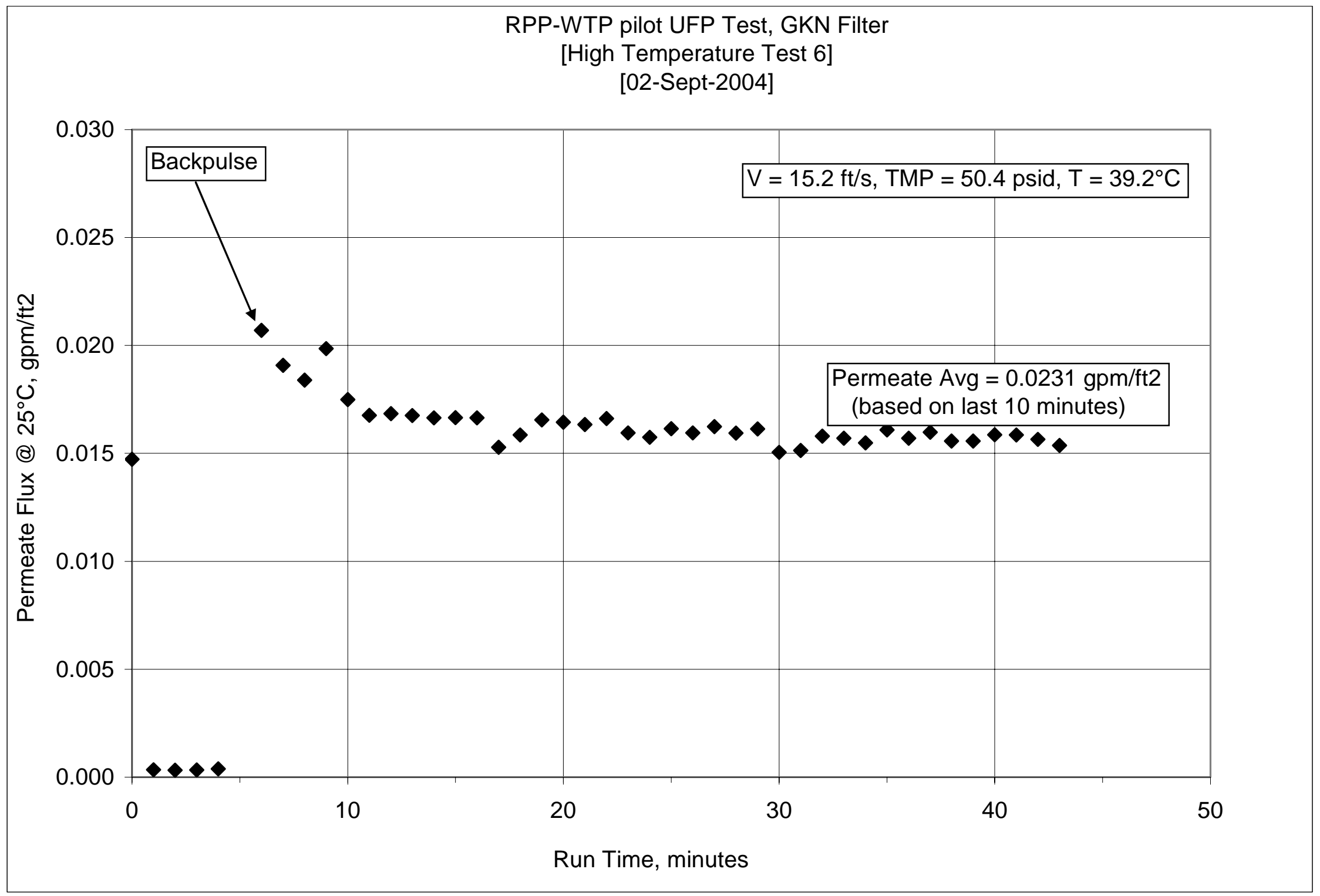


WSRC-TR-2005-00105, REVISION 0

SRNL-RPP-2005-00012, REVISION 0

RUN \# TEMP6; CAMPAIGN IV HIGH TEMPERATURE TEST

\begin{tabular}{|c|c|c|c|c|c|c|c|c|c|c|c|c|c|c|c|c|c|c|c|c|c|c|c|c|c|c|c|c|}
\hline \multirow{3}{*}{\begin{tabular}{|l|} 
\\
1 \\
\end{tabular}} & & & & & & & & & & & & & & & & & & & & & & & & & & & & \\
\hline & $\mathrm{A}$ & B & $\mathrm{D}$ & $E$ & \begin{tabular}{|l|} 
\\
\end{tabular} & \begin{tabular}{|l|l|} 
& $G$ \\
\end{tabular} & $\mathrm{H}$ & \begin{tabular}{l|l}
$\mathrm{J}$ \\
$\mathrm{y}$
\end{tabular} & \begin{tabular}{|l|l|}
$\mathrm{K}$ & \\
\end{tabular} & L & I & \begin{tabular}{|l|l|}
$\mathrm{N}$ & \\
\end{tabular} & 0 & $\mathrm{Q}$ & $\begin{array}{ll} \\
\end{array}$ & $\mathrm{s}$ & $\mathrm{T}$ & $\mathrm{v}$ & w & $\mathrm{x}$ & $\begin{array}{ll}\mid \mathrm{y} \\
\end{array}$ & $\mathrm{z}$ & $1 \mathrm{AA}$ & $A B$ & \begin{tabular}{|l|l|} 
& $A C$ \\
\end{tabular} & \begin{tabular}{|l|l|} 
& $A D$ \\
\end{tabular} & $\mathrm{AE}$ & $\mathrm{AF}$ \\
\hline & & & $\begin{array}{l}\ll \ll<< \\
\text { Fitrate. }\end{array}$ & 《e Temp & & $\begin{array}{l}\text { Measureme } \\
\text { Hi Amb }\end{array}$ & 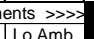 & $\begin{array}{l}\text { खerere } \\
\text { BotTMP }\end{array}$ & 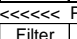 & $\begin{array}{l}\text { Pressure } \\
\text { FestiterdP }\end{array}$ & Measureme & & & & K<< Flow & Measurem & & & & & & & & & & & & \\
\hline \begin{tabular}{|l|}
3 \\
3 \\
\end{tabular} & DAIt & & & & \begin{tabular}{|l|} 
Slurry \\
dofor
\end{tabular} & \begin{tabular}{|l|} 
Hi Amb. \\
\end{tabular} & Lo Amb. & & & & & & Pulsepot & & & & & & & & & & & & & & & \\
\hline$\frac{3}{4}$ & & & & & & & $\operatorname{deg} C$ & psid & psig & psid & & psig & & gpm & gpm & gpm & & & & & & & & & & & & \\
\hline & & & & & & & & & & & & & & & & & & & & & & & & & & & & \\
\hline 6 & Seros- 04/19 & 2004 & & & & & & & & & & & & & & & & & & & & & & & & & & \\
\hline & 8/23/2004 & $8: 35: 38 \mathrm{AM}$ & 22.554 & 22.604 & 22.002 & 22.782 & 22.204 & -0.019 & 0.095 & -0.012 & 0.003 & $\begin{array}{l}-0.124 \\
\end{array}$ & $\begin{array}{ll}0.137 \\
\end{array}$ & -0.075 & 0.003 & 14.643 & 0.004 & & & & & $\sqrt{46.985}$ & FT2 & & & & & \\
\hline 8 & $8 / 24 / 2004$ & & 23.662 & 22.93 & 23.679 & \begin{tabular}{|l|}
22.998 \\
\end{tabular} & 22.735 & -0.021 & 0.122 & -0.014 & -0.001 & -0.124 & 0.134 & 23.176 & 1.205 & 14.643 & 0.003 & & & Convers & ision & 851 & $\mathrm{~m} 3 / \mathrm{m} 2$ & Idaylbarg & $\mathrm{rg} / \mathrm{gpm} / \mathrm{tt} 2 \mathrm{I}$ & $2 /$ barg & & \\
\hline & 6/2004 & 9:14:11 AM & & & & 25.316 & & & & & & & & & & 14.643 & & & & & & & & & & & & \\
\hline & $8 / 27 / 2004$ & 7:53:29 AM & 22.649 & 22.89 & & & & -0.017 & 0.14 & -0.008 & & -0.161 & & -0.084 & & 14.643 & & & & Note: & ug & & P1 is & prc & ly 1 . & & & \\
\hline 11 & 8/30/2004 & 7:28:55 AM & 22.511 & 22.848 & 22.397 & 22.669 & 22.914 & -0.017 & 0.138 & -0.009 & 0.003 & -0.147 & 0.122 & -0.082 & 0.003 & 14.643 & 0.006 & & & & & & & & & & & \\
\hline$\frac{12}{13}$ & & & & & & & & & & & & & & & & & & & & Wyy & & & & $\begin{array}{l}1.25 \text { inch } \\
<\text { Filtratte }\end{array}$ & ches of wat & $\begin{array}{l}\text { trubing } \\
\text { K PFPM }\end{array}$ & & \\
\hline 14 & & & & & & & & & & & & & & & & & & & & & & & & & Dat $25 \mathrm{C}$ & & 1000 & \\
\hline & & & & & & & & & & & & & & & & & & Time & Time & \begin{tabular}{|l|} 
Press. \\
\end{tabular} & $\begin{array}{l}\text { Vel. } \\
\end{array}$ & TMP & TMP & gpm & \begin{tabular}{|l|l|}
$\mathrm{gpm}$ & $\mathrm{g}$ \\
\end{tabular} & $\mathrm{gpm}$ & $\mathrm{nm}$ & \\
\hline & Jata - Per Mi & & & & & & & & & & & & & & & & & & Hour & psig & $\mathrm{ft} / \mathrm{s}$ & psi & & & & & & \\
\hline 17 & & $5: 08: 34$ PM & & 25.665 & 37.919 & 24.705 & 25.145 & 60.714 & 64.514 & 22.307 & 39.389 & & -6.096 & 61.044 & 0.126 & $\begin{array}{l}14.643 \\
\end{array}$ & & & & & & & 3.451 & 0.018 & & 0.0 & & \\
\hline 18 & $9 / 2 / 2004$ & 5:09:34 PM & 32.808 & 25.66 & 38.199 & 24.776 & 25.29 & 58.623 & $\begin{array}{ll}65.106 \\
\end{array}$ & 22.108 & 37.103 & 0.14 & $\begin{array}{ll}7.754 \\
\end{array}$ & 63.9 & 0.003 & $\begin{array}{l}14.643 \\
\end{array}$ & & 1.0 & 0.0166 & 64.0 & & 47.! & 3.300 & & & & & \\
\hline & $9 / 2 / 2004$ & 5:10:34 PM & & 25.66 & 38.341 & 24.966 & & 30.265 & 64.592 & 21.816 & & 28.778 & & \begin{tabular}{|l|l|l|}
57.184 \\
\end{tabular} & 0.003 & 14.643 & & 2.00 & & & & & 1.355 & & & & & \\
\hline 20 & $9 / 2 / 2004$ & 5:11:34 PM & 34.921 & 25.665 & 38.449 & 25.167 & 25.44 & \begin{tabular}{|l|l|}
17.4 \\
\end{tabular} & \begin{tabular}{|l|l|}
64.58 \\
\end{tabular} & 20.99 & $|-3.004|$ & \begin{tabular}{|l|l|}
41.973 \\
\end{tabular} & 19.971 & 62.484 & \begin{tabular}{l|l}
0.003 \\
\end{tabular} & 14.643 & & 3.00 & 0.05 & 63.5 & 14.9 & 7.2 & 0.496 & 0.000 & & & & \\
\hline 21 & $9 / 2 / 2004$ & 5:12:34 PM & 29.335 & 25.657 & 38.598 & 25.354 & 25.571 & -0.599 & \begin{tabular}{|c|}
63.003 \\
\end{tabular} & 16.018 & \begin{tabular}{|c|}
-16.043 \\
\end{tabular} & \begin{tabular}{|l|} 
\\
\end{tabular} & 59.48 & 59.78 & 0.003 & 14.643 & 0.779 & 4.00 & 0.06667 & \begin{tabular}{ll|l}
61.9 \\
\end{tabular} & 14.2 & -8.3 & \begin{tabular}{|c|c|}
-0.574 \\
\end{tabular} & 0.000 & 0.000 & 0.000 & -0.046 & \\
\hline & $9 / 2 / 2004$ & 5:13:34 PM & 36.583 & 25.659 & 38.737 & 25.481 & 25.708 & 55.587 & 63.56 & 18.125 & 38.195 & 2.019 & & 63.33 & 0.299 & $\begin{array}{l}14.643 \\
\end{array}$ & & 5.00 & 0.08333 & $\begin{array}{ll}62.4 \\
\end{array}$ & 15.1 & 46.9 & 3.233 & 0.043 & 0.031 & 0.001 & 0.667 & \\
\hline 23 & 9/2/2004 & 5:14:34 PM & 36.398 & 25.661 & 38.867 & 25.693 & 25.586 & 57.763 & 64.563 & 19.814 & 38.794 & 0.722 & 0.429 & 63.051 & 0.197 & 14.643 & & 6.00 & & 63.4 & 15. & 48.3 & 3.329 & 0.028 & & & & \\
\hline 24 & $9 / 2 / 2004$ & 5:15:34 PM & 36.061 & 25.673 & 38.933 & & 25.528 & 57.678 & & 20.124 & 8.275 & & 0.49 & 59.309 & 0.18 & 14.643 & & & 0.11667 & 63.3 & & 48. & 3.308 & & & & & \\
\hline & $9 / 2 / 2004$ & 5:16:34 PM & & 25.674 & 38.968 & 26.1177 & 25.519 & & & & 38.222 & 0.887 & & 64.405 & 0.173 & 14.643 & & 8.00 & 0.1333 & & & & 3.326 & & & & & \\
\hline \begin{tabular}{|l|}
27 \\
27
\end{tabular} & 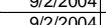 & 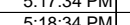 & $\begin{array}{l}35.194 \\
35123\end{array}$ & $\frac{25.680}{2571}$ & 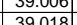 & $\frac{20.275}{26.198}$ & $\frac{25.634}{2572}$ & $\begin{array}{l}60.967 \\
60243\end{array}$ & & 20.354 & $\begin{array}{r}41.559 \\
4004 \\
\end{array}$ & -1.212 & $\begin{array}{l}\frac{-5.045}{5} \\
5708\end{array}$ & 64.369 & 0.183 & 14.643 & & 9.00 & 0.15 & 63. & $\frac{15.3}{1.5}$ & & 3.534 & & & & & \\
\hline 28 & 9. & 5:19:34 PM & $\begin{array}{l}35.105 \\
35.259 \\
\end{array}$ & 25.763 & 3900 & $\begin{array}{l}20.410 \\
26.566\end{array}$ & 25.783 & $\begin{array}{l}0.245 \\
60.39 \\
\end{array}$ & \begin{tabular}{|l|}
03.0000 \\
63.991 \\
\end{tabular} & $\frac{21.049}{20.787}$ & $\begin{array}{l}40.04 \\
40.45 \\
\end{array}$ & $\begin{array}{l}-1.215 \\
-1.215 \\
\end{array}$ & $\begin{array}{l}-5.190 \\
-5.896 \\
-10\end{array}$ & $\begin{array}{l}54.250 \\
58.466\end{array}$ & 0.171 & $\begin{array}{l}14.045 \\
14643\end{array}$ & & $\frac{10.00}{11.00}$ & 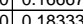 & $\frac{0<.0}{629}$ & 139 & 50.4 & 每.45t & 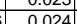 & 0.017 & 0.00 & & \\
\hline 29 & $9 / 2 / 2004$ & 5:20:34 PM & 35.435 & 25.788 & 39.133 & 26.63 & 25.807 & 6004 & \begin{tabular}{|l|}
63.624 \\
\end{tabular} & 20.735 & \begin{tabular}{|l|}
40.118 \\
40
\end{tabular} & \begin{tabular}{|l|} 
\\
-1.212 \\
\end{tabular} & -5.896 & 64.563 & $\frac{.1172}{0.172}$ & 14643 & & 1200 & 02 & 62.5 & 15.5 & 50.1 & $\frac{5.453}{3.453}$ & 0.025 & (17) & & & \\
\hline 30 & $9 / 2 / 2004$ & 5:21:34 PM & 36.669 & 25.792 & 39.123 & 26.705 & 25.811 & 59.739 & \begin{tabular}{|l|l|}
63.486 \\
\end{tabular} & 20.525 & 39.866 & \begin{tabular}{|l|}
-1.212 \\
\end{tabular} & $\frac{-5.902}{-5.502}$ & 60.769 & $\frac{0.171}{0.171}$ & 14.643 & & $\frac{13.00}{13.00}$ & $\begin{array}{ll}0 & 0.2166\end{array}$ & $\frac{26.4}{66.4}$ & $\frac{2.45}{14.5}$ & $\frac{0.8}{49.8}$ & $\frac{1.434}{3.434}$ & $\frac{0.024}{0.024}$ & $\frac{0.017}{0.017}$ & & 3.36 & \\
\hline 31 & & & & & & & & & & & & & & & & & & & & & & & 3.467 & & & & & \\
\hline 32 & 9/2/2004 & $5: 23: 34 \mathrm{PM}$ & 37.331 & 25.79 & 39.116 & 26.698 & 25.76 & $\mid 60.044$ & \begin{tabular}{|l|l|}
63.767 \\
\end{tabular} & .365 & 40.483 & $\frac{-1.212}{-1.212}$ & -5.694 & 65.283 & 0.17 & & & 15.00 & 0.25 & $\frac{6.6}{62.6}$ & 15.5 & 50.3 & $\frac{1.465}{3.465}$ & $\frac{0.024}{0.024}$ & $\frac{0.017}{0.017}$ & 0.000 & 0.331 & \\
\hline & $9 / 2 / 2004$ & $5: 24: 3$ & & 25.806 & 39.127 & 26.649 & 25.7 & & & & & & & 62.4 & $\begin{array}{l}0.17 \\
\end{array}$ & & & & 0.26667 & & & & 498 & & & & & \\
\hline 34 & $9 / 2 / 2004$ & 5:25:34 PM & 37.426 & \begin{tabular}{|l|l|}
25.817 \\
\end{tabular} & \begin{tabular}{|l|l|l}
39.123 \\
\end{tabular} & \begin{tabular}{|l|l|}
26.63 \\
\end{tabular} & & & \begin{tabular}{|l|l|}
63.815 \\
\end{tabular} & 20.132 & \begin{tabular}{|l|l|}
40.869 \\
\end{tabular} & -1.212 & & 67.819 & 0.156 & & & 17.00 & 0.28333 & \begin{tabular}{|l|l|}
62.7 \\
\end{tabular} & & & $\begin{array}{l}3.480 \\
\end{array}$ & & & & 303 & \\
\hline & 9/2/2004 & 5:26:34 PM & 37.446 & 25.842 & 39.147 & 26.665 & & & \begin{tabular}{|c|}
63.738 \\
\end{tabular} & & & & & 68.856 & $\begin{array}{l}0.162 \\
\end{array}$ & & & & & 62.6 & 16.4 & 50.4 & 3.475 & & 016 & & & \\
\hline 36 & $9 / 2 / 2004$ & 5:27:34 PM & 37.48 & 25.832 & 39.137 & 26.645 & & & \begin{tabular}{|c|c|c|}
63.48 \\
\end{tabular} & 20.159 & \begin{tabular}{|l}
40.666 \\
\end{tabular} & \begin{tabular}{|l|} 
\\
\end{tabular} & -5.876 & 62.949 & 0.169 & & & 19 & 0.31667 & 62.4 & 15.0 & 50.3 & 3.465 & & & & & \\
\hline 37 & $9 / 2 / 2004$ & 5:28:34 PM & 37.474 & 25.841 & 39.151 & 26.604 & 25.586 & 59.81 & 63.434 & 20.17 & 40.465 & $|-1.212|$ & -5.795 & 62.467 & 0.168 & & & 20.00 & 0.0 .33333 & 62. & 14.9 & 50.1 & 3.457 & & 016 & 0.000 & 0.328 & \\
\hline & 9/2/200 & 0.29 .5 & 37.468 & & 39.164 & & & & 64.225 & & & 212 & & & 0.167 & & & 21 & 0.00 & . & . & & 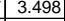 & & & & & \\
\hline 39 & 9/2/2004 & 5:30:3 & 37.534 & & 39.196 & & & & 64.072 & & & 212 & & 66.1 & & & & & & & & & & & & & & \\
\hline & $\begin{array}{l}91212004 \\
0.12004\end{array}$ & & 37.536 & 25.856 & 39.159 & 20.4891 & & & 63.556 & & & $\frac{121}{20}$ & & 61.814 & 0.163 & & & 2.8 & & 62.4. & 14.7 & & 3.468 & & & & 0.311 & \\
\hline & (1/210004 & $\frac{3.32 .34 \mathrm{~F}}{5 \cdot 33 \cdot 34 \mathrm{P}}$ & 年 & & & & & & 03.5 & & & & & $\frac{01.541}{63006}$ & & & & & & $0<.4$ & 14.1. & & & & & & & \\
\hline 43 & 9/2/2004 & 5:34:34 PM & 37.563 & 25.827 & $\begin{array}{l}3.1 .047 \\
39.171\end{array}$ & 26364 & 25. & 59 & 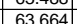 & 20.168 & $\mid$ & \begin{tabular}{|l|}
$\mid-1.212$ \\
-1.21
\end{tabular} & & 65.742 & 0.163 & & & $\frac{2.000}{2600}$ & 0.4333 & 6.5 & 157 & 503 & 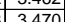 & & & & 317 & \\
\hline 44 & 9/2/2004 & $5: 35: 34$ & 37.55 & 25.813 & 39.173 & & & & \begin{tabular}{|l|}
63.678 \\
\end{tabular} & & & \begin{tabular}{|l|} 
\\
-1.209 \\
\end{tabular} & & 61.733 & 0.166 & & & 27 & 0.45 & $\frac{62.6}{66.6}$ & $\frac{14.7}{14.7}$ & 50.3 & $\mid 3.466$ & 0.024 & $\frac{0.016}{0.016}$ & 0.000 & 323 & \\
\hline & & & & & & & & & & & & & & & & & & & & & & & & & & & & \\
\hline 46 & 9/2/2004 & $5: 37: 34 \mathrm{P}$ & 37.592 & 25.821 & \begin{tabular}{|c|c|c|}
39.1999 \\
\end{tabular} & 26.323 & & & \begin{tabular}{|l|}
63.268 \\
\end{tabular} & & 40.67 & \begin{tabular}{|l|l|l|}
$\mid 1.209$ \\
\end{tabular} & & 63.3 & 0.165 & & & 29. & 0.48333 & 62 & & 50. & $\begin{array}{l}3.458 \\
\end{array}$ & & 016 & & & \\
\hline 47 & & & & & & & & & & & & & & & & & & & & SL.v & 14.9 & & & & & & & \\
\hline 40 & 9/2/2004 & 5:39:3 & 37.636 & 25 & 39.253 & 26.363 & & & 63.6 & 19.9 & 40.6 & -1.215 & & 62.54 & 0.155 & & & 31 & 0.51667 & 62.6 & 14.9 & 50.2 & 3.463 & & & 0.000 & 0.301 & \\
\hline 49 & 9/2/2004 & & 37.645 & 25 & 39.3 & & & & 63. & & & -1.209 & & 63.5 & 0.162 & & & 32 & 0.53 & 62.4 & 15.1 & 50.1 & 3 & & & & 0.316 & \\
\hline 50 & 9/2/2004 & 5:41: & 37.619 & $25.8 \mathrm{r}-\mathrm{P}$ & 39.275 & & & & 63.4 & & & -1.212 & & & & & & 33 & & 62.3 & 16.4 & . & & & & & 313 & \\
\hline 51 & 9/2/2004 & 5:42:3 & 37.661 & 25.816 & 39.327 & 26. & & $60.6 \quad-6>$ & 64.066 & 20.113 & & & & 59.298 & 0.159 & & & 34 & 0.56667 & 62. & 14.1 & 50.3 & & & & & 0.308 & \\
\hline & 9/2/200 & & & & & & & & & & & & & 57 & & & & 35 & & & 13 & & & & & & & \\
\hline 53 & $9 / 2 / 200$ & & 37.662 & 25.1 & 39.299 & & & & & & & -1.215 & & & & & & & & & 14. & & & & & & & \\
\hline $5^{5}$ & G/2/20004 & & 37.692 & $25.1 / 6$ & 39.334 & & & & & & & -1.212 & & 68 & 0.164 & & & & 0.61667 & 63. & 16. & (n) & & & & & & \\
\hline 5 & 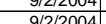 & 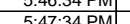 & (37.102 & 年. & (39.303 & 20.4 & & & $\mid$ & & & $-{ }^{-1.212}$ & & 60.64 & 0.16 & & & & 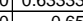 & & $\frac{14}{11}$ & 50.4 & & & & & 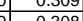 & \\
\hline 57 & 9/2/2004 & 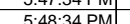 & 37746 & 25803 & 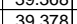 & 26.404 & 20.44 & 5.5976 & $\mid 63796$ & 10.929 & 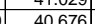 & $\mid$ & & 7.5030 & 0.163 & & & 400 & 0.0667 & 6,2, & 175 & $50^{2}$ & & & 0.0016 & & 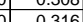 & \\
\hline$\frac{58}{58}$ & 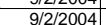 & $\frac{3.469 .34 \mathrm{P}}{5.49}$ & 3773 & $\frac{25.002}{25.802}$ & (39) & 26.445 & & F.9.797 & 8.5566 & $\frac{1.96}{19.9}$ & & & & 63. & & & & 4100 & 0.06833 & & & & & & & & & \\
\hline 50 & 9/2/2004 & & 37.748 & & 39.41 & 26.403 & & 59.847 & & & & $\mid \begin{array}{ll}\mid-1.212 \\
\mid 1212\end{array}$ & & 640 & & & & 4200 & & 628 & 152 & $502+2$ & & & & & & \\
\hline 60 & 9/2/2004 & 5:51:3 & 37.734 & 25 & 39.386 & & & & & & & -1.212 & & 63.9 & 0.158 & & & & $\mid 0.71667$ & 62.8 & ? & 50.4 & & & & & 0.305 & \\
\hline & & & 37.737 & 25.744 & 39.35 & 26.377 & $25.3 \mathrm{r}-\mathrm{C}$ & & 634 & & & -1215 & & 64.344 & & & & & & & & & & & & & & \\
\hline & 9/2/2004 & $5: 53: 34$ & 37.729 & 25.756 & 39.342 & 26.418 & 25. & & 64.31 & 19.9 & 41.074 & -1.218 & & 65.229 & 0.162 & 14 & & 45.00 & 0.75 & 63.2 & & & & & & & & \\
\hline 63 & 9/2/2004 & 5:54:34 PM & 37.766 & 25.768 & 39.373 & 26.5 & 25.6 & 60.135 & 64.06 & 19.80 & 41.16 & $\mid-1.212$ & & 64.8 & 0.162 & 14.6 & & 46.00 & 0.76 & $62-2>3$ & 154 & & & & & & & \\
\hline 64 & 9/2/2004] & 5:55:34 PM & 37.744 & 25.786 & 39.405[ & $26.468 \mid$ & 25.7 & 59.668 & 63.504 & 19.759 & 40.789 & -1.212 & -5.96 & 59.459 & 0.162 & 14.643 & 0.00 & 47.00 & 0.78333 & 624 & 142 & & \begin{tabular}{|l|l|}
3.463 \\
\end{tabular} & 0.023 & 0.016 & 0.000 & 0.314 & 4 \\
\hline 65 & $9 / 2 / 2004$ & 5:56:34 PM & 37.719 & 25.765 & 39.405 & 26.408 & 25.844 & 59.7 & 63.848 & $\begin{array}{l}3 \quad 19.74 \\
3\end{array}$ & 40.705 & $\mid-1.212$ & -5.922 & 66.476 & 0.16 & 14.643 & & 48.00 & 0.8 & 62.7 & $\begin{array}{l}7 \\
7\end{array}$ & \begin{tabular}{l|l} 
& 50.2 \\
\end{tabular} & 3.461 & 0.023 & 0.016 & 0.000 & 0.310 & 0.2 \\
\hline
\end{tabular}


WSRC-TR-2005-00105, REVISION 0 SRNL-RPP-2005-00012, REVISION 0

RUN \# TEMP6; CAMPAIGN IV HIGH TEMPERATURE TEST - CONT.

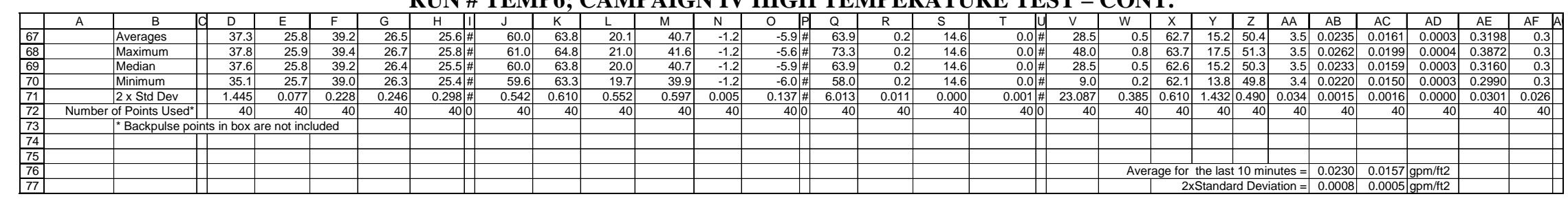


WSRC-TR-2005-00105, REVISION 0

SRNL-RPP-2005-00012, REVISION 0

RUN \# TEMP7; CAMPAIGN IV HIGH TEMPERATURE TEST

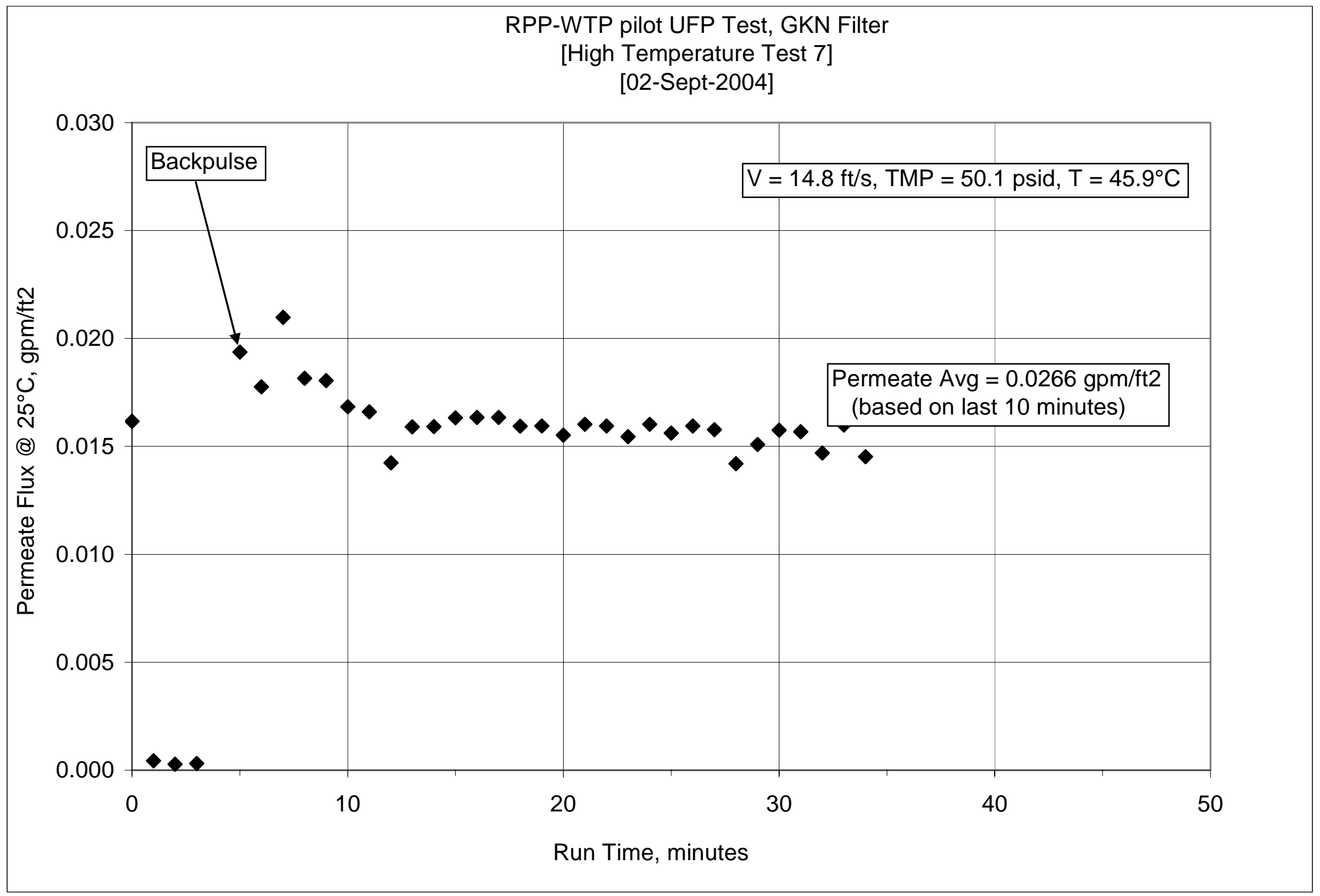


WSRC-TR-2005-00105, REVISION 0

SRNL-RPP-2005-00012, REVISION 0

RUN \# TEMP7; CAMPAIGN IV HIGH TEMPERATURE TEST

\begin{tabular}{|c|c|c|c|c|c|c|c|c|c|c|c|c|c|c|c|c|c|c|c|c|c|c|c|c|c|c|c|c|}
\hline & $\mathrm{A}$ & $B$ & D & $E$ & $\mathrm{~F}$ & G & $\mathrm{H}$ & $\mathrm{J}$ & $\mathrm{K}$ & $L^{\prime}$ & $\mathrm{M}$ & $\mathrm{N}$ & 0 & Q & \begin{tabular}{|l|} 
\\
\end{tabular} & $\mathrm{s}$ & $\mathrm{T}$ & $\mathrm{v}$ & W & $x$ & & & & & & & $\mathrm{AE}$ & AF $[A$ \\
\hline$\frac{1}{2}$ & DATE & TIME & Filtrate & $\begin{array}{c}<<\text { Temp } \\
\text { Cleaning }\end{array}$ & $\begin{array}{l}\text { berature } \\
\text { islury }\end{array}$ & Measureme & ints >>? & & Fiter & $\begin{array}{l}\text { Pressure 1 } \\
\text { Filter dP }\end{array}$ & $\begin{array}{l}\text { neasureme } \\
\text { TopTTPP }\end{array}$ & $\begin{array}{l}\text { nths >>> } \\
\text { Fitrate }\end{array}$ & & 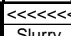 & 《e Flow & $\begin{array}{l}\text { Measurem } \\
\end{array}$ & ents $\gg \gg>>$ & & & & & & n RawD & & & & & \\
\hline & DAIE & IIME & & & & & Lo Amb. & Botlaip & Finter & & & & Pulsepot & & & & Backpulse & & & & & & & & & & & \\
\hline \begin{tabular}{|l}
4 \\
\end{tabular} & & & $\frac{\operatorname{deg} C}{\mathrm{~T} 2}$ & $\frac{\operatorname{deg} C}{\text { T3 }}$ & \begin{tabular}{|l|}
$\operatorname{deg} C$ \\
T1
\end{tabular} & $\frac{\operatorname{deg} \mathrm{C}}{\mathrm{T} 4}$ & $\frac{\operatorname{deg} C}{T 5}$ & $\begin{array}{l}\text { psid } \\
\text { dP2 }\end{array}$ & $\frac{p s i g}{P 1}$ & $\begin{array}{l}\text { psid } \\
\text { dP1 }\end{array}$ & $\begin{array}{l}\text { psid } \\
\text { dP3 }\end{array}$ & $\frac{p \text { psig }}{\text { P2 }}$ & $\begin{array}{l}\text { psig } \\
\text { P3 }\end{array}$ & $\frac{\mathrm{gpm}}{\mathrm{O} 1}$ & $\frac{\mathrm{gpm}}{\mathrm{O} 2}$ & $\frac{\mathrm{gpm}}{\mathrm{O} 3}$ & $\frac{\mathrm{gpm}}{\mathrm{Obp}}$ & & & & & & & & & & & \\
\hline 6 & $200=041$ & 201 & & & & & & & & & & & & & & & & & & & & & & & & & & \\
\hline$\frac{6}{7}$ & $\frac{e r o s-04 / 19}{8 / 23 / 2004}$ & 0004 & 22.554 & 22.60 & 22.002 & \begin{tabular}{l|l|}
22.782 \\
\end{tabular} & 22.204 & $\begin{array}{c}-0.019 \\
\end{array}$ & 0.095 & $\begin{array}{c}-0.012 \\
\end{array}$ & \begin{tabular}{|l|l|}
0.003 \\
\end{tabular} & -0.12 & 0.137 & -0.075 & 0.003 & $\begin{array}{l}14.643 \\
\end{array}$ & 0.004 & & & Filter S & Surface $A$ & \begin{tabular}{|c|c|}
6.985 \\
\end{tabular} & FT2 & & & & & \\
\hline 8 & $8 / 24 / 2004$ & 8:10:33 AM & 23.662 & 22.93 & \begin{tabular}{|l|l|}
23.679 \\
\end{tabular} & 22.998 & 22.735 & -0.021 & 0.122 & -0.014 & -0.001 & -0.12 & 0.134 & 23.176 & 1.205 & 14.643 & 0.003 & & & Conver & & $\frac{0.05}{851}$ & $\mathrm{~m} 3 / \mathrm{m} 2 /$ & |day/barg & / gpm/tt2/ & t2/barg & & \\
\hline 9 & $8 / 26 / 2004$ & 9:14:11 AM & & 23.352 & 24.932 & 25.316 & 23.242 & $\begin{array}{l}-0.008 \\
\end{array}$ & 0.126 & -0.008 & 0.011 & -0.10 & & & & & & & & & & & & & & & & \\
\hline & & & & & & & & & & & & & & -0.084 & 80027 & $\begin{array}{l}14.643 \\
\end{array}$ & & & & & & & & & & & & \\
\hline & 8/30/2004 & $7: 28: 55 \mathrm{AM}$ & 22.511 & 22.848 & 22.397 & 22.669 & $\overline{22.914}$ & $\begin{array}{l}-0.017 \\
\end{array}$ & 0.138 & $\begin{array}{l}-0.009 \\
\end{array}$ & $\begin{array}{l}0.003 \\
\end{array}$ & -0.14 & 0.122 & -0.082 & 0.003 & 14.643 & 0.006 & & & & & & & & & & & \\
\hline & & & & & & & & & & & & & & & & & & & & & $\begin{array}{l}\text { Plis } \\
\end{array}$ & rrect & $\frac{d \text { for } 3}{3}$ & & & & & \\
\hline 13 & & & & & & & & & & & & & & & & & & & & $\frac{v w V}{V W v}$ & & & & < Filtrate & e Flux $>$ & $\mid<<$ PERI & | & $\gg$ \\
\hline 14 & & & & & & & & & & & & & & & & & & & & & & & & & at $25 \mathrm{C}$ & & 1000 & \\
\hline$\frac{15}{15}$ & & & & & & & & & & & & & & & & & & Time & ime & Press. & el. & MPP & TMP & spm & ppm & $\mathrm{pm}$ & $\mathrm{pm}$ & eter \\
\hline $\begin{array}{l}\frac{16}{17} \\
\end{array}$ & 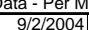 & ute $6: 07: 36 \mathrm{PM}$ & 40.979 & 25.814 & \begin{tabular}{|l|l|}
44.719 \\
\end{tabular} & 25.591 & 25.963 & 59.287 & 64.06 & 20.214 & 39.9 & $-1.21 !$ & -5.925 & 63.662 & 0.173 & 14.643 & 0.003 & & our & sig & 15.6 & $\begin{array}{ll}51 \\
49.6\end{array}$ & 3.419 & 0.025 & 0.016 & 0.000 & 0.326 & 0.28 \\
\hline & 9/2/2004 & 6:08:36 PM & & & & & & 46.41 & 64.288 & 20.268 & 26.62 & 11.94 & 21.009 & 66.491 & 0.005 & 14.643 & 00003 & 1.0 & 0.01667 & & 16.3 & 36.5 & 2.518 & \begin{tabular}{|l|}
0.001 \\
\end{tabular} & 0.000 & 0.000 & 0.012 & 0.01 \\
\hline 19 & 9/2/2004 & 6:09:36 PM & 41.505 & 25.838 & 45.117 & 25.485 & 25.833 & 14.841 & $63.92 \mathrm{~s}$ & 19.808 & -4.446 & 43.74 & 20.688 & 57.025 & $\begin{array}{l}0.003 \\
\end{array}$ & 14.643 & 0.004 & 2.0 & 0.03333 & 62.8 & 14.0 & 5.2 & 0.358 & 0.000 & 0.000 & 0.000 & 0.053 & 0.05 \\
\hline & 9/2/2004 & 6:10:36 PM & 36.743 & 25.837 & 45.271 & 25.559 & 25.726 & -1.945 & $62.22 \mathrm{G}$ & 15.492 & -16.948 & 59.58 & 60.663 & 60.433 & 0.003 & 14.643 & 0.946 & 3.0 & 0.05 & 61.1 & $14.8 \mathrm{C}$ & -9.4 & 0.651 & 0.000 & 0.000 & 0.000 & & \\
\hline & |l/2004 & 6:11:36 PM & 43.035 & 25.847 & 45.417 & 25.619 & 25.961 & 55.075 & 62.721 & 18.159 & 37.711 & 1.866 & 0.38 & 66.756 & 0.317 & 14.643 & & & .06667 & 61.6 & 16.4 & & 3.199 & 0.045 & 0.028 & 0.001 & & \\
\hline 22 & $\begin{array}{ll}9 / 2120004 \\
0 / 21004\end{array}$ & $6: 12: 36 \mathrm{PM}$ & 44.231 & 20.848 & 45.597? & 25.09 & 26.032 & 56.145 & 6 & 19.311 & 37.546 & 1.03 & 0.455 & 62.125 & 0.225 & 14.643 & 0.00 & 5.0 & 0.08333 & 62.0 & $1 \frac{15.2}{13 .}$ & $\frac{4.8}{471}$ & 3.230 & 0.032 & 0.019 & & 0.413 & 0.35 \\
\hline 24 & 年 & 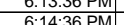 & $\begin{array}{l}44.144 \\
43.953\end{array}$ & $\begin{array}{l}25.064 \\
25891\end{array}$ & 年 & & $\frac{20.080}{26099}$ & $\begin{array}{l}50.461+ \\
59695\end{array}$ & 然. & $\frac{19.491}{1958}$ & 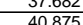 & 1.072 & $\begin{array}{l}0.53 \\
-5078\end{array}$ & $\frac{60.144}{66128}$ & o. & 14.045 & & & 0.1 & & & & 3.240 & 0 & 0.018 & & & 0.32 \\
\hline 25 & $9 / 2 / 2004$ & $6.15 .36 \mathrm{PM}$ & 年 430850 & 25.917 & 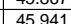 & 25.005 & 26201 & 60.245 & 64.155 & 19.696 & 41.372 & $-\frac{-1.21}{-1.21}$ & - & 60.556 & . & 14643 & $\begin{array}{l}0.003 \\
0.003\end{array}$ & 800 & 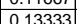 & 630 & 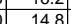 & 50. & 3.403 & 然 0.029 & 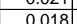 & 0.000 & 0 & 0.30 \\
\hline 25 & 9/2/2004 & $6: 16: 36$ PM & 43.71 & 25.93 & 46.041 & 26.058 & $\frac{2.201}{26.288}$ & 6.60 .6 & 64.414 & 19.844 & 41.386 & -1.21 & \begin{tabular}{|c|c|}
-5.714 \\
\end{tabular} & 57.98 & 0.207 & 14.643 & 0.004 & 9.0 & $\frac{1.15}{0.15}$ & & 14.2 & 510 & $\frac{3.516}{3.516}$ & 0.030 & 0 & & & 0.30 \\
\hline & 9/2/2004 & 6:17:36 PM & 43.892 & 25.948 & 46.107 & 26.121 & 26.336 & 59.22 & & 19.356 & 40.98 & $\frac{-1.21}{-1.21}$ & -5.682 & 53.882 & 0.194 & 14.643 & 0.006 & 10.0 & 16667 & $\frac{61.4}{61.4}$ & & 50.1 & 3.454 & 0.028 & & & & . \\
\hline 28 & $9 / 2 / 2004$ & $6: 18: 36 \mathrm{PM}$ & 43.895 & 25.961 & 46.1 & 26.284 & 25.98 & 58.928 & \begin{tabular}{|l|l|}
62.632 \\
\end{tabular} & 19.428 & 40.395 & -1.20 & -5.792 & 59.716 & 0.202 & 14.643 & 0.004 & 11.00 & 0.18333 & 61.5 & 14.6 & 49.7 & 3.424 & 0.029 & 017 & 000 & 334 & 0.28 \\
\hline & 9/2/2004 & & & & 46.053 & & & & & & 40.725 & & & 58.266 & 0.173 & 14.643 & & 12.0 & & & 14.3 & 49.9 & 3.442 & 0.025 & 0.014 & & 285 & $\frac{0.28}{0.24}$ \\
\hline 30 & 9/2/2004 & 6:20:36 PM & 44.101 & 25.962 & 46 & 26.265 & 25.082 & 59.428 & 63.16 & $\begin{array}{l}19.357 \\
\end{array}$ & 40.758 & -1.21 .2 & -5.833 & $\begin{array}{ll}61.028 \\
6\end{array}$ & 0.193 & $\begin{array}{l}14.643 \\
\end{array}$ & 0.0 & 13.00 & 0.21667 & 62.0 & 15.0 & 50.1 & 3.454 & 0.028 & & & & 0.27 \\
\hline 31 & 9/2/2004 & $6: 21: 36 \mathrm{PM}$ & 44.109 & 25.971 & 45.984 & 26.189 & 25.261 & 58.943 & 62.739 & 19.319 & $\begin{array}{l}40.454 \\
\end{array}$ & -1.21 & -5.599 & 61.028 & 0.193 & 14.643 & 0.003 & 14.00 & 0.23333 & 61.6 & 15.0 & $49.7 \quad$ & 3.427 & 0.028 & 0.016 & 000 & 0.320 & 0.27 \\
\hline 32 & 9/2/2004 & 6:22:36 PM & 44.072 & 25.968 & 45.971 & 26.106 & 25.198 & 59.193 & \begin{tabular}{|l|l|l|}
62.816 \\
\end{tabular} & 19.314 & 40.756 & -1.212 & -5.804 & 59.397 & 0.198 & 14.643 & 0.00 & 15.0 & 0.25 & 61.7 & 14.6 & 50.0 & 3.446 & 0.028 & 0.016 & & & 0.28 \\
\hline & 9/2/2004 & 6:23:36 PM & 44.087 & 25.964 & & & & 59.096 & & 19.304 & 40.711 & -1.212 & & 61.203 & 0.198 & & & $16.0 \mathrm{Cl}$ & & 61.4 & 15.0 & 49.9 & 3.441 & 0.028 & & & & 028 \\
\hline$\frac{34}{325}$ & 9 & 6:24:36 PM & 44.221 & 25.971 & 45.925 & 26.008 & 25.39 & 58.922 & 62.458 & $19.3 / 1$ & 40.567 & -1.212 & -5.636 & 65.051 & 0.198 & 14.643 & 0.003 & 17.00 & 0.28333 & 61.3 & 15.9 & 49.7 & 3.430 & 0.028 & 116 & .000 & 329 & 0.2 \\
\hline$\frac{35}{36}$ & 2 & $\begin{array}{l}6.253 .36 \mathrm{PM} \\
6.620 .36 \mathrm{PM}\end{array}$ & $\begin{array}{l}44.154 \\
44.048\end{array}$ & $\frac{25.961}{25058}$ & 45.902 & 25.915 & 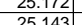 & & $\frac{0.928}{6.978}$ & $\begin{array}{l}19.416 \\
19212\end{array}$ & $\begin{array}{l}40.809 \\
40708\end{array}$ & -1.212 & $\begin{array}{l}-5.81 \\
-581\end{array}$ & 51.809 & 0.193 & 14.643 & & 18. & 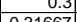 & $\frac{6.8}{6.8}$ & $\frac{1.24}{1.47}$ & S & 3.453 & & & & & 0.2 \\
\hline 年 & (1) & 0.20 .36 & $\begin{array}{l}44.040 \\
440104\end{array}$ & 20.950 & 4.0954 & 20.911 & $\frac{25.143}{2192}$ & 59.144 & 0 & 19.210 & 40.148 & -1.21 & $-\frac{-5.81}{-81}$ & S9.901 & 0.193 & 14.643 & & 19.00 & 0.31067 & 61.8 & 14.7. & 49.9 & 3.444 & $\frac{028}{027}$ & 0.016 & 然 & 0.319 & 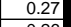 \\
\hline \begin{tabular}{|l|l|}
38 \\
\end{tabular} & $9 / 2 / 2004$ & $\frac{6.6}{6 \cdot 28: 36 ~ P M}$ & 44.168 & $\frac{25.966}{25.966}$ & $\frac{4.906}{45.906}$ & $\frac{25.079}{25.79}$ & $\frac{24.796}{24.796}$ & $\begin{array}{l}5.2579 \\
59.279\end{array}$ & 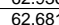 & $\frac{19.129}{19.208}$ & & $\frac{-1.21}{-1.21}$ & $\frac{0.056}{-5.76}$ & $\frac{5.918}{59.18}$ & $\frac{0.180}{0.194}$ & $\frac{14043}{14643}$ & 0.0 & $\frac{20.0}{21.0}$ & $\frac{0.035}{0.35}$ & 01.0 & 14.5. & 50.2 & 0 & & & & & $\frac{0.26}{0.27}$ \\
\hline \begin{tabular}{|l|l|}
39 \\
\end{tabular} & 9/2/2004 & 6:22:36 PM & 44.168 & 25.957 & 45.888 & 25.784 & 25.052 & 59.405 & $\mid 62.92$ & 19.176 & 40.992 & $\frac{-1.21}{-1.21}$ & -5.755 & 60.039 & 0.193 & 14.643 & 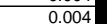 & $\frac{22.0}{22.0}$ & 0.36667 & $\frac{61.8}{61.8}$ & 14.7 & $\frac{0.2}{0.2}$ & 3461 & 0.028 & 0.016 & 0000 & $\frac{118}{318}$ & 0.27 \\
\hline 40 & $9 / 2 / 2004$ & $6: 30: 36 \mathrm{PM}$ & 44.144 & & 45.888 & & & 59.171 & & 19.219 & & & & & & & & & & & & & & & & & & \\
\hline 41 & $9 / 2 / 2004$ & 6:31:36 PM & 44.116 & 25.943 & 45.904 & 25.821 & 25023 & 59.418 & $\frac{63.055}{63.055}$ & 19.239 & 40.937 & -1.21 & -5.78 & 70.366 & 0.194 & 14.643 & & $\frac{24.0}{24.0}$ & 0.4 & 61.9 & 17.2 & 502 & 3.460 & 0.028 & 0.016 & 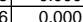 & $\frac{319}{319}$ & $\frac{0.2}{0.2}$ \\
\hline $\begin{array}{ll}42 \\
\end{array}$ & $9 / 2 / 2004$ & 6:32:36 PM & 44.312 & 25.94 & $\begin{array}{ll}45.9 \\
\end{array}$ & 25.772 & 25.36 & $\begin{array}{l}58.897 \\
\end{array}$ & & $\begin{array}{l}19.124 \\
\end{array}$ & 40.574 & $-1.21 !$ & & $\begin{array}{l}60.327 \\
6\end{array}$ & 189 & & & 25 & $\overline{0.41667}$ & 61.5 & 14.8 & 49.7 & 429 & & & & 314 & 0.2 \\
\hline \begin{tabular}{|l|l|}
43 \\
\end{tabular} & 9/2/2004 & $6: 33: 36 \mathrm{PM}$ & 44.222 & 25.932 & \begin{tabular}{|l|l|} 
& 45.892 \\
\end{tabular} & 25.799 & 25.606 & 59.364 & $62.52 \xi$ & 19.297 & 41 & -1.21 & -5.84 & 62.734 & 0.193 & 14.643 & 0.003 & 26.00 & $\mid 0.43333$ & 61.4 & 15.4 & 50.2 & 3.460 & & | & 0.000 & $\mid$ & 0.2 \\
\hline 44 & $9 / 2 / 2004$ & $6: 34: 36$ PM & 44.337 & 25.941 & 45.901 & 25.883 & 25.89 & 59.538 & 62.926 & 19.237 & 41.175 & -1.212 & -5.71 & 62.292 & 0.191 & 14.643 & & 27.0 & 0.45 & 61.8 & 15.3 & 0.4 & 3.472 & 027 & 116 & 0.000 & 0.313 & 0.2 \\
\hline 45 & |2/2004 & $6: 35: 36$ & 44.294 & 25.946 & 45.935 & 25.949 & & 59.573 & 63. & 19.328 & & -1.212 & & 65.904 & & & & 28.0 & & 62.2 & 16.2 & 0.3 & 3.470 & & & & & 0.2 \\
\hline 46 & $9 / 2 / 20004$ & $\begin{array}{l}6: 363: 36 \text { PM } \\
6: 3736\end{array}$ & 44.202 & $\frac{25.956}{25092}$ & 45.96 & 25.934 & 25.815 & $\begin{array}{l}59.196 \\
59.171\end{array}$ & 62.61 & 19.075 & 40.896 & -1.212 & 5.00 & 57.167 & 0.183 & 14.643 & & 29.00 & . & 61.5 & 14.0 & 5.0 & 3.450 & 026 & 4 & & 0.4 & 0.2 \\
\hline 41 & $\begin{array}{ll}9 / 212004 \\
012004\end{array}$ & 6.37 .36 & 44.46 & & 45.965 & & & & & 19.067 & & & & 58.4 & 0.191 & & & 30.6 & & & 些. & & & & & & & \\
\hline 48 & 年 & $0.38 .30 \mathrm{PM}$ & $\begin{array}{ll}44.200 \\
4.231\end{array}$ & $\frac{25.942}{25941}$ & 45.931 & 25.929 & $\frac{25.926}{25845}$ & $\frac{5.04}{58.092}$ & $\frac{02.44}{62.45}$ & 19.114 & 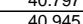 & -1.21 & 年. & 13.3000 & 0.198 & $\begin{array}{l}14.043 \\
1.63\end{array}$ & & 3.00 & $\frac{0.51001}{0.5232}$ & 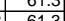 & 10.0 & t.9.9 & 3.442 & & & & 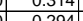 & 0.2 \\
\hline 50 & $9 / 2 / 2004$ & $6: 5.50 .36$ PM & 44.484 & 25.946 & 年5.549 & $\frac{2.514}{25.914}$ & $\begin{array}{l}25.040 \\
25.765\end{array}$ & 59.561 & (3) & 1935 & $\frac{74.545}{41.048}$ & -120 & $\begin{array}{l}-5.021 \\
-5.769\end{array}$ & 6.728 & 0 & $\frac{14.043}{14.643}$ & & 33.00 & 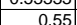 & 62.1 & 1..64 & 50.3 & \begin{tabular}{|l|}
.4450 \\
3468
\end{tabular} & & & & 势 & 0.2 \\
\hline 51 & 9/2/2004 & 6:41:36 PM & 44.265 & 25.946 & \begin{tabular}{|l|l|}
45.955 \\
\end{tabular} & 25.839 & 25.74 & 59.005 & 62.82 & 19.229 & 40.645 & -1.21 & -5.674 & 62.913 & 0.176 & 14.643 & .003 & 34.00 & 0.56667 & 61.7 & 15.4 & 49.8 & \begin{tabular}{|l|l|}
3.435 \\
\end{tabular} & 0.025 & 0.015 & 0.000 & 0.291 & $\frac{0.2}{0.2}$ \\
\hline 5 & & & & & & & & & & & & & & & & & & & & & & & & & & & & \\
\hline & & Averages & 44.1 & 26.0 & 45.9 & 26.0 & $25.5 \mid$ & 59.3 & $\begin{array}{ll}62.5 \\
\end{array}$ & $\begin{array}{ll}19.3 \\
\end{array}$ & 40.9 & -1.2 & -5.7 & 62.0 & $\begin{array}{ll}0.2 \\
\end{array}$ & $\begin{array}{ll}14.6 \\
\end{array}$ & $\overline{0.0}$ & 20.5 & 0.3 & $\begin{array}{ll}61.8 \\
\end{array}$ & 15.2 & 50.1 & 3.5 & 0276 & 0161 & & 3207 & \\
\hline & & Maximum & 44.5 & & $\begin{array}{l}46.1 \\
\end{array}$ & \begin{tabular}{|l|}
26.3 \\
\end{tabular} & & $\begin{array}{ll}60.6 \\
\end{array}$ & ST & $\begin{array}{ll}19.8 \\
\end{array}$ & & 5 & & 73.4 & 0.2 & & & & & $\begin{array}{ll}63.3 \\
\end{array}$ & $\overline{18.0}$ & $\overline{51.0}$ & & & & & & \\
\hline & & Median & 44.1 & 26.0 & 45.9 & 25.9 & & 59.2 & 62.8 & $1 \mathrm{~s}$ & 40.9 & -1.2 & -5 & 60 & 0.2 & $\begin{array}{l}14.6 \\
\end{array}$ & & 20.5 & 0. & & 14.9 & 50.1 & 3.5 & & & 0.0003 & 0.31 & \\
\hline 5 & & Minimum & 43.1 & 25.9 & 45.9 & 25.8 & 24 & 58.9 & 62.4 & 19 & 40.4 & -1.2 & & 5 & 0.2 & 14.6 & 0.0 & 7.0 & 0.1 & 61.3 & 13.2 & 49.7 & 3.4 & & 0142 & & 0.2821 & 0. \\
\hline & & $2 \times \operatorname{Std} \mathrm{Dev}$ & 0.514 & 0.035 & 0.123 & 0.306 & 0.918 & 0.752 & 0.956 & 0.362 & 0.479 & 0.00 & 0.298 & 8.378 & 0.025 & 0.000 & 0.001: & 16.155 & 0.269 & 0.956 & \begin{tabular}{|l|l|} 
\\
\end{tabular} & 30.586 & 0.040 & 0.0036 & 0.0026 & 0.0001 & 0.0500 & .043 \\
\hline 58 & Number $\mathrm{C}$ & ff Points Used & 28 & & 28 & & & & $2 \varepsilon$ & & 28 & $2 \varepsilon$ & & 28 & 28 & 28 & $28 \mid c>>>$ & 28 & 28 & 28 & 28 & 28 & 28 & 28 & 28 & 28 & 28 & $2 \varepsilon$ \\
\hline & & & n box & are not 1 & & & & & & & & & & & & & & & & & & & & & & & & \\
\hline & & & & & & & & & & & & & & & & & & & & & & & & & & & & \\
\hline 6 & & & & & & & & & & & & & & & & & & & Aver & rage for & $r$ the last & it $10 \mathrm{mir}$ & nutes $=$ & 0.0272 & 0.0157 & $\mathrm{ppm} / \mathrm{tt2}$ & & \\
\hline 63 & & & & & & & & & & & & & & & & & & & & & $2 x \operatorname{xtandar}$ & ard Devi & iation $=$ & \begin{tabular}{|l|}
0.0021 \\
\end{tabular} & \begin{tabular}{|l|}
0.0012 \\
\end{tabular} & $2 \mathrm{gpm} / \mathrm{tt} 2$ & & \\
\hline
\end{tabular}




\section{RUN \# WASH1 AND WASH 2; CAMPAIGN IV SLURRY WASHING}

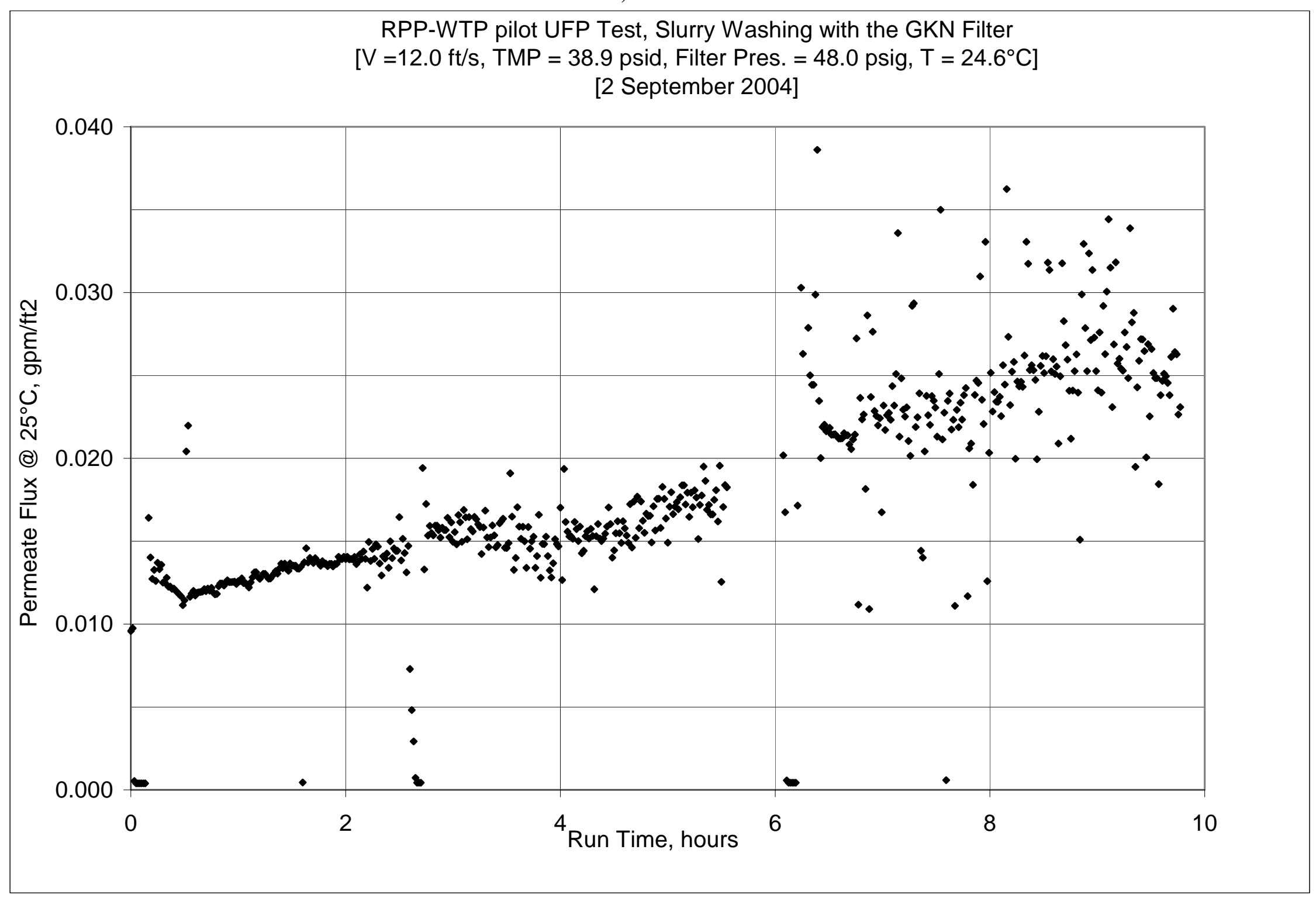


WSRC-TR-2005-00105, REVISION 0

SRNL-RPP-2005-00012, REVISION 0

RUN \# WASH1 AND WASH 2; CAMPAIGN IV SLURRY WASHING

\begin{tabular}{|c|c|c|c|c|c|c|c|c|c|c|c|c|c|c|c|c|c|c|c|c|c|c|c|c|c|c|c|c|}
\hline & A & $B$ & D & $\mathrm{E}$ & $F$ & G & $\mathrm{H}$ & \begin{tabular}{l|l}
$\mathrm{J}$ & 1 \\
\end{tabular} & $\mathrm{~K}$ & \begin{tabular}{|l|l|}
$L$ \\
\end{tabular} & \begin{tabular}{l|l}
$\mathrm{M}$ \\
\end{tabular} & $\mathrm{N}$ & $\mathrm{O}$ & \begin{tabular}{l|l} 
\\
\end{tabular} & $\mathrm{R}$ & $\mathrm{s} \mid$ & $\mathrm{T}$ & $\mathrm{v}$ & \begin{tabular}{l|l|} 
& $w$ \\
\end{tabular} & $|x|$ & & & & & & & & $\mathrm{AF}$ \\
\hline$\frac{1}{2}$ & & & $\begin{array}{l}\text { ¿ } \\
\text { Filtrate }\end{array}$ & $\begin{array}{l}\text { « Tempe } \\
\text { Cleaning }\end{array}$ & & पeasureme & ents >>> & BotTMP & $\begin{array}{l}2<\ll<<< \\
\text { Filter }\end{array}$ & $\begin{array}{l}\text { Pressure } N \\
\text { Fiteter } \mathrm{P} \mid\end{array}$ & $\begin{array}{l}\text { neasuremen } \\
\text { TopTMP }\end{array}$ & $\begin{array}{l}\text { ntsts > >>> } \\
\text { Fitrate }\end{array}$ & & 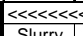 & 《< Flown & Measureme & & & & & & & & & & & & \\
\hline 2 & DAIE & & $\frac{\text { Fillrate }}{\operatorname{deg} C}$ & $\begin{array}{l}\text { Cleaning } \\
\operatorname{deg} C\end{array}$ & $\frac{\text { Slurry }}{\operatorname{deg} C}$ & \begin{tabular}{|l|} 
Hi Amb. \\
$\operatorname{deg} \mathrm{C}$
\end{tabular} & $\mid \frac{\text { Lo Amb. }}{\operatorname{deg} \mathrm{C}}$ & $\frac{\text { BotTMP }}{\text { psid }}$ & \begin{tabular}{|l|} 
Filter \\
psig
\end{tabular} & $\begin{array}{l}\text { Filter dP } \\
\text { psid }\end{array}$ & $\begin{array}{l}\text { TopTMP } \\
\text { psid }\end{array}$ & & \begin{tabular}{|l} 
Pulsepot \\
psig
\end{tabular} & \begin{tabular}{l|l} 
Slurry \\
gnm
\end{tabular} & \begin{tabular}{|l|l|} 
Filtrate \\
gom
\end{tabular} & Hi Filtate & \begin{tabular}{|l|} 
Backpulse \\
\end{tabular} & & & & & & & & & & & \\
\hline 4 & & & $\mathrm{~T} 2$ & T3 & T1 & $\mathrm{T4}$ & T5 & $\mathrm{dP2}$ & P1 & $\frac{p \text { psia }}{\text { dP1 }}$ & $\begin{array}{l}\text { Psid } \\
\text { dP3 }\end{array}$ & $\begin{array}{l}\text { Psig } \\
\text { P2 }\end{array}$ & $\begin{array}{l}\text { pssig } \\
\text { P3 }\end{array}$ & Qp1 & $\frac{\mathrm{gpm}}{\mathrm{Q} 2}$ & $\frac{\mathrm{gpm}}{\mathrm{Q} 3}$ & $\frac{\mathrm{gpm}}{\mathrm{Qbp}}$ & & & & & & & & & & & \\
\hline & & & & & & & & & & & & & & & & & & & & & & & & & & & & \\
\hline & eros- 04/19 & $1 / 2004$ & & & & & & & & & & & & & & & & & & & & & & & & & & \\
\hline 8 & $\frac{8 / 23 / 2004}{8 / 24 / 2004}$ & $\begin{array}{l}8.35 .30 \mathrm{AMT} \\
8 \cdot 10: 33 \mathrm{AM}\end{array}$ & $\frac{2.5354}{23662}$ & & $\frac{22.002}{23679}$ & & & $\begin{array}{l}-0.019 \\
-0.021 \\
\end{array}$ & 0.095 & $\frac{-0.012}{-0.014}$ & & \begin{tabular}{|c|}
-0.124 \\
-0.124 \\
\end{tabular} & & & 0.003 & $\frac{14.643}{14643}$ & 0.004 & & & $\begin{array}{ll}\text { Filter St } \\
\text { Congers }\end{array}$ & Surface $A$ & 2.96 .985 & $\mathrm{Fl}$ & dday/hard & $r / g n m^{\prime / t r}$ & ditz/harch & & \\
\hline 9 & $8 / 26 / 2004$ & 9:14:11 AM & 告.519 & 23.352 & 24.932 & 25.316 & 23.242 & $\mid-0.008$ & $\frac{1.126}{0.126}$ & $\mid-0.008$ & 0.011 & -0.106 & o. 0.091 & 48.1735 & $\mid$ & 14643 & 0.007 & & & furver: & & & & taylivary & 要 & thefinary & & \\
\hline & $8 / 27 / 2004$ & & 22.649 & 22.89 & 22.298 & & & \begin{tabular}{|c|c|c|}
-0.017 \\
\end{tabular} & $\begin{array}{l}0.14 \\
\end{array}$ & -0.008 & | 0.005 & -0.161 & 0.11 & \begin{tabular}{|c|c|}
-0.084 \\
\end{tabular} & 0.003 & 14.643 & & & & & Gauge & & P1 is & ippros & ately & psig & & \\
\hline & 8/30/2004 & 7:28:55 AM & 22.511 & 22.848 & 22.397 & 22.669 & 22.914 & -0.017 & 0.138 & -0.009 & 0.003 & -0.147 & 0.12 & -0.082 & 0.003 & 14.643 & 0.00 & & & & & & & & & & & \\
\hline & & & & & & & & & & & & & & & & & & & & & & & ed for 31 & 1.25 inch & ches of wat & Nater tubin & & \\
\hline 4 & & & & & & & & & & & & & & & & & & & & 促 & & & & & 250 & & x1000 & \\
\hline & & & & & & & & & & & & & & & & & & Time & Time & Press. & el. & TMP & TMP & $\overline{\mathrm{gpm}}$ & $\mathrm{pm}$ & $\mathrm{gpm}$ & & \\
\hline & ata - Per M & inute & & & & & & & & & & & & & & & & Min. & Hour & psig & & psi & bar & $\frac{1+12}{f t 2}$ & & ffr/psi & $\mathrm{t} 2 / \mathrm{psi}$ & $\frac{\text { meler }}{\text { daylbar }}$ \\
\hline 17 & 9/2/2004 & & $\begin{array}{l}29.094 \\
20050\end{array}$ & 26.23 & 27.277 & 26.282 & 25.826 & 47.571 & 50.674 & 18.147 & \begin{tabular}{|c|}
30.397 \\
\end{tabular} & -1.212 & & 51.558 & 0.075 & 14.643 & 0.00 & & & $\begin{array}{l}0 \quad 49.5 \\
7\end{array}$ & 12.3 & & 2.688 & 0.011 & 0.010 & & 0.246 & 0.21 \\
\hline 18 & 9/2/2004 & 7:27:34 PM & $\begin{array}{l}28.955 \\
20277 \\
2077\end{array}$ & 26.231 & \begin{tabular}{|l|}
27.288 \\
27021
\end{tabular} & 26.293 & 25.795 & $\begin{array}{r}47.476 \\
2.763\end{array}$ & 50.687 & 18.204 & 30.212 & -1.215 & & $\begin{array}{r}48.4 \\
50.92]\end{array}$ & 0.076 & 14.643 & & 1.0 & 0.01667 & 49.6 & & 38.8 & 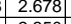 & & & & 0.251 & \\
\hline 留 & $\begin{array}{l}91212004 \\
0.120004\end{array}$ & 7:28:34 PM & 28.277 & $\frac{26.24}{2623}$ & $\mid$\begin{tabular}{|l|l|}
27.281 \\
\end{tabular} & 26.256 & 25.837 & 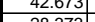 & & 17.965 & $\begin{array}{l}25.496 \\
257\end{array}$ & .2.071 & & 50.8827 & 0.004 & 14.643 & & $\frac{2.00}{2.00}$ & 0.03333 & - & & & Le.350 & 0.001 & & & & \\
\hline & 多 & $\frac{7: 29: 34}{730.34}$ & $\frac{2.0 .021}{28561}$ & $\frac{2.2534}{26224}$ & $\frac{27.286}{27386}$ & 26.247 & 250.030 & $\begin{array}{l}28.373 \\
1252\end{array}$ & S1.1661 & $\mid$ & $\mid 11.214$ & $\begin{array}{l}1.041 \\
31563\end{array}$ & & 年 & 0.0003 & $\begin{array}{l}14.045 \\
1.637\end{array}$ & & 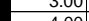 & 0.05 & 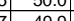 & $\frac{11.0}{126}$ & 19. & 年 & & & , & & \\
\hline & 9.1/2/2004 & $7.531 .34 \mathrm{E}$ & 28.076 & $\frac{2.244}{26224}$ & 2730 & (2.2010 & & | & 50.643 & 16817 & $\mid$ & & & 53.489 & & 14.045 & & 50 & . & 年, & 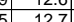 & & & & & & & 0.0 \\
\hline & $9 / 2 / 2004$ & $\begin{array}{l}7: 32: 34 \\
\end{array}$ & $\frac{2.410}{28.793}$ & 26.203 & \begin{tabular}{|l|l|}
27.285 \\
\end{tabular} & 26.155 & 25.662 & 年.1.136 & 49.675 & $\frac{1.014}{12.624}$ & \begin{tabular}{|l|}
-2.747 \\
\end{tabular} & 35.24 & & 55.546 & 0.003 & $\begin{array}{ll}14.0453 \\
14.643\end{array}$ & & 6.00 & 0.000 .1 & $\frac{7.8 .6}{48.6}$ & $\frac{\frac{1.2 .}{13.2}}{13.2}$ & 3 & 1020 & & 000 & 0.000 & & $\frac{0.9}{0 .}$ \\
\hline & 9/2/2004 & 7:33:34 PM & 28.453 & 26.201 & 27.258 & 26.103 & 25.555 & 9.152 & 50.092 & 12.723 & -2.872 & 35.416 & & 52.868 & 0.003 & 14.643 & 0.00 & 7.00 & 0.11667 & 49.0 & $\frac{12.6}{12.6}$ & & $\frac{1.216}{0.216}$ & & & 0.000 & $\frac{124}{124}$ & 0.1. \\
\hline & 9/2/2004 & 7:34:34 PM & 28.329 & 26.196 & 27.208 & 26.023 & 25.395 & 9.175 & 49.961 & 12.802 & -3.055 & 35.416 & & 51.485 & 0.003 & 14.643 & .00 & 8.00 & 0.13333 & $\begin{array}{ll}48.8 \\
\end{array}$ & 12.3 & & 0.211 & . & & .000 & 0.128 & \\
\hline & 2/2004 & & & 26.194 & & & & & & & & 6.15 & & \begin{tabular}{|l|l|}
49.727 \\
\end{tabular} & 0.365 & $\begin{array}{l}14.643 \\
\end{array}$ & & 9.00 & 0.15 & & & & & & & & & \\
\hline & 9/2/2004 & $7: 36: 3$ & 28.103 & 26.169 & \begin{tabular}{|l|l|}
27.167 \\
\end{tabular} & 25.892 & & 43.78 & 50.509 & 15.057 & 29.596 & $\begin{array}{l}0.725 \\
\end{array}$ & & 50.085 & 0.125 & $\begin{array}{ll}14.643 \\
\end{array}$ & & 10.00 & 0.16667 & 49.4 & $\overline{11 . !}$ & 36. & 2.530 & .018 & $\overline{0.016}$ & 0.000 & 0.447 & 0.38 \\
\hline & 9/2/2004 & & & 26.168 & 27.155 & 25.885 & & $\begin{array}{l}44.301 \\
\end{array}$ & 51.081 & 15.759 & 29.297 & 0.603 & & 51.819 & 0.104 & $\begin{array}{l}14.643 \\
\end{array}$ & & $\overline{11.0}$ & 0.18333 & & 12. & & & & & .000 & & 0.32 \\
\hline & $9 / 2 / 2004$ & $7: 38: 3$ & 28.014 & 26.165 & \begin{tabular}{|l|l|}
27.013 \\
\end{tabular} & 25.897 & & 44.218 & 51.02 & 16.065 & 28.859 & 0.557 & & 49.4 & 0.094 & $\begin{array}{l}14.643 \\
\end{array}$ & & 12.0 & & & & & & & & & & \\
\hline & $9 / 2 / 2004$ & $7: 39: 34 \mathrm{~F}$ & & & 266.991 & 25.96 & 25.452 & 44.052 & 50.732 & 15.891 & \begin{tabular}{|c|}
29.001 \\
\end{tabular} & 0.597 & & 54.123 & 0.098 & 14.643 & & 13.0 & 0.21667 & & 2.9 & 36.5 & & & & & & \\
\hline 31 & $9 / 2 / 2004$ & $7: 40: 34$ & 27.816 & 26.146 & 26.969 & 25.938 & 25.475 & 43.934 & \begin{tabular}{|l|}
50.718 \\
\end{tabular} & 15.927 & 28.839 & 0.722 & & 50.778 & 0.093 & 14.643 & & 14.00 & 0.23333 & $\begin{array}{l}49.6 \\
\end{array}$ & 12.1 & 36.4 & 2.509 & & | 0.013 & 0.000 & 0.346 & 0.29 \\
\hline & 9/2/2004 & & 27.721 & 26.145 & 26.943 & 25.963 & & & \begin{tabular}{|l|}
50.6 \\
\end{tabular} & & & & & 50.859 & 0.101 & 14.643 & & 15.0 & 0.25 & 49.5 & 12. & & 2.511 & 114 & .014 & & & 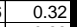 \\
\hline 33 & 9/2/2004 & 7::22:34 & 27.565 & 26.134 & 26.897 & 25.921 & 25.498 & 48.015 & \begin{tabular}{|l|}
50.78 \\
\end{tabular} & 16.036 & $33.017 \mid$ & -1.212 & & 53.506 & 0.098 & 14.643 & & 16.00 & 0.26667 & 49.7 & 12. & 40 & .793 & 0.014 & .013 & 0.000 & 0.328 & 0.2 \\
\hline 泣 & $9 / 2 / 2004$ & & & 26.148 & $\mid$\begin{tabular}{|l|l|}
26.896 \\
\end{tabular} & 25.99 & & & 51.087 & 16.05 & 33.368 & -1.209 & & 51.076 & 0.1 & 14.643 & & 17.00 & 0.28333 & 50.0 & $x$ & & 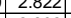 & 014 & . & 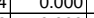 & (2) & 02 \\
\hline & & & 20.539 & & & 25.01 & & & & & 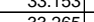 & & & & & & & & & & $\frac{1.24}{120}$ & & & & & & & \\
\hline & & & $\frac{2.481}{2745}$ & & \begin{tabular}{|l|l|}
2.86411 \\
\end{tabular} & $\frac{25.9881}{25051}$ & & & $\frac{51.128}{51317}$ & & & $-\frac{1.2 .}{1.2}$ & & $\frac{50}{409}$ & & $\frac{14.06}{146}$ & & & 0 & $\frac{5.6}{503}$ & $\frac{1.6}{114 .}$ & & 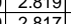 & & 013 & & & $i$ \\
\hline 38 & $9 / 2 / 2004$ & & $\frac{2.349}{27.419}$ & 26.123 & \begin{tabular}{|l|l|l|}
26796 \\
\end{tabular} & | & & & & & & & & & & 146 & & & 0 & 498 & 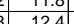 & & & & & & & \\
\hline 39 & $\frac{1 / 2 / 2004}{9}$ & & 27.375 & 26.114 & $\mid 26.772$ & 25.986 & & & 51.151 & 16.266 & 33.247 & $\frac{-1.21}{-1.21}$ & & $\frac{09.4}{49.4}$ & & 14.643 & & $\frac{22}{22}$ & 0.3666 & 500 & 118 & & 58 & & 012 & & & 0.4 \\
\hline 40 & 9/2/2004 & & 27.335 & $\frac{26.114}{226}$ & \begin{tabular}{|l|l|}
26.767 \\
\end{tabular} & 25.986 & & 48.606 & \begin{tabular}{|l|l|}
51.105 \\
\end{tabular} & 16.169 & & \begin{tabular}{|l|l|}
-1.215 \\
\end{tabular} & & 53.304 & 0.0 & 14.643 & & 23.9 & 0.38333 & 50.0 & 127 & & & & $\frac{012}{012}$ & & & \\
\hline & $9 / 2 / 2004$ & & & & & 26.037 & & $\begin{array}{lll}48.4 \\
\end{array}$ & & & & -1.209 & & 50507 & & & & 24.06 & & & 15 & & & & & & 0.297 & in \\
\hline & 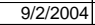 & $7: 51: 3$ & 27.266 & 26.119 & $\begin{array}{l}26.672 \\
\end{array}$ & 26.062 & & & 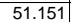 & \begin{tabular}{ll|l}
16.164 \\
\end{tabular} & 33.304 & -1.212 & & 53.0 & & $\begin{array}{ll}14.643 \\
\end{array}$ & & & o.41667 & 50.6 & 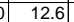 & & 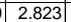 & & .012 & & $\frac{0.294}{0.294}$ & t \\
\hline 43 & & & & 26.10 & & & & & & & & & & & & & & & 0.43333 & & 12.3 & & & & & & & 4 \\
\hline 44 & 9/2/2004 & & 27.172 & 26.1 & 26.538 & & & & 51.412 & & & & & & & & & & $\begin{array}{l}0.45 \\
\end{array}$ & 50.3 & 12.1 & & 2.839 & & 0.012 & & & to \\
\hline 45 & & & 27.137 & 26.1 & & & & & & & & -1.2 & & & & & & & 0.46667 & 49.9 & $12.2^{2}$ & & 24 & & 0.012 & & 0.285 & 5 \\
\hline 46 & 9/2/2004 & 7:55:34 & 27.091 & 26.094 & 26.433 & 26.037 & & 48.2 & \begin{tabular}{|l|}
50.567 \\
\end{tabular} & 17.074 & 32.069 & -1.212 & & 52.6 & 0. & 14.643 & & 29.00 & 0.48333 & 49.4 & $12.5 \mathrm{r}$ & & 2.770 & & 0.011 & 0.000 & & 0.2 \\
\hline 47 & 9/2/2004 & & 27.047] & 26.085 & 26.378 & 26.032 & & & & & 32.141 & -1.21 & & 52.25 & 0.083 & 14.643 & & & & 49.8 & 12. & & & & . & & 85 & 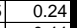 \\
\hline 48 & 9/2/212004 & 7:57:34 PM & 26.973 & 26.081 & 26.319 & 26.038 & & 47.268 & 50.629 & 16.8699 & 31.269 & -1.215 & & 51.201 & 0.148 & 14.643 & & 31.0 & 0.51667 & 49.5 & 12.2 & & & 021 & 0.02 & 0.001 & 0.520 & 0.4 \\
\hline 49 & 9/2/2004 & & 26.946 & 26.07 & 26. & 26.046 & & & & & & & & 52.5 & & 14. & & 32.00 & 0.53333 & 49.6 & . & & & 023 & & . & 0.505 & 0.4 \\
\hline 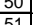 & $\begin{array}{l}91212004 \\
0 / 21004\end{array}$ & & 26.892 & 26.064 & \begin{tabular}{|l|l|}
26.153 \\
\end{tabular} & 26.056 & & & \begin{tabular}{|l|l|l|l|l} 
\\
5.25
\end{tabular} & & 32.018 & $-\frac{1.1215}{1215}$ & & & & 14.6 & & 2000 & 0.55 & 49 & 12. & 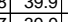 & & & & & & 0 \\
\hline & & & & & & & & & & & & & & & & & & & & & 12. & & & & & & & \\
\hline & & & $\frac{2.01}{2676}$ & 26.06 & $\mid 25$ & 260 & & & & & & -1.12 & & & & & & & . & & 127 & & & & & & & 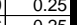 \\
\hline & & & & 260 & & & & & & & & & & & & & & & & & . & & & & & & & t \\
\hline 55 & & & & & & & & & & & & & & & & & & & 0.63 & & $x=$ & & & & & & & t \\
\hline 56 & 9/2/2004 & & 26.613 & 26.06 & \begin{tabular}{|l|}
25.683 \\
\end{tabular} & & & & & & & -1.215 & & & & 14.6 & & & & 49.4 & 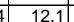 & & & & & & & \\
\hline & & & & & & & & & & & & & & & & & & & & & & & & & & & & \\
\hline 58 & & & 26.51 & & & & & & & & & & & & & & & & $\mid 0.68$ & & & & & & & & & \\
\hline & & & & & & & & & & & & & & & & & & & & & & & & & & & & \\
\hline 60 & $9 / 2 / 20$ & $8: 09: 34$ & 26.411 & 26.0 & 25.476 & 26.03 & & 47.7 & & 16.1 & 32.531 & -1.212 & & 52.5 & 0.1 & 14.6 & & 43.00 & 0.71 & \begin{tabular}{ll|l}
49.4 \\
\end{tabular} & 12 & & & & & & 2 & th \\
\hline 61 & 9/2/2004 & & 26.37 & 26. & 25.436 & & & & & 16. & 3 & -1.2 & & & & 14. & & & 0.73 & & & & & & & & & $f$ \\
\hline 62 & $9 / 2 / 200$ & & 26.32 & & 25. & & & 47.818 & & & & & & & & 14. & & & 1.75 & 49.4 & 12 & & & & & & 3 & s. \\
\hline 63 & $9 / 2 / 26$ & & 26.2 & 26. & \begin{tabular}{|l} 
\\
\end{tabular} & 26.0 & & & & 15.9 & & -1.21 & & & & & & & & & 12.2 & & 14 & 12 & & & & ti \\
\hline 64 & $9 / 2 / 26$ & & 26.242 & & & 26.0 & & 47.1 & 50. & 16. & 31.9 & -1.21 & & & & 14.6 & & 47 & 0.78333 & 48.9 & & & & & & & & 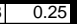 \\
\hline & & & 26.2 & & & & & & & & & & & & & & & 48.00 & & .4 & & & & & & & & \\
\hline 60 & 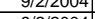 & 8.15 .6 & 20.147 & 20.033 & 20.101 & 20.001 & & & 49.028 & 13.0 & 32.2000 & -1.213 & & & & & & 49.00 & 0.81007 & 48.5 & 12.0 & & & & & & & \\
\hline & & & & & 25.041 & & & & & & & & & & & & & & 0.83333 & 48.5 & 12. & & & & & & & \\
\hline & & & $\frac{2.001}{26016}$ & $\frac{2.0261}{26027}$ & 2.01 & 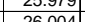 & $\frac{2.5}{25.5}$ & 40.003 & 4.2 & $\begin{array}{l}15.000 \\
15607\end{array}$ & 31.904 & -1.14 & & 32.0906 & 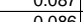 & 1.0 & & 31.00 & 0.85 & 年 & & & 2.708 & 0122 & & 0.000 & 0.317 & 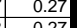 \\
\hline 70 & $9 / 2 / 2004$ & 8:19:34 PM & 25.966 & 26.006 & \begin{tabular}{|l|l|}
24.8999 \\
\end{tabular} & \begin{tabular}{|l|}
26.009 \\
\end{tabular} & \begin{tabular}{|l|}
25.50 \\
\end{tabular} & 47.171 & \begin{tabular}{|l|l|}
49.976 \\
\end{tabular} & \begin{tabular}{|l|}
15.45 \\
\end{tabular} & 32.635 & $|-1.215|$ & & \begin{tabular}{|c|}
49.328 \\
\end{tabular} & $\begin{array}{l}0.087 \\
0.00\end{array}$ & \begin{tabular}{|l|}
14.643 \\
\end{tabular} & 0.00 & 53.06 & 0.88333 & \begin{tabular}{|l|l|}
$\mid 3$ & 48.9 \\
\end{tabular} & $11.7 \mid$ & 7) 39.9] & 2.751 & \begin{tabular}{|l|}
0.012 \\
\end{tabular} & 0.012 & 0.000 & \begin{tabular}{|c|c|c|}
0.313 \\
\end{tabular} & \\
\hline
\end{tabular}


WSRC-TR-2005-00105, REVISION 0

SRNL-RPP-2005-00012, REVISION 0

RUN \# WASH1 AND WASH 2; CAMPAIGN IV SLURRY WASHING - CONT.

\begin{tabular}{|c|c|c|c|c|c|c|c|c|c|c|c|c|c|c|c|c|c|c|c|c|c|c|c|c|c|c|c|c|}
\hline & A & $\mathrm{B}$ & D & $\mathrm{E}$ & $\mathrm{F}$ & $G_{G}$ & $\mathrm{H}$ & $\mathrm{J}$ & $\mathrm{K}$ & $\mathrm{L}$ & $\begin{array}{ll} \\
\end{array}$ & $\mathrm{N}$ & $\mathrm{O}$ & Q & $\mathrm{R}$ & \begin{tabular}{l|l} 
\\
\end{tabular} & $\mathrm{T}$ & $\mathrm{v}$ & w & $x$ & \begin{tabular}{l|l}
$\mathrm{Y}$ & \\
\end{tabular} & $\mathrm{z}$ & AA & $A B$ & $A C$ & AD & $\mathrm{AE}$ & $\mathrm{AF}=$ \\
\hline 71 & 9/2/2004 & 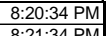 & $\begin{array}{l}25.926 \\
25875\end{array}$ & $\begin{array}{l}26.011 \\
26001\end{array}$ & $\frac{24.864}{24708}$ & $\begin{array}{l}25.964 \\
25.958\end{array}$ & \begin{tabular}{|l}
25.555 \\
25535
\end{tabular} & \begin{tabular}{r|r|}
46.74 \\
46.877
\end{tabular} & \begin{tabular}{|l|l|}
49.404 \\
49563
\end{tabular} & \begin{tabular}{|l|l|}
15.534 \\
\end{tabular} & \begin{tabular}{|l|l|}
32.153 \\
32272 \\
\end{tabular} & $\begin{array}{l}-1.212 \\
\end{array}$ & $\begin{array}{l}-8.027 \\
\end{array}$ & \begin{tabular}{|l|l|}
50.029 \\
5
\end{tabular} & $\begin{array}{l}0.088 \\
097\end{array}$ & \begin{tabular}{|l|l|}
14.643 \\
\end{tabular} & \begin{tabular}{|c|c|c|}
0.003 \\
\end{tabular} & 54.00 & $\begin{array}{r}0.9 \\
\end{array}$ & \begin{tabular}{|l|l|}
48.3 \\
18.9
\end{tabular} & & & & & $\begin{array}{ll}0.013 \\
\end{array}$ & 0.000 & 0.321 & \\
\hline & 9/2/2004 & $8: 21: 34$ PM & $\begin{array}{l}25.875 \\
25815\end{array}$ & $\begin{array}{l}26.001 \\
26.011\end{array}$ & $\begin{array}{l}124.798 \\
\end{array}$ & $\begin{array}{l}25.958 \\
25.063\end{array}$ & \begin{tabular}{|r|}
25.535 \\
25525
\end{tabular} & \begin{tabular}{|l|l|}
46.877 \\
4689
\end{tabular} & \begin{tabular}{|l|l|}
49.563 \\
\end{tabular} & \begin{tabular}{|l|l|}
15.608 \\
15.131
\end{tabular} & \begin{tabular}{|l|}
32.227 \\
32342
\end{tabular} & $\begin{array}{l}-1.212 \\
.1212\end{array}$ & $\begin{array}{l}-8.03 \\
\end{array}$ & \begin{tabular}{|l|l|}
50.494 \\
5062
\end{tabular} & \begin{tabular}{|l|l|}
0.087 \\
0087
\end{tabular} & \begin{tabular}{|l|l|l|}
14643 \\
\end{tabular} & & & & $\begin{array}{l}48.4 \\
4.9\end{array}$ & & & & & & 0.000 & 0.317 & \\
\hline 73 & $\frac{9 / 2 / 2004}{9 / 2 / 2004}$ & $8: 22: 34 \mathrm{PM}$ & $\frac{25.845}{2585}$ & $\frac{26.011}{26.006}$ & \begin{tabular}{|l|l|l|l|}
524.798 \\
524.733
\end{tabular} & $\begin{array}{l}25.963 \\
25.978\end{array}$ & \begin{tabular}{|r|}
25.525 \\
2544
\end{tabular} & \begin{tabular}{|l|l|}
46.868 \\
46779
\end{tabular} & \begin{tabular}{|l|}
49.563 \\
79.652 \\
9
\end{tabular} & \begin{tabular}{|l|}
15.431 \\
15.38
\end{tabular} & \begin{tabular}{|l|}
32.342 \\
32.256
\end{tabular} & $\begin{array}{ll}12 & -1.212 \\
6 & 121\end{array}$ & $\begin{array}{r}-8.03 \\
\end{array}$ & \begin{tabular}{|l|}
50.628 \\
50277 \\
\end{tabular} & \begin{tabular}{|l|l|}
0.087 \\
0087
\end{tabular} & \begin{tabular}{|l|}
14.643 \\
1466
\end{tabular} & $\begin{array}{l}0.004 \\
0003\end{array}$ & & $\begin{array}{ll}0.93333 \\
0\end{array}$ & $\begin{array}{l}48.4 \\
48.5 \\
\end{array}$ & & & $\frac{2.73}{272}$ & & \begin{tabular}{|l|l|}
0.013 \\
\end{tabular} & 0.000 & 0.316 & \\
\hline $\begin{array}{l}74 \\
75 \\
\end{array}$ & $\begin{array}{l}9 / 2 / 2004 \\
9 / 2 / 2004\end{array}$ & $8: 23: 34 \mathrm{PM}$ & 25.8055 & $\frac{26.006}{26.006}$ & $\begin{array}{l}524.733 \\
5 \\
5\end{array}$ & 25.978 & \begin{tabular}{|l|}
25.47 \\
25.52
\end{tabular} & \begin{tabular}{|c|}
46.779 \\
46.796
\end{tabular} & $\begin{array}{l}49.652 \\
\end{array}$ & \begin{tabular}{|c|}
15.382 \\
15.288
\end{tabular} & \begin{tabular}{|}
32.256 \\
32.243 \\
\end{tabular} & 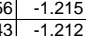 & $\begin{array}{r}-8.03 \\
-80.21\end{array}$ & \begin{tabular}{|c|}
50.077 \\
490.13 \\
\end{tabular} & \begin{tabular}{|c|}
0.087 \\
0.087
\end{tabular} & \begin{tabular}{|l|}
14.643 \\
14.643
\end{tabular} & $\begin{array}{l}0.003 \\
0003\end{array}$ & 57.00 & \begin{tabular}{|r|r|}
0 & 0.95 \\
\end{tabular} & $\begin{array}{r}48.5 \\
48.5 \\
\end{array}$ & & & & & \begin{tabular}{|c|}
0.013 \\
0.013
\end{tabular} & $\begin{array}{l}0.000 \\
0.000\end{array}$ & $\begin{array}{l}0.318 \\
0.318\end{array}$ & $\begin{array}{l}0.27 \\
0.27\end{array}$ \\
\hline 76 & 9/2/2/2004 & $\begin{array}{l}8.24 .34 \mathrm{PM} \\
8: 25: 34 \mathrm{PM}\end{array}$ & $\frac{25.715}{25.734}$ & $\begin{array}{l}26.006 \\
25.999\end{array}$ & $\begin{array}{l}524.713 \\
5 \\
5\end{array}$ & $\begin{array}{l}26.003 \\
25.987\end{array}$ & $\begin{array}{r}25.52 \\
25.498\end{array}$ & \begin{tabular}{|}
46.796 \\
47.003
\end{tabular} & $\frac{49.65}{49.81}$ & $\begin{array}{l}15.288 \\
15.353 \\
\end{array}$ & $\begin{array}{l}32.245 \\
32.596\end{array}$ & \begin{tabular}{c|c}
3 & -1.212 \\
36 & -1.212
\end{tabular} & $\begin{array}{l}-8.012 \\
-8.018\end{array}$ & \begin{tabular}{|}
44.013 \\
52.985 \\
\end{tabular} & $\begin{array}{l}0.087 \\
0.086\end{array}$ & $\begin{array}{l}14.643 \\
14.643\end{array}$ & 0.0 & $\begin{array}{l}55.00 \\
59.00\end{array}$ & $\begin{array}{l}0 \\
\\
\end{array}$ & $\begin{array}{l}48.5 \\
48.7\end{array}$ & & & $\begin{array}{l}2.725 \\
2.744\end{array}$ & $\frac{0.012}{0.012}$ & $\mid \begin{array}{l}0.013 \\
0.012\end{array}$ & $\begin{array}{l}0.000 \\
0.000\end{array}$ & $\begin{array}{l}0.318 \\
0.312\end{array}$ & $\frac{0.27}{0.27}$ \\
\hline 77 & & 8:26:34 PM & 25.698 & 25.993 & \begin{tabular}{|l|l|}
3 & 24.626 \\
\end{tabular} & & 25.513 & 47.439 & 50.573 & 15.48 & $\begin{array}{l}32.679 \\
\end{array}$ & \begin{tabular}{l|l}
9 & -1.215 \\
\end{tabular} & -8.015 & 49.741 & 0.087 & 14.643 & & 60.00 & & 49.4 & & & $\frac{2.144}{2.762}$ & 0.012 & 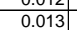 & 0.000 & $\begin{array}{l}0.312 \\
0.314\end{array}$ & 0.27 \\
\hline 78 & 9/2/2004 & 8:27:34 PM & 25.662 & 25.988 & \begin{tabular}{|l|l|}
324.605 \\
\end{tabular} & 25.93 & 25.482 & 47.19 & \begin{tabular}{|l|l|}
50.09 \\
\end{tabular} & 15.381 & & & $\begin{array}{l}-8.015 \\
\end{array}$ & 47.901 & $\begin{array}{l}0.087 \\
\end{array}$ & \begin{tabular}{|l|l|}
14.643 \\
\end{tabular} & 0.003 & 61.00 & \begin{tabular}{l|l}
0 & 1.01667 \\
\end{tabular} & 49.0 & & & & & $\begin{array}{l}0.013 \\
\end{array}$ & & & \\
\hline 79 & & 8:28:34 PM & & 25.983 & \begin{tabular}{|l|l|}
3 & 24.55 \\
\end{tabular} & 25.985 & \begin{tabular}{|l|l|}
25.437 \\
\end{tabular} & $\begin{array}{l}47.362 \\
\end{array}$ & \begin{tabular}{|l|l|} 
& 50.181 \\
\end{tabular} & & \begin{tabular}{|l|l|}
32.919 \\
\end{tabular} & \begin{tabular}{l|l}
9 & -1.212 \\
\end{tabular} & & $\begin{array}{l}49.339 \\
\end{array}$ & & \begin{tabular}{|l|l|}
14.643 \\
\end{tabular} & & 62.00 & & 49.1 & & 40.1 & 2.768 & & & & 0.318 & \\
\hline 80 & 9/2/2004 & $8: 29: 34 \mathrm{PM}$ & 25.602 & 25.988 & $\begin{array}{ll}32.545 \\
\end{array}$ & 25.99 & 25.472 & $\begin{array}{l}47.526 \\
\end{array}$ & \begin{tabular}{|l|l|} 
& 50.531 \\
\end{tabular} & $\begin{array}{l}15.363 \\
\end{array}$ & & -1.212 & -8.004 & 49.977 & 0.086 & 14.643 & & & 1.05 & & & 40. & 2.776 & & & 0.000 & 0.310 & \\
\hline & & & & & & & & & & & & & & & & & & & & & & & 2751 & & (1) & & & \\
\hline 82 & 2/2004 & $1: 34 \mathrm{PM}$ & 25.542 & 25.973 & $\begin{array}{l}324.495 \\
3\end{array}$ & 25.965 & 25.432 & 47.099 & 50.021 & 15.42 & 32.551 & -1.215 & \begin{tabular}{|c|c|}
-8.018 \\
\end{tabular} & 45.428 & .085 & 14.64 & & & 1.08333 & 48. & & 398 & \begin{tabular}{|l|l|l|}
2.746 \\
\end{tabular} & & D.012 & .000 & 0.310 & \\
\hline 83 & 2/2004 & 8:32:34 PM & 25.512 & 25.978 & 24.47 & 25.955 & 25.447 & 47.181 & 50.25 & 15.464 & 32.475 & -1.212 & -8.024 & 49.989 & 0.084 & 14.64 & & & 1.1 & 49.1 & & 39.8 & 2.746 & 0.012 & 0.012 & 0.00 & 0.306 & \\
\hline & 9//2/2004| & $34 \mathrm{PM}$ & & & 24.404 & 25.934 & & & & 15.158 & 32.713 & & & 50.081 & & 14.64 & & & & & & & 2.747 & & & & & \\
\hline 85 & |2/2004 & 8:34:34 PM & 25.461 & 25.966 & 24.369 & 25.959 & 25.471 & 46.617 & 49.667 & 15.012 & 32.412 & -1.215 & $\begin{array}{r}-7.998 \\
-\end{array}$ & 52.444 & 0.088 & 14.643 & & 68.00 & 1.13333 & 48.5 & 12 & 39.5 & 2.724 & 0.013 & 0.013 & 0.000 & 0.325 & \\
\hline $\begin{array}{ll}86 \\
87\end{array}$ & 9/2/2004 & 8:35:34 PM & 25.431 & 25.961 & 24.353 & 25.929 & 25.486 & 46.53 & 49.418 & 15.042 & 32.4 & -1.212 & -7.955 & 48.704 & 0.09 & 14.643 & & 69.00 & 1.15 & 48.3 & 11. & & 2.721 & 0.013 & 0.013 & 0.000 & 0.333 & \\
\hline$\frac{81}{80}$ & 22004 & $6: 34$ PM & 25.405 & 25.955 & 24.332 & 25.937 & 25.464 & 46.545 & 49.833 & 15.096 & 32.319 & -1.212 & -7.978 & 50.932 & 0.09 & 14.643 & & 70.00 & 16667 & 48.7 & & & 2.719 & & 0.013 & & & \\
\hline 年8 & 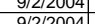 & 7:34 PM & 2.5 .35 & & 24.297 & $25.931]$ & 25.464 & 46.634 & 49.686 & 15.059 & 32.399 & & $=0.7 .989$ & 48.8960 & 0.088 & 14.643 & & & 18333 & & & & & 013 & 0.013 & & 0.325 & \\
\hline$\frac{\partial 9}{90}$ & $\frac{212004 \mid}{21204}$ & $\frac{8.34 \mathrm{FM}}{2.2104}$ & | & $\frac{23.949}{25051}$ & 24.240 & 25.917 & 250.409 & 40.090 & $\frac{49.191}{5005}$ & $\begin{array}{l}13.080 \\
15259\end{array}$ & $\frac{32.303}{32525}$ & $-\frac{1.218}{1.1212}$ & & $\begin{array}{l}50.065 \\
19501\end{array}$ & $\begin{array}{ll}0.081 \\
0.089\end{array}$ & $\begin{array}{l}1.044 \\
1.642\end{array}$ & & & $\frac{1.2}{1067}$ & & & & & & 0.013 & & & \\
\hline 91 & $9 / 2 / 2004$ & $8 \cdot 3.34 \mathrm{PM}$ & 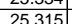 & $\begin{array}{l}2.3054 \\
25.95\end{array}$ & 524207 & 年 25.967 & $\frac{25.409}{25.444}$ & $\frac{46.982}{46.532}$ & $\frac{50.05}{49.613}$ & $\frac{15.259}{15275}$ & $\begin{array}{l}32.525 \\
32.024\end{array}$ & $\frac{-1.1212}{-1.212}$ & $\begin{array}{l}-7.986 \\
-7.955 \\
\end{array}$ & $\begin{array}{l}49.591 \\
50.85\end{array}$ & $\frac{0.000}{0.089}$ & $\frac{14.64}{14.643}$ & & & 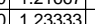 & $\frac{48 .}{48}$ & & & 270 & & & & & \\
\hline 92 & 9/2/2004 & 41:34 PM & 25.29 & 25.94 & $\begin{array}{lll}44 & 24.212\end{array}$ & 25.947 & 25.444 & 46.995 & 50.388 & 15.133 & 32.613 & -1.215 & -7.958 & 48.481 & 0.089 & 14.643 & & 75.00 & 1.25 & 49.3 & 11.5 & 39.8 & 2.744 & 0.013 & 0.013 & 0.000 & 0.0327 & \\
\hline & 9/2/2004 & 42:34 PM & 25.27 & 25.945 & \begin{tabular}{|l|l|}
5 & 24.212 \\
\end{tabular} & 25.937 & 25.384 & 46.794 & 49.827 & 15.067 & 32.566 & -1.212 & -7.958 & & 0.088 & 14.643 & & 76.00 & .26667 & 48.7 & 11. & & 2.736 & 0.013 & & & & \\
\hline 94 & 2/2004 & 8:43:34 PM & 25.25 & 25.945 & \begin{tabular}{|l|l|}
5.192 \\
\end{tabular} & 25.952 & 25.414 & \begin{tabular}{|l|l}
46.763 \\
\end{tabular} & \begin{tabular}{|l|l|}
49.907 \\
\end{tabular} & \begin{tabular}{|l|l|}
15.087 \\
\end{tabular} & \begin{tabular}{|l|l|}
32.574 \\
\end{tabular} & \begin{tabular}{l|l}
4 & -1.212 \\
\end{tabular} & \begin{tabular}{|l|l|}
-7.958 \\
\end{tabular} & $\begin{array}{l}49.418 \\
\end{array}$ & \begin{tabular}{l|l}
0.087 \\
\end{tabular} & $\begin{array}{l}14.643 \\
\end{array}$ & & 77.0 & 1.28333 & 48. & 11 & 39. & 2.735 & 0.012 & 0.013 & .00 & 0.321 & \\
\hline & | & & 25.235 & 25.935 & 24.172 & 25.912 & 25.464 & 46.848 & $\begin{array}{l}3 \quad 49.999 \\
\end{array}$ & 15.169 & 32.473 & -1.212 & $\begin{array}{l}-7.96 \\
\end{array}$ & 52.461 & 0.087 & 14.643 & & & 1.3 & & & & & & & & & \\
\hline & 22004 & & & & & 25.912 & & & & 15.335 & & & -7.955 & 9.353 & 0.088 & 14.643 & & & 1.31667 & 49 & & & 2.743 & 0.013 & 0.013 & & 0.324 & \\
\hline & |2004] & & 25.19 & 925 & $\begin{array}{ll}5 & 24.172\end{array}$ & & 25.384 & $47.634]$ & 50.884 & 15.435 & 32.982 & -1.215 & \begin{tabular}{|l|l|} 
& -7.94 \\
\end{tabular} & 117 & 0.089 & 14.643 & & & & 49 & & & & & & & & \\
\hline & $9 / 2 / 2004$ & & 25.18 & 25 & 24.157 & 25.952 & 25.4 & & 51.18 & 15.462 & 33.085 & -1.215 & & .29 & 0.09 & 14.64 & & & 1.35 & & & & & & 0.013 & & & \\
\hline & & 4 P PM & 25.164 & & $\begin{array}{l}9 \\
9\end{array}$ & & 25.4 & & & 15.141 & 32.991 & & & & 0.089 & & & & & & & & & & & & & \\
\hline & 9/2/2004 & B4 PM & 25.154 & 25.919 & $\begin{array}{l}9 \\
\text { 9) } 24.141\end{array}$ & 25.912 & 25.41 & .273 & $\begin{array}{l}350.446 \\
\end{array}$ & 15.248 & 32.755 & -1.212 & -7.94 & 49.795 & 0.091 & 14.643 & & 83.0 & 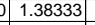 & & 11 & & 2.759 & & & & 0.334 & \\
\hline 101 & 9/2/2004 & 34 PM & 25.138 & 25.914 & $\begin{array}{l}4 \\
4\end{array}$ & 25.936 & 25.4 & 47.179] & 50.337 & 15.146 & 32.822 & 1.610 & -7.91 & 50.834 & 0.093 & 14.643 & & 84 & \begin{tabular}{|l} 
\\
\end{tabular} & 49.2 & & & 2.100 & & & & & \\
\hline$\frac{102}{122}$ & 9/2/2004| & 51:34 PM & 25.118 & 25.898 & $\begin{array}{l}324.099 \\
\end{array}$ & 25.89 & 25.387 & 47.407| & 50.817 & 15.259 & $\begin{array}{l}32.966 \\
20.702\end{array}$ & -1.215 & -7.923 & 50.843 & 0.091 & 14.643 & & 85. & .41667 & 49. & 12 & 40.2 & $2.771 \mid$ & 0.013 & 0.013 & 000 & 0.333 & \\
\hline$\sqrt{104}$ & 9/2/20004 & 4 PM & 25.123 & & 24.135 & $\begin{array}{l}25.922 \\
\end{array}$ & 25.4 & & 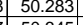 & 15.152 & 32.703 & -1.209 & & 51.364 & 0.093 & 14.643 & & & .43333 & 499 & & & 2.747 & & 0.014 & & 0.042 & \\
\hline & & & 250.0974 & & 2.109 & $\frac{2.8015}{2503}$ & & (4).097) & $\frac{50.345}{50.521}$ & 15.160 & & -1.1212 & & 30.9 & & 14.64 & & & 1.45 & & & & & & & & & \\
\hline & 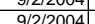 & 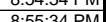 & 25071 & $\frac{23.091}{2507}$ & $\frac{24.050}{24003}$ & | & $\frac{2.4}{252}$ & 416.0 & $\begin{array}{l}50.521 \\
50253\end{array}$ & 10 & $\frac{32.594}{3275}$ & $-\frac{1.212}{1.212}$ & & 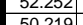 & 0.09 & 14.04 & & & 先66061 & 年 & & & 2.14 & 0.01 & 0 & & 年 & \\
\hline 107 & 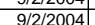 & $48 \mathrm{PM}$ & 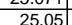 & & 24.067 & 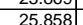 & & & $\frac{1.02}{50.22}$ & 15.216 & 32.64 & $\frac{1.42}{-1.212}$ & & $.7 \mathrm{C}$ & 0.092 & 年 14.64 & & & $\frac{4}{4 \frac{40350}{15}}$ & & 11 & & $\frac{2.144}{2744}$ & & & & & \\
\hline 108 & 9/2/2004 & 7:34 PM & 25.055 & 25.891 & 124.092 & 25.878 & 25.3 & 46.939 & 50.305 & 15.05 & 32.759 & $\frac{-1.212}{-1.212}$ & -7.906 & 50.563 & 0.092 & 14.64 & & & 1.51667 & 49 & & & 2.747 & 0.013 & 0.014 & & 0.339 & \\
\hline & & 8:58:34 PM & 25.05 & 25.886 & \begin{tabular}{|l|l|}
5 & 24.087 \\
\end{tabular} & 25.918 & 25 & & 750000 & 15.135 & 32.941 & \begin{tabular}{|l|l|}
$1-1.212$ \\
\end{tabular} & & $\frac{47.6}{47.6}$ & & 14.6 & & & & & - & & & & & & & \\
\hline & 9/2/2004 & 8:59:34 PM & 25.04 & 25.885 & \begin{tabular}{|l|l|}
54.122 \\
\end{tabular} & 25.883 & 25.405 & 47.127 & 50.623 & 15.164 & \begin{tabular}{|l|l|}
32.707 \\
\end{tabular} & -1.212 & -7.88 & 49.564 & 0.091 & 14.6 & & & 1.55 & 49 & & & 2757 & & & & & \\
\hline$\ldots$ & |2/2004 & & 25.025 & & 24.167 & & & 47.196 & 50.78 & 15.069 & & & & 48.909 & 0.091 & 14.6 & & & 1.56667 & 49 & & & 2.760 & & & & & \\
\hline & |2/2004 & & \begin{tabular}{|l|l|}
25.03 \\
\end{tabular} & 25.875 & 24.217 & 25.878 & 25.3 & 47.103 & 50.546 & 15.172 & 32.691 & -1.212 & & & & 14.6 & & & 1.58333 & $\overline{4 !}$ & & & & & & & & \\
\hline 113 & 9/2/2004 & 9:02:34 F & 25.08 & 25.8 & 824.257 & 25.868 & 25. & 45.348 & 50.869 & 15.226 & 30. & & & 51.7 & & 14.6 & & & 1.6 & 49 & 12 & & 2.624 & 0.000 & & & 0.012 & \\
\hline & & & & & & & & & & 15.409 & & & & & & & & & 61667 & & & & & & & & & \\
\hline 115 & 9/2/212004 & 9:04:34 PM & 25.04 & 25 & \begin{tabular}{|l|l|} 
& 24.347 \\
\end{tabular} & $25.838 \mid$ & 25. & & 50.49 & 15.006 & 32. & -1.2 & -7.6 & 47.618 & 0.1 & 14.6 & & & 1.63333 & & 11 & & 2.742 & 0.014 & & & 0.367 & \\
\hline & 2004 & 9:05:34 & 25.034 & 25.8 & $\begin{array}{ll}9 & 24.327 \\
\end{array}$ & 25.787 & 25. & 46.5 & 350.065 & 14.879 & 32. & -1.2 & & 50.9 & 0.05 & 14.6 & & & 1.65 & & 12 & & 2.72 & & & & 0.347 & \\
\hline & & & 25.048 & 25 & 4) 24.356 & 25.816 & & & $\begin{array}{l}450.351 \\
\end{array}$ & 14.885 & & & & & & 14.6 & & & 1.66667 & & & & & & & & & \\
\hline $1 \pm$ & 004[ & & 25.052 & 25.832 & $\begin{array}{l}2 \mid 24.37 \\
\end{array}$ & 25.79 & & & 50.355 & 14.936 & & -1.212 & & 48 & & $14.6 \mathrm{C}$ & & & 1.68333 & 49 & -2 & & 2.1 & & & & & \\
\hline & & & 25.05 & & & & & & & & & & & & & & & & 4.4 & & & & & & & & & \\
\hline & & & $\frac{23.00}{2507}$ & & $\frac{2.446}{2.123}$ & $\frac{23.00}{2570}$ & & & 50.1 & $\frac{14.91}{1.92}$ & & & & & & & & & & & & & & & & & & \\
\hline & & $\frac{1.3 .54}{9 \cdot 11.34}$ & 25005 & 25815 & 5 & 25803 & $\frac{2.5}{25}$ & & 5043 & 1.4.5017 & & $\frac{1.212}{-1.212}$ & & & 0.094 & 146 & & & 175 & 49 & & & 17 & & & & 年 & \\
\hline 123 & 9/2/2004 & & 25.0 & & \begin{tabular}{|l|l|}
24.461 \\
\end{tabular} & 25.79 & & & 50.14 & 14.946 & 32.344 & & & & & 14.6 & & & 1.76667 & & & & & & & & & \\
\hline & & & 25.104 & 25.814 & $\begin{array}{l}424.486 \\
\end{array}$ & 25.801 & & & 50.3 & 14.9 & & & & & & & & & 1.78333 & & & & & & & & & \\
\hline & & & \begin{tabular}{|l|}
25.107 \\
\end{tabular} & 25.808 & 324.5 & 25.81 & & & & & & & & & & & & & 1.8 & & & & & & & & & \\
\hline 126 & & & & & 24 & & & & & & & & & & & & & & .81667 & & & & & & & & & \\
\hline 127 & & & 25.132 & & \begin{tabular}{l|l|} 
& 24.53 \\
\end{tabular} & 25. & & 46.6 & 50.4 & 14.9 & & -1.206 & & & & & & & 1.83333 & & & & & & & & & \\
\hline & & & 25.132 & & $\begin{array}{l}324.555 \\
3\end{array}$ & 25.7 & & 46 & & & & -1.2 & & & & & & & & & & & & & & & & \\
\hline & 9/2/2004 & $9: 18: 34 \mathrm{~F}$ & 25.13 & 25.79 & 24.5 & 25.79 & & & \begin{tabular}{|l|l|} 
& 49.9 \\
\end{tabular} & 14.8 & & & & & & 14.6 & & & & $\overline{4}$ & & & 26 & & & & & \\
\hline & 2004 & & 25.14 & 25 & 24.574 & 25.7 & & 46.6 & & & & & & & & 14.6 & & & 383 & & & & & & & & & \\
\hline & 9/2/2004 & 9:20:34 F & 25.15 & 25.7 & 24.55 & 25.7 & 25. & 46.404 & 50.27 & 14. & 32.3 & -1.2 & & & & 14.6 & & & 1.9 & & 12 & & & & & & 0.346 & \\
\hline & $9 / 2 / 2$ & $9: 21: 34 \mathrm{P}$ & 25.1 & 25. & & 25.787 & & & 49.5 & 14.701 & & & & & & 14.6 & & & 1.91667 & 48 & 11 & & & & & & 351 & \\
\hline 1333 & $9 / 2 / 2004$ & & 25.169 & & $\begin{array}{l}924.552 \\
9\end{array}$ & 25.792 & 25. & 45.96 & 49.91 & 14.71 & & -1.212 & & & & 14.6 & & & 1.93333 & & 12 & & 2.0 & & & & 361 & \\
\hline & & & & & & & & & & & & & & & & & & & & & & & & & & & & \\
\hline & 2004 & & 2.102 & 23.182 & 24.545 & 23. & & 45.8044 & & $\begin{array}{l}14.131 \\
1.780\end{array}$ & & & & & & 14.6 & & & & & & & & & & & & \\
\hline 势 & 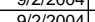 & 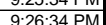 & 25166 & $\frac{25.101}{25771}$ & $\frac{24.550}{24543}$ & $\frac{25.118}{25758}$ & 25295 & 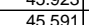 & 年 43.06 & 14.1564 & 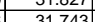 & $\mid$ & & 490 & 0.0 & 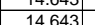 & & & & 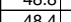 & 11 & 38 & 6 & & & & 0.036 & \\
\hline 138 & $9 / 2 / 2004$ & $\frac{3 \cdot 27}{9 \cdot 27}$ & 25.171 & 25782 & 2564 & 25749 & 25.34 & 45.972 & 49.94 & 1479 & 31899 & -1212 & & & & 1464 & & & 201667 & 48 & & & & & & & 036 & \\
\hline & $9 / 2 / 2004$ & $9.28 \cdot 34 \mathrm{PM}$ & 25.16 & 2577 & 24.569 & 2573 & 25.2 & 46.283 & 50.39 & 14893 & 32.049 & -1.212 & & 516 & 0.09 & 14.643 & & 22. & 203333 & 49.3 & & & & 0.14 & 4 & & 0.355 & \\
\hline 440 & 9/2/2004 & 9:29:34 PM & 25.16 & 25.765 & & 25.722 & & 466.124 & 49.854 & 14.648 & 32.34 & -1.215 & & 01.0 & 0.09 & 14.643 & & 3.0 & & $48.7 \quad$ & & & & & $\frac{0.014}{0.014}$ & & 0.355 & \\
\hline
\end{tabular}


WSRC-TR-2005-00105, REVISION 0

SRNL-RPP-2005-00012, REVISION 0

RUN \# WASH1 AND WASH 2; CAMPAIGN IV SLURRY WASHING - CONT

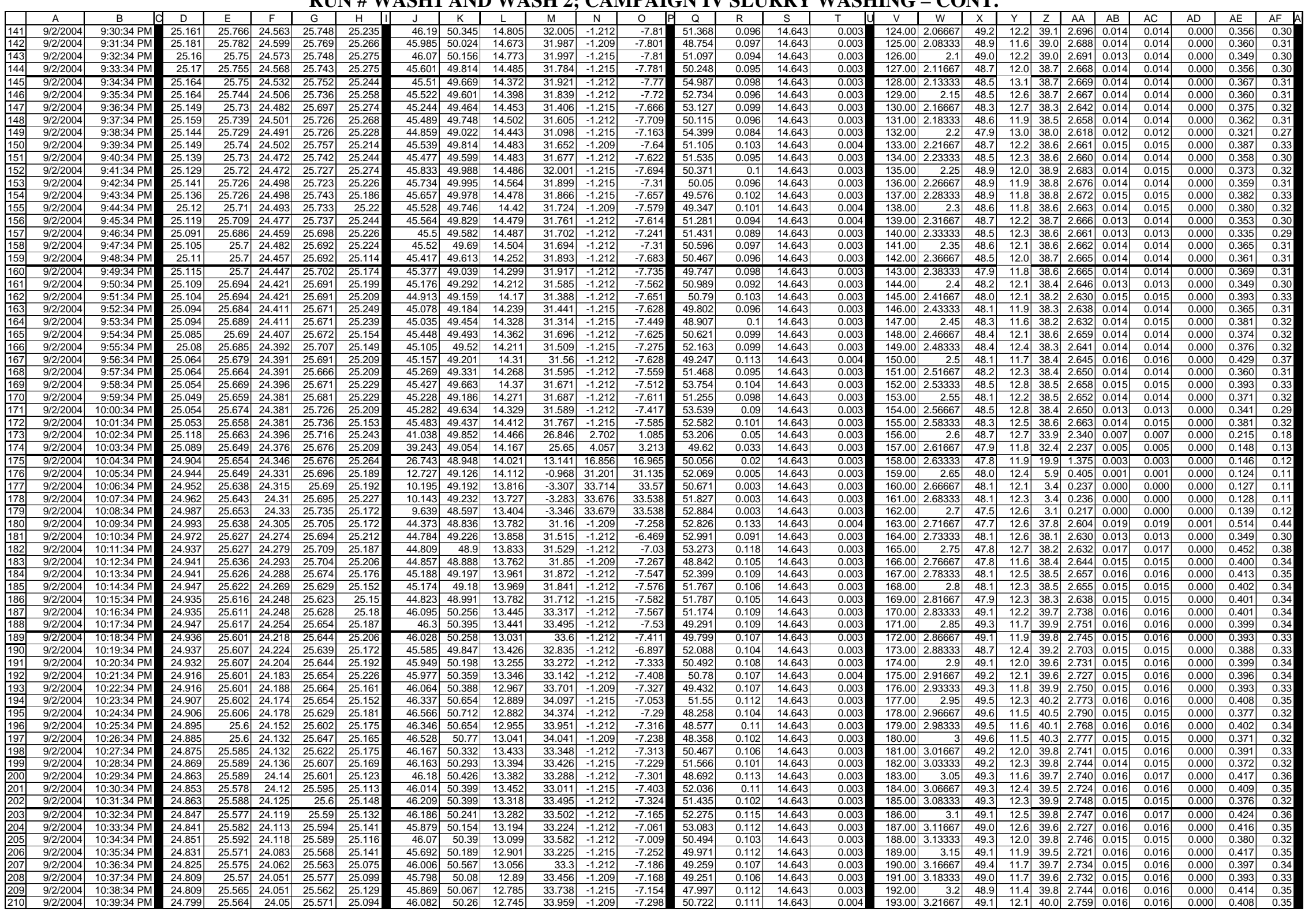


WSRC-TR-2005-00105, REVISION 0

SRNL-RPP-2005-00012, REVISION 0

RUN \# WASH1 AND WASH 2; CAMPAIGN IV SLURRY WASHING - CONT.

\begin{tabular}{|c|c|c|c|c|c|c|c|c|c|c|c|c|c|c|c|c|c|c|c|c|c|c|c|c|c|c|c|}
\hline$\square$ & A & \begin{tabular}{|c|}
$\mathrm{B}$ \\
\end{tabular} & $\mathrm{D}$ & $E$ & $\mathrm{~F}$ & G & $\mathrm{H}$ & $\mathrm{J}$ & $\mathrm{K}$ & \begin{tabular}{l|l|}
$\mathrm{L}$ \\
\end{tabular} & $\mathrm{M}$ & $\mathrm{N}$ & $\mathrm{O}$ & Q & \begin{tabular}{ll|l}
$R$ & \\
\end{tabular} & $\mathrm{~s}$ & $T$ & $\mathrm{v}$ & w & \begin{tabular}{|l|}
$x$ \\
\end{tabular} & \begin{tabular}{l|l}
$Y$ & $Z$ \\
\end{tabular} & $\mathrm{AA}$ & $A B$ & AC & $\mathrm{AD}$ & AE & AF $A$ \\
\hline 211 & 9/2/2004 & \begin{tabular}{|l|}
$10: 40: 34 \mathrm{PM}$ \\
\end{tabular} & $\frac{\square .783}{24.3}$ & 25.558 & \begin{tabular}{|l|l|}
3 & 24.07 \\
\end{tabular} & 25.515 & \begin{tabular}{|l|} 
\\
\end{tabular} & 46.194 & 50.422 & 12.67 & 34.045 & -1.212 & -7.32 & $\begin{array}{ll}47.847 \\
\end{array}$ & 0.109 & 14.643 & 0.004 & 194.00 & 3.23333 & \begin{tabular}{|l|}
49.3 \\
\end{tabular} & & 2.766 & & 0.016 & 0.000 & 0.399 & 0.34 \\
\hline & & \begin{tabular}{|l}
$10: 41: 34$ PM \\
\end{tabular} & 24.799 & 25.569 & 24.096 & & 25.114 & & \begin{tabular}{|l|l|}
49.9944 \\
\end{tabular} & 12.994 & 33.255 & \begin{tabular}{|l|l|}
-1.212 \\
\end{tabular} & & & 0.108 & 14.643 & & 195.00 & 3.25 & 48.9 & & 2.721 & & 0.016 & 0.000 & 0.402 & 0.34 \\
\hline & 9/2/2004 & \begin{tabular}{|l|}
$10: 42: 34$ PM \\
\end{tabular} & 24.799 & 25.559 & 24.136 & 25.511 & 25.094 & $\begin{array}{l}46.078 \\
\end{array}$ & 50.413 & 13.223 & 33.327 & \begin{tabular}{|l|l|}
-1.212 \\
\end{tabular} & $\begin{array}{r}-7.053 \\
\end{array}$ & 50.515 & 0.097 & $\begin{array}{ll}14.643 \\
\end{array}$ & 0.003 & $\begin{array}{l}196.00 \\
\end{array}$ & \begin{tabular}{|l|}
3.26667 \\
\end{tabular} & \begin{tabular}{l|l}
49.3 \\
\end{tabular} & \begin{tabular}{|l|}
12.0 \\
\end{tabular} & \begin{tabular}{|l|l|}
2.737 \\
\end{tabular} & 0.014 & 0.014 & 0.000 & 0.358 & 0.31 \\
\hline$\frac{214}{215}$ & $\begin{array}{l}9 / 2 / 2004 \\
9 / 2 / 2004\end{array}$ & \begin{tabular}{|l|}
$10: 43: 34$ PM \\
$10: 44: 34$ PM
\end{tabular} & $\begin{array}{l}24.793 \\
24793 \\
\end{array}$ & $\frac{25.553}{25.548}$ & \begin{tabular}{|r|}
24.16 \\
324.185
\end{tabular} & $\begin{array}{r}25.515 \\
25.55 \\
\end{array}$ & 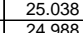 & $\begin{array}{l}45.881 \\
46086 \\
\end{array}$ & \begin{tabular}{|l|}
50.038 \\
50216
\end{tabular} & \begin{tabular}{|c|}
12.927 \\
1289
\end{tabular} & $\begin{array}{l}33.658 \\
33869 \\
\end{array}$ & \begin{tabular}{|l|}
-1.215 \\
-1215 \\
\end{tabular} & $\begin{array}{r}-7.122 \\
-726 \\
\end{array}$ & $\begin{array}{ll}49.462 \\
49.497\end{array}$ & $\begin{array}{l}0.108 \\
0.115\end{array}$ & $\begin{array}{l}14.643 \\
14633 \\
\end{array}$ & $\begin{array}{l}0.003 \\
0.003\end{array}$ & \begin{tabular}{|l|l|}
197.00 \\
198.00
\end{tabular} & \begin{tabular}{|r|}
3.28333 \\
33
\end{tabular} & $\begin{array}{l}48.9 \\
49.1\end{array}$ & \begin{tabular}{|l|l|l|}
11.8 & 39.8 \\
118 & 400 \\
\end{tabular} & \begin{tabular}{|l|}
2.742 \\
2756
\end{tabular} & 0.015 & 0.016 & 0.000 & 0.398 & $\begin{array}{l}0.34 \\
0.36\end{array}$ \\
\hline & 9/2/2004 & \begin{tabular}{|l|l|}
$10: 45: 34 \mathrm{PM}$ \\
\end{tabular} & 24.792 & 25.538 & 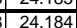 & 25.485 & \begin{tabular}{|l|}
24.980 \\
25.022 \\
\end{tabular} & $\begin{array}{l}46.429 \\
460\end{array}$ & \begin{tabular}{|l|}
50.643 \\
50.45
\end{tabular} & $\frac{12.849}{12.714}$ & 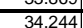 & & & \begin{tabular}{|l|}
48.882 \\
48.8
\end{tabular} & 0.1104 & $\begin{array}{l}14.6434 \\
14.643 \\
\end{array}$ & & & & & & & & 0.017 & & 0.421 & 0.36 \\
\hline 217 & 9/2/2004 & \begin{tabular}{|l|}
$10: 46: 34 \mathrm{PM}$ \\
\end{tabular} & 24.807 & 25.538 & $\begin{array}{l}\mid 24.205 \\
3\end{array}$ & 25.505 & 25.002 & \begin{tabular}{|c|}
45.906 \\
45.45
\end{tabular} & \begin{tabular}{|l|l|}
50.026 \\
5026
\end{tabular} & $\mid \begin{array}{l}12.14 \\
12.705\end{array}$ & $\begin{array}{l}34 .<44 \\
33.791\end{array}$ & \begin{tabular}{|l|}
-1.212 \\
-1.212 \\
\end{tabular} & & $\begin{array}{l}40.002 \\
49.793 \\
\end{array}$ & $\begin{array}{l}0.104 \\
0.1 \\
\end{array}$ & $\begin{array}{l}14.045 \\
14.643 \\
\end{array}$ & $\frac{0.0}{0.0}$ & $\begin{array}{l}129.00 \\
200.00\end{array}$ & \begin{tabular}{|l|}
$\mid 3.31667$ \\
3.33333 \\
\end{tabular} & $\frac{49.5}{48.9}$ & $\begin{array}{l}\frac{11.6}{11.9} \\
110 . \\
33 .\end{array}$ & $\frac{2.781}{2.747}$ & $\frac{0.015}{0.014}$ & $\mid 0.015$ & $\frac{0.000}{0.000}$ & $\begin{array}{l}0.378 \\
0.367\end{array}$ & $\begin{array}{l}0.32 \\
0.31\end{array}$ \\
\hline 218 & 9/2/2004 & $10: 47: 34 \mathrm{PM}$ & 24.812 & 25.542 & 24.209 & 25.524 & 25.047 & 46.101 & \begin{tabular}{|l|}
50.529 \\
\end{tabular} & 12.533 & 34.072 & \begin{tabular}{|l|}
-1.212 \\
\end{tabular} & -7.168 & 49.161 & 0.104 & 14.643 & & 201.00 & \begin{tabular}{|r|}
3.35 \\
\end{tabular} & 40.94 & \begin{tabular}{l|l}
11.9 & 30. \\
11.7 & 40. \\
\end{tabular} & 2.764 & & 0.015 & 0.000 & 0.380 & 0.32 \\
\hline 219 & 9/2/2004 & $10: 48: 34$ PM & 24.827 & 25.537 & 24.229 & 25.514 & 25.047 & 46.012 & 50.312 & 12.584 & 33.879 & \begin{tabular}{|l|}
-1.209 \\
\end{tabular} & & 47.09 & 0.109 & 14.643 & & & \begin{tabular}{|l|}
3.36667 \\
\end{tabular} & 49.2 & 11.2 & 2.754 & & & & 0.399 & 0.34 \\
\hline & 9/2/2004 & 10:49:34 PM & 24.842 & 25.552 & 24.249 & 25.529 & 25.037 & 46.089 & \begin{tabular}{|l|l|}
50.31 \\
\end{tabular} & 12.563 & 34.109 & -1.212 & -7.30 & 52.609 & 0.105 & 14.643 & & 203.00 & \begin{tabular}{|l|}
3.38333 \\
\end{tabular} & 49.2 & 12.5 & 2.765 & 0.015 & 0.015 & 0.000 & 0.383 & 0.33 \\
\hline 221 & 9/2/2004 & \begin{tabular}{|l|}
$10: 50: 34$ PM \\
\end{tabular} & 24.831 & 25.531 & 24.228 & 25.508 & 25.001 & 46.453 & 50.571 & 12.631 & 34.407 & -1.212 & & 51.308 & 0.1 & \begin{tabular}{|l|}
14.643 \\
\end{tabular} & & 204.00 & 3.4 & 49.4 & 12.2 & 2.788 & 0.014 & 0.015 & 0.000 & 0.362 & 0.31 \\
\hline$\frac{222}{223}$ & 9/2/2004 & 10:51:34 PM & 24.835 & 25.52 & 24.232 & $\begin{array}{ll}25.487 \\
25.077\end{array}$ & 25.035 & 45.943 & $\begin{array}{l}50.446 \\
50.619\end{array}$ & 12.601 & 33.836 & \begin{tabular}{|l|l|}
-1.215 \\
\end{tabular} & $\begin{array}{r}-6.74 \\
\end{array}$ & 49.603 & 0.101 & $\begin{array}{l}14.643 \\
1.613\end{array}$ & & 500 & \begin{tabular}{|l|}
3.41667 \\
\end{tabular} & 49.3 & $\begin{array}{ll}11.8 \\
11.6\end{array}$ & 2.750 & 0.014 & 0.015 & 0.000 & 0.370 & 0.32 \\
\hline & $\begin{aligned} 9 / 2 / 2004 \\
9 / 2 / 2004\end{aligned}$ & \begin{tabular}{|l|}
$10: 52: 34$ PM \\
$10.53 .34 \mathrm{PM}$
\end{tabular} & $\frac{24.845}{24849}$ & $\frac{25.53}{25.524}$ & 24.272 & \begin{tabular}{|l|}
25.497 \\
25.491 \\
\end{tabular} & 25.045 & 46.234 & $\begin{array}{ll}50.614 \\
49.992 \\
\end{array}$ & $\begin{array}{l}12.452 \\
12386\end{array}$ & $\begin{array}{l}34.255 \\
34.207 \\
\end{array}$ & \begin{tabular}{|l|l|}
-1.209 \\
\end{tabular} & $\begin{array}{l}-7.177 \\
-7259 \\
\end{array}$ & $\begin{array}{ll}48.673 \\
49668\end{array}$ & \begin{tabular}{|l|l|}
0.11 \\
0.111
\end{tabular} & \begin{tabular}{|l|l|}
14.643 \\
14.643
\end{tabular} & 0.00 & 206.00 & $\begin{array}{l}3.43333 \\
345 \\
\end{array}$ & 49.5 & & 2.775 & 0.016 & 0.016 & 0.000 & $\begin{array}{l}0.399 \\
0.05 \\
\end{array}$ & 0.34 \\
\hline \begin{tabular}{|c|}
225 \\
\end{tabular} & $\begin{array}{l}99 / 2 / 2004 \\
9 / 2 / 2004\end{array}$ & \begin{tabular}{|l|}
$10: 53: 34$ PM \\
$10: 54: 34$ PM \\
\end{tabular} & 24.8464 & $\frac{25.524}{25.524}$ & \begin{tabular}{|l|}
$24.2 / 1$ \\
24.286
\end{tabular} & \begin{tabular}{r|}
25.491 \\
25486 \\
\end{tabular} & $\begin{array}{l}25.044 \\
25.059 \\
\end{array}$ & \begin{tabular}{|l|}
45.8499 \\
4656
\end{tabular} & \begin{tabular}{|l|}
49.992 \\
50.641 \\
\end{tabular} & $\frac{12.386}{12.613}$ & 34.207 34.357 & $\begin{array}{l}-1.215 \\
-1.215\end{array}$ & $\begin{array}{l}-7.258 \\
-7.226 \\
\end{array}$ & $\begin{array}{l}49.668 \\
50.267 \\
\end{array}$ & 0.1112 & $\begin{array}{l}14.643 \\
14.633 \\
\end{array}$ & & 207.00 & \begin{tabular}{r|}
3.45 \\
3.4666
\end{tabular} & $\frac{48.9}{49.5}$ & 120 & $2.761 \mid$ & $\begin{array}{l}0.016 \\
0.016\end{array}$ & $\begin{array}{ll}0.016 \\
0.006\end{array}$ & $\frac{0.000}{0.000}$ & 0.405 & 0.34 \\
\hline & 9/2/2004 & 10:55:34 PM & & 25.513 & \begin{tabular}{|l|l|}
3 & 24.29 \\
\end{tabular} & & $\begin{array}{l}25.059 \\
25.073 \\
\end{array}$ & 45.344 & \begin{tabular}{|l|}
49.594 \\
\end{tabular} & 12.871 & & -1.212 & $\frac{-1.220}{-6.842}$ & 48.715 & 0.1 & 14.643 & & 208000 & $\begin{array}{l}3.4606 / 1 \\
3.48333 \\
\end{array}$ & 48.5 & & $\begin{array}{l}2.786 \\
2.702\end{array}$ & & $\begin{array}{l}0.015 \\
0.015 \\
\end{array}$ & & $\mid \begin{array}{l}0.405 \\
0.373\end{array}$ & 0.34 \\
\hline 227. & 9/2/2004 & 10:56:34 PM & 24.863 & 25.513 & 24.325 & 25.46 & $\begin{array}{ll}25.048 \\
\end{array}$ & 45.763 & \begin{tabular}{|l|}
49.903 \\
\end{tabular} & 13.084 & 33.249 & -1.212 & -6.94 & 50.066 & $\begin{array}{ll} \\
\end{array}$ & 14.643 & 0.003 & 210.00 & 3.5 & 48.8 & \begin{tabular}{|l|l|}
11.9 & 39.5 \\
\end{tabular} & 2.724 & 0.014 & 0.015 & 0.000 & 0 & $\frac{0.32}{0.31}$ \\
\hline & 9/2/2004 & 10:57:34 PM & & 25.508 & 324.345 & & & & 50.359 & 12.987 & & -1.209 & & & & & & & & & & & & & & & $\frac{0.31}{0.32}$ \\
\hline & $9 / 2 / 2004$ & & 24.848 & 25.503 & 24.355 & 25.46 & & 45.236 & 50.556 & 12.945 & 32.804 & -0.535 & & 50.788 & 0.131 & 14.643 & & & 53333 & 49.4 & & 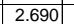 & & & .000 & 0 & $\frac{0.32}{0.42}$ \\
\hline & & & & & 24.33 & 25.454 & & 45.748 & \begin{tabular}{|l|l|}
49.845 \\
\end{tabular} & 12.577 & 33.797 & -1.215 & & 47.874 & 0.113 & 14.643 & & & & 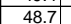 & & & & & & & 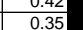 \\
\hline 23. & |l/2004 & $34 \mathrm{PM}$ & 24.901 & 25.487 & 24.354 & 25.439 & 25.116 & 455.512 & 50.063 & 12.575 & 33.497 & -1.212 & & 50.18. & 0.091 & $\begin{array}{ll}14.643 \\
\end{array}$ & & & 3.56667 & 48.9 & & & & & $\overline{000}$ & & \\
\hline & & & & 25.492 & & 25.464 & & & 5002 & 12.007 & 33.854 & -1.215 & & & & & & & 3.58333 & & & & & & & & \\
\hline 23 & $9 / 2 / 2004$ & 11:02:34 PM & 24.911 & 25.487 & 24.364 & 25.469 & 25.051 & 46.526 & \begin{tabular}{|l|l|} 
& 50.374 \\
\end{tabular} & 12.554 & 34.593 & -1.215 & -7.16 & $\begin{array}{ll}48.859 \\
\end{array}$ & 0.117 & 14.643 & & 216.00 & 3.6 & $49.8 \mathrm{r}>\mathrm{C}$ & \begin{tabular}{|c|c|}
11.6 \\
\end{tabular} & 2.796 & & & 0.000 & 0.420 & 0.36 \\
\hline & $9 / 2 / 2$ & 11:03:34 PM & 24.916 & 25.487 & 24.384 & 25.479 & 25. & 46.36 & 50.492 & 12.561 & 34.263 & -1.212 & & 49.512 & 0.109 & 14.643 & & 217.0 & 3.61667 & $\begin{array}{lll}49.4 \\
\end{array}$ & $\begin{array}{lll}11.8 \\
\end{array}$ & 2.779 & & 16 & & 0.394 & 0.34 \\
\hline & & 34 PM & 24.916 & 25.476 & 24.383 & 25.473 & 25. & 46.491 & 50.662 & 12.525 & 34.476 & -1.212 & & 48.913 & 0.104 & 14.643 & & & 3.633 & 49. & & & & & & 374 & \\
\hline 236 & - > & 11:05:34 PM & & 25.476 & 24.398 & 25.458 & & 46.539 & 50.962 & 12.372 & 34.567 & -1.209 & & 49.91 & & 14.643 & & & & & & 2.796 & & 0.016 & 0.000 & & \\
\hline (23) & 9/2/2/2004 & | 11:06: & 24.936 & 25.481 & 24.408 & 25.443 & & $46.4 / 8$ & 50.153 & $12.2 / 3$ & 34.649 & -1.212 & & 51.141 & 0.103 & 14.643 & & & 66667 & & 12.2 & 2.199 & & 0.015 & & & .31 \\
\hline & $\frac{9 / 212004}{90213}$ & 11:00:34 PMM & 24.934 & 25.469 & 24.411 & 25.4424 & & 45.69 & \begin{tabular}{|c|}
49.9090 \\
\end{tabular} & 12.4711 & 33.816 & -1.209 & & 50.44 & 0.099 & 14.64 & & & 3.683333 & 48. & $\frac{12.0}{12.6}>0$ & 2.74 & & & & & 0.29 \\
\hline$\frac{239}{240}$ & $\begin{array}{l}9 / 2 / 2004 \\
9 / 2 / 2004\end{array}$ & 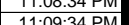 & 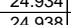 & 年5.409 & $\begin{array}{l}24.421 \\
22431\end{array}$ & $\begin{array}{l}25.456 \\
25445\end{array}$ & 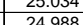 & $\begin{array}{r}45.9 \\
45.85 \\
\end{array}$ & 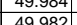 & $\begin{array}{c}12.099 \\
12.949\end{array}$ & 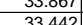 & $\frac{-1.212}{-1212}$ & & (50.294 & 0.109 & 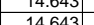 & & & $\begin{array}{r}3.7 \\
371667\end{array}$ & 年 & $\frac{12.0}{126}$ & $\frac{2.150}{2733}$ & & & & 0.398 & 0.34 \\
\hline 241 & 9/2/2004 & 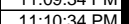 & 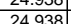 & 25.457 & | 24.452 & 25.425 & 24.4 & 46.032 & 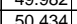 & 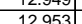 & 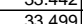 & -1215 & & $=48.93$ & $\frac{1.13}{0.13}$ & 14.043 & & 224 & 年 & 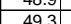 & 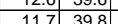 & 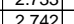 & & | & 0.000 & & (152 \\
\hline 2012 & 9/2/2004 & 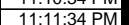 & 24.943 & 25.457 & | 24.435 & 25.44 & 24.4 & (45.489 & 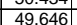 & 12839 & 33.253 & $\frac{1.215}{-1215}$ & & 48.984 & 0.105 & 14.643 & & $\frac{22}{22}$ & 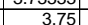 & 48.5 & $\frac{\pi}{117}$ & 2714 & & & & 0 & . \\
\hline 243 & & & 24.938 & 25.437 & 24.415 & 25.42 & 24. & 45.8251 & 50.156 & 12.642 & 33.707 & $\frac{-1.212}{-1.212}$ & & 51.249 & $\frac{0.0922}{0.092}$ & 14.643 & & & 3.76667 & 49.0 & x- & & & $\frac{10}{133}$ & & 0 & 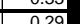 \\
\hline & 9 & & & 25.463 & 24.446 & & & & 49.491 & & & & & $\frac{51.47}{554}$ & & & & & & & & & & & & & \\
\hline & & $11: 14: 34 \mathrm{PM}$ & 24.943 & 25.442 & 24.415 & $\frac{25.455}{25.385}$ & & 45.439 & 49.702 & 12,488 & $\frac{13.654}{33.374}$ & -1.215 & & 49.30 & 0.0 .14 & 14.64 & & & 3.8 & 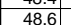 & $11.7-7.7$ & & & D17 & 0.000 & $\frac{0.031}{0.421}$ & 036 \\
\hline & & & & & & 25,404 & & & $498 \mathrm{C}$ & & & & & & & & & & & & & & & & & & \\
\hline 247 & 9/2/2004 & \begin{tabular}{|l|l|}
$11: 16: 34 \mathrm{PM}$ \\
\end{tabular} & 24.946 & 25.435 & 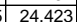 & 25.378 & & 45.45 & \begin{tabular}{|l|l|}
49.7009 \\
\end{tabular} & 12.454 & 33.612 & -1.215 & & & 0.102 & & & & 3.83333 & 48.6 & 11.7 & 2726 & & 0.015 & 0.000 & 0.375 & 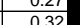 \\
\hline 248 & & & & 25.451 & 24.459 & 25.388 & & 45.363 & 49.7 & \begin{tabular}{|c|c|c|}
12.643 \\
\end{tabular} & & -1.206 & & & & 14.6 & & & 3.85 & 48.6 & 11.8 & 2.7 & & & & \begin{tabular}{|c|c|c|}
0.378 \\
\end{tabular} & \\
\hline 24 & 9/2/2004 & 11:18:34 PM & 24.956 & 25.441 & 24.439 & 25.378 & & 45.728 & \begin{tabular}{|l|l|} 
& 49.957 \\
\end{tabular} & \begin{tabular}{|c|c|}
12.644 \\
\end{tabular} & 33.516 & -1.212 & & $52.09 \varepsilon$ & 0.105 & \begin{tabular}{|c|c|c|}
14.643 \\
\end{tabular} & & & 3.86667 & 48.8 & 12.4 & 2.73 & & & & 0.385 & 0.33 \\
\hline & 9//2/2 & 11:19:34 PM & 24.956 & 25.435 & 24.438 & 25.368 & 24. & & \begin{tabular}{|l|l|}
49.814 \\
\end{tabular} & 12.554 & 33.475 & -1.212 & & & & 14. & & & 3.88333 & 48. & 12.4 & & & & & & \\
\hline 25 & 9/2/2004 & 11:20: & 24.954 & 25.429 & 24.432 & 25.381 & & 45. & 50.075 & 12.561 & & $-1.2 .2>0$ & & $48 .{ }^{4} \mathrm{r}-\mathrm{r}$ & & 14. & & & 3.9 & 48. & 11.5 & & & & & & $0.28 \mathrm{P}>\mathrm{l}$ \\
\hline & & & & & 3) 24.426 & 25.401 & & 45.796 & 50.1 & & & & & & & & & & & & & & & & & & \\
\hline & & & 24.953 & 25.423 & 24.44 & 25.425 & & & 49.8 & 12.681 & 33 & -1.212 & & & & & & & & & & & & & & & $0.2-2>$ \\
\hline 255 & $\begin{array}{l}91 / 21004 \\
0 / 2004\end{array}$ & & 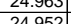 & 告5.428 & 2. 24.454 & 25.4155 & & & $\begin{array}{l}4.933 \\
50.26\end{array}$ & $\begin{array}{l}12.5871 \\
12821\end{array}$ & 33.680 & $\frac{-1.212}{1.212}$ & & & & 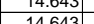 & & & & 年 & 122 & & & & & 0 & {$\left[n^{21}+2\right.$} \\
\hline & & $\frac{11.4 .34 \mathrm{rMM}}{11.253 \mathrm{PM}}$ & 24.950 & & 24.454 & 年 & & 40.039 & 5.420 & 12.021 & 年 30.970 & -1.212 & & & & 年 & & & 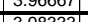 & & 12.4 & & & & & & 0.31 \\
\hline$\frac{25}{257}+3$ & $\frac{1}{9 / 2 / 20044}$ & 11.25: & 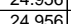 & $\frac{25.410}{25.426}$ & $\mid$ & (25.305 & & $\mid$ & $\mid \frac{3.526}{4928}$ & 12.046 & 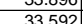 & $\frac{-1.42}{-1212}$ & & & $\frac{0.11}{0171}$ & 年 & & & | & 年 & 119 & & & & (2) & 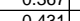 & \\
\hline$\frac{258}{258}>-2$ & & & 2240 & 25.415 & 524,427 & 25.402 & & & $|49.34|$ & 1227 & & -1212 & & & & & & & 401667 & & 124 & & & & & & \\
\hline$\frac{259}{259}$ & $\frac{1}{9 / 2 / 2004}$ & \begin{tabular}{|l|l|}
$1: 28: 34 \mathrm{PM}$ \\
\end{tabular} & 24.949 & 25.404 & 24.416 & 25.396 & & $\mid 45.524$ & $\mid$\begin{tabular}{|l|l|}
$\mid 49.64$ \\
\end{tabular} & 12.281 & 33.756 & -1.212 & & & 0.13 & 14.6 & & & \begin{tabular}{|l|l|}
4.03333 \\
4
\end{tabular} & 48. & $\frac{12.1}{12.1}$ & & & 19 & & 0.488 & \\
\hline & & & & & & & & & & & & & & & & & & & & & & & & & & & \\
\hline 261 & & & & 25,406 & & 25.40 & & & & & & & & & & & & & & & & & & & & & \\
\hline 26 & $9 / 2 / 2 / 2$ & & 24. & $25.4 \mathrm{~F}-\mathrm{C}$ & 24.414 & 25.393 & & 45.6 & \begin{tabular}{|l|l|}
49.858 \\
\end{tabular} & 12294 & 33. & -1209 & & & & 14.6 & & & 4.08333 & & 11.4 & & & & & & \\
\hline 2 & $9 / 2 / 2$ & $11: 32: 34 \mathrm{PI}$ & 24.941 & 25.396 & $\mid 24.398$ & 25.363 & & 45.47 & \begin{tabular}{|l|}
49.567 \\
\end{tabular} & 12.23 & 33.738 & -1.215 & & & & 14.6 & & & $\begin{array}{ll}4.1 \\
\end{array}$ & & 11. & 27. & & & & 6 & \\
\hline & 9/2/2004 & & 24.941 & 25.396 & 24.403 & 25.378 & & 45.765 & 49.8 & 12.2 & 34.4 & -1.212 & & & & 14.6 & & & 4.11667 & & $12.6>-7$ & & & 0.015 & & 79 & \\
\hline 265 & 9/2/2004 & & 24.94 & 25.39 & 24.407 & 25.372 & & 45.715 & 49. & $\frac{12.3}{3}$ & & -1.212 & & & & & & & 4.133 & & $10+2+3$ & & & & & & \\
\hline 266 & 9/2/2004 & $11: 35$ & 24.924 & 25.374 & 24.397 & 25.341 & & 45.734 & 49.88 & 12.21 & 34.0 & -1.212 & & 52.839 & & 14. & & & 4.15 & $48.8 \mathrm{~s}-\mathrm{r}$ & 12. & & & & & 94 & \\
\hline |267 & & 11:36: & 24.934 & 25.379 & & 25.32 & & & & & & -1.2 & & & & & & & & & & & & & & & \\
\hline 年68 & 9/2/2/2004 & & 24.9299 & $25.3 / 4$ & 24.387 & 25.326 & & & 49.4 & & 33. & -1.212 & & & & 14. & & & 4.18333 & 48.4. & & & & & & & \\
\hline & & 11:38:34 & 资4.999 & $25.3 / 4$ & 24.381 & $\frac{25.301}{2.301}$ & & 45.68 & 49.874 & $12.2344 \mathrm{Y}$ & $33.6 \mathrm{rat}$ & -1.212 & & & & & & & 4.2 & 48. & tre & & & & & & \\
\hline$\frac{15}{271}$ & 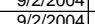 & 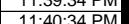 & 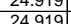 & 年3.309 & 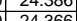 & 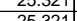 & & $\mid$ & 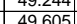 & 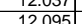 & 33.011 & 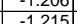 & & $\frac{49.11}{456}$ & & $\mid \frac{\mid 4.045}{11643}$ & & & 4.21001 & & $\frac{11}{10}>>>$ & & & & & 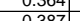 & \\
\hline & & $\frac{1.1 .4 .044}{11.41 .34}$ & 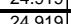 & 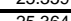 & 24.000 & $\frac{2.021}{25250}$ & & 年 & 49.0005 & & 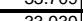 & -1.210 & & & & 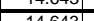 & & & 50035 & & & & & & & & \\
\hline$\frac{153}{273}>3$ & & $\mid$\begin{tabular}{|l|l|l|}
11.42 \\
11.42
\end{tabular} & 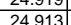 & $\frac{23.304}{25258}$ & $\frac{24.4}{24}$ & (25050 & & 年 45.3015 & $\frac{4.0}{49.8}$ & 年 & 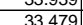 & $\frac{1.203}{-1212}$ & & & & $\frac{14.6}{14.6}$ & & & 4264 & & $16 \mathrm{c}-2$ & 271 & & & & & \\
\hline 274 & $9 / 2 / 2004$ & & 24.897 & 25342 & 24.344 & 25.369 & & & 4974 & & & -1.212 & & 51.45 & & & & & 28333 & & $1232+>$ & & & & & & \\
\hline $278-27$ & & & 24.902 & $\frac{25.3}{25.3}-2$ & & $\frac{25.464}{25}$ & & 45. & 494 & & & $\frac{-1212}{-1212}$ & & & & & & & & & & & & & & & \\
\hline 276 & $9 / 2 / 2004$ & & 24.9 & 25.34 & $\mid 24.348$ & 25.523 & & & 49.551 & 12.164 & 33.59 & -1.218 & & 50.194 & 0.083 & & & & & & & & & & & & \\
\hline 27 & & & & $25355 \mathrm{~S}$ & 24.372 & 25557 & & 45.52 & 49.748 & & 34.049 & -1.212 & & & & 146 & & & & & & & & & & & \\
\hline 278 & $9 / 2 / 2004$ & $\overline{11.4}$ & 24.92 & 25.365 & 24.373 & 25.633 & & 45.416 & 49.655 & 12.6 & 33.912 & -1.212 & & 47.244 & 0. & 14.6 & & & 4.35 & 48.5 & & & & & & & \\
\hline 270 & & & & $5367>$ & 2120 & 25654 & & 45.545 & 49.696 & 12064 & 34087 & 1212 & & 49835 & 0104 & 14643 & & $262+2>$ & 36667 & 186 & & & & & & & \\
\hline 280 & 9/2/2004 & 11:49:34 PM & 24.912 & 25.362 & 24.364 & 25.619 & & 45.275 & \begin{tabular}{|l|l|}
449.547 \\
\end{tabular} & 11.974 & 33.776 & -1.212 & & 50.081 & 0.103 & 14.643 & & 63.0 & & & & & & . 015 & & 0.380 & \\
\hline
\end{tabular}


WSRC-TR-2005-00105, REVISION 0

SRNL-RPP-2005-00012, REVISION 0

RUN \# WASH1 AND WASH 2; CAMPAIGN IV SLURRY WASHING - CONT.

\begin{tabular}{|c|c|c|c|c|c|c|c|c|c|c|c|c|c|c|c|c|c|c|c|c|c|c|c|c|c|c|c|c|}
\hline & A & $\mathrm{B}$ & D & $E$ & $\mathrm{~F}$ & $\mathrm{G}^{\mathrm{G}}$ & $\mathrm{H}$ & $\mathrm{J}$ & $\mathrm{K}$ & $\mathrm{L}$ & $\mathrm{M}$ & $\mathrm{N}$ & \begin{tabular}{l|l} 
\\
\end{tabular} & Q & $\mathrm{R}$ & \begin{tabular}{l|l} 
\\
\end{tabular} & & $\mathrm{v}$ & w & $x$ & & & & & & & & AF $A A$ \\
\hline $28-28$ & 9/2/2004 & 11:50:34 PM & 24.912 & 25.367 & 24.364 & 25.594 & 25.157 & 45.827 & 50.009 & $\begin{array}{l}12.075 \\
\end{array}$ & 34.224 & \begin{tabular}{|l|}
-1.212 \\
\end{tabular} & $\begin{array}{l}-6.949 \\
\end{array}$ & 49.533 & 0.104 & 14.643 & 0.003 & 264.00 & 4.4 & 48.9 & 11.8 & & 2.760 & 0.015 & 0.015 & 0.000 & 0.379 & \\
\hline & $9 / 2 / 2004$ & 11:51:34 PM & 24.907 & 25.357 & 24.359 & 25.564 & \begin{tabular}{|l|}
25.157 \\
\end{tabular} & 45.224 & \begin{tabular}{|l|l|}
49.466 \\
\end{tabular} & 11.978 & $\begin{array}{l}33.69 \\
\end{array}$ & \begin{tabular}{|l|}
-1.212 \\
\end{tabular} & $\begin{array}{l}-6.888 \\
\end{array}$ & 50.644 & 0.106 & \begin{tabular}{|l|}
14.643 \\
\end{tabular} & & 265.00 & & 48.3 & & & & & 0.015 & & & \\
\hline & $99 / 2 / 2004$ & 11:52:34 PM & 24.897 & 25.352 & \begin{tabular}{|r|}
24.37 \\
\end{tabular} & 25.535 & \begin{tabular}{|l|}
25.137 \\
2.18 \\
\end{tabular} & 45.773 & \begin{tabular}{|l|l|}
49.905 \\
\end{tabular} & $\begin{array}{l}12.097 \\
1.207 \\
\end{array}$ & \begin{tabular}{|l|l|}
34.129 \\
3.270
\end{tabular} & \begin{tabular}{|l|l|}
-1.212 \\
\end{tabular} & -6.833 & 50.017 & 0.109 & \begin{tabular}{|l|}
14.643 \\
1.67
\end{tabular} & & $\begin{array}{l}266.00 \\
2600\end{array}$ & \begin{tabular}{l|l|}
04.43333 \\
\end{tabular} & $\begin{array}{ll}48.8 \\
\end{array}$ & 11.9 & 40.0 & 2.754 & \begin{tabular}{|l|l|}
0.016 \\
\end{tabular} & 0.016 & 0.000 & 0.398 & \\
\hline 284 & 9/2/2004 & 11:53:34 PM & 24.899 & 25.364 & \begin{tabular}{|l|}
24.386 \\
\end{tabular} & 25.526 & 25.189 & 45.394 & 50.206 & $\begin{array}{ll}12.007 \\
\end{array}$ & 33.789 & \begin{tabular}{|l|l|}
-0.969 \\
\end{tabular} & -5.891 & $\begin{array}{l}48.26 \\
\end{array}$ & 0.117 & $\begin{array}{l}14.643 \\
\end{array}$ & & 267.00 & 4.45 & 49.1 & 11.5 & - & 2.730 & & 0.017 & 0.000 & 0.430 & 0.37 \\
\hline & 9/2/2004 & 11:54:34 PM & 24.899 & 25.349 & 24.351 & 25.491 & 25.169 & 45.456 & \begin{tabular}{|l|l|}
49.733 \\
\end{tabular} & $\begin{array}{ll}11.892 \\
1.70\end{array}$ & $\begin{array}{ll}33.978 \\
3\end{array}$ & -1.212 & -6.952 & 48.93 & 0.11 & $\begin{array}{l}14.643 \\
\end{array}$ & & 268.00 & \begin{tabular}{l|l}
0.46667 \\
0
\end{tabular} & 48.6 & & & \begin{tabular}{|l|}
2.738 \\
\end{tabular} & 0.016 & 0.016 & 0.000 & 0.404 & \\
\hline$\frac{286}{287}$ & $\begin{array}{l}9 / 2 / 2004 \\
9 / 2 / 2004\end{array}$ & $\begin{array}{l}\text { 11:55:34 PM } \\
11.56 \cdot 34 \text { PM }\end{array}$ & $\begin{array}{l}24.914 \\
24.904\end{array}$ & $\begin{array}{l}25.354 \\
25349 \\
\end{array}$ & \begin{tabular}{|l|}
24.346 \\
24.321
\end{tabular} & $\begin{array}{l}25.451 \\
25.441 \\
\end{array}$ & \begin{tabular}{|l|}
25.164 \\
25.109 \\
\end{tabular} & $\begin{array}{l}45.317 \\
45083 \\
\end{array}$ & 5.547 & \begin{tabular}{|l|l|}
11.798 \\
11.891
\end{tabular} & \begin{tabular}{|l|}
34.049 \\
33651
\end{tabular} & \begin{tabular}{|c|}
-1.212 \\
-1.215 \\
\end{tabular} & -6.57 & \begin{tabular}{|l|}
50.434 \\
47.974
\end{tabular} & 0.096 & \begin{tabular}{|l|l|}
14.643 \\
14634
\end{tabular} & 0.0023 & 269.00 & \begin{tabular}{|l|r|}
0 & 4.48333 \\
\end{tabular} & 48.4 & \begin{tabular}{|l|l|}
12.0 \\
114
\end{tabular} & \begin{tabular}{l|l}
0 & 39.7 \\
1 & 39.4
\end{tabular} & | 2714 & & $\begin{array}{l}0.014 \\
\end{array}$ & 0.000 & 0.353 & 0.30 \\
\hline & $\begin{array}{l}99 / 2 / 2004 \\
9 / 2 / 2004\end{array}$ & $\begin{array}{l}\text { 11:5:5:3 PM } \\
11: 57: 34 \text { PM }\end{array}$ & $\begin{array}{l}24.904 \\
24.894 \\
\end{array}$ & $\begin{array}{l}25.349 \\
25.349\end{array}$ & \begin{tabular}{|l|}
$\mid 24.321$ \\
24.312 \\
\end{tabular} & $\begin{array}{l}25.441 \\
25.442\end{array}$ & \begin{tabular}{|l|}
25.109 \\
24.984 \\
\end{tabular} & $\begin{array}{l}45.083 \\
45.497\end{array}$ & \begin{tabular}{|l|}
49.495 \\
49.688 \\
\end{tabular} & \begin{tabular}{|l|}
11.891 \\
11.899 \\
\end{tabular} & \begin{tabular}{|l|}
33.651 \\
3.3974
\end{tabular} & \begin{tabular}{|c|}
$\mid-1.215$ \\
-1.212 \\
\end{tabular} & $\begin{array}{l}-6.544 \\
-6.868 \\
\end{array}$ & \begin{tabular}{|l|}
47.974 \\
50.755 \\
\end{tabular} & $\begin{array}{l}0.099 \\
0.106\end{array}$ & $\begin{array}{l}14.643 \\
14.463 \\
\end{array}$ & 0.0003 & $\begin{array}{l}270.00 \\
27.00 \\
\end{array}$ & \begin{tabular}{l|r|}
0 & 4.5 \\
0 & 4.51667
\end{tabular} & $\begin{array}{l}48.4 \\
48.6\end{array}$ & $\frac{11.4}{12.1}$ & $\frac{39.4}{397}$ & \begin{tabular}{|l|}
2.714 \\
2.740 \\
\end{tabular} & \begin{tabular}{|l|}
0.014 \\
0.015 \\
\end{tabular} & $\begin{array}{l}0.014 \\
0.015\end{array}$ & $\begin{array}{l}0.000 \\
0.000\end{array}$ & 0.367 & 0.31 \\
\hline 289 & 9/2/2004 & 11:58:34 PM & 24.884 & 25.339 & & 25.427 & & & & 11.982 & & & & 51.579 & 0.1111 & $\begin{array}{l}\frac{14.643}{14.643} \\
\end{array}$ & $\begin{array}{l}0.004 \\
0.003\end{array}$ & $\begin{array}{l}217.1 .00 \\
272.00 \\
\end{array}$ & \begin{tabular}{|l|l|}
0.531001 \\
0 & 4.53333 \\
\end{tabular} & $\frac{48.6}{48.2}$ & $\frac{11.1}{12.3}$ & $\frac{39.1}{39.3}$ & $\left|\frac{2.40 \mid}{2.711}\right|$ & \begin{tabular}{|l|}
0.015 \\
0.016 \\
\end{tabular} & 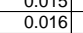 & $\begin{array}{l}0.000 \\
0.000 \\
\end{array}$ & $\begin{array}{l}0.3899 \\
0.412\end{array}$ & $\frac{0.33}{0.35}$ \\
\hline 290 & 9/2/2004 & 11:59:34 PM & & & 24.297 & 25.427 & & 45.226 & 49.425 & 11.824 & & -1.215 & & 48.721 & 0.106 & 14.643 & & 273.00 & \begin{tabular}{l|l}
0 & 4.55 \\
\end{tabular} & 48.3 & 11.6 & & 2.728 & & 0.015 & 0.000 & 0.391 & $\begin{array}{l}0.35 \\
0.33 \\
\end{array}$ \\
\hline & \begin{tabular}{|l|}
$9 / 3 / 2004$ \\
\end{tabular} & 12:00:34 AM & 24.876 & 25.33 & 24.298 & 25.418 & 25.036 & 45.193 & 49.57 & 11.946 & 33.729 & -1.212 & & 50.924 & 0.102 & \begin{tabular}{ll|}
14.643 \\
\end{tabular} & & 274.00 & \begin{tabular}{l|l|}
0 & 4.56667 \\
\end{tabular} & 48.4 & 12.1 & & & 0.015 & 0.015 & 0.000 & & $\begin{array}{l}0.33 \\
0.32 \\
\end{array}$ \\
\hline 292 & $9 / 3 / 2004$ & & & & & & & & & & & & & & & & & & & & & & & & & & & $\begin{array}{l}0.32 \\
0.35 \\
\end{array}$ \\
\hline 293 & 9/3/2004 & $12: 02: 3$ & 24.866 & 25.33 & 24.303 & 25.448 & & & 49.197 & 11.86 & 33.686 & & & 50.619 & 0.108 & 14.643 & & & & & & & & & 0.016 & & 401 & \\
\hline 294 & 9/3/2004 & & 24.861 & & & & & & 49.427 & 11.866 & & & & & & & & & & & & & & & & & & \\
\hline 295 & 9/3/2004 & 12:04:34 AM & 24.865 & 25.325 & 24.287 & 25.407 & 24.975 & 45.45 & & 11.941 & 33.967 & -1.212 & -6.304 & 48.579 & 0.102 & 14.643 & & 278.00 & 4.6333 & 48.9 & 11.6 & & 2.738 & .015 & 0.015 & 0.000 & 0.375 & \\
\hline 296 & 9/3/2004 & 12:05:34 AM & 24.85 & 25.33 & 24.287 & 25.412 & 24.96 & 45.161 & 49.425 & 11.802 & 33.772 & -1.215 & $-6.7 \mathrm{x}>\mathrm{x}$ & 50.434 & 0.118 & 14.643 & & 279.0 & 4.65 & $48.3 \mathrm{r}-\mathrm{l}$ & & & 2.72 & (1) & 0.017 & - & 0.437 & \\
\hline 2970 & 9/3/2004 & 12:06:34 AM & 24.854 & 25.324 & 24.266 & 25.412 . & 24.994 & 44.765 & 49.114 & 11.602 & 33.571 & -1.212 & -6.7 & 50.165 & 0.1 & 14.643 & & & 4.66667 & 48.0 & 11. & 39.2. & 2.700 & 0.014 & 0.015 & 0.000 & 0.373 & \\
\hline & 9/3/2004 & 12:07:34 AM & 24.854 & 25.328 & 24.266 & 25.406 & 25.029 & 44.966 & 49.433 & 11.64 & 33.787 & -1.212 & -6.6911 & 52.78 & 0.119 & 14.643 & & 281.0 & 4.68333 & 48.3 & $12.6 \mathrm{r}-\mathrm{r}$ & & 2.715 & 017 & 0.017 & & 0.442 & \\
\hline & 9/3/2004 & 12:00:34 AM & 24.847] & 25.322 & 24.264| & $\frac{25.384}{20.7}$ & 25.027 & 44.931 & 49.281 & 11.703 & 33.649 & -1.212 & & 50.8977 & 0.104 & 14.643 & & 282.0 & 4.7 & 48.2 & 12 & & $2.70 \mathrm{~s}$ & & 0.015 & 0.000 & .387 & \\
\hline 301 & (15004 & $\frac{12: 09: 3}{12: 032}$ & 24.8353 & & $\frac{24.253}{24.250}-2$ & 25.347 & 24.99 & 45.083 & 49.493 & $11.7 / 7$ & 33.645 & & & 50.357 & 0.121 & 14.643 & & & 17667 & 48 & & & & & 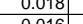 & & 4499 & \\
\hline & 212004 & $\frac{12: 1013}{12 \cdot 1 \cdot 3}$ & $\frac{24.831}{24825}$ & $\frac{25.321}{253}$ & $\begin{array}{ll}24.258 \\
22.252\end{array}$ & 告5.338 & 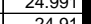 & $\begin{array}{ll}44.863 \\
45.255\end{array}$ & 年9.431 & $\frac{11.142}{1176}$ & 年33.592 & - 1.212 & & إ) & 0.108 & 14.643 & & & 1.13333 & 48 & & & & & 0.016 & & 0.042 & \\
\hline & (1/3/2004 & 12:15:34 AM & 2 & 25.31 & 24.252 & 年 25317 & $\frac{24.9}{249}$ & 45 & $\begin{array}{ll}49.005 \\
491\end{array}$ & 11.728 & (33.945 & - & & 6956 & & $\frac{14.64}{14.64}$ & & & 47665 & & & & 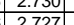 & & & & - & \\
\hline 304 & 9/3/2004 & $12: 13: 34 \mathrm{AM}$ & $\frac{24.819}{24}$ & 25.299 & 24246 & 25.291 & 2490 & 44.684 & 49.234 & 11.621 & 33491 & -1212 & & 49387 & & 14.64 & & & 478333 & & & & & & & & & \\
\hline & 9/3/2004 & 12:14:34 AM & 24.824 & 25.319 & 24.276 & 25.321 & 24.979 & 45.107 & 49.398 & 11.672 & 33.879 & -1.209 & -6.723 & 49.009 & 0.114 & 14.643 & & 288.00 & 4.8 & 48.3 & 11.7 & $7 \quad 39.5$ & 2.723 & \begin{tabular}{|l|l|}
0.016 \\
\end{tabular} & 0.017 & 0.000 & 0.422 & 0.36 \\
\hline & 2004 & & 24.823 & 25.308 & 24.261 & 25.325 & 24.918 & 45.17 & 49.4 & \begin{tabular}{|l|l|}
11.799 \\
\end{tabular} & 33.887 & -1.206 & & 53.02 & 0.113 & 14.643 & 0.004 & & 4.81667 & 48.3 & 12.6 & & 2.725 & 0.016 & $\begin{array}{l}0.017 \\
\end{array}$ & 0.000 & 0.418 & 0.36 \\
\hline 308 & 9/3/2004 & 12:16:34 AM & 24.803 & & 24.22 & 25.335 & 24.93 & 45.4199 & 49.858 & 11.824 & 34.047 & -1.212 & & 52.622 & 0.113 & 14.643 & & 290.00 & 0000 & 48 & & & 2.739 & & 0.017 & & 0.416 & \\
\hline 308 & 9/3/2004] & 12:17:3 & 24.813 & 25.288 & 24.2355 & 25.36 & 24.9 & 45.4411 & 50.046 & 11.917 & 33.9 & -1.212 & & 51.036 & 0.102 & 14.643 & & & 4.85 & 48.9 & 12.2 & & & & 0.015 & & 0.376 & \\
\hline & & $12: 18$ & & & & $\frac{25.35}{25.251}$ & $24.5>>3$ & & 49.8 & 11.742 & & & & 52.459 & & 14.643 & & & & & & & & & & & .431 & \\
\hline & 32004 & 12:19:34 AM & 24.8017 & $\frac{25.292}{25202}$ & $\frac{2.259}{2.241}$ & $\frac{25.354}{25329}$ & $\frac{24 .}{24 .}$ & 45.091 & $\frac{49.38}{49.16}-x$ & 111.7 & $\frac{33.172}{33.37}$ & $\begin{array}{l}-1.209 \\
1.212\end{array}$ & & $\frac{52.207}{48.099}$ & 0.107 & $\frac{14.64}{1464}$ & & & 88333 & 48 & & & & & & & 年 & 0.34 \\
\hline 312 & 9/3/3/2004 & $\frac{12.20 .3}{12: 21: 3}$ & $\frac{24.016}{24.796}$ & & $\frac{24.244}{24.223}$ & $\frac{25.539}{25.323}$ & 24.9 & $\begin{array}{l}44.097 \\
44.637\end{array}$ & 年. & 11./.573 & 33.53 & $\begin{array}{l}-1.1212 \\
-1.212 \\
\end{array}$ & & | & $\frac{.12}{0.12}$ & 14.043 & & & 4916.97 & & 123 & & 695 & & | & & $\mid 0.456$ & \\
\hline & 9/3/2004 & 12:22:34 AM & \begin{tabular}{|l|l|l}
24.796 \\
\end{tabular} & 25.286 & 24.229 & 25.309 & 24.9 & 45.267 & \begin{tabular}{|l|}
49.704 \\
\end{tabular} & 11.779 & 33.785 & -1.212 & & 52.709 & 0.108 & 14.643 & & & 4.93333 & 48 & 12. & & & & & & 0.400 & \\
\hline 314 & 9/3/2004 & 12:23:34 AM & 24.791 & 25.281 & 24.244 & 25.283 & 24.9 & 44.788 & 49.02 & 11.757 & 33.428 & -1.212 & & 53.133 & 0.125 & 14.643 & & & 4.95 & & & & 2.696 & 018 & 0.018 & & 467 & \\
\hline & & $12: 24: 3$ & 24.786 & & 24.243 & 25.273 & & & & & $\begin{array}{ll}33.454 \\
\end{array}$ & & & & 0.12 & & & & & & & & & & & & & \\
\hline & 9/3/2004 & $12: 25$ & 24.781 & 25.271 & 24.253 & 25.258 & 24.9 & 44.811 & & 11.784 & 33.362 & & & 51.1 & 0.112 & 14.6 & & & 4.98333 & & & & & & 0.016 & & 419 & \\
\hline & & & 24.789 & 25.259 & 24.276 & 25.291 & & & & 11.598 & 33.547 & -1.209 & & & & & & & & & & & & & & & & \\
\hline 318 & & 12:27: & 24.798 | & & 24.31 & 25.295 & & & & $\begin{array}{ll}11.697 \\
\end{array}$ & 33.292 & -1.21 .25 & & 52.6 & & & & & 5.01667 & & 12 & & 2.685 & & & & 0.439 & \\
\hline & & & 24.798 & & & 25.295 & & & & 11.698 & & & & & & & & & .0333 & & & & & & & & & \\
\hline & $9 / 3 / 20$ & 12:29: & 24.808 & 25.267 & 24.335 & 25.32 & $24.8 \mathrm{r}-\mathrm{T}$ & & & 11.623 & 33.733 & -1.209 & & 50.861 & & & & & 5.05 & & 12 & & & & & & & 36 \\
\hline & & & 24.7911 & 25.241 & 24.303 & 25.278 & $24.8 \mathrm{~s}$ & 44.998] & & 11.723 & 33.649 & -1.215 & & 50.19 & & & & & 5.06667 & & & & & & & & 134 & \\
\hline & 9/3/2004 & 12:31: & 24.806 & & 24.338 & 25.303 & & & & 11.625 & & & & 49 & & & & & 5.0833 & & 11 & & & & & & & \\
\hline & 9/3/2004 & 12:32: & 24.815 & & 24.337 & 25.312 & & & & 11.5 & 33.662 & -1.212 & & 50.5 & 0.116 & & & & 5.1 & & 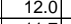 & & & & & & & \\
\hline 324 & & & 24.8099 & & 24.331 & 25.216 & & & & 11.399 & 33.54 & & & & 0.121 & & & & 5.11667 & & & & & & & & 0.452 & .38 \\
\hline & & & $\frac{24.831}{2482}$ & 25.2506 & & $25.29 y^{2}$ & & & & 11.474 & 33.469 & -1.20 & & & & & & & 然5030 & & & & & & & & & \\
\hline & & & 24.8 & 25.25 & 24.352 & 25.2524 & $24.8 \mathrm{r}-\mathrm{l}$ & & & 11.542 & 33.504 & & & & & & & & 5.15 & & $11.8 \mathrm{r}-\mathrm{p}$ & & & & & & & \\
\hline$\frac{\mid 328}{328}$ & 9/3/2004 & 12.37.3 & 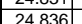 & & \begin{tabular}{|l|l|}
24.353 \\
253
\end{tabular} & 25.218 & & 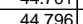 & & 势1.617 & & $\frac{1.250}{-1.212}$ & & $\frac{5.2}{50}$ & & & & & 5 & & $\frac{1}{12}$ & & & & & & & \\
\hline 329 & & $12: 38: 3$ & 24.83 & & 24.363 & 25.212 & & & & 11.542 & 33.444 & & & & & & & & 0.10000 & & & & & & & & & \\
\hline 33 & 9/3/2004 & 12:39: & 24.83 & 25.224 & 24.357 & 25.167 & 24 & & & 11.412 & 33.551 & -1.215 & & & & & & & $5.21667 \mathrm{C}$ & & & & & & & & 0.459 & \\
\hline & & & 24.8 & & & & & & & & & & & & & & & & & & & & & & & & & \\
\hline & & & 24.83 & & & 25.1 & & & & & & & & & & & & & 5.25 & & & & & & & & & \\
\hline$[333$ & 9/3/2004 & 12:42: & 24.84 & & \begin{tabular}{|l|l|}
24.376 \\
\end{tabular} & 25.1 & & & & 11.564 & & & & & & & & & 5.26667 & & & & & & & & & \\
\hline & 9/3/2004 & $12: 43: 3$ & 24.85 & 25.223 & \begin{tabular}{|l|l|}
24.416 \\
\end{tabular} & 25.19 & $24 . \mathrm{S}>\mathrm{C}$ & & & 11.52 & $\begin{array}{l}33.467 \\
\end{array}$ & -1.20 & & 52. & 0.11 & 14.64 & & & 5.28333 & & 12 & & 6 & 01 & & & 0.388 & \\
\hline & 9/3/20 & 12:44 & 24.8 & & 24. & 25.2 & & & & 11.5 & & & & & & & & & 5.3 & & & & & & & & & \\
\hline 336 & 9/3/2004 & 12:45:3 & 24.865 & 25.21 & 24. & 25.2 & 25.0 & 44.469 | & & $11.35 \mathrm{~s}$ & & & & & & & & & 5.31667 & & 11. & & & & & & 0.456 & \\
\hline & & 12:46: & 24.87 & 25 & 24.5 & 25.3 & & & & 11.521 & 33.452 & & & & & & & & . 333 & & 11 & & & & & & & \\
\hline 338 & & & 24.8 & & 24.39 & 25.294 & & 44. & & 11.269 & & -1.212 & & & & & & & 5.35 & & 12 & & & & & & 482 & \\
\hline$\sqrt{321}+3$ & & & & & & & & & & & & & & & & & & & 5.36667 & & & & & & & & & \\
\hline & & & 24.86 & & 24.371 & 25.28 & & & & 11.327 & & & & & & & & & 5.383353 & & & & & & & & & \\
\hline & & & $\frac{24.05}{218}$ & & 24. & $\frac{25.2}{25}>2$ & & & & $\frac{11.3}{1.1}$ & & -1.14 & & & & & & & & & & & & & & & & \\
\hline$\frac{\mid 343}{343}>3$ & 年 & & 2485 & & \begin{tabular}{|l|}
24.35 \\
2455
\end{tabular} & 25. & 249 & | & & $\frac{11.45}{11.36}>>$ & & 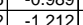 & & 486 & & & & & 543 & & & & & & & & & \\
\hline 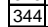 & & & 24.85 & 25.197 & 24.3 & 25.2 & & & & 11.41 & & & & & & & & & 545 & & 12. & & & & & & 146 & \\
\hline 345 & & & $\frac{24.85}{24 .}$ & & 24.349 & & & 44.3 & & 11.483 & & & & & & & & & 5.46667 & & $12.1+3$ & & & & & & & \\
\hline & & & & & & 25.1 & & & & & & & & & & & & & & & & & & & & & & \\
\hline & & & 24.85 & 25.197 & & 25.214 & & 45.145 & & & & & & & & & & & & & & & & & & & & \\
\hline & & & 24.8 & & 24.332 & 25.22 & & 44.78 & & 11.491 & 33.6 & -0. & & & & & & & & & & & & & & & & \\
\hline & & & 2485 & 2516 & & 5524 & & 45.5 & $145,-2$ & 1140 & & & & 30 & & & & & & 20 & & & & & & & 459 & 0.39 \\
\hline 350 & 9/3/2004 & 12:59:34 AM & 24.841 & 25.181 & 24.309 & \begin{tabular}{|l|l|}
25.223 \\
\end{tabular} & \begin{tabular}{l|l|}
3 & 24.991 \\
\end{tabular} & 45.363 & 50.417 & 11.381 & 34.285 & \begin{tabular}{|c|c|}
5 & -0.76 \\
\end{tabular} & -5.411 & 50.454 & 0.125 & \begin{tabular}{|l|l}
14.643 \\
\end{tabular} & & 333.00 & 5.55 & 49.3 & 12.0] & & \begin{tabular}{|l|l|}
2.746 \\
\end{tabular} & \begin{tabular}{|l|l|}
0.018 \\
\end{tabular} & 0.018 & 0.000 & 0.458 & 0.39 \\
\hline
\end{tabular}


WSRC-TR-2005-00105, REVISION 0

SRNL-RPP-2005-00012, REVISION 0

RUN \# WASH1 AND WASH 2; CAMPAIGN IV SLURRY WASHING - CONT.

\begin{tabular}{|c|c|c|c|c|c|c|c|c|c|c|c|c|c|c|c|c|c|c|c|c|c|c|c|c|c|c|c|c|}
\hline & A & B & \begin{tabular}{l|l|} 
\\
\end{tabular} & $E$ & $F$ & G & $\begin{array}{ll}\mathrm{H} \\
\mathrm{H}\end{array}$ & $\mathrm{J}$ & $\mathrm{K}$ & $\mathrm{L}$ & $\mathrm{M}$ & $\mathrm{N}$ & 0 & Q & $\mathrm{R}$ & \begin{tabular}{l|l} 
\\
\end{tabular} & \begin{tabular}{l|l|l|} 
\\
\end{tabular} & $\mathrm{v}$ & w & $x$ & $\mathrm{Y}$ & $z$ & AA & $\mathrm{AB}$ & $\mathrm{AC}$ & $\mathrm{AD}$ & $\mathrm{AE}$ & \\
\hline$\frac{351}{352}$ & $9 / 3 / 2004$ & $\begin{array}{l}1: 31: 05 \mathrm{AM} \\
1.3205 \mathrm{AM}\end{array}$ & $\frac{25.221}{25221}$ & $\begin{array}{l}25.16 \\
2515\end{array}$ & \begin{tabular}{|l|}
25.009 \\
25009
\end{tabular} & $\frac{25.212}{25.217}$ & 24.92 & $\begin{array}{l}46.501 \\
46379\end{array}$ & \begin{tabular}{|l|}
51.363 \\
51.418 \\
\end{tabular} & \begin{tabular}{|l|l|l|l}
12.775 \\
12703
\end{tabular} & \begin{tabular}{|l|l|l|l|}
34.117 \\
33945
\end{tabular} & \begin{tabular}{|r|}
-0.81 \\
-0.758 \\
\end{tabular} & $\begin{array}{r}-5.33 \\
-5.428 \\
\end{array}$ & \begin{tabular}{|c|}
50.371 \\
48.396
\end{tabular} & \begin{tabular}{ll|}
0.141 \\
0.117
\end{tabular} & \begin{tabular}{|l|l|l|l|}
14.643 \\
14.643
\end{tabular} & 0.003 & $\begin{array}{l}364.52 \\
365.52\end{array}$ & \begin{tabular}{ll|}
6.07528 \\
6.09194 \\
\end{tabular} & $\begin{array}{l}50.2 \\
50.3\end{array}$ & 12. & 40.3 & $\frac{2.779}{2.769}$ & $\begin{array}{l}0.020 \\
0.017\end{array}$ & \begin{tabular}{|l|}
0.020 \\
0.017
\end{tabular} & 0.001 & 0.501 & $\begin{array}{l}0.43 \\
0.35\end{array}$ \\
\hline$\frac{352}{353}$ & $\begin{array}{l}9 / 3 / 2004 \\
9 / 3 / 2004\end{array}$ & $\begin{array}{l}1: 32: 05 \mathrm{AM} \\
1: 33: 05 \mathrm{AM}\end{array}$ & $\frac{25.211}{25.396}$ & $\begin{array}{r}25.15 \\
25.151\end{array}$ & \begin{tabular}{|l|}
25.009 \\
25.025 \\
\end{tabular} & $\begin{array}{l}25.217 \\
25.203\end{array}$ & $\begin{array}{r}24.991 \\
24.931\end{array}$ & $\begin{array}{l}46.3919 \\
23.049\end{array}$ & \begin{tabular}{|l|}
51.418 \\
51.959 \\
\end{tabular} & $\begin{array}{l}12.03 \\
12.783\end{array}$ & $\begin{array}{l}33.945 \\
10.336\end{array}$ & \begin{tabular}{|l|}
-0.588 \\
23.477 \\
\end{tabular} & $\begin{array}{l}-5.428 \\
-3.763\end{array}$ & $\begin{array}{l}48.396 \\
51.439 \\
\end{array}$ & $\begin{array}{l}0.111 \\
0.004\end{array}$ & $\begin{array}{l}\frac{14.643}{14.643} \\
\end{array}$ & & $\begin{array}{l}365.52 \\
366.52 \\
\end{array}$ & $\mid \begin{array}{l}\mid .699194 \\
6.10861\end{array}$ & $\begin{array}{l}50.3 \\
50.8\end{array}$ & & & $\begin{array}{l}2.169 \\
1.151\end{array}$ & \begin{tabular}{|c|}
0.011 \\
0.001
\end{tabular} & $\begin{array}{l}0.011 \\
0.001 \\
\end{array}$ & $\begin{array}{l}0.000 \\
0.000\end{array}$ & $\begin{array}{l}0.417 \\
0.034\end{array}$ & \\
\hline$\frac{350}{354}$ & $9 / 3 / 2004$ & $1: 34: 05 \mathrm{AM}$ & 25.353 & 25.157 & \begin{tabular}{|l|}
25.025 \\
\end{tabular} & $\frac{25.205}{25.234}$ & 24.951 & 告. & \begin{tabular}{|l|}
51.9514 \\
51.514
\end{tabular} & $\frac{12.109}{12.229}$ & $\begin{array}{l}-2.5000 \\
-2.909\end{array}$ & \begin{tabular}{|l|}
2.471 \\
37.535 \\
\end{tabular} & 年 & $\begin{array}{l}51.459 \\
51.082\end{array}$ & $\begin{array}{l}0.003 \\
0.003\end{array}$ & $\begin{array}{l}4.040 \\
14.643\end{array}$ & & $\begin{array}{l}300.52 \\
367.52\end{array}$ & 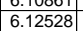 & $\begin{array}{l}50.4 \\
50.4\end{array}$ & & & & 0.000 & $\mid 0.000$ & 0.0000 & $\begin{array}{l}0.138 \\
0.138\end{array}$ & \\
\hline 355 & $\begin{array}{l}/ 3 / 2004 \\
9 / 3 / 2004\end{array}$ & $\frac{1.54 .04 \mathrm{AM}}{1: 35: 05 \mathrm{Am}}$ & $\begin{array}{l}25.353 \\
25.308\end{array}$ & 25.137 & \begin{tabular}{|l|}
25.041 \\
25.036 \\
\end{tabular} & $\frac{25.254}{25.224}$ & 24.9047 & $\begin{array}{l}9.144 \\
8.706 \\
\end{array}$ & \begin{tabular}{|l|}
51.314 \\
51.333 \\
\end{tabular} & $\begin{array}{l}\frac{12.249}{11.983} \\
\end{array}$ & \begin{tabular}{|c|}
-2.0999 \\
-3.147 \\
\end{tabular} & \begin{tabular}{|l|}
37.535 \\
37.634 \\
\end{tabular} & $\begin{array}{l}11.148 \\
10.969\end{array}$ & $\begin{array}{c}31.064 \\
50.29 \\
\end{array}$ & $\begin{array}{l}0.003 \\
0.003 \\
\end{array}$ & \begin{tabular}{|l|}
14.64543 \\
14.6
\end{tabular} & & $\begin{array}{l}301.52 \\
368.52 \\
\end{array}$ & \begin{tabular}{|l|}
.125286 \\
6.14194 \\
\end{tabular} & $\begin{array}{l}50.4 \\
50.2\end{array}$ & $\begin{array}{l}11.2 .0 \\
12.0\end{array}$ & 2. & 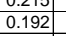 & $\begin{array}{l}0.000 \\
0.000\end{array}$ & 0 & 0.000 & $\begin{array}{l}0.158 \\
0.154 \\
\end{array}$ & 0.13 \\
\hline & 9/3/2004 & & 25.298 & 25.153 & \begin{tabular}{|l|}
25.050 \\
\end{tabular} & $\begin{array}{r}25.27 \\
25.27\end{array}$ & 24.933 & $\begin{array}{l}.180 \\
9.189 \\
\end{array}$ & \begin{tabular}{|l|}
51.704 \\
\end{tabular} & $\begin{array}{l}1.500 \\
12.012 \\
\end{array}$ & \begin{tabular}{|}
$\mid .141$ \\
-2.655 \\
\end{tabular} & \begin{tabular}{|l|}
37.637 \\
\end{tabular} & $\begin{array}{l}11.509 \\
11.57\end{array}$ & $\begin{array}{r}50.786 \\
5\end{array}$ & 0.003 & \begin{tabular}{|l|}
14.6433 \\
14.643
\end{tabular} & & $\begin{array}{l}30.52 \\
369.52 \\
\end{array}$ & \begin{tabular}{|l|}
.0 .15894 \\
6.15861
\end{tabular} & 50.6 & & & 0.0 .225 & 0.000 & 0.000 & 0.000 & - 0.134 & \\
\hline & $9 / 3 / 2004$ & $1: 37: 05 \mathrm{AM}$ & & 25.148 & 25.057 & 25.246 & 24.929 & 0.44 & \begin{tabular}{|l|l|}
51.746 \\
\end{tabular} & 11.865 & $\begin{array}{l}-13.679 \\
\end{array}$ & \begin{tabular}{|l|}
37.836 \\
\end{tabular} & 87.577 & 49.77 & 0.003 & 14.643 & & 370.52 & 6.17528 & 50.6 & & & -0.456 & & 0.000 & 0.000 & -0.065 & \\
\hline 358 & $9 / 3 / 2004$ & $1: 38: 05 \mathrm{AM}$ & 25.17 & 25.139 & 25.064 & 25.212 & 24.945 & 6.844 & \begin{tabular}{|l|l|}
50.848 \\
\end{tabular} & 9.528 & $\begin{array}{r}-2.646 \\
\end{array}$ & \begin{tabular}{|l|}
39.006 \\
\end{tabular} & 25.062 & 51.12 & $\begin{array}{l}0.003 \\
\end{array}$ & \begin{tabular}{|l|l|}
14.643 \\
\end{tabular} & & 371.52 & 6.19194 & 49.7 & & & 0.145 & 0.000 & 0.000 & 0.000 & 0.204 & \\
\hline 359 & 9/3/2004 & $1: 39: 05 \mathrm{AM}$ & $\begin{array}{l}25.144 \\
25363\end{array}$ & $\begin{array}{l}25.128 \\
251220\end{array}$ & \begin{tabular}{|l|}
25.062 \\
2567 \\
\end{tabular} & $\begin{array}{r}25.19 \\
25.85\end{array}$ & $\begin{array}{l}24.929 \\
2413\end{array}$ & \begin{tabular}{ll|}
11.261 \\
255
\end{tabular} & \begin{tabular}{|l|l|}
51.176 \\
5.220 \\
\end{tabular} & \begin{tabular}{r|}
9.75 \\
\end{tabular} & \begin{tabular}{|r|}
1.515 \\
1.20
\end{tabular} & \begin{tabular}{|l|}
34.797 \\
2.49
\end{tabular} & $\begin{array}{l}0.27 \\
0.230\end{array}$ & $\begin{array}{r}52.62 \\
\end{array}$ & $\begin{array}{l}0.12 \\
0.23\end{array}$ & $\begin{array}{l}14.643 \\
\end{array}$ & & \begin{tabular}{|l|l|}
372.52 \\
37252
\end{tabular} & \begin{tabular}{|l|l|}
6.20861 \\
\end{tabular} & \begin{tabular}{|l|l|}
50.1 \\
5.2
\end{tabular} & 12.5 & 6. & 0.440 & $\begin{array}{ll}0.017 \\
\end{array}$ & $\begin{array}{l}0.017 \\
\end{array}$ & & 2.685 & \\
\hline$\frac{360}{361}$ & \begin{tabular}{|c|}
$9 / 3 / 2004$ \\
$9 / 2 / 2004$
\end{tabular} & $\begin{array}{l}1: 40: 05 \mathrm{AM} \\
1: 1405 \mathrm{AM}\end{array}$ & $\begin{array}{l}25.363 \\
25.38\end{array}$ & $\begin{array}{l}25.122 \\
25.127\end{array}$ & \begin{tabular}{|l|}
25.067 \\
25.071 \\
\end{tabular} & $\begin{array}{l}25.185 \\
25.199\end{array}$ & \begin{tabular}{l|}
24.913 \\
22.867
\end{tabular} & \begin{tabular}{|c|}
24.541 \\
43.346
\end{tabular} & \begin{tabular}{|l|}
51.325 \\
51866 \\
\end{tabular} & \begin{tabular}{|c|}
10.587 \\
12812
\end{tabular} & \begin{tabular}{|l|}
13.942 \\
30.938 \\
\end{tabular} & \begin{tabular}{|r|}
21.468 \\
2867
\end{tabular} & \begin{tabular}{|l|l|}
0.322 \\
0.469
\end{tabular} & \begin{tabular}{|l|l|l|l|}
49.716 \\
8.898
\end{tabular} & \begin{tabular}{|l|l|}
0.313 \\
0.212
\end{tabular} & \begin{tabular}{|l|l|}
14.643 \\
14643
\end{tabular} & & \begin{tabular}{|l|}
373.52 \\
374.52
\end{tabular} & \begin{tabular}{|l|}
6.22528 \\
6224194
\end{tabular} & $\frac{50.2}{50.7}$ & & $\frac{19.2}{372}$ & \begin{tabular}{|l|l|}
1.327 \\
2564
\end{tabular} & $\begin{array}{l}0.045 \\
003 n\end{array}$ & $\begin{array}{l}0.045 \\
0.030\end{array}$ & 0.002 & $\begin{array}{l}2.324 \\
0815\end{array}$ & \\
\hline 362 & $9 / 3 / 2004$ & $\frac{1.41 .05 \mathrm{AlV}}{1.42 \cdot 05 \mathrm{AM}}$ & & & & & & & & & & 2.0146 & & & & $\mid \frac{\mid 14.643}{14.643}$ & & & & & & & & & & & & \\
\hline 363 & 9/3/2004 & $1: 43: 05 \mathrm{AM}$ & 25.392 & 25.131 & \begin{tabular}{|l|}
25.0045 \\
\end{tabular} & 25.219 & 24.957 & |46.188 & \begin{tabular}{|l|}
51.541 \\
\end{tabular} & 13.485 & 33.058 & $\begin{array}{r}-.140 \\
-0.3\end{array}$ & $\begin{array}{l}0.003 \\
-4.37\end{array}$ & 50.84 & $\begin{array}{l}.104 \\
0.281\end{array}$ & $\begin{array}{l}\mid 4.045 \\
14.643\end{array}$ & & 376.52 & \begin{tabular}{|l|}
6.275001 \\
6.27528
\end{tabular} & 50.4 & & 39.6 & 2.732 & 0.040 & 0.040 & 0.00 & $\begin{array}{l}0.104 \\
1.014\end{array}$ & \\
\hline 364 & $9 / 3 / 2004$ & $1: 44: 05 \mathrm{AM}$ & 25.376 & 25.136 & $\begin{array}{r}25.06 \\
\end{array}$ & 25.243 & 24.891 & 48.782 & \begin{tabular}{|l|}
51.957 \\
\end{tabular} & 13.79 & 35.353 & -1.212 & $\begin{array}{l}-3.916 \\
\end{array}$ & 49.58 & 0.308 & 14.643 & & 377.52 & \begin{tabular}{|l|l|}
6.29194 \\
\end{tabular} & 50.8 & 11.8 & & 2.900 & 0.044 & 0.044 & 0.00 & 1.046 & \\
\hline & $9 / 3 / 2004$ & $1: 45: 05 \mathrm{AM}$ & & 25.136 & & 25.193 & 24.946 & 48.776 & \begin{tabular}{|l|l|}
52.049 \\
\end{tabular} & 13.926 & & -1.212 & & 50.375 & & 14.643 & & & \begin{tabular}{|l|l|}
6.30861 \\
\end{tabular} & 50.9 & & & & & & & & \\
\hline 366 & $9 / 3 / 2004$ & 1:46:05 AM & 25.406 & $\begin{array}{l}25.145 \\
25.130\end{array}$ & \begin{tabular}{|l|}
25.064 \\
2562
\end{tabular} & 25.197 & 24.92 & \begin{tabular}{|l|}
49.06 \\
\end{tabular} & \begin{tabular}{|l|l|}
51.9977 \\
\end{tabular} & $\begin{array}{l}14.068 \\
\end{array}$ & 35.412 & $\begin{array}{l}-1.212 \\
-21\end{array}$ & & 47.307 & $\begin{array}{l}0.175 \\
\end{array}$ & 14.643 & & & 6.32528 & 50.9 & & 42.2 & 2.912 & & & 0.001 & 0.592 & \\
\hline$\frac{367}{368}$ & 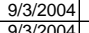 & $1: 47: 05 \mathrm{AM}$ & 25.405 & $\begin{array}{l}25.139 \\
25.144\end{array}$ & \begin{tabular}{|l|l|}
25.063 \\
25073
\end{tabular} & $\begin{array}{l}25.182 \\
25.221\end{array}$ & $\begin{array}{r}24.945 \\
22.999\end{array}$ & \begin{tabular}{|l|l|}
49.177 \\
49220
\end{tabular} & \begin{tabular}{|l|}
52.063 \\
5.20 \\
\end{tabular} & $\begin{array}{l}14.054 \\
1.113\end{array}$ & 35.546 & \begin{tabular}{|c|}
-1.212 \\
1209
\end{tabular} & & 50.2 & $\begin{array}{l}0.171 \\
\end{array}$ & \begin{tabular}{|l|l|}
14.643 \\
1.62
\end{tabular} & & & 6.34194 & 50.9 & & 42. & 2.921 & 0.024 & 0.024 & 0.001 & $\begin{array}{l}0.577 \\
\end{array}$ & \\
\hline & $9 / 3 / 2004$ & $\frac{1.480 .05 \mathrm{AM}}{1.49 .05}$ & 25.444 & 25.143 & \begin{tabular}{|l|}
$25.0 / 3$ \\
25062
\end{tabular} & $\frac{25.221}{25215}$ & $\begin{aligned} 24.929 \\
24.973\end{aligned}$ & $\frac{49.222}{48.411}$ & \begin{tabular}{|l|}
52.148 \\
51818
\end{tabular} & $\frac{14.131}{14.155}$ & $\begin{array}{r}35.49 \\
34743\end{array}$ & \begin{tabular}{|c|}
-1.209 \\
1218 \\
\end{tabular} & $\begin{array}{l}-6.631 \\
-4.225 \\
\end{array}$ & 51.664 & 0.171 & $\frac{14.643}{14643}$ & & & 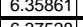 & 51.0 & & & 2.920 & 0.024 & $\frac{0.024}{0.027}$ & & & 0.49 \\
\hline & 9/3/2004 & $1: 50: 05 \mathrm{AM}$ & 25.379 & & & 25.216 & 24.934 & & & $\begin{array}{l}14.150 \\
13.807\end{array}$ & 35.371 & $\frac{-1.212}{-1.212}$ & $\begin{array}{l}-4.225 \\
-5.029 \\
\end{array}$ & $\begin{array}{l}\frac{49.509}{51.086} \\
\end{array}$ & $\begin{array}{c}0.27 \\
0.27\end{array}$ & $\begin{array}{l}14.045 \\
14.643\end{array}$ & & & $\mid$\begin{tabular}{|l|}
0.33528 \\
639194
\end{tabular} & $\frac{50 .}{50.1}$ & & $\frac{41}{42}$ & $\frac{2.867}{2.896}$ & $\frac{0.030}{0.039}$ & $\mid \begin{array}{l}0.030 \\
0.039\end{array}$ & 0.00 & $\begin{array}{l}0.718 \\
0.919\end{array}$ & \\
\hline 37 & $9 / 3 / 2004$ & $1: 51: 05 \mathrm{AM}$ & $\frac{25.309}{25}$ & 25.143 & \begin{tabular}{|l|l|}
25.032 \\
\end{tabular} & 25.21 & 24.933 & $\mid 49.996$ & \begin{tabular}{|l|}
51.406 \\
\end{tabular} & 13.767 & 36.769 & -1.215 & -6.423 & 51.1.193 & $\begin{array}{l}0.2164 \\
0.164\end{array}$ & 14.0443 & & 384.52 & \begin{tabular}{|l|l|}
6.40861 \\
\end{tabular} & 50.3 & $\frac{11.2}{12.2}$ & 43. & $\frac{2.0501}{2.991}$ & $\begin{array}{l}0.023 \\
0.02\end{array}$ & $\begin{array}{l}.023 \\
0.03\end{array}$ & 0.001 & 0.541 & 0.46 \\
\hline & $9 / 3 / 2004$ & 1:52:05 AM & 25.308 & 25.142 & \begin{tabular}{|l|}
25.051 \\
\end{tabular} & & 24.928 & 47.138 & \begin{tabular}{|l|}
48.937 \\
\end{tabular} & 15.026 & 32.434 & -1.212 & -6.463 & 53.304 & 0.14 & 14.643 & & & & 47.8 & & & 2.743 & 0.020 & 0.020 & 0.001 & & \\
\hline & 9/3/2004 & 1:53:05 AM & 25.274 & 25.133 & \begin{tabular}{|l|}
25.027 \\
\end{tabular} & 25.19 & \begin{tabular}{|l|l|l|}
24.918 \\
\end{tabular} & 46.796 & \begin{tabular}{|l|l|} 
& 48.502 \\
\end{tabular} & $\begin{array}{ll}13.829 \\
\end{array}$ & 33.495 & -1.212 & $\begin{array}{r}-6.7 \\
\end{array}$ & 49.349 & 0.153 & \begin{tabular}{|l|l|}
14.643 \\
\end{tabular} & & 386.52 & 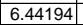 & \begin{tabular}{|l|l|}
47.4 \\
\end{tabular} & $11 . \varepsilon$ & 40.1 & \begin{tabular}{|l|l|}
2.768 \\
\end{tabular} & 0.022 & $\mid 0.022$ & 0.001 & 0.545 & \\
\hline 374 & \begin{tabular}{|c|}
$9 / 3 / 2004$ \\
\end{tabular} & 1:54:05 AM & 25.295 & 25.154 & 25.038 & 25.237 & 24.905 & 47.495 & 49.342 & 13.658 & 34.306 & -1.209 & -6.79 & & 0.154 & \begin{tabular}{|l|l|}
14.643 \\
\end{tabular} & & & & 48.2 & 12 & 40. & 2.820 & 0.022 & 0.022 & & 0.538 & \\
\hline 375 & 9/3/2004 & 1:55:05 AM & 25.294 & 25.134 & \begin{tabular}{|l|l|}
24.983 \\
\end{tabular} & 25.231 & 24.889 & $\begin{array}{l}47.196 \\
\end{array}$ & \begin{tabular}{|l|l|}
49.197 \\
\end{tabular} & 13.293 & 34.277 & -1.212 & -6.856 & 51.351 & 0.151 & 14.643 & & & & 48.1 & & 40. & 2.809 & 0.022 & 0.022 & 0.001 & 0.531 & \\
\hline \begin{tabular}{|l|l|}
376 \\
377
\end{tabular} & 9/3/2004 & 1:56:05 AM & 25.304 & & \begin{tabular}{|l|}
24.917 \\
2.996 \\
\end{tabular} & $\begin{array}{l}25.215 \\
25.05\end{array}$ & & $\begin{array}{l}46.717 \\
\end{array}$ & $\begin{array}{l}48.6 \\
\end{array}$ & $\begin{array}{l}13.087 \\
\end{array}$ & \begin{tabular}{|l|l|}
34.183 \\
2.17
\end{tabular} & \begin{tabular}{|l|l|}
-1.209 \\
\end{tabular} & $\begin{array}{l}-6.876 \\
.6832\end{array}$ & & $\begin{array}{l}0.151 \\
.152\end{array}$ & $\begin{array}{l}14.643 \\
14.0\end{array}$ & & & & 47.5 & & 40. & 2.789 & & $\begin{array}{l}0.022 \\
\end{array}$ & & 0.536 & 46 \\
\hline 377 & $9 / 3 / 2004$ & 1:57:05 AM & \begin{tabular}{|l|l|}
25.293 \\
25283
\end{tabular} & $\begin{array}{l}25.137 \\
25137\end{array}$ & \begin{tabular}{|l|l|}
24.886 \\
2.86
\end{tabular} & $\frac{25.195}{25.10}$ & $\begin{array}{l}24.918 \\
2.023\end{array}$ & \begin{tabular}{|l|l|}
46.563 \\
\end{tabular} & 48.6 & \begin{tabular}{l|l|}
12.947 \\
12.909
\end{tabular} & \begin{tabular}{|l|l|}
34.117 \\
3.119
\end{tabular} & \begin{tabular}{|l|}
-1.212 \\
.212
\end{tabular} & \begin{tabular}{|l|l|}
-6.833 \\
6.871
\end{tabular} & $\begin{array}{l}50.911 \\
5.236\end{array}$ & $\begin{array}{l}0.152 \\
\end{array}$ & \begin{tabular}{|l|l|}
14.643 \\
1.61
\end{tabular} & & & \begin{tabular}{|l|l|}
6.50861 \\
\end{tabular} & 47.5 & & 40.5 & $\begin{array}{l}2.781 \\
2781 \\
\end{array}$ & 0.022 & $\begin{array}{l}0.022 \\
\end{array}$ & 0.001 & 0.541 & \\
\hline$\frac{510}{379}$ & $9 / 3 / 2004$ & $\frac{15: 58: 0}{1.59 \cdot 0}$ & $\begin{aligned} 25.283 \\
25258\end{aligned}$ & $\frac{25.137}{25.137}$ & \begin{tabular}{|l|}
24.866 \\
24841
\end{tabular} & $\begin{array}{r}25.19 \\
25.195\end{array}$ & $\frac{24.923}{24.918}$ & 46.557 & $\begin{array}{l}48.523 \\
48.881\end{array}$ & 12.969 & $\begin{array}{l}34.119 \\
34.197\end{array}$ & $\frac{-1.212}{-1.212}$ & $\begin{array}{l}-6.871 \\
-6.682 \\
-12\end{array}$ & $\begin{array}{r}52.346 \\
52.034\end{array}$ & $\begin{array}{l}0.149 \\
0.149\end{array}$ & $\frac{14.643}{14643}$ & & & 1104 & 47.8 & 12. & & 2.78 & & & & 0.531 & 0.45 \\
\hline & $9 / 3 / 2004$ & $2: 00: 0$ & 25.242 & 25.137 & \begin{tabular}{|r|}
24.841 \\
24.81
\end{tabular} & $\frac{25.195}{25.189}$ & $\frac{24.9}{24.8}$ & & $\frac{48.081}{48.882}$ & $\begin{array}{l}\frac{13.017}{13.145} \\
\end{array}$ & & $\mid-1.212$ & & & $\begin{array}{l}0.149 \\
0.149 \\
\end{array}$ & & & & & 47 & & & & & & & & 0.45 \\
\hline & 9/3/2004 & 2:01:05 AM & 25.202 & 25.121 & \begin{tabular}{|l|}
24.01 \\
2455
\end{tabular} & 25.133 & 24.867 & 46.692 & 48.93 & 12.955 & 34.156 & -1.212 & -6.862 & 48.229 & 0.148 & 1464 & & 394.5 & & 47 & & & 78 & & & & & \\
\hline & & & & & 24.755 & & & & & & 34.142 & & & 48.024 & $\frac{0.140}{0.147}$ & $\frac{14.04}{14.64}$ & & & & & & & & & & & & \\
\hline & 9/3/2004 & & 25.201 & & & & & & & & & & & & & & & & & & & & & & & & & \\
\hline & 9 & & 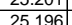 & 2515 & \begin{tabular}{|l|l|}
24.153 \\
434
\end{tabular} & $\begin{array}{l}25.100 \\
25193\end{array}$ & 24831 & 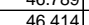 & \begin{tabular}{|l|l|}
48.069 \\
4869
\end{tabular} & $\mid$ & $\begin{array}{l}53.351 \\
33848\end{array}$ & -1212 & $\begin{array}{r}-6.19 \\
-6.781\end{array}$ & \begin{tabular}{|l|}
50.438 \\
50.329 \\
\end{tabular} & $\begin{array}{l}0.147 \\
0.147\end{array}$ & $\frac{14.643}{14.643}$ & & $\begin{array}{l}396.52 \\
397.52 \\
\end{array}$ & $\mid$\begin{tabular}{|l|}
0.068611 \\
6.62528
\end{tabular} & $\begin{array}{l}48.0 \\
47.6\end{array}$ & $\frac{12.00}{120}$ & 401 & $\frac{2.96}{2.767}$ & $\begin{array}{l}0.021 \\
0.021\end{array}$ & $\mid$ & $\frac{0.001}{0.001}$ & \begin{tabular}{|c|c|c|c|c|}
0.528 \\
\end{tabular} & \\
\hline & $9 / 3 / 2004$ & & 25.186 & 25.145 & \begin{tabular}{|l|l|}
24.689 \\
\end{tabular} & 25.213 & & 46.835 & \begin{tabular}{|l|}
49.207 \\
\end{tabular} & 12.875 & & & & & 0.1 & & & & & & & & & & 0.022 & & 0.530 & \\
\hline & $9 / 3 / 2004$ & & 25.14 & 25.134 & & 25.216 & & & \begin{tabular}{|l|l|}
48.64 \\
\end{tabular} & 12.716 & & & & 50.6 & 0.1 & & & & & & & & & & & & & \\
\hline 38 & 9/3/2004 & & 25.119 & 25.133 & \begin{tabular}{|l|l|}
24.652 \\
\end{tabular} & 25.211 & 24.8 & 46.153 & \begin{tabular}{|l|l|} 
& 48.641 \\
\end{tabular} & 12.667 & 33.848 & -1.209 & -6.8 & 52.409 & 0.148 & 14.643 & & & & & 12. & & 2758 & & & & & \\
\hline & & & 25.088 & 25.118 & \begin{tabular}{|l|}
24.621 \\
\end{tabular} & 25.18 & & \begin{tabular}{ll|l}
46.117 \\
\end{tabular} & \begin{tabular}{|l|l|l|} 
& 48.68 \\
\end{tabular} & 12.553 & & & & 52.991 & 0.144 & & & & & & & & & & & & & \\
\hline 386 & 9/3/2004 & & 25.073 & 25.128 & 24.611 & 25.195 & & 45.96 & 48.6 & 12.528 & 33.86 & -1.209 & & 50. & 0.1 & & & & & & & & & & & & & \\
\hline & & & 25.073 & & & 25.2 & & & & 12.511 & & & & & & & & & & & & & & & & & & \\
\hline & & & 25.077 & 25.141 & \begin{tabular}{|l|l|}
24.604 \\
\end{tabular} & 25.213 & & & & 12.534 & & & & & & & & & & & & & & & & & & \\
\hline & & & $25.05 / 7$ & & 24.58 & 25.194 & & & & & & & & & & & & & & & & & & & & & & \\
\hline & & & $\frac{25.0824}{2025}$ & & & 25.169 & & & & 12.654 & & & & & & & & & & & & & & & & & & \\
\hline & $\frac{91321204}{0.1200}$ & & $\frac{25.051}{25041}$ & $\frac{25.125}{2512}$ & \begin{tabular}{|l|l|}
24.538 \\
\end{tabular} & $\frac{25.153}{25.27}$ & & & & $\frac{12.3}{122}$ & & & & & & & & & & & & & & & & & & \\
\hline & & & $\frac{25.041}{25016}$ & & $\begin{array}{l}24.3060 \\
22503\end{array}$ & 25.151 & & & & 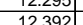 & & & & & & & & & & & & & & & & & & \\
\hline 397 & $9 / 3 / 2004$ & & 24.995 & 25.129 & \begin{tabular}{|l|l|}
24,487 \\
\end{tabular} & 25.141 & & 47.79 & 49.05 & 12417 & & & -64 & 49 & & & & & & & & & & & & & & \\
\hline & & & & & & & & & & & & & & & & & & & & & & & & & & & & \\
\hline & 9 & & 24,923 & 2513 & 24,4 & 25.189 & $\frac{2.60}{248}$ & 46.15 & & 12.28 & & $\frac{1.24}{-122}$ & & & & & & & & & & & & & & & & \\
\hline & & & & & & & & & & & & & & & & & & & & & & & & & & & & \\
\hline$\sqrt{44}$ & $\mid 9 / 3 / 2004$ & $\frac{1: 21: 0}{2: 21}$ & 24.857 & 25.126 & 24.444 & 25.174 & 24.8 & $\mid 47.6$ & \begin{tabular}{|l|l|}
48.4 \\
\end{tabular} & 12.434 & 35.638 & $\frac{-1.209}{-1.209}$ & & & 0. & 14 & & & & 47 & 12 & & & & & & 0 & \\
\hline$\sqrt{44}$ & 9/3/2004 & & 24.8 & & 24. & 25.15 & & & & 12. & & & & & & & & & & & & & & & & & & \\
\hline & $9 / 3 / 2004$ & & 24.8 & 25.1 & 24.419 & 25.194 & & 47.6 & 48 & 12.4 & & & & & & & & & & & 12 & & & & & & 0.541 & \\
\hline 44 & 9/3/2004 & & 24.874 & 25.116 & 24.3 & 25.173 & & 47. & 48.7 & 12.425 & & & & & & & & & & & & & & & & & & \\
\hline 4055 & 9/3/2004 & & 24.92 & & 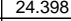 & 25.167 & & 47.3 & 48.881 & 12.511 & & & & & & & & & & & 12 & & & & & & & \\
\hline 406 & & & 24.93 & & \begin{tabular}{|l|l|} 
& 24.394 \\
\end{tabular} & 25.194 & & & & 12.516 & & & & & & & & & & & 12 & & & & & & & \\
\hline & 9/3/2004 & & 24.91 & 25.10 & 24.359 & 25.188 & & & & 12.355 & 35.106 & & & & & & & & & 47 & & & & & & & & \\
\hline & 9/3/2004 & & 24.91 & & \begin{tabular}{|l|}
24.37 \\
24.250
\end{tabular} & 25.19 & & & & 12.4 & & & & & & & & & & & & & & & & & & \\
\hline & $9 / 3 / 2004$ & & & & 24.35 & $\frac{25.17}{2.17}$ & & 4.1 .1 & 48.7 & 12.4 & & & & & & & & & & & & & & & & & & \\
\hline & 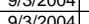 & & & & & $\frac{23.10}{2512}$ & & & & $\frac{12.4}{123}$ & & & & & & & & & & & & & & & & & & \\
\hline$\overline{41}$ & & & & & & $\frac{25.14}{2515}$ & & & & & & & & & & & & & & & & & & & & & & \\
\hline & & & 2481 & & & & & & & & & & & & & & & & & & & & & & & & & \\
\hline 414 & & & & & & & & & & & & & & & & & & & & & & & & & & & & \\
\hline & & & & & & & & 45.865 & & 12285 & & & & & & & & & & & & & & & & & & \\
\hline & & & 24.77 & & 24325 & 25164 & & & 48 & & & & & & & & & & & & & & & & & & & \\
\hline & $9 / 3 / 20$ & & 24748 & 25 & 24295 & 25.16 & 24.7 & 47.32 & 48.5 & $\begin{array}{ll}12.19 \\
\end{array}$ & 35.5 & & & & & & & & & & & & & & & & & \\
\hline 418 & $9 / 3 / 2004$ & & 24763 & 25.098 & 24.28 & 25.13 & & 46.75 & 48.41 & 12.28 & 347. & -1212 & & 48.7 & 0.1 & 14.6 & & & & & & & & & & & & \\
\hline & 9/3/2004 & $2 \cdot 39.05$ & 2477 & & 24.26 & 25099 & & 46.848 & 48.272 & 12154 & 35.174 & -1216 & & 51 & 0.11 & 14 & & & & 7 & & & & & & & & \\
\hline & 9/3/2004 & $2: 40: 0$ & 803 & 25.093 & 24.29 & 25.115 & & & 488.566 & 12.217 & 35.256 & -1.212 & & 0.17 & 0.158 & 14.643 & & & & 47.4. & & & & & & & 0.561 & \\
\hline
\end{tabular}


WSRC-TR-2005-00105, REVISION 0

SRNL-RPP-2005-00012, REVISION 0

RUN \# WASH1 AND WASH 2; CAMPAIGN IV SLURRY WASHING - CONT.

\begin{tabular}{|c|c|c|c|c|c|c|c|c|c|c|c|c|c|c|c|c|c|c|c|c|c|c|c|c|c|c|c|c|}
\hline & $\begin{array}{ll} \\
\end{array}$ & B & D & $E$ & $\mathrm{~F}$ & G & $\mathrm{H}$ & $\mathrm{J}$ & $\mathrm{K}$ & \begin{tabular}{l|l|l|l|} 
L & \\
\end{tabular} & $\mathrm{M}$ & \begin{tabular}{ll|}
$\mathrm{N}$ \\
\end{tabular} & 0 & Q & $\begin{array}{ll}R \\
\end{array}$ & \begin{tabular}{|c|} 
\\
\end{tabular} & $T$ & $\mathrm{~V}$ & w & $x$ & $\mathrm{Y}$ & $z$ & AA & $\mathrm{AB}$ & \begin{tabular}{ll|}
$A C$ \\
$A C$
\end{tabular} & $A D$ & AE & AF $[A$ \\
\hline$\frac{42}{42}$ & $\begin{array}{l}9 / 3 / 2004 \\
9 / 3 / 2004\end{array}$ & $\begin{array}{l}2: 41: 05 \mathrm{AM} \\
2.4205 \mathrm{AM}\end{array}$ & $\frac{24.787}{2479}$ & $\begin{array}{l}25.071 \\
25074\end{array}$ & \begin{tabular}{|l|}
24.284 \\
24207
\end{tabular} & $\begin{array}{l}25.109 \\
25077\end{array}$ & \begin{tabular}{|l|}
24.712 \\
24645 \\
\end{tabular} & $\begin{array}{l}46.719 \\
46.628\end{array}$ & \begin{tabular}{|r|}
48.43 \\
\end{tabular} & $\begin{array}{l}12.215 \\
12404\end{array}$ & \begin{tabular}{|l|l|}
35.004 \\
34612
\end{tabular} & \begin{tabular}{|l|l|}
-1.209 \\
.215
\end{tabular} & \begin{tabular}{|l|l|}
-6.428 \\
-6518
\end{tabular} & $\begin{array}{l}48.636 \\
50308\end{array}$ & \begin{tabular}{|l|l|}
0.144 \\
0138 \\
\end{tabular} & \begin{tabular}{|l|}
14.643 \\
14643
\end{tabular} & $\begin{array}{l}0.003 \\
0002\end{array}$ & 434.52 & \begin{tabular}{|l|l|}
7.24194 \\
.25964
\end{tabular} & 47.3 & $\begin{array}{l}11.6 \\
1.0\end{array}$ & 40.9 & \begin{tabular}{|l|}
2.817 \\
\end{tabular} & \begin{tabular}{|l|}
0.021 \\
0020
\end{tabular} & \begin{tabular}{|c|}
0.021 \\
0.020
\end{tabular} & 0.001 & 0.515 & 0.44 \\
\hline & 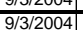 & $\frac{2: 42: 05 \mathrm{AM}}{2: 43: 05 \mathrm{AM}}$ & $\frac{24.99}{24.774}$ & $\frac{25.074}{25.079}$ & \begin{tabular}{|l|}
24.2917 \\
24317 \\
\end{tabular} & $\frac{25.071}{25.106}$ & $\begin{array}{l}24.645 \\
24.749\end{array}$ & $\frac{46.638}{47.403}$ & \begin{tabular}{|l|}
48.511 \\
48.492
\end{tabular} & $\frac{12.4044}{12.2911}$ & $\frac{34.612}{35.572}$ & & $\frac{-6.518}{-6342}$ & $\frac{50.208}{49.843}$ & & $\mid \frac{\mid \frac{\mid 4.643}{14.643}}{\mid}$ & & $\frac{435.52}{43652}$ & & $\frac{-4.4}{47.4}$ & $\frac{12.0}{11.9}$ & $\frac{40.6}{41.5}$ & & \begin{tabular}{|l|l|}
0.020 \\
0.020
\end{tabular} & & & $\frac{0.496}{0.704}$ & \\
\hline & & & & 25.079 & & 25.106 & & 46.501 & 48.39 & & & & & & & & & & & & & & & & & & & \\
\hline & & & & & & $\overline{25.1}$ & & & & 12. & & & & & 0.15 & & & & & 46.6 & & & & & & & 541 & \\
\hline & 2004 & $2: 46: 05 \mathrm{AM}$ & 24.749 & 25.079 & 24.327 & 25.121 & & & & & & & & & & & & & & & & & & & & & & \\
\hline & $9 / 3 / 2004$ & 2:47:05 AM & 24.744 & 25.078 & 24.341 & 25.12 & 24.754 & 46.58 & \begin{tabular}{|l|l|} 
& 48.086 \\
\end{tabular} & 12.328 & 34.632 & -1.212 & & 50.651 & 0.164 & 14.643 & & 440.5 & 7.34194 & 47.0 & 12.1 & & & & & & & \\
\hline 428 & 9/3/2004 & 2:48:05 AM & 24.788 & 25.082 & 24.365 & 25.135 & 24.718 & 44.63 & \begin{tabular}{|l|l|} 
& 47.882 \\
\end{tabular} & 12.138 & 32.871 & -1.209 & -4.463 & 55.719 & 0.099 & 14.643 & & 441.5 & 7.35861 & 46.8 & 13.3 & & 2.672 & & & & 0.372 & \\
\hline & & 2:49:05 AM & 24.686 & & 24.348 & & 24.721 & 46.534 & \begin{tabular}{|l|l|}
48.397 \\
\end{tabular} & 12.263 & 34.696 & & -5.596 & 48.644 & 0.096 & 14.643 & & 4442.5 & 7.37528 & 47.3 & 11.6 & 40.6 & & 0.014 & 0.014 & & 0.345 & \\
\hline & 9/3/2004 & 2:50:05 AM & & 25.061 & 24.349 & 25.088 & 24.767 & 46.872 & \begin{tabular}{|l|l}
7.839 \\
\end{tabular} & 12.202 & 35.182 & & -6.42 & 47.563 & 0.14 & 14.643 & & 44 & 39194 & 46.7 & 11.3 & & & & & & & \\
\hline$\frac{43}{2}$ & 2004 & 2:51:05 AM & 24.667| & 25.061 & 24.344| & 25.113 & .767 & 634 & 47.797 & 12.282 & 34.807 & & & & 0.163 & 14.643 & & 4444.5 & 40861 & 46.7 & 12.1 & 40.7 & & & & 0.00 & $0.584]$ & \\
\hline 先 & 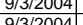 & 2:52:05 AM & 24.08 & $\frac{25.061}{25072}$ & 24.35 & $\frac{2.11}{25.115}$ & & 46.6844 & $\frac{40.204}{47036}$ & 1.2.356 & 34513 & 1212 & & 1037 & 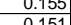 & $\begin{array}{l}14.643 \\
1.4643\end{array}$ & & & (1) & 16. & $\begin{array}{lll}x-4 \\
110\end{array}$ & & 786 & 21007 & & & 5 & \\
\hline & 9 & $2 \cdot 54: 05 \mathrm{AM}$ & $\frac{2.75}{248}$ & 25069 & 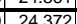 & $\frac{2.115}{25121}$ & $\frac{24.144}{2.715}$ & $\frac{4.01}{46.061}$ & $\begin{array}{l}4.9500 \\
1777\end{array}$ & $\frac{|1.241|}{12455}$ & $\frac{3.5313}{348}$ & $\frac{1.14}{1.209}$ & -0.101 & $\frac{49.31}{50.56}$ & $\frac{0.151}{0.12}$ & $\frac{\mid 4.6431}{14643}$ & & 44752 & $\frac{7.44944)}{7.4596}$ & 40.00 & $\begin{array}{ll}1.1 .6 \\
120\end{array}$ & & $\frac{2.1001}{2777}$ & $\frac{0.0<1}{0.023}$ & & & 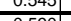 & \\
\hline & 9//3/2004 & 2:55:05 AM & 24.805 & 25.064 & \begin{tabular}{|l|}
24.342 \\
\end{tabular} & 25.132 & 24.76 & 45.879 & \begin{tabular}{|l|}
47.779 \\
\end{tabular} & 1..1.955 & 34.271 & \begin{tabular}{|c|}
-1.209 \\
\end{tabular} & -0.041 & 49.864 & 0.161 & $\begin{array}{l}4.6453 \\
14.643\end{array}$ & & 448.52 & 7.47528 & 46.7 & 11.9 & \begin{tabular}{|l|l|}
40.1 \\
\end{tabular} & 2.763 & \begin{tabular}{|l|}
0.023 \\
\end{tabular} & 0.023 & 0.001 & $\begin{array}{l}0.590 \\
0.586\end{array}$ & \\
\hline 436 & 9/3/2004 & 2:56:05 AM & 24.804 & 25.049 & \begin{tabular}{|l|}
24.317 \\
\end{tabular} & 25.126 & 24.794 & $\begin{array}{l}45.947 \\
\end{array}$ & \begin{tabular}{|l|l|}
47.712 \\
\end{tabular} & 12.025 & 34.314 & $\mid-1.218$ & -6.651 & 49.57 & 0.158 & 14.643 & & 449.52 & 7.49194 & 46.6 & 11.8 & 40. & 2.767 & 0.023 & & 0.001 & 0.575 & \\
\hline 4377 & 9/3/2004 & 2:57:05 AM & 24.809 & 25.058 & 24.326 & 25.191 & 24.844 & 45.902 & \begin{tabular}{|l|l} 
& 47.715 \\
\end{tabular} & 12.029 & 34.224 & -1.212 & -6.66 & 48.629 & 0.146 & 14.643 & & 450.52 & 7.50861 & 46.6 & 11.6 & 40.1 & 2.762 & 0.021 & & 0.00 & 0.532 & \\
\hline & 9/3/2004 & 2:58:05 AM & 24.804 & 25.068 & 24.341 & 25.246 & 24.909 & 46.279 & 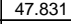 & 12.091 & 34.597 & 212 & -6.48 & 1.188 & 0.172 & 14.643 & & 451.5 & 52528 & 46.7 & 11.7 & & & & & & & \\
\hline 439 & 9/3/2004 & 2:59:05 AM & 24.8099 & 25.079 & 24.347 & 25.311 & 25.009 & 45.41 & 47.75 & 12.182 & 33.518 & & -5.344 & & 0.24 & 14.643 & & & 54194 & 46.6 & & & & & & & & \\
\hline 440 & & & 24.805 & 25.084 & & & 25.035 & 45.182 & 47.851 & 12.104 & & -1.212 & & & 0.145 & 14.643 & & 453. & & 46.7 & & & & & & & 530 & \\
\hline & 9/3/2004] & 3:01:05 AM & 24.7911 & 25.086 & 24.349 & 25.414 & 250206 & $\begin{array}{l}46.375 \\
4.3911\end{array}$ & $\begin{array}{l}47.858 \\
47802\end{array}$ & $\frac{12.101}{1207}$ & $\begin{array}{l}34.692 \\
3292\end{array}$ & - 1.206 & -5.012 & 49.791 & 0.150 & $\begin{array}{l}14.663 \\
103\end{array}$ & & & 7.57528 & 46.7 & 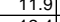 & & & & & & & \\
\hline 443 & 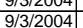 & $\begin{array}{l}3.02 .05 \mathrm{Al} \\
3: 03: 05 \mathrm{AM}\end{array}$ & $\frac{24.024}{24.811}$ & $\frac{23.090}{25.085}$ & $\frac{24.501}{24.363}$ & $\begin{array}{l}25.420 \\
25.383\end{array}$ & 25.0026 & $\begin{array}{l}45.011 \\
46.144\end{array}$ & \begin{tabular}{|l|}
47.902 \\
4754
\end{tabular} & $\frac{1.100}{12.07}$ & 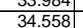 & $\begin{array}{l}-1.212 \\
-1.212\end{array}$ & $\begin{array}{l}-5.124 \\
-6.454\end{array}$ & $\begin{array}{l}52.194 \\
48.673\end{array}$ & $\begin{array}{l}0.044 \\
0.161 \\
\end{array}$ & $\frac{14.049}{14.643}$ & & $\frac{450.52}{456.52}$ & T. & 4 & $\frac{12.4}{11.6}$ & 40.4 & 2.7.152 & | & $\mid$ & 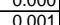 & $\mid$ & \\
\hline & $9 / 3 / 2004$ & 3:04:05 AM & 24.817 & 25.091 & 24.349 & 25.379 & 24.997 & 455.829 & \begin{tabular}{|l|}
47.543 \\
\end{tabular} & 11.914 & 34.253 & -1.212 & -6.564 & 48.786 & $\begin{array}{l}.104 \\
0.164\end{array}$ & $\mid 14.643$ & & 45752 & 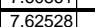 & 464 & $\begin{array}{ll}11.6 \\
11.6\end{array}$ & & 2761 & | & 年 & & 0.597 & \\
\hline 445 & 9/3/2004 & 3:05:05 AM & 24.808 & 25.087 & 24.33 & 25.35 & 24.943 & 46.136 & \begin{tabular}{|l|l|}
47.799 \\
\end{tabular} & 11.999 & 34.45 & -1.212 & -6.521 & & 0.149 & 14.643 & & 458.5 & 7.64194 & 46.7 & 11.9 & 40.3 & 2.778 & & & & 0.540 & \\
\hline & 9/3/2004 & 3:06:05 AM & 24.804 & 25.083 & 24.331 & 25.331 & 24.904 & 46.01 & \begin{tabular}{|l|l}
47.542 \\
\end{tabular} & 12.03 & 34.35 & -1.209 & -6.59 & 49.409 & $\begin{array}{l}0.153 \\
\end{array}$ & 14.643 & & 459.5 & 65861 & $\overline{46.4}$ & $\overline{11.8}$ & 40.2 & 2.770 & & & $\overline{0.0}$ & & \\
\hline 447 & 9/3/2004] & 3:07:05 AM & 24.805 & 25.079 & 24.312 & 25.277 & 24.89 & 45.987 & 47.578 & $11.979 \mid$ & 34.392 & & & 0.473 & 0.076 & 14.643 & & 460.5 & $\begin{array}{l}7.67528 \\
\end{array}$ & 46.5 & 2.0 & $\frac{40.2}{40.2}$ & 2.771 & $\mid 0.011$ & & & & \\
\hline & & & & & & & & & & & & & & & & & & 461.52 & 7.69194 & 46.5 & 11.8 & & & & & & (560 & \\
\hline 449 & 9/3/2004 & & 24.765 & 25.094 & 24.332 & 25.252 & 24.91 & 46.294 & \begin{tabular}{|l|l|}
47.903 \\
\end{tabular} & 12.008 & 34.725 & -1.209 & & 49.597 & 0.15 & $14.643 \mid$ & & 462.52 & 7.70861 & 46.8 & $11.8 \mathrm{~g}$ & & & & & & 540 & \\
\hline & $9 / 3 / 2004$ & $3: 10: 05 \mathrm{AM}$ & 24.775 & 25.094 & 24.322 & 25.232 & 24.92 & 466.03 & \begin{tabular}{|l|l|}
47.799 \\
\end{tabular} & 12.049 & 34.275 & $\mid-1.209$ & \begin{tabular}{c|c|c|}
-6.33 \\
\end{tabular} & 49.629 & $\begin{array}{l}0.16 \\
\end{array}$ & 14.643 & & 463.5 & 7.72528 & \begin{tabular}{l|l}
46.7 \\
\end{tabular} & 11.8 & 40.2 & 2.768 & 0.023 & & & & \\
\hline & 9/3/2004| & & 24.779 & 25.088 & 24.306 & 25.216 & 24.90 & 45.981 & 477.692 & $11.924 \mid$ & 34.413 & & & 50.402 & 0.153 & 14.6 & & 464. & $7.74194 \mid$ & & & & & & & & & \\
\hline & 9/3/2004| & & 24.783 & 25.088 & 24.29 & 25.195 & & 45.862 & 47.59 & 11.978 & 34.251 & -1.215 & & 49.706 & 0.163 & \begin{tabular}{|c|c|}
14.643 \\
\end{tabular} & & & 7.75861 & 46.5 & $\overline{111.8}$ & & 2.762 & & 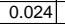 & 0.00 & 0.594 & \\
\hline & & 13:05 AM & & 25.087 & 24.295 & 25.2 & 24.883 & 45.761 & 47.592 & 111.77 & 34.392 & -1.21 & -6.454 & 48.692 & 0.166 & 14.643 & & 46 & & 46.5 & 11.6 & & & & & & & \\
\hline 454 & 9/3/2004 & 3:14:05 AM & 24.793 & 25.097 & 24.3 & 25.19 & 24.923 & 45.479 & 47.565 & 11.896 & 33.877 & & -5.4 & 50.83 & 0.08 & 14.643 & & 467.52 & 7.79194 & 46.4 & 12.1 & 39.7 & 2.736 & & 12 & & 94 & \\
\hline 45 & 3/2004 & & 24.783 & 25.093 & 24.295 & 25.195| & 24. & 46.468 & 47.511 & $11.987]$ & 34.887 & 20 & & 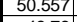 & 0.141 & 14.6 & & & & 46.4 & 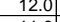 & 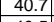 & (7) & & & & 0.506 & \\
\hline & & & 24.762 & & & & & & & & & & & & & & & & & & & & 2.191 & & & & & \\
\hline$\frac{451}{458}$ & 9131212004 & & 24.767 & 25.096 & 24.284 & 25.174 & 24.88 & 45.995 & 47.543 & 11.867 & 34.579 & -1.215 & -6.0 & 5 & 0.126 & 14.643 & & 470.5 & 7.84194 & 46.4 & 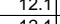 & . & & 0.018 & & & 457 & \\
\hline 459 & 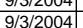 & & $24.156 \mid$ & $\frac{25.011}{25101}$ & $\frac{\mid 24.24}{24284}$ & 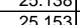 & 24.671 & $\begin{array}{l}45.965 \\
46.178\end{array}$ & \begin{tabular}{|l|l|}
47.653 \\
\end{tabular} & $\mid$ & 34.456 & $\mid$ & $\begin{array}{l}-\frac{0.329}{-6509} \\
-6\end{array}$ & $\begin{array}{l}50.941 \\
53.431\end{array}$ & o.1.35 & 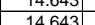 & & 年47.52 & $\mid$ & $\frac{40.35}{46.5}$ & $\frac{1.1}{127}$ & $\frac{4.1}{403}$ & $20.100 \mid$ & 0 & 0.025 & & $\mid$ & \\
\hline 460 & $9 / 3 / 2004$ & 3:20:05 AM & 24.761 & 25.101 & 24.268 & 25.153 & 24.891 & 46.105 & 47.339 & $\frac{12.032}{12.032}$ & 34.526 & -1.215 & -6.266 & 5 & $\begin{array}{l}.168 \\
0.168\end{array}$ & $\mid$ & & 473.52 & \begin{tabular}{|l}
7.89194 \\
\end{tabular} & 46.2 & $\frac{12.0}{12.0}$ & 40.3 & 2.780 & \begin{tabular}{|l|}
0.024 \\
\end{tabular} & & & 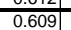 & \\
\hline 461 & & 3:21:05 AM & 24.766 & 25.101 & 24.279 & 25.158 & 24.851 & 45.473 & \begin{tabular}{|l|l|}
47.399 \\
\end{tabular} & 11.917 & 33.885 & \begin{tabular}{|c|}
-1.212 \\
\end{tabular} & -5.80 & \begin{tabular}{|l|l}
54.87 \\
\end{tabular} & 0.212 & 14.6 & & 47. & 361 & 46.3 & 13.1 & 39. & & & & & & \\
\hline 462 & 9/3/2004 & & 24.751 & 25.09 & 24.263 & 25.158 & 24.821 & 45.755 & 47.19 & 11.805 & 34.312 & -1.212 & & 47.964 & 0.161 & 14.6 & & & 7.92528 & 46.1 & 11.4 & 40.0| & 2.760 & 0.023 & $\mid .024$ & & 0.588 & \\
\hline 463 & 2004 & & 24.751 & 25.095 & 24.258 & 25.132 . & & & 47.37 & 11.84 & 34.575 & 209 & & & 0.151 & & & & & & the & & 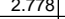 & & & & 0.040 & \\
\hline 464 & & & 24.736 & & & & & & & 11.85 & & & & 52.2 & & & & & & 46.4 & & & & & & & & \\
\hline & 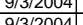 & & 24.725 & 250.085 & \begin{tabular}{|l|l|l|} 
\\
\end{tabular} & 25.107 & 24.845 & & & $\mid \frac{11 . / 44}{1.97 \mid}$ & 34.214 & - -1.215 & & 50,5 & 0.080 & & & & & 15 & 120 & & 2007 & & & & 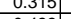 & \\
\hline & Ylot & & 24.110 & 25.09 & $\mid$\begin{tabular}{|l|l|}
$\mid 2031$ \\
\end{tabular} & 20.124 & & & 47.543 & 11.015 & & & & 50.50 & 0 & & & & & & 12.0 & & & & & & & \\
\hline$\frac{407}{468}$ & 年 & & $\frac{24.058}{24698}$ & $\frac{25}{25}$ & 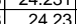 & $\frac{2.513}{25.13}$ & 2481 & & \begin{tabular}{|l|l|}
47.249 \\
\end{tabular} & $\frac{1.545}{12015}$ & $\frac{34.056}{34.474}$ & $\frac{\mid c .42}{-1212}$ & & $\frac{40.016}{48.446}$ & 0.176 & 14.66 & & & $\frac{3.00251}{8.0258}$ & $\frac{7.4}{46.4}$ & $\frac{\frac{1.0}{11.5}}{11}$ & & (7) & & & & | & \\
\hline 469 & 9/3/2004 & $3: 29$ & $\frac{24.718}{24.78}$ & $\frac{25}{25}$ & 24.225 & 25.105 & 24.8 & 45.784 & \begin{tabular}{|l|}
47.688 \\
\end{tabular} & 11.963 & 34.156 & -1.212 & & $\frac{78.5}{48.5}$ & $\begin{array}{l}.164 \\
0.164\end{array}$ & 14.643 & & & & & 11.6 & & & & & & & \\
\hline 470 & $9 / 3 / 2004$ & & 24.712 & & 24.204 & 25.079 & & 45.769 & & 11.798 & 34.3 & & & 50.7 & 0.16 & & & & 8.05 & & & & & & & & & \\
\hline 471 & & & 24.737 & & 24.225 & 25.124 & & & & 11.801 & 34.244 & -1.212 & & & & & & & & $\begin{array}{l}46.2 \\
\end{array}$ & 11.4 & & & & & & 36 & \\
\hline $4 \sqrt{72}$ & & & 24.716 & 25 & 24.208 & 25.078 & & & & 11.68 & 34.2 & -1.212 & & 47.76 & 0.16 & & & & & & 11.4 & 39.9 & 2.7. & & & & & \\
\hline 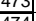 & & & 24.701 & 25.081 & 24.2 & & & & & 11.796 & & -1.212 & & & & & & 48 & & & 11.0 & 39.7 & & & & & 0.568 & \\
\hline 475 & (1) & & & $\frac{25}{25}$ & & 20.146 & & 45.846 & & 12.06 & & & & & & & & & & & 12.3 & & 2.756 & & & & & \\
\hline$\frac{176}{476}$ & (3/3/2004 & $\begin{array}{l}0.54 .53 t \\
3.35 .53 \AA\end{array}$ & 2472 & 年3.045 & $\mid \begin{array}{l}24.21 \\
24268\end{array}$ & 25.238 & & & $\frac{4}{48.00}$ & $\frac{11.96}{11.9}$ & $\begin{array}{l}3.5 \\
338\end{array}$ & $\begin{array}{l}-1.219 \\
-1.209\end{array}$ & & & 0.12 & $\frac{14.6}{146}$ & & & & & 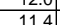 & & & & & & 19. & \\
\hline 477 & 9/3/2004 & $3: 36: 53 \mathrm{~A}$ & 24.716 & & $\mid 24.273$ & 25.22 & 25.6 & 45.3 & & 11.986 & 33.627 & -1.212 & & & 0.187 & 14.6 & & 490.3 & 8.17 & 46.8 & 11.8 & & & & & & 0.692 & \\
\hline 478 & 9/3/2004 & & & & & 25.36 & & & & 12.052 & & & & & & & & & & & & & & & & & & \\
\hline 479 & 9/3/2004 & $3: 38: 5$ & 24.734 & $\begin{array}{l}25.104 \\
\end{array}$ & \begin{tabular}{|l|l|}
24.342 \\
\end{tabular} & 25.367 & 25.12 & 45.56 & \begin{tabular}{|l|l|}
48.001 \\
\end{tabular} & 11.963 & 33.787 & -1.212 & & . & $\begin{array}{l}0.173 \\
\end{array}$ & 14. & & 492.32 & 28 & $\frac{\pi 6.9}{46.9}$ & 12.1 & 39 & 273 & & & & $\begin{array}{l}6.636 \\
\end{array}$ & \\
\hline 480 & $9 / 3 / 2004$ & & \begin{tabular}{|l|l}
24.734 \\
\end{tabular} & 25.094 & 24.342 & 25357 & & 46.08 & 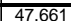 & 11.899 & 34.454 & -1.212 & & & & & & & & & & & & & & & & \\
\hline & & & 24.756 & & & 25.348 & & & & 11.91 & & & & & & & & & & & & & & & & & & \\
\hline 482 & $9 / 3 / 2004$ & $3: 41: 53 \mathrm{~A}$ & 24.751 & 25.095 & $\mid 24.368$ & 25.298 & 24.9 & 46.234 & 47.986 & 12.01 & 34.4 & -1.215 & & 486 & 0.1 & 14.6 & & & 8.25 & 46.9 & 116 & & & & & & 10 & \\
\hline 483 & 9/3/2004 & & 24.751 & 25.086 & $\mid 24.374$ & 25.253 & & & 47.729 & 11.89 & 34.4 & -1.21 & & & 0.1 & 14.6 & & 496 & 4 & 46 & 12.0 & 4 & & & & & 6 & \\
\hline 484 & 9/3/2004 & 3:43:5 & 24.771 & 25.09 & 24.383 & 25.238 & 24. & 45.852 & 47. & 12.046 & 34.14 & -1.212 & & 52.3 & 0.1 & 14.6 & & & 8.28 & 46.8 & 12.5 & 46 & 275 & & & & 0.616 & \\
\hline 48 & 9/3/2 & 3:44:5 & 24.791 & 25.095 & 24. & 25.188 & & 45.931 & 48.295 & $12.007 \mid$ & 34.099 & -1.209 & & & 0.1 & 14.6 & & & & $47 \quad$ & & & & & & & 0.608 & \\
\hline 486 & 9/3/2004 & & 24.792 & 25.096 & \begin{tabular}{|l|l|} 
& 24.409 \\
\end{tabular} & 25.194 & & 46. & & 12.08 & & & & & & & & & & 46.7 & 12.0 & & & & & & & \\
\hline$\frac{487}{80}$ & 004 & & 24 & & 24.399 & 25.179 & & & $47.9 / 4$ & 12.186 & $34.87 \%$ & & & & & & & 500.32 & & 46.8 & 11.9 & & & & & & & \\
\hline & 91/3/2004 & $3: 47: 53$ & 24.832 & 25.081 & 24.404 & 25.184 & 24.8 & & 47.636 & 11.962 & 34.166 & -1.212 & & 49.98 & 0.218 & 14.643 & & 501.3 & & 46.5) & 11.9 & 40 & 2.194 & 0.001 & & 0.001 & 0.183 & \\
\hline $\begin{array}{l}489 \\
490 \\
\end{array}$ & $\begin{array}{l}9 / 3 / 2004 \\
9 / 3 / 2004\end{array}$ & $\begin{array}{l}3: 48: 53 \mathrm{AM} \\
3: 49.53 \mathrm{AM}\end{array}$ & $\frac{24.821}{24.815}$ & $\frac{25.081}{25.075}$ & $\mid$\begin{tabular}{|l|}
24.409 \\
24.413
\end{tabular} & $\begin{array}{l}25.173 \\
25.167\end{array}$ & $\frac{24.86}{24.8}$ & $\begin{array}{r}45.85 \\
45.643 \\
\end{array}$ & \begin{tabular}{|l|}
47.547 \\
47.779 \\
\end{tabular} & $\begin{array}{l}12.087 \\
12.055\end{array}$ & $\begin{array}{l}34.086 \\
33.797\end{array}$ & 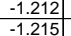 & $\begin{aligned}-6.122 \\
-5.986 \\
\end{aligned}$ & 54.024 & 0.174 & \begin{tabular}{|l|l|l|l|l}
14.643 \\
14.643
\end{tabular} & & & 8.38861 & $\begin{array}{l}46.4 \\
46.7 \\
\end{array}$ & \begin{tabular}{|c|}
12.7 \\
13.0
\end{tabular} & \begin{tabular}{|l|}
43.0 \\
39.7 \\
\end{tabular} & 2.739 & \begin{tabular}{|l|l|}
0.025 \\
\end{tabular} & $\begin{array}{l}0.025 \\
0.026\end{array}$ & $\frac{0.001}{0.001}$ & $\begin{array}{l}0.634 \\
0.645\end{array}$ & \\
\hline
\end{tabular}


WSRC-TR-2005-00105, REVISION 0

SRNL-RPP-2005-00012, REVISION 0

RUN \# WASH1 AND WASH 2; CAMPAIGN IV SLURRY WASHING - CONT.

\begin{tabular}{|c|c|c|c|c|c|c|c|c|c|c|c|c|c|c|c|c|c|c|c|c|c|c|c|c|c|c|c|c|}
\hline & A & $B$ & D & $E$ & $F$ & \begin{tabular}{l|l} 
\\
\end{tabular} & $\mathrm{H}$ & $\mathrm{J}$ & $\mathrm{K}$ & $\mathrm{L}$ & $\mathrm{M}$ & $\mathrm{N}$ & 0 & $Q$ & $\mathrm{R}$ & \begin{tabular}{l|l}
$\mathrm{s}$ \\
\end{tabular} & & $\mathrm{v}$ & w & $\mathrm{x}$ & & & AA & & & & & AF $\quad A$ \\
\hline & \begin{tabular}{|c|} 
\\
\end{tabular} & 3:50:53 AM & \begin{tabular}{l|l|}
24.835 \\
\end{tabular} & 25.085 & \begin{tabular}{|l|}
24.428 \\
\end{tabular} & 25.132 & 24.81 & 45.844 & 47.756 & \begin{tabular}{|l|l|}
11.954 \\
\end{tabular} & 34.136 & -1.212 & -6.379 & 50.651 & 0.174 & 14.643 & 0.003 & 504.32 & \begin{tabular}{l|l}
2 & 8.40528 \\
\end{tabular} & 46.6 & 12.1 & 40.0 & 2.757 & \begin{tabular}{|l|l|}
0.025 \\
\end{tabular} & 0.025 & 0.001 & 0.633 & \\
\hline & $\begin{array}{l}9 / 3 / 2004 \\
\end{array}$ & & & 25.09 & \begin{tabular}{|l|}
24.438 \\
\end{tabular} & 25.132 & 24.83 & 45.657 & \begin{tabular}{|l|l|}
47.472 \\
\end{tabular} & $\begin{array}{l}12.011 \\
\end{array}$ & 33.941 & \begin{tabular}{|l|}
-1.212 \\
\end{tabular} & $\begin{array}{r}-6.316 \\
\end{array}$ & \begin{tabular}{|l|}
49.291 \\
\end{tabular} & 0.17 & \begin{tabular}{|l}
14.643 \\
\end{tabular} & & \begin{tabular}{ll|}
505.32 \\
\end{tabular} & \begin{tabular}{l|l}
2 & 8.42194 \\
\end{tabular} & 46.3 & & & \begin{tabular}{|l|}
2.7444 \\
\end{tabular} & & 0.025 & & & \\
\hline \begin{tabular}{|l|l|}
4933 \\
\end{tabular} & $\begin{array}{l}9 / 3 / 2004 \\
0 / 200\end{array}$ & $3: 52: 53 \mathrm{AM}$ & \begin{tabular}{|l|}
24.824 \\
2.874
\end{tabular} & 25.063 & \begin{tabular}{|l|l|}
24.416 \\
2.491
\end{tabular} & 25.095 & 24.834 & 45.464 & \begin{tabular}{|r|}
47.37 \\
\end{tabular} & \begin{tabular}{|l|l|}
11.923 \\
121
\end{tabular} & $\begin{array}{l}33.861 \\
3.059\end{array}$ & 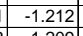 & -6.064 & 51.354 & 0.137 & \begin{tabular}{|l|}
14.643 \\
\end{tabular} & & \begin{tabular}{|l|}
506.32 \\
5032
\end{tabular} & \begin{tabular}{l|l}
28.43861 \\
2
\end{tabular} & 46.2 & & 39.7 & \begin{tabular}{|l|l|}
2.735 \\
\end{tabular} & \begin{tabular}{|l|l|}
0.020 \\
\end{tabular} & 0.020 & 0.001 & $\begin{array}{l}0.503 \\
\end{array}$ & 0.43 \\
\hline 494 & $9 / 3 / 2004$ & 3:53:53 AM & 24.874 & 25.098 & 24.481 & 25.136 & 24.824 & $\begin{array}{l}44.747 \\
\end{array}$ & \begin{tabular}{|l|l|}
47.582 \\
\end{tabular} & \begin{tabular}{|l|l|l|}
12.113 \\
\end{tabular} & $\begin{array}{l}32.958 \\
\end{array}$ & \begin{tabular}{|c|}
-1.209 \\
\end{tabular} & $\begin{array}{r}-4.39 \\
\end{array}$ & \begin{tabular}{|l|l|}
49.416 \\
\end{tabular} & $\begin{array}{l}0.157 \\
\end{array}$ & \begin{tabular}{|l|l|}
14.643 \\
\end{tabular} & & $\begin{array}{l}507.32 \\
\end{array}$ & \begin{tabular}{l|l|}
2 & 8.45528 \\
\end{tabular} & 46.5 & & 38.9 & 2.679 & & \begin{tabular}{|l|l|}
0.023 \\
\end{tabular} & 0.001 & 0.587 & \\
\hline 495 & $9 / 3 / 2004$ & $3: 54: 53 \mathrm{AM}$ & 24.843 & 25.083 & 24.466 & 25.11 & 24.843 & 46.018 & \begin{tabular}{|l|}
47.731 \\
\end{tabular} & $\begin{array}{l}12.205 \\
1.074\end{array}$ & 34.146 & $\begin{array}{l}-1.209 \\
\end{array}$ & & \begin{tabular}{|l|l|}
50.162 \\
\end{tabular} & $\begin{array}{l}0.176 \\
\end{array}$ & \begin{tabular}{|l|l|}
14.643 \\
\end{tabular} & & \begin{tabular}{|l|l|}
508.32 \\
\end{tabular} & \begin{tabular}{l|l|}
2 & 8.47194 \\
\end{tabular} & 46.6 & & 40.1 & \begin{tabular}{|l|l|}
2.764 \\
\end{tabular} & 0.025 & 0.026 & 0.001 & 0.638 & \\
\hline $\begin{array}{ll}496 \\
497\end{array}$ & $\begin{array}{l}9 / 3 / 2004 \\
9 / 3 / 2004\end{array}$ & $\begin{array}{l}3: 55: 53 \mathrm{AM} \\
3.56553 \mathrm{AM}\end{array}$ & \begin{tabular}{|l|}
24.838 \\
24.868
\end{tabular} & $\begin{array}{l}25.077 \\
25.072\end{array}$ & \begin{tabular}{|l|}
24.445 \\
24.455 \\
\end{tabular} & $\begin{array}{l}25.089 \\
25.084\end{array}$ & $\begin{array}{l}24.833 \\
22848 \\
\end{array}$ & $\begin{array}{l}46.101 \\
45.342\end{array}$ & \begin{tabular}{|l|}
47.706 \\
47387 \\
\end{tabular} & $\begin{array}{l}11.971 \\
11.973\end{array}$ & \begin{tabular}{|l|}
34.421 \\
33668
\end{tabular} & \begin{tabular}{|c|}
-1.215 \\
-1.215 \\
\end{tabular} & $\begin{array}{l}-6.185 \\
5558\end{array}$ & \begin{tabular}{|l|}
49.013 \\
51898 \\
\end{tabular} & \begin{tabular}{|c|c|}
0.18 \\
0.173
\end{tabular} & \begin{tabular}{|l|l|}
14.643 \\
14634
\end{tabular} & 然 & \begin{tabular}{|l|}
509.32 \\
51032
\end{tabular} & \begin{tabular}{c|c|}
2 & 8.48861 \\
2
\end{tabular} & $\begin{array}{l}46.6 \\
46.3\end{array}$ & & $\begin{array}{l}7 \\
7\end{array}$ & \begin{tabular}{|l|}
2.776 \\
2724
\end{tabular} & \begin{tabular}{|l|l|}
0.026 \\
0025
\end{tabular} & \begin{tabular}{|l|l}
0.026 \\
\end{tabular} & $\begin{array}{l}0.001 \\
0.001\end{array}$ & 0.650 & \\
\hline$\frac{497}{498}$ & $\begin{array}{l}9 / 3 / 2004 \\
9 / 3 / 2004\end{array}$ & $\begin{array}{l}3: 565: 53 \mathrm{AM} \\
3: 57: 53 \mathrm{AM}\end{array}$ & \begin{tabular}{|l|}
24.868 \\
24.871
\end{tabular} & $\begin{array}{l}25.072 \\
25.071\end{array}$ & \begin{tabular}{|l|}
24.455 \\
24.474 \\
\end{tabular} & $\begin{array}{l}25.084 \\
25.093\end{array}$ & $\begin{array}{r}24.848 \\
24.866\end{array}$ & $\begin{array}{l}45.342 \\
45.755 \\
\end{array}$ & $\begin{array}{r}47.387 \\
47.65 \\
\end{array}$ & \begin{tabular}{ll|l}
11.973 \\
12.026
\end{tabular} & $\begin{array}{l}33.668 \\
34.027 \\
\end{array}$ & $\begin{array}{l}-1.215 \\
-1.212\end{array}$ & $\begin{array}{l}-5.558 \\
-6.188 \\
\end{array}$ & $\begin{array}{l}51.885 \\
50.152\end{array}$ & $\begin{array}{r}0.173 \\
0.18\end{array}$ & \begin{tabular}{|l|l|}
14.643 \\
14.643
\end{tabular} & $\begin{array}{l}0.0033 \\
0.003\end{array}$ & $\begin{array}{l}510.32 \\
511.32\end{array}$ & $\begin{array}{l}8.50528 \\
852194\end{array}$ & $\begin{array}{l}46.3 \\
46.5\end{array}$ & & $\begin{array}{ll}39.5 \\
39.9\end{array}$ & \begin{tabular}{|l|}
2.7724 \\
2.750
\end{tabular} & \begin{tabular}{|l|}
0.025 \\
0.023
\end{tabular} & $|0.025|$ & $\begin{array}{l}0.001 \\
0.001\end{array}$ & \begin{tabular}{|l|l|}
0.637 \\
0.566
\end{tabular} & 0.54 \\
\hline 499 & 9/3/2004 & 3:58:53 AM & 24.861 & 25.076 & 24.484 & 25.133 & $\begin{array}{l}24.0021 \\
2.921\end{array}$ & & \begin{tabular}{|l|l|}
47.712 \\
\end{tabular} & 11.928 & 34.4 & & $\begin{array}{l}-0.1000 \\
-6.07\end{array}$ & 52.1016 & $\begin{array}{l}0.18 \\
0.219 \\
\end{array}$ & \begin{tabular}{|l|}
4.645 \\
14.643
\end{tabular} & $\begin{array}{l}.0003 \\
0.03\end{array}$ & \begin{tabular}{|l|}
512.32 \\
512.32
\end{tabular} & \begin{tabular}{l|l|}
2 & 8.52194 \\
2 & 8.53861
\end{tabular} & $\begin{array}{l}46.5 \\
6.6\end{array}$ & $\frac{11.9}{12.6}$ & $\frac{39.9}{40.2}$ & \begin{tabular}{|l}
$\mid 2.50$ \\
2.773
\end{tabular} & \begin{tabular}{|l|}
0.026 \\
0.031 \\
\end{tabular} & $\begin{array}{l}0.026 \\
0.032\end{array}$ & $\frac{0.001}{0.001}$ & \begin{tabular}{|c|}
0.766 \\
0.791 \\
\end{tabular} & $\frac{0.56}{0.67}$ \\
\hline & & & 24.867 & & \begin{tabular}{|l|}
24.505 \\
\end{tabular} & 25.194 & 24.962 & 46.194 & & 11.957 & & -1.215 & & 51.612 & 0.216 & 14.643 & & 513.32 & \begin{tabular}{|l|l|l}
2 & 8.55528 \\
\end{tabular} & 46.4 & & & & \begin{tabular}{|l|}
0.031 \\
\end{tabular} & & 0.001 & & $\frac{0.67}{0.66}$ \\
\hline & 9/3/2004 & 4:00:53 AM & 24.872 & 25.091 & \begin{tabular}{|r|}
24.52 \\
\end{tabular} & 25.239 & 25.002 & 46.254 & \begin{tabular}{|l|l|}
47.679 \\
\end{tabular} & 12.002 & 34.599 & $\begin{array}{l}-1.212 \\
\end{array}$ & -6.029 & \begin{tabular}{|l|l|}
48.481 \\
\end{tabular} & 0.174 & \begin{tabular}{|l|l|}
14.643 \\
\end{tabular} & 0.003 & 514.32 & \begin{tabular}{l|l|}
2 & 8.57194 \\
\end{tabular} & 46.6 & & 40.4 & \begin{tabular}{|l|}
2.787 \\
\end{tabular} & \begin{tabular}{|l|l|}
0.025 \\
\end{tabular} & 0.025 & 0.001 & 0.625 & $\begin{array}{l}0.66 \\
0.53 \\
\end{array}$ \\
\hline 502 & & & & & & & & & & & & & & & & & & & & & & & & & & & & \\
\hline 503 & 9/3/2004 & & 24.927 & 25.096 & 24.54 & 25.374 & 25.112 & 45.788 & 63 & 12.097 & 23020 & -1.212 & & 50.048 & 0.173 & 14.643 & & & & & & & 2752 & & $\mid$ & & 0.629 & \\
\hline 504 & & & & & & 25.425 & & & & & 33.863 & & & 52.718 & & 14.643 & & & & & & & & .025 & & & & \\
\hline 505 & 9/3/2004 & AM & 24.939 & 25.103 & 24.542 & 25.436 & & 45.524 & 47.8 & \begin{tabular}{|c|c|}
11.994 \\
\end{tabular} & 33.807 & & & 49.7 & 0.144 & 14.64 & & & & & & & & & 0.021 & & & \\
\hline & $9 / 3 / 2004$ & & & 2509 & 24.528 & 25.397 & & & & 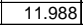 & 33 & & & & & \begin{tabular}{|c|c|}
14.643 \\
\end{tabular} & & & & 46. & & & 2.743 & & & & & \\
\hline 507 & 9/3/2004 & :53 AM & 24.916 & 25.101 & 24.539 & 25.394 & 25.101 & 45.135 & 47.147 & 11.949 & 33.569 & -1.212 & -5.214 & 50.594 & 0.219 & 14.643 & & 520.32 & 8.67194 & 46.0 & 12. & 39.4 & 2.713 & 0.031 & 0.032 & 0.001 & 0.807 & \\
\hline & $9 / 3 / 2004$ & 07:53 AM & 24.901 & 25.096 & 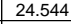 & 25.364 & 25.066 & 45.929 & 47.777 & 11.958 & 34.253 & -1.209 & -5.992 & 50.801 & 0.195 & 14.643 & & 521.32 & 8.68861 & 46. & 12 & & 2.764 & & 0.028 & & 0.705 & \\
\hline$\frac{50}{5 n^{2}}$ & 9/3/2004 & $4: 08: 53 \mathrm{AM}$ & 24.928 & 25.092 & 24.54 & 25.325 & 25.043 & 45.781 & 47.615 & 12.008 & 34.162 & -1.212 & -5.995 & 47.632 & 0.185 & 14.643 & & 522.32 & 8.70528 & 46.5 & & 40 & 2.756 & & 0.027 & 0.00 & & \\
\hline & 9/3/2004 & 4:09:53 AM & 24.934 & 25.098 & 24.547 & 25.301 & 25.02 & 46.072 & & & 34.261 & -1.212 & & 51.965 & 0.179 & 14.643 & & & 2194 & 46.7 & & & & & 0.026 & & 646 & \\
\hline$\frac{511}{512}$ & 9l/1/2004 & $\begin{array}{l}S A M \\
B A M\end{array}$ & 24.995 & $\frac{25.094}{25095}$ & 24.547 & 255286 & $\begin{aligned} 25.022 \\
252\end{aligned}$ & $\begin{array}{l}46.362 \\
45002\end{array}$ & 47.681 & $\frac{12.34}{12.34}$ & 34.4311 & $-\frac{-1.215}{1.1212}$ & & 50.45 & 0.166 & $\frac{14.643}{14643}$ & & 524.32 & 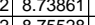 & 46.6 & & & 2.785 & . & 0.024 & & 0.056 & \\
\hline & 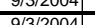 & $\frac{4.115}{4 \cdot 12.5}$ & $\frac{24.960}{2.021}$ & $\frac{23.095}{25019}$ & 24.534 & $\frac{25.268}{2527}$ & 25.011 & $\begin{array}{l}45.902 \\
5725\end{array}$ & 年 & 12.062 & $\frac{34.082}{32020}$ & $-\frac{1.212}{1.212}$ & & 51.8080 & 0.146 & 14.643 & & 525.32 & $\frac{8.15528}{87721}$ & 46.2 & & & 2.1519 & & $\frac{0.021}{0.12}$ & & & 0.45 \\
\hline 515 & $9 / 3 / 2004$ & & | & 25.09 & $\frac{24.524}{24.513}$ & $\frac{25.233}{25232}$ & $\frac{24.9}{24 .}$ & $\frac{45 . / 26}{45.473}$ & $\begin{array}{r}47.48 \\
47.426\end{array}$ & $\frac{12.079}{12011}$ & 33.928 & $\frac{-1.212}{-1.212}$ & & 50.692 & $\begin{array}{l}0.166 \\
0.174\end{array}$ & $\frac{14.643}{14643}$ & & 526.32 & & $\frac{46.4}{463}$ & & & 2.746 & 0.024 & 0.024 & & & 0.51 \\
\hline 515 & $9 / 3 / 2004$ & 14:53 AM & 24.94 & 25.095 & 24.508 & 25.207 & 24.975 & $\begin{array}{l}45.410 \\
45.392\end{array}$ & \begin{tabular}{|l|}
41.420 \\
47.348 \\
\end{tabular} & $\begin{array}{l}1.011 \\
11.969\end{array}$ & 33.777 & $\begin{array}{l}-1.212 \\
-1.212 \\
\end{array}$ & $\begin{array}{l}-0.245 \\
-6.145\end{array}$ & 50.621 & $\begin{array}{l}.181 \\
0.181\end{array}$ & $\begin{array}{l}14.045 \\
14.643\end{array}$ & & 528,32 & 880528 & 46 & 12 & & 2729 & 0.026 & $\mid$ & 0.001 & $\mid$ & \\
\hline & 9/3/2004 & 4:15:53 AM & 24.93 & 25.089 & 24.497 & 25.166 & 24.94 & 45.769 & 47.837 & 12.039 & 33.896 & -1.215 & -6.217 & 50.888 & 0.165 & 14.643 & & 529.32 & 8.82194 & 46. & 12 & & 2.746 & 0.024 & $\mid 0.024$ & & & \\
\hline & 9/3/2004 & 4:16:53 AM & 24.939 & 25.093 & 24.517 & 25.181 & 24.934 & 45.753 & \begin{tabular}{|l|} 
\\
\end{tabular} & $\begin{array}{l}12.045 \\
\end{array}$ & 34.095 & -1.215 & & $\begin{array}{l}50.578 \\
\end{array}$ & 0.104 & 14.643 & & 530.32 & 8.83861 & 46.3 & & & 2.753 & 0.015 & $\mid$ & & 378 & \\
\hline & & & 24.924] & 25.103 & & & 24.9 & 46.089 & 47.713 & 12.148 & 34.166 & & & $\begin{array}{l}477.712 \\
\end{array}$ & & 14.643 & & & & 46.6 & & & 2.16 & & 0.030 & & 745 & \\
\hline 5519 & $|2004|$ & & $24.903 \mid$ & 25.092 & 24.52 & 25.159 & & 46.134 & 47.686 & 12.085 & \begin{tabular}{|l|l|}
34.4 \\
\end{tabular} & $\begin{array}{c}-1.212 \\
\end{array}$ & & $\mid 47.597$ & 0.227 & 14.643 & & & 7194 & 46 & & & 2.776 & 032 & 0.033 & (2) & 0.818 & 0.00 \\
\hline & & & 24.946 & 25.101 & \begin{tabular}{|l|l|}
24.534 \\
\end{tabular} & 25.153 & 24.8 & & & & & & & \begin{tabular}{|l}
48.746 \\
\end{tabular} & 0.192 & 14.643 & & & & & & & & 027 & & & & \\
\hline & & & 24.936 & & & & & & & 11.896 & & & & 48.5 & & & & & & & & & & & & & & \\
\hline & $9 / 3 / 2004$ & & 24.931 & & 24.513 & 25.168 & 24.88 & 45.864 & & 11.962 & 34.154 & & & 51 & & & & & & 46 & & & $2.7 \quad-7$ & & & & & \\
\hline & 9/3/2004 & & 24.93 & 25.101 & 24.514 & 25.188 & & 45.763 & & 11.917 & 34.13 & 209 & & 50.281 & [87 & & & & & $\overline{4}$ & & & 2.75 & & & & 679 & \\
\hline $5<4$ & & & 24.911 & 25.09 & 24.503 & 25.158 & & & 47 & 12.014 & 34.314 & -1.212 & & 47.19 & & & & & & & & & & & & & & \\
\hline 525 & 9/3/2004 & 24:53 AM & 24.936 & 25.096 & 24.504 & 25.163 & 24.821 & 45.806 & 47.671 & 12.078 & 34.015 & -1.218 & & 49.985 & 0.188 & 14.643 & & 538.32 & 8.97194 & 46.5 & 11 & & 2.752 & 0.027 & 0.027 & 0.001 & 0.684 & \\
\hline 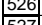 & 9 & $4: 2$ & 24.936 & 20.051 & 24.514 & 25.153 & 24.8 & 45.655 & 47.22 & 12.011 & 34.006 & 1010 & & 53.871 & 0.174 & 14.643 & & 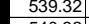 & 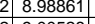 & 46. & & & 27, & - & 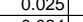 & $n$ & 年 & \\
\hline & & & & & & 25.128 & & & & & 33.694 & -1.218 & & & 0.166 & & & & & 46.7 & 12 & & 2.73 & 0.024 & & & 0.608 & \\
\hline 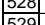 & 9 & & 24.906 & $\frac{25.07}{258}$ & 24.488 & 25.097 & 24.8 & 45. & 47.6 & $\frac{11.948}{11.982}$ & 33.857 & -1.218 & & 50.2 & 0.19 & 144 & & & & 45 & & & 2.1344 & & & & 0.0966 & \\
\hline & & & & & & $\frac{2.1}{251}$ & & & & 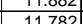 & & & & & & & & & & & & & & & & & & \\
\hline 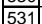 & $9 / 3 / 2004$ & & 24924 & 25088 & \begin{tabular}{|l|}
24.440 \\
\end{tabular} & 25.141 & $\frac{24}{247}$ & & & 11.922 & 33.974 & -121 & & & & & & & & & $\frac{12}{12}$ & & 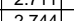 & . & & & 0.1466 & \\
\hline & 9/3/2004 & & 24.918 & $\frac{25.008}{25.088}$ & \begin{tabular}{|l|l|}
24.501 \\
\end{tabular} & $\frac{25.145}{25.105}$ & $\frac{2.4 .8}{24.8}$ & & & $\frac{1.563}{11.863}$ & & $\frac{1.212}{-1.212}$ & & & 0.107 & 14. & & & & & 11 & & 27 & & $\mid \frac{0.020}{0.030}$ & & $\frac{0.0758}{0.758}$ & \\
\hline & $9 / 3 / 2004$ & & 24.902 & 25.071 & 24.484 & 25.114 & & & & 11.926 & & & & & & & & & & & 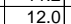 & & & & & & & \\
\hline (534) & & & 24.90 & 25.076 & 24.499 & & & & & \begin{tabular}{l|l|l|}
11.857 \\
\end{tabular} & & & & & & & & & & 46. & & & & & & & & \\
\hline [535] & $9 / 3 / 2$ & & 24.936 & 25.09 & \begin{tabular}{|l|l|}
24.503 \\
\end{tabular} & 25.253 & 24.4 & 45 & & \begin{tabular}{ll|}
12.062 \\
\end{tabular} & 33. & -1.212 & & 49.9 & 0.159 & 14. & & & & 46 & $\frac{11}{11}$ & & 2.71 & & & & 0.585 & \\
\hline 536 & 2004 & & & 25.08 & 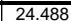 & 25.322 & 25.6 & & & 11.64 & & -1.209 & & 50. & $0.18:$ & & & & 9.15 & & & & 2.7 & 0.02 & 0.02 & & & \\
\hline & & & 24.9 & & 24.4 & 25.39 & & & & & & & & & & & & & & & & & & & & & & \\
\hline & $9 / 3 / 2004$ & & 24.90 & 25.09 & 24.48 & 25. & & & & 11.94 & 34.00 & & & & & & & & & $46 \quad$ & & & 2.74 & & & & 54 & \\
\hline & 9/3/2004 & & 24.90 & 25.08 & 24.476 & 25.3 & & 45. & & $11.7 \mathrm{~g}$ & & & & 4 & & 14. & & & & 4 & & &. .7 & & & & & \\
\hline & 9/3/2004 & & 24.92 & 25 & 24.493 & 25.3 & & 45. & & 11 & & & & & & & & & & & 12 & & & & & & & \\
\hline 541 & & & 24.90 & 25. & 24.473 & 25.293 & 25. & & & 11.917 & & -1.212 & & & & & & & & & 14 & & & & & & 0.644 & \\
\hline & & & 24.96 & & 24.485 & 25.28 & 24. & 44. & & 11.909 & & -1.21 & & & 0.1 & & & & & 46 & 11 & & 2.1 & & & & 0.105 & \\
\hline 340 & & & & & 24.4 & & & & & & & & & & & & & & & & & & & & & & & \\
\hline & & & 24.90 & & 24.48 & 25.2 & & & & 11.68 & & -1. & & & & & & & & & 12 & & & & & & & \\
\hline 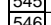 & 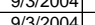 & & $\begin{array}{ll}24.86 \\
2488\end{array}$ & & \begin{tabular}{|l|l|}
24.455 \\
2457
\end{tabular} & 25. & $\frac{24 .}{24 .}$ & & & $\frac{11.817}{11727}$ & $\begin{array}{r}33.52 \\
33.276\end{array}$ & - & & & & & & & & 40 & $\frac{\frac{11}{11}}{11}$ & & & & & & & $\frac{73}{62}$ \\
\hline $\mid 547$ & $9 / 212004$ & & 24.06 & & 24461 & 252 & & & & 11.9 & & & & & & & & & & & & & & & & & & \\
\hline 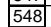 & $9 / 3 / 2004$ & & 24.87 & & \begin{tabular}{|l|l|}
24.445 \\
\end{tabular} & 25.1 & & & & & & $\frac{x}{-1 .}$ & & & & & & & & & & & & & & & & \\
\hline & & & 24.86 & & 24.45 & 25.185 & & 45.4 & & & & & & & & & & & & & & & & & & & & \\
\hline & & & 24.88 & & 24.456 & & & & & & & & & & & & & & & & & & & & & & & \\
\hline & & & 24.8 & & 24.455 & 25.16 & 24. & & & 11.9 & & & & & & & & & & & & & & & & & & \\
\hline & & & 24.8 & & 24.4 & & & & & & & & & & & & & & & & & & & & & & & \\
\hline & $9 / 3 / 2004$ & & 24.86 & & 24.444 & 25.13 & 24.8 & $\begin{array}{l}44.348 \\
\end{array}$ & & 12.0 & 32.5 & & & & & & & & & & & & & & & & 88 & \\
\hline & & & & & & & & 44 & & 12.1 & & & & & & & & & & & & & & & & & & \\
\hline & 9/3/2004 & & 24.8 & & 24.46 & 25.137 & 24.9 & 44.798 & & 12.243 & 32.8 & -1.212 & & & & 14.6 & & & & & & & & & & & 0.693 & \\
\hline & & & 24.88 & & 24. & 25.1 & 24.8 & 44. & & 12.11 & 32.7 & & & & & & & & & & & & & & & & & \\
\hline 55 & $9 / 3 / 2004$ & & 24.89 & & \begin{tabular}{|l|}
24.472 \\
\end{tabular} & 25.12 & 24 & 44.465 & & 12.222 & 32.5 & -1.212 & & & & & & & & 46.1 & & & & & & 0.001 & 691 & \\
\hline & 2004 & & $24.8 \mathrm{~s}$ & & 24.4 & 25.1 & 24. & 44. & & 12.144 & 32.385 & -1.22 & & & & & & & & 40.4 & & & & & & & & \\
\hline 560| & $9 / 3 / 2004$ & $4.59 \cdot 53 \mathrm{AM}$ & $\begin{array}{l}4913 \\
4913\end{array}$ & 25.078 & 24,491 & $\begin{array}{l}25.146 \\
25.155 \\
\end{array}$ & $\begin{array}{l}24.824 \\
24848\end{array}$ & $\begin{array}{r}44.29 \\
44.313\end{array}$ & \begin{tabular}{|l|}
47.1111 \\
\end{tabular} & $\begin{array}{r}12.1 \\
12.134 \\
\end{array}$ & $\begin{array}{r}32.59 \\
32.416 \\
\end{array}$ & \begin{tabular}{|l|}
-1.2121 \\
-1215 \\
\end{tabular} & -5096 & 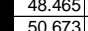 & 0171 & 14643 & & & & 46.4 & & & & & & 0.001 & $\begin{array}{l}0.646 \\
0.647\end{array}$ & \\
\hline & & & & & & & & & & & & & & & & & & $573.32 \mid$ & & & & & & & & & & \\
\hline
\end{tabular}


WSRC-TR-2005-00105, REVISION 0

SRNL-RPP-2005-00012, REVISION 0

RUN \# WASH1 AND WASH 2; CAMPAIGN IV SLURRY WASHING - CONT.

\begin{tabular}{|c|c|c|c|c|c|c|c|c|c|c|c|c|c|c|c|c|c|c|c|c|c|c|c|c|c|c|c|c|}
\hline & & & & & & & & & & & & & & & & & & & & & & & & & & & & \\
\hline & A & & $\mathrm{D}$ & $E$ & $F$ & G & $\mathrm{H}$ & $\mathrm{J}$ & $\mathrm{K}$ & $\mathrm{L}$ & $\mathrm{M}$ & $\mathrm{N}$ & 0 & Q & $R$ & $\mathrm{~s}$ & \begin{tabular}{l|l}
$\mathrm{T}$ & $\mathrm{s}$ \\
\end{tabular} & v & w & $\mathrm{x}$ & & $\mathrm{z}$ & $\mathrm{AA}$ & $\mathrm{AB}$ & & & & AF $[A$ \\
\hline 561 & $99 / 3 / 2004$ & 5:00:53 AM & $\begin{array}{ll}24.902 \\
24.996 \\
\end{array}$ & $\begin{array}{l}25.066 \\
2505\end{array}$ & $\begin{array}{r}24.49 \\
\end{array}$ & 25.124 & $\begin{array}{l}24.817 \\
2491 \\
\end{array}$ & \begin{tabular}{|l|l|}
44.726 \\
4.54
\end{tabular} & \begin{tabular}{|l|l|}
47.478 \\
4755 \\
\end{tabular} & $\begin{array}{l}12.173 \\
\end{array}$ & $\begin{array}{l}32.835 \\
325\end{array}$ & \begin{tabular}{|l|l|}
-1.2099 \\
\end{tabular} & -4.572 & \begin{tabular}{|l|l|}
50.77 \\
\end{tabular} & $\begin{array}{ll}0.127 \\
\end{array}$ & $\begin{array}{ll}14.643 \\
\end{array}$ & 0.003 & $\begin{array}{l}574.32 \\
57.32\end{array}$ & 9.57194 & 46.4 & 12.1 & 38.8 & 2.674 & \begin{tabular}{|l|l|}
0.018 \\
\end{tabular} & 0.018 & 0.000 & 0.476 & $\begin{array}{l}0.40 \\
0.53\end{array}$ \\
\hline 504 & $\begin{array}{l}9 / 3 / 2004 \\
9 / 3 / 2004\end{array}$ & $\begin{array}{l}\text { 5:01:53 AM } \\
\text { 5:02:53 AM }\end{array}$ & \begin{tabular}{|l|}
24.8966 \\
24.916 \\
\end{tabular} & $\begin{array}{r}25.056 \\
25.06\end{array}$ & \begin{tabular}{|l|}
24.494 \\
24.508 \\
\end{tabular} & $\begin{array}{l}25.088 \\
25.077\end{array}$ & $\begin{array}{l}24.781 \\
24.796\end{array}$ & $\begin{array}{l}44.332 \\
4432\end{array}$ & \begin{tabular}{|l|}
47.533 \\
47.532 \\
\end{tabular} & $\frac{12.195}{12.06}$ & $\begin{array}{r}32.56 \\
326\end{array}$ & \begin{tabular}{|l|}
-1.215 \\
-1.212 \\
\end{tabular} & \begin{tabular}{|r|}
-4.639 \\
-4.639 \\
\end{tabular} & $\frac{50.229}{49875}$ & $\frac{0.164}{0.17}$ & \begin{tabular}{|l|l|}
14.6443 \\
14643
\end{tabular} & $\begin{array}{l}0.0033 \\
0.03\end{array}$ & \begin{tabular}{|l|}
575.32 \\
576.32 \\
\end{tabular} & \begin{tabular}{|l|l|}
9.58061 \\
960528
\end{tabular} & $\begin{array}{ll}46.4 \\
46.4\end{array}$ & \begin{tabular}{|l|}
12.0 \\
119
\end{tabular} & $\frac{38.5}{38.3}$ & 2.656 & \begin{tabular}{|l|}
0.023 \\
0.024 \\
\end{tabular} & $\begin{array}{l}0.024 \\
0025 \\
\end{array}$ & 0.001 & $\begin{array}{ll}0.618 \\
0641\end{array}$ & 0.53 \\
\hline $\begin{array}{l}503 \\
564 \\
\end{array}$ & $\begin{array}{l}9 / 3 / 2004 \\
9 / 3 / 2004\end{array}$ & 5:03:53 AM & $\begin{array}{r}24.910 \\
24.92 \\
\end{array}$ & $\begin{array}{r}25.005 \\
25.059 \\
\end{array}$ & \begin{tabular}{|l|l|}
24.513 \\
\end{tabular} & $\frac{25.041}{25.042}$ & 24.81 & $\begin{array}{l}44.352 \\
44.707 \\
\end{array}$ & \begin{tabular}{|l|l|}
47.563 \\
47.53
\end{tabular} & $\begin{array}{l}12.200 \\
12.05 \\
\end{array}$ & $\begin{array}{l}32.200 \\
32.902 \\
\end{array}$ & -1.212 & \begin{tabular}{|l|}
-4.639 \\
-5.107 \\
\end{tabular} & \begin{tabular}{|l|}
$49.8 / 5$ \\
48.634 \\
\end{tabular} & $\begin{array}{l}0.17 \\
0.173 \\
\end{array}$ & $\begin{array}{l}14.643 \\
14.643 \\
\end{array}$ & $\begin{array}{l}.003 \\
0.003 \\
\end{array}$ & $\begin{array}{l}56 / 6.32 \\
577.32 \\
\end{array}$ & $\begin{array}{l}9.60528 \\
9.62194 \\
\end{array}$ & $\begin{array}{l}46.4 \\
46.4 \\
\end{array}$ & \begin{tabular}{|l|}
11.9 \\
11.6
\end{tabular} & $\begin{array}{l}38.3 \\
38.8\end{array}$ & $\frac{2.640}{2.675}$ & $\begin{array}{l}0.024 \\
0.025 \\
\end{array}$ & $\begin{array}{l}0.025 \\
0.025 \\
\end{array}$ & 0.001 & $\begin{array}{l}.6644 \\
0.647 \\
\end{array}$ & $\begin{array}{l}0.55 \\
0.55 \\
\end{array}$ \\
\hline ret & $9 / 3 / 2004$ & 5:04:53 AM & 24.93 & 25.064 & $\mid 24.528$ & 25.062 & 24.8 & 44.539 & .661 & 12.265 & 32.465 & $\frac{1.1 .10}{-1.212}$ & & 50.59 & 0.172 & $\begin{array}{l}14.045 \\
14.643\end{array}$ & 0.004 & & .61948 & $\begin{array}{l}46.4 \\
46.5\end{array}$ & $\begin{array}{l}11.0 \\
12.0\end{array}$ & 8.5 & $\frac{2.075}{2.655}$ & 些25 & 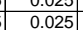 & & $\begin{array}{l}0.647 \\
0.648 \\
\end{array}$ & 0.55 \\
\hline 566 & $9 / 3 / 2004$ & 5:05:53 AM & 24.919 & 25.048 & \begin{tabular}{|l|l|}
24.486 \\
\end{tabular} & 25.045 & 24.7 & 44.255 & & 12.128 & 32.377 & -1.215 & & 48.9 & 0.169 & 14.6 & & & & 46. & 11.7 & 38. & 2.642 & $\mid 0.024$ & & 0.001 & 0.641 & 0.55 \\
\hline 567 & $9 / / 2 / 2004$ & 5:06:53 AM & 24.923 & 25.058 & 24.506 & 25.07 & 24.813 & $\begin{array}{ll}44.199 \\
\end{array}$ & 47.657 & 12.175 & 32.205 & -1.212 & & 51.013 & 0.164 & 14.6 & 0.003 & & & & & & .634 & & $\mid 0.024$ & 0.001 & & 0.53 \\
\hline 568 & 9/3/2004 & 5:07:53 AM & 24.928 & 25.058 & 24.521 & 25.065 & 24.803 & 44.077 & $\begin{array}{lll}47.387 \\
\end{array}$ & 12.199 & 32.135 & -1.209 & -5.046 & 50.496 & $\begin{array}{ll}0.18 \\
\end{array}$ & 14.643 & 0.003 & & 6.68861 & 46. & & 38.1 & 2.627 & 0.026 & 0.026 & 0.001 & 0.685 & 0.58 \\
\hline & 9/3/2004 & 5:08:53 AM & 24.903 & 25.047 & 24.51 & 25.034 & 24.768 & 44.834 & \begin{tabular}{|l|l|}
47.47 \\
\end{tabular} & 12.138 & 32.923 & -1.212 & -4.65 & 49.566 & 0.2 & 14.643 & 0.003 & & & 46. & 11.8 & 38.9 & 2.681 & 0.029 & 0.029 & 0.001 & 0.747 & 0.64 \\
\hline 570 & 9/3/2004 & 5:09:53 AM & 24.897 & 25.046 & 24.515 & 25.033 & 24.792 & 44.498 & $\begin{array}{l}47.252 \\
\end{array}$ & 12.089 & 32.699 & -1.212 & 861 & 48.513 & 0.182 & 14.643 & 0.003 & & .72194 & 46.1 & 11.6 & 38.6 & 6.661 & 0.026 & 226 & 0.001 & 0.684 & 0.58 \\
\hline $5 / 1$ & 2004 & 5:10:53 AM & 24.917 & 25.046 & 24.52 & 25.053 & & 44.724 & & 12.3 & 32.66 & -1.215 & & & 0.181 & 14.64 & & & & 46.5 & & & 668 & 026 & & .001 & & \\
\hline $5 / 2$ & (9/2/20044 & 5:11:53 AM & 24.921 & $25.04]_{1}$ & 24.515 & 25.018 & & 44.533 & 44.625 & & 32.428 & & & 48.184 & 0.156 & & & & & & & & & & & & 0.5888 & .5 \\
\hline & 9/3/2004 & 5:12:53 AM & 24.916 & 25.02 & 24.493 & 24.987 & 24.796 & 44.442 & 47.251 & 12.138 & 32.596 & -1.212 & -5.041 & 50.432 & 0.159 & 14.643 & & 586.32 & 1771 & 46.1 & 12.0 & 38. & 2.65 & 0.023 & 0.023 & 0.001 & 0.599 & \\
\hline 575 & Jash 1 & & & & & & & & & & & & & & & & & & & & & & & & & & & \\
\hline 576 & & Averages & 25.3 & 25.6 & 24.6 & 25.6 & 25.2 & 45.5 & 49.9 & 13.7 & 32.4 & -0.7 & -6.5 & 50.5 & 0.1 & 14.6 & 0.0 & & & $\begin{array}{lll}48.8 \\
\end{array}$ & 12.0 & 38.9 & 2.7 & 0.014 & 0.014 & 0.000 & 0.4 & \\
\hline 577 & & Maximum & 28.0 & 26.2 & 27.0 & 26.1 & & 48.8 & 51.4 & & 35.5 & 33.7 & 33.6 & 55.0 & 0.2 & & 0.0 & & & 50.3 & 13.1 & 41.2 & 2.8 & & & & 0.6 & \\
\hline 578 & & Median & 25.0 & 25.6 & 24.4 & 25.6 & & 45.8 & $\begin{array}{ll}49.9 \\
\end{array}$ & & 33.2 & -1.2 & -7.2 & 50.5 & 0.1 & 14.6 & 0.0 & & & 48.8 & 128 & 39.6 & 2.7 & 0.014 & & & 0.4 & $c$ \\
\hline 579 & & Minimum & 24.8 & 25.2 & 24.1 & 25.1 & 24.8 & 9.6 & 48.4 & 10.9 & -3.3 & -1.2 & -8.2 & 44.8 & 0.0 & 14.6 & 0 & & & 47.3 & 10.7 & 3.1 & 0.2 & 000 & & 0.000 & & \\
\hline 准 & Numbers & $1 \times x \sin 2$ & $\frac{1.404}{324} \mathrm{~s}$ & 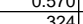 & 1.302 & 0.523 & 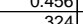 & 0.304 & 1.224 & 3.20 & 0.421 & 1.033 & 9.000 & (3.290 & 0.037 & 0.000 & 0.00 & & & & & & $0.5 / 2$ & . & 0.005 & 0.000 & 125 & \\
\hline 年 & Number & 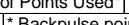 & 324 & 324 & 324 & 324 & 324 & 324 & 324 & 324 & 324 & 324 & 324 & 324 & 324 & 324 & 32 & & & 324 & 324 & 324 & 324 & 324 & 324 & 324 & 324 & \\
\hline 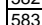 & & Backpulse po & (1) & . & & & & & & & & & & & & & & & & & & & & & & & & \\
\hline & & & & & & & & & & & & & & & & & & & & & & & & & & & & \\
\hline 585 & Wash 2 & & & & & & & & & & & & & & & & & & & & & & & & & & & \\
\hline & & Averag & 24.9 & 25.1 & 24.5 & 25.2 & & & & & & -1.2 & -6.0 & & & & & & & 46.9 & 12.0 & & 2.8 & & & & & \\
\hline 587 & & & 25.4 & 25.2 & 25.1 & 25.4 & 25.1 & & 52.1 & & & -1.2 & & & 0.3 & & & & & 51.0 & 13.3 & & 3.0 & & & 0.001 & & \\
\hline 5888 & & & & & & & & & & & & & & & & & & & & 46.6 & & & 2.0 & & & & 0.6 & \\
\hline 589 & & Minit & $\begin{array}{r}24.7 \\
\end{array}$ & 25.0 & 24.2 & 25.0 & 2 & 43.4 & 46.9 & & & -1.2 & & 24.3. & 0.0 & & & & & 45.8 & 10.5 & & 2.6 & .001 & & 00 & 0.0 & \\
\hline 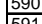 & Nempor & $2 \times$ Std & 0.317 & $\begin{array}{r}0.048 \\
208 \\
\end{array}$ & \begin{tabular}{|l|l|} 
& 0.361 \\
& 08 \\
\end{tabular} & $\frac{0.114}{208}$ & $\frac{0.190}{208}$ & $\frac{1.920}{208}$ & 1.723 & 0.971 & 1.599 & 0.004 & 1.304 & 3.209 & 0.062 & 0.0 & 0.00 & & & 1.723 & 0.779 & 1.706 & 0.118 & \begin{tabular}{|l|l|} 
\\
\end{tabular} & 0.0 & 0.000 & 0.230 & 0.196 \\
\hline 年 & Tumber & of Points Used & 208 & 281 & 208 & 208 & 208 & 2006 & 208 & 208 & 208 & 200 & 208 & 208 & 208 & 208 & 20 & & & 208 & 208 & 208 & 208 & 208 & 208 & 208 & 208 & 208 \\
\hline & & |Backpulse poI & & 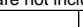 & & & & & & & & & & & & & & & & & & & & & & & & \\
\hline 594 & & & & & & & & & & & & & & & & & & & & & & & & & & & & \\
\hline 595 & Wash $1 \& 2$ & & & & & & & & & & & & & & & & & & & & & & & & & & & \\
\hline $\begin{array}{l}596 \\
596\end{array}$ & & Averag & 25.1 & 25.4 & 24.5 & 25.5 & 2 & 45.7 & 49.2 & $\begin{array}{l}13.1 \\
\end{array}$ & 33.1 & -0.9 & -6.3 & 50.4 & 0.1 & 14 & 0 & & & 48.0 & 12.0 & \begin{tabular}{|l|} 
\\
\end{tabular} & 2.7 & .018 & \begin{tabular}{|c|}
0.018 \\
\end{tabular} & 0.0 & 0.5 & 0.4 \\
\hline 5997 & & & 28.0 & 26.2 & & & & & & & & 33.7 & & & & & & & & & & & & & & & & \\
\hline 598 & & Media & 24.9 & 25.3 & 24.4 & & & $\begin{array}{l}45.9 \\
\end{array}$ & & & 33 & -1.2 & & & & & & & & 48.3 & 12.0 & 39.7 & & & & 0.0 & 0.4 & \\
\hline 599 & & & 24.7 & 25.0 & 24.1 & & & 9.6 & 46 & & -3.3 & 2 & -8 & 44 & & 1 & & & & 45.8 & 10.5 & 3.1 & & & & & 0.0 & \\
\hline 600 & & & 1.165 & 0.688 & 1.048 & 0.606 & 0.485 & $\begin{array}{l}6.673 \\
\end{array}$ & 2.382 & 2.938 & 6.875 & 6.151 & 7.555 & 3.296 & 0.081 & 0.000 & 0.00 & & & 2.382 & 0.785 & 6.650 & 0.459 & 0.012 & 0.012 & 0.000 & 0.289 & 0.246 \\
\hline $6 \frac{601}{602}$ & Number & 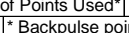 & $\begin{array}{r}532 \\
\text { ts in box }\end{array}$ & $\begin{array}{l}532 \\
\text { are notincli }\end{array}$ & $\begin{array}{l}1 \\
\text { Iuded }\end{array}$ & 532 & 532 & 532 & 532 & 532 & 532 & 532 & 532 & 532 & & & 532 & & & 532 & & 532 & 532 & 532 & 532 & 532 & 532 & 532 \\
\hline
\end{tabular}


WSRC-TR-2005-00105, REVISION 0

SRNL-RPP-2005-00012, REVISION 0

RUN \# 4.18 AND 4.19; ACID CLEANING AND CAUSTIC RINSING

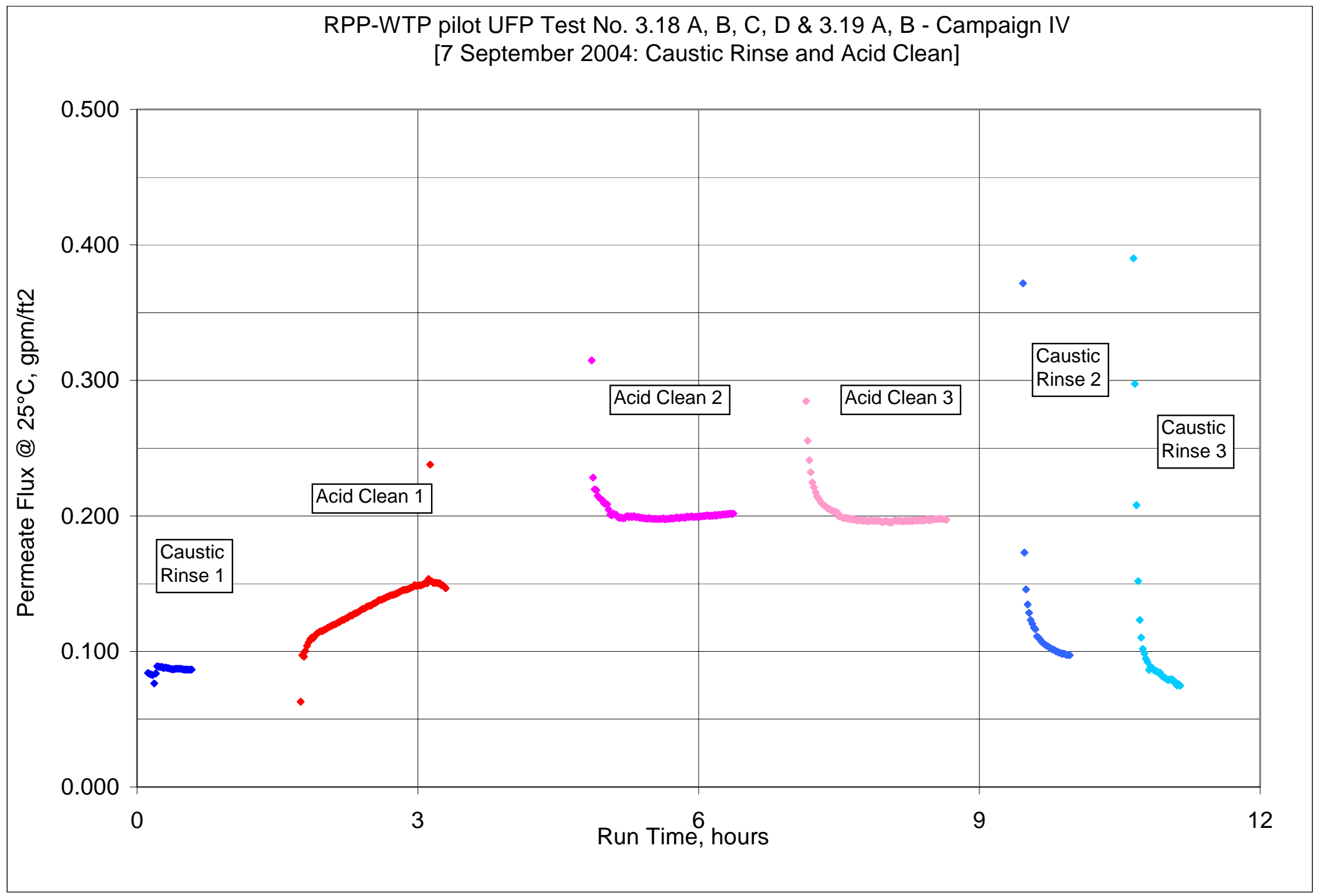


WSRC-TR-2005-00105, REVISION 0

SRNL-RPP-2005-00012, REVISION 0

RUN \# 4.18 AND 4.19; ACID CLEANING AND CAUSTIC RINSING - CONT.

\begin{tabular}{|c|c|c|c|c|c|c|c|c|c|c|c|c|c|c|c|c|c|c|c|c|c|c|c|c|c|c|c|c|}
\hline & A & B & $\mathrm{D} /$ & \begin{tabular}{|l|l|l|} 
& \\
\end{tabular} & $\mid \mathrm{F}$ & \begin{tabular}{|l|l|} 
& $G$ \\
\end{tabular} & $\mathrm{H}$ & \begin{tabular}{l|l}
$\mathrm{J}$ & 1 \\
\end{tabular} & \begin{tabular}{l|l}
$\mathrm{K}$ & \\
\end{tabular} & $\mathrm{L}$ & \begin{tabular}{|l|l|}
$\mathrm{M}$ & \\
\end{tabular} & \begin{tabular}{|l|l|}
$\mathrm{N}$ \\
\end{tabular} & \begin{tabular}{|l|l|}
0 \\
\end{tabular} & \begin{tabular}{l|l} 
& \\
\end{tabular} & \begin{tabular}{|l|}
$\mathrm{R}$ \\
\end{tabular} & $\mid \mathrm{s}$ & $\mathrm{T}$ & $\mathrm{v} / \mathrm{I}$ & W & \begin{tabular}{|c|}
$x$ \\
\end{tabular} & $\mathrm{Y}$ & 12 & AA & $\mathrm{AB}$ & $\mathrm{AC}$ & $\mathrm{AD}$ & $\mathrm{AE}$ & AF \\
\hline & & & 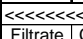 & $\begin{array}{l}\text { << Tempe } \\
\text { Cleaninal }\end{array}$ & erature $\mathrm{Mt}$ & 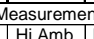 & & 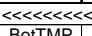 & 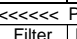 & $\begin{array}{l}\text { Pressure } \\
\text { Fiverter| }\end{array}$ & Measuremen & & Pulcent & 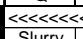 & $\frac{1<\text { Flow } N}{k}$ & Measureme & & & & & & & & & & & & \\
\hline & DAIt & & $\frac{\text { Filfrate }}{\text { deg } C}$ & $\begin{array}{l}\text { Cleaning } \\
\text { deg C }\end{array}$ & \begin{tabular}{|l|} 
Slury \\
deg C \\
\end{tabular} & \begin{tabular}{|c|} 
HIAmb. \\
$\operatorname{deg} C$
\end{tabular} & \begin{tabular}{|c|} 
Lo Amb. \\
$\operatorname{deg} C$
\end{tabular} & $\frac{\text { BotIMP }}{\text { psid }}$ & $\begin{array}{l}\text { Filter } \\
\text { psiq }\end{array}$ & 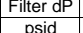 & $\begin{array}{l}\text { TopTMP } \\
\text { psid }\end{array}$ & \begin{tabular}{|l|l} 
Filtrate \\
psig
\end{tabular} & \begin{tabular}{|l|} 
Pulsepot \\
psia
\end{tabular} & $\begin{array}{l}\text { Slurry } \\
\text { gpm }\end{array}$ & \begin{tabular}{|l|} 
Filtrate \\
gam
\end{tabular} & Hi Filtate & & & & & & & & & & & & \\
\hline & & & $\mathrm{T} 2$ & & T1 & T4 & & $\frac{d P 2}{d P 2}$ & P1 & dP1 & $\begin{array}{l}\text { PSId } \\
d P 3\end{array}$ & P2 & P3 & Q1 & $\frac{\mathrm{gpm}}{\mathrm{Q} 2}$ & $\begin{array}{l}\mathrm{gpm} \\
\mathrm{O} 3\end{array}$ & gapp & & & & & & & & & & & \\
\hline & & & & & & & & & & & & & & & & & & & & & & & & & & & & \\
\hline & os- 04/19/ & & & & & & & & & & & & & & & & & & & & & & & & & & & \\
\hline & $8 / 23 / 2004$ & 8:35:38 AM & 22.554 & 22.604 & 422.002 & 22.782 & \begin{tabular}{l|l|}
22.204 \\
\end{tabular} & -0.019 & 0.095 & -0.012 & 0.003 & 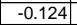 & $\begin{array}{ll}0.137 \\
\end{array}$ & -0.075 & 0.003 & 14.643 & 0004 & & & & Surface $A$ & & FT2 & & & & & \\
\hline & 8/24/2004 & $8: 10: 33 \mathrm{AM}$ & & 222.93 & 23.679 & & 22.735 & -0.021 & 0.122 & -0.014 & -0.001 & \begin{tabular}{|c|c|c|}
-0.124 \\
\end{tabular} & 0.134 & 23.176 & 1.205 & 14.643 & 0.0 & & & Convers & rsion & 851 & $\mathrm{~m} 3 / \mathrm{m} 2$ & day/barc & $\mathrm{arg} / \mathrm{gpm}$ & $2 /$ barg & & \\
\hline & & 9:14:11 AM & & & 24.932 & & 3.242 & -0.008 & 0.126 & -0.008 & 0.011 & & & 48.335 & 0.043 & 14.643 & & & & & & & & & & & & \\
\hline & & & 22.649 & 22.89 & 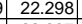 & & 2.071 & & 0.14 & & & & & -0.084 & 0.003 & 14.643 & & & & & & & & & & & & \\
\hline & & 7:28:55 & $22.511 \mid$ & 22.848 & 2.397 & 22.669 & 22.914 & -0.017 & 0.138 & -0.009 & 0.003 & -0.147 & & -0.08 & 0.003 & 14.643 & & & & & & & & & & & & \\
\hline 13 & & & & & & & & & & & & & & & & & & & & Pressi & re Plis & :orrect & ted for & 5 Fituter & 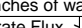 & ertubing & & \\
\hline 14 & & & & & & & & & & & & & & & & & & & & & & & & Fillicic & at $25 \mathrm{C}=$ & PEK & (1) & \\
\hline 15 & & & & & & & & & & & & & & & & & & Time & Time & Press. & elt. & TMP & TMP & $\mathrm{gpm}$ & $\mathrm{gpm}$ & gpm & $\mathrm{gpm}$ & neter \\
\hline 16 & ata - Per Mii & inute & & & & & & & & & & & & & & & & & Hour & sig $f$ & & psi & & Itit2 & & $\mathrm{ft} / \mathrm{psi}$ & $\mathrm{t} / 2 / \mathrm{psi}$ & daylbar \\
\hline 17 & $\begin{array}{l}\text { 9/7/2004 } \\
\end{array}$ & \begin{tabular}{|c|} 
9:20:45 AM \\
\end{tabular} & 24.052 & 23.276 & 23.813 & 24.076 & 23.481 & 22.946 & 25.607 & 6.149 & \begin{tabular}{l|l|}
16.95 \\
\end{tabular} & -1.209 & -3.572 & 45.035 & 0.388 & 14.643 & & & & 24.5 & $\begin{array}{ll}10.7 \\
10\end{array}$ & 19.9 & 1.375 & 0.056 & \begin{tabular}{|l|l|}
6 & 0.057 \\
\end{tabular} & & & 2.45 \\
\hline 18 & 9/7/2004 & 9:21:45 AM & 24.055 & 23.284 & \begin{tabular}{|l|l|} 
& 23.837 \\
\end{tabular} & 24.095 & $\begin{array}{l}23.489 \\
22.526\end{array}$ & 22.917 & 25.568 & 6.024 & \begin{tabular}{l|l|l|}
17.053 \\
\end{tabular} & \begin{tabular}{|l|}
-1.212 \\
\end{tabular} & -3.532 & 46.022 & 0.392 & 14.643 & 0.0 & 1.00 & 0.01667 & 24.4 & & & 1.378 & 0.056 & 0.058 & 0.003 & 2.902 & 2.47 \\
\hline 20 & $9 / 7 / 2004$ & $\begin{array}{l}9: 22: 45 \mathrm{AM} \\
9 \cdot 23.45 \mathrm{AM}\end{array}$ & $\begin{array}{l}24.062 \\
24.039 \\
\end{array}$ & $\begin{array}{ll}23.301 \\
23303\end{array}$ & \begin{tabular}{|l|}
23.879 \\
23.876
\end{tabular} & 24.147 & $\begin{array}{l}23.526 \\
23.520\end{array}$ & $\begin{array}{r}5.836 \\
\end{array}$ & $\begin{array}{l}25.551 \\
25.952 \\
\end{array}$ & $\begin{array}{l}6.047 \\
5.926 \\
\end{array}$ & \begin{tabular}{|r|}
-0.348 \\
\end{tabular} & \begin{tabular}{|l|}
14.702 \\
16.639 \\
\end{tabular} & $\begin{array}{r}20.41 \\
20.055\end{array}$ & \begin{tabular}{ll|l}
46.022 \\
46.162
\end{tabular} & 0.003 & \begin{tabular}{|l|l|l|}
14.643 \\
1.643
\end{tabular} & 0.0 & 2.00 & 0.03333 & 24.4 & 11.0 & & $\begin{array}{l}.189 \\
2099 \\
209\end{array}$ & 0.000 & 0.000 & 0.000 & 0.162 & 0.14 \\
\hline & $\frac{9 / 1 / 7004}{9 / 7 / 2004}$ & $\begin{array}{l}9: 32: 45 \mathrm{AM} \\
9.24: 45 \mathrm{AM}\end{array}$ & 24.039 & & & 24.149 & & $\begin{array}{r}4.31 \\
427 \\
\end{array}$ & \begin{tabular}{l|l}
25.852 & \\
25.837 &
\end{tabular} & $\frac{5.926}{5.828}$ & $\begin{array}{r}-1.741 \\
1.754\end{array}$ & \begin{tabular}{|l|}
16.639 \\
16.64 \\
\end{tabular} & 20.055 & 46.162 & 0.003 & 14.6433 & & & & 24.7 . 24. & & & & & 0.000 & & & 0.29 \\
\hline 22 & 9/7/2004 & $\begin{array}{l}\frac{9.44 .45 \mathrm{AMT}}{9: 25: 45 \mathrm{AM}} \\
\end{array}$ & $\frac{24.041}{24.054}$ & $\frac{20.318}{23.318}$ & \begin{tabular}{|l|l|}
20.940 \\
23.931 \\
\end{tabular} & $\frac{24.220}{24.204}$ & 23.591 & 18.20199 & 25.375 & $\begin{array}{l}3.020 \\
5.283 \\
\end{array}$ & \begin{tabular}{|c|}
1.1542 \\
13.312
\end{tabular} & \begin{tabular}{|l|}
1.044 \\
1.541 \\
\end{tabular} & $\frac{00.479}{0.319}$ & $\begin{array}{ll}40.454 \\
48692\end{array}$ & . 0.0053 & $\frac{14.045}{14.643}$ & & $\frac{4.0}{5.0}$ & 0.08333 & $\frac{24.1}{24.2}$ & $\frac{11.1}{11.6}$ & $\frac{1.5}{16.0}$ & $\frac{0.001}{1.00}$ & 0.076 & 0.079 & 0.005 & $\frac{0.049}{4.929}$ & 0.30 \\
\hline & $9 / 7 / 2004$ & 9:26:45 AM & 24.136 & 23.325 & 23.953 & 24.231 & 23.635 & 18.584 & 25.52 & 5.269 & $\begin{array}{l}10.016 \\
13.162\end{array}$ & \begin{tabular}{|l|}
1.625 \\
\end{tabular} & 0.475 & $\begin{array}{l}40.054 \\
45.857\end{array}$ & 0.516 & $\begin{array}{l}14.045 \\
14.643\end{array}$ & & $\frac{3.00}{6.00}$ & $\frac{0.00053}{0.1}$ & 24.4. & 10.9 & 告. & $\frac{1.1094}{10.04}$ & $\frac{0.074}{0.074}$ & 0.076 & 0.005 & 告.795 & $\begin{array}{l}4.19 \\
4.08 \\
\end{array}$ \\
\hline 24 & $9 / 7 / 2004$ & 9:27:45 AM & \begin{tabular}{|l|l|l|}
24.178 \\
\end{tabular} & 23.337 & 23.975 & 24.273 & $\begin{array}{l}23.617 \\
\end{array}$ & 20.474 & $\begin{array}{l}25.444 \\
\end{array}$ & & 15.356 & \begin{tabular}{|l|l|}
-0.416 \\
\end{tabular} & -1.78 & 46.529 & 0.571 & 14.643 & & 7.00 & 0.11667 & $\frac{24.3}{24}$ & & & 1.235 & & 0.084 & & 4.697 & 08 \\
\hline & $9 / 7 / 2004$ & & & & & & & & & & & & -1.835 & 47.194 & 0.567 & & & & & & & & & & & 0.005 & & \\
\hline 26 & & & & & & \begin{tabular}{|l|l|}
24.287 \\
\end{tabular} & & 20.53 & 25.359 & & 15.467 & $\mid-0.538$ & -1.878 & 44.274 & 0.564 & 14.643 & & 9.00 & 0.15 & $\frac{24.2}{24.2}$ & & & & 0.081 & 0.083 & & 4.611 & 3.93 \\
\hline & $9 / 7 / 2004$ & & & 23.368 & \begin{tabular}{|l|l|}
24.071 \\
\end{tabular} 47.071 & 24.309 & 23.679 & 20.814 & & 5.112 & & $\mid-0.627$ & & & & 14.6 & & & & & 10.6 & & & & 0.083 & & & \\
\hline 28 & $9 / 7 / 2004$ & $9: 31: 45$ & 24.267 & & & 24.327 & 23.701 & 21.247 & 25.545 & 5.041 & 16.37 & -1.166 & -2.274 & 49.397 & 0.52 & \begin{tabular}{|c|c|}
14.643 \\
\end{tabular} & & 11.00 & \begin{tabular}{|l|l|l|l}
0.18333 \\
\end{tabular} & 24.4 & $\frac{11.8}{11.8}$ & & & 0.074 & 0.076 & 0.004 & 4.060 & 3.46 \\
\hline 29 & $9 / 7 / 2004$ & 9:32:45 АM & 24.294 & 23.398 & 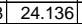 & 24.364 & 23.733 & \begin{tabular}{|l|}
21.39 \\
\end{tabular} & 26.382 & 5.015 & 16.441 & -0.468 & -1.809 & 47.951 & \begin{tabular}{|l|l|}
0.571 \\
\end{tabular} & 14.643 & & 12.00 & 0.2 & 25.3 & 11.4 & 18.9 & \begin{tabular}{|l|}
1.304 \\
\end{tabular} & $\begin{array}{l}0.082 \\
\end{array}$ & 0.084 & 0.004 & 4.428 & 3.77 \\
\hline 30 & $9 / 7 / 2004$ & & \begin{tabular}{|l|l|}
24.3 \\
\end{tabular} & 23.395 & 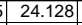 & 24.336 & & 22.243 & \begin{tabular}{|l|l}
27.79 \\
\end{tabular} & 4. & 17.404 & \begin{tabular}{|l|l|} 
\\
\end{tabular} & -1.38 & $\begin{array}{l}44.599 \\
\end{array}$ & 607 & & & 13.0 & 0.21667 & 26.7 & to. & & 367 & \begin{tabular}{|l|l|}
0.087 \\
\end{tabular} & 0.089 & 0.004 & 4.493 & 33 \\
\hline & $9 / 7 / 2004$ & 9:34:45 AM & 24.333 & 23.407 & 24.175 & 24.343 & 23.768 & 22.284 & 27.819 & 4.908 & 17.472 & 0.209 & -1.398 & 43.728 & 0.605 & 14.643 & & 14.00 & 0.23333 & 26.7 & 10.4 & 19.9 & \begin{tabular}{|l|l|} 
& 1.371 \\
\end{tabular} & 0.087 & 0.089 & 004 & 4.460 & 3.80 \\
\hline (2) & $9 / 7 / 2004$ & & 24.37 & 23.419| & 24.197 & 24.36 & 20,10 & 22.093| & 27.605 & 4.921 & 17.141 & 0.149 & -1 & 45.991 & 0.605 & & & 15.00 & \begin{tabular}{|l}
0.25 \\
\end{tabular} & 26.5 & (1) & 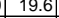 & 1.000 & . & 0.089 & 0.005 & 4.517 & 3.84 \\
\hline 33 & & & & & & & & & & & & & & & & & & & & & & & & & & & & 3.83 \\
\hline $\begin{array}{ll}\frac{34}{325} \\
5\end{array}$ & $\begin{array}{l}91 / 2004 \\
0.172004\end{array}$ & & $24.4 \angle 5$ & 23.449 & 24.237 & 24.415 & 23.82 & 22.120 & 27.622 & 4.7. & 11.511 & 0.14 & -1.4 & $44 .{ }_{4.2}$ & & & & 17.00 & 28333 & 20.5 & t10.5 & 19.0 & & 0.086 & 0.088 & 0.004 & 4.421 & 3.76 \\
\hline & 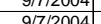 & & 24.440 & $\frac{2.4460}{23458}$ & 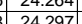 & 24.424 & & $\frac{22.250}{22386}$ & 20.101 & 4..984 & $\frac{\mid 1.320}{1756}$ & (.145 & & & & & & & & $\frac{2.6}{26.7}$ & & & & & & & & 3.79 \\
\hline 地 & $9 / 7 / 2004$ & $9: 40:$ & $\begin{array}{r}r 4.49 \\
24.5\end{array}$ & 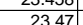 & 7) 24.2513 & $\begin{array}{l}24.4545 \\
2456\end{array}$ & 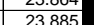 & 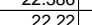 & 27.009 & $\begin{array}{r}4.444 \\
5.09\end{array}$ & 17236 & $\mid$ & $\frac{-1.44}{-146}$ & 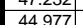 & 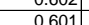 & 14. & & 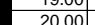 & $\begin{array}{l}0.5031 \\
0.3333\end{array}$ & $\frac{20.1}{266}$ & 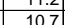 & . & 360 & 0.086 & $\begin{array}{l}0.000 \\
0.088\end{array}$ & . & 年.4067 & 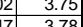 \\
\hline 38 & $9 / 7 / 2004$ & & 24.518 & 23.472 & 24.331 & 24.428 & 23.847 & 22.218 & 27.659 & 4.979 & 17.207 & 0.082 & & 46.502 & 0.599 & 14. & & 2100 & 0.0 .35 & 26.5 & 111 & & 1.359 & 0.086 & 0.087 & 0.004 & 4.433 & 3.18 \\
\hline t & & & & & & & & & & & & & & & & & & & & & & & & & & & & \\
\hline & $9 / 7 / 2004$ & & \begin{tabular}{|l|}
24.566 \\
\end{tabular} & 23.485 & \begin{tabular}{|l|}
24.368 \\
\end{tabular} & $\mid 24.34$ & & 22.276 & \begin{tabular}{|l|l}
27.73 \\
\end{tabular} & $\begin{array}{l}4.93 \\
\end{array}$ & 17.324 & 0.114 & & 48. & \begin{tabular}{l|l}
0.596 \\
\end{tabular} & & & 23.0 & 0.38333 & 26. & & & & 085 & $\overline{0.087}$ & & 4.387 & $\frac{0.00}{3.73}$ \\
\hline & & & 24.592 & & 24.385 & 24.302 & & 22.1 & 27.622 & & & 0.125 & & & & & & & & & & & & & & & & \\
\hline 42 & $9 / 7 / 2004$ & & 24.603 & 23.492 & \begin{tabular}{|r|r|}
24.39 \\
\end{tabular} & 24.227 & 23.737 & 22.415 & 27.991 & 5.064 & $\begin{array}{l}17.264 \\
\end{array}$ & 0.178 & & & 0.6 & & & 25. & 0.41667 & 26.9 & 109 & 198 & 368 & . & 0.087 & 0.004 & 4.405 & 3.75 \\
\hline 43 & $9 / 7 / 2004$ & & 24.6 & & \begin{tabular}{|l|}
24.415 \\
\end{tabular} & & & 22.444 & 28.059 & & & 0.2 & & & \begin{tabular}{|l|l|}
0.599 \\
\end{tabular} & & & & 0.43 & & & & & & & & 62 & 52 \\
\hline 44 & $9 / 7 / 2004$ & & 24.653 & 23.498 & 24.446 & 24.182 & & 22.398 & \begin{tabular}{|l|}
27.962 \\
\end{tabular} & 336 & \begin{tabular}{|l|l|}
17.482 \\
\end{tabular} & \begin{tabular}{l|l}
0.207 \\
\end{tabular} & & & \begin{tabular}{|l|l|}
0.6 \\
\end{tabular} & & & 27.0 & & 26. & 10.3 & & & & 0.087 & & 4.376 & 3.72 \\
\hline 45 & $9 / 7 / 2004$ & & 24.658 & 23.493 & $\begin{array}{l}324.436 \\
\end{array}$ & 24.147 & & 22.212 & 27.753 & & 17.346 & 0.166 & & & & & & & 0.46 & & 11 & & & & & & 4.405 & 3.75 \\
\hline 46 & $9 / 7 / 2 \mathrm{C}$ & & 24.689 & 23.508 & 324. & 24.2 & & 22.435 & & & & 0.149 & & & & & & & 0.48333 & & 0.0 & & & & & & 4.373 & \\
\hline 47 & $9 / 7 / 20$ & & 24.7 & & & 24 & & & & & & & & & & & & & & & 0.6. & & & & & & & 12 \\
\hline & & & 24.14 & 23.5266 & & & & 22.506 & 28.016 & & & 0.19 & & & & & & & 0.51667 & & & & & & & & 4.346 & 16] \\
\hline 49 & & & & & 24.5 & & & & & & & & & & & & & & & & & & & & & & & 3.6 \\
\hline & & & & & & & & & 27.82 & & & & & & & & & & & & & & & & & & & \\
\hline 51 & & & & & & & & & & & & & & & & & & & & & & & & & & & & \\
\hline & & & & & & & & & 28.16 & & & & & & & & & & & & & & & & & & & \\
\hline 㬝 & & & & 20.80 & 25.5 & & & $\frac{42.2}{42.5}$ & $\frac{42.91}{43.37}$ & & & -1.22 & & & & & & & & & 11 & & & & & & $\frac{2.130}{2.900}$ & 30 \\
\hline & $9 / 7 / 20$ & & | & & & & & $\begin{array}{l}4.5 .517 \\
3.34]\end{array}$ & $\mid$ & & 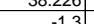 & $|-1.209|$ & & & & & & & & & 1111 & & & & & & & \\
\hline 56 & $9 / 7 / 2004$ & 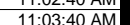 & $\frac{25.065}{25815}$ & $\frac{2.0 .046}{23.916}$ & 42536 & & & $\mid$ & $\frac{45.26}{4324}$ & & -1.325 & \begin{tabular}{|l|}
3.000 \\
\end{tabular} & & 4673 & & & & & & . & 11.1 & & & & & & 1.005 & 5 \\
\hline & $9 / 7 / 2004$ & & 25.441 & 23.906 & 25.309 & & & -0.886 & $\mid$\begin{tabular}{|c|c|}
43.48 \\
\end{tabular} & & & 3975 & & & & & & & & 424 & $11 .$. & & & & & & & 4 \\
\hline & & & 25.678 & & & & & & 42.927 & & & & & & & & & & & & & & & & & & & \\
\hline 59 & & & & & & & & & & & & & & & & & & & & & & & & & & & 2383 & \\
\hline & & & 25.65 & & \begin{tabular}{|l|l|}
25209 \\
\end{tabular} & & & & 42384 & & & & & & & & & & & & & & & & & & & \\
\hline & $9 / 7 / 2$ & & 25.622 & 23.943 & 25. & 24.9 & & & 42.62 & & & -1.2 & & & & & & & & & & & 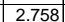 & & & & & \\
\hline & $9 / 7 / 200$ & & 25.584 & 23.944 & 25.1 & 25 & & 42.306 & \begin{tabular}{|c|}
42.854 \\
\end{tabular} & & & -1.2 & & & & & & & & & & & & & & & & \\
\hline & $9 / 7 / 2$ & & 25.566 & 23.966 & 25.6 & 25.0 & & 42.138 & 42.802 & & & -1.2 & & & & & & & & 41. & & & & & & & 2.6 & \\
\hline 64 & $9 / 7 / 2$ & 11:11: & 25.533 & 23.973| & 25. & & & 42.5 & 42.68 & & & -1.2 & & & & & & & & 41.6 & 10 & & & & & & 2.7 & \\
\hline & 9/7/2 & & 25.488 & & 25. & & & & & & & & & & & & & & & & & & & & & & & \\
\hline 66 & 9/7/2004 & & 25.481 & 23.982 & 25.029 & 25.062 & & 42.177 & 42.9977 & & 37.906 & -1.215 & & & & & & & & & & & & & & & 2.740 & \\
\hline 69 & $9 / 7 / 2004$ & & 25.448 & 23.994 & \begin{tabular}{|l|}
24.991 \\
\end{tabular} & 25.0699 & & 42.503 & 43.128 & & 38.49 & -1.215 & & & & & & & & 42.0 & 11.00 & & & & & & 2.748 & \\
\hline & 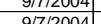 & 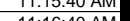 & 20.434 & 2.0015 & \begin{tabular}{|l|l|l|}
24.930 \\
\end{tabular} & 2 & & 42.150 & 42.931 & 4.0 & 30.0101 & - & ne & & & & & 115 & & $\begin{array}{l}41.8 \\
1.87\end{array}$ & 11 & 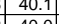 & 2 & 1011 & 0.113 & 0.003 & 2.813 & 3. \\
\hline 70 & $9 / 7 / 2004$ & $11: 17: 40 A$ & $\frac{25.401}{25.387}$ & 24.023 & 24.935 & 25.113 & & $\frac{42.104}{42.482}$ & \begin{tabular}{|l|l|l|l|l}
43.316 \\
\end{tabular} & 4.639 & \begin{tabular}{|}
31.91 \\
38.339
\end{tabular} & \begin{tabular}{|l|l|l|} 
\\
\end{tabular} & & 45.042 & 0.003 & 0798 & & 116.92 & & & & & & & & & 28 & \\
\hline & $9 / 7 / 2004$ & & & & & & & & & & & & & & & & & 116.92 & & 42.2 & & & & & & & & \\
\hline
\end{tabular}


WSRC-TR-2005-00105, REVISION 0

SRNL-RPP-2005-00012, REVISION 0

RUN \# 4.18 AND 4.19; ACID CLEANING AND CAUSTIC RINSING - CONT.

\begin{tabular}{|c|c|c|c|c|c|c|c|c|c|c|c|c|c|c|c|c|c|c|c|c|c|c|c|c|c|c|c|c|}
\hline & A & B & D & $E$ & $\mathrm{~F}$ & G & $\mathrm{H}$ & $\mathrm{J}$ & $\mathrm{K}$ & $\mathrm{L}$ & \begin{tabular}{l|l}
$M$ & \\
\end{tabular} & $\mathrm{~N}$ & 0 & \begin{tabular}{l|l} 
\\
\end{tabular} & $\mathrm{R}$ & $\mathrm{s}$ & $\mathrm{T}$ & $\mathrm{v}$ & w & $\mathrm{x}$ & $\mathrm{Y}$ & $z$ & $\mathrm{AA}$ & $A B$ & $A C$ & AD & $\mathrm{AE}$ & \\
\hline 7. & 9/7/2004 & 11:18:40 AM & 25.389 & 24.045 & 24.947 & 25.15 & \begin{tabular}{|l|}
24.309 \\
\end{tabular} & 42.065 & \begin{tabular}{|l|l|}
42.865 \\
\end{tabular} & 4.652 & $\begin{array}{r}37.84 \\
\end{array}$ & \begin{tabular}{|l|l|}
-1.209 \\
\end{tabular} & 0.524 & $\begin{array}{ll}46.443 \\
\end{array}$ & 0.005 & 0.802 & 0.005 & 117.92 & 1.96528 & 41.7 & 11.1 & & & 0.115 & 0.115 & 0.003 & 2.878 & 2.45 \\
\hline & $9 / 7 / 2004$ & & 25.354 & 24.045 & & 25.125 & & 42.293 & & 4.684 & & \begin{tabular}{|l|}
-1.209 \\
\end{tabular} & & & & & & & 1.98194 & \begin{tabular}{|l|l|} 
\\
\end{tabular} & 11.2 & 40.1 & & 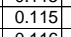 & & & & \\
\hline 74 & $\begin{array}{l}9 / 7 / 2004 \\
0 / 7 / 2004\end{array}$ & 11:20:40 AM & \begin{tabular}{|l|}
25.34 \\
2531
\end{tabular} & $\begin{array}{ll}24.061 \\
24062\end{array}$ & \begin{tabular}{l|l|l|}
24.908 \\
24.859 \\
\end{tabular} & $\begin{array}{l}25.166 \\
25147 \\
251\end{array}$ & \begin{tabular}{|l|l|}
24.314 \\
\end{tabular} & $\begin{array}{ll}42.328 \\
2.250\end{array}$ & \begin{tabular}{|l|l|}
43.028 \\
\end{tabular} & $\begin{array}{r}4.61 \\
\end{array}$ & $\begin{array}{ll}38.261 \\
20201\end{array}$ & \begin{tabular}{|l|l|}
-1.209 \\
\end{tabular} & & 46.131 & 0.003 & 0.808 & $\begin{array}{l}0.005 \\
0.6\end{array}$ & & 1.99861 & 41.9 & 11.0 & & 2.170 & 0.116 & 0.116 & $\begin{array}{ll}0.003 \\
\end{array}$ & 2.878 & \\
\hline $\begin{array}{l}74 \\
755 \\
\end{array}$ & $9 / 7 / 2004$ & 11:21:40 AM & $\begin{array}{l}25.321 \\
252.27\end{array}$ & $\begin{array}{ll}24.062 \\
24063\end{array}$ & $\begin{array}{l}24.859 \\
24.841 \\
\end{array}$ & $\begin{array}{l}25.147 \\
25.179\end{array}$ & \begin{tabular}{|l|}
24.346 \\
2.327
\end{tabular} & $\begin{array}{ll}42.258 \\
42.287\end{array}$ & \begin{tabular}{|l|l|}
43.034 \\
42.972
\end{tabular} & \begin{tabular}{|l|l}
4.603 \\
4709
\end{tabular} & $\begin{array}{l}38.222 \\
337977\end{array}$ & \begin{tabular}{|l|l|}
-1.212 \\
-12212 \\
\end{tabular} & & \begin{tabular}{|l|}
45.471 \\
45.77 \\
\end{tabular} & $\begin{array}{l}0.003 \\
0.003\end{array}$ & $\begin{array}{l}0.81 \\
0.816\end{array}$ & 0.006 & $\begin{array}{l}120.92 \\
10102\end{array}$ & \begin{tabular}{|l|}
2.01528 \\
20204
\end{tabular} & \begin{tabular}{|l|}
41.9 \\
41.8
\end{tabular} & 109 & tant & \begin{tabular}{|l|}
2.774 \\
2764
\end{tabular} & \begin{tabular}{|l|l|}
0.116 \\
0.117 \\
\end{tabular} & $\begin{array}{l}0.116 \\
\end{array}$ & $\begin{array}{ll}0.003 \\
\end{array}$ & $\begin{array}{ll}2.893 \\
2028\end{array}$ & \\
\hline 76 & $\begin{array}{l}9 / 7 / 2004 \\
9 / 72004\end{array}$ & $11.22 .240 \mathrm{AM}$ & 25.278 & 24.003 & 24.8416 & & & $\frac{42.181}{42.125}$ & $\begin{array}{l}42.972 \\
42.908 \\
\end{array}$ & $\begin{array}{l}4.109 \\
4.637 \\
\end{array}$ & $\begin{array}{l}37.971 \\
38.049\end{array}$ & $\mid$\begin{tabular}{|c|}
$\mid-1.212$ \\
-1.215 \\
\end{tabular} & & \begin{tabular}{|r|}
45.77 \\
49.201 \\
\end{tabular} & $\begin{array}{l}0.003 \\
0.003 \\
\end{array}$ & $\begin{array}{r}0.816 \\
0.82 \\
\end{array}$ & $\begin{array}{l}0.006 \\
0.005\end{array}$ & $\frac{121.92}{122.92}$ & \begin{tabular}{|l|}
2.03194 \\
20.04861
\end{tabular} & $\begin{array}{l}41.8 \\
41.8 \\
\end{array}$ & & $\begin{array}{l}40.1 \\
40.1\end{array}$ & 2.7664 & \begin{tabular}{|l|}
0.117 \\
0.177 \\
\end{tabular} & $\begin{array}{l}0.117 \\
0.118\end{array}$ & 0.003 & & $\begin{array}{l}2.49 \\
2.50\end{array}$ \\
\hline \begin{tabular}{|l|}
77 \\
\end{tabular} & 9/7/2004 & & 25.27 & 24.081 & 24.833 & 25.182 & 24.375 & 42.167 & 42.788 & 4.584 & 38.195 & \begin{tabular}{|r|}
$\mid-1.209$ \\
\end{tabular} & & \begin{tabular}{|c|}
46.218 \\
\end{tabular} & 0.003 & 0.823 & & & 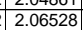 & \begin{tabular}{|l|}
41.0 \\
41.7 \\
\end{tabular} & 11.0 & & & & $\begin{array}{l}.118 \\
0.118 \\
\end{array}$ & & $\frac{2.941}{2.946}$ & $\frac{2.50}{2.51}$ \\
\hline 78 & 9/7/2004 & & & 24.093 & 24.835 & 25.184 & & 42.169 & 43.012 & 4.646 & 38.041 & \begin{tabular}{|l|}
-1.212 \\
\end{tabular} & & \begin{tabular}{|l|l|}
44.994 \\
\end{tabular} & & 0.828 & 0.006 & & 2.08194 & 41.9 & 10.7 & & & 0.119 & & & $\frac{2.940}{2.970}$ & 2.2 .51 \\
\hline & 9/7/2004 & & 25.249 & 24.11 & 24.832 & 25.181 & 24.384 & 42.156 & & 4.7 & 37.883 & \begin{tabular}{|l|}
-1.212 \\
\end{tabular} & & 45.887 & 0.003 & 0.831 & & & 2.09861 & 41.9 & 10.9 & 40.0 & 2.759 & 0.119 & 0.120 & 0.003 & & $\frac{2.53}{2.54}$ \\
\hline \begin{tabular}{|l|l|}
80 \\
81
\end{tabular} & 9/7/2004 & 11:27:40 AM & 25.235 & 24.111 & 24.793 & 25.216 & & 42.15 & 42.865 & & & & & 46.498 & 0.003 & 0.833 & & & 1528 & & & & & & & & 994 & \\
\hline \begin{tabular}{|l|l}
81 \\
02
\end{tabular} & & & & & & & & & & 4.682 & & -1.212 & & & & & & & & 42.0 & & & & & & & 992 & \\
\hline & $9 / 7 / 2004$ & 11:29:40 AM & 25.208 & 24.129 & 24.771 & 25.135 & & 42.138 & 42.893 & 4.584 & & -1.212 & & & & & & & 14861 & 41.8 & 8 & & & & & & & \\
\hline 라 & & 11:30:40 AM & 25.214 & 24.136 & 24.773 & 25.146 & & & & & $\mid 37.94$ & -1.209 & & & & & & 129.96 & & 41.6 & 11 & & 2.75 & & & & & \\
\hline 84 & $9 / 7 / 2004$ & 11:31:40 AM & 25.189 & 24.125 & 24.737 & 25.065 & 24.1 & 42.289 & 43.146 & 4.599 & 38.22 & $\begin{array}{ll}-1.212 \\
\end{array}$ & & 46.058 & 0.003 & 0.852 & 0.006 & 130.92 & 2.18194 & 42.0 & 11.0 & 40.3 & 2.775 & D.122 & 0.123 & 0.003 & 3.053 & \\
\hline & 9/7/2004 & 11:32:40 AM & 25.179 & 24.13 & 24.727 & 25.025 & 24. & 42.293 & 43.15 & 4.658 & 38.174 & -1.209 & & 45.98 & 0 & 0.854 & 0.006 & 131.92 & 2.19861 & 42.0 & 11.0 & 40. & 2.774 & & 0.123 & 0.003 & 3.062 & \\
\hline 86 & $9 / 7 / 2004$ & 11:33:40 AM & 25.184 & 24.1355 & 24.732 & 25.02 & & 42.2 & 43.173 & 4.703 & 37.971 & -1.209 & & 43.315 & 0.003 & 0.857 & 0.004 & 132.9 & 2.21528 & 42.0 & 10.5 & 40 & 2.76 & 123 & & 0.003 & 3.081 & \\
\hline $807+2$ & . & 11:34:40 AN & 25.154 & 24.126 & 24.688 & 24.981 & & & 43.043 & 4.659 & 8.043 & -1.218 & & 46.1999 & & & & & 23194 & 41.9 & & & & & & & & \\
\hline 88 & & $11: 35: 40 \mathrm{AM}$ & 25.159 & 24.146 & 24.728 & 25.021 & & 42.11 & 42.983 & 4.548 & 38.088 & -1.212 & & 44.579 & & 0.865 & & 134 & 248611 & 41.99 & & 4 & 76 & .124 & .125 & 0.003 & 3.112 & \\
\hline & 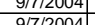 & $\begin{array}{l}11.30 .40 \mathrm{AN} \\
11.170 \mathrm{AN}\end{array}$ & (25.145) & 24.144 & 24.118 & & & $\frac{}{42.021}$ & 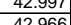 & 年. 4253 & $\frac{3.684}{30.79}$ & -1.209 & & 年14.64 & & & & 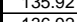 & .6558 & 41.9 & & & & & .126 & & 3.161 & \\
\hline 01 & $\begin{array}{l}9 / 1 / 20044 \\
9 / 72004\end{array}$ & 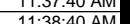 & $\frac{23.141}{25147}$ & 24.1404 & 24.075 & $\frac{25.020}{25043}$ & & 年 & (2.006 & (4.545 & 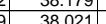 & $\frac{-1.2124}{-1206}$ & & 4971 & & 0 & & 年 & 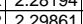 & (4t.6) & & & $\frac{11}{76}$ & & 然120 & 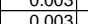 & 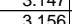 & \\
\hline 92 & 9/7/2004 & $11: 39: 40 \mathrm{AM}$ & 25.133 & 24.104 & 24.676 & 25.099 & & $\begin{array}{l}42.006 \\
42.077 \\
\end{array}$ & $4 \frac{42.900}{43.155}$ & $\frac{4.775}{4.746}$ & 㐌0.021 & $\begin{array}{l}-1.200 \\
-1.212 \\
\end{array}$ & & $\begin{array}{l}4.9511 \\
46.934\end{array}$ & & 0.070 & 0.004 & 1389 & $\frac{2.25001}{2.31528}$ & $\begin{array}{ll}41.0 \\
42.0 \\
\end{array}$ & $\frac{11.1}{11.2}$ & $\frac{40 .}{39}$ & 75 & $\frac{.120}{126}$ & 0.128 & 003 & $\frac{0.100}{3.198}$ & \\
\hline 93 & $9 / 7 / 2004$ & 11:40:40 AM & 25.118 & 24.164 & 24.666 & 25.109 & 24 & 41.972 & 42.802 & 4.587 & 37.902 & -1.215 & & 45.549 & 0.003 & 0.087 & $\frac{0.005}{0.005}$ & 139.92 & $\frac{2.3524}{23394}$ & 41.7 & 10.8 & $\frac{25.6}{39.5}$ & . 2.754 & . & $\frac{0.128}{0.128}$ & $\frac{0.003}{0.003}$ & $\frac{2.250}{3.210}$ & \\
\hline 94 & 9/7/2004 & $11: 41: 40 \mathrm{AM}$ & 25.114 & 24.176 & 24.677 & 25.161 & & 41.966 & & 4.632 & & -1.209 & & 45.204 & & & & & 2.34861 & $\begin{array}{lll}41.7 \\
\end{array}$ & & & & & & & & \\
\hline 95 & 7/2004 & $11: 42$ & 25.106 & 24.172 & 24.659 & 25.117 & & 42.03 & 42.831 & 4.644 & 37.891 & $\begin{array}{ll}-1.212 \\
\end{array}$ & & 51.037 & 003 & 0.894 & & 141.92 & 2.36528 & 41.7 & 12.2 & 40 & 75 & .128 & 0.129 & 0.003 & 3.234 & \\
\hline 96 & & $11: 43: 40 \mathrm{~A}$ & & & & & & 41.943 & \begin{tabular}{|l|l|l|l|}
42.848 \\
\end{tabular} & $\begin{array}{ll}4.629 \\
\end{array}$ & & -1.212 & & 43.84 & & 0.9 & & & 2.38194 & $\begin{array}{lll}41.7 \\
\end{array}$ & & & & & 130 & & & \\
\hline & & 11:44:40 & 25.108 & 24.194 & 24.676 & 25.149 & & 41.744 & 42.676 & 4.604 & 37.592 & -1.212 & & & & & & 143 & 2.39861 & \begin{tabular}{ll|l}
41.6 \\
\end{tabular} & & & & & & & & \\
\hline \begin{tabular}{|c|c|} 
\\
\end{tabular} & & 11:45:40 AM & 25.109 & 24.211 & 24.672 & 25.196 & & 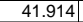 & 42.966 & 4.633 & 37.692 & -1.209 & & 43.846 & & 909 & & & & \begin{tabular}{ll|l}
41.8 \\
\end{tabular} & & & & & . .131 & 0.003 & 3.300 & \\
\hline & 9/7/2004 & 11:46:40 AM & 25.105 & 24.216 & 24.653 & 25.211 & 24. & 41.681 & 42.514 & 4.54 & 37.653 & -1.209 & & 45.766 & & 0.911 & 0.004 & $145.92 \mathrm{r}$ & 2.43194 & 41.4 & 10.9 & 39. & $2.735^{4}$ & 0.130 & 0.132 & & 3.320 & \\
\hline & 004 & 11:47:40 AM & 25.102 & 24.2233 & 24.655 & 25.208 & 24. & 41.963 & 43.047 & 4.561 & $37.848 \mid$ & -1.212 & & 45.572 & & 0.918 & 0.00 & 146 & 2.44861 & 41.99 & 9 & & & 131 & 133 & & & \\
\hline & $9 / 7 / 20044$ & $11: 48: 40$ & 25.102 & 24.2499 & 24.676 & 25.244 & & 42.073 & & 4.7122 & 37.834 & -1.2099 & & 46.548 & & & & 147 & & 41.9 & & & & & & & 334 & \\
\hline & 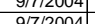 & $\frac{11}{11}$ & $\frac{25.08}{2508}$ & 2.2435 & 24.652 & $\frac{25.205}{25.236}$ & & $\begin{array}{ll}41.756 \\
42.212\end{array}$ & 42.79 & 4.5 & $\begin{array}{ll}37.608 \\
38.117\end{array}$ & $\frac{-1.215}{1.212}$ & & & & 0.924 & & & & 41.7 & & & & & 0.134 & & 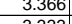 & \\
\hline 10 & & & 25084 & & & 年5.250 & & 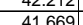 & 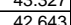 & & & & & & & & & & & & & & & & & & & \\
\hline & 9/7/2004 & 11:52:40 AM & 25.088 & 24265 & 24641 & 25.32 & 24 & 4 & 4304 & 4645 & 37.8 & $\mid$\begin{tabular}{|c|}
$\mid-1.212$ \\
\end{tabular} & & 45567 & & & & 151 & 253194 & 41.9 & 105 & 39 & & \begin{tabular}{|l|}
0.135 \\
0.134
\end{tabular} & 0.136 & & $\begin{array}{ll}3.390 \\
3396\end{array}$ & \\
\hline & $9 / 7 / 2 / 2$ & 11:53:40 AM & 25.084 & 24.271 & 24.632 & 25.316 & & 41.795 & $\begin{array}{ll}42.688 \\
\end{array}$ & 4.54 & 37.791 & \begin{tabular}{|c|}
-1.215 \\
\end{tabular} & & 444.756 & & 0.939 & & 152.92 & 2.5 & 41.6 & $\frac{10.7}{10.7}$ & 39 & & $\frac{134}{134}$ & 0.136 & 0.003 & 3.414 & \\
\hline 10 & & $11: 54$ & 25.08 & 24.282 & 24.628 & 25.322 & & 41.93 & 43.01 & 4.632 & 37.787 & -1.2 & & 45. & & & & & & \begin{tabular}{|c|c|}
41.9 \\
\end{tabular} & & & & & & & & \\
\hline & |2004 & & 25.086 & 24.298 & 24.644 & 25.378 & & 42.107 & 43.119 & 4.588 & 37.977 & \begin{tabular}{|l|l|} 
& -1.212 \\
\end{tabular} & & 47. & & & & 154. & 2.58194 & 42.0 & 0 & & & 136 & .1.138 & & & \\
\hline & & & $25.098 \mathrm{~g}$ & 24.315 & 24.667 & 25.405 & & 41 & th & 4.6 & & & & & & & & & & & & & & & & & & \\
\hline & & & & & & & & 41.957 & 42.902 & & & & & & & & & & & & & & & & & & & \\
\hline & $\begin{array}{l}9 / 71 / 2004 \\
9 / 7 / 2004\end{array}$ & 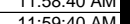 & 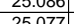 & 24.318 & 24.644 & 255.418 & & 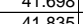 & 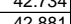 & & $\begin{array}{ll}37.383 \\
37807\end{array}$ & $\frac{-1.212}{1.200}$ & & & & & & 15. & 2.63194 & 年14.60 & ne & & & & & & $\begin{array}{l}3.506 \\
3.905 \\
\end{array}$ & \\
\hline 113 & $\begin{array}{l}9 / 1 / 20044 \\
9 / 72004\end{array}$ & 12.00 & 25.067 & 24.314 & 24626 & $\begin{array}{l}25.009 \\
25.349\end{array}$ & & & $\begin{array}{l}42.001 \\
43.024\end{array}$ & & 37. & \begin{tabular}{|l|}
-1.209 \\
-1212 \\
\end{tabular} & & $\frac{45 .}{45 .}$ & & & & & & (4t. & $108,-3$ & & & 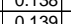 & 0140 & & 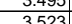 & \\
\hline & & $12: 01$ & 25.063 & & 24616 & 25.31 & & & 年 & & & -1.212 & & & & & & & & 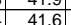 & & & & & & & 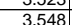 & \\
\hline & 9/7/2004 & $12: 02: 4$ & 25.047 & 24.299 & 24.601 & 25.259 & & $41.8, \quad 3$ & 43.032 & & 37.62 & \begin{tabular}{|l|l|}
-1.212 \\
\end{tabular} & & 51.679 & & & & & & & & & & & & & 3.549 & \\
\hline & & & 25.052 & & & & & & & & & & & & & & & & & & & & & & & & & \\
\hline & & 12:04: & 25.062 & 24.32 & 24.601 & 25.254 & & $\begin{array}{lll}41.783 \\
\end{array}$ & 42.848 & 4.554 & 37.791 & -1.212 & & 46.9 & & & & & & 41.7 & 11.2 & & & 0.140 & 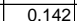 & & 3.563 & \\
\hline & & & 25.06 & 24.32 & 24. & 25.2 & & & 42 & & & $-1.2 .2>3$ & & 46. & & & & & & 41 & & & & & & & & \\
\hline & $9 / 7 / 2004$ & $12: 06$ & 25.062 & 24.33 & 24.606 & 25.2. & & & 42.7 & & 37.508 & $\mid-1.21$ & & 45. & & & & & & & 10 & & & 141 & & & 599 & \\
\hline & $9 / 7 / 2004$ & 12:07 & 25.05 & 24.3 & 24. & 25.174 & & 41. & 42.6 & & & -1.2. & & & & & & & & & & & & & & & & \\
\hline & 9/7/2 & $12: 08$ & 25.06 & 24.3 & 24. & 25.206 & & 41. & 42.6 & & & & & & & & & & & & & & & & & & & \\
\hline & & & 25.059 & 24.346 & 24.612 & 25.221 & & 41. & 42.923 & 4.4 & & & & & & & & & & 41.8 & 11.9 & & & & & & 16 & \\
\hline & $9 / 7 / 22$ & & 25.05 & 24.352 & & 25.226 & & & 42.8 & 4. & & & & & & & & & & & & & & & & & & \\
\hline & & & 25.061 & 24.353 & 24.5999 & 25.253 & & & & 4.6 & & & & & & & & & & & & & & & & & & \\
\hline & (9/7/2004 & & 255047 & $\frac{24.359}{2437}$ & 24.59 & 25.253 & & $\begin{array}{ll}41.806 \\
1.625\end{array}$ & 42.979 & $\frac{4.522}{4.59}$ & $\begin{array}{l}37.748 \\
37.95\end{array}$ & $\begin{array}{l}-1.209 \\
-1.209\end{array}$ & & 46.4 & & & & & & 41 & & & & & & & & \\
\hline & 年 & & 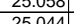 & $\frac{24.31}{24.32}$ & 24.000 & 253.3 & & & $42, .035$ & 4. & & $\frac{-1.21}{1.12}>0$ & & & & & & & & & & & & & & & & \\
\hline$\frac{151}{128}$ & 9/7/7/2004 & \begin{tabular}{|l|}
$12: 15: 40 \mathrm{PM}$ \\
\end{tabular} & 25.055 & (24.5064 & $\frac{24.5}{246}$ & 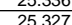 & & $\begin{array}{l}41.920 \\
41.891\end{array}$ & $\frac{45.1}{430}$ & $\frac{4.4}{45}-3$ & 337.955 & $\frac{-1.4}{-121}$ & & & & & & & & & & & & & & & & \\
\hline & 9/7/2004 & 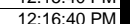 & $\frac{2.0005}{25045}$ & 24.372 & $\frac{2.45}{24.5}$ & 25317 & & 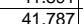 & 43.043 & $\frac{7.46}{46}-2$ & $\frac{37}{37}$ & $|-1.215|$ & & 43. & & & & & & & & & & & & & 3706 & \\
\hline & & & & & & 25.338 & & 41.661 & & & & & & & & & & & & & & & & & & & & \\
\hline 1131 & & & 25.0 & & & 25.3 & & 42.2 & 43.5 & & & & & & & & & & & 42.4 & & & & & & & 3.726 & \\
\hline & & & 25.046 & 24.34 & 24.6 & & & $\begin{array}{lll}41.974 \\
\end{array}$ & 43.354 & 4.7 - $>3$ & & & & & & & & & & 42.2 & & & & & & & & \\
\hline |133 & $9 / 7 / 2004$ & $12: 2$ & 25.062 & 24.409 & 24.635 & 25.40 & & 42.098 & 43.3 & 4.8 & 37. & $-1.2 .2>>3$ & & 45.6 & & & & & & 4 & & & & & & & & \\
\hline & $9 / 7 / 20$ & $12: 211$ & 25. & $24.44-5>$ & 24 & 25.414 & & 42.09 & 43.329 & 4 & 37.64 & -1.21 & & & & & & 180 & & & & & & & & & & \\
\hline & 9/7/2004 & & 25. & 24.426 & 24.6 & 25.461 & & 41.968 & 43.117 & 4.6 & 37. & & & & & 1. & & & & & & & & & & & & \\
\hline 36 & & & 25. & 24.4. & 24. & 25.501 & & & 43.2 & 4.74 & & & & & & & & & & & & & & & & & & \\
\hline & 9/7/2004 & & 25.11 & 24.442 & 24.668 & 25.52 & & 42.306 & 43.548 & 4.81 & 37.844 & -1.215 & & 47.7 & 0.003 & 1.037 & & & & 42.4 & & & $2.8 \div-1$ & 0.148 & & 0.004 & 3.739 & \\
\hline & & & 25.116 & 24. & & & & 42. & 43.4 & 4.62 & & & & & & & & & & 42.4 & & & & 49 & & & & \\
\hline 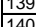 & $9 / 7 / 2004$ & \begin{tabular}{|l|l|}
12.20 .470 \\
$12.27 .40 \mathrm{PM}$
\end{tabular} & 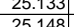 & $\begin{array}{r}24.46 \\
24.411\end{array}$ & 24.740 & 25.555 & 2458 & $\begin{array}{l}42.33 \\
4302\end{array}$ & 43.53 & $\begin{array}{l}4.639 \\
4724\end{array}$ & & -1.212 & & (45.4565 & 0.003 & 1.04 & & & 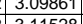 & 42.4 & & & & | 0155 & 0.153 & & $3.733 \mathrm{r}$ & \\
\hline & & & 25.148 & & & & & & & & & $\mid-1.206$ & & 44.746] & 0.003 & 1.064 & & & 3.11528 & 42.3 & & & & 0.152 & 0.153 & 0.004 & 3.820 & \\
\hline
\end{tabular}


WSRC-TR-2005-00105, REVISION 0

SRNL-RPP-2005-00012, REVISION 0

\begin{tabular}{|c|c|c|c|c|c|c|c|c|c|c|c|c|c|c|c|c|c|c|c|c|c|c|c|}
\hline \multicolumn{24}{|c|}{. Uivi. } \\
\hline & \begin{tabular}{c|c} 
A & \\
$9 / 7 / 2004$ & 1 \\
\end{tabular} & $\frac{\mathrm{B}}{12: 28: 40 \mathrm{PM}}$ & & & \begin{tabular}{c|c}
$G$ \\
25.507 \\
2507
\end{tabular} & & $\frac{\mathrm{J}}{41.99}$ & \begin{tabular}{|c|}
$K$ \\
43.466 \\
\end{tabular} & $\begin{array}{ll}\mathrm{L} \\
4.64 \\
\end{array}$ & \begin{tabular}{l|l|}
$\mathrm{M}$ \\
38.033 \\
\end{tabular} & $\begin{array}{ll}-1.212 \\
-12\end{array}$ & \begin{tabular}{|l|}
0 \\
0.637 \\
\end{tabular} & \begin{tabular}{|c|}
$Q$ \\
46.954 \\
\end{tabular} & $\begin{array}{l}\mathrm{R} \\
0.003 \\
\end{array}$ & $\begin{array}{ll}3 & s \\
3 & 1.64 \\
\end{array}$ & \begin{tabular}{l|l}
649 & $T .004$ \\
649
\end{tabular} & \begin{tabular}{|c|c|}
$V$ & $W$ \\
187.92 & 3.13194 \\
\end{tabular} & \begin{tabular}{|c|c|c}
$X$ & $Y$ \\
42.3 & 11.2 & 40.0 \\
\end{tabular} & \begin{tabular}{|l|l|}
$A A$ & $A B$ \\
0.759 & 0.236 \\
\end{tabular} & & \begin{tabular}{ll|}
$\mathrm{AD}$ & 0.006 \\
0.00
\end{tabular} & $\begin{array}{ll}\mathrm{AE} \\
5.945 \\
\end{array}$ & \begin{tabular}{|l|l|} 
AF \\
5.06 \\
\end{tabular} \\
\hline & 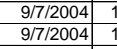 & $\begin{array}{l}\text { 12:292:40 PM } \\
\text { 12:30:40 PM }\end{array}$ & 25.165 & 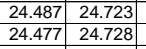 & $\begin{array}{l}25.477 \\
25.427 \\
\end{array}$ & 24.4255 & $\begin{array}{l}42.096 \\
4.912 \\
4.912\end{array}$ & 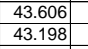 & $\begin{array}{l}4.733 \\
4.677 \\
4\end{array}$ & $\begin{array}{l}37.817 \\
3.863 \\
\end{array}$ & $\begin{array}{l}-\frac{-1.212}{-1.212} \\
\end{array}$ & \begin{tabular}{|l}
0.631 \\
0.634 \\
\end{tabular} & $\frac{46.81}{46.186}$ & $\begin{array}{l}0.003 \\
0.003 \\
\end{array}$ & $\begin{array}{ll}3 & 1.05 \\
3 & 1.04 \\
\end{array}$ & $\begin{array}{l}0.004 \\
0.005 \\
\end{array}$ & 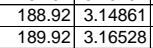 & $\frac{42.5}{42.1}$ & $\frac{2.755}{2.750} 0.151$ & 152 & $\begin{array}{l}0.004 \\
0.004 \\
0\end{array}$ & $\frac{3.799}{3.776}$ & $\frac{3.23}{3.21}$ \\
\hline & \begin{tabular}{l|l}
$9 / 7 / 2004$ & 1 \\
$9 / 7 / 2004$ & 1 \\
\end{tabular} & $\begin{array}{l}\frac{12: 31: 40 \mathrm{PM}}{12: 32: 40 \mathrm{PM}} \\
\end{array}$ & $\begin{array}{l}25.165 \\
25.169\end{array}$ & $\begin{array}{l}24.482 \\
24.482 \\
24.733 \\
24.753\end{array}$ & $\begin{array}{l}25.412 \\
25.381\end{array}$ & $\begin{array}{l}24.415 \\
24.349 \\
\end{array}$ & $\begin{array}{l}42.339 \\
42.092\end{array}$ & \begin{tabular}{|l|}
43.72 \\
43.459 \\
\end{tabular} & $\begin{array}{l}4.884 \\
4.758 \\
4\end{array}$ & $\begin{array}{l}38.002 \\
37.941 \\
\end{array}$ & $\begin{array}{l}-1.215 \\
-1.209 \\
\end{array}$ & \begin{tabular}{|l|}
0.628 \\
0.634 \\
\end{tabular} & $\begin{array}{l}44.773 \\
47.705 \\
\end{array}$ & $\begin{array}{l}.003 \\
0.003 \\
\end{array}$ & $\begin{array}{ll}3 & 1.044 \\
3 & 1.04 \\
\end{array}$ & $\begin{array}{l}0.005 \\
0.005 \\
\end{array}$ & 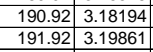 & $\begin{array}{l}42.6 \\
42.3 \\
\end{array}$ & $\begin{array}{ll}2.770 & 0.150 \\
2.759 & 0.149 \\
\end{array}$ & \begin{tabular}{|c|}
151 \\
151 \\
\end{tabular} & $\begin{array}{l}0.004 \\
0.004\end{array}$ & $\begin{array}{l}3.752 \\
3.761 \\
\end{array}$ & $\begin{array}{l}3.19 \\
3.20 \\
\end{array}$ \\
\hline & \begin{tabular}{l|l|l}
$9 / 7 / 2004$ & 1 \\
$9 / 7 / 2004$ & 1
\end{tabular} & $\begin{array}{l}12: 33: 40 \mathrm{PM} \\
12: 3440 \mathrm{PM}\end{array}$ & $\begin{array}{l}25.173 \\
25.178\end{array}$ & $\begin{array}{l}24.486 \\
244.732 \\
224.48 \\
24.731\end{array}$ & $\begin{array}{l}25.335 \\
25.325\end{array}$ & $\begin{array}{l}24.423 \\
224.443 \\
\end{array}$ & $\begin{array}{l}42.019 \\
42.073\end{array}$ & \begin{tabular}{|l|l|}
43.327 \\
43.434 \\
\end{tabular} & $\begin{array}{l}4.749 \\
4.726 \\
\end{array}$ & \begin{tabular}{|l|l|}
37.846 \\
37.91
\end{tabular} & $\begin{array}{l}-\frac{-1.212}{-1.212} \\
\end{array}$ & \begin{tabular}{|l|l}
0.634 \\
0.637
\end{tabular} & $\begin{array}{l}44.994 \\
46.821\end{array}$ & $\begin{array}{l}0.003 \\
0.03 \\
\end{array}$ & $\begin{array}{ll}3 & 1.044 \\
3 & 1.04 \\
3\end{array}$ & \begin{tabular}{|l|}
0.007 \\
0.005 \\
\end{tabular} & $\begin{array}{l}923.21526 \\
922.23196\end{array}$ & & 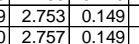 & 50 & $\begin{array}{l}0.004 \\
0.004 \\
\end{array}$ & $\begin{array}{l}3.764 \\
3.759 \\
\end{array}$ & $\begin{array}{l}3.20 \\
3.20 \\
\end{array}$ \\
\hline & \begin{tabular}{ll|l}
$9 / 72004$ & 1 \\
0.72004 & 1
\end{tabular} & 12:35:40 PM & & \begin{tabular}{r|r|}
24.48 & 24.75 \\
\end{tabular} & & & & 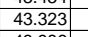 & 4.728 & & -1.212 & & 47.009 & 0.003 & , & 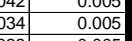 & 3.2486 & & 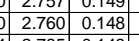 & & & & $\frac{3.26}{3.17}$ \\
\hline & $\begin{array}{ll}99 / 7 / 2004 & 1 \\
9 / 7 / 2004 & 1\end{array}$ & $\begin{array}{l}12: 36640 \mathrm{PM} \\
21237.40 \mathrm{PM}\end{array}$ & & & $\begin{array}{l}255384 \\
22535\end{array}$ & $\begin{aligned} 24.532 \\
22.563\end{aligned}$ & $\begin{array}{l}42.566 \\
42965\end{array}$ & \begin{tabular}{|l|l|}
43.986 \\
33271
\end{tabular} & $\begin{array}{l}4.818 \\
4.704\end{array}$ & $\begin{array}{l}33.2655 \\
3.762\end{array}$ & & & $\begin{array}{l}45.957 ? \\
48033\end{array}$ & $\begin{array}{l}0.003 \\
0.003\end{array}$ & & $\begin{array}{l}0.005 \\
0.006 \\
0.0\end{array}$ & 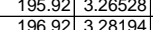 & & $\frac{2.775}{2788}$ & & & 685 & 3.14 \\
\hline & \begin{tabular}{|l|l|l|l|l|l|l|l|}
$9 / 7004$ & 1
\end{tabular} & 12:38:40 PM & & & 25.421 & & & | & 4.636 & & & & 48.254 & & \begin{tabular}{|l|l|}
3 & 1.01 \\
\end{tabular} & 0.006 & & $\begin{array}{l}11.5 \\
11.5 \\
40.0 \\
\end{array}$ & 0 & $\mid \begin{array}{l}0.1184 \\
0.147 \\
\end{array}$ & o. 0.004 & $\begin{array}{l}3.707 \\
3.663\end{array}$ & \begin{tabular}{|l|l|}
3.15 \\
.12 \\
\end{tabular} \\
\hline & $\begin{array}{l}/ 7 / 2004 \\
\end{array}$ & $2: 07: 06 \mathrm{PM}$ & 3.303 & \begin{tabular}{|l|l|}
24.614 & 23.104 \\
\end{tabular} & 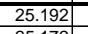 & 24.55 & 41.754 & \begin{tabular}{|l|l|l|l|l|}
43.642 \\
\end{tabular} & 4.779 & $\begin{array}{l}77.518 \\
7018 \\
\end{array}$ & $\begin{array}{ll}-1.212 \\
-1.200\end{array}$ & $\begin{array}{r}-4.856 \\
\end{array}$ & 45.023 & 0.003 & & 0.003 & 554.772 & $\begin{array}{l}40.739 .6 \\
10.739 .6\end{array}$ & $\begin{array}{l}6.7330 .171 \\
6.730 .171\end{array}$ & 0.180 & 0.005 & 4.539 & 3.86 \\
\hline & $\frac{97 / 1 / 2004}{97 / 2004}$ & $\begin{array}{l}2: 08: 00 \mathrm{PM} \\
20.0906 \mathrm{PM}\end{array}$ & & $\begin{array}{l}24.608 \\
24614 \\
24323396 \\
23296\end{array}$ & $\begin{array}{l}25.172 \\
25353\end{array}$ & $\begin{array}{r}24.43 \\
24.446 \\
\end{array}$ & $\frac{41.972}{3.21}$ & \begin{tabular}{|l|r|}
43.58 \\
441199
\end{tabular} & $\begin{array}{l}4.773 \\
49044\end{array}$ & \begin{tabular}{|l|l|}
37.881 \\
-1241 \\
\end{tabular} & 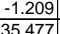 & $\begin{array}{l}-4.856 \\
2026\end{array}$ & $\begin{array}{l}45.342 \\
44.939\end{array}$ & $\begin{array}{l}0.003 \\
0.03 \\
\end{array}$ & \begin{tabular}{|c|c|}
3 & 1.18 \\
3 & -0.01 \\
\end{tabular} & 0.003 & & & 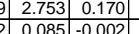 & 1.178 & 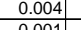 & . & \begin{tabular}{|l|l|}
.80 \\
1.23
\end{tabular} \\
\hline & & & & & & & & & & & & & & & & & & & & & & & \\
\hline & & 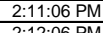 & & & 5.1177 & & & $\begin{array}{l}44.9525 \\
33355 \\
\end{array}$ & $\begin{array}{l}4.677 \\
4816\end{array}$ & $\begin{array}{l}-8.309 \\
34524\end{array}$ & & & $\frac{44.3}{473}$ & 0.003 & $\frac{.011}{12}$ & $\frac{1.75}{0.03}$ & 8391 & $\frac{-5 .}{26}$ & 404 & & 些00 & $\frac{0.305}{9612}$ & 0.26 \\
\hline & & & . .034 & & & & & 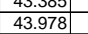 & 4.801 & $\begin{array}{l}34.524 \\
37.161 \\
\end{array}$ & & & $\begin{array}{l}4.3 .32 \\
46.131\end{array}$ & $\frac{0.003}{0.003}$ & 1.54 & 543 & & & & & & & \\
\hline & & & & & & & & 44.28 & & & & & & & & & & & & & & & \\
\hline & $\frac{97 / 212044}{972004}$ & $\begin{array}{l}\frac{11506 \mathrm{PM}}{1066 \mathrm{PM}} \\
\end{array}$ & & $\begin{array}{l}\frac{24.6292}{24.0332} \\
244636 \\
244144\end{array}$ & 5.172 & & $\frac{42.768}{42864}$ & $\frac{45.265}{45.518}$ & $\begin{array}{l}4.488 \\
4585\end{array}$ & & & & $\frac{46.9}{44.78}$ & 0 & $\frac{4.485}{46^{2}}$ & 4899| & & $40 .{ }_{40.5}$ & & & & 5.359 & 4.5 .5 \\
\hline & & 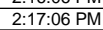 & & $\begin{array}{l}1 \\
1124 \\
1124\end{array}$ & 5 & & & & 4.564 & & & & 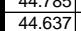 & & & 势 & & 等4 & & & & 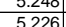 & \\
\hline & & 1:06 PM & & & & & & 45.25 & 4.586 & & & & & & & 454 & & 44. & & & & & \\
\hline & & $1: 190.06 \mathrm{PM}$ & & $\begin{array}{ll}4.401 \\
4.52\end{array}$ & 5.276] & 1. & $\frac{43 .}{4286}$ & 45.574 & $\frac{4.615}{4606}$ & $\frac{39.164}{385252}$ & & & $\frac{45.317}{44969}$ & 0.0003 & $\frac{4.45}{44}$ & 453 & 4.972. & & & & & .5.144 & 4.36 \\
\hline & $9 / 7 / 2004$ & 2:21:06 PM & & & 25.382 & 24.789 & 42.857 & & 4.514 & 39.063 & -1.21 & & 44.672 & 0. & 1.44 & & & 44. & & & & & \\
\hline & & & & & & & & 45.698 & & & & & 455.09 & & & & & 44.6 & & & & 5.041 & \\
\hline & & $24: 06 \mathrm{PM}$ & & & 5448 & & $\begin{array}{l}42.677 \\
42323\end{array}$ & $\begin{array}{l}455196 \\
44307\end{array}$ & $\begin{array}{l}4.757 \\
4.757\end{array}$ & 38.632 & $\begin{array}{l}1.221 \\
1.212\end{array}$ & $\begin{array}{l}-1.17 \\
-1.18\end{array}$ & 48.9434 & 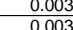 & $\frac{1.41}{1.39}$ & 418 & 50 & 40.7 & 2750 & & & & 4.28 \\
\hline & 9/7/2004 & 25:06 PI & & & 5.479 & & 41.8 & 44.125 & 4.622 & & & -1.176 & 46.0. & 0 & .39: & & & & & & & & \\
\hline & & & & & & & & 44.456 & & & & & 45.968 & 0 & .4:40: & 403 & & 10. & & & & & \\
\hline & & $28.06 \mathrm{PD}$ & & & 5 & & 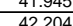 & $\begin{array}{l}4.4206 \\
4466\end{array}$ & 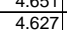 & & & & 45.549 & & 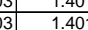 & 401 & & & & & & & \\
\hline & & & & & & & & 44.129 & & & & & & & $\sqrt{3}$ & 389 & & & & & & & \\
\hline & & :30:06 & & & 25.407 & & & 44.148 & & & & & & & & & & & & & & .980 & $\frac{4.2}{4.2}$ \\
\hline & $\frac{97 / 72004}{997204}$ & 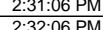 & & 5.0.038 & 25.4.4212 & & 41.6 & 44.266 & 4.754 & 37.8 & & & 46.59 & 0.003 & (3. & 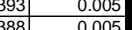 & 5.100 & & & & & 1.990 & \\
\hline & & & & & & & & & & & & & & & & & & & & & & & \\
\hline & 9 & 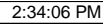 & & & $\frac{5.30}{5.30}$ & & & $\begin{array}{l}44.558 \\
\end{array}$ & $\begin{array}{ll}4.806 \\
\end{array}$ & & & & & & 1.398 & 398 & & & & & & & $\frac{3.2}{4.2}$ \\
\hline & 97/7/2004] & $\begin{array}{l}3: 35: 06 \mathrm{PN} \\
306 \mathrm{PM}\end{array}$ & & th & . 2.566 & & $\begin{array}{l}42.078 \\
4.276\end{array}$ & $\begin{array}{l}44.547 \\
44579\end{array}$ & $\begin{array}{l}4.707 \\
4.907 \\
-1\end{array}$ & 37.9. & & & 45.65 & 0.0033 & . 40.0 & 望3 & 5 & & & & & 9998 & 4.2. \\
\hline & & & & & | & & 年 & & & & & & & & 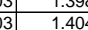 & 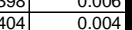 & & & & & & & \\
\hline & $97 / 1 / 200$ & :38:06 PN & & 24.7. & 25.39 & & 42. & 44.529 & 4.676 & 38.0 & & & 45.23. & 0.6 & 1.2 & 1.4 & 52091 & & & & & .966 & $\frac{4.2}{4.2}$ \\
\hline & & 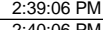 & & & 5.431 & & 42 & 44.458 & $\begin{array}{l}4.614 \\
4.612\end{array}$ & & & & & & 4.40 & 407 & & & & & & 990 & \\
\hline & & $\begin{array}{ll}\mathrm{PN} \\
\mathrm{PN}\end{array}$ & & & & & 42 42 & & 年.1.13 & & & & & & & & & & & & & & \\
\hline & & & & & 5.5 & & 42. & & 4.621 & & & & 45.934 & & .40. & 404 & & & & & & 962 & \\
\hline & & $: 43: 06$ & & 2.7 .7 & 5.5 & & $\frac{42.1}{42.12}$ & 44.326] & 4.62 & & & & & & 4.40 & 整1 & & & & & & 337 & 4.2 \\
\hline & & & & & & & & & & & & & & & & & & & & & & & \\
\hline & & :46:06 PN & & & 5 & & 41. & 44.021 & 4.574 & & & & & & 1.4 & 1.4 & & & & & & $\begin{array}{l}9047 \\
9.477\end{array}$ & \\
\hline & & :47:06 & & & 5.59 & & 42.1 & 44. & 4.70 & & & & & & .399. & 399 & & & & & & 227 & 4. \\
\hline & & & & & & & & & & & & & & & & & & & & & & & \\
\hline & & & & \begin{tabular}{ll|l}
4.834 & 25.44
\end{tabular} & 5602 & & 42.03 & \begin{tabular}{|l|l|}
44.288 \\
\end{tabular} & 4.6966 & & & & & & 1. & 1.4 & & & & & & 951 & \\
\hline$\frac{19}{10}$ & $9 / 7 / 120$ & 15:06 & & 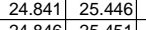 & 5.604 & & $\begin{array}{ll}42.177 \\
4.75\end{array}$ & 44.612 & 4.704 & 38.1 & & & 46. & & 1. & 1.44 & & & & & & .926 & \\
\hline & & & & & & & . & & & & & & & & & & & & & & & & \\
\hline & & 54.0 & & & & & 42.1 & & 4.6 & & & & & & $1.39 !$ & 399 & & & & & & & \\
\hline & & & & & & & $\frac{42.05}{42.17}$ & & 4. & 38. & & & & & 1. & 1.4 .4 & & & & & & 938 & \\
\hline & & & $585 \mathrm{I}$ & 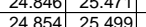 & & & $\begin{array}{lll}42.1 \\
420\end{array}$ & & $\frac{4}{46}$ & & & & & & & & & & & & & & \\
\hline & & & & & & & & & & & & & & & & $\frac{405}{405}$ & & & & & & & \\
\hline & & $\mathrm{PM}$ & & 要677 25. & 25.45 & 24.848 & 42.55 & & $4.77^{4}$ & 38.4 & & & & & 1. & 1.4 & & & & & & 4.878 & \\
\hline & & & $.887 \mathrm{I}$ & & 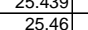 & & 42.227 & 44,496 & 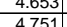 & 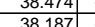 & & & & & . & $\frac{41}{402}$ & & & & & & & \\
\hline & & & & & & & & & & & & & & & & $\frac{{ }^{40}}{2}$ & & & & & & & \\
\hline & & 103:06 PN & & & & & 411.926 & & \begin{tabular}{|l|l|l|l|l|}
4.678 \\
\end{tabular} & 38.04 & & & & & . 40. & 404 & & & & & & 4.961 & 4. \\
\hline & & & & & & & & & & & & & & & & & & & & & & & \\
\hline
\end{tabular}


WSRC-TR-2005-00105, REVISION 0

SRNL-RPP-2005-00012, REVISION 0

RUN \# 4.18 AND 4.19; ACID CLEANING AND CAUSTIC RINSING - CONT.

\begin{tabular}{|c|c|c|c|c|c|c|c|c|c|c|c|c|c|c|c|c|c|c|c|c|c|c|c|c|c|c|c|c|}
\hline & A & B & D & $E$ & $F$ & G & $\mathrm{H}$ & $\mathrm{J}$ & $\mathrm{K}$ & $\mathrm{L}$ & $M$ & $\mathrm{~N}$ & 0 & Q & $\mathrm{R}$ & $\mathrm{s}$ & $\mathrm{T}$ & $\mathrm{V}$ & w & $x$ & $\mathrm{Y}$ & $z$ & AA & $A B$ & $A C$ & $A D$ & $\mathrm{AE}$ & AF $A$ \\
\hline 2 & 9/7/2004 & 3:06:06 PM & 25.805 & $24.91 \mathrm{~s}$ & 25.374 & 25.608 & \begin{tabular}{|l|} 
\\
\end{tabular} & 42.22 & 44.62 & 4.657 & 38.269 & \begin{tabular}{|l|}
-1.212 \\
\end{tabular} & -1.025 & 46.108 & 0.003 & 1.403 & 0.003 & 345.35 & 5.75583 & 43.5 & 11.0 & 40. & & 0.201 & 0.199 & 0.005 & 4.939 & 4.20 \\
\hline$\frac{211}{211}$ & $\begin{array}{l}9 / 7 / 2004 \\
9 / 7 / 2004\end{array}$ & $\begin{array}{l}\text { 3:07:06 PM } \\
\text { 3:08:06 PM }\end{array}$ & $\frac{25.775}{25.736}$ & $\begin{array}{l}24.919 \\
24.925 \\
\end{array}$ & \begin{tabular}{r|r}
25.329 \\
25.29
\end{tabular} & $\begin{array}{l}25.612 \\
25.644 \\
\end{array}$ & \begin{tabular}{|l|}
25.005 \\
24.946
\end{tabular} & $\begin{array}{r}42.31 \\
42.206 \\
\end{array}$ & \begin{tabular}{|l|}
44.527 \\
44.597 \\
\end{tabular} & \begin{tabular}{|c|}
4.645 \\
4.777
\end{tabular} & $\begin{array}{l}38.472 \\
38.049 \\
\end{array}$ & \begin{tabular}{|c|}
-1.206 \\
-1.212 \\
\end{tabular} & & $\begin{array}{l}45.782 \\
45936\end{array}$ & $\begin{array}{l}0.003 \\
0.003\end{array}$ & $\begin{array}{l}1.402 \\
1.396\end{array}$ & $\begin{array}{l}0.004 \\
0.006\end{array}$ & \begin{tabular}{|l|}
346.35 \\
34.35
\end{tabular} & \begin{tabular}{|l|l|}
5.7725 \\
5.789817
\end{tabular} & \begin{tabular}{|l|l}
43.4 \\
43.5
\end{tabular} & $\frac{10.9}{10.9}$ & \begin{tabular}{|l|l}
40.4 \\
40.1
\end{tabular} & 2767 & 0.200 & \begin{tabular}{|c|}
0.199 \\
0.198
\end{tabular} & $\begin{array}{l}0.005 \\
0.005\end{array}$ & $\begin{array}{l}4.924 \\
4.940\end{array}$ & $\begin{array}{l}4.19 \\
420\end{array}$ \\
\hline$\frac{21}{21}$ & $\begin{array}{l}91 / 2004 \\
9 / 7 / 2004\end{array}$ & 3:00:06 PM & $\frac{25.156}{25.702}$ & $\frac{24.925}{24.921}$ & $\begin{array}{l}25.29 \\
25.26\end{array}$ & 25.644 & $\begin{array}{r}24.946 \\
24.962\end{array}$ & $\begin{array}{l}42.206 \\
42.185 \\
\end{array}$ & \begin{tabular}{|l|}
44.59 \\
4.531 \\
\end{tabular} & $\begin{array}{l}.188 \\
4.643 \\
\end{array}$ & $\begin{array}{l}38.049 \\
38.226 \\
\end{array}$ & \begin{tabular}{|l|}
-1.212 \\
-1.212 \\
\end{tabular} & & $\begin{array}{l}45.9360 \\
44.948\end{array}$ & $\begin{array}{l}0.0003 \\
0.003\end{array}$ & $\begin{array}{r}1.359 \\
1.4 \\
\end{array}$ & & $\begin{array}{l}344.35 \\
348.35 \\
\end{array}$ & \begin{tabular}{|l|l|}
5.18911 \\
5.80583
\end{tabular} & $\begin{array}{l}43.5 \\
43.4 \\
\end{array}$ & & $\begin{array}{l}40.1 \\
40.2\end{array}$ & & 0.200 & $\begin{array}{l}0.1989 \\
0.199 \\
\end{array}$ & 0.005 & $\begin{array}{l}4.940 \\
4.949 \\
\end{array}$ & \\
\hline & $9 / 7 / 2004$ & 3:10:06 PM & 25.678 & 24.927 & 25.252 & 25.631 & 24.928 & 41.88 & \begin{tabular}{|l|l|}
44.129 \\
\end{tabular} & 4.61 & 37.961 & -1.212 & & 46.193 & 0.003 & $\begin{array}{r}1.399 \\
\end{array}$ & & 349.35 & \begin{tabular}{|l|l|}
5.8225 \\
\end{tabular} & 43.0 & 11.0 & 39.9 & 2.75 & 0.200 & 0.199 & 0.005 & 4.982 & 4.2 \\
\hline 216 & $9 / 7 / 2004$ & 3:11:06 PM & 25.649 & 24.933 & 25.207 & 25.611 & 24.979 & 42.127 & \begin{tabular}{|l|l|}
44.508 \\
\end{tabular} & 4.696 & 38.008 & \begin{tabular}{|l|l|}
-1.212 \\
\end{tabular} & & 45.811 & 0.003 & 1.398 & & 350.35 & 5 & 43.4 & \begin{tabular}{|l|l}
10.9 \\
\end{tabular} & 40.1 & 2.763 & 0.200 & 0.199 & 0.005 & & \\
\hline$\frac{217}{217}$ & 9/7/2004 & 3:12:06 PM & 25.623 & 24.932 & 25.192 & 25.641 & 24.998 & 42.185 & \begin{tabular}{|l|l|}
44.489 \\
\end{tabular} & 4.712 & $\begin{array}{l}38.187 \\
3.107\end{array}$ & \begin{tabular}{|l|}
-1.209 \\
\end{tabular} & & $\begin{array}{l}46.06 \\
\end{array}$ & 0.003 & 1.395 & & $\begin{array}{l}351.35 \\
\end{array}$ & 5.85583 & $\begin{array}{l}43.4 \\
\end{array}$ & 11.0 & 40.2 & 2.77 & 0.200 & $\begin{array}{l}0.199 \\
\end{array}$ & 0.005 & 4.943 & \\
\hline & 9/7/2004 & 3:13:06 PM & 25.598 & 24.942 & 25.162 & 25.631 & 25.013 & 42.134 & \begin{tabular}{|l|l|}
44.529 \\
\end{tabular} & $\begin{array}{ll}4.673 \\
\end{array}$ & 38.107 & \begin{tabular}{|l|l|}
-1.212 \\
\end{tabular} & & 46.06 & 0.003 & 1.4 & & 352.35 & \begin{tabular}{|l|l}
5.8725 \\
\end{tabular} & 43.4 & & 40.1 & 2.766 & 0.200 & & 0.005 & & \\
\hline$\frac{211}{221}$ & $\begin{array}{l}9 / 7 / 1 / 2004 \\
9 / 2004\end{array}$ & $\begin{array}{l}3: 14: 06 \mathrm{PM} \\
\text { 3:15:06 PM }\end{array}$ & $\frac{25.579}{25.56}$ & $\begin{array}{l}24.948 \\
24.959\end{array}$ & $\begin{array}{l}25.133 \\
25.139\end{array}$ & $\begin{array}{l}25.622 \\
25.633 \\
\end{array}$ & $\begin{aligned} 25.004 \\
25.03\end{aligned}$ & $\begin{array}{l}41.692 \\
42.65\end{array}$ & \begin{tabular}{|l|l|}
43.922 \\
44.496
\end{tabular} & \begin{tabular}{|l|}
4.579 \\
4701
\end{tabular} & \begin{tabular}{|l|l|}
37.848 \\
38.17
\end{tabular} & \begin{tabular}{|l|l|} 
& -1.215 \\
\end{tabular} & \begin{tabular}{|c|c|}
-1.017 \\
-1019
\end{tabular} & \begin{tabular}{|c|c|}
46.654 \\
45.59
\end{tabular} & \begin{tabular}{|l}
0.003 \\
0.003
\end{tabular} & $\begin{array}{l}1.397 \\
1.397\end{array}$ & & \begin{tabular}{|l|l|}
353.35 \\
35.35 \\
\end{tabular} & \begin{tabular}{|l|l|}
5.88917 \\
5
\end{tabular} & \begin{tabular}{|l|l|}
42.8 \\
434 \\
\end{tabular} & $\frac{11.1}{11.0}$ & 39.8 & $\begin{array}{l}2.742 \\
2769 \\
\end{array}$ & 0.200 & \begin{tabular}{|c|}
0.199 \\
\end{tabular} & $\begin{array}{l}0.005 \\
0.05\end{array}$ & $\begin{array}{l}5.010 \\
4960\end{array}$ & $\begin{array}{l}4.26 \\
.222 \\
\end{array}$ \\
\hline$\frac{220}{221}$ & $\begin{array}{c}9 / 7 / 2004 \\
97 / 2004\end{array}$ & $\begin{array}{l}\text { 3:11:06 PM } \\
3: 16: 06 \mathrm{PM}\end{array}$ & $\frac{25.56}{25.535}$ & 24.959 & $\begin{array}{l}25.139 \\
25.114\end{array}$ & $\begin{array}{l}25.633 \\
25.653 \\
\end{array}$ & \begin{tabular}{|l|}
25.03 \\
25.03 \\
\end{tabular} & $\begin{array}{r}42.165 \\
41.874 \\
\end{array}$ & \begin{tabular}{|l|}
44.496 \\
44.011 \\
\end{tabular} & $\mid$\begin{tabular}{|l|}
4.701 \\
4.638
\end{tabular} & $\begin{array}{r}38.17 \\
38.014 \\
\end{array}$ & \begin{tabular}{|l|l|}
-1.209 \\
-1.212 \\
\end{tabular} & $\begin{array}{r}-1.019 \\
-1.014 \\
\end{array}$ & $\begin{array}{r}45.59 \\
46.116\end{array}$ & \begin{tabular}{|l}
0.003 \\
0.003
\end{tabular} & $\begin{array}{r}1.397 \\
1.4\end{array}$ & 0.003 & \begin{tabular}{|l|}
355.35 \\
355.35
\end{tabular} & $\begin{array}{l}5.90583 \\
5.9225 \\
5\end{array}$ & \begin{tabular}{|l|}
43.4 \\
42.9 \\
\end{tabular} & $\frac{10.9}{11.0}$ & \begin{tabular}{|l|l|}
40.2 \\
39.9
\end{tabular} & \begin{tabular}{r|r|}
2.769 \\
2.754
\end{tabular} & $\begin{array}{l}0.200 \\
0.200\end{array}$ & $\begin{array}{l}0.199 \\
0.200\end{array}$ & $\begin{array}{l}0.005 \\
0.005\end{array}$ & $\begin{array}{l}4.960 \\
5.002\end{array}$ & $\begin{array}{l}4.22 \\
4.26\end{array}$ \\
\hline & $9 / 7 / 2004$ & 3:17:06 PM & 25.516 & 24.965 & & 25.673 & & & \begin{tabular}{|l|}
44.608 \\
\end{tabular} & 4.694 & & & & 46.153 & 0.003 & 1.396 & & & 5 & & 11.0 & & & & & & & $\frac{4.26}{4.21}$ \\
\hline 223 & 9/7/2004 & 3:18:06 PM & 25.501 & & \begin{tabular}{|l|l|}
25.084 \\
\end{tabular} & 25.673 & 25.046 & 42.198 & \begin{tabular}{|l|l|}
44.558 \\
\end{tabular} & 4.685 & 38.133 & \begin{tabular}{|l|}
-1.209 \\
\end{tabular} & & 45.567 & 0.003 & 1.395 & 0.004 & 357.35 & 5.95583 & $\begin{array}{r}43.4 \\
\end{array}$ & 10. & 40.2 & 2.769 & 0.200 & $\mid 0.199$ & .005 & & \\
\hline 224 & & & 25.486 & & & & & & \begin{tabular}{|l|l}
4.686 \\
\end{tabular} & & & & & 45.912 & 0.003 & 1.395 & & & 5.9725 & \begin{tabular}{l|l}
43.6 \\
\end{tabular} & & & & 0.200 & 0.199 & & 4.978 & $\frac{4.2}{4.2}$ \\
\hline & & & 25.46 & 24.975 & 25.045 & 25.674 & & 42.187 & $\begin{array}{l}44.576 \\
\end{array}$ & 4.688 & 38.199 & -1.212 & & 45.571 & 0.003 & 1.395 & & & 0.0091 & & & & & & & & 4.963 & \\
\hline & 9/7/2004 & 3:21:06 PM & 25.45 & 24.981 & & 25.675 & \begin{tabular}{|l|l|l|l|}
25.047 \\
\end{tabular} & 42.283 & \begin{tabular}{|l|l|l|}
44.707 \\
\end{tabular} & 4.765 & & & & 46.345 & 0.003 & 1.393 & & & 60058 & & & & & & & & 4.956 & \\
\hline & 9/7/2004 & 3:22:06 PM & 25.443 & 24.997 & 25.031 & 25.685 & 25.093 & 42.2 & $\begin{array}{l}44.572 \\
\end{array}$ & 4.68 & 38.15 & $\begin{array}{l}-1.209 \\
\end{array}$ & -1.01 & |46.425 & 0.003 & 1.396 & & 361.35 & $\begin{array}{l}6.0225 \\
\end{array}$ & 43.4 & 11.1 & 40.2 & 2.770 & 0.200 & 0.200 & 0.005 & 4.970 & \\
\hline & $9 / 7 / 2004$ & 3:23:06 PM & 25.438 & 25.007 & 25.027 & 25.711 & & 41.791 & 44.185 & 4.62 & 37.741 & -1.212 & & 44.871 & 0.003 & 1.396 & & 362.3. & 6.03917 & 43.1 & 10. & & & & 0.200 & & 5.022 & 4. \\
\hline 告地 & $9 / 7 / 2004$ & 3:24:06 PM & 25.418 & 25.002 & 24.992 & 25.681 & 25.108 & 41.87 & 44.164 & 4.566 & 38.025 & -1.1212 & & 45.753 & 0.003 & 1.396 & & 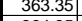 & 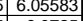 & 43.0 & & & & & & & 5.004 & \\
\hline & 9l/2/2004 & $\begin{array}{ll}3: 52: 06 \mathrm{PM} \\
\end{array}$ & 25.41 & 25.004 & & & 25.089 & 42.005 & 44.212 & 4.616 & $\begin{array}{r}38.15 \\
20.262\end{array}$ & & & 45.901 & 0.003 & 1.396 & & 364.35 & 6.0725 & 43.1 & & & & & & & 4.991 & 4.25 \\
\hline & $\begin{array}{l}9 / 1 / 20004 \\
/ 7 / 2004\end{array}$ & $\begin{array}{l}3.26 .06 \mathrm{PM} \\
3.27 .06 \mathrm{PM}\end{array}$ & 25.38 & $\frac{25.015}{25.026}$ & $\begin{array}{r}24.979 \\
24.99\end{array}$ & $\frac{25.688}{25.714}$ & $\frac{2.506}{25.056}$ & $\frac{42.339}{42.171}$ & $\begin{array}{l}44.738 \\
44.465\end{array}$ & $\begin{array}{r}\mid 4.73 \\
4.634 \\
\end{array}$ & $\begin{array}{l}38.263 \\
38.304\end{array}$ & $\frac{-1.21}{-1.212}$ & & $\begin{array}{l}45.149 \\
45.736\end{array}$ & $\frac{0.003}{0.003}$ & $\begin{array}{l}1.399 \\
1.399\end{array}$ & & $\frac{30.55}{366.35}$ & $\mid$ & $\frac{43.6}{43.3}$ & 10 & 40 & 2774 & & $\frac{0.200}{0.200}$ & & $\frac{4.973}{4.979}$ & 4.23 \\
\hline 2 & 9/7/2004 & 3:28:06 PM & 25.36 & 25.021 & 24.97 & 25.719 & 25.066 & 42.09 & \begin{tabular}{|l|}
44.303 \\
\end{tabular} & 4.641 & 38.215 & \begin{tabular}{|l|}
-1.2121 \\
\end{tabular} & -1.002 & 46.266 & 0.003 & 1.395 & & 367.35 & \begin{tabular}{|l|l|}
6.1225 \\
\end{tabular} & 43.2 & $\frac{21.0}{11.0}$ & 40.2 & $\frac{2.768}{2.768}$ & $\frac{200}{200}$ & 0.200 & 0.005 & $\frac{4.975}{4.978}$ & 4.2 \\
\hline & 9/7/2004 & 3:29:06 PM & 25.362 & 25.016 & 24.95 & 25.709 & 25.061 & 42.243 & \begin{tabular}{|l|l|l|}
44.682 \\
\end{tabular} & 4.73 & 38.179 & 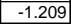 & & 45.864 & 0.003 & 1.397 & & 368.35 & \begin{tabular}{|l|l|}
56.13917 \\
\end{tabular} & 43.6 & 10.9 & 40. & 77 & 0.200 & 0.200 & 0.005 & 4.981 & \\
\hline & & 06 PM & 25.36 & & 24.947 & & & & & & 38.259 & -1.21 & & 45.924 & & & & & & & & & & & & & & \\
\hline & $9 / 7 / 2004$ & & & 25.043 & 24.947 & 25.756 & & 42.04 & 44.214 & 4 & 38.006 & -1.21 & & 45.849 & $\begin{array}{l}0.003 \\
\end{array}$ & 1.4 & & 370.35 & 6.1725 & $\overline{43.1}$ & & & & & 0.201 & & & \\
\hline & $9 / 7 / 2004$ & 3:32: & 25.344 & & & 25.747 & 25.139 & 42.291 & 444.637 & 4.748 & & & & 45.914 & $\begin{array}{l}0.003 \\
\end{array}$ & 1.395 & & & & 43.5 & & & & & 0.200 & & 4.967 & \\
\hline & $9 / 7 / 2004$ & & & 25.043 & & 25.697 & 25.159 & 42.011 & $\begin{array}{l}44.518 \\
\end{array}$ & 4.645 & 37.941 & & & 45.793 & & 1.4 & & & 6.20583 & 43.4 & & & & & & & & \\
\hline 236 & $9 / 7 / 2004$ & 3:34:06 PM & 25.32 & 25.048 & 24.922 & 25.687 & 25.124 & $\begin{array}{l}41.968 \\
\end{array}$ & $\begin{array}{l}44.394 \\
\end{array}$ & $\begin{array}{l}4.662 \\
\end{array}$ & 38.025 & -1.212 & -0.5 & 45.644 & 0.003 & 1.399 & & 373.35 & \begin{tabular}{|l|l|}
56.2225 \\
\end{tabular} & 43.3 & 10.9 & 40 & 2.758 & 0.200 & 0.201 & 0.005 & 5.019 & 4.2 \\
\hline$\frac{240}{12}$ & 9/7/2004 & 3:35:06 PM & 25.315 & & 24.912 & 25.657 & & 42.187 & 44.604 & 4.704 & 38.14 & -1.212 & & 45.738 & 0.003 & 1.398 & & 374.35 & & 43.5 & 10.5 & 40. & & 0.200 & & 0.005 & 4.996 & $\sqrt{42}$ \\
\hline 241 & 9//21/2004 & 3:36:06 PM & & 25.039 & 24.873 & 25.592 & 24.8 & 42.03 & 44.094 & 4.61 & 38.174 & -1.212 & & 46.074 & 0.003 & 1.4 & & & & 43.0 & & & & & & & & \\
\hline$\frac{2}{2}$ & 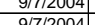 & $\begin{array}{l}3: 37: 06 \mathrm{PM} \\
30: 060\end{array}$ & 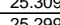 & $\begin{array}{l}25.045 \\
25052\end{array}$ & $\begin{array}{l}24.882 \\
24877\end{array}$ & 255581 & 24.818 & $\begin{array}{l}42.013 \\
4094\end{array}$ & $\begin{array}{l}44.469 \\
44372\end{array}$ & $\begin{array}{l}4.608 \\
4777\end{array}$ & $\begin{array}{l}38.057 \\
37.062\end{array}$ & $\frac{-1.212}{-1212}$ & & 45.151 & 0.003 & 1.4 & & $\frac{3 / 6.35}{37725}$ & $\frac{6.2725}{6.625}$ & 43.3 & 10. & & 2.16 & 0.200 & 0.201 & 0.005 & 5.019 & 4.2 \\
\hline$\frac{2.44}{244}$ & 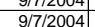 & & 2528 & 25.041 & 24.875 & 25.341 & 24. & 年14.964 & $\begin{array}{l}44.575 \\
44.498\end{array}$ & $\begin{array}{l}4.104 \\
4.642\end{array}$ & $\begin{array}{l}38.903 \\
38.002\end{array}$ & $\frac{-1.24}{-1.212}$ & & 年 45.0000 & $\begin{array}{l}0.0003 \\
0.003\end{array}$ & $\begin{array}{l}1.599 \\
1.402\end{array}$ & & $\begin{array}{l}37.35 \\
37.355 \\
\end{array}$ & 5.28017 & 43.44 & 100 & 40, & 2757 & 201 & 0.201 & 0.0005 & S. 5020 & 2 \\
\hline & $9 / 7 / 2004$ & 3:40:06 PM & $25.2 \varepsilon$ & 25.044 & 24.879 & $\frac{2.414}{25.442}$ & 24.81 & 41.963 & 44.121 & 4.685 & 38.047 & -1.212 & & 45.263 & 0.003 & $\frac{1.402}{1.402}$ & & & & 43.0 & $\frac{10 .}{10.8}$ & 40 & & & $\frac{1.201}{0.201}$ & & 5.034 & $\frac{4.2}{4.2}$ \\
\hline$\sqrt{24}$ & 9/7/2004 & 3:41:06 PM & 25.26 & 25.038 & 24.847 & 25.416 & 24.799 & 41.93 & 44.336 & $\mid 4.604$ & 37.988 & -1.215 & -0.95 & 45.757 & 0.003 & 1.403 & & $380.3^{3}$ & \begin{tabular}{|l|l|}
6.33917 \\
\end{tabular} & 43.2 & $\frac{10.5}{10.5}$ & & 2.755 & 0.201 & 0.202 & 0.005 & 5.048 & $\frac{4.4}{43}$ \\
\hline & $9 / 7 / 2004$ & & & & & & & & 44.085 & & & & & & & 1.4 & & & & & & & & & & & & \\
\hline 248 & $9 / 7 / 2004$ & 06 PM & $\frac{25.273}{25.273}$ & 25.048 & 24.841 & $\frac{25.456}{25.456}$ & & 42.192 & \begin{tabular}{|l|l|}
44.554 \\
\end{tabular} & \begin{tabular}{|l|l|}
4.682 \\
\end{tabular} & 38.244 & $\frac{-1.215}{-1.215}$ & & $\mid$ & 0.003 & 1.403 & & 382.35 & 6.3725 & 43.4 & $\frac{10.8}{10.8}$ & 40.8 & 2.773 & $\frac{0.201}{0.201}$ & $\frac{1.202}{0.202}$ & $\frac{0.005}{0.005}$ & 5.017 & 42 \\
\hline $24 !$ & $9 / 7 / 2004$ & 4:23:40 PM & 23.63 & 24.859 & 23.364 & 25.136 & 24.5 & 42.828 & \begin{tabular}{|l|l|}
45.285 \\
\end{tabular} & 4.638 & 38.977 & -1.212 & & $\begin{array}{l}46.518 \\
\end{array}$ & 0.003 & 1.485 & & $\mid 422.92$ & 27.04861 & 44.2 & 11 & 40. & 2.826 & 0.213 & 0.223 & 0.005 & 5.444 & \\
\hline 25 & $9 / 7 / 2004$ & & & 24.862 & 23.593 & & & 42.942 & 45.607 & & & -1212 & & & & 1.485 & & & 7.06528 & 44.5 & 10 & & & 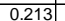 & 0.021 & 0.005 & 5,404 & 46 \\
\hline 25 & $9 / 7 / 2004$ & & 23.894 & 24.845 & 23.771 & 25.012 & 24.60 & 5.199 & \begin{tabular}{|l|}
45.768 \\
\end{tabular} & \begin{tabular}{|l|l|}
4.576 \\
\end{tabular} & $\begin{array}{l}0.612 \\
\end{array}$ & 34.895 & & 45.186 & $\begin{array}{l}0.003 \\
\end{array}$ & -0.012 & & \begin{tabular}{|l|l|}
424.92 \\
\end{tabular} & 7.08194 & 44.6 & 10.8 & & & & & -0.001 & -0.612 & -0.5 \\
\hline 25 & $9 / 7 / 2004$ & 4:26:40 PM & 23 & 24.859 & 23.956 & 25.091 & 24 & 3.515 & \begin{tabular}{|l|l|}
45.913 \\
\end{tabular} & 4.667 & -1.07 & 37.66 & 20 & 47.261 & 0.003 & -0.013 & & 425 & 7.09861 & 44.8 & 11. & 1. & 0.084 & & -0.002 & -0.002 & -1.568 & -1.3 \\
\hline$\frac{253}{253}$ & 9/7/2004 & & 23.942 & 24.847 & 24.1 & 25.1 & & 2.961 & 46.061 & 4.616 & -1.563 & 38.389 & & 44.664 & & & & & & 44.9 & & & & & & & & \\
\hline 254 & 9//2/2004 & & 24.347 & & & & & 27.357 & \begin{tabular}{|l|l} 
\\
\end{tabular} & 4.774 & 24.505 & 11.011 & & 48.022 & 0.007 & 4.261 & & 427.92 & 7.13194 & 43.6 & & 25. & 1.788 & 0.610 & 0.624 & 0.024 & 44.046 & 20.4 \\
\hline$\frac{25}{25}$ & 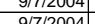 & & 24.55 & 24.833 & 24.371 & 25.066 & 24 & 41.559 & 46.119 & 4.809 & 37.569 & $\frac{-1.212}{-120}$ & & 46.142 & 0.0003 & 1..505 & & 428.9 & $\frac{1.4001}{7.652}$ & 45.0 & 111 & nat & 278 & ET & 2055 & 0.007 & 7.194 & 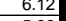 \\
\hline$\frac{256}{25}$ & 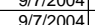 & & & $\frac{24.041}{24831}$ & & $\frac{25.105}{25169}$ & & $\frac{42.30}{42.31}$ & \begin{tabular}{|l|l|}
46.093 \\
453
\end{tabular} & $\mid$ & & & & $\frac{46.128}{45.267}$ & & $\frac{1.158}{1.662}$ & & & & $\frac{44.0}{44.3}$ & & & & & & & $\frac{6.321}{5.968}$ & 50 \\
\hline 25 & $9 / 7 / 2004$ & $4 \cdot 32 \cdot 40$ & 24.848 & 24.837 & 24.581 & 25.23 & & 42843 & \begin{tabular}{|l|l|}
45.777 \\
\end{tabular} & 4.682 & 38.954 & -1.212 & & 45.063 & 0.003 & 1.603 & & & \begin{tabular}{|l|l|l|} 
& 1.9861 \\
\end{tabular} & 44.7 & & & & & & & & $\frac{5.0}{48}$ \\
\hline & $917 / 2004$ & & & 24833 & & 25246 & & 42602 & 45627 & 484 & 3838 & -10200 & & 46.95 & & & & & & . & & 40 & & & & & & 4.8 \\
\hline 26 & $9 / 7 / 2004$ & $4 \cdot 34: 40$ PM & 249 & 24839 & 24.648 & 25282 & & 42428 & 45327 & 4785 & 38.267 & -1212 & & 47.198 & 0.003 & 1.529 & & 433 & 7.23 & 442 & $\frac{x+1}{11}$ & & 2782 & 2019 & & 0.005 & 5.479 & 4.16 \\
\hline & & & & & & 25.304 & & 42.401 & 44.999 & & 38.367 & -1.212 & & & & & & & & 43.9 & & & & & & & & $\frac{4.0}{45}$ \\
\hline $26>$ & $9 / 7 / 2004$ & $4: 36: 40 \mathrm{PM}$ & 25.03 & 24.847 & 24.721 & 25.346 & & 42.318 & & \begin{tabular}{|l|l|}
4.72 \\
\end{tabular} & 38.248 & -1.212 & & 47.1 & 0.003 & 1.486 & & 435 & 7.26 & 43.9 & 11 & 40.3 & 2777 & 0.213 & 214 & 005 & & 4.5 \\
\hline & 9/7/2004 & 4:37:40 & 25.07 & 24.849 & 24.748 & 25.377 & & 42.49 & 45.302 & 4.819 & 38.31 & -1.21 & & 46.7 & 0.003 & 1.475 & & 436 & $7.2 \varepsilon$ & $\begin{array}{lll}44.2 \\
\end{array}$ & & & & & 0.213 & $\frac{0.005}{2.05}$ & 5.264 & \\
\hline & 9/7/2004 & & 25 & 24.854 & 24.803 & 25.443 & & 42.5 & 45.223 & 4.745 & 38.406 & & & & & 1.465 & & & & 44.1 & & & & & & & & 4.4 \\
\hline 26 & $9 / 7 / 264$ & 4:39:40 PM & 25 & 24 & & 25.448 & & 42.77 & \begin{tabular}{|l|l|} 
& 45.364 \\
\end{tabular} & 4.845 & 38.5 & -1.21 & & 47.3 & & 1.456 & & & & 44.2 & 11. & & & & & & 5.148 & \\
\hline$\frac{26}{36}$ & $9 / 7 / 2004$ & & 25.17 & 24.867 & 24.866 & $25.4 / 5$ & & 42.399 & \begin{tabular}{|l|l|}
45.016 \\
\end{tabular} & 4.997? & 38.255 & -1.212 & & 46.206 & 0.003 & 1.45 & & & 1.35 & 43.9 & $\pm x$ & & & 0.208 & 0.208 & .005 & $5.16 \%$ & \\
\hline$\frac{1018}{268}$ & 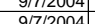 & 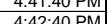 & & $\frac{2.0}{248}$ & 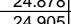 & $\frac{2.3524}{2556}$ & & 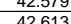 & $\frac{45.010}{45066}$ & $\frac{4.063}{4.834}$ & $\begin{array}{l}30.309 \\
38.476\end{array}$ & $\frac{-1.1212}{4-1212}$ & & 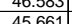 & & $\frac{1.444}{1438}$ & & & & $\frac{43.9}{439}$ & & & & & & & & . \\
\hline$\frac{206}{269}$ & $9 / 7 / 2004$ & $4 \cdot 4 \cdot 4 \cdot 40$ & & 24888 & 24.922 & 25621 & & 42783 & 4 & 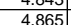 & $\begin{array}{l}30.470 \\
38.452\end{array}$ & $\frac{1.212}{-1212}$ & & $\frac{4.0}{468}$ & & $\frac{1.450}{1431}$ & & 441 & & $\frac{45.5}{442}$ & & & & & & & & \\
\hline 27 & $9 / 7 / 2004$ & & & 24.9 & 24.949 & 25.673 & & 44.708 & 4501 & 4.758 & 38.71 & -1.206 & & 47.1 & & 1.431 & & & & 439 & & & & & & & & \\
\hline 271 & $9 / 7 / 12004$ & & & & 24.97 & 25.686 & & 42.5 & \begin{tabular}{|l|l|}
44.97 \\
\end{tabular} & & & -1.209 & & & & 1.424 & & & & 43.8 & & & & & & & & \\
\hline 272 & & & & & 24.99 & 25.67 & & & & & & & & & & & & & & & & & & & & & & \\
\hline 273 & $9 / 7 / 2004$ & & 25. & 24.91 & 25.01 & 25.709 & & 42.569 & 44.972 & \begin{tabular}{|l|l|}
4.749 \\
\end{tabular} & 38.482 & -1.212 & & 47.228 & 0 & 1.42 & & 446 & 7.4 & 43.8 & & & & 203 & & 005 & & \\
\hline 274 & $9 / 7 / 2004$ & $4: 48: 4$ & & 24. & 25.0 & 25.7 & & 44 & 44.8 & 4.7 & 38.4 & & & 47.2 & & 1.418 & & 44 & & & & & & & & & & \\
\hline 275 & $9 / 7 / 2004$ & 4:49:40 & & 24.935 & 25.05 & 25.774 & & 42.97 & 45.287 & 4.844 & 38.8 & -1.206 & & 46.5 & & & & 448 & & 44.2 & & & & & & & 54 & \\
\hline 276 & 9/7/2004 & & 25.409 & 24.947 & 25.052 & 25.831 & & 42.463 & 44.825 & 4.778 & 38.349 & -1.212 & & 47.949 & 0.003 & 1.396 & & 449 & 27.49 & 43.7 & 11.4 & & & 0.200 & 0.200 & 0.005 & 4.939 & 4.2 \\
\hline $2 \frac{277}{270}$ & 9//212004 & 4:51:40 PM & 25.415 & 24.949 & 25.054 & 25.82 & & 42.256 & 44.558 & 4.729 & 38.197 & -1.215 & & 46.164 & & 1.398 & & & & 43.4 & & & & & & & 4.968 & \\
\hline$\frac{128}{379}$ & 9 & 4.52.40 PM & 25.453. & 24.956 & 25.011 & 25.841 & & 42.453 & 44.9606 & 4.6818 & 38.474 & -1.209 & & 46.101 & 0.003 & 1.392 & & 451.92 & 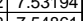 & 43.8 & 11.0 & 40.5 & $2 . .990$ & 0.199 & 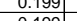 & 0.005 & 4.915 & 4.1 \\
\hline$\frac{275}{280}$ & $9 / 7 / 2004$ & $\begin{array}{l}4.50 .44 \mathrm{PM} \\
: 54: 40 \mathrm{PM}\end{array}$ & $\frac{20.44}{25.443}$ & 24.9096 & \begin{tabular}{|l|}
25.099 \\
\end{tabular} & \begin{tabular}{|l|}
25.030 \\
2515 \\
\end{tabular} & & $\begin{array}{r}\frac{42.494}{42.165} \\
\end{array}$ & \begin{tabular}{|l|}
44.995 \\
44.751 \\
\end{tabular} & \begin{tabular}{|l|}
4.0100 \\
4.81 \\
\end{tabular} & $\begin{array}{l}30.201 \\
37.887\end{array}$ & \begin{tabular}{|l|}
-1.209 \\
-1.215 \\
\end{tabular} & -2.144 & 46.001 & 0.003 & $\frac{1.591}{1.391}$ & & $\begin{array}{l}453.92 \\
453.92\end{array}$ & \begin{tabular}{|l|l|}
7.54601 \\
7.56528
\end{tabular} & \begin{tabular}{|l|l|}
43.6 \\
\end{tabular} & \begin{tabular}{|l|}
11.0 \\
\end{tabular} & & 2.760 & \begin{tabular}{|c|}
0.199 \\
\end{tabular} & \begin{tabular}{|c|}
0.199 \\
0.199
\end{tabular} & 0.005 & \begin{tabular}{|c|}
4.9103 \\
4.963
\end{tabular} & 4.1 .2 \\
\hline
\end{tabular}


WSRC-TR-2005-00105, REVISION 0

SRNL-RPP-2005-00012, REVISION 0

RUN \# 4.18 AND 4.19; ACID CLEANING AND CAUSTIC RINSING - CONT.

\begin{tabular}{|c|c|c|c|c|c|c|c|c|c|c|c|c|c|c|c|c|c|c|c|c|c|c|c|c|c|c|c|c|}
\hline & A & & D & $E$ & $F$ & $G$ & $\mathrm{H}$ & $\mathrm{J}$ & $\begin{array}{ll}\mathrm{K} \\
\end{array}$ & $\mathrm{L}$ & M & $\mathrm{N}$ & 0 & $\begin{array}{ll} \\
\end{array}$ & $\mathrm{R}$ & $\mathrm{s}$ & \begin{tabular}{|lll}
$\mathrm{T}$ & $\mathrm{L}$ \\
\end{tabular} & $\mathrm{v}$ & w & \begin{tabular}{|l|l|}
$x$ & \\
\end{tabular} & & $z$ & & $A B$ & $\mathrm{AC}$ & & & AF \\
\hline & 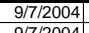 & $4: 55: 40 \mathrm{PM}$ & $\frac{25.449}{25.56}$ & 24.973 & \begin{tabular}{|l|}
25.087 \\
2.58 \\
\end{tabular} & 25.836 & 24.989 & 42.136 & \begin{tabular}{|l|l|}
44.576 \\
\end{tabular} & 4.766 & \begin{tabular}{|l|}
37.977 \\
\end{tabular} & -1.212 & $\begin{array}{l}-2.138 \\
\end{array}$ & $\begin{array}{l}46.79 \\
\end{array}$ & 0.003 & 1.39 & $\begin{array}{l}.004 \\
\end{array}$ & 454.92 & $\begin{array}{l}7.58194 \\
7.5194\end{array}$ & \begin{tabular}{|l|}
43.5 \\
\end{tabular} & & 40.1 & 2.762 & 0.199 & 0.199 & 0.005 & 4.956 & \\
\hline & & 4:56:40 PM & & 24.98 & \begin{tabular}{|l|}
25.09 \\
\end{tabular} & 25.809 & 24.941 & & \begin{tabular}{|l|l|l|l|}
442 \\
\end{tabular} & $\begin{array}{l}4.78 \\
\end{array}$ & $\begin{array}{l}37.932 \\
\end{array}$ & -1.212 & -2.138 & & $\begin{array}{l}0.003 \\
\end{array}$ & $\begin{array}{l}1.384 \\
\end{array}$ & 0.003 & 455.9 & 7.59861 & 43.2 & & 40.6 & 2.756 & & $\begin{array}{l}0.198 \\
\end{array}$ & 0.005 & 4.944 & 4.21 \\
\hline 28 & $\begin{array}{l}9 / 7 / 2004 \\
9 / 7 / 2004\end{array}$ & 4:57:40 PM & $\frac{25.458}{25.453}$ & \begin{tabular}{|l|l|}
24.977 \\
24971
\end{tabular} & \begin{tabular}{|l|}
25.071 \\
25077
\end{tabular} & $\begin{array}{r}25.81 \\
25.786\end{array}$ & $\begin{array}{l}24.833 \\
24858\end{array}$ & \begin{tabular}{|l|l|}
42.007 \\
422076
\end{tabular} & 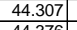 & $\begin{array}{l}4.638 \\
4.763\end{array}$ & \begin{tabular}{|c|}
38.09 \\
38.790
\end{tabular} & \begin{tabular}{|c|}
-1.212 \\
1.212
\end{tabular} & $\begin{array}{l}-2.135 \\
2125 \\
\end{array}$ & \begin{tabular}{|l|l|}
46.733 \\
46598
\end{tabular} & $\begin{array}{l}0.003 \\
\end{array}$ & \begin{tabular}{|l|l|}
1.387 \\
1381
\end{tabular} & $\begin{array}{l}0.003 \\
003\end{array}$ & 456.92 & 7.01020 & 43.2 & & 10.0 & $\begin{array}{l}2.761 \\
.771 \\
\end{array}$ & 0.199 & $\begin{array}{l}0.198 \\
\end{array}$ & 0.005 & $\begin{array}{l}4.948 \\
\end{array}$ & \\
\hline & 9/7//20004 & $\begin{array}{l}\text { 4:58:40 PM } \\
\text { 4:59:40 PM }\end{array}$ & $\frac{25.453}{25.468}$ & $\begin{array}{l}24.977 \\
24.987\end{array}$ & \begin{tabular}{|l|}
25.077 \\
25.087
\end{tabular} & $\begin{array}{l}25.786 \\
25.776\end{array}$ & $\begin{array}{l}24.858 \\
24.798 \\
\end{array}$ & \begin{tabular}{|c|}
42.216 \\
42.281
\end{tabular} & \begin{tabular}{|r|}
44.376 \\
44.608
\end{tabular} & $\begin{array}{l}4.763 \\
4.772 \\
4.72\end{array}$ & $\begin{array}{l}38.179 \\
38.234\end{array}$ & \begin{tabular}{|c|}
-1.212 \\
-1.209
\end{tabular} & $\begin{array}{l}-2.135 \\
-2.132\end{array}$ & \begin{tabular}{|c|}
$\mid 46.598$ \\
46.858
\end{tabular} & \begin{tabular}{|c|c|}
0.003 \\
0.003
\end{tabular} & \begin{tabular}{|l|}
1.381 \\
1.333
\end{tabular} & $\begin{array}{l}0.003 \\
0.003\end{array}$ & $\begin{array}{l}457.92 \\
458.92 \\
\end{array}$ & $\begin{array}{l}7.63194 \\
7.64861 \\
\end{array}$ & $\begin{array}{l}43.3 \\
43.5\end{array}$ & $\begin{array}{l}11.1 \\
11.2\end{array}$ & $\begin{array}{l}40.2 \\
40.3\end{array}$ & \begin{tabular}{|l|l|}
2.771 \\
2.776
\end{tabular} & $\begin{array}{l}0.198 \\
0.198 \\
\end{array}$ & $\begin{array}{l}0.197 \\
0.198\end{array}$ & $\begin{array}{l}0.005 \\
0.005\end{array}$ & $\begin{array}{r}4.908 \\
4.906 \\
\end{array}$ & $\begin{array}{l}4.18 \\
4.18\end{array}$ \\
\hline 286 & $9 / 7 / 2004$ & & 25.469 & & & & 24.774 & & & 4.824 & & & $\begin{array}{l}-2.132 \\
-2.132\end{array}$ & & & 1.38 & $\begin{array}{l}0.003 \\
0.003\end{array}$ & $\begin{array}{l}458.92 \\
459.92 \\
\end{array}$ & $\begin{array}{l}7.04601 \\
7.66528 \\
\end{array}$ & $\begin{array}{l}43.5 \\
43.6 \\
\end{array}$ & & 40.3 & & $\begin{array}{l}0.198 \\
0.198 \\
\end{array}$ & $\begin{array}{l}0.198 \\
0.197 \\
\end{array}$ & $\begin{array}{l}0.005 \\
0.005\end{array}$ & $\begin{array}{l}4.906 \\
4.890\end{array}$ & $\begin{array}{l}4.18 \\
4.16\end{array}$ \\
\hline & & 5:01:40 PM & 25.473 & 24.992 & \begin{tabular}{|l|}
25.106 \\
\end{tabular} & 25.7 & 24.853 & 42.148 & \begin{tabular}{|l|l|}
44.63 \\
\end{tabular} & 4.861 & \begin{tabular}{|l|l|}
37.836 \\
\end{tabular} & -1.212 & \begin{tabular}{|l|}
-2.13 \\
\end{tabular} & \begin{tabular}{|l|l|}
46.299 \\
\end{tabular} & $\begin{array}{l}0.003 \\
\end{array}$ & 1.381 & 0.003 & 460.92 & \begin{tabular}{|l|l|}
7.68194 \\
\end{tabular} & 43.5 & & & & 0.198 & \begin{tabular}{|c|}
0.197 \\
\end{tabular} & & & $\frac{4.10}{4.19}$ \\
\hline 288 & 9/7/2004 & & 25.478 & 24.997 & & 25.736 & & & & & & & & & & & & & & & & & & & & & & $\frac{4.19}{4.16}$ \\
\hline 289 & & 5:03:40 PM & & 24.997 & 2.001 & & & & 44.744 & 4.849 & 38.117 & & & & & & & & & & & & & & 0.197 & & & \\
\hline$\frac{290}{291}$ & $9 / 7 / 2004$ & 5:04:40 PM & 25.494 & & 25.107 & 25772 & & $\begin{array}{lll}42.6 \\
\end{array}$ & \begin{tabular}{|l|l|l|l|} 
\\
\end{tabular} & & 38.638 & & & & & & & & 73194 & 43 & & & & & 0.197 & & & 442 \\
\hline & 9 & 5:05:40 PM & 25.499 & 25.023 & 25.123 & 25.822 & 25.024 & 42.246 & \begin{tabular}{|l|l}
44.736 \\
\end{tabular} & 4.772 & 38.016 & -1.209 & -2.118 & 46.333 & $\mid 0.003$ & 1.377 & & & 74861 & 43.6 & & 40.1 & 2.767 & 5.197 & 0.196 & 0.005 & 4.895 & \\
\hline & & 5:06:40 PM & 25.499 & $\frac{25.028}{25021}$ & 25.123 & 25.827 & 25.064 & 42.071 & 44.203 & 4.637 & $\begin{array}{l}38.179 \\
-2.152\end{array}$ & & -2.118 & 46.5 & 0.003 & 1.376 & & & .76528 & 43.1 & & & 2.766 & & 0.196 & 0.005 & & 4.16 \\
\hline & $\frac{9 / 7 / 200}{9 / 7 / 200}$ & 5:07:40 PM & $\begin{array}{r}25.5 \\
25.51\end{array}$ & 25.024 & \begin{tabular}{|l|}
25.118 \\
25.123
\end{tabular} & 25.797 & $\frac{25.084}{25.104}$ & 42.266 & $\begin{array}{l}44.691 \\
14.423\end{array}$ & 4.727 & $\begin{array}{l}38.152 \\
37.975 \\
37\end{array}$ & & $\frac{-2.118}{2.118}$ & 46.166 & 0.003 & 1.375 & & & & 43.6 & & & & & 0.197 & & 4.8944 & \\
\hline & & 5.060 .40 & $\frac{23.51}{2531}$ & 250.046 & | & $\begin{array}{l}23.002 \\
25829\end{array}$ & & 年 & \begin{tabular}{|}
$\mid 4.4435$ \\
4482
\end{tabular} & & $\frac{51.975}{3809}$ & & & & & & & & & & & & & & & & 4.899 & 4.17 \\
\hline & $9 / 7 / 2004$ & 5:10:40 PM & $\frac{2.031}{25.522}$ & 25.061 & 25.135 & 25.059 & & 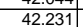 & \begin{tabular}{|l|}
44.6162 \\
44.616
\end{tabular} & $\begin{array}{l}4.070 \\
4.857\end{array}$ & 37.988 & $\frac{-1.209}{-1.209}$ & & & o.003 & & & & & 12 & & & 765 & & 0.197 & & 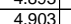 & $\frac{4.16}{4.17}$ \\
\hline & $9 / 7 / 2004$ & 5:11:40 PM & 25.522 & 25.061 & \begin{tabular}{|l|l|l|}
25.135 \\
\end{tabular} & 255.839 & 25.166 & 42.185 & \begin{tabular}{|l|}
44.411 \\
\end{tabular} & 4.694 & 38.257 & -1.212 & -2.109 & \begin{tabular}{|l|l|}
46.794 \\
\end{tabular} & 0.003 & 1.378 & & & 84861 & 43.3 & & & .773 & 197 & 0.197 & & 4.886 & $\frac{4.11}{4.16}$ \\
\hline & & 5:12:40 PM & & 25.077 & & & & & \begin{tabular}{|l|l|}
44.69 \\
\end{tabular} & 4.791 & & & & & & & & & & & & & & & & & & $\frac{4.16}{4.12}$ \\
\hline & $9 / 7 / 2004$ & 5:13:40 PM & 25.534 & 25.078 & 25.162 & 25.881 & 25.223 & 42.347 & \begin{tabular}{|l|l} 
\\
44.73
\end{tabular} & 4.802 & \begin{tabular}{|l|l|}
38.115 \\
\end{tabular} & & -2.1 & \begin{tabular}{|l|l|l|l|}
4621 \\
\end{tabular} & \begin{tabular}{|c|}
0.003 \\
\end{tabular} & 1.378 & & & 88194 & 43.6 & & & & & 0.196 & 005 & 4.881 & $\frac{4.12}{4.15}$ \\
\hline & & & & & & & & & & & & & & & & & & & & & & & & & & & & \\
\hline & $9 / 7 / 2004$ & 5:15:40 PM & 25.536 & 25.09 & \begin{tabular}{|c|}
25.154 \\
\end{tabular} & 25.908 & 25.2 & $|42.387|$ & \begin{tabular}{|l||}
44.626 \\
\end{tabular} & 4.709 & 38.443 & $\mid-1.212$ & -2.104 & 45.738 & 0.003 & 1.379 & & 474.92 & 7.91528 & \begin{tabular}{|l|}
43.5 \\
\end{tabular} & 10. & 40.4 & 2.786 & 0.197 & 0.197 & 0.005 & 4.864 & \\
\hline & $9 / 7 / 2004$ & 5:16:40 PM & 25.542 & 25.097 & \begin{tabular}{|l|l|}
25.156 \\
\end{tabular} & 25.915 & \begin{tabular}{|l|l|}
25.077 \\
\end{tabular} & \begin{tabular}{|l|l|}
42.227 \\
\end{tabular} & \begin{tabular}{|l|l|}
44.583 \\
\end{tabular} & 4.738 & 38.183 & \begin{tabular}{|c|}
-1.209 \\
\end{tabular} & $\begin{array}{r}-2.098 \\
\end{array}$ & 46.637 & 0.003 & 1.377 & 0.004 & 475.92 & 7.93194 & \begin{tabular}{|l|}
43.5 \\
\end{tabular} & 11.1 & 40.2 & 2.772 & 0.197 & 0.196 & 0.005 & 4.882 & 4.15 \\
\hline 303 & $9 / 7 / 2004$ & 5:17:40 PM & 25.539 & 25.098 & \begin{tabular}{|l|}
25.147 \\
\end{tabular} & 25.856 & 25.009 & $\begin{array}{l}42.281 \\
\end{array}$ & \begin{tabular}{|l|l|}
44.572 \\
\end{tabular} & 4.796 & 38.179 & \begin{tabular}{|l|}
-1.212 \\
\end{tabular} & -2.101 & $\begin{array}{l}46.483 \\
\end{array}$ & $\begin{array}{l}0.003 \\
\end{array}$ & $\begin{array}{l}1.373 \\
\end{array}$ & 0.004 & 476.92 & \begin{tabular}{|l|l|}
7.94861 \\
7
\end{tabular} & \begin{tabular}{|l|}
43.4 \\
\end{tabular} & 11.1 & 40.2 & 2.774 & 0.197 & $\begin{array}{l}0.196 \\
\end{array}$ & 0.005 & $\begin{array}{l}4.866 \\
\end{array}$ & 4.14 \\
\hline 304 & 9/7/2004 & 5:18:40 PM & 25.535 & 25.099 & \begin{tabular}{|l|}
25.149 \\
25.2
\end{tabular} & 25.793 & 24.88 & $\begin{array}{r}42.048 \\
\end{array}$ & \begin{tabular}{|l|l|}
44.349 \\
\end{tabular} & 4.707 & 37.99 & \begin{tabular}{|l|l|}
-1.212 \\
\end{tabular} & $\begin{array}{r}-2.101 \\
.21\end{array}$ & 46.627 & 0.003 & $\begin{array}{l}1.371 \\
\end{array}$ & 0.003 & & & 43.2 & & & 2.759 & & $\begin{array}{l}0.195 \\
\end{array}$ & $\begin{array}{l}0.005 \\
\end{array}$ & 4.884 & 4.16 \\
\hline & $\begin{array}{l}9 / 7 / 2004 \\
9 / 7 / 2004\end{array}$ & $\begin{array}{l}5: 19: 40 \mathrm{PM} \\
5 \\
5.20: 40 \mathrm{PM}\end{array}$ & $\begin{array}{l}25.526 \\
25.512\end{array}$ & 25.091 & \begin{tabular}{|l|}
25.135 \\
25126 \\
\end{tabular} & $\begin{array}{l}25.739 \\
2573\end{array}$ & $\begin{array}{l}24.586 \\
24.407\end{array}$ & \begin{tabular}{|l|}
42.264 \\
42214 \\
\end{tabular} & \begin{tabular}{|l|l|}
44.576 \\
44.46 \\
\end{tabular} & 4.685 & $\begin{array}{l}38.254 \\
38.043\end{array}$ & $\begin{array}{l}-1.212 \\
-1.212 \\
\end{array}$ & $\begin{array}{r}-2.101 \\
-2098\end{array}$ & $\begin{array}{l}46.738 \\
46141\end{array}$ & \begin{tabular}{|c|}
0.003 \\
0.03
\end{tabular} & \begin{tabular}{|l|l|}
1.373 \\
1375
\end{tabular} & 0.003 & $\begin{array}{l}478.92 \\
479.92\end{array}$ & \begin{tabular}{|c|}
7.98194 \\
7798861
\end{tabular} & 43.5 & $\frac{11.1}{110}$ & $\begin{array}{l}40.3 \\
40.1\end{array}$ & \begin{tabular}{l|l|l|}
2.776 \\
2.767
\end{tabular} & \begin{tabular}{|c|c|}
0.197 \\
0.197
\end{tabular} & $\begin{array}{l}0.196 \\
0.196\end{array}$ & $\begin{array}{l}0.005 \\
0.05\end{array}$ & \begin{tabular}{|l|l|l|l|l|l|l|}
4.884 & \\
\end{tabular} & $\begin{array}{l}4.14 \\
4.6\end{array}$ \\
\hline 307 & $99 / 7 / 2004$ & $\begin{array}{l}5: 20.40 P M \\
5: 21: 40 \mathrm{PM}\end{array}$ & $\frac{25.512}{25.508}$ & $\frac{25.07 .4}{25.072}$ & \begin{tabular}{|l|}
25.126 \\
25.131
\end{tabular} & $\begin{array}{l}25.73 \\
25.76\end{array}$ & $\frac{24.401}{24.342}$ & $\begin{array}{r}42.214 \\
42.09\end{array}$ & \begin{tabular}{|l|}
44.4460 \\
44.404 \\
\end{tabular} & $\begin{array}{l}4.771 \\
4.732 \\
\end{array}$ & & $\begin{array}{l}-\frac{-1.212}{-1.212} \\
\end{array}$ & $\frac{-2.09}{-2.09}$ & $\begin{array}{l}46.141 \\
46.577\end{array}$ & \begin{tabular}{|c|}
0.003 \\
0.003
\end{tabular} & $\begin{array}{l}1.375 \\
1.374\end{array}$ & & & $\begin{array}{ll}11 \\
8 \\
\end{array}$ & & & & & & & & & $\begin{array}{l}4.16 \\
4.16\end{array}$ \\
\hline 308 & & 5:22:40 PM & 25.518 & 25.072 & 25.111 & 25.805 & 24.282 & 42.034 & \begin{tabular}{|l|}
44.33 \\
\end{tabular} & 4.747 & 37.977 & -1.212 & -2.095 & 46.706 & 0.003 & 1.369 & 0.003 & & 8.03194 & $\begin{array}{l}43.0 \\
43.2\end{array}$ & & 46 & & & 0.195 & 0.005 & $\begin{array}{l}4.054 \\
4.884\end{array}$ & $\frac{4.16}{4.16}$ \\
\hline 210 & & & 25.496 & 25.046 & 25.095 & 25.814 & & $\begin{array}{l}41.816 \\
\end{array}$ & \begin{tabular}{|l|l|} 
\\
44.15 \\
\end{tabular} & 4.701 & 37.768 & -1.212 & -2.0 & 46.245 & \begin{tabular}{|c|c|}
0.003 \\
\end{tabular} & & & & & & & & & & & & & \\
\hline & $9 / 7 / 200$ & $5: 24: 40$ PM & & & & & 24.2 & & & & & & & 46.475 & & $1.36 \mathrm{C}$ & & & & 43 & & & & & 0.195 & & 4.888 & 4.16 \\
\hline & $9 / 7 / 206$ & 5:25:40 PM & & 25.04 & & 25.863 & 24.3 & 42.289 & \begin{tabular}{|l|}
44.628 \\
\end{tabular} & 4.6 & 38.41 & -1.209 & & 46 & & & & & 08194 & & & & & & & & & \\
\hline & 9/7/2004 & 5:26:40 PM & & 25.04 & 25.101 & 25.92 & & 42.474 & \begin{tabular}{|l|l|}
44.908 \\
\end{tabular} & 4.76 & 38.398 & & & 45.9 & 0.003 & 1.37 & & & 8.098 & & & & & & & & 4.865 & \\
\hline & $\begin{array}{l}9 / 7 / 200 \\
0.7200\end{array}$ & 5:27:40 PM & 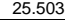 & 25.043| & 25.102 & 25.986 & 24.42 & 42.196 & \begin{tabular}{|l|l|} 
\\
44.494
\end{tabular} & 4.749 & 38.137] & 209 & -2.0 & 46.825 & 0.003 & $1.37 \mathrm{rt}$ & & & 3.115 & 43.4. & & 4 & & & 0.196 & 0.005 & 4.887 & 4.16 \\
\hline & 90 & $5: 28: 40$ & 25.499 & & \begin{tabular}{|l|}
25.018 \\
\end{tabular} & & & 41.8999 & 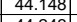 & 4.58 & & & & & & & & & & & & & & & 1.196 & & & \\
\hline & & 5:29:40 PM & & & 25.102 & 26.081 & & & \begin{tabular}{|l|l|}
4.2433 \\
\end{tabular} & & & & & & & & & & & & & & & & & & & \\
\hline & 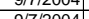 & 5:30:40 PM & 20.5956 & & 25.103 & 20.098 & & 41.8 & 44.167 & 4.64 & & & 2.2. & 45.0 & & $\frac{1.3}{1.32}$ & & & 8 & & & & & & & & & 4.1 \\
\hline $318 \mathrm{~s}$ & 然 & 0 & $\frac{2.450}{20-57}$ & & 2.030 & & & & . & & & & & & & & & & 181 & & & & & & & & & \\
\hline & $90,7 / 20$ & 5:32:40 PM & 25.507 & 25.02 & 25.095 & 26.145 & 24.43 & 42.067 & 44.247 & 4.58 & 38.238 & -1.212 & -2.0 & 46.174 & 0.003 & $1.37 \mathrm{rt}$ & & & 8.19861 & 43.1 & $1.6 \mathrm{c}$ & 40 & & & 0.196 & & 4.886 & 4.11 \\
\hline 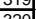 & $9 / 7 / 2004$ & $\begin{array}{l}5: 33: 40 \\
5.3: 40\end{array}$ & 25.497 & 25.016 & 25.1011 & 26.19 & & 42.131 & \begin{tabular}{|l|l}
44.456 \\
\end{tabular} & & & & & 40.11 & 0.0 & 1.07 & & & & & & & & & 年 & nח & 4.8977 & \\
\hline & & 5:34:40 & & & 25.1277 & & 24.4 & & 44.489 & 4.7 & 37.967 & -1.2. & & 46.055 & & & & & 8.23194 & 43.4 & & & & & 0.196 & & 4.904 & 4.17 \\
\hline & 9l/7212004 & 5:35:40 PM & 25.504 & $\frac{25.01}{25.01}$ & 25.112 & 26.212 & 24.4 & $\frac{42.0}{4.8}$ & 44.475 & 4.6 & 37.9 & -1.212 & & 45.6 & & 1.3. & & & & 43. & & & & & & & & \\
\hline & & & & & & & & & & & & & & & & & & & & & & & & & & & & \\
\hline & 917120 & 5:37:40 PM & 25.4499 & 25.013 & 25.098 & 26.267 & 24.5 & 42. & 44.373 & & 38. & & & 46. & & & & & 8.28. & 43 & & & & & 0.196 & & 4.874 & 4.15 \\
\hline 2 & $9 / 7 / 2004$ & $5: 38: 40$ & 25.506 & 25.0 & 25.1099 & 26.254 & & 42. & 44.4 & 4.7. & & & & 46.2 & & & & & & & & & & & & & 4.898 & \\
\hline & & & 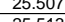 & $\frac{25.6}{256}$ & $\frac{25.12}{25.12}$ & 26.275 & & & & & & & & & & & & & & & & & & & & & & 4.1 \\
\hline & & & & & & & & & 44.2 & 4. & & & & & & & & & & & & & & & & & & \\
\hline & $9 / 7 / 200$ & & & & \begin{tabular}{|l|l|l|l|}
25096 \\
\end{tabular} & & & & & & & & & & & & & & & & & & & & & & & \\
\hline & $9 / 7 / 2004$ & & and & 25.01 & $\mid$\begin{tabular}{|l|l|}
25.11 \\
25
\end{tabular} & $\frac{26.284}{2284}$ & $\frac{2.24}{24.2}$ & 42.05 & 44.311 & 4.635 & & & & & & & & & & & & & & & & & & \\
\hline & $9 / 72004$ & $5: 44: 40 \mathrm{PM}$ & 25.506 & 25015 & \begin{tabular}{|l|l|}
25119 \\
\end{tabular} & 26.234 & & & 44,156 & 457 & & & & & & & & & & 430 & & & & & & & 4890 & \\
\hline & & & & $\frac{25.009}{25}$ & & 26.178 & & 42.324 & \begin{tabular}{|l|}
44.494 \\
\end{tabular} & & & & & & & & & & & & & & & & & & 4 & \\
\hline & & & 25.509 & 24.998 & $25.10 \varepsilon$ & 26.14 & & 42.25 & \begin{tabular}{|l|l|}
44.481 \\
\end{tabular} & 4.6 & & & & & & & & & & & & & & & & & 4.8 & \\
\hline & $9 / 7 / 200$ & & & & 25.1 & 26.1 & & $42.2 \mathrm{~s}$ & 44 & 4.7 & & & & & & $1.3 \varepsilon$ & & & & $\overline{43}$ & & $46-2$ & & & & & & \\
\hline 334 & $9 / 7 / 200$ & 5:48:40 P & 25.5 & 24.95 & \begin{tabular}{|l|}
25.12 \\
\end{tabular} & 26.11 & 24.0 & 42.02 & 44.291 & 4.66 & 38.057 & & & & & & & & & & & & & & & & \begin{tabular}{|c|}
4.914 \\
\end{tabular} & \\
\hline & & & & & 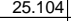 & & & & 44.433 & & & & & & & & & & & & & & & & & & & \\
\hline $33 t$ & & & & & & 20.129 & & & 44.309 & & & & & & & & & & & & & & & & & & 4.930 & \\
\hline & $\frac{9}{91 / 2120}$ & & & 24.989 & 25.125 & 26.183 & 24.2 & 42.0 & 44.36 & 4.6 .6 & 38.043 & -1.209 & & & & 1.3 & & & & 43. & & & & & & & & \\
\hline $339^{2}$ & & & & & & & & & & & & & & & & & & & & \begin{tabular}{|l|}
43.5 \\
3.
\end{tabular} & & & & & & & 4.9 & \\
\hline & & & & & & & & 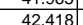 & 447 & & & & & & & & & 512.92 & \begin{tabular}{|}
8.54861 \\
856528 \\
\end{tabular} & \begin{tabular}{|l|l}
43.2 \\
136
\end{tabular} & & & & & & & & $\begin{array}{l}4.20 \\
4.6\end{array}$ \\
\hline 341 & $9 / 7 / 200$ & & 25.525 & & \begin{tabular}{|l|l|}
25.124 \\
\end{tabular} & 26.278 & & & \begin{tabular}{|l|l|}
4.858 \\
\end{tabular} & & & & & & & & & & & & & & & & & & & \\
\hline 342 & $9 / 7 / 20$ & & & & & & & 41.9 & \begin{tabular}{|l|l|}
44.235 \\
\end{tabular} & 4.6 & & & & & & & & & & & & & & & & & & \\
\hline 343 & $9 / 7 / 20$ & & & & & 26.3 & & 42.1 & 44.2 & & 38.144 & & & & & & & & & & & & & & & & & \\
\hline 344 & 9/7/2 & & & & & $26.3 \mathrm{~s}$ & & 42. & 44. & & & & & & & & & & & & & & & & & & & \\
\hline 345 & 9/7/120 & 5:59:40 & 25.527 & 24.98 & 25.131 & 26.34 & & 42.285 & \begin{tabular}{|l|l|} 
& 44.804 \\
\end{tabular} & 4.75 & 38.121 & -1.212 & & & & & & & & \begin{tabular}{|l|l|} 
& 43.7 \\
\end{tabular} & 11.6 & & & & & & 4.907 & \\
\hline 346 & $9 / 7 / 2$ & & & 24.4 & & & & 22.1 & 22.8 & 4.6 & & & & & & & & & & & & & & & & & 11 & \\
\hline & 9/7/2004 & 6:43: & 24.932 & 24.439 & \begin{tabular}{|l|}
24.654 \\
\end{tabular} & 25.604 & 24. & 22.025 & $\mid 22.6$ & 4.5 & 17.823 & -1.209 & & & \begin{tabular}{|l|l|l|l}
0.003 \\
\end{tabular} & $\begin{array}{l}0.702 \\
\end{array}$ & & & & & & $\begin{array}{ll}19.9 \\
\end{array}$ & & 0.101 & & & 5.094 & \\
\hline & & & & 24.4 .4 & & 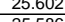 & & $\frac{4.996}{3.526}$ & 23.05 & 4.614 & 0.3 & & & 46.14 & $\begin{array}{l}0.003 \\
\end{array}$ & -0.013 & & 563.90 & & \begin{tabular}{|l|}
22.0 \\
\end{tabular} & 11.1 & & 0.185 & 02 & & \begin{tabular}{|c|c|}
-0.001 \\
\end{tabular} & 0.702 & $\begin{array}{l}-0.60 \\
-121\end{array}$ \\
\hline & & 6:46:39 PM & 4.928 & 24,406 & & 25.601 & & 3.613 & 23.433 & 4.578 & $-0.988]$ & & & 6.197 & 0.003 & 0.012 & & & \begin{tabular}{|l|l|l|l|} 
\\
\end{tabular} & 223 & & & & & $\begin{array}{l}-0.002 \\
-0.002 \\
\end{array}$ & -0.00 & & \\
\hline & & & & & & & & & & & & & & & & & & & & & & & & & & & $-1.326 \mid$ & \\
\hline
\end{tabular}


WSRC-TR-2005-00105, REVISION 0

SRNL-RPP-2005-00012, REVISION 0

RUN \# 4.18 AND 4.19; ACID CLEANING AND CAUSTIC RINSING - CONT.

\begin{tabular}{|c|c|c|c|c|c|c|c|c|c|c|c|c|c|c|c|c|c|c|c|c|c|c|c|c|c|c|c|c|}
\hline & A & B & D & & & G & $\mathrm{H}$ & $\mathrm{J}$ & $\mathrm{K}$ & $\mathrm{L}$ & M & $\mathrm{N}$ & 0 & \begin{tabular}{l|l} 
\\
\end{tabular} & $\mathrm{R}$ & $\mathrm{s}$ & $\mathrm{T}$ & v & w & $x$ & & & & & & & & AF $\mathrm{AA}$ \\
\hline & 9/7/2004 & $6: 47: 39 \mathrm{PM}$ & 24.841 & 24.383 & 24.499 & 25.568 & 23.885 & 7.553 & 23.159 & 4.586 & $\begin{array}{l}.147 \\
\end{array}$ & 10.304 & \begin{tabular}{|l|}
0.417 \\
\end{tabular} & $\begin{array}{c}47.034 \\
\end{array}$ & 1.205 & -0.013 & 0. & 566.90 & \begin{tabular}{l|l}
90 & 9.44833 \\
\end{tabular} & 22.0 & 11. & 5.4 & \begin{tabular}{|l|}
0.369 \\
\end{tabular} & $\frac{-0.00}{-0.00}$ & $\frac{-0.002}{-0.02}$ & 0.00 & $\frac{-0.353}{-0.53}$ & -0.30 \\
\hline \begin{tabular}{|l|l|}
352 \\
52 \\
\end{tabular} & $9 / 7 / 2004$ & 6:18:39 PM & 24.733 & 24.365 & 24.466 & $\begin{array}{r}25.52 \\
25.57\end{array}$ & \begin{tabular}{|l|}
23.747 \\
\end{tabular} & 15.869 & \begin{tabular}{|l|}
22.641 \\
2.621 \\
\end{tabular} & 4.534 & $\begin{array}{l}12.187 \\
1.570\end{array}$ & $\begin{array}{l}0.765 \\
0.765 \\
\end{array}$ & $\begin{array}{r}-4.029 \\
\end{array}$ & \begin{tabular}{|l|}
48.419 \\
8.719
\end{tabular} & 0.004 & $\begin{array}{l}2.557 \\
\end{array}$ & & 5667.90 & \begin{tabular}{l|l}
90.465 \\
90
\end{tabular} & 21.5 & & & \begin{tabular}{|l|l|}
0.967 \\
\end{tabular} & \begin{tabular}{|l|l|l}
0.366 \\
\end{tabular} & 0.372 & 0.026 & 26.492 & \\
\hline 353 & 9/7/2004 & 6:49:39 PM & 24.699 & $\begin{array}{l}24.362 \\
21.321\end{array}$ & $\begin{array}{l}24.452 \\
2.287\end{array}$ & 25.497 & \begin{tabular}{|l|}
23.678 \\
\end{tabular} & 21.438 & 23.238 & 4.522 & 17.506 & $\begin{array}{l}-1.206 \\
\end{array}$ & $\begin{array}{r}-4.486 \\
\end{array}$ & $\begin{array}{l}47.146 \\
\end{array}$ & 0.003 & 1.189 & & & 48167 & & & & 1.343 & & $\begin{array}{l}0.173 \\
\end{array}$ & 0.009 & 8.878 & \\
\hline $\begin{array}{l}354 \\
355\end{array}$ & $9 / 7 / 2004$ & 6:50:39 PM & $\begin{array}{r}24.654 \\
2463 \\
\end{array}$ & $\begin{array}{l}24.321 \\
24308\end{array}$ & \begin{tabular}{l|l|}
1 & 24.387 \\
& 24348
\end{tabular} & \begin{tabular}{|l|}
25.406 \\
25.363 \\
\end{tabular} & $\begin{array}{l}23.588 \\
23609\end{array}$ & \begin{tabular}{|l|}
21.809 \\
2181
\end{tabular} & \begin{tabular}{|l|}
23.087 \\
23045 \\
\end{tabular} & $\begin{array}{r}4.56 \\
4515\end{array}$ & $\begin{array}{l}17.652 \\
17777\end{array}$ & $\begin{array}{l}-1.209 \\
.212\end{array}$ & $\begin{array}{r}-4.497 \\
\end{array}$ & \begin{tabular}{|l|l|}
47.142 \\
46.979 \\
\end{tabular} & \begin{tabular}{|l|l|}
0.003 \\
0.003
\end{tabular} & $\begin{array}{l}1.001 \\
0.923\end{array}$ & & $\begin{array}{l}569.90 \\
5700\end{array}$ & $\begin{array}{rl}90 & 9.49833 \\
90 & 9515\end{array}$ & 22.0 & & \begin{tabular}{|l|}
19.7 \\
198 \\
\end{tabular} & 1.360 & \begin{tabular}{|l|l|l|} 
\\
\end{tabular} & \begin{tabular}{|l|l|l|l|l|}
0.345 \\
\end{tabular} & 0.007 & $\begin{array}{l}7.390 \\
6800\end{array}$ & \\
\hline \begin{tabular}{|l|}
355 \\
356
\end{tabular} & $\begin{array}{l}9 / 7 / 2004 \\
9 / 7 / 2004\end{array}$ & $\begin{array}{ll}6: 55: 39 \mathrm{PM} \\
6: 52: 39 \mathrm{PM}\end{array}$ & $\begin{array}{r}24.63 \\
24.607 \\
\end{array}$ & $\frac{24.308}{24.284}$ & \begin{tabular}{l|l|}
4 & 24.348 \\
4 & 24.349
\end{tabular} & $\begin{array}{l}25.563 \\
25.359 \\
\end{array}$ & $\begin{array}{r}23.609 \\
23.621\end{array}$ & \begin{tabular}{|c|}
21.811 \\
22.554
\end{tabular} & \begin{tabular}{|r|}
23.045 \\
23.16
\end{tabular} & $\begin{array}{l}\mid 4.5515 \\
4.563\end{array}$ & $\begin{array}{l}\frac{17.777}{17.861} \\
\end{array}$ & $\frac{-1.212}{-1.212}$ & $\begin{array}{l}-4.52 \\
-4.52 \\
-1\end{array}$ & \begin{tabular}{|c|}
$\mid 46.9797$ \\
47.175 \\
\end{tabular} & \begin{tabular}{|l}
0.003 \\
0.003
\end{tabular} & $\begin{array}{l}0.923 \\
0.881 \\
\end{array}$ & $\begin{array}{l}0.003 \\
0.003\end{array}$ & $\begin{array}{r}577.90 \\
571.90 \\
\end{array}$ & \begin{tabular}{c|c}
90 & 9.515 \\
90 & 9.5316
\end{tabular} & 21.9 & 112 & \begin{tabular}{|l|}
19.8 \\
200 \\
\end{tabular} & $\mid$\begin{tabular}{|l|}
$\mid$ \\
$\mid$
\end{tabular} & \begin{tabular}{|l|l|l} 
\\
0.126
\end{tabular} & \begin{tabular}{|c|c|}
0.135 \\
0.129
\end{tabular} & $\begin{array}{l}0.007 \\
0.006\end{array}$ & $\begin{array}{c}6.800 \\
6.442\end{array}$ & $\begin{array}{r}5.79 \\
58\end{array}$ \\
\hline \begin{tabular}{|l|}
357 \\
\end{tabular} & & & & & & & 23.661 & & & 4.552 & 17.73 & \begin{tabular}{|l|}
-1.14 \\
-1.212 \\
\end{tabular} & $\begin{array}{r}-4.523 \\
-4.523\end{array}$ & \begin{tabular}{|r|}
46.745 \\
\end{tabular} & 0.003 & 0.844 & & $\begin{array}{l}571.90 \\
572.90\end{array}$ & $\begin{array}{llll}90 & 9.5316 \\
0 & 9.54833\end{array}$ & $\begin{array}{ll}22.0 \\
21.7\end{array}$ & $\begin{array}{l}11.2 \\
11.1\end{array}$ & $\begin{array}{l}20.0 \\
19.8\end{array}$ & $\begin{array}{l}1.3 / 6 \\
1.364\end{array}$ & \begin{tabular}{|l|l|} 
& 0.126 \\
0.121
\end{tabular} & \begin{tabular}{|l|l|l|l|}
0.123 \\
\end{tabular} & $\begin{array}{l}0.006 \\
0.006\end{array}$ & & $\begin{array}{l}5.48 \\
5.30\end{array}$ \\
\hline \begin{tabular}{|l|}
358 \\
\end{tabular} & $9 / 7 / 2004$ & 6:54:39 PM & & 24.243 & & & 23.685 & 22.417 & $\begin{array}{l}23.236 \\
\end{array}$ & 4.332 & 18.715 & -1.209 & & \begin{tabular}{|l|}
46.26 \\
\end{tabular} & 0.003 & 0.824 & & & 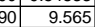 & 22. & & & & & & 0.006 & $\frac{0.202}{5.854}$ & $\begin{array}{l}5.30 \\
4.98 \\
\end{array}$ \\
\hline 359 & $9 / 7 / 2004$ & 6:55:39 PM & $\begin{array}{l}24.547 \\
\end{array}$ & 24.229 & $\begin{array}{l}9 \\
9\end{array}$ & 25.325 & $\begin{array}{l}23.686 \\
\end{array}$ & 22.346 & \begin{tabular}{|l|l|}
23.302 \\
\end{tabular} & $\begin{array}{l}4.45 \\
\end{array}$ & 18.294 & -1.212 & -4.52 & 43.235 & & 0.805 & & & & & & & & & & & & $\begin{array}{l}4.98 \\
4.93\end{array}$ \\
\hline $360 \mid$ & & & & 24.22 & & & & & 23.35 & & & & & & & & & & & & & & & & & & & \\
\hline 361 & $9 / 7 / 2004$ & 6:57:39 PM & 24.544 & 24.226 & 24.302 & 25.316 & $\begin{array}{l}23.743 \\
\end{array}$ & 22.197 & 23.191 & 4.68 & 17.87 & -1.212 & -4.5 & \begin{tabular}{|l|l|}
46.84 \\
\end{tabular} & & 0.761 & & 576.90 & 9.615 & 22.1 & & & 17 & 0.10 & 0 & 0.006 & 5.547 & $\begin{array}{l}4.02 \\
4.72 \\
\end{array}$ \\
\hline 362 & $9 / 7 / 2004$ & 6:58:39 PM & 24.544 & 24.216 & 24.312 & 25.311 & 23.754 & 22.417 & 23.12 & 4.586 & 18.294 & -1.212 & -4.52 & 46.552 & & 0.755 & & 577.90 & $\begin{array}{l}90 \\
96.63167\end{array}$ & 22.1 & 11.1 & 20. & 1.403 & 0.108 & 0.110 & 0.005 & 5.414 & \\
\hline $36-8$ & 9/7/2004 & 6:59:39 PM & 24.53 & 24.202 & 24.302 & 25.302 & 23.759 & 22.137 & 22.946 & 4.476 & 18.038 & -1.212 & -4.52 & $\begin{array}{l}46.934 \\
\end{array}$ & 0.6 & 0.745 & & 578.90 & \begin{tabular}{l|l}
90 & 9.64833 \\
\end{tabular} & 21.8 & & 20. & 1.385 & 0.10 & 0.109 & 0.005 & 5.415 & 4.61 \\
\hline & $9 / 7 / 2004$ & 7:00:39 PM & & & & & & & & & 18.42 & & & & 0.003 & 0.734 & & 579.90 & 9.665 & & & & & & & & & 4.47 \\
\hline 36 & 9/7/2004 & 7:01:39 PM & $\begin{aligned} 24.52 \\
24.532\end{aligned}$ & 24.193 & \begin{tabular}{|l|}
24.293 \\
2.20
\end{tabular} & 25.363 & $\begin{array}{r}23.795 \\
2.770\end{array}$ & \begin{tabular}{ll|}
22.056 \\
2.025
\end{tabular} & 22.842 & $\begin{array}{l}4.388 \\
\end{array}$ & 18.112 & -1.212 & $\begin{array}{r}-4.52 \\
\end{array}$ & 46.671 & 0.003 & $\begin{array}{l}0.726 \\
\end{array}$ & & 580.90 & $\begin{array}{l}90 \\
90\end{array}$ & 21.7 & & & 1.385 & 0.104 & .106 & 0.005 & 5.279 & 4.49 \\
\hline 367 & $\frac{9 / 7 / 72004}{97 / 2004}$ & $\begin{array}{l}7: 02: 39 \mathrm{PM} \\
7: 03: 39 \mathrm{PM}\end{array}$ & $\frac{24.532}{24.522}$ & $\frac{24.189}{24.174}$ & \begin{tabular}{l|l|}
4 & 24.289 \\
4 & 24.274
\end{tabular} & \begin{tabular}{|l|}
25.384 \\
25.409 \\
\end{tabular} & $\begin{array}{r}23.776 \\
23.851\end{array}$ & 22.425 & $\frac{23.128}{23.24}$ & $\begin{array}{r}4.564 \\
4.396\end{array}$ & $\frac{18.297}{18.323}$ & $\frac{-1.212}{-1.212}$ & $\begin{array}{l}-4.517 \\
-4.517 \\
\end{array}$ & $\begin{array}{l}46.151 \\
46.665\end{array}$ & 0.003 & $\begin{array}{r}0.72 \\
0.714\end{array}$ & & $\begin{array}{l}581.90 \\
582.90\end{array}$ & $\frac{0.00715}{9.715}$ & 22. & $\frac{11.0}{11 .}$ & 20.4 & $\begin{array}{l}1.404 \\
1.404\end{array}$ & \begin{tabular}{|l|l|} 
\\
\end{tabular} & 0.105 & \begin{tabular}{|l}
0.005 \\
0.005
\end{tabular} & \begin{tabular}{|l|l|}
5.165 \\
5.23
\end{tabular} & $\begin{array}{l}4.40 \\
436\end{array}$ \\
\hline$\frac{01}{368}$ & $9 / 7 / 72004$ & 7:04:39 PM & $\frac{24.524}{24.513}$ & 24.165 & \begin{tabular}{l|l|} 
& 24.274 \\
5 & 24.256
\end{tabular} & \begin{tabular}{|l|}
25.409 \\
25.436 \\
\end{tabular} & 23.838 & 22.4095 & \begin{tabular}{|l|}
23.244 \\
23.039 \\
\end{tabular} & 4.28 & $\begin{array}{l}10.023 \\
18.666\end{array}$ & $\frac{-1.242}{-1.212}$ & $\begin{array}{l}-4.511 \\
-4.512\end{array}$ & 46.291 & 0. & 0.114 & & $\begin{array}{l}582.90 \\
583.90\end{array}$ & & & & $\frac{2.4}{20.5}$ & $\begin{array}{l}\frac{1.404}{1.415} \\
\end{array}$ & & \begin{tabular}{|c|c|c|} 
\\
0.104
\end{tabular} & $\frac{0.005}{0.005}$ & $\begin{array}{l}5.123 \\
5.059 \\
\end{array}$ & $\begin{array}{l}4.36 \\
430\end{array}$ \\
\hline 369 & $9 / 7 / 2004$ & 7:05:39 PM & \begin{tabular}{|l|l|}
24.518 \\
\end{tabular} & 24.17 & \begin{tabular}{|l|l|}
7 & 24.271 \\
\end{tabular} & 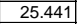 & 23.858 & 22.421 & 23.12 & $\begin{array}{l}4.462 \\
\end{array}$ & 18.292 & -1.212 & -4.509 & 46.241 & 0.0 & 0.704 & & 584.90 & $\begin{array}{lll}90 & 9.74833\end{array}$ & 21. & & 20.4 & $\frac{1.410}{1.404}$ & \begin{tabular}{|l|l|}
0.10 \\
\end{tabular} & $\begin{array}{l}0.104 \\
0.103 \\
\end{array}$ & 0.005 & 5 & $\frac{4.30}{4.30}$ \\
\hline 370 & $9 / 7 / 2004$ & 7:06:39 PM & 24.514 & 24.166 & \begin{tabular}{|l|l|}
6 & 24.267 \\
\end{tabular} & 25.412 & 23.909 & 22.502 & \begin{tabular}{|l|}
23.228 \\
\end{tabular} & $\begin{array}{r}4.49 \\
\end{array}$ & 18.356 & -1.212 & -4.486 & \begin{tabular}{|l|l|}
46.548 \\
\end{tabular} & & & & 585.90 & \begin{tabular}{l|l}
90 & 9.765
\end{tabular} & & & & & & & & & \\
\hline 371 & & 7:07:39 PM & 24.514 & 24.172 & 24.272 & 25.427 & 23.899 & 22.39 & 23.122 & $\begin{array}{l}4.468 \\
\end{array}$ & 18.264 & -1.212 & & | 45.813 & & 0.695 & & & & 22 & & & & & $\frac{0.102}{0.102}$ & & 4.997 & \\
\hline & $9 / 7 / 2004$ & 7:08:39 PM & 24.51 & 24.167 & 24.278 & 25.443 & & & 23.184 & \begin{tabular}{|l|l|}
4.39 \\
\end{tabular} & $\begin{array}{l}18.407 \\
\end{array}$ & -1.215 & -4.463 & 45.498 & & o. 0.692 & & 587.90 & 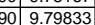 & $\frac{22}{22}$ & & & & & 0.101 & & 4.956 & \\
\hline 373 & $9 / 7 / 2004$ & $7: 09: 39 \mathrm{PM}$ & & 24.183 & \begin{tabular}{l|l}
24.293 \\
\end{tabular} & 25.448 & 23.94 & 22.463 & 23.075 & 4.477 & 18.475 & -1.212 & & $\begin{array}{l}46.573 \\
\end{array}$ & & 0.686 & & 588 & 9.815 & & & & 1.41 & & & & 4.895 & \\
\hline 374 & $9 / 7 / 2004$ & 7:10:39 PM & 24.51 & 24.172 & 24.293 & 25.443 & 23.935 & 22.664 & 23.365 & 4.505 & 18.578 & -1.209 & -4.46 & 46.106 & & 0.685 & & 589.90 & \begin{tabular}{l|l}
9.83167 \\
\end{tabular} & & & & & 0.098 & 0.100 & & 4.852 & 4.13 \\
\hline 375 & & 7:11:39 PM & 24.501 & 24.163 & 24.273 & 25.413 & 23.95 & 22.504 & 23.182 & $\begin{array}{l}4.498 \\
\end{array}$ & 18.387 & -1.215 & -4.46 & | 46.343 & 0.00 & $\begin{array}{l}0.678 \\
\end{array}$ & & 590.90 & $\begin{array}{l}909.84833 \\
\end{array}$ & 22. & & 20. & 1.410 & 0.097 & 0.099 & 0.005 & 4.846 & 4.12 \\
\hline $\begin{array}{ll}376 \\
377\end{array}$ & 9/7/2004 & 39PM & 24.508 & 24.175 & 24.28 & 25.435 & 23.947 & 22.481 & 23.18 & 4.51 & & -1.2 & -4.45 & 46.16 & & 0.677 & & 591.9 & 9.865 & 22. & & & 1.40 & 0.09 & 0.099 & & 4.846 & 4. \\
\hline & - & $=1.13 .39 \mathrm{PM}$ & $\begin{array}{r}24.51 \\
24511\end{array}$ & 24.183 & $\begin{array}{ll}3 \\
3\end{array}$ & 25.4653 & $\begin{array}{r}23.965 \\
23.986\end{array}$ & $\frac{22.444}{22086}$ & 22.979 & $\begin{array}{l}4.433 \\
\end{array}$ & 18.518 & $\frac{-1.212}{-200}$ & -4.45 & 45.957 & & 0.073 & & 592.90 & $\begin{array}{ll}90 \\
0\end{array}$ & 21. & & & & & 0.098 & & 4.800 & \\
\hline$\frac{150}{370}$ & 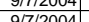 & $7.145 .39 \mathrm{PM}$ & 24.511 & & & & & & & 4.084 & & & & 40.459 & & & & & & & & & & & & & & 4.11 \\
\hline 380 & $97 / 7004$ & 7.1.15.39 PM & 24497 & 24.170 & 24.241 & 25.400 & 23. & 20703 & 20.034 & 4.416 & 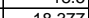 & 121 & -4.4 & (1).001 & & 0.061 & & & $\begin{array}{lll}90 & 9.915 \\
0\end{array}$ & 21 & 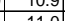 & & 1.41 & , & 0 & 0 & 4.1808 & 1002 \\
\hline $\mid$ & $9 / 7 / 2004$ & 7:17:39 PM & 24.503 & 24.18 & $8 \mid 24.266$ & 25.426 & 23.783 & 22.386 & 22.963 & 4.427 & $\frac{10.044}{18.44}$ & -1212 & -4.45 & 45.465 & 0.00 & 0.666 & & 5596.90 & 909.94833 & 218 & 10,8 & 20.4 & $\frac{1.414}{1.407}$ & 0.095 & 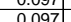 & 0.005 & 年. & $\begin{array}{l}4.03 \\
406\end{array}$ \\
\hline \begin{tabular}{|l|l|l|l|}
382 \\
\end{tabular} & $9 / 7 / 2004$ & $7: 18: 39 \mathrm{PM}$ & 24.499 & 24.177 & \begin{tabular}{|l|l|}
7 & 24.262
\end{tabular} & 25.347 & 23.704 & 22.726 & 23.303 & 4.441 & 18.781 & -1.215 & -4.45 & 46.208 & 0.00 & 0.665 & & 59790 & 90 & & & 20 & 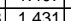 & & (7) & 0005 & 4684 & 4.00 \\
\hline & & & & & & & & & & & & & & & & & & & & & & & & & & & & 3.99 \\
\hline$\sqrt{38}+4$ & $9 / 7 / 2004$ & $7 \cdot 54 \cdot 29$ & 25.182 & 23.973 & 24.92 & 289 & & 21.938 & 20449 & 4.484 & 17.70 & & & 46168 & & & & & & 21. & & & & & 081 & 000 & 4405 & $\begin{array}{l}3.48 \\
3.49\end{array}$ \\
\hline 385 & $9 / 7 / 2004$ & 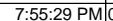 & 25.139 & 23.97 & 24.882 & 25.291 & 23. & 18.284 & 22.818 & 4.508 & 14.081 & -1.212 & -4 . & 45 & & & & & $\begin{array}{lll}773 & 10.5789\end{array}$ & 21. & 10.6 & 16.2 & 1116 & & 002 & 0.000 & -0.115 & $\begin{array}{c}-0.49 \\
-0.10\end{array}$ \\
\hline & & & & & & & 23.914 & & 22.731 & & -1.206 & 14.294 & & & & & & & & & & & & & & & & 16 \\
\hline 38 & $9 / 7 / 2004$ & 7:57:29 PM & 25.147 & 23.988 & $\begin{array}{c}8 \\
8\end{array}$ & 25.309 & 23.96 & 3.335 & 22.556 & 4.439 & -1.101 & 14.274 & 48.36 & j.131 & & -0.01 & & 636.73 & \begin{tabular}{l|l|l}
73 & 10.6122
\end{tabular} & 21. & 11. & & 0.077 & & & & .289 & 110 \\
\hline 38 & $9 / 7 / 2004$ & 7:58:29 PMI & $\begin{array}{ll}0 & 25.069\end{array}$ & 23.99 & $\begin{array}{l}9 \mid 24.767 \\
\end{array}$ & 25.316 & 23.988 & 7.929 & 22.417 & 4.39 & 3.806 & & 0.38 & 46.836 & 1.26 & 14.643 & & 63 & $\begin{array}{lll}73 & 10.6289\end{array}$ & 21. & 11 & -5 & 0.405 & & 110 & $0.36 c$ & 59.638 & \\
\hline 年 & $9 / 7 / 20004$ & 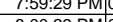 & 25.011 & 24.002 & 24.749 & 25.378 & $\frac{23.95}{2309}$ & $\begin{array}{l}14.84 \\
1.8017\end{array}$ & 年 & 4.467| & $\frac{11.19}{11.19}$ & 10 & & 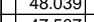 & & 2.106 & & & & 21. & & & 100 & (2) & & 0 & 0.010 & \\
\hline & & & & & & & & & & & & & & & & & & & & & & & & & & & & \\
\hline$\frac{5 x}{30}$ & $9 / \pi 2004$ & 8:01:29 PM & 24.933 & 24.024 & 24.101 & 25.4 & 2.3 .9 & 20.187 & 22.699 & 4.512 & $\frac{16.189}{17.56}$ & -1.21 & & 41.5 & & 1.44 & & & 10.6 & 21. & & 18 & 1.254 & & 208 & 0.01 & 111.431 & 9.1 \\
\hline & $9 / 7 / 72004$ & 8.03 .29 PMC & 24896 & & 24639 & 25.438 & 23.94 & 21712 & 22602 & 4517 & 17.617 & & -38 & & & 1.0512 & & & 1070 & & & & 1..54t & & & 0 & & 6.65 \\
\hline & & & & & & 2542 & & & & 4 & 17804 & & & & & & & & & & & & & & & & 0.250 & 5.33 \\
\hline 395 & & & 24859 & & & 25.401 & & 21.93 & 22664 & 4468 & & 1.200 & & & & & & & & & & & & & & 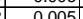 & 5.113 & $\begin{array}{l}4.14 \\
4.35\end{array}$ \\
\hline $\begin{array}{l}396 \\
3\end{array}$ & $9 / 7 / 2004$ & $8: 06: 2$ & 24.835 & 24.0 & 24.573 & 25.382 & 23.8 & 22.183 & 22.784 & 4.286 & 18.364 & -1.20 & & 44. & & 0.6 & & & 10 & 21 & & & 1020 & & & & 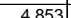 & $\frac{4}{4}$ \\
\hline 397 & & & 24.811 & 24.0 & & 25.388 & & \begin{tabular}{|l|l|l|}
22.183 \\
\end{tabular} & \begin{tabular}{|l|l|}
22.809 \\
\end{tabular} & 4.344 & & -1.2 & & & & & & & & & & & 1.5 & & & & 4.6 & \\
\hline $33^{3}$ & $9 / 7 / 2004$ & $8: 0 €$ & 24.78 & 24.0 & 24.528 & 25.352 & & 22.039 & 22 & 4.41 & & & & & & & & & & & 1 & & & & & & 4.612 & \\
\hline 33 & $9 / 7 / 2004$ & 8:09:29 PM[ & 24.781 & 24.052 & 24.524 & 25.328 & 23 & 22.351 & 22.973 & 4.342 & 18.397 & -1.212 & -3.8 & 46.2 & & & & & & 21. & 11 & & & & & 0.0 & 4.237 & \\
\hline 400 & 9/7/2004 & & 24.756 & 24.0 & 24.5 & 25.298 & & 22.012 & 24 & 4.381 & & $-1.20 \mathrm{c}$ & & & & 0.61 & & & & 21 & & & & & & & 4.437 & \\
\hline$\frac{401}{4012}$ & $9 / 1 / 2004$ & 8.11 .29 & 24.714 & 24.016 & 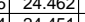 & $\frac{25.171}{2.115}$ & 23. & 22.289 & 22.822 & 4.436 & $\frac{1}{10}$ & -1.1215 & & & & & & & & 211 & & & & & & & 4.3 & \\
\hline & & & 24.105 & 24.014 & 4) 24.451 & 250.110 & & 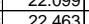 & \begin{tabular}{|l|l|l} 
\\
\end{tabular} & & & & & & & & & & & & & & & & & & 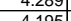 & \\
\hline & & & & & & 250005 & & 22005 & & $4.4 \pi$ & & & & & & & & & & & & & & & & & & \\
\hline & & & & & & & & & & 4.4075 & & & & & & & & & & & 10.6 & 20. & & & & & & \\
\hline & 20 & & & 2007 & & & & & & 40.72 & & & & & & 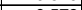 & & & & 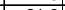 & & & & & & & & 3.47 \\
\hline $4^{4}$ & IIIIZOU4 & $0.10 .29 \mathrm{~F}$ & 24.040 & $23.9 / 4$ & & 24.965 & & 22.500 & 23.029 & & & & & & & & & & & & & & & & & & & \\
\hline & & & & $\frac{23.978}{2072}$ & & & & 2.2.3517 & 22.859 & 4.374 & 18.393 & & & & & & & & & 21. & & 20.4 & & & & & [3] & \\
\hline$\frac{44}{44}$ & & 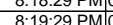 & $\frac{24.031}{24.617}$ & $\frac{23.972}{23969}$ & $\begin{array}{l}2.3045 \\
2455\end{array}$ & $\frac{24.5}{249}$ & $\frac{23.5}{236}$ & $\frac{21.212}{22183}$ & & $\begin{array}{l}4.645 \\
4.524\end{array}$ & $\frac{11.644}{18098}$ & $\frac{1.21}{-121}$ & -38 & $\frac{46.596}{46.464}$ & & & & & 100 & $\frac{21 .}{21 .}$ & & & & & & & $\frac{4}{4}$ & \\
\hline$\frac{45}{41}$ & $9 / 7 / 720$ & $8.2029 \mathrm{PN}$ & 24.602 & 23.963 & \begin{tabular}{|c|c|c|}
3 & 24.349 \\
\end{tabular} & 24.988 & 23. & 21.954 & 22.293 & | & & -1.212 & & 46.6 & & 0.5 & & $\frac{65}{65}$ & $\begin{array}{ll}73 & 10.9956\end{array}$ & 212 & 11. & 19.9 & & & & & 4.009 & \\
\hline 4 & & 8:21:229F & 2459 & 23.964 & 24.346 & 25.005 & & 21.826 & & 4.428 & & & $\frac{-3.8}{-3.8}$ & & & 0.5 & & & 110122 & 21. & 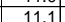 & & & & & & 3988 & \\
\hline & & & & 23.959 & & 24.99 & & 21.62 & 22.04 & & & & & & & & & & & & & & & & & & 35 & \\
\hline & & $8: 23: 29 \mathrm{P}$ & & 23.956 & 24.342 & 25.011 & & 22.062 & 22.469 & 4.627 & & -1.209 & & & & & & & & 21.3 & & & & & & & 4 & \\
\hline & & 8:24:2 & 24.566 & 9 & 24.328 & 24.99 & & 21.849 & & 4.34 & & -1.209 & & & & & & & 11. & & 1 & & & & & & 3. & \\
\hline 4 & 17/2004 & $8: 25: 29 \mathrm{PM}$ & 24.541 & 23.947 & 24.2 & 24.997 & 23 & 21.857] & 22.386 & 4.444 & 17. & -1.209 & $-3-3$ & 46.8 & & & & 66 & \begin{tabular}{|l|l|}
11.0 \\
\end{tabular} & 21. & 1 & & & & & & 3.895 & 3 \\
\hline 416 & 9/7/200 & $8: 26: 29 \mathrm{PM}$ & 24.547 & 23.963 & \begin{tabular}{l|l|}
3 & 24.289 \\
\end{tabular} & 24.984 & 23. & 21.874 & 22.347 & 4.437 & 17.794 & -1.209 & & 46.3 & & & & 66 & 11.0 & 21.8 & & & 1.3 & & & & 3.896 & \\
\hline 41 & $9 / 7 / 2004$ & 8:27:29 PMC & 24.542 & 23.968 & $\begin{array}{l}8 \quad 24.299 \\
\end{array}$ & 24.9999 & 23.6 & 21.892 & 22.291 & 4.429| & 17.896 & -1.209 & -3.841 & 46.418 & & 0.51 & & 666 & $\begin{array}{ll}73 & 11.1122\end{array}$ & 21.2 & 11. & 19.5 & & & & & 3.766 & \\
\hline & $9 / 1 / 20044$ & 8:28:20 PMV & $\begin{array}{l}0.24 .522 \\
02502\end{array}$ & 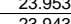 & $\frac{5}{30.2464}$ & $\begin{array}{r}24.979 \\
2967\end{array}$ & 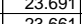 & $21.1 / 14$ & 22.165 & (.4.424 & $\frac{11.171}{17091}$ & 年-1.2995 & $-\frac{-3.384}{3.89}$ & $\frac{46.426}{4.462}$ & 0.0002 & 0.519 & & & 73 & $\frac{21.6}{21 .}$ & $\frac{11.1}{110}$ & & & & & & & \\
\hline & 9/7/2004 & 8:22:29 & & & & 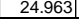 & 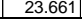 & & 22.291 & 4.312 & 17.981 & & $-3.85 \mid$ & $\begin{array}{r}46.36 \\
\end{array}$ & 0.003 & 0.51 & & & $\begin{array}{lll}73 & 11.1456\end{array}$ & 21.2 & & & & 0.073 & 0.075 & & 3.739| & $3.18 \mid$ \\
\hline
\end{tabular}


WSRC-TR-2005-00105, REVISION 0

SRNL-RPP-2005-00012, REVISION 0

RUN \# 4.18 AND 4.19; ACID CLEANING AND CAUSTIC RINSING - CONT.

\begin{tabular}{|c|c|c|c|c|c|c|c|c|c|c|c|c|c|c|c|c|c|c|c|c|c|c|c|c|c|c|c|c|}
\hline & A & $\mathrm{B}$ & $\mathrm{D}$ & $E$ & $F$ & G & \begin{tabular}{l|l}
$\mathrm{H}$ & $\mathrm{H}$ \\
\end{tabular} & $\mathrm{J}$ & $\mathrm{K}$ & $\mathrm{L}$ & $M$ & $\mathrm{~N}$ & \begin{tabular}{l|l}
$\mathrm{O}$ & $\mathrm{P}$ \\
\end{tabular} & \begin{tabular}{l|l|l|}
$P$ & $Q$ \\
\end{tabular} & $\mathrm{R}$ & $\mathrm{s}$ & \begin{tabular}{l|l}
$\mathrm{T}$ & $\mathrm{U}$ \\
\end{tabular} & U $\quad v$ & w & $\mathrm{x}$ & $\mathrm{Y}$ & $z$ & $\mathrm{AA}$ & $A B$ & $\mathrm{AC}$ & $A D$ & $\mathrm{AE}$ & AF $A$ \\
\hline \begin{tabular}{|l|l|}
425 \\
26
\end{tabular} & \begin{tabular}{|l} 
Caustic Rin \\
\end{tabular} & & & & & & & & & & & & & & & & & & & & & & & & & & & \\
\hline $\mid$\begin{tabular}{|l|}
427 \\
427
\end{tabular} & & Averages & 24.5 & 23.5 & 24.3 & 24.3 & 23.8 & 22.0 & 27.4 & 5.0 & 17.1 & 0.0 & -1.5 & 45.9 & 0.6 & 14.6 & 0.0 & & & 26.3 & 10.9 & 19.6 & \begin{tabular}{|l|l|}
5 & 1.349 \\
\end{tabular} & 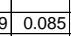 & 0.086 & 0.004 & 4.4 & 3.8 \\
\hline & & Maximum & & & 24.6 & 24.5 & & & 28.2 & & 17.8 & 0.2 & $\begin{array}{r}1.4 \\
\end{array}$ & 49.4 & 0.6 & 14.6 & 0.0 & & & 27.0 & 11.8 & \begin{tabular}{|l|}
20.2 \\
\end{tabular} & 21.393 & 30.087 & 0.089 & 0.005 & 4.7 & $\begin{array}{l}3.0 \\
4.0\end{array}$ \\
\hline \begin{tabular}{|l|l|}
429 \\
\end{tabular} & & Median & 24.5 & 23.5 & 24.3 & 24.3 & 23.8 & 22.2 & 27.8 & 5.0 & 17.3 & 0.1 & -1.5 & $\begin{array}{l}4.4 .0 \\
4\end{array}$ & 0.6 & 14.6 & 0.0 & & & 26.6 & 11.0 & 19.8 & \begin{tabular}{|l|l|}
3.367 \\
\end{tabular} & 70.086 & 0.087 & 0.004 & 4.4 & 3.7 \\
\hline 430 & & Minimum & 24.2 & 23.3 & 24.0 & 24.1 & 23.6 & 20.5 & 25.4 & 4.8 & 15.4 & -1.2 & -2.3 & 43.0 & 0.5 & 14.6 & 0.0 & & & 24.2 & 10.2 & 17.9 & 9.235 & $\begin{array}{ll}5 & 0.074 \\
\end{array}$ & 0.076 & 0.004 & 4.1 & 3.5 \\
\hline \begin{tabular}{|l|l|}
431 \\
\end{tabular} & & $2 \times \operatorname{Std}$ Dev & 0.388 & 0.123 & $\begin{array}{l}0.344 \\
\end{array}$ & 0.156 & 0.167 & 1.283 & 1.854 & 0.192 & 1.370 & 0.677 & 0.416 & 3.292 & $\begin{array}{ll}0.037 \\
\end{array}$ & 0.000 & 0.000 & & & 1.854 & \begin{tabular}{|l|l|}
0.784 \\
\end{tabular} & & \begin{tabular}{|l|}
20.091 \\
\end{tabular} & $\begin{array}{ll}10 & 0.005 \\
\end{array}$ & 0.005 & 0.000 & 0.231 & 0.197 \\
\hline \begin{tabular}{|l|l|}
432 \\
\end{tabular} & Number & of Points Used ${ }^{*}$ & \begin{tabular}{|l|}
$\quad 29$ \\
\end{tabular} & 29 & 29 & 29 & 29 & 29 & 29 & 29 & 29 & 29 & 29 & 29 & 29 & 29 & 29 & & & 29 & 29 & \begin{tabular}{|l|}
29 \\
\end{tabular} & \begin{tabular}{|l|}
$9 \quad 29$ \\
\end{tabular} & 29 & 29 & 29 & 29 & 29 \\
\hline \begin{tabular}{|l|l|}
433 \\
421
\end{tabular} & & * Backpulse po & ints in box & are not incli & uded & & & & & & & & & & & & & & & & & & & & & & & \\
\hline $\mid \frac{344}{435}$ & Acid Clean & & & & & & & & & & & & & & & & & & & & & & & & & & & \\
\hline \begin{tabular}{|l|l|}
436 \\
\end{tabular} & & & & & & & & & & & & & & & & & & & & & & & & & & & & \\
\hline \begin{tabular}{|l|}
437 \\
438 \\
\end{tabular} & & \begin{tabular}{|l} 
Averages \\
Maximum
\end{tabular} & $\begin{array}{l}25.2 \\
25.7 \\
\end{array}$ & $\begin{array}{l}24.2 \\
24.5 \\
\end{array}$ & \begin{tabular}{|l|}
24.7 \\
25.3
\end{tabular} & $\begin{array}{l}25.2 \\
25.6 \\
\end{array}$ & $\begin{array}{l}24.4 \\
24.7 \\
\end{array}$ & $\begin{array}{l}41.9 \\
42.9 \\
\end{array}$ & $\begin{array}{l}43.0 \\
44.0\end{array}$ & $\begin{array}{l}4.6 \\
4.9 \\
\end{array}$ & \begin{tabular}{|l|}
37.8 \\
38.8 \\
\end{tabular} & $\begin{array}{r}-1.1 \\
5.5 \\
\end{array}$ & $\begin{array}{r}0.6 \\
0.6\end{array}$ & $\begin{array}{l}46.2 \\
517\end{array}$ & 0.0 & 0.9 & 0.0 & & & $\begin{array}{l}41.9 \\
429\end{array}$ & $\begin{array}{ll}11.0 \\
123\end{array}$ & \begin{tabular}{|l|l|}
39.9 \\
34.8
\end{tabular} & \begin{tabular}{|l|l|}
9 & 2.748 \\
3 & 285 \\
\end{tabular} & $\begin{array}{ll}8 & 0.132 \\
5 & 0236\end{array}$ & \begin{tabular}{|l|l|}
0.133 \\
0238
\end{tabular} & $\begin{array}{l}0.003 \\
0.006\end{array}$ & $\begin{array}{l}3.3 \\
5.9 \\
\end{array}$ & $\begin{array}{l}2.8 \\
51\end{array}$ \\
\hline $\begin{array}{l}430 \\
439 \\
\end{array}$ & & Median & $\begin{array}{l}25.1 \\
25.1\end{array}$ & $\begin{array}{r}24.3 \\
24.3 \\
\end{array}$ & $\begin{array}{r}24.3 \\
24.7\end{array}$ & 25.2 & 24.1 & $\frac{-24.9}{42.1}$ & $\begin{array}{l}44.0 \\
33.0\end{array}$ & $\begin{array}{l}4.9 \\
4.6 \\
\end{array}$ & $\begin{array}{l}38.8 \\
37.9 \\
\end{array}$ & $\begin{array}{r}5.5 \\
-1.2 \\
\end{array}$ & $\begin{array}{l}0.6 \\
0.6\end{array}$ & \begin{tabular}{|l|}
51.7 \\
46.1
\end{tabular} & $\begin{array}{l}1.0 \\
0.0\end{array}$ & $\begin{array}{l}1.6 \\
0.9 \\
\end{array}$ & $\begin{array}{l}0.0 \\
0.0\end{array}$ & & & 42.9 & $\frac{12.3}{11.0}$ & \begin{tabular}{|c|}
3 \\
50.0 \\
40.0
\end{tabular} & \begin{tabular}{|l|l|}
30.2815 \\
0.755
\end{tabular} & \begin{tabular}{|l|l|}
5 & 0.236 \\
5 & 0.134
\end{tabular} & $\begin{array}{l}0.238 \\
0.135 \\
\end{array}$ & $\begin{array}{l}0.006 \\
0.003\end{array}$ & $\frac{5.9}{3.4}$ & $\frac{5.1}{29}$ \\
\hline $\mid$\begin{tabular}{|l|}
440 \\
440 \\
\end{tabular} & & Minimum & 25.0 & $\begin{array}{l}24.5 \\
23.9 \\
\end{array}$ & $\begin{array}{l}24.1 \\
2.6 \\
\end{array}$ & 24.9 & $\begin{array}{l}24.4 \\
24.1 \\
\end{array}$ & $\begin{array}{l}42.1 \\
32.2 \\
\end{array}$ & $\begin{array}{l}4.0 .4 \\
42.4\end{array}$ & $\begin{array}{l}4.0 \\
4.5 \\
\end{array}$ & 28.0 & -1.2 & 0.5 & \begin{tabular}{|l|}
40.1 \\
43.0 \\
\end{tabular} & 0.0 & 0.4 & 0.0 & & & 41.3 & \begin{tabular}{|l|}
11.0 \\
10.2 \\
\end{tabular} & 20.1 & \begin{tabular}{|l|l|}
12.075 \\
\end{tabular} & \begin{tabular}{|l|l|}
5 & 0.063 \\
\end{tabular} & 0.063 & 0.002 & $\begin{array}{l}0.4 \\
2.1 \\
\end{array}$ & $\begin{array}{l}2.5 \\
1.8 \\
\end{array}$ \\
\hline 441 & & $2 \times$ Std Dev & 0.323 & 0.337 & 0.323 & 0.311 & 0.307 & 2.069 & 0.552 & 0.178 & 2.082 & 1.367 & 0.083 & 3.039 & 0.206 & 0.266 & 0.002 & & & 0.552 & \begin{tabular}{|l|l|}
2 & 0.724 \\
\end{tabular} & & 20.143 & $\begin{array}{ll}30.038 \\
\end{array}$ & 0.039 & 0.001 & 0.954 & 0.812 \\
\hline \begin{tabular}{|l|l|}
442 \\
\end{tabular} & Number & of Points Used & \begin{tabular}{|r|}
$\quad 94$ \\
\end{tabular} & 94 & 94 & 94 & 94 & 94 & 94 & 94 & 94 & 94 & 94 & 94 & 94 & 94 & 94 & & & 94 & 94 & \begin{tabular}{|l|l|}
4 & 94 \\
\end{tabular} & \begin{tabular}{|l|l|}
4 & 94 \\
\end{tabular} & $\begin{array}{l}4 \\
4\end{array}$ & 94 & 94 & 94 & 94 \\
\hline \begin{tabular}{|l|}
443 \\
4
\end{tabular} & & ${ }^{*}$ Backpulse po & ints in box & are not incli & uded & & & & & & & & & & & & & & & & & & & & & & & \\
\hline \begin{tabular}{|l|l|}
444 \\
45
\end{tabular} & & & & & & & & & & & & & & & & & & & & & & & & & & & & \\
\hline$\left|\frac{445}{446}\right|$ & Acid Clean & & & & & & & & & & & & & & & & & & & & & & & & & & & \\
\hline \begin{tabular}{|l|}
4467 \\
\end{tabular} & & Averages & 25.4 & 24.9 & 25.0 & 25.5 & 24.9 & 42.1 & 44.5 & 4.7 & 38.1 & -1.2 & \begin{tabular}{c|c|c|}
-1.1 \\
\end{tabular} & 45.9 & 0.0 & 1.4 & 0.0 & & & 43.4 & 10.9 & & 2.8 & 0.2 & 0.2 & 0.0 & 5.0 & 4.3 \\
\hline \begin{tabular}{|l|}
448 \\
\end{tabular} & & Maximum & 25.9 & 25.1 & 25.5 & 25.8 & 25.2 & 43.3 & 45.7 & 4.8 & 39.5 & -1.1 & $\begin{array}{r}-1.0 \\
\end{array}$ & 48.7 & 0.0 & 2.1 & 0.0 & & & 44.6 & \begin{tabular}{|l|l|}
11.6 \\
\end{tabular} & \begin{tabular}{|l|l|}
5 & 41.4 \\
\end{tabular} & 2.9 & 0.3 & 0.3 & 0.0 & 8.6 & 7.3 \\
\hline 4449 & & Median & 25.5 & 24.9 & 25.1 & 25.5 & 24.9 & 42.1 & 44.5 & 4.7 & 38.1 & -1.2 & -1.0 & 45.9 & 0.0 & 1.4 & 0.0 & & & 43.4 & \begin{tabular}{|l|l|}
10.9 \\
\end{tabular} & 40.1 & $\begin{array}{l}1 \quad 2.8 \\
\end{array}$ & 0.2 & 0.2 & 0.0 & 5.0 & \\
\hline \begin{tabular}{|c|}
450 \\
51
\end{tabular} & & $\begin{array}{l}\text { Minimum } \\
\text { 2. }\end{array}$ & \begin{tabular}{|r|}
23.8 \\
0872
\end{tabular} & $\begin{array}{r}24.6 \\
0275\end{array}$ & \begin{tabular}{|l|}
23.7 \\
0774
\end{tabular} & $\begin{array}{r}25.1 \\
0309\end{array}$ & 24.4 & $\begin{array}{l}38.6 \\
0.61\end{array}$ & $\begin{array}{r}43.4 \\
0746\end{array}$ & $\begin{array}{r}4.5 \\
0.30\end{array}$ & $\begin{array}{r}34.5 \\
1012\end{array}$ & $\begin{array}{r}-1.2 \\
0.20\end{array}$ & $\begin{array}{r}-1.3 \\
0.25\end{array}$ & $\begin{array}{l}44.2 \\
1327 \\
\end{array}$ & $\begin{array}{r}0.0 \\
0.06\end{array}$ & $\begin{array}{r}1.4 \\
015\end{array}$ & $\begin{array}{r}0.0 \\
0.02\end{array}$ & & & 42.3 & \begin{tabular}{r|}
10.5 \\
0316
\end{tabular} & \begin{tabular}{|l|l|}
36.6 \\
\end{tabular} & \begin{tabular}{|l|l|}
5 & 2.5 \\
\end{tabular} & $\begin{array}{ll}5 & 0.2 \\
0.22\end{array}$ & 0.2 & 0.0 & 4.9 & 4.2 \\
\hline$\left|\frac{451}{452}\right|$ & Number & $2 \times$ Std Dev & 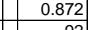 & 0.275 & 0.774 & 0.309 & 0.362 & 0.961 & 0.746 & 0.130 & 1.012 & 0.016 & 0.125 & \begin{tabular}{|l|}
1.327 \\
\end{tabular} & 0.006 & 0.156 & 0.002 & & & 0.746 & | 0.316 & 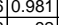 & $\mid$ & 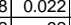 & 0.026 & 0.001 & 0.789 & 0.671 \\
\hline 45 & Number & $\begin{array}{l}\text { of Points Used* } \\
\text { |* B Backulse pe }\end{array}$ & ints in box & (a) & gle 2 & & & & & & & & & & & & & & & & & & & & & & & 92 \\
\hline 454 & & & . & 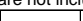 & & & & & & & & & & & & & & & & & & & & & & & & \\
\hline 455 & Acid Clean & & & & & & & & & & & & & & & & & & & & & & & & & & & \\
\hline & & & & & & & & & & & & & & & & & & & & & & & & & & & & \\
\hline 457 & & Averages & 25.4 & 25.0 & 25.0 & 25.9 & 24.7 & 42.1 & 44.7 & 4.7 & 38.1 & -1.1 & -2.1 & 46.3 & 0.0 & 1.4 & 0.0 & & & 43.5 & 11.0 & 40.1 & & 0.206 & 0.206 & 0.005 & 5.2 & 4.4 \\
\hline 458 & & Maximum & 25.5 & 25.1 & 25.2 & 26.4 & 25.2 & 43.0 & 46.1 & 4.9 & 39.0 & 11.0 & -0.9 & 48.0 & 0.0 & 4.3 & $0.6>$ & & & 45.0 & 11.4 & 40.9 & 2.820 & 0.610 & 0.624 & 0.024 & 24.0 & 20.5 \\
\hline |459 & & Median & 25.5 & 25.0 & 25.1 & 25.8 & 24.6 & $\begin{array}{ll}42.2 \\
\end{array}$ & 44.6 & 4.7 & 38.2 & -1.2 & -2.1 & 46.3 & 0.0 & 1.4 & 0.0 & & & 43.5 & 11.0 & 40.2 & & 0.198 & 0.197 & 0.005 & 4.9 & 42 \\
\hline 4660 & & Minimum & 24.3 & 24.8 & 24.2 & 25.1 & 24.1 & 27.4 & 44.0 & 4.5 & 24.5 & -1.2 & -2.2 & 45.1 & 0.0 & 1.4 & 0.0 & & & 42.9 & 10.7 & 25.9 & 1.788 & $\begin{array}{llll}8 & 0.196 \\
\end{array}$ & 0.195 & 0.005 & 4.8 & 4.1 \\
\hline \begin{tabular}{|l|}
61 \\
62
\end{tabular} & Numbre & $2 \times$ Std Dev & 0.465 & 0.147 & 0.370 & 0.642 & 0.659 & 3.133 & 0.849 & 0.157 & 2.883 & 2.535 & 0.253 & 1.053 & 0.001 & 0.615 & 0.001 & & & 0.848 & 0.251 & 3.0055 & 0.207 & $0.08 \varepsilon$ & 0.091 & 0.004 & 4.000 & .404 \\
\hline$\left|\frac{442}{463}\right|$ & Number & $\mid$ & $\frac{11}{92}$ & a & 92 & & & & 92 & & 92 & 92 & 92 & & 92 & 92 & 92 & & & & 92 & 92 & & 92 & 92 & 92 & $92>2>$ & 92 \\
\hline \begin{tabular}{|c|}
464 \\
454
\end{tabular} & & Dackpulse po & . & are not Incli & & & & & & & & & & & & & & & & & & & & & & & & \\
\hline 465 & Acid Cleans & & & & & & & & & & & & & & & & & & & & & & & & & & & \\
\hline \begin{tabular}{|l|l|}
466 \\
467
\end{tabular} & & Aिरrack & & & & & 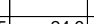 & & 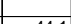 & 57 & 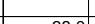 & & & & & & & & & & & & & & & & & \\
\hline 4667 & & Averages & 25.3 & 24.7 & 24.9 & 25.5 & 24.6 & 42.0 & $\frac{44.1}{44.1}$ & 4.7 & 38.0 & $\frac{-1.1}{1.1}$ & -0.88 & 46.2 & 0.0 & 1.3 & 0.0 & & & 42.9 & 11.0 & & D. 2.759 & 0.186 & 0.180 & 0.005 & $\frac{4.5}{2.5}$ & 3.9 \\
\hline $\mid \frac{400}{469}$ & & $\begin{array}{l}\text { Meaximum } \\
\text { Median }\end{array}$ & 25.9 & $\frac{24.1}{24.8}$ & $\frac{25.5}{25.0}$ & $\frac{2.4}{25.5}$ & 24.6 & $\frac{34.3}{42.1}$ & $\frac{46.1}{44.3}$ & $\frac{4.9}{4.7}$ & 39.5 & $\begin{array}{c}11.0 \\
-1.2\end{array}$ & \begin{tabular}{|c|}
-1.0 \\
-1.0
\end{tabular} & $\frac{51.1}{46.1}$ & 1.0 & $\begin{array}{c}4.3 \\
1.4 \\
\end{array}$ & 0.0 & & & 43.0 & \begin{tabular}{|l|}
12.3 \\
11.0 \\
\end{tabular} & \begin{tabular}{|l|}
41.4 \\
40.1 \\
\end{tabular} & $\begin{array}{l}4.2 .835 \\
1\end{array}$ & $\begin{array}{l}\frac{3}{3} 0.010 \\
3\end{array}$ & $\begin{array}{l}0.624 \\
0.197 \\
\end{array}$ & 0.024 & $\frac{24.0}{4.9}$ & $\frac{4.2}{4.2}$ \\
\hline 470 & & Minimum & 23.8 & 23.9 & 23.7 & 24.9 & 24.1 & 27.4 & 42.4 & 4.5 & 24.5 & -1.2 & -2.2 & 43.0 & 0.0 & 0.4 & 0.0 & & & 41.3 & \begin{tabular}{|l|}
10.2 \\
\end{tabular} & 25.9 & 1.788 & $\begin{array}{ll}80.063 \\
\end{array}$ & 0.063 & 0.002 & 2.1 & 1.8 \\
\hline 471 & & $2 \times \operatorname{Std}$ Dev & 0.631 & 0.701 & 0.596 & 0.684 & 0.600 & 2.242 & 1.639 & 0.171 & 2.155 & 1.664 & 2.206 & 2.044 & 0.120 & 0.621 & 0.002 & & & 1.639 & \begin{tabular}{|l|l|}
0.487 \\
\end{tabular} & 2.193 & 0.151 & \begin{tabular}{ll|l}
1 & 0.089 \\
\end{tabular} & 0.090 & 0.003 & 2.952 & 2.512 \\
\hline \begin{tabular}{|l|l|}
472 \\
\end{tabular} & Number & of Points Used & \begin{tabular}{|l|l|} 
& 278 \\
\end{tabular} & 278 & 278 & 278 & 278 & 278 & 278 & 278 & 278 & 278 & 278 & 278 & 278 & 278 & 278 & & & 278 & \begin{tabular}{l|l|}
8 & 278 \\
\end{tabular} & \begin{tabular}{|l|}
3278 \\
\end{tabular} & \begin{tabular}{|l|}
8 \\
\end{tabular} & \begin{tabular}{l|l}
8 & 278 \\
\end{tabular} & 278 & 278 & 278 & 278 \\
\hline - 473 & & * Backpulse po & ints in box & are not incli & & & & & & & & & & & & & & & & & & & & & & & & \\
\hline $\mid$\begin{tabular}{|l|}
475 \\
\end{tabular} & Caustic Rin: & $\frac{152}{s e}$ & & & & & & & & & & 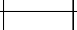 & 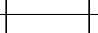 & & & & & & & 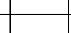 & 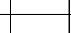 & & & & & & 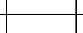 & - \\
\hline \begin{tabular}{|l|}
476 \\
\end{tabular} & & & & & & & & & & & & & & & & & & & & & & & & & & & & \\
\hline |477 & & Averages & 24.5 & 24.2 & 24.3 & 25.4 & 23.8 & 22.1 & 23.1 & 4.5 & 18.1 & -1.1 & -4.5 & 46.3 & 0.0 & 0.8 & 0.0 & & & 22.0 & 11.0 & 20. & \begin{tabular}{|c|c|}
1.385 \\
\end{tabular} & $\begin{array}{l}5 \\
5\end{array}$ & 0.119 & 0.006 & 6.1 & 5.2 \\
\hline \begin{tabular}{|c|}
478 \\
470
\end{tabular} & & Maximum & 24.7 & 24.4 & 24.5 & 25.5 & 24.0 & 22.7 & 23.4 & 4.7 & 18.8 & 0.8 & -4.0 & 48.4 & 0.0 & 2.6 & 0.0 & & & 22.3 & 11.5 & 20.8 & $\begin{array}{ll}1.431 \\
\end{array}$ & $\begin{array}{l}10.366 \\
50.10\end{array}$ & 0.372 & 0.026 & 26.5 & 22.5 \\
\hline & & Median & 24.5 & 24.2 & 24.3 & 25.4 & & & 23.1 & 4.5 & 18.3 & -1.2 & -4.5 & 46.3 & 0.0 & 0.7 & & & & & & & & & & & 5.1 & 4.4 \\
\hline$\left|\frac{480}{481}\right|$ & & $\begin{array}{l}\frac{\text { Minimum }}{2 \times \text { Std Dev }} \\
\end{array}$ & $\begin{array}{r}24.5 \\
0.117 \\
\end{array}$ & \begin{tabular}{r|}
24.2 \\
0.115 \\
\end{tabular} & $\begin{array}{r}24.2 \\
0.101 \\
\end{array}$ & \begin{tabular}{r|}
25.3 \\
0.14 \\
\end{tabular} & $\begin{array}{r}23.6 \\
0.229 \\
\end{array}$ & $\begin{array}{r}15.9 \\
234\end{array}$ & $\begin{array}{r}22.6 \\
0.340\end{array}$ & $\begin{array}{r}4.3 \\
0.374\end{array}$ & $\frac{12.2}{2241}$ & $\begin{array}{r}-1.2 \\
0699 \\
\end{array}$ & $\begin{array}{r}-4.5 \\
0173 \\
\end{array}$ & $\frac{43.2}{1678}$ & 0.0 & 0.1 & 0.6 & & & 21.5 & 10.33 & 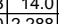 & $0.96 r$ & (1) 10.095 & 0.091 & 0.005 & $\frac{4.1}{76.9}$ & $\begin{array}{r}4.0 \\
\end{array}$ \\
\hline$\left|\frac{814}{482}\right|$ & Number & $\frac{12 \times \text { SStd Dev }}{\text { of Points Used* }}$ & 0.117 & 0.1151 & 0.1011 & $\begin{array}{r}0.114 \\
31\end{array}$ & $\frac{0.29}{31}$ & $\begin{array}{r}2.344 \\
31\end{array}$ & 0.340 & $\begin{array}{r}0.174 \\
31 \\
\end{array}$ & $\frac{2.241}{31}$ & 0.699 & $0.1 / 31$ & 1.678 & $\frac{0.000}{31}$ & $\frac{0.660}{31}$ & $\frac{0.001}{31}$ & & & $\frac{0.340}{31}$ & $\begin{array}{ll}0.40 \\
0.31\end{array}$ & $\mid 2.286$ & $\mid \begin{array}{l}\mid \\
1 \\
1\end{array}$ & $\begin{array}{ll}10.099 \\
11\end{array}$ & 0.098 & 0.000 & 1.648 & 6.509 \\
\hline \begin{tabular}{|c|}
483 \\
48
\end{tabular} & & ${ }^{*}$ Backpulse po & ints in box & are not incl & uded & & & & & & & & & & & & & & & & & & & & & & & 31 \\
\hline 484 & & & & & & & & & & & & & & & & & & & & & & & & & & & & \\
\hline \begin{tabular}{|l|l}
485 \\
196
\end{tabular} & Caustic Rin & se 3 & & & & & & & & & & & & & & & & & & & & & & & & & & \\
\hline $\mid$\begin{tabular}{|l|}
486 \\
87 \\
\end{tabular} & & Averages & 24.7 & 24.0 & 24.5 & 25.2 & & 21.6 & 226 & 44 & 176 & -11 & -38 & 462 & 00 & 08 & 00 & & & 215 & 110 & 196 & 1252 & $0.107 \mathrm{r}-\mathrm{s}$ & 0109 & & 50 & 51 \\
\hline 488 & & Maximum & 25.0 & 24.1 & $\begin{array}{l}24.7 \\
24.7\end{array}$ & 25.4 & 24.0 & 22.5 & 23.0 & 4.6 & 19.0 & 1.5 & -3.2 & 48.0 & 0.0 & 2.7 & 0.0 & & & 21.9 & 11.4 & $\begin{array}{l}19.0 \\
20.7\end{array}$ & $\begin{array}{l}1.052 \\
1.427 \\
\end{array}$ & 0.387 & 0.390 & 0.030 & 30.0 & $\begin{array}{l}5.1 \\
25.5\end{array}$ \\
\hline 4899 & & Median & 24.7 & 24.0 & 24.4 & 25.0 & \begin{tabular}{l|l|l|}
23.7 \\
\end{tabular} & 22.0 & 22.7 & 4.4 & 17.9 & -1.2 & -3.9 & 46.3 & 0.0 & 0.6 & 0.0 & & & 21.5 & 11.0 & 19.9 & \begin{tabular}{|l|l|}
9 & 1.373 \\
\end{tabular} & $\begin{array}{lll}3 & 0.084\end{array}$ & 0.085 & $\begin{array}{l}0.004 \\
\end{array}$ & 4.2 & 3.6 \\
\hline 490 & & Minimum & 24.5 & 23.9 & 24.2 & 24.9 & 23.4 & 14.8 & 22.0 & 4.0 & 11.2 & -1.2 & -3.9 & 44.6 & 0.0 & 0.5 & 0.0 & & & & 10.6 & 13.0 & 0.896 & \begin{tabular}{l|l|}
6 & 0.073 \\
\end{tabular} & 0.075 & 0.004 & 3.7 & 3.2 \\
\hline 491| & & $2 \times$ Std Dev & 0.285 & 0.073 & 0.285 & 0.378 & 0.318 & 3.003 & 0.540 & & 2.878 & 0.949 & 0.241 & 1.282 & 0.000 & 0.946 & 0.001 & & & 0.540 & 0.305 & $\frac{2.935}{2.935}$ & \begin{tabular}{|l|l|}
50.202 \\
\end{tabular} & \begin{tabular}{l|l|}
2 & 0.135
\end{tabular} & 0.136 & 0.011 & 10.522 & 8.954 \\
\hline \begin{tabular}{|l|l|}
492 \\
182
\end{tabular} & Number & of Points Used & \begin{tabular}{|r|}
131 \\
\end{tabular} & 31 & 31 & 31 & 31 & 31 & 31 & 31 & 31 & 31 & 31 & 31 & 31 & 31 & 31 & & & 31 & 31 & 31 & 31 & 31 & 31 & 31 & 31 & 31 \\
\hline
\end{tabular}


WSRC-TR-2005-00105, REVISION 0 SRNL-RPP-2005-00012, REVISION 0

RUN \# 4.18 AND 4.19; ACID CLEANING AND CAUSTIC RINSING - CONT.

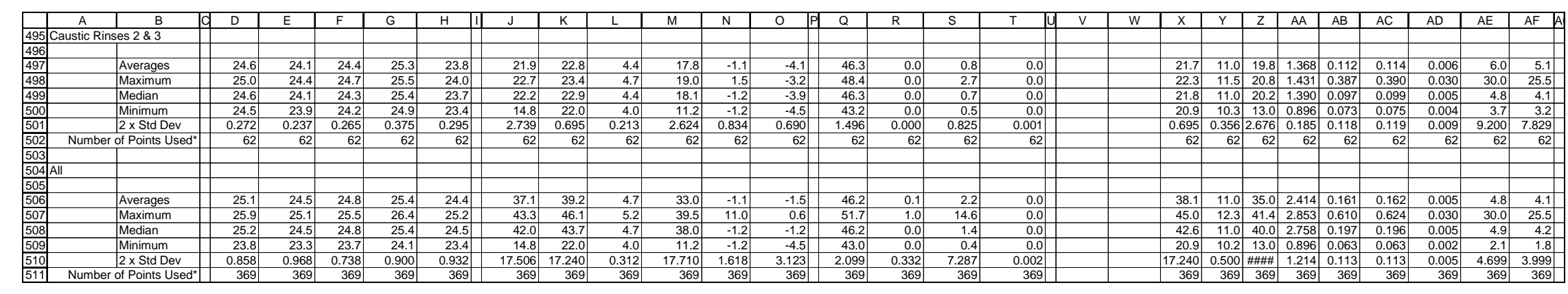


WSRC-TR-2005-00105, REVISION 0 SRNL-RPP-2005-00012, REVISION 0

RUN \# 4.21 A, B AND C; PRE-TEST BASELINE, CAUSTIC RINSE 3 SOLUTION AND 0.1 M NAOH

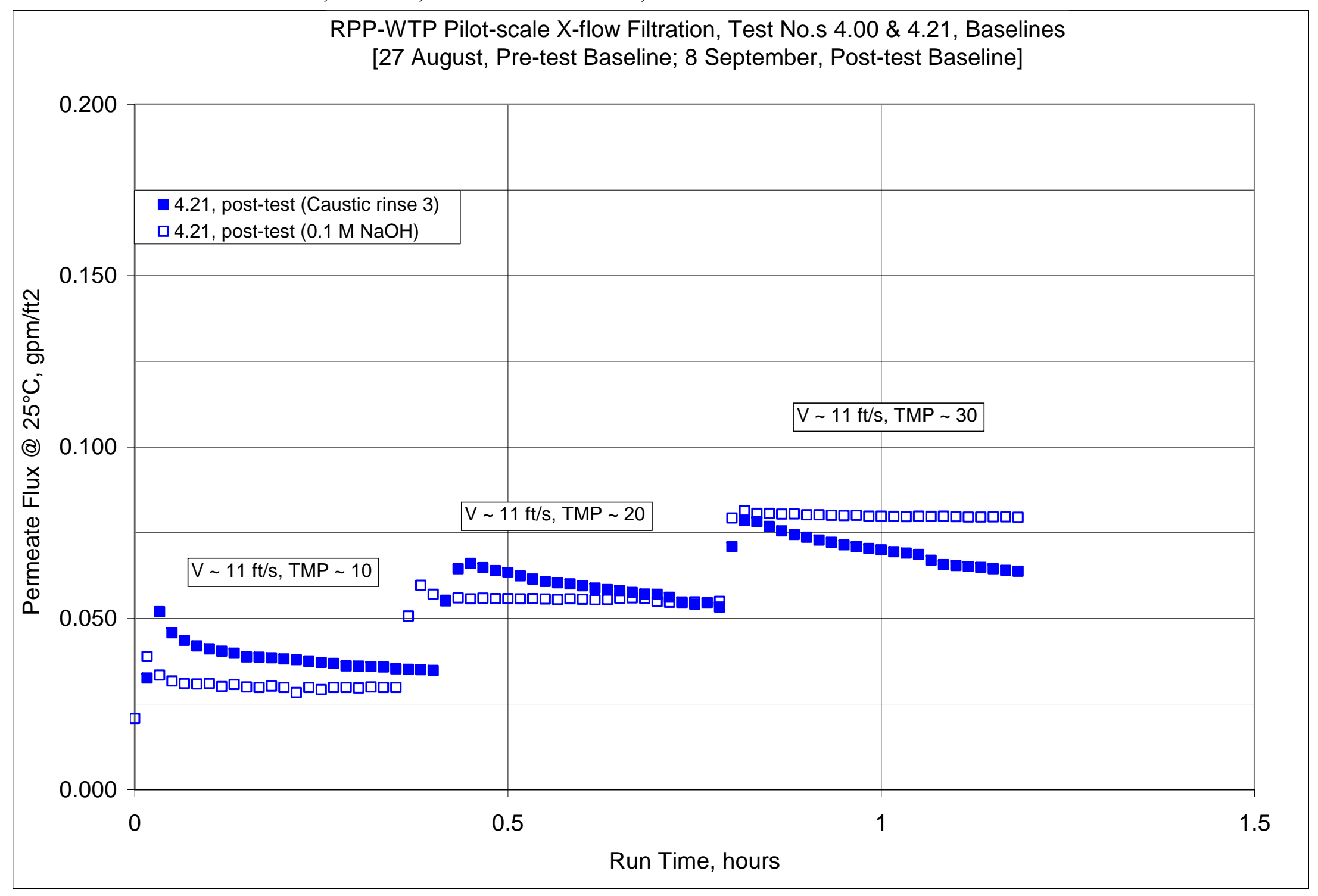


WSRC-TR-2005-00105, REVISION 0

SRNL-RPP-2005-00012, REVISION 0

RUN \# 4.21 A, B AND C; PRE-TEST BASELINE WITH CAUSTIC RINSE 3 SOLUTION

\begin{tabular}{|c|c|c|c|c|c|c|c|c|c|c|c|c|c|c|c|c|c|c|c|c|c|c|c|c|c|c|c|c|}
\hline & $\mathrm{A}$ & $\mathrm{B}$ & $\frac{D}{1<<<3}$ & $E$ & $1 F$ & \begin{tabular}{c|c} 
\\
$G$
\end{tabular} & $\frac{H}{H}$ & 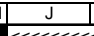 & $\frac{1}{1} \mathrm{~K}$ & $\frac{L}{S P}$ & M M & I N & $\begin{array}{ll}1 & 0 \\
y & 0\end{array}$ & $\frac{Q}{Q} \mid$ & 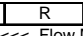 & $\mathrm{s}$ & $1 \frac{T}{1}$ & $\frac{v}{v<<<<}$ & $\frac{w}{1}$ & $\frac{x}{1<x<<}$ & Y Y & $\mathrm{z}$ & AA & $\mid A B$ & $A C$ & 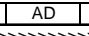 & $\mathrm{AE}$ & ${ }^{A F}$ \\
\hline 2 & DATE & TIME & $\begin{array}{l}\text { Fittrate } \\
\text { Fese }\end{array}$ & $\begin{array}{l}\frac{k<\text { Tem }}{\text { Cleanin }} \\
\end{array}$ & $\begin{array}{l}\text { inperature } \\
\text { g| Slurry }\end{array}$ & $\frac{2 \text { Measuremer }}{\text { y Hi Amb. IL }}$ & 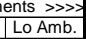 & BotTMP & $\begin{array}{l}\ll<\ll< \\
\text { Filter } \\
\end{array}$ & $\begin{array}{l}\text { <e Pressure } \\
\text { |Filiter dF }\end{array}$ & $\begin{array}{l}\text { Measuremen } \\
\text { DTopMP }\end{array}$ & $\begin{array}{l}\text { ents } \gg \gg> \\
\text { | Filtrate }\end{array}$ & $\begin{array}{l}\text { plp>>>>> } \\
\text { | Pulsepot }\end{array}$ & $\begin{array}{l}k<<<<< \\
\text { Slurry }\end{array}$ & $\begin{array}{l}\text { e< Flow| } \\
\text { J Fittrate }\end{array}$ & $\begin{array}{l}\text { Measureme } \\
\text { |Hi Fittate }\end{array}$ & 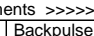 & & & & Calculat & ated From & m Raw D & & & & & \\
\hline 20 & & & $\operatorname{deg} C$ & $\operatorname{deg} \mathrm{C}$ & deg C & \begin{tabular}{|l|l|}
$c$ & $\operatorname{deg} C$ \\
\end{tabular} & $\operatorname{deg} C$ & psid & psig & psid & psid & \begin{tabular}{|l|} 
psigute \\
\end{tabular} & psig & gpm & \begin{tabular}{|l|} 
gpm \\
\end{tabular} & gpm & gachouse & & & & & & & & & & & \\
\hline & & & $\mathrm{T} 2$ & & & $\mathrm{~T} 4$ & & & $\mathrm{P} 1$ & $\mathrm{dP1}$ & $\mathrm{dP3}$ & $\mathrm{P2}$ & P3 & Q1 & Q2 & $\mathrm{Q}^{3}$ & $\mathrm{Qbp}$ & & & & & & & & & & & \\
\hline 5 & & & & & & & & & & & & & & & & & & & & & & & & & & & & \\
\hline & eroso-01/26/i & & & & & & & & & & & & & & & & & & & & & & & & & & & \\
\hline$\frac{1}{8}$ & $\begin{array}{l}8 / 23 / 2004 \\
8 / 24 / 2004\end{array}$ & \begin{tabular}{|l|l} 
& $8: 35: 38 \mathrm{AM}$ \\
& $8: 10: 33 \mathrm{AM}$ \\
\end{tabular} & $\frac{22.554}{23.662}$ & & \begin{tabular}{|l|}
22.00 \\
23.67 \\
\end{tabular} & \begin{tabular}{|c|c|c|c|c|}
022.782 & 22.998 \\
79 & 22.998
\end{tabular} & \begin{tabular}{|l|}
22.204 \\
322.735 \\
\end{tabular} & $\mid \begin{array}{l}-0.019 \\
-0.021\end{array}$ & $\begin{array}{l}9 \\
1\end{array}$ & $\begin{array}{l}-0.01 \\
-0.01 \\
\end{array}$ & $\begin{array}{r}0.003 \\
-0.001\end{array}$ & \begin{tabular}{|l|l|}
-0.124 \\
-0.124 \\
\end{tabular} & $\begin{array}{l}0.137 \\
0.134 \\
\end{array}$ & $\frac{-0.075}{23.176}$ & & \begin{tabular}{|l|l|}
14.643 \\
& 14.643 \\
\end{tabular} & & & & $\begin{array}{ll}\text { ers } \\
\text { iver }\end{array}$ & & 85 & & & & & & \\
\hline \begin{tabular}{|l|l}
9 \\
\end{tabular} & $8 / 26 / 2004$ & 9:14:11 AM & & & 24.93 & \begin{tabular}{ll|}
32 & 22.390 \\
32 & 25.316
\end{tabular} & $\frac{2.150}{23.242}$ & $\begin{array}{l}-0.021 \\
-0.008\end{array}$ & 0.126 & $\begin{array}{ll}-0.01 \\
-0.008\end{array}$ & 年 & \begin{tabular}{|l|l|}
-0.124 \\
\end{tabular} & & 48.335 & & $\begin{array}{l}14.643 \\
14.643\end{array}$ & & & & & & & & & & & & \\
\hline 10 & $8 / 27 / 2004$ & 7:53:29 AM & 22.649 & 22.8 & \begin{tabular}{|l|}
22.29 \\
\end{tabular} & \begin{tabular}{|l|l|}
98 & 22.872 \\
\end{tabular} & 22.871 & -0.017 & 0.1 & -0.00 & \begin{tabular}{l|l}
0.005 \\
\end{tabular} & \begin{tabular}{|l|} 
\\
\end{tabular} & 0.111 & -0.084 & 0.003 & 14.643 & 0.00 & & & Note: C & Gauge F & esssure & re $\mathrm{P} 1$ is ap & approxim & mately $1.4 \mathrm{p}$ & psig & & \\
\hline 11 & 8/30/2004 & 7:28:55 AM & 22.511 & 22.84 & 22.39 & \begin{tabular}{ll|l}
97 & 22.669 \\
\end{tabular} & 22.914 & -0.017 & $0.13 \varepsilon$ & -0.00 & $\begin{array}{l}0.003 \\
\end{array}$ & \begin{tabular}{|l|}
-0.147 \\
\end{tabular} & 0.12. & -0.082 & 0.003 & $\begin{array}{l}14.643 \\
\end{array}$ & 0.000 & & & & & & & & & & & \\
\hline & & & & & & & & & & & & & & & & & & & & & & & & & & ter tubing & & \\
\hline$\frac{13}{14}$ & & & & & & & & & & & & & & & & & & & & WVWV & & & & $<$ Filtrat & Frate Flux > & « PERM & & \\
\hline 15 & & & & & & & & & & & & & & & & & & & Time & Bress. & . Vel. & TMP & TMP & $\mathrm{nm}$ & $\frac{a t}{\mathrm{at} 25 \mathrm{C}}$ & & $\frac{x 1000}{\mathrm{gpm}}$ & \\
\hline 16 & & & & & & & & & & & & & & & & & & Min. & Hour & psig & ftts & psi & bar & |lit2 & & 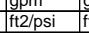 & $\begin{array}{l}g \mathrm{pm} \\
\mathrm{ft} / \mathrm{psi}\end{array}$ & day/bar \\
\hline & ednesday, & $1,9 / 8 / 04$ & & & & & & & & & & & & & & & & & & & & & & & & & & \\
\hline & Iseline 4.21 & & & & & & & & & & & & & & & & & & & & & & & & & & & \\
\hline$\frac{19}{20}$ & $\begin{array}{l}98 / 8 / 20044 \\
9 / 8 / 2004\end{array}$ & $\begin{array}{l}\text { 8:39:00 AM } \\
840: 06 \mathrm{AM}\end{array}$ & $\frac{23.316}{23.438}$ & $\frac{22.62}{22.6}$ & 23.63 & $\begin{array}{ll}23.018 \\
23.024\end{array}$ & $\begin{array}{r}22.56 \\
22.496 \\
\end{array}$ & $\begin{array}{l}12.367 \\
12.309 \\
\end{array}$ & $\frac{13.246}{13.20}$ & $\frac{4.43}{4.46}$ & \begin{tabular}{|l|l|}
8.064 \\
7.927
\end{tabular} & $\begin{array}{l}-1.209 \\
-1.209\end{array}$ & $\begin{array}{r}-4.402 \\
-4.405 \\
\end{array}$ & $\frac{45.847}{46055}$ & 0.26 & -0.008 & & 0.00 & & 12.1 & & 10. & 0.704 & 0.03 & $\begin{array}{l}0.039 \\
0.039\end{array}$ & 0.004 & $\begin{array}{l}3.787 \\
3.813 \\
\end{array}$ & \\
\hline 21 & 9/8/2004 & 8:41:06 AM & 23.428 & 22.64 & \begin{tabular}{|l|}
23.15 \\
23.86 \\
\end{tabular} & 23.029 & \begin{tabular}{|l|}
22.450 \\
\end{tabular} & $\begin{array}{r}11.3 .9935 \\
2.935\end{array}$ & \begin{tabular}{l|l}
5 & 13.23 \\
5 & 13.35 \\
\end{tabular} & 4.52. & $\begin{array}{l}.1 .354 \\
-1.354 \\
\end{array}$ & $\begin{array}{l}-1.204 \\
5.047 \\
\end{array}$ & $\begin{array}{r}-4.405 \\
20.488 \\
\end{array}$ & 45.972 & 0.003 & $\begin{array}{l}-0.011 \\
-0.013 \\
\end{array}$ & & 2.00 & 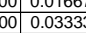 & $\begin{array}{ll}12.1 \\
12.2\end{array}$ & & 0.8 & \begin{tabular}{|l|}
0.0955 \\
0.055 \\
\end{tabular} & 0.000 & $\begin{array}{l}0.039 \\
0.000\end{array}$ & $\begin{array}{l}0.004 \\
0.001\end{array}$ & $\begin{array}{l}3.813 \\
0.561\end{array}$ & $0.4 \varepsilon$ \\
\hline & & 8:42:06 AM & & & 23.94 & $\begin{array}{ll}48 & 23.056 \\
6\end{array}$ & 22.472 & $\begin{array}{l}3.171 \\
\end{array}$ & \begin{tabular}{l|l|}
1 & 13.575 \\
\end{tabular} & 4.65. & $\begin{array}{l}-1.364 \\
\end{array}$ & & 37.368 & 46.251 & & -0.013 & & 3.00 & \begin{tabular}{l|l}
00 & 0.05 \\
0
\end{tabular} & 12.4 & & & 0.062 & 0.000 & 0.000 & 0.000 & 0.490 & 42 \\
\hline & 9/8/2004 & 8:43:06 AM & & & & \begin{tabular}{ll|}
59 & 23.072 \\
\end{tabular} & 22.483 & & & 4.5 & 2.453 & 1.278 & 0.44 & & 0.497 & & & & $\begin{array}{lll}00 & 0.0666\end{array}$ & 12.3 & & & 0.316 & & 0.073 & 0.016 & 15.940 & \\
\hline 24 & 9/8/2004 & 8:44:06 AM & $\begin{aligned} 23.826 \\
23.057\end{aligned}$ & $\frac{22.66}{22.67}$ & \begin{tabular}{|l|}
24.12 \\
242 \\
\end{tabular} & \begin{tabular}{|l|l|}
29 & 23.127 \\
206 & 23113
\end{tabular} & $\begin{aligned} 22.408 \\
22.468\end{aligned}$ & $\begin{array}{l}7.06 \\
736\end{array}$ & $\begin{array}{l}6 \quad 13.246 \\
6 \\
6\end{array}$ & 4.62 & $\begin{array}{l}2.587 \\
285 \\
\end{array}$ & $\begin{array}{l}0.791 \\
0.673\end{array}$ & $\begin{array}{l}0.573 \\
0.640 \\
\end{array}$ & $\begin{array}{l}46.181 \\
46.156\end{array}$ & 0.323 & $\begin{array}{r}14.643 \\
14643\end{array}$ & & 5.00 & \begin{tabular}{l|l}
00 & 0.0833 \\
0
\end{tabular} & \begin{tabular}{l|l}
3 & 12.1 \\
1 & 12.2
\end{tabular} & 11 & & 0.333 & 0.046 & 0.047 & 0.010 & $\begin{array}{l}9.826 \\
7.225 \\
-125\end{array}$ & 8.36 \\
\hline 26 & $\begin{array}{l}9 / 8 / 2004 \\
9 / 8 / 2004\end{array}$ & & $\frac{23.957}{24.118}$ & $\frac{22.67}{22.67}$ & $\frac{24.2}{2432}$ & $\begin{array}{l}23.143 \\
23.164 \\
\end{array}$ & $\frac{22.468}{22.465}$ & $\begin{array}{r}1.36 \\
7.163 \\
\end{array}$ & 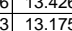 & $\frac{4.59}{4.36}$ & $\begin{array}{l}2.858 \\
3059 \\
\end{array}$ & $\frac{0.663}{0.67}$ & & $\frac{46.156}{46162}$ & & $\frac{14.643}{14.643}$ & & $\frac{6.00}{700}$ & \begin{tabular}{|l|}
0.1 \\
0.1166 \\
\end{tabular} & $\frac{12.3}{120}$ & & & & & & & $\frac{7.325}{6.367}$ & $\frac{6.2 .}{5.4}$ \\
\hline 27 & 9/8/2004 & & 24.145 & 226 & 24.43 & \begin{tabular}{l|l}
33 & 23.22
\end{tabular} & 20.526 & 11.564 & 4 & 4.57 & 7.241 & -1.212 & & 46.4 & & 14.643 & & 80 & 0.1333 & 124 & & & 0.648 & & & 0.006 & 5523 & $\frac{5.42}{473}$ \\
\hline 28 & 9/8/2004 & & 24.255 & 22.69 & 24.47 & 23.276 & & 11.935 & 13.43 & 4.43 & 7.832 & $\frac{-1.206}{-1.206}$ & & 46.518 & 0.315 & 14.643 & & 9.0 & 0.15 & $\frac{12.3}{12.3}$ & & & .681 & & .046 & 0.005 & 4.631 & 9 \\
\hline & 9/8/2004 & & 24.311 & & & & & 11.981 & & 4.47 & & & & 46.494 & & 14.643 & & & & & & & & & & & 4.401 & th \\
\hline 30 & 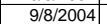 & $8: 50: 0$ & 24.357 & 22.69 & \begin{tabular}{|l|l|}
24.61 \\
\end{tabular} & 23.252 & & 11.95 & 13.178 & 4.36 & & -1.212 & & 46.443 & $\sigma_{0}$ & $\begin{array}{l}14.643 \\
\end{array}$ & & $\frac{11.0}{11.0}$ & 183 & & & & & & & & 4.243 & \\
\hline & 9/8/2004 & & 24.447 & 22.6 & 24.64 & & & & 13.4 & & & -1.215 & & $46 .{ }^{4}$ & 0.2 & & & & & 12.4 & & & & & & & & \\
\hline 32 & 9/8/2004 & & 24.493 & 22.70 & 24.69 & \begin{tabular}{l|l|}
6 & 23.218 \\
\end{tabular} & & 12.201 & 13.43 & & 7.861 & -1.209 & & 46.26 & 0 & & & 13.0 & 0.2166 & 12.3 & & & & & 0.040 & & 4.031 & \\
\hline & 9/8/2004 & & 24.538 & 22.70 & 24.74 & 23.238 & 22.55 & 12.095 & 13.188 & 4.47 & 7.902 & -1.209 & & 46.381 & 0.2 & 14.643 & & $14.0 \mathrm{Cl}$ & 0.2333 & 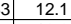 & & & & & & & 3.980 & \\
\hline 34 & 9/8/2004 & & & & 24.79 & & & & & & 7.181 & & & 46.42 & & & & 15. & 0.24 & 12.2 & & & 0.687 & & & 0.004 & 890 & \\
\hline$\frac{35}{36}$ & & & 24.669 & $\frac{22.72}{20.73}$ & \begin{tabular}{|l|l|}
24.86 \\
\end{tabular} & 23.35 & 22.57 & 12.423 & 13.44 & 4.42 & 8.249 & -1.209 & & 46. & & 14.643 & & & 206067 & 12.4 & & & & & & & 3.740 & \\
\hline & & & $\frac{24.118}{2478}$ & & \begin{tabular}{|l|l|l|}
24.96 \\
\end{tabular} & $\frac{2.300}{23.366}$ & & 12.445 & 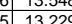 & & (1) & $\frac{1.209}{-1209}$ & & & & & & & & $\frac{1.4}{121}$ & & & & & & & $\frac{3.150}{3792}$ & \\
\hline \begin{tabular}{|l|l}
38 \\
\end{tabular} & 12004 & & 24.806 & 22 & 24.99 & \begin{tabular}{l|l}
93 & 23.342
\end{tabular} & & 12317 & $\begin{array}{ll}7 & 1.3 .378 \\
\end{array}$ & & 959 & \begin{tabular}{|l|l|}
-1.209 \\
\end{tabular} & & & & & & & 031667 & 123 & & & & & & & & \\
\hline 39 & & & 24.852 & 22.79 & \begin{tabular}{|l|}
25.00 \\
\end{tabular} & 23.387 & & $\begin{array}{l}12.389 \\
12.389\end{array}$ & \begin{tabular}{|l|l|l|l|}
9 & 13.401
\end{tabular} & 4.5 & & & & & & & & & & $\frac{12.3}{12.3}$ & & & & & & & & \\
\hline 40 & 9/8/2004 & & 24902 & 22.7 & \begin{tabular}{|l|}
25.07 \\
\end{tabular} & 23.373 & & & & & & & & & & & & & 0.00 & $\frac{1.4}{12.4}$ & & & & & 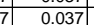 & & $\frac{3.604}{3.604}$ & \\
\hline 41 & & 9:01: & 24.953 & 22.74 & 25.10 & 23.363 & & 12.362 & 13.3 & & 8.076 & -1.209 & & & & 14 & & & 0.36667 & $\frac{\frac{\pi}{12.3}}{12.3}$ & & & & & & & 3.604 & \\
\hline & & & 24.9 & & 2 & $\begin{array}{ll}96 & 23.363 \\
\end{array}$ & & 12.1 & & & & & & & & & & & 0.38333 & & & & & & & & & \\
\hline 43 & 9/8/2004 & & 25.003 & 22.76 & 25.16 & 23.348 & & 12.4 & $\begin{array}{ll}5 & 13.3 \\
5\end{array}$ & & & & & & & & & 24 & & 12.3 & 1 & & & & & & 3.471 & \\
\hline 44 & 9/8/2004 & & 25.059 & 22.77 & \begin{tabular}{|l|l|}
25.18 \\
\end{tabular} & $\begin{array}{ll}88 & 23.335 \\
\end{array}$ & & 12.15 & & 4. & & -1.209 & & & & & & & 0.41667 & 11.9 & & & & & & & & \\
\hline 45 & & & 25.084 & & 25.18 & \begin{tabular}{ll|}
83 & 23.365 \\
\end{tabular} & & 12.2. & & & & & & & & & & & 0.4 & 22.0 & & & & & & & & \\
\hline$\frac{40}{17}$ & & & 25.105 & & $\mid 25.27$ & 23.421 & & & & & & & & & & & & & & $\frac{1.2}{1.2}$ & & & & & & & & \\
\hline $48 \quad-1$ & & & $\frac{25.104}{25202}$ & & & \begin{tabular}{|l|l|l|l|}
23569 \\
\end{tabular} & & & & & & & & & & & & & 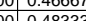 & & & & & & & & & \\
\hline (4) & & & & & & 21 & & & & & & $\frac{59}{10}$ & & & & & & & & & & & & & & & & \\
\hline & & & 25.249 & & & & & & & & & & & & & & & & & & & & & & & & & \\
\hline & & & 25.314 & & 25.36 & 23.565 & & 21085 & & 431 & 1709 & & & & & & & & & & & & & & & & & \\
\hline 5 & & & 25.369 & 22 & 25.38 & 8323.566 & & 22. & $\begin{array}{l}725.5 \\
\end{array}$ & 4 & 18.2 & -1. & & & & & & & & 24.5 & & & & & & & & \\
\hline & & & 25.38 & 22.8 & 25.34 & $\begin{array}{l}23.66 \\
\end{array}$ & & 21.8 & & & & & & & & & & & & 23.9 & & & & & & & & \\
\hline 5 & 9/8/2004 & & 25.414 & 22.81 & 25.35 & 23.721 & & 22.2 & & & 18.182 & & & 45. & & & & & 0.58 & 24.2 & & & & & & & & \\
\hline 5 & & & 25.444 & 22.8 & 25.3 & \begin{tabular}{|l|l|} 
& 23.736 \\
\end{tabular} & & 22.5 & & 4.3 & 18.5 & & & 45 & & & & & 0.6 & 24.4 & & & & & & & & \\
\hline - & & & 25.449 & & & & & 22.801 & & & & & & & & & & & & 4.7. & & & & & & & & \\
\hline 5 & & & 2554356 & 222.83 & $\mid 2.30$ & 23.882 & & 22.4 & 25 & & & -1 & & & & & & & 0.63333 & 24.0 & & & & & & & & \\
\hline & & & $\frac{20.450}{25.457}$ & $\frac{2.0}{228}$ & 2520 & $\frac{2.5344}{2394}$ & & $\frac{2.3}{225}$ & 252 & & & 年. & & & & & & & & 24.4 & & & & & & & & \\
\hline & & & & & & $\mid$ & & 22.776 & $\frac{14.5}{625.4}$ & $\frac{1.52}{4.2}$ & & & & & & & & & 0 & & & & & & & & & \\
\hline 6 & & & 25.457 & 22.87 & \begin{tabular}{|l|l|}
25.30 \\
\end{tabular} & 23.985 & 22. & 22.666 & \begin{tabular}{l|l}
6 & 25. \\
\end{tabular} & 4.34 & & \begin{tabular}{|c|} 
\\
\end{tabular} & & & & & & & & 243 & & & 418 & & & & & \\
\hline & & & & & & & & & & & & & & & & & & & & & & & & & & & & \\
\hline 6 & & & $25.4 !$ & & $\begin{array}{l}25.29 \\
\end{array}$ & 23.906 & & 22.794 & 253 & & & & & & & & & & & & & & & & & & & \\
\hline & & & 25.449 & & 25.29 & 23.8 & & 22.798 & 25. & & & -1.2 & & & & & & & & & & & 430 & & & & & \\
\hline & 9/8/2004 & & 25. & 22.89 & 25.2 & 23.5 & 22. & 22.73 & $\begin{array}{ll}3 & 25.3\end{array}$ & 4.34 & 18.5 & -1.2. & & & & & & & 0.7 & & & & 42 & & & & & \\
\hline & 9/8/2004 & & 25 & & 25.27. & 23.9 & & $22.761 \mid$ & & 4.27 & & & & & & & & & & & & & & & & & 2.747 & \\
\hline & & & 25.44 & & & & & 23.0 & & & & -1.212 & & & & & & & & 24.2 & & & & & & & & \\
\hline 6 & .004 & 9:28:06 & 25.435 & 22.91 & $\frac{25.26}{25.23}$ & 24.024 & & 22.863 & $\begin{array}{llll}3 & 25.261 \\
\end{array}$ & 4.26 & $\begin{array}{r}18.908 \\
\end{array}$ & 1.209 & & & 0.39 & 14.643 & & & 66 & 24. & & & .44 & & & & 688 & \\
\hline & $9 / 8 / 2004$ & $\begin{array}{r}99: 29: 06 \mathrm{AM} \\
9: 30: 06 \mathrm{AM}\end{array}$ & $\frac{25.441}{25.432}$ & \begin{tabular}{|l|l|}
2 & 22.93 \\
\end{tabular} & 25.2 & $\begin{array}{l}39 \\
25 \\
25\end{array}$ & & $\begin{array}{r}22.166 \\
22.5 \\
\end{array}$ & $\begin{array}{l}524.69 \\
24\end{array}$ & $\begin{array}{l}4.50 \\
.408\end{array}$ & 18.338 & $.20 \Omega$ & & 6.41 & 0.38 & 14.64 & & & & & & & & & & & & \\
\hline
\end{tabular}


WSRC-TR-2005-00105, REVISION 0

SRNL-RPP-2005-00012, REVISION 0

RUN \# 4.21 A, B AND C; PRE-TEST BASELINE WITH CAUSTIC RINSE 3 SOLUTION - CONT.

\begin{tabular}{|c|c|c|c|c|c|c|c|c|c|c|c|c|c|c|c|c|c|c|c|c|c|c|c|c|c|c|c|c|}
\hline & & & & & & & & & & & & & & & & & & & & & & & & & & & & \\
\hline - & A & $\mathrm{B} \quad \mathrm{C}$ & $\begin{array}{ll} \\
\end{array}$ & $\mathrm{E}$ & \begin{tabular}{|l|} 
\\
\end{tabular} & \begin{tabular}{|l|} 
\\
\end{tabular} & \begin{tabular}{l|l}
$\mathrm{H}$ \\
\end{tabular} & $\mathrm{J}$ & $\mathrm{K}$ & $\mathrm{L}$ & \begin{tabular}{|l|}
$\mathrm{M}$ \\
\end{tabular} & \begin{tabular}{|l|} 
\\
\end{tabular} & 0 & \begin{tabular}{l|l} 
\\
\end{tabular} & \begin{tabular}{|l|l|}
$\mathrm{R}$ \\
\end{tabular} & \begin{tabular}{l|l} 
\\
\end{tabular} & \begin{tabular}{l|l|l}
$T$ & $U$ \\
\end{tabular} & $\mathrm{v}$ & w & $x$ & $\mathrm{Y}$ & $\mathrm{z}$ & AA & $A B$ & \begin{tabular}{ll|}
$A C$ \\
\end{tabular} & $\mathrm{AD}$ & $\mathrm{AE}$ & $\mathrm{AF} \quad \mathrm{A}$ \\
\hline 71 & $9 / 8 / 2004$ & $9: 31: 06 \mathrm{AM}$ & $\begin{array}{l}25.432 \\
5.42\end{array}$ & 22.944 & \begin{tabular}{|l|}
25.251 \\
\end{tabular} & \begin{tabular}{|l|l|}
24.116 \\
\end{tabular} & \begin{tabular}{|l|}
22.8899 \\
\end{tabular} & 22.763 & 24.988 & 4.464 & \begin{tabular}{|l|}
18.654 \\
\end{tabular} & \begin{tabular}{|l|}
-1.209 \\
\end{tabular} & -3.598 & 45.949 & \begin{tabular}{|l|}
0.383 \\
\end{tabular} & \begin{tabular}{r|}
14.643 \\
\end{tabular} & 0.003 & 52.00 & \begin{tabular}{|l|l|}
0.86667 \\
\end{tabular} & 23.9 & 10.9 & & 1.428 & 0.055 & 0.054 & 0.003 & 2.629 & 2.24 \\
\hline t & $9 / 8 / 2004$ & $9: 32: 06 \mathrm{AM}$ & $\begin{array}{l}25.444 \\
5.49\end{array}$ & 22.955 & \begin{tabular}{|l|}
25.267 \\
5.277 \\
\end{tabular} & 24.127 & $\begin{array}{r}22.96 \\
\end{array}$ & 22.494 & 24.644 & 4.489 & \begin{tabular}{|l|l|}
18.276 \\
2.55
\end{tabular} & \begin{tabular}{|l|l|}
-1.209 \\
\end{tabular} & -3.653 & 46.569 & $\begin{array}{l}0.375 \\
\end{array}$ & $\begin{array}{l}14.643 \\
\end{array}$ & 0.003 & & 0.88333 & 23.5 & 11.1 & 20.4 & 1.405 & & & & 2.614 & 2.22 \\
\hline 73 & 9/8/2004 & 9:33:06 AM & $\begin{array}{l}25.439 \\
2550\end{array}$ & 22.955 & \begin{tabular}{|l|l|}
25.278 \\
2518
\end{tabular} & 24.088 & \begin{tabular}{|l|}
22.981 \\
2.232
\end{tabular} & 31.173 & $\begin{array}{l}34.735 \\
3.503\end{array}$ & 5.948 & $\begin{array}{l}25.551 \\
27091\end{array}$ & \begin{tabular}{|l|l|}
-1.209 \\
\end{tabular} & $\begin{array}{l}-2.633 \\
.034\end{array}$ & \begin{tabular}{|c|}
55.118 \\
\end{tabular} & $\begin{array}{l}0.499 \\
\end{array}$ & \begin{tabular}{|l|l|}
14.643 \\
\end{tabular} & 0.003 & 54.00 & 0.9 & 33.6 & $\begin{array}{l}13.1 \\
\end{array}$ & 28.4 & 1.955 & 0.071 & 0.071 & 0.002 & 2.499 & 2.13 \\
\hline \begin{tabular}{|l|}
74 \\
75
\end{tabular} & $\begin{array}{l}9 / 8 / 2004 \\
98 / 82004\end{array}$ & $9: 34: 06 \mathrm{AM}$ & 25.505 & \begin{tabular}{|l|l|}
22.961 \\
22077
\end{tabular} & \begin{tabular}{|l|l|}
25.408 \\
25515 \\
\end{tabular} & $\begin{array}{l}24.123 \\
2.125\end{array}$ & \begin{tabular}{|l|}
23.032 \\
23029
\end{tabular} & $\begin{array}{l}32.048 \\
32322\end{array}$ & $\begin{array}{l}36.503 \\
36.866\end{array}$ & $\begin{array}{l}4.474 \\
4.464\end{array}$ & \begin{tabular}{|l|}
27.961 \\
28086
\end{tabular} & \begin{tabular}{|c|}
-0.873 \\
\end{tabular} & $\begin{array}{l}-2.046 \\
257\end{array}$ & \begin{tabular}{|l|l|}
46.779 \\
46525
\end{tabular} & \begin{tabular}{|l|l|}
0.555 \\
\end{tabular} & \begin{tabular}{|r|}
14.643 \\
1.643
\end{tabular} & 0.003 & 55.00 & \begin{tabular}{|l|l|}
0.91667 \\
0.02232
\end{tabular} & $\begin{array}{l}35.4 \\
357 \\
3\end{array}$ & $\begin{array}{l}11.1 \\
1.1\end{array}$ & $\begin{array}{ll}30.0 \\
302\end{array}$ & \begin{tabular}{|l|}
2.069 \\
2080
\end{tabular} & \begin{tabular}{|l|}
0.079 \\
0.079 \\
\end{tabular} & \begin{tabular}{|c|}
0.079 \\
0.078
\end{tabular} & 0.003 & $\begin{array}{l}2.618 \\
\end{array}$ & 2.23 \\
\hline 76 & 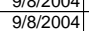 & $\begin{array}{l}9: 35: 06 \mathrm{AM} \\
93: 36: 06 \mathrm{AM}\end{array}$ & \begin{tabular}{|c|}
25.571 \\
25646
\end{tabular} & $\begin{array}{l}22.977 \\
22.982 \\
\end{array}$ & \begin{tabular}{|}
25.515 \\
25.51 \\
\end{tabular} & \begin{tabular}{|l|}
24.155 \\
24.185 \\
\end{tabular} & $\begin{array}{l}23.028 \\
22.948\end{array}$ & $\begin{array}{l}32.322 \\
32.291 \\
\end{array}$ & $\begin{array}{rl}36.866 & 36.52 \\
362\end{array}$ & $\begin{array}{l}4.464 \\
4.469 \\
\end{array}$ & \begin{tabular}{|l|}
28.086 \\
28.139
\end{tabular} & \begin{tabular}{|c|}
$\mid-0.873$ \\
-1.088 \\
\end{tabular} & $\begin{array}{l}-2.057 \\
-2.258\end{array}$ & \begin{tabular}{|l|l|}
46.625 & 46.746
\end{tabular} & 0.5544 & $\begin{array}{l}14.643 \\
14643 \\
\end{array}$ & 0.003 & $\begin{array}{l}56.00 \\
57.00\end{array}$ & $\mid \begin{array}{l}0.93333 \\
0.95\end{array}$ & $\begin{array}{l}35.7 \\
35.4\end{array}$ & $\begin{array}{l}11.1 \\
11.1 \\
\end{array}$ & $\begin{array}{ll}30.2 \\
30.22\end{array}$ & \begin{tabular}{|l|}
2.082 \\
2.083 \\
\end{tabular} & \begin{tabular}{|l|}
0.079 \\
0.078
\end{tabular} & \begin{tabular}{|l|l|l|}
0.078 & 077 \\
\end{tabular} & $\begin{array}{l}0.003 \\
0.003\end{array}$ & $\begin{array}{r}2.5888 \\
2541\end{array}$ & $\begin{array}{l}2.20 \\
2.16\end{array}$ \\
\hline \begin{tabular}{|l|}
77 \\
\end{tabular} & 9/8/2004 & 9:37:06 AM & 25.677 & 22.988 & \begin{tabular}{|l|}
25.495 \\
\end{tabular} & 24.236 & \begin{tabular}{|l|l|}
23.014 \\
\end{tabular} & 32.073 & 36.205 & $\frac{4.409}{4.349}$ & 28.069 & \begin{tabular}{|r|}
-1.209 \\
\end{tabular} & $\begin{array}{l}-2.245 \\
-24\end{array}$ & 46.227 & 0 & 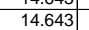 & $\frac{0.003}{0.003}$ & & \begin{tabular}{|c|}
0.96667 \\
\end{tabular} & 35.1 & $\begin{array}{ll}11 .+1 \\
11.0\end{array}$ & $\frac{30.2}{30.1}$ & \begin{tabular}{|l|}
2.083 \\
2.073 \\
\end{tabular} & \begin{tabular}{|l|}
0.078 \\
0.077 \\
\end{tabular} & 0 & $\frac{0.003}{0.003}$ & $\frac{2.541}{2.512}$ & $\frac{2.16}{2.14}$ \\
\hline 78 & $9 / 8 / 2004$ & & 25.702 & 23.009 & \begin{tabular}{|l|}
25.486 \\
\end{tabular} & \begin{tabular}{|l|}
24.286 \\
\end{tabular} & 22.939 & 32.245 & 36.236 & 4.345 & 28.119 & \begin{tabular}{|l|l|}
-1.209 \\
\end{tabular} & -2.323 & 45.96 & \begin{tabular}{|l|}
0.527 \\
\end{tabular} & 14.643 & 0.003 & 59.00 & \begin{tabular}{|l|l|}
0.98333 \\
\end{tabular} & 35.1 & 10.9 & 30.2 & 2.081 & 0.075 & 0.074 & 0.002 & 2.466 & $\frac{2.14}{2.10}$ \\
\hline 79 & 9/8/2004 & 9:39:06 AM & 25.702 & 23.004 & \begin{tabular}{|l|}
25.461 \\
\end{tabular} & 24.302 & 22.934 & & \begin{tabular}{|l|l|}
36.404 \\
\end{tabular} & 4.401 & 28.051 & \begin{tabular}{|l|}
-1.209 \\
\end{tabular} & -2.387 & 45.486 & 0.521 & \begin{tabular}{|l|l|}
14.643 \\
\end{tabular} & 0.00 & 60.00 & & 35.3 & 10.8 & & 2.080 & & 0.074 & & 2.441 & \\
\hline 80 & 9/8/2004 & 9:40:06 AM & $\begin{array}{l}25.698 \\
\end{array}$ & 23.01 & \begin{tabular}{|l|}
25.431 \\
\end{tabular} & 24.342 & 22.975 & 32.411 & & 4.329 & 28.371 & & 2.436 & 45.966 & & \begin{tabular}{|l|l|}
14.643 \\
\end{tabular} & & & $\mid$ & $\overline{35.1}$ & & & & & 0.073 & & & \\
\hline $81 \mid$ & & 9:41:06 AM & 25.688 - & & 25.421 & & & & 36.356 & 4.365 & 28.449 & -1.212 & & 45.538 & 0.51 & 14.6 & & & 3333 & 35.2 & & & & & & & 366 & .01 \\
\hline \begin{tabular}{|l|l|}
82 \\
83 \\
\end{tabular} & 9/8/2004 & 9:42:06 AM & 25.693 | & 23.03 & \begin{tabular}{|l|}
25.437 \\
\end{tabular} & 24.453 & 22.91 & 32.677 & 36.501 & 4.33 & 28.648 & -1.206 & & 45.557 & 0.505 & & & & 1.05 & 35.4 & & & & & 0.071 & & 329 & 1.98 \\
\hline (2) & & 9:43:06 AM & 25.685 & 23.042 & $\mid 25.413$ & 24.45 & 22.982 & 32.728 & 36.503 & 4.373 & 28.65 & $|-1.209|$ & -2.575 & 46.105 & 0.501 & 14.643 & & 64.00 & 1.06667 & 35.4 & 11.0 & & & & 0.071 & & 2.310 & $\begin{array}{l}1.98 \\
1.97\end{array}$ \\
\hline 84 & 9/8/2004 & 9:44:06 AM & 25.675 & 23.042 & 25.404 & 24.45 & 23.022 & 32.954 & 36.727 & 4.411 & 28.736 & $\mid-1.209$ & -2.609 & 45.824 & 0.497 & 14.643 & . 0003 & $65.0 \mathrm{C5}$ & 1.08333 & 35.6 & 10.9 & $30.8 \mathrm{z}$ & 2.127 & & 0.070 & 0.002 & 2.281 & 1.94 \\
\hline 35 [ & 9/8/2004 & 9:45:06 AM & 25.672 & 23.053 & 25.39 & 24.451 & 23.009 & 32.753 & 36.479 & 4.19 & 28.861 & $\mid-1.209$ & -2.638 & 45.688 & 0.494 & 14.643 & & 66.00 & & 35.4 & & & & .071 & 0.070 & & 2.271 & 1.93 \\
\hline & 9/8/2004 & 9:46:06 AM & 25.662 & 23.064 & 25.376 & 24.462 & 23.014 & 33.023 & 36.736 & 4.358 & 28.95 & -1.212 & -2.682 & 45.909 & 0.49 & 14.643 & & 67.00 & & & 10.5 & & & & 0.069 & & & 1.91 \\
\hline 87 & 9 9/8/2004 & & 25.652 & 23.0644 & 25.356 & 24.462 & & 33.075 & 36.64 & 4.365 & $28.987 \mid$ & & & 45.044 & 0.487 & 14.643 & & & 1.13333 & 35.5 & & & & & 069] & & & 1.89 \\
\hline 88 & & 9:48:06 AM & 25.654 & 23.075 & \begin{tabular}{|l|}
25.342 \\
\end{tabular} & 24.428 & 22.986 & 33 & 36.441 & 4.287 & 29.013 & -1.209 & & 45.553 & 0.484 & 14.64 & & 69. & & 35.3 & 10. & & & & & & 2.213 & 1.88 \\
\hline 年 & & 9:49:06 AM & \begin{tabular}{|l|l|l|}
25.655 \\
25625
\end{tabular} & $\frac{23.092}{23087}$ & \begin{tabular}{|l|}
25.348 \\
25333 \\
\end{tabular} & 24.4655 & $\frac{22.987}{23097}$ & $\begin{array}{l}32.56 \\
332026 \\
\end{array}$ & \begin{tabular}{|c|c|}
35.867 \\
3
\end{tabular} & 4.591 & $\begin{array}{l}28.264 \\
28.182\end{array}$ & $\mid$\begin{tabular}{|c|}
-1.209 \\
-1209
\end{tabular} & $\begin{array}{l}-2.841 \\
-2.927 \\
-290\end{array}$ & 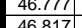 & 0.472 & $\frac{14.643}{14643}$ & & $\begin{array}{l}70.00 \\
71.00\end{array}$ & $\begin{array}{l}1.16667 \\
1.8333\end{array}$ & $\begin{array}{l}34.7 \\
34.6\end{array}$ & $\frac{1.1}{112}>>>$ & 30.4 & & .068 & $0.067 \mid$ & & $2.200 \mid$ & 1.87 \\
\hline \begin{tabular}{|l|l|l|l|l|l|l|}
91 & \\
\end{tabular} & 9/8/2004 & 9:51:06 AM & 25.62 & 23.097 & $\mid$\begin{tabular}{|c|}
$\mid 2.350$ \\
25.314 \\
\end{tabular} & $\frac{24.405}{24.465}$ & 23.033 & $\begin{array}{l}\frac{0.420}{32.475} \\
\end{array}$ & 35.857 & 4.476 & 20 & \begin{tabular}{|c|}
$\mid-1.205$ \\
-1.209 \\
\end{tabular} & & 势 & - & $\mid$ & & 年 & $\mid$ & 34.0 & 11.42 & 302 & 2084 [ & $\mid 0.066$ & 0.006 & 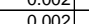 & 2.1.166 & 1.84 \\
\hline 92 & $9 / 8 / 2$ & 9:52:06 AM & 25.621 & 23.108 & $|25.31|$ & 24.491 & 23.048 & 32.589 & 35.792 & 4.478 & 28.369 & $\mid$ & -2.965 & 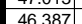 & $\begin{array}{l}0.459 \\
0.459 \\
\end{array}$ & 14.643 & & (72.04 & $\mid \begin{array}{l}121.667 \\
\mid\end{array}$ & 347 & $\frac{11.6}{110}$ & & $\mid$\begin{tabular}{|l|l|}
2101 \\
2101
\end{tabular} & & & & 213 & $\frac{1.84}{182}$ \\
\hline 93 & $9 / 8 / 2004$ & 9:53:06 AM & $\frac{25.606}{2006}$ & 23.113 & \begin{tabular}{|l|}
25.305 \\
\end{tabular} & 24.501 & \begin{tabular}{|l|}
23.058 \\
\end{tabular} & 32.515 & $\frac{2.623}{35.623}$ & 4.449 & 28.314 & $|-1.209|$ & & $\frac{6.391}{46.391}$ & $\mid \frac{\mid .457}{0.457}$ & 14.643 & & 74.00 & $\mid$ & $\frac{34.5}{34.5}$ & $\frac{\frac{\pi 1 .}{11 .}}{11 .}>0$ & 30.4 & & $\frac{0.065}{0.065}$ & $\frac{0.065}{0.065}$ & & $\frac{2.133}{2.133}$ & $\frac{1.82}{1.82}$ \\
\hline & 9/8/2004 & & & 23.108 & $\mid$\begin{tabular}{|r|}
25.3 \\
\end{tabular} & 24.516 & & $\frac{32.513}{32.513}$ & 35.598 & 4.471 & & $|-1.209|$ & & 46.494 & 0.454 & & & 7500 & & 34.5 & & & & & 0.064 & & & $\frac{1.82}{1.80}$ \\
\hline 95 & & & $25.593 \mid$ & 23.135 & 25.306 & 24.513 & & 32.72 & 5 & 4.523 & & $|-1.212|$ & & $\frac{45.874}{44.8}$ & 0.451 & & & & $\frac{26667}{2667}$ & 34.8 & $\frac{\frac{10.9}{109}}{2}$ & & & & 0.064 & & 2099 & $\frac{1.80}{179}$ \\
\hline 96 & 9/8/2004 & 9:56:06 AM & $\begin{array}{l}5.583 \\
5.583\end{array}$ & 23.13 & 25.296 & 24.508 & 23.055 & 32.465 & 35.554 & 4.361 & 28.439 & $|-1.209|$ & & 46.12 & 0.449 & 14.643 & & & & 34.4 & $\frac{11.0}{11.0}$ & $30.5=2$ & 2.100 & 064 & 0.064 & 0.002 & 2.093 & \\
\hline & & & & & & & & & & & & & & & & & & & & & & & & & & & & \\
\hline 98 & & & & & & & & & & & & & & & & & & & & & & & & & & & & \\
\hline 99 & & & & & & & & & & & & & & & & & & & & & & & & & & & & \\
\hline & & & & & & & & & & & & & & & & & & & & & & & & & & & & \\
\hline .01 01 & & Average & & 22.7 & 25.0 & 23.4. & 22.0 & 12.3 & 13.3 & 4.5 & 8.1 & -1.2 & -4.4. & 46.3. & 0.3 & 14.6 & & & & 12.2. & 11.0 & & & & 0.000 & & 3.7 & \\
\hline & & Maximum & 25.2 & $22.8 \mathrm{~T}$ & 25.3 & 23.6 & 22.7 & 12.5 & 13.5 & 4.7 & 8.3 & -1.2 & -4.2 & 46.7] & 0.3 & 14.6 & & & & 12.4 & 11.1 & 10.4 & & 042 & 0.042 & & 4.2 & 3.6. \\
\hline 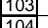 & & Median & 24.9 & 22.8 & 25.0 & 23.4 & 22.6 & 12.3 & 13.3 & 4.5 & 8.1 & -1.22 & -4.4 .4 & 46.3 & 0.3 & 14.6 & & & & 12.2 & 111.0 & $10.2]$ & & & (3) & & . & 3.1 \\
\hline 105 & & $2 \times$ Std Dev & $\frac{2.44}{0515}$ & 0.10 & 24.09 & $\frac{2.47}{0.91}$ & 0105 & 1.2.0 & $\frac{1.06}{0.727}$ & 2.4.2 & 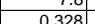 & $\mid-1.4$ & 0165 & 年, & 0026 & (4.600 & & & & $\frac{1.5}{0272}$ & (10.5 087 & 0 & & & & & & \\
\hline 106 & Number o & f Points Used & $\frac{0.010}{20}$ & 20 & - $\frac{0.429}{20}$ & $\frac{0.151}{20}$ & $\begin{array}{l}0.100 \\
20\end{array}$ & 20 & $\frac{0.272}{20}$ & 0.200 & $\frac{0.020}{20}$ & $\frac{0.000}{20}$ & $\frac{0.100}{20}$ & 20 & $\frac{0.00}{20}$ & $\frac{0.00}{20}$ & $\frac{0.07}{20}$ & & & 20 & $\frac{0.067}{20}$ & $\frac{1.20}{20}$ & 20 & $\frac{0.04 \mid}{20}$ & 20.00 & 20 & 0.400 & 0.414 \\
\hline 107 & & * Backpulse poin & sin boxa & re not inc & Eluded & & & & & & & & & & & & & & & & & & & & & & & \\
\hline (2) & & & & & & & & & & & & & & & & & & & & & & & & & & & & \\
\hline & & & & & & & & & & & & & & & & & & & & & & & & & & & & \\
\hline $110 \mid \mathrm{E}$ & & Averages & 25.4 & 22.9 & 25.3 & $\begin{array}{llll}23.9 & & \end{array}$ & & 22.6 & 25.2 & 4.3 & \begin{tabular}{ll|l|}
18.6 \\
\end{tabular} & \begin{tabular}{cc|c|}
-1.2 &
\end{tabular} & -3.3 & 45.8 & 0.4 & 14.6 & 0.0 & & & 24.1 & \begin{tabular}{ll|l}
10.9 &
\end{tabular} & \begin{tabular}{ll|}
20.6 \\
\end{tabular} & 1.420 & 059 & & & 2.9 & \\
\hline & & Maximum & 25.5 & 23.0 & 25.4 & 24.1 & 23.0 & 23.0 & 25.8 & 4.5 & $\begin{array}{ll}19.1 \\
\end{array}$ & \begin{tabular}{|c|c|}
-1.2 \\
\end{tabular} & & $\begin{array}{lll}46.8 \\
\end{array}$ & 0.5 & 14.6 & & & & 24.7 & 11.1 & 21.0 & & 0.065 & 0.065 & & 3.3 & \\
\hline & & Median & 25.4 & 22. & 25.3 & 23.9 & $22.8 \mathrm{Y}-\mathrm{T}$ & 22.7 & 25.3 & 4.3 & & $\begin{array}{lll}-1.2 & & \end{array}$ & $-3.4 \quad<>3$ & (45.8 & 0.4 & 14. & & & & 24. & 10. & $0.7[3$. & 1.4 & & & & 2.8 & \\
\hline 13 & & Minimum & 25.4 & 22.8 & 25.2 & 23.7 & & 21.8 & 24.6 & 4.2 & 18.0 & $\begin{array}{ll}-1.2 \\
\end{array}$ & -3.7 & 45.0 & 0.4 & 14.6 & $0.8 \quad-3$ & & & 23.5 & \begin{tabular}{ll|}
10.7 \\
\end{tabular} & $\begin{array}{ll}19.9 \\
\end{array}$ & \begin{tabular}{|c|c|}
1.373 \\
\end{tabular} & 0.054 & 0.053 & 0.003 & 2.6 & \\
\hline 114 & & $2 \times \operatorname{Std} \mathrm{Dev}$ & 0.031 & 0.084 & 0.067 & 0.240 & 0.152 & 0.510 & 0.530 & 0.174 & 0.541 & | & 0.392 & $\begin{array}{lll}0.862 & & \\
\end{array}$ & $\mid 0.047$ & 0.000 & 0.00 & & & 0.530 & 0.2050 & 0.514 & \begin{tabular}{|l|l|} 
\\
\end{tabular} & \begin{tabular}{|l|l|} 
\\
\end{tabular} & | 0.007 & 0.000 & 0.361. & 0.307 \\
\hline & Number o & f Points Used ${ }^{*}$ & 20 & 20 & 20 & & & 20 & 20 & 20 & 20 & 20 & 20 & 20 & 20 & 20 & 2 & & & 20 & 20 & 20 & 20 & 20 & 20 & 20 & 20 & 20 \\
\hline$=$ & & * Backpulse poin & is in box a & re not inc & Eluded & & & & & & & & & & & & & & & & & & & & & & & \\
\hline & & & & & & & & & & & & & & & & & & & & & & & & & & & & \\
\hline $119 \mathrm{C}$ C & & Averages & 25.7 & 23.1 & 25.4 & 24.4 & 230 & 32.6 & 36.2 & 4.4 & 28.5 & -12 & $-2,7$ & 46.0 & 05 & 146 & & & & & & 305 & 2105 & & 00699 & & & \\
\hline & & & 25.7 & 23.1 & 25.5 & 24.5 & & & $\begin{array}{ll}0.4 \\
36.7\end{array}$ & $\begin{array}{l}4.4 \\
4.6 \\
\end{array}$ & 29.0 & $\frac{-1.2}{-1.2}$ & 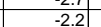 & 47.0 & 0.5 & $\frac{14.0}{14.6}$ & 0.6 & & & 35.6 & $\frac{11.0}{11.2}$ & 31.0 & \begin{tabular}{|l|}
2.139 \\
2.139 \\
\end{tabular} & & 0 & 0.003 & 2.5 & \\
\hline 211 & & Median & 25.7 & 23.1 & 25.4 & 24.5 & 23.0 & 32.5 & 36.2 & 4.4 & 28.4 & \begin{tabular}{|l|}
-1.2 \\
\end{tabular} & -2.7 & 46.0 & 0.5 & 14.6 & 0.0 & & & 35.1 & 10.9 & 30.5 & \begin{tabular}{|l|}
2.100 \\
\end{tabular} & \begin{tabular}{|l|} 
\\
\end{tabular} & \begin{tabular}{|l|l|l|}
0.069 \\
\end{tabular} & 0.002 & 2.2 & 1.9 \\
\hline & & & 25.6 & 23.0 & \begin{tabular}{|l|}
25.3 \\
\end{tabular} & 24.2 & 22.9 & 32.1 & 35.6 & 4.2 & 28.0 & $\begin{array}{r}-1.2 \\
\end{array}$ & $\begin{array}{ll}-3.1 \\
\end{array}$ & 45.0 & 0.4 & 14.6 & 0.0 & & & 34.4 & 10.7 & 30.1 & \begin{tabular}{|l|l|}
2.073 \\
\end{tabular} & 0.064 & 0.064 & 0.002 & 2.1 & 1.8 \\
\hline & & $2 \times$ Std Dev & 0.076 & 0.088 & \begin{tabular}{|l|}
0.127 \\
\end{tabular} & 0.157 & 0.103 & 0.524 & 0.770 & 0.189 & $\begin{array}{l}0.634 \\
\end{array}$ & 0.003 & 0.496 & 0.984 & 0.053 & 0.000 & 0.000 & & & 0.770 & $0.234 \mid 0$ & 0.562 & \begin{tabular}{|l|l|} 
\\
\end{tabular} & 508 & 0.007 & 0.000 & 0.248 & 0.21 \\
\hline 1244 & Number o & f Points Used ${ }^{*}$ & 20 & 20 & 20 & 20 & & & 20 & & 20 & 20 & 20 & 20 & 20 & 20 & 20 & & & 20 & 20 & 20 & 20 & 20 & 20 & 20 & 20 & \\
\hline & & * Backpulse poin & is in box a & re not inc & Eluded & & & & & & & & & & & & & & & & & & & Cald & & & & \\
\hline$\frac{12}{12}$ & & & & & & & & & & & & & & & & & & & & & & Avgs & $\mathrm{gStDv}(\mathrm{c}$ & $\begin{array}{l}\left|\frac{\mid}{\text { Call pla }}\right| \\
\text { (alls }\end{array}$ & $\begin{array}{l}\mid 0.006 \\
0.006\end{array}$ & & & \\
\hline 128 & & & & & & & & & & & & & & & & & & & & & & $\operatorname{MaxS}$ & 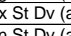 & $\begin{array}{l}\text { (all pts) } \\
\text { (ants) }\end{array}$ & $\begin{array}{l}0.007 \\
004\end{array}$ & & & \\
\hline & & & & & & & & & & & & & & & & & & & & & & & & & & & & \\
\hline
\end{tabular}


WSRC-TR-2005-00105, REVISION 0

SRNL-RPP-2005-00012, REVISION 0

RUN \# 4.21 A, B AND C; PRE-TEST BASELINE WITH 0.1 M NAOH

\begin{tabular}{|c|c|c|c|c|c|c|c|c|c|c|c|c|c|c|c|c|c|c|c|c|c|c|c|c|c|c|c|c|c|}
\hline & A & B & $\mathrm{D}$ & $E$ & $\mathrm{~F}$ & $G$ & $\mathrm{H}$ & $\mathrm{J}$ & $\mathrm{K}$ & $\mathrm{L}$ & $M$ & $\mathrm{~N}$ & 0 & $Q$ & $\mathrm{R}$ & & $\mathrm{s}$ & $\mathrm{T}$ & $\mathrm{V}$ & w & \begin{tabular}{l|l|l|}
$x$ & \\
\end{tabular} & $\underline{Y}$ & $\mathrm{z}$ & $\mathrm{AA}$ & $A B$ & $A C$ & $A D$ & $\mathrm{AE}$ & AF $A$ \\
\hline & $\begin{array}{l}\text { SWednesday, } \\
\text { Baseline } 4.21\end{array}$ & & & & & & & & & & & & & & & & & & & & & & & & & & & & \\
\hline & $998 / 2004$ & & & & & & & & & & & & & & & & & & & & & & & & & & & & \\
\hline & 9/8/2004 & 12:27:56 PM & 24.821 & 23.465 & 24.914 & 24.14 & 23.149 & 12.17 & 13.395 & 4.41 & 8.031 & -1.212 & $\begin{array}{l}-4.763 \\
\end{array}$ & 46.174 & 0.1 & 198 & 14.643 & 0.003 & 1.00 & 0.01667 & \begin{tabular}{|l|}
12.4 \\
12.3
\end{tabular} & $\frac{1}{11}$ & \begin{tabular}{|l|}
10.1 \\
10.1
\end{tabular} & 0.697 & 028 & 0.028 & $\begin{array}{l}0.003 \\
0.003\end{array}$ & $\frac{2.161}{2.812}$ & 2.39 \\
\hline & 9/8/2004 & & & & & \begin{tabular}{|l|}
24.176 \\
\end{tabular} & & & 13.36 & 4.44 & & -1.212 & & 46.183 & 0.1 & & 14.643 & 0.004 & & 0.03333 & & & 10.0 & & & 0.028 & & & \\
\hline 138 & 9/8/2004 & 12:29:56 PM & 24.857 & 23.476 & 24.906 & 24.186 & 23.09 & 12.174 & 13.435 & 4.4 & \begin{tabular}{|l|l|}
8.046 \\
\end{tabular} & \begin{tabular}{|l|}
-1.206 \\
\end{tabular} & -4.737 & 45.966 & 0.1 & 193 & $\begin{array}{l}14.643 \\
\end{array}$ & 0.004 & 3.00 & 0.05 & 12.3 & & 10.1 & \begin{tabular}{|c|c|}
0.697 \\
\end{tabular} & 0.028 & $\begin{array}{ll}0.028 \\
\end{array}$ & 0.003 & 2.740 & \\
\hline$\frac{139}{140}$ & 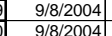 & $\begin{array}{l}12: 30: 56 \text { PM } \\
12.31 .56 \text { PM }\end{array}$ & $24.857 \mid$ & $\frac{23.471}{23.492}$ & 24.885 & $\begin{array}{ll}24.181 \\
24.237\end{array}$ & $\begin{array}{r}23.084 \\
23.11 \\
\end{array}$ & $\frac{12.267}{1205}$ & 13.546 & $\begin{array}{l}4.61 \\
4.34 \\
\end{array}$ & $\begin{array}{l}7.927 \\
7.968 \\
\end{array}$ & \begin{tabular}{|l|l|}
-1.212 \\
-1.206
\end{tabular} & $\begin{array}{r}-4.783 \\
-4.778 \\
\end{array}$ & $\begin{array}{l}45.987 \\
46.099 \\
\end{array}$ & $\begin{array}{ll}0.1 \\
0.1\end{array}$ & $\begin{array}{l}193 \\
195 \\
\end{array}$ & \begin{tabular}{|l}
14.643 \\
14.643 \\
\end{tabular} & $\begin{array}{l}0.003 \\
0.003\end{array}$ & 5.00 & \begin{tabular}{|l|l|}
0.06667 \\
0.08333
\end{tabular} & $\begin{array}{l}12.4 \\
123\end{array}$ & 11.0 & \begin{tabular}{|l|l|}
10.1 \\
10.0
\end{tabular} & \begin{tabular}{|l|l|}
0.696 \\
0.692
\end{tabular} & & \begin{tabular}{|l|l}
0.028 \\
0.028
\end{tabular} & $\begin{array}{l}0.003 \\
0.003 \\
\end{array}$ & $\frac{2.745}{2.786}$ & $\begin{array}{l}2.34 \\
2.37 \\
\end{array}$ \\
\hline$\frac{14}{141}$ & | $9 / 8 / 2004$ & $\begin{array}{l}12.31 .56 \mathrm{PM} \\
12: 32: 56 \mathrm{PM}\end{array}$ & 24.888 & $\begin{array}{l}23.492 \\
23.487 \\
\end{array}$ & \begin{tabular}{|l|l|}
24.946 \\
24.941 \\
\end{tabular} & \begin{tabular}{|l|}
$\mid 24.23$ \\
24.242 \\
\end{tabular} & $\begin{array}{l}23.11 \\
23.156\end{array}$ & $\frac{12.105}{12.12}$ & 13.385 & 4.38 & 8.013 & \begin{tabular}{|l|}
-1.200 \\
-1.209 \\
\end{tabular} & $\begin{array}{l}-4.178 \\
-4.757 \\
\end{array}$ & \begin{tabular}{|l|l|l|l|l|l|}
46.018 \\
\end{tabular} & 0.1 & 198 & $\begin{array}{l}14.643 \\
14.643 \\
\end{array}$ & 0.003 & $\begin{array}{l}5.00 \\
6.00 \\
\end{array}$ & 0.1 & $\begin{array}{l}11.0 \\
12.3 \\
\end{array}$ & & $\frac{10.6}{10.1}$ & $\begin{array}{l}0.092 \\
0.694\end{array}$ & & $\frac{0.028}{0.028}$ & $\frac{0.003}{0.003}$ & $\frac{2.786}{2.821}$ & 2.37 \\
\hline & $9 / 8 / 2004$ & 12:33:56 PM & 24.904 & 23.498 & 24.952 & 24.308 & 23.201 & 12.205 & \begin{tabular}{|l|l|l|l|l}
5 & 13.544
\end{tabular} & 4.4 & 7.97 & -1.206 & -4.691 & 46.227 & 0.1 & 193 & 14.643 & 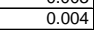 & & 0.11667 & 12.4 & & $\frac{10.1}{10.1}$ & & 0.028 & 0.028 & & $\frac{2.84}{2.74}$ & $\begin{array}{r}2.40 \\
2.33 \\
\end{array}$ \\
\hline 143 & 9/8/2004 & 12:34:56 P & 24.914 & 23.498 & & 24.394 & & & & & & $\mid-1.212$ & & & & & & & 8. & & & & & & & & & & \\
\hline & & & & & 24.929 & 24.415 & & 12.178 & 13.453 & 4.49 & 7.998 & & & 46.116 & & & 14.643 & & & 0.15 & 12.3 & & 10.1 & & & $\frac{0.028}{0.028}$ & 0.003 & 271 & \\
\hline $14 !$ & $9 / 8 / 2004$ & 12:36:56 PM & 24.981 & $\frac{23.495}{23}$ & 24.935 & 24.411 & 23.25 & 3.31 & 13.52 & 4.47 & -1.025 & 4.807 & & & & & ( & & & & 12.4 & & 1.: & 0.079 & & 0.000 & & & \\
\hline 146 & 9/8/2004 & 12:37:56 PM & 24.938 & 23.512 & 24.961 & 24.478 & & 2.513 & 13.437 & 4. & -1.737 & 5.562 & & 45.761 & 0.0 & & $\begin{array}{ll}14.643 \\
\end{array}$ & & 11.00 & 0.18333 & 12.3 & & 0.4 & & & 0.000 & 0.001 & 1.108 & \\
\hline 144 & 9/8/2004 & 12:38:56 PM & 24.793 & 23.517 & 24.957 & 24.483 & 23.241 & 7.398 & 13.453 & 4.43 & 3.215 & 0.487 & 0.33 & 46.032 & 0.2 & & 14.643 & 0.0 & 12.6 & 0.2 & 12.3 & 11 & 5. & 0.36 & 0.045 & 0.043 & 0.008 & & \\
\hline $1 \frac{148}{48}$ & 9/8/2004 & 12:39:56 PM & 24.925 & 23.529 & 24.953 & 24.5 & & 7.564 & 13.493 & 4.37 & 3.44 & . & & 46.35 & & & 14.643 & & & .21667 & & & 5.5 & & & 0.02 & & & \\
\hline & $\begin{array}{l}98 / 2004 \\
0 / 2004\end{array}$ & $12: 40: 56 \mathrm{PM}$ & 24.945 & 23.529 & 24.938 & 24.51 & & $\frac{1.545}{5.73}$ & $\frac{13.42}{13.20}$ & 4.45 & 3.309 & 0.331 & 0 & 45.855 & & & 14.643 & & 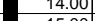 & .23333 & $\frac{12.3}{12.3} 5>$ & & & & & 0.024 & & & \\
\hline & 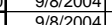 & $\frac{1.24 .1}{120}$ & 24.900 & 年3.500 & $\begin{array}{l}24.950 \\
24982\end{array}$ & 24.527 & (23.28 208 & 7.435 & 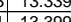 & $\frac{1.35}{434}$ & 3.3524 & 0.334 & & 40.161 & & & 14.0 1.0 & & 13. & 0.25 & & & 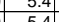 & & & 0.022 & 0.044 & & \\
\hline 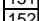 & 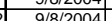 & $12.42 .507 \mathrm{PM}$ & & $\frac{2.0545}{23543}$ & 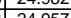 & 24.054 & & 1.451 & 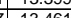 & 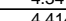 & 3.0254 & 0.005 & & 40.00 & & & 14.045 & & $\begin{array}{l}10 \\
17\end{array}$ & 0.20007 & $\begin{array}{ll}1.5 \\
1.20\end{array}$ & & 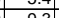 & nfth & & 0.021 & 0.004 & 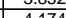 & \\
\hline & & 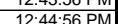 & & & & $\frac{24.525}{24601}$ & & (11786 & & $\frac{4.41}{441}$ & & 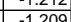 & - & (40.151 & & & 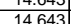 & & 17. & 0.2035 & $\frac{12.3}{124}$ & & $\frac{7.0}{97}+20$ & & & & & & \\
\hline 1 & 9/8/2004 & $\begin{array}{l}\frac{13: 405}{12.56 \mathrm{PM}} \\
\end{array}$ & 24.951 & 23.545 & 24.924 & 24.566 & $\frac{25.369}{23.369}$ & $\frac{1.1 .648}{11.1848}$ & $\frac{10.0 .48}{13.48}$ & $\frac{1.40}{4.50}$ & 7.709 & $\frac{-1.212}{-1.212} \mathrm{Cl}$ & & 46.026 & 0.8 & & 14.643 & & 19. & 0.31667 & $\frac{1.24}{12.4}$ & 11 & $\frac{9.8}{9.8}$ & 0.674 & & $\frac{0.032}{0.032}$ & $\frac{0.003}{0.003}$ & $\frac{5.424}{3.24}$ & \\
\hline & & & & & & & & 11.969 & 13.615 & 4.45 & 7.81 & & & 45.999 & 0.2 & & 14.643 & & & & & & 9.4 & & & 0.031 & & & \\
\hline & 9/8/2004 & 12:47:56 PM & 24.984 & 23.583 & 24.992 & 24.694 & 23.4 & $12.0 \varepsilon$ & 13.706 & 4.46 & 7.912 & $-1.212 \quad>>$ & & 46.2 & & & 14.643 & & & 0.35 & 12.6 & & & & & & & & \\
\hline 15 & 9/8/2004 & 12:48:56 PM & 24.98 & 23.574 & 24.973 & 24.731 & 23.438 & 11.95 & 13.476 & 4.36 & 7.894 & -1.212 & & 46.172 & 0.2 & & 14.643 & & 22 & 0.36667 & 12.4 & & 9.9 & 1.68 & & 0.031 & .003 & 3.119 & \\
\hline & & & 24.98 & 23.586 & 24.945 & & & 11.896 & 13.424 & & 8.078 & -1.215 & & 46.535 & & & 14.643 & & 23.0 & 0.38333 & 12.3 & & 10. & 6.689 & 0.036 & 0.030 & 0.003 & 3.015 & \\
\hline & 9.8/2004 & 12:50:56 PM & 24.999 & 23.603 & 24.967 & 24.754] & & 11.971 & 13.4222 & 4.44 & 7.842 & -1.209 & -4.6 & 46.143 & & & 14.643 & & 24 & 0.4 & 12.3 & & 9.8 & & & 0.033 & & 3.096 & \\
\hline & $\begin{array}{l}9.8 / 2004 \\
0 / 2004\end{array}$ & $\begin{array}{l}12.51 .56 \mathrm{MM} \\
2.52 .56 \mathrm{PM}\end{array}$ & 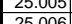 & $\frac{2.602}{23.621}$ & $\frac{24.963}{2499}$ & 24.121 & & $\frac{12.076}{11098}$ & 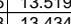 & $\frac{4.45}{432}>>$ & 7.5035 & 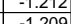 & & 40.164 & & & $\frac{14.643}{14643}$ & & & . 4.1606 & $\begin{array}{ll}1.2 .4 \\
123\end{array}$ & & 0.040 & & & & & . & \\
\hline & $9 / 210004$ & $\begin{array}{l}12.52 .30 \\
12.53 .56\end{array}$ & 25019 & $\frac{2.024}{23639}$ & 24.997 & $\frac{24.141}{2472}$ & & $\frac{11.950}{1210}$ & & $\frac{1.32}{445}$ & 8. & 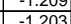 & & $\frac{40.00}{16.141}$ & & & $\begin{array}{l}14.045 \\
14612\end{array}$ & & & . & $\frac{12.35}{124}$ & & & & & & & & \\
\hline$\frac{106}{16}$ & $\frac{17 / 20404}{9 / 8 / 2004}$ & $\frac{13.546}{125.56}$ & & $\frac{23.034}{23.634}$ & 24.973 & $\frac{24.1}{24.71}$ & $\frac{25}{23}$ & $\frac{12.19}{12.199}$ & $\begin{array}{l}13.567 \\
13.607\end{array}$ & $\frac{2.45}{4.44}$ & $\begin{array}{l}0.1594 \\
7.984\end{array}$ & - & $\frac{-4.6}{-4}$ & $\begin{array}{l}40.141 \\
46.139\end{array}$ & & & $\frac{14.043}{14.643}$ & & $\frac{21}{28}$ & $\begin{array}{rl}0.45 & 0.4667 \\
0.5\end{array}$ & $\frac{12.4}{12.5}$ & & $\frac{1.1}{10.1}$ & 0.65 & & $\frac{0.030}{0.030}$ & & 它 & \\
\hline & $9 / 8 / 2004$ & & & & 24.965 & 24.73 & & 12.209 & & 4.56 & 7957 & -1.212 & & 46.1 & & & 14. & & & 0.4833 & 125 & & 10 & & & & & 814 & \\
\hline 165 & 2004 & & & 23.653 & 24.961 & 24.7 & & 12. & 13.482 & 4.37 & & $\frac{-1.212}{-1.212}$ & & 46.0 & & & & & 30 & 0.5 & 12.4 & & 10. & & & & & & \\
\hline 166 & $\mid$ & & & 23.653 & 24.972 & 24.7 & & & 3.536 & 4.4 & 8 & -1.212 & & 46.2 & & & & & & 0.51667 & 12.4 & & 10.1 & & & & & 2.892 & \\
\hline $\begin{array}{l}167 \\
\end{array}$ & 9/8/2004 & & & 23.664 & 24.988 & & & & 3.451 & 4.33 & \begin{tabular}{|c|}
8.074 \\
\end{tabular} & -1.215 & & 46.5 & & & & & & 153333 & $\begin{array}{ll}12.3 \\
\end{array}$ & & & & & & & & \\
\hline 168 & 9/8/2004 & 12:59: & 25.024 & 23.669 & 24.973 & 24.87 & & 12.2 & 13.611 & 4.4 & 8.177 & -1.212 & & $\begin{array}{l}46.091 \\
\end{array}$ & & 208 & $\begin{array}{l}14.643 \\
\end{array}$ & & & 0.55 & 12.5 & & 10.2 & 0.7 & & 0.030 & & 2.918 & \\
\hline 165 & & & 25.028 & & & & & & & 4.3 & & & & 46.1 & & & & & & 56667 & 12.4 & & 0. & & & & & 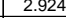 & \\
\hline$\frac{176}{17}$ & 9 & & & & & & & & $\frac{13.522}{13.52}$ & 4.48 & & & & 46.3 & & & 14.6 & & & 0.58333 & 12.4 & & 10. & & & 0.030 & & 2.972 & \\
\hline & & & & & 24.95 & & & & $\frac{1.3 .486}{1320}$ & & & & & 46. & & & & & & 0. & 12.4 & & & & & & & & \\
\hline$\frac{112}{177}$ & $\frac{91012004}{0.1204}$ & & & & & & & & & & & & & 40.4 & & & & & & & $\frac{12.5}{12.5}$ & & & & & & & & \\
\hline$\frac{114}{174}$ & $\mid$ & & & $\frac{23.677}{23.677}$ & 25.106 & & & 23.4 & 25.831 & $\frac{1.52}{4.52}$ & 18.814 & \begin{tabular}{|l|l|}
-1.209 \\
\end{tabular} & & $\frac{46}{46}$ & & $\frac{518}{418}$ & & & & 0.0005 & 24.7 & & 200 & & & & & 8.45 & \\
\hline & & & & & 25.084 & & & & 25.027 & 4.54 & 18.286 & \begin{tabular}{|l|l|}
-1.209 \\
\end{tabular} & & & & & & & & 0.66667 & 23.9 & & & & & & & & \\
\hline \begin{tabular}{|l|}
17 \\
\end{tabular} & $1 / 2004$ & & 5.205 & 23.695 & 250.069 & 24.96 & & 22 & 24.685 & 4.5 & 18.18 & -1.209 & & 46.4 & $\frac{1.3}{0.3}$ & 387 & 14. & & & 6833 & 236 & & 20. & & & 0.055 & & 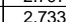 & \\
\hline & 9/8/2004 & & & 23.7 & 25.033 & 24. & & 22. & 68 & 4.66 & 17.884 & -1.212 & & 46. & & & & & & 0.7 & 23.9 & & 20.1 & & & & & 2.776 & \\
\hline 178 & 9/8/2004 & & & 23.721 & 25.025 & 25. & & 22.4 & 24.834 & & 18.299 & -1.212 & & 46. & & & & & & 0.716 & 23.7 & & & & & & & & \\
\hline 17 & 9/8/2004 & & 25.19 & 23.711 & 24.98 & 25. & & 22.55 & 25.077 & 4.43 & 18.516 & -1.212 & & 46.5 & & & 14. & & & 0.7333 & 24.0 & & 20.6 & & & & & 2.718 & 2 \\
\hline & & & & & & 25.0 & & 22 & & & & -1.212 & & & & & & & & 0.7 & & & 20.4 & & & & & & \\
\hline 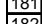 & & & & & & & & & & & & & & & & & & & & & 23.6 & & & & & & & & \\
\hline$\frac{102}{183}$ & $9 / 182044$ & & & & & & & & & & & & & & & & & & & 0.18356 & 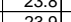 & & 0.5 & & & & & 2716 & \\
\hline 184 & $9 / 8 / 2004$ & & & & & & & & 250 & & & & & & & & & & & 0816 & 20.04 & & & & & & & & \\
\hline & & & & & & & & & & & & & & & & & & & & & 2339 & & $20 .-1$ & & & & & 270 & \\
\hline 186 & 9/8/2004 & & 25.107 & 23.752 & 24.83 & 25.2 & & 22.3 & 24.928 & & & & & 46. & & & & & & 0.8 & 23.8 & & 20. & & & & & & \\
\hline & & & & & & & & & & 4.4 & & & & & & & & & & & 24.0 & & & & & & & & \\
\hline \begin{tabular}{|l|l} 
\\
\end{tabular} & $9 / 8 / 20$ & & & 23.7 & & & & & $\overrightarrow{24.8}$ & & 18.223 & -1. & & 45. & & & & & & 883 & 23.7 & & & & & & & & \\
\hline $\begin{array}{lll}1 & \end{array}$ & 9/8/20 & & & 23. & & & & & 24.9 & & & & & 46.2 & & & 14.6 & & & & 23.8 & & & & & & & & \\
\hline 19 & & & 25.0 & 23.781 & 24.769 & 25. & & 22.601 & & 4.33 & 18.608 & -1.212 & & & & & & & & 0.91667 & 23.9 & & 20. & & & & & 2.71 & \\
\hline$\sqrt{10}$ & 98/212004 & & 25.047 & 23.792 & 24.7 & 25.309 & & 22.3 & 24.87 & & 18.239 & -1.212 & & 45.6 & & & & & & 1.500 & 23.7 & & 20. & & & & & & \\
\hline & $\frac{918 / 20}{91 / 20}$ & & & $\frac{2.1933}{2781}$ & 24.746 & 25.28 & & & & & & & & & & & & & & & 24.3 & & & & & & & & \\
\hline & 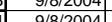 & 立.24.5 & 1500 & $\frac{3.01}{2380}$ & & & & 22.4 & $24.0=$ & $4.5 \%$ & to.000 & & & $\begin{array}{ll}40.4 \\
4.63\end{array}$ & & & & & & 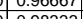 & 23.5 & & & & & & & & \\
\hline$\frac{13}{19}$ & $9 / 2 / 200$ & & & 23.583 & 24756 & 2524 & & 22.058 & 243 & $\frac{1.3}{4.3}$ & 18.07 & -1209 & & 463 & & & & & & & 年, & & 201 & & & & & & \\
\hline & & & & & & & & & & & & & & & & & & & & & & & & & & & & & \\
\hline & & & & & & 25 & & & 248 & 4.4 & & & & & & & & & & & 23.7 & & & & & & & & \\
\hline & 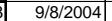 & & & 23.8 & 24.7 & & & & 24.54 & 4. & 18.2 & \begin{tabular}{|l|}
-1.212 \\
\end{tabular} & & & & & & & & & 23.4 & & & & & & & 718 & 2 \\
\hline 199 & $9 / 8 / 2004$ & & 249 & 23.863 & 24.765 & 25.184 & & 33.1 & 375 & & 28.459 & $\mid-1$, & & 49.9 & 6 & & 14.6 & & & 106 & 36.4 & & & & & & & 2.573 & 2 \\
\hline 200 & 9/8/2004 & 1:31:5 & 25.044 & 23.874 & 24.947 & 25.23 & & 32.473 & 37. & 4.69 & 28.219 & -0.763 & & 48.218 & & & 14.6 & & 65.06 & 1.0833 & 36.1 & & 30.3 & & & 0.081 & & 2.684 & 2.2 \\
\hline & & $1: 32$ & & & 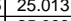 & 25. & & 32.056 & & 4.37 & 28.2011 & -0.81 & & 46.201 & & 50. & & & & 1 & 35.5 & & 30.1 & & & 0.081 & .003 & 2.674 & 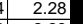 \\
\hline & 9/8/2004 & $1: 33: 5$ & .19 & 3.88 & 5.06 & & & 32.12 & 6.816 & 4.48 & 27.96] & 1807 & & 46.087 & & 56 & 14.643 & & & 1166 & 35.7 & & 30. & & & 0.08 & 0.003 & 2.683 & $2.8 \mathrm{C}$ \\
\hline
\end{tabular}


WSRC-TR-2005-00105, REVISION 0

SRNL-RPP-2005-00012, REVISION 0

RUN \# 4.21 A, B AND C; PRE-TEST BASELINE WITH 0.1 M NAOH - CONT.

\begin{tabular}{|c|c|c|c|c|c|c|c|c|c|c|c|c|c|c|c|c|c|c|c|c|c|c|c|c|c|c|c|c|}
\hline & A & B & $\mathrm{D}$ & $E$ & $F$ & G & $\mathrm{H}$ & $\mathrm{J}$ & $\mathrm{K}$ & $\mathrm{L}$ & $\begin{array}{ll} \\
\end{array}$ & \begin{tabular}{|l|}
$N$ \\
$N$
\end{tabular} & 0 & \begin{tabular}{l|l} 
\\
\end{tabular} & \begin{tabular}{|l|l|l|}
$R$ & \\
\end{tabular} & $s$ & \begin{tabular}{l|l}
$\mathrm{T}$ & $\mathrm{K}$
\end{tabular} & $\mathrm{v}$ & $\mathrm{w}$ & \begin{tabular}{|l|}
$x$ \\
\end{tabular} & $\mathrm{Y}$ & $z_{2}$ & AA & $A B$ & $\mathrm{AC}$ & AD & $\mathrm{AE}$ & AF \\
\hline 20 & 9/8/2004 & 1:34:56 PM & 25.235 & 23.88 & 25.099 & 25.412 & 23.664 & 32.361 & 37.146 & 4.468 & 28.188 & \begin{tabular}{|c|}
-0.807 \\
\end{tabular} & $\begin{array}{r}-1.956 \\
\end{array}$ & 46.174 & 0.563 & 14.643 & 0.003 & 68.00 & \begin{tabular}{|l|l|}
1.13333 \\
\end{tabular} & 36.0 & 11.0 & 30.3 & 2.087 & 0.081 & 0.080 & 0.003 & 2.655 & 2.26 \\
\hline & $9 / 8 / 2004$ & 1:35:56 PM & 25.284 & 23.875 & \begin{tabular}{|l|l|}
25.108 \\
\end{tabular} & 25.411 & 23.648 & & & 4.465 & 27.977 & & -1.959 & & 0.564 & 14.643 & & 69.00 & 1.15 & & 11.0 & 201 & & & & & 2.675 & 2.28 \\
\hline 205 & 9/8/2004 & $1: 36: 56 \mathrm{PM}$ & $\begin{array}{l}25.348 \\
2.303\end{array}$ & 23.889 & \begin{tabular}{|l|l|}
25.187 \\
.51
\end{tabular} & 25.47 & $\begin{array}{l}23.526 \\
2.775\end{array}$ & $\begin{array}{r}31.92 \\
\end{array}$ & $\begin{array}{l}36.762 \\
3.71\end{array}$ & 4.363 & 27.788 & \begin{tabular}{|l|l|}
-0.804 \\
\end{tabular} & $\begin{array}{r}-1.95 \\
\end{array}$ & \begin{tabular}{|l|l|}
46.354 \\
\end{tabular} & $\begin{array}{l}0.563 \\
\end{array}$ & 14.643 & $\begin{array}{l}0.004 \\
\end{array}$ & 70.00 & 1.16667 & 35.6 & 11.0 & 29.9 & \begin{tabular}{|l|l}
2.058 \\
\end{tabular} & \begin{tabular}{|l|l|}
8 & 0.08 \\
\end{tabular} & 0.080 & 0.003 & 2.686 & 2.29 \\
\hline 206 & $9 / 8 / 2004$ & $\begin{array}{l}1: 37: 56 \mathrm{PM} \\
1.38565 \mathrm{PM}\end{array}$ & $\begin{array}{l}25.372 \\
25.436\end{array}$ & 23.882 & $\begin{array}{r}25.19 \\
25.5\end{array}$ & $\begin{array}{r}25.429 \\
25.443\end{array}$ & $\begin{array}{r}23.475 \\
23.424\end{array}$ & \begin{tabular}{|l|}
31.988 \\
32.129
\end{tabular} & $\begin{array}{l}36.715 \\
37175 \\
\end{array}$ & 4.374 & 27.903 & \begin{tabular}{|c|c|} 
\\
\end{tabular} & $\begin{array}{r}1.959 \\
1050\end{array}$ & $\begin{array}{l}45.755 \\
5.097\end{array}$ & 0.563 & 14.643 & 0.003 & 71.00 & 1.18333 & 35.6 & $\begin{array}{l}10.9 \\
110\end{array}$ & 29.9 & 2006 & \begin{tabular}{ll|l}
5 & 0.08 \\
3
\end{tabular} & 0.080 & $\begin{array}{l}0.003 \\
0.032\end{array}$ & 2.677 & 2.28 \\
\hline & $\begin{array}{l}9 / 8 / 2004 \\
9 / / 8 / 2004\end{array}$ & $\begin{array}{l}1: 335: 56 \mathrm{PM} \\
1: 3956 \mathrm{PM}\end{array}$ & $\begin{array}{r}25.436 \\
25.45\end{array}$ & $\frac{23.907}{23.895}$ & $\begin{array}{r}25.25 \\
25268 \\
\end{array}$ & $\begin{array}{r}25.443 \\
25.412\end{array}$ & $\frac{23.424}{23.498}$ & \begin{tabular}{r|r}
32.129 \\
32.17
\end{tabular} & \begin{tabular}{|c|}
37.175 \\
37028
\end{tabular} & $\begin{array}{l}4.474 \\
4.409\end{array}$ & \begin{tabular}{|l|}
27.702 \\
27.95 \\
\end{tabular} & \begin{tabular}{|c|}
$\mid-0.804$ \\
-0.824
\end{tabular} & $\begin{array}{l}-1.959 \\
-1.956\end{array}$ & $\begin{array}{l}45.987 \\
46.402 \\
\end{array}$ & $\begin{array}{l}0.563 \\
0.563\end{array}$ & $\begin{array}{l}14.643 \\
14.643 \\
\end{array}$ & 0.004 & $\begin{array}{l}72.00 \\
73.00\end{array}$ & \begin{tabular}{r|r}
1.21667 \\
1.1667
\end{tabular} & $\begin{array}{r}36.0 \\
35.9 \\
\end{array}$ & $\begin{array}{l}\frac{11.0}{11 .} \\
11 .\end{array}$ & \begin{tabular}{|l|}
29.9 \\
$\mid 33.1$
\end{tabular} & & $\begin{array}{ll}3 & 0.088 \\
3 & 0.088 \\
\end{array}$ & $\begin{array}{l}0.080 \\
0.000\end{array}$ & $\begin{array}{l}0.003 \\
0.003\end{array}$ & $\begin{array}{r}2.675 \\
2661\end{array}$ & $\begin{array}{l}2.28 \\
2.26\end{array}$ \\
\hline 209 & $9 / 8 / 2004$ & 1:40:56 PM & 25.49 & 23.906 & \begin{tabular}{|l|}
25.304 \\
\end{tabular} & 25.427 & $\frac{20.490}{23.559}$ & $\mid$\begin{tabular}{|c|}
32.114 \\
32.148
\end{tabular} & 37.057 & $\frac{4.495}{4.384}$ & $\begin{array}{l}27.955 \\
27.975\end{array}$ & \begin{tabular}{|c|}
-0.044 \\
-0.81
\end{tabular} & $\begin{array}{l}-1.950 \\
-1.956\end{array}$ & $\begin{array}{l}40.404 \\
45.765\end{array}$ & $\begin{array}{l}.503 \\
0.564\end{array}$ & $\frac{14.643}{14.643}$ & 0.003 & $\begin{array}{l}73.00 \\
74.00\end{array}$ & \begin{tabular}{|l|l|}
1.2000 \\
1.23333 \\
\end{tabular} & $\begin{array}{l}35.9 \\
35.9\end{array}$ & $\frac{11.1}{10.9}$ & \begin{tabular}{|l|l|} 
& 30.1 \\
30.1 & 30.1 \\
\end{tabular} & \begin{tabular}{|l|}
2.073 \\
2.073
\end{tabular} & 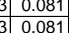 & 0.080 & & $\frac{2.661}{2.663}$ & $\begin{array}{l}2.26 \\
2.27\end{array}$ \\
\hline 210 & $9 / 8 / 2004$ & 1:41:56 PM & 25.536 & 23.922 & 25.355 & 25.428 & 23.565 & 31.959 & 36.682 & 4.255 & 28.065 & \begin{tabular}{|c|}
-0.804 \\
\end{tabular} & -1.95 & 46.214 & 0.563 & 14.643 & 0.004 & 75.00 & & 35.6 & 11.0 & 30.0 & \begin{tabular}{|l|l|} 
& 0.069 \\
\end{tabular} & $\begin{array}{lll}9 & 0.081 \\
\end{array}$ & 0.080 & 0.003 & 2.059 & $\frac{2.27}{2.26}$ \\
\hline 211 & $9 / 8 / 2004$ & 1:42:56 PM & & & & & 23.544 & & 37.316 & 4.293 & 28.272 & \begin{tabular}{|l|}
-0.807 \\
\end{tabular} & -1.965 & & 0.563 & 14.643 & 0.00 & 76.00 & \begin{tabular}{|l|l|}
1.26667 \\
\end{tabular} & & 10.9 & & & & & & & \\
\hline \begin{tabular}{|l|}
212 \\
\end{tabular} & $9 / 8 / 2004$ & 1:43:56 PM & 25.58 & 23.911 & \begin{tabular}{|l|}
25.384 \\
\end{tabular} & 25.382 & \begin{tabular}{|l|l|}
23.624 \\
\end{tabular} & 32.096 & 36.932 & 4.401 & \begin{tabular}{|l|l|}
27.924 \\
\end{tabular} & -0.833 & -1.959 & \begin{tabular}{|l|l|l|l|}
46.049 \\
\end{tabular} & 0.563 & 14.643 & & 77.00 & 1.28333 & $\begin{array}{ll}35.8 \\
\end{array}$ & & & $.06 !$ & .08 & 0.080 & & $\frac{2.050}{2.657}$ & $\frac{2.24}{2.26}$ \\
\hline$\angle 13$ & 9/8/2004 & & & & & 25.403 & & & \begin{tabular}{|c|}
36.847 \\
\end{tabular} & 4.3 & 28.125 & -0.85 & & 45.082 & 0.563 & 14.643 & & 78.00 & 1.3 & & & & & & & & & $\frac{2.26}{2.25}$ \\
\hline 214 & $9 / 8 / 2004$ & 1:45:56 PM & 25.646 & 23.932 & 25.43 & 25.428 & 23.76 & 32.488 & 37.082 & 4.366 & 28.533 & -0.81 & -1.965 & 45.889 & 0.564 & 14.643 & & 79.00 & .31667 & 36.0 & 10.9 & 30. & 2.104 & \begin{tabular}{|l|l|}
4 & 0.08 \\
\end{tabular} & 0.080 & 0.003 & 2.615 & $\frac{2.25}{2.23}$ \\
\hline 215 & $9 / 8 / 2004$ & 1:46:56 PM & 25.661 & 23.932 & \begin{tabular}{|l|}
25.44 \\
\end{tabular} & 25.438 & \begin{tabular}{|l|l}
23.77 \\
\end{tabular} & 32.224 & 37.142 & 4.416 & 28.022 & -0.81 & -1.959 & 45.895 & 0.564 & 14.643 & 1.0 & 80.00 & 1.33333 & 36.0 & $\mid 10.9$ & \begin{tabular}{|l|l|}
40.1 \\
9
\end{tabular} & \begin{tabular}{|l|} 
\\
\end{tabular} & $\begin{array}{l}7 \\
0.08\end{array}$ & 0.080 & 0.003 & 2.648 & 2.50 \\
\hline 216 & $9 / 8 / 2004$ & 1:47:56 PM & 25.668 & 23.934 & \begin{tabular}{|l|}
25.432 \\
\end{tabular} & 25.46 & 23.797 & 31.982 & 36.541 & 4.301 & 28.153 & -0.81 & -1.962 & \begin{tabular}{|l|l|l|}
46.028 \\
\end{tabular} & 0.564 & 14.643 & 0.00 & 81.00 & 1.35 & 35.4 & 11.0 & 30.1 & 2.073 & & 0.080 & 0.003 & 2.653 & 2.26 \\
\hline 21 & 9/8/2004 & 1:48:56 PM & 25.709 & 23.959 & \begin{tabular}{|l|l|}
25.478 \\
\end{tabular} & 25.491 & 23.828 & 32.199 & $\begin{array}{l}36.831 \\
\end{array}$ & 4.294 & 28.342 & -0.804 & -1.95 & 45.97 & $\begin{array}{l}0.564 \\
\end{array}$ & 14.643 & 0.004 & 82.00 & .36667 & 35.7 & & & & $\overline{0.0 \varepsilon}$ & & & & 2.24 \\
\hline & 9/8/2004 & 1:49:56 PM & & & & 25.517 & 23.839 & & 36.783 & 4.33 & 28.121 & -0.807 & -1.956 & 45.651 & 0.563 & 14.643 & & & & & 10.9 & & & & & & & 2.25 \\
\hline 219 & $9 / 8 / 2004$ & 1:50:56 PM & 25.746 & 23.962 & 25.485 & 25.504 & 23.865 & 32.098 & 36.775 & 4.284 & 28.131 & -0.81 & -1.968 & 46.089 & 0.563 & 14.643 & & 84.00 & 1.4 & 35.6 & 11.0 & & & & 0.080 & 0.003 & 2.640 & 2.25 \\
\hline & $9 / 8 / 2004$ & 1:51:56 PM & 25.756 & $\begin{array}{l}23.967 \\
23090\end{array}$ & $\begin{array}{r}25.51 \\
\end{array}$ & $\begin{array}{r}25.544 \\
25.56\end{array}$ & $\begin{array}{r}23.895 \\
22007\end{array}$ & 32.434 & $\begin{array}{r}37.38 \\
\end{array}$ & 4.37 & 28.275 & -0.81 & -1.953 & 46.016 & 0.564 & 14.643 & & 85.00 & 1.41667 & 36.3 & 11.0 & 30.4 & & & & & 622 & 2.23 \\
\hline$\frac{221}{222}$ & $\begin{array}{l}9 / 8 / 2 / 204 \\
9 / / 2004\end{array}$ & $\begin{array}{l}1: 52: 5 \mathrm{PM} \\
1.55: 56 \mathrm{PM}\end{array}$ & $\frac{25.768}{25793}$ & $\frac{23.968}{23984}$ & $\begin{array}{l}25.522 \\
25.57\end{array}$ & $\begin{array}{r}25.56 \\
25.631\end{array}$ & $\begin{array}{r}23.901 \\
23.902\end{array}$ & $\begin{array}{l}32.233 \\
32.573\end{array}$ & $\begin{array}{l}37.096 \\
37351\end{array}$ & $\begin{array}{l}4.402 \\
4.407\end{array}$ & 28.116 & -0.807 & - $-\frac{1.959}{.95}$ & $\begin{array}{ll}45.899 \\
46.833\end{array}$ & 0.564 & $\frac{14.643}{14643}$ & & 86.00 & 1.43333 & $\frac{36.0}{36.2}$ & 10.9 & & & 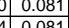 & & 0.003 & 2.637 & \\
\hline 223 & & & & & & & & & & & & & & & & & & & & & 11.0 & & & & & & & \\
\hline & & & & & & & & & & & & & & & & & & & & & & & & & & & & \\
\hline & & & & & & & & & & & & & & & & & & & & & & & & & & & & \\
\hline$\frac{226}{227}$ & & & & & & & & & & & & & & & & & & & & & & & & & & & & \\
\hline & & & & & & & & & & & & & & & & & & & & & & & & & & & & \\
\hline & & Averages & 25.0 & 23.6 & 25.0 & 24.7 & 23.4 & 12.1 & 13.5 & 4.4 & 8.0 & -1.2 & -4.7 & 462 & 0.2 & 146 & & & & 124 & 1110 & & & & & & & \\
\hline 230 & & Maximum & 25.0 & 23.7 & 25.0 & 24.9 & 23.5 & $\begin{array}{l}12.3 \\
\end{array}$ & \begin{tabular}{|l|l|}
13.7 \\
\end{tabular} & 4.6 & 8.2 & -1.2 & -4.6 & 46.6 & 0.2 & 14.6 & 0 & & & 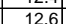 & 11.1. & 10.6 & 0707 & & & & 3. & \\
\hline & & Median & 25.0 & 23.6 & 25.0 & 24.7 & 23.4 & 12.1 & \begin{tabular}{|l|l|}
13.5 \\
\end{tabular} & 4.5 & 8.0 & -1.2 & -4.7 & 46.1 & \begin{tabular}{l|l}
0.2 \\
\end{tabular} & 14.6 & 0.0 & & & 12.4 & \begin{tabular}{|l|}
11.0 \\
\end{tabular} & 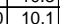 & 0.695 & \begin{tabular}{|ll}
5 & 0.036 \\
\end{tabular} & 0.030 & 0.003 & 3.0 & 2. \\
\hline 232 & & Minimum & 24.9 & 23.5 & 24.9 & 24.6 & \begin{tabular}{|l|l|}
23.3 \\
\end{tabular} & 11.8 & $\mid 13.4$ & 4.2 & 7.7 & -1.2 & -4.7 & 46.0 & 0.2 & 14.6 & 0.0 & & & 12.3 & 11.0 & 9.7 & \begin{tabular}{|l|l|} 
\\
\end{tabular} & & 0.028 & 0.003 & 2.8 & 2.4 \\
\hline & N & $2 \times$ Std Dev & 0.050 & 0.083 & 0.039 & 0.157 & 0.181 & 0.286 & $\begin{array}{ll}0.154 \\
\end{array}$ & 0.152 & 0.273 & 0.005 & 0.091 & 0.296 & \begin{tabular}{|l|l|l|}
0.014 \\
\end{tabular} & 0.000 & 0.001 & & & \begin{tabular}{|l|l|}
0.154 \\
\end{tabular} & 0.070 & 0.265 & \begin{tabular}{|l|l|} 
\\
\end{tabular} & \begin{tabular}{|l|l|}
0.002 \\
\end{tabular} & 0.002 & 0.000 & $\begin{array}{l}0.271 \\
\end{array}$ & 0.230 \\
\hline & Number & of Points Used & $\begin{array}{l}1 \\
1\end{array}$ & 20 & 20 & 20 & & 20 & & 20 & 20 & 20 & 20 & 20 & 20 & 20 & 2 & & & 20 & 20 & \begin{tabular}{|l|l|} 
\\
\end{tabular} & 26 & 20 & & 20 & & 20 \\
\hline & & ${ }^{*}$ Backpulse poir & ts in box & re not in & Iuded & & & & & & & & & & & & & & & & & & & & & & & \\
\hline & & & & & & & & & & & & & & & & & & & & & & & & & & & & \\
\hline 238 & & Averages & 25.1 & 23.8 & 24.8 & 25.2 & 23.6 & 22.4 & 24.9 & 4.4 & 18.3 & -1.2 & -3.6 & 46.1 & $\mid 0.4$ & 14.6 & 0.0 & & & 238 & 110 & & & $\begin{array}{l}0.055 \\
\end{array}$ & 0.055 & & & \\
\hline \begin{tabular}{|l|}
239 \\
\end{tabular} & & Maximum & 25.2 & 23.8 & 25.0 & 25.3 & 23.8 & 22.8 & 25.4 & 4.6 & 18.6 & -1.2 & -3.5 & 46.7 & $\begin{array}{l}0.4 \\
0.4\end{array}$ & 14.6 & & & & 243 & 11.1 & 20.7 & & & & & $\frac{28}{28}$ & \\
\hline 240 & & Median & 25.1 & 23.8 & \begin{tabular}{|l|l|}
24.8 \\
\end{tabular} & 25.2 & 23.6 & 22.4 & $\begin{array}{l}24.9 \\
\end{array}$ & 4.4 & 18.3 & -1.2 & -3.6 & 46.1 & 0.4 & 14.6 & & & & 23.8 & 11.0 & 204 & & & & & $\frac{2.7}{2.7}$ & \\
\hline 241 & & Minimum & 25.0 & 23.7 & 24.7 & 25.0 & \begin{tabular}{|l|l|}
23.4 \\
\end{tabular} & 22.1 & 24.4 & 4.3 & 181 & -1.12 & -36 & 456 & 0.4 & & & & & 23.3 & $\mid 10.9$ & 20.1 & 1.383 & & & 003 & & \\
\hline 242 & & $2 \times \operatorname{Std} \mathrm{Dev}$ & $\begin{array}{l}0.135 \\
\end{array}$ & 0.083 & 0.170 & 0.157 & 0.176 & 0.337 & $\begin{array}{l}0.453 \\
\end{array}$ & 0.148 & 0.316 & 0.004 & 0.071 & 0.606 & 0.008 & 0.000 & 0.001 & & & 0.453 & 0.144 & 0.301 & 0.02 & 0.001 & 0.001 & 0.000 & 0.034 & 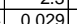 \\
\hline 24 & Number & of Points Used & 20 & 20 & 20 & 20 & 20 & 20 & 20 & 20 & 20 & 20 & 20 & 20 & 20 & 20 & 20 & & & 20 & 20 & \begin{tabular}{|l|l|}
5 & 20 \\
\end{tabular} & 2 & 20 & 20 & 20 & 26 & $26>>3$ \\
\hline & & |* Backpulse poil & its in box & ire not in & linded & & & & & & & & & & & & & & & & & & & & & & & \\
\hline & & & & & & & & & & & & & & & & & & & & & & & & & & & & \\
\hline & & & & & & & & & & & & & & & & & & & & & & & & & & & & \\
\hline 247 & & Averages & 25.6 & 23.9 & 25.4 & 25.5 & 23.7 & 32.2 & 37.0 & 4.4 & 28.1 & -0.8 & -2.0 & 46.0 & 0.6 & 14.6 & 0 & & & 35.9 & 11.0 & 30.1 & 2.078 & 0.08 & 0.0 & 0.003 & 2.6 & \\
\hline 2 & & Maximum & 25.8 & 24.0 & 25.6 & 25.6 & 23.9 & 32.6 & 37.4 & 4.5 & 28.5 & -0.8 & -2. & 46. & 0.6 & & & & & 36.3 & 11.1 & 30.5 & $2.104 \mathrm{~s}$ & 0.08 & & 003 & 2.7 & \\
\hline$\frac{22}{22}$ & & Whedian & $\frac{2.6}{252}$ & $\frac{2.3 .9}{320}>$ & $\frac{25.4}{251}-1$ & $\frac{25.4}{2.4}$ & 23.7 & $\frac{32.2}{310}$ & $\begin{array}{l}33.0 \\
33.5\end{array}$ & 4.4 & $\frac{28.1}{277}$ & -0.8 & $=-2.00$ & 46.0 & 0.6 & $\frac{14.6}{146}$ & 0 & & & 3.9 & $\begin{array}{l}11.0 \\
109\end{array}$ & | $30.1 \mid$ & 2.076 & (5) 0.08 & & (2) & 2.7 & \\
\hline$\frac{250}{251}$ & & $2 \times \mathrm{Std} \mathrm{Dev}$ & $\begin{array}{r}2.4 \\
0.331 \\
\end{array}$ & 0.064 & $\begin{array}{r}25.1 \\
0.270\end{array}$ & $\begin{array}{l}2.4 .4 \\
0.126\end{array}$ & $\begin{array}{r}23.4 \\
0.307 \\
\end{array}$ & $\begin{array}{l}31.9 \\
0.345 \\
\end{array}$ & $\begin{array}{l}30.5 \\
0.463 \\
\end{array}$ & 0.127 & $\begin{array}{ll}27.1 \\
0.408\end{array}$ & $\begin{array}{r}-0.9 \\
0.022\end{array}$ & $\begin{array}{r}-2.0 \\
0.010\end{array}$ & $\begin{array}{r}45.7 \\
0.392\end{array}$ & $\begin{array}{r}0.6 \\
0.001\end{array}$ & $\begin{array}{r}11.6 \\
0.000 \\
\end{array}$ & 0.001 & & & \begin{tabular}{|l|}
35.4 \\
0.463 \\
\end{tabular} & $\begin{array}{r}10.9 \\
0.093 \\
\end{array}$ & $\begin{array}{l}29.9 \\
30.349 \\
\end{array}$ & \begin{tabular}{|l|l|}
.058 \\
0.024
\end{tabular} & 0.001 & $\begin{array}{l}0.079 \\
0.001\end{array}$ & $\begin{array}{l}0.003 \\
0.000\end{array}$ & $\begin{array}{r}2.6 \\
0.042\end{array}$ & \begin{tabular}{|l|} 
\\
\end{tabular} \\
\hline 252 & Number & of Points Used & & & & & & & 20 & 20 & 20 & 20 & 20 & 20 & 20 & 20 & $\frac{.01}{20}$ & & & 20 & 20 & \begin{tabular}{|c|}
5 \\
5
\end{tabular} & 20 & 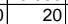 & 20 & 20 & 20 & 20 \\
\hline & & ${ }^{\star}$ Backpulse poir & ts in box & re not in & luded & & & & & & & & & & & & & & & & & & & & & & & \\
\hline 254 & & & & & & & & & & & & & & & & & & & & & & & & 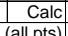 & 0.001 & & & \\
\hline$\frac{2 !}{2 !}$ & & & & & & & & & & & & & & & & & & & & & & Avg & $\frac{g S t D v}{x S t P y y}$ & (all pts) & 0.0011 & & & \\
\hline$\frac{257}{257}>2>$ & & & & & & & & & & & & & & & & & & & & & & Min & in St Dv & $\begin{array}{l}(\text { aalp pist } \\
\text { (all pts) }\end{array}$ & $\begin{array}{l}0.002 \\
0.001\end{array}$ & & & \\
\hline
\end{tabular}


WSRC-TR-2005-00105, REVISION 0

SRNL-RPP-2005-00012, REVISION 0

\section{Section H3: Pilot Cesium Ion Exchange Process (UFP) Raw Data}

The CIX was only operated for four cycles, Cycles 1 and 2 during Campaign II and Cycles 3 and 4 during Campaign III. Because each cycle data file is more than 6000 lines long, with data taken ever 60 seconds, the full data stream would occupy more than 75 pages per cycle. The full set is not published here, but is available upon request. However, since for most of the testing periods of each stage the data are similar, therefore, an abridged data set are given in the follow manner:

For each cycle the data for all six cycle steps have been condensed to fit onto 9 pages in equal time intervals to fit on the following number of pages.

LAW Treatment Data pages 1 to 4 of a given cycle

For the Regeneration Process

$0.1 \mathrm{M} \mathrm{NaOH}$ Displacement Data page 5 of a given cycle

DI Water Pre-Elution Rinse Data page 6 of a given cycle

$0.5 \mathrm{M} \mathrm{HNO}_{3}$ Eluent Data page 7 of a given cycle

DI Water Post-Elution Rinse Data page 8 of a given cycle

$0.25 \mathrm{M} \mathrm{NaOH}$ Regeneration Data page 9 of a given cycle

Notes on data:

1. The raw differential pressures were periodically adjusted by adding or subtracting an offset so that the corrected differential pressures would be zero when flow was zero. Those correction are shown in columns 27 to 32 of the tables.

2. The flowmeter reading was corrected using flow checks with a graduated cylinder and stopwatch. 
The short headings for used in SIPP-CIX tables are described below

\begin{tabular}{|c|c|}
\hline Column Heading & Description \\
\hline Date & Day data point was taken \\
\hline Time & Time data point was taken \\
\hline P1 & Gage pressure at the top of IX Column 1 \\
\hline P2 & Gage pressure at the top of IX Column 2 \\
\hline P3 & Pump Discharge Pressure \\
\hline DP1 & Pressure drop across resin bed in IX Column 1 \\
\hline DP2 & Pressure drop across resin bed in IX Column 2 \\
\hline DP3 & Redundant pressure drop across resin bed in IX Col. 1 \\
\hline DP4 & Redundant pressure drop across resin bed in IX Col. 2 \\
\hline DP5 & Pressure drop across bottom half of bed for Col. 1 \\
\hline DP6 & Pressure drop across bottom half of bed for Col. 1 \\
\hline DP7 & Pressure drop across bottom Flow Distributer for Col. 1 \\
\hline DP8 & Pressure drop across bottom Flow Distributer for Co. 2 \\
\hline DP9 & Pressure drop across top Flow Distributer for Col. 1 \\
\hline DP10 & Pressure drop across top Flow Distributer for Col. 2 \\
\hline DP11 & Pressure drop across filters \\
\hline Flow Meter & Measured chemical supply flows to IX Columns \\
\hline flow cor. & Period correction to flow meter after checking accuracy \\
\hline Cond1 & Conductivity probe for Lead Column \\
\hline $\mathrm{pH}$ & $\mathrm{pH}$ probe \\
\hline T1 & Temperature of at top of Column 1 \\
\hline $\mathrm{T} 2$ & Temperature of at top of Column 2 \\
\hline T3 & Temperature of at bottom of Column 1 \\
\hline T4 & Temperature of at bottom of Column 2 \\
\hline T5 & Temperature of chemical supply flows to both columns \\
\hline elapsed time & Hours of testing \\
\hline DP1 cor. & DP1 correction for zero drift \\
\hline DP2 cor. & DP2 correction for zero drift \\
\hline DP3 cor. & DP3 correction for zero drift \\
\hline DP4 cor. & DP4 correction for zero drift \\
\hline DP5 cor. & DP5 correction for zero drift \\
\hline DP6 cor. & DP6 correction for zero drift \\
\hline Resin H1 & Measured resin height in Column 1 \\
\hline Resin H2 & Measured resin height in Column 2 \\
\hline Differential BV & Inlet Flow as a function of Bed Volumes \\
\hline Cummulative BV & Total volume of flow thru the IX Columns in BV \\
\hline Cummulative BV & Total volume of flow thru the IX Columns in BV \\
\hline
\end{tabular}


WSRC-TR-2005-00105, REVISION 0

SRNL-RPP-2005-00012, REVISION 0

Table H3-1-1. CIX Campaign II, Cycle 1, LAW Treatment

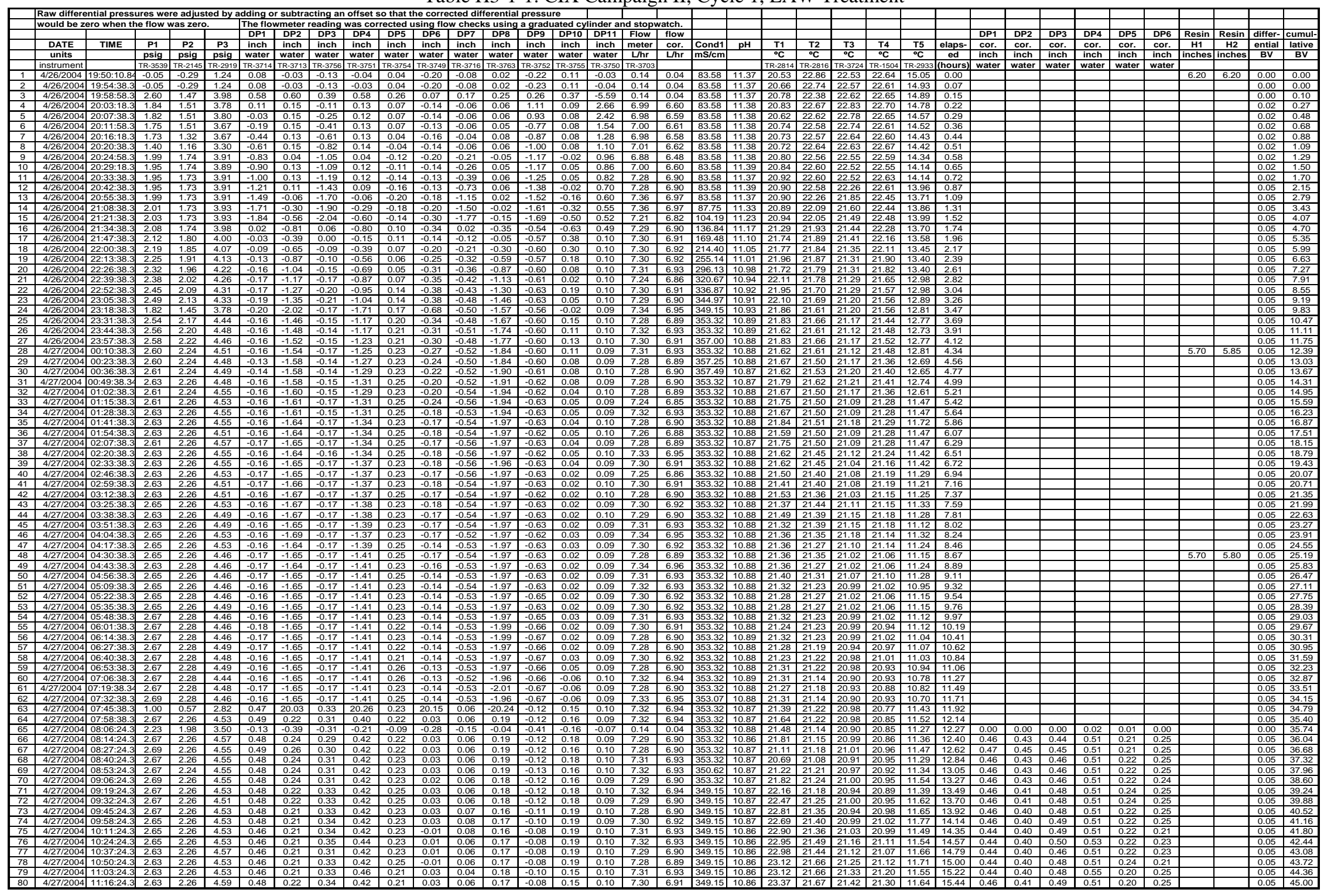


WSRC-TR-2005-00105, REVISION 0

SRNL-RPP-2005-00012, REVISION 0

Table H3-1-2. CIX Campaign II, Cycle 1, LAW Treatment

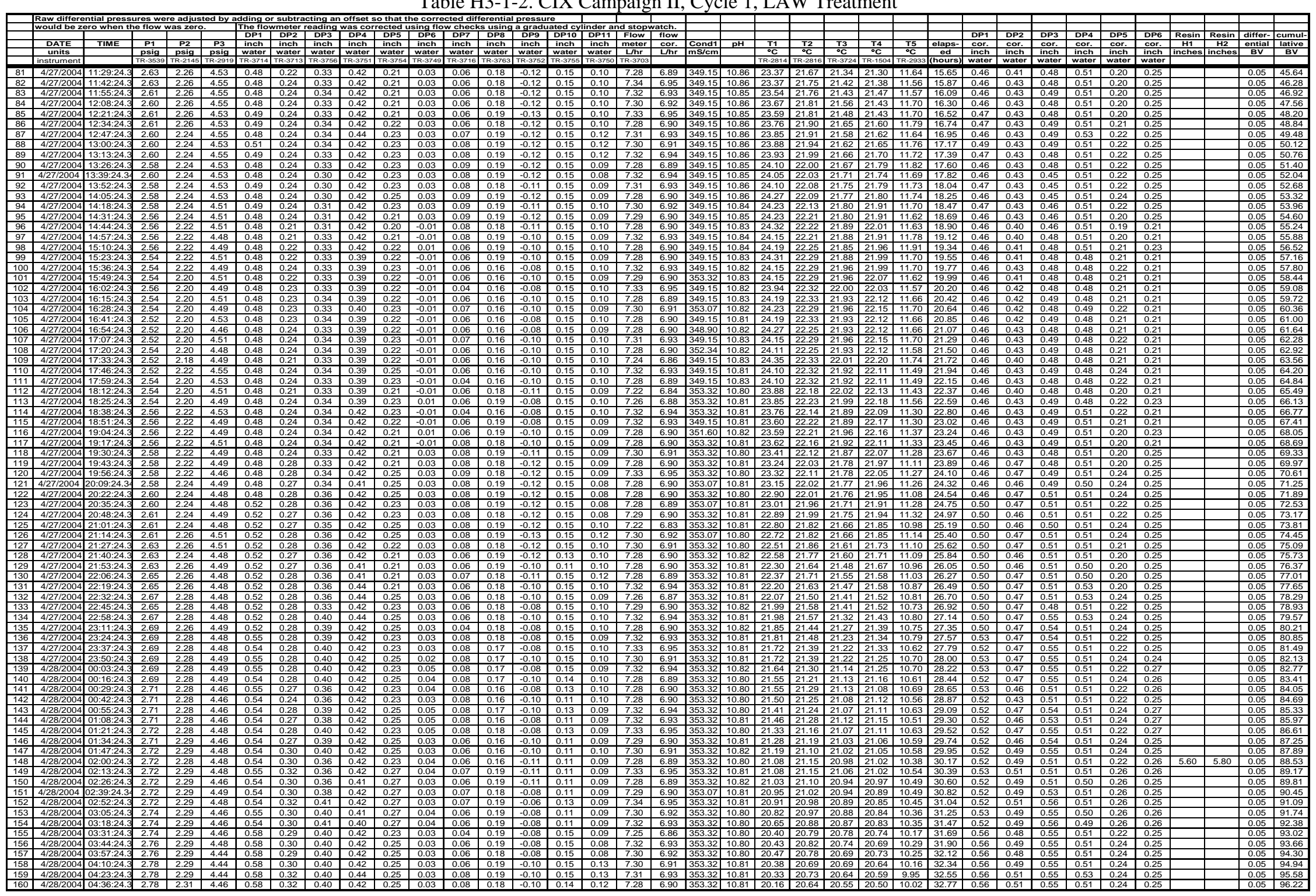


WSRC-TR-2005-00105, REVISION 0

SRNL-RPP-2005-00012, REVISION 0

Table H3-1-3. CIX Campaign II, Cycle 1, LAW Treatment

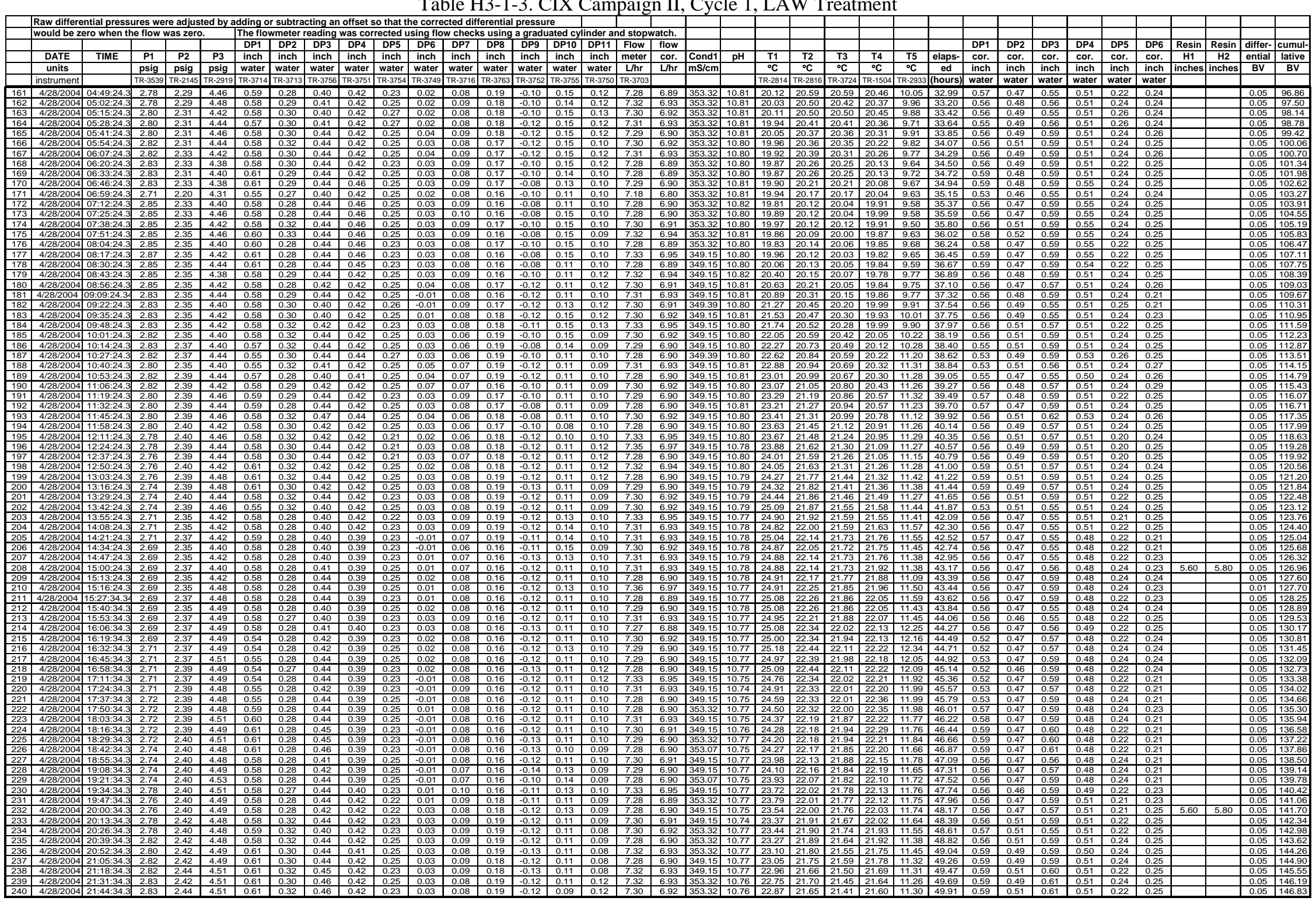


WSRC-TR-2005-00105, REVISION 0

SRNL-RPP-2005-00012, REVISION 0

Table H3-1-4. CIX Campaign II, Cycle 1, LAW Treatment

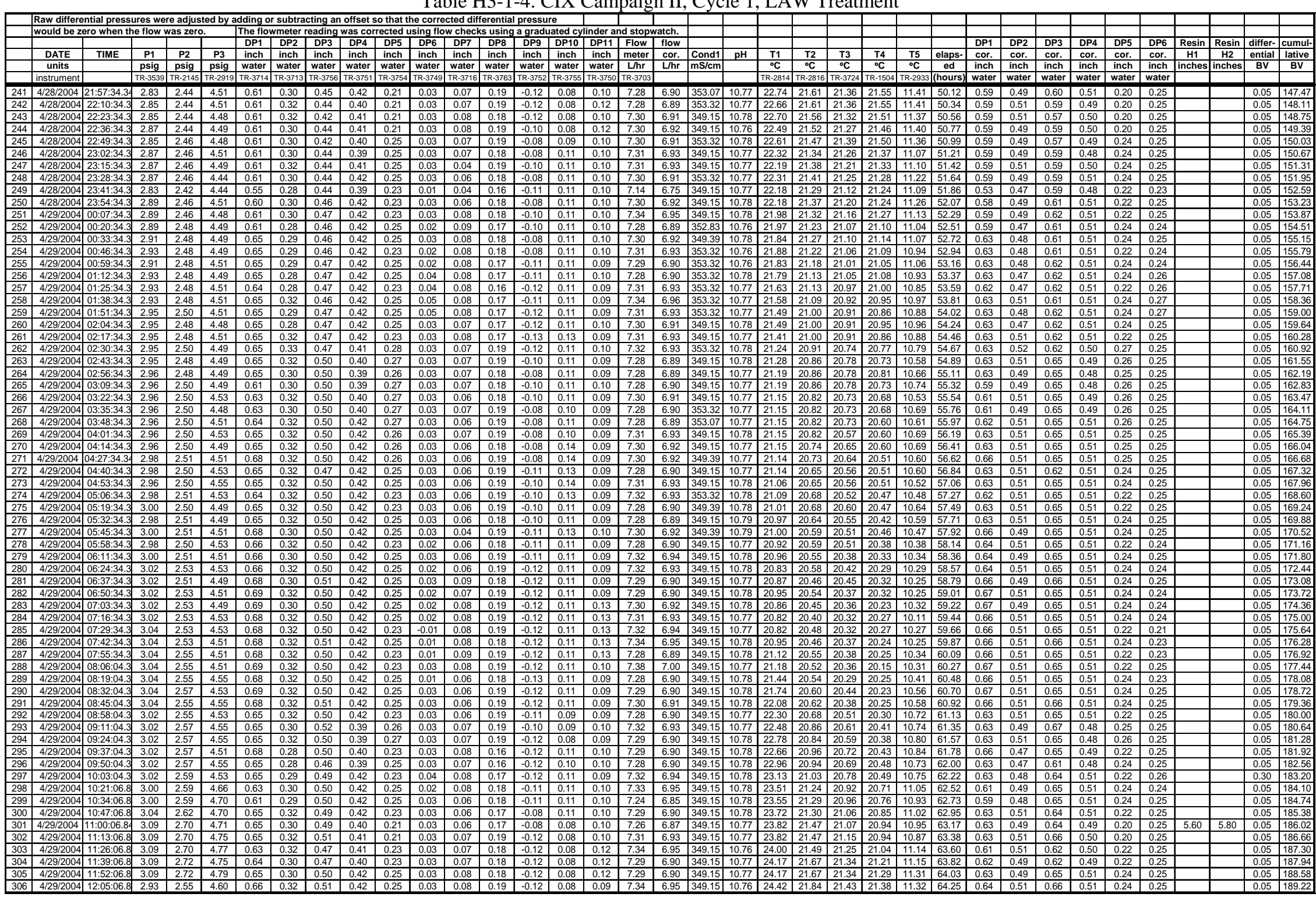


WSRC-TR-2005-00105, REVISION 0

SRNL-RPP-2005-00012, REVISION 0

Table H3-1-5. CIX Campaign II, Cycle 1, 0.1 M NaOH Displacement

\begin{tabular}{|c|c|c|c|c|c|c|c|c|c|c|c|c|c|c|c|c|c|c|c|c|c|c|c|c|c|c|c|c|}
\hline & \multirow{2}{*}{\multicolumn{4}{|c|}{$\begin{array}{l}\text { Raw differential pressures were adjusted } \\
\text { would be zero when the flow was zero. }\end{array}$}} & \multirow{2}{*}{\multicolumn{9}{|c|}{$\begin{array}{l}\text { y adding or subtracting an offset so that the corrected differential pressure } \\
\text { The flowmeter reading was corrected using flow checks using a graduu }\end{array}$}} & \multicolumn{2}{|c|}{ ated cylinder and $s$} & $\begin{array}{l} \\
\text { stopwatch } \\
\end{array}$ & 1 & & & & & & & & & & & \\
\hline & & & & & & & & & & & & & & $\begin{array}{l}\text { tated cylin } \\
\text { DPP }\end{array}$ & $\frac{d \text { der and s }}{\text { DPP10 }}$ & $\begin{array}{l}\text { stopwathh } \\
\text { DDP11 }\end{array}$ & Flow- & Flow & & & & & & & & & & \\
\hline & DATE & TIME & P1 & P2 & P3 & inch & inch & inch & inch & inch & inch & inch & inch & inch & inch & inch & meter & Cor. & Cond1 & $\mathrm{pH}$ & $\mathrm{T}_{1}$ & T2 & $T 3$ & T4 & T5 & lapsed & intial & lative \\
\hline & units & & psig & psig & psig & & & & & & & ater & & & & water & L/hr & L/hr & $\mathrm{mSIcm}$ & & ${ }^{\circ} \mathrm{C}$ & ${ }^{\circ} \mathrm{C}$ & ${ }^{\circ} \mathrm{C}$ & ${ }^{\circ} \mathrm{C}$ & ${ }^{\circ} \mathrm{C}$ & ours & BV & BV \\
\hline & instrument & & \begin{tabular}{|l} 
TR-3539 \\
205
\end{tabular} & $\frac{T R-2145}{235}$ & $\frac{\text { TR-2919 }}{354}$ & & $\frac{T R-3713}{-0.20}$ & $\begin{array}{l}\text { TR-3756 } \\
-0.26\end{array}$ & $\begin{array}{l}\text { TR-3751 } \\
-0.09\end{array}$ & $\begin{array}{l}\frac{T R-3754}{-0.02} \\
-02\end{array}$ & $\frac{T R-3749}{-0.20}$ & $\frac{T R-3716}{0.10}$ & \begin{tabular}{|l|} 
TR-3763 \\
0.01
\end{tabular} & TR-3752 & TR-3755 & $\begin{array}{ll}\text { TR-3750 } \\
012\end{array}$ & \begin{tabular}{|l|} 
TR-3703 \\
247
\end{tabular} & & & & TR-2814 & \begin{tabular}{|l|} 
TR-2816 \\
2102 \\
\end{tabular} & \begin{tabular}{|l|l|} 
TR-3724 \\
15151
\end{tabular} & TR-1504 & TR-2933 & & & \\
\hline & $\begin{array}{l}4 / 29 / 2004 \\
4 / 29 / 2004\end{array}$ & $\frac{12: 16: 20.34}{12.1700 .34}$ & \begin{tabular}{|l|}
2.05 \\
241
\end{tabular} & $\frac{2.35}{2.35}$ & $\begin{array}{l}3.54 \\
4.11 \\
\end{array}$ & & $\frac{-0.20}{-0.20}$ & $\begin{array}{l}-0.26 \\
0.47 \\
\end{array}$ & $\begin{array}{l}-0.09 \\
-0.09\end{array}$ & $\frac{-0.02}{0.25}$ & $\frac{-0.20}{-0.20}$ & 0.10 & \begin{tabular}{|l|}
0.01 \\
0.01
\end{tabular} & $\begin{array}{l}-0.07 \\
-0.12 \\
-12\end{array}$ & $\begin{array}{l}-0.12 \\
-0.12 \\
-12\end{array}$ & $\begin{array}{l}0.12 \\
-0.58\end{array}$ & \begin{tabular}{|l|}
2.47 \\
780 \\
\end{tabular} & $\frac{2.17}{7.42}$ & 439.1 & $\frac{10.77}{10.77}$ & \begin{tabular}{l|}
24.02 \\
24.02
\end{tabular} & $\frac{21.92}{21.92}$ & $\frac{21.51}{21.51}$ & $\frac{21.47}{21.47}$ & $\frac{11.49}{11.57}$ & 64.44 & $\frac{0.00}{0.01}$ & $\frac{189.29}{189.32}$ \\
\hline 3 & $4 / 29 / 2004$ & $12: 17: 46.34$ & 2.63 & 2.35 & 4.33 & 0.55 & -0.20 & 0.42 & -0.09 & 0.21 & -0.20 & 0.07 & 0.01 & $\frac{-0.12}{-0.12}$ & $\begin{array}{l}-0.12 \\
-0.12\end{array}$ & $\begin{array}{l}-0.006 \\
-0.86\end{array}$ & 6.00 & $\frac{7.46}{6.36}$ & $\frac{349.15}{349.15}$ & $\frac{10.17}{10.77}$ & $\frac{24.02}{23.86}$ & $\frac{21.92}{21.92}$ & 21.51 & $\frac{12.47}{21.47}$ & $\frac{11.57}{11.49}$ & $\frac{64.45}{64.46}$ & 0.01 & $\frac{189.32}{189.35}$ \\
\hline 4 & & & & & 4.35 & & & 0.44 & -0.09 & & -0.20 & 0.08 & 0.01 & -0.12 & -0.12 & -2.11 & & & 349.1 & 10.77 & & & 21.43 & 21.38 & 11.24 & 64.47 & & \\
\hline 5 & & 12:19:06.34 & 2.65 & 2.35 & & 0.58 & -0.20 & 0.45 & -0.09 & 0.23 & -0.20 & 0.09 & 0.01 & -0.12 & -0.12 & -2.45 & 7.32 & 6.93 & 349. & 10.77 & 23.70 & 21.92 & 21.43 & 21.38 & 11.24 & 64.48 & .01 & 8189.41 \\
\hline & $4 / 29 / 2004$ & 12:19:46.34 & 2.63 & & & & & 0.42 & -0.09 & & & 0.08 & & -0.07 & -0.12 & -2.65 & 7.19 & & 349.1 .1 & 10.77 & & 21.97 & 21.48 & 21.43 & 11.04 & 64.49 & .01 & 89.45 \\
\hline$\frac{7}{3}-2+3$ & $4 / 29 / 2004$ & $\begin{array}{ll}12: 20: 26.34 \\
12 \cdot 21: 0624\end{array}$ & $\frac{2.65}{2.65}$ & 2.35 & $\frac{05}{02}$ & 0.58 & & 0.41 & -0.08 & 0.25 & -0.20 & .09 & 0.01 & 0.04 & -0.12 & -2.71 & 7.30 & 6.91 & 349.1 & 10.77 & 24.23 & 21.97 & 21.40 & 21.43 & 10.88 & 64.50 & 0.01 & 899.48 \\
\hline$\frac{0}{9}+2-3$ & $4 / 29 / 2004$ & $\begin{array}{l}12: 21: 06.34 \\
12 \cdot 21: 4624\end{array}$ & $\frac{2.65}{2.65}$ & 2.35 & & & -0.20 & 0.44 & -0.08 & $\begin{array}{l}0.23 \\
0.23\end{array}$ & & & 0.01 & 0.13 & -0.12 & $\frac{-2.78}{280}$ & 7.30 & 6.92 & $\frac{349.15}{349.15}$ & 10.77 & 24.47 & 21.88 & 21.40 & 21.43 & 10.88 & 64.52 & 0.01 & 89.51 \\
\hline$\frac{9}{10}$ & $4 / 29 / 2004$ & 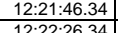 & $\frac{2.65}{267}$ & $\frac{2.35}{2.35}$ & $\frac{00}{02}$ & 0. & $\begin{array}{l}-0.20 \\
-20\end{array}$ & 0.44 & -0.09 & 0.23 & & 0.09 & 0.02 & $\begin{array}{l}0.21 \\
0^{323}\end{array}$ & -0.12 & $\frac{-2.80}{283}$ & $\begin{array}{l}7.30 \\
733\end{array}$ & $\frac{6.92}{6.94}$ & $\frac{349.1}{349.1}$ & $\frac{10.77}{10.77}$ & 24.63 & $\frac{21.97}{2201}$ & $\begin{array}{l}21.48 \\
2.152\end{array}$ & $\frac{21.43}{21.48}$ & $\begin{array}{l}10.96 \\
11109\end{array}$ & $\frac{64.53}{6.54}$ & 0.01 & 189.54 \\
\hline$\overline{11}$ & $\begin{array}{l}44 / 2992504 \\
4 / 29 / 2004\end{array}$ & $\frac{12: 222: 2.34}{12: 23: 06}$ & $\begin{array}{l}2.67 \\
2.67\end{array}$ & $\frac{2.35}{2.35}$ & $\frac{4.02}{4.02}$ & $\begin{array}{l}0.58 \\
0.58\end{array}$ & $\frac{-0.20}{-0.21}$ & $\begin{array}{l}0.45 \\
0.42\end{array}$ & $\frac{-0.09}{-0.08}$ & $\begin{array}{l}0.23 \\
0.23\end{array}$ & $\frac{-0.20}{-0.20}$ & 0.09 & $\begin{array}{l}0.01 \\
0.02\end{array}$ & $\frac{0.32}{0.40}$ & $\frac{-0.12}{-0.12}$ & $\begin{array}{l}-2.83 \\
-2.85\end{array}$ & $\begin{array}{l}7.32 \\
7.29\end{array}$ & $\frac{6.94}{6.90}$ & $\frac{349.1}{349.1}$ & $\frac{10.77}{10.76}$ & $\frac{24.91}{24.99}$ & $\frac{22.01}{22.01}$ & 21.44 & $\frac{21.48}{21.48}$ & $\frac{11.09}{11.09}$ & $\frac{64.54}{64.55}$ & $\frac{0.01}{0.01}$ & $\frac{189.58}{189.61}$ \\
\hline 12 & $4 / 29 / 2004$ & $\begin{array}{ll}12: 23: 46.34 \\
\end{array}$ & 2.67 & 2.35 & & & & 0.42 & -0.09 & & -0.20 & 0.09 & 0.00 & 0.46 & -0.12 & -2.85 & 7.32 & & 349.1 & 10.77 & 24.99 & 22.01 & 21.44 & 21.56 & 11.01 & 64.56 & & 189.64 \\
\hline & & 12:24:26.34 & 2.67 & 2.35 & 00 & & & 0.40 & -0.08 & 0.22 & & 0.09 & 0.01 & 0.52 & -0.12 & -2.85 & $\begin{array}{l}7.26 \\
\end{array}$ & 6.88 & & 10.78 & 24.99 & 21.93 & 21.44 & 21.40 & & 64.57 & 0.01 & 189.68 \\
\hline$\frac{14}{15}>>2>$ & & \begin{tabular}{ll|l}
$12: 25: 06.34$ \\
\end{tabular} & & 2.35 & & & & $\begin{array}{l}0.42 \\
\end{array}$ & -0.09 & & & & 0.01 & 0.57 & -0.12 & -2.87 & 7.32 & & $\frac{349.15}{20.10}$ & 10.78 & 24.99 & & 21.44 & 21.40 & 11.01 & 84.58 & 0.01 & 89.71 \\
\hline 15 & & 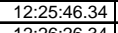 & 2.69 & 2.35 & 4.04 & & & 0.41 & $\begin{array}{l}-0.09 \\
0.09\end{array}$ & $\begin{array}{l}0.23 \\
0.23\end{array}$ & & & 0.02 & 0.62 & $\begin{array}{l}-0.12 \\
\end{array}$ & -2.87 & 7.28 & 6.90 & $\frac{349.39}{2.39}$ & 10.78 & 25.12 & 22.06 & 21.49 & 21.52 & 11.14 & 84.59 & 0.01 & 89.74 \\
\hline 16 & $4 / 29 / 2004$ & $12: 26: 26.34$ & 2.69 & 2.37 & & & -0.2 & 0.40 & -0.09 & 0.23 & & & 0.01 & 0.68 & -0.12 & -2.88 & 7.26 & 6.8 & 49.15 & 10.77 & & $\frac{22.06}{2006}$ & 21.49 & 21.52 & $\frac{11.14}{1007}$ & & 0.01 & 39.77 \\
\hline 17 & $4 / 29 / 2004$ & $\begin{array}{ll}12: 27: 36.34 \\
120.28\end{array}$ & $\begin{array}{l}2.69 \\
271\end{array}$ & $\frac{2.37}{239}$ & & & & 0.41 & -0.09 & & & & 0.01 & 0.76 & & $\begin{array}{l}-2.88 \\
-288\end{array}$ & $\begin{array}{l}7.34 \\
733 \\
3\end{array}$ & & & 10.77 & 24. & 22.06 & 21.49 & & 10.97 & & & 39.83 \\
\hline & $4 / 2912004$ & $\frac{12: 28: 56.34}{12: 30: 16.34}$ & $\frac{2.71}{2.71}$ & $\frac{2.39}{2.39}$ & $\frac{02}{04}$ & & $\frac{-0}{-0}$ & $\frac{0.40}{0.40}$ & $\frac{-0.09}{-0.08}$ & $\frac{0}{0}$ & & & 0.01 & 0.86 & & $\begin{array}{r}-2.88 \\
-2.88\end{array}$ & $\begin{array}{l}7.30 \\
7.32 \\
729\end{array}$ & $\frac{3 c}{6 c}>0$ & $\frac{349.1}{349.1}$ & $\frac{10.77}{1077}$ & $\frac{25 .}{25 .}$ & $\frac{22.06}{2206}$ & $\frac{21.41}{21.49}$ & $2 x$ & $\frac{11.14}{1114}$ & $\frac{64.65}{64.67}$ & $\frac{0.02}{0.02}$ & 189.90 \\
\hline & $4 / 29 / 2004$ & $\begin{array}{l}12.30 .10 .34 \\
12: 31: 36.34\end{array}$ & 2.112 & $\frac{2.39}{2.39}$ & $\begin{array}{l}4.04 \\
4.02\end{array}$ & 0. & $\begin{array}{l}-0.2 \\
-0.2\end{array}$ & $\begin{array}{l}0.40 \\
0.40\end{array}$ & $\begin{array}{l}-0.08 \\
-0.08 \\
-\end{array}$ & $\begin{array}{l}0.25 \\
0.23\end{array}$ & $\begin{array}{l}-0.20 \\
-0.20\end{array}$ & 0.09 & 0.02 & $\begin{array}{l}0.96 \\
1.07\end{array}$ & $\begin{array}{l}-0.12 \\
-0.12 \\
\end{array}$ & $\begin{array}{r}-2.88 \\
-2.88 \\
\end{array}$ & $\begin{array}{l}7.29 \\
7.26 \\
\end{array}$ & & $\frac{349.1}{349.1}$ & $\frac{10.17}{10.77}$ & 25. & $\frac{22.06}{22.06}$ & 21.49 & 21.52 & $\frac{11.14}{11.06}$ & $\begin{array}{l}64.67 \\
64.69\end{array}$ & & $\begin{array}{l}189.96 \\
190.03 \\
\end{array}$ \\
\hline & & 12:32:56.34 & 2.72 & 2.39 & 4. & 0. & & 0.40 & -0.09 & 0.23 & 20 & 0.09 & 0.02 & & & -2.88 & 7.29 & 6.5 & & 10.77 & 25. & & & 21.60 & & & & \\
\hline & & 12:34:16.34 & & 2.40 & & & & & -0.09 & & & & & 1.26 & & & & & & & & & 21.49 & & & & & 190.16 \\
\hline & & 3 & 2.72 & 2.40 & & & & & -0.09 & & & & 0.02 & & & -2.88 & 7.32 & & & 10.77 & 25. & 21.98 & & 21.52 & & & .02 & \\
\hline 24 & $4 / 29 / 2004$ & $12: 36: 56.34$ & 2.72 & 2.40 & & & & 0.40 & $\begin{array}{r}-0.08 \\
0.08\end{array}$ & 0.25 & & & 0.02 & 1.46 & & -2.89 & 7.29 & 6. & $\frac{349.1}{20.1}$ & 10.77 & 25.12 & 22.06 & 21.49 & 21.52 & 11.06 & & 0.02 & 0.29 \\
\hline & $4 / 29 / 2004$ & $12: 38: 16.34$ & 2.74 & $\frac{2.42}{2.2}$ & 4. & & -0.11 & 0.40 & - -0.09 & 0.20 & & & 0.02 & 1.56 & & -2.89 & 7.28 & 6. & 349.1 & 10.77 & 25.12 & 22.06 & 21.41 & 21.52 & 10.9 & 64.80 & 0.02 & 30.36 \\
\hline & $4 \frac{4 / 29 / 2}{4 / 29 / 2}$ & $\frac{12: 39: 3}{12: 40: 5}$ & $\frac{2.14}{2.76}$ & $\frac{2.42}{2.42}$ & & & & & $\begin{array}{l}-\frac{-0.09}{-0.09} \\
\end{array}$ & 0 & & & & & & $\frac{-2}{-2}$ & $\frac{7.2}{7.3}$ & & & & & $\frac{2 .}{2 .}$ & & & & & & 0.42 \\
\hline 28 & $4 / 29 / 2004$ & $\frac{1<: 40.5}{12: 42: 1}$ & 2.76 & $\frac{2.42}{2.42}$ & & & & 0.41 & -0.09 & 0 & & & & $1.8 \mathrm{r}$ & & -2. & 7.28 & & & $10.7>-7$ & 25 & & 21 & & & & & $\frac{190.49}{190.55}$ \\
\hline 29 & $4 / 29 / 2004$ & & 2.76 & 2.40 & & & & 0.45 & -0.09 & 0 & & & & & & -2.88 & 7.32 & & & 10.77 & & 22. & & & & & & \\
\hline & & & & & & & & & -0.09 & & & & & & & & 7.30 & & & & & & & & & & & \\
\hline 31 & & & 2.76 & 2.35 & & & & & & & & & & & & -2.8 & 7.31 & & & & & 22. & & 21. & & & & 0.75 \\
\hline 32 & $4 / 29 / 2004$ & $12: 47: 36.34$ & 2.7 & 2.35 & & & -0.2 & 0.71 & -0.09 & 0. & & & & & & -2. & 7.2 & & 349. & & 25. & 22.3 & 21. & 21. & & & & 0.82 \\
\hline 33 & & & $\begin{array}{l}2.78 \\
280\end{array}$ & $\frac{2.35}{235}$ & & & $\frac{-0.2}{-0.2}$ & $0.8 \mathrm{c}$ & -0.08 & & & & 0.0 & 1. & & -2. & 7.3 & 6. & & & 25. & & $2115+4$ & & & & & $\begin{array}{l}90.88 \\
0095\end{array}$ \\
\hline & $\frac{4 / 29 /}{4 / 20 / 6}$ & $\frac{12: 50: 1}{1251: 3}$ & 2.80 & $\frac{2.35}{2.37}$ & & & -0.2 & 0.94 & $\begin{array}{r}-0.09 \\
-0.08\end{array}$ & & & & & & & -2 & 7.30 & & & & & & 21.5 & & & & & $\frac{190.95}{10101}$ \\
\hline 36 & & & & $\frac{2.31}{2.35}$ & & & & & $\frac{-0.08}{-0.09}$ & & & & & & & & & & & & & & & & & & & $\frac{191.01}{19108}$ \\
\hline 37 & $4 / 29 / 2004$ & & 2.82 & 2.39 & & 1. & & 1.25 & -0.08 & & & & & $\frac{1.0}{1.8}$ & & $\begin{array}{l}-2.09 \\
-2.89\end{array}$ & $\frac{7.20}{7.30}$ & 6. & & & & & & & & & & \\
\hline & & & & 2.42 & & & & & & & & & & & & & & & & & & & & & & & & \\
\hline 39 & & 12:56 & 2. $\quad \mathrm{C}$ & 2.4 & & & & & -0 & & & & & & & & & & & & & & & & & & & \\
\hline 40 & & & & & & & & & & & & & & & & & & & & & & & & & & & & \\
\hline 41 & & & 2.83 & 2.5 & & & & & & & & & & & & & & & & & & & & & & & & \\
\hline & & & & & & & & & & & & & & & & & & & & & & & & & & & & \\
\hline 43 & & & & & & & & & & & & & & & & & & & & & & & & & & & & \\
\hline 44 & & & & & & & & & & & & & & & & & & & & & & & & & & & & \\
\hline $45-2>3$ & & & 2.85 & 2.55 & & & & 2.0 & -0.1 & & & & & & & & & & & & & & & & & & & \\
\hline & & & & & & & & & & & & & & & & & & & & & & & & & & & & \\
\hline & & & & & & & & & & & & & & & & & & & & & & & & & & & & \\
\hline $4 c$ & & & & & & & & & & & & & & & & & & & & & & & & & & & & \\
\hline & & & 2. & 2. & & & & & & & & & & & & & & & & & & & & & & & & \\
\hline & & & & & & & & & & & & & & & & & & & & & & & & & & & & \\
\hline & & & & & & & & & 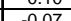 & & & & & & & & & & & & & & & & & & & \\
\hline & 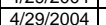 & & $\frac{2.50}{280}$ & $\frac{2.51}{261}$ & & $\frac{2.37}{2.73}$ & $-0.2>2-3$ & $\frac{2.56}{256}$ & $\frac{-1.1}{-0.1}$ & & & & & & & & & & & & & & & & & & & \\
\hline & & & & & & & & & & & & & & & & & & & & & & & & & & & & \\
\hline 5 & & & & $\frac{26}{2 .}$ & & & & & & & & & & & & & & & & & & & & & & & & \\
\hline & & & & 26 & & & & & & & & & & & & & & & & & & & & & & & & \\
\hline & & 13:20:25:34 & 29. & 26 & & $3.6-2>$ & & 29 & -0.0 & & & & & & & & & & & & & & & & & & & \\
\hline & & 13:22:25. & $\overline{2.93} \mathrm{Y}$ & 2.64 & 4.2 & 3. & & 2.9 & -0.0 & & & & & & & & & & & & & & & & & & & \\
\hline & 4/29/2004 & & & 2.6 & 4.3 & 3. & & 2.95 & -0.0 & & & & & & & -2.4 & & & & & & & & & & & & \\
\hline 60 & 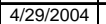 & \begin{tabular}{|l|}
$13: 26: 25.34$ \\
\end{tabular} & \begin{tabular}{|l|l|}
2.58 \\
\end{tabular} & 2.66 & 3.74 & 2.81 & -0.21 & $\begin{array}{ll}2.63 \\
\end{array}$ & -0.09 & 0.69 & \begin{tabular}{c|c|c|}
-0.24 \\
\end{tabular} & 0.39 & $\begin{array}{ll}-0.02 \\
\end{array}$ & $\begin{array}{ll}1.68 \\
\end{array}$ & $\begin{array}{ll}-0.12 \\
\end{array}$ & \begin{tabular}{|l|l|} 
& -3.02 \\
\end{tabular} & 2.49 & \begin{tabular}{l|l}
2.18 \\
\end{tabular} & \begin{tabular}{|l|l|}
349.15 \\
\end{tabular} & \begin{tabular}{|l|l|}
10.75 \\
\end{tabular} & 25.26 & 22.52 & 21.79 & 21.82 & \begin{tabular}{|l|}
11.11 \\
\end{tabular} & \begin{tabular}{|l|l|}
65.60 \\
\end{tabular} & 0.01 & 192.65 \\
\hline
\end{tabular}


WSRC-TR-2005-00105, REVISION 0

SRNL-RPP-2005-00012, REVISION 0

Table H3-1-6. CIX Campaign II, Cycle 1, DI Water Pre-Elution Rinse

\begin{tabular}{|c|c|c|c|c|c|c|c|c|c|c|c|c|c|c|c|c|c|c|c|c|c|c|c|c|c|c|c|c|c|c|c|c|c|c|}
\hline Raw differe & titial pressur & tessive & ajuste & & ng of subtrac & ting an offt & Ist & 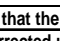 & 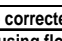 & tontreter & thalapre & & & & & & & & & & & & & & & & & & & & & & & \\
\hline & & & & & & & & & & & & & & & & & & & & & & & & & & & & & & $\overline{D P 6}$ & & & & \\
\hline & & & & & \begin{tabular}{|l|l|l|} 
inch & inch \\
\end{tabular} & inch & & & inch & & & inch & \begin{tabular}{|l|l} 
inch \\
\end{tabular} & inch & meter & $\begin{array}{l} \\
\end{array}$ & & $\mathrm{pH}$ & T1 & T2 & $\mathrm{T3}$ & & & ed & inch & & inch & inch & & inch & & $\mathrm{H} 2$ & ential & \\
\hline $\begin{array}{l}\text { units } \\
\text { ntrtimen }\end{array}$ & & & psig & psig & \begin{tabular}{|l|l|} 
water & wate \\
\end{tabular} & $\begin{array}{l}\text { Water } \\
\text { p. }\end{array}$ & later & ter & water & water & water & water & water & \begin{tabular}{|l|} 
water \\
\end{tabular} & L/hr & Lhr & mslcm & & ${ }^{\circ} \mathrm{C}$ & & ${ }^{\circ} \mathrm{C}$ & & & & & & & & & & & & & \\
\hline $4 / 221 / 2004$ & & & 83 & & 2.85 & 2.67 & -0.08 & & & & & & -0.16 & & & & & & & & & & & & & & & & & & & & & \\
\hline & & & & & & & & & & & & & & & & & & & .4.0. & & & & & & & & & & & & & & & \\
\hline 4292/2004 & 14:16:25.34 & & & & & & & & & & & & & & & & & & & & & & & & & & & & & & & & & \\
\hline 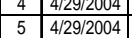 & $\begin{array}{ll}14.17 .5 .54 \\
14: 19: 25.34\end{array}$ & & $\frac{2.81}{2.81}$ & $\begin{array}{l}4.37 \\
4.35 \\
\end{array}$ & \begin{tabular}{|l|l|l|}
3.02 & -0.20 \\
\end{tabular} & \begin{tabular}{|l|l|}
2.87 \\
\end{tabular} & $\begin{array}{l}-0.08 \\
\end{array}$ & & -0.24 & & -0.02 & & & & & & & & & & & & & & & & & & & & & & & \\
\hline & & & & & & & & & & & & & & & & & & & & & & & & & & & & & & & & & & \\
\hline & & & & & & & & & & & & & & & & & & & & & & & & & & & & & & & & & & \\
\hline 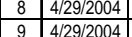 & 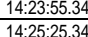 & & 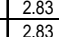 & 4 & \begin{tabular}{l|l}
03 & -0.20
\end{tabular} & & & & & & & & & & & \begin{tabular}{|l|l|l|}
729 \\
\end{tabular} & & & & & & & & & & & & & & & & & & \\
\hline $42 / 2912004$ & $14: 26: 55.34$ & & 2.83 & $\begin{array}{l}4.29 \\
4.29\end{array}$ & \begin{tabular}{|l|l}
3.04 & -0.20 \\
\end{tabular} & 2.88 & & & & 1.46 & & 1. & & & 7.3 & \begin{tabular}{|l|}
1.25 \\
6.93 \\
\end{tabular} & & & $\frac{25.01}{25.09}$ & 22.60 & $\mid$\begin{tabular}{|l|}
21.47 \\
21.47
\end{tabular} & \begin{tabular}{|l|}
22.06 \\
\end{tabular} & & & & & & & & & & & & \\
\hline $\begin{array}{l}292 / 2004 \\
292004\end{array}$ & & & & & \begin{tabular}{|l|l|l|l|l|}
7 & -020
\end{tabular} & & & & & & & & & & & & & & & & & & & & & & & & & & & & & \\
\hline & & & & & & & & & & & & & & & 7.2 & 6.87 & 316 & \begin{tabular}{|l|l|}
11.28 \\
\end{tabular} & $24.97 \mathrm{H}$ & & 21.11 & & & & & & & & & & & & & \\
\hline & & & & & & & & & & & & & & & 7.34 & & & & & & & & & & & & & & & & & & & \\
\hline $\begin{array}{l}922004 \\
192004\end{array}$ & & & & & & & & & & & & & & & & & & & & & & & & & & & & & & & & & & \\
\hline & $\frac{14: 335.55 .54}{14372.2534}$ & & $\frac{2.68}{288}$ & & $=0.020$ & \begin{tabular}{|l|l|}
2.94 \\
\end{tabular} & & & & & & & & $\frac{-2.98}{-20}$ & $\frac{7.26}{720}$ & & 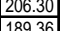 & & & & & 20.02 & & & & & & & & & & & & \\
\hline & 14:38. & 2.17 & 2.88 & & \begin{tabular}{|l|l|}
4 & -0.20 \\
\end{tabular} & & & & & & & & & & & & & & & & & 22.10 & & & & & & & & & & & & \\
\hline 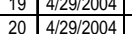 & 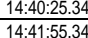 & $\frac{2 .}{2 .}$ & $\frac{2.88}{2.86}$ & & $\frac{-.20}{-0.20}$ & 209 & & & & & & & & & & & & & & & 21.10 & & & & & & & & & & & & & \\
\hline & & 2.16 & & & & & & & & & & & & & & & & & & & & & & & & & & & & & & & & \\
\hline $29 / 2004$ & 14:44:40.34 & 41.82 & & & & & & & & & & & & & & & & & & & & & & & & & & & & & & & & \\
\hline 2912004| & & 1.42 & 2.00 & & , & & & & & & & & & & 0.97 & 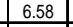 & & & & & & & & & & & & & & & & & & \\
\hline & & & & & & & & & & & & & & & & & & & & & & & & & & & & & & & & & & \\
\hline (19004) & . & & & & 0.20 & & & & & & & & & & & $\frac{0.34}{746}$ & & & . & 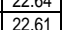 & & & & & & & & & & & & & & \\
\hline & 14:48:25.34 & & & & & & & & & & & & & & & 3 & & & & & & & & & & & & & & & & & & \\
\hline & & & 288 & & & & & & & & & & & & & & & & & & & & & & & & & & & -0.02 & 6.00 & & & \\
\hline & & & & & & & & & & & & & & & & & & & & & & & & & & & & & & & & & & \\
\hline & & & & & & & & & & & & & & & & . & & & & & & & & & & & & & & & & & & \\
\hline & & & & & & & & & & & & & & & 6.9 & 5 & & & & & & & & & & & & & & & & & & \\
\hline & & & 50 & & -0 & & & & & & & & & & & 6.86 & & & & & & & & & & & & & & & & & & \\
\hline & & & & & & & & & & & & & & & & & & & & & & & & & & & & & & & & & & \\
\hline (1) & 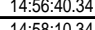 & $\mid \begin{array}{l}2.12 \\
310\end{array}$ & -9.92 & & $=0.20$ & & & & & & & & 0.0 .16 & & $\frac{7.27}{720}$ & . & & & & & 1.24 & & & & & & & & & . & & & & 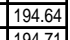 \\
\hline & & & 294 & & & & & & & & & & & & & & & & & & & & & & & & & & & & & & & \\
\hline gl|2004 & 14.00 .40 .04 & & 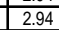 & & \begin{tabular}{|c|c|c|}
5 & -2.21 \\
\end{tabular} & & & & & & & & & & $\frac{1.26}{7.26}$ & & & & & & & & & & & & & & & & & & & \\
\hline 922004 & 15:02:40.34 & & & & \begin{tabular}{|l|l|l|}
0.25 \\
\end{tabular} & & & & & & & & & & 7.32 & & & & & & & & & & & & & & & & & & & \\
\hline & :04:10.34 & 2.10 & 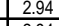 & 5 & $\begin{array}{ll}25 & -0.20 \\
5\end{array}$ & $\mid 0.06$ & & & & & & & & & 7.06 & 6.67 & & & & - 0.04 & 1.43 & & & & & & & & & & & & & \\
\hline & & & & & & & & & & & & & & & $\frac{32}{13}$ & 弚.44 & & & & & & & & & & & & & & & & & & \\
\hline & & & & & $\frac{-2.21}{0.21}$ & & & & & & & & 6 & & 0.14 & & & & & & & & & & & & & & & & & & & 195. \\
\hline & 0:10.34 & 1.02 & 2.95 & & & & & .00 & & & & & & & 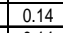 & 0.04 & & & & & & & & & & & & & & & & & & \\
\hline & & & 2.95 & \begin{tabular}{|l|}
2.42 \\
\end{tabular} & $\begin{array}{ll}0.29 & -0.20 \\
\end{array}$ & & & 0.12 & & \begin{tabular}{|c|c|} 
\\
\end{tabular} & & & & & \begin{tabular}{|c|c|}
.14 \\
\end{tabular} & \begin{tabular}{|l|}
0.04 \\
5.77
\end{tabular} & & & 年5.355 & 22.69 & & & & & & $=0.0 .0$ & & & & & & & 0.00 & \\
\hline$\frac{46}{47} 4 / 2 / 2$ & $\frac{15: 10: 4.4984}{1550.5984}$ & $\frac{1.42}{170}$ & \begin{tabular}{|l|}
2.95 \\
297
\end{tabular} & \begin{tabular}{|l|}
2.88 \\
314
\end{tabular} & \begin{tabular}{c|c|c|c|}
0.0 .20 & -0.38 & -0.20
\end{tabular} & \begin{tabular}{|l|l|} 
& 0.27 \\
0.23
\end{tabular} & \begin{tabular}{|c|c|}
-0.09 \\
-0.088
\end{tabular} & \begin{tabular}{|l|}
0.20 \\
0.17
\end{tabular} & \begin{tabular}{|c|c|c|}
-0.23 \\
-0.24
\end{tabular} & \begin{tabular}{|l|}
-0.52 \\
-0.52 \\
\end{tabular} & & $\frac{0.44}{0.42}$ & \begin{tabular}{|l|l|}
-0.16 \\
0.14
\end{tabular} & \begin{tabular}{|l|l|}
0.14 \\
0.12
\end{tabular} & \begin{tabular}{|l|l|}
11.98 \\
1195
\end{tabular} & \begin{tabular}{|l|}
5.47 \\
1189
\end{tabular} & & $\begin{array}{l}\frac{11.55}{11.55} \\
\end{array}$ & $\begin{array}{l}26.55 \\
26.55\end{array}$ & 22.69 & $\mid \frac{22.1 .7}{21.24}$ & 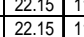 & $\begin{array}{l}11.62 \\
1137 \\
1137\end{array}$ & $6 \frac{6.74}{6735}$ & $\frac{0.39}{0.33}$ & & 0.45 & & 0.23 & 0.002 & & & & $\frac{195.8}{195.8}$ \\
\hline $292 / 2004$ & 15:11:14.84 & 1.90 & 2.95 & & \begin{tabular}{c|c}
0.30 & -0.21 \\
\end{tabular} & 0.11 & \begin{tabular}{|c|}
-0.09 \\
\end{tabular} & \begin{tabular}{|l|l|}
0.12 \\
\end{tabular} & \begin{tabular}{|l|}
-0.22 \\
\end{tabular} & & & & \begin{tabular}{|c|}
-0.16 \\
\end{tabular} & & 9.29 & 8.99 & & & & & & & & & & & & .00 & & .0.0 & & & & \\
\hline & & & & & & 0 & & & & & & & & & & & & & & & & & & & & & & & & & & & & \\
\hline & & & 2.95 & 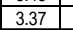 & $\begin{array}{c}0.15 \\
0.20\end{array}$ & & & 0.06 & & & & & 0.16 & & 5.78 & 5.37 & & & & & 21.64 & & & & & & & & & & & & & 195. \\
\hline & & & & & \begin{tabular}{c|c|c|}
0.08 & -0.20
\end{tabular} & $\frac{-0.14}{-0.14}$ & & & & & & & & & & & & & & & & & & & & & & & & & & & & \\
\hline & & & $9 t$ & 30 & \begin{tabular}{l|l}
0.08 & -0.20 \\
\end{tabular} & \begin{tabular}{|c|c|} 
& -0.11 \\
\end{tabular} & 0.09 & 0.03 & .24 & & & & & & 2.46 & & & & & & 21.88 & & & & & & & & & .02 & & & & \\
\hline & & & & & & & & & & & & & & & & & & & & & & & & & & & & & & & & & & \\
\hline & & & & & & & & & & & & & & & & & & & & & & & & & & & & & & & & & & \\
\hline
\end{tabular}


WSRC-TR-2005-00105, REVISION 0

SRNL-RPP-2005-00012, REVISION 0

Table H3-1-7. CIX Campaign II, Cycle 1, 0.5 M Nitric Acid Eluent

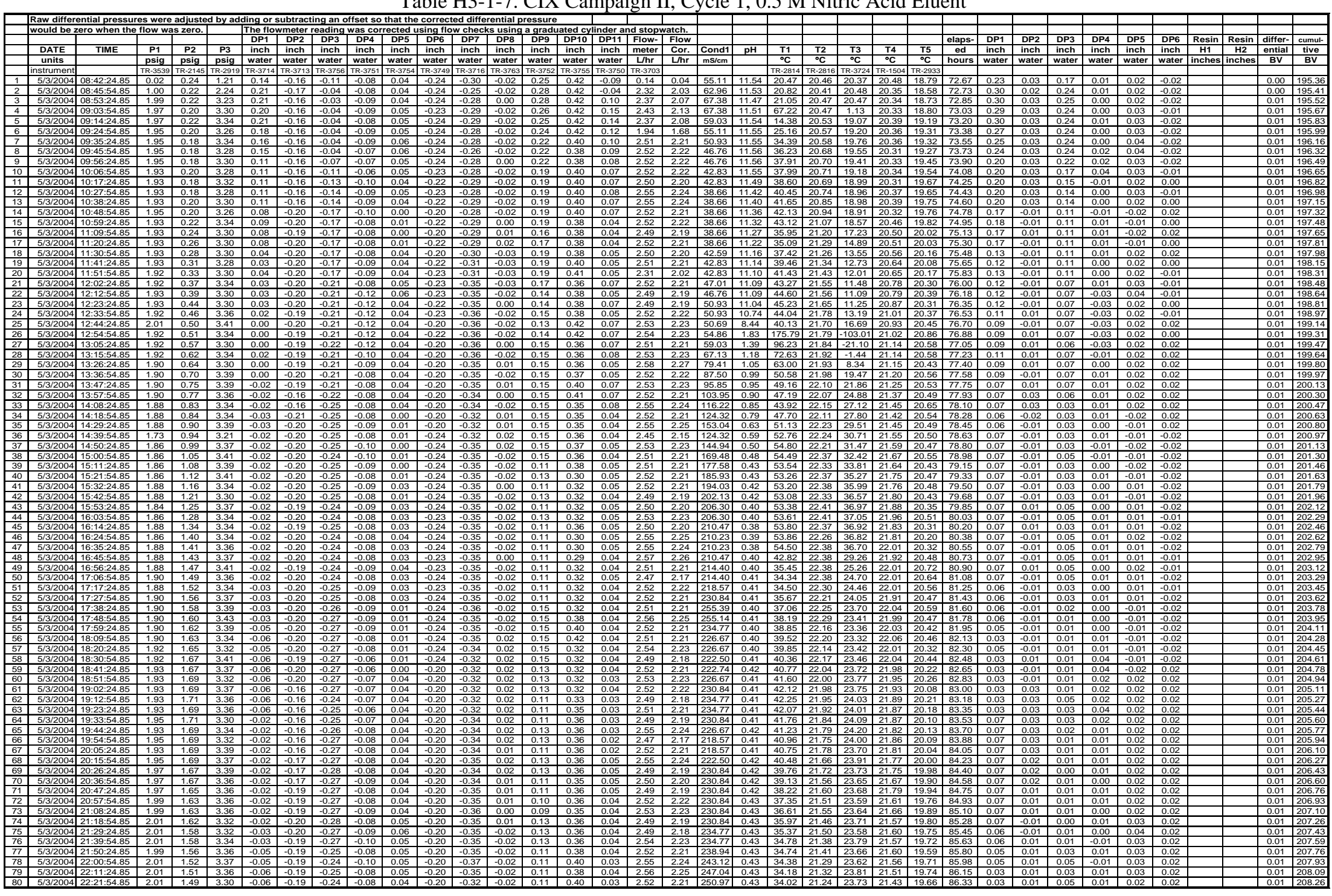


WSRC-TR-2005-00105, REVISION 0

SRNL-RPP-2005-00012, REVISION 0

Table H3-1-8. CIX Campaign II, Cycle 1, DI Water Post-Elution Rinse

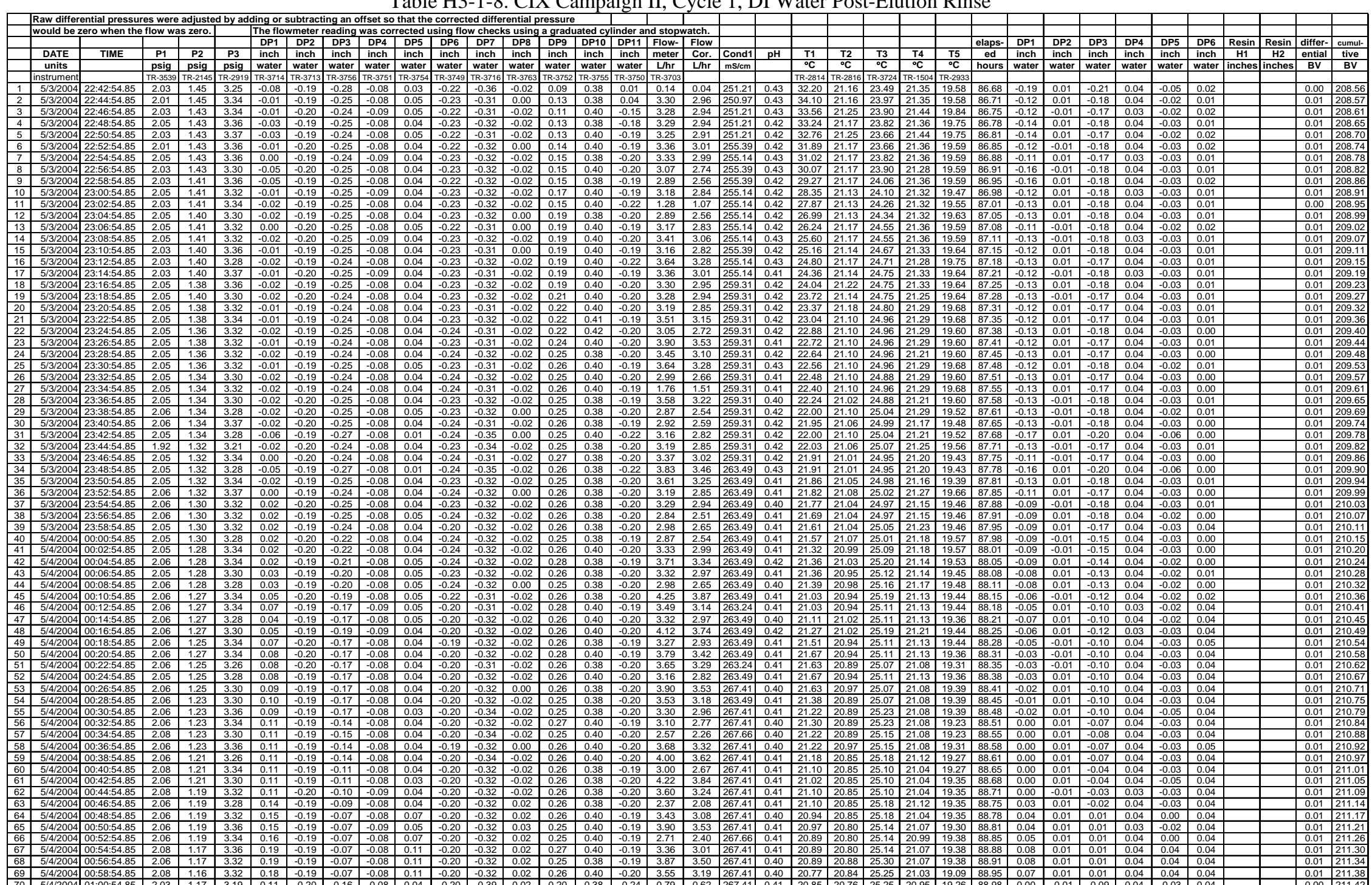


WSRC-TR-2005-00105, REVISION 0

SRNL-RPP-2005-00012, REVISION 0

Table H3-1-9. CIX Campaign II, Cycle 1, 0.25M NaOH Regenerant

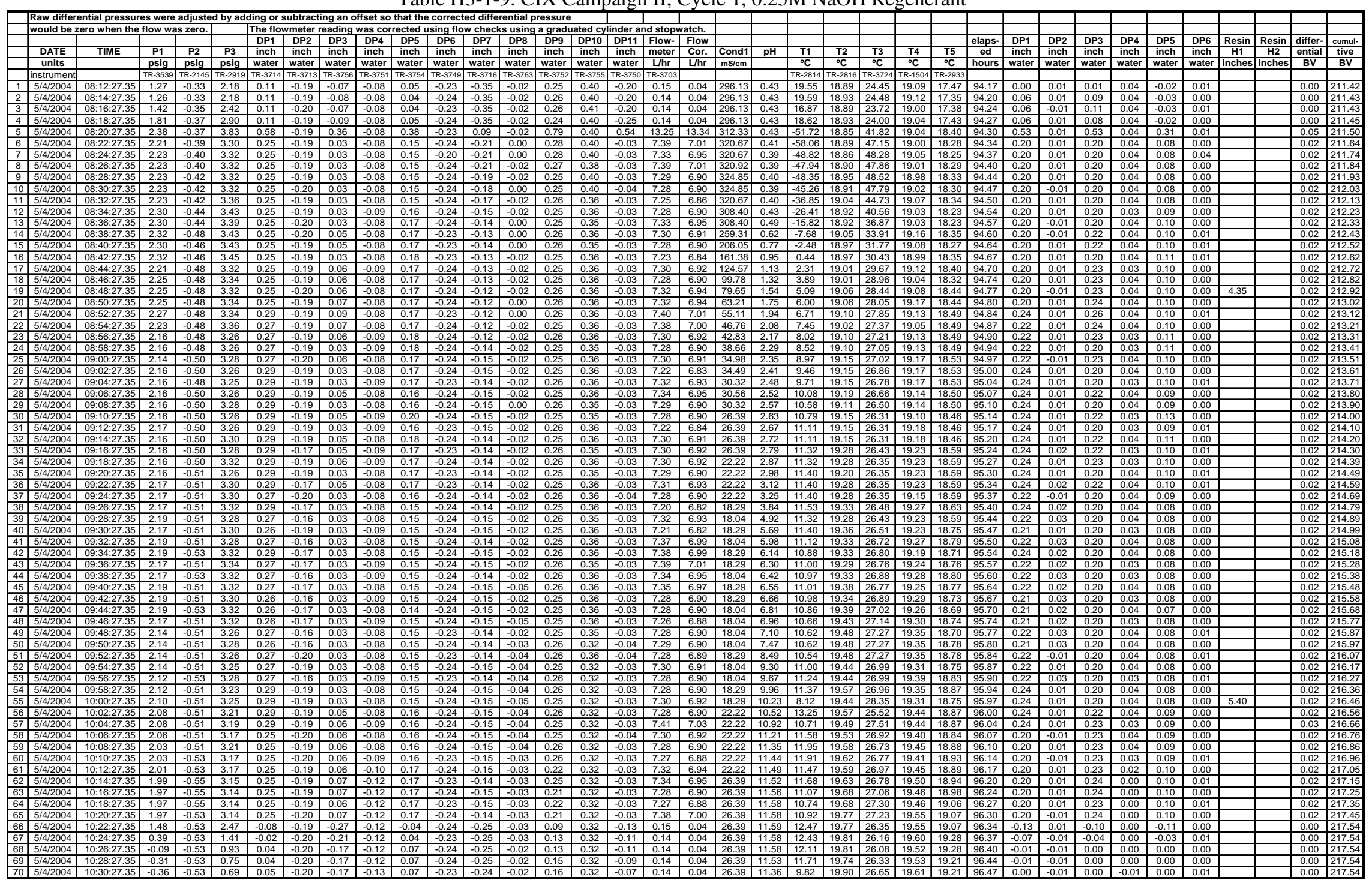


WSRC-TR-2005-00105, REVISION 0

SRNL-RPP-2005-00012, REVISION 0

Table H3-2-1. CIX Campaign II, Cycle 2, LAW Treatment

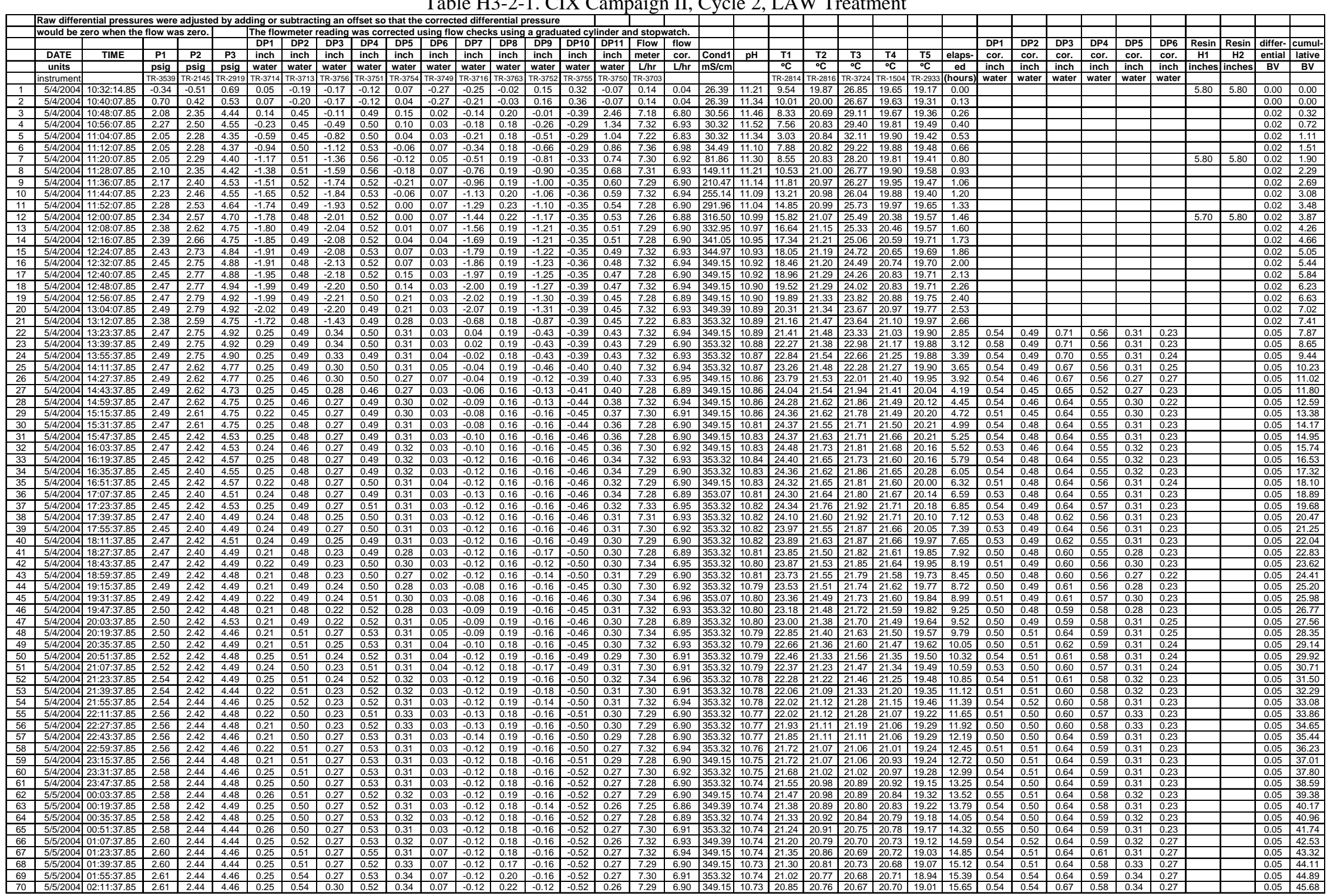


WSRC-TR-2005-00105, REVISION 0

SRNL-RPP-2005-00012, REVISION 0

Table H3-2-2. CIX Campaign II, Cycle 2, LAW Treatment

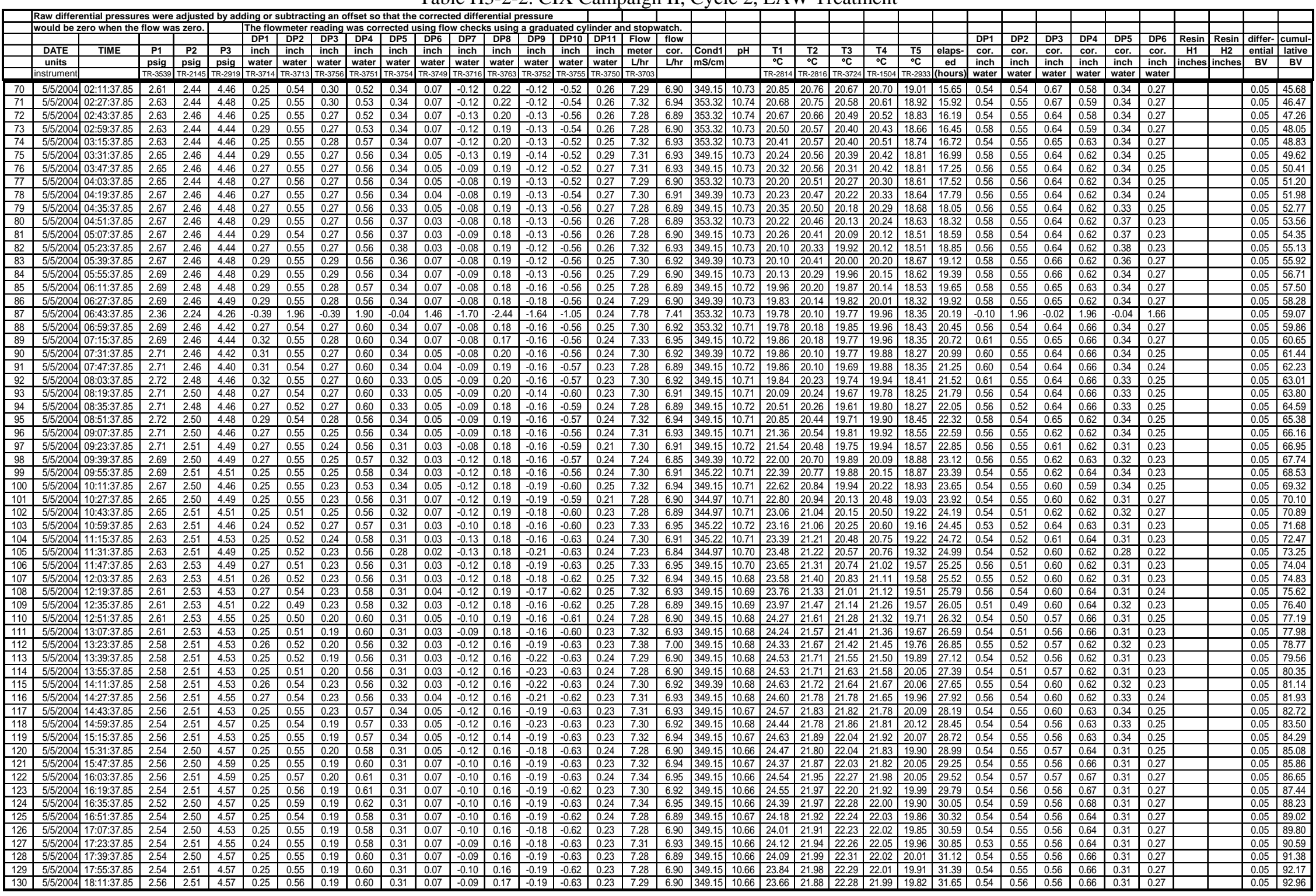


WSRC-TR-2005-00105, REVISION 0

SRNL-RPP-2005-00012, REVISION 0

Table H3-2-3. CIX Campaign II, Cycle 2, LAW Treatment

\begin{tabular}{|c|c|c|c|c|c|c|c|c|c|c|c|c|c|c|c|c|c|c|c|c|c|c|c|c|c|c|c|c|c|c|c|c|c|c|c|}
\hline & $\begin{array}{l}\text { 年aw dififer } \\
\text { would be }\end{array}$ & $\begin{aligned} \text { rential pressus } \\
\text { zero when the }\end{aligned}$ & $\begin{array}{l}\text { res wer } \\
\text { fllown }\end{array}$ & $\begin{array}{l}\text { ad zero } \\
\text { as ero }\end{array}$ & ed by a & The of & & & & & & & & & & & & ratcn. & & & & & & & & & & & & & & & & & \\
\hline & & & & & & $\begin{array}{l}\text { the eft } \\
\text { DP1 } \\
\end{array}$ & DP2 & $\frac{\text { DP3 }}{\text { DP }}$ & DP4 & DP5 & DP6 & DP7 & $\frac{1}{D}$ & DP9 & DP10 & DP11 & Flow & flow & & & & & & & & & DP1 & DP2 & \begin{tabular}{|l|} 
DP3 \\
\end{tabular} & \begin{tabular}{|l|} 
DP4 \\
\end{tabular} & DP5 & DP6 & & & differ- \\
\hline & DATE & TIME & $\mathrm{P1}$ & $P 2$ & ro & inch & inch & inch & inch & inch & inch & inch & inch & inch & inch & inch & meter & \begin{tabular}{|l|} 
cor. \\
\end{tabular} & Cond? & $\mathrm{pH}$ & T1 & T2 & $\mathrm{T3}$ & $\mathrm{T} 4$ & $\mathrm{~T} 5$ & | elaps- & \begin{tabular}{|l|} 
cor. \\
\end{tabular} & \begin{tabular}{|c|} 
cor. \\
\end{tabular} & \begin{tabular}{|c|} 
cor. \\
\end{tabular} & cor. & cor. & cor. & $\mathrm{H} 1$ & & ential \\
\hline & $\begin{array}{l}\text { units } \\
\text { instrument }\end{array}$ & & $\frac{\text { psig }}{\text { TR-3533 }}$ & $\frac{\frac{p s i g}{\text { TS-214 }}}{4}$ & $\frac{\text { psig }}{\text { TS-291 }}$ & $\frac{\text { wate }}{\text { TR-371 }}$ & $\frac{\text { water }}{\text { TR-371 }}$ & 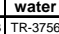 & \begin{tabular}{|l|l|} 
water \\
TR-3751
\end{tabular} & \begin{tabular}{|l|l|} 
water \\
TR-3754
\end{tabular} & \begin{tabular}{|l|l|} 
Water \\
TR-3749
\end{tabular} & \begin{tabular}{|l|l|} 
Water \\
TR-3716
\end{tabular} & 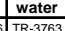 & \begin{tabular}{|l|l|} 
water \\
TRR-3752
\end{tabular} & \begin{tabular}{|l|l|} 
& anter \\
2 TR-3755
\end{tabular} & \begin{tabular}{|c|} 
water \\
TR.3750
\end{tabular} & 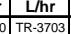 & & $\mathrm{mS} / \mathrm{cn}$ & & $\frac{{ }^{\circ} \mathrm{C} C}{\mathrm{TR}-281}$ & $\frac{{ }^{\circ} \mathrm{C} C}{\mathrm{TR}-281}$ & $\begin{array}{l}{ }^{\circ} \mathrm{C}-\mathrm{C} \\
\mathrm{T} R \mathrm{~T}\end{array}$ & $\begin{array}{l}{ }^{\circ} \mathrm{C}-\mathrm{T} \\
\mathrm{TR}-150\end{array}$ & $\frac{{ }^{\circ} \mathrm{C}}{\mathrm{TR}-293}$ & \begin{tabular}{|c|} 
ed \\
(yhours \\
\end{tabular} & \begin{tabular}{|c|} 
inch \\
water
\end{tabular} & \begin{tabular}{|l|} 
inch \\
water \\
\end{tabular} & \begin{tabular}{|l|} 
inch \\
water
\end{tabular} & \begin{tabular}{|l|} 
inch \\
water \\
\end{tabular} & $\begin{array}{l}\text { inch } \\
\text { wwater }\end{array}$ & $\begin{array}{l}\text { inch } \\
\text { water }\end{array}$ & & & \\
\hline & & & 258 & 2.51 & 4.59 & 0.25 & 0.55 & 0.18 & 0.57 & 0.34 & 0.05 & -0.12 & 0.16 & -0.19 & -0.66 & 2.24 & 728 & 6.90 & & 10.66 & & & & 21.95 & & & & & & & & & & & \\
\hline$\overline{32}$ & $5 / 5 / 2004$ & $18: 43: 37.85$ & 2.58 & 2.53 & 4.59 & 0.24 & 0.55 & 0.17 & 0.58 & 0.34 & 0.05 & -0.12 & 0.16 & -0.19 & -0.66 & 0.24 & 7.28 & 6.89 & \begin{tabular}{|l|}
349.1 \\
\end{tabular} & $\begin{array}{l}\frac{10.00}{10.66} \\
\end{array}$ & $\frac{2.10}{23.70}$ & & $\frac{2 . .24}{22.32}$ & $\frac{2.50}{22.03}$ & $\frac{1.00}{19.78}$ & $\frac{31.52}{32.19}$ & $\begin{array}{l}0.54 \\
0.53\end{array}$ & 0.55 & 0.54 & 0.04 & 0.044 & 0.25 & & & 0.05 \\
\hline 133 & $5 / 5 / 2004$ & $18: 59: 37.85$ & 2.56 & 2.51 & 4.55 & 0.24 & 0.55 & 0.19 & 0.60 & 0.33 & 0.07 & & $\overline{0.16}$ & -0.19 & -0.66 & 23 & 7.30 & $\begin{array}{ll}6.91 \\
\end{array}$ & 349.15 & 0.66 & 23.36 & 1.83 & 2.23 & 21.94 & 19.76 & 32.45 & 0.53 & 0.55 & 0.56 & 0.66 & 0.33 & 0.27 & & & 0.05 \\
\hline 134 & $5 / 5 / 200$ & 19:15:37.85 & 2.58 & 2.51 & $\begin{array}{ll}4.57 \\
\end{array}$ & 0.24 & 0.55 & 0.20 & 0.60 & 0.32 & 0.6 & & $\overline{0.16}$ & -0.18 & -0.66 & 0.23 & 7.28 & 6.89 & 449.1 & 1.66 & 23.48 & 1.86 & 2.18 & 21. & 19.80 & 32.72 & 0.53 & 0.55 & 0.57 & 0.66 & $\overline{0.32}$ & 0.25 & & & $\overline{.05}$ \\
\hline 35 & $5 / 5 / 200$ & 19:31:37.85 & 2.58 & 2.53 & 4.55 & 0.26 & 0.55 & 0.20 & 0.60 & 0.31 & & & 0.16 & & 0.66 & 0.2 & 7.30 & 6.92 & 499.1 & & & & & & 9.79 & 32.99 & 0.55 & & & 0.66 & & & & & \\
\hline & $5 / 5 / 200$ & 19:47:37.85 & 2.60 & 2.53 & 4.55 & 0.25 & 0.56 & 0.20 & 0.60 & 0.31 & 0.04 & & 0.16 & -0.18 & -0.66 & & 7.28 & 6.89 & 349.1 & & 3.1. & & & & 19.74 & 3325 & 0.54 & 056 & & 0.66 & & 0.24 & & & \\
\hline & $5 / 5 / 200$ & $20: 03: 37.85$ & 2.60 & 2.51 & 4.57 & 0.29 & 0.55 & 0.20 & 0.58 & 0.31 & 0.0 & & 0.16 & -0.19 & -0.66 & & 7.32 & 6.94 & 349. & 1.6 & 3.0 & 1.7 .7 & 2. & & 19.7 & 33.52 & 0.58 & & & 0.64 & 0.31 & 0.24 & & & 10 \\
\hline 138 & $5 / 5 / 2004$ & $20: 19: 37.85$ & 2.60 & 2.53 & 4.57 & 0.27 & 0.56 & 0.20 & 0.58 & 0.31 & 0.04 & -0. & 0.16 & -0.19 & -0.67 & 0.8 & 7.33 & 6.95 & 349.1 & 0.66 & 22.94 & 1.7 & 2.0 & & 19.5 & 33.79 & 0.56 & 0.56 & & 0.64 & 0.31 & 0.24 & & & \\
\hline & $5 / 5 / 200$ & $20: 35: 37.85$ & 2.61 & 2.53 & 4.62 & 0.25 & 0.56 & 0.19 & 0.58 & 0.31 & 0.0 & -0. & 0.16 & -0.18 & -0.66 & 0.2 & 7.28 & 6.90 & 349. & 0.6 & 22.8 & 1.7. & & 1. & $19.57 \mathrm{Z}$ & 34.05 & 0.54 & 0.56 & 0.56 & 0.64 & 0.31 & 0.23 & & & \\
\hline 140 & $5 / 5 / 200$ & 20:51:37.85 & 2.63 & 2.55 & 4.55 & 0.25 & 0.57 & 0.19 & 0.60 & 0.31 & 0.03 & -0.12 & 0.16 & -0.16 & -0.66 & 0.2 & 7.34 & 6.95 & 349.3 & 10.66 & $22.8 \mathrm{C}$ & 1.75 & & 21. & 19.52 & 34.32 & 0.54 & & 0.56 & 0.66 & 0.31 & 0.23 & & & \\
\hline & $5 / 5 / 200$ & $21: 07: 37.85$ & 2.41 & 2.31 & 4.37 & 0.27 & 0.57 & 0.20 & 0.60 & 0.31 & 0.03 & -0.12 & 0.16 & -0.16 & -0.63 & 0.20 & 7.36 & 6.98 & 349.1 & 10.66 & 22.63 & 21.74 & 21.9 & 21.7 & 19.51 & 34.59 & 0.56 & 0.57 & 0.57 & 0.66 & 0.31 & 0.23 & & & \\
\hline 142 & $5 / 5 / 200$ & 21:23:37.85 & 2.43 & 2.33 & 4.35 & 0.25 & 0.56 & 0.19 & 0.60 & 0.32 & 0.03 & -0.12 & 0.19 & -0.17 & -0.62 & 0.20 & 7.31 & 6.93 & 349.1 & 10.66 & 22.43 & & 22.0 & $21.72 \mathrm{PC}$ & 19.47 & 34.85 & 0.54 & 0.56 & 0.56 & 0.66 & 0.32 & 0.23 & & & \\
\hline 143 & $5 / 5 / 2004$ & 21:39:37.85 & 2.43 & 2.33 & 4.38 & 0.25 & 0.55 & 0.19 & 0.60 & 0.31 & 0.03 & -0.08 & 0.19 & -0.18 & -0.62 & 0.20 & 7.28 & 6.90 & 349.15 & 10.66 & 22.49 & 21.76 & $22.00 \mathrm{C}$ & 21.7 & 19.54 & 35.12 & 0.54 & 0.55 & 0.56 & 0.66 & 0.31 & 0.23 & & & \\
\hline 144 & $5 / 5 / 200$ & $21: \frac{1}{2}: 37.8{ }$ & 2.43 & 2.33 & 4.35 & 0.25 & 0.55 & 0.20 & 0.62 & 0.31 & 0.07 & -0.08 & 0.19 & -0.18 & -0.63 & 0.26 & 7.28 & 6.90 & 349.15 & 10.65 & 22.4 & 016 & 21.86 & 1. & 19.48 & 35.39 & 0.54 & & & 0.68 & 0.31 & & & & \\
\hline 145 & $5 / 5 / 200$ & $22: 11: 37.85$ & 2.45 & 2.35 & 4.38 & 0.25 & 0.61 & 0.19 & 0.62 & 0.34 & 0.07 & -0.05 & 0.19 & -0.18 & -0.63 & 0.19 & 7.30 & 6.92 & 349.15 & 10.66 & 22.3 & 1.66 & $21.82 \mathrm{~s}$ & $21.6 ؟$ & 19.51 & 35.65 & 0.54 & 0.61 & 0.56 & 0.68 & 0.34 & & & & \\
\hline 146 & $5 / 5 / 200$ & $22: 27: .37 .8$ & 2.45 & 2.35 & 4.38 & 0.25 & 0.61 & 0.23 & 0.62 & 0.34 & 0.05 & -0.0 & 0.19 & -0.19 & -0.63 & 0.2 & 7.31 & 6.93 & 349.15 & \begin{tabular}{|l|l|}
10.65 \\
\end{tabular} & 22.19 & 1.61 & 1.7 & 21.6 & 19.47 & 35.92 & 0.54 & 0.61 & 0.60 & 0.68 & 0.34 & 0.25 & & & \\
\hline & 5/200 & $22: 43: 37.85$ & 2.45 & 2.35 & 4.38 & 0.25 & 0.62 & 0.23 & 0.62 & 0.33 & 0.07 & -0.1 & 0.19 & -0.19 & -0.65 & 0.1 & 7.30 & 6.92 & 349.35 & & 22.14 & & 1.8. & & 9.4 & 36.19 & 0.54 & 0.62 & 0.60 & 0.68 & 0.33 & 0.27 & & & \\
\hline 148 & $5 / 5 / 200$ & 22:59:37.85 & 2.47 & 2.35 & 4.37 & 0.29 & 0.62 & 0.23 & 0.62 & 0.31 & 0.04 & -0.1 & 0.19 & -0.19 & -0.66 & & 7.28 & 6.89 & |349.1 & & & & & & & 36.45 & 0.5 & 0.62 & 0.60 & 0.68 & .31 & 0.24 & & & .05 \\
\hline 149 & $5 / 5 / 200$ & 3:15:37.85 & 2.47 & 2.37 & 4.38 & 0.27 & 0.57 & 0.23 & 0.60 & 0.31 & 0.04 & -0.1 & 0.19 & -0.19 & -0.66 & & 7.28 & 6.89 & 349.1 & & & & & & 9.4 & 6.7 & 0.56 & 0.57 & 0.60 & 0.66 & 0.31 & 0.24 & & & 05 \\
\hline & $5 / 5 / 200$ & :31:37.85 & 2.49 & 2.35 & 4.37 & 0.27 & 0.61 & 0.23 & 0.60 & 0.31 & 0.03 & -0. & 0.19 & -0.19 & -0.66 & & 7.27 & 6.88 & 349. & & & & & & 9.3 & 36.9 & 0.56 & 0.6 & 0.60 & 0.66 & 0.31 & 0.23 & & & 05 \\
\hline 151 & $5 / 5 / 200$ & $23: 47: 37.85$ & 2.49 & 2.37 & 4.35 & 0.27 & 0.63 & 0.23 & 0.61 & 0.31 & 0.05 & -0.1 & 0.19 & -0.19 & -0.66 & & 7.32 & 6.94 & $349.3 \mathrm{C}$ & & & & & 1. & 9.2 & 37.25 & 0.56 & 0.63 & 0.60 & 0.67 & 0.31 & 0.25 & & & .05 \\
\hline & $5 / 6 / 6200$ & 03:37.8 & 2.49 & 2.35 & 4.40 & 0.27 & 0.6 & 0.2 & 0.62 & 0.31 & 0.0 & & 0.19 & & -0.6 & & 7.29 & 6.9 & & & & & & & & & 0.5 & & 0.6 & 0.68 & & 0.23 & & & \\
\hline & & 0:19:37.8 & 2.49 & 2.35 & 4.35 & 0.26 & & 0.2 & 0.62 & & & & 0.18 & 0.19 & & & $7.28 \mathrm{x}$ & & B49. & & & & & & & & & & & & & & & & \\
\hline 154 & 1200 & 00:35:37.85 & 2.49 & 2.37 & 4.38 & 0.27 & 0.63 & 0.23 & 0.62 & 0.3 & 0.0 & & 0.19 & -0.19 & 0.6 & & 7.28 & 6.90 & 349. & & & & & & & 8.0 & 0.5 & 0.6. & 0.66 & 0.68 & 0.3. & 0.23 & & & \\
\hline & & $00: 51: 37.85$ & 2.50 & 2.37 & 4.37 & 0.27 & 0.62 & 0.2 & 0.61 & & 0.04 & & 0.19 & & & & 7.32 & & & & & & & & & 8.3 & & & & & & .24 & & & \\
\hline 156 & & $01: 07: 37.85$ & 2.50 & 2.37 & 4.35 & 0.26 & 0.63 & 0.23 & 0.60 & 0. & 0.6 & & 0.18 & -0.18 & & & 7.28 & 190 & & & & & & & & & & & & 0.6 & & & & & \\
\hline 157 & $5 / 6 / 2000$ & $01: 23: 37.85$ & 2.50 & 2.37 & 4.37 & 0.25 & 0.62 & 0.23 & 0.60 & 0.32 & 0.03 & & 0.18 & -0.19 & -0.70 & & 7.28 & 6.90 & & & & 1.46 & & & & 38.85 & 0.54 & 0.6 & 0.6 & 0.6 & 0.32 & $0.2 \mathrm{r}$ & & & \\
\hline$\frac{158}{50}$ & & & 2.50 & 2.37 & 4.38 & 0.26 & 0.62 & 0.2 & 0.62 & 0.31 & 0.0 & & 0.19 & -0.18 & 10 & & 7.28 & 6.90 & & & & & 1. & & & 9.1 & & & & 0.6 & & & & & \\
\hline 159 & 1800 & $01: 55: 37.85$ & 2.50 & 2.39 & 4.38 & 0.25 & 0.62 & 0.2 & 0.62 & & & & & & & & 7.30 & & & & & & & & & & 0.54 & & & & & & & & \\
\hline 160 & . & $02: 11: 37.85$ & 2.50 & 2.37 & 4.38 & 0.26 & & 0.22 & 0.63 & & & & & 1.14 & & & 7.32 & & & & & & & & 9.1 & .65 & 0. & & & 0.6 & & 0 & & & \\
\hline & 5 & & 2.52 & 2.37 & 4.38 & & & 0.25 & 0.62 & & & & & & & & 7.3 & & & & & & & & & 9.92 & & & & & & & & & \\
\hline & & 2:43: & 2.52 & 2.37 & 4.40 & 0.2 & & 0.2 & 0.61 & & & & & & & & & & & & & & & & & & & & & & & & & & \\
\hline & & & 2.52 & 2.39 & 4.38 & 0.2 & 0.6 & 0.2 & 0.61 & & & & & & & & & & & & & & & & & & & & & & & & & & \\
\hline 104 & 200 & & 2.52 & 2.39 & 4.38 & 0.2 & 0.6 & 0.2 & 0.65 & & & & 0.1 & & & & & & & & & & & & & & & & & & & & & & \\
\hline & 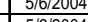 & & 2.52 & 2.39 & 4.37 & 0.25 & 0.65 & 0.2 & 0.66 & 0.3 & & & & & & & & & & & & & & & & 100 & & & & & & & & & \\
\hline$\frac{100}{167}$ & 年 & & 2.54 & 2.39 & 4.31 & 0.25 & 0.05 & 0.6 & 0.06 & 0. & 0.0 & & & & & & & & & & & & & & & & 0. & & & & & & & & \\
\hline$\frac{110}{160}$ & 5 & & 2.52 & 2.39 & & 0.25 & & & 0.61 & & & & & & & & & & & & & & & & & & & & & & & & & & \\
\hline 168 & & & 2.39 & 2.44 & 4.24 & 0.64 & & & 0.6 & 0. & & & & & & & & & & & & & & & 9.1. & & & & & & & & & & \\
\hline$\frac{109}{170}$ & $5 / 1012$ & & 2.54 & 2.40 & 4.37 & 0.25 & & & 0 & 0.5 & & & & & & & & & & & & & & & 19.1. & & & & & & & & & & \\
\hline 100 & 年 & & 2.54 & 2.40 & 4.42 & 0.25 & $\frac{0.6}{0.0}$ & & 0.65 & 0.3 & & & & & & & & & & & & & & & & & & & & & & & & & \\
\hline & & & 2.30 & 2.40 & 4.30 & 0.2 & & & & & & & & & & & & & & & & & & & & & & & & & & & & & \\
\hline & & 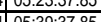 & 2.30 & 2.42 & $\begin{array}{c}4.30 \\
.308\end{array}$ & & & & 0.01 & & & & & & & & & & & & & & & & & & & & & & & & & & \\
\hline 174 & $\frac{512100}{55(1200}$ & 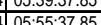 & 2.30 & 2.42 & $\begin{array}{ll}4.30 \\
.309\end{array}$ & 0.44 & & 0.4 & 0.00 & & & & & & & & & & & & & & & & & & & & & & & & & & \\
\hline & $5 / 16200$ & 03.35 .30 .05 & 2.30 & 2.46 & 先, & $\frac{0.65}{0.20}$ & 0.0 & 0.25 & 0.015 & & & -4. & & & & & & & & & & & & & & & & & & & & & & & \\
\hline 10 & $5 / 515200$ & & $\frac{2.30}{256}$ & 2.44 & $\frac{1.50}{440}$ & 0.29 & 0.0 & 0.4 & . & 0. & 0 & 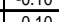 & & & & & & & & & & & & & & & & & & & & & & & \\
\hline 177 & 55162004 & 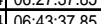 & $\frac{2.00}{258}$ & $\frac{2.44}{244}$ & $\frac{4.48}{438}$ & $\frac{0.50}{0.29}$ & 0.0 & 0.47 & 0.06 & 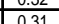 & 0.03 & -0 & & & & & & $\frac{0 .}{6}$ & & & & & & & & & & & & & & & & & \\
\hline 178 & $5 / 6 / 2004$ & & $\frac{258}{258}$ & 2.42 & 4.42 & 0.29 & 0.6 & 0.20 & 0.66 & 0. & & & & & & & & 6. & & & & & & & & & & & & & & & & & 0.05 \\
\hline \begin{tabular}{|l|l|}
179 \\
\end{tabular} & $\overline{6 / 2 C}$ & 107:15: & 2.60 & 2.44 & 4.38 & 0.27 & 0.65 & $\begin{array}{ll}0.24 \\
\end{array}$ & $\begin{array}{ll}0.67 \\
\end{array}$ & 0. & \begin{tabular}{|l|} 
\\
\end{tabular} & -0. & & 18 & $\begin{array}{l}-0.70 \\
-10\end{array}$ & & 31 & 6. & & & & & & & & & & & & & & 0.2 & & & 0.05 \\
\hline \begin{tabular}{|l|l|}
180 \\
\end{tabular} & $5 / 6 / 2$ & & 2.60 & 2.44 & 4.38 & 0.29 & 0.65 & $\begin{array}{ll}0.25 \\
\end{array}$ & 0.1 & 0. & \begin{tabular}{|l|} 
\\
\end{tabular} & -0. & & -0.18 & $\begin{array}{l}-0.70 \\
\end{array}$ & & 7.34 & 6.9 & & & 35 & & & & & & & & & & & & & & 0.05 \\
\hline \begin{tabular}{|l|l|}
181 \\
\end{tabular} & $5 / 6 / 2$ & & 2.61 & 2.46 & 4.42 & 0.27 & 0.65 & 0.2 & $\begin{array}{ll}0.66 \\
\end{array}$ & & \begin{tabular}{|l|l|l|l}
0.03 \\
\end{tabular} & -0. & & \begin{tabular}{|c|}
-0.19 \\
\end{tabular} & $\begin{array}{l}-0.70 \\
\end{array}$ & 0. & 7.31 & 6.9 & & & 21 & & & & & & & 0.65 & & & & & & & 0.05 \\
\hline \begin{tabular}{|l|l|}
182 \\
\end{tabular} & & \begin{tabular}{|l|l|l|l|l}
$08: 03$ \\
\end{tabular} & 2.60 & 2.46 & 4.37 & 0.25 & 0.65 & 0.2 & & & \begin{tabular}{|l|l|}
0.03 \\
\end{tabular} & -0 & & -0.19 & -0.70 & 0. & 7.31 & 6. & & & & & & & & & & & & & & 0. & & & 0.05 \\
\hline 183 & & & 2.60 & 2.46 & 4.37 & 0.25 & 0.6 & & & 0. & 0. & & 0 & 19 & & & 28 & 6.8 & & & & & & & & & & & 0 & & & 0.2 & & & 0.05 \\
\hline \begin{tabular}{|l|l|}
184 \\
\end{tabular} & & & 2.61 & 2.46 & 4.38 & 0.25 & 0.6 & 0.2 & 0.66 & 0.31 & 0.03 & & 0 & 18 & 70 & & 7.30 & 6.92 & & & & & & & & & & & & & & 0.2 & & & 0.05 \\
\hline 185 & & & 2.61 & 2.46 & 4.40 & 0.25 & 0.6 & 0.1 & 0. & 0. & 0.03 & -0. & & .18 & & & 28 & 6. & & & & & & & & & & 0. & & 0.73 & & 0.23 & & & 0.05 \\
\hline 186 & & & 2.60 & 2.48 & & 0.25 & 0.65 & 0.20 & 0.68 & 0. & 0.03 & -0. & 18 & 19 & 70 & & & 6. & & & 22.22 & & 21.08 & & 19.26 & & 0.54 & 0.65 & & 74 & 0.33 & 0.23 & & & 0.05 \\
\hline 187 & & & 2.60 & 2.48 & 4.40 & 0.25 & 0.65 & 0.22 & 0.66 & 0.31 & 0.03 & -0.13 & 0.18 & -0.19 & -0.73 & 18 & .28 & 6.8 & & & 36 & & 21.14 & & $\frac{3.32}{21}$ & 46.85 & 0.54 & 0.65 & & 0.72 & 0.31 & 0.23 & & & 0.05 \\
\hline 188 & $5 / 6 / 2 C$ & & 2.60 & 2.48 & 4.42 & 0.26 & 0.65 & 0.23 & 0.65 & 0.32 & 0.03 & -0.12 & 0.19 & -0.22 & -0.72 & 0.19 & 7.34 & 6. & & & 22. & & 21.1 & 21.18 & 19.25 & 47.12 & 0.55 & 0.65 & 0.60 & 0.71 & 0.32 & 0.23 & & & 0.05 \\
\hline 189 & $5 / 6 / 2004$ & 09:55:37.85 & 2.63 & 2.53 & 4.33 & 0.31 & 0.51 & 0.23 & 0.52 & 0.34 & 0.03 & \begin{tabular}{|l|l|}
-0.12 \\
\end{tabular} & 0.19 & 0.49 & 0.63 & 0.18 & 7.28 & 6.90 & 349.15 & \begin{tabular}{|l|}
10.64 \\
\end{tabular} & 22.70 & 21.65 & 21.24 & 21.27 & 19.34 & 47.39 & 0.64 & 0.56 & 0.60 & 0.58 & 0.34 & 0.23 & & & \begin{tabular}{|l|}
0.05 \\
\end{tabular} \\
\hline 190 & $5 / 6 / 2004$ & \begin{tabular}{|l|l}
$10: 11: 37.85$ \\
\end{tabular} & 2.63 & 2.55 & \begin{tabular}{|l|l|} 
\\
\end{tabular} & \begin{tabular}{|l}
0.32 \\
\end{tabular} & \begin{tabular}{|l|l|}
0.51 \\
\end{tabular} & \begin{tabular}{|l|l|} 
& 0.25 \\
\end{tabular} & \begin{tabular}{|l|l|}
0.52 \\
\end{tabular} & 0.34 & \begin{tabular}{|l|l|} 
& 0.03 \\
\end{tabular} & \begin{tabular}{|l|l|} 
& -0.12 \\
\end{tabular} & \begin{tabular}{|l|l|} 
& 0.19 \\
\end{tabular} & \begin{tabular}{|l|l|} 
& 0.52 \\
\end{tabular} & \begin{tabular}{|l|}
0.63 \\
\end{tabular} & \begin{tabular}{|l|l|} 
& 0.18 \\
\end{tabular} & \begin{tabular}{|l|l|} 
& \\
\end{tabular} & \begin{tabular}{|l|}
6.93 \\
\end{tabular} & 349.15 & \begin{tabular}{|l|l|}
10.63 \\
\end{tabular} & \begin{tabular}{|l|}
22.92 \\
\end{tabular} & \begin{tabular}{|l|l|}
21.78 \\
\end{tabular} & \begin{tabular}{|l|l|}
21.30 \\
\end{tabular} & \begin{tabular}{|l|l|}
21.41 \\
\end{tabular} & \begin{tabular}{|l|l|}
19.48 \\
\end{tabular} & \begin{tabular}{|l|}
47.65 \\
\end{tabular} & \begin{tabular}{|l|l|}
0.65 \\
\end{tabular} & \begin{tabular}{|l|}
0.56 \\
\end{tabular} & \begin{tabular}{|l|}
0.62 \\
\end{tabular} & \begin{tabular}{|l|}
0.58 \\
\end{tabular} & \begin{tabular}{|l|}
0.34 \\
\end{tabular} & \begin{tabular}{|l|l|} 
& 0.23 \\
\end{tabular} & & & \begin{tabular}{|l|l|}
0.05 \\
\end{tabular} \\
\hline
\end{tabular}


WSRC-TR-2005-00105, REVISION 0

SRNL-RPP-2005-00012, REVISION 0

Table H3-2-4. CIX Campaign II, Cycle 2, LAW Treatment

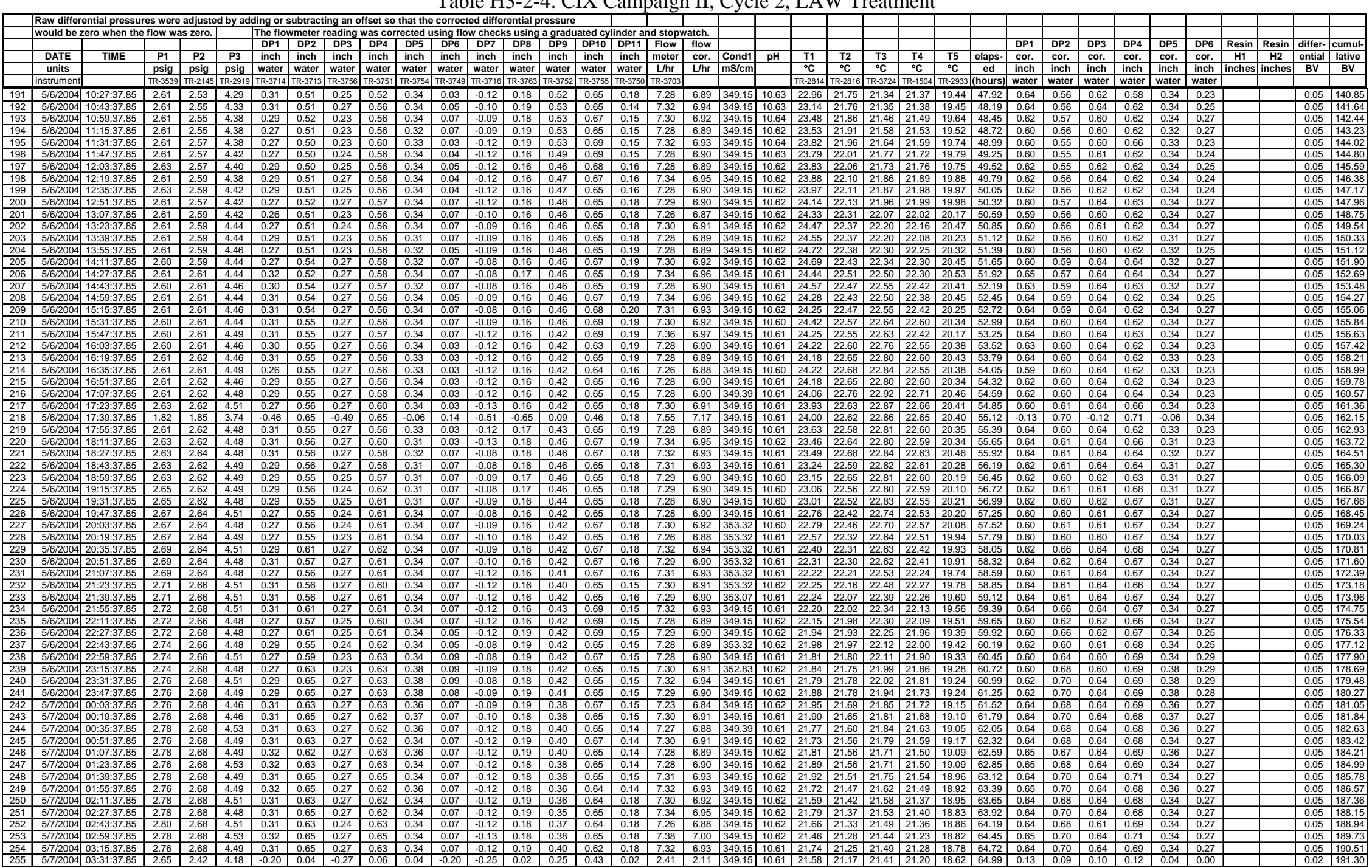


WSRC-TR-2005-00105, REVISION 0

SRNL-RPP-2005-00012, REVISION 0

Table H3-2-5. CIX Campaign II, Cycle 2, 0.1 M NaOH Displacement

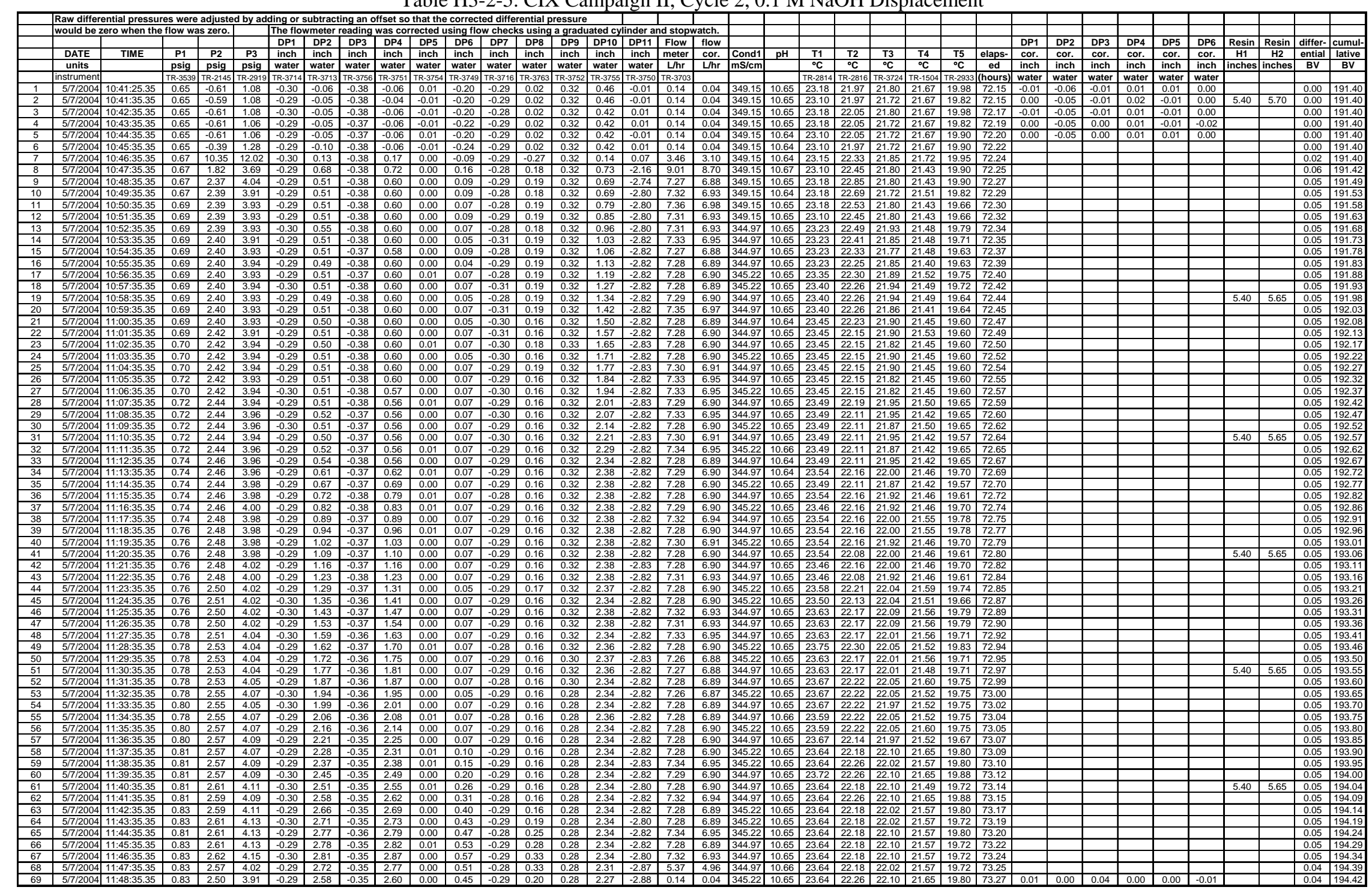


WSRC-TR-2005-00105, REVISION 0

SRNL-RPP-2005-00012, REVISION 0

Table H3-2-6. CIX Campaign II, Cycle 2, DI Water Pre-Elution Rinse

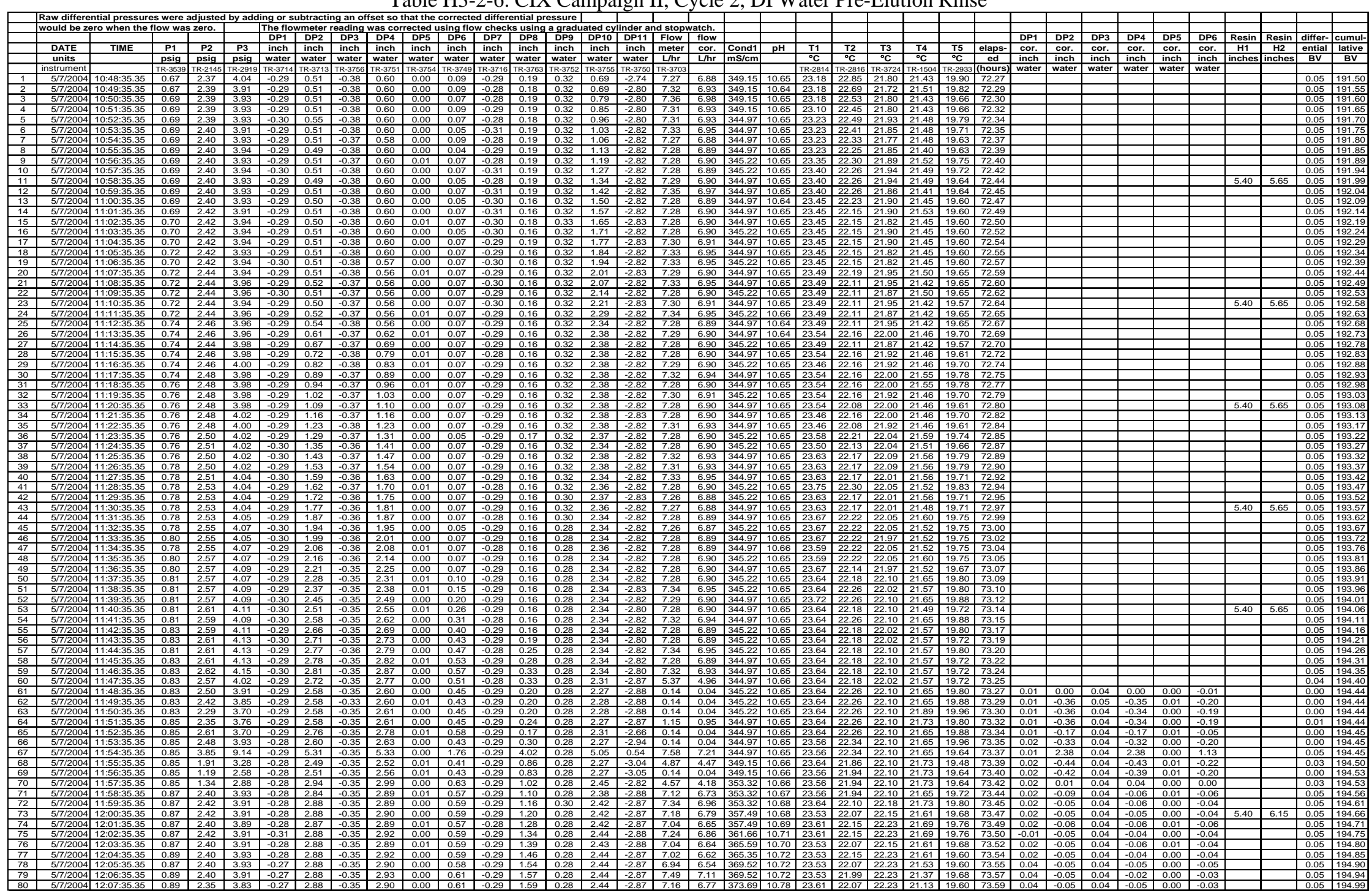


WSRC-TR-2005-00105, REVISION 0

SRNL-RPP-2005-00012, REVISION 0

Table H3-2-7. CIX Campaign II, Cycle 2, 0.5 M Nitric Acid Eluent

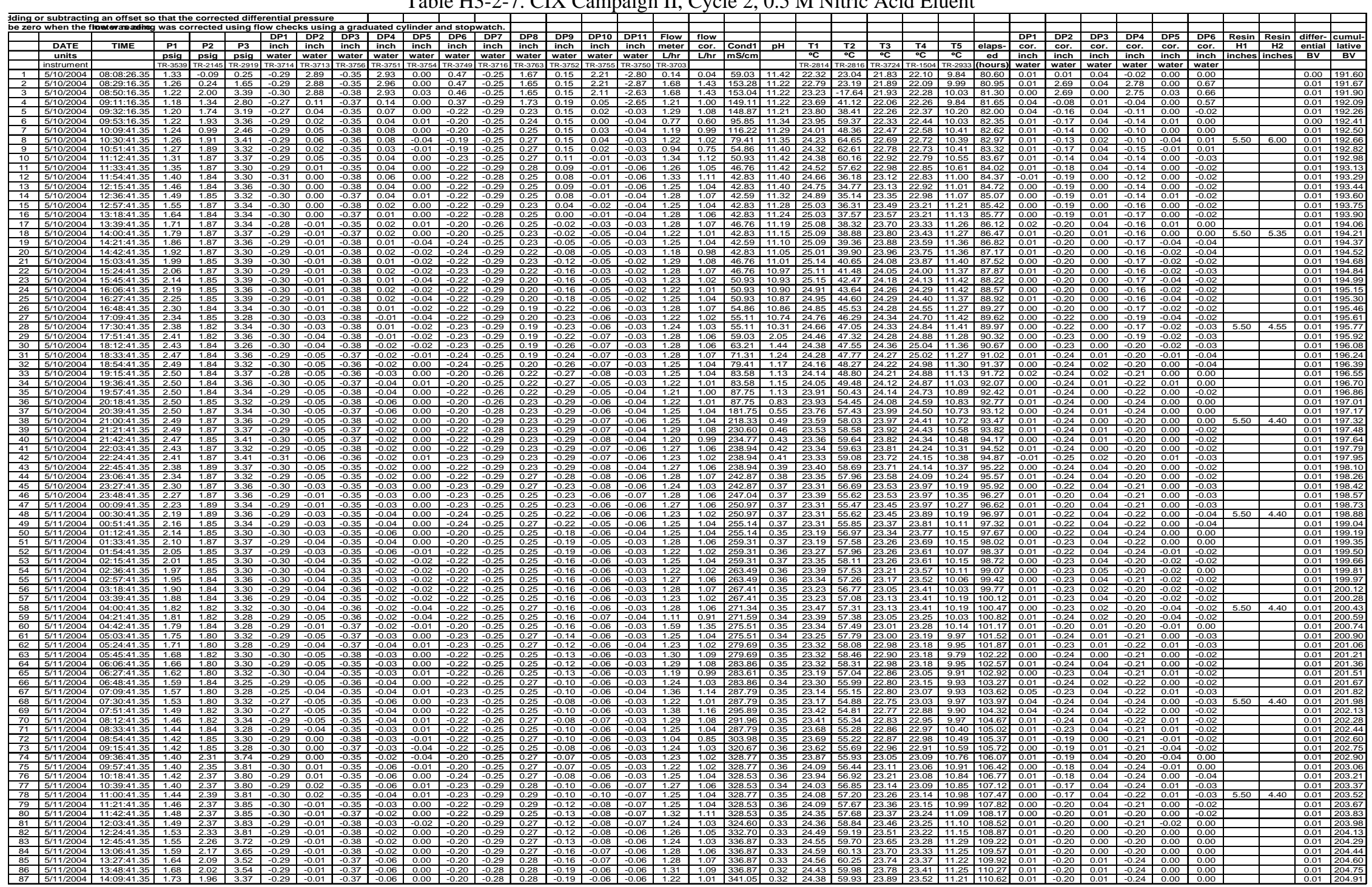


WSRC-TR-2005-00105, REVISION 0

SRNL-RPP-2005-00012, REVISION 0

Table H3-2-8. CIX Campaign II, Cycle 2, DI Water Post-Elution Rinse

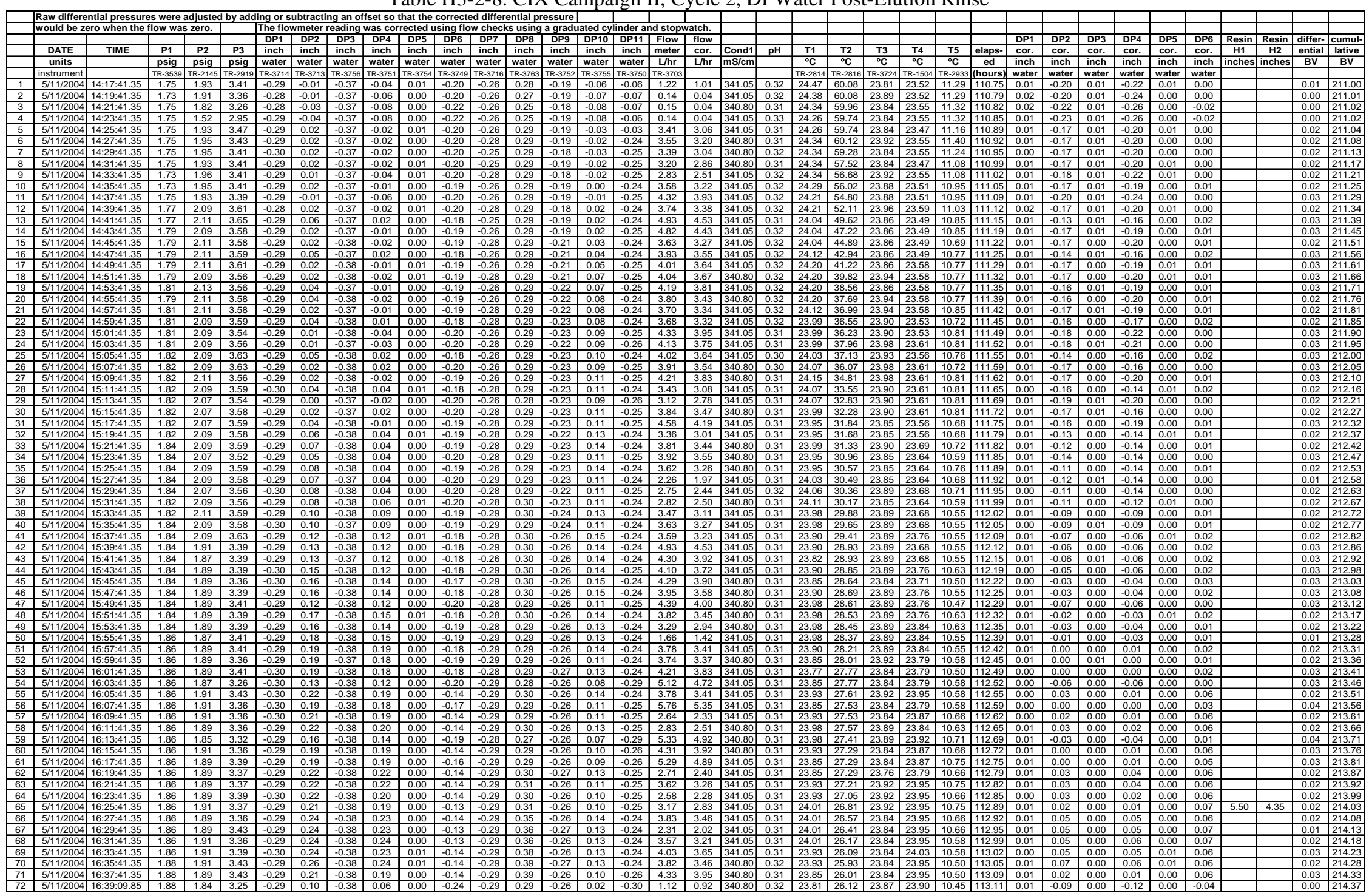


WSRC-TR-2005-00105, REVISION 0

SRNL-RPP-2005-00012, REVISION 0

Table H3-2-9. CIX Campaign II, Cycle 2, 0.25M NaOH Regenerant

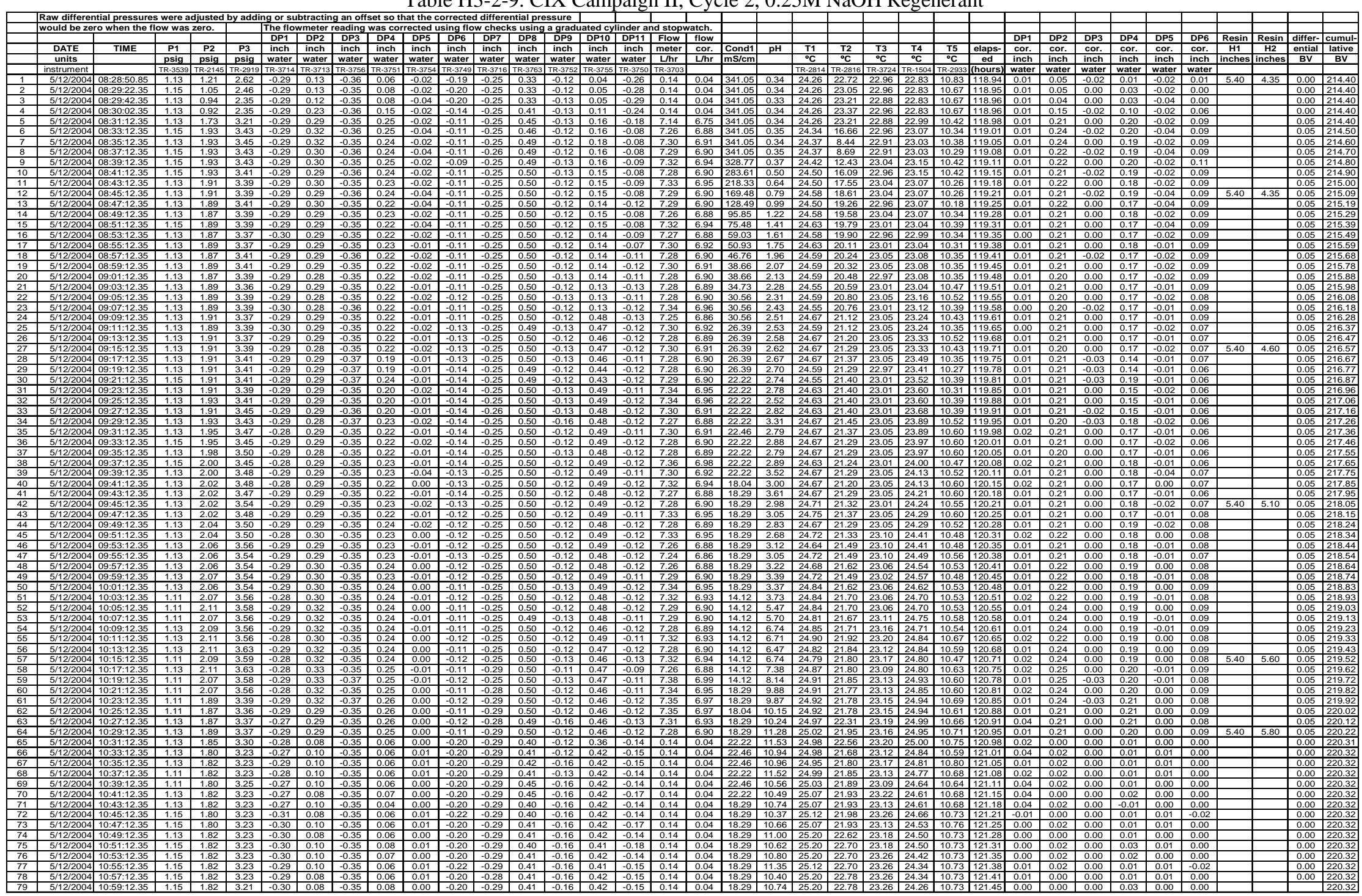


WSRC-TR-2005-00105, REVISION 0

SRNL-RPP-2005-00012, REVISION 0

Table H3-3-1. CIX Campaign II, Cycle 3, LAW Treatment

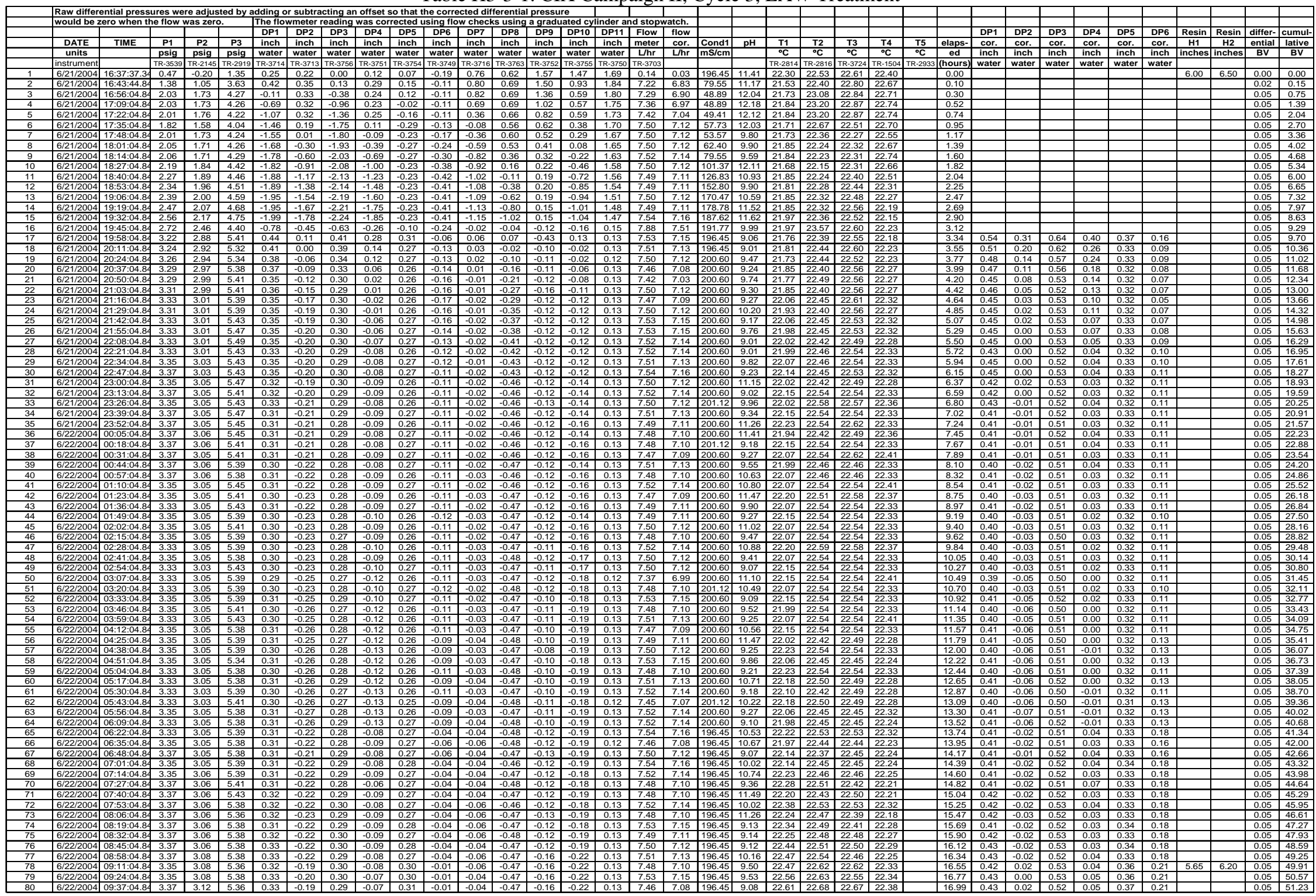


WSRC-TR-2005-00105, REVISION 0

SRNL-RPP-2005-00012, REVISION 0

Table H3-3-2. CIX Campaign II, Cycle 3, LAW Treatment

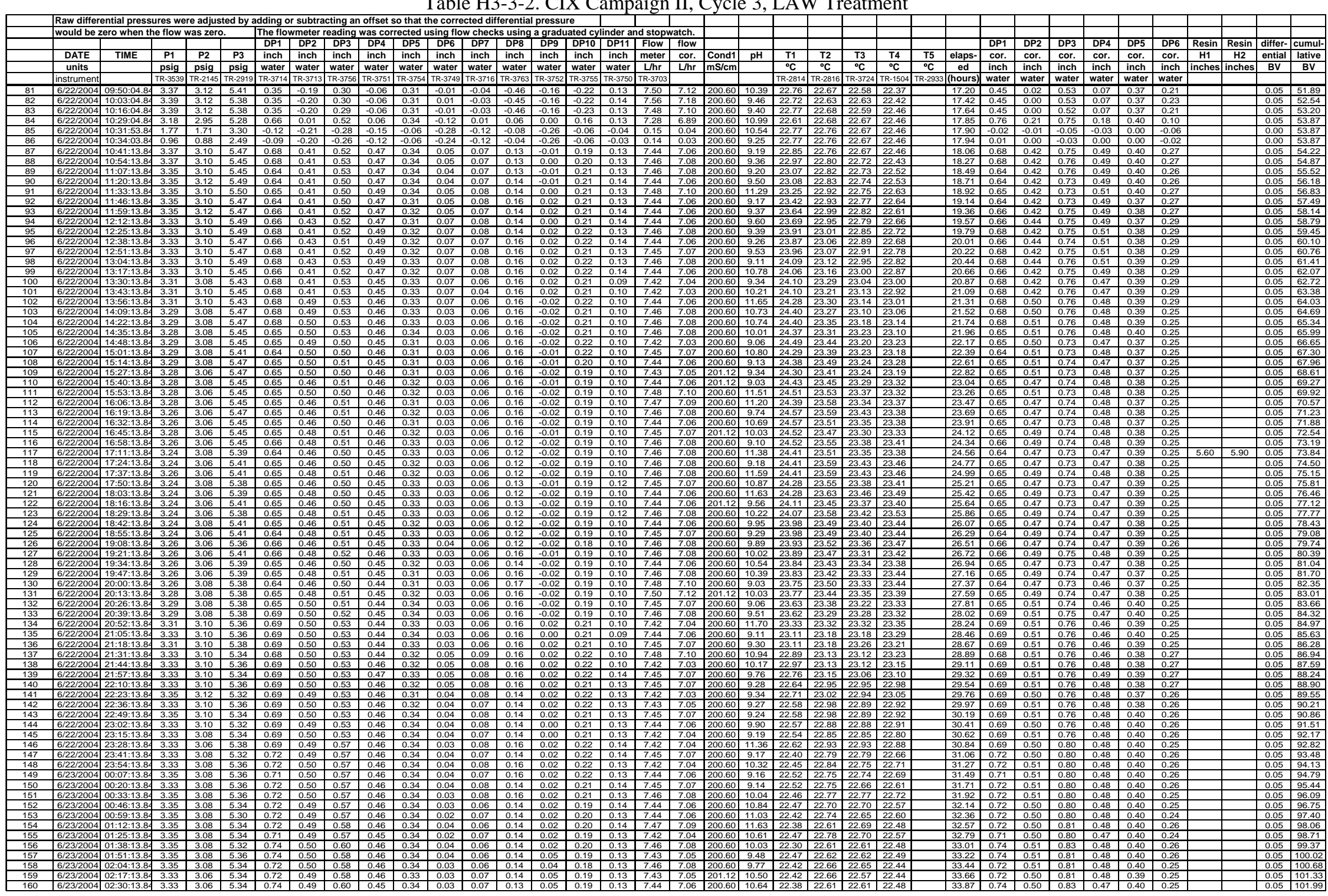


WSRC-TR-2005-00105, REVISION 0

SRNL-RPP-2005-00012, REVISION 0

Table H3-3-3. CIX Campaign II, Cycle 3, LAW Treatment

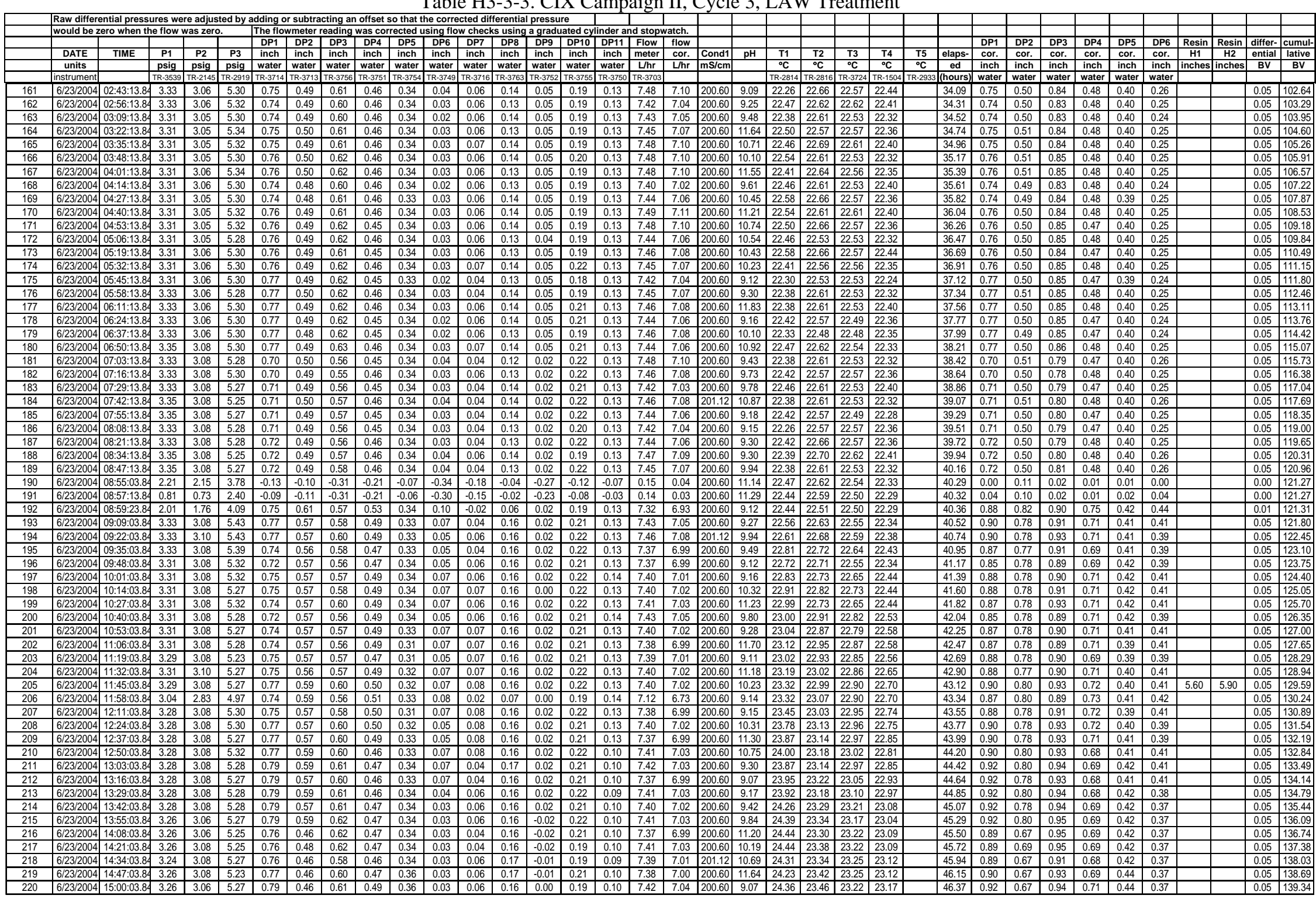


WSRC-TR-2005-00105, REVISION 0

SRNL-RPP-2005-00012, REVISION 0

Table H3-3-4. CIX Campaign II, Cycle 3, LAW Treatment

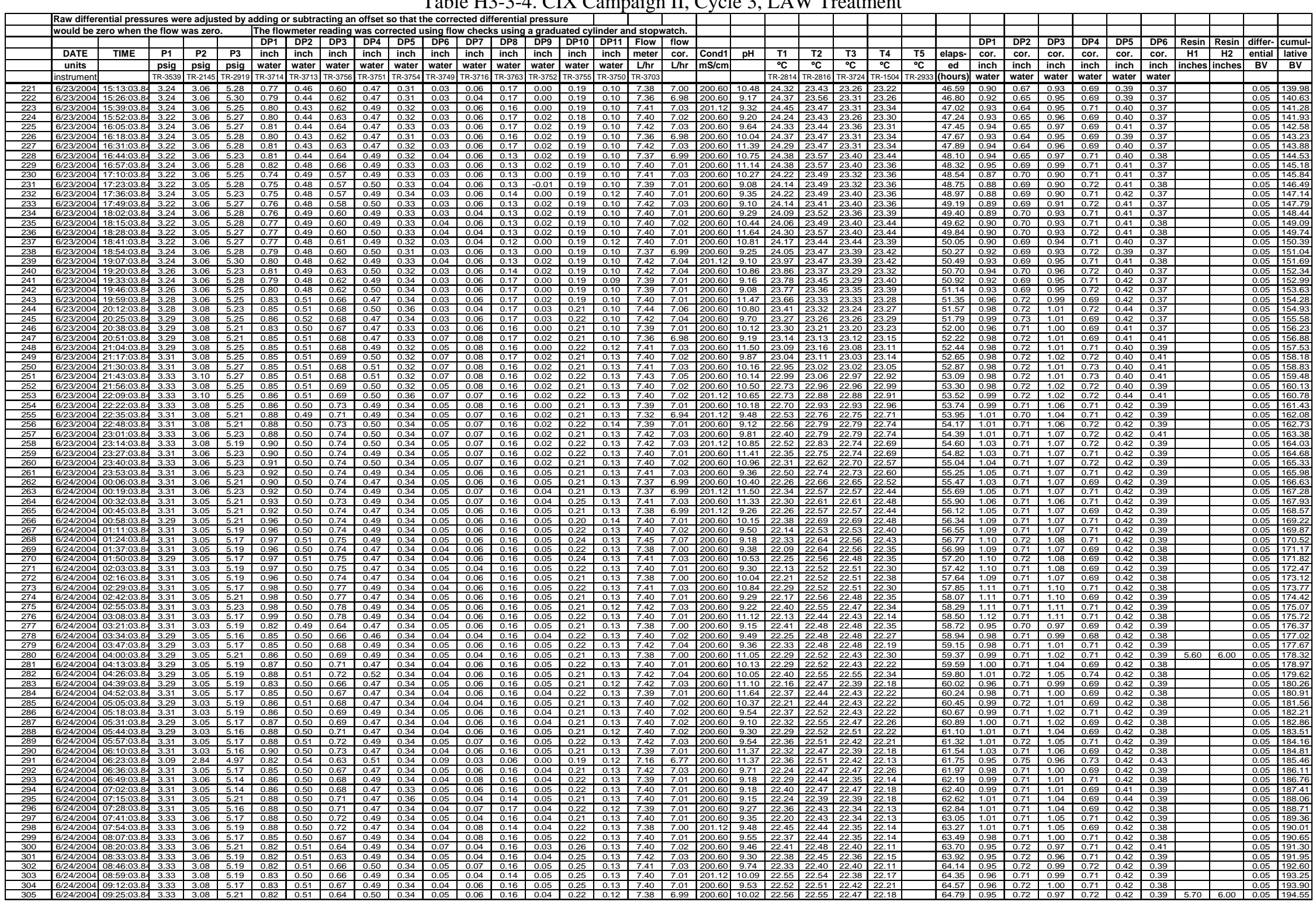


WSRC-TR-2005-00105, REVISION 0

SRNL-RPP-2005-00012, REVISION 0

Table H3-3-5. CIX Campaign II, Cycle 3, 0.1 M NaOH Displacement

\begin{tabular}{|c|c|c|c|c|c|c|c|c|c|c|c|c|c|c|c|c|c|c|c|c|c|c|c|c|c|c|c|c|c|c|c|c|c|c|c|}
\hline & $\begin{array}{l}\text { Raw diffe } \\
\text { would be }\end{array}$ & ro when the ff & & & . & The flo & & ang ant & 15 & & & & & & & & & & & & & & & & & & & & & & & & & & \\
\hline & DATE & TIME & $P 1$ & P2 & $P 3$ & \begin{tabular}{|l|} 
DP1 \\
inch
\end{tabular} & & $\begin{array}{l}\text { DP3 } \\
\text { inch }\end{array}$ & \begin{tabular}{|l|l|} 
PP4 \\
inch
\end{tabular} & inch & $\begin{array}{l}\text { DP6 } \\
\text { inch }\end{array}$ & $\begin{array}{l}\text { DP7 } \\
\text { inch }\end{array}$ & $\begin{array}{l}\text { DP8 } \\
\text { inch }\end{array}$ & $\begin{array}{l}\text { DP9 } \\
\text { inch }\end{array}$ & $\begin{array}{l}\text { DP10 } \\
\text { Dinch } \\
\text { int }\end{array}$ & & 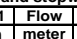 & $\begin{array}{l}\text { flow } \\
\text { cor. }\end{array}$ & Cond1 & & & & & & & & $\mathrm{DP1}$ & DP2 & $\begin{array}{ll}\mathrm{DP3} 3 \\
\text { cor. }\end{array}$ & $\frac{\text { DP4 }}{\text { cor. }}$ & & DP6 & $\frac{\text { Resin }}{\mathrm{H}}$ & & \\
\hline & $\begin{array}{l}\text { Lnits } \\
\text { units }\end{array}$ & & psig & \begin{tabular}{|l}
$\mathrm{psig}$ \\
$\mathrm{psig}$
\end{tabular} & sig & \begin{tabular}{|l|} 
water \\
\end{tabular} & 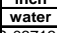 & & \begin{tabular}{|l|} 
incher \\
water
\end{tabular} & & & \begin{tabular}{|l|} 
inche \\
water
\end{tabular} & \begin{tabular}{|l|} 
incher \\
water
\end{tabular} & vater & $\begin{array}{l}\text { inch } \\
\text { water } \\
\text { water }\end{array}$ & & 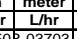 & \begin{tabular}{|l|} 
cor. \\
Lhr \\
\end{tabular} & $\mathrm{mSI} / \mathrm{cm}$ & & $T 1$ & 12 & & & & $\frac{\text { elaps }}{\text { ed }}$ & inch & inch & $\begin{array}{l}\text { cor. } \\
\text { inch }\end{array}$ & $\begin{array}{l}\text { core } \\
\text { inch }\end{array}$ & inch & $\begin{array}{l}\text { Cor. } \\
\text { inch } \\
\end{array}$ & & & $\frac{\text { ential }}{\mathrm{BV}}$ \\
\hline & $\frac{\text { instrum }}{6 / 24 / 22}$ & 7:48.84 & $\begin{array}{l}-0.053939 \\
-0.25 \\
\end{array}$ & $\frac{p-02145}{-0.17}$ & & $\begin{array}{l}\frac{9-03714}{2.51} \\
\end{array}$ & $\frac{0.0713}{0.00}$ & $\begin{array}{l}3 \frac{3-03756}{2.29} \\
\end{array}$ & 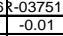 & $\frac{k-0354}{0.34}$ & 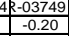 & 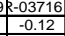 & $0=0.0363$ & $\begin{array}{l}\frac{3-03752}{1.85} \\
1\end{array}$ & $\begin{array}{l}\frac{2-03755}{-0.02} \\
2\end{array}$ & $\frac{5-03550}{2-2.88}$ & $\begin{array}{l}3.1 \\
0.12\end{array}$ & & & & & & & & & & & & & & & & & & \\
\hline & $\frac{6 / 24 / 200}{6 / 24 / 200}$ & \begin{tabular}{|c|}
$10: 51: 48.84$ \\
10.55484
\end{tabular} & \begin{tabular}{l|l|}
2.39 \\
252 \\
\end{tabular} & \begin{tabular}{|l|l|}
-0.15 \\
-0.13
\end{tabular} & \begin{tabular}{|l|}
4.02 \\
15
\end{tabular} & \begin{tabular}{|l|}
2.91 \\
297
\end{tabular} & \begin{tabular}{|l|l|}
0.00 \\
0.00 \\
\end{tabular} & \begin{tabular}{|l|l|}
2.73 \\
281
\end{tabular} & \begin{tabular}{|l|l|}
-0.01 \\
-0.01
\end{tabular} & \begin{tabular}{|l|l|}
0.75 \\
0.85 \\
\end{tabular} & 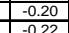 & \begin{tabular}{|l|l|}
0.18 \\
0.41 \\
\end{tabular} & \begin{tabular}{|l|l|}
-0.02 \\
0.02
\end{tabular} & \begin{tabular}{|l|}
2.10 \\
12 \\
\end{tabular} & \begin{tabular}{|l|l|}
-0.02 \\
-0.02
\end{tabular} & \begin{tabular}{|l|l|}
2 & -2.87 \\
2 & -287
\end{tabular} & $\frac{1.22}{734}$ & 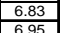 & $\left\{\begin{array}{l}200.60 \\
20060\end{array}\right.$ & $\mid$ & 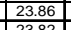 & \begin{tabular}{|l|l|}
2.230 \\
\end{tabular} & $\frac{22.64}{22.69}$ & $\frac{22.43}{22.48}$ & & $\begin{array}{l}66.23 \\
66.30 \\
66.30\end{array}$ & & & & & & & & & \begin{tabular}{c|c|}
0.05 & 197.69 \\
0.05 & 197.89 \\
\end{tabular} \\
\hline & & \begin{tabular}{|l|}
10.353 .468 .04 \\
$10: 49.84$ \\
\end{tabular} & 2.52 & \begin{tabular}{|l|l|}
-1.13 \\
-0.13 \\
\end{tabular} & & 2.98 & \begin{tabular}{|l|l|}
0.00 \\
\end{tabular} & \begin{tabular}{|l|}
2.81 \\
2.81 \\
\end{tabular} & \begin{tabular}{|l|l|}
-0.011 \\
0.01
\end{tabular} & \begin{tabular}{|l|l|}
0.85 \\
\end{tabular} & \begin{tabular}{|c|c|c|c|c|}
-0.24 \\
-0.19
\end{tabular} & \begin{tabular}{|l|}
0.41 \\
0.71 \\
\end{tabular} & \begin{tabular}{|l|l|}
$=.062$ \\
-0.02 \\
\end{tabular} & \begin{tabular}{|l|l|}
2.12 \\
\end{tabular} & $\begin{array}{l}\mid-0.02 \\
-0.02 \\
\end{array}$ & \begin{tabular}{l|l|} 
& -2.87 \\
2 & -2.87 \\
\end{tabular} & $\frac{1.34}{7.32}$ & 6.95 & & $\begin{array}{l}9.35 \\
9.48 \\
\end{array}$ & $\begin{array}{lll}20.7 .74 \\
23.74\end{array}$ & & $\frac{2.09}{22.69}$ & $\frac{22.46}{22.48}$ & & $\frac{6.30}{66.37}$ & & & & & & & & & 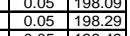 \\
\hline & & & $\frac{2.52}{2.54}$ & & & & & & & & & & \begin{tabular}{|l|l|}
-0.02 \\
-0.02
\end{tabular} & & \begin{tabular}{|l|l|}
-0.01 \\
-0.02
\end{tabular} & & $\begin{array}{l}7.42 \\
7702 \\
\end{array}$ & \begin{tabular}{|l|}
7.03 \\
6.62 \\
\end{tabular} & & & & & & & & & & & & & & & & & $\begin{array}{l}\frac{198.49}{198.49} \\
10868\end{array}$ \\
\hline & $6 \frac{6 / 24 / 2}{6 / 24 / 2}$ & |lint:11:48.84 & $\begin{array}{l}2.54 \\
2.45 \\
\end{array}$ & \begin{tabular}{|l|l|l|l|l|}
-0.09 \\
-0.09
\end{tabular} & \begin{tabular}{|l|}
4.09 \\
4.09 \\
\end{tabular} & $\begin{array}{l}3.00 \\
2.98 \\
\end{array}$ & \begin{tabular}{|l|}
0.00 \\
0.00 \\
\end{tabular} & \begin{tabular}{|l|l|}
2.83 \\
2.83 \\
\end{tabular} & & \begin{tabular}{|l|l|}
0.82 \\
0.82 \\
\end{tabular} & 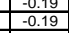 & \begin{tabular}{|l|l|}
1.28 \\
1.46
\end{tabular} & \begin{tabular}{|l|l|}
-0.022 \\
-0.02 \\
\end{tabular} & \begin{tabular}{|l|l|}
2.12 \\
\end{tabular} & \begin{tabular}{|l|l|}
-0.02 \\
-0.01 \\
\end{tabular} & & $\begin{array}{l}7.02 \\
7.32 \\
\end{array}$ & \begin{tabular}{|l|l|}
6.92 \\
6.94
\end{tabular} & \begin{tabular}{|l|l|}
2000.60 \\
222.43 \\
\end{tabular} & $\frac{1.14}{102}$ & 39 & & 229 & $\frac{2.4}{225}$ & & (6.5.5. & & & & & & & & & 198.68 \\
\hline & & & & & & & & & & & & $\begin{array}{l}1.52 \\
153\end{array}$ & $\begin{array}{l}-0.02 \\
-0.02\end{array}$ & & \begin{tabular}{|l|l|}
-0.01 \\
\end{tabular} & & & & & & & & & & & & & & & & & & & & 199.08 \\
\hline & $6 / 2 / 24 / 200$ & \begin{tabular}{|l|l|}
$111: 1: 988.84$ \\
$11: 23: 48.84$ \\
\end{tabular} & $\begin{array}{l}\frac{2.06}{1.82} \\
\end{array}$ & $\begin{array}{l}-0.07 \\
-0.07 \\
-\end{array}$ & \begin{tabular}{|l}
3.49 \\
3.41 \\
\end{tabular} & $\begin{array}{r}3.04 \\
-2.39 \\
\end{array}$ & & \begin{tabular}{|l|l|} 
& 2.87 \\
-1.15 \\
\end{tabular} & $\begin{array}{l}0.02 \\
0.02 \\
\end{array}$ & \begin{tabular}{|l|l|}
0.81 \\
\end{tabular} & \begin{tabular}{|l|}
-0.18 \\
-0.18 \\
\end{tabular} & \begin{tabular}{|l|l|}
1.53 \\
1.29 \\
\end{tabular} & \begin{tabular}{|l|l|}
-0.02 \\
-0.02 \\
\end{tabular} & \begin{tabular}{|l|}
2.12 \\
2.02 \\
\end{tabular} & \begin{tabular}{|l|l|}
-0.01 \\
0.00 \\
\end{tabular} & 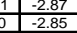 & $\begin{array}{l}5 \\
5.32 \\
7.06 \\
\end{array}$ & \begin{tabular}{|l|}
6.94 \\
6.66 \\
\end{tabular} & \begin{tabular}{|l|l|l|l|l|l|l}
18.61 \\
96.69
\end{tabular} & $\begin{array}{l}9.83 \\
12.07 \\
\end{array}$ & \begin{tabular}{|l|}
24.01 \\
23.61 \\
\end{tabular} & \begin{tabular}{|l|}
23.11 \\
23.11 \\
\end{tabular} & $2 \frac{21.34}{21.42}$ & $\frac{2.56}{22.5}$ & & $\begin{array}{l}66.70 \\
66.77 \\
-10\end{array}$ & & & & & & & & & $\begin{array}{ll}0.05 & 199.28 \\
0.05 & 199.48 \\
\end{array}$ \\
\hline & & $\begin{array}{l}\mid 11: 27: 48.84 \\
1133.3684\end{array}$ & & \begin{tabular}{|c|c|}
-0.06 \\
-04
\end{tabular} & & & & & & & & & \begin{tabular}{|l|l|}
-0.02 \\
0.00
\end{tabular} & & \begin{tabular}{|l|l|}
0.00 \\
0.00
\end{tabular} & & $\begin{array}{l}7.50 \\
0.14 \\
\end{array}$ & & & & & & & & & 66.83 & & & & & & & & & $\begin{array}{ll}0.05 & 199.68 \\
0.007 & 10.72 \\
\end{array}$ \\
\hline & $6 / 2 / 24 / 4$ & 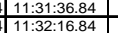 & $\begin{array}{l}-1.15 \\
0.91\end{array}$ & $\begin{array}{l}-0.04 \\
-0.04 \\
-0.04\end{array}$ & \begin{tabular}{|l|}
0.34 \\
2.55 \\
\end{tabular} & \begin{tabular}{|l|l|}
0.11 \\
0.42 \\
\end{tabular} & \begin{tabular}{|l|}
0.01 \\
0.02 \\
\end{tabular} & \begin{tabular}{|l|l|}
-0.14 \\
0.19
\end{tabular} & 0.022 & \begin{tabular}{|l|}
0.04 \\
0.17 \\
\end{tabular} & \begin{tabular}{|c|}
-0.18 \\
-0.17
\end{tabular} \mid & \begin{tabular}{|l|}
-0.25 \\
-0.21 \\
\end{tabular} & \begin{tabular}{|l|}
0.00 \\
-0.02 \\
\end{tabular} & + & \begin{tabular}{|l|}
0.00 \\
0.02 \\
\end{tabular} & $\begin{array}{l}-2.94 \\
-2.284 \\
-x^{2}\end{array}$ & \begin{tabular}{l|l|} 
& 0.14 \\
& 4.31 \\
\end{tabular} & \begin{tabular}{|l|}
0.03 \\
3.92 \\
\end{tabular} & $\begin{array}{l}101.17 \\
101.37 \\
\end{array}$ & $12.37 \mathrm{C}$ & 23.897 & & 21.79 & & & $\frac{66.90}{66.91}$ & & & & 0.00 & & $\begin{array}{l}-0.01 \\
0.00\end{array}$ & & & $\begin{array}{ll}0.00 & 199.73 \\
0.00 & 199.74 \\
\end{array}$ \\
\hline & & & & & & & & & & & & & & & & & & & & & & & & & & & & & & & & & & & \\
\hline & & 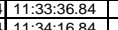 & 1.79 & $\frac{-0.02}{0.00}$ & & & & & 0.0 .0 & & & & -0.02 & & & & & & & & & & & & & & & & & & & & & & 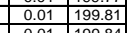 \\
\hline & 6/241/1 & 11:34:56.84 & 1.93 & \begin{tabular}{|l|l|}
0.00 \\
\end{tabular} & \begin{tabular}{|l|}
3.52 \\
3.52 \\
\end{tabular} & \begin{tabular}{|l|l|}
0.38 \\
0.38 \\
\end{tabular} & & \begin{tabular}{|l|l|}
0.16 \\
0.16 \\
\end{tabular} & & \begin{tabular}{|l|l|}
0.15 \\
\end{tabular} & \begin{tabular}{|l|l|l|l|l|l|}
-0.17 \\
-0.17
\end{tabular} & & $\begin{array}{l}-0.02 \\
-0.02 \\
\end{array}$ & & \begin{tabular}{|l|}
0.02 \\
0.02 \\
\end{tabular} & \begin{tabular}{|l|} 
\\
\\
$2-2.85$ \\
\end{tabular} & $\frac{7.12}{7.22}$ & \begin{tabular}{|l|l|}
0.82 \\
\end{tabular} & 74.87 & \begin{tabular}{|l|l|}
12.09 \\
12.09
\end{tabular} & $\frac{23.97}{24.05}$ & 23.16 & $\begin{array}{l}21.79 \\
12.79\end{array}$ & & & $\frac{66.94}{66.95}$ & & & & & & 0.001 & & & $\frac{199.84}{199.87}$ \\
\hline & & 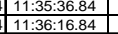 & $\frac{1.92}{1.92}$ & \begin{tabular}{|l|}
0.00 \\
0.00 \\
\end{tabular} & \begin{tabular}{|l|l|}
3.52 \\
.52 \\
\end{tabular} & $\begin{array}{l}0.37 \\
0.37\end{array}$ & \begin{tabular}{|l|}
0.02 \\
0.02 \\
\end{tabular} & \begin{tabular}{|l|}
0.14 \\
0.16 \\
\end{tabular} & 0.022 & \begin{tabular}{|l|l|}
0.15 \\
0.15 \\
\end{tabular} & \begin{tabular}{|c|}
-0.18 \\
-0.17 \\
\end{tabular} & \begin{tabular}{|l|}
-0.25 \\
-0.25 \\
\end{tabular} & \begin{tabular}{|l|l|}
-0.02 \\
-0.02 \\
\end{tabular} & \begin{tabular}{|l|l|}
0.11 \\
0.11 \\
\end{tabular} & \begin{tabular}{|l|}
0.02 \\
0.00
\end{tabular} & \begin{tabular}{|l|l|} 
& -2.25 \\
& -2.87 \\
\end{tabular} & 7.24 & \begin{tabular}{|l|l|l}
6.85 \\
6.99 \\
\end{tabular} & $\begin{array}{l}70.72 \\
70.72\end{array}$ & \begin{tabular}{|l|}
12.03 \\
11.98 \\
\end{tabular} & \begin{tabular}{|l|}
24.21 \\
24.37 \\
\end{tabular} & \begin{tabular}{|l|}
23.24 \\
23.16
\end{tabular} & $\frac{2.1 .}{21.7}$ & 22.6 & & \begin{tabular}{|l|l|l|l|}
66.96 \\
66.97 \\
\end{tabular} & & 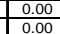 & & $\frac{0.00}{0.00}$ & & $\begin{array}{l}-0.01 \\
0.00\end{array}$ & & & $\begin{array}{l}\frac{199.90}{1994} \\
\end{array}$ \\
\hline & & 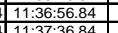 & 1.90 & \begin{tabular}{|l|l|}
0.00 \\
\end{tabular} & & \begin{tabular}{|l|}
0.37 \\
038
\end{tabular} & \begin{tabular}{|l|l|}
0.02 \\
\end{tabular} & \begin{tabular}{|l|l|}
0.16 \\
0.16
\end{tabular} & & \begin{tabular}{|l|l|} 
\\
\end{tabular} & \begin{tabular}{|l|} 
\\
\end{tabular} & \begin{tabular}{|l|} 
\\
\end{tabular} & \begin{tabular}{|l|} 
\\
\end{tabular} & \begin{tabular}{|l|}
0.11 \\
0.11
\end{tabular} & \begin{tabular}{|l|l|}
0.02 \\
0
\end{tabular} & \begin{tabular}{|l|l|} 
& -2.87 \\
& -2.87 \\
\end{tabular} & 7.25 & \begin{tabular}{|l|l|}
6.86 \\
6.97
\end{tabular} & \begin{tabular}{|l|l|}
66.56 \\
6656
\end{tabular} & $\begin{array}{ll}11.18 \\
1260\end{array}$ & \begin{tabular}{|l|}
24.62 \\
2478 \\
\end{tabular} & \begin{tabular}{|l|l|}
23.24 \\
2316
\end{tabular} & 21.87 & 22 & & \begin{tabular}{|l|l|}
66.99 \\
6700
\end{tabular} & 0.23 & 0 & & 0.00 & & 0.00 & & & \begin{tabular}{|l|}
199.99 \\
199.97 \\
\end{tabular} \\
\hline & & & & & & & & & & $\frac{0.14}{0.14}$ & & & 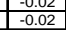 & & & & & & & & & & & & & 67.0 & & & & & & & & & $\begin{array}{l}2200.00 \\
200.04 \\
\end{array}$ \\
\hline & & $\begin{array}{ll}11: 38: 56.84 \\
113238\end{array}$ & 1.88 & \begin{tabular}{|l|l|}
0.02 \\
\end{tabular} & \begin{tabular}{|l|}
.47 \\
\end{tabular} & \begin{tabular}{|l|}
0.38 \\
\end{tabular} & \begin{tabular}{|l|l|}
0.01 \\
\end{tabular} & \begin{tabular}{|l|l|}
0.16 \\
\end{tabular} & & \begin{tabular}{|l|l|}
0.15 \\
0.14
\end{tabular} & \begin{tabular}{|l|l|} 
& -0.17 \\
0.017
\end{tabular} & \begin{tabular}{|l|l|}
-0.25 \\
\end{tabular} & $\frac{-0.02}{-0.02}$ & \begin{tabular}{|l|l|}
0.11 \\
0.14
\end{tabular} & \begin{tabular}{|l|l|} 
& 0.02 \\
\end{tabular} & \begin{tabular}{|l|r|} 
& -2.85 \\
\end{tabular} & \begin{tabular}{|l|l|}
7.44 \\
746
\end{tabular} & \begin{tabular}{|l|l|}
7.06 \\
778
\end{tabular} & \begin{tabular}{|l}
66.56 \\
6656 \\
\end{tabular} & $\frac{0.23}{0.23}$ & $\begin{array}{ll}25.02 \\
2518\end{array}$ & 3.1.16 & $\frac{2.17}{219}$ & & & 67.02 & \begin{tabular}{|l|l|}
0.24 \\
\end{tabular} & $\frac{-0.0}{0.0}$ & . & 0.00 & 0.10 & 0.00 & & & \begin{tabular}{c|ccc}
0.01 & 200.07 \\
\end{tabular} \\
\hline & 6/24/200 & 11:40:16.84 & 1..06 & \begin{tabular}{|l|l|}
0.02 \\
0.02
\end{tabular} & \begin{tabular}{|l|}
3.468 \\
3.48
\end{tabular} & 0.37 & \begin{tabular}{|l|l|}
0.02 \\
02
\end{tabular} & \begin{tabular}{|l|l|}
0.16 \\
0.16
\end{tabular} & 0.02 & \begin{tabular}{|l|l|}
0.14 \\
0.14
\end{tabular} & $\begin{array}{l}-0.11 \\
-0.18 \\
\end{array}$ & $\begin{array}{l}-0.25 \\
-0.25 \\
\end{array}$ & $\frac{-0.02}{-0.02}$ & \begin{tabular}{|l|}
0.14 \\
0.14
\end{tabular} & \begin{tabular}{|l|l|}
0.02 \\
0.02
\end{tabular} & \begin{tabular}{|l|l|} 
& -2.87 \\
\end{tabular} & $\begin{array}{l}1.40 \\
7.32\end{array}$ & \begin{tabular}{|l|}
6.04 \\
\end{tabular} & \begin{tabular}{|l}
60.30 \\
62.40
\end{tabular} & $\frac{1.45}{12.36}$ & 25.10 & 23.24 & 11.01 & .0 & & 67.04 & & & & 0.00 & & $\frac{0.01}{-0.01}$ & & & 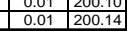 \\
\hline & & & & & & & & 0.16 & & & & & & & & & 7.30 & 6.91 & & & & & & & & 67.05 & & .00 & & 0.00 & & -0.01 & & & $\begin{array}{lll}0.01 & 200.17 \\
0.17\end{array}$ \\
\hline & & 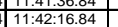 & & $\frac{0.02}{0.02}$ & & $\frac{2.37}{0.37}$ & \begin{tabular}{|l|l|}
0.02 \\
0.02
\end{tabular} & $\frac{0.14}{0.16}$ & $\frac{\frac{0.02}{0.02}}{4}$ & 0.15 & & $\frac{-0.25}{-0.25}$ & $\frac{-0.02}{-0.02}$ & & & & $\frac{1.18}{7.22}$ & & & & & & & & & $\frac{67.06}{6707}$ & & & & 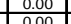 & & 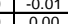 & & & 0.01 \\
\hline & & & 1.8 & & & & & & & & $\begin{array}{l}-0.17 \\
0.17 \\
\end{array}$ & -0.25 & -0.02 & & 0.02 & & 7.1 & & 61.88 & & & & 2.0 & 2.5 & & $\begin{array}{l}67.07 \\
67.09 \\
\end{array}$ & 24 & 0.00 & .24 & 0.00 & .10 & 0.00 & & & $\begin{array}{ll}0.01 \\
0.01 & 20.24 \\
0.01\end{array}$ \\
\hline & 2412 & 1:44:34:16.8. & $\frac{1.88}{1.88}$ & $\begin{array}{l}0.04 \\
0.04\end{array}$ & \begin{tabular}{|l|}
3.47 \\
3.47
\end{tabular} & \begin{tabular}{|l|}
0.38 \\
0.38 \\
\end{tabular} & & & $\frac{0.02}{0.02}$ & 0.15 & \begin{tabular}{|l|l|}
-0.17 \\
\end{tabular} & \begin{tabular}{|l|l|}
-0.25 \\
-0.25
\end{tabular} & $\frac{-0.02}{-0.02}$ & & \begin{tabular}{|l|}
0.02 \\
0.02 \\
\end{tabular} & & \begin{tabular}{|l|l}
77.43 \\
\end{tabular} & \begin{tabular}{|l|}
7.05 \\
\end{tabular} & 62.40 & 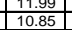 & 25.06 & & & & & $\frac{67.10}{67.11}$ & & & & $\frac{0.00}{0.00}$ & & & & & $\begin{array}{ll}0.01 & 20.30 .30 \\
0.01 & 200.33\end{array}$ \\
\hline & & $\begin{array}{l}556.84 \\
36.84\end{array}$ & 1.86 & 0.04 & $\frac{47}{47}$ & 0.38 & .04 & & $\frac{0.02}{0.02}$ & & & $\begin{array}{l}-0.25 \\
-0.25 \\
-0.0\end{array}$ & $\begin{array}{l}-0.02 \\
-0.02\end{array}$ & & $\frac{0.02}{0.02}$ & & & & & $\frac{32}{33}$ & & & & & & $\frac{67.12}{67.13}$ & & $\frac{0.02}{0.00}$ & 0.25 & 0.00 & 0.10 & 0.00 & & & $\begin{array}{lll}0.01 & 20.0 .37 \\
0.01 & 27\end{array}$ \\
\hline & 6/24//2 & $11: 46: 16.84$ & 1.86 & 0.04 & 3.45 & 0.38 & \begin{tabular}{|l|l|}
0.02 \\
\end{tabular} & \begin{tabular}{|l|l|}
0.17 \\
\end{tabular} & 0.02 & \begin{tabular}{l|l|l|l|}
0.14 \\
\end{tabular} & -0.1 & -0.25 & -0.02 & 0.16 & \begin{tabular}{|l|l|}
0.02 \\
\end{tabular} & \begin{tabular}{|l|l|} 
& -2.85 \\
\end{tabular} & 7.28 & 6.89 & 58.25 & 10.02 & 25.06 & \begin{tabular}{|l|}
23.21 \\
\end{tabular} & 122.24 & 22.67 & & 67.14 & & 0.00 & & 0.00 & 0.09 & 0.00 & & & $\begin{array}{ll}0.01 & 20.4 \\
0.01 & 200.4\end{array}$ \\
\hline & & 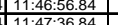 & 1.86 & 0.06 & $\begin{array}{l}3.45 \\
3.45 \\
\end{array}$ & 0.038 & & & $\begin{array}{l}0.02 \\
0.02\end{array}$ & $\begin{array}{l}0.15 \\
0.14\end{array}$ & & - -0.25 & $\frac{-0.02}{-0.02}$ & & $\begin{array}{l}0.02 \\
0.02\end{array}$ & $\begin{array}{l}-2.85 \\
-2.85 \\
\end{array}$ & \begin{tabular}{|c|c|}
$\mid 7.26$ \\
7
\end{tabular} & . & & & & & & & & $\begin{array}{l}67.15 \\
67.16\end{array}$ & 24 & & & 0.000 & 0.10 & & & & 0.01 \\
\hline & & & & 0.04 & \begin{tabular}{|l|}
3.45 \\
\end{tabular} & & & & & & & & & & & & & & & & & & & & & & & & & & & & & & \\
\hline & & 48.50. & & 0.06 & & & & & 0.0 & & & -0.2 & -0.02 & & & & & & & & & & & & & 67.1 & & & & & & 0.00 & & & \\
\hline & & 1684 & 18 & & & & & 0.17 & & & & & & & & & & & & 240 & & & & & & & & & & & & 0.00 & & & $\begin{array}{ccc}0.01 & 200.60 \\
\end{array}$ \\
\hline & & (1) & & 0.04 & & 0.38 & & & 0.02 & & 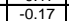 & $\frac{-0.25}{-0.25}$ & $\frac{-0.02}{-0.02}$ & & O.022 & & & & & & & & & & & 6722 & & & & & & & & & 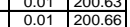 \\
\hline & & $\frac{51: 36.84}{4}$ & 1.86 & 0.04 & & 0.38 & 0.01 & 0.16 & 0.02 & & & $\frac{-0.2}{-0.2}$ & -0.02 & & 0.02 & -2.87 & 7.48 & 718 & 53.57 & $\frac{12.16}{12.16}$ & 24.87 | & \begin{tabular}{|l|}
23.17 \\
\end{tabular} & 224 & 256 & & 67.23 & & -0.01 & & 0.00 & & 0.00 & & & $\begin{array}{ll}0.01 & 20.00 \\
0.01 & 200.70\end{array}$ \\
\hline & & & & 0.06 & & & & & . & & & $\begin{array}{l}-0.25 \\
-0.22\end{array}$ & $\begin{array}{l}-0.02 \\
-002\end{array}$ & & $\frac{0.02}{0.02}$ & & & 7 & & & & & & & & $\frac{67.2}{67^{2}}$ & & & & & & 0.00 & & & \\
\hline & & & & $\frac{0.06}{0.06}$ & & & & & & & & & $\begin{array}{l}-0.02 \\
-0.02\end{array}$ & & 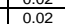 & & & & & & & & & & & $\frac{6.72}{67.26}$ & & & & & & & & & \\
\hline & 6/2/24/4 & 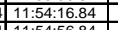 & 1.86 & \begin{tabular}{|l|l|}
0.06 \\
0.06
\end{tabular} & \begin{tabular}{|l|l|}
3.43 \\
3.47
\end{tabular} & $\begin{array}{l}0.38 \\
0.38\end{array}$ & \begin{tabular}{|l|l|}
0.02 \\
0.02
\end{tabular} & \begin{tabular}{|l|l|}
0.17 \\
0.17
\end{tabular} & $\frac{0.02}{0.02}$ & 0.15 & \begin{tabular}{|l|}
-0.17 \\
0.17
\end{tabular} & \begin{tabular}{|l|l|}
-0.25 \\
\end{tabular} & \begin{tabular}{|l|l|}
-0.02 \\
0.02
\end{tabular} & \begin{tabular}{|l|l}
0.19 \\
\end{tabular} & \begin{tabular}{|l|l|}
0.02 \\
002
\end{tabular} & & $\frac{7.30}{7.732}$ & \begin{tabular}{|l|l|}
6.9 \\
6.9
\end{tabular} & \begin{tabular}{|l}
53.5 \\
53.5
\end{tabular} & $\frac{12.09}{12.09}$ & $\frac{24.8}{24.9}$ & & $\mid$ & & & \begin{tabular}{|l|l|}
67.27 \\
6.20 \\
\end{tabular} & & & & 0.00 & & 0.00 & & & $\begin{array}{ll}0.01200 .8 \\
0.01\end{array}$ \\
\hline & & 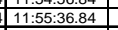 & 1.04 & \begin{tabular}{|l|}
0.006 \\
0.06
\end{tabular} & \begin{tabular}{|l|}
3.47 \\
\end{tabular} & \begin{tabular}{|l|}
0.38 \\
0.38 \\
\end{tabular} & & $\begin{array}{l}0.16 \\
0.16\end{array}$ & 0.062 & \begin{tabular}{|l|l|}
0.14 \\
\end{tabular} & \begin{tabular}{|l|l|}
-0.17 \\
-0.17 \\
\end{tabular} & \begin{tabular}{|l|l|l}
-0.25 \\
-0.26 \\
\end{tabular} & & 0.19 & & \begin{tabular}{|l|l|} 
& -2.87 \\
\end{tabular} & $\begin{array}{l}7.38 \\
7.38 \\
\end{array}$ & \begin{tabular}{|l|}
6.93 \\
6.99 \\
\end{tabular} & & 2.65 & $\frac{24.99}{24.91}$ & & & 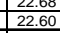 & & $\begin{array}{ll}67.29 \\
67.30\end{array}$ & & & & & & 0.00 & & & \\
\hline & & $1: 56: 16.84$ & 1.84 & & & 0.38 & & & & & & & - 0.02 & & 0.00 & & 7.30 & 6.92 & & & 4.91 & & 2.49 & & & 67.31 & & & & 0.00 & & & & & \\
\hline & 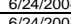 & 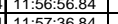 & 1.86 & 0.06 & (3.45 & $\frac{0.38}{0.38}$ & $\frac{0.02}{0.02}$ & 0.16 & 0.02 & 014 & 0.18 & 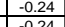 & $\begin{array}{l}-0.02 \\
-002 \\
-0.02\end{array}$ & & 0.02 & 85 & 7 & & & 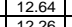 & & & 年 & 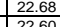 & & $\frac{6.32}{673^{2}}$ & & 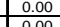 & 3 & 0.00 & 10 & 0.00 & & & 0.01 \\
\hline & & 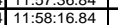 & 淟.84 & $=0.06$ & & 年 & 势. & & 然. & 0.14 & 0 & $\begin{array}{l}-0.23 \\
-0.23\end{array}$ & $=0.022$ & & \begin{tabular}{|l|}
0.02 \\
0.02
\end{tabular} & & & & & & & & & & & $\frac{16.34}{67.34}$ & & & & & & & & & \\
\hline & $\frac{61}{61}$ & $\begin{array}{ll}11: 59: 18.84 \\
100: 00\end{array}$ & $\frac{1.82}{1.92}$ & 0.06 & 2.14 & & & & 0.02 & .044 & & \begin{tabular}{|l|l|}
-0.29 \\
\end{tabular} & & & \begin{tabular}{|l|l|}
0.02 \\
0.02
\end{tabular} & & & & & & 23.76 & & $\frac{22.7}{22.7}$ & 2 & & 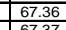 & & & & 0.00 & & 0.00 & & & 0.02 \\
\hline & & 0.06 .84 & & 0.06 & $\frac{0.27}{0.27}$ & 0.15 & 0.02 & . & & 0.04 & \begin{tabular}{|l|l|}
-0.17 \\
-0.17
\end{tabular} & $\begin{array}{l}-0.28 \\
-0.29 \\
\end{array}$ & $\begin{array}{l}-0.02 \\
-0.02 \\
-0.0\end{array}$ & & $\begin{array}{l}0.02 \\
0.02 \\
\end{array}$ & \begin{tabular}{|l|l|}
-2.93 \\
\end{tabular} & 0.14 & 0.03 & & & 24. & & 22.6 & 22.4 & & $\frac{67.3 t}{67.38}$ & & & 0.00 & 0.00 & 0.01 & & & & $\begin{array}{l}0.00 \\
0.00 \\
0.00 \\
201.0\end{array}$ \\
\hline & & : & & 0.07 & & & & & 0.02 & 50 & & -0.24 & -0.02 & & 0.02 & & & & & & & & & & & 67.39 & & & & & & & & & \\
\hline & & $\begin{array}{l}4.55 .87 \\
0.5787\end{array}$ & & $\begin{array}{l}-0.68 \\
-0.68\end{array}$ & & 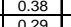 & 2.04 & & 0.01 & 0.42 & $\begin{array}{l}-0.20 \\
0.18\end{array}$ & $\frac{-0.06}{-0.06}$ & $\frac{0.02}{0.02}$ & .17 & $\frac{-0.02}{-0.01}$ & .78 & $\frac{1.14}{15}$ & & & & & & 1.5 & $\frac{21}{21}$ & & 剓.2.28 & & & & & & 0.00 & & & 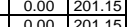 \\
\hline & & $8: 020.50 .87$ & $1.97 \mathrm{Cl}$ & $\begin{array}{l}-.068 \\
-0.68\end{array}$ & 3.04 & & 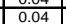 & & $\begin{array}{l}0.01 \\
0.02 \\
\end{array}$ & .07 & - & $\frac{.03}{0.03}$ & $\frac{.02}{0.02}$ & 0.25 & -0.01 & & & & 4.00 & & & & & & & & & & & & & & & & \\
\hline & & & 178 & -0.70 & & & & & & & & & & & & & & & & & & & & & & & & & & & & & & & \\
\hline & & $0: 50.8$ & & $\begin{array}{l}-0.70 \\
-0.70\end{array}$ & & & & & $\frac{0.02}{0.02}$ & & & -0.05 & 0.02 & & -0.02 & & & & & & & & & & & & & & & & & & & & \\
\hline & & : $: 18: 50.87$ & $\frac{1.9}{1.9}$ & $\begin{array}{l}-\frac{-1.10}{-0.70} \\
\end{array}$ & 3.63 & $\frac{0.41}{0.40}$ & $\frac{0.04}{0.04}$ & $\frac{0.17}{0.17}$ & $\frac{0.02}{0.01}$ & & & $\frac{-0.0}{-0.0}$ & $\frac{0.02}{0.02}$ & \begin{tabular}{|l|l|l|}
0.31 \\
\end{tabular} & $\frac{-0.02}{-0.02}$ & & 4.59 & 4.20 & 26. & 9.32 & & & \begin{tabular}{|l|l|}
7.97 \\
\end{tabular} & $\frac{21.8}{21.8}$ & & $\frac{72.62}{72.68}$ & & & & & & & & & \\
\hline & & & & & & & & 0.14 & & \begin{tabular}{|l|l|} 
\\
\end{tabular} & & & & 0.30 & & \begin{tabular}{|l|l|l|} 
& -2.67 \\
\end{tabular} & \begin{tabular}{|l|l|}
4.65 \\
\end{tabular} & \begin{tabular}{|l|l|} 
\\
\end{tabular} & & & & & & 11.09 & & & & & & & & & & & \\
\hline & & :26:50.87 & 1.88 & 0.68 & 3.63 & 0.38 & 0.04 & 0.16 & 0.02 & \begin{tabular}{|l|l|} 
\\
\end{tabular} & -0.17 & 0.01 & 0.02 & 0.28 & -0.01 & & 4.78 & \begin{tabular}{|l|l|l} 
& \\
\end{tabular} & 26.09 & .50 & & & & 21.87 & & 72.82 & & & & & & & & & \\
\hline & & & 1.88 & -0.70 & & $\frac{0.36}{0.36}$ & 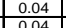 & 0.11 & 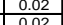 & 0.14 & -0.18 & o. & o. 0.02 & 0.28 & $\frac{-0.01}{-0.01}$ & $\frac{2.67}{2.67}$ & $\begin{array}{l}4.76 \\
4.94\end{array}$ & 告.36 & $\frac{21.94}{21.94}$ & . & & & & & & $\frac{72.8}{770}$ & & & & & & & & & \\
\hline & & & & & & & & & & & & & & & & & & & 1.68 & & & & & & & & & & & & & 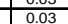 & & & \\
\hline & & $\begin{array}{l}8: 42: 50.87 \\
8: 40.5087\end{array}$ & $\begin{array}{l}1.88 \\
1.88\end{array}$ & \begin{tabular}{|l|l|} 
& -0.68 \\
0.68
\end{tabular} & $\begin{array}{l}3.58 \\
3.58\end{array}$ & $\begin{array}{l}0.35 \\
0355\end{array}$ & $\begin{array}{l}0.04 \\
0.04 \\
\end{array}$ & $\begin{array}{ll}0.06 \\
006\end{array}$ & \begin{tabular}{|l|l|}
0.02 \\
002
\end{tabular} & & \begin{tabular}{|l|}
-0.18 \\
0.020
\end{tabular} & \begin{tabular}{|l|l|}
0.01 \\
0.03 \\
\end{tabular} & \begin{tabular}{|l|l|}
0.02 \\
002
\end{tabular} & 0.28 & \begin{tabular}{|l|l|}
-0.02 \\
-0.01
\end{tabular} & & $\frac{4.97}{4.95}$ & $\frac{4.57}{455}$ & \begin{tabular}{|l|}
21.68 \\
2194 \\
\end{tabular} & \begin{tabular}{|l|}
9.40 \\
9.32 \\
\end{tabular} & \begin{tabular}{|l|l|}
38.58 \\
3780
\end{tabular} & & & $\begin{array}{l}21.91 \\
21.92 \\
\end{array}$ & & $\begin{array}{l}73.08 \\
73.15\end{array}$ & 0.27 & \begin{tabular}{|c|c|c|}
-0.03 \\
\end{tabular} & 0.26 & & & 0.02 & & & \begin{tabular}{c|c}
.03 & 202.2. \\
03 & 202.
\end{tabular} \\
\hline & & & & & & & & & & & & & & & & & & & & & & & & & & & & & & & & & & & 202.49 \\
\hline & & : $: 54: 50.87$ & 1.86 & - & $\begin{array}{l}3.58 \\
\end{array}$ & 0.33 & 0.00 & 0.03 & 0.02 & 10 & -0.2 & $\begin{array}{l}-0.03 \\
-0.03\end{array}$ & $\frac{0.02}{0.02}$ & 0.25 & $\frac{-0.01}{-0.01}$ & 2.67 & \begin{tabular}{|l}
5.02 \\
\end{tabular} & & $\frac{21.94}{21.94}$ & 61 & 36.26 & & 16.50 & $\frac{1.95}{1.95}$ & & 73.28 & 0.25 & -0.06 & .23 & $\frac{04}{04}$ & 0.05 & 0.00 & & & \begin{tabular}{|l|l|l|}
.03 & 202.63
\end{tabular} \\
\hline \begin{tabular}{ll|}
773 & \\
74 &
\end{tabular} & $\frac{6 / 26 / 200}{6 / 28 / 200}$ & 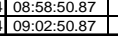 & $\begin{array}{l}1.88 \\
1.88 \\
\end{array}$ & \begin{tabular}{|l|l|}
-0.66 \\
-0.66 \\
\end{tabular} & \begin{tabular}{|l|l|}
3.56 \\
3.58 \\
\end{tabular} & \begin{tabular}{|l|l|}
0.32 \\
0.32 \\
\end{tabular} & \begin{tabular}{|l|l|}
0.00 \\
0.00 \\
\end{tabular} & \begin{tabular}{|l|}
0.03 \\
0.02 \\
\end{tabular} & \begin{tabular}{|l|l|}
0.02 \\
0.02 \\
\end{tabular} & \begin{tabular}{|l|l|}
0.10 \\
0.11 \\
\end{tabular} & $\mid \begin{array}{l}\mid-0.20 \\
\mid-0.20 \\
\mid\end{array}$ & \begin{tabular}{|l|l|}
-0.04 \\
-0.06
\end{tabular} & \begin{tabular}{|l|l|}
-0.02 \\
0.01 \\
\end{tabular} & \begin{tabular}{|l|l|}
0.25 \\
0.25 \\
\end{tabular} & \begin{tabular}{|l|l|}
-0.01 \\
-0.02 \\
\end{tabular} & \begin{tabular}{|c|}
-2.67 \\
\end{tabular} & \begin{tabular}{|l|l|}
4.91 \\
4.99 \\
\end{tabular} & \begin{tabular}{|l|}
4.59 \\
4.59
\end{tabular} & $\mid \frac{21.94}{21.68}$ & \begin{tabular}{|l|}
9.23 \\
9.24
\end{tabular} & \begin{tabular}{|l|}
35.52 \\
34.93 \\
\end{tabular} & $\frac{22.45}{22.42}$ & \begin{tabular}{|l|l|}
16.63 \\
16.92
\end{tabular} & \begin{tabular}{|l|}
22.00 \\
21.96 \\
\end{tabular} & & \begin{tabular}{|l|}
73.35 \\
73.42 \\
\end{tabular} & \begin{tabular}{|l|}
0.24 \\
0.24
\end{tabular} & \begin{tabular}{|l|l|}
-0.06 \\
-0.06 \\
\end{tabular} & \begin{tabular}{|l|}
0.23 \\
0.22 \\
\end{tabular} & \begin{tabular}{|l|}
0.04 \\
0.04 \\
\end{tabular} & \begin{tabular}{|l|l|}
0.05 \\
0.06 \\
\end{tabular} & \begin{tabular}{|l|l|}
0.00 \\
0.00
\end{tabular} & & & \begin{tabular}{|l|l|}
0.03 & 202.7 \\
0.03 & 202.8 \\
\end{tabular} \\
\hline
\end{tabular}


WSRC-TR-2005-00105, REVISION 0

SRNL-RPP-2005-00012, REVISION 0

Table H3-3-6. CIX Campaign II, Cycle 3, DI Water Pre-Elution Rinse

\begin{tabular}{|c|c|c|c|c|c|c|c|c|c|c|c|c|c|c|c|c|c|c|c|c|c|c|c|c|c|c|c|c|c|c|c|c|c|c|c|}
\hline & 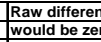 & owhen the & & $\begin{array}{l}\text { ajusted } \\
\text { is zero. }\end{array}$ & tadain & no or su & . & & 500 & & & & & & & 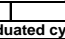 & & & & & & & & & & & & & & & & & & & \\
\hline & & & & & & & & \begin{tabular}{|l} 
ine \\
$P \mathrm{P}$ \\
\end{tabular} & & & & & & & & & & & & & & & & & & & & & DP4 & DP5 & & & & & \\
\hline & $\begin{array}{l}\text { DATET } \\
\text { units }\end{array}$ & TIME & $\begin{array}{l}\text { P1 } \\
\text { psig }\end{array}$ & $\begin{array}{l}\frac{\text { P2 }}{\text { psig }} \\
\end{array}$ & $\begin{array}{l}\text { P3 } \\
\text { psig } \\
\end{array}$ & \begin{tabular}{|l|l|} 
inch \\
water \\
\end{tabular} & $\begin{array}{l}\text { inch } \\
\text { water } \\
\text { wat }\end{array}$ & \begin{tabular}{|l} 
inch \\
water \\
\end{tabular} & \begin{tabular}{|l|} 
water \\
\end{tabular} & $\begin{array}{l}\text { inch } \\
\text { water } \\
\end{array}$ & \begin{tabular}{|l|} 
inch \\
water \\
\end{tabular} & $\begin{array}{l}\text { inch } \\
\text { water } \\
\end{array}$ & $\begin{array}{l}n \text { inch } \\
\text { ir } \\
\text { water }\end{array}$ & $\begin{array}{l}1 \\
\begin{array}{l}\text { inch } \\
\text { water }\end{array} \\
\text { wate }\end{array}$ & 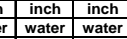 & $\begin{array}{l}\text { meter } \\
\text { Lhr }\end{array}$ & 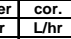 & $\begin{array}{l}\text { ir } \\
\text { ir }\end{array}$ & $\mathrm{pH}$ & $\begin{array}{l}\mathrm{T} 1 \\
{ }^{\circ} \mathrm{C} \\
\end{array}$ & $\begin{array}{l}\mathrm{T} 2 \\
{ }^{\circ} \mathrm{C} \\
\end{array}$ & $\begin{array}{l}\mathrm{T} 3 \\
{ }^{\circ} \mathrm{C} \\
\end{array}$ & $\begin{array}{l}\mathrm{T} 4 \\
{ }^{\circ} \mathrm{C} \\
\end{array}$ & $\begin{array}{l}\mathrm{T} 5 \\
{ }^{\circ} \mathrm{C} \\
\end{array}$ & $\begin{array}{l}\text { elaaps- } \\
\text { ed }\end{array}$ & & inch & $\begin{array}{l}\text { cor. } \\
\text { nch } \\
\text { nats }\end{array}$ & $\begin{array}{l}\text { cor. } \\
\text { inch } \\
\end{array}$ & $\begin{array}{l}\text { cor. } \\
\text { inch } \\
\text { inctis }\end{array}$ & $\begin{array}{l}\text { cor. } \\
\text { inch } \\
\text { int }\end{array}$ & $\begin{array}{l}\text { H1 } \\
\text { inches } \\
\text { int }\end{array}$ & $\begin{array}{l}\mathrm{H}_{2} \\
\text { inches } \\
\end{array}$ & $\begin{array}{l}\text { ential } \\
\text { BV }\end{array}$ & lative \\
\hline & $\begin{array}{l}\text { instrument } \\
6 / 2 / 20004 \\
\end{array}$ & & 1.88 & & & & & & & & \begin{tabular}{|l|l|} 
TR-3749 \\
-0.17
\end{tabular} & & & & & 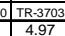 & & & & & 2.28 & & 1.91 & & $\begin{array}{l}8.03 \\
303\end{array}$ & & vater & rater & $\begin{array}{l}\text { water } \\
0.04\end{array}$ & vater & rater & & & & \\
\hline & $6 / 6 / 288 / 20$ & $08: 39: 50$ & $\frac{1.88}{1.88}$ & 然 & & & & & & & -0.17 & & & & & \begin{tabular}{|l|l|}
4.97 \\
\end{tabular} & 4.57 & & & & & & $\frac{1.91}{1.97}$ & & & & . 0.03 & & 0.04 & 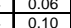 & & & & & 02.00 \\
\hline & & & & & & & & & & & & & & & & \begin{tabular}{|l|l|}
5.04 \\
\end{tabular} & 64 & & & & & & & & & & & & & & & & & & \\
\hline & & & & & & & & & & & -0.20 & & & & & \begin{tabular}{|l|l|}
4.98 \\
\end{tabular} & 4.58 & & & & & & & & & & & & & & & & & & 202.20 \\
\hline & $\begin{array}{l}6 / 1 / 28 / 2 / 2 \\
6\end{array}$ & $\begin{array}{l}08: 4: 50.5 .8 \\
08: 49: 50 .\end{array}$ & $\begin{array}{l}7 \\
7\end{array}$ & $\begin{array}{l}-0.68 \\
-0.68 \\
-1\end{array}$ & $\begin{array}{l}3.59 \\
3.58 \\
-15\end{array}$ & \begin{tabular}{|l|l|}
0.35 \\
0.33 \\
\end{tabular} & \begin{tabular}{|l|l|}
0.00 \\
0.04 \\
\end{tabular} & \begin{tabular}{|l|l|}
0.06 \\
0.05 \\
\end{tabular} & \begin{tabular}{|l|l|}
0.02 \\
0.02 \\
\end{tabular} & \begin{tabular}{|l}
0. \\
0. \\
\end{tabular} & \begin{tabular}{|l|l|}
-0.20 \\
-0.17 \\
\end{tabular} & \begin{tabular}{|c|c|}
-0.03 \\
-0.03 \\
\end{tabular} & & & \begin{tabular}{l|l}
02 & -2.67 \\
\end{tabular} & $\begin{array}{l}4.97 \\
4.88 \\
\end{array}$ & $\begin{array}{l}4.57 \\
.48 \\
\end{array}$ & 1.94 & \begin{tabular}{|l|l|}
10.78 \\
9.32 \\
\end{tabular} & 7.11 & $\frac{22.34}{22.30}$ & 5.05 & $\overline{21.8}$ & & $\begin{array}{l}73.17 \\
73.20\end{array}$ & & $\frac{0.06}{0.03}-150$ & 0.26 & $\begin{array}{l}0.04 \\
0.04\end{array}$ & 0.06 & $\frac{.00}{.03}$ & & & \begin{tabular}{|l|l|}
0.03 \\
0.03 \\
\end{tabular} & 202.26 2023 \\
\hline & 8/2004 & $\begin{array}{l}08: 51: 50.8 \\
08: 5: 50.8\end{array}$ & \begin{tabular}{l|l}
7 & 1.88 \\
7 & 1.86
\end{tabular} & $\begin{array}{l}-0.68 \\
-0.68\end{array}$ & & $\begin{array}{l}0.33 \\
0.32\end{array}$ & 0.00 & \begin{tabular}{|l|l|}
0.05 \\
0.03
\end{tabular} & & & \begin{tabular}{|l|l|}
-0.20 \\
-0.20
\end{tabular} & \begin{tabular}{|c|c|}
-0.03 \\
0.04
\end{tabular} & & & & \begin{tabular}{|l|l|}
4.92 \\
495
\end{tabular} & 4.52 & $\begin{array}{l}21.68 \\
21.94 \\
\end{array}$ & \begin{tabular}{|l|l|}
10.68 \\
11.48
\end{tabular} & & $\frac{22.35}{2236}$ & $\frac{6.20}{6.37}$ & 2.t. & & 73.23 & & $\begin{array}{l}-0.06 \\
-0.05\end{array}$ & & $\frac{0.04}{0.04}$ & $\frac{0.05}{0.06}$ & .00 & & & & $\begin{array}{l}202.39 \\
202.36 \\
2026\end{array}$ \\
\hline & & & & & & & & & & & $\frac{-0.20}{-0.20}$ & $\begin{array}{l}-0.04 \\
-0.03 \\
\end{array}$ & & & & \begin{tabular}{|l|l|}
4.95 \\
4.82 \\
\end{tabular} & $\begin{array}{l}4.55 \\
4.42 \\
-12\end{array}$ & & & & & & & & & & & & & & & & & & 202.46 \\
\hline & & 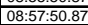 & & & & & & & & & & & & & & & & & & & & & & & & & & & & & & & & & $\begin{array}{l}0.2 .52 \\
0.258\end{array}$ \\
\hline & 82000 & $08: 59: 50.8$ & 1.88 & \begin{tabular}{c|c}
8 & -0.66 \\
\end{tabular} & & & 0.00 & \begin{tabular}{|l|l|} 
\\
\end{tabular} & & & $\begin{array}{l}-0.20 \\
-0.0\end{array}$ & -0.06 & & & & $\begin{array}{ll}4.87 \\
\end{array}$ & $\begin{array}{ll}4.48 \\
\end{array}$ & 21.68 & 10.47 & 35.44 & 22.37 & 7.16 .7 & 1 & & $\sqrt{3.3}$ & & & & $\overline{0.02}$ & & & & & & 202.65 \\
\hline & $\frac{66 / 2882004}{66282004}$ & 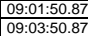 & $\begin{array}{l}7 \\
7\end{array}$ & \begin{tabular}{l|l}
8 & -0.66 \\
8 & -0.66 \\
\end{tabular} & \begin{tabular}{|l|l|}
3.58 \\
3.56 \\
\end{tabular} & \begin{tabular}{|l|l|}
0.32 \\
0.31 \\
\end{tabular} & \begin{tabular}{|l|l|}
0.00 \\
0.01 \\
\end{tabular} & \begin{tabular}{|l|}
0.03 \\
0.03 \\
\end{tabular} & \begin{tabular}{|l|l|}
0.02 \\
0.02 \\
\end{tabular} & \begin{tabular}{|l|l|}
0.10 \\
0.10 \\
\end{tabular} & \begin{tabular}{|l|l|l}
-0.20 \\
-0.20
\end{tabular} & \begin{tabular}{|c|c|}
-0.04 \\
-0.06 \\
\end{tabular} & $\frac{-0.02}{0.00}$ & & \begin{tabular}{|l|l|}
-2.66 \\
-2.66 \\
\end{tabular} & \begin{tabular}{|l|l|}
4.93 \\
.82
\end{tabular} & 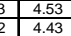 & $\begin{array}{l}3 \\
3 \\
3\end{array}$ & \begin{tabular}{|l|l|}
9.98 \\
10.02 \\
\end{tabular} & 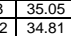 & 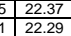 & $\begin{array}{l}7.76 .79 \\
99.87 \\
97\end{array}$ & 势. & & $\frac{73.46}{73.43}$ & & $\begin{array}{l}-0.06 \\
-0.05 \\
\end{array}$ & & 0.04 & 0.05 & 0.00 & & & & $\begin{array}{l}02.71 \\
02.78\end{array}$ \\
\hline & & . & \begin{tabular}{|l}
1.88 \\
1.86 \\
\end{tabular} & $\begin{array}{l}-0.66 \\
-0.66 \\
-0.6\end{array}$ & & \begin{tabular}{|l|l|}
0.31 \\
.31
\end{tabular} & 0.00 & & & & & & & & & \begin{tabular}{|l|l}
4.86 \\
\end{tabular} & $\frac{46}{48}$ & $\begin{array}{l}21.68 \\
2.294\end{array}$ & \begin{tabular}{|l|l|l|l|l|}
0.49 \\
0.13
\end{tabular} & & $\frac{2.42}{20.38}$ & 17.00 & $\frac{2.9}{2.0}$ & & 73.47 & & -0.0 & & $\frac{0.04}{0.04}$ & 0.05 & .00 & & & & $\begin{array}{l}0.84 \\
0.22 .84 \\
0.291\end{array}$ \\
\hline & & & & & & & & & & & & & & & & & & & & & & & & & & & & & & & & & & & \\
\hline & & & & & & & & & & & & & & & & $\frac{4.14}{4.83}$ & & & & & & & & & & & & & & & & & & & 202.93. \\
\hline & 8/2004 & \begin{tabular}{|l|l|l}
$09: 13: 50.87$ \\
\end{tabular} & $\frac{1.18}{1.88}$ & -0.68 & 356 & 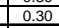 & 0.01 & 0.03 & \begin{tabular}{|l|l|}
0.02 \\
\end{tabular} & & -0.20 & \begin{tabular}{|l|l|}
-0.07 \\
\end{tabular} & & & & 4.85 & 4.45 & $\frac{1.94}{1.94}$ & 9.54 & 33.8 & 2.44 & $\begin{array}{lll}4 & 17.51 \\
\end{array}$ & & & 73.6 & & & & & & & & & & $\begin{array}{l}203.10 \\
203.10 \\
\end{array}$ \\
\hline & & & & & & & & & & & & & & & & 4.90 & 4.56 & 6 & 10.6 & & & & & & & & & & & & & & & & .1.16 \\
\hline & $8 / 200$ & :19:50 & 1.00 & & & & & & & & & & & & & $\frac{2.91}{4.91}$ & & & $11.2 \mathrm{r}$ & & & & & & & & & & & & & & & & 3..22 \\
\hline & & & 1.88 & -0.66 & 3.56 & & & & \begin{tabular}{|l|l|}
-0.02 \\
\end{tabular} & 0.11 & \begin{tabular}{|c|c|}
-0.20 \\
\end{tabular} & \begin{tabular}{|l|l|}
-0.08 \\
\end{tabular} & & & & 4.85 & 4.46 & \begin{tabular}{|l|l|}
6 & 21.68 \\
\end{tabular} & 9.42 & & 2.36 & & 21. & & & & & & & 0.0 & & & & & 法.35 \\
\hline & & $9: 2.2$ & 1.8 & & & & & & & & & & & & & \begin{tabular}{|l|l|}
5.01 \\
\end{tabular} & . & 1.94 & \begin{tabular}{|l|l|}
11.76 \\
1009
\end{tabular} & & $\frac{22.36}{22.44}$ & & & & 738 & & 500 & & 50 & & & & & 0.03 & $\begin{array}{l}203.42 \\
23.48\end{array}$ \\
\hline & $8 / 2$ & & 1.8 & & & & & & & & & & & & & & & & & & & & & & & & & & & & & & & & \\
\hline & & & & & & & & & & & & & & & & $\frac{4.86}{4.94}$ & & & & & & & & & & & & & & & & & & & \\
\hline & & & & & & & & & & & & & & & & $\frac{4.94}{4.94}$ & $\begin{array}{l}4.54 \\
455\end{array}$ & & & & & & & & & & & & & & & & & & $\begin{array}{l}203.68 \\
023.74\end{array}$ \\
\hline & $6 / 2$ & $8 \cdot 3$ & & & & & 0.00 & \begin{tabular}{|l|l|}
0.01 \\
\end{tabular} & -0.01 & & -0.20 & & & & & 4.88 & & 21.68 & & & & & & & 75 & & 0.06 & & .07 & & & & & & \\
\hline & 88127 & : & $\begin{array}{ll}1.88 \\
\end{array}$ & -0.68 & & 0.29 & 0.00 & $\mid$\begin{tabular}{|c|}
$\mid$ \\
$\mid$
\end{tabular} & $\mid \begin{array}{l}-0.6 \\
-0.6\end{array}$ & & \begin{tabular}{|l|l|} 
& -0.20 \\
0.20
\end{tabular} & \begin{tabular}{|c|c|}
-0.08 \\
0.08
\end{tabular} & & & & \begin{tabular}{|l|l|}
4.97 \\
.87
\end{tabular} & 4.57 & $\begin{array}{l}7 \\
72.94 \\
72.169\end{array}$ & 9.25 & & 38 & & & & & & & & & 000 & & & & 0.03 & \\
\hline & & & $\frac{7.8}{1.8}$ & & & & 0.00 & 0.00 & & & & -0.08 & & & & 4.99 & 4.59 & & & & & & & & & & & & & & & & & & \\
\hline & & - & & & & & & & & & & & & & & 4.99 & & & & & & & & & & & & & & & & & & & \\
\hline & & & 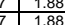 & & & & & & & & & & & & & & & & & & & & & & & & & & & & & & & & \\
\hline & 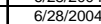 & 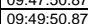 & $\frac{1.188}{1.88}$ & & & & & & & & & & & & & 告.950 & & & & & & & & & & & & & & & & & & & \\
\hline & $8 / 2$ & 0.51 .50 & $\frac{1.5}{1.5}$ & 66 & & & 0.00 & -0 & & & & & & & & 4.98 & 4. & & & & & & & & & & & & & & & & & & \\
\hline & . & t.535.50 & 1 & & & & & -0 & & & & & & & & & & & & & & & & & & & & & & & & & & & \\
\hline & & $0.57 \cdot 50.8$ & $\frac{1.8}{19}$ & -0.66 & & & & & & & & & & & & 年.955 & & $\frac{21.94}{2168}$ & & & & & & & & & & & & & & & & & \\
\hline & . & 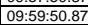 & $\frac{1.8}{1.8}$ & 0 & & & & & & & & & & & & $\frac{4.99}{4.99}$ & & & & & & & & & & & & & & & & & & & \\
\hline & $88 / 20$ & $10: 01: 50.8$ & 1.88 & -0.64 & & & 0.01 & -0.04 & & & -0.20 & & & & & 5.02 & 4.62 & 26.05 & 9.8 & & 355 & & 22 & & & & & & & & & & & & \\
\hline & & & & & & & & & & & & & & & & 4.94 & & & & & & & & & & & & & & & & & & & \\
\hline & 8126 & 7.50 & $\frac{1.88}{1.90}$ & & & & 0.01 & $\mid \begin{array}{l}-0.04 \\
-0.04\end{array}$ & & & & $\frac{-0}{-0}$ & & & & $\frac{5.04}{4.94}$ & $\begin{array}{l}4.64 \\
4.54\end{array}$ & $\frac{26.09}{26.09}$ & & & & 22 & & & & & & & & & & & & & \\
\hline & $6 / 2$ & 10:09:50.87 & 1.88 & $\frac{-0.64}{-0.64}$ & & 0.21 & 0.01 & \begin{tabular}{|c|c|}
-0.04 \\
\end{tabular} & \begin{tabular}{|l|l|}
-0.02 \\
\end{tabular} & 0.07 & -0.20 & -0.10 & \begin{tabular}{l|l}
0 & -0.02 \\
0
\end{tabular} & & -2.66 & 4.88 & 4.48 & 26.09 & 1.08 & 27.1 & 22.52 & 221.95 & 2,12 & & 74. & & $=0.05$ & & 0.0 & & & & & & \\
\hline & & $\frac{\frac{10: 11: 50.8}{10: 13: 50.8}}{\frac{1}{10}}$ & \begin{tabular}{l|l}
7 \\
7
\end{tabular} & 0.64 & & & & \begin{tabular}{|l|}
-0.04 \\
-0.04 \\
\end{tabular} & & & $\begin{array}{l}-0.19 \\
-0.20 \\
\end{array}$ & & & & & \begin{tabular}{|l|}
4.99 \\
4.95 \\
\end{tabular} & \begin{tabular}{l|l|}
5 & 4.5
\end{tabular} & & & & & & & & & & & & & & & & & & \\
\hline & & 0 & 1.90 & \begin{tabular}{l|l}
0 & -0.64 \\
\end{tabular} & & & & \begin{tabular}{|l|l|}
-0.04 \\
\end{tabular} & & & & & & & & 4.94 & & & & & & & & & & & & & & & & & & & \\
\hline & 6/28/20 & & 1.88 & -0.64 & & & & & & & -0.20 & & & & & & & 26.09 & & & & & & & & & & & & & & & & & \\
\hline & $\begin{array}{l}6 / 288 / 200 \\
66 / 28 / 200\end{array}$ & 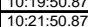 & $\begin{array}{l}\frac{1.90}{1.88} \\
1.88\end{array}$ & $\begin{array}{l}-0.64 \\
-0.64 \\
\end{array}$ & $\begin{array}{l}3.52 \\
3.50 \\
\end{array}$ & \begin{tabular}{|l|l|}
0.21 \\
0.21 \\
\end{tabular} & $\begin{array}{l}0.01 \\
0.00\end{array}$ & \begin{tabular}{|l|}
-0.04 \\
-0.04
\end{tabular} & $\mid$\begin{tabular}{|c|}
-0.01 \\
0.02
\end{tabular} & 0.07 & $\begin{array}{l}-0.20 \\
-0.20 \\
\end{array}$ & & & & $\begin{array}{l}-2.66 \\
-2.66 \\
\end{array}$ & $\begin{array}{l}4.93 \\
4.97\end{array}$ & $\begin{array}{l}4.53 \\
4.57 \\
4.57\end{array}$ & $\begin{array}{l}\frac{30.51}{30.51} \\
30\end{array}$ & \begin{tabular}{|l|l|}
1.57 \\
.72
\end{tabular} & & \begin{tabular}{|l|}
22.53 \\
22.49 \\
\end{tabular} & \begin{tabular}{l|l}
3 & 21 \\
9 & 22 \\
\end{tabular} & $\frac{22.15}{22.12}$ & & & & \begin{tabular}{|l|l|} 
& -0.06 \\
\end{tabular} & & & & & & & & (2) \\
\hline & 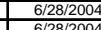 & $\begin{array}{l}10: 2 \\
10: 2 \\
102\end{array}$ & \begin{tabular}{l|l}
7 & 1.88 \\
7 & 1.08
\end{tabular} & $\begin{array}{l}-0.64 \\
-0.64\end{array}$ & & \begin{tabular}{|l|l|}
0.20 \\
020
\end{tabular} & 0.01 & \begin{tabular}{|l|l|} 
& -0.04 \\
\end{tabular} & \begin{tabular}{|l|l|} 
& -0.01 \\
\end{tabular} & 0. & \begin{tabular}{|c|}
-0.20 \\
\end{tabular} & \begin{tabular}{|l|l|}
-0.10 \\
\end{tabular} & & & & \begin{tabular}{|l|l} 
& 4.95 \\
\end{tabular} & & 30.51 & $\begin{array}{l}0.32 \\
0.23\end{array}$ & & \begin{tabular}{|l|}
22.49 \\
2262
\end{tabular} & 9 & 2 & & 74 & & (0) & & & & & & & & \\
\hline & & & $\frac{1.88}{1.88}$ & & & & & & & & & & & & & & & $\frac{20.51}{30.51}$ & & & & & & & & & & & & & & & & & \\
\hline & $6 / 2$ & $10: 29: 50.87$ & 1.62 & -0.62 & 25 & 0.20 & & & \begin{tabular}{|l|l|}
-0.01 \\
\end{tabular} & 0.09 & -0.20 & & & & & 5.05 & & & 1.32 & & 22.62 & \begin{tabular}{|l|l|}
2 & 22.29 \\
\end{tabular} & $\frac{22.25}{22.25}$ & & 74.8 & 0.12 & & & .01 & 0.04 & & & & & \\
\hline & $\frac{6 / 28 / 21}{6 / 281 / 20}$ & & & & & & & & & 0.07 & \begin{tabular}{|l|l|}
-0.20 \\
-0.20
\end{tabular} & \begin{tabular}{|l|l|l|}
-0.10 \\
-0.12
\end{tabular} & & & & \begin{tabular}{|l}
4.90 \\
4.98 \\
\end{tabular} & & & $\frac{0.19}{0.92}$ & & 222.54 & & & & 74.9 & & & & & & & & & & \\
\hline & $\frac{6 / 28 / 2004}{6 / 62804}$ & 10:35:50.87 & $\begin{array}{l}1.00 \\
1.88 \\
\end{array}$ & $\begin{array}{l}-0.62 \\
-0.62 \\
\end{array}$ & \begin{tabular}{|l|l|}
3.54 \\
\end{tabular} & \begin{tabular}{|l|l|}
0.10 \\
\end{tabular} & 0.02 & $\begin{array}{c}-0.00 \\
-0.07 \\
\end{array}$ & \begin{tabular}{|l|l|l|}
0.02 \\
\end{tabular} & $\begin{array}{l}0.07 \\
\end{array}$ & \begin{tabular}{|l|l}
-0.20 \\
\end{tabular} & & & & 2.66 & 4.97 & & & 1.57 & & $\frac{22.62}{22.62}$ & $\begin{array}{l}\frac{1}{2} \\
222.62 \\
22.62\end{array}$ & & & 749 & & & & & & & & & & $\begin{array}{l}3.2050 .00 \\
0.00\end{array}$ \\
\hline & & & 1.8 & & & & $\frac{0.02}{0.02}$ & & & & & & & & & 4. & & & & & & & & & & & & & & & & & & & \\
\hline & & & 1 & $\frac{-0.01}{-0.61}$ & & & & & & & & & & & & & & & & & & & & & & & & & & & & & & & \\
\hline & $6 / 28 / 2$ & & 1. & & & & & & & & & & & & & & & & -6 & & $\frac{22.67}{22.67}$ & & & & & & & & & & & & & & \\
\hline & $6 / 28 / 200$ & ;:50.87 & 1.8 & & & & & & & & & & & & & 4.94 & & & -0.1 & & & & & & & & & & & & & & & & \\
\hline & & & 1 & - & & & 0.6 & & & & & & & & & 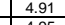 & & & 0. & & & & & & & & & & & & & & & & \\
\hline & & & $\frac{1}{1}$ & & & & & & & & & & & & & & & & & & & & & & & & & & & & & & & & \\
\hline & & & & & & 81 & & & & & & & & & & 4.89 & & & - & & & & 2.21 & & & & & & & & & & & & \\
\hline & & & & & & & & & & & & & & & & 4.92 & & & 0.96 & & & & . & & & & & & & & & & & & \\
\hline & & & $\frac{1.8}{4.8}$ & $\frac{0.57}{0.57}$ & & & & & & & & & & & & 4.91 & & & & & & & $\frac{2.34}{230}$ & & & & & & & & & & & & \\
\hline & $\begin{aligned} 0.120120 \\
6 / 28120\end{aligned}$ & 11: & $\begin{array}{l}7 \\
7\end{array}$ & 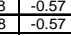 & \begin{tabular}{|l|}
3. \\
3
\end{tabular} & \begin{tabular}{|l|l|}
0.19 \\
0.18
\end{tabular} & & \begin{tabular}{|l|l|}
-0.07 \\
-0.08 \\
\end{tabular} & $\begin{array}{l}30 \\
30\end{array}$ & \begin{tabular}{|l|}
0.07 \\
0.07
\end{tabular} & \begin{tabular}{|l|}
-0.19 \\
\\
\end{tabular} & \begin{tabular}{|l|l|}
-0.099 \\
-0.10
\end{tabular} & $\begin{array}{ll}0 \\
0\end{array}$ & & \begin{tabular}{|l|}
-2.266 \\
\end{tabular} & \begin{tabular}{|l|l}
4.98 \\
4.92 \\
\end{tabular} & 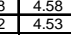 & & \begin{tabular}{|l|l|}
1.05 \\
0.15 \\
\end{tabular} & & \begin{tabular}{|l|l|l|}
922.71 \\
\end{tabular} & 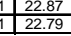 & $\begin{array}{l}22.26 \\
22.18\end{array}$ & & & & \begin{tabular}{|l|}
-0.04 \\
-0.03 \\
\end{tabular} & \begin{tabular}{|l|}
0.14 \\
0.12
\end{tabular} & 0.04 & 0.02 & 0.01 & & & 0.03 & $\begin{array}{l}3206.56 \\
\end{array}$ \\
\hline
\end{tabular}


WSRC-TR-2005-00105, REVISION 0

SRNL-RPP-2005-00012, REVISION 0

Table H3-3-7. CIX Campaign II, Cycle 3, 0.5 M Nitric Acid Eluent

\begin{tabular}{|c|c|c|c|c|c|c|c|c|c|c|c|c|c|c|c|c|c|c|c|c|c|c|c|c|c|c|c|c|c|c|c|c|c|c|c|c|}
\hline & Raw diff & al press & $w$ & & & & & gano & & & & & & & & & & & & & & & & & & & & & & & & & & & & \\
\hline & & & & & & $\begin{array}{l}\text { The } \\
\text { TP }\end{array}$ & 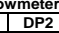 & & DP4 & & $\frac{\operatorname{sing16}}{\mathrm{DPC}}$ & DP7 & $\overline{D F}$ & & & & Flow & $\begin{array}{l}\sqrt{N} \\
\text { fllow }\end{array}$ & & & & & & & & & & & & & & & & & & \\
\hline & $\begin{array}{l}\text { DATE } \\
\text { units }\end{array}$ & TIME & psig & $\mathrm{P} 2^{2}$ & & $\begin{array}{l}\text { inch } \\
\text { water }\end{array}$ & $\begin{array}{l}\text { inch } \\
\text { water }\end{array}$ & $\begin{array}{l}\text { inch } \\
\text { water }\end{array}$ & $\begin{array}{l}\text { inch } \\
\text { water }\end{array}$ & $\begin{array}{l}\text { inch } \\
\text { water }\end{array}$ & $\begin{array}{l}\text { inch } \\
\text { water }\end{array}$ & $\begin{array}{l}\text { inch } \\
\text { water }\end{array}$ & $\begin{array}{l}\text { inch } \\
\text { water }\end{array}$ & $\begin{array}{l}\text { inch } \\
\text { water }\end{array}$ & $\begin{array}{l}\text { inch } \\
\text { water }\end{array}$ & incl & $\begin{array}{l}\text { meter } \\
\text { Lhhr }\end{array}$ & $\begin{array}{ll}r & \text { cor. } \\
& \text { Lhlhr }\end{array}$ & $\frac{\text { Cond1 }}{\text { mSlcm }}$ & $\mathrm{pH}$ & $\frac{\mathrm{T} 1}{{ }^{\circ} \mathrm{C}}$ & $\frac{\mathrm{T} 2}{{ }^{\circ} \mathrm{C}}$ & $\frac{13}{{ }^{\circ} \mathrm{C}}$ & $\begin{array}{l}\frac{T 4}{{ }^{\circ} \mathrm{C}} \\
\end{array}$ & 75 & $\begin{array}{l}\text { elaps- } \\
\text { ed }\end{array}$ & $\begin{array}{l}\text { cor. } \\
\text { inch } \\
\text { inch }\end{array}$ & cor. & $\begin{array}{l}\text { cor. } \\
\text { inch }\end{array}$ & $\begin{array}{l}\text { cor. } \\
\text { inch }\end{array}$ & $\begin{array}{l}\text { cor. } \\
\text { inch } \\
\text { inch }\end{array}$ & $\begin{array}{l}\text { cor. } \\
\text { inch } \\
\end{array}$ & $\begin{array}{l}\text { H1 } \\
\text { ches }\end{array}$ & $\begin{array}{l}\text { H2 } \\
\text { inches } \\
\text { ing }\end{array}$ & $\begin{array}{c}\text { ential } \\
\text { BV }\end{array}$ & $\begin{array}{l}\text { lative } \\
\text { BV }\end{array}$ \\
\hline & & & & 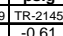 & & $\frac{\pi R-37}{T T-37}$ & Th837 & & 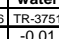 & & sac & T.8.3. & & & & & & & & & & & & & 4 & & $\begin{array}{l}\text { water } \\
0.11\end{array}$ & & $\begin{array}{c}\text { water } \\
0.12\end{array}$ & $\begin{array}{l}\text { water } \\
\text { water }\end{array}$ & vater & $\begin{array}{l}\text { water } \\
0.01\end{array}$ & & & & \\
\hline & & \begin{tabular}{|l|l|}
$10.515: 50.8$ \\
1050.8
\end{tabular} & \begin{tabular}{|l|}
1.88 \\
1.88 \\
\end{tabular} & $\begin{array}{l}-0.61 \\
-0.59 \\
\end{array}$ & \begin{tabular}{|l}
3.50 \\
3.50 \\
\end{tabular} & \begin{tabular}{|l|l|}
0.19 \\
0.19
\end{tabular} & \begin{tabular}{|l|l|}
0.02 \\
0.00
\end{tabular} & $\begin{array}{l}-0.08 \\
-0.07 \\
\end{array}$ & \begin{tabular}{|c|}
$-\frac{-0.01}{0.02}$ \\
\end{tabular} & \begin{tabular}{|l|l}
0.09 \\
0.07
\end{tabular} & \begin{tabular}{|l|l|l|l|}
-0.20 \\
-0.20
\end{tabular} & \begin{tabular}{l|l}
0 \\
0 \\
0 & -0.12 \\
& -0.10 \\
\end{tabular} & $\begin{array}{l}x-0.02 \\
0.020 \\
\end{array}$ & \begin{tabular}{|l|l|}
0.19 \\
0.19
\end{tabular} & \begin{tabular}{|l}
-0.02 \\
0.03 \\
-0.03
\end{tabular} & $\begin{array}{l}-2.66 \\
\end{array}$ & $\begin{array}{l}4.89 \\
4.94\end{array}$ & & & \begin{tabular}{|l|}
-0.078 \\
0.07 \\
0.77
\end{tabular} & $\frac{6.04}{6.04}$ & $\frac{22.67}{22.67}$ & 22.74 & & & 7.15.23 & $\frac{0.11}{0.11}$ & \begin{tabular}{|l|}
-0.04 \\
-0.06 \\
\end{tabular} & $\frac{0.12}{0.14}$ & $\frac{0.01}{0.04}$ & $\frac{0.04}{0.02}$ & $\frac{0.01}{0.00}$ & & & $\frac{0.03}{0.03}$ & \\
\hline & & & \begin{tabular}{|l|}
1.88 \\
1.88 \\
\end{tabular} & $\begin{array}{l}-0.57 \\
-0.55 \\
\end{array}$ & & & & $\begin{array}{l}-0.07 \\
-0.07 \\
\end{array}$ & \begin{tabular}{|l|l|}
0.02 \\
0.01 \\
\end{tabular} & & \begin{tabular}{|l|l|}
-0.19 \\
-0.18 \\
\end{tabular} & 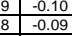 & & & & & \begin{tabular}{|l|}
4.09 \\
4.94 \\
\end{tabular} & & & $\begin{array}{l}0.17 \\
0.12 \\
0.12\end{array}$ & $\frac{25.77}{25.58}$ & & 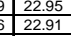 & & & $\begin{array}{l}75.35 \\
75.47 \\
\end{array}$ & & & & & & $\frac{0.01}{0.02}$ & & & & \\
\hline & & $\begin{array}{l}11: 12: 50.8 \\
11: 19: 50.8\end{array}$ & \begin{tabular}{|l|}
1.88 \\
1.88 \\
\end{tabular} & \begin{tabular}{|l|l|}
-0.55 \\
-0.53
\end{tabular} & & & & \begin{tabular}{|l|l|}
-0.07 \\
-0.08
\end{tabular} & & \begin{tabular}{|l|l|}
0.07 \\
0.07
\end{tabular} & \begin{tabular}{|l|l|}
-0.19 \\
-0.018
\end{tabular} & \begin{tabular}{l|l} 
\\
8 & -0.08 \\
-0.08
\end{tabular} & 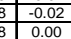 & \begin{tabular}{|l|l|}
0.19 \\
0.19
\end{tabular} & \begin{tabular}{|l|l|}
-0.02 \\
9 & -0.02 \\
\end{tabular} & & \begin{tabular}{|l|}
4.94 \\
4.98 \\
\end{tabular} & & & $\begin{array}{r}-0.17 \\
0.45 \\
\end{array}$ & $\begin{array}{l}25.14 \\
25.07\end{array}$ & & & & & $\begin{array}{l}7.51 \\
775.70\end{array}$ & & & & $\begin{array}{l}0.03 \\
0.04\end{array}$ & & $\begin{array}{l}0.01 \\
0.02 \\
0.02\end{array}$ & & & 0.03 & $\frac{06.5}{0.69}$ \\
\hline & & & \begin{tabular}{|l|l|l}
1.88 \\
1.88 \\
\end{tabular} & $\begin{array}{l}-0.53 \\
-0.53 \\
-0.51\end{array}$ & & \begin{tabular}{|l|}
0.15 \\
0.15 \\
0.15
\end{tabular} & \begin{tabular}{|l|l|}
0.04 \\
0.044
\end{tabular} & \begin{tabular}{|l}
-0.00 \\
-0.10 \\
-0.10
\end{tabular} & \begin{tabular}{|l|l|}
0.02 \\
0.02
\end{tabular} & & \begin{tabular}{|l|l|l}
-0.19 \\
-0.17
\end{tabular} & \begin{tabular}{l|l|l|l|l|l} 
& -0.08 \\
7 & -0.08 & -0.08
\end{tabular} & & \begin{tabular}{|l|l|}
0.19 \\
0.19 \\
0.19
\end{tabular} & $\begin{array}{l}-0.02 \\
-0.02 \\
-0.02\end{array}$ & & \begin{tabular}{|l|}
4.90 \\
5.02 \\
5.02
\end{tabular} & & & \begin{tabular}{|l|l|}
0.83 \\
0.56 \\
\end{tabular} & $\frac{24.88}{24.88}$ & & & & & $\begin{array}{l}75.82 \\
75.83 \\
5\end{array}$ & & & & $\frac{.04}{0.04}$ & $\frac{1.02}{.02}$ & $\frac{0.02}{0.01}$ & & & $\begin{array}{l}0.03 \\
0.03\end{array}$ & $\begin{array}{l}\frac{0.7 .36}{0.7676} \\
0.759\end{array}$ \\
\hline & & & $\frac{1.88}{1.86}$ & $\frac{-0.51}{-0.51}$ & & \begin{tabular}{|l|}
0.15 \\
0.15 \\
\end{tabular} & \begin{tabular}{|l|}
0.04 \\
0.04 \\
\end{tabular} & & \begin{tabular}{|l|}
0.02 \\
0.02 \\
\end{tabular} & & 0.17 & & & \begin{tabular}{|l|l|}
0.19 \\
0.19 \\
\end{tabular} & & & $\frac{5.02}{4.84}$ & & & & & & & & & $\begin{array}{l}\frac{75.93}{76.05} \\
\end{array}$ & & & & $\frac{0.04}{0.04}$ & & $\frac{0.03}{0.02}$ & & & & 0.07 .59 \\
\hline & & & & & & & $\frac{0.04}{0.04}$ & & & & & & & & & & & & & & & & & & & & & & & & & & & & & \\
\hline & & & & $\frac{-0.48}{-0.46}$ & & & & & & & & & & & & & & & & & & & & & & & & & & & & & & & & \\
\hline & & & 1.86 & -0.44 & \begin{tabular}{|l}
3.47 \\
\end{tabular} & \begin{tabular}{|l|l|}
0.15 \\
\end{tabular} & \begin{tabular}{|l|l|}
0.04 \\
0.04 \\
\end{tabular} & -0.10 & \begin{tabular}{|l|l|}
0.02 \\
0.02
\end{tabular} & \begin{tabular}{|l|l|} 
\\
\end{tabular} & & \begin{tabular}{|l|l|}
-0.08 \\
-0.08 \\
\end{tabular} & & \begin{tabular}{|l|l|}
0.19 \\
\end{tabular} & \begin{tabular}{|l|l|l|} 
& -0.02 \\
\end{tabular} & & 4.91 & & & 0.90 & $\begin{array}{l}42.34 \\
24.34 \\
\end{array}$ & & & & & $\begin{array}{l}70.40 \\
76.52 \\
\end{array}$ & .07 & & .10 & .04 & & & & & & $\begin{array}{l}0.49 \\
08.72 \\
0.72 \\
\end{array}$ \\
\hline & 年 & $\begin{array}{l}12: 15: 55.87 \\
12 \cdot 2 \cdot 250.87\end{array}$ & $\frac{1.86}{1.88}$ & $\begin{array}{l}-0.40 \\
-0.39 \\
\end{array}$ & \begin{tabular}{|l|}
3.48 \\
350
\end{tabular} & \begin{tabular}{|l|l|}
0.15 \\
0.16
\end{tabular} & \begin{tabular}{|l|l|}
0.04 \\
0.04
\end{tabular} & \begin{tabular}{|l|l|}
-0.10 \\
-0.09
\end{tabular} & \begin{tabular}{|l|}
0.02 \\
0.02
\end{tabular} & \begin{tabular}{|l|}
0.04 \\
0.05 \\
\end{tabular} & \begin{tabular}{|l|l|}
-0.18 \\
-0.17
\end{tabular} & 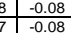 & $\begin{array}{l}-0.02 \\
-0.02 \\
-0.02\end{array}$ & \begin{tabular}{|l|}
0.17 \\
0.19
\end{tabular} & $\begin{array}{c}-0.02 \\
9-0.01\end{array}$ & $\begin{array}{l}-2.66 \\
-2.65 \\
\end{array}$ & $\begin{array}{l}4.95 \\
5.95 \\
\end{array}$ & & 6.49 & -0.17 & $\begin{array}{l}24.31 \\
24.35\end{array}$ & $\begin{array}{l}33.09 \\
3314\end{array}$ & & $\frac{2.40}{252}$ & & $\begin{array}{l}76.63 \\
76.75 \\
\end{array}$ & . & 0.03 & & $\frac{0.04}{0.04}$ & .010 & 0.02 & & & & $\begin{array}{l}8.95 \\
9.17\end{array}$ \\
\hline & & & & & & 0.10 & & & & & & & & & & & & & & & & & & & & & & & & & & & & & & 99.17 \\
\hline & & & & $\begin{array}{l}-0.33 \\
-0.33 \\
-3.0\end{array}$ & & 0.16 & & & & & & & & & & & & & & & & & & & & & & & & & & & & & & \\
\hline & & & $\frac{1.86}{186}$ & & \begin{tabular}{|l|}
3.48 \\
3.48 \\
\end{tabular} & \begin{tabular}{|l|l|}
0.16 \\
0.15
\end{tabular} & \begin{tabular}{|l|l|}
0.04 \\
0.04
\end{tabular} & $\begin{array}{l}-0.09 \\
-0.10\end{array}$ & $\frac{0.02}{0.02}$ & & & - 0.008 & & $\frac{0.19}{0.19}$ & & & $\frac{5.14}{480}$ & & & & & . & & & & $\frac{1.100}{17.0}$ & & & & & & & & & & 09.85 \\
\hline & & & 1.86 & $\begin{array}{l}-0.29 \\
-0.29\end{array}$ & & 0.15 & 0.04 & & \begin{tabular}{|l|l|}
0.02 \\
\end{tabular} & & & & & \begin{tabular}{|l|l|}
0.19 \\
\end{tabular} & & & 4.81 & & 年.89 & & & 3.28 & & 22.74 & & & & & & $\overrightarrow{0.0}$ & & $\frac{0.03}{0.03}$ & & & & $\frac{10}{10}$ \\
\hline & 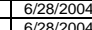 & $\begin{array}{l}3: 04: 50 . \\
33: 11: 50 .\end{array}$ & 1.84 & \begin{tabular}{|c|c|} 
& -0.26 \\
-0.24
\end{tabular} & \begin{tabular}{|l|l|}
3.48 \\
3.47
\end{tabular} & $\begin{array}{l}0.16 \\
0.15\end{array}$ & \begin{tabular}{|l|l|}
0.04 \\
0.04
\end{tabular} & \begin{tabular}{|l|l|} 
& -0.09 \\
\end{tabular} & \begin{tabular}{|l|l|}
0.02 \\
\end{tabular} & \begin{tabular}{|l|l|}
0.06 \\
0.05
\end{tabular} & \begin{tabular}{|l|l|} 
& -0.17 \\
\end{tabular} & \begin{tabular}{l|l}
7 & -0.08 \\
\end{tabular} & $\frac{8}{0}$ & \begin{tabular}{|l|l|} 
& 0.19 \\
\end{tabular} & \begin{tabular}{l|l}
$y^{\prime}$ & -0.0 \\
& -0.0
\end{tabular} & & $\begin{array}{l}5.11 \\
484\end{array}$ & $\frac{4.1}{4.4}$ & \begin{tabular}{|l|l|}
82.46 \\
86.62
\end{tabular} & 0.84 & 24.14 & 3.24 & 4 & 20.71 & & 77.45 & . & 0.0 & & 0.04 & & $\begin{array}{l}0.03 \\
0.03 \\
.03\end{array}$ & & & 0.03 & $\begin{array}{ll}10.5 \\
10.7\end{array}$ \\
\hline & & $1: 8: 50$. & & -0.18 & \begin{tabular}{|l|}
3.47 \\
\end{tabular} & 0.16 & \begin{tabular}{|l}
0.04 \\
\end{tabular} & -0.09 & -0.02 & 0.06 & & \begin{tabular}{|l|l|l}
7 & -0.12 \\
\end{tabular} & -0.02 & 0.19 & \begin{tabular}{|c|c|}
3 & -0.0 \\
\end{tabular} & & 4.964 & & \begin{tabular}{|l|l|}
95.4 \\
\end{tabular} & & & & & & & & & & & & & & & & & 10.7 10. \\
\hline & & & 1.86 & $\frac{-0.18}{-0.17}$ & \begin{tabular}{|l|}
3.45 \\
3.47
\end{tabular} & \begin{tabular}{|l|l|l|}
0.16 \\
0.16
\end{tabular} & \begin{tabular}{|l|l|}
0.04 \\
0.04
\end{tabular} & 0.09 & \begin{tabular}{|l|l|}
-0.02 \\
-0.01
\end{tabular} & & & & & \begin{tabular}{|l|l|}
0.19 \\
0.19
\end{tabular} & $\begin{array}{l}-0.02 \\
-0.02\end{array}$ & & $\frac{4.89}{4.83}$ & & $\frac{99.61}{10.03}$ & & $\begin{array}{ll}24.26 \\
20.31\end{array}$ & & & & & 77.80 & & & & $\frac{1.0}{10}$ & & $\frac{0.03}{203}$ & & & & \\
\hline & & & & & & 0.18 & & & & & & & & 0.19 & & & 4.76 & & & & & & & & & & & & & & & & & & & $\frac{11}{11}$ \\
\hline & & & & & & & & & & & & & & & & & & & 88.4 & & & & & & & & & & & & & & & & & \\
\hline & & $\begin{array}{l}13.533 .50 .08 \\
14: 00: 50.87\end{array}$ & $\begin{array}{l}\frac{1.84}{1.84} \\
1.8\end{array}$ & \begin{tabular}{|l|l|}
-0.07 \\
-0.06 \\
\end{tabular} & \begin{tabular}{|l|}
3.48 \\
3.47 \\
\end{tabular} & \begin{tabular}{|l|l|}
0.18 \\
0.16
\end{tabular} & $\begin{array}{l}0.04 \\
0.04\end{array}$ & \begin{tabular}{|l|l|}
-0.09 \\
-0.08 \\
\end{tabular} & \begin{tabular}{|l|l|}
-0.02 \\
-0.02
\end{tabular} & \begin{tabular}{|l|l|}
0.07 \\
0.07 \\
\end{tabular} & \begin{tabular}{|l|l|}
-0.18 \\
-0.20
\end{tabular} & $\begin{array}{l}8 \\
0 \\
0\end{array}$ & $\begin{array}{l}\frac{2}{2} \\
2 \\
\end{array}$ & \begin{tabular}{|l|l|}
0.16 \\
0.16
\end{tabular} & 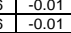 & \begin{tabular}{|l|}
-2.67 \\
-2.67 \\
\end{tabular} & $\begin{array}{l}4.89 \\
4.80\end{array}$ & & $\frac{1122.6}{112.6}$ & $\begin{array}{l}1.03 \\
0.95\end{array}$ & $\begin{array}{l}24.47 \\
24.45\end{array}$ & 3.39 & 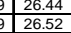 & 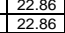 & & $\begin{array}{l}78.27 \\
78.38\end{array}$ & 0 & & & $\frac{0.00}{0.00}$ & & 0.02 & 4.40 & & & $2.3 \mathrm{C}$ \\
\hline & $281 / 200$ & 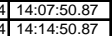 & $\frac{1.84}{1.84}$ & \begin{tabular}{|l|l|l|}
-0.04 \\
0.00
\end{tabular} & \begin{tabular}{|l|}
3.45 \\
\end{tabular} & $\begin{array}{l}0.18 \\
0.16\end{array}$ & \begin{tabular}{|l|l|}
0.04 \\
0.04
\end{tabular} & \begin{tabular}{|l|l|}
-0.09 \\
-0.08
\end{tabular} & \begin{tabular}{|l|l|}
-0.02 \\
-0.02
\end{tabular} & \begin{tabular}{|l|l|}
0.07 \\
0.07
\end{tabular} & \begin{tabular}{|l|l|}
-0.20 \\
-0.20
\end{tabular} & $\begin{array}{l}-0.12 \\
-0.12 \\
-\frac{1}{2}\end{array}$ & 0.000 & \begin{tabular}{|l|l|}
0.16 \\
0.16
\end{tabular} & $\begin{array}{l}-0.02 \\
-0.01 \\
-0.0\end{array}$ & & $\begin{array}{l}4.87 \\
4.92\end{array}$ & & $\begin{array}{ll}17.01 \\
77.01\end{array}$ & & $\begin{array}{ll}24.53 \\
24.49\end{array}$ & $\frac{23.39}{23.52}$ & & 22.86 & & $\begin{array}{l}78.50 \\
78.62 \\
\end{array}$ & 0.10 & $\begin{array}{l}-\frac{-0.0}{-0.0} \\
-x_{1}\end{array}$ & 0.11 & $\begin{array}{l}0.00 \\
0.00\end{array}$ & .02 & $\begin{array}{l}0.00 \\
0.00\end{array}$ & & & $\begin{array}{l}0.03 \\
0.03\end{array}$ & E12.7 \\
\hline & & & 1.84 & \begin{tabular}{|l|l|l|l}
0.02 \\
.029
\end{tabular} & \begin{tabular}{|l|l|}
3.47 \\
\end{tabular} & 0.18 & $\begin{array}{l}0.04 \\
0.04\end{array}$ & -0.08 & \begin{tabular}{|c|c|}
-0.02 \\
\end{tabular} & & \begin{tabular}{|l|l|}
-0.20 \\
\end{tabular} & \begin{tabular}{l|l}
0 & -0.12 \\
& 0.12 \\
\end{tabular} & & \begin{tabular}{|l|l|}
0.17 \\
\end{tabular} & 00 & & 4.97 & & $\frac{\frac{11.70}{117.01}}{11.06}$ & 0.19 & 24.45 & $\frac{23.36}{23.39}$ & & 2017 & & $\frac{10.06}{78.73}$ & $\begin{array}{l}0.00 \\
0.10 \\
\end{array}$ & & $\frac{0.12}{0.12}$ & $\begin{array}{l}0.00 \\
0.00 \\
0.00\end{array}$ & .02 & 200 & & & $\frac{0.03}{0.03}$ & \\
\hline & & & $\frac{1.84}{1.84}$ & $\frac{0.04}{0.09}$ & $\frac{3.47}{3.47}$ & 0.14 & 0.04 & & $\frac{-0.02}{-0.02}$ & & & & & & & & $\frac{4.84}{4.85}$ & & & & & & & & & & & & & & & & & & & \\
\hline & 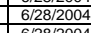 & 14:42:50.87 & 1.84 & \begin{tabular}{|l|l|}
0.11 \\
\end{tabular} & $\begin{array}{l}3.43 \\
3.45\end{array}$ & \begin{tabular}{|l|l|} 
& .14 \\
\end{tabular} & $\begin{array}{l}0.04 \\
0.04\end{array}$ & \begin{tabular}{|l|l|} 
& -0.08 \\
\end{tabular} & \begin{tabular}{|c|c|}
-0.02 \\
-0.01
\end{tabular} & \begin{tabular}{|l|l|} 
& 0.07 \\
\end{tabular} & \begin{tabular}{|l|l|}
-0.19 \\
\end{tabular} & \begin{tabular}{l|l}
9 & -0.12 \\
& -1.12
\end{tabular} & $\begin{array}{ll}2 & -0.02 \\
2 & -0.02 \\
\end{array}$ & \begin{tabular}{|l|l|}
0.16 \\
0.10
\end{tabular} & \begin{tabular}{|c|c|c|}
5 & -0.02 \\
\end{tabular} & $\frac{-2.68}{-2.67}$ & \begin{tabular}{|l|l}
4.83 \\
.521
\end{tabular} & 4.45 & $\frac{121.43}{1.2509}$ & -0.18 & 24.41 & 23.44 & $\begin{array}{l}4 \\
4 \\
569.96\end{array}$ & 22.91 & & 79.08 & ne & & & $\frac{0.0}{20}$ & & & & & 0.03 & 13. \\
\hline & & 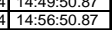 & $\frac{1.84}{1.82}$ & \begin{tabular}{|l|}
0.13 \\
0.15 \\
\end{tabular} & \begin{tabular}{|l|}
3.45 \\
3.45 \\
\end{tabular} & \begin{tabular}{|l|l|}
0.15 \\
\end{tabular} & \begin{tabular}{|l|l|}
0.04 \\
\end{tabular} & \begin{tabular}{|l|l|}
-0.07 \\
-0.08 \\
\end{tabular} & \begin{tabular}{|l|l|}
-0.01 \\
-0.02
\end{tabular} & \begin{tabular}{|l|}
0.07 \\
0.07 \\
\end{tabular} & \begin{tabular}{|l|l|}
-0.19 \\
-0.20
\end{tabular} & $\begin{array}{l}9 \\
0 \\
0\end{array}$ & $\frac{0.00}{-0.02}$ & $\begin{array}{l}0.19 \\
0.17\end{array}$ & $\begin{array}{c}-0.02 \\
7 \\
\end{array}$ & & $\frac{5.21}{4.93}$ & & $\frac{125.85}{125.59}$ & & 24.42 & & & $\frac{23.00}{22.98}$ & & & & & & .0 & & 0.0 & & & .03 & \\
\hline & 8012 & & $\frac{1.82}{184}$ & $\frac{0.18}{0.022}$ & \begin{tabular}{|l|}
3.43 \\
3.33
\end{tabular} & \begin{tabular}{|l|l|}
0.14 \\
0.15
\end{tabular} & 0.04 & & \begin{tabular}{|l|}
-0.02 \\
-0.02
\end{tabular} & & \begin{tabular}{|l|l|} 
& -0.20 \\
-020
\end{tabular} & \begin{tabular}{l|l} 
& -0.12 \\
0 & -0.12
\end{tabular} & & & -01 & & $\begin{array}{ll}4.86 \\
488\end{array}$ & & $\frac{125.59}{125.99}$ & & $\begin{array}{l}24.46 \\
22.40\end{array}$ & & & & & 79.44 & & & & & & & & & & \\
\hline & & & & 0.2 & & 0.14 & 0.04 & 0.05 & & & $\begin{array}{l}-0.20 \\
-0.20 \\
-10\end{array}$ & $\frac{-1.12}{-0.12}$ & & & $=0.6$ & & & & & & & & & 20.96 & & & & & & & & & & & & \\
\hline & & & & & & & 0. & & & & & & & 0. & & & & & & & & & & & & & & & & & & & & & & 155 \\
\hline & $8 / 2$ & $11: 3: 3: 150.80 .87$ & $\frac{1.84}{1.82}$ & $\frac{0.28}{0.29}$ & $\begin{array}{l}3.45 \\
3.45\end{array}$ & \begin{tabular}{|l|l|}
0.15 \\
0.16
\end{tabular} & \begin{tabular}{|l|l|}
0.04 \\
0.04
\end{tabular} & $\frac{-0.09}{-0.09}$ & \begin{tabular}{|l|l|}
-0.02 \\
-0.02
\end{tabular} & $\frac{2.07}{0.07}$ & \begin{tabular}{|l|l|}
-0.20 \\
-0.20
\end{tabular} & $\frac{-.12}{-0.12}$ & & $\frac{0.19}{0.16}$ & & & $\frac{2.57}{4.87}$ & & $\frac{125.85}{130.00}$ & & & 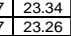 & & & & $\frac{79 .}{80 .}$ & $\frac{108}{0.8}$ & & & $\frac{.00}{0.0}$ & & 0.001 & & & $\frac{0.03}{0.03}$ & \\
\hline & & 15:42:22.87 & 1.31 & 0.28 & & \begin{tabular}{|l|l|}
0.08 \\
\end{tabular} & 0.04 & -0.20 & \begin{tabular}{|c|c|}
-0.02 \\
\end{tabular} & $\begin{array}{l}.00 \\
\end{array}$ & \begin{tabular}{|l|l|}
-0 \\
\end{tabular} & $\frac{-0.14}{-0.14}$ & & \begin{tabular}{|l|l|}
0.14 \\
\end{tabular} & 4 & $\frac{.16}{98}$ & 0.15 & & $\begin{array}{l}121.17 \\
123017 \\
\end{array}$ & & 24.199. & 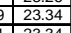 & & 22.96 & & 80.0 & . & & & 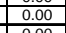 & & 0.0 & & & & \\
\hline & & & & & & \begin{tabular}{|l|l|}
0.11 \\
0.11
\end{tabular} & \begin{tabular}{|l|}
0.04 \\
0.04
\end{tabular} & & & & & & & & & & & & & & & & & & & & & & & & & & & & & \\
\hline & $28 / 2$ & 16:02:42.87 & 1.84 & 0.33 & \begin{tabular}{|l}
3.39 \\
\end{tabular} & \begin{tabular}{|l|l|}
0.11 \\
0.16
\end{tabular} & 0.05 & -0.11 & -0.02 & $\frac{0.05}{0.07}$ & \begin{tabular}{|l|l|} 
& -.020 \\
\end{tabular} & \begin{tabular}{l|l}
0 & -0.12 \\
\end{tabular} & 0.00 & 0.20 & $=0.02$ & -2.89 & 2.55 & 2.25 & 130.00 & 0.95 & 24.23 & 23.26 & $\begin{array}{l}626.78 \\
\end{array}$ & 23.04 & & 80.42 & 0.08 & & .03 & 00 & & .00 & & & 0.02 & \\
\hline & & & 1.82 & & & & & & & & & & & & & & & & & & & & & & & & & & & & & & & & & 16.1 \\
\hline & 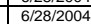 & 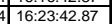 & 1.84 & $\frac{.0 .40}{0.40}$ & & $\frac{1.14}{0.14}$ & $\frac{0.04}{0.04}$ & & $\frac{0.02}{-0.02}$ & & & -0.1 & & $\frac{2.25}{0.25}$ & & & & & & & & & & & & & & & & & & & & & & 16. \\
\hline & & & & 0.4 & & 0. & 0. & & -0.02 & & & $-c$ & & 0.27 & & & & & & & & & & & & & & & & & & 0.0 & 4.40 & & & \\
\hline & t & & & $\frac{0.40}{0.42}$ & & \begin{tabular}{|l|l|}
0.11 \\
0.15
\end{tabular} & \begin{tabular}{|l|l|}
0.04 \\
\end{tabular} & & \begin{tabular}{|l|l|}
-0 \\
-0
\end{tabular} & & & $\frac{-0.12}{0.12}$ & & \begin{tabular}{|l|l|}
0.28 \\
0.30
\end{tabular} & & & $\frac{2.92}{2.92}$ & & $\frac{134.42}{134.42}$ & 0.47 & 44.40 & & & & & $\frac{81.00}{81.12}$ & $\frac{0.04}{-0.04}$ & & & & & & & & $\frac{.02}{.02}$ & 16. \\
\hline & (5) & $\begin{array}{c}16: 51: 42.87 \\
1051: 07\end{array}$ & $\frac{1.82}{1.82}$ & 0.46 & \begin{tabular}{|l|l}
3.41 \\
\end{tabular} & \begin{tabular}{|l|l|}
0.19 \\
020
\end{tabular} & \begin{tabular}{|l|l|}
0.04 \\
004
\end{tabular} & -0.10 & \begin{tabular}{|l|l|}
-0.02 \\
0.02
\end{tabular} & \begin{tabular}{|l|l|}
0.06 \\
0.06
\end{tabular} & \begin{tabular}{|l|l|} 
& -0.20 \\
\end{tabular} & \begin{tabular}{|l|l|}
-0.12 \\
12
\end{tabular} & & \begin{tabular}{|l|l|} 
\\
\end{tabular} & & $\frac{-2.88}{.89}$ & $\begin{array}{l}3.73 \\
3.38\end{array}$ & $\begin{array}{l}3.36 \\
3.36\end{array}$ & $\frac{134.42}{12.42}$ & $\frac{-0.08}{0.07}$ & & 3.47 & 烈 & & & & 0.00 & & -0.02 & 00 & & & & & & $\frac{17.7}{17.7}$ \\
\hline & & & & 0.48 & & & & & & & & & & & & & & & & & & & & & & & & & & & & & & & & \\
\hline & & & & $\frac{0.48}{0.48}$ & & 0 & & & & & & & & & & & & & & & & & & & & & & & & & & & & & & \\
\hline & & & & & & & 0.0 & & & & & & & & & & .25 & & 134.42 & & & & & & & & & & & & & & & & & \\
\hline & 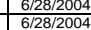 & $\begin{array}{ll}17: 26.42 .87 \\
1733: 42.87\end{array}$ & $\frac{1.82}{1.84}$ & $\frac{0.51}{0.55}$ & $\frac{5.43}{3.39}$ & $\frac{0.26}{0.29}$ & 0.08 & $\frac{0.00}{0.03}$ & \begin{tabular}{|l|l|}
-0.02 \\
-0.02
\end{tabular} & $\frac{.09}{0.09}$ & \begin{tabular}{|l|l|} 
& -0.20 \\
\end{tabular} & $\frac{-0.12}{-0.12}$ & -0.02 & $\frac{0.30}{0.31}$ & -0.01 & -2.88 & $\frac{2.55}{3.83}$ & & 134.16 & 0.89 & $\frac{24.65}{24.53}$ & $\frac{23.52}{23.47}$ & 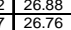 & 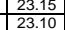 & & $\frac{81.82}{81.93}$ & $\frac{10}{10}$ & & $\frac{0.11}{0.11}$ & 0.000 & 0.02 & 0.00 & & & $\frac{0.02}{0.02}$ & 218. \\
\hline & & & & 0.55 & & & & & & & $\begin{array}{l} \\
\end{array}$ & -0.12 & & 0.31 & & & 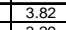 & & & & & & & & & & & & & & & & & & & \\
\hline & & & & 0.5 & & 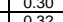 & & & & & & & & & & & & & & & & & & & & & & & & & & & & & & \\
\hline & & & & -0.4 & & 0.29 & & & & & & $\frac{-0.13}{-0.13}$ & & & & -2.90 & 0.14 & & 147.41 & & 2.34 & & & & & & & & & & & & & & 0.00 & \\
\hline & & & 0.006 & $\begin{array}{l}-0.42 \\
-0.044\end{array}$ & \begin{tabular}{|l|l|}
0.82 \\
0.86
\end{tabular} & \begin{tabular}{|l|l|}
0.29 \\
0.29
\end{tabular} & 0.05 & 0.000 & $\mid \begin{array}{l}\mid=0.02 \\
-0.02\end{array}$ & 0.04 & -0 & 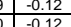 & \begin{tabular}{|c|c|}
2 & -0.02
\end{tabular} & \begin{tabular}{|l|l|l|}
026 \\
\end{tabular} & & $\begin{array}{l}-2.90 \\
-2.90 \\
\end{array}$ & \begin{tabular}{|l|l|}
0.14 \\
0.15
\end{tabular} & 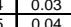 & $\frac{142.99}{13884}$ & 13 & $\frac{22.25}{2234}$ & $\frac{22.32}{2241}$ & $\begin{array}{l}25.05 \\
125.54 \\
1254\end{array}$ & 2212 & & $\begin{array}{l}87.46 \\
87.58\end{array}$ & 0.10 & $\begin{array}{l}-0.01 \\
-0.01\end{array}$ & 0.08 & \begin{tabular}{|l|l|} 
& 0.00 \\
0.00
\end{tabular} & .01 & . & & & 0.00 & \\
\hline & & & 0.83 & $\begin{array}{l}-.44 \\
-0.42 \\
-1\end{array}$ & 0.80 & \begin{tabular}{|l|l}
0.29 \\
0.38
\end{tabular} & & & & & & & & & & & \begin{tabular}{|l|l|l} 
\\
7.466
\end{tabular} & & & & & & & & & $\begin{array}{l}87.70 \\
87\end{array}$ & & & & & & & & & & \\
\hline & & 8:48:53.37 & 1.95 & -0.42 & 52 & 0.38 & 0.05 & & -0.02 & & -0 & -0.02 & & 0.32 & & -2.71 & 7.08 & & & & & & & & & & & & & & & & & & & \\
\hline & $99 / 20$ & :53.37 & 92 & $\begin{array}{l}-0.46 \\
-0.44 \\
-\frac{1}{2}\end{array}$ & & 0.38 & $\frac{0.05}{0.05}$ & $\frac{0.11}{0109}$ & \begin{tabular}{|l|l|}
-0.02 \\
-0.02 \\
\end{tabular} & $\frac{0.14}{0.14}$ & \begin{tabular}{|l|l|}
-0.20 \\
-0.19
\end{tabular} & \begin{tabular}{|l|l|}
0.01 \\
0.02
\end{tabular} & $\frac{0.00}{0.00}$ & 0.32 & \begin{tabular}{|l|l|}
-0.01 \\
-0.02
\end{tabular} & $\begin{array}{l}-2.69 \\
-2.69 \\
\end{array}$ & $\frac{7.28}{7.24}$ & \begin{tabular}{l|l} 
& 6.90 \\
& 6.86 \\
\end{tabular} & $\begin{array}{l}134.42 \\
52.07\end{array}$ & & $\begin{array}{l}\frac{9.98}{15.98} \\
\end{array}$ & $\frac{22.31}{22.36}$ & $\begin{array}{l}1 \\
\\
\end{array}$ & $\frac{22.10}{22.06}$ & & $\begin{array}{l}87.93 \\
88.05\end{array}$ & & & & .00 & $\frac{0.09}{0.09}$ & .01 & & & $\begin{array}{l}0.05 \\
0.05\end{array}$ & $\frac{19}{n}$ \\
\hline & & & & & & & & & & & & & & & & & & & & & & & & & & & & & & & & & & & & \\
\hline
\end{tabular}


WSRC-TR-2005-00105, REVISION 0

SRNL-RPP-2005-00012, REVISION 0

Table H3-3-8. CIX Campaign II, Cycle 3, DI Water Post-Elution Rinse

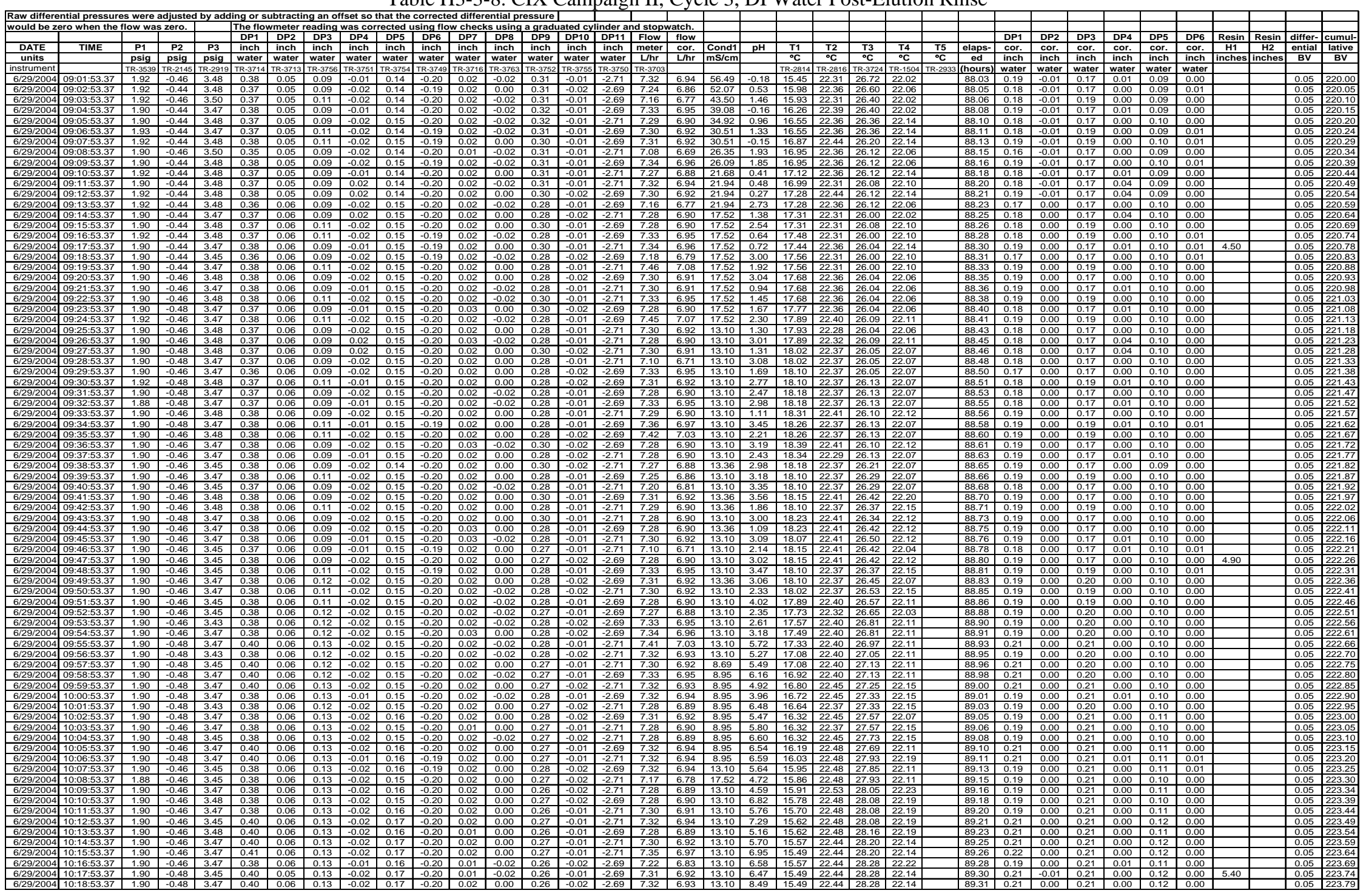


WSRC-TR-2005-00105, REVISION 0

SRNL-RPP-2005-00012, REVISION 0

Table H3-3-9. CIX Campaign II, Cycle 3, 0.25M NaOH Regenerant

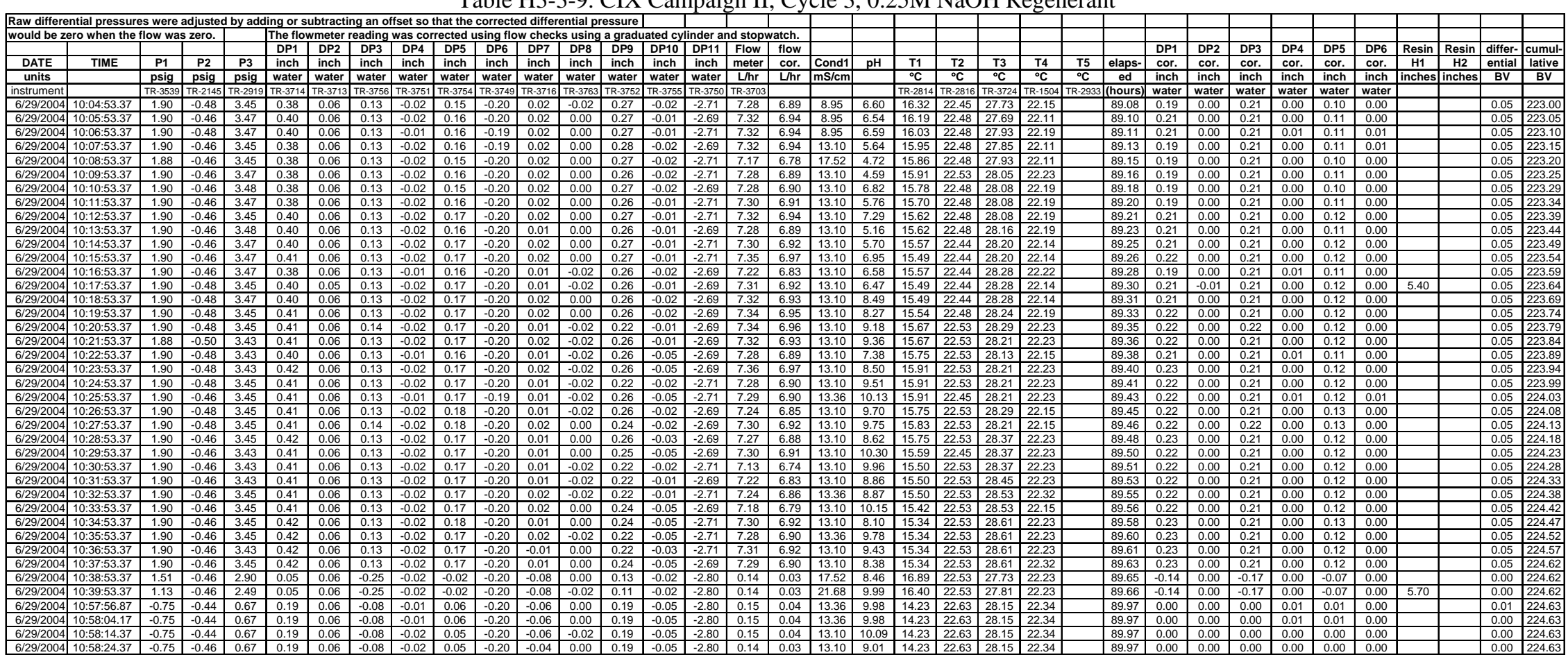


WSRC-TR-2005-00105, REVISION 0

SRNL-RPP-2005-00012, REVISION 0

Table H3-4-1. CIX Campaign II, Cycle 4, LAW Treatment

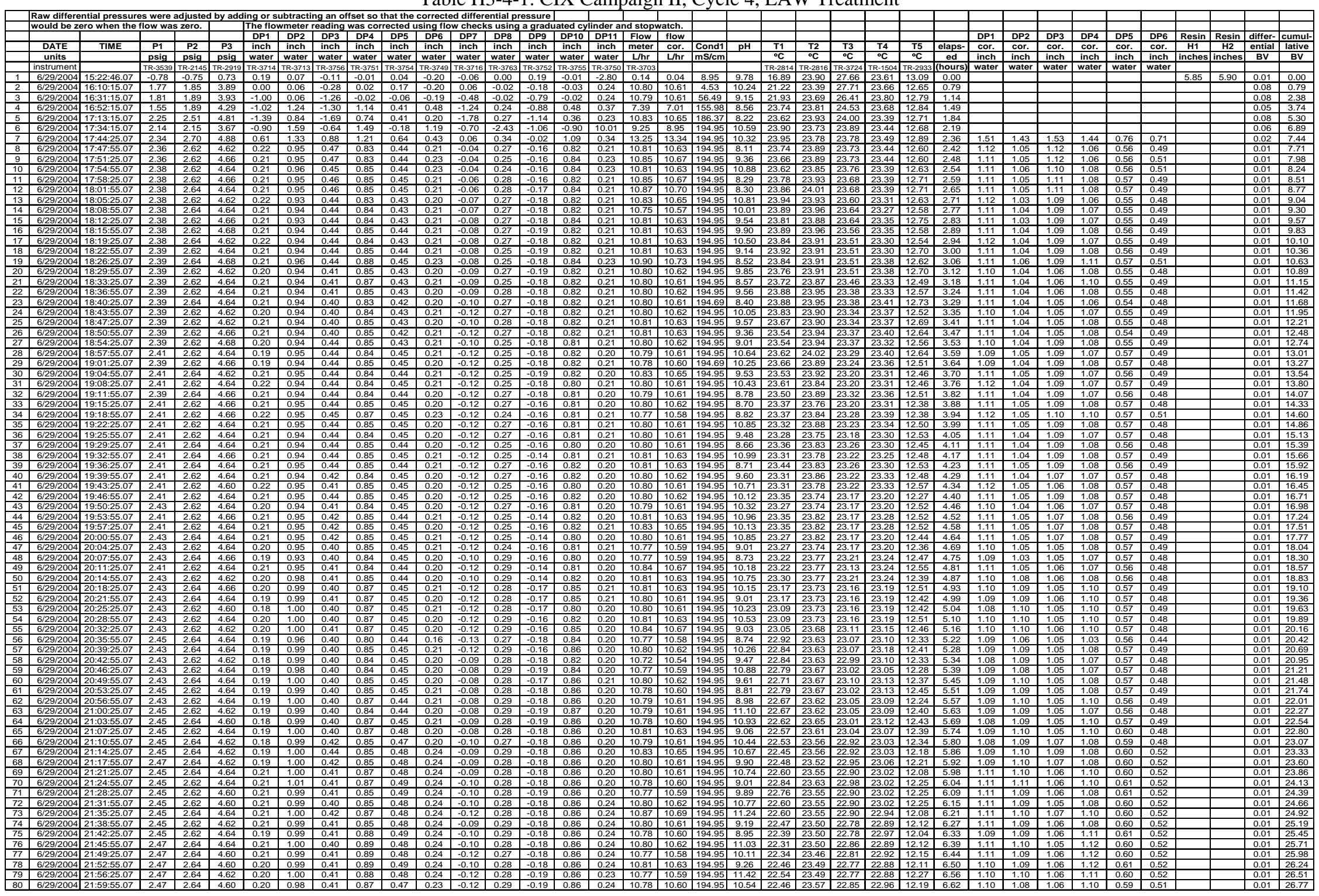


WSRC-TR-2005-00105, REVISION 0

SRNL-RPP-2005-00012, REVISION 0

Table H3-4-2. CIX Campaign II, Cycle 4, LAW Treatment

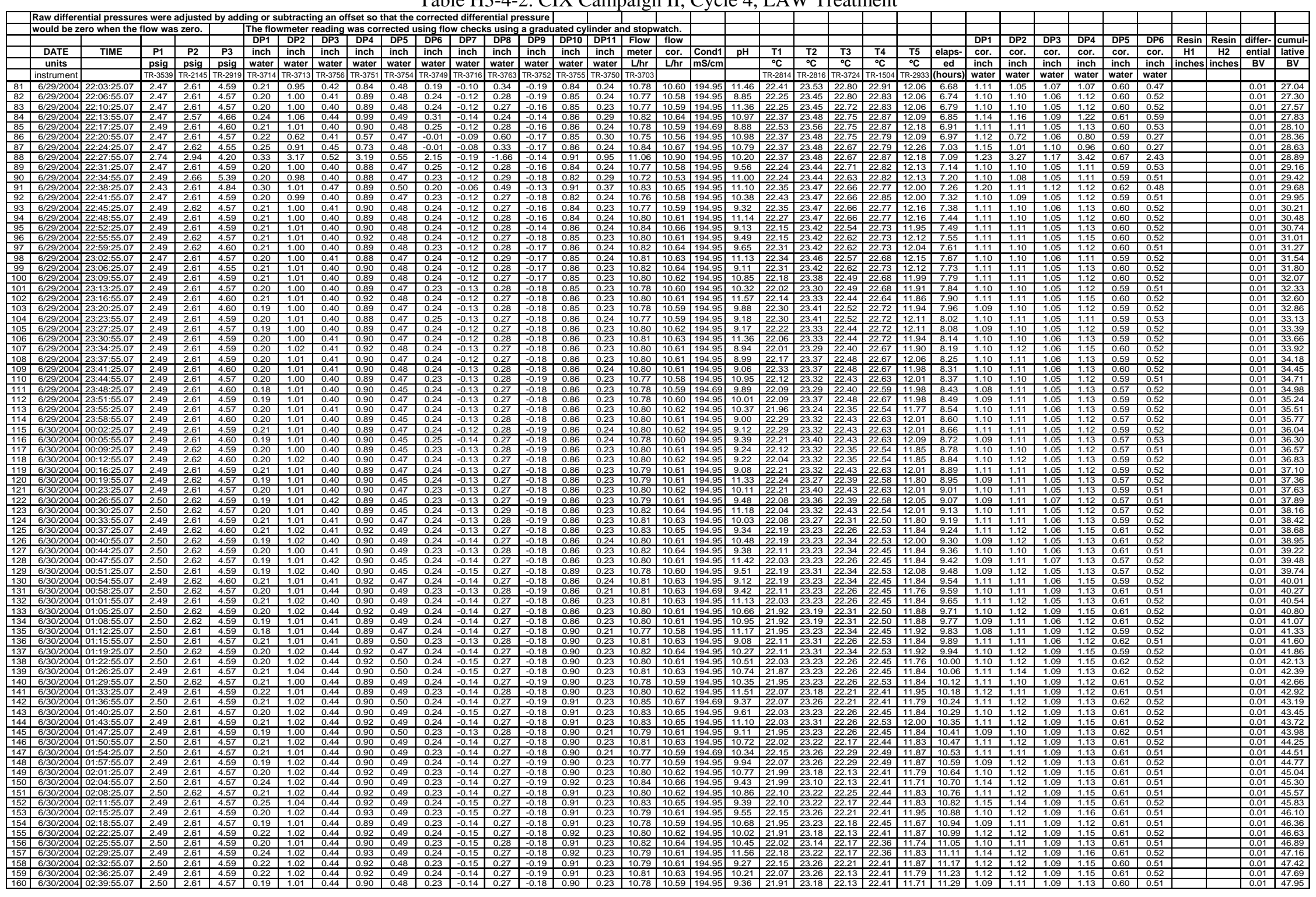


WSRC-TR-2005-00105, REVISION 0

SRNL-RPP-2005-00012, REVISION 0

Table H3-4-3. CIX Campaign II, Cycle 4, LAW Treatment

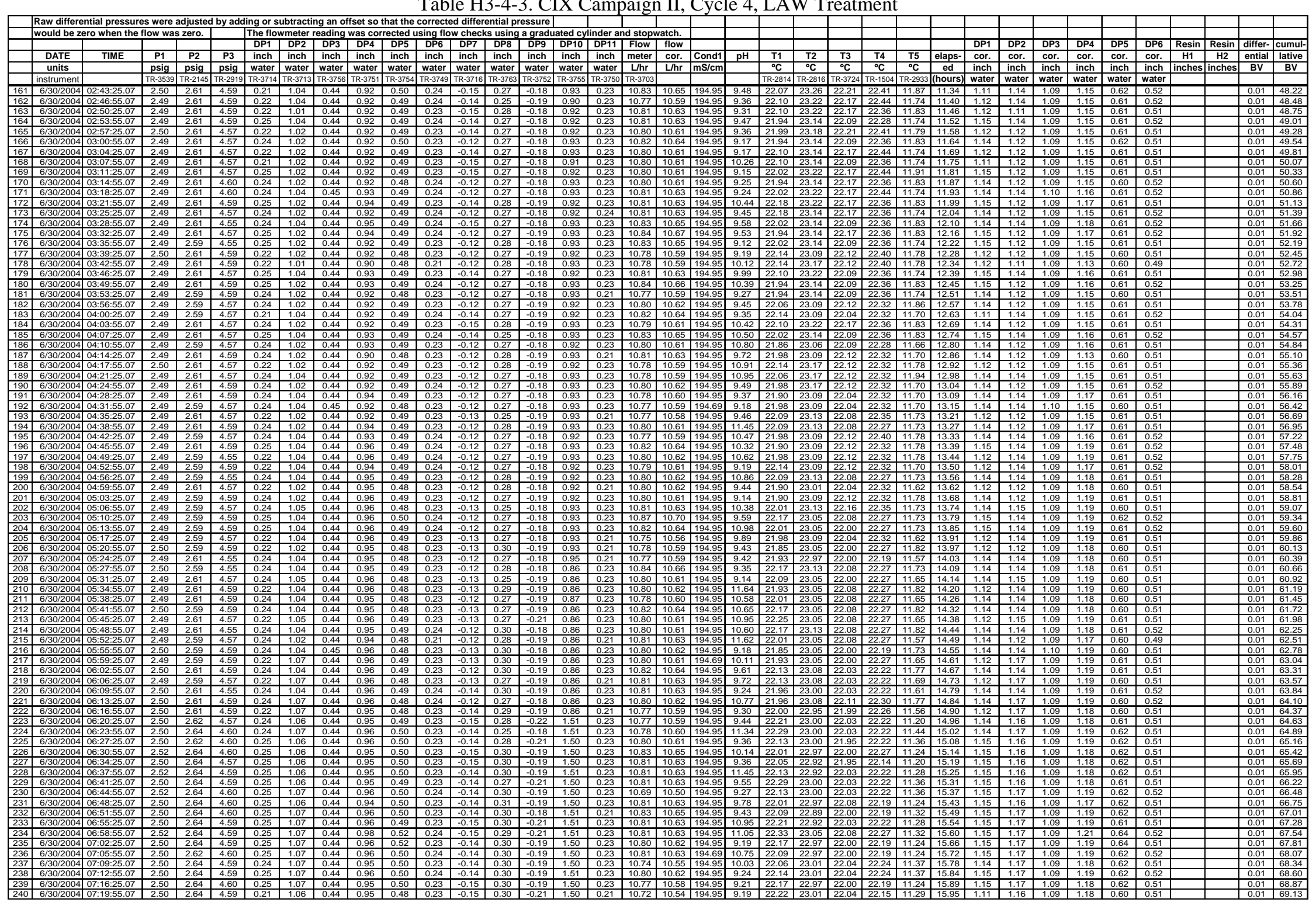


WSRC-TR-2005-00105, REVISION 0

SRNL-RPP-2005-00012, REVISION 0

Table H3-4-4. CIX Campaign II, Cycle 4, LAW Treatment

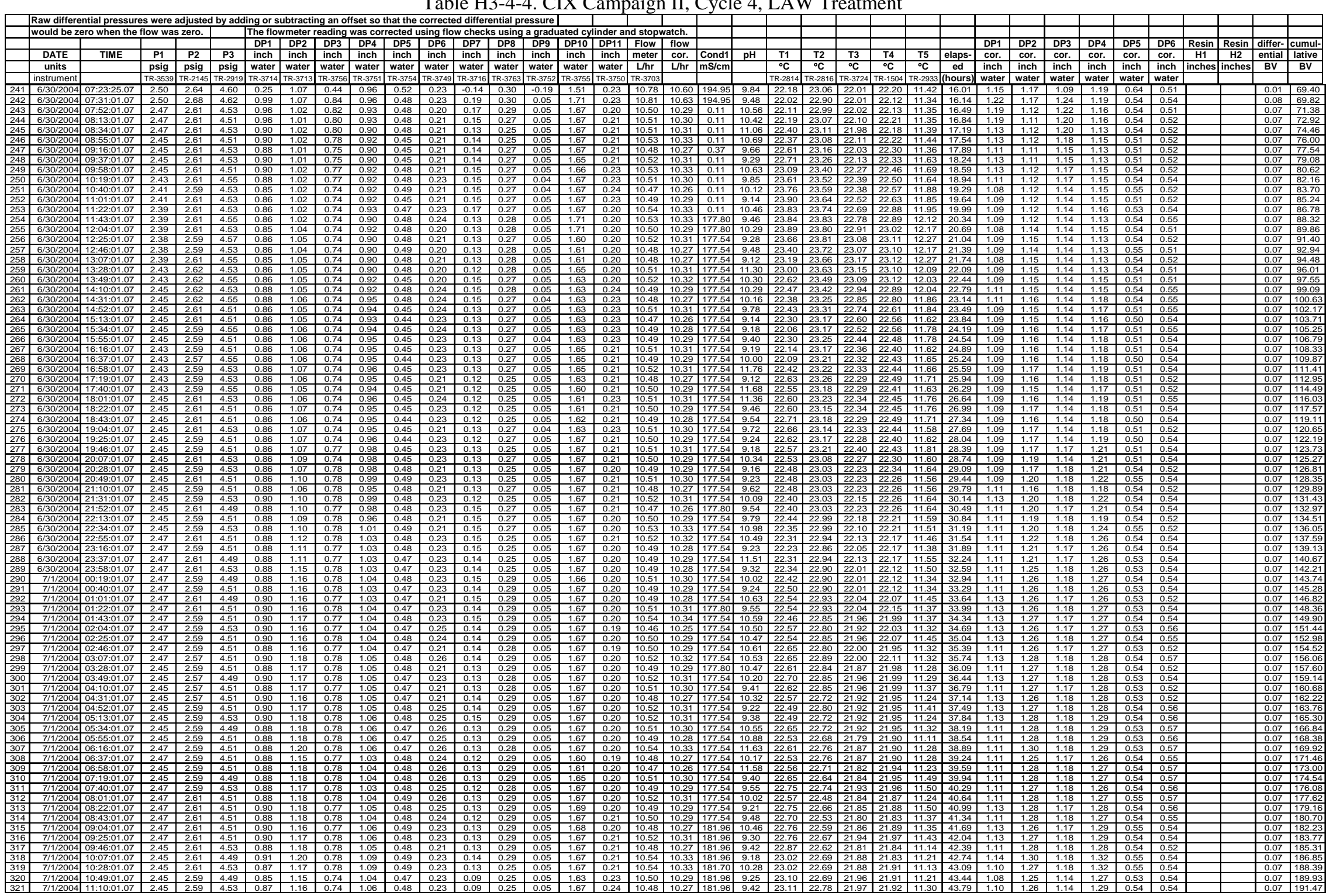


WSRC-TR-2005-00105, REVISION 0

SRNL-RPP-2005-00012, REVISION 0

Table H3-4-5. CIX Campaign II, Cycle 4, 0.1 M NaOH Displacement

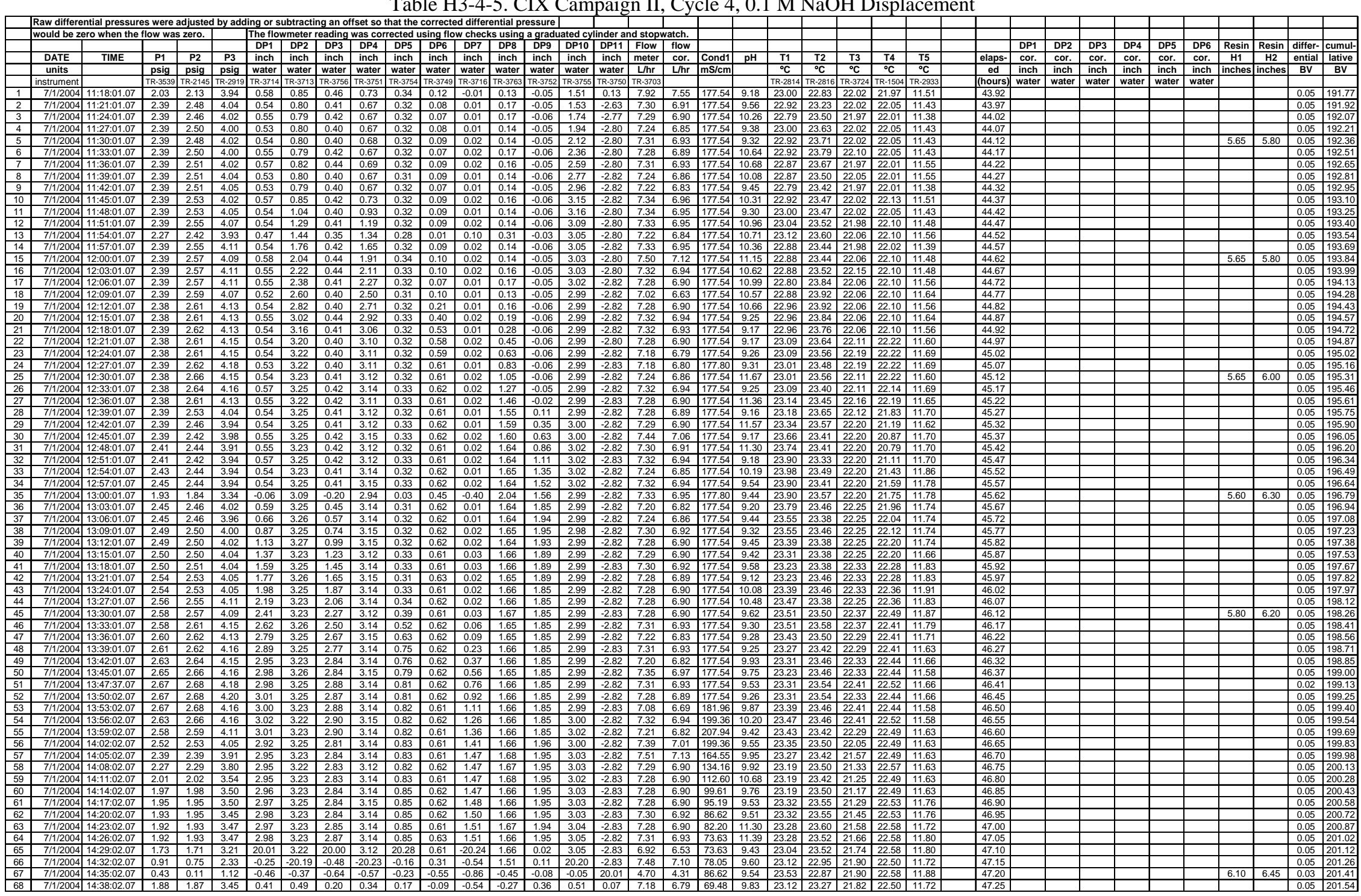


WSRC-TR-2005-00105, REVISION 0

SRNL-RPP-2005-00012, REVISION 0

Table H3-4-6. CIX Campaign II, Cycle 4, DI Water Pre-Elution Rinse

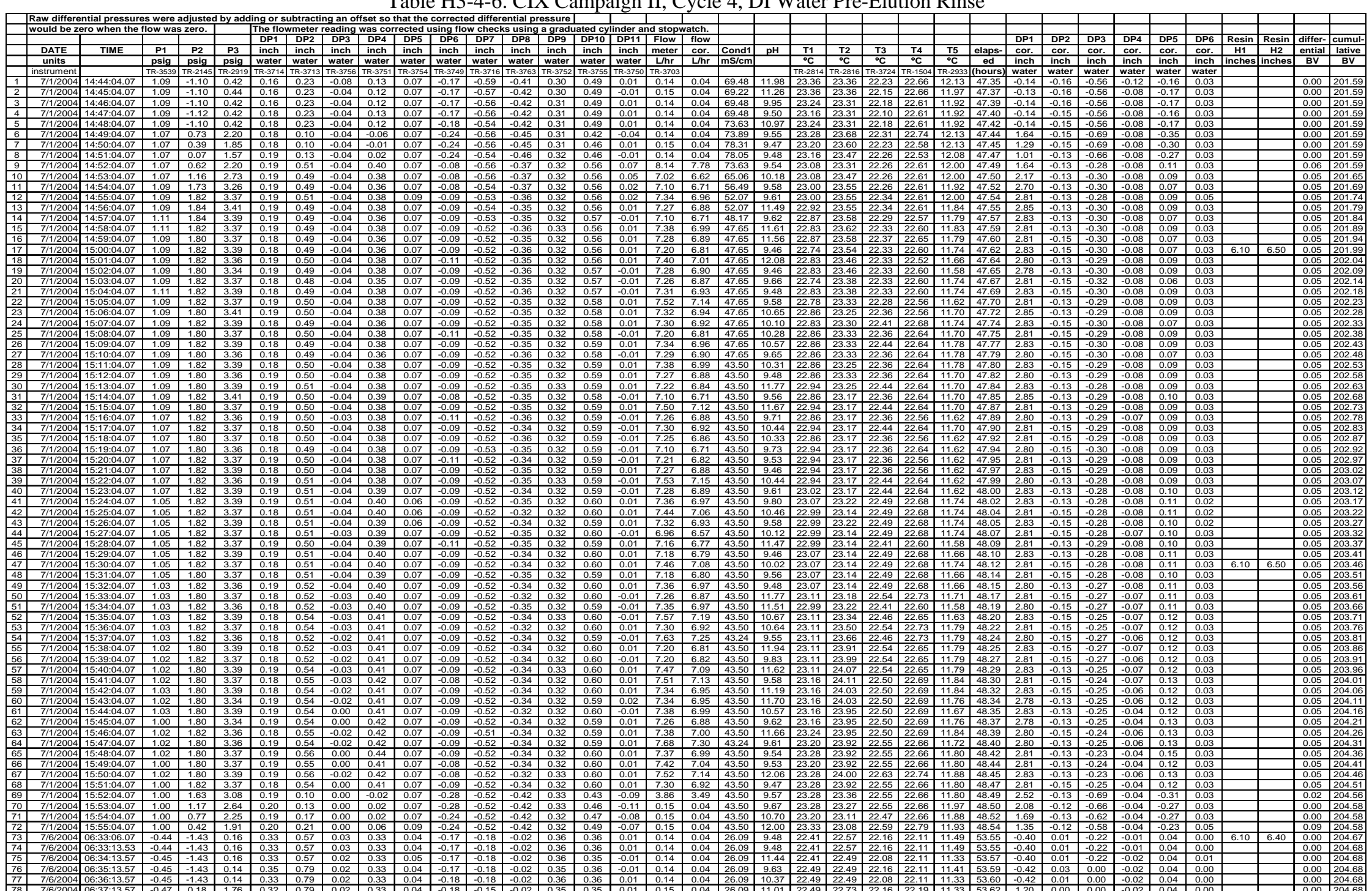


WSRC-TR-2005-00105, REVISION 0

SRNL-RPP-2005-00012, REVISION 0

Table H3-4-7. CIX Campaign II, Cycle 4, 0.5 M Nitric Acid Eluent

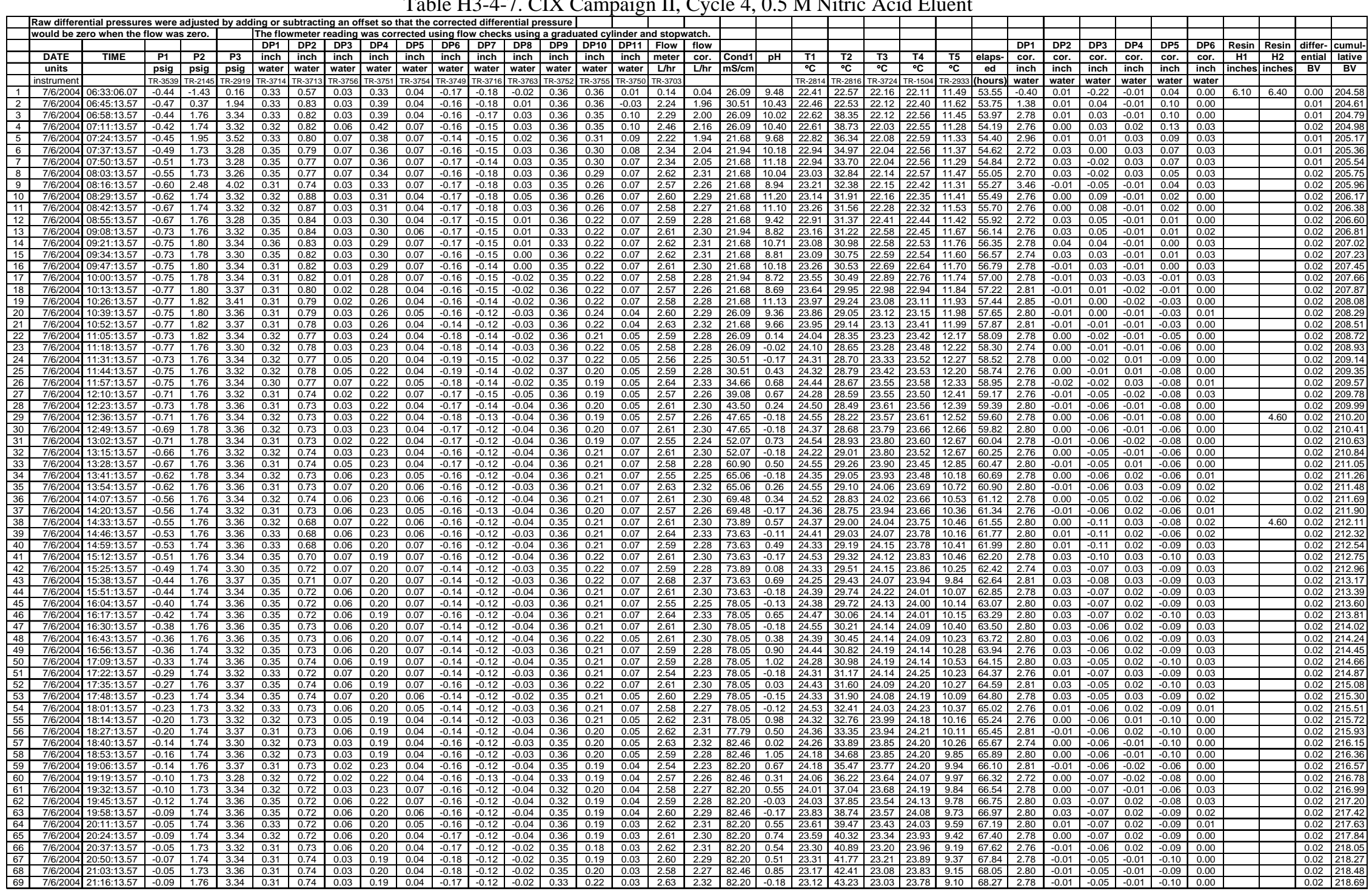


WSRC-TR-2005-00105, REVISION 0

SRNL-RPP-2005-00012, REVISION 0

Table H3-4-8. CIX Campaign II, Cycle 4, DI Water Post-Elution Rinse

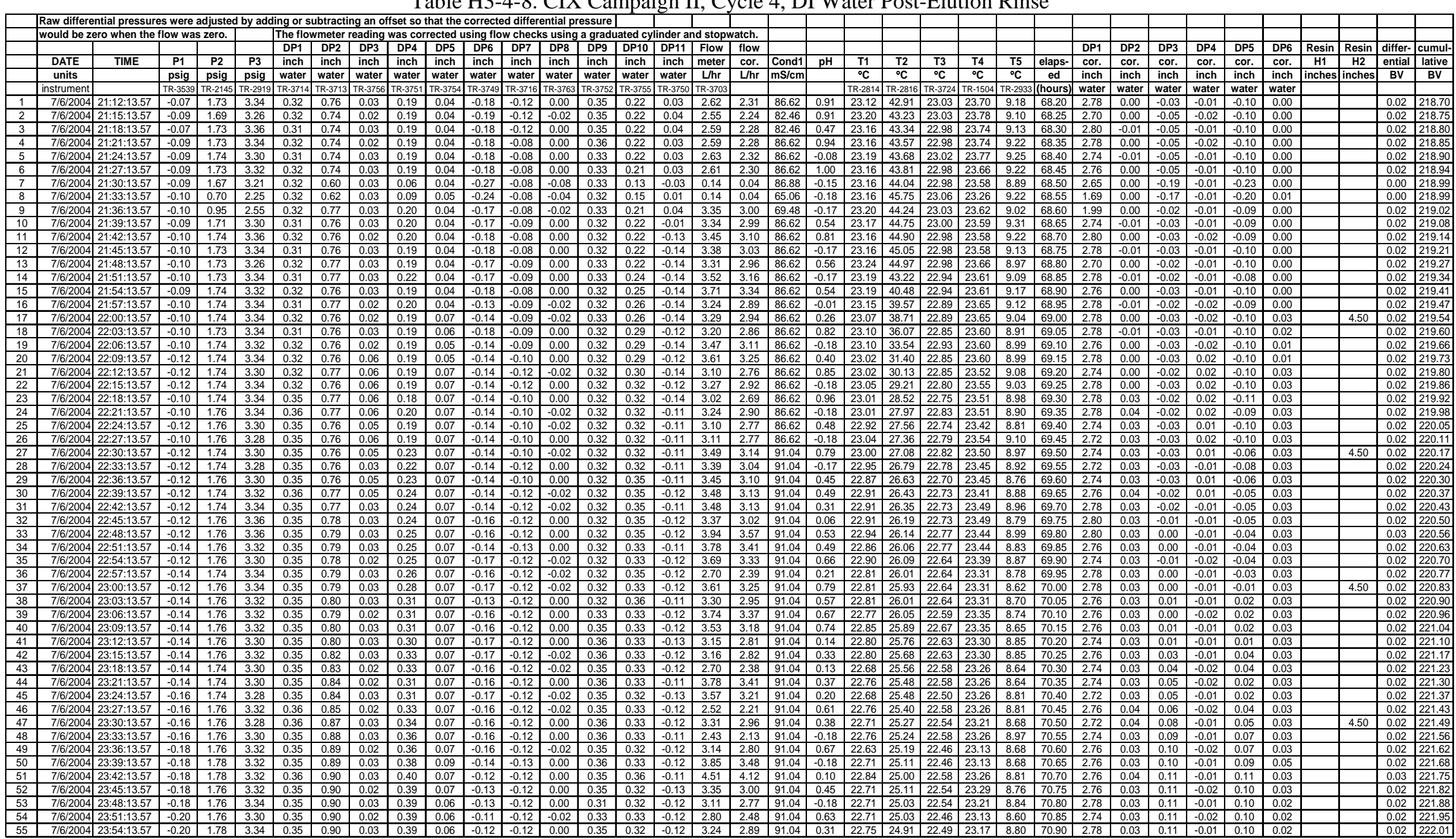


WSRC-TR-2005-00105, REVISION 0

SRNL-RPP-2005-00012, REVISION 0

Table H3-4-9. CIX Campaign II, Cycle 4, 0.25M NaOH Regenerant

\begin{tabular}{|c|c|c|c|c|c|c|c|c|c|c|c|c|c|c|c|c|c|c|c|c|c|c|c|c|c|c|c|c|c|c|c|c|c|c|c|c|c|}
\hline \multirow{3}{*}{\begin{tabular}{|l|l} 
& $R a$ \\
& Re \\
& \\
\end{tabular}} & Raw differ & ntial pressures & s were & adjuste & d by & addin & ng ors & ubtract & ting an of & ffset so & that the & & & & ressure & & & & & & & & & & & & & & & & & & & & & & \\
\hline & would be & ro when the fi & low wa & as zero. & & & the flo & veter & & & & using flo & ow check & ss using & a gradl & 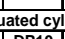 & linder an & ind stop & Owatch. & & & & & & & & & & & & & & & & & & \\
\hline & DATE & TIMEE & $\overline{\mathrm{P} 1}$ & P2 & $\bar{F}_{\mathbf{P}}$ & & $\begin{array}{l}\text { DP1 } \\
\text { inch }\end{array}$ & $\begin{array}{l}\text { DP2 } \\
\text { inch }\end{array}$ & \begin{tabular}{|l|} 
DP3 3 \\
inch
\end{tabular} & \begin{tabular}{|l|} 
DP4 \\
inch \\
\end{tabular} & $\frac{1 \text { D55 }}{\text { inch }}$ & \begin{tabular}{|l|} 
DP66 \\
inch
\end{tabular} & \begin{tabular}{|l|} 
DP7 \\
\end{tabular} & \begin{tabular}{|l|} 
DP8 \\
inch
\end{tabular} & \begin{tabular}{|l|} 
DP9 \\
inch
\end{tabular} & \begin{tabular}{|l|} 
DP10 \\
inch
\end{tabular} & \begin{tabular}{|l|} 
DP11 \\
inch
\end{tabular} & \begin{tabular}{|l|} 
Flow \\
meter \\
\end{tabular} & flow & $\overline{c o n}$ & $\mathrm{NH}$ & T1 & $T 2$ & $T^{3}$ & T4 & 75 & elans- & DP1 & DP2 & DP3 & DP4 & DP5 & DP6 & $\frac{\text { Resin }}{H 1}$ & Resin & differ- & cumul- \\
\hline & units & & psig & psig & & & water & water & Nater & water & Nater & & whater & $\mid$\begin{tabular}{|l|l|} 
water \\
\end{tabular} & \begin{tabular}{|l|} 
water \\
\end{tabular} & water & & L/hr & L/hr & & & & & & & & ed & inch & & & & & $\begin{array}{l}\text { ior. } \\
\text { inch }\end{array}$ & & & & \\
\hline & & & & 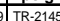 & & & & & & . & & & & | & Thent & Water & & 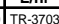 & & & & -28 & & -2 & & (2) & bour & & & & & & & & & & \\
\hline & $7 / 6 / 26$ & 56:13.5 & & 1.43 & 2. & & 0.35 & 0.79 & & 0.29 & 0.07 & & \begin{tabular}{|l|l|} 
& -0.12 \\
\end{tabular} & \begin{tabular}{|l|l|}
-0.04 \\
\end{tabular} & \begin{tabular}{|l|l|}
0.35 \\
\end{tabular} & 0.26 & $\begin{array}{ll}-0.19 \\
\end{array}$ & 0.14 & & & 0.14 & & & & & 8.67 & & & 0.03 & & & 0.00 & 0.03 & & & & 220.50 \\
\hline & $\frac{777 / 20}{717120}$ & $07: 52: 58.07$ & -0.75 & -1.27 & & & 0.32 & 0.89 & 03 & 0.31 & 0.07 & \begin{tabular}{|l|l|}
-0.17 \\
\end{tabular} & \begin{tabular}{|l|l|} 
& -0.12 \\
\end{tabular} & \begin{tabular}{|l|l|}
-0.04 \\
\end{tabular} & \begin{tabular}{|l|l|}
0.32 \\
\end{tabular} & \begin{tabular}{|l|l|}
0.36 \\
\end{tabular} & \begin{tabular}{l|l|}
-0.14 \\
\end{tabular} & \begin{tabular}{|l|l|}
0.14 \\
\end{tabular} & 0.04 & 6.62 & 0.80 & 22.74 & 8.50 & 22.08 & 22.03 & 8.21 & & 0.24 & & & 0.01 & 0.02 & 0.03 & & & & 220.57 \\
\hline & $777 / 70004$ & $07: 56: 58.07$ & -0.77 & & & $\frac{28}{39}$ & 0.31 & 1.01 & & 0.45 & 0.05 & & $\frac{-.12}{0.12}$ & & & $\begin{array}{l}0.40 \\
0.38\end{array}$ & & $\begin{array}{l}7.63 \\
7732\end{array}$ & & & 0.35 & & 70 & & & 8.06 & & & & & & & & & & & 220.58 \\
\hline 5 & $777 / 2004$ & 08:04:58.07 & -0.77 & 2.09 & & 74 & 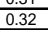 & $\frac{1.01}{1.01}$ & $\begin{array}{l}0.03 \\
0.03 \\
\end{array}$ & 0.46 & 0.05 & $\frac{-0.11}{-0.09}$ & $\frac{-0.12}{-0.12}$ & \begin{tabular}{|l|}
0.06 \\
0.07 \\
\end{tabular} & \begin{tabular}{|l|}
0.32 \\
0.32 \\
\end{tabular} & \begin{tabular}{|l|}
0.38 \\
0.37 \\
\end{tabular} & $\frac{0.04}{0.04}$ & $\frac{7.32}{7.34}$ & $\frac{0.94}{6.95}$ & $\frac{140.03}{112.60}$ & \begin{tabular}{|l|l|l|}
0.16 \\
0.59
\end{tabular} & $\frac{22.67}{22.67}$ & \begin{tabular}{|l|l|}
6.90 \\
7.93
\end{tabular} & $\frac{2.11}{22.17}$ & $\frac{22.45}{2.37}$ & 8.47 & $\frac{76.02}{76.08}$ & $\frac{2.83}{3.18}$ & 0.01 & $\frac{0.22}{0.22}$ & 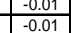 & 0.17 & $\frac{0.01}{0.00}$ & & & $\frac{0.05}{0.05}$ & 220.77 \\
\hline & & & & & & & & & & 0.46 & 0.04 & -0.11 & & & & & & & & & & & & & & & & & & & & & 0.00 & & & & \\
\hline & $\frac{777 / 2004}{7772004}$ & $\begin{array}{l}08: 12: 58.07 \\
08.16\end{array}$ & $\begin{array}{l}-0.77 \\
-0.077\end{array}$ & $\frac{1.74}{174}$ & & $\frac{41}{39}$ & $\begin{array}{l}0.31 \\
0.32\end{array}$ & $\frac{1.01}{102}$ & $\begin{array}{l}0.03 \\
0.03\end{array}$ & 0.46 & 0.05 & -0.11 & \begin{tabular}{|l|l|}
-0.12 \\
-012
\end{tabular} & \begin{tabular}{|l|l|}
0.12 \\
0.13
\end{tabular} & \begin{tabular}{|l|l|}
0.32 \\
032 \\
\end{tabular} & 0.36 & 0.04 & $\begin{array}{l}7.28 \\
734\end{array}$ & $\frac{6.90}{6.95}$ & 99.61 & \begin{tabular}{|c|c|}
0.64 \\
\end{tabular} & $\frac{22.67}{2.271}$ & \begin{tabular}{l|l|}
7 & 11.38 \\
\end{tabular} & 22.17 & $\frac{22.37}{2321}$ & 8.47 & $\begin{array}{l}76.22 \\
7629 \\
\end{array}$ & $\begin{array}{l}2.85 \\
.283\end{array}$ & $\frac{-0.01}{0.01}$ & 0.22 & -0.01 & 0.17 & 0.01 & & & 0.05 & 221.37 \\
\hline 9 & $777 / 72004$ & $\begin{array}{l}08: 16: 58.07 \\
088.20 .50 .07\end{array}$ & $\begin{array}{l}-0.77 \\
-0.78\end{array}$ & \begin{tabular}{|l|l|}
1.74 \\
173
\end{tabular} & & $\frac{39}{36}$ & 0.32 & $\frac{1.02}{1.04}$ & $\begin{array}{l}0.03 \\
0.03\end{array}$ & $\begin{array}{l}0.46 \\
0.46\end{array}$ & $\begin{array}{l}0.04 \\
0.05\end{array}$ & \begin{tabular}{|c|}
-0.11 \\
-0.11 \\
\end{tabular} & \begin{tabular}{|l|}
-0.12 \\
-0.12
\end{tabular} & \begin{tabular}{|l|}
0.13 \\
0.14 \\
\end{tabular} & \begin{tabular}{|l|}
0.32 \\
0.32 \\
\end{tabular} & $\begin{array}{l}0.36 \\
0.366\end{array}$ & $\begin{array}{l}0.04 \\
0.05\end{array}$ & $\begin{array}{l}7.34 \\
7755\end{array}$ & $\begin{array}{l}6.95 \\
717\end{array}$ & 4350 & $\begin{array}{l}-0.17 \\
0.08\end{array}$ & $\frac{2284}{2284}$ & $\frac{19.51}{1912}$ & $\frac{2.48}{2218}$ & 20.45 & $\begin{array}{l}8.43 \\
848\end{array}$ & 76.35 & $\frac{2.050}{280}$ & 0 & $\frac{0.23}{0.25}$ & $\begin{array}{l}-0.01 \\
-0.01\end{array}$ & 0.17 & 0.00 & & & 0.05 & 221.56 \\
\hline & & $08: 24: 58.07$ & & & & & 0.32 & 1.05 & $\begin{array}{l}0.03 \\
0.03\end{array}$ & $\frac{0.40}{0.46}$ & 0.05 & & & \begin{tabular}{|l|}
0.14 \\
0.14 \\
\end{tabular} & \begin{tabular}{|l|}
0.32 \\
0.32 \\
\end{tabular} & $\begin{array}{l}0.30 \\
0.35\end{array}$ & & & & & \begin{tabular}{|l}
0.00 \\
0.73
\end{tabular} & & & 22.18 & & $\frac{8.48}{8.33}$ & 76.35 & $\frac{2.80}{2.78}$ & & & & & $\frac{0.01}{0.00}$ & & & & 221.76 \\
\hline & $717 / 200$ & $08: 28: 58.07$ & -0.82 & 1.71 & & 34 & 0.32 & 0.99 & 0.02 & 0.46 & 0.04 & \begin{tabular}{|l|}
-0.09 \\
\end{tabular} & 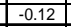 & \begin{tabular}{|l|}
0.16 \\
\end{tabular} & \begin{tabular}{|c|}
0.32 \\
\end{tabular} & \begin{tabular}{|l|}
0.36 \\
\end{tabular} & 0.05 & 7.48 & 7.10 & 17.52 & -0.08 & & .110 & & 2.43 & $\frac{0.35}{8.37}$ & $\begin{array}{l}\frac{10.44}{76.48} \\
768\end{array}$ & $\frac{2.10}{2.78}$ & & $\frac{0.20}{0.20}$ & & 0.17 & & & & & $\frac{21.97}{22.17}$ \\
\hline & & & & & & & 0.33 & 0.98 & 0.03 & 0.46 & 0.04 & & & $\begin{array}{l}0.16 \\
\end{array}$ & & & & & & & 0.06 & & & & & $\frac{8.46}{8.46}$ & & & & o.c. & & & & & & & 222.17 \\
\hline & $777 / 200$ & $8: 36: 58.07$ & -0.82 & 1.71 & & & 0.33 & 0.98 & & 0.46 & 0.04 & \begin{tabular}{|l|l|}
$\mid-0.09$ \\
\end{tabular} & \begin{tabular}{|l|l|} 
& -0.12 \\
\end{tabular} & \begin{tabular}{|l|l|}
0.16 \\
\end{tabular} & $\begin{array}{l}0.32 \\
\end{array}$ & 0.35 & 0.05 & 7.50 & 7.1. & 13.10 & 1.26 & 23.06 & \begin{tabular}{l|l|l|l|l|l|}
6212
\end{tabular} & $\overline{2.32}$ & 15 & 8.46 & 76.62 & 2.78 & & 0.19 & & 0.17 & 0.00 & & & & $\frac{222.31}{222.58}$ \\
\hline & $\begin{array}{ll}7 / 7 / 200 \\
717700\end{array}$ & 8:40:58.07 & -0.82 & 1.71 & & & 0.35 & 0.99 & 0.02 & 0.46 & 0.05 & \begin{tabular}{|c|c|} 
\\
\end{tabular} & \begin{tabular}{|l|l|} 
& -0.12 \\
\end{tabular} & $\begin{array}{l} \\
\end{array}$ & $\begin{array}{l}0.32 \\
\end{array}$ & 0.32 & 0.05 & 7.48 & 7.1 & 8.69 & 2.72 & 22.98 & 21.20 & 2.32 & & 8.37 & 76.68 & 2.76 & .0 & 0.20 & $\begin{array}{ll}-0.02 \\
\end{array}$ & 0.17 & 0.01 & & 4.50 & & 222.78 \\
\hline & $\frac{777 / 20}{77720}$ & & -0.84 & 1.71 & & & 0.35 & 1.00 & & 0.46 & & & $\mid-0.12$ & & 0.32 & 0.32 & & 7.5 & & & 2.81 & & & & & 54 & & & & & & 1.1 & 01 & & & & 22.98 \\
\hline & $\pi 1720$ & 8:48:58.07 & $\frac{-0.84}{-0.84}$ & $\frac{1.69}{179}$ & & & $\frac{0.35}{0.35}$ & 1.00 & $\frac{0.03}{0.032}$ & 0.46 & 0.05 & $\frac{-0 .}{-0.0}$ & -0.12 & 0.14 & 0.32 & 0.32 & & 7.53 & & $\begin{array}{l}3.95 \\
395\end{array}$ & 2.85 & & & & & 8.59 & & & & & & 0.1 & $\frac{0.01}{012}$ & & & & 223.19 \\
\hline 18 & $7 / 7 / 200$ & $08: 56: 58.07$ & $\begin{array}{l}-0.84 \\
-0.84\end{array}$ & \begin{tabular}{|l|l|}
.1 .73 \\
\end{tabular} & & 36 & 0.35 & 1.01 & 0.02 & 0.46 & 0.06 & -0.07 & \begin{tabular}{|l|l|}
-0.12 \\
\end{tabular} & \begin{tabular}{|l|}
0.10 \\
0.16 \\
\end{tabular} & \begin{tabular}{|l|}
0.32 \\
\end{tabular} & \begin{tabular}{|l|}
0.32 \\
\end{tabular} & 0.05 & 7.53 & 7.15 & 8.69 & \begin{tabular}{|l|l|}
2.91 \\
\end{tabular} & \begin{tabular}{|l|}
23.07 \\
\end{tabular} & $\begin{array}{l}7.21 .45 \\
21\end{array}$ & $\frac{2.49}{22.49}$ & 23.17 & $\frac{0.1}{8.63}$ & 76.95 & 2.80 & 0.03 & 0.22 & $\frac{-0.02}{-0.02}$ & o.17 & 0.02 & & & 0.05 & 223.39 \\
\hline & $777 / 2$ & & & & & & & 1.05 & & 0.46 & & & \begin{tabular}{|l|l|}
-0.12 \\
\end{tabular} & 0.14 & & & & & & & 3.24 & & & 22.46 & & 851 & & & & & & & & & & & 223.79 \\
\hline & 7772000 & $\begin{array}{l}9: 04: 58.07 \\
0.050 .07\end{array}$ & -0.86 & 1.74 & & 39 & 0.35 & $\frac{1.04}{1.02}$ & 0.03 & 0.46 & 0.07 & -0.07 & -0.12 & 0.16 & 0.32 & 0.32 & 0.05 & 7.51 & 7.1 & .5 & $\begin{array}{l}3.35 \\
1.44\end{array}$ & & & 2.46 & 3.29 & 8.43 & 77.08 & 2.83 & 0.0 & 0.25 & -0.01 & 0.17 & 0.03 & & & & 224.00 \\
\hline 22 & 71717200 & $\frac{09.08 \cdot 128.58}{09 \cdot 17}$ & $\frac{-0.86}{-0.88}$ & $\frac{1.88}{176}$ & & & $\frac{0.35}{0.35}$ & 1.02 & 0 & $\frac{0.46}{0.46}$ & 0.07 & -0.07 & -0.13 & $\frac{0.14}{0.16}$ & 0.30 & 031 & 005 & 744 & $\frac{1.1}{70}$ & 153 & $\frac{4.44}{5.97}$ & 5304 & 2183 & 255 & & $\frac{8.64}{853}$ & 8722 & 285 & & 025 & 801 & 017 & $\frac{0.03}{0.23}$ & & & & 224.20 \\
\hline & & & & & & & $\frac{0.35}{0.35}$ & $\frac{1.44}{106}$ & $\frac{0.05}{0.03}$ & $\frac{0.486}{0.46}$ & & $\frac{-0.07}{-0.07}$ & & & & & & & & & & & & & & & & & & & & & & & & & 224.40 \\
\hline 24 & $7 / 7 / 20$ & 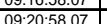 & $\begin{array}{l}-0.06 \\
-0.88\end{array}$ & 180 & & $\frac{41}{41}$ & 0.35 & 107 & 0.03 & 0.46 & 0.07 & -0.06 & $\frac{-.12}{-0.12}$ & 0.14 & 0.031 & 0.31 & & 750 & & 8.69 & 659 & 2301 & 21.87 & 2259 & 2359 & 849 & 7735 & 8.85 & 0.03 & 0.28 & -0.01 & 0.17 & 0.03 & & & 0.05 & $\frac{60}{81}$ \\
\hline & & & & 1.82 & & & 0.35 & 1.09 & $\frac{.0 .03}{0.03}$ & $\frac{.946}{0.46}$ & $\frac{.0 .07}{0.07}$ & -0.1. & & $\frac{0.14}{0.14}$ & & 0.31 & & & & & & & & & & 8.7 & & & & & & & & & & & \\
\hline & $\pi / 2$ & & & & & & 0.35 & 1.13 & & 0.46 & 0.07 & -0.06 & -0.12 & $\overline{0.14}$ & & 0.31 & & & & & $\begin{array}{l}4.64 \\
\end{array}$ & & & & & & & & & & & & & & & & 25.21 \\
\hline & 720 & :58 & -0.88 & 1.71 & & & 0.35 & 1.04 & 0.03 & 0.46 & & -0.06 & & 0.14 & 0.32 & 0.32 & & 750 & & & 5.40 & $2328 \mathrm{r}$ & 1.9 & 26. & $3.78 \quad$ & 869 & & & & & & & & & & & 25.41 \\
\hline & 7172004 & $99: 36: 58.07$ & -0.88 & 1.71 & 3. & & 0.35 & 1.02 & 0.02 & 0.47 & 0.07 & -0. & -0.12 & 0.14 & 0.32 & 0.31 & & 7.52 & & (1) & 50 & & 1.8 & 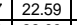 & 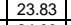 & 8.74 & & & & & & & & & & & 25.62 \\
\hline & 71717200 & 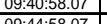 & - & $\frac{1.13}{1.71}$ & & & 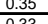 & 1.02 & $\frac{0.02}{0.02}$ & $\begin{array}{l}0.46 \\
0.077\end{array}$ & 0.01 & $\mid=0.03$ & $\mid$\begin{tabular}{|l|l|l|l|}
-0.12 \\
0.12
\end{tabular} & 0.14 & \begin{tabular}{|l|l|} 
\\
032 \\
\end{tabular} & 0.31 & & 71 & & & & & & & & $\frac{8.15}{970}$ & & & & & & & & & 5.10 & & \\
\hline & $7 / 717200$ & 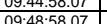 & $=0.098$ & \begin{tabular}{|l|l|}
1.17 \\
\end{tabular} & & & 0.33 & $\frac{1.11}{101}$ & 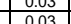 & 0 & & $\mid$\begin{tabular}{|c|c|}
$\mid-0.04$ \\
-0.06
\end{tabular} & $\mid$ & \begin{tabular}{|l|}
0.14 \\
0.14
\end{tabular} & \begin{tabular}{|l|}
0.32 \\
0.33 \\
\end{tabular} & 0.31 & & $\frac{1.51}{749}$ & & & $\frac{1.30}{961}$ & 356 & 01.94 & 资 & & & & & & & & & & & & & $\frac{26.02}{20232}$ \\
\hline & 7771200 & & $\frac{-0.88}{-0.88}$ & 1.71 & & $\frac{16}{36}$ & 0.32 & $\frac{0.99}{0.99}$ & 0.03 & 0.49 & 0 & \begin{tabular}{|c|}
-0.04 \\
\end{tabular} & \begin{tabular}{|c|}
-0.10 \\
\end{tabular} & \begin{tabular}{|l|l|}
0.14 \\
\end{tabular} & \begin{tabular}{|l|l|}
0.32 \\
\end{tabular} & 0 & & 7.48 & & & $\begin{array}{l}8.02 \\
\end{array}$ & $\frac{3.56}{3.56}$ & $\frac{21.94}{20.194}$ & 2.74 & $\frac{1.14}{4.14}$ & 8.81 & $\frac{77.88}{77.88}$ & & & 0.20 & -0.01 & 0.20 & .03 & & & 0.05 & 226.23 \\
\hline 33 & & & -0.88 & & & & & 0.99 & & & & & & & & & & & & & & 23.48 & & 22.66 & & 8.72 & & & & 0.20 & & & & & & 0.05 & 226.63 \\
\hline$\frac{34}{35}$ & 717172 & 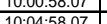 & 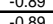 & $\frac{1.63}{0.033}$ & & $\frac{13}{91}$ & $\frac{0.32}{0.31}$ & $\frac{0.51}{0.63}$ & 0.00 & 0.095 & 0.07 & -0.24 & -0.10 & 0.03 & 0.32 & $\frac{0.15}{0.22}$ & & $\frac{0.01}{0.14}$ & & 69 & 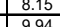 & & 203 & 27 & & $816 \quad-1$ & & & & & & 211 & .03 & & $6.05 \mathrm{~S}$ & & $\frac{226.83}{22.84}$ \\
\hline & 771712 & & & & & & & 0.98 & & 0.10 & & & & & & & & & & & & & & & & & & & & & & & & & & & \\
\hline 37 & $777 / 200$ & $0: 12: 58.07$ & -0.89 & 1.71 & 3. & 37 & 0.32 & 0.98 & 0.02 & 0.49 & 0.0 & -0. & -0.05 & 0. & 0.3 & 0.3 & & 7.50 & 7.12 & 8.95 & & $\begin{array}{l}23.66 \\
32.66\end{array}$ & 22.45 & 22.85 & 24.24 & 8.75 & 78.2 & $\frac{2.81}{2.81}$ & & 0.1 & 000 & $\frac{7.2}{1.2}$ & .00 & & & 0.00 & 27.20 \\
\hline (2) & 77777200 & 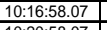 & -0.89 & 1.73 & & 等 & 0.31 & 0.99 & 0.02 & 0.50 & 0.04 & \begin{tabular}{|l|}
-0.04 \\
\end{tabular} & \begin{tabular}{|l|l|} 
& -0.09 \\
\end{tabular} & 0.14 & & 0.32 & -0.03 & 7.49 & & 8.95 & & & 2.41 & 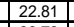 & 4..दI & 8.79 & 78.28 & 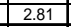 & $x_{1}$ & 年 & & & & & & 0.05 & 227.41. \\
\hline & & & & 1.15 & & & $\frac{0.51}{0.32}$ & 0.99 & & & & & & \begin{tabular}{|l|}
0.14 \\
0.14
\end{tabular} & \begin{tabular}{|l|}
0.32 \\
0.32 \\
\end{tabular} & $\begin{array}{l}0.32 \\
0.32\end{array}$ & & & & 8.95 & 10.14 & & & & & 8.16 & 78.35 & & & $\frac{0.20}{0.20}$ & -0.02 & 0.20 & .00 & & & & 227.61 \\
\hline & & & & & & & 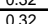 & 0 & & & . & & & 0.14 & & & & & & & & & & & & & & & & & & & & & & & 227.81 \\
\hline & & & & 1.74 & & & 0.32 & 1.00 & & 0.50 & 0.04 & & -0.08 & & 0 & 0.35 & & 749 & 7 & & & & & & & & & & & & & & & & & & 248.0 \\
\hline & $7 / 2$ & & -0.89 & $\begin{array}{l}1.74 \\
\end{array}$ & & & 0.31 & 1.01 & 0.0 & 0.5 & 0.04 & $\mid-0.1$ & \begin{tabular}{|l|l|} 
& -0.08 \\
\end{tabular} & \begin{tabular}{|l|l|} 
\\
\end{tabular} & 0. & & & & & & & & & & & & & & & & & & & & & & $228.42 \mathrm{C}$ \\
\hline 4 & $7 / 26$ & & -0.89 & 1.76 & & 43 & 0.31 & 1.01 & 0.02 & 0.52 & 0.05 & -0. & -0.08 & 0. & & & & & & & & & 1.95 & & & & & & & & & & & & & & $\frac{2.04}{20.49}$ \\
\hline & 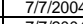 & & -0.89 & 1.78 & & 41 & 0.3 & 1.0 & & & 0.6 & & & & & & & & & & & & & & & & & & & & & & & & & & \\
\hline 40 & & & -0.89 & $\frac{1.78}{1.78}$ & 3. & $\frac{41}{12}$ & 0.31 & 1.04 & 0.02 & 0.56 & 0.04 & & -0 & 0. & & & & & & & & & & & 23.9 & & & & & & & & & & & & \\
\hline 48 & 771720 & $\begin{array}{l}10.52 .58 \\
10.56 .58\end{array}$ & $\begin{array}{l}-0.08 \\
-0.08\end{array}$ & 1.180 & & 43 & 0.32 & 1.05 & 0.03 & 0.50 & 0.04 & & & & & 0.035 & & $\frac{1.45}{7.48}$ & & & & & & & & & & & & 0.27 & & & & & & 05 & \\
\hline & & & & & & & & & & & & & & & & & & & & & & & & & & & & & & & & & & & & & \\
\hline & 7712 & & $=0.00$ & $\frac{1.00}{188}$ & 3 & $\frac{\pi 5}{155}$ & 0.04 & 1.00 & 0.06 & . & . & -0 & -0 & 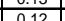 & & & & & 7 & & & & $\frac{2.00}{2213}$ & & 23 & 891 & $\frac{15}{79}$ & & & 030 & & 2 & & & & & 20.69 .6 \\
\hline & & & -0.88 & 1.82 & & & 0.32 & & 0.02 & & & -0 & & & & & & & & & & & & & & & & & & & & & & & & & \\
\hline & $777 / 20$ & 1:12:58.07 & -0.88 & 1.82 & 3. & 47 & 0.32 & 1.12 & 0.03 & $\begin{array}{l}0.53 \\
\end{array}$ & 0.04 & \begin{tabular}{|l|l|}
-0.03 \\
\end{tabular} & \begin{tabular}{|l|}
-0.08 \\
\end{tabular} & 0.13 & $\begin{array}{ll}0.33 \\
\end{array}$ & 0.36 & & & 7.2 & 10 & & & 22.17 & & & 9.04 & 7922 & 0.91 & & & & & & & & & 30.24 \\
\hline & & & -0.86 & 1.82 & 3. & & 0.31 & 1.09 & $\begin{array}{l}0.03 \\
\end{array}$ & 0.53 & 0.04 & -0.03 & -0.08 & $\begin{array}{l}0.13 \\
\end{array}$ & 0.3 & 0.35 & & 7.47 & & .36 & 0.92 & 08 & $\begin{array}{l}8 \quad 22.38 \\
\end{array}$ & 23.10 & & 9.01 & & & & & & & & & & & 0.45 \\
\hline & & & -0.88 & 1.84 & 3. & & 0.31 & 1.07 & 0.02 & 0.53 & 0.04 & -0.0 & -0.08 & 0. & 0.35 & 0.36 & & 7.49 & & & & & & & & 9.01 & & & & & & & & & & & \\
\hline & & & $\frac{0.88}{0.88}$ & 1.84 & 3. & $\frac{48}{4}$ & $\frac{0.31}{0.32}$ & $\frac{1.07}{107}$ & $\frac{0.03}{0.03}$ & 57 & 0.04 & -0.0 & -0.6 & 0.14 & 0.3 & 0.36 & & & & & & & $=2.5$ & & & & & & & & & & & & & & \\
\hline & & & $\begin{array}{l}0.08 \\
-0.08 \\
-0.08\end{array}$ & $\frac{1.14}{1.73}$ & . & 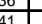 & 0 & 1.07 & 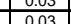 & & 0.04 & 列 & 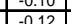 & 望4 & 0. & 0.36 & & & & & & & & & & & & & & & & & & & & & \\
\hline & $7 / 7 / 2$ & & $\frac{0.00}{-088}$ & 174 & & & & & & & $\frac{0.04}{0.04}$ & & $-0,12$ & 0.14 & 035 & & & & & & & & & & & & & & & & & & & & & & \\
\hline & & & -0.88 & 173 & & 37 & 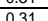 & 109 & 0.05 & 0.53 & $\frac{0.04}{0.04}$ & 0.0 & 0.12 & 0.144 & 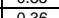 & & & & & & & & & & & & & & & & & & & & & & \\
\hline & $777 / 20$ & & -086 & 173 & & & & 109 & 005 & & 0.0 & $-0,0$ & & 0 & & & & & & & & 2 & & & & & & & & & & & & & & & \\
\hline 61 & $7 / 7 / 2004$ & 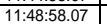 & -0.88 & $\begin{array}{ll}1.76 \\
\end{array}$ & 3. & 41 & 0.32 & 1.09 & 0.05 & 0.53 & 0.04 & -0.07 & $\begin{array}{c}-0.12 \\
-0.12\end{array}$ & 0.16 & 0.32 & 0.35 & -0.07 & 7.44 & 7.06 & 13.10 & 8.93 & 24.34 & \begin{tabular}{ll|l}
4422.81 \\
\end{tabular} & 23.21 & 23.16 & 9.03 & 79.82 & 2.85 & .00 & 0.30 & 0.01 & 0.24 & 0.00 & & & 0.05 & 232.07 \\
\hline & $77 / 12004$ & \begin{tabular}{|l|}
$11: 52: 58.07$ \\
1115658.07
\end{tabular} & $\begin{array}{l}-0.88 \\
-0.88\end{array}$ & \begin{tabular}{|l|l|} 
& -1.05 \\
\end{tabular} & & 56 & 0.32 & 0.79 & 0.05 & 0.29 & 0.04 & \begin{tabular}{|c|}
-0.17 \\
0.17
\end{tabular} & \begin{tabular}{|l|l|} 
& -0.12 \\
-012
\end{tabular} & 06 & \begin{tabular}{|l|l|}
0.32 \\
0.32 \\
\end{tabular} & $\frac{32}{32}$ & -0.11 & 0.15 & 0.04 & $\begin{array}{l}3.10 \\
3.10\end{array}$ & 1.98 & $\begin{array}{ll}24.39 \\
244\end{array}$ & 3.01 & $\begin{array}{l}3.17 \\
3.22 \\
\end{array}$ & $\begin{array}{l}3.12 \\
3.17 \\
3.17\end{array}$ & $\begin{array}{l}8.83 \\
896\end{array}$ & 79.88 & $\frac{0.00}{0.00}$ & $\begin{array}{c}0.00 \\
2000\end{array}$ & 0.00 & $\frac{0.01}{0.02}$ & $\frac{0.00}{0.00}$ & 0.00 & & & 0.00 & 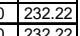 \\
\hline
\end{tabular}


WSRC-TR-2005-00105, REVISION 0

SRNL-RPP-2005-00012, REVISION 0

\section{Section H4: Pilot Feed Waste Evaporator Process (TLP) Raw Data}

Like for the FEP, the TLP data were obtained ever 60 seconds, however the following tables have been reduced in size to show only the data ever 300 seconds. The full data stream is available upon request.

The short headings for used in TLP tables the are described below

\section{Legend for TLP Data}

\begin{tabular}{|ll|}
\hline Conc Temp. Reboiler In & Concentrate Temperature at Reboiler Inlet \\
\hline Conc Temp. Reboiler out & Concentrate Temperature at Reboiler Outlet \\
\hline Reboiler Steam Supply Temp. & Reboiler Steam Supply Temperature \\
\hline Ejector Steam Supply Temp. & Ejector (Vacuum Pump) Steam Supply Temperature \\
\hline Cooling Water Supply Temp. & Cooling Water Supply (to all condensers) Temperature \\
\hline Evap. Vessel Temp. & Evaporator Vessel Concentrate Temperature \\
\hline Primary Condensate Temp. & Primary Condensate Temperature \\
\hline Inter. Cond. Outlet Temp. & Inter-condenser Outlet Temperature \\
\hline After Cond. Outlet Temp. & After-condenser Outlet Temperature \\
\hline Recirc. Flow Rate & Recirculation Flow Rate of Concentrate \\
\hline Feed Rate & Feed Rate to the Evaporator Recirculation Loop \\
\hline Prim. Condensate Flow & Primary Condensate Flow Rate \\
\hline Intercondenser Press. & Intercondenser Pressure \\
\hline Evaporator Press. & Evaporator Pressure \\
\hline
\end{tabular}


WSRC-TR-2005-00105, REVISION 0

SRNL-RPP-2005-00012, REVISION 0

Table H4-1a: Campaign II

\begin{tabular}{|c|c|c|c|c|c|c|c|c|c|c|c|c|c|c|c|c|}
\hline & DATE & TIME & $\begin{array}{c}\text { Conc } \\
\text { Temp. } \\
\text { Reboiler } \\
\text { In }\end{array}$ & $\begin{array}{l}\text { Conc } \\
\text { Temp. } \\
\text { Reboiler } \\
\text { out }\end{array}$ & $\begin{array}{l}\text { Reboiler } \\
\text { Steam } \\
\text { Supply } \\
\text { Temp. }\end{array}$ & $\begin{array}{l}\text { Ejector } \\
\text { Steam } \\
\text { Supply } \\
\text { Temp. }\end{array}$ & $\begin{array}{l}\text { Cooling } \\
\text { Water } \\
\text { Supply } \\
\text { Temp. }\end{array}$ & $\begin{array}{l}\text { Evap. } \\
\text { Vessel } \\
\text { Temp. }\end{array}$ & $\begin{array}{c}\text { Primary } \\
\text { Conden- } \\
\text { sate } \\
\text { Temp. }\end{array}$ & $\begin{array}{l}\text { Inter. } \\
\text { Cond. } \\
\text { Outlet } \\
\text { Temp. }\end{array}$ & $\begin{array}{l}\text { After } \\
\text { Cond. } \\
\text { Outlet } \\
\text { Temp. }\end{array}$ & $\begin{array}{c}\text { Recirc. } \\
\text { Flow } \\
\text { Rate }\end{array}$ & $\begin{array}{l}\text { Feed } \\
\text { Rate }\end{array}$ & $\begin{array}{l}\text { Prim. } \\
\text { Conden- } \\
\text { sate } \\
\text { Flow }\end{array}$ & $\begin{array}{c}\text { Intercondenser } \\
\text { Press. }\end{array}$ & $\begin{array}{c}\text { Evapo- } \\
\text { rator. } \\
\text { Press. }\end{array}$ \\
\hline & & & ${ }^{\circ} \mathrm{C}$ & ${ }^{\circ} \mathrm{C}$ & ${ }^{\circ} \mathrm{C}$ & ${ }^{\circ} \mathrm{C}$ & ${ }^{\circ} \mathrm{C}$ & ${ }^{\circ} \mathrm{C}$ & ${ }^{\circ} \mathrm{C}$ & ${ }^{\circ} \mathrm{C}$ & ${ }^{\circ} \mathrm{C}$ & GPM & GPM & GPM & PSIG & PSIG \\
\hline 1 & $5 / 12 / 2004$ & $6: 25: 05$ AM & 44.04 & 47.37 & 109.87 & 173.09 & 22.89 & 44.91 & 22.95 & 23.68 & 25.45 & 67.76 & 0.01 & 0.00 & -3.06 & -4.81 \\
\hline 2 & $5 / 12 / 2004$ & 6:35:07 AM & 65.26 & 67.10 & 135.69 & 174.69 & 20.82 & 64.97 & 23.24 & 21.74 & 23.72 & 84.05 & -0.01 & 0.00 & -9.19 & -10.92 \\
\hline 3 & $5 / 12 / 2004$ & 6:45:08 AM & 49.51 & 53.79 & 146.32 & 170.92 & 20.54 & 49.42 & 24.26 & 21.38 & 23.25 & 83.69 & -0.01 & 0.18 & -11.47 & -13.33 \\
\hline 4 & $5 / 12 / 2004$ & 6:55:09 AM & 46.52 & 50.07 & 147.14 & 174.60 & 20.43 & 46.37 & 23.76 & 21.37 & 23.36 & 83.30 & -0.01 & 0.66 & -11.71 & -13.40 \\
\hline 5 & $5 / 12 / 2004$ & 7:05:11 AM & 45.95 & 49.88 & 147.99 & 174.18 & 20.36 & 45.80 & 24.01 & 21.29 & 23.27 & 83.12 & 0.08 & 0.33 & -11.80 & -13.43 \\
\hline 6 & $5 / 12 / 2004$ & 7:15:12 AM & 48.32 & 52.70 & 147.94 & 172.93 & 20.32 & 48.52 & 23.84 & 21.21 & 23.16 & 83.24 & 0.79 & 0.23 & -11.54 & -13.42 \\
\hline 7 & $5 / 12 / 2004$ & $7: 25: 13$ AM & 56.13 & 60.36 & 147.98 & 170.89 & 20.29 & 56.31 & 23.72 & 21.12 & 23.01 & 83.55 & 0.73 & 0.16 & -10.94 & -12.67 \\
\hline 8 & $5 / 12 / 2004$ & 7:35:15 AM & 55.72 & 59.90 & 147.50 & 170.29 & 20.24 & 55.81 & 23.75 & 21.06 & 22.97 & 83.43 & 0.65 & 0.91 & -11.00 & -12.73 \\
\hline 9 & $5 / 12 / 2004$ & 7:45:16 AM & 55.44 & 59.63 & 147.90 & 170.61 & 20.24 & 55.49 & 23.91 & 21.05 & 23.00 & 83.32 & 0.61 & 0.55 & -11.07 & -12.79 \\
\hline 10 & $5 / 12 / 2004$ & 7:55:17 AM & 56.39 & 60.52 & 147.19 & 170.43 & 20.23 & 56.46 & 23.76 & 21.05 & 22.95 & 83.22 & 0.59 & 0.52 & -11.01 & -12.76 \\
\hline 11 & $5 / 12 / 2004$ & 8:05:19 AM & 57.16 & 61.25 & 146.67 & 170.34 & 20.23 & 57.24 & 23.71 & 21.04 & 22.93 & 83.14 & 0.57 & 0.51 & -10.97 & -12.74 \\
\hline 12 & $5 / 12 / 2004$ & $8: 15: 20$ AM & 57.24 & 61.03 & 147.59 & 170.03 & 20.22 & 57.30 & 23.40 & 21.02 & 22.92 & 83.05 & 0.55 & 0.50 & -10.98 & -12.74 \\
\hline 13 & $5 / 12 / 2004$ & $8: 25: 21$ AM & 52.48 & 55.90 & 147.85 & 174.59 & 20.22 & 52.39 & 23.57 & 21.10 & 23.18 & 82.62 & 0.42 & 0.45 & -11.39 & -13.16 \\
\hline 14 & $5 / 12 / 2004$ & 8:35:23 AM & 53.23 & 56.83 & 148.12 & 174.10 & 20.20 & 53.30 & 23.08 & 20.99 & 23.11 & 82.50 & 0.35 & 0.33 & -11.48 & -13.16 \\
\hline 15 & $5 / 12 / 2004$ & 8:45:24 AM & 59.60 & 62.42 & 147.72 & 174.84 & 20.19 & 59.56 & 22.69 & 20.73 & 23.17 & 82.67 & 0.33 & 0.24 & -11.60 & -12.51 \\
\hline 16 & $5 / 12 / 2004$ & 8:55:26 AM & 56.81 & 60.32 & 148.32 & 173.92 & 20.19 & 56.76 & 23.36 & 21.07 & 23.12 & 83.40 & 0.45 & 0.52 & -11.12 & -12.88 \\
\hline 17 & $5 / 12 / 2004$ & 9:05:27 AM & 55.69 & 59.26 & 148.45 & 173.41 & 20.18 & 55.70 & 23.31 & 21.05 & 23.07 & 84.31 & 0.47 & 0.52 & -11.22 & -13.01 \\
\hline 18 & $5 / 12 / 2004$ & 9:15:28 AM & 56.30 & 59.79 & 148.46 & 173.21 & 20.16 & 56.30 & 23.22 & 21.03 & 23.03 & 85.02 & 0.46 & 0.45 & -11.20 & -12.97 \\
\hline 19 & $5 / 12 / 2004$ & 9:25:30 AM & 56.77 & 60.22 & 148.50 & 173.28 & 20.15 & 56.74 & 23.28 & 21.02 & 23.03 & 85.26 & 0.45 & 0.44 & -11.19 & -12.95 \\
\hline 20 & $5 / 12 / 2004$ & 9:35:31 AM & 56.71 & 60.14 & 148.50 & 173.35 & 20.15 & 56.69 & 23.20 & 21.02 & 23.02 & 85.35 & 0.44 & 0.44 & -11.23 & -12.96 \\
\hline 21 & $5 / 12 / 2004$ & 9:45:33 AM & 57.24 & 60.62 & 148.53 & 173.26 & 20.14 & 57.21 & 23.19 & 21.01 & 23.01 & 85.24 & 0.43 & 0.44 & -11.22 & -12.96 \\
\hline 22 & $5 / 12 / 2004$ & 9:55:34 AM & 57.69 & 61.04 & 148.55 & 173.22 & 20.14 & 57.69 & 23.11 & 21.01 & 23.01 & 85.12 & 0.45 & 0.44 & -11.19 & -12.95 \\
\hline 23 & $5 / 12 / 2004$ & 10:05:35 AM & 57.86 & 61.17 & 148.54 & 173.31 & 20.14 & 57.85 & 23.12 & 21.01 & 23.01 & 85.35 & 0.45 & 0.43 & -11.21 & -12.96 \\
\hline 24 & $5 / 12 / 2004$ & 10:15:37 AM & 61.22 & 64.64 & 143.85 & 171.06 & 20.14 & 61.31 & 22.70 & 20.98 & 22.92 & 87.22 & 0.39 & 0.42 & -10.88 & -12.86 \\
\hline 25 & $5 / 12 / 2004$ & 10:25:38 AM & 59.07 & 62.44 & 144.46 & 171.56 & 20.14 & 58.83 & 24.73 & 21.00 & 22.94 & 86.84 & 0.40 & 0.36 & -11.24 & -13.04 \\
\hline 26 & $5 / 12 / 2004$ & 10:35:39 AM & 58.08 & 61.15 & 142.37 & 170.92 & 20.15 & 57.68 & 22.95 & 20.96 & 22.86 & 86.29 & 0.46 & 0.33 & -11.24 & -13.35 \\
\hline 27 & $5 / 12 / 2004$ & 10:45:41 AM & 60.33 & 62.84 & 136.50 & 170.39 & 20.13 & 59.72 & 22.82 & 20.94 & 22.80 & 87.29 & 0.52 & 0.72 & -10.94 & -13.33 \\
\hline 28 & $5 / 12 / 2004$ & 10:55:42 AM & 60.54 & 63.04 & 138.69 & 170.16 & 20.13 & 59.46 & 23.47 & 20.94 & 22.79 & 87.37 & 0.51 & 0.38 & -11.02 & -13.28 \\
\hline 29 & $5 / 12 / 2004$ & 11:05:44 AM & 60.44 & 62.95 & 141.51 & 170.16 & 20.12 & 59.32 & 23.60 & 20.94 & 22.81 & 87.28 & 0.49 & 0.48 & -11.06 & -13.28 \\
\hline
\end{tabular}


WSRC-TR-2005-00105, REVISION 0

SRNL-RPP-2005-00012, REVISION 0

Table H4-1b: Campaign II

TLP Campaign II Data (Contd...)

\begin{tabular}{|c|c|c|c|c|c|c|c|c|c|c|c|c|c|c|c|c|}
\hline & DATE & TIME & $\begin{array}{c}\text { Conc } \\
\text { Temp. } \\
\text { Reboiler } \\
\text { In }\end{array}$ & $\begin{array}{c}\text { Conc } \\
\text { Temp. } \\
\text { Reboiler } \\
\text { out }\end{array}$ & $\begin{array}{c}\text { Reboiler } \\
\text { Steam } \\
\text { Supply } \\
\text { Temp. }\end{array}$ & $\begin{array}{l}\text { Ejector } \\
\text { Steam } \\
\text { Supply } \\
\text { Temp. }\end{array}$ & $\begin{array}{l}\text { Cooling } \\
\text { Water } \\
\text { Supply } \\
\text { Temp. }\end{array}$ & $\begin{array}{l}\text { Evap. } \\
\text { Vessel } \\
\text { Temp. }\end{array}$ & $\begin{array}{c}\text { Primary } \\
\text { Conden- } \\
\text { sate } \\
\text { Temp. }\end{array}$ & $\begin{array}{l}\text { Inter. } \\
\text { Cond. } \\
\text { Outlet } \\
\text { Temp. }\end{array}$ & $\begin{array}{l}\text { After } \\
\text { Cond. } \\
\text { Outlet } \\
\text { Temp. }\end{array}$ & $\begin{array}{c}\text { Recirc. } \\
\text { Flow } \\
\text { Rate }\end{array}$ & $\begin{array}{l}\text { Feed } \\
\text { Rate }\end{array}$ & $\begin{array}{l}\text { Prim. } \\
\text { Conden- } \\
\text { sate } \\
\text { Flow }\end{array}$ & $\begin{array}{c}\text { Intercondenser } \\
\text { Press. }\end{array}$ & $\begin{array}{c}\text { Evapo- } \\
\text { rator. } \\
\text { Press. }\end{array}$ \\
\hline & & & ${ }^{\circ} \mathrm{C}$ & ${ }^{\circ} \mathrm{C}$ & ${ }^{\circ} \mathrm{C}$ & ${ }^{\circ} \mathrm{C}$ & ${ }^{\circ} \mathrm{C}$ & ${ }^{\circ} \mathrm{C}$ & ${ }^{\circ} \mathrm{C}$ & ${ }^{\circ} \mathrm{C}$ & ${ }^{\circ} \mathrm{C}$ & GPM & GPM & GPM & PSIG & PSIG \\
\hline 30 & $5 / 12 / 2004$ & $11: 15: 45$ AM & 60.76 & 63.25 & 140.24 & 170.35 & 20.12 & 59.64 & 23.47 & 20.93 & 22.80 & 87.21 & 0.43 & 0.49 & -11.06 & -13.29 \\
\hline 31 & $5 / 12 / 2004$ & $11: 25: 46$ AM & 61.27 & 63.72 & 140.28 & 170.38 & 20.12 & 60.06 & 23.48 & 20.94 & 22.81 & 86.86 & 0.07 & 0.47 & -11.08 & -13.28 \\
\hline 32 & $5 / 12 / 2004$ & $11: 35: 48$ AM & 61.72 & 64.01 & 140.12 & 170.12 & 20.12 & 60.66 & 23.26 & 20.94 & 22.77 & 86.03 & 0.57 & 0.49 & -11.02 & -13.27 \\
\hline 33 & $5 / 12 / 2004$ & $11: 45: 49$ AM & 73.68 & 62.59 & 141.21 & 170.60 & 20.11 & 59.14 & 23.32 & 20.94 & 22.80 & 86.37 & 0.81 & 0.45 & -11.09 & -13.26 \\
\hline 34 & $5 / 12 / 2004$ & $11: 55: 50$ AM & 177.30 & 57.58 & 143.58 & 173.53 & 20.11 & 54.59 & 23.12 & 20.99 & 22.98 & 86.18 & 0.51 & 0.46 & -11.43 & -13.39 \\
\hline 35 & $5 / 12 / 2004$ & 12:05:52 PM & 177.35 & 60.02 & 148.62 & 173.66 & 20.12 & 56.70 & 23.13 & 20.99 & 23.01 & 86.44 & 0.58 & 0.43 & -11.35 & -13.40 \\
\hline 36 & $5 / 12 / 2004$ & 12:15:53 PM & 177.43 & 61.35 & 148.57 & 173.17 & 20.11 & 58.14 & 22.84 & 20.97 & 22.96 & 86.59 & 0.60 & 0.46 & -11.21 & -13.39 \\
\hline 37 & $5 / 12 / 2004$ & 12:25:54 PM & 177.49 & 63.98 & 147.75 & 174.33 & 20.11 & 61.59 & 22.42 & 21.00 & 23.04 & 86.86 & 0.56 & 0.17 & -10.85 & -13.25 \\
\hline 38 & $5 / 12 / 2004$ & 12:35:56 PM & 65.82 & 60.06 & 147.57 & 175.34 & 20.12 & 57.86 & 22.00 & 21.03 & 23.11 & 86.79 & 0.56 & 0.32 & -11.16 & -13.28 \\
\hline 39 & $5 / 12 / 2004$ & 12:45:57 PM & 61.19 & 64.10 & 148.41 & 174.39 & 20.13 & 61.07 & 22.64 & 21.02 & 23.06 & 87.04 & 0.10 & 0.53 & -10.91 & -13.26 \\
\hline 40 & $5 / 12 / 2004$ & 12:55:59 PM & 63.06 & 66.00 & 148.56 & 174.33 & 20.13 & 63.07 & 22.78 & 21.03 & 23.10 & 86.61 & 0.19 & 0.22 & -10.77 & -12.83 \\
\hline 41 & $5 / 12 / 2004$ & 1:06:00 PM & 59.12 & 62.16 & 148.41 & 174.22 & 20.13 & 59.92 & 22.31 & 21.04 & 23.11 & 87.25 & 0.30 & 0.20 & -10.88 & -12.83 \\
\hline 42 & $5 / 12 / 2004$ & 1:16:01 PM & 59.33 & 62.47 & 148.31 & 174.27 & 20.15 & 59.19 & 23.19 & 21.04 & 23.04 & 88.19 & 0.04 & 0.24 & -10.87 & -12.84 \\
\hline 43 & $5 / 12 / 2004$ & 1:26:03 PM & 59.17 & 62.27 & 148.46 & 174.32 & 20.13 & 59.12 & 23.06 & 21.03 & 23.06 & 87.81 & 0.29 & 0.29 & -10.92 & -12.84 \\
\hline 44 & $5 / 12 / 2004$ & 1:36:04 PM & 59.50 & 62.57 & 148.46 & 174.36 & 20.15 & 59.48 & 22.93 & 21.03 & 23.07 & 87.86 & 0.41 & 0.81 & -10.91 & -12.84 \\
\hline 45 & $5 / 12 / 2004$ & 1:46:05 PM & 59.79 & 62.82 & 148.47 & 174.18 & 20.14 & 59.77 & 22.91 & 21.03 & 23.09 & 87.80 & 0.40 & 0.40 & -10.91 & -12.84 \\
\hline 46 & $5 / 12 / 2004$ & 1:56:07 PM & 60.08 & 63.08 & 148.48 & 174.29 & 20.16 & 60.06 & 22.96 & 21.05 & 23.14 & 87.75 & 0.40 & 0.39 & -10.92 & -12.86 \\
\hline 47 & $5 / 12 / 2004$ & 2:06:08 PM & 58.09 & 61.49 & 145.51 & 173.06 & 20.17 & 57.96 & 23.97 & 21.02 & 23.02 & 87.47 & 0.41 & 0.39 & -11.22 & -13.03 \\
\hline 48 & $5 / 12 / 2004$ & 2:16:10 PM & 60.19 & 63.72 & 136.60 & 170.20 & 20.16 & 60.19 & 23.05 & 20.96 & 22.80 & 87.45 & 0.39 & 0.36 & -10.92 & -12.91 \\
\hline 49 & $5 / 12 / 2004$ & 2:26:11 PM & 57.59 & 61.21 & 135.97 & 170.56 & 20.16 & 57.55 & 23.74 & 20.96 & 22.82 & 87.02 & 0.40 & 0.62 & -11.18 & -13.01 \\
\hline 50 & $5 / 12 / 2004$ & 2:36:12 PM & 58.98 & 62.54 & 136.99 & 170.46 & 20.18 & 58.96 & 23.49 & 20.98 & 22.82 & 86.74 & 0.46 & 0.53 & -11.10 & -12.91 \\
\hline 51 & $5 / 12 / 2004$ & 2:46:14 PM & 58.69 & 62.28 & 136.71 & 170.97 & 20.18 & 58.70 & 23.51 & 20.99 & 22.86 & 86.40 & 0.65 & 0.47 & -11.12 & -12.93 \\
\hline 52 & $5 / 12 / 2004$ & 2:56:15 PM & 56.35 & 60.00 & 140.48 & 170.31 & 20.20 & 56.32 & 23.76 & 20.98 & 22.83 & 86.08 & 0.67 & 0.48 & -11.31 & -13.10 \\
\hline 53 & $5 / 12 / 2004$ & 3:06:17 PM & 57.05 & 60.72 & 137.44 & 170.75 & 20.20 & 57.15 & 23.45 & 21.01 & 22.86 & 86.15 & 0.84 & 0.51 & -11.21 & -13.01 \\
\hline 54 & $5 / 12 / 2004$ & 3:16:18 PM & 56.71 & 60.41 & 138.63 & 170.64 & 20.20 & 56.81 & 23.58 & 21.00 & 22.85 & 86.46 & 0.87 & 0.47 & -11.19 & -13.01 \\
\hline 55 & $5 / 12 / 2004$ & 3:26:19 PM & 56.33 & 60.04 & 138.44 & 170.56 & 20.20 & 56.44 & 23.53 & 21.00 & 22.86 & 86.86 & 0.86 & 0.48 & -11.21 & -13.03 \\
\hline 56 & $5 / 12 / 2004$ & 3:36:21 PM & 56.40 & 60.12 & 138.93 & 170.63 & 20.21 & 56.47 & 23.69 & 21.01 & 22.85 & 87.03 & 0.74 & 0.48 & -11.24 & -13.03 \\
\hline 57 & $5 / 12 / 2004$ & 3:46:22 PM & 56.24 & 59.97 & 138.52 & 170.80 & 20.21 & 56.30 & 23.63 & 21.01 & 22.89 & 86.98 & 0.71 & 0.50 & -11.25 & -13.04 \\
\hline 58 & $5 / 12 / 2004$ & 3:56:23 PM & 56.42 & 60.14 & 139.05 & 170.95 & 20.21 & 56.40 & 23.75 & 21.01 & 22.89 & 86.97 & 0.45 & 0.49 & -11.28 & -13.05 \\
\hline
\end{tabular}


WSRC-TR-2005-00105, REVISION 0

SRNL-RPP-2005-00012, REVISION 0

Table H4-1c: Campaign II

\begin{tabular}{|c|c|c|c|c|c|c|c|c|c|c|c|c|c|c|c|c|}
\hline & DATE & TIME & $\begin{array}{c}\text { Conc } \\
\text { Temp. } \\
\text { Reboiler } \\
\text { In }\end{array}$ & $\begin{array}{l}\text { Conc } \\
\text { Temp. } \\
\text { Reboiler } \\
\text { out }\end{array}$ & $\begin{array}{l}\text { Reboiler } \\
\text { Steam } \\
\text { Supply } \\
\text { Temp. }\end{array}$ & $\begin{array}{l}\text { Ejector } \\
\text { Steam } \\
\text { Supply } \\
\text { Temp. }\end{array}$ & $\begin{array}{l}\text { Cooling } \\
\text { Water } \\
\text { Supply } \\
\text { Temp. }\end{array}$ & $\begin{array}{l}\text { Evap. } \\
\text { Vessel } \\
\text { Temp. }\end{array}$ & $\begin{array}{l}\text { Primary } \\
\text { Conden- } \\
\text { sate } \\
\text { Temp. }\end{array}$ & $\begin{array}{l}\text { Inter. } \\
\text { Cond. } \\
\text { Outlet } \\
\text { Temp. }\end{array}$ & $\begin{array}{l}\text { After } \\
\text { Cond. } \\
\text { Outlet } \\
\text { Temp. }\end{array}$ & $\begin{array}{c}\text { Recirc. } \\
\text { Flow } \\
\text { Rate }\end{array}$ & $\begin{array}{l}\text { Feed } \\
\text { Rate }\end{array}$ & $\begin{array}{l}\text { Prim. } \\
\text { Conden- } \\
\text { sate } \\
\text { Flow }\end{array}$ & $\begin{array}{l}\text { Intercondenser } \\
\text { Press. }\end{array}$ & $\begin{array}{c}\text { Evapo- } \\
\text { rator. } \\
\text { Press. }\end{array}$ \\
\hline & & & ${ }^{\circ} \mathrm{C}$ & ${ }^{\circ} \mathrm{C}$ & ${ }^{\circ} \mathrm{C}$ & ${ }^{\circ} \mathrm{C}$ & ${ }^{\circ} \mathrm{C}$ & ${ }^{\circ} \mathbf{C}$ & ${ }^{\circ} \mathrm{C}$ & ${ }^{\circ} \mathrm{C}$ & ${ }^{\circ} \mathrm{C}$ & GPM & GPM & GPM & PSIG & PSIG \\
\hline 59 & $5 / 12 / 2004$ & 4:06:25 PM & 56.39 & 60.06 & 139.73 & 170.71 & 20.20 & 56.33 & 23.71 & 21.01 & 22.90 & 86.63 & 0.31 & 0.50 & -11.31 & -13.10 \\
\hline 60 & $5 / 12 / 2004$ & 4:16:26 PM & 57.34 & 60.98 & 140.53 & 170.65 & 20.19 & 57.35 & 23.64 & 21.00 & 22.85 & 86.35 & 0.65 & 0.49 & -11.24 & -13.02 \\
\hline 61 & $5 / 12 / 2004$ & 4:26:28 PM & 56.05 & 59.77 & 139.13 & 170.88 & 20.18 & 56.16 & 23.59 & 20.99 & 22.87 & 86.31 & 0.99 & 0.49 & -11.26 & -13.05 \\
\hline 62 & $5 / 12 / 2004$ & 4:36:29 PM & 55.52 & 59.27 & 140.26 & 170.96 & 20.17 & 55.67 & 23.49 & 20.97 & 22.88 & 86.77 & 1.00 & 0.48 & -11.27 & -13.05 \\
\hline 63 & $5 / 12 / 2004$ & 4:46:30 PM & 55.33 & 59.13 & 140.38 & 170.95 & 20.19 & 55.48 & 23.58 & 20.98 & 22.87 & 87.00 & 0.95 & 0.48 & -11.27 & -13.05 \\
\hline 64 & $5 / 12 / 2004$ & 4:56:32 PM & 55.61 & 59.38 & 138.95 & 170.63 & 20.19 & 55.72 & 23.60 & 20.98 & 22.85 & 87.18 & 0.88 & 0.48 & -11.23 & -13.02 \\
\hline 65 & $5 / 12 / 2004$ & 5:06:33 PM & 55.54 & 59.34 & 140.05 & 170.94 & 20.19 & 55.64 & 23.61 & 20.98 & 22.88 & 87.20 & 0.85 & 0.49 & -11.23 & -13.02 \\
\hline 66 & $5 / 12 / 2004$ & 5:16:34 PM & 56.39 & 60.14 & 139.75 & 170.83 & 20.19 & 56.32 & 23.76 & 20.98 & 22.88 & 87.26 & 0.28 & 0.49 & -11.22 & -12.99 \\
\hline 67 & $5 / 12 / 2004$ & 5:26:36 PM & 57.24 & 60.94 & 140.09 & 171.20 & 20.19 & 57.06 & 23.83 & 20.99 & 22.90 & 86.90 & -0.01 & 0.51 & -11.27 & -13.00 \\
\hline 68 & $5 / 12 / 2004$ & 5:36:37 PM & 57.59 & 61.21 & 140.02 & 170.88 & 20.19 & 57.52 & 23.57 & 20.99 & 22.86 & 86.36 & 0.27 & 0.52 & -11.25 & -13.05 \\
\hline 69 & $5 / 12 / 2004$ & 5:46:39 PM & 57.91 & 61.52 & 140.81 & 171.10 & 20.19 & 57.94 & 23.57 & 20.99 & 22.86 & 86.31 & 0.74 & 0.49 & -11.27 & -13.03 \\
\hline 70 & $5 / 12 / 2004$ & 5:56:40 PM & 53.42 & 55.43 & 136.53 & 174.36 & 20.18 & 53.58 & 21.17 & 21.07 & 23.01 & 86.03 & 0.69 & 0.48 & -11.23 & -13.20 \\
\hline 71 & $5 / 12 / 2004$ & 6:06:41 PM & 52.99 & 54.54 & 132.01 & 174.54 & 20.17 & 52.69 & 22.99 & 20.95 & 22.64 & 86.23 & 0.12 & 0.20 & -10.46 & -12.32 \\
\hline
\end{tabular}


WSRC-TR-2005-00105, REVISION 0

SRNL-RPP-2005-00012, REVISION 0

Table H4-2a: Campaign III

\begin{tabular}{|c|c|c|c|c|c|c|c|c|c|c|c|c|c|c|c|c|}
\hline & DATE & TIME & $\begin{array}{c}\text { Conc } \\
\text { Temp. } \\
\text { Reboiler } \\
\text { In }\end{array}$ & $\begin{array}{c}\text { Conc } \\
\text { Temp. } \\
\text { Reboiler } \\
\text { out }\end{array}$ & $\begin{array}{l}\text { Reboiler } \\
\text { Steam } \\
\text { Supply } \\
\text { Temp. }\end{array}$ & $\begin{array}{l}\text { Ejector } \\
\text { Steam } \\
\text { Supply } \\
\text { Temp. }\end{array}$ & $\begin{array}{l}\text { Cooling } \\
\text { Water } \\
\text { Supply } \\
\text { Temp. }\end{array}$ & $\begin{array}{l}\text { Evap. } \\
\text { Vessel } \\
\text { Temp. }\end{array}$ & $\begin{array}{c}\text { Primary } \\
\text { Conden- } \\
\text { sate } \\
\text { Temp. }\end{array}$ & $\begin{array}{l}\text { Inter. } \\
\text { Cond. } \\
\text { Outlet } \\
\text { Temp. }\end{array}$ & $\begin{array}{l}\text { After } \\
\text { Cond. } \\
\text { Outlet } \\
\text { Temp. }\end{array}$ & $\begin{array}{l}\text { Recirc. } \\
\text { Flow } \\
\text { Rate }\end{array}$ & $\begin{array}{l}\text { Feed } \\
\text { Rate }\end{array}$ & $\begin{array}{l}\text { Prim. } \\
\text { Conden- } \\
\text { sate } \\
\text { Flow }\end{array}$ & $\begin{array}{c}\text { Intercondenser } \\
\text { Press. }\end{array}$ & $\begin{array}{c}\text { Evapo- } \\
\text { rator. } \\
\text { Press. }\end{array}$ \\
\hline & & & ${ }^{\circ} \mathrm{C}$ & ${ }^{\circ} \mathbf{C}$ & ${ }^{\circ} \mathrm{C}$ & ${ }^{\circ} \mathrm{C}$ & ${ }^{\circ} \mathrm{C}$ & ${ }^{\circ} \mathrm{C}$ & ${ }^{\circ} \mathrm{C}$ & ${ }^{\circ} \mathrm{C}$ & ${ }^{\circ} \mathbf{C}$ & GPM & GPM & GPM & PSIG & PSIG \\
\hline 1 & $7 / 8 / 2004$ & $6: 17: 45$ AM & 23.02 & 22.63 & 22.36 & 168.01 & 22.42 & 20.71 & 22.54 & 23.27 & 25.37 & 0.11 & -0.01 & 0.14 & -1.82 & -3.40 \\
\hline 2 & $7 / 8 / 2004$ & 6:27:46 AM & 23.89 & 24.07 & 40.64 & 175.43 & 21.92 & 23.26 & 21.90 & 22.90 & 25.10 & 40.56 & -0.01 & 0.01 & -8.36 & -10.12 \\
\hline 3 & $7 / 8 / 2004$ & $6: 37: 47$ AM & 50.70 & 52.66 & 143.53 & 174.96 & 21.75 & 51.11 & 21.69 & 22.69 & 24.87 & 90.62 & -0.01 & 0.03 & -9.39 & -11.22 \\
\hline 4 & $7 / 8 / 2004$ & 6:47:49 AM & 58.87 & 59.17 & 136.30 & 175.55 & 21.65 & 58.68 & 21.60 & 22.62 & 24.81 & 89.88 & -0.01 & 0.02 & -9.83 & -11.74 \\
\hline 5 & $7 / 8 / 2004$ & 6:57:50 AM & 57.33 & 59.56 & 131.53 & 174.77 & 21.57 & 57.14 & 23.71 & 22.52 & 24.65 & 90.17 & 0.15 & 0.14 & -10.33 & -12.11 \\
\hline 6 & $7 / 8 / 2004$ & 7:07:52 AM & 49.34 & 53.59 & 145.57 & 172.53 & 21.51 & 49.46 & 25.55 & 22.41 & 24.48 & 89.38 & 0.70 & 0.35 & -11.28 & -12.99 \\
\hline 7 & $7 / 8 / 2004$ & 7:17:53 AM & 50.90 & 55.25 & 142.48 & 170.54 & 21.47 & 51.00 & 25.60 & 22.34 & 24.29 & 89.10 & 0.60 & 0.86 & -11.16 & -12.93 \\
\hline 8 & $7 / 8 / 2004$ & 7:27:54 AM & 51.88 & 56.17 & 140.26 & 170.95 & 21.41 & 52.00 & 25.11 & 22.44 & 24.05 & 89.31 & 0.67 & 0.55 & -11.08 & -12.91 \\
\hline 9 & $7 / 8 / 2004$ & 7:37:56 AM & 52.00 & 56.24 & 141.57 & 170.37 & 21.34 & 52.16 & 24.73 & 22.30 & 23.91 & 89.08 & 0.76 & 0.54 & -11.10 & -12.87 \\
\hline 10 & $7 / 8 / 2004$ & 7:47:57 AM & 52.53 & 56.76 & 141.31 & 170.71 & 21.33 & 52.71 & 24.82 & 22.30 & 23.92 & 89.15 & 0.85 & 0.54 & -11.10 & -12.84 \\
\hline 11 & $7 / 8 / 2004$ & 7:57:58 AM & 52.50 & 56.71 & 144.03 & 170.73 & 21.30 & 52.67 & 24.77 & 22.27 & 23.91 & 89.21 & 0.82 & 0.55 & -11.13 & -12.85 \\
\hline 12 & $7 / 8 / 2004$ & 8:08:00 AM & 52.80 & 56.99 & 142.12 & 170.86 & 21.27 & 52.96 & 24.66 & 22.25 & 23.89 & 89.16 & 0.70 & 0.53 & -11.11 & -12.89 \\
\hline 13 & $7 / 8 / 2004$ & 8:18:01 AM & 53.33 & 57.47 & 141.00 & 170.51 & 21.27 & 53.42 & 24.77 & 22.24 & 23.87 & 89.21 & 0.56 & 0.55 & -11.11 & -12.87 \\
\hline 14 & $7 / 8 / 2004$ & 8:28:03 AM & 53.71 & 57.84 & 141.73 & 170.83 & 21.25 & 53.76 & 24.69 & 22.23 & 23.88 & 88.88 & 0.49 & 0.53 & -11.08 & -12.86 \\
\hline 15 & $7 / 8 / 2004$ & 8:38:04 AM & 54.25 & 58.32 & 141.42 & 170.61 & 21.24 & 54.30 & 24.73 & 22.21 & 23.84 & 88.89 & 0.47 & 0.55 & -11.07 & -12.86 \\
\hline 16 & $7 / 8 / 2004$ & 8:48:05 AM & 55.54 & 59.52 & 141.23 & 170.51 & 21.24 & 55.66 & 24.25 & 38.16 & 23.73 & 88.76 & 0.49 & 0.49 & -10.28 & -12.79 \\
\hline 17 & $7 / 8 / 2004$ & 8:58:07 AM & 56.58 & 60.19 & 144.92 & 170.94 & 21.24 & 56.63 & 24.58 & 30.33 & 23.85 & 88.81 & 0.51 & 0.55 & -10.72 & -12.68 \\
\hline 18 & $7 / 8 / 2004$ & 9:08:08 AM & 55.05 & 59.01 & 140.81 & 170.42 & 21.21 & 55.12 & 24.52 & 22.21 & 23.79 & 88.58 & 0.48 & 0.52 & -11.05 & -12.90 \\
\hline 19 & $7 / 8 / 2004$ & 9:18:09 AM & 55.70 & 59.64 & 139.96 & 170.90 & 21.21 & 55.74 & 24.57 & 22.22 & 23.81 & 88.38 & 0.47 & 0.52 & -11.00 & -12.91 \\
\hline 20 & $7 / 8 / 2004$ & 9:28:11 AM & 55.83 & 59.72 & 139.89 & 170.26 & 21.20 & 55.89 & 24.47 & 22.19 & 23.76 & 88.15 & 0.44 & 0.51 & -11.03 & -12.89 \\
\hline 21 & $7 / 8 / 2004$ & 9:38:12 AM & 56.02 & 59.89 & 140.09 & 170.49 & 21.18 & 56.07 & 24.51 & 22.19 & 23.78 & 88.19 & 0.49 & 0.52 & -11.05 & -12.87 \\
\hline 22 & $7 / 8 / 2004$ & 9:48:14 AM & 56.14 & 59.98 & 141.20 & 170.30 & 21.17 & 56.24 & 24.38 & 22.17 & 23.76 & 88.05 & 0.59 & 0.50 & -11.06 & -12.86 \\
\hline 23 & $7 / 8 / 2004$ & 9:58:15 AM & 56.25 & 60.10 & 141.91 & 170.51 & 21.19 & 56.32 & 24.52 & 22.20 & 23.78 & 88.00 & 0.58 & 0.51 & -11.07 & -12.84 \\
\hline 24 & $7 / 8 / 2004$ & 10:08:16 AM & 55.98 & 59.82 & 141.75 & 170.54 & 21.18 & 56.12 & 24.37 & 22.18 & 23.76 & 87.94 & 0.77 & 0.50 & -11.10 & -12.85 \\
\hline 25 & $7 / 8 / 2004$ & 10:18:18 AM & 56.34 & 60.15 & 140.59 & 170.31 & 21.14 & 56.46 & 24.31 & 22.15 & 23.73 & 88.00 & 0.68 & 0.49 & -11.06 & -12.87 \\
\hline 26 & $7 / 8 / 2004$ & 10:28:19 AM & 56.80 & 60.60 & 141.83 & 170.38 & 21.17 & 56.87 & 24.44 & 22.17 & 23.75 & 87.92 & 0.51 & 0.50 & -11.08 & -12.86 \\
\hline 27 & $7 / 8 / 2004$ & 10:38:20 AM & 56.92 & 60.70 & 141.81 & 170.48 & 21.14 & 56.98 & 24.35 & 22.15 & 23.76 & 87.87 & 0.53 & 0.50 & -11.07 & -12.90 \\
\hline 28 & $7 / 8 / 2004$ & 10:48:22 AM & 56.45 & 60.26 & 143.02 & 170.66 & 21.17 & 56.61 & 24.34 & 22.18 & 23.77 & 87.85 & 0.78 & 0.49 & -11.13 & -12.92 \\
\hline 29 & $7 / 8 / 2004$ & 10:58:23 AM & 57.18 & 60.91 & 141.91 & 170.21 & 21.14 & 57.25 & 24.18 & 22.14 & 23.73 & 87.76 & 0.41 & 0.47 & -11.08 & -12.92 \\
\hline
\end{tabular}


WSRC-TR-2005-00105, REVISION 0

SRNL-RPP-2005-00012, REVISION 0

Table H4-2b: Campaign III

TLP Campaign III Data (Contd..)

\begin{tabular}{|c|c|c|c|c|c|c|c|c|c|c|c|c|c|c|c|c|}
\hline & DATE & TIME & $\begin{array}{c}\text { Conc } \\
\text { Temp. } \\
\text { Reboiler } \\
\text { In }\end{array}$ & $\begin{array}{l}\text { Conc } \\
\text { Temp. } \\
\text { Reboiler } \\
\text { out }\end{array}$ & $\begin{array}{l}\text { Reboiler } \\
\text { Steam } \\
\text { Supply } \\
\text { Temp. }\end{array}$ & $\begin{array}{l}\text { Ejector } \\
\text { Steam } \\
\text { Supply } \\
\text { Temp. }\end{array}$ & $\begin{array}{l}\text { Cooling } \\
\text { Water } \\
\text { Supply } \\
\text { Temp. }\end{array}$ & $\begin{array}{l}\text { Evap. } \\
\text { Vessel } \\
\text { Temp. }\end{array}$ & $\begin{array}{c}\text { Primary } \\
\text { Conden- } \\
\text { sate } \\
\text { Temp. }\end{array}$ & $\begin{array}{l}\text { Inter. } \\
\text { Cond. } \\
\text { Outlet } \\
\text { Temp. }\end{array}$ & $\begin{array}{l}\text { After } \\
\text { Cond. } \\
\text { Outlet } \\
\text { Temp. }\end{array}$ & $\begin{array}{c}\text { Recirc. } \\
\text { Flow } \\
\text { Rate }\end{array}$ & $\begin{array}{l}\text { Feed } \\
\text { Rate }\end{array}$ & $\begin{array}{l}\text { Prim. } \\
\text { Conden- } \\
\text { sate } \\
\text { Flow }\end{array}$ & $\begin{array}{c}\text { Intercondenser } \\
\text { Press. }\end{array}$ & $\begin{array}{c}\text { Evapo- } \\
\text { rator. } \\
\text { Press. }\end{array}$ \\
\hline & & & ${ }^{\circ} \mathrm{C}$ & ${ }^{\circ} \mathrm{C}$ & ${ }^{\circ} \mathrm{C}$ & ${ }^{\circ} \mathrm{C}$ & ${ }^{\circ} \mathrm{C}$ & ${ }^{\circ} \mathrm{C}$ & ${ }^{\circ} \mathrm{C}$ & ${ }^{\circ} \mathrm{C}$ & ${ }^{\circ} \mathrm{C}$ & GPM & GPM & GPM & PSIG & PSIG \\
\hline 30 & $7 / 8 / 2004$ & 11:08:24 AM & 57.60 & 61.32 & 142.60 & 170.43 & 21.16 & 57.60 & 24.56 & 22.17 & 23.76 & 87.64 & 0.34 & 0.52 & -11.12 & -12.92 \\
\hline 31 & $7 / 8 / 2004$ & 11:18:26 AM & 57.01 & 60.77 & 143.99 & 170.60 & 21.14 & 57.11 & 24.37 & 22.16 & 23.76 & 87.07 & 0.69 & 0.50 & -11.13 & -12.91 \\
\hline 32 & $7 / 8 / 2004$ & 11:28:27 AM & 56.42 & 60.23 & 140.19 & 170.72 & 21.16 & 56.66 & 24.22 & 22.18 & 23.76 & 87.20 & 0.94 & 0.48 & -11.08 & -12.89 \\
\hline 33 & $7 / 8 / 2004$ & 11:38:29 AM & 56.40 & 60.19 & 142.42 & 170.53 & 21.13 & 56.55 & 24.23 & 22.14 & 23.74 & 87.36 & 0.66 & 0.48 & -11.10 & -12.90 \\
\hline 34 & $7 / 8 / 2004$ & 11:48:30 AM & 57.05 & 60.83 & 142.92 & 170.76 & 21.15 & 57.11 & 24.45 & 22.17 & 23.77 & 87.26 & 0.53 & 0.50 & -11.10 & -12.93 \\
\hline 35 & $7 / 8 / 2004$ & 11:58:31 AM & 57.40 & 61.11 & 142.43 & 170.16 & 21.13 & 57.47 & 24.29 & 22.14 & 23.73 & 87.17 & 0.51 & 0.49 & -11.09 & -12.92 \\
\hline 36 & $7 / 8 / 2004$ & 12:08:33 PM & 57.42 & 61.15 & 142.65 & 170.53 & 21.16 & 57.53 & 24.37 & 22.17 & 23.78 & 87.13 & 0.66 & 0.49 & -11.11 & -12.90 \\
\hline 37 & $7 / 8 / 2004$ & 12:18:34 PM & 57.32 & 61.04 & 142.90 & 170.82 & 21.14 & 57.44 & 24.32 & 22.16 & 23.78 & 87.33 & 0.68 & 0.49 & -11.12 & -12.89 \\
\hline 38 & $7 / 8 / 2004$ & 12:28:35 PM & 57.68 & 61.37 & 141.83 & 170.36 & 21.17 & 57.84 & 24.21 & 22.18 & 23.76 & 87.24 & 0.74 & 0.46 & -11.09 & -12.93 \\
\hline 39 & $7 / 8 / 2004$ & 12:38:37 PM & 56.94 & 60.67 & 143.06 & 170.71 & 21.14 & 57.12 & 24.25 & 22.16 & 23.77 & 87.40 & 0.90 & 0.49 & -11.13 & -12.94 \\
\hline 40 & $7 / 8 / 2004$ & 12:48:38 PM & 56.93 & 60.67 & 144.05 & 170.71 & 21.17 & 57.13 & 24.37 & 22.19 & 23.79 & 88.01 & 0.97 & 0.49 & -11.14 & -12.92 \\
\hline 41 & $7 / 8 / 2004$ & 12:58:40 PM & 57.52 & 61.17 & 141.17 & 170.00 & 21.15 & 57.65 & 24.08 & 22.16 & 23.74 & 88.99 & 0.66 & 0.45 & -11.06 & -12.91 \\
\hline 42 & $7 / 8 / 2004$ & 1:08:41 PM & 57.83 & 61.51 & 143.88 & 170.66 & 21.18 & 57.84 & 24.54 & 22.21 & 23.81 & 88.89 & 0.35 & 0.52 & -11.13 & -12.91 \\
\hline 43 & $7 / 8 / 2004$ & 1:18:42 PM & 57.25 & 60.94 & 144.19 & 170.96 & 21.16 & 57.33 & 24.42 & 22.19 & 23.81 & 88.52 & 0.64 & 0.50 & -11.15 & -12.90 \\
\hline 44 & $7 / 8 / 2004$ & 1:28:44 PM & 57.25 & 60.95 & 143.17 & 170.22 & 21.18 & 57.43 & 24.35 & 22.20 & 23.78 & 88.16 & 0.76 & 0.49 & -11.10 & -12.86 \\
\hline 45 & $7 / 8 / 2004$ & 1:38:45 PM & 57.12 & 60.81 & 142.10 & 170.45 & 21.17 & 57.32 & 24.29 & 22.19 & 23.79 & 88.55 & 0.90 & 0.48 & -11.06 & -12.86 \\
\hline 46 & $7 / 8 / 2004$ & 1:48:46 PM & 56.57 & 60.32 & 142.06 & 170.98 & 21.17 & 56.75 & 24.37 & 22.21 & 23.83 & 88.84 & 0.90 & 0.49 & -11.10 & -12.94 \\
\hline 47 & $7 / 8 / 2004$ & 1:58:48 PM & 56.92 & 60.64 & 141.12 & 170.55 & 21.19 & 57.12 & 24.32 & 22.22 & 23.80 & 88.99 & 0.88 & 0.48 & -11.05 & -12.92 \\
\hline 48 & $7 / 8 / 2004$ & 2:08:49 PM & 56.93 & 60.65 & 140.69 & 170.51 & 21.18 & 57.12 & 24.36 & 22.21 & 23.81 & 89.27 & 0.87 & 0.49 & -11.06 & -12.89 \\
\hline 49 & $7 / 8 / 2004$ & 2:18:51 PM & 57.64 & 61.34 & 141.41 & 170.41 & 21.21 & 57.73 & 24.45 & 22.23 & 23.81 & 89.30 & 0.53 & 0.49 & -11.02 & -12.86 \\
\hline 50 & $7 / 8 / 2004$ & 2:28:52 PM & 57.48 & 61.16 & 141.79 & 170.52 & 21.20 & 57.54 & 24.41 & 22.23 & 23.81 & 89.13 & 0.40 & 0.49 & -11.07 & -12.86 \\
\hline 51 & $7 / 8 / 2004$ & 2:38:53 PM & 57.13 & 60.81 & 144.17 & 170.85 & 21.19 & 57.19 & 24.57 & 22.22 & 23.84 & 88.91 & 0.54 & 0.52 & -11.14 & -12.90 \\
\hline 52 & $7 / 8 / 2004$ & 2:48:55 PM & 57.59 & 61.26 & 142.31 & 170.96 & 21.20 & 57.68 & 24.42 & 22.23 & 23.85 & 88.99 & 0.61 & 0.49 & -11.10 & -12.93 \\
\hline 53 & $7 / 8 / 2004$ & 2:58:56 PM & 57.51 & 61.17 & 142.25 & 170.66 & 21.23 & 57.64 & 24.30 & 22.25 & 23.82 & 88.66 & 0.67 & 0.47 & -11.08 & -12.91 \\
\hline 54 & $7 / 8 / 2004$ & 3:08:58 PM & 56.84 & 60.53 & 141.76 & 170.50 & 21.20 & 57.00 & 24.43 & 22.22 & 23.82 & 88.52 & 0.80 & 0.50 & -11.08 & -12.92 \\
\hline 55 & $7 / 8 / 2004$ & 3:18:59 PM & 56.23 & 59.97 & 143.54 & 170.89 & 21.20 & 56.39 & 24.44 & 22.23 & 23.85 & 88.68 & 0.86 & 0.50 & -11.13 & -12.91 \\
\hline 56 & $7 / 8 / 2004$ & 3:29:00 PM & 56.63 & 60.37 & 142.65 & 170.65 & 21.23 & 56.82 & 24.39 & 22.25 & 23.84 & 88.79 & 0.86 & 0.48 & -11.09 & -12.90 \\
\hline 57 & $7 / 8 / 2004$ & 3:39:02 PM & 56.85 & 60.59 & 142.24 & 170.88 & 21.22 & 57.04 & 24.33 & 22.26 & 23.86 & 89.02 & 0.85 & 0.48 & -11.06 & -12.85 \\
\hline 58 & $7 / 8 / 2004$ & 3:49:03 PM & 56.13 & 59.90 & 143.04 & 170.92 & 21.22 & 56.30 & 24.48 & 22.25 & 23.86 & 89.05 & 0.85 & 0.50 & -11.13 & -12.89 \\
\hline
\end{tabular}


WSRC-TR-2005-00105, REVISION 0

SRNL-RPP-2005-00012, REVISION 0

Table H4-2c: Campaign III

\begin{tabular}{|c|c|c|c|c|c|c|c|c|c|c|c|c|c|c|c|c|}
\hline & DATE & TIME & $\begin{array}{c}\text { Conc } \\
\text { Temp. } \\
\text { Reboiler } \\
\text { In }\end{array}$ & $\begin{array}{c}\text { Conc } \\
\text { Temp. } \\
\text { Reboiler } \\
\text { out }\end{array}$ & $\begin{array}{c}\text { Reboiler } \\
\text { Steam } \\
\text { Supply } \\
\text { Temp. }\end{array}$ & $\begin{array}{l}\text { Ejector } \\
\text { Steam } \\
\text { Supply } \\
\text { Temp. }\end{array}$ & $\begin{array}{l}\text { Cooling } \\
\text { Water } \\
\text { Supply } \\
\text { Temp. } \\
\end{array}$ & $\begin{array}{l}\text { Evap. } \\
\text { Vessel } \\
\text { Temp. }\end{array}$ & $\begin{array}{c}\text { Primary } \\
\text { Conden- } \\
\text { sate } \\
\text { Temp. }\end{array}$ & $\begin{array}{l}\text { Inter. } \\
\text { Cond. } \\
\text { Outlet } \\
\text { Temp. }\end{array}$ & $\begin{array}{l}\text { After } \\
\text { Cond. } \\
\text { Outlet } \\
\text { Temp. }\end{array}$ & $\begin{array}{c}\text { Recirc. } \\
\text { Flow } \\
\text { Rate } \\
\end{array}$ & $\begin{array}{l}\text { Feed } \\
\text { Rate }\end{array}$ & $\begin{array}{l}\text { Prim. } \\
\text { Conden- } \\
\text { sate } \\
\text { Flow } \\
\end{array}$ & $\begin{array}{c}\text { Intercondenser } \\
\text { Press. } \\
\end{array}$ & $\begin{array}{c}\text { Evapo- } \\
\text { rator. } \\
\text { Press. }\end{array}$ \\
\hline & & & ${ }^{\circ} \mathrm{C}$ & ${ }^{\circ} \mathrm{C}$ & ${ }^{\circ} \mathrm{C}$ & ${ }^{\circ} \mathrm{C}$ & ${ }^{\circ} \mathrm{C}$ & ${ }^{\circ} \mathrm{C}$ & ${ }^{\circ} \mathrm{C}$ & ${ }^{\circ} \mathrm{C}$ & ${ }^{\circ} \mathrm{C}$ & GPM & GPM & GPM & PSIG & PSIG \\
\hline 59 & $7 / 8 / 2004$ & 3:59:04 PM & 56.83 & 60.56 & 140.31 & 170.89 & 21.21 & 57.00 & 24.29 & 22.25 & 23.85 & 89.30 & 0.79 & 0.47 & -11.05 & -12.85 \\
\hline 60 & $7 / 8 / 2004$ & 4:09:06 PM & 56.46 & 60.14 & 143.73 & 170.30 & 21.21 & 56.45 & 24.56 & 22.24 & 23.84 & 89.19 & 0.27 & 0.52 & -11.17 & -12.91 \\
\hline 61 & $7 / 8 / 2004$ & 4:19:07 PM & 57.85 & 61.50 & 141.29 & 170.90 & 21.23 & 57.83 & 24.46 & 22.27 & 23.85 & 88.96 & 0.28 & 0.50 & -11.06 & -12.85 \\
\hline 62 & $7 / 8 / 2004$ & 4:29:08 PM & 57.81 & 61.44 & 143.04 & 170.96 & 21.23 & 57.88 & 24.39 & 22.27 & 23.86 & 88.78 & 0.66 & 0.48 & -11.10 & -12.88 \\
\hline 63 & $7 / 8 / 2004$ & 4:39:10 PM & 57.00 & 60.68 & 143.55 & 170.86 & 21.23 & 57.14 & 24.49 & 22.27 & 23.87 & 88.95 & 0.89 & 0.50 & -11.15 & -12.92 \\
\hline 64 & $7 / 8 / 2004$ & 4:49:11 PM & 57.03 & 60.70 & 140.80 & 170.65 & 21.24 & 57.14 & 24.37 & 22.26 & 23.84 & 88.71 & 0.69 & 0.49 & -11.11 & -12.88 \\
\hline 65 & $7 / 8 / 2004$ & 4:59:13 PM & 57.17 & 60.85 & 141.43 & 170.91 & 21.27 & 57.27 & 24.42 & 22.29 & 23.89 & 88.55 & 0.75 & 0.48 & -11.06 & -12.87 \\
\hline 66 & $7 / 8 / 2004$ & 5:09:14 PM & 56.35 & 60.06 & 141.55 & 170.82 & 21.26 & 56.53 & 24.44 & 22.28 & 23.89 & 88.72 & 0.91 & 0.49 & -11.11 & -12.88 \\
\hline 67 & $7 / 8 / 2004$ & 5:19:15 PM & 57.15 & 60.82 & 140.15 & 170.50 & 21.26 & 57.19 & 24.35 & 22.27 & 23.87 & 88.79 & 0.38 & 0.48 & -11.04 & -12.85 \\
\hline
\end{tabular}




\section{Appendix I: SIPP Interim Reports}

At the termination of each of the first three campaigns that made up the four-campaign SIPP task, an interim report was issued as per the Task Plan (Duignan, 2003c). Each of those interim reports discussed not only the campaign that had just ended, but those that preceded it. As such, this, the fourth report, not only discusses the last campaign, Campaign IV, but also contrasts the highlights of the results obtained in the preceding campaigns. Each of the interim reports was made to be stand alone and, therefore, contains a significant amount detail To include all of the detail contain in the interim reports into the body of this Final report would have made the report difficult to read.

The interim reports are referenced throughout this report and are listed in the Reference section. However, while those reports are accessible through the Westinghouse Savannah River Document Control their accessibility to the RPP-WTP project may be time consuming to obtain. Therefore, the three interim reports are annexed here in their entirety for ready reference.

Those reports are:

\section{Appendix Section: I-1}

Report Title: Interim Report: RPP-WTP Semi-Integrated Pilot Plant - Campaign I

Report Date: April 2004

WSRC Report Number: WSRC-TR-2004-00201, Rev. 0

RPP-WTP Report Number: SRT-RPP-2004-00034, Rev. 0

Number of Roman and Cardinal Numbered Pages: 46

\section{Appendix Section: I-2}

Report Title: Interim Report: RPP-WTP Semi-Integrated Pilot Plant - Campaign II Report Date: September 2004

WSRC Report Number: WSRC-TR-2004-00478, Rev. 0

RPP-WTP Report Number: SRNL-RPP-2004-00075, Rev. 0

Number of Roman and Cardinal Numbered Pages: 200

\section{Appendix Section: I-3}

Report Title: Interim Report: RPP-WTP Semi-Integrated Pilot Plant - Campaign III

Report Date: November 2004

WSRC Report Number: WSRC-TR-2004-00565, Rev. 0

RPP-WTP Report Number: SRNL-RPP-2004-00092, Rev. 0

Number of Roman and Cardinal Numbered Pages: 165 
WSRC-TR-2005-00105, REVISION 0

SRNL-RPP-2005-00012, REVISION 0

\section{Appendix Section: I-1}

Interim Report: RPP-WTP Semi-Integrated Pilot Plant - Campaign I

[For Information Only] 
WSRC-TR-2004-00201, REV. 0

SRT-RPP-2004-00034, REV. 0

\section{INTERIM REPORT: RPP-WTP SEMI-INTEGRATED PILOT PLANT - CAMPAIGN I}

April 2004

SAVANNAH RIVER NATIONAL LABORATORY

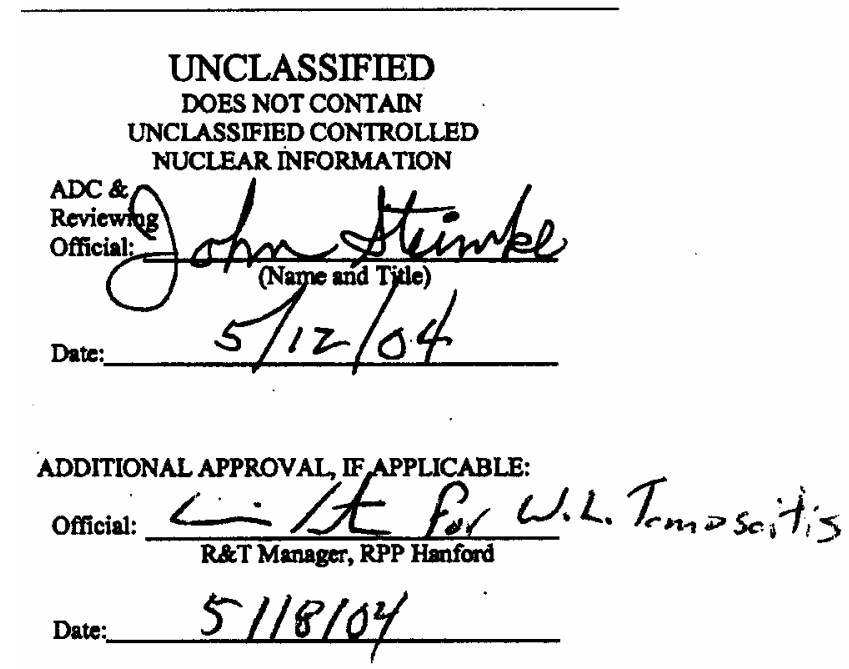

Westinghouse Savannah River Company Savannah River Site Aiken, SC 29808 
WSRC-TR-2004-00201, REV. 0

SRT-RPP-2004-00034, REV. 0

\section{DISCLAIMER}

This report was prepared for the United States Department of Energy under Contract No. DE-AC09-96SR18500 and is an account of work performed under that contract. Neither the United States Department of Energy, nor WSRC, nor any of their employees makes any warranty, expressed or implied, or assumes any legal liability or responsibility for accuracy, completeness, or usefulness, of any information, apparatus, or product or process disclosed herein or represents that its use will not infringe privately owned rights. Reference herein to any specific commercial product, process, or service by trade name, trademark, name, manufacturer or otherwise does not necessarily constitute or imply endorsement, recommendation, or favoring of same by Westinghouse Savannah River Company or by the United States Government or any agency thereof. The views and opinions of the authors expressed herein do not necessarily state or reflect those of the United States Government or any agency thereof.

Printed in the United States of America

Prepared For

U.S. Department of Energy 
WSRC-TR-2004-00201, REV. 0

SRT-RPP-2004-00034, REV. 0

Key Words:

Ultrafiltration

Hanford Tank 241 AY-102/C-106

Retention:

Permanent

Key WTP R\&T References:

Test Specifications:

24590-PTF-TSP-RT-02-015, Rev. 0, 15

Nov. 2002

24590-PTF-TSP-RT-03-002, Rev. 0, 05

Jun. 2003

Test Plan: WSRC-TR-2003-00338, Rev. 0,

04 Aug. 2003 (or SRT-RPP-2003000165)

Test Exceptions: None

R\&T Focus Area: Ultrafiltration \& SIPP

Test Scoping Statements: S-48 \& S-134

\title{
INTERIM REPORT: RPP-WTP SEMI-INTEGRATED PILOT PLANT - CAMPAIGN I
}

\author{
M.R. Duignan, SRNL \\ J.R. Zamecnik, SRNL \\ M.R. Williams, SRNL
}

Issue Date: 19 APRIL 2004

Westinghouse Savannah River Company

Savannah River Site

Aiken, SC 29808

Prepared for the U.S. Department of Energy Under Contract Number DE-AC09-96SR18500

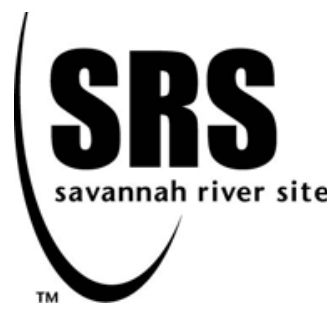


WSRC-TR-2004-00201, REV. 0

SRT-RPP-2004-00034, REV. 0

\section{REVIEWS AND APPROVALS}
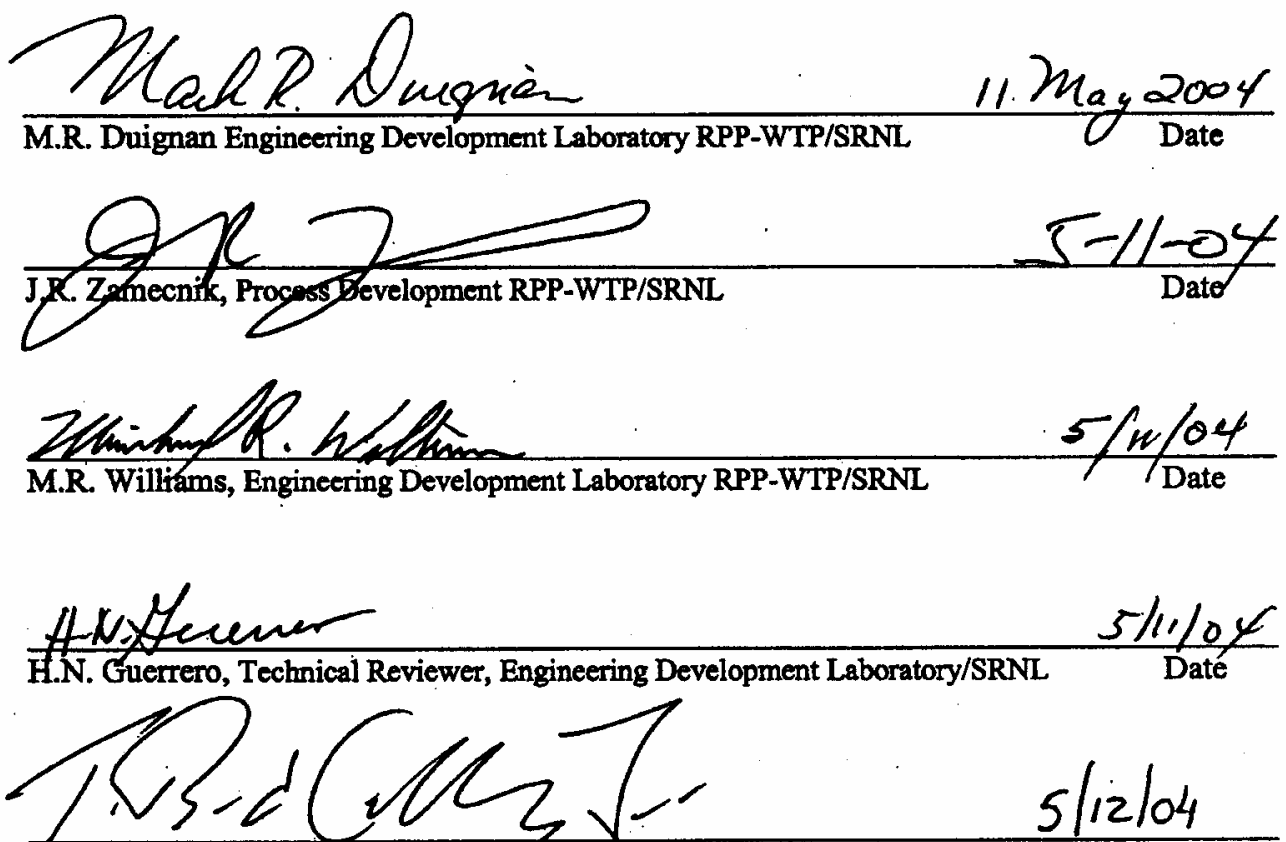

T.B. Calloway, Jr., Level 4 Manage, Process Development RPP-WTP/SRNL Date

Date

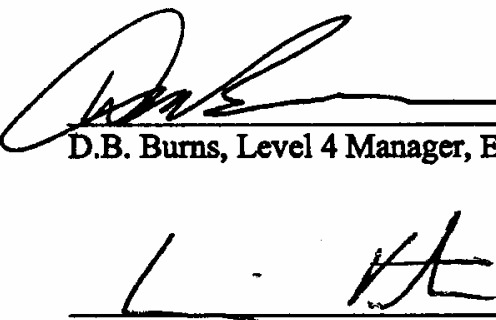

R.A. Peterson, RPP Customer

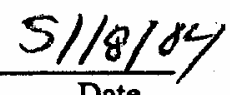




\section{TABLE OF CONTENTS}

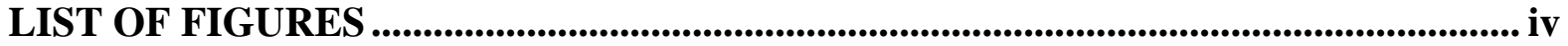

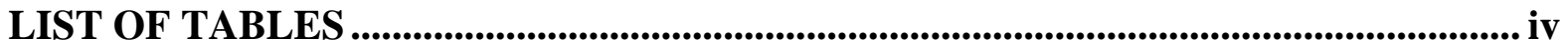

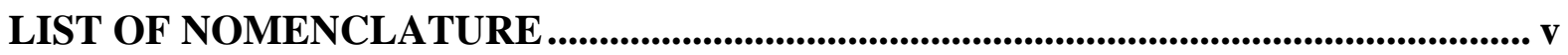

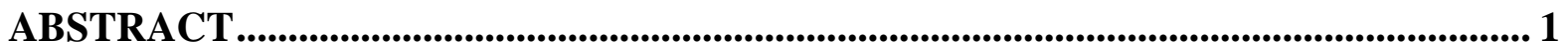

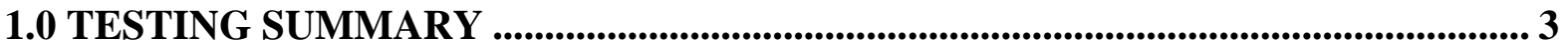

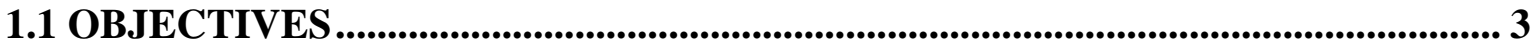

1.2 TEST EXCEPTIONS ..................................................................................... 3

1.3 RESULTS AND PERFORMANCE AGAINST SUCCESS CRITERIA ................. 3

1.4 QUALITY REQUIREMENTS.................................................................................... 4

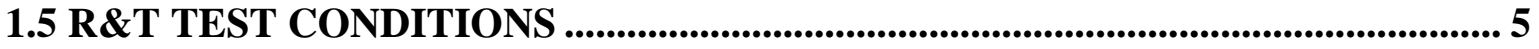

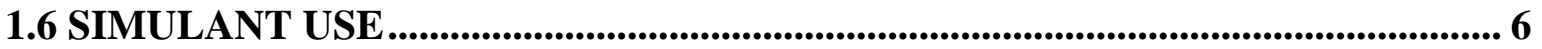

1.7 DISCREPANCIES AND FOLLOW-ON TESTS .................................................. 6

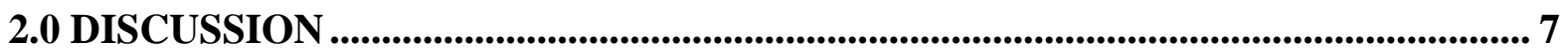

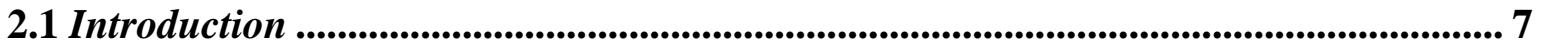

2.2 CAmpaign I Test Matrix and SIPP Modeling ................................................................ 8

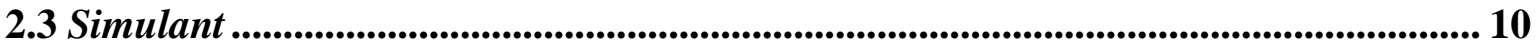

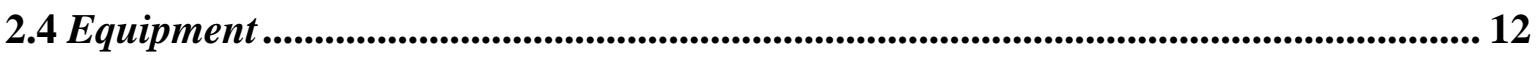

2.5 Measurement Uncertainty ................................................................................ 14

2.6 AY102/C106 concentration (Dewatering) .................................................................. 14

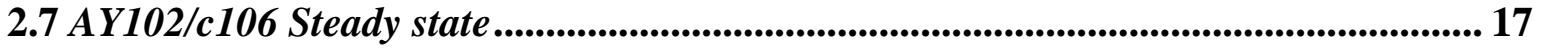

2.8 AY102/c106 Washing ........................................................................................ 19

2.9 AY102/c106 Final concentration .............................................................................. 30

2.10 filter Rinsing and cleaning .................................................................................. 32

2.11 filter baselining .................................................................................................................... 36

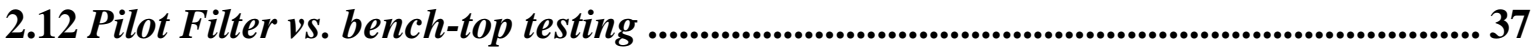

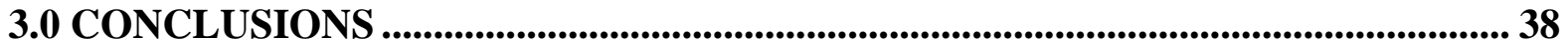

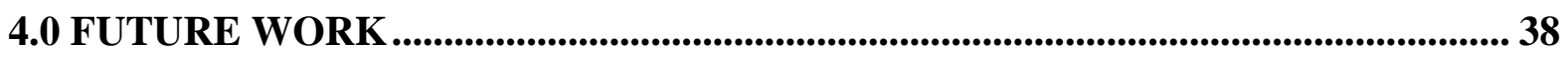

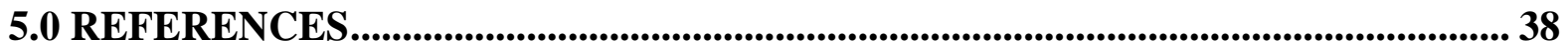




\section{LIST OF FIGURES}

Figure 1. A bench-top scale comparison of actual to simulated waste of AY102/C106 ...... 11

Figure 2. Cross-flow Ultrafilter .................................................................................. 13

Figure 3. Dewatering of AY102/C106 simulant.............................................................. 15

Figure 4. Dewatering of AY102/C106 simulant............................................................ 16

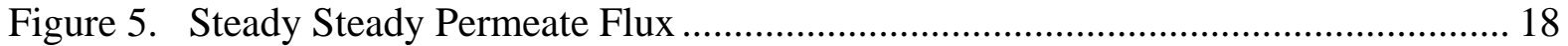

Figure 6. $1^{\text {st }}$ washing of concentrated AY102/C106 with 22 mini-batches.......................... 19

Figure 7. 2nd washing of concentrated AY102/C106 with 22 mini-batches....................... 20

Figure 8. Solids and rheological data throughout simulant washing ............................... 21

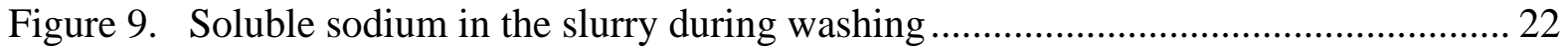

Figure 10. Soluble aluminum in the slurry during washing............................................... 23

Figure 11. Soluble phosphorus in the slurry during washing ......................................... 23

Figure 12. Soluble chromium in the slurry during washing .......................................... 24

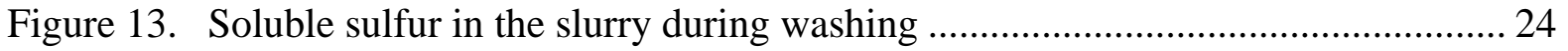

Figure 14. Soluble fluoride in the slurry during washing ............................................. 25

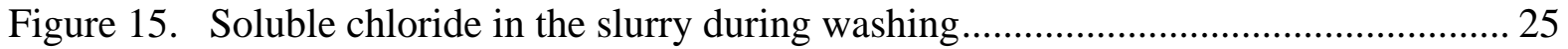

Figure 16. Soluble cesium in the slurry during washing ................................................ 26

Figure 17. Soluble oxalate in the slurry during washing .............................................. 26

Figure 18. Soluble formate in the slurry during washing ........................................... 27

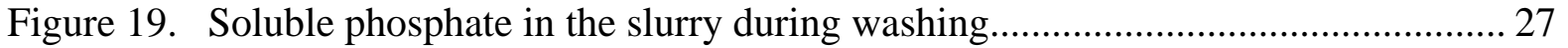

Figure 20. Soluble sulfate in the slurry during washing ................................................ 28

Figure 21. Soluble nitrate in the slurry during washing................................................. 28

Figure 22. Soluble nitrite in the slurry during washing ............................................... 29

Figure 23. Soluble carbonate in the slurry during washing ........................................... 29

Figure 24. Soluble total organic carbon in the slurry during washing.............................. 30

Figure 25. Post-wash dewatering ......................................................................... 31

Figure 26. Temperature of the post-wash slurry during final concentration ....................... 31

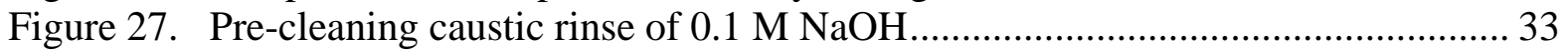

Figure 28. Three 90-minute periods of cleaning with 2 M HNO3 ..................................... 34

Figure 29. Two post-cleaning caustic rinses of $0.1 \mathrm{M} \mathrm{NaOH}$........................................ 35

Figure 30. Rinse and cleaning recycle streams .......................................................... 35

Figure 31. Pre- and post-test water $(0.1 \mathrm{M} \mathrm{NaOH})$ baseline test runs ............................... 36

Figure 32. Comparison of the pilot to bench-top (CUF) scale tests with AY102/C106 simulant

\section{LIST OF TABLES}

Table 1. Overall SIPP Campaign I Test Matrix ............................................................ 9

Table 2. AY-102 / C-106 Actual Waste Composition ...................................................... 10

Table 3. Rheological and other data during dewatering of AY102/C106 simulant.............. 17 
WSRC-TR-2004-00201, REV. 0

SRT-RPP-2004-00034, REV. 0

\section{LIST OF NOMENCLATURE}

\begin{tabular}{|c|c|}
\hline ADS & Analytical Development Section (part of WSRC/SRNL) \\
\hline AN102 & Department of Energy Hanford Site Tank 241-AN-102 \\
\hline AN107 & Department of Energy Hanford Site Tank 241-AN-107 \\
\hline AY102/C106 & $\begin{array}{l}\text { Department of Energy Hanford Site Combined Tanks 241-AY-102 and 241- } \\
\text { C-106 }\end{array}$ \\
\hline BNI & Bechtel National, Inc. \\
\hline CFD & Computational Fluid Dynamics \\
\hline CUF & $\begin{array}{l}\text { Cells Ultrafiltration facility (bench-top cross-flow filter used with both } \\
\text { radioactively cold and hot wastes) }\end{array}$ \\
\hline${ }^{\circ} \mathrm{C}$ & Degree Centigrade (or Celcius) \\
\hline $\mathrm{D}$ & Diameter \\
\hline DIF & Deionized and Filtered (0.1 micron) Water \\
\hline DOE & United States Department of Energy \\
\hline $\mathrm{dP}$ & Differential Pressure \\
\hline $\mathrm{ft}$ & Foot \\
\hline i.d. or ID & Inside Diameter \\
\hline in. & inch \\
\hline hr & Time: Hour \\
\hline HLW & High Lever (Activity) Waste \\
\hline l or L & Liter \\
\hline LAW & Low (Level) Activity Waste \\
\hline $\mathrm{m}$ & Meter \\
\hline M & Molar \\
\hline $\mathrm{Mg}$ & Milligram \\
\hline $\min$ & Time: Minute \\
\hline $\mathrm{ml}$ or $\mathrm{mL}$ & Milliliter \\
\hline $\mathrm{mm}$ & Millimeter \\
\hline nominal & $\begin{array}{l}\text { The word "nominal" for a filter rating is a vague term because its meaning is } \\
\text { manufacturer dependent. Further, a "nominal" rating does not give an exact } \\
\text { size to a filter medium, but rather an approximation to the expected } \\
\text { performance of a filter. In the case of Mott, a nominal rated } 0.1-\mu \mathrm{m} \text { filter } \\
\text { means that approximately } 95 \% \text { of particles greater than } 0.1 \mu \mathrm{m} \text { will not pass } \\
\text { the filter. }\end{array}$ \\
\hline NTU & Nephelometric Turbidity Unit \\
\hline $\mathrm{Pa}$ & Pascal \\
\hline PSD & Particle Size Distribution \\
\hline psi & Pounds Per Square Inch \\
\hline psig & Pounds Force Per Square Inch Gauge \\
\hline psid & Pounds Force Per Square Inch Difference \\
\hline QA & Quality Assurance \\
\hline RPP & River Protection Project (at the DOE Hanford Site) \\
\hline o.d. or OD & Outside Diameter \\
\hline
\end{tabular}


OLI Modeling software which is part of Environmental Simulation Program software package used in developing the SIPP task

s or sec Time: Second

SIPP Semi-Integrated Pilot Plant

SRNL Savannah River National Laboratory (part of WSRC)

Std Dev $\quad$ Standard Deviation

TS

Total Solids

EDL Engineering Development Laboratory (part of WSRC/SRNL)

TMP Transmembrane Pressure (the average pressure drop across the thickness of the filter medium - perpendicular to the slurry flow.)

TRU Transuranic

UDS Undissolved Solids

UF Ultrafiltration

UFP Ultrafiltration Process (part of the RPP-WTP Pretreatment Facility)

$\mathrm{V} \quad$ Velocity of the slurry flow along the length of the filter tubes

WGI Washington Group International

WTP Waste \& Immobilization Treatment Plant

WSRC Westinghouse Savannah River Company 


\begin{abstract}
The first step towards performing the Semi Integrated Pilot Plant (SIPP) task was to subject a simulant of Hanford Tank 241-AY-102/C-106 (AY102/C106) to cross-flow ultrafiltration. That task is comprised of four campaigns of which the first, Campaign I, is the subject of this interim report. The report is called interim because it is limited to the results obtained from a single campaign and further limited to only highlights of that single campaign. A final report will follow Campaign IV and its intent is to comprehensively cover the overall task activities, as well as the full database accumulated and the equipment used during the yearlong SIPP task.
\end{abstract}

The SIPP task is considered semi-integrated because it only deals with the pretreatment operations of the DOE River Protection Plan - Waste Treatment and Immobilization Plant (RPP-WTP). That is, the pilot plant starts by receiving waste from the tank farm and ends when waste is processed to the point of being sent for vitrification.

SIPP is a limited version of the plant's pretreatment facility in that it tests pilot scale unit operation of Feed Evaporation, Ultrafiltration, Cesium Ion Exchange, and finally Treated LAW Evaporation. However, an integral part of SIPP is to produce the many recycle streams that must also be processed with stored tank farm waste. That is, it is very important to know the impact that the recycle streams will have on the operation of the several unit operations so as to better model the plant's overall pretreatment throughput, leading to a production rate of stabilized waste.

While Campaign II, III, and IV will utilize all four-unit operations and incorporate the many recycle streams, Campaign I is limited to the ultrafiltration of a single waste stream (AY102/C106) without recycles. This was done for three reasons: 1. To obtain filter flux results on a single waste to compare to other single waste filter tests done in the past so that filter performance can be determined, 2. To obtain filter flux results on a single waste to compare to the filter performance when the many recycle streams are added to that waste to determine their effect on flux, and 3. To produce first generation recycle streams for subsequent campaigns. Ultrafiltration will be the single largest source of such streams ( $\sim 40 \%)$ and creating those streams by other means would lead to large uncertainties.

Campaign I started with a batch of AY102/C106 simulant at a prototypic undissolved solids (UDS) concentration of $12 \mathrm{wt} \%$, which was then filtered to $24 \mathrm{wt} \%$ UDS and a final volume of 37 gallons. This volume represents the $18,000^{\dagger}$ gallons for the full-scale RPP-WTP. The batch was then washed twice with a caustic solution $(0.01 \mathrm{M} \mathrm{NaOH})$. Each wash volume (45 gallons) was based on planned plant flowsheet and each washing batch was administered in 22 equal minibatches to mimic twenty-two 1000-gallons batches to be used for each washing cycle in the full-scale plant. Finally, the washed simulated waste was drained from the ultrafiltration unit and stored for eventual vitrification and the unit was prototypically rinsed and acid cleaned to show cleaning effectiveness.

\footnotetext{
${ }^{\dagger}$ After Campaign I began in January 2004, RPP-WTP increased the base filter batch size from 18,000 to 21,550 gallons.
} 
During concentration ${ }^{\ddagger}$, the simulated waste started at a permeate filter flux of $0.014 \mathrm{gpm} / \mathrm{ft}^{2}$ at $12.5 \mathrm{wt} \%$ UDS with a density of $1.27 \mathrm{~kg} / \mathrm{L}$, consistency of $13.4 \mathrm{mPa}-\mathrm{s}$, and yield stress of $4.9 \mathrm{~Pa}$. At the end of concentration' the permeate filter flux was $0.005 \mathrm{gpm} / \mathrm{ft}^{2}$ at $23.7 \mathrm{wt} \%$ UDS with a density of $1.36 \mathrm{~kg} / \mathrm{L}$, consistency of $23.8 \mathrm{mPa}-\mathrm{s}$, and yield stress of $34.8 \mathrm{~Pa}$. The overall mean permeate filter flux during the 12 hours of concentration was $0.008 \mathrm{gpm} / \mathrm{ft}^{2}$. Throughout concentration no solids were found in the separated permeate. The measured permeate turbidity remained below 0.5 NTU, which is significantly below the 2 NTU threshold set in the task plan to indicate significant solids.

To meet test requirements, before waste washing began, the solids concentration of the batch was reduced to $20 \mathrm{wt} \%$ UDS. During the first washing cycle the simulated waste started at a permeate filter flux of $0.015 \mathrm{gpm} / \mathrm{ft}^{2}$ at $19.6 \mathrm{wt} \%$ UDS with a density of $1.33 \mathrm{~kg} / \mathrm{L}$, consistency of $19.1 \mathrm{mPa}-\mathrm{s}$, and yield stress of $8.5 \mathrm{~Pa}$. At the end of the first washing the permeate filter flux was $0.023 \mathrm{gpm} / \mathrm{ft}^{2}$ at $20.1 \mathrm{wt} \%$ UDS with a density of $1.17 \mathrm{~kg} / \mathrm{L}$, consistency of $12.6 \mathrm{mPa}-\mathrm{s}$, and yield stress of $10.4 \mathrm{~Pa}$. The overall average permeate filter flux during the 7 hours of cycle 1 washing was $0.018 \mathrm{gpm} / \mathrm{ft}^{2}$. During the second washing cycle the simulated waste started at a permeate filter flux of $0.025 \mathrm{gpm} / \mathrm{ft}^{2}$. At the end of the second washing the permeate filter flux was $0.032 \mathrm{gpm} / \mathrm{ft}^{2}$ at $20.6 \mathrm{wt} \%$ UDS with a density of $1.16 \mathrm{~kg} / \mathrm{L}$, consistency of $9.0 \mathrm{mPa}-\mathrm{s}$, and yield stress of $8.2 \mathrm{~Pa}$. The overall average permeate filter flux during the 4 hours of cycle 2 washing was $0.029 \mathrm{gpm} / \mathrm{ft}^{2}$.

The filter cleaning sequence followed the RPP-WTP planned process of first rinsing the filter with caustic solution, then clean it three times with $2 \mathrm{M}$ nitric acid, and finally rinsing twice with caustic solution. Each of the three acid cleaning lasted 90 minutes long with a significant permeate flux improvement after each. At the start of cleaning the permeate flux of the $2 \mathrm{M}$ nitric acid was $0.10 \mathrm{gpm} / \mathrm{ft}^{2}$ and after 4.5 hours, the three acid cleanings increased that flux to $0.32 \mathrm{gpm} / \mathrm{ft}^{2}$.

Finally, the before and after water test to evaluate the level of cleanliness showed that the filter did not return to pre-test water permeate fluxes, but it was still more than an order of magnitude higher than when filtering waste. The average pre-test water permeate flux was $0.9 \mathrm{gpm} / \mathrm{ft}^{2}$ at $11 \mathrm{ft} / \mathrm{s}$ axial velocity and a TMP of $30 \mathrm{psid}$. The average post-test water permeate flux was $0.2 \mathrm{gpm} / \mathrm{ft}^{2}$ at $11 \mathrm{ft} / \mathrm{s}$ axial velocity and a TMP of 30 psid.

\footnotetext{
${ }^{\ddagger}$ Unless otherwise noted, all the data described in the Abstract were obtained with the pilot ultrafiltration test rig operating at a constant slurry axial velocity of $11 \mathrm{ft} / \mathrm{s}$, a transmembrane pressure of $40 \mathrm{psid}$, and a temperature of $25^{\circ} \mathrm{C}$.
} 
WSRC-TR-2004-00201, REV. 0

SRT-RPP-2004-00034, REV. 0

\subsection{TESTING SUMMARY}

\subsection{OBJECTIVES}

Campaign I, one of four planned campaigns for the SIPP task, is a completely separate body of work from Campaigns II to IV. This work was originally scoped as a stand alone task and as such had its own test specification (Townson, 2002; Scoping Statement S-48). That specification has since been incorporated into the larger SIPP task, which has a large set of objectives, many of which do not apply to this first campaign. However, the objectives listed in Scoping Statement S-48 are still applicable and therefore listed below:

\begin{tabular}{|l|l|l|}
\hline Test Objective & $\begin{array}{l}\text { Objective } \\
\text { Met (Y/N) }\end{array}$ & Discussion \\
\hline $\begin{array}{l}\text { 1. Collect data on performance of the } \\
\text { ultrafilter unit to dewater the simulant } \\
\text { derived from the tank 241-AY-102/C- } \\
106 \text { characterization. }\end{array}$ & Y & $\begin{array}{l}\text { Data were collected and discussed } \\
\text { throughout this report. }\end{array}$ \\
\hline $\begin{array}{l}\text { 2. Estimate the removal efficiency for } \\
\text { soluble species and aid calculations to } \\
\text { minimize the mass of waste oxides } \\
\text { vitrified as HLW. }\end{array}$ & Y & $\begin{array}{l}\text { Efficiency was determined from } \\
\text { periodic samples. See the last } \\
\text { column of Table 3. }\end{array}$ \\
\hline $\begin{array}{l}\text { 3. Multi-tube cross-flow filtration tests } \\
\text { will yield representative data on } \\
\text { equipment performance (permeate flux, } \\
\text { backpulse efficiency) and this will be } \\
\text { cross checked by separate work done } \\
\text { using the simulant and active waste at } \\
\text { single tube scale. }\end{array}$ & $\mathrm{Y}$ & $\begin{array}{l}\text { Pilot data obtained are compared to } \\
\text { bench-top simulant and active } \\
\text { waste test in section 2.12. }\end{array}$ \\
\hline
\end{tabular}

\subsection{TEST EXCEPTIONS}

\begin{tabular}{|l|l|}
\hline List Test Exceptions & Describe Test Exceptions \\
\hline 1.None & N/A \\
\hline
\end{tabular}

\subsection{RESULTS AND PERFORMANCE AGAINST SUCCESS CRITERIA}

\begin{tabular}{|l|l|}
\hline List Success Criteria & $\begin{array}{l}\text { Explain How the Tests Did or Did Not } \\
\text { Meet the Success Criteria }\end{array}$ \\
\hline $\begin{array}{l}\text { 1.The project flowsheet currently assumes } \\
\text { the concentrated slurry contains 20 wt\% }\end{array}$ & $\begin{array}{l}\text { The assumption was confirmed by } \\
\text { dewatering to greater than 20 wt\% UDS. } \\
\text { UDS, this test needs to confirm (or } \\
\begin{array}{l}\text { improve upon) that assumption. The target } \\
\text { for this test will be set at 25 wt\% UDS. }\end{array}\end{array}$ \\
\hline $\begin{array}{l}\text { 2. No solids must pass into the } \\
\text { ultrafiltration permeate (<0.001 wt\% UDS) } \\
\text { and/or permeate }<2 \text { NTU. }\end{array}$ & $\begin{array}{l}\text { Periodic turbidity checks consistency } \\
\text { indicated the permeate to be solids free } \\
\text { with a measurement of }<0.5 \text { NTU }\end{array}$ \\
\hline
\end{tabular}




\begin{tabular}{|c|c|}
\hline $\begin{array}{l}\text { 3. The mean flux throughout the complete } \\
\text { dewatering cycle should be greater than } \\
0.0277 \mathrm{gpm} / \mathrm{ft}^{2} \text { (24590-PTF-RPT-ENG-01- } \\
002 \text {, Rev. } 0 \text {. Impact of Study \#17 on the } \\
\text { Configuration of Ultrafiltration System) } \\
\text { and the TMP less than } 60 \text { psig. }\end{array}$ & $\begin{array}{l}\text { The mean flux for dewatering the AY- } \\
\text { 102/C-106 simulant was } 0.008 \text { gpm/ } / \mathrm{ft}^{2} \\
\text { over the } 12.6 \text {-hour test; concentrating } \\
\text { from } 12.5 \text { to } 24 \text { wt\% UDS. While this } \\
\text { flux is significantly below the desired } \\
\text { mean flux of } 0.0277 \mathrm{gpm} / \mathrm{ft}^{2} \text {, the simulant } \\
\text { does match the real waste behavior. There } \\
\text { was } 1 \text { real waste test and } 2 \text { simulant tests } \\
\text { at the bench-top scale. The solids } \\
\text { concentration starting and ending points } \\
\text { are all different, but a comparison can be } \\
\text { made from } 12.5 \text { to } 14 \text { wt\% UDS. The real } \\
\text { waste mean permeate flux was } 0.013 \\
\text { gpm/ } \mathrm{ft}^{2} \text {, the two bench-top test gave, } \\
0.012 \text { and } 0.016 \text { gpm } / \mathrm{ft}^{2} \text {. The pilot-scale } \\
\text { permeate flux, of this report, obtained } \\
0.011 \text { gpm/ } \mathrm{ft}^{2} \text {. }\end{array}$ \\
\hline $\begin{array}{l}\text { 4. Determination of de-watering flux vs. } \\
\text { wt\% UDS solids at an optimum flow/TMP } \\
\text { condition. }\end{array}$ & $\begin{array}{l}\text { Those data were obtained and discussed in } \\
\text { section 2.6. }\end{array}$ \\
\hline $\begin{array}{l}\text { 5. Performance data generated from this } \\
\text { simulant test matches data generated from } \\
\text { similar test with real AY-102/C-106 waste } \\
\text { samples. }\end{array}$ & Yes. See answer to item 3. \\
\hline $\begin{array}{l}\text { 6. The cross-flow test rig after chemical } \\
\text { cleaning can be returned to pre-test } \\
\text { operating levels. }\end{array}$ & $\begin{array}{l}\text { As shown in section } 2.10 \text { the post-test } \\
\text { mean water flux at a TMP = } 20 \text { psid was } \\
0.14 \mathrm{gpm} / \mathrm{ft}^{2} \text { and did not return to the } 0.54 \\
\text { gpm/ } \mathrm{ft}^{2} \text { obtained from the pre-test water } \\
\text { flux. However, the post-test water flux } \\
\text { was } 5 \text { times higher that the WTP dewater } \\
\text { flux of } 0.0277 \mathrm{gpm} / \mathrm{ft}^{2} \text { and was more than } \\
17 \text { times greater that the actual mean } \\
\text { dewater flux of } 0.008 \mathrm{gpm} / \mathrm{ft}^{2} \text {. }\end{array}$ \\
\hline
\end{tabular}

\subsection{QUALITY REQUIREMENTS}

This work was conducted in accordance with the RPP-WTP QA requirements specified for work conducted by SRNL as identified in DOE IWO MOSRLE60. SRNL has provided matrices to WTP, demonstrating compliance of the SRNL QA program with the requirements specified by WTP. Specific information regarding the compliance of the SRNL QA program with RW-0333P, Revision 10, NQA-1 1989, Part 1, Basic and Supplementary Requirements and NQA-2a 1990, Subpart 2.7 is contained in these matrices. 
WSRC-TR-2004-00201, REV. 0

SRT-RPP-2004-00034, REV. 0

\subsection{R\&T TEST CONDITIONS}

\begin{tabular}{|l|l|}
\hline List R\&T Test Conditions & \multicolumn{1}{|c|}{ Were Test Conditions Followed? } \\
\hline $\begin{array}{l}\text { 1.The target minimum reportable quantity } \\
\text { for each analyte in Table 1.2 (Townson, } \\
\text { 2002) shall be reviewed to confirm that the } \\
\text { minimum detection limit (MDL) is at least } \\
\text { 3X below the target minimum reportable } \\
\text { quantity. If the required MDL is not } \\
\begin{array}{l}\text { achievable for any particular analyte, then } \\
\text { that analyte must be identified with the } \\
\text { and approved before testing began. }\end{array}\end{array}$ & \\
corresponding MDL value in the test plan. & \\
\hline $\begin{array}{l}\text { 2. Permeate will be analyzed for solids by } \\
\text { turbidity and the particle size, if sufficient } \\
\text { solids make this possible, using a Lasentec } \\
\text { particle size analyzer. }\end{array}$ & $\begin{array}{l}\text { As listed in Success Criterion No. } 2 \text { the } \\
\text { turbidity indicated that no significant } \\
\text { undissolved solids were found, therefore } \\
\text { no particle size distribution measurements } \\
\text { were needed. }\end{array}$ \\
\hline $\begin{array}{l}\text { 3. The QC target requirements listed in } \\
\text { Tables 1.3, 1.4, and 1.5 (Townson, 2002) } \\
\text { shall be reviewed and any requirements } \\
\text { that are not expected to be met shall be } \\
\text { identified and the expected delta from the } \\
\text { criteria be identified. Permeate will be } \\
\text { analyzed for solids by turbidity and the } \\
\text { particle size, if sufficient solids make this } \\
\begin{array}{l}\text { possible, using a Lasentec particle size } \\
\text { analyzer. }\end{array}\end{array}$ & $\begin{array}{l}\text { As listed in Success Criterion No. } 2 \text { the } \\
\text { turbidity indicated that no significant } \\
\text { undissolved solids were found, therefore } \\
\text { no particle size distribution measurements } \\
\text { were needed. }\end{array}$ \\
\hline
\end{tabular}




\subsection{SIMULANT USE}

A simulant of Hanford tank 241-AY-102/C-106 was used. The simulant was developed by SRNL (Zamecnik, et al., 2004) and evaluated against an actual waste sample. See section 3.3.

\subsection{DISCREPANCIES AND FOLLOW-ON TESTS}

No discrepancies occurred during testing, but there will be follow-on work. This test, referred to as Campaign I, is the first of four Campaigns, which make up an overall task referred to as Semi Integrated Pilot Plant (SIPP) (Duignan, 2003c). 


\subsection{DISCUSSION}

\subsection{INTRODUCTION}

Bechtel National, Inc. (BNI) has been contracted by the Department of Energy (DOE) to design a Waste Treatment and Immobilization Plant (WTP) to stabilize liquid radioactive waste that is stored at the Hanford Site as part of the River Protection Project (RPP). Because of its experience with radioactive waste stabilization, the Savannah River National Laboratory (SRNL) of the Westinghouse Savannah River Company (WSRC) is working with BNI and Washington Group International (WGI), to help design and test certain parts of the waste treatment facility. One part of the process is the separation of radioactive solids from the liquid wastes by cross-flow ultrafiltration. This task tested a cross-flow filter, prototypic in porosity, length and diameter, with a simulated radioactive waste, made to prototypically represent the chemical and physical characteristics of a Hanford waste in tank 241-AY102/C-106 (AY102/C106)).

This technical baseline research and development work was initiated by a WGI test specification (Townson, 2002) to test a simulated radioactive waste in an existing pilot scale cross-flow filtration system in the Engineering Development Laboratory (EDL) of SRNL. This specification requested a similar test to those previously done on the same equipment (Duignan, 2000a, Duignan, 2000b, Duignan, 2003a). The main difference was the simulated wasted tested. As in previous simulant tests, the actual waste was first filtered at a bench-top scale (Poirier et al., 2003) and then an SRNL simulant was developed (Zamecnik et al., 2004). In that same bench-top simulant report, the simulant was found to have the same filter performance as the actual waste. This report deals with the pilot-scale testing of that proven simulant to determine full-scale filter performance data.

The chosen filter was manufactured by the Mott Metallurgical Corporation to meet RPP-WTP specifications (Townson, 2002, Longwell, 2003), as follows:

7 filter tubes with each having an inside diameter of 0.5 -inch

90 -inch porous length for each filter tube and made of stainless steel

Nominal rated 0.1 micron filter element ('nominal' is explained in the nomenclature)

and the pilot test rig was designed to have the following:

Maximum axial velocity through filter tubes of $15 \mathrm{ft} / \mathrm{s}(4.6 \mathrm{~m} / \mathrm{s})$

Maximum transmembrane pressure (TMP) of 60 psid (414 M Pa)

Maximum axial velocity to be achievable at the maximum TMP

Instrumentation to monitor the axial velocity, the permeate flow rate, the

TMP, and the slurry temperature

All materials to be compatible with the high-caustic simulants and the $2 \mathrm{M}$ nitric acid cleaning solution

All specifications were met or exceeded. 
Before testing began, a larger task emerged to include not only filtration, but also all of the principal unit operations that make up the RPP-WTP pretreatment facility. They include: Feed Evaporation, Ultrafiltration, Ion Exchange, and then Treated LAW Evaporation and this larger test is referred to as the Semi Integrated Pilot Plant (SIPP). It is referred to as "semiintegrated" because the test does not include the vitrification portion of the plant. Another Test Specification was issued (Longwell, 2003) which incorporated the filtration Test Specification as a first step in the process to test all the unit operations. In general, the requirements of the initial Test Specification did not significantly change, but were only supplemented with further testing that would follow the ultrafiltration test. In the context, of the SIPP task, the ultrafiltration test gained a larger role. This test was now referred to as Campaign I, since it is the first of four planned campaigns of SIPP. Campaign I was not only to obtain filter performance data of AY102/C106 to compare to other single-waste filter test, but it would also produce several recycle streams, e.g., solutions used to wash the waste stream, solutions used to rinse and clean the filter, etc. Once these recycle streams were generated they could be combined with other streams to be fed to the entire series of unit operations in order to learn how those streams affect plant operations. Further, after the filter recycle streams are produced and combined with plant waste to be processed, the single waste, i.e., AY102/C102, filter data could be compared to those data after recycle streams are added. Such information would be more useful for RPP-WTP plant design since under steady state operations the plant will always operate with some feedback secondary streams, making the test data much more prototypic.

This report will discuss the filter operation during dewatering, constant slurry solids concentration, slurry washing, rinsing and cleaning, and highlights of simulant changes undergone from concentration to washing.

A summary of EDL task activities is as follows:

WGI Test Specification for UF Test Received - December 2002

WGI Test Specification for SIPP Received - June 2003

WSRC Task Technical \& Quality Assurance Plan Approved - August 2003

Test Procedure Approved - October 2003

Received AY102/C106 Simulant - January 2004

Campaign I Test - 26 January to 12 February 2004

\subsection{CAMPAIGN I TEST MATRIX AND SIPP MODELING}

The ultrafiltration test specification (Townson, 2002) indicated the test matrix to be used and combined with the SIPP test specification (Longwell, 2003), several of the ultrafiltration test runs were eliminated. Table 1 shows the test matrix, which was summarized and condensed from both specifications. The number ordering of the test specifications has been maintained so that it could be compared to the original requirements. However, since several tests were eliminated by the second test specification, there are gaps between several of the test run numbers. 
WSRC-TR-2004-00201, REV. 0

SRT-RPP-2004-00034, REV. 0

Table 1. Overall SIPP Campaign I Test Matrix

\begin{tabular}{|c|c|c|c|c|c|}
\hline Test No. & Test Activity & $\begin{array}{c}\text { Trans- } \\
\text { membrane } \\
\text { Pressure } \\
\text { (psid) }\end{array}$ & $\begin{array}{c}\text { Slurry } \\
\text { Velocity } \\
\text { (ft/s) }\end{array}$ & $\begin{array}{c}\text { Slurry } \\
\text { Temp } \\
\left({ }^{\circ} \mathrm{C}\right)\end{array}$ & $\begin{array}{l}\text { Run } \\
\text { Time } \\
\text { (min.) }\end{array}$ \\
\hline $1.00 \mathrm{~A}$ & Water (1) & 10 & 11 & 25 & 20 \\
\hline $1.00 \mathrm{~B}$ & Water & 20 & 11 & 25 & 20 \\
\hline $1.00 \mathrm{C}$ & Water & 30 & 11 & 25 & 20 \\
\hline 1.03 & Dewater to 25 wt\% (2) & 40 & 12 & 25 & (3) \\
\hline 1.04 & 25 wt $\%$ & 40 & 11 & 25 & 120 \\
\hline 1.07 & $25 w t \%$ & 30 & 9 & 25 & 60 \\
\hline 1.08 & $25 w t \%$ & 30 & 13 & 25 & 60 \\
\hline 1.09 & 25 wt $\%$ & 50 & 13 & 25 & 60 \\
\hline 1.10 & $25 w t \%$ & 50 & 9 & 25 & 60 \\
\hline 1.11 & $25 w t \%$ & 40 & 11 & 25 & 120 \\
\hline 1.16 & 25 wt $\%$ & 40 & 11 & 25 & 120 \\
\hline Wash & $20 \mathrm{wt} \%(4)$ & 40 & 12 & 30 & $(5)$ \\
\hline 1.17 & $>20 \mathrm{wt} \%$ & 40 & 12 & 25 & (6) \\
\hline $1.18 \mathrm{~A}$ & Water Rinse & 40 & 12 & 25 & 30 \\
\hline $1.18 \mathrm{~B}$ & Acid clean (8) & 40 & 12 & 25 & 90 \\
\hline $1.18 \mathrm{C}$ & Acid clean & 40 & 12 & 25 & 90 \\
\hline $1.18 \mathrm{D}$ & Acid clean & 40 & 12 & 25 & 90 \\
\hline $1.19 \mathrm{~A}$ & Water Rinse & 20 & 12 & 25 & 30 \\
\hline 1.19B & Water Rinse & 20 & 12 & 25 & 30 \\
\hline $1.19 \mathrm{C}$ & Water Rinse & 20 & 12 & 25 & 30 \\
\hline 1.20 & Water (9) & $10,20,30$ & 11 & 25 & 60 \\
\hline \multicolumn{6}{|c|}{$\begin{array}{l}\text { Notes: } \\
\text { (1) - The water was deionized, filtered with a 0.1-micron absolute filter, } \\
\text { then made caustic to } 0.1 \mathrm{M} \mathrm{NaOH} \text { (but } 0.01 \mathrm{M} \mathrm{NaOH} \text { for washing). } \\
\text { (2) - wt } \%=\text { weight percent of undissolved solids } \\
\text { (3) - Run time is the time to dewater the simulant from the low wt } \% \text { to } \\
\text { the final concentration. The only test specification requirement } \\
\text { was to run for no less than } 12 \text { hours. } \\
\text { (4) - To meet the test spec. the slurry was diluted to } 20 \mathrm{wt} \% \mathrm{UDS} \text {, then } \\
\text { washed with two volumes of water (note } 1 \text { ) each equaling } 21 / 18 \mathrm{x} \\
\text { simulant volume. Each wash was broken down into } 22 \text { mini-washes. } \\
\text { (5) - The wash run time was the time necessary to introduce and } \\
\text { remove all } 22 \text { subvolumes. } \\
\text { (6) - Test } 1.17 \text { run time was not fixed. Filtering was to continue } \\
\text { until the simulant plugged the filter, or some test rig limit was reached. } \\
\text { (8) - } 2 \mathrm{M} \text { nitric acid was used. } \\
\text { (9) - The rinse water from run } 1.19 \mathrm{C} \text { was left in the test rig for this test. }\end{array}$} \\
\hline
\end{tabular}

This test matrix and, in general, all SIPP Campaign I work, was not impacted significantly by SIPP needs. That is, Campaign I is still the independent filter test as described by Townson, 2003. However, the following campaigns are highly dependent on resulting simulant streams from Campaign I and as such all four campaigns needed to be planned together. To plan for SIPP, SRNL did its own modeling to determine how to run Campaigns II, III, IV through Campaign I dependence. Using the RPP-WTP flowsheet (Lee and Binsfield), an OLI model was made of steady-state Pretreatment operation. This model was used to determine the operation of the pilot-scale unit operations of the Pretreatment system. Campaign I was used by the model to make the connection between pilot-scales and the full-size plant. A key parameter for the rate of glass production in the plant is the size of the Ultrafiltration washed batch of HLW. The pilot ultrafiltration batch size for waste is $140 \mathrm{~L}$ (or 37 gallons). The 
WSRC-TR-2004-00201, REV. 0

SRT-RPP-2004-00034, REV. 0

plant UFP batch size was $18,000^{\dagger}$ gallons. With this scale, the OLI model was used to scale all other pilot streams. Where the model was useful in directing some of the Campaign I testing is in the caustic and acidic streams used to rinse and clean the filter after dewatering. These streams would be stored after Campaign I to be recycled as part of the feed for Campaign II. The model indicated Campaign II recycle streams volumes and therefore solids concentrations could be estimated from expected waste heels in the plant. This will be discussed in more detail in the section on rinsing and cleaning.

\subsection{SIMULANT}

The principal reason for this test was to evaluate filter performance for a simulated waste from Hanford Tank 241-AY-102/C-106, therefore, the test is only as good as the simulant used. Previously, an actual waste sample was obtained from Hanford and cross-flow filter tested at a bench-top scale ${ }^{\ddagger}$ (Poirier et al., 2003). A simulant recipe was developed and within measurement uncertainty, matched the chemical breakdown of the actual waste shown in Table 2. The simulant was then tested with the bench-top scale, CUF Run 1, to evaluate its performance against the actual waste. Within measurement uncertainty the two filter results were the same (Zamecnik et al, 2004) as is shown in Fig. 1.

Table 2. AY-102 / C-106 Actual Waste Composition

\begin{tabular}{|c|c|c|c|c|c|}
\hline Species & $\mathrm{mg} / \mathrm{kg}$ & Species & $\mathrm{mg} / \mathrm{kg}$ & Species & $\mathrm{mg} / \mathrm{kg}$ \\
\hline \hline $\mathrm{Ag}$ & 254 & $\mathrm{Mg}$ & 217 & $\mathrm{Y}$ & 27.5 \\
\hline $\mathrm{Al}$ & 15579 & $\mathrm{Mn}$ & 4889 & $\mathrm{Zn}$ & 36.2 \\
\hline $\mathrm{B}$ & 13.9 & $\mathrm{Mo}$ & 47.3 & $\mathrm{Zr}$ & 708 \\
\hline $\mathrm{Ba}$ & 100 & $\mathrm{Na}$ & 76878 & $\mathrm{NO}-$ & 255 \\
\hline $\mathrm{Bi}$ & 5.69 & $\mathrm{Ni}$ & 594 & $\mathrm{NO} 2-$ & 4077 \\
\hline $\mathrm{Ca}$ & 693 & $\mathrm{Nd}$ & 211 & $\mathrm{PO}[-3]$ & 3618 \\
\hline $\mathrm{Cd}$ & 19.9 & $\mathrm{P}$ & 1670 & $\mathrm{SO} 4=$ & 1700 \\
\hline $\mathrm{Ce}$ & 146 & $\mathrm{~Pb}$ & 703 & $\mathrm{C} 2 \mathrm{O} 4-$ & 2882 \\
\hline $\mathrm{Co}$ & 5.97 & $\mathrm{Pr}$ & 59.9 & $\mathrm{Cl}$ & 125 \\
\hline $\mathrm{Cr}$ & 382 & $\mathrm{Rb}$ & 2.02 & $\mathrm{Br}$ & 84.8 \\
\hline $\mathrm{Cs}$ & 3.77 & $\mathrm{~S}$ & 716 & $\mathrm{~F}$ & 139 \\
\hline $\mathrm{Cu}$ & 44.4 & $\mathrm{Si}$ & 9075 & $\mathrm{COOH}-$ & 129 \\
\hline $\mathrm{Fe}$ & 22682 & $\mathrm{Sr}$ & 64.2 & $\mathrm{Citrate}$ & 47.5 \\
\hline $\mathrm{K}$ & 365 & $\mathrm{U}$ & 548 & $\mathrm{CH} 3 \mathrm{COO}-$ & 422 \\
\hline $\mathrm{La}$ & 102 & $\mathrm{~V}$ & 5.87 & $\mathrm{OH}-$ & 6235 \\
\hline $\mathrm{Li}$ & 92.6 & $\mathrm{~W}$ & 125 & $\mathrm{CO}=$ & 58474 \\
\hline
\end{tabular}

\footnotetext{
${ }^{\dagger}$ After Campaign I was complete the RPP-WTP UFP batch size was increased from 18,000 gallons to 21,550 gallons to account for waste heels in the tanks. That change is incorporated in future campaigns, but not for Campaign I.

‡ The bench-top test apparatus is referred to a CUF = Cells Unit Filter, because it was made to operate in the radioactive hot cells at SRNL.
} 
WSRC-TR-2004-00201, REV. 0

SRT-RPP-2004-00034, REV. 0

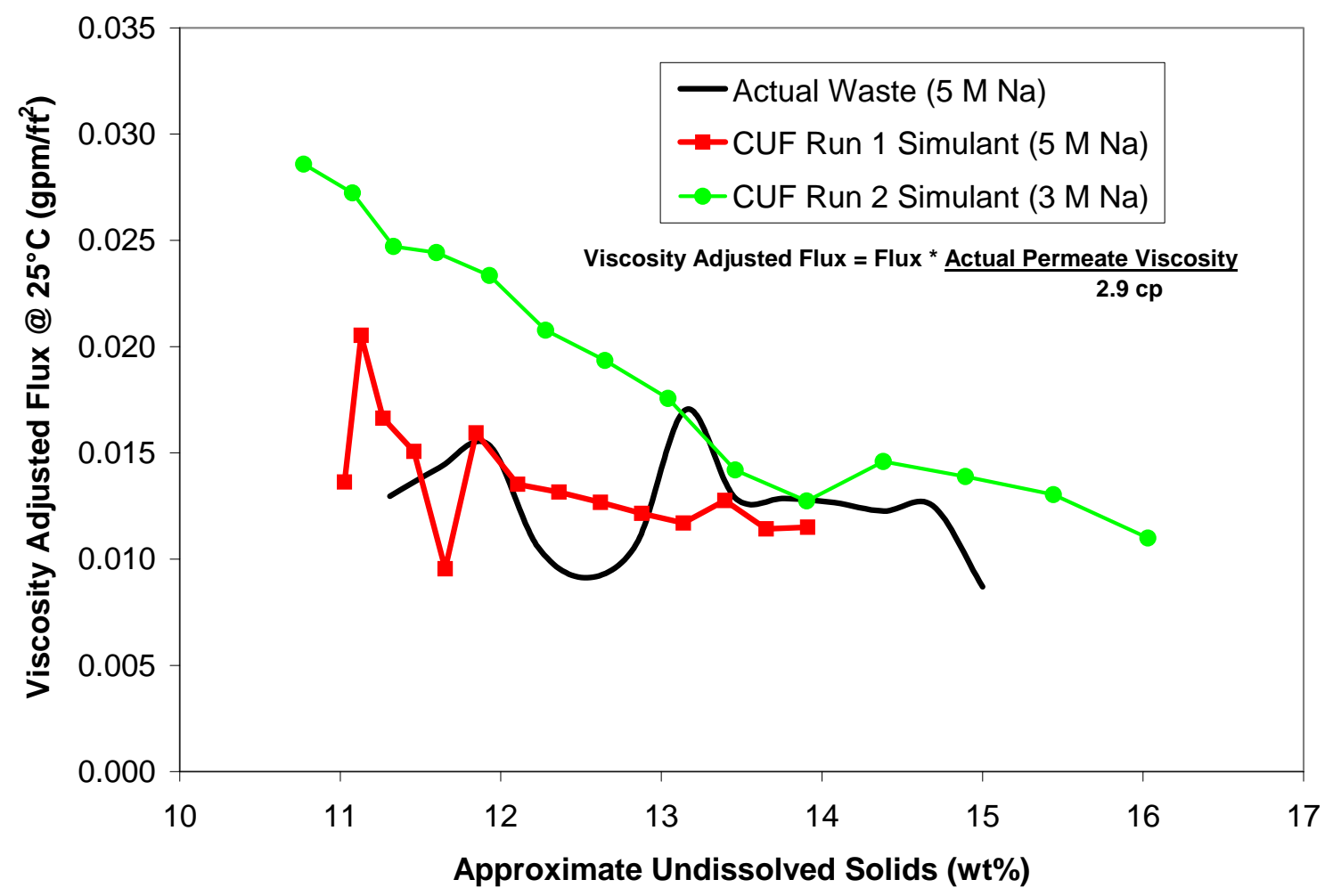

Figure 1. A bench-top scale comparison of actual to simulated waste of AY102/C106

Unfortunately, when both the actual waste and simulants were evaporated to attain a sodium molarity of $5 \mathrm{M}$, a significant amount of solids, basically calcium carbonate, precipitated causing pluggages throughout the filter test apparatus. This is the reason why the actual waste and CUF Run 1 curves in Fig. 1 fluctuate so much. A decision was made not to boil the simulant, but to leave it at the $3 \mathrm{M}$ sodium concentration that the actual waste had when it was first received. Another bench-top filter test, CUF Run 2, was done with a second simulant batch that was left at $3 \mathrm{M}$ sodium. Unfortunately, there was not another actual waste sample to repeat that test, but since permeate flux is strongly dependent upon the permeate viscosity all the data were adjusted, with the equation show in Fig. 1, to that of the actual waste, i.e., $2.9 \mathrm{cp}$, thus a better comparison can be made. Even with the viscosity adjustment, the $3 \mathrm{M}$ sodium simulant, from 11 to $15 \mathrm{wt} \%$ UDS, had a higher permeate flux. A relatively smooth descent in flux for CUF Run 2 indicated a significant reduction in precipitated solids in the feed.

The bench-top studies were accepted by WTP as sufficient to verify the AY102/C106 simulant and allow pilot-scale work to proceed. The actual simulant development is beyond the scope of this interim report and will be reported in final documentation. However, a short description of its preparation will help understanding the pilot-scale test. There are many steps in making the very complex simulant, including precipitations and washings. For the bench-top work, the simulant was made at SRNL. For the pilot scale work, the quantity was too large to be easily handled. In fact, since the filter test was combined with the SIPP task 
in which simulant would be needed for several test campaigns, it was more efficient to procure the simulant from an outside vendor. Even with the many steps to make the simulants the final act of joining the many parts together was very crucial to obtain a desired end product. To obtain a simulant in exactly the form needed it was purchased in three complex mixtures which would be finalized just before each pilot filter test was to begin. In fact, the selected vendor actually made small portions of the several simulant constituents, then shipped them to SRNL to be evaluated before the end products could be made. It consisted of three procured parts, which were: Supernatant, Precipitated sludge solids, and Wet Sodium Aluminosilicate (NAS). These parts were joined together in a very specific sequence, with several dry chemicals to obtain exactly the simulant needed. The general proportions were:

\begin{tabular}{|c|c|}
\hline Constituent & Percentage \\
\hline Supernate Simulant & 15.91 \\
\hline Precipitated Sludge Solids & 72.17 \\
\hline NAS & 8.44 \\
\hline Dry additions & \\
\hline $\mathrm{Al}(\mathrm{OH}) 3$ & 1.72 \\
\hline $\mathrm{Al} 2 \mathrm{O} 3$ & 0.61 \\
\hline Calcium Phosphate Tribasi & 0.04 \\
\hline $\mathrm{SiO} 2$ & 1.11 \\
\hline Total & $100 \%$ \\
\hline
\end{tabular}

The actual combination of all of the above was quite complex but percentages shown are what were necessary to have a starting feed of approximately $12 \mathrm{wt} \%$ UDS. To fine tune the solids concentration some of the supernatant was purposely left out of the mixture during preparation so the solids content could be sampled and then diluted with just enough liquid to achieve the target solids content. Even with all the care taken the target solids content of $11.8 \mathrm{wt} \%$ turned out to be $12.5 \mathrm{wt} \%$ when the experiment began.

Approximately, 80 gallon of the slurry was made during the week of 19 January 2004 and was complete on 22 January, approximately 116 hours before its first use on 27 January.

\subsection{EQUIPMENT}

For convenience a simple schematic of the test apparatus is shown in Fig. 2. Details of the overall system has been previously well documented (Duignan, 2000a, Duignan 2000b, Duignan 2003). A detailed description of the equipment will be included SIPP final report with highlights of changes specific for current testing. 
WSRC-TR-2004-00201, REV. 0

SRT-RPP-2004-00034, REV. 0

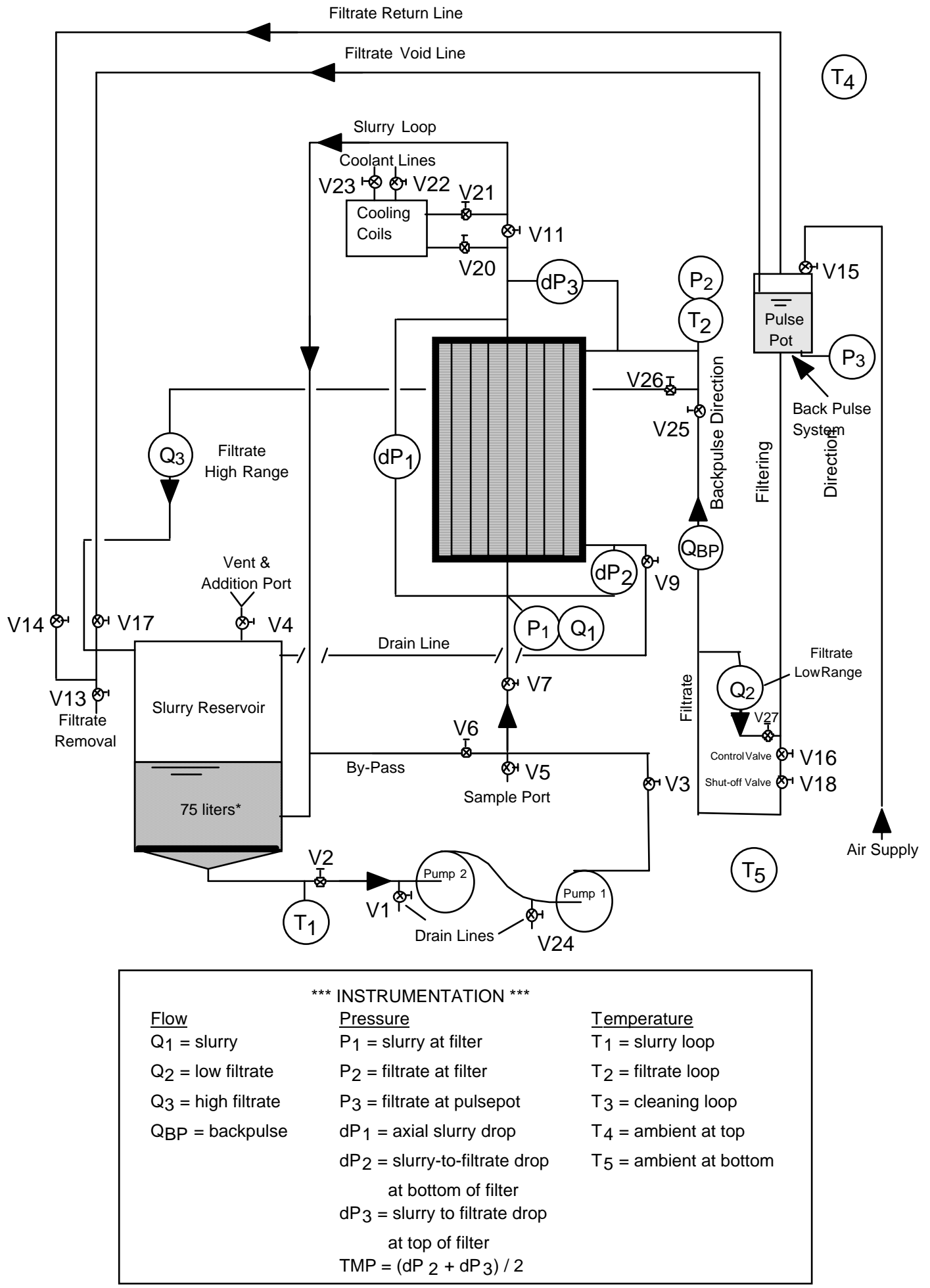

Figure 2. Cross-flow Ultrafilter 


\subsection{MEASUREMENT UNCERTAINTY}

The derivation of measurement uncertainty will not be shown in this single-campaign interim report, but will be included in the final report that will incorporate results from all four campaigns. The uncertainties listed here are based on past work, which was done, on the same equipment under similar circumstances and similar individual instrument uncertainties (Duignan 2003a). These uncertainties are to illustrate the expected magnitude of principal parameters measured throughout this test. They are instrumental in determining the significance in comparing any two datum points. The measurement uncertainties (at the $95 \%$ confidence level) for the important calculated quantities are:

$\begin{array}{lll}\text { Slurry Velocity in a Filter Tube } & = & \pm 6 \% \\ \text { Transmembrane Pressure } & = & \pm 2 \% \\ \text { Temperature Corrected Permeate Flux } & = & \pm 6 \%\end{array}$

For analytical results included herein the measurement uncertainty vary and sometimes can be large. Uncertainties for data obtain from outside organizations, i.e., Analytical Development Section or the Mobile Laboratory of SRNL are beyond the scope of this task but they are assumed to be no larger than $\pm 20 \%$ of any result shown. For properties measured in the Engineering Development Laboratory, the following uncertainties on a reading were obtained through calibration:

Slurry or Liquid Density $=\quad \pm 0.5 \%$

Liquid Viscosity $=\quad \pm 0.5 \%$

Turbidity $= \pm 0.1$ NTU

\subsection{AY102/C106 CONCENTRATION (DEWATERING)}

Clearly the principal reason to perform Campaign I is to obtain filter flux data which is assumed prototypic to full-scale plant operation. The filter is prototypic in most of its features. They are: porous tube length of 90 inches, porous tube inside diameter of 0.5 inches, outside diameter of 0.75 inches, nominal porous size of 0.1 micron, symmetric stainless steel sintered porous wall construction, axial slurry velocity of 11 to $12 \mathrm{ft} / \mathrm{s}$, and a transmembrane pressure of approximately 40 psid. It is not prototypic in three features: it only has 7 tubes instead of 241 tubes, it was mounted vertical instead of horizontal, and while the backpulse system functions prototypically, its design must be different because of scaling.

In general, filter performance data from the pilot test rig are assumed to well represent those that will be obtained in the full-scale unit. Differences in filter operation that will ultimately be realized during plant operation will likely result from differences between the simulant used and several very complex waste streams to be filtered. Little is known on how gross and especially subtle differences in the many waste-stream properties will affect filtration. However, this study should help to better understand filter operation that will be very useful plant operation. 
Figure 3 shows the temporal permeate flux data with the ordinate adjusted for temperature, as required by the RPP-WTP test specification to account for the effect of viscosity. The adjustment equation is:

$$
\text { Adjusted Flux = Flux }\left(\mathrm{gpm} / \mathrm{ft}^{2}\right) \times \exp (2500 \times((1 /(273+\mathrm{T}))-(1 / 298)))
$$

where $\mathrm{T}=$ slurry/permeate temperature in degrees Celcius

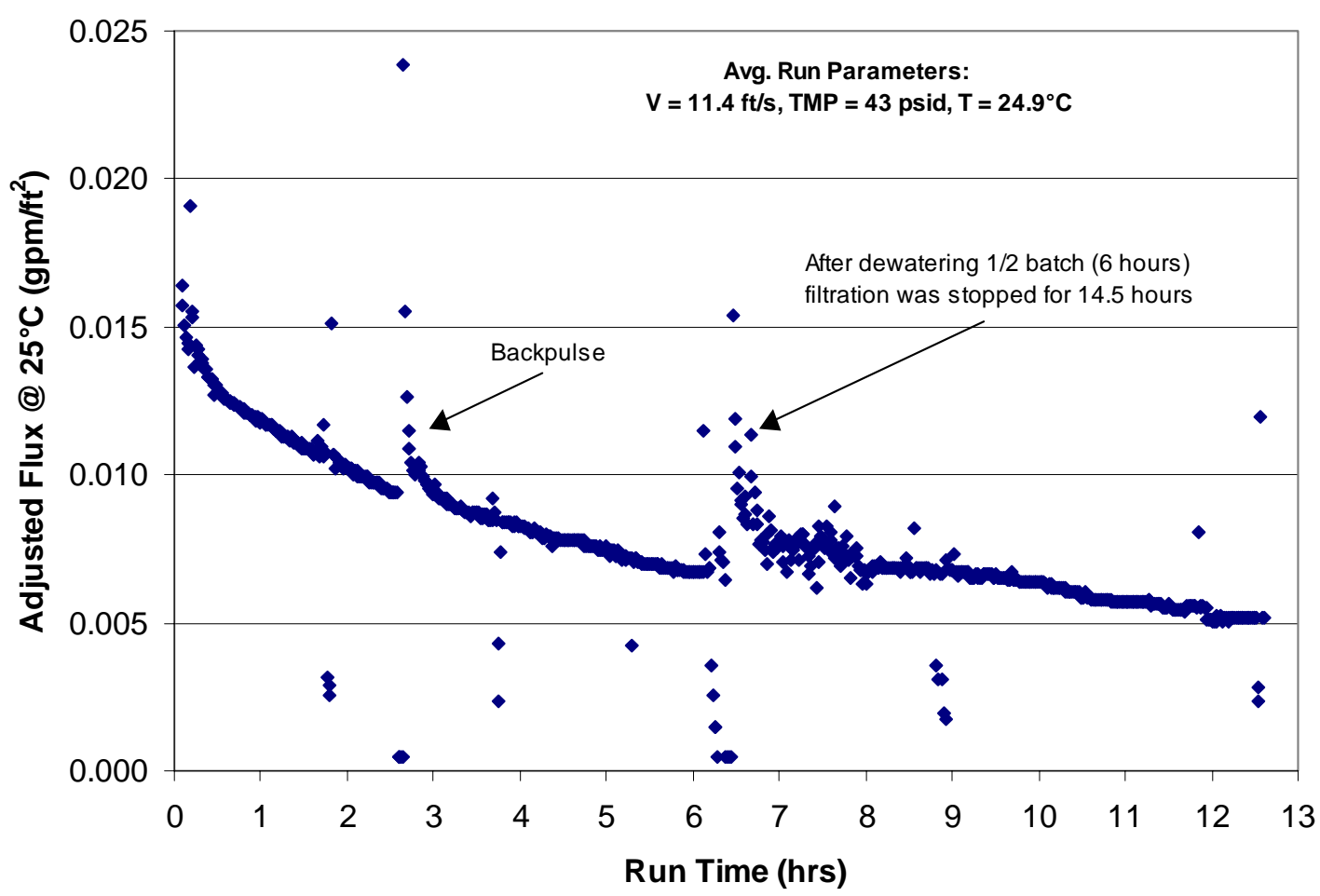

Figure 3. Dewatering of AY102/C106 simulant

The entire run time can be seen as approximately 12.5 hours. However, in an attempt to match plant operation, the dewatering was stopped at the one-half batch mark and allowed to sit for a minimum of 12 hours; it turned out to be 14.5 hours. This hold period was done to mimic the much longer time needed to filter in the plant during which some waste instabilities may cause changes, e.g., precipitation. The entire dewatering process took 27 hours but Fig. 3 only shows the actual filtering time. The data scatter in Fig. 3 was caused by two actions: sampling and backpulsing. At approximately every two hours samples were drawn. To draw permeate samples, it was necessary to divert the permeate flow for a few seconds into a sample bottle, including the time it took to rinse out the sampling port and bottle. At approximately two-hour intervals, there are data points below and above the main filter flux curve. These indicated the opening and closing of the permeate sampling valve. The two larger disturbances were caused be backpulsing. The criterion was backpulse when the flux dropped below $0.015 \mathrm{gpm} / \mathrm{ft}^{2}$. However, as is evident, the flux started very close to the criterion, therefore, it was changed to backpulse when the flux dropped below 0.010 $\mathrm{gpm} / \mathrm{ft}^{2}$. At 2.5 hours into the run, the flux dropped to $0.0095 \mathrm{gpm} / \mathrm{ft}^{2}$ and a backpulse was 
initiated, but within 30 minutes the flux returned to what it had been before the backpulse. Because of the poor recovery, a backpulse was not repeated during the first one-half batch of dewatering. On the next day, which is shown as the 6.5-hour point, the dewatering began once again and it was started with a backpulse. Once again, it only took a few minutes for the flux to return to the main flux curve. The further instability at the beginning of the first day was probably caused by the settling of the viscous slurry and it seems that between 60 to 90 minutes were needed to fully mix and bring the dewatering to steady state again.

Another way to understand the dewatering process is to see how the undissolved solids changed. Figure 4 repeats the data of Fig. 3, but the corresponding solids concentration has been substituted for time. These concentration data were obtained by the samples taken during the test and then extrapolation between the sample points by knowing how much permeate mass was removed with time. Some flux results are from $12.5 \mathrm{wt} \%$ UDS to:

$24 \mathrm{wt} \%$ UDS the mean flux was $0.008 \mathrm{gpm} / \mathrm{ft}^{2}$, $20 \mathrm{wt} \%$ UDS the mean flux was $0.009 \mathrm{gpm} / \mathrm{ft}^{2}$, $15 \mathrm{wt} \%$ UDS the mean flux was $0.011 \mathrm{gpm} / \mathrm{ft}^{2}$.

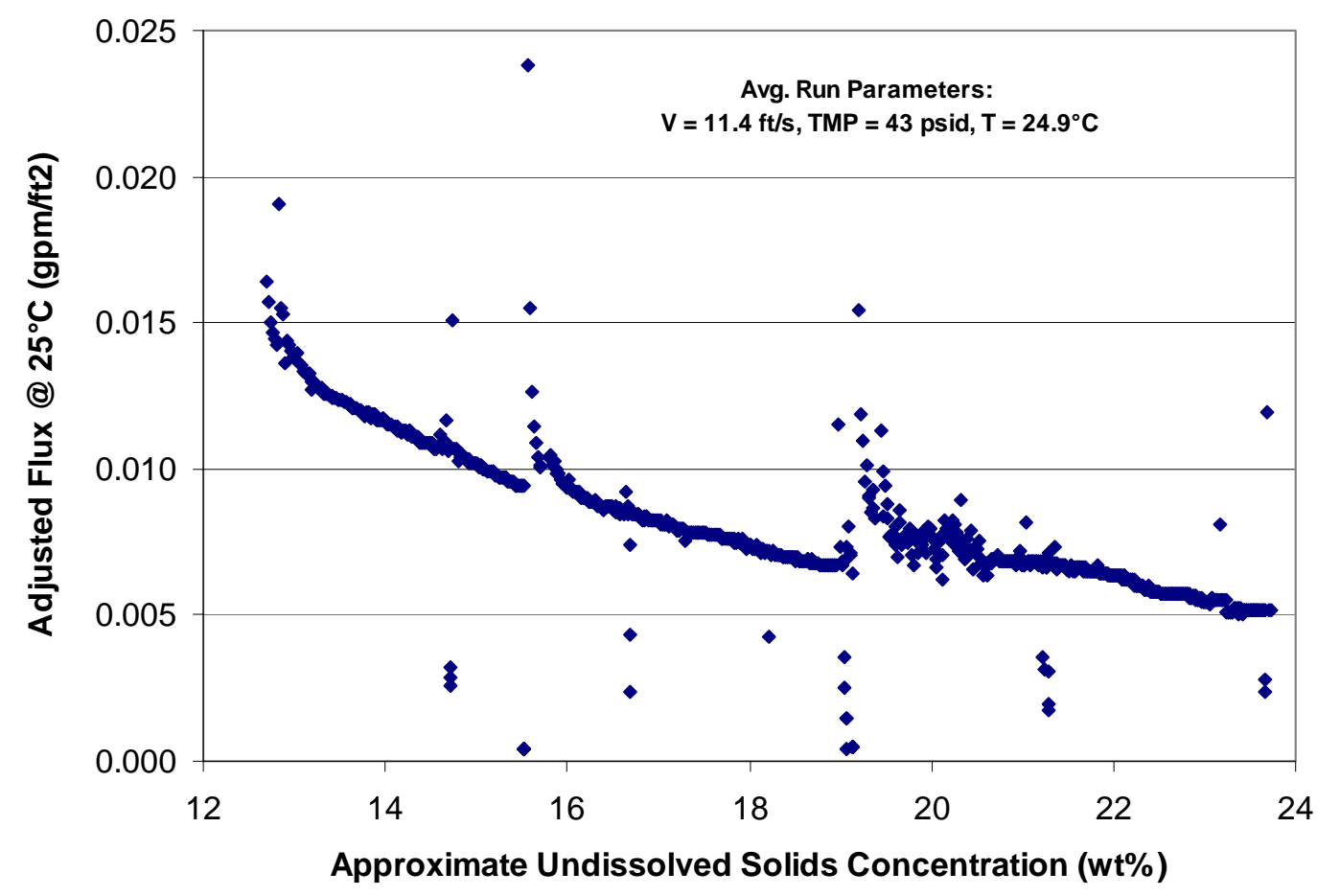

Figure 4. Dewatering of AY102/C106 simulant

The plant operation will concentrate the waste in the ultrafilter to approximately $17 \mathrm{wt} \%$ UDS, therefore all of the campaigns of SIPP have a target of $20 \mathrm{wt} \%$ UDS to obtain information at an upper bound. However, there is little filter data at the higher concentrations so the Test Specification for this filter work indicate a concentration of 25 wt\% UDS as the target if obtainable. The dewater test was stopped after 12.5 hours of 
WSRC-TR-2004-00201, REV. 0

SRT-RPP-2004-00034, REV. 0

filtering because the rough estimate ${ }^{\dagger}$ of concentration indicated $25 \mathrm{wt} \%$ UDS. The actual value was $23.7 \mathrm{wt} \%$ UDS. Table 3 shows rheological and other physical data of the slurry and permeate during the dewatering.

Table 3. Rheological and other data during dewatering of AY102/C106 simulant

\begin{tabular}{|c|c|c|c|c|c|c|c|c|c|}
\hline Approx. Run Time & \multicolumn{6}{|c|}{ 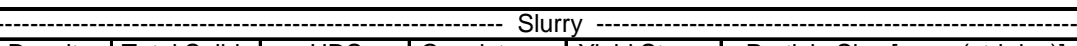 } & \multicolumn{3}{|c|}{ Permeate } \\
\hline When Sampled & Density & Total Solids & UDS & Consistency & Yield Stress & Particle Size [mean(std dev)] & Density & Viscosity & Turbidity \\
\hline hour & $\mathrm{g} / \mathrm{mL}$ & $w t \%$ & $w t \%$ & $\mathrm{mPa}-\mathrm{s}$ & $\mathrm{Pa}$ & $\overline{\text { Vol. Dist. | Num. Dist. (micron) }}$ & $\mathrm{g} / \mathrm{mL}$ & $\mathrm{mPa}-\mathrm{s}$ & NTU \\
\hline 0 & 1.27 & 27.4 & 12.5 & 13.4 & 4.9 & $2.2(1.8) \mid 0.7(0.3)$ & $\mathrm{n} / \mathrm{a}$ & $\mathrm{n} / \mathrm{a}$ & n/a \\
\hline 2 & 1.28 & 29.2 & 14.6 & 12.8 & 10.4 & & 1.17 & 2.50 & 0.30 \\
\hline 4 & 1.30 & 31.0 & 16.8 & 15.1 & 15.1 & & $\mathrm{n} / \mathrm{a}$ & $\mathrm{n} / \mathrm{a}$ & $\mathrm{n} / \mathrm{a}$ \\
\hline 6 & 1.33 & 32.9 & 19.1 & 17.6 & 22.4 & & 1.17 & $\mathrm{n} / \mathrm{a}$ & $\mathrm{n} / \mathrm{a}$ \\
\hline 8 & 1.33 & 34.7 & 21.3 & 19.6 & 30.9 & & $\mathrm{n} / \mathrm{a}$ & 2.48 & 0.25 \\
\hline 10 & 1.37 & 36.3 & 23.1 & 20.9 & 35.5 & & $\mathrm{n} / \mathrm{a}$ & $\mathrm{n} / \mathrm{a}$ & $\mathrm{n} / \mathrm{a}$ \\
\hline 12.6 & 1.36 & 36.9 & 23.7 & 23.8 & 34.8 & $1.8(1.3) \mid 0.6(0.2)$ & 1.17 & 2.50 & $\mathrm{n} / \mathrm{a}$ \\
\hline
\end{tabular}

As can be seen from the table, the consistency of the slurry simulant almost doubled from 13 to $24 \mathrm{mPa}-\mathrm{s}$, which coincidentally matched the concentration of undissolved solids. During the same time period, the yield stress increased a factor of 7 (i.e., 5 to $35 \mathrm{~Pa}$ ), while the density increase was $7 \%$ (i.e., 1.27 to $1.36 \mathrm{~g} / \mathrm{mL}$ ). The fact that there was a finite yield stress means the waste stream is rheologically non-Newtonian. More specifically the waste is shear thinning. The solids particle behavior makes sense in that there was very little change to the bulk of the particle sizes. The mean size of both number distributions reduced insignificantly, while the range of sizes became more uniform as seen be the small standard deviations after dewatering. The permeate rheology showed that the density and the viscosity did not change throughout the process. Since the turbidity measurements of the permeate were significantly lower than the test specification threshold of 2 NTU the permeate was considered free of solids.

\subsection{AY102/C106 STEADY STATE}

This portion of the Ultrafiltration Test Specification (Townson, 2002) is not incorporated in any of the subsequent filter tests in SIPP. For Campaign I, as in past filtration tests (Duignan, 2000a; Duignan, 2000b; Duignan, 2003a), a series of operational parameters, i.e., axial slurry velocity (V) and transmembrane pressure (TMP) were obtained to contrast to previous filter tests to further complete the filter database. However, the number of steadystate tests was limited since six of the original thirteen test runs were removed by the SIPP test specification (Longwell, 2003). As shown in Table 1, the remaining test numbers were: $1.04,1.07,1.08,1.09,1.10,1.11,1.16$. Figure 5 shows the average results for all of those tests. Three of those tests, i.e., 1.04, 1.11, and 1.16 had a duration of 120 minutes, while the rest were done for 1 hour, as per the filtration test specification (Townson, 2002). To have an equal comparison, all the test averages for the first 60 minutes of data from each test are shown. As expected the tests with the highest axial velocity had the highest filter flux. Tests

\footnotetext{
${ }^{\dagger}$ Real time concentration analysis was not available and only the initial samples taken on the previous day were used to extrapolate to estimate the $25 \mathrm{wt} \%$ UDS.
} 
$1.08,1.09,1.11,1.16$ all were statistically the same and $\mathrm{V}$ ranged from 11 to $13 \mathrm{ft} / \mathrm{s}$. The TMP ranged from 30 to 50 psid, but as in the past, the permeate flux was relatively insensitive to changes in TMP in that range of pressures. Runs 1.07 and 1.10 had significantly lower fluxes, which was a direct result of $\mathrm{V}$ being $9 \mathrm{ft} / \mathrm{s}$ or lower. The odd point was the first, Test 1.04. It was odd because it was lower than the tests with fluxes between 0.007 and $0.008 \mathrm{gpm} / \mathrm{ft}^{2}$ which had the same flow parameters of $\mathrm{V}$ and TMP (tests 1.11 and 1.16), but on the other hand the average filter flux of Test 1.04 was $0.0055 \mathrm{gpm} / \mathrm{ft}^{2}$, which was very close to the flux at the end of the dewatering test, i.e., $0.0052 \mathrm{gpm} / \mathrm{ft}^{2}$, as seen as in Figs. 3 or 4 . One explanation may be a change in particle size. Table 3 shows that at the end of dewatering, i.e., after 12.6 hours of filtering, the particle size had a Volume distribution mean of 1.8 micron and a Number distribution mean of 0.6 micron. By the end of steady state testing those means moved to slightly smaller sizes. The Volume distribution changed from uni-modal to bi-modal with $75 \%$ of the particles having a mean of 1.5 micron and 25\% with a mean of 0.5 micron. The Number distribution changed even less to a mono-modal mean of 0.5 micron. However, there is not enough information to definitively state that these small particle changes affected the filter flux.

Based on the steady-state results, the standing recommendation to operate the filter at an axial velocity of $11 \mathrm{ft} / \mathrm{s}$, or above, and a TMP above 30 psid remains the same. The higher the axial velocity, the faster permeate will be produced.

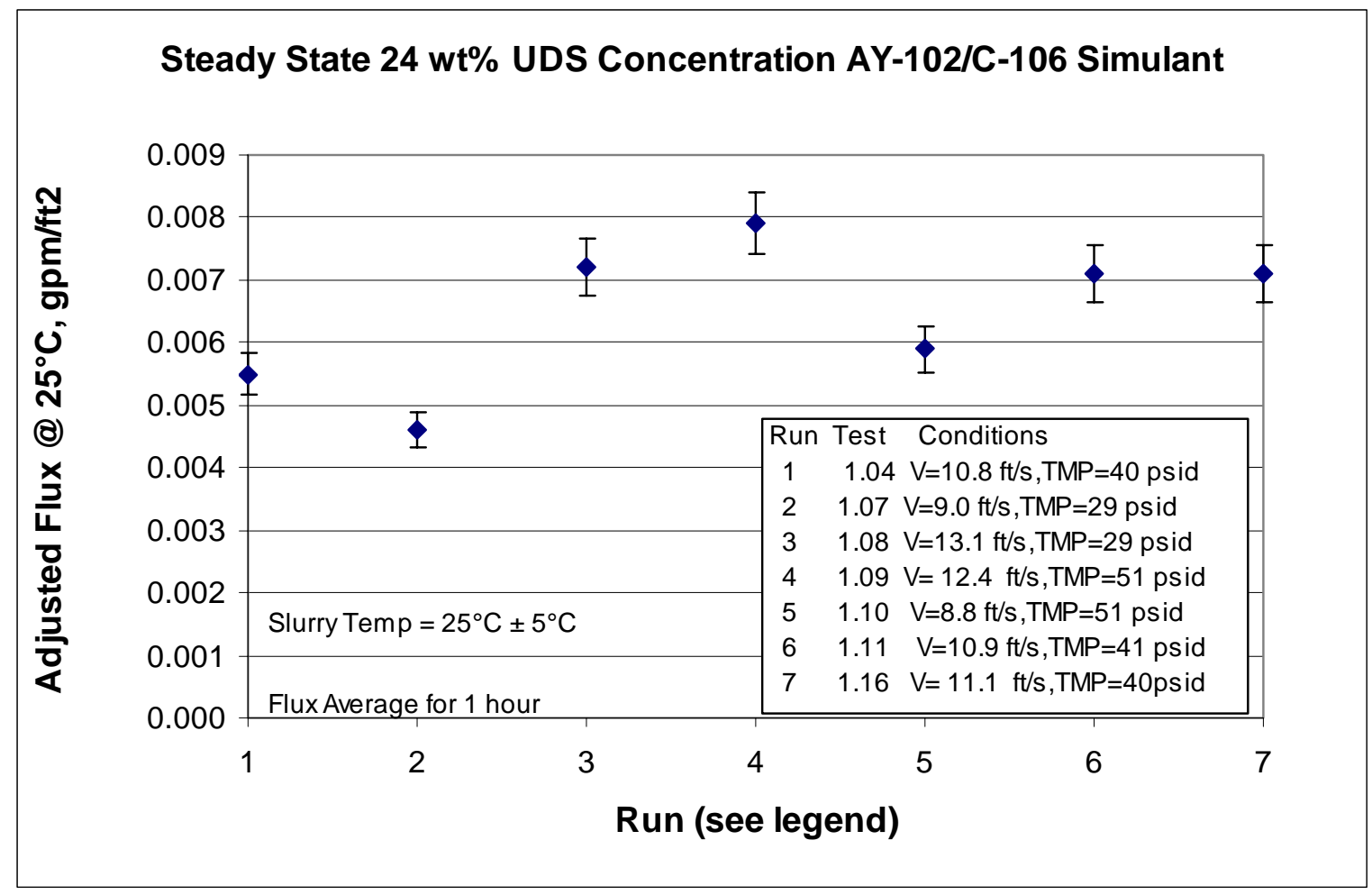

Figure 5. Steady Steady Permeate Flux 


\subsection{AY102/C106 WASHING}

One very import aspect of this filter task was to see the effects of washing the slurry. Initially this was combined with both washing and leaching, but according to the Test Specification (Longwell, 2003) either route could have been chosen by RPP-WTP. The RPP-WTP customer decided not to leach, which left only the two washing sequences. In the plant, the filtration batch size was set at 18,000 gallons (see the first footnote on page 10). Once all the waste that needs separation is fed through the filter and concentrated to a $25^{\dagger}$ wt\% UDS, the waste is washed twice with 21,770 gallons (this is the volume of one permeate storage tank). The WTP will transfer the wash water (actually it is process water, which for this test is made of $0.01 \mathrm{M} \mathrm{NaOH}$ ) in 1,000-gallon batches. To mimic plant operation, the wash water was transferred to the pilot test rig in 22 mini-batches. Since the batch size was 140 liters, batch of wash water was $140 \mathrm{~L} \mathrm{x} 21,770 / 18,000=169.3 \mathrm{~L}$, making each minibatch of wash water 7.7 liters. That is, to the concentrated slurry (it was at $24 \mathrm{wt} \%$ UDS) $7.7 \mathrm{~L}$ of $0.01 \mathrm{M} \mathrm{NaOH}$ were added and then dewatered until 7.7 $\mathrm{L}$ of permeate was removed. This was repeated 22 times. This was one complete wash cycle, which was repeated, for a total of 44 mini-batches of wash water. Figure 6 shows the first washing cycle results.

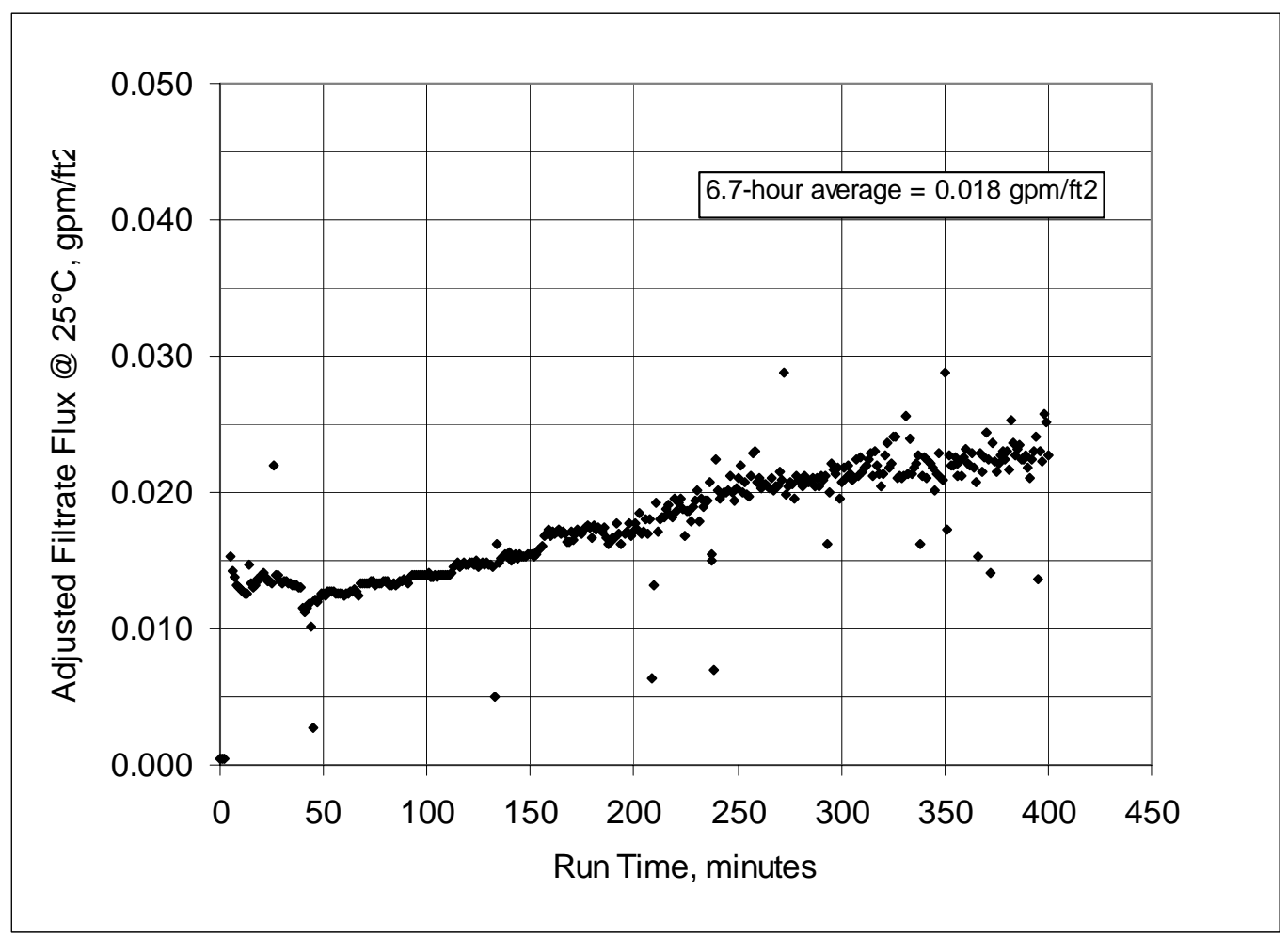

Figure 6. $1^{\text {st }}$ washing of concentrated AY102/C106 with 22 mini-batches [average operating parameters: $\mathrm{V}=12.1 \mathrm{ft} / \mathrm{s}, \mathrm{TMP}=41 \mathrm{psia}, \mathrm{T}=25^{\circ} \mathrm{C}$ ]

\footnotetext{
${ }^{\dagger}$ The plant value is actually closer to $17 \mathrm{wt} \%$ UDS. See the dewatering section for an explanation.
} 
As water was added to the concentrated simulant the permeate flux slowly increased from 0.015 to $0.023 \mathrm{gpm} / \mathrm{ft}^{2}$ during the first wash cycle. Figure 7 shows during the second washing cycle the flux continued to increase but at a much slower rate, from approximately 0.025 to $0.031 \mathrm{gpm} / \mathrm{ft}^{2}$.

The flux data in Fig. 6 start steady but becomes a more erratic, with the transition somewhere between 150 and 200 minutes. The 150-minute mark is when approximately 35\% of the first entire washing batch is processed (at around the $8^{\text {th }}$ mini-batch). This instability may always occur when reducing the supernatant density with water, but some of it may have been a systematic problem. All liquid flow rates in the test rig were measured with a magnetic flow meter, which relies on ions in the stream to measure flow. As the ions are being washed away the meters have to rely on a smaller population to make measurements.

Another feature of the data is what appears to be outliers; the data points above and below the main filter flux data. Those for the most part indicate when a sample was taken. To take a sample of permeate that stream is temporarily redirected to a sampling station, which stops the flow for a very short period of time. Then when the sample is taken, there is a very short surge as permeate pressure reestablishes steady state flow.

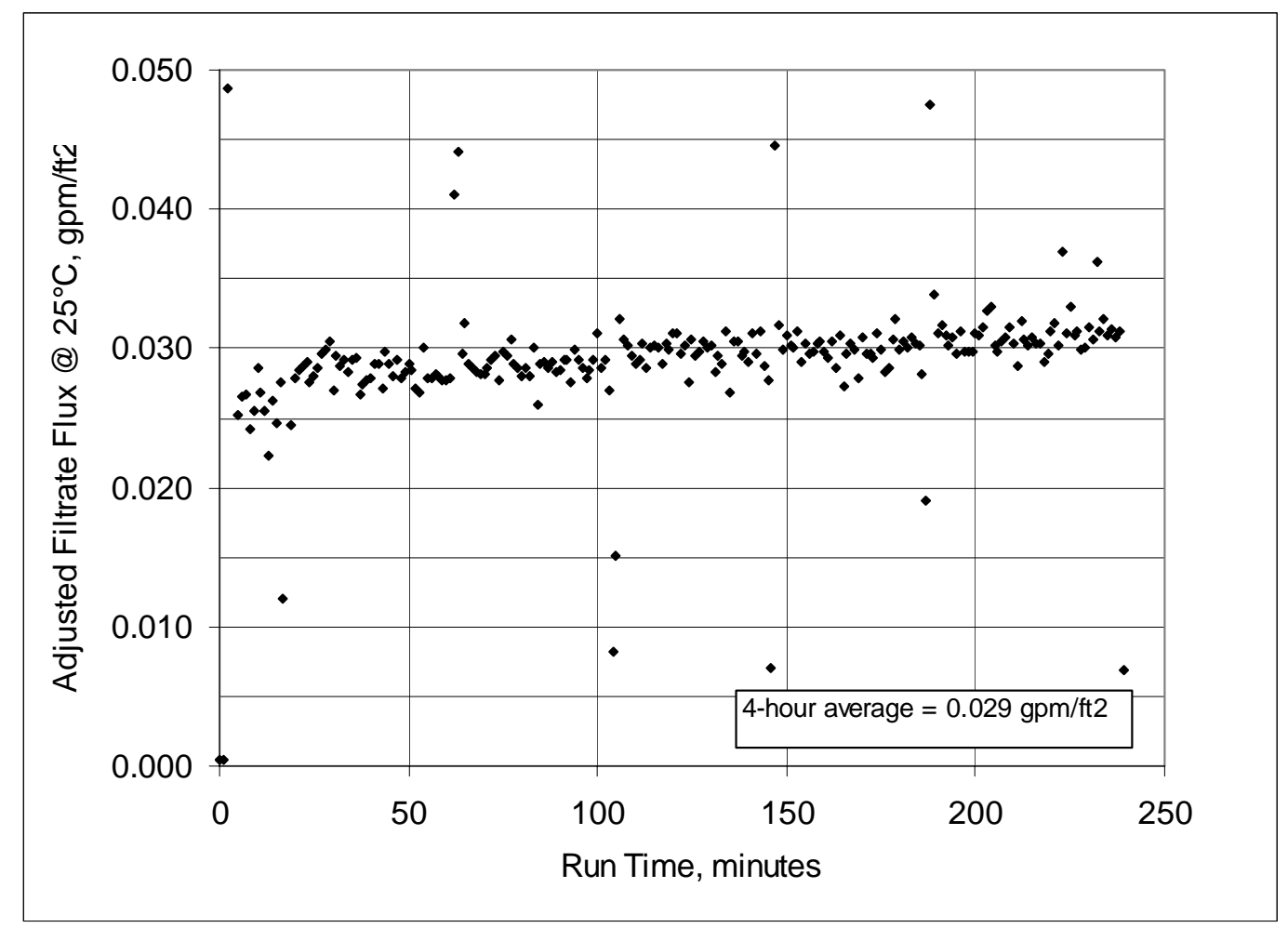

Figure 7. 2nd washing of concentrated AY102/C106 with 22 mini-batches [average operating parameters: $\mathrm{V}=12.1 \mathrm{ft} / \mathrm{s}, \mathrm{TMP}=40 \mathrm{psia}, \mathrm{T}=25^{\circ} \mathrm{C}$ ] 
The solids data shown in Fig. 8 seems straight forward. The total solids decrease quickly in the beginning, from 33 to $27 \mathrm{wt} \%$, and conversely the undissolved solids increased, from 19 to $20 \mathrm{wt} \%$, which was expected as the supernatant was being replaced by more and more water. Between the $13^{\text {th }}$ and $44^{\text {th }}$ wash, the total solids decreased asymptotically to $23 \mathrm{wt} \%$, but the undissolved solids basically remained the same at 20 to $21 \mathrm{wt} \%$. The only way that the "undissolved" solids concentration could remain constant, while the supernatant was becoming less dense, because of washing, is if some of the solids actually did dissolve. In fact, this did happen as evidenced from the temporary increases in some of the analytes like fluoride, Fig. 14 and especially oxalate, Fig. 17. The oxalate dissolution is also the reason the total organic carbon peaked approximately midway through washing, see Fig. 24. A measure of the insoluble solids before and after washing indicated that approximately $7 \%$ of the solids did dissolve.

The rheological data in Fig. 8 were generally as expected. As the number of washes increased, the consistency of the simulated waste slurry was cut in half; it decreased from a high of $19 \mathrm{mPa}-\mathrm{s}$ at $19.6 \mathrm{wt} \%$ UDS then dropped to $9 \mathrm{mPa}-\mathrm{s}$ at $20.6 \mathrm{wt} \%$ UDS. However, the yield stress stayed the same (i.e., 8.5 to $8.2 \mathrm{~Pa}$ ), which was unexpected since a past test (see Fig. 64 of Duignan, 2003a) with an Envelope C waste showed that the yield stress doubled for similar slurry washing. This is good news since avoiding a higher yield stress would not challenge the pumps more. Finally, the density of the slurry dropped from 1.33 $\mathrm{g} / \mathrm{ml}$ before washing to $1.16 \mathrm{~g} / \mathrm{ml}$ after the first washing cycle. During the second washing cycle (mini-washes 23 to 44 ) the slurry density remained basically constant, indicating that the majority of the soluble solids were removed during the first 22 mini-washes.

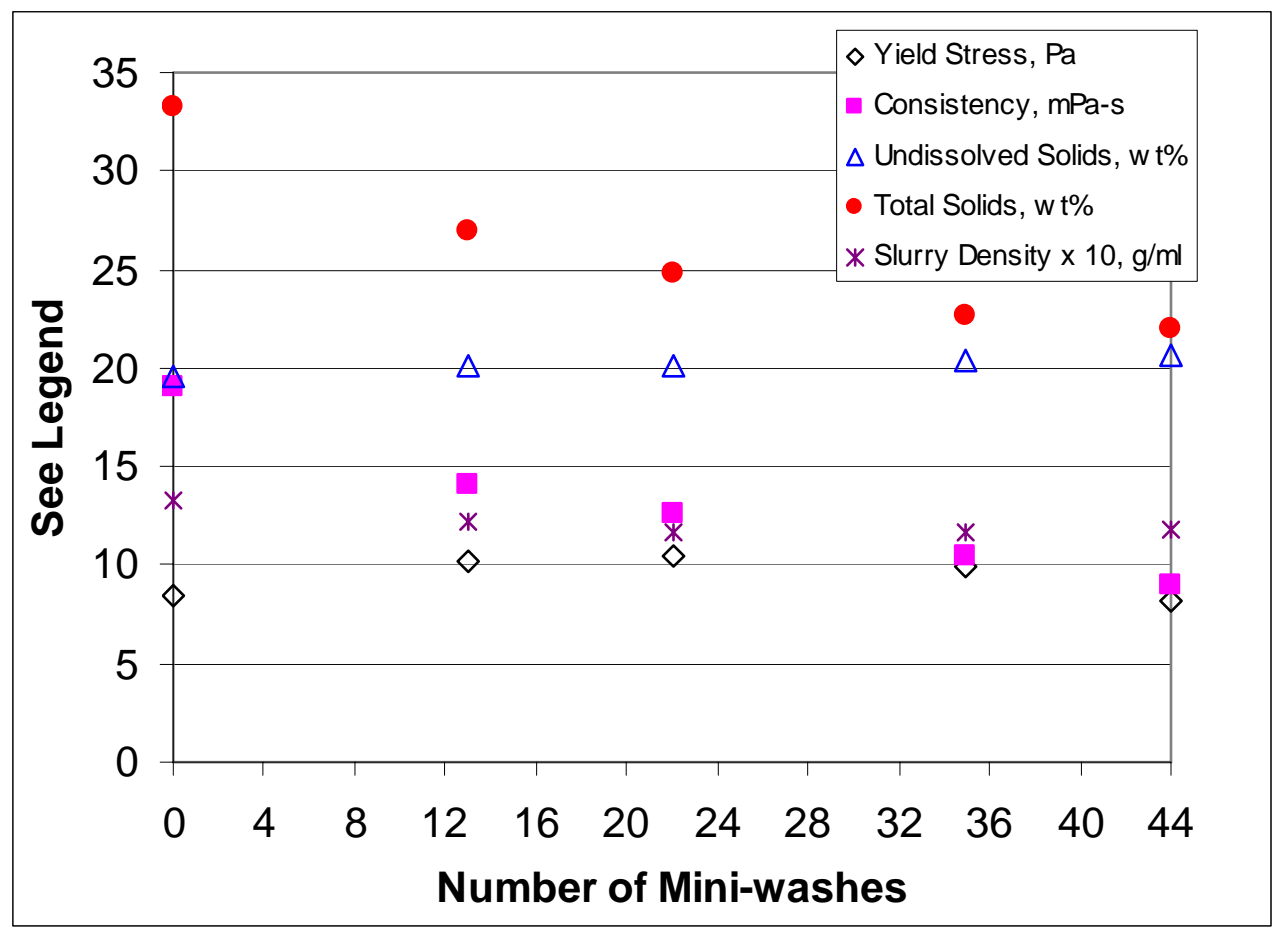

Figure 8. Solids and rheological data throughout simulant washing 
Throughout washing, permeate samples were taken at approximately every $4^{\text {th }}$ washing. (The washing number sampling points were: $1,5,9,13,17,22$ ). These numbers are important because while both slurry and permeate samples were taken at the same time, they are not matched samples. Since the permeate flux is very low it takes approximately 30 minutes for permeate created at the filter to flow to the sampling point. With this knowledge, it is easy to relate slurry and permeate samples by matching the time difference. For this interim report, the following figures show the effect of washing on a particular analyte from the slurry. However, it is important to realize that the very first sample of permeate does not reflect the result of washed slurry, it is actually permeate of unwashed slurry, since the washed slurry permeate was in the permeate line at that time.

The next sixteen figures (i.e., Figs. 9 to 24) show analytical results of the analyte concentrations in the slurry being washed. To obtain these concentrations the appropriate concentration was measured in each one of the thirteen (13) permeate samples during washing (i.e., twelve were taken during the washing process and the first one, before the washing began). Then with the measured concentrations from the initial slurry sample, the intermediary slurry concentration could be calculated.

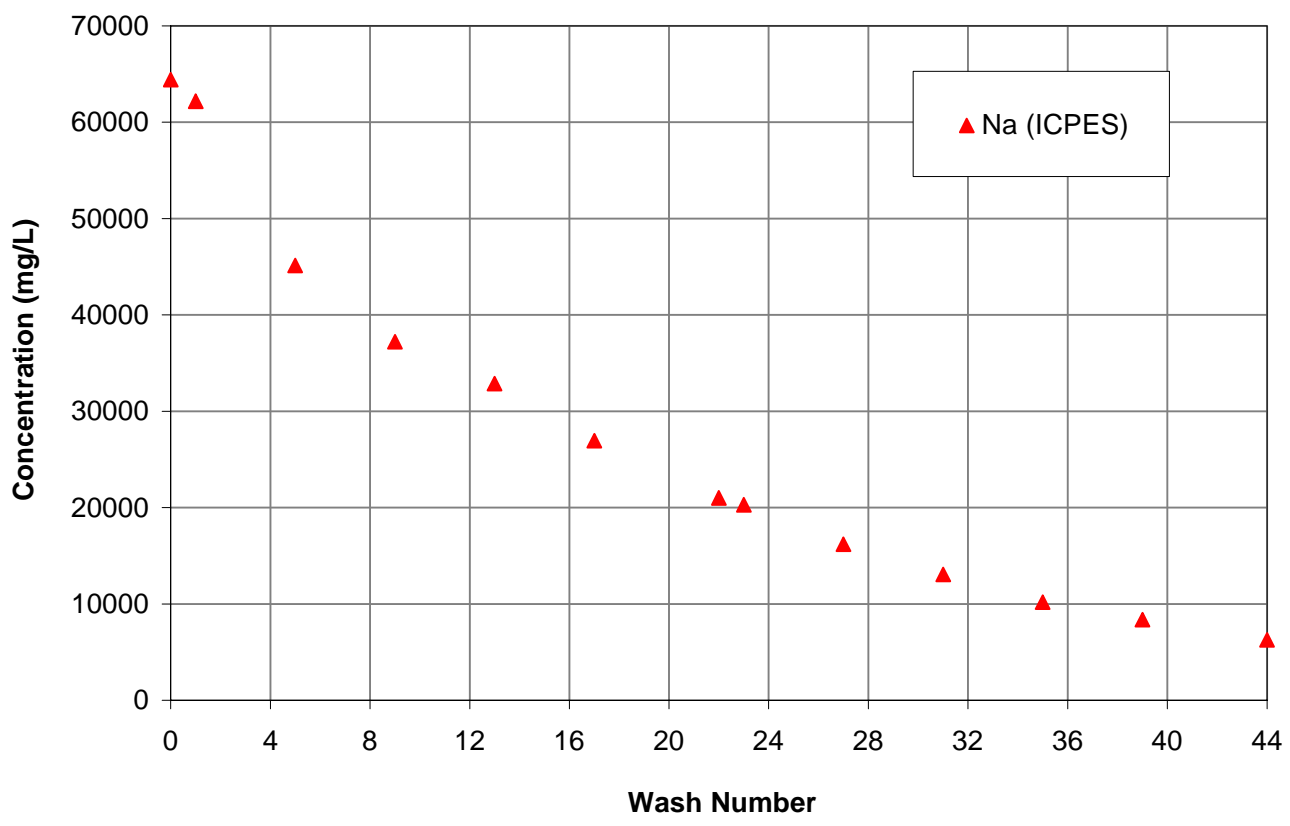

Figure 9. Soluble sodium in the slurry during washing 
WSRC-TR-2004-00201, REV. 0

SRT-RPP-2004-00034, REV. 0

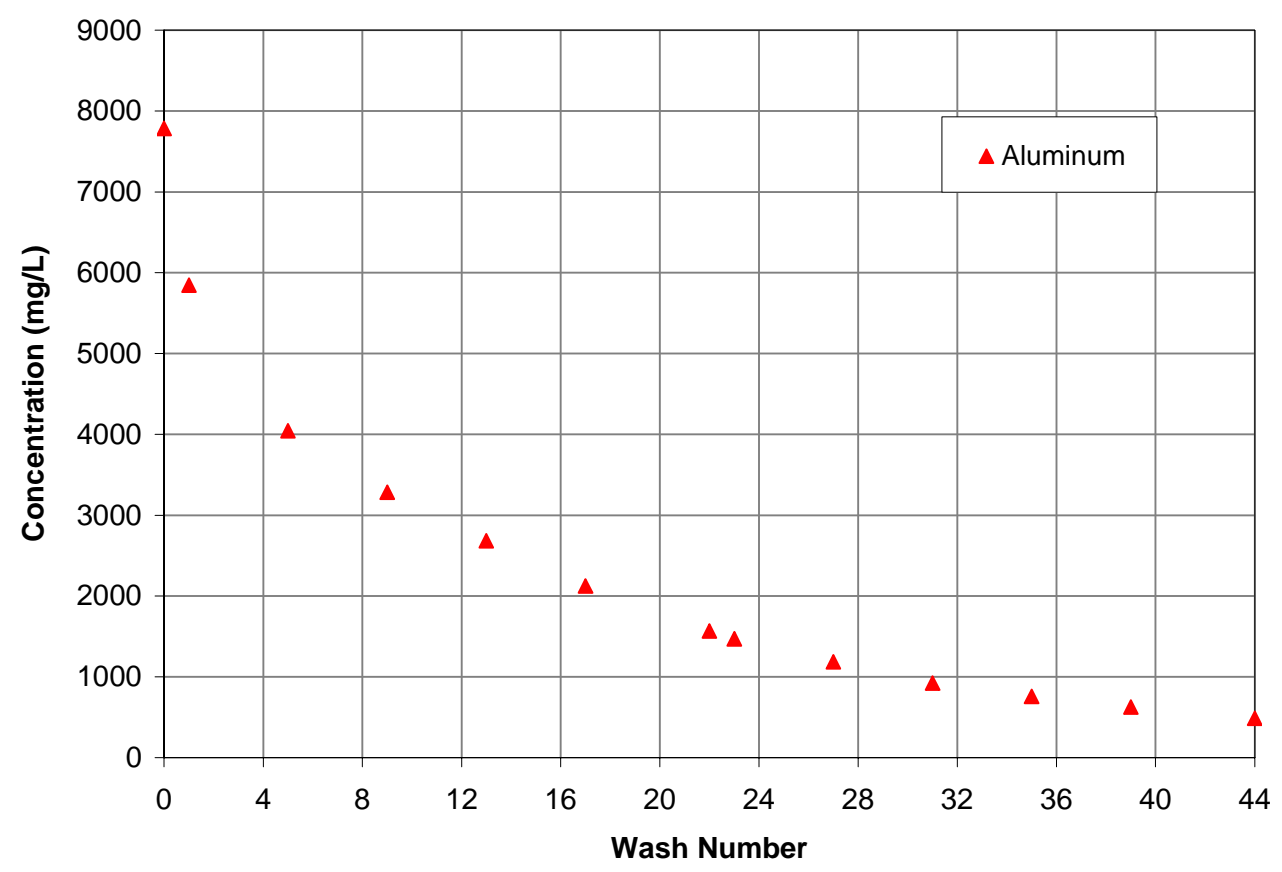

Figure 10. Soluble aluminum in the slurry during washing

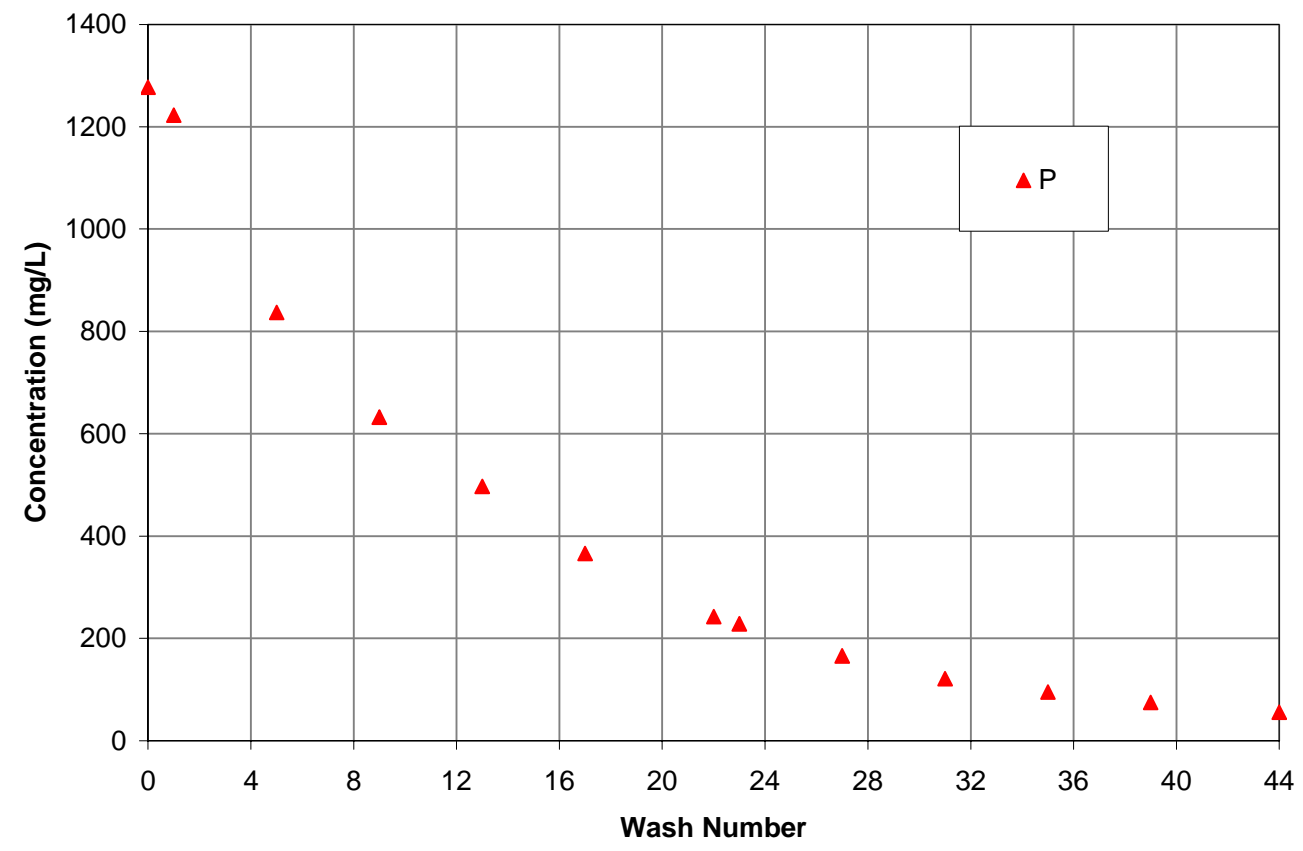

Figure 11. Soluble phosphorus in the slurry during washing 
WSRC-TR-2004-00201, REV. 0

SRT-RPP-2004-00034, REV. 0

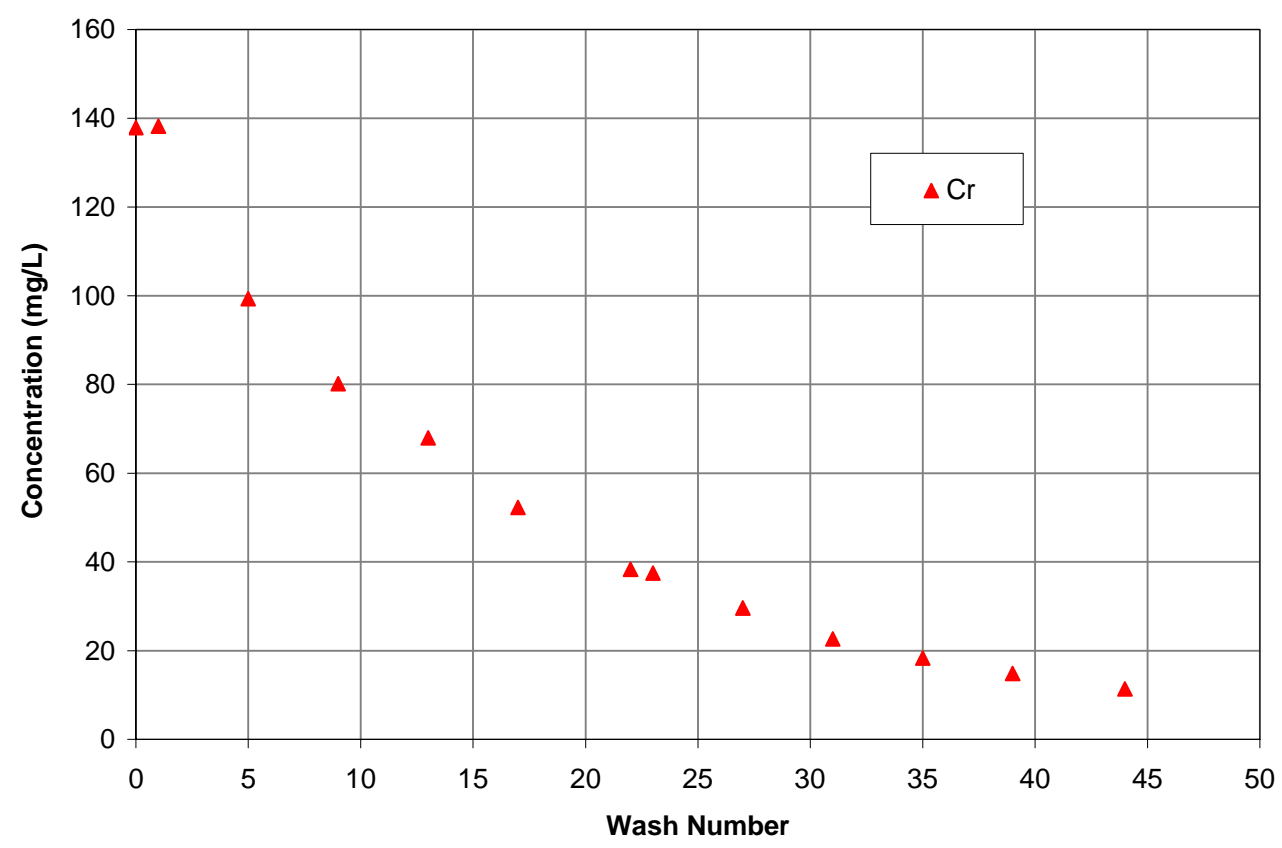

Figure 12. Soluble chromium in the slurry during washing

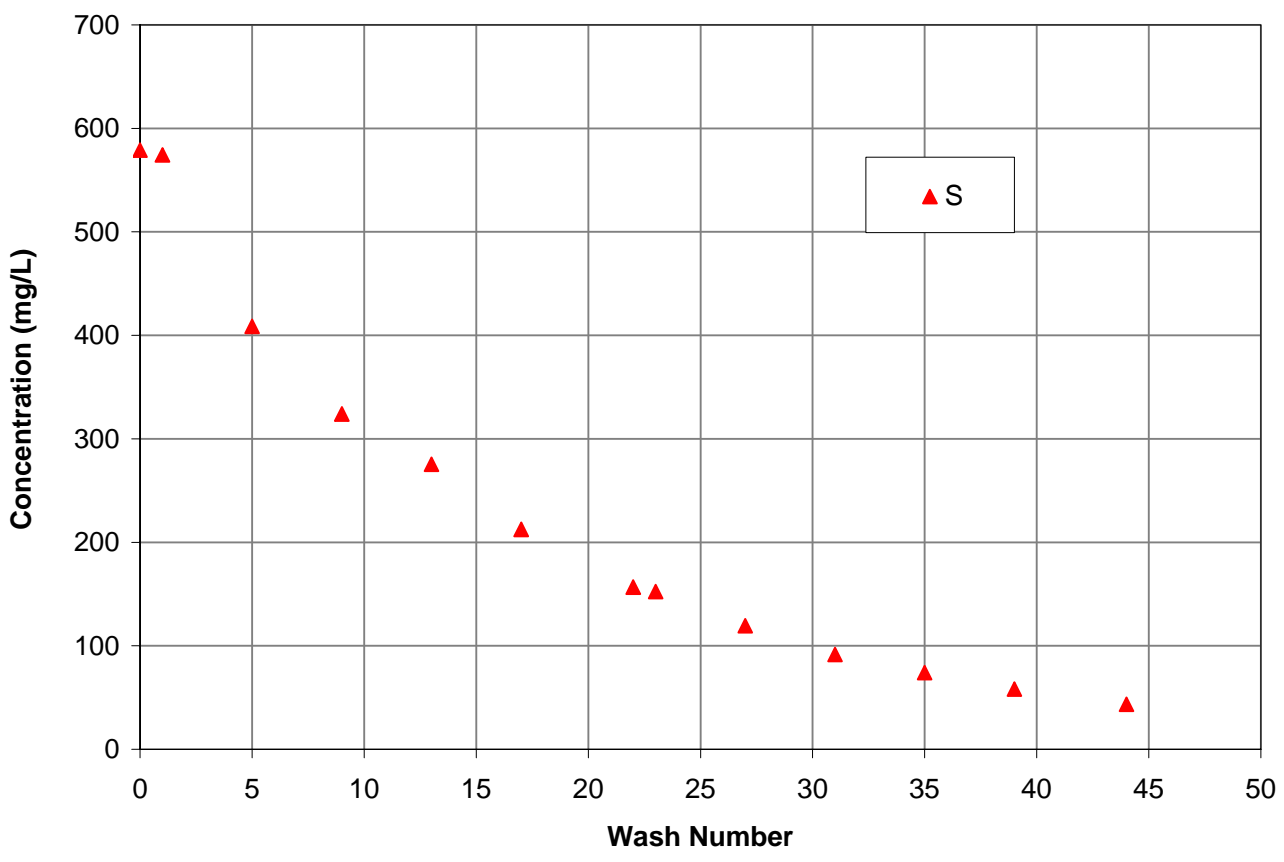

Figure 13. Soluble sulfur in the slurry during washing 
WSRC-TR-2004-00201, REV. 0

SRT-RPP-2004-00034, REV. 0

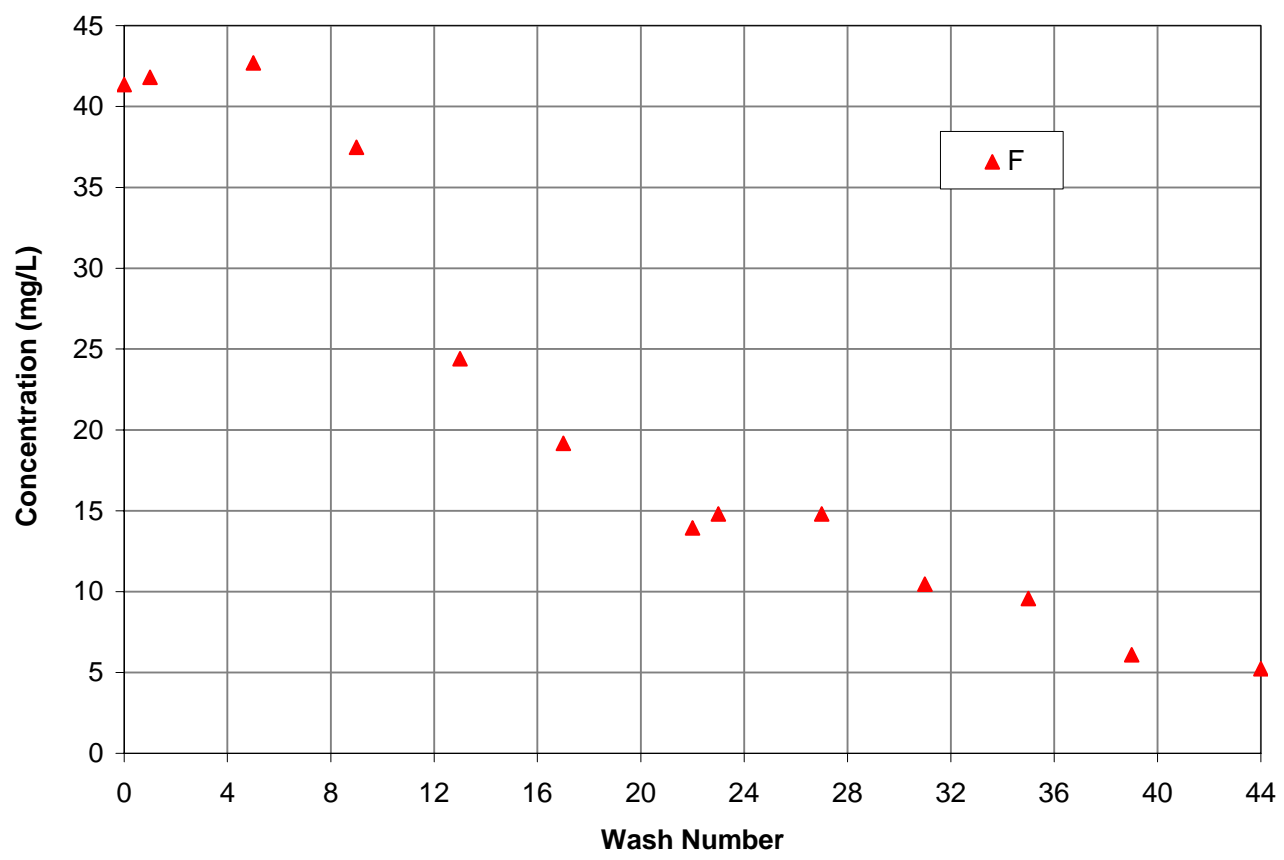

Figure 14. Soluble fluoride in the slurry during washing

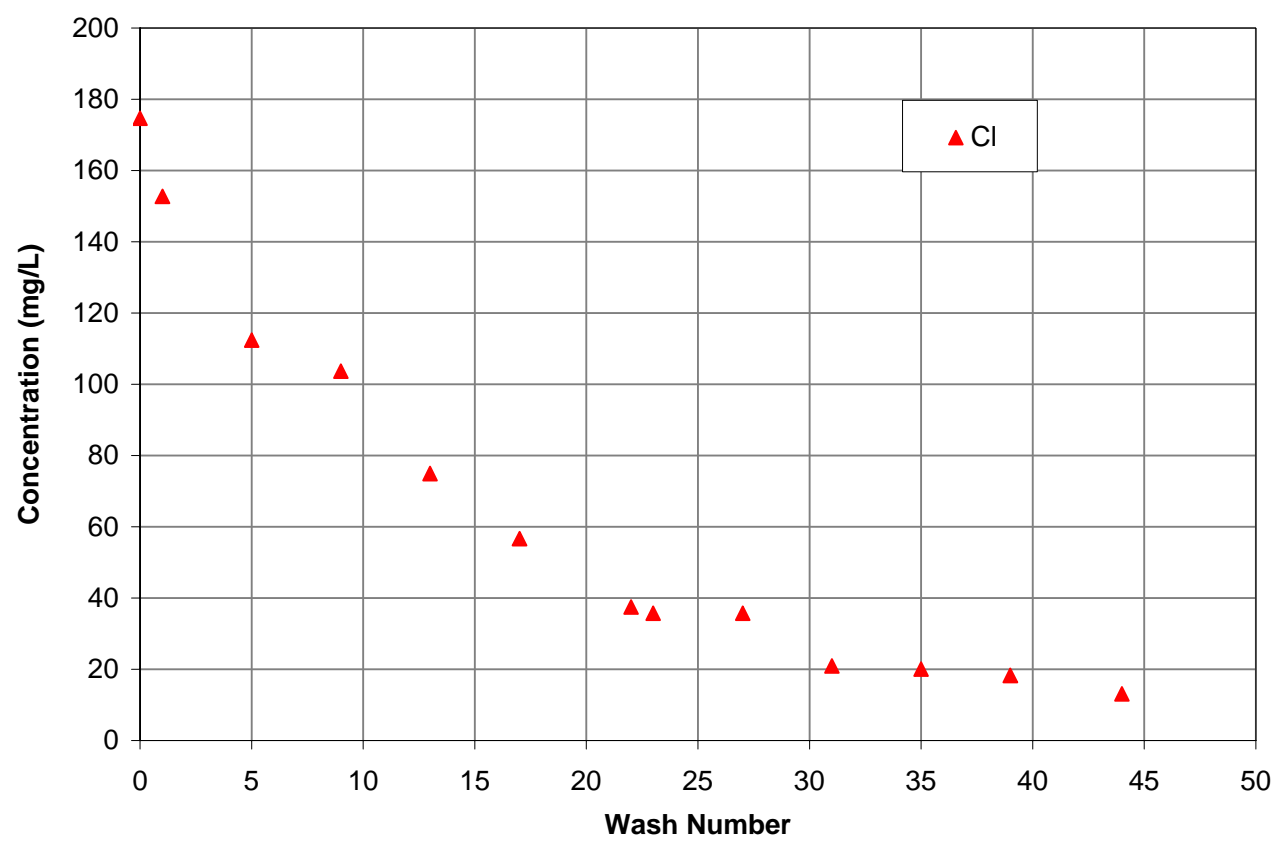

Figure 15. Soluble chloride in the slurry during washing 
WSRC-TR-2004-00201, REV. 0

SRT-RPP-2004-00034, REV. 0

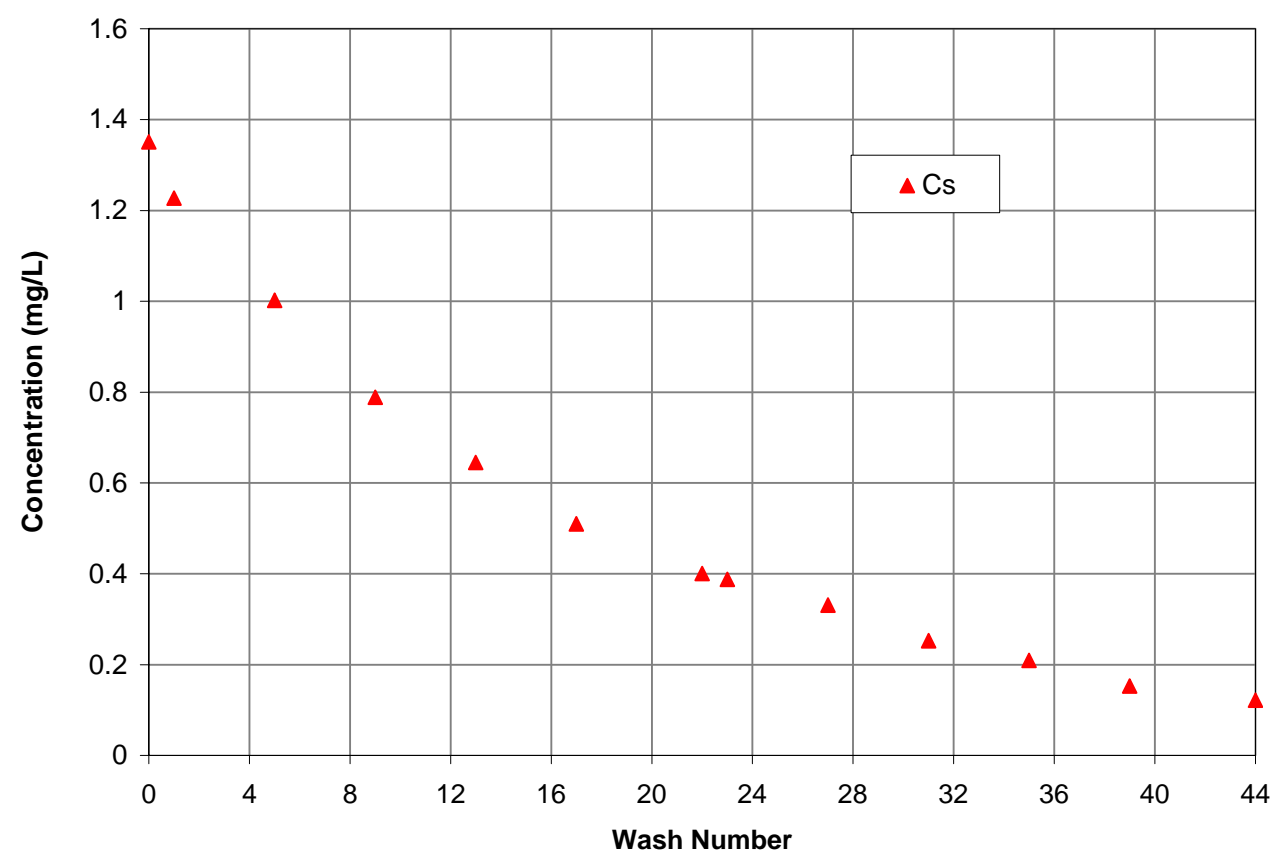

Figure 16. Soluble cesium in the slurry during washing

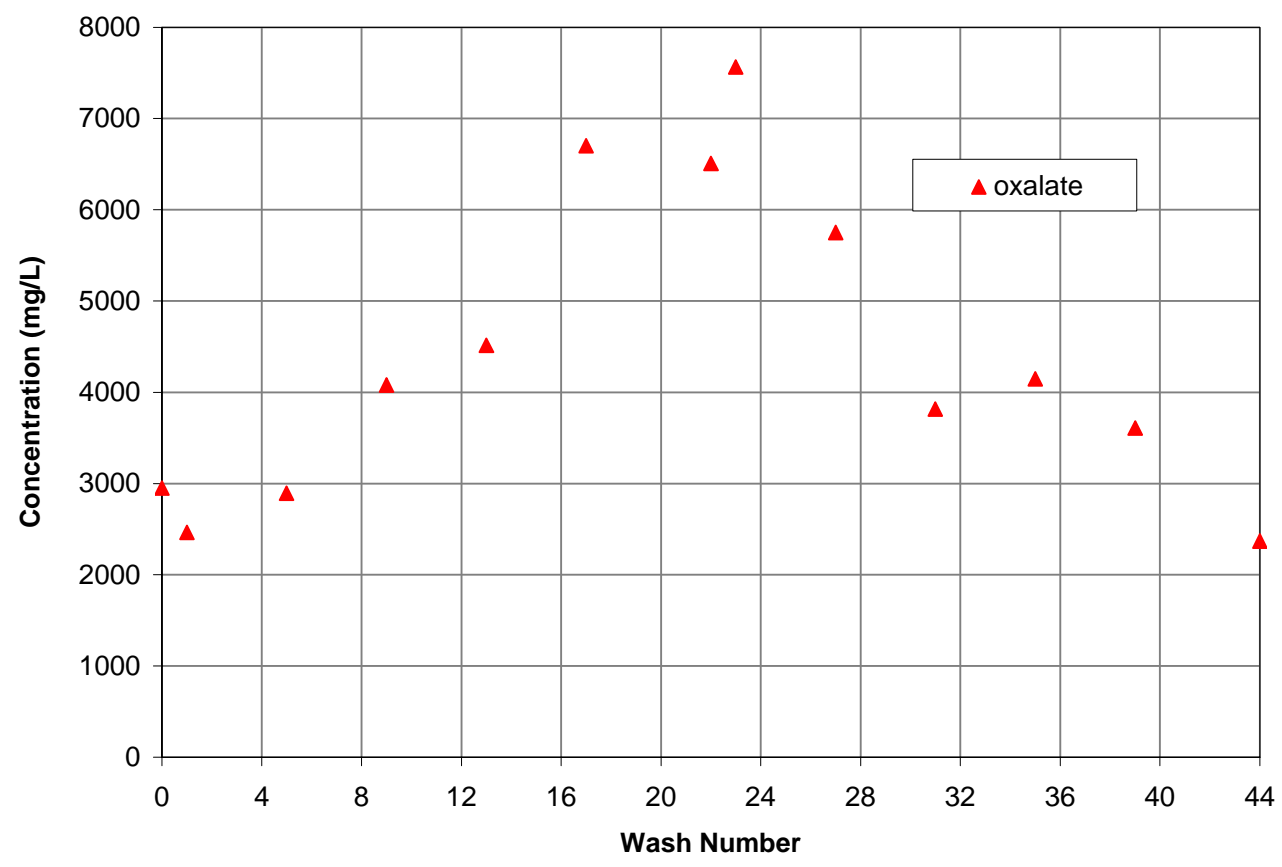

Figure 17. Soluble oxalate in the slurry during washing 
WSRC-TR-2004-00201, REV. 0

SRT-RPP-2004-00034, REV. 0

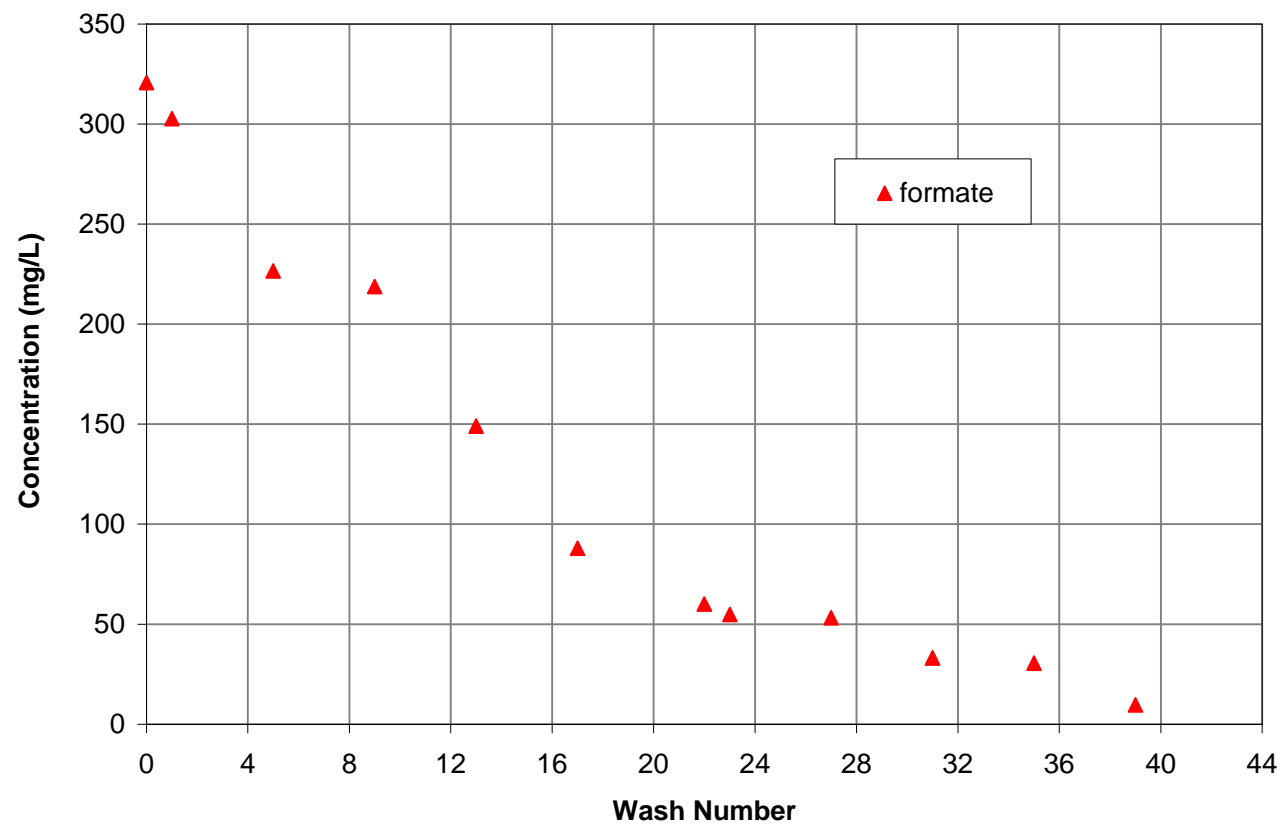

Figure 18. Soluble formate in the slurry during washing

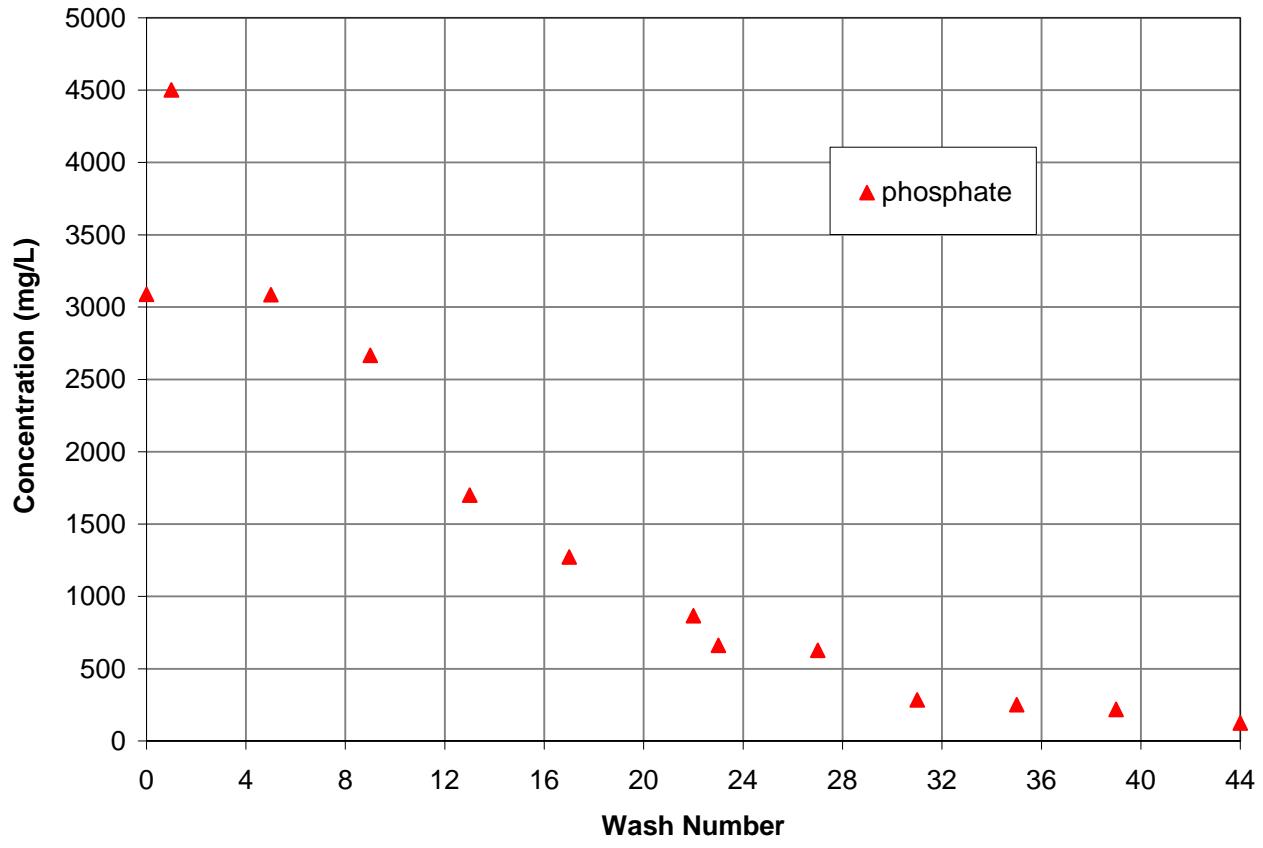

Figure 19. Soluble phosphate in the slurry during washing 
WSRC-TR-2004-00201, REV. 0

SRT-RPP-2004-00034, REV. 0

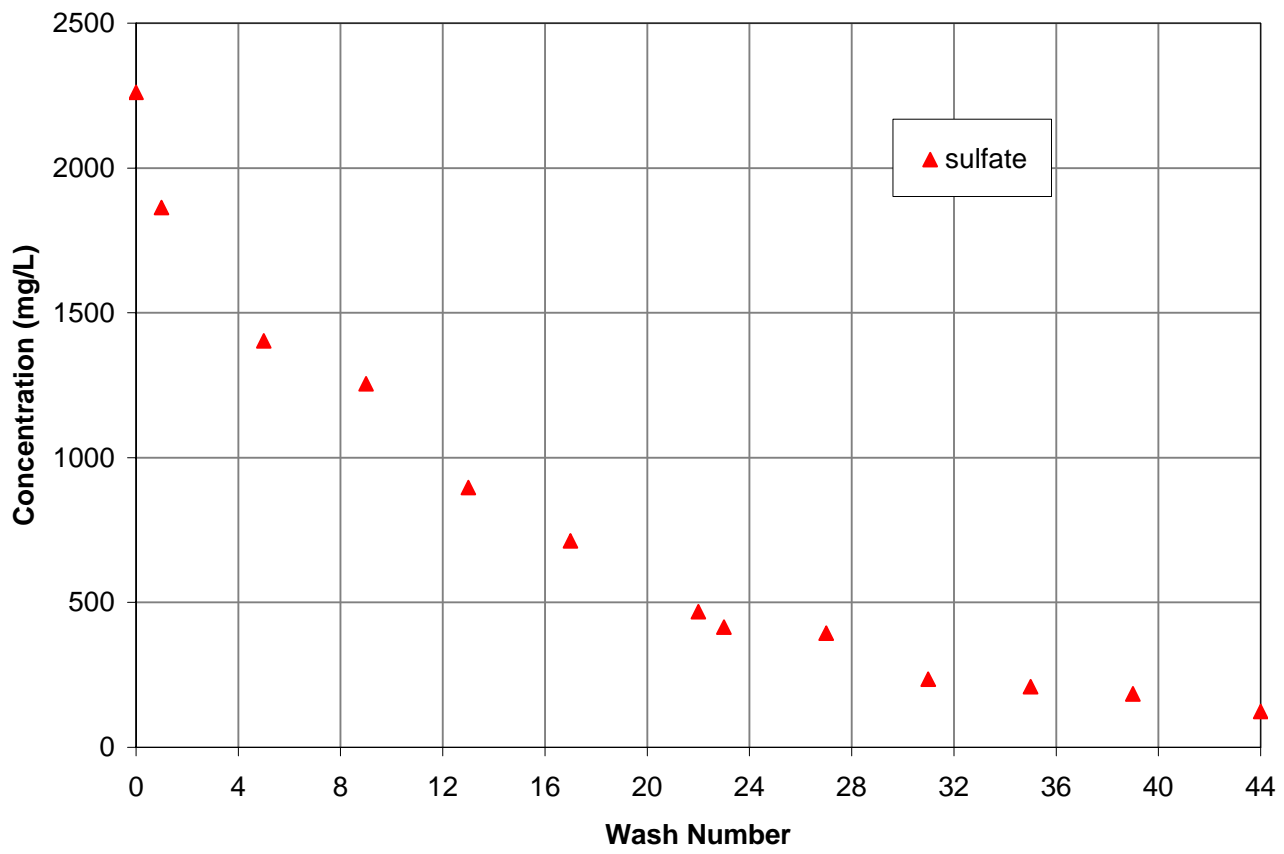

Figure 20. Soluble sulfate in the slurry during washing

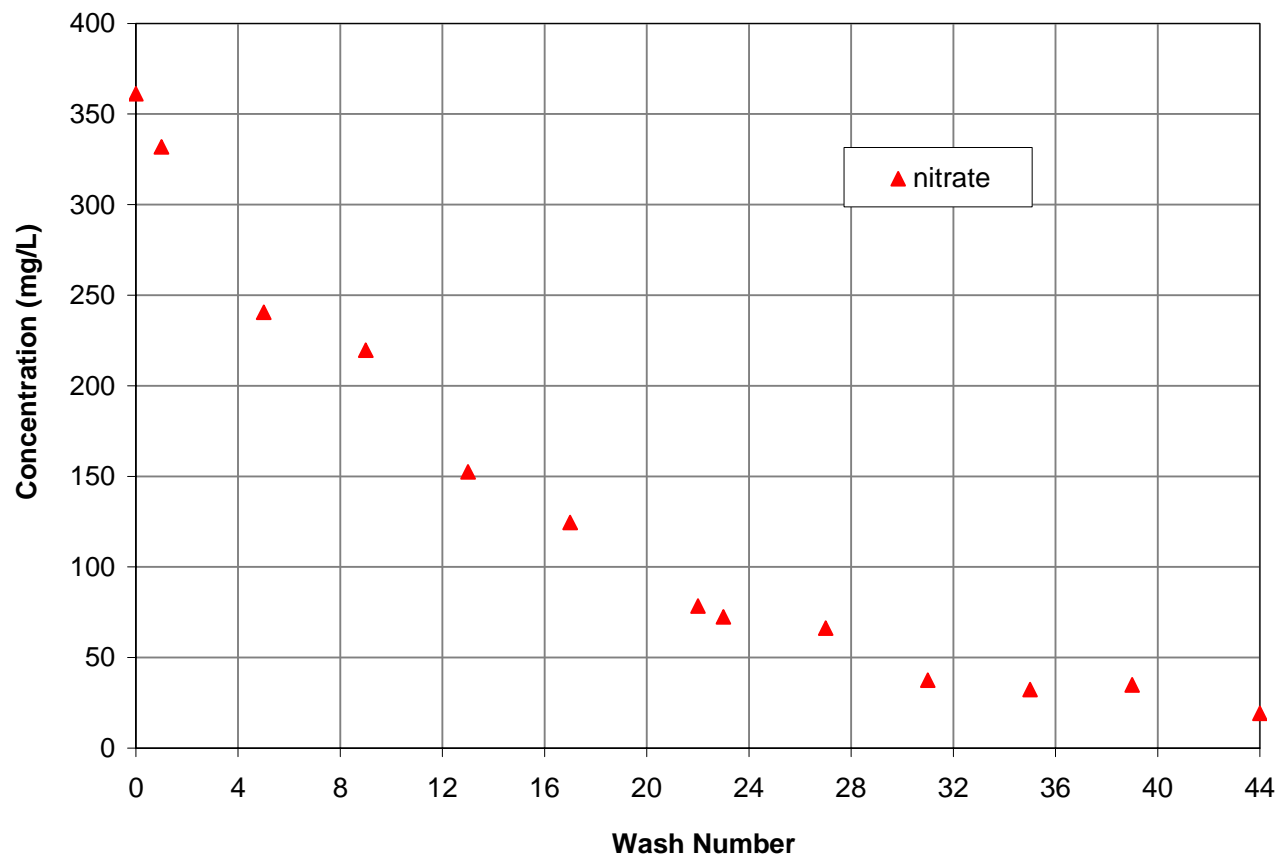

Figure 21. Soluble nitrate in the slurry during washing 
WSRC-TR-2004-00201, REV. 0

SRT-RPP-2004-00034, REV. 0

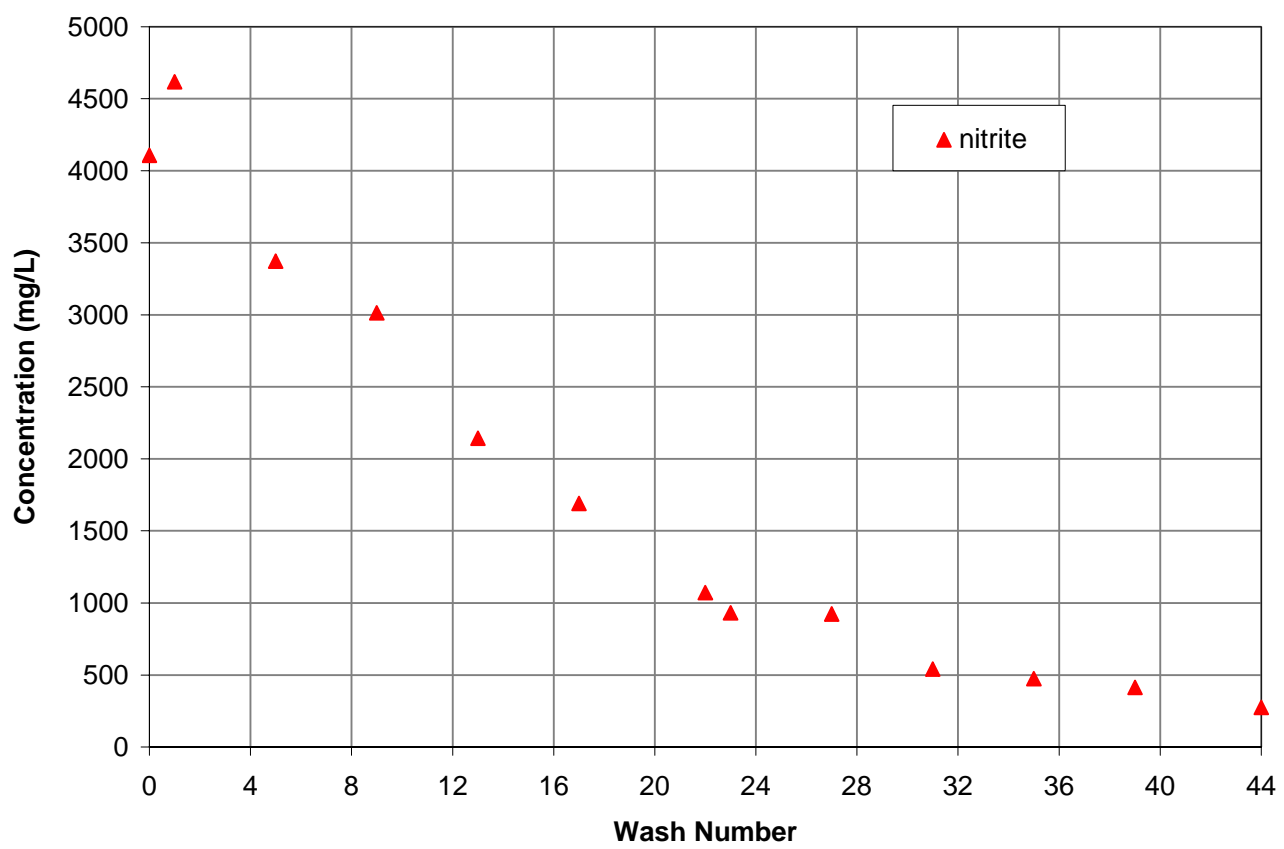

Figure 22. Soluble nitrite in the slurry during washing

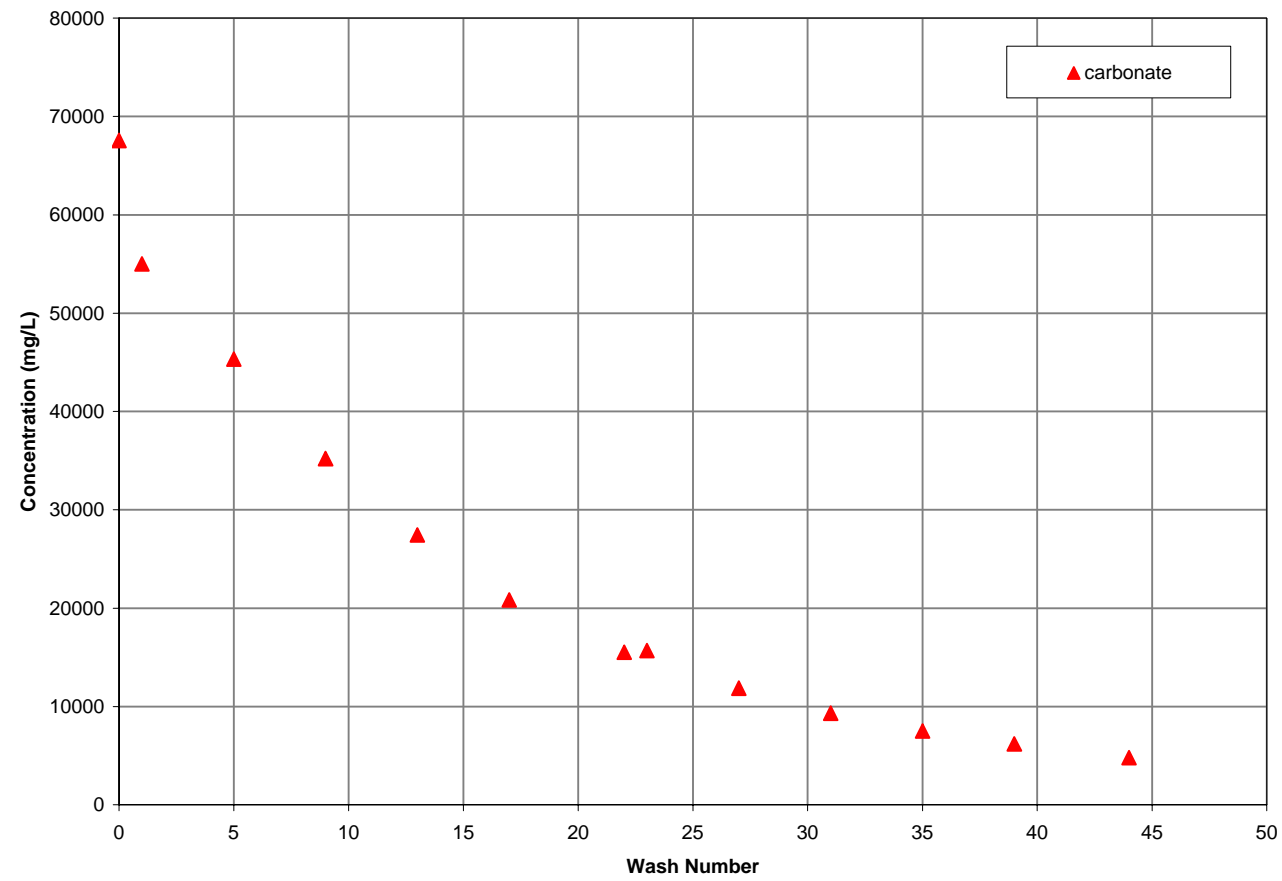

Figure 23. Soluble carbonate in the slurry during washing 
WSRC-TR-2004-00201, REV. 0

SRT-RPP-2004-00034, REV. 0

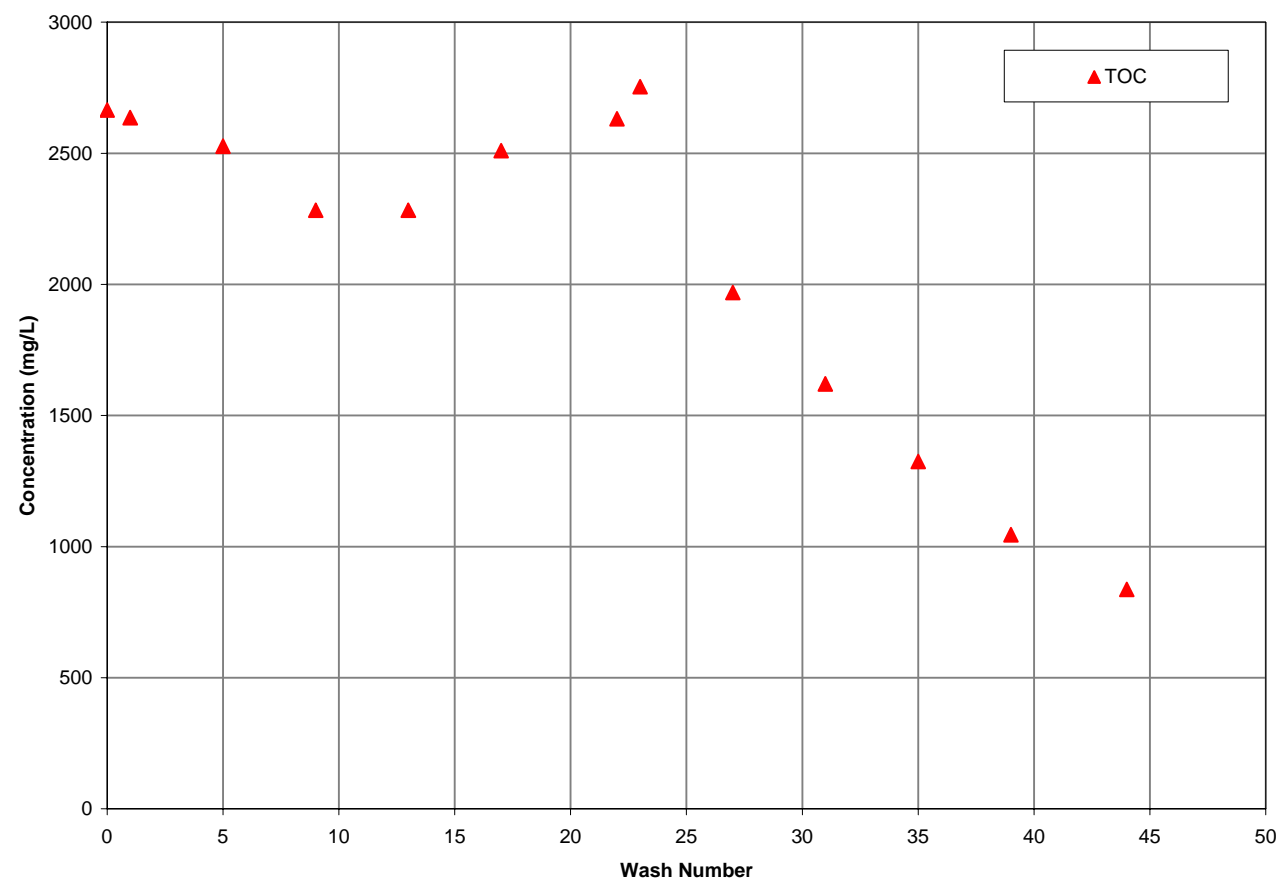

Figure 24. Soluble total organic carbon in the slurry during washing

\subsection{AY102/C106 FINAL CONCENTRATION}

As with the steady-state tests, there were no surprises during final concentration of the washed slurry when it was concentrated from 20.6 to approximately $29 \mathrm{wt} \%$ UDS. Once again, this portion of the Ultrafiltration Test Specification (Townson, 2002) is not incorporated in any of the subsequent filter tests in SIPP and the principal reason for concentrating the washed slurry was to try to plug the filter and then attempt a recovery. Unfortunately for the pilot test, as in the past tests, the filter did not plug. However, this is good news for the plant. Figure 25 shows that during the 2 hours of dewatering, the washed slurry filter flux dropped steadily from 0.03 to $0.01 \mathrm{gpm} / \mathrm{ft}^{2}$, while the solids concentration increased from 21 to $29 \mathrm{wt} \%$ UDS. 
WSRC-TR-2004-00201, REV. 0

SRT-RPP-2004-00034, REV. 0

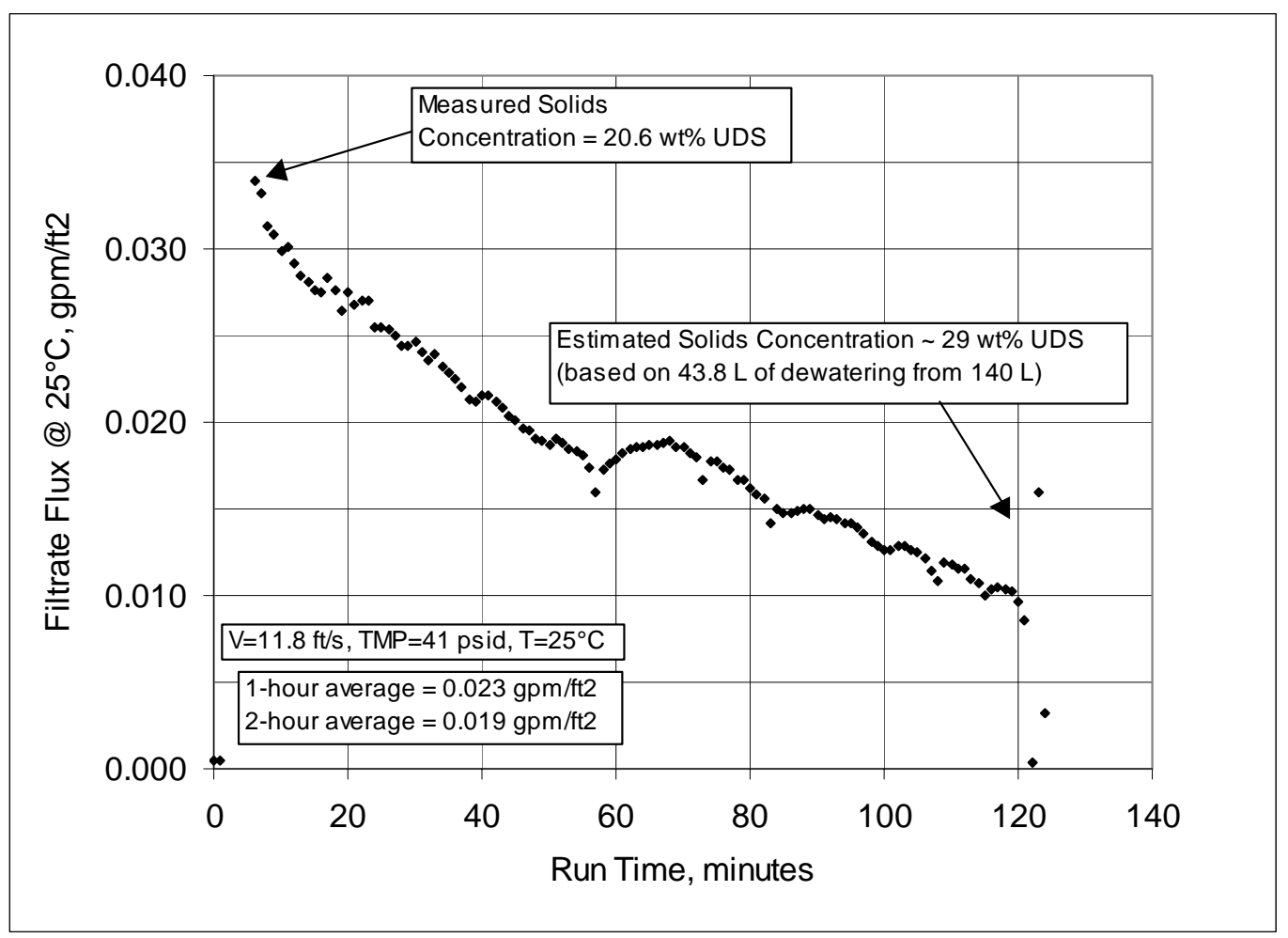

Figure 25. Post-wash dewatering

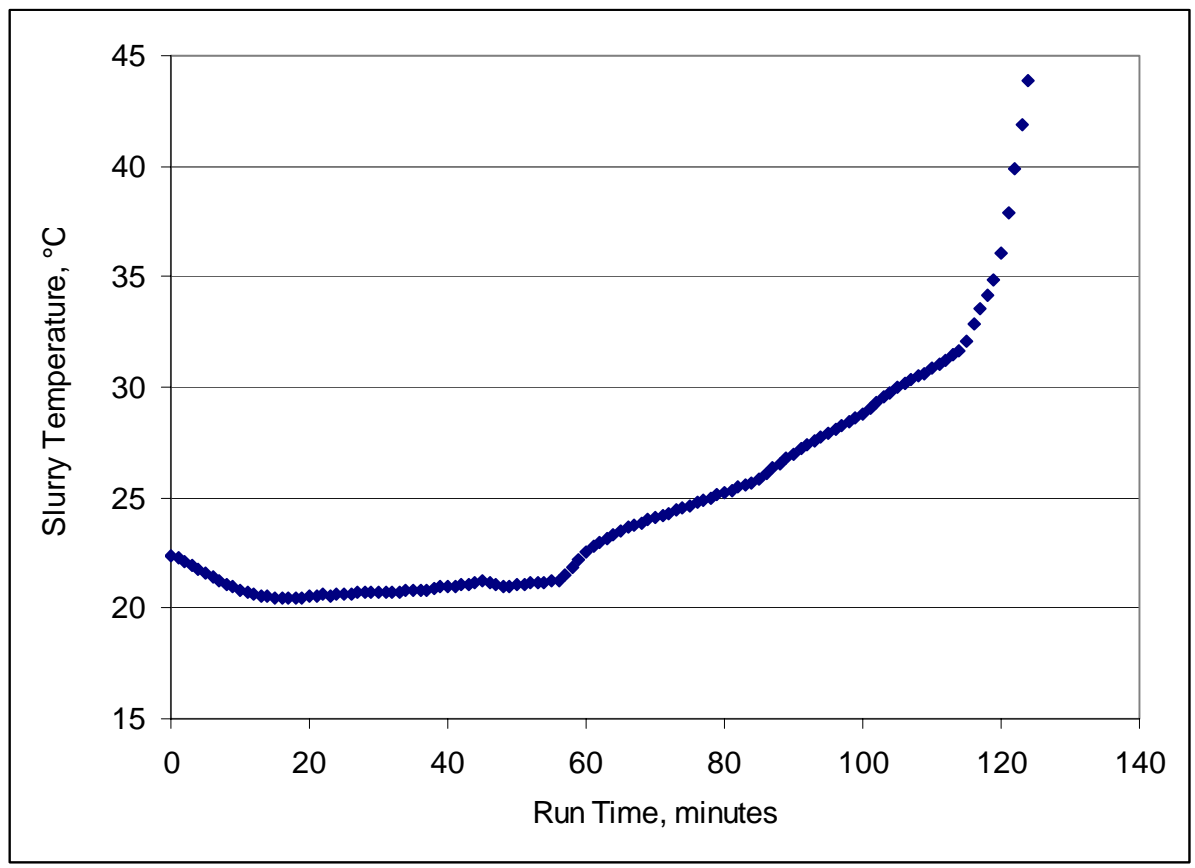

Figure 26. Temperature of the post-wash slurry during final concentration 
As in the past, the dewatering had to be stopped because of the slurry temperature. Figure 26 shows the temperature history. To increase the time of dewatering, the temperature of the slurry was cooled down to approximate $20^{\circ} \mathrm{C}$, which was the lower limit of the Test Specified (Townson, 2002) of $25^{\circ} \mathrm{C} \pm 5^{\circ} \mathrm{C}$. After about 100 minutes of concentration, the upper operating temperature of $30^{\circ} \mathrm{C}$ was reached, but for this test concentration could continue until the safe limits of the test rig, which was set at $50^{\circ} \mathrm{C}$ temperature. After approximately 120 minutes of operation the temperature was increasing at approximately $2^{\circ} \mathrm{C}$ per minute. At $44^{\circ} \mathrm{C}$, the test rig was shut down because even after shut down it takes a few minutes for the thermal momentum to stop and the cooling system to reverse the temperature rise, which was caused by pump heat.

Plugging of the filter did occur, but only after the test was over. The filter test rig was shut down for two hours to allow the temperature to decrease. When it was restarted slurry would not flow through the filter. In an attempt to break up the pluggage, the filter was disconnected from the pump recirculation loop and all of the permeate, which was previously removed during the concentration, was replaced. The idea was to bring all the thick slurry in the pumps back to its initial consistency, then allow the slurry to push through the filter tubes. However, even after dilution the filter remained plugged. Another attempt was made by closing the valves downstream of the filter (see valves V11 and V20 in Fig. 2) and backpulse through the filter. With the downstream valves closed, the only way for slurry, liquid, and air to leave the system was through the filter entrance back through the pumps and into the feed tank. This was done and since the liquid level in the feed tank increased significantly during the backpulse, this meant something flowed through the porous filter walls and out the filter entrance. By accident the backpulse air valve was left open a little too long and some air was also fed through the filter wall and into the slurry. Next, the main valve downstream of the filter (V11) was opened and then the pumps were very slowly powered up. The idea was that if the backpulse stream only left small fissures in the slurry, which was plugging the filter, then a very slow flow of slurry from the pumps would move through those fissures and erode the pluggage. That is, it was feared that a strong pulse of slurry from the pumps being on at full force may close those fissures. In any case, the slow flowing slurry worked and within 10 minutes, the filter pressure dropped to expected values, thus indicating the pluggage was removed and flow reestablished. A fast check of the filter permeate flux after the recovery confirmed that the filter returned to the same state it had before the post-wash final concentration began.

\subsection{FILTER RINSING AND CLEANING}

To follow the RPP-WTP flowsheet after slurry washing, the filter system was deinventoried of the slurry and the process of rinsing and cleaning begun. It involved first rinsing the system with a mild caustic solution $(0.1 \mathrm{M} \mathrm{NaOH})$ for 30 minutes, then the filter was cleaned three times with $2 \mathrm{M}$ nitric acid. Each acid cleaning was done for 90 minutes. Finally the system was rinsed two more times with mild caustic rinses at 30 minutes each. The filter was checked with the caustic solution to measure "water" flux to compare to the pre-test caustic water baseline test, i.e., test number 1.00 . 
Figure 27 shows the permeate flux history of the pre-cleaning caustic rinse after a backpulse and Fig. 30 (a) shows a settled sample of the rinse solution, which had $\mathrm{pH} 13$. Notice the large amount of undissolved solids.

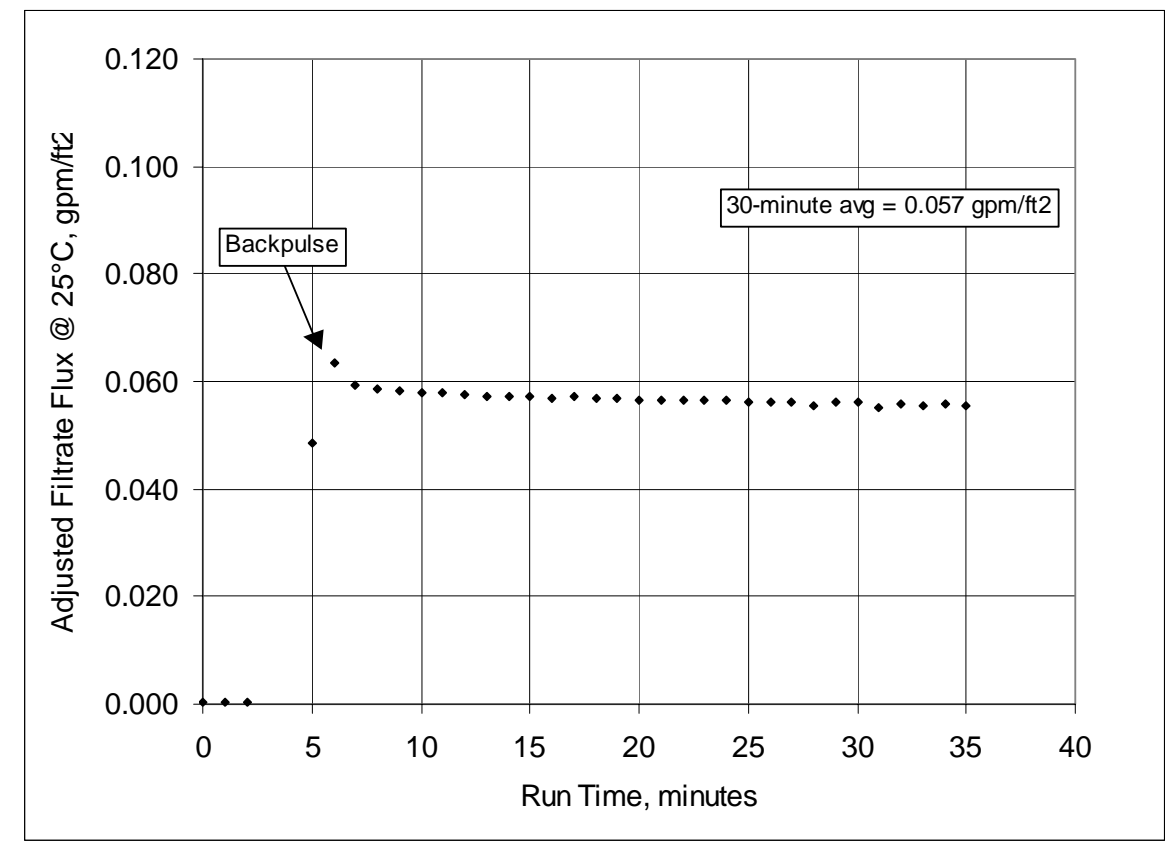

Figure 27. Pre-cleaning caustic rinse of $0.1 \mathrm{M} \mathrm{NaOH}$

[average operating parameters: $\mathrm{V}=10.9 \mathrm{ft} / \mathrm{s}, \mathrm{TMP}=21 \mathrm{psia}, \mathrm{T}=24^{\circ} \mathrm{C}$ ]

The concentration of those solids turned out to be approximately $8 \mathrm{wt} \%$ and represented the expected heel left in the filter loop and feed tank during the first rinsing. To obtain this mixture, 7.5 liters of the previously washed slurry at $21 \mathrm{wt} \%$ UDS was added to the 100 liters of $0.01 \mathrm{M} \mathrm{NaOH}$ rinse solution. After Campaign I ended, SRNL found out from WTP that most of the heel in the feed tank will be steam lifted out of the filter feed tank, therefore, the first rinse solution in the full scale unit will have closer to $2 \mathrm{wt} \%$ UDS than $8 \mathrm{wt} \%$ UDS.

Figure 28 shows the filter flux results for all three nitric acid cleanings. The time gap between each two cleanings was the time necessary to deinventory the filter system and put in another charge of acid. It is interesting to see that the curve almost looks continuous when the gaps are kept. This implies that while the filter system was stopped and emptied of the dirty acid stream and replaced with the new one, the acid left in, and on, the filter porous tube continued to dissolve solids. Unfortunately, since the flux data were still increasing at the end of the last 90-minutes cleaning, further cleaning may be warranted. Fig. 30 (b), (c), and (d) show the dirty cleaning solutions, which all have a $\mathrm{pH} 1$. 
WSRC-TR-2004-00201, REV. 0

SRT-RPP-2004-00034, REV. 0

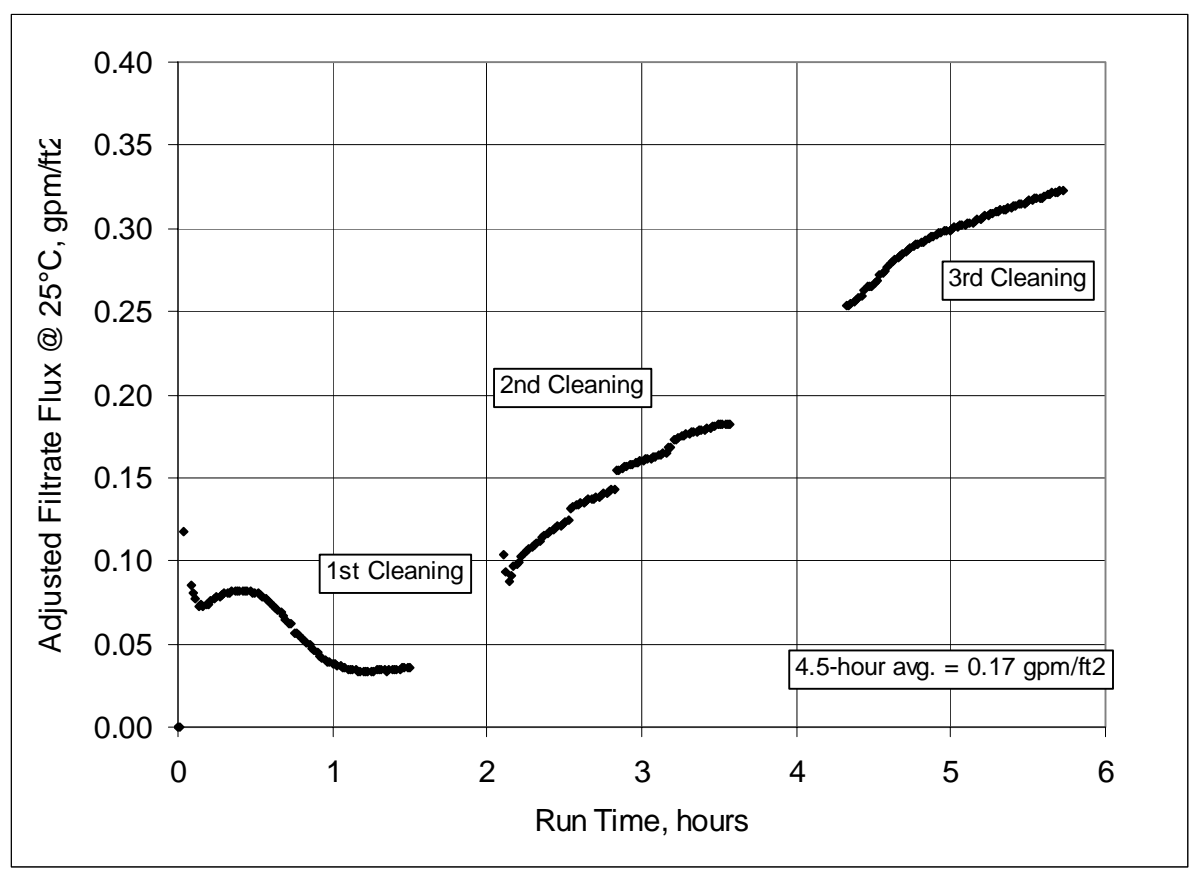

Figure 28. Three 90-minute periods of cleaning with 2 M HNO3

[average operating parameters: $\mathrm{V}=11.0 \mathrm{ft} / \mathrm{s}, \mathrm{TMP}=40 \mathrm{psia}, \mathrm{T}=25^{\circ} \mathrm{C}$ ]

Notice that Fig. 30 (b) is much whiter, or cloudier, than the following two samples of cleaning solution. The first acid cleaning had significant solids and the first data set in Fig. 28 shows the filter flux actually dropping. It appears that while the solids were dissolving they were forming a sticky mass on the filter surface, which caused the flux to drop. After about 1 hour, the flux stopped dropping and began to increase. While the flux continued to increase for the remaining cleaning periods there were still solids present and it was really only until the last cleaning, Fig.30 (d), that no solids were visible.

Following the cleaning, there were two more caustic rinses. As is clear from Fig. 29 the permeate flux dropped very fast during the first caustic rinse. This has always been the result of adding a caustic rinse after acid cleaning because after draining all the acid solution there are always trapped pockets of acid in the filter system, as well as all the acid solution that coat the system walls. Many of the dissolved solids in the remaining acid precipitates and causes a reduction in filter flux. There were even enough acid to make the first caustic rinse acidic. The measured $\mathrm{pH}$ level in the first post-clean rinse solution was $\mathrm{pH} 1$. However, it appeared to clear in the last acid cleaning solution, see Fig. 30 (e). The final $0.1 \mathrm{M} \mathrm{NaOH}$ rinse remained caustic. It had a $\mathrm{pH}$ of 12, which means that most of the acid was flushed out of the system. However, in the last rinse the precipitated solids became visible. 
WSRC-TR-2004-00201, REV. 0

SRT-RPP-2004-00034, REV. 0

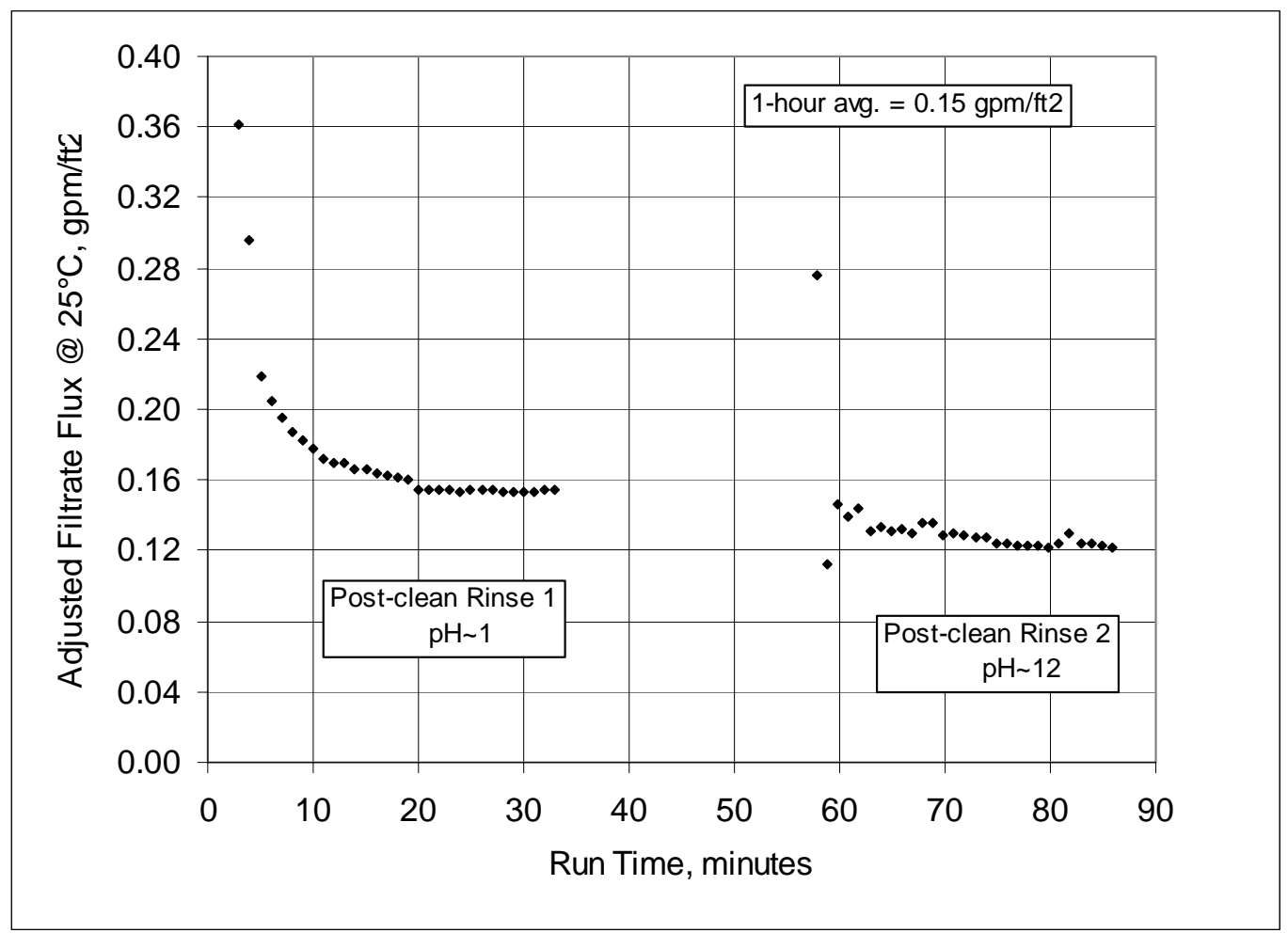

Figure 29. Two post-cleaning caustic rinses of $0.1 \mathrm{M} \mathrm{NaOH}$

[average operating parameters: $\mathrm{V}=11.0 \mathrm{ft} / \mathrm{s}, \mathrm{TMP}=20 \mathrm{psia}, \mathrm{T}=24^{\circ} \mathrm{C}$ ]

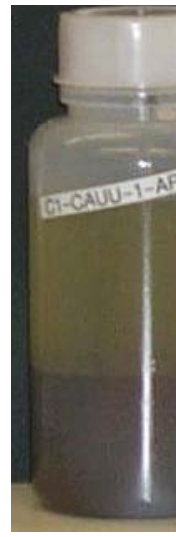

(a) (b)

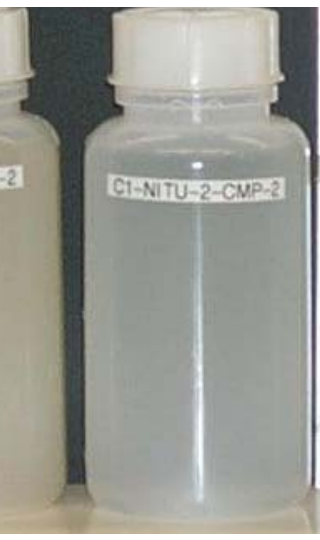

(c)

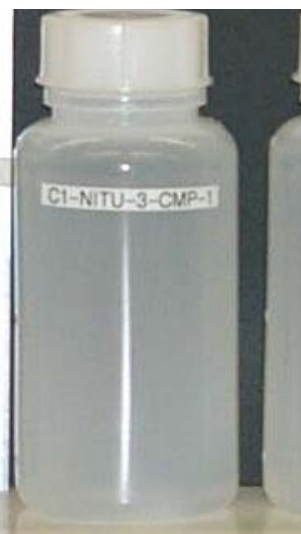

(d)

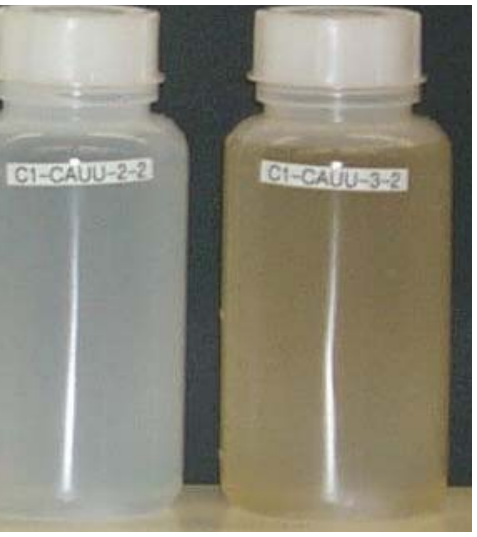

(e)

(f)

Figure 30. Rinse and cleaning recycle streams

(a) pre-clean caustic rinse, $\mathrm{pH} 13$, (b) first $2 \mathrm{M}$ nitric cleaning, $\mathrm{pH} 1$, (c) second $2 \mathrm{M}$ nitric cleaning, $\mathrm{pH} 1$, (d) third $2 \mathrm{M}$ nitric cleaning, $\mathrm{pH} 1$, (e) first post-clean caustic rinse, $\mathrm{pH} 1$,

(f) second post-clean caustic rinse, $\mathrm{pH} 12$ 
Those solids appear reddish, see Fig. 30 (f), which implied some percentage of iron. At the end of the last caustic rinse the permeate flux had asymptotically dropped to $0.12 \mathrm{gpm} / \mathrm{ft}^{2}$. This caustic flux was still more than 15 times higher than the average dewatering permeate flux of $0.007 \mathrm{gpm} / \mathrm{ft}^{2}$.

\subsection{FILTER BASELINING}

As seen in the past (Duignan, 2000a), the filter flux for the post-test water run always is lower than the initial water runs, see Fig. 31. It is not known why the filter always give much better results before testing. One reason may be that while the pilot test rig sits idle for many months, and in this case it was approximately 1 year since its last use, the filter remains submerged in a mild caustic solution to keep it wet and prevent the growth of algae. During that time, any solids lodged in the filter walls may be slowly dissolving, giving higher flux results for its next use. This fact should become clearer between Campaign I and II of the SIPP because the idle period for the filter was only approximately 10 weeks. In any case, the lower post-test water flux should not be a problem because it is still more than an order of magnitude higher than when filtering slurry wastes. There is some past evidence that the level of cleanliness of the filter may not affect the permeate flux of the waste as long as the clean water flux is significantly higher. What significant means is not known, but performance from one campaign to the next should indicate how the partially clean filter substrate performs. That is, does it get progressively worse or does it reach some repeatable clean condition above which it is not efficient to spend more time or money to clean.

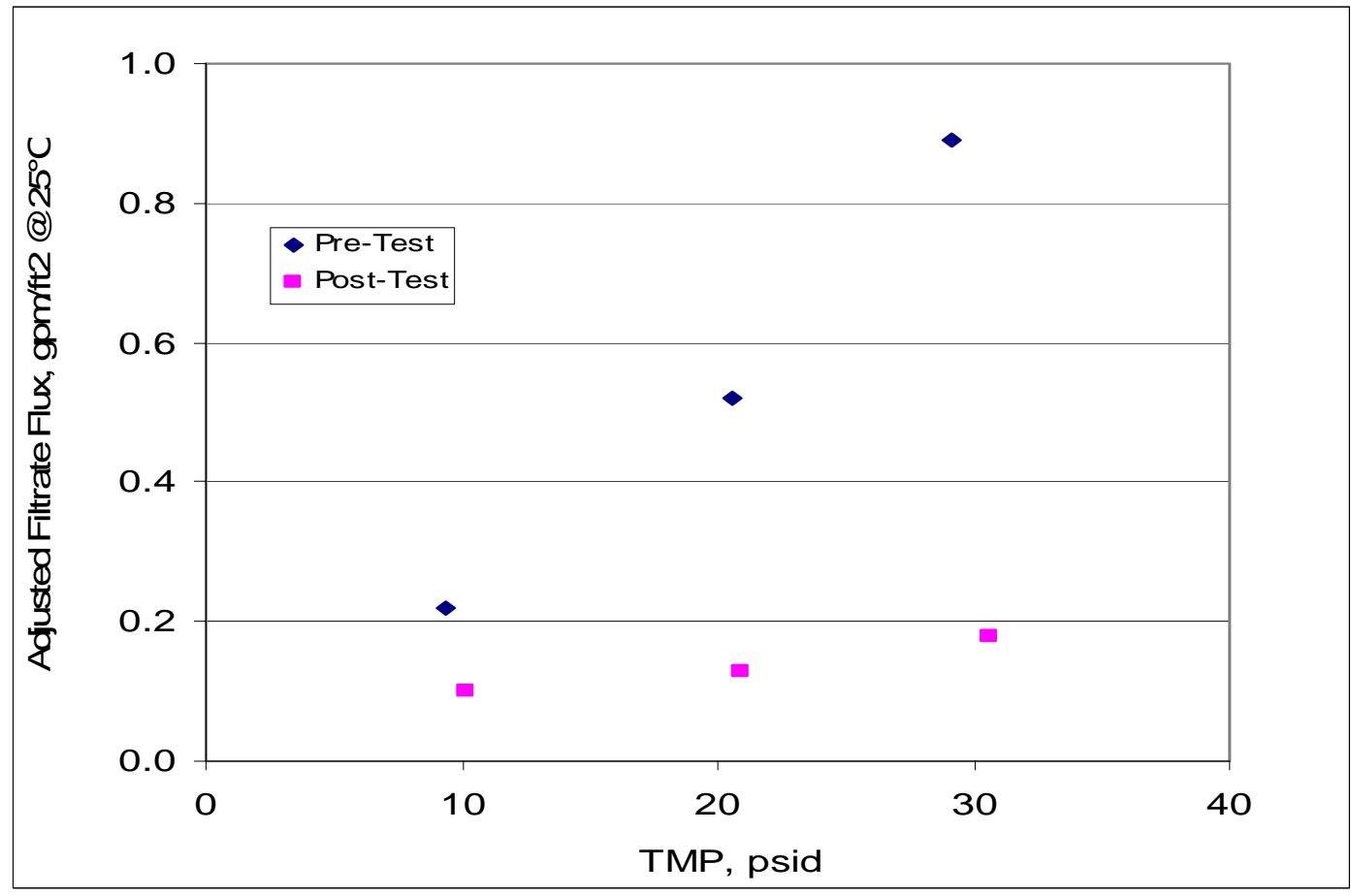

Figure 31. Pre- and post-test water $(0.1 \mathrm{M} \mathrm{NaOH})$ baseline test runs

[average operating parameters: $\mathrm{V}=11.0 \mathrm{ft} / \mathrm{s}, \mathrm{T}=26^{\circ} \mathrm{C}$ ] 


\subsection{PILOT FILTER VS. BENCH-TOP TESTING}

After completing Campaign I, the filter results were compared to bench-top (CUF) work. While the comparison is not exact due to differences in scale (see Duignan, 2003a, section 3.3.3.2, 3.3.3.3, and 3.3.3.4), it is instructive to contrast differences. Figure 32 is a repeat of CUF Run 2 data, see Fig. 1, with the data from Fig. 4 superimposed.

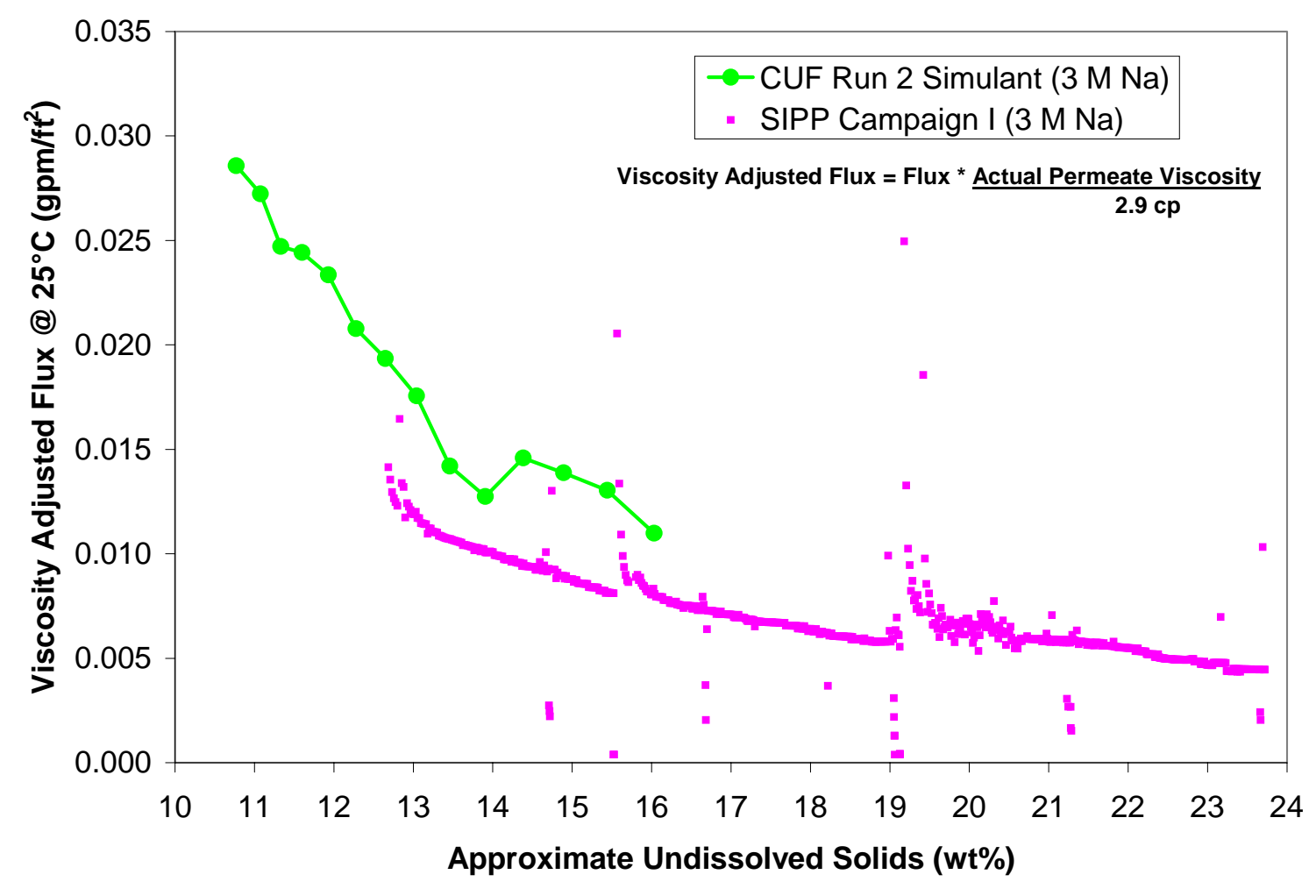

Figure 32. Comparison of the pilot to bench-top (CUF) scale tests with AY102/C106 simulant

[Compare with Fig. 1]

Note, that like the bench-top data, the pilot data were adjusted for differences in permeate viscosity. As has been seen in the past (e.g., see section 3.3.3.3 in Duignan, 2003a), the bench-top data are above the pilot data. Past estimates have indicated differences of at least $30 \%$, which would bring the two curves very close to each other. The bench-top (CUF) data had to be stopped at $16 \mathrm{wt} \%$ UDS due to system limitations. However, as the slurry becomes more concentrated the two curves would expect to be even closer when the filter cake becomes very thick and differences in scale become smaller. 


\subsection{CONCLUSIONS}

The average pilot ultrafiltration permeate flux data for AY-102/C-106 simulant was less than the expected WTP design of $0.0277 \mathrm{gpm} / \mathrm{ft}^{2}$, but was considered to be representative of the actual Hanford waste. Therefore, the flux obtained in this test is that which may be expected during actual operation. A summary of the overall conclusions follows:

- Average permeate flux when dewatering from 12 to $24 \mathrm{wt} \%$ was $0.008 \mathrm{gpm} / \mathrm{ft}^{2}$.

- At no time did the permeate turbidity measure to be higher than $0.5 \mathrm{NTU}$ and therefore the permeate was considered always free of solids.

- Steady-state solids concentration confirmed that the best permeate flux is obtained with the slurry axial velocity was at or above $11 \mathrm{ft} / \mathrm{s}$.

- When washing a batch of waste, which was concentrated to $20 \mathrm{wt} \%$ UDS, with two equal batches of $0.01 \mathrm{M} \mathrm{NaOH}$ solution, the consistency was reduced by one-half, but the yield stress remained statistically constant.

- When washing, many elements like sodium and aluminum were reduced by almost an order of magnitude. Some species in the undissolved solids actually did dissolve, like oxalate and fluoride, causing the concentration of undissolved solids to decrease as the dense supernatant was replaced with caustic water.

- When the filter became plugged with highly concentrated solids, recovery was possible for the vertically mounted filter by backpulsing after the downstream slurry valve was closed.

- Cleaning the filter with $2 \mathrm{M}$ nitric acid was sufficient to increase the $0.1 \mathrm{M} \mathrm{NaOH}$ permeate flux to an order of magnitude larger than that of waste flux. However, it was not sufficient to return the filter to pretest cleanliness.

\subsection{FUTURE WORK}

As per the SIPP test specification (Longwell, 2003), the work discussed in this interim report is for Campaign I, which was only the first of four campaigns. Future work will be reported in other interim reports to culminate in a final report near the end of this calendar year. The final report will mention any future work outside of the current task, which may be the result of information elicited from the SIPP task.

\subsection{REFERENCES}

Duignan, M. R. (2000a) "Final Report: Pilot-scale Cross-flow Ultrafiltration Test Using a Hanford Site Tank 241-AN-105 Waste Simulant - Envelope A + Entrained Solids,” Westinghouse Savannah River Company Document No. BNF-003-98-0221, Rev. 0, (23 February).

Duignan, M. R. (2000b), "Final Report: Pilot-scale Cross-flow Ultrafiltration Test Using a Hanford Site Tank 241-AN-107 Waste Simulant - Envelope C + Entrained Solids + Strontium-Transuranic Precipitation,” Westinghouse Savannah River Company Document No. BNF-003-0226, Rev. 0. (24 March) 
Duignan, M. R. (2003a). "Final Report: Pilot-scale cross-flow ultrafiltration test using a Hanford Site tank 241-AN-102 waste simulant.” Westinghouse Savannah River Document No. WSRC-TR-2003-00204, Rev. 0. (6 May) [also SRT-RPP-2003-00087]

Duignan, M. R. (2003b), “RPP-WTP Pilot Scale Cross Flow Filtration,” Westinghouse Savannah River Company Notebook No. WSRC-NB-2003-000149. (began on 12 June)

Duignan, M. R. (2003c). “Pilot-scale Integrated Pretreatment System Test,” (Task Technical and QA Plan) Westinghouse Savannah River Document No. WSRC-TR-2003-00338, Rev. 0. (4 August) [also SRT-RPP-2003-00165]

Duignan, M. R. (2004), “RPP-WTP Pilot-scale Cross-flow Ultrafiltration Test Procedure,” Westinghouse Savannah River Company Field Procedure No FP-975, (26 January)

Lee, E. and M. Binsfield, 2002 "Flowsheet Bases, Assumptions, and Requirements," Doc. No. 24590-WTP-RPT-PT-02-005, Rev. 1. (December)

Longwell. R. L. (2003). Pilot Scale Integration of Pretreatment Process System. Waste Treatment Plant - River Protection Project Document No. 24590-PTF-TSP-RT-03-002, Rev. 0. (5 June)

Poirier, M. R., P. R. Burket, J. L. Siler, and J. R. Zamecnik (2003), "Filtration, washing and leaching of a Hanford AY-102/C-106 sample,” Westinghouse Savannah River Company Document No. WSRC-TR-2003-00240, Rev. 0 (or SRT-RPP-2003-00110, Rev. 0). (June)

Stallings, M. E., M. R. Poirier, and R. E. Eibling (2003). Task Technical and Quality Assurance Plan for AY-102/C-106 Simulant Ultrafiltration and Washing (Including AY102/C-106 Simulant Development). Westinghouse Savannah River Document No. WSRCTR-2003-00217, Rev. 0. (31 May) [also SRT-RPP-2002-00235]

Townson, P. S. (2002). AY-102/C-106 Simulant Pilot Scale Ultrafiltration and Washing Test Specification. Waste Treatment Plant - River Protection Project Document No. 24590-PTFTSP-RT-02-015, Rev. 0. (15 November)

Zamecnik, J. R., P. R. Burket, R. E. Eibling, and M. R. Poirier (2004), “Tank 241-AY-102 Simulant development, ultrafiltration, and washing,” Westinghouse Savannah River Company Document No. WSRC-TR-2003-00547, Rev. 0 (or SRT-RPP-2003-00240, Rev. 0). (March) 
R. E. Edwards, 773-A

H. F. Sturm, 773-A

J. C. Marra, 773-42A

S. T. Wach, 773-42A

D. B. Burns, 786-5A

T. B. Calloway, 999-W

H. N. Guerrero, 786-5A

M. R. Duignan, 786-5A

M. R. Williams, 786-5A

J. R. Zamecnik, 773-41A

RPP File, c/o M. F. Jones, 773-43A, Room 109

\section{OFF-SITE}

W. L. Tamosaitis, Waste Treatment Plant, 3000 George Washington Way, Richland, WA99352

R. A. Peterson, Waste Treatment Plant, 3000 George Washington Way, Richland, WA 99352

P. S. Sundar, Waste Treatment Plant, 3000 George Washington Way, Richland, WA 99352 
WSRC-TR-2005-00105, REVISION 0

SRNL-RPP-2005-00012, REVISION 0

\section{Appendix Section: I-2}

Interim Report: RPP-WTP Semi-Integrated Pilot Plant - Campaign II

[For Information Only] 
WSRC-TR-2004-00478, REVISION 0

SRNL-RPP-2004-00075, REVISION 0

\section{INTERIM REPORT: RPP-WTP SEMI-INTEGRATED PILOT PLANT CAMPAIGN II}

September 2004

SAVANNAH RIVER NATIONAL LABORATORY

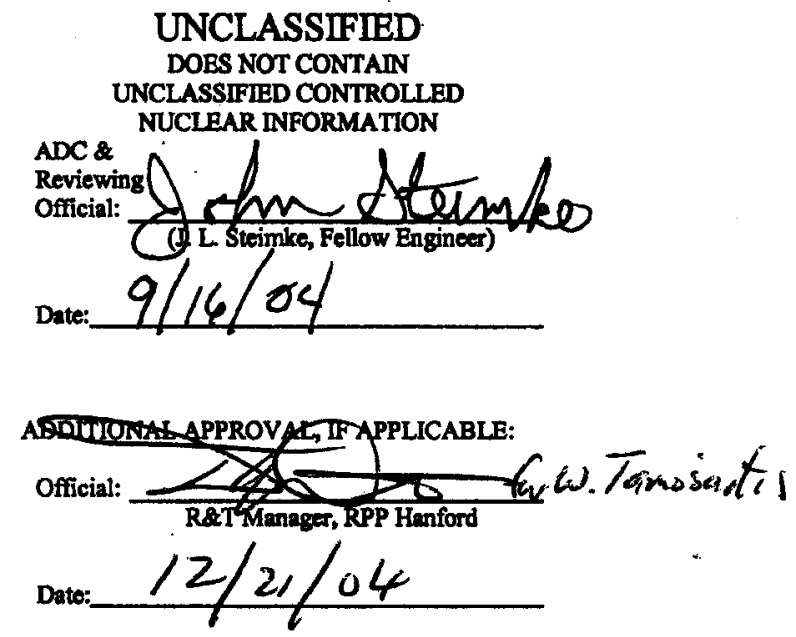

Westinghouse Savannah River Company

Savannah River Site

Aiken, SC 29808

Prepared for the U.S. Department of Energy Under Contract Number DEAC09-96SR18500

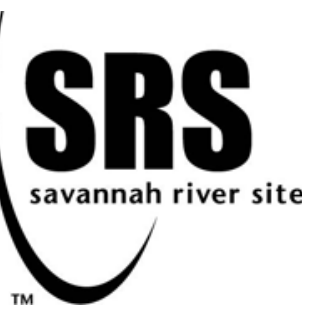


WSRC-TR-2004-00478, REVISION 0

SRNL-RPP-2004-00075, REVISION 0

DISCLAIMER

This report was prepared for the United States Department of Energy under Contract No. DE-AC09-96SR18500 and is an account of work performed under that contract. Neither the United States Department of Energy, nor WSRC, nor any of their employees makes any warranty, expressed or implied, or assumes any legal liability or responsibility for accuracy, completeness, or usefulness, of any information, apparatus, or product or process disclosed herein or represents that its use will not infringe privately owned rights. Reference herein to any specific commercial product, process, or service by trade name, trademark, name, manufacturer or otherwise does not necessarily constitute or imply endorsement, recommendation, or favoring of same by Westinghouse Savannah River Company or by the United States Government or any agency thereof. The views and opinions of the authors expressed herein do not necessarily state or reflect those of the United States Government or any agency thereof.

Printed in the United States of America

Prepared For

U.S. Department of Energy 
WSRC-TR-2004-00478, REVISION 0

SRNL-RPP-2004-00075, REVISION 0

WSRC Job Number: EES-23041

Key Words:

Ultrafiltration

Hanford Tank 241 AY-102/C-106

\title{
Retention: Permanent
}

Key WTP R\&T References:

Test Specifications:

24590-PTF-TSP-RT-02-015, Rev. 0,

15 Nov. 2002

24590-PTF-TSP-RT-03-002, Rev. 0,

05 Jun. 2003

24590-PTF-TSP-RT-01-003, Rev. 0,

20 Nov. 2001 (CIX chemical testing only)

Test Plans:

WSRC-TR-2003-00338, Rev. 0, 04 Aug. 2003

(SRT-RPP-2003-000165) and WSRC-TR-

2001-00340, Rev. 0, 06 Feb. 2002 (or SRT-

RPP-2001-000116) (CIX chemical testing only)
Test Exceptions:

All of the following test exceptions only affect the CIX chemical testing sub-task:

WSRC-TR-2001-00340

24590-WTP-TEF-RT-02-025, Rev. 0, 20 May 2002

24590-WTP-TEF-RT-02-046, Rev. 0, 01 Aug. 2002

24590-WTP-TEF-RT-03-004, Rev. 0, 07 Feb. 2003

24590-WTP-TEF-RT-03-037, Rev. 0, 30 Jul. 2003

24590-WTP-TEF-RT-03-037, Rev. 1, 17 Sept. 2003

24590-WTP-TEF-RT-03-054, Rev. 0, 17 Sept. 2003

24590-WTP-TEF-RT-03-069, Rev. 0, 14 Nov. 2003

R\&T Focus Area: RPP-WTP Pretreatment

Test Scoping Statements: S-48 \& S-134

\section{INTERIM REPORT: RPP-WTP SEMI-INTEGRATED PILOT PLANT - CAMPAIGN II}

\author{
M.R. Duignan*, D.J. Adamson, C.D. Barnes, M.D. Fowley, \\ Z.H Qureshi, J.L. Steimke, M.R. Williams, and J.R. Zamecnik
}

*Principal Author

Savannah River National Laboratory

Issue Date: 10 SEPTEMBER 2004

Westinghouse Savannah River Company

Savannah River Site

Aiken, SC 29808

Prepared for the U.S. Department of Energy Under Contract Number DE-AC09-96SR18500

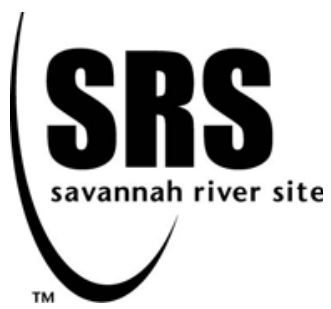


WSRC-TR-2004-00478, REVISION 0

SRNL-RPP-2004-00075, REVISION 0

\section{REVIEWS AND APPROVALS}

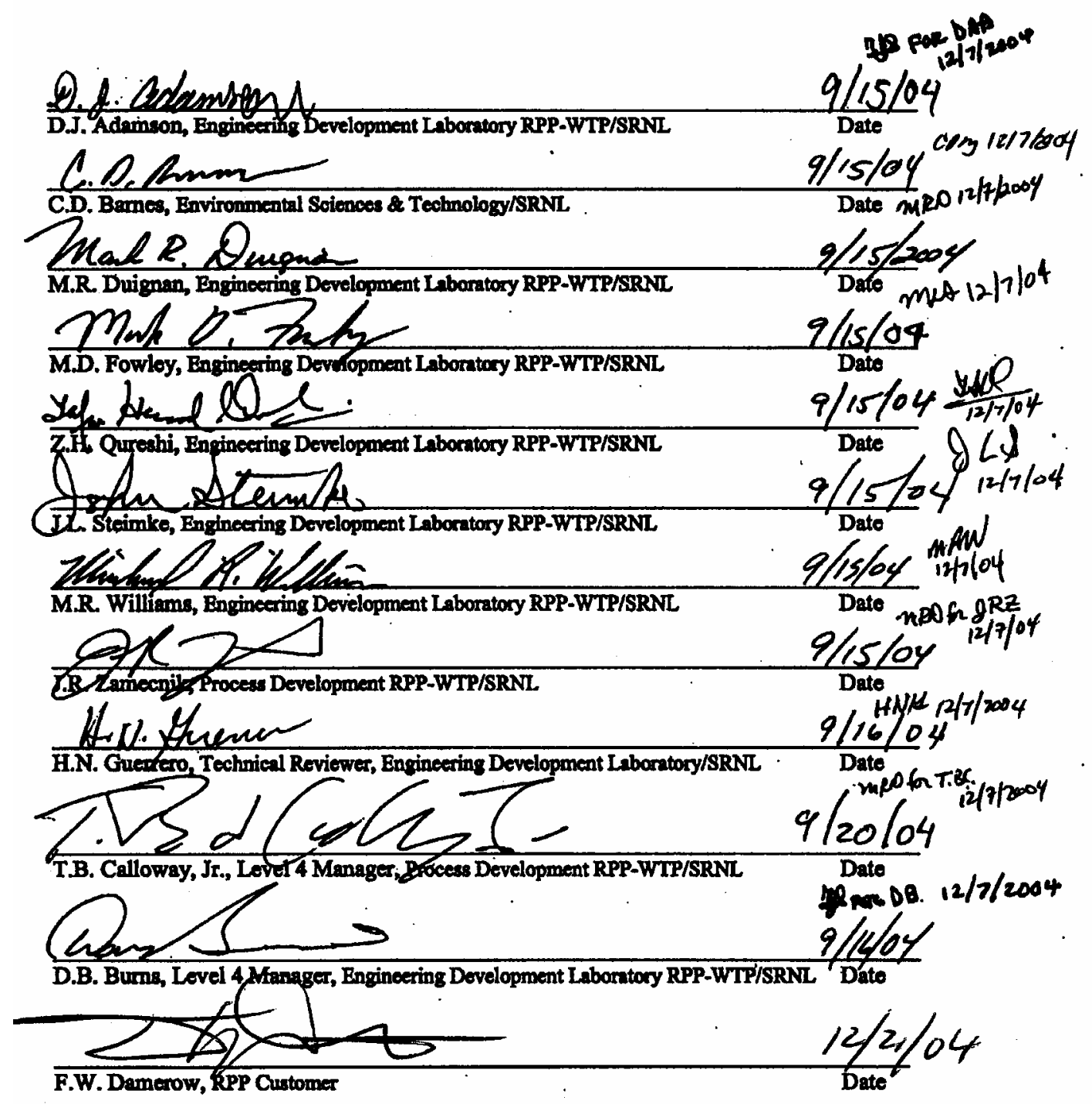




\section{TABLE OF CONTENTS}

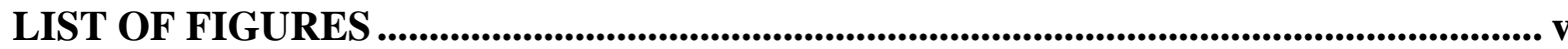

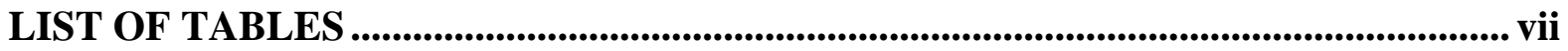

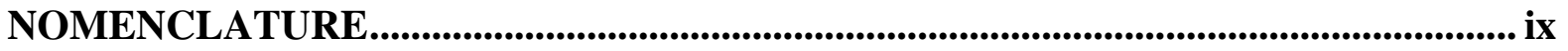

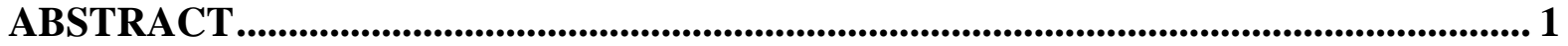

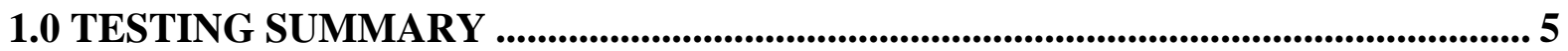

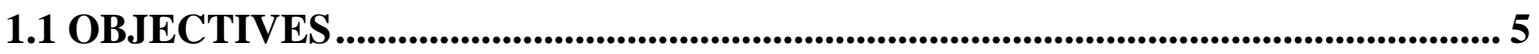

1.2 TEST EXCEPTIONS ...................................................................................... 8

1.3 RESULTS AND PERFORMANCE AGAINST SUCCESS CRITERIA ................. 9

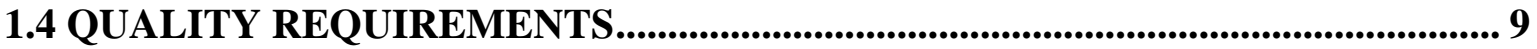

1.5 R\&T TEST CONDITIONS ................................................................................. 10

1.6 SIMULANT USE .......................................................................................... 11

1.7 DISCREPANCIES AND FOLLOW-ON TESTS ............................................... 11

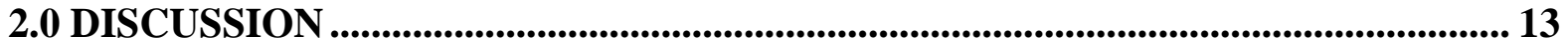

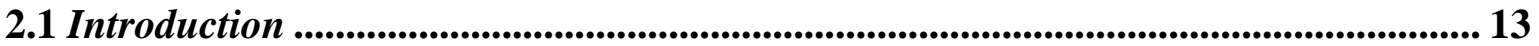

2.2 The Plan ........................................................................................................... 14

2.3 The Computational Model....................................................................................... 19

2.4 The Overall SIPP Test Matrix.............................................................................................. 25

2.5 The Principal Waste Simulants: AY102 and AP101 (Author - Zamecnik)................. 33

2.6 Pilot Waste Feed Evaporator (Authors - Qureshi, Williams \& Zamecnik) ................. 34

2.6.1 Chemical Description of the Pilot FEP Feed Stream (Author - Zamecnik) ...... 35

2.6.2 Simulant Handling (Author - Williams)........................................................... 43

2.6.3 Pilot FEP Equipment (Author - Qureshi).............................................................. 45

2.6.4 Pilot FEP Operation (Author - Qureshi) ..................................................................... 48

2.6.5 Pilot FEP Discussion of Results................................................................... 51

2.6.6 Pilot FEP Conclusions.......................................................................................... 57

2.7 Pilot Ultrafiltration (Authors - Fowley, Williams \& Zamecnik)................................... 58

2.7.1 Chemical Description of the Pilot-Scale UFP Feed Stream (Author - Zamecnik)

2.7.2 Simulant Handling (Author - Williams)..........................................................64 64

2.7.3 Pilot UFP Operation and Equipment (Author - Fowley) ...................................68

2.7.4 Pilot UFP Discussion of Results ............................................................................. 73

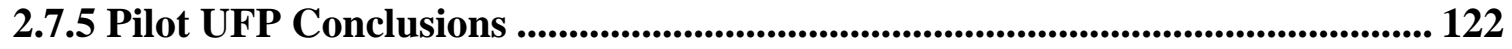

2.8 Pilot Cesium ion exchange (Authors - Steimke \&Williams) ................................. 123

2.8.1 Chemical Description of the Pilot-Scale CIX Feed Stream ............................ 124

2.8.2 Simulant Handling (Author - Williams).................................................................. 124

2.8.3 Pilot CIX Equipment (Author - Steimke) ............................................................ 125

2.8.4 Pilot CIX Operation (Author - Steimke) ........................................................... 129

2.8.5 Pilot CIX Discussion of Results (Author - Steimke)............................................. 131

2.8.6 Pilot CIX Conclusions (Author - Steimke)..................................................... 131

2.9 PILOT TREATED LAW EVAPORATOR (Authors - Adamson, Williams \&

Zamecnik) ............................................................................................................... 131

2.9.1 Chemical Description of the Pilot TLP Feed Stream (Author - Zamecnik).... 131

2.9.2 Simulant Handling (Author - Williams)........................................................ 133

2.9.3 Pilot TLP Equipment (Author - Adamson).......................................................... 134

2.9.4 Pilot TLP Operation (Author - Adamson) ....................................................... 134 
2.9.5 Pilot TLP Discussion of Results (Author - Adamson)............................................ 138

2.9.6 Pilot TLP Conclusions .................................................................................... 140

2.10 Glass ProductIon based on SIPP results (AUTHOR - zamecnik) .............................. 141

2.10.1 Extrapolated Glass Production Rates .................................................................... 141

2.10.2 Increasing Glass Production Rates..................................................................... 143

3.0 CONCLUSIONS ............................................................................................................. 147

3.1 PILOT-SCALE FEP UNIT OPERATION ................................................................... 147

3.2 PILOT-SCALE UFP UNIT OPERATION .................................................................. 147

3.3 PILOT-SCALE CIX UNIT OPERATION.................................................................... 148

3.4 PILOT-SCALE TLP UNIT OPERATION ................................................................ 148

4.0 FUTURE WORK ........................................................................................................... 149

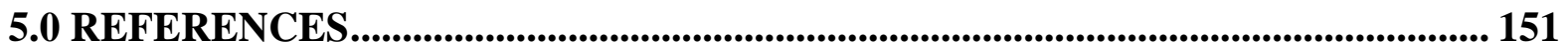

APPENDICES:

A: Computational Model (Author - Barnes)

B: WTP Discussion of Pretreatment Facility Waste Processing Residence Times

C: Anti-Foam Subcontractor Analysis

D: Campaign II Sampling Matrix 


\section{LIST OF FIGURES}

Figure 1. Schematic of the overall task operation taken from the task plan ........................... 18

Figure 2. Basis for Generation of Recycles ....................................................................... 36

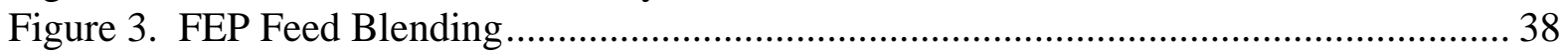

Figure 4. Mixing of Recycles.......................................................................................... 39

Figure 5. Picture of the Settled Contents of the Mixtures Shown in Fig. 3: They Are

Simulants of (a) PWD-VSL-16, (b) PWD-VSL-15, (c) PWD-VSL-33, (d) PWD-VSL-44,

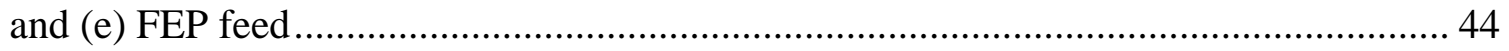

Figure 6. Pilot-scale FEP Unit Operation Feed Tank ……………………................................ 44

Figure 7. SIPP Pilot-Scale FEP/TLP Unit .......................................................................... 46

Figure 8. SIPP Pilot-Scale FEP/TLP Unit: 15-foot Acrylic Section with Ongoing

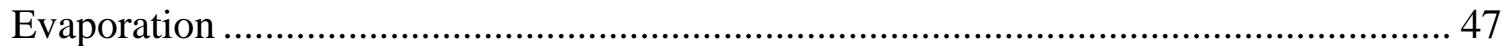

Figure 9. SIPP Pilot-Scale FEP/TLP Unit: Feed Ready for Evaporation to Commence........ 47

Figure 10. SIPP Pilot-Scale FEP/TLP Unit: Evaporating....................................................... 48

Figure 11. Total \& Undissolved Solids in pilot-scale FEP unit operation............................... 53

Figure 12. Schematic of Antifoam Fate in the SIPP ............................................................ 57

Figure 13. Schematic of the Planned Ultrafiltration Process - Page 1 ………………......... 59

Figure 14. Schematic of Ultrafiltration Process - Page 2 2...................................................... 60

Figure 15. Simulants of (a) AY102, (b) FEP Bottoms, (c) AP101, and (d) UFP feed which is

a mixture of (a), (b), and (c) - These are Samples after Solids Had Settled.................... 66

Figure 16. UFP Primary Mixing Tank (Volume 1000 liters) which represents the RPP-

WTP UFP Preparation Feed Tank................................................................................ 67

Figure 17. UFP Slurry Reservoir (Volume 100 liters) which represents the RPP-WTP UFP

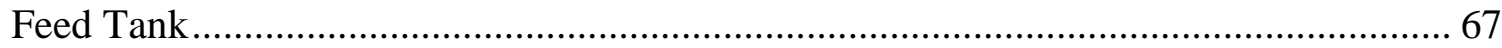

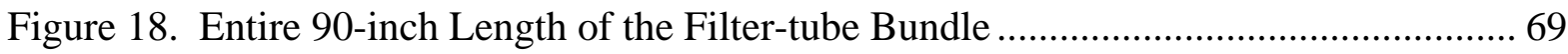

Figure 19. Close-up of the Tubes' Surfaces and Bulkhead Stabilizing Plate .......................... 69

Figure 20. The Exit (Left) and Entrance (Right) Tube Sheets of the Filter Tube Bundle..... 70

Figure 21. Campaign II Ultrafiltration Test Rig .................................................................... 72

Figure 22. Dewatering of UF Feed, Permeate Flux vs. Run Time ......................................... 75

Figure 23. Dewatering of UF Feed, Permeate Flux vs. Concentration.................................... 76

Figure 24. $1^{\text {st }}$ Washing of Concentrated Slurry with 22 Wash Additions (or mini-batches). 78

Figure 25. 2nd Washing of Concentrated Slurry with 22 Wash Additions (or mini-batches) 80

Figure 26. Solids and Rheological Data throughout Simulant Washing ................................ 80

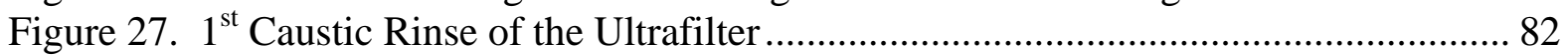

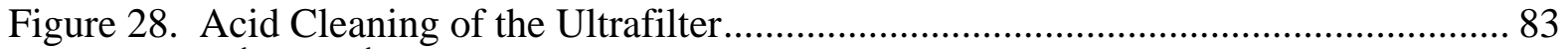

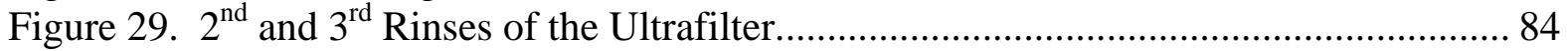

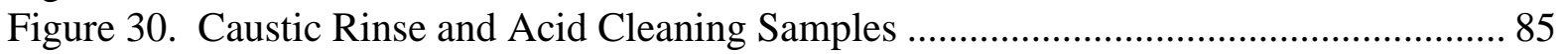

Figure 31. Pre- and Post-Test Baselines for Campaign II ....................................................... 86

Figure 32. Comparison of Pre- and Post-Test Baselines, Campaign I and II ......................... 86

Figure 33. XRD Spectrum of Solids Precipitated from Campaign I Ultrafilter Permeate .... 90

Figure 34. Solids Content and Density of Slurry During Washing ........................................ 96

Figure 35. Total Solids and Density of Permeate During Washing........................................ 97

Figure 36. Yield Stress versus Total Solids for Campaign I Filtration.................................... 99

Figure 37. Yield Stress versus Total Solids for Campaign II Filtration .................................. 99

Figure 38. Graphic Representation of Leaching \& Washing Data ...................................... 103

Figure 39. Measured and Predicted Solids Compositions in Washed Slurry and Permeate 104 
WSRC-TR-2004-00478, REVISION 0 SRNL-RPP-2004-00075, REVISION 0

Figure 40. Measured and Predicted Compositions of Selected Species in Washed Slurry and

Permeate. 104

Figure 41. Measured and Predicted Compositions of Just the Sodium in Washed Slurry and

Permeate shown in Fig. 40 ..................................................................................... 105

Figure 42. Campaign I Ultrafilter Rinsing and Cleaning Solutions...................................... 113

Figure 43. Campaign II Ultrafiltration Rinsing and Cleaning Solutions (Fig. 30 repeated) 113 Figure 44. XRD Spectrum of Solids from Campaign I First Nitric Acid Cleaning Solution

Figure 45. Particle Size Distribution of Campaign I AY102 Simulant ............................... 115

Figure 46. Particle Size Distribution of Washed Campaign I Slurry..................................... 115

Figure 47. Comparison of Ultrafiltration Feed Particle Size Distributions ........................... 116

Figure 48. Particle Size Distribution of Washed Campaign II Slurry …………................... 116

Figure 49. Schematic of Antifoam Fate in the SIPP - UFP ................................................ 118

Figure 50. Fit of Flux vs. Elapsed Processing Time for the Simulated AY102/AP101 HLW

Figure 51. Permeate Volume vs. Elapsed Processing Time for the Simulated AY102/AP101

HLW

Figure 52. Undissolved Solids vs Processing Time for the simulated AY102/AP101 HLW

Figure 53. Fit of Flux versus Undissolved Solids for the simulated AY102/AP101 HLW. 121

Figure 54. Time-Averaged Flux vs. Undissolved Solids for the simulated AY102/AP101

HLW ………................................................................................................. 122

Figure 55. Schematic of Ion Exchange Facility for Integrated Testing............................... 127

Figure 56. Pilot-scale CIX columns: The left column (COLUMN 1) is undergoing resin

regeneration during Cycle 1 operation .......................................................................... 126

Figure 57. Flowrate During Cycle 1 …………………................................................... 132

Figure 58. Pressure Drop Across Resin Bed for Lead Column and Cycle 1........................ 133

Figure 59. Pressure Drop Across Resin Bed for Lag Column and Cycle 1.......................... 133

Figure 60. Resin Bed Height During Cycle 1 ………..................................................... 134

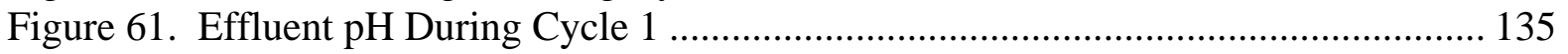

Figure 62. Effluent Conductivity During Cycle 1 ............................................................... 135

Figure 63. Test of Ability of ICP-MS to Measure Cesium.................................................. 137

Figure 64. Cesium Concentration in Simulant Exiting Lead Column ................................... 138

Figure 65. Cesium Concentration Exiting Lag Column ................................................. 139

Figure 66. Elution Plot, Linear ....................................................................................... 130

Figure 67. Elution Plot, Logarithmic .................................................................................. 130

Figure 68. Simulated TLP Feed and Resulting Bottoms Concentrate ................................... 133

Figure 69. TLP Evaporator Pot Temperature History ………………………………............ 135

Figure 70. TLP Evaporator Pot Pressure History ……………………………………........ 135

Figure 71. TLP Simulant Recirculation Flow in Evaporator System .................................... 136

Figure 72. TLP Simulant Boiling in Clear Acrylic Section of Evaporator........................... 138

Figure 73. Material Balance on TLP Evaporator of Culmlative Volume Fed...................... 140

Figure 74. Average Flux to Achieve Rates versus Undissolved Solids................................ 143

Figure 75. Average Flux to Achieve Rates versus Temperature .......................................... 145

Figure 76. Predicted Combination of Filtration Temperature and Undissolved Solids

Endpoint to Meet Glass Production Targets ...................................................................... 145 


\section{LIST OF TABLES}

Table 1. Feeds and products for planning SIPP developed for OLI/ESP computer model ... 20

Table 2. Percent contribution for each of the recycle streams to the total FEP feed .............. 23

Table 3. Percent contribution for each of the UFP feed streams ............................................ 24

Table 4. Percent contribution for each of the TLP feed streams ............................................ 25

Table 5. Hold period before pilot-scale FEP begins .............................................................. 26

Table 6. Pilot-scale FEP test matrix with the colored columns representing Campaign II ... 27

Table 7. Hold period before pilot-scale UFP begins.................................................................. 27

Table 8. Pilot-scale UFP test matrix with the colored columns representing Campaign II... 28

Table 9. Hold period before pilot-scale CIX begins .............................................................. 29

Table 10. Pilot-scale CIX (1st half) test matrix with the colored columns representing Campaign II............................................................................................................ 29

Table 11. Pilot-scale CIX (2nd half) test matrix with the colored columns representing

Campaign II.............................................................................................................. 30

Table 12. Hold period before pilot-scale TLP begins.............................................................. 30

Table 13. Pilot-scale TLP test matrix with the colored columns representing Campaign II. 31

Table 14. Pilot-scale schedule for Campaign II.................................................................... 32

Table 15. Measured Simulant Compositions .............................................................................. 33

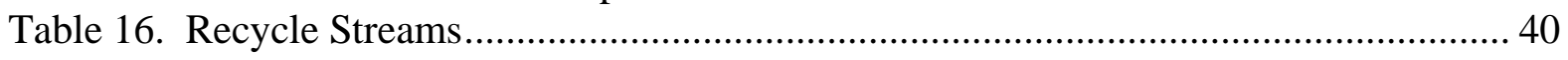

Table 17. Measured Recycle Compositions........................................................................... 41

Table 18. Chronology of the evaporation campaign............................................................... 51

Table 19. Material Balance on FEP Evaporator …………….................................................. 53

Table 20. Analyses of FEP Feed \& Bottoms \& Material Balance............................................ 54

Table 21. Masses of Total and Dissolved Species in FEP Evaporator Streams ..................... 55

Table 22. Measured Pilot-Scale UFP Feeds …………......................................................... 61

Table 23. Comparison of Measured and Calculated UFP Feed Concentrations...................... 63

Table 24. Campaign II UFP Test Matrix ${ }^{7}$................................................................................. 71

Table 25. Measured Rheological and Other Data during Dewatering of Slurry .................... 77

Table 26. Periodic Turbidity Measurements of Dewatering Permeate.................................... 77

Table 27. Measured Total Solids (TS) and Undissolved Solids (UDS) Concentrations of the

Spent Rinse and Cleaning Solutions ............................................................................... 85

Table 28. Measured and Calculated Concentrated UFP Slurry Compositions ........................ 88

Table 29. Measured Composition of Permeate and Supernate from UFP Feed Slurry .......... 89

Table 30. Comparison of OLI Model and Measured UFP Solids Contents ........................... 91

Table 31. Comparison of Model and Measured UFP-Stream Compositions .......................... 92

Table 32. Hypothetical Material \& Charge Balance on Solid Phase....................................... 93

Table 33. Dilution of Concentrated Slurry Back to Approximately $20 \mathrm{wt} \%$ Undissolved Solids

Table 34. Measured Compositions of Concentrated \& Washed Slurry and Wash Permeates

Table 35. Measured Solids, Density, and Rheological Data for Ultrafiltration...................... 98

Table 36. Chemical Compositions After Leaching \& Washing - Percent Retained in Slurry

Table 37. Percent of Several Insoluble Species in Slurry 
Table 38. Campaign II - Measured Composition of Filter Rinsing and Cleaning Solutions 107

Table 39. Campaign I - Measured Composition of Filter Rinsing and Cleaning Solutions 109

Table 40. Comparison of Campaigns I \& II Cleaning Solutions ........................................... 111

Table 41. Measured Composition of the Filtered Solids ..................................................... 112

Table 42. Hydraulic Summary ............................................................................................. 132

Table 43. Probe Responses for Standard Solutions ............................................................ 134

Table 44. Comparison of the Volume for Each of the CIX Regeneration Process Solutions to the Internal Volume of Pilot-scale CIX Column......................................................... 136

Table 45. Mass Balances for Cycles 1 and 2 ................................................................. 129

Table 46. Measured Composition of TLP Feeds .................................................................. 132

Table 47. TLP Simulant and Concentrate........................................................................ 137

Table 48. Measured TLP Products..................................................................................... 139

Table 49. Glass Production Rates Based on Ultrafilter Throughput .................................... 142 


\section{NOMENCLATURE}

AA Atomic absorption (spectroscopy)

ACTL Aiken County Technology Laboratory

ADS

Analytical Development Section (part of WSRC/SRNL)

AP101

DOE Hanford Site Tanks 241-AP-101 simulated waste

AN102

DOE Hanford Site Tank 241-AN-102

AN107

AY102

DOE Hanford Site Tank 241-AN-107

$\mathrm{BNI}$

DOE Hanford Site Combined Tanks 241-AY-102 and 241-C-106

CIX

Bechtel National, Inc.

CFD

Cesium Ion Exchange (Process) (part of the RPP-WTP Pretreatment Facility)

CUF Cells Unit Filter

Computational Fluid Dynamics

${ }^{\circ} \mathrm{C} \quad$ Degree Centigrade (or Celsius)

D Diameter

DIF Deionized and Filtered (0.1 micron) Water

DOE United States Department of Energy

dP Differential Pressure

DS Dissolved solids

EDL Engineering Development Laboratory (part of WSRC/SRNL)

EPDM Ethylene Propylene Diene Monomer

FEP

ft $\quad$ Foot

gm Gram

gpm Gallons per Minute

HLP High Level (Activity Waste) Process

HLW High Level (Activity) Waste

hr Hour

i.d. or ID Inside Diameter

ICPES Inductively coupled plasma emission spectroscopy

ICPMS Inductively coupled plasma mass spectrometry

IHLW Immobilized high level waste (glass)

ILAW Immobilized low activity waste (glass)

in. Inch

IX Ion Exchange

hr Time: Hour

l or L Liter

LAW Low (Level) Activity Waste

m Meter

M Molar (mol/L)

Mg Milligram

min Minute

$\mathrm{ml}$ or $\mathrm{mL} \quad$ Milliliter

mm Millimeter 


\begin{tabular}{|c|c|}
\hline MTG/d & Metric tons glass/day \\
\hline NAS & Sodium Aluminosilicate \\
\hline nominal & $\begin{array}{l}\text { The word "nominal" for a filter rating is a vague term. Its meaning is } \\
\text { manufacturer dependent. Further, a "nominal" rating does not give an exact } \\
\text { size to a filter medium, but rather an approximation to the expected performance } \\
\text { of a filter. In the case of Mott, a nominal rated } 0.1-\mu \mathrm{m} \text { filter means that } \\
\text { approximately } 95 \% \text { of particles greater than } 0.1 \mu \mathrm{m} \text { will not pass the filter. }\end{array}$ \\
\hline NTU & Nephelometric Turbidity Unit \\
\hline o.d. or OD & Outside Diameter \\
\hline OLI & $\begin{array}{l}\text { Modeling software which is part of Environmental Simulation Program } \\
\text { software package used in developing the SIPP task }\end{array}$ \\
\hline $\mathrm{Pa}$ & Pascal \\
\hline PSD & Particle Size Distribution \\
\hline psi & Pounds Per Square Inch \\
\hline psig & Pounds Force Per Square Inch Gauge \\
\hline psid & Pounds Force Per Square Inch Difference \\
\hline PWD & Plant Wash and Disposal (System) \\
\hline QA & Quality Assurance \\
\hline RLD & Radioactive Liquid (Waste) Disposal (System) \\
\hline RPP & River Protection Project (at the DOE Hanford Site) \\
\hline s or sec & Second \\
\hline SBS & Submerged Bed Scrubber - source of LAW and HLW melter recycle streams \\
\hline SIPP & Semi-Integrated Pilot Plant \\
\hline SRNL & Savannah River National Laboratory (part of WSRC) \\
\hline SS & Suspended solids \\
\hline SSM & Steady-State Matrix (Applies to Campaign I only - see Fig. 33) \\
\hline Std Dev, $\sigma$ & Standard Deviation \\
\hline TLP & Treated (Feed) LAW Process (part of the RPP-WTP Pretreatment Facility) \\
\hline TS & Total Solids \\
\hline TMP & $\begin{array}{l}\text { Transmembrane Pressure (the average pressure drop across the thickness } \\
\text { of the filter medium - perpendicular to the slurry flow.) }\end{array}$ \\
\hline TRU & Transuranic \\
\hline UDS & Undissolved Solids \\
\hline UF & Ultrafiltration \\
\hline UFP & Ultrafiltration Process (part of the RPP-WTP Pretreatment Facility) \\
\hline V & Velocity of the slurry flow along the length of the filter tubes \\
\hline VSL & Vitreous State Laboratory (of Catholic University of America, Wash. DC) \\
\hline WGI & Washington Group International \\
\hline WTP & Waste \& Immobilization Treatment Plant \\
\hline WSRC & Westinghouse Savannah River Company \\
\hline XRD & $\mathrm{X}$-ray diffraction \\
\hline
\end{tabular}

Also note: Within this report, supernate refers to the liquid (undissolved solids-free) portion of a slurry sample. When referring to the ultrafiltration process, the permeate is synonymous with the slurry supernate; either term can thus be used, but permeate will be the preferred term herein. For the evaporation operations, the liquid fraction of the slurry feed or bottoms product will be called the supernate. 


\begin{abstract}
Campaign II, the second step towards the Semi Integrated Pilot Plant (SIPP) task, done by the Savannah River National Laboratory (SRNL) at the Savannah River Site, was to take the several recycle streams produced in Campaign I, the first step of the task, and combine them with other simulated recycle and chosen waste streams. The combined stream was processed in a fashion that mimicked the pretreatment operations of the DOE River Protection Project - Waste Treatment and Immobilization Plant (RPP-WTP). The SIPP task is considered semi-integrated because it only deals with the pretreatment operations of the RPP-WTP. That is, the pilot plant starts by receiving waste from the tank farm and ends when waste is processed to the point of being sent for vitrification. The resulting pretreated LAW and HLW simulants produced by the SIPP are shipped to $\mathrm{VSL}^{\dagger}$ for vitrification in pilot WTP melters.

Within the SIPP task these steps are referred to as Campaigns and there are four Campaigns in all. Campaign I, which is completely different than other campaigns, subjected a simulant of Hanford Tank 241-AY-102/C-106 (AY102) waste to cross-flow ultrafiltration only and in that process several important recycle streams were produced as a result of washing the simulant and cleaning the cross-flow filter. These streams are to feed subsequent campaigns and that work was the subject of the issued Campaign I interim report (Duignan et al. 2004).
\end{abstract}

The operation of each of the subsequent campaigns, i.e., II, III, and IV, while different from Campaign I, are very similar to each other, and can be best understood as the process of operating a series of Pretreatment Unit Operations in a somewhat prototypic manner. That is, while Campaign I studied the operation of a single, albeit important, Pretreatment Unit Operation, i.e., Ultrafiltration, all subsequent campaigns are to study the four major unit operations that make-up the RPP-WTP Pretreatment Facility. They are: Waste Feed Evaporation Process (FEP), Ultrafiltration Process (UFP), Cesium Ion Exchange Process (CIX), and the Treated LAW Evaporation Process (TLP). Each of the campaigns operate basically as a separate subtask, but as with Campaign I, the recycle streams produced in one campaign feeds into the subsequent campaign. Therefore, all four campaigns are chemically connected through these recycle streams, which carry over effects of the preceding campaign.

The results of Campaign II operations are the subject of this second interim report. The reports are called interim because they are limited to the results obtained from a single campaign (or past campaigns) and further limited to only highlights of that single campaign. A final report will follow Campaign IV and its intent is to comprehensively cover the overall task activities, as well as the full database accumulated and the equipment used during the yearlong SIPP task.

SIPP is a limited version of the plant's pretreatment facility in that it tests pilot-scale unit operations of FEP, UFP, CIX, and TLP only. However, an integral part of SIPP is to produce the many recycle streams that must also be processed with stored tank farm

\footnotetext{
${ }^{\dagger}$ Vitreous State Laboratory of the Catholic University of America
} 
waste. That is, it is very important to know the impact that the recycle streams will have on the operation of the several unit operations so as to better model the plant's overall pretreatment throughput, leading to a production rate of stabilized waste.

This report will begin with a summary of the overall operation of the task done during Campaign II to understand the layout of this report. This summary is then followed with highlights of the test results.

With input and review from Hanford, SRNL developed a steady-state OLI/ESP model of the RPP-WTP Pretreatment Facility operation. That model's principal role was to determine the amounts of simulated wastes needed for each of the four unit operations. In general, it did well, but was found to be off by approximately $15 \%$ in predicted SIPP volumes due to unknowns in the undissolved solids of the several simulants. For example, the UF feed composition calculated from the model inputs, assuming no dissolution occurs upon mixing, was $2.97 \mathrm{wt} \%$ UDS versus the model value of $2.45 \mathrm{wt} \%$ where dissolution occurred. When this $0.52 \mathrm{wt} \%$ of solids in the slurry is added to the supernate as soluble solids, the supernate dissolved solids content increases from 28.1 to $29.2 \mathrm{wt} \%$, exactly as shown in the model. (This example is explained in detail in Section 2.7.4.2.1 and with Table 30.)

\section{FEP Evaporation}

The entire evaporation process proceeded as designed and a total of 1,125 liters of feed was concentrated to 147 liters. During evaporation a vacuum of $1 \mathrm{psia}(6.9 \mathrm{kPa})$ at approximately $50^{\circ} \mathrm{C}$ was maintained and the average evaporation rate was approximately $0.43 \mathrm{gpm}(1.5 \mathrm{lpm})$. No significant foaming was observed prior to the addition of antifoam agent and during the evaporation process; the froth height remained around $1 \mathrm{ft}$ $(0.3 \mathrm{~m})$. At start-up and unsteady conditions, significant flashing resulted in feed splashing on the lower demister pad. Overall, the composition of the FEP feed was predicable by mass balance of the individual feed streams. The dissolved metals concentrations paralleled the ratios in the AY102 simulant present in the recycles. Finally, most of the oxalate present in the feed was precipitated beginning when approximately $53 \%$ of the feed by volume had been evaporated. The oxalate appears to have precipitated as the $\mathrm{Na}$ salt $\mathrm{Na}_{2} \mathrm{C}_{2} \mathrm{O}_{4}$.

\section{UFP}

The average permeate flux when dewatering from $\sim 4$ to $\sim 24 \mathrm{wt} \%$ was $0.011 \mathrm{gpm} / \mathrm{ft}^{2}$ and when cleaning the filter, $2 \mathrm{M}$ nitric acid was sufficient to increase the $0.1 \mathrm{M} \mathrm{NaOH}$ permeate flux to an order of magnitude larger than that of waste flux. The turbidity of the permeate from dewatering and slurry washing was always lower than $0.5 \mathrm{NTU}$, indicating that the permeate was free of significant solids. Even after 14 days the permeate turbidity increase to 0.56 NTU (see Table 26), which indicated that there was no significant post-filtration precipitation. (However, AY102-only permeate from Campaign I did post-precipitate after 2 months.). Washing of concentrated slurry raised its yield stress from about 10 to $15 \mathrm{~Pa}$, but lowered the consistency from about 23 to 13 $\mathrm{mPa} \cdot \mathrm{s}$ and from the Campaign I data it was found that the yield stress of unwashed slurry is reduced upon extended pumping. The washing data did match previous SRNL and literature leaching and washing data for soluble washable species (see Table 36). 
WSRC-TR-2004-00478, REVISION 0

SRNL-RPP-2004-00075, REVISION 0

Because of substantial amount of heel material during rinsing and cleaning of the filter, the use of a more concentrated $\mathrm{NaOH}$ solution for the third caustic flush during filter cleaning may be warranted since the $\mathrm{pH}$ of the spent third wash was acidic. Finally, at least $92 \%$ of the antifoam remained in the HLW sludge slurry after filtration. However, due to Dow Corning's difficulties in measuring true concentrations of antifoam the measurement uncertainties were very large and submitted samples are being rechecked and will be reported in future SIPP reports.

CIX

The ion exchange resin SuperLig ${ }^{\circledR} 644$ effectively removed cesium from simulated waste and the analytical ability to measure cesium concentrations in five-molar sodium did allow verification of the waste acceptance criterion of 50\% breakthrough from the lead column after 100 Bed Volumes of waste were treated. However, the analytical inability to measure very low cesium concentrations in five-molar sodium did not allow verification of the waste acceptance criterion of $5.2 \mu \mathrm{g} / \mathrm{L}$ from the lag column after 100 $\mathrm{BV}$ of waste was treated. On performing a mass balance, the masses of cesium in and out of the resin beds for Cycles 1 and 2 balanced to within measurement uncertainty. Hydraulically, the pressure drops throughout the two cycles were low and the resin beds permeabilities were approximately the same as were measured for the 24-inch Test (Fowley et al, 2004).

\section{TLP Evaporation}

The Pilot-scale evaporator operated well for the TLP evaporation in concentrating the TLP simulant from an initial density of $1.14 \mathrm{~kg} / \mathrm{L}$ to $1.395 \mathrm{~kg} / \mathrm{L}$ at $25^{\circ} \mathrm{C}(1.360 \mathrm{~kg} / \mathrm{L}$ at $50^{\circ} \mathrm{C}$ ). There was no significant foaming observed prior to the addition of antifoam agent and during the evaporation process, the froth height remained around $1 \mathrm{ft}(0.3 \mathrm{~m})$. The two Demisters, in series, operated well with no significant differential pressure buildup across the Demisters during the TLP Campaign II. However, condensation had to be purged from the instrumentation lines during the campaign. The composition of the products from the TLP matched what was expected from the mass balance. Finally, no precipitation was noted of additional solids beyond those in the feed. 
WSRC-TR-2004-00478, REVISION 0 SRNL-RPP-2004-00075, REVISION 0

This page intentionally left blank. 


\subsection{TESTING SUMMARY}

\subsection{OBJECTIVES}

Campaign II, one of four planned campaigns for the SIPP task, is the first test that includes all four WTP Pretreatment Facility unit operations, along with the incorporation of recycle waste streams. The objectives listed below are those given in the RPP-WTP Test Specification (Longwell, 2003) for completeness, however, only the body of work approved in the WSRC Technical Task and Quality Assurance Plan (Duignan, 2003c) is required to satisfy this task. Objectives not met because they were not agreed to in the Task Plan are so noted.

\begin{tabular}{|c|c|c|}
\hline Overall Test Objectives & $\begin{array}{l}\text { Objective } \\
\text { Met (Y/N) }\end{array}$ & Discussion \\
\hline $\begin{array}{l}\text { 1. Modify the design and operation of } \\
\text { the existing pretreatment pilot facilities } \\
\text { to extent necessary to mimic the WTP } \\
\text { Pretreatment Facilities. }\end{array}$ & $\mathrm{Y}$ & $\begin{array}{l}\text { Each of the four unit operations that } \\
\text { comprise the SIPP test were either modified } \\
\text { or built to meet planned operation. }\end{array}$ \\
\hline $\begin{array}{l}\text { 2. Operate the (pilot) integrated } \\
\text { facilities using simulated AY102 } \\
\text { (Envelope A/D) and AP101 (Envelope } \\
\text { A) waste feeds. }\end{array}$ & $\mathrm{Y}$ & $\begin{array}{l}\text { Campaign II involved the use of simulated } \\
\text { AY102, AP101, as well as other waste } \\
\text { streams, which were recycle streams. }\end{array}$ \\
\hline $\begin{array}{l}\text { 3. Collect chemical and physical } \\
\text { property data to be defined in the test } \\
\text { plan then refine and validate the } \\
\text { thermodynamic model(s) developed by } \\
\text { SRNL. }\end{array}$ & $\mathrm{Y}$ & $\begin{array}{l}\text { Sufficient property data were obtained to } \\
\text { satisfy project needs as is evidenced by the } \\
\text { customer approval of the Campaign II } \\
\text { sample plan before testing began. }\end{array}$ \\
\hline $\begin{array}{l}\text { 4. Demonstrate pretreatment production } \\
\text { rates required to produce } 80 \text { metric tons } \\
\text { per day of LAW glass and } 5.4 \text { metric } \\
\text { tons of HLW glass. These are contract } \\
\text { defined "expected" production } \\
\text { throughputs for 5-day sprint runs listed } \\
\text { in Table C.6-5.1 of the contract (DOE } \\
2003 \text { ). These required flow rates will } \\
\text { be determined before testing begins by } \\
\text { application of the appropriate scale } \\
\text { factors to the unit operations that } \\
\text { represent an equivalence of } \\
\text { pretreatment throughput to meet } \\
\text { maximum glass production. }\end{array}$ & $\mathrm{N}$ & $\begin{array}{l}\text { The unit production rates were measured but } \\
\text { the targets were not met. The SIPP task was } \\
\text { limited to four separate unit operations that } \\
\text { comprise the principal operations of the } \\
\text { RPP-WTP Pretreatment Facility. The pilot } \\
\text { FEP and TLP evaporation units exceeded } \\
\text { the plant-modeled rate of } 0.395 \text { gpm. The } \\
\text { pilot CIX was subjected to a test exception } \\
\text { (described in Section 1.2), which changed } \\
\text { this objective. The pilot CIX goal was no } \\
\text { longer to show production rate, but to } \\
\text { demonstrate cesium removal efficiency. } \\
\text { The prototypic LAW resin bed residence } \\
\text { time took precedence over production rate, } \\
\text { and thus production throughput rate was not } \\
\text { demonstrated. As shown in Campaign I, the } \\
\text { pilot ultrafiltration permeate rate during } \\
\text { dewatering from 3.4 to } 20 \text { wt\% UDS was } \\
0.015 \text { gpm/ft2. This rate is below the target } \\
\text { rate of } 0.02 \text { gpm/ft2, but is representative of } \\
\text { the filter process since a similar flux rate } \\
\text { was obtained for both Campaign I, with no } \\
\text { recycle streams, and Campaign II, which } \\
\text { included several recycle streams. }\end{array}$ \\
\hline
\end{tabular}


WSRC-TR-2004-00478, REVISION 0 SRNL-RPP-2004-00075, REVISION 0

\begin{tabular}{|c|c|c|}
\hline FEP/TLP Test Objectives & $\begin{array}{l}\text { Objective } \\
\text { Met (Y/N) }\end{array}$ & Discussion \\
\hline $\begin{array}{l}\text { 1. Operate the Waste Feed Evaporator } \\
\text { and the Treated LAW Evaporator using } \\
\text { simulants derived from the integrated } \\
\text { operation of the pretreatment pilot } \\
\text { plant. }\end{array}$ & $\mathrm{Y}$ & $\begin{array}{l}\text { Feeds for both the FEP and TLP evaporators } \\
\text { included derived simulants or recycle } \\
\text { streams produced during SIPP operations. }\end{array}$ \\
\hline $\begin{array}{l}\text { 2. Recycle secondary waste generated } \\
\text { from the ultrafiltration pilot operations } \\
\text { and the ion exchange unit operation to } \\
\text { the evaporators. }\end{array}$ & $\mathrm{Y}$ & $\begin{array}{l}\text { Recycle streams from the ultrafiltration pilot } \\
\text { operation from Campaign I were fed to the } \\
\text { FEP evaporator. However, the test } \\
\text { specification indicated that no pilot CIX } \\
\text { secondary streams will be processed. } \\
\text { [Longwell, 2003: section } 6.2,6^{\text {th }} \text { paragraph]. }\end{array}$ \\
\hline $\begin{array}{l}\text { 3. Operate the pilot evaporator using } \\
\text { simulated HLW Submerged Bed } \\
\text { Scrubber solution, simulated } \\
\text { Pretreatment Caustic Scrubbers, ion } \\
\text { exchange recycle solutions, and } \\
\text { ultrafiltration recycles generated from } \\
\text { pilot operations. }\end{array}$ & $\mathrm{Y}$ & $\begin{array}{l}\text { Campaign II used all the streams indicated } \\
\text { except those produced from the pilot CIX. } \\
\text { Pilot CIX recycle streams will be used for } \\
\text { Campaign III. }\end{array}$ \\
\hline $\begin{array}{l}\text { 4. Operate the pilot evaporator using } \\
\text { simulated LAW Submerged Bed } \\
\text { Scrubber solution and ion exchange } \\
\text { treated feed to produce LAW feed } \\
\text { solution. }\end{array}$ & $\mathrm{Y}$ & See the preceding response. \\
\hline $\begin{array}{l}\text { 5. Compare the results (solubility, } \\
\text { chemical, and physical properties, } \\
\text { which will be indicated in the test plan) } \\
\text { developed on the pilot scale with } \\
\text { results developed by experiments } \\
\text { conducted at the bench scale. }\end{array}$ & $\mathrm{N}$ & $\begin{array}{l}\text { However, any extant bench-scale data will } \\
\text { be used for such comparisons in the final } \\
\text { report. }\end{array}$ \\
\hline $\begin{array}{l}\text { 6. Determine the chemical and physical } \\
\text { properties of simulants evaporated at } \\
\text { the pilot scale as a function of sodium } \\
\text { concentration and temperature. }\end{array}$ & $\mathrm{N}$ & $\begin{array}{l}\text { The approved task plan did not require these } \\
\text { property vs. temperature evaluations. } \\
\text { However, densities and sodium } \\
\text { concentrations were measured and included } \\
\text { in this report. }\end{array}$ \\
\hline $\begin{array}{l}\text { 7. Evaluate the selected antifoam } \\
\text { reagent performance in the evaporators. }\end{array}$ & $\mathrm{Y}$ & $\begin{array}{l}\text { Antifoam was added to both evaporators } \\
\text { and those results are discussed herein. }\end{array}$ \\
\hline $\begin{array}{l}\text { 8. Evaluate the effect of in-leakage on } \\
\text { air emissions. }\end{array}$ & $\mathrm{N}$ & $\begin{array}{l}\text { This work was not included in the approved } \\
\text { task plan due to cost. }\end{array}$ \\
\hline $\begin{array}{l}\text { 9. Determine the chemical and physical } \\
\text { properties of concentrated recycle and } \\
\text { waste blends, which will be indicated } \\
\text { in the test plan, to be sent for } \\
\text { precipitation/ultrafiltration. }\end{array}$ & $\mathrm{Y}$ & $\begin{array}{l}\text { The concentrated bottoms streams were } \\
\text { analyzed and discussed herein. }\end{array}$ \\
\hline $\begin{array}{l}\text { 10. Operate the pilot evaporator unit } \\
\text { assuming a full-scale WTP } \\
\text { Pretreatment process flow rate of } \\
\text { 80/5.4 MTG/d. }\end{array}$ & $\mathrm{Y}$ & $\begin{array}{l}\text { Both evaporators were run at a rate of better } \\
\text { than the plant rate of } 0.395 \text { gpm. Which was } \\
\text { based on a production rate of } 80 / 6 \text { MTG/d } \\
\text { (Calloway, 2004). }\end{array}$ \\
\hline
\end{tabular}


WSRC-TR-2004-00478, REVISION 0 SRNL-RPP-2004-00075, REVISION 0

\begin{tabular}{|c|c|c|}
\hline UFP Test Objectives & $\begin{array}{l}\text { Objective } \\
\text { Met (Y/N) }\end{array}$ & Discussion \\
\hline $\begin{array}{l}\text { 1. Operate with prototypical } \\
\text { wash/leach/wash/steps using simulated } \\
\text { AY102 and AP101 and concentrated } \\
\text { evaporator recycles. }\end{array}$ & $\mathrm{Y}$ & $\begin{array}{l}\text { The approved task plan made the leach } \\
\text { step optional, pending RPP-WTP guidance. } \\
\text { Before testing began RPP-WTP decided } \\
\text { against doing the leach step. All other } \\
\text { objectives were met. }\end{array}$ \\
\hline $\begin{array}{l}\text { 2. Operate with } \\
\text { prototypical/recommended } \\
\text { Ultrafiltration cleaning method. }\end{array}$ & $\mathrm{Y}$ & $\begin{array}{l}\text { RPP-WTP recommended continued use of } \\
2 \text { M nitric acid for cleaning, which was } \\
\text { done. }\end{array}$ \\
\hline $\begin{array}{l}\text { 3. Determine the chemical and physical } \\
\text { properties of the concentrated solids, } \\
\text { washed solids, leached solids, and final } \\
\text { solids product derived from each } \\
\text { campaign. }\end{array}$ & $\mathrm{Y}$ & $\begin{array}{l}\text { Properties of the concentrates were } \\
\text { measured. However no leaching was } \\
\text { performed as requested by the RPP-WTP } \\
\text { customer. }\end{array}$ \\
\hline $\begin{array}{l}\text { 4. Determine the chemical and physical } \\
\text { properties of permeate, recycles, and } \\
\text { cleaning solutions, which will be } \\
\text { indicated in the test plan, derived from } \\
\text { each campaign. }\end{array}$ & $\mathrm{Y}$ & $\begin{array}{l}\text { Properties of those streams were measured } \\
\text { and discussed herein. }\end{array}$ \\
\hline $\begin{array}{l}\text { 5. Compare the results developed on } \\
\text { the pilot scale with results developed } \\
\text { by experiments conducted at the bench } \\
\text { scale to assess the effect of recycles. }\end{array}$ & $\mathrm{N}$ & $\begin{array}{l}\text { No bench scale experiments were done to } \\
\text { study the effects of recycles. }\end{array}$ \\
\hline $\begin{array}{l}\text { 6. Provide pump data (pump curves) } \\
\text { for the high solid content slurries. }\end{array}$ & $\mathrm{N}$ & $\begin{array}{l}\text { This requirement was not included in the } \\
\text { approved task plan. }\end{array}$ \\
\hline $\begin{array}{l}\text { 7. Demonstrate operation of the } \\
\text { Ultrafiltration unit assuming a full- } \\
\text { scale WTP Pretreatment process flow } \\
\text { rate corresponding to production of } \\
80 / 5.4 \text { MTG/d. }\end{array}$ & $\mathrm{N}$ & $\begin{array}{l}\text { See response } 4 \text { to the Overall Test } \\
\text { Objectives, above. }\end{array}$ \\
\hline $\begin{array}{l}\text { 8. Evaluate the downstream effects of } \\
\text { antifoam reagent. Determine the fate } \\
\text { of the reagent by tracking its } \\
\text { concentration through the integrated } \\
\text { pilot. }\end{array}$ & $\mathrm{N}$ & $\begin{array}{l}\text { The effect on filter flux seemed to be } \\
\text { insignificant, but it is discussed herein } \\
\text { along with that data used to track the } \\
\text { antifoam. }\end{array}$ \\
\hline
\end{tabular}

\begin{tabular}{|c|c|c|}
\hline CIX Test Objectives & $\begin{array}{l}\text { Objective } \\
\text { Met (Y/N) }\end{array}$ & Discussion \\
\hline $\begin{array}{l}\text { 1. Operate the pilot ion exchange unit } \\
\text { using ultrafiltration permeate produced } \\
\text { using simulated AY102 and AP101. }\end{array}$ & $\mathrm{Y}$ & $\begin{array}{l}\text { This was done along with the bottoms } \\
\text { produced by evaporating the several } \\
\text { recycle streams. }\end{array}$ \\
\hline $\begin{array}{l}\text { 2. Operate the pilot ion exchange unit } \\
\text { assuming a full-scale WTP } \\
\text { Pretreatment process flow rate } \\
\text { corresponding to production of } 80 / 5.4 \\
\text { MTG/d. }\end{array}$ & $\mathrm{N}$ & $\begin{array}{l}\text { Test Exception 24590-WTP-TEF-RT-03- } \\
069 \text { required the pilot CIX show prototypic } \\
\text { cesium removal. This required matching } \\
\text { the Plant's treated waste residence time in } \\
\text { the resin and not the full-scale flow rate. } \\
\text { Therefore, this objective could not be met. }\end{array}$ \\
\hline $\begin{array}{l}\text { 3. Demonstrate ion exchange column } \\
\text { operating parameters using the } \\
\text { recommended ion exchange resin. }\end{array}$ & $\mathrm{Y}$ & $\begin{array}{l}\text { The currently planned resin, } \\
\text { SuperLig }{ }^{\circledR} 644 \text { was employed. }\end{array}$ \\
\hline
\end{tabular}


WSRC-TR-2004-00478, REVISION 0

SRNL-RPP-2004-00075, REVISION 0

\begin{tabular}{|l|l|l|}
\hline CIX Test Objectives & $\begin{array}{l}\text { Objective } \\
\text { Met (Y/N) }\end{array}$ & Discussion \\
\hline $\begin{array}{l}\text { 4. Determine the chemical and physical } \\
\text { properties, which will be indicated in } \\
\text { the test plan, of all ion exchange } \\
\text { effluents. }\end{array}$ & Y & $\begin{array}{l}\text { Properties of those streams were measured } \\
\text { and discussed herein. }\end{array}$ \\
\hline $\begin{array}{l}\text { 5. Compare the results developed on } \\
\text { the pilot scale with results developed } \\
\text { by experiments conducted at the bench } \\
\text { scale to assess the effect of recycles. }\end{array}$ & $\mathrm{N}$ & $\begin{array}{l}\text { No bench scale experiments were done to } \\
\text { study the effects of recycles. }\end{array}$ \\
\hline $\begin{array}{l}\text { 6. Determine the chemical and physical } \\
\text { properties, which will be indicated in } \\
\text { the test plan, of blended ion exchange } \\
\text { secondary wastes (pre-elution rinse and } \\
\text { spent regenerant) that are recycled back } \\
\text { to the waste feed evaporation system. }\end{array}$ & $\mathrm{Y}$ & $\begin{array}{l}\text { No pilot CIX recycle streams were } \\
\text { required for Campaign II pilot waste feed } \\
\text { evaporation, as per the approved Task } \\
\text { Plan, but samples were taken from the } \\
\text { streams that will be used for Campaign III. } \\
\text { Those results are discussed herein. }\end{array}$ \\
\hline $\begin{array}{l}\text { 7. Determine the chemical and physical } \\
\text { properties of ion exchange eluates, } \\
\text { which will be indicated in the test plan. }\end{array}$ & $\mathrm{Y}$ & $\begin{array}{l}\text { Properties of those streams were measured } \\
\text { and discussed herein. }\end{array}$ \\
\hline $\begin{array}{l}\text { 8. Evaluate the potential effects of the } \\
\text { evaporator antifoam reagent on ion } \\
\text { exchange performance. }\end{array}$ & $\mathrm{Y}$ & $\begin{array}{l}\text { The effect on ion exchanger seemed to be } \\
\text { insignificant, based on good cesium } \\
\text { removal. }\end{array}$ \\
\hline
\end{tabular}

\subsection{TEST EXCEPTIONS}

\begin{tabular}{|l|l|}
\hline List Test Exceptions & Describe Test Exceptions \\
\hline $\begin{array}{l}\text { 1. 24590-WTP-TEF-RT-02-025, } \\
\text { Rev. 0, 20 May 2002 }\end{array}$ & See Test Exception No. 7. \\
\hline $\begin{array}{l}\text { 2. 24590-WTP-TEF-RT-02-046, } \\
\text { Rev. 0, 01 Aug. 2002 }\end{array}$ & See Test Exception No. 7. \\
\hline $\begin{array}{l}\text { 3. 24590-WTP-TEF-RT-03-004, } \\
\text { Rev. 0, 07 Feb. 2003 }\end{array}$ & See Test Exception No. 7. \\
\hline $\begin{array}{l}\text { 4. 24590-WTP-TEF-RT-03-037, } \\
\text { Rev. 0, 30 Jul. 2003 }\end{array}$ & See Test Exception No. 7. \\
\hline $\begin{array}{l}\text { 5. 24590-WTP-TEF-RT-03-037, } \\
\text { Rev. 1, 17 Sept. 2003 }\end{array}$ & See Test Exception No. 7. \\
\hline $\begin{array}{l}\text { 6. 24590-WTP-TEF-RT-03-054, } \\
\text { Rev. 0, 17 Sept. 2003 }\end{array}$ & See Test Exception No. 7. \\
\hline $\begin{array}{l}\text { 7. 24590-WTP-TEF-RT-03-069, } \\
\text { Rev. 0, 14 Nov. 2003 }\end{array}$ & $\begin{array}{l}\text { As of the completion of Campaign II there was no test exception to } \\
\text { the SIPP task plan (Duignan et al., 2004). All of the listed test } \\
\text { exceptions, 1 though 7, affect only a task plan dealing solely with } \\
\text { ion exchange operation (Steimke, 2002). For completeness, all the } \\
\text { test exceptions were listed but only this seventh test exception } \\
\text { impacts SIPP test. As with all the other pilot unit operation the pilot } \\
\text { CIX was to operate in a fashion to demonstrate the rate of glass } \\
\text { production. However, RPP-WTP thought it was more important to } \\
\text { demonstrate cesium removal efficiency. Since prototypic column } \\
\text { flow rates and prototypic resin residence time must be different for } \\
\text { other than full-scale sizes. The pilot unit was not used at a LAW } \\
\text { flow rate that would demonstrate glass production rates. This } \\
\text { seventh test exception describes how the pilot scale IX columns } \\
\text { would operate to demonstrate cesium removal. That removal } \\
\text { efficiency is what is discussed herein. }\end{array}$ \\
\hline
\end{tabular}




\subsection{RESULTS AND PERFORMANCE AGAINST SUCCESS CRITERIA}

\begin{tabular}{|l|l|}
\hline List Success Criteria & $\begin{array}{l}\text { Explain How the Tests Did or Did Not } \\
\text { Meet the Success Criteria }\end{array}$ \\
\hline $\begin{array}{l}\text { 1. The integrated operation of the pretreatment } \\
\text { process produces vitrification feed streams similar } \\
\text { in chemical composition to those produced in bench } \\
\text { scale experiments. }\end{array}$ & $\begin{array}{l}\text { Bench scale experiments were not part of the SIPP } \\
\text { scope. However, the SIPP task did produce both } \\
\text { LAW and HLW feed streams with simulated waste } \\
\text { and recycle streams that were processed in the } \\
\text { same manner expected for the RPP-WTP } \\
\text { Pretreatment Facility. The chemical composition } \\
\text { obtained is that which is expected for the full-scale } \\
\text { facility and therefore this criterion was met. }\end{array}$ \\
\hline $\begin{array}{l}\text { 2. The integrated pretreatment process demonstrates } \\
\text { a scaled throughput of 80 MTG/d of LAW and 5.4 } \\
\text { MTG/d of HLW. }\end{array}$ & $\begin{array}{l}\text { This success criterion was partially met in that both } \\
\text { the pilot FEP and TLP operated at, or above the } \\
\text { evaporation rate (0.395 gpm) necessary to meet the } \\
\text { stated glass production rates. The pilot UFP } \\
\text { realized a dewatering filter flux rate of 0.015 } \\
\text { gpm/ft }{ }^{2} \text { This value is below the rate needed meet } \\
\text { the glass production rates, but is representative of } \\
\text { rate obtained from bench scale test with actual } \\
\text { waste. The pilot CIX rate purposely did not meet } \\
\text { the full-scale plant rate, because it was required to } \\
\text { operate at a slower rate that matched the residence } \\
\text { time the plant waste will have in the resin. }\end{array}$ \\
\hline
\end{tabular}

\subsection{QUALITY REQUIREMENTS}

This work was conducted in accordance with the RPP-WTP QA requirements specified for work conducted by SRNL as identified in DOE IWO MOSRLE60. SRNL has provided matrices to WTP, demonstrating compliance of the SRNL QA program with the requirements specified by WTP. Specific information regarding the compliance of the SRNL QA program with RW-0333P, Revision 10, NQA-1 1989, Part 1, Basic and Supplementary Requirements and NQA-2a 1990, Subpart 2.7 is contained in these matrices. 
WSRC-TR-2004-00478, REVISION 0

SRNL-RPP-2004-00075, REVISION 0

\subsection{R\&T TEST CONDITIONS}

\begin{tabular}{|c|c|}
\hline List R\&T Test Conditions & Were Test Conditions Followed? \\
\hline $\begin{array}{l}\text { 1. SRNL shall prepare a specific test plan } \\
\text { containing detailed information needed to } \\
\text { implement (the) test specification. The test plan } \\
\text { shall also include, as appropriate, references to } \\
\text { documents that drive test documentation } \\
\text { requirements to assure data integrity and } \\
\text { reproducibility. This would include such items as } \\
\text { detailed test procedures, instrument calibration, } \\
\text { methods of data gathering, etc. The test plan shall } \\
\text { indicate what methodologies will be employed to } \\
\text { verify or validate data produced during testing } \\
\text { activities. } \\
\text { A draft test plan shall be submitted to WTP R \& T } \\
\text { for review and comment. Comments labeled } \\
\text { "Mandatory" by the reviewer require a disposition. } \\
\text { The changes in the test plan shall be provided } \\
\text { electronically and clearly identified by } \\
\text { redline/strikeout or highlighting to facilitate the } \\
\text { review process. } \\
\text { The final test plan must be approved by a WTP R \& } \\
\text { T representative prior to performing work. }\end{array}$ & $\begin{array}{l}\text { As required a test plan was developed (Duignan, } \\
\text { 2003c) to meet the test specification. All analytes } \\
\text { that could not meet the listed criterion were } \\
\text { indicated in the task plan and approved before } \\
\text { testing began. Documents that were available } \\
\text { before and when the test plan was completed were } \\
\text { referenced therein, but those that were not, e.g. } \\
\text { procedures, calibration, will be references as they } \\
\text { become available in the subsequent task reports. } \\
\text { The test plan was submitted to WTP R\&T for } \\
\text { review and approval. All reviewers' comments } \\
\text { were addressed and disposition, after which the } \\
\text { report was approved by WTP R\&T prior to testing, } \\
\text { thus it became the principal document to carry out } \\
\text { testing. }\end{array}$ \\
\hline $\begin{array}{l}\text { 2. Volumetric waste-to-recycle ratios will be } \\
\text { calculated using appropriate WTP's Aspen Custom } \\
\text { Modeler (ACM) Model runs as a basis for the } \\
\text { combined feed stream. The block diagram to be } \\
\text { used for this work is depicted in Figure } 1 \text {. Simulant } \\
\text { AY102 feed rate will be established based on the } \\
\text { requirement for scaled } 6 \text { MTG/d HLW production, } \\
\text { and AP101 feed rate will be based on the additional } \\
\text { requirement to produce scaled } 80 \text { MTG/d LAW } \\
\text { production. Tankage required for execution of the } \\
\text { work will be provided and incorporated as required } \\
\text { by [SRNL]. }\end{array}$ & $\begin{array}{l}\text { As required, SRNL developed an OLI model based } \\
\text { on several sources, included ACM and sources for } \\
\text { ACM prior to any SIPP testing. The modeling } \\
\text { results were compared to ACM results by both } \\
\text { SRNL and RPP-WTP personnel. The OLI results } \\
\text { were scaled to SIPP operation to assist in } \\
\text { completing a test plan and to determine waste } \\
\text { stream and recycle stream volumetric needs. The } \\
\text { model was also used to determine scaled flowrates } \\
\text { of each of the four unit operation in order to know } \\
\text { what operation rate were necessary to meet full- } \\
\text { scale plant glass production rates of } 80 \text { MTG/d } \\
\text { from LAW and } 5.4 \text { MTG/d from HLW. }\end{array}$ \\
\hline $\begin{array}{l}\text { Prior to initiation of the test campaigns, the system } \\
\text { will be simulated using the OLI Environmental } \\
\text { Simulation Program (ESP) models developed in } \\
\text { previous completed evaporation tasks. The } \\
\text { expanded waste feed evaporation model, which } \\
\text { includes ultrafiltration and recycles, along with the } \\
\text { treated feed evaporation model will simulate the } \\
\text { pilot operations under minimum (48/3.2 MTG/d) } \\
\text { and expected (80/5.4 MTG/d) operating conditions. } \\
\text { Output of the models will then be compared to } \\
\text { above referenced ACM model runs and the data } \\
\text { obtained during the pilot operations. Results of this } \\
\text { modeling effort will be discussed with WTP R\&T } \\
\text { personnel and approval of the path forward obtained } \\
\text { PRIOR TO START of testing. }\end{array}$ & \\
\hline
\end{tabular}


WSRC-TR-2004-00478, REVISION 0

SRNL-RPP-2004-00075, REVISION 0

\begin{tabular}{|l|l|}
\hline List R\&T Test Conditions & Were Test Conditions Followed? \\
\hline $\begin{array}{l}\text { 3. The LAW and HLW SBS recycles will be } \\
\text { simulants generated from VSL/Duratek pilot melter } \\
\text { testing. }\end{array}$ & $\begin{array}{l}\text { Both submerged bed scrubber (SBS) recycle } \\
\text { streams were obtained from VSL and used in } \\
\text { Campaign II testing. }\end{array}$ \\
\hline $\begin{array}{l}\text { 4. The simulant waste feeds will be based on } \\
\text { approved formulations developed under the WTP's } \\
\text { waste simulant program participated in by [SRNL]. }\end{array}$ & $\begin{array}{l}\text { The simulated waste feed AY102 was developed } \\
\text { and validated by SRNL (Zamecnik, et al., 2004). } \\
\text { It was filter tested at the bench scale against an } \\
\text { actual waste sample. The formulation was } \\
\text { approved by WTP R\&T before its use. The } \\
\text { formulation for the simulated feed AP101 was } \\
\text { developed by Battelle (Russell, et al., 2003) and } \\
\text { approved by WTP R\&T. All other waste streams } \\
\text { used for SIPP are either recycle streams or derived } \\
\text { from recycle streams. }\end{array}$ \\
\hline $\begin{array}{l}\text { 5. Generation of initial ultrafiltration recycles will } \\
\text { be accomplished by performing the work specified } \\
\text { in AY102 Simulant Pilot Scale Ultrafiltration and } \\
\begin{array}{l}\text { Washing Test, 24590-PTF-TSP-RT-02-015 } \\
\text { (Townson, 2002). }\end{array}\end{array}$ & $\begin{array}{l}\text { The production of these recycle streams was done } \\
\text { during Campaign I (Duignan et al., 2004). }\end{array}$ \\
\hline $\begin{array}{l}\text { 6. An exception to the test matrix defined in (the } \\
\text { Campaign I test) specification 24590-PTF-TSP-RT- } \\
\text { 02-015 will be to not perform certain tests specified } \\
\text { in Appendix A, Table 1.1 (of that specification). } \\
\begin{array}{l}\text { The tests that will not be performed are (listed in } \\
\text { the SIPP Test Specification, Longwell, 2003.) }\end{array}\end{array}$ & $\begin{array}{l}\text { The tests indicated in the SIPP Test Specification } \\
\text { were not performed. }\end{array}$ \\
\hline
\end{tabular}

\subsection{SIMULANT USE}

Many simulated waste streams were either used or produced during Campaign II testing. Those include Hanford tank farm stored wastes, i.e., tank 241-AY-102/C-106, herein referred to as AY102, and tank 241-AP-101, herein referred to as AP101. During the process of making glass many recycle streams will be generated and processed. Those include streams from the: HLW and LAW Submerged Bed Scrubbers, Vessel Vent System, HLW Glass Canister Decontamination process, FEP and TLP condensate solutions, UFP wash permeate, UFP rinse solutions, UFP acid cleaning solutions, UFP loop flush solutions, CIX caustic LAW displacement solutions, CIX post elution rinse solutions, and CIX caustic regeneration solutions. These streams are discussed throughout this report.

\subsection{DISCREPANCIES AND FOLLOW-ON TESTS}

No discrepancies occurred during testing, but there will be follow-on work. This test, referred to as Campaign II, is the second of four Campaigns, which make up an overall task referred to as Semi Integrated Pilot Plant (SIPP) (Duignan, 2003c). 
WSRC-TR-2004-00478, REVISION 0 SRNL-RPP-2004-00075, REVISION 0

This page intentionally left blank. 


\subsection{DISCUSSION}

\subsection{INTRODUCTION}

Bechtel National, Inc. (BNI) has been contracted by the Department of Energy (DOE) to design a Waste Treatment and Immobilization Plant (WTP) to stabilize liquid radioactive wastes that are stored at the Hanford Site as part of the River Protection Project (RPP). Because of its experience with radioactive waste stabilization, the Savannah River National Laboratory (SRNL) of the Westinghouse Savannah River Company (WSRC) is working with BNI and Washington Group International (WGI), to help design and test certain parts of the waste treatment facility. A major part of the waste stabilization process is the pretreatment of the wastes before vitrification, which will occur in the Pretreatment Facility where both HLW and LAW product streams will be handled. SRNL has constructed a Semi Integrated Pilot Plant (SIPP), which imitates important unit operations of the pretreatment process. However, since the actual pilot-scale vitrification is not handled by SRNL, the pilot plant is referred to as semi integrated. After SIPP pretreats the waste streams into vitrification feed streams, they will be shipped to the Vitreous State Laboratory (VSL) of Catholic University of America complete the entire process.

The subject of this report is the testing of one phase, or campaign, of all of the four principal unit operations that comprise the RPP-WTP Pretreatment Facility, i.e., Waste feed Evaporation Process (FEP), Ultrafiltration Process (UFP), Cesium Ion Exchange Process (CIX), and Treated LAW Evaporation Process (TLP). The operation and results of each of these unit operations are explained herein.

This technical baseline research and development work was initiated by a WGI test specification (Longwell, 2003) to test simulated radioactive wastes in pilot scale pretreatment unit operations in the Engineering Development Laboratory (EDL) of SRNL. This report deals with the second of a four-campaign task, but it is the first campaign to use all four unit operations. Campaign I was at one time a completely separate task (Townson, 2003) to filter a specific waste. Subsequently, that first campaign was incorporated into the SIPP task to eliminate duplication of effort and to produce several important recycle streams. While, Campaign I was limited to a single unit operation, ultrafiltration, and a single waste stream, i.e., AY102, the process of operating the filter unit allowed the production of many of the important recycle streams needed to carry out the more comprehensive Campaign II test. After dewatering the single waste stream, the slurry was washed to produce one recycle stream. Then the filter unit was flushed, cleaned, and rinsed, thus creating other important recycle streams. Besides producing recycles, the Campaign I single-waste filter results were then used to compare with filter results obtained after mixing that waste stream with others, i.e., AP101 and the many recycle streams. A further benefit to limiting Campaign I to a single waste stream was that the filter results could be compared to the pilot filter tests done with other single waste streams, (see Duignan, 2000a, Duignan, 2000b, Duignan, 2003a). 
This report will discuss the four pilot unit operations during Campaign II. There were many individuals involved and the information for each section was provided primarily by each of the principal investigators. They are:

Simulant development and sample analyses John R. Zamecnik

Simulant and sample handling - Michael R. Williams

SIPP Computational OLI/ESP Modeling - C. Dan Barnes

FEP operation - Zafar H. Qureshi

UFP operation - Mark D. Fowley

CIX operation - John L. Steimke

TLP operation - Duane J. Adamson

A summary of EDL task activities is as follows:

WGI Test Specification for UF Test Received - December 2002

WGI Test Specification for SIPP Received - June 2003

WSRC Task Technical \& Quality Assurance Plan Approved - August 2003

Test Procedure Approved - October 2003

Received AY102/C106 Simulant - January 2004

Campaign I - 26 January to 12 February 2004

Campaign II - 12 April to 14 May 2004

\subsection{THE PLAN}

Initial planning for the Semi Integrated Pilot Plant (SIPP) was to decide what to study. One of the first decisions was to limit the task to the RPP-WTP Pretreatment Facility since pilot melter facilities already existed at another site ${ }^{\dagger}$. This is the reason that this task is referred to as "Semi Integrated," i.e., to indicate that vitrification is not part of the pilot operations at the Savannah River National Laboratory (SRNL). The next step was to decide which simulant(s) to test. Since the waste types at Hanford were broadly classified as envelopes A, B, C, and D (Eibling and Nash, 2001), therefore three campaigns were planned, one for each envelope, except D. Envelope D is basically a sludge, which would not be processed alone, but would be mixed with some of the other envelopes. As such, each campaign would process a chosen simulated waste through the principal unit processes of the Pretreatment Facility. Those units are the Feed (Waste) Evaporator Process (FEP), Ultrafiltration Process (UFP), Cesium Exchange (Process) (CIX), the Treated LAW Evaporation Process (TLP), and for Envelope C-types wastes, the Strontium-Transuranics (Sr/TRU) Precipitation Process.

Having a task that would test a range of possible waste types was a good comprehensive approach; however, to obtain meaningful operational data for the plant it was not just a matter of processing each of the different wastes once through each of the unit process. Steady state operation of the Pretreatment Facility would also have to include all the recycle waste streams. That is, in the process of separating the wastes into necessary components for further processing, other waste streams are produced. A few of those

\footnotetext{
${ }^{\dagger}$ The Vitreous State Laboratory at Catholic University of America in Washington, DC.
} 
streams are: waste washings, filter cleaning and rinsing solutions, CIX regeneration solutions, HLW canister decontamination solutions, vessel vent solutions, submerged bed scrubber solutions from both the HLW and LAW vitrification facilities, etc. In steady state these recycle streams will be recycled to different locations in the Pretreatment Facility. Those streams need to be included to simulate the actual plant operation. This means that each of the campaigns would have to run many times until the effects of the recycle streams stabilized so that steady state operation is realized. After reevaluating the task needs, available funds and time, it was clear that the scope had to be reduced. It would be limited to processing a key waste stream with all the recycle streams and then processed enough times that steady-state operation would be attained. Focus was then directed to the wastes that the plant would use for commissioning, i.e., 241-AY-102/ C-106, here referred to as AY102, and 241-AP-101, herein referred to as AP101. The first waste is the result of two waste tanks that have already been mixed together, AY-102 and C-106 and is classified as an Envelope A/D mixture. The second waste, AP101, is an Envelope A waste. With this waste, all of the principal WTP Pretreatment Processes would be tested except the Sr/TRU Process because it did not include an Envelope C-type waste.

The SIPP task was dedicated to perform integrated processing of one selected waste stream combination that included recycle streams produced during the processing of waste through the four pilot-scale unit operations. As such, the testing of an "integrated" process is integrated in the sense of capturing the salient chemical processes, that is, it is important to note that the SIPP is not integrated with respect to time. The operation of the Pretreatment Facility is complex in both chemistry and time, but the many interconnected systems and supporting processes that will exist in the plant to allow the four main unit operation to function were not included in SIPP because of schedule and expense. Just the scaling issues alone, for an entire Pretreatment facility, would have been an almost impossible task to accomplish. In short, running the four principal unit operations with simulated waste and recycle streams as chemically close as to what will result in the plant was accepted as the most efficient use of the time and money available. Time of operation was limited to the operation of each individual unit operation only, and not to their temporal interdependence on the supporting Pretreatment Facility operations, e.g. vessel fillings, waste testing and adjusting, waste transfers, line flushings, etc. However, prototypical hold times (see Appendix B) between the four unit operations were included to provide sufficient time for precipitation of slow forming salts, e.g., Sodium Aluminosilicate and $\mathrm{NaF}_{7}\left(\mathrm{PO}_{4}\right)_{2}$.

To reiterate, the SIPP task is the operation of four individual and separate pilot-scale unit operations, each successively processing a single waste stream combination, which is chemically connected through the feedback of recycle streams. The plan is described in the RPP-WTP test specification (Longwell, 2003) and in the WSRC technical task plan (Duignan, 2003c). The task's original series of three campaigns was maintained, but instead of processing different waste types, only one type would be used. Each successive campaign introduced recycle streams produced from the preceding stream. To feed the first campaign an extra, initial, campaign was added. This addition was actually an already planned ultrafiltration test (Townson, 2002). That test became known as Campaign I and it only processed a simulant of AY102. In this way the filtration results 
of Campaign I served three purposes: 1 . To generate a filtration data set for a singlewaste type that could be compared to past single-waste filtration tests, 2. to become a baseline for filtration of AY102 in order to determine how additions of AP101 and recycles to that waste effect filtration, and 3. to produce a series of recycle streams to feed to Campaign II. The last reason was very important to SIPP because the UFP produces several important recycle streams, i.e., slurry washing permeate, sludge loop flushing, loop acid cleaning, and loop caustic rinsings. Campaign I was performed in January and February of 2004 and the results were published in Duignan et al., 2004. As summary of all the campaigns is as follows:

Campaign I UFP only with simulate of (AY102)

Campaign $\mathrm{II}^{\dagger}$ All four unit operations with simulant of (AY102) + (AP101) + recycle streams (normally the CIX also has recycle streams but since Campaign I did not test the CIX they were not included in Campaign II).

Campaign III All four unit operations with simulant of (AY102) $+\left(\right.$ AP101) $+2^{\text {nd }}$ generation recycle streams (but $1^{\text {st }}$ generation CIX recycle streams).

Campaign IV $V^{\ddagger}$ Two unit operations (FEP and UFP) with simulant of (AY102) + (AP101) $+3^{\text {rd }}$ generation recycle streams (but $2^{\text {nd }}$ generation CIX recycle streams).

As shown in the preceding Section 1.1, on "Test Objectives," there were many objectives; the details of which will not be repeated here, but will be discussed in the appropriate later sections. However, the major goals were to better understand how each of the four pilot-scale unit operation process waste, to study how the processes change with successively complex recycle streams, and to produce throughput data to benchmark computer codes being developed by Bechtel and others, and to predict and plan for, plant operation.

Figure 1 is a schematic of the overall SIPP operation. It is broken into the four unit operations and indicates both the salient chemical and time steps. The operations are listed in a chronological order, at least with respect to the SIPP test, with the top (FEP) section being first. Of course the plant will be much more complex. A very short summary of the overall operation is as follows:

1. FEP evaporates a combined mixture of recycles.

2. UFP filters a combined mixture of the FEP concentrated product and waste from the tank farm until the mixture reaches a target concentration. The UFP produces two streams: the permeate which is sent to the CIX for LAW processing, and the concentrated slurry. That slurry is then washed to remove many of the soluble solids after which it is sent to the HLW melter facility.

3. CIX takes the permeate from the UFP and removes most of the soluble, but radioactive, cesium.

\footnotetext{
${ }^{\dagger}$ This report deals specifically with Campaign II results.

₹ As of May 2004 RPP-WTP informed SRNL to change the scope of Campaign IV to test a new filter made by GKN, which was chosen instead of the Mott for the RPP-WTP Pretreatment Facility Ultrafiltration

Process. The number of the unit operations was reduced from four to two for Campaign IV, those being the pilot-scale FEP and UFP so that GKN performance could be compared to the Mott.
} 
4. TLP takes the CIX product, which still has very low levels of radiation, and evaporates most of the water before it is sent to the LAW melter facility.

The evaporation of the many combined recycle streams in the FEP will be operated in a continuous sense, in that after it fills the UFP preparation tank for one filter train (there will be two UFPs), it will feed the preparation tank for the other filter train. However, the FEP must ultimately be a batch operation because it will be receiving waste from recycle tanks (PWD = Plant Wash and Disposal System) that will be batched through the FEP only after the feed is adjusted for $\mathrm{pH}$ and tested for acceptance.

When the UFP feed and preparation tanks are full and ready for processing, the filtration can commence. This process is also batch, but the feed tank must be continuously fed with new feed as dewatering is ongoing. The feed tank will have a base volume of 21,550 gallons into which approximately 1000 gallons will be transferred from the preparation tank. Each time the feed tank level drops to 21,550 gallons another batch of 1000 gallons will be transferred until the dewatered slurry reaches its target solids concentration. For this task, the target was $20 \mathrm{wt} \%$ insoluble solids. This dewatering process is shown in the UFP section of Figure 1 as the top filtration loop. The middle loop illustrates the washing and leaching of the final concentrated slurry batch. (Note: For SIPP, the leaching process was not performed as requested by RPP-WTP (Calloway, 2004)). After the concentrated waste is washed, it is then sent to the HLW melter for further processing, but for the SIPP, the washed slurry is simply drummed to be shipped for processing in a pilot HLW melter. With the loop de-inventoried, the final loop shows that the UFP undergoes caustic rinsing and acid cleaning, in preparation for the next batch.

For the plant, the CIX does not necessarily have to wait for the entire filtration process to end. There are several filter permeate tanks to feed the CIX and they receive permeate from each of the two filter trains. However, for SIPP the CIX can begin after a fixed amount of permeate is produced from the UFP, which is approximately 200 bed volumes. A bed volume is the volume of space occupied in an IX column by the resin, including the interstitial spaces among the resin particles. The plant will process a minimum of 100 bed volumes ( 30,000 gallons) of permeate before a IX column needs regeneration. Originally, the SIPP IX was to demonstrate the design throughput rate of treated waste, like the other pilot unit operations, but it was thought more important to use it for chemical testing to show cesium removal efficiency. This meant the important parameter would be a prototypic residence time for permeate in the resin, or 3 bed volumes per hour. For the 6-inch diameter pilot scale column, this resulted in a very low flow rate ( $\sim 0.031 \mathrm{gpm}$ ), more than two orders of magnitude slower than the plant design flowrate of 15 gpm. SIPP used 200 bed volumes because several past tests had indicated that the resin may last longer than the original plant design of 100 bed volumes. It was very important to show how long the resin truly lasts before needing to regenerate it. The third section of Fig. 1 shows two columns because of the two processes that occur within CIX: the first process is the removal of cesium, the second processes is a series of steps to regenerate the resin. 
WSRC-TR-2004-00478, REVISION 0

SRNL-RPP-2004-00075, REVISION 0

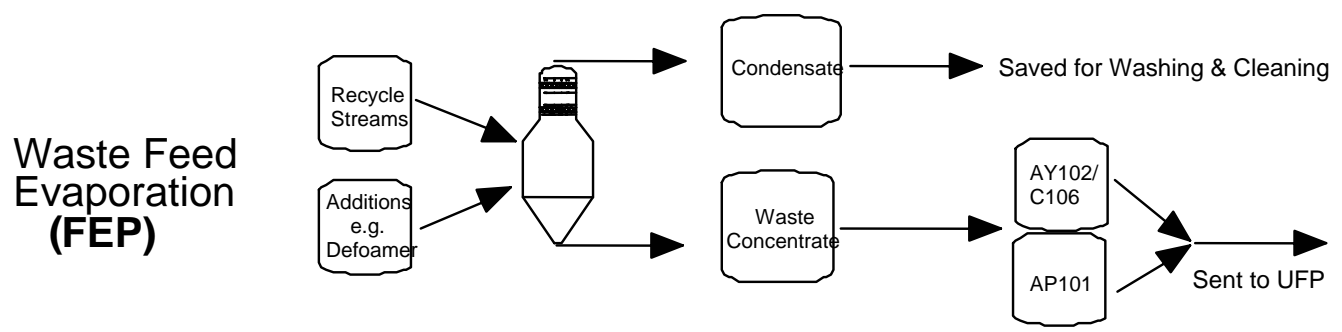

Recycle Streams: HLW SBS, UFP Wash Water, UFP Cleaning Acid, UFP Rinses \& Flushes, HLW Canister Decon, Vessel Vent, and CIX recycles
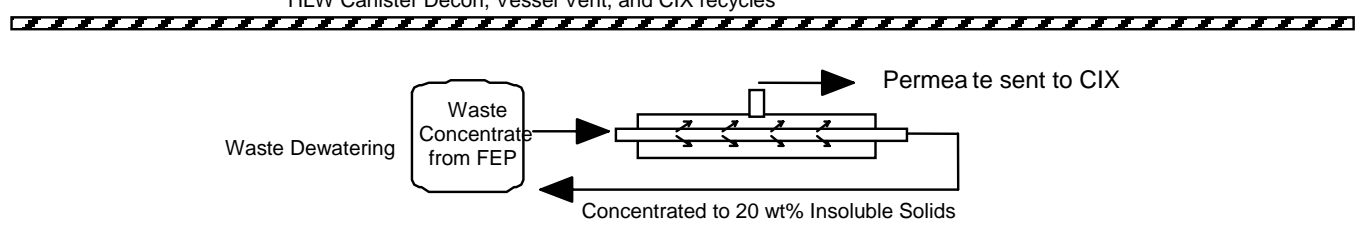

Cross-flow Ultrafiltration waste Washing (UFP)
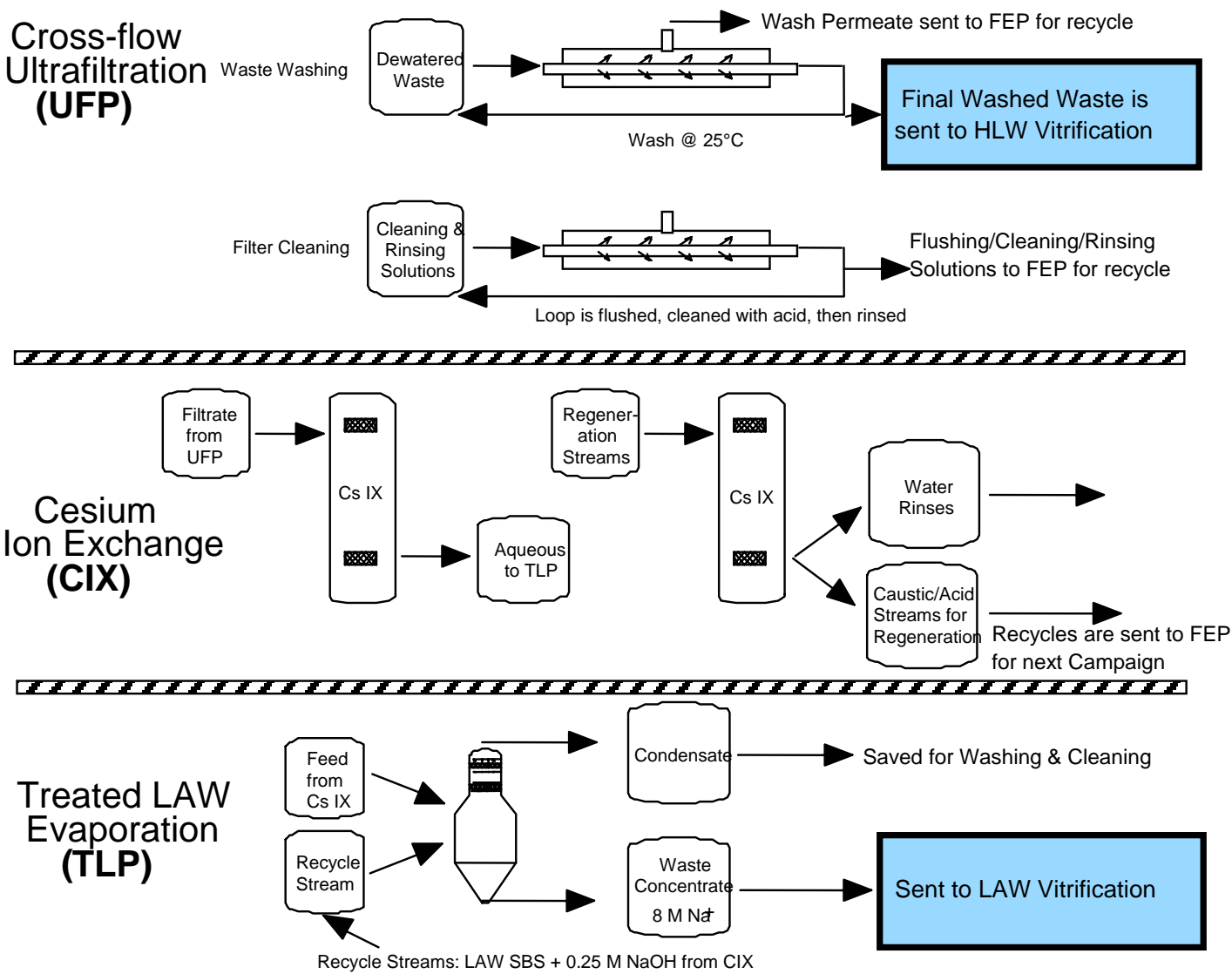

Figure 1. Schematic of the overall task operation taken from the task plan 
The final section of Fig. 1 shows the TLP operation. The CIX product is mostly water and the TLP removes it while concentrating the sodium up to $8 \mathrm{M}$. This is the last step before the treated LAW stream is sent to the LAW melter facility.

Along the way, several recycle streams are created in the UFP and the CIX, as well as from other sources, like the submerged bed scrubber quencher streams from both the HLW and LAW melter facilities. These recycle streams are then fed back in the process, which then repeat. For the SIPP, this repeating results in the different campaigns.

\subsection{THE COMPUTATIONAL MODEL}

In general, this section will not explain the details in how the modeling for SIPP was done, but why it was done and what was done. The how is explained in Appendix A, and the complete set of modeling results can be found in Barnes, 2004. It is important to note that the computational assistance brought to bear for SIPP was only to help in planning, as requested by the Test Specification (Longwell, 2003; in the first two paragraphs of section 6.2 of that document.). There were several RPP extant models of certain aspects of the WTP Pretreatment Facility, but no single model or package that incorporated both dynamic mechanical and chemical operation. Since SIPP was basically four quasisteady-state unit operations and since it was important to obtain fairly accurate chemical compositions as simulated waste was being processed, a steady-state model was therefore developed that could handle the complex chemistries of the treated wastes.

In concept, the operation of the SIPP is straightforward, but determining the exact simulant needs is quite complicated because of the differing scales of each pilot-scale unit operation and the chemical interaction among the recycle streams from all the campaigns. A very important part in developing the test matrix of the task plan was knowing how much simulant to use and how should all the different waste and recycle streams be mixed together. That need was satisfied by computationally predicting SIPP streams for all four campaigns. As an added benefit, once the model was made, it would be possible to evaluate changes in simulant volumes and mixing ratios to determine if the entire SIPP process comes to steady state. Steady state could be shown, at least chemically, by demonstrating that the products produced in SIPP change insignificantly from one campaign to the next.

From the beginning it was clear that an accurate model of the entire operation of Pretreatment Facility was not possible, but to show how SIPP was to operate, model accuracy was really not necessary. For SIPP, the model was to serve two purposes: determine how much slurry to use and what would be the mix ratio of feeds (simulated wastes and recycle streams) for each of the unit operations. The Test Specification requested that the WTP Aspen Custom Modeler (ACM) Model be used to obtain the bases for the combined feed streams, i.e., the feed mix ratios. With the feed stream obtained in this manner an OLI Environmental Simulation Program (OLI/ESP) was used to determine the steady-state operation for each unit operation. 


\section{WSRC-TR-2004-00478, REVISION 0 SRNL-RPP-2004-00075, REVISION 0}

Figure 2 shows the results of the OLI/ESP model that includes WTP accepted mixture ratios for combined feed streams from the ACM model. Once again, details can be found in Appendix A, but some of the salient features are described here.

The principal value in the entire figure is the initial "batch" size of the UFP feed tank. In Table 1 this batch size for the SIPP is shown in red as 140 liters and is listed as "UF1 SLURRY (batch at $20 \mathrm{wt} \%$ UDS).” This waste volume is very significant because it will be the batch size of HLW sent for HLW vitrification. When this batch is dewatered to some predetermined concentration of insoluble solids (it was $20 \mathrm{wt} \%$ for SIPP), then the permeate produced during dewatering will be the batch size of LAW sent for LAW vitrification.

Table 1. Feeds and products for planning SIPP developed for OLI/ESP computer model

\begin{tabular}{|c|c|c|c|c|c|c|}
\hline \multirow{2}{*}{$\begin{array}{l}\text { WTP } \\
\text { Process }\end{array}$} & \multirow[b]{2}{*}{ Stream Type } & \multirow[b]{2}{*}{ Stream Name } & \multicolumn{4}{|c|}{ Campaign (in Liters) } \\
\hline & & & I & II & III & IV \\
\hline \multirow{6}{*}{ FEP } & Feed & UFP \& CIX RECYCLEs (previous campaign) & $\mathrm{N} / \mathrm{A}$ & 583.1 & 602.8 & 602.8 \\
\hline & Recycle & HLW (Submerged Bed Scrubber) & $\mathrm{N} / \mathrm{A}$ & 190.6 & 190.6 & 189.9 \\
\hline & Streams & PVP (Vessel Vent System) & N/A & 220.7 & 220.7 & 220.0 \\
\hline & & HLW DECON & $\mathrm{N} / \mathrm{A}$ & 114.6 & 114.7 & 114.3 \\
\hline & Products & FEP CONDENSATE (to be process wash) & $\mathrm{N} / \mathrm{A}$ & 996.0 & 980.6 & 984.9 \\
\hline & Produced & FEP COOLED BOTTOMS (UFP feed) & N/A & 114.4 & 150.8 & 144.9 \\
\hline \multirow{2}{*}{$\begin{array}{l}\text { UFP } \\
\text { Feeds }\end{array}$} & Feed & AY102 WASTE (at 12 wt\% UDS) & 253.5 & 252.4 & 242.2 & 241.4 \\
\hline & Feed & AP101 WASTE $\left(\right.$ at $\left.\sim 8 \mathrm{M} \mathrm{Na}^{+}\right)$ & $\mathrm{N} / \mathrm{A}$ & 671.3 & 674.4 & 672.1 \\
\hline \multirow{13}{*}{ UFP } & Products & UF1 FILTRATE (routed to CIX, then TLP) & 113.5 & 922.3 & 952.2 & 943.7 \\
\hline & Produced & UF1 SLURRY (batch at 20 wt\% UDS) & 140 & 116.8 & 116.4 & 116.1 \\
\hline & $\begin{array}{l}\text { Batch } \\
\text { Waste }\end{array}$ & $\begin{array}{l}\text { FEP COND WASH (0.01 M NaOH) } \\
19 \mathrm{M} \mathrm{NaOH} \text { (to adjust FEP COND) }\end{array}$ & & $\begin{array}{c}281.1 \\
1.8\end{array}$ & $\begin{array}{c}281.5 \\
0.2\end{array}$ & $\begin{array}{c}280.7 \\
0.2\end{array}$ \\
\hline & Washing & TOTAL CAUSTIC WASH & 338.6 & 282.5 & 281.6 & 280.8 \\
\hline & \multirow{7}{*}{$\begin{array}{c}\text { Filter } \\
\text { Rinsing } \\
\& \\
\text { Cleaning }\end{array}$} & FEP COND ACID (2 M HNO3) & & 86.1 & 85.8 & 85.6 \\
\hline & & $12 \mathrm{M}$ HNO3 (to adjust FEP COND) & & 17.0 & 17.0 & 16.9 \\
\hline & & TOTAL ACID CLEANING & 122.5 & 102.2 & 101.9 & 101.6 \\
\hline & & FEP CONDENSATE TO CAUSTIC RINSE & & 101.8 & 101.5 & 101.2 \\
\hline & & $19 \mathrm{M} \mathrm{NaOH}$ (to adjust FEP COND) & & 0.5 & 0.5 & 0.5 \\
\hline & & TOTAL CAUSTIC RINSE (0.1 M NaOH) & & 102.2 & 101.8 & 101.5 \\
\hline & & TOTAL CAUSTIC RINSE (for FEP recycle)) & 117.1 & 97.6 & 97.3 & 97.0 \\
\hline & Final Filter & HLW SLURRY (to HLW melter) & 138.1 & 110.8 & 110.3 & 110.0 \\
\hline & Products & TOTAL FEP RECYCLE (for next campaign) & 583.1 & 602.8 & 604.8 & 602.3 \\
\hline \multirow{4}{*}{$\mathrm{CIX}$} & Column & CIX 0.1 M NaOH RINSE (for FEP recycle) & $\mathrm{N} / \mathrm{A}$ & 36.9 & 38.1 & 37.8 \\
\hline & Regeneration & CIX DI WATER (for FEP recycle) & & 36.9 & 38.1 & 37.8 \\
\hline & Products & $\mathrm{CIX} 0.25 \mathrm{M} \mathrm{NaOH}$ (for FEP recycle) & $\mathrm{N} / \mathrm{A}$ & 40.0 & 41.3 & 40.9 \\
\hline & Produced & $\mathrm{CIX} 0.25 \mathrm{M} \mathrm{NaOH}$ (for TLP recycle) & $\mathrm{N} / \mathrm{A}$ & 36.9 & 38.1 & 37.8 \\
\hline \multirow{3}{*}{ TLP } & Feeds wFiltrate & LAW SBS (Submerged Bed Scrubber) & $\mathrm{N} / \mathrm{A}$ & 833.0 & 832.9 & 830.2 \\
\hline & Products & TLP CONDENSATE (to be process wash) & N/A & 1214.1 & 1231.4 & 1224.9 \\
\hline & Produced & TLP COOLED BOTTOMS (to LAW melter) & $\mathrm{N} / \mathrm{A}$ & 589.3 & 603.4 & 598.1 \\
\hline
\end{tabular}


This SIPP batch of 140 liters $^{\dagger}$ for Campaign I was arbitrarily chosen, but once chosen all other volumes were the result of SIPP processing. The choice was made by using existing test facilities, where the pilot UFP could contain 140 liters and still have enough room to feed in more slurry until dewatering is complete. Knowing this batch size, using the feed stream ratios from the ACM model, and the chemical makeup of the base waste slurry, i.e., AY102/C106 and AP101, the OLI/ESP model could then be iterated among all of the campaigns to result in the numbers shown in Table 1. Note that the batch size of 140 liters, with $20 \mathrm{wt} \%$ insoluble solids, changes from Campaign I to a value of approximately 116 liters (with $20 \mathrm{wt} \%$ insoluble solids) for all the other campaigns, and as such, the 116-liter volume was taken as equivalent to the full scale UFP batch ${ }^{\ddagger}$ size in those campaigns. The difference between the batch size for Campaign I (140 L) and the subsequent campaigns (116 L) was not well understood when the task was planned but because the sophisticated OLI/ESP software includes all the steady state chemistry of the processed wastes, which included dozens of compounds, it was assumed that differences in the starting waste slurries resulted in this "discrepancy." That is, Campaign I only processed a single waste, AY102, while the other campaigns included also AP101 and the concentrated recycle streams from the FEP. The inputs for the known waste chemistries, of the starting feeds, were checked many times to verify accuracy, therefore the difference was assumed to be real. In any case, the purpose of the model was to be used as a tool to plan the task and procure needed quantities of simulant, therefore further investigations on the ( $15 \%)$ difference ${ }^{*}$ were not thought necessary. The simulated waste products were procured with enough safety margin to cover any problems if, for instance, shortages occurred during experimentation.

As shown in Fig. 1, once the UFP is finished concentrating, the waste is washed with approximately $0.01 \mathrm{M} \mathrm{NaOH}$. The plant will hold the slurry volume constant and feed in two volumes of the caustic solution, each volume approximately equal to the volume of slurry, before sending the waste to the HLW Vitrification Facility. While the plant will hold the UFP volume constant, the model resulted in slightly smaller volumes, see the line in Table 1 entitled, "HLW Slurry (to HLW melter).” due to computational requirements. These are the simulated processed feeds for HLW vitrification. The other highlighted lines in Table 1 are: "AY102 WASTE (at 12 wt\% UDS)," which was the

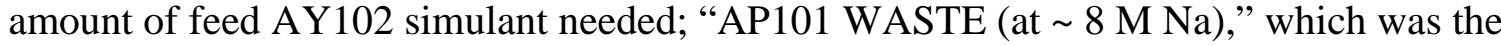
amount of feed AP101 simulant needed; "UFP \& CIX RECYCLEs (previous campaign)," which was the portion of recycle streams carried over from the previous campaign to be mixed with other recycle streams that did not come directly from one of the four unit

\footnotetext{
${ }^{\dagger}$ For WTP this batch size was 18,000 gallons when this task began, but it currently stands at 21,550 gallons. In fact, for Campaign I operation, the 18,000-gallons size was valid and used for scaling purposes, but with Campaign II and all subsequent campaigns the latter volume was used.

${ }^{\ddagger}$ Same as the above footnote

* In fact, the actual discrepancy was not understood until after Campaign III was complete and analysis of the SIPP data was underway. The problem turned out to be something that could not be easily resolved a priori because of the OLI/ESP. For the software package to converge on a solution the anion and cation charges of ALL simulant analytes must balance. Unfortunately, the simulated wastes streams contained many unknown complex insoluble solids compounds for which ion charge balances had to be assumed. Those assumptions caused the resulting volumetric difference once a slurry was concentrated to $20 \mathrm{wt} \%$ insoluble solids. This is a clear example of the current limitations of computational models and the need for benchmarking to experimental results of complex interactions.
} 
operations; finally, the last line contains the volumes of LAW that are treated through all four unit operation and are ready as feed for LAW vitrification.

An important goal of the OLI/ESP SIPP model was to show if steady state operation is attained. That is, if after four campaigns the unit operation volumetric needs change significantly, it would have meant that more campaigns would be necessary. When doing the model it was not clear what would be a significant difference, but fortunately steady state was attained very fast. The volumetric flow rates differences between Campaigns II and III turned out to be small and even smaller between Campaigns III and IV. In fact, as described in Appendix A, the difference in the recycle volumetric flow rates for the last two campaigns was less than $0.09 \%$.

Another feature included in Table 1, but not obvious, is the feed proportions used for each unit operation, which were supplied by the ACM model. For each of the campaigns, the proportions are slightly different, but not significantly so. The feed for the FEP was made up from only recycle streams and the volumetric proportion of each of the streams used is shown in Table 2.

While the proportions in Table 2 are close to the recycle stream values in the planning table shown as Table 1, they will not be exactly the same because of last minute changes, round off numbers, and uncertainties in some of the streams. For example, the amount of recycle stream (7) was changed several times by WTP because the exact amount of material that will come from the UFP loop during flushing is not well characterized. Moreover, for a time the fate of UFP loop flush material was uncertain. Also note, that while Table 2 contains what was believed to be the brunt of the recycle stream sources, the design of the plant was (and still is) continually evolving, which may have a significant impact on those streams in both composition and volume. For instance, recycle streams that were not considered, but will likely have an impact on future operations of the WTP, were the slurries that will be generated during equipment and vessel maintenance activities in the vitrification facilities. These waste streams will be much higher in silica and other glass former chemicals, e.g., Li, than the simulated recycles streams tested in the SIPP. Compounds that contain lithium are very insoluble in solutions containing aluminum and could impact filtration.

One of the most useful aspects of the results in Table 1 was to know the amount of simulated waste slurry to procure for the SIPP task. With the initial pilot-scale UFP feed tank volume fixed at 140 liters, then all other volumes could be calculated after the effect of recycle streams were computationally considered. Once the amount of the AY102 and AP101 were known, their procurement could begin. Their makeup, especially the former, is very complex and expensive (it was discussed in Duignan, et al., 2004; see Section 2.3) and the coordination of simulant procurement was a major effort for the task. 
Table 2. Percent contribution for each of the recycle streams to the total FEP feed

\begin{tabular}{|c|c|c|c|c|}
\hline \multirow{3}{*}{$\begin{array}{l}\text { List of Recycle Streams Used for } \\
\text { SIPP and Verified by WTP }\end{array}$} & \multicolumn{4}{|c|}{$\begin{array}{l}\text { Percentage of Recycle to FEP } \\
<-----C a m p a i g n \text { Number------> }\end{array}$} \\
\hline & $I^{*}$ & $I^{\star \star}$ & III & IV \\
\hline & Vol. \% & Vol. \% & Vol. \% & Vol. \% \\
\hline (1) HLW SBS & $\mathrm{n} / \mathrm{a}$ & $17.1 \%$ & $17.5 \%$ & $17.4 \%$ \\
\hline (2) Vessel Vent & $\mathrm{n} / \mathrm{a}$ & $19.7 \%$ & $20.3 \%$ & $20.2 \%$ \\
\hline (3) HLW Can. Decon & $\mathrm{n} / \mathrm{a}$ & $10.3 \%$ & $10.5 \%$ & $10.5 \%$ \\
\hline (4) UFP Recycle (0.01 M NaOH) & $\mathrm{n} / \mathrm{a}$ & $30.3 \%$ & $21.7 \%$ & $21.6 \%$ \\
\hline (5) UFP Recycle (0.1 M NaOH) & $\mathrm{n} / \mathrm{a}$ & $10.5 \%$ & $9.0 \%$ & $8.9 \%$ \\
\hline (6) UFP Recycle (2 M HNO3) & $\mathrm{n} / \mathrm{a}$ & $11.0 \%$ & $9.4 \%$ & $9.4 \%$ \\
\hline (7) UFP Recycle (3 Loop Flushes) & $\mathrm{n} / \mathrm{a}$ & $1.2 \%$ & $1.2 \%$ & $1.2 \%$ \\
\hline (8) $\mathrm{CIX}$ Recycle (0.1 M NaOH) & $\mathrm{n} / \mathrm{a}$ & $0.0 \%$ & $3.4 \%$ & $3.5 \%$ \\
\hline (9) CIX Recycle (DI Water) & $\mathrm{n} / \mathrm{a}$ & $0.0 \%$ & $3.4 \%$ & $3.5 \%$ \\
\hline (10) CIX Recycle (0.25 M NaOH) & $\mathrm{n} / \mathrm{a}$ & $0.0 \%$ & $3.7 \%$ & $3.8 \%$ \\
\hline Total Feed to the FEP & $\mathrm{n} / \mathrm{a}$ & $100 \%$ & $100 \%$ & $100 \%$ \\
\hline \multicolumn{5}{|c|}{$\begin{array}{l}\text { Notes: } \\
\text { Source of Streams: (1) was made by an outside vendor: VSL*** } \\
\qquad \text { (2) and (3) were made for each campaign } \\
\text { (4) to (10) were products of unit operations } \\
\text { * Campaign I only used a single simulated waste with no } \\
\text { recycles in a single unit operation, i.e., UFP. } \\
\text { ** Since Campaign I was only a filter operation, therefore, no CIX } \\
\text { recycle streams were produced for Campaign II, as indicated } \\
\text { by the RPP-WTP Test Specification (Longwell, 2003). } \\
\text { *** VSL = Vitreous State Lab. of Catholic University of America }\end{array}$} \\
\hline
\end{tabular}

There were many routes to operating the UFP, but for SIPP, and initial plant operation, it was thought prudent to only use the FEP to process recycle streams and the UFP would be the starting point for tank farm wastes. One major benefit of this method was that it minimizes insoluble solids in the FEP and thus hopefully minimizes condenser fouling that would affect operational efficiency. This means that the feed for the UFP had three waste sources, one of which came from the FEP after the recycle streams were concentrated to a target density. The other two were mentioned above. Their individual contributions to the UFP feed are shown in Table 3. 
Table 3. Percent contribution for each of the UFP feed streams

\begin{tabular}{|c|c|c|c|c|}
\hline \multirow{2}{*}{$\begin{array}{l}\text { Feed Streams Used for SIPP } \\
\text { in the UFP and Verified by WTP }\end{array}$} & \multicolumn{4}{|c|}{$\begin{array}{l}\text { Percentage of Streams to UFP } \\
<-----C a m p a i g n \text { Number----> }\end{array}$} \\
\hline & $\frac{I^{*}}{\text { Vol. } \%}$ & \begin{tabular}{c|}
$I^{* *}$ \\
Vol. $\%$
\end{tabular} & $\frac{\text { III }}{\text { Vol. \% }}$ & $\frac{\text { IV }}{\text { Vol. \% }}$ \\
\hline (1) FEP Bottc & $0 \%$ & $11.1 \%$ & $14.3 \%$ & $13.8 \%$ \\
\hline (2) $A Y-1$ & $100 \%$ & $24.3 \%$ & $22.6 \%$ & $22.8 \%$ \\
\hline (3) AP-101 & $0 \%$ & $64.6 \%$ & $63.1 \%$ & $63.4 \%$ \\
\hline Total Feed to the UFP & $100 \%$ & $100 \%$ & $100 \%$ & $100 \%$ \\
\hline \multicolumn{5}{|c|}{$\begin{array}{l}\text { Notes: } \\
\text { Source of Streams: (1) was concentrated from recycles in the FEP } \\
\qquad \begin{array}{l}\text { (2) outside vendor, but finalized in EDL** } \\
\text { (3) came from an outside vendor }\end{array} \\
\text { * Campaign I only used a single simulated waste. } \\
\text { ** EDL = Engineering Development Laboratory of the Savannah } \\
\text { River National Laboratory of the Westinghouse Savannah } \\
\text { River Company where SIPP pilot-scale testing was performed. }\end{array}$} \\
\hline
\end{tabular}

As with the recycle percentages in Table 2, the percentages in Table 3 are slightly different than what is shown in Table 1 . Those differences are mostly attributed to volumes actually created during experimentation. The computational volumes were not expected to be exact and while the values in Table 3, were (Campaigns I and II) or are to be (Campaigns III and IV) used for SIPP, the actual plant mixture may still be different as its design continues to evolve.

One product of the pilot-scale UFP is the permeate produced during dewatering. This waste stream is then sent on to the CIX in its preparation for LAW vitrification. As this task evolved, the pilot-scale CIX operation was disassociated from measuring volumetric throughput to measure glass production, because WTP thought it more important to understand the cesium removal efficiency of the chosen resin, SuperLig ${ }^{\circledR} 644$. This requirement made the amount of permeate important, since cesium removal was to be studied. It was necessary to have 400 "bed volumes" of feed for each campaign, excluding Campaign I. A bed volume is the space occupied in a ion exchange column by settled resin. For the pilot-scale CIX that volume was approximately 2.3 liters, making the produced number of bed volumes 401, 414, and 410 for Campaigns II, III, and IV, respectively. This can be seen in the Table 1 line entitled, "UF1 FILTRATE (routed to CIX, then TLP)". For example, in Campaign II the permeate produced form the UFP was 922.3 liters, or $922.3 / 2.3=401$ bed volumes.

Finally, the proportions of different feeds for the pilot-scale TLP were needed. Those are shown in Table 4. 
Table 4. Percent contribution for each of the TLP feed streams

\begin{tabular}{|c|c|c|c|c|}
\hline \multirow{3}{*}{$\begin{array}{l}\text { Feed Streams Used for SIPP } \\
\text { in the TLP and Verified by WTP }\end{array}$} & \multicolumn{4}{|c|}{$\begin{array}{l}\text { Waste Stream Vol. \% to TLP } \\
<------C a m p a i g n \text { Number------> }\end{array}$} \\
\hline & $I^{\star}$ & $I^{\star \star}$ & III & $\mathrm{IV}$ \\
\hline & Vol. \% & Vol. \% & Vol. \% & Vol. \% \\
\hline (1) LAW SBS (from LAW melter) & $\mathrm{n} / \mathrm{a}$ & $47.4 \%$ & $45.7 \%$ & $45.8 \%$ \\
\hline (2) $0.25 \mathrm{M} \mathrm{NaOH}$ (recycle from CIX) & $\mathrm{n} / \mathrm{a}$ & $0.0 \%$ & $2.1 \%$ & $2.1 \%$ \\
\hline (3) Treated LAW from CIX & $\mathrm{n} / \mathrm{a}$ & $52.6 \%$ & $52.2 \%$ & $52.1 \%$ \\
\hline Total Feed to the TLP & $\mathrm{n} / \mathrm{a}$ & $100 \%$ & $100 \%$ & $100 \%$ \\
\hline \multicolumn{5}{|c|}{$\begin{array}{l}\text { Notes: } \\
\text { Source of Streams: (1) was made by an outside vendor: VSL*** } \\
\qquad \begin{array}{l}\text { (2) comes from a regenerated IX column } \\
\text { (3) comes from CIX after Cs is removed }\end{array} \\
\text { * Campaign I did employ the pilot-scale TLP } \\
\text { ** Since Campaign I was only a filter operation, therefore, no CIX } \\
\text { recycle streams were produced for Campaign II, as indicated } \\
\text { by the RPP-WTP Test Specification (Longwell, 2003). } \\
\text { *** VSL = Vitreous State Lab. of Catholic University of America }\end{array}$} \\
\hline
\end{tabular}

As with Tables 2 and 3, the percentages in Table 4 differ slightly than those from Table 1 because of limitations in the model, but all the values were sufficient to plan and begin testing. With the plan and model in place then the test matrix could be established and the plans finalized.

\subsection{THE OVERALL SIPP TEST MATRIX}

With the plan in place and the planning results from the computational model, an overall test matrix was established. The following matrix was not the last word on how each of the unit operation was to run, but it was the starting point for each of the unit operations to develop individual test plans, as well as the simulant mixing and sample plans. As the task began with Campaign I (Duignan, et al., 2004), and then on to Campaign II the matrix continued to evolve as WTP needs changed, therefore, this test matrix may be slightly different than the individual test plans, but is instructive here to illustrate the overall operation of SIPP. See the overall test matrix, then some global explanation can be given to better understand SIPP in its "integrated" form before discussing each of the individual unit operation and their results. 
That matrix is shown in pieces in the following series of figures:

[Note that , Table $\mathrm{X}>>$ Table Y means: table $\mathrm{x}$ indicates the wait time before using slurry in the table $\mathrm{y}$ unit operation.]

Table 5 (simulant aging) $\quad>>\quad$ Table 6 (pilot FEP unit operation)

Table 7 (simulant aging) $\quad>\quad$ Table 8 (pilot UFP unit operation)

Table 9 (simulant aging) $\quad>\quad$ Table 10 (pilot CIX unit operation - first cycle)

$>\quad$ Table 11 (pilot CIX unit operation - second cycle)

Table 12 (simulant aging) $\quad>\quad$ Table 13 (pilot TLP unit operation)

These nine figures are in chronological order of performance.

First, Figs. 5, 7, 9, and 12 were included to show hold-up time intervals between unit operations, especially because they were not part of the computational model, which was done at steady state. In an attempt to mimic the periods that waste "waits" before being processed in each unit operation, some prototypic times were included in SIPP

Table 5. Hold period before pilot-scale FEP begins ${ }^{\dagger}$

\begin{tabular}{|l|l|l|l|l|l|l|l|}
\hline & Pre-FEP Hold Times (stipulated by WTP) & & & & & \\
\hline & Minimum hold time between the time recycle stream mixture and beginning evaporation. & & & & & \\
\hline
\end{tabular}

The actual hold times for process feeds were controversial and, in fact, were not available when Campaign I was performed in January 2004. Actually, since Campaign I was a single-waste filter test, the concept of wait time to plant operation made little sense. However, this is not true for the other three campaigns, which deal with waste and recycle mixing and adjusting before each processing step. Waste aging may affect many processing steps, for example, the post-UFP precipitation was a key issue for the ion exchange system. Using the appropriate WTP plant hold times allow this issue to be resolved. At approximately three weeks before Campaign II, WTP sent information to SRNL to make rough determinations of those periods (see the e-mail in Appendix B). For example, Table 5 allows time for the mixed multiple recycle streams to age. Recycle streams are fed from several different sources to the Plant Wash and Disposal System tanks that will feed the Waste feed Evaporation Process. The recycle streams come in at different rates and then need to be adjusted for $\mathrm{pH}$ as the acid and alkaline streams merge in order to mitigate the precipitation of solids or gels. After the adjustment, the FEP is fed, but because of the large scale, the transfer times are not insignificant. As explained in Appendix B, the time associated in the pre-evaporation process is approximately 2 days and therefore, once the SIPP FEP feed of recycle streams is adjusted properly it is held for that number of days before evaporation begins.

For each of the unit operations, a hold time is set after the appropriate feed is made ready. In summary those simulated minimum hold times are:

After joining recycle feeds and before processing in the FEP - 2 days After making FEP bottoms and before joining it with other UFP feeds -2 days

${ }^{\dagger}$ Blanks in all tables in this report are intentional. 


\section{WSRC-TR-2004-00478, REVISION 0 SRNL-RPP-2004-00075, REVISION 0}

After joining waste feeds and before processing in the UFP - 2 days After producing UFP permeate and before processing in the CIX - 4.5 days After joining treated LAW with recycle streams and before processing in the TLP - 2 days

Table 6. Pilot-scale FEP test matrix with the colored columns representing Campaign II

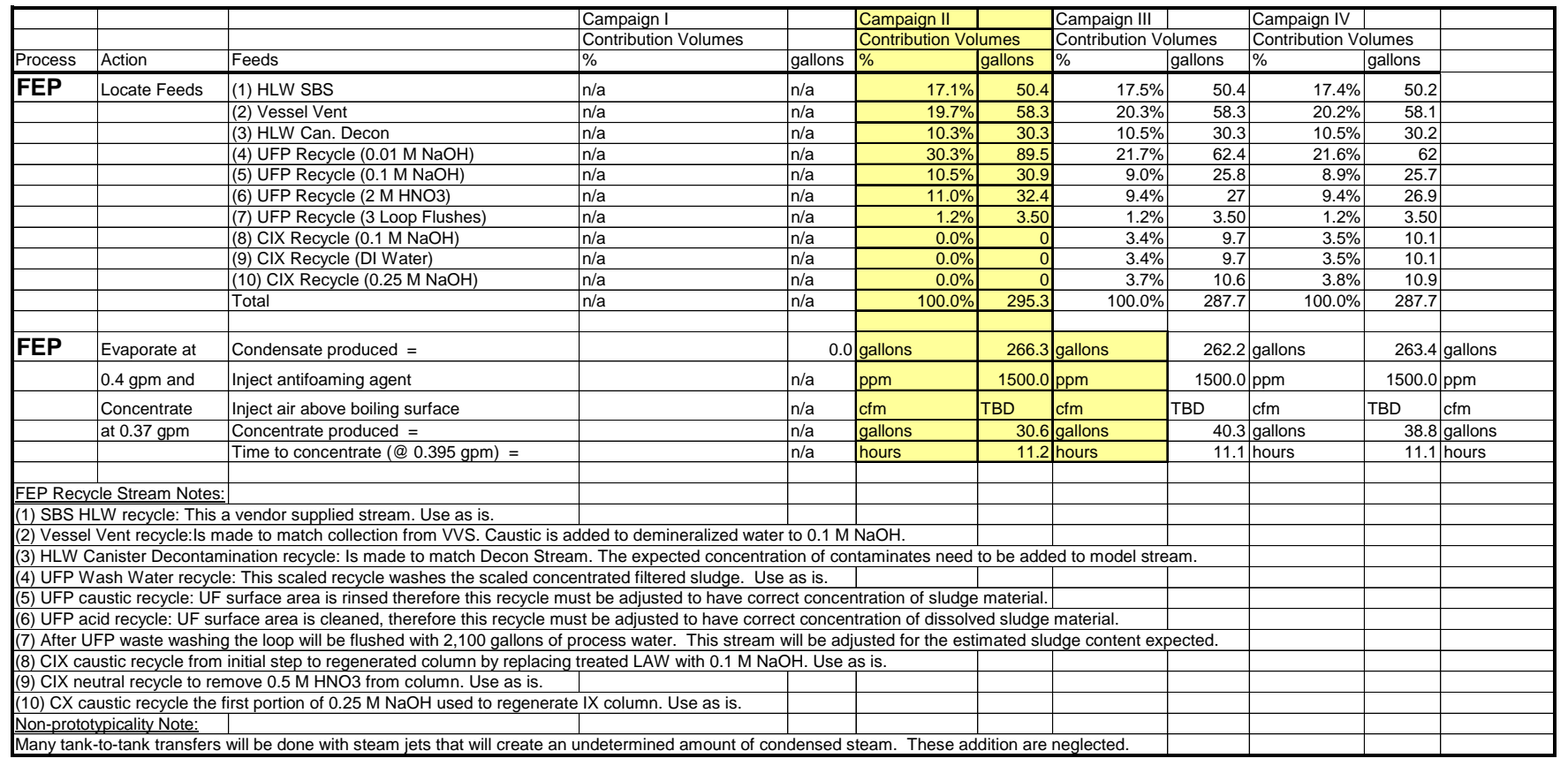

Once the recycle streams were mixed in the planned proportions, Table 2, and the appropriate hold time was realized, then the FEP test matrix, shown in Table 6, was performed. The goal was to concentrate the feed stream so that it had a sodium molarity of 5, but the "visible" target was an evaporated slurry density of $1.266 \mathrm{~g} / \mathrm{cc}$ at $50^{\circ} \mathrm{C}$ and 1 psia (which is $1.252 \mathrm{~g} / \mathrm{cc}$ at $1 \mathrm{~atm}$ ). Density was used because the WTP will only be able to control concentration by measuring density.

Table 7. Hold period before pilot-scale UFP begins

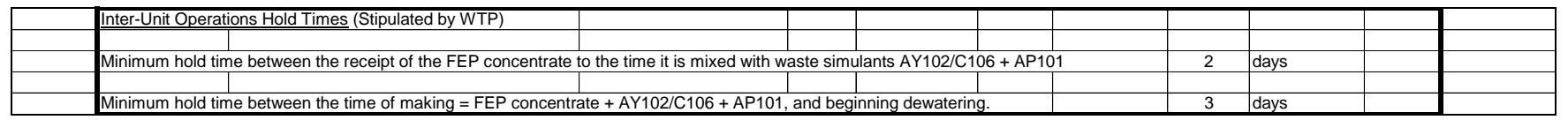

When the FEP was complete its slurry product was held for 2 days, Table 7, before being mixed in the planned proportions, Table 3, with the simulated wastes of AY102 and AP101. Completing this mixture started another 3-day clock before starting the UFP. While processing the waste through the UFP is complicated with several activities involving the washing of the concentrated slurry to the cleaning of the filter loop (see Table 8), the basic operation is to separate the waste feed stream into a HLW product, containing all of the insoluble solids, and its supernatant, containing most of the cesium. This was done by dewatering the feed from a concentration less than $5 \mathrm{wt} \%$ to $20 \mathrm{wt} \%$ insoluble solids. The details of the UFP operation will be discussed in the appropriate 


\section{WSRC-TR-2004-00478, REVISION 0 SRNL-RPP-2004-00075, REVISION 0}

subsection of this report. One feature that is not immediately obvious is the process containing an internal hold time. Dewatering in WTP will be a relatively lengthy process because filtration is very slow and the volumes are very large. While the waste is waiting to be processed through the UFP, it will be sitting in the feed preparation tank, which feeds the feed tank. During this process period, chemical reactions may still be occurring within the waste and crystallization or precipitation may result. In an attempt to capture these transient phenomena, a waiting period was inserted in the dewatering process of the pilot UFP. The period is a 12-hour hold time, which is arbitrary, but is loosely connected to the planned plant operational time of the UFP, and was acceptable to the WTP project.

Table 8. Pilot-scale UFP test matrix with the colored columns representing Campaign II

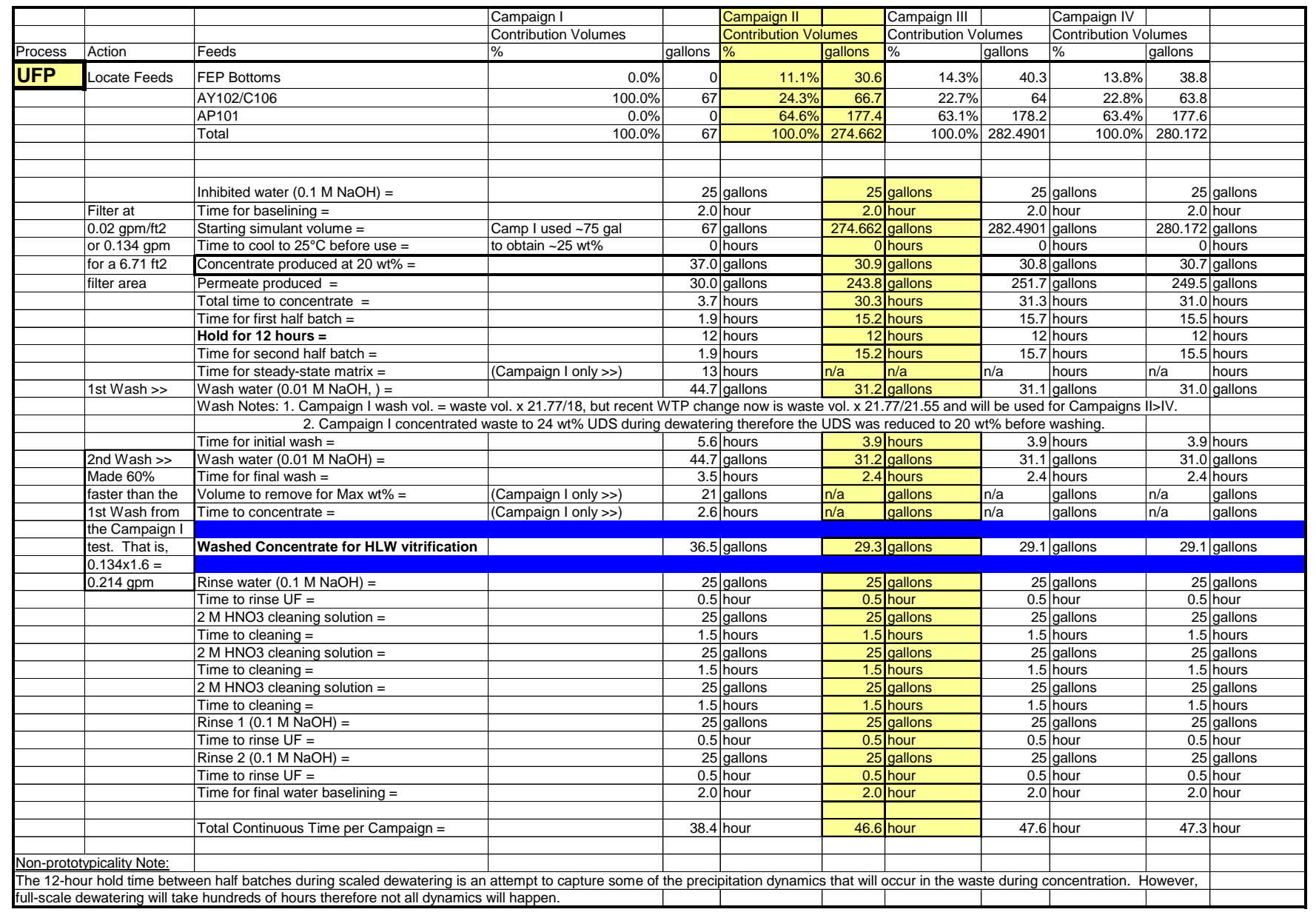

The pilot-scale UFP produced permeate that was needed for the next step in the SIPP operation, i.e., pilot CIX. While the UFP continued to process the dewatered slurry with washing and cleaning the UFP loop with both caustic and acid flushes, the permeate sat in the pilot CIX feed tank to age at least 4.5 days, as shown in Table 9. 
WSRC-TR-2004-00478, REVISION 0

SRNL-RPP-2004-00075, REVISION 0

Table 9. Hold period before pilot-scale CIX begins

\begin{tabular}{|l|l|l|l|l|l|l|l|}
\hline & Inter-Unit Operations Hold Time (stipulated by WTP) & & & & \\
\hline & Minimum hold time between the receipt of the UFP permeate to the time it is processed by the CIX & & 4.5 & days & & \\
\hline \\
\hline
\end{tabular}

As it turned out it was convenient to produce the first permeate on a Monday and then to begin pilot CIX operation the following Monday. There were approximately 6.5 days between the end of the first half of permeate production, sometime after midnight of that Monday, the planned start of the CIX. Pilot CIX operation was also broken in two parts because it was necessary to run with two complete ion exchange cycles, which allowed both IX columns to take turns as the lead or the lag column. This is the reason there are two CIX tables, i.e., Tables 10 and 11. The basic difference between them is that the lead column in the first cycle of operation, Table 10, became the lag column in the second cycle, Table 11. The permeate produced during the pilot UFP was sufficient to satisfy the CIX cycle need of 400 bed volumes of feed ( 900 liters). Each cycle included the processing of 200 bed volumes of UFP permeate and then the lead column would undergo regeneration for use in the next cycle as the lag column.

The colored portion of Table 10 highlights the work done for this report, that is, Campaign II. Campaign II included pilot CIX cycles 1 and 2. Once again, Campaign I did not include any ion exchange work, which explains why cycle 1 began in Campaign II.

Table 10. Pilot-scale CIX (1st half) test matrix with the colored columns representing Campaign II

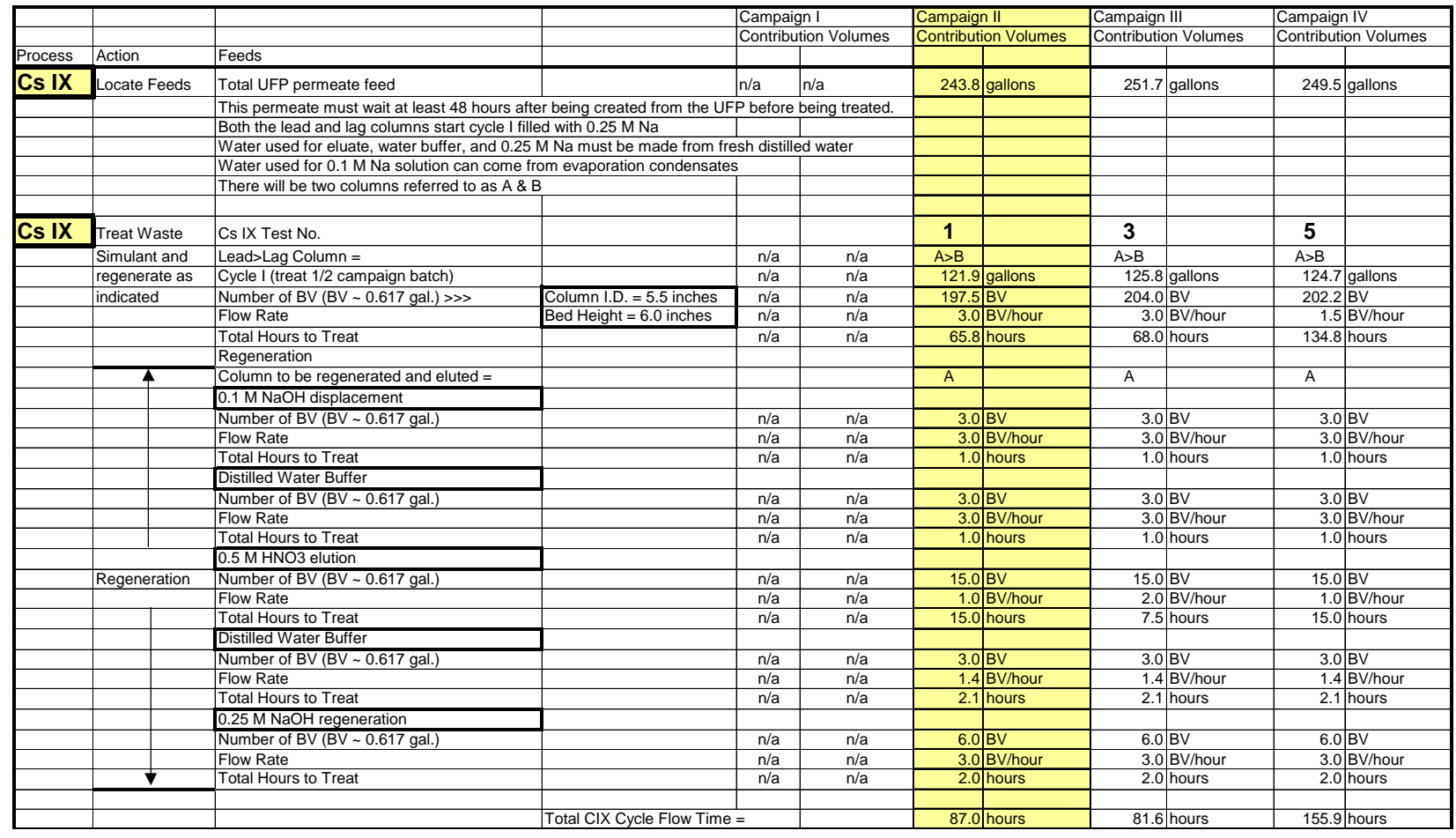




\section{WSRC-TR-2004-00478, REVISION 0 SRNL-RPP-2004-00075, REVISION 0}

Once both cycles of a Campaign are complete then both columns are filled with $0.1 \mathrm{M} \mathrm{NaOH}$ to keep the resin stable for the next campaign. This inter-campaign period would be no less than 5 weeks.

Table 11. Pilot-scale CIX (2nd half) test matrix with the colored columns representing Campaign II

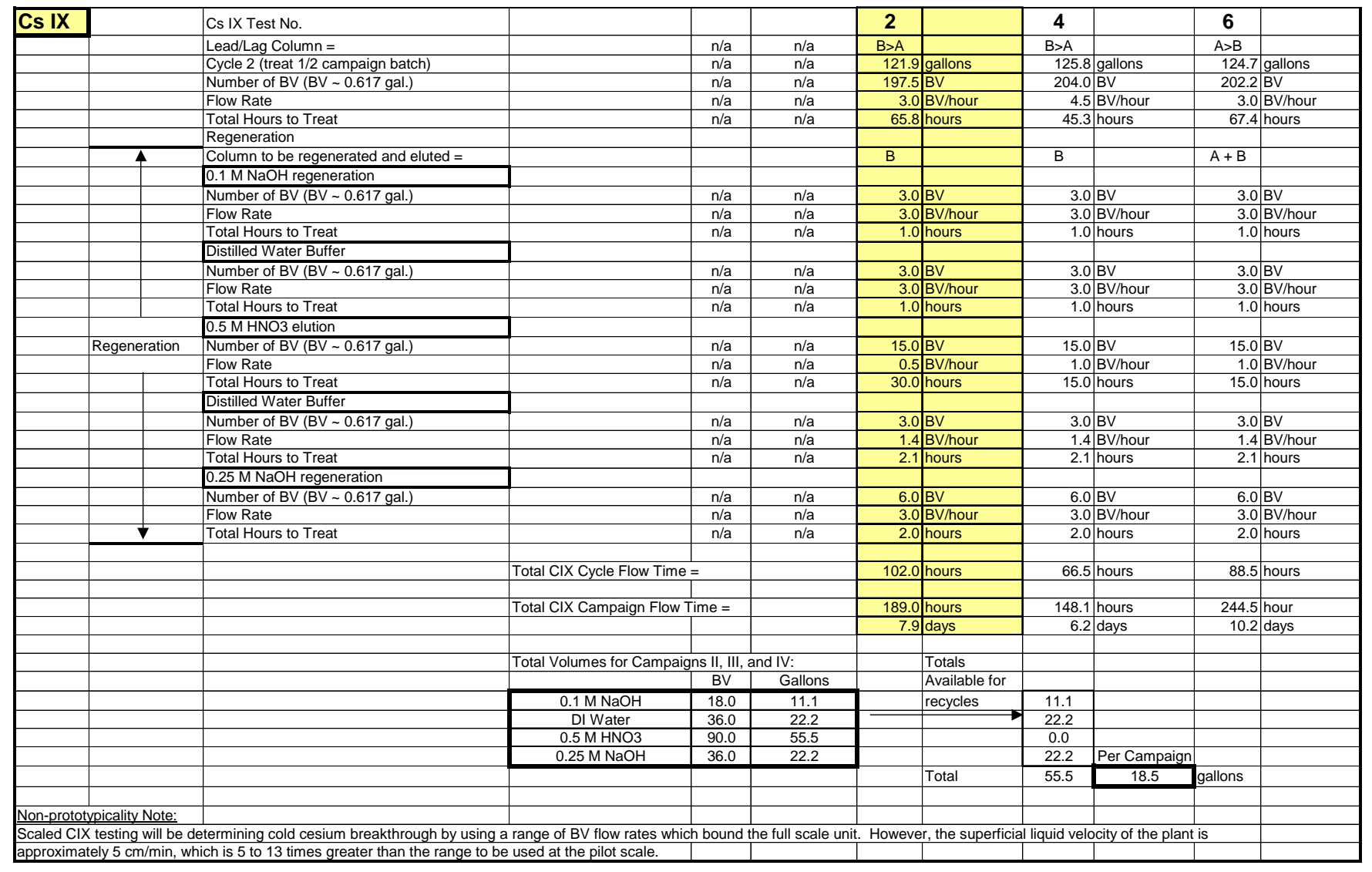

The final step in the SIPP was once again to evaporate the waste. The now treated simulated LAW product from the CIX was mixed with two recycle streams shown in Table 4. After mixing, the resulting solution was held before processing, Table 12.

Table 12. Hold period before pilot-scale TLP begins

The operation of the pilot TLP, Table 13 was similar to the pilot FEP, Table 6 . However, in the TLP the goal was to concentrate the feed stream so that it had a sodium molarity of 8. Once again the "visible" target was an evaporated slurry density of $1.360 \mathrm{~g} / \mathrm{cc}$ at $50^{\circ} \mathrm{C}$ and 1 psia (which is $1.395 \mathrm{~g} / \mathrm{cc}$ at $1 \mathrm{~atm}$ ). Density was used because the WTP will only be able to control concentration by measuring density. A large difference between the pilot FEP and TLP operations was the quantity of concentrated product produced. For the FEP, the final volume of the concentrate could be completely contained within the 


\section{WSRC-TR-2004-00478, REVISION 0 SRNL-RPP-2004-00075, REVISION 0}

confines of the evaporator ( $<50$ gallons), but for the TLP the concentrated volume was approximately 3 times larger. That is, the FEP was shut down just when steady-state waste concentration was reached. For the TLP, steady-state waste concentration was reached well before the waste feed was exhausted. This meant the TLP produced a continuous waste stream after the TLP contents reached the steady-state density target. The only significant impact this difference had on operation was to the defoamer chemical added to the waste. The concentration of defoamer was to be maintained at $1500 \mathrm{ppm}$, therefore, once the TLP reached steady state and concentrated waste was being removed, defoamer was periodically added.

Table 13. Pilot-scale TLP test matrix with the colored columns representing Campaign II

\begin{tabular}{|c|c|c|c|c|c|c|c|c|c|c|c|}
\hline & & & Campaign I & & Campaign II & & Campaign III & & Campaign IV & & \\
\hline & & & Contribution Volumes & & Contribution Vol & lumes & Contribution Vo & olumes & Contribution Vo & olumes & \\
\hline Process & Action & Feeds & $\%$ & gallons & $\%$ & Tgallons & $\%$ & gallons & $\%$ & gallons & \\
\hline TLP & Locate Feeds & Simulant & n/a & n/a & $52.6 \%$ & 243.8 & $52.2 \%$ & 251.7 & $52.1 \%$ & 249.5 & \\
\hline & & (Recycle 1) SBS LAW & n/a & n/a & $47.4 \%$ & 220.1 & $45.7 \%$ & 220.1 & $45.8 \%$ & 219.3 & \\
\hline & & (Recycle 2) $0.25 \mathrm{M} \mathrm{NaOH}$ from $\mathrm{CIX}$ & n/a & $\mathrm{n} / \mathrm{a}$ & $0.0 \%$ & 0 & $2.1 \%$ & 10 & $2.1 \%$ & 10.1 & \\
\hline & & Total & n/a & n/a & $100.0 \%$ & 463.862 & $100.0 \%$ & 481.7901 & $100.0 \%$ & 478.872 & \\
\hline TID & Evanorate at & Condencete nroduch - & & 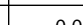 & a pallone & 2008 & ano & 3252 & agllong & 3236 & Aa \\
\hline & & & & & & & & & & & \\
\hline & $0.4 \mathrm{gpm}$ and & Inject antifoaming agent & & n/a & ppm & 1500.0 & $\mathrm{ppm}$ & 1500.0 & $\mathrm{ppm}$ & 1500.0 & $\mathrm{ppm}$ \\
\hline & Concentrate & Inject air above boiling surface & & n/a & $\mathrm{cfm}$ & TBD & $\mathrm{cfm}$ & TBD & $\mathrm{cfm}$ & TBD & $\mathrm{cfm}$ \\
\hline & at $0.37 \mathrm{gpm}$ & & & & & & & & & & \\
\hline & & Concentrate produced for LAW vitrification & & n/a & gallons & 155.7 & 7 gallons & 159.4 & gallons & 158.0 & gallons \\
\hline & & Time to concentrate $=$ & & $n / a$ & hours & 135 & 5 hours & 13.7 & hours & 137 & 7 hours \\
\hline & & & & & & & & & & & \\
\hline TLP Rec & Stream Note & & & & & & & & & & \\
\hline (1) SBS & AW recycle: This & a vendor supplied stream. Use as is. & & & & & & & & & \\
\hline (2) Caus & IX regeneratiol & ecycle: This stream should have the appropriat & te concentration of cal & e., $0.25 \mathrm{~N}$ & $\mathrm{M} \mathrm{NaOH}$. Use as & & & & & & \\
\hline
\end{tabular}

The timetable for Campaign II is listed in Table 14. It started on the day indicated, i.e., Monday, 12 April, 2004, but since this schedule was published before the test began minor changes occurred. However, those changes were insignificant.

The only other aspect of the test not shown in all the matrix tables is the disposition of the water products. That is, the treated waste from the TLP was drummed to be sent to VSL for LAW melter operations and the washed concentrated slurry from the UFP was drummed to be sent to VSL for HLW melter operation. The other product is all the boiled off and condensed water from both evaporation operations. Those waters were mixed together, as they will be in the plant and then a portion is reused to make rinse solutions for the UFP and displacement solution for the CIX. 


\section{WSRC-TR-2004-00478, REVISION 0 SRNL-RPP-2004-00075, REVISION 0}

Table 14. Pilot-scale schedule for Campaign II

\begin{tabular}{|c|c|c|c|c|c|c|c|c|c|c|c|c|}
\hline Start Date & & Personnel Nomeclature: & & & & & Notes: & \multirow{2}{*}{\multicolumn{5}{|c|}{$\begin{array}{l}\text { (1) Hold times are included to mimick times that } \\
\text { the plant is filling/transfer between unit operations. }\end{array}$}} \\
\hline 26-Jan-04 & & \multicolumn{5}{|c|}{$\mathrm{da}=\mathrm{D}$. Adamson, ma=M.Armstrong, db=D.Burns, vb=V.Bush, } & & & & & & \\
\hline 12-Apr-04 & & \multicolumn{5}{|c|}{ jc=J.Corbett, md=M.Duignan, df=D.Foreman, mf=M.Fowley, } & & \multicolumn{5}{|c|}{ Those times begin when a mixture is FIRST made, } \\
\hline 6-Jun-04 & & $\mathrm{sh}=\mathrm{S}$. Hatcher, jm=J.Mills, $\mathrm{zq}=\mathrm{Z}$. & Quresh & $\mathrm{i}, \mathrm{mr}=\mathrm{M} \cdot \mathrm{R}$ & estivo & & & \multicolumn{4}{|c|}{ therefore mixing times are also included. } & \\
\hline 26-Jul-04 & & \multicolumn{5}{|c|}{ ts=T.Steeper, js=J.Steimke, mw=M.Williams, jz=J.Zamecnik } & & \multicolumn{5}{|c|}{ (2) CIX \# of days is only valid for Campaign II } \\
\hline \multicolumn{13}{|l|}{ Campaign II } \\
\hline Day & Unit Op & Activity & Expt & Backup & Non-E. & Backup & Unit Op & Activity & Expt & Backup & Non-E. & Backup \\
\hline 12-Apr-04 & FEP & Mix Recycles & $\mathrm{jz} / \mathrm{mw}$ & $\mathrm{mw} / \mathrm{jz}$ & df & $\mathrm{vb}$ & & & & & & \\
\hline 13-Apr-04 & FEP & Admin Hold (1) & & & & & & & & & & \\
\hline 14-Apr-04 & FEP & Evaporation & $\mathrm{zq}$ & da & jm & $\mathrm{vb}$ & UFP & Baseline & $\mathrm{mf}$ & md & ma & df \\
\hline 15-Apr-04 & FEP & Product Transfers & $\mathrm{zq}$ & da & $\mathrm{jm}$ & $\mathrm{vb}$ & UFP & Admin Hold (1) & & & & \\
\hline 16-Apr-04 & FEP & Clean Equipment & $\mathrm{zq}$ & da & $\mathrm{jm}$ & $\mathrm{vb}$ & UFP & Mix UF feeds & $\mathrm{jz} / \mathrm{mw}$ & $\mathrm{mw} / \mathrm{jz}$ & df & $\mathrm{vb}$ \\
\hline 17-Apr-04 & & & & & & & UFP & Admin Hold (1) & & & & \\
\hline 18-Apr-04 & & & & & & & UFP & Admin Hold (1) & & & & \\
\hline 19-Apr-04 & & & & & & & UFP & Dewater & $\mathrm{mf}$ & $\mathrm{md}$ & $\mathrm{ma}$ & df \\
\hline 20-Apr-04 & & & & & & & UFP & Dewater & $\mathrm{mf}$ & $\mathrm{md}$ & $\mathrm{ma}$ & df \\
\hline 21-Apr-04 & CIX (2) & Admin Hold (1) & & & & & UFP & Transfers/Wash & $\mathrm{mf}$ & $\mathrm{md}$ & $\mathrm{ma}$ & df \\
\hline 22-Apr-04 & CIX & Admin Hold (1) & & & & & UFP & Wash & $\mathrm{mf}$ & $\mathrm{md}$ & $\mathrm{ma}$ & df \\
\hline 23-Apr-04 & $\mathrm{CIX}$ & Admin Hold (1) & & & & & & & & & & \\
\hline 24-Apr-04 & $\mathrm{CIX}$ & Admin Hold (1) & & & & & & & & & & \\
\hline 25-Apr-04 & $\mathrm{CIX}$ & Admin Hold (1) & & & & & & & & & & \\
\hline 26-Apr-04 & $\mathrm{CIX}$ & Treat Simulant & js & da & df & $\mathrm{vb}$ & UFP & Rinse/Clean & $\mathrm{mf}$ & $\mathrm{md}$ & ma & df \\
\hline 27-Apr-04 & $\mathrm{CIX}$ & Treat Simulant & js & da & df & $\mathrm{vb}$ & UFP & Baseline & $\mathrm{mf}$ & $\mathrm{md}$ & $\mathrm{ma}$ & df \\
\hline 28-Apr-04 & $\mathrm{CIX}$ & Treat Simulant & js & da & df & $\mathrm{vb}$ & & & & & & \\
\hline 29-Apr-04 & $\mathrm{CIX}$ & 0.1 M Caustic/DI Water Flush & js & da & df & $\mathrm{vb}$ & & & & & & \\
\hline \multicolumn{13}{|l|}{ 30-Apr-04 } \\
\hline \multicolumn{13}{|l|}{ 1-May-04 } \\
\hline \multicolumn{13}{|l|}{ 2-May-04 } \\
\hline 3-May-04 & $\mathrm{CIX}$ & $0.5 \mathrm{M} \mathrm{HNO3}$ & js & da & df & $\mathrm{vb}$ & & & & & & \\
\hline 4-May-04 & $\mathrm{CIX}$ & DI Water Flush & js & da & df & $\mathrm{vb}$ & & & & & & \\
\hline 5-May-04 & $\mathrm{CIX}$ & $0.25 \mathrm{M} \mathrm{NaOH}$ Regeneration & js & da & df & $\mathrm{vb}$ & & & & & & \\
\hline 6-May-04 & $\mathrm{CIX}$ & Treat Simulant & js & da & df & $\mathrm{vb}$ & & & & & & \\
\hline 7-May-04 & $\mathrm{CIX}$ & Treat Simulant & js & da & $\mathrm{df}$ & $\mathrm{vb}$ & & & & & & \\
\hline 8-May-04 & CIX & Treat Simulant & js & da & df & $\mathrm{vb}$ & & & & & & \\
\hline \multicolumn{13}{|l|}{ 9-May-04 } \\
\hline 10-May-04 & $\mathrm{CIX}$ & $0.1 \mathrm{M}$ Caustic/DI Water Flush & js & da & df & $\mathrm{vb}$ & TLP & Make Feed & $\mathrm{jz} / \mathrm{mw}$ & $\mathrm{mw} / \mathrm{jz}$ & df & $\mathrm{vb}$ \\
\hline 11-May-04 & $\mathrm{CIX}$ & $0.5 \mathrm{M} \mathrm{HNO3}$ & js & da & df & $\mathrm{vb}$ & TLP & Admin Hold (1) & & & & \\
\hline 12-May-04 & $\mathrm{CIX}$ & DI Water Flush & js & da & df & $\mathrm{vb}$ & TLP & Evaporation & da & $\mathrm{zq}$ & $\mathrm{vb}$ & jm \\
\hline 13-May-04 & $\mathrm{CIX}$ & $0.25 \mathrm{M} \mathrm{NaOH}$ Regeneration & js & da & df & $\mathrm{vb}$ & TLP & Product Transfers & da & $\mathrm{zq}$ & $\mathrm{vb}$ & $\mathrm{jm}$ \\
\hline 14-May-04 & & & & & & & TLP & Clean Equipment & da & $\mathrm{zq}$ & $\mathrm{vb}$ & $\mathrm{jm}$ \\
\hline
\end{tabular}




\subsection{THE PRINCIPAL WASTE SIMULANTS: AY102 AND AP101 (AUTHOR-ZAMECNIK)}

Together with the pilot-scale FEP concentrated recycle streams, the waste feed for the pilot-scale UFP contained two simulated Hanford waste streams: tank 241-AY-102/C106 referred herein as AY102, and tank 241-AP-101, referred herein as AP101.

The composition and properties of AY102, including the distribution of species between the solid and supernate phases, has been previously described (Duignan, et al., 2004; Zamecnik, et al., 2004). This simulant was blended at SRNL from fractions manufactured by Optima Chemical (Douglas, GA) and additional reagents, as described in the references. The AP101 simulant formulation was defined by Pacific Northwest National Laboratory (Russell, et al., 2003) and the simulant was manufactured by Optima Chemical. The measured total slurry compositions of both of these simulants are shown in Table 15.

Table 15. Measured Simulant Compositions

\begin{tabular}{|c|c|c|c|c|}
\hline All values $\mathrm{mg} / \mathrm{L}$ except as noted. & \multicolumn{2}{|c|}{ AY102 Slurry } & \multicolumn{2}{|c|}{ AP101 } \\
\hline Total Solids (wt\%) & 26.80 & & 31.94 & \\
\hline Undissolved Solids (wt\%) & 11.75 & & 0.00 & \\
\hline Supernate Solids (wt\%) & 17.05 & & 31.94 & \\
\hline Density kg/L & 1.240 & & 1.279 & \\
\hline $\mathrm{Ag}$ & 624 & & 37.3 & \\
\hline $\mathrm{Al}$ & 15996 & 17484* & 7469 & $6932 *$ \\
\hline $\mathrm{B}$ & $<165$ & & $<142$ & \\
\hline $\mathrm{Ba}$ & 293 & 255 & $<1.53$ & \\
\hline $\mathrm{Bi}$ & 6.93 & & 0.0576 & \\
\hline $\mathrm{Ca}$ & 1131 & 1500 & 23.0 & \\
\hline $\mathrm{Cd}$ & 46.8 & & $<3.9$ & \\
\hline $\mathrm{Ce}$ & 366 & & $<127$ & \\
\hline $\mathrm{Co}$ & 151 & & $<15.6$ & \\
\hline $\mathrm{Cr}$ & 632 & 634 & 179 & 175 \\
\hline $\mathrm{Cs}$ & 18.7 & & 6.02 & \\
\hline Cs & $<17$ & & $<19.6$ & \\
\hline $\mathrm{Cu}$ & 77.9 & 81.8 & $<5.86$ & \\
\hline $\mathrm{Fe}$ & 35836 & 35216 & 76.4 & 68.4 \\
\hline $\mathrm{K}$ & 373 & & 30568 & \\
\hline $\mathrm{La}$ & 305 & & 13.9 & \\
\hline $\mathrm{Li}$ & $<24.8$ & & $<21.1$ & \\
\hline $\mathrm{Mg}$ & 379 & 373 & 5.51 & \\
\hline $\mathrm{Mn}$ & 7812 & 7638 & 3.00 & \\
\hline Mo & $<17$ & & $<10.6$ & \\
\hline $\mathrm{Na}$ (ICPES) & 84283 & & 127900 & \\
\hline $\mathrm{Na}(\mathrm{AA})$ & 91760 & & 129179 & \\
\hline Na average & 88021 & & 128540 & \\
\hline $\mathrm{Na}(\mathrm{M})$ & 3.83 & & 5.59 & \\
\hline $\mathrm{Nd}$ & 847 & & $<72.9$ & \\
\hline
\end{tabular}


WSRC-TR-2004-00478, REVISION 0

SRNL-RPP-2004-00075, REVISION 0

Table 15 continued

\begin{tabular}{|r|cc|c|}
\hline All values mg/L except as noted. & \multicolumn{2}{|c|}{ AY102 Slurry } & AP101 \\
\hline $\mathrm{Ni}$ & 1151 & 357 \\
$\mathrm{P}$ & $1748 \quad 1600$ & 269 \\
$\mathrm{~Pb}$ & 1662 & 1593 & $<156$ \\
\hline $\mathrm{Pr}$ & 170 & 0.0223 \\
$\mathrm{Rb}$ & 4.60 & 6.06 \\
$\mathrm{~S}$ & $892 \quad 899$ & 1599 \\
$\mathrm{Si}$ & 11016 & 329 \\
$\mathrm{Sr}$ & 279 & $<26$ \\
$\mathrm{~W}$ & $<84.7$ & $<1.18$ \\
$\mathrm{Y}$ & 50.8 & $<97.7$ \\
$\mathrm{Zn}$ & 81.3 & 0.0728 \\
$\mathrm{Zr}$ & 484 & 5.95 \\
\hline bromide & 69.4 & $<5.86$ \\
\hline chloride & 165 & $<128$ \\
\hline fluoride & 43.4 & 1530 \\
\hline formate & 389 & 28.0 \\
\hline nitrate & 319 & 1380 \\
nitrite & 4228 & 105000 \\
\hline oxalate & 9213 & 33400 \\
\hline phosphate & 3807 & 1550 \\
\hline sulfate & 1860 & 1150 \\
\hline acetate & 415 & 3740 \\
\hline citric acid & $<81.8$ & 1727 \\
formate & 264 & $<104$ \\
\hline$*$ Some rows two measurements. Those are the result of using two different dissolution methods. & & \\
\hline
\end{tabular}

2.6 PILOT WASTE FEED EVAPORATOR (AUTHORS - QURESHI, WILLIAMS \& ZAMECNIK)

The Waste Feed Evaporator Process, or FEP, is the first unit operation in the RPP-WTP Pretreatment Facility that a waste $(<5 \mathrm{M} \mathrm{Na}$ ) will see on start up. However, if the incoming wastes or waste blends are greater than $5 \mathrm{M} \mathrm{Na}$, the FEP evaporators are used to concentrate WTP recycle streams for blending with the fresh tank waste. Since the AY102 and AP101 have sufficient sodium concentration, the SIPP FEP operations only included evaporation of SIPP recycles. The SIPP FEP bottoms stream was then mixed with simulated waste streams, i.e., AY102 and AP101 that would normally come from the Hanford Tank farm to produce a feed stream for the Ultrafiltration Process (UFP) unit operation.

This section will explain the pilot-scale FEP unit operation that was done as part of Campaign II testing. The process started with the preparation of the FEP feed product, which was a mixture of many recycle streams in a predetermined fashion, see Table 2. A chemical explanation of the feed (recycle) stream formation will be followed by a short description on how feed formation was handled just before FEP operation and then how the waste stream was handled during and after its concentration. The following section will discuss the physical equipment used to evaporate the waste and how the test was run. Finally, the results will be discussed and conclusions made for the FEP unit operation. 


\subsubsection{Chemical Description of the Pilot FEP Feed Stream (Author-Zamecnik)}

The feed for the FEP was a series of recycle streams to be produced from operating the RPP-WTP Pretreatment Facility. The basis for the quantities of each recycle used was the modeling work done for the SIPP (Barnes, 2003), see Table 2. The basis for creating each recycle is shown in Fig. 2. This basis uses the information provided by WTP at the time this work was done; the current WTP operational plans may be different from what is described below.

The process was assumed to create 21,550 gal of concentrated HLW slurry per batch, with 16,750 gal going on to the HLW melter, 4300 gal "recycle" returning back to the feed tank, and a 500-gal heel remaining in the UFP feed tank. This 500-gal volume is referred to as the ejector heel, because it is what remains after the 4300 gallons of slurry is steam "ejected" from the tank. At the time of Campaign II testing the exact placement of the ejected slurry was still not well determined, therefore, for the SIPP the recycle of the 4,300 gal was not considered; this material was sent on to the HLW melter. Note that the 300 gallons of sludge estimated to be in the inlet of the pump is included in the 4,800 gal in the tank.

For the plant, the pump loop contains $~ 700$ gal of washed concentrated slurry. The loop is first drained to the Plant Overflow Vessel (PWD-VSL-33; the hyphenated tank numbers used in this section corresponds to those in the actual WTP plant) and then it is rinsed with three additions of $\sim 700$ gal each of condensate or water. Since all of the thick slurry will not drain out, we approximated the un-drained slurry as $10 \%$ of the volume, or 70 gal. (If it all drained out, the rinses would not be needed.) The first rinse was then 630 gal and subsequent draining and rinsing assumed much less residual slurry. The final rinse was assumed to leave about $0.25 \mathrm{wt} \%$ undissolved solids. With this sequence of rinsing, the material transferred to PWD-33 would be about 2,005 gal of slurrycondensate mixture with about $7.7 \mathrm{wt} \%$ undissolved solids.

In the SIPP, this process was approximated by mixing 4.6L of washed concentrated slurry with 8.6L of condensate from the FEP evaporator. This condensate represents that which would be collected in plant Radioactive Liquid Disposal (RLD) process and used for rinsing purposes. This made-up the recycle that would go to Campaign III. The same recycle stream for Campaign II was made up from Campaign I washed concentrated slurry and deionized and filtered (DIF) water (because Campaign I did not include evaporation and thus no condensate was available). The residual rinse in the filter loop that would be present at the start of the first $0.1 \mathrm{M} \mathrm{NaOH}$ rinse was made from $\sim 0.1 \mathrm{~L}$ of washed concentrated slurry and 12.5L of FEP condensate.

The tank heel prior to each rinse and acid cleaning step was estimated to give the correct undissolved solids loading expected for plant operation. Note that in the SIPP, the amount of rinse and acid used is greater, on a proportional basis, than in the WTP. To account for using more rinse or acid, a proportionally larger heel is left. 
WSRC-TR-2004-00478, REVISION 0

SRNL-RPP-2004-00075, REVISION 0

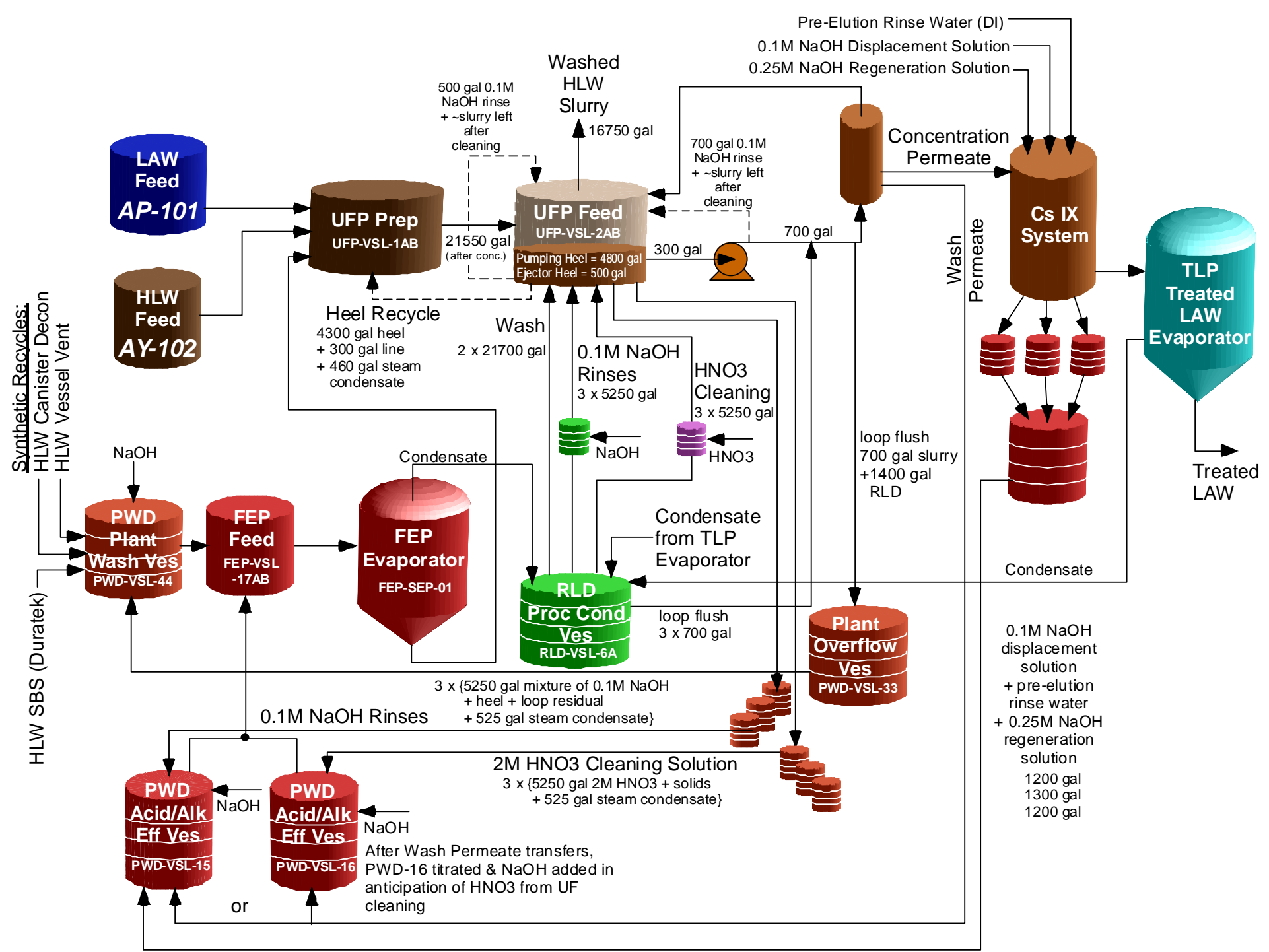

Figure 2. Basis for Generation of Recycles 
The ejector heel of 500 gal of washed concentrated slurry was simulated in the SIPP by adding $4 \mathrm{~L}$ of washed concentrated slurry back into the filter feed tank. Based on previous experience, we assumed that about 5L of slurry was held up in the filter loop, giving a total slurry inventory prior to rinsing of about 9L. The amount of slurry actually retained in the filter loop was found to be closer to 22L in Campaign III, so the amount of solids in the feed tank at the start of the rinse for Campaign II was much higher than expected or planned.

In the subsequent rinsing and cleaning steps, the ejector heel was approximated by putting back about 20L of the drained solution. With dilute slurries, the amount of holdup in the UF system was about 1.6L. The 21.6L of heel in each step, mixed with 94.6L of caustic rinse or acid was the same ratio as the heel + filter loop $(500+700$ gal $)$ remaining in the WTP to the rinse amount (5,250 gal); both ratios were 0.228 .

For Campaign I the first $0.1 \mathrm{M} \mathrm{NaOH}$ rinse recycle generated for Campaign II contained too much undissolved solids; the UDS was $7.4 \mathrm{wt} \%$ versus the desired $1.8 \mathrm{wt} \%$. Due to a misunderstanding of the WTP flowsheet, the steam ejection of the pump heel (from 4,800 gal to 500 gal) was not accounted for, so the solids content in this first rinse recycle was much too high. To account for this, the amount of first rinse used was less than the amount of the second and third rinses to give the correct undissolved solids content. Unfortunately, a spreadsheet error resulted in still adding too much of the first rinse. The first rinse added was 18.2L, with 49.5L each of the other two rinses; the correct amounts should have been 3.3L of first rinse and 56.9L each of rinses two and three. This error resulted in there being approximately $4.46 \mathrm{~kg}$ of undissolved solids in the FEP feed versus the desired approximately $3.34 \mathrm{~kg}$. (The measurement of the undissolved solids content of recycle streams, which was less than $1 \mathrm{wt} \%$ undissolved solids, is subject to significant error, which may be greater than $\pm 100 \%$.)

Figure 3 shows a schematic of the FEP feed blending and Fig. 4 shows the amounts of recycle streams mixed to give the FEP feed. The amount of the combined HLW recycles and the UFP loop flush, or PWD-44, used is shown by (4) in Fig. 4. The measured total solids and density of this mixture matches the prediction well, but the UDS measured was 0.09 vs. $0.40 \mathrm{wt} \%$. The FEP feed composition is shown as (7) in Fig. 4. In both the actual mixing and in a lab test, the HLW SBS solids, which are easily suspended and remain suspended for days, were flocculated and quickly precipitated when mixed with the synthetic recycles.

In making the SIPP UFP recycles, they were arbitrarily split between the PWD-VSL-15 and PWD-VSL-16 tanks. Into PWD-15 was added the second batch of UFP wash permeate (from the second 22 wash sequence) and the three caustic UFP flushes; all were caustic, except for the second flush, which was acidic due to the acid cleaning heel. The amount of caustic flush 1 added was less than that of flushes $2 \& 3$ to give closer to the correct undissolved solids in the recycle stream since the flush 1 UDS was too high. The resulting solution was predicted to be caustic, as it was eventually found to be. These four solutions were added in the order given above, and at no time did the bulk solution pH drop below 12. 
WSRC-TR-2004-00478, REVISION 0
SRNL-RPP-2004-00075, REVISION 0

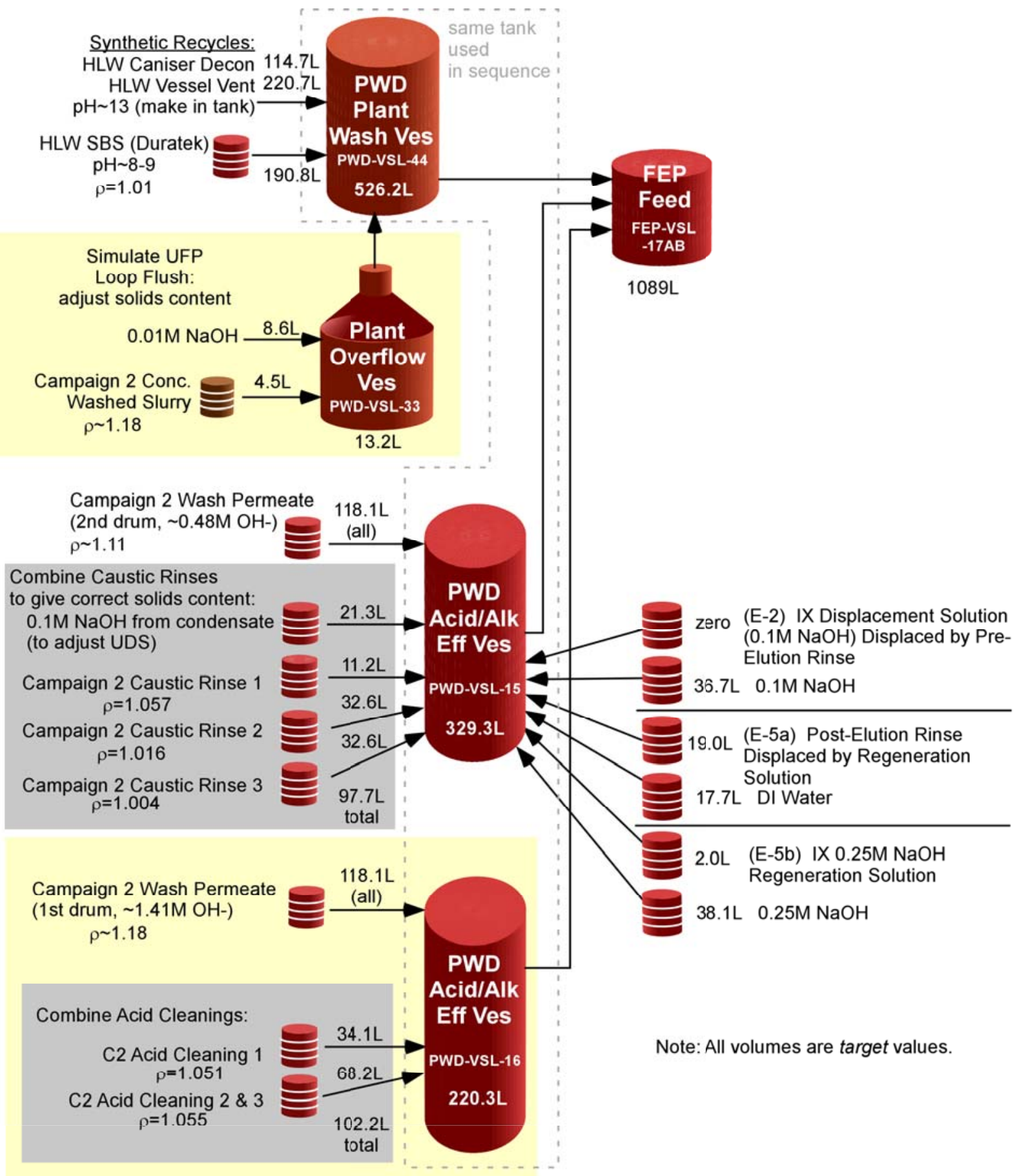

Figure 3. FEP Feed Blending 
WSRC-TR-2004-00478, REVISION 0

SRNL-RPP-2004-00075, REVISION 0
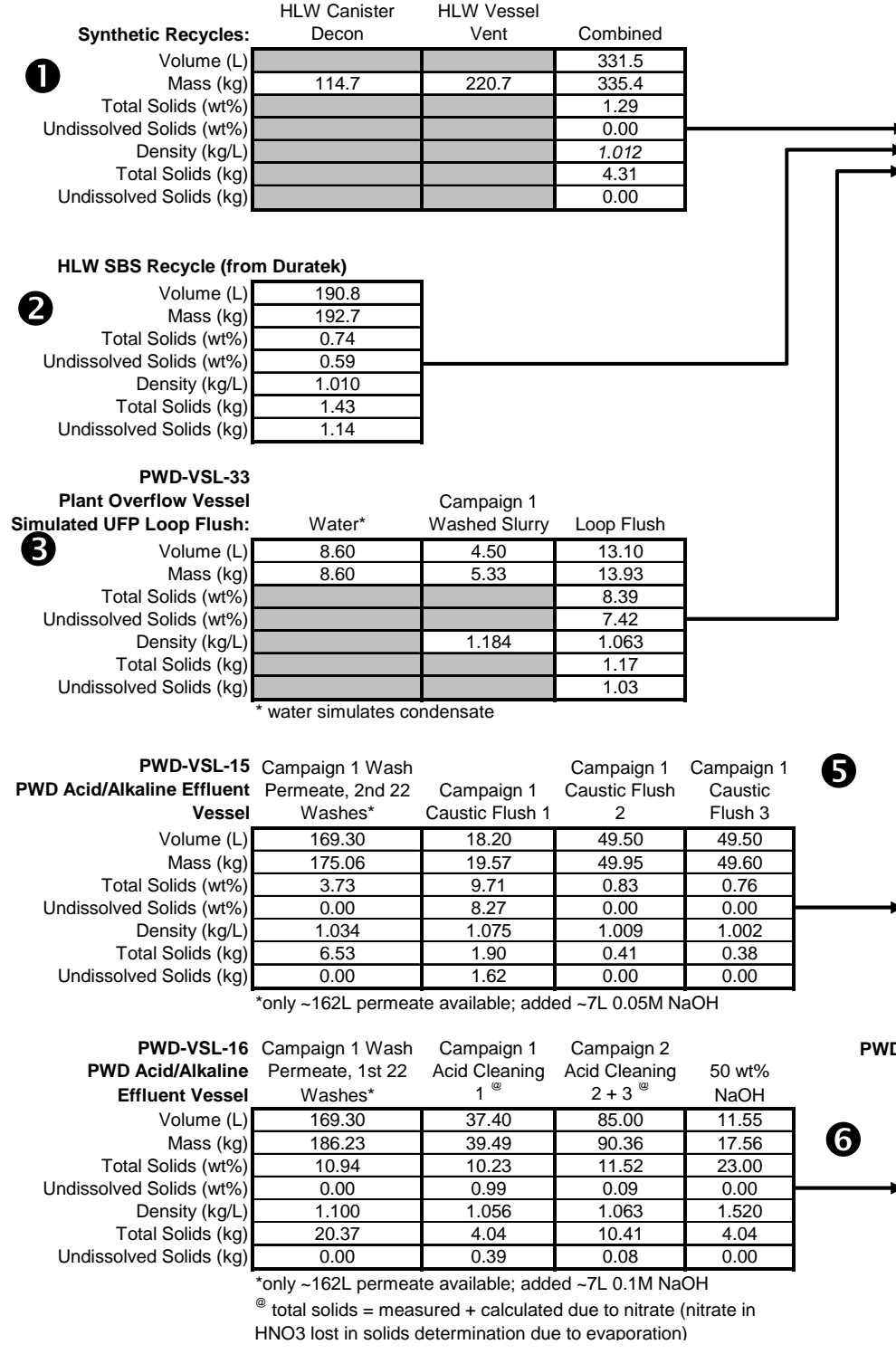

Figure 4. Mixing of Recycles

The PWD-16 tank recycles were the first 22 washes permeate, the three acid cleanings, and $\mathrm{NaOH}$ for $\mathrm{pH}$ adjustment. The first 22 washes permeate was used with the acid since it contained more caustic than the second 22 washes permeate, and thus less $\mathrm{NaOH}$ would be required to neutralize the acid. The addition order was the wash permeate, then the $\mathrm{NaOH}$, then the acid solutions. The amount of $\mathrm{NaOH}$ had been previously calculated and also tested in a small bench-scale test. An excess of $10 \%$ of $\mathrm{NaOH}$ was actually added to account for discrepancies in the actual volumes used. Again, the $\mathrm{pH}$ never dropped below 12 during mixing.

The measured solids loadings for the combined PWD-15 and -16 recycle streams were both slightly less than predicted from addition of the individual components. For the acid cleanings, the total solids content was estimated as the measured value plus the amount 
that would be due to the nitrate present; the nitrate in the $\mathrm{HNO}_{3}$ was evaporated during the solids determination. Table 16 summarizes the amounts of each of the recycle steams.

Table 16. Recycle Streams

\begin{tabular}{|c|c|c|}
\hline Stream & Source & Volume (L) \\
\hline HLW SBS Liquid & Duratek & 190.8 \\
\hline Vessel Vent Liquid & Synthetic & 220.7 \\
\hline HLW Canister Decon Liquid & Synthetic & 114.7 \\
\hline UFP Recycle - Wash Permeate & Campaign I & 338.8 \\
\hline $1^{\text {st }} 22$ Washes Permeate & & 162.3 \\
\hline $0.1 \mathrm{M} \mathrm{NaOH}$ & & 7.0 \\
\hline $2^{\text {nd }} 22$ Washes Permeate & & 162.3 \\
\hline $0.05 \mathrm{M} \mathrm{NaOH}$ & & 7.0 \\
\hline UFP Recycle - 0.1 M NaOH Rinse & Campaign I & 117.0 \\
\hline $1^{\text {st }}$ Rinse & & 18.2 \\
\hline $2^{\text {nd }}$ Rinse & & 49.5 \\
\hline $3^{\text {rd }}$ Rinse & & 49.5 \\
\hline UFP Recycle - 2M HNO $\mathrm{HN}_{3}$ Cleaning & Campaign I & 122.6 \\
\hline $1^{\text {st }}$ Cleaning & & 37.5 \\
\hline $2^{\text {nd }}$ Cleaning & & 37.5 \\
\hline $3^{\text {rd }}$ Cleaning & & 37.5 \\
\hline UFP Recycle - UF Loop Flushes & Campaign I & 13.2 \\
\hline $0.01 \mathrm{M} \mathrm{NaOH}$ (to approximate condensate) & & 8.6 \\
\hline Campaign I Washed Concentrated Slurry & & 4.6 \\
\hline CIX Recycles & none & 0 \\
\hline $50 \mathrm{wt} \% \mathrm{NaOH}$ to Adjust $\mathrm{pH}$ & reagents & 11.6 \\
\hline TOTAL & & 1129 \\
\hline
\end{tabular}

The synthetic HLW recycles were made up from reagents to approximate the expected compositions. The vessel vent liquid was $0.1 \mathrm{M} \mathrm{NaOH}$, while the HLW canister decon liquid was $0.15 \mathrm{M} \mathrm{NaOH}$ and $0.29 \mathrm{M} \mathrm{NaNO}_{3}$. The wash permeate, $0.1 \mathrm{M} \mathrm{NaOH}$ rinses, and the $2 \mathrm{M} \mathrm{HNO}_{3}$ cleaning liquids were generated from Campaign I. The amount of wash permeate available was slightly less than needed, so about 7L of $0.1 \mathrm{M} \mathrm{NaOH}$ and $7 \mathrm{~L}$ of $0.05 \mathrm{M} \mathrm{NaOH}$ were added to approximate the permeates from the first 22 and second 22 washes, respectively, from Campaign I. Note that the amount of each $\mathrm{NaOH}$ rinse and $\mathrm{HNO}_{3}$ cleaning solution available from Campaign I was greater than that needed.

The composition of the recycles is shown in Table 17. The three simulated recycle tanks were combined to make the FEP evaporator feed. These recycles were combined in the order: PWD-44, PWD-15, and then PWD-16. Nothing unusual was observed when these were mixed. 
WSRC-TR-2004-00478, REVISION 0

SRNL-RPP-2004-00075, REVISION 0

Table 17. Measured Recycle Compositions

\begin{tabular}{|c|c|c|c|c|c|c|c|c|c|c|c|c|c|}
\hline & & HLW SBS & $\begin{array}{l}\text { HLW } \\
\text { SBS }\end{array}$ & $\begin{array}{l}\text { HLW } \\
\text { SBS }\end{array}$ & $\begin{array}{l}\text { Loop } \\
\text { Flush }\end{array}$ & $\begin{array}{c}\text { Permeate } \\
\text { from } 1 \text { st } \\
22 \\
\text { Washes } \\
\end{array}$ & $\begin{array}{c}\text { Permeate } \\
\text { from } 2 \text { nd } \\
22 \\
\text { Washes } \\
\end{array}$ & $\begin{array}{l}\text { Caustic } \\
\text { Flush } 1 \\
\end{array}$ & $\begin{array}{c}\text { Caustic } \\
\text { Flushes } \\
2+3\end{array}$ & $\begin{array}{c}\text { Acid } \\
\text { Cleaning } \\
1 \\
\end{array}$ & $\begin{array}{c}\text { Acid } \\
\text { Cleaning } \\
2 \\
\end{array}$ & $\begin{array}{c}\text { Acid } \\
\text { Cleanin } \\
\text { g } 3 \\
\end{array}$ & $\begin{array}{c}\text { Acid } \\
\text { Cleaning } \\
\text { Composit } \\
\mathrm{e}\end{array}$ \\
\hline Total Solids & $\mathrm{wt} \%$ & 0.74 & & & 8.39 & 10.9 & 3.73 & 9.71 & 0.800 & 1.07 & 0.18 & 0 & NM \\
\hline Undissolved Solids & wt $\%$ & 0.59 & & & 7.42 & 0 & 0 & 8.27 & 0 & 0.99 & 0 & 0 & NM \\
\hline Supernate Solids & wt $\%$ & 0.15 & & & 1.05 & 10.9 & 3.73 & 1.57 & 0.800 & 0 & 0 & 0 & NM \\
\hline Density & $\mathrm{kg} / \mathrm{L}$ & $1.01-1.03$ & & & 1.063 & 1.10 & 1.03 & 1.09 & 1.01 & 1.06 & 1.06 & 1.06 & 1.06 \\
\hline $\mathrm{pH}$ & & & & & & $\sim 13$ & $\sim 12.7$ & 12.7 & $\begin{array}{l}0.80 \\
11.2 \\
\end{array}$ & $<1$ & $<1$ & $<1$ & $<1$ \\
\hline $\begin{array}{c}\text { Elemental Analysis } \\
\text { Sample Preparation } \\
\end{array}$ & & None & Filtered & $\begin{array}{c}\text { Dissol } \\
\text { ution }\end{array}$ & & None & None & $\begin{array}{l}\text { Dissolut } \\
\text { ion }\end{array}$ & $\begin{array}{l}\text { Dissolut } \\
\text { ion } \\
\end{array}$ & Filtered & Filtered & Filtered & $\begin{array}{c}\text { Dissoluti } \\
\text { on }\end{array}$ \\
\hline \multicolumn{14}{|c|}{ Elements by ICPES (unless otherwise noted) } \\
\hline $\mathrm{Ag}$ & $\mathrm{mg} / \mathrm{L}$ & $<0.3$ & $<0.3$ & $<7.58$ & & $<0.6$ & $<0.6$ & 410 & $<15.0$ & 12.8 & 2.82 & 0.727 & 21.0 \\
\hline $\mathrm{Al}$ & $\mathrm{mg} / \mathrm{L}$ & 16.6 & 0.669 & 19.1 & & 3860 & 1040 & 6680 & $<25.0$ & 256 & 42.0 & 8.00 & 302 \\
\hline $\mathrm{B}$ & $\mathrm{mg} / \mathrm{L}$ & 522 & 506 & 605.0 & & $<0.12$ & 0.621 & 50.0 & & 4.00 & 0 & $<0.12$ & \\
\hline $\mathrm{Ba}$ & $\mathrm{mg} / \mathrm{L}$ & 0.145 & 1.81 & $<0.505$ & & $<0.04$ & $<0.04$ & 191 & $<1.00$ & 11.4 & 1.73 & 0.321 & 9.00 \\
\hline Bi (ICPMS) & $\mathrm{mg} / \mathrm{L}$ & & & & & NM & $<0.01$ & $<0.01$ & $<0.01$ & & & & $<0.01$ \\
\hline $\mathrm{Ca}$ & $\mathrm{mg} / \mathrm{L}$ & 9.01 & 5.89 & 9.3 & & $<0.06$ & $<0.06$ & 1070 & $<1.00$ & 37.7 & 6.14 & 0.155 & 32.0 \\
\hline $\mathrm{Cd}$ & $\mathrm{mg} / \mathrm{L}$ & 0.380 & 0.0377 & 0.7 & & $<0.04$ & $<0.04$ & 30.0 & $<1.00$ & 1.35 & 0.239 & 0.0414 & 2.00 \\
\hline $\mathrm{Ce}$ & $\mathrm{mg} / \mathrm{L}$ & $<0.7$ & $<0.7$ & $<17.7$ & & $<1.4$ & $<1.4$ & 250 & $<35.0$ & 11.6 & 1.82 & $<1.4$ & $<33.0$ \\
\hline Co & $\mathrm{mg} / \mathrm{L}$ & $<0.08$ & $<0.08$ & $<2.02$ & & $<0.16$ & $<0.16$ & 30.0 & $<4.00$ & 0.377 & $<0.16$ & $<0.16$ & $<4.00$ \\
\hline $\mathrm{Cr}$ & $\mathrm{mg} / \mathrm{L}$ & 5.96 & 3.69 & 12.4 & & 94.0 & 26.0 & 310 & $<2.00$ & 16.0 & 3.00 & 1.00 & 15.0 \\
\hline Cs (AA) & $\mathrm{mg} / \mathrm{L}$ & & & & & 0.860 & 0.27 & $<16.4$ & $<4.95$ & $<0.1$ & $<0.1$ & $<0.1$ & $<4.72$ \\
\hline Cs (ICPMS) & $\mathrm{mg} / \mathrm{L}$ & & & & & NM & 0.263 & 0.635 & 0.138 & & & & 0.120 \\
\hline $\mathrm{Cu}$ & $\mathrm{mg} / \mathrm{L}$ & 1.46 & 1.09 & $<0.758$ & & $<0.06$ & $<0.06$ & 50.0 & $<1.00$ & 2.40 & 0.505 & 0.108 & 2.00 \\
\hline $\mathrm{Fe}$ & $\mathrm{mg} / \mathrm{L}$ & 83.2 & 1.42 & 114.8 & & $<0.08$ & $<0.08$ & 21700 & 9.00 & 1050 & 200 & 40.0 & 1020 \\
\hline $\mathrm{K}(\mathrm{AA})$ & $\mathrm{mg} / \mathrm{L}$ & $<3.75$ & & & & 1000 & 344 & 39.2 & $<7.43$ & 5.00 & 1.00 & 0.170 & $<7.08$ \\
\hline $\mathrm{La}$ & $\mathrm{mg} / \mathrm{L}$ & 2.51 & $<0.1$ & 2.6 & & $<0.2$ & $<0.2$ & 190 & $<5.00$ & $<12$ & $<12$ & $<12$ & 7.00 \\
\hline $\mathrm{Li}$ & $\mathrm{mg} / \mathrm{L}$ & 109 & 106 & 110.4 & & $<0.4$ & $<0.4$ & $<33.0$ & $<10.0$ & 8.91 & 1.60 & 0.257 & $<9.00$ \\
\hline $\mathrm{Mg}$ & $\mathrm{mg} / \mathrm{L}$ & 34.7 & 28.6 & 36.1 & & $<0.01$ & $<0.01$ & 230 & 0.250 & $<0.4$ & $<0.4$ & $<0.4$ & 10.0 \\
\hline $\mathrm{Mn}$ & $\mathrm{mg} / \mathrm{L}$ & 5.31 & 0.253 & 6.0 & & $<0.02$ & $<0.02$ & 4810 & 2.00 & 10.9 & 2.02 & 0.376 & 212 \\
\hline Mo & $\mathrm{mg} / \mathrm{L}$ & $<0.1$ & $<0.1$ & $<2.53$ & & $<0.2$ & $<0.2$ & $<16.0$ & $<5.00$ & 219 & 42.0 & 8.00 & 5.00 \\
\hline $\mathrm{Na}$ & $\mathrm{mg} / \mathrm{L}$ & 992 & 978 & 988.7 & & 44200 & 14100 & 9280 & 2110 & $<0.2$ & $<0.2$ & $<0.2$ & 1240 \\
\hline $\mathrm{Na}(\mathrm{AA})$ & $\mathrm{mg} / \mathrm{L}$ & 918 & & & & 52400 & 16800 & 9090 & 2070 & 1200 & 206 & 40.2 & 1180 \\
\hline
\end{tabular}


WSRC-TR-2004-00478, REVISION 0 SRNL-RPP-2004-00075, REVISION 0

\begin{tabular}{|c|c|c|c|c|c|c|c|c|c|c|c|c|c|}
\hline Table 17 (continued) & & HLW SBS & $\begin{array}{c}\text { HLW } \\
\text { SBS }\end{array}$ & $\begin{array}{c}\text { HLW } \\
\text { SBS }\end{array}$ & $\begin{array}{l}\text { Loop } \\
\text { Flush }\end{array}$ & $\begin{array}{c}\text { Permeate } \\
\text { from 1st } \\
22 \\
\text { Washes }\end{array}$ & $\begin{array}{c}\text { Permeate } \\
\text { from 2nd } \\
22 \\
\text { Washes }\end{array}$ & $\begin{array}{l}\text { Caustic } \\
\text { Flush } 1\end{array}$ & $\begin{array}{c}\text { Caustic } \\
\text { Flushes } \\
2+3\end{array}$ & $\begin{array}{c}\text { Acid } \\
\text { Cleaning } \\
1\end{array}$ & $\begin{array}{c}\text { Acid } \\
\text { Cleaning } \\
2\end{array}$ & $\begin{array}{c}\text { Acid } \\
\text { Cleanin } \\
\text { g } 3\end{array}$ & $\begin{array}{c}\text { Acid } \\
\text { Cleaning } \\
\text { Composit } \\
\text { e }\end{array}$ \\
\hline $\mathrm{Nd}$ & $\mathrm{mg} / \mathrm{L}$ & 1.45 & $<0.9$ & $<22.7$ & & $<1.8$ & $<1.8$ & 538 & $<45.0$ & 1230 & 224 & 41.0 & $<42.0$ \\
\hline $\mathrm{Ni}$ & $\mathrm{mg} / \mathrm{L}$ & 0.468 & 0.255 & 1.6 & & 0.387 & $<0.12$ & 713 & 3.00 & 25.6 & 4.65 & $<1.8$ & 35.0 \\
\hline $\mathrm{P}$ & $\mathrm{mg} / \mathrm{L}$ & 0.932 & $<0.8$ & $<20.2$ & & 740 & 139 & 351 & $<40.0$ & 33.0 & 6.00 & 1.00 & $<38.0$ \\
\hline $\mathrm{Pb}$ & $\mathrm{mg} / \mathrm{L}$ & 1.67 & $<0.8$ & $<20.2$ & & $<1.6$ & $<1.6$ & 1110 & $<40.0$ & 17.0 & 5.00 & 2.00 & $<38.0$ \\
\hline Pr (ICPMS) & $\mathrm{mg} / \mathrm{L}$ & & & & & & $<0.01$ & 124 & 0.0620 & & & & 5.56 \\
\hline Rb (ICPMS) & $\mathrm{mg} / \mathrm{L}$ & 0.670 & & & & & 0.0110 & 0.352 & 0.0160 & & & & $<0.01$ \\
\hline $\operatorname{Re}$ & $\mathrm{mg} / \mathrm{L}$ & 3.69 & 3.74 & 3.8 & & & & & & 47.0 & 9.00 & $<1.6$ & \\
\hline $\mathrm{S}$ & $\mathrm{mg} / \mathrm{L}$ & 117 & 121 & 123.0 & & 385 & 102 & 260 & $<50.0$ & 8.47 & $<2$ & $<2$ & $<47.0$ \\
\hline $\mathrm{Si}$ & $\mathrm{mg} / \mathrm{L}$ & 70.0 & 14.6 & 59.5 & & 8.18 & 2.70 & 6310 & 722 & 98.0 & 20.0 & 5.00 & 878 \\
\hline $\mathrm{Sr}$ & $\mathrm{mg} / \mathrm{L}$ & 17.5 & 8.38 & 17.8 & & 0.0767 & 0.0188 & 190 & 0 & 9.00 & 4.00 & 1.00 & 8.00 \\
\hline $\mathrm{Ti}$ & $\mathrm{mg} / \mathrm{L}$ & 1.80 & $<0.03$ & 1.7 & & $<0.06$ & $<0.06$ & 10.0 & $<1.00$ & 0.113 & $<0.06$ & $<0.06$ & $<1.00$ \\
\hline W & $\mathrm{mg} / \mathrm{L}$ & & & & & 44.0 & 12.0 & $<82.0$ & $<25.0$ & $<1$ & $<1$ & $<1$ & $<24.0$ \\
\hline Y (ICPMS) & $\mathrm{mg} / \mathrm{L}$ & & & & & & $<0.01$ & 36.1 & 0.0220 & & & & 1.64 \\
\hline $\mathrm{Zn}$ & $\mathrm{mg} / \mathrm{L}$ & 18.9 & 0.751 & 20.6 & & $<0.04$ & $<0.04$ & 180 & 1.00 & 3.61 & 0.643 & 0.0943 & 2.00 \\
\hline $\mathrm{Zr}$ & $\mathrm{mg} / \mathrm{L}$ & 0.336 & $<0.03$ & 0.8 & & $<0.06$ & $<0.06$ & 300 & 1.00 & 0.246 & 1.83 & 0.971 & 13.0 \\
\hline \multicolumn{14}{|l|}{ Anions by IC: } \\
\hline bromide & $\mathrm{mg} / \mathrm{L}$ & $<10$ & & $<10$ & & 27.0 & $<100$ & $<79$ & $<100$ & & & & \\
\hline chloride & $\mathrm{mg} / \mathrm{L}$ & 332 & & 332 & & 97.0 & 21.0 & 12.0 & $<20$ & & & & \\
\hline fluoride & $\mathrm{mg} / \mathrm{L}$ & 43.0 & & 43 & & 36.0 & 7.00 & 7.00 & $<20$ & & & & \\
\hline formate & $\mathrm{mg} / \mathrm{L}$ & $<10$ & & $<10$ & & 165 & 10.0 & 11.0 & $<100$ & & & & \\
\hline nitrate & $\mathrm{mg} / \mathrm{L}$ & $<10$ & & $<10$ & & 216 & 36.0 & 23.0 & 12500 & & & & \\
\hline nitrite & $\mathrm{mg} / \mathrm{L}$ & $<10$ & & $<10$ & & 3170 & 528 & 198 & $<100$ & & & & \\
\hline oxalate & $\mathrm{mg} / \mathrm{L}$ & 34.0 & & 34 & & 5530 & 4440 & 1400 & $<100$ & & & & \\
\hline phosphate & $\mathrm{mg} / \mathrm{L}$ & 991 & & 991 & & 2740 & 266 & 328 & $<100$ & & & & \\
\hline sulfate & $\mathrm{mg} / \mathrm{L}$ & 287 & & 287 & & 1250 & 225 & 98.0 & $<50$ & & & & \\
\hline \multicolumn{14}{|l|}{ Anions by IEC: } \\
\hline acetate & $\mathrm{mg} / \mathrm{L}$ & & & & & 35.0 & 101 & & & & & & \\
\hline citric acid & $\mathrm{mg} / \mathrm{L}$ & & & & & $<10$ & $<10$ & & & & & & \\
\hline formate & $\mathrm{mg} / \mathrm{L}$ & & & & & 189 & 46.0 & & & & & & \\
\hline Carbonate (from TIC) & $\mathrm{mg} / \mathrm{L}$ & & & & & 5900 & 1775 & & & & & & \\
\hline Oxalate (from TOC) & $\mathrm{mg} / \mathrm{L}$ & & & & & 754 & 745 & & & & & & \\
\hline
\end{tabular}




\subsubsection{Simulant Handling (Author - Williams)}

For the following description of how the recycle FEP feed stream was made at the experimental site, see Fig. 3. A 760-liter tank was used to mix pilot-scale quantities of simulated HLW recycles that were then transferred to the pilot-scale FEP Feed Tank, which represented the plant tank: FEP-VSL-17AB. On 7 April 2004, pilot-scale quantities of HLW Canister Decon and HLW Vessel Vent solutions were synthesized by combining 330 liters of deionized and filtered (DIF) water with $3 \mathrm{~kg}$ of $50 \mathrm{wt} \% \mathrm{NaOH}$, $2.77 \mathrm{~kg}$ of sodium nitrate, and $192.7 \mathrm{~kg}$ of HLW SBS Quencher solution from VSL. At this stage, the mixing tank represented the plant tank: PWD-VSL-44. To this mixture, the contents of mock plant tank PWD-VSL-33 (Plant Overflow Vessel) were added, which contained $5.31 \mathrm{~kg}$ of Campaign I concentrated washed HLW slurry from drum No. RM-03-280, 8.6 liters of DIF water and 6.88 grams of $50 \mathrm{wt} \% \mathrm{NaOH}$. After mixing the entire contents of the mock PWD-VSL-44, they were transferred to the FEP Feed Tank, also on 7 April.

On 8 April, the then empty 760-liter mixing tank was used as a plant mock PWD-VSL-15 tank and was filled with caustic recycle streams from Campaign I. To that tank, the second of two drums of wash permeate was added, drum No. RM-03-270, which contained $175.1 \mathrm{~kg}$ of material. Then the $0.1 \mathrm{M}$ caustic flush recycle stream was added from three drums referred to as caustic flush 1,2 , and 3 . They were from drums Nos. RM-03-281, RM-03-285, and RM-03-284, which contained $19.6 \mathrm{~kg}, 49.9 \mathrm{~kg}$, and 49.6 $\mathrm{kg}$, respectively. The target $\mathrm{pH}$ for this mixture was to be greater than or equal to 12 , and since the $\mathrm{pH}$ was measured at 13 no additional $\mathrm{NaOH}$ was added. The contents of this mock PWD-VSL-15 mixing vessel were then added to the mixture in the FEP Feed Tank, also on 8 April.

Once again the 760-liter mixing tank was empty and this time it would represent the plant PWD-VSL-16 tank to receive the acidic recycle streams. That solution had to be adjusted to be alkaline before being joined to the caustic contents of the FEP feed tank. Specifically, the empty tank was first filled with $186.2 \mathrm{~kg}$ of $0.1 \mathrm{M} \mathrm{NaOH}$ wash permeate from the first of two drums of wash permeate recycle, drum No. RM-03-269. (This drum actually had only $179.3 \mathrm{~kg}$, so an additional $6.9 \mathrm{~kg}$ of fresh $0.1 \mathrm{M} \mathrm{NaOH}$ were added to make up the difference.) While the tank agitator was running, $17.6 \mathrm{~kg}$ of fresh $50 \mathrm{wt} \%$ $\mathrm{NaOH}$ were added. This amount of the added caustic was based on the amount of acidic recycle streams to be neutralized before being introduced to the FEP. Into the waiting mixture of caustic were added two drums of spent $2 \mathrm{M}$ nitric acid UFP cleaning solution with constant stirring. From the drum (referred to as acid cleaning 1) No. RM-03-307 an amount of $39.5 \mathrm{~kg}$ was transferred and from RM-03-305 (referred to as acid cleaning 2 \& 3) an amount of $90.4 \mathrm{~kg}$ was transferred. The $\mathrm{pH}$ of the solution after mixing was measured to be 12.8. The acid/caustic mixture was transferred to the FEP Feed Tank on 12 April to start the clock on a 2-day holding period before evaporation began. To roughly see what each of these mixtures looked like Fig. 5 shows the line-up of each after the solids have settled. To understand Fig. 5 compare those pictures with Fig. 3, which 
schematically shows the four individual mixtures that eventually were joined to make the final FEP feed.

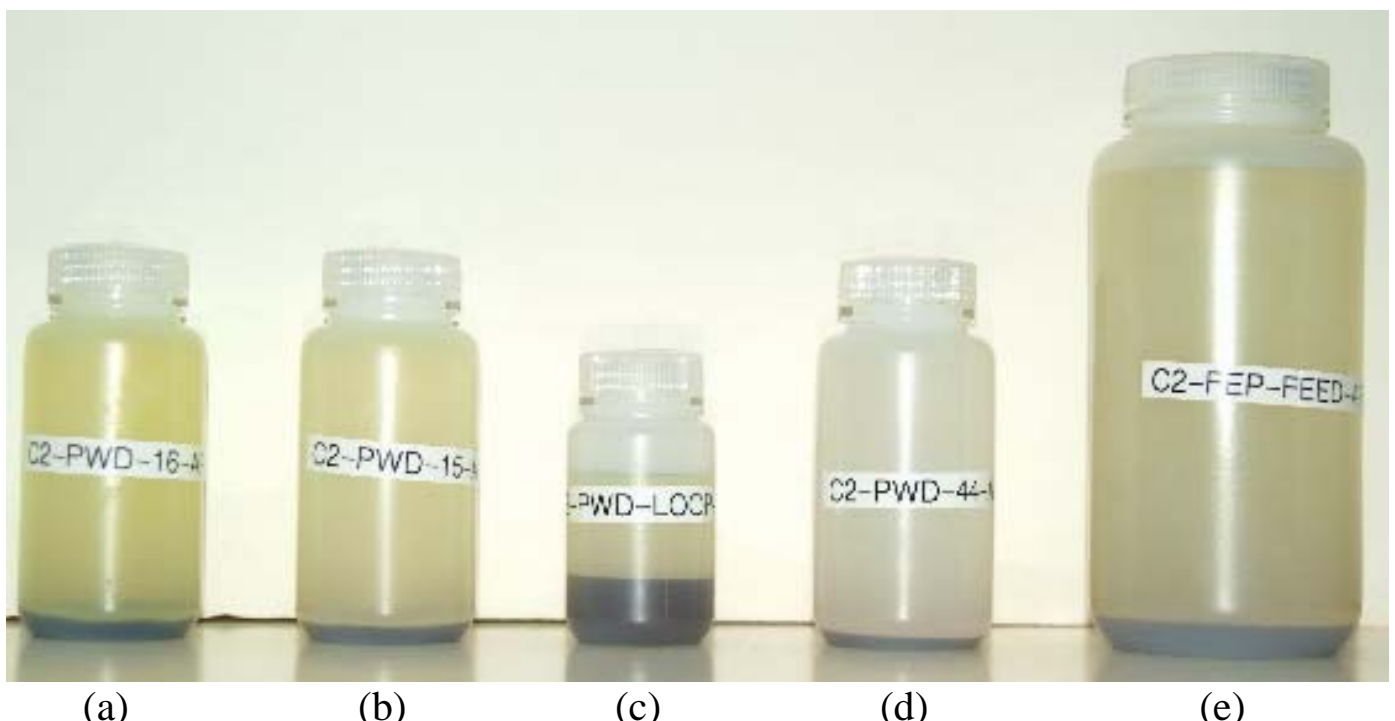

Figure 5. Picture of the Settled Contents of the Mixtures Shown in Fig. 3: They Are Simulants of (a) PWD-VSL-16, (b) PWD-VSL-15, (c) PWD-VSL-33, (d) PWD-VSL-44, and (e) FEP feed

The total material mixed in the pilot-scale FEP unit operation Feed Tank, Fig. 6, and subsequently sent to the FEP Evaporator, was 1,170.3 kg.

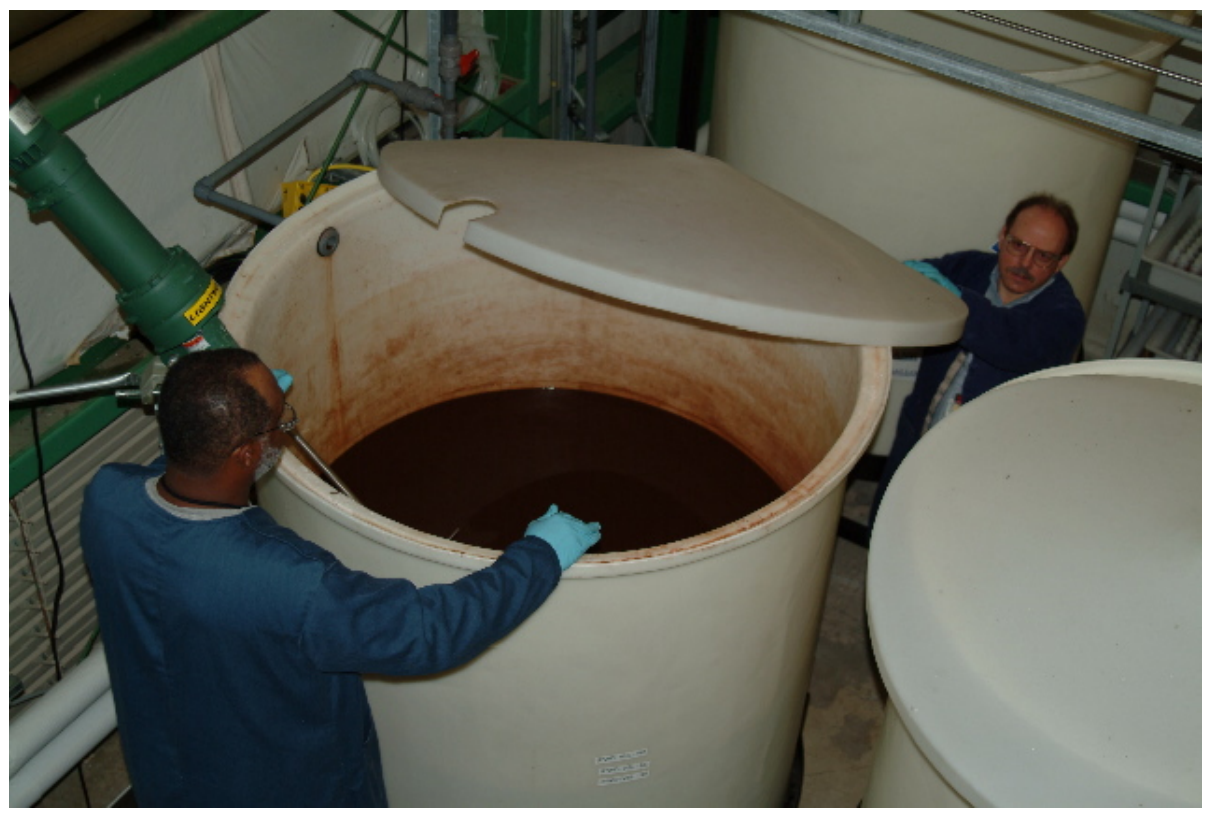

Figure 6. Pilot-scale FEP Unit Operation Feed Tank 
After evaporation started on 14 April, and during the first 30 minutes of operation, 278.4 g of Dow Corning Q2-3183A antifoam were added in three batches of $92.8 \mathrm{~g}$ each. This was done to attain a target concentration of $1500 \mathrm{ppm}$ of antifoam that the WTP plans to utilize. Note that, per the vendor's recommendation the antifoam was diluted to a 10:1 ratio with deionized and filtered water (DIF) before the addition was made. At the end of FEP testing, a total of $183.7 \mathrm{~kg}$ of concentrate "FEP bottoms" were produced with a density of $1.256 \mathrm{gm} / \mathrm{ml}$, as measured by volumetric flask at $25.5^{\circ} \mathrm{C}$, and $1.263 \mathrm{gm} / \mathrm{ml}$, as measured by densitometer.

\subsubsection{Pilot FEP Equipment (Author - Qureshi)}

All evaporation campaigns were performed in the Pilot-Scale Evaporator designed and constructed for the RPP Project. The evaporator is located in the Engineering Development Laboratory of the Savannah River National Laboratory (SRNL).

Figure 7 shows the Process and Instrumentation Diagram (P\&ID). The Pilot Scale Evaporator System consists of many sub-systems provided below alphabetically.

- Antifoam System

- Concentrate Loop

- Evaporator Recirculation

- Feed Loop

- House Air

- Jet Pump

- Primary Condensate

- Process Water

- Secondary Condensate

- Steam Generator

- Spray Water

Typically, each of the SIPP campaigns was to evaporate about 1,000 liters of waste simulant. For this campaign, Campaign II, 1,170 kg were used which was approximately 1,125 liters. The pilot-scale SIPP evaporator uses forced circulation where heat is added in the reboiler that is essentially a shell and tube heat exchange with steam on the shell side. No evaporation occurs in the reboiler due to hydrostatic pressure head. The evaporator vessel is kept under vacuum ( $\sim 1$ psia or $6.9 \mathrm{kPa})$ and the boiling temperature for water is about $104^{\circ} \mathrm{F}\left(40^{\circ} \mathrm{C}\right)$ at this pressure. The simulant has a boiling point elevation of about $11^{\circ} \mathrm{F}\left(\sim 6^{\circ} \mathrm{C}\right)$. (Thus the entire evaporation process occurs at moderate temperatures, $\left.100-120 \mathrm{~F}\left(44^{\circ}-49^{\circ} \mathrm{C}\right)\right)$. The evaporator vessel is about 27 feet $(8.2 \mathrm{~m})$ tall with a nominal inside diameter of $17^{\prime \prime}(0.43 \mathrm{~m})$. The bottom 12 feet of the vessel is stainless steel and the top 15 feet is acrylic, see Fig. 8. 
WSRC-TR-2004-00478, REVISION 0

SRNL-RPP-2004-00075, REVISION 0

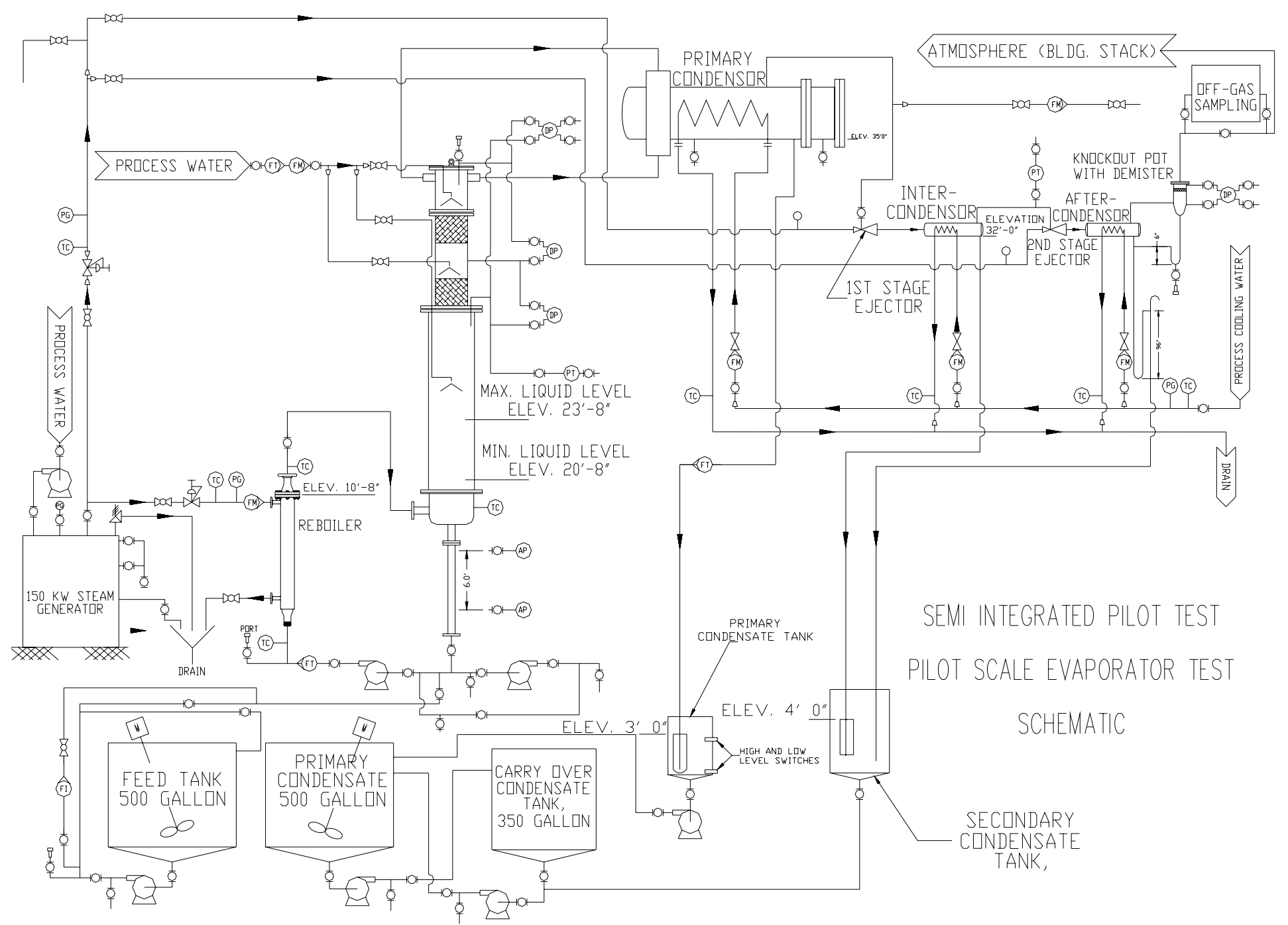

Figure 7. SIPP Pilot-Scale FEP/TLP Unit 


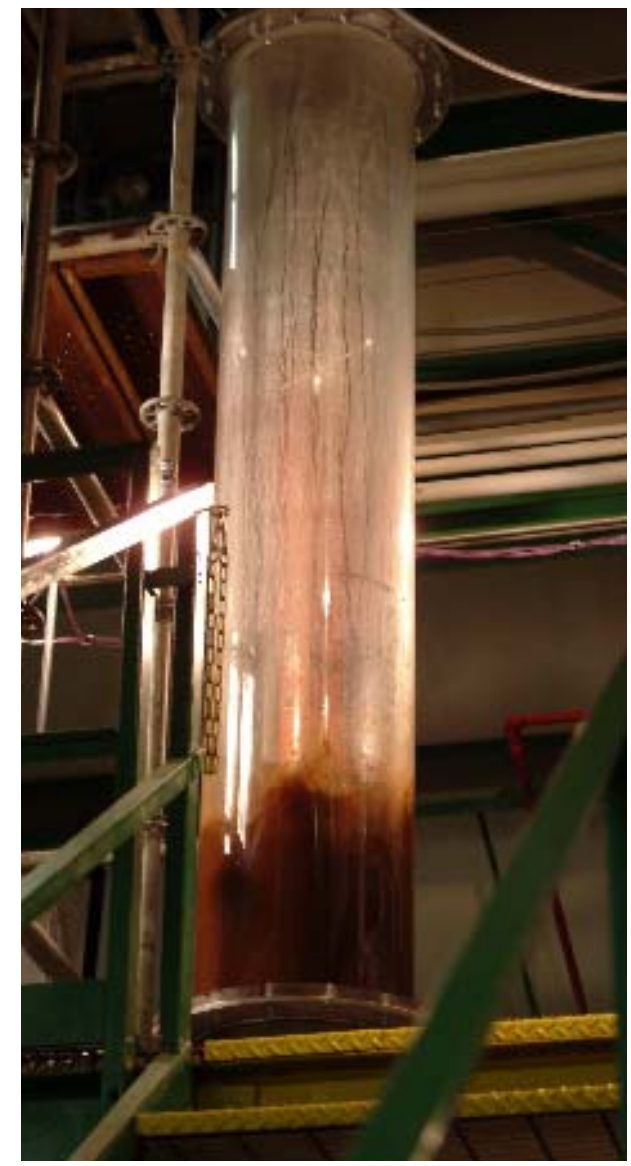

Figure 8. SIPP Pilot-Scale FEP/TLP Unit: 15-foot Acrylic Section with Ongoing Evaporation

Of the bottom 12 feet $(3.7 \mathrm{~m})$, the stainless steel vessel is only 2 feet $(0.6 \mathrm{~m})$ high and the remaining height of 10 feet $(3 \mathrm{~m})$ is taken up by a 2 " $(0.05 \mathrm{~m})$ stainless steel pipe. The boiling surface is maintained in the lower part of the acrylic section. The minimum and maximum liquid levels in the acrylic section are 5" (0.13 m) and 40" (1.0 m), respectively, see Fig 9.

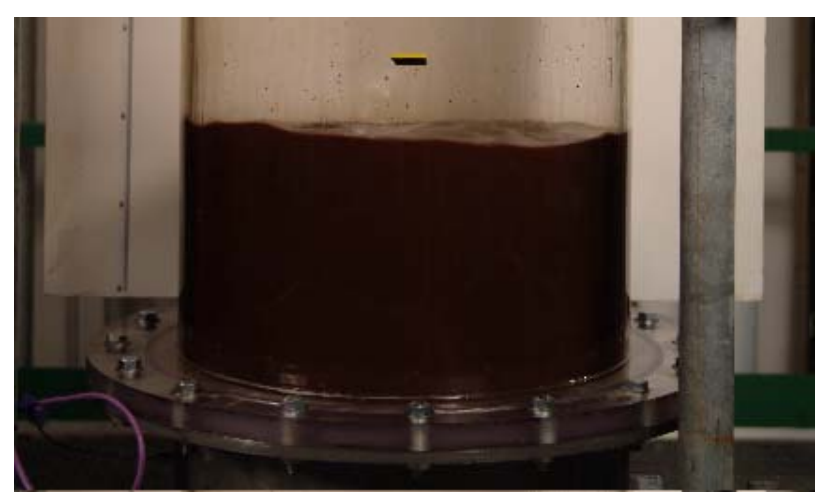

Figure 9. SIPP Pilot-Scale FEP/TLP Unit: Feed Ready for Evaporation to Commence 
The designed capacity of evaporator vessel is about 40 gallons (151 liters) and a feed tank supplies the simulant to the evaporator. The evaporator is designed to evaporate $0.4-0.5 \mathrm{gpm}$ $(1.5-1.9 \mathrm{lpm})$ of water. Figure 10 shows the pilot-scale FEP at approximately the 30-gallons level while evaporating at approximately $0.5 \mathrm{gpm}$, before and after defoamer was added.

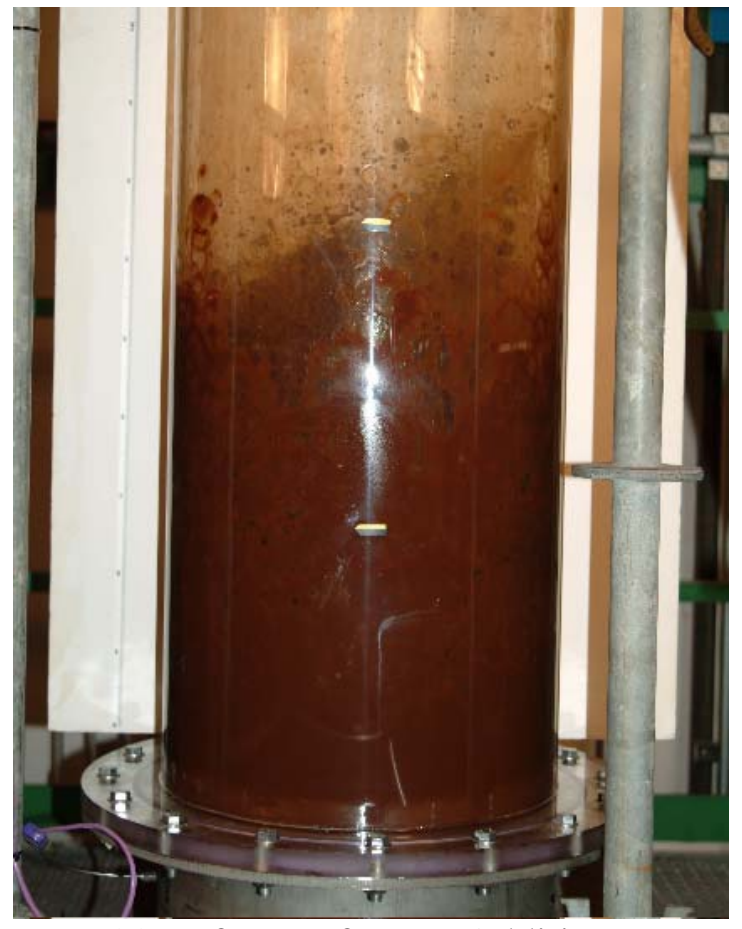

(a) Before Defoamer Addition

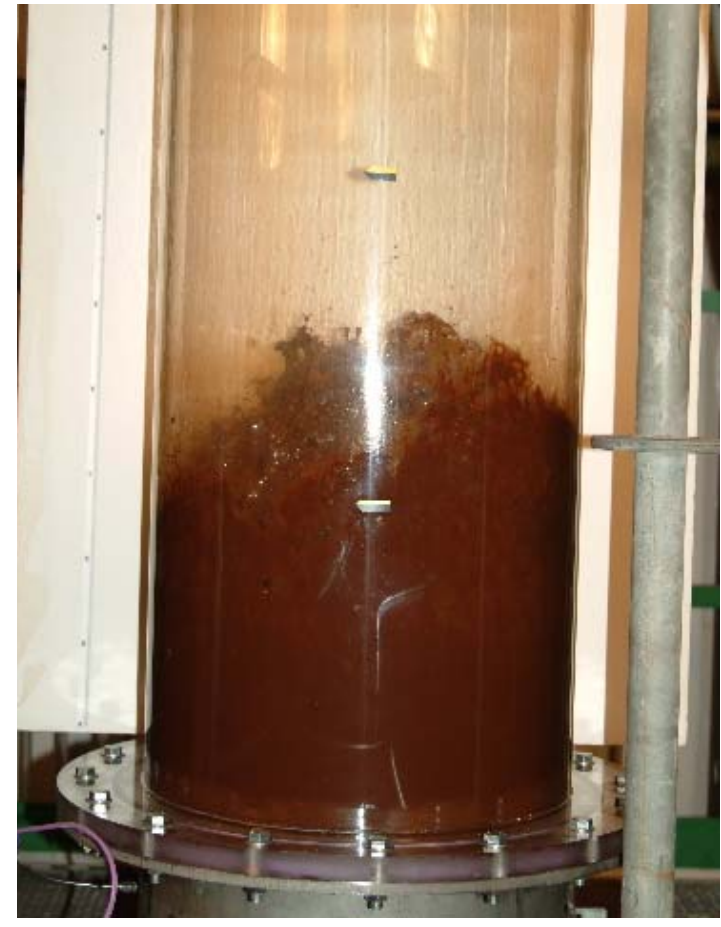

(b) After Defoamer Addition

Figure 10. SIPP Pilot-Scale FEP/TLP Unit: Evaporating

The water vapor is condensed in the primary condenser. Under steady conditions, the concentrate is removed from the recirculation line and collected in a drum. The condensate from the primary condenser is collected in the primary condensate tank. A two-stage steam driven jet pump maintains desired vacuum in the evaporator vessel. The jet pump has its own set of condensers, inter and after-condenser. A separate tank catches the condensate from the jet pump. Several sample and injection ports are provided to draw samples and inject antifoam agent as needed. All test data was acquired by a data acquisition system (DAS). Additionally, manual data and observations were recorded in the lab notebook issued for this task.

\subsubsection{Pilot FEP Operation (Author - Qureshi)}

Prior to the SIPP campaigns, the evaporator system was disassembled, cleaned, inspected and reassembled. The system was found to be free of any material defects, fouling and malfunction from previous evaporation runs. After assembly of the system the following tests were performed to ensure proper operation of the evaporator. 


\subsubsection{Experimental Facility Leak Testing}

Objective - Determine if the system can hold vacuum and the air in-leakage rate is acceptable

\section{Test Sequence}

i) Isolated the Evaporator Recirculation (ER) Loop from rest of the system by closing appropriate valves.

ii) Disconnected the First Stage Ejector line from the primary condenser and connected a vacuum pump at the primary condenser discharge.

iii) Filled water in Evaporator vessel to the maximum operating liquid level (about 40" (1.02 m)) in the lower acrylic section.

iv) Filled up the primary dip tube reservoir with water.

v) Started and operated the vacuum pump until the water level in the dip tube is about 31 feet $(9.4 \mathrm{~m})$ of water.

vi) Verified that there was no air bubbling into the system.

vii) Verified that the system can hold vacuum for about 30 minutes.

\subsubsection{Evaporation Test with Water}

Objective - To shake down the system and its components using water as test fluid prior to the chemical runs.

\section{Test Sequence}

i) Isolated the Reboiler and the Jet Pump from the Steam Generator.

ii) Started the Steam Generator.

iii) Filled the Feed Tank with Process Water to about 25\% level (about 125 gallons (743 liters)).

iv) Using the Feed Loop pump FL-PU1, transferred water from the Feed Tank to the evaporator vessel to a level about $40 ”(1.02 \mathrm{~m})$ in the acrylic section.

v) Filled the primary condensate and secondary condensate dip tubes with water.

vi) Started the Jet Pump Vacuum System.

vii) Started the main recirculation pump and started supplying steam to the Reboiler. 


\section{WSRC-TR-2004-00478, REVISION 0}

SRNL-RPP-2004-00075, REVISION 0

viii) Adjusted steam to the Reboiler to limit the system temperature below $140^{\circ} \mathrm{F}\left(60^{\circ} \mathrm{C}\right)$.

ix) Once the system pressure dropped to about 1 psia (6.9 kPa), boiled water for about 30 minutes.

x) After completing the water boiling test, turned off the steam supply to the Jet Pump and the Reboiler and shut down the Steam Generator.

\subsubsection{SIPP-Feed Evaporation Process (FEP)Tests}

Objective - To perform pilot scale tests of the Feed Evaporation Process.

This evaporation campaign used about 1125 liters (1170 kg) of FEP Feed. The feed composition and mixing process is described in sections 2.6.1 and 2.6.2.

The FEP Evaporation Campaign II took about 12 hours to complete. During this time period, several samples were drawn as outlined in SIPP Sample Matrix Campaign II (see Appendix D).

\section{Test Sequence}

i) Started Steam Generator and the Data Acquisition System.

ii) Ran the Feed Tank mixer for about 5 minutes.

iii) Using the Feed Loop Pump, transferred feed from the Feed Tank to the evaporator vessel to about 24 ” $(0.61 \mathrm{~m})$ level in the clear section.

iv) Filled the primary condensate and secondary condensate dip tubes with water.

v) Supplied process water to the Jet Pump condensers and started the Jet Pump.

vi) Supplied process water to the Primary Condenser to about 30 gpm (114 lpm).

vii) Started the main recirculation pump and turned on the steam to the Reboiler.

viii) Once the feed reached the saturation temperature and boiling started, maintained the liquid level in the evaporator by feeding the appropriate amount of feed using the feed pump.

ix) Recorded all the temperature, pressure and flow date via DAS. Additionally, video taped the action near the boiling surface as needed at different times

x) Antifoam agent was added to the recirculation loop at the beginning of the evaporation campaign.

xi) Drew liquid samples according the sample matrix. 
xii) Continued evaporation process until the target density of $1.25 \mathrm{~g} / \mathrm{ml} @ 25^{\circ} \mathrm{C}$ was reached.

xiii) Once the evaporation campaign was complete, the steam generator was shut down and all the concentrate was transferred into a 55-gal drum.

xiv) After removing all the concentrate, filled the system with water ( 30 gallons (114 liters)) and rinsed it thoroughly by running the recirculation pump.

\subsubsection{Pilot FEP Discussion of Results}

\subsubsection{Experimental Observations (Author-Qureshi))}

The SIPP FEP Campaign II was run on Wednesday, 14 April 2004 in SRNL's Engineering Development Lab. A total of about 1,125 liters of feed was concentrated down to about 147 liters. The entire evaporation process proceeded without any glitch or equipment failure. The evaporator was designed to evaporate around $0.4 \mathrm{gpm}(1.5 \mathrm{lpm})$ of water. The average evaporation rate during this campaign was around $0.43 \mathrm{gpm}$.

Table 18 provides a chronology of the evaporation campaign. Time recorded below follows a military format.

Table 18. Chronology of the evaporation campaign

\begin{tabular}{|l|l|l|}
\hline Time & Event & Comments \\
\hline 0600 & Started Steam Generator & \\
\hline 0615 & Started Feed Mixing by pump recirculation and agitator & \\
\hline 0640 & Pulled Feed sample & \\
\hline 0642 & Started Feed transfer to evaporator vessel & \\
\hline 0644 & Started Jet Pump to pull vacuum in the evaporator & \\
\hline 0647 & Stopped Feed transfer & \\
\hline 0648 & Started main circulation pump & \\
\hline 0649 & Started steam supply to reboiler & Density 1.015 g/ml @65C \\
\hline 0700 & Pulled 200 ml sample from the evaporator circulation loop & No significant foaming \\
\hline 0715 & Steady boiling taking place & Addition time 1 minute \\
\hline 0739 & 500 ppm of antifoam agent added to the recirc. loop & Stable froth height 1 ft. \\
\hline 0744 & Additional 500 ppm of antifoam added to the recirc. loop & \\
\hline 0749 & Additional 500 ppm of antifoam added to the recirc. loop & \\
\hline 1000 & Pulled all samples at 400 liter total transfer & $1.09 \mathrm{~g} / \mathrm{ml} \mathrm{@} \mathrm{50C}$ \\
\hline 1010 & Pulled 50 ml from evaporator for density check & \\
\hline 1145 & Pulled all samples at 600 liter total transfer & $1.13 \mathrm{~g} / \mathrm{ml} @$ 50C \\
\hline 1215 & Pulled 50 ml from evaporator for density check & $1.16 \mathrm{~g} / \mathrm{ml} @$ 20C \\
\hline 1254 & Pulled $10 \mathrm{ml}$ sample for density check with density meter & \\
\hline 1330 & Pulled all samples at 800 liter total transfer & $1.211 \mathrm{~g} / \mathrm{ml} @$ @ 20C \\
\hline 1415 & Pulled $10 \mathrm{ml}$ sample for density check with density meter & \\
\hline 1600 & Pulled $10 \mathrm{ml}$ sample for density check with density meter & $1.224 \mathrm{~g} / \mathrm{ml} @ 20 \mathrm{C}$ \\
\hline
\end{tabular}


WSRC-TR-2004-00478, REVISION 0

SRNL-RPP-2004-00075, REVISION 0

\begin{tabular}{|l|l|l|}
\hline 1632 & Pulled $10 \mathrm{ml}$ sample for density check with density meter & $\begin{array}{l}1.25 \mathrm{~g} / \mathrm{ml} @ \text { @ 20C Target } \\
\text { value reached }\end{array}$ \\
\hline 1645 & System shut down. Concentrate made 147.2 liters $(38.9$ gal) & \\
\hline 1700 & Pulled all end samples & Density in drum $1.248 \mathrm{~g} / \mathrm{ml}$ \\
\hline 1710 & Transferred all concentrate to a 55 gal drum & \multicolumn{2}{|l}{} \\
\hline
\end{tabular}

\subsubsection{FEP Waste Products (Author - Zamecnik)}

Table 19 shows that the predicted and measured solids concentrations in the FEP feed matched well. Table 20 shows the FEP feed and bottoms compositions. The feed was concentrated from 1,125L to 147.2L final volume. For the bottoms sample, elemental analyses from both the microwave and sodium peroxide dissolutions are shown for selected elements. The IC analyses were performed on filtered samples, so these analyses represent only the soluble portion of the total species. The "Total Mass" columns show the calculated total mass of each species based on the measured concentration and the calculated total mass. The "Bottoms/Feed \%" column shows that for most of the more abundant species, the material balance closes reasonably well. The total mass that was calculated was very consistent, with the bottoms being $99.5 \%$ of the feed. A notable exception is oxalate, which was present in the bottoms at only $10 \%$ of its initial amount, indicating that $90 \%$ of the dissolved oxalate precipitated during the evaporation process. The elemental compositions of the supernate portion of the feed and bottoms supernates are also shown in Table 20. Note that the supernate portion is the liquid (undissolved solids-free) part of the stream.

Table 21 summarizes the masses of selected dissolved and total species in the FEP evaporator feed and bottoms. Species expected to be and found to be mostly undissolved were Ag, Ba, Ca, Fe, Mg, Mn, Nd, Na, and Si. Aluminum was about $45 \%$ undissolved, sodium was perhaps $8 \%$ undissolved, and $\mathrm{Cr}$ was about $38 \%$ undissolved. These values parallel the relative amount of soluble to total of each species in the AY102 slurry, which was the major solids-containing component of the recycle. The small amounts of dissolved $\mathrm{Ag}, \mathrm{Ca}, \mathrm{Nd}$, and $\mathrm{Ni}$, which were initially present in the supernate portion of the feed, appeared to have precipitated. The dissolved Si content did not decrease. Table 21 also shows that about 1,536 g of oxalate apparently precipitated. When this oxalate is combined with $\mathrm{Na}$ as its cation, the total predicted amount of sodium oxalate precipitated is 2,339 g, which is extremely close to the measured increase in undissolved solids of 2,281 g. Therefore, precipitation of sodium oxalate accounts for the increase in undissolved solids seen.

Figure 11 shows the mass of total solids, undissolved solids, and soluble sodium oxalate during the evaporation as a function of concentrate sodium. The dotted line for the oxalate is hypothetical since there are no intermediate data. The graph shows that the precipitation of oxalate appears to have started when the bottoms sodium concentration reached $2.6 \mathrm{M}$, which corresponded to when $50 \%$ of the total volume of feed was evaporated. 
Table 19. Material Balance on FEP Evaporator

\section{FEP Feed}

(7a)

(7b)

(Combined Recycles)

Calculated

(7c)

(7d)

(7e)

Calculated (from Calculated

rom Measured alculated FEP (using calc. Measured, Measured, FEP Feed) $\quad$ Feed) $\quad$ PWD-44) $\quad$ Sample $1{ }^{@}$ Sample $2{ }^{\circledR}$

\begin{tabular}{l|lllll}
\cline { 2 - 4 } Volume (L) & 1125.1 & 1125.1 & 1125.1 & 1125.1 & 1125.1
\end{tabular}

Mass (kg)

Total Solids (wt\%)

Undissolved Solids (wt\%)

Density (kg/L)

Total Solids (kg)

1165.6

4.64

1170.1

4.70

1168.0

4.63

0.36

0.29

1166.7

0.14

1.040

1.038

54.99

54.08

0.17

1.037

54.09

4.26

3.34

54.72

1.98

1166.7

Undissolved Solids (kg)

1.66

\section{Evaporator Bottoms}

\begin{tabular}{|r|ccccc|}
\cline { 2 - 6 } Volume Fed (L) & 200 & 400 & 600 & 800 & 1125 \\
Mass Fed (kg) & 207 & 415 & 622 & 830 & 1167 \\
Volume (L) & 147.6 & 147.6 & 147.6 & 147.6 & 147.6 \\
Mass (kg) & 158.9 & 164.8 & 170.7 & 176.5 & 186.0 \\
Total Solids (wt\%) & 7.88 & 11.88 & 16.33 & 21.02 & 28.95 \\
ved Solids (wt\%) & 0.37 & 0.59 & 0.99 & 1.93 & 3.03 \\
Density (kg/L) & 1.077 & 1.116 & 1.156 & 1.196 & 1.261 \\
Total Solids (kg) & 12.52 & 19.58 & 27.87 & 37.10 & 53.85 \\
\hline
\end{tabular}

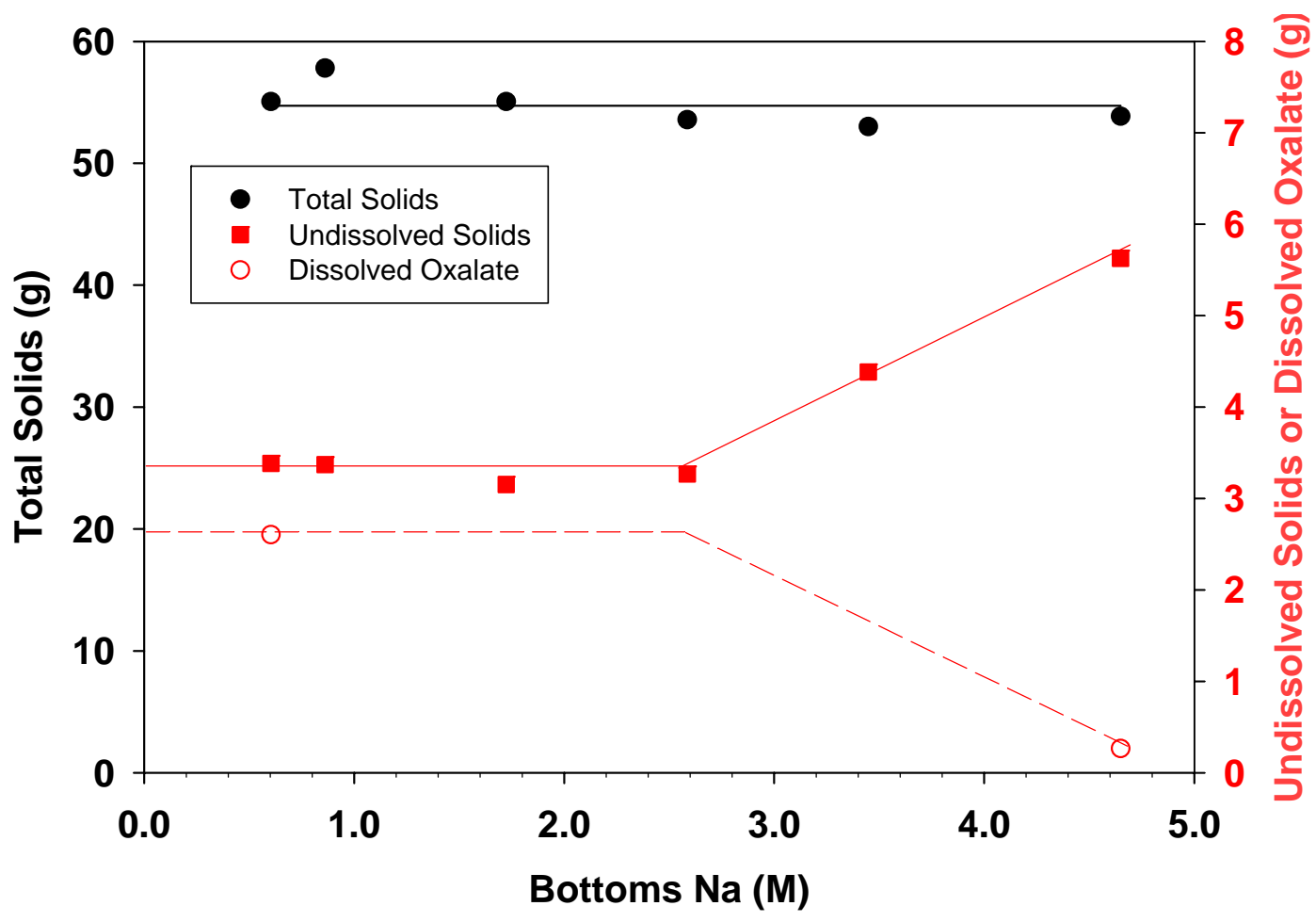

Figure 11. Total \& Undissolved Solids in pilot-scale FEP unit operation 
WSRC-TR-2004-00478, REVISION 0 SRNL-RPP-2004-00075, REVISION 0

Table 20. Analyses of FEP Feed \& Bottoms \& Material Balance

\begin{tabular}{|c|c|c|c|c|c|c|c|c|}
\hline & $\begin{array}{c}\text { FEP } \\
\text { Feed } \\
\text { Slurry }\end{array}$ & $\begin{array}{c}\text { FEP } \\
\text { Bottoms } \\
\text { Slurry }\end{array}$ & & $\begin{array}{c}\text { FEP } \\
\text { Feed } \\
\text { Slurry }\end{array}$ & $\begin{array}{c}\text { FEP } \\
\text { Bottoms } \\
\text { Slurry }\end{array}$ & $\begin{array}{l}\text { Bottoms/ } \\
\text { Feed \% }\end{array}$ & $\begin{array}{l}\text { FEP Feed } \\
\text { Supernate }\end{array}$ & $\begin{array}{c}\text { FEP } \\
\text { Bottoms } \\
\text { Supernate }\end{array}$ \\
\hline Total Solids (wt\%) & 4.72 & 28.9 & Volume (L) & 1125 & 147.6 & NA & & \\
\hline Undissolved Solids (wt\%) & 0.290 & 3.03 & Mass (kg) & 1168 & 186.0 & NA & & \\
\hline Supernate Solids (wt\%) & 4.44 & 26.7 & $\begin{array}{r}\text { Undissolved } \\
\text { Solids (g) }\end{array}$ & 3340 & 5630 & NC & & \\
\hline Density (kg/L) & 1.04 & 1.26 & $\begin{array}{r}\text { Total Solids } \\
(\mathrm{kg})\end{array}$ & 54.08 & 53.83 & 99.5 & & \\
\hline $\mathrm{pH}$ & NM & 13.0 & & & & & & \\
\hline Elements: & $\mathrm{mg} / \mathrm{L}$ & $\mathrm{mg} / \mathrm{L}$ & \multicolumn{4}{|c|}{ Total Mass (g) } & \multicolumn{2}{|c|}{$\mathrm{mg} / \mathrm{L}$} \\
\hline $\mathrm{Ag}$ & $<34.2$ & 112 & 95.0 & $<38.5$ & 16.6 & & 0.827 & $<0.6$ \\
\hline $\mathrm{Al}$ & 954 & 6900 & 6590 & 1070 & 1020 & $95 \%$ & 567 & 4640 \\
\hline $\mathrm{B}$ & $<146$ & 698 & & $<164$ & 103 & & 83.0 & 690 \\
\hline $\mathrm{Ba}$ & 5.38 & 47.7 & & 6.05 & 7.04 & $116 \%$ & $<0.024$ & $<0.04$ \\
\hline $\mathrm{Ca}$ & 214 & 447 & & 240 & 66.0 & $27 \%$ & 1.17 & $<0.06$ \\
\hline $\mathrm{Cd}$ & $<4.15$ & 7.78 & & $<4.67$ & 1.15 & & $<0.028$ & $<0.04$ \\
\hline $\mathrm{Ce}$ & $<131$ & $<127$ & & $<147$ & $<18.7$ & & $<1.54$ & $<1.4$ \\
\hline Co & 231 & 147 & & 260 & 21.8 & $8 \%$ & $<0.088$ & $<0.16$ \\
\hline $\mathrm{Cr}$ & 52.9 & 237 & 212 & 59.5 & 35.0 & $59 \%$ & 20.3 & 150 \\
\hline $\mathrm{Cu}$ & 16.6 & 16.9 & 16.5 & 18.7 & 2.50 & $13 \%$ & $<0.1$ & 0.139 \\
\hline Cs (ICPMS) & 0.580 & 15.3 & & 0.652 & 2.25 & $345 \%$ & & \\
\hline Cs (AA) & $<20.7$ & $<18.2$ & & $<23.3$ & $<2.69$ & & & 13.4 \\
\hline $\mathrm{Fe}$ & 866 & 5800 & 5620 & 974 & 856 & $88 \%$ & $<0.088$ & $<0.08$ \\
\hline K (AA) & $<31.1$ & 305 & & $<35$ & 45.0 & & 41.4 & 305 \\
\hline $\mathrm{La}$ & $<12.5$ & 60.0 & & $<14.1$ & 8.86 & & $<1.4$ & $<0.2$ \\
\hline $\mathrm{Li}$ & 28.8 & 140 & 126 & 32.4 & 20.6 & $64 \%$ & 0.230 & 8.86 \\
\hline $\mathrm{Mg}$ & 14.6 & 104 & 102 & 16.4 & 15.3 & $93 \%$ & $<0.168$ & 0.0226 \\
\hline $\mathrm{Mn}$ & 162 & 1190 & 1190 & 182 & 175 & $96 \%$ & $<0.018$ & $<0.02$ \\
\hline Mo & $<20.7$ & $<18.2$ & & $<23.3$ & $<2.69$ & & $<0.2$ & 0.479 \\
\hline $\mathrm{Na}$ & 16385 & 118739 & & 18434 & 17526 & $95 \%$ & 13878 & 107000 \\
\hline $\mathrm{Na}$ (AA) & 161074 & 116596 & & 18084 & 17210 & $95 \%$ & 15300 & 114000 \\
\hline Na (average) & 16229 & 117668 & & 18259 & 17368 & $95 \%$ & 14589 & 110500 \\
\hline $\mathrm{Nd}$ & $<74.7$ & 106 & & $<84$ & 15.7 & & $<0.52$ & $<1.8$ \\
\hline $\mathrm{Ni}$ & 216 & 1150 & & 243 & 169 & $70 \%$ & 0.398 & 0.357 \\
\hline $\mathrm{P}$ & $<166$ & 1121 & & $<187$ & 165 & & 128 & 1100 \\
\hline $\mathrm{Pb}$ & $<166$ & 223 & & $<187$ & 32.9 & & $<1.38$ & $<1.6$ \\
\hline $\operatorname{Pr}(\mathrm{ICPMS})$ & 3.80 & 30.3 & & 4.27 & 4.47 & $105 \%$ & & \\
\hline $\mathrm{Rb}$ (ICPMS) & 0.385 & 1.26 & & 0.433 & 0.186 & $43 \%$ & & \\
\hline $\mathrm{S}$ & 227 & 908 & 768 & 256 & 134 & $52 \%$ & 95.2 & 790 \\
\hline $\mathrm{Si}$ & 385 & 1990 & & 433 & 294 & $68 \%$ & 5.48 & 37.9 \\
\hline $\mathrm{Sr}$ & 14.1 & 86.9 & 80.5 & 15.8 & 12.8 & $81 \%$ & $<0.01$ & 0.402 \\
\hline $\mathrm{Ti}$ & 7.64 & 11.5 & 5.77 & 8.59 & 1.70 & $20 \%$ & $<0.28$ & $<0.06$ \\
\hline V & $<83.1$ & $<72.6$ & & $<93.5$ & $<10.7$ & & & \\
\hline W & $<104$ & $<90.8$ & & $<117$ & $<13.4$ & & & 90.0 \\
\hline Y (ICPMS) & 1.21 & 8.97 & & 1.37 & 1.32 & $97 \%$ & & \\
\hline Zn & 5.08 & 46.1 & & 5.71 & 6.81 & $119 \%$ & $<0.74$ & 1.86 \\
\hline $\mathrm{Zr}$ & 24.1 & 85.7 & 78.5 & 27.1 & 12.7 & $47 \%$ & $<0.096$ & 0.0820 \\
\hline
\end{tabular}


WSRC-TR-2004-00478, REVISION 0 SRNL-RPP-2004-00075, REVISION 0

\begin{tabular}{|c|c|c|c|c|c|c|c|}
\hline Table 20 (continued) & $\begin{array}{l}\text { FEP } \\
\text { Feed }\end{array}$ & $\begin{array}{c}\text { FEP } \\
\text { Bottoms }\end{array}$ & $\begin{array}{c}\text { FEP } \\
\text { Feed } \\
\text { Slurry }\end{array}$ & $\begin{array}{c}\text { FEP } \\
\text { Bottoms } \\
\text { Slurry } \\
\end{array}$ & $\begin{array}{l}\text { Bottoms/ } \\
\text { Feed \% }\end{array}$ & \multirow[t]{2}{*}{$\begin{array}{l}\text { FEP Feed } \\
\text { Supernate }\end{array}$} & \multirow[t]{2}{*}{$\begin{array}{c}\text { FEP } \\
\text { Bottoms } \\
\text { Supernate }\end{array}$} \\
\hline Anions (Soluble) & $\mathrm{mg} / \mathrm{L}$ & $\mathrm{mg} / \mathrm{L}$ & \multicolumn{3}{|c|}{ Total Mass (g) } & & \\
\hline bromide & 32.0 & 53.0 & 36.0 & 7.82 & $22 \%$ & & \\
\hline chloride & 83.0 & 609 & 93.4 & 89.9 & $96 \%$ & & \\
\hline fluoride & 11.0 & 145 & 12.4 & 21.4 & $173 \%$ & & \\
\hline formate & 39.0 & 408 & 43.9 & 60.2 & $137 \%$ & & \\
\hline nitrate & 15000 & 119000 & 16875 & 17600 & $104 \%$ & & \\
\hline nitrite & 495 & 3740 & 548 & 552 & $101 \%$ & & \\
\hline oxalate & 1520 & 1180 & 1710 & 174 & $10 \%$ & & \\
\hline phosphate & 563 & 4240 & 633 & 626 & $99 \%$ & & \\
\hline sulfate & 257 & 1960 & 289 & 289 & $100 \%$ & & \\
\hline Total Carbonate & 1500 & 9500 & 1690 & 1400 & $83 \%$ & & \\
\hline
\end{tabular}

* Elements measured on Microwave or Na Peroxide dissolution; highest or best value shown; selected comparison values shown for Bottoms

Table 21. Masses of Total and Dissolved Species in FEP Evaporator Streams

\section{Total Slurry Supernate Only}

Mass in Mass in Mass in Mass in

Feed (g) Bottoms (g) Feed (g) Bottoms (g) Comments

\begin{tabular}{|r|cccc|l|}
\hline Undissolved Solids & 3345 & 5626 & & & precipitation occurred \\
$\mathrm{Ag}$ & $<38.5$ & 16.6 & 0.930 & $<0.089$ & $\begin{array}{l}\text { mostly undissolved; dissolved portion may } \\
\text { have precipitated }\end{array}$ \\
$\mathrm{Al}$ & 1073 & 1018 & 638 & 685 & 45\% undissolved \\
$\mathrm{B}$ & $<164$ & 103 & 93.4 & 102 & soluble \\
$\mathrm{Ba}$ & 6.05 & 7.04 & $<0.027$ & $<0.006$ & undissolved \\
$\mathrm{Ca}$ & 240 & 66.0 & 1.32 & $<0.009$ & mostly undissolved; dissolved portion may \\
have precipitated
\end{tabular}


The condensate from the FEP evaporator was analyzed for metals and anions. The only species detected above the detection limits were $\mathrm{Na}$ (4-20 mg/L), $\mathrm{Cr}$ (2 mg/L), B (7 mg/L), and Si (17 $\mathrm{mg} / \mathrm{L}$ ); no anions were detected. The $\mathrm{pH}$ was found to be 9.4 , and the density 1.00. Note that 20 $\mathrm{mg} / \mathrm{L}$ of $\mathrm{Na}$ as $\mathrm{NaOH}$ would give a $\mathrm{pH}$ of 10.9 , so the $9.4 \mathrm{pH}$ is consistent with this upper bound.

The fate of the added Dow Corning Q2-3183A antifoam was monitored by measuring the concentration of the antifoam in the initial charge to the evaporator, in the final bottoms product, and in the condensate. The analyses were performed by Dow Corning Analytical Services, as shown in Appendix C. The antifoam concentrations were measured by Fourier Transform Infrared Spectroscopy (FTIR) on hexane extracts of the samples. Actually, only the silicone portion, which is $40 \mathrm{wt} \%$ of the total mass, of the antifoam was quantified; the antifoam concentration was then calculated as 2.5 times the measured value. The FTIR was calibrated on the silicone portion of the antifoam molecule rather than on the actual antifoam. The antifoam was added to the evaporator in three batches after boil-up had been initiated. After all of the antifoam was added, the first sample was taken. The calculated concentration of antifoam in the bottoms at $25^{\circ} \mathrm{C}$ was $1,887 \mathrm{mg} / \mathrm{L}\left(1,500 \mathrm{mg} / \mathrm{kg} @ 50^{\circ} \mathrm{C}\right)$. The measured concentration in a sample immediately after addition was $3,950 \mathrm{mg} / \mathrm{L}$. Figure 12 is a schematic of the SIPP system showing the amount of antifoam in several streams. The final bottoms product was found to have an antifoam concentration of $6,025 \mathrm{mg} / \mathrm{L}$. Both of these values are significantly higher than the calculated addition. Dow-Corning is currently rerunning these samples and also standard antifoam solutions. The wide variation in these values indicates that it is both difficult to sample the slurry representatively and difficult to quantitatively analyze the samples for this antifoam agent. The analysis of the condensate sample is pending; however the antifoam concentration is expected to be below the detection limit of $25 \mathrm{mg} / \mathrm{L}$ since the condensate contained very little of any solutes when analyzed by ICPES and IC. 


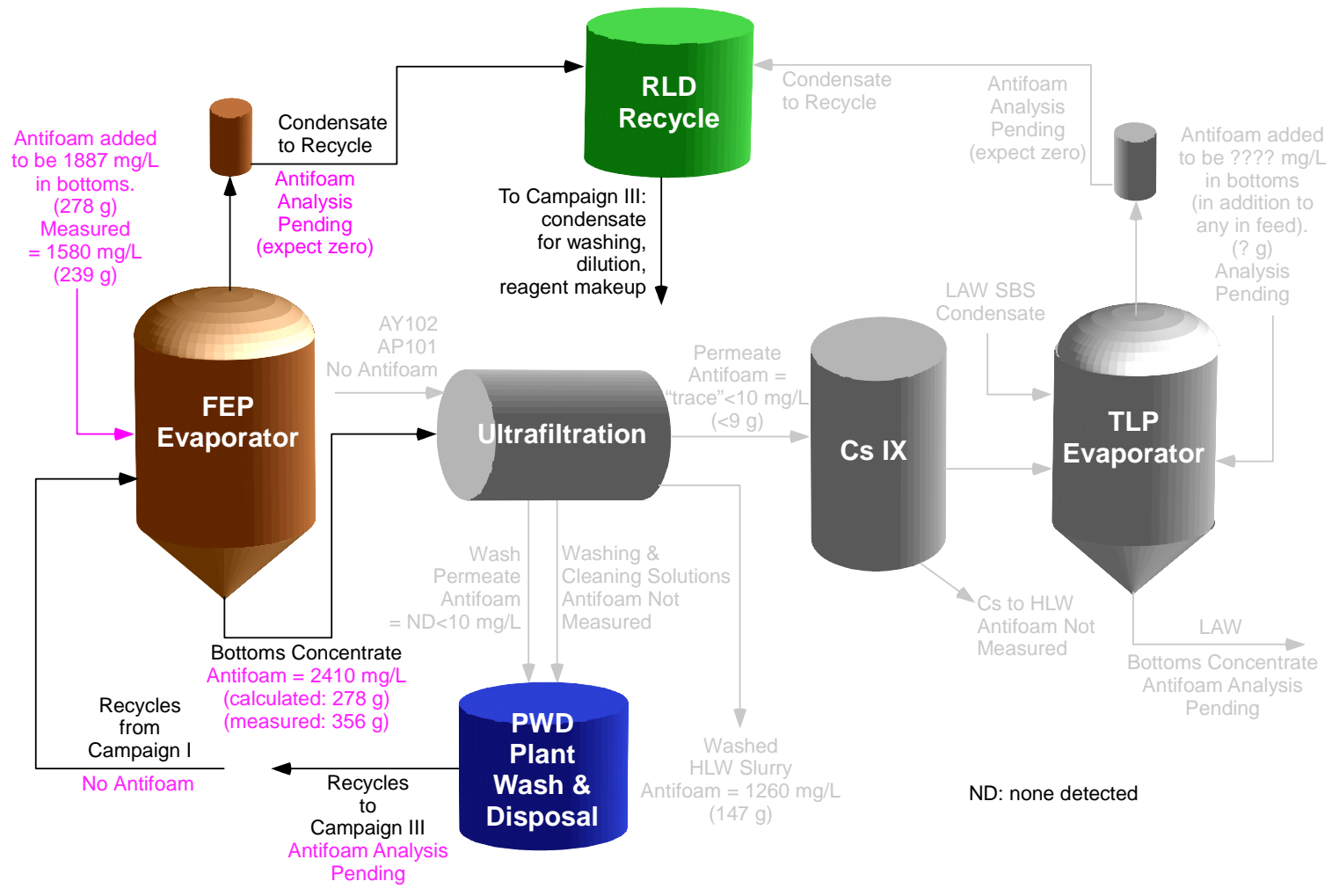

Figure 12. Schematic of Antifoam Fate in the SIPP

\subsubsection{Pilot FEP Conclusions}

\subsubsection{Experimental Observations (Author - Qureshi)}

During SIPP FEP Campaign II, a total of about 1,125 liters of feed was concentrated down to about 147 liters. The feed was evaporated under vacuum $(1$ psia $(6.9 \mathrm{kPa}))$ at moderate temperatures $\left(\sim 50^{\circ} \mathrm{C}\right)$. The entire evaporation process proceeded as designed. The evaporator was designed to evaporate around $0.4 \mathrm{gpm}(1.5 \mathrm{lpm})$ of water. The average evaporation rate during this campaign was around $0.43 \mathrm{gpm}$.

No significant foaming was observed prior to the addition of antifoam agent. During the evaporation process, the froth height remained around $1 \mathrm{ft}(0.3 \mathrm{~m})$ that is significantly less than the $5 \mathrm{ft}(1.5 \mathrm{~m})$ distance to the lower demister pad. However, during start-up and unsteady conditions, significant flashing resulted in feed splashing on the lower demister pad. These conditions are associated with much larger vapor flux and vapor velocities at the interface.

The concentrate density was measured by three methods during the evaporation process. In the first method, warm concentrate $\left(\sim 50^{\circ} \mathrm{C}\right)$ was drawn in a 50 or $200 \mathrm{ml}$ flask and weighed immediately on a scale accurate to $0.1 \mathrm{~g}$. The second method employed a density meter with an accuracy of $0.001 \mathrm{~g} / \mathrm{ml}$. The density meter would cool the sample and the density was reported at $20 \mathrm{C}$. The third method was online density measurement using two absolute pressure 
transducers placed $6 \mathrm{ft}(1.82 \mathrm{~m})$ apart in a vertical leg of the evaporator recirculation loop. This method resulted in large uncertainties in density measurement since two large pressure signals were subtracted. Thus samples were drawn periodically for density measurement by the more accurate density meter.

\subsubsection{Waste Products Observations (Author - Zamecnik)}

- The composition of the FEP feed was predictable by mass balance of the individual feed streams.

- Dissolved metals concentrations parallel the ratios in the AY102 simulant present in the recycles.

- Dissolved oxalate, present in the feed, began to precipitate when approximately 53\% (see Fig. 11) of the feed had been evaporated. A total of about $90 \mathrm{wt} \%$ (see Figs. 20 and 21) of that oxalate precipitated at the end of evaporation. The oxalate appears to have precipitated as the $\mathrm{Na}$ salt $\mathrm{Na}_{2} \mathrm{C}_{2} \mathrm{O}_{4}$.

\subsection{PILOT ULTRAFILTRATION (AUTHORS - FOWLEY, WILLIAMS \& ZAMECNIK)}

The Ultrafiltration Process, or UFP, is the second separation technology unit operation in the RPP-WTP that a waste will see on start up. This is true for SIPP, since the chosen waste type, Envelope A/D, is not subjected to precipitation before filtration. However, in steady-state operation the idea of first, second, third, etc. loses meaning since the many systems throughout the plant could be operating simultaneously on the many stages of waste processing. With respect to SIPP, UFP is the second separation operation in line and filters a mixture of FEP bottoms mixed with simulated wastes of AY102 and AP101 to produce two products: 1. A concentrated slurry HLW product, and 2. A liquid LAW product in preparation for vitrification.

This section will explain the pilot UFP unit operation that was done as part of Campaign II testing. The process started with the preparation of the feed product, which was a mixture of FEP bottoms and simulated tank farm waste streams in a predetermined fashion, see Table 3. The chemical explanation of the feed stream formation will be followed by a short description on how feed formation was handled just before UFP operation and then how the waste stream was handled during and after its separation. The following section will discuss the physical equipment used to filter the waste and how the test was run. Finally, the results will be discussed and conclusions made.

\subsubsection{Chemical Description of the Pilot-Scale UFP Feed Stream (Author - Zamecnik)}

Figure 13 and Figure 14 show schematically how the concentration, washing, rinsing and cleaning steps were performed. The pilot-scale UFP feed slurry was made by combining the FEP evaporator bottoms, AY102 slurry simulant, and AP101 supernate simulant. Table 22 summarizes the compositions of these feed streams and Table 23 compares calculated versus measured compositions. 
WSRC-TR-2004-00478, REVISION 0

SRNL-RPP-2004-00075, REVISION 0

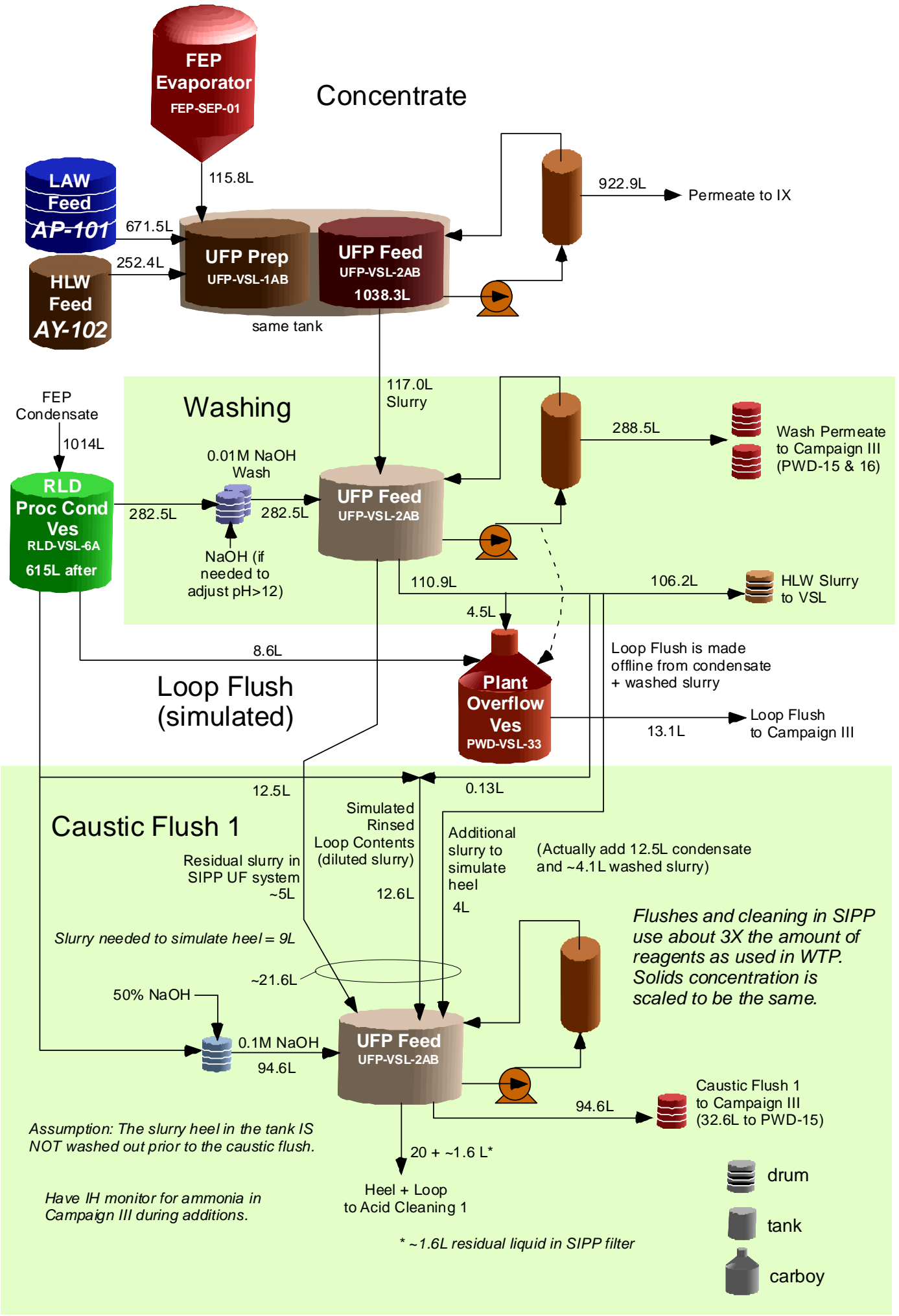

Figure 13. Schematic of the Planned Ultrafiltration Process - Page 1

(Note: The expected "residual slurry" of $5 \mathrm{~L}$ during the Caustic Flush 1 step turned out to be approximately 22 liters and the existing flush stream was closer to $112 \mathrm{~L}$ instead of 94.6 L, but all other steps followed the plan.) 
WSRC-TR-2004-00478, REVISION 0

SRNL-RPP-2004-00075, REVISION 0

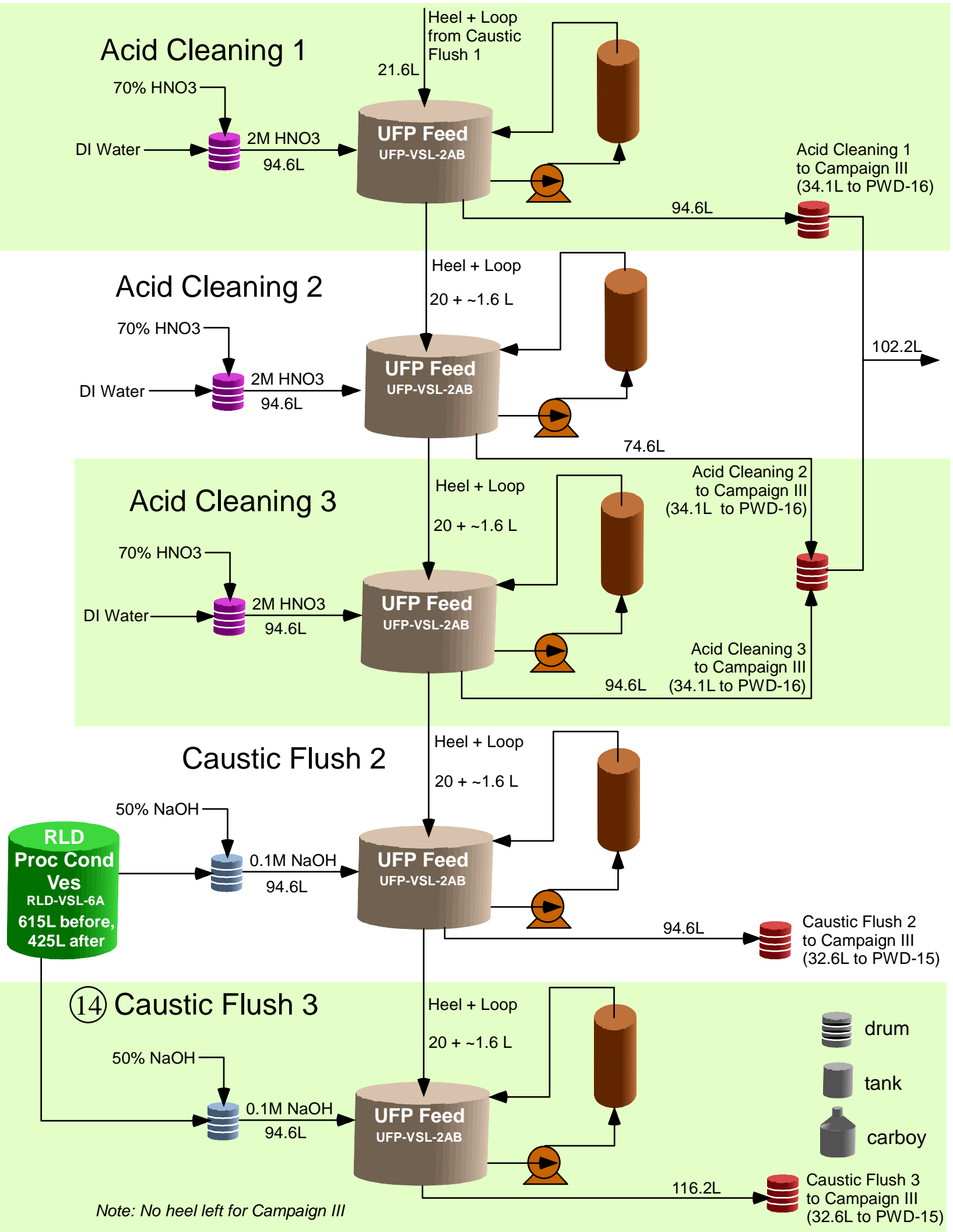

Figure 14. Schematic of Ultrafiltration Process - Page 2 
WSRC-TR-2004-00478, REVISION 0 SRNL-RPP-2004-00075, REVISION 0

Table 22. Measured Pilot-Scale UFP Feeds

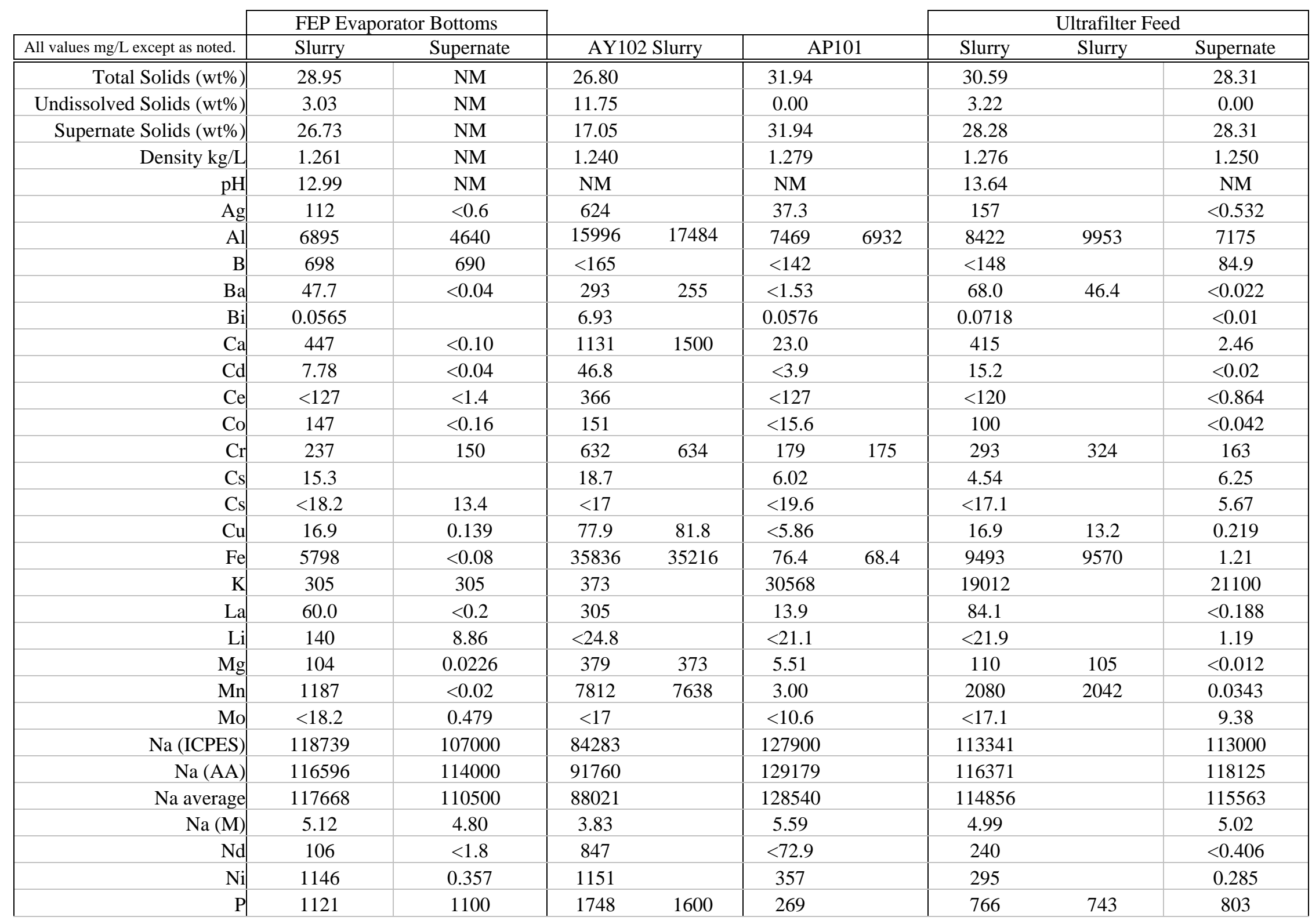


WSRC-TR-2004-00478, REVISION 0 SRNL-RPP-2004-00075, REVISION 0

Table 22 continued

\begin{tabular}{|c|c|c|c|c|c|c|c|c|c|}
\hline & \multicolumn{2}{|c|}{ FEP Evaporator Bottoms } & & & & & \multicolumn{3}{|c|}{ Ultrafilter Feed } \\
\hline All values mg/L except as noted. & Slurry & Supernate & \multicolumn{2}{|c|}{ AY102 Slurry } & \multicolumn{2}{|c|}{ AP101 } & Slurry & Slurry & Supernate \\
\hline $\mathrm{Pb}$ & 223 & $<1.6$ & 1662 & 1593 & $<156$ & & 475 & 419 & 1.77 \\
\hline $\operatorname{Pr}$ & 30.3 & NM & 170 & & 0.0223 & & 34.1 & & $<0.01$ \\
\hline $\mathrm{Rb}$ & 1.26 & NM & 4.60 & & 6.06 & & 4.16 & & 5.12 \\
\hline $\mathrm{S}$ & 908 & 790 & 892 & 899 & 1599 & 1426 & 1340 & 1128 & 1140 \\
\hline $\mathrm{Si}$ & 1992 & 37.9 & 11016 & & 329 & & 3247 & & 56.7 \\
\hline $\mathrm{Sr}$ & 86.9 & 0.402 & 279 & 292 & $<1.18$ & & 60.2 & 83.0 & $<0.436$ \\
\hline $\mathrm{W}$ & $<90.8$ & 90.0 & $<84.7$ & & $<97.7$ & & $<85.7$ & & 38.8 \\
\hline $\mathrm{Y}$ & 8.97 & $<0.01$ & 50.8 & & 0.0728 & & 10.1 & & $<0.01$ \\
\hline $\mathrm{Zn}$ & 46.1 & 1.86 & 81.3 & & 5.95 & & 28.6 & & 3.04 \\
\hline $\mathrm{Zr}$ & 85.7 & 0.0820 & 484 & & $<5.86$ & & 125 & & 0.613 \\
\hline bromide & & 53.0 & 69.4 & & $<128$ & & $<53.6$ & & $<10$ \\
\hline chloride & & 609 & 165 & & 1530 & & 1242 & & 1175 \\
\hline fluoride & & 145 & 43.4 & & 28.0 & & 81.7 & & 33.5 \\
\hline formate & & 408 & 389 & & 1380 & & 1108 & & 1170 \\
\hline nitrate & & 119000 & 319 & & 105000 & & 93914 & & 85600 \\
\hline nitrite & & 3740 & 4228 & & 33400 & & 51678 & & 24050 \\
\hline oxalate & & 1180 & 9213 & & 1550 & & 4440 & & 740 \\
\hline phosphate & & 4240 & 3807 & & 1150 & & 2412 & & 2430 \\
\hline sulfate & & 1960 & 1860 & & 3740 & & 3483 & & 5280 \\
\hline acetate & & NA & 415 & & 1727 & & 1314 & & 1210 \\
\hline citric acid & & NA & $<81.8$ & & $<104$ & & $<241$ & & $<100$ \\
\hline formate & & NA & 264 & & 1432 & & 1072 & & 941 \\
\hline unbound hydroxide & & 6103 & NM & & NM & & 38930 & & NM \\
\hline total organic carbon & & 1000 & NM & & NM & & 3280 & & 3005 \\
\hline carbonate & & 9500 & NM & & NM & & 40600 & & 31350 \\
\hline
\end{tabular}


WSRC-TR-2004-00478, REVISION 0

SRNL-RPP-2004-00075, REVISION 0

Table 23. Comparison of Measured and Calculated UFP Feed Concentrations

\begin{tabular}{|c|c|c|c|c|c|c|c|c|c|}
\hline & \multicolumn{5}{|c|}{ INPUTS } & \multicolumn{4}{|c|}{ ULTRAFILTER FEED } \\
\hline & \multirow{2}{*}{$\begin{array}{l}\text { FEP Bottoms } \\
\text { Slurry }\end{array}$} & \multirow{2}{*}{$\begin{array}{l}\text { FEP Bottoms } \\
\text { Supernate }\end{array}$} & \multicolumn{3}{|c|}{ AY102 AY102 } & \multicolumn{2}{|c|}{ Slurry } & \multicolumn{2}{|c|}{ Slurry Supernate } \\
\hline & & & Slurry & Supernate & AP101 & Calculated & Measured & Calculated & Measured \\
\hline \multirow{4}{*}{$\begin{array}{r}\text { Total Solids wt\% } \\
\text { Undissolved Solids wt\% } \\
\text { Supernate Solids wt\% } \\
\text { Density kg/L }\end{array}$} & 28.95 & 26.73 & 26.80 & 17.00 & 31.94 & 30.39 & 30.59 & 28.12 & 28.31 \\
\hline & 3.03 & 0.00 & 11.75 & 0.00 & 0.00 & 3.13 & 3.22 & 0.00 & 0.00 \\
\hline & 26.73 & 26.73 & 17.05 & 17.00 & 31.94 & 28.14 & 28.28 & 28.12 & 28.31 \\
\hline & 1.261 & 1.246 & 1.240 & 1.168 & 1.279 & 1.280 & 1.276 & 1.259 & 1.250 \\
\hline \multirow{25}{*}{$\begin{array}{rr}\text { Concentration in } & \mathrm{Ag} \\
\text { mg/L. }<\text { indicates no } & \mathrm{Al} \\
\text { data or value below } & \mathrm{B} \\
\text { detection limit. } & \mathrm{Ba} \\
& \mathrm{Ca} \\
\mathrm{Cd} \\
\mathrm{Co} \\
\mathrm{Cr} \\
\mathrm{Cu} \\
\mathrm{Fe} \\
\mathrm{K} \\
\mathrm{La} \\
\mathrm{Li} \\
\mathrm{Mg} \\
\mathrm{Mn} \\
\mathrm{Na} \\
\mathrm{Nd} \\
\mathrm{Ni} \\
\mathrm{P} \\
\mathrm{Pb} \\
\mathrm{S} \\
\mathrm{Si} \\
\mathrm{Sr} \\
\mathrm{Zn} \\
\mathrm{Zr}\end{array}$} & 112 & $<$ & 624 & $<$ & 37.3 & 190 & 157 & 24.7 & $<$ \\
\hline & 6895 & 4640 & 16740 & 8030 & 7200 & 9573 & 9187 & 7161 & 7175 \\
\hline & 698 & 690 & & $<$ & $<$ & 78.5 & $<$ & 77.2 & 84.9 \\
\hline & 47.7 & $<$ & 274 & $<$ & $<$ & 72.5 & 57.2 & $<$ & $<$ \\
\hline & 447 & $<$ & 1316 & 1.09 & 23.0 & 388 & 415 & 15.5 & 2.46 \\
\hline & 7.78 & $<$ & 46.8 & $<$ & $<$ & 12.3 & 15.2 & $<$ & $<$ \\
\hline & 147 & $<$ & 151 & $<$ & $<$ & 53.7 & 100 & $<$ & $<$ \\
\hline & 237 & 150 & 633 & 147 & 177 & 297 & 309 & 168 & 163 \\
\hline & 16.9 & 0.139 & 79.9 & $<$ & $<$ & 21.5 & 15.0 & 0.0156 & 0.219 \\
\hline & 5798 & $<$ & 35526 & 0.191 & 72.4 & 9406 & 9532 & 47.9 & 1.21 \\
\hline & 305 & 305 & 373 & 358 & 30568 & 20057 & 19012 & 20342 & 21100 \\
\hline & 60.0 & $<$ & 305 & $<$ & 13.9 & 90.6 & 84.1 & 9.20 & $<$ \\
\hline & 140 & 8.86 & $<$ & $<$ & $<$ & 15.7 & $<$ & 0.992 & 1.19 \\
\hline & 104 & 0.0226 & 376 & $<$ & 5.51 & 107 & 107 & 3.65 & $<$ \\
\hline & 1187 & $<$ & 7725 & $<$ & 3.00 & 2029 & 2061 & 1.98 & 0.0343 \\
\hline & 117668 & 110500 & 88021 & 73575 & 128540 & 118613 & 114856 & 114620 & 115563 \\
\hline & 106 & $<$ & 847 & $<$ & $<$ & 220 & 240 & $<$ & $<$ \\
\hline & 1146 & 0.357 & 1151 & 0.602 & 357 & 644 & 295 & 236 & 0.285 \\
\hline & 1121 & 1100 & 1674 & 1366 & 269 & 711 & 754 & 620 & 803 \\
\hline & 223 & $<$ & 1628 & $<$ & $<$ & 424 & 447 & $<$ & 1.77 \\
\hline & 908 & 790 & 895 & 628 & 1512 & 1308 & 1234 & 1236 & 1140 \\
\hline & 1992 & 37.9 & 11016 & 19.1 & 329 & 3138 & 3247 & 226 & 56.7 \\
\hline & 86.9 & 0.402 & 286 & 0.204 & $<$ & 79.8 & 71.6 & 0.0927 & 0.0691 \\
\hline & 46.1 & 1.86 & 81.3 & $<$ & 5.95 & 29.0 & 28.6 & 4.15 & 3.04 \\
\hline & 85.7 & 0.0820 & 484 & $<$ & $<$ & 128 & 125 & 0.00918 & 0.613 \\
\hline \multirow{9}{*}{$\begin{array}{r}\text { bromide } \\
\text { chloride } \\
\text { fluoride } \\
\text { formate } \\
\text { nitrate } \\
\text { nitrite } \\
\text { oxalate } \\
\text { phosphate } \\
\text { sulfate } \\
\end{array}$} & \multirow{9}{*}{$\mathrm{NM}$} & 53.0 & 69.4 & 50.0 & $<$ & 23.0 & $<$ & 17.6 & $<$ \\
\hline & & 609 & 165 & 170 & 1530 & 1385 & 1242 & 1403 & 1175 \\
\hline & & 145 & 43.4 & 44.0 & 28.0 & 50.3 & 81.7 & 50.2 & 33.5 \\
\hline & & 408 & 389 & 290 & 1380 & 1292 & 1108 & 1281 & 1170 \\
\hline & & 119000 & 319 & 361 & 105000 & 101022 & 81154 & 102257 & 85600 \\
\hline & & 3740 & 4228 & 4790 & 33400 & 29310 & 25775 & 29802 & 24050 \\
\hline & & 1180 & 9213 & 2810 & 1550 & 3683 & 4440 & 2101 & 740 \\
\hline & & 4240 & 3807 & 4010 & 1150 & 2369 & 2412 & 2386 & 2430 \\
\hline & & 1960 & 1860 & 2225 & 3740 & 3795 & 3483 & 3905 & 5280 \\
\hline
\end{tabular}

$-63-$




\section{WSRC-TR-2004-00478, REVISION 0 SRNL-RPP-2004-00075, REVISION 0}

Replicate values from microwave and sodium peroxide fusion dissolutions are shown for some of the analyses. The actual total volume of products was 916L of permeate and 117L of concentrated slurry, for a total of 1,033L versus the recorded total (from the feed tanks) of 1037L, which is an error of only $0.4 \%^{\dagger}$.

Using the input compositions and volumes, the compositions of the combined feed slurry and the supernate from this slurry were calculated. As already mentioned, those input data and the calculated values are shown in Table 23. For most of the more abundant species ( $>5000 \mathrm{mg} / \mathrm{L}$, or $>0.5 \%$ ), the calculated values matched the measured values well. Exceptions are the nitrate and nitrite, which had higher than expected error. This comparison shows that most of the data are sufficiently accurate so that the composition of the pilot-scale UFP feed can be predicted from the individual components.

\subsubsection{Simulant Handling (Author - Williams)}

Before testing began the simulants were prepared, which would be needed to create recycle streams from the UFP. Preparations of the simulated waste feed were then performed so as to have the accepted proportions of the constituents as per the OLI/ESP model (see Table 3).

After the dewatering portion of pilot-scale UFP testing, the slurry was adjusted in order to have a target solids concentration of $20 \mathrm{wt} \%$ undissolved solids (UDS) before washing began, as required by

\footnotetext{
${ }^{\dagger}$ Note on pilot-scale UFP volumes: The measured total volumes of concentrated slurry and permeate, based on different assumptions, are shown in the footnote table below. The initial amount of feed, based on the amounts of the simulants and the FEP bottoms, was about 1038L. The final slurry volume was measured from the tank level and the permeate was measured from the total of the drum masses. Applying estimated uncertainties to these measurements, the final permeate volume range was calculated as shown. The volume of samples are added back into the totals. The effect of the slurry sample removal was accounted for by adding back the amount of permeate that would have been produced from the samples to the measured permeate. The volume of concentrated slurry from the samples was ignored $(<0.2 \mathrm{~L})$. The average calculated amount of permeate was then 916L, while the total slurry + permeate average was 1033L, which is within $0.5 \%$ of the initial estimated amount. Subtracting off the amount of permeate used to dilute the slurry back to 20 wt\% UDS (21L), the final volume of permeate available to be sent to IX was then 881-909L. The estimated amount of permeate actually transferred to the IX system was 870L, and the amount of permeate treated in the IX was about 864L.
}

Footnote Table: Measured Product Volumes

\begin{tabular}{|c|c|c|c|c|c|c|c|}
\hline & $\begin{array}{l}\text { Total } \\
\text { (L) }\end{array}$ & $\begin{array}{l}\text { Concentrated } \\
\text { Slurry ( 23.5 } \\
\text { wt\% UDS) } \\
\text { Volume } \\
\text { (L) }\end{array}$ & $\begin{array}{l}\text { Permeate } \\
\text { Collected } \\
(\mathrm{kg})\end{array}$ & $\begin{array}{l}\text { Permeate } \\
\text { Volume } \\
\text { Collected } \\
\text { (L) }\end{array}$ & $\begin{array}{l}\text { Permeate } \\
\text { Volume "Lost" } \\
\text { Due to Samples } \\
\text { (L) }\end{array}$ & $\begin{array}{l}\text { Permeate + } \\
\text { Samples } \\
\text { (L) }\end{array}$ & $\begin{array}{l}\text { Slurry + } \\
\text { Permeate } \\
\text { (L) }\end{array}$ \\
\hline Method: & $\begin{array}{l}\text { Summation of } \\
\text { feeds (AY102, } \\
\text { AP101, FEP } \\
\text { Bottoms) }\end{array}$ & Tank Volume & Mass of Drums & $\begin{array}{l}\text { Mass of Drums } \\
(\text { density }= \\
1.2455 \pm 0.0035)\end{array}$ & $\begin{array}{l}\text { Permeate } \\
\text { Samples }+ \\
\text { Permeate in } \\
\text { Slurry Samples }\end{array}$ & Summation & Summation \\
\hline & $\begin{array}{l}1038 \pm 10(1 \%)^{*} \\
1028 \\
1048\end{array}$ & $\begin{array}{l}117 \pm 2(\sim 2 \%)^{*} \\
115 \\
119\end{array}$ & $\begin{array}{l}1136 \pm 11(1 \%)^{*} \\
1125 \\
1147\end{array}$ & $\begin{array}{l}911.5 \pm 11.5 \\
900 \\
923\end{array}$ & $\begin{array}{l}4.5 \pm 0.5 \\
4 \\
5\end{array}$ & $\begin{array}{l}916 \pm 12 \\
904 \\
928\end{array}$ & $\begin{array}{l}1033 \pm 14 \\
1019 \\
1047\end{array}$ \\
\hline
\end{tabular}

* estimated 
the Test Specification (Longwell, 2003). The UFP produced $117 \mathrm{~L}$ of concentrated slurry at $24 \mathrm{wt} \%$ UDS and approximately $916 \mathrm{~L}$ of permeate. Therefore, some of the $24 \mathrm{wt} \%$ slurry was removed and permeate was added back to the slurry to maintain the slurry volume at $117 \mathrm{~L}$ while attaining $20 \mathrm{wt} \%$ UDS. This section describes how the simulants were made and adjusted at the experimental site.

\subsubsection{Preparing for UFP Recycle Streams}

Refer to Fig. 3 (recycle streams ) and Figs. 13 and 14 (UFP unit operation) to better understand the following descriptions of feed preparations and use.

In preparation to create the UFP wash-water recycle stream on 15 April 2004, two 55-gallon drums, labeled "Campaign 2-UFP First or Second Wash,” were each filled with 141.3 liters of FEP condensate into which were added 226 grams of $50 \mathrm{wt} \% \mathrm{NaOH}$ to attain a caustic concentration of $0.01 \mathrm{M} \mathrm{NaOH}^{\dagger}$. The loop flush recycle stream, that would be made in Campaign II for use in Campaign III, was made in one carboy labeled "PWD-VSL-33" and it was filled with 8.6 liters of FEP condensate. This carboy would receive 4.5 liters of washed HLW slurry after washing. The UFP "Heel" sludge recycle stream was created in another carboy by adding 12.5 liters of FEP condensate. The rinse water recycle was created in three 55-gallon drums, labeled "Campaign 2-0.1M NaOH for UFP Caustic Flush 1, 2 or 3.” Each was filled with 94.6 liters of FEP condensate, which was made caustic to a concentration of $0.1 \mathrm{M} \mathrm{NaOH}$ by adding 766.8 grams of $50 \mathrm{wt} \% \mathrm{NaOH}$ into each. The final UFP recycle stream was created in three 55-gallon drums labeled "Campaign 2-UFP Acid Cleaning 1, 2 or 3.” Each drum was filled with 94.6 liters of DI water, which was made acidic to a concentration of $2 \mathrm{M} \mathrm{HNO}_{3}$, by adding $17 \mathrm{~kg}$ of $70 \% \mathrm{HNO}_{3}$.

\subsubsection{Preparing UFP Feed}

In preparation for pilot-scale UFP unit operation, AP101 simulated waste, which was prepared by Optima, was mixed with the FEP Evaporator Bottoms and AY102 waste simulant in the UFP Primary Mixing Tank. This tank represented the plant tank UFP-VSL-1AB. Three major components of the AY102 simulant were also made by Optima, but those components were joined, and its final preparation was made, at the test location in a separate mixing tank on 14 April in order to have just the right mix.

\footnotetext{
${ }^{\dagger}$ The concentration of $0.01 \mathrm{M} \mathrm{NaOH}$ was nominal. The real target was to have a pH of at least 12 . Due to the high acid content of the EDL water supply, the initial addition of 113 grams of the $50 \mathrm{wt} \% \mathrm{NaOH}$ to the 141 liters of water still indicated a pH below 12. To be safe the amount of caustic was doubled to 226 grams which theoretically gave a concentration of $0.02 \mathrm{M} \mathrm{NaOH}$ and a $\mathrm{pH}=12.3$. After the addition to the recycle stream it measured $\mathrm{pH}>12$.
} 
The final adjustment included the following:

\begin{tabular}{|c|c|}
\hline Material & Mass Added (g) \\
\hline \multicolumn{2}{|l|}{ Wet Additions } \\
\hline AY102 Supernate Simulant by Optima & 68,500 \\
\hline Precipitated Sludge Solids by Optima & 238,200 \\
\hline NAS, sodium aluminosilicate (wet) by Optima & 27,950 \\
\hline \multicolumn{2}{|l|}{ Dry Additions } \\
\hline $\mathrm{Al}(\mathrm{OH})_{3}$ & 5,661 \\
\hline $\mathrm{Al}_{2} \mathrm{O}_{3}$ & $2,015.2$ \\
\hline Calcium Phosphate Tribasic & 135.0 \\
\hline $\mathrm{SiO}_{2}$ & 3,655 \\
\hline $\mathrm{RbNO}_{3}$ & 0.46 \\
\hline Total & $346,116.66$ \\
\hline
\end{tabular}

After samples were taken, AY102 simulant in excess of the target volume of 66.7 gallons was removed. That volume was indicated by the OLI/ESP model for Campaign II and is shown at the top of Table 8. With AY102 ready, the final UFP feed preparation could be completed. The 66.7 gallons (320.6 kg) of AY102 simulant was transferred to the UFP Primary Mixing Tank. To this slurry, 147.4 $\mathrm{kg}$ of the $184 \mathrm{~kg}$ FEP bottoms made in the pilot-scale FEP were transferred to the UFP Primary Mixing Tank on 16 April. The last addition was $860.9 \mathrm{~kg}$ of AP101 simulant, which was also completed on 16 April. The total feed in the UFP Primary Mixing Tank was 1,328.9 kg before samples were removed for analysis. Figure 15 shows each of the constituents that went into making the UFP feed and the final feed mixture.

After the UFP Primary Mixing Tank contents had settled over the weekend of 17 to 18 April, 15.8 liters of supernatant was decanted from the tank surface on 19 April and was used to fill the permeate system of the pilot-scale UFP test facility on start up. Subsequently, the UFP Primary Mixing Tank, Fig. 16, was mixed for one hour and samples taken before initiating the transfer to the UFP Slurry Reservoir, Fig. 17, to begin dewatering operations on 19 April.

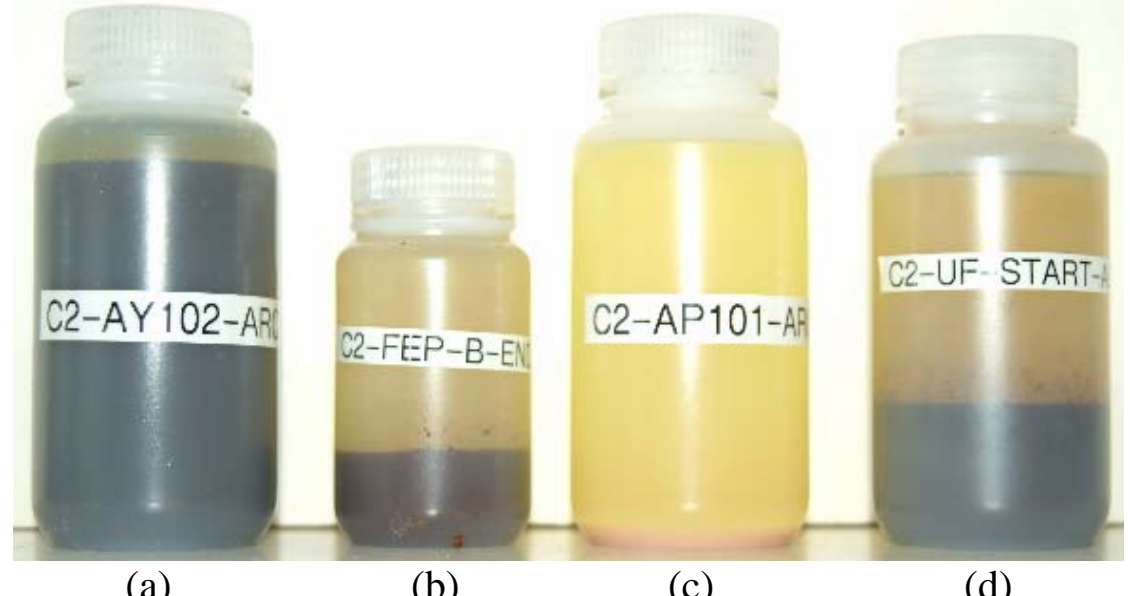

(a)

(b)

(c)

(d)

Figure 15. Simulants of (a) AY102, (b) FEP Bottoms, (c) AP101, and (d) UFP feed which is a mixture of (a), (b), and (c) - These are Samples after Solids Had Settled 
WSRC-TR-2004-00478, REVISION 0 SRNL-RPP-2004-00075, REVISION 0

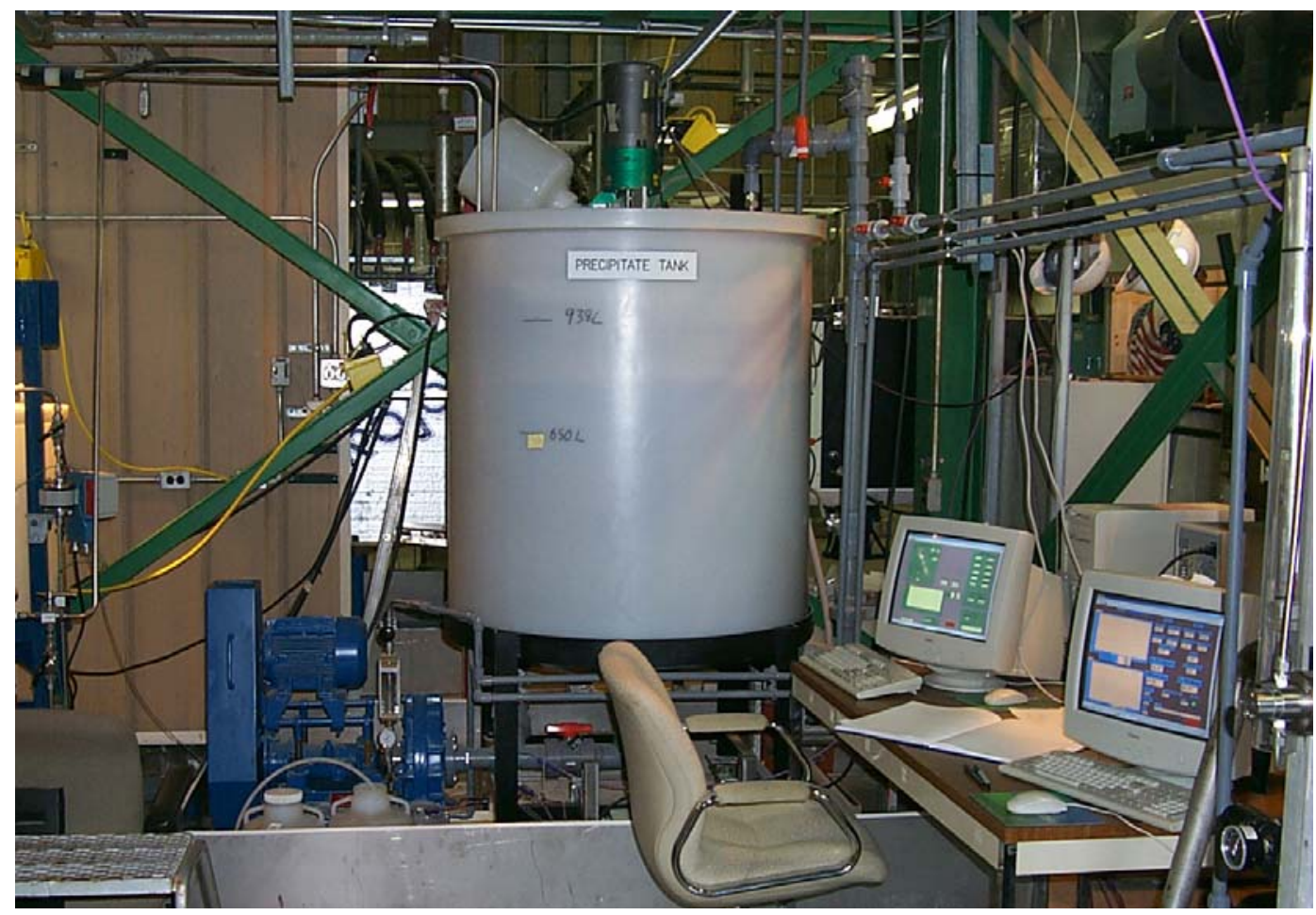

Figure 16. UFP Primary Mixing Tank (Volume 1000 liters) which represents the RPP-WTP UFP Preparation Feed Tank

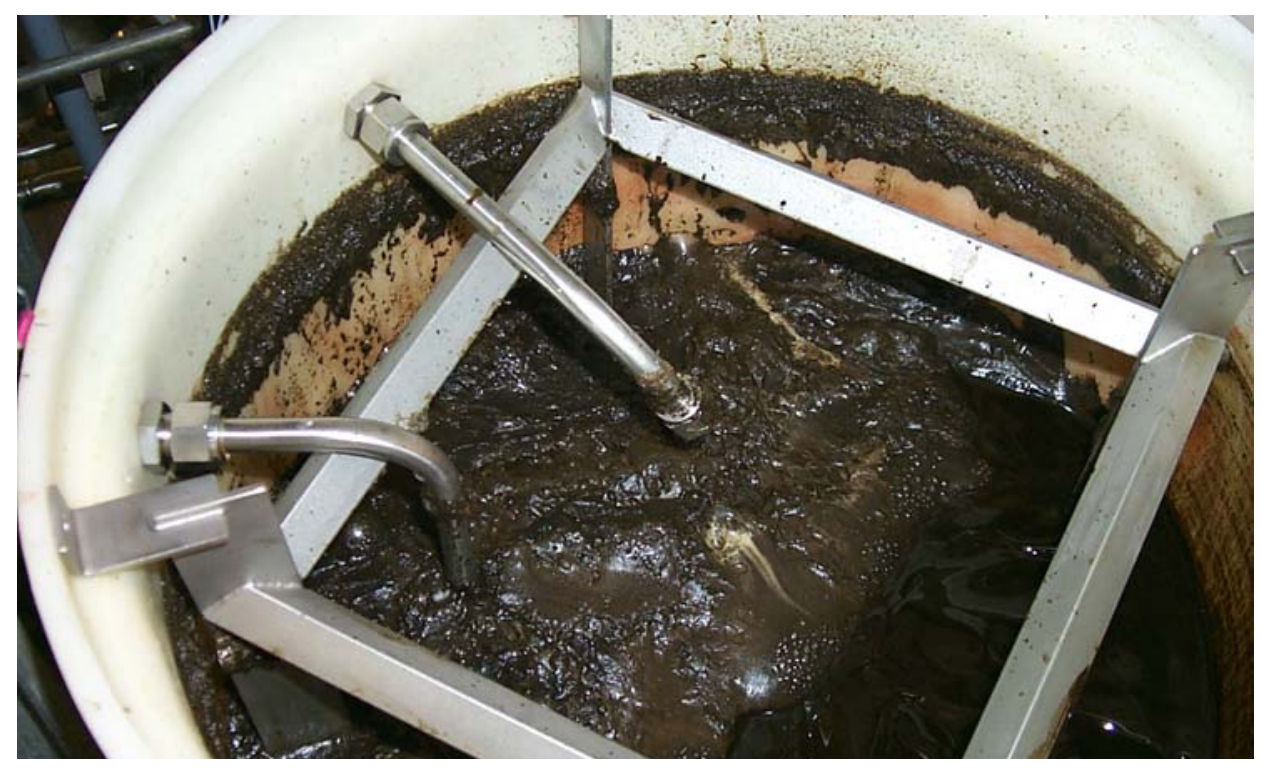

Figure 17. UFP Slurry Reservoir (Volume 100 liters) which represents the RPP-WTP UFP Feed Tank 


\subsubsection{Final UFP Products}

The slurry produced by the UFP filter was concentrated to approximately $24 \mathrm{wt} \%$ UDS by 22 April. To bring the fixed-volume batch of slurry back to washing target of $20 \mathrm{wt} \%$ UDS, $27.91 \mathrm{~kg}$ of slurry were removed and 19.6 liters (or $24.6 \mathrm{~kg}$ ) of permeate were added. After the newly diluted slurry was well mixed, samples were taken. The concentrated slurry remaining in the UFP test rig was then washed in two phases using the nominal $0.01 \mathrm{M} \mathrm{NaOH}$ wash water from drums labeled "Campaign 2UFP First or Second Wash" on 23 April. The permeate from slurry washing was collected in 55gallon drums for use in Campaign III as Wash Permeate recycle. The washed HLW concentrated slurry had a density of $1.22 \mathrm{gm} / \mathrm{ml}$. An amount of 4.5 liters of concentrated slurry were added to the carboy labeled "PWD-VSL-33" to be held for Campaign III as Loop Flush recycle. An additional amount of 4.13 liters of concentrated slurry were added to the carboy labeled "UFP Heel," to be added back into the UFP test rig to represent a heel.

The remaining material, about 100 liters, was transferred to a 55-gallon drum labeled "Campaign 2 Concentrated Washed HLW Slurry" for shipment to VSL on 26 April for its eventual vitrification. The permeate produced by the UFP was placed into either of two drums labeled "Campaign 2-CsIX Permeate Feed” and transported to a CIX Simulant Supply Tank. About 863 liters of permeate was transferred to the CIX Simulant Supply Tank on 23 April.

On 26 April the filter rinse-clean-rinse sequence began. The carboy labeled "UFP Heel” was returned to the empty slurry reservoir as well as the contents from drum marked "Caustic Flush 1" to rinse the UFP flow loop. The resulting volume was 134 liters of material. This volume was larger than the expected 117 liters. This indicated that there was already a substantial heel remaining in the flow loop (of approximately 22 liters of material) rather than the expected heel amount of 5 liters. After the first rinse, all of the contents of the slurry reservoir were pumped to a drum labeled "Campaign 2-Caustic Flush 1 to Campaign 3 (PWD-VSL-15)" and later stored for Campaign III. An amount of 20 liters were returned to the slurry reservoir to represent the next "UFP Heel. . This cycle repeated itself for all the Acid Cleanings 1, 2, 3 and Caustic Flush 2 and 3, that is, 20 liters of each of the preceding rinse/cleanings, simulated heel material, were mixed with the next batch of cleaning or rinsing. The cleaning and rinses were completed on 27 April. The used cleaning and rinsing solutions were stored in drums labeled "Campaign 2-Acid Cleaning 1 or 2 \& 3 to Campaign 3 (PWD-VSL-16)" and "Campaign 2-Caustic Flush 2 or 3 to Campaign 3 (PWD-VSL-15)” for Campaign III, respectively.

\subsubsection{Pilot UFP Operation and Equipment (Author - Fowley)}

The test rig for the Semi-Integrated Pilot Plant (SIPP) is a pilot scale representation of the Ultrafiltration Process (UFP) of the River Protection Project (RPP) Waste \& Immobilization Treatment Plant (WTP). The UFP will remove and concentrate solids from combined simulated waste feed streams. The solids are removed to protect downstream ion exchange (IX) beds and to meet the specifications for LAW product. The UFP will also minimize storage requirements for HLW and enhance HLW vitrification efficiency.

The UFP filter unit, see Figs. 18, 19, and 20, was manufactured by the Mott Metallurgical Corporation to meet RPP-WTP specifications (Townson, 2002, Longwell, 2003), as follows: 
- 7 filter tubes with each having an inside diameter of 0.5 -inch

- 90-inch porous length for each filter tube and made of stainless steel

- Nominal rated 0.1 micron filter element

The pilot-scale test rig was designed to have the following:

- Maximum axial velocity through filter tubes of $4.6 \mathrm{~m} / \mathrm{s}(15 \mathrm{ft} / \mathrm{s})$

- Maximum transmembrane pressure (TMP) of $60 \mathrm{psid}$

- Maximum axial velocity to be achievable at the maximum TMP

- Instrumentation to monitor the axial velocity, the permeate flow rate, the TMP, and the slurry temperature

- All materials to be compatible with the high-caustic simulants and the $2 \mathrm{M}$ nitric acid cleaning solution

All filter specifications were met or exceeded.

Figure 18. Entire 90-inch Length of the Filter-tube Bundle

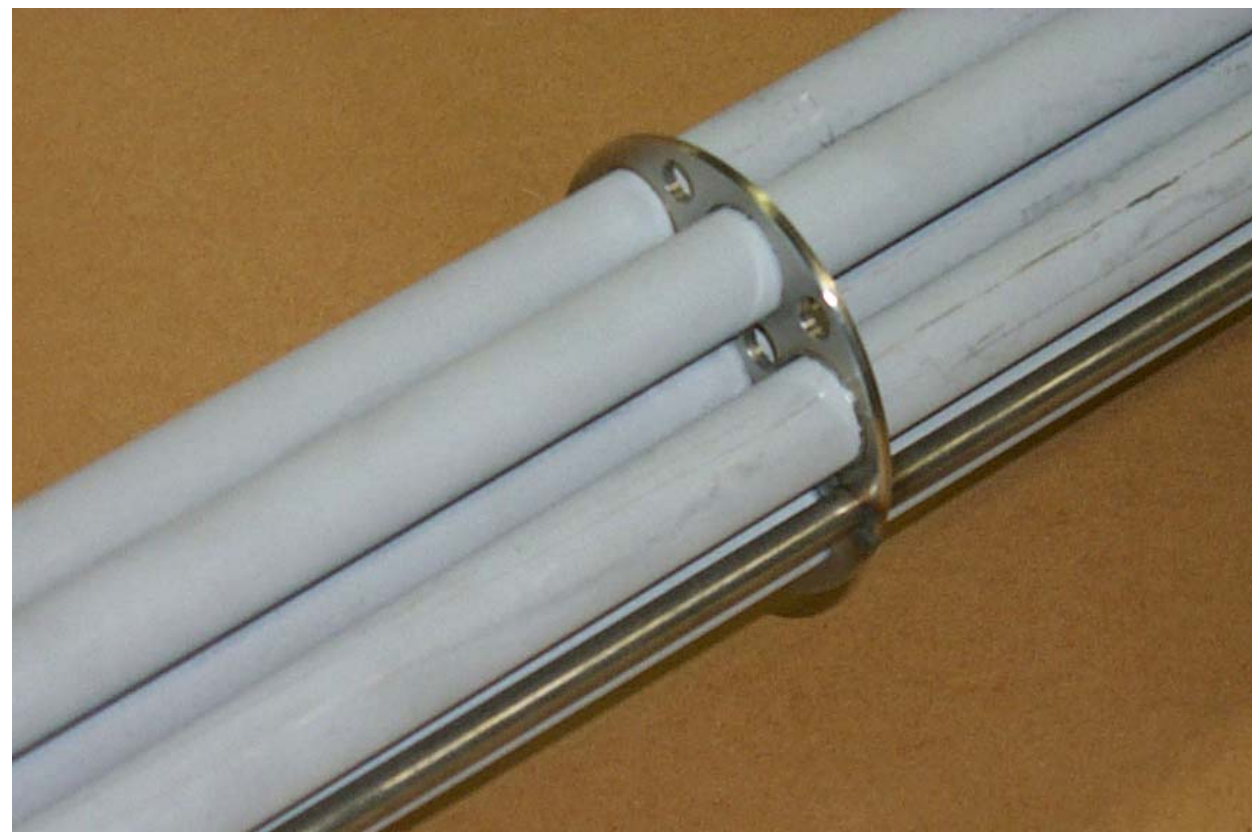

Figure 19. Close-up of the Tubes’ Surfaces and Bulkhead Stabilizing Plate 

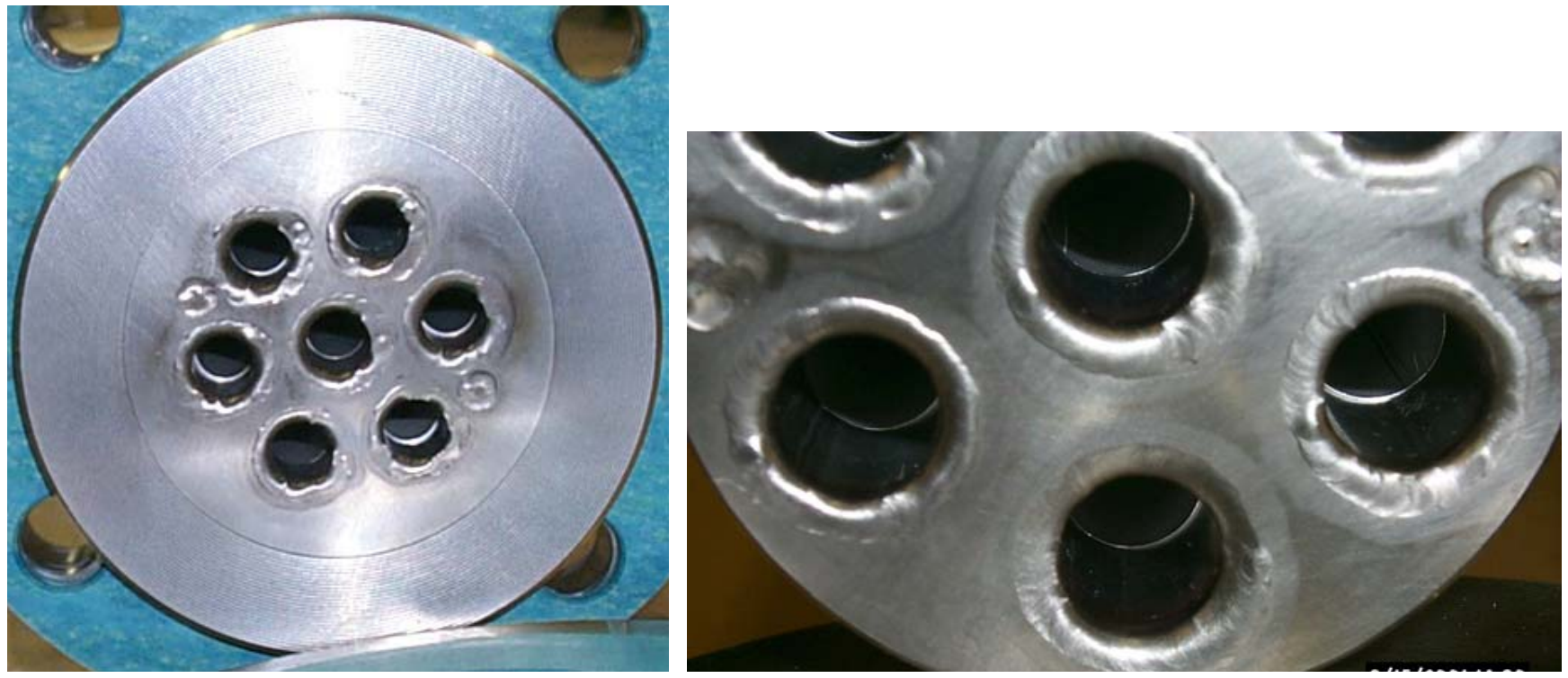

Figure 20. The Exit (Left) and Entrance (Right) Tube Sheets of the Filter Tube Bundle

The filter was prototypic in most of its features. The tubes were symmetric and made from porous stainless steel sintered material with a nominal pore size of 0.1 micron. The porous tube length was 90 inches, the tube inside diameter was 0.488 inches, and the outside diameter was 0.654 inches ${ }^{\dagger}$. Tests were conducted at an axial slurry velocity of 11 to $12 \mathrm{ft} / \mathrm{s}$, and a TMP of approximately 40 psid.

The filter was not prototypic in three features: it only had 7 tubes instead of 241 tubes, it was mounted vertical instead of horizontal, and while the backpulse system functioned prototypically, the system design must be different in its dynamic hydraulic response because of scaling. In general, filter performance data from the pilot test rig was assumed to well represent those that will be obtained in the full-scale unit.

\subsubsection{Test Matrix}

Table 24 shows the Campaign II UFP test matrix. The test number ordering has been maintained and sequentially adjusted from Campaign I (Duignan, et al., 2004). Several tests were eliminated prior to Campaign I and between Campaign I and II, which produced numerical gaps between several of the test numbers.

\footnotetext{
${ }^{\dagger}$ These inside and outside diameters are averages obtained from the many measurements made on the tube before testing began. The tubes ordered from the Mott Corporation were to have a 0.5-inch inside diameter and a 0.75-inch outside diameter to match the then current design of the WTP UFP filters.
} 
Table 24. Campaign II UFP Test Matrix ${ }^{7}$

\begin{tabular}{|c|c|c|c|c|c|}
\hline $\begin{array}{l}\text { Test } \\
\text { No. }\end{array}$ & Test Activity & $\begin{array}{l}\text { Trans- } \\
\text { Membrane } \\
\text { Pressure } \\
\text { (psid) }\end{array}$ & $\begin{array}{c}\text { Slurry } \\
\text { Velocity } \\
(\mathrm{ft} / \mathrm{s})\end{array}$ & $\begin{array}{l}\text { Slurry } \\
\text { Temp. } \\
\left({ }^{\circ} \mathrm{C}\right)\end{array}$ & $\begin{array}{l}\text { Run } \\
\text { Time } \\
\text { (min) }\end{array}$ \\
\hline $2.00 \mathrm{~A}$ & Pre-test baseline $\mathrm{A}^{1}$ & 10 & 11 & 25 & 20 \\
\hline $2.00 \mathrm{~B}$ & Pre-test baseline $\mathrm{B}^{1}$ & 20 & 11 & 25 & 20 \\
\hline $2.00 \mathrm{C}$ & Pre-test baseline $\mathrm{C}^{1}$ & 30 & 11 & 25 & 20 \\
\hline $2.03 \mathrm{~A}$ & Dewater first half of slurry $^{2}$ & 40 & 12 & 25 & 948 \\
\hline $2.03 \mathrm{~B}$ & Dewater second half of slurry $^{2}$ & 40 & 12 & 25 & 2452 \\
\hline Wash 1 & Slurry wash $1^{3}$ & 40 & 12 & 25 & 379 \\
\hline Wash 2 & Slurry wash $2^{3}$ & 40 & 12 & 25 & 246 \\
\hline $2.18 \mathrm{~A}$ & Caustic rinse $1^{4}$ & 20 & 11 & 25 & 30 \\
\hline $2.18 \mathrm{~B}$ & Acid clean $1^{5}$ & 40 & 11 & 25 & 90 \\
\hline $2.18 \mathrm{C}$ & Acid clean $2^{5}$ & 40 & 11 & 25 & 90 \\
\hline $2.18 \mathrm{D}$ & Acid clean $3^{5}$ & 40 & 11 & 25 & 90 \\
\hline $2.19 \mathrm{~A}$ & Caustic rinse $2^{4}$ & 20 & 11 & 25 & 30 \\
\hline $2.19 \mathrm{~B}$ & Caustic rinse $3^{4}$ & 20 & 11 & 25 & 30 \\
\hline $2.21 \mathrm{~A}$ & Post-test baseline $\mathrm{A}^{6}$ & 10 & 11 & 25 & 20 \\
\hline $2.21 \mathrm{~B}$ & Post-test baseline $\mathrm{B}^{6}$ & 20 & 11 & 25 & 20 \\
\hline $2.21 \mathrm{C}$ & Post-test baseline $C^{6}$ & 30 & 11 & 25 & 20 \\
\hline
\end{tabular}

Notes:

1. The pre-test baseline tests were conducted with de-ionized, filtered $(0.1 \mu \mathrm{m})$ water made caustic to $0.1 \mathrm{M} \mathrm{NaOH}$.

2. The slurry was dewatered to $20 \mathrm{wt} \%$ undissolved solids or greater.

3. The slurry was washed with $0.01 \mathrm{M} \mathrm{NaOH}$ solution made from FEP condensate.

4. The caustic rinse consisted of $0.1 \mathrm{M} \mathrm{NaOH}$ solution made from FEP condensate.

5. The acid clean consisted of $2 \mathrm{M} \mathrm{HNO}_{3}$ solution.

6. The post-test baseline tests were conducted with FEP condensate made caustic to $0.1 \mathrm{M} \mathrm{NaOH}$.

7. Flow parameters: TMP, Velocity, and Temperature, were required by the test specification (Townson, 2002)

Recycle streams are included in the SIPP UFP (to a limited extent) to mimic actual WTP operation. The caustic wash solutions and caustic rinse solutions were made from Campaign II FEP condensate. The permeate from the slurry washing and the spent solution from the rinsing and cleaning of the filter unit were recycled as part of the Campaign III FEP feed.

All UF testing activities were performed using an approved field procedure (Duignan, 2004). The field procedure described generic activities regarding the operation of the test rig (filling and draining the test rig, dewatering, backpulsing, sampling, etc.). Work guidances were used for daily operation of the test rig. The work guidances referenced the field procedure and specified the order of activities for completing a test number or series of test numbers in the test matrix. Pertinent test information such as observation, non-critical calculations, chronological events, etc. was recorded in the laboratory notebook (Fowley, 2004). All documents for this test activity are maintained by the Engineered 
Equipment and Systems (EES) Department Document Control System per EES procedure L9.1-2006 in Job Folder \# EES-23041.

\subsubsection{Test Equipment}

A schematic diagram of the cross-flow filter test rig and support equipment is shown in Figure 21. AY102 simulant was mixed in the Secondary Mixing Tank and then pumped to the Primary Mixing Tank, which contained the AP101 supernatant and the FEP Evaporator concentrate. Both tanks were mechanically agitated and had product recirculation loops to provide sufficient agitation during the mixing process. Sample lines were available in the recirculation lines to take representative samples for analysis.

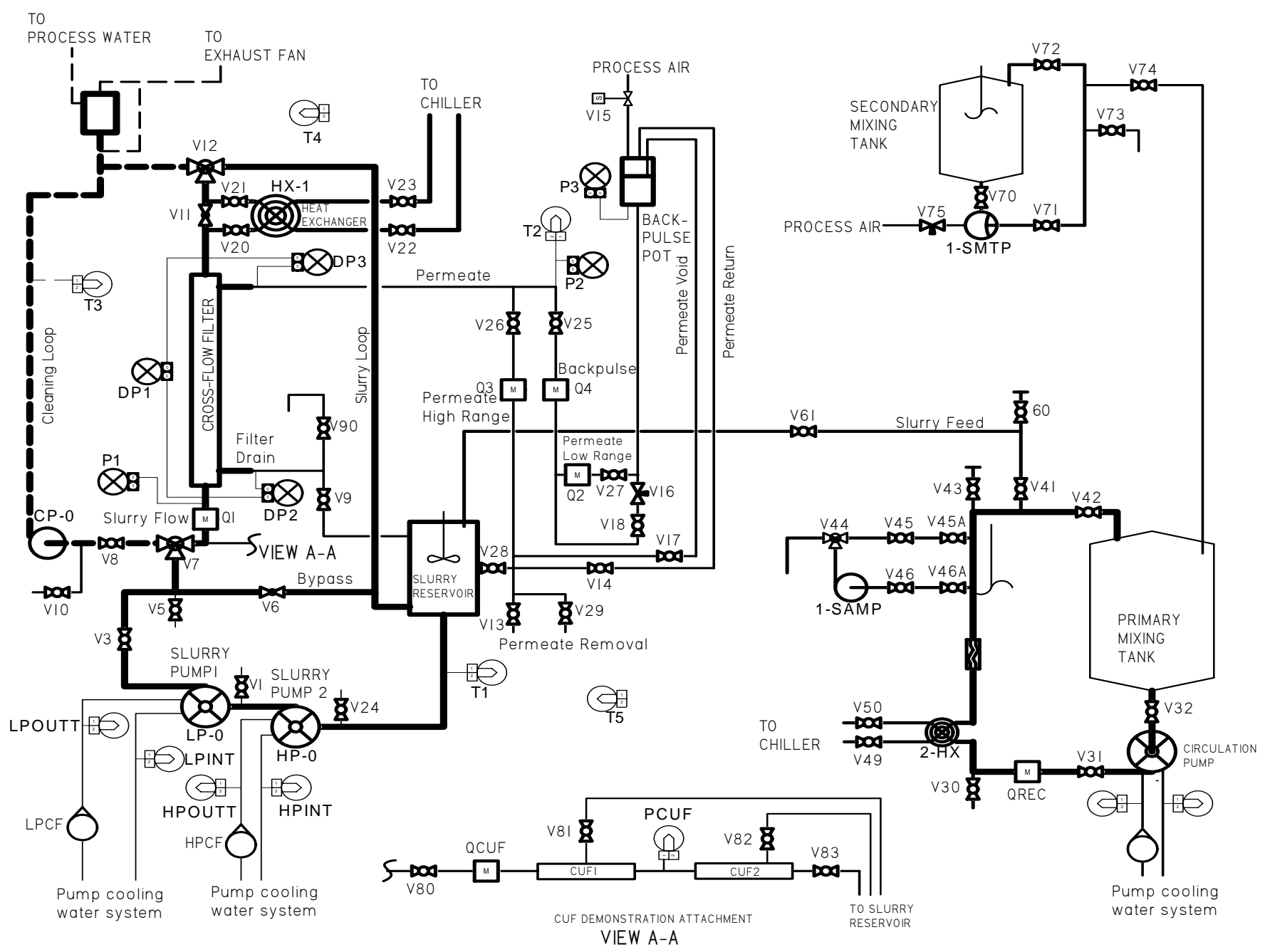

Figure 21. Campaign II Ultrafiltration Test Rig

The slurry in the Primary Mixing Tank was transferred to the test rig Slurry Reservoir using a bypass in the recirculation line on the Primary Mixing Tank. Once a batch of slurry was transferred to the Slurry Reservoir the test rig pumping system would circulate the slurry through the filter. The test rig pumping system consisted of two 10-hp Galigher ${ }^{\circledR}$ slurry pumps in series. The impeller and impeller 
housing were lined with EPDM to be compatible with both the high caustic slurry and the $2 \mathrm{M}$ nitric acid cleaning solution.

The stainless steel test rig is composed of three basic flow loops; the slurry loop, the permeate loop, and the cleaning loop (not used for SIPP and not described in this report). The slurry loop is the primary flow path and contains the cross-flow filter. The slurry loop valves may be positioned to bypass the cross-flow filter. The permeate loop starts at the filter housing, contains a backpulse pot and ends at the slurry reservoir. The backpulse pot and associated tubing provides a pulse of permeate flow in the reverse direction to knock off built-up slurry cake on the inside of the filter tubes. A more detailed description of the test rig is documented in (Duignan, 2000a, Duignan 2000b, Duignan 2003a) and will be provided in the SIPP final report.

\subsubsection{Measurement Uncertainty}

The derivation of measurement uncertainty will not be shown in this report, but will be included in the final report, which will incorporate results from all four campaigns. The uncertainties listed here are based on past work done on the same equipment under similar circumstances and similar individual instrument uncertainties (Duignan 2003a). The uncertainties listed here are to illustrate the expected magnitude of principal parameters measured throughout this test. The measurement uncertainties (at the 95\% confidence level) for the important calculated quantities are:

$\begin{array}{ll}\text { Slurry Velocity in a Filter Tube } & = \pm 6 \% \\ \text { TMP } & = \pm 2 \% \\ \text { Temperature Corrected Permeate Flux } & = \pm 6 \%\end{array}$

For analytical results included herein, the measurement uncertainty varies and sometimes can be large. Uncertainties for data obtained from outside organizations, i.e., Analytical Development Section (ADS), the Mobile Laboratory of Savannah River National laboratory (SRNL), or the Aiken County Technical laboratory (ACTL) are beyond the scope of this task but they are assumed to be no larger than $\pm 15 \%$ of any result shown. For properties measured in the EDL, the following uncertainties on a reading were obtained through calibration:

$\begin{array}{ll}\text { Slurry or Liquid Density }= & \pm 0.5 \% \\ \text { Liquid Viscosity }= & \pm 0.5 \% \\ \text { Turbidity }= & \pm 0.1 \mathrm{NTU}\end{array}$

\subsubsection{Pilot UFP Discussion of Results}

\subsubsection{Experimental Observations(Author - Fowley)}

\subsection{Slurry Concentration (Dewatering)}

Dewatering started Monday, 19 April. The simulant in the Primary Mixing Tank was not agitated during the weekend before the 19th so that solids would settle out and allow supernate to be drawn from the top of the mixture. After the supernate was removed, the simulant was thoroughly agitated for one hour prior to transfer to the test rig. The supernate was added back to the test rig prior to the 


\section{WSRC-TR-2004-00478, REVISION 0 SRNL-RPP-2004-00075, REVISION 0}

initiation of dewatering to account for the volume of permeate necessary to fill the permeate loop. Doing so would assure the correct solids weight \% of the simulant in the test rig after the slurry and permeate loops were primed and ready.

Per the test matrix, the dewatering was conducted in two stages. The delineation between the stages was approximately one-half of the expected permeate removed from the simulant (461 liters). A hold period, a minimum of 12 hours, was inserted between the stages to purposely extend the dewatering process, making it more comparable with the duration of the dewatering process of the RPP-WTP plant, during which some waste instabilities could cause changes, e.g., precipitation. The first stage was completed on 20 April, the second stage was completed on 22 April. The hold period turned out to be 20 hours. The entire dewatering process, including the hold, and downtime for sampling, took approximately 77 hours. Excluding the hold, and sampling downtime, dewatering took 53 hours and 8 minutes. (This 77-hour period is approximately one half of the dewatering time that WTP will take, which was estimated to be 138 hours, as shown in Table A9 in Appendix A. However, any UFP-feed reactions are expected to occur during first half of the dewatering period.)

Approximately $916 \mathrm{~L}$ of permeate was collected during dewatering. $21 \mathrm{~L}$ of permeate were added back to the slurry to achieve the correct weight \% solids for the following slurry washing test. The remaining permeate was used in the Campaign II Ion Exchange process.

Figure 22 shows the adjusted permeate flux for the entire slurry dewatering process. The flux is adjusted for temperature, as required by the RPP-WTP test specification to account for the effect of viscosity. The adjustment equation is:

$$
\begin{gathered}
\text { Adjusted Flux }=\text { Flux }\left(\mathrm{gpm} / \mathrm{ft}^{2}\right) \times \exp (2500 \times((1 /(273+\mathrm{T}))-(1 / 298))) \\
\text { where } \mathrm{T}=\text { slurry/permeate temperature in degrees Celsius }
\end{gathered}
$$

Dewatering started with a backpulse. Another backpulse was conducted at approximately 11.5 hours into the first stage of dewatering. Per procedure a backpulse was conducted when the permeate flux dropped below $0.015 \mathrm{gpm} / \mathrm{ft}^{2}$. When dewatering resumed after the backpulse the permeate flux was higher ( $>0.030 \mathrm{gpm} / \mathrm{ft}^{2}$ ) but quickly ( 18 minutes) returned to the pre-backpulse flux. Because of the poor recovery, a backpulse was not repeated for the remainder of the dewatering.

The majority of the data scatter shown in Figure 22 (and in Figure 23) was caused by process disruptions: backpulsing, sampling and exchanging the permeate collection drums. Slurry and permeate samples were drawn approximately every two hours. The permeate sample was taken from a sample port in the permeate loop. Prior to sampling, permeate was recirculated back to the slurry reservoir. This action changed the hydraulic resistance of the permeate loop and affected the flux measurement. Exchanging the permeate drums also required switching the permeate loop to recirculation mode.

Samples of concentrated slurry and permeate were taken during the dewatering process in accordance with the sample matrix, Appendix D. Samples were taken at three periods during each dewatering stage (at approximately 154 liters, 307 liter, and 461 liters of permeate removed). Permeate density, viscosity, and turbidity, as well as slurry density, were measured in the EDL soon after the samples 
were taken. Separate permeate and slurry samples were sent to ACTL for measurements of density, yield stress, consistency, and weight \% solids [total solids (TS), undissolved solids (UDS), dissolved solids (DS), and suspended solids (SS)] analysis.

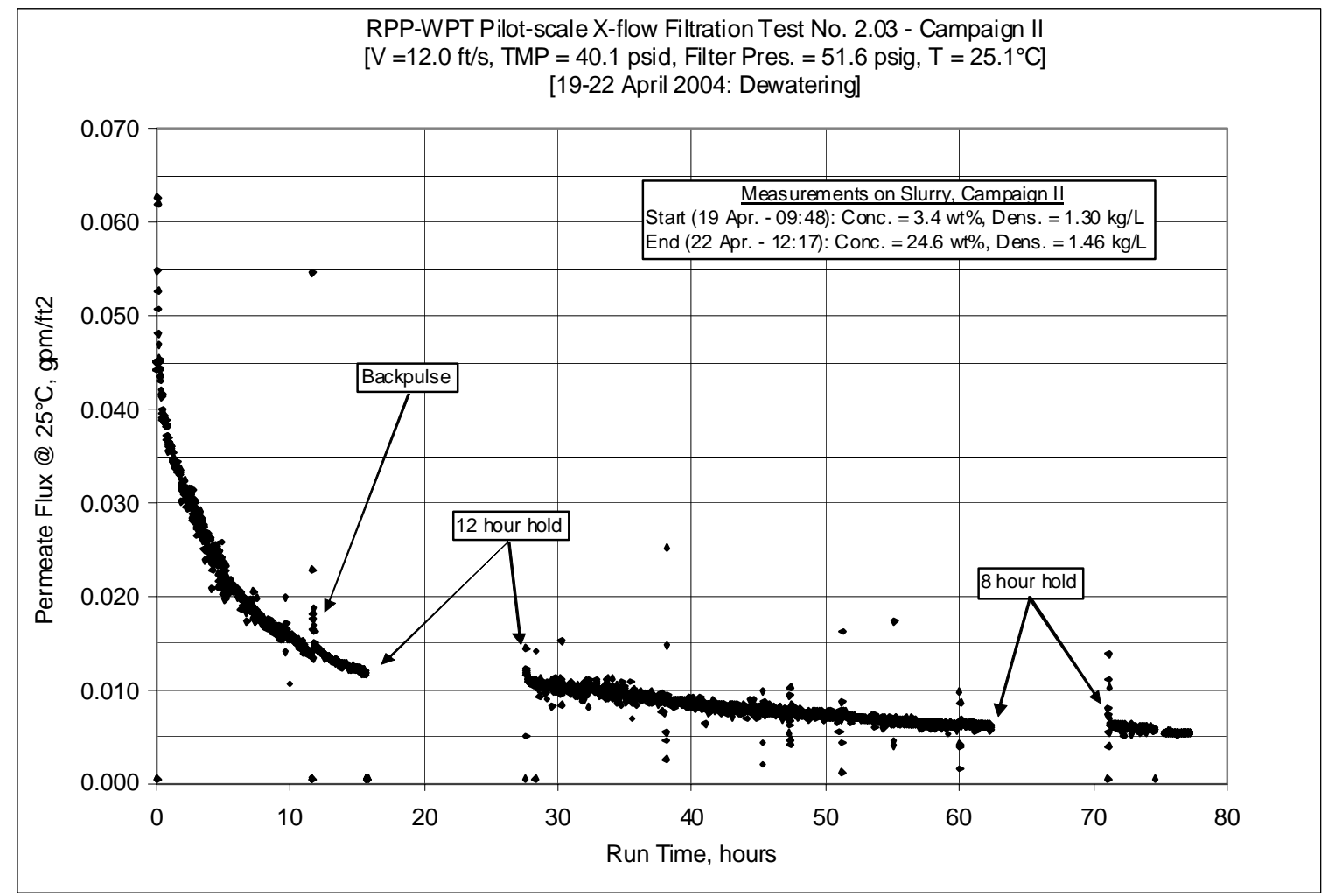

Figure 22. Dewatering of UF Feed, Permeate Flux vs. Run Time 


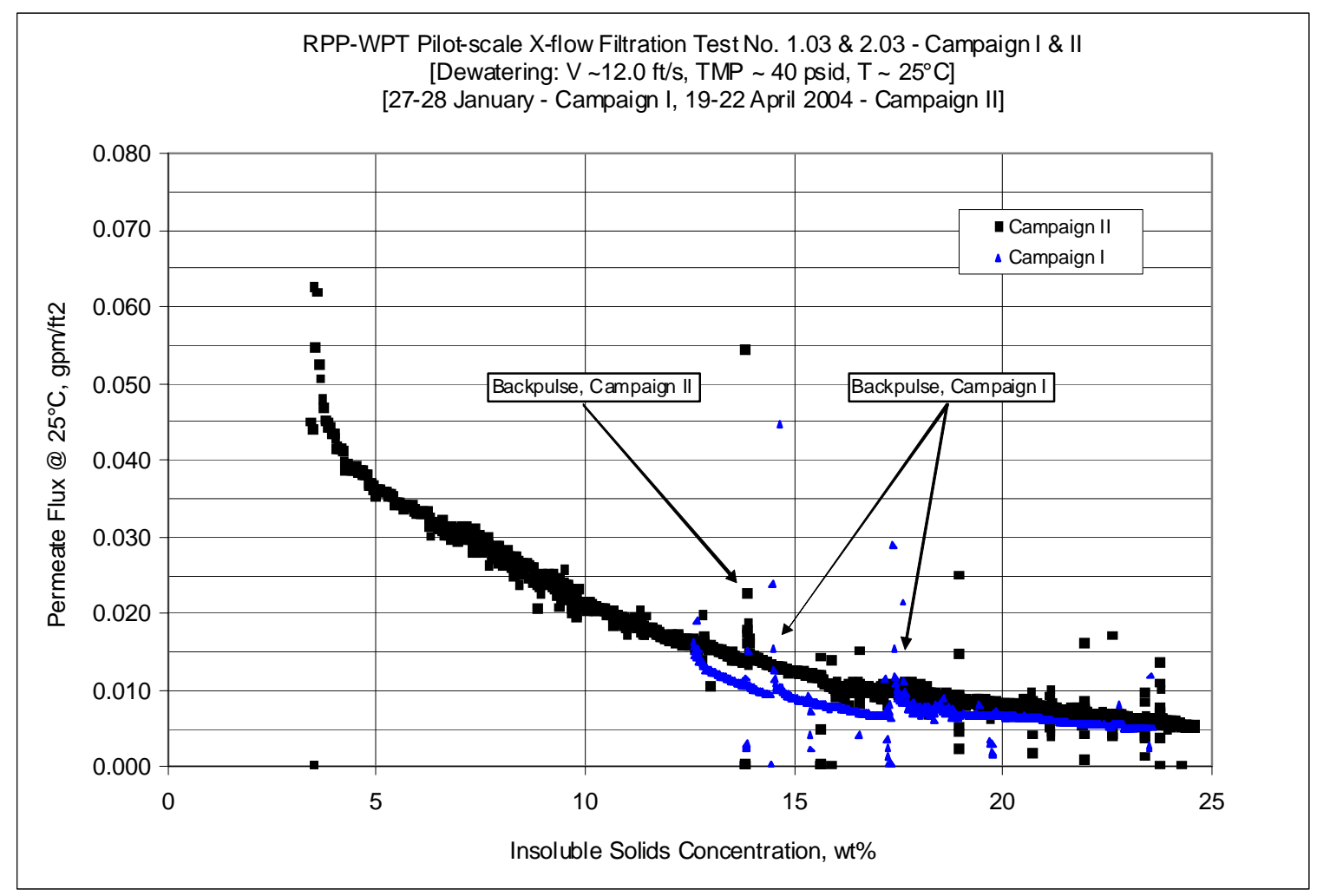

Figure 23. Dewatering of UF Feed, Permeate Flux vs. Concentration

Another way to visualize the dewatering process is to track permeate flux against the concentration of UDS. Figure 23 shows the adjusted permeate flux versus UDS concentration. These concentration data, shown in the figure, were derived by interpolating between the six slurry samples results acquired during dewatering and based on the mass of permeate removed over time (see Section 2.7.4.2.6). For comparison, the dewatering data from Campaign I are also shown in Fig. 23. The simulant for Campaign I contained only AY102 and was $12.5 \mathrm{wt} \%$ at the beginning of dewatering. Note that the data trends are similar for both Campaign I and II. This consistent performance indicates that; 1) the UFP unit was successfully cleaned after Campaign I, 2) filter performance can be predictable, and 3) the addition of AP101 and FEP bottoms to the AY102 had little effect on filter performance. As indicated on the chart, two backpulses were performed in the Campaign. Poor recovery was evident in both campaigns.

The RPP-WTP operation will concentrate the waste in the UFP unit to approximately 17 wt\% UDS, therefore all of the campaigns of SIPP have a target of $20 \mathrm{wt} \%$ UDS to obtain information at an upper bound. However, there is little filter data at the higher concentrations so the Test Specification for this filter work indicated a concentration of $25 \mathrm{wt} \%$ UDS as the target if obtainable. The dewatering test was stopped after 53 hours and 8 minutes when a rough estimate ${ }^{\dagger}$ of concentration indicated 25 wt $\%$ UDS. The actual ending value was $24.6 \mathrm{wt} \%$ UDS.

Table 25 shows rheological and other physical data of the slurry and permeate during the dewatering.

\footnotetext{
${ }^{\dagger}$ Real time concentration analysis was not available and only the initial samples taken on the previous day were used to extrapolate to estimate the $25 \mathrm{wt} \%$ UDS.
} 
Table 25. Measured Rheological and Other Data during Dewatering of Slurry

\begin{tabular}{|c|c|c|c|c|c|c|c|c|c|}
\hline \multirow{2}{*}{$\begin{array}{c}\text { Target } \\
\text { Volume of } \\
\text { Permeate } \\
\text { Produced }\end{array}$} & \multirow{2}{*}{$\begin{array}{l}\text { Cumulative } \\
\text { Weight of } \\
\text { Permeate } \\
\text { Produced }\end{array}$} & \multicolumn{4}{|c|}{ Slurry ${ }^{1}$} & \multicolumn{4}{|c|}{ Permeate $^{2}$} \\
\hline & & Density & TS & UDS & Consistency & Density & Viscosity $^{3}$ & Temp & Turbidity \\
\hline $\mathrm{L}$ & $\mathrm{Kg}$ & $\mathrm{gm} / \mathrm{mL}$ & $\mathrm{wt} \%$ & $\mathrm{wt} \%$ & $\mathrm{mPa}-\mathrm{s}$ & $\mathrm{gm} / \mathrm{mL}$ & $\mathrm{mPa}-\mathrm{s}$ & ${ }^{\circ} \mathrm{C}$ & NTU \\
\hline 0 & 0 & 1.28 & 30.6 & 3.2 & 4.74 & $\mathrm{n} / \mathrm{a}$ & $\mathrm{n} / \mathrm{a}$ & $\mathrm{n} / \mathrm{a}$ & $\mathrm{n} / \mathrm{a}$ \\
\hline $\begin{array}{l}\text { Day1 } \\
153.7 \\
\end{array}$ & 179.00 & 1.29 & 33.4 & 7.0 & $\mathrm{n} / \mathrm{a}$ & 1.25 & 2.56 & 22 & 0.22 \\
\hline 307.4 & 358.05 & 1.32 & 35.8 & 11.2 & $\mathrm{n} / \mathrm{a}$ & 1.25 & 2.52 & 22 & 0.22 \\
\hline 461.2 & 576.50 & 1.36 & 38.7 & 14.9 & $\mathrm{n} / \mathrm{a}$ & 1.25 & 2.59 & 23 & 0.20 \\
\hline $\begin{array}{l}\text { Day } 2 \\
153.7\end{array}$ & 768.50 & 1.38 & 42.0 & 19.5 & $\mathrm{n} / \mathrm{a}$ & 1.25 & 2.37 & 25 & 0.23 \\
\hline 307.4 & 960.50 & 1.40 & 43.1 & 21.3 & $\mathrm{n} / \mathrm{a}$ & 1.25 & 2.37 & 25 & 0.23 \\
\hline 461.2 & 1136.05 & 1.47 & 45.4 & 23.5 & 22.89 & 1.25 & 2.45 & 24 & 0.25 \\
\hline
\end{tabular}

Notes:

1 Data from ACTL.

2 Data from EDL.

3 Viscosity measurement of permeate prior to start of dewatering by ACTL was $3.01 \mathrm{mPa}$-s. EDL used a Cannon-Fenske viscometer, ACTL used a Haake Rheometer.

As expected, the density, TS, UDS and consistency of the slurry increased during dewatering. The consistency increased by a factor of 5, while the wt\% of UDS increased by a factor of 7 . Density increased $15 \%$ and wt $\%$ TS increased $5 \%$. The permeate rheology showed that the density and viscosity remained constant throughout the process (the viscosity variations are due to temperature). The turbidity measurements of the permeate were significantly lower than the test specification threshold of 2 NTU and, therefore, was considered free of solids.

To determine if post-precipitation occurs in the separated waste, supernatant samples were taken of permeate at the end of first and second dewatering tests and were set aside, at ambient temperature, to periodically monitor turbidity with time. That is, turbidity was measured when the sample was first taken and at different days over a two week period. Table 26 shows the results

Table 26. Periodic Turbidity Measurements of Dewatering Permeate

\begin{tabular}{|c|c|c|c|c|}
\hline \multirow{2}{*}{ Date } & \multicolumn{4}{|c|}{ Turbidity } \\
\cline { 2 - 5 } & \multicolumn{2}{|c|}{$\begin{array}{c}\text { Sample from the } \\
\text { First dewatering }\end{array}$} & \multicolumn{2}{c|}{$\begin{array}{c}\text { Sampler from the } \\
\text { Second dewatering }\end{array}$} \\
\cline { 2 - 5 } & Day & (NTU) & Day & (NTU) \\
\hline $4 / 20 / 04$ & 1 & 0.200 & - & - \\
\hline $4 / 22 / 04$ & 3 & 0.245 & 1 & 0.250 \\
\hline $4 / 23 / 04$ & 4 & 0.300 & 2 & 0.375 \\
\hline $4 / 26 / 04$ & 7 & 0.300 & 5 & 0.475 \\
\hline $5 / 3 / 04$ & 14 & 0.375 & 12 & 0.560 \\
\hline
\end{tabular}


While the sample remained visually clear the increasing turbidity indicates that there was some precipitation, but even after two weeks the measurement was well below the 2 NTU threshold of significant solids. Further chemical analyses of the permeate and the slurry are provided in section 2.7.4.2.

\subsection{Slurry Washing}

One important aspect of the UFP unit operation was the investigation of the effects of washing the concentrated slurry. In WTP, the filtration batch size was set at 21,550 gallons. Once the UFP slurry is concentrated, it is washed twice with 21,770 gallons (this represents the volume of one permeate storage tank) using 22 mini-batches of 1,000-gallon each. To mimic plant operation (noting that the Campaign II pilot-scale batch size was 116.6 liters) the batch of wash solution was $116.6 \mathrm{~L} \mathrm{x}$ 21,770/21,550 = 117.8 L, making each mini-batch of wash solution $\sim 5.4$ liters. The pilot-scale process for each mini-batch of wash was to add $5.4 \mathrm{~L}$ of wash solution $(0.01 \mathrm{M} \mathrm{NaOH})$ and then dewater until $5.4 \mathrm{~L}$ of permeate was removed. This was repeated 21 more times to complete one wash cycle, and then the wash cycle was repeated, for a total of 44 mini-batches of wash solution. The two washes occurred on separate but consecutive days. Figure 24 shows the first washing results.

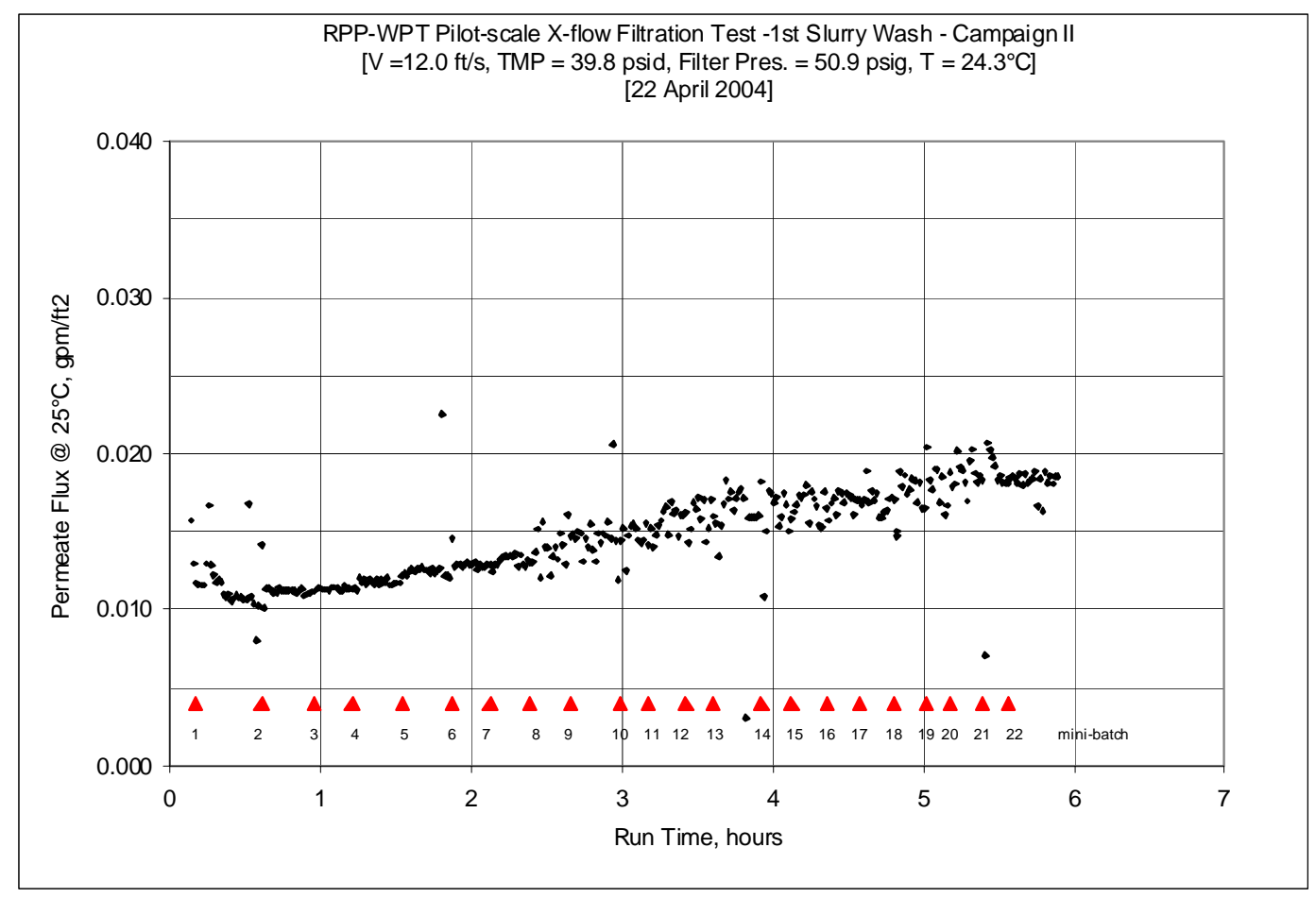

Figure 24. $1^{\text {st }}$ Washing of Concentrated Slurry with 22 Wash Additions (or mini-batches)

As wash solution was added to the concentrated simulant, the adjusted flux slowly increased from 0.011 to $0.018 \mathrm{gpm} / \mathrm{ft}^{2}$ during the first wash cycle (64\% increase). The flux increase in Campaign I was a comparable 53\%. Figure 25 shows that during the second washing cycle, the adjusted flux continued to increase but at a much slower rate, from approximately 0.020 to $0.025 \mathrm{gpm} / \mathrm{ft}^{2}$ (25\% increase). The flux increase in Campaign I was 32\%. In both Campaign I and II, the adjusted flux 
increased from the end of the first day of washing to the beginning of the second day of washing, while the test rig was idle. This increase was 11\% in Campaign II and $8 \%$ in Campaign I. It is believed that some solids, which were entrained in the filter media, continued to dissolve in the residual wash solution during this idle time, creating an improved flux when the test rig was restarted. The permeate flux, for both Campaigns I and II, increased by a factor of 2 over the entire washing process as the soluble solids were washed out and replaced with water causing the density and viscosity of the supernate to decrease.

The adjusted flux data in the first wash started steady but became more erratic, with the transition somewhere around 2.5 hours $\left(8^{\text {th }}\right.$ mini-batch). Interestingly enough, this is approximately the same point in the first wash of Campaign I where the flux data became erratic. A similar trend is seen in the second wash (Figure 25$)$ with the transition somewhere at 1.5 hours ( $31^{\text {st }}$ mini-batch). This initial, steady behavior of the data, was not seen in the second wash of Campaign I.

The flux measurement is based on the permeate flow rate. The erratic behavior is seen in the permeate flow rate data and a similar behavior is seen in the gauge pressure data at the backpulse pot (P3), which was part of the filtrate loop (see Fig. 21). The data scatter of the P3 reading may be systematic of the pilot-scale UFP and caused by air (or gas) entering the filtrate loop. Pockets of gas were seen in the filtrate discharge line (entering the filtrate collection drum) during slurry washing and also during the lower fluxes of dewatering. However, the magnitude of the data scatter at the lower fluxes of dewatering was not as great as the magnitude of the scatter during slurry washing. The gas pockets probably affected the hydraulic resistance of the permeate loop enough to cause the erratic behavior of the $\mathrm{P} 3$ reading and the filtrate flow rate. The backpulse pot and a significant portion of the filtrate loop are pressurized during the preparation of a backpulse. During this period there was no evidence of a leak in the filtrate loop.

Note that at the end of the first wash (directly after the $22^{\text {nd }}$ mini-wash) the flux measurement becomes relatively stable. The significance of this point is that the permeate is re-routed from the collection drum back to the slurry reservoir (recirculation mode). This could further indicate that the effect is systematic of the pilot-scale UFP.

There is also the possibility that the erratic permeate flow rate is due to the inability of the magnetic flow meter to acquire a steady signal in the permeate. The ions necessary to produce a signal were being continuously depleted by the wash solution (primarily DIF water). This would not, however, explain the steady behavior during the first half of the second wash. The erratic behavior of the flux measurement is, at this time, inexplicable and will be investigated further during subsequent campaigns.

Rheological data from the first and second slurry washes are shown in Fig. 26. The consistency decreases slightly during the washings and the yield stress increases slightly. The wt\% TS decreases and the wt\% UDS slightly increases as dissolved solids are washed away or precipitate. 


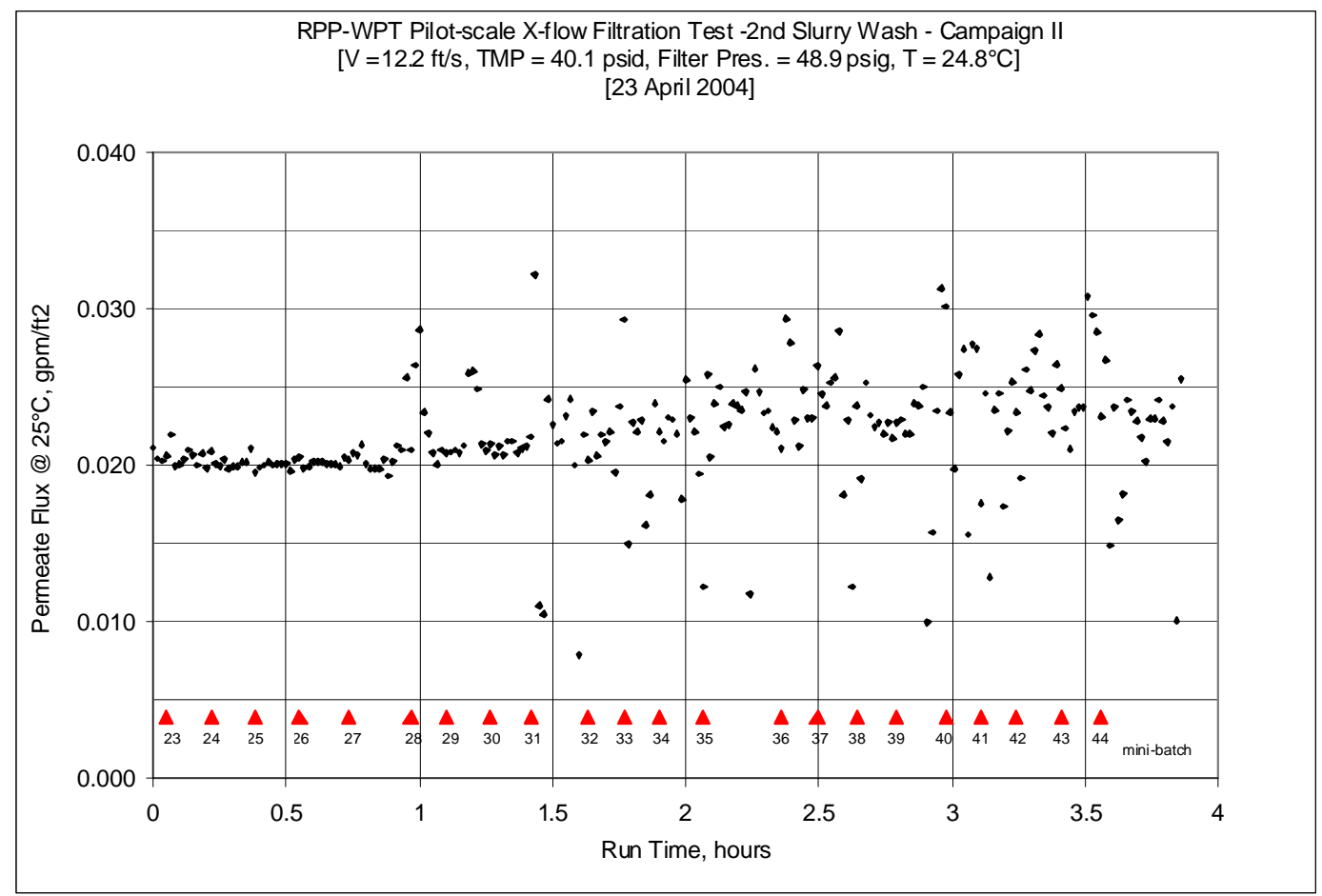

Figure 25. 2nd Washing of Concentrated Slurry with 22 Wash Additions (or mini-batches)

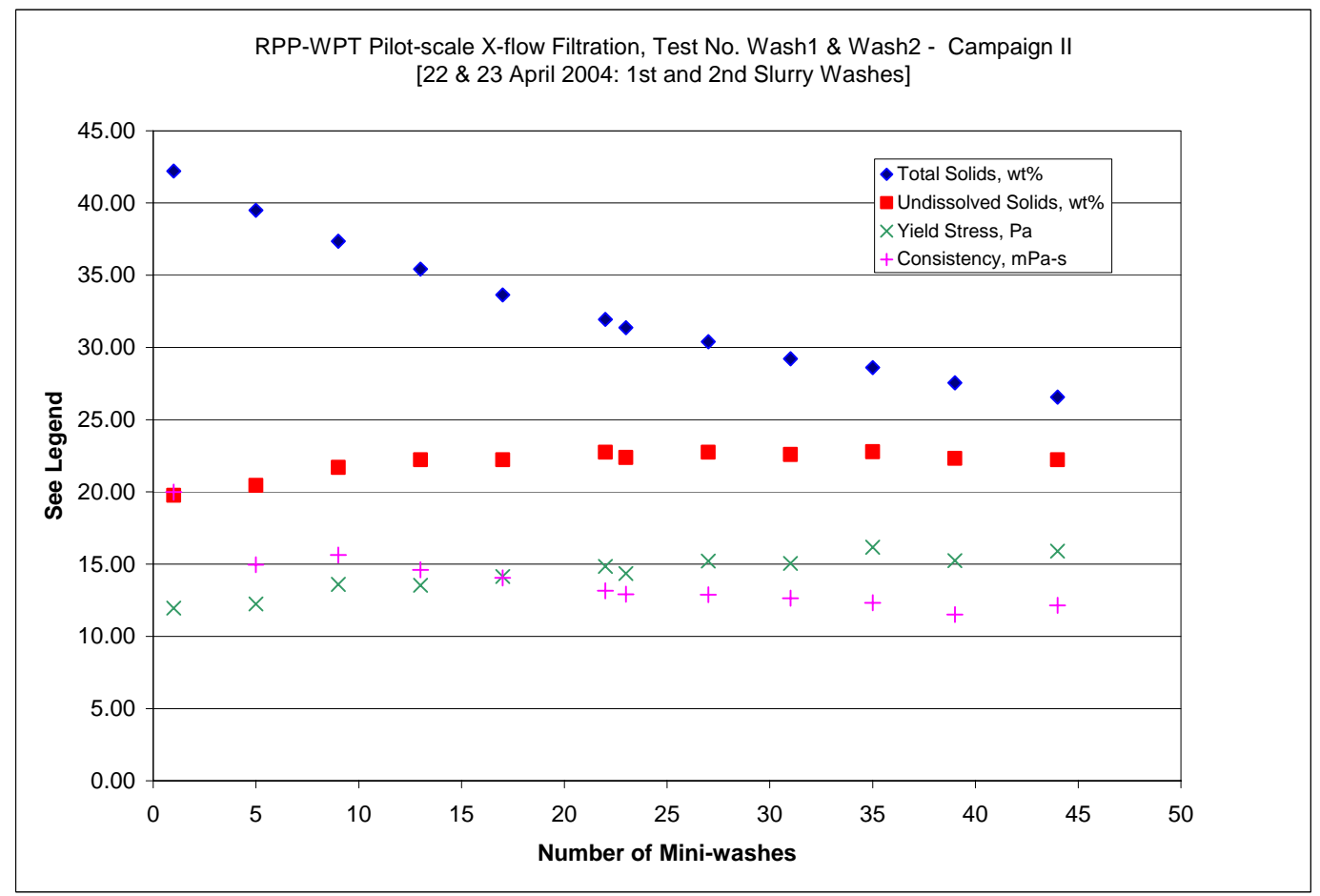

Figure 26. Solids and Rheological Data throughout Simulant Washing 


\section{WSRC-TR-2004-00478, REVISION 0 SRNL-RPP-2004-00075, REVISION 0}

Throughout the washing, permeate samples were taken at approximately every $4^{\text {th }}$ washing (see the Sampling Plan in Appendix D). These numbers are important because while both slurry and permeate samples were taken at the same time, they are not matched samples. The permeate flux was very low. It took approximately 30 minutes for permeate created at the filter to flow to the sampling point. By knowing the delay time then the slurry and permeate samples can be appropriately matched.

As already mentioned in the simulant handling section, the wash permeate was collected in drums for use as Campaign III FEP feed. The washed slurry was removed from the test rig and stored in a drum. The washed slurry would eventually be vitrified in the Vitreous State Laboratory (VSL) at Catholic University of America located in Washington DC.

\subsection{Filter Rinsing and Cleaning}

Following slurry washing, the slurry was removed and the process of rinsing and cleaning the filter and test rig began, as shown schematically in Figs. 13 and 14. Rinsing and cleaning involve pumping a mild caustic rinsing solution through the test rig, followed by three acid cleaning solutions, followed by two more mild caustic rinsing solutions. The sequence of the solutions and durations are provided below:

- $\quad 0.1 \mathrm{M} \mathrm{NaOH}$ for 30 minutes

- $2.0 \mathrm{M} \mathrm{HNO}_{3}$ for 90 minutes

- $2.0 \mathrm{M} \mathrm{HNO}_{3}$ for 90 minutes

- $2.0 \mathrm{M} \mathrm{HNO}_{3}$ for 90 minutes

- $0.1 \mathrm{M} \mathrm{NaOH}$ for 30 minutes

- $0.1 \mathrm{M} \mathrm{NaOH}$ for 30 minutes

Each solution was drained from the test rig prior to the addition of the next solution. The rinse solutions were made using condensate from the FEP Evaporator. The acid solutions were made from deionized and filtered (DIF) water.

As with any piping system, a heel remained in the system after it was drained. During rinsing and cleaning the heel consisted of the previous solution (see Figs. 13 and 14). For each of the rinsing and cleaning steps, it was necessary and important to have a heel, representative of what is expected in the full-scale plant, since it affects the chemistry of each subsequent solution. Therefore, a quantity of the previous solution was added to each solution to represent a heel that would be left in the RPP-WTP filter system. The heel for the first caustic rinse consisted of a mixture of washed slurry $(4.1 \mathrm{~L})$ and mild caustic solution (12.5 L), representing the filter-loop rinse (see the Caustic Flush 1 section of Fig. 13). It was estimated that an actual heel of $5 \mathrm{~L}$ of washed slurry remained in the test rig after slurry washing, yielding a total of 9.1 L of washed slurry and $12.5 \mathrm{~L}$ of mild caustic solution (21.6 L total) to represent the RPP-WTP heel. The heel for each subsequent solution consisted of 20 liters of the spent, previous solution (see Fig. 14). The actual heel of rinsing or cleaning solution left in the test rig was estimated at $1.6 \mathrm{~L}$, yielding a total heel of $21.6 \mathrm{~L}$.

After the washed slurry was removed from the test rig, it was noticed that the volume removed was lower than the expected value. Likewise, after the first rinse solution was put into the test rig, the resulting volume (as indicated by the slurry reservoir) was greater than expected. This implied that the 
test rig retained more slurry as a heel than expected. With the available information, the actual heel of washed slurry was estimated to be $22 \mathrm{~L}$, instead of $5 \mathrm{~L}$. (See the last paragraph of Section 2.7.2.3.)

The adjusted permeate flux for the first caustic rinse is shown in Fig. 27. A backpulse preceded the first mild caustic rinse. The slowly diminishing flux indicates that the filter cake removed by the backpulse was rebuilt with residual slurry during the rinse period. A similar trend was seen in the first slurry wash of Campaign I. Figure 30 (a) shows a settled sample of the first rinse solution (pH 13). Note the large amount of UDS. The concentration of the undissolved solids was approximately 5 wt\%. Further chemical analyses of the rinse (and cleaning) solutions are provided in section 2.7.4.2.

The first caustic rinse solution was removed from the test rig after the 30 minute rinsing step. An amount of $20 \mathrm{~L}$ was returned to the slurry reservoir for the representative heel and the rest was stored in a drum for use as Campaign III FEP feed.

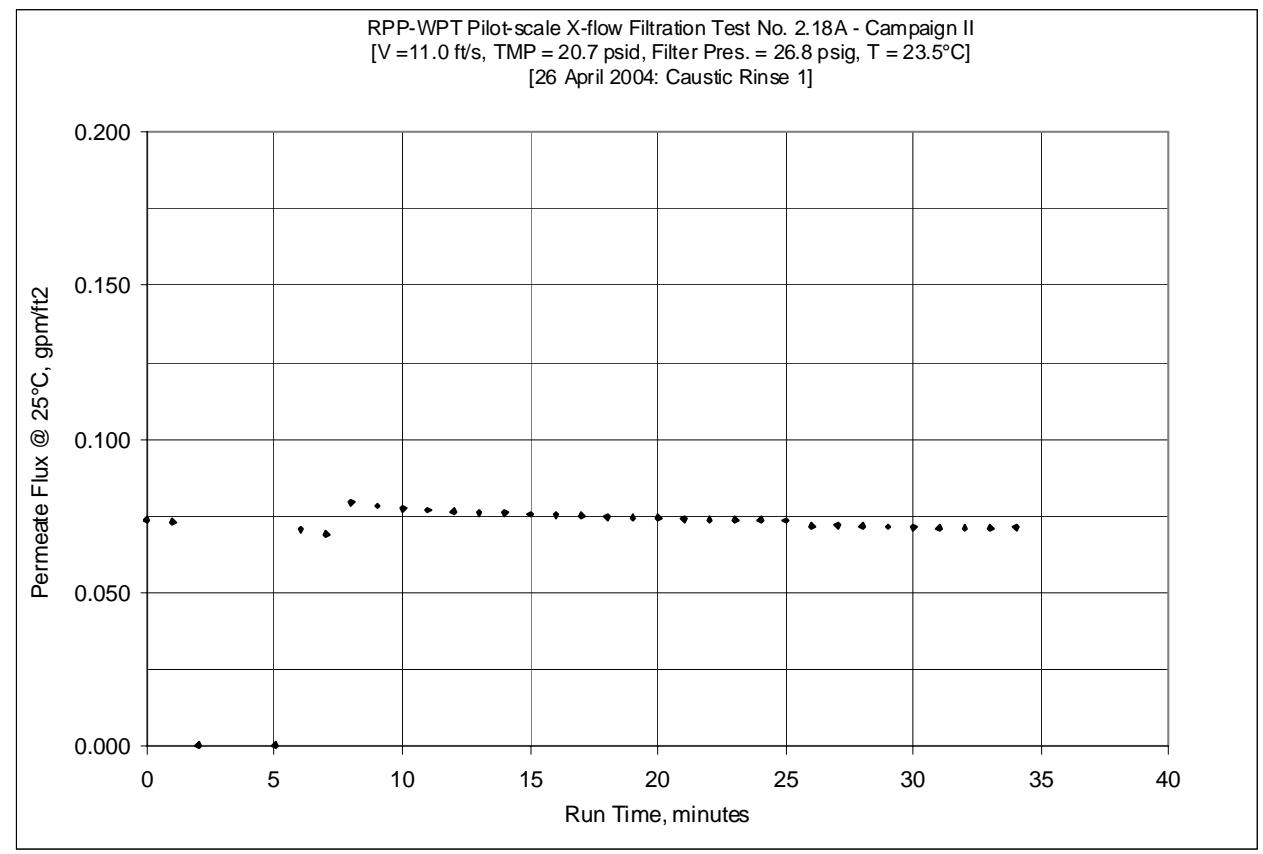

Figure 27. $1^{\text {st }}$ Caustic Rinse of the Ultrafilter

Three acid cleanings followed the first caustic rinse. Figure 28 shows the adjusted filter flux results for all three acid cleanings. A backpulse preceded each cleaning, which accounts for the higher initial flux at the beginning of each cleaning. The time gaps between the cleanings represent the times necessary to remove the old cleaning solution from the test rig and add a new cleaning solution. The flux improved during each cleaning as solids were dissolved by the acid. 


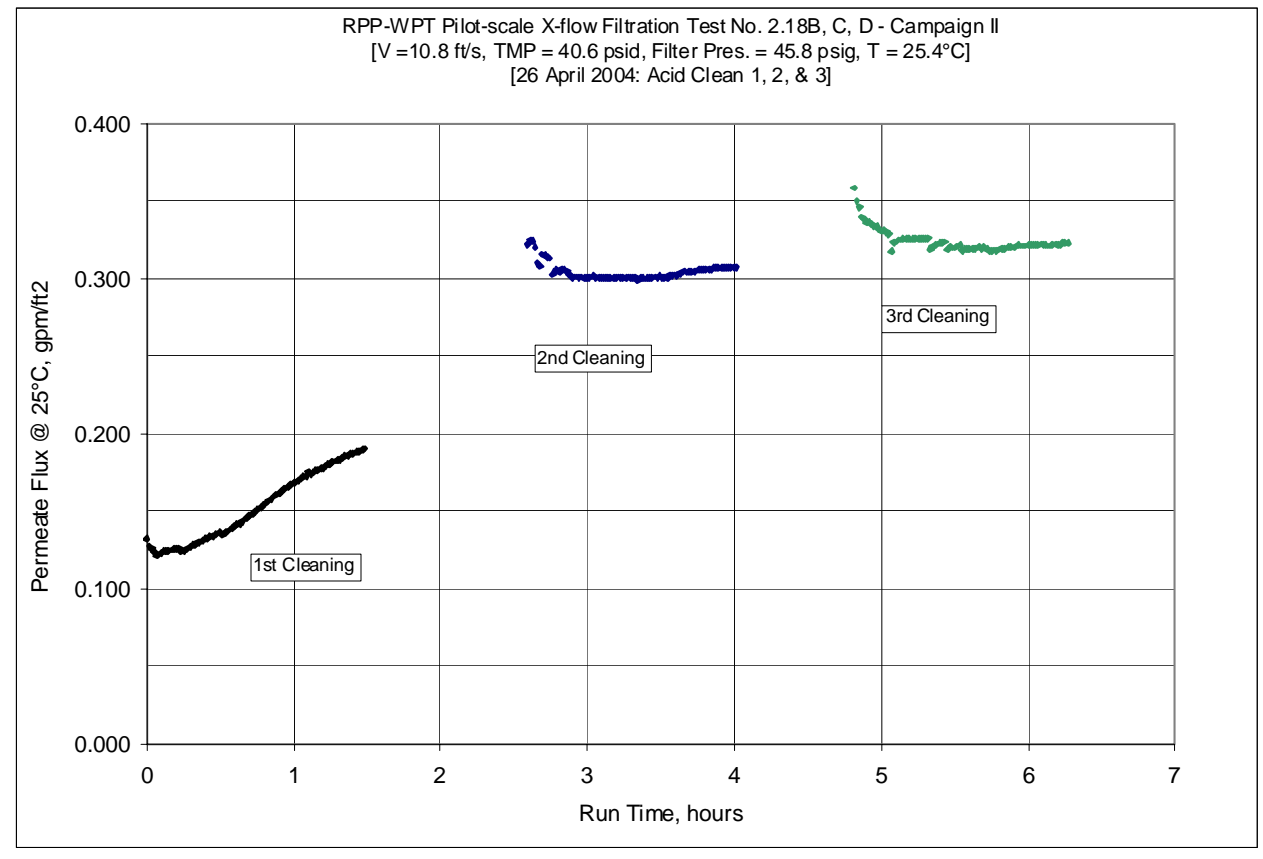

Figure 28. Acid Cleaning of the Ultrafilter

The flux also improved between cleanings, which implies two things: 1) that residual acid solution in and on the filter media continued to dissolve solids and 2) backpulsing prior to cleaning removed stuck-on or lodged material.

Figures 30 (b), (c), and (d) are photographs of the spent acid cleaning solutions. All of the cleaning solutions have a $\mathrm{pH}<1$. Note that the three acid cleaning solutions are stained with the first solution containing small amounts of brown sludge and a whitish solid. The stained color diminishes from the first to the third cleaning, but does not completely disappear. The whitish solids were minimal in the second spent cleaning solution and not apparent in the third. Table 27 shows that the TS wt\% of the acid cleaning solutions was relatively significant in the first cleaning solution, but was greatly reduced by the third cleaning. The UDS wt\% of the cleaning solutions was insignificant.

The acid cleaning flux results from Campaign I were, in many ways, different from the Campaign II results. 1) The first cleaning flux from Campaign I decreased during the first 60 minutes after which it increased for over a 30-minute period; in Campaign II this flux increased over the entire 90-minute period, with only an initially short decrease. This was attributed to significant amounts of suspended solids generated during the Campaign I cleaning. The solids were evident in a sample of the spent solution as white cloudy substance. This substance was significantly reduced in the Campaign II spent solution. 2) The flux of the entire acid cleaning for Campaign I never reached, or approached, a maximum. At the end of the third cleaning the flux was still increasing, implying that further cleaning was necessary. In Campaign II, the flux appears to approach a maximum. The flux was increasing slightly in the third cleaning, but probably not enough to warrant further cleaning.

The acid cleaning solutions were removed from the test rig after each 90-minute cleaning. An amount of $20 \mathrm{~L}$ of spent cleaning solution from each step was retained for the representative heel, and the rest of the solutions were stored in drums for use as Campaign III FEP feed. 
The acid cleanings were followed by two mild caustic rinses. Figure 29 shows the adjusted flux of the last two rinses and reveals that the permeate flux of the second caustic rinse (or the first rinse after acid cleaning) was notably less than that of the third acid cleaning. This trend was seen in Campaign I and was attributed to the caustic solution precipitating solids from the acid heel. (See Tables 27 and 38 for compositions found in the Campaign II rinsing and cleaning solutions.) Further, the volume and concentration of the acid heel was enough to make the second caustic rinse acidic ( $\mathrm{pH} 1)$. Morever, the volume and concentration of the second cautic rinse heel was also enough to maintain the third caustic rinse acidic ( $\mathrm{pH}$ 1.2). The temperature adjusted flux during the third caustic rinse continued to decline as more solids precipitated from the $\mathrm{pH} 1.2$ solution. At the end of the last rinse the flux had dropped to $0.14 \mathrm{gpm} / \mathrm{ft}^{2}$. However, note that this flux was still more than an order of magnitude higher than the average dewatering flux of $0.011 \mathrm{gpm} / \mathrm{ft}^{2}$.

The second caustic rinse solution (first post-clean caustic rinse) was removed from the test rig after the 30 minute rinsing step. An amount of $20 \mathrm{~L}$ was returned to the slurry reservoir for the representative heel and the rest was stored in a drum for use as Campaign III FEP feed. The third caustic rinse solution (second post-clean caustic rinse) was left in the test rig and was used for the post-test baseline test. After the baseline test the caustic solution was removed from the test rig and stored in a drum for use as Campaign III FEP feed. The rinsing solutions are shown in Fig. 30 (e) and (f) and they appear to be relatively clear solutions with minimal staining, (f) being most clear. Table 27 indicates the the wt\% TS and wt\% UDS increased slightly.

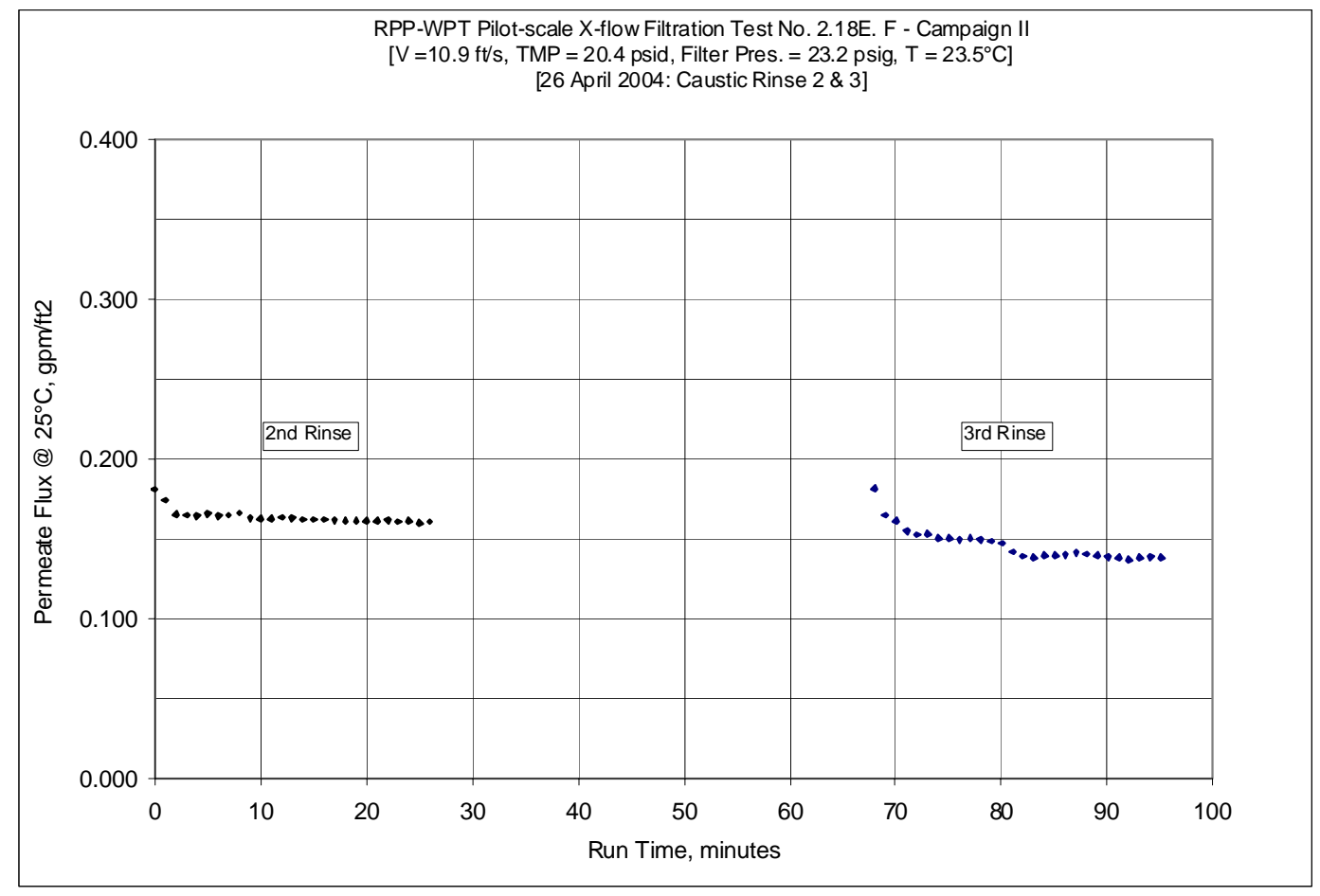

Figure 29. $2^{\text {nd }}$ and $3^{\text {rd }}$ Rinses of the Ultrafilter 


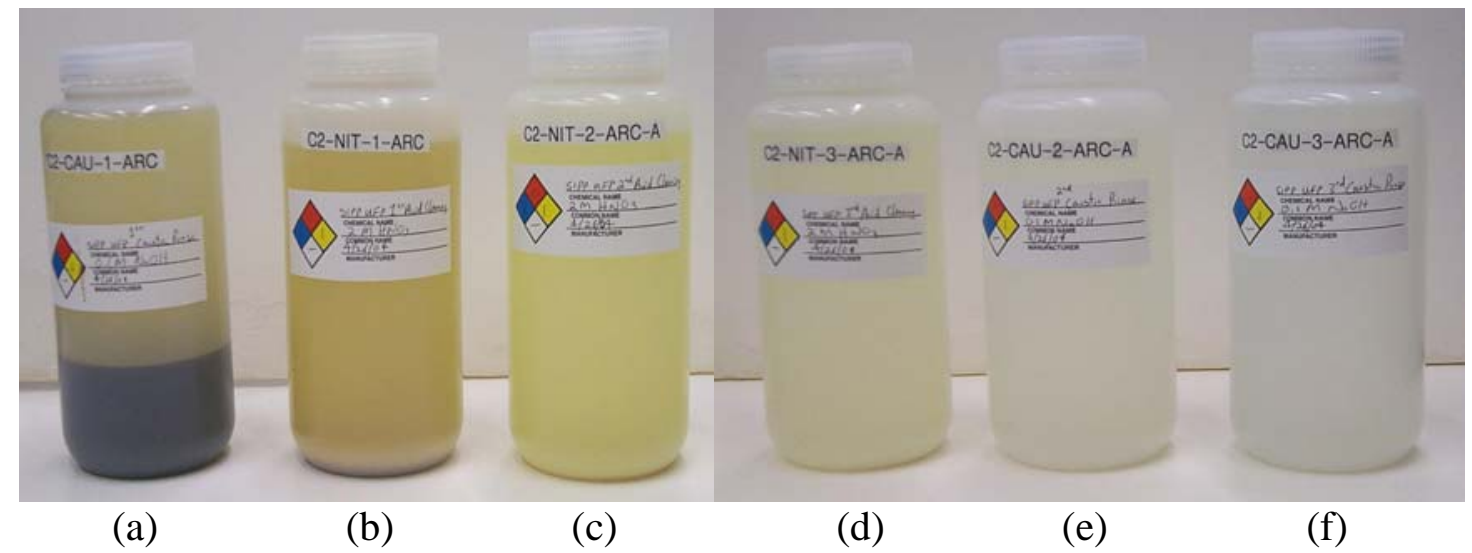

Figure 30. Caustic Rinse and Acid Cleaning Samples

(a) pre-clean caustic rinse, $\mathrm{pH} 13$, (b) first $2 \mathrm{M}$ nitric cleaning, $\mathrm{pH}$ 1, (c) second $2 \mathrm{M}$ nitric cleaning, $\mathrm{pH} 1$, (d) third $2 \mathrm{M}$ nitric cleaning, $\mathrm{pH} \sim 1$, (e) first post-clean caustic rinse, $\mathrm{pH} \sim 1$, (f) second postclean caustic rinse, $\mathrm{pH} \sim 1.2$

Table 27. Measured Total Solids (TS) and Undissolved Solids (UDS) Concentrations of the Spent Rinse and Cleaning Solutions

\begin{tabular}{|c|c|c|}
\hline \multirow{2}{*}{ Step } & TS & UDS \\
\cline { 2 - 3 } & wt\% & wt $\%$ \\
\hline First Caustic Rinse $0.1 \mathrm{M} \mathrm{NaOH}$ & 7.46 & 5.09 \\
\hline First Acid Cleaning $2.0 \mathrm{M} \mathrm{HNO}_{3}$ & 2.36 & 0.12 \\
\hline Second Acid Cleaning $2.0 \mathrm{M} \mathrm{HNO}_{3}$ & 0.67 & 0.00 \\
\hline Third Acid Cleaning $2.0 \mathrm{M} \mathrm{HNO}_{3}$ & 0.13 & 0.00 \\
\hline Second Caustic Rinse $0.1 \mathrm{M} \mathrm{NaOH}$ & 0.66 & 0.13 \\
\hline Third Caustic Rinse $0.1 \mathrm{M} \mathrm{NaOH}$ & 0.81 & 0.13 \\
\hline
\end{tabular}

\subsection{Filter Baselining}

A baseline test was conducted after the test rig was rinsed and cleaned, using the mild caustic rinse solution $(0.1 \mathrm{M} \mathrm{NaOH})$ from the third rinse step. The post-test baseline test data had a lower flux than the pre-test baseline test data (Fig. 31), indicating that the filter was not completely free of solids in the filter media. This has been the case in previous cross-flow filtration tests (Duignan, 2003a). Although lower, the adjusted permeate flux for the post-test baseline was still more than an order of magnitude higher than the adjusted flux during dewatering of the waste simulant.

It is believed that the pre-test flux is always better than the post-test flux because the filter is submerged in a mild caustic solution for an extended period of time prior to each campaign. The filter is submerged in a mild caustic solution between campaigns to keep the filter wet and to prevent the growth of algae. The mild caustic solution may continue to dissolve solids lodged in the filter media, allowing them to migrate out. The longer the filter is submerged in the solution, the better the flux. 
WSRC-TR-2004-00478, REVISION 0

SRNL-RPP-2004-00075, REVISION 0

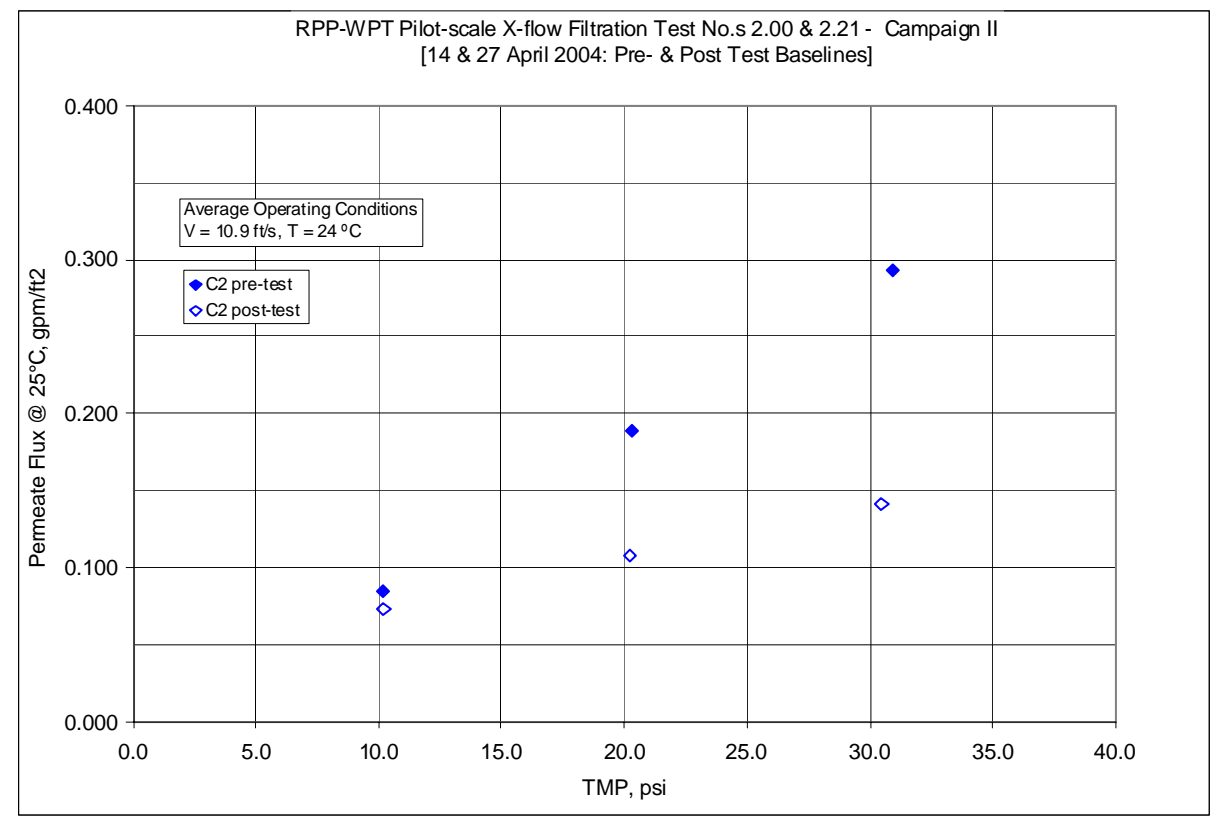

Figure 31. Pre- and Post-Test Baselines for Campaign II

Figure 32 compares the pre-and post-test baseline tests from Campaign I and II. Prior to Campaign I, the filter was submerged in a mild caustic for approximately 1 year. Prior to Campaign II, the filter was submerged in mild caustic for approximately a little over 2 months.

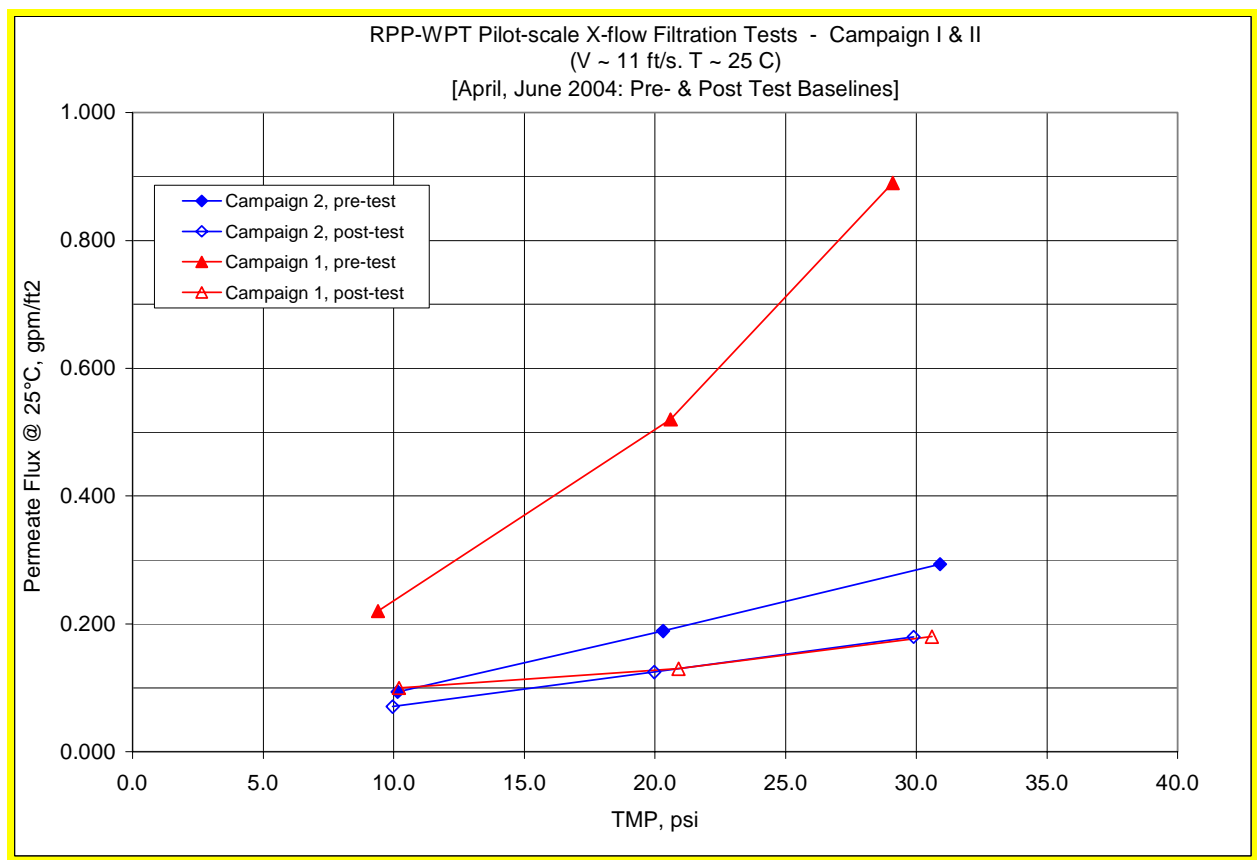

Figure 32. Comparison of Pre- and Post-Test Baselines, Campaign I and II 
The pre-test flux of Campaign I is noticeably greater than that of Campaign II, implying that the time that the filter is submerged in a mild caustic solution between campaigns may affect the pre-test flux.

The post-test baselines are comparable, with the Campaign II baseline data having slightly less flux than the Campaign I data. At this point it is difficult to determine whether the reduced flux is a trend.

\subsubsection{UFP Waste Products - Chemical Analysis (Author - Zamecnik)}

In this section, parts of the chemical analysis discussion on the pilot-scale UFP waste stream products will repeat some of the material discussed in Section 2.7.4.1. In many cases the repetition is unavoidable, but it also facilitates better understanding of the many aspects of the UFP unit operation.

\subsection{Dewatering}

Table 28 compares the composition of UFP feed slurry to those calculated and measured values of the slurry after it was dewatered from $3 \mathrm{wt} \%$ UDS to $24 \mathrm{wt} \%$ UDS. The calculation of the concentrated slurry composition was based on the total feed volume of 1033L and a final concentrated slurry volume of 117L. The calculation was done using the equation below:

$$
\begin{aligned}
\mathrm{C}_{\mathrm{C}} & =\frac{\mathrm{C}_{\mathrm{F}} \times \mathrm{V}_{\mathrm{F}}-\mathrm{C}_{\mathrm{P}} \times \mathrm{V}_{\mathrm{P}}}{\mathrm{V}_{\mathrm{C}}} \\
\text { where } \mathrm{C}_{\mathrm{C}} & =\text { Concentrated Slurry Composition }(\mathrm{mg} / \mathrm{L}) \\
\mathrm{C}_{\mathrm{F}} & =\text { Feed Slurry }(\mathrm{mg} / \mathrm{L}) \\
\mathrm{V}_{\mathrm{F}} & =\text { Feed Volume }(\mathrm{L}) \\
\mathrm{C}_{\mathrm{P}} & =\text { Permeate Composition }(\mathrm{mg} / \mathrm{L}) \\
\mathrm{V}_{\mathrm{P}} & =\text { Permeate Volume }(\mathrm{L}) \\
\mathrm{V}_{\mathrm{C}} & =\text { Concentrated Slurry Volume }(\mathrm{L})
\end{aligned}
$$

Most of the calculated and measured compositions of mainly insoluble species values agreed well (Fe, $\mathrm{La}, \mathrm{Mg}, \mathrm{Mn}, \mathrm{Nd}, \mathrm{Ni}, \mathrm{Pb}, \mathrm{Si}, \mathrm{Sr}, \mathrm{Zn}, \mathrm{Zr}$ ). The corresponding agreement for the soluble species is not as good as for the insoluble species. The partly soluble Al values agreed well, but the predicted $\mathrm{Cr}$ value was higher than the measured values. The values for K, Na, P (phosphate), S (sulfate), chloride, nitrate, and nitrite all had larger differences. For many of these soluble species, the concentration of the species in the slurry was very close to the composition in the supernate, so the numerator in the equation above was greatly affected by the uncertainty in the compositions (it was a small difference between large numbers, relatively). Some values could not be calculated because the input values were below detection limit values. For Rb and Cs, the predicted values were less than zero due to their concentrations in the supernate and slurry being similar. 
WSRC-TR-2004-00478, REVISION 0

SRNL-RPP-2004-00075, REVISION 0

Table 28. Measured and Calculated Concentrated UFP Slurry Compositions

\begin{tabular}{|c|c|c|c|c|c|c|c|}
\hline $\begin{array}{c}\text { All concentrations in } \\
\text { mg/L unless } \\
\text { otherwise noted }\end{array}$ & $\begin{array}{l}\text { Feed } \\
\text { Slurry }\end{array}$ & $\begin{array}{c}\text { Concentrated } \\
\text { Slurry } \\
\text { Measured } \\
\end{array}$ & $\begin{array}{l}\text { Concentrated } \\
\text { Slurry } \\
\text { Calculated } \\
\end{array}$ & & Feed Slurry & \begin{tabular}{|c|} 
Concentrated \\
Slurry \\
Measured \\
\end{tabular} & $\begin{array}{c}\text { Concentrated } \\
\text { Slurry } \\
\text { Calculated } \\
\end{array}$ \\
\hline Total Solids wt\% & 30.6 & 45.4 & 45.3 & $\mathrm{Nd}$ & 240 & 2014 & 2038 \\
\hline $\begin{array}{r}\text { Undissolved Solids } \\
\text { wt\% }\end{array}$ & 3.22 & 23.5 & 24.0 & $\mathrm{Ni}$ & 295 & 2705 & 2502 \\
\hline $\begin{array}{r}\text { Supernate Solids } \\
\text { wt\% }\end{array}$ & 28.3 & 28.6 & 28.0 & $\mathrm{P}$ & $\begin{array}{l}766 \\
743\end{array}$ & $\begin{array}{c}941 \\
1033\end{array}$ & 701 \\
\hline Density kg/L & 1.28 & 1.47 & 1.47 & $\mathrm{~Pb}$ & $\begin{array}{l}475 \\
419\end{array}$ & $\begin{array}{l}3998 \\
3719\end{array}$ & 3782 \\
\hline $\mathrm{Ag}$ & 157 & 1455 & 1334 & $\operatorname{Pr}$ & 34.1 & 420 & 289 \\
\hline $\mathrm{Al}$ & $\begin{array}{l}8422 \\
9953\end{array}$ & $\begin{array}{l}23079 \\
28518\end{array}$ & 24272 & $\mathrm{Rb}$ & 4.16 & 2.48 & $<0$ \\
\hline $\mathrm{B}$ & $<148$ & $<222$ & & S & $\begin{array}{l}1340 \\
1128\end{array}$ & $\begin{array}{l}1357 \\
1177\end{array}$ & 1938 \\
\hline $\mathrm{Ba}$ & $\begin{array}{l}68.0 \\
46.4\end{array}$ & $\begin{array}{l}631 \\
673\end{array}$ & 486 & $\mathrm{Si}$ & 3247 & $\begin{array}{l}20640 \\
25872\end{array}$ & 27169 \\
\hline $\mathrm{Ca}$ & 415 & $\begin{array}{l}2661 \\
2940\end{array}$ & 3505 & $\mathrm{Sr}$ & $\begin{array}{l}60.2 \\
83.0\end{array}$ & $\begin{array}{l}623 \\
703\end{array}$ & 608 \\
\hline $\mathrm{Cd}$ & 15.2 & 89.7 & 129 & $\mathrm{Y}$ & 10.1 & 129 & 85.9 \\
\hline $\mathrm{Ce}$ & $<120$ & 810 & & $\mathrm{Zn}$ & 28.6 & $\begin{array}{l}231 \\
213\end{array}$ & 220 \\
\hline $\mathrm{Co}$ & 100 & $<22.2$ & 851 & $\mathrm{Zr}$ & 125 & $\begin{array}{l}1070 \\
1120\end{array}$ & 1060 \\
\hline $\mathrm{Cr}$ & $\begin{array}{l}293 \\
324\end{array}$ & $\begin{array}{l}1147 \\
1123\end{array}$ & 1403 & bromide & $<53.6$ & $<42.6$ & \\
\hline $\mathrm{Cs}$ & 4.54 & 4.37 & $<0$ & chloride & 1242 & 1085 & 1747 \\
\hline $\mathrm{Cu}$ & $\begin{array}{l}16.9 \\
13.2\end{array}$ & $\begin{array}{l}187 \\
207\end{array}$ & 126 & fluoride & 81.7 & 76.4 & 448 \\
\hline $\mathrm{Fe}$ & $\begin{array}{l}9493 \\
9570\end{array}$ & $\begin{array}{l}82614 \\
81291\end{array}$ & 80985 & formate & 1108 & 1029 & 533 \\
\hline $\mathrm{K}$ & 19012 & 19110 & 17677 & nitrate & 93914 & 73059 & 52974 \\
\hline $\mathrm{La}$ & 84.1 & 703 & 715 & nitrite & 51678 & 20727 & 16705 \\
\hline $\mathrm{Li}$ & $<21.9$ & $\begin{array}{l}133 \\
134\end{array}$ & & oxalate & 4440 & 34986 & 32562 \\
\hline $\mathrm{Mg}$ & $\begin{array}{l}110 \\
105\end{array}$ & $\begin{array}{l}956 \\
922\end{array}$ & 911 & phosphate & 2412 & 1573 & 2272 \\
\hline $\mathrm{Mn}$ & $\begin{array}{l}2080 \\
2042\end{array}$ & $\begin{array}{l}18228 \\
17934\end{array}$ & 17510 & sulfate & 3483 & 3058 & 5050 \\
\hline $\mathrm{Mo}$ & $<17.1$ & $<27.8$ & & total organic carbon & 3280 & 8780 & 483 \\
\hline $\mathrm{Na}$ & 113341 & $\begin{array}{l}131168 \\
134505\end{array}$ & 109559 & carbonate & 40600 & 24600 & 94315 \\
\hline
\end{tabular}

Compositions of the supernate from the feed slurry, the Ultrafiltration permeate, and the IX product are shown in Table 29. Except for Cs, and possibly K, the concentration of each species should be the same, so comparison of the values for each species gives a measure of the uncertainty in the analyses. For the major species, the variation in the average, as given by two standard deviations, was generally less than $10 \%$. 
WSRC-TR-2004-00478, REVISION 0

SRNL-RPP-2004-00075, REVISION 0

Table 29. Measured Composition of Permeate and Supernate from UFP Feed Slurry

\begin{tabular}{|c|c|c|c|c|c|c|c|c|c|c|c|}
\hline & $\begin{array}{c}\text { Supernate } \\
\text { from Feed } \\
\text { Slurry }\end{array}$ & Permeate & $\begin{array}{c}\text { Ion } \\
\text { Exchange } \\
\text { Product } \\
\end{array}$ & Average & $\begin{array}{c}2 \times \text { Std } \\
\text { Dev }\end{array}$ & & $\begin{array}{c}\text { Supernate } \\
\text { from Feed } \\
\text { Slurry }\end{array}$ & Permeate & $\begin{array}{c}\text { Ion } \\
\text { Exchange } \\
\text { Product } \\
\end{array}$ & Average & $\begin{array}{c}2 \times \text { Std } \\
\text { Dev }\end{array}$ \\
\hline $\begin{array}{r}\text { total solids } \\
\text { (wt\%) }\end{array}$ & 28.31 & 28.15 & 27.93 & $28.13^{*}$ & 0.19 & $\mathrm{P}$ & 805 & 800 & 775 & 793 & 16.0 \\
\hline $\begin{array}{r}\text { density } \\
(\mathrm{kg} / \mathrm{L})\end{array}$ & 1.250 & 1.243 & 1.247 & 1.247 & 0.003 & $\mathrm{~Pb}$ & 1.86 & 1.68 & 15.3 & 6 & 0.13 \\
\hline $\mathrm{Ag}$ & $<0.53$ & $<0.6$ & $<0.6$ & $<0.53$ & & $\mathrm{Pr}$ & $<0.01$ & $<0.01$ & $<0.01$ & $<0.01$ & \\
\hline $\mathrm{Al}$ & 7300 & 7050 & 7190 & 7180 & 125 & $\mathrm{Rb}$ & 5.12 & 4.93 & 4.86 & 4.97 & 0.13 \\
\hline $\mathrm{B}$ & 82.6 & 87.1 & 79.5 & 83.1 & 3.9 & $\mathrm{~S}$ & 1080 & 1200 & 1152 & 1144 & 60 \\
\hline $\mathrm{Ba}$ & $<0.022$ & $<0.04$ & $<0.044$ & $<0.022$ & & Si & 59.3 & 54.2 & 57.0 & 57 & 2.6 \\
\hline $\mathrm{Bi}$ & $<0.01$ & $<0.01$ & $<0.01$ & $<0.01$ & & $\mathrm{Sr}$ & $<0.436$ & 0.0691 & 0.0715 & 0.0703 & 0.0017 \\
\hline $\mathrm{Ca}$ & 2.46 & $<0.06$ & 0.382 & 1.42 & 1.47 & $\mathrm{Ti}$ & 0.0685 & 0.0887 & $<0.116$ & 0.0786 & 0.0143 \\
\hline $\mathrm{Cd}$ & $<0.02$ & $<0.04$ & $<0.04$ & $<0.02$ & & $\mathrm{~W}$ & 38.8 & 50.3 & NM & 44.5 & 8.1 \\
\hline $\mathrm{Ce}$ & $<0.86$ & $<1.4$ & $<17.28$ & $<0.86$ & & $\mathrm{Y}$ & $<0.01$ & $<0.01$ & $<0.01$ & $<0.01$ & \\
\hline $\mathrm{Co}$ & $<0.042$ & $<0.16$ & $<0.086$ & $<0.042$ & & $\mathrm{Zn}$ & 3.03 & 3.05 & 3.05 & 3.04 & 0.01 \\
\hline $\mathrm{Cr}$ & 161 & 165 & 160 & 162 & 2 & $\mathrm{Zr}$ & 0.363 & 0.863 & 0.514 & 0.580 & 0.257 \\
\hline Cs (ICPMS) & 6.25 & 5.91 & 0.00301 & NC & & chloride & 1140 & 1210 & 1090 & 1147 & 60 \\
\hline $\mathrm{Cu}$ & 0.0649 & 0.373 & 0.279 & 0.239 & 0.158 & fluoride & 38.0 & 29.0 & 48.0 & 38 & 9.5 \\
\hline $\mathrm{Fe}$ & 0.646 & 1.76 & 1.33 & 1.25 & 0.56 & formate & 1120 & 1220 & 1020 & 1120 & 100 \\
\hline $\mathrm{K}$ & 21000 & 21200 & 18900 & 20367 & 1274 & nitrate & 85900 & 85300 & 74400 & 81867 & 6473 \\
\hline $\mathrm{La}$ & $<0.188$ & $<0.2$ & $<0.376$ & $<0.188$ & & nitrite & 22900 & 25200 & 22400 & 23500 & 1493 \\
\hline $\mathrm{Li}$ & 1.23 & 1.15 & 1.07 & 1.15 & 0.08 & oxalate & 733 & 746 & 690 & 723 & 29 \\
\hline $\mathrm{Mg}$ & $<0.012$ & $<0.01$ & $<0.026$ & $<0.01$ & & phosphate & 2390 & 2470 & 2210 & 2357 & 133 \\
\hline $\mathrm{Mn}$ & 0.0397 & 0.0288 & $<0.034$ & 0.0343 & 0.0077 & sulfate & 3170 & 3390 & 2860 & 3140 & 266 \\
\hline Mo & 9.38 & 9.31 & 9.74 & 9.47 & 0.23 & acetate & 1210 & 1280 & 1280 & 1257 & 40 \\
\hline $\mathrm{Na}$ (ICPES) & 111000 & 119000 & 110000 & 113333 & 4933 & citric acid & $<100$ & $<100$ & $<100$ & $<100$ & \\
\hline $\mathrm{Na}(\mathrm{AA})$ & 122000 & 106500 & 109000 & 112500 & 8322 & formate & 941 & 1010 & 1020 & 990 & 43 \\
\hline $\mathrm{Na}$ average & 116500 & 112750 & 109500 & 112917 & 3503 & $\begin{array}{r}\text { unbound } \\
\text { hydroxide }\end{array}$ & NM & NM & 2346 & 2346 & \\
\hline $\mathrm{Na}(\mathrm{M})$ & 5.07 & 4.90 & 4.76 & 4.91 & 0.15 & $\begin{array}{r}\text { total organic } \\
\text { carbon }\end{array}$ & 4010 & 2000 & NM & 3005 & 1421 \\
\hline $\mathrm{Nd}$ & $<0.406$ & $<1.8$ & $<0.812$ & $<0.406$ & & carbonate & 23950 & 38750 & NM & 31350 & 10465 \\
\hline
\end{tabular}


Permeate samples were observed regularly over one month to determine if any post-filtration precipitation occurred. No significant precipitation was found to occur, even after three months. (See a one-month evaluation of the turbidity in Table 26.) This observation is contrary to what was observed in Campaign I. The AY102 simulant permeate showed some precipitation of solids after two months, but no precipitation at one month. Since the precipitated solids from Campaign I were not described in the Campaign I interim report (Duignan, et al., 2004), they are described here. Figure 33 shows an XRD spectrum of the precipitated solids. The crystalline solids found were aluminum hydroxide, sodium carbonate, and a zeolite, which are typical of solids precipitated from the AY102 simulant upon standing for extended periods of time. Postfiltration precipitation with the AY102 simulant does not necessarily indicate precipitation will occur with the actual waste; this and other simulants appear to be slightly unstable with respect to precipitation of aluminum species. Also, the AY102 simulant, being saturated in carbonate, is subject to precipitation of carbonates if the temperature is reduced for a period of time.

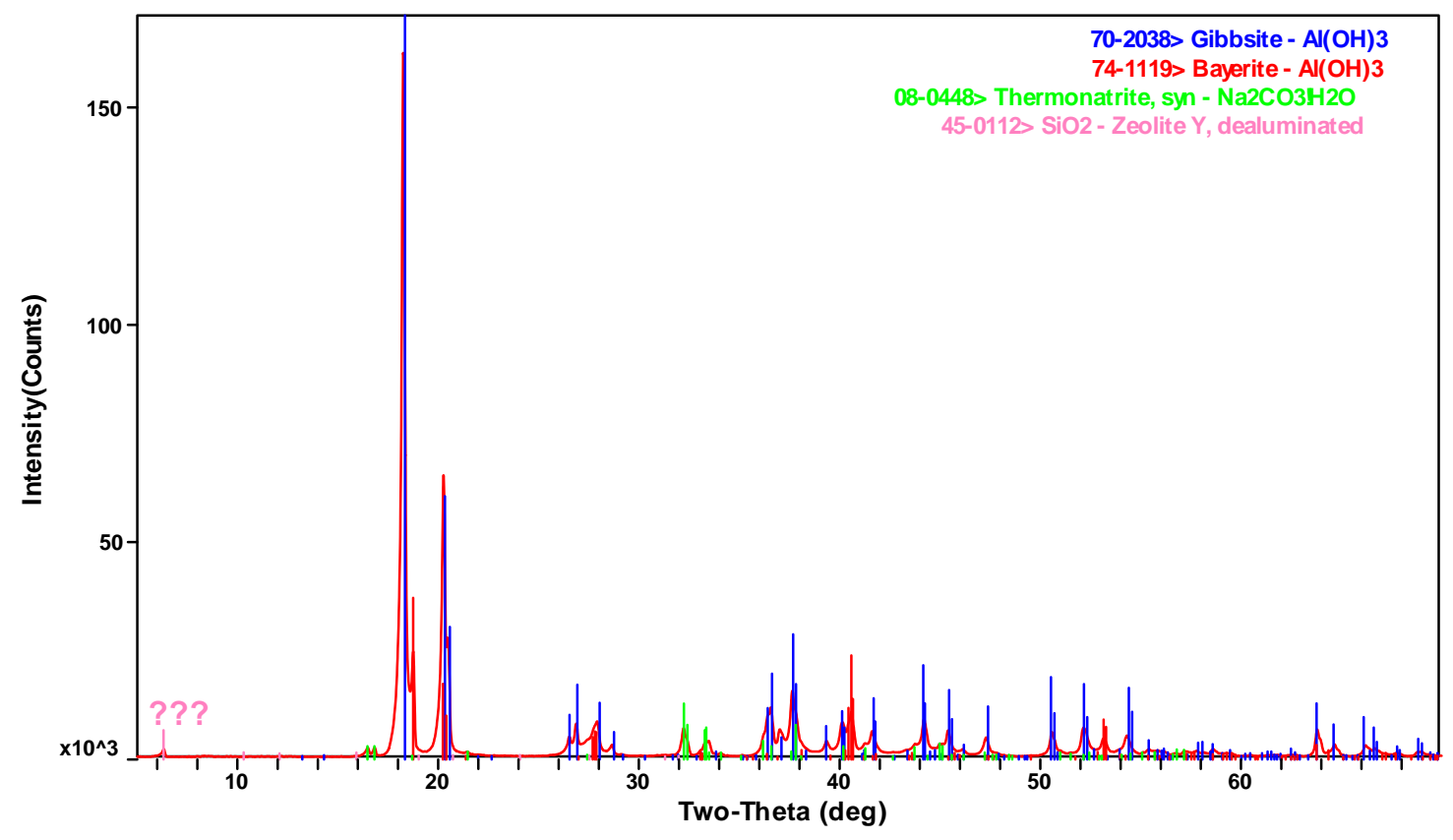

Figure 33. XRD Spectrum of Solids Precipitated from Campaign I Ultrafilter Permeate

Upon completion of slurry concentration, the final undissolved solids (UDS) content was measured from multiple samples and found to range from 23.5 to $24.7 \mathrm{wt} \%$ UDS, versus the target of $20.0 \mathrm{wt} \%$. The target was exceeded because the amount of solids in the Ultrafiltration slurry feed was greater than the model (Barnes, 2004) prediction, so the endpoint was also higher. The target values were calculated from the OLI model in which the FEP bottoms, AY102 and AP101, were mixed. Compared to the actual material, the AY102 slurry was assumed to have slightly less undissolved solids, while the FEP bottoms stream also had less solids.

In the model, the FEP bottoms stream was close to neutral in $\mathrm{pH}$ compared to the actual bottoms at $\mathrm{pH} 13$. The lower $\mathrm{pH}$, used in the model, resulted in some of the carbonate in the FEP feed being lost as $\mathrm{CO}_{2}$, which resulted in lower solids in the FEP bottoms. Also due to the $\mathrm{pH}$, some 
of the partially soluble species (carbonate, oxalate) in the AY102 portion of the feed were predicted to dissolve upon mixing. However, the actual FEP feed streams were $\mathrm{pH}$ adjusted so that the combined $\mathrm{pH}$ was $>12$, which resulted in more solids in the FEP bottoms and actual ultrafilter feed than in the model. Table 30 compares the model predictions with the actual measured values.

Table 30. Comparison of OLI Model and Measured UFP Solids Contents

\begin{tabular}{|c|c|c|c|c|c|c|c|}
\hline MODEL & AY102 & AP101 & $\begin{array}{c}\text { FEP } \\
\text { Bottoms }\end{array}$ & $\begin{array}{l}\text { UF Feed } \\
\text { (Model) }\end{array}$ & $\begin{array}{l}\text { UF Feed } \\
\text { (Calc)* }\end{array}$ & $\begin{array}{c}\text { UF } \\
\text { Concentrate }\end{array}$ & $\begin{array}{c}\text { UF } \\
\text { Permeate }\end{array}$ \\
\hline Total Solids (wt\%) & 26.31 & 32.85 & 29.88 & 30.95 & 30.97 & 43.38 & 29.22 \\
\hline Undissolved Solids (wt\%) & 11.50 & 0.01 & 2.18 & 2.45 & 2.97 & 20.00 & 0 \\
\hline Supernate Solids (wt\%) & 16.73 & 32.84 & 28.32 & 29.22 & 28.85 & 29.22 & 29.22 \\
\hline Slurry Density (kg/L) & 1.236 & 1.278 & 1.252 & 1.264 & 1.285 & 1.376 & 1.250 \\
\hline MEASURED & & & & & (Calc) & & \\
\hline Total Solids (wt\%) & 26.80 & 31.94 & 28.95 & 30.59 & 30.36 & 45.37 & 28.13 \\
\hline Undissolved Solids (wt\%) & 11.75 & $\sim 0$ & 3.03 & 3.22 & 3.19 & 23.51 & 0 \\
\hline Supernate Solids (wt\%) & 17.05 & 31.94 & 26.73 & 28.28 & 28.07 & 28.58 & 28.13 \\
\hline Slurry Density (kg/L) & 1.276 & 1.279 & 1.261 & 1.276 & 1.279 & 1.470 & 1.247 \\
\hline
\end{tabular}

* Calculated assuming no dissolution of any undissolved solids in feeds

Given the measured AY102, AP101 and FEP bottoms compositions, the calculated UF feed undissolved solids was $3.19 \mathrm{wt} \%$ versus the actual $3.22 \mathrm{wt} \%$, which is very good agreement. The UF feed composition calculated from the model inputs, assuming no dissolution occurs upon mixing, was $2.97 \mathrm{wt} \%$ UDS versus the model value of $2.45 \mathrm{wt} \%$ where dissolution occurred. When this difference of $0.52 \mathrm{wt} \%$ of solids in the slurry is added to the permeate as soluble solids, the permeate dissolved solids content increases from 28.1 to $29.2 \mathrm{wt} \%$, exactly as shown in the model.

The model and measured solids and chemical compositions of these streams (the concentrated UF slurry, UF permeate, and the washed slurry) are given in Table 31. The model predictions for the AY102 slurry were calculated for a UDS of $11.50 \mathrm{wt} \%$, while the actual material was $11.75 \mathrm{wt} \%$. Mostly, undissolved species such as $\mathrm{Fe}, \mathrm{Ca}, \mathrm{Ba}, \mathrm{Ag}, \mathrm{Mg}, \mathrm{Nd}, \mathrm{Ni}, \mathrm{Pb}$, and $\mathrm{Si}$ were about $25 \%$ higher in the actual slurry than in the predictions. The discrepancies are caused by the input data assumptions made for the OLI model. The composition data that the model needs to represent consist of elemental and carbon analyses for the solids phase of the waste and elemental, anion, and carbon (inorganic, organic) analyses for the supernate portion. Some information was also available about the undissolved "anions" in the solids (such as phosphate, sulfate). Unbound hydroxide and total base measurements were also available. Note that the carbon and the hydroxide/ base analyses can have significant uncertainty. Matching the composition data and balancing the charge is relatively easy for the supernate phase, but is significantly more difficult for the solids phase. Different assumptions can be made about what form each of the metals (cations) exists as in the solids. 
WSRC-TR-2004-00478, REVISION 0 SRNL-RPP-2004-00075, REVISION 0

Table 31. Comparison of Model and Measured UFP-Stream Compositions

\begin{tabular}{|c|c|c|c|c|c|c|c|c|c|c|c|c|c|c|}
\hline \multirow{2}{*}{$\begin{array}{l}\text { All concentrations in mg/L } \\
\text { unless otherwise noted }\end{array}$} & \multicolumn{2}{|c|}{ AY102 } & \multicolumn{2}{|c|}{ AP101 } & \multicolumn{2}{|c|}{ FEP Bottoms } & \multicolumn{2}{|c|}{ UF Feed } & \multicolumn{2}{|c|}{ UF Conc. Slurry } & \multicolumn{2}{|c|}{ UF Permeate } & \multicolumn{2}{|c|}{ UF Washed Slurry } \\
\hline & Model & Campaign 2 & Model & Campaign 2 & Model & Campaign 2 & Model & Campaign 2 & Model & Campaign 2 & Model & Campaign 2 & Model & Campaign 2 \\
\hline \begin{tabular}{|r|} 
Total Solids (wt\%) \\
\end{tabular} & 26.31 & 26.80 & 32.85 & 31.94 & 29.88 & 28.95 & 30.95 & 30.59 & 43.38 & 45.37 & 29.22 & 28.13 & 24.82 & 26.56 \\
\hline Undissolved Solids (wt\%) & 11.50 & 11.75 & 0.01 & $0-0.29$ & 2.18 & 3.03 & 2.45 & 3.22 & 20.00 & 23.51 & 0 & 0 & 21.42 & 22.22 \\
\hline Supernate Solids (wt\%) & 16.73 & 17.05 & 32.84 & 31.94 & 28.32 & 26.73 & 29.22 & 28.28 & 29.22 & 28.58 & 29.22 & 28.13 & 4.33 & 5.59 \\
\hline Slurry Density (kg/L) & 1.236 & 1.276 & 1.278 & 1.279 & 1.252 & 1.261 & 1.264 & 1.276 & 1.376 & 1.470 & 1.250 & 1.247 & 1.159 & 1.214 \\
\hline Supernate Density $(\mathrm{kg} / \mathrm{L})$ & 1.169 & 1.170 & 1.278 & NA & 1.231 & NA & 1.250 & 1.250 & 1.250 & 1.247 & 1.250 & 1.247 & 1.036 & 1.124 \\
\hline $\mathrm{Ag}$ & 491 & 624 & 0 & 37 & 0.007 & 112 & 119 & 157 & 1055 & 1455 & 0.7 & $<0.5$ & 1068 & 976 \\
\hline $\mathrm{Al}$ & 16252 & 16740 & 7816 & 7469 & 1719 & 6895 & 9187 & 9187 & 21696 & 25799 & 7603 & 7180 & 17131 & 15472 \\
\hline $\mathrm{B}$ & 9 & $<165$ & 16 & $<142$ & 10 & 698 & 14 & $<148$ & 12 & $<222$ & 14 & 83 & 0.8 & $<182$ \\
\hline $\mathrm{Ba}$ & 177 & 274 & 0.324 & $<1.5$ & 3 & 48 & 43 & 57 & 378 & 652 & 1.0 & $<0.02$ & 380 & 481 \\
\hline $\mathrm{Ca}$ & 970 & 1316 & 8 & 23 & 274 & 447 & 271 & 415 & 2385 & 2800 & 3.1 & 1.4 & 2408 & 2360 \\
\hline $\mathrm{Ce}$ & 248 & 366 & 0 & $<127$ & 210 & NA & 83 & $<120$ & 74 & 810 & 84 & $<1$ & 5 & 603 \\
\hline $\mathrm{Cr}$ & 548 & 633 & 170 & 179 & 585 & 237 & 307 & 309 & 274 & 1135 & 312 & 162 & 19 & 724 \\
\hline $\mathrm{Cs}$ & 6.3 & 18.7 & 0 & 6.0 & 6.7 & & 2.3 & 4.5 & 2.0 & & 2.3 & 5.9 & 0.14 & \\
\hline $\mathrm{Cu}$ & 68 & 80 & 1.6 & $<6$ & 67 & 16.9 & 25 & 15 & 22 & 197 & 25 & 0.2 & 1.5 & 149 \\
\hline $\mathrm{Fe}$ & 27922 & 35526 & 2.5 & 76 & 73 & 5798 & 6792 & 9532 & 58830 & 81953 & 204 & 1.2 & 59460 & 61160 \\
\hline $\mathrm{K}$ & 429 & 373 & 31176 & 30568 & 5211 & 305 & 20820 & 19012 & 18580 & 19110 & 21103 & 20367 & 1272 & 3859 \\
\hline $\mathrm{Li}$ & 86 & $<25$ & 0.3 & $<21$ & 92 & 140 & 31 & $<22$ & 28 & 134 & 32 & 1 & 1.9 & 105 \\
\hline $\mathrm{Mg}$ & 256 & 376 & 0 & 6 & 49 & 104 & 68 & 107 & 601 & 939 & 0 & $<0.01$ & 609 & 697 \\
\hline $\mathrm{Mn}$ & 38 & 7725 & 0 & 3 & 1 & 1187 & 9 & 2061 & 8.2 & 18081 & 9 & 0.03 & 2.8 & 13531 \\
\hline $\mathrm{Na}$ & 83976 & 88021 & 128777 & 128540 & 112614 & 117668 & 115997 & 114856 & 124774 & 132837 & 114885 & 112917 & 24694 & 33256 \\
\hline $\mathrm{Nd}$ & 361 & 847 & 0 & $<73$ & 0 & 106 & 88 & 240 & 780 & 2014 & 0 & $<0.4$ & 790 & 1396 \\
\hline $\mathrm{Ni}$ & 914 & 1151 & 14 & 357 & 0.3 & 1146 & 231 & 295 & 2040 & 2705 & 2 & 0.3 & 2065 & 2039 \\
\hline $\mathrm{P}$ & 2117 & 1674 & 496 & 269 & 1839 & 1121 & 1037 & 754 & 1076 & 987 & 1032 & 793 & 295 & 339 \\
\hline $\mathrm{Pb}$ & 1278 & 1628 & 15 & $<156$ & 1366 & 223 & 470 & 447 & 420 & 3859 & 477 & 6 & 29 & 2937 \\
\hline S & 806 & 895 & 1339 & 1599 & 932 & 908 & 1164 & 1234 & 1118 & 1267 & 1170 & 1144 & 159 & 593 \\
\hline $\mathrm{Si}$ & 8418 & 11016 & 137 & 329 & 360 & 1992 & 2173 & 3247 & 18330 & 23256 & 127 & 57 & 18483 & 17727 \\
\hline $\mathrm{Sr}$ & 226 & 286 & 0 & $<1$ & 0.07 & 87 & 55 & 72 & 489 & 663 & 0.05 & 0.07 & 495 & 519 \\
\hline $\mathrm{W}$ & 162 & $<85$ & 25 & $<98$ & 173 & & 74 & $<86$ & 66 & $<139$ & 75 & 45 & 4.54 & $<101$ \\
\hline $\mathrm{Zn}$ & 59 & 81 & 6 & 6 & 301 & 46 & 51 & 29 & 45 & 222 & 52 & 3.0 & 3.1 & 169 \\
\hline $\mathrm{Zr}$ & 998 & 484 & 0 & $<6$ & 7 & 86 & 243 & 125 & 1972 & 1095 & 24 & 1 & 1978 & 831 \\
\hline bromide & 124 & 69 & 0 & $<128$ & 133 & 53 & 45 & $<54$ & 40 & $<43$ & 45 & $<10$ & 2.74 & $<28$ \\
\hline chloride & 159 & 165 & 1624 & 1957 & 13486 & 609 & 2572 & 1211 & 2296 & 1085 & 2607 & 1147 & 157 & 99 \\
\hline fluoride & 225 & 43 & 50 & 36 & 379 & 145 & 135 & 75 & 121 & 76 & 137 & 38 & 8.3 & 19 \\
\hline nitrate & 218 & 319 & 86614 & 134295 & 112967 & 119000 & 68449 & 81154 & 61086 & 73059 & 69381 & 58600 & 4181 & 7875 \\
\hline nitrite & 6512 & 4228 & 49090 & 42719 & 10708 & 3740 & 34477 & 25775 & 30768 & 20727 & 34946 & 28133 & 2106 & 2315 \\
\hline oxalate & 10321 & 9213 & 1754 & 1982 & 16370 & 1180 & 5443 & 4253 & 27199 & 34986 & 2688 & 723 & 11762 & 15749 \\
\hline sulfate & 2416 & 1860 & 4012 & 4783 & 2793 & 1960 & 3487 & 3381 & 3349 & 3058 & 3504 & 3140 & 477 & 338 \\
\hline carbonate & 73317 & NA & 29970 & & 47667 & 9500 & 42420 & 40600 & 41631 & 24600 & 42520 & 31350 & 7574 & 5100 \\
\hline unbound hydroxide & 6962 & NA & 36457 & & 444 & 6103 & 23726 & 38930 & 23726 & & 23726 & 2346 & 1952 & \\
\hline
\end{tabular}


The simple example in Table 32 shows how the predicted concentration of Fe could be higher or lower than what really results when the simulant slurry is made. In this example, assume that the actual form of $\mathrm{Fe}$ in the solid is $\mathrm{FeOOH}$ (Case 1) and that $\mathrm{Na}$ and carbonate are present as sodium carbonate.

Table 32. Hypothetical Material \& Charge Balance on Solid Phase

\begin{tabular}{|c|c|c|c|c|c|c|c|}
\hline Case 1 & $\begin{array}{c}\text { Species } \\
\text { Concentration } \\
\mathrm{mg} / \mathrm{kg}\end{array}$ & $\begin{array}{c}\text { Species } \\
\text { Concentration } \\
\mathrm{mmol} / \mathrm{kg}\end{array}$ & Compound & $\begin{array}{c}\text { Compound } \\
\text { Concentration } \\
\mathrm{mg} / \mathrm{kg}\end{array}$ & $\begin{array}{c}\text { Species } \\
\text { Concentration } \\
\text { Normalized to } \\
100 \% \mathrm{mg} / \mathrm{kg}\end{array}$ & $\begin{array}{c}\text { Charge } \\
\mathrm{mmol} / \mathrm{kg}\end{array}$ & \\
\hline $\mathrm{Fe}^{+3}$ & 198070 & 3546.7 & $\mathrm{FeOOH}$ & 315134 & 198070 & 3546.7 & $" \mathrm{FeO}^{+} \mathrm{OH}^{-1 "}$ \\
\hline $\mathrm{Na}^{+}$ & 297105 & 12923.4 & $\mathrm{Na}_{2} \mathrm{CO}_{3}$ & 684866 & 297105 & 12923.4 & \\
\hline $\mathrm{CO}_{3}^{-2}$ & 387761 & 6461.7 & & & 387761 & -12923.4 & \\
\hline $\mathrm{OH}^{-}$ & 60319 & 3546.7 & & & 60319 & -3546.7 & \\
\hline $\mathrm{O}$ & 56744 & 3546.7 & & & 56744 & & \\
\hline Total & 1000000 & & & $\begin{array}{c}1000000 \\
100.0 \%\end{array}$ & & 0 & \\
\hline $\begin{array}{l}\text { Case } 2 \\
\text { Species }\end{array}$ & $\begin{array}{c}\text { Species } \\
\text { Concentration } \\
\mathrm{mg} / \mathrm{kg}\end{array}$ & $\begin{array}{c}\text { Species } \\
\text { Concentration } \\
\mathrm{mmol} / \mathrm{kg}\end{array}$ & Compound & $\begin{array}{c}\text { Compound } \\
\text { Concentration } \\
\mathrm{mg} / \mathrm{kg}\end{array}$ & $\begin{array}{c}\text { Species } \\
\text { Concentration } \\
\text { Normalized to } \\
100 \% \mathrm{mg} / \mathrm{kg}\end{array}$ & $\begin{array}{l}\text { Charge } \\
\mathrm{mmol} / \mathrm{kg}\end{array}$ & \\
\hline $\mathrm{Fe}^{+3}$ & 198070 & 3546.7 & $\mathrm{Fe}_{2} \mathrm{O}_{3}$ & 283187 & 204607 & 0 & $\mathrm{Fe}_{2} \mathrm{O}_{3}$ has \\
\hline $\mathrm{Na}^{+}$ & 297105 & 12923.4 & $\mathrm{Na}_{2} \mathrm{CO}_{3}$ & 684866 & 306910 & 12923.4 & no charge \\
\hline $\mathrm{CO}_{3}^{-2}$ & 387761 & 6461.7 & & & 400557 & -12923.4 & \\
\hline $\mathrm{OH}^{-}$ & 0 & 0.0 & & & 0 & 0.0 & \\
\hline $\mathrm{O}$ & 85117 & 5320.0 & & & 87926 & & \\
\hline Total & 968053 & & & $\begin{array}{c}968053 \\
96.8 \%\end{array}$ & & 0 & \\
\hline $\begin{array}{l}\text { Case } 3 \\
\text { Species }\end{array}$ & $\begin{array}{c}\text { Species } \\
\text { Concentration } \\
\mathrm{mg} / \mathrm{kg}\end{array}$ & $\begin{array}{c}\text { Species } \\
\text { Concentration } \\
\text { mmol/kg }\end{array}$ & Compound & $\begin{array}{c}\text { Compound } \\
\text { Concentration } \\
\mathrm{mg} / \mathrm{kg}\end{array}$ & $\begin{array}{c}\text { Species } \\
\text { Concentration } \\
\text { Normalized to } \\
100 \% \mathrm{mg} / \mathrm{kg}\end{array}$ & $\begin{array}{l}\text { Charge } \\
\mathrm{mmol} / \mathrm{kg}\end{array}$ & \\
\hline $\mathrm{Fe}^{+3}$ & 198070 & 3546.7 & $\mathrm{Fe}(\mathrm{OH})_{3}$ & 379028 & 186175 & 10639.97 & \\
\hline $\mathrm{Na}^{+}$ & 297105 & 12923.4 & $\mathrm{Na}_{2} \mathrm{CO}_{3}$ & 684866 & 279262 & 12923.4 & \\
\hline $\mathrm{CO}_{3}^{-2}$ & 387761 & 6461.7 & & & 364473 & -12923.4 & \\
\hline $\mathrm{OH}^{-}$ & 180958 & 10640.0 & & & 170090 & -10640.0 & \\
\hline Total & 1063894 & & & 1063894 & & 0 & \\
\hline
\end{tabular}

The "Species Concentrations" have been specified so that the total amount of solids is $100 \%$. If Fe is assumed to be present as $\mathrm{Fe}_{2} \mathrm{O}_{3}$ rather than $\mathrm{FeOOH}$ (Case 2), then the sum of the solids is less than $100 \%$; normalizing the concentrations is required to make the total $100 \%$. Similarly, if the Fe is assumed to be present as $\mathrm{Fe}(\mathrm{OH})_{3}$ (Case 3), then the total mass is greater than $100 \%$. The equivalences between these forms are shown below:

$2 \mathrm{Fe}(\mathrm{OH})_{3} \rightleftharpoons 2\left[\mathrm{FeO}(\mathrm{OH}) \cdot \mathrm{H}_{2} \mathrm{O}\right] \rightleftharpoons 2 \mathrm{FeO}(\mathrm{OH})+2 \mathrm{H}_{2} \mathrm{O} \rightleftharpoons \mathrm{Fe}_{2} \mathrm{O}_{3}+3 \mathrm{H}_{2} \mathrm{O} \rightleftharpoons \mathrm{Fe}_{2} \mathrm{O}_{3} \cdot 3 \mathrm{H}_{2} \mathrm{O}$ 
Note that the presence of hydroxide in the solids is assumed for the $\mathrm{FeOOH}$ and $\mathrm{Fe}(\mathrm{OH})_{3}$ cases. In actual analyses, the amount of "bound" hydroxide is not known very accurately, so it essentially becomes a variable in the material and charge balance. In the OLI model for the solids, all of the metals present were assumed to be associated with a corresponding anion (as in Case 3). None was assumed to be present as an oxide such as $\mathrm{Fe}_{2} \mathrm{O}_{3}$ or a partial oxide such as $\mathrm{FeOOH}$, so the total predicted mass for a given set of species concentrations was higher than if oxides were assumed. Therefore, to get a particular amount of undissolved solids in the model, the amount of elements needed was smaller than if the metal oxides were assumed. For the actual waste, it appears that some portion of the metals were present as oxides; for the simulant, some of the solids were added as oxides (e.g., $\mathrm{Al}_{2} \mathrm{O}_{3}, \mathrm{SiO}_{2}$ ). The result of these differences was that at the target undissolved solids concentration, the concentrations of the undissolved elements in the actual AY102 simulant was higher than the predicted concentrations.

The higher solids in the actual FEP bottoms is also reflected in the elemental analyses. The predominantly undissolved species $\mathrm{Al}, \mathrm{Fe}, \mathrm{Mn}, \mathrm{Ni}$, and $\mathrm{Si}$ are significantly higher than the model values. Note that Mn was inadvertently left out of the OLI model. The oxalate and carbonate values do not agree well, probably due to inaccurate measurements. The potassium in the model was significantly higher than in the actual bottoms product.

\subsection{Washing the Concentrated Slurry}

Because the final concentrated slurry was at greater than $20 \mathrm{wt} \%$ UDS, the 117L of slurry was diluted by first removing a calculated amount of slurry and then adding back permeate. Table 33 shows the amounts of slurry removed and permeate added and the predicted and actual diluted slurry solids contents. The slurry was actually diluted back to approximately 19.6 to $21.5 \mathrm{wt} \%$.

Table 33. Dilution of Concentrated Slurry Back to Approximately 20 wt\% Undissolved Solids

\begin{tabular}{|r|ccccc|}
\multicolumn{1}{c|}{} & $\begin{array}{c}\text { Over- } \\
\text { Concentrated } \\
\text { Slurry }\end{array}$ & $\begin{array}{c}\text { Slurry } \\
\text { Removed }\end{array}$ & $\begin{array}{c}\text { Permeate } \\
\text { Added }\end{array}$ & $\begin{array}{c}\text { Final } \\
\text { Measured }\end{array}$ & $\begin{array}{c}\text { Final } \\
\text { Predicted }\end{array}$ \\
\hline Total Solids (wt\%) & $44.94-45.80$ & 45.37 & 28.06 & $42.13-42.93$ & $42.1-42.9$ \\
\hline Undissolved Solids (wt\%) & $23.50-24.66$ & $23.50-24.66$ & 0 & $19.56-21.49$ & $19.9-20.5$ \\
\hline Supernate Solids* (wt\%) & 28.06 & 28.06 & 28.06 & 28.06 & 28.06 \\
\hline Density (kg/L) & 1.470 & 1.470 & 1.244 & 1.410 & 1.394 \\
\hline Volume (L) & $117 \pm 4$ & $\sim 19.0$ & $\sim 19.6$ & 121 & $117 \pm 4$ \\
\hline Mass (kg) & 172 & 27.9 & 24.6 & 168 & NC \\
\hline
\end{tabular}

* average of multiple samples used

After the dilution, the slurry was washed with 22 discrete additions of $\sim 5.37 \mathrm{~L}$ of $0.01 \mathrm{M} \mathrm{NaOH}$ ("wash water"), followed by an additional 22 similar washes. The total amount of wash water used was about 236.2L. Leaching of the solids with $\mathrm{NaOH}$ was not performed per WTP instructions. Table 34 summarizes the composition of the washed slurry after 22 and 44 washes and also shows the composite composition of the permeate from the first and second 22 washes. The soluble species are reduced to about $10 \%$ of their concentrations in the unwashed slurry. 
Table 34. Measured Compositions of Concentrated \& Washed Slurry and Wash Permeates

\begin{tabular}{|c|c|c|c|c|c|c|}
\hline $\begin{array}{l}\text { All values mg/L unless } \\
\text { otherwise noted }\end{array}$ & $\begin{array}{c}\text { Concentrated } \\
\text { Slurry } \\
\text { (diluted } \\
\text { to } \\
\sim 20 \mathrm{wt} \% \\
\text { UDS) } \\
\end{array}$ & $\begin{array}{c}\text { Washed } \\
\text { Slurry } \\
-22 \\
\text { Washe } \\
\text { s } \\
\end{array}$ & $\begin{array}{c}\text { Washed } \\
\text { Slurry } \\
-44 \\
\text { Wash } \\
\text { es } \\
\end{array}$ & $\begin{array}{c}\text { First } 22 \\
\text { Washes } \\
\text { Permeate } \\
\text { Composit } \\
\text { e }\end{array}$ & $\begin{array}{c}\text { Second } 22 \\
\text { Washes } \\
\text { Permeat } \\
\text { e } \\
\text { Compo } \\
\text { site } \\
\end{array}$ & $\begin{array}{c}\text { Washed Slurry / } \\
\text { Concentrat } \\
\text { ed Slurry } \\
(\%)\end{array}$ \\
\hline Total Solids (wt\%) & 42.86 & 31.94 & 26.56 & 20.66 & 8.89 & \\
\hline Undissolved Solids (wt\%) & 20.56 & 22.75 & 22.22 & 0 & 0 & \\
\hline Supernate Solids (wt\%) & 28.06 & 11.90 & 5.59 & 20.66 & 8.89 & 19.9 \\
\hline Density $(\mathrm{kg} / \mathrm{L})$ & 1.432 & 1.23 & 1.21 & 1.177 & 1.071 & \\
\hline Ag & 1212 & 1158 & 976 & $<0.6$ & $<0.6$ & 80.5 \\
\hline $\mathrm{Al}$ & 22661 & 18773 & 15472 & 4950 & 1850 & 68.3 \\
\hline B & 14.6 & $<144$ & $<182$ & 63.0 & 20.0 & \\
\hline $\mathrm{Ba}$ & 543 & 556 & 481 & $<0.04$ & $<0.04$ & 88.6 \\
\hline $\mathrm{Bi}$ & $<0.01$ & $<0.01$ & $<0.01$ & $<0.01$ & $<0.01$ & \\
\hline $\mathrm{Ca}$ & 2332 & 2337 & 2360 & $<0.06$ & $<0.06$ & 101.2 \\
\hline $\mathrm{Cd}$ & 86.4 & 87.4 & 73.5 & $<0.04$ & $<0.04$ & 85.1 \\
\hline Ce & 674 & 536 & 603 & $<1.4$ & $<1.4$ & 89.5 \\
\hline Co & 0 & $<17.8$ & $<16.1$ & $<0.16$ & $<0.16$ & \\
\hline $\mathrm{Cr}$ & 972 & 855 & 724 & 120 & 50.0 & 74.5 \\
\hline Cs (ICPMS) & 4.62 & 0.213 & $<0.01$ & 4.01 & 1.41 & \\
\hline Cs (AA) & 1.23 & $<22.2$ & $<20.3$ & 5.43 & 1.69 & \\
\hline $\mathrm{Cu}$ & 164 & 158 & 149 & 0.190 & $<0.06$ & 90.9 \\
\hline $\mathrm{Fe}$ & 68240 & 65829 & 66743 & 0.979 & 0.185 & 97.8 \\
\hline K (AA) & 19460 & 7080 & 3859 & 12000 & 4000 & 19.8 \\
\hline $\mathrm{La}$ & 585 & 500 & 468 & $<0.2$ & $<0.2$ & 80.0 \\
\hline $\mathrm{Li}$ & 111 & 107 & 105 & 1.48 & 1.71 & 94.6 \\
\hline $\mathrm{Mg}$ & 782 & 754 & 697 & $<0.01$ & $<0.01$ & 89.1 \\
\hline $\mathrm{Mn}$ & 15056 & 14479 & 14805 & $<0.02$ & $<0.02$ & 98.3 \\
\hline Mo & 1.56 & $<22.2$ & $<20.3$ & 6.88 & 2.35 & \\
\hline $\mathrm{Na}$ & 129132 & 57914 & 33019 & 73700 & 30400 & 25.6 \\
\hline $\mathrm{Na}(\mathrm{AA})$ & 129819 & 57792 & 33493 & 77000 & 27800 & 25.8 \\
\hline Na average & 129476 & 57853 & 33256 & 75350 & 29100 & 25.7 \\
\hline $\mathrm{Na} \mathrm{M}$ & 5.63 & 2.52 & 1.45 & 3.28 & 1.27 & \\
\hline $\mathrm{Nd}$ & 1677 & 1404 & 1396 & $<1.8$ & $<1.8$ & 83.2 \\
\hline $\mathrm{Ni}$ & 2252 & 2172 & 2039 & 0.217 & 0.145 & 90.5 \\
\hline $\mathrm{P}$ & 956 & 418 & 339 & 540 & 140 & 35.5 \\
\hline $\mathrm{Pb}$ & 3213 & 3153 & 3240 & 1.70 & $<1.6$ & 100.8 \\
\hline $\operatorname{Pr}$ & 349 & 364 & 375 & $<0.01$ & $<0.01$ & 107.4 \\
\hline $\mathrm{Rb}$ & 2.89 & 1.64 & $<0.01$ & 3.30 & 1.13 & \\
\hline $\mathrm{S}$ & 1256 & 598 & 593 & 840 & 290 & 47.2 \\
\hline $\mathrm{Si}$ & 19374 & 18767 & 17727 & 41.8 & 22.1 & 91.5 \\
\hline $\mathrm{Sr}$ & 552 & 559 & 519 & 0.0475 & 0.0174 & 94.0 \\
\hline $\mathrm{W}$ & 8.41 & $<107$ & $<101$ & 40.0 & 10.0 & \\
\hline $\mathrm{Y}$ & 108 & 110 & 113 & $<0.01$ & $<0.01$ & 104.6 \\
\hline $\mathrm{Zn}$ & 185 & 184 & 169 & 1.43 & 0.0818 & 91.4 \\
\hline $\mathrm{Zr}$ & 912 & 837 & 831 & 0.436 & 0.0872 & 91.1 \\
\hline
\end{tabular}


WSRC-TR-2004-00478, REVISION 0

SRNL-RPP-2004-00075, REVISION 0

\begin{tabular}{|r|ccccc|c|} 
Table 34 continued & $\begin{array}{c}\text { Concentrated } \\
\text { Slurry }\end{array}$ & $\begin{array}{c}\text { Washed } \\
\text { Slurry - 22 } \\
\text { Washes }\end{array}$ & $\begin{array}{c}\text { Washed } \\
\text { Slurry - 44 } \\
\text { Washes }\end{array}$ & $\begin{array}{c}\text { First 22 } \\
\text { Washes } \\
\text { Permeate } \\
\text { Composite }\end{array}$ & $\begin{array}{c}\text { Second 22 } \\
\text { Washes } \\
\text { Permeate } \\
\text { Composite }\end{array}$ & $\begin{array}{c}\text { Washed Slurry / } \\
\text { Concentrated } \\
\text { Slurry (\%) }\end{array}$ \\
\hline bromide & $<42.6$ & $<46.6$ & $<27.9$ & $<10$ & $<10$ & \\
\hline chloride & 1106 & 347 & 99.4 & 878 & 284 & 9.0 \\
\hline fluoride & 68.5 & 28.2 & 19.0 & 26.0 & $<2$ & 27.7 \\
\hline formate & 1061 & 421 & 154 & 949 & 236 & 14.5 \\
\hline nitrate & 75107 & 24049 & 7875 & 74000 & 19100 & 10.5 \\
\hline nitrite & 21475 & 7313 & 2315 & 19800 & 5480 & 10.8 \\
\hline oxalate & 29257 & 25399 & 15749 & 2030 & 6060 & 53.8 \\
\hline phosphate & 1723 & 488 & 150 & 1700 & 430 & 8.7 \\
\hline sulfate & 3113 & 1050 & 338 & 2430 & 803 & 10.9 \\
\hline acetate & 1099 & & & 825 & 257 & \\
\hline citric acid & & & & $<100$ & $<100$ & \\
\hline formate & 930 & & 6070 & 4390 & 2680 & \\
\hline carbonate & 26968 & & 5100 & 12850 & 10100 & 18.9 \\
\hline
\end{tabular}

Figure 34 shows the total, undissolved, and supernate solids weight percent and the density of the slurry as a function of the volume of wash water used, normalized to the UFP slurry batch. The total and supernate solids decrease as the soluble salts are removed, while the UDS increase slightly and the density stays fairly constant at about $1.20-1.25 \mathrm{~kg} / \mathrm{L}$.

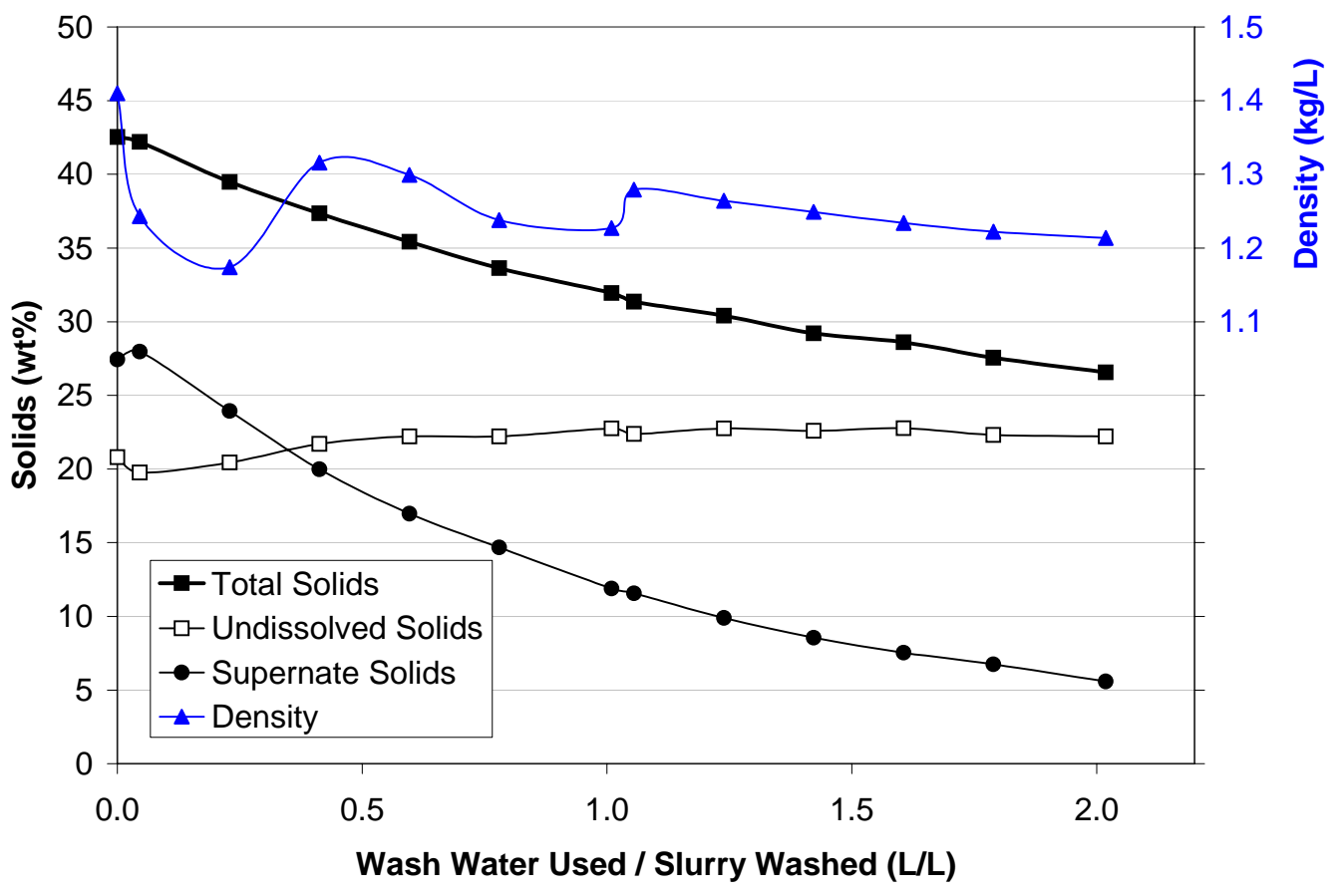

Figure 34. Solids Content and Density of Slurry During Washing 
The permeate total solids and density are shown in Fig. 35. The data for Figs. 34 and 35 are summarized in Table 35, along with rheological data and data for the Campaign.

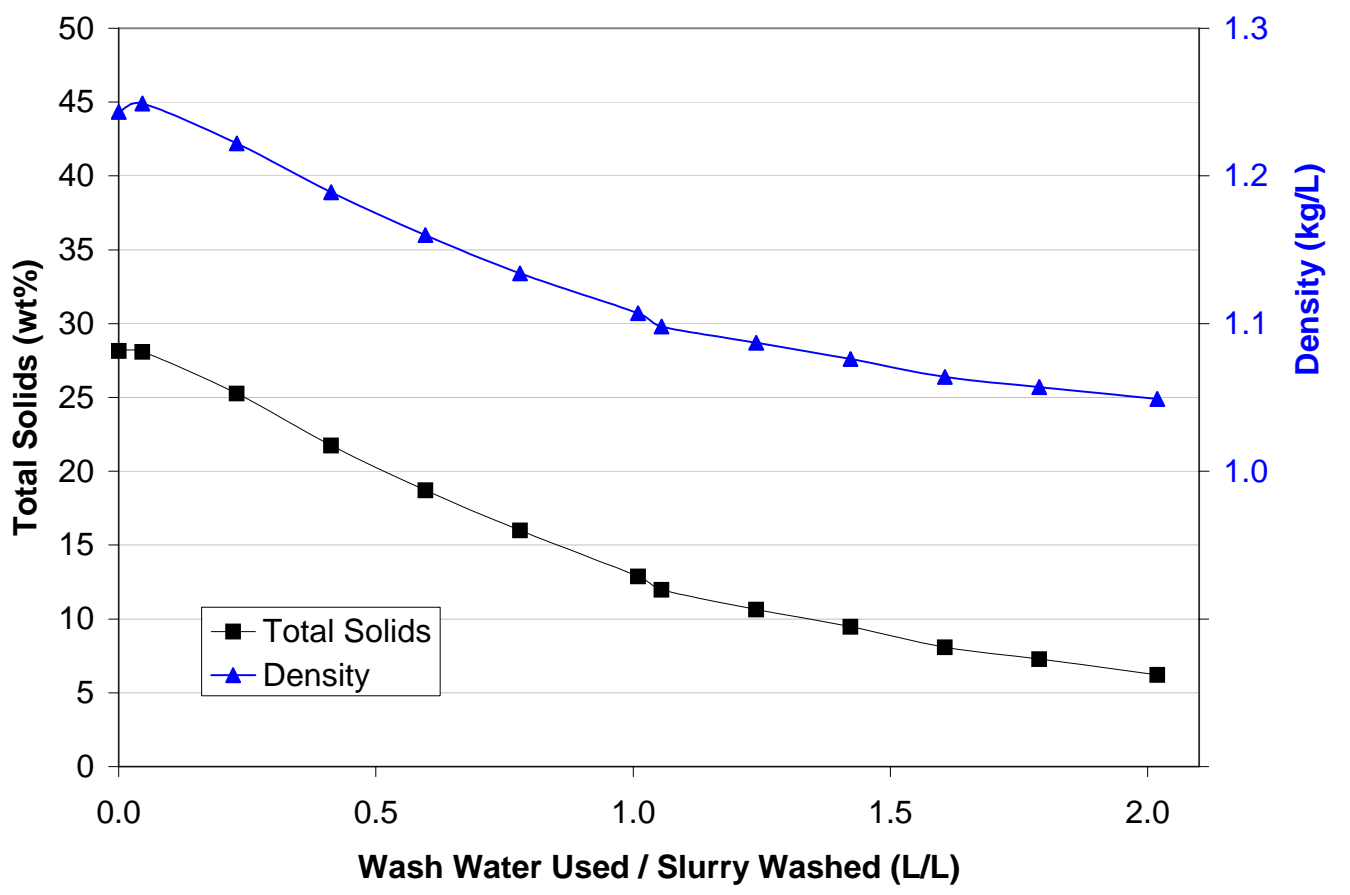

Figure 35. Total Solids and Density of Permeate During Washing 
WSRC-TR-2004-00478, REVISION 0 SRNL-RPP-2004-00075, REVISION 0

Table 35. Measured Solids, Density, and Rheological Data for Ultrafiltration Total Supernate

Solids Undissolved Solids Density Yield Consistency

Sample $\begin{array}{lllll}(\mathrm{wt} \%) & \text { Solids }(\mathrm{wt} \%) & (\mathrm{wt} \%) & (\mathrm{kg} / \mathrm{L}) & \text { Stress }(\mathrm{Pa}) \\ (\mathrm{mPa} \cdot \mathrm{s})\end{array}$

\begin{tabular}{|c|c|c|c|c|c|c|}
\hline \multicolumn{7}{|l|}{ CAMPAIGN I } \\
\hline Starting Slurry & 27.35 & 12.53 & 16.94 & 1.269 & 4.85 & 13.38 \\
\hline During Concentration & 29.21 & 14.63 & 17.08 & 1.279 & 10.42 & 12.75 \\
\hline During Concentration & 30.98 & 16.80 & 17.04 & 1.302 & 15.11 & 15.05 \\
\hline During Concentration & 32.92 & 19.06 & 17.12 & 1.326 & 22.43 & 17.55 \\
\hline During Concentration & 34.72 & 21.26 & 17.09 & 1.333 & 30.88 & 19.58 \\
\hline During Concentration & 36.30 & 23.12 & 17.14 & 1.367 & 35.48 & 20.90 \\
\hline Concentrated & 36.71 & 23.60 & 17.16 & 1.351 & 34.38 & 23.38 \\
\hline Concentrated & 36.97 & 23.72 & 17.37 & 1.371 & 34.63 & 24.00 \\
\hline Concentrated & 37.04 & 23.83 & 17.34 & 1.350 & 35.43 & 24.13 \\
\hline After Steady-State Matrix & 36.91 & 23.90 & 17.10 & 1.271 & 25.28 & 25.55 \\
\hline Diluted back to $20 \%$ & 33.19 & 19.63 & 16.87 & 1.329 & 8.53 & 19.05 \\
\hline After Wash 1 & 32.52 & 19.42 & 16.26 & 1.319 & 8.65 & 18.20 \\
\hline After Wash 5 & 30.46 & 20.10 & 12.97 & 1.284 & 9.23 & 16.03 \\
\hline After Wash 9 & 28.54 & 20.16 & 10.50 & 1.254 & 9.45 & 15.48 \\
\hline After Wash 13 & 27.03 & 20.11 & 8.66 & 1.220 & 10.15 & 14.13 \\
\hline After Wash 17 & 25.88 & 20.02 & 7.33 & 1.206 & 10.23 & 13.53 \\
\hline After Wash 22 & 24.78 & 20.12 & 5.83 & 1.165 & 10.38 & 12.63 \\
\hline After Wash 23 & 24.45 & 19.95 & 5.62 & 1.210 & 10.13 & 12.33 \\
\hline After Wash 27 & 23.90 & 20.31 & 4.50 & 1.157 & 10.45 & 11.75 \\
\hline After Wash 31 & 23.09 & 20.12 & 3.72 & 1.187 & 10.30 & 11.28 \\
\hline After Wash 35 & 22.74 & 20.42 & 2.92 & 1.163 & 9.90 & 10.50 \\
\hline After Wash 39 & 22.34 & 20.50 & 2.31 & 1.125 & NA & NA \\
\hline After Wash 44 & 21.99 & 20.55 & 1.81 & 1.183 & 8.15 & 8.95 \\
\hline \multicolumn{7}{|l|}{ CAMPAIGN II } \\
\hline $\begin{array}{ll}\text { Starting Slurry } \\
\end{array}$ & 30.59 & 3.22 & 28.28 & 1.276 & 0.00 & 4.74 \\
\hline Concentrated & 45.37 & 23.51 & 28.58 & 1.470 & 26.60 & 22.89 \\
\hline Diluted back to $20 \%$ & 42.53 & 20.50 & 27.71 & 1.410 & NA & NA \\
\hline After Wash 1 & 42.20 & 19.76 & 27.97 & 1.243 & 11.95 & 19.98 \\
\hline After Wash 5 & 39.49 & 20.45 & 23.93 & 1.174 & 12.26 & 14.96 \\
\hline After Wash 9 & 37.35 & 21.70 & 19.99 & 1.316 & 13.60 & 15.63 \\
\hline After Wash 13 & 35.42 & 22.22 & 16.97 & 1.299 & 13.53 & 14.60 \\
\hline After Wash 17 & 33.64 & 22.22 & 14.68 & 1.238 & 14.14 & 14.05 \\
\hline After Wash 22 & 31.94 & 22.75 & 11.90 & 1.227 & 14.84 & 13.16 \\
\hline After Wash 23 & 31.36 & 22.38 & 11.57 & 1.279 & 14.35 & 12.91 \\
\hline After Wash 27 & 30.40 & 22.75 & 9.90 & 1.264 & 15.22 & 12.88 \\
\hline After Wash 31 & 29.21 & 22.59 & 8.55 & 1.249 & 15.05 & 12.65 \\
\hline After Wash 35 & 28.60 & 22.78 & 7.54 & 1.234 & 16.17 & 12.32 \\
\hline After Wash 39 & 27.56 & 22.31 & 6.76 & 1.222 & 15.24 & 11.51 \\
\hline After Wash 44 & 26.56 & 22.22 & 5.59 & 1.214 & 15.90 & 12.14 \\
\hline
\end{tabular}

\subsection{Rheology Behavior During UFP Operation}

The average yield stress and consistency (average of up and down curves) during filtration for Campaigns I and II are shown in Fig. 36 and Fig. 37, respectively. The scales for both figures are the same, for comparison. The Campaign I data in Fig. 36 show that the yield stress increased from about $5 \mathrm{~Pa}$ to $35 \mathrm{~Pa}$ during concentrating (- to 2 ). The consistency also 
increased from about $13 \mathrm{mPa} \cdot \mathrm{s}$ to $25 \mathrm{mPa} \cdot \mathrm{s}$. The slurry was concentrated from about $27 \mathrm{wt} \%$ total solids and $12.5 \mathrm{wt} \%$ UDS up to $37 \mathrm{wt} \%$ total solids and $24 \mathrm{wt} \%$ UDS.

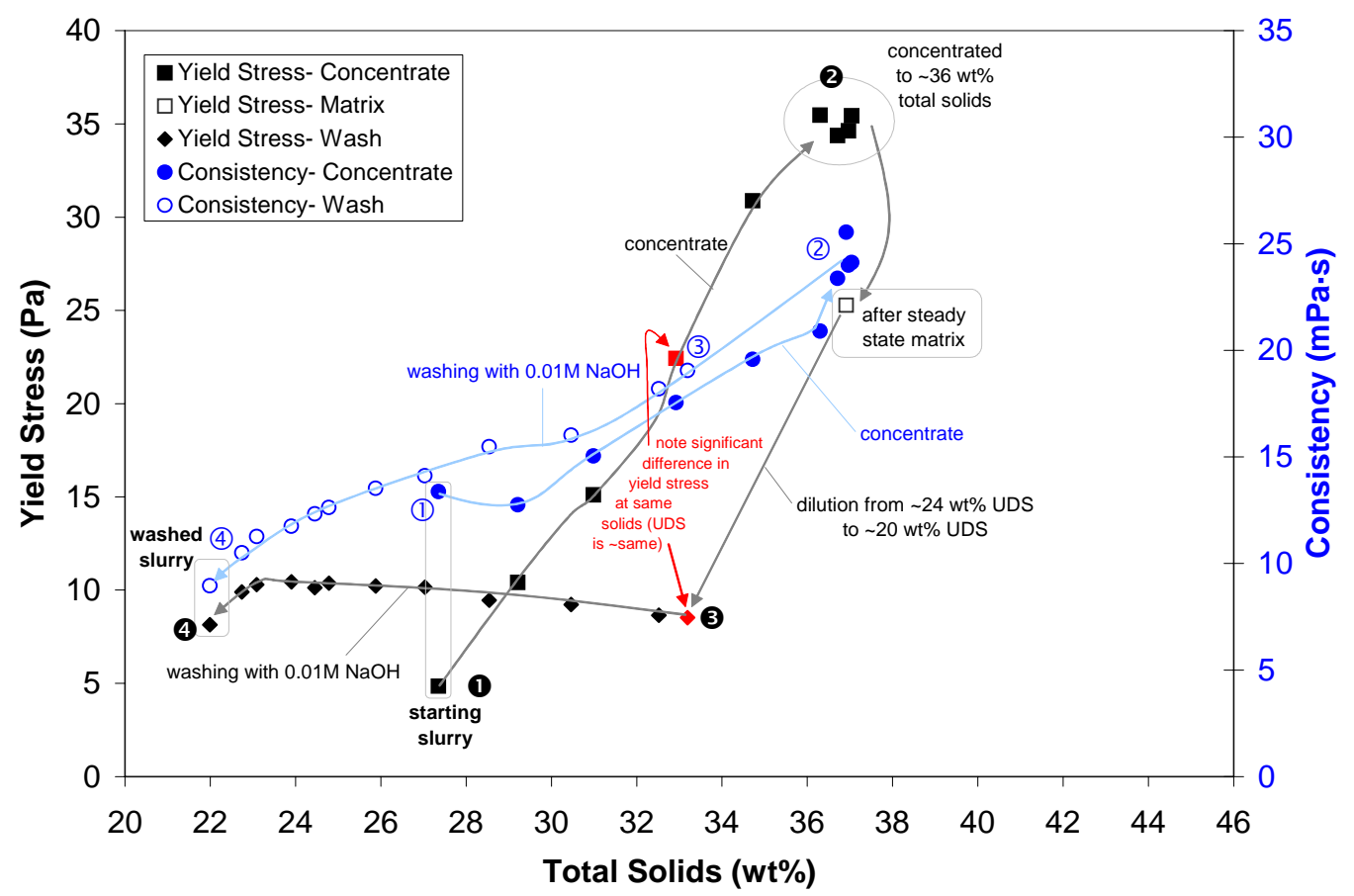

Figure 36. Yield Stress versus Total Solids for Campaign I Filtration

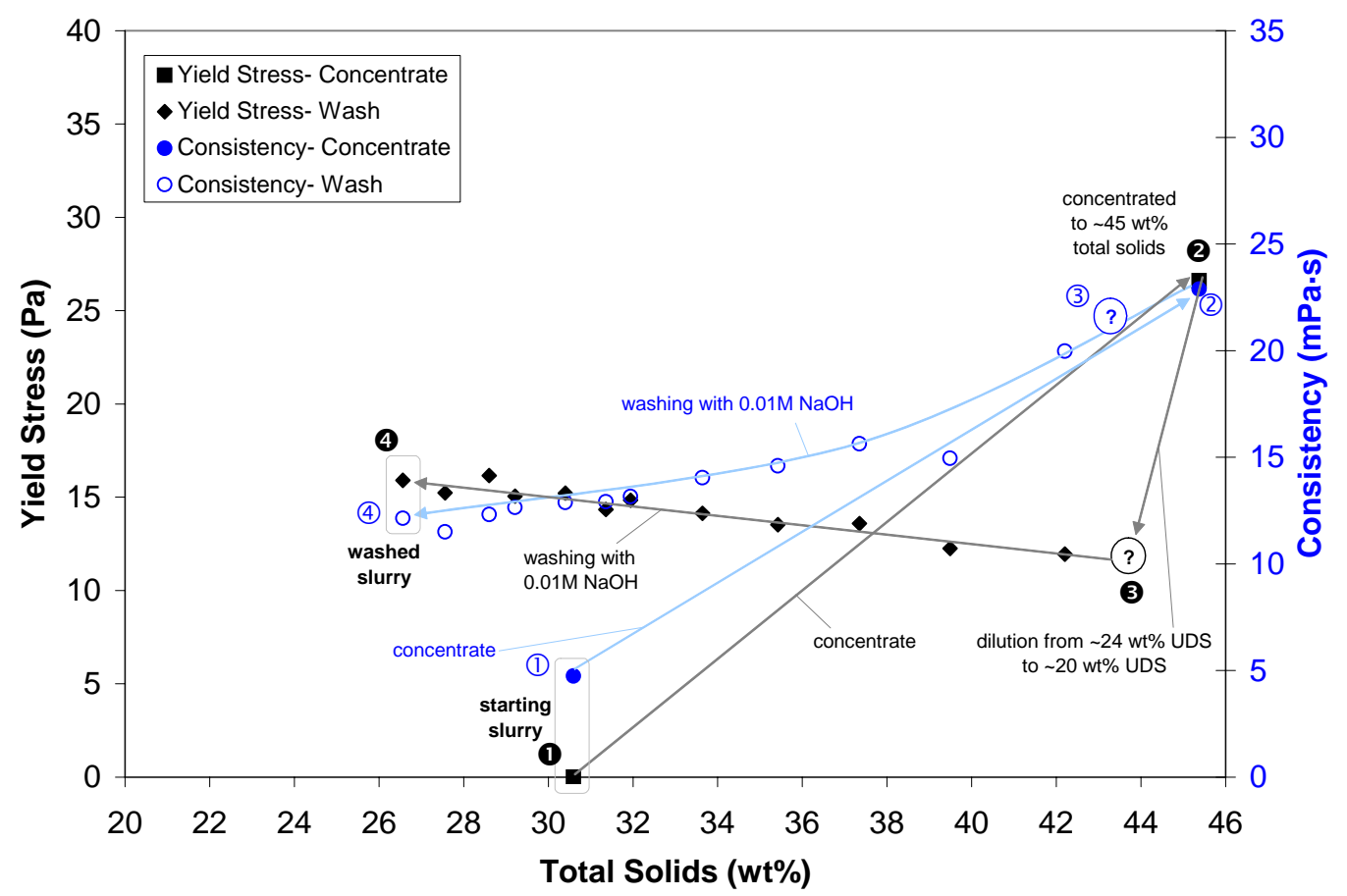

Figure 37. Yield Stress versus Total Solids for Campaign II Filtration 
At this time, the steady-state matrix of filtration conditions was run over the course of about eight hours (Duignan et al., 2004). At the end of this time, the yield stress had decreased to about $25 \mathrm{~Pa}$, while the consistency remained about the same, indicating a possible change in particle size or morphology. The slurry was then diluted back with permeate to about $20 \mathrm{wt} \%$ UDS since it had been over-concentrated to about $24 \mathrm{wt} \%$ UDS (to 3). This dilution significantly reduced the yield stress to about $8 \mathrm{~Pa}$ and slightly reduced the consistency to about $20 \mathrm{mPa} \cdot \mathrm{s}$. Note that the yield stress of the diluted slurry at about $20 \mathrm{wt} \%$ UDS and $33 \mathrm{wt} \%$ total solids is much lower than the yield stress found at the same solids loadings during concentrating up to $\sim 24 \mathrm{wt} \%$. This difference may be due to the apparent difference in particle size or morphology noted above.

Washing initially increased the yield stress slightly, then dropped it to about the same value as at the beginning of washing (3 to (4). During washing, the consistency dropped from 20 $\mathrm{mPa} \cdot \mathrm{s}$ to $9 \mathrm{mPa} \cdot \mathrm{s}$. These data show that the consistency is a function of both the dissolved and undissolved solids contents, while the yield stress is mostly a function of the undissolved solids content.

The Campaign II concentrating step started at a much lower UDS concentration. The total solids was similar to Campaign I, $31 \mathrm{wt} \%$, but the UDS was only $3.3 \mathrm{wt} \%$. This dilute slurry was Newtonian. Concentration to $45 \mathrm{wt} \%$ total solids and $24 \mathrm{wt} \%$ UDS increased the consistency from about $5 \mathrm{mPa} \cdot \mathrm{s}$ to $23 \mathrm{mPa} \cdot \mathrm{s}$ and the yield stress from zero to about $23 \mathrm{~Pa}$ ((0) to 2 ). The Campaign II yield stress of the concentrated slurry, $23 \mathrm{~Pa}$, was less than the Campaign I value of $35 \mathrm{~Pa}$, but was about the same as the Campaign I value after the steady state matrix was performed. The steady-state matrix was not performed in Campaign II.

Dilution with permeate (2 to (3) again reduced the yield stress significantly, going from $23 \mathrm{~Pa}$ to about $10 \mathrm{~Pa}$ (the rheology of the diluted sample was not measured; the likely properties are indicated by the ? on the graph). The consistency did not drop appreciably. Again, it appears that there was a change in particle morphology or size that resulted in a different yield stress after dilution.

Washing increased the yield stress from about 10 to $16 \mathrm{~Pa}$ and reduced the consistency from 23 $\mathrm{mPa} \cdot \mathrm{s}$ to about $11 \mathrm{mPa} \cdot \mathrm{s}$ (3 to $\mathbf{4}$ ). The final washed slurry for Campaign II had both higher yield stress and consistency than for Campaign I.

\subsection{Chemical Composition After Washing}

The results of washing the solids in Campaigns I and II are shown in Table 36. For both campaigns, the compositions of the solids washed are approximately the same since neither the recycle streams, nor the AP101, in Campaign II, contributed significantly to the total solids. These data are compared to previous work done on AY102 simulants at SRNL (Zamecnik, et al., 2003), on the SRNL AY102 radioactive sample (Coleman, 2003), the SRNL radioactive sample processed in the radioactive CUF filter (Poirier, 2003), and literature references (Lumetta, 1996; Brooks, 1997). These reference data are for a combination of washing, leaching, and rewashing, so the results should not be expected to agree since leaching was not done in the SIPP. 
WSRC-TR-2004-00478, REVISION 0

SRNL-RPP-2004-00075, REVISION 0

Table 36. Chemical Compositions After Leaching \& Washing - Percent Retained in Slurry

\begin{tabular}{|c|c|c|c|c|c|c|c|c|c|c|c|c|c|c|}
\hline & \multicolumn{7}{|c|}{ Values Based on Slurry Analyses } & \multicolumn{5}{|c|}{ Values Based on Permeate Analyses } & \multirow{2}{*}{\multicolumn{2}{|c|}{$\begin{array}{c}\text { Literature } \\
\text { Leached \& } \\
\text { Washed } \\
\end{array}$}} \\
\hline & \multicolumn{5}{|c|}{ Leached \& Washed } & \multicolumn{2}{|c|}{ Washed Only } & \multicolumn{3}{|c|}{ Leached \& Washed } & \multicolumn{2}{|c|}{$\begin{array}{c}\text { Washed } \\
\text { Only }\end{array}$} & & \\
\hline & 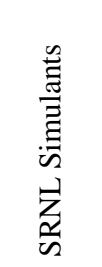 & 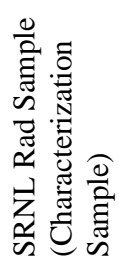 & 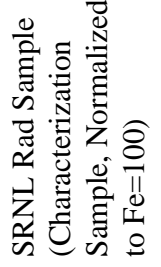 & 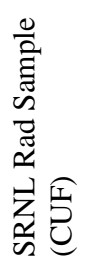 & 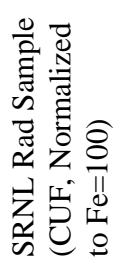 & 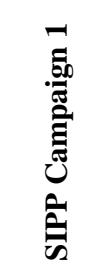 & 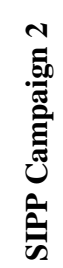 & 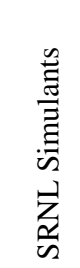 & 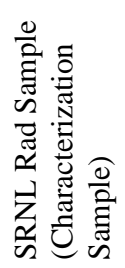 & 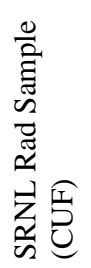 & 总 & 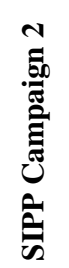 & 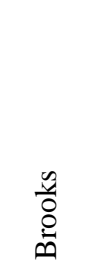 & 芭 \\
\hline $\mathrm{Al}$ & 31 & 54 & 61 & 56 & 63 & 72 & 68 & 53 & 64 & 60 & 74 & 70 & 69 & 53 \\
\hline $\mathrm{Cr}$ & $54-71$ & 74 & 83 & 80 & 90 & 87 & 77 & 62 & 90 & 87 & 85 & 82 & 51 & 68 \\
\hline $\mathrm{Na}$ & $9-28$ & 28 & 31 & 37 & 42 & 20 & 26 & 16 & 48 & 18 & 13 & 19 & & 39 \\
\hline $\begin{array}{r}\mathrm{K} \\
\text { carbonate } \\
\text { nitrate } \\
\text { nitrite } \\
\text { fluoride } \\
\text { oxalate }\end{array}$ & & & & & & $\begin{array}{c}10 \\
4 \\
5 \\
14 \\
11\end{array}$ & \begin{tabular}{l|}
20 \\
19 \\
12 \\
10 \\
28 \\
54
\end{tabular} & & & & $\begin{array}{c}7 \\
0 \\
0 \\
24\end{array}$ & \begin{tabular}{|c|c|}
17 & \\
14 \\
0 \\
0 \\
62 \\
72
\end{tabular} & & \\
\hline $\mathrm{P}$ & $8-11$ & 45 & 51 & 42 & 47 & 46 & 35 & 46 & 64 & 50 & 36 & 28 & 65 & 32 \\
\hline phosphate & & & & & & 13 & 9 & & & & $\mathbf{0}$ & 0 & & \\
\hline sulfate & $28-51$ & 42 & 47 & & & $\begin{array}{c}31 \\
5\end{array}$ & $\begin{array}{l}47 \\
11\end{array}$ & 73 & 51 & & $\begin{array}{l}32 \\
14\end{array}$ & $\begin{array}{l}9 \\
0\end{array}$ & & \\
\hline $\mathrm{Fe}$ & 100 & 89 & 100 & 89 & 100 & 98 & 98 & 100 & 99.2 & 100 & 100 & 100 & 100 & 100 \\
\hline $\mathrm{Mn}$ & $91-100$ & 89 & 100 & 73 & 82 & 100 & 98 & 100 & & 100 & 100 & 100 & $>99$ & $>98$ \\
\hline $\mathrm{Pb}$ & $58-100$ & 96 & 100 & 94 & 100 & 92 & 100 & 99.6 & & 100 & 100 & 100 & $91-92$ & 94 \\
\hline $\mathrm{Ba}$ & 100 & 76 & 85 & & & 100 & 89 & 100 & & 64 & 100 & 100 & $>98$ & $>98$ \\
\hline $\mathrm{Ca}$ & $80-97$ & 94 & 100 & 73 & 82 & 99 & 100 & 100 & & 100 & 100 & 100 & $>96$ & $>95$ \\
\hline $\mathrm{Cd}$ & 100 & 74 & 83 & 56 & 63 & 100 & 85 & 100 & & 100 & 100 & 100 & $>90$ & $>86$ \\
\hline $\mathrm{Cu}$ & $60-100$ & & & 100 & 100 & 100 & 91 & 100 & 100 & 98 & 100 & 100 & $82-84$ & \\
\hline $\mathrm{Mg}$ & 100 & 88 & 99 & 72 & 81 & 98 & 89 & 100 & & 100 & 100 & 100 & $>95$ & $>89$ \\
\hline $\mathrm{Nd}$ & & & & & & 75 & 83 & & & & 100 & 100 & & \\
\hline $\mathrm{Ni}$ & 100 & 90 & 100 & 89 & 100 & 100 & 91 & 100 & & & 100 & 100 & 99 & $>98$ \\
\hline $\mathrm{Si}$ & $75-82$ & & & 97 & 100 & 93 & 92 & 98.7 & 99 & 99.4 & 100 & 100 & & 91 \\
\hline $\mathrm{Sr}$ & 94 & 98 & 100 & & & 93 & 94 & 100 & & 99.6 & 100 & 100 & $>83$ & $>76$ \\
\hline $\mathrm{Zn}$ & $54-100$ & 56 & 63 & 86 & 97 & & 91 & 99.8 & & 93 & 100 & 99 & & $>65$ \\
\hline $\mathrm{Zr}$ & 100 & 81 & 91 & & & 96 & 91 & 99.8 & & & 100 & 100 & $95-97$ & 99 \\
\hline total mass & $46-55$ & & & & & 59 & 53 & & 52 & & & 43 & & \\
\hline
\end{tabular}


The data presented show the percent retained of the given species in the slurry, calculated both from the permeate (permeate) analyses and the leached or leached/washed slurry compositions.

$$
\begin{aligned}
\% \text { Retained (slurry basis) } & =\frac{\text { Amount in Leached/Washed Slurry }}{\text { Amount in Initial Slurry }} \\
\% \text { Retained (permeate basis) } & =\frac{\text { Amount in Initial Slurry - Amount in Permeate }}{\text { Amount in Initial Slurry }}
\end{aligned}
$$

The retention based on the permeate analyses is more accurate since it does not rely on quantifying the amounts in two slurry samples that require dissolutions to be analyzed. The permeate analyses are much more straightforward. For the SRNL radioactive samples, the retention based on the slurry analyses only showed low values for metals such as iron that were retained at $100 \%$ based on the permeate analyses. These data were normalized to an iron retention of $100 \%$, as shown in the table.

For the SIPP work, transition metals (Fe, Mn, Pb, Cd, Cu, Mg, Ni, Si, Zn, Zr), rare earth (Nd), and alkaline earth metals ( $\mathrm{Ca}, \mathrm{Ba}, \mathrm{Sr})$, had amounts retained that were generally greater than $90 \%$, with many such as iron at or near $100 \%$. Most of these results are slightly or somewhat higher than the reference results, which makes sense since no leaching was performed. The more easily washed and leached species were $\mathrm{Al}, \mathrm{Cr}, \mathrm{Na}, \mathrm{K}$, and the anions, which include phosphate and sulfate. The amounts retained for $\mathrm{Al}$ and $\mathrm{Cr}$ in the SIPP were higher than the previous results that included leaching, as expected. The Na retained was similar to the previous simulant work, but again lower than the radioactive sample work. The lower simulant values indicate that the simulants do not contain as much insoluble $\mathrm{Na}$ as the actual waste. Undissolved sodium is present as the carbonate, oxalate, and aluminosilicate in the simulant. Some of the carbonate and oxalate and all of the aluminosilicate is expected to remain.

The aluminum in the simulant is present as an aluminate ion or its hydrate, gibbsite $\mathrm{Al}(\mathrm{OH})_{3}$, alumina $\mathrm{Al}_{2} \mathrm{O}_{3}$, and amorphous aluminosilicate $\mathrm{NaAlSiO}_{4}$ (simplified formula). Without leaching, only the aluminate is expected to be washed out. Some of the chromium was also washed out; chromium is present both as undissolved hydroxide or oxalate and as soluble chromate. Potassium showed washing behavior similar to $\mathrm{Na}$ as expected. The soluble anions (nitrate, nitrite) were essentially all washed out. The fluoride values are inconclusive since the uncertainty in the concentrations was high due to the low concentration. Both oxalate and carbonate were retained at percentages similar to $\mathrm{Na}$, except for the oxalate results for Campaigns II which are higher than expected. The data are not sufficiently conclusive to determine if this is an effect of the recycle stream.

The retention of phosphorus was similar to the reference work. All of the phosphate in the simulant was as added as phosphates; some was added to the metals precipitation to produce metal phosphates and some was added as hydroxyapatite $\mathrm{Ca}_{10}\left(\mathrm{PO}_{4}\right)_{6}(\mathrm{OH})_{2}$, which is very insoluble. The phosphate analysis, which is done by ion chromatography, is a measure of the soluble phosphate. The phosphate analyses showed little or no soluble phosphate in the washed sample, so the P found should be due to the hydroxyapatite. Sulfur was added as sulfate to the 
simulant, and based on the sulfate retention being less than the sulfur $\mathrm{S}$ retention, it appears that relatively insoluble sulfates were formed in the simulant (possibly $\mathrm{PbSO}_{4}, \mathrm{BaSO}_{4}$ or similar). The total mass retention in the SIPP work was similar to that in the simulant studies; no total mass data for the SRNL radioactive work was available. Figure 38 shows the Percent Retained in the slurry values for each species, plotted in a way that the values can be visually compared.

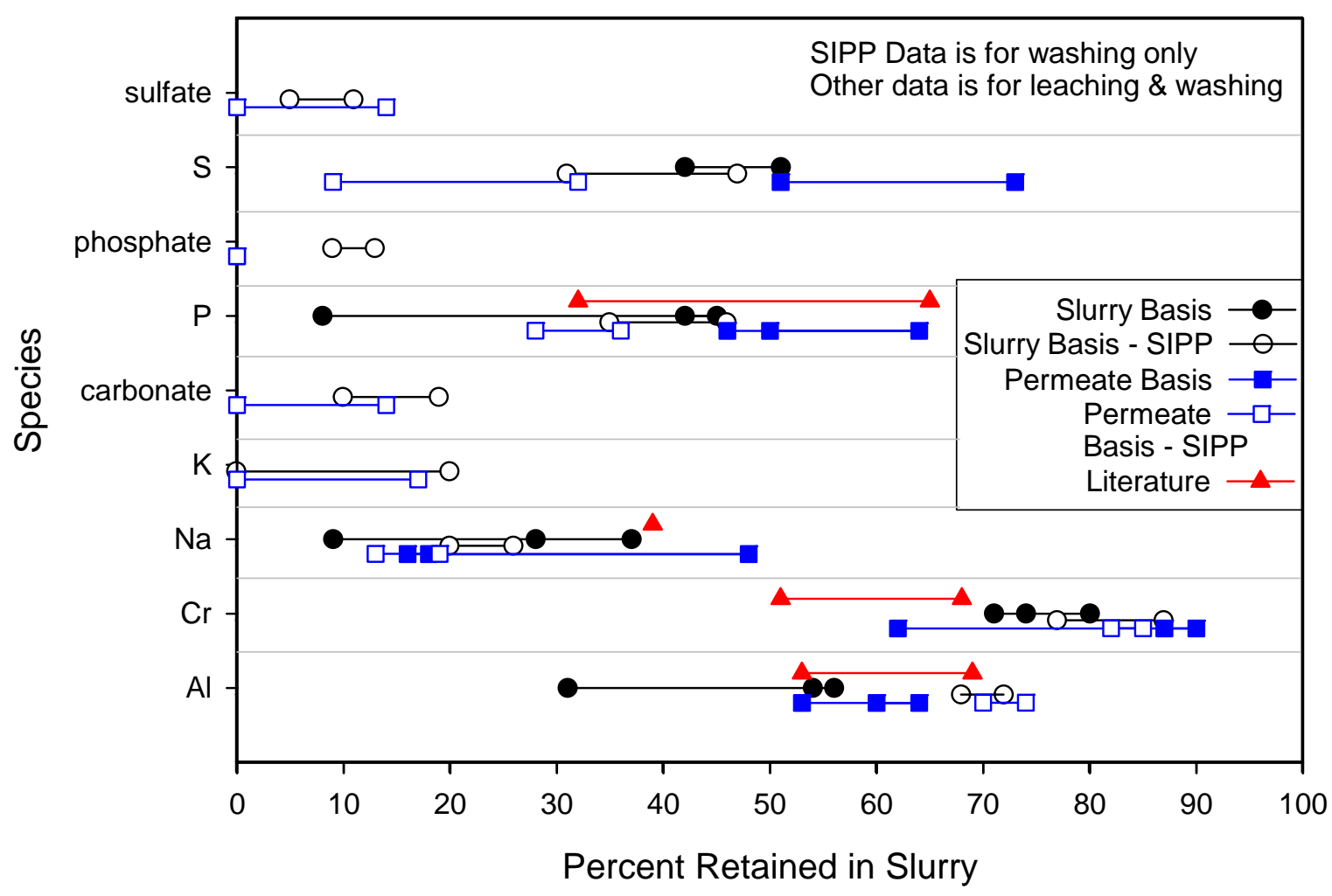

Figure 38. Graphic Representation of Leaching \& Washing Data

\subsection{Slurry Washing Model}

A simple washing model was also used to model the washing data from Campaign II. Forty-four washes were simulated for the given starting concentrations. The amount of insoluble species was adjusted to match the measured data. Figure 39 shows the predicted concentrations of total solids, undissolved solids, and supernate solids along with the measured values. The agreement is generally good. The total solids and UDS values predicted are slightly higher than the measured values, but this may be due to the final volume of washed slurry being slightly greater than the initial volume, that is, more dilute. Figure 40 shows the predicted and measured concentrations of $\mathrm{Na}, \mathrm{Al}, \mathrm{Cr}$, and nitrate that occurred during washing. Figure 41 repeats the data shown in Fig. 40 to highlight just sodium due to its importance to glass formulation. To match the data, the amounts of unwashed ( insoluble) $\mathrm{Na}, \mathrm{Al}$, and $\mathrm{Cr}$ were set to the values shown in Table 37. 
WSRC-TR-2004-00478, REVISION 0

SRNL-RPP-2004-00075, REVISION 0

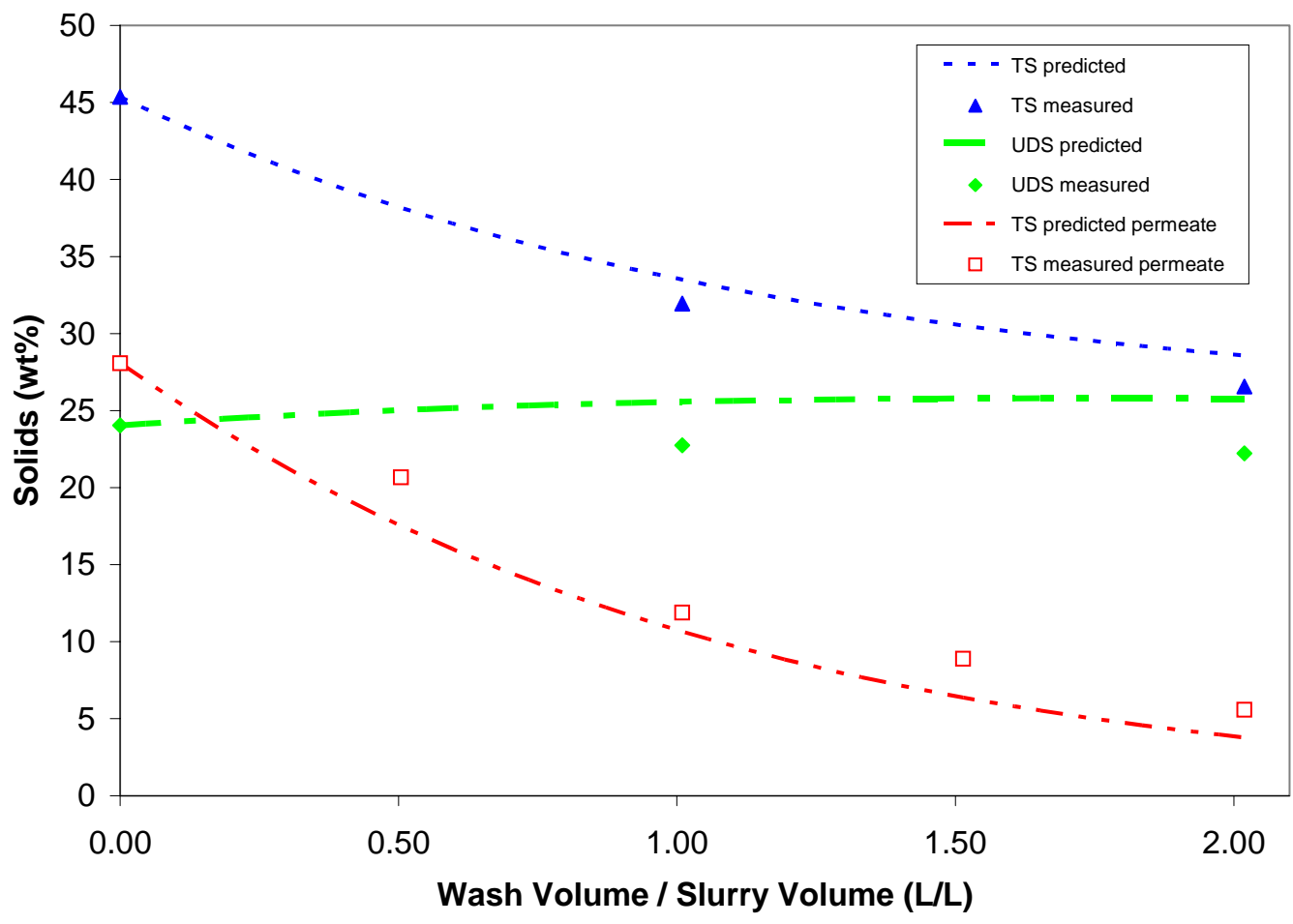

Figure 39. Measured and Predicted Solids Compositions in Washed Slurry and Permeate

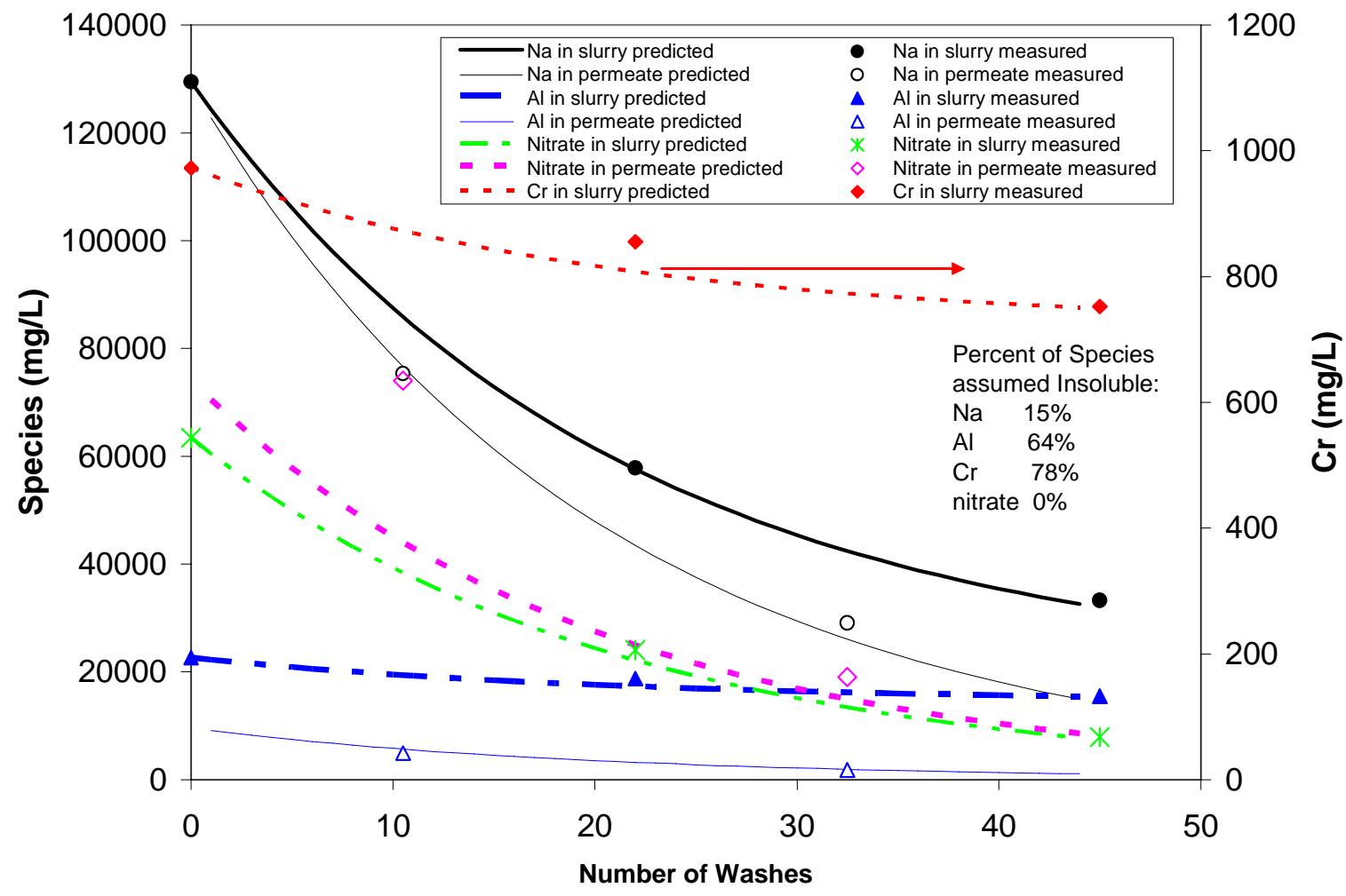

Figure 40. Measured and Predicted Compositions of Selected Species in Washed Slurry and Permeate 
WSRC-TR-2004-00478, REVISION 0

SRNL-RPP-2004-00075, REVISION 0

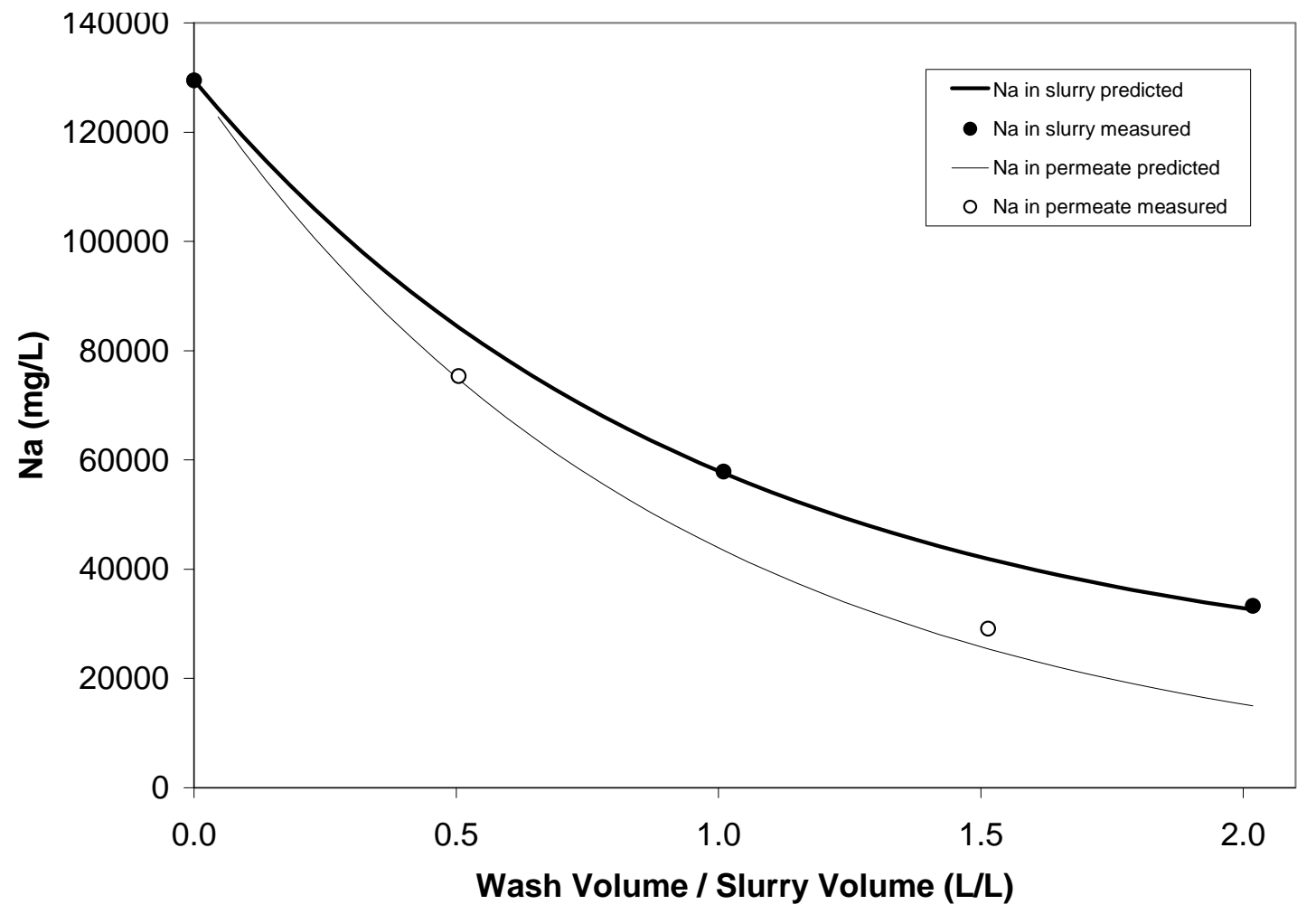

Figure 41. Measured and Predicted Compositions of Just the Sodium in Washed Slurry and Permeate shown in Fig. 40

Table 37. Percent of Several Insoluble Species in Slurry

Percent Retained

\begin{tabular}{|c|cc|}
\hline Species & Model & $\begin{array}{c}\text { From Permeate } \\
\text { Analysis }\end{array}$ \\
\hline $\mathrm{Al}$ & 64 & 70 \\
$\mathrm{Cr}$ & 78 & 82 \\
$\mathrm{Na}$ & 15 & 19 \\
\hline
\end{tabular}




\subsection{Filter Rinsing and Cleaning}

Upon completion of the slurry washing, the filtration unit was drained and then cleaned. Table 38 shows the composition of the rinsing and cleaning solutions. Since the composition of the cleaning solutions from Campaign I were not reported in the interim report of that campaign, they are tabulated in Table 39. The composition of Campaigns I and II solutions are compared in Table 40. Part of each of these solutions became a recycle stream for the next campaign. The first caustic flush in Campaign I was more concentrated than the Campaign II flush, and both were more concentrated than the target UDS of approximately $1.8 \mathrm{wt} \%$. The Campaign I recycles were discussed in the FEP Evaporator section. The caustic flush 1 recycle from Campaign II was higher in solids than the target because the amount of slurry remaining in the UF equipment was greater than expected. The volume of slurry remaining in the filter loop after draining (referred to as holdup) had been estimated at $\sim 5 \mathrm{~L}$, based on previous work with the system, but the actual amount turned out to be about 22L, so the amount of solids left in the system was significantly higher than expected. The first caustic flush was simulated in the following way. First, the filter loop rinse was simulated by adding about 12.5L of RLD condensate. Four liters of the concentrated slurry were then added, and then 94.6L of the 0.1M $\mathrm{NaOH}$ rinse solution was added. If the Ultrafiltration system holdup had been 5L, the resulting solids concentration would have been about $1.8 \mathrm{wt} \%$ UDS. However, with about 22L holdup, the calculated solids is $5.2 \mathrm{wt} \%$ UDS, which agrees well with the measured $5.09 \mathrm{wt} \%$. Therefore, the $\sim 22 \mathrm{~L}$ holdup volume is accurate. 
WSRC-TR-2004-00478, REVISION 0

SRNL-RPP-2004-00075, REVISION 0

Table 38. Campaign II - Measured Composition of Filter Rinsing and Cleaning Solutions

\begin{tabular}{|c|c|c|c|c|c|c|c|c|c|}
\hline All units mg/L unless as noted & $\begin{array}{l}\text { Caustic } \\
\text { Flush } 1\end{array}$ & $\begin{array}{l}\text { Caustic } \\
\text { Flush } 1\end{array}$ & $\begin{array}{c}\text { Acid } \\
\text { Cleaning } 1\end{array}$ & $\begin{array}{c}\text { Acid } \\
\text { Cleaning } 1\end{array}$ & $\begin{array}{c}\text { Acid } \\
\text { Cleaning } 2\end{array}$ & $\begin{array}{c}\text { Acid } \\
\text { Cleaning } 2\end{array}$ & $\begin{array}{c}\text { Acid } \\
\text { Cleaning } 3\end{array}$ & $\begin{array}{l}\text { Caustic } \\
\text { Flush } 2\end{array}$ & $\begin{array}{l}\text { Caustic } \\
\text { Flush } 3\end{array}$ \\
\hline Total Solids (wt\%) & & 7.46 & & 2.36 & & 0.67 & 0.13 & 0.66 & 0.81 \\
\hline Undissolved Solids (wt\%) & & 5.09 & & 0.12 & & 0.00 & 0.00 & 0.13 & 0.13 \\
\hline Supernate Solids (wt\%) & & 2.50 & & 2.24 & & 0.67 & 0.13 & 0.53 & 0.68 \\
\hline Density $(\mathrm{kg} / \mathrm{L})$ & & 1.057 & & 1.051 & & 1.055 & 1.055 & 1.016 & 1.004 \\
\hline Elements from: & Dissolution & Filtered & Dissolution & Filtered & Dissolution & Filtered & Filtered & Filtered & Filtered \\
\hline Ag & 135 & $<0.532$ & 183 & 2.03 & 102 & 0.770 & 1.69 & 0.676 & $<0.6$ \\
\hline $\mathrm{Al}$ & 3615 & 404 & 686 & 476 & 168 & 129 & 36.9 & 12.0 & 3.60 \\
\hline $\mathrm{B}$ & NM & 3.01 & NM & 8.73 & NM & 1.82 & $<0.984$ & $<0.18$ & $<0.18$ \\
\hline $\mathrm{Ba}$ & 123 & $<0.022$ & 21.7 & 22.4 & 6.27 & 5.58 & 1.60 & 0.536 & 0.164 \\
\hline $\mathrm{Ca}$ & 366 & 2.62 & $<5.15$ & 98.3 & $<4.43$ & 30.6 & 9.38 & 8.33 & 7.03 \\
\hline $\mathrm{Cd}$ & 21 & $<0.02$ & 3.64 & 3.70 & $<1.48$ & 0.918 & 0.280 & 0.0758 & $<0.04$ \\
\hline $\mathrm{Ce}$ & $<124$ & $<0.864$ & $<120$ & 26.6 & $<63.3$ & 6.73 & 1.69 & $<17.28$ & $<17.28$ \\
\hline $\mathrm{Co}$ & $<14.2$ & $<0.042$ & $<13.7$ & $<0.042$ & $<3.17$ & $<0.042$ & $<0.042$ & $<0.086$ & 0.102 \\
\hline $\mathrm{Cr}$ & 180 & 12.9 & 35.0 & 34.0 & 6.71 & 8.70 & 2.61 & 0.928 & 0.271 \\
\hline $\mathrm{Cs}$ & $<17.6$ & NM & $<17.1$ & 0.100 & NM & $<0.1$ & $<0.1$ & 0.135 & $<0.1$ \\
\hline $\mathrm{Cu}$ & 38 & $<0.03$ & 8.07 & 6.13 & $<2.22$ & 1.66 & 0.496 & 0.135 & $<0.06$ \\
\hline $\mathrm{Fe}$ & 14766 & $<0.074$ & 2596 & 2490 & 656 & 655 & 181 & 63.4 & 18.4 \\
\hline $\mathrm{K}$ & 1068 & NM & 254 & 272 & NM & 79.3 & 20.9 & 7.66 & 3.01 \\
\hline $\mathrm{La}$ & $<17.7$ & $<0.188$ & $<17.1$ & 21.2 & $<302$ & 5.68 & 1.58 & $<0.376$ & $<0.376$ \\
\hline $\mathrm{Li}$ & $<35.2$ & 2.11 & $<34.2$ & 4.07 & $<13.8$ & 1.09 & 0.318 & $<0.19$ & $<0.19$ \\
\hline $\mathrm{Mg}$ & 164 & 0.0271 & 30.0 & 29.3 & $<7.02$ & 8.80 & 2.72 & 2.03 & 1.49 \\
\hline $\mathrm{Mn}$ & 3198 & $<0.018$ & 552 & 533 & 4.88 & 141 & 39.2 & 13.3 & 3.93 \\
\hline $\mathrm{Mo}$ & $<17.7$ & $<1.78$ & $<17.1$ & $<1.78$ & 142 & $<1.78$ & $<0.3$ & $<0.356$ & $<0.356$ \\
\hline $\mathrm{Na}$ (ICPES) & 10411 & 5860 & 2533 & 2480 & $<132$ & 730 & 213 & 1320 & 1820 \\
\hline $\mathrm{Na}(\mathrm{AA})$ & 10570 & NM & 2470 & 2570 & NM & 766 & 201 & 1380 & 1930 \\
\hline $\mathrm{Na}$ average & 10491 & NC & 2501 & 2525 & $\mathrm{NC}$ & 748 & 207 & 1350 & 1875 \\
\hline $\mathrm{Na}(\mathrm{M})$ & 0.456 & 0.255 & 0.109 & 0.110 & $\mathrm{NC}$ & 0.0325 & 0.009 & 0.0587 & 0.0815 \\
\hline $\mathrm{Nd}$ & $<159$ & $<0.406$ & $<154$ & 61.8 & 621 & 16.3 & 4.50 & 2.01 & $<0.812$ \\
\hline $\mathrm{Ni}$ & 489 & $<0.1$ & 91.4 & 83.7 & $<30.1$ & 21.3 & 6.23 & 2.12 & 0.703 \\
\hline $\mathrm{P}$ & $<142$ & 19.3 & $<137$ & 6.25 & 21.9 & 3.82 & 1.49 & $<1.246$ & $<1.246$ \\
\hline $\mathrm{Pb}$ & 698 & $<1.7$ & $<137$ & 121 & $<45.6$ & 29.5 & 8.82 & 2.82 & $<1.6$ \\
\hline $\mathrm{S}$ & $<175$ & 43.5 & $<170$ & 16.4 & $<124$ & 5.01 & 2.50 & $<2.078$ & $<2.078$ \\
\hline Si & 3319 & 18.6 & $<1240$ & 247 & $<76$ & 89.3 & 26.3 & 18.4 & 15.4 \\
\hline $\mathrm{Sr}$ & 114 & 0.0184 & 20.6 & 21.7 & $<1400$ & 5.84 & 1.65 & $<0.87$ & $<0.87$ \\
\hline $\mathrm{Zn}$ & 39 & $<0.05$ & 5.04 & 7.00 & $<3.69$ & 2.18 & 0.617 & 0.261 & 0.102 \\
\hline $\mathrm{Zr}$ & 202 & $<0.112$ & 25.5 & 12.9 & 20.1 & 6.59 & 2.25 & $<0.16$ & $<0.16$ \\
\hline
\end{tabular}


WSRC-TR-2004-00478, REVISION 0 SRNL-RPP-2004-00075, REVISION 0

\begin{tabular}{|c|c|c|c|c|c|c|}
\hline Table 38 continued & $\begin{array}{c}\text { Caustic Flush } 1 \\
\text { Filtered }\end{array}$ & $\begin{array}{c}\text { Acid Cleaning } 1 \\
\text { Filtered }\end{array}$ & $\begin{array}{c}\text { Acid Cleaning } 2 \\
\text { Filtered }\end{array}$ & $\begin{array}{c}\text { Acid } \\
\text { Cleaning } 3 \\
\text { Filtered }\end{array}$ & $\begin{array}{l}\text { Caustic } \\
\text { Flush } 2 \\
\text { Filtered }\end{array}$ & $\begin{array}{l}\text { Caustic } \\
\text { Flush } 3 \\
\text { Filtered }\end{array}$ \\
\hline bromide & $<10$ & & & & $<10$ & $<10$ \\
\hline chloride & 37.0 & & & & $<2$ & $<2$ \\
\hline fluoride & 7.40 & & & & $<2$ & $<2$ \\
\hline formate & 57.1 & & & & $<10$ & $<10$ \\
\hline nitrate & 9217 & & & & 31100 & 8900 \\
\hline nitrite & 774 & & & & $<10$ & $<10$ \\
\hline oxalate & 4545 & & & & $<10$ & $<10$ \\
\hline phosphate & 60.2 & & & & $<10$ & $<10$ \\
\hline sulfate & 116 & & & & $<5$ & $<5$ \\
\hline Total Acid (M) & & 1.20 & 1.61 & 1.74 & 0.449 & 0.0623 \\
\hline
\end{tabular}


WSRC-TR-2004-00478, REVISION 0

SRNL-RPP-2004-00075, REVISION 0

Table 39. Campaign I - Measured Composition of Filter Rinsing and Cleaning Solutions

\begin{tabular}{|c|c|c|c|c|c|c|c|c|c|}
\hline \multicolumn{4}{|c|}{ Caustic Flush } & $\begin{array}{l}\text { Acid Cleaning } \\
2\end{array}$ & $\begin{array}{c}\text { Acid } \\
\text { Cleaning } 3\end{array}$ & $\begin{array}{l}\text { Acid Cleaning } \\
\text { Composite }\end{array}$ & $\begin{array}{l}\text { Caustic } \\
\text { Flush } 2 \\
\end{array}$ & \multicolumn{2}{|c|}{$\begin{array}{cc}\text { Caustic Flush Caustic Flushes } \\
3 & 2+3 \text { Composite } \\
\end{array}$} \\
\hline Total Solids wt\% & 9.71 & 1.07 & & 0.18 & 0.05 & 0.43 & 0.83 & 0.76 & 0.75 \\
\hline Undissolved Solids wt\% & 8.27 & 0.09 & & 0 & 0 & 0.09 & 0 & 0 & 0 \\
\hline Supernate Solids wt\% & 1.57 & 0.98 & & 0.18 & 0.05 & 0.34 & 0.83 & 0.760 & 0.75 \\
\hline Density kg/L & 1.075 & 1.056 & & 1.062 & 1.063 & 1.060 & 1.009 & 1.003 & 1.006 \\
\hline $\mathrm{pH}$ & 12.7 & & & & & & 0.80 & 11.2 & 1.04 \\
\hline Elements from: & Dissolution & Permeate & $\begin{array}{l}\text { Dissolution of } \\
\text { Filtered Solids }\end{array}$ & Permeate & Permeate & Dissolution & & & Dissolution \\
\hline $\mathrm{Ag}$ & 441 & 12.8 & 2280 & 2.82 & 0.73 & 22.3 & & & $<15$ \\
\hline $\mathrm{Al}$ & 6477 & 256 & 38600 & 42.0 & 8.00 & 320 & & & $<25$ \\
\hline B & 53.8 & 4.00 & & $<1$ & $<0.12$ & 0 & & & 0 \\
\hline $\mathrm{Ba}$ & 205 & 11.4 & 20 & 1.73 & 0.32 & 9.54 & & & $<1$ \\
\hline $\mathrm{Ca}$ & 994 & 37.7 & 28 & 6.14 & 0.16 & 33.9 & & & $<1$ \\
\hline $\mathrm{Cd}$ & 26.9 & 1.35 & 19 & 0.239 & 0.041 & 2.12 & & & $<1$ \\
\hline $\mathrm{Ce}$ & 231 & 11.6 & $<660$ & 1.82 & $<1.4$ & $<35$ & & & $<35$ \\
\hline Co & 32.3 & 0.377 & $<75$ & $<0.16$ & $<0.16$ & $<4.2$ & & & $<4$ \\
\hline $\mathrm{Cr}$ & 324 & 16.0 & 156 & 3.00 & 1.00 & 15.9 & & & $<2$ \\
\hline Cs & 0.683 & & & & 0.12 & 0.127 & & & \\
\hline $\mathrm{Cu}$ & 53.8 & 2.40 & $<30$ & 0.505 & 0.11 & 2.12 & & & $<1$ \\
\hline $\mathrm{Fe}$ & 23220 & 1050 & 2920 & 200 & 40.0 & 1082 & & & 9 \\
\hline K (AA) & 42.1 & 5.00 & & 1.00 & 0.17 & $<7.5$ & & & $<7.4$ \\
\hline $\mathrm{La}$ & 195 & 8.91 & $<95$ & 1.60 & 0.26 & 7.42 & & & $<5$ \\
\hline $\mathrm{Li}$ & $<35.5$ & $<0.4$ & $<190$ & $<0.4$ & $<0.4$ & $<9.5$ & & & $<10$ \\
\hline $\mathrm{Mg}$ & 247 & 10.9 & 151 & 2.02 & 0.38 & 10.6 & & & 0.25 \\
\hline Mn & 5139 & 219 & 142 & 42.0 & 8.00 & 225 & & & 2 \\
\hline Mo & $<17.2$ & $<0.2$ & $<95$ & $<0.2$ & $<0.2$ & 5.30 & & & $<5$ \\
\hline $\mathrm{Na}$ (ICPES) & 9976 & 1230 & 832 & 224 & 41.0 & 1315 & & & 2110 \\
\hline $\mathrm{Na}(\mathrm{AA})$ & 9772 & 1200 & & 206 & 40.2 & 1251 & & & 2070 \\
\hline Na (average) & 9874 & 1215 & & 215 & 41 & 1283 & & & 2090 \\
\hline $\mathrm{Na}(\mathrm{M})$ & 0.353 & 0.032 & & 0.0054 & 0.0011 & 0.034 & & & 0.09 \\
\hline $\mathrm{Nd}$ & 558 & 25.6 & $<845$ & 4.65 & $<1.8$ & $<44.5$ & & & $<45$ \\
\hline $\mathrm{Ni}$ & 766 & 33.0 & 289 & 6.00 & 1.00 & 37.1 & & & 3 \\
\hline $\mathrm{P}$ & 377 & 17.0 & 11300 & 5.00 & 2.00 & $<40.3$ & & & $<40$ \\
\hline $\mathrm{Pb}$ & 1091 & 47.0 & $<750$ & 9.00 & $<1.6$ & $<40.3$ & & & $<40$ \\
\hline $\mathrm{S}$ & 280 & 8.47 & 1160 & $<2$ & $<2$ & $<49.8$ & & & $<50$ \\
\hline Si & 7552 & 98.0 & 236000 & 20.0 & 5.00 & 931 & & & 722 \\
\hline $\mathrm{Sr}$ & 199 & 9.00 & 3 & 4.00 & 1.0 & 8.48 & & & $<1$ \\
\hline $\mathrm{Zn}$ & 194 & 3.61 & 33 & 0.643 & 0.094 & 2.12 & & & 1 \\
\hline $\mathrm{Zr}$ & 321 & 0.246 & 15647 & 1.83 & 0.97 & 13.8 & & & 1 \\
\hline
\end{tabular}


WSRC-TR-2004-00478, REVISION 0

SRNL-RPP-2004-00075, REVISION 0

\begin{tabular}{|c|c|c|c|c|c|c|c|c|}
\hline Table 39 continued & $\begin{array}{l}\text { Caustic } \\
\text { Flush } 1\end{array}$ & $\begin{array}{c}\text { Acid } \\
\text { Cleaning } 1\end{array}$ & $\begin{array}{c}\text { Acid } \\
\text { Cleaning } 2\end{array}$ & $\begin{array}{c}\text { Acid } \\
\text { Cleaning } 3\end{array}$ & $\begin{array}{l}\text { Acid Cleaning } \\
\text { Composite }\end{array}$ & $\begin{array}{l}\text { Caustic } \\
\text { Flush } 2\end{array}$ & $\begin{array}{l}\text { Caustic } \\
\text { Flush } 3\end{array}$ & $\begin{array}{l}\text { Caustic Flushes } \\
2+3 \text { Composite }\end{array}$ \\
\hline & Permeate & & & & & & & Permeate \\
\hline bromide & $<79$ & & & & & & & $<100$ \\
\hline chloride & 12.0 & & & & & & & $<20$ \\
\hline fluoride & 7.00 & & & & & & & $<20$ \\
\hline formate & 11.0 & & & & & & & $<100$ \\
\hline nitrate & 23.0 & & & & & & & 12500 \\
\hline nitrite & 198 & & & & & & & $<100$ \\
\hline oxalate & 1400 & & & & & & & $<100$ \\
\hline phosphate & 328 & & & & & & & $<100$ \\
\hline sulfate & 98.0 & & & & & & & $<50$ \\
\hline Total Acid M & & 1.56 & 1.95 & 1.98 & & & & \\
\hline
\end{tabular}


WSRC-TR-2004-00478, REVISION 0

SRNL-RPP-2004-00075, REVISION 0

Table 40. Comparison of Campaigns I \& II Cleaning Solutions

All values mgL except as noted $\quad$ Caustic Flush $1 \quad$ Acid Cleaning 1 Acid Cleaning 2 Acid Cleaning $3 \quad$ Caustic Flush $2 \quad$ Caustic Flush 3

\begin{tabular}{|c|c|c|c|c|c|c|c|c|c|c|c|c|}
\hline Campaign: & II & I & II & $\mathrm{I}$ & \multicolumn{2}{|c|}{ II $\quad$ I } & \multicolumn{2}{|l|}{ II } & II & $\mathrm{I}$ & $\mathrm{I}$ & II \\
\hline Total Solids (wt\%) & 7.46 & 9.71 & 2.36 & 1.07 & 0.67 & 0.18 & 0.13 & 0.05 & 0.66 & 0.83 & 0.76 & 0.81 \\
\hline Undissolved Solids (wt\%) & 5.09 & 8.27 & 0.12 & 0.09 & 0 & 0 & 0 & 0 & 0.13 & 0 & 0 & 0.13 \\
\hline Supernate Solids (wt\%) & 2.50 & 1.57 & 2.24 & 0.98 & 0.67 & 0.18 & 0.13 & 0.05 & 0.53 & 0.83 & 0.76 & 0.68 \\
\hline Density $(\mathrm{kg} / \mathrm{L})$ & 1.057 & 1.075 & 1.051 & 1.056 & 1.055 & 1.062 & 1.055 & 1.063 & 1.016 & 1.009 & 1.003 & 1.004 \\
\hline Elements from: & \multicolumn{2}{|c|}{ Dissolution } & \multicolumn{2}{|c|}{ Permeate } & \multicolumn{2}{|c|}{ Permeate } & \multicolumn{2}{|c|}{ Permeate } & Permeate & \multicolumn{2}{|c|}{ Dissolution } & Filtered \\
\hline Ag & 135 & 441 & 2.03 & 12.8 & 0.77 & 2.82 & 1.69 & 0.73 & 0.68 & \multicolumn{2}{|c|}{$<15$} & $<0.6$ \\
\hline $\mathrm{Al}$ & 3615 & 6477 & 476 & 256 & 129 & 42.0 & 36.9 & 8.00 & 12.0 & \multicolumn{2}{|c|}{$<25$} & 3.60 \\
\hline B & & 53.8 & 8.73 & 4.00 & 1.82 & $<1$ & $<0.98$ & $<0.12$ & $<0.18$ & & & $<0.18$ \\
\hline $\mathrm{Ba}$ & 123 & 205 & 22.4 & 11.4 & 5.58 & 1.73 & 1.60 & 0.32 & 0.54 & \multicolumn{2}{|c|}{$<1$} & 0.16 \\
\hline $\mathrm{Ca}$ & 366 & 994 & 98.3 & 37.7 & 30.6 & 6.14 & 9.38 & 0.16 & 8.33 & \multicolumn{2}{|c|}{$<1$} & 7.03 \\
\hline $\mathrm{Cd}$ & 21.0 & 26.9 & 3.70 & 1.35 & 0.92 & 0.24 & 0.28 & 0.04 & 0.08 & \multicolumn{2}{|c|}{$<1$} & $<0.04$ \\
\hline $\mathrm{Ce}$ & $<124$ & 231 & 26.6 & 11.6 & 6.73 & 1.82 & 1.69 & $<1.4$ & $<17$ & \multicolumn{2}{|c|}{$<35$} & $<17.3$ \\
\hline Co & $<14.2$ & 32.3 & $<0.04$ & 0.38 & $<0.04$ & $<0.16$ & $<0.04$ & $<0.16$ & $<0.09$ & \multicolumn{2}{|c|}{$<4$} & 0.10 \\
\hline $\mathrm{Cr}$ & 180 & 324 & 34.0 & 16.0 & 8.70 & 3.00 & 2.61 & 1.00 & 0.93 & \multicolumn{2}{|c|}{$<2$} & 0.27 \\
\hline Cs & $<17.6$ & 0.68 & 0.10 & & $<0.1$ & & $<0.1$ & 0.12 & 0.14 & & $<0.1$ \\
\hline $\mathrm{Cu}$ & 38.0 & 53.8 & 6.13 & 2.40 & 1.66 & 0.50 & 0.50 & 0.11 & 0.14 & \multicolumn{2}{|c|}{$<1$} & $<0.06$ \\
\hline $\mathrm{Fe}$ & 14766 & 23220 & 2490 & 1050 & 655 & 200 & 181 & 40.0 & 63.4 & \multicolumn{2}{|c|}{9.00} & 18.4 \\
\hline $\mathrm{K}$ & 1068 & 42.1 & 272 & 5.00 & 79.3 & 1.00 & 20.9 & 0.17 & 7.66 & \multicolumn{2}{|c|}{$<7.4$} & 3.01 \\
\hline $\mathrm{La}$ & $<17.7$ & 195 & 21.2 & 8.91 & 5.68 & 1.60 & 1.58 & 0.26 & $<0.38$ & \multicolumn{2}{|c|}{$<5$} & $<0.37$ \\
\hline $\mathrm{Li}$ & $<35.2$ & $<35.5$ & 4.07 & $<0.4$ & 1.09 & $<0.4$ & 0.32 & $<0.4$ & $<0.19$ & & & $<0.19$ \\
\hline $\mathrm{Mg}$ & 164 & 247 & 29.3 & 10.9 & 8.80 & 2.02 & 2.72 & 0.38 & 2.03 & & & 1.49 \\
\hline $\mathrm{Mn}$ & 3198 & 5139 & 533 & 219 & 141 & 42.0 & 39.2 & 8.00 & 13.3 & & & 3.93 \\
\hline Mo & $<17.7$ & $<17.2$ & $<1.78$ & $<0.2$ & $<1.78$ & $<0.2$ & $<0.3$ & $<0.2$ & $<0.36$ & & & $<0.35$ \\
\hline Na average & 10491 & 9874 & 2525 & 1215 & 748 & 215 & 207 & 41.0 & 1350 & & & 1875 \\
\hline $\mathrm{Nd}$ & $<159$ & 558 & 61.8 & 25.6 & 16.3 & 4.65 & 4.50 & $<1.8$ & 2.01 & & & $<0.81$ \\
\hline $\mathrm{Ni}$ & 489 & 766 & 83.7 & 33.0 & 21.3 & 6.00 & 6.23 & 1.00 & 2.12 & & & 0.70 \\
\hline $\mathrm{P}$ & $<142$ & 377 & 6.25 & 17.0 & 3.82 & 5.00 & 1.49 & 2.00 & $<1.25$ & & & $<1.24$ \\
\hline $\mathrm{Pb}$ & 698 & 1091 & 121 & 47.0 & 29.5 & 9.00 & 8.82 & $<1.6$ & 2.82 & & & $<1.6$ \\
\hline $\mathrm{S}$ & $<175$ & 280 & 16.4 & 8.47 & 5.01 & $<2$ & 2.50 & $<2$ & $<2.08$ & & & $<2.08$ \\
\hline $\mathrm{Si}$ & 3319 & 7552 & 247 & 98.0 & 89.3 & 20.0 & 26.3 & 5.00 & 18.4 & & & 15.4 \\
\hline $\mathrm{Sr}$ & 114 & 199 & 21.7 & 9.00 & 5.84 & 4.00 & 1.65 & 1.00 & $<0.87$ & & & $<0.87$ \\
\hline $\mathrm{Zn}$ & 39.0 & 194 & 7.00 & 3.61 & 2.18 & 0.64 & 0.62 & 0.09 & 0.26 & & & 0.102 \\
\hline $\mathrm{Zr}$ & 202 & 321 & 12.9 & 0.24 & 6.59 & 1.83 & 2.25 & 0.97 & $<0.16$ & & & $<0.16$ \\
\hline Total Acid (M) & & & 1.20 & 1.56 & 1.61 & 1.95 & 1.74 & 1.98 & 0.45 & & & 0.06 \\
\hline
\end{tabular}


Figure 42 and Fig. 43 are pictures of the cleaning solutions from Campaigns I and II, respectively. The first caustic flush contained the majority of the undissolved solids. The UDS in the acid cleaning solutions is expected to be low since the acid dissolved many of the undissolved species. The Campaign II acid cleaning solution 1 contained more brown (sludge) undissolved solids than the Campaign I solution; both contained fluffy whitish solids. The Campaign I material had very little color, while the Campaign II solution had some yellowish color. Figure 44 shows an XRD spectrum of solids filtered from the Campaign I solution. The major components are an alumina (corundum), silica (quartz), and what was identified as diaoyudaoite $\left(\mathrm{NaAl}_{11} \mathrm{O}_{17}\right)$. Table 41 shows the approximate composition of the filtered solids. This composition was determined by dissolving a filter paper with the solids and a filter paper that had contacted the liquid portion only. The concentrations in the liquid only filter paper were subtracted off as a blank. The main components in the elemental analysis agreed with the XRD analysis: Al and Si. Little Na was found, while Ag, Fe, P, S, and Zr were found in relatively significant amounts.

Table 41. Measured Composition of the Filtered Solids

\begin{tabular}{|ccc|} 
Element & $\begin{array}{c}\text { Sample - Blank } \\
(\mathrm{mg} / \mathrm{kg})\end{array}$ & $\begin{array}{c}\text { Sample - Blank } \\
(\mathrm{mmol} / \mathrm{kg})\end{array}$ \\
\hline $\mathrm{Ag}$ & 2280 & 21.1 \\
\hline $\mathrm{Al}$ & 38600 & 1431 \\
\hline $\mathrm{Ba}$ & 20.0 & 0.146 \\
$\mathrm{Ca}$ & 28.0 & 0.699 \\
\hline $\mathrm{Cd}$ & 19.0 & 0.169 \\
\hline $\mathrm{Cr}$ & 156 & 3.00 \\
\hline $\mathrm{Fe}$ & 2920 & 52.3 \\
\hline $\mathrm{Mg}$ & 151 & 6.21 \\
\hline $\mathrm{Mn}$ & 142 & 2.58 \\
\hline $\mathrm{Na}$ & 832 & 36.2 \\
\hline $\mathrm{Ni}$ & 289 & 4.92 \\
\hline $\mathrm{P}$ & 11300 & 365 \\
\hline $\mathrm{S}$ & 1160 & 36.2 \\
\hline $\mathrm{Si}$ & 236000 & 8403 \\
\hline $\mathrm{Sr}$ & 3.00 & 0.0342 \\
\hline $\mathrm{Ti}$ & 108 & 2.25 \\
\hline $\mathrm{Zn}$ & 33.0 & 0.505 \\
\hline $\mathrm{Zr}$ & 15647 & 172 \\
\hline
\end{tabular}

The Campaign I and II first acid cleaning solutions contained about the same concentration of undissolved solids, but the Campaign II material contained about twice the dissolved solids than the Campaign I solution, which is consistent with the fact that the Campaign II solution contained more undissolved solids than the Campaign I slurry. The small UDS values of around 0.13 in acid solutions 2 and 3 are so close to zero that the presence of undissolved solids is not conclusive. Visibly, there were slight solids in both second cleaning solutions, and no visible solids in the third cleaning solutions. 


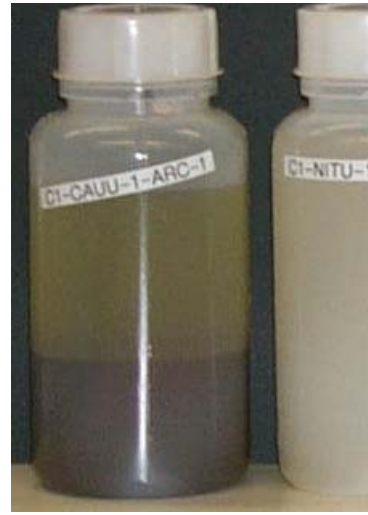

(a) (b)

(a) first caustic rinse

(b) first $2 \mathrm{M}$ nitric cleaning

(c) second $2 \mathrm{M}$ nitric cleaning

(d) third $2 \mathrm{M}$ nitric cleaning

(e) second caustic rinse

(f) third caustic rinse

(b-f) have non-colored supernate

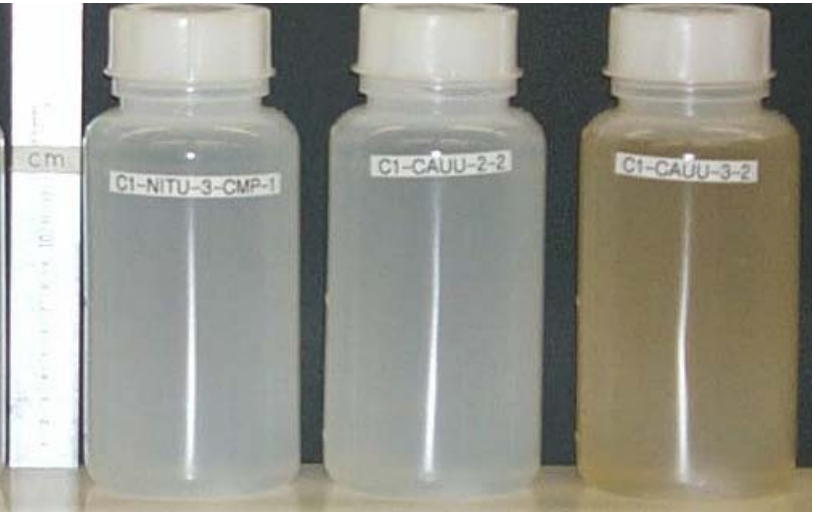

(d)

(e)

(f)

Figure 42. Campaign I Ultrafilter Rinsing and Cleaning Solutions

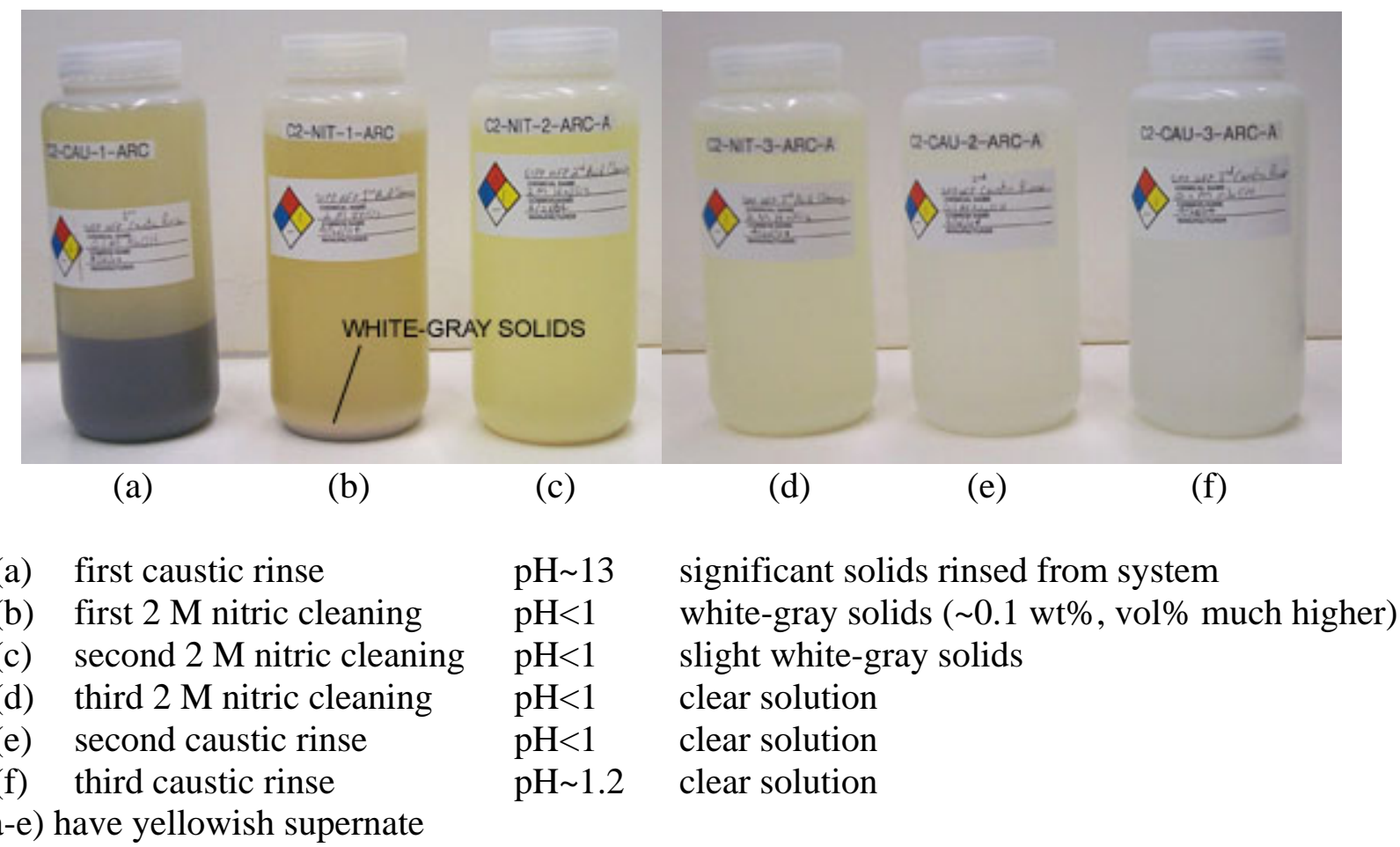

Figure 43. Campaign II Ultrafiltration Rinsing and Cleaning Solutions (Fig. 30 repeated) 


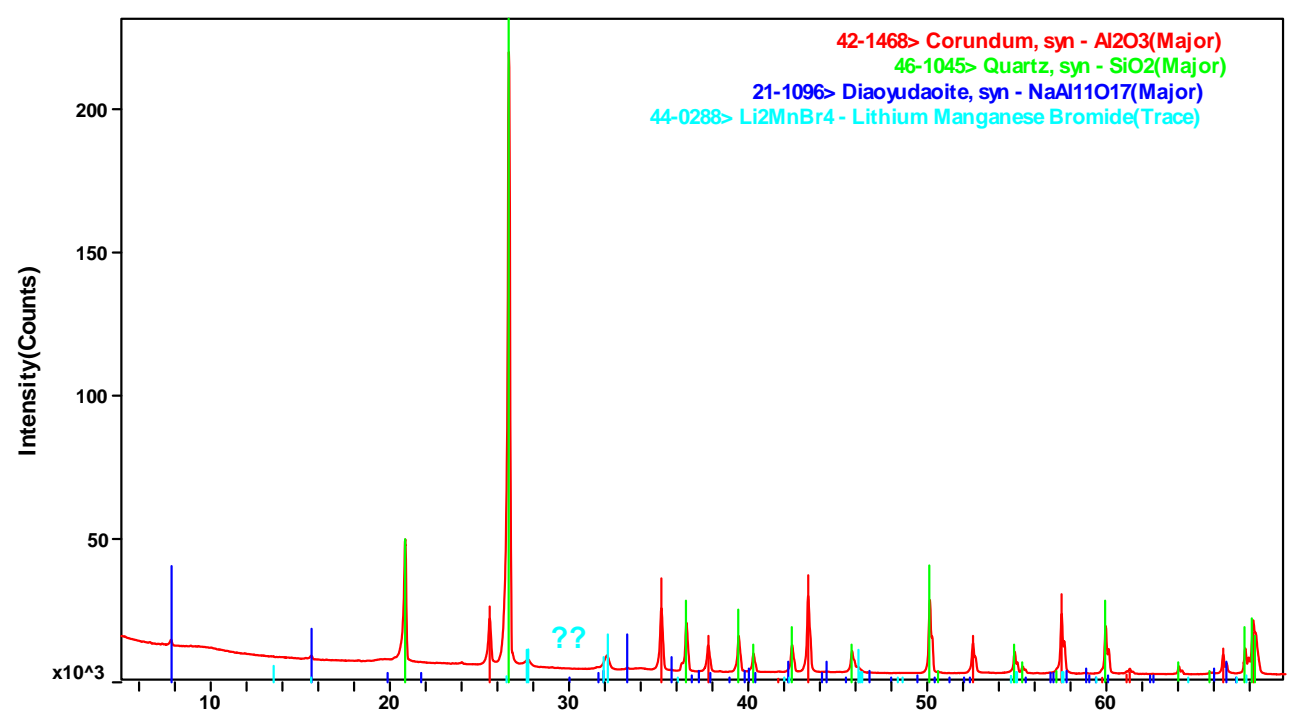

Figure 44. XRD Spectrum of Solids from Campaign I First Nitric Acid Cleaning Solution

The third caustic rinse in Campaign I contained a small amount of brownish solids, which appear to possibly be precipitated iron. This precipitation on the third caustic rinse has been observed in previous RPP Filtration Work. The Campaign II third rinse did not contain these solids. In Campaign II, the third caustic rinse had a total acid concentration of $0.0623 \mathrm{M}$, or a pH of about 1.2. It appears that because the $\mathrm{pH}$ remained acidic, precipitation such as that seen in Campaign I was not seen in Campaign II. It was expected that this sample would be slightly caustic with a $\mathrm{pH}$ of around 12, as was seen in Campaign I. The cleaning steps were conducted with prototypic ratios of cleaning solution to heel, so the low final $\mathrm{pH}$ was a surprise. Use of a $0.2 \mathrm{M} \mathrm{NaOH}$, or a larger quantity of $0.1 \mathrm{M} \mathrm{NaOH}$, may be prudent for the last rinse so that the final rinsing heel is caustic. The acidic heel has the potential to cause unwanted reactions with the next batch of waste feed (this was not studied as no heel was left between campaigns).

\subsection{Particle Size Distribution of Ultrafiltration Samples}

Particle size distributions of both Campaign I and II samples were measured with a Microtrac S3000 particle size analyzer. Figure 45 shows the particle size distributions of Campaign I slurry for the following conditions: initial (feed), after concentrating, after running a steady-state matrix (SSM) of processing conditions (additional processing), and after over-concentration (additional processing). The slurry was initially unimodal, but became bimodal during processing. Further processing continued to reduce the particle size. Note that the Campaign I slurry is AY102 simulant only. 
WSRC-TR-2004-00478, REVISION 0

SRNL-RPP-2004-00075, REVISION 0

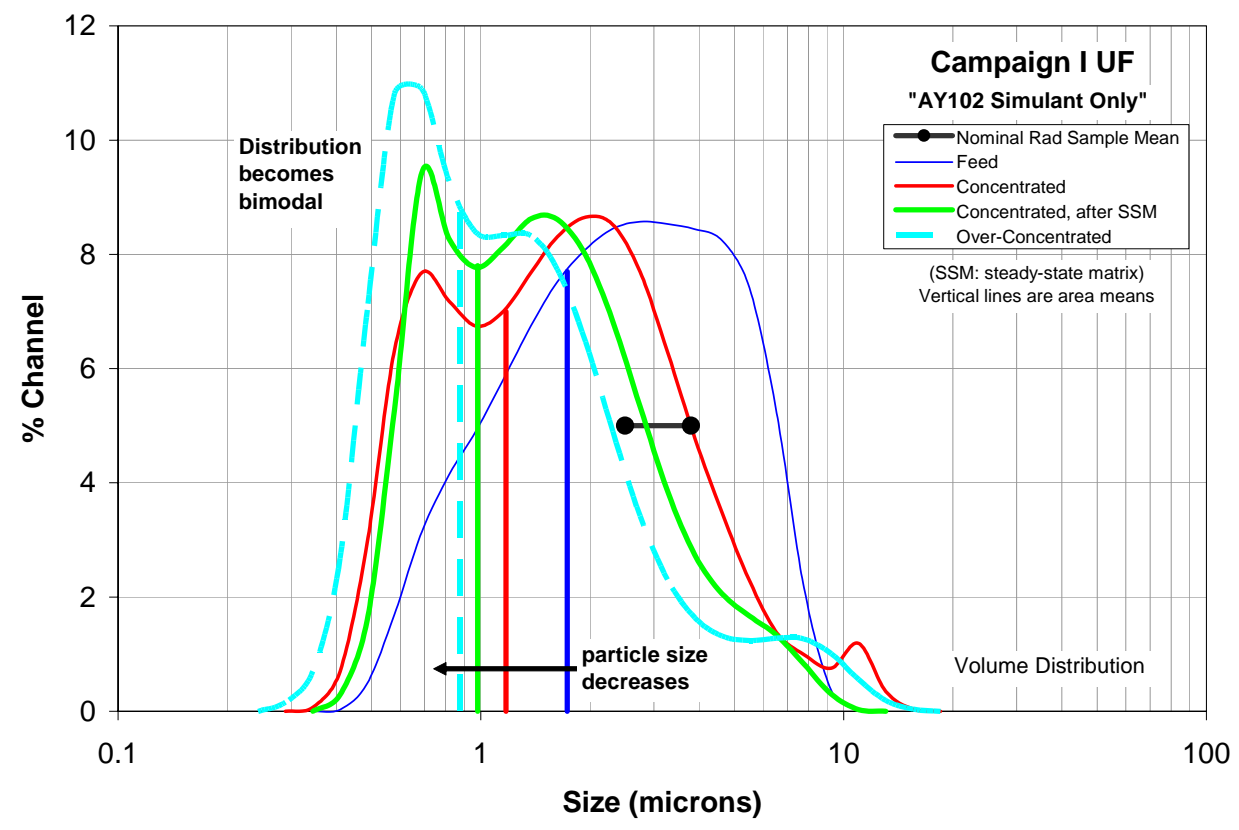

Figure 45. Particle Size Distribution of Campaign I AY102 Simulant

The changes in particle size distribution due to slurry washing are shown in Fig. 46. The area mean particle size does not change, but the distribution changes such that the relative amount of the smaller particles $(\sim 0.7 \mu \mathrm{m})$ decreases and the larger particles $(\sim 1.5 \mu \mathrm{m})$ increases.

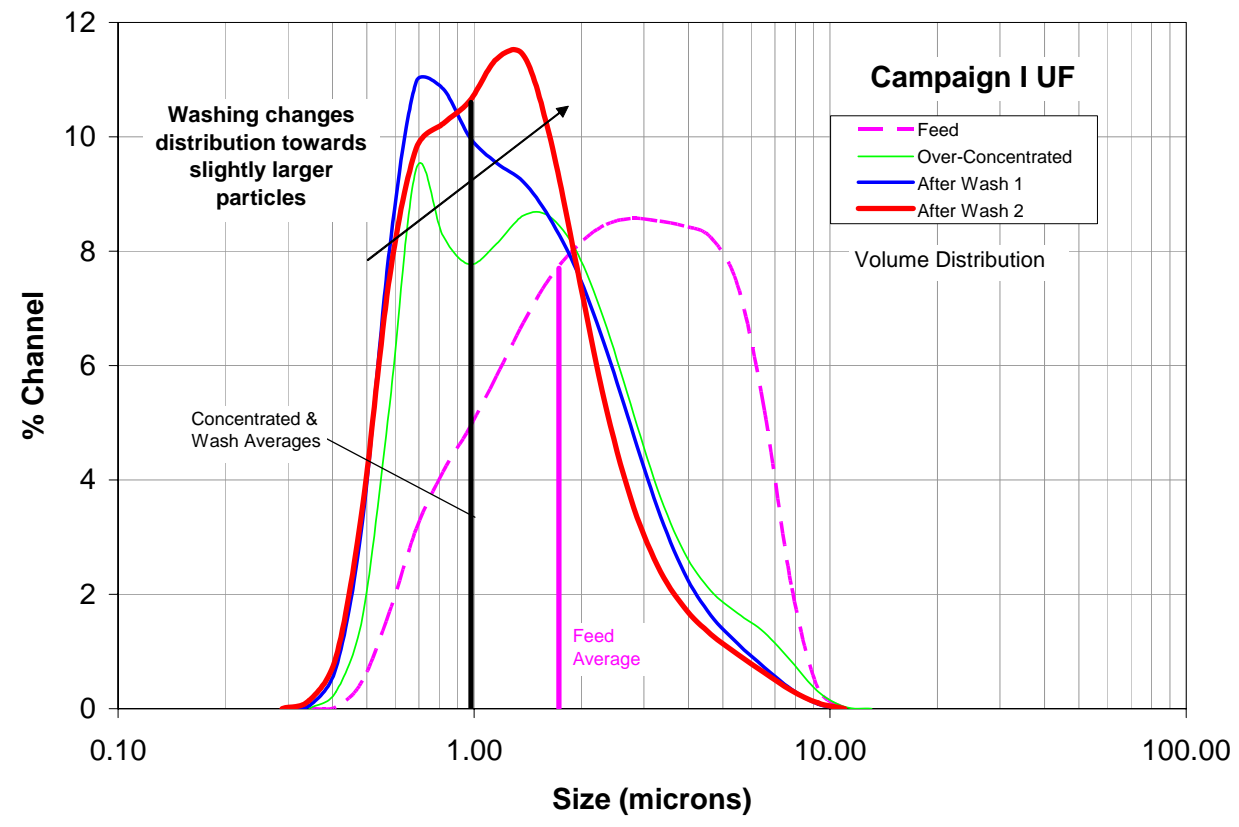

Figure 46. Particle Size Distribution of Washed Campaign I Slurry 
The Ultrafiltration feed particle size distributions are compared in Fig. 47. The Campaign II feed has a bimodal distribution compared to the Campaign I unimodal distribution. The area mean particle sizes are comparable. Figure 48 shows that washing the Campaign II slurry reduced the area mean particle size slightly.

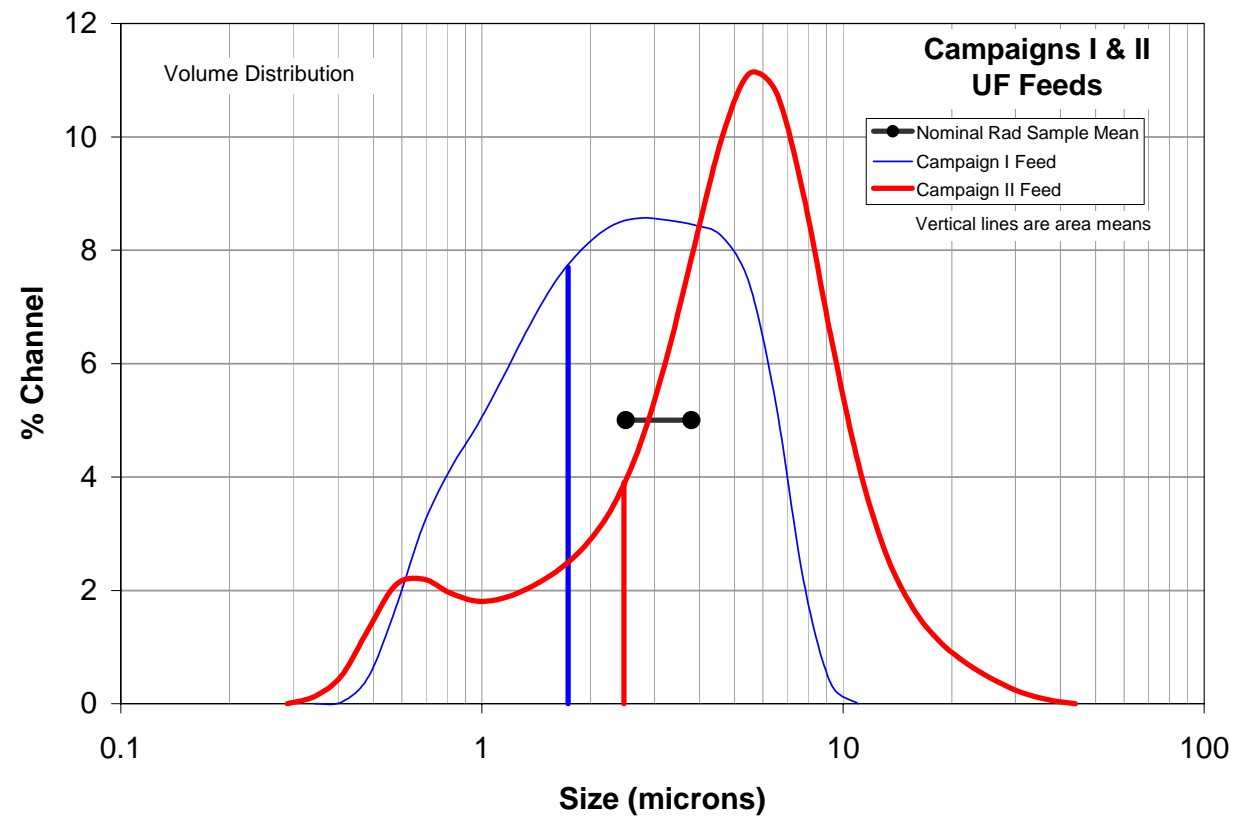

Figure 47. Comparison of Ultrafiltration Feed Particle Size Distributions

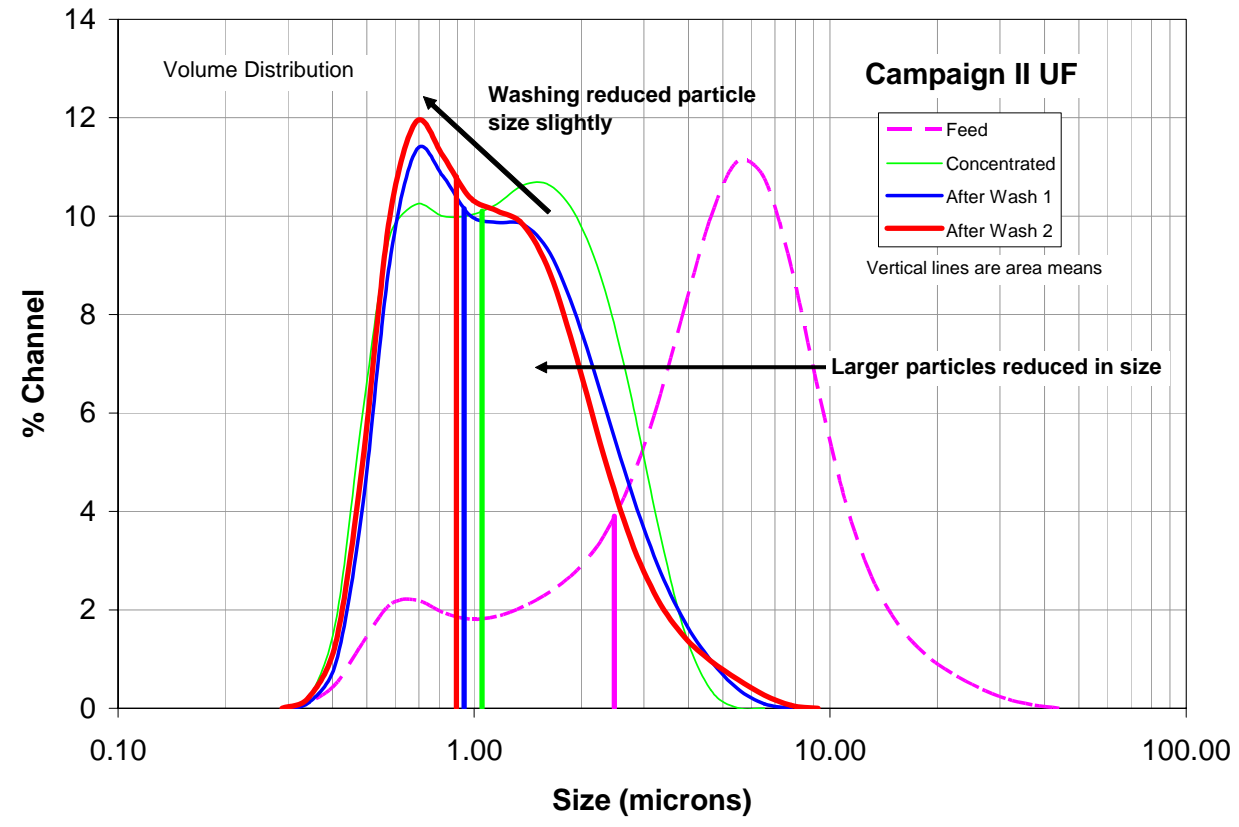

Figure 48. Particle Size Distribution of Washed Campaign II Slurry 


\subsection{Fate of Antifoam in the UFP System}

The fate of the Dow-Corning Q2-3183A antifoam, added to the FEP feed, was monitored by measuring the concentration of the antifoam in the FEP evaporator bottoms, the washed HLW slurry, the permeate to the IX system, the wash permeate, and the cleaning solutions. A schematic of the fate of the antifoam in the UFP process is given in Fig. 49. The initial amount of antifoam added in the FEP evaporator was $278 \mathrm{~g}$ into the pilot-scale FEP volume of $147.7 \mathrm{~L}$ of feed, which was calculated to give a final bottoms concentration of $1,887 \mathrm{mg} / \mathrm{L}$. (As mentioned in section 2.6.2, this amount of antifoam was based on the 1500 ppm that WTP plans to utilize.) However, from an initial sample Dow Corning measured the concentration at $4060 \mathrm{mg} / \mathrm{L}$, which indicated the amount of antifoam was $598 \mathrm{~g}$. Since the $278 \mathrm{~g}$ and $147.7 \mathrm{~L}$ were known quantities, the much larger mass based on the measured higher concentration indicate the difficulty in properly measuring the antifoam concentration. After all the feed was evaporated to the target density, the resulting bottoms slurry should still have had the initial 278 grams of antifoam. Once again, the Dow Corning measurement, from a bottoms sample, indicated the concentration was 6,025 mg/L, which implies a mass of 890 grams of antifoam. This is clearly much greater than the amount fed. Even after mixing the FEP bottoms with AY102 and AP101 to make the UFP feed and dewatering and washing the mixture, the washed slurry sample indicated a concentration of 3,105 $\mathrm{mg} / \mathrm{L}$ of antifoam, which translate to a mass of $368 \mathrm{~g}$. This is impossible since the starting value was $278 \mathrm{~g}$. As noted in the FEP discussion, these values are being rechecked with Dow Corning. Even with these high uncertain measurements, an estimate of how much antifoam remained in the washed slurry can be made. The permeate created in the UFP had an antifoam concentration of "trace $<25$ $\mathrm{mg} / \mathrm{L}$ ”, which means it was detected but was not quantifiable at the $25 \mathrm{mg} / \mathrm{L}$ minimum detection. Therefore, the maximum amount that could have been present in the permeate was $23 \mathrm{~g}$. It is concluded (based on the measured values, assuming any error affects all the same) that at least $92 \%^{\dagger}$ of the antifoam remains in the slurry. The wash permeate composite had no detectable antifoam present. The cleaning solutions were not analyzed, but analysis of the Campaign III FEP evaporator feed, which contains these recycles, is pending.

${ }^{\dagger}(278 \mathrm{~g}-23 \mathrm{~g}) / 278 \mathrm{~g}$ x $100 \sim 92 \%$ 


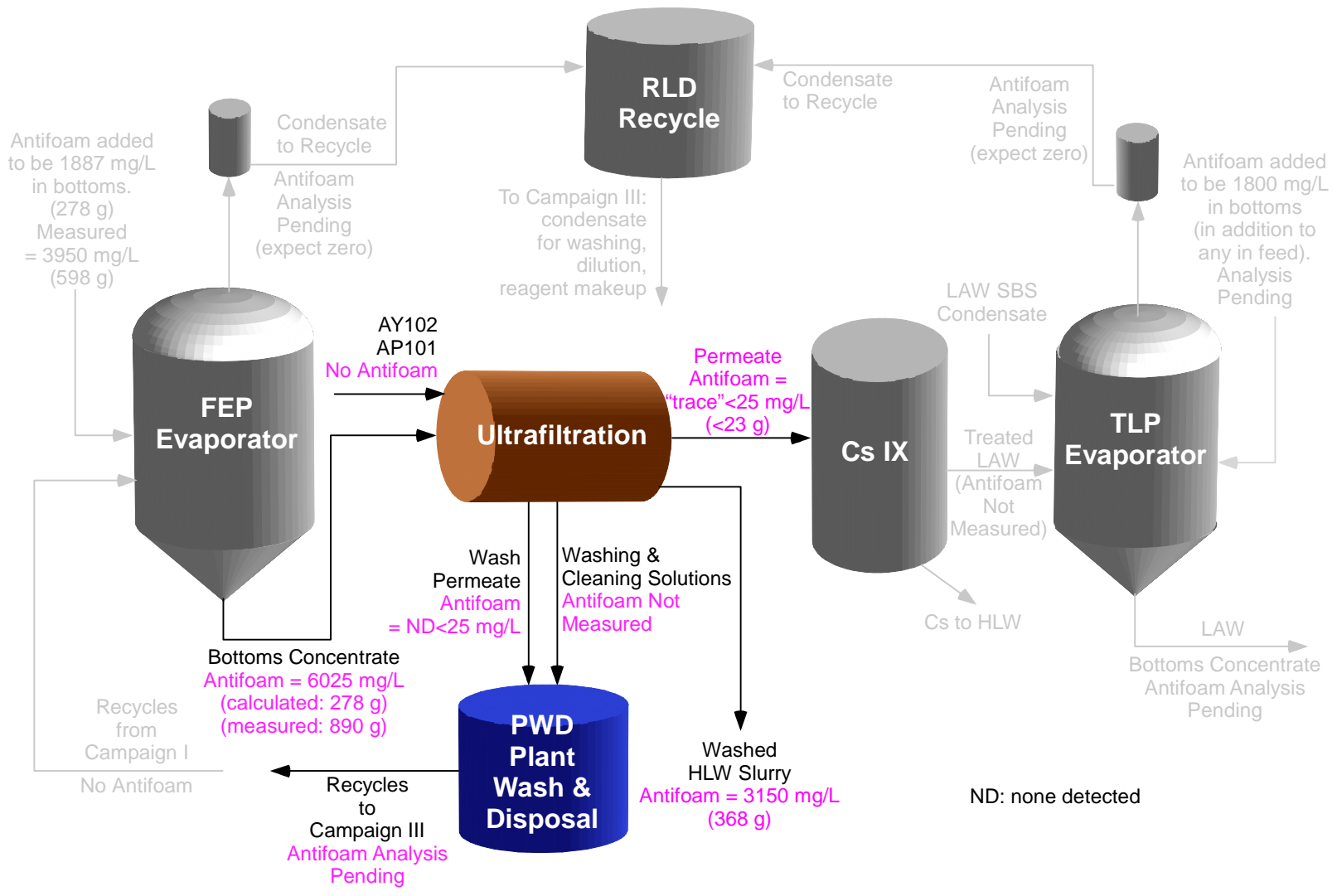

Figure 49. Schematic of Antifoam Fate in the SIPP - UFP

\subsection{Modeling of Pilot-scale UFP Permeate Flux for the simulated AY102/AP101 HLW}

The data taken during the concentration of the feed slurry was analyzed to determine the average flux during concentration and to correlate the flux versus the undissolved solids concentration. Figure 50 shows the flux plotted versus the elapsed processing time, where the elapsed processing time is defined as the actual time during which the slurry was undergoing concentration; down times for sampling, hold periods, or other pauses were subtracted out. The flux was calculated as the instantaneous flux from the measured permeate flowrate. The data were fit to the polynomial curve shown in Fig. 50. The total area under this curve was used to approximate the amount of permeate that was generated. This integration gave 936L of permeate compared to the measured 916L that was determined from the weights of the permeate drums. This difference is about $2 \%$. Figure 51 shows the measured permeate volume versus elapsed processing time, including the point at which $20 \mathrm{wt} \%$ UDS was achieved. A polynomial curve was fitted to these data. 
WSRC-TR-2004-00478, REVISION 0

SRNL-RPP-2004-00075, REVISION 0

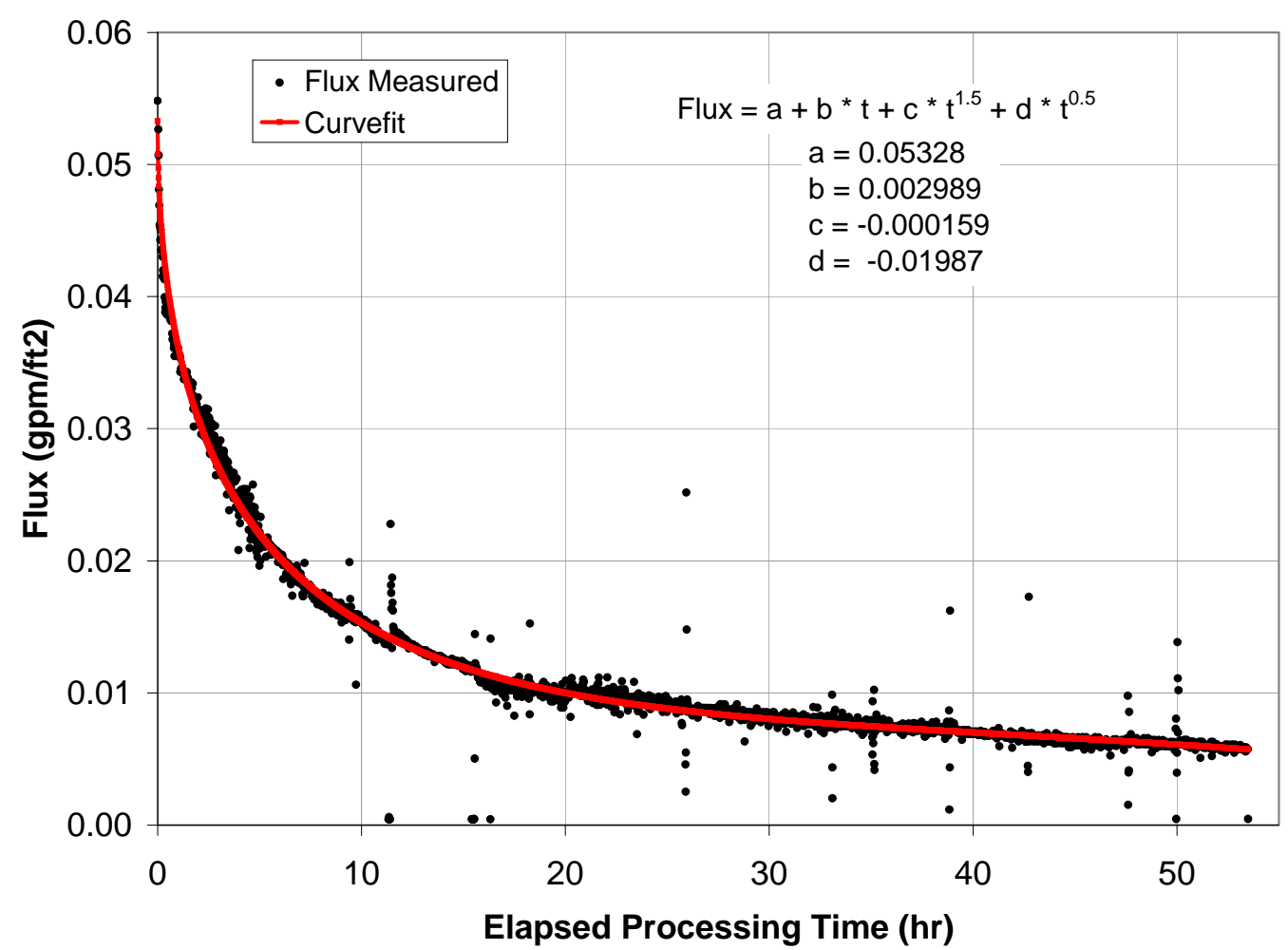

Figure 50. Fit of Flux vs. Elapsed Processing Time for the Simulated AY102/AP101 HLW

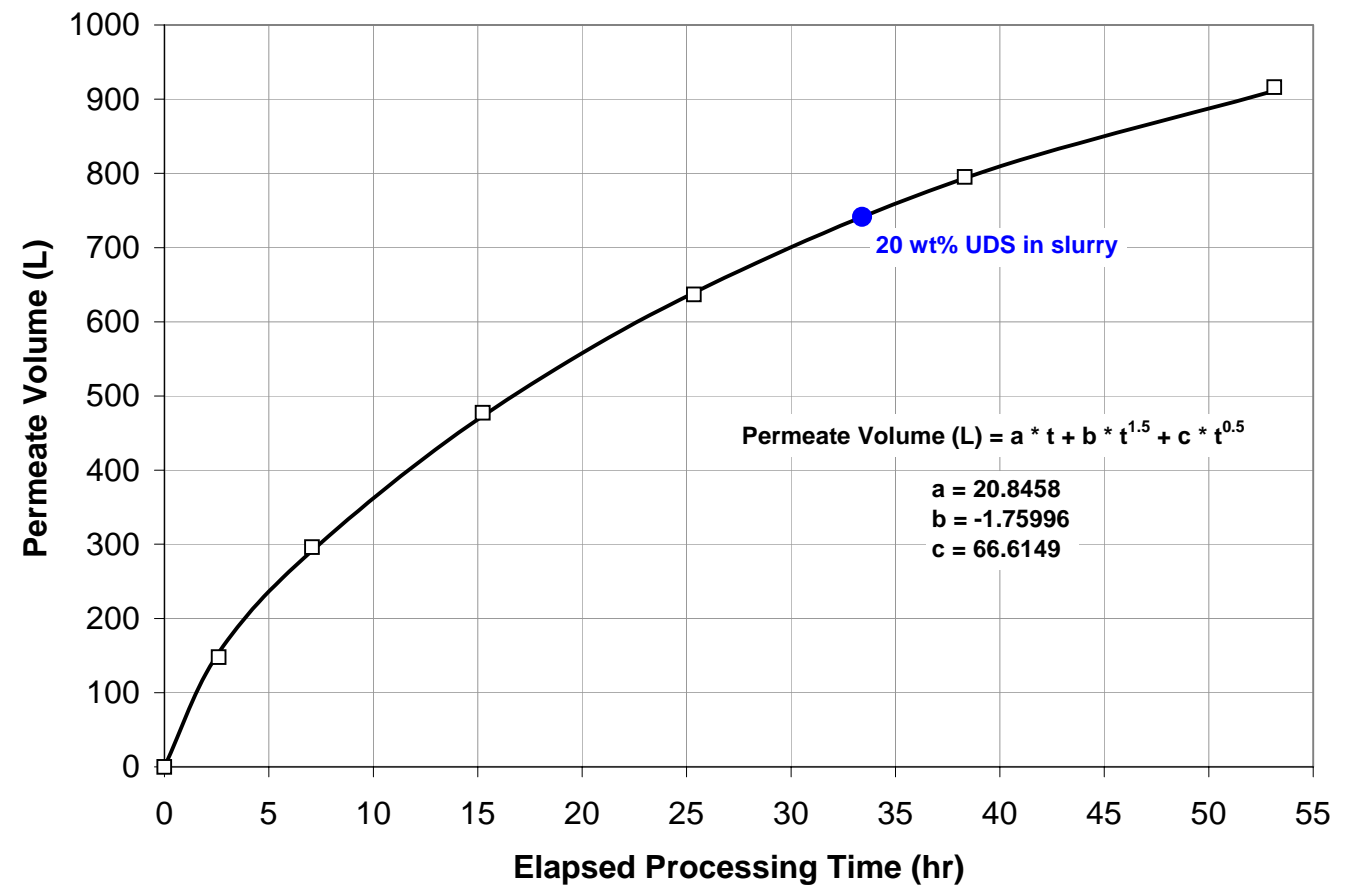

Figure 51. Permeate Volume vs. Elapsed Processing Time for the Simulated AY102/AP101 HLW 
Using the measurements of undissolved solids taken during concentrating, the UDS was correlated to the elapsed processing time, as shown in Fig. 52. Again, a polynomial fit was used.

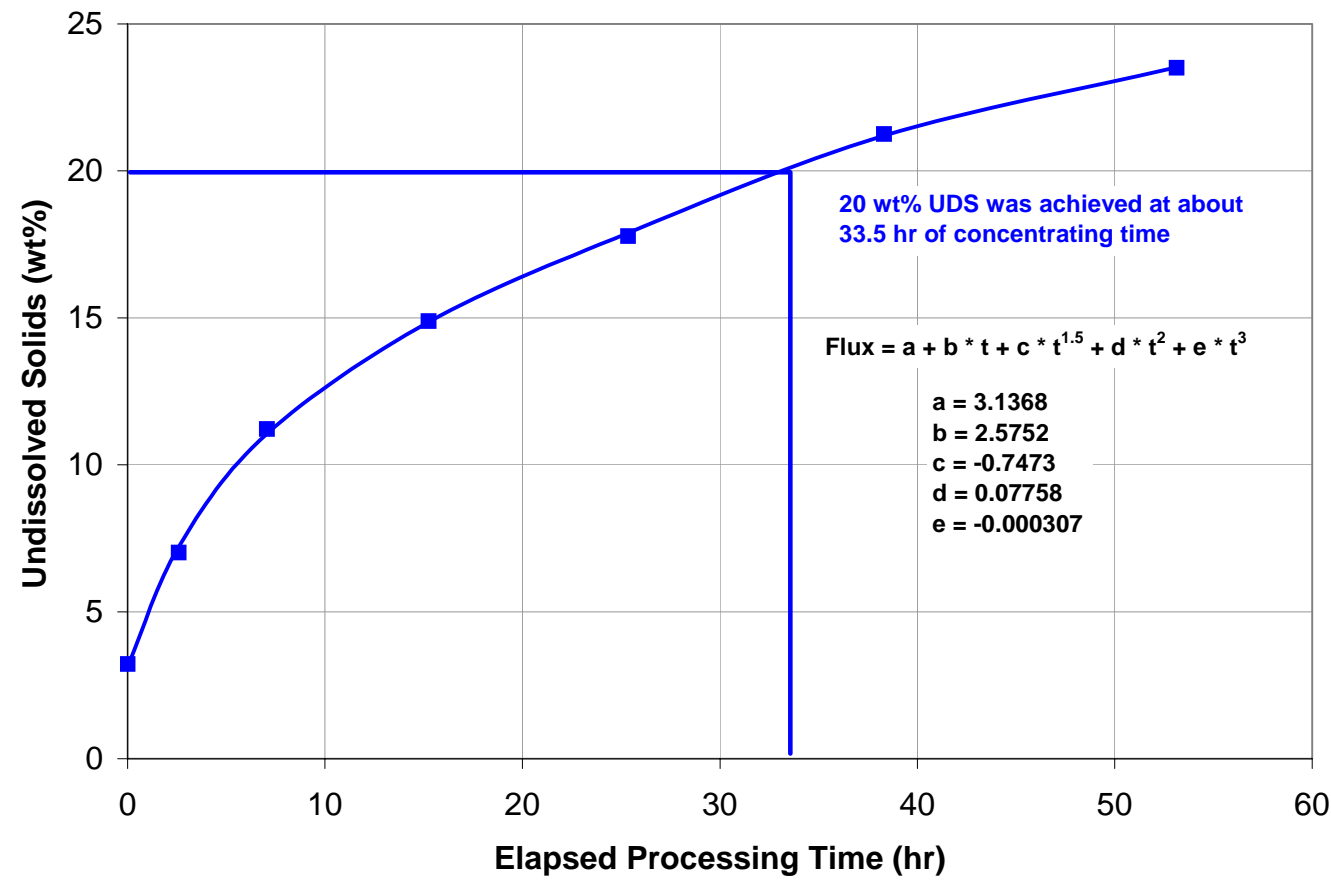

Figure 52. Undissolved Solids vs. Processing Time for the simulated AY102/AP101 HLW

The permeate flux and UDS concentration were obtained at each time step from correlating equations of Figs 50 and 52, and plotted in Fig. 53. The polynomial chosen should not be used for a UDS of less than $4 \mathrm{wt} \%$, nor above $24 \mathrm{wt} \%$. Basically, the section of the curve, which follows the data, is when the UDS concentration is increasing after the majority of the filter cake has been formed. However, on start up UDS concentration is increasing very slowly while the flux decreases very rapidly. This rapid flux decrease is primarily due to the filter being challenged. That is, the clean filter builds up a filter cake and during that initial period the empirical curve was not fit to the measured filter flux decline. The data were fit in this way so that the curve could be extrapolated back towards zero UDS and not predict the higher initial fluxes. Moreover, the fitted equation for this figure is only applicable up to $24 \mathrm{wt} \%$ UDS, since no data were available at higher concentrations. Figure 54 shows the integrated time averaged ${ }^{\dagger}$ flux at any UDS. This is the average flux from the initial UDS of $\sim 3 \mathrm{wt} \%$ to the UDS on the x-axis; e.g., at $10 \mathrm{wt} \%$, the average flux over the time period from $3 \mathrm{wt} \%$ to $10 \mathrm{wt} \%$ would be about $0.029 \mathrm{gpm} / \mathrm{ft}^{2}$, while at $20 \%$, it would be about $0.014 \mathrm{gpm} / \mathrm{ft}^{2}$. This graph allows the true time-averaged flux to be found from a graph that does not have time as an axis. Note that since the UDS versus time curve is not linear,

\footnotetext{
${ }^{\dagger}$ Note that the integrated time-averaged permeate flux shown in Fig. 54 is not the instantaneous flux, as shown in Fig. 53. For example, the averaged flux at $10 \mathrm{wt} \%$ UDS in Fig. 54 is $0.03 \mathrm{gpm} / \mathrm{ft}^{2}$ and represents average flux up to that point in TIME and does not include later fluxes, after that time, therefore, the average must be larger that the instantaneous flux at the same point. At $10 \mathrm{wt} \%$ UDS the instantaneous flux is $0.02 \mathrm{gpms}_{\mathrm{ft}}{ }^{2}$, as seen in Fig. 53.
} 
the average flux is not the area under the curve in a plot of flux versus UDS. Figure 54 shows the non-linearity of the permeate flux vs. elapsed processing time on a separate scale.

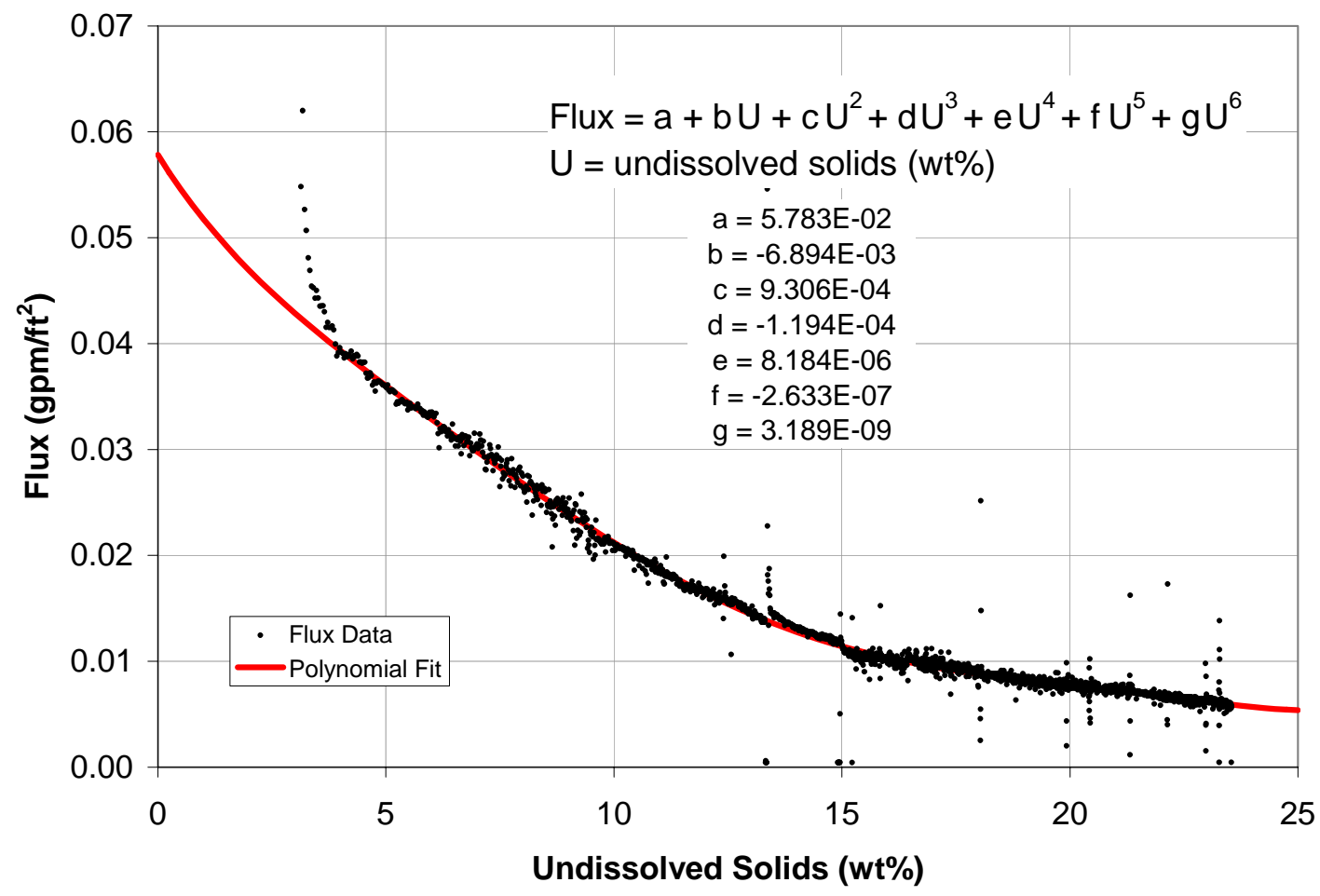

Figure 53. Fit of Flux versus Undissolved Solids for the simulated AY102/AP101 HLW 


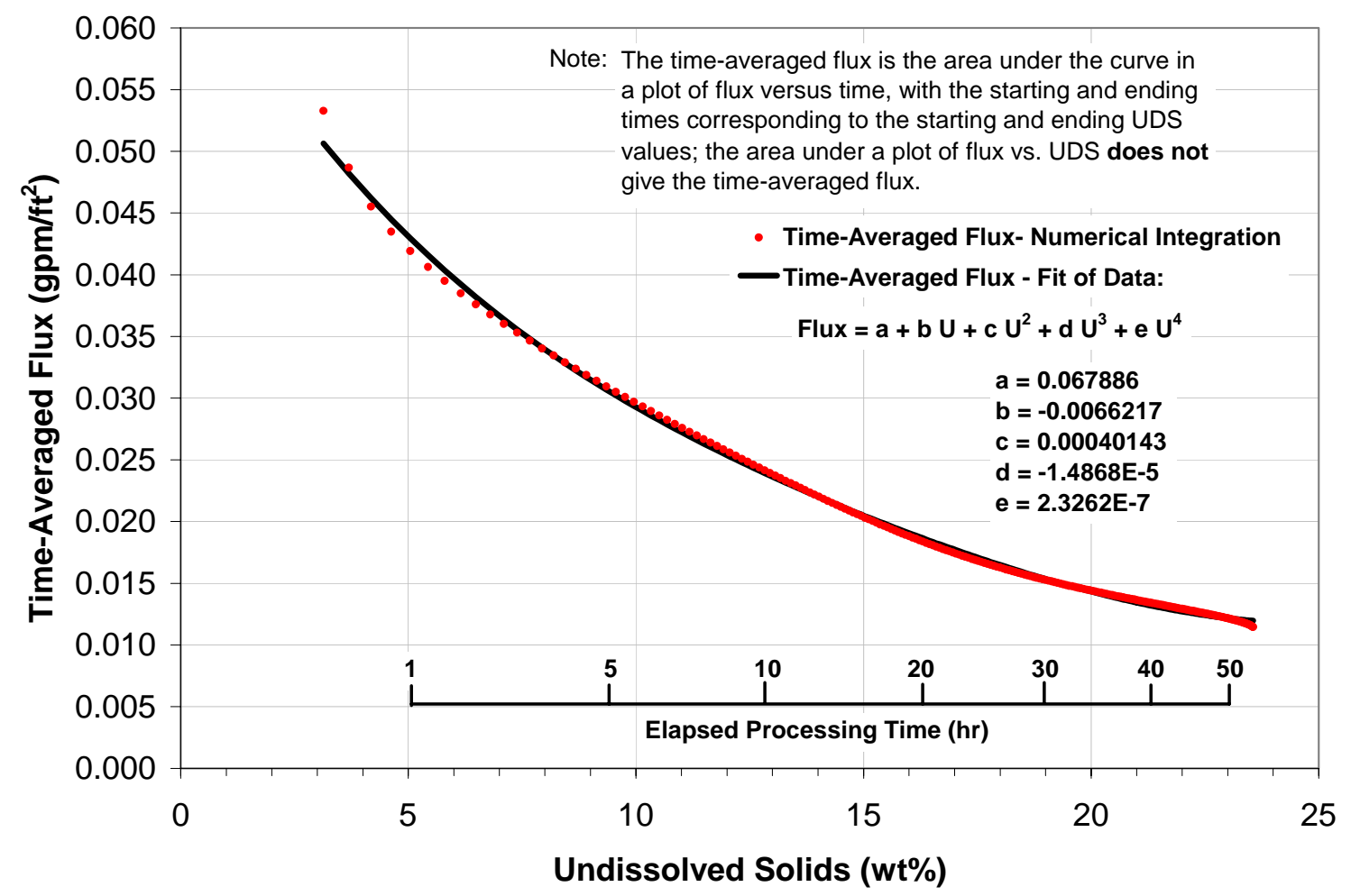

Figure 54. Time-Averaged Flux vs. Undissolved Solids for the simulated AY102/AP101 HLW

\subsubsection{Pilot UFP Conclusions}

\subsubsection{Experimental Observations (Author - Fowley)}

A summary of the overall conclusions are as follows:

- The average permeate flux when dewatering from $\sim 4$ to $\sim 24 \mathrm{wt} \%$ was $0.011 \mathrm{gpm} / \mathrm{ft}^{2}$.

- Cleaning the filter with $2 \mathrm{M}$ nitric acid was sufficient to increase the $0.1 \mathrm{M} \mathrm{NaOH}$ permeate flux to an order of magnitude larger than that of waste flux. However, it was not sufficient to return the filter to pre-test flux levels.

- The turbidity of the permeate from dewatering and slurry washing, when measured directly after the sample was taken, was always lower 0.5 NTU, indicating that the permeate was practically free of solids.

- The turbidity of the permeate from dewatering increased with time (days), indicating that solids were precipitating out of solution.

- Submerging the filter in a mild caustic solution over an extended period of time may improve flux. Likewise, it is probable that submerging the filter in a mild acid solution over an extended period of time will also improve flux. 


\subsubsection{Waste Products Observations (Author - Zamecnik)}

- UFP produced permeate showed no tendency for post-filtration precipitation. (However, AY102-only permeate from Campaign I did post-precipitate after 2 months.)

- OLI/ESP models of the AY102 sludge need to incorporate oxides, e.g., $\mathrm{Al}_{2} \mathrm{O}_{3}$, and hydrates, e.g., $\mathrm{FeOOH}$, in order to close mass and charge balances.

- Washing of concentrated slurry raised its yield stress from about 10 to $15 \mathrm{~Pa}$, but lowered the consistency from about 23 to $13 \mathrm{mPa} \cdot \mathrm{s}$.

- Campaign I data indicated that the yield stress of unwashed slurry is reduced upon extended pumping.

- Washing data matched previous SRNL and literature leaching and washing data for soluble washable species (see Table 36).

- Use of a more concentrated $\mathrm{NaOH}$ solution for the third caustic flush during filter cleaning may be warranted since the $\mathrm{pH}$ of the spent third wash was acidic.

- At least $96 \%$ of the antifoam remained in the HLW sludge slurry after filtration.

\subsection{PILOT CESIUM ION EXCHANGE (AUTHORS - STEIMKE \&WILLIAMS)}

The Cesium Ion Exchange Process, or CIX, is the third separation technology unit operation in the RPP-WTP Pretreatment Facility that a waste will see on start up. This is true for SIPP since the chosen waste type, Envelope A/D, is not subjected to precipitation before filtration. The SIPP CIX operation removes cesium from a mixture of the liquid LAW stream coming from the UFP before it is sent to the TLP for further evaporation and eventual vitrification.

The ion exchange resin used in this test was SuperLig ${ }^{\circledR} 644$, which is designed to remove cesium from caustic radioactive waste that is 5 molar concentration in sodium. It is manufactured by IBC Advanced Technologies. Pilot scale testing of this resin was first performed in the Engineering Development Laboratory in the year 2000 (Fowley et al., 2004). The testing was intended to be primarily that of the chemical performance of the resin on a pilot scale (more than one liter of resin); however, the pressure drop across the resin beds was unexpectedly high. Testing for the next three years was redirected to the hydraulic performance of the resin. The hydraulic issues were resolved to the satisfaction of RPP-WTP (River Protection Program - Waste \& Immobilization Treatment Plant) (Fowley et al., 2004). In 2003, Test Exception 24590-WTPTEF-RT-03-069 called for resumed chemical testing of SuperLig ${ }^{\circledR} 644$ resin at a pilot-scale for six chemical cycles. A cycle is the process whereby the Cesium Ion Exchange (CIX) unit operation treats enough LAW solution until the lead CIX column accumulates enough cesium to need regeneration, then it is regenerated. Later, the goals of the testing were modified to include Integrated Testing and the number of ion exchange cycles were reduced to four. This is an interim report on the first two cycles of testing.

For the actual RPP-WTP a cycle would entail the treating of a minimum of 100 Bed Volumes (BV) [BV = a resin bed volume of 415 gallons (1571 liters), under nominal operating conditions, which is subject to change] until the lead column of the three-column ion exchanger will contain enough accumulated cesium to require its regeneration. That lead column is then taken off-line to be regenerated while a waiting fourth column is valved-in for the CIX to continue further LAW 
treatment. That fourth column, containing reconditioned resin, is put in the last position (called the polishing position) of the three-column CIX unit and the column that had been in the second position (referred to the lag position) becomes the new lead column, and so forth. The pilot-scale CIX unit operation, of the SIPP task, only had two columns, the lead and lag positions. The pilot operation consisted of a treatment with approximately double the RPP-WTP minimum bed volumes, i.e., $200 \mathrm{BV}$ (pilot-scale BV = 2.34 liters) of simulated LAW, which was then followed by the regeneration of the lead column. A new cycle begins with the newly regenerated lead column in the lag position. Each of the SIPP campaigns consists of two CIX cycles. For Campaign II the pilot-scale CIX unit operation performed cycles 1 and 2. There were no CIX operations during Campaign I, since it only dealt with pilot-scale Ultrafiltration Process (UFP) operations.

This section of the interim report will explain the pilot CIX unit operation that was done as part of Campaign II testing. The process started with the preparation of the feed product, which is the permeate created in the UFP. An explanation of the chemical makeup of the feed stream will be followed by a short description on how feed was handled just before CIX operation and then how the waste stream was handled during and after ion exchange. That section will be followed by a discussion of the physical equipment and how the test was run. Finally, the results will be discussed and conclusions made.

\subsubsection{Chemical Description of the Pilot-Scale CIX Feed Stream}

The feed for the pilot-scale CIX unit operation is the permeate produced by simulant waste dewatering in the pilot-scale UFP unit operation. The chemical make-up of that feed was described in detail in Section 2.7.4.2.1 and will not be repeated here. Refer to that section and specifically to Tables. 29, 30, 31, and 32 for information on the CIX feed (simulated LAW) stream.

\subsubsection{Simulant Handling (Author - Williams)}

\subsubsection{Initial Preparations}

Before beginning the CIX test, the solutions to be used to regenerate the lead column were made ready, as shown in the CIX portion of the Fig. 2 schematic. Those solutions were:

1. $0.1 \mathrm{M} \mathrm{NaOH} \quad$ - Displaced the LAW in IX column

2. Deionized Water - Pre-elution rinse - Displaced the $0.1 \mathrm{M} \mathrm{NaOH}$ from IX column

3. $0.5 \mathrm{M} \mathrm{HNO}_{3} \quad$ - Elution - Displaced water and strips Cs ions from resin

4. Deionized Water - Post-elution rinse - Displaced acid from column

5. $0.25 \mathrm{M} \mathrm{NaOH} \quad$ - Displaced water and regenerated the resin

The regeneration caustic, $0.25 \mathrm{M} \mathrm{NaOH}$, needed a volume of 47.8 liters and it was made from deionized water and $50 \mathrm{wt} \% \mathrm{NaOH}$. Then it was stored in the Caustic Supply Tank on 26 April 2004. A large quantity of displacement caustic solution, $0.1 \mathrm{M} \mathrm{NaOH}$, had previously been made and it was stored in carboy labeled "Campaign 2-CsIX 0.1M NaOH Displace Sol." From this carboy 28.7 liters were removed and made ready for Campaign II on 28 April. To strip the ion- 
exchange resin of Cs ions, 70.1 liters of $0.5 \mathrm{M} \mathrm{HNO}_{3}$ were made from deionized water and $70 \%$ $\mathrm{HNO}_{3}$ and was placed in the Eluent Supply Tank on 28 April.

\subsubsection{Handling of Pilot-scale CIX feed LAW Simulant}

The simulant supply that was produced during pilot-scale UFP operation was split into two equal parts for Cycle 1 and 2 of CIX operations. Cycle 1 processed about 182 Bed Volumes (BV) (427 liters) of simulant. This first part of the simulant was processed on 3 May (which would then become TLP Evaporator feed ) had a measured density of $1.247 \mathrm{~kg} / \mathrm{L}$ and produced $532.2 \mathrm{~kg}$ of material. About one liter of the treated material was provided for a bench top TLP Evaporator operation to ensure that the process produced the desired product. Cycle 2 processed about 186 BV (436 liters) of simulant and produced $543.9 \mathrm{~kg}$ of treated simulant at $1.247 \mathrm{~kg} / \mathrm{L}$ on 7 May. Thus a total of $1076 \mathrm{~kg}$ of treated simulant was transferred to the pilot-scale TLP Evaporator Feed Tank from CIX operations.

\subsubsection{Handling CIX Regeneration and Campaign III CIX Recycle Streams}

During the two CIX cycles of Campaign II, the plan was to collect separately the simulant and the five solutions, used in the regeneration process, by moving the column discharge hose from one collection container to another, at the appropriate time. The appropriate time was to be determined by monitoring the $\mathrm{pH}$ and the conductivity of the discharge stream. This method proved unexpectedly difficult to implement, and in response, Campaign III discharge hose moves will be based on a plug-flow calculation with no mixing or chemical reaction of when a solution front would reach the column outlet. In hindsight, some Campaign II hose moves were made at appropriate times, while others were not, as explained in the Results Section 2.8.5.

Because of the small volumes of the CIX columns, most of the volumes of the regeneration process solutions were insufficient for SIPP recycle needs. As previously mentioned, the CIX columns were designed for Cs chemical testing and not to support SIPP overall testing. The different scales of testing (SIPP vs. chemical testing) meant that the volume of other CIX recycle streams generally had to be increased with simulants to meet SIPP needs

\subsubsection{Pilot CIX Equipment (Author - Steimke)}

Figure 55 is a picture of the two columns during Cycle 1 Regeneration and Fig. 56 is a schematic of the two ion exchange columns and peripheral equipment. The design is also documented in WSRC drawing EES-23041-R4-001. Each column had a liquid filled length of 23.75” (or a internal volume of 9.24 liters $\sim 4 \mathrm{BV}$ ). The following hardware is positioned inside the column with the following elevations with respect to the wedge screen (also known as: V-wire screen) that supports the resin and note that the total liquid filled length of each column is 23.75”, i.e., 16.75”$(-7.00 ”)$. 
Elevation

$16.75 ”$

$16.00 ”$

$14.75 ”$

$13.25 ”$

0 "

$-2.0 ”$

$-3.50$

$-7.00 ”$

\section{Column Hardware Description}

Top of liquid filled space

Flow distributor with six perforated arms

Perforated PVC plate with thirty seven 3/64" holes

Perforated stainless steel plate with 3/32” holes on a 5/32” triangular pitch Wedge screen with 0.010 ” slots to support resin

Perforated stainless steel plate with 3/32” holes on a 5/32” triangular pitch

Perforated PVC plate with thirty seven 3/64" holes

Bottom of the liquid filled space

The purpose of the flow distributor and the two plates below it was to generate a more gradual transition of density when relatively dense simulant was introduced to the top of the column on top of lighter liquid. The purpose of the wedge screen was to support the resin while resisting plugging. The purpose of the bottom two plates was to generate a more uniform flow field in the event upflow operations were needed, however, the normal flow direction was down.
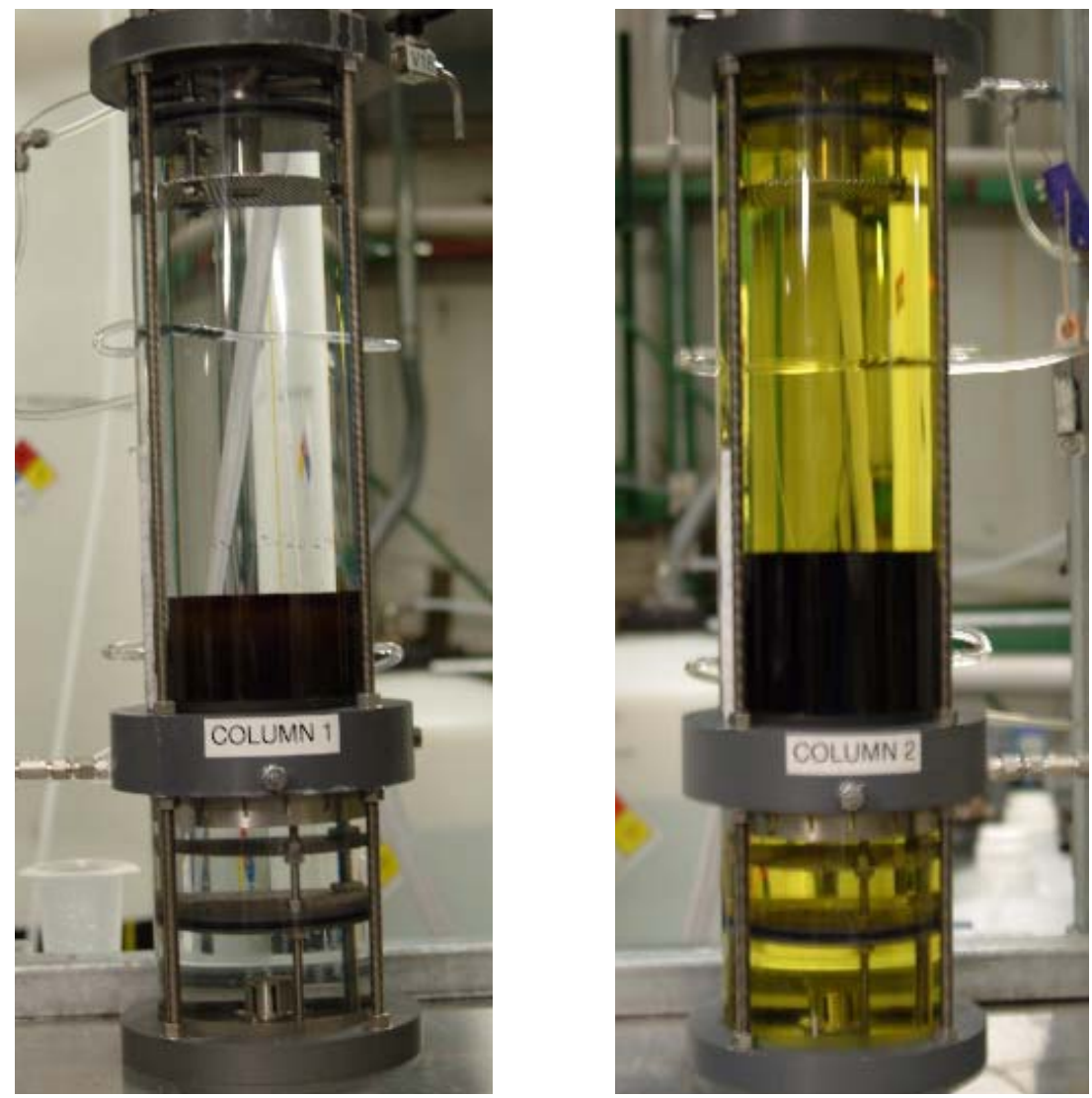

Figure 55. Pilot-scale CIX columns: The left column (COLUMN 1) is undergoing resin regeneration during Cycle 1 operation 
WSRC-TR-2004-00478, REVISION 0

SRNL-RPP-2004-00075, REVISION 0

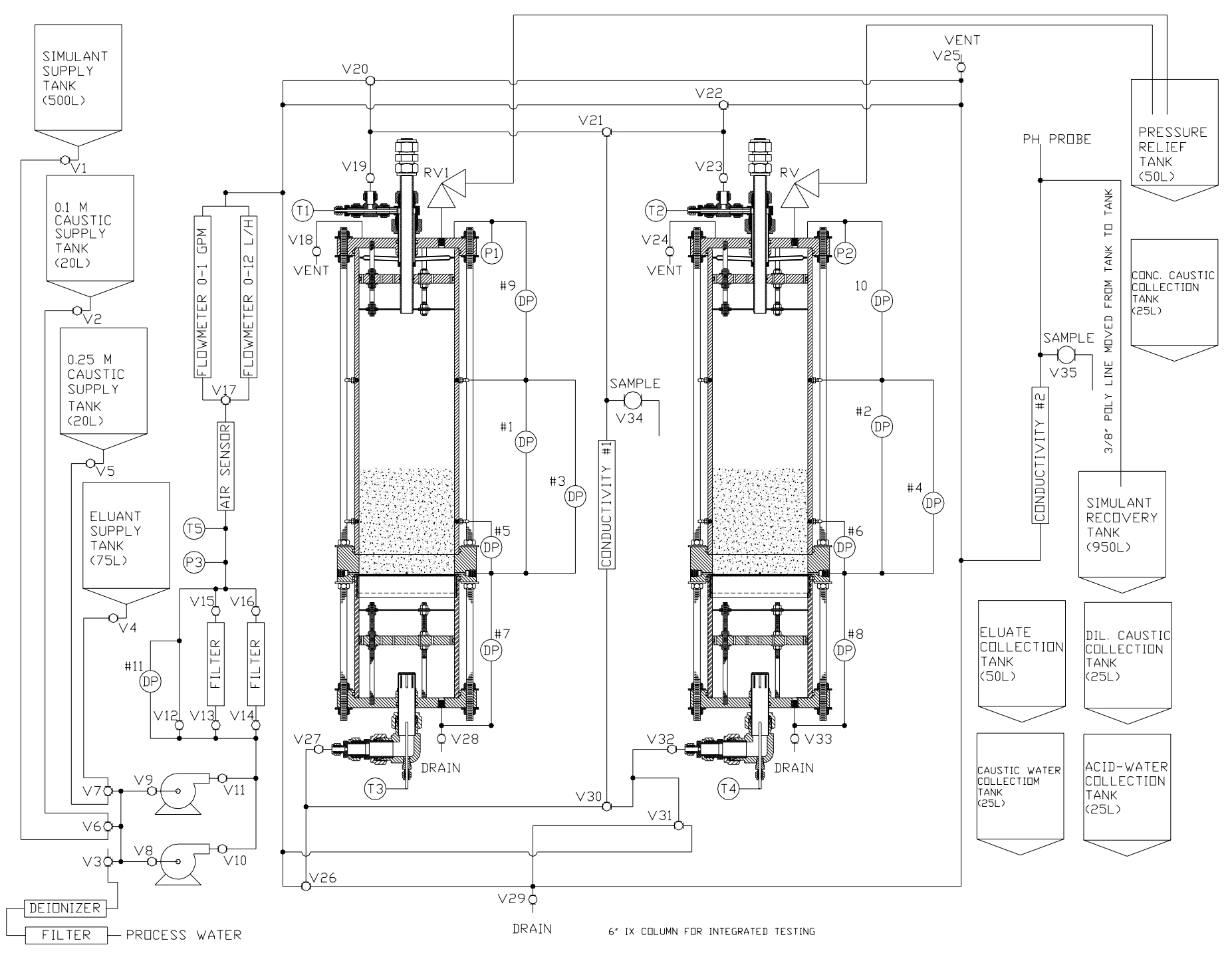

Figure 56. Schematic of Ion Exchange Facility for Integrated Testing 
By opening the proper valve, feed could be drawn from one of five supply tanks, which contained either: LAW simulant, displacement $0.1 \mathrm{M} \mathrm{NaOH}$ solution, deionized water for rinsing, eluent $0.5 \mathrm{M}$ nitric acid solution, or regeneration $0.25 \mathrm{M} \mathrm{NaOH}$ solution. The solutions were pumped by one or two gear pumps connected in parallel. Most of the time only one pump was sufficient, but two-pump operation was required for upflow. The facility contained two flowmeters in parallel, a high-range flowmeter for upflow with a range of $0-1 \mathrm{gpm}(227 \mathrm{~L} / \mathrm{hr})$ and a low-range flowmeter for normal operation with a range of $0-0.05 \mathrm{gpm}(12 \mathrm{~L} / \mathrm{hr})$. Valves allowed upflow or downflow through one column or two columns in series. The effluent line from the columns contained a $\mathrm{pH}$ probe, a conductivity probe and a thermocouple. A hose connected to the effluent line was placed in the appropriate collection jug for every step of the cycle. There were six collection jugs, one for each of the six steps of a CIX cycle.

\subsubsection{Pilot CIX Operation (Author-Steimke)}

\subsubsection{Summarized Test Procedure}

After construction of the test facility, shakedown was performed to check for leaks, to exercise all equipment and valve lineups, and to exercise safety equipment and circuitry. With the hardware ready, resin was deposited in the columns and both beds were hydraulically preconditioned with a downflow of $0.25 \mathrm{M} \mathrm{NaOH}$ for thirty minutes at a velocity of $15 \mathrm{~cm} / \mathrm{min}$. This was followed by chemical testing.

Each cycle of chemical testing had the following six chemical steps, all in downflow: LAW simulant, 0.1 M NaOH displacement solution, deionized water, $0.5 \mathrm{M}$ nitric acid eluent solution, deionized water, and $0.25 \mathrm{M} \mathrm{NaOH}$ regeneration solution. The total volume of LAW simulant used for each cycle was about $200 \mathrm{BV}$ (to reiterate $1 \mathrm{BV}=2.34$ liters for SIPP) and the simulant was always passed through both ion exchange columns in series. All steps, other than the simulant step, were conducted through one column only, which was the lead column for the simulant step. The $0.1 \mathrm{M} \mathrm{NaOH}$ step and the subsequent deionized water step (pre-elution rinse) were conducted at a target flowrate of $3 \mathrm{BV} / \mathrm{hr}$ for one hour. The volume used for the elution step was $15 \mathrm{BV}$. (Cycle $1 \mathrm{had}$ a flowrate of $1.0 \mathrm{BV} / \mathrm{hr}$ and a duration of 15 hours. Cycle 2 had a flowrate of $0.5 \mathrm{BV} / \mathrm{hr}$ and a duration of 30 hours). The post-elution rinse was run at a target flowrate of 1.4 $\mathrm{BV} / \mathrm{hr}$ for 2.25 hours. Finally, the regeneration step was run at a target flowrate of 3 $\mathrm{BV} / \mathrm{hr}$ for 2 hours. The lengths of the various steps differed from the target lengths by no more than three minutes.

The six different chemical solutions were collected in separate containers by moving the discharge hose from the columns. In Campaign II, for Cycles 1 and 2, the plan was to move the hose based on observing the change in the $\mathrm{pH}$ and/or conductivity probes. As previously mentioned, this method proved to be difficult to implement, therefore, in Campaign III, for Cycles 3 and 4, the timing for moving the hose to a different container will be based on a plug flow calculation of when the liquid front would reach the column discharge. 
There was a two-week period between the end of Cycle 2, part of Campaign II testing, and the start of Cycle 3, part of Campaign III testing. At the end of Cycle 2, the lag column was Column 1 and it contained simulant and the lead column, Column 2, which was regenerated, contained $0.25 \mathrm{M} \mathrm{NaOH}$. At the request of RPP-WTP, both columns were displaced with $0.1 \mathrm{M} \mathrm{NaOH}$ and the resin soaked in that solution until the start of Cycle 3 for Campaign III testing.

\subsubsection{Test Matrix Highlights}

The entire CIX test matrix is shown in Tables 10 and 11 and all simulant samples taken are listed in Appendix D, but for convenience some key parameters are listed below:

1. Cycles 1 and 2 had a simulant target flowrate of $3 \mathrm{BV} / \mathrm{hr}$.

2. Cycles 1 and 2 had eluent target flowrates of $1 \mathrm{BV} / \mathrm{hr}$ and $0.5 \mathrm{BV} / \mathrm{hr}$, respectively.

3. Columns 1 and 2 were the lead columns for Cycles 1 and Cycles 2, respectively.

The two cycles were run on the following dates in 2004.

\begin{tabular}{|c|c|c|}
\hline Cycle & Start date & Finish date \\
\hline 1 & 26 April & 4 May \\
\hline 2 & 4 May & 12 May \\
\hline
\end{tabular}

\subsubsection{Data Acquisition and Safety Circuitry}

A Dell computer with LabVIEW ${ }^{\mathrm{TM}}$ software was used to acquire data, control the flowrate, and shut down the flow if any measured pressure exceeded the setpoint of 20 psig. There was also a pressure relief valve set at 45 psig connected to the top of each of the two columns. The discharge line of the pressure relief valves was a hose leading to a jug. There was also a spill detector with a sensor placed below the columns on the floor of the containment for the facility. Any spill would disconnect power to the pumps. There was also an air detector in the line leading from the solution supply tanks to the pump. Any air in the line would disconnect power from the pumps. A manual bypass switch was used to start the pump/s when there was initially air in the line. The air detector was used because oxygen can damage the wet resin, particularly when it is in a caustic state.

\subsubsection{Resin Pre-Treatment, Deposition in Columns and Pre-Conditioning}

SuperLig ${ }^{\circledR} 644$ resin was taken from the 250-Gallon Batch (C-01-11-05-02-35-60). The resin was chemically pre-treated by washing with water, treating it in $0.5 \mathrm{M}$ nitric acid, washing with water, treating it with $0.25 \mathrm{M}$ sodium hydroxide and washing it with deionized water. The pre-treated resin was then wet sieved under a nitrogen atmosphere to remove particles finer than 40 mesh. The resin was mixed with $0.25 \mathrm{M} \mathrm{NaOH}$ solution to form a $30 \%$ by volume slurry. With a pump connected to the bottom of the columns, the slurry was drawn into and through each column, one at a time, at a velocity of 14 $\mathrm{cm} / \mathrm{min}(0.58 \mathrm{gpm})$ to deposit the resin beds. The initial resin deposition resulted in a 
crater about 1 inch deep in both columns. An upflow for two minutes at $13 \mathrm{~cm} / \mathrm{min}$ was used to level the tops of the two resin beds. This was followed by hydraulic preconditioning of both beds for 30 minutes with a flowrate of $15 \mathrm{~cm} / \mathrm{min}$ of $0.25 \mathrm{M} \mathrm{NaOH}$ solution. At this time, both beds had a resin height of 6.25 ”.

\subsubsection{Pilot CIX Discussion of Results (Author-Steimke)}

Two ion exchange columns were designed and constructed, as previously shown in Figs. 55 and 56. The columns had an inside diameter of 5.5 inches and a nominal resin height of 6 inches ${ }^{\dagger}$, giving a height to diameter ratio of 1.1. Other hardware included pumps, tanks, flowmeters, pressure transducers, other instruments, and a data acquisition system (DAS).

The facility was used to run four ion exchange cycles (two for Campaign II and two for Campaign III), where a cycle consists of six chemical steps. For Cycles 1 and 2 respectively, 427 liters and 436 liters or 182 bed volumes (BV) and $186 \mathrm{BV}$ of simulated high level waste was pumped through the two beds connected in series. For Cycle 1, Column 1 was the lead column and Column 2 was the lag column. The column positions were reversed for Cycle 2. For Cycles 1 and 2, the lead ion exchange bed removed 99.6 $\pm 0.2 \%$ of the cesium. For Cycles 1 and 2, the lead and lag ion exchange beds together removed $99.9 \pm 0.2 \%$ of the cesium. During operation, the pressure drop was measured for both resin beds. The maximum pressure drop occurred during the simulant flow step. For Cycles 1 and 2, Column 1 resin bed pressure drops were 0.6 and 0.5 inches of water, respectively. For Cycles 1 and 2, Column 2 resin bed pressure drops were 0.6 and 0.7 inches of water, respectively. The uncertainty in all four pressure drops was \pm 0.1 inch of water; therefore, the pressure drop differences between columns were insignificant. Subsequently, a cesium mass balance was performed. The cesium masses in and out of the columns agreed within the accuracy of ICP-MS (inductively coupled plasma mass spectroscopy) for measuring relatively low concentrations of cesium in 5-molar sodium solutions. During elution of each of the lead columns fifteen bed volumes of $0.5 \mathrm{M}$ nitric acid were used. After only the first three BV, about $90 \%$ of the cesium was eluted from the bed.

\subsubsection{Hydraulic Observations}

Table 42 summarizes some important hydraulic results from the Campaign II. Note, that the simulant had a density of $1.25 \mathrm{~kg} / \mathrm{L}$.

\footnotetext{
${ }^{\dagger}$ The actual resin height is not fixed. As explained in the following sections, and shown in Table 42, the height resin in the columns changes from pre-conditioned height of 6.25 inches, when the columns are initially filled, to as short as 5.4 inches after multiple resin regenerations.
} 
WSRC-TR-2004-00478, REVISION 0

SRNL-RPP-2004-00075, REVISION 0

Table 42. Hydraulic Summary

\begin{tabular}{|c|c|c|c|c|c|c|}
\hline Cycle & $\begin{array}{c}\text { Simulant } \\
\text { DP Col. 1 } \\
\text { Inches } \\
\text { H2O }\end{array}$ & $\begin{array}{c}\text { Simulant } \\
\text { DP Col. 2 } \\
\text { Inches } \\
\text { H2O }\end{array}$ & $\begin{array}{c}\text { Resin } \\
\text { height in } \\
\text { simulant, } \\
\text { Col. 1, } \\
\text { inches) }\end{array}$ & $\begin{array}{c}\text { Resin } \\
\text { height in } \\
\text { simulant, } \\
\text { Col. 2, } \\
\text { (inches) }\end{array}$ & $\begin{array}{c}\text { Simulant } \\
\text { superficial } \\
\text { velocity, } \\
\text { Col. 1, } \\
\text { (cm/hr) }\end{array}$ & $\begin{array}{c}\text { Simulant } \\
\text { superficial } \\
\text { velocity, } \\
\text { Col. 2, } \\
\text { (cm/hr) }\end{array}$ \\
\hline 1 & 0.6 (Lead) & 0.5 (Lag) & 5.6 & 5.8 & 46 & 46 \\
\hline 2 & $0.6(\mathrm{Lag})$ & 0.7 (Lead) & 5.4 & 5.7 & 46 & 46 \\
\hline
\end{tabular}

Figure 57 plots the flowrates during Cycle 1 for all six solutions used. The large fluctuations in the measured flowrate for the two water rinses is an instrument artifact because the deionized water used lacked sufficient ions for the magnetic flowmeter to achieve a steady reading. However, the readings were checked with a volumetric cylinder that showed the measured average flow to be correct. Figures 58 and 59 plot the pressure drops across the lead and lag column resin beds during Cycle 1 . There were duplicate measurements of the pressure drop across the entire resin bed and one measurement of the pressure drop across the bottom 3 inches of the resin bed. The duplicate pressure drop measurements agree. The highest pressure drops are for the simulant step and the pressure drop across the bottom 3 inches about half of the total pressure drop, as expected. The total pressure drop across the bed for simulant was about 0.6 inch of water. By way of comparison, the 24-inch Column Test (Fowley et al., 2004) had a pressure drop for Cycle 1 of 16 inches of water for a resin height that was four times as great and a superficial velocity that was seven times as great $(5 \mathrm{~cm} / \mathrm{min}$ vs. 0.75 $\mathrm{cm} / \mathrm{min}$ ). The 24-inch Test pressure drop was 27 times as large, which agrees well with the factors of four and seven.

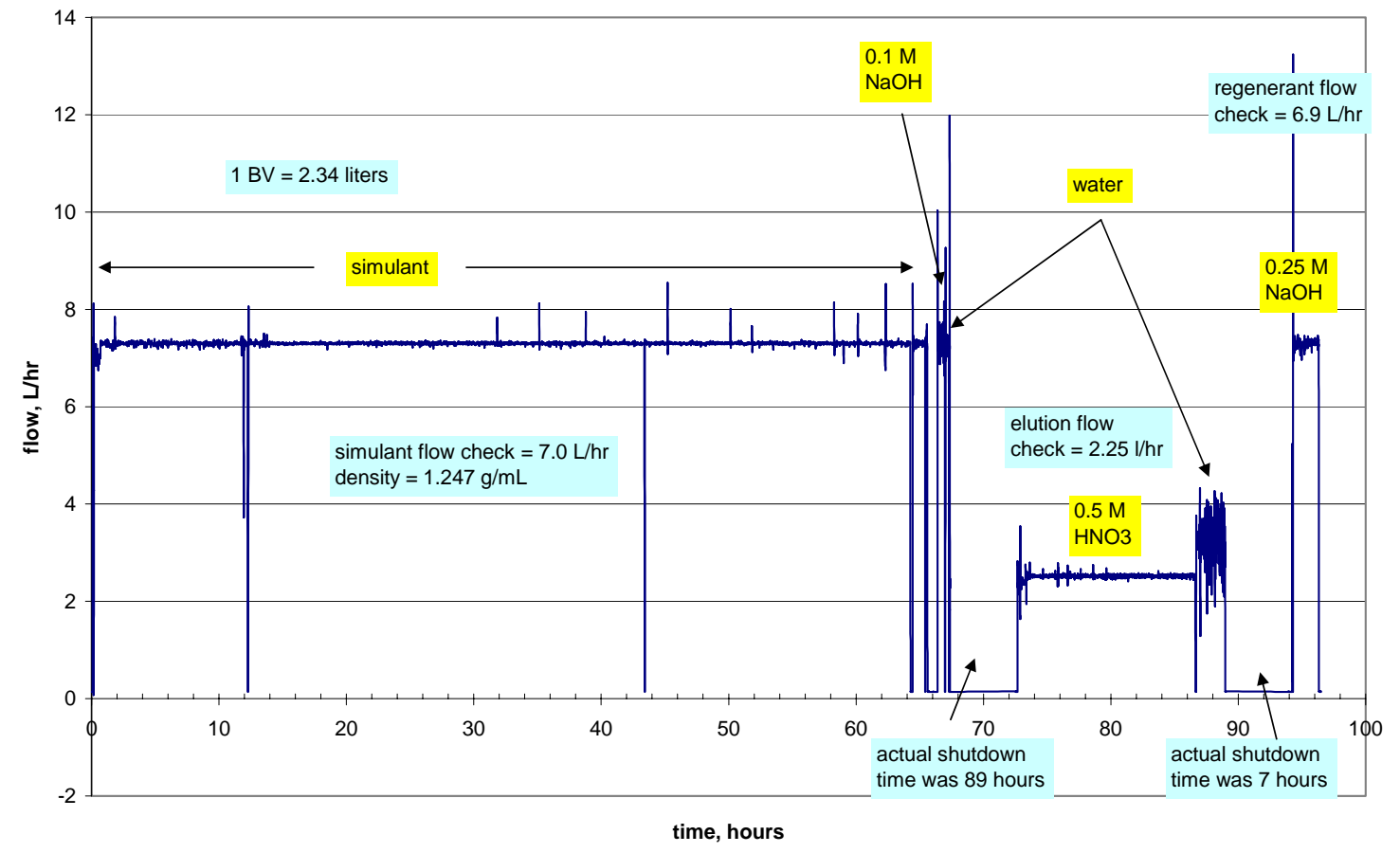

Figure 57. Flowrate During Cycle 1 
WSRC-TR-2004-00478, REVISION 0

SRNL-RPP-2004-00075, REVISION 0

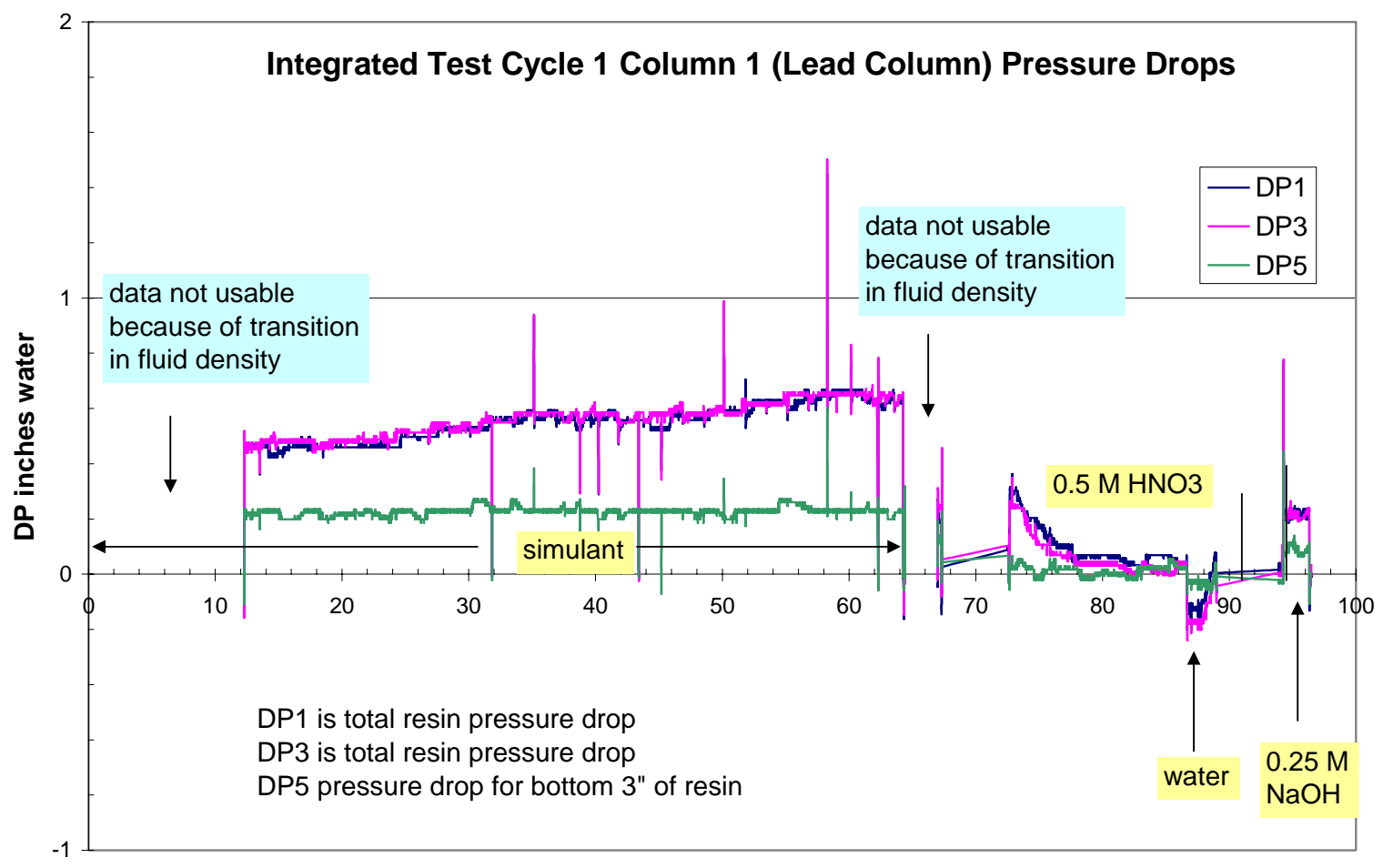

time, hours

Figure 58. Pressure Drop Across Resin Bed for Lead Column and Cycle 1

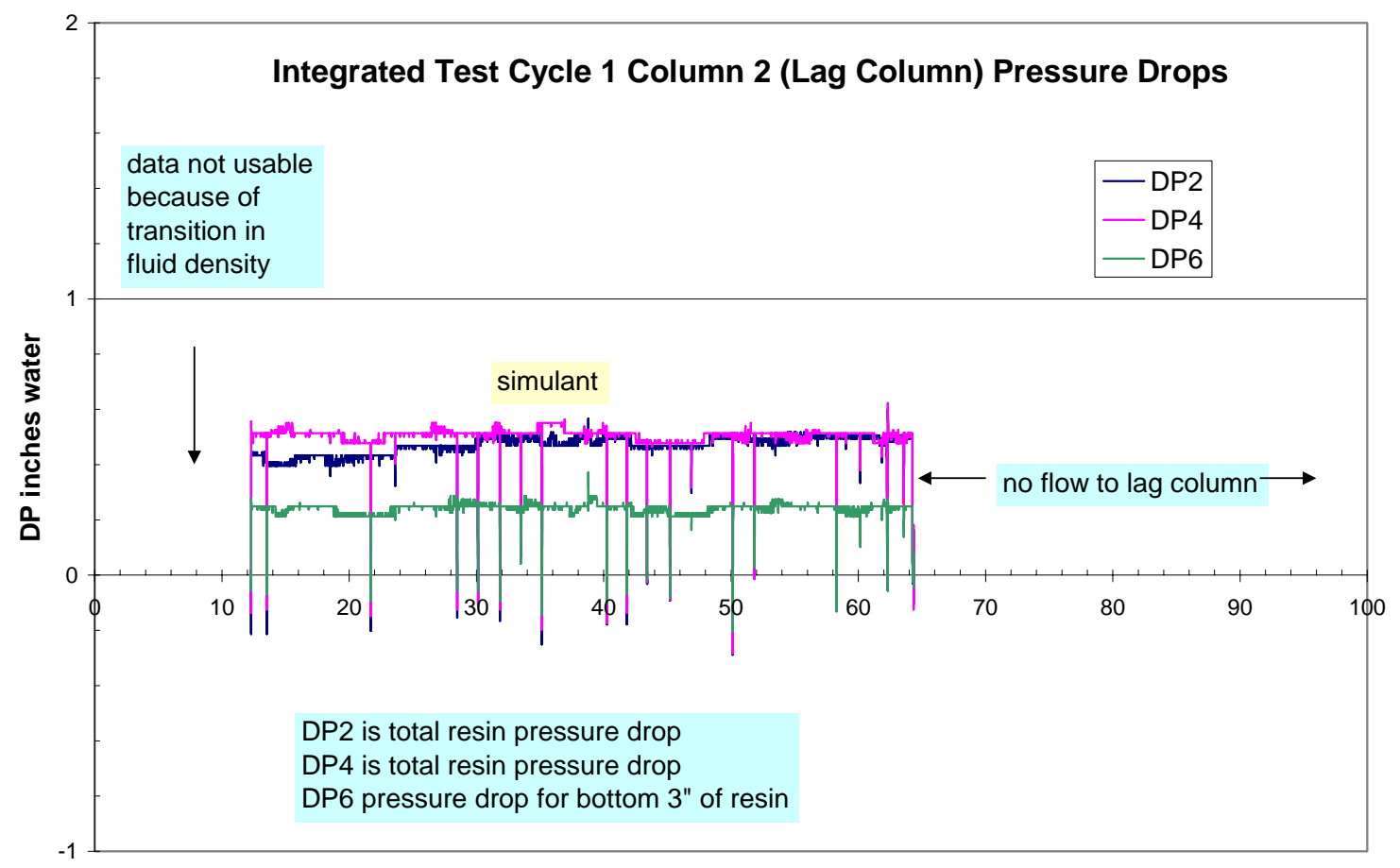

time, hours

Figure 59. Pressure Drop Across Resin Bed for Lag Column and Cycle 1 
Resin bed heights during Cycle 1 are plotted in Fig. 60. The plot shows the expected variation with the smallest resin bed height during the elution step.

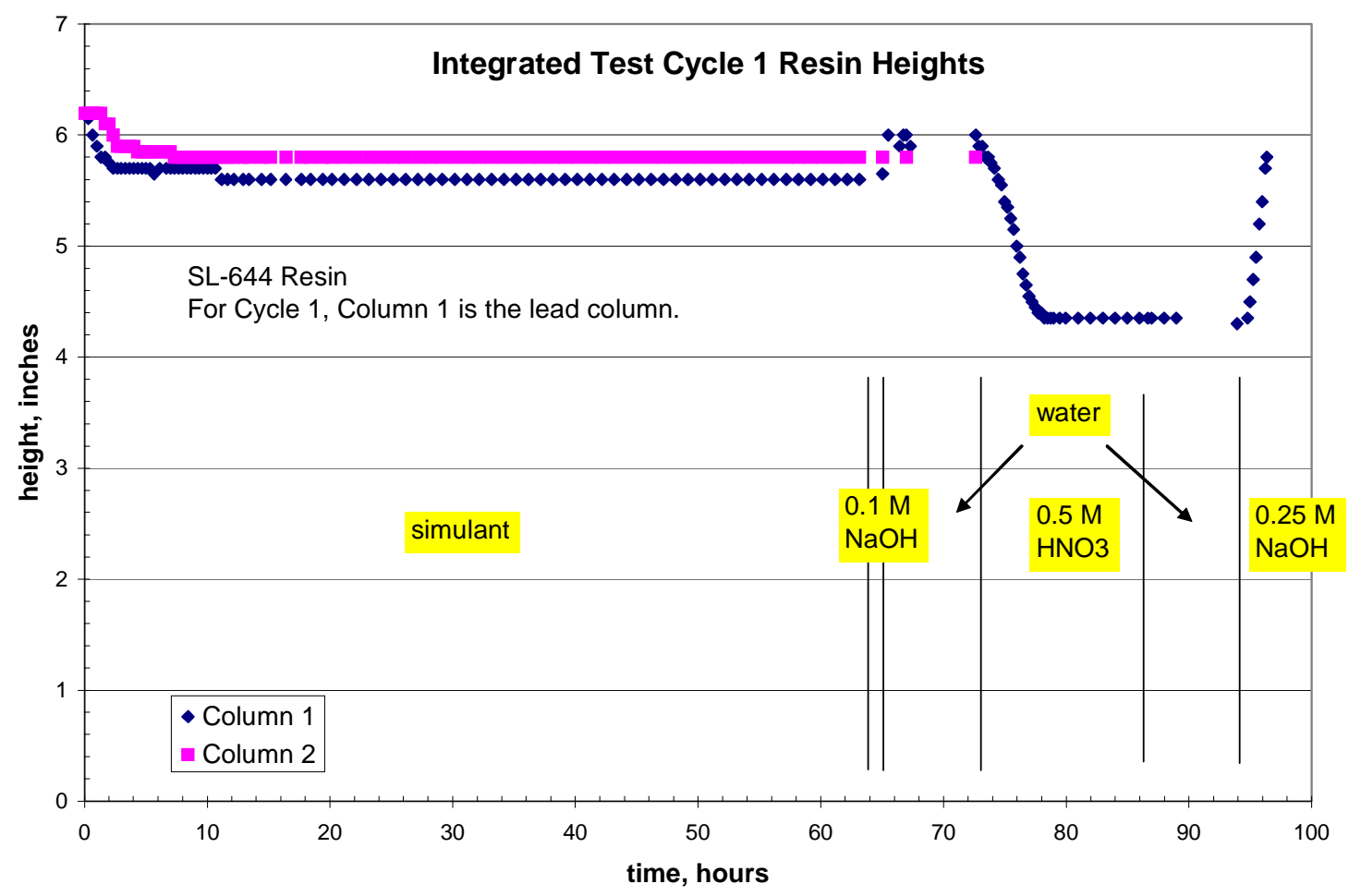

Figure 60. Resin Bed Height During Cycle 1

As mentioned in Section 2.8.2.3, during column regeneration, the $\mathrm{pH}$ and conductivity of the simulant exiting the lag column, or other solutions exiting the lead (only) column, were measured to determine the transition between solutions and also to give an indication of mixing and reaction of the different solutions in the column. To determine steady readings of the two probes, they were inserted in beakers of different solutions. The readings are listed in Table 43, which shows distinctly different values for each of the solutions. The theoretical $\mathrm{pH}$ of the two caustic solutions, 13.0 and 13.4, is higher than indicated in Table 43 because the $\mathrm{pH}$ probe was designed to have a range from 0 11.

Table 43. Probe Responses for Standard Solutions

\begin{tabular}{|c|c|c|}
\hline solution & conductivity & $\mathrm{pH}$ \\
\hline $0.1 \mathrm{M} \mathrm{NaOH}$ & 35 & 11.1 \\
\hline $0.25 \mathrm{M} \mathrm{NaOH}$ & 76 & 10.9 \\
\hline $0.5 \mathrm{M} \mathrm{HNO3}$ & 250 & 0.42 \\
\hline
\end{tabular}

Plots of $\mathrm{pH}$ and conductivity as a function of cumulative liquid volume for Cycle 1 are plotted in Figures 61 and 62. 
WSRC-TR-2004-00478, REVISION 0

SRNL-RPP-2004-00075, REVISION 0

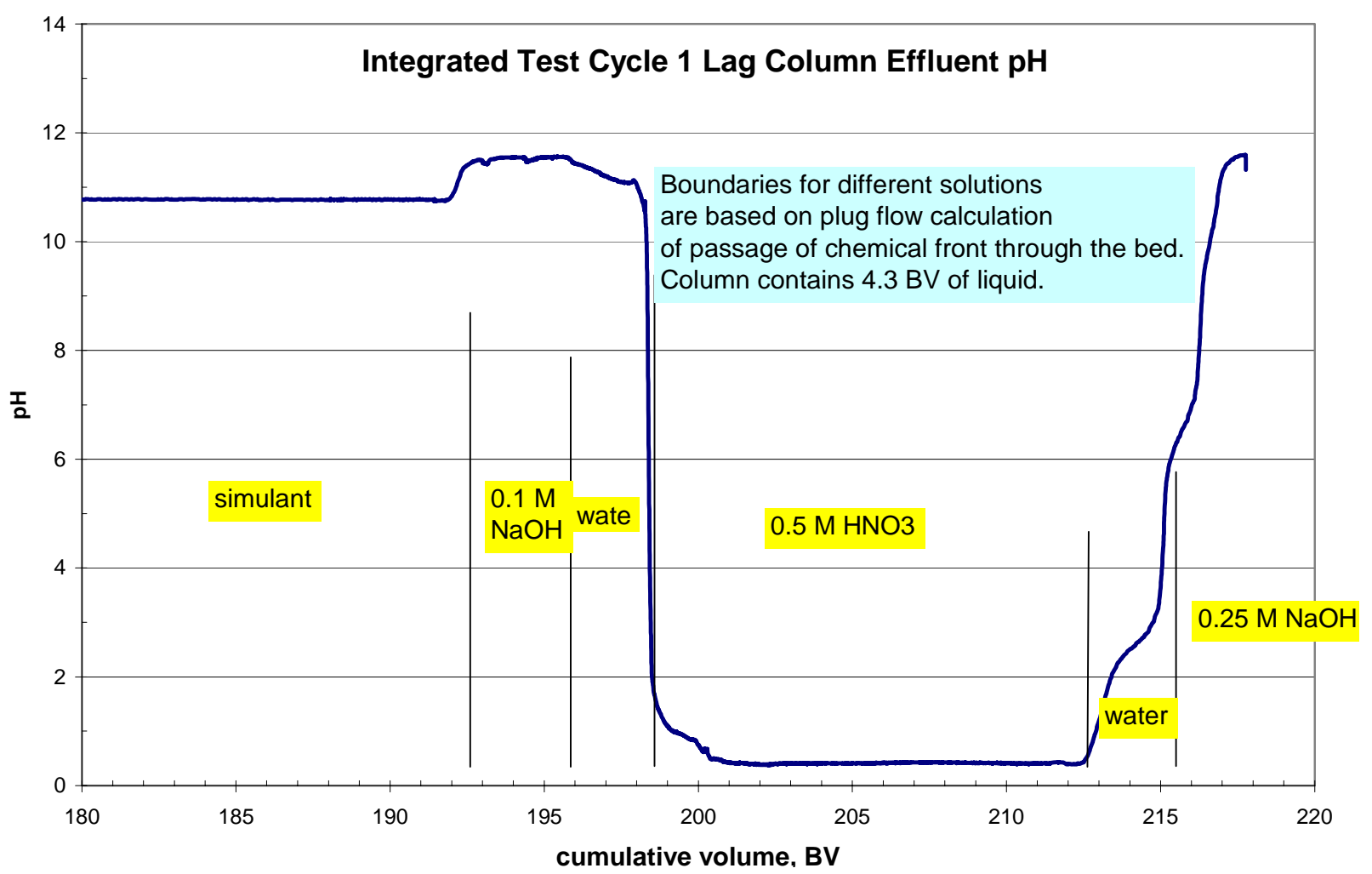

Figure 61. Effluent pH During Cycle 1

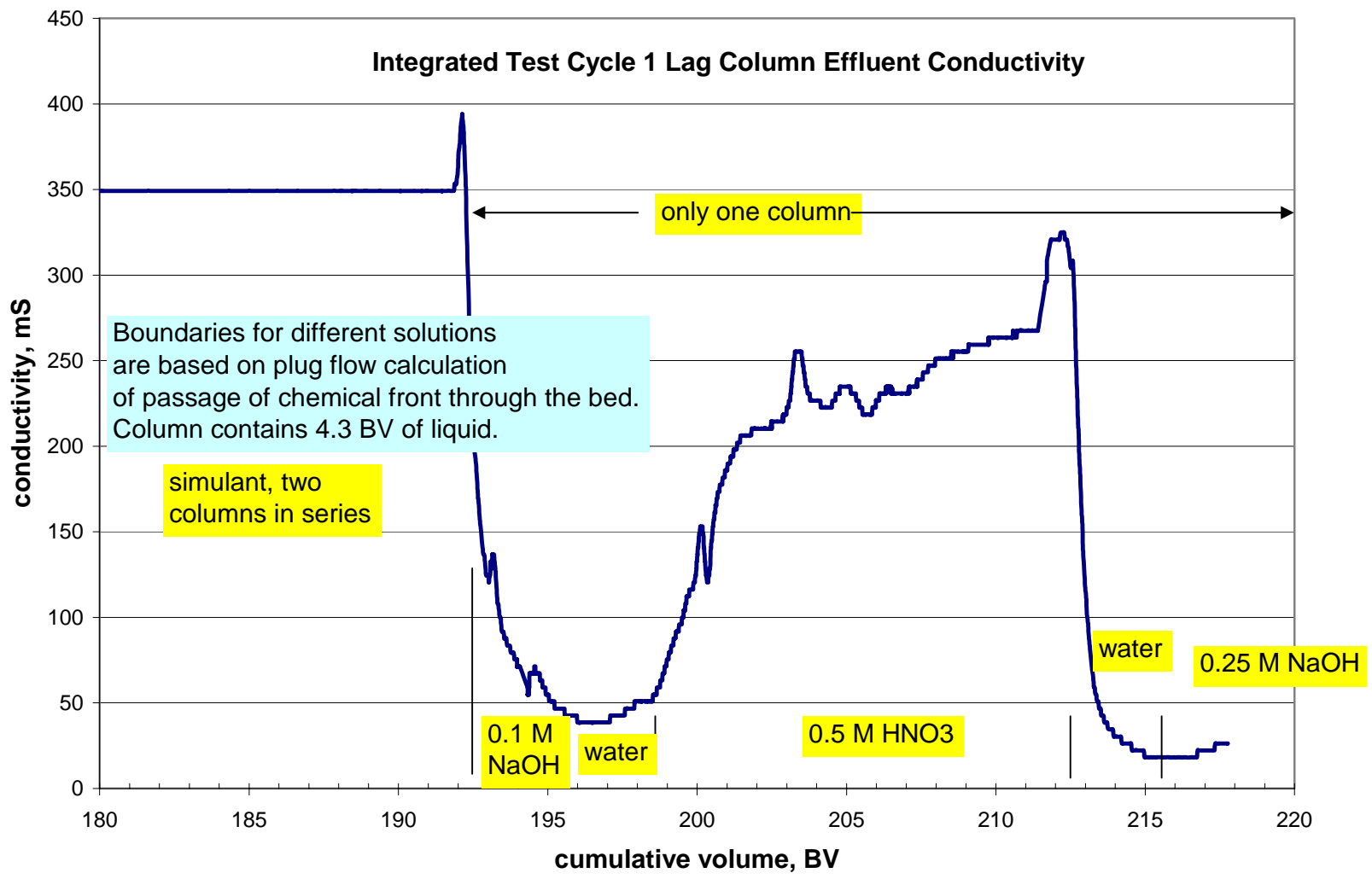

Figure 62. Effluent Conductivity During Cycle 1 
During testing, the $\mathrm{pH}$ and conductivity readings of the effluent streams did not always reach the steady state values of the standard solutions listed in Table 43. Also, during some transitions from one solution to the next solution mixing that occurred made the demarcation between each of the solutions difficult to discern. This was especially true between the $0.1 \mathrm{M} \mathrm{NaOH} /$ water and water/0.25 M NaOH interfaces, as seen in Figs. 61 and 62 for Cycle 1. Furthermore, SIPP was required by RPP-WTP to use prototypic volumes of each of the solutions, see Table 44, even though the SIP IX columns had proportionately more liquid freeboard than the latest WTP design. Because of the volumes used to transition from one to another solution were smaller than the internal volume of the columns, and because mixing occurred between the different density solutions, three solutions $(0.1 \mathrm{M} \mathrm{NaOH}$, pre-elution rinse water, and post-elution rinse water) did not have sufficient volume to fully displace the preceding solution.

Table 44. Comparison of the Volume for Each of the CIX Regeneration Process Solutions to the Internal Volume of Pilot-scale CIX Column

\begin{tabular}{|l|c|c|}
\hline Process solution & $\begin{array}{l}\text { Volume } \\
\text { pumped into } \\
\text { column, BV }\end{array}$ & $\begin{array}{l}\text { Internal } \\
\text { volume of } \\
\text { SIPP IX } \\
\text { column, BV }\end{array}$ \\
\hline $0.1 \mathrm{M} \mathrm{NaOH}$ & 3 & 4 \\
\hline Deionized water & 3 & 4 \\
\hline $0.5 \mathrm{M}$ HNO3 & 15 & 4 \\
\hline Deionized water & 3 & 4 \\
\hline $0.25 \mathrm{M} \mathrm{NaOH}$ & 6 & 4 \\
\hline
\end{tabular}

\subsubsection{CIX Chemical Observations}

\subsection{Cesium RPP-WTP Breakthrough Criterion}

When SIPP was in its planning stages during the Summer of 2003 (Duignan, 2003c), the breakthough criterion was defined as when the cesium concentration of the LAW exiting the lead column increased to $50 \%$ of that of the incoming feed stream. For Campaign II, the prototypic $5 \mathrm{M}$ sodium feed stream had a concentration of 5,900 $\mu \mathrm{g} / \mathrm{L}$ of cesium (See Tables 29 or 31). This implied measuring cesium concentrations of at least 2,950 $\mu \mathrm{g} / \mathrm{L}$. Such concentrations are not a problem to measure, and in fact subsequent tests submitted to the SRNL Analytical Laboratory, using an ICP-MS technique, demonstrated a minimum detection limit of $20 \mu \mathrm{g} / \mathrm{L}$. Even more sensitive measurements could be made if it were not for the high concentration of sodium, which requires heavy dilution of the sample. This ability was discussed during the pre-Campaign II test meeting held at SRNL on 23 March 2004 with the RPP-WTP customer. However, eleven days before SIPP Campaign II testing began, on 1 April 2004, the RPP-WTP customer informed SRNL that a new criterion (Toth, 2003) was to be used, which required the detection of the $0.3 \mathrm{Ci} / \mathrm{m}^{3}$ of Cs-137 from the CIX polishing column. Since SIPP had only lead and 
lag columns, then this criterion was to be applied to the lag column (Sundar, 2004). As given in Table 2 of Toth (2003), $0.3 \mathrm{Ci} / \mathrm{m}^{3}$ is equivalent to 3.7E-8 $\mathrm{M}$ in cesium or 5.2 $\mu \mathrm{g} / \mathrm{L}$. This magnitude of cesium presented a challenge to measure and, as described in the following section, was not overcome when tests with spiked samples were made. While cesium concentrations were reported down to $5 \mu \mathrm{g} / \mathrm{L}$, the uncertainty was large and lag column results may be more qualitative, than quantitative.

\subsection{Measurement Uncertainty}

The purpose of the resin is to remove cesium from LAW solutions that contain a mixture of constituents and that are $5 \mathrm{M}$ in sodium. Evaluation of the effectiveness of the resin requires the measurement of cesium concentration under challenging conditions, that is, with a high concentration of sodium. The Analytical Development Section (ADS) of SRNL claims an accuracy of $\pm 20 \%$ of the stated value. ADS accuracy includes the sum of bias and random error of two standard deviations. Because of the large sodium concentration, we decided to check that accuracy using spiked samples that were $5 \mathrm{M}$ in sodium. The results are shown in Fig. 63.

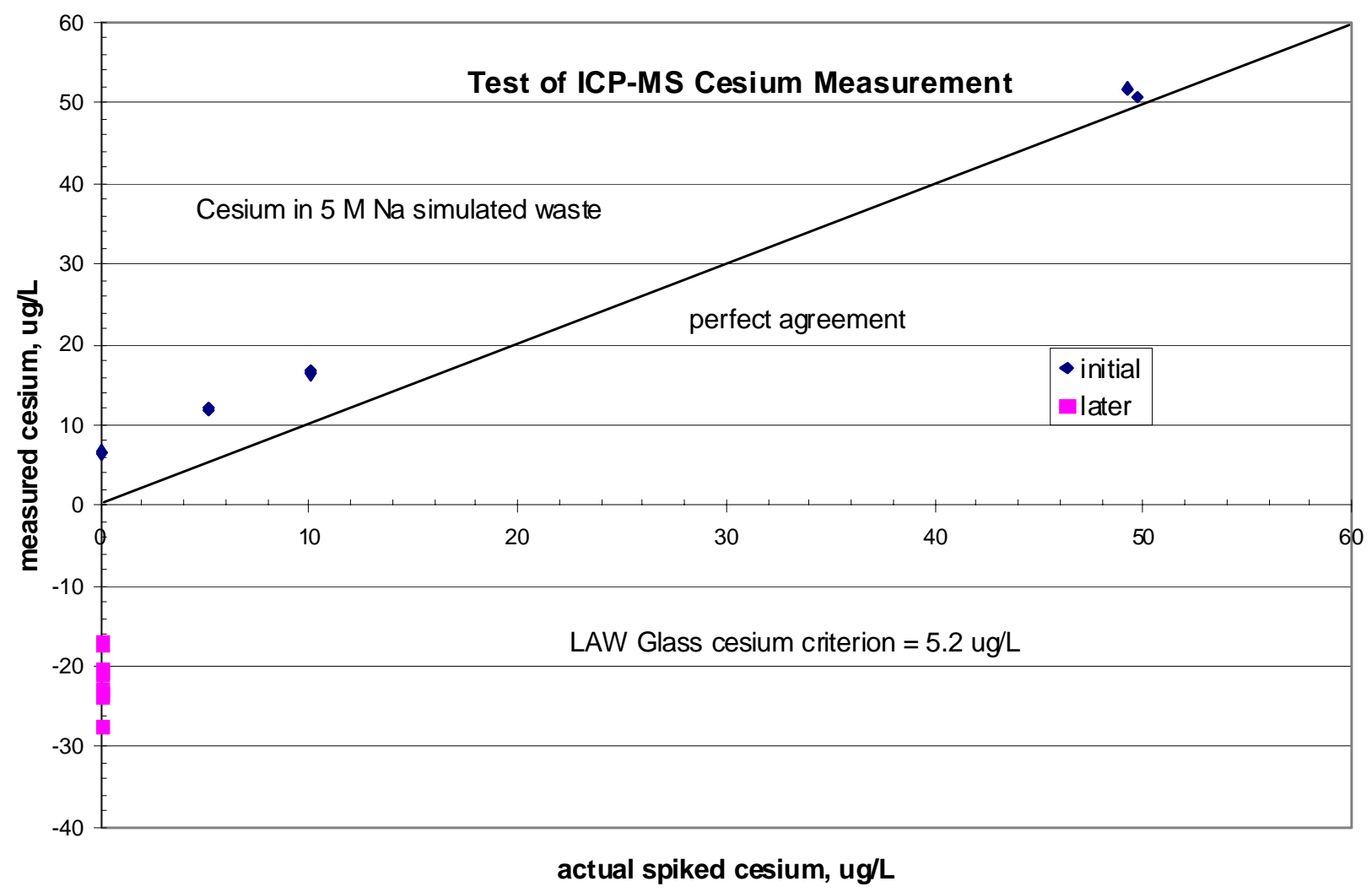

Figure 63. Test of Ability of ICP-MS to Measure Cesium

The initial results were encouraging and indicated a bias of less than $10 \mu \mathrm{g} / \mathrm{L}$ of cesium concentration for very low concentrations and less than $10 \%$ random error for concentrations of $50 \mu \mathrm{g} / \mathrm{L}$. Unfortunately, some later samples were analyzed to have 
negative concentrations as low as $-27 \mu \mathrm{g} / \mathrm{L}$. Of course, negative concentrations are physically impossible, but provide useful information about the uncertainty of the measurement. Since both positive and negative measurements errors were detected, it was decided to not use the concept of bias; that is, the entire error was considered to be random. Note that, even a random error of $\pm 10 \mu \mathrm{g} / \mathrm{L}$ would be insufficient to meet the new, more stringent, CIX criterion.

Previous measurements (Steimke, 2002), not plotted in Figure 63, for concentrations greater than $7000 \mu \mathrm{g} / \mathrm{L}$ confirmed a $2 \sigma$ random error of $\pm 10 \%$ for higher concentrations. Therefore, a conservative estimate for the random error of cesium concentration measurements for this data set is: $\pm(10 \%$ reading $+30 \mu \mathrm{g} / \mathrm{L})$. Thus, for this data set, the minimum detection limit for cesium in 5 molar sodium solution is $30 \mu \mathrm{g} / \mathrm{L}$. This random error must be kept in mind when evaluating such low concentrations. After we analyze data from Cycles 3 and 4 we will be able to reassess the magnitude of random error.

\subsection{Cesium Removal Effectiveness}

Simulant enters the lead column where more than $99 \%$ of the cesium is removed and then enters the lag column, where more cesium is removed. Figure 64 shows the cesium concentration exiting the lead column in Cycles 1 and 2, as a function of bed volumes passed.

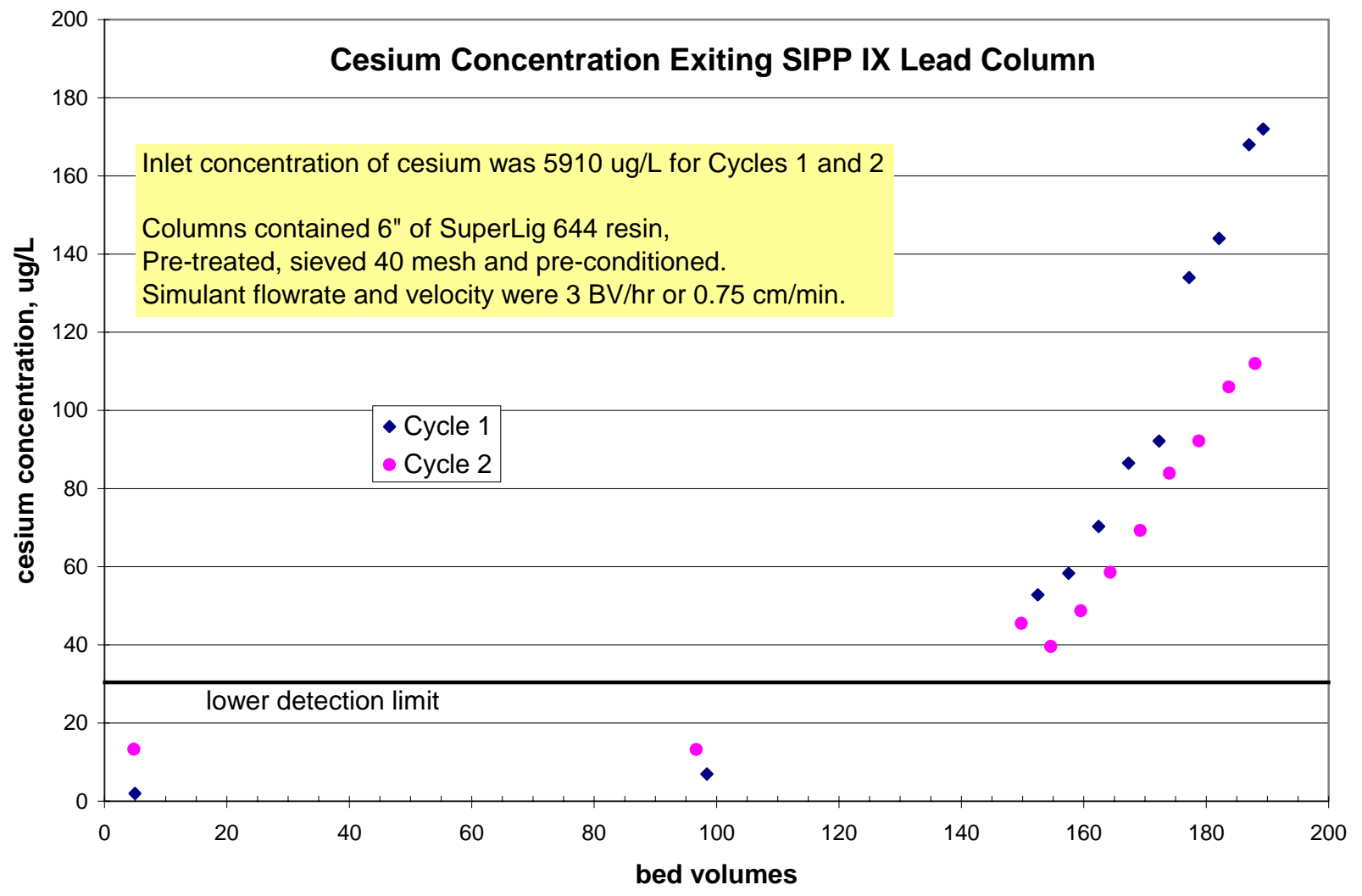

Figure 64. Cesium Concentration in Simulant Exiting Lead Column 
Initially, the cesium concentration was low, or perhaps really zero when considering analytical uncertainty, and then increased. Note that the effluent concentration in Column 1 (Cycle 1 lead column) increased sooner than in Column 2 (Cycle 2 lead column). As a possible explanation, both columns had the same volume of resin, but Column 2 may have had more mass, and therefore more capacity for removing cesium. In filling the columns with resin the requirement was to fill both columns to the same height using the same procedure, but it was possible that each column had slightly different packings, leading to slightly different masses. The Work Instruction for this activity calls for measurement of resin mass on a dry basis for both columns at the end of SIPP testing, i.e. after the Cycle 4 test. For both cycles the final effluent concentration did not exceed $3 \%$ of the inlet simulant feed concentration after processing at least 180 BV of simulant feed.

Figure 65 shows the cesium concentration exiting the lag column. Considering the uncertainty in the analytical measurement of cesium, all of the measurements may really be zero. Thus, given the measurement uncertainty and minimum detection limit, it is not possible to determine if the pilot scale IX unit operation met the RPP-WTP criterion for acceptance, i.e., less than $5.2 \mu \mathrm{g} / \mathrm{L}$ of cesium from the lag column. However, Fig. 64 clearly shows that at $180 \mathrm{BV}$ the lead column was still well within the old criterion, i.e., $175 \mu \mathrm{g} / \mathrm{L} / 5900 \mu \mathrm{g} / \mathrm{L}=0.03$ or $3 \%$, which is much less than the $50 \%$ breakthrough point.

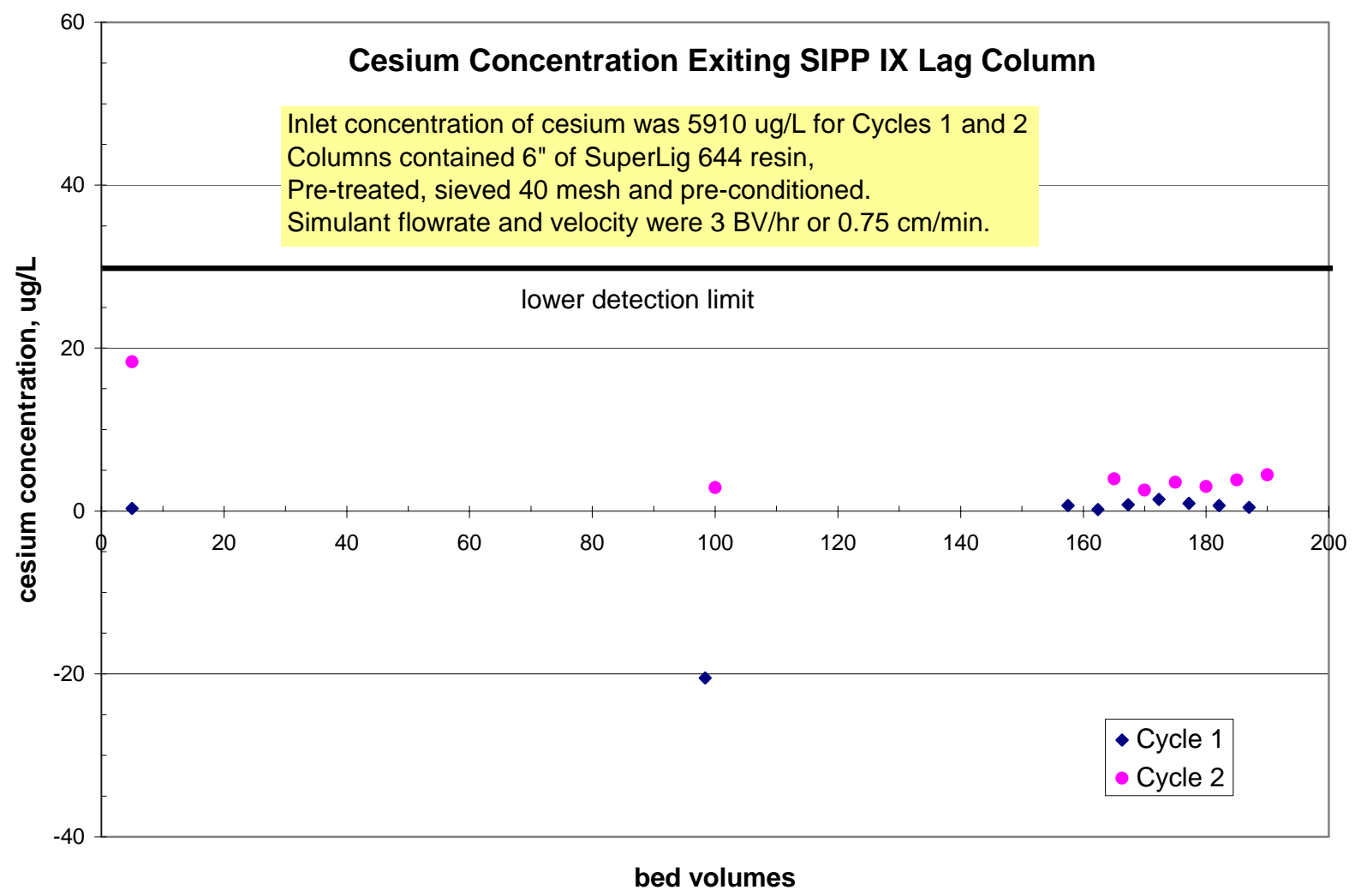

Figure 65. Cesium Concentration Exiting Lag Column 


\subsection{Cesium Mass Balance}

In the CIX unit operation, cesium enters the two IX columns dissolved in simulant and leaves the columns in simulant at a much lower concentration and also in eluate and other solutions. The total cesium mass in should equal the total cesium mass out plus any accumulation. For these IX columns, accumulation should be small. To perform this calculation, the LAW feed and receipt tanks were calibrated for volume. The mass balances for Cycles 1 and 2 are shown in Table 45 .

The rows of the table labeled "fresh simulant in" and "Cycle 1 spent" refer to inputs of cesium to the columns. The latter entry is the last simulant exiting the column during Cycle 1, which was set aside following WTP instructions and added to the simulant supply tank for Cycle 2. The rows labeled "simulant out” through "regenerant” are cesium outputs. The row labeled "final simulant" was the last simulant exiting the column and was collected separately from the rest of the simulant.

The volumes of simulant listed were measured using calibrated marks on the side of the simulant supply tank. Most of the rest of the volumes were computed using the measured flowrate and the elapsed time. Some of the volumes were computed from the mass of a solution and the measured density. The row labeled "0.1 M NaOH" for Cycle 2 includes pre-elution rinse and some of the eluate because the column discharge hose was moved too late. The row labeled "eluate" for Cycle 2 contains only the last part of the eluate.

The mass of cesium in a tank or jug is equal to the measured cesium concentration in the tank or jug multiplied by the volume. The biggest source of uncertainty in the mass of cesium was the uncertainty in the concentration. Total uncertainty for a particular solution was computed as the root sum square of the contributions from uncertainty in volume and uncertainty in concentration. The uncertainty of the simulant volume was \pm 3 liters and \pm 1 liter for all other solutions. The uncertainty in total mass of cesium in or cesium out was also computed using root sum square. Table 45 shows that the mass of cesium agreed with mass of cesium out, to within the stated uncertainty. 
WSRC-TR-2004-00478, REVISION 0

SRNL-RPP-2004-00075, REVISION 0

Table 45. Mass Balances for Cycles 1 and 2

\begin{tabular}{|c|c|c|c|c|c|c|c|c|c|c|c|}
\hline $\begin{array}{l}\text { Cycle } 1 \\
\text { Solution }\end{array}$ & $\begin{array}{l}\text { volume*, } \\
\text { liters }\end{array}$ & $\begin{array}{l}\text { cesium } \\
\text { conc., } \\
\text { mg/L }\end{array}$ & $\begin{array}{l}\text { ADS } \\
\#\end{array}$ & $\begin{array}{l}\text { cesium } \\
\text { mass, } \\
\text { mg }\end{array}$ & $\begin{array}{l}\text { mass } \\
\text { uncert- } \\
\text { ainty, mg }\end{array}$ & $\begin{array}{l}\text { Cycle } 2 \\
\text { Solution }\end{array}$ & $\begin{array}{l}\text { volume*, } \\
\text { liters }\end{array}$ & $\begin{array}{l}\text { cesium } \\
\text { conc., } \\
\text { mg/L }\end{array}$ & $\begin{array}{l}\text { ADS } \\
\#\end{array}$ & $\begin{array}{l}\text { cesium } \\
\text { mass, } \\
\text { mg }\end{array}$ & $\begin{array}{l}\text { mass } \\
\text { uncert- } \\
\text { ainty, mg }\end{array}$ \\
\hline $\begin{array}{l}\text { fresh } \\
\text { simulant in }\end{array}$ & 428 & 5.91 & 7820 & 2529 & 284 & $\begin{array}{l}\text { fresh simulant } \\
\text { in }\end{array}$ & 437 & 5.91 & 7820 & 2583 & 289 \\
\hline & & & & & & Cycle 1 spent & 9.4 & 0.12 & 8198 & 1 & 0 \\
\hline & & & & & & total Cs in & & & & 2584 & 289 \\
\hline & & & & & & & & & & & \\
\hline simulant out & 408 & 0.0213 & 9494 & 9 & 2 & simulant out & 427 & 0.00897 & 9495 & 4 & 1 \\
\hline $\begin{array}{l}\text { final } \\
\text { simulant }\end{array}$ & 9.4 & 0.12 & 8198 & 1 & 1 & final simulant & 20 & 0.102 & 8358 & 2 & 1 \\
\hline $\begin{array}{l}0.1 \mathrm{M} \\
\mathrm{NaOH}\end{array}$ & 12.2 & 0.344 & 8509 & 4 & 1 & $0.1 \mathrm{M} \mathrm{NaOH}$ & 44.5 & 50.6 & 8359 & 2252 & 277 \\
\hline $\begin{array}{l}\text { pre-elution } \\
\text { rinse }\end{array}$ & 0.7 & 70.8 & 8497 & 50 & 76 & $\begin{array}{l}\text { pre-elution } \\
\text { rinse }\end{array}$ & 0 & 0 & 8515 & 0 & 0 \\
\hline eluate & 31.5 & 87.2 & 8200 & 2747 & 363 & eluate & 6.8 & 2.47 & 8360 & 17 & 4 \\
\hline $\begin{array}{l}\text { post-elution } \\
\text { rinse }\end{array}$ & 7.5 & 0.887 & 8201 & 7 & 2 & $\begin{array}{l}\text { post-elution } \\
\text { rinse }\end{array}$ & 5.2 & 0 & 8361 & 0 & 0 \\
\hline regenerant & 4 & 0.0336 & 8510 & 0 & 0 & regenerant & 11 & 0.0109 & 8362 & 0 & 0 \\
\hline & & & & & & & & & & & \\
\hline total Cs out & & & & 2817 & 371 & total Cs out & & & & 2274 & 277 \\
\hline
\end{tabular}

* Volumetric measurement uncertainty for simulant is \pm 3 liters and \pm 1 liter for all other solutions 
An additional check on the cesium mass out was available for Cycle 1, i.e., the mass of cesium in the eluate was measured a second way. During Cycle 1 testing, samples were periodically taken during elution with the $0.5 \mathrm{M}$ nitric acid solution to measure how much cesium would be stripped from the column with time. That is, the cesium concentration spikes just after the front of acid reaches the bottom of the column, followed by a sharply decreasing concentration. Figures 66 and 67 plot the cesium concentrations of eluate samples discharging from Column 1. The Trapezoidal Rule and Simpson's Rule were used to numerically integrate the data in Fig. 66. In principal, these integrals should give the same mass of cesium as the mixed sample, but with more uncertainty because both Simpson's Rule and the Trapezoidal Rule are approximations. Simpson's Rule was applied two different ways. Recall that the equation for Simpson's Rule is:

$$
\int(x) d x=h / 3\left(f\left(x_{0}\right)+4 f\left(x_{1}\right)+2 f\left(x_{2}\right)+4 f\left(x_{3}\right)+\ldots .+4 f\left(x_{n-1}\right)+\left(f\left(x_{n}\right)\right)\right.
$$

where $\mathrm{h}$ is the difference in $\mathrm{x}$ between adjacent points. First, Simpson's Rule was applied to the data in Figure 66. Then, an additional point with concentration equal to zero was added to the beginning of the data set and another was added at the end of the data set. Then Simpson's Rule was applied again. The two applications of Simpson's Rule give different results depending on whether the data point with the largest concentration is multiplied by two or four. The results for cesium mass in the eluate follow.

Trapezoidal Rule 2,911 mg

Simpson's Rule \#1 2,679 mg

Simpson's Rule \#2 3,136 mg

Therefore, these three masses are consistent with cesium mass in the mixed eluate 2,747 $\pm 294 \mathrm{mg}$. 
WSRC-TR-2004-00478, REVISION 0

SRNL-RPP-2004-00075, REVISION 0

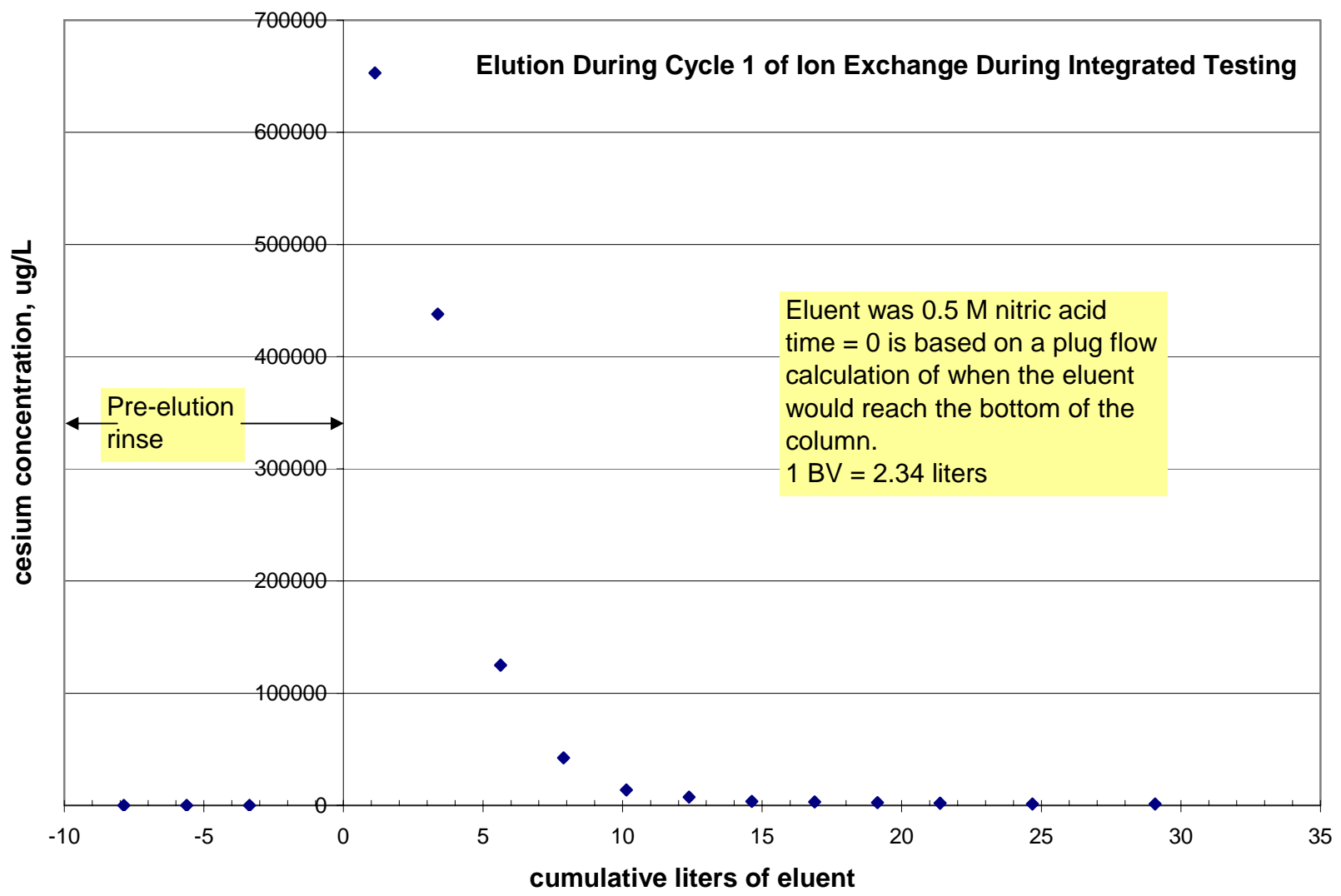

Figure 66. Elution Plot, Linear

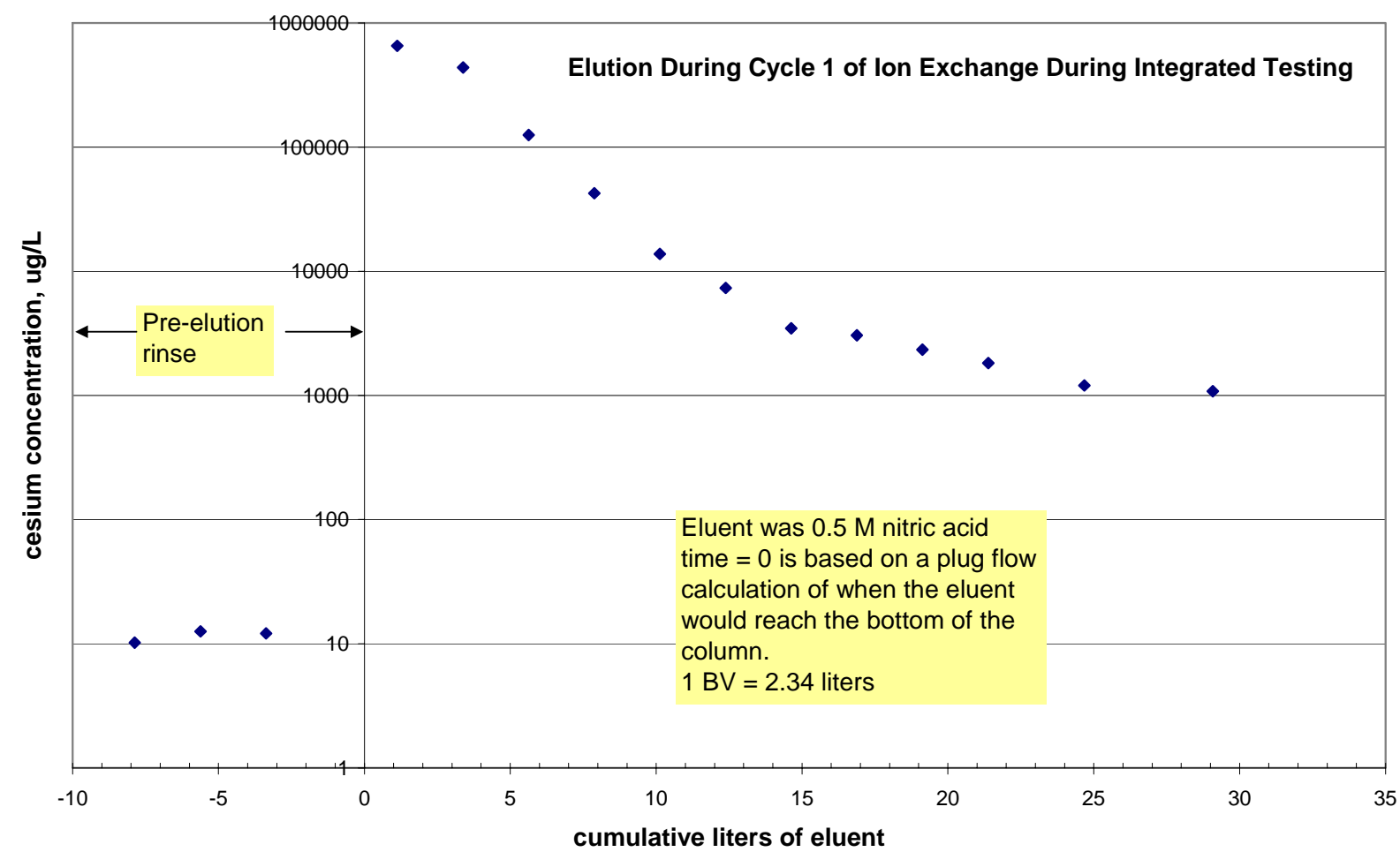

Figure 67. Elution Plot, Logarithmic 


\subsubsection{Pilot CIX Conclusions (Author - Steimke)}

- $\quad$ SuperLig ${ }^{\circledR} 644$ effectively removed cesium from simulated waste and the analytical ability to measure cesium concentrations in five molar sodium did allow verification of the waste acceptance criterion of $50 \%$ breakthrough from the lead column after 100 Bed Volumes of waste was treated. In fact, as shown in Fig. 51, in the worst case, Cycle 1, even after 180 BV of treated waste only the lead column exit cesium concentration was only $3 \%$ of the feed waste.

- $\quad$ SuperLig ${ }^{\circledR} 644$ effectively removed cesium from simulated waste, however, the analytical ability to measure very low cesium concentrations in five molar sodium did not allow verification of the waste acceptance criterion of $5.2 \mu \mathrm{g} / \mathrm{L}$ from the lag column after $100 \mathrm{BV}$ of waste was treated.

- The masses of cesium in and out of the resin beds for Cycles 1 and 2 balanced to within measurement uncertainty.

- $\quad$ Pressure drops throughout the two cycles were low and the resin beds permeabilities were about the same as were measured for the 24-inch Test (Fowley et al, 2004).

\subsection{PILOT TREATED LAW EVAPORATOR (AUTHORS - ADAMSON, WILLIAMS \& ZAMECNIK)}

The Treated LAW Evaporator Process, or TLP, is the last separation technology unit operation in the RPP-WTP Pretreatment Facility that a waste will see on start up. With respect to SIPP, it will be the last separation operation in line because it evaporates the treated LAW products that comes from the pilot CIX unit operation. This treated LAW product is then mixed with recycle streams to produce a feed stream for the TLP unit operation.

This section will explain the pilot TLP unit operation that was done as part of Campaign II testing. The process started with the preparation of the TLP feed product in a predetermined fashion, see Table 4 . That chemical explanation of the feed stream formation will be followed by a short description on how feed formation was handled just before TLP operation and then how the waste stream was handled during and after its concentration. The following section will discuss the physical equipment used to evaporate the waste and how the test was run. Finally, the results will be discussed and conclusions made.

\subsubsection{Chemical Description of the Pilot TLP Feed Stream (Author-Zamecnik)}

The TLP evaporator feeds consisted of the Ion Exchange product (Treated LAW) and the LAW SBS Condensate supplied by VSL. The compositions of these two feeds and the combined feed composition are listed in Table 46. Note that the calculated feed composition is the total composition including undissolved solids, whereas the measured feed composition is exclusive of solids. The insoluble metals Fe, Mg, and $\mathrm{Zr}$ are found to be higher in the calculated (total) value than in the supernate-only measurement, as expected. The undissolved solids content of the feed was only about $0.09 \mathrm{wt} \%$. At this level, the UDS measurement is subject to significant error, which could be as high as $\pm 100 \%$. However, there were visible solids in the feed. 
WSRC-TR-2004-00478, REVISION 0

SRNL-RPP-2004-00075, REVISION 0

Table 46. Measured Composition of TLP Feeds

\begin{tabular}{|c|c|c|c|c|}
\hline & IX Product & LAW SBS & $\begin{array}{l}\text { TLP Feed } \\
\text { Calculated }\end{array}$ & $\begin{array}{l}\text { TLP Feed } \\
\text { Measured }\end{array}$ \\
\hline Total Solids (wt\%) & 27.93 & 0.26 & 16.30 & 16.24 \\
\hline Undissolved Solids (wt\%) & 0.00 & 0.17 & 0.07 & 0.09 \\
\hline Supernate Solids (wt\%) & 27.93 & 0.09 & 16.24 & 16.16 \\
\hline Density (kg/L) & 1.247 & 1.002 & 1.131 & 1.136 \\
\hline Dissolution/Prep & None & Dissolution & Total & Supernate \\
\hline $\begin{array}{ll}\text { Elements/Anions } & \mathrm{Ag}\end{array}$ & $<0.6$ & $<4.01$ & & $<0.6$ \\
\hline in $\mathrm{mg} / \mathrm{L} \quad \mathrm{Al}$ & 7190 & 7.75 & 3782 & 3770 \\
\hline B & 79.5 & 136 & 106 & 91.7 \\
\hline $\mathrm{Ba}$ & $<0.04$ & $<0.27$ & & $<0.04$ \\
\hline $\mathrm{Ca}$ & 0.38 & $<0.40$ & 0.20 & 2.22 \\
\hline $\mathrm{Cd}$ & $<0.04$ & $<0.27$ & & $<0.04$ \\
\hline $\mathrm{Ce}$ & $<17.3$ & $<10.2$ & & $<17.3$ \\
\hline Co & $<0.09$ & $<1.07$ & & $<0.086$ \\
\hline $\mathrm{Cr}$ & 160 & 5.42 & 86.7 & 89.8 \\
\hline Cs & 0.0025 & 0.075 & 0.037 & 0.17 \\
\hline $\mathrm{Cu}$ & 0.28 & $<0.40$ & 0.15 & 0.16 \\
\hline $\mathrm{Fe}$ & 1.33 & 12.0 & 6.41 & 0.558 \\
\hline $\mathrm{K}$ & 18900 & 127 & 9992 & 9530 \\
\hline $\mathrm{La}$ & $<0.38$ & $<1.33$ & & $<0.38$ \\
\hline $\mathrm{Li}$ & 1.07 & 11.9 & 6.19 & 4.65 \\
\hline $\mathrm{Mg}$ & $<0.03$ & 3.67 & 1.74 & $<0.026$ \\
\hline $\mathrm{Mn}$ & $<0.03$ & $<0.12$ & & $<0.034$ \\
\hline Mo & 9.74 & $<1.33$ & 5.12 & 5.44 \\
\hline $\mathrm{Na}$ & 110000 & 605 & 58094 & 59000 \\
\hline $\mathrm{Na}$ (AA) & 109000 & 607 & 57569 & 62400 \\
\hline $\mathrm{Nd}$ & $<0.81$ & $<11.9$ & & $<0.81$ \\
\hline $\mathrm{Ni}$ & 0.302 & 0.35 & 0.33 & 0.28 \\
\hline $\mathrm{P}$ & 775 & $<10.7$ & 407 & 403 \\
\hline $\mathrm{Pb}$ & 15.3 & $<10.7$ & 8.02 & 8.09 \\
\hline $\mathrm{Rb}$ & 4.72 & & 2.48 & 2.73 \\
\hline $\mathrm{Re}$ & 0.572 & 7.19 & 3.71 & 9.41 \\
\hline $\mathrm{S}$ & 1152 & 80.3 & 644 & 667 \\
\hline $\mathrm{Si}$ & 57.0 & 22.5 & 40.6 & 41.7 \\
\hline $\mathrm{Sr}$ & 0.072 & $<0.072$ & 0.038 & 0.016 \\
\hline $\mathrm{Ti}$ & $<0.12$ & 1.18 & 0.56 & $<0.12$ \\
\hline Zn & 3.05 & 1.32 & 2.23 & 12.6 \\
\hline $\mathrm{Zr}$ & 0.51 & 1.32 & 0.90 & $<0.16$ \\
\hline bromide & $<100$ & $<88.6$ & 879 & $<100$ \\
\hline chloride & 1090 & 646 & 96.6 & 753 \\
\hline fluoride & 48.0 & 150 & 552 & 70.0 \\
\hline formate & 1020 & 34.0 & 39164 & 626 \\
\hline nitrate & 74400 & 139 & 13032 & 42600 \\
\hline nitrite & 22400 & 2657 & 363 & 13500 \\
\hline oxalate & 690 & $<88.6$ & 1161 & 389 \\
\hline phosphate & 2210 & $<88.6$ & 1617 & 1290 \\
\hline sulfate & 2860 & 240 & 973 & 1720 \\
\hline ammonium & & 2050 & 1233 & 998 \\
\hline unbound hydroxide & 2346 & & & 1081 \\
\hline carbonate & & & & 20150 \\
\hline total organic carbon & & & & 1360 \\
\hline
\end{tabular}




\subsubsection{Simulant Handling (Author - Williams)}

To make feed for the pilot-scale TLP, on 05/10/04 the 1,075 kg of treated simulant processed through the CIX were mixed with $800.1 \mathrm{~kg}$ of LAW SBS Quencher from VSL, a recycle stream. For Campaign II, no $0.25 \mathrm{M} \mathrm{NaOH}$ recycle stream from the CIX was used as per the Test Specification (Longwell, 2003). However, the mixing was done only intermittently as the ammonia smell was quite strong. Additional ventilation was employed for all operations subsequent to LAW SBS Quencher addition due to strong ammonia smell. Samples were pulled for analysis during and following TLP Evaporator operations to measure the ammonia concentration. Figure 68 (a) and (b) shows both the feed to the evaporator and the resulting bottoms after concentration. Figure 68 (c) show the bottom of the TLP Bottoms sample bottle, i.e., Fig. 68 (b), to highlight color and small amount of undissolved solids.

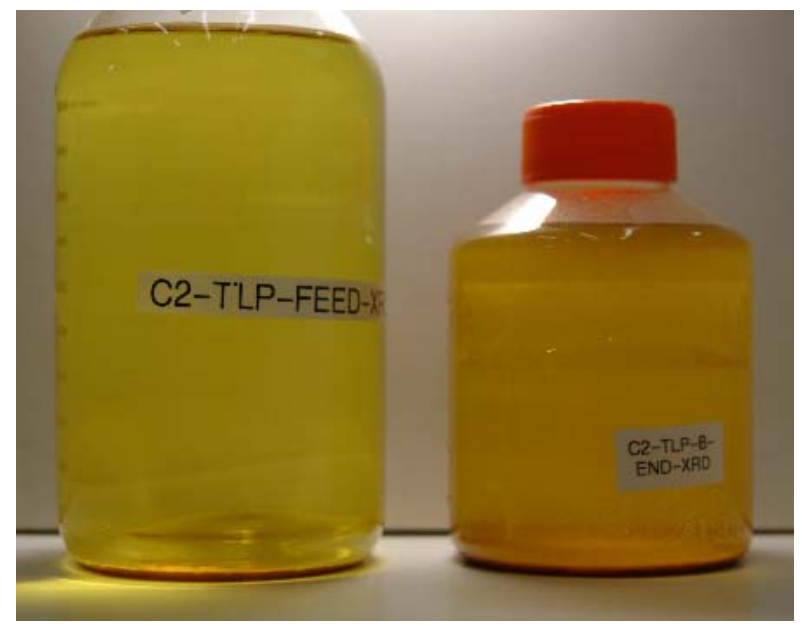

(a) TLP Feed (b) TLP Bottoms

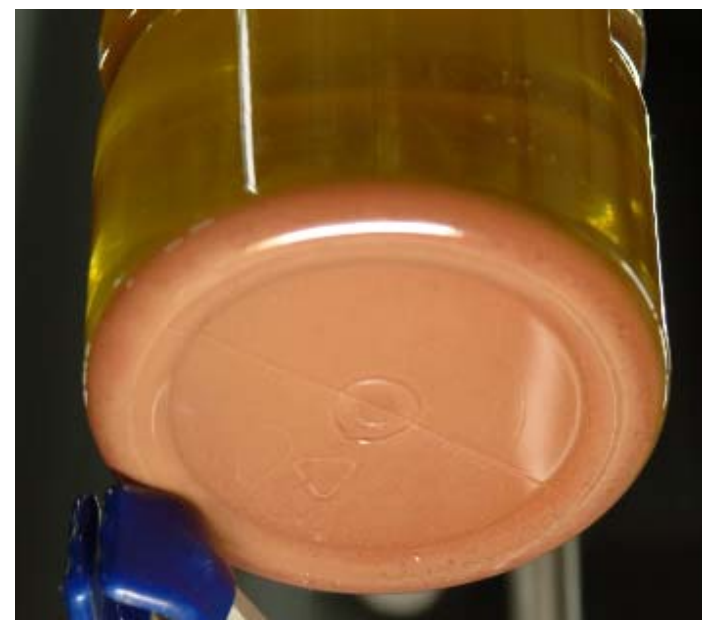

(c) Bottom view of Fig. TLP1 (b)

Figure 68. Simulated TLP Feed and Resulting Bottoms Concentrate

Upon startup, on 12 May, 2004 of TLP operations, a total of 309 g of DOW Corning Q23183A Antifoam was added directly into the evaporator in three batches of $103 \mathrm{~g}$ each in a 10:1 ratio with DIF water. The dilution was necessary to fully incorporate the antifoam because of its thick consistency. Despite having fewer recycle streams, the total volume of the TLP feed was larger than that for the FEP. Considering that the concentration target was different from the FEP bottoms, then the final concentrated TLP volume would be much larger than that for the FEP, in fact it was approximately 3.5 times larger. This meant that a steady-state evaporated concentration would be attained before exhausting the feed. Once the target slurry concentration was realized, then the TLP bottoms would have to be removed while more feed was being sent into the TLP at a rate that would keep the antifoam concentration at the target level of $1500 \mathrm{ppm}$. Of course, removing concentrated slurry meant removing its antifoam too. Therefore, antifoam had to be reintroduced periodically to maintain its concentration constant. Additional 
amounts of antifoam were added in batches and after approximately each 76 liters of TLP bottoms were removed, 154 grams of antifoam were added.

A total of $713 \mathrm{~kg}$ of TLP Evaporator bottoms were produced at 8M Na and placed in three drums labeled "Campaign II LAW Concentrated Slurry (Drum 1 of 3, 2 of 3, and 3 of 3)" for shipment to VSL on 13 May. After Campaign II was complete, the condensate collected from both the TLP and FEP operations were combined. This mixing is how WTP will handle its condensate streams, which will then be made available to produce wash and caustic solutions. For SIPP the combined condensate streams will be used for Campaign III wash and caustic solutions.

\subsubsection{Pilot TLP Equipment (Author - Adamson)}

One of the requirements for SIPP as per the Test Specification (Longwell, 2003) was to utilize and modify existing equipment in the Engineering Development Laboratory of SRNL. To that end only one pilot-scale evaporator was constructed to be used for both FEP and TLP testing because the design of those RPP-WTP Pretreatment Facility evaporators is the same. The task plan for those two evaporators for SIPP did not require simultaneous operation, therefore, once one unit operation test completed the test rig could be made ready for the subsequent test. As such, the description of the pilot-scale TLP is the same as for the pilot-scale FEP and therefore will not be repeated here. Please refer to equipment description for the FEP in section 2.6.3.

\subsubsection{Pilot TLP Operation (Author - Adamson)}

Prior to the TLP evaporation campaign, the evaporator system was cleaned and inspected. The evaporator system was cleaned and then operated with water for approximately two hours to make sure it was completely free of material from its last use. Subsequently, a turbidity test was conducted on the spent water in the system to ensure the system was clean and it had a value of $0.95 \mathrm{NTU}$, which indicated it was free of measurable solids.

The TLP evaporator was operated using Work Instructions, I-EDL-132 "SIPP Treated LAW Process (TLP) Evaporation Test”. Manually recorded information was recorded in pilot-scale TLP notebook (Adamson, 2004).

The pilot-scale TLP started with 1,638.8 liters (432.9 gallons) of simulated treated LAW feed. It took approximately 12 hours to boil off 1,115.7 liters (394.7 gallons) of water from the feed that had an initial feed density of $1.14 \mathrm{~kg} / \mathrm{L}$ to obtain the target density of $1.395 \mathrm{~kg} / \mathrm{L}$ at $25^{\circ} \mathrm{C}$. The total volume of the TLP Campaign II concentrated product was 523.1 liters (138.2 gallons).

The average boil off rate (condensate) for the TLP campaign was $1.58 \mathrm{~L} / \mathrm{min}(0.42$ GPM), which compared well to the design boil-off target rate of the RPP Pilot-scale Evaporator system of $1.5 \mathrm{~L} / \mathrm{min}(0.40 \mathrm{GPM})$. 
WSRC-TR-2004-00478, REVISION 0

SRNL-RPP-2004-00075, REVISION 0

Evaporation of the TLP simulant feed occurred at approximately $55^{\circ} \mathrm{C}$ under -13 psig vacuum (1.65 psia), since the atmospheric pressure was approximately 14.65 psia during the campaign. However, due to periodic removals of the TLP concentrate, followed by additions of cooler simulant feed, there were fluctuations in the evaporator pot temperature and pressure. The time histories of those fluctuations are shown in Figs. 69 and 70, respectively.

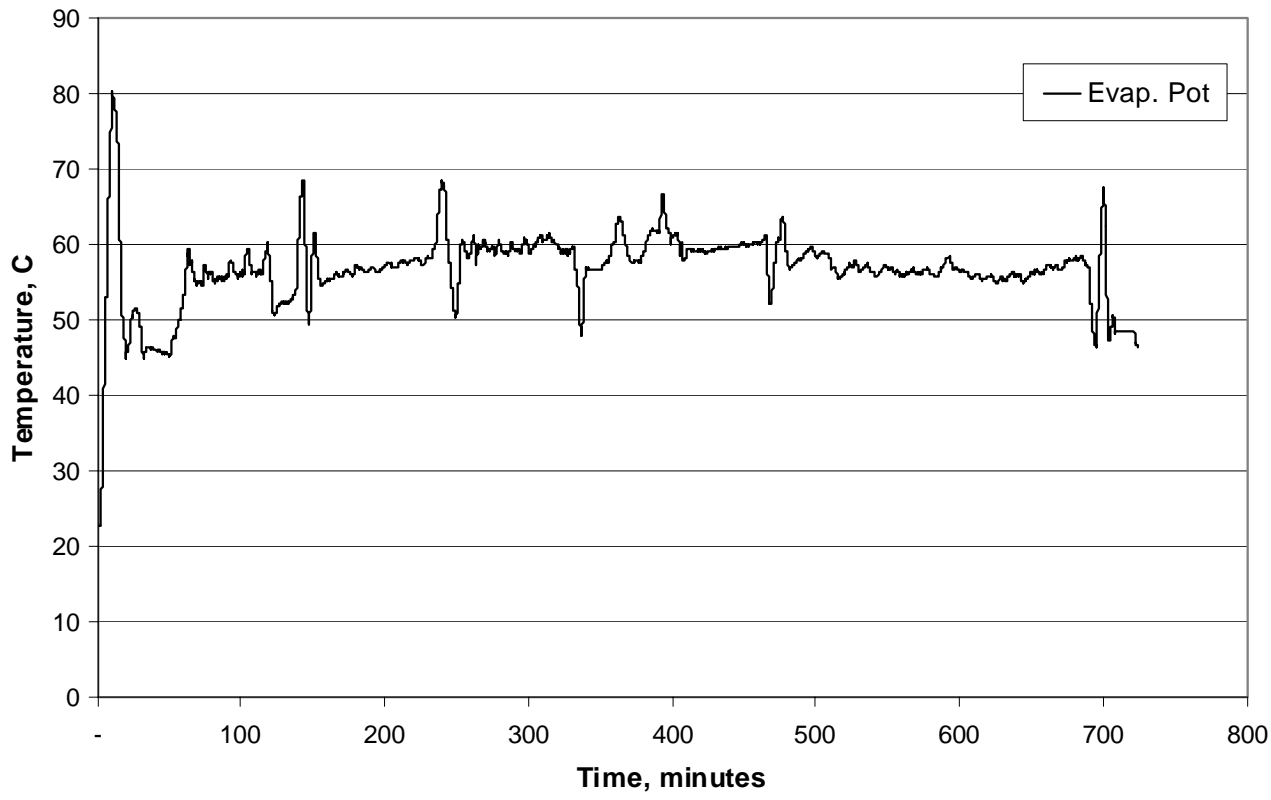

Figure 69. TLP Evaporator Pot Temperature History

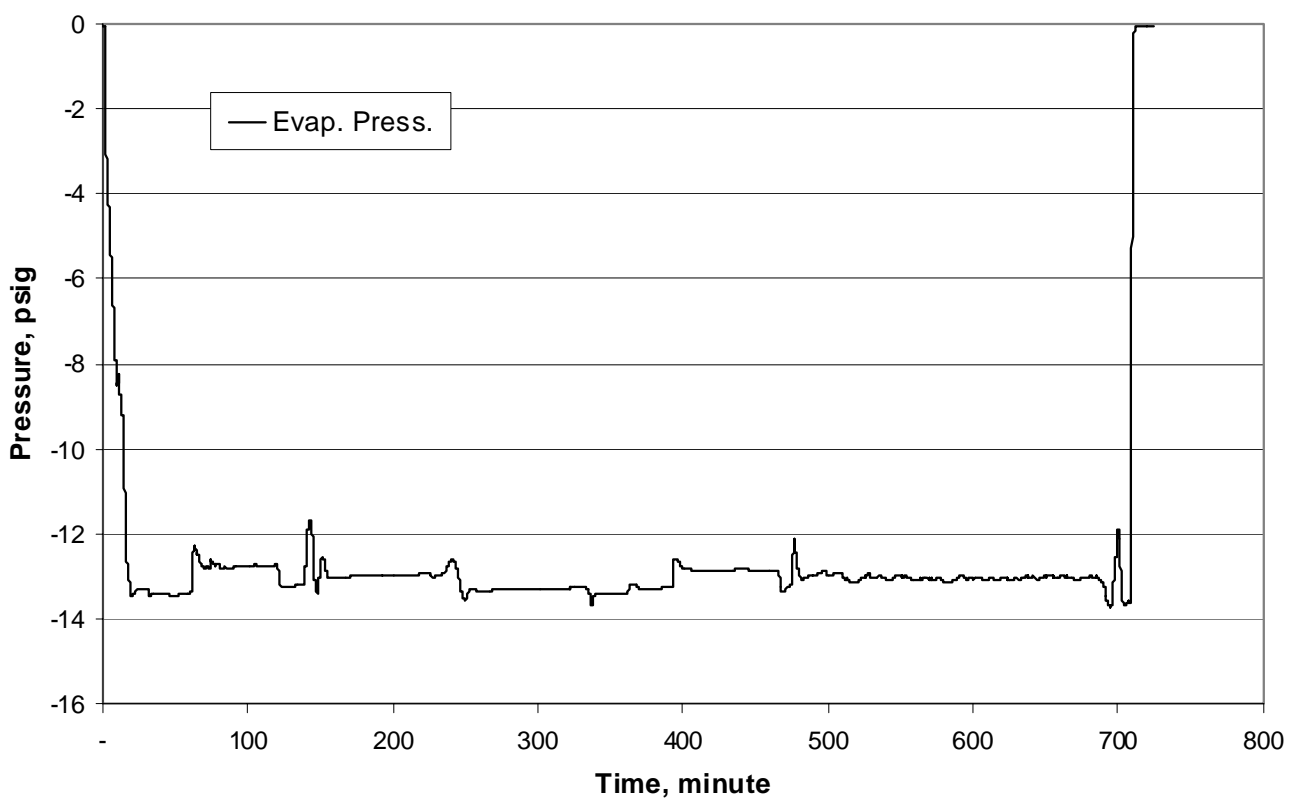

Figure 70. TLP Evaporator Pot Pressure History 
The TLP simulant was re-circulated through the reboiler and evaporator system at approximately 85 gpm as shown in Fig. 71 below. The recirculation of the simulant was continuous for the duration of the campaign.

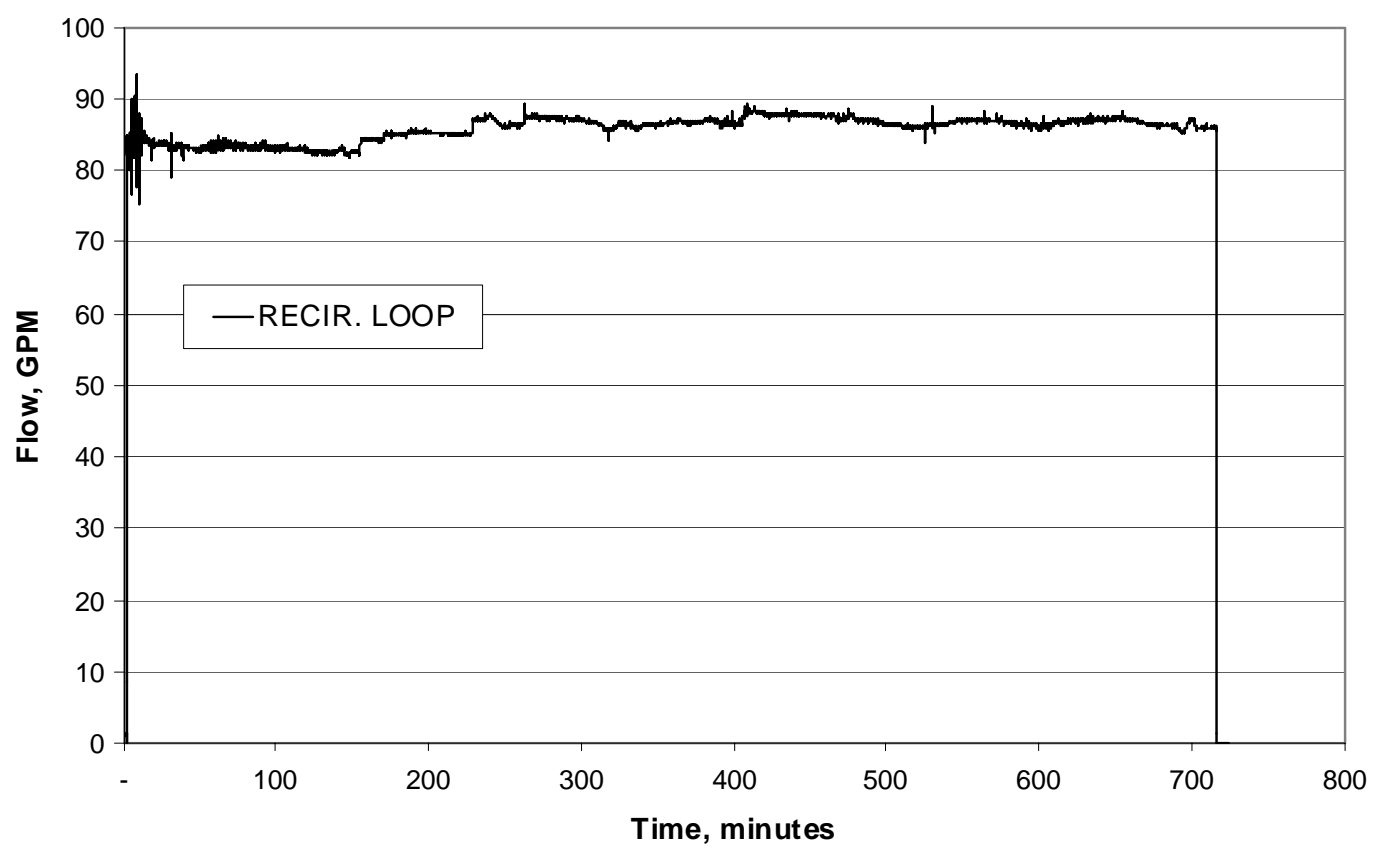

Figure 71. TLP Simulant Recirculation Flow in Evaporator System

A total of 926.4 grams of Q2 antifoam was added to the simulant during the TLP campaign II evaporation process. A dilute mixture of antifoam was pulled into the system via vacuum through valve CL-BV13 at the bottom of the evaporator pot. Because the antifoam chemical was relatively thick, the manufacturer recommends diluting it with DI water to a mixture of 10:1 before use. Initially 309 grams of Q2 were added to the simulant and then, as concentrate was removed additional Q2 was added, in four subsequent equal batches of 154 grams as concentrated bottoms was removed. While the goal was an antifoam concentration of $1500 \mathrm{ppm}$ the final concentration ended up being $1300 \mathrm{ppm}$ because of the pilot-scale TLP batch operation. That is, the experiment started at $1500 \mathrm{ppm}$, with a fixed evaporator volume of approximately 150 liters, and stayed that way until the feed, within the evaporator, reached the target density of 1.36 $\mathrm{kg} / \mathrm{L}$ at $50^{\circ} \mathrm{C}$. In this phase, the feed rate matched the evaporation rate. Once at density, the batch mode operation began with slowly allowing feed to enter the evaporator slightly faster than the evaporation rate to maintain the density while allowing the evaporator volume to increase to approximately 225 liters. At this point the antifoam concentration dropped to $1500 \mathrm{ppm} \times(150 / 225)=1000 \mathrm{ppm}$. No increased foaming was noted and previous work (Qureshi et al., 2004) has shown that antifoam beyond $300 \mathrm{ppm}$ is not effective. Once at 225 liters, then 75 liters of bottoms were removed to begin the next batch. This process continued until all the feed was consumed. However, a further drop in antifoam concentration was not desired, therefore enough antifoam was added so that the next 75 liters of concentrated feed would also have a $1500 \mathrm{ppm}$ concentration. When 
the process was finished the antifoam concentration of the composite product was 1300 ppm, based on the 926 grams of antifoam in 523 liters $(711 \mathrm{~kg})$ of bottoms produced. During the entire evaporation period very little foaming was witnessed.

To evaluate the pilot-scale TLP unit operation, samples from primary condensate, secondary condensate, and evaporator bottoms were pulled every 200 liters of simulant feed pumped into the evaporator. Along with those samples, other, more frequent samples, were pulled of the evaporator bottoms to check the density of the concentrate to determine when the target slurry density was attained. The density was checked using a density meter (Anton Paar, DMA 4500) and verified using a $200 \mathrm{ml}$ calibrated flask and a scale (M\&TE: DWB-514). All density measurements were recorded in the lab notebook.

After all the feed was evaporated the remaining bottoms in the pilot-scale TLP were removed and added to the concentrate previously removed. As already mentioned the final volume of concentrate produced was 523.1 liters (138.2 gallons), which was put into three 55-gal drums. As the drums were being filled from the evaporator, hot samples (at $50^{\circ} \mathrm{C}$ ) were taken to measure the densities to verify that the target bottoms density of 1.36 $\mathrm{g} / \mathrm{ml}$ was being maintained. Table 47 shows the average values of those density measurements, as well as the volume and mass of each drum's contents. The table also includes values for the initial feed simulant at $25^{\circ} \mathrm{C}$.

Table 47. TLP Simulant and Concentrate

\begin{tabular}{|l|c|c|c|}
\hline TLP Simulant & Volume, liter (gallon) & Density ( $\mathbf{0 . 0 1} \mathbf{~ k g / L )}$ & Weight $(\mathbf{k g})$ \\
\hline TLP Feed Simulant & 1,638.8 (432.9) & $1.14^{*}$ & $1,875.1$ \\
\hline Drum \# 1 concentrate & 155.4L (41.1) & $1.36^{* *}$ & 211.4 \\
\hline Drum \# 2 concentrate & 154.3L (40.8) & $1.37^{* *}$ & 211.4 \\
\hline Drum \# 3 concentrate & 213.4L (56.4) & $1.36^{* *}$ & 290.2 \\
\hline
\end{tabular}

* Temperature at $25^{\circ} \mathrm{C}$.

** Temperature at $50^{\circ} \mathrm{C}$. Also note that the target concentrate (bottoms) density of $1.36 \mathrm{~kg} / \mathrm{L}$ was at the evaporator pressure of 1 psia. While the densities for the concentrate going to the drums were measured at the correct temperature, the pressure was atmospheric, i.e., 14.65 psia. However, because of the very small compressibility of liquids, the effect of pressure on the slurry density is not significant in values given above.

A total of 1,115.7 liters (294.7 gal) of clean condensate (water) was removed from the simulant via evaporation to obtain 523.1 liters (138.2 gal) of concentrate with a density of $1.36 \mathrm{~kg} / \mathrm{L}$ at $50^{\circ} \mathrm{C}$ or $1.39 \mathrm{~kg} / \mathrm{L}$ at $25^{\circ} \mathrm{C}$. The concentrate was stored for uses at the Vitreous State Laboratory (VSL) of Catholic University of America in Washington, D.C. for further testing/processing in the pilot-scale LAW melter for the RPP Project. 


\subsubsection{Pilot TLP Discussion of Results (Author - Adamson)}

\subsubsection{Experimental Observations}

The SIPP TLP evaporation Campaign II was conducted on Wednesday, May 12, 2004 in SRNL's Engineering Development Laboratory. A total of 1,638.8 liters of TLP simulant feed was concentrated down to about 523.1 liters in a continuous run. The evaporation process was completed without any glitch or equipment failure. However, the Demister ${ }^{\circledR}$ instrumentation had to be purged during the campaign due to moisture in the lines causing inaccurate readings.

Essentially no foaming was witnessed during the TLP campaign, however frothing was seen throughout the campaign as shown in Fig. 72. The picture depicts the simulant boiling inside the acrylic section of the evaporator pot. Makeup antifoam was added but visual inspection would suggest that antifoam was not required to prevent foaming during the TLP evaporation process.

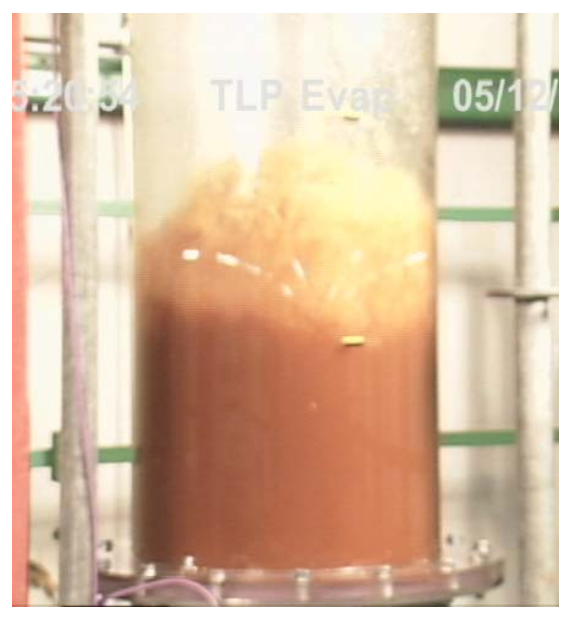

Figure 72. TLP Simulant Boiling in Clear Acrylic Section of Evaporator

\subsubsection{TLP Waste Products}

The TLP evaporator product and the predicted composition are shown in Table 48. The values for the various streams agree reasonably well, but a number of the concentrations found for the dissolution sample were significantly off from what was expected, and therefore were not shown here. This sample is being reanalyzed and results will be reported in the final SIPP report.

Figure 73 shows the measured total solids, undissolved solids, and density of the evaporator bottoms plotted versus the approximate amount of feed added. A material balance model was applied to the evaporator, and the results are shown as the solid lines. There was significant scatter in the solids measurements, and the final mass of concentrated bottoms plus condensate calculated was about $22 \mathrm{~kg}$ lower than the feed amount. This minor discrepancy may be due to a change in total volume upon 
evaporation - that is, if the condensate were $1,138 \mathrm{~L}$ vs. the calculated 1,116 L, the balance would be close. These data do not indicate any precipitation of additional solids beyond the amount present in the feed. Analyses of the Dow Corning Q2-3183A antifoam are pending.

Table 48. Measured TLP Products

Bottoms Bottoms Bottoms

$\begin{array}{lllll}\text { All values mg/L except as noted } & \text { (Calculated) } & \text { (Measured) } & \text { (Supernate) } & \text { Condensate }\end{array}$

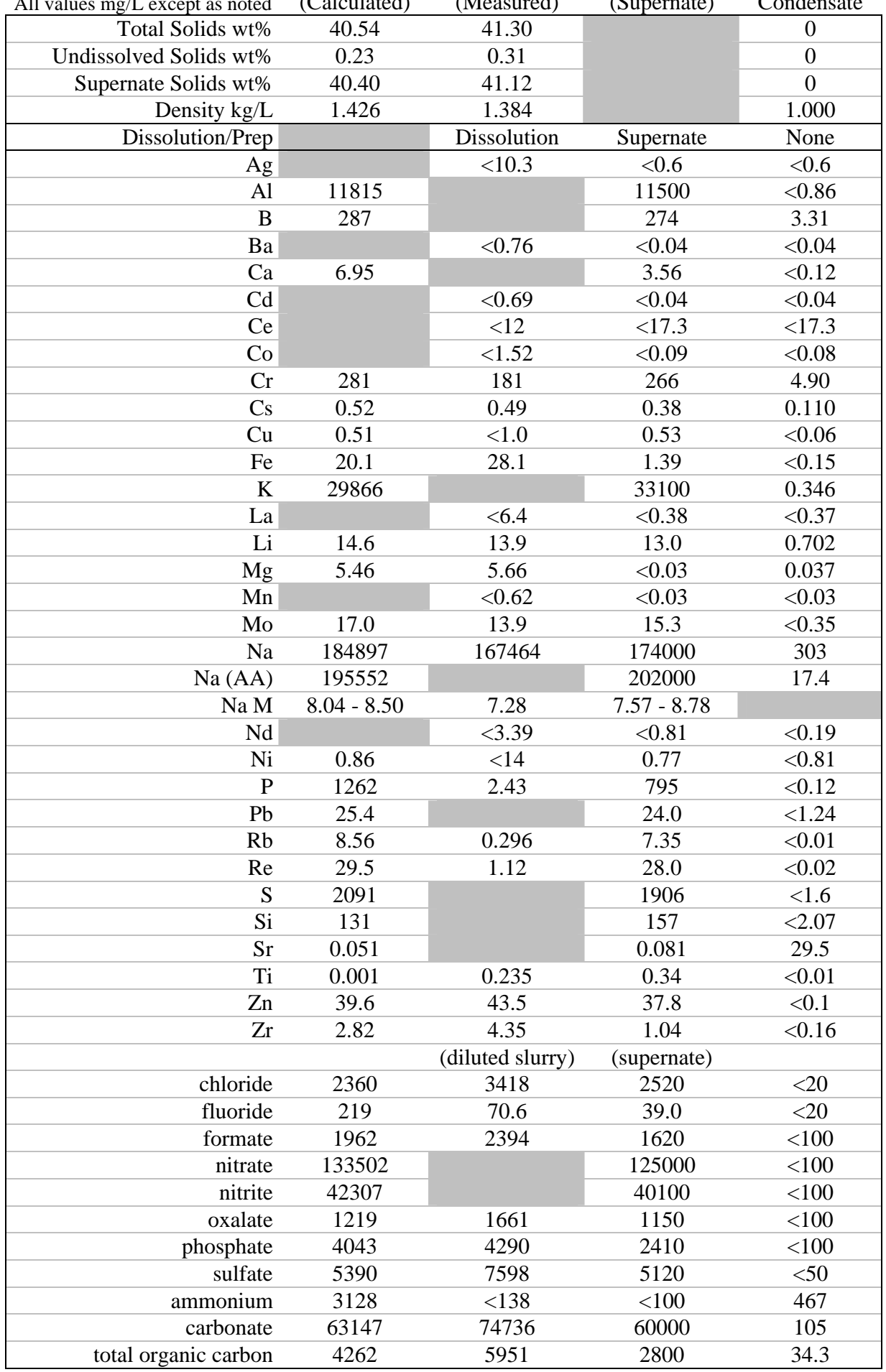


WSRC-TR-2004-00478, REVISION 0

SRNL-RPP-2004-00075, REVISION 0

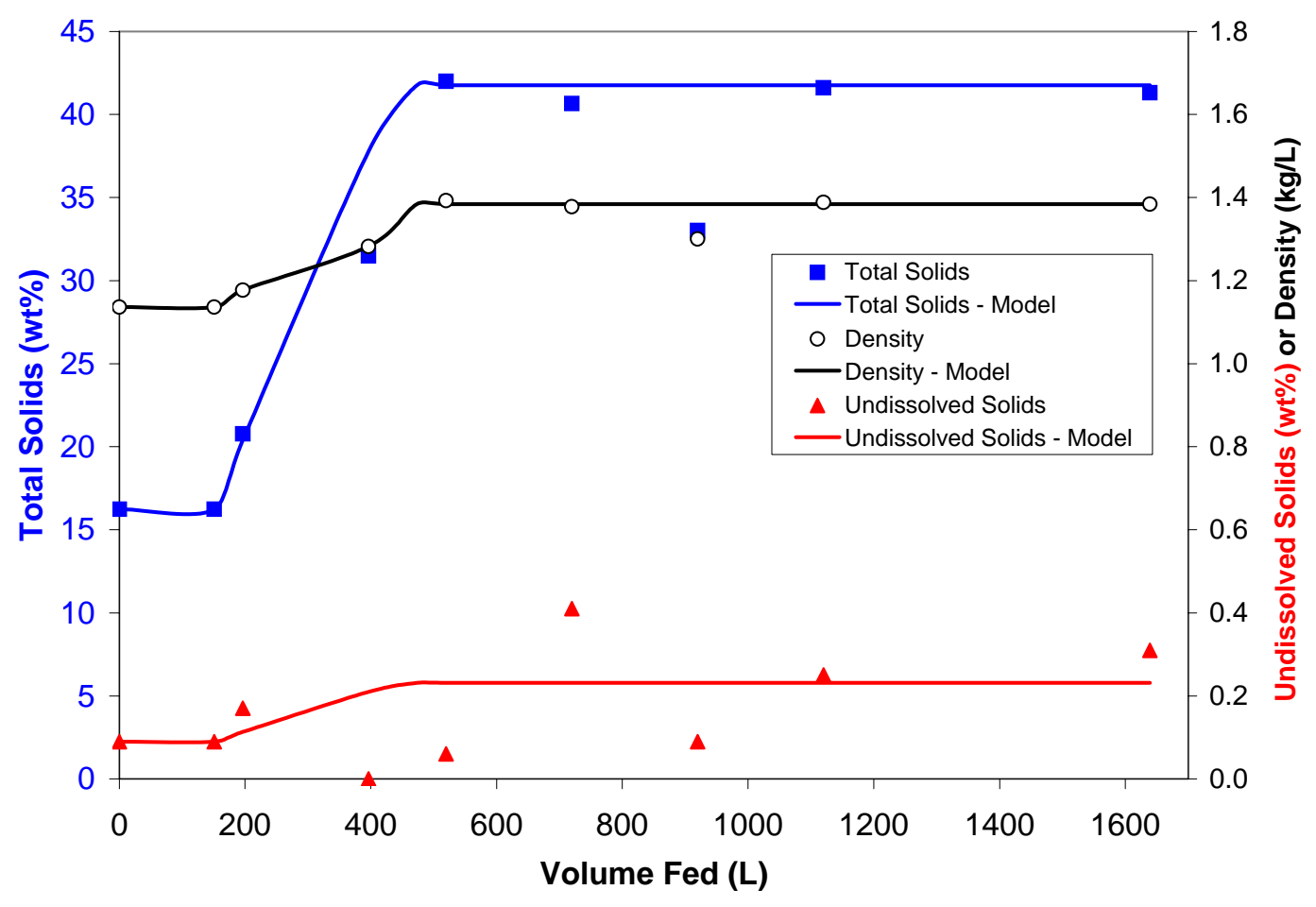

Figure 73. Material Balance on TLP Evaporator of Cumulative Volume Fed

\subsubsection{Pilot TLP Conclusions}

\subsubsection{Experimental Observations (Author - Adamson)}

- The Pilot-scale evaporator operated well for the TLP evaporation Campaign II via concentrating the TLP simulant from an initial density of $1.14 \mathrm{~kg} / \mathrm{L}$ to $1.395 \mathrm{~kg} / \mathrm{L}$ at $25^{\circ}$ $\mathrm{C}\left(1.360 \mathrm{~kg} / \mathrm{L}\right.$ at $\left.50^{\circ} \mathrm{C}\right)$.

- No significant foaming was observed prior to the addition of antifoam agent. During the evaporation process, the froth height remained around $1 \mathrm{ft}(0.3 \mathrm{~m})$ that is significantly less than the $5 \mathrm{ft}(1.5 \mathrm{~m})$ distance to the lower demister pad.

- The two Demisters in series operated well with no significant differential pressure build-up across the Demisters during the TLP campaign II. However, condensation had to be purged from the instrumentation lines during the campaign.

\subsubsection{Waste Products Observations (Author - Zamecnik)}

- The composition of the products from the TLP matched what was expected from the mass balance.

- No precipitation of additional solids beyond those in the feed was noted. 


\subsection{GLASS PRODUCTION BASED ON SIPP RESULTS (AUTHOR - ZAMECNIK)}

\subsubsection{Extrapolated Glass Production Rates}

Using the UFP flux data discussed in Section 2.7, the production rates of HLW and LAW glass were estimated. Rates were also estimated by "extrapolation" to different feed compositions. For the Campaign II UFP feed processed, the rates of HLW and LAW glass production were calculated assuming the following:

1. The concentration step in ultrafiltration is the rate limiting step in the production of glass.

2. ILAW glass $\mathrm{Na}_{2} \mathrm{O}$ loading of $14 \mathrm{wt} \%$.

3. IHLW glass $\mathrm{Fe}_{2} \mathrm{O}_{3}$ loading of $12.5 \mathrm{wt} \%$ (limiting condition). (Calloway, 2004)

4. WTP production rate is scaled to the SIPP production rate by the ratio of the filter surface areas $\left(710 \mathrm{ft}^{2} / 6.7 \mathrm{ft}^{2}\right)$.

5. Flux vs. time and flux vs. UDS curve-fits shown previously are used (see Figs 50 and 53).

6. Average flux is determined by integrating the area under the flux vs. time curve (see Fig 54).

7. Endpoint UDS $=20 \mathrm{wt} \%$.

8. All dissolved Na that goes to ILAW is "waste" Na.

9. WTP filter area of $710 \mathrm{ft}^{2}$ (no reduction in area assumed).

The "extrapolated" glass production rates were determined by calculating the blending of different quantities of the FEP Bottoms, AY102 slurry, and AP101 together. Here it was assumed that the flux curves at these calculated compositions would be the same as the measured curves, i.e., the flux curves are only dependent on the undissolved solids content and not on the actual chemical composition of the feed slurry. This assumption is reasonable so long as the relative amounts of the three components are not changed significantly from the actual amounts tested. For these extrapolated situations, the composition of the FEP Bottoms was assumed to be the same even though the recycle streams would actually have slightly different compositions since the UFP feed composition would be slightly different.

For assumption No. 8, above, "waste” Na (sodium) was defined as any Na in the actual wastes, plus any $\mathrm{Na}$ added during washing $(0.01 \mathrm{M} \mathrm{NaOH})$ and leaching (which was not done in SIPP). Any other Na added was "non-waste" and was not counted towards the ILAW rate. However, in the calculations done for the SIPP (see Appendix A), this distinction was ignored since the only $\mathrm{Na}$ additions were small amounts of $\mathrm{NaOH}$ added to the recycle streams to maintain the $\mathrm{pH}>12$. These amounts were so small that their effect on the Na concentration in the feed was smaller than the uncertainty in the measurement of the $\mathrm{Na}$.

Table 49 gives the glass production rates for several situations. The "Predicted Feed" column gives the production rates for the feed composition predicted from the mixing of the two simulated waste feed streams with the concentrated recycle stream, i.e., FEP 


\section{WSRC-TR-2004-00478, REVISION 0 SRNL-RPP-2004-00075, REVISION 0}

bottoms. The "Actual Feed" column contains the results from using the actual measured feed composition obtain from samples after the entire mixture was made. The glass rates for these two cases are essentially the same. The target glass rates were 6 MTG/d of IHLW and $80 \mathrm{MTG} / \mathrm{d}$ of ILAW (Longwell, 2003), but the actual rates at WTP scale were about 6.6 MTG/d of IHLW and 59.8 MTG/d of ILAW. The target rates were not reached because the ratio of undissolved solids (or Fe) to supernate (or dissolved $\mathrm{Na}$ ) was too high. The target ILAW/IHLW ratio was 13.3, but the experimentally obtained ratio turned out to be 9.1.

Table 49. Glass Production Rates Based on Ultrafilter Throughput

\begin{tabular}{|c|c|c|c|c|c|c|}
\hline Process Stream & Property & $\begin{array}{l}\text { Predicted } \\
\text { Feed }^{3}\end{array}$ & $\begin{array}{l}\text { Actual } \\
\text { Feed }^{4}\end{array}$ & $\begin{array}{l}\text { ILAW/IHLW }^{5} \\
=80 / 6(13.3)\end{array}$ & $\begin{array}{l}\mathrm{IHLW}^{6}= \\
6 \mathrm{MTG} / \mathrm{d}\end{array}$ & $\begin{array}{c}\text { ILAW/IHLW = 80/6, } \\
\text { flux adjusted so ILAW }{ }^{7}= \\
80 \mathrm{MTG} / \mathrm{d}\end{array}$ \\
\hline FEP Bottoms & Volume \% & 11.1 & 11.1 & 9.5 & 10.2 & 9.6 \\
\hline AY102 & Volume \% & 24.3 & 24.3 & 17.6 & 22.2 & 17.7 \\
\hline AP101 & Volume \% & 64.6 & 64.6 & 72.2 & 67.7 & 72.7 \\
\hline \multirow[t]{3}{*}{ Feed } & UDS (wt\%) & 3.13 & 3.22 & 2.32 & 2.85 & 2.32 \\
\hline & $\mathrm{Fe}(\mathrm{mg} / \mathrm{L})$ & 9315 & 9532 & 6900 & 8510 & 6890 \\
\hline & $\mathrm{Na}(\mathrm{mg} / \mathrm{L})$ & 117500 & 114900 & 120300 & 118500 & 120300 \\
\hline Concentrated & UDS (wt\%) & 20 & 20 & 20 & 20 & 20 \\
\hline Slurry & $\mathrm{Fe}(\mathrm{mg} / \mathrm{L})$ & 64700 & 66500 & 64600 & 64800 & 64600 \\
\hline Supernate/Permeate & $\begin{array}{l}\mathrm{Na}(\mathrm{mg} / \mathrm{L}) \\
(\mathrm{M})\end{array}$ & $\begin{array}{c}111900 \\
4.86\end{array}$ & $\begin{array}{c}114600 \\
4.98\end{array}$ & $\begin{array}{l}116100 \\
5.05\end{array}$ & $\begin{array}{c}113300 \\
4.93\end{array}$ & $\begin{array}{l}116100 \\
5.05\end{array}$ \\
\hline Avg. UFP Flux & $\mathrm{gpm} / \mathrm{ft}^{2}$ & 0.0144 & 0.0144 & 0.0148 & 0.0146 & 0.0185 \\
\hline ILAW Glass ${ }^{1}$ & MTG/d ${ }^{8}$ & 60.1 & 61.3 & 64.2 & 61.4 & 80.0 \\
\hline IHLW Glass $^{2}$ & MTG/d & 6.6 & 6.8 & 4.8 & 6.0 & 6.0 \\
\hline ILAW/IHLW & ratio & 9.1 & 9.1 & 13.33 & 10.2 & 13.33 \\
\hline
\end{tabular}

1. ILAW glass rate based on $14 \mathrm{wt} \% \mathrm{Na}_{2} \mathrm{O}$ loading

2. IHLW glass rate based on $12.5 \mathrm{wt} \% \mathrm{Fe}_{2} \mathrm{O}_{3}$ loading

3. Feed composition predicted from blending of FEP bottoms, AY102, and AP101

4. Actual ultrafilter feed composition used

5. Feed blend adjusted to meet this ratio (feed calculated)

6. Feed blend adjusted to meet this value (feed calculated)

7. See $5+$ flux adjusted to make rates (calculated model flux not used)

8. $\mathrm{MTG} / \mathrm{d}=$ metric tons glass / day (tonnes/day)

The "ILAW/IHLW" column, in Table 49, shows the predicted production rates if the feeds had been mixed such that the ILAW/IHLW ratio was 13.3. To achieve this ratio, more AP101 and less AY102 were required, while the FEP Bottoms amount stayed about the same. The UDS solids content of the feed was lowered to $2.33 \mathrm{wt} \%$ in this blending scheme. The glass rate ratio was met, but both glass rates would still be less than the targets, indicating that these rates cannot be met at the specified operating conditions. In the "IHLW" column, the blend was recalculated to force the IHLW rate to be $6.0 \mathrm{MTG} / \mathrm{d}$. The resulting ILAW rate was slightly higher than calculated for the predicted feed. For both of these calculated rates, the average permeate flux is slightly higher due to starting at lower UDS concentration.

The last column of Table 49 shows the blending required to meet the ILAW/IHLW ratio of 13.3. The glass rates require the same blending as column 3. Only a higher UFP flux 
is required. Note that the flux shown is higher than predicted from the experimental data. This is the flux that would be required to meet the rates, if it could be achieved.

\subsubsection{Increasing Glass Production Rates}

In the SIPP, the feed slurry to Ultrafiltration was concentrated to greater than $20 \mathrm{wt} \%$ UDS. As the UDS concentration gets higher, the filtration flux decreases. Even at an endpoint of $20 \mathrm{wt} \%$ UDS, the average flux is not high enough to make the glass rate goals. Two potential ways to improve the rate were proposed:

1. Decrease the undissolved solids concentration endpoint (e.g., 17 or $15 \mathrm{wt} \%$ ).

2. Increase the filtration temperature.

Decreasing the UDS endpoint has the effect of increasing the average flux, since time spent operating at lower flux is eliminated. A lower UDS endpoint will always result in a higher average UFP flux. Starting at a lower UDS also has the effect of increasing the average flux, but this is not a variable that can be changed if the ILAW/IHLW ratio is to be maintained. (The flux would be very high if only LAW with no solids were processed.) Figure 74 shows curves describing the production rates of ILAW and IHLW as a function of the endpoint UDS at a constant processing temperature of $25^{\circ} \mathrm{C}$. The target rates of 6 MTG/d IHLW and 80 MTG/d ILAW are met when the endpoint UDS is $16.7 \mathrm{wt} \%$; the flux is $0.0185 \mathrm{gpm} / \mathrm{ft}^{2}$. Note that this is the concentration at the end of the concentration step, not the concentration at the end of the washing step.

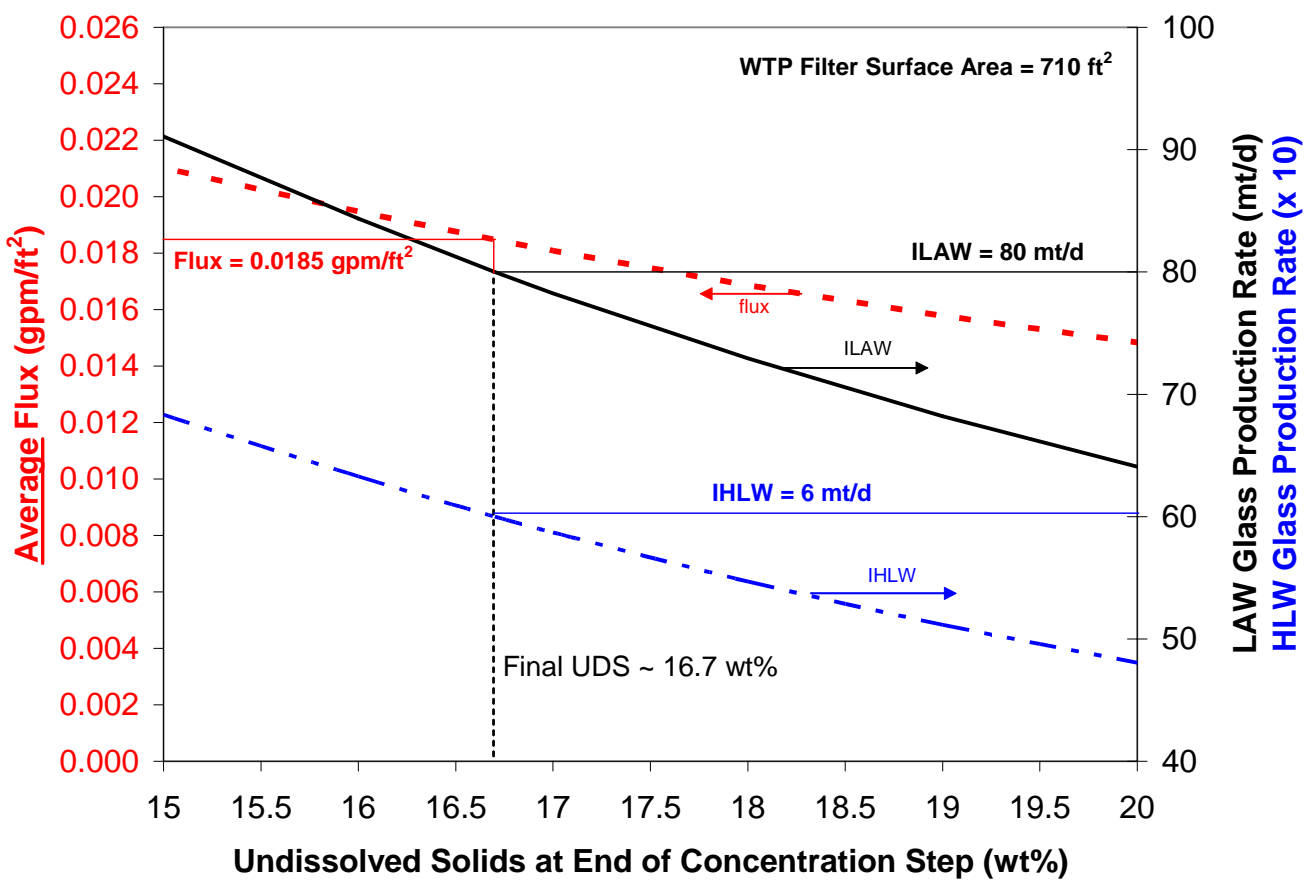

Figure 74. Average Flux to Achieve Rates versus Undissolved Solids 
WSRC-TR-2004-00478, REVISION 0

SRNL-RPP-2004-00075, REVISION 0

The concentration at the end of the washing step (i.e., the feed to the melter) can be higher than that at the end of the concentrating step (20\%, for example) because the rate at which the washed slurry is produced is significantly higher than the rate at which the unwashed slurry is concentrated (LAW production). That is, the flux during washing is high enough that $20 \mathrm{wt} \%$ can be achieved even if it was not achieved during the concentrating step. Therefore, concentrating to lower UDS will not necessarily have the effect of producing a more dilute feed to the HLW melter system. Concentrating to lower UDS does result in producing less LAW feed per unit of UF feed and does result in slightly more LAW (supernate) being recycled to the Plant Wash and Disposal (PWD) System.

Figure 75 shows the predicted effect of increasing the temperature during filtration. The increase in flux due to temperature was predicted using the following WTP-supplied equation $^{\dagger}$ :

$$
\text { Flux@T=Flux @25ํㅜ } \times \text { exp }\left[2500\left(\frac{1}{298}-\frac{1}{\mathrm{~T}+273}\right)\right]
$$

The minimum flux of $0.0185 \mathrm{gpm} / \mathrm{ft}^{2}$ is predicted at a temperature of about $33^{\circ} \mathrm{C}$. Figure 76 shows the combination of temperatures and ending UDS concentrations that result in predicted glass production rates meeting the target criteria.

\footnotetext{
† A cautionary note: The "supplied" equation is required by the RPP-WTP Test Specification as a "temperature correction (that) corrects flux back to an equivalent flux at $25^{\circ} \mathrm{C}$ and accounts for changes in fluid viscosity and surface tension." This correction has been applied to the filter data in this task, as well as all other past RPP-WTP filter tests, e.g., Duignan, 2000a, 2000b, 2003a, and Duignan et al., 2004. It is important to note that, the Test Specification also required filter testing to be carried out at $25^{\circ} \mathrm{C} \pm 5^{\circ} \mathrm{C}$, therefore the flux in that limited temperature range would only incur a correction of at most $\pm 15 \%$. In general, most data were measured within the range of $25^{\circ} \mathrm{C} \pm 2^{\circ} \mathrm{C}$, which means the correction was at most $6 \%$, therefore any error in the correction itself was assumed small. This assumption may not be valid as the flux temperature differs substantially from $25^{\circ} \mathrm{C}$. How well the supplied equation actually "corrects" for viscous and surface tension is not known and may be substantially in error. Applying the equation at temperatures substantially different than $25^{\circ} \mathrm{C}$ is not recommended unless it is verified. Its use here was only to illustrate the effect of temperature changes on flux. That is, the listed flux magnitudes may contain substantial error and should not be used for actual design
} 
WSRC-TR-2004-00478, REVISION 0

SRNL-RPP-2004-00075, REVISION 0

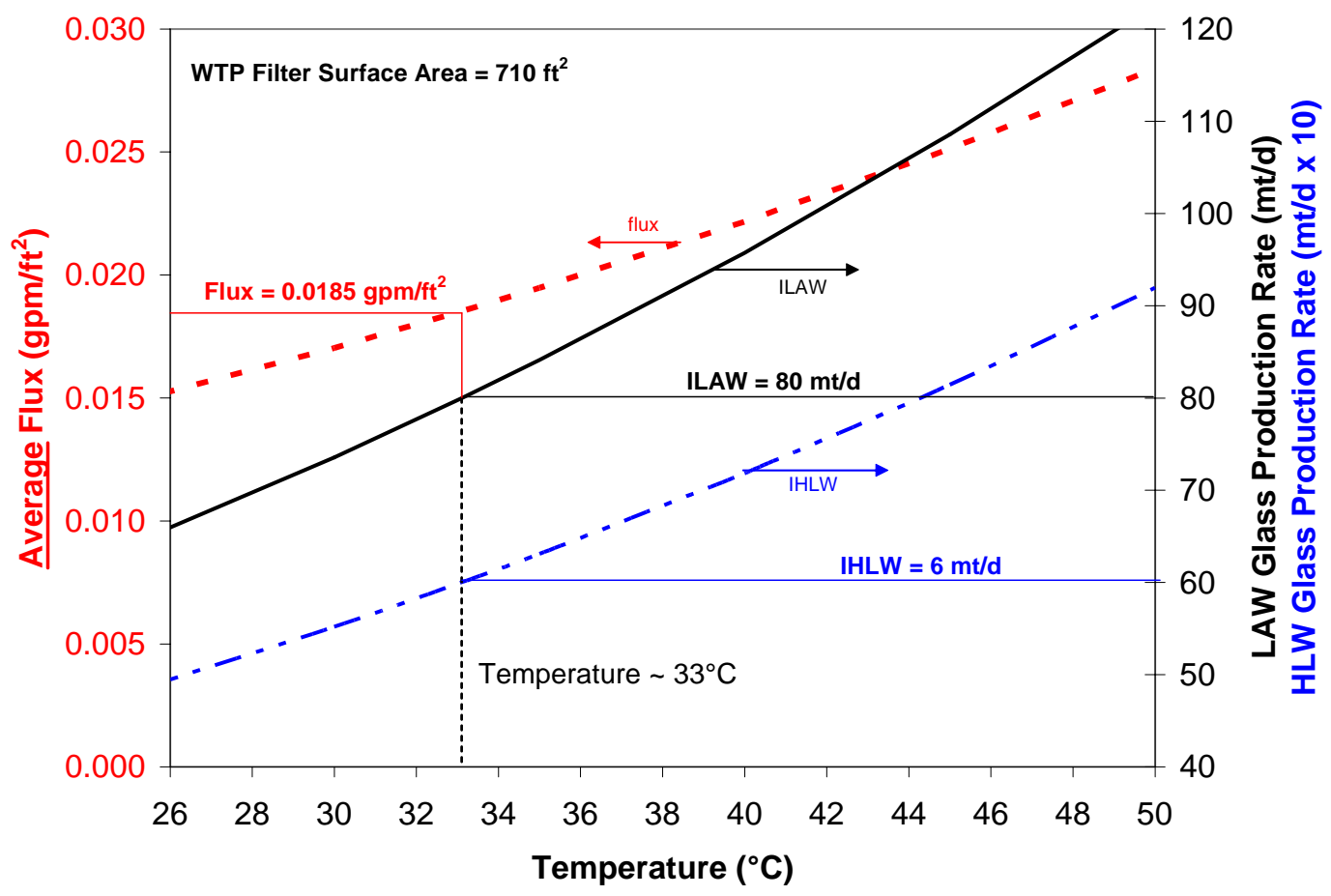

Figure 75. Average Flux to Achieve Rates versus Temperature

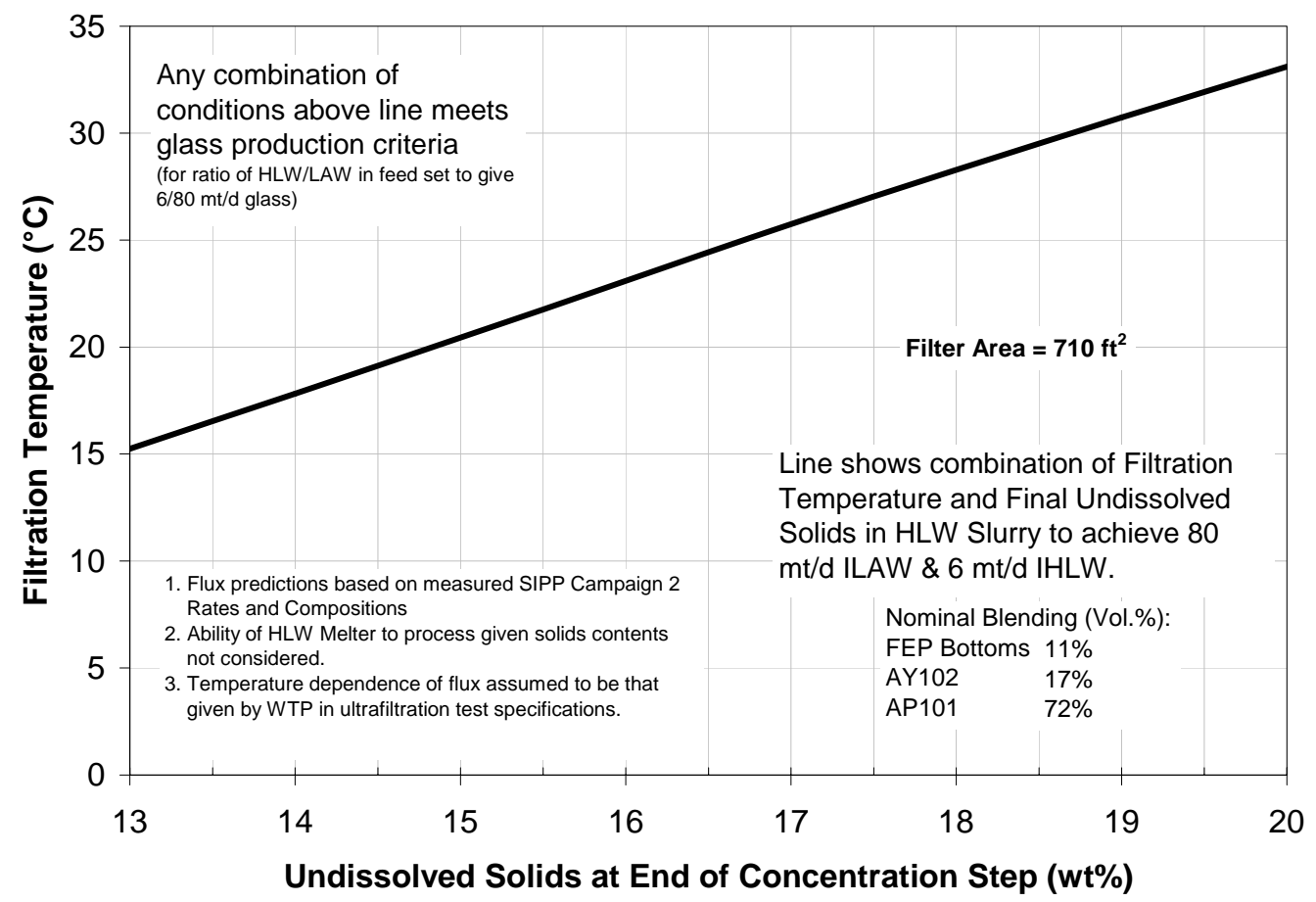

Figure 76. Predicted Combination of Filtration Temperature and Undissolved Solids Endpoint to Meet Glass Production Targets 
WSRC-TR-2004-00478, REVISION 0 SRNL-RPP-2004-00075, REVISION 0

This page intentionally left blank. 


\subsection{CONCLUSIONS}

\subsection{PILOT-SCALE FEP UNIT OPERATION}

- Entire evaporation process proceeded as designed.

- A total of 1,125 liters of feed was concentrated 147 liters.

- Evaporator operated at a vacuum of 1 psia $(6.9 \mathrm{kPa})$ and at $\sim 50^{\circ} \mathrm{C}$.

- The average evaporation rate was approximately $0.43 \mathrm{gpm}(1.5 \mathrm{lpm})$.

- No significant foaming was observed prior to the addition of antifoam agent.

- During the evaporation process, the froth height remained around $1 \mathrm{ft}(0.3 \mathrm{~m})$.

- At start-up and unsteady conditions, significant flashing resulted in feed splashing on the lower demister pad.

- The composition of the FEP feed was predictable by mass balance of the individual feed streams.

- Dissolved metals concentrations paralleled the ratios in the AY102 simulant present in the recycles.

- Most of the oxalate present in the feed was precipitated beginning when approximately $53 \%$ of the feed had been evaporated. The oxalate appears to have precipitated as the salt $\mathrm{Na}_{2} \mathrm{C}_{2} \mathrm{O}_{4}$.

\subsection{PILOT-SCALE UFP UNIT OPERATION}

- The average permeate flux when dewatering from $\sim 4$ to $\sim 24 \mathrm{wt} \%$ was $0.011 \mathrm{gpm} / \mathrm{ft}^{2}$.

- Cleaning the filter with $2 \mathrm{M}$ nitric acid was sufficient to increase the $0.1 \mathrm{M} \mathrm{NaOH}$ permeate flux to an order of magnitude larger than that of waste flux. However, it was not sufficient to return the filter to pre-test flux levels.

- The turbidity of the permeate from dewatering and slurry washing, when measured directly after the sample was taken, was always lower than $0.5 \mathrm{NTU}$, indicating that the permeate was free of significant solids.

- The turbidity of the permeate from dewatering increased with time, indicating that solids were precipitating out of solution, but after 14 days the turbidity was still only 0.56 NTU with now visible solids (see Table 26). However, the AY102-only permeate from Campaign I did post-precipitate after 2 months.

- Washing of concentrated slurry raised its yield stress from about 10 to $15 \mathrm{~Pa}$, but lowered the consistency from about 23 to $13 \mathrm{mPa} \cdot \mathrm{s}$.

- Campaign I data indicated that the yield stress of unwashed slurry is reduced with extended pumping.

- Washing data matched previous SRNL and literature leaching and washing data for soluble washable species (see Table 36).

- Use of a more concentrated $\mathrm{NaOH}$ solution for the third caustic flush during filter cleaning may be warranted since the $\mathrm{pH}$ of the spent third wash was acidic.

- At least $92 \%$ of the antifoam remained in the HLW sludge slurry after filtration. 


\subsection{PILOT-SCALE CIX UNIT OPERATION}

- SuperLig ${ }^{\circledR} 644$ effectively removed cesium from simulated waste and the analytical ability to measure cesium concentrations in five-molar sodium did allow verification of the waste acceptance criterion of $50 \%$ breakthrough from the lead column after 100 Bed Volumes of waste were treated.

- SuperLig ${ }^{\circledR} 644$ effectively removed cesium from simulated waste, however, the analytical ability to measure very low cesium concentrations in five-molar sodium did not allow verification of the waste acceptance criterion of $5.2 \mu \mathrm{g} / \mathrm{L}$ from the lag column after $100 \mathrm{BV}$ of waste were treated.

- The masses of cesium in and out of the resin beds for Cycles 1 and 2 balanced to within measurement uncertainty.

- Pressure drops throughout the two cycles were low and the resin beds permeabilities were approximately the same as were measured for the 24-inch Test (Fowley et al, 2004).

\subsection{PILOT-SCALE TLP UNIT OPERATION}

- The Pilot-scale evaporator operated well for the TLP evaporation in concentrating the TLP simulant from an initial density of $1.14 \mathrm{~kg} / \mathrm{L}$ to $1.395 \mathrm{~kg} / \mathrm{L}$ at $25^{\circ} \mathrm{C}(1.360 \mathrm{~kg} / \mathrm{L}$ at $\left.50^{\circ} \mathrm{C}\right)$.

- No significant foaming was observed prior to the addition of antifoam agent. During the evaporation process, the froth height remained around $1 \mathrm{ft}(0.3 \mathrm{~m})$.

- The two Demisters in series operated well with no significant differential pressure build-up across the Demisters during the TLP campaign II. However, condensation had to be purged from the instrumentation lines during the campaign.

- The composition of the products from the TLP matched what was expected from the mass balance.

- No precipitation of additional solids beyond those in the feed was noted. 


\subsection{FUTURE WORK}

As per the SIPP test specification (Longwell, 2003), the work discussed in this interim report is for Campaign II, which was only the second of four campaigns. Further work to be included in the immediate task will be the testing of a different filter media, made by GKN. See the second footnote on page 16. Other work that could follow may include the testing of other simulated wastes, UFP filter cleaning, small and pilot-scale comparisons, etc. However, details on any future work will be reported in the final report, which follows the completion of Campaign IV and the issuance of the Campaign III report. The final report will mention any future work outside of the current task, which may be the result of information elicited from the SIPP task.

As per the SIPP test specification (Longwell, 2003), the work discussed in this interim report is for Campaign II, which was only the second of four campaigns. Further work to be included the immediate task will be the testing of a different filter media, made by GKN. See the second footnote on page 24 .

Given the specific scope of the SIPP (i.e., demonstrating pretreatment performance and the influences of recycle streams using AY102/C106 and AP101 simulants) several key findings of the SIPP workshop (Edwards, 2003) were deferred to future programs or tasks. These items are:

$>$ Preliminary calculations indicate the SIPP will produce only enough HLW glass to turnover one DM-100 melter volume. Similar R\&D efforts conducted for DOE (e.g., Integrated DWPF Melter System, IDMS) typically produced sufficient glass to turnover the melter at a minimum of three volumes. This typically allows the melter system to achieve steady state operations with respect to glass composition, and production rates.

$>$ The transition of various Hanford waste feeds (e.g. mixing of Envelope D heels with Envelope C supernate) through an integrated system is not being tested by the SIPP. Future programs or tasks should consider testing additional sludge compositions in an integrated system. Similar R\&D efforts conducted for DOE (e.g. IDMS, DWPF Cold Commissioning, and Duratek LAW Pilot Melter) tested a variety of waste chemistries and conducted testing that demonstrated these facilities could operate successfully during waste transitions.

Additionally, since only one Envelope D simulant (AY102/C106) has been tested in the SIPP, the filtration flux at a pilot scale with other wastes (e.g. AZ101, AZ102, C104, and single-shell tank sludges) is unknown and likely to be different than the filtration flux measured with in the SIPP. Because bench-top cross-flow filter fluxes, using AY102/C106 Hanford waste, are higher than pilot-scale filter fluxes using AY102/C106 simulant (see Fig. 32 in Section 2.12 of Duignan et al., 2004), it is recommended that additional pilot-scale filtration tests be conducted to determine the filter flux with other Hanford sludges. Furthermore, since it will not be practical or cost effective to conduct 
WSRC-TR-2004-00478, REVISION 0

SRNL-RPP-2004-00075, REVISION 0

pilot-scale testing using all known sludge types, a correlation between bench-top radioactive filter experiments and pilot-scale data should be developed to predict the filter flux of future WTP sludge batches.

Leaching of aluminum or oxidative leaching of other metals (e.g. Cr) was not tested by the SIPP. Leaching was determined to be unnecessary for the AY102/C106 tank composition (Calloway, 2004). However, other Hanford wastes require leaching of aluminum and other metals to reduce the number of HLW canisters produced from a given waste type. While aluminum leaching and oxidative leaching of $\mathrm{Cr}$ have been demonstrated in the laboratory, pilot-scale demonstration is needed to assess the actual efficiency of these processes. Additionally, post precipitation of solids after high temperature leaching and subsequent cooling down could become a significant issue in these processes and should be assessed at a pilot scale.

Since Campaigns III and IV data have not been completely evaluated at this point in time, other recommendations for future work maybe included in these reports. 
WSRC-TR-2004-00478, REVISION 0

SRNL-RPP-2004-00075, REVISION 0

\subsection{REFERENCES}

Adamson, D. J. (2004), “SIPP Pilot TLP,” Westinghouse Savannah River Company Notebook No. WSRC-NB-2004-00038. (began on 4 March)

Barnes, C. D. (2004) “OLI/ESP Modeling of the Semi-Integrated Pilot Plant for Estimate of Campaigns I-IV Simulant Volumes,” Westinghouse Savannah River Document No. WSRC-TR-2004-00403, Rev. 0. (August). [also SRNL-RPP-2004-00063]

Brooks, K. P., R. L. Myers, and K. G. Rappe (1997), “Bench-Scale Enhanced Sludge Washing and Gravity Settling of Hanford Tank C-106 Sludge,” Battelle Pacific Northwest National Laboratory Document No. PNNL-11432.

Calloway, Jr., T. B. (2004) "OLI Modeling Estimate of SIPP Run Times and Leaching Effectiveness,” Westinghouse Savannah River Company Document No. SRT-RPP-200300241, (24 March). [This is an inter-office memorandum from Calloway to Reid Peterson.]

Coleman, C. J., M. S. Hay, and K. B. Martin (2003), “Compositing and Characterization of Samples from Hanford Tank 241-AY-102/C-106,” Westinghouse Savannah River Document No. WSRC-TR-2003-00205, Rev. 0. (October) [also SRT-RPP-2003-00086]

Duignan, M. R. (2000a), "Final Report: Pilot-scale Cross-flow Ultrafiltration Test Using a Hanford Site Tank 241-AN-105 Waste Simulant - Envelope A + Entrained Solids,” Westinghouse Savannah River Company Document No. BNF-003-98-0221, Rev. 0, (23 February).

Duignan, M. R. (2000b), "Final Report: Pilot-scale Cross-flow Ultrafiltration Test Using a Hanford Site Tank 241-AN-107 Waste Simulant - Envelope C + Entrained Solids + Strontium-Transuranic Precipitation,” Westinghouse Savannah River Company Document No. BNF-003-0226, Rev. 0. (24 March)

Duignan, M. R. (2003a). "Final Report: Pilot-scale cross-flow ultrafiltration test using a Hanford Site tank 241-AN-102 waste simulant.” Westinghouse Savannah River Document No. WSRC-TR-2003-00204, Rev. 0. (6 May) [also SRT-RPP-2003-00087]

Duignan, M. R. (2003b), “RPP-WTP Pilot Scale Cross Flow Filtration,” Westinghouse Savannah River Company Notebook No. WSRC-NB-2003-000149. (began on 12 June)

Duignan, M. R. (2003c). "Pilot-scale Integrated Pretreatment System Test,” (Task Technical and QA Plan) Westinghouse Savannah River Document No. WSRC-TR-200300338, Rev. 0. (4 August) [also SRT-RPP-2003-00165] 
WSRC-TR-2004-00478, REVISION 0

SRNL-RPP-2004-00075, REVISION 0

Duignan, M. R. (2004), "RPP-WTP Pilot-scale Cross-flow Ultrafiltration Test

Procedure,” Westinghouse Savannah River Company Field Procedure No FP-975, (26 January)

Duignan, M. R., J. R. Zamecnik, and M. R. Williams (2004). “Interim Report: RPP-WTP Semi-integrated Pilot Plant - Campaign I,” Westinghouse Savannah River Document No. WSRC-TR-2004-00201, Rev. 0. (19 April) [also SRT-RPP-2004-00034]

Edwards, R. E., (2003) "Semi-Integrated Pilot Plant (SIPP) Workshop - Meeting Minutes,” SRT-RPP-2003-00227, Westinghouse Savannah River Company, Aiken SC 29808 (5 November)

Eibling, R. E., and C. A. Nash (2001), "Hanford Waste Simulants Created to Support the Research and Development on the River Protection Project - Waste Treatment Plant,” Westinghouse Savannah River Document No. WSRC-TR-2001-00338, Rev. 0. (22 February) [also SRT-RPP-2000-00017]

Fowley, M. D. (2004), “SIPP Pilot UFP,” Westinghouse Savannah River Company Notebook No. WSRC-NB-2004-00036. (began on 4 March)

Fowley, M. D., D. J. Adamson, C. A. Nash, M. A. Shadday, S. E. Aleman, J. L. Steimke, L. L. Hamm, M. L. Restivo, T. J. Steeper, and W. D. King (2004), "Ion Exchange Testing With SuperLig ${ }^{\circledR} 644$ Resin (U),” Westinghouse Savannah River Document No. WSRCTR-2003-00514, Rev. 0. (July) [also SRT-RPP-2003-00233]

Lumetta, G. J., M. J. Wagner, F. V. Hoopes, and R. T. Steele (1996), “Washing and Caustic Leaching of Hanford Tank C-106 Sludge,” Battelle Pacific Northwest National Laboratory Document No. PNNL-11381.

Lee, E. and M. Binsfield (2002). "Flowsheet Bases, Assumptions, and Requirements," Doc. No. 24590-WTP-RPT-PT-02-005, Rev. 1. (December)

Longwell. R. L. (2003), “Pilot Scale Integration of Pretreatment Process System,” Waste Treatment Plant - River Protection Project Document No. 24590-PTF-TSP-RT-03-002, Rev. 0. (5 June)

Poirier, M. R., P. R. Burket, J. L. Siler, and J. R. Zamecnik (2003), "Filtration, washing and leaching of a Hanford AY-102/C-106 sample,” Westinghouse Savannah River Company Document No. WSRC-TR-2003-00240, Rev. 0 (or SRT-RPP-2003-00110, Rev. 0). (June)

Qureshi, Z. H., D. J. Adamson, W. E. Daniel, M. E. Stone, and M. R. Williams (2004), "WTP Pilot-scale Evaporation Tests (U)," Westinghouse Savannah River Company Document No. WSRC-TR-2003-00561, Rev. 0 (or SRT-RPP-2003-00244, Rev. 0). (March) 
WSRC-TR-2004-00478, REVISION 0

SRNL-RPP-2004-00075, REVISION 0

Russell, R. L., S. K. Fiskum, L. K. Jagoda, and A. P. Polaski (2003), “AP-101 Diluted Feed (Envelope A) Simulant Development Report," Battelle Pacific Northwest Division Document No. PNWD-3248, Rev. 0 (February) [also WTP-RPT-057]

Stallings, M. E., M. R. Poirier, and R. E. Eibling (2003). “Task Technical and Quality Assurance Plan for AY-102/C-106 Simulant Ultrafiltration and Washing (Including AY102/C-106 Simulant Development).” Westinghouse Savannah River Document No. WSRC-TR-2003-00217, Rev. 0. (31 May) [also SRT-RPP-2002-00235]

Steimke, J. L. (2002). "Pilot Scale Ion Exchange Testing with SuperLig ${ }^{\circledR} 639$ and SuperLig ${ }^{\circledR} 644$ Resin,” (Task Technical and QA Plan) Westinghouse Savannah River Document No. WSRC-TR-2001-00340, Rev. 0. (6 February) [also SRT-RPP-200100116]

Sundar, P.S. (2004) “Comments and Questions on Test Exception,” E-mail transmission to J. L. Steimke. (1 April at 07:10 PM EDT). [Excerpt of interest: "Since criterion for switching columns is the breakthrough in the lag column effluent, Cycle 1 (of SIPP) on the lead column will be terminated when the effluent from the lag column reaches the maximum LAW limit of cesium in glass. The value is $0.03 \mathrm{Ci} / \mathrm{m}^{3}$ (sic) of glass. One would use a corresponding concentration of cesium in the effluent. Frankly, I do not have this value but can get it to you, if that is an issue.”]

Toth, J. (2003). "Ion exchange feed compositions and minimum required Cesium removal from low activity waste solutions," RPP-WTP Memorandum to R. A. Peterson. Document No. CNN 076606 (or 24590-PADC-F00029, Rev. 1). (2 December)

Townson, P. S. (2002). "AY-102/C-106 Simulant Pilot Scale Ultrafiltration and Washing Test Specification,” Waste Treatment Plant - River Protection Project Document No. 24590-PTF-TSP-RT-02-015, Rev. 0. (15 November)

Zamecnik, J. R., P. R. Burket, R. E. Eibling, and M. R. Poirier (2004), “Tank 241-AY102 Simulant development, ultrafiltration, and washing,” Westinghouse Savannah River Company Document No. WSRC-TR-2003-00547, Rev. 0 (or SRT-RPP-2003-00240, Rev. 0). (March) 
WSRC-TR-2004-00478, REVISION 0 SRNL-RPP-2004-00075, REVISION 0

This page intentionally left blank. 


\section{Appendix A: Computational Model (aUtHor-BaRnes)}

The volumes of AY-102 and AP-101 simulant required for the four SIPP campaigns were estimated by modeling each campaign as a steady-state process using the software package, OLI/ESP version 6.6. In addition, the minimum SIPP flow rates/fluxes necessary to prove a production rate of 80 metric tons glass per day (MTG/d) for LAW glass and 6 MTG/d HLW glass were estimated based on Campaign IV stream compositions and flow rates and the SIPP unit operation scale factors relative to the current WTP design basis. The OLI/ESP PUBLIC databook, in addition to the private databooks CARBONAT, HNO3DB, SILICA, and ZEOLITE were used to generate the chemistry model (Zamecnik, 2004) for these simulations.

The model for Campaign I simulated the concentration and wash steps of simulant AY102 (Zamecnik et al., 2004) waste feed only, while the model for Campaigns II-IV, using a blend of simulant AY-102 and AP-101 (Russell et al., 2003) waste feed. Campaigns IIIV simulated most of the pre-treatment process including the FEP evaporator, ultrafiltration, slurry wash, cesium ion-exchange column recycles, and the TLP evaporator. The estimated SIPP volumes for each campaign were based on producing 140L of washed sludge from Campaign I and the ratio of AP-101:AY-102 waste feed predicted to give a glass production ratio of 80/6 MTG/d LAW/HLW glass for each of the Campaigns II-IV.

The structures of the models for Campaigns II-IV are identical. Each of the three campaigns represent an iteration towards steady-state of the ultra-filtration and Cs-IX recycle streams, with the predicted output recycle from one campaign being used as the input recycle to the subsequent campaign. The difference in the predicted volumetric recycle flows between campaigns III and IV was less than $0.09 \%$, indicating the campaign IV output recycle had essentially reached steady-state.

The major assumptions used in the model are discussed in the first section below, followed by the criteria used to determine several stream flow rates. Then the model structure is described with the help of simplified flow diagrams; detailed block diagrams are used to describe certain portions of the model. Finally, the modeling results of Campaigns I and IV are given, along with estimates of the minimum SIPP flow/flux rates and process times necessary to prove rate based on the results of Campaign IV. A complete OLI/ESP listing of the properties and composition of key streams for Campaigns I and IV are given in Appendix A of Barnes (2004).

\section{A.1. MODEL ASSUMPTIONS}

Although the WTP pre-treatment process will have two FEP evaporator/ultra-filtration trains, only one train was included in the model. The current design basis allows only one train to operate in the concentration mode (concentrating the waste feed blend to 
18,000 gals. at $20 \mathrm{wt} \%$ UDS while generating permeate for the Cs IX column) at any one time. Because this is the rate-limiting mode for this process, train " $A$ " will complete its treat-clean mode and the stand idle while train " $\mathrm{B}$ " completes its concentration mode (the treat-clean mode consists of washing the 18,000 gal batch of 20wt\% UDS slurry, heel and treated sludge transfers, and filter cleaning). At no time will both trains perform the same function simultaneously. Therefore, a single train must be capable of sustaining a production rate of $80 \mathrm{MTG} / \mathrm{d}$ LAW glass when in the concentration mode, or $6 \mathrm{MTG} / \mathrm{d}$ HLW glass when in the treat and clean mode.

LAW glass production rate was based on $14 w t \%$ loading of $\mathrm{Na}_{2} \mathrm{O}$ (Lee et al., 2003a) derived from "waste" sodium only, i.e. sodium from waste feed alone ("waste" sodium is defined to include sodium addition from the leach step, however, this step was not included in these particular models). The amount of sodium in the LAW stream considered to be "waste" sodium was calculated as the difference between the sodium in the waste feed streams and the sodium sent to HLP in the washed sludge (all sodium to HLP is assumed to be waste sodium). This is conservative, the calculated LAW glass rate would be higher for identical flow rates if some or all of the sodium going to the HLP were considered to be non-waste sodium, resulting in a corresponding increase in the amount of "waste" sodium to the LAW glass. The total amount of sodium sent to the LAW (or HLP) process is the same in either case.

HLW glass production was based on $12.5 \mathrm{wt} \%$ loading of $\mathrm{Fe}_{2} \mathrm{O}_{3}$. This was the first metal oxide to meet contract minimum, all other metal oxides and combinations thereof fell below the contract minimums (Lee et al., 2003b).

While the Cs-IX column recycle streams generated during the elution and resin regeneration sequence are modeled, the removal of Cs from the UF permeate by the column is not. This results in Cs being sent to the TLP evaporator that, in practice, would be removed from the stream and sent to the Cs eluate pot (the pot is not part of the SIPP) and eventually the HLP system. However, the concentration of Cs is small and felt to have no significant effect on the TLP bottoms with respect to the properties of interest, i.e. flow rates, density, precipitated solids, etc. No other pre-treatment process of the model is affected by this approximation.

With the exception of the filter loop, no approximations of tank heels or line volumes were included in the models. This is not likely to affect the relative feed rates of AY-102 and AP-101 a great deal; it would take a very large heel to impact the chemistry of a stream so dramatically as to shift the split of output to the LAW and HLP systems. But the lack of heels does increase the apparent efficiency of the process. Any treated sludge or Cs-IX effluent that is recycled due to heels and line volumes reduces the plant efficiency and glass production rates. Heels will also generate more recycle per unit of glass as compared to the model predictions. But because these recycles are largely dilute caustic, the only major impact is likely to be an increased load to the evaporators and increased condensate per unit of glass. 


\section{A.2. BASES FOR MODEL INPUT/RECYCLE STREAM FLOW RATES AND COMPOSITIONS}

In general, the models of each campaign are steady-state approximations of the pretreatment process and do not introduce any dynamics. The exception is the iteration on the ultra-filtration and Cs-IX recycle stream as it changes value between Campaigns II, III, and IV (reflected in the difference between the fixed input UF and Cs-IX recycles, and the calculated output UF and Cs-IX recycles). All input streams for one particular campaign (HLW and LAW SBS, vessel vent system, recycle inputs from the previous campaign) are set to an average flow rate and do not reflect the cyclic nature of inputs that will exist under actual plant conditions. These average flow rates are based on either the glass production rate, slurry batch size, or the Cs-IX effluent batch size, and the calculations are described in the following paragraphs. The corresponding flows are listed in Tables. A1, A2, and A3.

HLW and LAW SBS recycles generated from actual Duratek melter runs are to be used in the SIPP campaigns. The analytical compositions of these streams were used as input to the model, while the flow rates were based on the most recent ACM model available at the time (dated 11/04/2003 11:42:35am, for streams RDL62 and RDL21 for HLW and LAW SBS, respectively). Because the ACM model HLW glass production rate was 5.4 MTG/d, the given HLW SBS flow rate was adjusted by a ratio of 6:5.4 to represent a flow corresponding to $6 \mathrm{MTG} / \mathrm{d}$. The vessel vent system (PVP04 and PVP06 combined) and the HLW canister decon. (HDH06) stream compositions and flow rates were also based on the ACM model, and the stream compositions given in the ACM model were approximated as a $0.15 \mathrm{M} \mathrm{NaOH}-0.3 \mathrm{M} \mathrm{NaNO} 3$ stream for the canister decon., and a $0.1 \mathrm{M} \mathrm{NaOH}$ stream for the combined PVP04-06 vessel vent system streams. The vessel vent system flow rate is fixed as tank ventilation is not affected by the glass production rates. The flow rates for these streams are listed in Table A1.

Table A1. Campaigns II-IV Input Recycle Flow Rates as a Function of Glass Production Rate

\begin{tabular}{|c|c|c|}
\hline Stream & $\begin{array}{c}\text { Flow Rate - relative to 80/6 MTG/d } \\
\text { LAW/HLW glass production }\end{array}$ & Destination \\
\hline \hline HLW SBS (based on HLW glass) & $2.79 \mathrm{gpm}$ & FEP evaporator \\
\hline LAW SBS (based on LAW glass) & $12.2 \mathrm{gpm}$ & TLP evaporator \\
\hline PVP04 and PVP06 (vessel vent system - fixed rate) & $3.23 \mathrm{gpm}$ & FEP evaporator \\
\hline Canister decon. (based on HLW glass) & $1.68 \mathrm{gpm}$ & FEP evaporator \\
\hline
\end{tabular}

The filter is cleaned once for every 18,000 gal batch of $20 \mathrm{wt} \%$ UDS concentrated slurry, produced using three $-5,250$ gal flushes of $2 \mathrm{M} \mathrm{HNO3}$, and three $-5,250$ gal flushes of $0.01 \mathrm{M} \mathrm{NaOH}$ (Lee et al., 2003c). These acid and caustic rinse streams are each modeled using an average volumetric flow ratio relative to the slurry of $3 * 5,250 / 18,000$, or 0.875 . Following the transfer of treated sludge to the HLP, 700 gals. of sludge remains in the filter loop, which gets pushed to the FEP recycle. This is modeled using an average volumetric flow ratio relative to the slurry of 700/18,000. This same ratio is used for the 


\section{WSRC-TR-2004-00478, REVISION 0 SRNL-RPP-2004-00075, REVISION 0}

$0.01 \mathrm{M} \mathrm{NaOH}$ caustic rinse remaining in the filter loop after cleaning, this volume gets pushed to the Cs-IX at the start of the concentration step. These streams are listed in Table 2.

Table A2. Input/Recycle Stream Flow Rates as a Function of the 20wt\% UDS Concentrated Slurry Flow Rate (slurry batch size of 18,000 gals)

\begin{tabular}{|c|c|c|}
\hline Stream & $\begin{array}{c}\text { Volume - relative to 18,000 gals. slurry } \\
\text { (volumetric fraction relative to slurry) }\end{array}$ & Destination \\
\hline \hline Washed Sludge (in filter loop prior to cleaning) & 700 gals. $(0.03889)$ & FEP evaporator \\
\hline Acid Cleaning $\left(2 \mathrm{M} \mathrm{HNO}_{3}\right)$ & $3 * 5,250$ gals. $(0.8750)$ & FEP evaporator \\
\hline Caustic Rinse $(0.01 \mathrm{M} \mathrm{NaH})^{\text {NaOH }}$ & $(3 * 5,250-700)$ gals. $(0.8361)$ & FEP evaporator \\
\hline $\begin{array}{c}\text { Caustic Rinse (0.01M NaOH, remaining in filter } \\
\text { loop prior to concentration of blended waste feed. }\end{array}$ & 700 gals. $(0.03889)$ & Cs-IX column \\
\hline
\end{tabular}

The Cs-IX effluent batch size is 30,000 gals (Lee et al., 2003d). During the elution cycle, one column volume ( $\mathrm{CV}=1,200$ gals.) of $0.1 \mathrm{M} \mathrm{NaOH}$ is used to displace the feed, followed by one $\mathrm{CV}$ of pre-elution DI water. Both of these streams are recycled to the FEP Evaporator and modeled using a flow ratio relative to the Cs-IX effluent of $1,200 / 30,000$. The acid elution and post-elution DI rinse streams are sent to the Cs eluate pot which is not part of the SIPP, so these streams are not relevant to these models. Finally, the resin is regenerated with 2,500 gals. $0.25 \mathrm{M} \mathrm{NaOH}, 1,300$ gals of which is recycled to the FEP Evaporator, while the 1,200 gals. remaining in the column is pushed to the TLP Evaporator by the incoming feed. These streams are modeled using a flow ratio relative to the Cs-IX effluent of 1,300/30,000 and 1,200/30,000 for the FEP and TLP streams, respectively, and are listed in Table A3.

Table A3. Input/Recycle Stream Flow Rates as a Function of the Cs-IX Effluent Flow Rate

(effluent batch size of 30,000 gals, column volume of 1,200gals, liquid bed volume of 300 gals.)

\begin{tabular}{|l|c|c|}
\hline \multicolumn{1}{|c|}{ Stream } & $\begin{array}{l}\text { Volume - relative to 30,000 gals. effluent } \\
\text { (volumetric fraction relative to effluent) }\end{array}$ & Destination \\
\hline \hline $0.1 \mathrm{M} \mathrm{NaOH}$ used as feed displacement & 1,200 gals. (0.040) & FEP evaporator \\
\hline DI water - pre-elution rinse & 1,200 gals. (0.040) & FEP evaporator \\
\hline $0.25 \mathrm{M} \mathrm{NaOH}$ resin regeneration & 1,300 gals. (0.043) & FEP evaporator \\
\hline $0.25 \mathrm{M} \mathrm{NaOH}$ resin regeneration & 1,200 gals. (0.040) & TLP evaporator \\
\hline
\end{tabular}

\section{A.3. MODEL FLOW-DIAGRAMS}

A simplified flow diagram of the model for Campaign I is shown in Fig. 1, and a simplified flow diagram of the model for Campaigns II-IV is shown in Fig. 2. The model for Campaigns II-IV consisted of 294 OLI/ESP blocks (mixer, filter, etc.), of which 220 were used to represent the 44 wash steps. To simplify the block-diagram and yet convey a sense of the model's functionality, only the block diagrams detailing a single wash step (Fig. A3) and the FEP evaporator - waste feed blend concentration sequence (Fig. A4) were included here. Feed-Back and/or "Manipulate" controllers were used to set the input stream flow rates described above. Note that an OLI/ESP feed-back controller is not capable of reading the density of a stream directly, instead, it can calculate the $\mathrm{g} / \mathrm{m} 3$ 
of a collection of up to 100 species; 84 were used to define the permeate density and are listed (using the OLI/ESP naming convention) in Table A4.

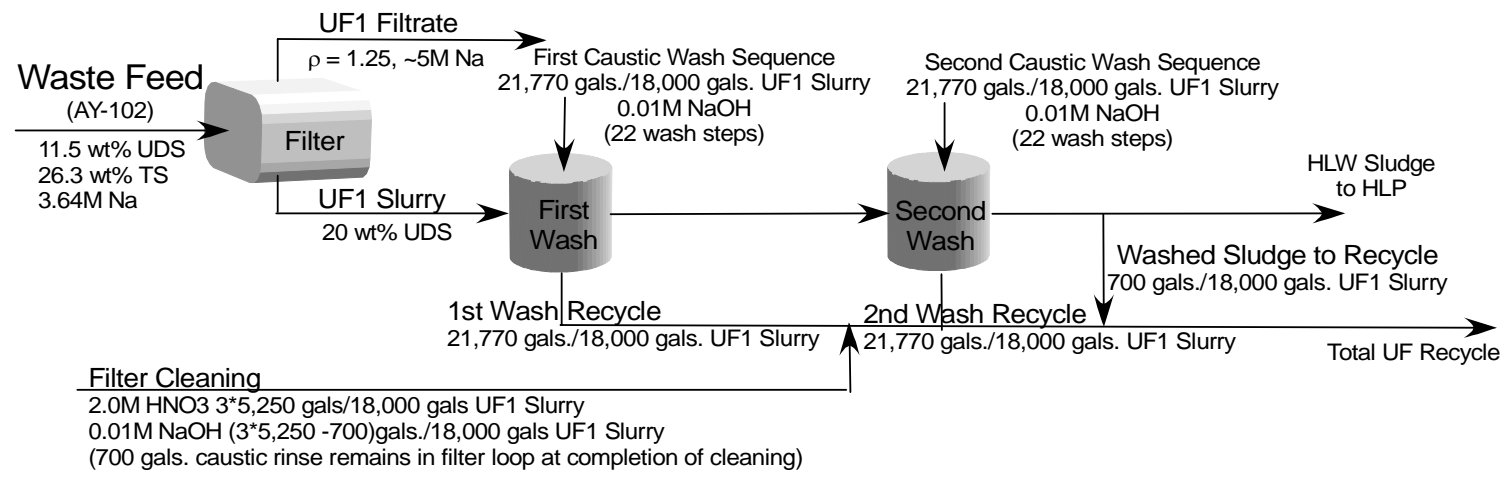

Figure A1. Simplified Flow-Diagram of Campaign I

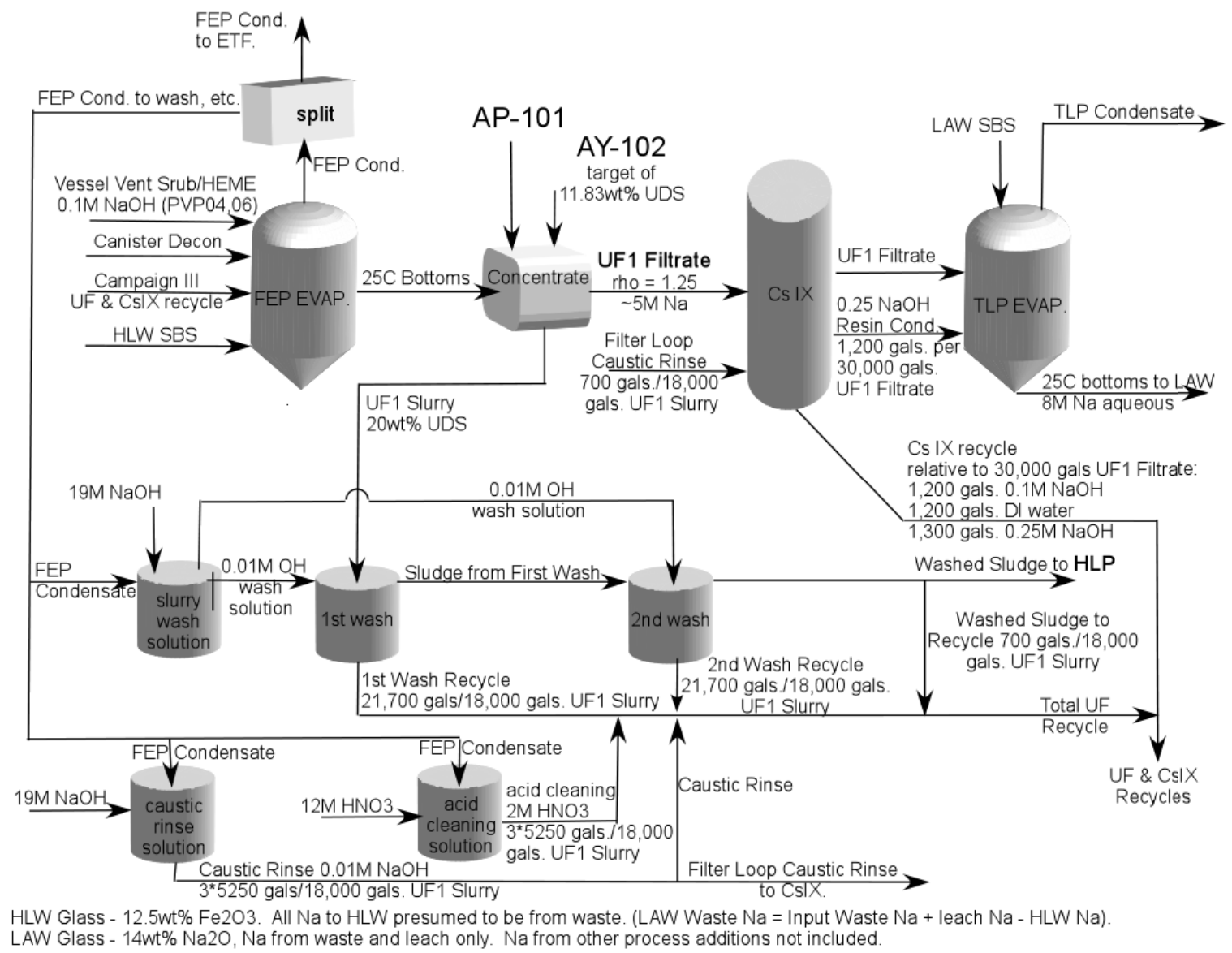

Figure A2. Simplified Flow-Diagram of Campaigns II-IV 
WSRC-TR-2004-00478, REVISION 0

SRNL-RPP-2004-00075, REVISION 0

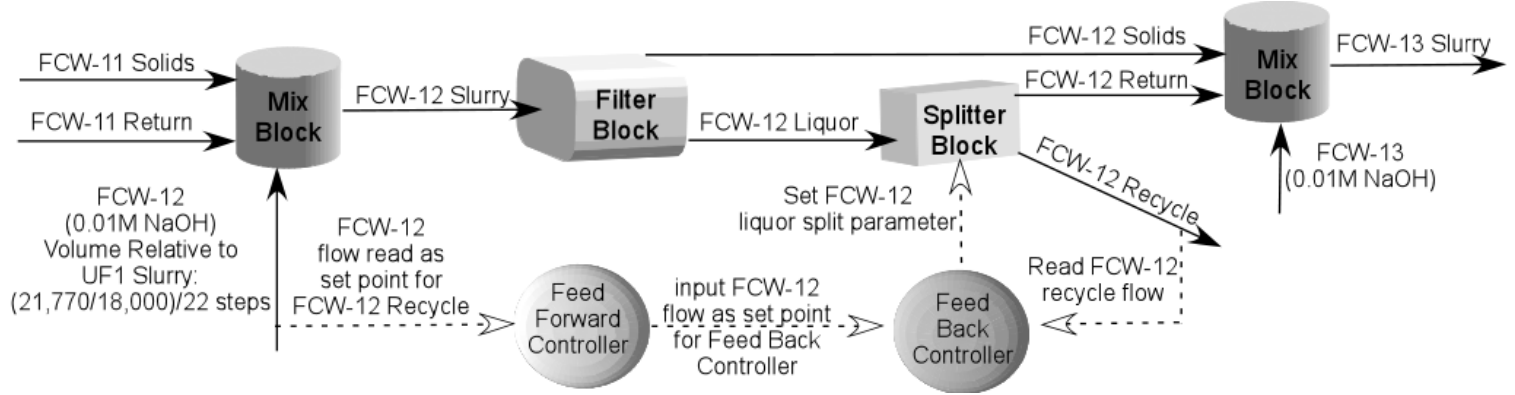

Figure A3. Example Wash Step - Step 12 of First Wash Sequence

(Sets volumetric flow of "FCW-12 Recycle" equal to "FCW-12)

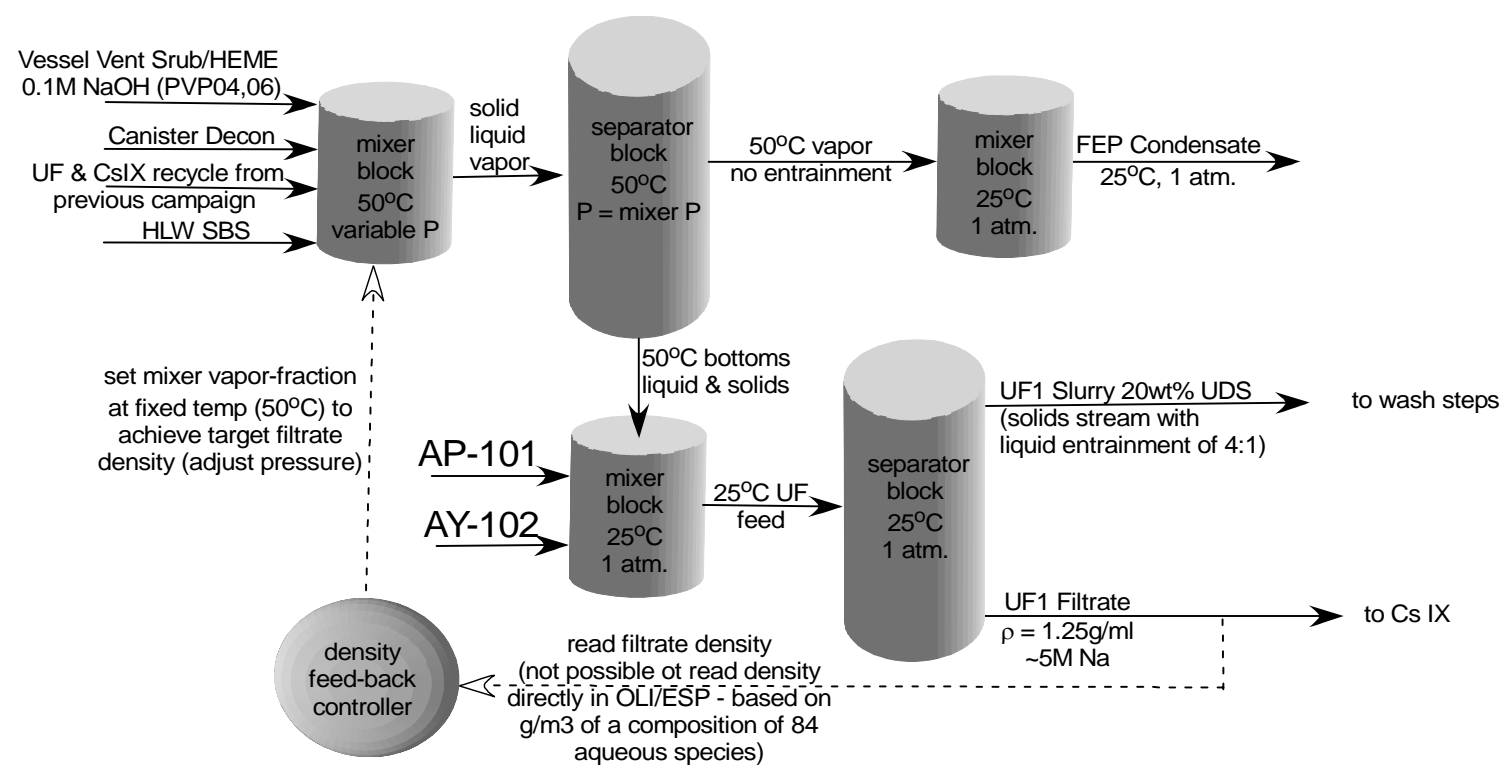

Figure A4. OLI/ESP Representation of FEP Evaporator and Slurry Concentration 
WSRC-TR-2004-00478, REVISION 0

SRNL-RPP-2004-00075, REVISION 0

Table A4. OLI/ESP Species used to Estimate Aqueous Phase Density

A suffix of AQ indicates an aqueous phase molecule; a suffix of ION indicates an aqueous phase anion/cation.

\begin{tabular}{|c|c|c|c|c|}
\hline H2O & BOH3AQ & CAC2O4AQ & CACO3AQ & CAH2SIO4AQ \\
\hline CEOH3AQ & CSCLAQ & CSNO3AQ & FEIIIOH3AQ & KACETAQ \\
\hline KCLAQ & KCOOHAQ & KNO3AQ & LIACETAQ & LIH2BO3AQ \\
\hline LIOHAQ & NAACETAQ & NAALOH4AQ & NABOH4AQ & NABRAQ \\
\hline NACOOHAQ & NAFAQ & NAHCO3AQ & NAHSIO3AQ & NANO3AQ \\
\hline BACO3AQ & OHION & AGCL3ION & AGCL4ION & AGOH2ION \\
\hline ALACETION & ALOH4ION & ALSIO3OHION & B2OOH5ION & B4O5OH4ION \\
\hline BAION & BAOHION & BOH4ION & BRION & CAH2BO3ION \\
\hline CAION & CANO3ION & CAOHION & CAPO4ION & CEOH4ION \\
\hline CLION & CO3ION & COOHION & CRIIC2O42ION & CRIIIION \\
\hline CRO4ION & CSION & CUOH3ION & CUOH4ION & FEIIIOH4ION \\
\hline FION & H2SIO4ION & H3SIO4ION & HCO3ION & HPBO2ION \\
\hline HPO4ION & KION & KSO4ION & LIION & LISO4ION \\
\hline MNOH3ION & MNOH4ION & NA2FION & NACO3ION & NAION \\
\hline NASO4ION & NIOH3ION & NO2ION & NO3ION & ACETATEION \\
\hline OXALATION & PO4ION & RUIIIOH2ION & SO4ION & SROHION \\
\hline WO4ION & ZNOH3ION & ZNOH4ION & ZROH5ION & \\
\hline
\end{tabular}

The structures of the models for Campaigns II-IV are identical. The campaigns are related to each other through the UF and Cs-IX recycles, with the recycle generated from one campaign being used directly, with no modifications, as input recycle to the subsequent campaign. The waste feed rates for each of these campaigns was adjusted to give the 80/6 MTG/d LAW/HLW glass ratio. The model for Campaign I was unique. The AY-102 waste feed flow rate was completely arbitrary, only the relative flow rates (acid cleaning flow, etc.) were of importance. Before using it as input to Campaign II, the (arbitrary) Campaign I UF recycle flow rate had to be adjusted to some approximation of a steady-state flow corresponding to the 80/6 MTG/d production rates of Campaigns II-IV. The was done by assuming that the amount of UF recycle generated in Campaign II would only be a function the AY-102 waste feed, and not of the AP-101 waste feed. Then the ratio of generated UF recycle to AY-102 for campaign II could be calculated by using the same ratio of UF recycle to AY-102 from Campaign I (input UF recycle-II = AY-102-II x (output UF recycle-I)/(AY-102-I) ). This turned out to be satisfactory since the difference between the Campaign III and IV recycle volumetric flow rates was less than $0.09 \%$.

\section{A.4. MODEL RESULTS}

Campaign I was intended to serve only as a filtration baseline for the remaining campaigns and does not represent the pre-treatment process, no comparisons between the model flow rates and glass production rates were done here. The simulation results for key streams from Campaign I are summarized in Tables. A5 and A6. A complete OLI/ESP listing of the properties and composition of key streams for Campaign I is given in Appendix A of Barnes (2004). 
WSRC-TR-2004-00478, REVISION 0

SRNL-RPP-2004-00075, REVISION 0

Table A5. Composition of Key Streams from the Model of Campaign I

\begin{tabular}{|c|c|c|c|c|c|c|c|}
\hline $\begin{array}{c}\mathrm{mg} / \mathrm{L} \\
\text { solution } \\
\text { @25C }\end{array}$ & $\begin{array}{c}\text { Waste Feed } \\
\text { (AY-102) }\end{array}$ & $\begin{array}{l}\text { UF1 Filtrate } \\
\text { to Cs-IX }\end{array}$ & $\begin{array}{l}\text { UF1 Slurry } \\
\text { 20wt } \% \text { UDS }\end{array}$ & $\begin{array}{l}1^{\text {st }} \text { Wash } \\
\text { Recycle }\end{array}$ & $\begin{array}{l}2^{\text {nd }} \text { Wash } \\
\text { Recycle }\end{array}$ & $\begin{array}{l}\text { Total UF } \\
\text { Recycle }\end{array}$ & $\begin{array}{c}\text { Sludge to } \\
\text { HLP }\end{array}$ \\
\hline $\mathrm{K}$ & 427.34 & 458.61 & 402.00 & 245.68 & 64.18 & 89.97 & 27.65 \\
\hline $\mathrm{Na}$ & 83620.84 & 80851.08 & 85865.68 & 45663.74 & 13618.32 & 17675.21 & 14929.21 \\
\hline $\mathrm{Ba}$ & 175.81 & 1.29 & 317.26 & 1.06 & 0.85 & 0.55 & 319.25 \\
\hline $\mathrm{Ca}$ & 965.97 & 3.44 & 1746.09 & 3.65 & 2.69 & 1.84 & 1762.15 \\
\hline $\mathrm{Zn}$ & 58.36 & 62.63 & 54.90 & 33.55 & 8.77 & 12.29 & 3.78 \\
\hline $\mathrm{Cu}$ & 67.23 & 72.15 & 63.24 & 38.65 & 6.63 & 13.15 & 8.60 \\
\hline $\mathrm{Mg}$ & 254.97 & 0.00 & 461.62 & 0.01 & 0.03 & 0.01 & 467.88 \\
\hline $\mathrm{Pb}$ & 1272.50 & 1365.61 & 1197.03 & 731.56 & 191.11 & 267.91 & 82.33 \\
\hline Al & 16182.77 & 2062.48 & 27627.02 & 862.61 & 163.30 & 297.89 & 26746.65 \\
\hline $\mathrm{Mn}$ & 37.42 & 2.54 & 65.69 & 0.55 & 0.09 & 0.18 & 65.81 \\
\hline $\mathrm{Fe}$ & 27804.11 & 45.56 & 50301.99 & 18.77 & 3.76 & 6.54 & 50961.18 \\
\hline $\mathrm{Ni}$ & 910.21 & 0.41 & 1647.59 & 0.17 & 0.03 & 0.06 & 1669.83 \\
\hline $\mathrm{F}$ & 224.30 & 240.71 & 210.99 & 128.95 & 33.69 & 47.22 & 14.51 \\
\hline $\mathrm{Cl}$ & 157.97 & 169.53 & 148.60 & 90.82 & 23.72 & 33.26 & 10.22 \\
\hline $\mathrm{Br}$ & 123.67 & 132.72 & 116.34 & 71.10 & 18.57 & 26.04 & 8.00 \\
\hline $\mathrm{Li}$ & 85.34 & 91.59 & 80.28 & 49.06 & 12.82 & 17.97 & 5.52 \\
\hline c & 0.00 & 0.00 & 0.00 & 0.00 & 0.00 & 0.00 & 0.00 \\
\hline $\mathrm{P}$ & 2108.50 & 2179.93 & 2050.60 & 1085.24 & 157.47 & 360.84 & 555.23 \\
\hline $\mathrm{s}$ & 803.08 & 818.09 & 790.90 & 438.34 & 114.64 & 160.57 & 123.83 \\
\hline $\mathrm{Cr}$ & 545.25 & 585.15 & 512.91 & 313.46 & 81.89 & 114.80 & 35.28 \\
\hline Si & 8382.35 & 28.08 & 15153.32 & 17.72 & 9.28 & 7.84 & 15327.15 \\
\hline $\mathrm{Sr}$ & 225.34 & 0.03 & 407.96 & 0.03 & 0.02 & 0.01 & 413.47 \\
\hline $\mathrm{B}$ & 8.96 & 9.62 & 8.43 & 5.15 & 1.35 & 1.89 & 0.58 \\
\hline $\mathrm{Cs}$ & 6.23 & 6.68 & 5.86 & 3.58 & 0.94 & 1.31 & 0.40 \\
\hline W & 161.10 & 172.89 & 151.55 & 92.62 & 24.19 & 33.92 & 10.42 \\
\hline $\mathrm{Ce}$ & 246.65 & 264.70 & 232.02 & 135.31 & 6.71 & 41.24 & 61.10 \\
\hline $\mathrm{Nd}$ & 359.53 & 0.00 & 650.93 & 0.00 & 0.00 & 0.00 & 659.82 \\
\hline $\mathrm{Zr}$ & 993.32 & 7.39 & 1792.40 & 3.75 & 0.98 & 1.37 & 1811.08 \\
\hline $\mathrm{Ag}$ & 488.67 & 0.02 & 884.71 & 0.00 & 0.00 & 0.00 & 896.79 \\
\hline $\mathrm{Ru}$ & 87.20 & 93.58 & 82.03 & 50.13 & 13.10 & 18.36 & 5.64 \\
\hline $\begin{array}{r}\text { density } \\
(\mathrm{g} / \mathrm{L})\end{array}$ & 1231 & 1169 & 1281 & 1098 & 1029 & 1041 & 1137 \\
\hline wt\% UDS & 11.50 & & 20.00 & 0.09 & 0.00 & 0.19 & 21.39 \\
\hline
\end{tabular}


WSRC-TR-2004-00478, REVISION 0

SRNL-RPP-2004-00075, REVISION 0

Table A6. Solids Composition of Key Streams from the Model of Campaign I

\begin{tabular}{|c|c|c|c|c|c|c|}
\hline $\begin{array}{c}\text { Solids } \\
\text { (wt } \% \text { of total UDS) }\end{array}$ & $\begin{array}{l}\text { AY-102 } \\
\text { WASTE FEED }\end{array}$ & $\begin{array}{l}\text { 20wt\% UDS } \\
\text { UF1 SLURRY }\end{array}$ & $\begin{array}{l}\text { 1st WASH } \\
\text { SEQUENCE }\end{array}$ & \begin{tabular}{|l|} 
\\
2nd WASH \\
SEQUENCE
\end{tabular} & $\begin{array}{l}\text { TOTAL UF } \\
\text { Cs-IX } \\
\text { RECYCLE }\end{array}$ & $\begin{array}{l}\text { SLUDGE } \\
\text { to HLP }\end{array}$ \\
\hline $\mathrm{Ba}(\mathrm{SO} 4)$ & 0.2097 & 0.2097 & 0.0022 & 0.0107 & 0.0134 & 0.2227 \\
\hline $\mathrm{Fe}(\mathrm{OH}) 3$ & 37.5385 & 37.5385 & 0.2297 & 0.7329 & - & 40.1081 \\
\hline $\mathrm{Al}(\mathrm{OH}) 3$ & 15.0762 & 15.0762 & 14.4437 & 65.3800 & - & 16.7198 \\
\hline $\mathrm{Mn}(\mathrm{OH}) 2$ & 0.0401 & 0.0401 & 0.0297 & 0.1011 & - & 0.0438 \\
\hline $\mathrm{Ni}(\mathrm{OH}) 2$ & 1.0149 & 1.0149 & 0.0017 & 0.0055 & - & 1.0842 \\
\hline $\mathrm{AGg} 2(\mathrm{CO} 3)$ & 0.4413 & 0.4413 & 0.0001 & 0.0002 & - & 0.4714 \\
\hline $\mathrm{Sr}(\mathrm{CO} 3)$ & 0.2683 & 0.2683 & - & - & - & 0.2865 \\
\hline $\mathrm{ZrO} 2$ & 0.9416 & 0.9416 & 0.0069 & 0.0319 & 0.0933 & 1.0060 \\
\hline $\mathrm{Mg}(\mathrm{OH}) 2$ & 0.4322 & 0.4322 & 0.0003 & 0.0273 & - & 0.4616 \\
\hline NASGEL.15.5H2O & 34.8581 & 34.8581 & 0.1099 & - & - & 37.2011 \\
\hline $\mathrm{Na} 2(\mathrm{C} 2 \mathrm{O} 4)$ & 6.9956 & 6.9956 & 85.1755 & - & - & - \\
\hline $\mathrm{Ca}(\mathrm{CO} 3)$ & 1.6988 & 1.6988 & - & - & - & 1.1021 \\
\hline $\mathrm{Nd}(\mathrm{PO} 4) \cdot 2 \mathrm{H} 2 \mathrm{O}$ & 0.4848 & 0.4848 & - & - & - & 0.5179 \\
\hline $\mathrm{Ca}(\mathrm{C} 2 \mathrm{O} 4) \cdot 1 \mathrm{H} 2 \mathrm{O}$ & - & - & - & - & 0.2793 & - \\
\hline $\mathrm{Ce}(\mathrm{PO} 4)$ & - & - & - & 24.7292 & 3.3897 & 0.0416 \\
\hline $\mathrm{Pb} 3(\mathrm{PO} 4) 2$ & - & - & - & - & 16.2916 & - \\
\hline $\mathrm{NaAl}(\mathrm{CO} 3)(\mathrm{OH}) 2$ & - & - & - & - & 79.9325 & - \\
\hline $\mathrm{Cu}(\mathrm{OH}) 2$ & - & - & - & 8.9813 & - & 0.0043 \\
\hline $\mathrm{Ca}(\mathrm{PO} 4) 2$ & - & - & - & - & - & 0.7291 \\
\hline
\end{tabular}

Campaigns II and III were intermediary steps towards the UF and Cs-IX recycle stream state-state flows of Campaign IV. As such, only the model results of Campaign IV are reported here and used to estimate the SIPP minimum flux/flow rates necessary to prove rate. These minimum rates, along with the estimated SIPP process volumes are used estimate the corresponding maximum SIPP run times as described below.

In the case of the FEP and TLP evaporators, the overhead flows predicted by the model for the $80 / 6 \mathrm{MTG} / \mathrm{d}$ glass production rates were scaled by a factor 0.13 (Table A7) to estimate the minimum SIPP overhead flows needed to prove rate.

The minimum LAW permeate flux rate necessary for the SIPP to prove rate was calculated by dividing the flow rate of the LAW permeate (going to the Cs-IX) predicted by the model for the $80 / 6 \mathrm{MTG} / \mathrm{d}$ glass production rates by the design basis filter area of $590 \mathrm{ft}^{2}$ (Table A7).

The flux rate for the wash permeate was calculated in a similar manner. However, as described earlier, the concentration mode is the rate-limiting step. One train will have completed the treat-clean mode and remain idle while the other finishes the concentration mode, assuming identical flux rates for the LAW and wash permeates. Instead of using identical fluxes, the calculation for the minimum wash permeate flux rate applied this idle time toward a lower wash permeate flux rate, resulting in equal process times for the 
concentration and treat-clean modes. The flux rate was calculated as volume of wash permeate produced by one treat-clean sequence (the washing of 18,000 gals. 20wt\% UDS slurry) was divided by the filter area and the time required for one concentration mode, less the time needed for filter cleaning, sludge and heel transfers (i.e. the time available for washing before the train must begin sludge transfer and cleaning in preparation for the next concentration mode).

Table A7. Design Basis and SIPP Capacities

\begin{tabular}{|c|c|c|c|}
\hline Unit Operation & Design Basis & SIPP & Scale Factor \\
\hline $\begin{array}{c}\text { FEP/TLP } \\
\text { Evaporator Cond. Capacity }\end{array}$ & $30 \mathrm{gpm}$ & $0.39 \mathrm{gpm}$ & 0.13 \\
\hline Ultra-filtration Area & $590 \mathrm{ft}^{2}$ & $6.7 \mathrm{ft}^{2}$ & based only on flux rate \\
\hline
\end{tabular}

These minimum SIPP flux/flow rates where then used to calculate the time required to process the SIPP volumes estimated by the model at these rates. These volumes were based on producing 140L of 20wt\% UDS slurry in Campaign I. All flows predicted by the Campaign I model were scaled by the ratio of 140L / (model flow of the $20 \mathrm{wt} \%$ UDS slurry) to estimate the volumes produced by Campaign I of the SIPP. The calculated volume of the Campaign I SIPP UF recycle (which is also the input UF Recycle for the Campaign II SIPP) was then used to scale the flows predicted by the Campaign II model by the ratio of (Campaign I SIPP UF recycle) / (Campaign II model UF Recycle input). This was done for Campaigns III and IV in the same manner, and is depicted schematically in Fig. A5. 
WSRC-TR-2004-00478, REVISION 0

SRNL-RPP-2004-00075, REVISION 0

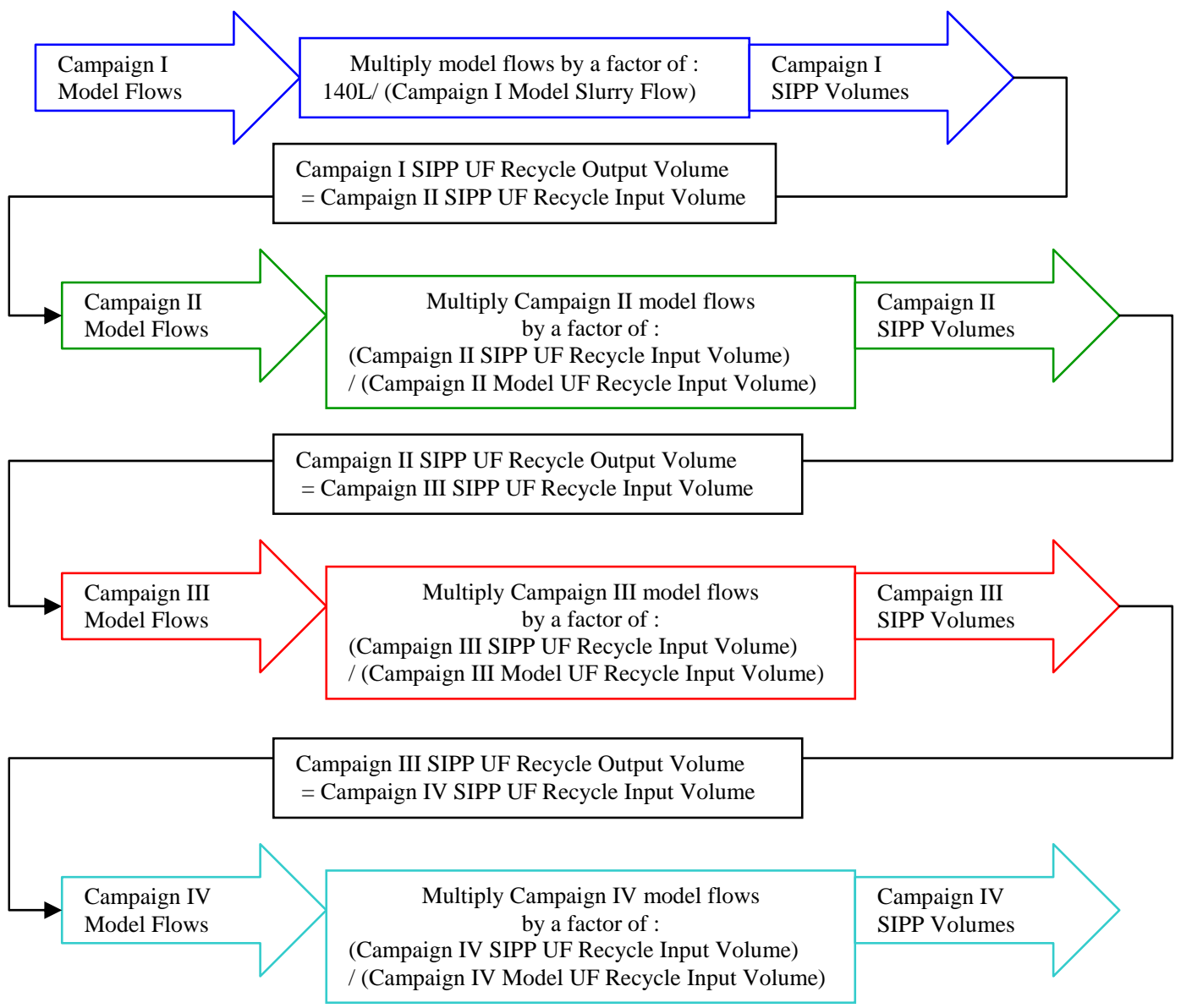

Figure A5. Algorithm for Estimation of SIPP Volumes based on Model Flow Rates

The model flow rates and corresponding SIPP volumes for Campaign IV are listed in Table A8. Table A9 shows the estimated Campaign IV SIPP run time based on values in Tables. A7 and A8. The Cs column will not be operated prototypically, but in a manner to determine the break-through curve, so an estimated run-time for the Cs-IX was not included. Also, it is not possible to scale the filter cleaning or the sludge transfer times; the duration for these operations for the SIPP were set at the design basis with the understanding that they will have to be adjusted based on the actual SIPP time for these operations. Finally, these times are only estimates based on the model compositions, the actual Campaign IV SIPP stream compositions, flow, and flux rates will be used to prove or disprove the glass production rates. The model predictions of the composition for key streams of Campaign IV are given in Tables A10 and A11. A complete OLI/ESP listing of the properties and composition of key streams for Campaign IV is given in Appendix B of Barnes (2004). 
WSRC-TR-2004-00478, REVISION 0 SRNL-RPP-2004-00075, REVISION 0

Table A8. Campaign IV SIPP Volumes and Model Flow Rates

\begin{tabular}{|l|r|r|}
\hline \multicolumn{1}{|c|}{ stream name } & $\begin{array}{c}\text { SIPP Campaign IV } \\
\text { Volumes (liters) }\end{array}$ & $\begin{array}{c}\text { Model Flow Rates for } \\
80 / 6 \text { MTG/d LAW/HLW } \\
\text { glass (gpm) }\end{array}$ \\
\hline AY102 & 241.39 & 3.545 \\
\hline AP101 & 672.10 & 9.870 \\
\hline RECYCLE (previous campaign) & 602.79 & 8.852 \\
\hline HLW SBS & 189.94 & 2.789 \\
\hline PVP04/06 (Vessel Vent System) & 219.98 & 3.230 \\
\hline HLW DECON & 114.26 & 1.678 \\
\hline FEP CONDENSATE & 984.86 & 14.462 \\
\hline FEP COOLED BOTTOMS & 144.93 & 2.128 \\
\hline UF1 FILTRATE to CIX & 943.65 & 13.857 \\
\hline UF1 SLURRY 20wt\% UDS & 116.09 & 1.705 \\
\hline TOTAL CAUSTIC WASH & 280.81 & 4.124 \\
\hline HLW SLURRY to HLP & 109.98 & 1.615 \\
\hline TOTAL ACID CLEANING & 101.59 & 1.492 \\
\hline TOTAL CAUSTIC RINSE & 101.54 & 1.491 \\
\hline CAUSTIC RINSE TO FEP EVAP & 97.03 & 1.425 \\
\hline CIX DI WATER & 37.75 & 0.554 \\
\hline CIX CAUSTIC RINSE & 37.75 & 0.554 \\
\hline CIX 0.25M NAOH FEP & & 0.600 \\
RECYCLE & 40.89 & 0.554 \\
\hline CIX 0.25M NAOH TLP & 37.75 & 8.844 \\
\hline TOTAL UF \& CIX RECYCLE & 602.27 & 12.191 \\
\hline LAW SBS & 830.20 & 17.987 \\
\hline TLP CONDENSATE & 1224.90 & 8.783 \\
\hline TLP COOLED BOTTOMS & 598.10 & \\
\hline
\end{tabular}


WSRC-TR-2004-00478, REVISION 0

SRNL-RPP-2004-00075, REVISION 0

Table A9. Estimated Filtration Batch Cycle Times Necessary to Make WTP Glass

Production Rate of 80/6 MTG/d LAW/HLW

\begin{tabular}{|c|c|c|c|c|}
\hline $\begin{array}{c}\text { Filtration Step } \\
\text { (hrs.) }\end{array}$ & \begin{tabular}{|} 
WTP Batch (18,000 gals.) \\
Cycle Time (hrs.) \\
Design Basis \\
(0.03 gpm/ft2)
\end{tabular} & \begin{tabular}{|c|} 
WTP Batch (18,000 gals.) \\
Cycle Time (hrs.) \\
Minimum Required Filtration Rates: \\
0.0235 and 0.00808 gpm/ft2 for the \\
concentration and wash modes \\
respectively
\end{tabular} & \begin{tabular}{|c|} 
SIPP Batch \\
Cycle Time (hrs.) \\
Design Filtration Rate \\
$\left(0.03 \mathrm{gpm} / \mathrm{ft}^{2}\right)$ \\
\end{tabular} & $\begin{array}{c}\text { SIPP Batch } \\
\text { Cycle Time (hrs) } \\
\text { Minimum Required } \\
\text { Filtration Rates: } \\
0.0235 \text { and } 0.00808 \\
\text { gpm/ft2 for the } \\
\text { concentration and wash } \\
\text { modes respectively }\end{array}$ \\
\hline 1st concentration & 137.8 & 176.0 & 20.7 & 26.4 \\
\hline LAW subtotal & 137.8 & 176.0 & 20.7 & 26.4 \\
\hline 1st wash & 20.5 & 76.1 & 3.1 & 11.4 \\
\hline 2nd wash & 20.5 & 76.1 & 3.1 & 11.4 \\
\hline filter cleaning & 19.8 & 19.8 & 19.8 & 19.8 \\
\hline sludge heel return & 2.0 & 2.0 & 2.0 & 2.0 \\
\hline sludge trans. to HLP & 2.0 & 2.0 & 2.0 & 2.0 \\
\hline idle time & 73.0 & 0.0 & 10.9 & 0.0 \\
\hline Treat \& Clean subtotal & 137.8 & 176.0 & 40.9 & 46.6 \\
\hline Total Batch Time & 275.5 & 352.0 & 61.6 & 73.0 \\
\hline
\end{tabular}




\section{WSRC-TR-2004-00478, REVISION 0 SRNL-RPP-2004-00075, REVISION 0}

Table A10. Composition of Key Streams from Campaign IV Model

\begin{tabular}{|c|c|c|c|c|c|c|c|c|c|c|c|c|}
\hline $\begin{array}{l}\text { mg/L } \\
\text { solution @ } \\
25 C\end{array}$ & AP101 & AY102 & $\begin{array}{c}\text { Input } \\
\text { UF\&CIX } \\
\text { Recycle } \\
\end{array}$ & $\begin{array}{c}\text { FEP } \\
\text { Bottoms }\end{array}$ & $\begin{array}{c}\text { UF } \\
\text { Blended } \\
\text { Feed } \\
\end{array}$ & $\begin{array}{l}\text { UF Filtrate } \\
\text { to Cs-IX }\end{array}$ & $\begin{array}{c}\text { UF Slurry } \\
\text { 20wt\% UDS } \\
\end{array}$ & $\begin{array}{l}\text { 1st Wash } \\
\text { Sequence }\end{array}$ & $\begin{array}{l}\text { 2nd Wash } \\
\text { Sequence }\end{array}$ & $\begin{array}{c}\text { Output } \\
\text { UF\&CIX } \\
\text { Recycle } \\
\end{array}$ & $\begin{array}{c}\text { HLW Sludge } \\
\text { to HLP }\end{array}$ & $\begin{array}{c}\text { TLP } \\
\text { Bottoms }\end{array}$ \\
\hline $\mathrm{K}$ & 31175.6 & 429.2 & 3560.4 & 18546.8 & 22406.3 & 22704.3 & 19983.4 & 12231.6 & 3190.6 & 3602.8 & 1363.9 & 37357.0 \\
\hline $\mathrm{Na}$ & 128776.8 & 83976.2 & 20822.7 & 104317.9 & 115067.1 & 113992.0 & 123803.0 & 62862.6 & 22113.6 & 20864.0 & 21965.4 & 184149.6 \\
\hline $\mathrm{Ba}$ & 0.3 & 176.6 & 3.2 & 13.4 & 42.3 & 1.0 & 377.4 & 0.9 & 0.8 & 3.2 & 380.7 & 1.6 \\
\hline $\mathrm{Zn}$ & 5.6 & 58.6 & 7.5 & 218.9 & 46.8 & 47.4 & 41.8 & 25.6 & 6.7 & 7.5 & 2.9 & 80.3 \\
\hline $\mathrm{Cu}$ & 1.6 & 67.5 & 3.0 & 12.4 & 18.1 & 18.3 & 16.1 & 9.9 & 2.6 & 2.9 & 1.1 & 28.9 \\
\hline $\mathrm{Mg}$ & & 256.1 & 4.5 & 57.2 & 66.1 & 0.0 & 603.8 & 0.0 & 0.0 & 4.6 & 612.5 & 1.2 \\
\hline $\mathrm{Pb}$ & 14.9 & 1277.9 & 54.9 & 228.3 & 331.8 & 336.2 & 295.9 & 181.1 & 47.2 & 53.3 & 20.2 & 530.4 \\
\hline $\mathrm{Al}$ & 7816.2 & 16251.5 & 1025.8 & 4424.2 & 9264.0 & 7717.1 & 21838.3 & 3334.5 & 580.5 & 1040.9 & 17351.1 & 12177.4 \\
\hline $\mathrm{Fe}$ & 2.5 & 27922.2 & 463.1 & 1957.6 & 6629.5 & 202.9 & 58869.4 & 77.0 & 13.0 & 463.6 & 59598.9 & 320.1 \\
\hline $\mathrm{Ni}$ & 13.7 & 914.1 & 15.6 & 64.8 & 225.8 & 1.8 & 2046.2 & 0.7 & 0.1 & 15.6 & 2074.4 & 2.9 \\
\hline $\mathrm{F}$ & 59.8 & 225.3 & 18.6 & 185.8 & 114.6 & 116.2 & 102.2 & 62.6 & 16.3 & 18.4 & 7.0 & 955.8 \\
\hline $\mathrm{Cl}$ & 1623.7 & 158.6 & 436.8 & 12291.8 & 2747.0 & 2783.5 & 2449.9 & 1499.6 & 391.2 & 441.7 & 167.2 & 5904.9 \\
\hline $\mathrm{Br}$ & & 124.2 & 5.2 & 21.5 & 31.2 & 31.6 & 27.9 & 17.1 & 4.4 & 5.0 & 1.9 & 49.9 \\
\hline $\mathrm{Li}$ & 0.3 & 85.7 & 3.6 & 15.0 & 21.8 & 22.1 & 19.4 & 11.9 & 3.1 & 3.5 & 1.3 & 36.9 \\
\hline $\mathrm{P}$ & 495.9 & 2117.5 & 128.1 & 533.0 & 869.7 & 862.6 & 927.0 & 409.4 & 123.0 & 126.2 & 287.6 & 1361.0 \\
\hline $\mathrm{S}$ & 1339.2 & 806.5 & 183.1 & 851.0 & 1149.4 & 1154.9 & 1104.4 & 622.3 & 162.5 & 184.0 & 158.2 & 2059.8 \\
\hline $\mathrm{Cr}$ & 170.2 & 547.6 & 41.7 & 173.3 & 256.4 & 259.8 & 228.6 & 139.9 & 36.5 & 41.2 & 15.6 & 409.9 \\
\hline $\mathrm{Si}$ & 136.5 & 8418.0 & 152.7 & 886.5 & 2125.3 & 125.3 & 18382.6 & 48.0 & 16.0 & 152.8 & 18566.4 & 265.4 \\
\hline $\mathrm{Sr}$ & & 226.3 & 3.7 & 15.4 & 53.7 & 0.1 & 489.4 & 0.1 & 0.1 & 3.7 & 496.2 & 0.1 \\
\hline W & 24.6 & 161.8 & 9.5 & 39.4 & 57.9 & 58.6 & 51.6 & 31.6 & 8.2 & 9.3 & 3.5 & 92.5 \\
\hline $\mathrm{Ce}$ & & 247.7 & 10.2 & 42.6 & 62.2 & 63.1 & 55.5 & 34.0 & 8.9 & 10.0 & 3.8 & 99.5 \\
\hline $\mathrm{Nd}$ & & 361.1 & 5.9 & 24.5 & 85.6 & 0.0 & 781.3 & 0.0 & 0.0 & 5.9 & 792.5 & 1.0 \\
\hline $\mathrm{Zr}$ & & 997.5 & 18.3 & 76.2 & 237.6 & 24.1 & 1973.1 & 12.5 & 3.1 & 18.4 & 1982.1 & 38.1 \\
\hline Ag & & 490.7 & 8.0 & 33.2 & 116.3 & 0.8 & 1055.0 & 0.1 & 0.0 & 8.0 & 1069.9 & 2.7 \\
\hline $\mathrm{Ru}$ & & 87.6 & 3.6 & 15.2 & 22.0 & 22.3 & 19.6 & 12.0 & 3.1 & 3.5 & 1.3 & 35.2 \\
\hline $\begin{array}{l}\text { density } \\
(\mathrm{g} / \mathrm{L})\end{array}$ & 1278 & 1236 & 1053 & 1253 & 1264 & 1250 & 1375 & 1142 & 1051 & 1053 & 1153 & 1398 \\
\hline wt\% UDS & 0.01 & 11.50 & 0.60 & 2.45 & 2.38 & 0.00 & 20.00 & 0.12 & 0.00 & 0.57 & 21.64 & 0.05 \\
\hline
\end{tabular}


WSRC-TR-2004-00478, REVISION 0

SRNL-RPP-2004-00075, REVISION 0

Table A11. Solids Composition of Key Streams from the Model of Campaign IV

\begin{tabular}{|c|c|c|c|c|c|c|c|c|c|c|c|}
\hline wt $\%$ of total UDS & AP101 & AY102 & $\begin{array}{c}\text { Input UF \& } \\
\text { CIX Recycle }\end{array}$ & $\begin{array}{c}\text { FEP } \\
\text { Bottoms }\end{array}$ & $\begin{array}{l}\text { UF Blended } \\
\text { Feed }\end{array}$ & $\begin{array}{l}\text { 20wt\% UDS } \\
\text { UF1 Slurry }\end{array}$ & $\begin{array}{l}\text { 1st Wash } \\
\text { Sequence }\end{array}$ & $\begin{array}{l}\text { 2nd Wash } \\
\text { Sequence }\end{array}$ & $\begin{array}{c}\text { UF\&CIX } \\
\text { Recycle }\end{array}$ & $\begin{array}{l}\text { HLP } \\
\text { Sludge }\end{array}$ & $\begin{array}{c}\text { TLP } \\
\text { Bottoms }\end{array}$ \\
\hline $\mathrm{Fe}(\mathrm{OH}) 3$ & - & 37.54 & 14.10 & 12.10 & 40.83 & 40.83 & 0.97 & 1.24 & 14.65 & 45.71 & - \\
\hline $\mathrm{Al}(\mathrm{OH}) 3$ & - & 15.08 & 9.43 & 29.19 & - & - & 35.77 & 98.35 & 15.81 & 2.15 & - \\
\hline $\mathrm{Ni}(\mathrm{OH}) 2$ & 12.60 & 1.01 & 0.39 & 0.33 & 1.17 & 1.17 & 0.01 & 0.01 & 0.41 & 1.31 & - \\
\hline Ag2(CO3) & - & 0.44 & 0.16 & - & 0.49 & 0.49 & 0.01 & 0.00 & 0.17 & 0.55 & - \\
\hline $\mathrm{ZrO} 2$ & - & 0.94 & 0.39 & 0.33 & 0.96 & 0.96 & 0.01 & 0.04 & 0.41 & 1.07 & - \\
\hline $\mathrm{Mg}(\mathrm{OH}) 2$ & - & 0.43 & - & 0.45 & 0.53 & 0.53 & 0.00 & 0.01 & - & 0.59 & 0.42 \\
\hline NASGEL.15.5H2O & - & 34.86 & 14.02 & 16.74 & 39.22 & 39.22 & 2.19 & - & 14.57 & 43.91 & - \\
\hline $\mathrm{Nd}(\mathrm{PO} 4) \cdot 2 \mathrm{H} 2 \mathrm{O}$ & - & 0.48 & 0.18 & 0.15 & 0.54 & 0.54 & 0.00 & - & 0.19 & 0.61 & 0.28 \\
\hline $\mathrm{Na} 2(\mathrm{C} 2 \mathrm{O} 4)$ & 77.69 & 7.00 & - & 38.21 & 13.55 & 13.55 & 61.05 & - & - & - & 97.91 \\
\hline $\mathrm{Ba}(\mathrm{SO} 4)$ & - & 0.21 & 0.07 & 0.07 & 0.23 & 0.23 & - & 0.14 & 0.08 & 0.26 & 0.08 \\
\hline $\mathrm{Ca}(\mathrm{CO} 3)$ & 9.71 & 1.70 & 0.32 & 2.33 & 2.18 & 2.18 & - & - & 0.57 & - & - \\
\hline $\mathrm{Mn}(\mathrm{OH}) 2$ & - & 0.04 & - & 0.02 & - & - & - & 0.19 & - & 0.00 & - \\
\hline $\mathrm{Sr}(\mathrm{CO} 3)$ & - & 0.27 & 0.10 & 0.08 & 0.30 & 0.30 & - & 0.01 & 0.10 & 0.34 & 0.01 \\
\hline $\mathrm{Cu}(\mathrm{OH}) 2$ & - & - & 0.06 & - & - & - & - & - & 0.06 & - & - \\
\hline $\mathrm{Zn}(\mathrm{OH}) 2$ & - & - & 0.13 & - & - & - & - & - & 0.13 & - & - \\
\hline $\mathrm{Ca} 3(\mathrm{PO} 4) 2$ & - & - & 0.37 & - & - & - & - & 0.02 & 0.15 & 0.09 & - \\
\hline $\mathrm{Ce}(\mathrm{PO} 4)$ & - & - & 0.27 & - & - & - & - & - & 0.28 & - & - \\
\hline Pb3(PO4)2 & - & - & 1.13 & - & - & - & - & - & 1.14 & - & - \\
\hline $\mathrm{NaAl}(\mathrm{CO} 3)(\mathrm{OH}) 2$ & - & - & 58.86 & - & - & - & - & - & 51.30 & - & - \\
\hline $\mathrm{CaF} 2$ & - & - & - & - & - & - & - & - & - & - & 1.30 \\
\hline $\mathrm{Ca}(\mathrm{C} 2 \mathrm{O} 4) \cdot 1 \mathrm{H} 2 \mathrm{O}$ & - & - & - & - & - & - & - & - & - & 3.41 & - \\
\hline
\end{tabular}




\section{A.5. MODEL RESULTS}

Analytical results of the stream compositions from the SIPP Campaign II run indicate a higher glass production rate than estimated by the model of about $15 \%$. This difference is due to the adjustment made to the solids portion of the simulant recipe used in the model. While it is not necessary, even undesirable, to base the solids portion of a simulant recipe on a charge balanced analysis, the model requires all input streams to be neutral. The simulant solids recipe had an excess of cations and hydroxide was added to achieve a charge balance for the model input. This led to an inconsistency between the charge balance and the concentration of the constituent species, adding hydroxide caused the mass basis to go from $1000 \mathrm{~kg}$ to $1138 \mathrm{~kg}$ (i.e. the solids composition was given in moles/1000kg dry solids). The simulant recipe was still in development at the time of the model runs, and instead of modifying the ratio of the solids species to achieve the correct mass basis, the composition was used as given. The result was to dilute the metals concentration in the solids of the model relative to that of the final simulant composition.

\section{A.6. REFERENCES FOR APPENDIX A}

Barnes, C. D. OLI/ESP Modeling of the Semi-Integrated Pilot Plant for Estimate of Campaigns I-IV Simulant Volumes (U). WSRC-TR-2004-0403, Rev. 0, Westinghouse Savannah River Co., Aiken, 2004.

Lee, E., et al. Flowsheet Bases, Assumptions, and Requirements. 24590-WTP-RPT-PT02-005, Rev. 1, pp 3.1-9, Section-3.1.3.5.1 - LAW Glass $\mathrm{Na}_{2}$ Loading, Washington Group International, Richland, WA, 2003a

Lee, E., et al. Flowsheet Bases, Assumptions, and Requirements. 24590-WTP-RPT-PT02-005, Rev. 1, pp 4.1-2, Table TS-1.1 Minimum Component Limits in High-Level Waste Glass, Washington Group International, Richland, WA, 2003b

Lee, E., et al. Flowsheet Bases, Assumptions, and Requirements. 24590-WTP-RPT-PT02-005, Rev. 1, pp 2.3-1 - 2.3.6, Ultra-Filtration Assumptions, Washington Group International, Richland, WA, 2003c

Lee, E., et al. Flowsheet Bases, Assumptions, and Requirements. 24590-WTP-RPT-PT02-005, Rev. 1, pp 2.5-33 Table 2.5-9 Cesium Removal System Process Stream Descriptions for Material Balance Calculations, Washington Group International, Richland, WA, 2003d

Russel, R.L., et al. AP-101 Diluted Feed (Envelope A) Simulant Development Report. PNWD-3248 WTP-RPT-057, Rev. 0, Battelle, Pacific Northwest Division, Richland, WA, 2003

Zamecnik, J.R., Burket, P.R., and Eibling, R.E. Tank 241-AY-102 Simulant Development, Ultrafiltration, and Washing. WSCR-TR-2003-0547, Rev. 0, Westinghouse Savannah River Co., Aiken, 2004 
WSRC-TR-2004-00478, REVISION 0

SRNL-RPP-2004-00075, REVISION 0

\title{
Appendix B: WTP Discussion on Pretreatment Facility Waste Processing Residence Times
}

----- Forwarded by Bond Calloway/WSRC/Srs on 03/16/2004 07:22 PM -----

\author{
"Harty, David" \\ < dharty@bechtel.com> \\ 03/16/2004 06:11 PM
}

To: Bond Calloway bond.calloway@srs.gov

Cc: Harty, David dharty@bechtel.com

Olander, Alan arolande@bechtel.com

Slaathaug, Eric ejslaath@bechtel.com

Lehrman, scott slehrman@bechtel.com

Peterson, Reid rpeterso@bechtel.com

Sundar, Parameshwaran S pssundar@bechtel.com

Tadlock, Johnjatadloc@bechtel.com

\section{SUBJECT: PLANT RESIDENCE TIME DISCUSSION}

$<<\mathrm{PT}$ Flow Diagram Rev 1b.vsd $>>\quad<$ PT Tank Vol_App B.doc $>>$

Attached (they have been removed) above are the handouts from the discussion this afternoon. Four different holdup times were discussed during the meeting.

Note: The defined production rates for SIPP are 6.0 MT IHLW/day and $80 \mathrm{MT}$ ILAW/day. Steady-state flowrates defined below are from the WTP Steady State Flowsheet.

I. Holdup Time For Permeate After Ultrafiltration.

Two permeate vessel tank volumes $(21,800$ each) plus the CXP Feed Vessel available volume $(78,000$ batch $-25,000$

contingency) are used for a total available holdup volume of 96,600 gallons. At a steady-state permeate flowrate of 14.8 gpm, the residence time is $96,600 /(14.8 * 60)=109$ hours $(\sim 4$ $1 / 2$ days).

II. Holdup Time For Blended Feed With Concentrated Recycles Prior To Ultrafiltration. 
WSRC-TR-2004-00478, REVISION 0

SRNL-RPP-2004-00075, REVISION 0

Two UFP Preparation vessel tank volumes $(48,300$ each) are used for a total available holdup volume of 96,600 gallons. At a steady-state feed to UFP Feed vessels of $16.3 \mathrm{gpm}$, the residence time is $96,600 /(16.3 * 60)=99$ hours ( 4 days). This value is overly conservative and the residence time of blending the concentrated recycles with the blended feed is dependent on the dynamic nature of recycle generation, feed blending, and the processing of feed form the preparation vessel to the feed vessel. If the online UFP Feed vessel is considered full and the UF Prep vessels are both filled with blended feed and concentrated recycle, then the residence time of $\sim 4$ days will result. Further consideration suggests that the residence time using one UFP Preparation vessel tank volume should be used. This would give a residence time of $48,300 /(16.3 * 60)=49$ hours ( 2 days). Use the second value.

III. Holdup Time for Blended Recycles (HLW-SBS with UFP recycles) Prior to Evaporation.

Two evaporator feed tanks with an available volume of (50,000 batch - 10,000 contingency) are used for a total available holdup volume of 80,000 gallons. At a steadystate evaporator feed flowrate of $18.5 \mathrm{gpm}$, the residence time is $80,000 /(18.5 * 60)=72$ hours $(\sim 3$ days $)$.

IV. Holdup Time For Recycles in the Evaporator.

One evaporator with a holdup volume of 5500 gallons is used. At a steady-state concentrate flowrate of $1.8 \mathrm{gpm}$, the residence time is 5500/(1.8*60) = 51 hours ( 2 days). It was noted that without the leaching process, there is no recycle leachate. Inclusion of the leaching recycle reduces the residence time in the evaporator because the concentrate flowrate is higher.

Comments are welcome.

Dave Harty

Pretreatment Process Operations, 371-3360 


\section{Appendix C: Anti-Foam Subcontractor Analysis}

The information contained on the following two pages of this appendix is a portion of the data to be analyzed by Dow Corning for samples of simulated waste processed during Campaign II.

Notes:

1. The unit shown in the Dow Corning results, "by wt in ppm," is wrong. The actual unit is $\mathrm{mg} / \mathrm{L}$.

2. No accuracy is stated in the Dow Corning document, but the results may be in error by as much as $50 \%$ because of the difficulty in isolating the analyte that implies the presence of antifoam.

3. As stated above, only a portion of the SIPP samples sent for analysis was complete at the time this report was issued. Those from the CIX and TLP operations were outstanding, however, those results may not be important because more than $99 \%$ of the antifoam, introduced in the FEP feed, was shown to have stayed with the slurry after UFP dewatering. That is, the concentrated UFP slurry had $1260 \mathrm{mg} / \mathrm{L}$ of antifoam (sample:C2-UF-W2-22-Antf) and the permeate removed had less than $10 \mathrm{mg} / \mathrm{L}$ (sample:C2-1X-Feed-Antf). Since the CIX and the TLP only processed the permeate waste stream, then the small amount of antifoam carried through may not have been measurable. 
WSRC-TR-2004-00478, REVISION 0

SRNL-RPP-2004-00075, REVISION 0

\section{DOW CORNING}

DOW consing

Customer Confidential

ISO9001:2000 Registered

\section{Analytical Solutions Report}

\begin{tabular}{|ll|}
\hline Customer: & $\begin{array}{l}\text { Savannah River } \\
\text { National Laboratory } \\
\text { Jack Zamecnik }\end{array}$ \\
E-Mail: & john.zamecnik@srs.gov \\
Phone: & $\mathbf{8 0 3 - 7 2 5 - 4 5 3 5}$
\end{tabular}

Phone:

\begin{tabular}{|ll|}
\hline Report No.: & 19763 \\
Quote Reference: & $\begin{array}{l}\text { Description of SRNL } \\
\text { Pilot System }\end{array}$ \\
& $\begin{array}{l}\text { Samples.pdf } \\
07 / 22 / 2004\end{array}$ \\
Sample Receipt Date: & $08 / 10 / 04$ \\
Report Sent Date: & 084 \\
Customer Reference: & AC37127T \\
\hline
\end{tabular}

Description of Request:

Feasibility to determine if Fourier Transform Infrared Spectroscopy (FTIR) can be used to quantify silicone in processed initial, diluted, sludge and liquid samples with and without Q2$3183 \mathrm{~A}$. If interferences are not present calibration curves will be generated for each material to quantify Q2-3183A.

\section{Sample Description:}

\begin{tabular}{|c|c|}
\hline Sample Identifier: & Comments: \\
\hline $\begin{array}{l}\text { C2-FEP-B-END- } \\
\text { ANTF }\end{array}$ & Concentrated FEP Bottoms: should contain \\
\hline $\begin{array}{l}\text { C2-FEP-B-Start- } \\
\text { ANTF }\end{array}$ & FEP Evaporator Feed Slurry \\
\hline $\begin{array}{l}\text { C2-FEP-B-Start- } \\
\text { Blank }\end{array}$ & FEP Evaporator Feed Slurry; does not contain antifoam \\
\hline C2-IX-Feed-ANTF & Ultrafilteration Permeate \\
\hline C2-UF-W2-22-ANTF & $\begin{array}{l}\text { Concentrated wash slurry; matches undissolved solids content, but } \\
\text { soluble salt composition is different }\end{array}$ \\
\hline C2-WPE-CMP-ANTF & wash water \\
\hline IX-Feed-Blank & Ultrafilteration Permeate; made from AY102+AP101 simulants filtered) \\
\hline Q2-3183A control & Will arrive week of $7 / 26 ;$ provided by DC \\
\hline $\begin{array}{l}\text { UF-Wash-Slurry- } \\
\text { Blank (C1) }\end{array}$ & Concentrated wash slurry: blank \\
\hline WPE-Blank (C1) & wash water; blank \\
\hline
\end{tabular}




\section{WSRC-TR-2004-00478, REVISION 0}

SRNL-RPP-2004-00075, REVISION 0

Dow Corning Analytical Solutions

Customer Confidential

ISO9001:2000 Registered

\section{Results:}

The feasibility study was successful. Silicone can be measured down to $10 \mathrm{ppm}$ in the process samples using the developed extraction method. The n-Heptane solvent spectrum was subtracted from each sample and standard spectrum. Peak height absorbances of the SiMethyl band near $1260 \mathrm{~cm}^{-1}$ were measured using the baseline technique. Calculations were performed using information from the standards and samples. The silicone concentrations are listed in the following table.

\begin{tabular}{|c|c|}
\hline Sample Reference Description & Silicone, by wt in ppm \\
\hline WPE-Blank (C1) & none detected $<10$ \\
\hline 1X-Feed-Blank & none detected $<10$ \\
\hline C2-FEP-B-Start-Blank & none detected $<10$ \\
\hline UF-Wash-Slumy-Blank (C1) & none detected $<10$ \\
\hline C2-WPE-CMP Antf & none detected $<10$ \\
\hline C2-1X-Feed-Antf & trace $<10$ \\
\hline C2-FEP-B-Start-Antf & 1580 \\
\hline C2-FEP-B-End-Antf & 2410 \\
\hline C2-UF-W2-22-Antf & 1260 \\
\hline
\end{tabular}

\section{Experimental:}

10.0 grams of each blank and antifoam sample were added to a $1 / 2$ ounce glass vial. All nine samples were diluted with $2.0 \mathrm{ml}$ of HPLC grade $n$-Heptane solvent. The vials were capped, agitated on a wrist action shaker for 15 minutes, and centrifuged at $3000 \mathrm{rpms}$ for 15 minutes. Fourier Transform Infrared (FTIR) spectrum was collected on the heptane solvent in a $0.2 \mathrm{~mm}$ fixed pathlength sealed liquid cell using a Thermo Nicolet Magna 750 FTIR spectrometer. The clear heptane extractables from the blank and antifoam samples were subsequently added to the sealed liquid cell and FTIR spectra were acquired. Five standards of polydimethylsiloxane were prepared in HPLC grade n-Heptane solvent. FTIR spectra were collected on these standard solutions as well. The cell was flushed with n-Heptane and dried with a low flow of nitrogen gas between each sample and standard.

Statement of Accuracy and Precision:

Precision and accuracy have not been determined for this analysis. 
WSRC-TR-2004-00478, REVISION 0 SRNL-RPP-2004-00075, REVISION 0

This page intentionally left blank.

$$
\text { - C4 - }
$$




\section{Appendix D: Campaign II Sampling Matrix}

The following 12 pages of this appendix contain a list of all the samples taken during Campaign II. Because of the large number of samples and the importance to document those that were taken, the tables have been put in a form, which is condensed. However, the tables are readable and their placement here should be useful to those wanting to better understand the data discussed in the body of this report. Because of the length of each list of samples with their corresponding test made, the original Excel speadsheet pages have been split in two and contain:

1. The top of each appendix page lists the specifics of each sample as to name, where in the test matrix it was taken, sample size, if it was expedited for fast processing, and if the sample needed special handing, like when solids had to be dissolved.

2. The bottom of each appendix page lists the specific analytic measurement history on the corresponding sample. The sample name is repeated to relate the history to the sample indicated in the top half.

\section{Notes on the following Sample Tables}

\begin{tabular}{|c|c|c|c|c|}
\hline Time Dep Sample & $\begin{array}{l}\text { Time dependent sa } \\
\text { If solids form, they }\end{array}$ & $\begin{array}{l}\text { These samples are to be observed } \\
\text { analyzed by XRD. A filtrate blank wi }\end{array}$ & out 5 & for evidence of precipitation of solids \\
\hline xplanation of ana & lyses: & & & \\
\hline & $\mathrm{pH} \mathrm{pH}$ & & ICC & Ion chrom. cations \\
\hline & ICPES includes all element & sent that can be analyzed by ICPES & IEC & Ion exclusion chrom. \\
\hline & ICPMS sludges & $\mathrm{Cs}, \mathrm{Rb}, \mathrm{Bi}, \mathrm{Y}, \mathrm{Pr}$ & $\mathrm{AA}$ & atomic absorption \\
\hline & supernates & $\mathrm{Cs}, \mathrm{Rb}$ & TS & total solids wt $\%$ \\
\hline & ICA Ion chrom - anions & normal suite & UDS & undissolved solids wt $\%$ \\
\hline
\end{tabular}

Explanation of analyses:

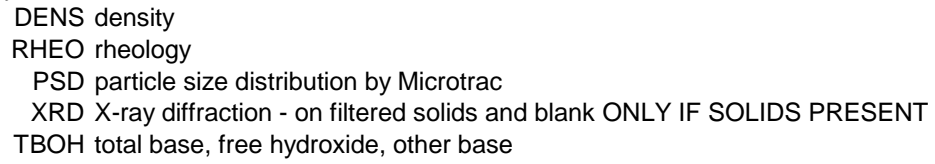

DISMW: microwave acid dissolution

DISPER: sodium peroxide fusion / HCL uptake dissolution 
WSRC-TR-2004-00478, REVISION 0

SRNL-RPP-2004-00075, REVISION 0

\section{Samples Taken During Pilot-Scale Recycle Blending}

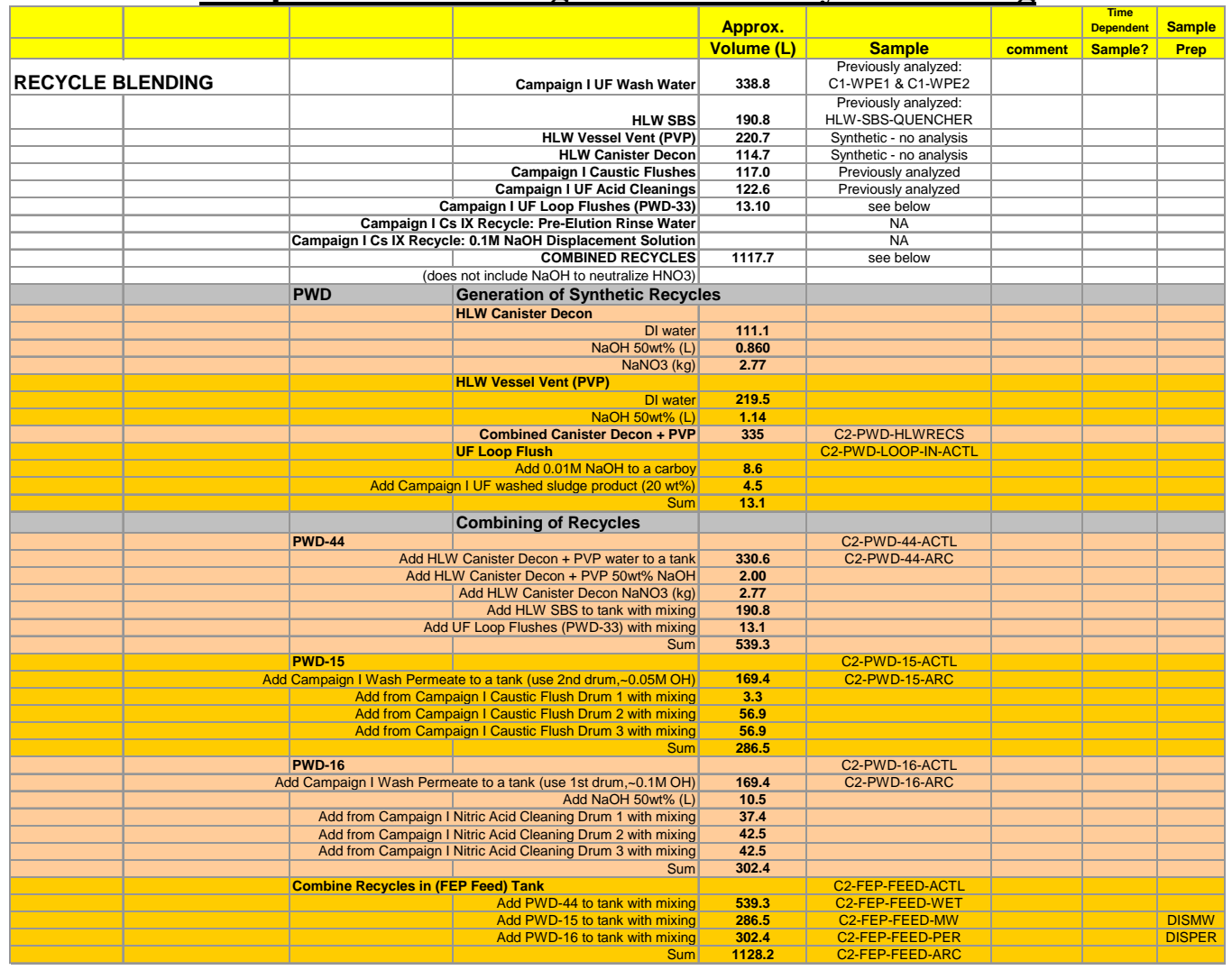

\section{Analytical Measurements On Samples Taken During Pilot-ScaleRecycle Blending}

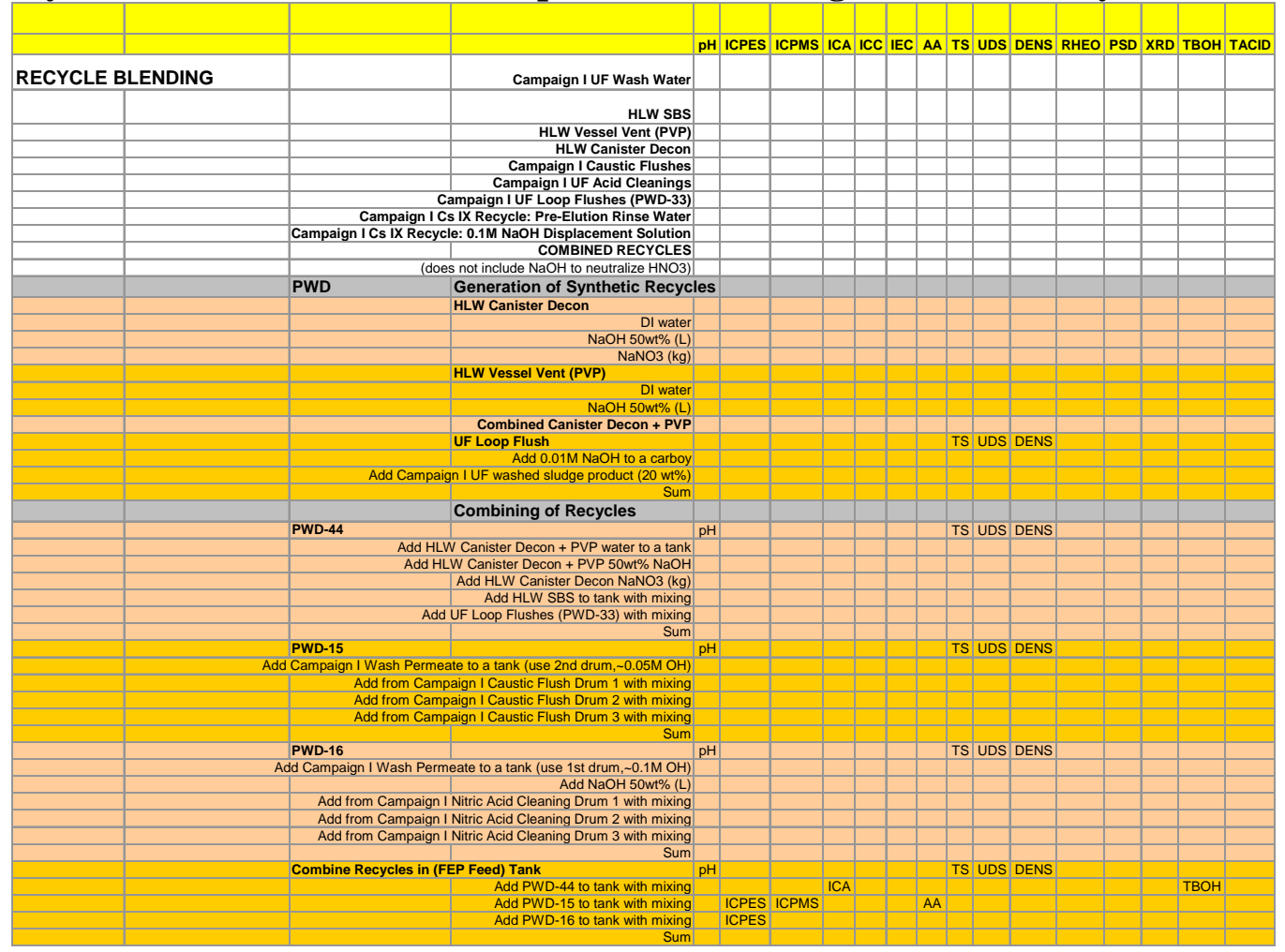


WSRC-TR-2004-00478, REVISION 0

SRNL-RPP-2004-00075, REVISION 0

\section{Samples Taken During Pilot-Scale FEP Testing}

\begin{tabular}{|c|c|c|c|c|c|c|c|}
\hline & & & Approx. & & & Time Dep & Sample \\
\hline & & & & Sample & comment & & Prep \\
\hline FEP EVAPORATOR & & Starting Volume & 1128.2 & & & & \\
\hline & Bottoms & After all antifoam additions & & C2-FEP-B-START-ACTL & & & \\
\hline & & & & C2-FEP-B-START-ANTF & & & \\
\hline & & & & C2-FEP-B-START-ARC & & & \\
\hline & Bottoms & At $\sim 200$ L evaporated & & C2-FEP-B-200-ACTL & & & \\
\hline & & & & C2-FEP-B-200-WET & & & \\
\hline & & & & C2-FEP-B-200-ARC & & & \\
\hline & Primary Condensate & & & C2-FEP-PC-200-ARC & & & \\
\hline & Secondary Condensate & & & C2-FEP-SC-200-ARC & & & \\
\hline & Bottoms & At $\sim 400$ L evaporated & & C2-FEP-B-400-ACTL & & & \\
\hline & & & & C2-FEP-B-400-ARC & & & \\
\hline & Primary Condensate & & & C2-FEP-PC-400-ARC & & & \\
\hline & Secondary Condensate & & & C2-FEP-SC-400-ARC & & & \\
\hline & Bottoms & At $\sim 600$ L evaporated & & C2-FEP-B-600-ACTL & & & \\
\hline & & & & C2-FEP-B-600-ARC & & & \\
\hline & Primary Condensate & & & C2-FEP-PC-600-ARC & & & \\
\hline & Secondary Condensate & & & C2-FEP-SC-600-ARC & & & \\
\hline & Bottoms & At -800 L evaporated & & C2-FEP-B-800-ACTL & & & \\
\hline & & & & C2-FEP-B-800-ARC & & & \\
\hline & Primary Condensate & & & C2-FEP-PC-800-ARC & & & \\
\hline & Secondary Condensate & & & C2-FEP-SC-800-ARC & & & \\
\hline \multirow[t]{3}{*}{ RUSH } & Bottoms & Evaporation completed & & C2-FEP-B-END-SOL & & RUSH & \\
\hline & & & & C2-FEP-B-END-ACTL & & & \\
\hline & & & & C2-FEP-B-END-WET & & & \\
\hline \multirow[t]{4}{*}{ RUSH } & & & & C2-FEP-B-END-SUP & & RUSH & SPECIAL \\
\hline & & & & C2-FEP-B-END-MW & & & DISMW \\
\hline & & & & C2-FEP-B-END-PER & & & DISPER \\
\hline & & & & C2-FEP-B-END-ANTF & & & \\
\hline & & & & C2-FEP-B-END-ARC & & & \\
\hline & Primary Condensate & Evaporation completed & & C2-FEP-PC-END-ARC & & & \\
\hline & Secondary Condensate & & & C2-FEP-SC-END-ARC & & & \\
\hline \multicolumn{2}{|c|}{ COMBINE ALL FEP CONDENSATES } & & & & & & \\
\hline & \multicolumn{2}{|c|}{ Combined FEP Condensate = RLD Condensate } & 1012.3 & $\begin{array}{l}\text { C2-RLD-FROM-FEP-ADS } \\
\text { C2-RLD-FROM-FEP-ACTL } \\
\text { C2-RLD-FROM-FEP-ARC }\end{array}$ & & & \\
\hline
\end{tabular}

\section{Analytical Measurements On Samples Taken During Pilot-Scale FEP Testing}

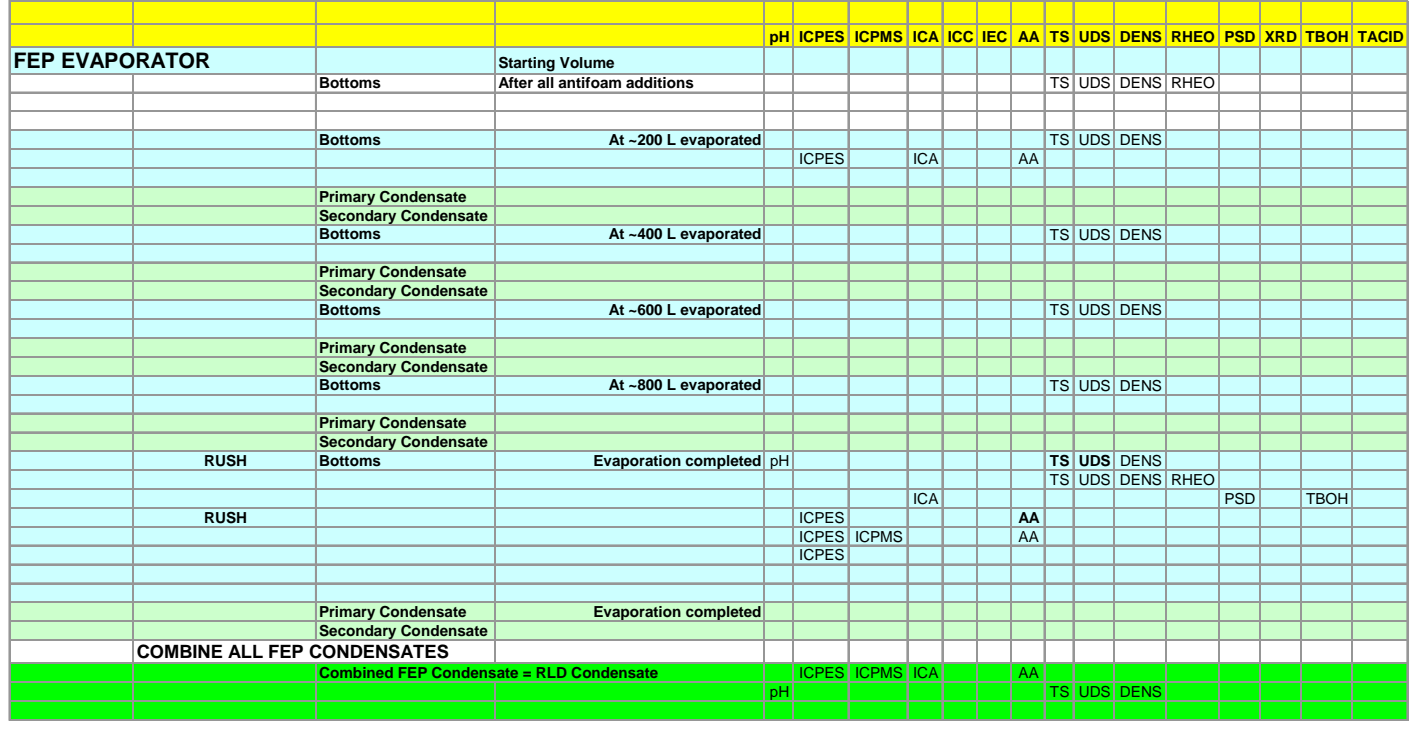


WSRC-TR-2004-00478, REVISION 0

SRNL-RPP-2004-00075, REVISION 0

\section{Samples Taken During Pilot-Scale UFP Testing}

\begin{tabular}{|c|c|c|c|c|c|c|c|c|}
\hline & & & & Approx. & & & Time Dep & Sample \\
\hline & & & & Volume (L) & Sample & comment & Sample? & Prep \\
\hline \multicolumn{2}{|c|}{ ULTRAFILTRATION } & UFP & FEP Bottoms (L) & 115.8 & see above for sample & & & \\
\hline & RUSH & & AY102 before Supernate Addition & & C2-AY102-SOL-NOSUP & & RUSH & \\
\hline & RUSH & & AY102 (L) & 252.5 & C2-AY102-SOL & & RUSH & \\
\hline & & & & & C2-AY102-WET & & & \\
\hline & & & & & C2-AY102-MW & & & DISMW \\
\hline & & & & & C2-AY102-PER & & & DISPER \\
\hline & RUSH & & & & C2-AY102-SUP & & RUSH & SPECIAL \\
\hline & & & & & C2-AY102-ARC & & & \\
\hline & & & AP101 (L) & 671.5 & C2-AP101-ACTL & & & \\
\hline & & & & & C2-AP101-WET & & & \\
\hline & & & & & C2-AP101-MW & & & DISMW \\
\hline & & & & & C2-AP101-PER & & & DISPER \\
\hline & & & & & C2-AP101-ARC & & & \\
\hline & & & $\begin{array}{r}\text { UFP Heel } \\
\end{array}$ & & - & & & \\
\hline & & & COMBINED UFP FEEDS & 1039.8 & - & & & \\
\hline & & & & & & & & \\
\hline & CONCENTRATE & & Starting volume (L) & 1039.8 & & & & \\
\hline & & & Fill feed tank to: & 140 & v & & & \\
\hline & & UFP Feed & before start of run & & $\begin{array}{l}\text { C2-UF-START-AS-ACTL } \\
\text { C2-UF-START-AS-MWW }\end{array}$ & & & DISMW \\
\hline & & & & & C2-UF-START-AS-PER & & & DISPER \\
\hline & & & & & C2-UF-START-AS-WET & & & \\
\hline & & & & & C2-UF-START-SUP-ACTL & & & SPECIAL \\
\hline & & & & & C2-UF-START-SUP-ADS & & & SPECIAL \\
\hline & & & & & C2-UF-START-ARC & & & \\
\hline & & & Total time to concentrate $(\mathrm{hr})$ & 30.1 & & & & \\
\hline & 1st $1 / 2$ of Batch & & Time for first half batch (hr) & 15.1 & & & & \\
\hline & RUSH & Concentrate Sample & at this vol permeate removed $(L)$ & 153.7 & C2-UF-CON1-154 & & RUSH & \\
\hline & RUSH & Concentrate Sample & at $\sim$ this vol permeate removed (L) & 307.4 & C2-UF-CON1-307 & & RUSH & \\
\hline & RUSH & Concentrate Sample & at $\sim$ this vol permeate removed ( $L$ ) & 461.2 & C2-UF-CON1-461 & & RUSH & \\
\hline & Note: EDL to measure & Permeate Sample & at $\sim$ this vol permeate removed ( $L$ ) & 153.7 & C2-PE-CON1-154-XRD & only if solids & $\mathrm{Y}$ & \\
\hline & turbidity throughout & & & & C2-PE-CON1-154-XRD-BLK & blank & & \\
\hline & filtration. & Permeate Sample & at $\sim$ this vol permeate removed (L) & 307.4 & C2-PE-CON1-307-XRD & only if solids & $\mathbf{Y}$ & \\
\hline & & & & & C2-PE-CON1-307-XRD-BLK & blank & & \\
\hline & & Permeate Sample & at $\sim$ this vol permeate removed (L) & 461.2 & C2-PE-CON1-461-XRD & only if solids & $\mathrm{Y}$ & \\
\hline & & & & & C2-PE-CON1-461-XRD-BLK & blank & & \\
\hline & & & & & C2-PE-CON1-461-ACTL & & & \\
\hline & Hold & & Hold for $12 \mathrm{hr}$ & & & & & \\
\hline
\end{tabular}

Analytical Measurements On Samples Taken During Pilot-Scale UFP Testing

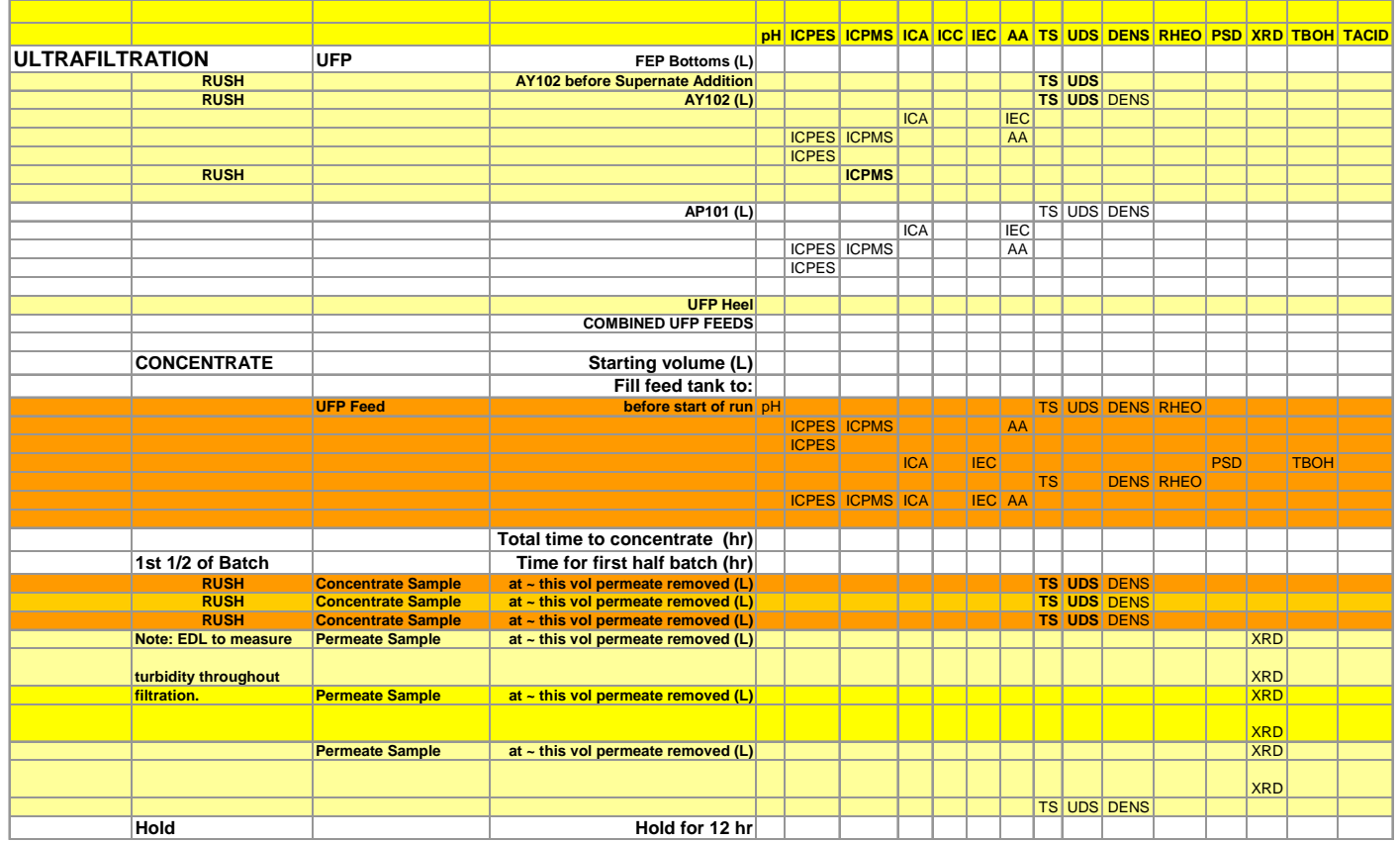


WSRC-TR-2004-00478, REVISION 0

SRNL-RPP-2004-00075, REVISION 0

\section{Samples Taken During Pilot-Scale UFP Testing (continued)}

\begin{tabular}{|c|c|c|c|c|c|c|c|c|}
\hline & & & & Approx. & & & Time Dep & Sample \\
\hline & & & & Volume (L) & Sample & comment & Sample? & Prep \\
\hline & 2nd $1 / 2$ of Batch & & Time for second half batch (hr) & 15.1 & & & & \\
\hline & RUSH & Concentrate Sample & at - this vol permeate removed $(L)$ & 153.7 & C2-UF-CON2-154 & RUSH & & \\
\hline & RUSH & Concentrate Sample & at this vol permeate removed (L) & 307.4 & C2-UF-CON2-307 & RUSH & & \\
\hline & & Concentrate Sample & all permeate removed, @ 20wt\% solids & 461.2 & C2-UF-CON2-461-MW & & & DISMW \\
\hline & & & & & C2-UF-CON2-461-PER & & & DISPER \\
\hline & & & & & C2-UF-CON2-461-WET & & & \\
\hline & RUSH & & & & C2-UF-CON2-461-SOL & RUSH & & \\
\hline & & & & & C2-UF-CON2-461-ACTL & & & \\
\hline & & & & & C2-UF-CON2-461-ARC & & & \\
\hline & & Permeate Sample & at this vol permeate removed (L) & 153.7 & C2-PE-CON2-154-XRD & only if solids & $\mathrm{Y}$ & \\
\hline & & & & & C2-PE-CON2-154-XRD-BLK & blank & & \\
\hline & & Permeate Sample & at $\sim$ this vol permeate removed (L) & 307.4 & C2-PE-CON2-307-XRD & only if solids & $\mathrm{Y}$ & \\
\hline & & & & & C2-PE-CON2-307-XRD-BLK & blank & & \\
\hline & & Permeate Sample & all permeate removed, @ 20wt\% solids & 461.2 & C2-PE-CON2-461-XRD & only if solids & $\mathrm{Y}$ & \\
\hline & & & & & C2-PE-CON2-461-XRD-BLK & blank & & \\
\hline & CONCENTRATION & Concentrate & volume in feed tank (L) & 117.0 & & & & \\
\hline & COMPLETE & Permeate Composite & ALL PERMEATE COMPOSITE & & C2-IX-FEED-ADS & & & \\
\hline & & to IX & total volume of permeate (L) & 922.3 & C2-IX-FEED-ACTL & & & \\
\hline & & & & & C2-IX-FEED-ANTF & & & \\
\hline & & & & & C2-IX-FEED-XRD & only if solids & $\mathrm{Y}$ & \\
\hline & & & & & C2-IX-FEED-XRD-BLK & blank & & \\
\hline & DILUTE SLURRY B & ICK TO 20WT\% WI & I PERMEATE IF NECESSARY & & & & & \\
\hline & RUSH & & & & C2-UF-CON2-ADJ & RUSH & & \\
\hline & FIRST WASH & & RLD Wash water (L) & 114.7 & & & & \\
\hline WASHING & & Concentrate Sample & after addition \# & 1 & C2-UF-W1-1 & & & \\
\hline & & Concentrate Sample & after addition \# & 5 & C2-UF-W1-5 & & & \\
\hline & & Concentrate Sample & after addition \# & 9 & C2-UF-W1-9 & & & \\
\hline & & Concentrate Sample & after addition \# & 13 & C2-UF-W1-13 & & & \\
\hline & & Concentrate Sample & after addition \# & 17 & C2-UF-W1-17 & & & \\
\hline & & Concentrate Sample & after addition \# (last) & 22 & C2-UF-W1-22-MW & & & DISMW \\
\hline & & & & & C2-UF-W1-22-PER & & & DISPER \\
\hline & & & & & C2-UF-W1-22-WET & & & \\
\hline & & & & & C2-UF-W1-22-ACTL & & & \\
\hline & & & & & C2-UF-W1-22-ARC & & & \\
\hline & & Wash Permeate & after addition \# & 1 & C2-WPE1-1-ACTL & & & \\
\hline & & & & & C2-WPE1-1-XRD & only if solids & $Y$ & \\
\hline & & & & & C2-WPE1-1-XRD-BLK & blank & & \\
\hline & & Wash Permeate & after addition \# & 5 & C2-WPE1-5-ACTL & & & \\
\hline & & & & & C2-WPE1-5-XRD & only if solids & $\mathrm{Y}$ & \\
\hline & & & & & C2-WPE1-5-XRD-BLK & blank & & \\
\hline & & Wash Permeate & after addition \# & 9 & C2-WPE1-9-ACTL & & & \\
\hline & & & & & C2-WPE1-9-XRD & only if solids & $\mathrm{Y}$ & \\
\hline & & & & & C2-WPE1-9-XRD-BLK & blank & & \\
\hline & & Wash Permeate & after addition \# & 13 & C2-WPE1-13-AC & & & \\
\hline & & & & & C2-WPE1-13-XRD & only if solids & $Y$ & \\
\hline & & & & & C2-WPE1-13-XRD-BLK & blank & & \\
\hline & & Wash Permeate & after addition \# & 17 & C2-WPE1-17-ACTL & & & \\
\hline & & & & & C2-WPE1-17-XRD & only if solids & $\mathrm{Y}$ & \\
\hline & & & & & C2-WPE1-17-XRD-BLK & blank & & \\
\hline
\end{tabular}

\section{Analytical Measurements On Samples Taken During Pilot-Scale UFP Testing (continued)}

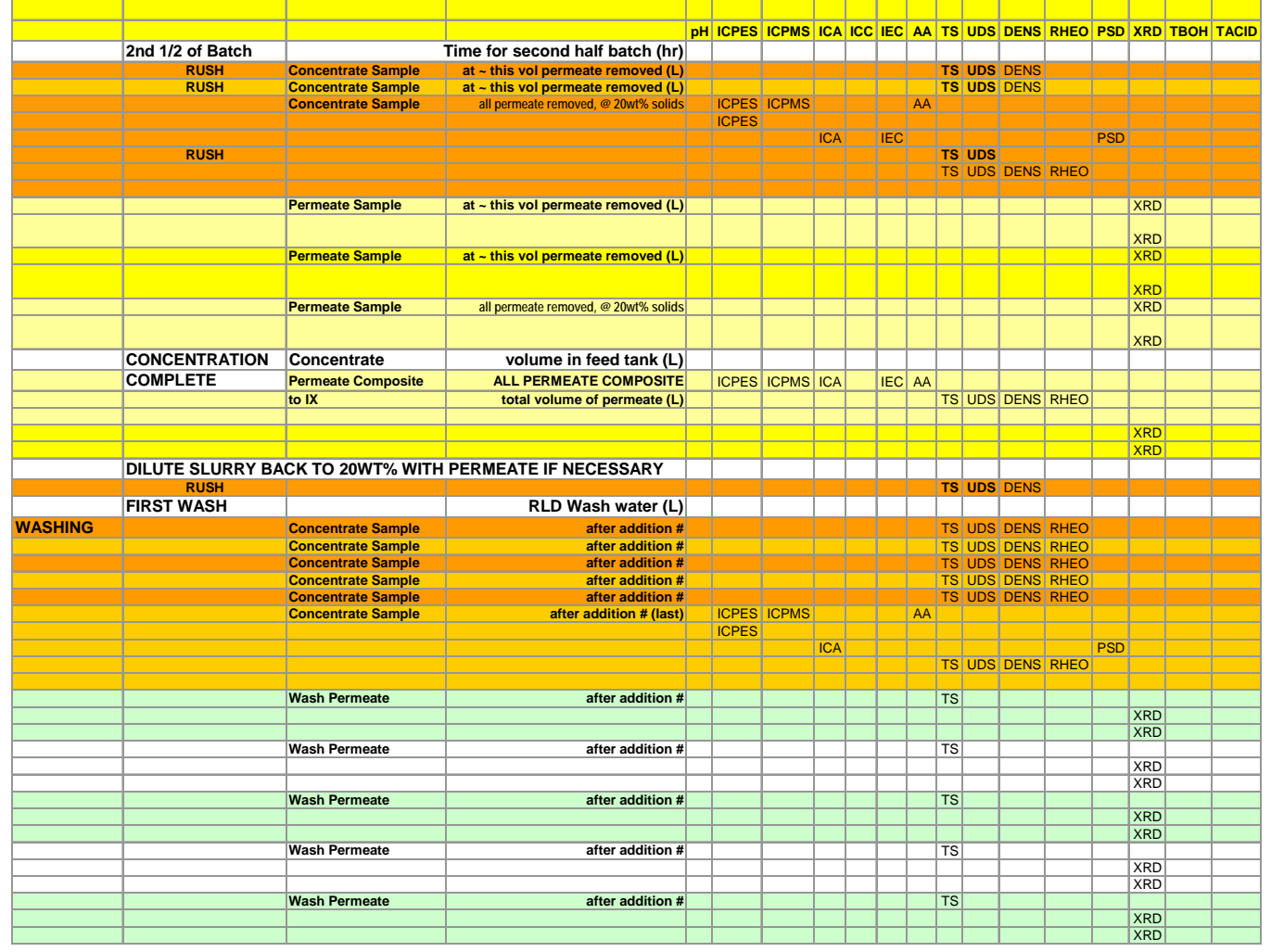


WSRC-TR-2004-00478, REVISION 0

SRNL-RPP-2004-00075, REVISION 0

\section{Samples Taken During Pilot-Scale UFP Testing (continued)}

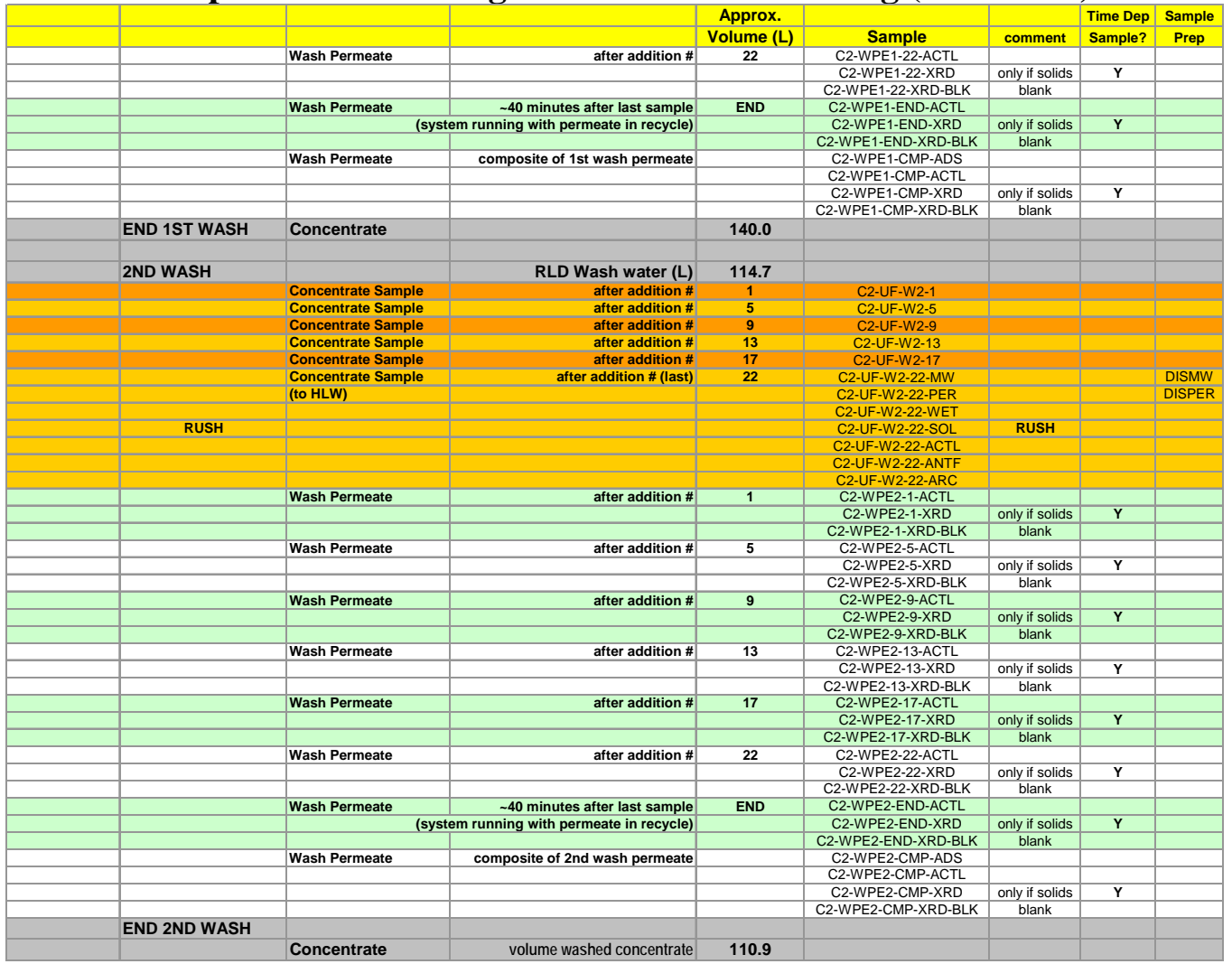

\section{Analytical Measurements On Samples Taken During Pilot-Scale UFP Testing (continued)}

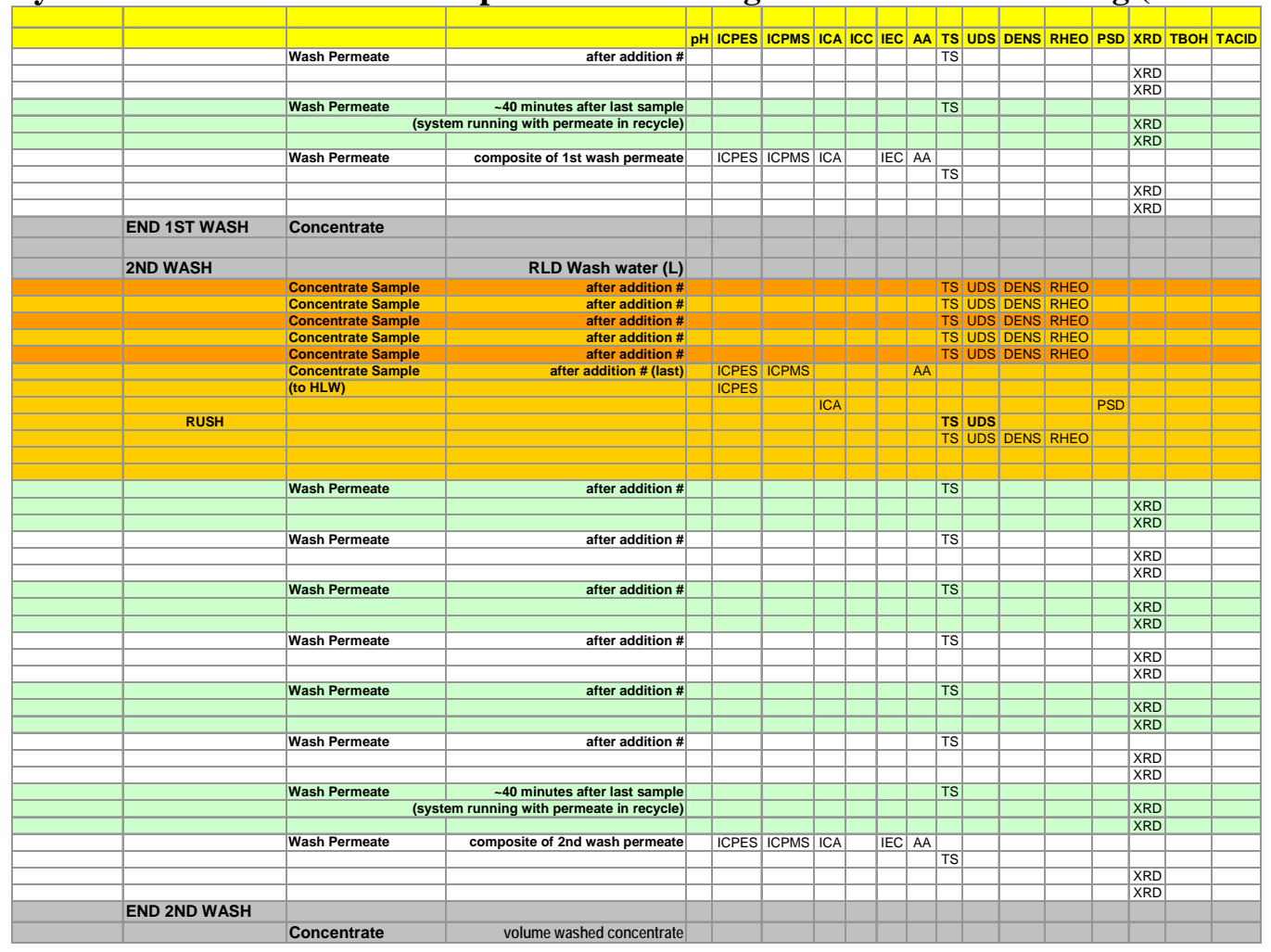


WSRC-TR-2004-00478, REVISION 0

SRNL-RPP-2004-00075, REVISION 0

\section{Samples Taken During Pilot-Scale UFP Testing (continued)}

\begin{tabular}{|c|c|c|c|c|c|c|c|c|}
\hline & & & & Approx. & & & Time Dep & Sample \\
\hline & & & & Volume (L) & Sample & comment & Sample? & Prep \\
\hline \multicolumn{9}{|l|}{ CLEANING } \\
\hline & Loop Flush & \multicolumn{2}{|c|}{ "Flush" UF Feed Loop- offline } & & C2-LOOP-TO-C3 & & & \\
\hline & & RLD Condensate & volume needed & 8.59 & & & & \\
\hline & & Concentrated Slurry & volume needed & 4.51 & & & & \\
\hline & & Save for Campaign III & & & & & & \\
\hline & UF Caustic Flush 1 & Flush UF System & & & & & & \\
\hline & & \multicolumn{2}{|c|}{ Caustic Flushing Solution } & & & & & \\
\hline & & RLD Dilution Water & volume needed for 3 flushes & 94.1 & & & & \\
\hline & & $\mathrm{NaOH}$ & add to $0.1 \mathrm{M}(\mathrm{L} 50 \mathrm{wt} \%)$ & 0.490 & & & & \\
\hline \multirow[t]{31}{*}{ CLEANING } & & \multicolumn{2}{|c|}{ Simulated Heel + Filter Loop Residue } & & & & & \\
\hline & & RLD Dilution Water & volume needed & 12.5 & & & & \\
\hline & & Concentrated Slurry & volume needed & 4.1 & & & & \\
\hline & & Caustic Flush 1 & after flushing & & C2-CAU-1-ACTL & & & \\
\hline & & & & & C2-CAU-2-WET & & & SPECIAL \\
\hline & & & & & C2-CAU-1-MW & & & DISMW \\
\hline & & & & & C2-CAU-1-ARC & & & \\
\hline & HNO3 Cleaning & $2 \mathrm{M}$ HNO3 cleaning & & & & & & \\
\hline & & Caustic Flush Heel & volume needed & 20.00 & & & & \\
\hline & & HNO3 Reagent & & & & & & \\
\hline & & & RLD Dilution Water & 247.5 & & & & \\
\hline & & & HNO3 (70 wt\%) & 36.3 & & & & \\
\hline & & RLD Dilutio & Vater + HNO3 total for 3 cleanings & 283.8 & & & & \\
\hline & Cleaning \#1 & Acid Cleaning 1 & after cleaning & 114.6 & C2-NIT-1-ACTL & & & \\
\hline & & & & & C2-NIT-1-WET & & & $\begin{array}{l}\text { SPECIAL } \\
\text { DISMW }\end{array}$ \\
\hline & & 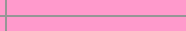 & ${ }^{2}$ & & $\begin{array}{l}\text { C2-NIT-1-MW } \\
\text { C2-NIT-1-PPT }\end{array}$ & collect solids & & \\
\hline & & & & & C2-NIT-1-PPT-BLK & $\begin{array}{l}\text { collect solids } \\
\text { blank }\end{array}$ & & \\
\hline & & & & & C2-NIT-1-ARC & & & \\
\hline & & Cleaning Heel & volume needed & 20.00 & & & & \\
\hline & Cleaning \#2 & Acid Cleaning 2 & after cleaning & 114.6 & C2-NIT-2-ACTL & & & \\
\hline & & & & & C2-NIT-2-WET & & & SPECIAL \\
\hline & & & & & C2-NIT-2-MW & only if solids & & DISMW \\
\hline & & & & & C2-NIT-2-ARC-A & & & \\
\hline & +5 & & & & C2-NIT-2-ARC-B & & & \\
\hline & & Cleaning Heel & volume needed $(\mathrm{L})$ & 20.00 & & & & \\
\hline & Cleaning \#3 & Acid Cleaning 3 & after cleaning (L) & 114.6 & C2-NIT-3-ACTL & & & \\
\hline & & & & & C2-NIT-3-WET & Only if solids & & $\begin{array}{l}\text { SPECIAL } \\
\text { DISMW }\end{array}$ \\
\hline & & & & & $\begin{array}{l}\text { C2-NIT-3-MW } \\
\text { C2-NIT-3RC-A }\end{array}$ & Onily il somids & & \\
\hline & & & & & C2-NIT-3-ARC-B & & & \\
\hline & & Cleaning Heel & volume needed (L) & 20.00 & & & & \\
\hline & Caustic Flushes $2 \& 3$ & Flush UF System & & & & & & \\
\hline & & \multicolumn{2}{|c|}{ Caustic Flushing Solution } & & & & & \\
\hline & & RLD Dilution Water & volume needed for 3 flushes (L) & & & & & \\
\hline & & $\mathrm{NaOH}$ & add to $0.1 \mathrm{M} \mathrm{(L} 50 \mathrm{wt} \%)$ & 0.490 & & & & \\
\hline & & Flush water & after Flush & 114.6 & C2-CAU-2-ACTL & & & \\
\hline & & & & & C2-CAU-2-WET & SPECIAL & & \\
\hline & & & & & C2-CAU-2-ARC-A & & & \\
\hline & & & & & C2-CAU-2-ARC-B & & & \\
\hline & & Flush 2 Heel & volume needed (L) & 20.00 & & & & \\
\hline
\end{tabular}

\section{Analytical Measurements On Samples Taken During Pilot-Scale UFP Testing (continued)}

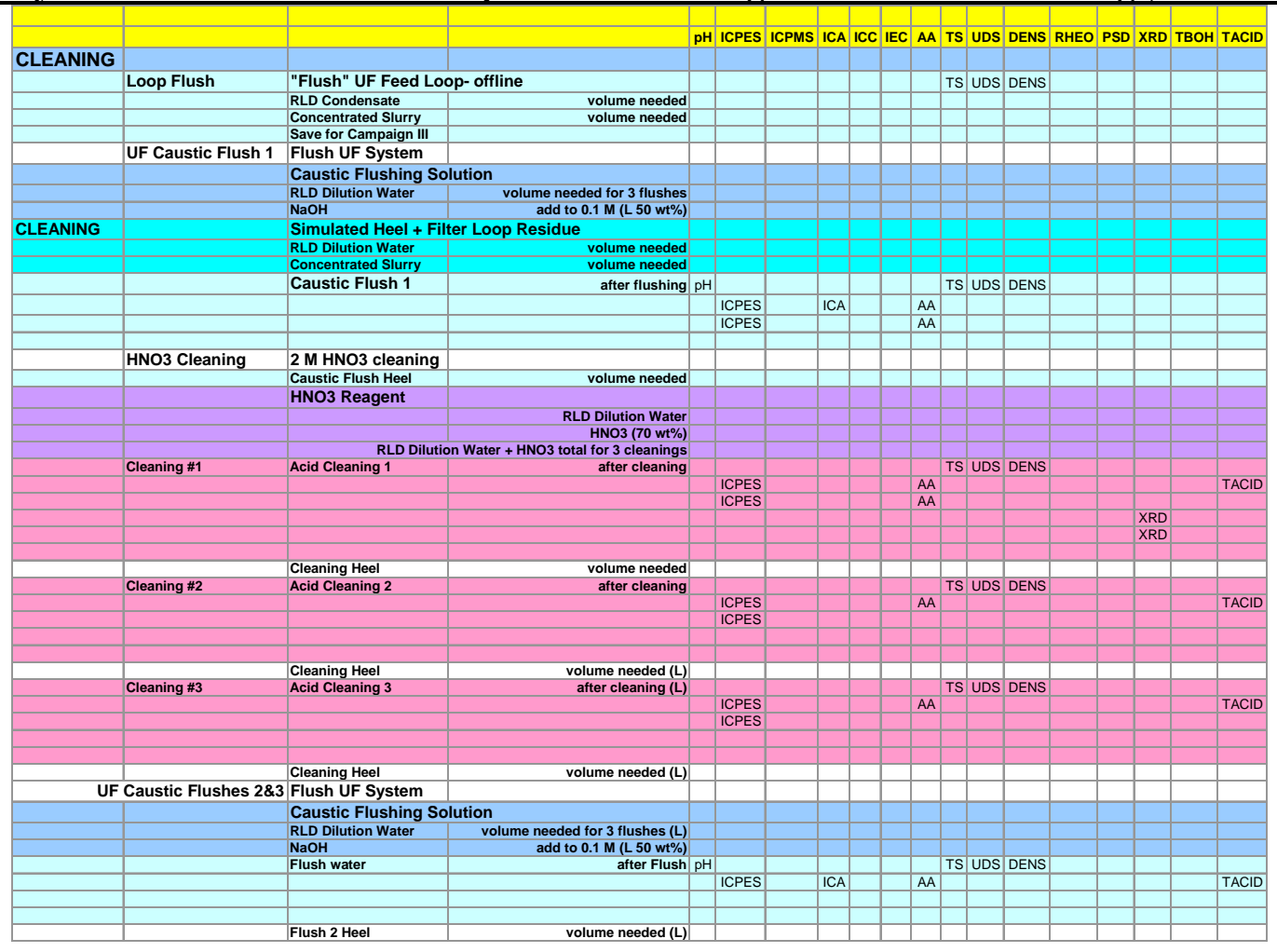


WSRC-TR-2004-00478, REVISION 0

SRNL-RPP-2004-00075, REVISION 0

Samples Taken During Pilot-Scale UFP Testing (continued)

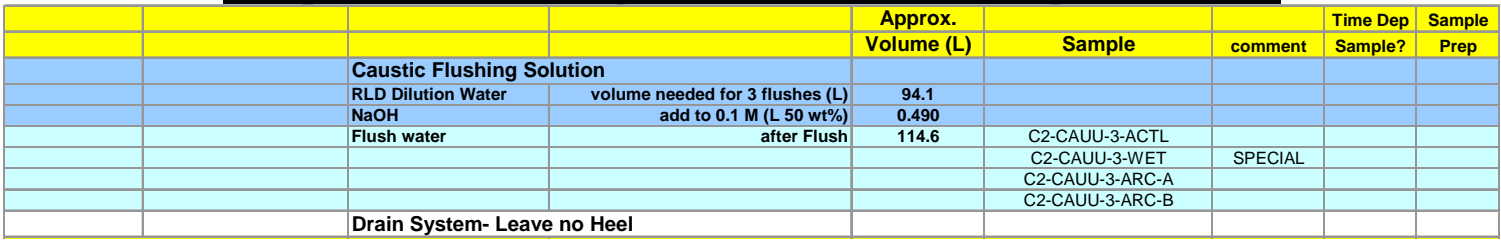

Analytical Measurements On Samples Taken During Pilot-Scale UFP Testing (continued)

\begin{tabular}{|c|c|c|c|c|c|c|c|c|c|c|c|c|c|c|c|c|c|c|}
\hline & & Caustic Flushing & & & & & & & & & & & & & & & & \\
\hline & & RLD Dilution Water & volume needed for 3 flushes $(L)$ & & & & & & & & & & & & & & & \\
\hline & & $\mathrm{NaOH}$ & add to $0.1 \mathrm{M}(\mathrm{L} 50 \mathrm{Wt} \%)$ & & & & & & & & & & & & & & & \\
\hline & & Flush water & arter Flush & $\mathrm{pH}$ & ICDES & & Ir & & & 010 & 15 & UDS & DENS & & & & TROH & \\
\hline & & & & & & & & & & & & & & & & & & \\
\hline & & & & & & & & & & & 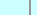 & & & & & & & \\
\hline & & Drain System- Le & Heel & & & & & & & & & & & & & & & \\
\hline
\end{tabular}


WSRC-TR-2004-00478, REVISION 0
SRNL-RPP-2004-00075, REVISION 0

Samples Taken During Pilot-Scale CIX Testing

\begin{tabular}{|c|c|c|c|c|}
\hline ION EXCHANGE & ION EXCHANGE & & & \\
\hline RUSH & Cycle 1 & Lead Column@ @ BV =5 & C2-IX-CY1-LEAD-5 & RUSH \\
\hline & & Lead Column@ BV=10 & C2-IX-CY1-LEAD-10 & \\
\hline & & Lead Column@ BV=15 & C2-IX-CY1-LEAD-15 & \\
\hline & & Lead Column@ BV =20 & C2-IX-CY1-LEAD-20 & \\
\hline & & Lead Column@ BV =25 & C2-IX-CY1-LEAD-25 & \\
\hline & & Lead Column@ BV = 30 & C2-IX-CY1-LEAD-30 & \\
\hline & & Lead Column @ BV = 35 & C2-IX-CY1-LEAD-35 & \\
\hline & & Lead Column @ BV = 40 & C2-IX-CY1-LEAD-40 & \\
\hline & & Lead Column @ BV =45 & C2-IX-CY1-LEAD-45 & \\
\hline & & Lead Column@ $@ B V=50$ & C2-IX-CY1-LEAD-50 & \\
\hline & & Lead Column @ BV = 55 & C2-IX-CY1-LEAD-55 & \\
\hline & & Lead Column@ BV=60 & C2-IX-CY1-LEAD-60 & \\
\hline & & Lead Column @ BV =65 & C2-IX-CY1-LEAD-65 & \\
\hline & & Lead Column@ BV =70 & C2-IX-CY1-LEAD-70 & \\
\hline & & Lead Column @ BV =75 & C2-IX-CY1-LEAD-75 & \\
\hline & & Lead Column@ $@$ BV = 80 & C2-IX-CY1-LEAD-80 & \\
\hline & & Lead Column@ BV =85 & C2-IX-CY1-LEAD-85 & \\
\hline & & Lead Column@ BV=90 & C2-IX-CY1-LEAD-90 & \\
\hline & & Lead Column@ BV=95 & C2-IX-CY1-LEAD-95 & \\
\hline RUSH & & Lead Column@ BV = 100 & C2-IX-CY1-LEAD-100 & RUSH \\
\hline & & Lead Column@ @ BV= 105 & C2-IX-CY1-LEAD-105 & \\
\hline & & Lead Column@ BV =110 & C2-IX-CY1-LEAD-110 & \\
\hline & & Lead Column@ BV = 115 & C2-IX-CY1-LEAD-115 & \\
\hline & & Lead Column@ @ BV =120 & C2-IX-CY1-LEAD-120 & \\
\hline & & Lead Column@ BV=125 & C2-IX-CY1-LEAD-125 & \\
\hline & & Lead Column@ BV = 130 & C2-IX-CY1-LEAD-130 & \\
\hline & & Lead Column@ @ BV = 135 & C2-IX-CY1-LEAD-135 & \\
\hline & & Lead Column@ BV=140 & C2-IX-CY1-LEAD-140 & \\
\hline & & Lead Column@ BV = 145 & C2-IX-CY1-LEAD-145 & \\
\hline & & Lead Column@BV =150 & C2-IX-CY1-LEAD-150 & \\
\hline RUSH & & Lead Column@BV=155 & C2-IX-CY1-LEAD-155 & \\
\hline RUSH & & Lead Column@BV=160 & C2-IX-CY1-LEAD-160 & \\
\hline RUSH & & Lead Column@ BV = 165 & C2-IX-CY1-LEAD-165 & \\
\hline RUSH & & Lead Column@BV=170 & C2-IX-CY1-LEAD-170 & \\
\hline RUSH & & Lead Column@ BV = 175 & C2-IX-CY1-LEAD-175 & RUSH \\
\hline RUSH & & Lead Column@ @ BV =180 & C2-IX-CY1-LEAD-180 & RUSH \\
\hline RUSH & & Lead Column@ BV=185 & C2-IX-CY1-LEAD-185 & RUSH \\
\hline RUSH & & Lead Column@ BV=190 & C2-IX-CY1-LEAD-190 & RUSH \\
\hline RUSH & & BLANK & C2-IX-CY1-LEAD-176 & RUSH \\
\hline & & Lead Column@ BV=END & C2-IX-CY1-LEAD-END & RUSH \\
\hline
\end{tabular}

Analytical Measurements On Samples Taken During Pilot-Scale CIX Testing ION EXCHANGE

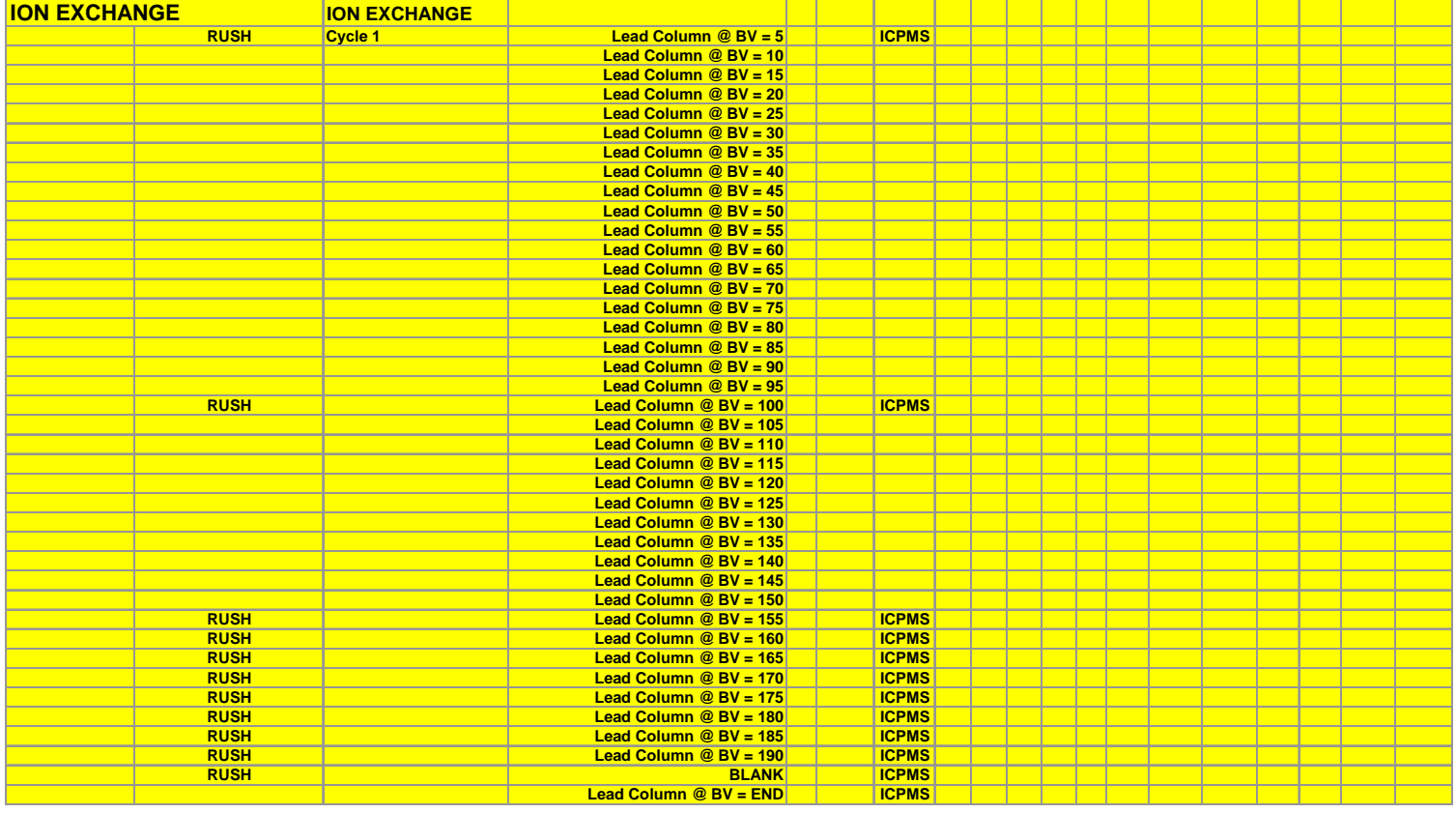


WSRC-TR-2004-00478, REVISION 0

SRNL-RPP-2004-00075, REVISION 0

\section{Samples Taken During Pilot-Scale CIX Testing (continued)}

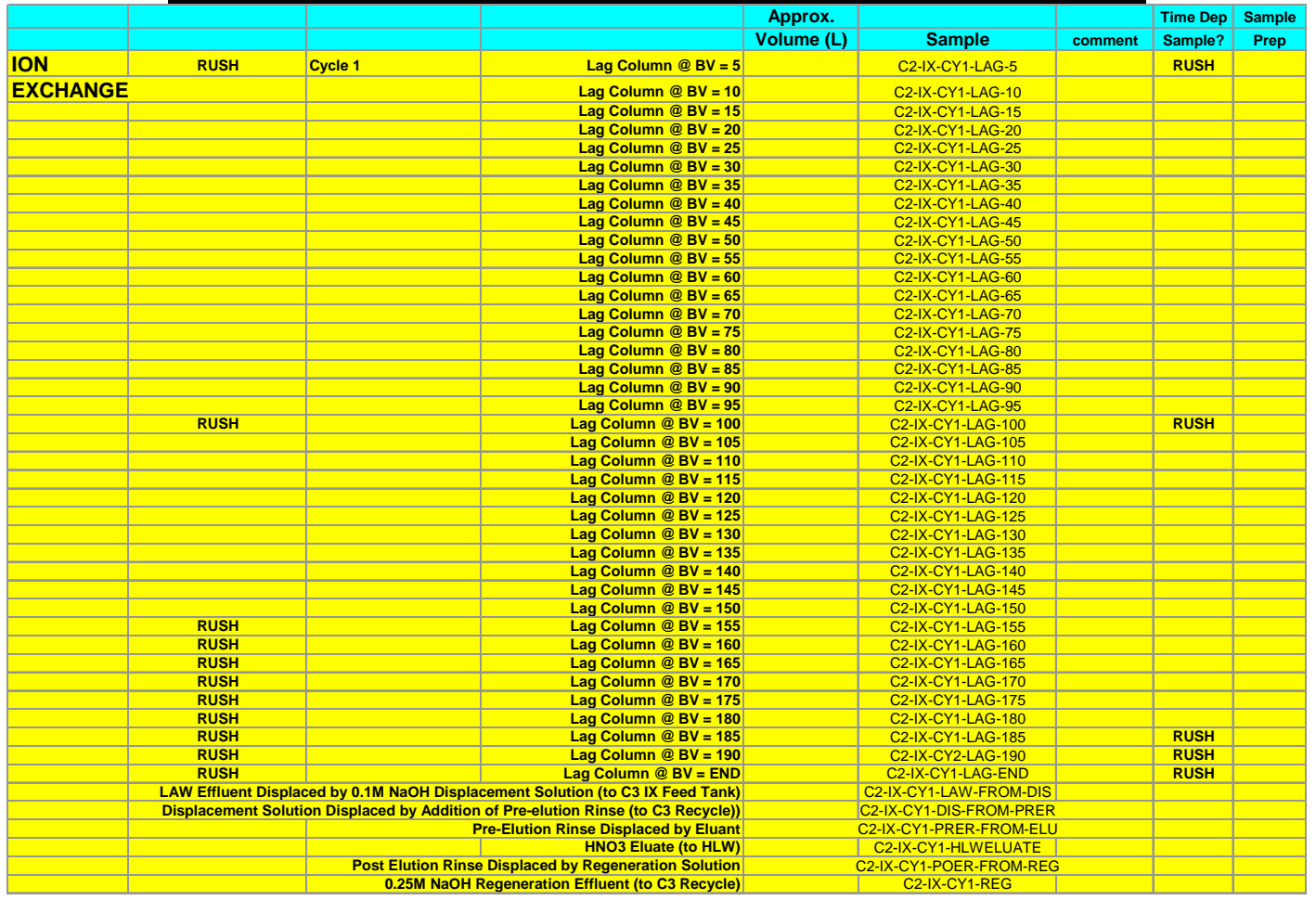

\section{Analytical Measurements On Samples Taken During Pilot-Scale CIX Testing (continued)}

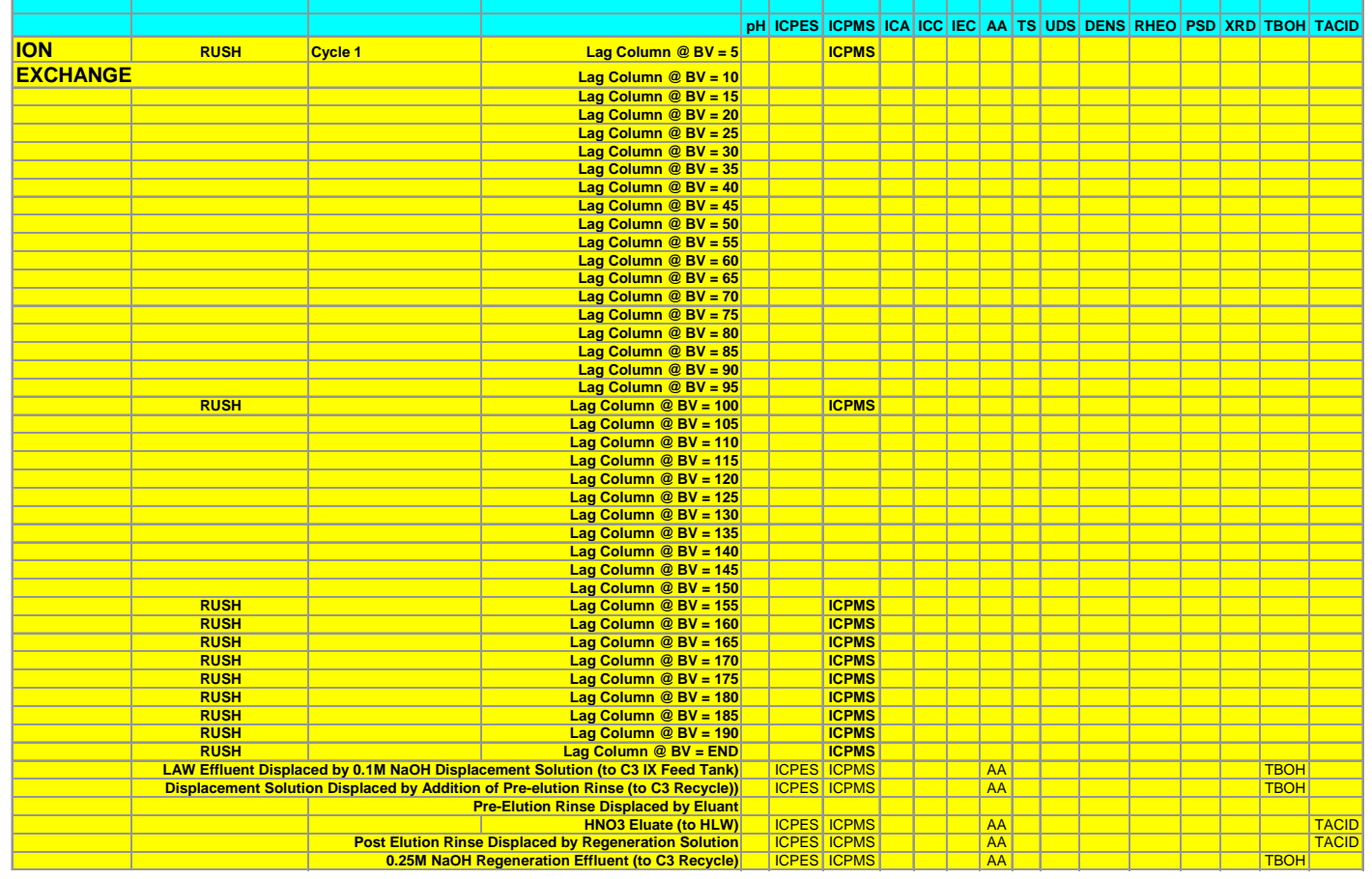


WSRC-TR-2004-00478, REVISION 0
SRNL-RPP-2004-00075, REVISION 0

\section{Samples Taken During Pilot-Scale CIX Testing (continued)}

\begin{tabular}{|c|c|c|c|c|c|c|c|c|}
\hline & & & & $\begin{array}{l}\text { Approx. } \\
\text { Volume (L) }\end{array}$ & Sample & comment & $\begin{array}{l}\text { Time Dep } \\
\text { Sample? }\end{array}$ & $\begin{array}{c}\text { Sample } \\
\text { Prep }\end{array}$ \\
\hline ION & RUSH & Cycle 2 & Lead Column@ BV =5 & & C2-IX-CY2-LEAD-5 & & RUSH & \\
\hline \multicolumn{2}{|l|}{ EXCHANGE } & & Lead Column@ @BV=10 & & C2-IX-CY2-LEAD-10 & & & \\
\hline & & & Lead Column@ BV=15 & & C2-IX-CY2-LEAD-15 & & & \\
\hline & & & Lead Column@ BV = 20 & & C2-IX-CY2-LEAD-20 & & & \\
\hline & & & Lead Column@ BV = 25 & & C2-IX-CY2-LEAD-25 & & & \\
\hline & & & Lead Column @ BV = 30 & & C2-IX-CY2-LEAD-30 & & & \\
\hline & & & Lead Column@ @ BV = 35 & & C2-IX-CY2-LEAD-35 & & & \\
\hline & & & Lead Column@ BV=40 & & C2-IX-CY2-LEAD-40 & & & \\
\hline & & & Lead Column@ @ BV = 45 & & C2-IX-CY2-LEAD-45 & & & \\
\hline & & & Lead Column@ @ B = 50 & & C2-IX-CY2-LEAD-50 & & & \\
\hline & & & Lead Column @ BV = 55 & & C2-IX-CY2-LEAD-55 & & & \\
\hline & & & Lead Column@ @ BV =60 & & C2-IX-CY2-LEAD-60 & & & \\
\hline & & & Lead Column @ BV=65 & & C2-IX-CY2-LEAD-65 & & & \\
\hline & & & Lead Column@ BV =70 & & C2-IX-CY2-LEAD-70 & & & \\
\hline & & & Lead Column@ $@$ BV =75 & & C2-IX-CY2-LEAD-75 & & & \\
\hline & & & Lead Column@ BV=80 & & C2-IX-CY2-LEAD-80 & & & \\
\hline & & & Lead Column @ BV=85 & & C2-IX-CY2-LEAD-85 & & & \\
\hline & & & Lead Column@ BV=90 & & C2-IX-CY2-LEAD-90 & & & \\
\hline & & & Lead Column@ BV=95 & & C2-IX-CY2-LEAD-95 & & & \\
\hline & RUSH & & Lead Column@ @ BV = 100 & & C2-IX-CY2-LEAD-100 & & RUSH & \\
\hline & & & Lead Column@ @ BV = 105 & & C2-IX-CY2-LEAD-105 & & & \\
\hline & & & Lead Column@ BV =110 & & C2-IX-CY2-LEAD-110 & & & \\
\hline & & & Lead Column@ BV =115 & & C2-IX-CY2-LEAD-115 & & & \\
\hline & & & Lead Column@ BV = 120 & & C2-IX-CY2-LEAD-120 & & & \\
\hline & & & Lead Column@ BV = 125 & & C2-IX-CY2-LEAD-125 & & & \\
\hline & & & Lead Column@ BV=130 & & C2-IX-CY2-LEAD-130 & & & \\
\hline & & & Lead Column@ BV = 135 & & C2-IX-CY2-LEAD-135 & & & \\
\hline & & & Lead Column@ BV = 140 & & C2-IX-CY2-LEAD-140 & & & \\
\hline & & & Lead Column@ BV=145 & & C2-IX-CY2-LEAD-145 & & & \\
\hline & & & Lead Column@ BV = 150 & & C2-IX-CY2-LEAD-150 & & & \\
\hline & RUSH & & Lead Column@ BV = 155 & & C2-IX-CY2-LEAD-155 & & & \\
\hline & RUSH & & Lead Column@ BV=160 & & C2-IX-CY2-LEAD-160 & & & \\
\hline & RUSH & & Lead Column@ @ BV = 165 & & C2-IX-CY2-LEAD-165 & & & \\
\hline & RUSH & & Lead Column@ BV $=170$ & & C2-IX-CY2-LEAD-170 & & & \\
\hline & RUSH & & Lead Column@ BV = 175 & & C2-IX-CY2-LEAD-175 & & RUSH & \\
\hline & RUSH & & Lead Column@ BV = 180 & & C2-IX-CY2-LEAD-180 & & RUSH & \\
\hline & RUSH & & Lead Column@ BV = 185 & & C2-IX-CY2-LEAD-185 & & RUSH & \\
\hline & RUSH & & Lead Column@ BV = 190 & & C2-IX-CY2-LEAD-190 & & RUSH & \\
\hline & RUSH & & Lead Column@ @ BV=END & & C2-IX-CY2-LEAD-END & & RUSH & \\
\hline
\end{tabular}

Analytical Measurements On Samples Taken During Pilot-Scale CIX Testing (continued)

\begin{tabular}{|c|c|c|c|c|c|c|c|c|c|c|c|c|c|c|c|c|c|}
\hline & & & & pH ICPES I & ICPMS & ICA & ICC I & IEC & AA T & TS U & UDS & DENS & RHEO & PSD & XRD & TBOH & TACID \\
\hline ION & RUSH & Cycle 2 & Lead Column@ BV = 5 & & ICPMS & & & & & & & & & & & & \\
\hline \multirow[t]{38}{*}{ EXCHANGE } & & & Lead Column @ BV= 10 & & & & & & & & & & & & & & \\
\hline & & & Lead Column @ BV=15 & & & & & & & & & & & & & & \\
\hline & & & Lead Column @ BV = 20 & & & & & & & & & & & & & & \\
\hline & & & Lead Column @ BV = 25 & & & & & & & & & & & & & & \\
\hline & & & Lead Column @ BV = 30 & & & & & & & & & & & & & & \\
\hline & & & Lead Column @ BV = 35 & & & & & & & & & & & & & & \\
\hline & & & Lead Column @ BV = 40 & & & & & & & & & & & & & & \\
\hline & & & Lead Column @ BV = 45 & & & & & & & & & & & & & & \\
\hline & & & Lead Column @ BV = 50 & & & & & & & & & & & & & & \\
\hline & & & Lead Column @ BV = 55 & & & & & & & & & & & & & & \\
\hline & & & Lead Column@ BV=60 & & & & & & & & & & & & & & \\
\hline & & & Lead Column @ BV = 65 & & & & & & & & & & & & & & \\
\hline & & & Lead Column@ BV=70 & & & & & & & & & & & & & & \\
\hline & & & Lead Column @ BV =75 & & & & & & & & & & & & & & \\
\hline & & & Lead Column@ BV=80 & & & & & & & & & & & & & & \\
\hline & & & Lead Column@ BV=85 & & & & & & & & & & & & & & \\
\hline & & & Lead Column @ BV = 90 & & & & & & & & & & & & & & \\
\hline & & & Lead Column @ BV = 95 & & & & & & & & & & & & & & \\
\hline & RUSH & & Lead Column@ BV $=100$ & & ICPMS & & & & & & & & & & & & \\
\hline & & & Lead Column@ BV = 105 & & & & & & & & & & & & & & \\
\hline & & & Lead Column@ @ BV = 110 & & & & & & & & & & & & & & \\
\hline & & & Lead Column@ BV = 115 & & & & & & & & & & & & & & \\
\hline & & & Lead Column@ BV = 120 & & & & & & & & & & & & & & \\
\hline & & & Lead Column@ BV = 125 & & & & & & & & & & & & & & \\
\hline & & & Lead Column@ @ BV = 130 & & & & & & & & & & & & & & \\
\hline & & & Lead Column@ BV = 135 & & & & & & & & & & & & & & \\
\hline & & & Lead Column@BV=140 & & & & & & & & & & & & & & \\
\hline & & & Lead Column@BV=145 & & & & & & & & & & & & & & \\
\hline & & & Lead Column@ BV $=150$ & & & & & & & & & & & & & & \\
\hline & RUSH & & Lead Column@ BV=155 & & ICPMS & & & & & & & & & & & & \\
\hline & RUSH & & Lead Column@ BV = 160 & & ICPMS & & & & & & & & & & & & \\
\hline & RUSH & & Lead Column@ BV = 165 & & ICPMS & & & & & & & & & & & & \\
\hline & RUSH & & Lead Column@ BV = 170 & & ICPMS & & & & & & & & & & & & \\
\hline & RUSH & & Lead Column@ @ BV= 175 & & ICPMS & & & & & & & & & & & & \\
\hline & RUSH & & Lead Column@ BV = 180 & & ICPMS & & & & & & & & & & & & \\
\hline & RUSH & & Lead Column@ BV = 185 & & ICPMS & & & & & & & & & & & & \\
\hline & RUSH & & Lead Column @ BV = 190 & & ICPMS & & & & & & & & & & & & \\
\hline & RUSH & & Lead Column@ @BV=END & & ICPMS & & & & & & & & & & & & \\
\hline
\end{tabular}


WSRC-TR-2004-00478, REVISION 0

SRNL-RPP-2004-00075, REVISION 0

\section{Samples Taken During Pilot-Scale CIX Testing (continued)}

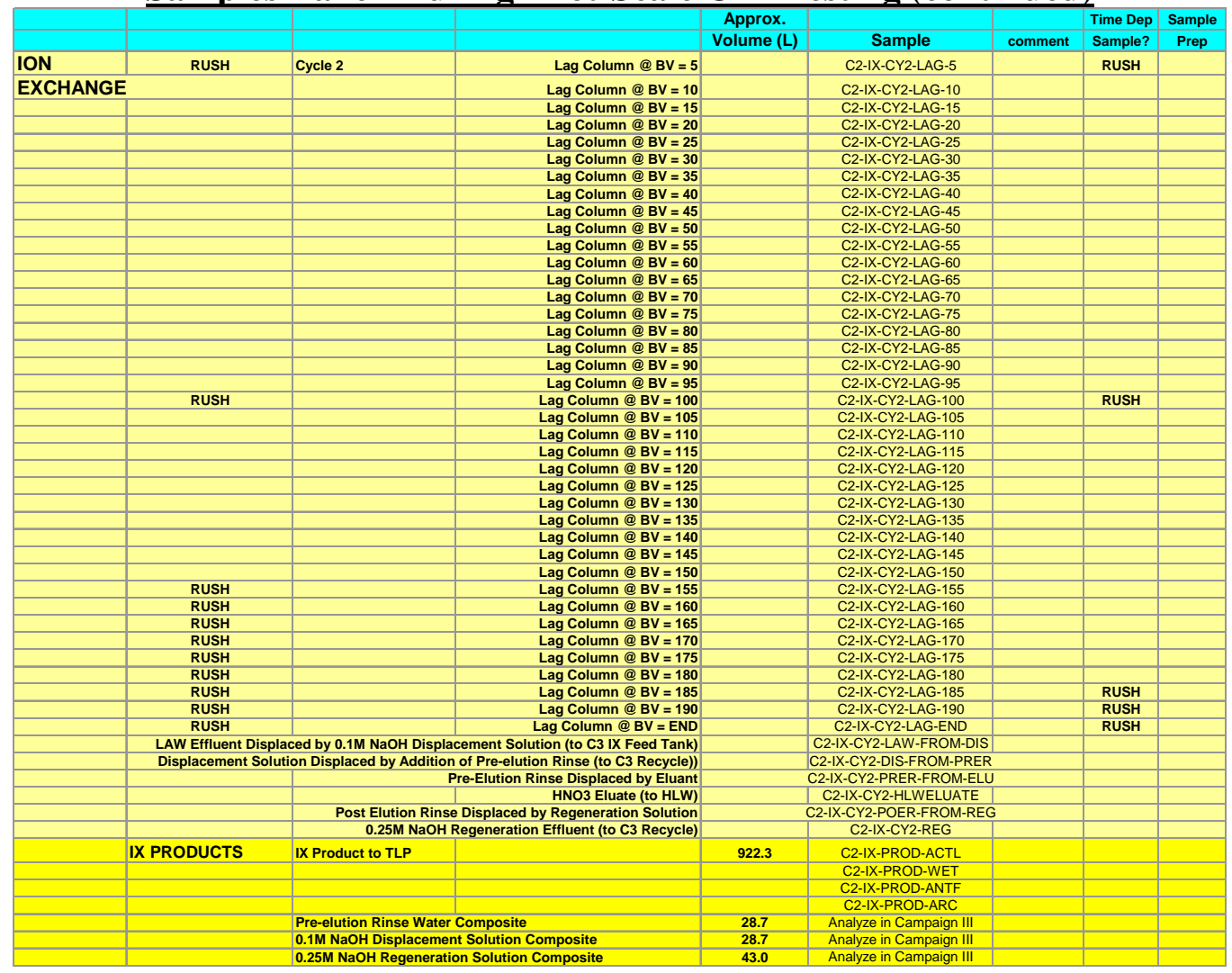

\section{Analytical Measurements On Samples Taken During Pilot-Scale CIX Testing (continued)}

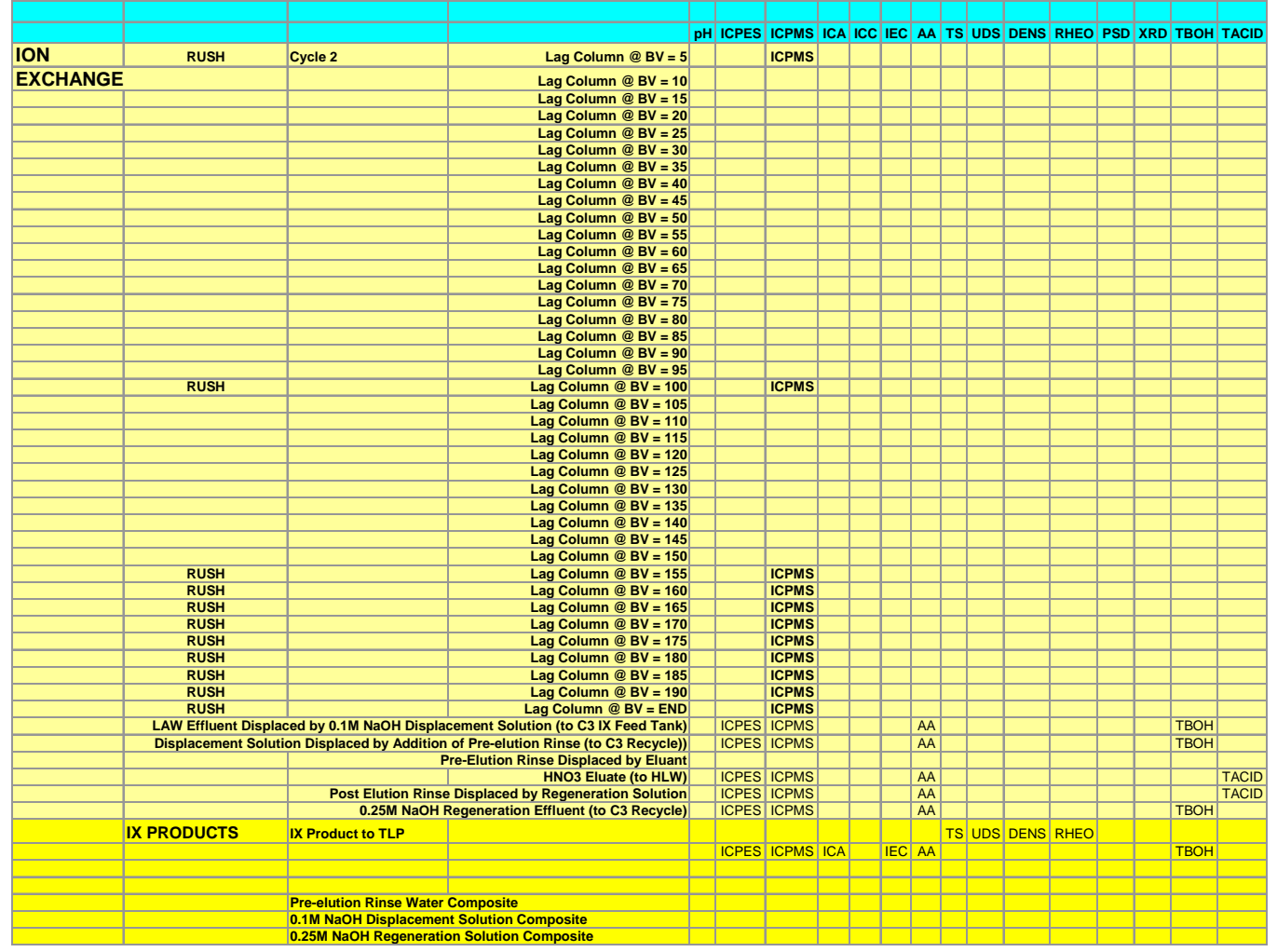


WSRC-TR-2004-00478, REVISION 0

SRNL-RPP-2004-00075, REVISION 0

\section{Samples Taken During Pilot-Scale TLP Testing}

\begin{tabular}{|c|c|c|c|c|c|c|c|c|}
\hline & & & & Approx. & & & Time Dep & Sample \\
\hline & & & & Volume (L) & Sample & comment & Sample? & Prep \\
\hline \multirow{2}{*}{\multicolumn{2}{|c|}{ TLP EVAPORATOR }} & LAW Feed (IX product) & & 922.3 & & & & \\
\hline & & \multirow{2}{*}{\multicolumn{2}{|c|}{ IX $0.25 \mathrm{M} \mathrm{NaOH}$ Regeneration Solution from Campaign I }} & 833.2 & & & & \\
\hline & & & & & & & & \\
\hline & EVAPORATE & \multicolumn{2}{|c|}{ Evaporator Feed Mixture } & 1755.5 & C2-TLP-FEED-ACTL & & & \\
\hline & & & & & C2-TLP-FEED-ADS & & & \\
\hline & & & & & $\begin{array}{l}\text { C2-ILPF-FEE-ARC } \\
\text { C2-TLP-FEED-XRD }\end{array}$ & only if solids & $\mathrm{Y}$ & \\
\hline & & & & & C2-TLP-FEED-XRD-BLK & blank & & \\
\hline & & Bottoms & After all antifoam additions & & C2-TLP-B-START-ACTL & & & \\
\hline & & & & & C2-TLP-B-START-ANTF & & & \\
\hline & & & & & C2-TLP-B-START-ARC & & & \\
\hline & & Bottoms & At $-200 \mathrm{~L}$ evaporated & & C2-TLP-B-200-ACTL & & & \\
\hline & & & & & C2-TLP-B-200-XRD & only if solids & $\mathrm{Y}$ & \\
\hline & & & & & C2-TLP-B-200-XRD-BLK & blank & & \\
\hline & & Primary Condensate & & & C2-TLP-PC-200-ARC & & & \\
\hline & & Secondary Condensate & & & C2-TLP-SC-200-ARC & & & \\
\hline & & Bottoms & At -400 L evaporated & & C2-TLP-B-400-ACTL & & & \\
\hline & & & & & C2-TLP-B-400-XRD & only if solids & $\mathrm{Y}$ & \\
\hline & & & & & C2-TLP-B-400-XRD-BLK & blank & & \\
\hline & & Primary Condensate & & & C2-TLP-PC-400-ARC & & & \\
\hline & & Secondary Condensate & At -600 L evaporated & & C2-TLP-SC-400-ARC & & & \\
\hline & & Bottoms & At $-600 \mathrm{~L}$ evaporated & & $\begin{array}{l}\text { C2-TLP-B-600-ACTL } \\
\text { C-TP-B-600-XRD }\end{array}$ & only if solids & $\mathrm{Y}$ & \\
\hline & & & & & C2-TLP-B-600-XRD-BLK & blank & & \\
\hline & & & & & C2-TLP-PC-600-ARC & & & \\
\hline & & Secondary Condensate & & & C2-TLP-SC-600-ARC & & & \\
\hline & & Bottoms & At $-800 \mathrm{~L}$ evaporated & & C2-TLP-B-800-ACTL & & & \\
\hline & & & & & C2-TLP-B-800-XRD & only if solids & $\mathrm{Y}$ & \\
\hline & & & & & C2-TLP-B-800-XRD-BLK & blank & & \\
\hline & & Primary Condensate & & & C2-TLP-PC-800-ARC & & & \\
\hline & & Secondary Condensate & At -800 L evaporated & & C2-TLP-SC-800-ARC & & & \\
\hline & & Bottoms & Al -800 L evaporated & & $\begin{array}{l}\text { C2-ILP-B-B-1000-ACIL } \\
\text { C2-TLP-B-1000-XRD }\end{array}$ & only if solids & $\mathrm{Y}$ & \\
\hline & & & & & C2-TLP-B-1000-XRD-BLK & blank & & \\
\hline & & Primary Condensate & & & C2-TLP-PC-1000-ARC & & & \\
\hline & & Secondary Condensate & & & C2-TLP-SC-1000-ARC & & & \\
\hline & & Bottoms & Evaporation completed & 589.4 & C2-TLP-B-END-ACTL & & & \\
\hline & & & & & C2-TLP-B-END-SUP & SPECIAL & & \\
\hline & & & & & C2-TLP-B-END-MW & only if solids & & DISMW \\
\hline & & & & & C2-TLP-B-END-PSD & only if solids & & \\
\hline & & & & & C2-TLP-B-END-ANTF & & & \\
\hline & & & & & C2-TLP-B-END-ARC & & & \\
\hline & & & & & C2-TLP-B-END-XRD & only if solids & $\mathrm{Y}$ & \\
\hline & & & & & C2-TLP-B-END-XRD-BLK & blank & & \\
\hline & & Primary Condensate & Evaporation almost completed & & C2-TLP-PC-END-ARC & & & \\
\hline & & Secondary Condensate & & & C2-TLP-SC-END-ARC & & & \\
\hline & COMBINE TLP & DENSATES & & & & & & \\
\hline & & $\begin{array}{l}\text { Combined TLP Conden } \\
\text { (Primary + Secondary) }\end{array}$ & sate = RLD Condensate & 1166.1 & $\begin{array}{l}\text { C2-RLD-FROM-TLP-ADS } \\
\text { C2-RLD-FROM-TLP-ACTL } \\
\text { C2-RLD-FROM-TLP-ARC }\end{array}$ & & & \\
\hline & COMBINE FEF & P CONDENSATES & & & & & & \\
\hline & & Combined FEP + TLP C & ondensate $=$ RLD Condensate & & $\begin{array}{l}\text { C2-RLD-TOTAL-ADS } \\
\text { C2-RLD-TOTAL-ACTL } \\
\text { C2-RLD-TOTAL-ARC }\end{array}$ & & & \\
\hline
\end{tabular}

\section{Analytical Measurements On Samples Taken During Pilot-Scale TLP Testing}

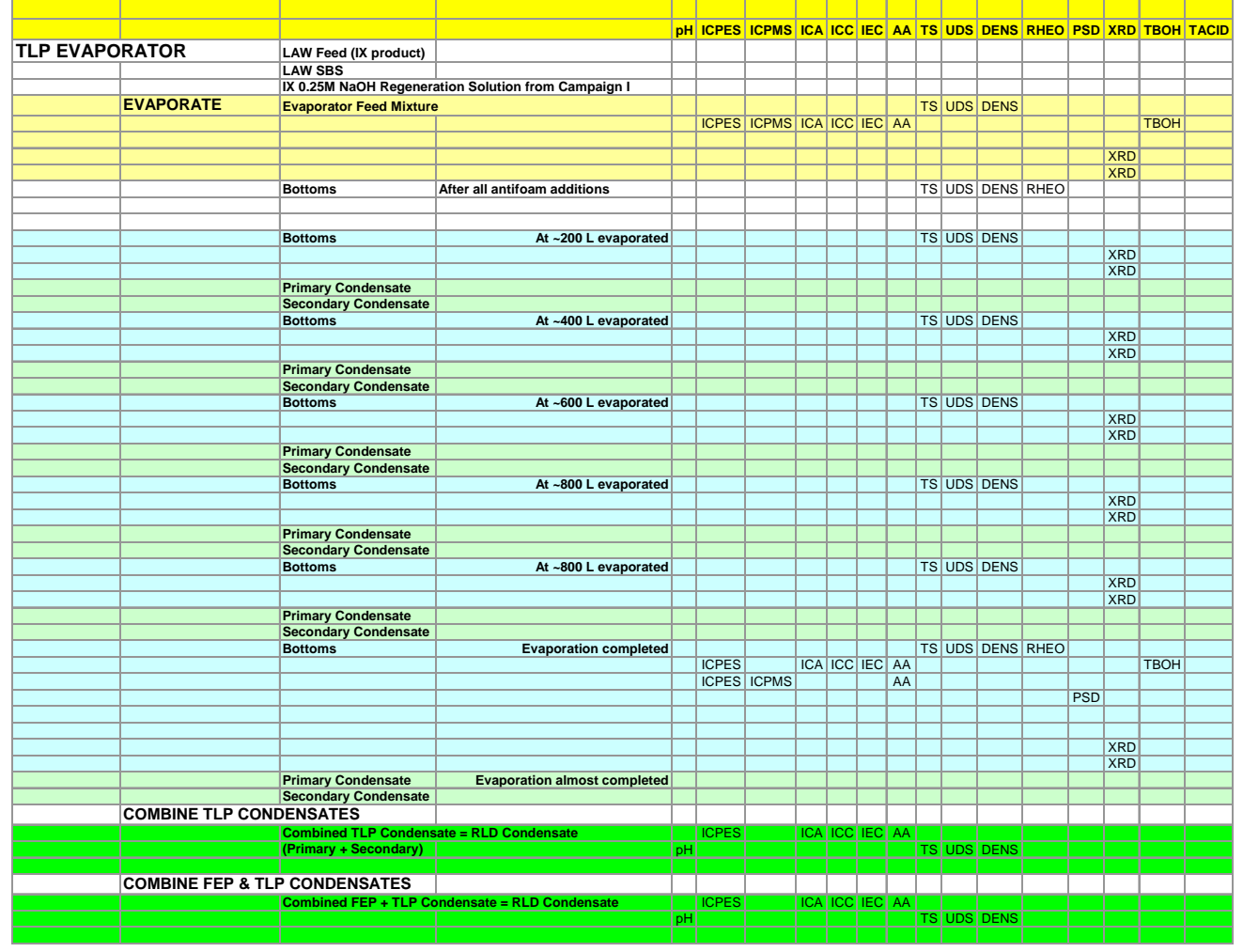


G. T. Wright, 773A

D. J. Green, 773-A

D. B. Moore-Shedrow, 773-A

L. M. Papouchado, 773-A

C. R. Wolfe, 773-A

H. A. Woltermann, 773-A

D. A. Crowley, 773-A

R. E. Edwards, Jr., 773-A

J. C. Griffin, 773-A

E. W. Holtsheiter, Jr., 773-A

H. F. Sturm, Jr., 773-A

S. T. Wach, 773-42A

D. B. Burns, 786-5A

T. B. Calloway, 999-W

S. D. Fink, 773-A

J. C. Marra, 773-42A

S. L. Marra, 999-W

W. R. Wilmarth, 773-42A

D. J. Adamson, 786-5A

C. D. Barnes, 773-42A

M. R. Duignan, 786-5A

M. D. Fowley, 786-5A

H. N. Guerrero, 786-5A

Z. H. Qureshi, 786-5A

J. L. Steimke, 786-5A

M. R. Williams, 786-5A

J. R. Zamecnik, 773-41A

RPP File, c/o M. F. Jones, 773-43A, Room 109

\section{OFFSITE}

W. L. Tamosaitis, Waste Treatment Plant, 3000 George Washington Way, Richland, WA99352

F. W. Damerow, Waste Treatment Plant, 3000 George Washington Way, Richland, WA 99352

P. S. Sundar, Waste Treatment Plant, 3000 George Washington Way, Richland, WA 99352 
WSRC-TR-2005-00105, REVISION 0

SRNL-RPP-2005-00012, REVISION 0

\section{Appendix Section: I-3}

Interim Report: RPP-WTP Semi-Integrated Pilot Plant - Campaign III

[For Information Only] 
WSRC-TR-2004-00565, REVISION 0

SRNL-RPP-2004-00092, REVISION 0

\section{INTERIM REPORT: RPP-WTP SEMI-INTEGRATED PILOT PLANT CAMPAIGN III}

November 2004

SAVANNAH RIVER NATIONAL LABORATORY
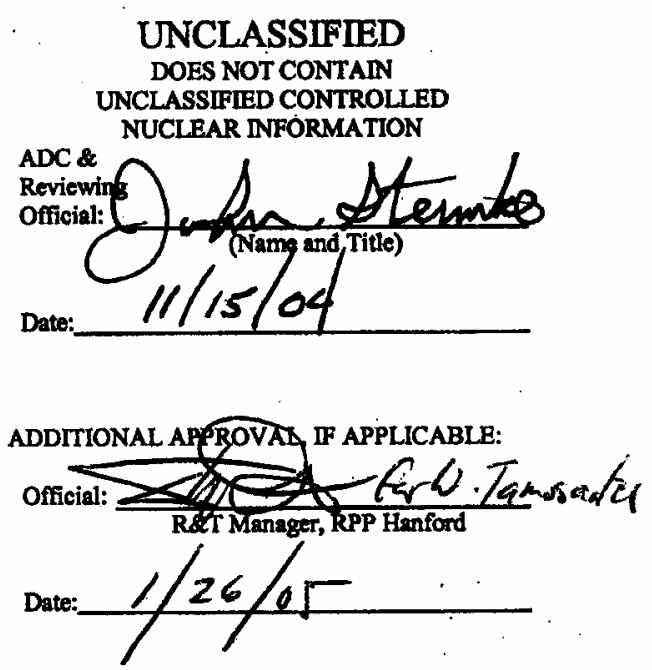

Westinghouse Savannah River Company

Savannah River Site

Aiken, SC 29808

Prepared for the U.S. Department of Energy Under Contract Number DEAC09-96SR18500

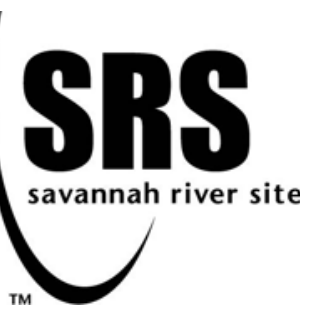


WSRC-TR-2004-00565, REVISION 0

SRNL-RPP-2004-00092, REVISION 0

\section{DISCLAIMER}

This report was prepared for the United States Department of Energy under Contract No. DE-AC09-96SR18500 and is an account of work performed under that contract. Neither the United States Department of Energy, nor WSRC, nor any of their employees makes any warranty, expressed or implied, or assumes any legal liability or responsibility for accuracy, completeness, or usefulness, of any information, apparatus, or product or process disclosed herein or represents that its use will not infringe privately owned rights. Reference herein to any specific commercial product, process, or service by trade name, trademark, name, manufacturer or otherwise does not necessarily constitute or imply endorsement, recommendation, or favoring of same by Westinghouse Savannah River Company or by the United States Government or any agency thereof. The views and opinions of the authors expressed herein do not necessarily state or reflect those of the United States Government or any agency thereof.

Printed in the United States of America

Prepared For

U.S. Department of Energy 
WSRC-TR-2004-00565, REVISION 0

SRNL-RPP-2004-00092, REVISION 0

WSRC Job Number: EES-23041

Key Words:

Ultrafiltration

Hanford Tank 241 AY-102/C-106

\section{Retention: Permanent}

Key WTP R\&T References:

Test Specifications:

24590-PTF-TSP-RT-02-015, Rev. 0, 15

Nov. 2002

24590-PTF-TSP-RT-03-002, Rev. 0, 05

Jun. 2003

24590-PTF-TSP-RT-01-003, Rev. 0, 20

Nov. 2001 (CIX chemical testing only)

Test Plans: WSRC-TR-2003-00338, Rev. 0, 04 Aug. 2003 (or SRT-RPP-2003-000165) and WSRC-TR-2001-00340, Rev. 0, 06 Feb. 2002 (or SRT-RPP-2001-000116) (CIX chemical testing only)

Test Exceptions: All of the following test exceptions only affect the CIX chemical testing sub-task: WSRC-TR-2001-00340 24590-WTP-TEF-RT-02-025, Rev. 0, 20 May 2002 24590-WTP-TEF-RT-02-046, Rev. 0, 01 Aug. 2002 24590-WTP-TEF-RT-03-004, Rev. 0, 07 Feb. 2003 24590-WTP-TEF-RT-03-037, Rev. 0, 30 Jul. 2003 24590-WTP-TEF-RT-03-037, Rev. 1, 17 Sept. 2003 24590-WTP-TEF-RT-03-054, Rev. 0, 17 Sept. 2003 24590-WTP-TEF-RT-03-069, Rev. 0, 14 Nov. 2003 R\&T Focus Area: RPP-WTP Pretreatment Test Scoping Statements: S-48 \& S-134

\section{INTERIM REPORT: RPP-WTP SEMI-INTEGRATED PILOT PLANT - CAMPAIGN III}

\section{M.R. Duignan*, D.J. Adamson, M.D. Fowley, Z.H Qureshi, J.L. Steimke, M.R. Williams, and J.R. Zamecnik, SRNL}

\section{*Principal Author}

Issue Date: 15 NOVEMBER 2004

Westinghouse Savannah River Company

Savannah River Site

Aiken, SC 29808

Prepared for the U.S. Department of Energy Under Contract Number DE-AC09-96SR18500

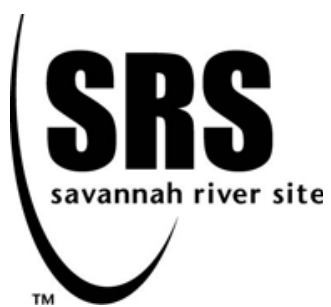


WSRC-TR-2004-00565, REVISION 0

SRNL-RPP-2004-00092, REVISION 0

\section{REVIEWS AND APPROVALS}

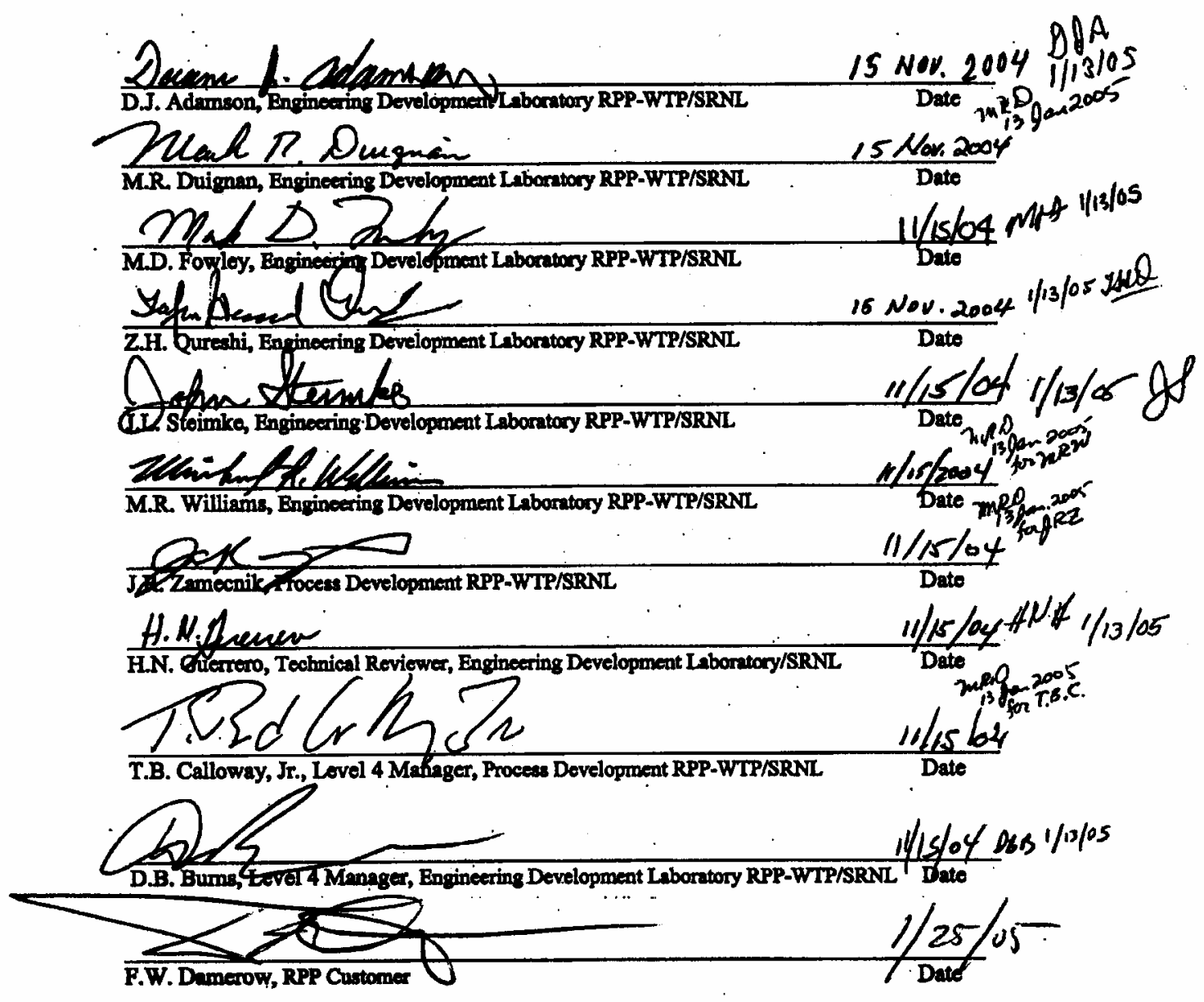




\section{TABLE OF CONTENTS}

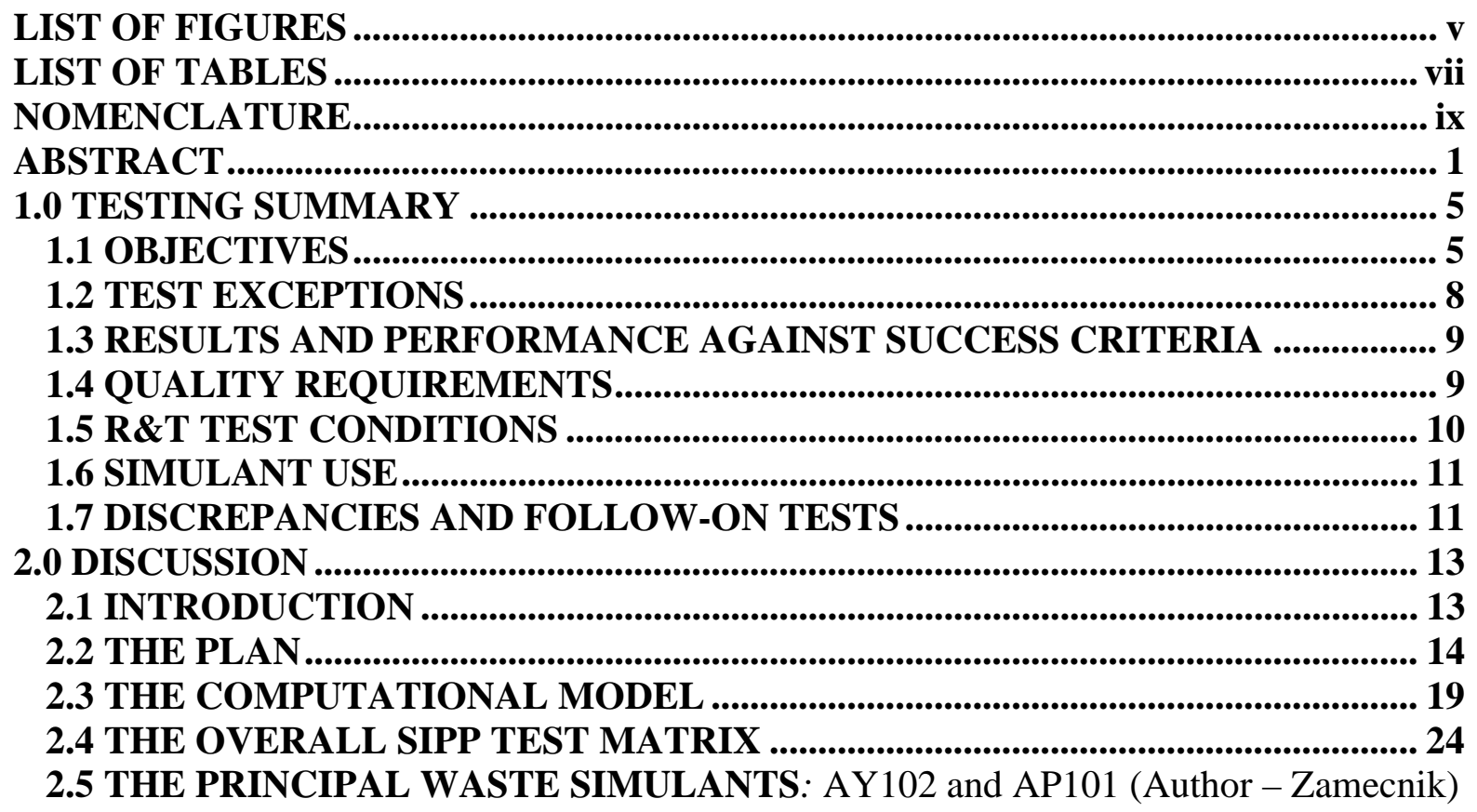

………................................................................................................................... 32

2.6 PILOT WASTE FEED EVAPORATOR (Authors - Qureshi, Williams \& Zamecnik)

2.6.1 Chemical Description of the Pilot FEP Feed Stream (Author - Zamecnik) ...... 34

2.6.2 Simulant Handling (Author - Williams)...................................................................... 42

2.6.3 Pilot FEP Equipment (Author - Qureshi)................................................................... 43

2.6.4 Pilot FEP Operation (Author - Qureshi) ..................................................................... 48

2.6.5 Pilot FEP Discussion of Results........................................................................ 51

2.6.6 Pilot FEP Conclusions....................................................................................................... 61

2.7 PILOT ULTRAFILTRATION (Authors - Fowley, Williams \& Zamecnik) ............ 62

2.7.1 Chemical Description of the Pilot-Scale UFP Feed Stream (Author - Zamecnik)

2.7.2 Simulant Handling (Author - Williams)......................................................................... 68

2.7.3 Pilot UFP Operation and Equipment (Author - Fowley) ......................................... 72

2.7.4 Pilot UFP Discussion of Results .............................................................................. 76

2.7.5 Pilot UFP Conclusions ............................................................................................. 102

2.8 PILOT CESIUM ION EXCHANGE (Authors - Steimke \&Williams)................... 103

2.8.1 Chemical Description of the Pilot-Scale CIX Feed Stream................................ 104

2.8.2 Simulant Handling (Author - Williams)...................................................................... 104

2.8.3 Pilot CIX Equipment (Author - Steimke)................................................................. 105

2.8.4 Pilot CIX Operation (Author - Steimke) ................................................................. 109

2.8.5 Pilot CIX Discussion of Results (Author - Steimke)................................................ 110

2.8.6 Pilot CIX Conclusions (Author - Steimke)................................................................ 125

2.9 PILOT TREATED LAW EVAPORATOR (Authors - Adamson, Williams \&

Zamecnik).................................................................................................................. 126

2.9.1 Chemical Description of the Pilot TLP Feed Stream (Author - Zamecnik).... 126

2.9.2 Simulant Handling (Author - Williams)..................................................................... 128 
2.9.3 Pilot TLP Equipment (Author - Adamson)................................................................. 128

2.9.4 Pilot TLP Operation (Author - Adamson) .................................................................. 129

2.9.5 Pilot TLP Discussion of Results .......................................................................... 132

2.9.6 Pilot TLP Conclusions ............................................................................................... 136

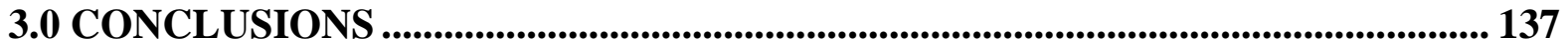

3.1 PILOT-SCALE FEP UNIT OPERATION .................................................................... 137

3.2 PILOT-SCALE UFP UNIT OPERATION ................................................................... 137

3.3 PILOT-SCALE CIX UNIT OPERATION................................................................... 138

3.4 PILOT-SCALE TLP UNIT OPERATION ................................................................. 138

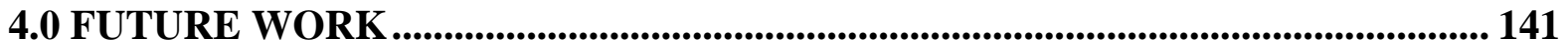

5.0 REFERENCES................................................................................................................... 143

APPENDIX A: WTP DISCUSSION ON PRETREATMENT FACILITY WASTE

PROCESSING RESIDENCE TIMES ........................................................... 147

APPENDIX B: CAMPAIGN III SAMPLING MATRIX................................................... 149 


\section{LIST OF FIGURES}

Figure 1. Schematic of the overall task operation taken from the task plan ........................... 17

Figure 2. Generation of Caustic Rinse 1 Recycle ..................................................................... 35

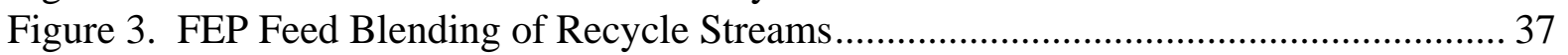

Figure 4. Pilot-scale FEP Unit Operation Feed Tank …………………….............................. 43

Figure 5. SIPP Pilot-Scale FEP/TLP Unit ............................................................................. 45

Figure 6. SIPP Pilot-Scale FEP/TLP Unit: The bottom half of the 15-foot tall Acrylic

Section with Ongoing Evaporation ................................................................................ 46

Figure 7. SIPP Pilot-Scale FEP/TLP Unit: Feed Ready for Evaporation to Commence....... 47

Figure 8. SIPP Pilot-Scale FEP/TLP Unit: Evaporating......................................................... 48

Figure 9. Schematic of Ultrafiltration Process - Page 1 …………………………….............. 64

Figure 10. Schematic of Ultrafiltration Process - Page 2 2...................................................... 65

Figure 11. UFP Primary Mixing Tank (Volume 1000 liters) which represents the RPP-

WTP UFP Preparation Feed Tank................................................................................. 70

Figure 12. UFP Slurry Reservoir (Volume 100 liters) which represents the RPP-WTP UFP

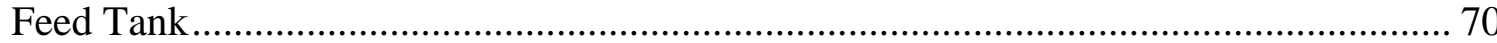

Figure 13. Entire 90-inch Length of the Filter-tube Bundle (Upstream end in Foreground) 72

Figure 14. Closeup of the Tubes' Surfaces and Tube Sheets .................................................. 73

Figure 15. The Exit (Left) and Entrance (Right) Tube Sheets of the Filter Tube Bundle..... 73

Figure 16. Campaign II Ultrafiltration Test Rig .................................................................. 74

Figure 17. Pre-Test Acid Cleaning of Filter Unit, Permeate Flux vs. Run Time .................. 77

Figure 18. . Dewatering of UF Feed, Permeate Flux vs. Run Time ....................................... 78

Figure 19. Dewatering of UF Feed - Campaigns I, II, and III, Permeate Flux vs.

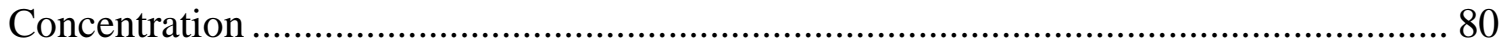

Figure 20. 1st Washing of Concentrated Slurry with 22 Wash Mini-batches ........................ 81

Figure 21. 2nd Washing of Concentrated Slurry with 22 Wash Mini-batches ....................... 82

Figure 22. 1st and 2nd Washing of Concentrated Slurry, Campaigns I, II and III ................. 83

Figure 23. 1st Caustic Rinse of the Ultrafilter ......................................................................... 85

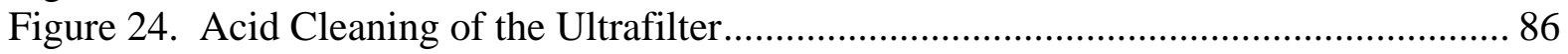

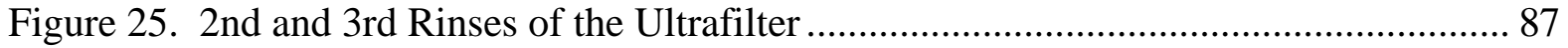

Figure 26. Filter Rinse and Clean, Campaigns I, II, and III ................................................ 88

Figure 27. Caustic Rinse and Acid Cleaning Samples .......................................................... 88

Figure 28. Baselines, Campaign II Post-test and Campaign III Pre-test ................................. 89

Figure 29. Pre- and Post-Test Baselines for Campaign III ..................................................... 91

Figure 30. Comparison of Pre- and Post-Test Baselines, Campaign I, II, and III .................. 92

Figure 31. Tracking ammonium through the pilot-scale UFP operation for Campaign III. 100

Figure 32. Pilot-scale CIX columns: The left column (COLUMN 1) is undergoing resin

regeneration during Cycle 3 operation ......................................................................... 106

Figure 33. Schematic of Ion Exchange Facility for Integrated Testing ............................... 107

Figure 34. Flowrate During Cycle 1 ……..................................................................... 112

Figure 35. Pressure Drop Across Resin Bed for Lead Column and Cycle 1........................ 113

Figure 36. Pressure Drop Across Resin Bed for Lag Column and Cycle 1........................ 113

Figure 37. Resin Bed Height During Cycle 1 …………..................................................... 114

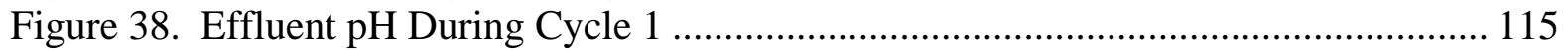

Figure 39. Effluent Conductivity During Cycle 1 ................................................................. 116

Figure 40. Test of Ability of ICP-MS to Measure Cesium................................................... 117 
WSRC-TR-2004-00565, REVISION 0

SRNL-RPP-2004-00092, REVISION 0

Figure 41. Cesium Concentration in Simulant Exiting Lead Column ................................. 119

Figure 42. Cesium Concentration Exiting Lag Column ....................................................... 120

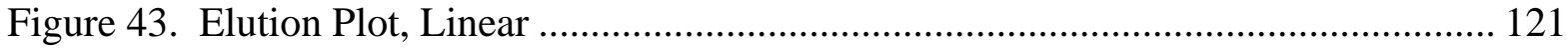

Figure 44. Elution Plot, Logarithmic ............................................................................... 121

Figure 45. TLP Evaporator Pot Temperature during Campaign III..................................... 130

Figure 46. TLP Evaporator Pot Pressure during Campaign III ............................................. 130

Figure 47. TLP Simulant Recirculation during Campaign III .............................................. 131

Figure 48. TLP Simulant Boiling in Clear Acrylic Section of Evaporator........................... 133 


\section{LIST OF TABLES}

Table 1. Feeds and products for planning SIPP developed for OLI/ESP computer model ... 20

Table 2. Percent contribution for each of the recycle streams to the total FEP feed .............. 22

Table 3. Percent contribution for each of the UFP feed streams ............................................. 23

Table 4. Percent contribution for each of the TLP feed streams ............................................ 24

Table 5. Hold period before pilot-scale FEP begins .............................................................. 25

Table 6. Pilot-scale FEP test matrix with the colored columns representing Campaign III.. 26

Table 7. Hold period before pilot-scale UFP begins................................................................. 26

Table 8. Pilot-scale UFP test matrix with the colored columns representing Campaign III . 27

Table 9. Hold period before pilot-scale CIX begins .................................................................. 28

Table 10. Pilot-scale CIX (1st half) test matrix with the colored columns representing Campaign III .......................................................................................................... 28

Table 11. Pilot-scale CIX (2nd half) test matrix with the colored columns representing

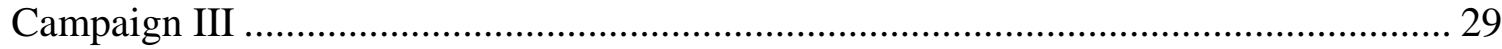

Table 12. Hold period before pilot-scale TLP begins............................................................ 29

Table 13. Pilot-scale TLP test matrix with the colored columns representing Campaign III 30

Table 14. Pilot-scale schedule for Campaign III ................................................................... 31

Table 15. Measured Simulant Compositions ............................................................................. 32

Table 16. Recycle Streams*......................................................................................... 38

Table 17. Composition of Campaign II Wash Permeate Recycles to FEP Evaporator .......... 39

Table 18. Measured Recycle Compositions............................................................................. 40

Table 19. Chronology of the Campaign III FEP operations .................................................. 52

Table 20. Composition of Campaign III FEP Evaporator Streams ........................................... 53

Table 21. Comparison of Campaign II and III FEP Compositions........................................... 54

Table 22. Campaign II Predicted and Measured FEP Evaporator Feed Compositions.......... 56

Table 23. Campaign III Predicted and Measured FEP Evaporator Feed Compositions......... 57

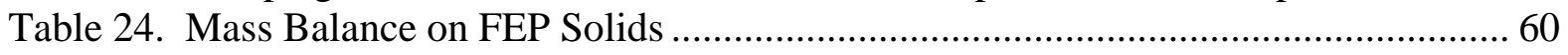

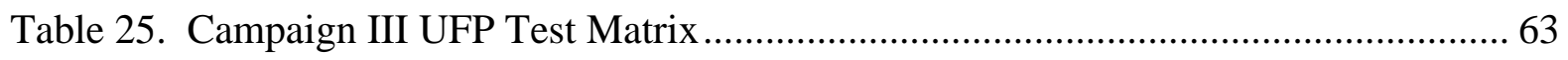

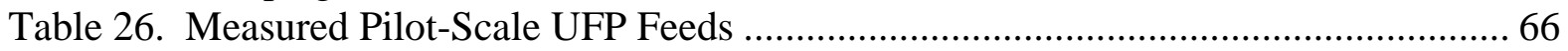

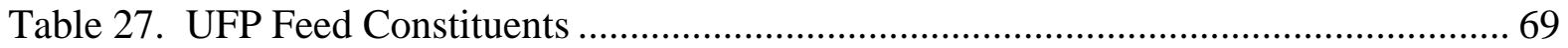

Table 28. EDL Measured Data during Dewatering of the AY102+AP101+FEP Bottoms

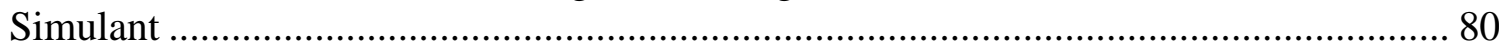

Table 29. Total Solids (TS) and Undissolved Solids (UDS) Concentrations of the Spent

Rinse and Cleaning Solutions .................................................................................... 89

Table 30. Campaign III Solids Content of Slurry During Ultrafiltration ................................ 93

Table 31. Concentrated Slurry and Permeate Compositions ...................................................... 94

Table 32. Comparison of Campaigns I-III Washed Slurry Compositions............................... 96

Table 33. Comparison of Wash Permeate Composites from Campaigns I-III ........................ 97

Table 34. Estimation of Slurry Concentrations for Campaign III (Values in italics are for Campaign II) .................................................................................................. 98

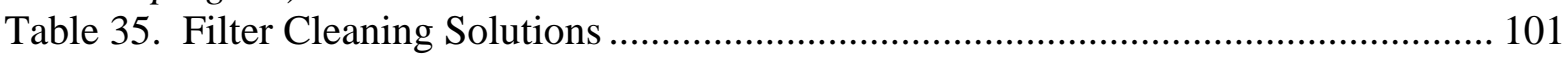

Table 36. Cycle Dates of Operation.............................................................................. 110

Table 37. Highlights of the Pilot-Scale CIX Cs removal efficiency.................................... 111

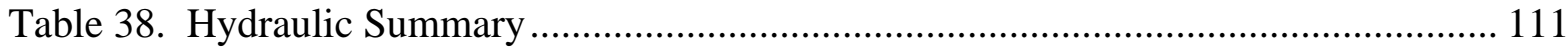


WSRC-TR-2004-00565, REVISION 0

SRNL-RPP-2004-00092, REVISION 0

Table 39. Comparison of the Volume for Each of the CIX Regeneration Process Solutions to the Internal Volume of Pilot-scale CIX Column........................................................ 114

Table 40. Probe Responses for Standard Solutions ....................................................... 116

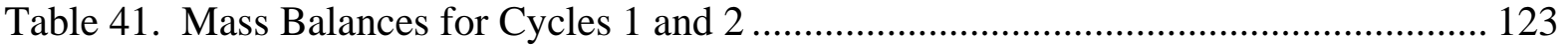

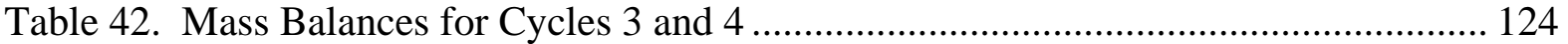

Table 43. Mass of Eluate Cesium as a function of Integration Method ............................. 125

Table 44. Measured Composition of TLP Feeds ......................................................... 127

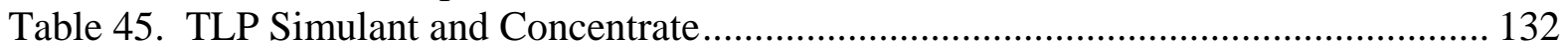

Table 46. Comparison of Campaigns II and III TLP Compositions.................................. 135 
WSRC-TR-2004-00565, REVISION 0

SRNL-RPP-2004-00092, REVISION 0

\section{NOMENCLATURE}

\begin{tabular}{|c|c|}
\hline AA & Atomic absorption (spectroscopy) \\
\hline ACTL & Aiken County Technology Laboratory \\
\hline ADS & Analytical Development Section (part of WSRC/SRNL) \\
\hline AP101 & DOE Hanford Site Tanks 241-AP-101 simulated waste \\
\hline AN102 & DOE Hanford Site Tank 241-AN-102 \\
\hline AN107 & DOE Hanford Site Tank 241-AN-107 \\
\hline AY102 & DOE Hanford Site Combined Tanks 241-AY-102 and 241-C-106 \\
\hline BNI & Bechtel National, Inc. \\
\hline CIX & Cesium Ion Exchange (Process) (part of the RPP-WTP Pretreatment Facility) \\
\hline CFD & Computational Fluid Dynamics \\
\hline CUF & $\begin{array}{l}\text { Cells Unit Filter (bench-top cross-flow filter used with both radioactively cold and } \\
\text { hot wastes) }\end{array}$ \\
\hline${ }^{\circ} \mathrm{C}$ & Degree Centigrade (or Celsius) \\
\hline $\mathrm{D}$ & Diameter \\
\hline DIF & Deionized and Filtered (0.1 micron) Water \\
\hline DOE & United States Department of Energy \\
\hline $\mathrm{dP}$ & Differential Pressure \\
\hline DS & See Solids \\
\hline EDL & Engineering Development Laboratory (part of WSRC/SRNL) \\
\hline EPDM & Ethylene Propylene Diene Monomer \\
\hline FEP & (Waste) Feed Evaporation Process (part of the RPP-WTP Pretreatment Facility) \\
\hline $\mathrm{ft}$ & Foot \\
\hline gm & Gram \\
\hline gpm & Gallons per Minute \\
\hline HLP & High Level (Activity Waste) Process \\
\hline HLW & High Level (Activity) Waste \\
\hline $\mathrm{hr}$ & Hour \\
\hline i.d. or ID & Inside Diameter \\
\hline IC & Ion Chromatography \\
\hline ICPES & Inductively coupled plasma emission spectroscopy \\
\hline ICPMS & Inductively coupled plasma mass spectrometry \\
\hline IHLW & Immobilized high level waste (glass) \\
\hline ILAW & Immobilized low activity waste (glass) \\
\hline in. & Inch \\
\hline IX & Ion Exchange \\
\hline $\mathrm{hr}$ & Hour \\
\hline l or $\mathrm{L}$ & Liter \\
\hline LAW & Low (Level) Activity Waste \\
\hline $\mathrm{m}$ & Meter \\
\hline M & Molar (mol/L) \\
\hline $\mathrm{Mg}$ & Milligram \\
\hline $\min$ & Minute \\
\hline $\mathrm{ml}$ or $\mathrm{mL}$ & Milliliter \\
\hline $\mathrm{mm}$ & Millimeter \\
\hline MTG/d & Metric tons glass/day \\
\hline NAS & Sodium Aluminosilicate \\
\hline
\end{tabular}


WSRC-TR-2004-00565, REVISION 0

SRNL-RPP-2004-00092, REVISION 0

nominal The word "nominal" for a filter rating is a vague term. Its meaning is manufacturer dependent. Further, a "nominal" rating does not give an exact size to a filter medium, but rather an approximation to the expected performance of a filter. In the case of Mott, a nominal rated $0.1-\mu \mathrm{m}$ filter means that approximately $95 \%$ of particles greater than $0.1 \mu \mathrm{m}$ will not pass the filter.

NTU Nephelometric Turbidity Unit

o.d. or OD Outside Diameter

OLI/ESP Modeling software which is part of Environmental Simulation Program software package used in developing the SIPP task

Pa Pascal

PSD Particle Size Distribution

psi Pounds Per Square Inch

psig Pounds Force Per Square Inch Gauge

psid Pounds Force Per Square Inch Difference

PWD Plant Wash and Disposal (System)

QA Quality Assurance

RLD Radioactive Liquid (Waste) Disposal (System)

RPP River Protection Project (at the DOE Hanford Site)

s or sec Time: Second

SBS Submerged Bed Scrubber - source of LAW and HLW melter recycle streams

SIPP Semi-Integrated Pilot Plant

SRNL Savannah River National Laboratory (part of WSRC)

Solids

TS $=$ Total Solids $=$ Mass percent of all solids in a stable slurry whether dissolved or not

UDS = Undissolved Solids = Mass percent of solids in a stable slurry that are not dissolved

DS = Dissolved Solids $=$ TS - UDS $=$ Mass percent of solids in a stable slurry that are dissolved

SS = Soluble Solids $=($ TS - UDS $) /(100 \%$ - UDS $) \times 100 \%=$ Mass percent of solids in only the supernate portion of a stable slurry

SS See $\underline{\text { Solids }}$

SSM Steady-State Matrix (Applies to Campaign I only - see Fig. 33)

Std Dev Standard Deviation

Supernate Supernatant

TLP Treated (Feed) LAW Process (part of the RPP-WTP Pretreatment Facility)

TS See Solids

TMP Transmembrane Pressure (the average pressure drop across the thickness of the filter medium - perpendicular to the slurry flow.)

TRU Transuranic

UDS See $\underline{\text { Solids }}$

UF Ultrafiltration

UFP Ultrafiltration Process (part of the RPP-WTP Pretreatment Facility)

$\mathrm{V} \quad$ Velocity of the slurry flow along the length of the filter tubes

VSL Vitreous State Laboratory (of Catholic University of America, Wash. DC)

WGI Washington Group International

WTP Waste \& Immobilization Treatment Plant

WSRC Westinghouse Savannah River Company

XRD X-ray diffraction

Also note: Within this report, supernate refers to the liquid (undissolved solids-free) portion of a slurry sample. When referring to the ultrafiltration process, the permeate is synonymous with the slurry supernate; either term can thus be used, but permeate will be the preferred term herein. For the evaporation operations, the liquid fraction of the slurry feed or bottoms product will be called the supernate. 


\begin{abstract}
Campaign III, the third step towards the Semi Integrated Pilot Plant (SIPP) task, conducted by the Savannah River National Laboratory (SRNL) at the Savannah River Site, was to take the several recycle streams produced in Campaign II, the second step of the task, and combine them with other simulated recycle and chosen waste streams. (Campaign II was fed recycles from Campaign I.) The combined stream was processed in a fashion that mimicked the pretreatment operations of the DOE River Protection Project - Waste Treatment and Immobilization Plant (RPP-WTP). The SIPP task is considered semi-integrated because it only deals with the pretreatment operations of the RPP-WTP. That is, the pilot plant starts by receiving waste from the tank farm and ends when waste is processed to the point of being sent for vitrification. The resulting pretreated LAW and HLW simulants produced by the SIPP are shipped to VSL (Vitreous State Laboratory) ${ }^{\dagger}$ for vitrification in pilot WTP melters.

Within the SIPP task these steps are referred to as Campaigns and there are four Campaigns in all. Campaign I, which is completely different than other campaigns, subjected a simulant of Hanford Tank 241-AY-102/C-106 (AY102) waste to cross-flow ultrafiltration only and in that process several important recycle streams were produced as a result of washing the simulant and cleaning the cross-flow filter. These streams were fed to subsequent campaigns and that work was the subject of the issued Campaign I interim report (Duignan et al., 2004a). The streams created in Campaign I were used for Campaign II, and during Campaign II more of the same recycle streams were produced, with the addition of recycle streams created during the pilot-scale ion exchange unit operation (Duignan et al., 2004b)
\end{abstract}

The operation of each of the subsequent campaigns, i.e., II, III, and IV, while different from Campaign I, are very similar to each other, and can be best understood as the process of operating a series of Pretreatment Unit Operations in a somewhat prototypic manner. That is, while Campaign I studied the operation of a single, albeit important, Pretreatment Unit Operation, i.e., Ultrafiltration, all subsequent campaigns are to study the four major unit operations that make-up the RPP-WTP Pretreatment Facility. They are: Waste Feed Evaporation Process (FEP), Ultrafiltration Process (UFP), Cesium Ion Exchange Process (CIX), and the Treated LAW Evaporation Process (TLP). Each of the campaigns operate basically as a separate subtask, but as with Campaign I, the recycle streams produced in one campaign feed into the subsequent campaign. Therefore, all four campaigns are chemically connected through these recycle streams, which carry over effects of the preceding campaign.

The results of Campaign III operations are the subject of this third interim report. The reports are called interim because they are limited to the results obtained from a single campaign (or past campaigns) and further limited to only highlights of that single campaign. A final report will follow Campaign IV and its intent is to comprehensively

\footnotetext{
${ }^{\dagger}$ Part of the Catholic University of America
} 
cover the overall task activities, as well as the full database accumulated and the equipment used during the yearlong SIPP task.

SIPP is a limited version of the plant's pretreatment facility in that it tests pilot-scale unit operations of FEP, UFP, CIX, and TLP only. However, an integral part of SIPP is to produce the many recycle streams that must also be processed with stored tank farm waste. That is, it is very important to know the impact that the recycle streams will have on the operation of the several unit operations so as to better model the plant's overall pretreatment throughput, leading to a production rate of stabilized waste.

Like the Campaign II report, this report will begin with a summary of the overall operation of the task done during Campaign III to understand the layout of this report. This summary is then followed with highlights of the test results.

With input and review from Hanford, SRNL developed a steady-state OLI/ESP model of the RPP-WTP Pretreatment Facility operation. That model's principal role was to determine the amounts of simulated wastes needed for each of the four unit operations. In general, it did well, but was found to be off by approximately 15\% in predicted SIPP volumes due to unknowns in the undissolved solids of the several simulants. (For a detailed discussion of model predictions differences see the Campaign II Interim Report, Duignan et al., 2004b; Section 2.7.4.2.1).

Campaign III conclusions are:

\section{FEP Evaporation}

- A total of about $1112 \mathrm{~kg}$ of FEP feed, at a density of $1.04 \mathrm{~g} / \mathrm{ml}$ and $0.6 \mathrm{M} \mathrm{Na}$, was concentrated down to $170 \mathrm{~kg}$, at a density of $1.26 \mathrm{~g} / \mathrm{ml}$ and $4.3 \mathrm{M} \mathrm{Na}$.

- The feed was evaporated under vacuum (1 psia $(6.9 \mathrm{kPa}))$ at moderate temperatures $\left(\sim 50^{\circ} \mathrm{C}\right)$ and the entire evaporation process proceeded as designed.

- The evaporator was designed to evaporate around $0.4 \mathrm{gpm}(1.5 \mathrm{lpm})$ of water and for this campaign the average evaporation rate was $0.52 \mathrm{gpm}$.

- No significant foaming was observed prior to the addition of antifoam agent. During the evaporation process, the froth height remained around $1 \mathrm{ft}(0.3 \mathrm{~m})$ that is significantly less than the $5 \mathrm{ft}(1.5 \mathrm{~m})$ distance to the lower demister pad.

- During start-up and unsteady conditions, significant flashing resulted in feed splashing on the lower demister pad. These conditions are associated with much larger vapor flux and vapor velocities at the interface.

- As seen in Campaign II, a large portion of the dissolved oxalate in the feed was precipitated, probably as sodium oxalate.

- The oxalate concentration in the FEP feed in Campaign III was less than that in Campaign II, which is consistent with the higher oxalate concentration in the Campaign I slurry.

- About $25-40 \%$ of the dissolved $\mathrm{Al}$ in the feed to the FEP evaporator precipitated during mixing of the recycles, assumed to occur because of localized regions of low $\mathrm{pH}$. Vigorous mixing when joining caustic and acidic recycle streams in the Plant Wash and Disposal System would help to minimize Al precipitation. 
- The fraction of dissolved aluminum in Campaign III FEP concentrate (bottoms) was less than in Campaign II, resulting in more undissolved Al fed to the UFP.

\section{UFP}

- $\quad$ The average temperature-adjusted permeate flux when dewatering from 3 to 24 wt\% UDS was $0.011 \mathrm{gpm} / \mathrm{ft}^{2}$. The average temperature-adjusted permeate flux when dewatering from $\sim 4$ to $20 \mathrm{wt} \%$ UDS was $0.014 \mathrm{gpm} / \mathrm{ft}^{2}$. The average, temperatureadjusted permeate flux when dewatering from $\sim 4$ to $17 \mathrm{wt} \%$ UDS was $0.018 \mathrm{gpm} / \mathrm{ft}^{2}$.

- The turbidity of the permeate from dewatering and slurry washing, when measured directly after the sample was taken, was always lower than 0.5 NTU. The Test Specification indicated a solids-free threshold of 2 NTU, therefore the measured values indicated the permeate was free of significant undissolved solids.

- Cleaning the filter with $2 \mathrm{M}$ nitric acid was sufficient to increase the $0.1 \mathrm{M} \mathrm{NaOH}$ permeate flux to an order of magnitude larger than that of waste flux. However, it was not sufficient to return the filter to pre-test flux levels.

- The composition of the undissolved solid species in the washed sludges in Campaigns I-III were similar, as expected. The supernate portion species $\mathrm{Na}$ and $\mathrm{K}$ were higher in concentration in Campaigns II and III than in Campaign I due to the presence of the AP101 simulant.

- The aluminum concentration in the washed slurry in Campaign III was higher than in Campaign II due to there being more undissolved aluminum in the FEP concentrate.

- Because of higher undissolved aluminum in the FEP concentrate, the Campaign III permeate from the concentration step (IX feed) had less aluminum than in Campaign II.

- Ammonium in the first 0.01M NaOH UFP wash of Campaign III was mostly retained in the slurry, indicating that a slurry component has an affinity for ammonium. Most of the ammonium in the second wash was not retained.

\section{CIX}

- Pressure drops for the four cycles were low and the resin bed permeability was approximately the same as were measured for the 24-Inch Column Test (Fowley et al, 2004).

- The pressure drop across the columns for flow of simulant generally increased during the four cycles. [0.5 to 1.3 in. $\mathrm{H}_{2} 0$ ]

- $\quad$ SuperLig ${ }^{\circledR} 644$ effectively removed cesium from simulated waste and the analytical ability to measure cesium concentrations in five-molar sodium did allow verification of the waste acceptance criterion of 50\% breakthrough from the lead column after 100 Bed Volumes of simulated waste were treated.

- $\quad$ SuperLig ${ }^{\circledR} 644$ effectively removed cesium from simulated waste, however, the analytical difficulty of measuring very low cesium concentrations in five-molar sodium did not allow verification of the waste acceptance criterion of $5.2 \mu \mathrm{g} / \mathrm{L}$ from the lag column after $100 \mathrm{BV}$ of simulated waste were treated. This fact was known before the start of testing.

- The fraction of the original cesium that was removed by the lead column decreased from 99.6\% for Cycle 1 (in Campaign II) to 98.2\% for Cycle 4 (in Campaign III). 
- The masses of cesium in and out of the resin beds for all four cycles balanced to within measurement uncertainty.

\section{TLP Evaporation}

- The pilot-scale TLP evaporator operated as designed and concentrated TLP feed simulant from a density of $1.135 \mathrm{Kg} / \mathrm{L}$ at $20^{\circ} \mathrm{C}$ and a sodium concentration of $2.4 \mathrm{M}$, to a final density of $1.365 \mathrm{Kg} / \mathrm{L}$ at $20^{\circ} \mathrm{C}$ and a sodium concentration of $7.1 \mathrm{M}$.

- A small amount of foaming was observed before and after the addition of antifoam agent to the evaporator. During the evaporation process, the foaming/froth height remained around $1 \mathrm{ft}(0.3 \mathrm{~m})$ or less for the duration of the campaign. That is significantly less than the $5 \mathrm{ft}(1.5 \mathrm{~m})$ distance to the lower demister pad.

- The two Demisters in series operated well with no significant differential pressure build-up across the Demisters and there were no noticeable solids on the lower Demister after the 11-hour test.

- The composition of the products from the TLP matched what was expected from the mass balance.

- The concentration of aluminum in the TLP product was less than the result of Campaign II since the UF permeate contained less dissolved aluminum.

- No precipitation of additional solids beyond those in the feed was noted. 


\subsection{TESTING SUMMARY}

\subsection{OBJECTIVES}

Campaign III, one of four planned campaigns for the SIPP task, is the second test that includes all four WTP Pretreatment Facility unit operations, along with the incorporation of recycle waste streams. However, it is the first test to include recycle streams from the ion exchange unit operation to the large mixture of streams. For completeness, the objectives listed below are all those given in the RPP-WTP Test Specification (Longwell, 2003). However, only the body of work approved in the WSRC Technical Task and Quality Assurance Plan (Duignan, 2003c) is required to satisfy this task. Objectives not met because they were not agreed to in the Task Plan are so noted. With minor changes these Objectives and Discussion are similar to those given in the Campaign II report (Duignan, 2004b).

\begin{tabular}{|c|c|c|}
\hline Overall Test Objectives & $\begin{array}{l}\text { Objective } \\
\text { Met (Y/N) }\end{array}$ & Discussion \\
\hline $\begin{array}{l}\text { 1. Modify the design and operation of } \\
\text { the existing pretreatment pilot facilities } \\
\text { to extent necessary to mimic the WTP } \\
\text { Pretreatment Facilities. }\end{array}$ & $\mathrm{Y}$ & $\begin{array}{l}\text { Each of the four unit operations that } \\
\text { comprise the SIPP test were either modified } \\
\text { or built to meet planned operation. }\end{array}$ \\
\hline $\begin{array}{l}\text { 2. Operate the (pilot) integrated } \\
\text { facilities using simulated AY102 } \\
\text { (Envelope A/D) and AP101 (Envelope } \\
\text { A) waste feeds. }\end{array}$ & $\mathrm{Y}$ & $\begin{array}{l}\text { Campaign III involved the use of simulated } \\
\text { AY102, AP101, and recycle streams. }\end{array}$ \\
\hline $\begin{array}{l}\text { 3. Collect chemical and physical } \\
\text { property data to be defined in the test } \\
\text { plan then refine and validate the } \\
\text { thermodynamic model(s) developed by } \\
\text { SRNL. }\end{array}$ & $\mathrm{Y}$ & $\begin{array}{l}\text { Sufficient property data were obtained to } \\
\text { satisfy project needs as is evidenced by the } \\
\text { customer approval of the Campaign III } \\
\text { sample plan (Appendix B) before testing } \\
\text { began. }\end{array}$ \\
\hline $\begin{array}{l}\text { 4. Demonstrate pretreatment production } \\
\text { rates required to produce } 80 \text { metric tons } \\
\text { per day of LAW glass and } 5.4 \text { metric } \\
\text { tons of HLW glass. These are contract } \\
\text { defined "expected" production } \\
\text { throughputs for 5-day sprint runs listed } \\
\text { in Table C.6-5.1 of the contract (DOE } \\
\text { 2003). These required flow rates will } \\
\text { be determined before testing begins by } \\
\text { application of the appropriate scale } \\
\text { factors to the unit operations that } \\
\text { represent an equivalence of } \\
\text { pretreatment throughput to meet } \\
\text { maximum glass production. }\end{array}$ & $\mathrm{N}$ & $\begin{array}{l}\text { The unit production rates were measured but } \\
\text { the targets were not met. The SIPP task was } \\
\text { limited to four separate unit operations that } \\
\text { comprise the principal operations of the } \\
\text { RPP-WTP Pretreatment Facility. The pilot } \\
\text { FEP and TLP evaporation units exceeded } \\
\text { the plant-modeled rate of } 0.395 \text { gpm. The } \\
\text { pilot CIX was subjected to a test exception } \\
\text { (described in Section } 1.2 \text { ), which changed } \\
\text { this objective. As shown in Campaign II, } \\
\text { the pilot ultrafiltration permeate rate during } \\
\text { Campaign III dewatering from } 3.2 \text { to } 20 \\
\text { wt\% UDS was } 0.014 \text { gpm/ftt. This rate is } \\
\text { below the target rate of } 0.02 \text { gpm/ft }{ }^{2} \text {, but is } \\
\text { representative of the filter process since a } \\
\text { similar flux rate was obtained for Campaign } \\
\text { I, with no recycle streams, and Campaigns } \\
\text { II and III, which included several recycle } \\
\text { streams. }\end{array}$ \\
\hline
\end{tabular}


WSRC-TR-2004-00565, REVISION 0

SRNL-RPP-2004-00092, REVISION 0

\begin{tabular}{|c|c|c|}
\hline FEP/TLP Test Objectives & $\begin{array}{l}\text { Objective } \\
\text { Met }(\mathbf{Y} / \mathbf{N})\end{array}$ & Discussion \\
\hline $\begin{array}{l}\text { 1. Operate the Waste Feed Evaporator } \\
\text { and the Treated LAW Evaporator using } \\
\text { simulants derived from the integrated } \\
\text { operation of the pretreatment pilot } \\
\text { plant. }\end{array}$ & $\mathrm{Y}$ & $\begin{array}{l}\text { Feeds for both the FEP and TLP evaporators } \\
\text { included derived simulants or recycle } \\
\text { streams produced during SIPP operations. }\end{array}$ \\
\hline $\begin{array}{l}\text { 2. Recycle secondary waste generated } \\
\text { from the ultrafiltration pilot operations } \\
\text { and the ion exchange unit operation to } \\
\text { the evaporators. }\end{array}$ & $\mathrm{Y}$ & $\begin{array}{l}\text { Recycle streams from the ultrafiltration pilot } \\
\text { operation from Campaign II were fed to the } \\
\text { FEP evaporator. }\end{array}$ \\
\hline $\begin{array}{l}\text { 3. Operate the pilot evaporator using } \\
\text { simulated HLW Submerged Bed } \\
\text { Scrubber solution, simulated } \\
\text { Pretreatment Caustic Scrubbers, ion } \\
\text { exchange recycle solutions, and } \\
\text { ultrafiltration recycles generated from } \\
\text { pilot operations. }\end{array}$ & $\mathrm{Y}$ & Campaign III used all the streams indicated. \\
\hline $\begin{array}{l}\text { 4. Operate the pilot evaporator using } \\
\text { simulated LAW Submerged Bed } \\
\text { Scrubber solution and ion exchange } \\
\text { treated feed to produce LAW feed } \\
\text { solution. }\end{array}$ & $\mathrm{Y}$ & See the preceding response. \\
\hline $\begin{array}{l}\text { 5. Compare the results (solubility, } \\
\text { chemical, and physical properties, } \\
\text { which will be indicated in the test plan) } \\
\text { developed on the pilot scale with } \\
\text { results developed by experiments } \\
\text { conducted at the bench scale. }\end{array}$ & $\mathrm{N}$ & $\begin{array}{l}\text { However, any extant bench-scale data will } \\
\text { be used for such comparisons in the final } \\
\text { report. }\end{array}$ \\
\hline $\begin{array}{l}\text { 6. Determine the chemical and physical } \\
\text { properties of simulants evaporated at } \\
\text { the pilot scale as a function of sodium } \\
\text { concentration and temperature. }\end{array}$ & $\mathrm{N}$ & $\begin{array}{l}\text { The approved task plan did not require these } \\
\text { property-vs.-temperature evaluations. } \\
\text { However, densities and sodium } \\
\text { concentrations were measured and included } \\
\text { in this report. }\end{array}$ \\
\hline $\begin{array}{l}\text { 7. Evaluate the selected antifoam } \\
\text { reagent performance in the evaporators. }\end{array}$ & $\mathrm{Y}$ & $\begin{array}{l}\text { Antifoam was added to both evaporators } \\
\text { and those results are discussed herein. }\end{array}$ \\
\hline $\begin{array}{l}\text { 8. Evaluate the effect of in-leakage on } \\
\text { air emissions. }\end{array}$ & $\mathrm{N}$ & $\begin{array}{l}\text { This work was not included in the approved } \\
\text { task plan due to cost. }\end{array}$ \\
\hline $\begin{array}{l}\text { 9. Determine the chemical and physical } \\
\text { properties of concentrated recycle and } \\
\text { waste blends, which will be indicated } \\
\text { in the test plan, to be sent for } \\
\text { precipitation/ultrafiltration. }\end{array}$ & $\mathrm{Y}$ & $\begin{array}{l}\text { The concentrated bottoms streams were } \\
\text { analyzed and discussed herein. }\end{array}$ \\
\hline $\begin{array}{l}\text { 10. Operate the pilot evaporator unit } \\
\text { assuming a full-scale WTP } \\
\text { Pretreatment process flow rate of } 80 \\
\text { metric tons of LAW glass per day and } \\
5.4 \text { metric tons of HLW glass per day } \\
\text { (herein written as } 80 / 5.4 \text { MTG/d). }\end{array}$ & $\mathrm{Y}$ & $\begin{array}{l}\text { Both evaporators were run at a rate of better } \\
\text { than the plant rate of } 0.395 \text { gpm, which was } \\
\text { based on a production rate of } 80 / 6 \mathrm{MTG} / \mathrm{d} \\
\text { (Calloway, 2004). }\end{array}$ \\
\hline
\end{tabular}


WSRC-TR-2004-00565, REVISION 0 SRNL-RPP-2004-00092, REVISION 0

\begin{tabular}{|c|c|c|}
\hline UFP Test Objectives & $\begin{array}{l}\text { Objective } \\
\text { Met (Y/N) }\end{array}$ & Discussion \\
\hline $\begin{array}{l}\text { 1. Operate with prototypical } \\
\text { wash/leach/wash/steps using simulated } \\
\text { AY102 and AP101 and concentrated } \\
\text { evaporator recycles. }\end{array}$ & $\mathrm{Y}$ & $\begin{array}{l}\text { The approved task plan made the leach } \\
\text { step optional, pending RPP-WTP guidance. } \\
\text { Before testing began RPP-WTP decided } \\
\text { against doing the leach step. All other } \\
\text { objectives were met. }\end{array}$ \\
\hline $\begin{array}{l}\text { 2. Operate with } \\
\text { prototypical/recommended } \\
\text { Ultrafiltration cleaning method. }\end{array}$ & $\mathrm{Y}$ & $\begin{array}{l}\text { RPP-WTP recommended continued use of } \\
2 \text { M nitric acid for cleaning, which was } \\
\text { done. }\end{array}$ \\
\hline $\begin{array}{l}\text { 3. Determine the chemical and physical } \\
\text { properties of the concentrated solids, } \\
\text { washed solids, leached solids, and final } \\
\text { solids product derived from each } \\
\text { campaign. }\end{array}$ & $\mathrm{Y}$ & $\begin{array}{l}\text { Properties of the concentrates were } \\
\text { measured. However no leaching was } \\
\text { performed as requested by the RPP-WTP } \\
\text { customer. }\end{array}$ \\
\hline $\begin{array}{l}\text { 4. Determine the chemical and physical } \\
\text { properties of permeate, recycles, and } \\
\text { cleaning solutions, which will be } \\
\text { indicated in the test plan, derived from } \\
\text { each campaign. }\end{array}$ & $\mathrm{Y}$ & $\begin{array}{l}\text { Properties of those streams were measured } \\
\text { and discussed herein. }\end{array}$ \\
\hline $\begin{array}{l}\text { 5. Compare the results developed on } \\
\text { the pilot scale with results developed } \\
\text { by experiments conducted at the bench } \\
\text { scale to assess the effect of recycles. }\end{array}$ & $\mathrm{N}$ & $\begin{array}{l}\text { No bench scale experiments were done to } \\
\text { study the effects of recycles. }\end{array}$ \\
\hline $\begin{array}{l}\text { 6. Provide pump data (pump curves) } \\
\text { for the high solid content slurries. }\end{array}$ & $\mathrm{N}$ & $\begin{array}{l}\text { This requirement was not included in the } \\
\text { approved task plan. }\end{array}$ \\
\hline $\begin{array}{l}\text { 7. Demonstrate operation of the } \\
\text { Ultrafiltration unit assuming a full- } \\
\text { scale WTP Pretreatment process flow } \\
\text { rate corresponding to production of } \\
80 / 5.4 \text { MTG/d. }\end{array}$ & $\mathrm{N}$ & $\begin{array}{l}\text { See response } 4 \text { to the Overall Test } \\
\text { Objectives, above. }\end{array}$ \\
\hline $\begin{array}{l}\text { 8. Evaluate the downstream effects of } \\
\text { antifoam reagent. Determine the fate } \\
\text { of the reagent by tracking its } \\
\text { concentration through the integrated } \\
\text { pilot. }\end{array}$ & $\mathrm{N}$ & $\begin{array}{l}\text { The effect on filter flux seemed to be } \\
\text { insignificant, but it is discussed herein } \\
\text { along with that data used to track the } \\
\text { antifoam. }\end{array}$ \\
\hline
\end{tabular}

\begin{tabular}{|c|c|c|}
\hline CIX Test Objectives & $\begin{array}{l}\text { Objective } \\
\text { Met (Y/N) }\end{array}$ & Discussion \\
\hline $\begin{array}{l}\text { 1. Operate the pilot ion exchange unit } \\
\text { using ultrafiltration permeate produced } \\
\text { using simulated AY102 and AP101. }\end{array}$ & $\mathrm{Y}$ & $\begin{array}{l}\text { This was done along with the bottoms } \\
\text { produced by evaporating the several } \\
\text { recycle streams. }\end{array}$ \\
\hline $\begin{array}{l}\text { 2. Operate the pilot ion exchange unit } \\
\text { assuming a full-scale WTP } \\
\text { Pretreatment process flow rate } \\
\text { corresponding to production of } 80 / 5.4 \\
\text { MTG/d. }\end{array}$ & $\mathrm{N}$ & $\begin{array}{l}\text { Test Exception 24590-WTP-TEF-RT-03- } \\
069 \text { required the pilot CIX show prototypic } \\
\text { cesium removal. This required matching } \\
\text { the Plant's treated waste residence time in } \\
\text { the resin and not the full-scale flow rate. } \\
\text { Therefore, this objective could not be met. }\end{array}$ \\
\hline $\begin{array}{l}\text { 3. Demonstrate ion exchange column } \\
\text { operating parameters using the } \\
\text { recommended ion exchange resin. }\end{array}$ & $\mathrm{Y}$ & $\begin{array}{l}\text { The currently planned resin, } \\
\text { SuperLig }{ }^{\circledR} 644 \text { was employed. }\end{array}$ \\
\hline
\end{tabular}


WSRC-TR-2004-00565, REVISION 0

SRNL-RPP-2004-00092, REVISION 0

\begin{tabular}{|l|l|l|}
\hline CIX Test Objectives & $\begin{array}{l}\text { Objective } \\
\text { Met (Y/N) }\end{array}$ & Discussion \\
\hline $\begin{array}{l}\text { 4. Determine the chemical and physical } \\
\text { properties, which will be indicated in } \\
\text { the test plan, of all ion exchange } \\
\text { effluents. }\end{array}$ & $\mathrm{N}$ & $\begin{array}{l}\text { Only Lead and Lag column effluents were } \\
\text { measured for Cs concentrations to } \\
\text { minimize cost since Campaign II had } \\
\text { already made the complete set of } \\
\text { measurements. }\end{array}$ \\
\hline $\begin{array}{l}\text { 5. Compare the results developed on } \\
\text { the pilot scale with results developed } \\
\text { by experiments conducted at the bench } \\
\text { scale to assess the effect of recycles. }\end{array}$ & $\mathrm{N}$ & $\begin{array}{l}\text { No bench scale experiments were done to } \\
\text { study the effects of recycles. }\end{array}$ \\
\hline $\begin{array}{l}\text { 6. Determine the chemical and physical } \\
\text { properties, which will be indicated in } \\
\text { the test plan, of blended ion exchange } \\
\text { secondary wastes (pre-elution rinse and } \\
\text { spent regenerant) that are recycled back } \\
\text { to the waste feed evaporation system. }\end{array}$ & $\mathrm{N}$ & $\begin{array}{l}\text { See response to Item No. 4 of CIX Test } \\
\text { Objectives. }\end{array}$ \\
\hline $\begin{array}{l}\text { 7. Determine the chemical and physical } \\
\text { properties of ion exchange eluates, } \\
\text { which will be indicated in the test plan. }\end{array}$ & $\mathrm{N}$ & $\mathrm{Y}$ \\
\hline $\begin{array}{l}\text { 8. Evaluate the potential effects of the } \\
\text { evaporator antifoam reagent on ion } \\
\text { exchange performance. }\end{array}$ & $\begin{array}{l}\text { See response to Item No. 4 of CIX Test } \\
\text { Objectives. }\end{array}$ \\
\hline
\end{tabular}

\subsection{TEST EXCEPTIONS}

\begin{tabular}{|l|l|}
\hline List Test Exceptions & Describe Test Exceptions \\
\hline $\begin{array}{l}\text { 1. 24590-WTP-TEF-RT-02-025, Rev. 0, 20 May } \\
\text { 2002 }\end{array}$ & $\begin{array}{l}\text { See the description for Test Exception No. 24590- } \\
\text { WTP-TEF-RT-03-069, which is No. 7 in this list. }\end{array}$ \\
\hline $\begin{array}{l}\text { 2. 24590-WTP-TEF-RT-02-046, Rev. 0, 01 Aug. } \\
\text { 3. } 24590 \text {-WTP-TEF-RT-03-004, Rev. 0, 07 Feb. } \\
2003\end{array}$ & $\begin{array}{l}\text { See the description for Test Exception No. 24590- } \\
\text { WTP-TEF-RT-03-069, which is No. 7 in this list. }\end{array}$ \\
\hline $\begin{array}{l}\text { S. } 24590 \text {-WTP-TEF-RT-03-037, Rev. 0, 30 Jul. } \\
\text { WTP-TEF-RT-03-069, which is No. 7 in this list. }\end{array}$ \\
\hline $\begin{array}{l}\text { W. } 24590 \text {-WTP-TEF-RT-03-037, Rev. 1, 17 Sept. } \\
2003\end{array}$ & $\begin{array}{l}\text { See the description for Test Exception No. 24590- } \\
\text { WTP-TEF-RT-03-069, which is No. 7 in this list. }\end{array}$ \\
\hline $\begin{array}{l}\text { 6ee the description for Test Exception No. 24590- } \\
\text { WTP-TEF-RT-03-069, which is No. 7 in this list. }\end{array}$ \\
\hline $\begin{array}{l}\text { W.2450-WTP-TEF-RT-03-054, Rev. 0, 17 Sept. } \\
2003\end{array}$ & $\begin{array}{l}\text { See the description for Test Exception No. 24590- } \\
\text { WTP-TEF-RT-03-069, which is No. 7 in this list. }\end{array}$ \\
& $\begin{array}{l}\text { As of the completion of Campaign III there was no } \\
\text { test exception to the SIPP task plan (Duignan et } \\
\text { al., 2004). All of the listed test exceptions, 1 } \\
\text { though 7, affect only a task plan dealing solely } \\
\text { with ion exchange operation (Steimke, 2002). For } \\
\text { completeness, all the test exceptions were listed } \\
\text { but only this seventh test exception impacts SIPP } \\
\text { test. As with all the other pilot unit operations, the } \\
\text { pilot-scale CIX was to operate in a fashion to } \\
\text { demonstrate the rate of glass production. } \\
\text { However, RPP-WTP thought it was more } \\
\text { important to demonstrate cesium removal } \\
\text { efficiency. Since prototypic column flow rates and }\end{array}$ \\
\hline
\end{tabular}


WSRC-TR-2004-00565, REVISION 0

SRNL-RPP-2004-00092, REVISION 0

\begin{tabular}{|c|c|c|}
\hline & & $\begin{array}{l}\text { prototypic resin residence time must be different } \\
\text { for other than full-scale sizes. The pilot unit was } \\
\text { not used at a LAW flow rate that would } \\
\text { demonstrate glass production rates. This seventh } \\
\text { test exception describes how the pilot scale IX } \\
\text { columns would operate to demonstrate cesium } \\
\text { removal. That removal efficiency is what is } \\
\text { discussed herein. }\end{array}$ \\
\hline
\end{tabular}

\subsection{RESULTS AND PERFORMANCE AGAINST SUCCESS CRITERIA}

\begin{tabular}{|l|l|}
\hline List Success Criteria & $\begin{array}{l}\text { Explain How the Tests Did or Did Not } \\
\text { Meet the Success Criteria }\end{array}$ \\
\hline $\begin{array}{l}\text { 1. The integrated operation of the pretreatment } \\
\text { process produces vitrification feed streams similar } \\
\text { in chemical composition to those produced in bench } \\
\text { scale experiments. }\end{array}$ & $\begin{array}{l}\text { Bench scale experiments were not part of the SIPP } \\
\text { scope. However, the SIPP task did produce both } \\
\text { LAW and HLW feed streams with simulated waste } \\
\text { and recycle streams that were processed in the } \\
\text { same manner expected for the RPP-WTP } \\
\text { Pretreatment Facility. The chemical composition } \\
\text { obtained is that which is expected for the full-scale } \\
\text { facility and therefore this criterion was met. }\end{array}$ \\
\hline $\begin{array}{l}\text { 2. The integrated pretreatment process demonstrates } \\
\text { a scaled throughput of } 80 \text { MTG/d of LAW and 5.4 } 5 \text { MTG/d of HLW. }\end{array}$ & $\begin{array}{l}\text { This success criterion was partially met in that both } \\
\text { the pilot FEP and TLP operated at, or above the } \\
\text { evaporation rate (0.395 gpm) necessary to meet the } \\
\text { stated glass production rates. The pilot UFP } \\
\text { realized a dewatering filter flux rate of 0.014 } \\
\text { gpm/ft. This value is below the rate needed meet } \\
\text { the glass production rates, but is representative of } \\
\text { rate obtained from bench scale test with actual } \\
\text { waste. The pilot CIX rate purposely did not meet } \\
\text { the full-scale plant rate as explained in Test } \\
\text { Exception No 7 above. }\end{array}$ \\
\hline
\end{tabular}

\subsection{QUALITY REQUIREMENTS}

This work was conducted in accordance with the RPP-WTP QA requirements specified for work conducted by SRNL as identified in DOE IWO MOSRLE60. SRNL has provided matrices to WTP, demonstrating compliance of the SRNL QA program with the requirements specified by WTP. Specific information regarding the compliance of the SRNL QA program with RW-0333P, Revision 10, NQA-1 1989, Part 1, Basic and Supplementary Requirements and NQA-2a 1990, Subpart 2.7 is contained in these matrices. 
WSRC-TR-2004-00565, REVISION 0

SRNL-RPP-2004-00092, REVISION 0

\subsection{R\&T TEST CONDITIONS}

\begin{tabular}{|c|c|}
\hline List R\&T Test Conditions & Were Test Conditions Followed? \\
\hline $\begin{array}{l}\text { 1. SRNL shall prepare a specific test plan } \\
\text { containing detailed information needed to } \\
\text { implement (the) test specification. The test plan } \\
\text { shall also include, as appropriate, references to } \\
\text { documents that drive test documentation } \\
\text { requirements to assure data integrity and } \\
\text { reproducibility. This would include such items as } \\
\text { detailed test procedures, instrument calibration, } \\
\text { methods of data gathering, etc. The test plan shall } \\
\text { indicate what methodologies will be employed to } \\
\text { verify or validate data produced during testing } \\
\text { activities. } \\
\text { A draft test plan shall be submitted to WTP R \& T } \\
\text { for review and comment. Comments labeled } \\
\text { "Mandatory" by the reviewer require a disposition. } \\
\text { The changes in the test plan shall be provided } \\
\text { electronically and clearly identified by } \\
\text { redline/strikeout or highlighting to facilitate the } \\
\text { review process. } \\
\text { The final test plan must be approved by a WTP R \& } \\
\text { T representative prior to performing work. }\end{array}$ & $\begin{array}{l}\text { As required, a test plan was developed (Duignan, } \\
\text { 2003c) to meet the test specification. All analytes } \\
\text { that could not meet the listed criterion were } \\
\text { indicated in the task plan and approved before } \\
\text { testing began. Documents that were available } \\
\text { before and when the test plan was completed were } \\
\text { referenced therein, but those that were not, e.g. } \\
\text { procedures, calibration, will be references as they } \\
\text { become available in the subsequent task reports. } \\
\text { The test plan was submitted to WTP R\&T for } \\
\text { review and approval. All reviewers' comments } \\
\text { were addressed and dispositioned, after which the } \\
\text { report was approved by WTP R\&T prior to testing, } \\
\text { thus it became the principal document to carry out } \\
\text { testing. }\end{array}$ \\
\hline $\begin{array}{l}\text { 2. Volumetric waste-to-recycle ratios will be } \\
\text { calculated using appropriate WTP's Aspen Custom } \\
\text { Modeler (ACM) Model runs as a basis for the } \\
\text { combined feed stream. The block diagram to be } \\
\text { used for this work is depicted in Fig. 1. Simulant } \\
\text { AY102 feed rate will be established based on the } \\
\text { requirement for scaled } 6 \text { MTG/d HLW production, } \\
\text { and AP101 feed rate will be based on the additional } \\
\text { requirement to produce scaled } 80 \text { MTG/d LAW } \\
\text { production. Tankage required for execution of the } \\
\text { work will be provided and incorporated as required } \\
\text { by [SRNL]. } \\
\text { Prior to initiation of the test campaigns, the system } \\
\text { will be simulated using the OLI Environmental } \\
\text { Simulation Program (ESP) models developed in } \\
\text { previous completed evaporation tasks. The } \\
\text { expanded waste feed evaporation model, which } \\
\text { includes ultrafiltration and recycles, along with the } \\
\text { treated feed evaporation model will simulate the } \\
\text { pilot operations under minimum (48/3.2 MTG/d) } \\
\text { and expected (80/5.4 MTG/d) operating conditions. } \\
\text { Output of the models will then be compared to } \\
\text { above referenced ACM model runs and the data } \\
\text { obtained during the pilot operations. Results of this } \\
\text { modeling effort will be discussed with WTP R\&T } \\
\text { personnel and approval of the path forward obtained } \\
\text { PRIOR TO START of testing. }\end{array}$ & $\begin{array}{l}\text { As required, SRNL developed an OLI model based } \\
\text { on several sources, including ACM and sources for } \\
\text { ACM prior to any SIPP testing. The modeling } \\
\text { results were compared to ACM results by both } \\
\text { SRNL and RPP-WTP personnel. The OLI results } \\
\text { were scaled to SIPP operation to assist in } \\
\text { completing a test plan and to determine waste } \\
\text { stream and recycle stream volumetric needs. The } \\
\text { model was also used to determine scaled flow rates } \\
\text { of each of the four unit operation in order to know } \\
\text { what operation rate were necessary to meet full- } \\
\text { scale plant glass production rates of } 80 \text { MTG/d } \\
\text { from LAW and 5.4 MTG/d from HLW. Those } \\
\text { model results are discussed in Duignan et al., } \\
\text { (2004b) and Barnes (2004). }\end{array}$ \\
\hline
\end{tabular}




\begin{tabular}{|l|l|}
\hline List R\&T Test Conditions & Were Test Conditions Followed? \\
\hline $\begin{array}{l}\text { 3. The LAW and HLW SBS recycles will be } \\
\text { simulants generated from VSL/Duratek pilot melter } \\
\text { testing. }\end{array}$ & $\begin{array}{l}\text { Both submerged bed scrubber (SBS) recycle } \\
\text { streams were obtained from VSL and used in } \\
\text { Campaign III testing. }\end{array}$ \\
\hline $\begin{array}{l}\text { 4. The simulant waste feeds will be based on } \\
\text { approved formulations developed under the WTP's } \\
\text { waste simulant program participated in by SRNL. }\end{array}$ & $\begin{array}{l}\text { The simulated waste feed AY102 was developed } \\
\text { and validated by SRNL (Zamecnik, et al., 2004). } \\
\text { It was filter tested at the bench scale against an } \\
\text { actual waste sample. The formulation was } \\
\text { approved by WTP R\&T before its use. The } \\
\text { formulation for the simulated feed AP101 was } \\
\text { developed by Battelle (Russell et al., 2003) and } \\
\text { approved by WTP R\&T. All other waste streams } \\
\text { used for SIPP are either recycle streams or derived } \\
\text { from recycle streams. }\end{array}$ \\
\hline $\begin{array}{l}\text { 5. Generation of initial ultrafiltration recycles will } \\
\text { be accomplished by performing the work specified } \\
\text { in AY102 Simulant Pilot Scale Ultrafiltration and } \\
\begin{array}{l}\text { Washing Test, 24590-PTF-TSP-RT-02-015 } \\
\text { (Townson, 2002). }\end{array}\end{array}$ & $\begin{array}{l}\text { The production of these recycle streams was done } \\
\text { during Campaign I (Duignan et al., 2004a). }\end{array}$ \\
\hline $\begin{array}{l}\text { 6. An exception to the test matrix defined in (the } \\
\text { Campaign I test) specification 24590-PTF-TSP-RT- } \\
\text { 02-015 will be to not perform certain tests specified } \\
\text { in Appendix A, Table 1.1 (of that specification). } \\
\begin{array}{l}\text { The tests that will not be performed are (listed in } \\
\text { the SIPP Test Specification, Longwell, 2003.) }\end{array}\end{array}$ & $\begin{array}{l}\text { This condition only applied to Campaign I } \\
\text { operation, which had pilot-scale UFP test runs at } \\
\text { steady-state UDS concentration. It was not } \\
\text { applicable to subsequent campaigns. }\end{array}$ \\
\hline
\end{tabular}

\subsection{SIMULANT USE}

Many simulated waste streams were either used or produced during Campaign III testing. Those include Hanford tank farm stored wastes, i.e., tank 241-AY-102/C-106, herein referred to as AY102, and tank 241-AP-101, herein referred to as AP101. During the process of making glass many recycle streams will be generated and processed. Those include streams from the: HLW and LAW Submerged Bed Scrubbers, Vessel Vent System, HLW Glass Canister Decontamination process, FEP and TLP condensate solutions, UFP wash permeate, UFP rinse solutions, UFP acid cleaning solutions, UFP loop flush solutions, CIX caustic LAW displacement solutions, CIX post elution rinse solutions, and CIX caustic regeneration solutions. These streams are discussed throughout this report.

\subsection{DISCREPANCIES AND FOLLOW-ON TESTS}

No discrepancies occurred during testing, but there will be follow-on work. This test, referred to as Campaign III, is the third of four Campaigns, which make up an overall task referred to as Semi Integrated Pilot Plant (SIPP) (Duignan, 2003c). 
WSRC-TR-2004-00565, REVISION 0 SRNL-RPP-2004-00092, REVISION 0

This page intentionally left blank. 


\subsection{DISCUSSION}

\subsection{INTRODUCTION}

Bechtel National, Inc. (BNI) has been contracted by the Department of Energy (DOE) to design a Waste Treatment and Immobilization Plant (WTP) to stabilize liquid radioactive wastes that are stored at the Hanford Site as part of the River Protection Project (RPP). Because of its experience with radioactive waste stabilization, the Savannah River National Laboratory (SRNL) of the Westinghouse Savannah River Company (WSRC) is working with BNI and Washington Group International (WGI), to help design and test certain parts of the waste treatment facility. A major part of the waste stabilization process is the pretreatment of the wastes before vitrification, which will occur in the Pretreatment Facility where both HLW and LAW product streams will be handled. SRNL has constructed a Semi Integrated Pilot Plant (SIPP), which imitates important unit operations of the pretreatment process. However, since the actual pilot-scale vitrification is not handled by SRNL, the pilot plant is referred to as semi integrated. After SIPP pretreats the waste streams into vitrification feed streams, they will be shipped to the Vitreous State Laboratory (VSL) of Catholic University of America to complete the entire process.

The subject of this report is the testing of one phase, called Campaign III, of all of the four principal unit operations that comprise the RPP-WTP Pretreatment Facility, i.e., Waste Feed Evaporation Process (FEP), Ultrafiltration Process (UFP), Cesium Ion Exchange Process (CIX), and Treated LAW Evaporation Process (TLP). The operation and results of each of these unit operations are explained herein.

This technical baseline research and development work was initiated by a WGI test specification (Longwell, 2003) to test simulated radioactive wastes in pilot scale pretreatment unit operations in the Engineering Development Laboratory (EDL) of SRNL. This report deals with the third phase of a four-campaign task, and it is the second campaign to use all four unit operations. The first phase, Campaign I, was at one time a completely separate task (Townson, 2002) to filter a specific waste. Subsequently, that first campaign was incorporated into the SIPP task to eliminate duplication of effort and to produce several important recycle streams. While, Campaign I was limited to a single unit operation, ultrafiltration, and a single waste stream, i.e., AY102, the process of operating the filter unit allowed the production of many of the important recycle streams needed to carry out the more comprehensive phases, i.e., Campaigns II, III, and IV. After the filter dewatered the single waste stream, the slurry was washed to produce one recycle stream. Then the filter unit was flushed, cleaned, and rinsed, thus creating other important recycle streams. Besides producing recycles, the Campaign I single-waste filter results were then used to compare with filter results obtained after mixing that waste stream with others, i.e., AP101 and the many recycle streams. A further benefit to limiting Campaign I to a single waste stream was that the filter results could be compared to the pilot filter tests done with other single waste streams, (see Duignan, 2000a, Duignan, 2000b, Duignan, 2003a). 
This report will discuss the four pilot unit operations during Campaign III. There were many individuals involved and the information for each section was provided primarily by each of the principal investigators. They are:

Simulant development and sample analyses - John R. Zamecnik

Simulant and sample handling - Michael R. Williams

FEP operation - Zafar H. Qureshi

UFP operation - Mark D. Fowley

CIX operation - John L. Steimke

TLP operation - Duane J. Adamson

A summary of EDL task activities is as follows:

WGI Test Specification for UF Test Received - December 2002

WGI Test Specification for SIPP Received - June 2003

WSRC Task Technical \& Quality Assurance Plan Approved - August 2003

Test Procedure Approved - October 2003

Received AY102/C106 Simulant - January 2004

Campaign I - 26 January to 12 February 2004

Campaign II - 12 April to 14 May 2004

Campaign III - 7 June to 9 July 2004

Campaign IV - 2 to 6 August 2004 (Pretreatment of GKN filter)

Campaign IV - 9 to 26 August 2004 (only SIPP FEP and UFP operations were tested)

\subsection{THE PLAN}

Initial planning for the Semi Integrated Pilot Plant (SIPP) was to decide what to study. One of the first decisions was to limit the task to the RPP-WTP Pretreatment Facility since pilot melter facilities already existed at another site ${ }^{\dagger}$. This is the reason that this task is referred to as "Semi Integrated," i.e., to indicate that vitrification is not part of the pilot operations at the Savannah River National Laboratory (SRNL). The next step was to decide which simulant(s) to test. Since the waste types at Hanford were broadly classified as envelopes A, B, C, and D (Eibling and Nash, 2001), therefore three campaigns were planned, one for each envelope, except D. Envelope D is basically a sludge, which would not be processed alone, but would be mixed with some of the other envelopes. As such, each campaign would process a chosen simulated waste through the principal unit processes of the Pretreatment Facility. Those units are the Feed (Waste) Evaporator Process (FEP), Ultrafiltration Process (UFP), Cesium Exchange (Process) (CIX), the Treated LAW Evaporation Process (TLP), and for Envelope C-types wastes, the Strontium-Transuranics (Sr/TRU) Precipitation Process.

Having a task that would test a range of possible waste types was a good comprehensive approach; however, to obtain meaningful operational data for the plant it was not just a

\footnotetext{
${ }^{\dagger}$ The Vitreous State Laboratory at Catholic University of America in Washington, DC.
} 
matter of processing each of the different wastes once through each of the unit process. Steady-state operation of the Pretreatment Facility would also have to include all the recycle waste streams. That is, in the process of separating the wastes into necessary components for further processing, other waste streams are produced. A few of those streams are: waste washings, filter cleaning and rinsing solutions, CIX regeneration solutions, HLW canister decontamination solutions, vessel vent solutions, submerged bed scrubber solutions from both the HLW and LAW vitrification facilities, etc. In steady state these recycle streams will be recycled to different locations in the Pretreatment Facility. Those streams need to be included to simulate the actual plant operation. This means that each of the campaigns would have to run many times until the effects of the recycle streams stabilized so that steady-state operation is realized. After reevaluating the task needs, available funds and time, it was clear that the scope had to be reduced. It would be limited to processing a key waste stream with all the recycle streams and then processed enough times that steady-state operation would be attained. Focus was then directed to the wastes that the plant would use for commissioning, i.e., 241-AY-102/C106, herein referred to as AY102, and 241-AP-101, herein referred to as AP101. The first waste is the result of two waste tanks that have already been mixed together, AY-102 and C-106 and is classified as an Envelope A/D mixture. The second waste, AP101, is an Envelope A waste. With this waste, all of the principal WTP Pretreatment Processes would be tested except the Sr/TRU Process because it did not include an Envelope C-type waste.

The SIPP task was dedicated to perform integrated processing of one selected waste stream combination that included recycle streams produced during the processing of waste through the four pilot-scale unit operations. As such, the testing of an "integrated" process is integrated in the sense of capturing the salient chemical processes, that is, it is important to note that the SIPP is not integrated with respect to time. The operation of the Pretreatment Facility is complex in both chemistry and time, but the many interconnected systems and supporting processes that will exist in the plant to allow the four main unit operation to function were not included in SIPP because of schedule and expense. Just the scaling issues alone, for an entire Pretreatment facility, would have been an almost impossible task to accomplish. In short, running the four principal unit operations with simulated waste and recycle streams as chemically close as to what will result in the plant was accepted as the most efficient use of the time and money available. Time of operation was limited to the operation of each individual unit operation only, and not to their temporal interdependence on the supporting Pretreatment Facility operations, e.g. vessel fillings, waste testing and adjusting, waste transfers, line flushings, etc.

However, prototypical hold times (see Appendix A) between the four unit operations were included to provide sufficient time for precipitation of slow forming salts, e.g., Sodium Aluminosilicate and $\mathrm{NaF}_{7}\left(\mathrm{PO}_{4}\right)_{2}$.

To reiterate, the SIPP task is the operation of four individual and separate pilot-scale unit operations, each successively processing a single waste stream combination, which is chemically connected through the feedback of recycle streams. The plan is described in the RPP-WTP test specification (Longwell, 2003) and in the WSRC technical task plan (Duignan, 2003c). The task's original series of three campaigns was maintained, but 
instead of processing different waste types, only one type was used. Each successive campaign introduced recycle streams produced from the preceding stream. To feed the first campaign an extra, initial, campaign was added. This addition was actually an already planned ultrafiltration test (Townson, 2002). That test became known as Campaign I and it only processed a simulant of AY102. In this way the filtration results of Campaign I served three purposes: 1 . To generate a filtration data set for a singlewaste type that could be compared to past single-waste filtration tests, 2 . to become a baseline for filtration of AY102 in order to determine how additions of AP101 and recycles to that waste affect filtration, and 3. to produce a series of recycle streams to feed to Campaign II. The last reason was very important to SIPP because the UFP produces several important recycle streams, i.e., slurry washing permeate, sludge loop flushing, loop acid cleaning, and loop caustic rinsings. Campaign I was performed in January and February of 2004 and the results were published in Duignan et al., 2004a and Campaign II was performed in April and May of 2004 and the results were published in Duignan et al., 2004b. A summary of all the campaigns is as follows:

Campaign I UFP only with simulant of AY102.

Campaign II All four unit operations with simulant of AY102 + AP101 + recycle streams (normally the CIX also has recycle streams but since Campaign I did not test the CIX they were not included in Campaign II).

Campaign $\mathrm{III}^{\dagger}$ All four unit operations with simulant of AY102 + AP101 $+2^{\text {nd }}$ generation recycle streams (but with $1^{\text {st }}$ generation CIX recycle streams).

Campaign IV IV $^{\ddagger}$ wo unit operations (FEP and UFP) with simulant of AY102 + AP101 + $3^{\text {rd }}$ generation recycle streams (but with $2^{\text {nd }}$ generation CIX recycle streams).

As shown in the preceding Section 1.1, in “Test Objectives," there were many objectives; the details of which will not be repeated here, but will be discussed in the appropriate later sections. However, the major goals were to better understand how each of the four pilot-scale unit operations processes waste ${ }^{*}$, to study how the processes change with successively complex recycle streams, and to produce throughput data to benchmark computer codes being developed by Bechtel and others, and to predict and plan for, plant operation.

Figure 1 is a schematic of the overall SIPP operation. It is broken into the four unit operations and indicates both the salient chemical and time steps. The operations are listed in a chronological order, at least with respect to the SIPP test, with the top (FEP) section being first. Of course the plant will be much more complex. A very short summary of the overall operation is as follows:

\footnotetext{
${ }^{\dagger}$ This report deals specifically with Campaign III results.

‡ As of May 2004 RPP-WTP informed SRNL to change the scope of Campaign IV to test a new filter made by GKN, which was chosen instead of the Mott for the RPP-WTP Pretreatment Facility Ultrafiltration

Process. The number of the unit operations was reduced from four to two for Campaign IV, those being the pilot-scale FEP and UFP, so that GKN performance could be compared to the Mott.

${ }^{*}$ For example, the recycle streams for this campaign, Campaign III, contained chemicals carried over from Campaigns I and II.
} 
WSRC-TR-2004-00565, REVISION 0

SRNL-RPP-2004-00092, REVISION 0

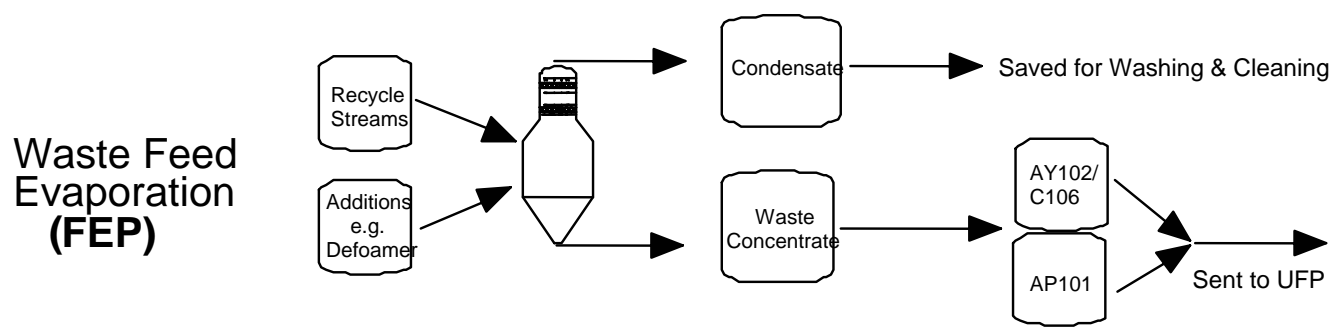

Recycle Streams: HLW SBS, UFP Wash Water, UFP Cleaning Acid, UFP Rinses \& Flushes, HLW Canister Decon, Vessel Vent, and CIX recycles
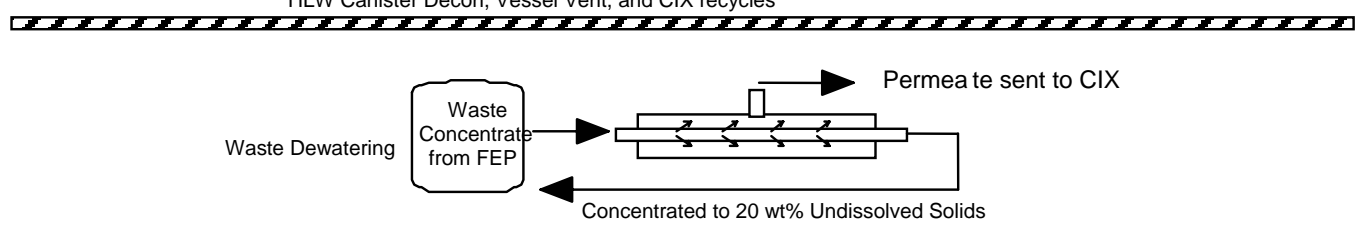

Cross-flow Ultrafiltration waste Washing (UFP)
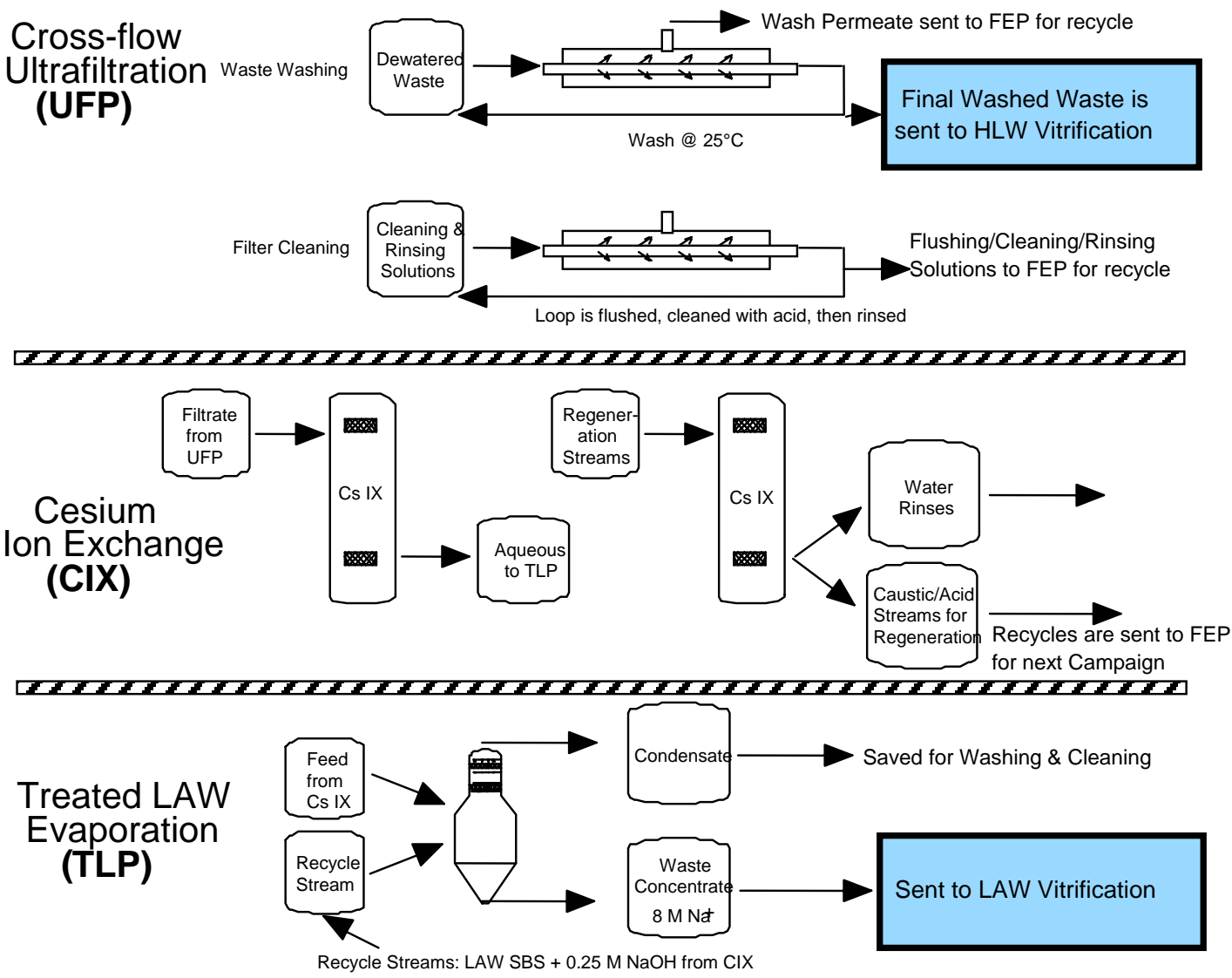

Figure 1. Schematic of the overall task operation taken from the task plan 
1. FEP evaporates a combined mixture of recycles.

2. UFP filters a combined mixture of the FEP concentrated product and waste from the tank farm until the mixture reaches a target concentration. The UFP produces two streams: the permeate which is sent to the CIX for LAW processing, and the concentrated slurry. That slurry is then washed to remove many of the soluble solids after which it is sent to the HLW melter facility. The slurry washing and subsequent UFP filter cleaning produces a series of recycle streams that are sent back to the FEP for processing.

3. CIX takes the permeate from the UFP and removes most of the soluble, but radioactive, cesium. When the resin in the lead IX column is regenerated, recycle streams are created which are either sent back to the FEP or forward to the TLP for processing.

4. TLP takes the CIX product, which still has very low levels of radiation, and evaporates most of the water before it is sent to the LAW melter facility.

The evaporation of the many combined recycle streams in the FEP will be operated in a continuous sense, in that after it fills the UFP preparation tank for one filter train (there will be two UFPs), it will feed the preparation tank for the other filter train. However, the FEP must ultimately be a batch operation because it will be receiving waste from recycle tanks (PWD = Plant Wash and Disposal System) that will be batched through the FEP only after the feed is adjusted for $\mathrm{pH}$ and tested for acceptance.

When the UFP feed and preparation tanks are full and ready for processing, the filtration can commence. This process is also batch, but the feed tank must be continuously fed with new feed as dewatering is ongoing. The feed tank will have a base volume of 21,550 gallons into which approximately 1000 gallons will be transferred from the preparation tank. Each time the feed tank level drops to 21,550 gallons another batch of 1000 gallons will be transferred until the dewatered slurry reaches its target solids concentration. For this task, the target was $20 \mathrm{wt} \%$ undissolved solids. This dewatering process is shown in the UFP section of Fig. 1 as the top filtration loop. The middle loop illustrates the washing and leaching of the final concentrated slurry batch. (Note: For SIPP, the leaching process was not performed as requested by RPP-WTP (Calloway, 2004)). After the concentrated waste is washed, it is then sent to the HLW melter for further processing, but for the SIPP, the washed slurry is simply drummed to be shipped for processing in a pilot HLW melter. With the loop de-inventoried, the final loop shows that the UFP undergoes caustic rinsing and acid cleaning, in preparation for the next batch.

For the plant, the CIX does not necessarily have to wait for the entire filtration process to end. There are several filter permeate tanks to feed the CIX and they receive permeate from each of the two filter trains. However, for SIPP the CIX can begin after a fixed amount of permeate is produced from the UFP, which is approximately 200 bed volumes. A bed volume is the volume of space occupied in an IX column by the resin, including the interstitial spaces among the resin particles. The plant will process a minimum of 100 bed volumes ( 30,000 gallons) of permeate before an IX column needs regeneration. 
Originally, the SIPP IX was to demonstrate the design throughput rate of treated waste, like the other pilot unit operations, but it was thought more important ${ }^{\dagger}$ to use it for chemical testing to show cesium removal efficiency. This meant the important parameter would be a prototypic residence time for permeate in the resin, or 3 bed volumes per hour. For the 6-inch diameter pilot-scale column, this resulted in a very low flow rate of $\sim 0.031 \mathrm{gpm}$, which is more than two orders of magnitude slower than the plant design flow rate of 15 gpm and approximately one order of magnitude slower than scaled flow rate of $\sim 0.2$ gpm. SIPP used 200 bed volumes because several past tests had indicated that the resin may last longer than the original plant design of 100 bed volumes. It was very important to show how long the resin truly lasts before needing to regenerate it. The third section of Fig. 1 shows two columns because of the two processes that occur within CIX: the first process is the removal of cesium, the second process is a series of steps to regenerate the resin.

The final section of Fig. 1 shows the TLP operation. The CIX product is mostly water and the TLP removes it while concentrating the sodium up to $8 \mathrm{M}$. This is the last step before the treated LAW stream is sent to the LAW melter facility.

Along the way, several recycle streams are created in the UFP and the CIX, as well as from other sources, like the submerged bed scrubber quencher streams from both the HLW and LAW melter facilities. These recycle streams are then fed back in the process, which then repeat. For the SIPP, this repeating results in the subsequent campaigns.

\subsection{THE COMPUTATIONAL MODEL}

A full explanation of the computation assistance obtained at the beginning of SIPP has already been described in detail elsewhere (Duignan et al., 2004b and Barnes, 2004) and will not be repeated here. However, it is important to know how the computational modeling was used for this task to understand its role for Campaign III. The model served two purposes: 1 . To determine how much slurry to use and, 2 . To estimate the mix ratio of feeds (simulated wastes and recycle streams) for each of the unit operations. The Test Specification (Longwell, 2003) requested that the WTP Aspen Custom Modeler (ACM) Model be used to obtain the bases for the combined feed streams, i.e., the feed mix ratios. With the feed stream obtained in this manner an OLI Environmental Simulation Program (OLI/ESP) was used to determine the steady-state operation for each unit operation in each of the campaigns.

In concept, the operation of the SIPP is straightforward, but determining the exact simulant needs was quite complicated because of the differing scales of each pilot-scale unit operation and because of the chemical interaction among the recycle streams from all the campaigns. A very important part in developing the test matrix of the task plan was knowing how much simulant to use and how should all the different waste and recycle streams be mixed together. That need was satisfied by computationally predicting SIPP streams for all four campaigns. As an added benefit, once the model was made, it would

\footnotetext{
† Test Exception No. 24590-WTP-TEF-RT-03-069, Rev. 0, 14 Nov. 2003
} 


\section{WSRC-TR-2004-00565, REVISION 0 SRNL-RPP-2004-00092, REVISION 0}

be possible to evaluate changes in simulant volumes and mixing ratios to determine if the entire SIPP process comes to steady state. Steady state could be shown, at least chemically, by demonstrating that the products produced in SIPP change insignificantly from one campaign to the next.

Table 1 shows the results of the OLI/ESP model that includes WTP accepted mixture ratios for combined feed streams from the ACM model. The principal value in the entire figure is the initial "batch" size of the UFP feed tank. In Table 1 this batch size for the SIPP is shown in red as 140 liters and is listed as "UF1 SLURRY (batch at $20 \mathrm{wt} \%$ UDS).” This waste volume is very significant because for this pilot-scale task it represents the batch size of HLW sent for HLW vitrification. When this batch is dewatered to some predetermined concentration of undissolved solids (it was $20 \mathrm{wt} \%$ for SIPP), then the permeate produced during dewatering will be the batch size of LAW sent for LAW vitrification.

Table 1. Feeds and products for planning SIPP developed for OLI/ESP computer model

\begin{tabular}{|c|c|c|c|c|c|c|}
\hline \multirow{2}{*}{$\begin{array}{c}\text { WTP } \\
\text { Process }\end{array}$} & \multirow[b]{2}{*}{ Stream Type } & \multirow[b]{2}{*}{ Stream Name } & \multicolumn{4}{|c|}{ Campaign (in Liters) } \\
\hline & & & $\mathrm{I}$ & II & III & IV \\
\hline \multirow{5}{*}{ FEP } & Feed & UFP \& CIX RECYCLEs (previous campaign) & N/A & 583.1 & 602.8 & 602.8 \\
\hline & Recycle & HLW (Submerged Bed Scrubber) & N/A & 190.6 & 190.6 & 189.9 \\
\hline & Streams & PVP (Vessel Vent System) & N/A & 220.7 & 220.7 & 220.0 \\
\hline & & HLW DECON & N/A & 114.6 & 114.7 & 114.3 \\
\hline & $\begin{array}{l}\text { Products } \\
\text { Produced }\end{array}$ & $\begin{array}{l}\text { FEP CONDENSATE (to be process wash) } \\
\text { FEP COOLED BOTTOMS (UFP feed) }\end{array}$ & $\begin{array}{l}\text { N/A } \\
\text { N/A }\end{array}$ & $\begin{array}{l}996.0 \\
114.4\end{array}$ & $\begin{array}{l}980.6 \\
150.8\end{array}$ & $\begin{array}{l}984.9 \\
144.9\end{array}$ \\
\hline & & & & & & \\
\hline \multirow{2}{*}{$\begin{array}{l}\text { UFP } \\
\text { Feeds }\end{array}$} & Feed & AY102 WASTE (at 12 wt\% UDS) & 253.5 & 252.4 & 242.2 & 241.4 \\
\hline & Feed & AP101 WASTE $\left(\right.$ at $\left.\sim 8 \mathrm{M} \mathrm{Na}^{+}\right)$ & N/A & 671.3 & 674.4 & 672.1 \\
\hline \multirow{10}{*}{ UFP } & Products & UF1 FILTRATE (routed to CIX, then TLP) & 113.5 & 922.3 & 952.2 & 943.7 \\
\hline & Produced & UF1 SLURRY (batch at 20 wt\% UDS) & 140 & 116.8 & 116.4 & 116.1 \\
\hline & $\begin{array}{l}\text { Batch } \\
\text { Waste }\end{array}$ & $\begin{array}{l}\text { FEP COND WASH (0.01 M NaOH) } \\
19 \mathrm{M} \mathrm{NaOH} \text { (to adjust FEP COND) }\end{array}$ & & $\begin{array}{c}281.1 \\
1.8\end{array}$ & $\begin{array}{c}281.5 \\
0.2\end{array}$ & $\begin{array}{c}280.7 \\
0.2\end{array}$ \\
\hline & Washing & TOTAL CAUSTIC WASH & 338.6 & 282.5 & 281.6 & 280.8 \\
\hline & \multirow{4}{*}{$\begin{array}{l}\text { Filter } \\
\text { Rinsing } \\
\quad \& \\
\text { Cleaning }\end{array}$} & $\begin{array}{l}\text { FEP COND ACID (2 M HNO3) } \\
12 \mathrm{M} \text { HNO3 (to adjust FEP COND) }\end{array}$ & & $\begin{array}{l}86.1 \\
17.0\end{array}$ & $\begin{array}{l}85.8 \\
17.0\end{array}$ & $\begin{array}{l}85.6 \\
16.9\end{array}$ \\
\hline & & TOTAL ACID CLEANING & 122.5 & 102.2 & 101.9 & 101.6 \\
\hline & & $\begin{array}{l}\text { FEP CONDENSATE TO CAUSTIC RINSE } \\
19 \mathrm{M} \mathrm{NaOH} \text { (to adjust FEP COND) } \\
\text { TOTAL CAUSTIC RINSE (0.1 M NaOH) }\end{array}$ & & $\begin{array}{c}101.8 \\
0.5 \\
102.2 \\
\end{array}$ & $\begin{array}{c}101.5 \\
0.5 \\
101.8 \\
\end{array}$ & $\begin{array}{c}101.2 \\
0.5 \\
101.5 \\
\end{array}$ \\
\hline & & TOTAL CAUSTIC RINSE (for FEP recycle)) & 117.1 & 97.6 & 97.3 & 97.0 \\
\hline & Final Filter & HLW SLURRY (to HLW melter) & 138.1 & 110.8 & 110.3 & 110.0 \\
\hline & Products & TOTAL FEP RECYCLE (for next campaign) & 583.1 & 602.8 & 604.8 & 602.3 \\
\hline \multirow{4}{*}{ CIX } & Column & CIX 0.1 M NaOH RINSE (for FEP recycle) & N/A & 36.9 & 38.1 & 37.8 \\
\hline & Regeneration & CIX DI WATER (for FEP recycle) & & 36.9 & 38.1 & 37.8 \\
\hline & Products & $\mathrm{CIX} 0.25 \mathrm{M} \mathrm{NaOH}$ (for FEP recycle) & $\mathrm{N} / \mathrm{A}$ & 40.0 & 41.3 & 40.9 \\
\hline & Produced & $\mathrm{CIX} 0.25 \mathrm{M} \mathrm{NaOH}$ (for TLP recycle) & $\mathrm{N} / \mathrm{A}$ & 36.9 & 38.1 & 37.8 \\
\hline \multirow[b]{2}{*}{ TLP } & Feeds wFiltrate & LAW SBS (Submerged Bed Scrubber) & $\mathrm{N} / \mathrm{A}$ & 833.0 & 832.9 & 830.2 \\
\hline & $\begin{array}{l}\text { Products } \\
\text { Produced }\end{array}$ & $\begin{array}{l}\text { TLP CONDENSATE (to be process wash) } \\
\text { TLP COOLED BOTTOMS (to LAW melter) }\end{array}$ & $\begin{array}{l}\mathrm{N} / \mathrm{A} \\
\mathrm{N} / \mathrm{A}\end{array}$ & $\begin{array}{l}1214.1 \\
589.3\end{array}$ & $\begin{array}{c}1231.4 \\
603.4\end{array}$ & $\begin{array}{c}1224.9 \\
598.1\end{array}$ \\
\hline
\end{tabular}


This SIPP batch of 140 liters $^{\dagger}$ for Campaign I was arbitrarily chosen, but once chosen all other volumes were the result of SIPP processing. The choice was made by using existing test facilities, where the pilot UFP could contain 140 liters and still have enough room to feed in more slurry until dewatering is complete. Knowing this batch size, using the feed stream ratios from the ACM model, and the chemical makeup of the base waste slurry, i.e., AY102/C106 and AP101, the OLI/ESP model could then be iterated among all of the campaigns to result in the numbers shown in Table 1. Note that the batch size of 140 liters, with $20 \mathrm{wt} \%$ undissolved solids, changes from Campaign I to a value of approximately 116 liters (with $20 \mathrm{wt} \%$ undissolved solids) for all the other campaigns, and as such, the 116-liter volume was taken as equivalent to the full scale UFP batch ${ }^{\ddagger}$ size in those campaigns. The difference between the batch size for Campaign I (140 L) and the subsequent campaigns $(116 \mathrm{~L})$ was not well understood when the task was planned but because the sophisticated OLI/ESP software includes all the steady-state chemistry of the processed wastes, which included dozens of compounds, it was assumed that differences in the starting waste slurries resulted in this "discrepancy." That is, Campaign I only processed a single waste, AY102, while the other campaigns included also AP101 and the concentrated recycle streams from the FEP. The inputs for the known waste chemistries, of the starting feeds, were checked many times to verify accuracy, therefore the difference was assumed to be real. In any case, the purpose of the model was to be used as a tool to plan the task and procure needed quantities of simulant, therefore further investigations on the ( $15 \%)$ difference ${ }^{*}$ were not thought necessary. The simulated waste products were procured with enough safety margin to cover any problems if, for instance, shortages occurred during experimentation.

As shown in Figure 1, once the UFP is finished concentrating, the waste is washed with approximately $0.01 \mathrm{M} \mathrm{NaOH}$. The plant will hold the slurry volume constant and feed in two volumes of the caustic solution, each volume approximately equal to the volume of slurry, before sending the waste to the HLW Vitrification Facility. While the plant will hold the UFP volume constant, the model resulted in slightly smaller volumes, see the line in Table 1 entitled, "HLW Slurry (to HLW melter).” due to computational requirements. These are the simulated processed feeds for HLW vitrification. The other highlighted lines in Table 1 are: "AY102 WASTE (at 12 wt\% UDS)," which was the amount of feed AY102 simulant needed; "AP101 WASTE (at $8 \mathrm{M} \mathrm{Na),"} \mathrm{which} \mathrm{was} \mathrm{the}$ amount of feed AP101 simulant needed; "UFP \& CIX RECYCLEs (previous campaign)," which was the portion of recycle streams carried over from the previous

\footnotetext{
${ }^{\dagger}$ For WTP this batch size was 18,000 gallons when this task began, but it currently stands at 21,550 gallons. In fact, for Campaign I operation, the 18,000-gallons size was valid and used for scaling purposes, but with Campaign II and all subsequent campaigns the latter volume was used.

₹ Same as the above footnote

* In fact, the actual discrepancy was not understood until after Campaign III was complete and analysis of the SIPP data was underway. The problem turned out to be something that could not be easily resolved a priori because of the OLI/ESP. For the software package to converge on a solution the anion and cation charges of ALL simulant analytes must balance. Unfortunately, the simulated wastes streams contained many unknown complex undissolved solids compounds for which ion charge balances had to be assumed. Those assumptions caused the resulting volumetric difference once a slurry was concentrated to $20 \mathrm{wt} \%$ undissolved solids. This is a clear example of the current limitations of computational models and the need for benchmarking to experimental results of complex interactions.
} 
campaign to be mixed with other recycle streams that did not come directly from one of the four unit operations; finally, the last line contains the volumes of LAW that are treated through all four unit operations and are ready as feed for LAW vitrification.

Another feature included in Table 1, but not obvious, is the feed proportions used for each unit operation, which were supplied by the ACM model. For each of the campaigns, the proportions are slightly different, but not significantly so. The feed for the FEP was made up from only recycle streams and the volumetric proportion of each of the streams used is shown in Table 2.

While the proportions in Table 2 are close to the recycle stream values in the planning table shown as Table 1, they will not be exactly the same because of last minute changes, round off numbers, and uncertainties in some of the streams.

Table 2. Percent contribution for each of the recycle streams to the total FEP feed

\begin{tabular}{|c|c|c|c|c|}
\hline \multirow{3}{*}{$\begin{array}{l}\text { List of Recycle Streams Used for } \\
\text { SIPP and Verified by WTP }\end{array}$} & \multicolumn{4}{|c|}{$\begin{array}{l}\text { Percentage of Recycle to FEP } \\
<------C a m p a i g n \text { Number------> }\end{array}$} \\
\hline & $I^{*}$ & $1 I^{\star \star}$ & III & IV \\
\hline & Vol. \% & Vol. \% & Vol. \% & Vol. \% \\
\hline (1) HLW SBS & $\mathrm{n} / \mathrm{a}$ & $17.1 \%$ & $17.5 \%$ & $17.4 \%$ \\
\hline (2) Vessel Vent & $\mathrm{n} / \mathrm{a}$ & $19.7 \%$ & $20.3 \%$ & $20.2 \%$ \\
\hline (3) HLW Can. Decon & $\mathrm{n} / \mathrm{a}$ & $10.3 \%$ & $10.5 \%$ & $10.5 \%$ \\
\hline (4) UFP Recycle (0.01 M NaOH) & $\mathrm{n} / \mathrm{a}$ & $30.3 \%$ & $21.7 \%$ & $21.6 \%$ \\
\hline (5) UFP Recycle (0.1 M NaOH) & $\mathrm{n} / \mathrm{a}$ & $10.5 \%$ & $9.0 \%$ & $8.9 \%$ \\
\hline (6) UFP Recycle (2 M HNO3) & $\mathrm{n} / \mathrm{a}$ & $11.0 \%$ & $9.4 \%$ & $9.4 \%$ \\
\hline (7) UFP Recycle (3 Loop Flushes) & $\mathrm{n} / \mathrm{a}$ & $1.2 \%$ & $1.2 \%$ & $1.2 \%$ \\
\hline (8) CIX Recycle (0.1 M NaOH) & $\mathrm{n} / \mathrm{a}$ & $0.0 \%$ & $3.4 \%$ & $3.5 \%$ \\
\hline (9) CIX Recycle (DI Water) & $\mathrm{n} / \mathrm{a}$ & $0.0 \%$ & $3.4 \%$ & $3.5 \%$ \\
\hline (10) CIX Recycle (0.25 M NaOH) & $\mathrm{n} / \mathrm{a}$ & $0.0 \%$ & $3.7 \%$ & $3.8 \%$ \\
\hline Total Feed to the FEP & $\mathrm{n} / \mathrm{a}$ & $100 \%$ & $100 \%$ & $100 \%$ \\
\hline \multicolumn{5}{|c|}{$\begin{array}{l}\text { Notes: } \\
\text { Source of Streams: (1) was made by an outside vendor: VSL*** } \\
\qquad \text { (2) and (3) were made for each campaign } \\
\text { (4) to (10) were products of unit operations } \\
\text { * Campaign I only used a single simulated waste with no } \\
\text { recycles in a single unit operation, i.e., UFP. } \\
\text { ** Since Campaign I was only a filter operation, therefore, no CIX } \\
\text { recycle streams were produced for Campaign II, as indicated } \\
\text { by the RPP-WTP Test Specification (Longwell, 2003). } \\
\text { *** VSL = Vitreous State Lab. of Catholic University of America }\end{array}$} \\
\hline
\end{tabular}

There were many routes to operating the UFP, but for SIPP, and initial plant operation, it was thought prudent to only use the FEP to process recycle streams and the UFP would 
be the starting point for tank farm wastes. One major benefit of this method was that it minimizes undissolved solids in the FEP and thus hopefully minimizes condenser fouling that would affect operational efficiency. This means that the feed for the UFP had three waste sources, one of which came from the FEP after the recycle streams were concentrated to a target density. The other two were mentioned above. Their individual contributions to the UFP feed are shown in Table 3.

Table 3. Percent contribution for each of the UFP feed streams

\begin{tabular}{|c|c|c|c|c|}
\hline \multirow{3}{*}{$\begin{array}{l}\text { Feed Streams Used for SIPP } \\
\text { in the UFP and Verified by WTP }\end{array}$} & \multicolumn{4}{|c|}{$\begin{array}{l}\text { Percentage of Streams to UFP } \\
<-----C a m p a i g n \text { Number------> }\end{array}$} \\
\hline & $I^{*}$ & II & III & IV \\
\hline & Vol. \% & Vol. \% & Vol. \% & Vol. \% \\
\hline (1) FEP Bottoms & $0 \%$ & $11.1 \%$ & $14.3 \%$ & $13.8 \%$ \\
\hline (2) AY-102/C-106 & $100 \%$ & $24.3 \%$ & $22.6 \%$ & $22.8 \%$ \\
\hline (3) AP-101 & $0 \%$ & $64.6 \%$ & $63.1 \%$ & $63.4 \%$ \\
\hline Total Feed to the UFP & $100 \%$ & $100 \%$ & $100 \%$ & $100 \%$ \\
\hline \multicolumn{5}{|c|}{$\begin{array}{l}\text { Notes: } \\
\text { Source of Streams: (1) was concentrated from recycles in the FEP } \\
\qquad \begin{array}{l}\text { (2) outside vendor, but finalized in EDL** } \\
\text { (3) came from an outside vendor }\end{array} \\
\text { * Campaign I only used a single simulated waste. } \\
\text { ** EDL = Engineering Development Laboratory of the Savannah } \\
\text { River National Laboratory of the Westinghouse Savannah } \\
\text { River Company where SIPP pilot-scale testing was performed. }\end{array}$} \\
\hline
\end{tabular}

As with the recycle percentages in Table 2, the percentages in Table 3 are slightly different than what is shown in Table 1. Those differences are mostly attributed to volumes actually created during experimentation. The computational volumes were not expected to be exact and while the values in Table 3, were (Campaigns I, II, and III) or are to be (Campaign IV) used for SIPP, the actual plant mixture may still be different as its design continues to evolve.

One product of the pilot-scale UFP is the permeate produced during dewatering. This waste stream is then sent on to the CIX in its preparation for LAW vitrification. As this task evolved, the pilot-scale CIX operation was disassociated from measuring volumetric throughput to measure glass production, because WTP thought it more important to understand the cesium removal efficiency of the chosen resin, SuperLig ${ }^{\circledR} 644$. This requirement made the amount of permeate important, since cesium removal was to be studied. It was necessary to have 400 "bed volumes" of feed for each campaign, excluding Campaign I. A bed volume is the space occupied in an ion exchange column by settled resin. For the pilot-scale CIX that volume was approximately 2.3 liters, making the produced number of bed volumes 401, 414, and 410 for Campaigns II, III, and IV, respectively. This can be seen in the Table 1 line entitled, "UF1 FILTRATE (routed to CIX, then TLP).” 
Finally, the proportions of different feeds for the pilot-scale TLP were needed. Those are shown in Table 4.

Table 4. Percent contribution for each of the TLP feed streams

\begin{tabular}{|c|c|c|c|c|}
\hline \multirow{3}{*}{$\begin{array}{l}\text { Feed Streams Used for SIPP } \\
\text { in the TLP and Verified by WTP }\end{array}$} & \multicolumn{4}{|c|}{$\begin{array}{l}\text { Waste Stream Vol. \% to TLP } \\
<------C a m p a i g n \text { Number------> }\end{array}$} \\
\hline & $\mathrm{I}^{\star}$ & $1 I^{* *}$ & III & IV \\
\hline & Vol. \% & Vol. \% & Vol. \% & Vol. \% \\
\hline (1) LAW SBS (from LAW melter) & $\mathrm{n} / \mathrm{a}$ & $47.4 \%$ & $45.7 \%$ & $45.8 \%$ \\
\hline (2) $0.25 \mathrm{M} \mathrm{NaOH}$ (recycle from CIX) & $\mathrm{n} / \mathrm{a}$ & $0.0 \%$ & $2.1 \%$ & $2.1 \%$ \\
\hline (3) Treated LAW from CIX & $\mathrm{n} / \mathrm{a}$ & $52.6 \%$ & $52.2 \%$ & $52.1 \%$ \\
\hline Total Feed to the TLP & $\mathrm{n} / \mathrm{a}$ & $100 \%$ & $100 \%$ & $100 \%$ \\
\hline \multicolumn{5}{|c|}{$\begin{array}{l}\text { Notes: } \\
\text { Source of Streams: (1) was made by an outside vendor: VSL*** } \\
\qquad \begin{array}{l}\text { (2) comes from a regenerated IX column } \\
\text { (3) comes from CIX after Cs is removed }\end{array} \\
\text { * Campaign I did employ the pilot-scale TLP } \\
\text { ** Since Campaign I was only a filter operation, therefore, no CIX } \\
\text { recycle streams were produced for Campaign II, as indicated } \\
\text { by the RPP-WTP Test Specification (Longwell, 2003). } \\
\text { *** VSL = Vitreous State Lab. of Catholic University of America }\end{array}$} \\
\hline
\end{tabular}

As with Tables 2 and 3, the percentages in Table 4 differ slightly than those from Table 1 because of limitations in the model, but all the values were sufficient to plan and begin testing.

\subsection{THE OVERALL SIPP TEST MATRIX}

To allow the reader to understand how Campaign III was done without having to refer to any of the other SIPP reports, a portion of the overall test plan explanation is repeated here from the Campaign II report. With the plan in place and the planning results from the computational model, an overall test matrix was established. The following matrix was not the last word on how each of the unit operation was to run, but it was the starting point for each of the unit operations to develop individual test plans, as well as the simulant mixing and sample plans. As the task began with Campaign I (Duignan, et al., 2004a), and then on to other campaigns, the matrix continued to evolve as WTP needs changed, therefore, this test matrix may be slightly different than the individual test plans. However, it is instructive here to illustrate the overall operation of SIPP. That is, a global explanation is first given to better understand SIPP in its "integrated" form, which is then followed by discussions on each of the individual unit operations and their results. 
That matrix is shown in pieces in the following series of figures:

[Note that , Table $\mathrm{X}>>$ Table $\mathrm{Y}$ means: table $\mathrm{x}$ indicates the wait time before using slurry in the table $\mathrm{y}$ unit operation.]

Table 5 (simulant aging) $\quad>>\quad$ Table 6 (pilot FEP unit operation)

Table 7 (simulant aging) $\quad>>\quad$ Table 8 (pilot UFP unit operation)

Table 9 (simulant aging) $\quad>\quad$ Table 10 (pilot CIX unit operation - first cycle)

$>\quad$ Table 11 (pilot CIX unit operation - second cycle)

Table 12 (simulant aging) $\quad>\quad$ Table 13 (pilot TLP unit operation)

These nine figures are in chronological order of performance.

First, Figs. 5, 7, 9, and 12 were included to show hold-up time intervals between unit operations, especially because they were not part of the computational model, which was done at steady state. In an attempt to mimic the periods that waste "waits" before being processed in each unit operation, some prototypic times were included in SIPP.

Table 5. Hold period before pilot-scale FEP begins ${ }^{\dagger}$

\begin{tabular}{|l|l|l|l|l|l|l|}
\hline Pre-FEP Hold Times (stipulated by WTP) & & & & \\
\hline & Minimum hold time between the time recycle stream mixture and beginning evaporation. & 2 & & & & \\
\hline
\end{tabular}

Waste aging may affect many processing steps, for example, the post-UFP precipitation was a key issue for the ion exchange system. Using the appropriate WTP plant hold times allowed this issue to be resolved. The many reasons for specific hold times are given in Appendix A. As an example, Table 5 allows time for the mixed multiple recycle streams to age. Recycle streams are fed from several different sources to the Plant Wash and Disposal System tanks that will feed the Waste feed Evaporation Process. The recycle streams come in at different rates and then need to be adjusted for $\mathrm{pH}$ as the acid and alkaline streams merge in order to mitigate the precipitation of solids or gels. After the adjustment, the FEP is fed, but because of the large scale, the transfer times are not insignificant. As explained in Appendix A, the time associated in the pre-evaporation process is approximately 2 days and therefore, once the SIPP FEP feed of recycle streams is adjusted properly it is held for that number of days before evaporation begins.

For each of the unit operations, a hold time is set after the appropriate feed is made ready. In summary those simulated minimum hold times are:

After joining recycle feeds and before processing in the FEP - 2 days

After making FEP bottoms and before joining it with other UFP feeds -2 days

After joining waste feeds and before processing in the UFP - 2 days

After producing UFP permeate and before processing in the CIX - 4.5 days

After joining treated LAW with recycle streams and before processing in the TLP - 2 days

\footnotetext{
${ }^{\dagger}$ Blanks in all tables in this report are intentional.
} 
WSRC-TR-2004-00565, REVISION 0

SRNL-RPP-2004-00092, REVISION 0

Table 6. Pilot-scale FEP test matrix with the colored columns representing Campaign III

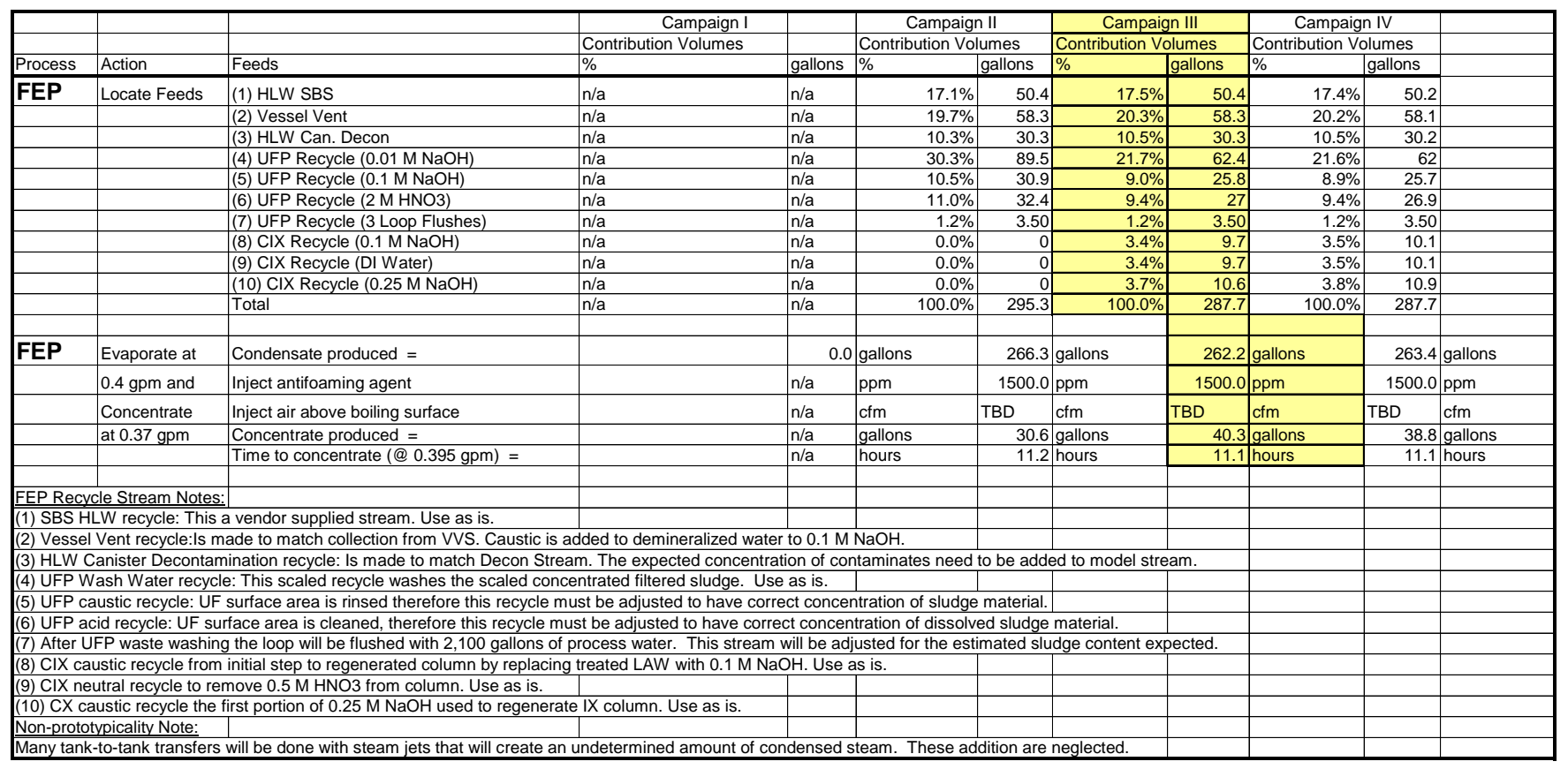

Once the recycle streams were mixed in the planned proportions, Table 2, and the appropriate hold time was realized, then the FEP test matrix, shown in Table 6, was performed. The goal was to concentrate the feed stream so that it had a sodium molarity of 5, but the "visible" target was an evaporated slurry density of $1.266 \mathrm{~g} / \mathrm{cc}$ at $50^{\circ} \mathrm{C}$ and 1 psia (which is $1.252 \mathrm{~g} / \mathrm{cc}$ at $20^{\circ} \mathrm{C}$ and $1 \mathrm{~atm}$ ). Density was used because the WTP will only be able to control concentration by measuring density.

Table 7. Hold period before pilot-scale UFP begins

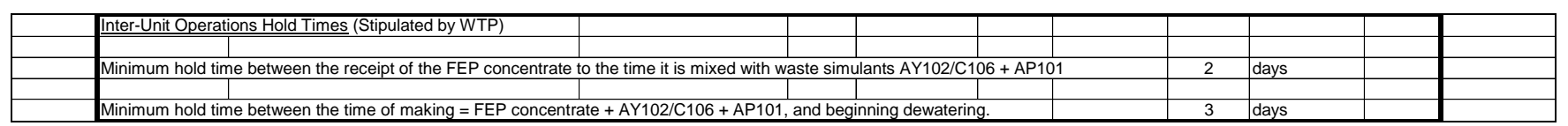

When feed evaporation was completed, its slurry product was held for 2 days, Table 7 , before being mixed in the planned proportions, Table 3, with the simulated wastes of AY102 and AP101. Completing this mixture started another 3-day clock before starting the UFP.

While processing the waste through the UFP is complicated with several activities involving the washing of the concentrated slurry to the cleaning of the filter loop (see Table 8), the basic operation is to separate the waste feed stream into a HLW product, containing all of the undissolved solids, and its supernatant, containing most of the cesium. This was done by dewatering the feed from a concentration less than $5 \mathrm{wt} \%$ to $20 \mathrm{wt} \%$ undissolved solids. The details of the UFP operation will be discussed in the appropriate subsection of this report. One feature that is not immediately obvious is the process containing an internal hold time. Dewatering in WTP will be a relatively lengthy 


\section{WSRC-TR-2004-00565, REVISION 0 SRNL-RPP-2004-00092, REVISION 0}

process because filtration is very slow and the volumes are very large. While the waste is waiting to be processed through the UFP, it will be sitting in the feed preparation tank, which feeds the feed tank. During this process period, chemical reactions may still be occurring within the waste and crystallization or precipitation may result. In an attempt to capture these transient phenomena, a waiting period was inserted in the dewatering process of the pilot UFP. The period is a minimum 12-hour hold time, which is arbitrary, but is loosely connected to the planned plant operational time of the UFP, and was acceptable to the WTP project.

Table 8. Pilot-scale UFP test matrix with the colored columns representing Campaign III

\begin{tabular}{|c|c|c|c|c|c|c|c|c|c|c|c|}
\hline \multirow{3}{*}{\begin{tabular}{|l|} 
\\
Process \\
\end{tabular}} & \multirow[b]{3}{*}{ Action } & \multirow[b]{3}{*}{ Feeds } & \multirow{3}{*}{\begin{tabular}{|l|}
\multicolumn{1}{|c|}{ Campaign I } \\
Contribution Volumes \\
$\%$ \\
\end{tabular}} & \multirow[b]{3}{*}{ gallons } & \multirow{2}{*}{\multicolumn{2}{|c|}{\begin{tabular}{|c|} 
Campaign II \\
Contribution Volumes
\end{tabular}}} & \multirow{2}{*}{\multicolumn{2}{|c|}{\begin{tabular}{|c|} 
Campaign III \\
Contribution Volumes
\end{tabular}}} & \multirow{2}{*}{\multicolumn{2}{|c|}{\begin{tabular}{|c|} 
Campaign IV \\
Contribution Volumes
\end{tabular}}} & \\
\hline & & & & & & & & & & & \\
\hline & & & & & $\%$ & gallons & $\%$ & gallons & $\%$ & gallons & \\
\hline \multirow[t]{43}{*}{ UFP } & Locate Feeds & FEP Bottoms & $0.0 \%$ & 0 & $11.1 \%$ & 30.6 & $14.3 \%$ & 40.3 & $13.8 \%$ & 38.8 & \\
\hline & & AY102/C106 & $100.0 \%$ & 67 & $24.3 \%$ & 66.7 & $22.7 \%$ & 64 & $22.8 \%$ & 63.8 & \\
\hline & & AP101 & $0.0 \%$ & 0 & $64.6 \%$ & 177.4 & $63.1 \%$ & \begin{tabular}{|r|}
178.2 \\
\end{tabular} & $63.4 \%$ & 177.6 & \\
\hline & & Total & $100.0 \%$ & 67 & $100.0 \%$ & 274.662 & $100.0 \%$ & 282.4901 & $100.0 \%$ & 280.172 & \\
\hline & & & & & & & & & & & \\
\hline & & & & & & & & & & & \\
\hline & & Inhibited water $(0.1 \mathrm{M} \mathrm{NaOH})=$ & & 25 & gallons & 25 & gallons & 25 & gallons & 25 & gallons \\
\hline & Filter at & Time for baselining $=$ & & 2.0 & hour & 2.0 & hour & 2.0 & hour & 2.0 & hour \\
\hline & $0.02 \mathrm{gpm} / \mathrm{ft} 2$ & Starting simulant volume $=$ & Camp I used $\sim 75$ gal & 67 & gallons & 274.662 & gallons & 282.4901 & gallons & 280.172 & gallons \\
\hline & or $0.134 \mathrm{gpm}$ & Time to cool to $25^{\circ} \mathrm{C}$ before use $=$ & to obtain $25 \mathrm{wt} \%$ & 0 & hours & 0 & hours & 0 & hours & 0 & hours \\
\hline & for a $6.71 \mathrm{ft} 2$ & Concentrate produced at $20 \mathrm{wt} \%=$ & & 37.0 & gallons & 30.9 & gallons & 30.8 & gallons & 30.7 & gallons \\
\hline & filter area & Permeate produced $=$ & & 30.0 & gallons & 243.8 & gallons & 251.7 & gallons & 249.5 & gallons \\
\hline & & Total time to concentrate $=$ & & 3.7 & hours & 30.3 & hours & 31.3 & hours & 31.0 & hours \\
\hline & & Time for first half batch $=$ & & 1.9 & hours & 15.2 & hours & 15.7 & hours & 15.5 & hours \\
\hline & & Hold for 12 hours $=$ & & 12 & hours & 12 & hours & 12 & hours & 12 & hours \\
\hline & & Time for second half batch = & & 1.9 & hours & 15.2 & hours & 15.7 & hours & 15.5 & hours \\
\hline & & Time for steady-state matrix $=$ & (Campaign I only >>) & 13 & hours & $n / a$ & n/a & $\mathrm{n} / \mathrm{a}$ & hours & $\mathrm{n} / \mathrm{a}$ & hours \\
\hline & 1st Wash >> & Wash water $(0.01 \mathrm{M} \mathrm{NaOH})=$, & & 44.7 & gallons & 31.2 & gallons & 31.1 & gallons & 31.0 & gallons \\
\hline & & Wash Notes: 1. Campaign I wash vol. = waste & vol. $\times 21.77 / 18$, but recent $\mathrm{H}$ & NTP chan & nge now is waste & vol. $\times 21$. & $77 / 21.55$ and wi & vill be used & for Campaigns II & II>IV. & \\
\hline & & 2. Campaign I concentrated & Naste to $24 \mathrm{wt} \%$ UDS during & dewaterin & ng therefore the $t$ & UDS was & reduced to $20 \mathrm{w}$ & $\overline{\mathrm{w}} \mathrm{t} \%$ before & washing. & & \\
\hline & & Time for initial wash $=$ & & 5.6 & \begin{tabular}{|l|l|} 
hours & \\
\end{tabular} & 3.9 & \begin{tabular}{|l|} 
hours \\
\end{tabular} & 3.9 & hours & 3.9 & hours \\
\hline & 2nd Wash >> & Wash water $(0.01 \mathrm{M} \mathrm{NaOH})=$ & & 44.7 & gallons & 31.2 & gallons & 31.1 & gallons & 31.0 & gallons \\
\hline & Made 60\% & Time for final wash $=$ & & 3.5 & hours & 2.4 & hours & 2.4 & hours & 2.4 & hours \\
\hline & faster than the & Volume to remove for Max wt $\%=$ & (Campaign I only >>) & 21 & gallons & n/a & gallons & $n / a$ & gallons & $\mathrm{n} / \mathrm{a}$ & gallons \\
\hline & 1st Wash from & Time to concentrate $=$ & (Campaign I only $\gg>$ ) & 2.6 & hours & $n / a$ & gallons & $\mathrm{n} / \mathrm{a}$ & gallons & $\mathrm{n} / \mathrm{a}$ & gallons \\
\hline & the Campaign I & & & & & & & & & & \\
\hline & test. That is, & Washed Concentrate for HLW vitrification & & 36.5 & gallons & 29.3 & gallons & 29.1 & gallons & 29.1 & gallons \\
\hline & $0.134 \times 1.6=$ & & & & & & & & & & \\
\hline & $0.214 \mathrm{gpm}$ & Rinse water $(0.1 \mathrm{M} \mathrm{NaOH})=$ & & 25 & gallons & 25 & gallons & 25 & gallons & 25 & gallons \\
\hline & & Time to rinse UF $=$ & & 0.5 & hour & 0.5 & hour & 0.5 & hour & 0.5 & hour \\
\hline & & $2 \mathrm{M} \mathrm{HNO3}$ cleaning solution = & & 25 & gallons & 25 & gallons & 25 & gallons & 25 & gallons \\
\hline & & Time to cleaning $=$ & & 1.5 & hours & 1.5 & hours & 1.5 & hours & 1.5 & hours \\
\hline & & $2 \mathrm{M} \mathrm{HNO} 3$ cleaning solution = & & 25 & gallons & 25 & gallons & 25 & gallons & 25 & gallons \\
\hline & & Time to cleaning $=$ & & 1.5 & hours & 1.5 & hours & 1.5 & hours & 1.5 & hours \\
\hline & & $2 \mathrm{M} \mathrm{HNO} 3$ cleaning solution = & & 25 & gallons & 25 & gallons & 25 & gallons & 25 & gallons \\
\hline & & Time to cleaning $=$ & & 1.5 & hours & 1.5 & hours & 1.5 & hours & 1.5 & hours \\
\hline & & Rinse $1(0.1 \mathrm{M} \mathrm{NaOH})=$ & & 25 & gallons & 25 & gallons & 25 & gallons & 25 & gallons \\
\hline & & Time to rinse UF $=$ & & 0.5 & hour & 0.5 & hour & 0.5 & hour & 0.5 & hour \\
\hline & & Rinse $2(0.1 \mathrm{M} \mathrm{NaOH})=$ & & 25 & gallons & 25 & gallons & 25 & gallons & 25 & gallons \\
\hline & & Time to rinse UF = & & 0.5 & hour & 0.5 & hour & 0.5 & hour & 0.5 & hour \\
\hline & & Time for final water baselining $=$ & & 2.0 & hour & 2.0 & hour & 2.0 & hour & 2.0 & hour \\
\hline & & & & & & & & & & & \\
\hline & & Total Continuous Time per Campaign = & & 38.4 & hour & 46.6 & hour & 47.6 & hour & 47.3 & hour \\
\hline \multicolumn{12}{|c|}{\begin{tabular}{|l|l|l|l|l|l|l|} 
Non-prototypicality Note: & & & & & & \\
\end{tabular}} \\
\hline \multicolumn{12}{|c|}{ The 12-hour hold time between half batches during scaled dewatering is an attempt to capture some of the precipitation dynamics that will occur in the waste during concentration. However, } \\
\hline full-scale & ewatering will tak & e hundreds of hours therefore not all dynamics & \begin{tabular}{l|l} 
will happen. & . \\
\end{tabular} & & & & & & & & \\
\hline
\end{tabular}

The pilot-scale UFP produced permeate that was needed for the next step in the SIPP operation, i.e., pilot CIX. While the UFP continued to process the dewatered slurry with washing and cleaning the UFP loop with both caustic and acid flushes, the permeate sat in the pilot CIX feed tank to age at least 4.5 days, as shown in Table 9. 
WSRC-TR-2004-00565, REVISION 0

SRNL-RPP-2004-00092, REVISION 0

Table 9. Hold period before pilot-scale CIX begins

\begin{tabular}{|l|l|l|l|l|l|l|}
\hline & Inter-Unit Operations Hold Time (stipulated by WTP) & & & & \\
\hline & Minimum hold time between the receipt of the UFP permeate to the time it is processed by the CIX & & 4.5 & days & & \\
\hline
\end{tabular}

As was done for Campaign II and then for Campaign III, it was convenient to produce the first permeate on a Monday and then to begin pilot CIX operation the following Monday. There were approximately 6.5 days between the end of the first half of permeate production, sometime after midnight of that Monday, and the planned start of the CIX. Pilot CIX operation was also broken in two parts because it was necessary to run with two complete ion exchange cycles, which allowed both IX columns to take turns as the lead or the lag column. This is the reason there are two CIX tables, i.e., Tables 10 and 11. The basic difference between them is that the lead column in the first cycle of operation, Table 10, became the lag column in the second cycle, Table 11. The permeate produced during the pilot UFP was sufficient to satisfy the CIX cycle need of 400 bed volumes of feed ( 900 liters). Each cycle included the processing of 200 bed volumes of UFP permeate and then the lead column would undergo regeneration for use in the next cycle as the lag column.

The colored portion of Tables 10 and 11 highlights the work done for this report, that is, Campaign III.

Table 10. Pilot-scale CIX (1st half) test matrix with the colored columns representing Campaign III

\begin{tabular}{|c|c|c|c|c|c|c|c|c|c|c|c|}
\hline \multirow{3}{*}{\begin{tabular}{|l|} 
\\
Process \\
\end{tabular}} & \multirow{3}{*}{\begin{tabular}{|l|} 
\\
Action \\
\end{tabular}} & \multirow[b]{3}{*}{ Feeds } & & \multirow{2}{*}{\multicolumn{2}{|c|}{$\begin{array}{r}\text { Campaign I } \\
\text { Contribution Volumes }\end{array}$}} & \multirow{2}{*}{\multicolumn{2}{|c|}{\begin{tabular}{|c|} 
Campaign II \\
Contribution Volumes
\end{tabular}}} & \multicolumn{2}{|c|}{ Campaign III } & \multicolumn{2}{|c|}{ Campaign IV } \\
\hline & & & & & & & & \multicolumn{2}{|c|}{ Contribution Volumes } & \multicolumn{2}{|c|}{ Contribution Volumes } \\
\hline & & & & & & & & & & & \\
\hline \multirow[t]{6}{*}{ Cs IX } & Locate Feeds & Total UFP permeate feed & & n/a & n/a & 243.8 & gallons & 251.7 & gallons & 249.5 & gallons \\
\hline & & \multicolumn{4}{|c|}{ This permeate must wait at least 48 hours after being created from the UFP before being treated. } & & & & & & \\
\hline & & \multirow{2}{*}{\multicolumn{4}{|c|}{\begin{tabular}{|l|l|l} 
Both the lead and lag columns start cycle I filled with $0.25 \mathrm{M} \mathrm{Na}$ & & \\
Water used for eluate, water buffer, and $0.25 \mathrm{M} \mathrm{Na}$ must be made from fresh distilled water \\
\end{tabular}}} & & & & & & \\
\hline & & & & & & & & & & & \\
\hline & & \multicolumn{3}{|c|}{ Water used for $0.1 \mathrm{M}$ Na solution can come from evaporation condensates } & & & & & & & \\
\hline & & \multicolumn{2}{|c|}{ There will be two columns referred to as A \& B } & & & & & & & & \\
\hline \multirow{30}{*}{ Cs IX } & & & & & & & & & & & \\
\hline & Treat Waste & Cs IX Test No. & & & & 1 & & 3 & & 5 & \\
\hline & Simulant and & Lead $>$ Lag Column $=$ & & n/a & $\mathrm{n} / \mathrm{a}$ & $A>B$ & & $A>B$ & & $A>B$ & \\
\hline & regenerate as & Cycle I (treat $1 / 2$ campaign batch) & & $\mathrm{n} / \mathrm{a}$ & $\mathrm{n} / \mathrm{a}$ & 121.9 & gallons & 125.8 & gallons & 124.7 & gallons \\
\hline & indicated & Number of BV $(B V \sim 0.617$ gal. $) \gg>>$ & Column I.D. $=5.5$ inches & $\mathrm{n} / \mathrm{a}$ & $\mathrm{n} / \mathrm{a}$ & 197.5 & BV & 204.0 & BV & 202.2 & BV \\
\hline & & Flow Rate & Bed Height $=6.0$ inches & $n / a$ & $\mathrm{n} / \mathrm{a}$ & 3.0 & BV/hour & 3.0 & BV/hour & 1.5 & BV/hour \\
\hline & & Total Hours to Treat & & $\mathrm{n} / \mathrm{a}$ & $\mathrm{n} / \mathrm{a}$ & 65.8 & hours & 68.0 & hours & 134.8 & hours \\
\hline & & Regeneration & & & & & & & & & \\
\hline & 4 & Column to be regenerated and eluted $=$ & & & & A & & A & & A & \\
\hline & & $0.1 \mathrm{M} \mathrm{NaOH}$ displacement & & & & & & & & & \\
\hline & & Number of BV (BV 0.617 gal.) & & $\mathrm{n} / \mathrm{a}$ & $\mathrm{n} / \mathrm{a}$ & 3.0 & BV & 3.0 & BV & 3.0 & BV \\
\hline & & Flow Rate & & $\mathrm{n} / \mathrm{a}$ & $\mathrm{n} / \mathrm{a}$ & 3.0 & BV/hour & 3.0 & BV/hour & 3.0 & BV/hour \\
\hline & & Total Hours to Treat & & $n / a$ & $\mathrm{n} / \mathrm{a}$ & 1.0 & hours & 1.0 & hours & 1.0 & hours \\
\hline & & Distilled Water Buffer & & & & & & & & & \\
\hline & & Number of BV (BV 0.617 gal.) & & $\mathrm{n} / \mathrm{a}$ & $\mathrm{n} / \mathrm{a}$ & 3.0 & BV & 3.0 & BV & 3.0 & BV \\
\hline & & Flow Rate & & $\mathrm{n} / \mathrm{a}$ & $\mathrm{n} / \mathrm{a}$ & 3.0 & BV/hour & 3.0 & BV/hour & 3.0 & BV/hour \\
\hline & & Total Hours to Treat & & $n / a$ & $\mathrm{n} / \mathrm{a}$ & 1.0 & hours & 1.0 & hours & 1.0 & hours \\
\hline & & $0.5 \mathrm{M} \mathrm{HNO}$ elution & & & & & & & & & \\
\hline & \multirow[t]{2}{*}{ Regeneration } & Number of BV (BV 0.617 gal.) & & $\mathrm{n} / \mathrm{a}$ & $\mathrm{n} / \mathrm{a}$ & 15.0 & BV & 15.0 & BV & 15.0 & BV \\
\hline & & Flow Rate & & $n / a$ & $\mathrm{n} / \mathrm{a}$ & 1.0 & BV/hour & 2.0 & BV/hour & 1.0 & BV/hour \\
\hline & & Total Hours to Treat & & $\mathrm{n} / \mathrm{a}$ & $\mathrm{n} / \mathrm{a}$ & 15.0 & hours & 7.5 & hours & 15.0 & hours \\
\hline & & Distilled Water Buffer & & & & & & & & & \\
\hline & & Number of BV (BV 0.617 gal.) & & $\mathrm{n} / \mathrm{a}$ & $\mathrm{n} / \mathrm{a}$ & 3.0 & BV & 3.0 & BV & 3.0 & BV \\
\hline & & Flow Rate & & $n / a$ & $\mathrm{n} / \mathrm{a}$ & 1.4 & BV/hour & 1.4 & BV/hour & 1.4 & BV/hour \\
\hline & & Total Hours to Treat & & $\mathrm{n} / \mathrm{a}$ & $\mathrm{n} / \mathrm{a}$ & 2.1 & hours & 2.1 & hours & 2.1 & hours \\
\hline & & $0.25 \mathrm{M} \mathrm{NaOH}$ regeneration & & & & & & & & & \\
\hline & & Number of BV (BV 0.617 gal.) & & $\mathrm{n} / \mathrm{a}$ & $\mathrm{n} / \mathrm{a}$ & 6.0 & BV & 6.0 & BV & 6.0 & BV \\
\hline & & Flow Rate & & $\mathrm{n} / \mathrm{a}$ & $\mathrm{n} / \mathrm{a}$ & 3.0 & BV/hour & 3.0 & BV/hour & 3.0 & BV/hour \\
\hline & $\downarrow$ & Total Hours to Treat & & $\mathrm{n} / \mathrm{a}$ & $\mathrm{n} / \mathrm{a}$ & 2.0 & hours & 2.0 & hours & 2.0 & hours \\
\hline & & & Total clX Cycle Flow Time = & & & 870 & hours & 816 & hours & 1559 & \\
\hline
\end{tabular}




\section{WSRC-TR-2004-00565, REVISION 0 SRNL-RPP-2004-00092, REVISION 0}

Campaign III included pilot CIX cycles 3 and 4. Once again, Campaign I did not include any ion exchange work, which explains why Cycle 1 began in Campaign II and Cycle 3 began in Campaign III.

Once both cycles of a campaign are complete then both columns are filled with $0.1 \mathrm{M}$ $\mathrm{NaOH}$ to keep the resin stable for the next campaign. This inter-campaign period would be no less than 5 weeks.

Table 11. Pilot-scale CIX (2nd half) test matrix with the colored columns representing Campaign III

\begin{tabular}{|c|c|c|c|c|c|c|c|c|c|c|c|}
\hline Cs IX & & Cs IX Test No. & & & & 2 & & 4 & & 6 & \\
\hline & & Lead/Lag Column $=$ & & $\mathrm{n} / \mathrm{a}$ & $\mathrm{n} / \mathrm{a}$ & $B>A$ & & $B>A$ & & $A>B$ & \\
\hline & & Cycle 2 (treat $1 / 2$ campaign batch) & & $\mathrm{n} / \mathrm{a}$ & $\mathrm{n} / \mathrm{a}$ & 121.9 & gallons & 125.8 & gallons & 124.7 & gallons \\
\hline & & Number of BV (BV 0.617 gal. $)$ & & $\mathrm{n} / \mathrm{a}$ & $\mathrm{n} / \mathrm{a}$ & 197.5 & BV & 204.0 & BV & 202.2 & BV \\
\hline & & Flow Rate & & $\mathrm{n} / \mathrm{a}$ & $\mathrm{n} / \mathrm{a}$ & 3.0 & BV/hour & 4.5 & BV/hour & 3.0 & BV/hour \\
\hline & & Total Hours to Treat & & $\mathrm{n} / \mathrm{a}$ & $\mathrm{n} / \mathrm{a}$ & 65.8 & hours & 45.3 & hours & $67.4 \mid$ & hours \\
\hline & & Regeneration & & & & & & & & & \\
\hline & $\bar{T}$ & Column to be regenerated and eluted $=$ & & & & $\mathrm{B}$ & & $\mathrm{B}$ & & $A+B$ & \\
\hline & & $0.1 \mathrm{M} \mathrm{NaOH}$ regeneration & & & & & & & & & \\
\hline & & Number of BV (BV 0.617 gal.) & & $\mathrm{n} / \mathrm{a}$ & $\mathrm{n} / \mathrm{a}$ & 3.0 & BV & 3.0 & BV & 3.0 & BV \\
\hline & & Flow Rate & & $\mathrm{n} / \mathrm{a}$ & $\mathrm{n} / \mathrm{a}$ & 3.0 & BV/hour & 3.0 & BV/hour & 3.0 & BV/hour \\
\hline & & Total Hours to Treat & & $\mathrm{n} / \mathrm{a}$ & $\mathrm{n} / \mathrm{a}$ & 1.0 & hours & 1.0 & hours & 1.01 & hours \\
\hline & & Distilled Water Buffer & & & & & & & & & \\
\hline & & Number of BV (BV 0.617 gal. $)$ & & $\mathrm{n} / \mathrm{a}$ & $\mathrm{n} / \mathrm{a}$ & 3.0 & BV & 3.0 & $\mathrm{BV}$ & 3.0 & BV \\
\hline & & Flow Rate & & $\mathrm{n} / \mathrm{a}$ & $\mathrm{n} / \mathrm{a}$ & 3.0 & BV/hour & 3.0 & BV/hour & 3.0 & BV/hour \\
\hline & & Total Hours to Treat & & $\mathrm{n} / \mathrm{a}$ & $\mathrm{n} / \mathrm{a}$ & 1.0 & hours & 1.0 & hours & $1.0 \mid$ & hours \\
\hline & & $0.5 \mathrm{M} \mathrm{HNO3} \mathrm{elution}$ & & & & & & & & & \\
\hline & Regeneration & Number of BV (BV 0.617 gal. $)$ & & $\mathrm{n} / \mathrm{a}$ & $\mathrm{n} / \mathrm{a}$ & 15.0 & BV & 15.0 & BV & 15.0 & BV \\
\hline & & Flow Rate & & $\mathrm{n} / \mathrm{a}$ & $\mathrm{n} / \mathrm{a}$ & 0.5 & BV/hour & 1.0 & BV/hour & 1.0 & BV/hour \\
\hline & & Total Hours to Treat & & $\mathrm{n} / \mathrm{a}$ & $\mathrm{n} / \mathrm{a}$ & 30.0 & hours & 15.0 & hours & 15.0 & hours \\
\hline & & Distilled Water Buffer & & & & & & & & & \\
\hline & & Number of BV (BV 0.617 gal. $)$ & & $\mathrm{n} / \mathrm{a}$ & $\mathrm{n} / \mathrm{a}$ & 3.0 & BV & 3.0 & BV & 3.0 & BV \\
\hline & & Flow Rate & & $\mathrm{n} / \mathrm{a}$ & $\mathrm{n} / \mathrm{a}$ & 1.4 & BV/hour & 1.4 & BV/hour & $1.4 \mathrm{t}$ & BV/hour \\
\hline & & Total Hours to Treat & & $\mathrm{n} / \mathrm{a}$ & $\mathrm{n} / \mathrm{a}$ & 2.1 & hours & 2.1 & hours & 2.1 & hours \\
\hline & & $0.25 \mathrm{M} \mathrm{NaOH}$ regeneration & & & & & & & & & \\
\hline & & Number of BV (BV 0.617 gal. $)$ & & $\mathrm{n} / \mathrm{a}$ & $\mathrm{n} / \mathrm{a}$ & 6.0 & BV & 6.0 & $\mathrm{BV}$ & 6.0 & BV \\
\hline & & Flow Rate & & $\mathrm{n} / \mathrm{a}$ & $\mathrm{n} / \mathrm{a}$ & 3.0 & BV/hour & 3.0 & BV/hour & 3.0 & BV/hour \\
\hline & $\downarrow$ & Total Hours to Treat & & $\mathrm{n} / \mathrm{a}$ & $\mathrm{n} / \mathrm{a}$ & 2.0 & hours & 2.0 & hours & 2.0 & hours \\
\hline & & & & & & & & & & & \\
\hline & & & Total CIX Cycle Flow Time $=$ & & & 102.0 & hours & 66.5 & hours & 88.5 & hours \\
\hline & & & & & & & & & & & \\
\hline & & & Total CIX Campaign Flow Tin & $\mathrm{ne}=$ & & 189.0 & hours & 148.1 & hours & 244.5 & hour \\
\hline & & & & & & 7.9 & days & 6.2 & days & 10.2 & days \\
\hline & & & & & & & & & & & \\
\hline & & & Total Volumes for Campaigns & SII, III, & $\mathrm{dIV}:$ & & Totals & & & & \\
\hline & & & & BV & Gallons & & Available for & & & & \\
\hline & & & $0.1 \mathrm{M} \mathrm{NaOH}$ & 18.0 & 11.1 & & recycles & 11.1 & & & \\
\hline & & & DI Water & 36.0 & 22.2 & & & 22.2 & & & \\
\hline & & & $0.5 \mathrm{M} \mathrm{HNO3}$ & 90.0 & 55.5 & & & 0.0 & & & \\
\hline & & & $0.25 \mathrm{M} \mathrm{NaOH}$ & 36.0 & 22.2 & & & 22.2 & Per Campaign & & \\
\hline & & & & & & & Total & 55.5 & 18.5 & gallons & \\
\hline \multirow{2}{*}{\multicolumn{11}{|c|}{ Non-prototypicality Note: }} & \\
\hline & & & & & & & & & & & \\
\hline \multicolumn{12}{|c|}{ Scaled CIX testing will be determining cold cesium breakthrough by using a range of BV flow rates which bound the full scale unit. However, the superficial liquid velocity of the plant is } \\
\hline approxim & tely $5 \mathrm{~cm} / \mathrm{min}, \mathrm{w}$ & ich is 5 to 13 times greater than the range & used at the pilot scale. & & & & & & & & \\
\hline
\end{tabular}

The final step in the SIPP was once again to evaporate the waste. The now treated simulated LAW product from the CIX was mixed with two recycle streams shown in Table 4. After mixing, the resulting solution was held before processing, Table 12.

Table 12. Hold period before pilot-scale TLP begins

\begin{tabular}{|l|l|l|l|l|l|l|}
\hline & Pre-TLP Hold Times (stipulated by WTP) & & & & \\
\hline & & & & \\
\hline \\
\hline
\end{tabular}


The operation of the pilot TLP, Table 13 was similar to the pilot FEP, Table 6. However, in the TLP the goal was to concentrate the feed stream so that it had a sodium molarity of 8. Once again the "visible" target was an evaporated slurry density of $1.360 \mathrm{~g} / \mathrm{cc}$ at $50^{\circ} \mathrm{C}$ and $1 \mathrm{psia}$ (which is $1.395 \mathrm{~g} / \mathrm{cc}$ at $20^{\circ} \mathrm{C}$ and $1 \mathrm{~atm}$ ). Density was used because the WTP will only be able to control concentration by measuring density. A large difference between the pilot FEP and TLP operations was the quantity of concentrated product produced. For the FEP, the final volume of the concentrate could be completely contained within the confines of the evaporator ( $<50$ gallons), but for the TLP the concentrated volume was approximately 3 times larger. That is, the FEP was shut down just when steady-state waste concentration was reached. For the TLP, steady-state waste concentration was reached well before the waste feed was exhausted. This meant the TLP produced a continuous waste stream after the TLP contents reached the steady-state density target. The only significant impact this difference had on operation was to the defoamer chemical added to the waste. The concentration of defoamer was to be maintained at $1500 \mathrm{ppm}$, therefore, once the TLP reached steady state and concentrated waste was being removed, defoamer was periodically added.

Table 13. Pilot-scale TLP test matrix with the colored columns representing Campaign III

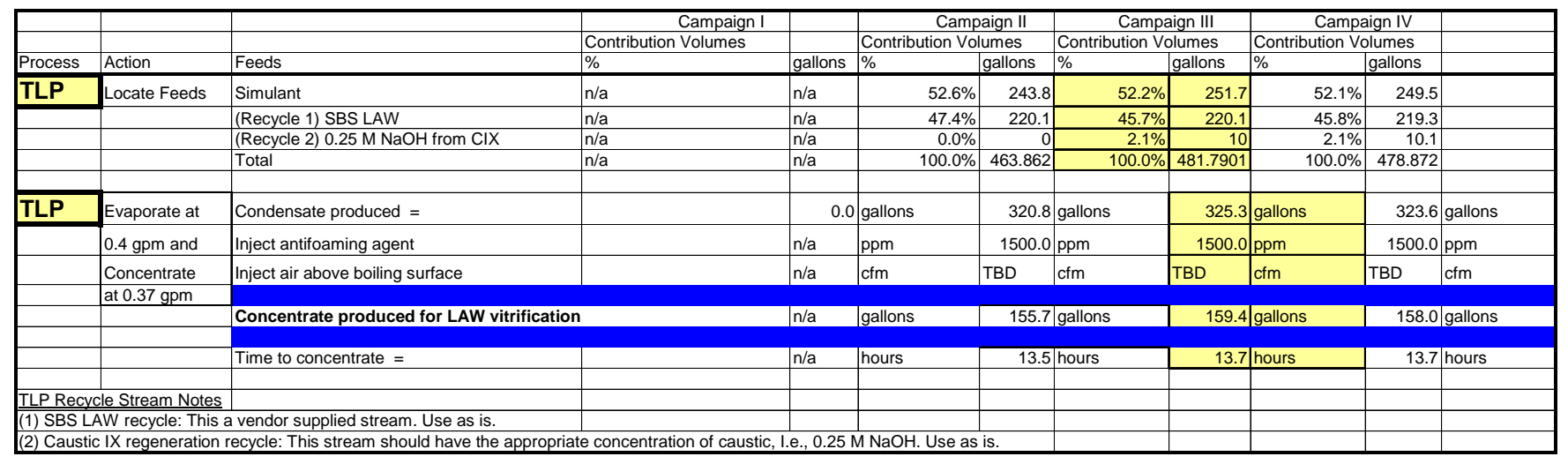

The timetable for Campaign III is listed in Table 14. It started on the day indicated, i.e., Monday, 7 June, 2004, but since this schedule was published before the test began minor changes occurred. However, those changes were insignificant.

The only other aspect of the test not shown in all the matrix tables is the disposition of the waste products. That is, the treated waste from the TLP was drummed to be sent to VSL for LAW melter operations and the washed concentrated slurry from the UFP was drummed to be sent to VSL for HLW melter operation. The other product is all the boiled off and condensed water from both evaporation operations. Those waters were mixed together, as they will be in the plant and then a portion is reused to make rinse solutions for the UFP and displacement solution for the CIX. 


\section{WSRC-TR-2004-00565, REVISION 0 SRNL-RPP-2004-00092, REVISION 0}

Table 14. Pilot-scale schedule for Campaign III

\begin{tabular}{|c|c|c|c|c|c|c|c|c|c|c|c|c|c|}
\hline Campaign & Start Date & & Personnel Nomeclature: & & & & & Notes: & \multirow{2}{*}{\multicolumn{5}{|c|}{$\begin{array}{l}\text { (1) Hold times are included to mimick times that } \\
\text { the plant is filling/transfer between unit operations. }\end{array}$}} \\
\hline $\mathrm{I}$ & 26-Jan-04 & & \multicolumn{5}{|c|}{$\mathrm{da}=\mathrm{D}$. Adamson, $\mathrm{ma}=\mathrm{M}$. Armstrong, $\mathrm{db}=\mathrm{D}$. Burns, $\mathrm{vb}=\mathrm{V}$. Bush, } & & & & & & \\
\hline II & 12-Apr-04 & & \multicolumn{5}{|c|}{ jc=J.Corbett, md=M.Duignan, df=D.Foreman, mf=M.Fowley, } & & \multicolumn{5}{|c|}{ Those times begin when a mixture is FIRST made, } \\
\hline III & 7-Jun-04 & & \multirow{2}{*}{\multicolumn{5}{|c|}{\begin{tabular}{ll|l} 
sh=S. Hatcher, jm=J.Mills, $z q=Z$.Qureshi, $\mathrm{mr}=\mathrm{M}$. Restivo & \\
\end{tabular}}} & & \multicolumn{4}{|c|}{ therefore mixing times are also included. } & \\
\hline \multirow[t]{2}{*}{ IV } & 23-Aug-04 & & & & & & ts=T.Steeper, js=J.Steimke, mw=M.Williams, jz=J.Zamecnik & & & & & & \\
\hline & Campaign III & & & & & & & & & & & & \\
\hline Day & Day & Unit Op & Activity & Expt & Backup & Non-E. & Backup & Unit Op & Activity & Expt & Backup & Non-E. & Backup \\
\hline Mon. & 7-Jun-04 & FEP & Mix Recycles & $\mathrm{jz} / \mathrm{mw}$ & $\mathrm{mw} / \mathrm{jz}$ & $\mathrm{df}$ & $\mathrm{vb}$ & & & & & & \\
\hline Tues. & 8-Jun-04 & FEP & Admin Hold (1) & & & & & & & & & & \\
\hline Wed. & 9-Jun-04 & FEP & Evaporation & $\mathrm{zq}$ & $\mathrm{da}$ & $\mathrm{jm}$ & $\mathrm{vb}$ & UFP & Baseline & $\mathrm{mf}$ & md & $\mathrm{ma}$ & df \\
\hline Thurs. & 10-Jun-04 & FEP & Product Transfers & $\mathrm{zq}$ & $\mathrm{da}$ & $\mathrm{jm}$ & $\mathrm{vb}$ & UFP & Admin Hold (1) & & & & \\
\hline Fri. & 11-Jun-04 & FEP & Clean Equipment & $z q$ & da & $\mathrm{jm}$ & $\mathrm{vb}$ & UFP & Mix UF feeds & $\mathrm{jz} / \mathrm{mw}$ & $\mathrm{mw} / \mathrm{jz}$ & $d f$ & $\mathrm{vb}$ \\
\hline Sat. & 12-Jun-04 & & & & & & & UFP & Admin Hold (1) & & & & \\
\hline Sun & 13-Jun-04 & & & & & & & UFP & Admin Hold (1) & & & & \\
\hline Mon. & 14-Jun-04 & & & & & & & UFP & Dewater & $\mathrm{mf}$ & md & $\mathrm{ma}$ & $d f$ \\
\hline Tues. & 15-Jun-04 & & & & & & & UFP & Dewater & $\mathrm{mf}$ & md & $\mathrm{ma}$ & df \\
\hline Wed. & 16-Jun-04 & $\mathrm{CIX}$ & Admin Hold (1) & & & & & UFP & Transfers/Wash & $\mathrm{mf}$ & $\mathrm{md}$ & $\mathrm{ma}$ & df \\
\hline Thurs. & 17-Jun-04 & $\mathrm{CIX}$ & Admin Hold (1) & & & & & UFP & Wash & $\mathrm{mf}$ & $\mathrm{md}$ & $\mathrm{ma}$ & $d f$ \\
\hline Fri. & 18-Jun-04 & $\mathrm{CIX}$ & Admin Hold (1) & & & & & & & & & & \\
\hline Sat. & 19-Jun-04 & $\mathrm{CIX}$ & Admin Hold (1) & & & & & & & & & & \\
\hline Sun & 20-Jun-04 & $\mathrm{CIX}$ & Admin Hold (1) & & & & & & & & & & \\
\hline Mon. & 21-Jun-04 & $\mathrm{CIX}$ & Treat Simulant & js & $\mathrm{da}$ & df & $\mathrm{vb}$ & UFP & Rinse/Clean & $\mathrm{mf}$ & $\mathrm{md}$ & $\mathrm{ma}$ & df \\
\hline Tues. & 22-Jun-04 & $\mathrm{CIX}$ & Treat Simulant & js & da & df & $\mathrm{vb}$ & UFP & Baseline & $\mathrm{mf}$ & md & $\mathrm{ma}$ & df \\
\hline Wed. & 23-Jun-04 & $\mathrm{CIX}$ & Treat Simulant & js & $\mathrm{da}$ & df & $\mathrm{vb}$ & & & & & & \\
\hline Thurs. & 24-Jun-04 & $\mathrm{CIX}$ & 0.1 M Caustic/DI Water Flush & js & $\mathrm{da}$ & $\mathrm{df}$ & $\mathrm{vb}$ & & & & & & \\
\hline Fri. & 25-Jun-04 & & & & & & & & & & & & \\
\hline Sat. & 26-Jun-04 & & & & & & & & & & & & \\
\hline Sun & 27-Jun-04 & & & & & & & & & & & & \\
\hline Mon. & 28-Jun-04 & $\mathrm{CIX}$ & $0.5 \mathrm{M} \mathrm{HNO3}$ & js & $\mathrm{da}$ & df & $\mathrm{vb}$ & & & & & & \\
\hline Tues. & 29-Jun-04 & $\mathrm{CIX}$ & DI Water Flush & js & da & df & $\mathrm{vb}$ & & & & & & \\
\hline Wed. & 30-Jun-04 & $\mathrm{CIX}$ & $0.25 \mathrm{M} \mathrm{NaOH}$ Regeneration & js & da & $\mathrm{df}$ & $\mathrm{vb}$ & & & & & & \\
\hline Thurs. & 1-Jul-04 & $\mathrm{CIX}$ & Treat Simulant & js & da & $\mathrm{df}$ & $\mathrm{vb}$ & & & & & & \\
\hline Fri. & 2-Jul-04 & $\mathrm{CIX}$ & Treat Simulant & js & $\mathrm{da}$ & $\mathrm{df}$ & $\mathrm{vb}$ & & & & & & \\
\hline Sat. & 3-Jul-04 & & & & & & & & & & & & \\
\hline Sun & 4-Jul-04 & & & & & & & & & & & & \\
\hline Mon. & 5-Jul-04 & 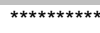 & 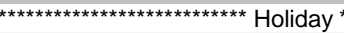 & 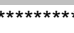 & 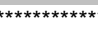 & 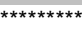 & 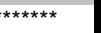 & 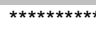 & 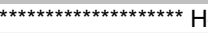 & oliday * & 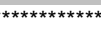 & 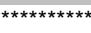 & 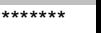 \\
\hline Tues. & 6-Jul-04 & $\mathrm{CIX}$ & 0.1 M Caustic/DI Water Flush & js & da & $\mathrm{df}$ & $\mathrm{vb}$ & TLP & Make Feed & $\mathrm{jz} / \mathrm{mw}$ & $\mathrm{mw} / \mathrm{jz}$ & $\mathrm{df}$ & $\mathrm{vb}$ \\
\hline Wed. & 7-Jul-04 & $\mathrm{CIX}$ & $0.5 \mathrm{M} \mathrm{HNO} 3$ & js & da & $\mathrm{df}$ & $\mathrm{vb}$ & TLP & Admin Hold (1) & & & & \\
\hline Thurs. & 8-Jul-04 & $\mathrm{CIX}$ & DI Water Flush & js & da & $\mathrm{df}$ & $\mathrm{vb}$ & TLP & Evaporation & da & $\mathrm{zq}$ & $\mathrm{vb}$ & $\mathrm{jm}$ \\
\hline Fri. & 9-Jul-04 & $\mathrm{CIX}$ & $0.25 \mathrm{M} \mathrm{NaOH}$ Regeneration & js & da & df & $\mathrm{vb}$ & TLP & Product Transfers & da & $\mathrm{zq}$ & $\mathrm{vb}$ & jm \\
\hline Mon. & 12-Jul-04 & & & & & & & TLP & Clean Equipment & da & $\mathrm{zq}$ & $\mathrm{vb}$ & jm \\
\hline
\end{tabular}




\subsection{THE PRINCIPAL WASTE SIMULANTS: AY102 AND AP101 (AUTHOR- ZAMECNIK)}

Together with the pilot-scale FEP concentrated recycle streams, the waste feed for the pilot-scale UFP contained two simulated Hanford waste streams: tank 241-AY-102/C106 referred herein as AY102, and tank 241-AP-101, referred herein as AP101.

The composition and properties of AY102, including the distribution of species between the solid and supernate phases, has been previously described (Duignan, et al., 2004a; Zamecnik, et al., 2004). This simulant was blended at SRNL from fractions manufactured by Optima Chemical (Douglas, GA) and additional reagents, as described in the references. The AP101 simulant formulation was defined by Pacific Northwest National Laboratory (Russell, et al., 2003) and the simulant was manufactured by Optima Chemical. The measured total slurry compositions of both of these simulants are shown in Table 15.

Table 15. Measured Simulant Compositions

\begin{tabular}{|c|c|c|c|c|}
\hline All values mg/L except as noted. & \multicolumn{2}{|c|}{ AY102 Slurry } & \multicolumn{2}{|c|}{ AP101 } \\
\hline Total Solids (wt\%) & 26.80 & & 31.94 & \\
\hline Undissolved Solids (wt\%) & 11.75 & & 0.00 & \\
\hline Supernate Solids (wt\%) & 17.05 & & 31.94 & \\
\hline Density kg/L & 1.240 & & 1.279 & \\
\hline $\mathrm{Ag}$ & 624 & & 37.3 & \\
\hline $\mathrm{Al}$ & 15996 & 17484* & 7469 & $6932 *$ \\
\hline $\mathrm{B}$ & $<165$ & & $<142$ & \\
\hline $\mathrm{Ba}$ & 293 & 255 & $<1.53$ & \\
\hline $\mathrm{Bi}$ & 6.93 & & 0.0576 & \\
\hline $\mathrm{Ca}$ & 1131 & 1500 & 23.0 & \\
\hline $\mathrm{Cd}$ & 46.8 & & $<3.9$ & \\
\hline $\mathrm{Ce}$ & 366 & & $<127$ & \\
\hline Co & 151 & & $<15.6$ & \\
\hline $\mathrm{Cr}$ & 632 & 634 & 179 & 175 \\
\hline Cs & 18.7 & & 6.02 & \\
\hline $\mathrm{Cs}$ & $<17$ & & $<19.6$ & \\
\hline $\mathrm{Cu}$ & 77.9 & 81.8 & $<5.86$ & \\
\hline $\mathrm{Fe}$ & 35836 & 35216 & 76.4 & 68.4 \\
\hline $\mathrm{K}$ & 373 & & 30568 & \\
\hline $\mathrm{La}$ & 305 & & 13.9 & \\
\hline $\mathrm{Li}$ & $<24.8$ & & $<21.1$ & \\
\hline $\mathrm{Mg}$ & 379 & 373 & 5.51 & \\
\hline $\mathrm{Mn}$ & 7812 & 7638 & 3.00 & \\
\hline Mo & $<17$ & & $<10.6$ & \\
\hline $\mathrm{Na}$ (ICPES) & 84283 & & 127900 & \\
\hline $\mathrm{Na}(\mathrm{AA})$ & 91760 & & 129179 & \\
\hline Na average & 88021 & & 128540 & \\
\hline $\mathrm{Na}(\mathrm{M})$ & 3.83 & & 5.59 & \\
\hline $\mathrm{Nd}$ & 847 & & $<72.9$ & \\
\hline
\end{tabular}


WSRC-TR-2004-00565, REVISION 0

SRNL-RPP-2004-00092, REVISION 0

Table 15 - continued

\begin{tabular}{|c|c|c|}
\hline All values mg/L except as noted. & AY102 Slurry & AP101 \\
\hline $\mathrm{Ni}$ & 1151 & 357 \\
\hline $\mathrm{P}$ & 1748 & 269 \\
\hline $\mathrm{Pb}$ & 1662 & $<156$ \\
\hline $\operatorname{Pr}$ & 170 & 0.0223 \\
\hline $\mathrm{Rb}$ & 4.60 & 6.06 \\
\hline $\mathrm{S}$ & 892 & 1599 \\
\hline $\mathrm{Si}$ & 11016 & 329 \\
\hline $\mathrm{Sr}$ & 279 & $<1.18$ \\
\hline $\mathrm{W}$ & $<84.7$ & $<97.7$ \\
\hline $\mathrm{Y}$ & 50.8 & 0.0728 \\
\hline $\mathrm{Zn}$ & 81.3 & 5.95 \\
\hline $\mathrm{Zr}$ & 484 & $<5.86$ \\
\hline bromide & 69.4 & $<128$ \\
\hline chloride & 165 & 1530 \\
\hline fluoride & 43.4 & 28.0 \\
\hline formate & 389 & 1380 \\
\hline nitrate & 319 & 105000 \\
\hline nitrite & 4228 & 33400 \\
\hline oxalate & 9213 & 1550 \\
\hline phosphate & 3807 & 1150 \\
\hline sulfate & 1860 & 3740 \\
\hline acetate & 415 & 1727 \\
\hline citric acid & $<81.8$ & $<104$ \\
\hline formate & 264 & 1432 \\
\hline
\end{tabular}

\subsection{PILOT WASTE FEED EVAPORATOR (AUTHORS - QURESHI, WILLIAMS \& ZAMECNIK)}

The Waste Feed Evaporator Process, or FEP, is the first unit operation in the RPP-WTP Pretreatment Facility that a waste $(<5 \mathrm{M} \mathrm{Na})$ will see on start up. However, if the incoming wastes or waste blends are greater than $5 \mathrm{M} \mathrm{Na}$, the FEP evaporators are used to concentrate WTP recycle streams for blending with the fresh tank waste. Since the AY102 and AP101 have sufficient sodium concentration, the SIPP FEP operations only included evaporation of SIPP recycles. The SIPP FEP bottoms stream was then mixed with simulated waste streams, i.e., AY102 and AP101, which would normally come from the Hanford Tank farm to produce a feed stream for the Ultrafiltration Process (UFP) unit operation.

This section will explain the pilot-scale FEP unit operation that was done as part of Campaign III testing. The process started with the preparation of the FEP feed product, which was a mixture of many recycle streams in a predetermined fashion, see Table 2. A chemical explanation of the feed (recycle) stream formation will be followed by a short description on how feed formation was handled just before FEP operation and then how the waste stream was handled during and after its concentration. The following section will discuss the physical equipment used to evaporate the waste and how the test was run. Finally, the results will be discussed and conclusions made for the FEP unit operation. 


\subsubsection{Chemical Description of the Pilot FEP Feed Stream (Author-Zamecnik)}

\subsubsection{Generation of the Recycle Streams}

The feed for the FEP was a series of recycle streams to be produced from operating the RPP-WTP Pretreatment Facility. The basis for the quantities of each recycle used was described in the Campaign II report (Duignan et al., 2004b). The condensate used (that in WTP would come from the Radioactive Liquid Waste Disposal System or RLD) to generate all of the recycles from Campaign II to Campaign III employed condensate from only the Campaign II FEP evaporator, except for two synthetic streams, which used a condensate mixture that came from combining the TLP and FEP products.

The UFP pump loop recycle was approximated by mixing 4.6L of washed concentrated slurry with 8.6L of RLD condensate from the FEP evaporator, both from Campaign II. The pump loop recycle from Campaign III to Campaign IV was made using the same proportions of Campaign III material. The residual rinse in the filter loop that would be present at the start of the first $0.1 \mathrm{M} \mathrm{NaOH}$ rinse was made from $\sim 0.1 \mathrm{~L}$ of washed concentrated slurry and 12.5L of FEP condensate. The UFP feed tank heel prior to each rinse and acid cleaning step was targeted to give the correct undissolved solids loading. Note, in the SIPP the amount of rinse and acid used is greater, on a proportional basis, than in the WTP. To account for using more rinse or acid, a proportionally larger heel is left.

The ejector heel of 500 gal of washed concentrated slurry left after slurry washing was simulated by the approximately 22L of washed concentrated slurry that remained in the system after draining. Note that this quantity of slurry holdup was much higher than previously assumed ( $\sim \mathrm{L})$. To this $22 \mathrm{~L}$ of slurry, $105.2 \mathrm{~L}$ of caustic rinse $1(\sim 0.1 \mathrm{M}$ $\mathrm{NaOH})$, made from RLD condensate, was added. The resulting diluted slurry still had more solids than would be representative of the UFP tank after caustic rinse 1, so the recycle to Campaign IV needed to be diluted 1:1 with fresh caustic rinse solution. The heel from caustic rinse 1 to acid cleaning 1 was made from three quantities 1.) 10L of the diluted slurry from caustic rinse 1,2 .) the $\sim 1.6 \mathrm{~L}$ of this slurry retained in the system, and 3.) $10 \mathrm{~L}$ of $0.1 \mathrm{M} \mathrm{NaOH}$ caustic flush. This scheme is shown in Fig. 2. The RLD condensate was essentially water, with the only significant impurity being ammonium ion at about $458 \mathrm{mg} / \mathrm{kg}$.

In the subsequent rinsing and cleaning steps, the ejector heel was approximated by putting back about $20 \mathrm{~L}$ of the drained solution plus the $\sim 1.6 \mathrm{~L}$ retained in the system. The 21.6L of heel in each step, mixed with 94.6L of caustic rinse or acid is the same ratio as the heel + filter loop $(500+700$ gal $)$ remaining in the WTP to the rinse amount (5250 gal); both ratios are 0.228 . 
WSRC-TR-2004-00565, REVISION 0

SRNL-RPP-2004-00092, REVISION 0

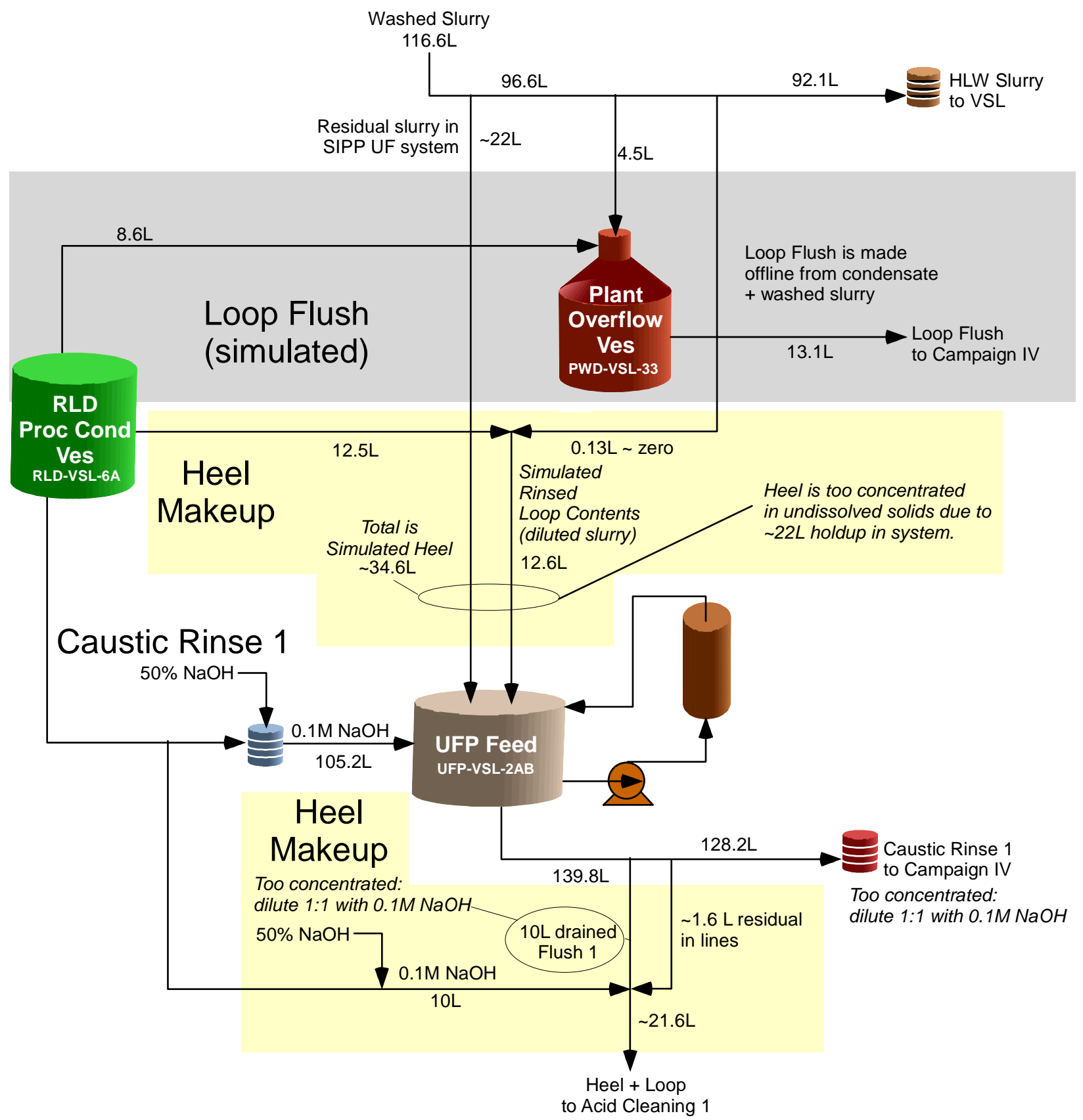

Figure 2. Generation of Caustic Rinse 1 Recycle

\subsubsection{FEP Feed Mixing}

Figure 3 shows a schematic of the FEP feed blending, and the amounts used are also shown in Table 16. The approximate actual volume of the combined feeds was 1075L versus the target of 1089L. After adding the contents of PWD-VSL-44 and -33 to the FEP Feed Tank, the UFP recycles were arbitrarily split between the PWD-VSL-15 and PWD-VSL-16 tanks. All of the recycles were generated in Campaign II or were made 
from reagents when insufficient material was available. Into the PWD-15 tank was added UFP wash permeate, which was the accumulated volume of the second 22 filter washes, then $0.1 \mathrm{M} \mathrm{NaOH}$ from condensate, then the three caustic rinses (i.e., 1, 2, and 3); all solutions were caustic, except for the second and third UFP rinses, which were acidic due to the acid cleaning heel. The amount of caustic rinse 1 added was less than that of rinses 2 and 3 to give closer to the correct undissolved solids in the recycle stream since the rinse 1 UDS was too high. The $21.3 \mathrm{~L}$ of $0.1 \mathrm{M}$ caustic from condensate was added to approximate what would have been added if rinse 1 would have had the correct undissolved solids content. This condensate came from the Campaign II TLP operation and contained ammonium. The wash permeates (each of the two 22-mini-batch washes totaled $118 \mathrm{~L}$ ) were to have been $141 \mathrm{~L}$ per the OLI model ${ }^{\dagger}$.

Next, the Campaign II Ion Exchange recycles were added. These consisted of the displacement solution $(\sim 0.1 \mathrm{M} \mathrm{NaOH})$ that was displaced from the column by the pre-elution rinse, the post-elution rinse displaced by the regeneration solution ( $\sim$ water), and the $0.25 \mathrm{M} \mathrm{NaOH}$ regeneration solution from the column. Because of scaling issues there were insufficient amounts of all of these recycles, so synthetic recycles were generated. The amounts of actual and synthetic recycles are shown in Fig. 3 and Table 16. Table 16 also shows the Campaign II quantities. The $36.7 \mathrm{~L}$ of $0.1 \mathrm{M} \mathrm{NaOH}$ synthetic recycle was made from the RLD condensate containing ammonium.

The final PWD-15 tank solution was predicted to be caustic and was found to be so. The recycle solutions were added in the order given above, and at no time did the bulk solution pH drop below 12.

The PWD-16 tank recycles were made from the first batch of wash permeate (comprised of the first 22 mini-washes) and the three acid cleanings. Because the mixture was sufficiently caustic; no additional $\mathrm{NaOH}$ addition was needed for $\mathrm{pH}$ adjustment. The first 22-washes permeate was used with the acid since it contained more caustic than the second 22-washes permeate, and thus less $\mathrm{NaOH}$ would be required to neutralize the acid. Again, the $\mathrm{pH}$ never dropped below 12 during mixing.

The measured solids loadings of the combined PWD-15 and -16 recycle were both slightly less than predicted from addition of the individual components. For the acid cleanings, the total solids content was estimated as the measured value plus the amount that would be due to the nitrate present; the nitrate in the $\mathrm{HNO}_{3}$ was evaporated during the solids determination. Table 16 summarizes the amounts of each of the recycle steams.

\footnotetext{
${ }^{\dagger}$ As mentioned in Section 2.3, after the modeling was done, and subsequent to completing Campaign I, the WTP UFP feed-tank batch size was increased from 18,000 gallons to 21,550 gallons. However, the criterion to determine the volume of washing water per batch was not changed, which was Volume = Volume of one Permeate Receipt tank $=21,770$ gallons. This means the amount of water used to wash the batch was relatively smaller, that is, $21,770 / 21,550=1.01$ instead of $21,770 / 18,000=1.209$. Since the pilot-scale UFP batch size was $\sim 117 \mathrm{~L}$, then the wash volume would be $117 \mathrm{x} 1.01=118 \mathrm{~L}$ instead of $117 \mathrm{x}$ $1.209=141 \mathrm{~L}$.
} 
WSRC-TR-2004-00565, REVISION 0

SRNL-RPP-2004-00092, REVISION 0

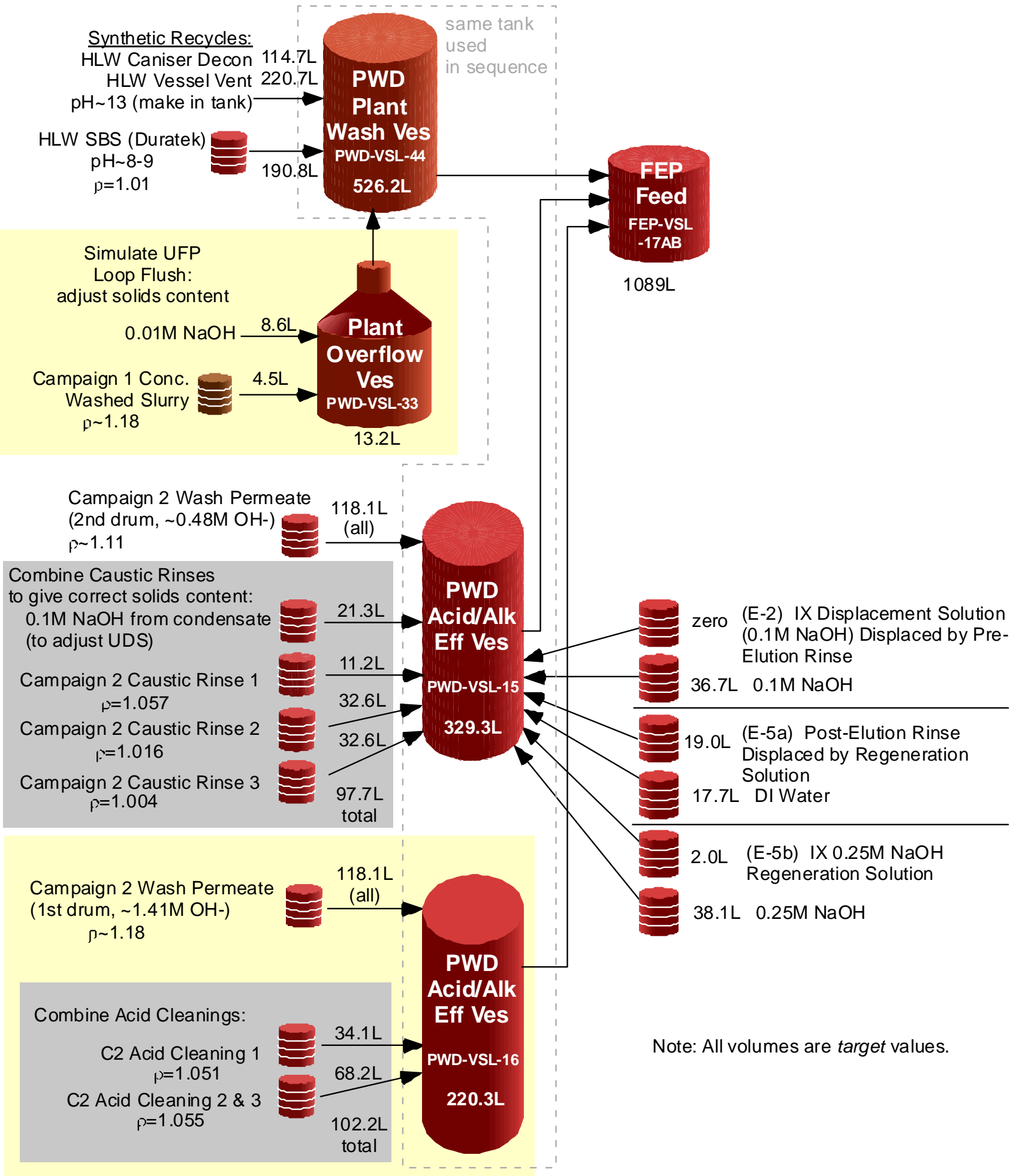

Figure 3. FEP Feed Blending of Recycle Streams 
Table 16. Recycle Streams*

\begin{tabular}{|c|c|c|c|}
\hline Stream & $\begin{array}{l}\text { Campaign } \\
\text { III Source }\end{array}$ & $\begin{array}{l}\text { Campaign } \\
\text { III Volume } \\
\text { (L) }\end{array}$ & $\begin{array}{l}\text { Campaign } \\
\text { II Volume } \\
\text { (L) }\end{array}$ \\
\hline HLW SBS Liquid & Duratek & 190.8 & 190.8 \\
\hline Vessel Vent Liquid & Synthetic & 220.7 & 220.7 \\
\hline HLW Canister Decon Liquid & Synthetic & 114.7 & 114.7 \\
\hline UFP Recycle - Wash Permeate & Campaign II & 236.2 & 338.8 \\
\hline $1^{\text {st } 22}$ Washes Permeate & & 118.1 & 169.3 \\
\hline $2^{\text {nd }} 22$ Washes Permeate & & 118.1 & 169.3 \\
\hline UFP Recycle - 0.1 M NaOH Rinse & Campaign II & 97.7 & 117.0 \\
\hline$x^{2}$ & & 11.2 & 18.2 \\
\hline $2^{\text {nd }}$ Rinse & & 32.6 & 49.5 \\
\hline $3^{\text {rd }}$ Rinse & & 32.6 & 49.5 \\
\hline $\begin{array}{ll}\text { Additional 0.1M Caustic } \\
\end{array}$ & & 21.3 & Not needed \\
\hline UFP Recycle - 2M HNO 3 Cleaning & Campaign II & 102.3 & 122.5 \\
\hline $1^{\text {st }}$ Cleaning & & 34.1 & 37.5 \\
\hline $2^{\text {nd }}$ Cleaning & & 34.1 & 37.5 \\
\hline $3^{\text {rd }}$ Cleaning & & 34.1 & 37.5 \\
\hline $50 \mathrm{wt} \%$ caustic to adjust $\mathrm{pH}$ & Synthetic & Not needed & 11.55 \\
\hline UFP Recycle - UF Loop Rinses & Campaign II & 13.1 & 13.2 \\
\hline RLD condensate & & 8.6 & 8.6 \\
\hline Campaign II Washed Concentrated Slurry & & 4.5 & 4.6 \\
\hline CIX Recycles & Campaign II & 113.5 & 0 \\
\hline Displacement Solution displaced by Pre-Elution Rinse & & 0 & 0 \\
\hline $0.1 \mathrm{M} \mathrm{NaOH}$ (insufficient Displacement Solution) & & 36.7 & 0 \\
\hline Post-Elution Rinse displaced by Regeneration Solution & & 19.0 & 0 \\
\hline DI Water (insufficient Post-Elution Rinse) & & 17.7 & 0 \\
\hline $0.25 \mathrm{M} \mathrm{NaOH}$ Regeneration Solution & & 2.0 & 0 \\
\hline $0.25 \mathrm{M} \mathrm{NaOH}$ (insufficient Regeneration Solution) & & 38.1 & 0 \\
\hline TOTAL & & 1089 & 1129 \\
\hline
\end{tabular}

* This table does not show the recycle streams organized by order added. See Fig. 3 for this information.

The synthetic HLW recycles were made up from reagents to approximate the expected compositions. The vessel vent liquid was $0.1 \mathrm{M} \mathrm{NaOH}$, while the $\mathrm{HLW}$ canister decon liquid was $0.15 \mathrm{M} \mathrm{NaOH}$ and $0.29 \mathrm{M} \mathrm{NaNO}$.

The composition of the recycles is shown in Tables 17 and 18. The HLW SBS recycle from VSL and the synthetic recycles have been described previously (Duignan et al., 2004b). The three simulated recycle tanks were combined to make the FEP evaporator feed. These recycles were combined in the order: PWD-44, PWD-33, PWD-15, and then PWD-16. Nothing unusual was observed when these were mixed. 
WSRC-TR-2004-00565, REVISION 0

SRNL-RPP-2004-00092, REVISION 0

Table 17. Composition of Campaign II Wash Permeate Recycles to FEP Evaporator

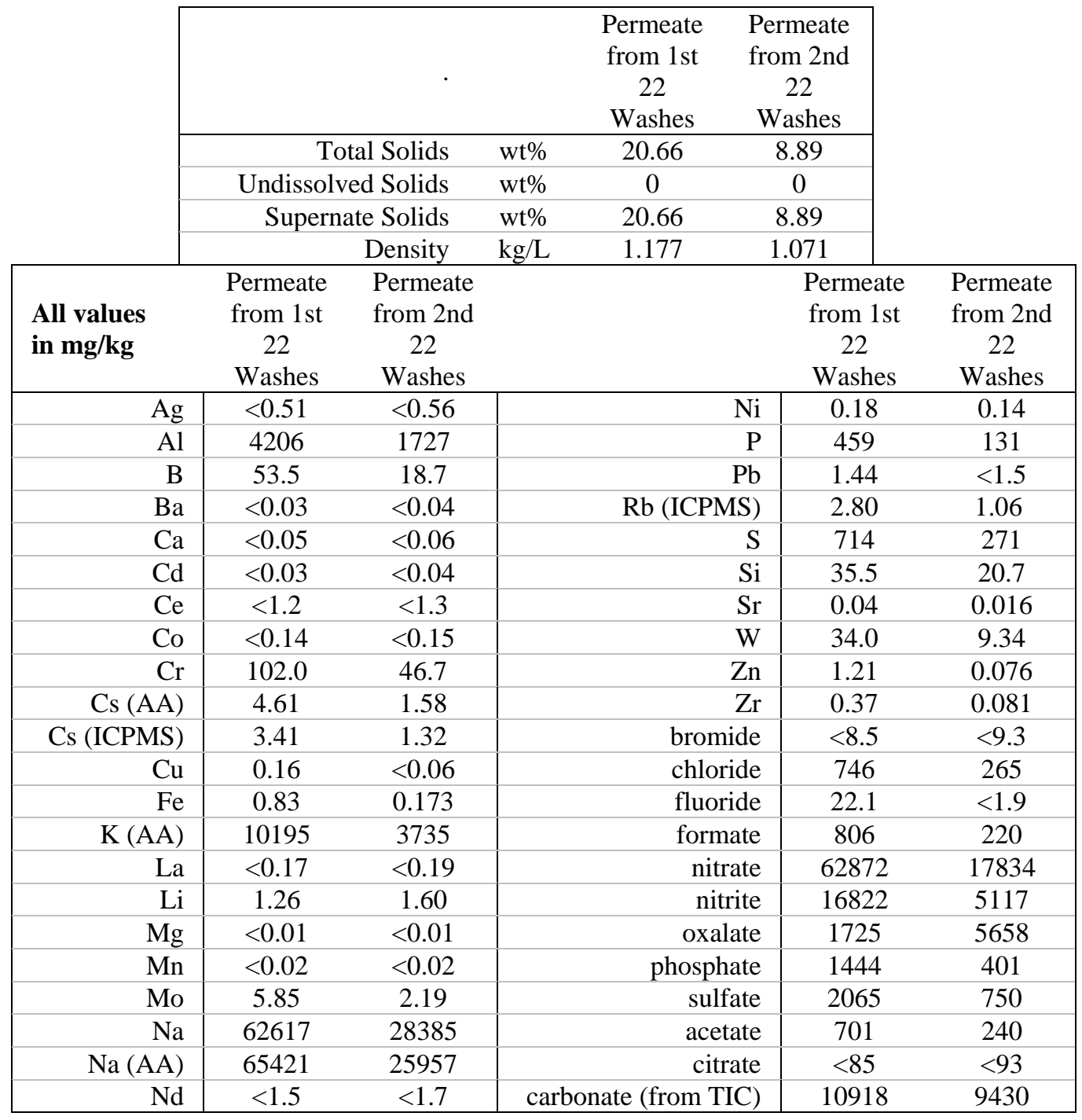


WSRC-TR-2004-00565, REVISION 0 SRNL-RPP-2004-00092, REVISION 0

Table 18. Measured Recycle Compositions

\begin{tabular}{|c|c|c|c|c|c|c|c|c|c|}
\hline $\begin{array}{l}\text { All units mg/kg unless } \\
\text { otherwise noted }\end{array}$ & $\begin{array}{l}\text { Caustic } \\
\text { Rinse } 1\end{array}$ & $\begin{array}{l}\text { Caustic } \\
\text { Rinse } 1\end{array}$ & $\begin{array}{c}\text { Acid } \\
\text { Cleaning } 1\end{array}$ & $\begin{array}{c}\text { Acid } \\
\text { Cleaning } 1\end{array}$ & $\begin{array}{c}\text { Acid } \\
\text { Cleaning } 2\end{array}$ & $\begin{array}{c}\text { Acid } \\
\text { Cleaning } 2\end{array}$ & $\begin{array}{c}\text { Nitric } \\
\text { Cleaning } 3\end{array}$ & $\begin{array}{l}\text { Caustic } \\
\text { Rinse } 2\end{array}$ & $\begin{array}{l}\text { Caustic } \\
\text { Rinse } 3\end{array}$ \\
\hline Volume Used (L) & 11.2 & & 34.1 & & 34.1 & & 34.1 & 32.6 & 32.6 \\
\hline Total Solids (wt\%) & 7.46 & & 2.36 & & 0.67 & & 0.13 & 0.66 & 0.81 \\
\hline Undissolved Solids (wt\%) & 5.09 & & 0.12 & & & & & 0.13 & 0.10 \\
\hline Supernate Solids (wt\%) & 2.50 & & 2.24 & & 0.67 & & 0.13 & 0.53 & 0.71 \\
\hline Density $(\mathrm{kg} / \mathrm{L})$ & 1.057 & & 1.051 & & 1.055 & & 1.055 & 1.016 & 1.004 \\
\hline Sample Preparation & Dissolution & Filtered & Dissolution & Filtered & Dissolution & Filtered & Filtered & Filtered & Filtered \\
\hline $\mathrm{Ag}$ & 128 & $<0.50$ & 174 & 1.93 & 96.9 & 0.73 & 1.60 & 0.665 & $<0.60$ \\
\hline $\mathrm{Al}$ & 3420 & 382 & 653 & 453 & 160 & 123 & 35.0 & 11.8 & 3.58 \\
\hline $\mathrm{B}$ & & 2.85 & & 8.31 & & 1.73 & $<0.93$ & $<0.18$ & $<0.18$ \\
\hline $\mathrm{Ba}$ & 116 & $<0.02$ & 21 & 21.3 & 5.94 & 5.29 & 1.51 & 0.528 & 0.163 \\
\hline $\mathrm{Ca}$ & 346 & 2.48 & $<4.9$ & 93.5 & $<4.2$ & 29.0 & 8.90 & 8.20 & 7.00 \\
\hline $\mathrm{Cd}$ & 20 & $<0.02$ & 3 & 3.52 & $<1.4$ & 0.87 & 0.265 & 0.0746 & $<0.04$ \\
\hline $\mathrm{Ce}$ & $<117$ & $<0.82$ & $<114$ & 25.3 & $<60$ & 6.38 & 1.60 & $<17$ & $<17$ \\
\hline Co & $<13.4$ & $<0.04$ & $<13.0$ & $<0.04$ & $<3.0$ & $<0.04$ & $<0.04$ & $<0.08$ & 0.102 \\
\hline $\mathrm{Cr}$ & 170 & 12.2 & 33 & 32.3 & 6.36 & 8.24 & 2.47 & 0.913 & 0.270 \\
\hline Cs & $<16.63$ & 0.241 & $<16$ & 0.095 & & $<0.09$ & $<0.09$ & 0.133 & $<0.10$ \\
\hline $\mathrm{Cu}$ & 36 & $<0.03$ & 8 & 5.84 & $<2.1$ & 1.57 & 0.470 & 0.133 & $<0.06$ \\
\hline $\mathrm{Fe}$ & 13949 & $<0.07$ & 2449 & 2369 & 622 & 621 & 172 & 62.4 & 18.3 \\
\hline K & 1010 & 721 & 242 & 259 & $<286$ & 75.2 & 19.8 & 7.54 & 3.00 \\
\hline $\mathrm{La}$ & $<16.7$ & $<0.18$ & $<16.3$ & 20.2 & $<13$ & 5.38 & 1.49 & $<0.37$ & $<0.37$ \\
\hline $\mathrm{Li}$ & $<33.3$ & 1.99 & $<32.5$ & 3.87 & $<6.6$ & 1.03 & 0.302 & $<0.19$ & $<0.19$ \\
\hline $\mathrm{Mg}$ & 155 & 0.026 & 25 & 27.9 & 4.62 & 8.34 & 2.57 & 2.00 & 1.49 \\
\hline Mn & 3026 & $<0.02$ & 525 & 507 & 135 & 134 & 37.2 & 13.0 & 3.91 \\
\hline Mo & $<16.7$ & $<1.7$ & $<16.3$ & $<1.7$ & $<125$ & $<1.69$ & $<0.28$ & $<0.35$ & $<0.35$ \\
\hline $\mathrm{Na}$ (ICPES) & 9850 & 5544 & 2410 & 2360 & 589 & 692 & 202 & 1299 & 1813 \\
\hline $\mathrm{Na}$ (AA) & 10000 & 8325 & 2350 & 2445 & & 726 & 191 & 1358 & 1922 \\
\hline $\mathrm{Nd}$ & $<150$ & $<0.38$ & $<147$ & 58.8 & $<28$ & 15.4 & 4.27 & 1.98 & $<0.81$ \\
\hline $\mathrm{Ni}$ & 463 & $<0.09$ & 87 & 79.7 & 20.8 & 20.2 & 5.90 & 2.09 & 0.700 \\
\hline $\mathrm{P}$ & $<134$ & 18.3 & $<130$ & 5.95 & $<43$ & 3.62 & 1.41 & $<1.2$ & $<1.2$ \\
\hline $\mathrm{Pb}$ & 660 & $<1.6$ & $<130$ & 115 & $<118$ & 28.0 & 8.36 & 2.77 & $<1.6$ \\
\hline $\mathrm{S}$ & $<166$ & 41.2 & $<162$ & 15.6 & $<72$ & 4.74 & 2.37 & $<2.0$ & $<2.1$ \\
\hline Si & 3140 & 17.6 & $<1180$ & 235 & $<1330$ & 84.6 & 24.9 & 18.1 & 15.3 \\
\hline $\mathrm{Sr}$ & 108 & 0.017 & 20 & 20.6 & 5.63 & 5.54 & 1.57 & $<0.9$ & $<0.87$ \\
\hline
\end{tabular}


WSRC-TR-2004-00565, REVISION 0 SRNL-RPP-2004-00092, REVISION 0

\begin{tabular}{|c|c|c|c|c|c|c|c|c|c|}
\hline $\mathrm{Ti}$ & $<6.7$ & $<0.05$ & $<53$ & 0.284 & $<4.1$ & $<0.05$ & $<0.06$ & 0.563 & 0.185 \\
\hline $\mathrm{Zn}$ & 37 & $<0.05$ & 5 & 6.66 & $<3.5$ & 2.07 & 0.584 & 0.257 & 0.102 \\
\hline $\mathrm{Zr}$ & 191 & $<0.11$ & 24 & 12.3 & 19.1 & 6.25 & 2.13 & $<0.16$ & $<0.16$ \\
\hline bromide & & $<9.5$ & & & & & & $<10$ & $<10$ \\
\hline chloride & & 33.1 & & & & & & $<2$ & $<2$ \\
\hline fluoride & & 6.62 & & & & & & $<2$ & $<2$ \\
\hline formate & & 51.1 & & & & & & $<10$ & $<10$ \\
\hline nitrate & & 8250 & & & & & & 30610 & 8865 \\
\hline nitrite & & 693 & & & & & & $<10$ & $<10$ \\
\hline oxalate & & 4068 & & & & & & $<10$ & $<10$ \\
\hline phosphate & & 53.9 & & & & & & $<10$ & $<10$ \\
\hline sulfate & & 104 & & & & & & $<5$ & $<5$ \\
\hline Total Acid M & & & 1.20 & & 1.61 & & 1.74 & 0.449 & 0.0623 \\
\hline
\end{tabular}




\subsubsection{Simulant Handling (Author - Williams)}

For the following description of how the recycle FEP feed stream was made at the experimental site, see Fig. 3. A 760-liter tank was used to mix pilot-scale quantities of simulated HLW recycles that were then transferred to the pilot-scale FEP Feed Tank, which was represented by the plant tank: FEP-VSL-17AB. On 18 May 2004, pilot-scale quantities of HLW Canister Decon and HLW Vessel Vent solutions were synthesized by combining 330 liters of deionized and filtered (DIF) water with $3 \mathrm{~kg}$ of $50 \mathrm{wt} \% \mathrm{NaOH}$, $2.77 \mathrm{~kg}$ of sodium nitrate, and $192.7 \mathrm{~kg}$ of HLW SBS Quencher solution from VSL. At this stage, the mixing tank represented the plant tank: PWD-VSL-44. To this mixture, the contents of mock plant tank PWD-VSL-33 (Plant Overflow Vessel) were added, which contained $5.31 \mathrm{~kg}$ of Campaign II concentrated washed HLW slurry and 8.6 liters of Campaign II FEP condensate. After mixing the entire contents of the mock PWDVSL-44, and -33, they were transferred to the FEP Feed Tank on 19 May, 2004. Before proceeding with the mock PWD-VSL-15 mixing, a beaker mixing recycle study of the proposed Evaporator Feed Tank contents was completed with samples from the respective recycle streams to ensure there were no unexpected consequences from the mixing sequence. The results were documented in the laboratory notebook (Williams, 2004).

On 24 May, the then empty 760-liter mixing tank was used as a plant mock PWD-VSL15 tank and was filled with caustic recycle streams from Campaign II. To that tank, the second of two drums of wash permeate was added, drum No. RM-03-270, which contained $119.3 \mathrm{~kg}$ of material. Then $21.3 \mathrm{~kg}$ of $0.1 \mathrm{M} \mathrm{NaOH}$ from condensate and the $0.1 \mathrm{M}$ caustic flush recycle streams were added from three drums referred to as caustic flush 1, 2, and 3. The amounts added from drums Nos. RM-03-298, RM-03-284, and RM-03-285, were $11.8 \mathrm{~kg}, 33.1 \mathrm{~kg}$, and $32.7 \mathrm{~kg}$, respectively. The material remaining in the drums was returned to storage. The IX recycles were added next including 36.7 liters of $0.1 \mathrm{M} \mathrm{NaOH}$ from condensate, 19.0 liters of Post-Elution Rinse displaced by Regen Solution, 17.7 liters of DI Water, 2.0 liters of $0.25 \mathrm{M} \mathrm{NaOH}$ Regen Solution and 38.1 liters of $0.25 \mathrm{M} \mathrm{NaOH}$ from DI Water. The target $\mathrm{pH}$ for this mixture was to be greater than or equal to 12, and since the $\mathrm{pH}$ was measured at 13 no additional $\mathrm{NaOH}$ was added. The contents of this mock PWD-VSL-15 mixing vessel were then added to the mixture in the FEP Feed Tank, also on 24 May.

Once again the 760-liter mixing tank was empty and this time it would represent the plant PWD-VSL-16 tank to receive the acidic recycle streams. That solution has to be adjusted to be alkaline before being joined to the caustic contents of the FEP feed tank.

Specifically, the empty tank was first filled with $130.3 \mathrm{~kg}$ of $0.1 \mathrm{M} \mathrm{NaOH}$ wash permeate from the first of two drums of wash permeate recycle, drum No. RM-03-269. While the tank agitator was running, two drums of spent $2 \mathrm{M}$ nitric acid UFP cleaning solution were added with constant stirring. From the drum (referred to as acid cleaning 1) No. RM-03297 an amount of $35.8 \mathrm{~kg}$ was transferred and from RM-03-301 (referred to as acid cleaning 2 \& 3) an amount of $72.0 \mathrm{~kg}$ was transferred. The acid/caustic mixture was transferred to the FEP Feed Tank on 25 May to start the clock on a 2-day holding period 
before evaporation began. The total material mixed in the pilot-scale FEP unit operation Feed Tank, Fig. 4, and subsequently sent to the FEP Evaporator, was 1,112.2 kg.

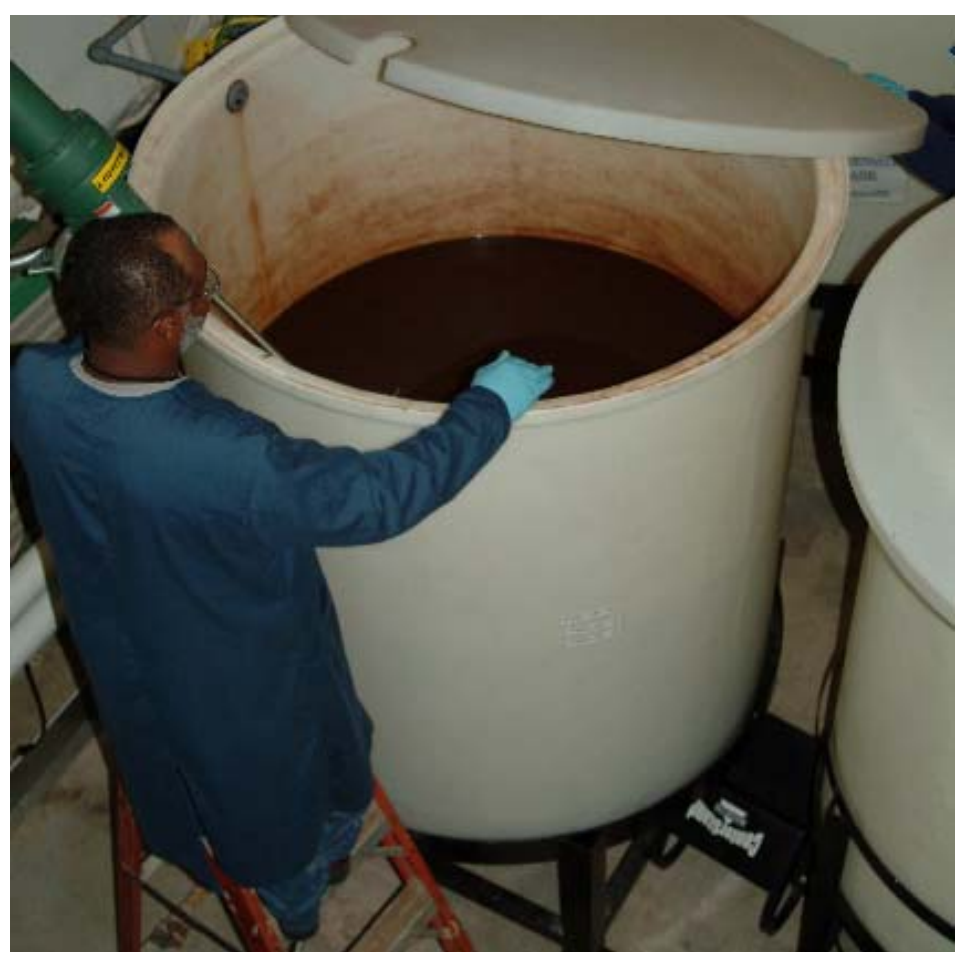

Figure 4. Pilot-scale FEP Unit Operation Feed Tank

After evaporation started on 7 June, and during the first 30 minutes of operation, $278.4 \mathrm{~g}$ of Dow Corning Q2-3183A antifoam were added in three batches of $92.8 \mathrm{~g}$ each. Note that, per the vendor's recommendation the antifoam was diluted to a 10:1 ratio with deionized and filtered water (DIF) before the addition was made. At the end of FEP testing, a total of $170.8 \mathrm{~kg}$ of concentrate "FEP bottoms" were produced with a density of $1.257 \mathrm{gm} / \mathrm{ml}$, as measured by volumetric flask at $25.5^{\circ} \mathrm{C}$, and $1.259 \mathrm{gm} / \mathrm{ml}$, as measured by densitometer at $20^{\circ} \mathrm{C}$.

\subsubsection{Pilot FEP Equipment (Author - Qureshi)}

All evaporation campaigns were performed in the Pilot-Scale Evaporator designed and constructed for the RPP Project. The evaporator is located in the Engineering Development Laboratory of the Savannah River National Laboratory (SRNL).

Figure 5 shows the Process and Instrumentation Diagram (P\&ID). The Pilot Scale Evaporator System consists of many sub-systems provided below alphabetically. 
- Antifoam System

- Concentrate Loop

- Evaporator Recirculation

- Feed Loop

- House Air

- Jet Pump

- Primary Condensate

- Process Water

- Secondary Condensate

- Steam Generator

- Spray Water

Typically, each of the SIPP campaigns was to evaporate about 1,000 liters of waste simulant. The pilot-scale SIPP evaporator uses forced circulation where heat is added in the reboiler that is essentially a shell and tube heat exchange with steam on the shell side. No evaporation occurs in the reboiler due to hydrostatic pressure head. The evaporator vessel is kept under vacuum ( 1 psia or $6.9 \mathrm{kPa}$ ) and the boiling temperature for water is about $104^{\circ} \mathrm{F}\left(40^{\circ} \mathrm{C}\right)$ at this pressure. The simulant has a boiling point elevation of about $11^{\circ} \mathrm{F}\left(\sim 6^{\circ} \mathrm{C}\right)$. (Thus the entire evaporation process occurs at moderate temperatures, 100 $\left.-120 \mathrm{~F}\left(44^{\circ}-49^{\circ} \mathrm{C}\right)\right)$. The evaporator vessel is about 27 feet $(8.2 \mathrm{~m})$ tall with a nominal inside diameter of 17 " $(0.43 \mathrm{~m})$. The bottom 12 feet of the vessel is stainless steel and the top 15 feet is acrylic, see Fig. 6 . 
WSRC-TR-2004-00565, REVISION 0

SRNL-RPP-2004-00092, REVISION 0

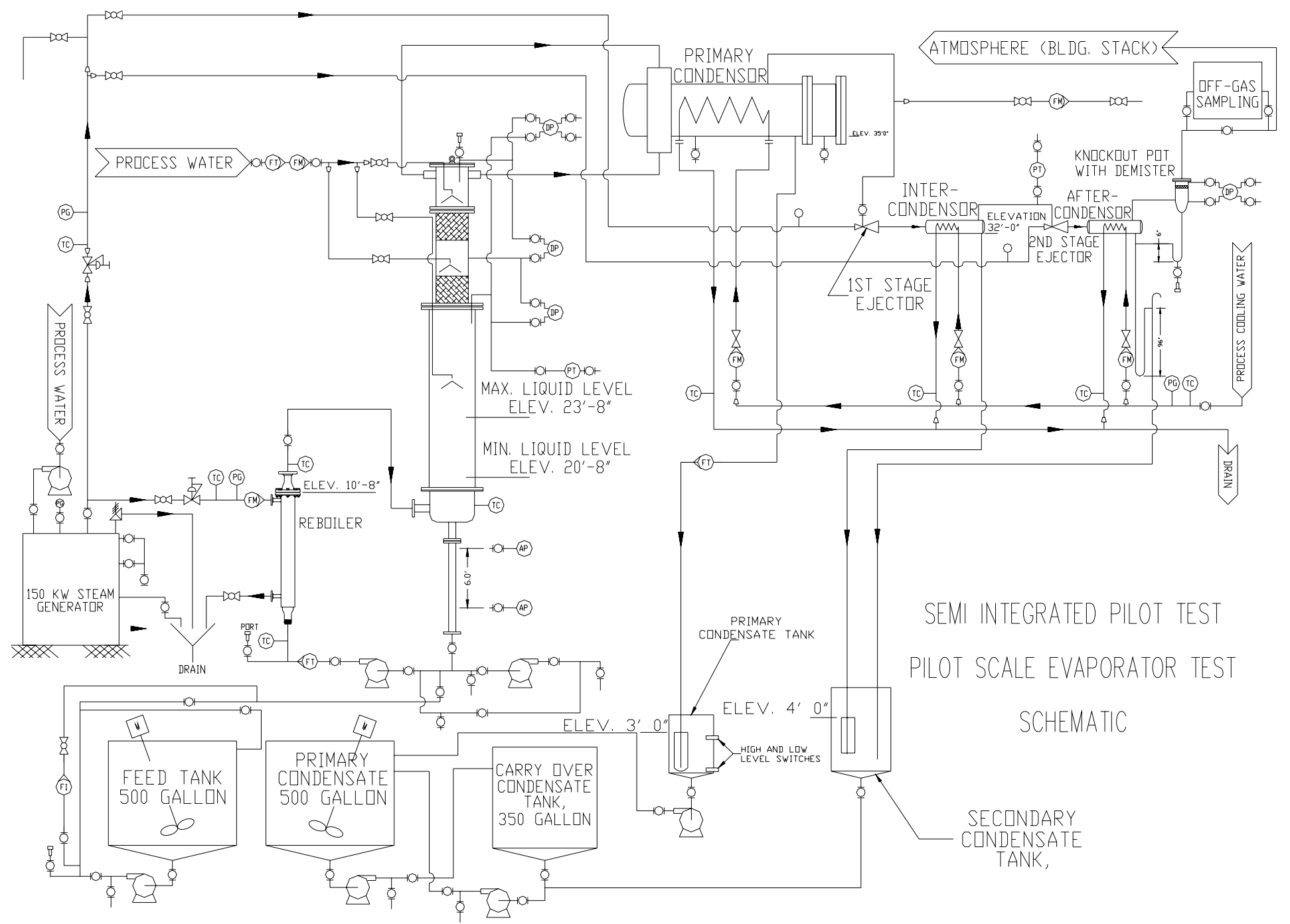

Figure 5. SIPP Pilot-Scale FEP/TLP Unit 
WSRC-TR-2004-00565, REVISION 0

SRNL-RPP-2004-00092, REVISION 0

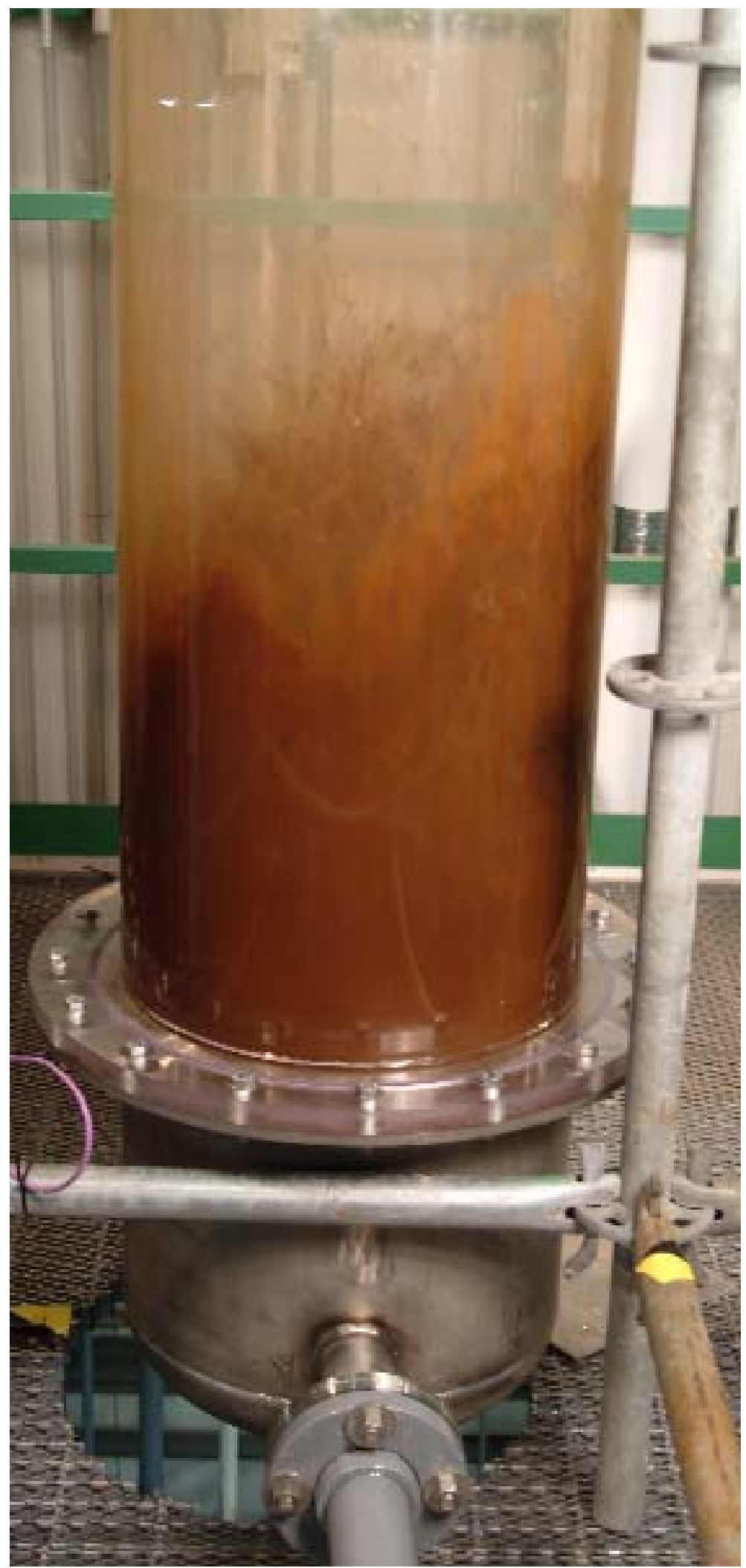

Figure 6. SIPP Pilot-Scale FEP/TLP Unit: The bottom half of the 15-foot tall Acrylic Section with Ongoing Evaporation 
Of the bottom 12 feet $(3.7 \mathrm{~m})$, the stainless steel vessel is only 2 feet $(0.6 \mathrm{~m})$ high and the remaining height of 10 feet $(3 \mathrm{~m})$ is taken up by a 2 " $(0.05 \mathrm{~m})$ stainless steel pipe. The boiling surface is maintained in the lower part of the acrylic section. The minimum and maximum liquid levels in the acrylic section are 5" $(0.13 \mathrm{~m})$ and $40 "(1.0 \mathrm{~m})$, respectively, see Fig 7.

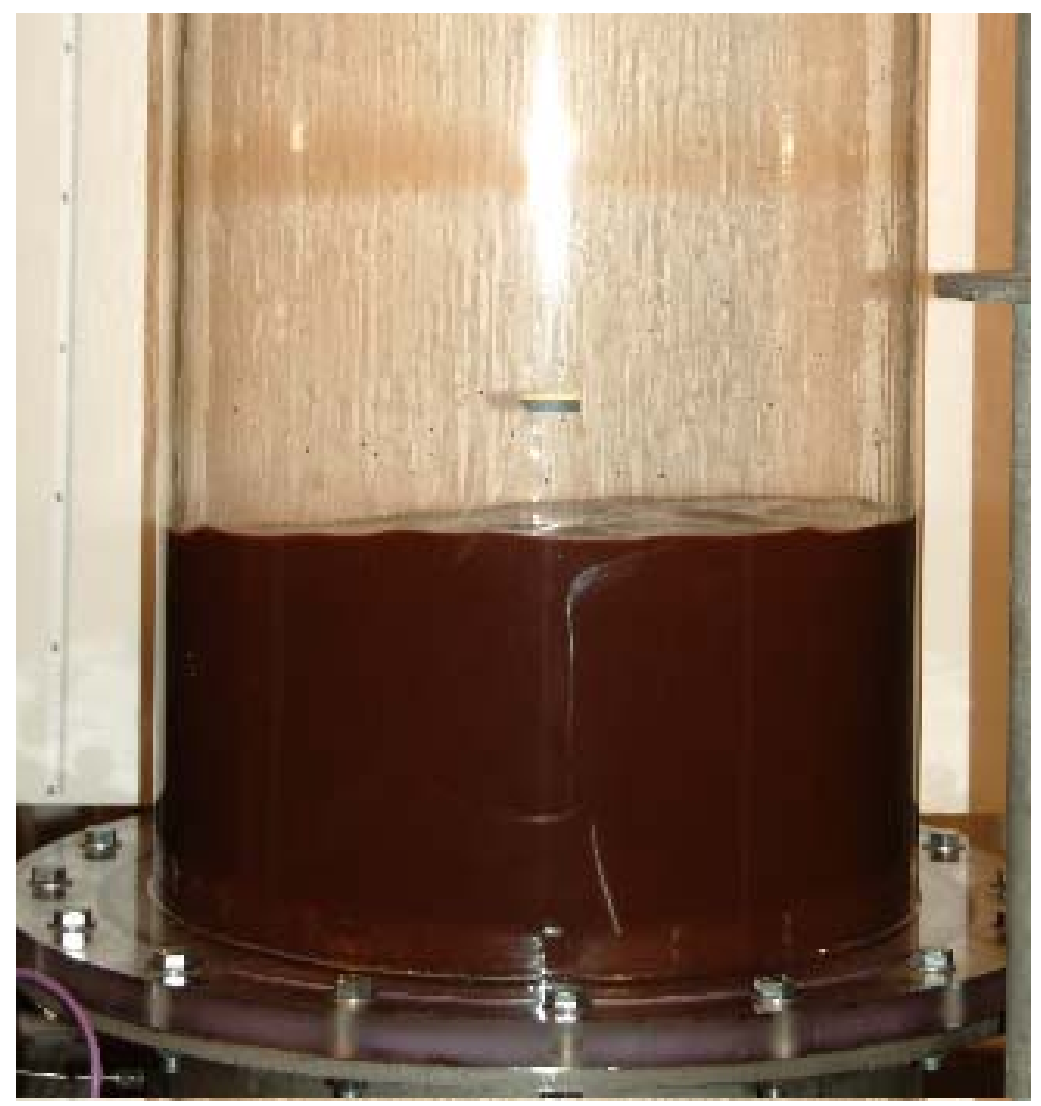

Figure 7. SIPP Pilot-Scale FEP/TLP Unit: Feed Ready for Evaporation to Commence

The designed capacity of evaporator vessel is about 40 gallons (151 liters) and a feed tank supplies the simulant to the evaporator. The evaporator is designed to evaporate $0.4-0.5$ gpm $(1.5-1.9 \mathrm{lpm})$ of water. Figure 8 shows the pilot-scale FEP at approximately the 30-gallons level while evaporating at approximately $0.5 \mathrm{gpm}$, before and after defoamer was added. 


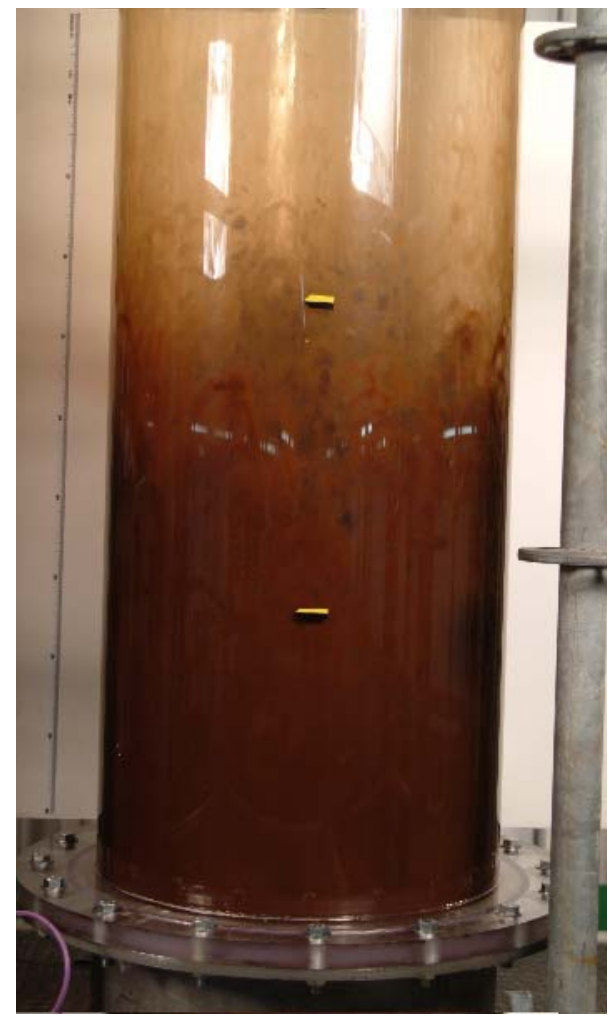

(a) Before Defoamer Addition

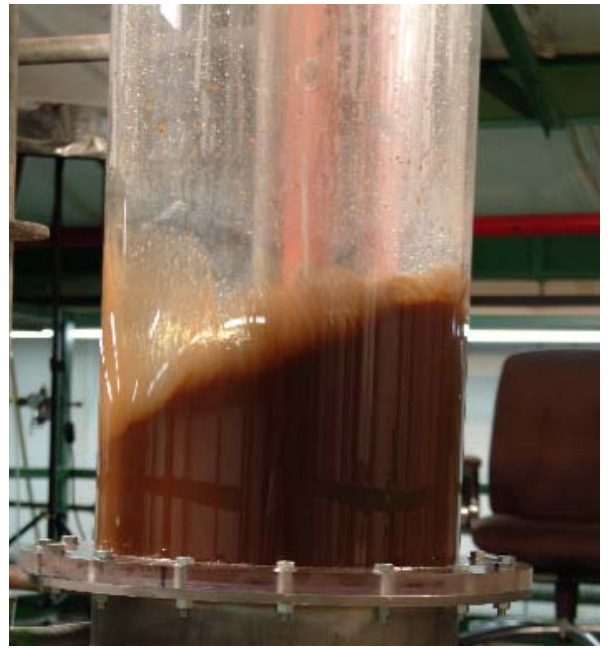

(b) After Defoamer Addition

Figure 8. SIPP Pilot-Scale FEP/TLP Unit: Evaporating

The water vapor is condensed in the primary condenser. Under steady conditions, the concentrate is removed from the recirculation line and collected in a drum. The condensate from the primary condenser is collected in the primary condensate tank. A two-stage steam driven jet pump maintains desired vacuum in the evaporator vessel. The jet pump has its own set of condensers, inter and after-condenser. A separate tank catches the condensate from the jet pump. Several sample and injection ports are provided to draw samples and inject antifoam agent as needed. All test data was acquired by a data acquisition system (DAS). Additionally, manual data and observations were recorded in the lab notebook issued for this task (Qureshi, 2004).

\subsubsection{Pilot FEP Operation (Author - Qureshi)}

Prior to the SIPP campaigns, the evaporator system was disassembled, cleaned, inspected and reassembled. The system was found to be free of any material defects, fouling and malfunction from previous evaporation runs. After assembly of the system the following tests were performed to ensure proper operation of the evaporator. 


\subsubsection{Experimental Facility Leak Testing}

Objective - Determine if the system can hold vacuum and the air in-leakage rate is acceptable.

\section{Test Sequence}

i) Isolated the Evaporator Recirculation (ER) Loop from rest of the system by closing appropriate valves.

ii) Disconnected the First Stage Ejector line from the primary condenser and connected a vacuum pump at the primary condenser discharge.

iii) Filled water in Evaporator vessel to the maximum operating liquid level (about 40" $(1.02 \mathrm{~m}))$ in the lower acrylic section.

iv) Filled up the primary dip tube reservoir with water.

v) Started and operated the vacuum pump until the water level in the dip tube is about 31 feet $(9.4 \mathrm{~m})$ of water.

vi) Verified that there was no air bubbling into the system.

vii) Verified that the system can hold vacuum for about 30 minutes.

\subsubsection{Evaporation Test with Water}

Objective - To shake down the system and its components using water as test fluid prior to the chemical runs.

\section{Test Sequence}

i) Isolated the Reboiler and the Jet Pump from the Steam Generator.

ii) Started the Steam Generator.

iii) Filled the Feed Tank with Process Water to about 25\% level (about 125 gallons (743 liters)).

iv) Using the Feed Loop pump FL-PU1, transferred water from the Feed Tank to the evaporator vessel to a level about 40 ” $(1.02 \mathrm{~m})$ in the acrylic section.

v) Filled the primary condensate and secondary condensate dip tubes with water.

vi) Started the Jet Pump Vacuum System. 
vii) Started the main recirculation pump and started supplying steam to the Reboiler.

viii) Adjusted steam to the Reboiler to limit the system temperature below $140^{\circ} \mathrm{F}\left(60^{\circ} \mathrm{C}\right)$.

ix) Once the system pressure dropped to about 1 psia (6.9 $\mathrm{kPa}$ ), boiled water for about 30 minutes.

x) After completing the water boiling test, turned off the steam supply to the Jet Pump and the Reboiler and shut down the Steam Generator.

\subsubsection{SIPP-Feed Evaporation Process (FEP) Tests}

Objective - To perform pilot scale tests of the Feed Evaporation Process.

This evaporation campaign used about $1112 \mathrm{~kg}$ of FEP Feed. The feed composition and mixing process is described in Sections 2.6.1.1 and 2.6.1.2.

The FEP Evaporation Campaign III took about 9 hours to complete. During this time period, several samples were drawn as outlined in SIPP Sample Matrix Campaign III (see Appendix B).

\section{Test Sequence}

i) Started Steam Generator and the Data Acquisition System.

ii) Ran the Feed Tank mixer for about 30 minutes.

iii) Using the Feed Loop Pump, transferred feed from the Feed Tank to the evaporator vessel to about 24 " $(0.61 \mathrm{~m})$ level in the clear section.

iv) Filled the primary condensate and secondary condensate dip tubes with water.

v) Supplied process water to the Jet Pump condensers and started the Jet Pump.

vi) Supplied process water to the Primary Condenser to about 30 gpm (114 lpm).

vii) Started the main recirculation pump and turned on the steam to the Reboiler.

viii) Once the feed reached the saturation temperature and boiling started, maintained the liquid level in the evaporator by feeding the appropriate amount of feed using the feed pump.

ix) Recorded all the temperature, pressure and flow date via DAS. Additionally, video taped the action near the boiling surface as needed at different times 
x) Antifoam agent was added to the recirculation loop at the beginning of the evaporation campaign.

xi) Drew liquid samples according the sample matrix.

xii) Continued evaporation process until the target density of $1.253 \mathrm{~g} / \mathrm{ml} @ 25^{\circ} \mathrm{C}$ and 1 atm (or $1.226 \mathrm{~g} / \mathrm{ml} @ 50^{\circ} \mathrm{C}$ and $1 \mathrm{psia}$ ) was reached. [Note: There were several types of density measurements made but the most accurate was done by a Anton Paar, DMA 4500 meter which maintains samples at $20^{\circ} \mathrm{C}$. The target density at $20^{\circ} \mathrm{C}$ was estimated to be $1.258 \mathrm{~g} / \mathrm{ml}$ and this was used during the test to determine when the FEP was properly concentrated.]

xiii) Once the evaporation campaign was complete, the steam generator was shut down and all the concentrate was transferred into a 55-gal drum.

xiv) After removing all the concentrate, filled the system with water ( $\sim 30$ gallons (114 liters)) and rinsed it thoroughly by running the recirculation pump.

\subsubsection{Pilot FEP Discussion of Results}

\subsubsection{Experimental Observations (Author-Qureshi))}

The SIPP FEP Campaign III process began on Wednesday, 7 June 2004 in SRNL's Engineering Development Lab. A total of about $1,112 \mathrm{~kg}$ of feed was concentrated down to about $170 \mathrm{~kg}$. The entire evaporation process proceeded without any glitch or equipment failure. The evaporator was designed to evaporate around $0.4 \mathrm{gpm}(1.5 \mathrm{lpm})$ of water. The average evaporation rate during this campaign was around $0.52 \mathrm{gpm}$.

Table 19 provides a chronology of the evaporation campaign. Time recorded below follows a military format. 
WSRC-TR-2004-00565, REVISION 0

SRNL-RPP-2004-00092, REVISION 0

Table 19. Chronology of the Campaign III FEP operations

\begin{tabular}{|c|c|c|}
\hline Time & Event (Evaporation occurred on 9 June 2004) & Comments \\
\hline 0600 & Started Feed Mixing by pump recirculation and agitator & \\
\hline 0615 & Started Steam Generator & \\
\hline 0630 & Started Feed transfer to evaporator vessel & \\
\hline 0635 & Started Jet Pump to pull vacuum in the evaporator & \\
\hline 0640 & Pulled Feed sample & 1.035 g/ml @25C \\
\hline 0642 & Stopped Feed transfer & \\
\hline 0658 & Started main circulation pump & \\
\hline 0700 & Started steam supply to reboiler & \\
\hline 0704 & Added $278.5 \mathrm{~g}$ of antifoam agent and 1:10 ratio DI water & Added in the recirc. loop \\
\hline 0722 & Steady boiling taking place & Stable froth height $\sim 1 \mathrm{ft}$. \\
\hline 0846 & Pulled all samples at 400 liter total transfer & \\
\hline 0919 & Pulled $200 \mathrm{ml}$ sample for density check & $1.077 \mathrm{~g} / \mathrm{ml} @ 40 \mathrm{C}$ \\
\hline 1008 & Pulled all samples at 600 liter total transfer & \\
\hline 1015 & Pulled $200 \mathrm{ml}$ sample for density check & $1.109 \mathrm{~g} / \mathrm{ml} @ 50 \mathrm{C}$ \\
\hline 1136 & Pulled all samples at 800 liter total transfer & \\
\hline 1136 & Pulled $200 \mathrm{ml}$ sample for density check & 1.147 g/ml @50C \\
\hline 1140 & Pulled $10 \mathrm{ml}$ sample for density check with density meter & $1.164 \mathrm{~g} / \mathrm{ml} @ 20 \mathrm{C}$ \\
\hline 1312 & Pulled $10 \mathrm{ml}$ sample for density check with density meter & 1.188 g/ml @ 20C \\
\hline 1400 & Transferred all feed to the evaporator & \\
\hline 1407 & Pulled $200 \mathrm{ml}$ sample for density check & $1.177 \mathrm{~g} / \mathrm{ml} @ 50 \mathrm{C}$ \\
\hline 1425 & Pulled $10 \mathrm{ml}$ sample for density check with density meter & $1.245 \mathrm{~g} / \mathrm{ml} @ 20 \mathrm{C}$ \\
\hline 1429 & Pulled $10 \mathrm{ml}$ sample for density check with density meter & $\begin{array}{l}1.257 \mathrm{~g} / \mathrm{ml} @ 20 \mathrm{C} \\
\text { Target value reached }\end{array}$ \\
\hline 1430 & System shut down. Pulled all end samples & \\
\hline 1455 & $\begin{array}{l}\text { Transferred all concentrate to a } 55 \text { gal drum Concentrate } \\
\text { produced } 170.1 \mathrm{~kg}\end{array}$ & \\
\hline
\end{tabular}

\subsubsection{FEP Waste Products (Author-Zamecnik)}

Table 20 shows the total and dissolved (supernate) compositions of the Campaign III FEP feed and concentrated bottoms. The concentrations of the more prevalent species in the FEP streams for Campaigns II and III are compared in Table 21. Note that complete ion analyses are not available for Campaign II. The Campaign II average UDS and total solids $(0.29,3.03 \mathrm{wt} \%)$ are both slightly higher than the respective values for Campaign III $(0.23,2.70 \mathrm{wt} \%)$. Similar trends are seen in the individual undissolved species, such as Fe (4600 vs. $3910 \mathrm{mg} / \mathrm{kg}$ ) or Mn (942 vs. $828 \mathrm{mg} / \mathrm{kg}$ ). Most of the soluble species such as Na were lower in Campaign III than in Campaign II. 
WSRC-TR-2004-00565, REVISION 0 SRNL-RPP-2004-00092, REVISION 0

Table 20. Composition of Campaign III FEP Evaporator Streams

\begin{tabular}{|c|c|c|c|c|}
\hline $\begin{array}{l}\text { All units } \mathrm{mg} / \mathrm{kg} \text { unless } \\
\text { otherwise noted }\end{array}$ & Feed Slurry & $\begin{array}{c}\text { Feed } \\
\text { Permeate }\end{array}$ & $\begin{array}{l}\text { Concentrate } \\
\text { Slurry }\end{array}$ & $\begin{array}{c}\text { Concentrate } \\
\text { Permeate }\end{array}$ \\
\hline Total Solids (wt\%) & 4.93 & 4.71 & 30.75 & 28.82 \\
\hline \multirow{2}{*}{ Undissolved Solids (wt\%) } & 0.23 & & 2.70 & \\
\hline & $(0.12-0.35)$ & 0 & $(2.55-2.86)$ & 0 \\
\hline Supernate Solids (wt\%) & 4.71 & 4.71 & 28.82 & 28.82 \\
\hline Density (kg/L) & 1.035 & 1.038 & 1.255 & $1.231 *$ \\
\hline $\mathrm{Ag}$ & 13.3 & 0.82 & 54.7 & 4.91 \\
\hline $\mathrm{Al}$ & 811 & 452 & 5255 & 2781 \\
\hline $\mathrm{B}$ & & 95.4 & 566 & 581 \\
\hline $\mathrm{Ba}$ & 5.25 & $<0.02$ & 24.5 & $<0.02$ \\
\hline $\mathrm{Ca}$ & 24.5 & 2.84 & 111 & 6.66 \\
\hline $\mathrm{Cd}$ & 1.20 & $<0.03$ & 6.00 & $<0.02$ \\
\hline $\mathrm{Ce}$ & $<15.4$ & $<1.5$ & $<115$ & $<1.2$ \\
\hline Co & $<0.88$ & $<0.08$ & $<7.3$ & $<0.07$ \\
\hline $\mathrm{Cr}$ & 25.4 & 20.4 & 156 & 126 \\
\hline Cs & & & 17.3 & \\
\hline $\mathrm{Cu}$ & $<1$ & $<0.1$ & 8.00 & $<0.08$ \\
\hline $\mathrm{Fe}$ & 635 & $<0.09$ & 3910 & $<0.07$ \\
\hline $\mathrm{K}$ & 1930 & & 11350 & \\
\hline $\mathrm{La}$ & $<14$ & $<1.4$ & $<104$ & $<1.1$ \\
\hline $\mathrm{Li}$ & 16.5 & 1.16 & 105 & $<0.16$ \\
\hline $\mathrm{Mg}$ & 13.4 & $<0.16$ & 70.0 & $<0.13$ \\
\hline $\mathrm{Mn}$ & 134 & $<0.02$ & 828 & $<0.01$ \\
\hline Mo & $<2$ & 0.97 & $<15$ & 6.08 \\
\hline $\mathrm{Na}$ & 13300 & 13623 & 79400 & 80159 \\
\hline $\mathrm{Nd}$ & 15.1 & $<0.50$ & 79.2 & $<0.41$ \\
\hline $\mathrm{Ni}$ & 15.1 & $<0.12$ & 128 & 0.72 \\
\hline $\mathrm{P}$ & 65.3 & 54.8 & 444 & 396 \\
\hline $\mathrm{Pb}$ & 31.3 & $<1.3$ & 225 & $<1.1$ \\
\hline $\mathrm{Rb}$ & & & 7.1 & \\
\hline S & 133 & 135 & 813 & 829 \\
\hline $\mathrm{Si}$ & 147 & 9.06 & 1560 & 65.9 \\
\hline $\mathrm{Sr}$ & 9.25 & 0.04 & 54.1 & 0.20 \\
\hline $\mathrm{Ti}$ & $<2.8$ & $<0.27$ & 34 & $<0.22$ \\
\hline $\mathrm{Zn}$ & $<7.41$ & $<0.71$ & 58.0 & $<0.59$ \\
\hline $\mathrm{Zr}$ & 8.41 & $<0.09$ & 53.6 & $<0.08$ \\
\hline bromide & $<86$ & $<96$ & $<96$ & $<79$ \\
\hline chloride & 147 & 132 & 1150 & 1100 \\
\hline fluoride & $<17$ & 11.6 & 93.0 & 35.9 \\
\hline formate & 86 & 78.3 & 732 & 667 \\
\hline nitrate & 19700 & 17101 & 120000 & 121912 \\
\hline nitrite & 2240 & 2058 & 14400 & 14422 \\
\hline oxalate & 1070 & 976 & 3860 & 924 \\
\hline phosphate & 363 & 303 & 2270 & 2279 \\
\hline sulfate & 350 & 318 & 2260 & 2191 \\
\hline total inorganic carbon (TIC) & & 812 & 5630 & 6020 \\
\hline total organic carbon (TOC) & & 378 & 3920 & $<500$ \\
\hline carbonate from TIC & & 4060 & 28150 & 30100 \\
\hline carbonate (titration) & & 4020 & & 30600 \\
\hline oxalate from TOC & & 1379 & 14314 & $<1902$ \\
\hline
\end{tabular}

* estimated 
WSRC-TR-2004-00565, REVISION 0

SRNL-RPP-2004-00092, REVISION 0

Table 21. Comparison of Campaign II and III FEP Compositions

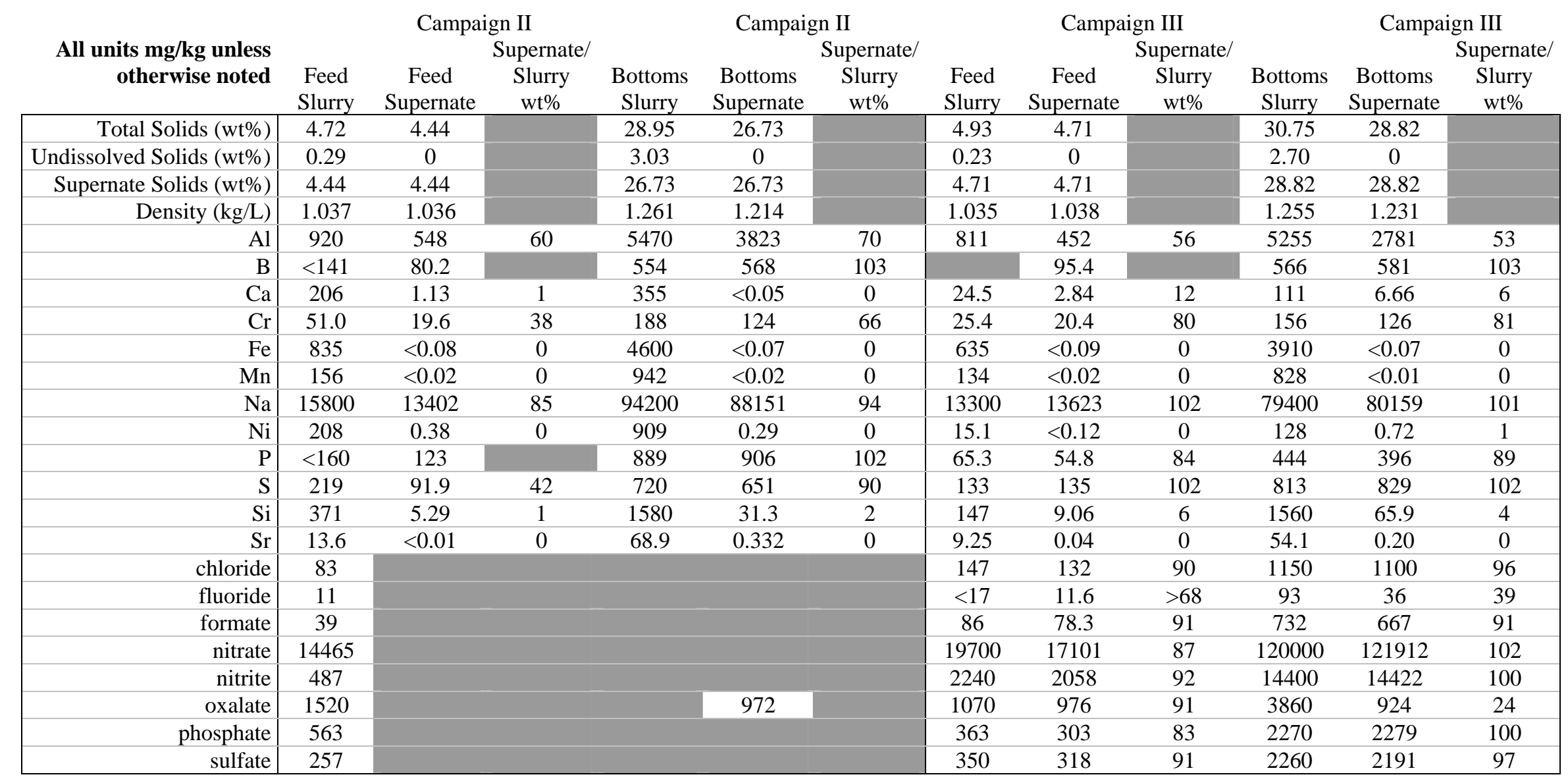


The final Na concentration in Campaign III was $\sim 3.5 \mathrm{M}$ versus the target of $\sim 4 \mathrm{M}$ at a density of $1.26 \mathrm{~kg} / \mathrm{L} @ 25^{\circ} \mathrm{C}$; the final Na in Campaign II was 3.96M. The final density in Campaign III was $1.255 \mathrm{~kg} / \mathrm{L}$, which is essentially the target value of 1.26 . Species such as K, nitrate, and nitrite, were more abundant in the recycles in Campaign III due to the AP101 washed from the slurry in Campaign II.

The $\mathrm{Al}$ in Campaign III was less dissolved than in Campaign II. About 53-56\% (feed/concentrate) was dissolved in Campaign III versus $60-70 \%$ in Campaign II. This result indicates that the distribution of the $\mathrm{Al}$ species in Campaign III was different than in Campaign II. Table 18 shows that the majority of the $\mathrm{Al}$ in the UF cleaning recycles was undissolved. The $\mathrm{Al}$ in the wash permeates was, of course, soluble. Based on the quantities added, roughly $50 \%$ of the $\mathrm{Al}$ would be assumed to be soluble, which is what was found. The exact distribution is difficult to predict from the mixing of the recycles. The total $\mathrm{Al}$ in the FEP streams in Campaign III were very similar to the values for Campaign II.

Table 21 also shows that only a portion of the $\mathrm{Cr}$ appears to be undissolved. About $80 \%$ was dissolved in Campaign III compared to 38-66\% in Campaign II. However, the concentrations are low and subject to significant relative uncertainty, so this difference may not be real. The phosphorus in the Campaign III streams appears to have been only about $90 \%$ dissolved based on the ICPES analyses; all of the P appears to have been dissolved in Campaign II. However, the phosphorus calculated from the IC analyses is consistently about twice as high as the P from elemental analyses, so this observation is uncertain. A small amount (6-12\%) of the calcium in Campaign III was dissolved versus virtually none for Campaign II.

Predictions of the FEP feed composition were made for both Campaign II and Campaign III. These predictions were made by mathematically combining each of the streams to the evaporator. A list of these streams was shown in Table 16. Some assumptions were made in performing these calculations:

- The total solids of $\mathrm{NaOH}$ synthetic streams could be approximated as the wt\% of $\mathrm{Na}$.

- The total solids of acid cleaning streams was the sum of the measured total solids plus the contribution of nitrate that would not be measured in acid solution by the solids analysis technique.

- The nitrate in the acid cleaning streams was the same molarity as the total acid measurement.

- Where permeate analyses were not available, the presence of no dissolved species was assumed.

- Where analyses of dissolved low-UDS samples were not available, the permeate analyses were used.

Tables 22 and 23 show the calculated and measured FEP feed compositions for Campaign II and Campaign III, respectively. For both Campaign II and Campaign III, the predicted and measured $\mathrm{Na}$ and nitrate agree well. Many less abundant species also agree reasonably well, and the undissolved solids predictions are good. 
WSRC-TR-2004-00565, REVISION 0

SRNL-RPP-2004-00092, REVISION 0

Table 22. Campaign II Predicted and Measured FEP Evaporator Feed Compositions

\begin{tabular}{|c|c|c|c|c|c|c|}
\hline $\begin{array}{l}\text { All units mg/kg unless } \\
\text { otherwise noted }\end{array}$ & $\begin{array}{c}\text { FEP Feed } \\
\text { Total } \\
\text { Predicted }\end{array}$ & $\begin{array}{c}\text { FEP Feed } \\
\text { Total } \\
\text { Measured }\end{array}$ & $\begin{array}{l}\text { FEP Feed } \\
\text { Permeate } \\
\text { Predicted }\end{array}$ & $\begin{array}{l}\text { FEP Feed } \\
\text { Permeate } \\
\text { Measured }\end{array}$ & $\begin{array}{l}\text { Permeate/ } \\
\text { Slurry } \\
\text { Predicted \% }\end{array}$ & $\begin{array}{c}\text { Permeate/ } \\
\text { Slurry } \\
\text { Measured \% }\end{array}$ \\
\hline Total Solids (wt\%) & 3.70 & 4.69 & 3.54 & & & \\
\hline Undissolved Solids (wt\%) & 0.33 & 0.29 & 0.00 & & & \\
\hline Supernate Solids (wt\%) & 3.38 & 4.41 & 3.54 & & & \\
\hline Density $(\mathrm{kg} / \mathrm{L})$ & 1.033 & 1.037 & 1.033 & & & \\
\hline $\mathrm{Ag}$ & 12.4 & $<<33$ & 0.53 & 0.800 & & \\
\hline $\mathrm{Al}$ & 918 & 920 & 736 & 549 & 80 & 60 \\
\hline B & 88 & $<141$ & 84.7 & 80.4 & & \\
\hline $\mathrm{Ba}$ & 5.68 & 5.19 & 0.74 & $<0.02$ & & \\
\hline $\mathrm{Ca}$ & 29.8 & 206 & 2.42 & 1.13 & & \\
\hline $\mathrm{Cd}$ & 1.013 & $<4.0$ & 0.060 & $<0.03$ & & \\
\hline $\mathrm{Ce}$ & 5.53 & $<126$ & 0.44 & $<1.5$ & & \\
\hline Co & 0.56208 & 223 & 0.01 & $<0.09$ & & \\
\hline $\mathrm{Cr}$ & 27.9 & 51.0 & 19.0 & 19.7 & 68 & 39 \\
\hline $\mathrm{Cu}$ & 1.73 & 16 & 0.28 & $<0.10$ & & \\
\hline $\mathrm{Fe}$ & 666 & 835 & 43 & $<0.09$ & & \\
\hline $\mathrm{K}(\mathrm{AA})$ & 200 & $<30$ & 199 & 40 & & \\
\hline $\mathrm{La}$ & 5.34 & $<12$ & 0 & $<1.4$ & & \\
\hline $\mathrm{Li}$ & 18.2 & 27.8 & 18.0 & 0.22 & & \\
\hline $\mathrm{Mg}$ & 12.6 & 14.1 & 4.79 & $<0.16$ & & \\
\hline $\mathrm{Mn}$ & 144.3 & 156 & 0.6 & $<0.02$ & & \\
\hline Mo & 0.49 & $<20$ & 8.80 & $<0.19$ & & \\
\hline $\mathrm{Na}$ & 16018 & 15800 & 15749 & 14815 & 98 & 94 \\
\hline $\mathrm{Nd}$ & 12.3 & $<72$ & 48.9 & $<0.50$ & & \\
\hline $\mathrm{Ni}$ & 22.0 & 208 & 1.35 & 0.385 & & \\
\hline $\mathrm{P}$ & 138.4 & $<160$ & 131.4 & 123.7 & & \\
\hline $\mathrm{Pb}$ & 27.2 & $<160$ & 0.78 & $<1.3$ & & \\
\hline $\mathrm{S}$ & 97 & 219 & 93 & 92 & 96 & 42 \\
\hline $\mathrm{Si}$ & 323 & 371 & 70.6 & 5.30 & & \\
\hline $\mathrm{Sr}$ & 8.43 & 13.6 & 1.87 & $<0.01$ & & \\
\hline $\mathrm{Ti}$ & 0.497 & 7.4 & 0.004 & $<0.27$ & & \\
\hline $\mathrm{Zn}$ & 6.89 & 4.9 & 0.354 & $<0.72$ & & \\
\hline $\mathrm{Zr}$ & 8.22 & 23.2 & 0.19 & $<0.09$ & & \\
\hline bromide & 4 & 32 & 4 & & & \\
\hline chloride & 73 & 83 & 73 & & 100 & \\
\hline fluoride & 13.7 & 11 & 13.5 & & 99 & \\
\hline formate & 26 & 39.0 & 26 & & 99 & \\
\hline nitrate & 13639 & 14465 & 13638 & & 100 & \\
\hline nitrite & 551 & 487 & 547 & & 99 & \\
\hline oxalate & 1510 & 1520 & 1482 & & 98 & \\
\hline phosphate & 617 & 563 & 610 & & 99 & \\
\hline sulfate & 268 & 257 & 266 & & 99 & \\
\hline carbonate (from TIC) & 1168 & & 1136 & & 97 & \\
\hline oxalate (from TOC) & 254 & & 222 & & 87 & \\
\hline
\end{tabular}


WSRC-TR-2004-00565, REVISION 0

SRNL-RPP-2004-00092, REVISION 0

Table 23. Campaign III Predicted and Measured FEP Evaporator Feed Compositions

All units mg/kg unless

otherwise noted

\begin{tabular}{|c|c|c|c|c|c|c|}
\hline & Predicted & Measured & Predicted & Measured & Predicted \% & Measured \% \\
\hline Total Solids (wt\%) & 5.19 & 4.93 & & 4.71 & & \\
\hline Undissolved Solids (wt\%) & 0.17 & 0.23 & & 0.00 & & \\
\hline Supernate Solids (wt\%) & 0.00 & 4.71 & & 4.71 & & \\
\hline Density $(\mathrm{kg} / \mathrm{L})$ & 1.038 & 1.035 & & 1.038 & & \\
\hline $\mathrm{Ag}$ & 13.2 & 12.9 & 3.22 & 0.79 & & \\
\hline $\mathrm{Al}$ & 828 & 783 & 736 & 436 & 89 & 56 \\
\hline B & 111 & & 94.4 & 92.0 & & \\
\hline $\mathrm{Ba}$ & 4.01 & 5.07 & 1.24 & $<0.02$ & & \\
\hline $\mathrm{Ca}$ & 13.7 & 23.7 & 4.71 & 2.73 & 34 & 12 \\
\hline $\mathrm{Cd}$ & 0.69 & 1.16 & 0.13 & $<0.03$ & & \\
\hline $\mathrm{Ce}$ & 1.29 & $<15$ & 0.85 & $<1.4$ & & \\
\hline Co & 0.003 & $<0.85$ & 0.003 & $<0.08$ & & \\
\hline $\mathrm{Cr}$ & 25.4 & 24.6 & 19.9 & 19.6 & 78 & 80 \\
\hline $\mathrm{Cu}$ & 1.40 & $<1.0$ & 0.41 & $<0.10$ & & \\
\hline $\mathrm{Fe}$ & 473 & 613 & 103 & $<0.09$ & & \\
\hline $\mathrm{K}(\mathrm{AA})$ & 1707 & 1865 & 1681 & & & \\
\hline $\mathrm{La}$ & 1.51 & $<14$ & 0.69 & $<1.3$ & & \\
\hline $\mathrm{Li}$ & 19.3 & 16.0 & 18.4 & 1.12 & & \\
\hline $\mathrm{Mg}$ & 11.1 & 12.9 & 5.90 & $<0.15$ & & \\
\hline $\mathrm{Mn}$ & 95.6 & 129 & 18.0 & $<0.02$ & & \\
\hline Mo & 5.25 & $<1.9$ & 5.25 & 0.93 & & \\
\hline $\mathrm{Na}$ & 13275 & 12850 & 13113 & 13129 & 99 & 102 \\
\hline $\mathrm{Nd}$ & 22.2 & 14.6 & 20.8 & $<0.48$ & & \\
\hline $\mathrm{Ni}$ & 14.9 & 14.6 & 2.88 & $<0.12$ & & \\
\hline $\mathrm{P}$ & 73.1 & 63.1 & 72.2 & 52.8 & 99 & 84 \\
\hline $\mathrm{Pb}$ & 17.4 & 30.3 & 4.19 & $<1.3$ & & \\
\hline $\mathrm{S}$ & 140 & 129 & 140 & 130 & 99 & 101 \\
\hline $\mathrm{Si}$ & 116 & 142 & 18.5 & 8.73 & 16 & 6 \\
\hline $\mathrm{Sr}$ & 6.52 & 8.93 & 2.13 & 0.038 & & \\
\hline $\mathrm{Ti}$ & 0.56 & $<2.7$ & 0.21 & $<0.26$ & & \\
\hline $\mathrm{Zn}$ & 4.75 & $<7.2$ & 0.52 & $<0.68$ & & \\
\hline $\mathrm{Zr}$ & 6.37 & 8.12 & 1.12 & $<0.09$ & & \\
\hline bromide & 0 & $<83$ & 0 & $<93$ & & \\
\hline chloride & 178 & 142 & 178 & 128 & 100 & 90 \\
\hline fluoride & 10.1 & $<16$ & 10.1 & 11.2 & & \\
\hline formate & 125 & 83.1 & 124 & 75.4 & 99 & 91 \\
\hline nitrate & 21014 & 19034 & 20979 & 16480 & 100 & 87 \\
\hline nitrite & 2659 & 2164 & 2650 & 1983 & 100 & 92 \\
\hline oxalate & 959 & 1034 & 896 & 940 & 93 & 91 \\
\hline phosphate & 391 & 351 & 391 & 292 & 100 & 83 \\
\hline sulfate & 389 & 338 & 387 & 306 & 100 & 91 \\
\hline carbonate (from TIC) & 2419 & & 2399 & 4061 & 100 & \\
\hline oxalate (from TOC) & 2781 & & 2692 & 1379 & 100 & \\
\hline
\end{tabular}

FEP Feed FEP Feed FEP Feed FEP Feed

Total Total Permeate

Permeate/ Permeate/

Slurry Slurry

redicted \% Measured \% 
The total solids predicted for Campaign II are low compared to the measured amount, but are in very good agreement for Campaign III. The sulfur value measured for the slurry in Campaign II had a potentially high blank, so this value is questionable; it also does not agree with the sulfate measurement. The Campaign III anion values for the permeate are all lower than the values measured for the slurry by around $10 \%$. (It was $17 \%$ for phosphate and there is no comparison for fluoride due to the lack of data.) Since there are no insoluble nitrite, nitrate, or formate compounds that could precipitate, we attribute the lower permeate anion values, except phosphate, to an analytical uncertainty. The analytical uncertainty of the anion analysis technique is at best $\pm 10 \%$, so the anion results except for phosphate, include $100 \%$ in the error bound for chloride, nitrite, oxalate, and sulfate; nitrate is within 13\%. The Campaign III phosphorus and phosphate both indicate that perhaps $16 \%$ of the $\mathrm{P}$ was undissolved versus the prediction of no undissolved $\mathrm{P}$. The Campaign II data for P are inconclusive, and no Ion Chromatography (IC) analyses were available for phosphate.

For both Campaign II and Campaign III, the measured ratio of permeate-to-slurry aluminum was lower than expected based on a simple material balance with no consideration of solubility changes. This lower ratio indicates that soluble $\mathrm{Al}$ in the feeds had precipitated. The higher than predicted undissolved Al indicates that $25-40 \%$ of the dissolved $\mathrm{Al}$ in the feeds to the FEP precipitated during feed mixing. Even though the bulk $\mathrm{pH}$ of the FEP feed mixtures was maintained at greater than 12, it appears that localized regions of lower $\mathrm{pH}$ must have resulted in precipitation of $\mathrm{Al}$ (possibly as gibbsite):

$$
\mathrm{Al}(\mathrm{OH})_{4}^{-}+\mathrm{HNO}_{3} \rightarrow \mathrm{Al}(\mathrm{OH})_{3}+\mathrm{NO}_{3}^{-}+\mathrm{H}_{2} \mathrm{O}
$$

This precipitated $\mathrm{Al}$ did not re-dissolve. Note that some formation of undissolved $\mathrm{Al}$ was also shown to occur in the first acid cleaning step in the UF process, but this precipitation would have already been accounted for in the recycle stream analyses, and hence, in the predictions. Therefore, the Al precipitation observed upon FEP feed mixing would be in addition to this prior precipitation.

Small-scale mixing tests done prior to Campaign III showed that rapid addition of the nitric acid recycle stream to streams with dissolved $\mathrm{Al}$ resulted in a gelatinous white precipitate that quickly re-dissolved upon vigorous mixing. However, the degree of redissolution was not determined. Therefore, even though the bulk $\mathrm{pH}$ was maintained where $\mathrm{Al}$ would not precipitate, precipitation did actually occur. Poor mixing when adding the acid stream to a caustic stream would exacerbate this situation since poor mixing would promote localized regions of lower $\mathrm{pH}$. This was observed in the FEP feed preparations. No special methods were planned on how to add one recycle stream to another apart from the indicated groupings and volumes shown in Fig. 3 and explained as Simulant Handling Section 2.6.2. However, before one completed batch was added to the next the batch it was required to be well mixed. When mixing the contents of the mock WTP tank PWD-VSL-16, as shown at the bottom of Fig. 3, the first acid cleaning solution was added to the caustic UFP wash permeate. The only mixing that occurred 
was from the action of joining the volumes together. As the milky white acid stream (its composition is listed in Table 18) was poured on top of the quiescent clear yellowish caustic stream (the wash permeate composition is listed in Table 17) there was an immediate reaction on the surface of the caustic, which turned the mixture brownish. This brown coloring was expected because the acid stream contained dissolved transition metals such as iron that precipitate as brownish hydroxides when the $\mathrm{pH}$ is changed to basic ( $\mathrm{pH} \sim 12$ ). Unfortunately, this precipitate visually masked any $\mathrm{Al}$ that may have also precipitated.

The batch was then mixed well and maintained mixed while the last two clear acid cleaning solutions were added. This brownish mixture was then vigorously mixed for approximately one hour before it was finally added to the FEP feed tank, as shown in Figure 3. As in the small scale, the $\mathrm{pH}$ of the joined mixture was maintained high enough to prevent $\mathrm{Al}$ precipitation in a well mixed environment, i.e., $\mathrm{pH}>12$. However, practically maintaining a batch well mixed at the same time that its level is constantly changing is not a simple task. As pockets of less-than-well-mixed solution occur there is the potential of precipitation. It is expected that this will also occur in the WTP PWD tanks and consideration should be given to providing a well-mixed environment.

The phosphorus in the FEP feed and bottoms was also measured by IC. The samples were analyzed by IC in two ways: the sample was filtered prior to analyses, resulting in the dissolved anion concentration; and the sample was diluted $>100 \mathrm{X}$ with DI water, then analyzed to give an approximate "total" anion concentration. This "total" is the amount of an anion easily dissolvable in water, so phosphate or fluoride in fluorophosphates or other relatively insoluble compounds would not be measured by this method. Oxalate may also not be completely measured by this method if some is present as heavy metal oxalates; however, this method should dissolve alkali oxalates present due to being above saturation. (Estimation of oxalate from total organic carbon analyses was not possible due to very large inaccuracies in these results.)

The IC analyses of the total bottoms concentrate and concentrate permeate (Table 21) gave virtually the same values for all species except oxalate and fluoride, indicating that a portion of the oxalate and fluoride were undissolved. The results for phosphate are inconclusive since the IC analysis indicates $100 \%$ solubility while the P analysis suggests $11 \%$ undissolved. The feed data indicated that there may have been undissolved phosphate.

Undissolved fluoride could be present due to two sources. Relatively insoluble $\mathrm{CaF}_{2}$ was added to the AY102 simulant in small quantities. The sodium fluorophosphate double salt $2 \mathrm{Na}_{3} \mathrm{PO}_{4} \cdot \mathrm{NaF}$ has also been found to precipitate from the AY102 simulant, so it could possibly have been formed during the evaporation.

The estimated total oxalate in the Campaign III feed was about 50\% less than the amount in the Campaign II feed (1070 vs. $1520 \mathrm{mg} / \mathrm{kg})$. This difference is consistent with the recycle solution compositions; the Campaign I recycles to Campaign II contained higher 
oxalate because the oxalate concentration in the Campaign I slurry was higher (oxalate in the AY102-only stream is higher than in the AY102+AP101 mixture).

The oxalate analyses show that the dissolved oxalate concentration remains essentially constant in these evaporator solutions. The values for Campaign II concentrate, and Campaign III feed and concentrate were 972, 976, and $924 \mathrm{mg} / \mathrm{kg}$, respectively. The consistency of these values suggests that this is the approximate solubility of oxalate in these streams. Table 24 presents the material balance on undissolved solids and oxalate. The data used to calculate the increase in UDS had significant relative error; the measured UDS values were 0.12 and $0.35 \mathrm{wt} \%$, which when converted to a total mass basis, is significant. The solids analysis technique is relatively inaccurate at concentrations below $1 \mathrm{wt} \%$, so wide variations in measured values result from analysis of a single sample. While the final report will treat uncertainties in more detail, the solids analyses of ten Campaign III samples that were done in duplicate, and ranged from 2 to $46 \mathrm{wt} \%$, had standard deviations of:

$$
\begin{aligned}
& \text { Mean } \sigma(\text { total solids, TS })= \pm 0.11 \mathrm{wt} \% \\
& \text { Mean } \sigma(\text { undissolved solids, UDS })= \pm 0.22 \mathrm{wt} \% \\
& \text { Mean } \sigma(\text { soluble solids, SS })= \pm 0.13 \mathrm{wt} \%
\end{aligned}
$$

If the standard deviation is assumed to be the uncertainty in the measurements, then the UDS values for the feed are $0.12 \pm 0.22$ and $0.35 \pm 0.22$, which overlap.

Similar absolute uncertainty applies to the concentrate also. The amount of UDS in the feed was 1285-3849 g, while that in the concentrate was 4333-4867 g. The undissolved oxalate, when assumed to be sodium oxalate $\left(\mathrm{Na}_{2} \mathrm{C}_{2} \mathrm{O}_{4}\right)$, was $1414 \mathrm{~g}$, which was obtained from difference of the dissolved sodium oxalate in the feed, $1653 \mathrm{~g}$, and the dissolved sodium oxalate in the bottoms, $239 \mathrm{~g}$. This amount of oxalate is reasonably close to the average increase in UDS of $2033 \mathrm{~g}$, so the precipitation of sodium oxalate may account for most of the increase in undissolved solids. This same behavior was seen in Campaign II. Also, note that the calculated amount of oxalate in the concentrate is only about $50 \%$ of the amount in the feed. This discrepancy is probably due to not all of the oxalate in the concentrate dissolving in the dilution for the IC analysis.

Table 24. Mass Balance on FEP Solids

\begin{tabular}{|r|ccc|ccc|ccc|}
\multicolumn{1}{c}{} & \multicolumn{2}{c}{ Feed } & \multicolumn{2}{c}{ Bottoms } & \multicolumn{3}{c|}{ Difference } \\
\multicolumn{1}{c}{} & Low & Mean & High & Low & Mean & High & Low & Mean & High \\
\hline Undissolved Solids (wt\%) & $0.12 \pm 0.22$ & 0.23 & $0.35 \pm 0.22$ & $2.55 \pm 0.22$ & 2.70 & $2.86 \pm 0.22$ & & & \\
\hline Undissolved Solids (g) & 1285 & 2567 & 3849 & 4333 & 4600 & 4867 & 3048 & 2033 & 1018 \\
\hline Total Oxalate (g) & & 1190 & & & 657 & & & & \\
Dissolved Oxalate (g) & & 1085 & & & 157 & & & \\
\hline Oxalate* as $\mathrm{Na}_{2} \mathrm{C}_{2} \mathrm{O}_{4}$ (g) & & 1653 & & \multicolumn{3}{c|}{239} & & 1414 \\
\hline
\end{tabular}

*Obtained from the dissolved oxalate in the preceding row

\section{* A note on Ammonium in the FEP *}

The total ammonium in the FEP feed was measured at $<69 \mathrm{mg} / \mathrm{kg}$. The calculated value, based on the two ammonium containing inputs, was $24 \mathrm{mg} / \mathrm{kg}$, or about $26.6 \mathrm{~g}$. The FEP 
condensate produced (939L) contained $65 \mathrm{mg} / \mathrm{kg}$ of ammonium, or a mass of $61 \mathrm{~g}$. This mass is more than twice the amount calculated in the FEP feed, but this difference is probably within the uncertainty of the measurements used for the calculation. It is also possible that some residual ammonium remained in the evaporator system from the Campaign II TLP evaporation. While the system was cleaned several time between the campaigns there may have been a hidden pocket of liquid. The measured ammonium in the FEP evaporator bottoms was $<51 \mathrm{mg} / \mathrm{kg}$ which means $170 \mathrm{~kg}$ produced contained less than $9 \mathrm{~g}$.

\subsubsection{Pilot FEP Conclusions}

\subsubsection{Experimental Observations (Author-Qureshi)}

During SIPP FEP Campaign III, a total of about $1112 \mathrm{~kg}$ of feed was concentrated down to $170 \mathrm{~kg}$. The feed was evaporated under vacuum (1 psia $(6.9 \mathrm{kPa}))$ at moderate temperatures $\left(\sim 50^{\circ} \mathrm{C}\right)$. The entire evaporation process proceeded as designed. The evaporator was designed to evaporate around $0.4 \mathrm{gpm}(1.5 \mathrm{lpm})$ of water. The average evaporation rate during this campaign was around $0.52 \mathrm{gpm}$.

No significant foaming was observed prior to the addition of antifoam agent. During the evaporation process, the froth height remained around $1 \mathrm{ft}(0.3 \mathrm{~m})$ that is significantly less than the $5 \mathrm{ft}(1.5 \mathrm{~m})$ distance to the lower demister pad. However, during start-up and unsteady conditions, significant flashing resulted in feed splashing on the lower demister pad. These conditions are associated with much larger vapor flux and vapor velocities at the interface.

The concentrate density was measured by three methods during the evaporation process. In the first method, warm concentrate $\left(\sim 50^{\circ} \mathrm{C}\right)$ was drawn in a 50 or $200 \mathrm{ml}$ flask and weighed immediately on a scale accurate to $0.1 \mathrm{~g}$. The second method employed a density meter with an accuracy of $0.001 \mathrm{~g} / \mathrm{ml}$. The density meter would cool the sample and the density was reported at $20^{\circ} \mathrm{C}$; this was the density measurement used for reporting. The third method was online density measurement using two absolute pressure transducers placed $6 \mathrm{ft}(1.82 \mathrm{~m})$ apart in a vertical leg of the evaporator recirculation loop. This method resulted in large uncertainties in density measurement since two large pressure signals were subtracted, but was useful in determining when the density target was near. Thus samples were drawn periodically for density measurement by the density meter.

\subsubsection{Waste Products Observations (Author - Zamecnik)}

- As seen in Campaign II, a large portion of the dissolved oxalate in the feed was precipitated, probably as sodium oxalate. 
- The oxalate concentration in the FEP feed in Campaign III was less than that in Campaign II, which is consistent with the higher oxalate concentration in the Campaign I slurry.

- About $25-40 \%$ of the dissolved $\mathrm{Al}$ in the feed to the FEP evaporator precipitated during mixing of the recycles, assumed to occur because of localized regions of low $\mathrm{pH}$. Vigorous mixing when joining caustic and acidic recycle streams in the Plant Wash and Disposal System would help to minimize Al precipitation.

- The fraction of dissolved aluminum in Campaign III FEP concentrate (bottoms) was less than in Campaign II, resulting in more undissolved Al fed to the UFP.

\subsection{PILOT ULTRAFILTRATION (AUTHORS - FOWLEY, WILLIAMS \& ZAMECNIK)}

The RPP-WTP Ultrafiltration Process (UFP) will remove undissolved solids (UDS) from a combined HLW and LAW feed. The solids are removed to protect downstream ion exchange (IX) beds and to meet the specifications for LAW product. In addition, the UFP will concentrate the removed solids as a slurry to minimize storage requirements for HLW and to enhance HLW vitrification efficiency.

The SIPP UFP will concentrate and remove solids from combined simulated waste feed streams. The SIPP UFP filters a mixture of FEP bottoms (which are the recycle streams concentrated in FEP) mixed with simulated wastes of AY102 and AP101 to produce two products: 1) A concentrated slurry HLW product for vitrification, and 2) A liquid LAW product for the ion exchange process. Recycle streams are included in the SIPP UFP to mimic actual WTP operation.

Table 25 shows the SIPP UFP Campaign III test matrix. The test number ordering has been maintained and sequentially adjusted from Campaign I (Duignan, et al., 2004a). Several tests were eliminated prior to Campaign I and between Campaign I and II, which produced numerical gaps between several of the test numbers.

The remainder of this section will explain the pilot UFP unit operation that was done as part of Campaign III testing. The process started with the preparation of the feed product. The chemical explanation of the feed stream formation will be followed by a short description on how feed formation was handled just before UFP operation and then how the waste stream was handled during and after its separation. The following section will discuss the physical equipment used to filter the waste and how the test was run. Finally, the results will be discussed and conclusions made. 
WSRC-TR-2004-00565, REVISION 0

SRNL-RPP-2004-00092, REVISION 0

Table 25. Campaign III UFP Test Matrix

\begin{tabular}{|c|c|c|c|c|c|}
\hline $\begin{array}{c}\text { Test } \\
\text { No. }\end{array}$ & Test Activity & $\begin{array}{c}\text { Trans-Membrane } \\
\text { Pressure } \\
(\mathrm{psid})\end{array}$ & $\begin{array}{c}\text { Slurry } \\
\text { Velocity } \\
(\mathrm{ft} / \mathrm{s})\end{array}$ & $\begin{array}{c}\text { Slurry } \\
\text { Temp. } \\
\left({ }^{\circ} \mathrm{C}\right)\end{array}$ & $\begin{array}{c}\text { Run } \\
\text { Time } \\
(\mathrm{min})\end{array}$ \\
\hline 3.00A & Pre-test baseline A $^{1}$ & 10 & 11 & 25 & 20 \\
\hline 3.00B & Pre-test baseline B $^{1}$ & 20 & 11 & 25 & 20 \\
\hline 3.00C & Pre-test baseline C $^{1}$ & 30 & 11 & 25 & 20 \\
\hline 3.03A & Dewater first half of slurry $^{2}$ & 40 & 12 & 25 & 960 \\
\hline 3.03B & Dewater second half of slurry $^{2}$ & 40 & 12 & 25 & 2300 \\
\hline Wash 1 & Slurry wash 1 $^{3}$ & 40 & 12 & 25 & 379 \\
\hline Wash 2 & Slurry wash 2 $^{3}$ & 40 & 12 & 25 & 229 \\
\hline 3.18A & Caustic rinse 1 $^{4}$ & 20 & 11 & 25 & 30 \\
\hline 3.18B & Acid clean 1 $^{5}$ & 40 & 11 & 25 & 90 \\
\hline 3.18C & Acid clean 2 $^{5}$ & 40 & 11 & 25 & 90 \\
\hline 3.18D & Acid clean 3 $^{5}$ & 40 & 11 & 25 & 90 \\
\hline 3.19A & Caustic rinse 2 $^{4}$ & 20 & 11 & 25 & 30 \\
\hline 3.19B & Caustic rinse 3 $^{4}$ & 20 & 11 & 25 & 30 \\
\hline 3.21A & Post-test baseline A $^{6}$ & 10 & 11 & 25 & 20 \\
\hline 3.21B & Post-test baseline B $^{6}$ & 20 & 11 & 25 & 20 \\
\hline 3.21C & Post-test baseline C $^{6}$ & 30 & 11 & 25 & 20 \\
\hline
\end{tabular}

Notes:

1. The pre-test baseline tests were conducted with de-ionized, filtered (absolute $0.1 \mu \mathrm{m}$ ) water made caustic to

$0.1 \mathrm{M} \mathrm{NaOH}$.

2. The slurry was dewatered to $20 \mathrm{wt} \%$ undissolved solids or greater.

3. The slurry was washed with $0.01 \mathrm{M} \mathrm{NaOH}$ solution made from Campaign II RLD condensate.

4. The caustic rinse consisted of $0.1 \mathrm{M} \mathrm{NaOH}$ solution made from Campaign II RLD condensate.

5. The acid clean consisted of $2 \mathrm{M} \mathrm{HNO}_{3}$ solution.

6. The post-test baseline tests were conducted with FEP condensate made caustic to $0.1 \mathrm{M} \mathrm{NaOH}$.

\subsubsection{Chemical Description of the Pilot-Scale UFP Feed Stream (Author - Zamecnik)}

Figures 9 and 10 show schematically how the concentration, washing, rinsing and cleaning steps were performed for Campaign III. The pilot-scale UFP feed slurry was made by combining the FEP evaporator bottoms, AY102 slurry simulant, and AP101 supernate simulant. The feed was not extensively characterized as it was for Campaign II, because it was basically the same. Only solids and density measurements were made. However, for convenience the composition of the Campaign II feed is summarized in Table 26. 
WSRC-TR-2004-00565, REVISION 0

SRNL-RPP-2004-00092, REVISION 0

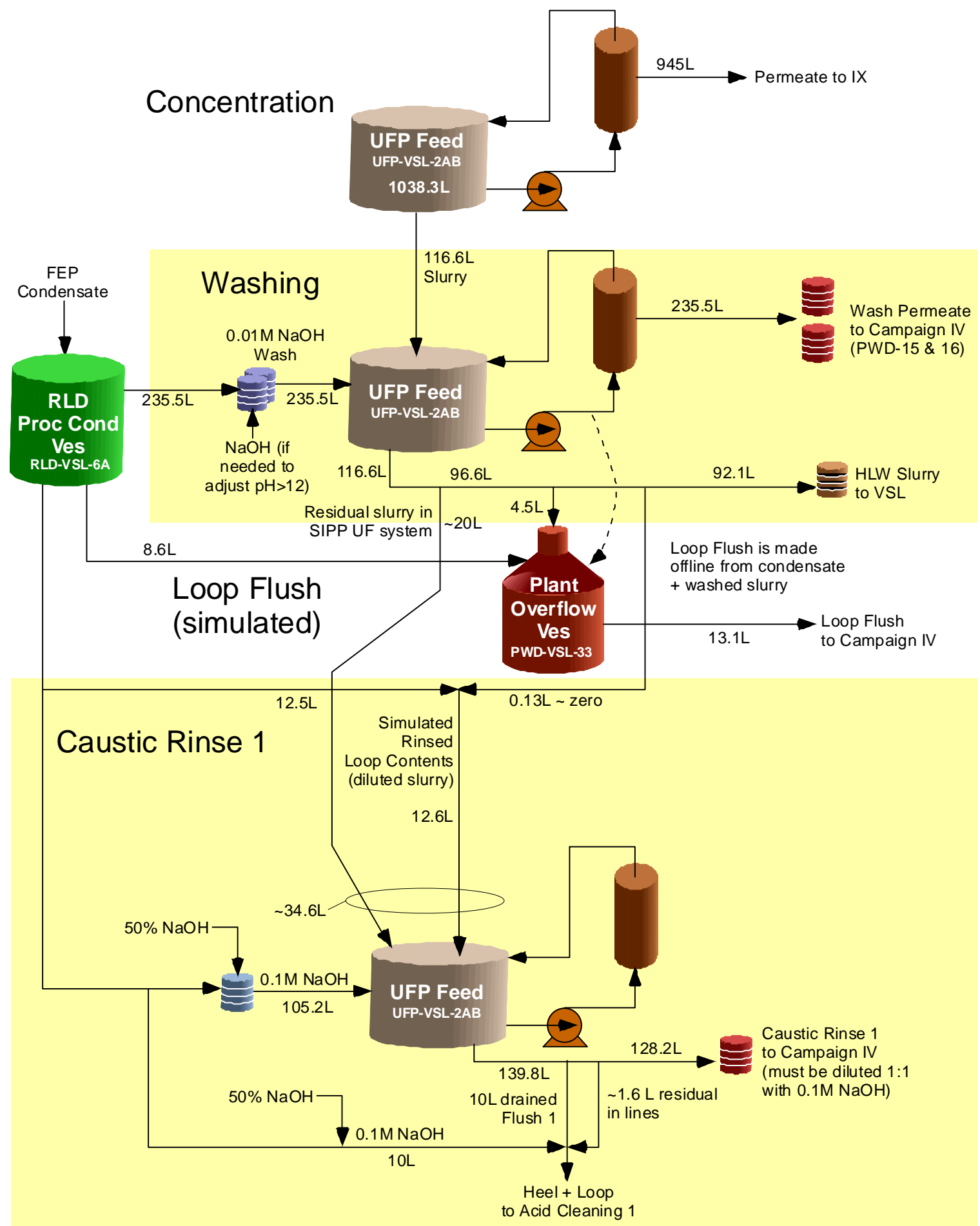

Figure 9. Schematic of Ultrafiltration Process - Page 1 


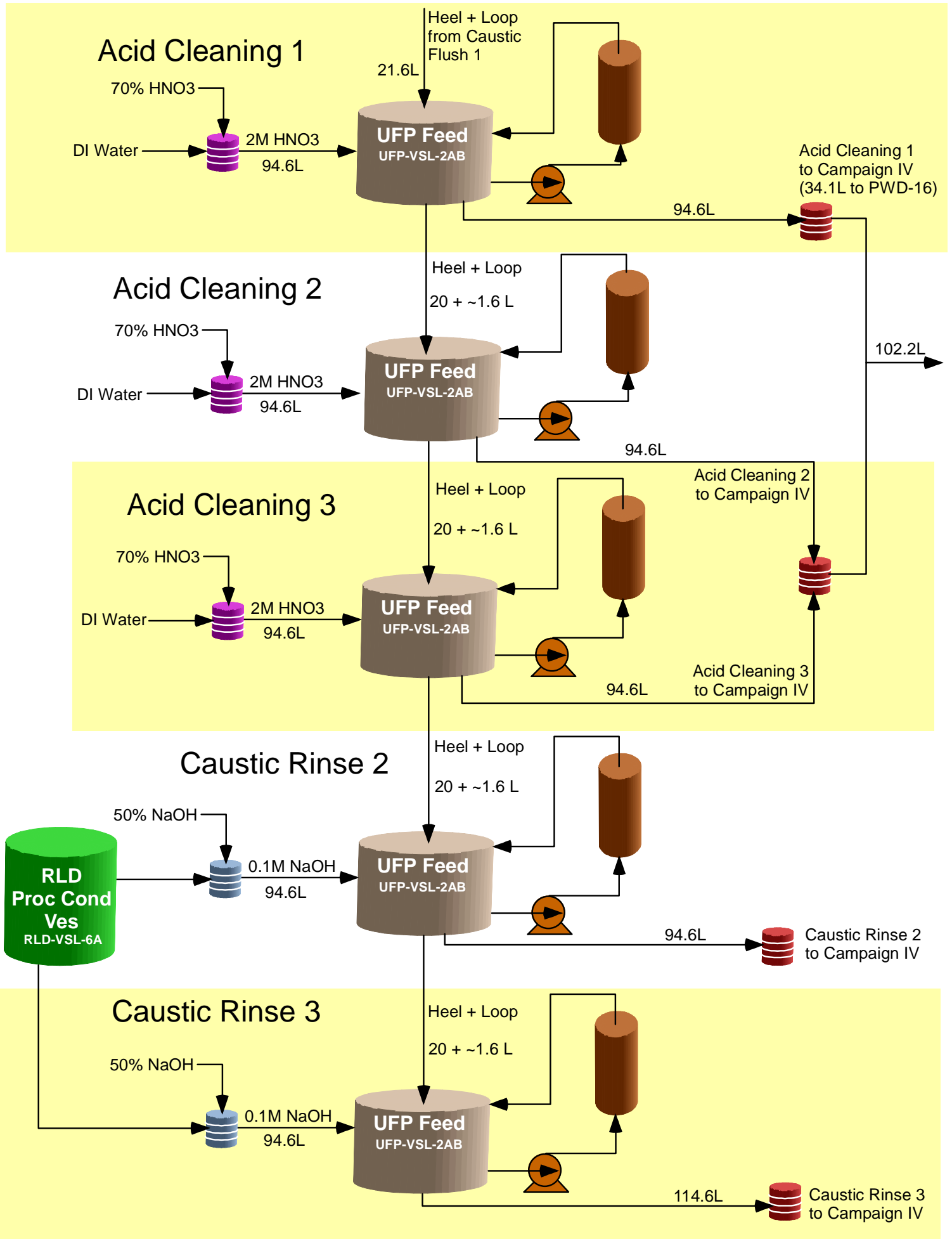

Figure 10. Schematic of Ultrafiltration Process - Page 2 
WSRC-TR-2004-00565, REVISION 0 SRNL-RPP-2004-00092, REVISION 0

Table 26. Measured Pilot-Scale UFP Feeds

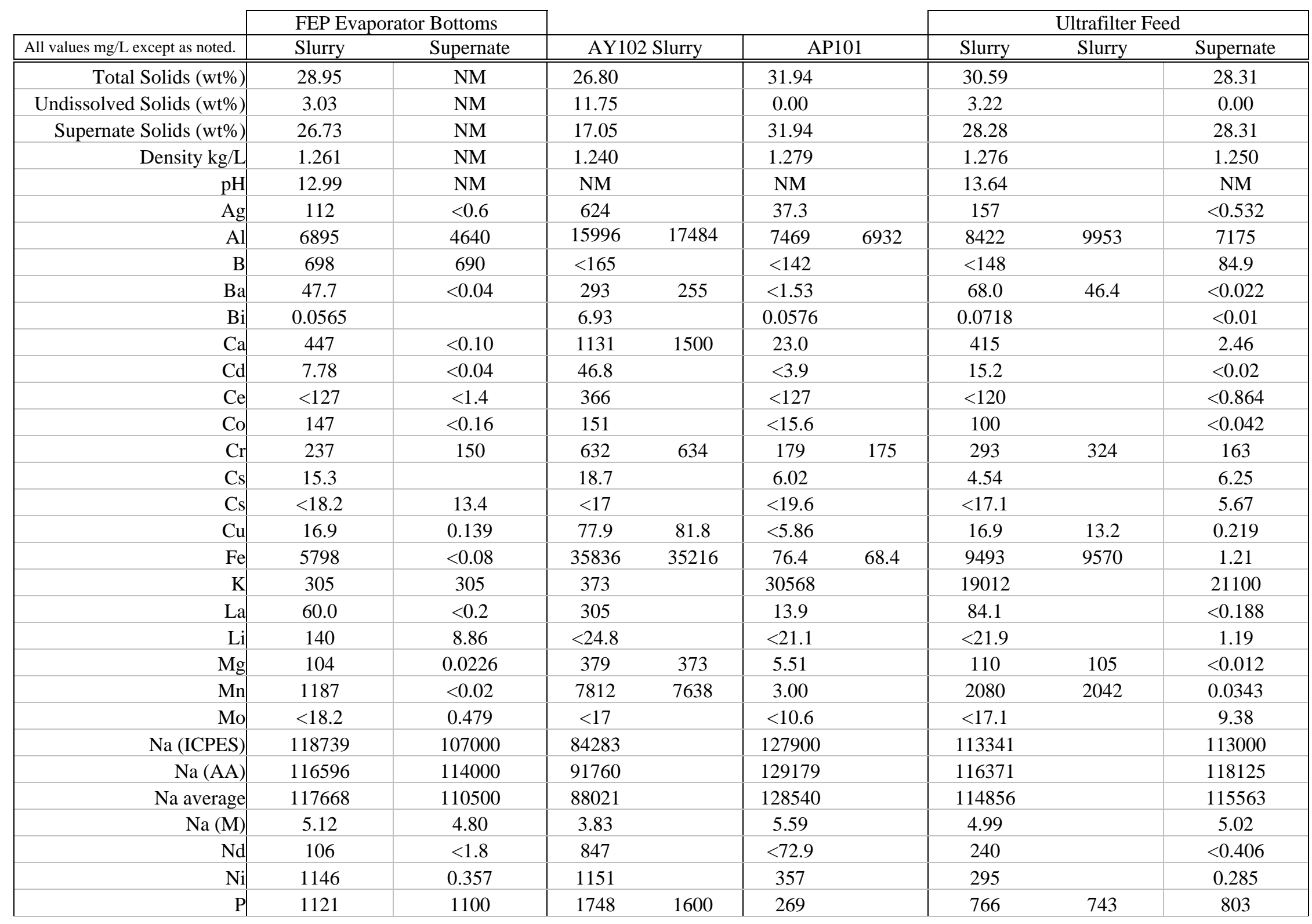

- 66 - 
WSRC-TR-2004-00565, REVISION 0 SRNL-RPP-2004-00092, REVISION 0

Table 26 continued

\begin{tabular}{|c|c|c|c|c|c|c|c|c|}
\hline & \multicolumn{2}{|c|}{ FEP Evaporator Bottoms } & & & & \multicolumn{3}{|c|}{ Ultrafilter Feed } \\
\hline All values mg/L except as noted. & Slurry & Supernate & \multicolumn{2}{|c|}{ AY102 Slurry } & AP101 & Slurry & Slurry & Supernate \\
\hline $\mathrm{Pb}$ & 223 & $<1.6$ & 1662 & 1593 & $<156$ & 475 & 419 & 1.77 \\
\hline $\operatorname{Pr}$ & 30.3 & NM & 170 & & 0.0223 & 34.1 & & $<0.01$ \\
\hline $\mathrm{Rb}$ & 1.26 & NM & 4.60 & & 6.06 & 4.16 & & 5.12 \\
\hline $\mathrm{S}$ & 908 & 790 & 892 & 899 & 1599 & 1340 & 1128 & 1140 \\
\hline $\mathrm{Si}$ & 1992 & 37.9 & 11016 & & 329 & 3247 & & 56.7 \\
\hline $\mathrm{Sr}$ & 86.9 & 0.402 & 279 & 292 & $<1.18$ & 60.2 & 83.0 & $<0.436$ \\
\hline $\mathrm{W}$ & $<90.8$ & 90.0 & $<84.7$ & & $<97.7$ & $<85.7$ & & 38.8 \\
\hline $\mathrm{Y}$ & 8.97 & $<0.01$ & 50.8 & & 0.0728 & 10.1 & & $<0.01$ \\
\hline $\mathrm{Zn}$ & 46.1 & 1.86 & 81.3 & & 5.95 & 28.6 & & 3.04 \\
\hline $\mathrm{Zr}$ & 85.7 & 0.0820 & 484 & & $<5.86$ & 125 & & 0.613 \\
\hline bromide & & 53.0 & 69.4 & & $<128$ & $<53.6$ & & $<10$ \\
\hline chloride & & 609 & 165 & & 1530 & 1242 & & 1175 \\
\hline fluoride & & 145 & 43.4 & & 28.0 & 81.7 & & 33.5 \\
\hline formate & & 408 & 389 & & 1380 & 1108 & & 1170 \\
\hline nitrate & & 119000 & 319 & & 105000 & 93914 & & 85600 \\
\hline nitrite & & 3740 & 4228 & & 33400 & 51678 & & 24050 \\
\hline oxalate & & 1180 & 9213 & & 1550 & 4440 & & 740 \\
\hline phosphate & & 4240 & 3807 & & 1150 & 2412 & & 2430 \\
\hline sulfate & & 1960 & 1860 & & 3740 & 3483 & & 5280 \\
\hline acetate & & NA & 415 & & 1727 & 1314 & & 1210 \\
\hline citric acid & & NA & $<81.8$ & & $<104$ & $<241$ & & $<100$ \\
\hline formate & & NA & 264 & & 1432 & 1072 & & 941 \\
\hline unbound hydroxide & & 6103 & NM & & NM & 38930 & & NM \\
\hline total organic carbon & & 1000 & NM & & NM & 3280 & & 3005 \\
\hline carbonate & & 9500 & NM & & NM & 40600 & & 31350 \\
\hline
\end{tabular}




\subsubsection{Simulant Handling (Author - Williams)}

Before testing began the simulants were prepared, which would be needed to create recycle streams from the UFP. Preparations of the simulated waste feed were then performed so as to have the accepted proportions of the constituents as per the OLI/ESP model (see Table 3).

After the dewatering portion of pilot-scale UFP testing, the slurry was adjusted in order to have a target solids concentration of $20 \mathrm{wt} \%$ undissolved solids (UDS) before washing began, as required by the Test Specification (Longwell, 2003). The UFP produced $117 \mathrm{~L}$ of concentrated slurry at $\sim 24 \mathrm{wt} \%$ UDS and approximately $915 \mathrm{~L}$ of permeate.

Therefore, some of the $24 \mathrm{wt} \%$ slurry was removed and approximately $25 \mathrm{~L}$ of permeate were added back to the slurry to maintain the slurry volume at $117 \mathrm{~L}$ while attaining 20 $w t \%$ UDS. This section describes how the simulants were made and adjusted at the experimental site.

\subsubsection{Preparing for UFP Recycle Streams}

Refer to Fig. 3 (recycle streams) and Figs. 9 and 10 (UFP unit operation) to better understand the following descriptions of feed preparations and use.

In preparation to create the UFP wash-water recycle stream on 20 May 2004, two 55-gallon drums, labeled "Campaign 3-UFP First or Second Wash,” were each filled with 141.3 liters of Campaign II RLD condensate into which were added 226 grams of $50 \mathrm{wt} \%$ $\mathrm{NaOH}$ to attain a caustic concentration of $0.01 \mathrm{M} \mathrm{NaOH}^{\dagger}$. From each drum, $23.5 \mathrm{~kg}$ of wash solution were removed per instructions so that the proper amount was available for Campaign III UFP washing activities. The loop rinse recycle stream, which would be made in Campaign III for use in Campaign IV, was made in one carboy labeled "PWDVSL-33” and it was filled with 8.6 liters of Campaign II RLD condensate. This carboy would receive 4.5 liters of washed HLW slurry after washing. To create the UFP "Heel" sludge recycle stream another carboy was filled with 12.5 liters of Campaign II RLD condensate to await receipt of washed slurry. To create rinse water recycle, three 55-gallon drums, labeled "Campaign 3-0.1M NaOH for UFP Caustic Rinse 1, 2 or 3," were each filled with 105.2 liters of FEP condensate in the Caustic Rinse 1 drum and 94.6 liters of FEP condensate in the Caustic Rinse 2 and 3 drums. Those drums were made to have a caustic concentration of $0.1 \mathrm{M} \mathrm{NaOH}$ by adding 852.7 grams of $50 \mathrm{wt} \%$ $\mathrm{NaOH}$ in the Caustic Rinse 1 drum and 766.8 grams of $50 \mathrm{wt} \% \mathrm{NaOH}$ into each of the Caustic Rinse 2 and 3 drums. The final UFP recycle stream created was for acid cleaning. It was made by using three 55-gallon drums labeled "Campaign 3-UFP Acid Cleaning 1, 2 or 3,” which were each filled with 94.6 liters of DI water. To make the acid concentration $2 \mathrm{M} \mathrm{HNO}_{3}, 17 \mathrm{~kg}$ of $70 \% \mathrm{HNO}_{3}$ were added into each of those drums.

\footnotetext{
${ }^{\dagger}$ The concentration of $0.01 \mathrm{M} \mathrm{NaOH}$ was nominal. The real target was to have a $\mathrm{pH}$ of at least 12 . Due to the high acid content of the EDL water supply the initial addition of 113 grams of the $50 \mathrm{wt} \% \mathrm{NaOH}$ to the 141 liters of water still indicated a pH below 12. To be safe the amount of caustic was doubled to 226 grams which theoretically gave a concentration of $0.02 \mathrm{M} \mathrm{NaOH}$ and a $\mathrm{pH}=12.3$. It measured as $\mathrm{pH}>12$.
} 


\subsubsection{Preparing UFP Feed}

In preparation for pilot-scale UFP unit operation, AP101 simulated waste, which was prepared by Optima Chemical Group LLC in Douglas, Georgia, was mixed with the FEP Evaporator Bottoms and the AY102 simulated waste in the UFP Primary Mixing Tank. This tank represented the plant tank UFP-VSL-1AB. Three major components of the AY102 simulant were also made by Optima, but those components were joined, and its final preparation was made, at the test location in a separate mixing tank on 24 May in order to have just the right mix. The final adjustment is shown in Table 27:

Table 27. UFP Feed Constituents

\begin{tabular}{|c|c|}
\hline Material & Mass Added (g) \\
\hline \multicolumn{2}{|l|}{ Wet Additions } \\
\hline AY102 Supernate Simulant by Optima & 72,100 \\
\hline Precipitated Sludge Solids by Optima & 238,200 \\
\hline NAS, sodium aluminosilicate (wet) by Optima & 27,871 \\
\hline \multicolumn{2}{|l|}{ Dry Additions } \\
\hline $\mathrm{Al}(\mathrm{OH})_{3}$ & $5,661.4$ \\
\hline $\mathrm{Al}_{2} \mathrm{O}_{3}$ & $2,015.2$ \\
\hline Calcium Phosphate Tribasic & 135.0 \\
\hline $\mathrm{SiO}_{2}$ & $3,655.6$ \\
\hline $\mathrm{RbNO}_{3}$ & 0.46 \\
\hline Total & $349,638.66$ \\
\hline
\end{tabular}

After samples were taken, AY102 simulant in excess of the target volume of 64 gallons was removed. That volume was indicated by the OLI/ESP model to be used for Campaign III and is shown at the top of Table 8. With AY102 ready, the final UFP feed preparation could be completed. The 64 gallons (323.9 kg) of AY102 simulant was transferred to the UFP Primary Mixing Tank. To this slurry, $171.5 \mathrm{~kg}$ of FEP bottoms made in the pilot-scale FEP were transferred to the UFP Primary Mixing Tank on 10 June. The last addition was $863.4 \mathrm{~kg}$ of AP101 simulant, which was also completed on 10 June. The total feed in the UFP Primary Mixing Tank was 1,358.8 kg before samples were removed for analysis.

After the UFP Primary Mixing Tank contents had settled over the weekend of 11 to 13 June, 15.8 liters of supernatant were decanted from the tank surface on 14 June, which were used to fill the permeate system of the pilot-scale UFP test facility on start up. Subsequently, the UFP Primary Mixing Tank, Fig. 11, was mixed for one hour and samples were taken before initiating the transfer to the UFP Slurry Reservoir, Fig. 12, to begin dewatering operations on 14 June. 


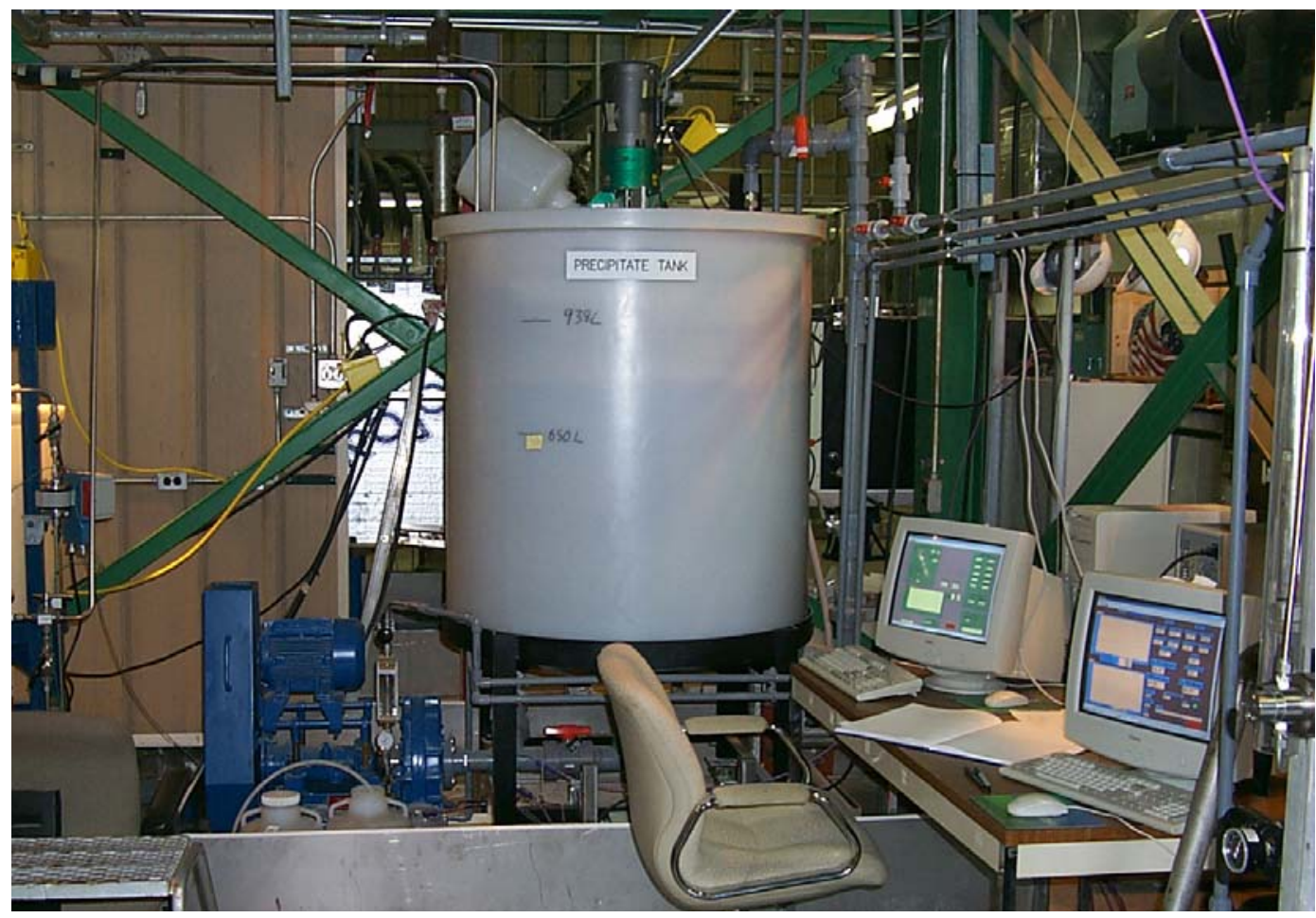

Figure 11. UFP Primary Mixing Tank (Volume 1000 liters) which represents the RPPWTP UFP Preparation Feed Tank

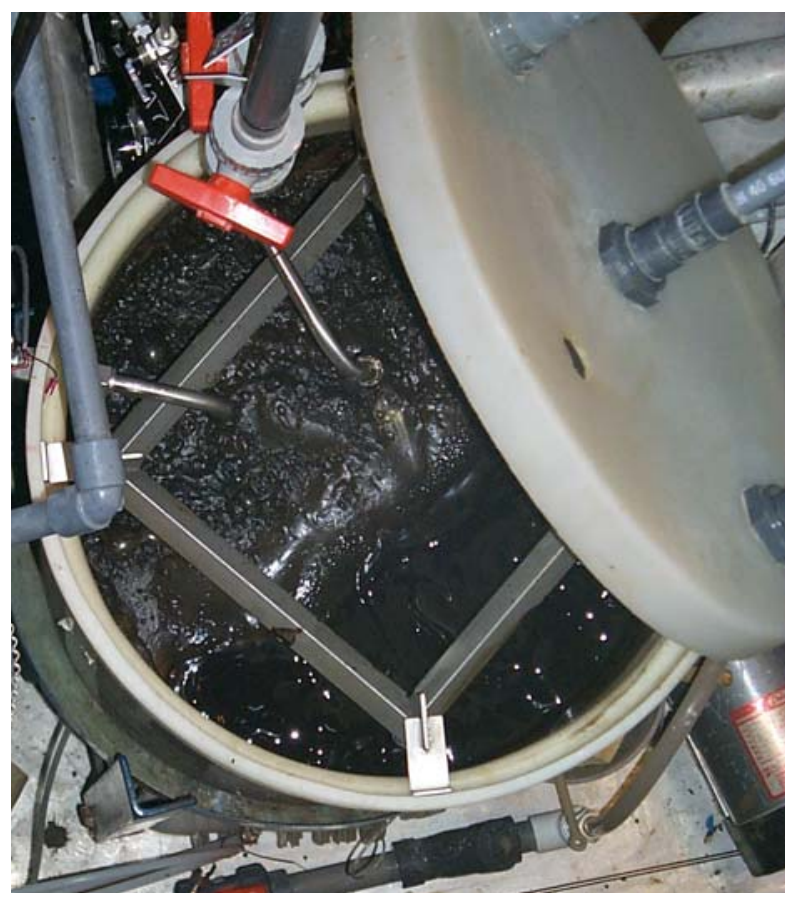

Figure 12. UFP Slurry Reservoir (Volume 100 liters) which represents the RPP-WTP UFP Feed Tank 


\subsubsection{Final UFP Products}

The slurry produced by the UFP filter was concentrated to greater than $24 \mathrm{wt} \%$ UDS by 17 June. To bring the fixed-volume batch of slurry back to washing target of $20 \mathrm{wt} \%$ UDS, $35.0 \mathrm{~kg}$ of $24-w \mathrm{t} \%$ slurry were removed and 24.8 liters (or $31.1 \mathrm{~kg}$ ) of permeate were added back. After the newly constituted slurry was well mixed, samples were taken. The concentrated slurry remaining in the UFP test rig was then washed in two phases using the $0.01 \mathrm{M} \mathrm{NaOH}$ wash water from drums labeled "Campaign 3-UFP First or Second Wash" on 21 June. The water used to wash the slurry was collected in 55gallon drums for use in Campaign IV as Wash Permeate recycle. The washed HLW concentrated slurry had a density of $1.22 \mathrm{gm} / \mathrm{ml}$. From that slurry 4.5 liters were added to the carboy labeled "PWD-VSL-33" to be held for Campaign IV as Loop Flush recycle and 0.13 liters were added to the carboy containing 12.5 liters of condensate to be added back into the UFP test rig to represent simulated rinsed loop contents.

The remaining concentrated and washed material, about 92.1 liters, was transferred to a 55-gallon drum labeled "Campaign 3 Concentrated Washed HLW Slurry” for shipment to VSL on 21 June for its eventual vitrification. The permeate produced by the UFP was placed into either of two drums labeled “Campaign 3-CsIX Permeate Feed" and transported to a CsIX Simulant Supply Tank. About 890 liters of permeate were transferred to the CsIX Simulant Supply Tank on 17 June.

On 21 June the filter rinse-clean-rinse sequence began. The contents of the carboy labeled "UFP Heel” were returned to the empty slurry reservoir as well as the contents from the drum marked "Caustic Flush 1" to rinse the UFP flow loop. The resulting volume was 134 liters of material. This volume was larger than the expected 117 liters. This indicated that there was already a substantial heel remaining in the flow loop of approximately 20 liters of material rather than the expected heel amount of 5 liters. After rinsing, all of the contents of the slurry reservoir were pumped to a drum, except 20 liters which were pumped to the carboy labeled "UFP Heel" to make the next heel. The drum was labeled "Campaign 3-Caustic Flush 1 to Campaign 3 (PWD-VSL-15)" and stored for Campaign IV. The 20 liters of "UFP Heel” were returned to the slurry reservoir along with first acid cleaning solution. This cycle repeated itself for all the Acid Cleanings 1, 2, 3 and Caustic Flush 2 and 3, that is, 20 liters of each of the preceding rinse/cleanings, simulated heel material, were fed with the next batch of cleaning or rinsing. The cleaning and rinsings were completed on 24 June. The drum contents produced were stored in drums labeled "Campaign 3-Acid Cleaning 1 or 2 \& 3 to Campaign 4 (PWD-VSL-16)" and “Campaign 3-Caustic Flush 2 or 3 to Campaign 4 (PWD-VSL-15)” for Campaign IV. 


\subsubsection{Pilot UFP Operation and Equipment (Author - Fowley)}

The SIPP UFP filter unit (see Figs. 13, 14, and 15) was manufactured by the Mott Metallurgical Corporation to meet RPP-WTP specifications (Townson, 2002; Longwell, 2003). The filter unit had the following physical characteristics:

- 7 filter tubes with each having an inside diameter of 0.5-inch

- 90-inch porous length for each filter tube

- Stainless steel (316L) construction

- Nominal rated 0.1 micron filter element

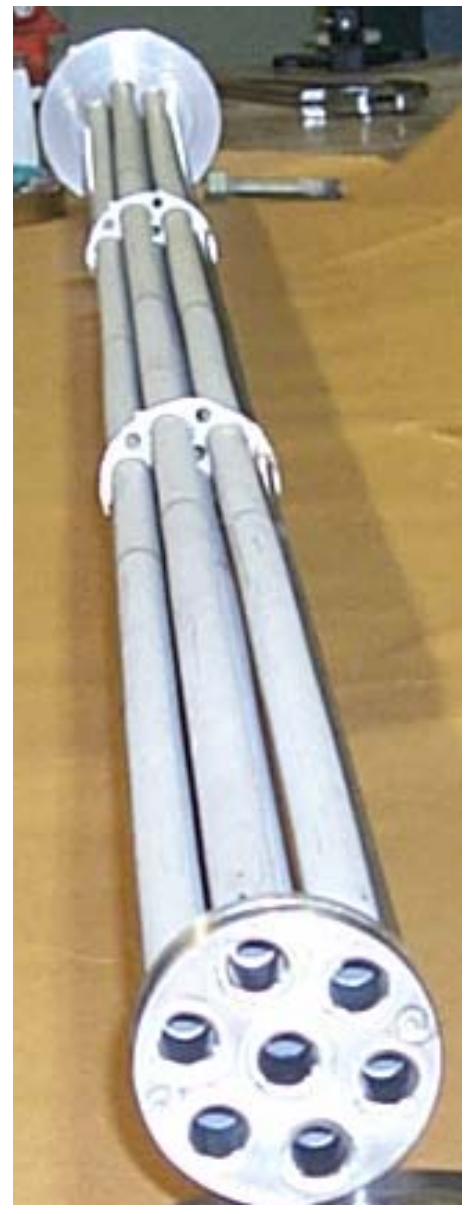

Figure 13. Entire 90-inch Length of the Filter-tube Bundle (Upstream end in Foreground) 


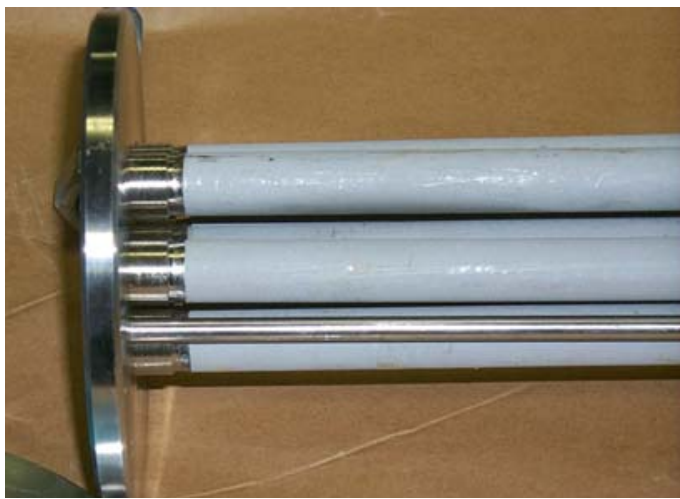

(a) Downstream (Exit) end of Filter Elements

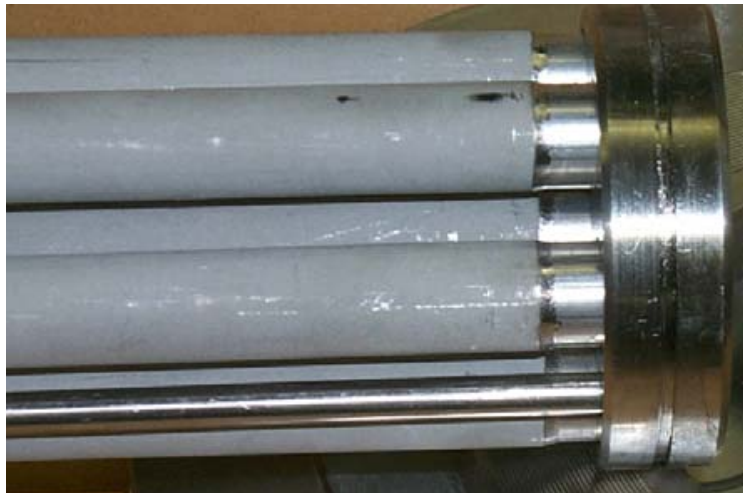

(b) Upstream (Entrance) end of Filter Elements

Figure 14. Closeup of the Tubes' Surfaces and Tube Sheets
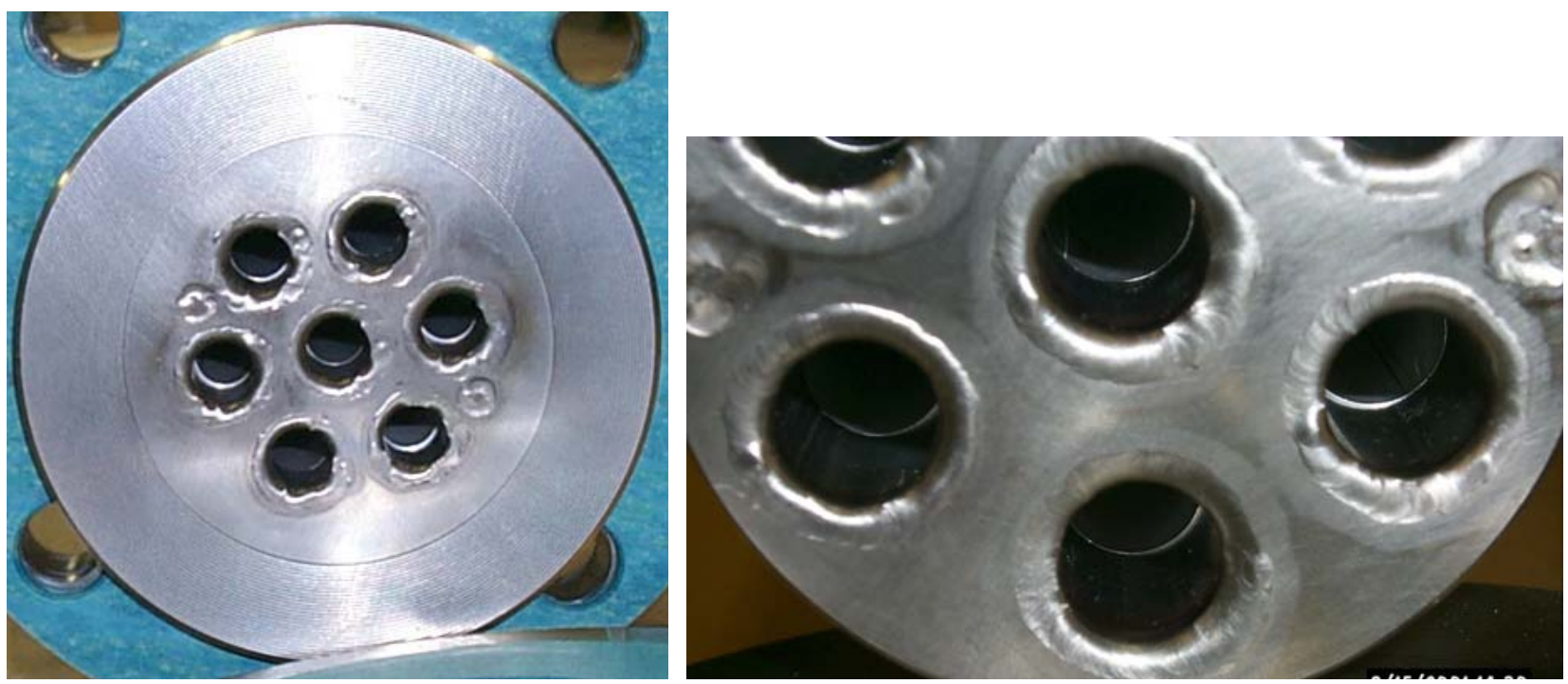

Figure 15. The Exit (Left) and Entrance (Right) Tube Sheets of the Filter Tube Bundle

The filter unit was prototypic in most of its features. The filter unit was not prototypic in three features: it only had 7 tubes instead of 241 tubes, it was mounted vertical instead of horizontal (which is considered an insignificant difference due the high velocity of the slurry), and while the backpulse system was prototypically designed, its dynamic response (momentum) must be different because of the smaller scale. In general, filter performance data from the pilot test rig were assumed to well represent those that will be obtained in the full-scale unit.

The filter unit and associated hardware represent the pilot-scale test rig. A schematic diagram of the test rig is shown in Fig. 16. The pilot-scale test rig was designed to have the following: 
- Maximum axial velocity through filter tubes of $4.6 \mathrm{~m} / \mathrm{s}(15 \mathrm{ft} / \mathrm{s})$

- Maximum transmembrane pressure (TMP) of 60 psid

- Maximum axial velocity to be achievable at the maximum TMP

- Instrumentation to monitor the axial velocity, the permeate flow rate, the TMP, and the slurry temperature

- All materials to be compatible with the high-caustic simulants and the $2 \mathrm{M}$ nitric acid cleaning solution

All specifications were met or exceeded.

The stainless steel pilot-scale test rig is composed of three basic flow loops; the slurry loop, the permeate loop, and the cleaning loop (not used for SIPP and not described in this report). The slurry loop is the primary flow path for circulating slurries and contains the filter unit and the pumping system. The pumping system consisted of two 10-hp Galigher ${ }^{\circledR}$ slurry pumps in series. The impeller and impeller housing were lined with EPDM to be compatible with both the high caustic slurry and the $2 \mathrm{M}$ nitric acid cleaning solution.

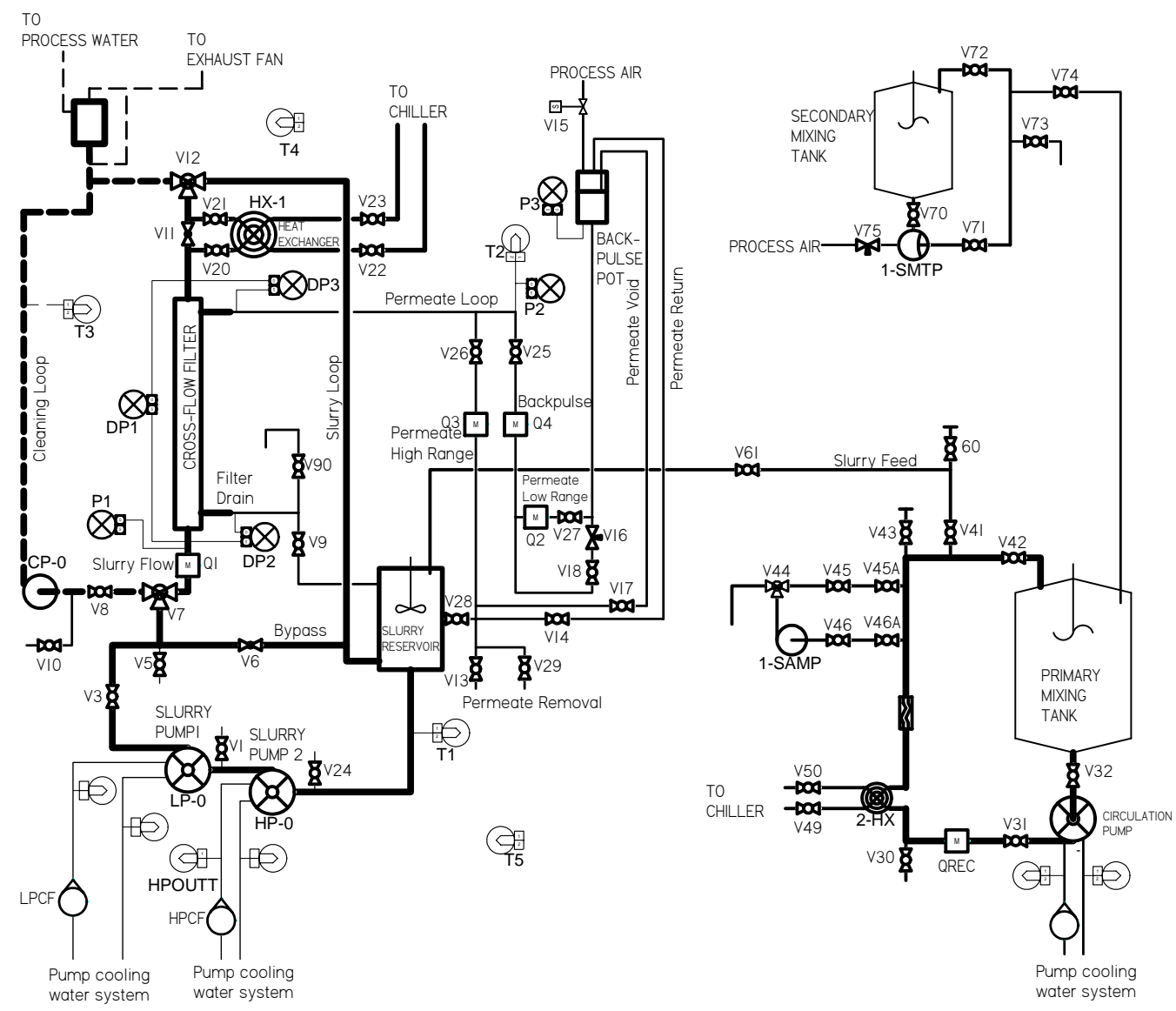

Figure 16. Campaign II Ultrafiltration Test Rig 
The permeate loop starts at the filter housing, contains a backpulse pot and ends at the Slurry Reservoir. The backpulse pot and associated tubing provides a pulse of permeate flow in the reverse direction to knock off built-up slurry cake on the inside of the filter tubes. Discharge valves were located in the permeate loop near the Slurry Reservoir to facilitate the removal of permeate from the system. A more detailed description of the flow loops is documented in (Duignan, 2000a, Duignan 2000b, Duignan 2003a) and will be provided in the SIPP final report.

The Primary Mixing Tank was used to mix the combined simulated wastes during feed preparation and to transfer the mixture to the Slurry Reservoir before and during dewatering. The tank was mechanically agitated and included a product recirculation loop to provide additional mixing and facilitate the transfer to the Slurry Reservoir. A sampling system was included in the recirculation loop to acquire a representative sample.

The Secondary Mixing Tank was used to prepare and transfer the AY102 simulated waste to the Primary Mixing Tank during feed preparation. The tank was mechanically agitated and included a product recirculation loop to provide additional mixing and facilitate the transfer to the Primary Mixing Tank. A sample port was located in the recirculation loop to acquire a representative sample.

\subsubsection{Measurement Uncertainty}

The derivation of measurement uncertainty will not be shown in this report, but will be included in the final report, which will incorporate results from all four campaigns. The uncertainties listed here are based on past work done on the same equipment under similar circumstances and are listed here are to illustrate the expected magnitude of principal parameters (at the 95\% confidence level).

$\begin{array}{ll}\text { Slurry Velocity in a Filter Tube } & = \pm 6 \% \\ \text { TMP } & = \pm 2 \% \\ \text { Temperature Corrected Permeate Flux } & = \pm 6 \%\end{array}$

The magnitude of the measurement uncertainty for analytical results can fluctuate significantly depending on the analytical method and equipment. Uncertainties for data obtained from outside organizations, i.e., Analytical Development Section (ADS), the Mobile Laboratory of Savannah River National laboratory (SRNL), or the Aiken County Technical laboratory (ACTL) are beyond the scope of this task but they are assumed to be no larger than $\pm 15 \%$ of any result shown, unless otherwise specified. For properties measured in the EDL, the following uncertainties on a reading were obtained through calibration:

$$
\begin{array}{ll}
\text { Slurry or Liquid Density } & = \pm 0.5 \% \\
\text { Liquid Viscosity } & = \pm 0.5 \% \\
\text { Turbidity } & = \pm 0.1 \mathrm{NTU}
\end{array}
$$


The density of the slurry and permeate were determined by mass and volume measurements from a calibrated platform scale and volumetric flask. The viscosity of the permeate was measured by a Cannon-Fenske viscometer. The turbidity by a turbidity meter calibrated with standards.

\subsubsection{Pilot UFP Discussion of Results}

\subsubsection{Experimental Observations (Author - Fowley)}

\subsection{Pre-test Activities}

Towards the end of Campaign II some plastic ties used to hold Slurry Reservoir tubing steady broke and entered the filter loop. Those ties did not have any noticeable effect on filter operation but prior to Campaign III the filter unit and pumping system were inspected. The filter unit was inspected in place and was quickly determined to be clear of foreign objects. The test rig was re-assembled and filled with DIF to keep the filter tubes submerged during the pump inspection. However, upon further inspection the ties were discovered in both pump housings. One of the pump impellers sustained light damage due to the fretting action of the ties. Both pump housing were removed and the impellers and liners were replaced.

The baseline tests (Test \# 3.00A, B, and C) were conducted on 8 June, after the pump housings were reinstalled. The baseline tests were conducted with $0.1 \mathrm{M} \mathrm{NaOH}$ and produced permeate flux results much lower than expected (lower than the post-test baseline results of Campaign II). This behavior was not unexpected because when the test loop had been disassembled and then reassembled in the past usually the permeate filter flux would be lower. The lower flux was assumed to be the result of hidden pockets of slurry being released because by adding a small amount of nitric acid the flux would increase immediately. To re-establish a post-Campaign II water flux, the filter was cleaned with 2.0 $\mathrm{M} \mathrm{HNO}_{3}$ for an extended period of time, and then thoroughly flushed with DIF water (with multiple backpulses). The acid cleaning operation lasted for 3 hours on 9 June and 2 hours on 10 June, with a 15 hour idle time overnight in acid (see Fig.17). The flux improved immediately when the acid cleaning was initiated and continued to improve for the duration of the cleaning. A significant improvement occurred during the idle time. After thoroughly flushing the system the baseline tests were repeated with $0.1 \mathrm{M} \mathrm{NaOH}$. The flux results were greatly improved. The results of both of these pre-test baselines are presented in Section 2.7.4.1.5 (Fig. 28). 
WSRC-TR-2004-00565, REVISION 0

SRNL-RPP-2004-00092, REVISION 0

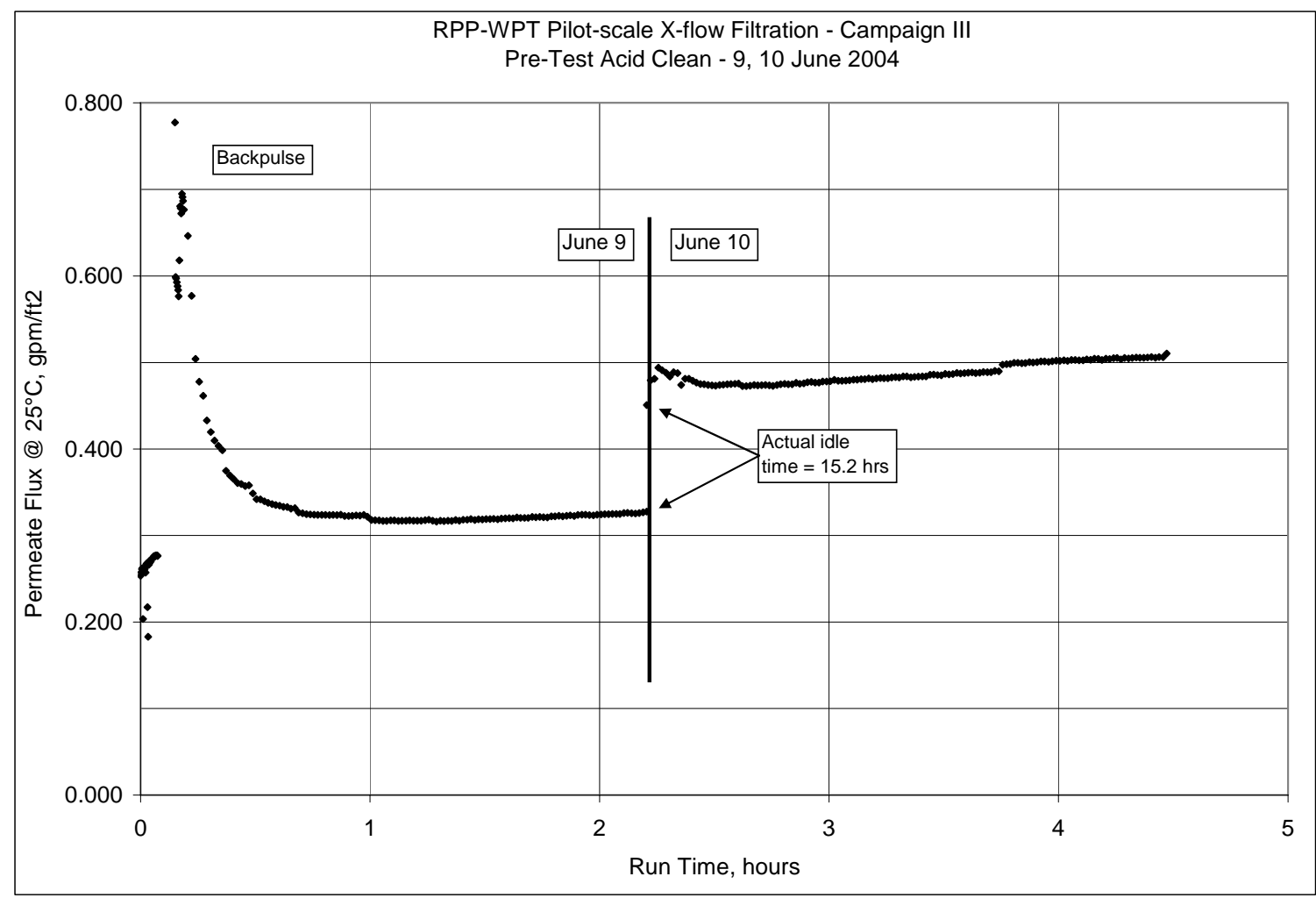

Figure 17. Pre-Test Acid Cleaning of Filter Unit, Permeate Flux vs. Run Time

\subsection{Slurry Concentration (Dewatering)}

Dewatering started Monday, 14 June. The waste simulant in the Primary Mixing Tank was allowed to settle over the weekend before the 14th so that supernate could be drawn from the top of the mixture. After the supernate was removed, the simulant was thoroughly agitated for one hour prior to the initial transfer of slurry to the slurry reservoir. That supernate was added to the slurry reservoir prior to the initiation of dewatering to account for the volume of permeate necessary to fill the permeate loop. Doing so assured that the solids concentration of the feed waste simulant did not change after filling the test rig so that the test rig also began with the slurry at feed conditions.

Per the test matrix, the dewatering was conducted in two stages. The delineation between the stages was approximately one-half of the expected permeate removed from the simulant (476 liters). A hold period, a minimum of 12 hours, was inserted between the stages to purposely extend the dewatering process, making it more comparable with the duration of the dewatering process of the RPP-WTP plant, to allow for waste reactions to occur, e.g., precipitation. The first stage was completed in about 16 hours and finished in the early morning of 15 June, the second stage, dewatering approximately 440 liters, was completed in about 39 hours and finished in the early morning of 17 June. The second period was much longer because by the end of the first period the permeate flux had dropped by more than a factor of 5 . The entire dewatering process, including the hold, 
and downtime for sampling, took approximately 67 hours. Excluding the hold, and sampling downtime, dewatering took 55 hours (Campaign II took 53 hours). (This 67hour period is approximately one half of the dewatering time that WTP will take, which was estimated to be 138 hours, as shown in Table A9 in Appendix A of Duignan et al., 2004b. However, any UFP-feed reactions are expected to occur during first half of the dewatering period.)

A volume of about $1049 \mathrm{~L}$ of waste simulant was dewatered to $117 \mathrm{~L}$ resulting in a final concentration of $24.6 \mathrm{wt} \%$ UDS. The RPP-WTP operation will concentrate the waste in the UFP unit to approximately $17 \mathrm{wt} \%$ UDS. However, all of the campaigns of SIPP have a target of at least $20 \mathrm{wt} \%$ UDS. The dewatering test was stopped after 55 hours when a rough estimate ${ }^{\dagger}$ of concentration indicated $25 \mathrm{wt} \%$ UDS. A volume of $915 \mathrm{~L}$ of permeate (excluding samples) was collected in drums during dewatering. Approximately $16 \mathrm{~L}$ of permeate remained in the permeate loop and about 1 liter of samples (slurry) were taken during dewatering.

Figure 18 shows the adjusted permeate flux for the entire slurry dewatering process. The flux is adjusted for temperature, as required by the RPP-WTP test specification, to account for the effects of viscosity and surface tension.

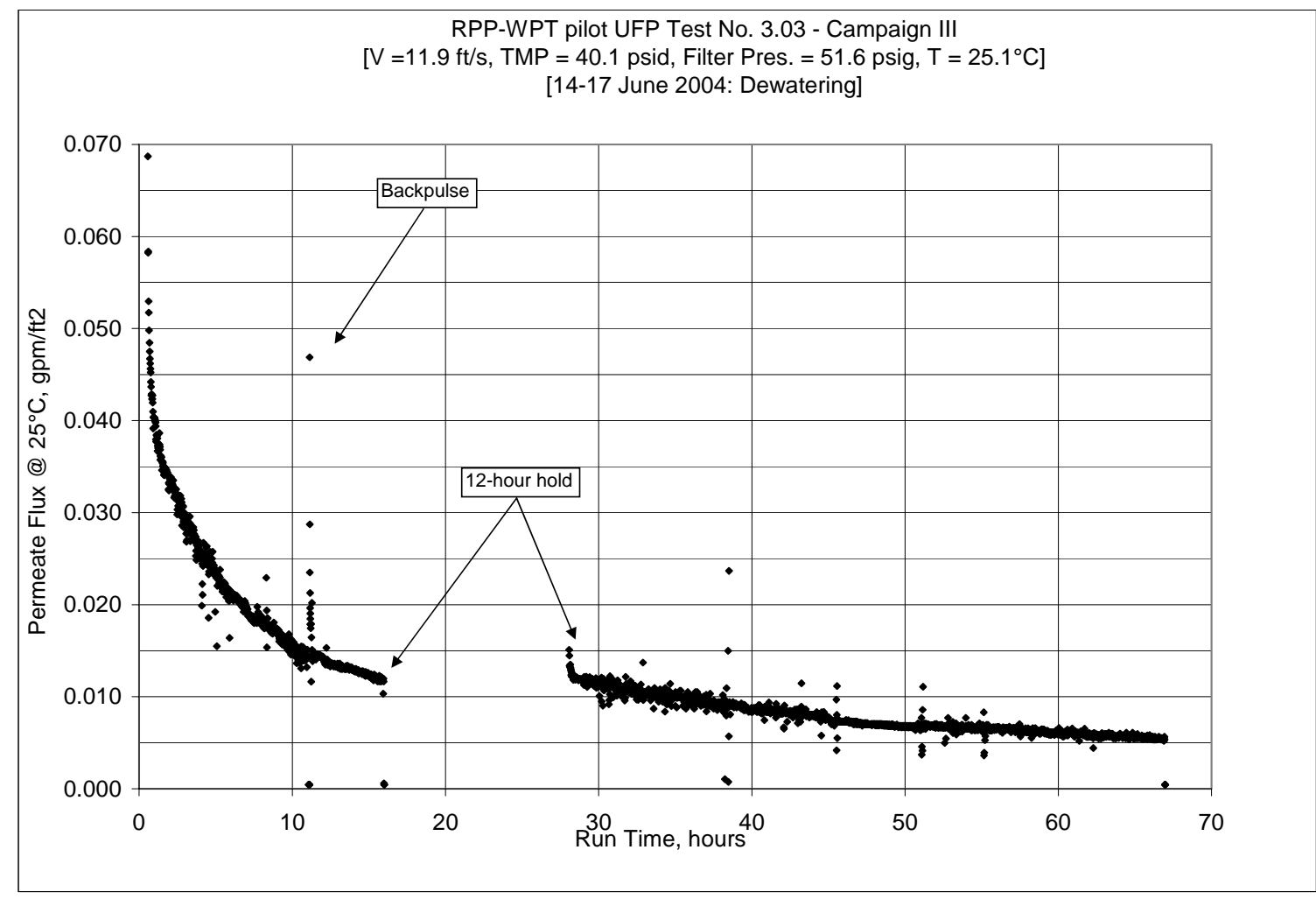

Figure 18. . Dewatering of UF Feed, Permeate Flux vs. Run Time

\footnotetext{
${ }^{\dagger}$ Real time concentration analysis was not available and only the initial samples taken on the previous day were used to extrapolate to estimate the $25 \mathrm{wt} \%$ UDS.
} 
The adjustment equation is:

$$
\begin{aligned}
& \text { Adjusted Flux = Flux }\left(\mathrm{gpm} / \mathrm{ft}^{2}\right) * \mathrm{e}^{(2500 \times((1 /(273+\mathrm{T}))-(1 / 298)))} \\
& \text { where } \mathrm{T}=\text { slurry/permeate temperature in degrees Celsius }
\end{aligned}
$$

Dewatering started with a backpulse. The backpulse pot and the method of backpulsing were designed to mimic plant operation. A backpulse is performed by first closing the permeate loop flow valve (V27, see Fig. 16) to stop dewatering. The backpulse pot is then partially evacuated to create a air pocket. The air pocket is pressurized to $40 \mathrm{psig}$ above the pressure of the slurry loop. The backpulse valve (V18) is opened very fast (less than a second), held open for a predetermined amount of time, then closed very fast (less than a second) to force permeate back through the filter media and remove filter cake. The permeate flow valve is then opened very slowly (approximately 60 seconds) to re-establish dewatering.

Another backpulse was conducted at approximately 11.1 hours into the first stage of dewatering. Per procedure a backpulse was conducted when the permeate flux dropped below $0.015 \mathrm{gpm} / \mathrm{ft}^{2}$. When dewatering resumed after the backpulse the permeate flux was higher ( $>0.030 \mathrm{gpm} / \mathrm{ft}^{2}$ ) but quickly ( 11 minutes) returned to the pre-backpulse flux. Because of the poor recovery, backpulsing was terminated for the remainder of the dewatering.

Samples of concentrated slurry and permeate were taken during the dewatering process in accordance to a Sampling Plan, Appendix B. Samples were taken at three periods during each dewatering stage (at approximately 159 liters, 317 liter, and 476 liters of permeate removed). Permeate density, viscosity, and turbidity, as well as slurry density, were measured in the EDL soon after the samples were taken. Separate permeate and slurry samples were sent to ACTL for measurements of density, yield stress, consistency, and weight \% solids [total solids (TS), undissolved solids (UDS), dissolved solids (DS), and supernate solids (SS)].

Another way to visualize the dewatering process results is to track permeate flux against the concentration of UDS. Figure 19 shows the temperature adjusted permeate flux versus UDS concentration. These concentration data were derived from a polynomial interpolation of six slurry samples acquired during dewatering by using the mass of permeate removed over time (see Section 2.7.4.2.6 in Duignan et al., 2004b). For comparison, the dewatering data from Campaigns I and II are also shown. The data trends of Campaigns II and III are almost identical, while the data trend of Campaign I is similar. The simulant for Campaign I contained only AY102 and had $12.5 \mathrm{wt} \%$ UDS at the beginning of dewatering. The consistent performance of the UFP indicates that; (1) the filter unit was successfully cleaned after Campaigns I and II, (2) filter performance can be predictable, and (3) the addition of AP101 and FEP bottoms to the AY102 had little effect on filter performance. Note also, that backpulsing was performed during all campaigns and a poor flux recovery was consistent. 
WSRC-TR-2004-00565, REVISION 0

SRNL-RPP-2004-00092, REVISION 0

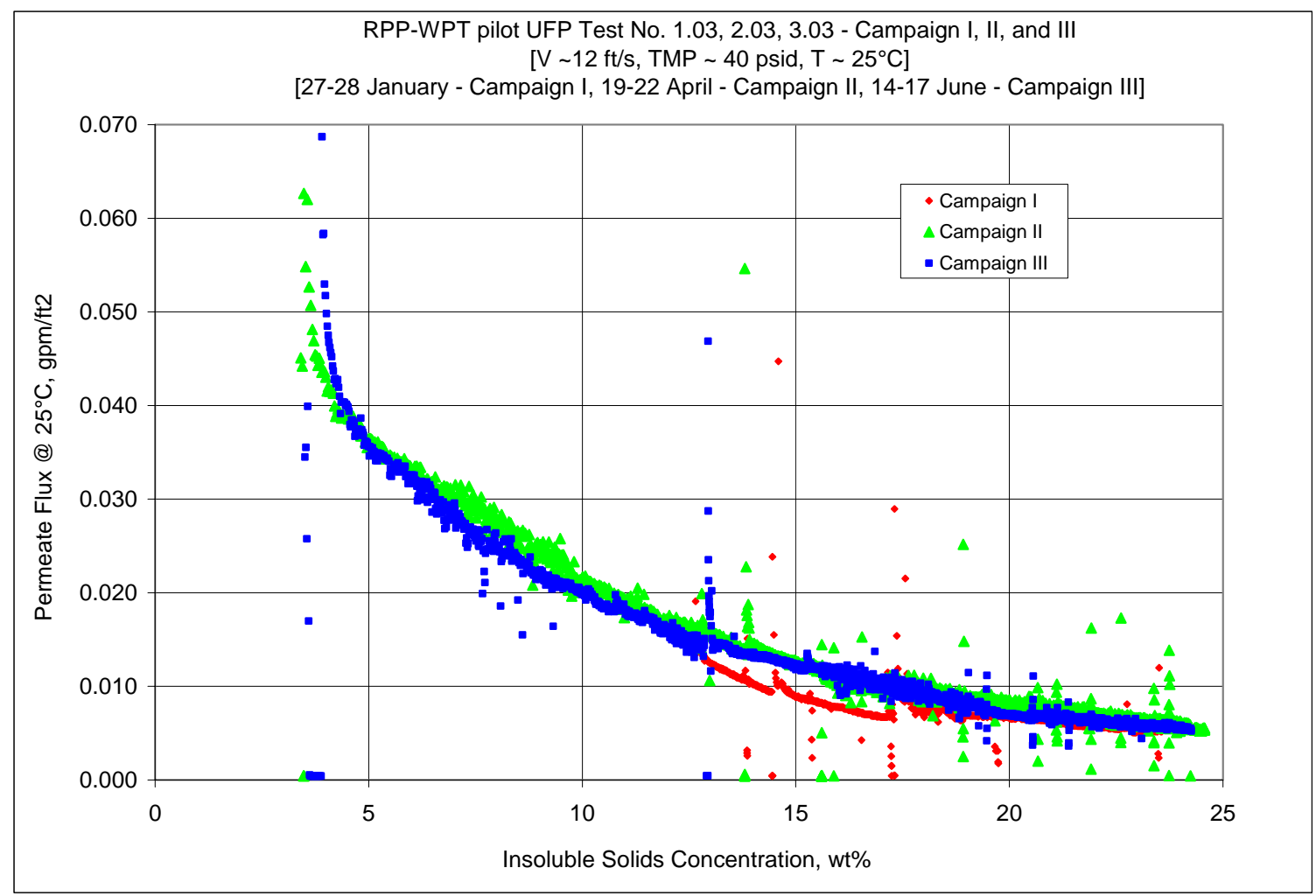

Figure 19. Dewatering of UF Feed - Campaigns I, II, and III, Permeate Flux vs. Concentration

Table 28 shows rheological and other physical data of the slurry and permeate measured at the EDL during the dewatering.

Table 28. EDL Measured Data during Dewatering of the AY102+AP101+FEP Bottoms Simulant

\begin{tabular}{|c|c|c|c|c|c|c|}
\hline \multirow{2}{*}{$\begin{array}{c}\text { Target } \\
\text { Volume of } \\
\text { Permeate } \\
\text { Produced (1) }\end{array}$} & $\begin{array}{c}\text { Cumulative } \\
\text { Weight of }\end{array}$ & $\begin{array}{c}\text { Permeate } \\
\text { Produced }\end{array}$ & Slurry & \multicolumn{4}{|c|}{ Permeate } & Tensity & Density & Viscosity & Temp & Turbidity (2) \\
\cline { 5 - 7 } & $\mathrm{Kg}$ & $\mathrm{gm} / \mathrm{mL}$ & $\mathrm{gm} / \mathrm{mL}$ & $\mathrm{mPa}-\mathrm{s}$ & ${ }^{\circ} \mathrm{C}$ & $\mathrm{NTU}$ \\
\hline $\mathrm{L}$ & 197.10 & 1.30 & 1.24 & $\mathrm{n} / \mathrm{a}$ & $\mathrm{n} / \mathrm{a}$ & 0.24 \\
\hline $\begin{array}{c}\text { Day1 } \\
158.7\end{array}$ & 394.10 & 1.33 & 1.25 & 2.15 & 26.5 & 0.27 \\
\hline 317.3 & 591.10 & 1.36 & 1.25 & 2.25 & 25.7 & 0.34 \\
\hline 476.0 & 788.50 & 1.40 & 1.25 & 2.23 & 26.0 & 0.17 \\
\hline $\begin{array}{c}\text { Day 2 } \\
158.7\end{array}$ & 985.50 & 1.43 & 1.25 & 2.17 & 26.4 & 0.34 \\
\hline 317.3 & 1141.70 & 1.46 & 1.25 & 2.39 & 23.0 & 0.35 \\
\hline 476.0 & & & & & & \\
\hline
\end{tabular}

(1) These were "target” and not actual volumes "produced.” The actual quantity produced is indicated by the mass in the second column.

(2) The Test Specification indicated a threshold of 2 NTU. The measured values indicated the permeate was free of significant undissolved solids. 
As expected, the density of the slurry increased during dewatering. The permeate density remained constant. The permeate viscosity (and permeate temperature) fluctuated slightly throughout the process (the highest viscosity is associated with the lowest temperature and vice versa). The turbidity measurements of the permeate were significantly lower than the test specification threshold of 2 NTU and, therefore, the permeate was considered free of solids.

Following dewatering, $35 \mathrm{~kg}$ of concentrated slurry were removed from the slurry reservoir and $31.1 \mathrm{~kg}$ of permeate were added back to the slurry reservoir to achieve $117 \mathrm{~L}$ of slurry at $20 \mathrm{wt} \%$ solids for the following slurry washing test. The remaining permeate was used in the Campaign III Ion Exchange process.

\subsection{Slurry Washing}

In RPP-WTP, the filtration batch size is 21,550 gallons. The concentrated slurry is washed twice with 21,770 gallons (this represents the volume of one permeate storage tank) using 22 mini-batches of approximately 1,000-gallon each. In SIPP, the pilot-scale filtration batch size is $116.8 \mathrm{~L}$. To mimic RPP-WTP, the concentrated slurry was washed twice with $118.0 \mathrm{~L}$ using 22 mini-batches of $5.4 \mathrm{~L}$ each.

Figure 20 shows the temperature adjusted permeate flux of the first washing. The flux increased from approximately 0.012 to $0.017 \mathrm{gpm} / \mathrm{ft}^{2}$ during the first wash cycle $(42 \%$ increase).

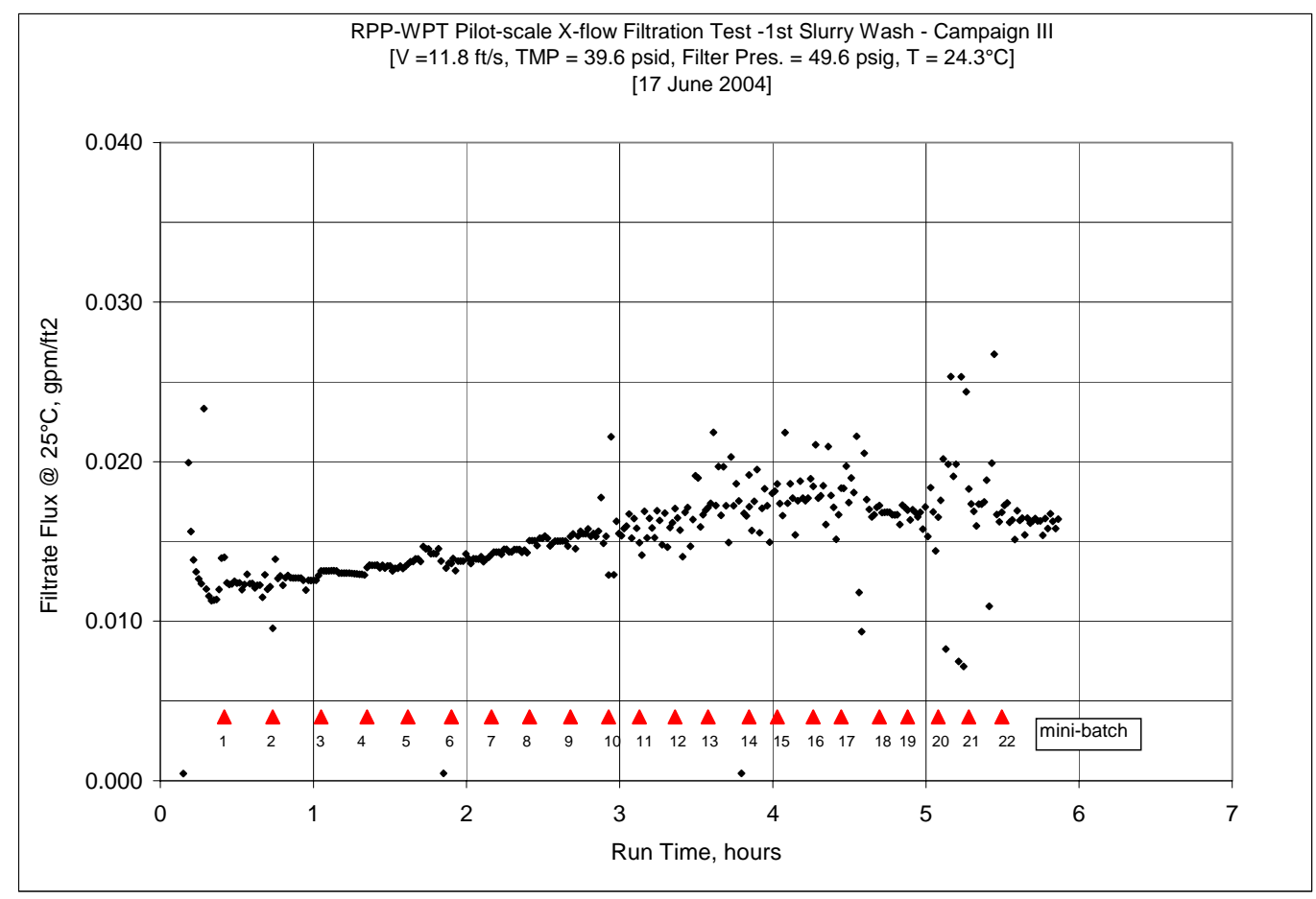

Figure 20. 1st Washing of Concentrated Slurry with 22 Wash Mini-batches 
The flux increased in the first wash of Campaigns I (Duignan et al, 2004a) and II (Duignan, et al., 2004b) by 53\% and 64\%, respectively. Figure 21 shows that the temperature adjusted flux continued to increase during the second wash, from approximately 0.025 to $0.029 \mathrm{gpm} / \mathrm{ft}^{2}$ (16\% increase). The flux increased in the second wash of Campaigns I and II by 32\% and 25\%, respectively.

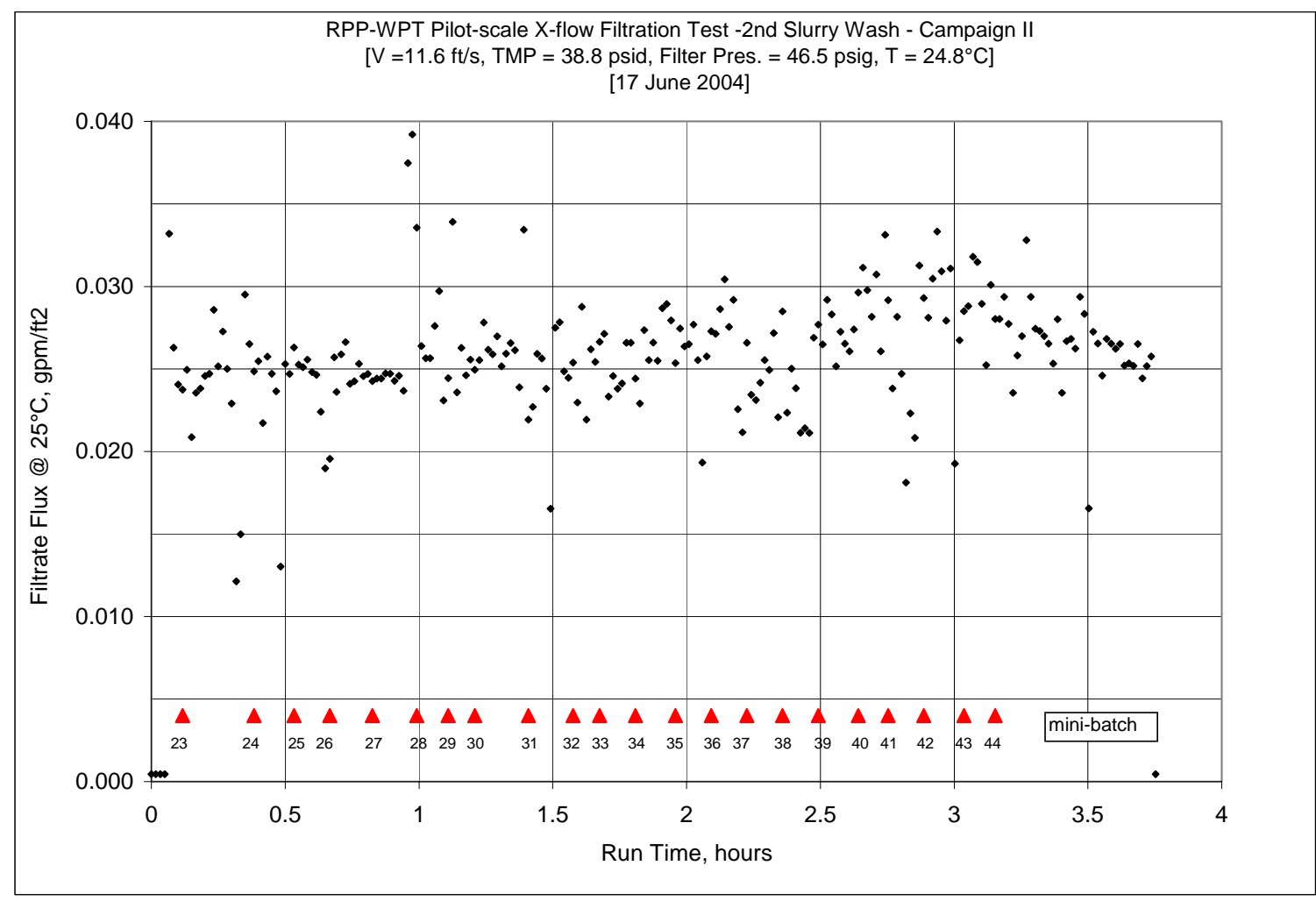

Figure 21. 2nd Washing of Concentrated Slurry with 22 Wash Mini-batches

In all of the campaigns to date, the temperature adjusted flux increased from the end of the first wash to the beginning of the second wash, while the test rig was idle. It is believed that solids entrained in the filter media continued to dissolve during this idle time, creating an improved flux when the test rig was restarted. The idle duration was 11 hours and 52 minutes in Campaign I, 10 hours and 42 minutes in Campaign II, and 1 hour and 37 minutes in Campaign III (the first and second washes of Campaigns I and II were conducted on consecutive days, the Campaign III washes were conducted on a single day). During these idle times the temperature adjusted flux increased 8\% in Campaign 1, $11 \%$ in Campaign II, and $47 \%$ in Campaign III. The flux increase was considerable after the 97-minute idle time of Campaign III, but was still the same order of magnitude as in the previous campaigns. If the flux does increase between the first and second washes because of continued dissolution of solids then most of the dissolving occurs during the first hour or so.

The total increase from the beginning of the first wash to the end of the second wash was $142 \%$ for Campaign III. The total increase was $107 \%$ and $127 \%$ for Campaigns I and II 
respectively. The greater total increase of Campaign III was mostly realized during the idle time between the washes.

The temperature adjusted flux data of Campaigns I, II, and III for the first and second washes are shown in Fig. 22 and in general the increasing flux trends are similar. As mentioned previously, the first and second washes of Campaigns I and II were conducted on consecutive days, whereas the two washes of Campaign III were on the same day. To facilitate comparison, the figure shows the washes of all three campaigns with the idle time between the washes of Campaigns I and II modified to match to the start of the second wash of Campaign III.

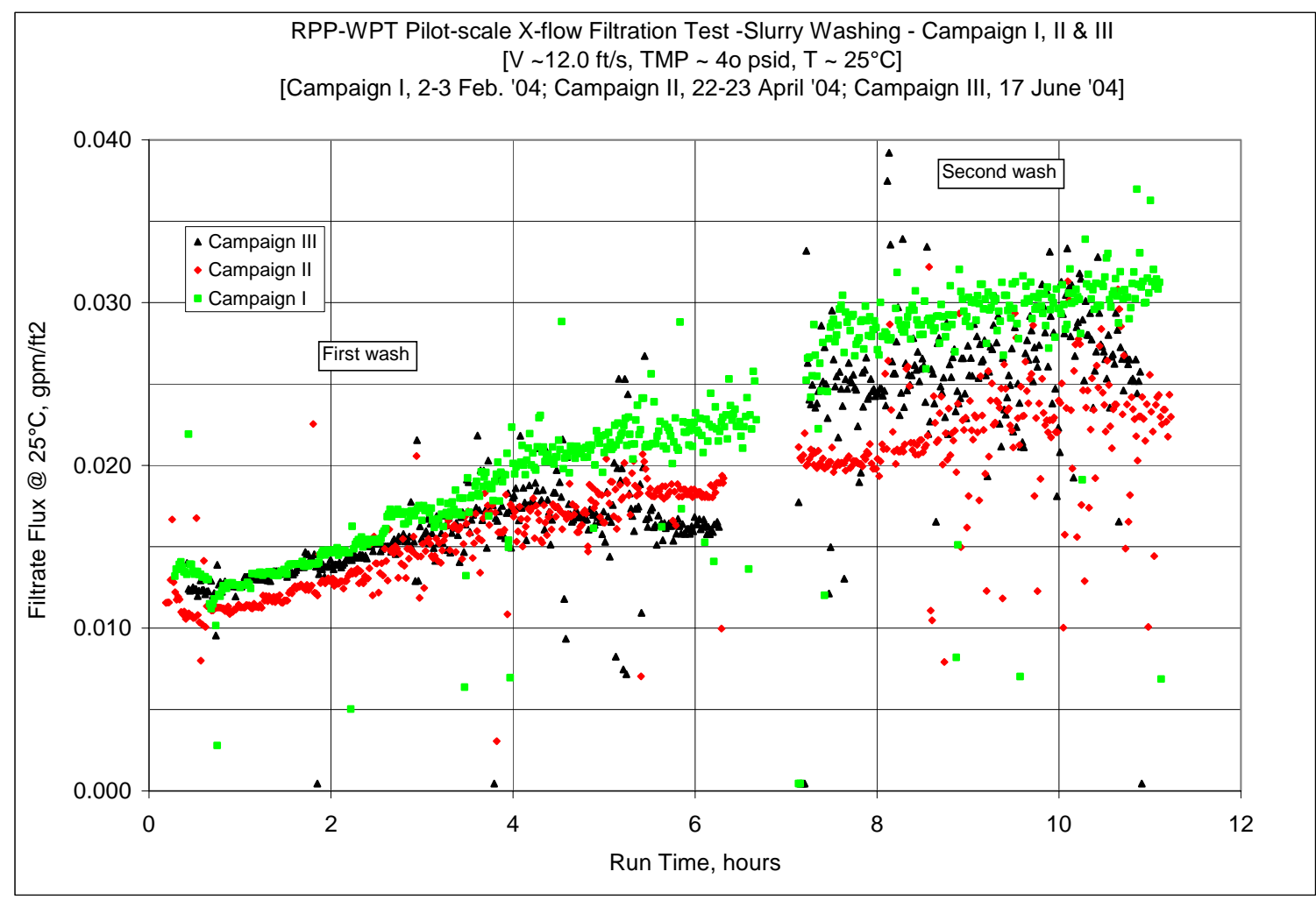

Figure 22. 1st and 2nd Washing of Concentrated Slurry, Campaigns I, II and III

Note in Fig. 20, the initial flux readings are relatively stable and become progressively unstable during the first wash, and more so in the second wash. This instability is systematic to the pilot-scale test rig only and was attributed to gas bubbles and the smalldiameter tubes in the permeate loop. The bubbles were small in volume at the start of the first wash but became larger and more frequent as the wash progressed probably because of the changing permeate viscosity and surface tension. Fluctuations in the permeate flow rate were noticed to correspond with the gas bubbles seen in the section of the permeate loop discharging to the receipt drums. Similar fluctuations were noticed in the pressure measured at the backpulse pot. The permeate loop from the backpulse pot to the Slurry reservoir (including the discharge line) was polyethylene. The gas bubbles were seen in the entire section of the polyethylene line, from the backpulse pot to the discharge 
line. Because of the very slow moving permeate (less than $1 \mathrm{ft} / \mathrm{s}$ in the discharge line) each time the flow was stopped for either sample taking, or to add another one of the 44 mini-washes, air that was trapped at the bottom of the line would migrate up the line. The discharge velocity was not sufficient to entrain the air once the flow was reestablished. This means the discharge system always had oscillating air bubbles that caused the indicated flux instabilities. The full-size WTP will have larger discharge pipes and higher permeate flows, which will continue to flow when a new charge of washing water is added. Such instabilities will not be as prominent, if at all.

Throughout the washing, permeate samples were taken at wash numbers 1, 5, 9, 13, 17, and 22, for both washes (see the Sampling Plan in Appendix B). These sample periods are important because while both slurry and permeate samples were taken at the same time, they are not matched samples. The permeate flux was very low and the permeate loop lengthy. It took approximately 30 minutes for permeate created at the filter to flow to the sampling point. The slurry and permeate samples can be appropriately matched by knowing the delay time. To match the slurry sample taken at the end of each wash, the test rig was operated (in permeate recirculation mode) for an additional 30 minutes. At the end of the 30 minutes a permeate sample was taken. The analysis of the slurry and permeate samples taken during the washing are presented in Section 2.7.4.2.2.

The wash permeate was collected in drums for use as Campaign IV FEP feed. The washed slurry was removed from the test rig and stored in a drum and would eventually be vitrified in the small-scale HLW melter, which is in the VSL at Catholic University of America.

\subsection{Filter Rinsing and Cleaning}

Following slurry washing, the slurry was removed and the process of rinsing and cleaning the filter began. The filter was rinsed with $0.1 \mathrm{M} \mathrm{NaOH}$ and cleaned with $2.0 \mathrm{M} \mathrm{HNO}_{3}$ in the following sequence:

- $\quad 0.1 \mathrm{M} \mathrm{NaOH}$ for 30 minutes

- $2.0 \mathrm{M} \mathrm{HNO}_{3}$ for 90 minutes

- $2.0 \mathrm{M} \mathrm{HNO}_{3}$ for 90 minutes

- $2.0 \mathrm{M} \mathrm{HNO}_{3}$ for 90 minutes

- $0.1 \mathrm{M} \mathrm{NaOH}$ for 30 minutes

- $0.1 \mathrm{M} \mathrm{NaOH}$ for 30 minutes

To match WTP planned RLD operations the rinse solutions were made using condensate from the Campaign II evaporator operations. The acid solutions were made from deionized and filtered (DIF) water.

At the end of a particular cleaning sequence step the solution was drained from the test rig and the subsequent solution was added. For each of the rinsing and cleaning steps it was necessary and important to have a heel, representative of what would be expected in the full-scale plant, since it affects the chemistry of each subsequent solution. Therefore, 
a WTP scaled quantity of the previous solution was added back to each new solution to represent a heel that would be left in the RPP-WTP filter system.

The heel for the first caustic rinse consisted of a mild caustic solution (12.6 L), representing the filter-loop rinse and the residual slurry remaining in the test rig after washing (estimated to be $20 \mathrm{~L}$ ). This produced a total heel of $32.6 \mathrm{~L}$. The heel for each subsequent solution consisted of 20 liters of the spent, previous solution and the residual solution remaining in the test rig (estimated to be $1.6 \mathrm{~L}$ ). This produced a total heel of 21.6 L.

The temperature adjusted permeate flux for the first caustic rinse is shown in Fig. 23. A backpulse preceded the first mild caustic rinse. The slowly diminishing flux indicates that the filter cake removed by the backpulse was rebuilt with residual slurry during the rinse period. A similar trend was seen in the first slurry wash of Campaign I (Duignan et al., 2004a). Fig. 27 (a) shows a settled sample of the first rinse solution (pH 13) that came from the heel. Note the large amount of UDS, which measured to be $6 \mathrm{wt} \%$ and similar to $5 \mathrm{wt} \%$ that was obtained in Campaign II (Duignan et al., 2004b). Further chemical analyses of the rinse (and cleaning) solutions are provided in Section 2.7.4.2.3.

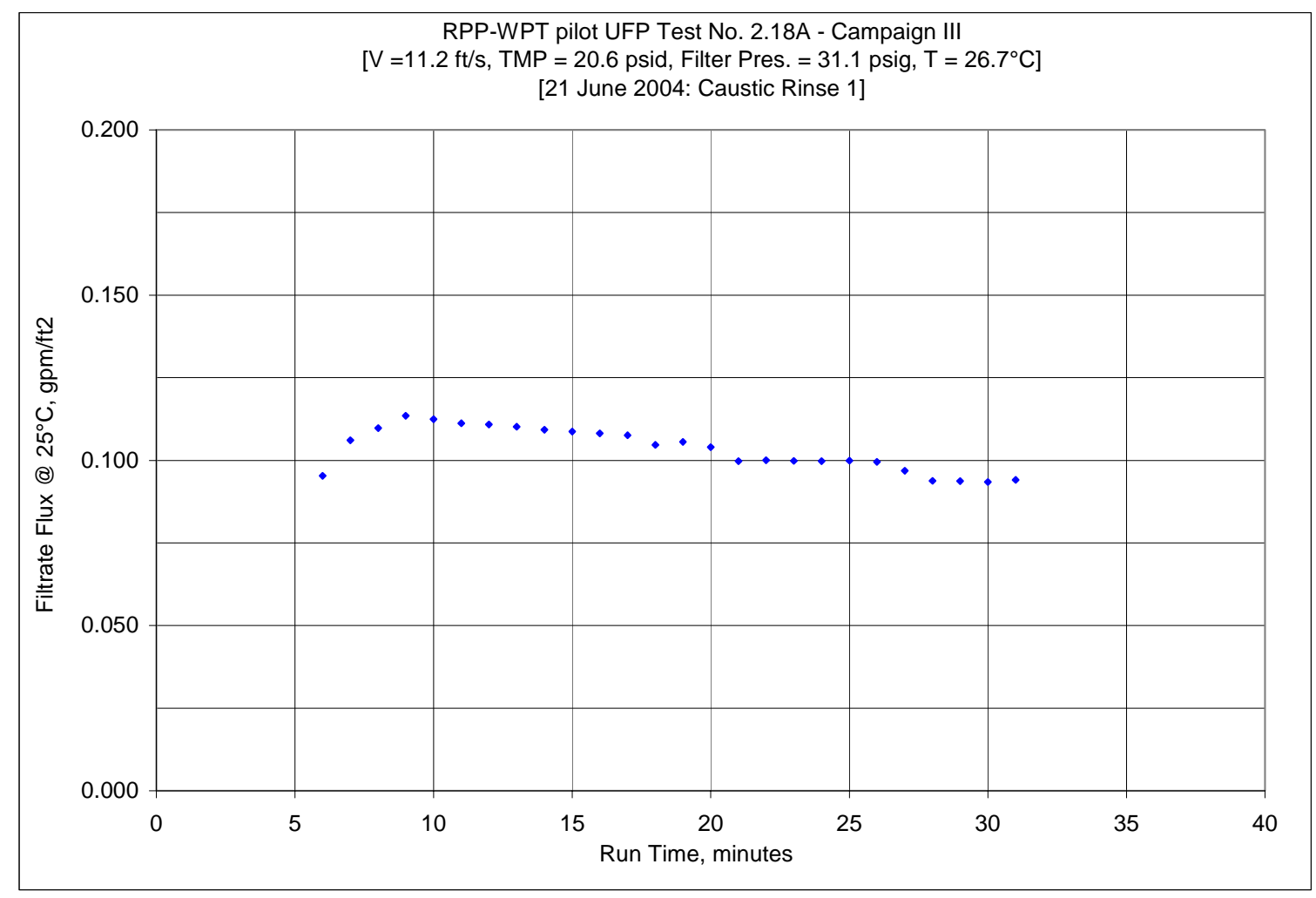

Figure 23. 1st Caustic Rinse of the Ultrafilter

Figure 24 shows the temperature adjusted filter flux results for all three acid cleanings that followed the first rinse. A backpulse preceded each cleaning, which accounts for the higher initial flux at the beginning of each cleaning. The time gaps between the cleanings 
represent the times necessary to remove the old cleaning solution from the test rig and add a new cleaning solution and heel.

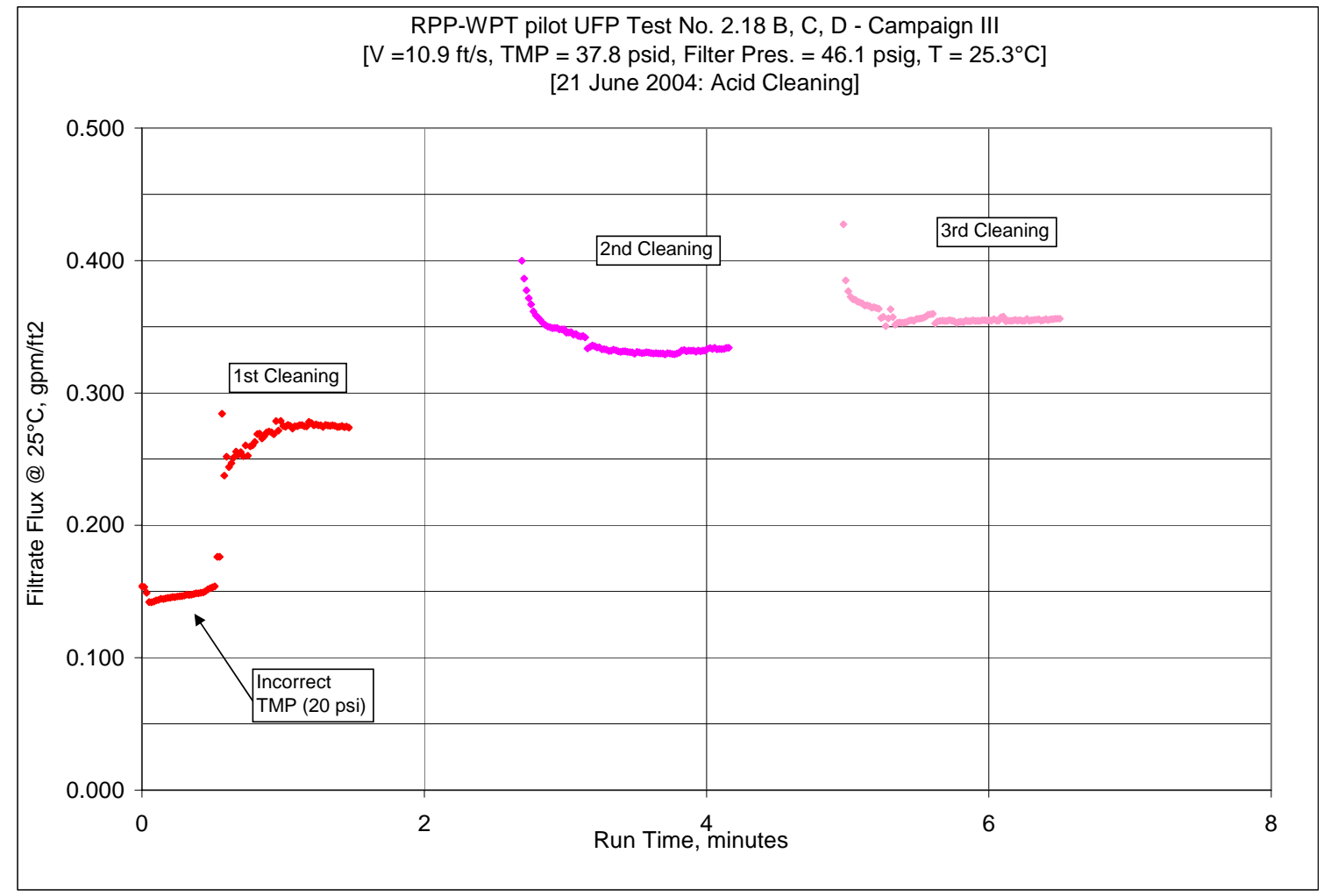

Figure 24. Acid Cleaning of the Ultrafilter

The flux also improved between cleanings, which implies two things: 1) that residual acid solution in and on the filter media continued to dissolve solids and 2) backpulsing prior to cleaning temporarily removed surface fouling.

Figure 27 (b), (c), and (d) show samples of the spent acid cleaning solutions. All of the cleaning solutions have a $\mathrm{pH}<1$. Note that the first acid cleaning solution, Fig. 27 (b) is slightly stained and that there is a small amount of whitish solids on the bottom of the bottle. In Campaign II those white solids were analyzed and a spectral analysis indicated a composition of alumina, silica, and diaoyudaoite (Duignan et al., 2004b). The $2^{\text {nd }}$ and $3^{\text {rd }}$ acid cleaning solutions are not noticeably stained, but still have small and diminishing amounts of the whitish solid. Table 29 shows that the weight percents of total solids of the acid cleaning solutions were relatively moderate in the first cleaning solution, but were greatly reduced by the third cleaning. The weight percents of undissolved solids of the cleaning solutions were insignificant, indicating that the solids had dissolved in the acid.

The acid cleanings steps were followed by two mild caustic rinses. Figure 25 shows the temperature adjusted flux of the last two rinses and reveals that the permeate flux of the second caustic rinse (or the first rinse after acid cleaning) was notably less than that of the 
third acid cleaning. This is a common trend seen in all filter cleanings, e.g., Campaign I and II, and was attributed to the caustic solution precipitating solids from the acid heel. The volume and concentration of the acid heel was enough to make the second caustic rinse acidic ( 0.4 molar acid). Furthermore, the volume and concentration of the second caustic rinse heel was also enough to make the third caustic rinse slightly acidic (0.04 molar acid). The temperature adjusted flux during the third caustic rinse continued to decline as more solids precipitated from the more-neutral solution. At the end of the last rinse the flux had dropped to $0.17 \mathrm{gpm} / \mathrm{ft}^{2}$, which is still more than an order of magnitude higher than the average dewatering flux of $0.011 \mathrm{gpm} / \mathrm{ft}^{2}$. Samples of the rinse solutions are shown in Fig. 27 (e) and (f) and they appear to be relatively clear.

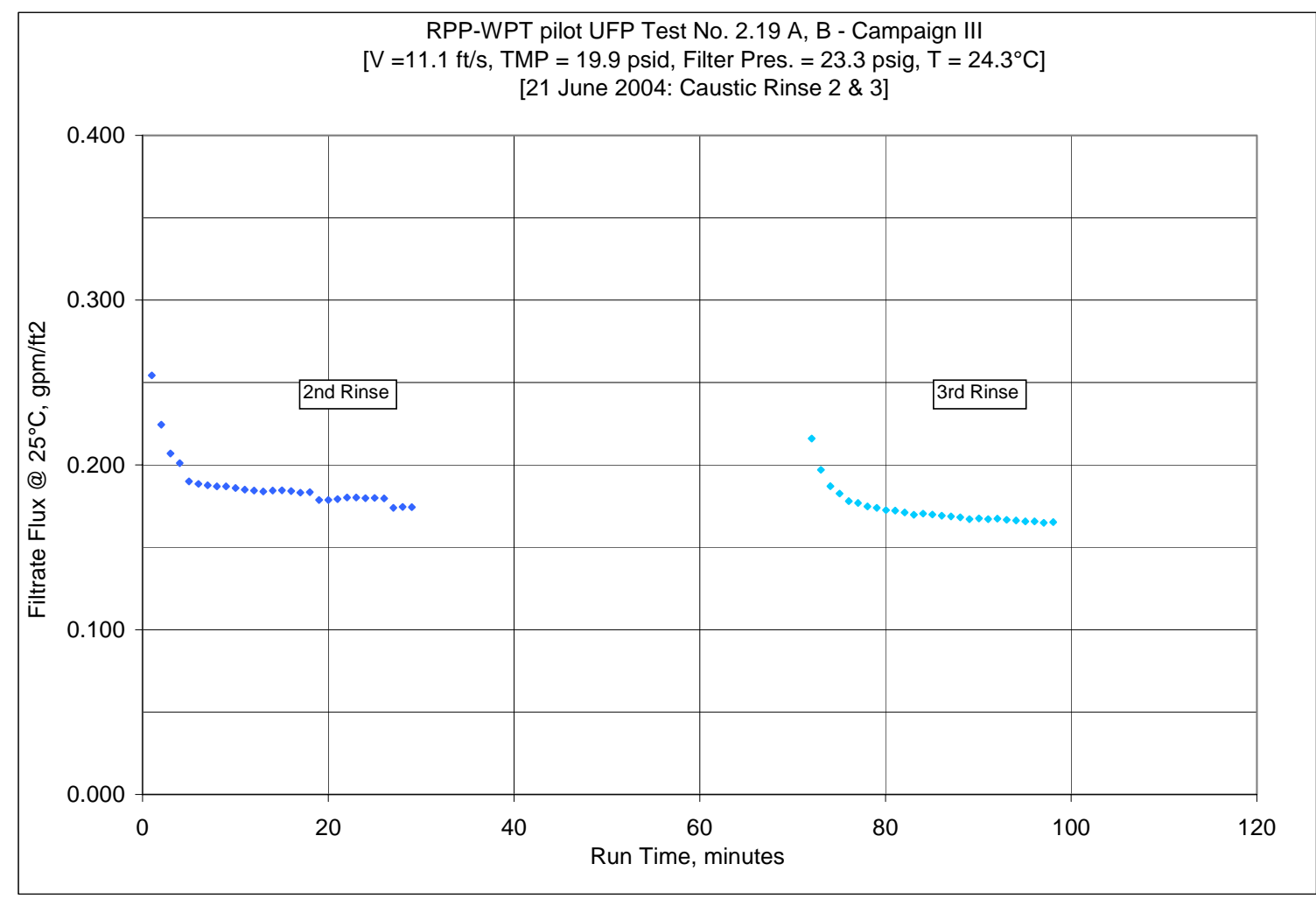

Figure 25. 2nd and 3rd Rinses of the Ultrafilter

The third caustic rinse solution was left in the test rig and, as per the WTP planned operation, was used for the post-test baseline test. After the baseline test the caustic solution was removed from the test rig and stored in a drum for use as Campaign IV FEP feed.

Figure 26 shows the permeate flux of the entire rinse and clean process for Campaigns I, II, and III. The Campaign II and III data are very similar in trend and magnitude. The Campaign I trend is different during the acid cleanings but similar during caustic rinses. As noted previously, the slurry of Campaign I was AY102 only, where the slurry of Campaigns II and III was a mixture of AY102, AP101 and FEP bottoms. Furthermore, the issue of tank heels was not resolved until after Campaign I was complete, therefore 
the heels were different. Despite the differences, after all acid cleanings and caustic rinses the ending "clean filter” fluxes for all campaigns was $0.15 \mathrm{gpm} / \mathrm{ft}^{2} \pm 0.02 \mathrm{gpm} / \mathrm{ft}^{2}$.

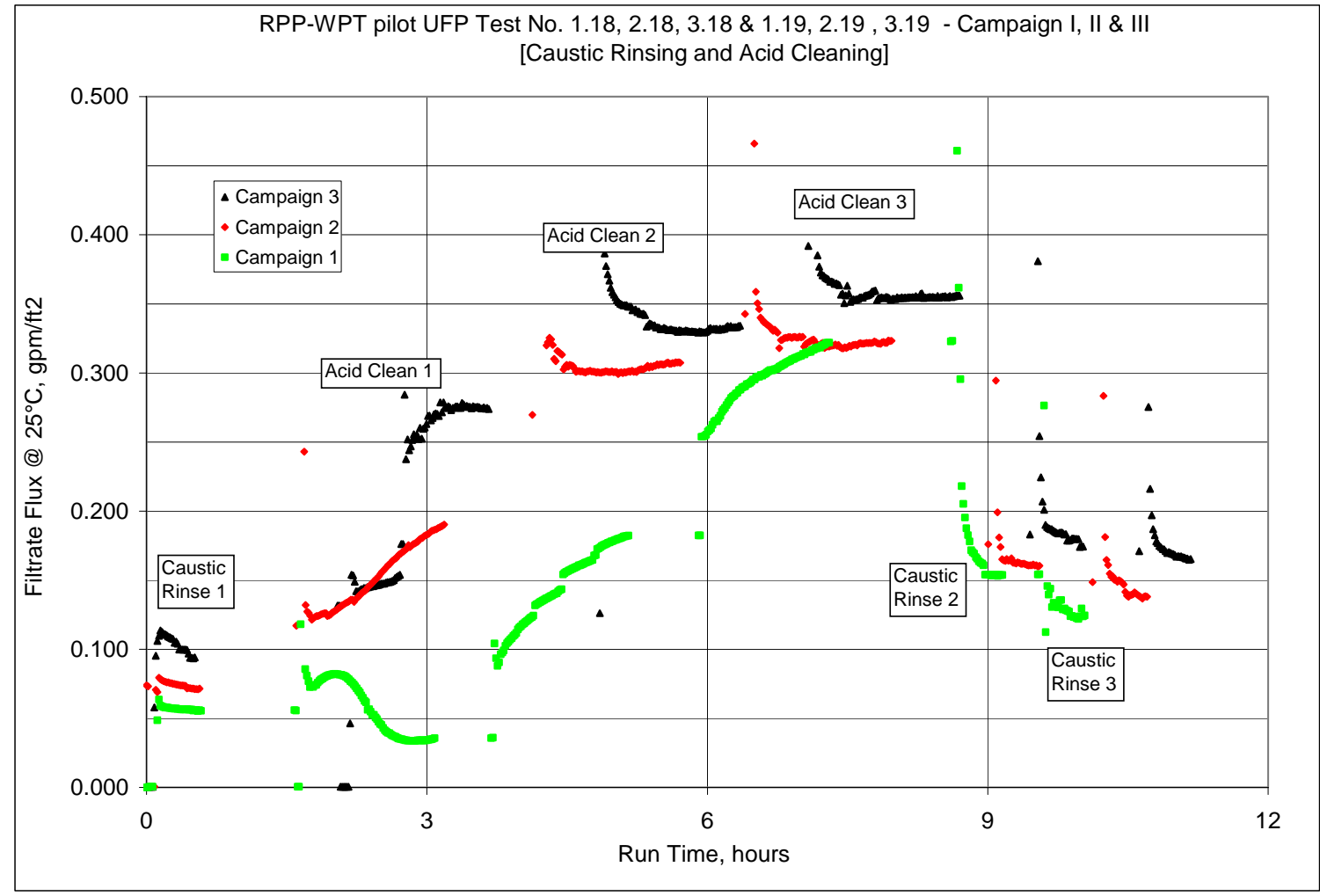

Figure 26. Filter Rinse and Clean, Campaigns I, II, and III

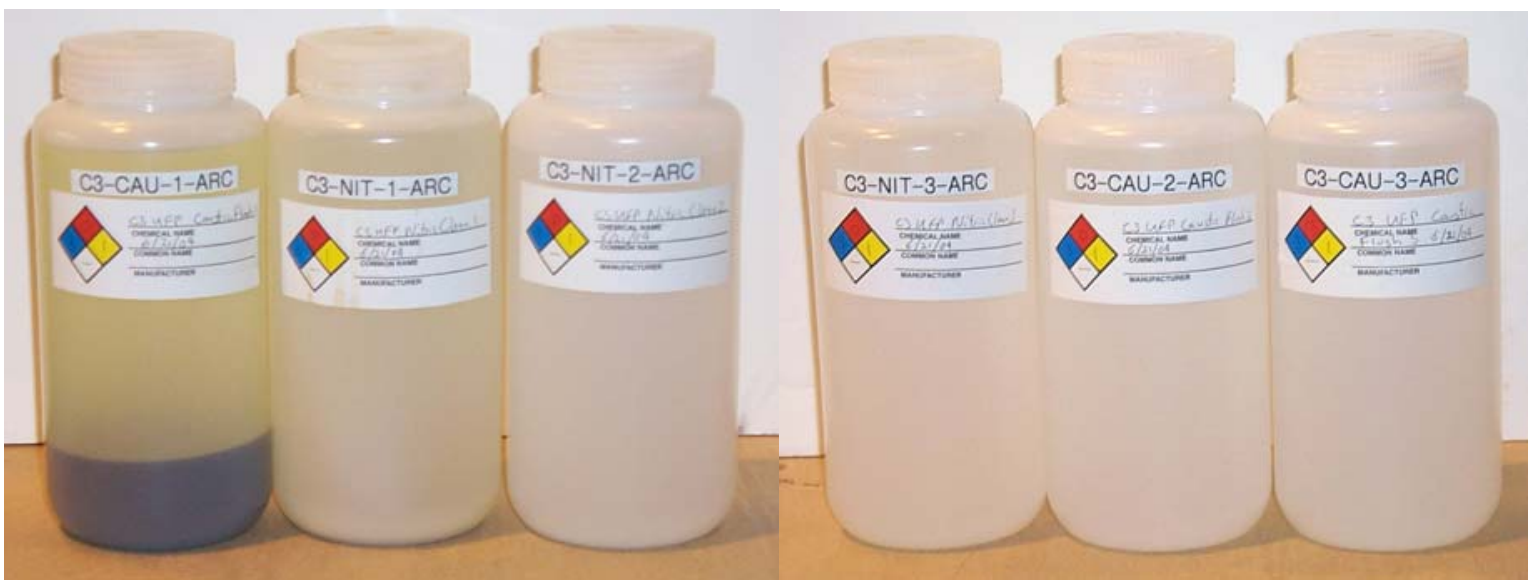

(a) (b)

(c)

(d)

(e)

(f)

Figure 27. Caustic Rinse and Acid Cleaning Samples

(a) pre-clean caustic rinse, $\mathrm{pH} 13$, (b) first $2 \mathrm{M}$ nitric cleaning, $\mathrm{pH} 1$, (c) second $2 \mathrm{M}$ nitric cleaning, $\mathrm{pH} 1$, (d) third $2 \mathrm{M}$ nitric cleaning, $\mathrm{pH} 1$, (e) first post-clean caustic rinse, $\mathrm{pH} 1$, (f) second post-clean caustic rinse, $\mathrm{pH} \sim 1.2$ 
WSRC-TR-2004-00565, REVISION 0

SRNL-RPP-2004-00092, REVISION 0

Table 29. Total Solids (TS) and Undissolved Solids (UDS) Concentrations of the Spent Rinse and Cleaning Solutions

\begin{tabular}{|c|c|c|}
\hline \multirow{2}{*}{ Step } & TS & UDS \\
\hline & $\mathrm{wt} \%$ & $\mathrm{wt} \%$ \\
\hline First Caustic Rinse $0.1 \mathrm{M} \mathrm{NaOH}$ & 6.25 & 4.27 \\
\hline First Acid Cleaning $2.0 \mathrm{M} \mathrm{HNO}_{3}$ & 1.50 & 0.12 \\
\hline Second Acid Cleaning $2.0 \mathrm{M} \mathrm{HNO}_{3}$ & 0.48 & 0.07 \\
\hline Third Acid Cleaning $2.0 \mathrm{M} \mathrm{HNO}_{3}$ & 0.10 & 0.00 \\
\hline Second Caustic Rinse $0.1 \mathrm{M} \mathrm{NaOH}$ & 0.75 & 0.12 \\
\hline Third Caustic Rinse 0.1 M NaOH & 0.86 & 0.03 \\
\hline
\end{tabular}

\subsection{Filter Baselining}

As was explained in Section 2.7.4.1.1, pretest maintenance work on the pilot-scale UFP pumps was needed before Campaign III and when pre-test baseline water test was done the flux was low. See the closed-diamond data in Fig. 28. This was not unexpected because work on the test rig always seems to affect filter flux, therefore, the filter was cleaned with nitric acid and then the pre-test baseline was repeated. The closed-square data in Fig. 28 shows the improved flux, which was even higher than the post-test baseline data from Campaign II, see the closed-triangle data.

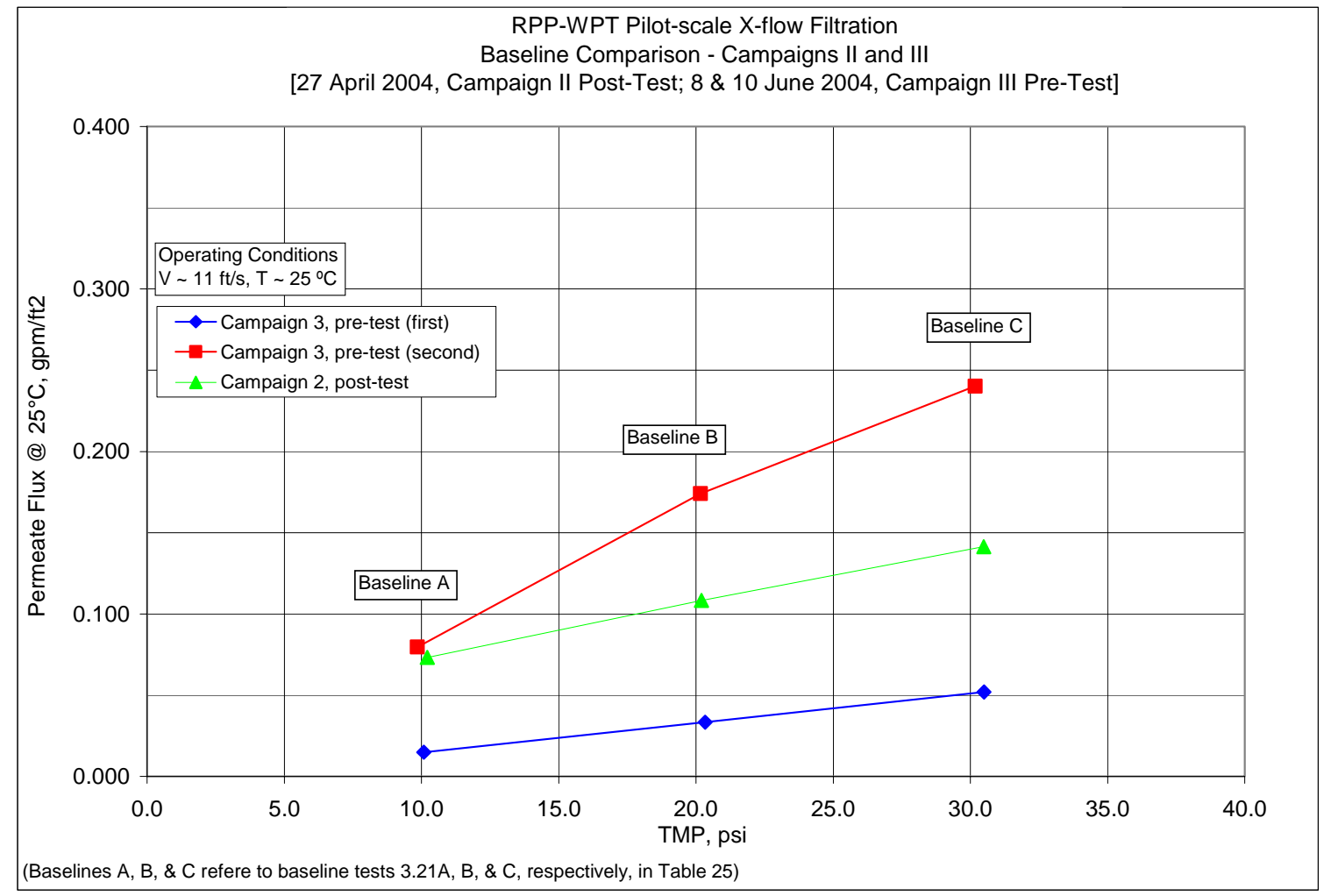

Figure 28. Baselines, Campaign II Post-test and Campaign III Pre-test 
The low flux of the initial pre-test baseline is assumed to be caused by one of, or a combination of, the following events: 1) air was entrained in the filter media during the filter inspection, 2) the filter was plugged by solids that were encrusted on the interior of, or trapped within dead zones of the slurry loop and loosened during the pump repair, or 3) solids were pushed farther into the filter media when the system was restarted after the pump repair.

The filter inspection prior to Campaign III was completed in approximately 30 minutes, after which the filter unit was re-assembled and submerged. It is unlikely that the filter media became dry during this short period. Furthermore, as was seen in Fig. 17 of Section 2.7.4.1.1, the immediate increase in the flux and the response to the backpulsing implies that events 2) and/or 3) may have been responsible for the low flux.

A post-test baseline was conducted after the test rig was rinsed and cleaned (tests \# 3.18 \& 3.19 in Table 25), using the mild caustic rinse solution $(0.1 \mathrm{M} \mathrm{NaOH})$ from the third rinse step. The post-test baseline had a lower temperature adjusted flux than the second pre-test baseline, indicating that the filter media was not completely free of solids. However, this has been the case in previous cross-flow filtration tests (e.g., Duignan, 2003a). Although the post-test was lower than the pre-test baseline flux, the temperature adjusted flux for the post-test baseline was still significantly higher than the adjusted flux during dewatering of the waste simulant $\left(0.011 \mathrm{gpm} / \mathrm{ft}^{2}\right)$.

As previously noted in the Campaign II report (Duignan et al., 2004b), the final caustic rinse, which is used for filter baselining purposes to determine cleaning effectiveness, was not caustic, but borderline acidic because of the heel material. That is, one effect of the tank heel, which is carried over through each of the cleaning steps, was maintaining the final rinses marginally acidic. Because of this effect the post-test baseline was repeated in a fresh $0.1 \mathrm{M} \mathrm{NaOH}$ and without adding in any heel. This extra rinse was used to see if the rinses were truly caustic, and without undissolved solids, how it would affect the baseline flux. The concern was that once the rinse turned caustic the filter flux would be significantly impacted by further precipitating dissolved solids. However, the post-“post-test”-baseline test, with a truly caustic rinse, did not significantly affect filter flux. Compare the two post-test data curves in Fig. 29. That is, the truly caustic data begin slightly below the final rinse (but acidic) data, but at TMP $=30$ psid the results are identical. This lack of significance was reinforced in Campaign II. In that campaign a similar post-“post-test”-baseline test was done and those data were slightly above the final Campaign II caustic rinse. It appears that the final caustic rinse, even though it was slightly acidic, does result in a stable baseline filter flux. 
WSRC-TR-2004-00565, REVISION 0

SRNL-RPP-2004-00092, REVISION 0

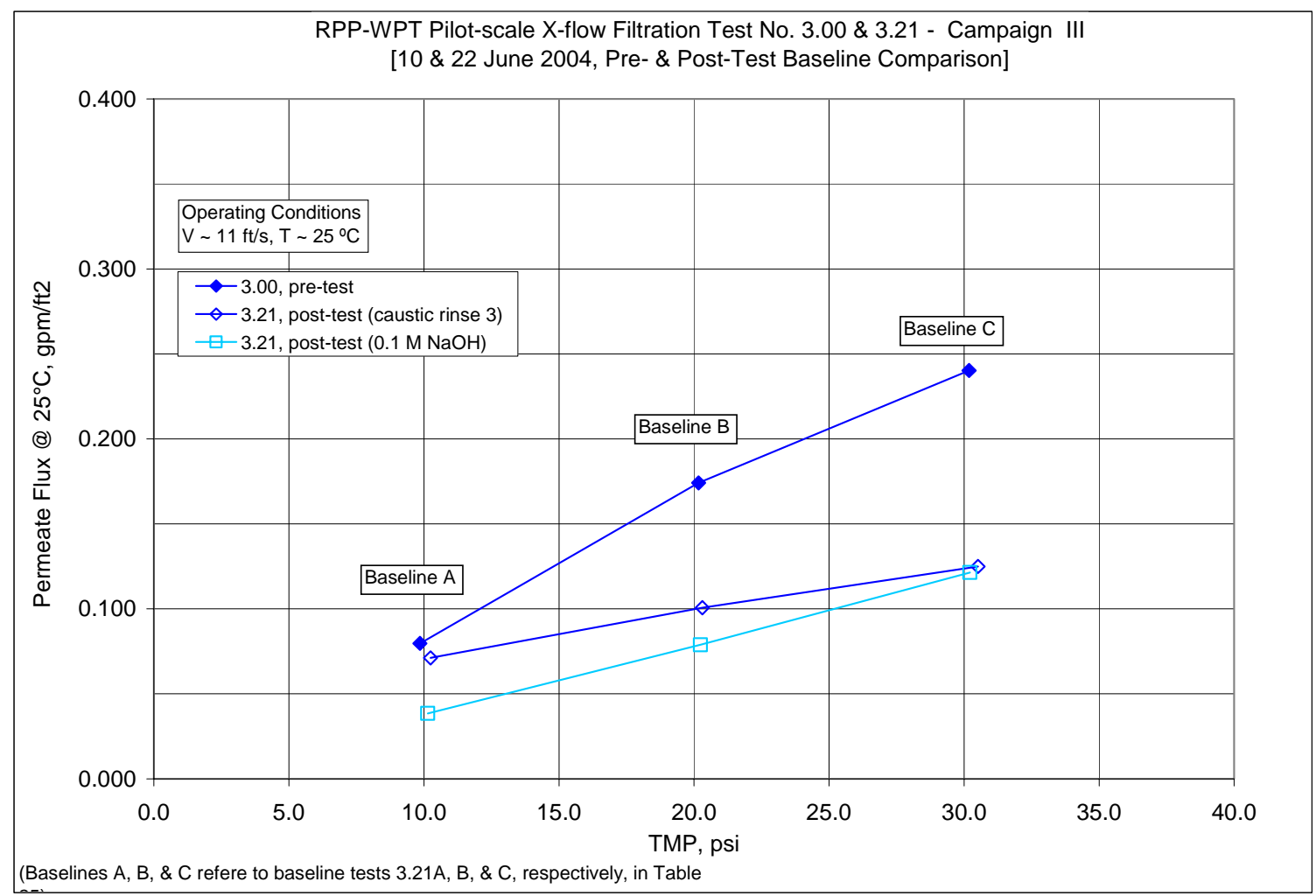

Figure 29. Pre- and Post-Test Baselines for Campaign III

One of the unavoidable non-prototypic aspects of SIPP was the time interval between each of the Campaigns. The Pretreatment Facility of WTP will operate continuously, therefore, the filter elements will be ready for use for the next batch of slurry as soon as it is cleaned and the UFP feed tank is full. For SIPP, the preparations for each Campaign led to intervals of approximately: 8 weeks between Campaigns I and II, 3 weeks between Campaigns II and III, and 4 weeks between Campaigns III and IV; as seen in the "summary of EDL activities" at the end of Section 2.1 of this report. However, because of the SIPP intervals it was possible to see the effect soaking the filter elements soaked in inhibited water $(0.1 \mathrm{M} \mathrm{NaOH})$ for finite lengths of time. To date, three campaigns have been completed, therefore, Fig. 30 compares the pre-and post-test baseline tests from Campaign I, II, and III. The pre-test flux data of Campaign I are noticeably greater than that of Campaign II and III. It is believed that the pre-test flux of Campaign I is greater because the filter was submerged in $0.1 \mathrm{M} \mathrm{NaOH}$ for an extended period of time prior to Campaign I (approximately 1 year), whereas prior to Campaign II and III, the filter was submerged in the mild caustic for the shorter time intervals just mention above. The mild caustic solution may continue to dissolve solids lodged in the filter media, allowing them to migrate out. 
The pre-test baselines of Campaigns II and III are comparable. In addition, the post-test baselines of Campaigns I, II, and III are comparable, but lower than the pre-test values. The lower flux of the post-test baselines indicates that the filter was not cleaned to the pre-test condition, i.e., some solids remained in the filter media. However, the similarity of the flux data from the Campaigns I, II, and III dewatering tests (see Fig. 19) indicate that the differences seen in the baseline results are not an accurate predictor of dewatering flux.

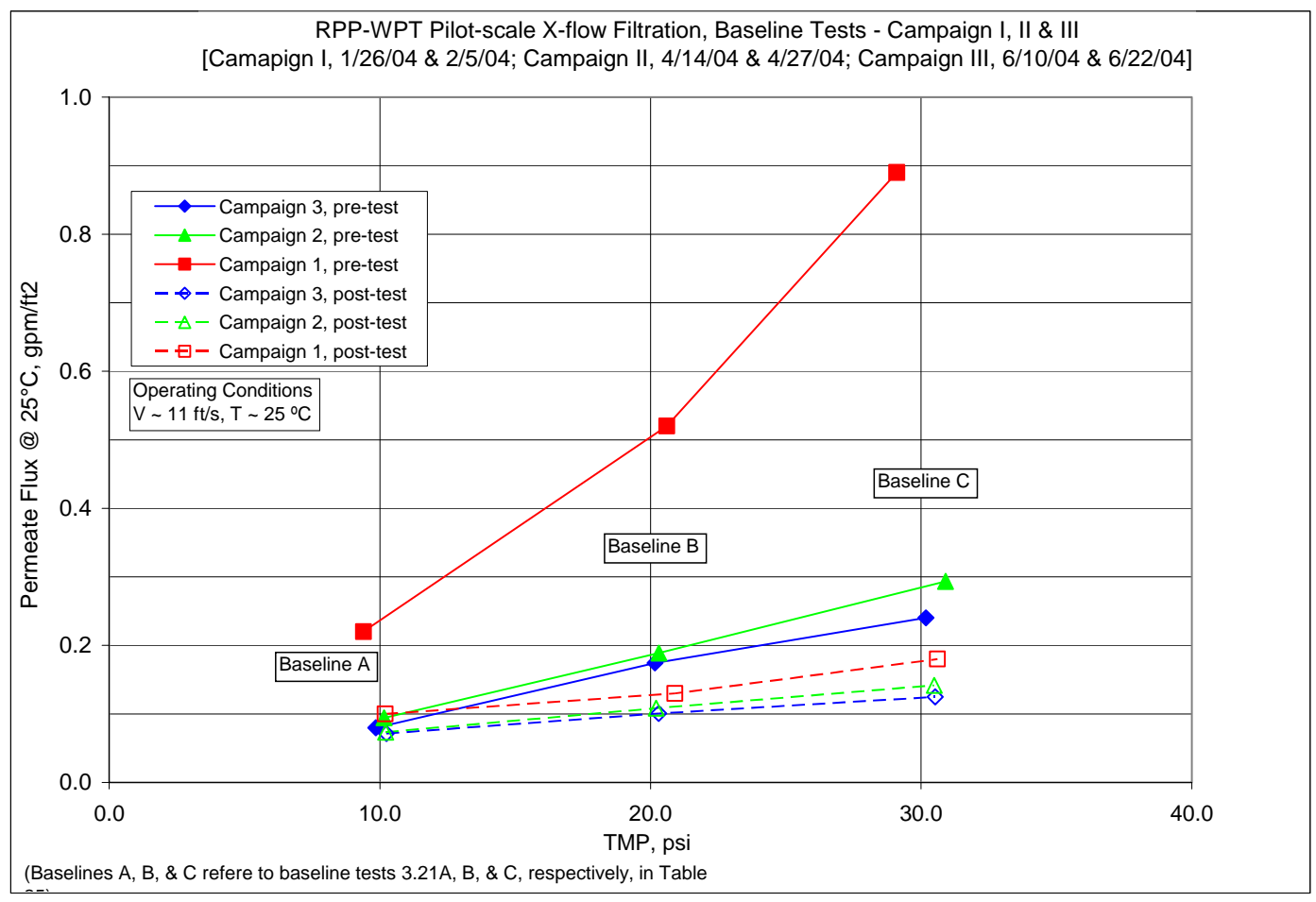

Figure 30. Comparison of Pre- and Post-Test Baselines, Campaign I, II, and III

\subsubsection{UFP Waste Products - Chemical Analysis (Author - Zamecnik)}

This section gives more in-depth chemical analyses for the results just presented in the preceding section, Section 2.7.4.1. In some cases the repetition is unavoidable, but it also facilitates better understanding of the many aspects of the UFP unit operation.

\subsection{Dewatering}

The total and undissolved solids contents during dewatering of the slurry are given in Table 30 as a function of the amount of permeate removed. The undissolved solids (UDS) value is calculated from the measured total and supernate solids. The table shows both the value calculated from the measured supernate solids for each sample and the value calculated from the mean supernate solids. The supernate solids content of any sample should be the same, so the variations shown are due to measurement error. 


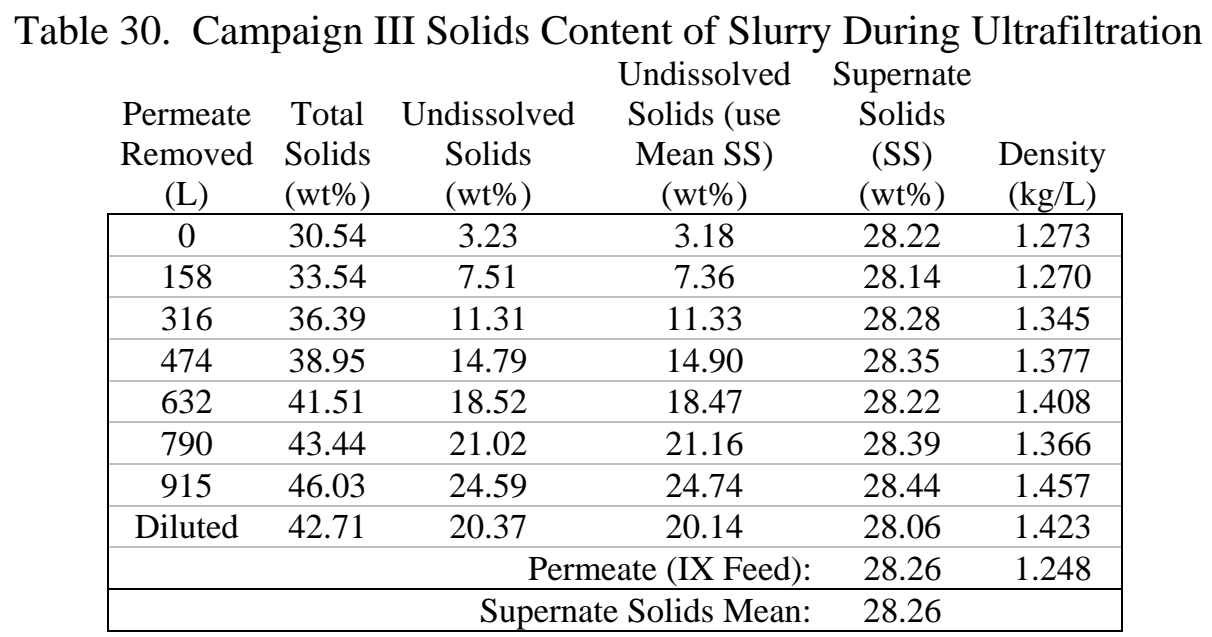

Upon completion of concentrating the slurry, the final undissolved solids (UDS) content was 24.6 to $24.7 \mathrm{wt} \%$ versus the target of $20 \mathrm{wt} \%{ }^{\dagger}$. Because the final concentrated slurry was at greater than $20 \mathrm{wt} \%$ UDS, the batch of $\sim 117 \mathrm{~L}$ of slurry was diluted by first removing a calculated amount of slurry and then adding back permeate. The slurry was actually diluted back to between 20.1 to $20.4 \mathrm{wt} \%$ UDS.

Table 31 shows the concentrations of the dewatered simulated waste and permeate just before washing for Campaigns I, II, and III (the Campaign III composition is calculated). Note that one of the most significant differences between Campaign II and Campaign III is the $\mathrm{Al}$ concentrations. For the permeate, most of the other major species had similar concentrations in Campaign II and Campaign III (K, Na, nitrite, nitrate, phosphate, sulfate). The major undissolved species in the Campaign II and Campaign III slurries were also of similar concentration (Ca, Fe, Mn, $\mathrm{Pb}, \mathrm{Si}, \mathrm{Cr}, \mathrm{Nd}$ ). The $\mathrm{Na}$ and $\mathrm{K}$ are higher in Campaign II and Campaign III, than in Campaign I, due to the contribution of the AP101 simulant. Recall that the Campaign I simulant was just of AY102/C106, without the additions of either AP101 nor the recycle streams.

\footnotetext{
${ }^{\dagger}$ As mentioned the Abstract, and discussed in detail in the Campaign II report (Duignan et al., 2004b), the OLI/ESP model was slightly off in predicting target values because of the difficulties in handling the complex chemical combinations of the simulated wastes. To produce the target amount of permeate during dewatering [see Table 1, Row "UF1 Filtrate (routed to CIX, than TLP)] all of the UFP feed had to be processed, which resulted in the solids content of the UFP concentrate to be $24.6 \mathrm{wt} \%$ UDS. However, the Test Specification (Longwell, 2003) required washing to be done on a UFP product at $20 \mathrm{wt} \%$ UDS and therefore, the concentrate was diluted to that value.
} 
WSRC-TR-2004-00565, REVISION 0

SRNL-RPP-2004-00092, REVISION 0

Table 31. Concentrated Slurry and Permeate Compositions

\begin{tabular}{|c|c|c|c|c|c|c|}
\hline \multirow[b]{2}{*}{ Campaign: } & \multicolumn{3}{|c|}{-------------Slurry------------- } & \multicolumn{3}{|c|}{------------Permeate----------- } \\
\hline & I & II & III* & I & II & III \\
\hline Total Solids (wt\%) & 36.91 & 45.37 & 46.27 & 17.09 & 28.15 & 28.27 \\
\hline Undissolved Solids (wt\%) & 23.90 & 23.51 & 24.90 & 0 & 0 & 0 \\
\hline Supernate Solids (wt\%) & 17.09 & 28.58 & 28.46 & 17.09 & 28.15 & 28.27 \\
\hline Density $(\mathrm{kg} / \mathrm{L})$ & 1.357 & 1.470 & 1.448 & 1.168 & 1.243 & 1.248 \\
\hline All units $\mathbf{m g} / \mathbf{k g} \quad \mathrm{Ag}$ & 1037 & 990 & 1070 & $<0.51$ & $<0.48$ & 0.761 \\
\hline unless stated otherwise $\mathrm{Al}$ & 19117 & 17550 & 22158 & 7252 & 5670 & 5136 \\
\hline B & 188 & $<151$ & & $<0.10$ & 70.1 & 85.7 \\
\hline $\mathrm{Ba}$ & 477 & 444 & 409 & $<0.03$ & $<0.03$ & $<1.3$ \\
\hline $\mathrm{Ca}$ & 2280 & 1905 & 2046 & 1.73 & $<0.05$ & $<3.6$ \\
\hline $\mathrm{Cd}$ & 63.5 & 70.6 & 71.6 & $<0.03$ & $<0.03$ & $<0.04$ \\
\hline $\mathrm{Ce}$ & 516 & 551 & 513 & $<1.2$ & $<1.1$ & $<1.0$ \\
\hline Co & 59.0 & $<15$ & 212 & $<0.14$ & $<0.13$ & $<0.11$ \\
\hline $\mathrm{Cr}$ & 845 & 772 & 799 & 128 & 132 & 139 \\
\hline $\mathrm{Cu}$ & 129 & 134 & 120 & $<0.05$ & 0.30 & $<0.03$ \\
\hline $\mathrm{Fe}$ & 58417 & 55750 & 53984 & 0.16 & 1.42 & 1.36 \\
\hline $\mathrm{K}$ & 330 & 13000 & 20885 & 297 & 17051 & 16346 \\
\hline $\mathrm{La}$ & 407 & 478 & 477 & $<0.17$ & $<0.16$ & 0.849 \\
\hline $\mathrm{Li}$ & 0 & 90.9 & 91.8 & $<0.34$ & 0.93 & 1.72 \\
\hline Mg & 617 & 639 & 618 & $<0.01$ & $<0.01$ & $<0.24$ \\
\hline Mn & 12650 & 12300 & 11669 & $<0.02$ & 0.02 & $<0.02$ \\
\hline $\mathrm{Na}$ & 60800 & 89230 & 109462 & 60017 & 90483 & 88141 \\
\hline $\mathrm{Nd}$ & 1288 & 1370 & 1267 & $<1.5$ & $<1.4$ & $<1.5$ \\
\hline $\mathrm{Ni}$ & 1750 & 1840 & 3135 & 0.58 & 0.303 & 0.247 \\
\hline $\mathrm{P}$ & 1263 & 672 & 103 & 1190 & 643 & 554 \\
\hline $\mathrm{Pb}$ & 2793 & 2625 & 2507 & $<1.4$ & 1.35 & $<0.36$ \\
\hline $\mathrm{S}$ & 680 & 862 & 1857 & 539 & 965 & 905 \\
\hline $\mathrm{Si}$ & 17983 & 15821 & 17999 & 17.0 & 43.6 & 42.1 \\
\hline $\mathrm{Sr}$ & 500 & 451 & 454 & 0.24 & 0.06 & 0.162 \\
\hline $\mathrm{Ti}$ & 22.0 & 10.9 & 66.4 & $<0.05$ & 0.07 & 0.10 \\
\hline $\mathrm{Zn}$ & 458 & 151 & 178 & $<0.03$ & 2.45 & 2.37 \\
\hline $\mathrm{Zr}$ & 797 & 745 & 722 & $<0.05$ & 0.69 & 1.43 \\
\hline bromide & 33.3 & $<29$ & 97.5 & 48.8 & $<8.0$ & $<80$ \\
\hline chloride & 98.0 & 738 & 45.9 & 163 & 973 & 1082 \\
\hline fluoride & 39.0 & 52.0 & 18.4 & 38.5 & 23.3 & 44.9 \\
\hline formate & 168 & 700 & 467 & 299 & 981 & 1066 \\
\hline nitrate & 224 & 49700 & 37079 & 336 & 68606 & 72917 \\
\hline nitrite & 3107 & 14100 & 10559 & 3827 & 20268 & 23077 \\
\hline oxalate & 14133 & 23800 & 18351 & 2748 & 600 & 658 \\
\hline phosphate & 2693 & 1070 & 846 & 2877 & 1987 & 1963 \\
\hline sulfate & 1427 & 2080 & 209 & 2106 & 2727 & 2853 \\
\hline total inorganic carbon (TIC) & 15133 & 3347 & 3456 & 12586 & 6233 & 6859 \\
\hline total organic carbon (TOC) & 15300 & 5973 & 13734 & 2483 & 1609 & 801 \\
\hline total carbon (TC) & 30433 & 9320 & 17190 & 15068 & 7842 & 7660 \\
\hline carbonate from TIC & 75667 & 16735 & 17279 & 62928 & 31166 & 34295 \\
\hline free hydroxide & & & & & & 19343 \\
\hline
\end{tabular}




\subsection{Washing the Concentrated Slurry}

After diluting the dewatered slurry to $20 \mathrm{wt} \%$ UDS, it was washed with 22 discrete additions of $\sim 5.35 \mathrm{~L}$ of approximately $0.01 \mathrm{M} \mathrm{NaOH}$, followed by an additional 22 similar washes. The target total amount of wash water used was about $2 \times 118 \mathrm{~L}=236 \mathrm{~L}$. (See the footnote in Section 2.6.1.2. FEP Feed Mixing). Table 32 summarizes the composition of the washed slurry after all 44 washes. Table 33 shows limited characterization of the permeate composites from the first and second 22 washes and compares these same analyses to Campaign I and II values. The Campaign II and III values for solids and density are similar, as expected. The total base values are different because the recycle compositions (FEP concentrate) to Campaign II and III were different.

The compositions of the Campaign II and III washed slurries, shown in Table 32, were expected to be essentially the same since the starting slurries were expected to have similar compositions. The majority of the undissolved components for both campaigns came from the AY102 simulant, with a smaller (and comparable amount) from the FEP evaporator bottoms. A trace amount of solids also came from the AP101 simulant for both campaigns. Examination of the data in Table 32 shows that the compositions are indeed similar, with the exception of aluminum, which was higher than expected in Campaign III.

The soluble species (Na, anions) were all lower in Campaign III than in Campaign II. This difference was due to the lower concentrations of these in the FEP concentrate. The amounts of AY102 slurry and AP101 simulant used in Campaign III were approximately the same as in Campaign II.

Because the amount of aluminum was higher than expected, mass balance calculations were performed to check if these higher values were reasonable. Note that to reduce costs, elemental and ion analyses of the ultrafilter feed and concentrate were not performed, so these compositions must be estimated. Table 34 shows the material balance compositions in tabular form. For several species, values shown in italics are from Campaign II and are shown for comparison. The measured FEP concentrate values were added to the estimated AY102 and AP101 streams. The estimates used were the analyses from the previous campaigns, but no significant differences were expected since the same materials were used. The sum of these streams was the "UF Slurry-Start". The amount of permeate generated was then mathematically subtracted from this slurry to give the "UF Slurry-Concentrated" stream. The calculated UDS and total solids content matched the measured values well. The permeate composition used here was from measured values. Note that for soluble species, this subtraction introduces significant error in the calculated concentrated slurry composition, so these values are not good as the predictions of the undissolved species. 
WSRC-TR-2004-00565, REVISION 0

SRNL-RPP-2004-00092, REVISION 0

Table 32. Comparison of Campaigns I-III Washed Slurry Compositions

Campaign I

\begin{tabular}{|c|c|c|c|c|c|c|c|c|c|}
\hline & & & & & & & & & \\
\hline Total Solids (wt\%) & \multicolumn{3}{|c|}{21.99} & \multicolumn{3}{|c|}{26.56} & \multicolumn{3}{|c|}{26.72} \\
\hline Undissolved Solids (wt\%) & \multicolumn{3}{|c|}{20.55} & \multicolumn{3}{|c|}{22.22} & \multicolumn{3}{|c|}{22.05} \\
\hline Supernate Solids (wt\%) & \multicolumn{3}{|c|}{1.81} & \multicolumn{3}{|c|}{5.59} & \multicolumn{3}{|c|}{5.99} \\
\hline Density $(\mathrm{kg} / \mathrm{L})$ & \multicolumn{3}{|c|}{1.183} & \multicolumn{3}{|c|}{1.214} & \multicolumn{3}{|c|}{1.192} \\
\hline $\begin{array}{l}\text { Dissolution: } \\
\text { Elements by ICPES mg/kg: }\end{array}$ & $\begin{array}{l}\text { Micro- } \\
\text { wave }\end{array}$ & $\begin{array}{l}\text { Sodium } \\
\text { Peroxide } \\
\text { Fusion }\end{array}$ & $\begin{array}{c}\text { Mean } \\
\text { or } \\
\text { Best }\end{array}$ & $\begin{array}{l}\text { Micro- } \\
\text { wave }\end{array}$ & $\begin{array}{c}\text { Sodium } \\
\text { Peroxide } \\
\text { Fusion }\end{array}$ & $\begin{array}{c}\text { Mean } \\
\text { or } \\
\text { Best }\end{array}$ & $\begin{array}{l}\text { Micro } \\
\text {-wave }\end{array}$ & $\begin{array}{l}\text { Sodium } \\
\text { Peroxide } \\
\text { Fusion }\end{array}$ & $\begin{array}{c}\text { Mean } \\
\text { or } \\
\text { Best }\end{array}$ \\
\hline $\mathrm{Ag}$ & & 970 & 970 & & 804 & 804 & & 904 & 904 \\
\hline $\mathrm{Al}$ & 12000 & 15500 & 13750 & 12400 & 13100 & 12750 & 16800 & 19300 & 18050 \\
\hline All units $\mathrm{mg} / \mathrm{kg}$ & & 130 & 130 & & $<150$ & $<150$ & & 149 & 149 \\
\hline unless otherwise noted $\mathrm{Ba}$ & 419 & 460 & 440 & 408 & 384 & 396 & 450 & 410 & 430 \\
\hline $\mathrm{Ca}$ & 1880 & 2240 & 2060 & 1890 & 2000 & 1945 & 1810 & 2070 & 1940 \\
\hline $\mathrm{Cd}$ & 70.0 & 70.0 & 70.0 & 74.2 & 47.0 & 60.6 & 72.0 & 51.0 & 61.5 \\
\hline $\mathrm{Ce}$ & 166 & 620 & 393 & 123 & 497 & 310 & 427 & 518 & 473 \\
\hline Co & 13.0 & 30.0 & 21.5 & $<13$ & $<45$ & $<13$ & $<8.4$ & $<8.0$ & $<8$ \\
\hline $\mathrm{Cr}$ & 689 & 700 & 695 & 620 & 573 & 597 & 583 & 567 & 575 \\
\hline $\mathrm{Cu}$ & 123 & 130 & 127 & 133 & 113 & 123 & 128 & 119 & 124 \\
\hline $\mathrm{Fe}$ & 52400 & 52000 & 52200 & 55000 & 45800 & 50400 & 50800 & 48900 & 49850 \\
\hline K & 84.0 & & 84.0 & 3859 & & 3859 & 3490 & 3890 & 3690 \\
\hline $\mathrm{La}$ & 152 & 460 & 306 & 128 & 386 & 257 & 378 & 421 & 400 \\
\hline $\mathrm{Li}$ & $<33$ & $<40$ & $<33$ & 95 & 78 & 87 & 76.0 & 104 & 90 \\
\hline Mg & 554 & 550 & 552 & 640 & 509 & 575 & 565 & 555 & 560 \\
\hline Mn & 11700 & 11600 & 11650 & 12200 & 10100 & 11150 & 11300 & 10900 & 11100 \\
\hline Mo & $<16$ & $<20$ & $<16$ & $<17$ & $<25$ & $<16$ & 19.0 & $<17$ & 0.90 \\
\hline $\mathrm{Na}$ & 14000 & & 14000 & 28300 & & 28300 & 25500 & & 25500 \\
\hline $\mathrm{Nd}$ & 500 & 1270 & 885 & 372 & 1150 & 761 & 1180 & & 1180 \\
\hline $\mathrm{Ni}$ & 1720 & & 1720 & 1680 & & 1680 & 1600 & & 1600 \\
\hline $\mathrm{P}$ & 822 & 470 & 646 & 240 & 319 & 280 & 370 & 337 & 354 \\
\hline $\mathrm{Pb}$ & 2320 & 2390 & 2355 & 2670 & 2170 & 2420 & 2900 & 2790 & 2845 \\
\hline $\mathrm{S}$ & 186 & 270 & 228 & 194 & 489 & 342 & 151 & 284 & 218 \\
\hline $\mathrm{Si}$ & 14900 & 15600 & 15250 & 17200 & 14600 & 15900 & 11670 & 16600 & 14135 \\
\hline $\mathrm{Sr}$ & 405 & 450 & 428 & 446 & 410 & 428 & 374 & 420 & 397 \\
\hline $\mathrm{Ti}$ & 9.0 & 10.0 & 9.5 & 14.8 & 11.0 & 12.9 & $<27$ & $<24$ & $<24$ \\
\hline $\mathrm{Zn}$ & 118 & 180 & 149 & 145 & 133 & 139 & 151 & 150 & 151 \\
\hline $\mathrm{Zr}$ & 697 & 330 & 514 & 718 & 652 & 685 & 693 & 690 & 692 \\
\hline Soluble Ions: & & & & & ndissolved & ample & & & \\
\hline bromide & & $<78$ & & & $<<27.9$ & & & 51.3 & \\
\hline chloride & & 8.00 & & & 99.4 & & & 69.5 & \\
\hline fluoride & & 6.00 & & & 19.0 & & & 17.4 & \\
\hline formate & & $<78$ & & & 154 & & & 106 & \\
\hline nitrate & & 12.0 & & & 7875 & & & 6457 & \\
\hline nitrite & & 172 & & & 2315 & & & 1714 & \\
\hline oxalate & & 1450 & & & 15749 & & & 11010 & \\
\hline phosphate & & 397 & & & 150 & & & 137 & \\
\hline sulfate & & 80.0 & & & 338 & & & 237 & \\
\hline Carbon: & & & & & Total Sa & & & & \\
\hline $\begin{array}{r}\text { total inorganic } \\
\text { carbon (TIC) }\end{array}$ & & 1630 & & & 1020 & & & 886 & \\
\hline total organic carbon (TOC) & & 2250 & & & 6070 & & & 3129 & \\
\hline carbonate (from TIC) & & 8150 & & & 5100 & & & 4429 & \\
\hline oxalate (from TOC) & & 8250 & & & 22245 & & & 11456 & \\
\hline
\end{tabular}


Table 33. Comparison of Wash Permeate Composites from Campaigns I-III

\begin{tabular}{|c|c|c|c|c|c|c|}
\hline & \multicolumn{2}{|c|}{ Campaign III } & \multicolumn{2}{|c|}{ Campaign II } & \multicolumn{2}{|c|}{ Campaign I } \\
\hline & $\begin{array}{c}1^{\text {st }} 22 \\
\text { Washes }\end{array}$ & $\begin{array}{l}2^{\text {nd }} 22 \\
\text { Washes }\end{array}$ & $\begin{array}{c}1^{\text {st }} 22 \\
\text { Washes }\end{array}$ & $\begin{array}{c}2^{\text {nd }} 22 \\
\text { Washes }\end{array}$ & $\begin{array}{c}1^{\text {st }} 22 \\
\text { Washes }\end{array}$ & $\begin{array}{c}2^{\text {nd }} 22 \\
\text { Washes }\end{array}$ \\
\hline Total Solids wt\% & 20.83 & 8.80 & 20.66 & 8.89 & 10.94 & 3.73 \\
\hline Undissolved Solids wt\% & 0.24 & 0 & 0 & 0 & 0 & 0 \\
\hline Supernate Solids wt\% & 20.64 & 8.80 & 20.66 & 8.89 & 10.94 & 3.73 \\
\hline Density kg/L & 1.176 & 1.070 & 1.177 & 1.071 & & \\
\hline ammonium & $<200$ & 1270 & & & & \\
\hline Total Base M & 0.79 & 0.62 & 1.41 & 0.48 & & \\
\hline
\end{tabular}

The concentrated stream composition was then diluted to $20.4 \mathrm{wt} \%$ UDS, as was done during processing. The "UF Slurry-Diluted to 20wt\%" stream was then adjusted to approximately represent the washing steps. An empirical "wash factor" was defined:

$$
\text { wash factor }=\frac{\text { concentration of species in washed slurry }(\mathrm{mg} / \mathrm{L})}{\text { concentration of species in unwashed slurry }(\mathrm{mg} / \mathrm{L})}
$$

Note that this factor is based on the $\mathrm{mg} / \mathrm{L}$ concentrations and not $\mathrm{mg} / \mathrm{kg}$. The data used for these factors were from Campaign II. The essentially insoluble species such as Fe have factors near unity as expected. The agreement between the calculated and measured washed slurry compositions is reasonably good. The calculated Al value is lower than the measured value, but it is still higher than the Campaign II value. Therefore, the higher Al values for the Campaign III concentrated and washed slurries are deemed to be correct. Note that the $\mathrm{Al}$ is higher in the washed slurry even though the total $\mathrm{Al}$ in the feed is about the same. The implication of this result is that more Al goes to the HLW stream in Campaign III than in Campaign II. Also, this higher amount of undissolved Al is consistent with the higher amount in the FEP concentrate. 
WSRC-TR-2004-00565, REVISION 0

SRNL-RPP-2004-00092, REVISION 0

Table 34. Estimation of Slurry Concentrations for Campaign III (Values in italics are for Campaign II)

\begin{tabular}{|c|c|c|c|c|c|c|c|c|c|c|}
\hline $\begin{array}{r}\text { All values mg/kg unless } \\
\text { otherwise noted }\end{array}$ & $\begin{array}{c}\text { FEP } \\
\text { Concentrate } \\
\text { (measured) }\end{array}$ & $\begin{array}{c}\text { AY102 } \\
\text { Slurry } \\
\text { (estimated) }\end{array}$ & $\begin{array}{c}\text { AP101 } \\
\text { (estimated) }\end{array}$ & $\begin{array}{c}\text { UF Slurry - } \\
\text { Start } \\
\text { (calculated) }\end{array}$ & $\begin{array}{l}\text { UF Slurry - } \\
\text { Concentrated } \\
\text { (calculated) }\end{array}$ & $\begin{array}{l}\text { UF Slurry - } \\
\text { Diluted to } \\
\sim 20 \mathrm{wt} \% \\
\text { (calculated) }\end{array}$ & $\begin{array}{c}\text { Permeate } \\
\text { (measured) }\end{array}$ & $\begin{array}{c}\text { UF Slurry - } \\
\text { Washed } \\
\text { (calculated) }\end{array}$ & $\begin{array}{c}\text { UF Slurry - } \\
\text { Washed } \\
\text { (measured) }\end{array}$ & $\begin{array}{l}\text { Wash } \\
\text { Factor }\end{array}$ \\
\hline Total Solids (wt\%) & 30.75 & 26.68 & 31.94 & 30.59 & 46.27 & 42.99 & 28.27 & 26.46 & 26.72 & \\
\hline Undissolved Solids (wt\%) & 2.70 & 11.91 & 0.25 & 3.21 & 24.90 & 20.37 & 0.00 & 22.05 & 22.05 & \\
\hline Supernate Solids (wt\%) & 28.82 & 16.77 & 31.77 & 28.28 & 28.46 & 28.41 & 28.27 & 5.65 & 5.99 & 0.20 \\
\hline Density $(\mathrm{kg} / \mathrm{L})$ & 1.255 & 1.256 & 1.279 & 1.273 & 1.448 & 1.407 & 1.248 & 1.212 & 1.192 & \\
\hline Volume (L) & 133 & 238 & 662 & 1032 & 117 & 117 & 915 & 117 & 117 & \\
\hline $\mathrm{Ag}$ & 55 & 497 & 29.2 & 139 & 1070 & 876 & 0.761 & 891 & 904 & 0.81 \\
\hline \multirow[t]{2}{*}{$\mathrm{Al}$} & 5255 & 13328 & 5629 & 7334 & 22158 & 19060 & 5136 & 16288 & 18050 & 0.68 \\
\hline & 5470 & & & 7200 & 17550 & 15824 & 5672 & & 12745 & \\
\hline B & 566 & 0 & 0 & 72.0 & 0 & 15.6 & 85.7 & 4.90 & 149 & 0.25 \\
\hline $\mathrm{Ba}$ & 24.5 & 218 & 0 & 52.8 & 417 & 341 & $<1.3$ & 381 & 430 & 0.89 \\
\hline $\mathrm{Ca}$ & 111 & 1048 & 18.0 & 264 & 2070 & 1693 & $<3.6$ & 2128 & 1940 & 1.00 \\
\hline $\mathrm{Cd}$ & 6.00 & 37.3 & 0 & 9.24 & 71.8 & 58.8 & $<0.04$ & 62.8 & 61.5 & 0.85 \\
\hline $\mathrm{Ce}$ & $<115$ & 291 & 0 & 66.3 & 520 & 426 & $<1.0$ & 476 & 473 & 0.89 \\
\hline Co & $<7.3$ & 120 & 0 & 27.4 & 213 & 174 & $<0.11$ & 219 & $<8.0$ & 1.00 \\
\hline $\mathrm{Cr}$ & 156 & 504 & 138 & 224 & 799 & 679 & 139 & 657 & 575 & 0.77 \\
\hline $\mathrm{Cu}$ & 8.00 & 63.6 & 0 & 15.5 & 120 & 98.3 & $<0.03$ & 112 & 124 & 0.91 \\
\hline \multirow[t]{2}{*}{$\mathrm{Fe}$} & 3910 & 28285 & 56.6 & 6971 & 53984 & 44161 & 1.36 & 54387 & 49850 & 0.98 \\
\hline & 4600 & & & 7470 & 55750 & 47654 & 1.41 & & 54978 & \\
\hline \multirow[t]{2}{*}{ K } & 11350 & 297 & 23900 & 16932 & 20885 & 20059 & 16346 & 4302 & 3890 & 0.20 \\
\hline & 242 & & & 14900 & 13000 & 13589 & 17056 & & 3179 & \\
\hline $\mathrm{La}$ & $<104$ & 243 & 10.9 & 62.3 & 477 & 390 & 0.85 & 392 & 400 & 0.80 \\
\hline $\mathrm{Li}$ & 105 & 0 & 0 & 13.4 & 91.8 & 75.4 & 1.72 & 89.1 & 90.0 & 0.94 \\
\hline Mg & 70.0 & 299 & 4.31 & 79.8 & 620 & 507 & $<0.24$ & 567 & 560 & 0.89 \\
\hline Mn & 828 & 6150 & 2.35 & 1507 & 11669 & 9546 & $<0.02$ & 11756 & 11100 & 0.98 \\
\hline \multirow[t]{2}{*}{$\mathrm{Na}$} & 79400 & 70080 & 100500 & 90894 & 109462 & 105582 & 88141 & 29439 & 25500 & 0.26 \\
\hline & 93350 & & & 90012 & 90365 & 90656 & & & 27694 & \\
\hline $\mathrm{Nd}$ & 79.2 & 674 & 0 & 164 & 1277 & 1044 & $<1.5$ & 1089 & 1180 & 0.83 \\
\hline
\end{tabular}


WSRC-TR-2004-00565, REVISION 0

SRNL-RPP-2004-00092, REVISION 0

\begin{tabular}{|c|c|c|c|c|c|c|c|c|c|c|}
\hline $\begin{array}{r}\text { Table } 34 \text { continued } \\
\text { All values } \mathbf{~ m g / k g ~ u n l e s s ~} \\
\text { otherwise noted }\end{array}$ & $\begin{array}{c}\text { FEP } \\
\text { Concentrate } \\
\text { (measured) }\end{array}$ & $\begin{array}{c}\text { AY102 } \\
\text { Slurry } \\
\text { (estimated) }\end{array}$ & $\begin{array}{c}\text { AP101 } \\
\text { (estimated) }\end{array}$ & $\begin{array}{l}\text { UF Slurry - } \\
\text { Start } \\
\text { (calculated) }\end{array}$ & $\begin{array}{l}\text { UF Slurry - } \\
\text { Concentrated } \\
\text { (calculated) }\end{array}$ & $\begin{array}{l}\text { UF Slurry - } \\
\text { Diluted to } \\
\sim 20 \mathrm{wt} \% \\
\text { (calculated) }\end{array}$ & $\begin{array}{c}\text { Permeate } \\
\text { (measured) }\end{array}$ & $\begin{array}{l}\text { UF Slurry - } \\
\text { Washed } \\
\text { (calculated) }\end{array}$ & $\begin{array}{l}\text { UF Slurry - } \\
\text { Washed } \\
\text { (measured) }\end{array}$ & $\begin{array}{l}\text { Wash } \\
\text { Factor }\end{array}$ \\
\hline $\mathrm{Ni}$ & 128 & 916 & 279 & 405 & 3135 & 2564 & 0.25 & 2932 & 1600 & 0.91 \\
\hline $\mathrm{P}$ & 444 & 1333 & 210 & 495 & 103 & 185 & 554 & 81.3 & 354 & 0.35 \\
\hline $\mathrm{Pb}$ & 225 & 1296 & 0 & 324 & 2509 & 2053 & $<0.36$ & 2580 & 2845 & 1.00 \\
\hline $\operatorname{Re}$ & 3.98 & 0 & 0 & 0.506 & 3.92 & 3.21 & & 1.01 & $<8.6$ & 0.25 \\
\hline $\mathrm{S}$ & 813 & 713 & 1182 & 1028 & 1857 & 1684 & 905 & 995 & 218 & 0.47 \\
\hline \multirow[t]{2}{*}{$\mathrm{Si}$} & 1560 & 8771 & 257 & 2360 & 17999 & 14731 & 42.1 & 17032 & 14135 & 0.92 \\
\hline & 1580 & & & 2545 & 15820 & 13529 & 43.6 & & 14602 & \\
\hline $\mathrm{Sr}$ & 54.1 & 228 & 0 & 58.7 & 454 & 371 & 0.16 & 438 & 397 & 0.94 \\
\hline $\mathrm{Ti}$ & 34.0 & 7.96 & 3.91 & 8.66 & 66.4 & 54.3 & 0.10 & 62.1 & $<24$ & 0.91 \\
\hline $\mathrm{Zn}$ & 58.0 & 64.5 & 4.69 & 25.1 & 178 & 146 & 2.37 & 167 & 151 & 0.91 \\
\hline $\mathrm{Zr}$ & 53.6 & 385 & 0 & 94.5 & 722 & 591 & 1.43 & 676 & 692 & 0.91 \\
\hline bromide & $<96$ & 55.3 & 0 & 12.6 & 97.5 & 65.1 & $<80$ & 0 & $<51$ & \\
\hline chloride & 1150 & 131 & 1196 & 948 & 45.9 & 234 & 1082 & 22.6 & 69.5 & 0.09 \\
\hline fluoride & 93.0 & 34.6 & 21.9 & 33.8 & 18.4 & 23.2 & 44.9 & 8.16 & 17.4 & 0.28 \\
\hline formate & 732 & 310 & 1079 & 860 & 467 & 576 & 1066 & 92.6 & 106 & 0.15 \\
\hline \multirow[t]{2}{*}{ nitrate } & 120000 & 254 & 82095 & 68290 & 37079 & 43600 & 72917 & 5611 & 6457 & 0.12 \\
\hline & 94370 & & & 63600 & 47900 & 51750 & 68624 & & 6487 & \\
\hline \multirow[t]{2}{*}{ nitrite } & 14400 & 3367 & 26114 & 19447 & 10559 & 12837 & 23077 & 1377 & 1714 & 0.10 \\
\hline & 2966 & & & 20200 & 14100 & 14996 & 20274 & & 1907 & \\
\hline \multirow[t]{2}{*}{ oxalate } & 3860 & 7335 & 1212 & 2942 & 18351 & 15132 & 658 & 10269 & 11010 & 0.54 \\
\hline & & & & 3545 & 23800 & 20619 & 600 & & 12750 & \\
\hline phosphate & 2270 & 3031 & 899 & 1559 & 846 & 1050 & 1963 & 119 & 137 & 0.09 \\
\hline sulfate & 2260 & 1481 & 2924 & 2511 & 209 & 690 & 2853 & 95.4 & 237 & 0.11 \\
\hline total inorganic carbon (TIC) & 5630 & 11863 & 4655 & 6420 & 3456 & 4075 & 6859 & 973 & 886 & 0.19 \\
\hline total organic carbon (TOC) & 3920 & 3981 & 1653 & 2471 & 13734 & 11380 & 801 & 11299 & 3129 & 0.79 \\
\hline total carbon & 9550 & 15844 & 6308 & 8891 & 17190 & 15455 & 7660 & 12272 & 4015 & \\
\hline carbonate from TIC & 28150 & 59315 & 23276 & 32098 & 17279 & 20375 & 34295 & 4865 & 4429 & 0.19 \\
\hline oxalate from TOC & 14314 & 14571 & 5973 & 8990 & 50400 & 41748 & 2851 & 41447 & 11465 & 0.79 \\
\hline
\end{tabular}




\section{* A note on Ammonium in the UFP (Compare to note at the end of Section 2.6.5) *}

The ammonium concentrations in several of the process streams were measured.

Figure 31 shows the streams, the concentrations, and the amount in grams in each stream.

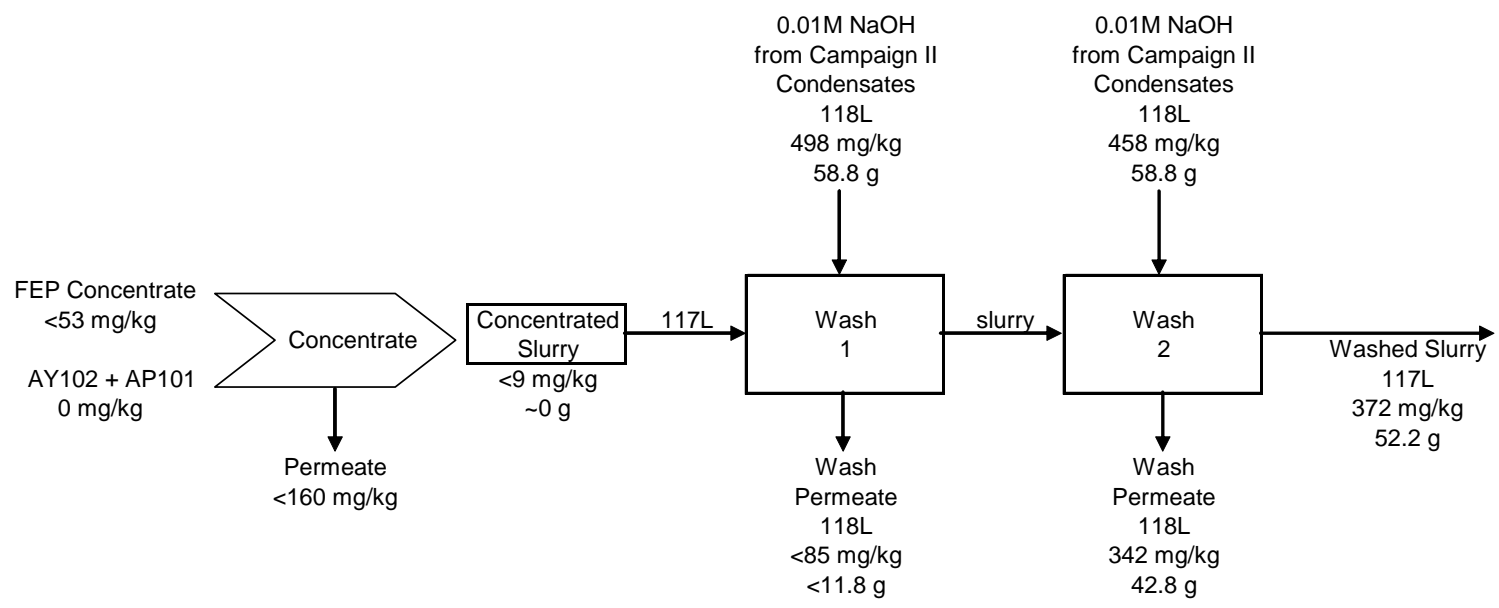

Figure 31. Tracking ammonium through the pilot-scale UFP operation for Campaign III

The combined amount of ammonium in the $2^{\text {nd }}$ wash permeate and washed slurry stream is about $80 \%$ of the inlet total that is all from the $0.01 \mathrm{M} \mathrm{NaOH}$, which is reasonable closure of the material balance since the ammonium analysis in the presence of high $\mathrm{Na}$ is difficult. Surprisingly, no detectable ammonium was found in the first wash permeate, so it was retained in the slurry. The washing process is essentially a dilution of the supernate portion of the slurry, so we expected that about $60 \%$ of the ammonium would have been in the wash permeate (118L wash, 100 L supernate). That this did not occur indicates that some component in the slurry had an affinity for ammonium. Most of the second wash permeate ammonium remained in the wash permeate, indicating that little, if any, ammonium was retained by the slurry.

\subsection{Filter Rinsing and Cleaning}

Upon completion of the slurry washing, the filtration unit was drained and then cleaned. These cleaning steps are shown in Figs. 9 and 10. Compared to Campaign II, for Campaign III only limited analyses of the rinsing and cleaning solutions were performed. Table 35 shows the solids content and acid or base content of the rinsing and cleaning solutions. These values are compared to the values from Campaigns I and II. Elemental and anion analyses for the Campaigns I and II streams were reported in the Campaign II report. 
Table 35. Filter Cleaning Solutions

\begin{tabular}{|c|c|c|c|c|c|c|c|c|c|}
\hline \multicolumn{3}{|c|}{ Caustic Rinse 1} & \multicolumn{3}{|c|}{ Acid Cleaning $1^{\mathrm{a}}$} & \multicolumn{3}{|c|}{ Acid Cleaning 2} & \multirow[b]{2}{*}{ III } \\
\hline Campaign: & I & II & III & $\mathrm{I}$ & II & III & I & II & \\
\hline Total Solids (wt\%) & 9.71 & 7.46 & 6.26 & 1.07 & 2.36 & 1.50 & 0.18 & 0.67 & 0.48 \\
\hline Undissolved Solids (wt\%) & 8.27 & 5.09 & 4.26 & 0.09 & 0.12 & 0.12 & 0.00 & 0.00 & 0.08 \\
\hline Supernate Solids (wt\%) & 1.57 & 2.50 & 2.08 & 0.98 & 2.24 & 1.38 & 0.18 & 0.67 & 0.41 \\
\hline Density $(\mathrm{kg} / \mathrm{L})$ & 1.075 & 1.057 & 1.046 & 1.056 & 1.051 & 1.046 & 1.062 & 1.055 & 1.055 \\
\hline Total or Free ${ }^{\mathrm{b}}$ Base (M) & $0.0450^{\mathrm{b}}$ & NM & 0.201 & & & & & & \\
\hline or $\quad$ Total Acid (M) & & & & 1.56 & 1.20 & 1.10 & 1.95 & 1.61 & 1.63 \\
\hline \multicolumn{3}{|c|}{ Acid Cleaning 3} & \multicolumn{3}{|c|}{ Caustic Rinse 2} & \multicolumn{3}{|c|}{ Caustic Rinse 3} & \\
\hline Campaign: & $\mathrm{I}$ & II & III & $\mathrm{I}$ & II & III & I & II & III \\
\hline Total Solids (wt\%) & 0.05 & 0.13 & 0.10 & 0.83 & 0.66 & 0.75 & 0.76 & 0.81 & 0.86 \\
\hline Undissolved Solids (wt\%) & 0 & 0 & 0 & 0.00 & 0.13 & 0.12 & $0^{\mathrm{c}}$ & 0.13 & 0.03 \\
\hline Supernate Solids (wt\%) & 0.05 & 0.13 & 0.10 & 0.83 & 0.53 & 0.63 & 0.76 & 0.68 & 0.83 \\
\hline Density $(\mathrm{kg} / \mathrm{L})$ & 1.063 & 1.055 & 1.057 & 1.009 & 1.016 & 1.017 & 1.003 & 1.004 & 1.005 \\
\hline Total Base (M) & & & & & & & $0.002^{b}$ & & \\
\hline or $\quad$ Total Acid (M) & 1.98 & 1.74 & 1.78 & 0.158 & 0.449 & 0.420 & & 0.0623 & 0.0393 \\
\hline \multicolumn{10}{|c|}{$\begin{array}{l}\text { acid cleaning solutions total and undissolved solids contents do not include nitrate from } \\
\text { nitric acid (evaporates during measurement) }\end{array}$} \\
\hline
\end{tabular}

The solids content of the Caustic Rinse 1 solution was decreased from Campaign I to Campaign III as the remaining slurry heel amount was adjusted to be closer to the correct amount. Each of the acid cleaning solutions was relatively similar, but overall, the Campaign I solutions had less total solids than the Campaign II and III solutions. This difference is attributed to the Campaign II and III final washed slurries having a higher total solids content due to the presence of more dissolved solids (see Table 30). The acid content during cleaning in Campaign I was also higher due to using smaller heels between cleaning steps. The Campaign I heels were each about 10.5-12 L, whereas in the remaining campaigns, the heels were approximately 22L. No undissolved solids were found in any of the acid cleaning 3 solutions, but small amounts were measured in the subsequent caustic rinse steps. As previously described in the Campaign II report (Duignan et al., 2004b), the solids found in Campaign II were identified by XRD as gibbsite, silica, and a sodium aluminate compound.

\subsection{Particle Size Distribution}

For Campaign III operation slurry particle size was not measured. Due to the very similar filter performance of Campaigns II and III, the particle morphology was assumed to be similar, too. Slurry particles were measured analyzed and for both Campaigns I and II and were discussed in the Campaign II report (Duignan et al., 2000b). In summary, by a volume distribution the mean particle sizes for UFP simulated feed ranged from approximately 1.5 microns (Campaign I) to 2.5 microns (Campaign II). This is comparable to the particle sizes in the actual AY102/C106 waste, which range from 2.5 to 4 microns. After dewatering, for both Campaigns, the mean size of the particles reduced to close to 1 micron. After washing the changes to the particle sizes were insignificant. 


\subsubsection{Pilot UFP Conclusions}

\subsubsection{Experimental Observations (Author - Fowley)}

A summary of the overall conclusions are as follows:

- $\quad$ The average temperature-adjusted permeate flux when dewatering from $\sim 3$ to 24 wt $\%$ UDS was $0.011 \mathrm{gpm} / \mathrm{ft}^{2}$. The average temperature-adjusted permeate flux when dewatering from $\sim 4$ to $20 \mathrm{wt} \%$ UDS was $0.014 \mathrm{gpm} / \mathrm{ft}^{2}$. The average, temperatureadjusted permeate flux when dewatering from $\sim 4$ to $17 \mathrm{wt} \%$ UDS was $0.018 \mathrm{gpm} / \mathrm{ft}^{2}$.

- Cleaning the filter with $2 \mathrm{M}$ nitric acid was sufficient to increase the $0.1 \mathrm{M} \mathrm{NaOH}$ permeate flux to an order of magnitude larger than that of waste flux. However, it was not sufficient to return the filter to pre-test flux levels.

- The turbidity of the permeate from dewatering and slurry washing, when measured directly after the sample was taken, was always lower than 0.5 NTU. The Test Specification indicated a solids-free threshold of 2 NTU, therefore the measured values indicated the permeate was free of significant undissolved solids.

- Submerging the filter in a $0.1 \mathrm{M} \mathrm{NaOH}$ solution over an extended period of time may improve flux since the water flux always improved after the filter was stored in the solution for periods of 6 weeks or more between tests.

\subsubsection{Waste Products Observations (Author-Zamecnik)}

- The composition of the undissolved solid species in the washed sludges in Campaigns I-III was similar, as expected. The supernate portion species $\mathrm{Na}$ and $\mathrm{K}$ were higher in concentration in Campaigns II and III than in Campaign I due to the presence of the AP101 simulant.

- The aluminum concentration in the washed slurry in Campaign III was higher than in Campaign II due to there being more undissolved aluminum in the FEP concentrate.

- Because of higher undissolved aluminum in the FEP concentrate, the Campaign III permeate from the concentration step (IX feed) had less aluminum than in Campaign II.

- Ammonium in the first $0.01 \mathrm{M} \mathrm{NaOH}$ UFP wash of Campaign III was mostly retained in the slurry, indicating that a slurry component has an affinity for ammonium. Most of the ammonium in the second wash was not retained. 


\subsection{PILOT CESIUM ION EXCHANGE (AUTHORS - STEIMKE \& WILLIAMS)}

The Cesium Ion Exchange Process, or CIX, is the third separation technology unit operation in the RPP-WTP Pretreatment Facility that the waste stream will pass through on start-up. This is true for SIPP because the chosen waste type, Envelope A/D, is not subjected to precipitation before filtration. The SIPP CIX operation removes cesium from a mixture of the liquid LAW stream coming from the UFP before it is sent to the TLP for further evaporation and eventual vitrification.

The ion exchange resin used in this test was SuperLig ${ }^{\circledR} 644$, which is designed to remove cesium from caustic radioactive waste that is at a 5-molar concentration in sodium. It is manufactured by IBC Advanced Technologies. Pilot scale testing of this resin was first performed in the Engineering Development Laboratory in the year 2000 (Fowley et al., 2004). That earlier testing was intended to be focused primarily on the chemical performance of the resin on a pilot scale (more than one liter of resin); however, the pressure drop across the resin beds was unexpectedly high. Testing for the next three years was redirected to the hydraulic performance of the resin. The hydraulic issues were resolved to the satisfaction of RPP-WTP (Fowley et al., 2004).

In 2003, Test Exception 24590-WTP-TEF-RT-03-069 called for resumed chemical testing of SuperLig ${ }^{\circledR} 644$ resin at a pilot-scale for six chemical cycles, which would be incorporated into the four campaigns of the SIPP task (Duignan, 2003c). A cycle is the process whereby the Cesium Ion Exchange unit operation treats LAW solution until the lead IX column accumulates enough cesium to require regeneration, then it is regenerated. Later, the goals of the SIPP task were modified that reduced the number of ion exchange cycles to four; Cycles 1 and 2 would be done in Campaign II and Cycles 3 and 4 in Campaign III. There were no CIX operations during Campaign I of SIPP. This report includes all four cycles of testing, but with primary emphasis on the last two cycles because the Campaign II interim report (Duignan et al., 2004b) has already described the first two cycles.

For the actual RPP-WTP a cycle would entail the treatment of a minimum of 100 Bed Volumes (BV = a resin bed volume of 415 gallons (1571 liters) under normal operating conditions, which is subject to change) until the lead column of the three-column ion exchanger will contain enough accumulated cesium to require its regeneration. That lead column is then taken off line to be regenerated while a waiting fourth column is valved-in for the CIX to continue further LAW treatment. That fourth column, containing reconditioned resin, is put in the last position (called the polishing position) of the threecolumn CIX unit and the column that had been in the second position (referred to the lag position) becomes the new lead column, and so forth. The pilot scale CIX unit operation, of the SIPP task, had only two columns, the lead and lag positions. The pilot operation consisted of the treatment with approximately $190 \mathrm{BV}(\mathrm{BV}=2.34$ liters $)$ of simulated LAW, which was then followed by the regeneration of the lead column. A new cycle begins with the newly regenerated lead column in the lag position. 
This section will explain the pilot CIX unit operation that was performed as part of Campaign III testing along with the previous reported Campaign II (Duignan et al., 2004b) results for comparison. The process started with the preparation of the feed product, which is permeate created by the UFP. An explanation of the chemical makeup of the feed stream will be followed by a short description on how feed was handled just before CIX operation and then how the waste stream was handled during and after ion exchange. That section will be followed by a discussion of the experimental hardware and how the test was run. Finally, the results will be discussed and conclusions made.

\subsubsection{Chemical Description of the Pilot-Scale CIX Feed Stream}

The feed for the pilot-scale CIX unit operation was the permeate produced by simulant waste dewatering in the pilot-scale UFP unit operation. The chemical make-up of that feed was described in detail in Section 2.7.4.2.1 and will not be repeated here. Refer to that section and specifically to Tables 30 and 31 for information on the CIX feed (simulated LAW) stream.

\subsubsection{Simulant Handling (Author - Williams)}

\subsubsection{Initial Preparations}

Before beginning the CIX test, the solutions to be used to regenerate the lead column were made ready. Those solutions were:

1. $0.1 \mathrm{M} \mathrm{NaOH}$ Displaced the LAW in IX column while maintaining high $\mathrm{pH}$ to prevent precipitation

2. Deionized Water Pre-elution rinse - Displaced the $0.1 \mathrm{M} \mathrm{NaOH}$ from IX column

3. $0.5 \mathrm{M} \mathrm{HNO}_{3} \quad$ Eluent - Displaced water and stripped Cs ions from resin by replacing them with hydrogen ions. Note: eluent is pumped into the column and eluate flows out of the column

4. Deionized Water Post-elution rinse - Displaced acid eluate from column

5. $0.25 \mathrm{M} \mathrm{NaOH}$ Displaced water and regenerated the resin by replacing hydrogen ions with sodium ions

The regeneration caustic, $0.25 \mathrm{M} \mathrm{NaOH}$, required a volume of 47.8 liters and it was made from DIF water and $50 \mathrm{wt} \% \mathrm{NaOH}$. Then it was stored in the Caustic Supply Tank before 25 May 2004. A large quantity of displacement caustic solution, $0.1 \mathrm{M} \mathrm{NaOH}$ was made with condensate and it was stored in a carboy labeled "Campaign 3-0.1 M $\mathrm{NaOH}$ for CsIX.” From this carboy 28.7 liters were removed and made ready for Campaign III before 2 June by adding it to the $0.1 \mathrm{M}$ Caustic Supply Tank. To strip the ion-exchange (IX) resin of Cs ions, 70.1 liters of $0.5 \mathrm{M} \mathrm{HNO}_{3}$ was made from deionized water and $70 \% \mathrm{HNO}_{3}$ and was placed in the Eluent Supply Tank before 2 June. 


\subsubsection{Handling of Pilot-scale CIX Feed LAW Simulant}

The Simulant Supply that was produced during pilot-scale UFP operation was split into two equal parts for Cycle 3 and 4 of CIX operations. Both cycles processed 194 Bed Volumes (BV) ( 453 liters) of fresh simulant. The treated simulant was transferred to the pilot-scale TLP Evaporator Feed Tank from CIX operations.

\subsubsection{Handling CIX Regeneration and Campaign III CIX Recycle Streams}

The flow rate and duration of each of the chemical streams, was set by communication from RPP-WTP. The different chemical effluents from the column/s were collected in different containers, either jugs or tanks. There is a transit time for a fluid to pass through a column or columns, depending on flow rate. For Campaign III, the effluent hose was moved from one container to another after the transit time had elapsed, where the transit time was based on a plug flow calculation through the columns.

\subsubsection{Pilot CIX Equipment (Author - Steimke)}

\subsubsection{IX Resin Columns}

Figure 32 is a picture of the two columns during Cycle 3 Regeneration and Fig. 33 is a schematic of the two ion exchange columns and peripheral equipment. The design is also documented in WSRC drawing EES-23041-R4-001.

Two ion exchange columns were designed and constructed for the SIPP task. The columns had an inside diameter of 5.5 inches and a target resin height of 6 inches ${ }^{\dagger}$, giving a height to diameter ratio of 1.1, the same ratio as for the WTP columns. Other hardware included pumps, tanks, flowmeters, pressure transducers, other instruments and a data acquisition system (DAS).

The hardware listed below was positioned inside the column with the following elevations with respect to the wedge screen (also known as: V-wire screen), that supported the resin. Note that the total liquid filled length of each column was 23.75”, i.e., 16.75”- (-7.00”), which is equivalent to an internal volume of 9.24 liters $~ 4 \mathrm{BV}$.

\footnotetext{
${ }^{\dagger}$ The actual resin height is not fixed. As explained in the following sections, and shown in Table 38, the height resin in the columns changes from 6.25 inches, when the columns are initially filled, to as short as 5.4 inches after multiple resin regenerations.
} 
WSRC-TR-2004-00565, REVISION 0 SRNL-RPP-2004-00092, REVISION 0

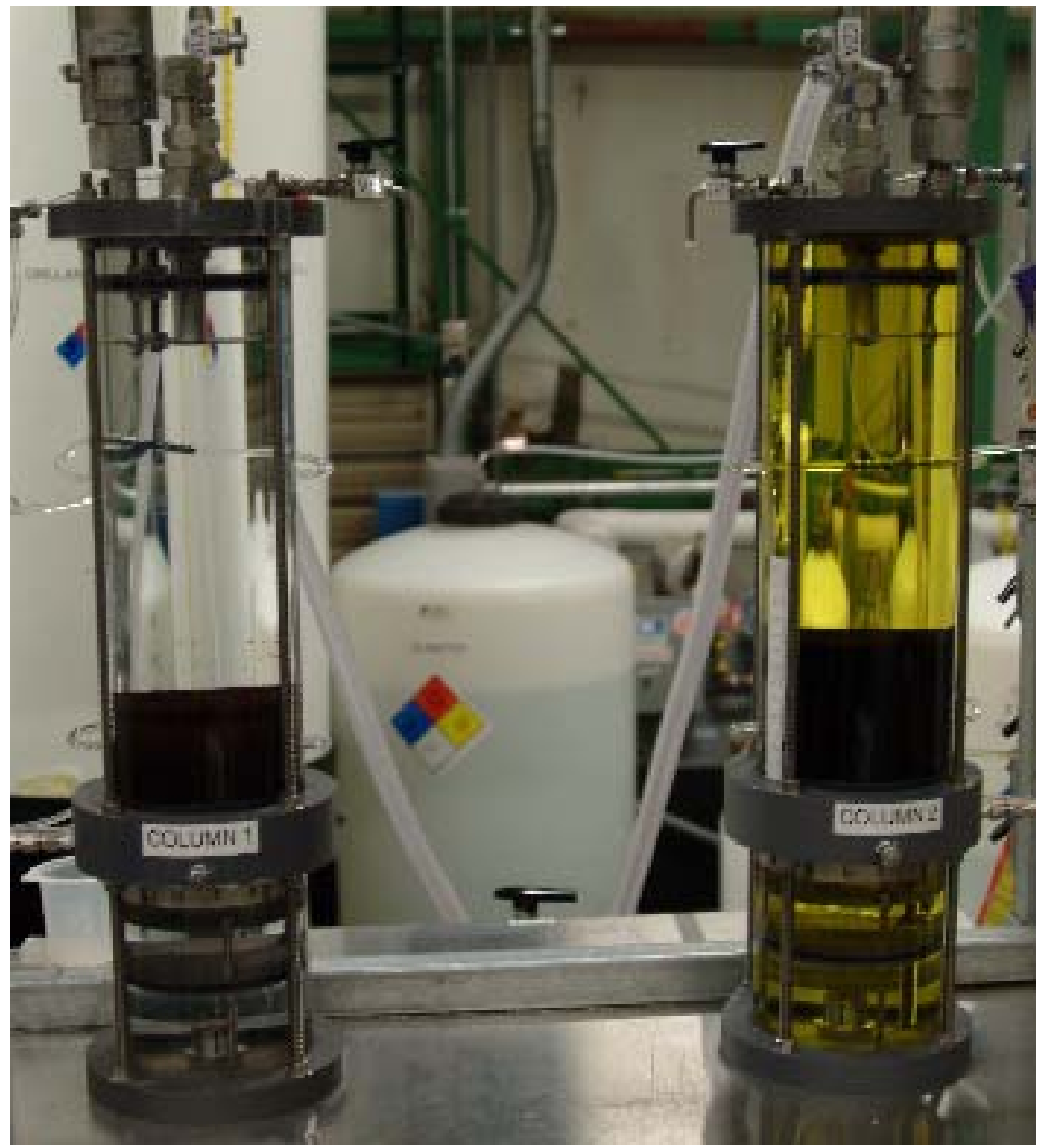

Figure 32. Pilot-scale CIX columns: The left column (COLUMN 1) is undergoing resin regeneration during Cycle 3 operation 
WSRC-TR-2004-00565, REVISION 0

SRNL-RPP-2004-00092, REVISION 0

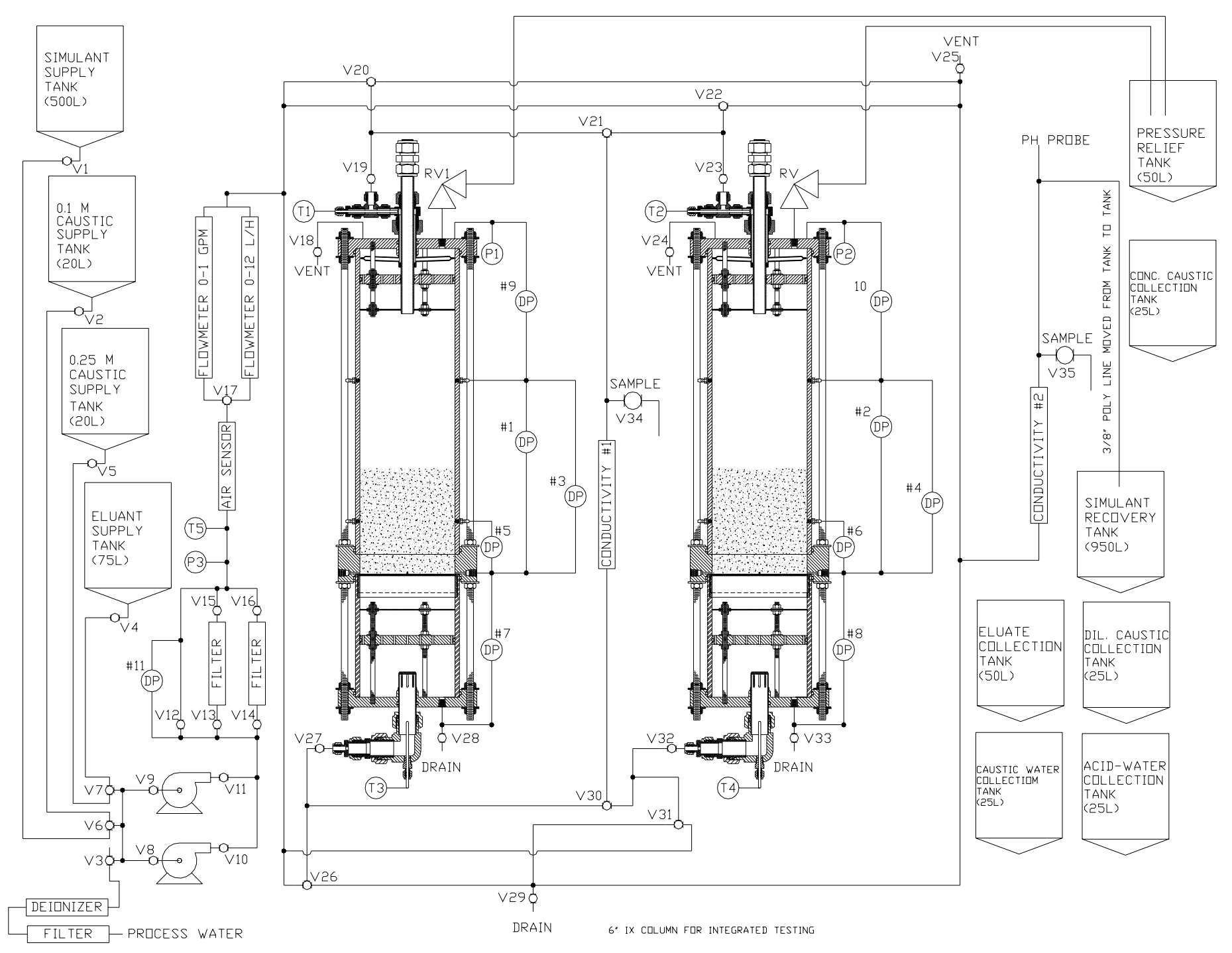

Figure 33. Schematic of Ion Exchange Facility for Integrated Testing 
Elevation

$16.75 ”$

$16.00 ”$

$14.75 ”$

$13.25 ”$

0 "

$-2.0 ”$

$-3.50$

$-7.00 ”$
Column Hardware Description

Top of liquid filled space

Flow distributor with six perforated arms

Perforated PVC plate with thirty seven $3 / 64$ " holes

Perforated stainless steel plate with 3/32" holes on a 5/32" triangular pitch

Wedge screen (Vee wire screen) with 0.010 " slots to support resin

Perforated stainless steel plate with 3/32” holes on a 5/32” triangular pitch

Perforated PVC plate with thirty seven 3/64" holes

Bottom of the liquid filled space

The purpose of the flow distributor and the two plates below it was to generate a more gradual transition of density when relatively dense simulant was introduced to the top of the column on top of lighter liquid. The purpose of the wedge screen was to support the resin while resisting plugging. The purpose of the bottom two plates was to generate a more uniform flow field in the event upflow operations were needed, however, the normal flow direction was down.

By opening the proper valve, feed could be drawn from one of five supply tanks, which contained either: LAW simulant, displacement 0.1 M NaOH solution, DIF water for rinsing, eluent $0.5 \mathrm{M}$ nitric acid solution, or regeneration $0.25 \mathrm{M} \mathrm{NaOH}$ solution. The solutions were pumped by one or two gear pumps connected in parallel. Most of the time only one pump was sufficient, but two-pump operation was required for upflow. The facility contained two flowmeters in parallel, a high range flowmeter for upflow with a range of 0 - $1 \mathrm{gpm}$ and a low range flowmeter for normal operation with a range of 0 - $12 \mathrm{~L} / \mathrm{hr}$ (0 - $0.053 \mathrm{gpm})$. Valves allowed upflow or downflow through one column or two columns in series. The effluent line from the columns contained a $\mathrm{pH}$ probe, a conductivity probe and a thermocouple. A hose connected to the effluent line was placed in the appropriate collection jug for every step of the cycle. There were six collection jugs, one for each of the six steps of the cycle.

\subsubsection{The Data Acquisition and Safety Circuitry}

A Dell computer with LabVIEW ${ }^{\mathrm{TM}}$ software was used to acquire data, control the flow rate, and shut down the flow if any measured pressure exceeded the setpoint of 20 psig. There was also a pressure relief valve set at 45 psig connected to the top of each of the two columns. The discharge line of the pressure relief valves was a hose leading to a jug. There was also a spill detector with a sensor placed below the columns on the floor of the containment for the facility. Any spill would disconnect power to the pumps. There was also an air detector in the line leading from the solution supply tanks to the pump. Any air in the line would disconnect power from the pumps. A manual bypass switch was used to start the pump/s when there was initially air in the line. The air detector was used because oxygen can damage the wet resin, particularly when it is in a caustic state. 


\subsubsection{The Resin}

To prepare the CIX resin, SuperLig ${ }^{\circledR} 644$, for use, it was taken from the 250-Gallon Batch (C-01-11-05-02-35-60). The resin was chemically pre-treated by washing with water, treating it in $0.5 \mathrm{M}$ nitric acid, washing with water, treating it with $0.25 \mathrm{M}$ sodium hydroxide and washing it with water. The pre-treated resin was then wet sieved under a nitrogen atmosphere to remove particles finer than 40 mesh.

The resin was added to the columns in the following way. A feed tank with an agitator was temporarily connected to the top of a column. The column was completely filled with $0.25 \mathrm{M} \mathrm{NaOH}$ solution, the feed tank was partially filled. The valve in the feed line between the tank and column was closed. Resin was added to the feed tank to form a $30 \%$ by volume slurry and the agitator was energized. The bottom of the column was connected to pump suction. Pump discharge was connected to a waste container. The valve in the feed line was opened and the pump was simultaneously energized to create a downward velocity of $14 \mathrm{~cm} / \mathrm{min}$ (a flow rate equal to $0.58 \mathrm{gpm}$ ) which deposited a resin bed with the target average resin height of 6 inches. However, the initial resin deposition resulted in a crater about 1 inch deep in both columns. An upflow for two minutes at $13 \mathrm{~cm} / \mathrm{min}$ was used to level the top of the resin beds. This process was repeated with the second resin bed. This was followed by pre-conditioning of both beds for thirty minutes with a flow rate of $15 \mathrm{~cm} / \mathrm{min}$ of $0.25 \mathrm{M} \mathrm{NaOH}$ solution. At this time, both beds had a resin height of 6.25 inches. The columns were then ready for SIPP operation.

\subsubsection{Pilot CIX Operation (Author-Steimke)}

\subsubsection{Chemical Testing}

Each cycle of chemical testing had the following six chemical steps all in downflow: 1) LAW simulant, 2) 0.1 M NaOH displacement solution, 3) deionized water, 4) $0.5 \mathrm{M}$ nitric acid eluent solution, 5) deionized water, and 6) $0.25 \mathrm{M} \mathrm{NaOH}$ regeneration solution. The total volume of LAW simulant used for each cycle was about $190 \mathrm{BV}$ and simulant was always passed through both ion exchange columns connected in series. To reiterate, one BV was equal to 2.34 liters. All steps other than the simulant step were conducted through one column only. The $0.1 \mathrm{M} \mathrm{NaOH}$ step and the subsequent deionized water step (pre-elution rinse) were conducted at a flow rate of $3 \mathrm{BV} / \mathrm{hr}$ for one hour. The volume used for the elution step was $15 \mathrm{BV}$. The post-elution rinse was run at a flow rate of $1.4 \mathrm{BV} / \mathrm{hr}$ for 2.25 hours. The regeneration step was run at a flow rate of 3 BV/hr for 2 hours. (See Table 10 for Cycle 3 and Table 11 for Cycle 4.)

The six different chemical solutions were collected in separate jugs or tanks by moving the discharge hose of the columns. In Campaign II, for Cycles 1 and 2, the plan was to move the hose based on observing the change in the $\mathrm{pH}$ and/or conductivity probes. This proved to be difficult to implement. Therefore, in Campaign III, for Cycles 3 and 4, the 
timing for moving the hose to a different container was based on a plug flow calculation of when the liquid front would reach the column discharge.

There was a 39-day period between the end of Cycle 2, part of Campaign II testing, and the start of Cycle 3, part of Campaign III testing. At the end of Cycle 2, the lag column was Column 1 and it contained simulant and the lead column, Column 2, which was regenerated, contained $0.25 \mathrm{M} \mathrm{NaOH}$. At the request of RPP-WTP, both columns were displaced with $0.1 \mathrm{M} \mathrm{NaOH}$ and the resin soaked in that solution until the start of Cycle 3 for Campaign III testing. At the conclusion of all testing, the contents of both columns were displaced with $0.1 \mathrm{M} \mathrm{NaOH}$ solution.

\subsubsection{Test Matrix Highlights}

The entire CIX test matrix is shown in Tables 10 and 11 with each cycle operation occurring on the dates listed in Table 36. For convenience some key parameters are listed below for Campaigns II and III:

- Cycles 1, 2 and 3 had a simulant flow rate of $3 \mathrm{BV} / \mathrm{hr}$. The simulant flow rate for Cycle 4 was $4.5 \mathrm{BV} / \mathrm{hr}$.

- Cycles 1 and 4 had an eluent flow rate of $1 \mathrm{BV} / \mathrm{hr}$. The eluent flow rates for Cycle 2 and 3 were $0.5 \mathrm{BV} / \mathrm{hr}$ and $2.0 \mathrm{BV} / \mathrm{hr}$, respectively.

- Column 1 was the lead column for Cycles 1 and 3. Column 2 was the lead column for Cycles 2 and 4.

Table 36. Cycle Dates of Operation

\begin{tabular}{|c|c|c|}
\hline Cycle & Start date & Finish date \\
\hline 1 & $4 / 26$ & $5 / 4$ \\
\hline 2 & $5 / 4$ & $5 / 12$ \\
\hline 3 & $6 / 21$ & $6 / 29$ \\
\hline 4 & $6 / 29$ & $7 / 7$ \\
\hline
\end{tabular}

\subsubsection{Pilot CIX Discussion of Results (Author - Steimke)}

\subsubsection{General Results}

This section presents an overview of the results. The following sections give the detailed results.

The IX facility was used to run four ion exchange cycles, where a cycle consisted of six chemical steps. Table 37 lists the volumes of fresh LAW simulant that were pumped through the two beds connected in series. At 2.34 liters per bed volume, these volumes translate to about $190 \mathrm{BV}$. In addition, the final 9 liters of simulant that were pumped into the lead column, Column 1, for Cycles 1 and 3, were added to the fresh simulant in the simulant supply tank for use in Cycles 2 and 4 to mimic WTP operation. 
WSRC-TR-2004-00565, REVISION 0

SRNL-RPP-2004-00092, REVISION 0

Table 37. Highlights of the Pilot-Scale CIX Cs removal efficiency

\begin{tabular}{|l|l|l|l|l|}
\hline Cycle & $\begin{array}{l}\text { Fresh simulant } \\
\text { volume, L }\end{array}$ & $\begin{array}{l}\text { Fresh simulant } \\
\text { volume, BV }\end{array}$ & $\begin{array}{l}\text { Percent cesium } \\
\text { removal by lead } \\
\text { column }\end{array}$ & $\begin{array}{l}\text { Percent cesium } \\
\text { removal by both } \\
\text { columns }\end{array}$ \\
\hline 1 & 428 & 183 & 99.6 & greater than 99.9 \\
\hline 2 & 437 & 187 & 99.7 & greater than 99.9 \\
\hline 3 & 453 & 194 & 98.5 & greater than 99.8 \\
\hline 4 & 453 & 194 & 98.2 & greater than 99.7 \\
\hline
\end{tabular}

The lead column removed the great majority of the cesium and the lag column removed most of the remaining cesium. Note that the percentage of cesium removed by the lead and lag columns decreased over the course of four cycles. The values in the last column are stated to be "greater than" the amount listed because of the measurement uncertainty discussed in Section 2.8.5.3.1.

During ion exchange operation, the axial pressure drop was measured for both resin beds. The maximum pressure drop occurred during the simulant step, because the simulant has about three times the viscosity of the other solutions. Pressure drops generally increased over the four cycles. The axial pressure drop across the bottom 3" of the resin column was also measured. That pressure drop was close to half of the total pressure drop for 6" of resin. This implies a resin bed with uniform permeability and no significant cracking or fissuring.

A cesium mass balance was performed for all four cycles. The cesium masses in and out of the columns agreed within the accuracy of the ICP-MS (inductively coupled plasma mass spectroscopy) used for measuring cesium in 5 molar sodium solution. During elution of each of the lead columns fifteen bed volumes of $0.5 \mathrm{M}$ nitric acid were used. After only the first three BV, about $90 \%$ of the cesium was eluted from the bed.

\subsubsection{Hydraulic Observations}

Table 38 summarizes some important hydraulic results from Campaign III (Cycles 3 and 4), but also includes the results from Campaign II (Cycles 1 and 2) so the complete set of cycles can be compared. Note, that the simulant had a density of $1.25 \mathrm{~g} / \mathrm{mL}$.

Table 38. Hydraulic Summary

\begin{tabular}{|c|c|c|c|c|c|c|}
\hline Cycle & $\begin{array}{c}\text { Simulant } \\
\Delta \text { P Col. 1 }\end{array}$ & $\begin{array}{c}\text { Simulant } \\
\Delta \text { P Col. 2 }\end{array}$ & $\begin{array}{c}\text { Resin } \\
\text { height in } \\
\text { simulant, } \\
\text { Col. 1, } \\
\text { inches) }\end{array}$ & $\begin{array}{c}\text { Resin } \\
\text { height in } \\
\text { simulant, } \\
\text { Col. 2, } \\
\text { (inches) }\end{array}$ & $\begin{array}{c}\text { Simulant } \\
\text { superficial } \\
\text { velocity, } \\
\text { Col. 1, } \\
\text { (cm/hr) }\end{array}$ & $\begin{array}{c}\text { Simulant } \\
\text { superficial } \\
\text { velocity, } \\
\text { Col. 2, } \\
\text { (cm/hr) }\end{array}$ \\
\hline 1 & $0.6(\mathrm{Lead})$ & $0.5(\mathrm{Lag})$ & 5.6 & 5.8 & 46 & 46 \\
\hline 2 & $0.6(\mathrm{Lag})$ & $0.7(\mathrm{Lead})$ & 5.4 & 5.7 & 46 & 46 \\
\hline 3 & $1.0(\mathrm{Lead})$ & $0.7(\mathrm{Lag})$ & 5.6 & 5.9 & 46 & 46 \\
\hline 4 & $1.2(\mathrm{Lag})$ & $1.3(\mathrm{Lead})$ & 5.7 & 5.8 & 69 & 69 \\
\hline
\end{tabular}


Figure 34 plots the flow rates during Cycle 1, typical results, for all six solutions used. The large fluctuations in flow rate for the two water rinses occurred because the deionized water used lacked sufficient ions for the magnetic flowmeter to achieve a steady reading. However, flow checks using a volumetric cylinder and stopwatch showed that the average flow reading was correct. Figures 35 and 36 plot the axial pressure drops across the lead and lag column resin beds during Cycle 1 . There were duplicate measurements of the pressure drop across the entire resin bed and one measurement of the axial pressure drop across the bottom 3 inches of the resin bed. The duplicate pressure drop measurements agree. The highest pressure drops were for the simulant step because of its higher viscosity. The pressure drop across the bottom 3 inches was about half of the total pressure drop, as expected. The total axial pressure drop across the bed for simulant was about 0.6 inches of water. By way of comparison, the 24-Inch Column Test (All-In Test) (Fowley et al., 2004) had an axial pressure drop for Cycle 1 of 16 inches of water for a resin height that was four times as great and a superficial velocity that was seven times as great $(5 \mathrm{~cm} / \mathrm{min}$ vs. $0.75 \mathrm{~cm} / \mathrm{min})$. That is, the 24-Inch Column Test axial pressure drop was 27 times as large, which agrees well with the product of the resin height ratio (4) and the velocity ratio (7).

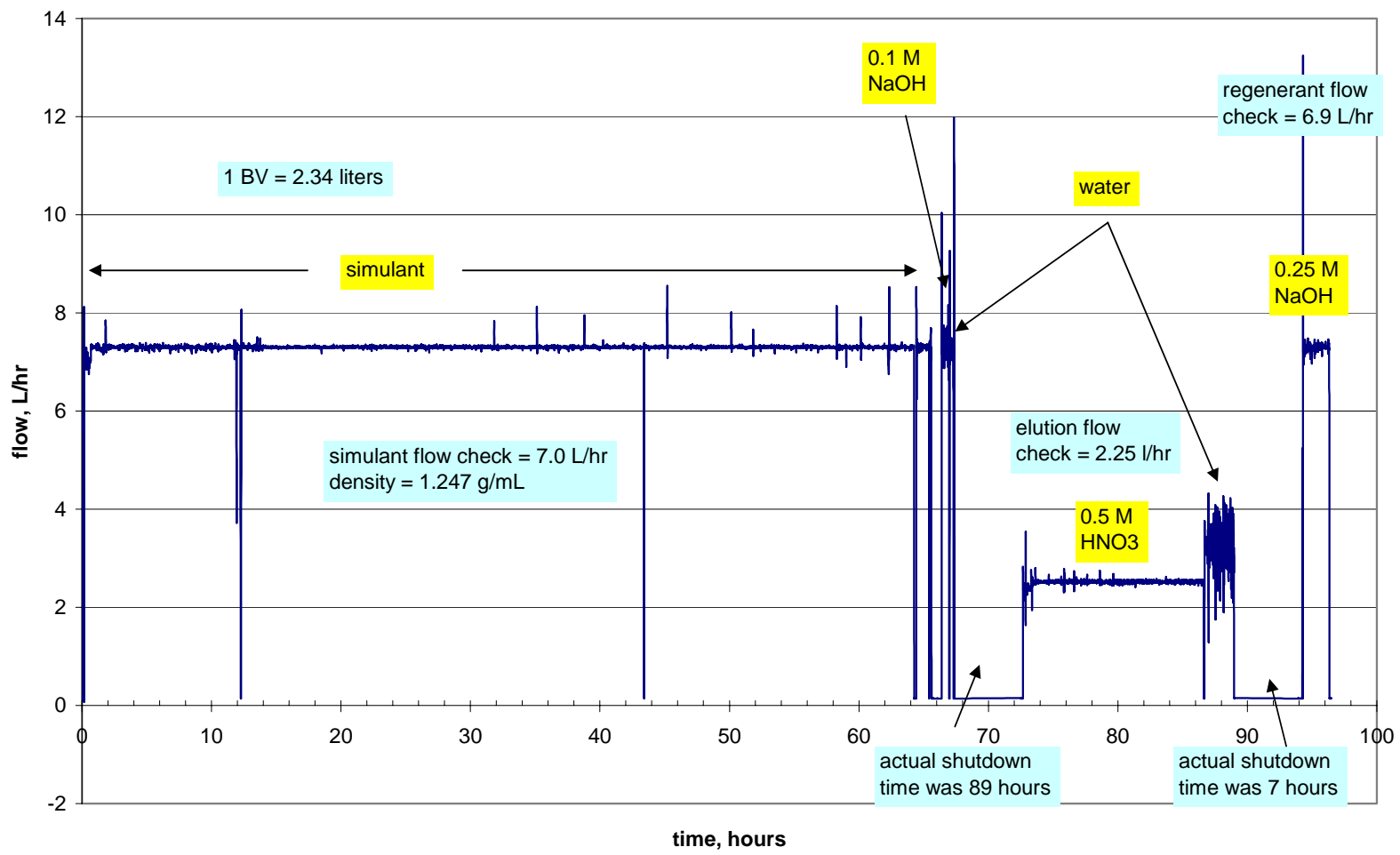

Figure 34. Flow rate During Cycle 1 
WSRC-TR-2004-00565, REVISION 0

SRNL-RPP-2004-00092, REVISION 0

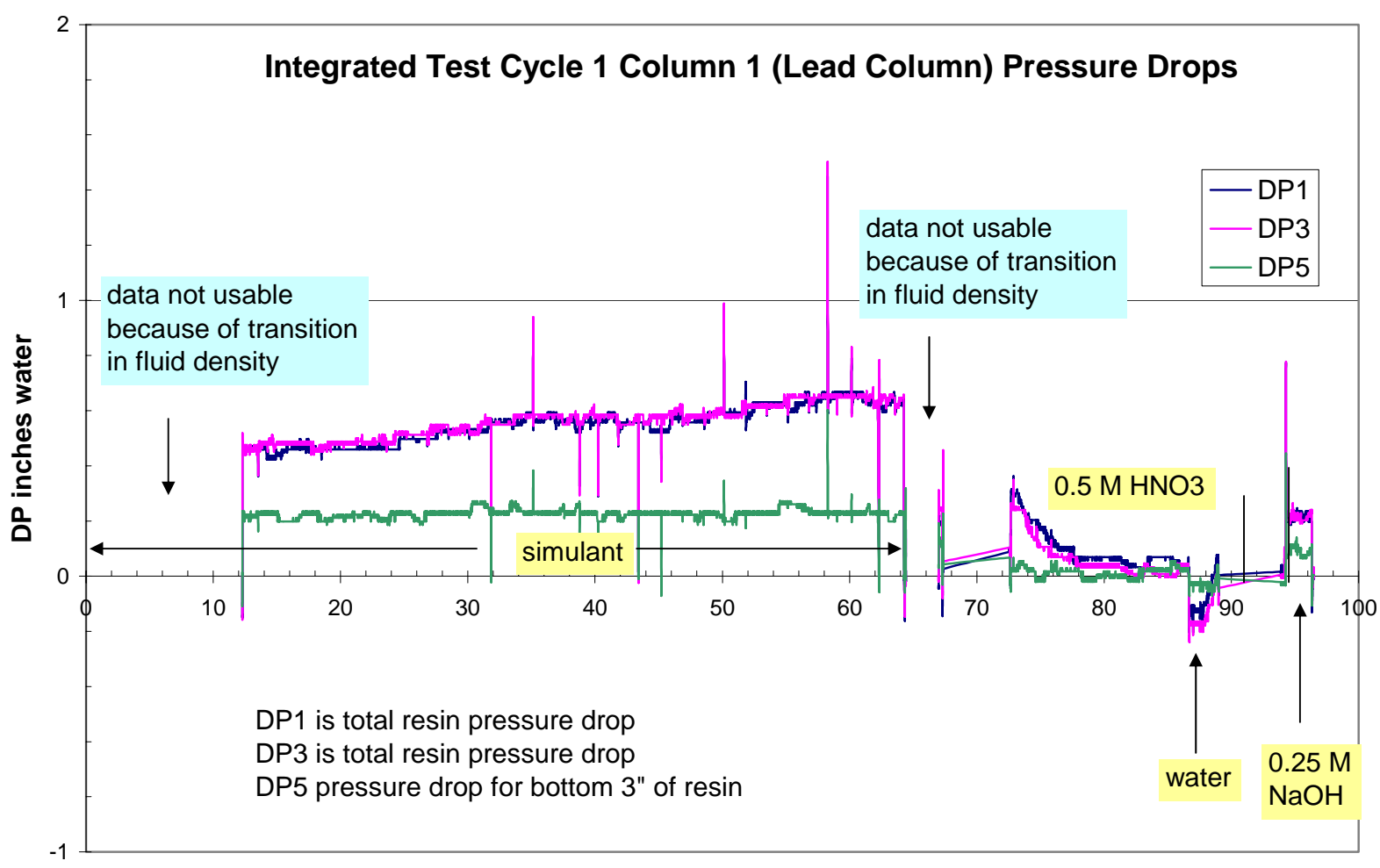

time, hours

Figure 35. Pressure Drop Across Resin Bed for Lead Column and Cycle 1

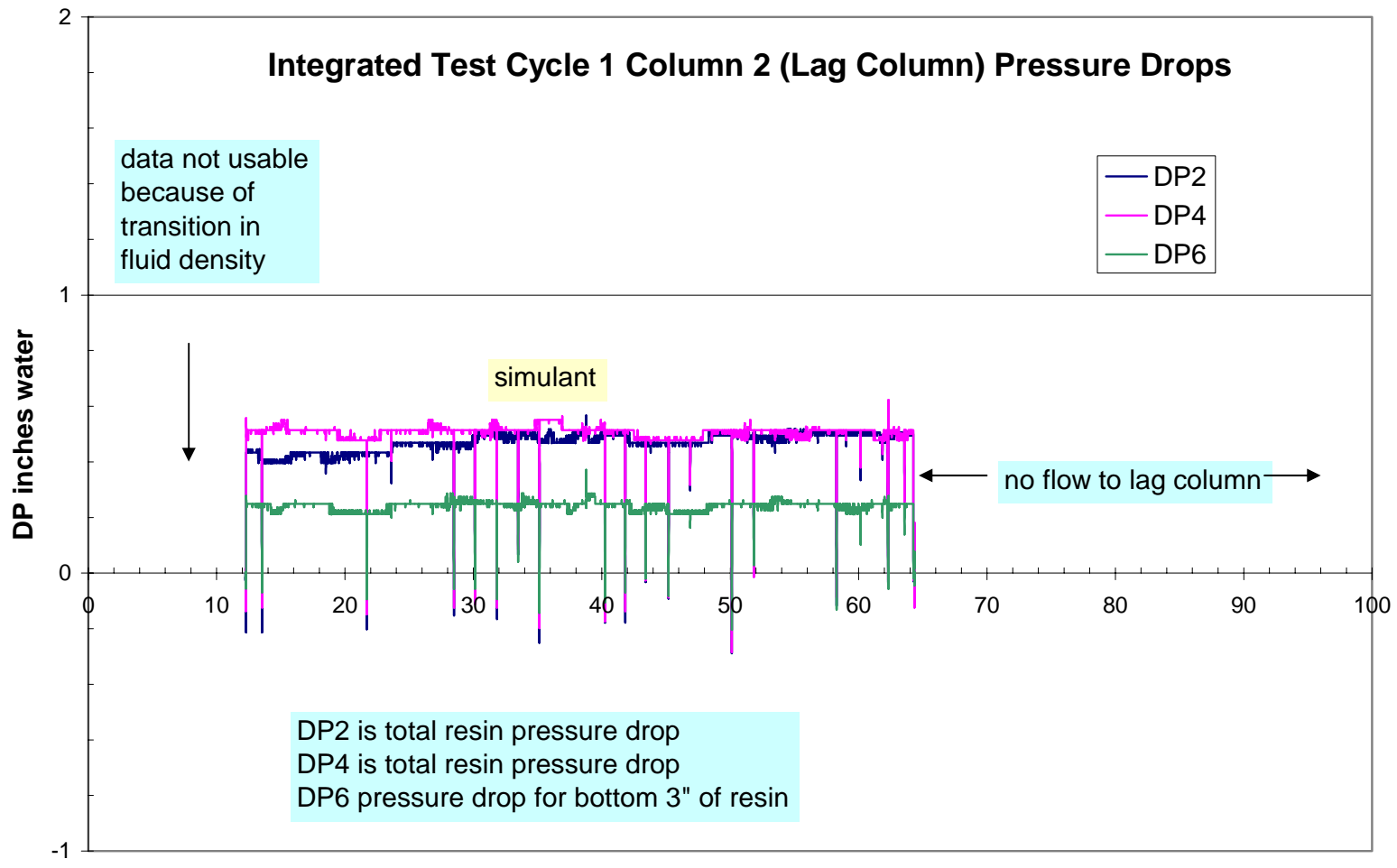

time, hours

Figure 36. Pressure Drop Across Resin Bed for Lag Column and Cycle 1 
Resin bed heights during Cycle 1 are plotted in Fig. 37. The plot shows the expected variation with the smallest resin bed height during the elution step.

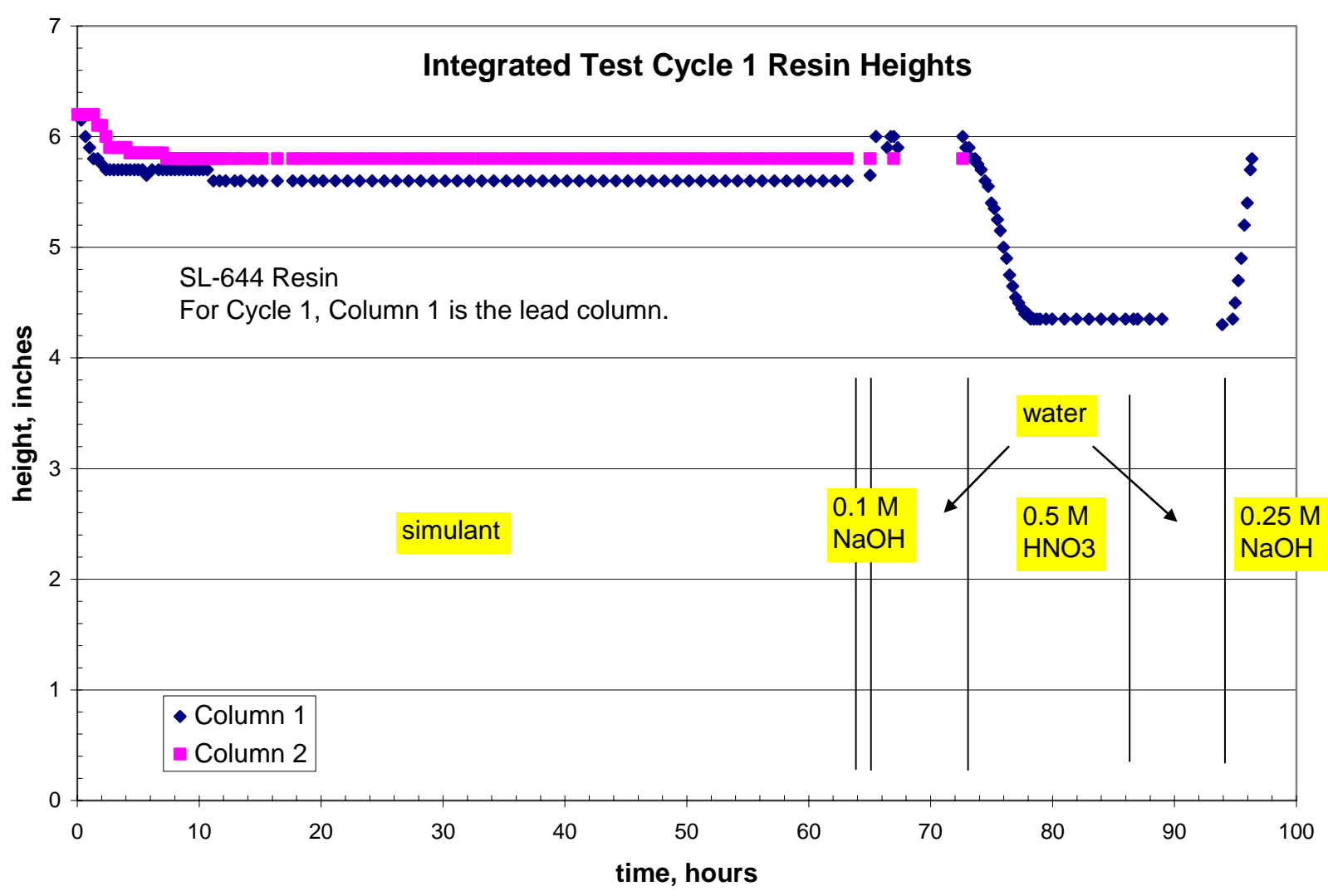

Figure 37. Resin Bed Height During Cycle 1

Table 39 lists the volumes of the different solutions. Note that some of the volumes are less than the internal volume of the column.

Table 39. Comparison of the Volume for Each of the CIX Regeneration Process Solutions to the Internal Volume of Pilot-scale CIX Column

\begin{tabular}{|c|c|c|}
\hline $\begin{array}{c}\text { Regeneration } \\
\text { Process } \\
\text { Solution }\end{array}$ & $\begin{array}{c}\text { Volume } \\
\text { Introduced } \\
\text { into Column }\end{array}$ & $\begin{array}{c}\text { Internal } \\
\text { Internal Volume } \\
\text { of the pilot-scale } \\
\text { CIX column }\end{array}$ \\
\hline & $\mathrm{BV}$ & $\mathrm{BV}$ \\
\hline $0.1 \mathrm{M} \mathrm{NaOH}$ & 3 & 4 \\
\hline Water & 3 & 4 \\
\hline $0.5 \mathrm{M} \mathrm{HNO3}$ & 15 & 4 \\
\hline Water & 3 & 4 \\
\hline $0.25 \mathrm{M} \mathrm{NaOH}$ & 6 & 4 \\
\hline
\end{tabular}


Figures 38 and 39 show the measured $\mathrm{pH}$ and conductivity of the column effluent as a function of bed volume for Cycle 1. The X axis for these plots is cumulative volume reaching the exit of the column expressed in $\mathrm{BV}$. The $\mathrm{pH}$ plot has the expected shape, with one exception. The particular $\mathrm{pH}$ probe used was designed to have a range from zero to 12; we did not find a $\mathrm{pH}$ probe with a wider range that would also withstand long immersion in our solutions. Therefore, the probe read about 12 for solutions with $\mathrm{pH}$ that high and higher. The timing for changes in $\mathrm{pH}$ agrees well with the times computed using a plug flow calculation for when a new solution would reach the column exit. During the regeneration step with $0.25 \mathrm{M} \mathrm{NaOH}$, the $\mathrm{pH}$ increased to almost 12, implying that the caustic wave had broken through the column.

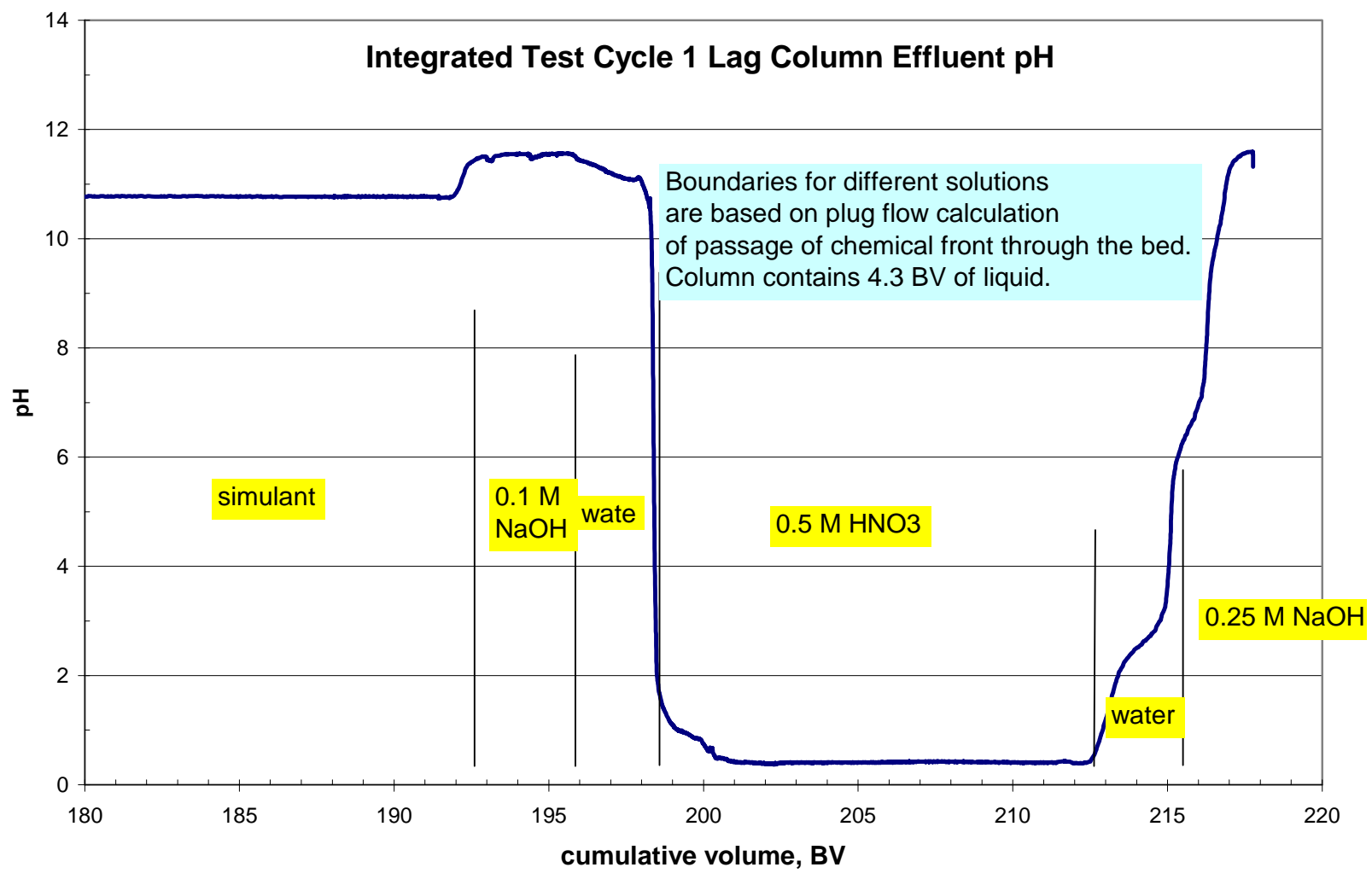

Figure 38. Effluent pH During Cycle 1

The conductivity probe reading, plotted in Fig. 39, told a somewhat different story than the $\mathrm{pH}$ probe. The conductivity plot has a noisier appearance than the $\mathrm{pH}$ plot because electrical conductivity is roughly proportional to ionic concentration and $\mathrm{pH}$ is proportional to the logarithm of hydrogen ion concentration. There are definite spikes in the measured conductivity at the simulant and $0.1 \mathrm{M} \mathrm{NaOH}$ transition and also at the acid and water transition. Conductivity of an aqueous solution increases with temperature. These spikes may have occurred as a result of heat of mixing. After acid is introduced to the column, most of the change in conductivity occurs in the first 3 to $5 \mathrm{BV}$. However, the conductivity appears to be changing slowly during the remaining period that acid flows through the column, which indicates that the resin may continue to consume small amounts of acid. When $0.25 \mathrm{M} \mathrm{NaOH}$ is introduced to the column, the conductivity 
reading never increased very much. This implies that nearly all of the sodium ions in the $\mathrm{NaOH}$ solution are loaded on the resin and nearly all of the hydroxide ions react with residual hydrogen ions on the resin to form water.

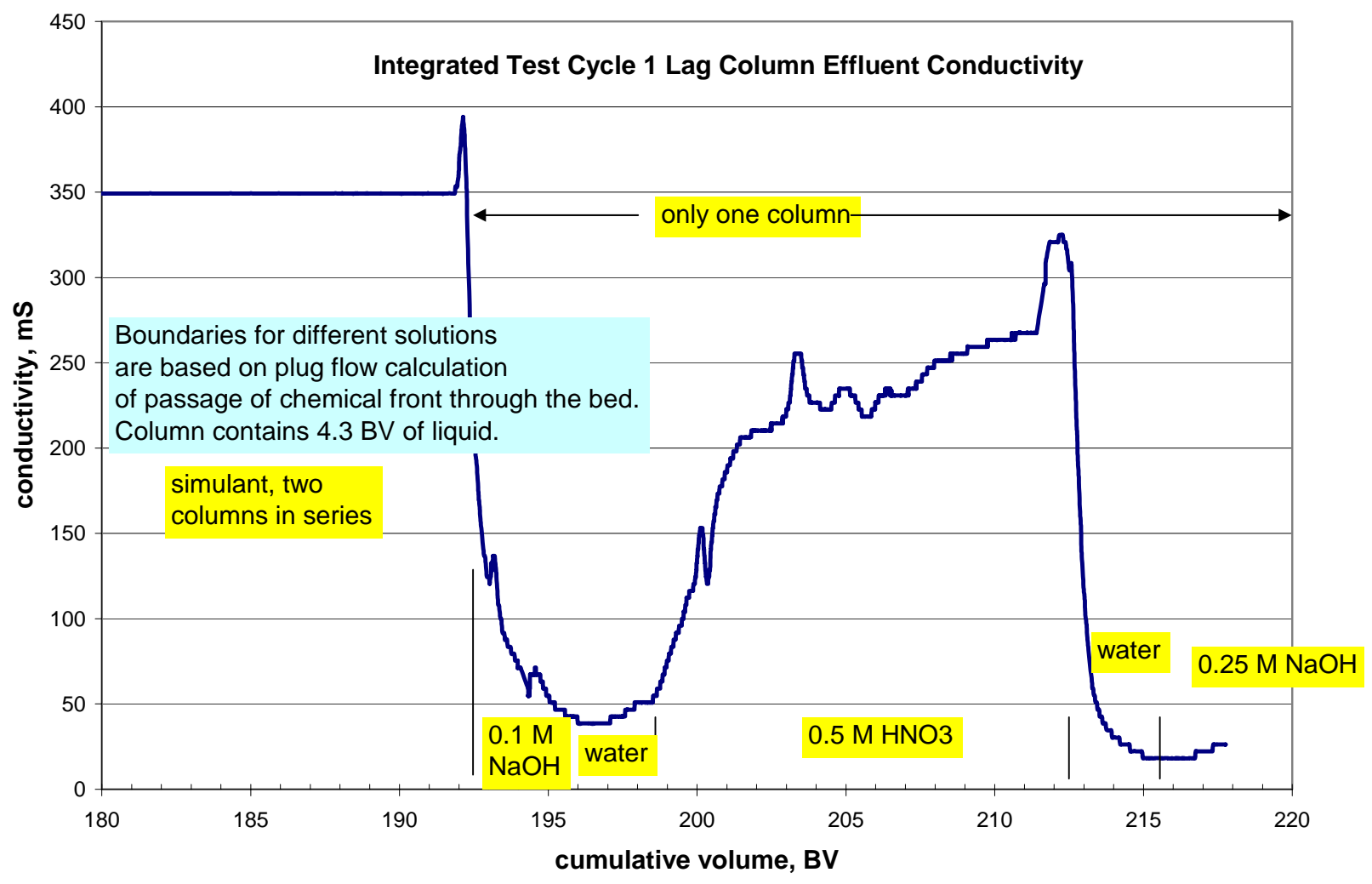

Figure 39. Effluent Conductivity During Cycle 1

Table 40 shows the response of the conductivity probe and $\mathrm{pH}$ probe when inserted into beakers of the various solutions. This table can be used to estimate how closely the transient conductivity and $\mathrm{pH}$ approached the steady-state values.

Table 40. Probe Responses for Standard Solutions

\begin{tabular}{|c|c|c|}
\hline solution & conductivity & $\mathrm{pH}$ \\
\hline DI water & $<5$ & 5 to 9 \\
\hline $0.1 \mathrm{M} \mathrm{NaOH}$ & 35 & 11.1 \\
\hline $0.25 \mathrm{M} \mathrm{NaOH}$ & 76 & 10.9 \\
\hline $0.5 \mathrm{M} \mathrm{HNO3}$ & 250 & 0.42 \\
\hline
\end{tabular}




\subsubsection{CIX Chemical Observations}

\subsection{Measurement Uncertainty}

The purpose of the resin is to remove cesium from LAW solutions that contain a mixture of constituents and are $5 \mathrm{M}$ in sodium. Evaluation of the effectiveness of the resin requires the measurement of cesium concentration under challenging conditions, that is, with a high concentration of sodium. The samples must be heavily diluted to reduce sodium concentration to a manageable level, which also dilutes cesium. The Analytical Development Section (ADS) of SRNL claimed an accuracy of $\pm 20 \%$ of the stated value for these cesium measurements. Because of the large sodium concentration the accuracy was checked using spiked samples a well as blanks that were $5 \mathrm{M}$ in sodium. The results are shown in Fig. 40.

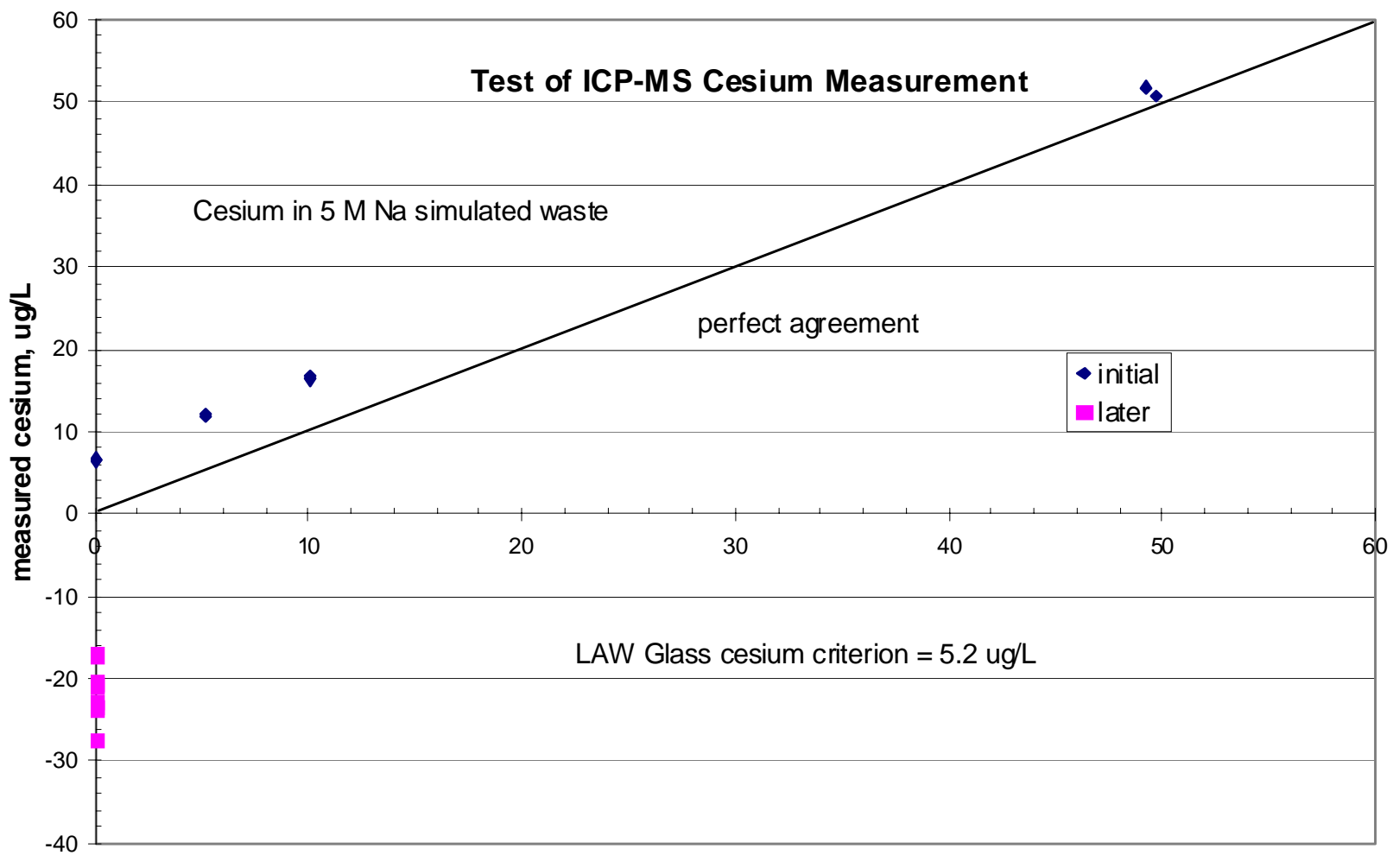

actual spiked cesium, ug/L

Figure 40. Test of Ability of ICP-MS to Measure Cesium 
The initial results were encouraging and indicated a bias of less than $10 \mu \mathrm{g} / \mathrm{L}$ of cesium concentration for very low concentrations and less than $10 \%$ uncertainty for concentrations of $50 \mu \mathrm{g} / \mathrm{L}$. Based on the initial results it appeared possible to correct for the bias. Unfortunately, later samples were analyzed to have negative concentrations as low as $-27 \mu \mathrm{g} / \mathrm{L}$. Of course, negative concentrations are physically impossible, but provide useful information about the accuracy of the measurement. Therefore, no effort was made to correct for bias and measurement uncertainty was treated as random. Previous measurements (Steimke, 2002), not plotted, for concentrations greater than $7000 \mu \mathrm{g} / \mathrm{L}$ confirmed a measurement uncertainty of $\pm 10 \%$ for higher concentrations. Therefore, the estimate for the uncertainty of cesium concentration measurements in this program is: \pm (10\% reading $+30 \mu \mathrm{g} / \mathrm{L})$, which is expressed for $95 \%$ confidence limits.

It should be noted that, even the originally indicated bias of $10 \mu \mathrm{g} / \mathrm{L}$ was insufficient to meet SIPP needs. Before SIPP began, the original criterion for when a Lead column would need regeneration was when the cesium concentration of the exiting LAW stream from the lead column would exceed one-half the incoming concentration, i.e., 50\% breakthrough. For Campaign III, the simulated LAW feed cesium concentration was $7760 \mu \mathrm{g} / \mathrm{L}$ (see Cs concentration of the UF (fresh) permeate in Table 42), therefore, detecting a concentration $3880 \mu \mathrm{g} / \mathrm{L}$ accurately would have been no problem. However, that criterion changed to a much more stringent one. The new criterion is based on when the exiting stream of a Lag column would contain a concentration of greater than $5.2 \mu \mathrm{g} / \mathrm{L}$ of cesium. Unfortunately, while some ADS cesium measurements appear to detect concentrations to better than $5 \mu \mathrm{g} / \mathrm{L}$, as already stated, the actual ADS uncertainty is estimated to be $\pm(10 \%$ of a reading $+30 \mu \mathrm{g} / \mathrm{L}$ of cesium $)$, implying that the detection limit for cesium is $30 \mathrm{ug} / \mathrm{L}$. This relatively large uncertainty must be kept in mind when evaluating such low concentrations. However, changes in cesium concentration throughout a cycle are expected to indicate when the cesium concentration is increasing in the exiting Lag column stream.

\subsection{Cesium Removal Effectiveness}

Simulant is pumped through the lead column where most of the cesium is removed and then enters the lag column, where additional cesium is removed. Figure 41 shows the cesium concentration exiting the lead column for the four cycles, as a function of bed volumes passed. 
WSRC-TR-2004-00565, REVISION 0

SRNL-RPP-2004-00092, REVISION 0

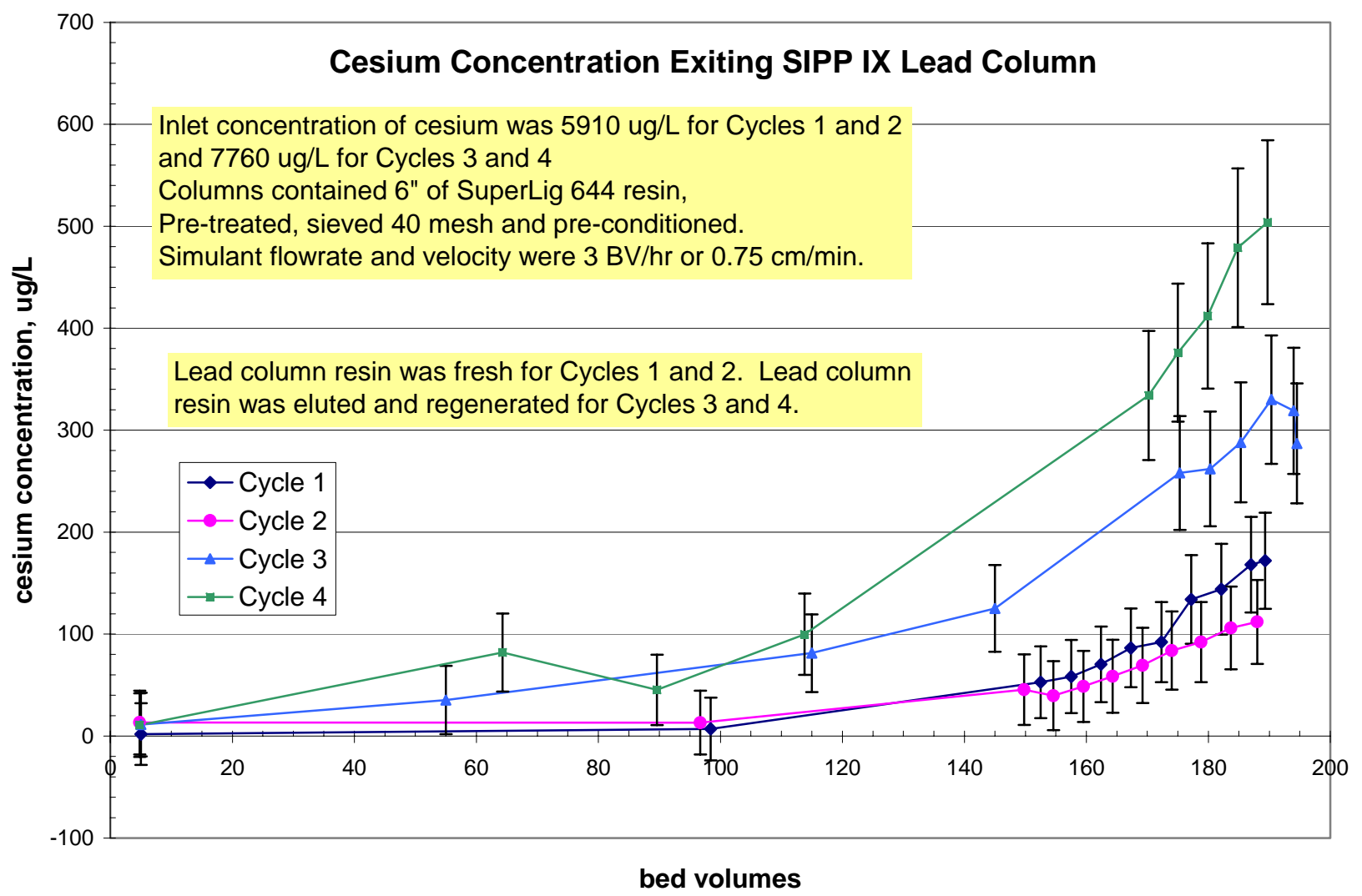

Figure 41. Cesium Concentration in Simulant Exiting Lead Column

Initially, the cesium concentration was low, or perhaps really zero when considering analytical uncertainty, and then increased. There is a general trend to more cesium escaping the lead column with additional cycles. Note that the lead column resin is fresh for Cycles 1 and 2 and has been eluted and regenerated for Cycles 3 and 4 . For all four cycles the final effluent cesium concentration was less than $7 \%$ of the inlet simulant feed concentration.

Figure 42 shows the cesium concentration exiting the lag column. Considering the uncertainty in the analytical measurement of cesium, all of the measurements may really be zero. However, the measurement uncertainty does not allow determination of whether the RPP-WTP criterion, i.e., cesium concentration less than $5.2 \mu \mathrm{g} / \mathrm{L}$ from the lag column, is satisfied. The concentration measured for one sample, the sample collected at $175 \mathrm{BV}$ for Cycle 4 was not plotted because it was so much higher than for the other samples. This sample was analyzed twice for cesium concentration and the results were $1190 \mu \mathrm{g} / \mathrm{L}$ and $1630 \mu \mathrm{g} / \mathrm{L}$. Note that the lead column resin is fresh for Cycle 1 and has been eluted and regenerated for Cycles 2, 3, and 4. 
WSRC-TR-2004-00565, REVISION 0

SRNL-RPP-2004-00092, REVISION 0

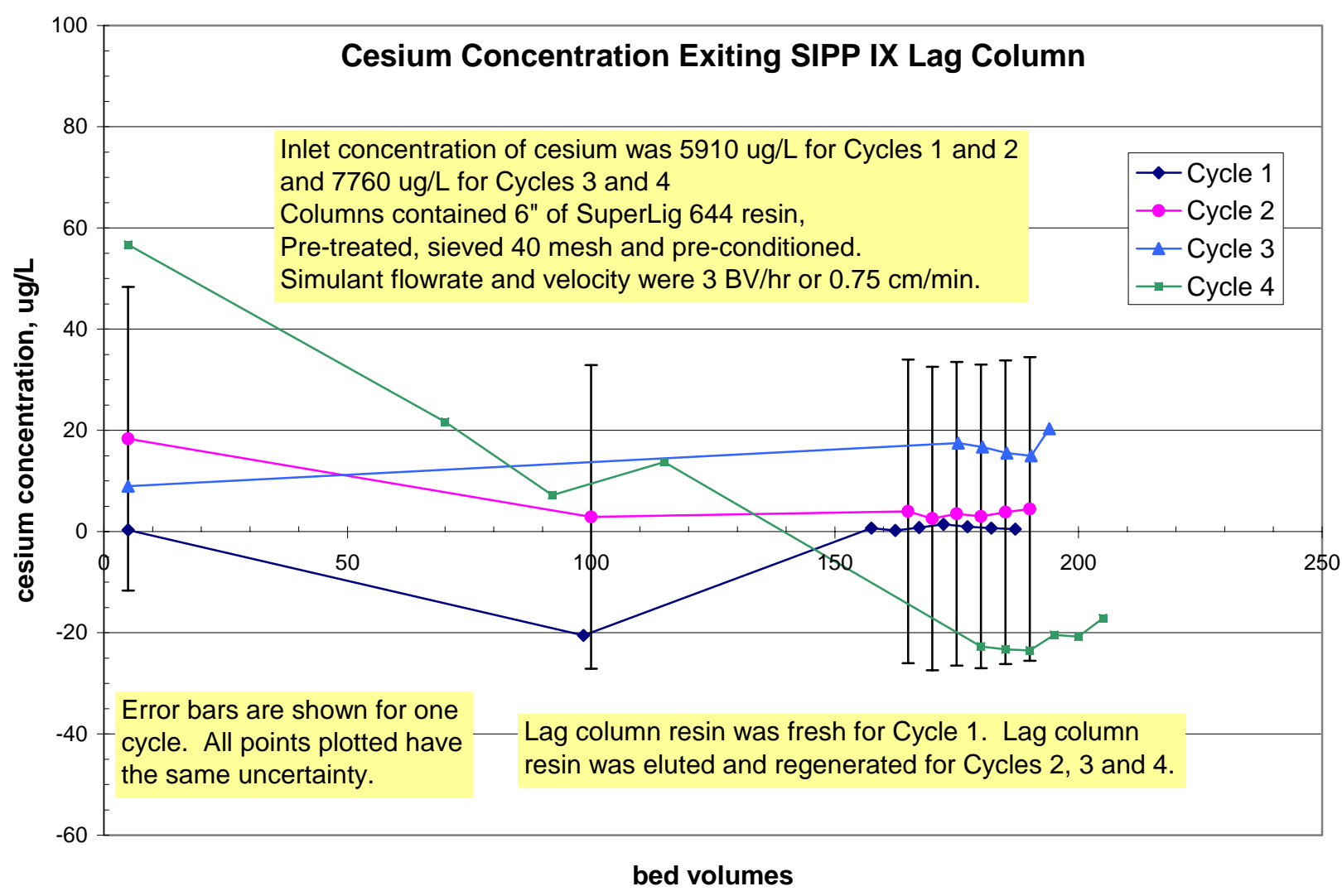

Figure 42. Cesium Concentration Exiting Lag Column

\subsection{Elutions of Resin Beds}

In all four cycles the lead column was eluted with $0.5 \mathrm{M}$ nitric acid followed by regeneration with $0.25 \mathrm{M} \mathrm{NaOH}$. For Cycles 1, 3, and 4 samples were collected of the eluate at regular intervals. Cesium concentrations are plotted in Figs. 43 and 44 as a function of cumulative flow.

Zero volume refers to the time when a plug flow calculation indicated that acid first reached the outlet of the column. There is no obvious difference in the elution profiles for Cycles 1, 3, and 4. Note that the concentration of cesium peaks when the front of acid reaches the outlet of the column. Also note that the great majority of the cesium is removed by the first $5 \mathrm{BV}$ of eluent. 
WSRC-TR-2004-00565, REVISION 0

SRNL-RPP-2004-00092, REVISION 0

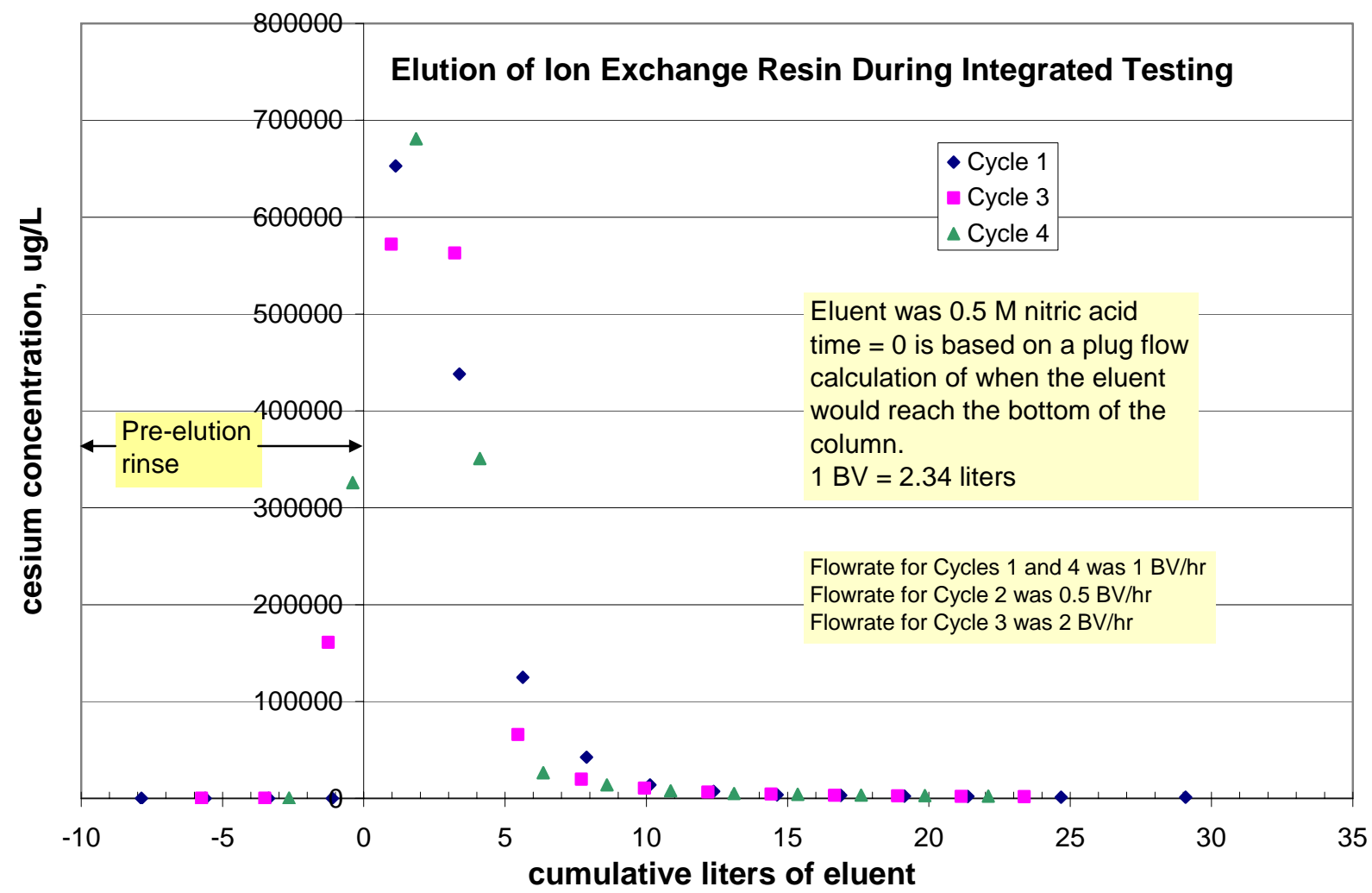

Figure 43. Elution Plot, Linear

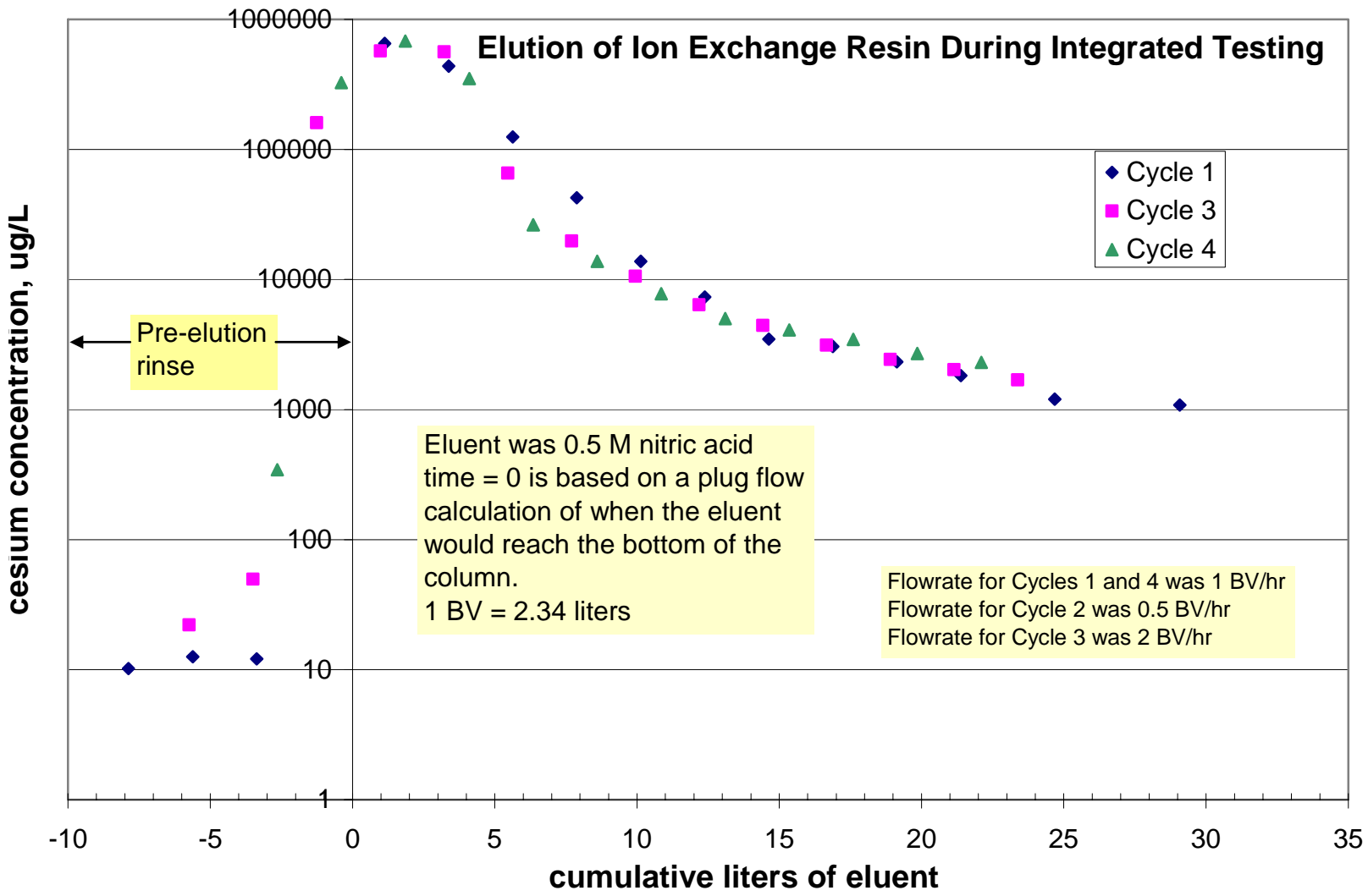

Figure 44. Elution Plot, Logarithmic 


\subsection{Cesium Mass Balance}

In the CIX unit operation, cesium enters the two IX columns dissolved in simulant and leaves the columns in simulant at a much lower concentration and also in eluate and other solutions. The cesium mass entering should equal the cesium mass leaving plus any accumulation. The accumulation or cesium inventory change on the resin was assumed to be small, as is implied by Table 37. To perform this calculation, the LAW feed and receipt tanks were calibrated for volume and the results are discussed below. For some solutions the volume was calculated based on the average calibrated flow rate and the elapsed time. Mass balances are listed in Tables 41 and 42. For all four cycles the cesium mass in agrees with the cesium mass out to within measurement uncertainty, which is expressed for 95\% confidence limits. As previously mentioned in Section 2.8.5.3.1, the uncertainty of measuring cesium concentration was $\pm(10 \%$ of reading + $30 \mu \mathrm{g} / \mathrm{L})$. The uncertainty in measuring volume was \pm 3 liters for simulant and \pm 1 liter for the other solutions. The mass of cesium in a solution is equal to the cesium concentration multiplied by the solution volume. The cesium mass out was the sum of the masses for the various solutions. The uncertainty on the cesium mass out was computed as the root sum square of the uncertainties for the various solutions. 
WSRC-TR-2004-00565, REVISION 0

SRNL-RPP-2004-00092, REVISION 0

Table 41. Mass Balances for Cycles 1 and 2

\begin{tabular}{|c|c|c|c|c|c|c|c|c|c|c|c|}
\hline $\begin{array}{l}\text { Cycle } 1 \\
\text { Solution }\end{array}$ & $\begin{array}{l}\text { volume } \\
\text { liters }\end{array}$ & $\begin{array}{l}\text { cesium } \\
\text { conc., } \\
\text { mg/L }\end{array}$ & $\begin{array}{l}\text { ADS } \\
\text { Sample } \\
\text { No. }\end{array}$ & $\begin{array}{l}\text { cesium } \\
\text { mass, } \\
\text { mg }\end{array}$ & $\begin{array}{l}\text { mass } \\
\text { uncert- } \\
\text { ainty, mg }\end{array}$ & $\begin{array}{l}\text { Cycle } 2 \\
\text { Solution }\end{array}$ & $\begin{array}{l}\text { volume } \\
\text { liters }\end{array}$ & $\begin{array}{l}\text { cesium } \\
\text { conc., } \\
\text { mg/L }\end{array}$ & $\begin{array}{l}\text { ADS } \\
\text { Sample } \\
\text { No. }\end{array}$ & $\begin{array}{l}\text { cesium } \\
\text { mass, } \\
\text { mg }\end{array}$ & $\begin{array}{l}\text { mass } \\
\text { uncert- } \\
\text { ainty, mg }\end{array}$ \\
\hline \multirow[t]{3}{*}{$\begin{array}{l}\text { fresh } \\
\text { simulant in }\end{array}$} & 428 & 5.91 & 207820 & 2529 & \pm 284 & $\begin{array}{l}\text { fresh simulant } \\
\text { in }\end{array}$ & 437 & 5.91 & 207820 & 2583 & \pm 289 \\
\hline & & & & & & $\begin{array}{l}\text { Cycle } 1 \text { spent } \\
\text { simulant }\end{array}$ & 9.4 & 0.12 & 208198 & 1 & \pm 0 \\
\hline & & & & & & total Cs in & & & & 2584 & \pm 289 \\
\hline simulant out & 408 & 0.0213 & 209494 & 9 & \pm 2 & simulant out & 427 & 0.00897 & 209495 & 4 & \pm 1 \\
\hline $\begin{array}{l}\text { final } \\
\text { simulant }\end{array}$ & 9.4 & 0.12 & 208198 & 1 & \pm 1 & final simulant & 20 & 0.102 & 208358 & 2 & \pm 1 \\
\hline $0.1 \mathrm{M} \mathrm{NaOH}$ & 12.2 & 0.344 & 208509 & 4 & \pm 1 & $0.1 \mathrm{M} \mathrm{NaOH}$ & 44.5 & 50.6 & 208359 & 2252 & \pm 277 \\
\hline $\begin{array}{l}\text { pre-elution } \\
\text { rinse }\end{array}$ & 0.7 & 70.8 & 208497 & 50 & \pm 76 & $\begin{array}{l}\text { pre-elution } \\
\text { rinse }\end{array}$ & 0 & 0 & 208515 & 0 & \pm 0 \\
\hline eluate & 31.5 & 87.2 & 208200 & 2747 & \pm 363 & eluate & 6.8 & 2.47 & 208360 & 17 & \pm 4 \\
\hline $\begin{array}{l}\text { post-elution } \\
\text { rinse }\end{array}$ & 7.5 & 0.887 & 208201 & 7 & \pm 2 & $\begin{array}{l}\text { post-elution } \\
\text { rinse }\end{array}$ & 5.2 & 0 & 208361 & 0 & \pm 0 \\
\hline regenerant & 4 & 0.0336 & 208510 & 0 & \pm 0 & regenerant & 11 & 0.0109 & 208362 & 0 & \pm 0 \\
\hline total Cs out & & & & 2817 & \pm 371 & total Cs out & & & & 2274 & \pm 277 \\
\hline
\end{tabular}


Table 42. Mass Balances for Cycles 3 and 4

\begin{tabular}{|c|c|c|c|c|c|c|c|c|c|c|c|}
\hline $\begin{array}{l}\text { Cycle } 3 \\
\text { solution }\end{array}$ & $\begin{array}{l}\text { volume } \\
\text { liters }\end{array}$ & $\begin{array}{l}\text { cesium } \\
\text { conc., } \\
\text { mg/L }\end{array}$ & $\begin{array}{l}\text { ADS } \\
\text { Sample } \\
\text { No. }\end{array}$ & $\begin{array}{l}\text { cesium } \\
\text { mass, } \\
\text { mg }\end{array}$ & $\begin{array}{l}\text { mass } \\
\text { uncert- } \\
\text { ainty, mg }\end{array}$ & $\begin{array}{l}\text { Cycle } 4 \\
\text { solution }\end{array}$ & $\begin{array}{l}\text { volume } \\
\text { liters }\end{array}$ & $\begin{array}{l}\text { cesium } \\
\text { conc., } \\
\text { mg/L }\end{array}$ & $\begin{array}{l}\text { ADS } \\
\text { Sample } \\
\text { No. }\end{array}$ & $\begin{array}{l}\text { cesium } \\
\text { mass, } \\
\text { mg }\end{array}$ & $\begin{array}{l}\text { mass } \\
\text { uncert- } \\
\text { ainty, mg }\end{array}$ \\
\hline $\begin{array}{l}\text { fresh } \\
\text { simulant in }\end{array}$ & 453 & 7.76 & 209229 & 3515 & \pm 388 & $\begin{array}{l}\text { fresh simulant } \\
\text { in }\end{array}$ & 453 & 7.76 & 209229 & 3515 & \pm 388 \\
\hline $\begin{array}{l}\text { Cycle } 2 \text { spent } \\
\text { simulant (1) }\end{array}$ & & & & & & $\begin{array}{l}\text { Cycle } 3 \text { spent } \\
\text { simulant }\end{array}$ & 9 & 0.363 & 211124 & 3 & \pm 0 \\
\hline & & & & & & total Cs mass in & & & & 3519 & \pm 388 \\
\hline simulant out & 434 & 0.0237 & 209496 & 10 & \pm 14 & simulant out & 439 & 0 & 209497 & 0 & \pm 13 \\
\hline final simulant & 9.4 & 0.363 & 211124 & 3 & \pm 1 & final simulant & 19.3 & 0 & 211130 & 0 & \pm 1 \\
\hline $0.1 \mathrm{M} \mathrm{NaOH}$ & 7.7 & 41.3 & 211125 & 318 & \pm 73 & $0.1 \mathrm{M} \mathrm{NaOH}$ & 8.8 & 0 & 211131 & 0 & \pm 0 \\
\hline $\begin{array}{l}\text { pre-elution } \\
\text { rinse }\end{array}$ & 7.1 & 39.8 & 211126 & 283 & \pm 68 & $\begin{array}{l}\text { pre-elution } \\
\text { rinse }\end{array}$ & 7.3 & 16.6 & 211132 & 121 & \pm 29 \\
\hline eluate & 31.3 & 87.3 & 211127 & 2732 & \pm 361 & eluate & 33.4 & 91.9 & 211133 & 3069 & \pm 400 \\
\hline $\begin{array}{l}\text { post-elution } \\
\text { rinse }\end{array}$ & 7.8 & 0.694 & 211128 & 5 & \pm 1 & $\begin{array}{l}\text { post-elution } \\
\text { rinse }\end{array}$ & 7.7 & 0.456 & 211134 & 4 & \pm 1 \\
\hline regenerant & 13.8 & 2.23 & 211129 & 31 & 6 & regenerant & 13.2 & 0.352 & 211135 & 5 & \pm 1 \\
\hline total Cs out & & & & 3383 & \pm 375 & total Cs out & & & & 3199 & \pm 401 \\
\hline
\end{tabular}

(1) The small volume of Cycle 2 spent simulant obtained during Cycle 2 regeneration is included fresh simulant. There was no separate concentration measurement made. That is, the $3515 \mathrm{mg}$ of Cs in the fresh simulant includes the few milligrams of Cs from the Cycle 2 spent simulant. 
As a consistency check on the outlet cesium, the total cesium in the composite eluate and the composite pre-elution rinse was compared with the integral of the data plotted in Figure 43. In principal, the integral should give the same mass as the composite samples. The integral was calculated numerically using three methods, the Trapezoidal Rule and two variations of Simpson's Rule. The Trapezoidal Rule approximates the integral as a series of trapezoids. The area of each trapezoid is computed as the length of the base of the trapezoid multiplied by the average height. Simpson's Rule approximates segments of the data as parabolas. Simpson's Rule was applied two different ways. Recall that the equation for Simpson's Rule is:

$$
\int(x) d x=h / 3\left(f\left(x_{0}\right)+4 f\left(x_{1}\right)+2 f\left(x_{2}\right)+4 f\left(x_{3}\right)+\ldots .+4 f\left(x_{n-1}\right)+\left(f\left(x_{n}\right)\right)\right.
$$

where $\mathrm{h}$ is the difference in $\mathrm{x}$ between adjacent points. First, Simpson's Rule was applied to the data in Fig. 43. Then, two additional points with concentration equal to zero were added to the beginning and end of the data set. Then Simpson's Rule was applied again. The two applications of Simpson's Rule give different results depending on whether the data point with the largest concentration is multiplied by two or four. The results for cesium mass in the eluate are shown in Table 43. No results are listed for Cycle 2 because no periodic eluate samples were collected during Cycle 2. The masses of cesium calculated using numerical integration agree reasonably well with the masses determined using composite samples.

Table 43. Mass of Eluate Cesium as a function of Integration Method

\begin{tabular}{|l|l|l|l|l|}
\hline $\begin{array}{l}\text { Cycle } \\
\text { number }\end{array}$ & $\begin{array}{l}\text { Trapezoidal } \\
\text { Rule, } \\
\text { mg Cs }\end{array}$ & $\begin{array}{l}\text { Simpson’s } \\
\text { Rule a, } \\
\text { mg Cs }\end{array}$ & $\begin{array}{l}\text { Simpson's } \\
\text { Rule b, } \\
\text { mg Cs }\end{array}$ & $\begin{array}{l}\text { Cs from } \\
\text { composite, } \\
\text { mg }\end{array}$ \\
\hline 1 & 2911 & 2679 & 3136 & 2797 \\
\hline 3 & 3164 & 3090 & 3236 & 3015 \\
\hline 4 & 3203 & 3188 & 3217 & 3190 \\
\hline
\end{tabular}

\subsubsection{Pilot CIX Conclusions (Author - Steimke)}

- Pressure drops for the four cycles were low and the resin bed permeability was approximately the same as were measured for the 24-Inch Column Test (Fowley et al, 2004).

- The pressure drop across the columns for flow of simulant generally increased during the four cycles. [0.5 to 1.3 in. $\mathrm{H}_{2} 0$, Table 38]

- SuperLig ${ }^{\circledR} 644$ effectively removed cesium from simulated waste and the analytical ability to measure cesium concentrations in five molar sodium did allow verification of the waste acceptance criterion of $50 \%$ breakthrough from the lead column after 100 Bed Volumes of simulated waste were treated. In fact, as shown in Fig. 41, in the worst case, Cycle 4, even after 190 BV of treated waste only the lead column exit cesium concentration was less than $7 \%$ of the feed waste. 
WSRC-TR-2004-00565, REVISION 0

SRNL-RPP-2004-00092, REVISION 0

- $\quad$ SuperLig ${ }^{\circledR} 644$ effectively removed cesium from simulated waste, however, the analytical difficulty of measuring very low cesium concentrations in five molar sodium did not allow verification of the waste acceptance criterion of $5.2 \mu \mathrm{g} / \mathrm{L}$ from the lag column after $100 \mathrm{BV}$ of simulated waste were treated. This fact was known before the start of testing.

- The fraction of the original cesium that was removed by the lead column decreased from 99.6\% for Cycle 1 (in Campaign II) to 98.2\% for Cycle 4 (in Campaign III). (Table 37).

- The masses of cesium in and out of the resin beds for all four cycles balanced to within measurement uncertainty.

\subsection{PILOT TREATED LAW EVAPORATOR (AUTHORS - ADAMSON, WILLIAMS \& ZAMECNIK)}

The Treated LAW Evaporator Process, or TLP, is the last separation technology unit operation in the RPP-WTP Pretreatment Facility that a waste will see on start up. With respect to SIPP, it will be the last separation operation in line because it evaporates the treated LAW products that come from the pilot CIX unit operation. This treated LAW product is then mixed with recycle streams to produce a feed stream for the TLP unit operation.

This section will explain the pilot TLP unit operation that was done as part of Campaign III testing. The process started with the preparation of the TLP feed product in a predetermined fashion, see Table 4. That chemical explanation of the feed stream formation will be followed by a short description on how feed formation was handled just before TLP operation and then how the waste stream was handled during and after its concentration. The following sections will discuss the physical equipment used to evaporate the waste and how the test was run. Finally, the results will be discussed and conclusions made.

\subsubsection{Chemical Description of the Pilot TLP Feed Stream (Author-Zamecnik)}

The TLP evaporator feeds consisted of the Ion Exchange product (Treated LAW), the LAW SBS condensate supplied by VSL, and IX regeneration solution displaced by feed. The compositions of the IX feed (similar to IX product except for Cs) and the LAW SBS Condensate are given in Table 44. The regeneration solution consisted of 20.5L of actual regeneration solution plus $17.3 \mathrm{~L}$ of simulated regeneration solution; both were essentially $0.25 \mathrm{M} \mathrm{NaOH}$. The calculated and measured TLP Feed compositions are also shown in Table 44. The composition of the IX product was not measured (except for Cs). The values in this table show that the measured TLP feed composition was slightly more dilute than expected from the calculation. A possible reason for this difference is that the IX product was likely slightly more dilute than the IX feed; this was also found to be true for Campaign II. 
WSRC-TR-2004-00565, REVISION 0

SRNL-RPP-2004-00092, REVISION 0

Table 44. Measured Composition of TLP Feeds

\begin{tabular}{|c|c|c|c|c|}
\hline $\begin{array}{l}\text { All values } \mathrm{mg} / \mathrm{kg} \text { unless } \\
\text { otherwise noted }\end{array}$ & $\begin{array}{l}\text { Ion Exchange } \\
\text { Feed } \\
\end{array}$ & $\begin{array}{l}\text { LAW SBS } \\
\text { Condensate }\end{array}$ & $\begin{array}{c}\text { Feed } \\
\text { Calculated } \\
\end{array}$ & $\begin{array}{c}\text { Feed } \\
\text { Measured }\end{array}$ \\
\hline Total Solids (wt\%) & 28.29 & 0.26 & 16.32 & 16.23 to 16.24 \\
\hline Undissolved Solids (wt\%) & 0.00 & 0.17 & 0.07 & 0.07 to 0.15 \\
\hline Supernate Solids (wt\%) & 28.29 & 0.09 & 16.26 & 16.10 to 16.18 \\
\hline Density $(\mathrm{kg} / \mathrm{L})$ & 1.248 & 1.002 & 1.130 & 1.134 \\
\hline $\mathrm{Ag}$ & 0.76 & $<3.9$ & 0.44 & $<50$ \\
\hline $\mathrm{Al}$ & 5136 & 7.53 & 2947 & 2900 \\
\hline $\mathrm{B}$ & 85.7 & 132 & 103 & \\
\hline $\mathrm{Ba}$ & $<1.3$ & $<0.26$ & 0 & $<2.0$ \\
\hline $\mathrm{Ca}$ & $<3.6$ & $<0.39$ & 0 & $<6.6$ \\
\hline $\mathrm{Cd}$ & $<0.04$ & $<0.26$ & 0 & $<2.4$ \\
\hline $\mathrm{Ce}$ & $<1.0$ & $<10$ & 0 & $<128$ \\
\hline Co & $<0.11$ & $<1.0$ & 0 & $<7.3$ \\
\hline $\mathrm{Cr}$ & 139 & 5.26 & 81.6 & 82.8 \\
\hline $\mathrm{Cu}$ & $<0.03$ & $<0.39$ & 0 & $<8.3$ \\
\hline $\mathrm{Fe}$ & 1.36 & 11.7 & 5.55 & 15.0 \\
\hline $\mathrm{K}$ & 16346 & 123 & 9420 & \\
\hline $\mathrm{La}$ & 0.85 & $<1.3$ & 0.49 & $<116$ \\
\hline $\mathrm{Li}$ & 1.72 & 11.5 & 5.68 & 1.00 \\
\hline $\mathrm{Mg}$ & $<0.24$ & 3.56 & 1.45 & $<14$ \\
\hline $\mathrm{Mn}$ & $<0.02$ & $<0.12$ & 0 & $<1.5$ \\
\hline Mo & 8.65 & $<1.3$ & 4.96 & $<17$ \\
\hline $\mathrm{Na}$ & 88141 & 587 & 50874 & 47684 \\
\hline $\mathrm{Nd}$ & $<1.5$ & $<12$ & 0 & $<43$ \\
\hline $\mathrm{Ni}$ & 0.25 & 0.34 & 0.28 & $<10$ \\
\hline $\mathrm{P}$ & 554 & $<10$ & 317 & 371 \\
\hline $\mathrm{Pb}$ & $<0.36$ & $<10$ & 0 & $<114$ \\
\hline $\operatorname{Re}$ & & 21.8 & 8.88 & \\
\hline $\mathrm{S}$ & 905 & 78.0 & 551 & 502 \\
\hline $\mathrm{Si}$ & 42.1 & 21.8 & 33.0 & 0 \\
\hline $\mathrm{Sr}$ & 0.16 & $<0.07$ & 0.09 & 8.00 \\
\hline $\mathrm{Ti}$ & 0.10 & 1.15 & 0.53 & $<23$ \\
\hline $\mathrm{Zn}$ & 2.37 & 1.29 & 1.88 & $<61$ \\
\hline $\mathrm{Zr}$ & 1.43 & 1.29 & 1.35 & $<8.0$ \\
\hline bromide & $<80$ & $<86$ & 0 & $<61$ \\
\hline chloride & 1082 & 626 & 875 & 795 \\
\hline fluoride & 44.9 & 146 & 85.1 & 56.0 \\
\hline formate & 1066 & 32.9 & 624 & 521 \\
\hline nitrate & 72917 & 135 & 41852 & 37200 \\
\hline nitrite & 23077 & 2575 & 14278 & 12300 \\
\hline oxalate & 658 & $<86$ & 377 & 374 \\
\hline phosphate & 1963 & $<86$ & 1125 & 1220 \\
\hline sulfate & 2853 & 233 & 1730 & 1570 \\
\hline ammonium & $<80$ & 990 & 827 & 785 \\
\hline
\end{tabular}




\subsubsection{Simulant Handling (Author - Williams)}

To make feed for the pilot-scale TLP, on 6 July 2004 the 1,132 kg of treated simulant processed through the CIX were mixed with $805.0 \mathrm{~kg}$ of LAW SBS Quencher from VSL, a recycle stream. For Campaign III, $21.4 \mathrm{~kg}$ of $0.25 \mathrm{M} \mathrm{NaOH}$ Regen Solution from Campaign II CIX was added to $17.8 \mathrm{~kg}$ of $0.25 \mathrm{M} \mathrm{NaOH}$ made with DI water and used as per the Test Specification (Longwell, 2003). However, the mixing was done only intermittently as the ammonia smell was quite strong. Additional ventilation was employed for all operations subsequent to LAW SBS Quencher addition due to strong ammonia smell. Samples were pulled for analysis during and following TLP Evaporator operations to measure the ammonia concentration (see Table 46).

Upon startup, on 8 July, 2004 of TLP operations, a total of 309 g of DOW Corning Q23183A Antifoam was added directly into the evaporator in three batches of $103 \mathrm{~g}$ each in a 10:1 ratio with DIF water. The dilution was necessary to fully incorporate the antifoam because of its thick consistency. Despite having fewer recycle streams, the total volume of the TLP feed was larger than that for the FEP. Considering that the concentration target was different from the FEP bottoms, then the final concentrated TLP volume would be much larger than that for the FEP, in fact it was approximately 3.5 times larger. This meant that a steady-state evaporated concentration would be attained before exhausting the feed. Once the target slurry concentration was realized, then the TLP bottoms would have to be removed while more feed was being sent into the TLP at a rate that would keep the antifoam concentration at the target level of $1500 \mathrm{ppm}$. Of course, removing concentrated slurry meant removing its antifoam too. Therefore, antifoam had to be reintroduced periodically to maintain its concentration constant. Additional amounts of antifoam were added in batches and after approximately each 95 liters of TLP bottoms were removed, 192.5 grams of antifoam were added.

A total of $775.4 \mathrm{~kg}$ of TLP Evaporator bottoms were produced and placed in three drums labeled "Campaign III LAW Concentrated Slurry (Drum 1 of 3, 2 of 3, and 3 of 3)" for shipment to VSL on 9 July 2004. After Campaign III was complete, the condensate collected from both the TLP and FEP operations were combined as RLD (as already explained in the first paragraph Section 2.6.1.1 of the FEP). This mixing is how WTP will handle its condensate streams, which will then be made available to produce wash and caustic solutions. For SIPP the combined condensate streams will be used for Campaign IV wash and caustic solutions.

\subsubsection{Pilot TLP Equipment (Author - Adamson)}

One of the requirements for SIPP as per the Test Specification (Longwell, 2003) was to utilize and modify existing equipment in the Engineering Development Laboratory of SRNL. To that end only one pilot-scale evaporator was constructed to be used for both FEP and Treated LAW Process (TLP) testing, because the design of those RPP-WTP Pretreatment Facility evaporators is the same. The plan for the use of those two 
WSRC-TR-2004-00565, REVISION 0

SRNL-RPP-2004-00092, REVISION 0

evaporators during SIPP testing did not require simultaneous operation, therefore, once one unit operation test completed the test rig could be made ready for the subsequent unit operation. As such, the description of the pilot-scale TLP is the same as for the pilotscale FEP and therefore will not be repeated here. Please refer to equipment description for the FEP in section 2.6.3.

\subsubsection{Pilot TLP Operation (Author - Adamson)}

Prior to the TLP evaporation campaign, the evaporator system was cleaned, rinsed and inspected. Clean water was then added to the evaporator pot and the system was operated for approximately two hours. The spent water was visually clear with a pH of 7 .

The TLP evaporator was operated using Work Instructions, I-EDL-132 "SIPP Treated LAW Process (TLP) Evaporation Test.” Manually recorded information was recorded in notebook (Adamson, 2004).

The TLP evaporation of Campaign III took place on 8 July 2004. It took approximately 11 hours to boil off 1,138.1 liters of water (condensate) from the TLP simulant feed (initial feed density $=1.135 \mathrm{~kg} / \mathrm{L}$ ) to obtain an average density of $1.365 \mathrm{~kg} / \mathrm{L}$ at $20^{\circ} \mathrm{C}$. The total volume of the TLP Campaign III concentrated product was 567.5 liters (149.9 gal).

The average condensate boil off rate for the TLP campaign III was $1.72 \mathrm{~L} / \mathrm{min}(0.45$ gpm). The design boil off target of the RPP Pilot-scale Evaporator system is $1.5 \mathrm{~L} / \mathrm{min}$ (0.40 gpm).

Evaporation of the TLP simulant feed occurred at approximately $57^{\circ} \mathrm{C}$ under $-12.80 \mathrm{psig}$ vacuum (1.71 psia). The atmospheric pressure was approximately 14.51 psia during the campaign. However, due to pulling off the TLP concentrate and adding the cooler simulant feed, there were small fluctuations in the evaporator pot temperature and pressure as depicted in Figs. 45 and 46. 
WSRC-TR-2004-00565, REVISION 0

SRNL-RPP-2004-00092, REVISION 0

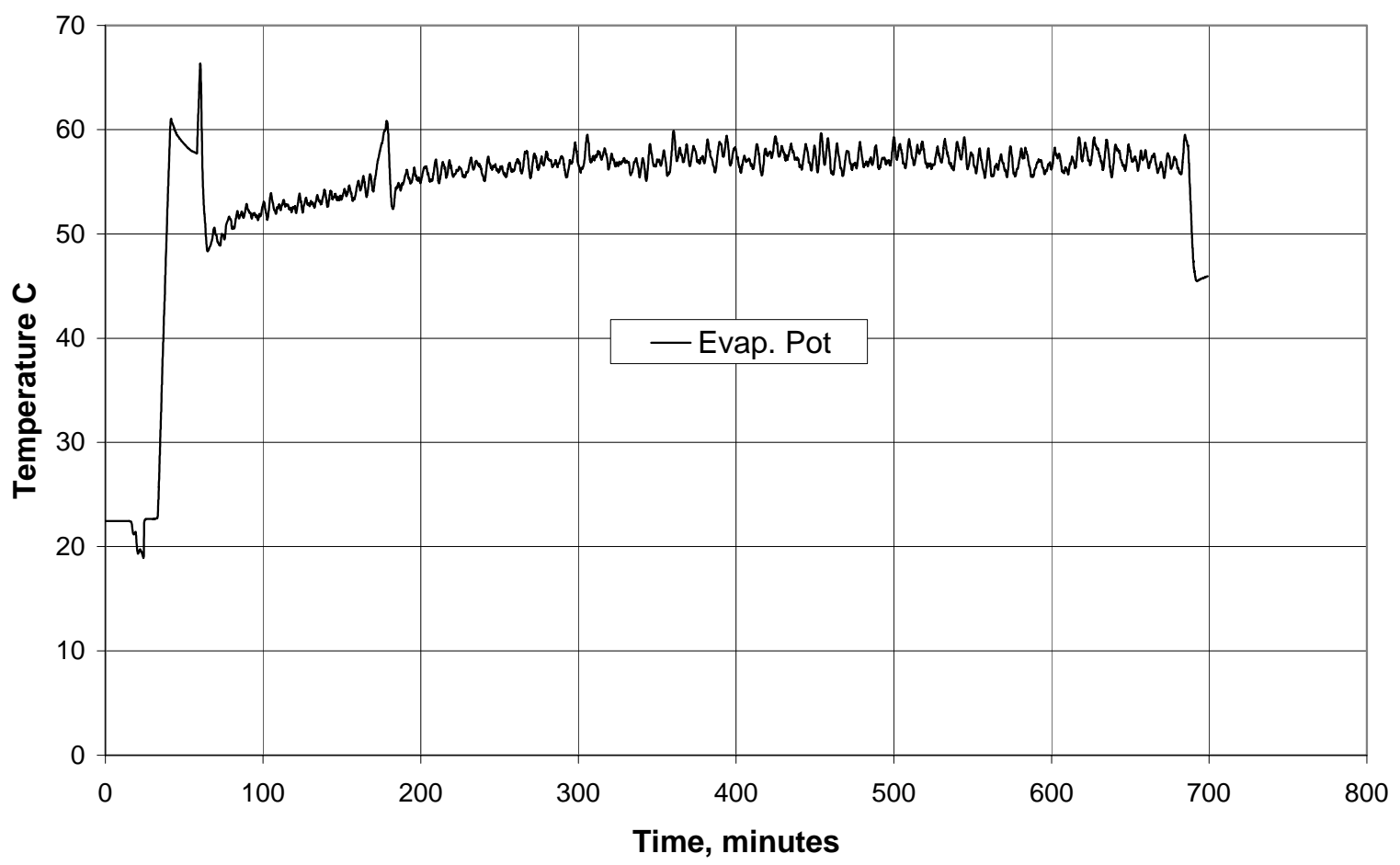

Figure 45. TLP Evaporator Pot Temperature during Campaign III

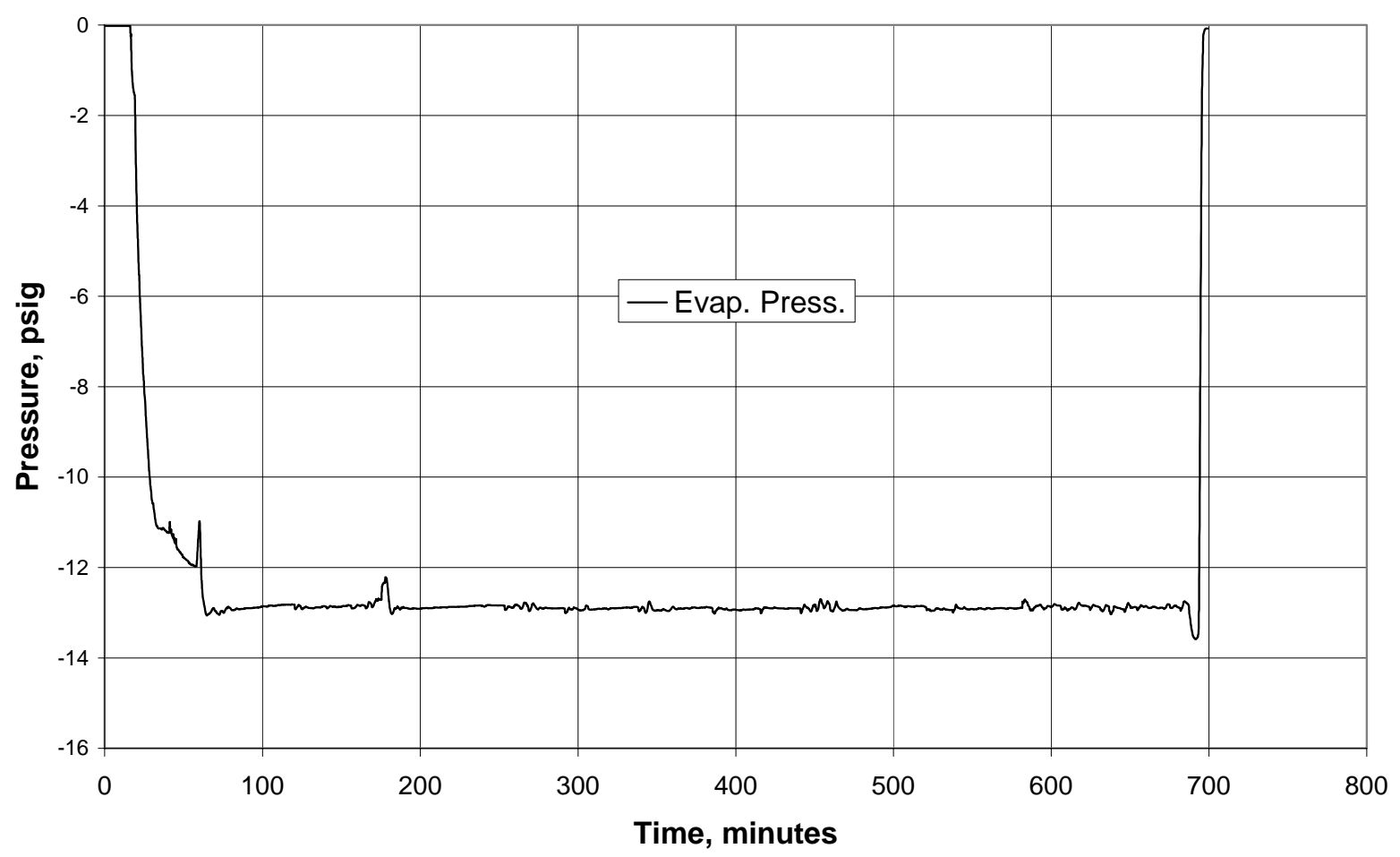

Figure 46. TLP Evaporator Pot Pressure during Campaign III 
The TLP simulant was re-circulated through the reboiler and evaporator system, see Fig. 5, at approximately $89 \mathrm{gpm}$, as shown in Fig. 47. The recirculation of the simulant was continuous for the duration of the campaign. Also recirculation continued when removing the concentrate from the evaporator system.

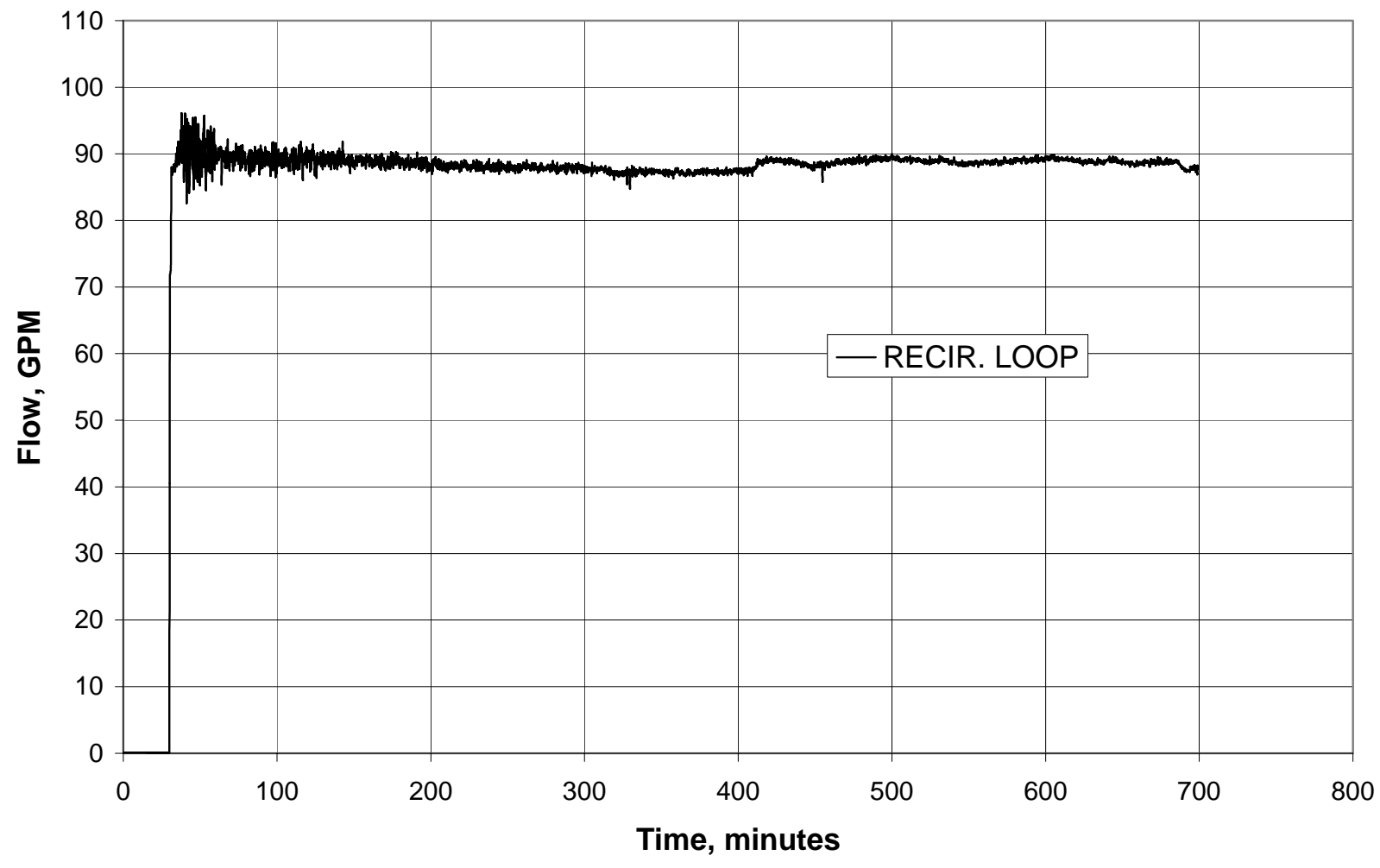

Figure 47. TLP Simulant Recirculation during Campaign III

A total of 888 grams of Q2 antifoam was added to the simulant during the TLP campaign III evaporation process. A dilute mixture of antifoam was pulled into the system via vacuum through valve CL-BV13 at the bottom of the evaporator pot. Because the antifoam chemical was relatively thick, the manufacturer recommends diluting it with DI water to a mixture of 10:1 before use. Initially 309 grams of Q2 were added to the simulant and then, as concentrate was removed additional Q2 was added, in three subsequent equal batches of 193 grams, as concentrated bottoms was removed. While the goal was an antifoam concentration of $1500 \mathrm{ppm}$ the final concentration ended up being 1150 ppm, because of the pilot-scale TLP batch operation. That is, the experiment started at $1500 \mathrm{ppm}$, with a fixed evaporator volume of approximately 150 liters, and stayed that way until the feed, within the evaporator, reached the target density of $1.365 \mathrm{~kg} / \mathrm{L}$ at $50^{\circ} \mathrm{C}$. In this phase, the feed rate matched the evaporation rate. Once at the target density, the batch mode operation began with slowly allowing feed to enter the evaporator slightly faster than the evaporation rate to maintain the density while allowing the evaporator volume to increase to approximately 225 liters. At this point the antifoam concentration dropped to $1500 \mathrm{ppm} \times(150 / 225)=1000 \mathrm{ppm}$. No increase in foaming was noted and previous work (Qureshi et al., 2004) has shown that antifoam greater than 
$300 \mathrm{ppm}$ is not effective. Once at 225 liters, then 95 liters of bottoms were removed to begin the next batch. This process continued until all the feed was consumed. However, a further drop in antifoam concentration was not desired, therefore enough antifoam was added so that the next 95 liters of concentrated feed would also have a $1500 \mathrm{ppm}$ concentration. Moreover, no antifoam was added to the final batch of feed because it was small, less than 40 liter. When the process was finished the antifoam concentration of the composite product was $1150 \mathrm{ppm}$, based on the 888 grams of antifoam in 567.5 liters (772 kg) of bottoms produced. During the entire evaporation period very little foaming was witnessed.

Samples from primary condensate, secondary condensate, and evaporator bottoms were pulled every 200 liters of simulant feed pumped into the evaporator. Other samples were pulled more often to check the density of the evaporator bottoms. The density was checked using a density meter (Anton Paar, DMA 4500) and measurements were recorded in the lab notebook (Adamson, 2004).

The supplied TLP simulant feed for evaporation had a volume of 1,706.6 liters with a density of $1.135 \mathrm{~kg} / \mathrm{L}$ at $20^{\circ} \mathrm{C}$. The simulant was concentrated down to three drums. Table 45 gives the volume, density and weight of the TLP simulant and the three drums of concentrate.

Table 45. TLP Simulant and Concentrate

\begin{tabular}{|l|l|l|l|}
\hline TLP Simulant & Volume & Density* & Weight \\
\hline TLP Supplied Simulant & $1,706.6 \mathrm{~L}(450.8 \mathrm{gal})$ & $1.135 \mathrm{~kg} / \mathrm{L}$ & $1,937 \mathrm{~kg}$ \\
\hline Drum \# 1 concentrate (\#03-254) & $194.1 \mathrm{~L} \quad(51.3 \mathrm{gal})$ & $1.362 \mathrm{~kg} / \mathrm{L}$ & $264.4 \mathrm{~kg}$ \\
\hline Drum \# 2 concentrate $(\# 03-257)$ & $193.3 \mathrm{~L} \quad(51.0 \mathrm{gal})$ & $1.366 \mathrm{~kg} / \mathrm{L}$ & $264.0 \mathrm{~kg}$ \\
\hline Drum \# 3 concentrate $(\# 03-255)$ & $180.1 \mathrm{~L} \mathrm{(47.7} \mathrm{gal)}$ & $1.370 \mathrm{~kg} / \mathrm{L}$ & $247.4 \mathrm{~kg}$ \\
\hline
\end{tabular}

* Temperature at $20^{\circ} \mathrm{C}$

A total of 1,139.1 liters (300.9 gal) of clean condensate (water) was removed from the simulant via evaporation to obtain 567.5 liters (149.9 gal) of concentrate with an average density of $1.365 \mathrm{~kg} / \mathrm{L}$ at $20^{\circ} \mathrm{C}$ for the three drums. The concentrate was transported to the Vitreous State Laboratory for further testing/processing for the RPP Project.

\subsubsection{Pilot TLP Discussion of Results}

\subsubsection{TLP Experimental Observations (Author-Adamson)}

The SIPP TLP evaporation Campaign III was conducted on Wednesday, 8 July, 2004 in SRNL's Engineering Development Laboratory. A total of 1,706.6 liters of TLP simulant feed was concentrated down to 567.5 liters in a continuous run. The evaporation process was completed without any glitch or equipment failure. However, as with TLP Campaign II, the Demister ${ }^{\circledR}$ instrumentation had to be purged during the campaign due to moisture in the lines causing inaccurate readings. 
As shown in Fig. 48, a small amount of foaming and frothing was witnessed throughout TLP campaign III. In fact, the amount of foaming/frothing before adding the defoamer was not much larger and the need of defoamer for the AY102+AP101+recycle mixture is questionable. The foaming/frothing maximum height above the liquid level was one foot or less. The picture depicts the simulant boiling inside the acrylic section of the evaporator pot.

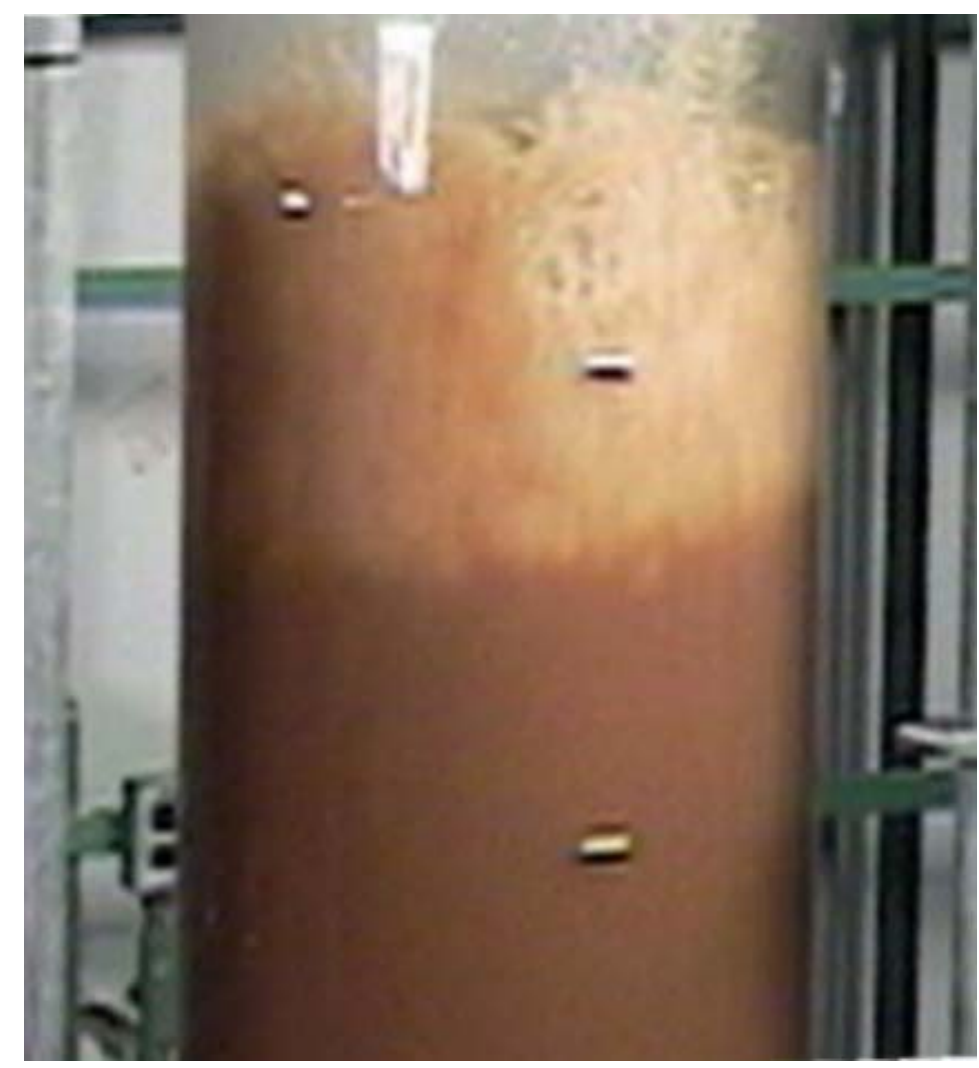

Figure 48. TLP Simulant Boiling in Clear Acrylic Section of Evaporator

The Campaign III TLP evaporation performed as well as the Campaign II operation, with respect to evaporation rates and times of operation. However, the evaporation during Campaign III was stopped earlier than required because a target density of $1.365 \mathrm{~kg} / \mathrm{L}$ at $20^{\circ} \mathrm{C}$ was used instead of the actual target of $1.397 \mathrm{~kg} / \mathrm{L}$, as determined by the OLI/ESP model (Duignan et al., 2004b). That is, the target was $1.365 \mathrm{~kg} / \mathrm{L}$, but this was at the evaporator operating conditions of $50^{\circ} \mathrm{C}$ and 1 psia. Since pilot-scale TLP bottoms density was being measured at laboratory ambient conditions, then the evaporation should have continued until the density was $1.397 \mathrm{~kg} / \mathrm{L}$ at $20^{\circ} \mathrm{C}$ and $1 \mathrm{~atm}$. The main impact of the early shut down was that the sodium concentration, expected to attain $8 \mathrm{M}$, was approximately 11\% under, i.e., 7.1 M. During Campaign II operation the pilot-scale TLP operation produced 523.1 liters of bottoms product at $8.2 \mathrm{M}$ (this value can be found in the penultimate column of Table 46 and the row entitled " $\mathrm{Na}$ (average in $\mathrm{M}$ )"), and, as stated in the preceding section, Campaign III produced 567.5 liters of bottoms at 7.1 M 
(see the third to the last column of Table 46). While the bottoms produced from each Campaign was kept separate when they were shipped to VSL for pilot-scale LAW melter testing, once the entire volume of bottoms is mixed together the resulting sodium concentration will be $7.6 \mathrm{M}$.

\subsubsection{TLP Waste Products (Author - Zamecnik)}

Table 46 shows the feed and bottoms concentrate compositions for both Campaigns II and III. The Campaign III concentrate composition was calculated by material balance and is compared to the material balance from Campaign II in the adjoining columns of the table. The Campaign II material balance results in a very good prediction of the concentrate composition, so the Campaign III material balance is also expected to be accurate. (Note that the Campaign II concentrate analysis from the microwave dissolution is not shown because these values have been determined to be suspect.) The Campaign III calculated concentrate is lower in $\mathrm{Na}$ and $\mathrm{Al}$, slightly higher in $\mathrm{K}$, and about the same in most of the other significant cations and anions. Therefore, the main difference in Campaign III was a lower concentration of $\mathrm{Na}$ and $\mathrm{Al}$, which would be present as $\mathrm{Na}^{+} \mathrm{Al}(\mathrm{OH})_{4}^{-}$. The sodium concentration in Campaign III was lower because the feed was only concentrated to a density of $1.365 \mathrm{~kg} / \mathrm{L}$, as explained in the preceding section. However, the aluminum would have been lower in Campaign III, than Campaign II, in any case because the feed concentration for Campaign III was lower.

Two measured values for the solids content of the Campaign III feed and concentrate are shown. As mentioned in Section 2.6.5.2, the accuracy of the analytical method for UDS is about $\pm 0.22 \mathrm{wt} \%$, both the feed and concentrate UDS values are less than the uncertainty. The bottoms values with the uncertainty are $0.07 \pm 0.22$ and $0.15 \pm 0.22 \mathrm{wt} \%$, which both include the estimated $0.28 \mathrm{wt} \%$. Therefore, the data are inconclusive and it cannot be determined if dissolution of solids occurred (which is indicated by the lack of increase in the UDS upon concentration). 
WSRC-TR-2004-00565, REVISION 0

SRNL-RPP-2004-00092, REVISION 0

Table 46. Comparison of Campaigns II and III TLP Compositions

\begin{tabular}{|c|c|c|c|c|c|c|}
\hline & \multicolumn{2}{|c|}{-------------Feed------------ } & \multicolumn{4}{|c|}{--------------------Bottoms-------------------- } \\
\hline & \multirow{2}{*}{$\begin{array}{c}\text { Campaign III } \\
\text { Measured }\end{array}$} & \multirow{2}{*}{$\begin{array}{c}\text { Campaign II } \\
\text { Measured }\end{array}$} & \multirow{2}{*}{$\begin{array}{l}\text { Campaign III } \\
\text { Measured }\end{array}$} & \multicolumn{3}{|c|}{ Campaign II } \\
\hline & & & & Calculated & Measured & Calculated \\
\hline Total Solids (wt $\% \pm 0.11)$ & $16.23 \quad 16.24$ & 16.24 & $\begin{array}{|ll|}40.06 & 40.04\end{array}$ & 40.42 & 41.30 & 40.54 \\
\hline Undissolved Solids (wt $\% \pm 0.22$ ) & $0.07 \quad 0.15$ & 0.09 & $0.15 \quad 0.07$ & 0.28 & 0.31 & 0.23 \\
\hline Supernate Solids (wt $\% \pm 0.13$ ) & 16.1016 .18 & 16.16 & $39.97 \quad 40.00$ & 40.25 & 41.12 & 40.41 \\
\hline Density $(\mathrm{kg} / \mathrm{L})$ & 1.134 & 1.136 & 1.368 & 1.368 & 1.384 & \\
\hline Volume (L) & 1706 & 1639 & & 568 & 523 & \\
\hline \multicolumn{7}{|l|}{ Concentrations in $\mathrm{mg} / \mathrm{kg}$ : } \\
\hline Sample Preparation & dissolution & filtered & & calc & filtered & calc \\
\hline $\mathrm{Ag}$ & $<50$ & $<0.5$ & & $<124$ & $<0.43$ & $<1.4$ \\
\hline $\mathrm{Al}$ & 2900 & 3319 & & 7220 & 8309 & 8537 \\
\hline B & & 80.7 & & & 198 & 208 \\
\hline $\mathrm{Ba}$ & $<2.0$ & $<0.04$ & & $<5.0$ & $<0.03$ & $<0.10$ \\
\hline $\mathrm{Ca}$ & $<6.6$ & 1.95 & & $<16$ & 2.57 & 5.02 \\
\hline $\mathrm{Cd}$ & $<2.4$ & $<0.04$ & & $<6.0$ & $<0.03$ & $<0.09$ \\
\hline $\mathrm{Ce}$ & $<128$ & $<15$ & & $<319$ & $<12$ & $<39$ \\
\hline Co & $<7.3$ & $<0.08$ & & $<18$ & $<0.06$ & $<0.19$ \\
\hline $\mathrm{Cr}$ & 82.8 & 79.0 & & 206 & 192 & 203 \\
\hline $\mathrm{Cu}$ & $<8.3$ & 0.143 & & $<21$ & 0.38 & 0.37 \\
\hline $\mathrm{Fe}$ & 21.0 & 0.492 & & 52.3 & 1.00 & 1.26 \\
\hline $\mathrm{K}$ & 9480 & 8389 & & 23603 & 23916 & 21579 \\
\hline $\mathrm{La}$ & $<116$ & $<0.33$ & & $<289$ & $<0.27$ & $<0.85$ \\
\hline $\mathrm{Li}$ & 1.0 & 4.09 & & 2.5 & 9.37 & 10.5 \\
\hline $\mathrm{Mg}$ & $<14$ & $<0.02$ & & $<35$ & $<0.02$ & $<0.06$ \\
\hline $\mathrm{Mn}$ & $<1.5$ & $<0.03$ & & $<3.7$ & $<0.02$ & $<0.08$ \\
\hline Mo & $<17$ & 4.79 & & $<41$ & 11.1 & 12.3 \\
\hline $\mathrm{Na}$ & 47684 & 51937 & & 118722 & 125723 & 133596 \\
\hline $\mathrm{Na}(\mathrm{AA})$ & & 54930 & & & 145954 & 141295 \\
\hline $\mathrm{Na}$ (average) & 47684 & 53433 & & 118722 & 135838 & 137445 \\
\hline $\mathrm{Na}$ (average in M) & 2.4 & 2.6 & & 7.1 & 8.2 & 8.3 \\
\hline $\mathrm{Nd}$ & $<43$ & $<0.71$ & & $<107$ & $<0.59$ & $<1.8$ \\
\hline $\mathrm{Ni}$ & $<10$ & 0.24 & & $<26$ & 0.56 & 0.62 \\
\hline $\mathrm{P}$ & 371 & 355 & & 924 & 574 & 912 \\
\hline $\mathrm{Pb}$ & $<114$ & 7.13 & & $<284$ & 17.3 & 18 \\
\hline $\mathrm{S}$ & 502 & 587 & & 1250 & 1377 & 1511 \\
\hline $\mathrm{Si}$ & 0 & 36.7 & & 0 & 113 & 94 \\
\hline $\mathrm{Sr}$ & 8.0 & 0.01 & & 19.92 & 0.06 & 0.04 \\
\hline $\mathrm{Ti}$ & $<23$ & $<0.10$ & & $<58$ & 0.25 & $<0.26$ \\
\hline $\mathrm{Zn}$ & $<61$ & 11.1 & & $<152$ & 27.3 & 29 \\
\hline $\mathrm{Zr}$ & $<8.0$ & $<0.14$ & & $<20$ & 0.749 & $<0.36$ \\
\hline bromide & $<61$ & $<88$ & & $<152$ & $<72$ & $<226$ \\
\hline chloride & 795 & 663 & & 1979 & 1821 & 1705 \\
\hline fluoride & 56.0 & 61.6 & & 139 & 28.2 & 159 \\
\hline formate & 521 & 551 & & 1297 & 1171 & 1417 \\
\hline nitrate & 37200 & 37500 & & 92619 & 90318 & 96461 \\
\hline nitrite & 12300 & 11884 & & 30624 & 28974 & 30569 \\
\hline oxalate & 374 & 342 & & 931 & 831 & 881 \\
\hline phosphate & 1220 & 1136 & & 3038 & 1741 & 2921 \\
\hline sulfate & 1570 & 1514 & & 3909 & 3699 & 3895 \\
\hline ammonium & 2730 & 879 & $<40$ & & $<72$ & \\
\hline
\end{tabular}




\subsubsection{Pilot TLP Conclusions}

\subsubsection{Experimental Observations (Author- Adamson)}

- The pilot-scale TLP evaporator operated as designed and concentrated the TLP feed simulant from a density of $1.135 \mathrm{Kg} / \mathrm{L}$ at $20^{\circ} \mathrm{C}$ and a sodium concentration of $2.4 \mathrm{M}$, to a final density of $1.365 \mathrm{Kg} / \mathrm{L}$ at $20^{\circ} \mathrm{C}$ and a sodium concentration of $7.1 \mathrm{M}$.

- A small amount of foaming was observed before and after the addition of antifoam agent to the evaporator. During the evaporation process, the foaming/froth height remained around $1 \mathrm{ft}(0.3 \mathrm{~m})$ or less for the duration of the campaign. That is significantly less than the $5 \mathrm{ft}(1.5 \mathrm{~m})$ distance to the lower demister pad.

- The two Demisters in series operated well with no significant differential pressure build-up across the Demisters during the TLP campaign III; there were no noticeable solids on the lower demister after the 11-hour test. However, condensation had to be purged from the instrumentation lines during the campaign.

\subsubsection{Waste Products Observations (Author-Zamecnik)}

- The composition of the products from the TLP matched what was expected from the mass balance.

- The concentration of aluminum in the TLP product from Campaign III was less than the result of Campaign II since the UF permeate contained less dissolved aluminum.

- No precipitation of additional solids beyond those in the feed was noted. 


\subsection{CONCLUSIONS}

\subsection{PILOT-SCALE FEP UNIT OPERATION}

- A total of about $1112 \mathrm{~kg}$ of FEP feed, at a density of $1.04 \mathrm{~g} / \mathrm{ml}$ and $0.6 \mathrm{M} \mathrm{Na}$, was concentrated down to $170 \mathrm{~kg}$, at a density of $1.26 \mathrm{~g} / \mathrm{ml}$ and $4.3 \mathrm{M} \mathrm{Na}$.

- The feed was evaporated under vacuum (1 psia $(6.9 \mathrm{kPa}))$ at moderate temperatures $\left(\sim 50^{\circ} \mathrm{C}\right)$ and the entire evaporation process proceeded as designed.

- The evaporator was designed to evaporate around $0.4 \mathrm{gpm}(1.5 \mathrm{lpm})$ of water and for this campaign the average evaporation rate was $0.52 \mathrm{gpm}$.

- No significant foaming was observed prior to the addition of antifoam agent. During the evaporation process, the froth height remained around $1 \mathrm{ft}(0.3 \mathrm{~m})$ that is significantly less than the $5 \mathrm{ft}(1.5 \mathrm{~m})$ distance to the lower demister pad.

- During start-up and unsteady conditions, significant flashing resulted in feed splashing on the lower demister pad. These conditions are associated with much larger vapor flux and vapor velocities at the interface.

- As seen in Campaign II, a large portion of the dissolved oxalate in the feed was precipitated, probably as sodium oxalate.

- The oxalate concentration in the FEP feed in Campaign III was less than that in Campaign II, which is consistent with the higher oxalate concentration in the Campaign I slurry.

- About $25-40 \%$ of the dissolved $\mathrm{Al}$ in the feed to the FEP evaporator precipitated during mixing of the recycles, assumed to occur because of localized regions of low $\mathrm{pH}$. Vigorous mixing when joining caustic and acidic recycle streams in the Plant Wash and Disposal System would help to minimize Al precipitation.

- The fraction of dissolved aluminum in Campaign III FEP concentrate (bottoms) was less than in Campaign II, resulting in more undissolved Al fed to the UFP.

\subsection{PILOT-SCALE UFP UNIT OPERATION}

- The average temperature-adjusted permeate flux when dewatering from $\sim 3$ to $\sim 24$ wt\% UDS was $0.011 \mathrm{gpm} / \mathrm{ft}^{2}$. The average temperature-adjusted permeate flux when dewatering from $\sim 4$ to $20 \mathrm{wt} \%$ UDS was $0.014 \mathrm{gpm} / \mathrm{ft}^{2}$. The average, temperatureadjusted permeate flux when dewatering from $\sim 4$ to $17 \mathrm{wt} \%$ UDS was $0.018 \mathrm{gpm} / \mathrm{ft}^{2}$.

- The turbidity of the permeate from dewatering and slurry washing, when measured directly after the sample was taken, was always lower 0.5 NTU. The Test Specification indicated a solids-free threshold of 2 NTU, therefore the measured values indicated the permeate was free of significant undissolved solids.

- Cleaning the filter with $2 \mathrm{M}$ nitric acid was sufficient to increase the $0.1 \mathrm{M} \mathrm{NaOH}$ permeate flux to an order of magnitude larger than that of waste flux. However, it was not sufficient to return the filter to pre-test flux levels. 
- The composition of the undissolved solid species in the washed sludges in Campaigns I-III were similar, as expected. The supernate portion species $\mathrm{Na}$ and $\mathrm{K}$ were higher in concentration in Campaigns II and III than in Campaign I due to the presence of the AP101 simulant.

- The aluminum in the washed slurry in Campaign III was higher due to there being more undissolved aluminum in the FEP concentrate than in Campaign II.

- Because of to higher undissolved aluminum in the FEP concentrate, the Campaign III permeate from the concentration step (IX feed) had less aluminum than in Campaign II.

- Ammonium in the first 0.01M NaOH UFP wash of Campaign III was mostly retained in the slurry, indicating that a slurry component has an affinity for ammonium. Most of the ammonium in the second wash was not retained.

\subsection{PILOT-SCALE CIX UNIT OPERATION}

- Pressure drops for the four cycles were low and the resin bed permeability was approximately the same as were measured for the 24-Inch Column Test (Fowley et al, 2004).

- The pressure drop across the columns for flow of simulant generally increased during the four cycles. [0.5 to 1.3 in. $\mathrm{H}_{2} 0$, Table 38]

- $\quad$ SuperLig ${ }^{\circledR} 644$ effectively removed cesium from simulated waste and the analytical ability to measure cesium concentrations in five-molar sodium did allow verification of the waste acceptance criterion of 50\% breakthrough from the lead column after 100 Bed Volumes of simulated waste were treated.

- SuperLig ${ }^{\circledR} 644$ effectively removed cesium from simulated waste, however, the analytical difficulty for measuring very low cesium concentrations in five-molar sodium did not allow verification of the waste acceptance criterion of $5.2 \mu \mathrm{g} / \mathrm{L}$ from the lag column after $100 \mathrm{BV}$ of simulated waste were treated. This fact was known before the start of testing.

- The fraction of the original cesium that was removed by the lead column decreased from 99.6\% for Cycle 1 to $98.2 \%$ for Cycle 4 .

- The masses of cesium in and out of the resin beds for all four cycles balanced to within measurement uncertainty.

\subsection{PILOT-SCALE TLP UNIT OPERATION}

- The pilot-scale TLP evaporator operated as designed and concentrated TLP feed simulant from a density of $1.135 \mathrm{Kg} / \mathrm{L}$ at $20^{\circ} \mathrm{C}$ and a sodium concentration of $2.4 \mathrm{M}$, to a final density of $1.365 \mathrm{Kg} / \mathrm{L}$ at $20^{\circ} \mathrm{C}$ and a sodium concentration of $7.1 \mathrm{M}$.

- A small amount of foaming was observed before and after the addition of antifoam agent to the evaporator. During the evaporation process, the foaming/froth height remained around $1 \mathrm{ft}(0.3 \mathrm{~m})$ or less for the duration of the campaign. That is significantly less than the $5 \mathrm{ft}(1.5 \mathrm{~m})$ distance to the lower demister pad. 
WSRC-TR-2004-00565, REVISION 0

SRNL-RPP-2004-00092, REVISION 0

- The two Demisters in series operated well with no significant differential pressure build-up across the Demisters and there were no noticeable solids on the lower Demister after the 11-hour test.

- The composition of the products from the TLP matched what was expected from the mass balance.

- The concentration of aluminum in the TLP product was less than the result of Campaign II, since the UF permeate contained less dissolved aluminum.

- No precipitation of additional solids beyond those in the feed was noted. 
WSRC-TR-2004-00565, REVISION 0 SRNL-RPP-2004-00092, REVISION 0

This page intentionally left blank. 


\subsection{FUTURE WORK}

As per the SIPP test specification (Longwell, 2003), the work discussed in this interim report is for Campaign III, which was only the third of four campaigns. Further work to be included in the immediate task will be Campaign IV, which will involve the testing of a different filter media, made by GKN. See the second footnote on page 16. Other work, not currently part of the SIPP scope, could include the testing of other simulated wastes, UFP filter cleaning, small and pilot-scale comparisons, etc.

Results of Campaign III indicate that aluminum precipitation will occur if the $\mathrm{pH}$ criterion for the PWD system remains at 12 . Additional investigation is needed to establish a $\mathrm{pH}$ criterion that will both minimize the soda hydroxide additions and prevent aluminum precipitation. This work should be conducted using prototypic plant mixing system since precipitation and dissolution kinetics are impacted by the degree of mixing employed in the plant. Additionally, if pulse jet systems are employed it is likely that localized regions of low $\mathrm{pH}$ will occur potentially causing aluminum to precipitate and not redissolve.

Details on any future work will be reported in the final report, which follows the completion of the last SIPP campaign, i.e., Campaign IV. 
WSRC-TR-2004-00565, REVISION 0 SRNL-RPP-2004-00092, REVISION 0

This page intentionally left blank. 
WSRC-TR-2004-00565, REVISION 0

SRNL-RPP-2004-00092, REVISION 0

\subsection{REFERENCES}

Adamson, D. J. (2004), “SIPP Pilot TLP,” Westinghouse Savannah River Company Notebook No. WSRC-NB-2004-00038. (began on 4 March)

Barnes, C. D. (2004) “OLI/ESP Modeling of the Semi-Integrated Pilot Plant for Estimate of Campaigns I-IV Simulant Volumes,” Westinghouse Savannah River Document No. WSRC-TR-2004-00403, Rev. 0. (August). [also SRNL-RPP-2004-00063]

Brooks, K. P., R. L. Myers, and K. G. Rappe (1997), “Bench-Scale Enhanced Sludge Washing and Gravity Settling of Hanford Tank C-106 Sludge,” Battelle Pacific Northwest National Laboratory Document No. PNNL-11432.

Calloway, Jr., T. B. (2004) "OLI Modeling Estimate of SIPP Run Times and Leaching Effectiveness,” Westinghouse Savannah River Company Document No. SRT-RPP-200300241, (24 March). [This is an inter-office memorandum from Calloway to Reid Peterson.]

Coleman, C. J., M. S. Hay, and K. B. Martin (2003), “Compositing and Characterization of Samples from Hanford Tank 241-AY-102/C-106,” Westinghouse Savannah River Document No. WSRC-TR-2003-00205, Rev. 0. (October) [also SRT-RPP-2003-00086]

Duignan, M. R. (2000a), “Final Report: Pilot-scale Cross-flow Ultrafiltration Test Using a Hanford Site Tank 241-AN-105 Waste Simulant - Envelope A + Entrained Solids," Westinghouse Savannah River Company Document No. BNF-003-98-0221, Rev. 0, (23 February).

Duignan, M. R. (2000b), "Final Report: Pilot-scale Cross-flow Ultrafiltration Test Using a Hanford Site Tank 241-AN-107 Waste Simulant - Envelope C + Entrained Solids + Strontium-Transuranic Precipitation,” Westinghouse Savannah River Company Document No. BNF-003-0226, Rev. 0. (24 March)

Duignan, M. R. (2003a). "Final Report: Pilot-scale cross-flow ultrafiltration test using a Hanford Site tank 241-AN-102 waste simulant.” Westinghouse Savannah River Document No. WSRC-TR-2003-00204, Rev. 0. (6 May) [also SRT-RPP-2003-00087]

Duignan, M. R. (2003b), “RPP-WTP Pilot Scale Cross Flow Filtration,” Westinghouse Savannah River Company Notebook No. WSRC-NB-2003-000149. (began on 12 June)

Duignan, M. R. (2003c). "Pilot-scale Integrated Pretreatment System Test,” (Task Technical and QA Plan) Westinghouse Savannah River Document No. WSRC-TR-200300338, Rev. 0. (4 August) [also SRT-RPP-2003-00165] 
WSRC-TR-2004-00565, REVISION 0

SRNL-RPP-2004-00092, REVISION 0

Duignan, M. R. (2004), "RPP-WTP Pilot-scale Cross-flow Ultrafiltration Test Procedure,” Westinghouse Savannah River Company Field Procedure No FP-975, (26 January)

Duignan, M. R., J. R. Zamecnik, and M. R. Williams (2004a). “Interim Report: RPPWTP Semi-integrated Pilot Plant - Campaign I,” Westinghouse Savannah River Document No. WSRC-TR-2004-00201, Rev. 0. (19 April) [also SRT-RPP-2004-00034]

Duignan, M. R., D. J. Adamson, C. Dan Barnes, M. D. Fowley, Z. N. Qureshi, J. L. Steimke, M. R. Williams, and J. R. Zamecnik (2004b). "Interim Report: RPP-WTP Semiintegrated Pilot Plant - Campaign II,” Westinghouse Savannah River Document No. WSRC-TR-2004-00478, Rev. 0. (10 September) [also SRT-RPP-2004-00075]

Edwards, R. E., (2003) "Semi-Integrated Pilot Plant (SIPP) Workshop - Meeting Minutes,” SRT-RPP-2003-00227, Westinghouse Savannah River Company, Aiken SC 29808 (5 November)

Eibling, R. E., and C. A. Nash (2001), "Hanford Waste Simulants Created to Support the Research and Development on the River Protection Project - Waste Treatment Plant," Westinghouse Savannah River Document No. WSRC-TR-2001-00338, Rev. 0. (22 February) [also SRT-RPP-2000-00017]

Fowley, M. D. (2004), “SIPP Pilot UFP,” Westinghouse Savannah River Company Notebook No. WSRC-NB-2004-00036. (began on 4 March)

Fowley, M. D., D. J. Adamson, C. A. Nash, M. A. Shadday, S. E. Aleman, J. L. Steimke, L. L. Hamm, M. L. Restivo, T. J. Steeper, and W. D. King (2004), "Ion Exchange Testing With SuperLig ${ }^{\circledR} 644$ Resin (U),” Westinghouse Savannah River Document No. WSRCTR-2003-00514, Rev. 0. (July) [also SRT-RPP-2003-00233]

Lumetta, G. J., M. J. Wagner, F. V. Hoopes, and R. T. Steele (1996), "Washing and Caustic Leaching of Hanford Tank C-106 Sludge,” Battelle Pacific Northwest National Laboratory Document No. PNNL-11381.

Lee, E. and M. Binsfield (2002). "Flowsheet Bases, Assumptions, and Requirements," Doc. No. 24590-WTP-RPT-PT-02-005, Rev. 1. (December)

Longwell. R. L. (2003), “Pilot Scale Integration of Pretreatment Process System,” Waste Treatment Plant - River Protection Project Document No. 24590-PTF-TSP-RT-03-002, Rev. 0. (5 June)

Poirier, M. R., P. R. Burket, J. L. Siler, and J. R. Zamecnik (2003), "Filtration, washing and leaching of a Hanford AY-102/C-106 sample,” Westinghouse Savannah River Company Document No. WSRC-TR-2003-00240, Rev. 0 (or SRT-RPP-2003-00110, Rev. 0). (June) 
Qureshi, Z. H. (2004), “SIPP Pilot FEP,” Westinghouse Savannah River Company Notebook No. WSRC-NB-2004-00034. (began on 4 March)

Russell, R. L., S. K. Fiskum, L. K. Jagoda, and A. P. Polaski (2003), “AP-101 Diluted Feed (Envelope A) Simulant Development Report,” Battelle Pacific Northwest Division Document No. PNWD-3248, Rev. 0 (February) [also WTP-RPT-057]

Stallings, M. E., M. R. Poirier, and R. E. Eibling (2003). “Task Technical and Quality Assurance Plan for AY-102/C-106 Simulant Ultrafiltration and Washing (Including AY-102/C-106 Simulant Development).” Westinghouse Savannah River Document No. WSRC-TR-2003-00217, Rev. 0. (31 May) [also SRT-RPP-2002-00235]

Steimke, J. L. (2002). "Pilot Scale Ion Exchange Testing with SuperLig ${ }^{\circledR} 639$ and SuperLig ${ }^{\circledR} 644$ Resin,” (Task Technical and QA Plan) Westinghouse Savannah River Document No. WSRC-TR-2001-00340, Rev. 0. (6 February) [also SRT-RPP-200100116]

Townson, P. S. (2002). “AY-102/C-106 Simulant Pilot Scale Ultrafiltration and Washing Test Specification,” Waste Treatment Plant - River Protection Project Document No. 24590-PTF-TSP-RT-02-015, Rev. 0. (15 November)

Zamecnik, J. R., P. R. Burket, R. E. Eibling, and M. R. Poirier (2004), “Tank 241-AY102 Simulant development, ultrafiltration, and washing,” Westinghouse Savannah River Company Document No. WSRC-TR-2003-00547, Rev. 0 (or SRT-RPP-2003-00240, Rev. 0). (March) 
WSRC-TR-2004-00565, REVISION 0 SRNL-RPP-2004-00092, REVISION 0

This page intentionally left blank. 
WSRC-TR-2004-00565, REVISION 0

SRNL-RPP-2004-00092, REVISION 0

\title{
APPENDIX A: WTP DISCUSSION ON PRETREATMENT FACILITY WASTE PROCESSING RESIDENCE TIMES
}

----- Forwarded by Bond Calloway/WSRC/Srs on 03/16/2004 07:22 PM -----

\author{
"Harty, David"

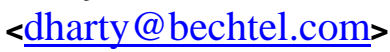 \\ 03/16/2004 06:11 PM
}

To: Bond Calloway bond.calloway@srs.gov

Cc: Harty, David dharty@bechtel.com

Olander, Alan arolande@bechtel.com

Slaathaug, Eric ejslaath@bechtel.com

Lehrman, scott slehrman@bechtel.com

Peterson, Reid rpeterso@bechtel.com

Sundar, Parameshwaran S pssundar@bechtel.com

Tadlock, Johnjatadloc@bechtel.com

\section{SUBJECT: PLANT RESIDENCE TIME DISCUSSION}

$<<\mathrm{PT}$ Flow Diagram Rev 1b.vsd $>>\quad<$ PT Tank Vol_App B.doc $>>$

Attached (they have been removed) above are the handouts from the discussion this afternoon. Four different holdup times were discussed during the meeting.

Note: The defined production rates for SIPP are 6.0 MT IHLW/day and $80 \mathrm{MT}$ ILAW/day. Steady-state flowrates defined below are from the WTP Steady-State Flowsheet.

I. Holdup Time For Permeate After Ultrafiltration.

Two permeate vessel tank volumes $(21,800$ each) plus the CXP Feed Vessel available volume $(78,000$ batch $-25,000$

contingency) are used for a total available holdup volume of 96,600 gallons. At a steady-state permeate flowrate of 14.8 gpm, the residence time is $96,600 /(14.8 * 60)=109$ hours ( $\sim 41 / 2$ days $)$. 
WSRC-TR-2004-00565, REVISION 0

SRNL-RPP-2004-00092, REVISION 0

II. Holdup Time For Blended Feed With Concentrated Recycles Prior To Ultrafiltration.

Two UFP Preparation vessel tank volumes $(48,300$ each) are used for a total available holdup volume of 96,600 gallons. At a steady-state feed to UFP Feed vessels of $16.3 \mathrm{gpm}$, the residence time is $96,600 /(16.3 * 60)=99$ hours ( 4 days). This value is overly conservative and the residence time of blending the concentrated recycles with the blended feed is dependent on the dynamic nature of recycle generation, feed blending, and the processing of feed form the preparation vessel to the feed vessel. If the online UFP Feed vessel is considered full and the UF Prep vessels are both filled with blended feed and concentrated recycle, then the residence time of $\sim 4$ days will result. Further consideration suggests that the residence time using one UFP Preparation vessel tank volume should be used. This would give a residence time of $48,300 /(16.3 * 60)=49$ hours ( 2 days). Use the second value.

III. Holdup Time for Blended Recycles (HLW-SBS with UFP recycles) Prior to Evaporation.

Two evaporator feed tanks with an available volume of (50,000 batch - 10,000 contingency) are used for a total available holdup volume of 80,000 gallons. At a steadystate evaporator feed flowrate of $18.5 \mathrm{gpm}$, the residence time is $80,000 /(18.5 * 60)=72$ hours ( 3 days).

IV. Holdup Time For Recycles in the Evaporator.

One evaporator with a holdup volume of 5500 gallons is used. At a steady-state concentrate flowrate of $1.8 \mathrm{gpm}$, the residence time is 5500/(1.8*60) $=51$ hours ( 2 days). It was noted that without the leaching process, there is no recycle leachate. Inclusion of the leaching recycle reduces the residence time in the evaporator because the concentrate flow rate is higher.

Comments are welcome.

Dave Harty

Pretreatment Process Operations, 371-3360 
WSRC-TR-2004-00565, REVISION 0

SRNL-RPP-2004-00092, REVISION 0

\section{APPENDIX B: CAMPAIGN III SAMPLING MATRIX}

This appendix contains a list of all the samples taken during Campaign III. Because of the large number of samples and the importance to document those that were taken, the tables have been put in a form, which is condensed. However, the tables are readable and their placement here should be useful to those wanting to better understand the data discussed in the body of this report.

\section{Notes on the following Sample Tables}

Time Dep Sample

Explanation of analyses:

$$
\mathrm{pH} \text { pH }
$$

ICPES includes all elements present that can be analyzed by ICPES

ICPMS sludges

supernates

ICA Ion chrom - anions

\section{Cs, Rb, Bi, Y, Pr}

$\mathrm{Cs}, \mathrm{Rb}$

normal suite

es are to be observed daily for about 5 days for evidence of precipitation of solids. Time dependent sample. These samples are to be observed daily for about 5 days
If solids form, they will be analyzed by XRD. A filtrate blank will also be analyzed.

Explanation of analyses:

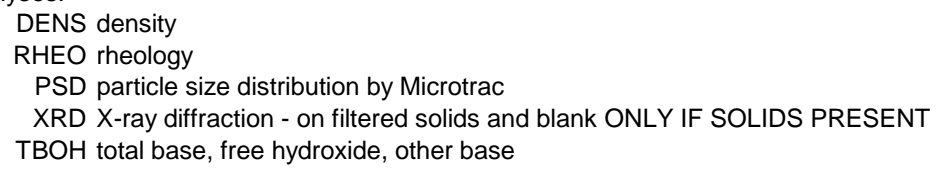

DISMW: microwave acid dissolution

DISPER: sodium peroxide fusion / HCL uptake dissolution 
WSRC-TR-2004-00565, REVISION 0

SRNL-RPP-2004-00092, REVISION 0

\section{$\underline{\text { Note on Analysis of Sample Compositions }}$}

All analyses except total solids, undissolved solids, supernate solids, density, and rheological measurements were performed by the WSRC/SRNL Analytical Development Section per the task plan specifications. The aforementioned analyses were performed by the SRNL Immobilization Technology Section at the Aiken County Technology Laboratory. Table B1 shows the methods used for analysis of each of the species measured.

Table B1 Analysis Methods Used

\begin{tabular}{|c|c|c|}
\hline Analysis or Preparation & Analytical Method & Preparation Method \\
\hline Slurry Sample Dissolution & \multicolumn{2}{|c|}{$\begin{array}{c}\text { Microwave acid (MW) or } \\
\text { Sodium peroxide (PER) fusion/ } \mathrm{HCl} \text { uptake }\end{array}$} \\
\hline $\begin{array}{r}\text { Ag, Al, B, Ba, Ca, Cd, Ce, Co, Cr, } \\
\mathrm{Cu}, \mathrm{Fe}, \mathrm{La}, \mathrm{Li}, \mathrm{Mg}, \mathrm{Mn}, \mathrm{Mo}, \mathrm{Nd}, \mathrm{P}, \\
\text { Pb, Re, S, Si, Sr, Ti, W, Zn, Zr }\end{array}$ & ICPES & both \\
\hline $\mathrm{Na}$ & ICPES, also AA & MW \\
\hline $\mathrm{Ni}$ & ICPES & MW \\
\hline $\mathrm{B}$ & ICPES & PER \\
\hline $\mathrm{K}$ & AA, also ICPES & MW \\
\hline $\mathrm{Cs}$ & ICPMS, also AA & MW \\
\hline $\mathrm{Bi}, \mathrm{Pr}, \mathrm{Rb}, \mathrm{Y}$ & ICPMS & MW \\
\hline $\begin{array}{r}\text { bromide, chloride, fluoride, formate, } \\
\text { nitrate, nitrite, phosphate, oxalate, } \\
\text { sulfate }\end{array}$ & Anion Chromatography (IC) & none \\
\hline acetate, citrate & Ion exclusion chromatography & none \\
\hline ammonium & Cation Chromatography & none \\
\hline $\begin{array}{r}\text { organic carbon, inorganic carbon } \\
\text { (TIC) }\end{array}$ & carbon analyzer & micronization \\
\hline "total” oxalate & $\begin{array}{l}\text { calculated from organic carbon } \\
\text { minus formate }\end{array}$ & none \\
\hline carbonate & $\begin{array}{l}\text { calculated from TIC, } \\
\text { also titration }\end{array}$ & none \\
\hline total base, free hydroxide, other base & titration & precipitation of carbonate \\
\hline total acid & titration & none \\
\hline $\begin{array}{r}\text { total solids, dissolved solids (solids } \\
\text { in supernate fraction of slurry) }\end{array}$ & halogen lamp solids analyzer & none \\
\hline undissolved solids & $\begin{array}{l}\text { calculated from total and supernate } \\
\text { solids }\end{array}$ & none \\
\hline density & $?$ & none \\
\hline rheology & Haake rheometer & none \\
\hline
\end{tabular}


WSRC-TR-2004-00565, REVISION 0

SRNL-RPP-2004-00092, REVISION 0

Table B2 Campaign III Sample Matrix (1 of 4 pages)

\begin{tabular}{|c|c|c|c|c|c|c|c|c|c|c|c|c|c|c|c|c|c|c|c|c|c|c|c|c|}
\hline & & & & & ADS & Sample & Sample & Sample & 2 & 3 & 4 & 5 & 6 & 7 & 8 & 9 & 10 & 11 & 12 & 13 & 14 & 15 & 16 & 17 \\
\hline \multirow{38}{*}{ RECYCLE BLE } & RIFNDING & & & Sample & Travel Copy & Location & Vol mL & & $\mathrm{pH}$ & & ICPMS & & & & & TS & UDS & DENS & RHEO & PSD & XRD & TB.. & TACID & TICITOC \\
\hline & BLENDING & & Campaign II UF Wash Water & & & & & & & & & & & & & & & & & & & & & \\
\hline & & & $\begin{array}{r}\text { HLW SBS } \\
\text { HLW Vessel Vent (PVP) }\end{array}$ & & & & & & - & & & & - & & & - & & & & & & & & \\
\hline & & & & & & & & & -2 & & & & 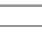 & & & - & & & & & & & & \\
\hline & & & $\begin{array}{l}\text { Campaign II Caustic Flushes } \\
\text { Campaing IUF Acid Cleaninins }\end{array}$ & & & & & & D & & & & Z & & & D & & & & & & & & \\
\hline & & & 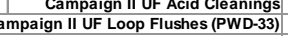 & & & & & & - & & & & - & & & - & & & & & & & & \\
\hline & Campaign II Cs IX & cle: Displacement Soluti & ion Displaced by Pre-Eution Rinse (2) & NO ANALYSIS & & & & & 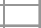 & & & & & & & 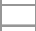 & & & & & & & & \\
\hline & Campaign II Cs IX & ycle: Post-Elution Rinse D & $\begin{array}{l}\text { Displaced + Regeneration Solutitin (5) } \\
\text { coMBBNED RECYCLES }\end{array}$ & NO ANALYSIS & & & & & - & & & & & & & 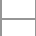 & & & & & & & & \\
\hline & & PWD & Generation of Synthetic Recyc & & & & & & 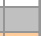 & & & & {[} & & & ב & & & & & & & & \\
\hline & & & HLW Canister Decon & NO SAMPLE & & & & & 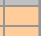 & & & & 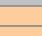 & & & - & & & & & & & & \\
\hline & & & $\begin{aligned} \text { Dl water } \\
\mathrm{NaOH} \text { 50utto }\end{aligned}$ & & & & & & 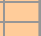 & & & & & & & - & & & & & & & & \\
\hline & & & NaNO3 (kg) & & & & & & 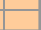 & & & & 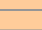 & & & 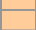 & & & & & & & & \\
\hline & & & HLW Vessel Vent (PVP) & NO SAMPLE & & & & & 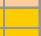 & & & & 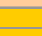 & & & 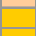 & & & & & & & & \\
\hline & & & $\begin{aligned} \text { Dl water } \\
\mathrm{NaOH} 5 \text { 5umto }\end{aligned}$ & & & & & & - & & & & & & & 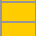 & & & & & & & & \\
\hline & & & Combined Canister Decon + PVP & & & & & & - & & & & - & & & - & & & & & & & & \\
\hline & & UF LO & oop Flush (PWD-33) from Campaign UI & NO SAMPLE & & & & & 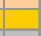 & & & & & & & 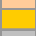 & & & & & & & & \\
\hline & & & Combining of Recycles & & & & & & & & & & & & & & & & & & & & & \\
\hline & & PWD-44 & & C3-PWD-44-ARC & NONE & mixing tank & 50 & & 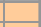 & & & & & & & 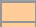 & & & & & & & & \\
\hline & & $\begin{array}{l}\text { Add HLV } \\
\text { Add HL }\end{array}$ & 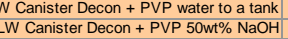 & & & & & & 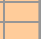 & & & & & & & 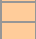 & & & & & & & & \\
\hline & & & Add HLW Canister Decon NaNO3 $(\mathrm{kg})$ & & & & & & 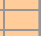 & & & & & & & 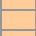 & & & & & & & & \\
\hline & & & $\begin{array}{l}\text { Add HLW SBS to tank } \\
\text { d }\end{array}$ & & & & & & 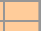 & & & & & & & 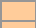 & & & & & & & & \\
\hline & & & $\begin{array}{l}\text { Add UF Loop Flush PWD-33 } \\
\text { Sum }\end{array}$ & & & & & & - & & & & & & & - & & & & & & & & \\
\hline & & PWD-15 & 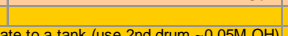 & C3-PWD-15-ARC & NONE & mixing tank & 250 & & & & & & & & & 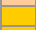 & & & & & & & & \\
\hline & & Campaign II Wash Permec & ate to a tank (use 2nd drum,, $0.05 \mathrm{O}$ OH) & & & & & & 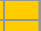 & & & & & & & E & & & & & & & & \\
\hline & & Add & 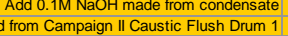 & & & & & & 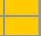 & & & & & & & - & & & & & & & & \\
\hline & & & d trom Campaign II C Cuustic Flush Drum 2 & & & & & & 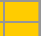 & & & & & & & 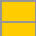 & & & & & & & & \\
\hline & & & Sum & & & & & & & & & & & & & 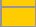 & & & & & & & & \\
\hline & & PWD-16 & & C3-PWD-16-ARC & NONE & mixing tank & 250 & & 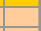 & & & & 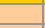 & & & 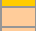 & & & & & & & & \\
\hline & & dd Campaign II Wash Perm & neate to a tank (use $1 \mathrm{st}$ drum,, $0.1 \mathrm{O} \mathrm{OH}$ ) & & & & & & & & & & & & & - & & & & & & & & \\
\hline & & Add from & 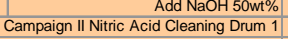 & & & & & & 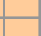 & & & & - & & & - & & & & & & & & \\
\hline & & Add from & Campaign II Nitric Acid Cleaning Drum 2 & & & & & & & & & & & & & & & & & & & & & \\
\hline & & Add from & Campaign II Nitric Acid Cleaning Drum 3 & & & & & & 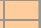 & & & & E & & & E & & & & & & & & \\
\hline & & Combine Recycles in (FE & EP Feed) Tank & C3-FEP-FEED-ACTL & NONE & mixing tank & 50 & & 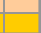 & & & & - & 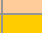 & 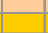 & TS & UDS & DENS & & & & & & \\
\hline & & & Add PWD-44 to tank & C3-FEP-FEED-ARC & NONE & mixing tank & 250 & & & & & & & & & & & & & & & & & \\
\hline & & & $\begin{array}{l}\text { Add PWD-15 to tank } \\
\text { Add PWD-16totank }\end{array}$ & $\begin{array}{l}\text { C3-FEP-FEED-ANTF } \\
\text { C3-FEP-FED-ADS }\end{array}$ & $\begin{array}{l}\text { NONE } \\
42007 \\
4207-3\end{array}$ & mixing tank & 100 & & - & & & & $1 \mathrm{CC}$ & & & & & & & & & & & \\
\hline & & & $\begin{array}{l}\text { Add PWDD-16 to tank } \\
\text { IX Recycles }\end{array}$ & & & & & & & & & & $\mathrm{ICC}$ & & & & & & & & & & & \\
\hline & & & $\begin{array}{r}\text { Sum } \\
\text { RID trom Campion }\end{array}$ & C2-RLD-FNAL-AGED & 42072 & & 100 & & & & & & ICC & & & & & & & & & & & \\
\hline \multirow{2}{*}{\multicolumn{2}{|c|}{ FEP EVAPORATOR }} & & RLD from Campaign II & C2-RLD-FINAL-AGED & $4<01 / 2$ & & 100 & & & & & & $\mathrm{KC}$ & & & & & & & & & & & \\
\hline & & & $\begin{array}{l}\text { Starting Volume } \\
\text { Atter all antifoam additions }\end{array}$ & C3-FEP-B-START-ARC & & bettoms tank & & & & & & & & & & & & & & & & & & \\
\hline & & Bottoms & After all antifoam additions & $\begin{array}{l}\text { C3-FEP-B-BSTART-ARC } \\
\text { C3-FEP-B-START-ANTFF }\end{array}$ & NONE & $\begin{array}{l}\text { Dottoms tank } \\
\text { bottoms tank }\end{array}$ & 100 & & - & & & & - & & & - & & & & & & & & \\
\hline & & Bottoms & At $-200 \mathrm{~L}$ fed & C3-FEP-B-200-ARC & NONE & bottoms tank & 100 & & 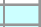 & & & 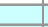 & 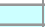 & & & 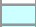 & & & & & & & & \\
\hline & & Bottoms & At $-400 \mathrm{~L}$ fed & C3-FEP-B-400-ARC & NONE & bottoms tank & 100 & & 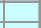 & & & & & & & 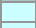 & & & & & & & & \\
\hline & & $\begin{array}{l}\text { Bottoms } \\
\text { Bottoms }\end{array}$ & At $-600 \mathrm{~L}$ fed & $\begin{array}{l}\text { C3-FEP-B-600-ARC } \\
\text { CS-BEP-B-80-ARC }\end{array}$ & NONE & $\begin{array}{l}\text { bottoms tank } \\
\text { pottoms tank }\end{array}$ & 100 & & - & & & & & & & 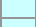 & & & & & & & & \\
\hline & & $\begin{array}{l}\text { Bottoms } \\
\text { Bottoms }\end{array}$ & $\begin{array}{l}\text { Evaporation completed } \\
\text { Eved }\end{array}$ & $\begin{array}{l}\text { C3-EPPB-8-800-ARC } \\
\text { C3-FEP-B-END-ACTL }\end{array}$ & $\begin{array}{l}\text { NONE } \\
\text { NONE }\end{array}$ & $\begin{array}{l}\text { bottoms tank } \\
\text { bottoms tank }\end{array}$ & $\begin{array}{l}100 \\
50\end{array}$ & & & & & & & & & TS & UDS & DENS & & & & & & \\
\hline & & & & $\begin{array}{l}\text { C3-FEP-B-END-WET } \\
\text { C3-FEP-B-END-MW }\end{array}$ & $\begin{array}{l}42088 \\
42089\end{array}$ & $\begin{array}{l}\text { bottoms tank } \\
\text { bottoms tank }\end{array}$ & $\begin{array}{l}50 \\
50\end{array}$ & DISMW & & & ICPMS & ICA & ICC & & AAK & & & & & & & TBOH & & TIC/TOC \\
\hline & & & & $\begin{array}{l}\text { C3-FEP-B-END-PER } \\
\text { CSFP-NOF }\end{array}$ & 42090 & bottoms tank & 50 & DISPER & & ICPES & & & & & & & & & & & & & & \\
\hline & & & & $\begin{array}{l}\text { C3-FEP-B-BND-AAC } \\
\text { C3-FEP-BENDANTF }\end{array}$ & $\begin{array}{l}\text { NNONE } \\
\text { NONE }\end{array}$ & $\begin{array}{l}\text { bottoms tank } \\
\text { bottoms tank }\end{array}$ & $\begin{array}{l}500 \\
100\end{array}$ & & & & & & & & & & & & & & & & & \\
\hline & & Primary Condensate & & C3-FEP-PC-END-ARC & NONE & & & & & & & & & & & & & & & & & & & \\
\hline & & Secondary Condensate & Composite & C3-FEP-SC-END-ARC & NONE & & 100 & & & & & & & & & & & & & & & & & \\
\hline \multirow{2}{*}{\multicolumn{4}{|c|}{$\begin{array}{l}\text { COMBINE ALL FEP CONDENSATES } \\
\text { Combined FEP Condensate }\end{array}$}} & & & & & & & & & & & & & & & & & & & & & \\
\hline & & & & $\begin{array}{l}\text { C3-RLDD-FOM-FEP-ARC } \\
\text { C3-RL-ROM-FEP-ADS }\end{array}$ & $\begin{array}{l}\text { NNNE } \\
42093\end{array}$ & & $\begin{array}{l}1000 \\
100\end{array}$ & & & & & & ICC & & & & & & & & & & & \\
\hline
\end{tabular}


WSRC-TR-2004-00565, REVISION 0

SRNL-RPP-2004-00092, REVISION 0

Table B2 Campaign III Sample Matrix (2 of 4 pages)

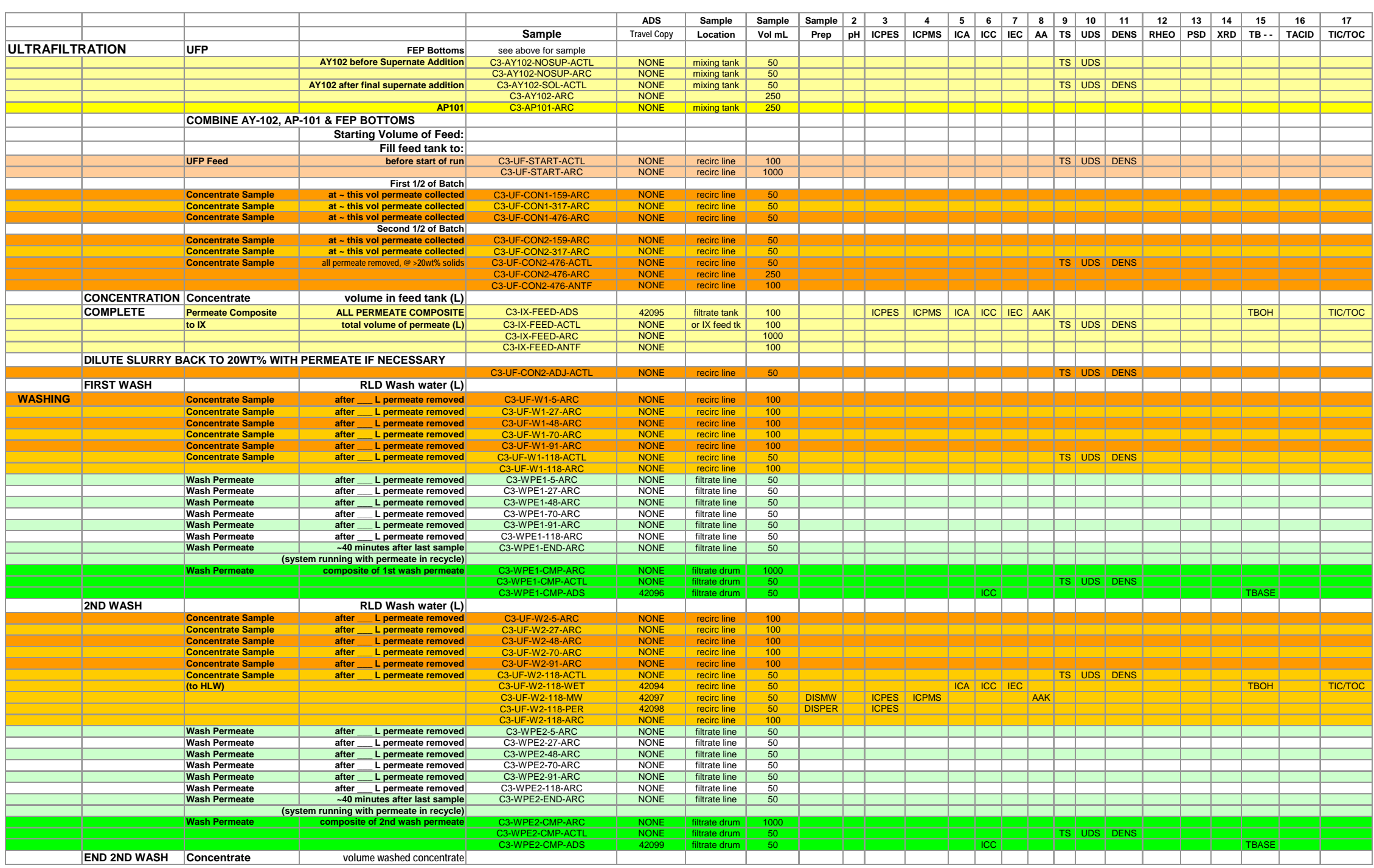


WSRC-TR-2004-00565, REVISION 0

SRNL-RPP-2004-00092, REVISION 0

Table B2 Campaign III Sample Matrix (3 of 4 pages)

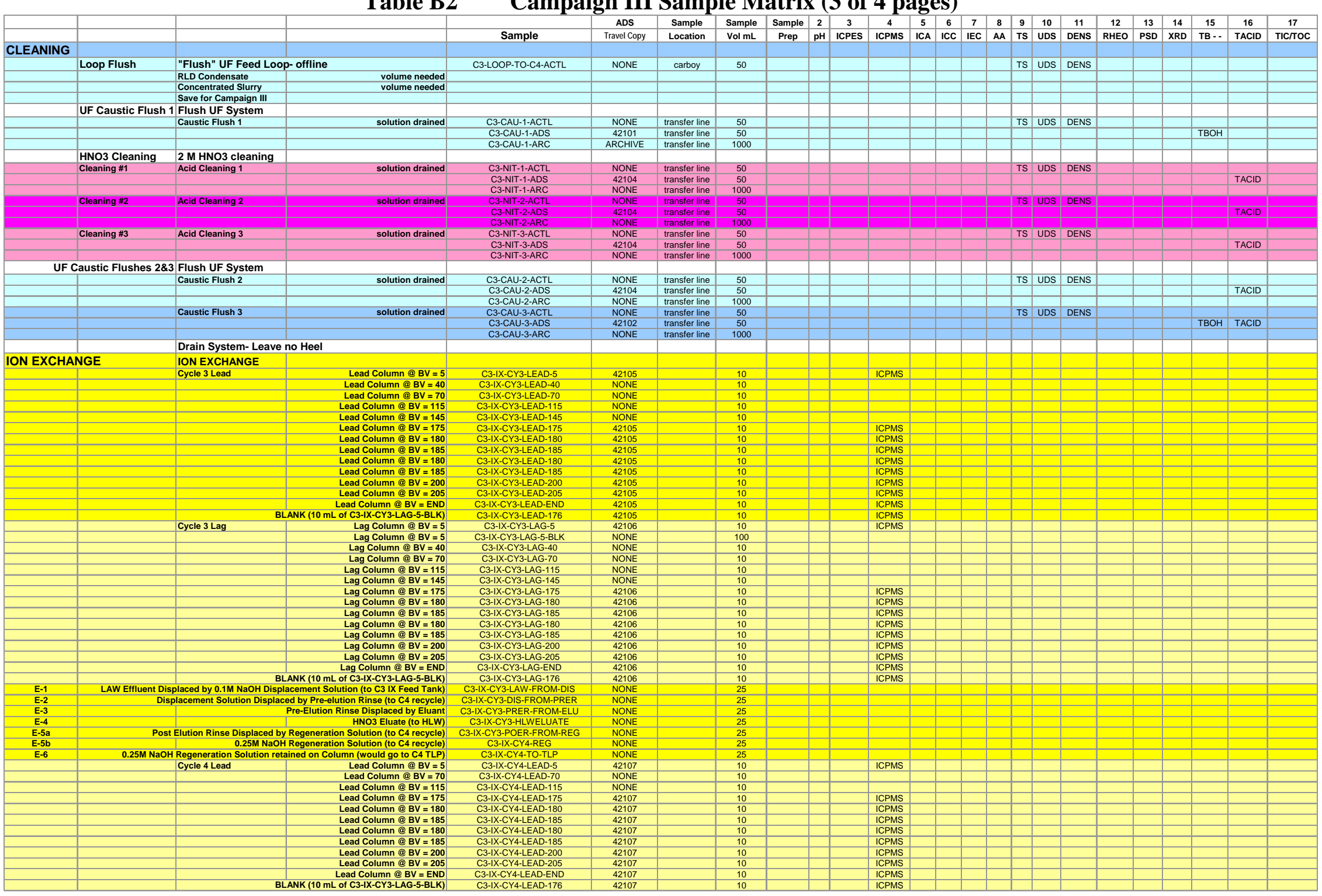


WSRC-TR-2004-00565, REVISION 0

SRNL-RPP-2004-00092, REVISION 0

\section{Table B2 Campaign III Sample Matrix (4 of 4 pages)}

\begin{tabular}{|c|c|c|c|c|c|c|c|c|c|c|c|c|c|c|c|c|c|c|c|c|c|c|c|c|}
\hline & & & & Sample & $\begin{array}{c}\text { ADS } \\
\text { Travel Copy }\end{array}$ & $\begin{array}{l}\text { Sample } \\
\text { Location }\end{array}$ & $\begin{array}{l}\text { Sample } \\
\text { Vol mL }\end{array}$ & $\begin{array}{c}\text { Sample } \\
\text { Prep }\end{array}$ & \begin{tabular}{|c|}
2 \\
$\mathrm{pH}$ \\
\end{tabular} & \begin{tabular}{c|}
3 \\
ICPES
\end{tabular} & $\begin{array}{c}4 \\
\text { ICPMS }\end{array}$ & \begin{tabular}{|c|}
5 \\
ICA
\end{tabular} & $\begin{array}{c}6 \\
\mathrm{ICC}\end{array}$ & \begin{tabular}{c|}
7 \\
IEC
\end{tabular} & $\begin{array}{c}8 \\
\mathrm{AA} \\
\end{array}$ & \begin{tabular}{|c|}
9 \\
TS
\end{tabular} & $\begin{array}{c}10 \\
\text { UDS }\end{array}$ & $\begin{array}{c}11 \\
\text { DENS }\end{array}$ & \begin{tabular}{c|}
12 \\
RHEO
\end{tabular} & \begin{tabular}{|l|l|}
13 \\
PSD
\end{tabular} & \begin{tabular}{|c|}
14 \\
XRD
\end{tabular} & $\begin{array}{c}15 \\
\text { TB.. }\end{array}$ & $\begin{array}{c}16 \\
\text { TACID }\end{array}$ & $\begin{array}{c}17 \\
\text { TIC/TOC } \\
\end{array}$ \\
\hline & & Cycle 4 Lag & $\begin{array}{l}\text { Lag Column @ BV }=5 \\
\text { Lag Column @B B }=70\end{array}$ & $\begin{array}{l}\text { C3-1X-CY4-AG-5 } \\
\text { C3-IX-CY4-LAG-70 }\end{array}$ & $\begin{array}{l}42108 \\
\text { NONE }\end{array}$ & & $\begin{array}{l}10 \\
10\end{array}$ & & & & & & & & & & & & & & & & & \\
\hline & & & Lag Column@ BV = 115 & C3-IX-CY4-LAG-115 & NONE & & 10 & & 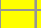 & & & & & & & & & & & & & & & \\
\hline & & & Lag Column @ BV = 175 & C3-IX-CY4-LAG-175 & NONE & & 10 & & 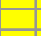 & & ICPMS & & 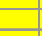 & & & 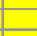 & & & & & & & & \\
\hline & & & $\begin{array}{l}\text { Lag Column @ BV }=180 \\
\text { B B }\end{array}$ & $\begin{array}{l}\text { C3-1-CY4-LAG-180 } \\
\text { C3-|-X-CY4-LA-185 }\end{array}$ & 42108 & & 10 & & 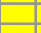 & & ICPMS & & ${ }^{2}$ & & & 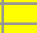 & & & & & & & & \\
\hline & & & $\begin{array}{l}\text { Lag Column @ BV V } 185 \\
\text { Lag Column @ BV }=180\end{array}$ & $\begin{array}{l}\text { C3-I-X-YY4-LAA-185 } \\
\text { C3-X-CY4-LG-180 }\end{array}$ & $\begin{array}{l}42108 \\
42108\end{array}$ & & $\begin{array}{l}10 \\
10\end{array}$ & & 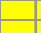 & & $\begin{array}{l}\text { IPPMS } \\
\text { IIPMS }\end{array}$ & & - & & & - & & & & & & & & \\
\hline & & & $\begin{array}{l}\text { Lag coumnn } @ B V=180 \\
\text { Lag Column } @ B V=185\end{array}$ & $\begin{array}{l}\text { C3-X-CYCA4-LAG-180 } \\
\text { C3-X-CY4-LAG-185 }\end{array}$ & 42108 & & 10 & & - & & $\begin{array}{l}\text { ICPMS } \\
\text { ICPMS }\end{array}$ & & & & & - & & & & & & & & \\
\hline & & & Lag column $@ B V=200$ & 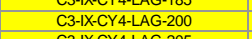 & 42108 & & 10 & & $\square$ & & ICPMS & & 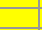 & & & - & & & & & & & & \\
\hline & & & & C3-IX-CY4-LAG-205 & & & 10 & & & & & & & & & & & & & & & & & \\
\hline & & & $\begin{array}{l}\text { Lag Column @ BV = END } \\
\text { ANK (10 mL of C3-1X-CY3-LAG-5-BLK }\end{array}$ & C3-IX-CY4-LAG-END & 42108 & & 10 & & 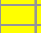 & & ICPMS & & 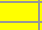 & & & 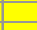 & & & & & & & & \\
\hline E-1 & LAW Effluent Displa & ced by $0.1 \mathrm{M} \mathrm{NaOH}$ Displace & Aement Solution (to C C I IX Feed Tank) & $\begin{array}{l}\text { C3-I-CYCY4-LAG-16-16-DIS } \\
\text { C3-I-CY4-LAW-FOM-DIS }\end{array}$ & $\begin{array}{l}42108 \\
\text { NONE }\end{array}$ & & $\frac{10}{25}$ & & - & & & & & & & & & & & & & & & \\
\hline$E-2$ & Displa & cement Solution Displaced & by Pre-elution Rinse (to C4 recycle) & C3-IX-CY4-DIS-FROM-PRER & NONE & & 25 & & 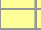 & & & & & & & & & & & & & & & \\
\hline$E-3$ & & & re-Elution Rinse Displaced by Eluant & C3-X-CY4-PRER-FROM-ELU & NONE & & 25 & & - & & & & 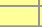 & & & 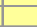 & & & & & & & & \\
\hline $\begin{array}{c}E-4 \\
E-5 a\end{array}$ & & Tion Pinse Dicoloced hy Pe & HNO3 Eluate (to HLW) & C3-1X-CY4-HLWELUATE & NONE & & 25 & & E & & & & ${ }^{-}$ & & & ${ }^{-}$ & & & & & & & & \\
\hline E-5a & Post El & $\begin{array}{l}\text { tion Rinse Displaced by Re } \\
0.25 \mathrm{M} \mathrm{NaOHRe}\end{array}$ & egenereration Solution (to C4 recycle) & $\begin{array}{l}\text { C3-1X-CY4-POER-FROM-REG } \\
\text { C3-1X-CYAREG }\end{array}$ & $\begin{array}{l}\text { NNNE } \\
\text { NONE }\end{array}$ & & 25 & & - & & & & - & & & 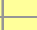 & & & & & & & & \\
\hline$E-6$ & $0.25 \mathrm{M} \mathrm{NaOHRe}$ & generation Solution retain & 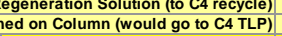 & $\begin{array}{l}\text { C3-1X-CY4-REG } \\
\text { C3-X-CY4-TO-TLP }\end{array}$ & NONE & & 25 & & - & & & & & & & & & & & & & & & \\
\hline & IX PRODUCTS & IX Product to TLP & IX PRODUCT & C3-X-PROD-ACTL & NONE & tank & 100 & & 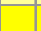 & & & & & & & TS & UDS & DENS & & & & & & \\
\hline & & & & C3-IX-PROD-ARC & NONE & $\operatorname{tank}$ & 500 & & 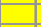 & & & & & & & & & & & & & & & \\
\hline & & & & C3-1X-PROD-ADS & 42110 & $\operatorname{tank}$ & 50 & & & & & & ICC & & & & & & & & & & & \\
\hline & & & & $\begin{array}{l}\text { C3-IX-PROD-ANTF } \\
\text { Analyze in Campaian N i f needed }\end{array}$ & NONE & tank & 100 & & & & & & & & & & & & & & & & & \\
\hline & & $\begin{array}{c}\text { Displacement solution } \\
\text { Post-elution rinse displa }\end{array}$ & $\begin{array}{l}n \text { displaced by pree-elution rinse }(E-2) \\
\text { aced by regeneration solution }(E-5)\end{array}$ & 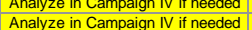 & & & & & & & & & & & & & & & & & & & & \\
\hline & & & $\begin{array}{r}\text { acea dy regenerauto solutron (E-5a) } \\
\text { Regeneration solution (E-5b) }\end{array}$ & Analyze in Campaign $\mathrm{N}$ if needed & & & & & & & & & & & & & & & & & & & & \\
\hline TLP EVA & ORATOR & & LAW Feed (IX product) & & & & & & 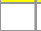 & & & & & & & 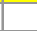 & & & & & & & & \\
\hline & & X0.25 NaOH Peoe & LAW SBS & & & & & & & & & & & & & 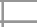 & & & & & & & & \\
\hline & & $\begin{array}{l}\text { IX } 0.25 \mathrm{M} \mathrm{N} \text { NoH Rege } \\
\text { Evaporator Feed Mixture }\end{array}$ & & & & & & & & & & & & & & & & & & & & & & \\
\hline & EVAPORATE & Evaporator Feed Mixture & & C3-TLP-FEED-ACTL & NONE & feed tank & 50 & & & & & & & & & TS & UDS & DENS & & & & & & \\
\hline & & & & $\begin{array}{l}\text { C3-TLL-FEED-WET } \\
\text { C3-TLP-FEED-MW }\end{array}$ & $\begin{array}{l}42111 \\
42112\end{array}$ & $\begin{array}{l}\text { feed tank } \\
\text { feed tank }\end{array}$ & $\begin{array}{l}50 \\
50\end{array}$ & DISMW & 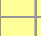 & ICPES & ICPMS & ICA & $\mathrm{ICC}$ & 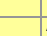 & AAK & & & & & & & TBOH & & TIC/TOC \\
\hline & & & & $\begin{array}{l}\text { C3-TLP-FEED-ARC } \\
\text { C3TP-ETAPTAC }\end{array}$ & NONE & feed tank & 1000 & & & & & & & & & & & & & & & & & \\
\hline & & Bottoms & After all antifoam additions & $\begin{array}{l}\text { C3-TLP-B-START-ARC } \\
\text { C3-TP-BSTTRTANTEF }\end{array}$ & NONE & bottoms tank & 100 & & $\square$ & & & & $\square$ & & & - & & & & & & & & \\
\hline & & Bottoms & At $-200 \mathrm{~L}$ fed & $\begin{array}{l}\text { C3-TLP-B-S-SART-ANTF } \\
\text { C3-TLP-B-200-ARC }\end{array}$ & $\begin{array}{l}\text { NONE } \\
\text { NONE }\end{array}$ & $\begin{array}{l}\text { bottoms tank } \\
\text { bottoms tank }\end{array}$ & $\frac{100}{50}$ & & 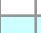 & & & & 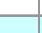 & & & & & & & & & & & \\
\hline & & Bottoms & & & NONE & & 50 & & & & & & & & & & & & & & & & & \\
\hline & & Bottoms & At $-600 \mathrm{~L}$ fed & C3-TLP-B-600-ARC & NONE & $\begin{array}{l}\text { Dotums salin } \\
\text { bottoms tank }\end{array}$ & 50 & & & & & & & & & & & & & & & & & \\
\hline & & Bottoms & At $-800 \mathrm{~L}$ fed & C3-TLP-B-800-ARC & NONE & bottoms tank & 50 & & & & & & & & & & & & & & & & & \\
\hline & & Bottoms & At $-1000 \mathrm{~L}$ fed & C3-TLP-B-1000-ARC & NONE & bottoms tank & 50 & & 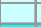 & & & & & & & & & & & & & & & \\
\hline & & Bottoms & Evaporation completed & $\begin{array}{l}\text { C3-TLP-B-END-ACTL } \\
\text { C3-TPP-B-END-ADS }\end{array}$ & $\begin{array}{l}\text { NONE } \\
42113\end{array}$ & $\begin{array}{l}\text { bottoms tank } \\
\text { bottoms tank }\end{array}$ & $\begin{array}{l}100 \\
50\end{array}$ & & & & & & ICC & & & TS & UDS & DENS & & & & & & \\
\hline & & & & C-TRP-B-END-ARC & NONE & $\begin{array}{l}\text { botoms tank } \\
\text { bottoms }\end{array}$ & 100 & & & & & & IC & & & & & & & & & & & \\
\hline & & 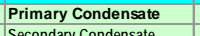 & Composite & $\begin{array}{l}\text { C3-TLP-PC-END-ARC } \\
\text { C3TP-SCENDAAC }\end{array}$ & NONE & & 100 & & -5 & & & & & & & & & & & & & & & \\
\hline & COMBINE TLP C & $\begin{array}{l}\text { Secondary Condensate } \\
\text { SNDENSATES }\end{array}$ & Composite & C3-TLP-SC-END-ARC & & & & & & & & & & & & & & & & & & & & \\
\hline & & Combined TLP Condensa & ate $=$ RLD Condensate & & & storage tank & & & & & & & & & & & & & & & & & & \\
\hline & & (Primary + Secondary) & & $\begin{array}{l}\text { C3-RLD-FROM-TLP-ACTLL } \\
\text { C3-RLD-FROM-TLP-ARC }\end{array}$ & $\begin{array}{l}\text { NONE } \\
\text { NONE }\end{array}$ & & $\begin{array}{c}50 \\
1000\end{array}$ & & & & & & & & & TS & & DENS & & & & & & \\
\hline & COMBINE FEP \& & TLP CONDENSATES & Combined condensate & $\begin{array}{l}\text { C3-RLD-COMP-ARC } \\
\text { C3-PID-CMMP-ADS }\end{array}$ & $\begin{array}{l}\text { NONE } \\
4215\end{array}$ & $\begin{array}{l}\text { storage tank } \\
\text { stroga tank }\end{array}$ & $\frac{1000}{50}$ & & & & & & ICC & & & & & & & & & & & \\
\hline$N^{2}$ & 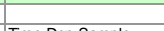 & $T$ Th & & & & & & & & & & & & & & & & & & & & & & \\
\hline Notes: & Time Dep Sample & Time dependent sample. Th & nese samples are to be observed dally for & rabout 5 days for evidence of precipi & itation of solids. & & & & & & & & & & & & & & & & & & & \\
\hline & Explanation of analyse & If solids form, they will be an & nnalyzed by XRD. A filtrate blank will also & be analyzed. & & & & & & & & & & & & & & & & & & & & \\
\hline & 年 & & & Ion chrom. catio & & & & & & & & & & & & & & & & & & & & \\
\hline & ICPES & includes all elements preser & ent that can be analyzed by ICPES & Ion exclusion chrom. & citrate, acetate & RHEO $n$ & heology & & & & & & & & & & & & & & & & & \\
\hline & ICPMS & sludges & $\mathrm{Cs}, \mathrm{Rb}, \mathrm{Bi}, \mathrm{Y}, \mathrm{Pr}$ & atomic absorption & $\mathrm{Cs}, \mathrm{K}, \mathrm{Na}$ & & varticle size & distributior & & Microtrac & & & & & & & & & & & & & & \\
\hline & $\mathrm{IC}$ & $\begin{array}{l}\text { supernates } \\
\text { lon chrom - anions }\end{array}$ & $\begin{array}{l}\mathrm{CS}, \mathrm{Rb} \\
\text { nomal suite }\end{array}$ & $\begin{array}{l}\text { total lalids thi\% } \\
\text { undissolved solids w\% }\end{array}$ & & $\begin{array}{l}\text { XRD } \times \\
\text { TBOH to }\end{array}$ & $\begin{array}{l}\text { raya difitacti } \\
\text { otal base, tit }\end{array}$ & $\begin{array}{l}\text { tion } \\
\text { tree hydroxic }\end{array}$ & ide, ot & her base & & & & & & & & & & & & & & \\
\hline
\end{tabular}


G. T. Wright, 773A

D. J. Green, 773-A

D. B. Moore-Shedrow, 773-A

J. E. Marra, 773-A

C. R. Wolfe, 773-A

H. A. Woltermann, 773-A

D. A. Crowley, 773-A

R. E. Edwards, Jr., 773-A

J. C. Griffin, 773-A

E. W. Holtsheiter, Jr., 773-A

H. F. Sturm, Jr., 773-A

S. T. Wach, 773-42A

D. B. Burns, 786-5A

T. B. Calloway, 999-W

S. D. Fink, 773-A

J. C. Marra, 773-42A

S. L. Marra, 999-W

W. R. Wilmarth, 773-42A

D. J. Adamson, 786-5A

C. D. Barnes, 773-42A

M. R. Duignan, 786-5A

M. D. Fowley, 786-5A

H. N. Guerrero, 786-5A

Z. H. Qureshi, 786-5A

J. L. Steimke, 786-5A

M. R. Williams, 786-5A

J. R. Zamecnik, 773-41A

RPP File, c/o M. F. Jones, 773-43A, Room 109

\section{$\underline{\text { OFF-SITE }}$}

W. L. Tamosaitis, Waste Treatment Plant, 3000 George Washington Way, Richland, WA99352

F. W. Damerow, Waste Treatment Plant, 3000 George Washington Way, Richland, WA 99352

P. S. Sundar, Waste Treatment Plant, 3000 George Washington Way, Richland, WA 99352 
WSRC-TR-2005-00105, REVISION 0

SRNL-RPP-2005-00012, REVISION 0

\section{Appendix J: Overall Chemical Compositions of SIPP Campaign II Process Streams}

As explained throughout this report, the SIPP task contained four campaigns, which the first was just the operation of the ultrafiltration process and the remaining three would operate all four unit operations, i.e., FEP, UFP, CIX, and TLP. As was also discussed, the pre-task steady-state OLI/ESP computational modeling of the SIPP process showed that in performing Campaign II, III, and IV the product streams became chemically equivalent very fast; the differences in stream composition between Campaign III and IV were less then 5\%. (Of course, this small difference cannot be fully shown experimentally because just before beginning the last campaign, the operation of Campaign IV was changed by the Hanford Project to specifically test the new GKN filter, therefore, the CIX and TLP unit operation were not used.)

The first full SIPP campaign was Campaign II and, as such, it was targeted to have its product streams chemically analyzed most extensively. Furthermore, since Campaigns III and IV were expected to be chemically similar, the number samples was greatly reduced to minimize cost to the project. For those campaigns only a minimum number of samples were taken to insure proper operation the plan tests. However, measurements made for Campaign II were extensive to capture inter-unit-operations chemically histories.

This Appendix indicates were taken and what were the results in the overall process. Figure J1 shows the overall SIPP operation for the FEP and UFP unit operations of Campaign II and sample points. Figure J2 shows the overall SIPP operation for the CIX and TLP unit operations of Campaign II and sample points. These figures are followed by a full table of analyzed results, Table J1. Matching the numbers in the figures to the columns in Table J1 will give the results at a particular sample point.

Table J1 shows the measured compositions of most of the process streams, including density and solids measurements, and the approximate stream volume. In some cases for slurry samples, only a total slurry (dissolved sample) analysis was performed; in other cases, analysis of the supernate liquid from a (lab) filtered sample was also performed.

Dissolutions were either by a microwave acid method or the sodium peroxide fusion / $\mathrm{HCl}$ uptake method or by both. For streams very dilute in undissolved solids (such as UFP cleaning solutions or TLP feed), only the supernate was analyzed because the dissolution method introduces more uncertainty in the elemental analyses than the small amount of undissolved solids adds to the total amount of an element. Anion and ammonium analyses were performed on either a supernate (lab filtered) sample or on the total sample (slurry) that was diluted then filtered prior to analysis. The first method measures only the anions actually dissolved in the supernate portion of the sample, whereas the second method can also measure some contribution of undissolved anions due to dissolution upon dilution. However, while this method will get some of the undissolved material, it is not designed or optimized to get all of an undissolved anion. An example is oxalate: sodium oxalate can be undissolved if present above its saturation concentration, but may dissolve when diluted with water prior to analysis. 
WSRC-TR-2005-00105, REVISION 0

SRNL-RPP-2005-00012, REVISION 0

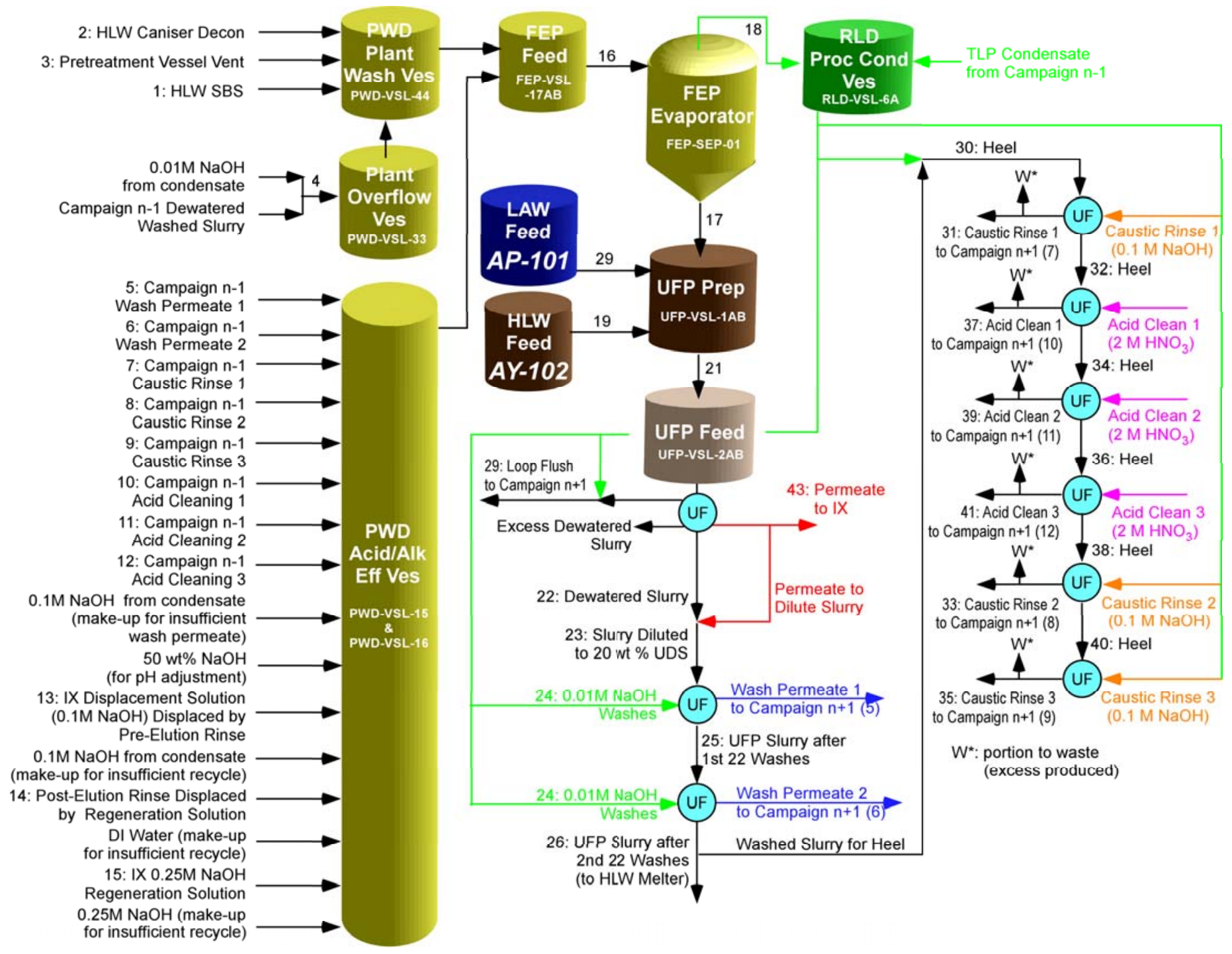

Figure J1. Product samples taken during Campaign II: FEP \& UFP. The numbers refer to column results in Appendix J 


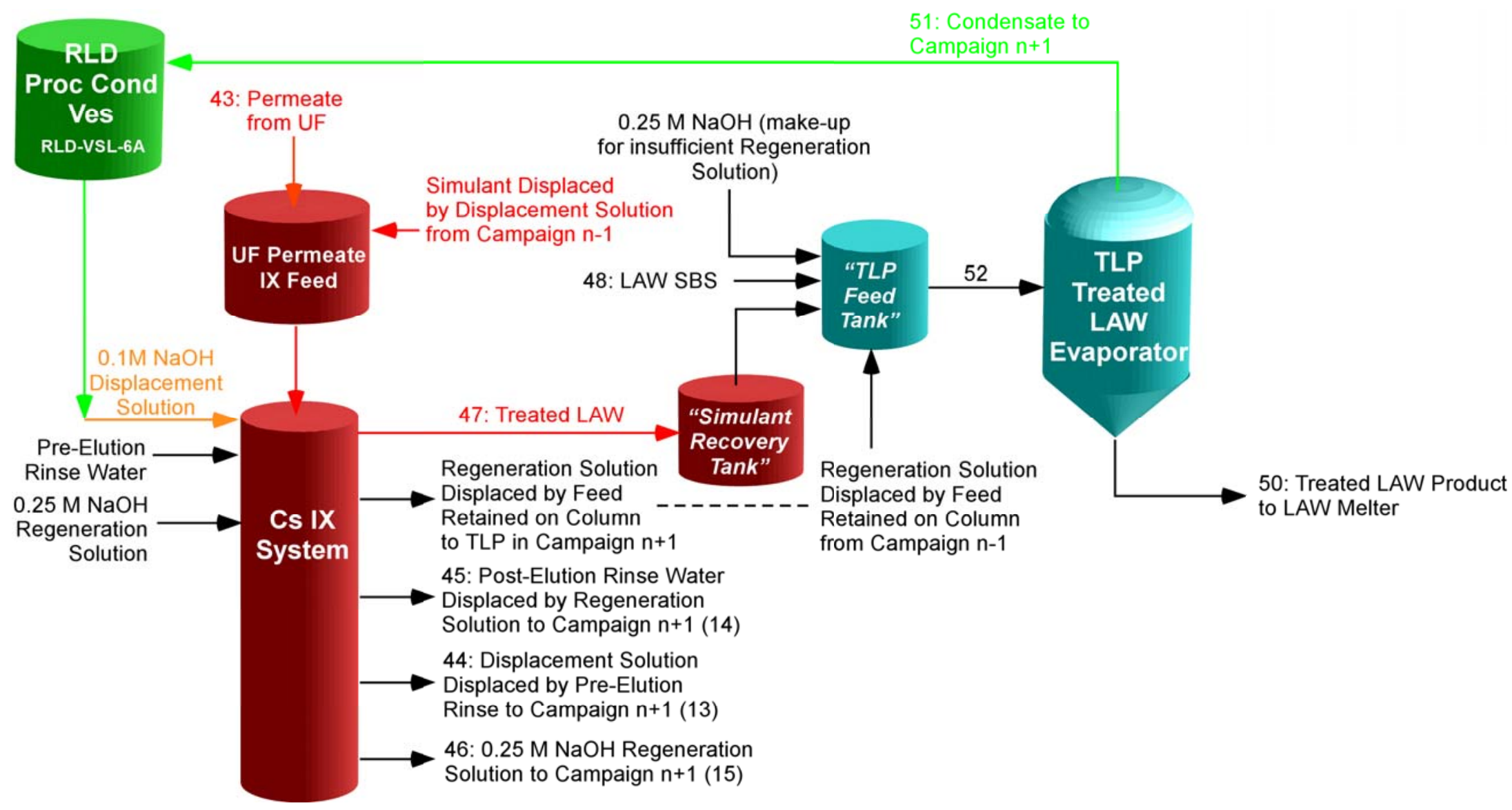

Figure J2. Product samples taken during Campaign II: CIX \& TLP. The numbers refer to column of results in Appendix J 
Table J1. Overall Chemical Compositions of SIPP Campaign II Process Streams

\begin{tabular}{|c|c|c|c|c|c|c|c|c|c|c|}
\hline & \multicolumn{10}{|c|}{ RECYCLES TO FEP EVAPORATOR } \\
\hline & 1 & 1 & 1 & 2 & & 3 & 4 & 5 & 6 & \\
\hline 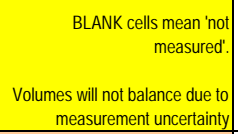 & $\begin{array}{l}\text { HLW-SBS- } \\
\text { QUENCHER } \\
\text { RECYCLE } \\
\end{array}$ & $\begin{array}{l}\text { HLW-SBS- } \\
\text { QUENCHER } \\
\text { RECYCLE } \\
\end{array}$ & $\begin{array}{l}\text { HLW-SBS- } \\
\text { QUENCHER } \\
\text { RECYCLE } \\
\end{array}$ & $\begin{array}{l}\text { HLW CANISTER DECON } \\
\text { SOLUTION }\end{array}$ & & $\begin{array}{l}\text { PRETREATMENT } \\
\text { VESSEL VENT } \\
\text { SOLUTION } \\
\end{array}$ & $\begin{array}{l}\text { LOOP FLUSH FROM PREVIOUS } \\
\text { CAMPAIGN }\end{array}$ & $\begin{array}{c}\text { WASH PERMEATE } \\
\text { FROM IST 22 WASHES } \\
\text { FROM PREVIOUS } \\
\text { CAMPAGN }\end{array}$ & $\begin{array}{c}\text { WASH PERMEATE } \\
\text { FROM N ND R2 22 WASHES } \\
\text { FROM PREVIOUS } \\
\text { CAPPAIGN } \\
\end{array}$ & \\
\hline 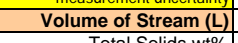 & $\frac{190.8}{10.8}$ & & & \begin{tabular}{|l|l|}
114.7 \\
11.7 \\
\end{tabular} & & $\begin{aligned} 220.7 \\
\end{aligned}$ & $\begin{array}{c}13.1 \\
839 \\
\end{array}$ & \begin{tabular}{|l|l}
169.3 \\
372 \\
\end{tabular} & \begin{tabular}{|l|l|l}
169.3 \\
1094
\end{tabular} & \\
\hline Undissolved Solids wt\% & $\frac{\mathrm{NM}}{\mathrm{NM}}$ & & & & & & $\frac{8.39}{7.42}$ & 3.00 & $\frac{10.94}{0.00}$ & \\
\hline $\begin{array}{l}\text { Supernate Solids wto } \\
\text { Solith }\end{array}$ & \begin{tabular}{|l}
$\mathrm{NM}$ \\
1010 \\
\end{tabular} & & & & & & 1.05 & 3.73 & $\begin{array}{c}10.94 \\
\end{array}$ & \\
\hline $\begin{array}{l}\text { Density kg/L } \\
\text { pH }\end{array}$ & \begin{tabular}{|l}
1.010 \\
NM
\end{tabular} & & & & & & $\frac{1.063}{\mathrm{NM}}$ & & & \\
\hline $\begin{array}{l}\text { Dissolution or } \\
\begin{array}{r}\text { Preparation Method (for } \\
\text { elements only) }\end{array} \\
\end{array}$ & As-Received & aqua regia & filtered & & & & & none & none & \\
\hline $\begin{array}{r}\text { Comments } \\
\text { Elements by ICPES }\end{array}$ & \begin{tabular}{|c}
$\begin{array}{c}\text { (Filtered after } \\
\text { diluting to } \\
\text { instrument range) }\end{array}$ \\
\end{tabular} & $\begin{array}{c}\text { Measurement } \\
\text { of total sample } \\
\text { composition }\end{array}$ & $\begin{array}{l}\text { Analysis is of } \\
\text { sample minus } \\
\text { undisolved } \\
\text { solids }\end{array}$ & Simulant used & & Simulant used & $\begin{array}{l}\text { Composition is diluted } \\
\text { washed slurry from previous } \\
\text { campaign, combine } 4.5 \mathrm{~L} \\
\text { slurry }+8.6 \mathrm{LL} 0.01 \mathrm{MaOH} \\
\end{array}$ & & & \\
\hline $\begin{array}{r}\text { Elements by ICPES } \\
\text { Units }\end{array}$ & $\mathrm{mg/L}$ & $\mathrm{mg} / \mathrm{kg}$ & $\mathrm{mg} / \mathrm{L}$ & & & & & $\mathrm{mg} / \mathrm{L}$ & $\mathrm{mg} / \mathrm{L}$ & \\
\hline $\mathrm{Ag}_{\mathrm{Al}}$ & $\frac{<0.3}{16.6}$ & $\begin{array}{c}<7.50 \\
18.9\end{array}$ & $\begin{array}{l}<0.3 \\
0.669 \\
\end{array}$ & & & & & $\begin{array}{c}<8.6 \\
3860 \\
\end{array}$ & $\begin{array}{l}<0.6 \\
1040\end{array}$ & \\
\hline$\frac{B}{B a}$ & $\frac{522}{0.145}$ & $\begin{aligned} 599 \\
<050\end{aligned}$ & $\begin{array}{ll}506 \\
181\end{array}$ & 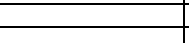 & & 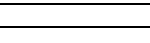 & & $<0.12$ & 0.621 & . \\
\hline $\begin{array}{ccc}\text { bal } \\
\text { Ca } \\
C a\end{array}$ & $\frac{0.145}{9.01}$ & $\begin{array}{c}<.50 \\
9.22 \\
\end{array}$ & $\begin{array}{l}1.81 \\
5.89 \\
\end{array}$ & & & & & $<0.04$ & $<0.04$ & \\
\hline $\begin{array}{ccc}\mathrm{cd} \\
\mathrm{Ce}\end{array}$ & 0.380 & $\begin{array}{l}0.738 \\
<17.5 \\
\end{array}$ & 0.0377 & 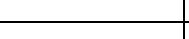 & & 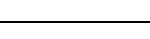 & 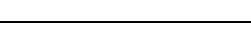 & $<0.04$ & $<0.04$ & \\
\hline col & $<0.08$ & $<2.00$ & $<0.08$ & & & & & $<0.16$ & $<0.16$ & \\
\hline$\frac{\mathrm{Cr} r}{\mathrm{Cu}}$ & $\frac{5.96}{1.46}$ & $\frac{12.3}{<0.75}$ & $\begin{array}{l}3.69 \\
1.09 \\
\end{array}$ & & & 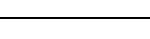 & & $\frac{94.0}{<0.06}$ & $\frac{26.0}{<0.06}$ & \\
\hline$\frac{\mathrm{Fe}}{\mathrm{Fe}}$ & 83.2 & 114 & 1.42 & & & & & $<0.08$ & $<0.08$ & \\
\hline$\frac{\mathrm{Lat}}{\mathrm{Li}}$ & $\frac{2.51}{109}$ & $\begin{array}{c}2.58 \\
109\end{array}$ & $\frac{<0.1}{106}$ & 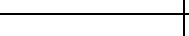 & 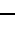 & 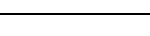 & & $\begin{array}{l}<0.2 \\
<0.4\end{array}$ & $\frac{0.2}{<0.4}$ & \\
\hline$\frac{\mathrm{Mg}}{\mathrm{Mn}}$ & $\begin{array}{ll}34.7 \\
5.31\end{array}$ & $\begin{array}{ll}35.7 \\
5.89\end{array}$ & $\begin{array}{ll}28.6 \\
0.253\end{array}$ & 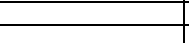 & & - & & \begin{tabular}{c|c|c|}
$<<01$ \\
$<<.02$
\end{tabular} & $\begin{array}{l}<<.01 \\
<.02 \\
\end{array}$ & \\
\hline Mo & $<0.1$ & $<2.50$ & $<0.1$ & & & & & $<0.2$ & $<0.2$ & \\
\hline $\begin{array}{ll}\mathrm{Nad} \\
\mathrm{NN} d \mathrm{~d}\end{array}$ & 9922 & $\begin{array}{l}979 \\
525\end{array}$ & 978 & 10166 & & 2300 & & 44200 & 14100 & \\
\hline $\begin{array}{l}\mathrm{Nd} \\
\mathrm{Ni} \\
\end{array}$ & $\begin{array}{l}1.45 \\
0.468\end{array}$ & $\begin{array}{l}22.5 \\
1.59\end{array}$ & $\begin{array}{l}<.9 \\
0.255\end{array}$ & & & & & $\begin{array}{l}<1.8 \\
0.387\end{array}$ & $<0.12$ & \\
\hline $\begin{array}{r}\mathrm{P} \\
\mathrm{Ph}\end{array}$ & $\begin{array}{ll}0.932 \\
167\end{array}$ & $\begin{array}{l}<20.0 \\
<20 \\
\end{array}$ & $\begin{array}{l}<0.8 \\
<08\end{array}$ & & & & & 740 & 139 & $\square$ \\
\hline$\frac{\mathrm{PD}}{\mathrm{Re}}$ & 1.67 & $\begin{array}{l}<2.0 .0 \\
3.73 \\
\end{array}$ & $\begin{array}{l}<.8 \\
3.74 \\
\end{array}$ & & & & & $<1.6$ & 1.6 & \\
\hline$\frac{S}{S i}$ & 117 & $\frac{122}{589}$ & 121 & & & & & $\frac{385}{88.8}$ & $\frac{102}{270}$ & $\square$ \\
\hline $\mathrm{Sr}$ & $\frac{70.0}{17.5}$ & $\begin{array}{r}58.9 \\
17.6\end{array}$ & $\begin{array}{ll}14.6 \\
8.38\end{array}$ & & & & & $\begin{array}{c}8.18 \\
0.0767\end{array}$ & $\frac{2.70}{0.0188}$ & \\
\hline Ti & 1.80 & 1.72 & $<0.03$ & & & & & $<0.06$ & $<0.06$ & \\
\hline $\begin{array}{c}\mathrm{v} \\
\mathrm{w} \\
\end{array}$ & $<0.4$ & $<10.0$ & $<0.4$ & & & & & & & \\
\hline $\begin{array}{r}\mathrm{Zn} \\
\mathrm{Zr}\end{array}$ & 18.9 & 20.4 & 0.751 & & & 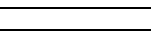 & 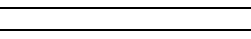 & $<0.04$ & $<0.04$ & \\
\hline $\begin{array}{r}\text { Elements by ICPMS } \\
\text { (same units as ICPES) }\end{array}$ & & & & & & & & & & \\
\hline $\begin{array}{l}\text { (same units as ICPES) } \\
R b\end{array}$ & 0.670 & & & & & & & & & \\
\hline \begin{tabular}{c|c|}
$\mathrm{Y}$ \\
$\mathrm{Cs}$
\end{tabular} & & & & & & & & & 0.0110 & $\square$ \\
\hline 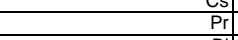 & & & & & & & & & 0.263 & \\
\hline $\begin{array}{r}\text { Bi } \\
\text { Re }\end{array}$ & 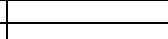 & & & & & & & - & & \\
\hline $\begin{array}{l}\text { Elements by Atomic } \\
\text { Absorption (same units } \\
\text { as IPEES) }\end{array}$ & & & & & & & & & & \\
\hline $\begin{array}{r}\text { Cs } \\
\mathrm{K}\end{array}$ & & & & & & & & 0.860 & & \\
\hline & $\begin{array}{l}\frac{<.75}{918} \\
918\end{array}$ & & & & & & & $\begin{array}{l}11000 \\
52400\end{array}$ & & \\
\hline $\begin{array}{l}\text { Anions by lon } \\
\text { Chromatography }\end{array}$ & & & & & & & & & & \\
\hline $\begin{array}{r}\text { units } \\
\text { hrmisd }\end{array}$ & $\frac{\mathrm{mglL}}{<10}$ & & & & & & & $\mathrm{mg/L}$ & $\frac{\mathrm{mglL}}{5100}$ & \\
\hline $\begin{array}{l}\text { Dromiace } \\
\text { chloride } \\
\end{array}$ & $\frac{10}{332}$ & & & & & & & $\frac{27.0}{97.0}$ & $\frac{100}{21.0}$ & $E$ \\
\hline fluoride & 43.0 & & & & & & & 36.0 & 7.00 & E \\
\hline $\begin{array}{l}\text { formate } \\
\text { nitrate }\end{array}$ & $\begin{array}{l}<10 \\
<10\end{array}$ & & & 17598 & & 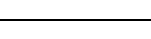 & & $\frac{165}{216}$ & $\frac{10.0}{36.0}$ & 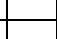 \\
\hline $\begin{array}{l}\text { nitrite } \\
\text { nxilatel }\end{array}$ & $<10$ & & & & & & & 3170 & 528 & \\
\hline $\begin{array}{r}\text { oxalate } \\
\text { phosphate }\end{array}$ & $\begin{aligned} 34.0 \\
999\end{aligned}$ & & & & & & & 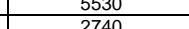 & $\frac{4440}{206}$ & \\
\hline $\begin{array}{c}\text { sulfate } \\
\end{array}$ & 287 & & & & & & & 1250 & 225 & \\
\hline $\begin{array}{c}\text { acetate } \\
\text { sitrate }\end{array}$ & NM & & & & & & & $\frac{35.0}{<10}$ & $\frac{101}{<10}$ & \\
\hline formate & NM & & & & & & & 189 & 46.0 & \\
\hline & NM & & & & & & & & & \\
\hline $\begin{array}{l}\text { Wet Chemistry } \\
\text { total base } \mathrm{M}\end{array}$ & & & & 0.149 & & & & & & \\
\hline $\begin{array}{c}\text { free hydroxide M } \\
\text { totiladidM }\end{array}$ & & & & 0.149 & & 0.1 & & & & \\
\hline $\begin{array}{l}\text { totalacadi M } \\
\text { carbonate by titration }\end{array}$ & & & & & & & & & & \\
\hline $\begin{array}{c}\text { Carbon Analyses } \\
\text { (unfiltered sample) }\end{array}$ & & & & & & & & & & \\
\hline & & & & & & & & $\mathrm{mglL}$ & $\mathrm{mglL}$ & \\
\hline $\begin{array}{l}\text { total inorganic carbon } \\
\end{array}$ & & & & & & & & 1180 & 355 & \\
\hline $\begin{array}{l}\text { total organic carbon } \\
\text { (oxalate + citrate + acetate }\end{array}$ & & & & & & & & 210 & 205 & \\
\hline
\end{tabular}


Table J1. Overall Chemical Compositions of SIPP Campaign II Process Streams

\begin{tabular}{|c|c|c|c|c|c|c|c|c|c|c|}
\hline & 7 & 7 & & 8 & & 9 & $8+9$ & 10 & 11 & 12 \\
\hline $\begin{array}{l}\text { BLANK cells mean 'not } \\
\text { measured'. } \\
\begin{array}{r}\text { Volumes will not balance due to to } \\
\text { measurement uncertainty }\end{array}\end{array}$ & $\begin{array}{c}\text { CAUSTIC RINSE } 1 \\
\text { FROM PREVIIOUS } \\
\text { CAMPAIGN } \\
\end{array}$ & $\begin{array}{l}\text { CAUSTIC RINSE } 1 \\
\text { FROM PREVIOUS } \\
\text { CAMPAIGN }\end{array}$ & & $\begin{array}{l}\text { CAUSTIC RINSE } 2 \\
\text { FROM PREVIOUS } \\
\text { CAMPAIGN }\end{array}$ & & $\begin{array}{l}\text { CAUSTIC RINSE } 3 \\
\text { FROM PREVIOUS } \\
\text { CAMPAIGN }\end{array}$ & $\begin{array}{l}\text { CAUSTIC RINSES } 1 \& 2 \\
\text { COMBINED FROM } \\
\text { PREVIOUS CAMPAIGN }\end{array}$ & \begin{tabular}{|l} 
NITRIC ACID CLEANING \\
1FROM PREVIOUS \\
CAMPAIGN
\end{tabular} & $\begin{array}{l}\text { NITRIC ACID CLEANING } \\
\text { 2FROM PREVIOUS } \\
\text { CAMPAIGN }\end{array}$ & $\begin{array}{l}\text { NTTRIC ACID CLEANING } \\
\text { 3 FROMPREVIOUS } \\
\text { CAMPAIGN }\end{array}$ \\
\hline Volume of Stream (L) & $\begin{array}{l}18.2 \\
071 \\
\end{array}$ & & & & & & $\begin{array}{l}99.0 \\
079\end{array}$ & \begin{tabular}{|l|l}
37.4 \\
\end{tabular} & 42.5 & \\
\hline $\begin{array}{c}\text { Thotal Soldis w th } \\
\text { Undissolved Solids wt\% }\end{array}$ & $\begin{array}{l}9.11 \\
8.27 \\
\end{array}$ & & & & & & 0.79 & 0.99 & 0.09 & \\
\hline $\begin{array}{l}\text { Supernate Solids wt\% } \\
\text { Density kgal }\end{array}$ & 1.57 & & & & & & 0.79 & 0.00 & 0.00 & \\
\hline $\mathrm{pH}$ & & & & & & & & & & - \\
\hline $\begin{array}{l}\text { Dissolution or } \\
\text { Preparation Method (for } \\
\text { elements only) }\end{array}$ & microwave acid & $\begin{array}{c}\text { Na peroxide fusion/ } \\
\text { HCl uptake }\end{array}$ & & & & & microwave acid & none & none & none \\
\hline & $\begin{array}{l}\text { Measurement of total } \\
\text { sample composition }\end{array}$ & $\begin{array}{l}\text { Measurement of total } \\
\text { sample composition }\end{array}$ & & $\begin{array}{c}\text { Composition not } \\
\text { measured }\end{array}$ & & $\begin{array}{c}\text { Composition not } \\
\text { measured }\end{array}$ & $\begin{array}{c}\text { Measurement of total } \\
\text { sample composition }\end{array}$ & & & \\
\hline $\begin{array}{l}\text { Elements by IICPES } \\
\text { Units }\end{array}$ & $\mathrm{mg} / \mathrm{kg}$ & $\mathrm{mg} / \mathrm{kg}$ & & & & & $\mathrm{mg} / \mathrm{kg}$ & $\mathrm{mg/L}$ & $\mathrm{mg} / \mathrm{L}$ & $\mathrm{mg} / \mathrm{L}$ \\
\hline $\begin{aligned} \mathrm{Ag} \\
\mathrm{Al}\end{aligned}$ & $\begin{array}{r}129 \\
5370 \\
\end{array}$ & $\frac{410}{6680}$ & & & & & $\begin{array}{l}<15.0 \\
<50\end{array}$ & $\frac{12.8}{256}$ & 2.82 & 0.727 \\
\hline$\frac{B}{B}$ & 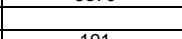 & 50.0 & & & & & 年 & 4.00 & 0 & $<0.12$ \\
\hline$\frac{\mathrm{Ba}}{\mathrm{Ca}}$ & $\frac{191}{780}$ & $\begin{array}{l}\frac{190}{1070} \\
\end{array}$ & & & 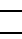 & & $\begin{array}{l}<.1 .00 \\
<1.00\end{array}$ & $\frac{11.4}{37.7}$ & $\frac{1.13}{6.14}$ & $\begin{array}{l}0.31 \\
0.155 \\
\end{array}$ \\
\hline 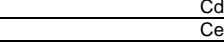 & $\begin{array}{l}\frac{30.0}{179} \\
\end{array}$ & $\frac{20.0}{250}$ & & & 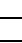 & & $\begin{array}{l}<1.00 \\
<35.0\end{array}$ & $\frac{1.35}{11.6}$ & 0.239 & 0.0414 \\
\hline$\frac{\mathrm{Co}}{\mathrm{Cr}}$ & $\frac{<13.0}{229}$ & $\frac{30.0}{330}$ & & & & & $\begin{array}{l}<4.00 \\
<200\end{array}$ & $\frac{0.377}{160}$ & $\frac{<.16}{300}$ & $<0.16$ \\
\hline 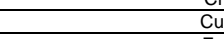 & 50.0 & 50.0 & & & 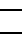 & & $<1.00$ & $\frac{16.0}{2.40}$ & 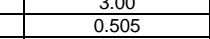 & $\begin{array}{l}1.00 \\
0.108 \\
\end{array}$ \\
\hline$\frac{\mathrm{Fe}}{\mathrm{La}}$ & $\frac{21700}{173}$ & $\frac{21500}{100}$ & & & & & 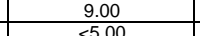 & $\frac{1050}{<2}$ & $\frac{200}{<12}$ & $\begin{array}{l}40.0 \\
<12\end{array}$ \\
\hline & $\frac{133.0}{<3.0}$ & $\frac{140.0}{<40.0}$ & & & - & & $\frac{1}{<10.0}$ & $\begin{array}{l}12 \\
8.91\end{array}$ & $\frac{12}{1.60}$ & $\begin{array}{l}<12 \\
0.257\end{array}$ \\
\hline 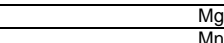 & 229 & 230 & - & 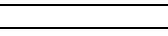 & ב & 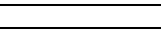 & 0.250 & $<0.4$ & $<0.4$ & $<0.4$ \\
\hline Mo & $\begin{array}{l}4810 \\
<16.0\end{array}$ & $\frac{450}{<20.0}$ & & & & & $<.000$ & $\frac{10.9}{219}$ & $\frac{2.02}{42.0}$ & $\frac{0.376}{8.00}$ \\
\hline 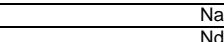 & 9280 & & & 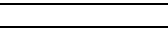 & & 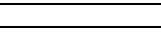 & 2110 & $<0.2$ & $<0.2$ & 0.2 \\
\hline$\frac{\mathrm{Na}}{\mathrm{Ni}}$ & 713 & 500 & & & -5 & & 3.00 & $\frac{1200}{25.6}$ & 4.65 & $\frac{41.0}{<1.8}$ \\
\hline$\frac{\mathrm{P}}{\mathrm{Pb}}$ & 351 & 350 & & & & & $<40.0$ & 33.0 & 6.00 & 1.00 \\
\hline$\frac{\mathrm{Pb}}{\mathrm{Re}}$ & 920 & 1110 & & & & & $<40.0$ & 17.0 & 5.00 & 2.00 \\
\hline $\mathrm{s}$ & $<164$ & 260 & & & & & $<50.0$ & $\begin{array}{ll}48.0 \\
8.47\end{array}$ & $\frac{9.00}{<2}$ & $\frac{<1.6}{<2}$ \\
\hline $\mathrm{Si}$ & 7740 & 6310 & & & 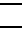 & & 722 & 98.0 & 20.0 & 5.00 \\
\hline $\begin{aligned} \mathrm{ST} \\
\mathrm{Ti}\end{aligned}$ & $\frac{180}{5.00}$ & $\frac{190}{10.0}$ & & & & & $\frac{0}{<1.00}$ & $\frac{9.00}{0.113}$ & $\frac{4.00}{<0.06}$ & $\frac{1.00}{<0.06}$ \\
\hline & & & & & & & & $<0.8$ & $<0.8$ & $<0.8$ \\
\hline $\begin{array}{c}W \\
\mathrm{Zn}\end{array}$ & $\frac{<2.0}{46.0}$ & $\begin{array}{l}100 \\
180\end{array}$ & & & - & & $<25.0$ & $\begin{array}{l}<1 \\
3.61\end{array}$ & $\begin{array}{l}<1 \\
0.643\end{array}$ & $\begin{array}{l}<1 \\
0.0943 \\
1\end{array}$ \\
\hline & 297 & & & & & & 1.00 & 0.246 & 1.83 & 0.971 \\
\hline $\begin{array}{l}\text { Elementst by IIPMS S } \\
\text { (same units as ICPES) }\end{array}$ & & & & & & & & & & \\
\hline $\begin{aligned} \mathrm{Rb} \\
\mathrm{Y}\end{aligned}$ & 0.352 & - & & & & & 0.0160 & 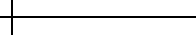 & 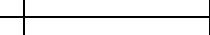 & \\
\hline Cs & 36.1 & & & & & & & 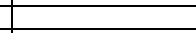 & & \\
\hline $\begin{aligned} \mathrm{Pr} \\
\mathrm{Bi}\end{aligned}$ & $\frac{0.635}{124}$ & - & & & & & 0.138 & 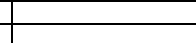 & & \\
\hline Flements & & & & & & & & & & \\
\hline $\begin{array}{l}\text { Absorption (same units } \\
\text { as ICPES) }\end{array}$ & & & & & & & & & & \\
\hline K & $\frac{<16.4}{39.2}$ & & & & & & $<4.95$ & $\begin{array}{l}<0.1 \\
5.00\end{array}$ & $\frac{0.1}{1.00}$ & $\begin{array}{l}<0.1 \\
0.170 \\
\end{array}$ \\
\hline $\begin{array}{r}\mathrm{Na} \\
\end{array}$ & 9090 & & & & & & 2070 & 1200 & 206 & 40.2 \\
\hline $\begin{array}{l}\text { Anions by lon } \\
\text { Chromatography }\end{array}$ & & & & & & & & & & \\
\hline units & $\frac{\mathrm{mg} / \mathrm{kg}}{<79}$ & & & & & & $\frac{\mathrm{mg} / \mathrm{kg}}{<10}$ & & & \\
\hline chloride & 12.0 & & & & & & $<20$ & & & - \\
\hline $\begin{array}{l}\text { fluoride } \\
\text { formate } \\
\end{array}$ & $\frac{7.00}{11.0}$ & & & & & & $\begin{array}{l}<<0 \\
<100\end{array}$ & & & \\
\hline nitrate & 23.0 & & & & & & 12500 & & & \\
\hline $\begin{aligned} \text { nitrite } \\
\text { nxalate }\end{aligned}$ & 198 & & & & & & $<100$ & & & \\
\hline $\begin{array}{c}\text { oxalate } \\
\text { phosphate }\end{array}$ & $\frac{1400}{328}$ & & & & & & $\begin{array}{l}<100 \\
<100 \\
<10\end{array}$ & 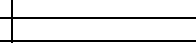 & 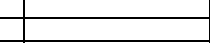 & - \\
\hline $\begin{array}{c}\text { sulfate } \\
\text { acetate }\end{array}$ & 98.0 & & & & & & & & & \\
\hline $\begin{array}{c}\text { acelale } \\
\text { citrate } \\
\end{array}$ & & & & & & & & & & \\
\hline $\begin{array}{r}\text { formate } \\
\text { rempnimm }\end{array}$ & & & & & & & & & & \\
\hline $\begin{array}{c}\text { ammonium } \\
\text { Wet Chemistry } \\
\end{array}$ & & & & & & & & & & \\
\hline $\begin{array}{c}\text { total base } \mathrm{M} \\
\text { free hydroxide } \mathrm{M}\end{array}$ & & & & & & & & & & \\
\hline total acid M & & & & & & & & 1.56 & 1.95 & 1.96 \\
\hline $\begin{array}{l}\text { carbonate by titration } \\
\text { Carbon Analyses }\end{array}$ & & & & & & & & & & \\
\hline $\begin{array}{c}\text { Larbon Anaryses } \\
\text { (unfiltered sample) }\end{array}$ & & & & & & & & & & \\
\hline total inorganic carbon & & & & & & & & & & \\
\hline $\begin{array}{r}\text { (carbonate) } \\
\text { total organic carbon }\end{array}$ & & & & & & & & & & \\
\hline $\begin{array}{l}(\text { oxalate }+ \text { citrate }+ \text { accatate } \\
+ \text { formate }\end{array}$ & & & & & & & & & & \\
\hline
\end{tabular}


Table J1. Overall Chemical Compositions of SIPP Campaign II Process Streams

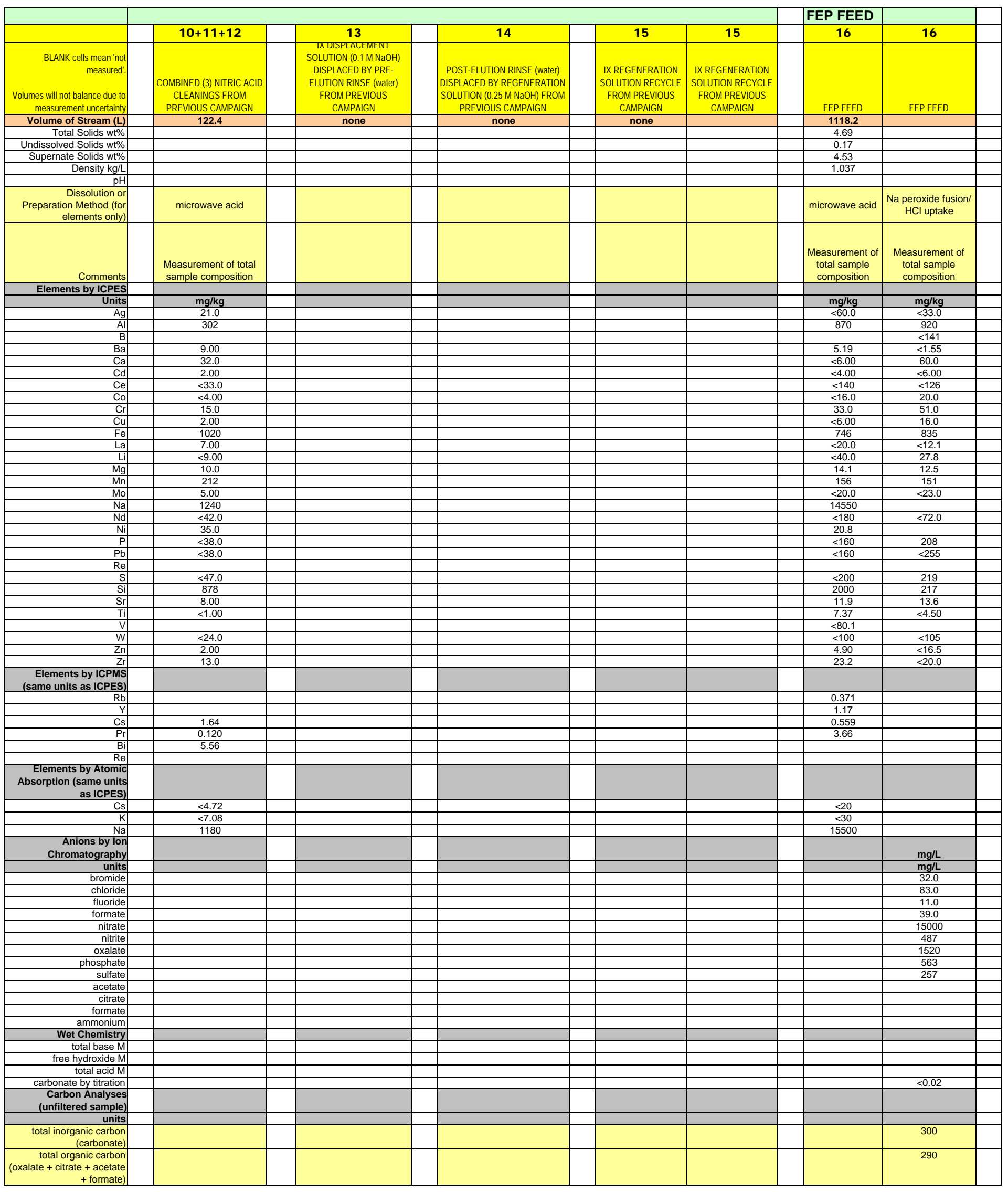


Table J1. Overall Chemical Compositions of SIPP Campaign II Process Streams

\begin{tabular}{|c|c|c|c|c|c|c|c|c|c|}
\hline & \multicolumn{3}{|c|}{ FEP BOTTOMS CONCENTRATE } & \multirow{2}{*}{$\begin{array}{c}\text { FEP COND } \\
18\end{array}$} & \multicolumn{4}{|l|}{ SIMULANTS } & \\
\hline & 17 & 17 & 17 & & 19 & 19 & 20 & 20 & \\
\hline $\begin{array}{r}\text { Volumes will not balance due to } \\
\text { measurement uncertainty }\end{array}$ & $\begin{array}{l}\text { FEP BOTTOMS } \\
\text { CONCENTRATE }\end{array}$ & $\begin{array}{l}\text { FEP BOTTOMS } \\
\text { CONCENTRATE }\end{array}$ & $\begin{array}{l}\text { FEP BOTTOMS } \\
\text { CONCENTRATE }\end{array}$ & $\begin{array}{c}\text { CONDENDATE FROM FEP } \\
\text { EVAPORATOR TO RLD } \\
\text { CONDENSATE }\end{array}$ & $\begin{array}{l}\text { AY-102 SIMULANT FEED } \\
\text { TO ULTRAFILTRATION }\end{array}$ & $\begin{array}{l}\text { AY-102 SIMULANT } \\
\text { FEED TO } \\
\text { ULTRAFILTRATION }\end{array}$ & $\begin{array}{l}\text { AP-101 SIMULANT } \\
\text { FEED TO } \\
\text { ULTRAFILTRATION }\end{array}$ & \begin{tabular}{|c|} 
AP-101 SIMULANT FEED \\
TO ULTRAFILTRATION
\end{tabular} & \\
\hline Volume of Stream (L) & 139.0 & & & 1014.0 & 252.4 & & 671.5 & & \\
\hline Total Solids wt $\%$ & 28.52 & 29.37 & & $<0.1$ & 26.85 & 26.75 & 31.94 & & \\
\hline Undissolved Solids wt $\%$ & 2.79 & 3.26 & to left are & $<0.1$ & 11.83 & 11.67 & 0.00 & & \\
\hline Supernate Solids wt $\%$ & 26.47 & 26.99 & duplicate & & 17.04 & 17.07 & 31.94 & & \\
\hline Density kg/L & 1.260 & 1.261 & measurements & 0.998 & 1.240 & & 1.279 & & \\
\hline $\mathrm{pH}$ & & 13.0 & & 9.4 & & & & & \\
\hline $\begin{array}{r}\text { Dissolution or } \\
\text { Preparation Method (for } \\
\text { elements only) }\end{array}$ & microwave acid & $\begin{array}{c}\text { Na peroxide fusion/ } \\
\mathrm{HCl} \text { uptake }\end{array}$ & filtered & & microwave acid & $\begin{array}{c}\text { Na peroxide fusion/ } \\
\mathrm{HCl} \text { uptake }\end{array}$ & microwave acid & $\begin{array}{l}\text { Na peroxide fusion/ } \\
\text { HCl uptake }\end{array}$ & \\
\hline Comments & \begin{tabular}{|l}
$\begin{array}{l}\text { Measurement of } \\
\text { total sample } \\
\text { composition }\end{array}$ \\
\end{tabular} & $\begin{array}{l}\text { Measurement of } \\
\text { total sample } \\
\text { composition }\end{array}$ & $\begin{array}{c}\text { Analysis is of } \\
\text { sample minus } \\
\text { undissolved solids }\end{array}$ & & $\begin{array}{l}\text { Measurement of total } \\
\text { sample composition }\end{array}$ & $\begin{array}{l}\text { Measurement of } \\
\text { total sample } \\
\text { composition }\end{array}$ & $\begin{array}{c}\text { Measurement of total } \\
\text { sample composition }\end{array}$ & $\begin{array}{c}\text { Measurement of total } \\
\text { sample composition }\end{array}$ & \\
\hline \multicolumn{9}{|c|}{\begin{tabular}{l|l|l|} 
Elements by ICPES & \\
\end{tabular}} & \\
\hline Units & $\mathrm{mg} / \mathrm{kg}$ & $\mathrm{mg} / \mathrm{kg}$ & $\mathrm{mg} / \mathrm{L}$ & $\mathrm{mg} / \mathrm{L}$ & $\mathrm{mg} / \mathrm{kg}$ & $\mathrm{mg} / \mathrm{kg}$ & $\mathrm{mg} / \mathrm{kg}$ & $\mathrm{mg} / \mathrm{kg}$ & \\
\hline $\mathrm{Ag}$ & 89.0 & 75.4 & $<0.6$ & $<0.6$ & 82.0 & 503 & $<45.8$ & 29.2 & \\
\hline Al & 5230 & 5470 & 4640 & $<1$ & 12900 & 14100 & 5840 & 5590 & \\
\hline $\mathrm{B}$ & & 554 & 690 & 7.00 & & $<133$ & & $<111$ & \\
\hline $\mathrm{Ba}$ & 37.8 & $<1.45$ & $<0.04$ & $<0.04$ & 236 & 206 & $<3.05$ & $<1.20$ & \\
\hline $\mathrm{Ca}$ & 56.0 & 220 & $<0.06$ & $<0.06$ & 912 & 1073 & $<4.58$ & 18.0 & \\
\hline $\mathrm{Cd}$ & 6.17 & $<5.75$ & $<0.04$ & $<0.04$ & 37.7 & 6.87 & $<3.05$ & $<4.75$ & \\
\hline $\mathrm{Ce}$ & $<101$ & $<120$ & $<1.4$ & $<1.4$ & $<96.0$ & 295 & $<107$ & $<99.0$ & \\
\hline Co & $<11.6$ & $<199$ & $<0.16$ & $<0.16$ & $<10.9$ & $<202$ & $<12.2$ & $<162$ & \\
\hline $\mathrm{Cr}$ & 168 & 188 & 150 & 2.28 & 510 & 511 & 140 & 141 & \\
\hline $\mathrm{Cu}$ & 13.1 & 6.52 & 0.139 & $<0.06$ & $\begin{array}{ll}62.8 \\
\end{array}$ & 66.0 & $<4.58$ & 0 & \\
\hline $\mathrm{Fe}$ & 4441 & 4532 & $<0.08$ & $<0.08$ & 28892 & 28400 & 59.7 & 55.2 & \\
\hline La & 18.6 & 47.6 & $<0.2$ & $<0.2$ & 79.3 & 246 & $<15.3$ & 10.9 & \\
\hline $\mathrm{Li}_{\mathrm{i}}$ & 100 & 111 & 8.86 & $<0.4$ & $<27.3$ & $<20.0$ & $<30.5$ & $<16.5$ & \\
\hline $\mathrm{Mg}$ & 77.4 & 82.3 & 0.0226 & 0.0210 & 306 & 301 & 5.60 & 4.31 & \\
\hline $\mathrm{Mn}$ & 942 & 940 & $<0.02$ & $<0.02$ & 6300 & 6160 & $<1.53$ & 2.35 & \\
\hline Mo & $<14.4$ & $<22.0$ & 0.479 & $<0.2$ & $<13.7$ & $<22.0$ & 18.3 & $<18.0$ & \\
\hline $\mathrm{Na}$ & 94200 & & 107000 & 30.0 & 67970 & & 98985 & & \\
\hline $\mathrm{Nd}$ & $<130$ & 84.4 & $<1.8$ & $<1.8$ & 245 & 683 & $<137$ & $<57.0$ & \\
\hline $\mathrm{Ni}$ & 161 & 909 & 0.357 & $<0.12$ & 928 & & 9.16 & 279 & \\
\hline $\mathrm{P}$ & 889 & $<245$ & 1100 & $<1.6$ & 1410 & 1290 & 210 & $<200$ & \\
\hline $\mathrm{Pb}$ & 177 & & $<1.6$ & $<1.6$ & 1340 & 1285 & $<122$ & & \\
\hline Re & $<14.4$ & & & & & & & & \\
\hline $\mathrm{s}$ & 465 & 720 & 790 & $<2$ & 719 & 725 & 1250 & 1150 & \\
\hline $\mathrm{Si}$ & 981 & 1394 & 37.9 & 17.2 & 6831 & 8884 & $<2234$ & 257 & \\
\hline $\mathrm{Sr}$ & 63.9 & 66.6 & 0.402 & $<0.012$ & 225 & 233 & $<0.92$ & 0.350 & \\
\hline $\mathrm{Ti}$ & 9.14 & 4.58 & $<0.06$ & $<0.06$ & 8.15 & 6.25 & 3.93 & $<3.55$ & \\
\hline $\mathrm{V}$ & $<57.6$ & & & & $<54.7$ & & $<61.1$ & $<83.0$ & \\
\hline $\mathrm{w}$ & $<72.0$ & $<100$ & 90.0 & $<1$ & $<68.3$ & $<99.0$ & $<76.4$ & & \\
\hline $\mathrm{Zn}$ & 36.6 & $<15.7$ & 1.86 & $<0.04$ & 65.6 & 29.5 & 4.65 & $<12.9$ & \\
\hline $\mathrm{Zr}$ & 68.0 & 62.2 & 0.0820 & $<0.06$ & 390 & 128 & $<4.58$ & $<16.0$ & \\
\hline $\begin{array}{r}\text { Elements by ICPMS } \\
\text { (same units as ICPES) }\end{array}$ & & & & & & & & & \\
\hline $\mathrm{Rb}$ & 1.00 & & & & 3.71 & & 4.74 & & \\
\hline $\mathrm{Y}$ & 7.12 & & & & 41.0 & & 0.0569 & & \\
\hline Cs & 12.1 & & & & 15.1 & & 4.71 & & \\
\hline $\mathrm{Pr}$ & 24.0 & & & & 137 & & 0.0174 & & \\
\hline $\mathrm{Bi}$ & 0.0448 & & & & 5.59 & & 0.0450 & & \\
\hline Re & & & & & & & & & \\
\hline $\begin{array}{r}\text { Elements by Atomic } \\
\text { Absorption (same units } \\
\text { as ICPES) }\end{array}$ & & & & & & & & & \\
\hline $\mathrm{Cs}$ & $<14.4$ & & 13.4 & $<0.1$ & $<13.7$ & & $<15.3$ & & \\
\hline $\mathrm{k}$ & 242 & & 305 & $<0.15$ & 301 & & 23900 & & \\
\hline $\mathrm{Na}$ & 92500 & & 114000 & 4.14 & 74000 & & 101000 & & \\
\hline $\begin{array}{r}\text { Anions by lon } \\
\text { Chromatography }\end{array}$ & & & $\mathrm{mg} / \mathrm{L}$ & $\mathrm{mg} / \mathrm{L}$ & & & & & \\
\hline $\begin{array}{r}\text { units } \\
\end{array}$ & $\mathrm{mg} / \mathrm{L}$ & & $\mathrm{mg} / \mathrm{L}$ & $\mathrm{mg} / \mathrm{L}$ & & & $\mathrm{mg} / \mathrm{L}$ & & \\
\hline bromide & & & 53.0 & $<10$ & & & $<100$ & & \\
\hline chloride & & & 609 & $<2$ & & & 1530 & & \\
\hline fluoride & & & 145 & $<2$ & & & 28.0 & & \\
\hline formate & & & 408 & $<10$ & & & 1380 & & \\
\hline nitrate & & & 119000 & $<10$ & & & 105000 & & \\
\hline nitrite & & & 3740 & $<10$ & & & 33400 & & \\
\hline oxalate & & & 1180 & $<10$ & & & 1550 & & \\
\hline phosphate & & & 4240 & $<10$ & & & 1150 & & \\
\hline sulfate & & & 1960 & $<5$ & & & 3740 & & \\
\hline acetate & & & & & & & 1350 & & \\
\hline citrate & & & & & & & $<81$ & & \\
\hline formate & & & & & & & 1120 & & \\
\hline ammonium & & & & & & & & & \\
\hline Wet Chemistry & & & & & & & & & \\
\hline total base M & 1.94 & & & & & & & & \\
\hline free hydroxide $\mathrm{M}$ & 0.359 & & & & & & & & \\
\hline total acid M & & & & & & & & & \\
\hline carbonate by titration & 0.346 & & & & & & & & \\
\hline $\begin{array}{r}\text { Carbon Analyses } \\
\text { (unfiltered sample) }\end{array}$ & & & & & & & & & \\
\hline units & $\mathrm{mg} / \mathrm{L}$ & & & & & & & & \\
\hline $\begin{array}{r}\text { total inorganic carbon } \\
\text { (carbonate) }\end{array}$ & 1900 & & & & & & & & \\
\hline $\begin{array}{r}\text { total organic carbon } \\
\text { (oxalate }+ \text { citrate }+ \text { acetate } \\
+ \text { formate) }\end{array}$ & 1000 & & & & & & & & \\
\hline
\end{tabular}


Table J1. Overall Chemical Compositions of SIPP Campaign II Process Streams

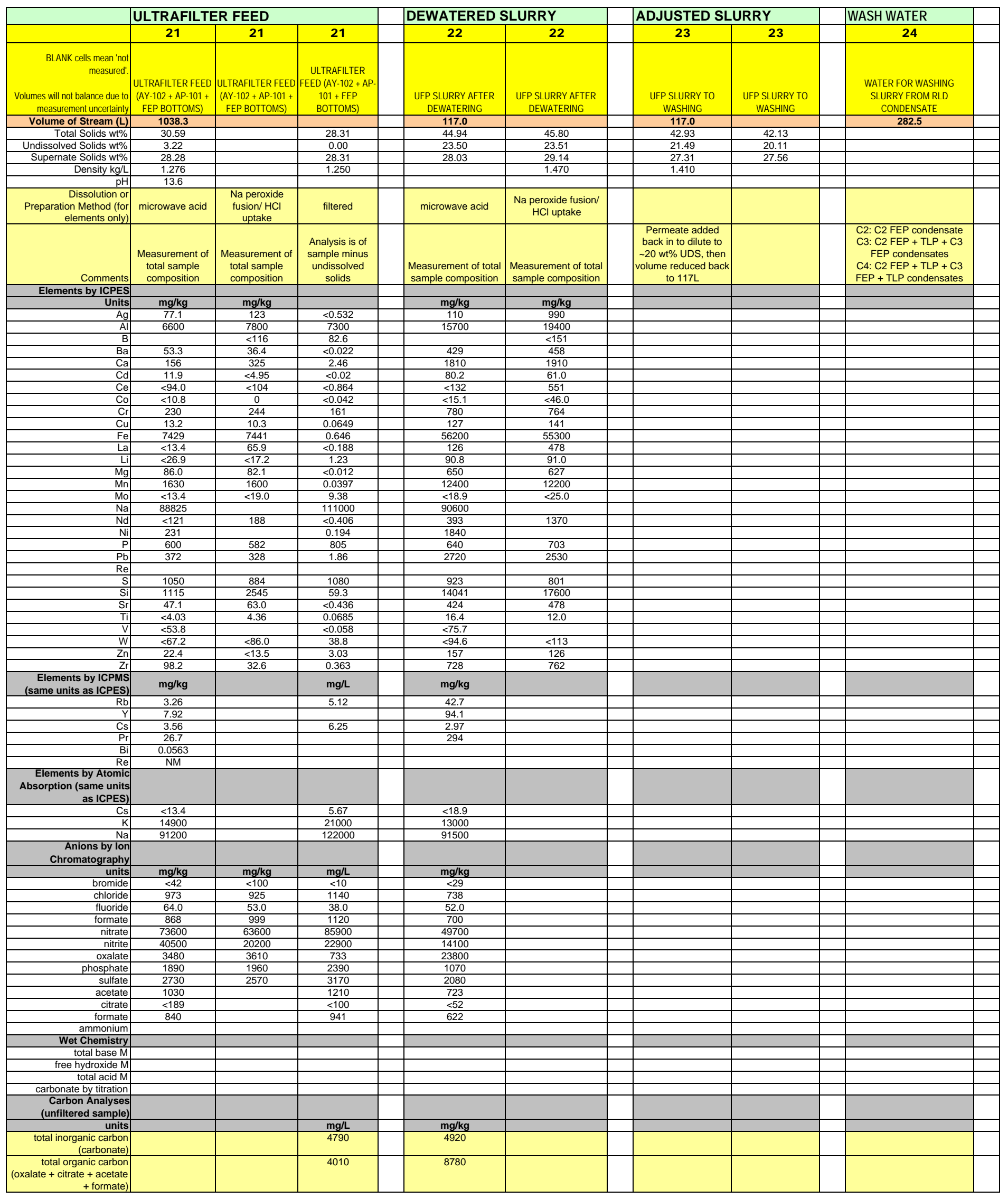


Table J1. Overall Chemical Compositions of SIPP Campaign II Process Streams

\begin{tabular}{|c|c|c|c|c|c|c|c|c|c|c|c|}
\hline & \multicolumn{2}{|c|}{ PARTIALLY WASHED SLURRY } & \multicolumn{3}{|c|}{ WASHED SLURRY } & \multicolumn{3}{|c|}{ WASH PERMEATES } & & \multirow{2}{*}{$\frac{\text { LOOP FLUSH }}{29}$} & \\
\hline & 25 & 25 & 26 & 26 & 26 & 27 & & 28 & & & \\
\hline $\begin{array}{l}\text { BLANK cells mean 'not } \\
\text { measured:" } \\
\text { Volumes will not balance due to } \\
\text { measurement uncertainty }\end{array}$ & $\begin{array}{l}\text { UFP SLURRY AFTER } \\
\text { FRST } 22 \text { WASHES } \\
\end{array}$ & $\begin{array}{l}\text { UFP SLURRY AFTER } \\
\text { FIRST } 22 \text { WASHES } \\
\end{array}$ & $\begin{array}{c}\text { UFP SLURRY AFTER } \\
\text { SECOND 22 WASHES } \\
\text { TO HLW } \\
\text { VITRRFICATION } \\
\end{array}$ & \begin{tabular}{|c} 
UFP SLURRY AFTER \\
SECOND 22 WASSES \\
TO HLW \\
VITRIFICATION \\
\end{tabular} & $\begin{array}{c}\text { UFP SLURRY AFTER } \\
\text { SECOON } 22 \text { WASHES } \\
\text { TO HLW } \\
\text { VITRIFICATION }\end{array}$ & $\begin{array}{l}\text { WASH PERMEATE } \\
\text { FROM MST } 22 \\
\text { WASHES TO NEXT } \\
\text { CAMPAIGN }\end{array}$ & & $\begin{array}{l}\text { WASH PERMEATE } \\
\text { FROOO } 2 \text { ND } 22 \text { WWSHHS } \\
\text { TO NEXT CAMPAIGN }\end{array}$ & & $\begin{array}{l}\text { LOOP FLUSH TO NEXT } \\
\text { CAMPAIGN }\end{array}$ & \\
\hline $\begin{array}{l}\text { Volume of Stream }(\mathrm{L}) \\
\text { Total Solids wo }\end{array}$ & & & \begin{tabular}{|l}
117.0 \\
26.0 \\
\end{tabular} & & & $\begin{array}{l}144.3 \\
20.66 \\
\end{array}$ & & $\begin{array}{ll}144.3 \\
89 \\
\end{array}$ & & $\begin{array}{ll}13.1 \\
10.42 \\
\end{array}$ & - \\
\hline 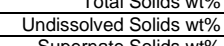 & & & $\begin{array}{ll}22.43 \\
22.24 \\
\end{array}$ & $\begin{array}{r}2.641 \\
22.18 \\
5.14\end{array}$ & $\begin{aligned} 2.685 \\
22.23 \\
\end{aligned}$ & $\frac{20.66}{0.00}$ & & 8.89 & & $\frac{10.42}{8.16}$ & - \\
\hline $\begin{array}{r}\text { Supernate Solids wto } \\
\text { Density kg/L }\end{array}$ & & & $\begin{array}{r}5.39 \\
1.200 \\
\end{array}$ & & & $\begin{array}{l}20.66 \\
1.177 \\
\end{array}$ & & $\begin{array}{r}8.89 \\
1.071 \\
\end{array}$ & & $\begin{array}{r}.46 \\
1.081 \\
\end{array}$ & $\square$ \\
\hline $\begin{array}{l}\text { Dissolutition or } \\
\text { Preparation Method (for } \\
\text { elements only) }\end{array}$ & microwave acid & $\begin{array}{l}\text { Na peroxide fusion/ } \\
\text { HCl uptake }\end{array}$ & microwave acid & $\begin{array}{l}\text { Na peroxide fusion } \\
\text { HCl uptake }\end{array}$ & & & & & & & \\
\hline $\begin{aligned} \text { Comments } \\
\text { Elements by IIPPES }\end{aligned}$ & $\begin{array}{l}\begin{array}{l}\text { Measurement of total } \\
\text { sample composition }\end{array} \\
\end{array}$ & $\begin{array}{l}\text { Measurement of total } \\
\text { sample composition }\end{array}$ & $\begin{array}{l}\text { Measurement of } \\
\text { total sample } \\
\text { composition }\end{array}$ & $\begin{array}{l}\text { Measurement of } \\
\text { total sample } \\
\text { composition }\end{array}$ & & & & & & 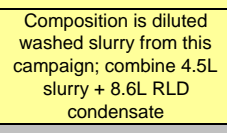 & \\
\hline Units & $\frac{\mathrm{mg} / \mathrm{kg}}{92 \mathrm{~g}}$ & mglkg & mglkg & mglkg & & $\mathrm{mg/L}$ & & $\mathrm{mg} / \mathrm{L}$ & & & - \\
\hline $\begin{array}{cl}\mathrm{Ag} \\
\mathrm{Al}\end{array}$ & $\begin{aligned} 9.9 \\
14300 \\
\end{aligned}$ & $\frac{944}{16300}$ & $\frac{90.3}{12400}$ & $\frac{804}{13100}$ & & $\begin{array}{l}<0.6 \\
4950\end{array}$ & & $\frac{<0.6}{1850}$ & & & - \\
\hline$\frac{\mathrm{B}}{\mathrm{Ba}}$ & 447 & $\frac{<117}{460}$ & $\begin{array}{ll}408 \\
\end{array}$ & $\begin{array}{c}<150 \\
384 \\
\end{array}$ & & $\frac{63.0}{<0.04}$ & & $\begin{array}{c}20.0 \\
<.04 \\
\end{array}$ & & & - \\
\hline Ca & $\frac{1810}{775}$ & 1923 & 1890 & 1914 & & $<0.06$ & & $<0.06$ & & & 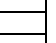 \\
\hline $\begin{array}{ll}\text { Cd } \\
C \mathrm{Ce}\end{array}$ & $\begin{array}{ll}77.5 \\
327\end{array}$ & $\frac{65.0}{546}$ & $\frac{74.2}{123}$ & $\begin{array}{ll}47.0 \\
497\end{array}$ & & $\begin{array}{c}<0.04 \\
<14\end{array}$ & & $\begin{array}{l}<0.04 \\
<14\end{array}$ & & & Z \\
\hline 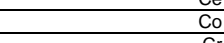 & $<14.5$ & $\begin{array}{c}546 \\
<36.0 \\
\end{array}$ & $\frac{123}{<13.3}$ & $\frac{497.0}{<45.0}$ & & $<0.16$ & & $<0.16$ & & & - \\
\hline$\frac{\mathrm{Cr}}{\mathrm{Cu}}$ & $\frac{690}{131}$ & $\frac{704}{126}$ & $\frac{620}{133}$ & $\frac{573}{113}$ & - & $\frac{120}{0.190}$ & & $\frac{50.0}{<0.06}$ & & & - \\
\hline & $\frac{52800}{353}$ & $\frac{54478}{462}$ & $\frac{55000}{128}$ & $\frac{45800}{386}$ & & $\frac{0.979}{<0.2}$ & & $\frac{0.185}{<0.2}$ & & & \\
\hline $\mathrm{Li}$ & 89.2 & 86.0 & 95.5 & 78.0 & & $\frac{.4 .8}{1.48}$ & & 1.71 & & & - \\
\hline$\frac{\mathrm{Mg}}{\mathrm{Mn}}$ & $\frac{610}{11700}$ & $\frac{619}{11900}$ & $\frac{640}{12200}$ & $\frac{509}{10100}$ & & $\frac{<0.01}{<0.02}$ & & $\frac{<0.01}{<0.02}$ & & & 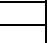 \\
\hline Mo & $\frac{1818.1}{<18.1}$ & $<20.0$ & $<16.7$ & $<25.0$ & & 6.88 & & 2.35 & & & - \\
\hline$\frac{\mathrm{Na}}{\mathrm{Nd}}$ & $\frac{47200}{988}$ & 1300 & $\frac{28300}{372}$ & 1150 & & $\frac{73700}{<1.8}$ & & 30400 & & & 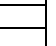 \\
\hline$\frac{\mathrm{Ni}}{\mathrm{P}}$ & $\frac{1770}{320}$ & 361 & $\frac{1680}{240}$ & 319 & & 0.217 & & $\frac{0.145}{140}$ & & & $\theta$ \\
\hline$\frac{\mathrm{P}}{\mathrm{Pb}}$ & $\frac{320}{3440}$ & $\frac{361}{2570}$ & 240 & $\frac{319}{2170}$ & & $\begin{array}{l}540 \\
1.70 \\
\end{array}$ & & $\frac{140}{<1.6}$ & & & - \\
\hline$\frac{\mathrm{Re}}{\mathrm{s}}$ & 493 & 482 & 194 & 489 & & 840 & & 290 & & & $\square$ \\
\hline & 13490 & 17100 & $\frac{194}{14917}$ & 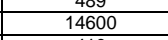 & & $\frac{84.8}{41.8}$ & & 22.1 & & & - \\
\hline $\begin{array}{c}\mathrm{Sr} \\
\mathrm{Ti} \\
\mathrm{Ti}\end{array}$ & $\frac{439}{10.2}$ & $\begin{array}{ll}471 \\
13.0 \\
\end{array}$ & $\begin{array}{ll}446 \\
148\end{array}$ & $\begin{array}{l}410 \\
110 \\
\end{array}$ & & $\frac{0.0475}{<0.06}$ & & $\frac{0.0174}{<<06}$ & & & Z \\
\hline $\mathrm{v}$ & $<72.5$ & & $\begin{array}{l}\frac{14.0}{666.7} \\
<6\end{array}$ & & & & & & & & - \\
\hline$w$ & $<90.7$ & $<87.0$ & $\frac{883.4}{81.5}$ & $<112$ & & 40.0 & & 10.0 & & & - \\
\hline $\begin{array}{ll}\mathrm{Zn} \\
\end{array}$ & $\frac{152}{712}$ & $\frac{131}{653}$ & $\frac{145}{718}$ & $\frac{133}{652}$ & & $\frac{1.43}{0.436}$ & & $\frac{0.0818}{0.0872}$ & & & \\
\hline $\begin{array}{r}\text { Elements by IIPMS } \\
\text { (same units a s ICPES) }\end{array}$ & & & & & & & & & & & \\
\hline & & & $\frac{31.8}{966}$ & & & 3.30 & & 1.13 & & & $\square$ \\
\hline$\frac{\mathrm{Cs}}{\mathrm{Cs}}$ & & & 90.6 & & & 4.01 & & 1.41 & & & - \\
\hline$\frac{\mathrm{Br}}{\mathrm{Bi}}$ & 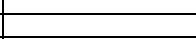 & & 311 & & & & & & & & - \\
\hline $\begin{array}{r}\mathrm{Re} \\
\text { Elements by Atomic }\end{array}$ & & & & & & & & & & & \\
\hline $\begin{array}{l}\text { Absorption (same units } \\
\text { as ICPES) }\end{array}$ & & & & & & & & & & & \\
\hline & $<18.1$ & & $<16.7$ & & & 5.43 & & 1.69 & & & \\
\hline & $\begin{array}{l}5770 \\
47100\end{array}$ & & $\frac{3380}{27600}$ & & & $\frac{12000}{77000}$ & & $\begin{array}{l}4000 \\
47800 \\
27800\end{array}$ & & & \\
\hline $\begin{array}{c}\text { Anions by lon } \\
\text { Chromatography }\end{array}$ & & & & & & & & & & & \\
\hline $\begin{array}{r}\text { units } \\
\text { uromide }\end{array}$ & & & & & & $\frac{\mathrm{mg} / \mathrm{L}}{-100}$ & & $\mathrm{mg/L}$ & & & ב \\
\hline $\begin{array}{l}\text { whlmide } \\
\text { chloride } \\
\end{array}$ & & & & & & 878 & & 284 & & & Z \\
\hline $\begin{array}{c}\text { flioorice } \\
\text { formate } \\
\end{array}$ & & & & & & $\frac{26.0}{949}$ & & $\frac{-2.00}{236}$ & & & - \\
\hline $\begin{array}{c}\text { nitrate } \\
\text { nitrite }\end{array}$ & & & & & & 74000 & & $\frac{19100}{5400}$ & & & 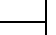 \\
\hline $\begin{array}{r}\text { numrte } \\
\text { oxalate }\end{array}$ & & & & & & 2030 & & 6060 & & & - \\
\hline $\begin{array}{r}\text { phosphate } \\
\text { suffate }\end{array}$ & & & & & & $\frac{1700}{2430}$ & & $\begin{array}{l}\frac{430}{803} \\
803\end{array}$ & & & \\
\hline acetate & & & & & & 825 & & 257 & & & - \\
\hline $\begin{array}{r}\text { cillate } \\
\text { formate } \\
\end{array}$ & & & & & & $\frac{-100}{654}$ & & $\frac{-100}{268}$ & & & \\
\hline $\begin{array}{r}\text { ammonium } \\
\text { Wet Chemistry }\end{array}$ & & & & & & & & & & & - \\
\hline $\begin{array}{r}r \text { total base M } \\
\text { free hydroxide M }\end{array}$ & & & & & & $\frac{1.25}{0.958}$ & & 0.071 & & & Z \\
\hline $\begin{array}{c}\text { total acid } \mathrm{M} \\
\end{array}$ & & & & & & & & & & & -1 \\
\hline $\begin{array}{l}\text { carbonate by tititati } \\
\text { Carbon Analys }\end{array}$ & & & & & & & & & & & \\
\hline (unfiltered s & & & & & & $\mathrm{mg} / \mathrm{kg}$ & & $\mathrm{mg/L}$ & & & \\
\hline total inorganic carbon & & & & & & 2570 & & 2020 & & & \\
\hline $\begin{array}{l}\text { total organic carbon } \\
\text { (oxalate + citrate + acetate }\end{array}$ & & & & & & 4390 & & 2680 & & & \\
\hline
\end{tabular}


Table J1. Overall Chemical Compositions of SIPP Campaign II Process Streams

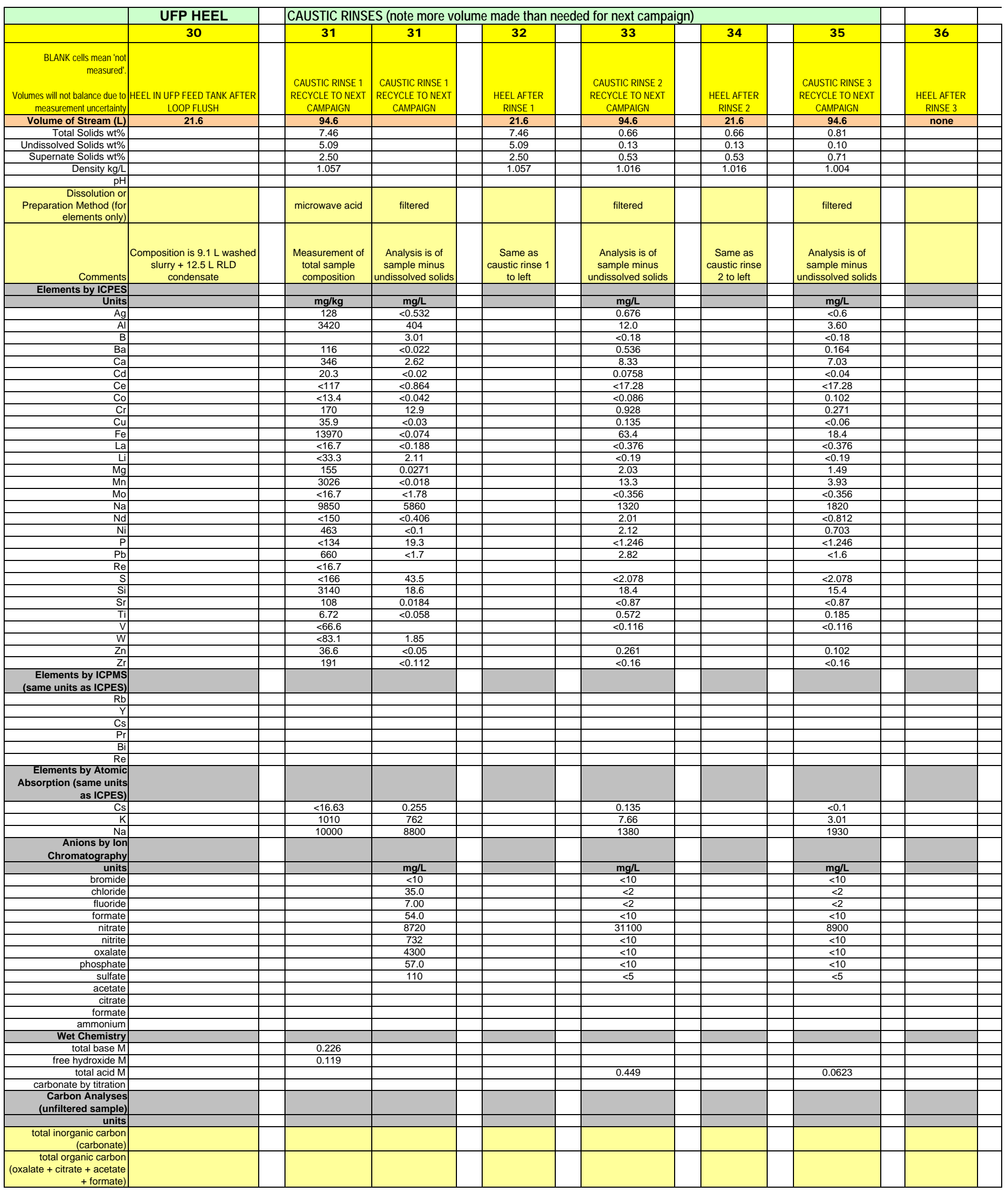


Table J1. Overall Chemical Compositions of SIPP Campaign II Process Streams

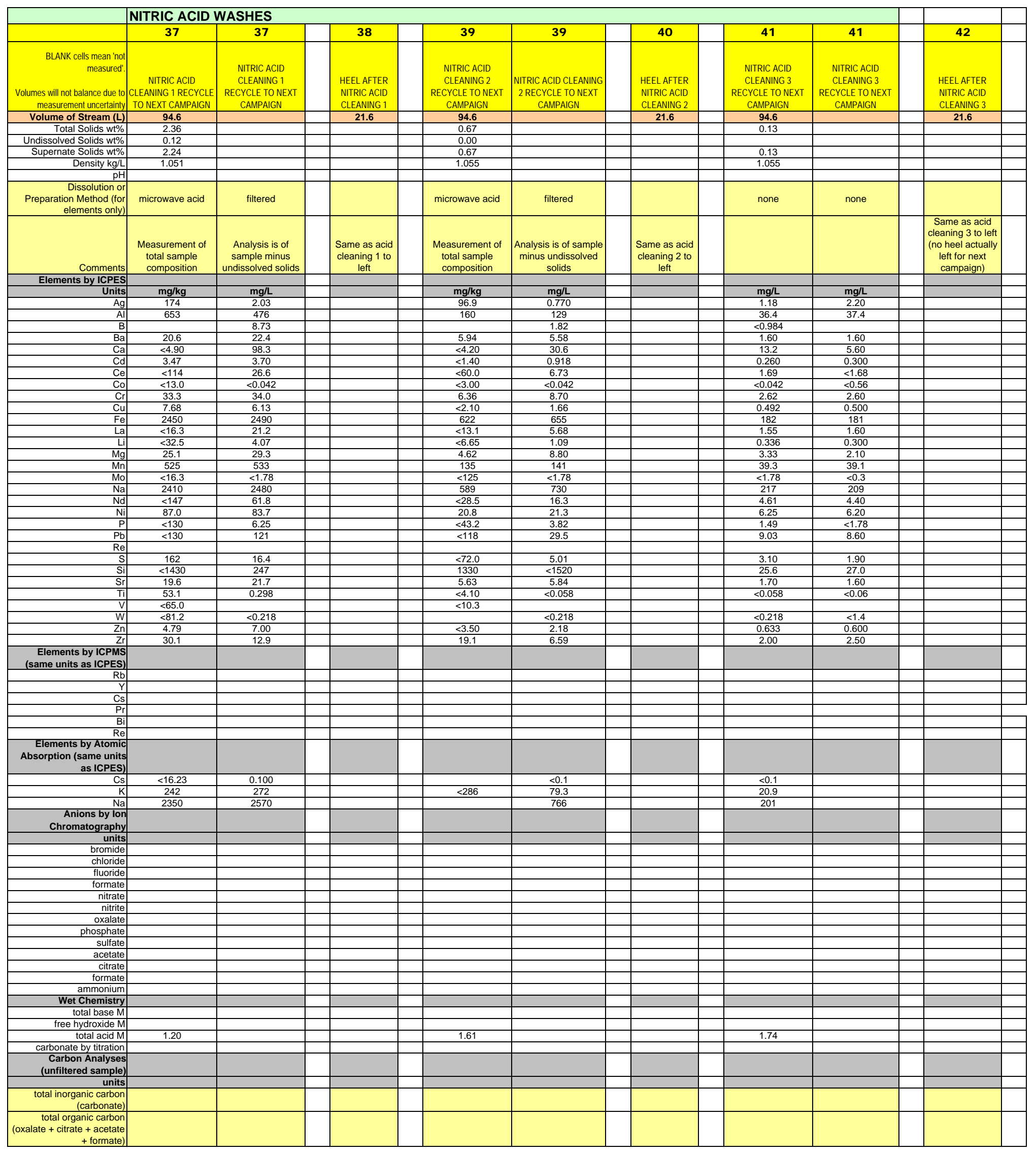


Table J1. Overall Chemical Compositions of SIPP Campaign II Process Streams

\begin{tabular}{|c|c|c|c|c|c|c|c|}
\hline & \multicolumn{2}{|c|}{ PERMEATE/IX FEED } & \multicolumn{4}{|c|}{ IX RECYCLES TO NEXT CAMPAIGN } & \multirow{2}{*}{\begin{tabular}{|c|} 
TLP EVAPOR \\
47 \\
\end{tabular}} \\
\hline & 43 & 43 & 44 & 45 & 46 & 46 & \\
\hline $\begin{array}{r}\text { BLANK cells mean 'not } \\
\text { measured'. } \\
\text { Volumes will not balance due to } \\
\text { measurement uncertainty }\end{array}$ & $\begin{array}{c}\text { PERMEATE FROM } \\
\text { SLURRY DEWATERING/ } \\
\text { IX FEED }\end{array}$ & $\begin{array}{c}\text { PERMEATE FROM } \\
\text { SLURRY DEWATERING/ } \\
\text { IX FEED }\end{array}$ & $\begin{array}{c}\text { IX DISPLACEMENT SOLUTION (0.1 } \\
\text { M NaOH) DISPLACED BY PRE- } \\
\text { ELUTION RINSE (water) TO NEXT } \\
\text { CAMPAIGN }\end{array}$ & $\begin{array}{c}\text { POST-ELUTION RINSE (water) } \\
\text { DISPLACED BY REGENERATION } \\
\text { SOLUTION (0.25 M NaOH) TO } \\
\text { NEXT CAMPAIGN }\end{array}$ & \begin{tabular}{|l|} 
IX REGENERATION \\
SOLUTION RECYCLE \\
TO NEXT CAMPAIGN \\
\end{tabular} & $\begin{array}{l}\text { IX REGENERATION } \\
\text { SOLUTION RECYCLE TO } \\
\text { NEXT CAMPAIGN }\end{array}$ & $\begin{array}{c}\text { IX PRODUCT TO TLP } \\
\text { EVAPORATOR } \\
\end{array}$ \\
\hline Volume of Stream (L) & 922.9 & & negligible produced & 19.0 & 22.5 & & 856.0 \\
\hline Total Solids wt\% & 28.36 & 28.09 & 0.70 & & 0.00 & & 27.93 \\
\hline Undissolved Solids wt\% & 0.00 & 0.00 & 0.00 & & 0.00 & & \\
\hline Supernate Solids wt $\%$ & 28.36 & 28.09 & 0.70 & & 0.00 & & 27.93 \\
\hline Density $\mathrm{kg} / \mathrm{L}$ & 1.230 & 1.250 & & & & & 1.247 \\
\hline \multicolumn{8}{|l|}{$\mathrm{pH}$} \\
\hline \multirow{2}{*}{$\begin{array}{r}\begin{array}{r}\text { Dissolution or } \\
\text { Preparation Method (for } \\
\text { elements only) }\end{array} \\
\text { Comments }\end{array}$} & none & & filtered & & filtered & & \\
\hline & 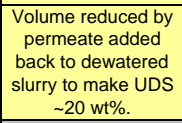 & \begin{tabular}{|c|}
$20 \mathrm{~L}$ used, so final \\
permeate volume $\sim 903$ \\
$\begin{array}{c}\text { L. Actual permeate to } \\
\text { IX } \sim 884 \mathrm{~L} \text { due to } \\
\text { losses. }\end{array}$ \\
\end{tabular} & $\begin{array}{l}\text { Volume needed for next } \\
\text { campaign was } 36.7 \mathrm{~L} .0 .1 \mathrm{M} \\
\mathrm{NaOH} \text { used instead. } \\
\text { Analysis is of sample minus } \\
\text { undissolved solids. }\end{array}$ & $\begin{array}{c}\text { Volume needed for next } \\
\text { campaign was } 36.7 \mathrm{~L} \text {. DI } \\
\text { water used to make up } \\
\text { extra needed. }\end{array}$ & $\begin{array}{c}\text { Analysis is of } \\
\text { sample minus } \\
\text { undissolved solids } \\
\end{array}$ & $\begin{array}{c}\text { Volume needed for } \\
\text { next campaign was } \\
43.0 \mathrm{~L} \text {. } 0.25 \mathrm{M} \mathrm{NaOH} \\
\text { used to make up extra } \\
\text { needed. }\end{array}$ & \\
\hline \multicolumn{8}{|c|}{\begin{tabular}{|l|l|} 
Elements by ICPES & \\
\end{tabular}} \\
\hline Units & $\mathrm{mg} / \mathrm{L}$ & & & & & & \\
\hline $\mathrm{Ag}$ & $<0.6$ & & $<0.532$ & $<0.532$ & $<0.532$ & & $<0.6$ \\
\hline Al & 7050 & & 96.6 & 2.85 & 7.24 & & 7190 \\
\hline $\mathrm{B}$ & 87.1 & & 5.13 & $<0.984$ & 2.20 & & 79.5 \\
\hline $\mathrm{Ba}$ & $<0.04$ & & $<0.022$ & $<0.022$ & $<0.022$ & & $<0.044$ \\
\hline $\mathrm{Ca}$ & $<0.06$ & & $<0.06$ & $<0.06$ & $<0.06$ & & 0.382 \\
\hline $\mathrm{Cd}$ & $<0.04$ & & $<0.02$ & $<0.02$ & $<0.02$ & & $<0.04$ \\
\hline $\mathrm{Ce}$ & $<1.4$ & & $<0.864$ & $<0.864$ & $<0.864$ & & $<17.28$ \\
\hline Co & $<0.16$ & & $<0.042$ & $<0.042$ & $<0.042$ & & $<0.086$ \\
\hline $\mathrm{Cr}$ & 165 & & 1.88 & 0.257 & 0.208 & & 160 \\
\hline $\mathrm{Cu}$ & 0.373 & & 0.0307 & $<0.03$ & $<0.03$ & & 0.279 \\
\hline $\mathrm{Fe}$ & 1.76 & & $<0.074$ & $<0.074$ & $<0.074$ & & 1.33 \\
\hline La & $<0.2$ & & $<0.188$ & $<0.188$ & $<0.188$ & & $<0.376$ \\
\hline $\mathrm{Li}$ & 1.15 & & $<0.096$ & $<0.096$ & $<0.096$ & & 1.07 \\
\hline $\mathrm{Mg}$ & $<0.01$ & & $<0.012$ & $<0.012$ & $<0.012$ & & $<0.026$ \\
\hline $\mathrm{Mn}$ & 0.0288 & & $<0.018$ & $<0.018$ & $<0.018$ & & $<0.034$ \\
\hline Mo & 9.31 & & $<0.178$ & $<0.178$ & $<0.178$ & & 9.74 \\
\hline $\mathrm{Na}$ & 119000 & & 4440 & 149 & 498 & & 110000 \\
\hline $\mathrm{Nd}$ & $<1.8$ & & $<0.406$ & $<0.406$ & $<0.406$ & & $<0.812$ \\
\hline $\mathrm{Ni}$ & 0.376 & & $<0.1$ & $<0.1$ & $<0.1$ & & 0.302 \\
\hline $\mathrm{P}$ & 800 & & 10.8 & $<0.624$ & 1.49 & & 775 \\
\hline $\mathrm{Pb}$ & 1.68 & & $<1.7$ & $<1.7$ & $<1.7$ & & 15.3 \\
\hline $\mathrm{Re}$ & 0.585 & & & & & & \\
\hline $\mathrm{s}$ & 1200 & & 39.6 & $<1.04$ & 1.49 & & 1152 \\
\hline $\mathrm{Si}$ & 54.2 & & 6.06 & 1.22 & 2.08 & & 57.0 \\
\hline $\mathrm{Sr}$ & 0.0691 & & $<0.006$ & $<0.006$ & $<0.006$ & & 0.0715 \\
\hline $\mathrm{Ti}$ & 0.0887 & & $<0.058$ & $<0.058$ & $<0.058$ & & $<0.116$ \\
\hline $\mathrm{V}$ & $<0.8$ & & & & & & \\
\hline $\mathrm{W}$ & 50.3 & & 0.476 & $<0.218$ & $<0.218$ & & \\
\hline $\mathrm{Zn}$ & 3.05 & & $<0.05$ & $<0.05$ & $<0.05$ & & 3.05 \\
\hline $\mathrm{Zr}$ & 0.863 & & $<0.112$ & $<0.112$ & $<0.112$ & & 0.514 \\
\hline $\begin{array}{r}\text { Elements by ICPMS } \\
\text { (same units as ICPES) }\end{array}$ & & & & & & & \\
\hline $\mathrm{Rb}$ & 4.93 & & 0.330 & 0.0420 & & & 4.86 \\
\hline $\begin{array}{r} \\
Y\end{array}$ & $<0.01$ & & & & & & \\
\hline Cs & 5.91 & & 0.324 & 0.887 & 0.0324 & 0.0336 & 0.00301 \\
\hline $\operatorname{Pr}$ & $<0.01$ & & & & & & \\
\hline $\mathrm{Bi}$ & $<0.01$ & & & & & & \\
\hline $\mathrm{Re}$ & & & & & & & 0.572 \\
\hline $\begin{array}{r}\text { Elements by Atomic } \\
\text { Absorption (same units } \\
\text { as ICPES) }\end{array}$ & & & & & & & \\
\hline $\mathrm{Cs}$ & 7.38 & & 0.415 & 0.785 & 0.140 & & 0.355 \\
\hline $\mathrm{K}$ & 21200 & & 1110 & 15.9 & 0.572 & & 18900 \\
\hline $\mathrm{Na}$ & 106500 & & 4320 & 97.9 & 400 & & 109000 \\
\hline $\begin{array}{r}\text { Anions by lon } \\
\text { Chromatography }\end{array}$ & & & & & & & \\
\hline units & $\mathrm{mg} / \mathrm{L}$ & & & & & & $\mathrm{mg} / \mathrm{L}$ \\
\hline bromide & $<10$ & & & & & & $<100$ \\
\hline chloride & 1210 & & & & & & 1090 \\
\hline fluoride & 29.0 & & & & & & 48.0 \\
\hline formate & 1220 & & & & & & 1020 \\
\hline nitrate & 25200 & & & & & & 74400 \\
\hline nitrite & 85300 & & & & & & 22400 \\
\hline oxalate & 746 & & & & & & 690 \\
\hline phosphate & 2470 & & & & & & 2210 \\
\hline sulfate & 3390 & & & & & & 2860 \\
\hline acetate & 1280 & & & & & & 1280 \\
\hline citrate & $<100$ & & & & & & $<100$ \\
\hline formate & 1010 & & & & & & 1020 \\
\hline ammonium & & & & & & & \\
\hline Wet Chemistry & & & & & & & \\
\hline total base $\mathrm{M}$ & & & 0.131 & & 0.00661 & & 0.309 \\
\hline free hydroxide $\mathrm{M}$ & & & 0.102 & & $<0.002$ & & 0.138 \\
\hline total acid M & & & & 0.0177 & & & 0.0363 \\
\hline carbonate by titration & & & & & & & 2346 \\
\hline $\begin{array}{r}\text { Carbon Analyses } \\
\text { (unfiltered sample) }\end{array}$ & & & & & & & \\
\hline units & $\mathrm{mg} / \mathrm{kg}$ & & & & & & \\
\hline $\begin{array}{r}\text { total inorganic carbon } \\
\text { (carbonate) } \\
\end{array}$ & 7750 & & & & & & \\
\hline $\begin{array}{r}\text { total organic carbon } \\
\text { (oxalate }+ \text { citrate }+ \text { acetate } \\
+ \text { formate) }\end{array}$ & 2000 & & & & & & \\
\hline
\end{tabular}


Table J1. Overall Chemical Compositions of SIPP Campaign II Process Streams

\begin{tabular}{|c|c|c|c|c|c|c|c|}
\hline \multicolumn{4}{|c|}{ :ATOR FEEDS } & \multirow{2}{*}{\begin{tabular}{|c|} 
TLP FEED \\
49 \\
\end{tabular}} & \multicolumn{2}{|c|}{ TLP PRODUCT } & \multirow{2}{*}{\begin{tabular}{|c|} 
TLP CONDENSATE \\
51 \\
\end{tabular}} \\
\hline & 47 & 48 & 48 & & 50 & 50 & \\
\hline $\begin{array}{r}\text { BLANK cells mean 'not } \\
\text { measured'. } \\
\text { Volumes will not balance due to } \\
\text { measurement uncertainty }\end{array}$ & $\begin{array}{l}\text { IX PRODUCT TO } \\
\text { TLP EVAPORATOR }\end{array}$ & $\begin{array}{c}\text { LAW SBS } \\
\text { CONDENSATE }\end{array}$ & $\begin{array}{c}\text { LAW SBS } \\
\text { CONDENSATE }\end{array}$ & $\begin{array}{c}\text { TLP EVAPORATOR } \\
\text { FEED }\end{array}$ & $\begin{array}{l}\text { TLP BOTTOMS } \\
\text { CONCENTRATE TO } \\
\text { LAW MELTER }\end{array}$ & $\begin{array}{c}\text { TLP BOTTOMS } \\
\text { CONCENTRATE TO } \\
\text { LAW MELTER } \\
\end{array}$ & TLP CONDENDATE TO RLD SYSTEM \\
\hline Volume of Stream (L) & & 783.0 & & 1639.0 & 523.0 & & 1116.0 \\
\hline Total Solids wt $\%$ & & 0.26 & & 16.24 & 41.30 & & 0.00 \\
\hline Undissolved Solids wt $\%$ & & 0.17 & & 0.09 & 0.31 & & 0.00 \\
\hline Supernate Solids wt $\%$ & & 0.09 & & 16.16 & 41.12 & & 0.00 \\
\hline Density kg/L & & 1.030 & 1.002 & 1.136 & 1.384 & & 0.997 \\
\hline pH & & 8.1 & & & & & 10.7 \\
\hline $\begin{array}{r}\text { Dissolution or } \\
\text { Preparation Method (for } \\
\text { elements only) }\end{array}$ & & aqua regia & & filtered & microwave acid & filtered & \\
\hline Comments & & $\begin{array}{c}\begin{array}{c}\text { Measurement of } \\
\text { total sample } \\
\text { composition }\end{array} \\
\end{array}$ & & $\begin{array}{c}\text { Analysis is of } \\
\text { sample minus } \\
\text { undissolved solids } \\
\end{array}$ & $\begin{array}{l}\text { Measurement of } \\
\text { total sample } \\
\text { composition }\end{array}$ & $\begin{array}{c}\text { Analysis is of } \\
\text { sample minus } \\
\text { undissolved solids }\end{array}$ & \\
\hline Elements by ICPES & & & & & & & \\
\hline Units & & $\mathrm{mg} / \mathrm{kg}$ & & $\mathrm{mg} / \mathrm{L}$ & $\mathrm{mg} / \mathrm{kg}$ & $\mathrm{mg} / \mathrm{L}$ & \\
\hline $\mathrm{Ag}$ & & $<3.89$ & & $<0.6$ & $<7.45$ & $<0.6$ & $<0.6$ \\
\hline $\mathrm{Al}$ & & 7.53 & & 3770 & 4810 & 11500 & $<0.862$ \\
\hline $\mathrm{B}$ & & 132 & & 91.7 & & 274 & 3.31 \\
\hline $\mathrm{Ba}$ & & $<0.26$ & & $<0.044$ & $<0.55$ & $<0.044$ & $<0.044$ \\
\hline $\mathrm{Ca}$ & & $<0.39$ & & 2.22 & 29.7 & 3.56 & $<0.12$ \\
\hline $\mathrm{Cd}$ & & $<0.26$ & & $<0.04$ & $<0.50$ & $<0.04$ & $<0.04$ \\
\hline $\mathrm{Ce}$ & & $<9.9$ & & $<17.28$ & $<8.65$ & $<17.28$ & $<17.28$ \\
\hline Co & & $<1.04$ & & $<0.086$ & $<1.10$ & $<0.086$ & $<0.086$ \\
\hline $\mathrm{Cr}$ & & 5.26 & & 89.8 & 131 & 266 & 4.90 \\
\hline $\mathrm{Cu}$ & & $<0.39$ & & 0.162 & $<0.75$ & 0.528 & $<0.06$ \\
\hline $\mathrm{Fe}$ & & 11.7 & & 0.558 & 20.3 & 1.39 & $<0.15$ \\
\hline $\mathrm{La}$ & & $<1.29$ & & $<0.376$ & $<4.65$ & $<0.376$ & $<0.376$ \\
\hline $\mathrm{Li}$ & & 11.5 & & 4.65 & 10.1 & 13.0 & 0.702 \\
\hline $\mathrm{Mg}$ & & 3.56 & & $<0.026$ & 4.09 & $<0.026$ & 0.0377 \\
\hline $\mathrm{Mn}$ & & $<0.12$ & & $<0.034$ & $<0.45$ & $<0.034$ & $<0.034$ \\
\hline Mo & & $<1.29$ & & 5.44 & 10.0 & 15.3 & $<0.356$ \\
\hline $\mathrm{Na}$ & & 587 & & 59000 & 121000 & 174000 & 303 \\
\hline $\mathrm{Nd}$ & & $<11.6$ & & $<0.812$ & $<2.45$ & $<0.812$ & $<0.196$ \\
\hline $\mathrm{Ni}$ & & 0.343 & & 0.275 & $<10.1$ & 0.769 & $<0.812$ \\
\hline $\mathrm{P}$ & & $<10.4$ & & 403 & 1.76 & 795 & $<0.12$ \\
\hline $\mathrm{Pb}$ & & $<10.4$ & & 8.09 & 980 & 24.0 & $<1.246$ \\
\hline $\mathrm{Re}$ & & 21.8 & & & & & \\
\hline $\mathrm{s}$ & & 78.0 & & 667 & $<19.9$ & 1906 & $<1.6$ \\
\hline $\mathrm{Si}$ & & 21.8 & & 41.7 & 1440 & 157 & $<2.078$ \\
\hline $\mathrm{Sr}$ & & $<0.07$ & & 0.0164 & 260 & 0.0806 & 29.5 \\
\hline $\mathrm{Ti}$ & & 1.15 & & $<0.116$ & 0.170 & 0.345 & $<0.012$ \\
\hline $\mathrm{V}$ & & $<5.19$ & & & 4.90 & & $<0.116$ \\
\hline W & & 25.2 & & & & & \\
\hline $\mathrm{Zn}$ & & 1.29 & & 12.6 & 31.4 & 37.8 & $<0.1$ \\
\hline $\mathrm{Zr}$ & & 1.29 & & $<0.16$ & 3.14 & 1.04 & $<0.16$ \\
\hline $\begin{array}{r}\text { Elements by ICPMS } \\
\text { (same units as ICPES) }\end{array}$ & & & & & & & \\
\hline $\mathrm{Rb}$ & 4.58 & & & 2.73 & 0.214 & 7.35 & $<$ \\
\hline $\mathrm{Y}$ & & & & & 0.00137 & $2.00 \mathrm{E}-05$ & $<$ \\
\hline $\mathrm{Cs}$ & 0.00204 & 0.0724 & & 0.168 & 0.357 & 0.380 & $<$ \\
\hline $\operatorname{Pr}$ & & & & & $4.90 \mathrm{E}-04$ & 0.0148 & $<$ \\
\hline $\mathrm{Bi}$ & & & & & & 0.0234 & $<$ \\
\hline $\mathrm{Re}$ & & 6.98 & & 9.41 & 0.810 & 28.0 & $<$ \\
\hline $\begin{array}{r}\text { Elements by Atomic } \\
\text { Absorption (same units } \\
\text { as ICPES) }\end{array}$ & & & & & & & \\
\hline $\mathrm{Cs}$ & & & & 0.505 & 0.985 & 1.03 & 0.110 \\
\hline $\mathrm{K}$ & & 123 & & 9530 & 34500 & 33100 & 0.346 \\
\hline $\mathrm{Na}$ & & 589 & & 62400 & 190000 & 202000 & 17.4 \\
\hline $\begin{array}{r}\text { Anions by lon } \\
\text { Chromatography }\end{array}$ & & & & & & & \\
\hline units & & $\mathrm{mg} / \mathrm{L}$ & & $\mathrm{mg} / \mathrm{L}$ & $\mathrm{mg} / \mathrm{L}$ & $\mathrm{mg} / \mathrm{L}$ & \\
\hline bromide & & $<86$ & & $<100$ & $<100$ & $<100$ & $<100$ \\
\hline chloride & & 627 & & 753 & 2470 & 2520 & $<20$ \\
\hline fluoride & & 146 & & 70.0 & 51.0 & 39.0 & $<20$ \\
\hline formate & & 33.0 & & 626 & 1730 & 1620 & $<100$ \\
\hline nitrate & & 135 & & 42600 & 135000 & 125000 & $<100$ \\
\hline nitrite & & 2580 & & 13500 & 43200 & 40100 & $<100$ \\
\hline oxalate & & $<86$ & & 389 & 1200 & 1150 & $<100$ \\
\hline phosphate & & $<86$ & & 1290 & 3100 & 2410 & $<100$ \\
\hline sulfate & & 233 & & 1720 & 5490 & 5120 & $<50$ \\
\hline acetate & & & & & & & $<10$ \\
\hline citrate & & & & & & & $<10$ \\
\hline formate & & & & & & & 5.00 \\
\hline ammonium & & 1990 & & 998 & $<100$ & $<100$ & 467 \\
\hline Wet Chemistry & & & & & & & \\
\hline total base $\mathrm{M}$ & & & & 0.162 & & & \\
\hline free hydroxide $\mathrm{M}$ & & & & 0.0636 & & & \\
\hline total acid $\mathrm{M}$ & & & & 0.0282 & & & \\
\hline carbonate by titration & & & & 1081 & & & \\
\hline $\begin{array}{r}\text { Carbon Analyses } \\
\text { (unfiltered sample) }\end{array}$ & & & & & & & \\
\hline units & & & & $\mathrm{mg} / \mathrm{L}$ & $\mathrm{mg} / \mathrm{kg}$ & $\mathrm{mg} / \mathrm{L}$ & $\mathrm{mg} / \mathrm{L}$ \\
\hline $\begin{array}{r}\text { total inorganic carbon } \\
\text { (carbonate) }\end{array}$ & & & & 4030 & 10800 & 12000 & 21.0 \\
\hline $\begin{array}{r}\text { total organic carbon } \\
(\text { oxalate }+ \text { citrate }+ \text { acetate } \\
+ \text { formate })\end{array}$ & & & & 1360 & 4300 & 2800 & 34.3 \\
\hline
\end{tabular}


G. T. Wright, 773A

D. J. Green, 773-A

D. B. Moore-Shedrow, 773-A

J. E. Marra, 773-A

C. R. Wolfe, 773-A

H. A. Woltermann, 773-A

D. A. Crowley, 773-A

R. E. Edwards, Jr., 773-A

J. C. Griffin, 773-A

E. W. Holtsheiter, Jr., 773-A

H. F. Sturm, Jr., 773-A

S. T. Wach, 773-42A

D. B. Burns, 786-5A

T. B. Calloway, 999-W

S. D. Fink, 773-A

J. C. Marra, 773-42A

S. L. Marra, 999-W

W. R. Wilmarth, 773-42A

D. J. Adamson, 786-5A

C. D. Barnes, $773-42 \mathrm{~A}$

M. R. Duignan, 786-5A

M. D. Fowley, 786-5A

H. N. Guerrero, 786-5A

Z. H. Qureshi, 786-5A

J. L. Steimke, 786-5A

M. R. Williams, 786-5A

J. R. Zamecnik, 773-41A

RPP File, c/o M. F. Jones, 773-43A, Room 109

\section{OFF-SITE}

W. L. Tamosaitis, Waste Treatment Plant, 3000 George Washington Way, Richland, WA99352

F. W. Damerow, Waste Treatment Plant, 3000 George Washington Way, Richland, WA 99352

P. S. Sundar, Waste Treatment Plant, 3000 George Washington Way, Richland, WA 99352 